NASA/TM-2013-218094

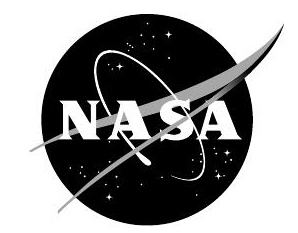

\title{
A Model to Assess the Risk of Ice Accretion Due to Ice Crystal Ingestion in a Turbofan Engine and its Effects on Performance
}

Philip C. E. Jorgenson and Joseph P. Veres

Glenn Research Center, Cleveland, Ohio

William B. Wright

ASRC Aerospace Corporation, Cleveland, Ohio

Peter M. Struk

Glenn Research Center, Cleveland, Ohio 


\section{NASA STI Program . . . in Profile}

Since its founding, NASA has been dedicated to the advancement of aeronautics and space science. The NASA Scientific and Technical Information (STI) program plays a key part in helping NASA maintain this important role.

The NASA STI Program operates under the auspices of the Agency Chief Information Officer. It collects, organizes, provides for archiving, and disseminates NASA's STI. The NASA STI program provides access to the NASA Aeronautics and Space Database and its public interface, the NASA Technical Reports Server, thus providing one of the largest collections of aeronautical and space science STI in the world. Results are published in both non-NASA channels and by NASA in the NASA STI Report Series, which includes the following report types:

- TECHNICAL PUBLICATION. Reports of completed research or a major significant phase of research that present the results of NASA programs and include extensive data or theoretical analysis. Includes compilations of significant scientific and technical data and information deemed to be of continuing reference value. NASA counterpart of peer-reviewed formal professional papers but has less stringent limitations on manuscript length and extent of graphic presentations.

- TECHNICAL MEMORANDUM. Scientific and technical findings that are preliminary or of specialized interest, e.g., quick release reports, working papers, and bibliographies that contain minimal annotation. Does not contain extensive analysis.

- CONTRACTOR REPORT. Scientific and technical findings by NASA-sponsored contractors and grantees.
- CONFERENCE PUBLICATION. Collected papers from scientific and technical conferences, symposia, seminars, or other meetings sponsored or cosponsored by NASA.

- SPECIAL PUBLICATION. Scientific, technical, or historical information from NASA programs, projects, and missions, often concerned with subjects having substantial public interest.

- TECHNICAL TRANSLATION. Englishlanguage translations of foreign scientific and technical material pertinent to NASA's mission.

Specialized services also include creating custom thesauri, building customized databases, organizing and publishing research results.

For more information about the NASA STI program, see the following:

- Access the NASA STI program home page at http://www.sti.nasa.gov

- E-mail your question to help@sti.nasa.gov

- Fax your question to the NASA STI Information Desk at 443-757-5803

- Phone the NASA STI Information Desk at 443-757-5802

- Write to: STI Information Desk NASA Center for AeroSpace Information 7115 Standard Drive Hanover, MD 21076-1320 
NASA/TM-2013-218094

\section{A Model to Assess the Risk of Ice Accretion Due to Ice Crystal Ingestion in a Turbofan Engine and its Effects on Performance}

Philip C. E. Jorgenson and Joseph P. Veres

Glenn Research Center, Cleveland, Ohio

William B. Wright

ASRC Aerospace Corporation, Cleveland, Ohio

Peter M. Struk

Glenn Research Center, Cleveland, Ohio

A revised and expanded version of AIAA-2012-3038, presented at the Atmospheric and Space Environments Conference

sponsored by the American Institute of Aeronautics and Astronautics

New Orleans, Louisiana, June 25-28, 2012

National Aeronautics and

Space Administration

Glenn Research Center

Cleveland, Ohio 44135 


\section{Acknowledgments}

This work is supported by the Atmospheric Environment Safety Technology Project (AEST) in the NASA Aviation Safety Program, and is in response to the Engine Icing Technical Challenge. A high level goal in that project is to provide a "First Generation Engine Icing Performance Degradation Parametric Simulation Capability." The APG milestone was due on 2Q2012 and the code that was developed is the deliverable. We would like to thank Dr. Renato Colantonio, manager of the NASA Atmospheric Environment Safety Technologies (AEST) project, under the Aviation Safety Program (ASP) for supporting this work. We would also like to acknowledge Dr. Jen-Ching Tsao, Ohio Aerospace Institute (OAI) for his insights and analyses of the NASA/National Research Council of Canada (NRCC) laboratory test data analyses. We also acknowledge the help and guidance we received from Mr. Scott Jones at NASA Glenn Research Center in the use of the NPSS engine cycle code.

This work was sponsored by the Fundamental Aeronautics Program at the NASA Glenn Research Center.

Level of Review: This material has been technically reviewed by technical management.

Available from

NASA Center for Aerospace Information 7115 Standard Drive

Hanover, MD 21076-1320
National Technical Information Service 5301 Shawnee Road Alexandria, VA 22312 


\title{
A Model to Assess the Risk of Ice Accretion Due to Ice Crystal Ingestion in a Turbofan Engine and its Effects on Performance
}

\author{
Philip C. E. Jorgenson and Joseph P. Veres \\ National Aeronautics and Space Administration \\ Glenn Research Center \\ Cleveland, Ohio 44135 \\ William B. Wright \\ ASRC Aerospace Corporation \\ Cleveland, Ohio 44135 \\ Peter M. Struk \\ National Aeronautics and Space Administration \\ Glenn Research Center \\ Cleveland, Ohio 44135
}

\begin{abstract}
The occurrence of ice accretion within commercial high bypass aircraft turbine engines has been reported under certain atmospheric conditions. Engine anomalies have taken place at high altitudes that were attributed to ice crystal ingestion, partially melting, and ice accretion on the compression system components. The result was one or more of the following anomalies: degraded engine performance, engine roll back, compressor surge and stall, and flameout of the combustor. The main focus of this research is the development of a computational tool that can estimate whether there is a risk of ice accretion by tracking key parameters through the compression system blade rows at all engine operating points within the flight trajectory. The tool has an engine system thermodynamic cycle code, coupled with a compressor flow analysis code, and an ice particle melt code that has the capability of determining the rate of sublimation, melting, and evaporation through the compressor blade rows. Assumptions are made to predict the complex physics involved in engine icing. Specifically, the code does not directly estimate ice accretion and does not have models for particle breakup or erosion. Two key parameters have been suggested as conditions that must be met at the same location for ice accretion to occur: the local wet-bulb temperature to be near freezing or below and the local melt ratio must be above $10 \%$. These parameters were deduced from analyzing laboratory icing test data and are the criteria used to predict the possibility of ice accretion within an engine including the specific blade row where it could occur. Once the possibility of accretion is determined from these parameters, the degree of blockage due to ice accretion on the local stator vane can be estimated from an empirical model of ice growth rate and time spent at that operating point in the flight trajectory. The computational tool can be used to assess specific turbine engines to their susceptibility to ice accretion in an ice crystal environment.
\end{abstract}




\section{Nomenclature}

\begin{tabular}{|c|c|}
\hline$A$ & $=$ Area, $\mathrm{ft}^{2}$ \\
\hline$c_{p_{w}}, c_{p_{a}}$ & $\begin{aligned}= & \text { Specific heat of water vapor, air, } \\
& \text { BTU } /\left(1 b_{m}-R\right)\end{aligned}$ \\
\hline$c_{p_{w e t}}$ & $\begin{aligned}= & \text { Specific heat air-water vapor mix, } \\
& \text { BTU } /\left(\mathrm{lb}_{\mathrm{m}}-\mathrm{R}\right)\end{aligned}$ \\
\hline$c_{p_{i}}$ & $=$ Specific heat of ice, BTU/( $\left.1 b_{m}-R\right)$ \\
\hline$C$ & $=$ Blade chord, in \\
\hline$C_{U}$ & $=$ Tangential velocity of air, $\mathrm{ft} / \mathrm{s}$ \\
\hline$D_{v}$ & $=$ Diffusion coeff. of water vapor into air \\
\hline$d$ & $=$ Ice particle size, diameter, $\mu \mathrm{m}$ \\
\hline$F_{w}, F_{a}$ & $\begin{aligned}= & \text { Molar flow rates of water vapor, air, } \\
& \text { mole } / \mathrm{s}\end{aligned}$ \\
\hline$G$ & $=$ Ice growth rate, $\mathrm{in} / \mathrm{s}$ \\
\hline$g_{c}$ & $=$ Unit conversion, $32.2 \mathrm{lb}-\mathrm{ft} /\left(\mathrm{lbf}-\mathrm{sec}^{2}\right)$ \\
\hline$h_{m}$ & $=$ Mass transfer coefficient, $\mathrm{ft} / \mathrm{hr}$ \\
\hline$h$ & $\begin{aligned}= & \text { Convective heat transfer coefficient, } \\
& \text { BTU } /\left(\mathrm{hr}-\mathrm{ft}^{2}-\mathrm{R}\right)\end{aligned}$ \\
\hline$\Delta H_{m e l t}$ & $=$ Enthalpy change of ice and air \\
\hline$I W C$ & $=$ Ice water content, $\mathrm{kg} / \mathrm{m}^{3}$ \\
\hline$J$ & $=$ Units conversion, $778.28 \mathrm{ft}-\mathrm{lb}_{\mathrm{f}} / \mathrm{BTU}$ \\
\hline$k$ & $=$ Thermal conductivity, BTU/(hr-ft-R) \\
\hline$L_{v}$ & $=$ Latent heat of evaporation, BTU/lb $\mathrm{m}$ \\
\hline$L e$ & $=$ Lewis Number \\
\hline$L_{f}$ & $=$ Latent heat of freezing, $\mathrm{BTU} / \mathrm{lb}_{\mathrm{m}}$ \\
\hline$L_{S}$ & $=$ Latent heat of sublimation, BTU $/ \mathrm{lb}_{\mathrm{m}}$ \\
\hline$L W C$ & $=$ Liquid Water Content, $\mathrm{kg} / \mathrm{m}^{3}$ \\
\hline$M_{a}, M_{w}$ & $=$ Molecular weight of air, water vapor \\
\hline$M_{w e t}$ & $=$ Molecular weight of air-water mixture \\
\hline$\dot{m}_{w}$ & $=$ Evaporation rate of water, $1 \mathrm{~b}_{\mathrm{m}} / \mathrm{hr}$ \\
\hline$N$ & $=$ Compressor rotational speed $(\mathrm{RPM})$ \\
\hline$n_{\text {melt }}$ & $=$ Melt fraction \\
\hline $\mathrm{Nu}$ & $=$ Nusselt number \\
\hline$P_{a} P_{w}$ & $=$ Partial pressure of air, water vapor, psia \\
\hline$P_{w, s}, P_{w, \infty}$ & $\begin{array}{l}=\text { Partial pressure of water vapor at surface, } \\
\text { and in free stream, psia }\end{array}$ \\
\hline$P_{S A T}$ & $=$ Saturation pressure, psia \\
\hline $\operatorname{Pr}$ & $=$ Prandtl number \\
\hline$\dot{q}$ & $=$ Heat flux, BTU/(sec-in $\left.{ }^{2}\right)$ \\
\hline$r_{2}$ & $=$ Blade tip radius, in \\
\hline$r_{C}$ & $=$ Arc radius of blade at mid-chord, in \\
\hline$R$ & $=$ Gas constant, $\mathrm{ft}-\mathrm{lb}_{\mathrm{f}} /\left(\mathrm{lb}_{\mathrm{m}}-\mathrm{R}\right)$ \\
\hline
\end{tabular}

$\mathfrak{R}$

$\mathrm{Re}$

$R_{\text {wet }}$

$S$

$S$,

Sc

$S h$

$T_{S}$

$T_{\text {Stat }}$

$T_{T o t}$

$T_{W B}$

$T_{\infty}$

$T_{M}$

$T W C$

$t$

$U$

$V_{d}$

$V_{\infty}$

$\widetilde{V}$

$W_{w}, W_{a}$

$w_{a}, w_{w}$

$w_{\infty}, w_{S}$

$X$,

$X_{0}, Y_{0}$

$X_{2}, Y_{2}$

$x_{0}, x$

$Y_{1}$

$Z$

Greek:

$\alpha$

$\beta_{1,} \beta_{2}$

$\gamma_{\text {wet }}$

$\theta$

$\mu$

$\tau$

$\phi$

$\rho_{i}, \rho_{w}, \rho_{a}$,

$\sigma$

$\chi_{a}, \chi_{w}$

$\omega$
$=$ Universal Gas constant, $1545.5 \mathrm{ft}-\mathrm{lb}_{\mathrm{f}} /(\mathrm{lb}-$ mole-R $)$

$=$ Reynolds number

$=$ Gas constant of air-water vapor mixture, $\mathrm{ft}-1 b_{\mathrm{f}} /\left(\mathrm{lb}_{\mathrm{m}}-\mathrm{R}\right)$

$=$ Blade spacing, baseline, in

$=$ Reduced blade spacing, in

$=$ Schmidt number

$=$ Sherwood Number

$=$ Surface temperature, $\mathrm{R}$

$=$ Static air temperature, $\mathrm{R}$

$=$ Total air temperature, $\mathrm{R}$

$=$ Wet-bulb temperature; surface, $\mathrm{R}$

$=$ Free-stream temperature, $\mathrm{R}$

$=$ Averaged temperature of control volume, $\mathrm{R}$

$=$ Total water content $=L W C+I W C$

$=$ Time, $\mathrm{s}$

$=$ Rotor peripheral velocity, $\mathrm{ft} / \mathrm{s}$

$=$ Ice particle velocity, $\mathrm{ft} / \mathrm{s}$

$=$ Free-stream air velocity, $\mathrm{ft} / \mathrm{s}$

$=$ Control volume

$=$ Flow rates of water vapor and air, $1 \mathrm{~b}_{\mathrm{m}} / \mathrm{s}$

$=$ Mass fraction of air, water vapor

$=$ Mass fraction of water vapor in dry air in the free-stream, and at the surface

$=$ Axial gap between rotor and stator, in

$=$ Blade circular arc center point $\mathrm{x}, \mathrm{y}$

$=$ Blade trailing edge coordinate, in

$=$ Initial location ice particle, current location of ice particle, in

$=$ Blade leading edge $\mathrm{y}$ coordinate, in

$=$ Axial distance, in

$=$ Thermal diffusivity, $\mathrm{ft}^{2} / \mathrm{s}$

$=$ Angles, leading; trailing edge, degrees

$=$ Ratio of specific heats of air-water mix

$=$ Blade arc angle, degrees

$=$ Viscosity of air, absolute, $1 \mathrm{bb}_{\mathrm{m}} /(\mathrm{ft}-\mathrm{s})$

$=$ Thermal response time, $\mathrm{s}$

$=$ Relative humidity

$=$ Density of ice, water vapor, air, $\mathrm{lb}_{\mathrm{m}} / \mathrm{ft}^{3}$

$=$ Blade tip solidity

$=$ Mole fraction of air, water vapor

$=$ Specific humidity 


\section{Introduction}

The purpose of this study is to document the development of a computational tool to estimate the risk of ice accretion and its effect on turbofan engine performance. Ice crystals ingested into the engines during the operation of an aircraft at high altitudes in high ice water content (HIWC) environments can result in ice accretion in the low pressure compression system. As ice crystals are ingested into the fan and low pressure compression system, the air temperature increases and a portion of the ice crystals melt. This allows the ice-water mixture to stick to the metal surfaces of the compressor components. The resulting accretion causes a blockage on stationary components such as the stator vanes, and subsequently results in the deterioration in performance of the compressor and engine. There have been numerous reported engine icing events ${ }^{1,2}$ that have occurred on commercial airlines (Figure 1). The total number of engine icing events per year, that were attributed to high ice water clouds, appear to be increasing since $2002^{3}$.

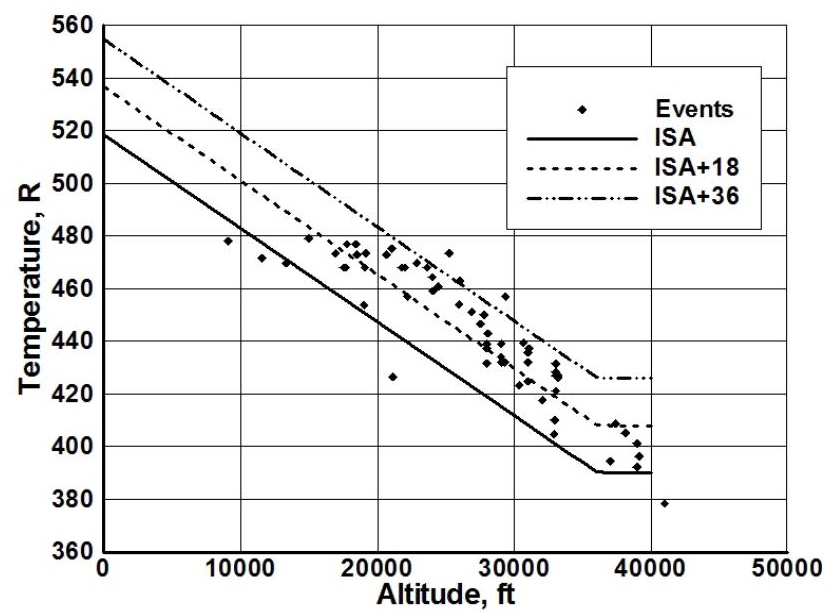

Figure 1: Reported engine icing events. The engine events occurred at altitudes between $10 \mathrm{~K}$ and $40 \mathrm{~K}$ feet and at temperatures between ISA and +36 R).

The computational tool that was developed in this study provides the capability to analyze a turbine engine through an ice cloud along a vehicle flight trajectory from takeoff, climb, cruise, and descent modes, and to evaluate the susceptibility of the engine to ice accretion. The tool has an engine system thermodynamic cycle code, coupled with a compressor flow analysis code, a fluid properties code, and an ice particle melt code. The mixed fidelity computational tool provides details of the flow conditions within each blade row of the fan and low pressure compression system during operation in the engine system environment. This work leverages from the previous code development effort and parametric study of the effects of blockage due to ice on engine and compressor performance reported in References 4, 5.

Ice accretion in the low pressure compression system can result in deteriorating the performance of the compressor, and consequently on the overall performance of the engine. In this study we utilize a representative notional engine in the $40 \mathrm{~K} \mathrm{lb}_{\mathrm{f}}$ thrust class and a typical flight trajectory that is appropriate for a commercial aircraft equipped with this class of engine.

In order to perform the study, it was necessary to have the details of the low pressure compressor (LPC) geometry to compute the flow conditions between each blade row with the mean line compressor flow analysis code. To that end, a conceptual design of a notional fan-core and LPC was completed to determine the key dimensions that define the blade and flow path. Details of the LPC design are provided in the appendix of this paper. The engine system analysis was performed by the Numerical Propulsion System Simulation (NPSS) code ${ }^{6}$. This code has been coupled with a mean line compressor analysis code $\left(\mathrm{COMDES}^{7}\right)$ such that blade row flow details of the LPC can be extracted. The baseline engine performance was computed with the NPSS-COMDES code, across the complete flight trajectory. The locations within the LPC where there is a risk of ice accretion were determined. At those operating points and locations within the LPC, a parametric analysis with additional blockage due to ice accretion could be performed, as was demonstrated in References 4, 5. These previous parametric analyses completed in 
References 4, 5 predicted that as the amount of blockage is increased, the LPC pressure ratio, choke margin, efficiency, and stall margin are reduced. All of these have a net detrimental effect on engine performance that can potentially cause an increase in turbine inlet temperature for a given thrust setting, or a reduced level of thrust, or rollback, for a given maximum turbine temperature. Additionally both of these possible scenarios result in an increase in the specific fuel consumption.

\section{Code Enhancements}

The computational tool that has been developed for this study leverages from the coupled Numerical Propulsion System Simulation engine thermodynamic cycle code and the mean line compressor flow analysis code (COMDES). A fluid properties model has been added to the COMDES code to account for supplemental water vapor in the air. Additionally, a code named MELT has been developed to model the melting of ice particles. This code leverages capabilities from the LEWICE2D code ${ }^{8,9}$. Modifications to account for water and ice crystals in the airflow have been made to the compressor code. These codes have been coupled together such that they exchange boundary conditions at each iteration. The compressor analysis code computes the detailed blade row by blade row flow conditions, as well as the overall compressor performance, thus the code is utilized by the system modeling code directly, and takes the place of a characteristic performance map. The mean line compressor code effectively eliminates the need for a map in the NPSS engine system modeling code for the fan-core and LPC components. A code named MELT has been developed that computes the melting rate of ice crystals, as they pass through the engine inlet, fan and low pressure compressor blade rows. The models for sublimation, melting, and evaporation in the MELT code have been adapted from the LEWICE2D code. However, there are no models for ice accretion, particle breakup, shedding, or erosion in MELT. The compressor code and MELT have been coupled to exchange boundary conditions at the blade leading and trailing edges. The compressor code has several additional capabilities, including the ability to calculate the effects of water vapor on the fluid properties of the air-water vapor mixture based on the mole fraction of air to water vapor. The specific humidity at the engine inlet is obtained from the NPSS code, and the local relative humidity is estimated through each component of the inlet-fan-LPC, taking the sublimation, melting, and evaporation into consideration, as well as the local air temperature. The resulting effect of the humid air on the performance of the compressor is computed.

\section{A. Numerical Propulsion System Simulation (NPSS)}

NPSS $^{6}$ is an advanced thermodynamic cycle code written in an object-oriented language for system modeling of gas turbine engines. The code provides the overall component-level performance and flow conditions of pressures and temperatures. However, the NPSS system modeling code alone does not have the fidelity to provide the flow conditions within the stages and blade rows. Therefore $\mathrm{COMDES}^{7}$, a mean line compressor flow code, was coupled to NPSS to provide the flow conditions at a higher fidelity, that is, within each blade row of the multi-stage fan-core and low pressure compressor. The block diagram in Figure 2 illustrates the turbofan engine model and its components as modeled with the NPSS-COMDES codes.

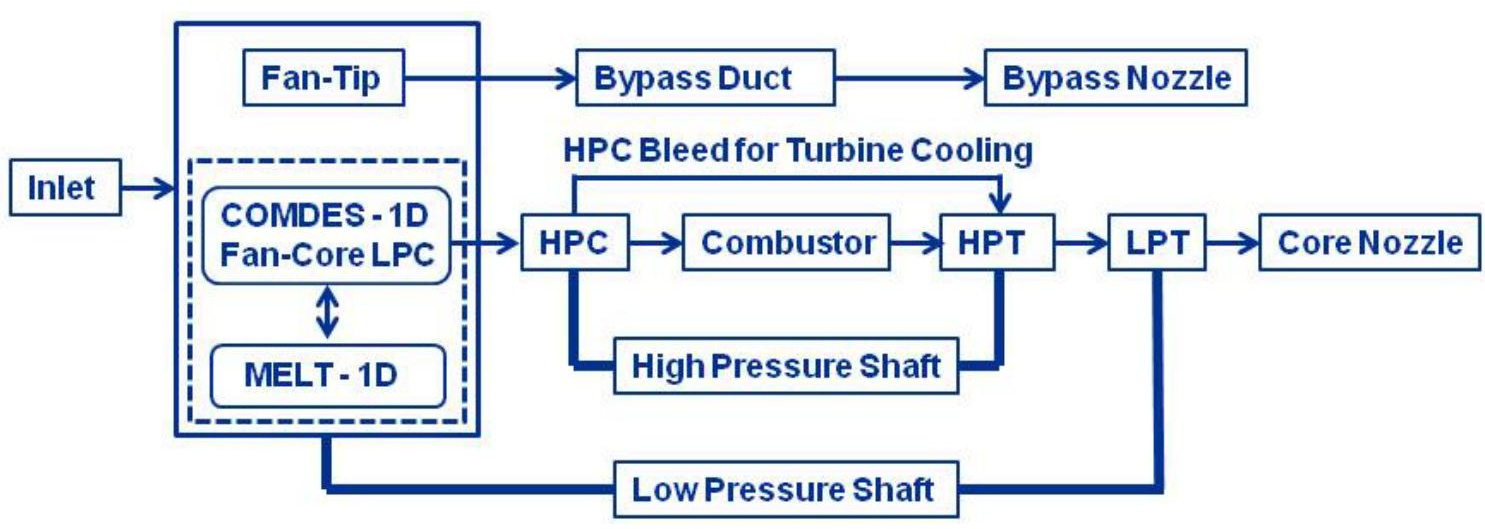

Figure 2: The NPSS engine system model of the two spool turbofan engine with the coupled COMDES compressor flow analysis code and MELT ice melting models. The fan-core performance (dashed box) is computed with the COMDES mean line flow code and the MELT code. 
The COMDES and the NPSS codes were coupled such that they exchange boundary conditions iteratively at each operating point through the flight trajectory. The fan-core and LPC performance illustrated in Figure 2 with the dashed box is not obtained from component characteristic maps, but rather is calculated with the COMDES mean line flow analysis code, and the MELT code. This process is illustrated in more detail in Figure 3, where the codes and their functionalities that have been developed for this study are described in more detail. The codes are outlined with the rectangular shaped border, while the boundary condition information passed between the codes have borders with rounded corners.

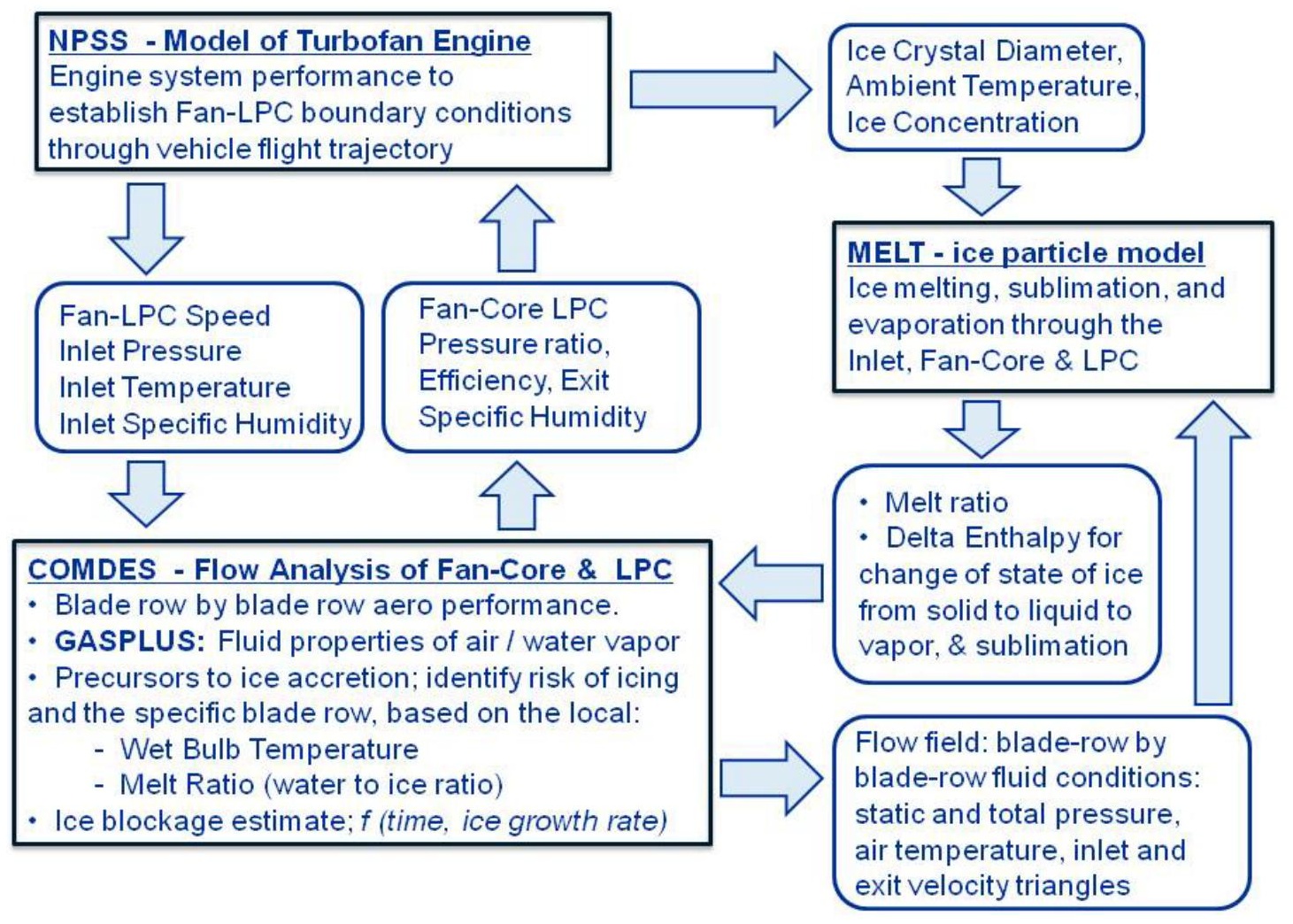

Figure 3: The NPSS engine system model, COMDES compressor flow model and MELT ice melting computer model boundary exchange strategy for the fan-core and LPC. The fan-core and LPC performance is computed with the COMDES code, and the MELT code.

While the fan-core and LPC performance is evaluated by means of the results from the COMDES code, the rest of the turbine engine components are modeled in NPSS with the use of traditional component characteristic performance maps. With this combined engine-compressor performance modeling tool, the detailed blade row conditions are determined while operating in an engine system environment. The notional engine is analyzed though the notional flight trajectory of a commercial carrier to determine the flow conditions, including the local pressure and temperature between each rotor and stator blade row of the multistage low pressure compressor (LPC).

Based on preliminary experimental results of ice accretion on a wedge shaped airfoil performed jointly by NASA and the National Research Council of Canada (NRCC) ${ }^{10}$, well-adhered ice accretions with rapid growth were observed to occur when the wet-bulb temperature was near freezing or below. Another key parameter was to have sufficient liquid water which is characterized in this study using the melt ratio, or the local value of the liquid water to the total water ratio (ice + water). For icing to occur, this study utilized a melt ratio of $10 \%$ based on observations that were made from the analysis of laboratory test data ${ }^{10}$. As a side note, the experiments conducted by NASA and NRCC showed that ice accretion could also occur at wet-bulb temperatures above freezing, particularly at higher ice water concentrations. However, this ice did not appear to be well-adhered and had a tendency to shed periodically. This shedding can also pose a hazard in the engine but is not considered in this study. 
The critical values of wet-bulb temperature and the melt ratio are the two key parameters that together are the necessary conditions which must be met simultaneously, at the same calculating station in the compressor, in order for there to be a high risk of ice accretion. Since the wet-bulb temperature and the melt ratio both change through each blade row, this technique also identifies the blade row where there is a high risk of ice accretion. However, it is recognized that other parameters such as the heat transfer rate through the blades may need to be considered as well, but are not included in the current version of the NPSS-COMDES codes. It is assumed in this study that accretion does not occur on the rotors, since the water/ice mixture would likely not have enough time to accrete on the high speed blade surface due to high centrifugal forces. Therefore the assumption is that accretion can only occur on the stator vanes and flow path walls.

Once a stator has been located in which the two parameters simultaneously indicate a risk of ice accretion, the amount of blockage at that stator vane location can be estimated as a function of time spent at that operating condition, and the measured ice accretion growth rate obtained from laboratory experiments ${ }^{10}$. The growth rate in terms of inches per second is converted to a value of additional blockage at that stator location, and the performance of the compressor with the additional blockage can then be computed with the compressor flow analysis code as a function of time spent at that operating condition. Parametric blockage is not addressed in this study, but was detailed in previous studies ${ }^{4,5}$. The updated performance of the iced compressor is then passed to the engine cycle code, and the engine performance with the iced compressor is aero-thermodynamically rebalanced.

\section{B. Compressor Flow Analysis Code Enhancements}

The compressor flow analysis code utilized in this study is the COMDES mean line flow code. The code computes the velocity, pressure, temperature, and flow angles at the leading edge and trailing edge of each blade row, at the hub, mean, and tip sections. The mean line compressor design and flow analysis code has been modified to include the effects of relative humidity on the fluid properties of air and water vapor mixture, and the subsequent effects on compressor performance. The capability of calculating the local relative humidity in each blade row is based on the initial value of specific humidity (mass of water/mass of air) at the compressor inlet. Additional modifications have been made to enable calculating the two key parameters which have been identified as early indicators of ice accretion: the local wet-bulb temperature and the percent of melt ratio within each blade row. If the limiting values of these key parameters are met, there is a risk that ice will accrete on the surfaces of the compressor. With these parameters as the precursors to ice accretion, the blade row within the compression system can be identified that is likely to experience ice buildup at a particular engine operating condition in the vehicle flight trajectory. The local wet-bulb temperature is calculated at each blade row. The geometry section of the code was improved with the addition of simple circular arc blades and vanes. An ice particle melting and evaporation model was coupled to the compressor code to compute the local melt ratio through each blade row.

\section{Water Vapor}

Additional modification to the COMDES code includes the capability of modeling the effects of water vapor on the gas properties of air. The model is based on a specified value of specific humidity at the inlet of the engine/compressor. The values for fluid properties of the water vapor and air are obtained in COMDES from the GASPLUS $^{11}$ code. The mole fraction of water vapor to air is used to compute the average mole weight of the fluid, the average gas constant, and the average specific heat ratios for water vapor $\left(c_{p_{w}}\right)$ and air $\left(c_{p_{a}}\right)$. Molar flow rates $\left(F_{w}, F_{a}\right)$ are given by:

$$
\begin{aligned}
& F_{w}=\frac{W_{w}}{M_{w}} \\
& F_{a}=\frac{W_{a}}{M_{a}}
\end{aligned}
$$


Mole fractions $\left(\chi_{w}, \chi_{a}\right)$ are given by:

$$
\begin{aligned}
& \chi_{w}=\frac{F_{w}}{\left(F_{w}+F_{a}\right)} \\
& \chi_{a}=1-\chi_{w}=\frac{F_{a}}{\left(F_{w}+F_{a}\right)}
\end{aligned}
$$

Mass fractions $\left(w_{w}, w_{a}\right)$ are given by:

$$
\begin{aligned}
& w_{w}=1-w_{a}=\frac{\chi_{w} M_{w}}{\chi_{a} M_{a}+\chi_{w} M_{w}} \\
& w_{a}=\frac{\chi_{a} M_{a}}{\chi_{a} M_{a}+\chi_{w} M_{w}}
\end{aligned}
$$

Average molar mass of the mixture:

$$
M_{w e t}=\chi_{a} M_{a}+\chi_{w} M_{w}
$$

The specific heat of the air-water vapor mixture $\left(c_{p_{\text {wet }}}\right)$ is calculated with the following formula:

$$
c_{p_{\text {wet }}}=w_{a} c_{p_{a}}+w_{w} c_{p_{w}}
$$

Average gas constant $\left(R_{\text {wet }}\right)$ of the air-water vapor mixture, in terms of the universal gas constant $(\mathfrak{R})$ :

$$
R_{\text {wet }}=\frac{\mathfrak{R}}{\left(\chi_{a} M_{a}+\chi_{w} M_{w}\right)}=\frac{\mathfrak{R}}{M_{\text {wet }}}
$$

The ratio of specific heats for the air-water vapor mixture:

$$
\gamma_{\text {wet }}=\frac{1}{\left(1-R_{\text {wet }} /\left(J c_{p_{\text {wet }}}\right)\right)}
$$

\section{Rotor and Stator Blade Geometry}

The geometry portion of the compressor code has been improved with the addition of a circular arc blade geometry generator. The code generates rotor blades and stator vanes based on the values of the blade angles at the leading and training edges, the tip solidity, and the flow path radii. The circular arc shaped mean camber lines of the blades are created at the hub, mean, and tip section of each rotor and stator of the stage. When used in the analysis mode, the circular arc blades act as reasonable approximations of the actual rotors and stators, Figure 4. This represents one stage of the five stage fan-core and LPC that are analyzed in this study, as will be illustrated in a later figure. With this method, the blade-to-blade distances and passage chord lengths can be computed with reasonable accuracy. 


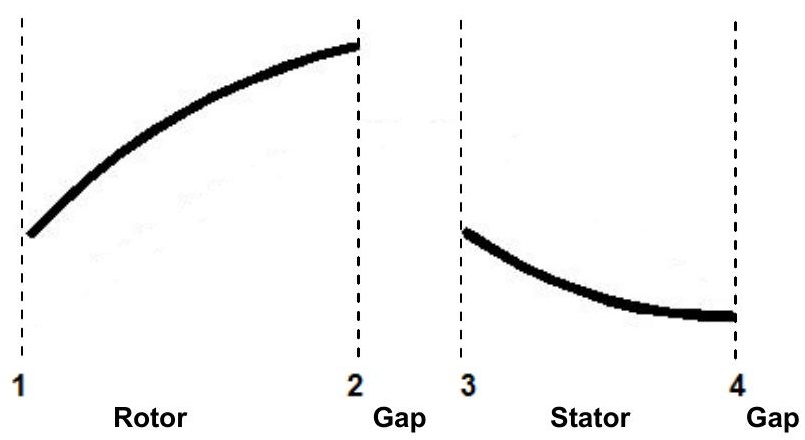

Figure 4: Rotor and stator blade mean camber line chords at the mid-span section for a typical stage. The station numbers 1 and 2 refer to the rotor leading and training edges respectively, while stations 3 and 4 refer to the stator leading and trailing edges respectively.

The lengths are used to estimate ice particle residence time and for calculating the sublimation, melt, and evaporation rates which will be addressed in the subsequent "Ice Particle Melting and Evaporation" section. The circular arc blade generation methodology that was used to determine the compressor rotor and stator blade dimensions are detailed below and the compressor geometry used in this study is provided in a later section on fan and low pressure compressor conceptual design.

The geometry section of the compressor code includes the capability to generate approximate blade shapes based on a simple circular arc blade camber line profile. The compressor code is run in the analysis mode, and the blade angles at the leading and trailing edges are specified. For rotors, the design point incidence, flow rate, and shaft rotational speed provides the blade angle at the leading edge. At the trailing edge, the work, or flow turning angle defines the blade angle at the blade exit. The tip solidity, number of blades, and flow path inner and outer radii are input parameters. The simple circular arc blade camber line is generated by the code at the hub, mean, and tip sections. The same procedure is followed to provide the stator vane camber line with simple circular arc shape. The following equations define the rotor blade and stator vane (referred to as airfoils in the following) camber geometric shape. The chord, $C$, is determined from the tip solidity, $\sigma$, the tip radius, $r_{2}$, and the number of airfoils, $N$, by the following equation.

$$
C=\frac{2 \pi r_{2} \sigma}{N}
$$

The true chord can be closely approximated by the following equation which is for the isosceles triangle in Figure 5 .

$$
C=2 r_{C} \operatorname{Sin} \frac{\theta}{2}
$$




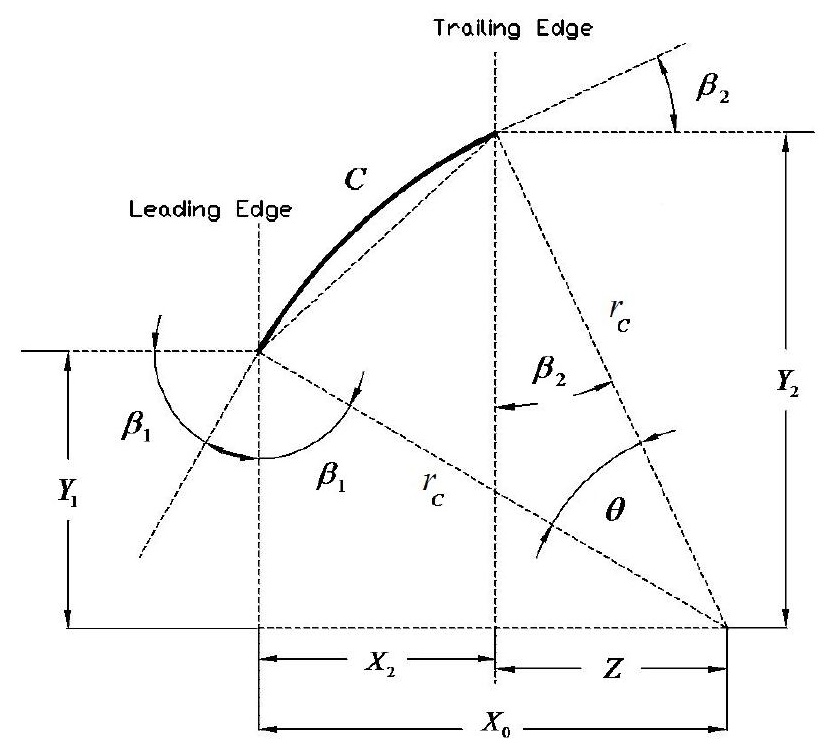

Figure 5: Compressor airfoil camber line geometry creation in COMDES. A simple circular arc camber line with radius $r_{c}$ is generated in the compressor code based on input values of tip solidity of the rotor and stator, and angles at the leading $\left(\beta_{1}\right)$ and trailing $\left(\beta_{2}\right)$ edges.

The following equations are used to solve for the coordinates of the airfoil leading and trailing edges and the coordinates of the center of the circular $\operatorname{arc}\left(X_{o}, Y_{o}\right)$ which defines the $\operatorname{arc}$ radius $r_{c}$ at the blade mid-chord.

$$
\begin{aligned}
& r_{C}{ }^{2}=X_{O}{ }^{2}+Y_{O}{ }^{2} \\
& r_{C}{ }^{2}=\left(X_{2}-X_{O}{ }^{2}\right)^{2}+Y_{2}^{2} \\
& \operatorname{Sin} \beta_{1}=\frac{X_{O}}{r_{C}} \\
& \operatorname{Cos} \beta_{2}=\frac{Y_{2}}{r_{C}}
\end{aligned}
$$

The airfoil section coordinates shown in Figure 5 are determined using the above equations for a circular arc. Both the rotor blades as well as the stator vanes are generated with a single circular arc.

\section{Energy Balance between Ice Particle and the Air}

As the ice passes through the inlet, fan, and LPC blade rows, it is continuously absorbing heat from the warmer air through the compressor blades. The amount of energy that the ice particle absorbs from the air while it experiences sublimation, melting, and evaporation will be described in the subsequent section named "Ice Particle Melting and Evaporation." The transfer of energy between the air and the ice particle can be expressed as a change in enthalpy. The enthalpy increase of the ice particle is equal to the enthalpy decrease of the air, which results in a decrease of the total temperature of the air. The energy balance between the ice particle and the air is determined iteratively with the results from the compressor code and the particle melt code exchanging boundary conditions of static temperature, static pressure and change in enthalpy until convergence. The change in total temperature of the air through the rotor is a function of the enthalpy rise due to the work input by the rotor, and the enthalpy that the ice particle removes from the air, as determined from the particle melt code. To account for effects of the energy exchange between the ice particle and the air through the compressor rotor, the Euler equation energy balance in the 
compressor code has been modified to account for the enthalpy exchange between the particle and the air, and is represented by the following equation, where the subscripts 1 and 2 represent the conditions at the rotor leading edge (station 1) and trailing edge (station 2) respectively (see Figure 4).

$$
J c_{p_{w e t}} g_{c}\left(T_{2}-T_{1}\right)=\left(U_{2} C_{U 2}-U_{1} C_{U 1}\right)-\Delta H_{m e l t(2-1)}
$$

The solution of flow conditions at the rotor exit is obtained by executing the compressor code and the particle melt code iteratively, by passing boundary conditions of static temperature and velocity from the compressor code to the MELT code, where the enthalpy change $\left(\Delta H_{\text {melt }}\right)$ of the ice particle is calculated, and returned to the compressor code where the change in air temperature due to the change in enthalpy is calculated. A similar iterative process is utilized to calculate the change in air temperature due to enthalpy exchange between the air and the ice particles through the gaps between the rotor and stator, and through the stator vanes, where the subscripts 1 and 2 represent the inlet and outlet of the gap, and subscripts 3 and 4 represent the conditions at the stator leading edge and trailing edge respectively (see Figure 4). Note that when calculating the air temperature in the gap, the subscripts 3 and 4 are replaced with subscripts 2 and 3, respectively, in the following equation.

$$
J c_{p_{\text {wet }}} g_{c}\left(T_{4}-T_{3}\right)=\Delta H_{\text {melt }(4-3)}
$$

\section{Wet-Bulb Temperature}

In this study, the wet-bulb temperature parameter is utilized as one of the key indicators of whether there is a risk of ice accretion within one of the compressor blade rows. A mathematical derivation of a wet-bulb temperature $\left(T_{W B}\right)$ for application in analysis of mixed-phase icing tests has been derived and implemented into the mean line compressor flow analysis code, thus enabling the multistage code to compute the local wet-bulb temperature at each rotor and stator leading and trailing edge and at the stator throat. In this derivation $T_{W B}$ is the temperature of an evaporating wet adiabatic surface and is a function of the surrounding air temperature (dry-bulb), air pressure, and moisture content. The $T_{W B}$ is obtained by equating the rate of heat transfer to a surface to the heat loss by evaporation. During this process, it is assumed that the surface remains wet and any water added to replace that lost by evaporation is at $T_{W B}$. The analysis is an adaptation of heat and mass transfer principles described in Incropera \& DeWitt $^{12}$ and thermodynamic concepts presented in Çengel and Boles ${ }^{13}$. It is assumed that the process is steady state, with adiabatic boundaries, except at the edge of the boundary layer. The surface (and water layer) is maintained at $T_{W B}$. The surface always remains wet (either evaporation is negligible from a mass loss perspective or water is replaced at $T_{S}$ via some process). The static pressure remains constant, and the partial pressure of water vapor at the surface is assumed to be the saturation value at the surface temperature. Figure 6 illustrates the conceptual representation of the control volume for the thermodynamic balance model that is utilized to derive the wet-bulb temperature.

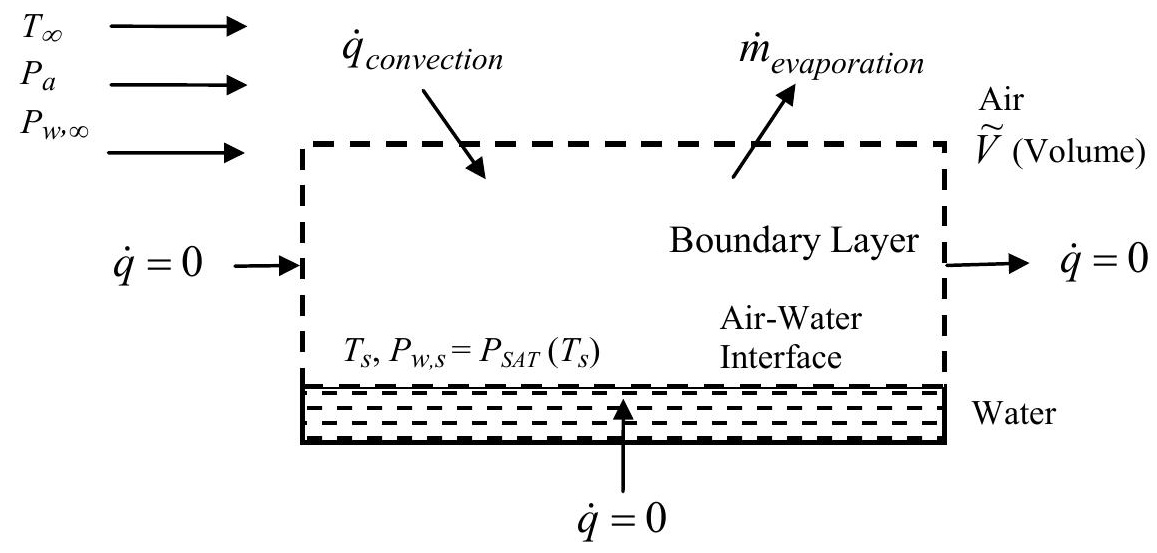

Figure 6: The control volume for the thermodynamic balance model. 
An energy balance performed on the above control volume with the given assumptions is shown in Eq. (19).

$$
\rho_{a} \tilde{V} c_{p_{a}} \frac{d T_{M}}{d t}=h A\left(T_{\infty}-T_{s}\right)-\dot{m}_{w} L_{v}
$$

Assuming a steady state process, the above equation can be rearranged to solve for the surface temperature as shown in Eq. (20).

$$
T_{s}=T_{\infty}-\frac{\dot{m}_{w} L_{v}}{h A}
$$

Examination of the above equation shows that $T_{W B}$ will be less than the $T_{\infty}$ when there is evaporation from the surface given the assumptions stated above.

To determine the amount of mass evaporated leaving the top of the boundary layer, it is assumed that the process is steady (i.e., no accumulation in the control volume). The evaporated water leaving the control volume is resupplied to the surface at $T_{W B}$ via some unspecified process. It is also assumed that saturated conditions exist at the water and interface. Under these assumptions, the mass flux leaving the control volume is shown in Eq. (21):

$$
\dot{m}_{w}=h_{m} A \rho_{a}\left(w_{s}-w_{\infty}\right)
$$

Combining the mass flux leaving the control volume, Eq. (21), with the energy balance, Eq. (20), provides another expression for the surface temperature, as shown in Eq. (22):

$$
T_{s}=T_{\infty}-\frac{h_{m}}{h} L_{v} \rho_{a}\left(w_{s}-w_{\infty}\right)
$$

Invoking the analogy of heat and mass transfer yields another expression for the surface temperature, equation. This analogy is valid for both local and bulk transfer coefficients as well as laminar or turbulent conditions. The properties values are evaluated at the average of the free-stream and the surface temperature. A more detailed derivation of this expression is presented in Reference 14.

$$
T_{s}=T_{\infty}-\frac{L_{v}}{c_{p_{a}} L e^{2 / 3}}\left(\frac{M_{w}}{M_{a}}\right) \frac{T_{M}}{P_{a}}\left[\frac{P_{w, s}}{T_{s}}-\frac{P_{w, \infty}}{T_{\infty}}\right]
$$

Eq. (23) can be solved iteratively. The surface temperature, $T s$, is the wet-bulb temperature, based on the above assumptions.

The partial pressure of water vapor at the surface is assumed to be the saturation value at the surface temperature, Eq. (24). The partial pressure of water vapor outside the boundary layer can be defined based on the ambient relative humidity, $\phi$, as shown in Eq. (25).

$$
\begin{aligned}
& P_{w, s}=\phi_{S} P_{s a t, s} ; \text { evaluated at } T_{W B} \quad\left(\phi_{S}=1.0, \text { fully saturated }\right) \\
& P_{w, \infty}=\phi_{\infty} P_{s a t, \infty} ; \text { evaluated at } T_{\infty}
\end{aligned}
$$

For this work, total conditions were used for $\mathrm{T}_{\infty}$ and $\mathrm{P}_{\infty}$ to calculate wet-bulb temperature to represent conditions at a stagnation point. 


\section{Ice Particle Melting and Evaporation}

The capability to model ice particle and evaporation has been added to the COMDES compressor analysis code. The appropriate equations were taken from the LEWICE2D code and were implemented into the compressor code in the subroutine named MELT. This enables the code to calculate the change of phase of an ice particle which sublimates, melts, and evaporates, as it passes through the fan and low pressure compressor blades and stator vanes. The ice particle residence times through the compressor of existing engines are estimated based on the velocities at the leading and trailing edge of each blade row, and the blade chords and the axial spacing of the gap between the rotors and stators. This provides a reasonable estimate of the actual residence times of the ice crystals through each blade passage and gap, resulting in an accurate estimate of the sublimation, melting, and evaporation physics. The inlet specific humidity and the ice particle concentration (grams per cubic meter) and size distributions are specified at the inlet to the engine. For this study, values for ice particle size and concentrations in the atmosphere are varied parametrically, as there is limited data currently available on the ice crystal content of high altitude convective clouds.

After the flow conditions through the blade rows have been calculated by the compressor code, the static temperatures, pressures, and velocities are passed to the ice melting subroutine to determine the rate of melting and evaporation, and thus, the local melt ratio in each blade row. The ice particle sublimation, melting, and evaporation equations are shown below. The calculations for sublimation, melting, and evaporation take into consideration the local temperatures, pressures and residence times as they traverse the blade passages and gaps between blades at their mid span location. Conservation of mass on each particle results in the following equation for the ice particle size as a function of position, where $w_{s}$ is the mass fraction of water vapor at the surface and $w_{\infty}$ is the mass fraction of the water vapor in the free-stream.

$$
d^{2}=d_{o}^{2}-\frac{4 S h \rho_{a} D_{v}}{\rho_{i} V_{\infty}}\left(x-x_{o}\right)\left(w_{s}-w_{\infty}\right)
$$

Similarly, the rise in specific humidity is related to the amount of mass lost by the particle and is given by the equation below.

$$
\omega=\omega_{o}+\frac{I W C}{\rho_{a}}\left(1-\left(\frac{d}{d_{o}}\right)^{3}\right)
$$

Equating the heat absorbed by the particles to a decrease in enthalpy in the airstream provides the decrease in air temperature.

$$
T_{a}=T_{a, o}-\frac{L_{s}}{c_{p_{a}}} \frac{I W C}{\rho_{a}}\left(1-\left(\frac{d}{d_{o}}\right)^{3}\right)
$$

The enthalpy increase of the particle through the compressor is equal to the heat absorbed from conduction plus the energy provided by evaporation, resulting in the following equation for the change in particle temperature prior to melt

$$
\frac{d T_{i}}{d x}=\frac{N u}{\tau_{T} V_{\infty}}\left(T_{a}-T_{i}\right)+\frac{L_{s} S h}{\tau_{T} c_{p_{a}} V_{\infty}} \frac{\operatorname{Pr}}{S c}\left(w_{\infty}-w_{s}\right)
$$

The mass fractions of water vapor in the above equation are given by the equations below.

$$
\begin{aligned}
& w_{\infty}=\phi_{\infty} \frac{M_{w}}{M_{a}} \frac{P_{w, \infty}}{P_{a}} \\
& w_{s}=\phi_{s} \frac{M_{w}}{M_{a}} \frac{P_{w, s}}{P_{a}} \quad\left(\phi_{s}=1.0, \text { fully saturated }\right)
\end{aligned}
$$


The non-dimensionalization of the energy equation above results in the following term for the thermal response time

$$
\tau_{T}=\frac{c_{p_{i}} \rho_{i} d^{2}}{6 k_{a}}
$$

The Nusselt number, Sherwood number, and the Prandtl number in these equations are provided by assuming spherical particles. If other particle geometries are of interest, the correlations can be easily modified for those cases.

$$
\begin{aligned}
& \operatorname{Pr}=\left(\frac{c_{p} \mu}{k}\right)_{a} \\
& N u=\frac{h d}{k_{a}} \\
& N u=2+0.6 \operatorname{Re}^{1 / 2} \operatorname{Pr}^{1 / 3}
\end{aligned}
$$

The Schmidt number is defined by the following equation.

$$
S c=\frac{\mu_{a}}{\rho_{a} D_{v}}
$$

The Sherwood number is defined by the following equation.

$$
\begin{aligned}
& S h=\frac{h_{m} d}{D_{v}} \\
& S h=2+0.6 \operatorname{Re}^{1 / 2} S c^{1 / 3}
\end{aligned}
$$

The Reynolds number in the above equation is based on the particle size and the relative velocity of the particle to the velocity of the air stream

$$
\operatorname{Re}=\frac{\rho_{a}\left|V_{\infty}-V_{d}\right| d}{\mu_{a}}
$$

where the particle/drop velocity, $V_{d}$, is obtained by integrating the equation of motion

$$
\frac{d V_{d}}{d t}=\frac{3}{4} C_{d} \operatorname{Re}^{2} \frac{\mu_{a}^{2}}{\rho_{a} \rho_{i} d^{3}}
$$

Once the particle starts to melt, its temperature no longer changes. In this case, the energy equation above can be written as a change in the enthalpy or a change in the melt fraction ${ }^{8}$ as shown below.

$$
\frac{d n_{m e l t}}{d x}=\frac{c_{p_{i}}}{L_{f}} \frac{N u}{\tau_{T} V_{\infty}}\left(T_{a}-T_{i}\right)+\frac{c_{p_{i}}}{L_{f}} \frac{L_{s} S h}{\tau_{T} c_{p_{a}} V_{\infty}} \frac{\operatorname{Pr}}{S c}\left(w_{\infty}-w_{s}\right)
$$

The local fluid conditions of temperature and pressure are used in the above equations to determine the rate of sublimation, ice melting, and evaporation. The distance the ice particle traverses through the compressor rotors and stators is determined from the length of the engine inlet, and the geometry of the rotor blades, stator vanes, and the axial gaps between the rotors and stators. The distance through the fan and LPC blade rows is estimated from the true blade chord of the rotors and stators at the mid span, and the axial distance through the gaps between the blades, as illustrated in Figure 7. Note that each stage is analyzed at the blade edges as shown in Figure 4. In this study, the 
simple circular arc blade camber line shapes are utilized and provide a means of easily estimating the geometry of the compressor blades and vanes. This facilitates estimating the distance traveled by the water/ice particle. The COMDES and MELT codes exchange boundary conditions of pressure, temperature and velocity, to estimate the residence time of the particle through each blade row, as well as to determine the rate of ice sublimation, melting, and evaporation.

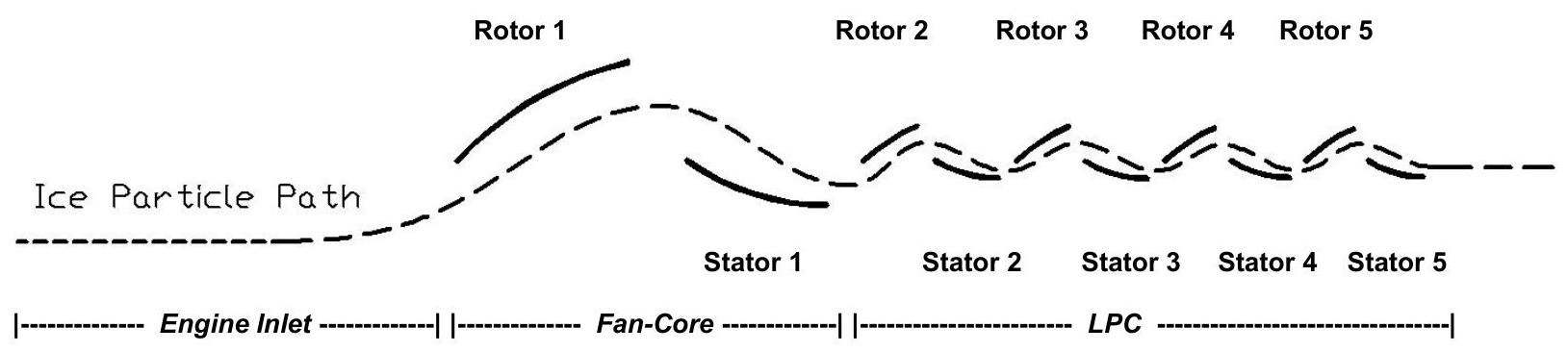

Figure 7: Ice particle path through the fan-core and low pressure compressor stages. Rotor and stator blade mean camber line chord at the mid-span section is used to estimate the distance traveled and residence time of the ice particles.

Figure 7 illustrates the ice particle path through the rotor blades in the relative frame of reference, while the path through the stator vanes is in the absolute frame of reference. The calculations of ice sublimation, melt, and evaporation are performed in increments from the leading to the trailing edge of each rotor and stator, as well as through the axial gap between the blades. As the engine is flown through the flight trajectory, the ice particle sublimation, melting, and evaporation is calculated in each rotor and stator blade row. The amount of water due to sublimation and evaporation is added to the local value of specific humidity, thus having an effect on the local relative humidity, and likewise on the local wet-bulb temperature calculation in the compressor code. The MELT code is also used to evaluate the particle conditions through the engine inlet which is then provided as ice particle initial conditions at the leading edge of the fan-core.

\section{Identifying the Risk of Ice Accretion}

When the wet-bulb temperature and melt ratio parameters indicate a risk of ice accretion at a particular location in the compressor, an additional blockage can be applied, which will result in a change in the performance of the compressor and engine. Though not implemented in the current study, the capability to simulate turbofan engine performance with additional blockage due to accretion has been previously demonstrated in References 4,5 . In that study, the blockage due to accretion was varied parametrically. The approach was to add blockage as a function of empirically derived ice growth rate, and time spent at that operating condition. However, the main focus of this paper was to determine the risk of ice accretion and its location, with an estimate of ice particle melt ratio, and the wet-bulb temperature calculation with no additional blockage due to ice accretion.

Using data from laboratory testing, ${ }^{10}$ Figure 8 shows the normalized ice accretion growth rate as a function of measured liquid water content $\left(L W C_{m}\right)$ to injected total water content $\left(T W C_{i n j}\right)$. In this figure, the measured ice growth rates $(G)$ are normalized by the theoretical maximum ice growth rate, $G_{\max }$, as shown in Eq. (42). The data from this experiment suggest that some liquid water needs to be present for accretion to occur. A non-zero growth rate, $G / G_{\max }$, was first observed when the ratio of $L W C$ to $T W C$ increased to $14 \%$ (Figure 8). Only the portion of the data with wet-bulb temperatures below freezing is represented in Figure 8. However, data for wet-bulb temperatures marginally above freezing have also resulted in ice accretion with shedding, as reported in Reference 14. The ice accretion growth rate as a function of the ratio of LWC to TWC data shown in Figure 8 is included in Appendix A.

In this study it is assumed that ice accretion can only occur at values of $T_{W B}$ that are below the temperature of $499 \mathrm{R}$ $\left(\sim 4^{\circ} \mathrm{C}\right)$. This assumption is based on observations ${ }^{10}$ that showed significant growth rates and buildup of ice at values of $T_{W B}$ below $499 \mathrm{R}$. However, at values just above freezing (between $492 \mathrm{R}$ and $499 \mathrm{R}$ ), the ice was slushy, and had a tendency to shed before a large buildup of ice could occur, but at values below freezing, the accreted ice did not shed and continued to grow as a function of time, provided that there was a positive melt ratio. Note that the effects 
of ice shedding have not been modeled in this study. However, the detrimental effects of shedding on engine performance cannot be ruled out. Therefore, in this study the upper limit of $499 \mathrm{R}$ is used as the maximum wet-bulb temperature ice accretion risk indicator. The criteria for the risk of ice accretion therefore are the wet-bulb temperature being less than 499R (i.e., slightly above freezing or below), and when the ratio of $L W C$ to $T W C$ exceeded $10 \%$, which includes some margin in comparison to the $14 \%$ ratio that was observed in the experiment to have significant growth rate. In this study, the ratio of $L W C$ to $T W C$ is an approximation to the melt ratio used elsewhere in this document.

It is important to note that the data in Figure 8 is preliminary as the values $L W C_{m}$ and $T W C_{i n j}$ require corrections which are not yet well understood. The subscripts $m$, referring to the $L W C$, denotes a measured value using a hotwire probe which has not yet been characterized in mixed-phase flow. The value used for $L W C$ in Figure 8 is the uncorrected value. The subscript $i n j$, referring to the $I W C$, denotes a bulk injected value using bulk water or ice flow rate data assuming a uniform cloud. The corrections to the $L W C$ and $I W C$ are topics of current research efforts. In addition, as higher fidelity, multi-disciplinary computational tools become available with accretion, erosion, particle breakup, and shedding models, they can result in more accurate predictive capability for the growth rate.

$$
G_{\max }=\left(\frac{T W C_{i n j}}{\rho_{i}}\right) V_{\infty}
$$

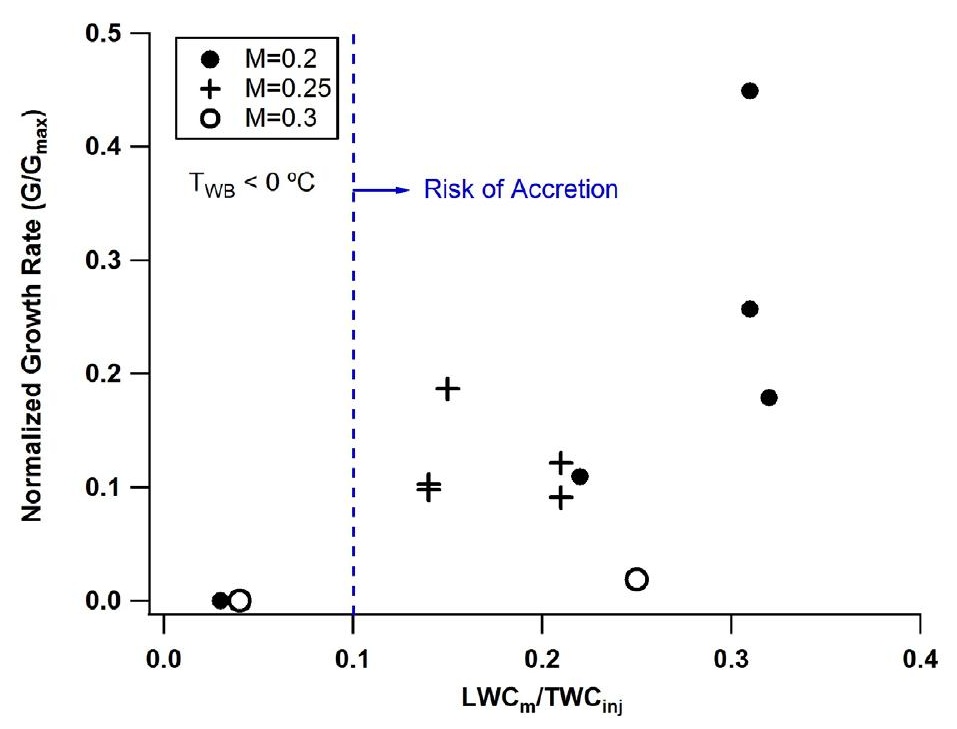

Figure 8: Ice accretion growth rate as a function of the liquid-water-to-total water content ratio (i.e., melt ratio) when the wet-bulb temperature was below freezing ${ }^{10}$. A finite rate of ice growth was observed when the melt ratio was above $10 \%$.

For completeness, an outline of how the additional blockage due to high growth rate of ice accretion can be utilized in the compressor flow analysis code. The amount of blockage due to ice accretion on the blades can be estimated from the maximum growth rates of ice accretion that have been observed during laboratory testing at the NRCC laboratory. ${ }^{10}$ The size of the ice growth is a function of time $(t)$ spent at that operating condition and the ice growth rate is specified in terms of length per time. Although this growth rate is measured at a single point (i.e., the leading edge) of a blade, it is used here to generate a first order estimate of the blockage within a compressor.

The amount of ice blockage in a blade row is estimated in the compressor code from the measured growth rate $(G)$ and the blade spacing parameter $(S)$. The ice accretion on the compressor vanes is illustrated in Figure 9, and shows how the blade spacing is reduced due to the estimated accretion. 


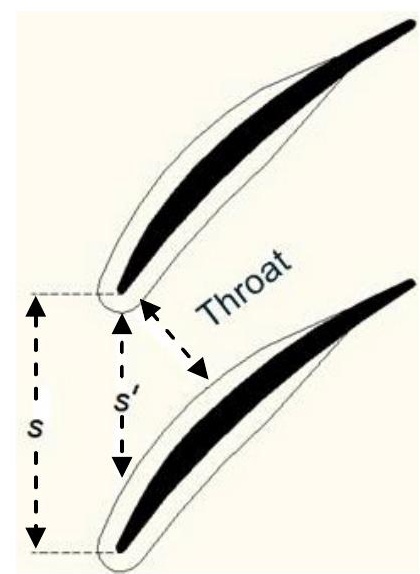

Figure 9: Estimating the blockage due to ice accretion in the stator blade row. The ice accretion can be modeled in the compressor code as an additional blockage at the stator leading edge and throat.

The blockage from ice accretion can be modeled in the compressor code as an additional blockage and added to the nominal value of aerodynamic blockage. The reduction in the circumferential blade spacing $\left(S^{\prime}\right)$ is estimated by Eq. (43), from the empirical value of ice growth rate $(G)$, the time spent at that operating condition $(t)$, and the nominal blade spacing $(S)$. The reduced blade spacing results in a reduced annular area at the stator inlet and exit, and also a reduced throat area.

$$
S^{\prime}=S-2 t G
$$

The blockage due to ice accretion is estimated from the reduction in blade spacing. The performance of the compressor and the engine system, computed with the additional blockage added parametrically (not as a function of time and growth rate) at a specific location in the compressor, has been demonstrated in References 4, 5.

\section{Analysis of Turbine Engine Performance in an Ice Crystal Environment}

\section{A. Fan and Low Pressure Compressor Conceptual Design}

In order to perform the engine icing study, it was necessary to have the geometry of the low pressure compressor (LPC) in adequate detail such that the flow conditions between each blade row can be computed with the mean line compressor flow code. The conceptual design task was performed for a notional turbofan engine in the $40000 \mathrm{lb}$ thrust class having a bypass ratio of 5.8 with the mean line compressor flow analysis code. The rotor and stator blade shapes were approximated with simple circular arcs. The conceptual design effort of the notional fan-core and LPC determined the key dimensions that define the blade and flow path geometry. Figure 10 illustrates the notional fan and low pressure compressor that was used in this study. The fan-core and LPC design point aerodynamic performance is included in Appendix B as output from the compressor code analysis. 


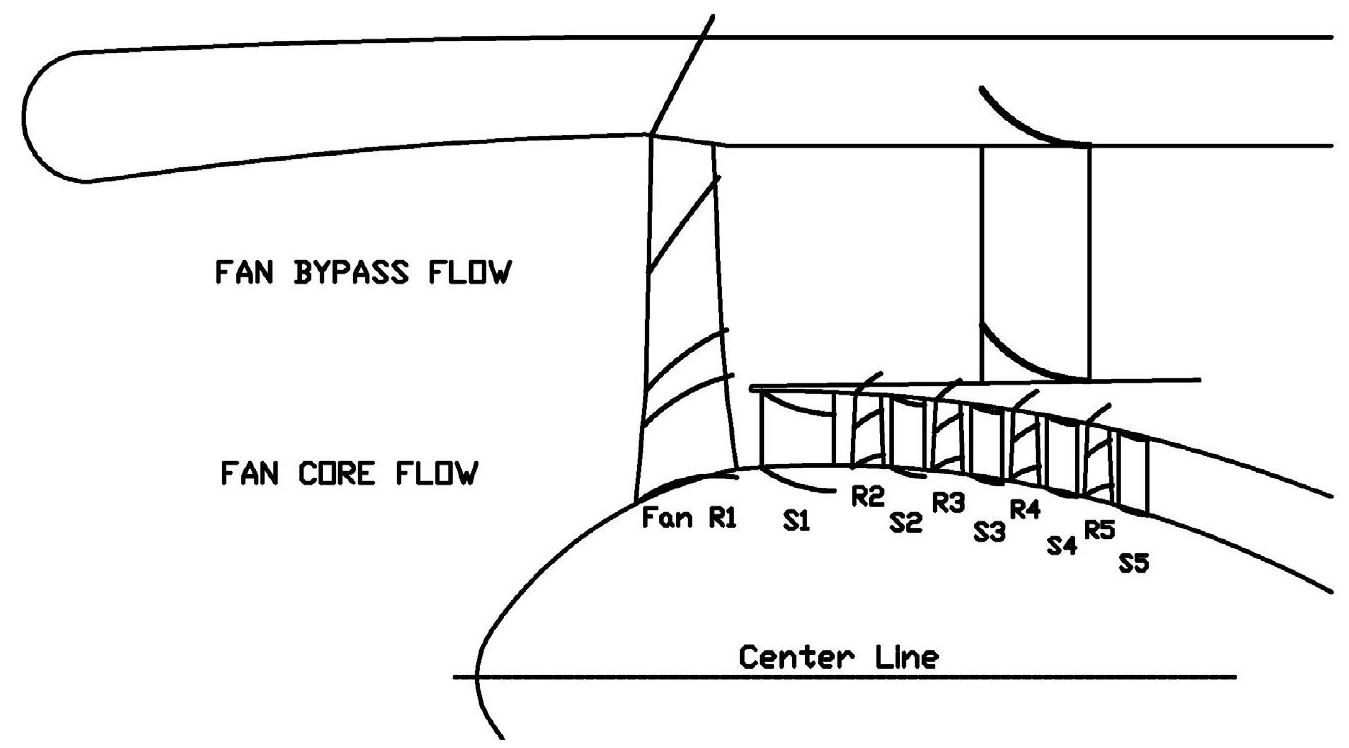

Figure 10: Fan and low pressure compressor of the notional high bypass ratio turbofan engine.

A compressor flow model with the COMDES code represents the fan-core and LPC in the NPSS engine system model. The radial view of the blade centerline sections are superimposed onto the flow path in Figure 10. Table I lists key aerodynamic and geometric design parameters at the root-mean-square (RMS) radius of each blade row and stator vane. The fan-core is considered the first stage in this study, followed by a four stage LPC.

Table I - The Fan-Core and LPC Design Point RMS Radius Geometric and Aerodynamic Parameters

\begin{tabular}{|l|l|l|l|l|l|l|l|l|l|l|}
\hline & $\begin{array}{l}\text { Fan-Core } \\
\text { Rotor1 }\end{array}$ & $\begin{array}{l}\text { Fan-Core } \\
\text { Stator1 }\end{array}$ & $\begin{array}{l}\text { LPC } \\
\text { Rotor2 }\end{array}$ & $\begin{array}{l}\text { LPC } \\
\text { Stator2 }\end{array}$ & $\begin{array}{l}\text { LPC } \\
\text { Rotor3 }\end{array}$ & $\begin{array}{l}\text { LPC } \\
\text { Stator3 }\end{array}$ & $\begin{array}{l}\text { LPC } \\
\text { Rotor4 }\end{array}$ & $\begin{array}{l}\text { LPC } \\
\text { Stator4 }\end{array}$ & $\begin{array}{l}\text { LPC } \\
\text { Rotor5 }\end{array}$ & $\begin{array}{l}\text { LPC } \\
\text { Stator5 }\end{array}$ \\
\hline Blade Chord, in. & 7.434 & 5.496 & 2.401 & 2.401 & 2.344 & 2.373 & 2.240 & 2.270 & 2.125 & 2.132 \\
\hline AxialChord, $X_{2}$ in & 6.804 & 5.235 & 2.130 & 2.130 & 2.066 & 2.284 & 1.969 & 2.180 & 1.858 & 2.044 \\
\hline Blade Gap, $X$, in. & 1.811 & 1.309 & 0.567 & 0.591 & 0.550 & 0.571 & 0.524 & 0.545 & 0.495 & 0.511 \\
\hline Radius, $r_{c}$, in. & 12.535 & 9.038 & 7.352 & 4.645 & 6.784 & 4.37 & 6.354 & 4.069 & 5.750 & 3.752 \\
\hline Leading Edge & & & & & & & & & & \\
\hline Press Static, psia & 13.67 & 14.67 & 16.61 & 18.785 & 20.12 & 22.45 & 23.91 & 26.41 & 27.88 & 30.04 \\
\hline Temp Static, R & 508.0 & 522.1 & 542.22 & 564.55 & 576.56 & 597.76 & 609.40 & 629.48 & 640.0 & 656.21 \\
\hline Mach no., abs & 0.38 & 0.638 & 0.46 & 0.534 & 0.42 & 0.499 & 0.39 & 0.449 & 0.34 & 0.417 \\
\hline Blade $\beta_{1}$ deg & 47.20 & 35.4 & 42.3 & 30.6 & 23.5 & 31.5 & 43.80 & 32.4 & 44.80 & 33.0 \\
\hline Abs Flow Angle & 0 & 36.15 & 0 & 31.22 & 0 & 32.18 & 0 & 33.00 & 0 & 0 \\
\hline Tip Radius, in. & 20.63 & 20.617 & 20.530 & 20.3433 & 20.096 & 19.6905 & 19.3131 & 18.8420 & 18.2524 & 17.6890 \\
\hline Hub Radius, in. & 12.51 & 15.102 & 15.211 & 15.2026 & 15.054 & 14.8484 & 14.3197 & 13.7204 & 13.0749 & 12.2606 \\
\hline Trailing Edge & & & & & & & & & & \\
\hline Press Static, psia & 14.69 & 16.35 & 18.76 & 19.79 & 22.40 & 23.56 & 26.19 & 27.53 & 30.06 & 31.22 \\
\hline Temp Static, R & 521.86 & 539.72 & 563.96 & 573.81 & 597.00 & 606.82 & 627.60 & 637.69 & 655.84 & 663.90 \\
\hline Mach no., abs. & 0.63 & 0.481 & 0.53 & 0.448 & 0.49 & 0.413 & 0.46 & 0.368 & 0.41 & 0.338 \\
\hline Blade $\beta_{2}$, deg & 12.7 & 0 & 23.5 & 0 & 23.5 & 0 & 23.5 & 0 & 23.5 & 0 \\
\hline Abs Flow Angle & 36.93 & 0 & 31.59 & 0 & 32.30 & 0 & 32.27 & 0 & 33.24 & 0 \\
\hline Tip Radius, in. & 20.63 & 20.573 & 20.445 & 20.1704 & 19.925 & 19.4117 & 19.0737 & 18.4850 & 17.9280 & 17.2148 \\
\hline Hub Radius, in. & 15.0 & 15.190 & 15.223 & 15.2026 & 14.848 & 14.4742 & 13.9111 & 13.2848 & 12.4811 & 11.6228 \\
\hline
\end{tabular}




\section{B. The Performance of the Notional Turbine Engine System Model through a Typical Commercial Aircraft Flight Trajectory}

In order to perform the engine icing study, the NPSS-COMDES engine model was executed at numerous operating conditions through a flight trajectory. The inlet conditions of ice crystal concentrations in grams per cubic meter, particle size, and the elevated ISA (International Standard Atmosphere) temperature were varied parametrically at ISA $+0 \mathrm{R},+18 \mathrm{R},+27 \mathrm{R}$, and $+36 \mathrm{R}$, since that is in the range of where ice accretion has been reported, as was illustrated in Figure 1. The detailed analysis of the fan-core and low pressure compressor with the COMDES code was obtained at each operating condition. The engine analyses were performed with the fully coupled computer models described in the above sections. The system model was utilized to determine the performance of the notional $40000 \mathrm{lb}_{\mathrm{f}}$ thrust class engine through a typical flight trajectory of a commercial aircraft (Figure 11).

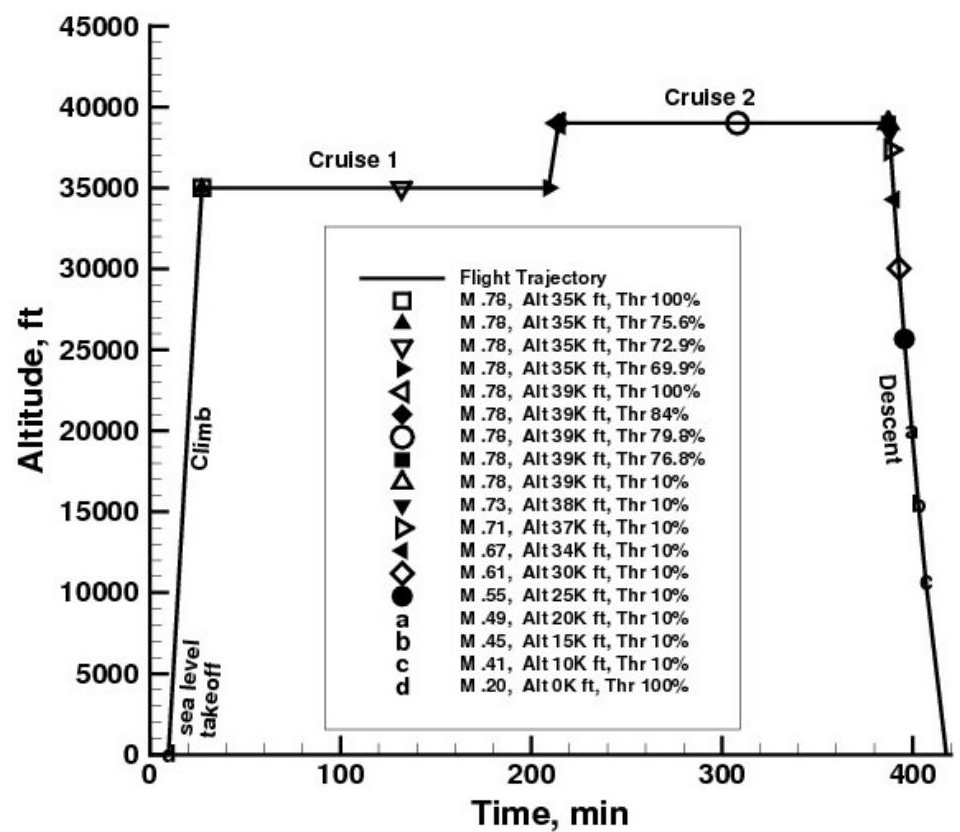

Figure 11: Flight trajectory for a typical commercial aircraft. The notional $40000 \mathrm{lb}$ thrust engine is "flown" through the vehicle flight trajectory to determine the blade row where conditions for ice accretion may be possible.

The notional flight trajectory ${ }^{4,5,15}$ used in this study includes takeoff, climb, two cruise altitudes, and one descent profile. The detailed output listing of the flight trajectory is included in Appendix C. The first cruise altitude is at 35000 feet, where the engine thrust is reduced from $100 \%$ down to $69.9 \%$ of maximum thrust at the end of the cruise. The aircraft then proceeds to 39000 feet where again the thrust reduces from $100 \%$ down to $76.8 \%$ maximum thrust at that altitude. The engine is then spooled back to $10 \%$ maximum thrust at 39000 feet as it prepares to descend in altitude. The engine is maintained at $10 \%$ maximum thrust as it descends to 10000 feet, which was the lowest altitude that was analyzed in this study.

Figures 12 and 13 illustrate the baseline pressure ratio and efficiency characteristic maps of the fan-core and LPC and the superimposed operating points through the flight trajectory. The baseline maps have no additional blockage due to ice accretion. These maps were generated by the compressor code executed in the stand-alone mode (independent of the NPSS system modeling code); however, the operating points along the map were obtained by running the fully coupled engine system model, the compressor flow analysis, and the melt codes as illustrated in Figure 3. The plot file of the overall compressor maps shown in Figures 12 and 13 are listed in Appendix D. The plot file of the fan-core and LPC performance along the engine operating line is listed in Appendix E. The complete output listing of the mean line compressor flow analysis code, along the entire baseline performance map and the flight trajectory, are listed in Appendices D and E of Reference 5 respectively. 
The risk of ice accretion on a stator is a function of where in the flight trajectory the engine is operating and the atmospheric conditions of temperature and pressure, as well as the ice particle concentration and size. As will be illustrated in a later section of this paper, at high altitudes, the rear stages of the LPC may be at risk for accretion, while at lower altitudes, the risk of accretion may be in the front stages.

Even though no additional blockage is added at any location to the compressor, the compressor analysis code was utilized in this study to determine which stage had a risk of ice accretion, based on the wet-bulb temperature and melt ratio criteria. This could not have been determined from an LPC compressor map showing overall performance alone.

In the previous study ${ }^{4}$ that included compressor flow analysis with parametric blockage, the LPC performance with ice accretion could not be represented by traditional compressor performance maps, since the amount of blockage due to ice, and the stage where there is blockage, was different along each speed line, and depended on the altitude and thrust level it is operating at in the flight trajectory. The solution that was chosen was to fully couple the NPSS and the COMDES codes, thus the compressor performance was passed to the system model for each unique point of operation, which included the unique amount of blockage due to ice accretion at a specific blade row.

In this study the performance of the engine with blockage is not addressed; as this was addressed in an earlier publication. The focus here is to more accurately predict the locations in the low pressure compression system where there is a risk of ice accretion, as well as the atmospheric conditions that could support it, along a flight trajectory. The fully coupled NPSS_COMDES_MELT code has been executed through the flight trajectory, and the results of the icing risk analyses, in each stage of the LPC, are shown graphically in the following figures.

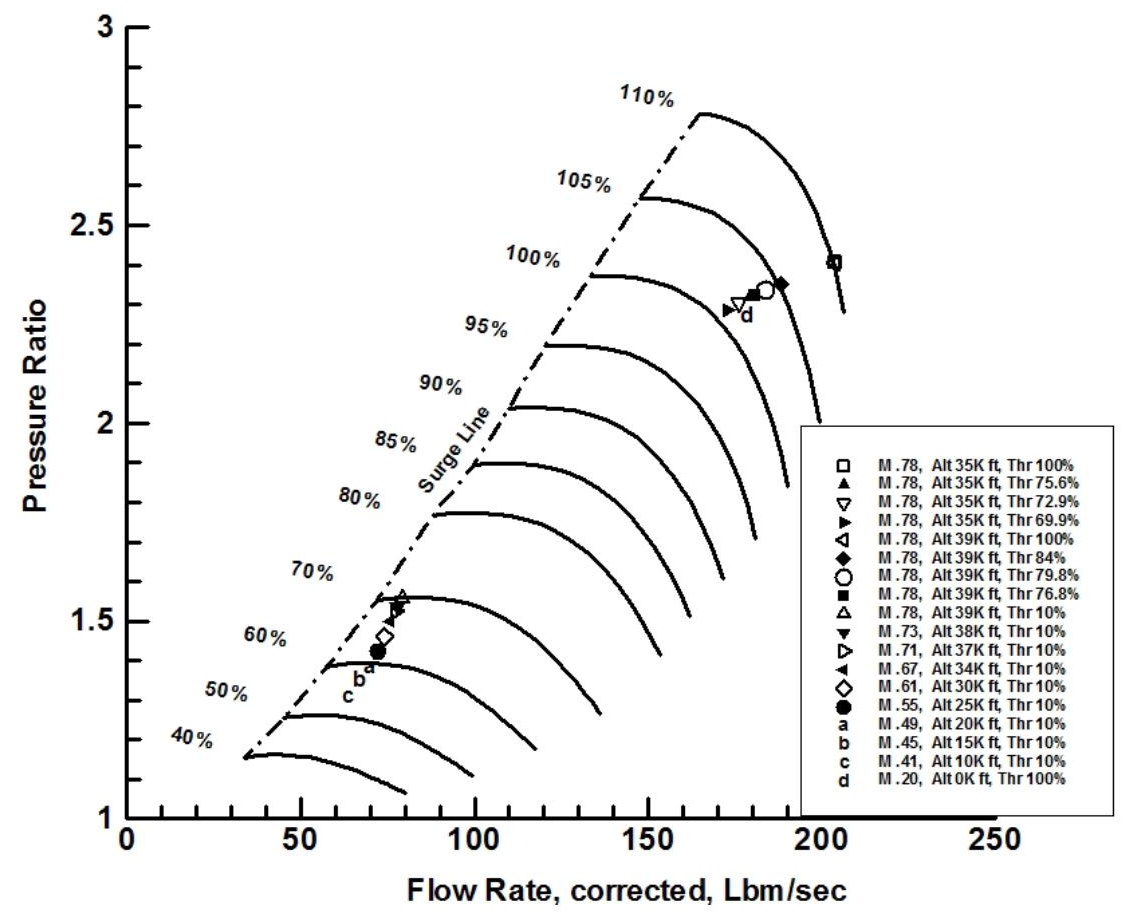

Figure 12: Fan-core and LPC pressure ratio showing the baseline performance. Operating points of the fancore and LPC throughout the flight trajectory are superimposed onto the baseline pressure ratio map. 


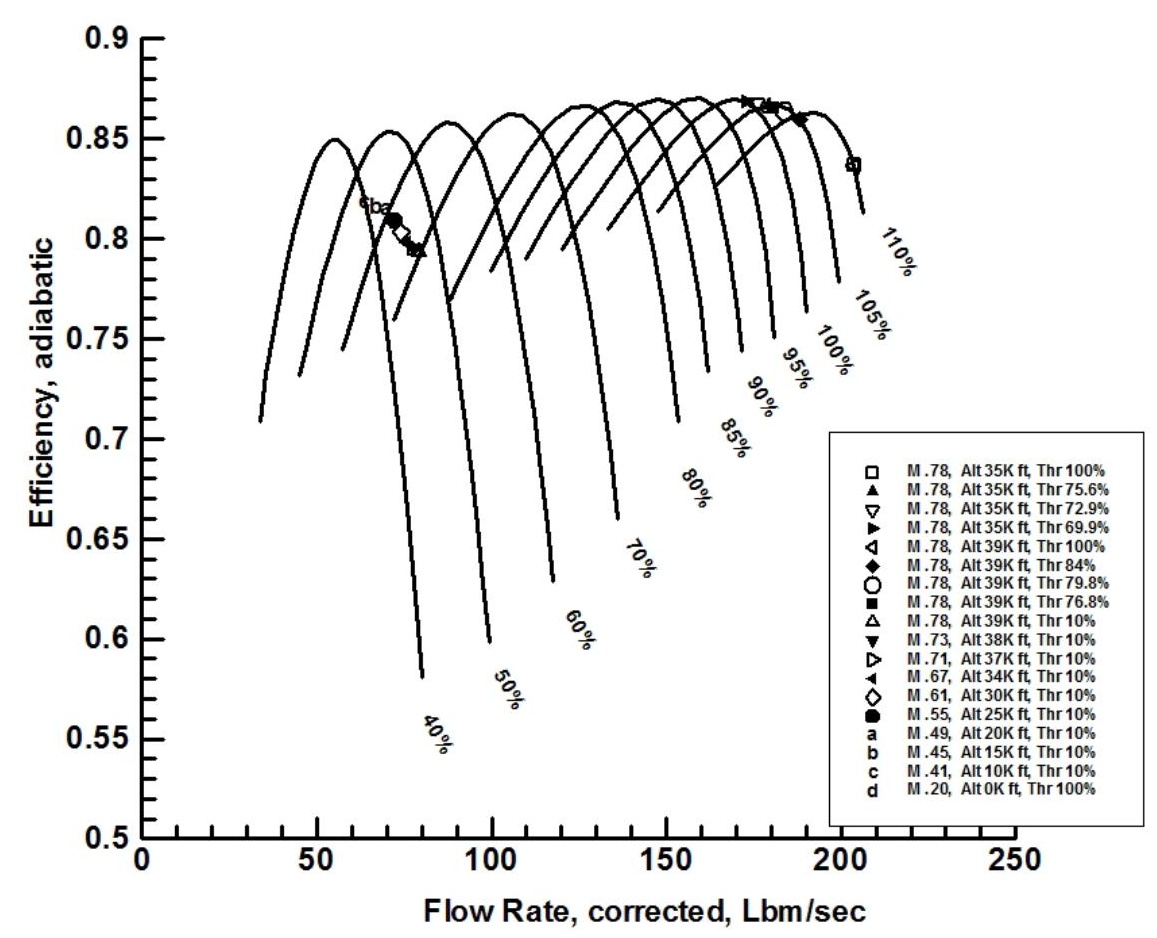

Figure 13: Fan-core and LPC performance showing the baseline efficiency map. Operating points of the fancore and LPC throughout the flight trajectory are superimposed onto the baseline efficiency maps.

A range of ice particle concentrations, and particle diameters were analyzed with the icing code, at various ambient temperatures above the ISA standard. The local values of wet-bulb temperature and melt ratio at each LPC blade and stator inlet and outlet, were computed for the engine operating conditions through the vehicle flight trajectory. The performance of the fan and LPC stages were analyzed.

\section{Ice Particle vs. Ambient Temperature}

In this study we assumed that ice crystals cannot form at temperatures above freezing. As illustrated in Figure 1, the range of temperatures where engine icing events occurred was between the ISA temperature and $36 \mathrm{R}$ above the ISA temperature. The altitude at which freezing occurs is a function of ISA offset temperature, as illustrated in Figure 14. For example, at an ISA offset temperature of $0 \mathrm{R}$, the minimum altitude where ice crystals can form in the atmosphere is $7500 \mathrm{ft}$, while with an offset of $+36 \mathrm{R}$, the minimum altitude is $17400 \mathrm{ft}$. In the study the ISA offset temperatures of $+18,+27,+36 \mathrm{R}$ were considered through a wide range of altitudes along the flight trajectory. However, at conditions below the $492 \mathrm{R}$ line in Figure 14, the assumption is that the particle is all liquid water; therefore the melt ratio even before entering the engine is equal to 1.0. Likewise, the calculated wet-bulb temperature at these conditions is above the range where ice accretion is a risk, based on the analysis. Therefore, no conditions exist that would support ice accretion at operating points below the $492 \mathrm{R}$ line shown in Figure 14 . 


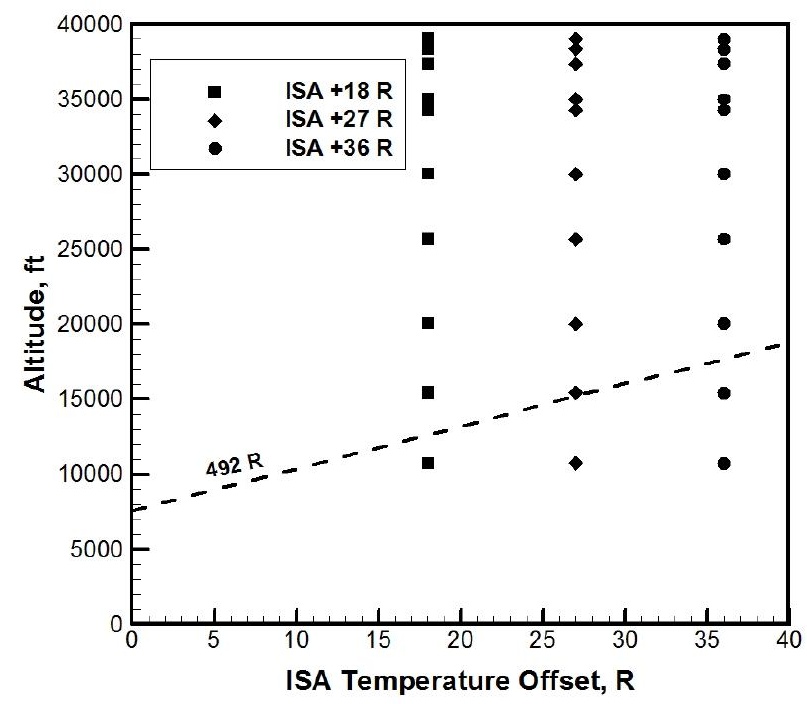

Figure 14: Altitude vs. ISA offset temperature indicating the assumed minimum altitude where ice crystals can form. Note that ice crystals are not expected to form at conditions below the $492 R$ line.

\section{Ice/Water Particle vs. Air Temperature through the Engine Inlet}

In the NPSS code, there is a model to estimate the aerodynamic performance of the engine inlet, from the entrance region through the plane of the fan face, based on the area ratio and length. The change in total temperature due to the forward velocity of the vehicle is estimated, and the static temperature is calculated from the entrance of the engine to the plane of the fan leading edge. At the entrance, the temperature of an ice crystal is equal to the ambient temperature. However, because the aircraft is flying at a moderately high Mach number, the total temperature of the air is increased above the ambient, and the change in temperature is calculated based on the Mach numbers at the entrance and at the fan face. As the air is diffused through the subsonic inlet, the static temperature of the air rises even before reaching the fan leading edge. At high altitude operating points, for example during the descent mode at an altitude of $38334 \mathrm{ft}$. and vehicle Mach .73 in Figure 11, the temperature of the ice particles in the engine inlet is typically well below freezing, until the particles are inside the fan-core and LPC, as illustrated in Figure 15. Through the inlet and most of the fan-core and LPC, the ice particle temperature rises and also sublimates. Note that throughout the flow path the enthalpy exchange between the ice particle and the air is iteratively computed.

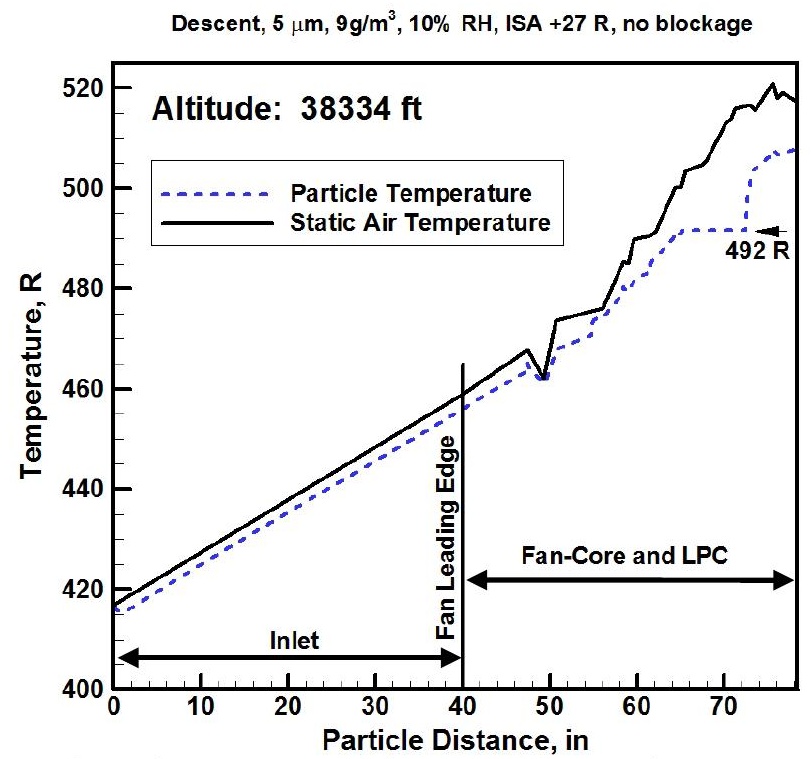

Figure 15: The temperature of the ice crystal particles lags the static temperature of the air through the engine inlet, the fan-core and the LPC at $38 \mathrm{~K}$ ft during the descent mode, with particle travel distance. 
At a distance traveled of 64 inches, the particle reaches the freezing temperature of water ( $492 \mathrm{R})$ and begins the melting and evaporation process. The ice-water particle remains at 492 degrees until it is fully melted at 72 inches of distance traveled. At 72 inches the particle is fully melted (melt ratio $=1.0$ ). Beyond 72 inches the fully melted particle continues to rise in temperature while continuing to evaporate. Although the computer code models the air flow and ice crystal physics through the inlet portion of the engine, the subsequent figures will illustrate the flow conditions of wet-bulb temperature and melt ratio only through the fan-core and LPC, since those are the areas where there can be risk of accretion due to ice crystals. Details of ice crystal temperature and static temperature of the air as a function of temperature data are provided in Appendix F.

\section{Methodology to Determine Ice Risk}

The methodology to determine whether there is a potential risk of ice accretion throughout the flight trajectory in the low pressure compression system involved tracking the wet-bulb temperature and the melt ratio through the flow path. The figures below illustrate how varying the ice particle size and temperature above ISA through the descent affected the local wet-bulb temperature and the melt ratio through each blade row in the fan-core and LPC. To illustrate this methodology Figure 16(a, b) is provided as an example. Figure 16(a) shows the seven descent mode altitudes that were analyzed, from 39000 to 20047 feet. In this and similar figures, the solid lines show the calculated wet-bulb temperature while the hatched lines show the melt ratio.

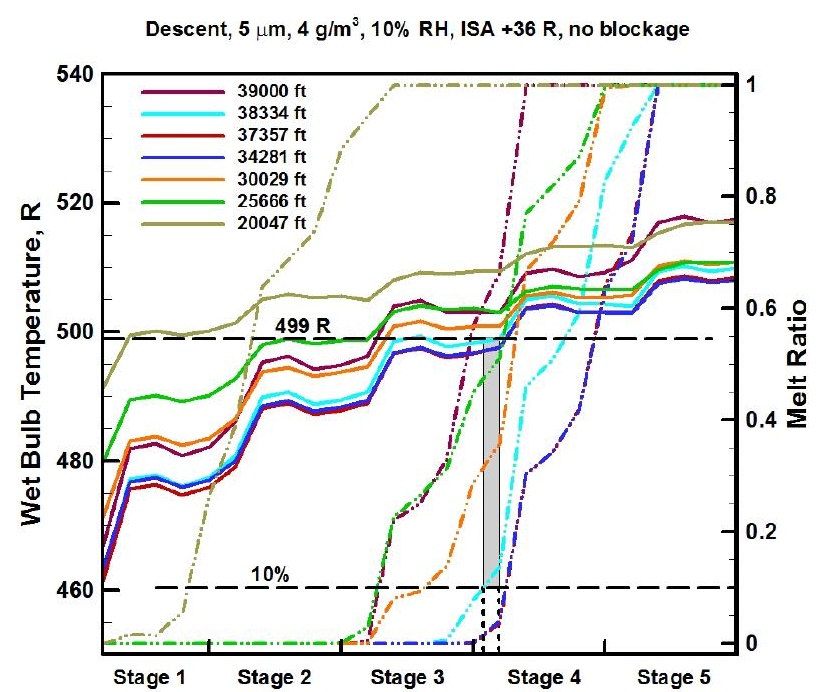

(a)

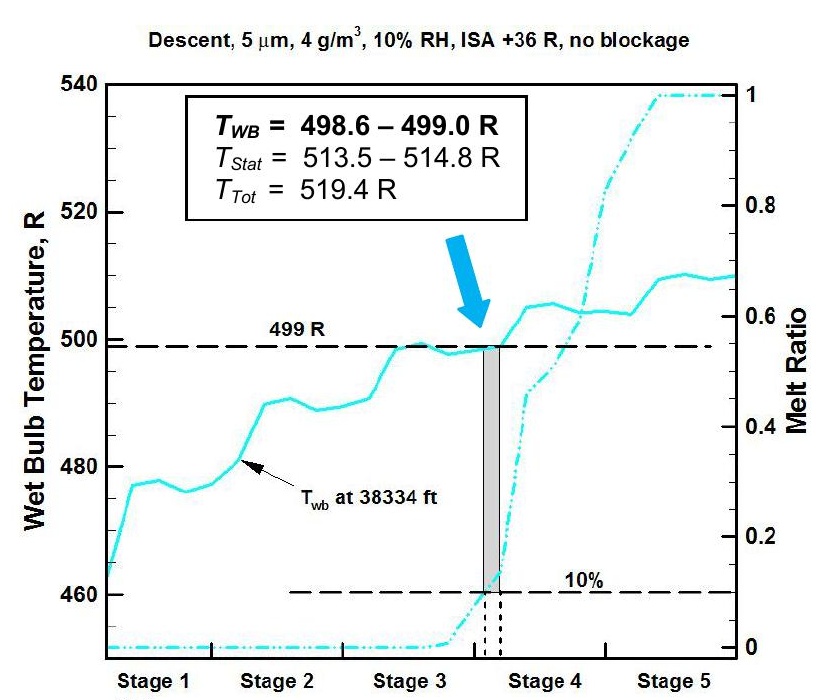

(b)

Figures 16(a, b): Wet-bulb temperature and melt ratio both indicate that there is a risk of ice accretion between stage-3 and stage-4 during descent. (a) All altitudes. (b) Risk of ice accretion at 38334 feet.

With an ice particle size of $5 \mu \mathrm{m}$, an IWC of $4 \mathrm{~g} / \mathrm{m}^{3}$, and the inlet temperature of $+36 \mathrm{R}$ offset from the ISA, it was found that at 38334 feet altitude, the wet-bulb temperature and the melt ratio criteria are both met at the same location, as indicated by the gray shaded area of Figure 16(a). Figure 16(b) shows the results at the 38334 feet extracted from Figure 16(a). This indicates that there is a risk of ice accretion in the stator gap of stage-3, specifically just past the stator trailing edge. The wet-bulb temperature in the stage- 3 stator gap is $498.6 \mathrm{R}$. The range of static temperature is 513.5-514.8 R, while the total temperature is 519.4 R. This methodology was used in the parametric icing risk analysis that follows, where particle size, IWC, and ISA offset temperature were varied through the flight trajectory (Figure 11), which includes two cruise altitudes and a descent mode.

\section{Parametric Icing Risk Analysis}

The parametric study of the engine performance was carried out by varying the specified inlet conditions of ice particle size, concentration, and ambient temperature offset above the ISA. This study extends the work from an earlier paper ${ }^{16}$ with updated COMDES and MELT analysis codes, as well as a broader range of IWC. Furthermore the complete analysis results are included in the Appendices. Each cruise altitude was studied at four separate operating points, representing different thrust settings, as shown in Figure 11. In Figures 18-23, the ice particle size studied were 5, 10, 25, 50, and $100 \mu \mathrm{m}$; the ISA temperature increase was varied from $+18 \mathrm{R}$ to $+36 \mathrm{R}$. The cruise conditions studied were at a range of engine thrust settings from $100 \%$ to $69.9 \%$ of maximum thrust for the given 
altitude. In the descent mode, the thrust was set at $10 \%$ of maximum thrust at each altitude analyzed. The ice concentrations in this study were 2,4 , and $9 \mathrm{~g} / \mathrm{m}^{3}$. The results shown in the following figures indicate whether there is a risk of ice accretion at the specific altitude operating condition, ice crystal concentration, size, and temperature above ISA. The scale in Figures 18-23 indicates the wet-bulb temperature variation through the blade rows and axial gaps in the fan-core and low pressure compressor. The melt ratio is also plotted on a scale from $0.0-1.0$, where 0.0 indicate no melting, and 1.0 indicates an ice particle that is $100 \%$ melted. Note that the inlet relative humidity was kept at $10 \%$, since it was noticed early in the analysis that it did not have a strong effect on the results. It was observed that even if the ambient relative humidity at the fan face was $80 \%$, the rise in temperature through the rotor blades quickly reduced the local relative humidity in each stage to significantly lower levels, as illustrated in Figure 17. The rate at which the relative humidity is reduced through the stages is also a function of engine thrust level, as shown in Figure 17. The local value of relative humidity has a direct effect on the local wet-bulb temperature determination, as calculated by the equations $(23,24$, and 25$)$.

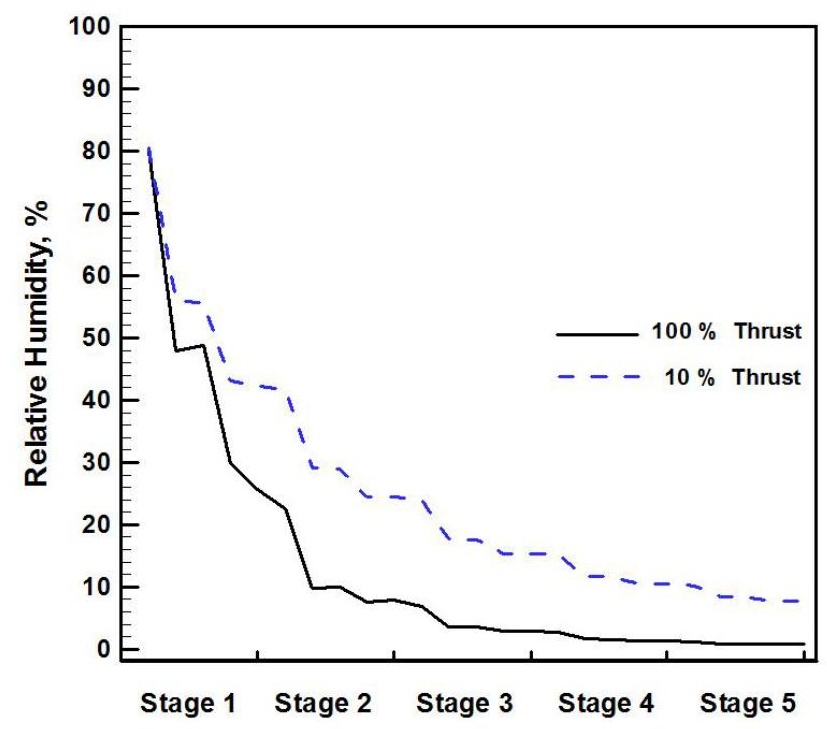

Figure 17: Change in relative humidity through the five stage fan-core and low pressure compressor, at $100 \%$ and $10 \%$ engine thrust levels. Note that the relative humidity is quickly reduced in each subsequent stage.

\section{Cruise at 35000 feet:}

The analysis was conducted at the altitude cruise condition of 35000 feet using the methodology described above. The engine thrust level was varied between $100 \%$ and $69.9 \%$ of max thrust at this cruise altitude. The particle size was varied from $5 \mu \mathrm{m}$ to $100 \mu \mathrm{m}$, and the IWC was varied between 2 and $9 \mathrm{~g} / \mathrm{m}^{3}$, but only the results for the $2 \mathrm{~g} / \mathrm{m}^{3}$ are presented below as representative at this cruise altitude. 


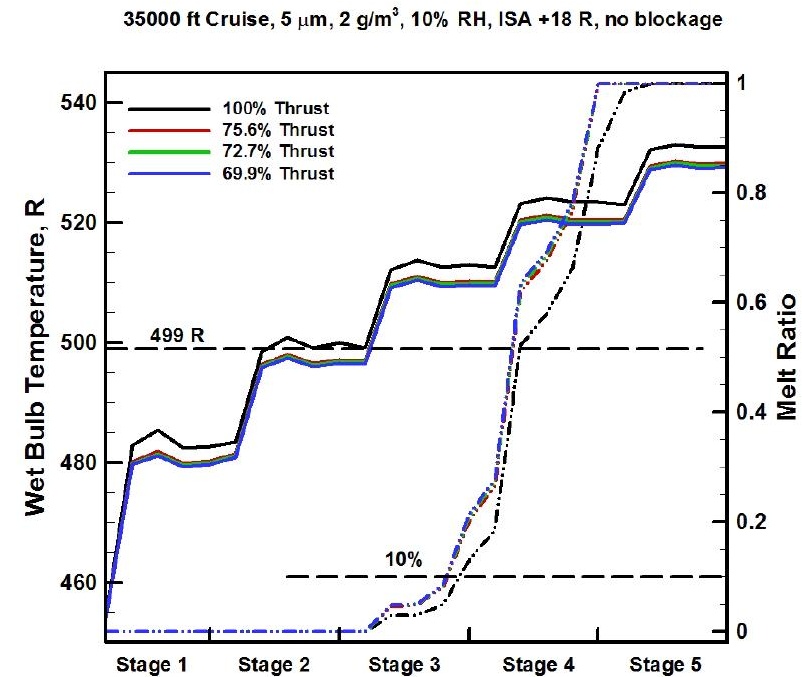

(a)

$35000 \mathrm{ft}$ Cruise, $10 \mu \mathrm{m}, 2 \mathrm{~g} / \mathrm{m}^{3}, 10 \% \mathrm{RH}$, ISA +18 R, no blockage

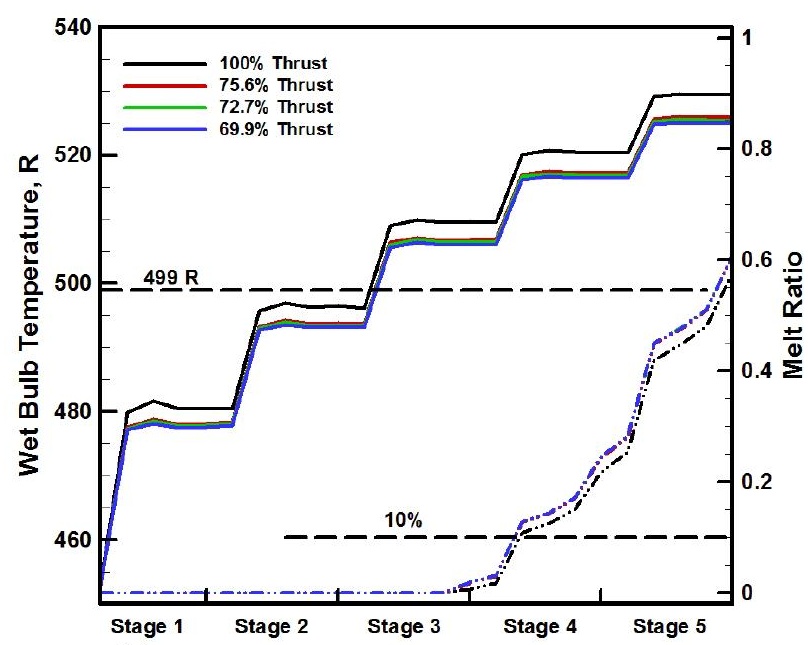

(c)

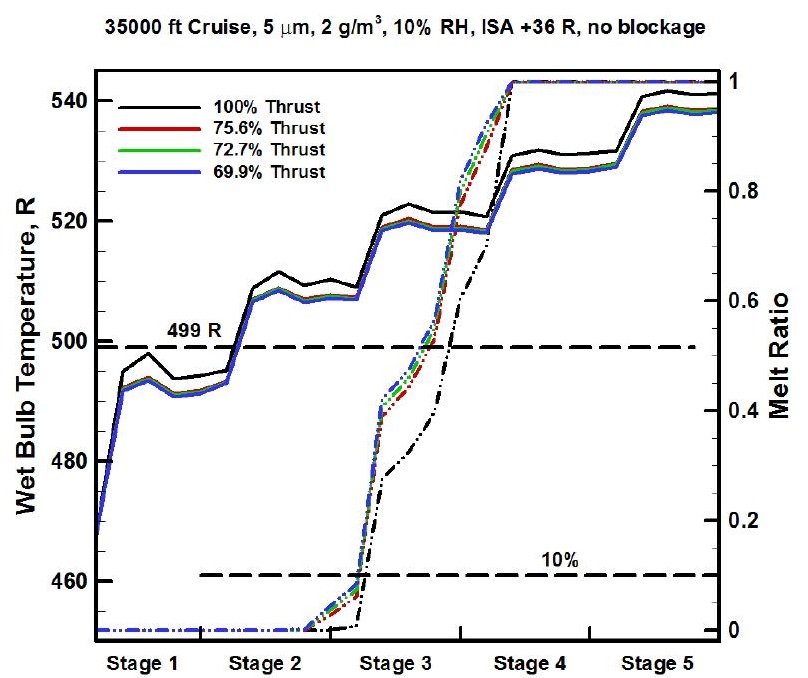

(b)

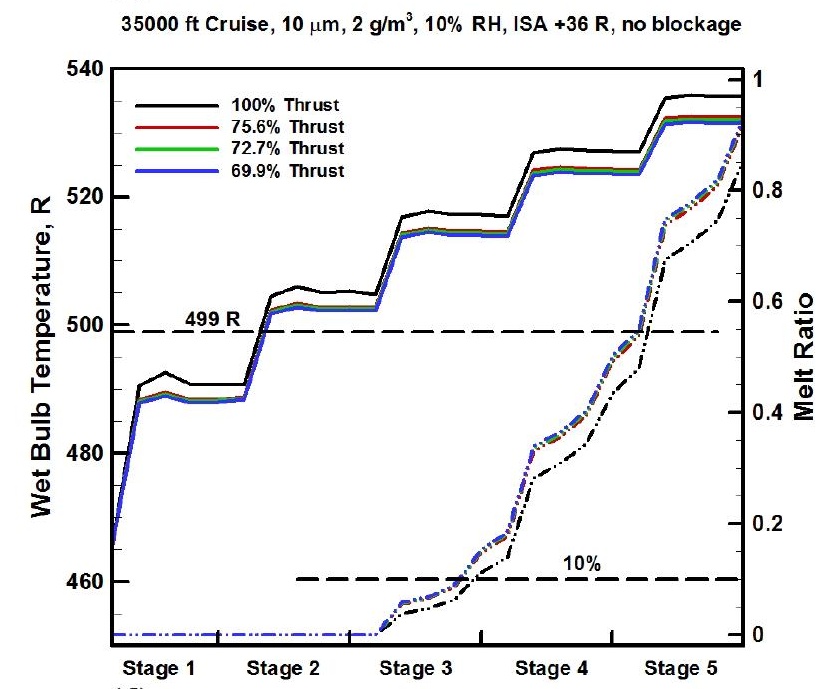

(d)

Figure 18(a-d): Cruise condition at 35000 feet altitude, with particle sizes of $5 \mu \mathrm{m}(\mathrm{a}, \mathrm{b})$ and $10 \mu \mathrm{m}(\mathrm{c}, \mathrm{d})$ and ISA temperatures of $+18 R(a, c)$ and $+36 R(b, d)$.

The results illustrated in Figure 18(a-d) show no risk of ice accretion is possible at this cruise condition with these particle sizes $(5$ and $10 \mu \mathrm{m})$, since the wet-bulb temperatures are excessively high in the compressor stages where the $10 \%$ melt ratio condition is met. For example, the $10 \%$ melt ratio criterion is reached in stage- 3 as shown in Figure 18(a), but the wet-bulb temperature there is $512 \mathrm{R}$, and is well above the $499 \mathrm{R}$ temperature limit. The effects of ice particle size and elevated ISA temperature can be seen in the location within the LPC where the minimum melt ratio criterion of $10 \%$ is reached. At all of these conditions, even though the melt ratio of $10 \%$ is achieved in the LPC, the wet-bulb temperature is well above the $499 \mathrm{R}$ limit, at that location. Therefore, there is no expected risk of ice accretion at these operating conditions.

The ice crystal particle size was further increased at this cruise altitude to determine the risk of ice accretion, as shown in Figure 19(a-f). The particle sizes were 25, 50, and $100 \mu \mathrm{m}$, with the same increment of temperatures $(+18$ $\mathrm{R}$ and $+36 \mathrm{R}$ ) above ISA. The range of engine thrust settings varied from $100 \%$ to $69.9 \%$ of maximum thrust. 
$35000 \mathrm{ft}$ Cruise, $25 \mu \mathrm{m}, 2 \mathrm{~g} / \mathrm{m}^{3}, 10 \% \mathrm{RH}$, ISA +18 R, no blockage

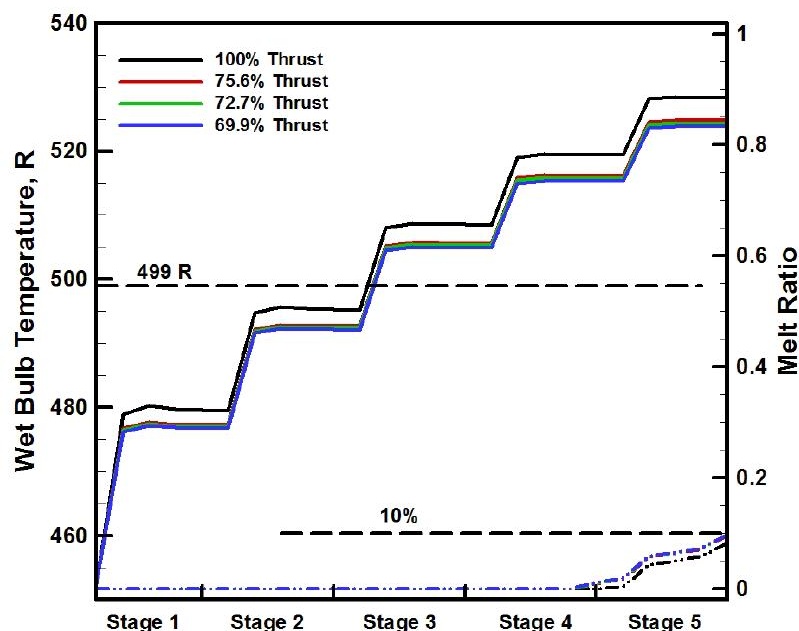

(a)

$35000 \mathrm{ft}$ Cruise, $50 \mu \mathrm{m}, 2 \mathrm{~g} / \mathrm{m}^{3}, 10 \% \mathrm{RH}$, ISA +18 R, no blockage

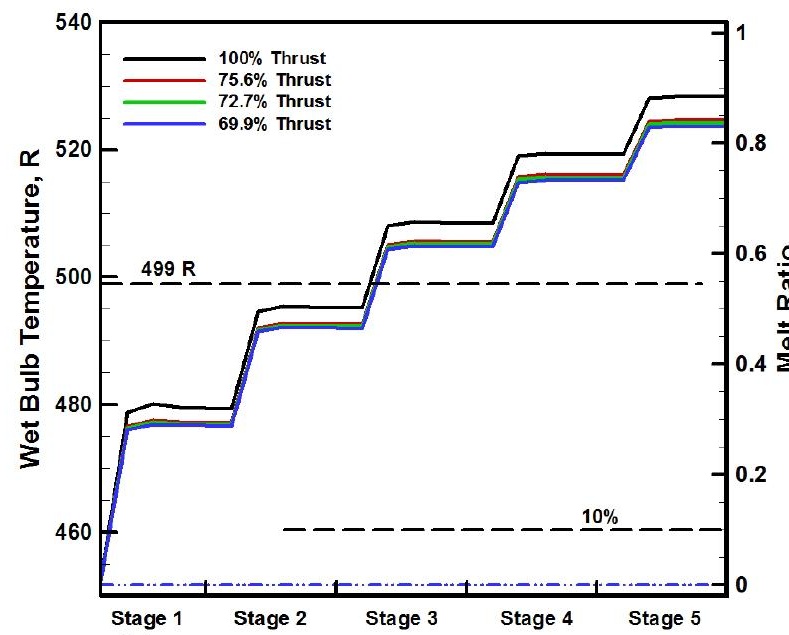

(c)

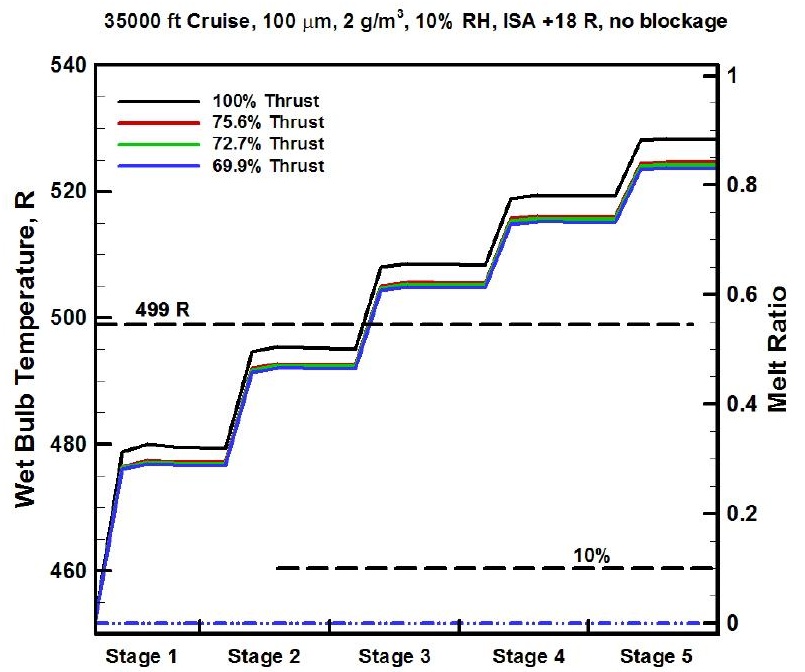

(e)

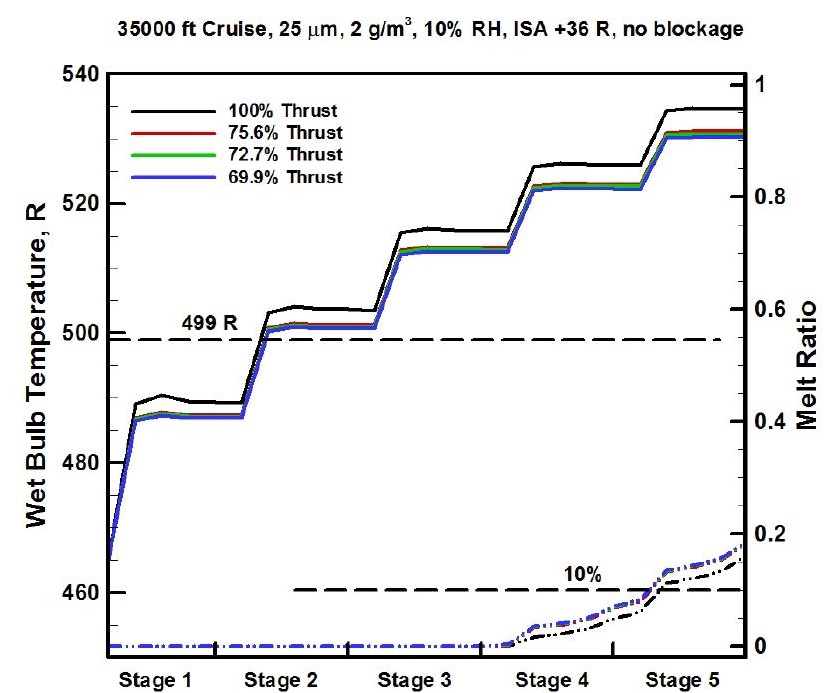

(b)

$35000 \mathrm{ft}$ Cruise, $50 \mu \mathrm{m}, 2 \mathrm{~g} / \mathrm{m}^{3}, 10 \% \mathrm{RH}$, ISA +36 R, no blockage

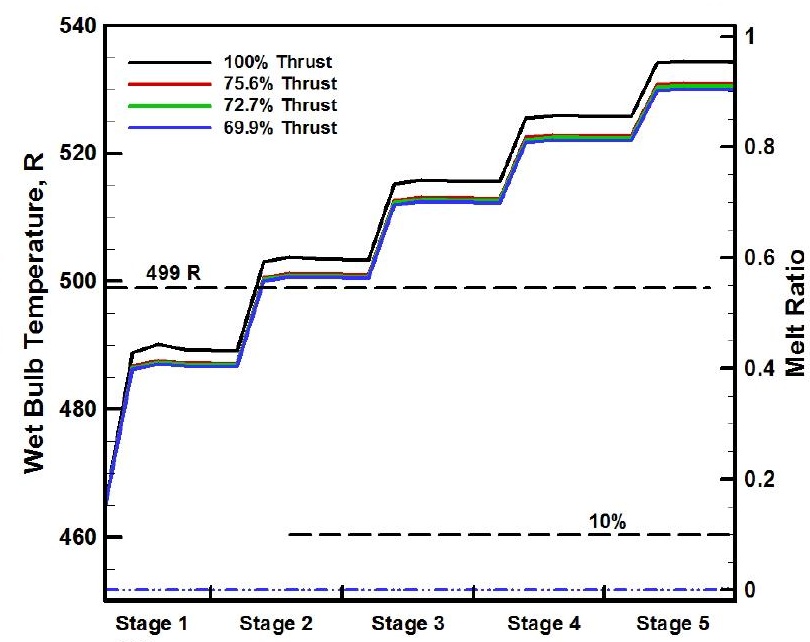

(d)

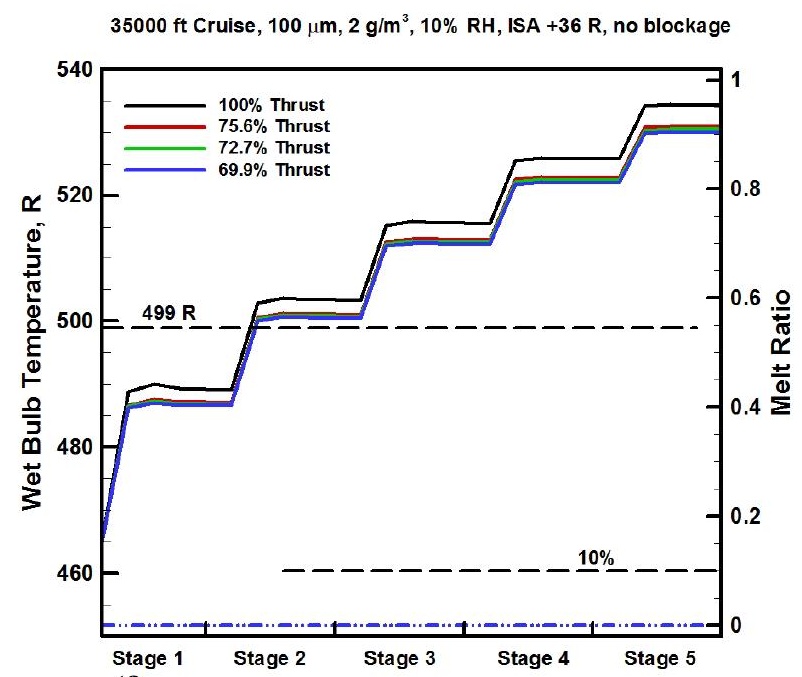

(f)

Figures 19(a-d): Cruise 1 condition, 35000 feet altitude, with particle sizes of $25 \mu \mathrm{m}(\mathrm{a}, \mathrm{b}), 50 \mu \mathrm{m}(\mathrm{c}, \mathrm{d})$ and $100 \mu \mathrm{m}(e, f)$ and ISA temperatures offsets of $+18 R(a, c, e)$ and $+36 R(b, d, f)$. 
The results illustrated in Figure 19(a-b) show that the 10\% melt ratio limit is reached only in stage-5 with the $25 \mu \mathrm{m}$ particle. However, the wet-bulb temperature in stage-5 is well above the $499 \mathrm{R}$ temperature limit. In Figure 19(c-f) the 50 and $100 \mu \mathrm{m}$ particles do not melt through the entire fan-core LPC, and the melt ratio remains zero. Therefore, there is no risk of ice accretion at any of the conditions illustrated in Figures 19(a-f). Note that even though the ice particle may not have melted, there was some degree of sublimation, adding to the specific humidity of the air and is accounted for in the flow analyses. Appendix G is a listing of the NPSS cycle analysis and Appendix H is the corresponding output listing of the compressor analysis code for the altitude cruise conditions at 35000 feet.

\section{Cruise at 39000 feet:}

The next points in the flight trajectory analyzed were at the cruise altitude of 39000 feet. The ice crystal particle size was varied from $5 \mu \mathrm{m}$ to $100 \mu \mathrm{m}$ in diameter, with the temperature offset at $+18 \mathrm{R}$ and $+36 \mathrm{R}$ above ISA. The results are illustrated in Figures 20(a-j). The range of engine thrust settings at this cruise condition was varied from $100 \%$ to $76.8 \%$ of maximum thrust.

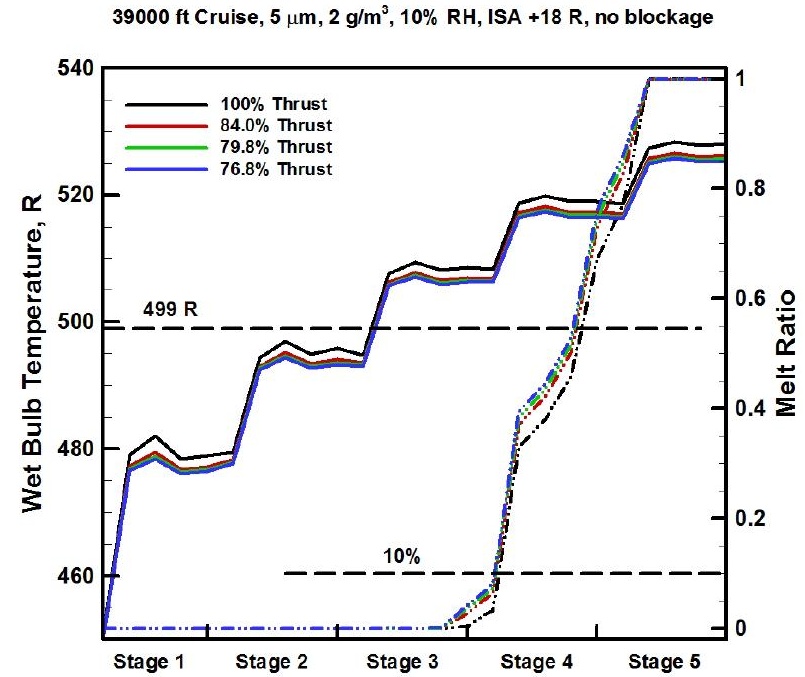

(a)

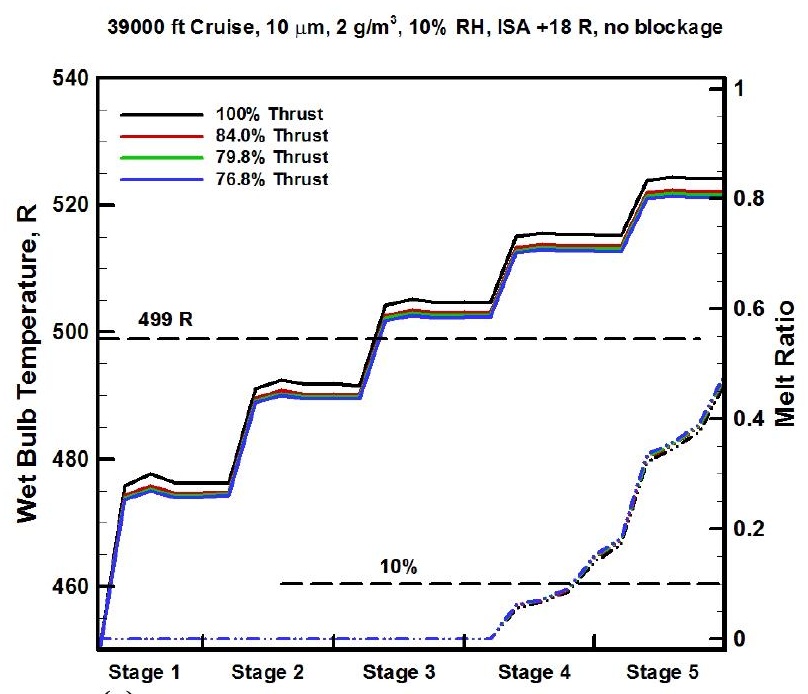

(c)

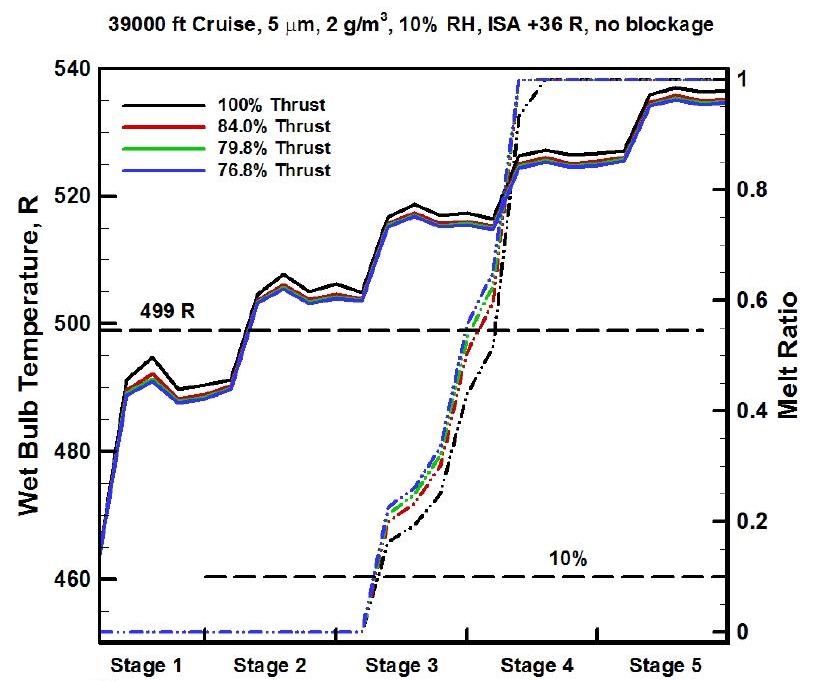

(b)

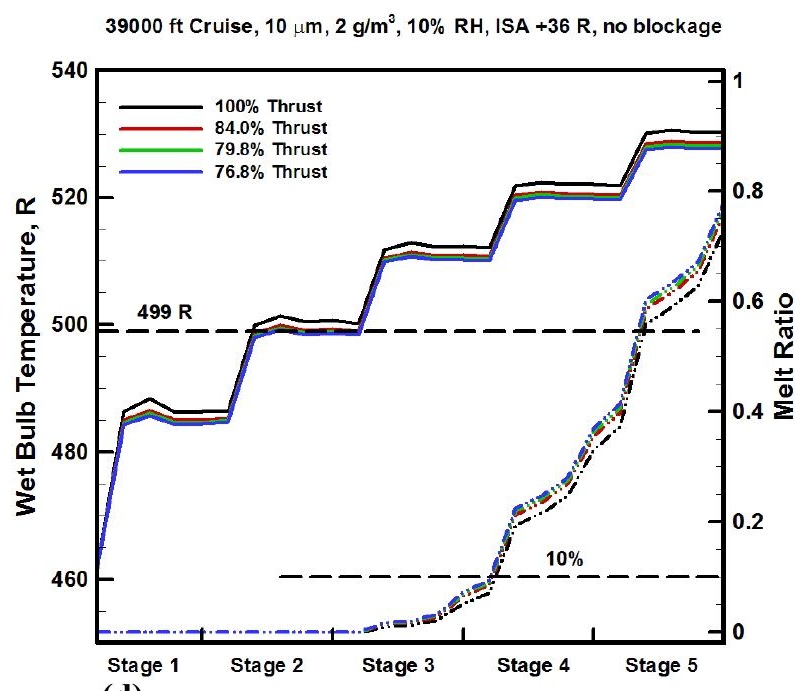

(d) 


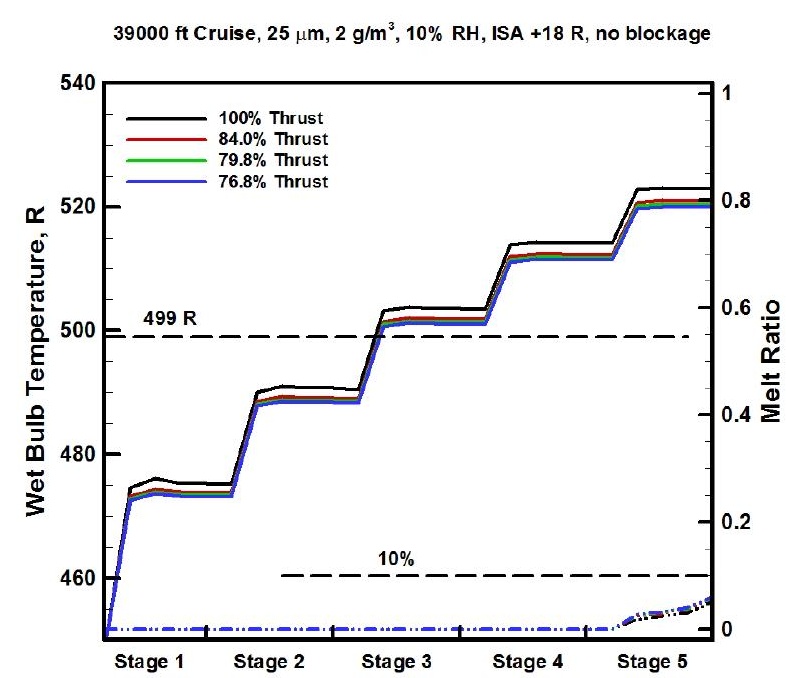

(e)

$39000 \mathrm{ft}$ Cruise, $50 \mu \mathrm{m}, 2 \mathrm{~g} / \mathrm{m}^{3}, 10 \% \mathrm{RH}$, ISA +18 R, no blockage

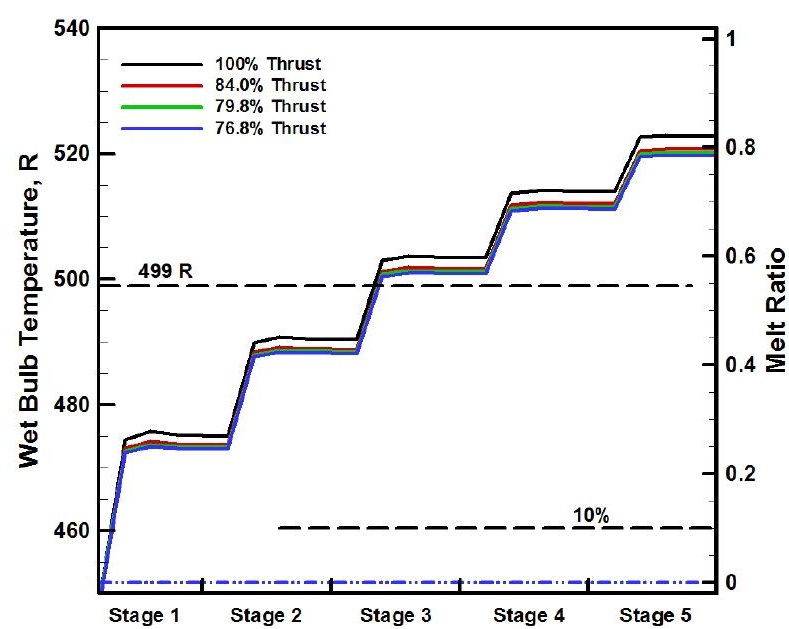

(g)

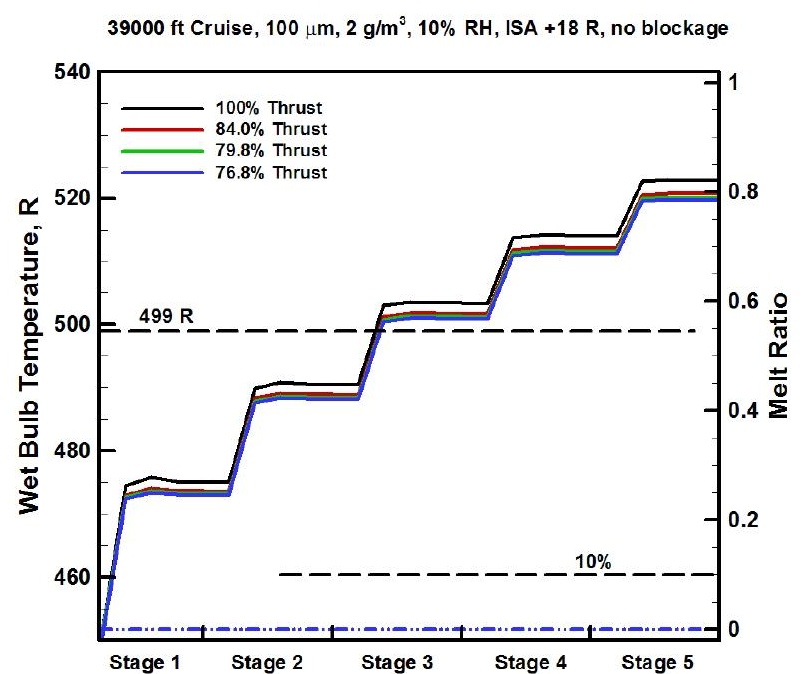

(i)

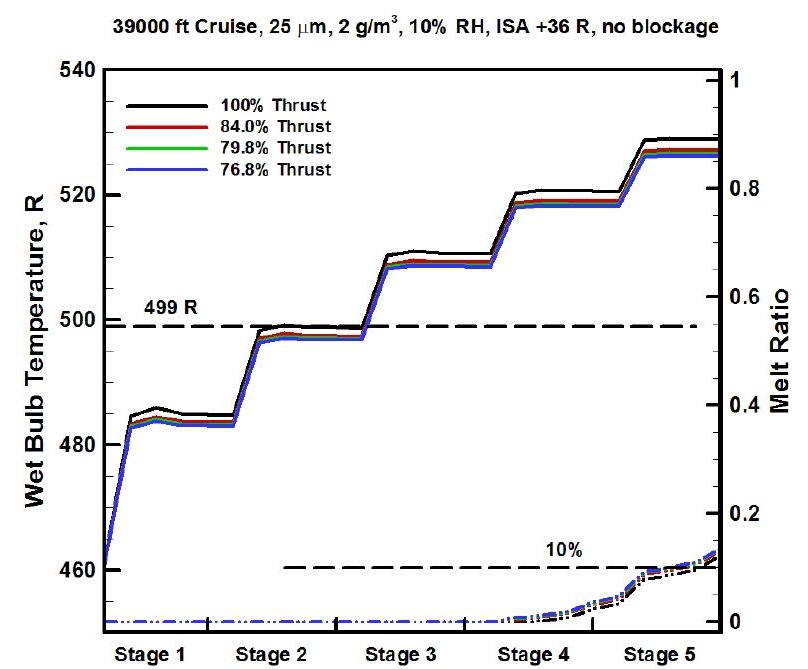

(f)

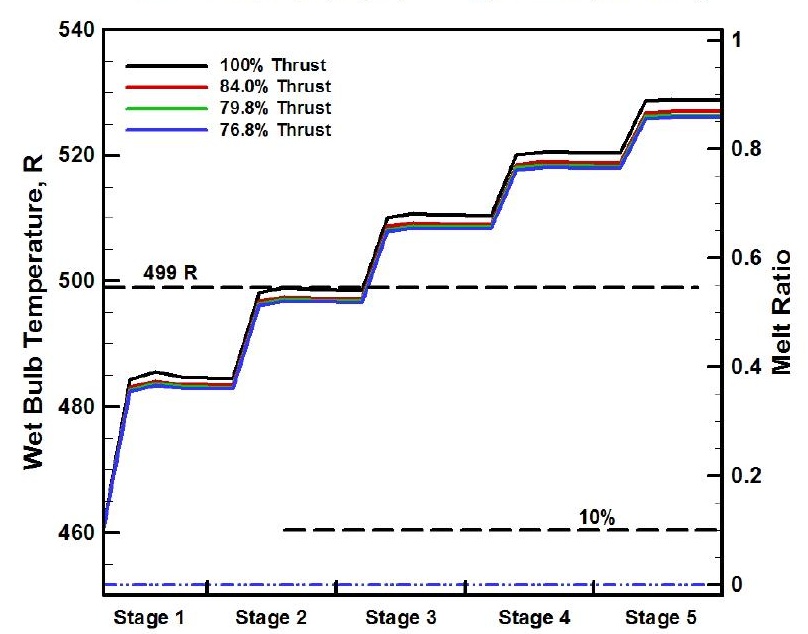

(h)

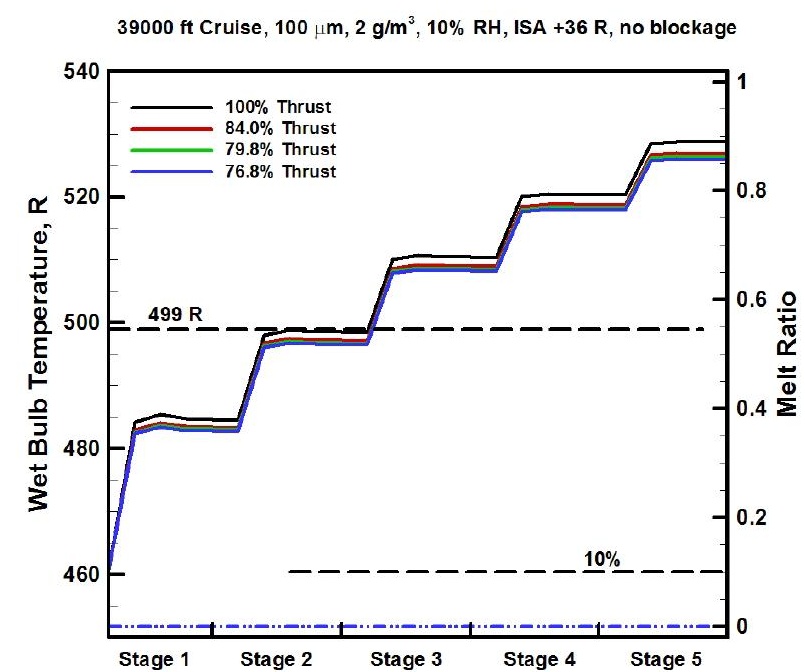

(j)

Figures 20 (a-j): The cruise condition at 39000 feet altitude, with particle sizes of $5 \mu \mathrm{m}(\mathrm{a}, \mathrm{b})$ and $10 \mu \mathrm{m}(\mathrm{c}, \mathrm{d})$ $25 \mu \mathrm{m}(\mathrm{e}, \mathrm{f}), 50 \mu \mathrm{m}(\mathrm{g}, \mathrm{h})$, and $100 \mu \mathrm{m}(\mathrm{i}, \mathrm{j})$ and ISA temperatures offsets of $+18 \mathrm{R}(\mathrm{a}, \mathrm{c}, \mathrm{e}, \mathrm{g}, \mathrm{i})$ and $+36 \mathrm{R}(\mathrm{b}$, $\mathbf{d}, \mathbf{f}, \mathbf{h}, \mathbf{j})$. 
The results illustrated in Figure 20(a) show that the 10\% melt ratio limit is reached in the axial gap at the leading edge of the rotor between stages 3 and 4 with the $5 \mu \mathrm{m}$ particle and ISA temperature offset of $+18 \mathrm{R}$. However, the wet-bulb temperature exceeds the maximum limit of $499 \mathrm{R}$ for accretion to occur. In Figure 20(b), at the engine inlet temperature of ISA $+36 \mathrm{R}$, the minimum melt ratio of above $10 \%$ is achieved in the stator of stage- 3 , while the wetbulb temperature exceeded the limit that would support ice accretion. In both of these cases, the melt ratio reaches the value of 1.0, indicating that the ice particle has completely melted before exiting the LPC. In Figure 20(c) with the $10 \mu \mathrm{m}$ particle and $+18 \mathrm{R}$ ISA offset, the results indicate that the minimum melt ratio is achieved in the stator of stage- 4 . At the elevated ISA temperature of +36 R, Figure 20 (d), the minimum melt ratio is reached at the leading edge of the stage- 4 rotor. In both cases the wet-bulb temperature is too high to support ice accretion. In Figures 20 (e) the minimum melt ratio is not reached at the lower temperature of ISA $+18 \mathrm{R}$, but is reached at ISA $+36 \mathrm{R}$ near the trailing edge of the stage-5 stator, as shown in Figure 20(f). However, the wet-bulb temperature is well above the limit that would support ice accretion. The results with the 50 and $100 \mu \mathrm{m}$ ice particle sizes as illustrated in Figures $20(\mathrm{~g}, \mathrm{~h}$, and $\mathrm{i}, \mathrm{j}$ ) show that the minimum melt ratio limit is not reached at any stage within the fan-core and LPC. Furthermore, the wet-bulb temperature at the end of the LPC is well above the maximum limit for accretion. Appendix I is a listing of the NPSS cycle analysis and Appendix $J$ is the corresponding output listing of the compressor analysis code for the altitude cruise conditions at 39000 feet.

\section{Descent:}

The next operating points in the flight trajectory that were analyzed were computed during the descent mode from the 39000 feet cruise altitude, as shown in Figure 11. These consisted of eight altitudes starting at 39000 feet and descending to 15435 feet, at $+18 \mathrm{R},+27 \mathrm{R}$ and $+36 \mathrm{R}$ ISA offset temperatures at reduced engine thrust level of $10 \%$ of maximum thrust at the given altitude. As before, the ice crystal particle sizes modeled were $5,10,25,50$, and $100 \mu \mathrm{ms}$ in diameter. Due to the ice particle formation limits that are illustrated in Figure 14, there were seven altitudes that were analyzed at the $+36 \mathrm{R}$ offset temperature between 39000 feet and 20047 feet. In a more refined parametric study, it is suggested that smaller increments of altitude, particle size, ice particle concentration, relative humidity, and elevated ISA temperatures should be used. The calculated results of wet-bulb temperature and melt ratio at each computing station within the fan-core and LPC stages are illustrated in Figures 21(a-f) with an initial ice crystal particle diameter of $5 \mu \mathrm{m}$ and IWC of 2 and 4 grams per cubic meter. Note that the analysis also included $9 \mathrm{~g} / \mathrm{m}^{3}$, but the results indicated no risk of ice accretion at any altitude, or ISA offset temperature.

Descent, $5 \mu \mathrm{m}, 2 \mathrm{~g} / \mathrm{m}^{3}, 10 \%$ RH, ISA +18 R, no blockage

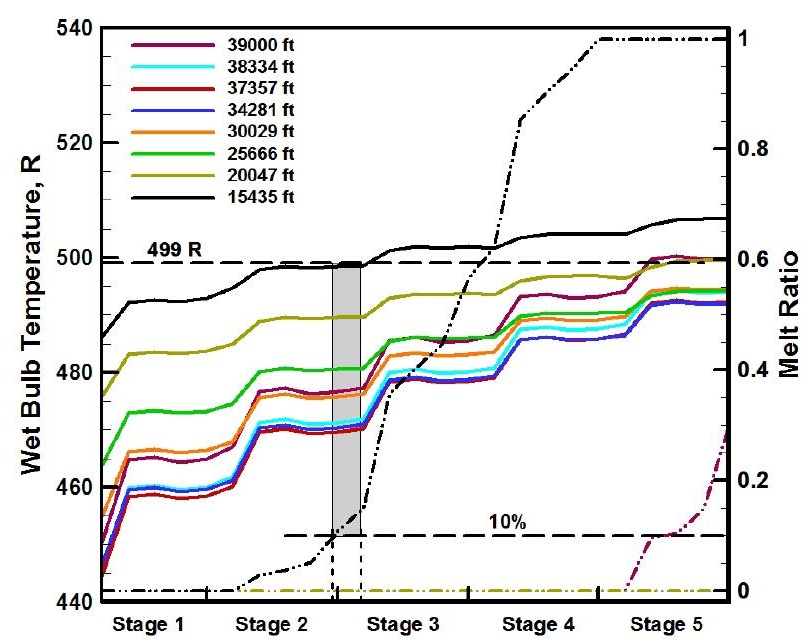

(a)

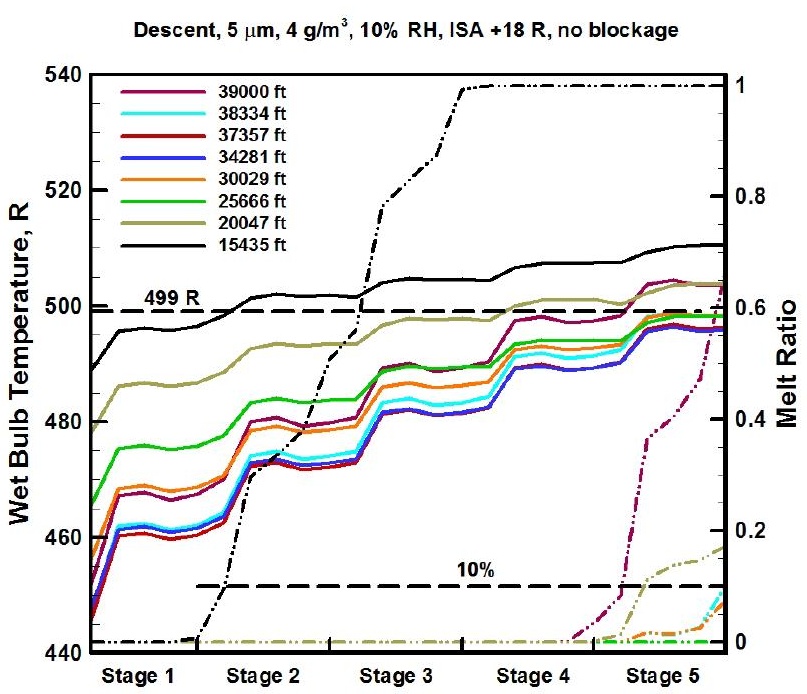

(b) 


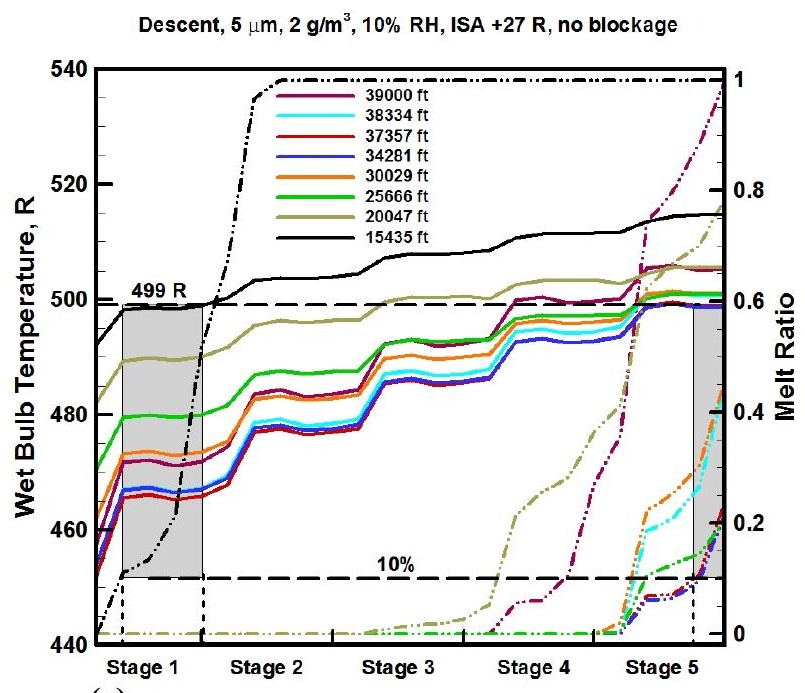

(c)

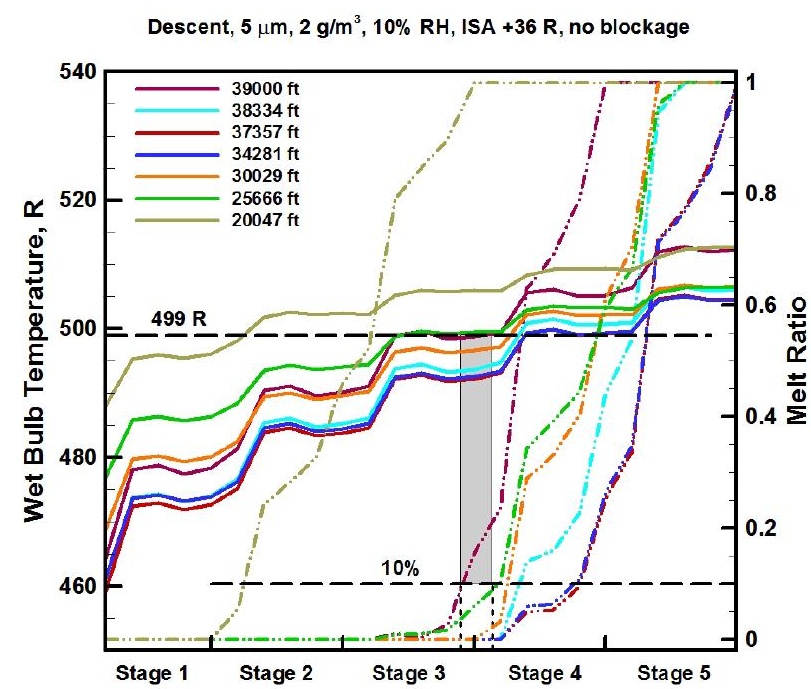

(e)

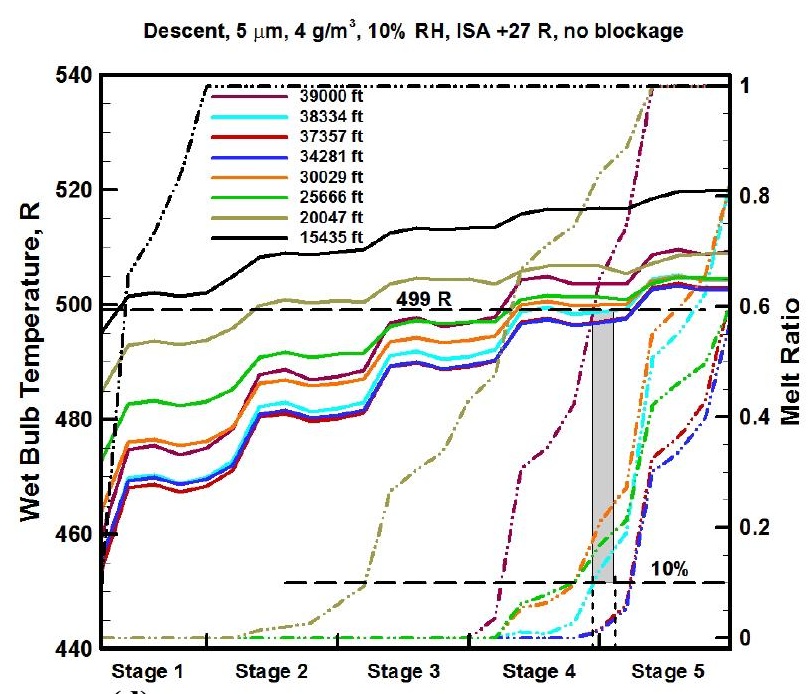

(d)

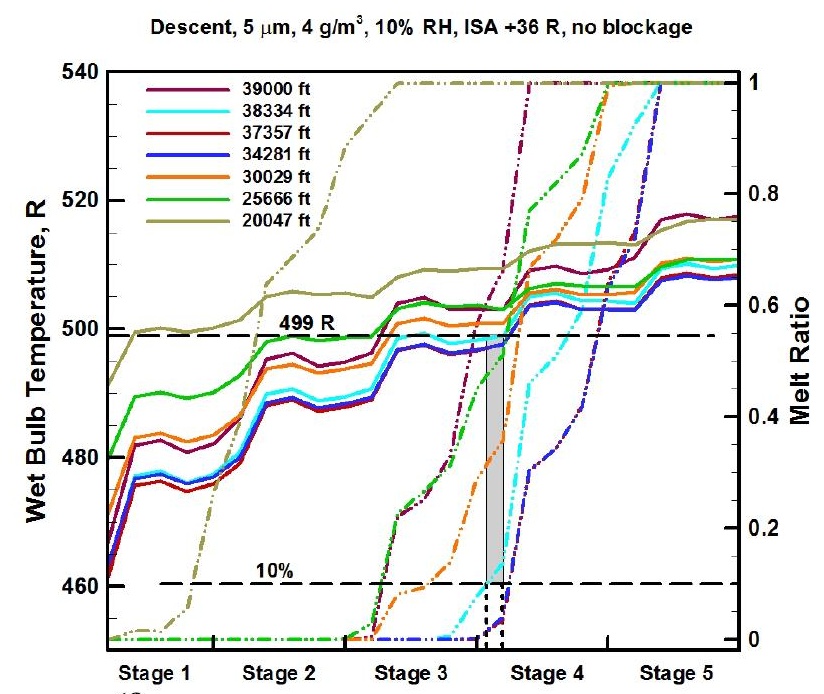

(f)

Figures 21(a-f): Descent mode. The ice particle size used in the analyses was $5 \mu \mathrm{m}$. The inlet temperature specified were ISA +18R (a, b), ISA + 27R (c, d), and ISA +36 R (e, f).

The results illustrated in Figure 21(a) show that the 10\% melt ratio limit is reached in the stator and gap after stage-2 with the $5 \mu \mathrm{m}$ particle and ISA $+18 \mathrm{R}$ at an altitude of 15435 feet. In addition, the maximum wet-bulb temperature limit criterion of $499 \mathrm{R}$ is also satisfied at this location. This would indicate that there is a potential risk for ice accretion. With the same values for particle size and ISA offset temperature, the IWC is increased to $4 \mathrm{~g} / \mathrm{m}^{3}$. However, in this case, the melt ratio criterion is not met where the wet-bulb temperature criterion is satisfied at any of the selected operating conditions in the descent flight trajectory, as shown in Figure 21(b). As the IWC is increased, the amount water vapor in the air due to sublimation and evaporation rises resulting in an increase in relative humidity as well as the wet-bulb temperature. Therefore there is no risk of ice accretion predicted for this case. In Figure 21(c), at the engine inlet temperature of ISA $+27 \mathrm{R}$, there are three altitudes where there is a risk of ice accretion during descent. The first occurs at an altitude of 37357 feet near the trailing edge section of the stage-5 stator and in the following gap. Similarly, at an altitude of 34281 feet the ice accretion risk coincides with that at 37357 feet. The altitude of 15435 feet there is an ice accretion risk predicted in the broad region of the stage-1 rotorstator gap and through the stage-1 stator, likewise illustrated in Figure 21(c). When the IWC is increased from 2 to $4 \mathrm{~g} / \mathrm{m}^{3}$ the region where there is a predicted risk of ice accretion shifts to the stage- 4 stator trailing edge and statorrotor gap as illustrated in Figure 21(d). At an ISA temperature offset of $+36 \mathrm{R}$ and the IWC of $2 \mathrm{~g} / \mathrm{m}^{3}$, there is a risk of ice accretion in the stage-3 stator trailing edge and stator-rotor gap in the descent from the cruise at an altitude of 
39000 feet, as shown in Figure 21(e). When the IWC is increased to $4 \mathrm{~g} / \mathrm{m}^{3}$, the icing region of risk is at the altitude of 38334 feet and is located in the gap between the stage-3 stator and the stage-4 rotor, as illustrated in Figure 21(f). The NPSS listings along with the corresponding compressor analysis for the descent conditions shown in Figure 21 (a-f) are provided in Appendices K and L, respectively.

As mentioned previously, the parametric study also included analyzing an ice particle size of $10 \mu \mathrm{m}$ with IWC of 2 , 4 , and $9 \mathrm{~g} / \mathrm{m}^{3}$ at the same range of ISA offset temperatures as in the previous descent analyses. For this particle size, a risk of ice accretion during descent was found only at an altitude of 15435 feet with an IWC of $9 \mathrm{~g} / \mathrm{m}^{3}$ and an ISA temperature offset of $+27 \mathrm{R}$. The accretion risk was located in the region of the stage- 1 stator trailing edge and a portion of the gap prior to rotor-2, as illustrated in Figure 22 (details of NPSS and compressor analysis output are provided in Appendix M).

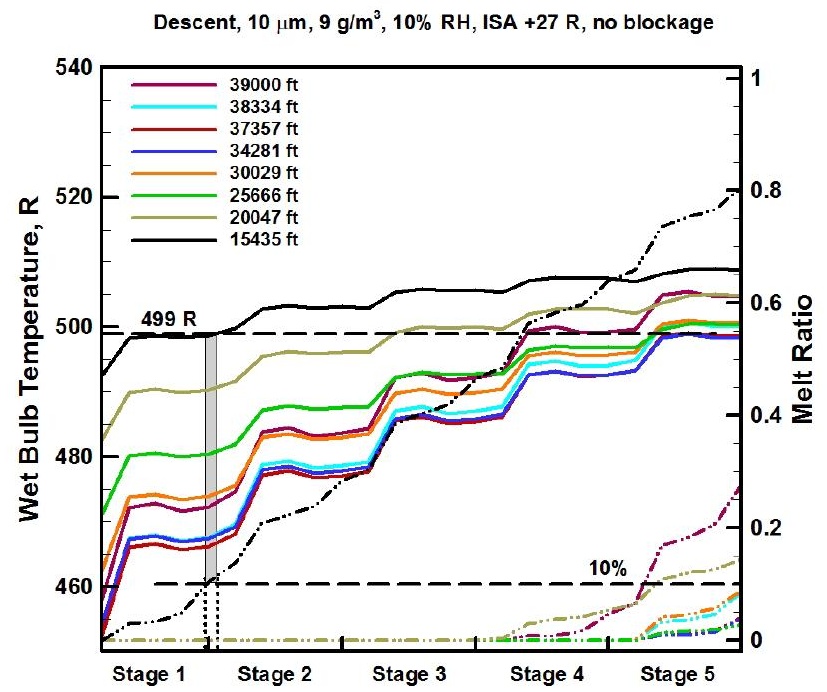

Figure 22: Descent condition, from 39000 feet to 15435 feet altitude. There is a risk of icing with an ice particle size of $10 \mu \mathrm{m}$ and a temperature offset of ISA $+27 \mathrm{R}$.

The parametric analysis of the descent mode was continued by further studying the effects of even larger particle sizes at a fixed IWC of $2 \mathrm{~g} / \mathrm{m}^{3}$ and at ISA offset temperatures of $+18 \mathrm{R}$ and $+36 \mathrm{R}$. As during the cruise conditions, the particles sizes utilized to analyze the descent portion of the flight trajectory were 10,25, 50 and $100 \mu \mathrm{m}$, and the results are presented in Figure 23(a-h).

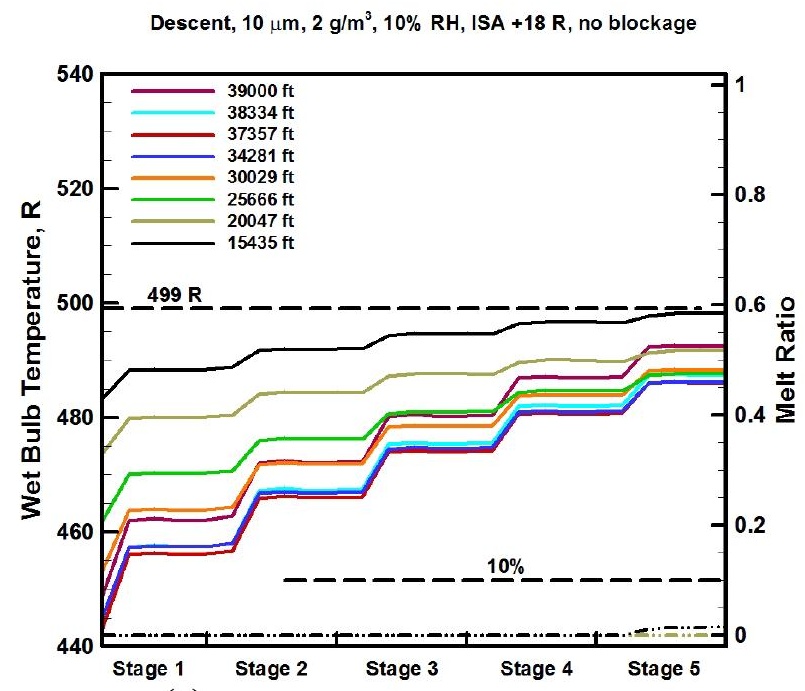

(a)

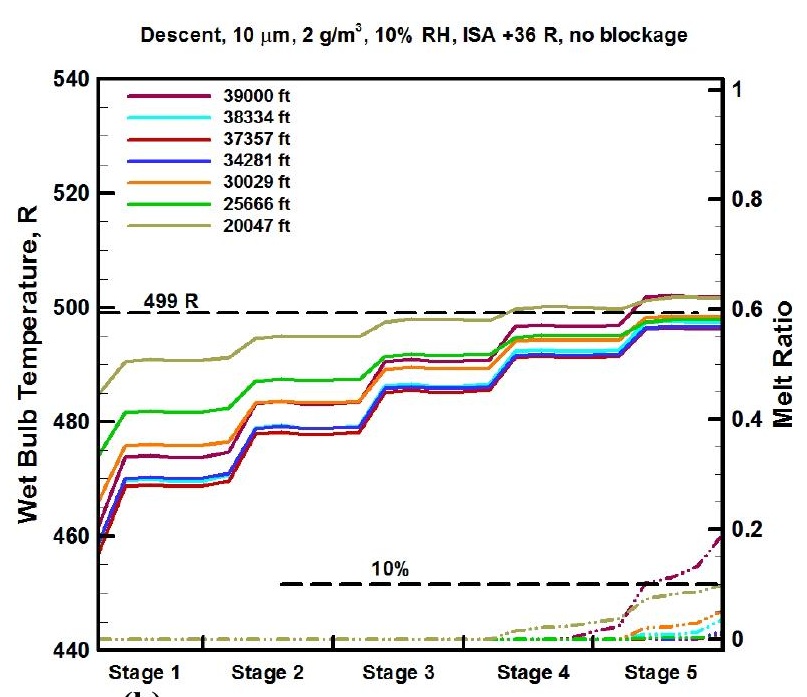

(b) 


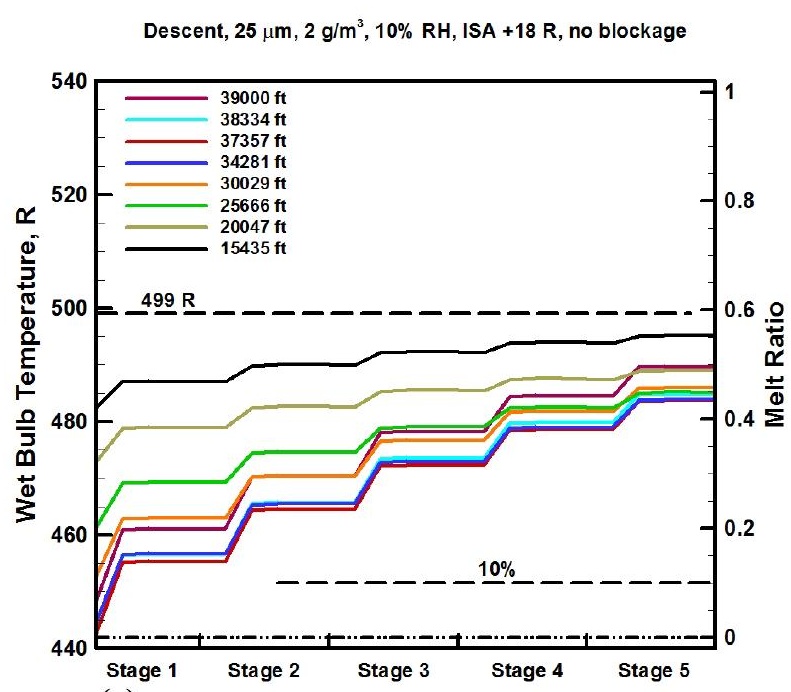

(c)

Descent, $50 \mu \mathrm{m}, 2 \mathrm{~g} / \mathrm{m}^{3}, 10 \% \mathrm{RH}$, ISA +18 R, no blockage

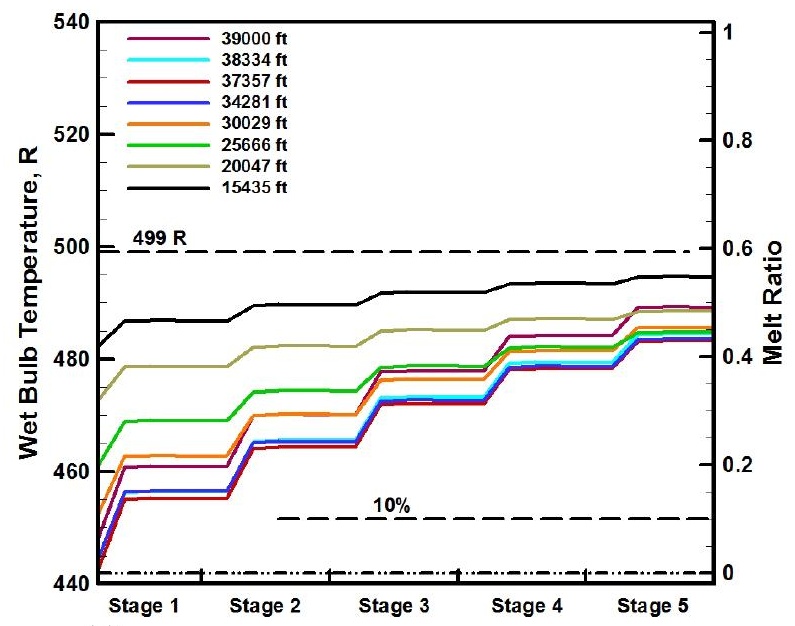

(e)

Descent, $100 \mu \mathrm{m}, 2 \mathrm{~g} / \mathrm{m}^{3}, 10 \% \mathrm{RH}$, ISA +18 R, no blockage

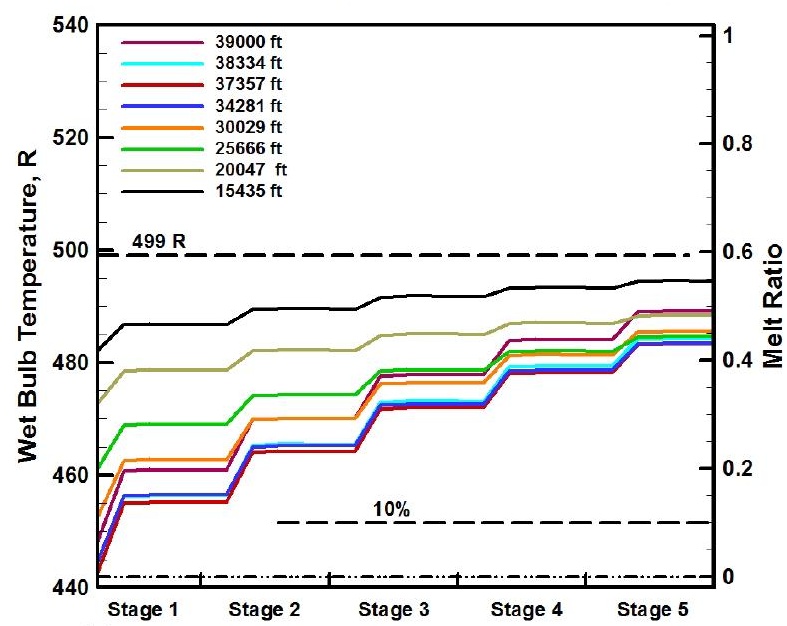

(g)

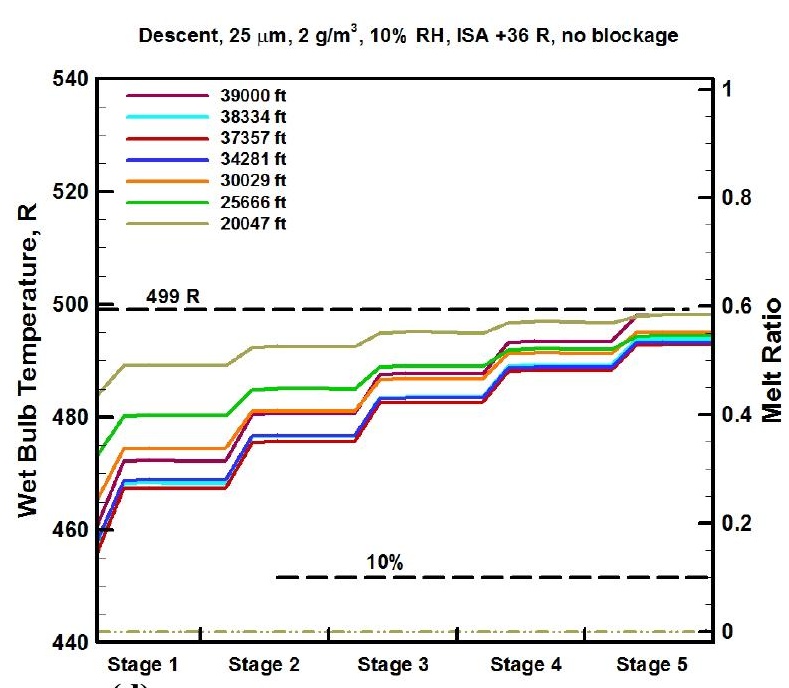

(d)

Descent, $50 \mu \mathrm{m}, 2 \mathrm{~g} / \mathrm{m}^{3}, 10 \% \mathrm{RH}$, ISA +36 R, no blockage

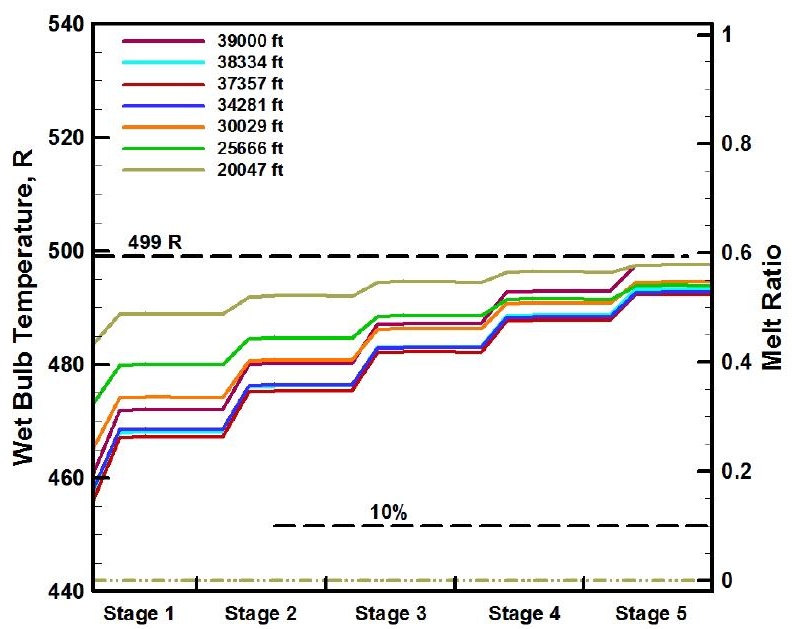

(f)

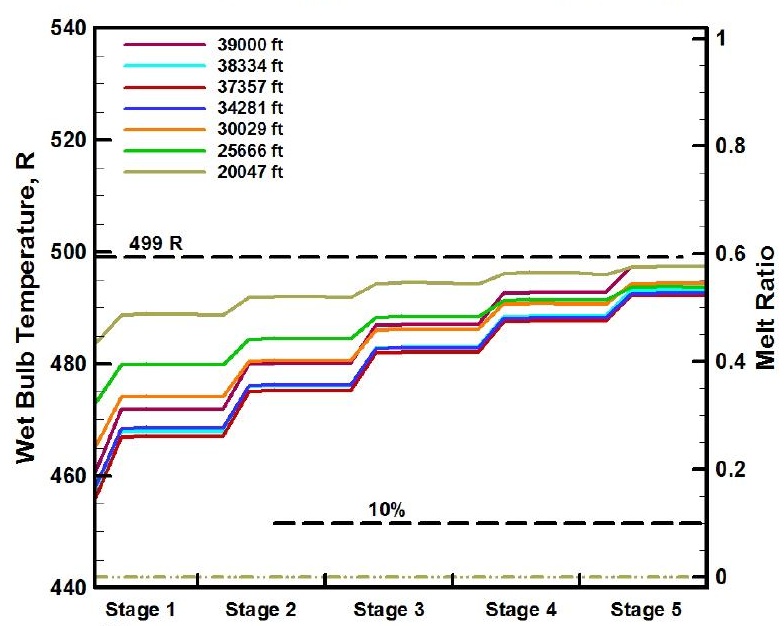

(h)

Figures 23(a-h): Descent mode, from 39000 feet to 15435 feet altitude (a, c, e, g) and 39000 feet to 20047 feet altitude $(b, d, f, h)$. The ice particle sizes used in the analyses were: $10 \mu \mathrm{m}(\mathrm{a}, \mathrm{b}), 25 \mu \mathrm{m}(\mathrm{c}, \mathrm{d}), 50 \mu \mathrm{m}(\mathrm{e}, \mathrm{f})$, and $100 \mu \mathrm{m}(\mathrm{g}, \mathrm{h})$. The inlet temperature offset specified were ISA +18R (a, c, e, g) and ISA +36 R (b, d, f, h). 
In Figures 23(a, c, e, g) with particle sizes of 10, 25, 50, and $100 \mu \mathrm{m}$, respectively, and an inlet temperature offset from ISA of $+18 \mathrm{R}$, and an IWC of $2 \mathrm{~g} / \mathrm{m}^{3}$, the melt ratio was well below the criterion limit minimum of $10 \%$. Similarly, in Figure 23(d, f, h) with the equivalent particle size and an inlet offset temperature of ISA $+36 \mathrm{R}$, the melt ratio was well below the criterion limit of $10 \%$ minimum. Therefore, no risk of ice accretion was expected at these conditions. The only case where the minimum melt ratio criterion was met is the $10 \mu \mathrm{m}$ and $+36 \mathrm{R}$ ISA temperature offset at the altitude of 39000 feet, in the stage-5 stator as shown in Figure 23(b). However the wetbulb temperature there was almost $3 \mathrm{R}$ above the specified maximum criterion. Therefore there was no risk of ice accretion. The NPSS listings for the descent conditions shown in Figure 23 (a-h) are provided in Appendix N with the corresponding compressor analysis detailed in Appendix O.

\section{Summary of Engine Icing Risk Analysis:}

Figures 24(a) and Table II illustrate where the risk of ice accretion was identified along the flight trajectory. Figure 24(b) shows where this notional engine may have a risk of ice accretion in comparison with the events that have been reported in the open literature. The engine events predicted in this study are also summarized in Table II. There were other conditions analyzed parametrically in this study, and the cases shown are a sample of the results to illustrate the method used to determine the icing risk based on the wet-bulb temperature and melt ratio criteria.

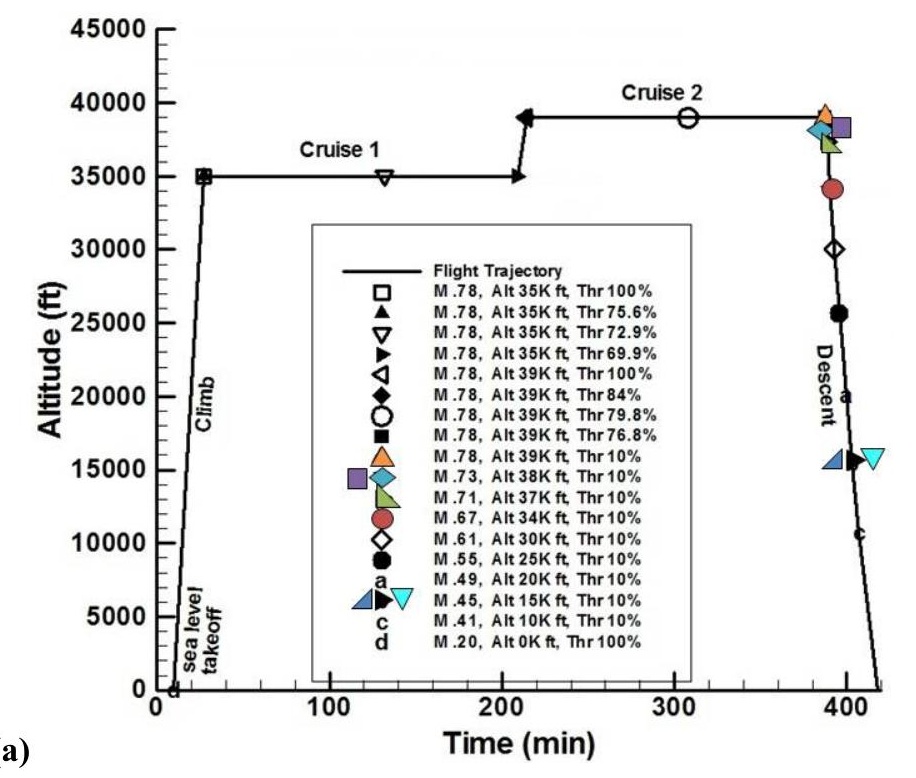

(a)

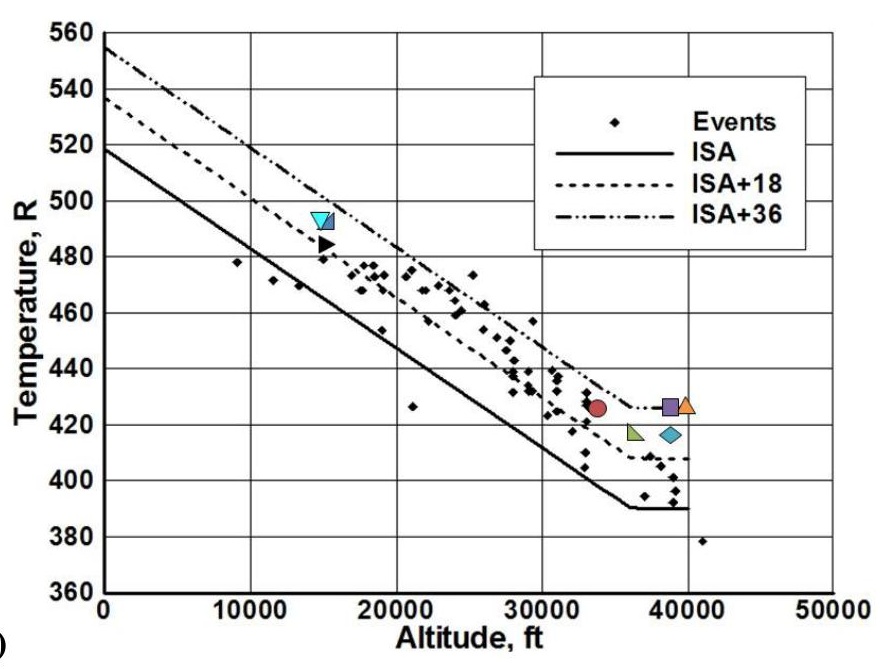

Figures 24(a, b): (a) Aircraft flight trajectory of the notional engine with icing risk identified at two particular operating points. (b) Icing risks of notional engine compared to known engine icing events. 
Table II - Summary of Engine Icing Risk at Selected Operating Points Along Flight Trajectory

\begin{tabular}{|c|c|c|c|c|c|c|c|c|c|}
\hline Altitude, $\mathbf{f t}$ & $\begin{array}{l}\text { Icing } \\
\text { Risk, } \\
\text { Stage }\end{array}$ & $\begin{array}{l}\text { Particle } \\
\text { Size, } \mu \mathrm{m}\end{array}$ & $\begin{array}{l}\text { IWC, } \\
\mathrm{g} / \mathrm{m}^{3}\end{array}$ & $\begin{array}{l}\text { ISA Offset } \\
\text { Temp, R }\end{array}$ & $\mathbf{T}_{\mathrm{wb}}, \mathbf{R}$ & $\mathbf{T}_{\text {Static }}, \mathbf{R}$ & $\mathbf{T}_{\text {Total }}, \mathbf{R}$ & $\begin{array}{l}\text { Ref. } \\
\text { Figure }\end{array}$ & $\begin{array}{l}\text { Symbol, } \\
\text { Figure } \\
24(a, b)\end{array}$ \\
\hline 39000 & 3 & 5 & 2 & +36 & 498.8 & $521.9-524.1$ & 529.1 & 20 (e) & $\triangle$ \\
\hline 38334 & 4 & 5 & 4 & +27 & 498.4 & 513.9-515.8 & 520.1 & $20(d)$ & $\diamond$ \\
\hline 38334 & 3 & 5 & 4 & +36 & 498.6 & $513.5-514.8$ & 519.4 & 20 (f) & $\square$ \\
\hline $\mathbf{3 7 3 5 7}$ & 5 & 5 & 2 & +27 & 498.9 & $519.8-520.2$ & 526.3 & $20(c)$ & $\Delta$ \\
\hline 34281 & 5 & 5 & 2 & +27 & 498.7 & 517.6-518.0 & 523.9 & 20 (c) & 0 \\
\hline 15435 & 1 & 5 & 2 & +27 & 498.2 & $510.3-512.2$ & $\mathbf{5 1 7 . 5}$ & 20 (c) & $\Delta$ \\
\hline 15435 & 2 & 5 & 2 & +18 & 498.5 & 505.9-508.0 & $\mathbf{5 1 3 . 0}$ & 20 (a) & D \\
\hline 15435 & 1 & 10 & 9 & +27 & 498.7 & 512.2-513.0 & 517.4 & 21 & $\nabla$ \\
\hline
\end{tabular}

\section{IV.Assumptions and Recommendations for Future Research}

This study is ongoing research and is intended to be a status report on the current state of the engine icing modeling tool development effort at NASA Glenn Research Center utilizing the NPSS-COMDES-MELT analysis tool. Simple empirical models have been used that were derived from test data to determine the aero-thermodynamic conditions within the fan and low pressure compressor that identified the risk of ice accretion. Two key parameters were identified to predict whether there is a risk of ice accretion within any turbofan engine. Numerous other simplifying assumptions were made that enabled studying the very complex physics involved in engine icing due to ice crystal ingestion. The actual IWC ice crystal concentration (grams of ice per cubic meter of air) in ice clouds is currently unknown. Likewise, the actual particle size existing in the atmosphere is also unknown, and is likely larger than was assumed in this study, before entering the fan and LPC, but may be broken up into much smaller particles upon impact with the high speed rotor blades and stator vanes of the compression system. Also, there may be a particle size distribution after impact, as opposed to a particle of a single size, as was assumed in this study. There is currently research in these areas that will attempt to quantify the ice particle impact dynamics, as well as the particle size distribution after impact. Therefore in this study it was assumed that the ice particles entering the engine have already been broken up into small particles due to impact with engine components. In addition it is assumed that the small particles are evenly distributed through the bypass duct and the engine core, as well as evenly distributed in the radial direction.

The low fidelity approach was selected for this study primarily to provide a fast capability to screen engines for potential icing risk in an ice crystal environment. It would be computationally expensive to model the three phase flow through the fan and low pressure compressor with a multidisciplinary, time-dependent code that simulates the aerodynamics, thermodynamics, ice particle physics, accretion, and heat transfer analyses through the blades and end walls. Additionally finer increments of altitude, ISA offset temperatures, and ice particle size can be considered for a more refined parametric study. Ranges of particle concentrations at altitude obtained from future flight tests may reduce the need to parametrically vary IWC.

Ice accretion is not addressed in the current study, but should be considered in the future. Likewise there is no radial distribution of blockage currently available in the low fidelity approach used in this study. However, a bulk value of blockage at a specific location in the compressor is available in the code and has been previously demonstrated. Further research is necessary to model the effects of shape and roughness of the accreted ice on the surfaces of the flow path walls and stator vanes. Unusual ice shapes that accrete on the compressor stators and walls will likely have a detrimental effect on the pressure recovery and losses and the cumulative effects on the downstream compressor stages, as well as the overall power balance of the engine system. Erosion of ice due to impact of incoming ice particles on the accreted ice was likewise not considered in this study, but is a topic for future research. Another area of research may be to study the dependence of accretion on the local Mach number and particle concentration. High fidelity compressor flow simulations in the future should consider these effects. There is no data available on the accreted ice shapes in turbomachinery, as the ice shapes may likely be influenced by the unsteady air flow fluctuations between the rotors and stators. Experiments that quantify the accreted ice shapes in rotating turbomachinery need to be conducted to determine the unsteady effects on ice accretion for high fidelity flow 
analysis and ice accretion code validation and calibration. These results can also be incorporated in the low fidelity modeling to improve its current capabilities.

The current study does not have an ice shedding model. Based on observed accretion behavior, the assumption in this study is that if the wet-bulb temperature is below freezing, then the ice that accretes is firm, and does not shed, but continues to grow as a function of time. Although laboratory testing has shown that there is some level of ice buildup at conditions that were at wet-bulb temperatures approximately $499 \mathrm{R}$, the ice accretion at these conditions was not firm, but was loose "slushy ice" that quickly shed before a substantial amount of ice could accrete. The current analysis was limited for this reason to wet-bulb temperatures less than or equal to $499 \mathrm{R}$. Even though this was not modeled, it is thought that the shedding of loose ice may cause a problem in the compressor by choking the downstream compressor stages due to the instantaneous addition of mass flow, thus it could possibly force the fan or other upstream stages to surge. Further research is needed in this area to study its effect on the compressor and engine system performance.

\section{V.Conclusion}

The purpose of this effort was to develop a system of codes to provide a first order predictive capability to estimate the risk of the occurrence of engine ice accretion during flight through high altitude convective ice crystal clouds. A first generation computational tool has been developed for estimating the susceptibility of engines to icing. The tool consists of an engine thermodynamic cycle model, a compressor mean line flow analysis model that calculates the aerodynamic performance of each blade row, and an ice particle sublimation, melting, and evaporation model that tracks the state of the ice/water particle through the compressor blade rows. Using this fully integrated capability, a notional turbofan engine was studied to predict the risk of ice accretion in the low pressure compressor, operating in a gas turbine engine system environment through a flight trajectory of a typical commercial aircraft. Two aerothermodynamic parameters were identified as the criteria for determining whether there is a risk of ice accretion when flying in a high altitude ice crystal environment: the local wet-bulb temperature and the ice melt ratio. A parametric approach was utilized to determine the risk of accretion in the low pressure compressor over a range of atmospheric conditions while varying ice crystal size, ice water content, and ambient temperature. With the assumptions that were made in this study, it was determined that the notional engine would experience a risk of ice accretion at multiple operating conditions during the descent mode of the flight trajectory, with ice particle sizes on the order of $5 \mu \mathrm{m}$ and ambient temperature offset from the ISA were in the range of $+18 \mathrm{R}$ to $+36 \mathrm{R}$. The results of this study indicate that the risk of ice accretion on this engine occurs between $39000-34000$ feet, and near 15000 feet. At the higher altitude near 39000 feet, the risk of accretion occurred in the rear LPC stages, while at the lower altitude near 15000 feet, the risk of accretion occurred in the front stages. All of the predicted icing risk operating points identified for this notional engine were within the envelope of the known icing events of commercial aircraft engines that have been reported in the literature. 


\section{APPENDICES}

The following appendixes can be accessed from the hyperlinks below or from the bookmarks panel located on the left-hand side of this document.

A. Normalized Growth Rate as a function of $\mathrm{LWC}_{\mathrm{m}} / \mathrm{TWC}_{\mathrm{inj}}$, Figure 8.

B. Design Point Performance; compressor code output listing for the Fan-Core and LPC.

C. Vehicle Flight Trajectory (FLOPS output listing), Figure 11.

D. Overall Performance Characteristic Maps; pressure ratio, efficiency vs. flow and RPM (with baseline aerodynamic blockages), Figures 12 and 13.

E. Fan-Core and LPC Performance along Engine Operating Line, Figures 12 and 13.

F. Ice Particle Temperature and Static Air Temperature from Inlet through LPC, Figure 15.

G. NPSS cycle analysis for the altitude cruise conditions at $35000 \mathrm{ft}$, Figure 18(a) to (d) and Figure 19(a) to (d).

H. Compressor code analysis for the altitude cruise conditions at 35000 feet, Figure 18(a-d) and Figure 19(a-d).

I. NPSS cycle analysis for the altitude cruise conditions at 39000 feet, Figure 20(a-j).

J. Compressor code analysis for the altitude cruise conditions at 39000 feet, Figure 20(a-j).

K. NPSS cycle analysis for the descent conditions, Figure 21(a-f).

L. Compressor code analysis for the descent conditions, Figure 21(a-f).

M. NPSS cycle analysis and compressor code analysis for the descent conditions, Figure 22.

N. NPSS cycle analysis for the descent conditions, Figure 23(a-h).

O. Compressor code analysis for the descent conditions, Figure 23(a-h).

\section{Nomenclature; Variable Description of COMDES Compressor Code Output}

Area1 Rotor leading edge annular area, in ${ }^{2}$

A1/A* Ratio of annular area at the rotor leading edge to the choke area

$\mathrm{A}^{*}$

Choke area of rotor, in ${ }^{2}$

AeroBl Aerodynamic blockage (e.g., $0.98=2 \%$ blockage)

AthrRotor Throat area of rotor, in ${ }^{2}$

Alfa Absolute flow angle at stator exit, degrees

Abs MACH Absolute Mach number

Beta BLADE Blade angle, degrees

B2 axial Rotor exit height in axial direction, in.

BetaFlo Relative flow angle, degrees

BetaBlade Rotor blade angle, degrees

Beta FLO Relative flow angle, degrees

C Absolute velocity, $\mathrm{ft} / \mathrm{s}$

ChokeMargin Choke margin of rotor

$\mathrm{Cu}$

Tangential component of absolute velocity at rotor leading edge, $\mathrm{ft} / \mathrm{s}$

$\mathrm{Cm} \quad$ Meridional component of velocity at rotor leading edge, $\mathrm{ft} / \mathrm{s}$

$\mathrm{C}_{\mathrm{p}} \quad$ Specific heat at constant pressure

CFM Volumetric flow rate, $\mathrm{ft}^{3} / \mathrm{min}$

$\mathrm{DelRCu} \quad$ Angular momentum change through blade

Del Enthalpy Enthalpy rise

Del_H/U^2 Head coefficient (Enthalpy rise / tip speed squared) 


\begin{tabular}{|c|c|}
\hline Del T & Total temperature rise, $\mathrm{R}$ \\
\hline Deviat & Flow deviation angle from blade trailing edge, degrees \\
\hline Diff Fct & Diffusion factor \\
\hline DIFF LOSS & Diffuser, or stator loss coefficient \\
\hline Efficiency & Efficiency, adiabatic \\
\hline EffAVG & Rotor average efficiency, adiabatic \\
\hline GAMMA & Ratio of specific heats $\left(\mathrm{C}_{\mathrm{p}} / \mathrm{C}_{\mathrm{v}}\right)$. \\
\hline GHP & Horsepower \\
\hline Incid & Rotor leading edge incidence angle, degrees \\
\hline NBLAD & Number of rotor blades \\
\hline Ns & Specific speed \\
\hline Ns nondim & Specific speed, non-dimensional \\
\hline OPR & Overall Pressure ratio, total-to-total \\
\hline PR & Stage pressure ratio, total-to-total \\
\hline Pdisch & Discharge pressure, psia \\
\hline $\mathrm{Pt}$ & Inlet total pressure, psia \\
\hline Ps & Static pressure, $1 \mathrm{bf} / \mathrm{ft}^{\wedge} 2$ \\
\hline POTH & $\begin{array}{l}\text { Potential ratio hub, absolute axial velocity at hub / axial velocity at mean radius radius } \\
\text { (typical value can be } 0.9 \text {, or } 10 \% \text { lower velocity at hub) }\end{array}$ \\
\hline POTS & $\begin{array}{l}\text { Potential ratio shroud, absolute axial velocity at tip / axial velocity at mean radius (typical } \\
\text { value can be } 1.1 \text {, or } 10 \% \text { higher velocity at tip) }\end{array}$ \\
\hline RPM cor & Shaft rotational speed, corrected to standard temperature conditions of $518.7 \mathrm{R}$ \\
\hline Rel Mach & Relative Mach number \\
\hline THK & Rotor blade thickness, in \\
\hline $\mathrm{R}$ & Radius from centerline at rotor leading edge, in \\
\hline $\mathrm{R}_{\mathrm{g}}$ & Gas constant, Universal gas constant / mole weight, for air $\mathrm{R}_{\mathrm{g}}=53.35 \mathrm{ft}-1 \mathrm{bf} / \mathrm{R}$ \\
\hline RPM act & Shaft rotational speed, rotations per minute \\
\hline Reynolds\# & Reynolds number \\
\hline Slip F & Slip factor \\
\hline Solidity & Solidity \\
\hline SCFM & Volumetric flow rate, standard cubic feet per minute (14.7 psia, $518.7 \mathrm{R})$ \\
\hline Stator & Setting angle at stator, or inlet guide vane exit, degrees \\
\hline THK & Blade normal thickness, in \\
\hline $\mathrm{Tt}$ & Inlet total temperature, degrees, $\mathrm{R}$ \\
\hline Ts & Static temperature, $\mathrm{R}$ \\
\hline TR & Temperature ratio, total-to-total \\
\hline Texit & Total temperature at last stage exit, $\mathrm{R}$ \\
\hline $\mathrm{U}$ & Blade peripheral velocity at rotor leading edge, $\mathrm{ft} / \mathrm{sec}$ \\
\hline U1 corrected & Rotor peripheral tip speed corrected to standard inlet temperature \\
\hline $\mathrm{W}$ act & Mass flow rate, $1 b_{\mathrm{m}} / \mathrm{s}$ \\
\hline W cor & $\begin{array}{l}\text { Mass flow rate corrected to standard pressure and temperature conditions (14.7 psia, } \\
518.7 \mathrm{R})\end{array}$ \\
\hline $\mathrm{Ws} 1 / \mathrm{W} 2$ & Inlet tip relative velocity / exit relative velocity \\
\hline $\mathrm{W}$ & Relative velocity, $\mathrm{ft} / \mathrm{s}$ \\
\hline
\end{tabular}




\section{Subscripts}

1 Rotor inlet (leading edge)

2 Rotor exit (trailing edge)

3 Radial diffuser vane inlet

$4 \quad$ Radial diffuser exit 

Appendix A: Normalized Growth Rate as a function of $\mathrm{LWC}_{\mathrm{m}} / \mathbf{T W C}_{\mathrm{inj}}$

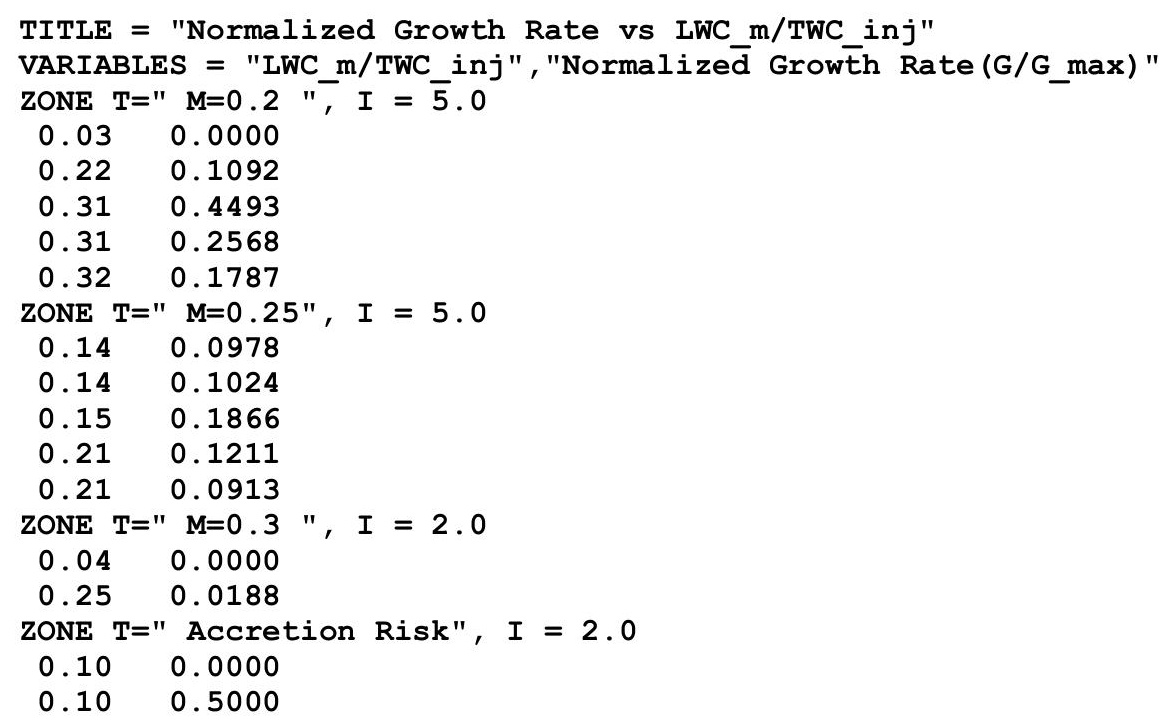



APPENDIX B: Design Point Performance; compressor code output listing for the Fan-Core and LPC.

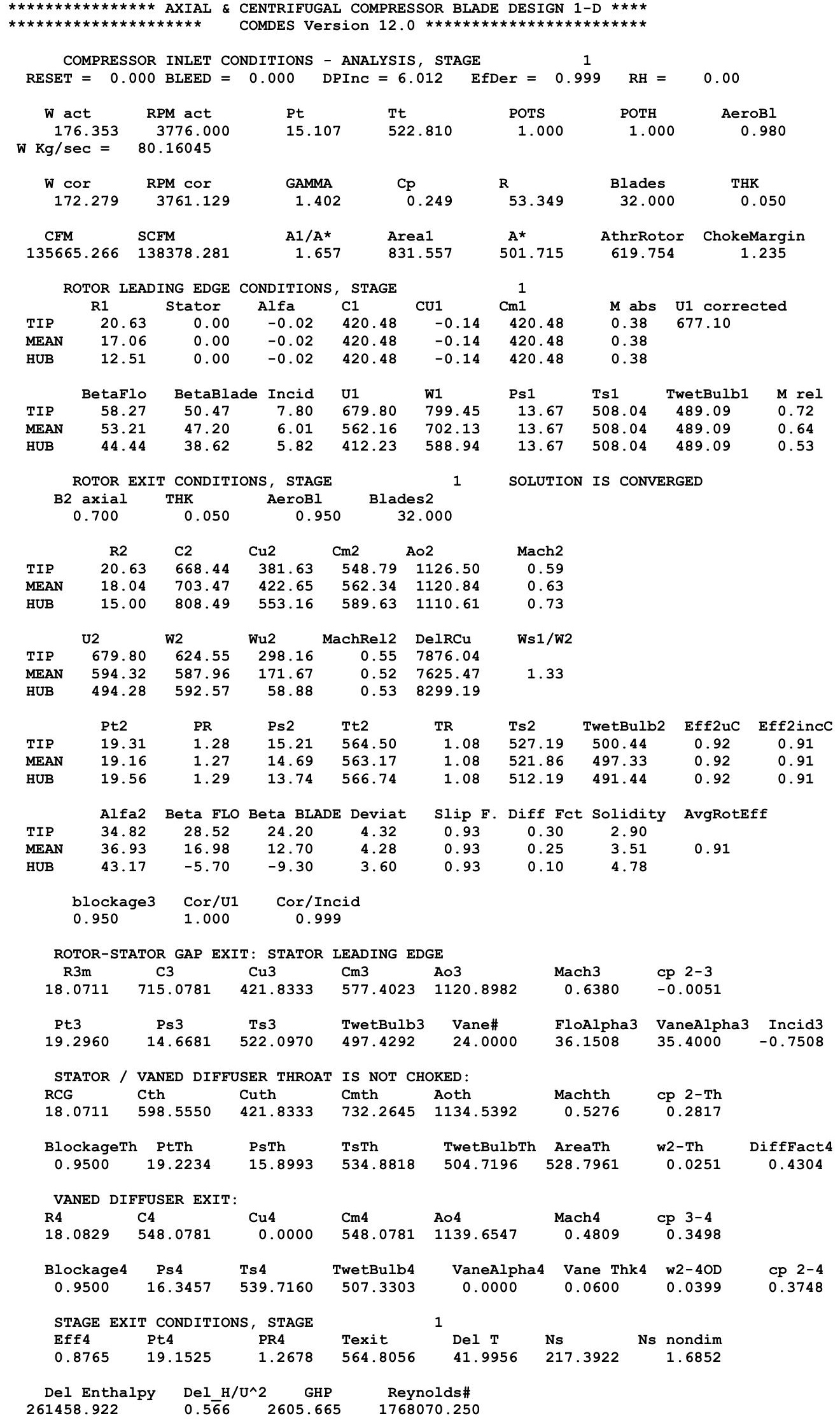




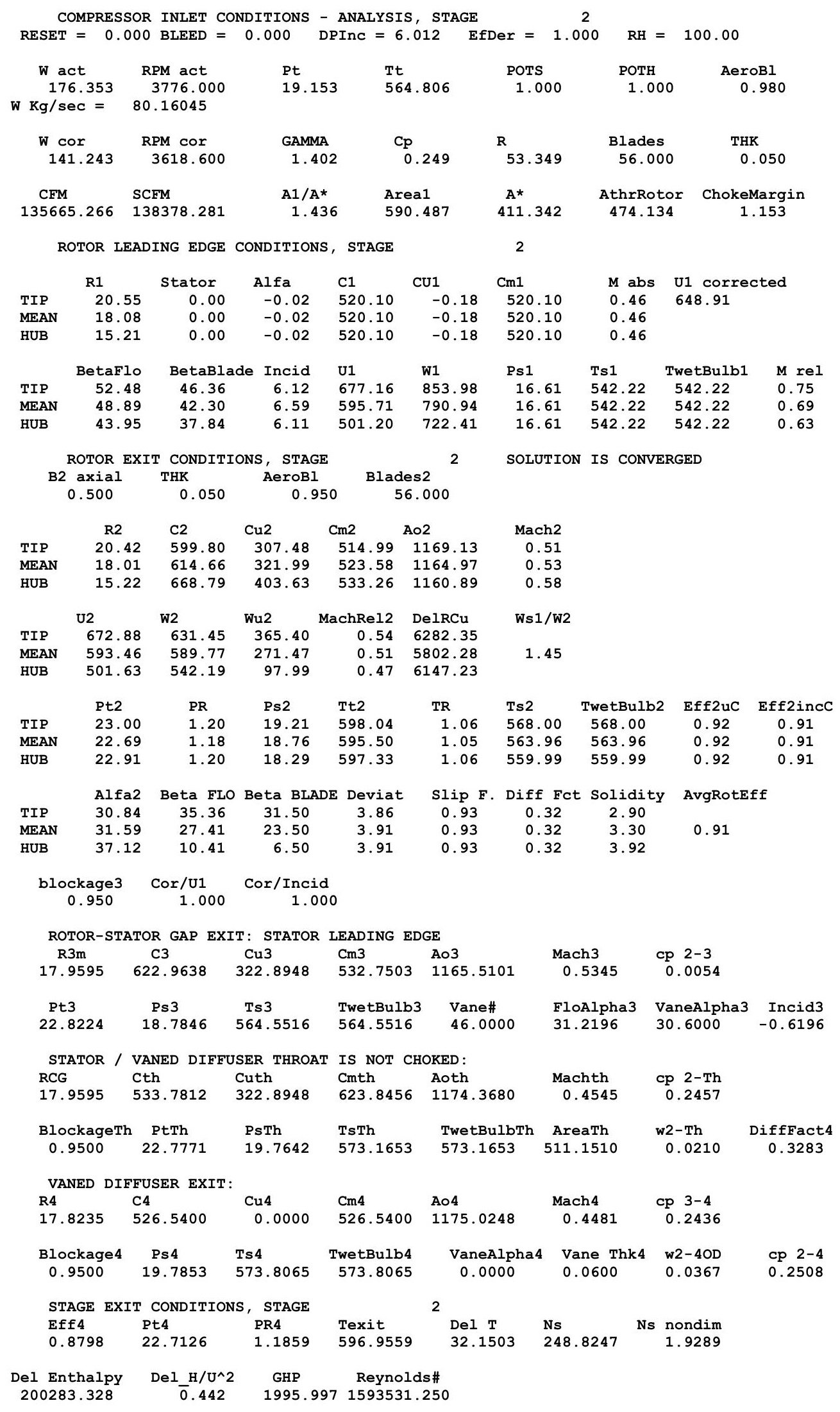




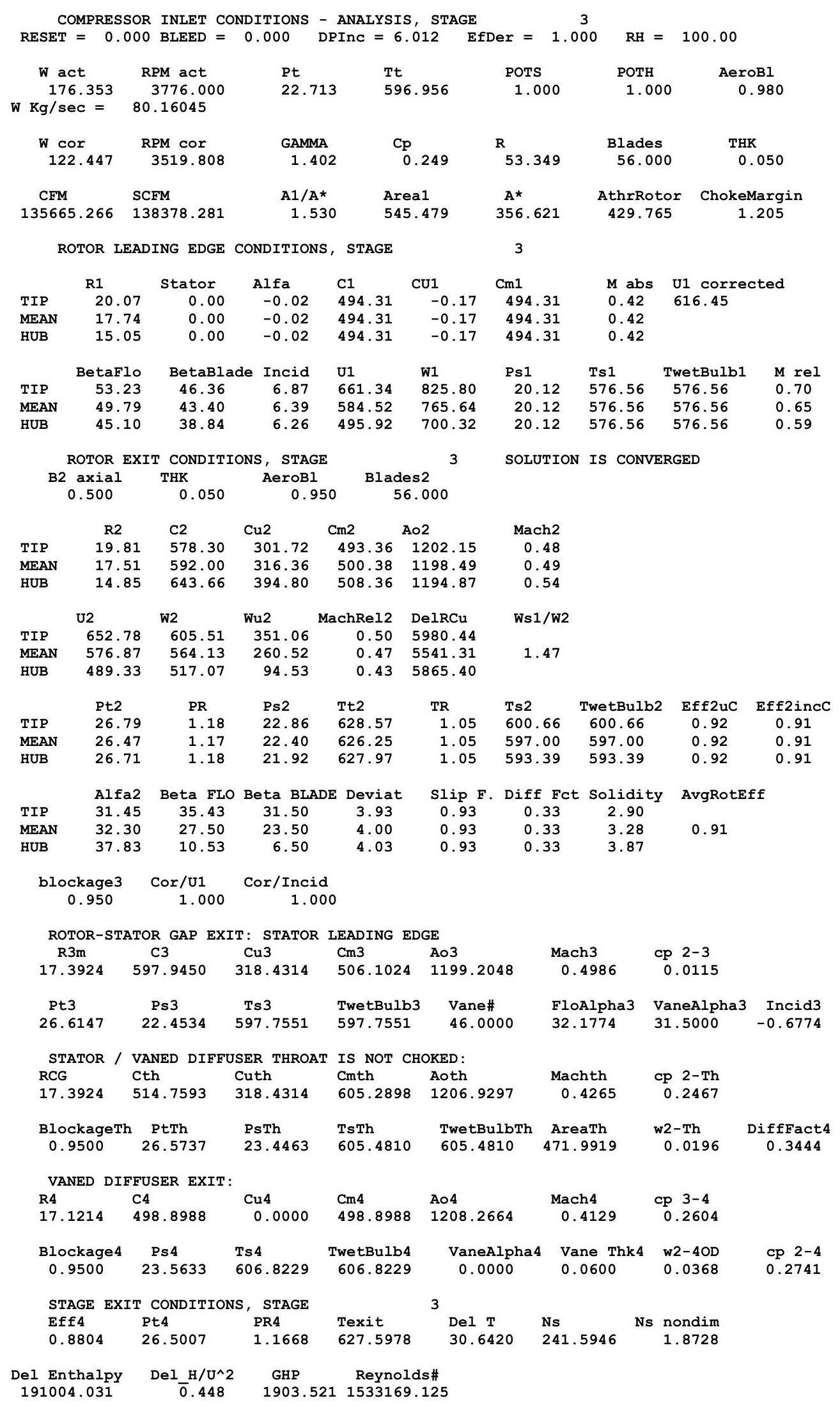




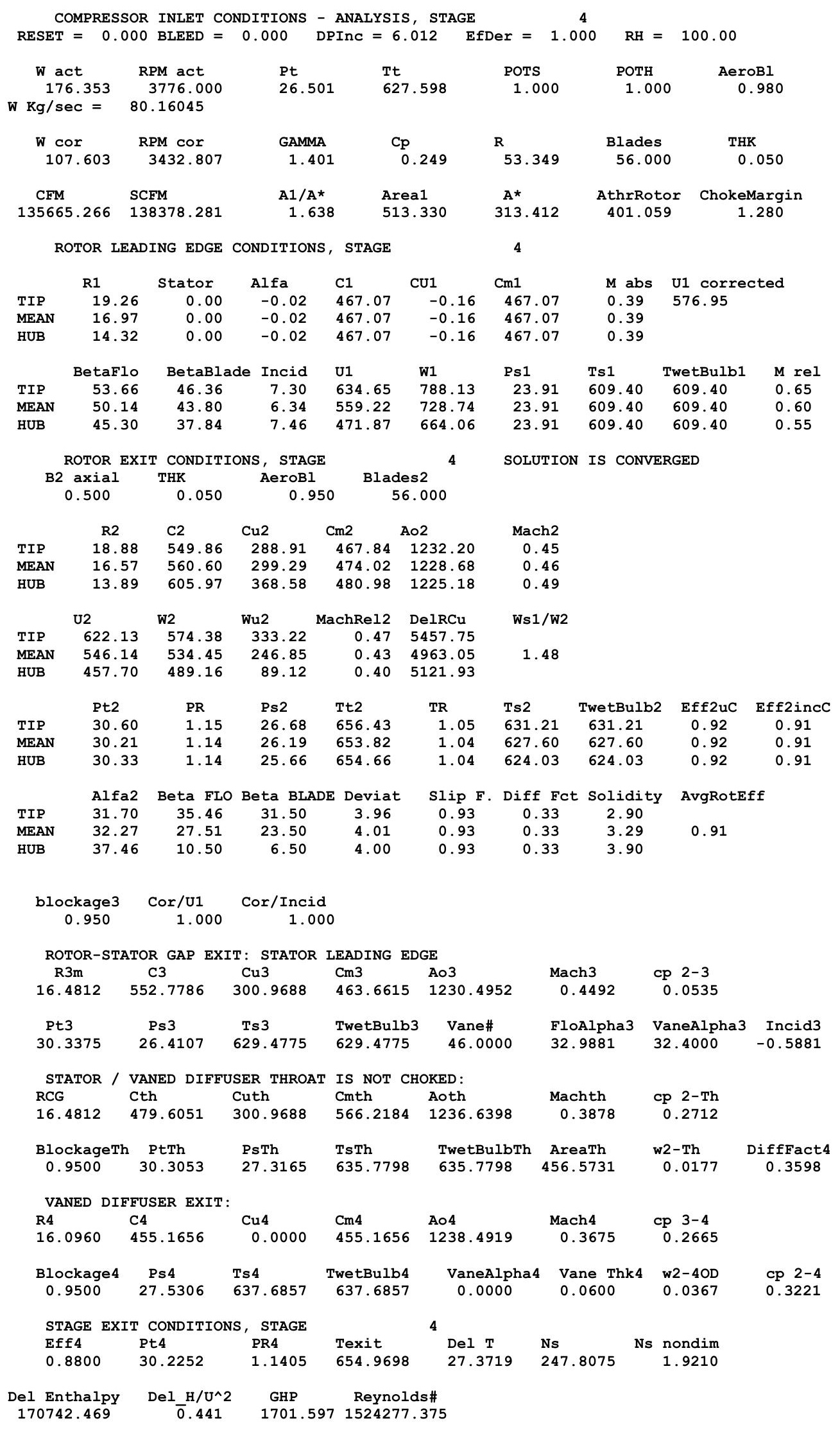




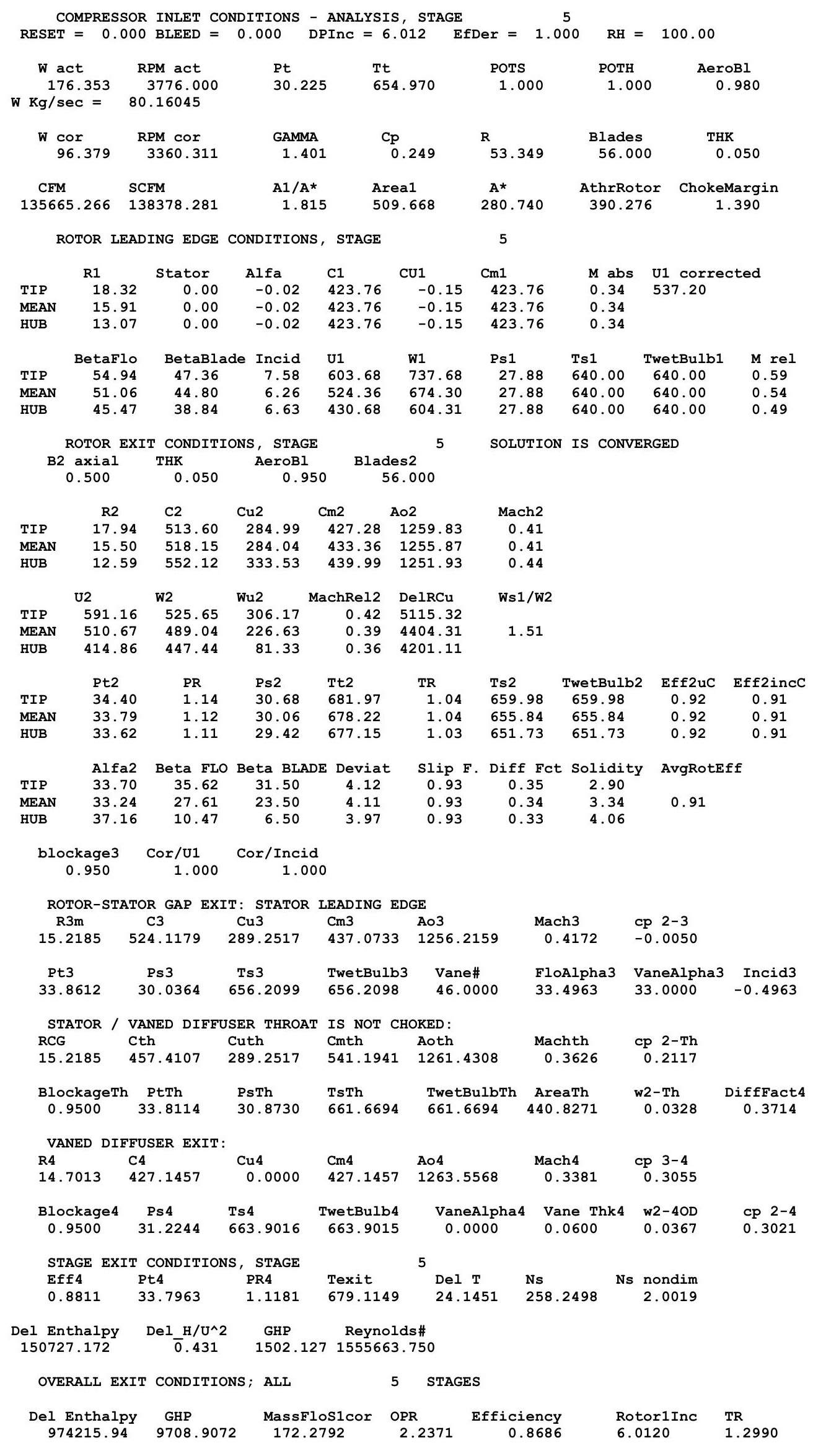





\section{APPENDIX C: Vehicle Flight Trajectory (FLOPS output listing)}

\begin{tabular}{|c|c|c|c|c|c|c|c|c|c|c|c|c|c|}
\hline WEIGHT & $\begin{array}{l}\text { ALTITUDE } \\
\text { ENERGY }\end{array}$ & $\begin{array}{l}\text { MACH NO. } \\
\text { VELOCITY }\end{array}$ & $\begin{array}{c}\text { DELTA T } \\
\text { TIME }\end{array}$ & $\begin{array}{c}\text { DELTA D } \\
\text { DIST }\end{array}$ & $\begin{array}{c}\text { DELTA F } \\
\text { FUEL }\end{array}$ & $\begin{array}{l}\text { THRUST } \\
\text { T/TMAX }\end{array}$ & $\begin{array}{l}\text { FUEL FLOW } \\
\text { SFC }\end{array}$ & $\begin{array}{l}\text { EX POW } \\
\text { DH/DT* }\end{array}$ & $\begin{array}{l}\text { CLIFT } \\
\text { CDRAG }\end{array}$ & $\begin{array}{l}\text { LIFT } \\
\text { DRAG }\end{array}$ & $\begin{array}{ll} & \text { L/D } \\
\text { SP } & \text { RANGE }\end{array}$ & $\begin{array}{c}Q \\
\text { NOX }\end{array}$ & $\begin{array}{c}\text { PCODE } \\
\text { ENG PAR }\end{array}$ \\
\hline 172183 & $\begin{array}{r}0 . \\
1743 .\end{array}$ & $\begin{array}{l}0.3000 \\
198.44\end{array}$ & $\begin{array}{l}0.00 \\
9.64\end{array}$ & $\begin{array}{l}0.0 \\
0.0\end{array}$ & $\begin{array}{r}0 . \\
526 .\end{array}$ & $\begin{array}{r}38219 \\
1.00000\end{array}$ & $\begin{array}{r}19137 \\
0.50072\end{array}$ & $\begin{array}{l}3205.2 \\
2047.8\end{array}$ & $\begin{array}{l}0.93275 \\
0.05828\end{array}$ & $\begin{array}{r}172183 . \\
10757 .\end{array}$ & $\begin{array}{r}16.006 \\
0.01037\end{array}$ & $\begin{array}{r}133.3 \\
0.0\end{array}$ & $\begin{array}{c}1 \\
28.231\end{array}$ \\
\hline 172053. & $\begin{array}{r}839 . \\
3154 .\end{array}$ & $\begin{array}{l}0.3467 \\
228.70\end{array}$ & $\begin{array}{r}0.41 \\
10.05\end{array}$ & $\begin{array}{l}1.5 \\
1.5\end{array}$ & $\begin{array}{l}130 . \\
655 .\end{array}$ & $\begin{array}{r}36279 \\
1.00000\end{array}$ & $\begin{array}{c}18935 \\
0.52194\end{array}$ & $\begin{array}{l}3685.2 \\
2047.8\end{array}$ & $\begin{array}{l}0.71920 \\
0.03721\end{array}$ & $\begin{array}{r}172053 . \\
8902 .\end{array}$ & $\begin{array}{r}19.327 \\
0.01208\end{array}$ & $\begin{array}{r}172.8 \\
3.3\end{array}$ & $\begin{array}{c}1 \\
28.385\end{array}$ \\
\hline 171961. & $\begin{array}{l}1500 . \\
4267 .\end{array}$ & $\begin{array}{r}0.3799 \\
250.00\end{array}$ & $\begin{array}{r}0.29 \\
10.34\end{array}$ & $\begin{array}{l}1.2 \\
2.6\end{array}$ & $\begin{array}{r}92 . \\
747 .\end{array}$ & $\begin{array}{r}35174 \\
1.00000\end{array}$ & $\begin{array}{r}18824 \\
0.53517\end{array}$ & $\begin{array}{l}3935.6 \\
2264.9\end{array}$ & $\begin{array}{l}0.61342 \\
0.03011\end{array}$ & $\begin{array}{r}171961 . \\
8442 .\end{array}$ & $\begin{array}{r}20.370 \\
0.01328\end{array}$ & $\begin{array}{r}202.5 \\
5.7\end{array}$ & $\begin{array}{c}1 \\
28.570\end{array}$ \\
\hline 171847 . & $\begin{array}{l}2922 . \\
5688 .\end{array}$ & $\begin{array}{r}0.3818 \\
250.00\end{array}$ & $\begin{array}{r}0.37 \\
10.71\end{array}$ & $\begin{array}{l}1.5 \\
4.1\end{array}$ & $\begin{array}{l}114 . \\
861 .\end{array}$ & $\begin{array}{r}34151 . \\
1.00000\end{array}$ & $\begin{array}{r}18246 \\
0.53427\end{array}$ & $\begin{array}{r}3772.3 \\
3853.9\end{array}$ & $\begin{array}{l}0.63946 \\
0.03180\end{array}$ & $\begin{array}{r}171847 \\
8545 .\end{array}$ & $\begin{array}{r}20.110 \\
0.01370\end{array}$ & $\begin{array}{r}194.1 \\
8.6\end{array}$ & $\begin{array}{c}1 \\
28.025\end{array}$ \\
\hline 171732 . & $\begin{array}{l}4343 . \\
7110 .\end{array}$ & $\begin{array}{l}0.3837 \\
250.00\end{array}$ & $\begin{array}{r}0.39 \\
11.10\end{array}$ & $\begin{array}{l}1.6 \\
5.7\end{array}$ & $\begin{array}{l}115 . \\
977 .\end{array}$ & $\begin{array}{r}33128 . \\
1.00000\end{array}$ & $\begin{array}{r}17666 . \\
0.53328\end{array}$ & $\begin{array}{l}3605.3 \\
3688.8\end{array}$ & $\begin{array}{l}0.66687 \\
0.03367\end{array}$ & $\begin{array}{r}171732 . \\
8672 .\end{array}$ & $\begin{array}{r}19.803 \\
0.01415\end{array}$ & $\begin{array}{r}186.0 \\
11.5\end{array}$ & $\begin{array}{c}1 \\
27.465\end{array}$ \\
\hline 171615 . & $\begin{array}{l}5765 . \\
8531 .\end{array}$ & $\begin{array}{l}0.3857 \\
250.00\end{array}$ & $\begin{array}{r}0.40 \\
11.50\end{array}$ & $\begin{array}{l}1.7 \\
7.4\end{array}$ & $\begin{array}{r}117 . \\
1094 .\end{array}$ & $\begin{array}{r}32118 \\
1.00000\end{array}$ & $\begin{array}{r}17122 . \\
0.53308\end{array}$ & $\begin{array}{l}3441.6 \\
3523.5\end{array}$ & $\begin{array}{l}0.69575 \\
0.03563\end{array}$ & $\begin{array}{r}171615 . \\
8790 .\end{array}$ & $\begin{array}{r}19.525 \\
0.01460\end{array}$ & $\begin{array}{r}178.1 \\
14.4\end{array}$ & $\begin{array}{c}1 \\
26.962\end{array}$ \\
\hline 171496 & $\begin{array}{l}7186 . \\
9953 .\end{array}$ & $\begin{array}{l}0.3876 \\
250.00\end{array}$ & $\begin{array}{r}0.42 \\
11.92\end{array}$ & $\begin{array}{l}1.7 \\
9.1\end{array}$ & $\begin{array}{r}119 . \\
1213 .\end{array}$ & $\begin{array}{r}31120 \\
1.00000\end{array}$ & $\begin{array}{c}16606 . \\
0.53361\end{array}$ & $\begin{array}{l}3274.5 \\
3358.0\end{array}$ & $\begin{array}{l}0.72618 \\
0.03785\end{array}$ & $\begin{array}{r}171496 . \\
8939 .\end{array}$ & $\begin{array}{r}19.185 \\
0.01505\end{array}$ & $\begin{array}{r}170.6 \\
17.3\end{array}$ & $\begin{array}{c}1 \\
26.506\end{array}$ \\
\hline 171374 & $\begin{array}{r}8608 \\
11374 .\end{array}$ & $\begin{array}{l}0.3896 \\
250.00\end{array}$ & $\begin{array}{r}0.45 \\
12.37\end{array}$ & $\begin{array}{r}1.8 \\
11.0\end{array}$ & $\begin{array}{r}121 . \\
1334 .\end{array}$ & $\begin{array}{r}30123 \\
1.00000\end{array}$ & $\begin{array}{c}16089 \\
0.53411\end{array}$ & $\begin{array}{l}3102.4 \\
3188.5\end{array}$ & $\begin{array}{l}0.75827 \\
0.04036\end{array}$ & $\begin{array}{r}171374 . \\
9122 .\end{array}$ & $\begin{array}{r}18.787 \\
0.01554\end{array}$ & $\begin{array}{r}163.2 \\
20.2\end{array}$ & $\begin{array}{c}1 \\
26.047\end{array}$ \\
\hline 171252 . & $\begin{array}{l}10000 \\
12767 .\end{array}$ & $\begin{array}{l}0.3916 \\
250.00\end{array}$ & $\begin{array}{r}0.46 \\
12.83\end{array}$ & $\begin{array}{r}1.9 \\
12.9\end{array}$ & $\begin{array}{r}122 . \\
1456 .\end{array}$ & $\begin{array}{r}29145 . \\
1.00000\end{array}$ & $\begin{array}{r}15580 . \\
0.53457\end{array}$ & $\begin{array}{r}2929.3 \\
3015.8\end{array}$ & $\begin{array}{l}0.79141 \\
0.04312\end{array}$ & $\begin{array}{r}171252 . \\
9331 .\end{array}$ & $\begin{array}{r}18.354 \\
0.01605\end{array}$ & $\begin{array}{r}156.3 \\
23.1\end{array}$ & $\begin{array}{c}1 \\
25.593\end{array}$ \\
\hline 171103 & $\begin{array}{l}10000 \\
14619\end{array}$ & $\begin{array}{l}0.5060 \\
323.00\end{array}$ & $\begin{array}{r}0.57 \\
13.40\end{array}$ & $\begin{array}{r}2.7 \\
15.6\end{array}$ & $\begin{array}{r}149 . \\
1605 .\end{array}$ & $\begin{array}{r}27729 \\
1.00000\end{array}$ & $\begin{array}{c}16107 \\
0.58087\end{array}$ & $\begin{array}{r}3624.8 \\
0.0\end{array}$ & $\begin{array}{l}0.47370 \\
0.02427\end{array}$ & $\begin{array}{r}171103 \\
8767\end{array}$ & $\begin{array}{r}19.516 \\
0.02005\end{array}$ & $\begin{array}{r}260.9 \\
26.6\end{array}$ & $\begin{array}{c}1 \\
26.792\end{array}$ \\
\hline 170999. & $\begin{array}{l}11212 . \\
16021\end{array}$ & $\begin{array}{l}0.5187 \\
329.60\end{array}$ & $\begin{array}{r}0.39 \\
13.79\end{array}$ & $\begin{array}{r}2.1 \\
17.7\end{array}$ & $\begin{array}{r}104 . \\
1709 .\end{array}$ & $\begin{array}{r}26853 \\
1.00000\end{array}$ & $\begin{array}{r}15718 \\
0.58533\end{array}$ & $\begin{array}{l}3527.8 \\
3090.0\end{array}$ & $\begin{array}{l}0.47234 \\
0.02425\end{array}$ & $\begin{array}{r}170999 . \\
8779 .\end{array}$ & $\begin{array}{r}19.477 \\
0.02097\end{array}$ & $\begin{array}{r}261.5 \\
29.1\end{array}$ & $\begin{array}{c}1 \\
26.429\end{array}$ \\
\hline 170895 & $\begin{array}{l}12423 \\
17423\end{array}$ & $\begin{array}{l}0.5312 \\
336.07\end{array}$ & $\begin{array}{r}0.40 \\
14.19\end{array}$ & $\begin{array}{r}2.2 \\
19.9\end{array}$ & $\begin{array}{r}104 . \\
1813 .\end{array}$ & $\begin{array}{r}25987 . \\
1.00000\end{array}$ & $\begin{array}{r}15322 . \\
0.58961\end{array}$ & $\begin{array}{l}3425.2 \\
3003.8\end{array}$ & $\begin{array}{l}0.47190 \\
0.02427\end{array}$ & $\begin{array}{r}170895 . \\
8788 .\end{array}$ & $\begin{array}{r}19.446 \\
0.02193\end{array}$ & $\begin{array}{r}261.6 \\
31.6\end{array}$ & $\begin{array}{c}1 \\
26.046\end{array}$ \\
\hline 170790 . & $\begin{array}{l}13635 \\
18826\end{array}$ & $\begin{array}{l}0.5438 \\
342.42\end{array}$ & $\begin{array}{r}0.42 \\
14.61\end{array}$ & $\begin{array}{r}2.3 \\
22.3\end{array}$ & $\begin{array}{r}105 . \\
1918 .\end{array}$ & $\begin{array}{r}25132 . \\
1.00000\end{array}$ & $\begin{array}{r}14920 . \\
0.59368\end{array}$ & $\begin{array}{l}3317.1 \\
2912.8\end{array}$ & $\begin{array}{l}0.47230 \\
0.02432\end{array}$ & $\begin{array}{r}170790 . \\
8794 .\end{array}$ & $\begin{array}{r}19.421 \\
0.02295\end{array}$ & $\begin{array}{r}261.2 \\
34.1\end{array}$ & $\begin{array}{c}1 \\
25.644\end{array}$ \\
\hline 170685 & $\begin{array}{l}14847 \text {. } \\
20228 \text {. }\end{array}$ & $\begin{array}{l}0.5562 \\
348.65\end{array}$ & $\begin{array}{r}0.43 \\
15.04\end{array}$ & $\begin{array}{r}2.5 \\
24.7\end{array}$ & $\begin{array}{r}105 . \\
2024 \text {. }\end{array}$ & $\begin{array}{r}24278 \\
1.00000\end{array}$ & $\begin{array}{r}14504 \\
0.59739\end{array}$ & $\begin{array}{l}3202.4 \\
2816.5\end{array}$ & $\begin{array}{l}0.47351 \\
0.02441\end{array}$ & $\begin{array}{r}170685 \\
8797 .\end{array}$ & $\begin{array}{r}19.402 \\
0.02404\end{array}$ & $\begin{array}{r}260.3 \\
36.5\end{array}$ & $\begin{array}{c}1 \\
25.207\end{array}$ \\
\hline 170578 & $\begin{array}{l}16059 \text {. } \\
21631 \text {. }\end{array}$ & $\begin{array}{l}0.5686 \\
354.78\end{array}$ & $\begin{array}{r}0.44 \\
15.48\end{array}$ & $\begin{array}{r}2.6 \\
27.3\end{array}$ & $\begin{array}{r}106 . \\
2130 \text {. }\end{array}$ & $\begin{array}{r}23705 \\
1.00000\end{array}$ & $\begin{array}{r}14330 . \\
0.60454\end{array}$ & $\begin{array}{l}3139.6 \\
2739.8\end{array}$ & $\begin{array}{l}0.47549 \\
0.02452\end{array}$ & $\begin{array}{r}170578 . \\
8798 .\end{array}$ & $\begin{array}{r}19.388 \\
0.02476\end{array}$ & $\begin{array}{r}259.1 \\
38.9\end{array}$ & $\begin{array}{c}1 \\
25.445\end{array}$ \\
\hline 170471 & $\begin{array}{l}17270 \\
23033\end{array}$ & $\begin{array}{l}0.5810 \\
360.79\end{array}$ & $\begin{array}{r}0.45 \\
15.93\end{array}$ & $\begin{array}{r}2.7 \\
30.0\end{array}$ & $\begin{array}{r}107 \\
2237\end{array}$ & $\begin{array}{r}23224 . \\
1.00000\end{array}$ & $\begin{array}{c}14223 . \\
0.61243\end{array}$ & $\begin{array}{l}3092.2 \\
2692.3\end{array}$ & $\begin{array}{l}0.47820 \\
0.02468\end{array}$ & $\begin{array}{r}170471 \\
8797\end{array}$ & $\begin{array}{r}19.378 \\
0.02537\end{array}$ & $\begin{array}{r}257.5 \\
41.3\end{array}$ & $\begin{array}{c}1 \\
25.877\end{array}$ \\
\hline 170363. & $\begin{array}{l}18482 \text {. } \\
24435 \text {. }\end{array}$ & $\begin{array}{l}0.5933 \\
366.72\end{array}$ & $\begin{array}{r}0.46 \\
16.39\end{array}$ & $\begin{array}{r}2.8 \\
32.8\end{array}$ & $\begin{array}{r}108 \\
2345 \text {. }\end{array}$ & $\begin{array}{r}22809 \\
1.00000\end{array}$ & $\begin{array}{r}14156 . \\
0.62063\end{array}$ & $\begin{array}{l}3055.0 \\
2655.7\end{array}$ & $\begin{array}{l}0.48164 \\
0.02486\end{array}$ & $\begin{array}{r}170363 . \\
8794\end{array}$ & $\begin{array}{r}19.373 \\
0.02591\end{array}$ & $\begin{array}{r}255.5 \\
43.8\end{array}$ & $\begin{array}{c}1 \\
26.432\end{array}$ \\
\hline 170227 & $\begin{array}{l}20000 \text {. } \\
26192 \text {. }\end{array}$ & $\begin{array}{l}0.6088 \\
374.00\end{array}$ & $\begin{array}{r}0.58 \\
16.96\end{array}$ & $\begin{array}{r}3.6 \\
36.3\end{array}$ & $\begin{array}{r}136 \text {. } \\
2481 \text {. }\end{array}$ & $\begin{array}{r}22385 . \\
1.00000\end{array}$ & $\begin{array}{r}14134 \\
0.63141\end{array}$ & $\begin{array}{l}3024.4 \\
2626.4\end{array}$ & $\begin{array}{l}0.48694 \\
0.02515\end{array}$ & $\begin{array}{r}170227 . \\
8792 .\end{array}$ & $\begin{array}{r}19.362 \\
0.02646\end{array}$ & $\begin{array}{r}252.5 \\
47.0\end{array}$ & $\begin{array}{c}1 \\
27.339\end{array}$ \\
\hline 170119. & $\begin{array}{l}21112 \text {. } \\
27567 \text {. }\end{array}$ & $\begin{array}{l}0.6243 \\
381.88\end{array}$ & $\begin{array}{r}0.47 \\
17.43\end{array}$ & $\begin{array}{r}2.9 \\
39.3\end{array}$ & $\begin{array}{r}108 \text {. } \\
2589 \text {. }\end{array}$ & $\begin{array}{r}21476 . \\
1.00000\end{array}$ & $\begin{array}{r}13663 . \\
0.63618\end{array}$ & $\begin{array}{l}2875.4 \\
2384.6\end{array}$ & $\begin{array}{l}0.48475 \\
0.02515\end{array}$ & $\begin{array}{r}170119 \\
8827\end{array}$ & $\begin{array}{r}19.272 \\
0.02795\end{array}$ & $\begin{array}{r}253.5 \\
49.4\end{array}$ & $\begin{array}{c}1 \\
26.515\end{array}$ \\
\hline 170009 . & $\begin{array}{l}22223 \text {. } \\
28943 \text {. }\end{array}$ & $\begin{array}{l}0.6398 \\
389.59\end{array}$ & $\begin{array}{r}0.49 \\
17.92\end{array}$ & $\begin{array}{r}3.2 \\
42.4\end{array}$ & $\begin{array}{r}110 . \\
2699 .\end{array}$ & $\begin{array}{r}20569 \\
1.00000\end{array}$ & $\begin{array}{r}13176 . \\
0.64059\end{array}$ & $\begin{array}{l}2716.6 \\
2260.2\end{array}$ & $\begin{array}{l}0.48353 \\
0.02521\end{array}$ & $\begin{array}{r}170009 \\
8863 .\end{array}$ & $\begin{array}{r}19.182 \\
0.02957\end{array}$ & $\begin{array}{r}253.9 \\
51.9\end{array}$ & $\begin{array}{c}1 \\
25.609\end{array}$ \\
\hline 169897. & $\begin{array}{l}23335 . \\
30318\end{array}$ & $\begin{array}{l}0.6552 \\
397.16\end{array}$ & $\begin{array}{r}0.52 \\
18.44\end{array}$ & $\begin{array}{r}3.4 \\
45.8\end{array}$ & $\begin{array}{r}113 \text {. } \\
2812 \text {. }\end{array}$ & $\begin{array}{r}19674 \\
1.00000\end{array}$ & $\begin{array}{r}12684 . \\
0.64468\end{array}$ & $\begin{array}{l}2551.0 \\
2129.1\end{array}$ & $\begin{array}{l}0.48322 \\
0.02531\end{array}$ & $\begin{array}{r}169897 . \\
8898 .\end{array}$ & $\begin{array}{r}19.093 \\
0.03131\end{array}$ & $\begin{array}{r}253.9 \\
54.3\end{array}$ & $\begin{array}{c}1 \\
24.656\end{array}$ \\
\hline 169781. & $\begin{array}{l}24446 . \\
31693 \text {. }\end{array}$ & $\begin{array}{l}0.6705 \\
404.58\end{array}$ & $\begin{array}{r}0.56 \\
19.00\end{array}$ & $\begin{array}{r}3.7 \\
49.6\end{array}$ & $\begin{array}{r}116 . \\
2927 .\end{array}$ & $\begin{array}{r}18801 \\
1.00000\end{array}$ & $\begin{array}{r}12192 . \\
0.64847\end{array}$ & $\begin{array}{l}2381.3 \\
1993.6\end{array}$ & $\begin{array}{l}0.48375 \\
0.02545\end{array}$ & $\begin{array}{r}169781 . \\
8933 .\end{array}$ & $\begin{array}{r}19.006 \\
0.03318\end{array}$ & $\begin{array}{r}253.5 \\
56.8\end{array}$ & $\begin{array}{c}1 \\
23.680\end{array}$ \\
\hline 169662 . & $\begin{array}{l}25558 \text {. } \\
33068 \text {. }\end{array}$ & $\begin{array}{l}0.6857 \\
411.87\end{array}$ & $\begin{array}{r}0.60 \\
19.60\end{array}$ & $\begin{array}{r}4.1 \\
53.6\end{array}$ & $\begin{array}{r}119 . \\
3047 .\end{array}$ & $\begin{array}{r}17993 . \\
1.00000\end{array}$ & $\begin{array}{r}11740 . \\
0.65245\end{array}$ & $\begin{array}{l}2219.0 \\
1859.3\end{array}$ & $\begin{array}{l}0.48508 \\
0.02564\end{array}$ & $\begin{array}{r}169662 . \\
8967 .\end{array}$ & $\begin{array}{r}18.920 \\
0.03508\end{array}$ & $\begin{array}{r}252.6 \\
59.2\end{array}$ & $\begin{array}{c}1 \\
22.833\end{array}$ \\
\hline 169538. & $\begin{array}{l}26670 \text {. } \\
34443 \text {. }\end{array}$ & $\begin{array}{l}0.7009 \\
419.04\end{array}$ & $\begin{array}{r}0.64 \\
20.24\end{array}$ & $\begin{array}{r}4.4 \\
58.1\end{array}$ & $\begin{array}{r}123 . \\
3170 .\end{array}$ & $\begin{array}{r}17249 . \\
1.00000\end{array}$ & $\begin{array}{r}11328 . \\
0.65674\end{array}$ & $\begin{array}{l}2064.7 \\
1731.4\end{array}$ & $\begin{array}{l}0.48718 \\
0.02586\end{array}$ & $\begin{array}{r}169538 . \\
9000 .\end{array}$ & $\begin{array}{r}18.837 \\
0.03699\end{array}$ & $\begin{array}{r}251.3 \\
61.6\end{array}$ & $\begin{array}{c}1 \\
22.136\end{array}$ \\
\hline 169410 & $\begin{array}{l}27781 \text {. } \\
35818 \text {. }\end{array}$ & $\begin{array}{l}0.7160 \\
426.08\end{array}$ & $\begin{array}{r}0.69 \\
20.93\end{array}$ & $\begin{array}{r}4.9 \\
62.9\end{array}$ & $\begin{array}{r}128 . \\
3298 .\end{array}$ & $\begin{array}{r}16516 . \\
1.00000\end{array}$ & $\begin{array}{r}10917 . \\
0.66098\end{array}$ & $\begin{array}{l}1906.8 \\
1605.2\end{array}$ & $\begin{array}{l}0.49002 \\
0.02612\end{array}$ & $\begin{array}{r}169410 \\
9029 .\end{array}$ & $\begin{array}{r}18.762 \\
0.03903\end{array}$ & $\begin{array}{r}249.7 \\
64.1\end{array}$ & $\begin{array}{c}1 \\
21.433\end{array}$ \\
\hline 169275 & $\begin{array}{l}28893 \text {. } \\
37193 \text {. }\end{array}$ & $\begin{array}{l}0.7311 \\
433.01\end{array}$ & $\begin{array}{r}0.75 \\
21.69\end{array}$ & $\begin{array}{r}5.4 \\
68.3\end{array}$ & $\begin{array}{r}134 . \\
3433 .\end{array}$ & $\begin{array}{r}15778 \\
1.00000\end{array}$ & $\begin{array}{r}10488 . \\
0.66475\end{array}$ & $\begin{array}{l}1741.0 \\
1474.4\end{array}$ & $\begin{array}{l}0.49357 \\
0.02641\end{array}$ & $\begin{array}{r}169275 . \\
9057 .\end{array}$ & $\begin{array}{r}18.691 \\
0.04129\end{array}$ & $\begin{array}{r}247.7 \\
66.6\end{array}$ & $\begin{array}{c}1 \\
20.642\end{array}$ \\
\hline 169133. & $\begin{array}{l}30004 \\
38568\end{array}$ & $\begin{array}{l}0.7462 \\
439.83\end{array}$ & $\begin{array}{r}0.83 \\
22.52\end{array}$ & $\begin{array}{r}6.0 \\
74.4\end{array}$ & $\begin{array}{r}142 . \\
3575 .\end{array}$ & $\begin{array}{r}15035 \\
1.00000\end{array}$ & $\begin{array}{c}10043 \\
0.66800\end{array}$ & $\begin{array}{l}1567.8 \\
1337.4\end{array}$ & $\begin{array}{l}0.49781 \\
0.02673\end{array}$ & $\begin{array}{r}169133 . \\
9082 .\end{array}$ & $\begin{array}{r}18.623 \\
0.04379\end{array}$ & $\begin{array}{r}245.4 \\
69.1\end{array}$ & $\begin{array}{c}1 \\
19.767\end{array}$ \\
\hline 168981 & $\begin{array}{l}31116 . \\
39944 \text {. }\end{array}$ & $\begin{array}{l}0.7613 \\
446.55\end{array}$ & $\begin{array}{r}0.92 \\
23.44\end{array}$ & $\begin{array}{r}6.8 \\
81.2\end{array}$ & $\begin{array}{r}152 . \\
3727 .\end{array}$ & $\begin{array}{r}14382 \\
1.00000\end{array}$ & $\begin{array}{r}9689 \\
0.67368\end{array}$ & $\begin{array}{l}1406.8 \\
1202.3\end{array}$ & $\begin{array}{l}0.50273 \\
0.02715\end{array}$ & $\begin{array}{r}168981 . \\
9125 .\end{array}$ & $\begin{array}{r}18.519 \\
0.04609\end{array}$ & $\begin{array}{r}242.8 \\
71.7\end{array}$ & $\begin{array}{c}1 \\
19.255\end{array}$ \\
\hline 168773 & $\begin{array}{l}32702 \text {. } \\
41656\end{array}$ & $\begin{array}{l}0.7720 \\
449.73\end{array}$ & $\begin{array}{r}1.33 \\
24.78\end{array}$ & $\begin{array}{l}10.0 \\
91.2\end{array}$ & $\begin{array}{r}208 . \\
3935 .\end{array}$ & $\begin{array}{r}13378 \\
1.00000\end{array}$ & $\begin{array}{r}9056 \\
0.67691\end{array}$ & $\begin{array}{l}1160.4 \\
1189.0\end{array}$ & $\begin{array}{l}0.52525 \\
0.02825\end{array}$ & $\begin{array}{r}168773 . \\
9078 .\end{array}$ & $\begin{array}{r}18.592 \\
0.04966\end{array}$ & $\begin{array}{r}232.1 \\
75.2\end{array}$ & $\begin{array}{c}1 \\
18.196\end{array}$ \\
\hline
\end{tabular}




\begin{tabular}{|c|c|c|c|c|c|c|c|c|c|c|c|c|c|}
\hline 168607 & $\begin{array}{l}33909 \\
42862 \text {. }\end{array}$ & $\begin{array}{l}0.7762 \\
449.73\end{array}$ & $\begin{array}{r}1.13 \\
25.91\end{array}$ & $\begin{array}{r}8.5 \\
99.7\end{array}$ & $\begin{array}{r}166 \\
4102 .\end{array}$ & $\begin{array}{r}12586 \\
1.00000\end{array}$ & $\begin{array}{r}8526 \\
0.67741\end{array}$ & $\begin{array}{r}966.3 \\
1063.3\end{array}$ & $\begin{array}{l}0.54923 \\
0.02934\end{array}$ & $\begin{array}{r}168607 \\
9008\end{array}$ & $\begin{array}{r}18.717 \\
0.05275\end{array}$ & $\begin{array}{r}221.7 \\
77.9\end{array}$ & $\begin{array}{c}1 \\
17.264\end{array}$ \\
\hline 168434 . & $\begin{array}{l}35000 \\
43954\end{array}$ & $\begin{array}{l}0.7800 \\
449.73\end{array}$ & $\begin{array}{r}1.25 \\
27.16\end{array}$ & $\begin{array}{r}9.4 \\
109.0\end{array}$ & $\begin{array}{r}172 . \\
4274 .\end{array}$ & $\begin{array}{r}11868 \\
1.00000\end{array}$ & $\begin{array}{r}8043 \\
0.67770\end{array}$ & $\begin{array}{l}781.7 \\
874.0\end{array}$ & $\begin{array}{l}0.57203 \\
0.03049\end{array}$ & $\begin{array}{r}168434 \\
8977\end{array}$ & $\begin{array}{r}18.764 \\
0.05592\end{array}$ & $\begin{array}{r}212.7 \\
80.5\end{array}$ & $\begin{array}{c}1 \\
16.404\end{array}$ \\
\hline SEGMENT & CRUISE & & & & & & & & & & & & \\
\hline WEIGHT & $\begin{array}{l}\text { ALTITUDE } \\
\text { ENERGY }\end{array}$ & $\begin{array}{l}\text { MACH NO. } \\
\text { VELOCITY }\end{array}$ & $\begin{array}{l}\text { DELTA T } \\
\text { TIME }\end{array}$ & $\begin{array}{l}\text { DELTA D } \\
\text { DIST }\end{array}$ & $\begin{array}{l}\text { DELTA } F \\
\text { FUEL }\end{array}$ & $\begin{array}{l}\text { THRUST } \\
\text { T/TMAX }\end{array}$ & $\begin{array}{l}\text { FUEL FLOW } \\
\text { SFC }\end{array}$ & $\begin{array}{l}\text { EX POW } \\
\text { DH/DT* }\end{array}$ & $\begin{array}{l}\text { CLIFT } \\
\text { CDRAG }\end{array}$ & $\begin{array}{l}\text { LIFT } \\
\text { DRAG }\end{array}$ & $\begin{array}{ll} & \mathrm{L} / \mathrm{D} \\
\text { SP } & \text { RANGE }\end{array}$ & $\begin{array}{c}Q \\
\text { NOx }\end{array}$ & $\begin{array}{l}\text { PCODE } \\
\text { ENG PAR }\end{array}$ \\
\hline 168434 . & $\begin{array}{l}35000 \\
43954\end{array}$ & $\begin{array}{l}0.7800 \\
449.73\end{array}$ & $\begin{array}{r}0.00 \\
27.16\end{array}$ & $\begin{array}{r}0.0 \\
109.0\end{array}$ & $\begin{array}{r}0 . \\
4274\end{array}$ & $\begin{array}{r}8977 \\
0.75639\end{array}$ & $\begin{array}{r}5889 . \\
0.65601\end{array}$ & $\begin{array}{r}781.7 \\
0.0\end{array}$ & $\begin{array}{l}0.57203 \\
0.03049\end{array}$ & $\begin{array}{r}168434 \\
8977\end{array}$ & $\begin{array}{r}18.764 \\
0.07637\end{array}$ & $\begin{array}{r}212.7 \\
80.5\end{array}$ & $\begin{array}{c}1 \\
12.730\end{array}$ \\
\hline 168000 & $\begin{array}{l}35000 \\
43954\end{array}$ & $\begin{array}{l}0.7800 \\
449.73\end{array}$ & $\begin{array}{r}4.42 \\
31.59\end{array}$ & $\begin{array}{r}33.2 \\
142.2\end{array}$ & $\begin{array}{r}434 \\
4708\end{array}$ & $\begin{array}{r}8958 \\
0.75480\end{array}$ & $\begin{array}{r}5876 \\
0.65598\end{array}$ & $\begin{array}{r}788.8 \\
0.0\end{array}$ & $\begin{array}{l}0.57055 \\
0.03042\end{array}$ & $\begin{array}{r}168000 \text {. } \\
8958 \text {. }\end{array}$ & $\begin{array}{r}18.755 \\
0.07654\end{array}$ & $\begin{array}{r}212.7 \\
86.1\end{array}$ & $\begin{array}{c}1 \\
12.713\end{array}$ \\
\hline 166400 & $\begin{array}{l}35000 . \\
43954 .\end{array}$ & $\begin{array}{l}0.7800 \\
449.73\end{array}$ & $\begin{array}{l}16.40 \\
47.99\end{array}$ & $\begin{array}{l}122.9 \\
265.1\end{array}$ & $\begin{array}{l}1600 \\
6308\end{array}$ & $\begin{array}{r}8889 \\
0.74901\end{array}$ & $\begin{array}{r}5830 . \\
0.65587\end{array}$ & $\begin{array}{r}815.2 \\
0.0\end{array}$ & $\begin{array}{l}0.56512 \\
0.03019\end{array}$ & $\begin{array}{r}166400 \\
8889\end{array}$ & $\begin{array}{r}18.720 \\
0.07714\end{array}$ & $\begin{array}{l}212.7 \\
106.4\end{array}$ & 12.650 \\
\hline 164800 & $\begin{array}{l}35000 \\
43954 .\end{array}$ & $\begin{array}{l}0.7800 \\
449.73\end{array}$ & $\begin{array}{l}16.53 \\
64.52\end{array}$ & $\begin{array}{l}123.9 \\
389.0\end{array}$ & $\begin{array}{l}1600 . \\
7908 .\end{array}$ & $\begin{array}{r}8821 \\
0.74332\end{array}$ & $\begin{array}{r}5785 \\
0.65576\end{array}$ & $\begin{array}{r}841.8 \\
0.0\end{array}$ & $\begin{array}{l}0.55968 \\
0.02996\end{array}$ & $\begin{array}{r}164800 \\
8821\end{array}$ & $\begin{array}{r}18.682 \\
0.07774\end{array}$ & $\begin{array}{l}212.7 \\
126.5\end{array}$ & ${ }^{1} 2^{1} .588$ \\
\hline 163200 . & $\begin{array}{l}35000 \\
43954\end{array}$ & $\begin{array}{l}0.7800 \\
449.73\end{array}$ & $\begin{array}{l}16.66 \\
81.18\end{array}$ & $\begin{array}{l}124.9 \\
513.9\end{array}$ & $\begin{array}{l}1600 . \\
9508 .\end{array}$ & $\begin{array}{r}8755 \\
0.73773\end{array}$ & $\begin{array}{r}5740 \\
0.65565\end{array}$ & $\begin{array}{r}868.6 \\
0.0\end{array}$ & $\begin{array}{l}0.55425 \\
0.02973\end{array}$ & $\begin{array}{r}163200 \\
8755\end{array}$ & $\begin{array}{r}18.641 \\
0.07835\end{array}$ & $\begin{array}{l}212.7 \\
146.6\end{array}$ & $\begin{array}{c}1 \\
12.528\end{array}$ \\
\hline 161600 & $\begin{array}{l}35000 \\
43954\end{array}$ & $\begin{array}{l}0.7800 \\
449.73\end{array}$ & $\begin{array}{l}16.78 \\
97.96\end{array}$ & $\begin{array}{l}125.8 \\
639.7\end{array}$ & $\begin{array}{r}1600 \\
11108\end{array}$ & $\begin{array}{r}8690 \\
0.73227\end{array}$ & $\begin{array}{r}5699 . \\
0.65575\end{array}$ & $\begin{array}{r}895.4 \\
0.0\end{array}$ & $\begin{array}{l}0.54882 \\
0.02951\end{array}$ & $\begin{array}{r}161600 \\
8690\end{array}$ & $\begin{array}{r}18.595 \\
0.07892\end{array}$ & $\begin{array}{l}212.7 \\
166.6\end{array}$ & $\begin{array}{c}1 \\
12.472\end{array}$ \\
\hline 160000 & $\begin{array}{l}35000 \\
43954\end{array}$ & $\begin{array}{l}0.7800 \\
449.73\end{array}$ & $\begin{array}{r}16.90 \\
114.86\end{array}$ & $\begin{array}{l}126.7 \\
766.4\end{array}$ & $\begin{array}{r}1600 \\
12708\end{array}$ & $\begin{array}{r}8628 \\
0.72704\end{array}$ & $\begin{array}{r}5661 . \\
0.65609\end{array}$ & $\begin{array}{r}922.1 \\
0.0\end{array}$ & $\begin{array}{l}0.54338 \\
0.02930\end{array}$ & $\begin{array}{r}160000 \\
8628\end{array}$ & $\begin{array}{r}18.544 \\
0.07944\end{array}$ & $\begin{array}{l}212.7 \\
186.6\end{array}$ & $\begin{array}{c}1 \\
12.424\end{array}$ \\
\hline 158400 & $\begin{array}{l}35000 \\
43954\end{array}$ & $\begin{array}{l}0.7800 \\
449.73\end{array}$ & $\begin{array}{r}17.01 \\
131.88\end{array}$ & $\begin{array}{l}127.5 \\
893.9\end{array}$ & $\begin{array}{r}1600 \\
14308\end{array}$ & $\begin{array}{r}8567 \\
0.72187\end{array}$ & $\begin{array}{r}5624 . \\
0.65643\end{array}$ & $\begin{array}{r}949.0 \\
0.0\end{array}$ & $\begin{array}{l}0.53795 \\
0.02909\end{array}$ & $\begin{array}{r}158400 \\
8567\end{array}$ & $\begin{array}{r}18.490 \\
0.07997\end{array}$ & $\begin{array}{l}212.7 \\
206.4\end{array}$ & $\begin{array}{c}1 \\
12.376\end{array}$ \\
\hline 156800 & $\begin{array}{l}35000 . \\
43954 .\end{array}$ & $\begin{array}{l}0.7800 \\
449.73\end{array}$ & $\begin{array}{r}17.13 \\
149.01\end{array}$ & $\begin{array}{r}128.4 \\
1022.3\end{array}$ & $\begin{array}{r}1600 \text {. } \\
15908 \text {. }\end{array}$ & $\begin{array}{r}8506 \\
0.71675\end{array}$ & $\begin{array}{r}5587 \\
0.65677\end{array}$ & $\begin{array}{r}976.4 \\
0.0\end{array}$ & $\begin{array}{l}0.53252 \\
0.02889\end{array}$ & $\begin{array}{r}156800 \\
8506\end{array}$ & $\begin{array}{r}18.434 \\
0.08050\end{array}$ & $\begin{array}{l}212.7 \\
226.2\end{array}$ & $\begin{array}{c}1 \\
12.329\end{array}$ \\
\hline 155200 & $\begin{array}{l}35000 \\
43954\end{array}$ & $\begin{array}{l}0.7800 \\
449.73\end{array}$ & $\begin{array}{r}17.24 \\
166.25\end{array}$ & $\begin{array}{r}129.2 \\
1151.5\end{array}$ & $\begin{array}{r}1600 \\
17508\end{array}$ & $\begin{array}{r}8446 \\
0.71168\end{array}$ & $\begin{array}{r}5550 \\
0.65712\end{array}$ & $\begin{array}{r}1004.1 \\
0.0\end{array}$ & $\begin{array}{l}0.52708 \\
0.02868\end{array}$ & $\begin{array}{r}155200 \\
8446\end{array}$ & $\begin{array}{r}18.376 \\
0.08103\end{array}$ & $\begin{array}{l}212.7 \\
245.8\end{array}$ & $\begin{array}{c}1 \\
12.283\end{array}$ \\
\hline 153600 & $\begin{array}{l}35000 \\
43954\end{array}$ & $\begin{array}{l}0.7800 \\
449.73\end{array}$ & $\begin{array}{r}17.35 \\
183.60\end{array}$ & $\begin{array}{r}130.1 \\
1281.6\end{array}$ & $\begin{array}{r}1600 \\
19108\end{array}$ & $\begin{array}{r}8386 \\
0.70667\end{array}$ & $\begin{array}{r}5514 . \\
0.65746\end{array}$ & $\begin{array}{r}1032.2 \\
0.0\end{array}$ & $\begin{array}{l}0.52165 \\
0.02848\end{array}$ & $\begin{array}{r}153600 \\
8386\end{array}$ & $\begin{array}{r}18.315 \\
0.08156\end{array}$ & $\begin{array}{l}212.7 \\
265.5\end{array}$ & $\begin{array}{c}1 \\
12.236\end{array}$ \\
\hline 152000 & $\begin{array}{l}35000 \\
43954\end{array}$ & $\begin{array}{l}0.7800 \\
449.73\end{array}$ & $\begin{array}{r}17.47 \\
201.07\end{array}$ & $\begin{array}{r}130.9 \\
1412.5\end{array}$ & $\begin{array}{r}1600 \\
20708\end{array}$ & $\begin{array}{r}8328 \\
0.70171\end{array}$ & $\begin{array}{r}5478 \\
0.65781\end{array}$ & $\begin{array}{r}1060.7 \\
0.0\end{array}$ & $\begin{array}{l}0.51621 \\
0.02828\end{array}$ & $\begin{array}{r}152000 \\
8328\end{array}$ & $\begin{array}{r}18.252 \\
0.08210\end{array}$ & $\begin{array}{l}212.7 \\
285.0\end{array}$ & $\begin{array}{c}1 \\
12.191\end{array}$ \\
\hline 151276 & $\begin{array}{l}35000 \\
43954\end{array}$ & $\begin{array}{l}0.7800 \\
449.73\end{array}$ & $\begin{array}{r}7.94 \\
209.01\end{array}$ & $\begin{array}{r}59.6 \\
1472.1\end{array}$ & $\begin{array}{r}724 \\
21432 .\end{array}$ & $\begin{array}{r}8301 \\
0.69949\end{array}$ & $\begin{array}{r}5462 . \\
0.65796\end{array}$ & $\begin{array}{r}1073.7 \\
0.0\end{array}$ & $\begin{array}{l}0.51375 \\
0.02819\end{array}$ & $\begin{array}{r}151276 \\
8301\end{array}$ & $\begin{array}{r}18.223 \\
0.08234\end{array}$ & $\begin{array}{l}212.7 \\
293.8\end{array}$ & $\begin{array}{c}1 \\
12.170\end{array}$ \\
\hline SEGMENT & CLIMB & & & & & & & & & & & & \\
\hline WEIGHT & $\begin{array}{l}\text { ALTITUDE } \\
\text { ENERGY }\end{array}$ & $\begin{array}{l}\text { MACH NO. } \\
\text { VELOCITY }\end{array}$ & $\begin{array}{c}\text { DELTA T } \\
\text { TIME }\end{array}$ & $\begin{array}{c}\text { DELTA D } \\
\text { DIST }\end{array}$ & $\begin{array}{c}\text { DELTA } F \\
\text { FUEL }\end{array}$ & $\begin{array}{l}\text { THRUST } \\
\text { T/TMAX }\end{array}$ & $\begin{array}{l}\text { FUEL FLOW } \\
\text { SFC }\end{array}$ & $\begin{array}{l}\text { EX POW } \\
\text { DH/DT* }\end{array}$ & $\begin{array}{l}\text { CLIFT } \\
\text { CDRAG }\end{array}$ & $\begin{array}{l}\text { LIFT } \\
\text { DRAG }\end{array}$ & $\begin{array}{ll} & \mathrm{L} / \mathrm{D} \\
\text { SP } & \text { RANGE }\end{array}$ & $\stackrel{Q}{\text { NOx }}$ & $\begin{array}{c}\text { PCODE } \\
\text { ENG PAR }\end{array}$ \\
\hline 151276 & $\begin{array}{l}35000 \\
43954\end{array}$ & $\begin{array}{l}0.7800 \\
449.73\end{array}$ & $\begin{array}{r}0.00 \\
209.01\end{array}$ & $\begin{array}{r}0.0 \\
1472.1\end{array}$ & $\begin{array}{r}0 . \\
21432 .\end{array}$ & $\begin{array}{r}11868 \\
1.00000\end{array}$ & $\begin{array}{r}8043 \\
0.67770\end{array}$ & $\begin{array}{l}1073.7 \\
1114.3\end{array}$ & $\begin{array}{l}0.51375 \\
0.02819\end{array}$ & $\begin{array}{r}151276 . \\
8301\end{array}$ & $\begin{array}{r}18.223 \\
0.05592\end{array}$ & $\begin{array}{l}212.7 \\
293.8\end{array}$ & $\begin{array}{c}1 \\
16.404\end{array}$ \\
\hline 151163. & $\begin{array}{l}35954 . \\
44815 .\end{array}$ & $\begin{array}{l}0.7793 \\
447.38\end{array}$ & $\begin{array}{r}0.86 \\
209.87\end{array}$ & $\begin{array}{r}6.4 \\
1478.5\end{array}$ & $\begin{array}{r}112 . \\
21545 .\end{array}$ & $\begin{array}{r}11328 \\
1.00000\end{array}$ & $\begin{array}{r}7696 . \\
0.67941\end{array}$ & $\begin{array}{r}937.6 \\
1114.3\end{array}$ & $\begin{array}{l}0.53820 \\
0.02919\end{array}$ & $\begin{array}{r}151163 . \\
8199\end{array}$ & $\begin{array}{r}18.436 \\
0.05813\end{array}$ & $\begin{array}{l}202.9 \\
295.5\end{array}$ & $\stackrel{1}{16.003}$ \\
\hline 150925 . & $\begin{array}{l}37534 \\
46395\end{array}$ & $\begin{array}{l}0.7800 \\
447.38\end{array}$ & $\begin{array}{r}1.93 \\
211.80\end{array}$ & $\begin{array}{r}14.4 \\
1492.8\end{array}$ & $\begin{array}{r}238 \\
21783\end{array}$ & $\begin{array}{r}10444 . \\
1.00000\end{array}$ & $\begin{array}{r}7138 . \\
0.68345\end{array}$ & $\begin{array}{l}702.6 \\
820.1\end{array}$ & $\begin{array}{l}0.57855 \\
0.03106\end{array}$ & $\begin{array}{r}150925 \\
8104\end{array}$ & $\begin{array}{r}18.624 \\
0.06267\end{array}$ & $\begin{array}{l}188.4 \\
298.9\end{array}$ & $\begin{array}{c}1 \\
15.369\end{array}$ \\
\hline 150637. & $\begin{array}{l}39000 . \\
47861 .\end{array}$ & $\begin{array}{l}0.7800 \\
447.38\end{array}$ & $\begin{array}{r}2.52 \\
214.31\end{array}$ & $\begin{array}{r}18.8 \\
1511.6\end{array}$ & $\begin{array}{r}288 \text {. } \\
22071 \text {. }\end{array}$ & $\begin{array}{r}9621 \\
1.00000\end{array}$ & $\begin{array}{r}6615 \\
0.68755\end{array}$ & $\begin{array}{l}462.2 \\
582.4\end{array}$ & $\begin{array}{l}0.61943 \\
0.03324\end{array}$ & $\begin{array}{r}150637 \\
8084\end{array}$ & $\begin{array}{r}18.633 \\
0.06763\end{array}$ & $\begin{array}{l}175.6 \\
302.9\end{array}$ & $\begin{array}{c}1 \\
14.771\end{array}$ \\
\hline SEGMENT & CRUIS & & & & & & & & & & & & \\
\hline WEIGHT & $\begin{array}{l}\text { ALTITUDE } \\
\text { ENERGY }\end{array}$ & $\begin{array}{l}\text { MACH NO. } \\
\text { VELOCITY }\end{array}$ & $\begin{array}{l}\text { DELTA T } \\
\text { TIME }\end{array}$ & $\begin{array}{c}\text { DELTA D } \\
\text { DIST }\end{array}$ & $\begin{array}{l}\text { DELTA F } \\
\text { FUEL }\end{array}$ & $\begin{array}{l}\text { THRUST } \\
\text { T/TMAX }\end{array}$ & $\begin{array}{l}\text { FUEL FLOW } \\
\text { SFC }\end{array}$ & $\begin{array}{l}\text { EX POW } \\
\text { DH/DT* }\end{array}$ & $\begin{array}{l}\text { CLIFT } \\
\text { CDRAG }\end{array}$ & $\begin{array}{l}\text { LIFT } \\
\text { DRAG }\end{array}$ & $\begin{array}{ll} & \mathrm{L} / \mathrm{D} \\
\text { SP } & \text { RANGE }\end{array}$ & $\stackrel{Q}{\text { NOx }}$ & $\begin{array}{c}\text { PCODE } \\
\text { ENG PAR }\end{array}$ \\
\hline 150637 & $\begin{array}{l}39000 . \\
47861\end{array}$ & $\begin{array}{l}0.7800 \\
447.38\end{array}$ & $\begin{array}{r}0.00 \\
214.31\end{array}$ & $\begin{array}{r}0.0 \\
1511.6\end{array}$ & $\begin{array}{r}0 \\
22071\end{array}$ & $\begin{array}{r}8084 \\
0.84026\end{array}$ & $\begin{array}{r}5410 . \\
0.66921\end{array}$ & $\begin{array}{r}462.2 \\
0.0\end{array}$ & $\begin{array}{l}0.61943 \\
0.03324\end{array}$ & $\begin{array}{r}150637 \\
8084\end{array}$ & $\begin{array}{r}18.633 \\
0.08270\end{array}$ & $\begin{array}{l}175.6 \\
302.9\end{array}$ & $\begin{array}{c}1 \\
12.406\end{array}$ \\
\hline 150400 . & $\begin{array}{l}39000 . \\
47861 .\end{array}$ & $\begin{array}{l}0.7800 \\
447.38\end{array}$ & $\begin{array}{r}2.62 \\
216.94\end{array}$ & $\begin{array}{r}19.6 \\
1531.2\end{array}$ & $\begin{array}{r}237 . \\
22308 \text {. }\end{array}$ & $\begin{array}{r}8071 \\
0.83893\end{array}$ & $\begin{array}{r}5401 . \\
0.66920\end{array}$ & $\begin{array}{r}466.8 \\
0.0\end{array}$ & $\begin{array}{l}0.61846 \\
0.03319\end{array}$ & $\begin{array}{r}150400 \\
8071\end{array}$ & $\begin{array}{r}18.634 \\
0.08283\end{array}$ & $\begin{array}{l}175.6 \\
305.8\end{array}$ & $\begin{array}{c}1 \\
12.394\end{array}$ \\
\hline 148800 & $\begin{array}{l}39000 . \\
47861 .\end{array}$ & $\begin{array}{l}800 \\
.38\end{array}$ & $\begin{array}{r}17.87 \\
234.81\end{array}$ & $\begin{array}{r}133.2 \\
1664.4\end{array}$ & $\begin{array}{r}1600 \text {. } \\
23908 \text {. }\end{array}$ & $\begin{array}{r}7986 \\
0.83010\end{array}$ & $\begin{array}{r}5344 \\
0.66915\end{array}$ & $\begin{array}{r}497.7 \\
0.0\end{array}$ & $\begin{array}{l}0.61188 \\
0.03284\end{array}$ & $\begin{array}{r}148800 \\
7986\end{array}$ & $\begin{array}{r}18.631 \\
0.08371\end{array}$ & $\begin{array}{l}175.6 \\
325.6\end{array}$ & $\begin{array}{c}1 \\
12.309\end{array}$ \\
\hline 147200 . & $\begin{array}{l}39000 . \\
47861 .\end{array}$ & $\begin{array}{l}0.7800 \\
447.38\end{array}$ & $\begin{array}{r}18.06 \\
252.86\end{array}$ & $\begin{array}{r}134.6 \\
1799.1\end{array}$ & $\begin{array}{r}1600 \\
25508\end{array}$ & $\begin{array}{r}7904 \\
0.82152\end{array}$ & $\begin{array}{r}5289 \\
0.66911\end{array}$ & $\begin{array}{r}528.5 \\
0.0\end{array}$ & $\begin{array}{l}0.60530 \\
0.03250\end{array}$ & $\begin{array}{r}147200 \\
7904\end{array}$ & $\begin{array}{r}18.624 \\
0.08459\end{array}$ & $\begin{array}{l}175.6 \\
345.2\end{array}$ & ${ }^{1}$ \\
\hline 145600 . & $\begin{array}{l}39000 \text {. } \\
47861 \text {. }\end{array}$ & $\begin{array}{l}0.7800 \\
447.38\end{array}$ & $\begin{array}{r}18.24 \\
271.11\end{array}$ & $\begin{array}{r}136.0 \\
1935.1\end{array}$ & $\begin{array}{r}1600 \text {. } \\
27108 \text {. }\end{array}$ & $\begin{array}{r}7825 \\
0.81327\end{array}$ & $\begin{array}{r}5235 \\
0.66906\end{array}$ & $\begin{array}{r}559.0 \\
0.0\end{array}$ & $\begin{array}{l}0.59872 \\
0.03218\end{array}$ & $\begin{array}{r}145600 \\
7825\end{array}$ & $\begin{array}{r}18.608 \\
0.08546\end{array}$ & $\begin{array}{l}175.6 \\
364.7\end{array}$ & $\begin{array}{c}1 \\
12.149\end{array}$ \\
\hline 144000 . & $\begin{array}{l}39000 \\
47861\end{array}$ & $\begin{array}{l}0.7800 \\
447.38\end{array}$ & $\begin{array}{r}18.43 \\
289.53\end{array}$ & $\begin{array}{r}137.4 \\
2072.5\end{array}$ & $\begin{array}{r}1600 \\
28708\end{array}$ & $\begin{array}{r}7750 \\
0.80551\end{array}$ & $\begin{array}{r}5185 . \\
0.66902\end{array}$ & $\begin{array}{r}588.7 \\
0.0\end{array}$ & $\begin{array}{l}0.59214 \\
0.03187\end{array}$ & $\begin{array}{r}144000 \\
7750 .\end{array}$ & $\begin{array}{r}18.581 \\
0.08629\end{array}$ & $\begin{array}{l}175.6 \\
384.1\end{array}$ & $\begin{array}{c}1 \\
12.075\end{array}$ \\
\hline 142400 . & $\begin{array}{l}39000 . \\
47861 .\end{array}$ & $\begin{array}{r}0.7800 \\
447.38\end{array}$ & $\begin{array}{r}18.60 \\
308.14\end{array}$ & $\begin{array}{r}138.7 \\
2211.2\end{array}$ & $\begin{array}{r}1600 \text {. } \\
30308 \text {. }\end{array}$ & $\begin{array}{r}7677 \\
0.79790\end{array}$ & $\begin{array}{r}5136 \\
0.66897\end{array}$ & $\begin{array}{r}618.6 \\
0.0\end{array}$ & $\begin{array}{l}0.58556 \\
0.03157\end{array}$ & $\begin{array}{r}142400 \\
7677 .\end{array}$ & $\begin{array}{r}18.550 \\
0.08712\end{array}$ & $\begin{array}{l}175.6 \\
403.4\end{array}$ & $\begin{array}{c}1 \\
12.002\end{array}$ \\
\hline 140800 & $\begin{array}{l}39000 \text {. } \\
47861 .\end{array}$ & $\begin{array}{l}0.7800 \\
447.38\end{array}$ & $\begin{array}{r}18.78 \\
326.92\end{array}$ & $\begin{array}{r}140.0 \\
2351.3\end{array}$ & $\begin{array}{r}1600 \\
31908\end{array}$ & $\begin{array}{r}7605 \\
0.79044\end{array}$ & $\begin{array}{r}5087 \\
0.66893\end{array}$ & $\begin{array}{r}648.8 \\
0.0\end{array}$ & $\begin{array}{l}0.57898 \\
0.03127\end{array}$ & $\begin{array}{r}140800 \\
7605\end{array}$ & $\begin{array}{r}18.514 \\
0.08794\end{array}$ & $\begin{array}{l}175.6 \\
422.5\end{array}$ & $\begin{array}{c}1 \\
11.931\end{array}$ \\
\hline 9200. & $\begin{array}{l}39000 . \\
47861 .\end{array}$ & $\begin{array}{l}0.7800 \\
447.38\end{array}$ & $\begin{array}{r}18.96 \\
345.88\end{array}$ & $\begin{array}{r}141.4 \\
2492.6\end{array}$ & $\begin{array}{r}1600 \\
33508\end{array}$ & $\begin{array}{r}7535 \\
0.78312\end{array}$ & $\begin{array}{r}5040 \\
0.66888\end{array}$ & $\begin{array}{r}679.1 \\
0.0\end{array}$ & $\begin{array}{l}0.57240 \\
0.03098\end{array}$ & $\begin{array}{r}139200 \\
7535 .\end{array}$ & $\begin{array}{r}18.475 \\
0.08877\end{array}$ & $\begin{array}{l}175.6 \\
441.5\end{array}$ & ${ }_{11}^{1} .861$ \\
\hline
\end{tabular}




\begin{tabular}{|c|c|c|c|c|c|c|c|c|c|c|c|c|c|}
\hline 137600 . & $\begin{array}{l}39000 \\
47861\end{array}$ & $\begin{array}{l}0.7800 \\
447.38\end{array}$ & $\begin{array}{r}19.14 \\
365.02\end{array}$ & $\begin{array}{r}142.7 \\
2635.3\end{array}$ & $\begin{array}{r}1600 \\
35108\end{array}$ & $\begin{array}{r}7466 \\
0.77595\end{array}$ & $\begin{array}{r}4993 \\
0.66884\end{array}$ & $\begin{array}{r}709.7 \\
0.0\end{array}$ & $\begin{array}{l}0.56582 \\
0.03070\end{array}$ & $\begin{array}{r}137600 \\
7466\end{array}$ & $\begin{array}{r}18.431 \\
0.08960\end{array}$ & $\begin{array}{l}175.6 \\
460.5\end{array}$ & $\begin{array}{c}1 \\
11.793\end{array}$ \\
\hline 136000 & $\begin{array}{l}39000 \\
47861\end{array}$ & $\begin{array}{l}0.7800 \\
447.38\end{array}$ & $\begin{array}{r}19.31 \\
384.33\end{array}$ & $\begin{array}{r}144.0 \\
2779.3\end{array}$ & $\begin{array}{r}1600 \\
36708\end{array}$ & $\begin{array}{r}7398 \\
0.76893\end{array}$ & $\begin{array}{r}4948 \\
0.66880\end{array}$ & $\begin{array}{r}740.6 \\
0.0\end{array}$ & $\begin{array}{l}0.55924 \\
0.03042\end{array}$ & $\begin{array}{r}136000 \\
7398\end{array}$ & $\begin{array}{r}18.383 \\
0.09042\end{array}$ & $\begin{array}{l}175.6 \\
479.3\end{array}$ & $\begin{array}{c}1 \\
11.726\end{array}$ \\
\hline 135762 . & $\begin{array}{l}39000 \\
47861\end{array}$ & $\begin{array}{l}0.7800 \\
447.38\end{array}$ & $\begin{array}{r}2.89 \\
387.22\end{array}$ & $\begin{array}{r}21.6 \\
2800.9\end{array}$ & $\begin{array}{r}238 \\
36946\end{array}$ & $\begin{array}{r}7388 \\
0.76790\end{array}$ & $\begin{array}{r}4941 \\
0.66879\end{array}$ & $\begin{array}{r}745.2 \\
0.0\end{array}$ & $\begin{array}{l}0.55826 \\
0.03038\end{array}$ & $\begin{array}{r}135762 \\
7388\end{array}$ & $\begin{array}{r}18.376 \\
0.09054\end{array}$ & $\begin{array}{l}175.6 \\
482.1\end{array}$ & $\begin{array}{c}1 \\
11.716\end{array}$ \\
\hline GMENT & DESCE & & & & & & & & & & & & \\
\hline WEIGHT & $\begin{array}{l}\text { ALTITUDE } \\
\text { ENERGY }\end{array}$ & $\begin{array}{l}\text { MACH NO. } \\
\text { VELOCITY }\end{array}$ & $\begin{array}{c}\text { DELTA T } \\
\text { TIME }\end{array}$ & $\begin{array}{c}\text { DELTA D } \\
\text { DIST }\end{array}$ & $\begin{array}{c}\text { DELTA F } \\
\text { FUEL }\end{array}$ & $\begin{array}{l}\text { THRUST } \\
\text { T/TMAX }\end{array}$ & $\begin{array}{l}\text { FUEL FLOW } \\
\text { SFC }\end{array}$ & $\begin{array}{l}\text { EX POW } \\
\text { DH/DT* }\end{array}$ & $\begin{array}{l}\text { CLIFT } \\
\text { CDRAG }\end{array}$ & $\begin{array}{l}\text { LIFT } \\
\text { DRAG }\end{array}$ & $\begin{array}{ll} & \text { L/D } \\
\text { SP } & \text { RANGE }\end{array}$ & $\underset{\text { NOX }}{Q}$ & $\begin{array}{c}\text { PCODE } \\
\text { ENG PAR }\end{array}$ \\
\hline 135762 . & $\begin{array}{l}39000 \\
47861\end{array}$ & $\begin{array}{l}0.7800 \\
447.38\end{array}$ & $\begin{array}{r}0.00 \\
387.22\end{array}$ & $\begin{array}{r}0.0 \\
2800.9\end{array}$ & $\begin{array}{r}0 . \\
36946 .\end{array}$ & $\begin{array}{r}-427 \\
-0.04441\end{array}$ & $\begin{array}{r}619 \\
0.00000\end{array}$ & $\begin{array}{r}-2608.1 \\
0.0\end{array}$ & $\begin{array}{l}0.55826 \\
0.03038\end{array}$ & $\begin{array}{r}135762 \\
7388\end{array}$ & $\begin{array}{r}18.376 \\
0.72280\end{array}$ & $\begin{array}{l}175.6 \\
482.1\end{array}$ & $\begin{array}{l}0 \\
2.182\end{array}$ \\
\hline 135760 & $\begin{array}{l}39000 \\
47383\end{array}$ & $\begin{array}{l}0.7587 \\
435.15\end{array}$ & $\begin{array}{r}0.19 \\
387.41\end{array}$ & $\begin{array}{r}1.4 \\
2802.3\end{array}$ & $\begin{array}{r}2 . \\
36948\end{array}$ & $\begin{array}{r}-398 \\
-0.04176\end{array}$ & $\begin{array}{r}613 . \\
0.00000\end{array}$ & $\begin{array}{r}-2476.9 \\
0.0\end{array}$ & $\begin{array}{l}0.59008 \\
0.03144\end{array}$ & $\begin{array}{r}135760 \\
7233\end{array}$ & $\begin{array}{r}18.770 \\
0.71018\end{array}$ & $\begin{array}{l}166.2 \\
482.1\end{array}$ & $\begin{array}{l}0 \\
1.969\end{array}$ \\
\hline 135755 . & $\begin{array}{l}38334 \\
46087\end{array}$ & $\begin{array}{l}0.7296 \\
418.48\end{array}$ & $\begin{array}{r}0.54 \\
387.95\end{array}$ & $\begin{array}{r}3.8 \\
2806.1\end{array}$ & $\begin{array}{r}6 . \\
36954 .\end{array}$ & $\begin{array}{r}-374 \\
-0.03825\end{array}$ & $\begin{array}{r}618 \\
0.00000\end{array}$ & $\begin{array}{l}-2348.1 \\
-1239.1\end{array}$ & $\begin{array}{l}0.61801 \\
0.03254\end{array}$ & $\begin{array}{r}135755 \\
7148\end{array}$ & $\begin{array}{r}18.991 \\
0.67666\end{array}$ & $\begin{array}{l}158.6 \\
482.1\end{array}$ & $\begin{array}{c}0 \\
1.740\end{array}$ \\
\hline 135749 & $\begin{array}{l}37357 \\
44791\end{array}$ & $\begin{array}{l}0.7144 \\
409.78\end{array}$ & $\begin{array}{r}0.56 \\
388.51\end{array}$ & $\begin{array}{r}3.9 \\
2810.0\end{array}$ & $\begin{array}{r}6 . \\
36960 .\end{array}$ & $\begin{array}{r}-377 \\
-0.03682\end{array}$ & $\begin{array}{r}629 \\
0.00000\end{array}$ & $\begin{array}{l}-2287.6 \\
-1747.3\end{array}$ & $\begin{array}{l}0.61504 \\
0.03220\end{array}$ & $\begin{array}{r}135749 \\
7106\end{array}$ & $\begin{array}{r}19.103 \\
0.65175\end{array}$ & $\begin{array}{l}159.4 \\
482.1\end{array}$ & $\begin{array}{c}0 \\
1.670\end{array}$ \\
\hline 135743 & $\begin{array}{l}36365 \\
43495\end{array}$ & $\begin{array}{l}0.6997 \\
401.32\end{array}$ & $\begin{array}{r}0.57 \\
389.08\end{array}$ & $\begin{array}{r}3.9 \\
2813.8\end{array}$ & $\begin{array}{r}6 . \\
36966\end{array}$ & $\begin{array}{r}-379 \\
-0.03538\end{array}$ & $\begin{array}{r}638 \\
0.00000\end{array}$ & $\begin{array}{l}-2229.2 \\
-1729.2\end{array}$ & $\begin{array}{l}0.61145 \\
0.03183\end{array}$ & $\begin{array}{r}135743 \\
7066\end{array}$ & $\begin{array}{r}19.209 \\
0.62901\end{array}$ & $\begin{array}{l}160.3 \\
482.1\end{array}$ & $\begin{array}{l}0 \\
1.600\end{array}$ \\
\hline 135736 & $\begin{array}{l}35310 \\
42199\end{array}$ & $\begin{array}{l}0.6852 \\
394.49\end{array}$ & $\begin{array}{r}0.59 \\
389.67\end{array}$ & $\begin{array}{r}3.9 \\
2817.7\end{array}$ & $\begin{array}{r}6 . \\
36972 .\end{array}$ & $\begin{array}{r}-387 \\
-0.03444\end{array}$ & $\begin{array}{r}652 \\
0.00000\end{array}$ & $\begin{array}{l}-2182.0 \\
-1796.1\end{array}$ & $\begin{array}{l}0.60629 \\
0.03139\end{array}$ & $\begin{array}{r}135736 \\
7027\end{array}$ & $\begin{array}{r}19.317 \\
0.60544\end{array}$ & $\begin{array}{l}161.7 \\
482.1\end{array}$ & $\begin{array}{l}0 \\
1.546\end{array}$ \\
\hline 135730 & $\begin{array}{l}34281 \\
40903\end{array}$ & $\begin{array}{l}0.6687 \\
386.78\end{array}$ & $\begin{array}{r}0.60 \\
390.27\end{array}$ & $\begin{array}{r}3.9 \\
2821.6\end{array}$ & $\begin{array}{r}7 \\
36979\end{array}$ & $\begin{array}{r}-380 \\
-0.03217\end{array}$ & $\begin{array}{r}672 . \\
0.00000\end{array}$ & $\begin{array}{l}-2127.7 \\
-1711.6\end{array}$ & $\begin{array}{l}0.60627 \\
0.03124\end{array}$ & $\begin{array}{r}135730 \\
6993\end{array}$ & $\begin{array}{r}19.409 \\
0.57600\end{array}$ & $\begin{array}{l}161.7 \\
482.1\end{array}$ & $\begin{array}{c}0 \\
1.575\end{array}$ \\
\hline 135723 & $\begin{array}{l}33222 \text {. } \\
39608\end{array}$ & $\begin{array}{l}0.6535 \\
379.78\end{array}$ & $\begin{array}{r}0.62 \\
390.89\end{array}$ & $\begin{array}{r}3.9 \\
2825.6\end{array}$ & $\begin{array}{r}7 . \\
36986\end{array}$ & $\begin{array}{r}-373 \\
-0.02993\end{array}$ & $\begin{array}{r}694 \\
0.00000\end{array}$ & $\begin{array}{l}-2078.8 \\
-1717.4\end{array}$ & $\begin{array}{l}0.60405 \\
0.03099\end{array}$ & $\begin{array}{r}135723 \\
6963\end{array}$ & $\begin{array}{r}19.491 \\
0.54718\end{array}$ & $\begin{array}{l}162.3 \\
482.1\end{array}$ & $\begin{array}{c}0 \\
1.665\end{array}$ \\
\hline 135715 & $\begin{array}{l}32176 \\
38312 \text {. }\end{array}$ & $\begin{array}{l}0.6376 \\
372.29\end{array}$ & $\begin{array}{r}0.63 \\
391.52\end{array}$ & $\begin{array}{r}4.0 \\
2829.5\end{array}$ & $\begin{array}{r}7 . \\
36993 .\end{array}$ & $\begin{array}{r}-352 \\
-0.02689\end{array}$ & $\begin{array}{r}715 \\
0.00000\end{array}$ & $\begin{array}{l}-2024.8 \\
-1657.0\end{array}$ & $\begin{array}{l}0.60430 \\
0.03089\end{array}$ & $\begin{array}{r}135715 \\
6937\end{array}$ & $\begin{array}{r}19.564 \\
0.52080\end{array}$ & $\begin{array}{l}162.2 \\
482.1\end{array}$ & $\begin{array}{c}0 \\
1.746\end{array}$ \\
\hline 135707 . & $\begin{array}{l}31106 \\
37016\end{array}$ & $\begin{array}{l}0.6228 \\
365.37\end{array}$ & $\begin{array}{r}0.65 \\
392.17\end{array}$ & $\begin{array}{r}4.0 \\
2833.5\end{array}$ & $\begin{array}{r}8 \\
37001\end{array}$ & $\begin{array}{r}-333 \\
-0.02420\end{array}$ & $\begin{array}{r}735 \\
0.00000\end{array}$ & $\begin{array}{l}-1975.5 \\
-1651.2\end{array}$ & $\begin{array}{l}0.60286 \\
0.03071\end{array}$ & $\begin{array}{r}135707 \\
6913\end{array}$ & $\begin{array}{r}19.631 \\
0.49741\end{array}$ & $\begin{array}{l}162.6 \\
482.2\end{array}$ & $\begin{array}{c}0 \\
1.839\end{array}$ \\
\hline 135699 & $\begin{array}{l}30029 \\
35720\end{array}$ & $\begin{array}{l}0.6084 \\
358.54\end{array}$ & $\begin{array}{r}0.66 \\
392.83\end{array}$ & $\begin{array}{r}4.0 \\
2837.5\end{array}$ & $\begin{array}{r}8 . \\
37009 .\end{array}$ & $\begin{array}{r}-316 \\
-0.02194\end{array}$ & $\begin{array}{r}753 \\
0.00000\end{array}$ & $\begin{array}{l}-1928.6 \\
-1622.4\end{array}$ & $\begin{array}{l}0.60159 \\
0.03055\end{array}$ & $\begin{array}{r}135699 \\
6891\end{array}$ & $\begin{array}{r}19.691 \\
0.47586\end{array}$ & $\begin{array}{l}162.9 \\
482.2\end{array}$ & $\begin{array}{c}0 \\
1.932\end{array}$ \\
\hline 135691 . & $\begin{array}{l}28950 \\
34424\end{array}$ & $\begin{array}{l}0.5939 \\
351.65\end{array}$ & $\begin{array}{r}0.68 \\
393.51\end{array}$ & $\begin{array}{r}4.0 \\
2841.5\end{array}$ & $\begin{array}{r}9 . \\
37018 .\end{array}$ & $\begin{array}{r}-301 \\
-0.01988\end{array}$ & $\begin{array}{r}776 \\
0.00000\end{array}$ & $\begin{array}{l}-1883.2 \\
-1587.3\end{array}$ & $\begin{array}{l}0.60114 \\
0.03045\end{array}$ & $\begin{array}{r}135691 \\
6874\end{array}$ & $\begin{array}{r}19.739 \\
0.45324\end{array}$ & $\begin{array}{l}163.0 \\
482.2\end{array}$ & $\begin{array}{c}0 \\
2.064\end{array}$ \\
\hline 135681. & $\begin{array}{l}27859 \text {. } \\
33128 \text {. }\end{array}$ & $\begin{array}{l}0.5800 \\
345.00\end{array}$ & $\begin{array}{r}0.70 \\
394.21\end{array}$ & $\begin{array}{r}4.0 \\
2845.6\end{array}$ & $\begin{array}{r}9 . \\
37027 .\end{array}$ & $\begin{array}{r}-277 \\
-0.01740\end{array}$ & $\begin{array}{r}799 \\
0.00000\end{array}$ & $\begin{array}{l}-1837.7 \\
-1566.0\end{array}$ & $\begin{array}{l}0.60029 \\
0.03035\end{array}$ & $\begin{array}{r}135681 \\
6860\end{array}$ & $\begin{array}{r}19.779 \\
0.43168\end{array}$ & $\begin{array}{l}163.2 \\
482.2\end{array}$ & $\begin{array}{c}0 \\
2.204\end{array}$ \\
\hline 135672 . & $\begin{array}{l}26764 \\
31832\end{array}$ & $\begin{array}{l}0.5662 \\
338.35\end{array}$ & $\begin{array}{r}0.71 \\
394.92\end{array}$ & $\begin{array}{r}4.1 \\
2849.6\end{array}$ & $\begin{array}{r}10 . \\
37037 .\end{array}$ & $\begin{array}{r}-249 \\
-0.01489\end{array}$ & $\begin{array}{r}824 \\
0.00000\end{array}$ & $\begin{array}{l}-1792.0 \\
-1533.1\end{array}$ & $\begin{array}{l}0.60001 \\
0.03028\end{array}$ & $\begin{array}{r}135672 \\
6847\end{array}$ & $\begin{array}{r}19.815 \\
0.41052\end{array}$ & $\begin{array}{l}163.3 \\
482.2\end{array}$ & $\begin{array}{l}0 \\
2.343\end{array}$ \\
\hline 135662 . & $\begin{array}{l}25666 \\
30536\end{array}$ & $\begin{array}{l}0.5524 \\
331.67\end{array}$ & $\begin{array}{r}0.73 \\
395.65\end{array}$ & $\begin{array}{r}4.1 \\
2853.7\end{array}$ & $\begin{array}{r}10 \\
37047\end{array}$ & $\begin{array}{r}-216 \\
-0.01236\end{array}$ & $\begin{array}{r}851 \\
0.00000\end{array}$ & $\begin{array}{l}-1745.9 \\
-1498.5\end{array}$ & $\begin{array}{l}0.60045 \\
0.03026\end{array}$ & $\begin{array}{r}135662 \\
6836\end{array}$ & $\begin{array}{r}19.846 \\
0.38982\end{array}$ & $\begin{array}{l}163.2 \\
482.3\end{array}$ & $\begin{array}{c}0 \\
2.480\end{array}$ \\
\hline 135651 & $\begin{array}{l}24561 \text {. } \\
29240 \text {. }\end{array}$ & $\begin{array}{r}0.5390 \\
325.11\end{array}$ & $\begin{array}{r}0.75 \\
396.40\end{array}$ & $\begin{array}{r}4.1 \\
2857.8\end{array}$ & $\begin{array}{r}11 . \\
37058\end{array}$ & $\begin{array}{r}-203 \\
-0.01110\end{array}$ & $\begin{array}{r}874 \\
0.00000\end{array}$ & $\begin{array}{l}-1705.9 \\
-1471.8\end{array}$ & $\begin{array}{l}0.60099 \\
0.03024\end{array}$ & $\begin{array}{r}135651 \\
6825\end{array}$ & $\begin{array}{r}19.874 \\
0.37209\end{array}$ & $\begin{array}{l}163.0 \\
482.3\end{array}$ & $\begin{array}{l}0 \\
2.630\end{array}$ \\
\hline 135639. & $\begin{array}{l}23440 \\
27944\end{array}$ & $\begin{array}{l}0.5265 \\
318.99\end{array}$ & $\begin{array}{r}0.77 \\
397.17\end{array}$ & $\begin{array}{r}4.1 \\
2861.9\end{array}$ & $\begin{array}{r}11 . \\
37069\end{array}$ & $\begin{array}{r}-200 \\
-0.01038\end{array}$ & $\begin{array}{r}897 \\
0.00000\end{array}$ & $\begin{array}{l}-1670.8 \\
-1461.2\end{array}$ & $\begin{array}{l}0.60020 \\
0.03016\end{array}$ & $\begin{array}{r}135639 \\
6815\end{array}$ & $\begin{array}{r}19.902 \\
0.35566\end{array}$ & $\begin{array}{l}163.2 \\
482.3\end{array}$ & $\begin{array}{c}0 \\
2.796\end{array}$ \\
\hline 135628 & $\begin{array}{l}22315 \\
26649\end{array}$ & $\begin{array}{r}0.5140 \\
312.87\end{array}$ & $\begin{array}{r}0.78 \\
397.96\end{array}$ & $\begin{array}{r}4.1 \\
2866.1\end{array}$ & $\begin{array}{r}12 . \\
37081 .\end{array}$ & $\begin{array}{r}-191 \\
-0.00942\end{array}$ & $\begin{array}{r}921 . \\
0.00000\end{array}$ & $\begin{array}{l}-1634.7 \\
-1434.5\end{array}$ & $\begin{array}{l}0.60001 \\
0.03011\end{array}$ & $\begin{array}{r}135628 \\
6807\end{array}$ & $\begin{array}{r}19.926 \\
0.33969\end{array}$ & $\begin{array}{l}163.3 \\
482.3\end{array}$ & $\begin{array}{c}0 \\
2.963\end{array}$ \\
\hline 135615 & $\begin{array}{l}21184 \\
25353\end{array}$ & $\begin{array}{r}0.5018 \\
306.85\end{array}$ & $\begin{array}{r}0.80 \\
398.76\end{array}$ & $\begin{array}{r}4.1 \\
2870.2\end{array}$ & $\begin{array}{r}12 . \\
37093 .\end{array}$ & $\begin{array}{r}-176 \\
-0.00828\end{array}$ & $\begin{array}{r}946 \\
0.00000\end{array}$ & $\begin{array}{l}-1598.0 \\
-1410.1\end{array}$ & $\begin{array}{l}0.60000 \\
0.03008\end{array}$ & $\begin{array}{r}135615 \\
6799\end{array}$ & $\begin{array}{r}19.948 \\
0.32426\end{array}$ & $\begin{array}{l}163.2 \\
482.4\end{array}$ & $\begin{array}{l}0 \\
3.132\end{array}$ \\
\hline 135602 . & $\begin{array}{l}20047 \\
24057\end{array}$ & $\begin{array}{l}0.4900 \\
300.95\end{array}$ & $\begin{array}{r}0.82 \\
399.58\end{array}$ & $\begin{array}{r}4.2 \\
2874.3\end{array}$ & $\begin{array}{r}13 . \\
37106\end{array}$ & $\begin{array}{r}-157 \\
-0.00705\end{array}$ & $\begin{array}{r}976 . \\
0.00000\end{array}$ & $\begin{array}{l}-1561.8 \\
-1386.6\end{array}$ & $\begin{array}{l}0.60000 \\
0.03006\end{array}$ & $\begin{array}{r}135602 \\
6793\end{array}$ & $\begin{array}{r}19.963 \\
0.30836\end{array}$ & $\begin{array}{l}163.2 \\
482.4\end{array}$ & $\begin{array}{l}0 \\
3.307\end{array}$ \\
\hline 135588. & $\begin{array}{l}18903 \\
22761\end{array}$ & $\begin{array}{l}0.4784 \\
295.19\end{array}$ & $\begin{array}{r}0.84 \\
400.42\end{array}$ & $\begin{array}{r}4.2 \\
2878.5\end{array}$ & $\begin{array}{r}14 . \\
37120\end{array}$ & $\begin{array}{r}-130 \\
-0.00570\end{array}$ & $\begin{array}{r}1011 \\
0.00000\end{array}$ & $\begin{array}{l}-1525.1 \\
-1362.3\end{array}$ & $\begin{array}{l}0.60000 \\
0.03003\end{array}$ & $\begin{array}{r}135588 \\
6787\end{array}$ & $\begin{array}{r}19.978 \\
0.29191\end{array}$ & $\begin{array}{l}163.2 \\
482.5\end{array}$ & $\begin{array}{l}0 \\
3.435\end{array}$ \\
\hline 135573. & $\begin{array}{l}17753 \\
21465\end{array}$ & $\begin{array}{l}0.4672 \\
289.56\end{array}$ & $\begin{array}{r}0.86 \\
401.28\end{array}$ & $\begin{array}{r}4.2 \\
2882.7\end{array}$ & $\begin{array}{r}15 \\
37135\end{array}$ & $\begin{array}{r}-103 \\
-0.00439\end{array}$ & $\begin{array}{r}1047 \\
0.00000\end{array}$ & $\begin{array}{l}-1489.1 \\
-1337.5\end{array}$ & $\begin{array}{l}0.60000 \\
0.03001\end{array}$ & $\begin{array}{r}135573 \\
6782\end{array}$ & $\begin{array}{r}19.991 \\
0.27649\end{array}$ & $\begin{array}{l}163.2 \\
482.5\end{array}$ & $\begin{array}{l}0 \\
3.568\end{array}$ \\
\hline 135558. & $\begin{array}{l}16597 \\
20169\end{array}$ & $\begin{array}{r}0.4562 \\
284.05\end{array}$ & $\begin{array}{r}0.88 \\
402.16\end{array}$ & $\begin{array}{r}4.2 \\
2886.9\end{array}$ & $\begin{array}{r}16 . \\
37151\end{array}$ & $\begin{array}{r}-76 \\
-0.00314\end{array}$ & $\begin{array}{r}1084 \\
0.00000\end{array}$ & $\begin{array}{l}-1454.1 \\
-1312.8\end{array}$ & $\begin{array}{l}0.60000 \\
0.02999\end{array}$ & $\begin{array}{r}135558 \\
6776\end{array}$ & $\begin{array}{r}20.005 \\
0.26208\end{array}$ & $\begin{array}{l}163.2 \\
482.6\end{array}$ & $\begin{array}{l}0 \\
3.706\end{array}$ \\
\hline 135541. & $\begin{array}{l}15435 \\
18873\end{array}$ & $\begin{array}{l}0.4456 \\
278.67\end{array}$ & $\begin{array}{r}0.90 \\
403.06\end{array}$ & $\begin{array}{r}4.2 \\
2891.1\end{array}$ & $\begin{array}{r}17 . \\
37167\end{array}$ & $\begin{array}{r}-47 \\
-0.00187\end{array}$ & $\begin{array}{r}1122 \\
0.00000\end{array}$ & $\begin{array}{l}-1419.5 \\
-1288.1\end{array}$ & $\begin{array}{l}0.60000 \\
0.02997\end{array}$ & $\begin{array}{r}135541 \\
6771\end{array}$ & $\begin{array}{r}20.018 \\
0.24826\end{array}$ & $\begin{array}{l}163.2 \\
482.6\end{array}$ & $\begin{array}{l}0 \\
3.852\end{array}$ \\
\hline 135523. & $\begin{array}{l}14268 \\
17577\end{array}$ & $\begin{array}{l}0.4352 \\
273.41\end{array}$ & $\begin{array}{r}0.92 \\
403.98\end{array}$ & $\begin{array}{r}4.2 \\
2895.4\end{array}$ & $\begin{array}{r}18 \\
37185\end{array}$ & $\begin{array}{r}-14 \\
-0.00055\end{array}$ & $\begin{array}{r}1171 \\
0.00000\end{array}$ & $\begin{array}{l}-1385.1 \\
-1263.2\end{array}$ & $\begin{array}{l}0.60000 \\
0.02995\end{array}$ & $\begin{array}{r}135523 \\
6766\end{array}$ & $\begin{array}{r}20.031 \\
0.23354\end{array}$ & $\begin{array}{l}163.1 \\
482.7\end{array}$ & $\begin{array}{l}0 \\
4.009\end{array}$ \\
\hline 135504 & $\begin{array}{l}13095 \\
16281\end{array}$ & $\begin{array}{l}0.4251 \\
268.27\end{array}$ & $\begin{array}{r}0.95 \\
404.93\end{array}$ & $\begin{array}{r}4.3 \\
2899.7\end{array}$ & $\begin{array}{r}19 . \\
37204\end{array}$ & $\begin{array}{r}26 . \\
0.00099\end{array}$ & $\begin{array}{r}1223 \\
0.00000\end{array}$ & $\begin{array}{l}-1350.1 \\
-1237.5\end{array}$ & $\begin{array}{l}0.60000 \\
0.02993\end{array}$ & $\begin{array}{r}135504 \\
6760\end{array}$ & $\begin{array}{r}20.044 \\
0.21930\end{array}$ & $\begin{array}{l}163.1 \\
482.8\end{array}$ & $\begin{array}{c}0 \\
4.167\end{array}$ \\
\hline 135484 . & $\begin{array}{l}11918 \\
14985\end{array}$ & $\begin{array}{l}0.4153 \\
263.24\end{array}$ & $\begin{array}{r}0.97 \\
405.90\end{array}$ & $\begin{array}{r}4.3 \\
2904.0\end{array}$ & $\begin{array}{r}20 . \\
37224\end{array}$ & $\begin{array}{r}75 \\
0.00274\end{array}$ & $\begin{array}{r}1276 \\
16.91116\end{array}$ & $\begin{array}{l}-1314.3 \\
-1210.7\end{array}$ & $\begin{array}{l}0.60000 \\
0.02992\end{array}$ & $\begin{array}{r}135484 \\
6755\end{array}$ & $\begin{array}{r}20.057 \\
0.20627\end{array}$ & $\begin{array}{l}163.1 \\
482.9\end{array}$ & $\begin{array}{c}0 \\
4.326\end{array}$ \\
\hline
\end{tabular}




$\begin{array}{rrrrrrrrrrrrrrr}135463 . & 10735 . & 0.4058 & 1.00 & 4.3 & 22 . & 133 . & 1329 . & -1277.9 & 0.60000 & 135463 . & 20.070 & 163.1 & 0 \\ & 13690 . & 258.33 & 406.90 & 2908.3 & 37246 . & 0.00466 & 10.02060 & -1182.7 & 0.02990 & 6750 . & 0.19432 & 483.0 & 4.484 \\ 135392 . & 7357 . & 0.3801 & 2.99 & 12.5 & 70 . & 276 . & 1499 . & -1182.9 & 0.60000 & 135392 . & 20.118 & 163.0 & 0 \\ & 10015 . & 245.02 & 409.89 & 2920.8 & 37316 . & 0.00885 & 5.43932 & -1131.0 & 0.02982 & 6730 . & 0.16346 & 483.3 & 4.893 \\ 135307 . & 3978 . & 0.3566 & 3.20 & 12.7 & 85 . & 440 . & 1689 . & -1092.1 & 0.60000 & 135307 . & 20.162 & 162.9 & 0 & \\ & 6375 . & 232.67 & 413.09 & 2933.5 & 37401 . & 0.01298 & 3.84134 & -1055.8 & 0.02976 & 6711 . & 0.13776 & 483.7 & 5.297 \\ 135167 . & 0 . & 0.3000 & 4.59 & 16.5 & 140 . & 874 . & 1980 . & -927.9 & 0.73223 & 135167 . & 18.998 & 133.3 & 0 \\ & 1743 . & 198.44 & 417.68 & 2950.0 & 37541 . & 0.02286 & 2.26642 & -867.5 & 0.03854 & 7115 . & 0.10020 & 484.5 & 5.551\end{array}$


Appendix D: Overall Performance Characteristic Maps; pressure ratio, efficiency vs. flow and RPM. (with baseline aerodynamic blockages)

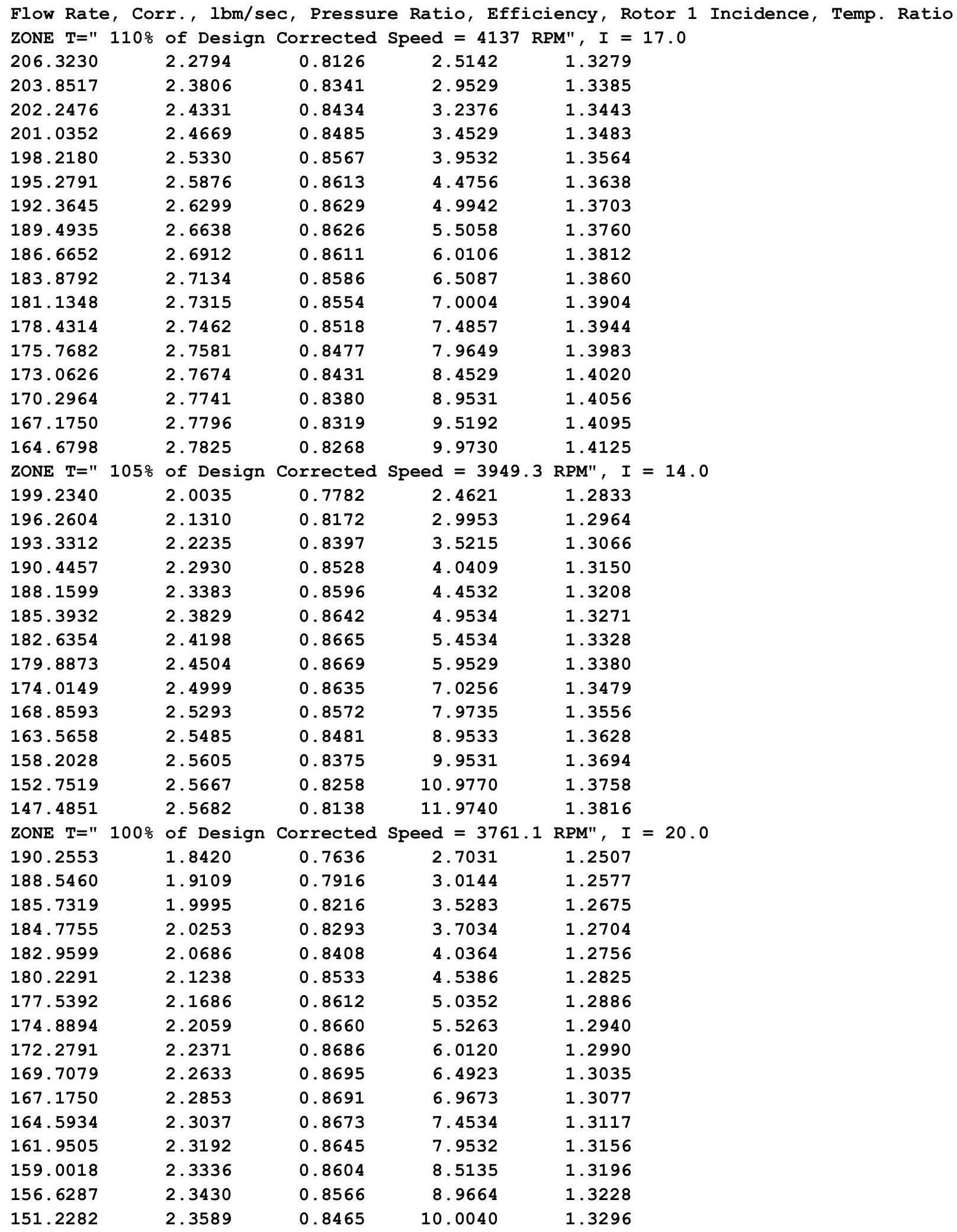




\begin{tabular}{|c|c|c|c|c|c|c|}
\hline 146.0140 & & 2.3680 & 0.8352 & 11.0152 & 1.3357 & \\
\hline 140.9795 & & 2.3722 & 0.8234 & 12.0004 & 1.3413 & \\
\hline 136.1186 & & 2.3728 & 0.8114 & 12.9602 & 1.3465 & \\
\hline 133.4166 & & 2.3719 & 0.8046 & 13.4974 & 1.3492 & \\
\hline ZONE $T="$ & $95 \%$ & of Design & Corrected & Speed $=3572.9$ & RPM", $I=$ & $=17.0$ \\
\hline 181.0216 & & 1.7094 & 0.7505 & 2.9530 & 1.2214 & \\
\hline 179.3280 & & 1.7675 & 0.7798 & 3.2685 & 1.2275 & \\
\hline 178.3378 & & 1.7961 & 0.7924 & 3.4534 & 1.2307 & \\
\hline 175.6672 & & 1.8635 & 0.8190 & 3.9536 & 1.2386 & \\
\hline 173.0107 & & 1.9184 & 0.8372 & 4.4535 & 1.2453 & \\
\hline 170.3689 & & 1.9648 & 0.8501 & 4.9531 & 1.2514 & \\
\hline 167.7381 & & 2.0045 & 0.8591 & 5.4531 & 1.2568 & \\
\hline 164.6798 & & 2.0437 & 0.8660 & 6.0376 & 1.2626 & \\
\hline 159.8008 & & 2.0923 & 0.8702 & 6.9773 & 1.2709 & \\
\hline 154.7835 & & 2.1290 & 0.8688 & 7.9532 & 1.2785 & \\
\hline 149.6973 & & 2.1560 & 0.8632 & 8.9529 & 1.2855 & \\
\hline 144.5575 & & 2.1749 & 0.8547 & 9.9739 & 1.2920 & \\
\hline 139.5732 & & 2.1869 & 0.8445 & 10.9747 & 1.2978 & \\
\hline 134.7507 & & 2.1938 & 0.8333 & 11.9530 & 1.3032 & \\
\hline 129.4638 & & 2.1971 & 0.8199 & 13.0371 & 1.3088 & \\
\hline 125.0000 & & 2.1971 & 0.8079 & 13.9618 & 1.3134 & \\
\hline 120.0866 & & 2.1944 & 0.7943 & 14.9898 & 1.3182 & \\
\hline $\mathrm{ZONE} T="$ & $90 \%$ & of Design & Corrected & Speed $=3384.6$ & RPM", $I=$ & $=16.0$ \\
\hline 171.4177 & & 1.6064 & 0.7440 & 3.2406 & 1.1957 & \\
\hline 170.3092 & & 1.6381 & 0.7634 & 3.4530 & 1.1991 & \\
\hline 167.7087 & & 1.6988 & 0.7951 & 3.9535 & 1.2064 & \\
\hline 164.6798 & & 1.7572 & 0.8209 & 4.5402 & 1.2137 & \\
\hline 162.2220 & & 1.7986 & 0.8367 & 5.0193 & 1.2191 & \\
\hline 157.4157 & & 1.8653 & 0.8569 & 5.9641 & 1.2284 & \\
\hline 151.9881 & & 1.9221 & 0.8676 & 7.0442 & 1.2375 & \\
\hline 147.4741 & & 1.9579 & 0.8697 & 7.9534 & 1.2443 & \\
\hline 142.3999 & & 1.9881 & 0.8670 & 8.9875 & 1.2512 & \\
\hline 137.4901 & & 2.0092 & 0.8606 & 10.0004 & 1.2574 & \\
\hline 132.7495 & & 2.0234 & 0.8517 & 10.9901 & 1.2629 & \\
\hline 128.1724 & & 2.0324 & 0.8414 & 11.9567 & 1.2680 & \\
\hline 123.1343 & & 2.0380 & 0.8286 & 13.0332 & 1.2733 & \\
\hline 118.8768 & & 2.0399 & 0.8169 & 13.9533 & 1.2776 & \\
\hline 114.2156 & & 2.0394 & 0.8034 & 14.9715 & 1.2822 & \\
\hline 109.7261 & & 2.0366 & 0.7899 & 15.9629 & 1.2864 & \\
\hline $\mathrm{ZONE} \quad \mathrm{T}="$ & $85 \%$ & of Design & Corrected & Speed $=3197.3$ & RPM", $I=$ & $=17.0$ \\
\hline 162.1044 & & 1.5115 & 0.7336 & 3.4535 & 1.1714 & \\
\hline 159.5892 & & 1.5634 & 0.7688 & 3.9533 & 1.1778 & \\
\hline 156.6287 & & 1.6157 & 0.7990 & 4.5460 & 1.1846 & \\
\hline 154.2909 & & 1.6521 & 0.8174 & 5.0175 & 1.1894 & \\
\hline 151.9881 & & 1.6845 & 0.8320 & 5.4850 & 1.1938 & \\
\hline 149.6973 & & 1.7136 & 0.8436 & 5.9530 & 1.1980 & \\
\hline 144.5575 & & 1.7687 & 0.8605 & 7.0142 & 1.2064 & \\
\hline 140.0713 & & 1.8063 & 0.8673 & 7.9532 & 1.2130 & \\
\hline 135.3568 & & 1.8370 & 0.8683 & 8.9530 & 1.2194 & \\
\hline 130.7028 & & 1.8597 & 0.8646 & 9.9532 & 1.2252 & \\
\hline 126.1111 & & 1.8761 & 0.8577 & 10.9531 & 1.2305 & \\
\hline 121.2965 & & 1.8880 & 0.8476 & 12.0155 & 1.2358 & \\
\hline 117.0996 & & 1.8946 & 0.8372 & 12.9533 & 1.2402 & \\
\hline 112.5109 & & 1.8986 & 0.8244 & 13.9913 & 1.2448 & \\
\hline 108.0884 & & 1.8997 & 0.8111 & 15.0041 & 1.2491 & \\
\hline
\end{tabular}




\begin{tabular}{|c|c|c|c|c|c|}
\hline 103.8398 & & 1.8987 & 0.7977 & 15.9884 & 1.2530 \\
\hline 99.7208 & & 1.8958 & 0.7841 & 16.9533 & 1.2567 \\
\hline $\mathrm{ZONE} \quad \mathrm{T}="$ & $80 \%$ & of Design & Corrected & Speed $=3009.1$ & RPM", $I=19.0$ \\
\hline 153.5195 & & 1.4133 & 0.7086 & 3.4810 & 1.1472 \\
\hline 151.2282 & & 1.4527 & 0.7418 & 3.9538 & 1.1524 \\
\hline 148.8259 & & 1.4898 & 0.7693 & 4.4533 & 1.1574 \\
\hline 146.0140 & & 1.5299 & 0.7961 & 5.0429 & 1.1629 \\
\hline 143.8347 & & 1.5585 & 0.8132 & 5.5035 & 1.1668 \\
\hline 141.6879 & & 1.5845 & 0.8273 & 5.9606 & 1.1705 \\
\hline 136.8026 & & 1.6357 & 0.8501 & 7.0126 & 1.1783 \\
\hline 132.0858 & & 1.6754 & 0.8622 & 8.0445 & 1.1850 \\
\hline 127.9930 & & 1.7029 & 0.8665 & 8.9529 & 1.1903 \\
\hline 123.1343 & & 1.7283 & 0.8656 & 10.0471 & 1.1962 \\
\hline 118.8887 & & 1.7448 & 0.8608 & 11.0174 & 1.2010 \\
\hline 114.7895 & & 1.7566 & 0.8534 & 11.9669 & 1.2054 \\
\hline 110.2775 & & 1.7656 & 0.8428 & 13.0264 & 1.2101 \\
\hline 106.3821 & & 1.7704 & 0.8320 & 13.9532 & 1.2139 \\
\hline 102.2312 & & 1.7731 & 0.8194 & 14.9531 & 1.2178 \\
\hline 97.7779 & & 1.7735 & 0.8047 & 16.0399 & 1.2219 \\
\hline 93.9346 & & 1.7721 & 0.7913 & 16.9895 & 1.2253 \\
\hline 89.7911 & & 1.7686 & 0.7762 & 18.0251 & 1.2288 \\
\hline 88.0087 & & 1.7665 & 0.7695 & 18.4744 & 1.2303 \\
\hline $\mathrm{ZONE} \quad \mathrm{T}="$ & $70 \%$ & of Design & Corrected & Speed $=2632.6$ & RPM", $I=20.0$ \\
\hline 136.1186 & & 1.2669 & 0.6602 & 3.4667 & 1.1063 \\
\hline 133.9698 & & 1.2921 & 0.6919 & 3.9529 & 1.1102 \\
\hline 131.4253 & & 1.3211 & 0.7258 & 4.5345 & 1.1145 \\
\hline 129.4638 & & 1.3427 & 0.7491 & 4.9872 & 1.1177 \\
\hline 127.4614 & & 1.3639 & 0.7704 & 5.4532 & 1.1208 \\
\hline 125.0000 & & 1.3885 & 0.7930 & 6.0315 & 1.1244 \\
\hline 121.1299 & & 1.4237 & 0.8213 & 6.9532 & 1.1298 \\
\hline 117.0002 & & 1.4564 & 0.8425 & 7.9535 & 1.1351 \\
\hline 112.9435 & & 1.4834 & 0.8554 & 8.9532 & 1.1400 \\
\hline 108.6316 & & 1.5069 & 0.8618 & 10.0345 & 1.1448 \\
\hline 104.8861 & & 1.5232 & 0.8623 & 10.9895 & 1.1487 \\
\hline 101.1634 & & 1.5360 & 0.8589 & 11.9532 & 1.1525 \\
\hline 97.2890 & & 1.5460 & 0.8520 & 12.9716 & 1.1562 \\
\hline 93.4649 & & 1.5531 & 0.8425 & 13.9922 & 1.1597 \\
\hline 89.7911 & & 1.5576 & 0.8314 & 14.9870 & 1.1630 \\
\hline 86.2617 & & 1.5601 & 0.8192 & 15.9559 & 1.1660 \\
\hline 82.4566 & & 1.5608 & 0.8048 & 17.0148 & 1.1692 \\
\hline 78.8194 & & 1.5599 & 0.7899 & 18.0407 & 1.1721 \\
\hline 75.3427 & & 1.5575 & 0.7747 & 19.0337 & 1.1749 \\
\hline 72.0193 & & 1.5536 & 0.7591 & 19.9940 & 1.1774 \\
\hline ZONE T=" & $60 \%$ & of Design & Corrected & Speed $=2257$ & RPM", $I=22.0$ \\
\hline 117.7028 & & 1.1757 & 0.6291 & 3.5438 & 1.0755 \\
\hline 115.9461 & & 1.1902 & 0.6569 & 3.9922 & 1.0779 \\
\hline 114.1585 & & 1.2048 & 0.6834 & 4.4529 & 1.0803 \\
\hline 111.9483 & & 1.2226 & 0.7137 & 5.0288 & 1.0831 \\
\hline 110.2775 & & 1.2358 & 0.7347 & 5.4688 & 1.0852 \\
\hline 108.4552 & & 1.2497 & 0.7556 & 5.9532 & 1.0874 \\
\hline 104.7498 & & 1.2766 & 0.7917 & 6.9532 & 1.0916 \\
\hline 101.1179 & & 1.3006 & 0.8189 & 7.9529 & 1.0956 \\
\hline 97.2890 & & 1.3228 & 0.8393 & 9.0280 & 1.0995 \\
\hline 93.9346 & & 1.3396 & 0.8507 & 9.9879 & 1.1028 \\
\hline 90.6187 & & 1.3536 & 0.8567 & 10.9534 & 1.1059 \\
\hline
\end{tabular}




\begin{tabular}{|c|c|c|c|c|c|}
\hline 87.1308 & & 1.3658 & 0.8579 & 11.9868 & 1.1090 \\
\hline 83.7060 & & 1.3754 & 0.8548 & 13.0194 & 1.1120 \\
\hline 80.4157 & & 1.3824 & 0.8483 & 14.0280 & 1.1147 \\
\hline 77.2548 & & 1.3873 & 0.8395 & 15.0123 & 1.1172 \\
\hline 74.2182 & & 1.3906 & 0.8290 & 15.9719 & 1.1196 \\
\hline 70.9444 & & 1.3926 & 0.8157 & 17.0217 & 1.1221 \\
\hline 67.8150 & & 1.3931 & 0.8016 & 18.0398 & 1.1244 \\
\hline 64.8237 & & 1.3924 & 0.7867 & 19.0262 & 1.1266 \\
\hline 61.9643 & & 1.3906 & 0.7714 & 19.9809 & 1.1286 \\
\hline 58.9348 & & 1.3874 & 0.7536 & 21.0048 & 1.1306 \\
\hline 57.4761 & & 1.3852 & 0.7444 & 21.5023 & 1.1316 \\
\hline $\mathrm{ZONE} \quad \mathrm{T}="$ & $50 \%$ & of Design & Corrected & Speed $=1880.5$ & RPM", $I=24.0$ \\
\hline 99.2594 & & 1.1100 & 0.5986 & 3.4637 & 1.0508 \\
\hline 97.6119 & & 1.1195 & 0.6276 & 3.9534 & 1.0524 \\
\hline 95.8370 & & 1.1298 & 0.6572 & 4.4873 & 1.0542 \\
\hline 94.3052 & & 1.1386 & 0.6813 & 4.9535 & 1.0557 \\
\hline 92.5326 & & 1.1488 & 0.7073 & 5.4992 & 1.0574 \\
\hline 91.0741 & & 1.1570 & 0.7272 & 5.9534 & 1.0587 \\
\hline 89.3421 & & 1.1666 & 0.7490 & 6.4986 & 1.0603 \\
\hline 87.9141 & & 1.1743 & 0.7654 & 6.9531 & 1.0616 \\
\hline 84.5493 & & 1.1914 & 0.7983 & 8.0419 & 1.0645 \\
\hline 81.6341 & & 1.2051 & 0.8204 & 9.0055 & 1.0670 \\
\hline 78.8194 & & 1.2169 & 0.8362 & 9.9539 & 1.0692 \\
\hline 75.7213 & & 1.2284 & 0.8475 & 11.0185 & 1.0717 \\
\hline 73.0520 & & 1.2368 & 0.8525 & 11.9530 & 1.0737 \\
\hline 70.2367 & & 1.2443 & 0.8535 & 12.9562 & 1.0758 \\
\hline 67.4759 & & 1.2503 & 0.8506 & 13.9573 & 1.0778 \\
\hline 64.7751 & & 1.2549 & 0.8444 & 14.9533 & 1.0797 \\
\hline 61.9643 & & 1.2584 & 0.8351 & 16.0072 & 1.0816 \\
\hline 59.4811 & & 1.2605 & 0.8246 & 16.9529 & 1.0832 \\
\hline 56.8929 & & 1.2617 & 0.8119 & 17.9529 & 1.0849 \\
\hline 54.1208 & & 1.2620 & 0.7962 & 19.0400 & 1.0867 \\
\hline 51.7336 & & 1.2614 & 0.7813 & 19.9894 & 1.0881 \\
\hline 49.2043 & & 1.2598 & 0.7637 & 21.0082 & 1.0896 \\
\hline 46.7987 & & 1.2572 & 0.7451 & 21.9893 & 1.0910 \\
\hline 45.1851 & & 1.2548 & 0.7314 & 22.6539 & 1.0920 \\
\hline ZONE T=" & $40 \%$ & of Design & Corrected & Speed $=1504$ & RPM", $I=25.0$ \\
\hline 80.0137 & & 1.0661 & 0.5805 & 3.4576 & 1.0319 \\
\hline 78.4253 & & 1.0725 & 0.6130 & 4.0348 & 1.0331 \\
\hline 77.2548 & & 1.0772 & 0.6359 & 4.4657 & 1.0339 \\
\hline 75.7213 & & 1.0835 & 0.6644 & 5.0372 & 1.0350 \\
\hline 74.5911 & & 1.0880 & 0.6842 & 5.4636 & 1.0358 \\
\hline 73.1105 & & 1.0940 & 0.7088 & 6.0289 & 1.0368 \\
\hline 72.0121 & & 1.0984 & 0.7258 & 6.4532 & 1.0376 \\
\hline 70.5897 & & 1.1040 & 0.7463 & 7.0089 & 1.0385 \\
\hline 68.1558 & & 1.1132 & 0.7772 & 7.9764 & 1.0402 \\
\hline 65.7516 & & 1.1218 & 0.8024 & 8.9529 & 1.0417 \\
\hline 63.2192 & & 1.1301 & 0.8230 & 10.0038 & 1.0434 \\
\hline 60.9785 & & 1.1367 & 0.8362 & 10.9531 & 1.0447 \\
\hline 58.6402 & & 1.1428 & 0.8452 & 11.9632 & 1.0462 \\
\hline 56.3352 & & 1.1481 & 0.8493 & 12.9783 & 1.0476 \\
\hline 54.1208 & & 1.1523 & 0.8493 & 13.9717 & 1.0488 \\
\hline 51.9714 & & 1.1556 & 0.8457 & 14.9530 & 1.0501 \\
\hline 49.7001 & & 1.1584 & 0.8386 & 16.0081 & 1.0513 \\
\hline 47.5078 & & 1.1603 & 0.8288 & 17.0439 & 1.0526 \\
\hline
\end{tabular}




$\begin{array}{lllll}45.6130 & 1.1614 & 0.8183 & 17.9529 & 1.0536 \\ 43.4090 & 1.1620 & 0.8038 & 19.0260 & 1.0547 \\ 41.4943 & 1.1620 & 0.7894 & 19.9719 & 1.0557 \\ 39.4656 & 1.1614 & 0.7723 & 20.9874 & 1.0567 \\ 37.5361 & 1.1601 & 0.7540 & 21.9660 & 1.0577 \\ 35.5225 & 1.1580 & 0.7323 & 23.0000 & 1.0587 \\ 33.6169 & 1.1550 & 0.7085 & 23.9903 & 1.0595\end{array}$





\section{Appendix E: Fan-Core and LPC Performance along Engine Operating Line}

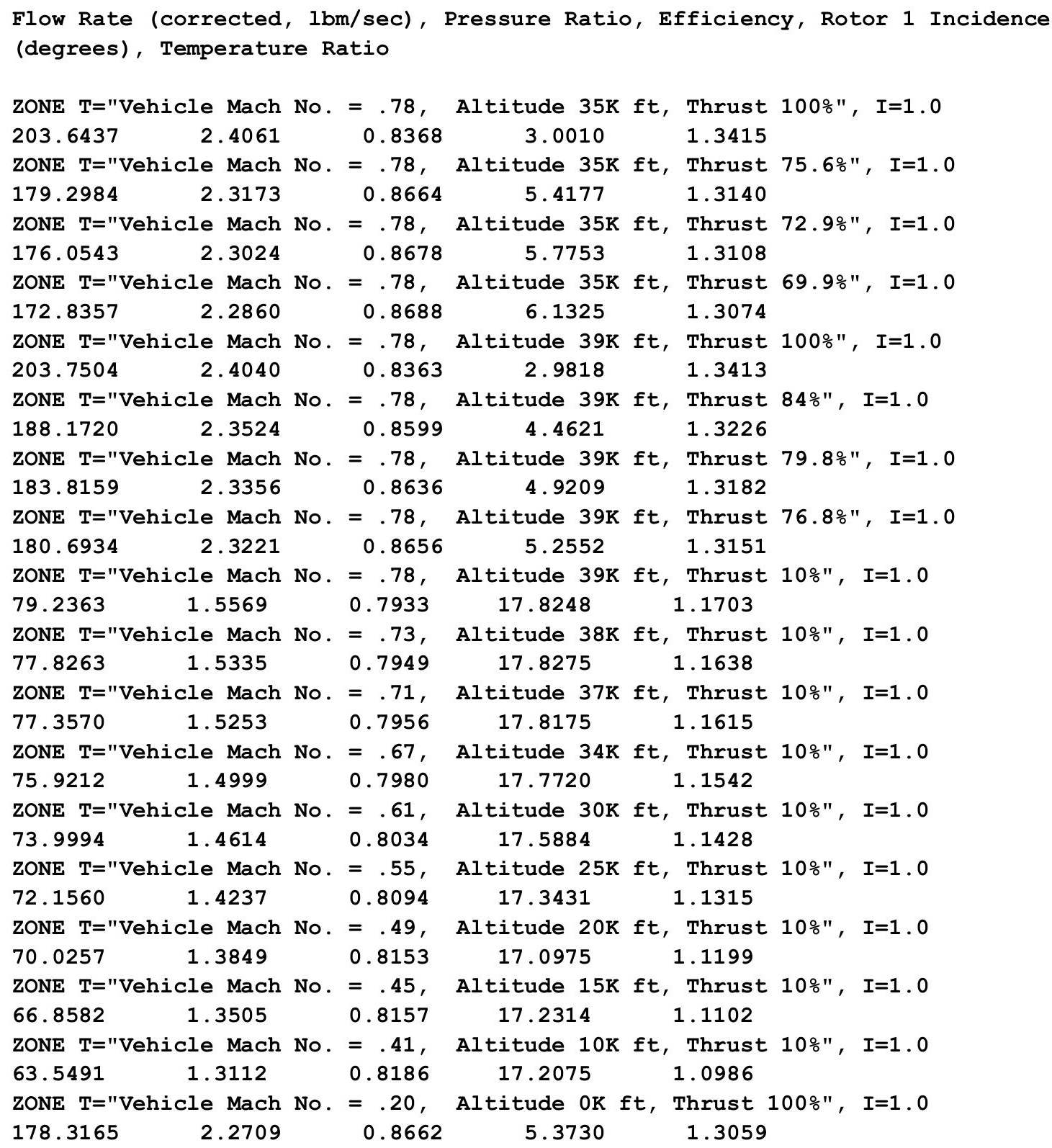





\section{Appendix F: Ice Particle Temperature and Static Air Temperature from Inlet through LPC}

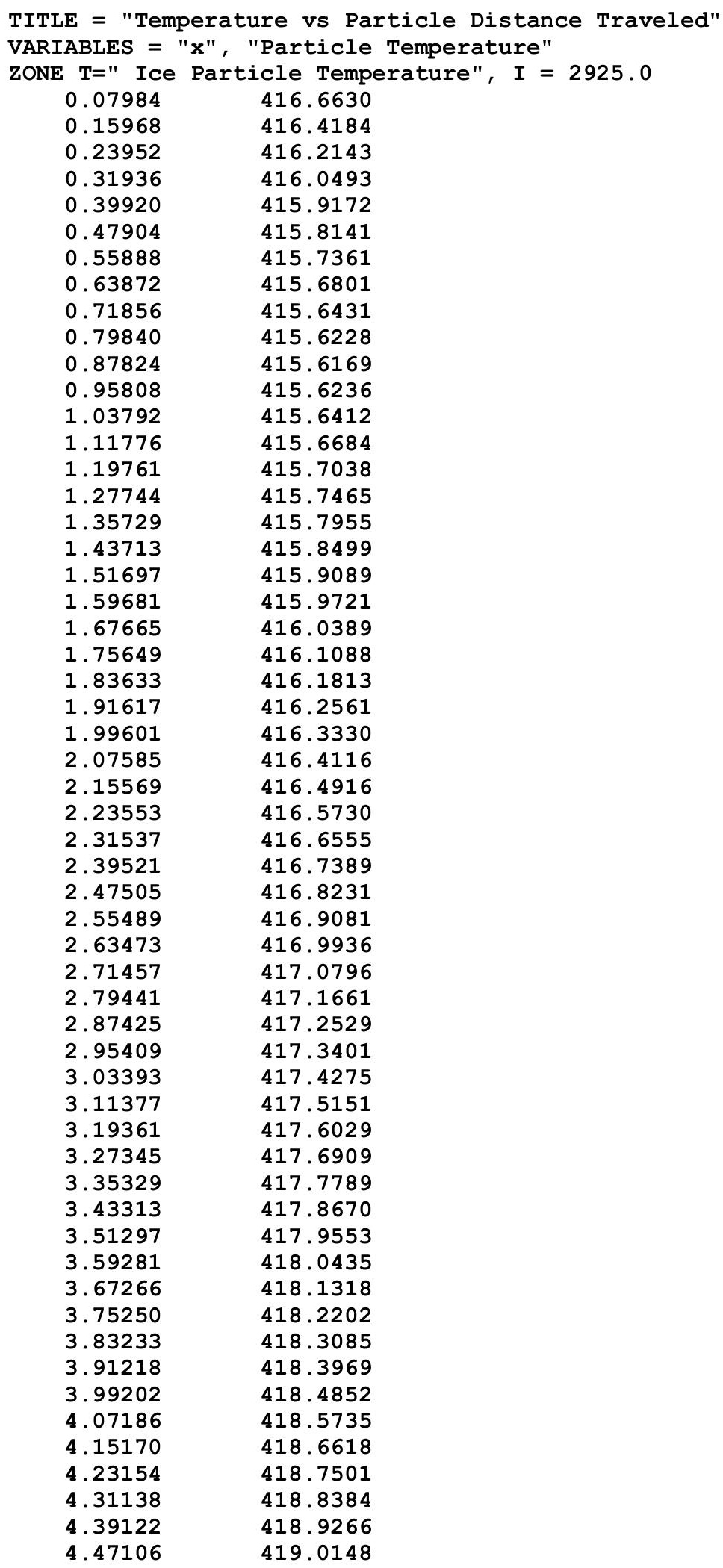




$\begin{array}{ll}4.55090 & 419.1029 \\ 4.63074 & 419.1910 \\ 4.71058 & 419.2791 \\ 4.79042 & 419.3671 \\ 4.87026 & 419.4551 \\ 4.95010 & 419.5431 \\ 5.02994 & 419.6310 \\ 5.10978 & 419.7188 \\ 5.18962 & 419.8066 \\ 5.26946 & 419.8943 \\ 5.34930 & 419.9820 \\ 5.42914 & 420.0696 \\ 5.50898 & 420.1571 \\ 5.58882 & 420.2447 \\ 5.66866 & 420.3321 \\ 5.74850 & 420.4196 \\ 5.82834 & 420.5069 \\ 5.90818 & 420.5942 \\ 5.98802 & 420.6815 \\ 6.06786 & 420.7687 \\ 6.14771 & 420.8558 \\ 6.22754 & 420.9429 \\ 6.30738 & 421.0300 \\ 6.38723 & 421.1170 \\ 6.46707 & 421.2039 \\ 6.54691 & 421.2908 \\ 6.62675 & 421.3777 \\ 6.70659 & 421.4644 \\ 6.78643 & 421.5512 \\ 6.86627 & 421.6379 \\ 6.94611 & 421.7246 \\ 7.02595 & 421.8112 \\ 7.10579 & 421.8978 \\ 7.18563 & 421.9843 \\ 7.26547 & 422.0707 \\ 7.34531 & 422.1572 \\ 7.42515 & 422.2436 \\ 7.50499 & 422.3299 \\ 7.58483 & 422.4162 \\ 7.66467 & 422.5024 \\ 7.74451 & 422.5886 \\ 7.82435 & 422.6747 \\ 7.90419 & 422.7608 \\ 7.98403 & 422.8469 \\ 8.06387 & 422.9329 \\ 8.14371 & 423.0189 \\ 8.22355 & 423.1048 \\ 8.30339 & 423.1907 \\ 8.38323 & 423.2766 \\ 8.46307 & 423.3623 \\ 8.54291 & 423.4481 \\ 8.62275 & 423.5338 \\ 8.70259 & 423.6195 \\ 8.78243 & 423.7051 \\ 8.86228 & 423.7907 \\ 8.94212 & 423.8763 \\ 9.02195 & 423.9618 \\ 9.10180 & 424.0472 \\ 9.18164 & 424.1327 \\ 9.26148 & 424.2181 \\ 9.34132 & 424.3034 \\ 9.42116 & 424.3887 \\ 9.50100 & 424.4740\end{array}$




$\begin{array}{rl}9.58084 & 424.5593 \\ 9.66068 & 424.6444 \\ 9.74052 & 424.7296 \\ 9.82036 & 424.8147 \\ 9.90020 & 424.8998 \\ 9.98004 & 424.9849 \\ 10.05988 & 425.0699 \\ 10.13972 & 425.1548 \\ 10.21956 & 425.2398 \\ 10.29940 & 425.3247 \\ 10.37924 & 425.4096 \\ 10.45909 & 425.4944 \\ 10.53893 & 425.5792 \\ 10.61877 & 425.6639 \\ 10.69861 & 425.7487 \\ 10.77845 & 425.8334 \\ 10.85829 & 425.9180 \\ 10.93813 & 426.0027 \\ 11.01797 & 426.0872 \\ 11.09781 & 426.1718 \\ 11.17765 & 426.2563 \\ 11.25749 & 426.3408 \\ 11.33734 & 426.4252 \\ 11.41718 & 426.5096 \\ 11.49702 & 426.5940 \\ 11.57686 & 426.6784 \\ 11.65670 & 426.7627 \\ 11.73654 & 426.8470 \\ 11.81638 & 426.9312 \\ 11.89622 & 427.0154 \\ 11.97606 & 427.0996 \\ 12.05590 & 427.1838 \\ 12.13575 & 427.2679 \\ 12.21559 & 427.3520 \\ 12.29543 & 427.4361 \\ 12.37527 & 427.5201 \\ 12.45511 & 427.6041 \\ 12.53495 & 427.6881 \\ 12.61479 & 427.7721 \\ 12.69463 & 427.8560 \\ 12.77447 & 427.9398 \\ 12.85431 & 428.0237 \\ 12.93415 & 428.1076 \\ 13.01400 & 428.1913 \\ 13.09384 & 428.2751 \\ 13.17368 & 428.3589 \\ 13.25352 & 428.4427 \\ 13.33336 & 428.5263 \\ 13.41320 & 428.6100 \\ 13.49304 & 428.6937 \\ 13.57288 & 428.7773 \\ 13.65272 & 428.8609 \\ 13.73256 & 428.9445 \\ 13.81240 & 429.0280 \\ 13.89224 & 429.1115 \\ 13.97209 & 429.1950 \\ 14.05193 & 429.2784 \\ 14.13177 & 429.3619 \\ 14.21161 & 429.4453 \\ 14.29145 & 429.5287 \\ 14.37129 & 429.6120 \\ 14.45113 & 429.6954 \\ 14.53097 & 429.7787\end{array}$




$\begin{array}{ll}14.61081 & 429.8619 \\ 14.69065 & 429.9452 \\ 14.77049 & 430.0284 \\ 14.85034 & 430.1116 \\ 14.93018 & 430.1948 \\ 15.01002 & 430.2779 \\ 15.08986 & 430.3611 \\ 15.16970 & 430.4442 \\ 15.24954 & 430.5273 \\ 15.32938 & 430.6103 \\ 15.40922 & 430.6933 \\ 15.48906 & 430.7764 \\ 15.56890 & 430.8594 \\ 15.64874 & 430.9423 \\ 15.72859 & 431.0253 \\ 15.80843 & 431.1082 \\ 15.88827 & 431.1911 \\ 15.96811 & 431.2740 \\ 16.04795 & 431.3569 \\ 16.12779 & 431.4397 \\ 16.20763 & 431.5225 \\ 16.28747 & 431.6053 \\ 16.36731 & 431.6881 \\ 16.44715 & 431.7709 \\ 16.52699 & 431.8536 \\ 16.60683 & 431.9363 \\ 16.68668 & 432.0190 \\ 16.76652 & 432.1017 \\ 16.84636 & 432.1844 \\ 16.92620 & 432.2670 \\ 17.00604 & 432.3497 \\ 17.08588 & 432.4323 \\ 17.16572 & 432.5148 \\ 17.24556 & 432.5974 \\ 17.32540 & 432.6799 \\ 17.40524 & 432.7625 \\ 17.48508 & 432.8450 \\ 17.56493 & 432.9275 \\ 17.64477 & 433.0100 \\ 17.72461 & 433.0924 \\ 17.80445 & 433.1749 \\ 17.88429 & 433.2573 \\ 17.96413 & 433.3397 \\ 18.04397 & 433.4221 \\ 18.12381 & 433.5045 \\ 18.20365 & 433.5868 \\ 18.28349 & 433.6692 \\ 18.36333 & 433.7515 \\ 18.44318 & 433.8338 \\ 18.52302 & 433.9161 \\ 18.60286 & 433.9983 \\ 18.68270 & 434.0806 \\ 18.76254 & 434.1628 \\ 18.84238 & 434.2451 \\ 18.92222 & 434.3272 \\ 19.00206 & 434.4095 \\ 19.08190 & 434.4916 \\ 19.16174 & 434.5738 \\ 19.24158 & 434.6559 \\ 19.32143 & 434.7381 \\ 19.40127 & 434.8202 \\ 19.48111 & 434.9023 \\ 19.56095 & 434.9844\end{array}$




$\begin{array}{ll}19.64079 & 435.0665 \\ 19.72063 & 435.1486 \\ 19.80047 & 435.2306 \\ 19.88031 & 435.3127 \\ 19.96015 & 435.3947 \\ 20.03999 & 435.4767 \\ 20.11983 & 435.5587 \\ 20.19967 & 435.6407 \\ 20.27951 & 435.7227 \\ 20.35935 & 435.8046 \\ 20.43919 & 435.8866 \\ 20.51903 & 435.9685 \\ 20.59887 & 436.0504 \\ 20.67871 & 436.1323 \\ 20.75855 & 436.2142 \\ 20.83839 & 436.2961 \\ 20.91823 & 436.3780 \\ 20.99807 & 436.4599 \\ 21.07791 & 436.5417 \\ 21.15775 & 436.6236 \\ 21.23759 & 436.7054 \\ 21.31742 & 436.7872 \\ 21.39726 & 436.8690 \\ 21.47710 & 436.9508 \\ 21.55694 & 437.0326 \\ 21.63678 & 437.1144 \\ 21.71662 & 437.1962 \\ 21.79646 & 437.2780 \\ 21.87630 & 437.3597 \\ 21.95614 & 437.4415 \\ 22.03598 & 437.5232 \\ 22.11582 & 437.6049 \\ 22.19566 & 437.6866 \\ 22.27550 & 437.7684 \\ 22.35534 & 437.8500 \\ 22.43518 & 437.9317 \\ 22.51502 & 438.0134 \\ 22.59486 & 438.0951 \\ 22.67470 & 438.1768 \\ 22.75454 & 438.2584 \\ 22.83438 & 438.3401 \\ 22.91422 & 438.4217 \\ 22.99406 & 438.5034 \\ 23.07390 & 438.5850 \\ 23.15374 & 438.6666 \\ 23.23358 & 438.7482 \\ 23.31342 & 438.8298 \\ 23.39326 & 438.9114 \\ 23.47310 & 438.9930 \\ 23.55294 & 439.0746 \\ 23.63278 & 439.1562 \\ 23.71262 & 439.2378 \\ 23.79246 & 439.3193 \\ 23.87230 & 439.4009 \\ 23.95214 & 439.4824 \\ 24.03197 & 439.5640 \\ 24.11181 & 439.6455 \\ 24.19165 & 439.7271 \\ 24.35133 & 439.8086 \\ 24.43117 & 439.8901 \\ 24.51101 & 439.9716 \\ 24.59085 & 440.0532 \\ & 440.1347\end{array}$




$\begin{array}{ll}24.67069 & 440.2162 \\ 24.75053 & 440.2977 \\ 24.83037 & 440.3792 \\ 24.91021 & 440.4607 \\ 24.99005 & 440.5422 \\ 25.06989 & 440.6237 \\ 25.14973 & 440.7052 \\ 25.22957 & 440.7867 \\ 25.30941 & 440.8681 \\ 25.38925 & 440.9496 \\ 25.46909 & 441.0311 \\ 25.54893 & 441.1125 \\ 25.62877 & 441.1940 \\ 25.70861 & 441.2755 \\ 25.78845 & 441.3569 \\ 25.86829 & 441.4384 \\ 25.94813 & 441.5199 \\ 26.02797 & 441.6013 \\ 26.10781 & 441.6828 \\ 26.18765 & 441.7643 \\ 26.26749 & 441.8457 \\ 26.34733 & 441.9272 \\ 26.42717 & 442.0086 \\ 26.50701 & 442.0900 \\ 26.58685 & 442.1715 \\ 26.66669 & 442.2529 \\ 26.74653 & 442.3344 \\ 26.82637 & 442.4158 \\ 26.90621 & 442.4973 \\ 26.98605 & 442.5787 \\ 27.06589 & 442.6602 \\ 27.14573 & 442.7416 \\ 27.22556 & 442.8231 \\ 27.30540 & 442.9045 \\ 27.38524 & 442.9860 \\ 27.46508 & 443.0674 \\ 27.54492 & 443.1489 \\ 27.62476 & 443.2304 \\ 27.70460 & 443.3119 \\ 27.78444 & 443.3933 \\ 27.86428 & 443.4748 \\ 27.94412 & 443.5563 \\ 28.02396 & 443.6378 \\ 28.10380 & 443.7192 \\ 28.18364 & 443.8007 \\ 28.26348 & 443.8822 \\ 28.34332 & 443.9637 \\ 28.42316 & 444.0452 \\ 28.50300 & 444.1267 \\ 28.58284 & 444.2082 \\ 28.66268 & 444.2896 \\ 28.74252 & 444.3712 \\ 28.82236 & 444.4526 \\ 28.90220 & 444.5341 \\ 28.98204 & 444.6157 \\ 29.06188 & 444.6972 \\ 29.14172 & 444.7787 \\ 29.22156 & 444.8603 \\ 29.30140 & 444.9418 \\ 29.38124 & 445.0233 \\ 29.46108 & 445.1049 \\ 29.54092 & 445.1864 \\ 29.62076 & 445.2680\end{array}$




$\begin{array}{ll}29.70060 & 445.3495 \\ 29.78044 & 445.4311 \\ 29.86028 & 445.5127 \\ 29.94011 & 445.5943 \\ 30.01995 & 445.6759 \\ 30.09979 & 445.7575 \\ 30.17963 & 445.8391 \\ 30.25947 & 445.9207 \\ 30.33931 & 446.0023 \\ 30.41915 & 446.0840 \\ 30.49899 & 446.1656 \\ 30.57883 & 446.2473 \\ 30.65867 & 446.3289 \\ 30.73851 & 446.4106 \\ 30.81835 & 446.4923 \\ 30.89819 & 446.5740 \\ 30.97803 & 446.6557 \\ 31.05787 & 446.7374 \\ 31.13771 & 446.8191 \\ 31.21755 & 446.9008 \\ 31.29739 & 446.9826 \\ 31.37723 & 447.0643 \\ 31.45707 & 447.1461 \\ 31.53691 & 447.2278 \\ 31.61675 & 447.3096 \\ 31.69659 & 447.3914 \\ 31.77643 & 447.4732 \\ 31.85627 & 447.5551 \\ 31.93611 & 447.6369 \\ 32.01595 & 447.7188 \\ 32.09579 & 447.8006 \\ 32.17563 & 447.8825 \\ 32.25547 & 447.9644 \\ 32.33531 & 448.0463 \\ 32.41515 & 448.1282 \\ 32.49499 & 448.2101 \\ 32.57483 & 448.2921 \\ 32.65467 & 448.3741 \\ 32.73450 & 448.4560 \\ 32.81435 & 448.5380 \\ 32.89418 & 448.6200 \\ 32.97403 & 448.7020 \\ 33.05386 & 448.7841 \\ 33.13371 & 448.8661 \\ 33.21354 & 448.9482 \\ 33.29338 & 449.0303 \\ 33.37322 & 449.1124 \\ 33.45306 & 449.1945 \\ 33.53290 & 449.2766 \\ 33.61274 & 449.3588 \\ 33.69258 & 449.4410 \\ 33.77242 & 449.5232 \\ 33.85226 & 449.6054 \\ 33.93210 & 449.6877 \\ 34.01194 & 449.7699 \\ 34.09178 & 449.8522 \\ 34.17162 & 449.9345 \\ 34.25146 & 450.0168 \\ 34.33130 & 450.0992 \\ 34.41114 & 450.1815 \\ 34.49098 & 450.2639 \\ 34.57082 & 450.3463 \\ 34.65066 & 450.4287\end{array}$




$\begin{array}{ll}34.73050 & 450.5112 \\ 34.81034 & 450.5936 \\ 34.89018 & 450.6761 \\ 34.97002 & 450.7586 \\ 35.04986 & 450.8411 \\ 35.12970 & 450.9236 \\ 35.20954 & 451.0062 \\ 35.28938 & 451.0888 \\ 35.36922 & 451.1714 \\ 35.44905 & 451.2541 \\ 35.52890 & 451.3368 \\ 35.60873 & 451.4195 \\ 35.68858 & 451.5022 \\ 35.76841 & 451.5850 \\ 35.84826 & 451.6678 \\ 35.92809 & 451.7506 \\ 36.00793 & 451.8334 \\ 36.08777 & 451.9163 \\ 36.16761 & 451.9992 \\ 36.24745 & 452.0821 \\ 36.32729 & 452.1651 \\ 36.40713 & 452.2481 \\ 36.48697 & 452.3311 \\ 36.56681 & 452.4142 \\ 36.64665 & 452.4973 \\ 36.72649 & 452.5803 \\ 36.80633 & 452.6635 \\ 36.88617 & 452.7466 \\ 36.96601 & 452.8299 \\ 37.04585 & 452.9131 \\ 37.12569 & 452.9964 \\ 37.20553 & 453.0797 \\ 37.28537 & 453.1630 \\ 37.36521 & 453.2464 \\ 37.44505 & 453.3298 \\ 37.52489 & 453.4133 \\ 37.60473 & 453.4967 \\ 37.68457 & 453.5803 \\ 37.76441 & 453.6638 \\ 37.84425 & 453.7475 \\ 37.92409 & 453.8311 \\ 38.00393 & 453.9148 \\ 38.08377 & 453.9985 \\ 38.16360 & 454.0822 \\ 38.24345 & 454.1660 \\ 38.32328 & 454.2498 \\ 38.40313 & 454.3337 \\ 38.48296 & 454.4177 \\ 38.56281 & 454.5016 \\ 38.64265 & 454.5856 \\ 38.72248 & 454.6696 \\ 38.80233 & 454.7538 \\ 38.88216 & 454.8379 \\ 38.96201 & 454.9221 \\ 39.04184 & 455.0063 \\ 39.12169 & 455.0906 \\ 39.20152 & 455.1749 \\ 39.28136 & 455.2592 \\ 39.36120 & 455.3437 \\ 39.44104 & 455.4281 \\ 39.52089 & 455.5126 \\ 39.60073 & 455.5972 \\ 39.68057 & 455.6818\end{array}$




$\begin{array}{ll}39.76041 & 455.7665 \\ 39.84026 & 455.8512 \\ 39.92010 & 455.9360 \\ 39.99994 & 456.0208 \\ 40.07360 & 456.0736 \\ 40.14721 & 456.0736 \\ 40.22081 & 456.1931 \\ 40.29442 & 456.2607 \\ 40.36802 & 456.3600 \\ 40.44163 & 456.4342 \\ 40.51523 & 456.5246 \\ 40.58884 & 456.6021 \\ 40.66244 & 456.6880 \\ 40.73605 & 456.7673 \\ 40.80965 & 456.8508 \\ 40.88326 & 456.9308 \\ 40.95686 & 457.0129 \\ 41.03046 & 457.0931 \\ 41.10407 & 457.1742 \\ 41.17767 & 457.2543 \\ 41.25128 & 457.3348 \\ 41.32489 & 457.4146 \\ 41.39849 & 457.4945 \\ 41.47210 & 457.5741 \\ 41.54570 & 457.6535 \\ 41.61930 & 457.7327 \\ 41.69291 & 457.8118 \\ 41.76651 & 457.8907 \\ 41.84012 & 457.9694 \\ 41.91372 & 458.0479 \\ 41.98733 & 458.1263 \\ 42.06093 & 458.2044 \\ 42.13454 & 458.2825 \\ 42.20814 & 458.3604 \\ 42.28175 & 458.4381 \\ 42.35535 & 458.5157 \\ 42.42896 & 458.5931 \\ 42.50256 & 458.6704 \\ 42.57616 & 458.7476 \\ 42.64977 & 458.8246 \\ 42.72337 & 458.9015 \\ 42.79698 & 458.9782 \\ 42.87058 & 459.0548 \\ 42.94419 & 459.1313 \\ 43.01779 & 459.2077 \\ 43.09140 & 459.2839 \\ 43.16500 & 459.3600 \\ 43.23861 & 459.4360 \\ 43.31221 & 459.5118 \\ 43.38581 & 459.5876 \\ 43.45942 & 459.6632 \\ 43.53302 & 459.7388 \\ 43.60663 & 459.8142 \\ 43.68024 & 459.8895 \\ 43.75384 & 459.9647 \\ 43.82745 & 460.0398 \\ 43.90105 & 460.1148 \\ 43.97466 & 460.1897 \\ 44.04826 & 460.2645 \\ 44.12186 & 460.3391 \\ 44.19547 & 460.4137 \\ 44.26907 & 460.4882 \\ 44.34268 & 460.5626\end{array}$




\begin{tabular}{|c|c|}
\hline 44.41628 & 460.6370 \\
\hline 44.48989 & 460.7112 \\
\hline 44.56349 & 460.7853 \\
\hline 44.63710 & 460.8593 \\
\hline 44.71070 & 460.9333 \\
\hline 44.78431 & 461.0071 \\
\hline 44.85791 & 461.0809 \\
\hline 44.93151 & 461.1546 \\
\hline 45.00512 & 461.2282 \\
\hline 45.07872 & 461.3017 \\
\hline 45.15233 & 461.3752 \\
\hline 45.22593 & 461.4485 \\
\hline 45.29954 & 461.5218 \\
\hline 45.37314 & 461.5950 \\
\hline 45.44675 & 461.6681 \\
\hline 45.52035 & 461.7412 \\
\hline 45.59396 & 461.8141 \\
\hline 45.66756 & 461.8870 \\
\hline 45.74117 & 461.9598 \\
\hline 45.81477 & 462.0326 \\
\hline 45.88837 & 462.1052 \\
\hline 45.96198 & 462.1779 \\
\hline 46.03559 & 462.2504 \\
\hline 46.10919 & 462.3229 \\
\hline 46.18280 & 462.3953 \\
\hline 46.25640 & 462.4676 \\
\hline 46.33001 & 462.5398 \\
\hline 46.40361 & 462.6120 \\
\hline 46.47721 & 462.6841 \\
\hline 46.55082 & 462.7562 \\
\hline 46.62442 & 462.8282 \\
\hline 46.69803 & 462.9001 \\
\hline 46.77163 & 462.9719 \\
\hline 46.84524 & 463.0437 \\
\hline 46.91884 & 463.1155 \\
\hline 46.99245 & 463.1871 \\
\hline 47.06605 & 463.2587 \\
\hline 47.13966 & 463.3303 \\
\hline 47.21326 & 463.4017 \\
\hline 47.28687 & 463.4731 \\
\hline 47.36047 & 463.5445 \\
\hline 47.43407 & 463.6158 \\
\hline 47.45212 & 465.2026 \\
\hline 47.47006 & 465.0741 \\
\hline 47.48800 & 464.9553 \\
\hline 47.50594 & 464.8462 \\
\hline 47.52387 & 464.7455 \\
\hline 47.54181 & 464.6525 \\
\hline 47.55975 & 464.5662 \\
\hline 47.57769 & 464.4858 \\
\hline 47.59562 & 464.4109 \\
\hline 47.61356 & 464.3408 \\
\hline 47.63150 & 464.2749 \\
\hline 47.64944 & 464.2129 \\
\hline 47.66737 & 464.1543 \\
\hline 47.68531 & 464.0987 \\
\hline 47.70325 & 464.0458 \\
\hline 47.72119 & 463.9954 \\
\hline 47.73912 & 463.9471 \\
\hline 47.75706 & 463.9007 \\
\hline 47.77500 & 463.8561 \\
\hline 47.79294 & 463.8130 \\
\hline 47.81087 & 463.7714 \\
\hline
\end{tabular}




\begin{tabular}{|c|c|}
\hline 47.82881 & 463.7309 \\
\hline 47.84675 & 463.6915 \\
\hline 47.86469 & 463.6531 \\
\hline 47.88263 & 463.6156 \\
\hline 47.90056 & 463.5789 \\
\hline 47.91850 & 463.5428 \\
\hline 47.93644 & 463.5075 \\
\hline 47.95438 & 463.4726 \\
\hline 47.97231 & 463.4383 \\
\hline 47.99025 & 463.4044 \\
\hline 48.00819 & 463.3709 \\
\hline 48.02613 & 463.3377 \\
\hline 48.04406 & 463.3049 \\
\hline 48.06200 & 463.2722 \\
\hline 48.07994 & 463.2399 \\
\hline 48.09788 & 463.2078 \\
\hline 48.11581 & 463.1758 \\
\hline 48.13375 & 463.1440 \\
\hline 48.15169 & 463.1123 \\
\hline 48.16963 & 463.0808 \\
\hline 48.18757 & 463.0494 \\
\hline 48.20551 & 463.0180 \\
\hline 48.22344 & 462.9868 \\
\hline 48.24138 & 462.9556 \\
\hline 48.25932 & 462.9244 \\
\hline 48.27726 & 462.8933 \\
\hline 48.29519 & 462.8623 \\
\hline 48.31313 & 462.8313 \\
\hline 48.33107 & 462.8003 \\
\hline 48.34901 & 462.7693 \\
\hline 48.36694 & 462.7383 \\
\hline 48.38488 & 462.7073 \\
\hline 48.40282 & 462.6764 \\
\hline 48.42076 & 462.6454 \\
\hline 48.43869 & 462.6144 \\
\hline 48.45663 & 462.5834 \\
\hline 48.47457 & 462.5524 \\
\hline 48.49251 & 462.5213 \\
\hline 48.51044 & 462.4903 \\
\hline 48.52839 & 462.4592 \\
\hline 48.54632 & 462.4281 \\
\hline 48.56426 & 462.3970 \\
\hline 48.58220 & 462.3658 \\
\hline 48.60014 & 462.3346 \\
\hline 48.61807 & 462.3034 \\
\hline 48.63601 & 462.2722 \\
\hline 48.65395 & 462.2409 \\
\hline 48.67189 & 462.2096 \\
\hline 48.68982 & 462.1783 \\
\hline 48.70776 & 462.1469 \\
\hline 48.72570 & 462.1155 \\
\hline 48.74364 & 462.0840 \\
\hline 48.76157 & 462.0526 \\
\hline 48.77951 & 462.0210 \\
\hline 48.79745 & 461.9895 \\
\hline 48.81539 & 461.9579 \\
\hline 48.83332 & 461.9263 \\
\hline 48.85126 & 461.8946 \\
\hline 48.86920 & 461.8629 \\
\hline 48.88714 & 461.8311 \\
\hline 48.90508 & 461.7993 \\
\hline 48.92301 & 461.7675 \\
\hline 48.94095 & 461.7357 \\
\hline
\end{tabular}




$\begin{array}{ll}48.95889 & 461.7037 \\ 48.97683 & 461.6718 \\ 48.99476 & 461.6398 \\ 49.01270 & 461.6078 \\ 49.03064 & 461.5757 \\ 49.04858 & 461.5437 \\ 49.06651 & 461.5115 \\ 49.08445 & 461.4793 \\ 49.10239 & 461.4471 \\ 49.12033 & 461.4149 \\ 49.13827 & 461.3825 \\ 49.15620 & 461.3502 \\ 49.17414 & 461.3178 \\ 49.19208 & 461.2854 \\ 49.21002 & 461.2529 \\ 49.22795 & 461.2204 \\ 49.24589 & 461.1879 \\ 49.25944 & 461.1688 \\ 49.27304 & 461.1559 \\ 49.28664 & 461.1484 \\ 49.30024 & 461.1459 \\ 49.31385 & 461.1482 \\ 49.32745 & 461.1550 \\ 49.34105 & 461.1659 \\ 49.35465 & 461.1808 \\ 49.36825 & 461.1994 \\ 49.38185 & 461.2215 \\ 49.39545 & 461.2470 \\ 49.40905 & 461.2755 \\ 49.42265 & 461.3070 \\ 49.43626 & 461.3412 \\ 49.44986 & 461.3781 \\ 49.46346 & 461.4173 \\ 49.47706 & 461.4588 \\ 49.49066 & 461.5025 \\ 49.50426 & 461.5482 \\ 49.51786 & 461.5959 \\ 49.53146 & 461.6452 \\ 49.54507 & 461.6963 \\ 49.55867 & 461.7489 \\ 49.57227 & 461.8030 \\ 49.58587 & 461.8585 \\ 49.59947 & 461.9153 \\ 49.61307 & 461.9733 \\ 49.62667 & 462.0324 \\ 49.64027 & 462.0927 \\ 49.65387 & 462.1539 \\ 49.66748 & 462.2161 \\ 49.68108 & 462.2791 \\ 49.69468 & 462.3430 \\ 49.70828 & 462.4077 \\ 49.72188 & 462.4731 \\ 49.73548 & 462.5391 \\ 49.74908 & 462.6058 \\ 49.76268 & 462.6731 \\ 49.77628 & 462.7409 \\ 49.78989 & 462.8092 \\ 49.80349 & 462.8781 \\ 49.81709 & 462.9473 \\ 49.83069 & 463.0170 \\ 49.84429 & 463.0871 \\ 49.85789 & 463.1576 \\ 49.87149 & 463.2284\end{array}$




$\begin{array}{ll}49.88509 & 463.2994 \\ 49.89869 & 463.3708 \\ 49.91230 & 463.4425 \\ 49.92590 & 463.5144 \\ 49.93950 & 463.5866 \\ 49.95310 & 463.6590 \\ 49.96670 & 463.7316 \\ 49.98030 & 463.8044 \\ 49.99390 & 463.8774 \\ 50.00750 & 463.9505 \\ 50.02110 & 464.0238 \\ 50.03471 & 464.0972 \\ 50.04831 & 464.1708 \\ 50.06191 & 464.2445 \\ 50.07551 & 464.3183 \\ 50.08911 & 464.3922 \\ 50.10271 & 464.4663 \\ 50.11631 & 464.5404 \\ 50.12991 & 464.6146 \\ 50.14351 & 464.6889 \\ 50.15712 & 464.7632 \\ 50.17072 & 464.8377 \\ 50.18432 & 464.9122 \\ 50.19792 & 464.9867 \\ 50.21152 & 465.0613 \\ 50.22512 & 465.1360 \\ 50.23872 & 465.2107 \\ 50.25232 & 465.2854 \\ 50.26593 & 465.3603 \\ 50.27953 & 465.4351 \\ 50.29313 & 465.5100 \\ 50.30673 & 465.5849 \\ 50.32033 & 465.6599 \\ 50.33393 & 465.7348 \\ 50.34753 & 465.8099 \\ 50.36113 & 465.8849 \\ 50.37473 & 465.9600 \\ 50.38834 & 466.0351 \\ 50.40194 & 466.1102 \\ 50.41554 & 466.1853 \\ 50.42914 & 466.2605 \\ 50.44274 & 466.3357 \\ 50.45634 & 466.4109 \\ 50.46994 & 466.4861 \\ 50.48354 & 466.5613 \\ 50.49714 & 466.6366 \\ 50.51075 & 466.7119 \\ 50.52435 & 466.7872 \\ 50.53795 & 466.8625 \\ 50.55155 & 466.9379 \\ 50.56515 & 467.0132 \\ 50.57875 & 467.0887 \\ 50.59235 & 467.1641 \\ 50.60595 & 467.2395 \\ 50.61955 & 467.3150 \\ 50.66056 & 467.4900 \\ 50.70137 & 467.6347 \\ 50.74218 & 467.7411 \\ 50.78298 & 467.8248 \\ 50.82379 & 467.8914 \\ 50.86460 & 467.9467 \\ 50.90541 & 467.9937 \\ 50.94622 & 468.0352\end{array}$




$\begin{array}{ll}50.98702 & 468.0727 \\ 51.02783 & 468.1075 \\ 51.06864 & 468.1404 \\ 51.10945 & 468.1719 \\ 51.15026 & 468.2026 \\ 51.19106 & 468.2326 \\ 51.23187 & 468.2622 \\ 51.27268 & 468.2914 \\ 51.31349 & 468.3205 \\ 51.35430 & 468.3493 \\ 51.39510 & 468.3780 \\ 51.43591 & 468.4067 \\ 51.47672 & 468.4353 \\ 51.51753 & 468.4638 \\ 51.55834 & 468.4923 \\ 51.59914 & 468.5208 \\ 51.63995 & 468.5491 \\ 51.68076 & 468.5775 \\ 51.72157 & 468.6059 \\ 51.76238 & 468.6342 \\ 51.80318 & 468.6625 \\ 51.84399 & 468.6907 \\ 51.88480 & 468.7189 \\ 51.92561 & 468.7471 \\ 51.96642 & 468.7753 \\ 52.00723 & 468.8035 \\ 52.04803 & 468.8316 \\ 52.08884 & 468.8597 \\ 52.12965 & 468.8878 \\ 52.17046 & 468.9159 \\ 52.21127 & 468.9439 \\ 52.25208 & 468.9719 \\ 52.29288 & 468.9998 \\ 52.33369 & 469.0278 \\ 52.37450 & 469.0558 \\ 52.41531 & 469.0837 \\ 52.45612 & 469.1116 \\ 52.49692 & 469.1394 \\ 52.53773 & 469.1673 \\ 52.57854 & 469.1951 \\ 52.61935 & 469.2229 \\ 52.66016 & 469.2507 \\ 52.70096 & 469.2784 \\ 52.74177 & 469.3062 \\ 52.78258 & 469.3338 \\ 52.82339 & 469.3615 \\ 52.86419 & 469.3892 \\ 52.90500 & 469.4168 \\ 52.94581 & 469.4445 \\ 52.98662 & 469.4720 \\ 53.02743 & 469.4996 \\ 53.06823 & 469.5272 \\ 53.10904 & 469.5547 \\ 53.14985 & 469.5822 \\ 53.19066 & 469.6096 \\ 53.23147 & 469.6371 \\ 53.27227 & 469.6646 \\ 53.31308 & 469.6920 \\ 53.35389 & 469.7194 \\ 53.39470 & 469.7468 \\ 53.43551 & 469.7741 \\ 53.47631 & 469.8015 \\ 53.51712 & 469.8288\end{array}$




$\begin{array}{ll}53.55793 & 469.8561 \\ 53.59874 & 469.8833 \\ 53.63955 & 469.9106 \\ 53.68036 & 469.9378 \\ 53.72116 & 469.9650 \\ 53.76197 & 469.9922 \\ 53.80278 & 470.0193 \\ 53.84359 & 470.0465 \\ 53.88440 & 470.0735 \\ 53.92521 & 470.1007 \\ 53.96601 & 470.1278 \\ 54.00682 & 470.1548 \\ 54.04763 & 470.1818 \\ 54.08844 & 470.2089 \\ 54.12925 & 470.2359 \\ 54.17005 & 470.2628 \\ 54.21086 & 470.2898 \\ 54.25167 & 470.3167 \\ 54.29248 & 470.3437 \\ 54.33329 & 470.3706 \\ 54.37409 & 470.3974 \\ 54.41490 & 470.4243 \\ 54.45571 & 470.4511 \\ 54.49652 & 470.4780 \\ 54.53733 & 470.5048 \\ 54.57813 & 470.5315 \\ 54.61894 & 470.5583 \\ 54.65975 & 470.5850 \\ 54.70056 & 470.6118 \\ 54.74137 & 470.6385 \\ 54.75445 & 470.6727 \\ 54.76741 & 470.6727 \\ 54.78036 & 471.0882 \\ 54.79332 & 471.4428 \\ 54.80628 & 471.7551 \\ 54.81924 & 472.0287 \\ 54.83220 & 472.2683 \\ 54.84516 & 472.4781 \\ 54.85812 & 472.6617 \\ 54.87107 & 472.8223 \\ 54.88403 & 472.9629 \\ 54.89699 & 473.0859 \\ 54.90995 & 473.1936 \\ 54.92290 & 473.2879 \\ 54.93586 & 473.3705 \\ 54.94882 & 473.4429 \\ 54.96178 & 473.5064 \\ 54.97474 & 473.5622 \\ 54.98770 & 473.6112 \\ 55.00066 & 473.6544 \\ 55.01361 & 473.6925 \\ 55.02657 & 473.7262 \\ 55.03953 & 473.7560 \\ 55.05249 & 473.7824 \\ 55.117434 & 473.8059 \\ 55.14320 & 473.8269 \\ 55.15615 & 473.8456 \\ 55 & 473.8626 \\ 55 & 473.8778 \\ 55 & 473.8916 \\ 55 & 473.9156 \\ 55 & 473.9261\end{array}$




$\begin{array}{ll}55.18207 & 473.9359 \\ 55.19503 & 473.9449 \\ 55.20798 & 473.9532 \\ 55.22094 & 473.9611 \\ 55.23390 & 473.9684 \\ 55.24686 & 473.9754 \\ 55.25982 & 473.9821 \\ 55.27278 & 473.9884 \\ 55.28574 & 473.9944 \\ 55.29869 & 474.0003 \\ 55.31165 & 474.0060 \\ 55.32461 & 474.0114 \\ 55.33757 & 474.0168 \\ 55.35052 & 474.0220 \\ 55.36348 & 474.0270 \\ 55.37644 & 474.0320 \\ 55.38940 & 474.0369 \\ 55.40236 & 474.0417 \\ 55.41532 & 474.0465 \\ 55.42827 & 474.0513 \\ 55.44123 & 474.0559 \\ 55.45419 & 474.0605 \\ 55.46715 & 474.0652 \\ 55.48011 & 474.0697 \\ 55.49306 & 474.0743 \\ 55.50602 & 474.0788 \\ 55.51898 & 474.0833 \\ 55.53194 & 474.0878 \\ 55.54490 & 474.0923 \\ 55.55785 & 474.0967 \\ 55.57081 & 474.1012 \\ 55.58377 & 474.1057 \\ 55.59673 & 474.1101 \\ 55.60969 & 474.1145 \\ 55.62265 & 474.1190 \\ 55.63560 & 474.1234 \\ 55.64856 & 474.1278 \\ 55.66152 & 474.1322 \\ 55.67448 & 474.1366 \\ 55.68744 & 474.1410 \\ 55.70039 & 474.1454 \\ 55.71335 & 474.1498 \\ 55.72631 & 474.1542 \\ 55.73927 & 474.1586 \\ 55.75223 & 474.1630 \\ 55.76519 & 474.1674 \\ 55.77814 & 474.1718 \\ 55.79110 & 474.1762 \\ 55.80406 & 474.1806 \\ 55.81702 & 474.1850 \\ 55.82998 & 474.1894 \\ 55.84293 & 474.1938 \\ 55.85589 & 474.1982 \\ 55.86885 & 474.2026 \\ 55.89181 & 474.2070 \\ 55.92068 & 474.2114 \\ 55.94660 & 474.2159 \\ 55.95956 & 474.2202 \\ 55.9854 & 474.2247 \\ 55 & 474.2291 \\ 55 & 474.2335 \\ 55 & 474.2424\end{array}$




$\begin{array}{ll}55.99843 & 474.2468 \\ 56.01139 & 474.2513 \\ 56.02435 & 474.2557 \\ 56.03731 & 474.2601 \\ 56.05027 & 474.2645 \\ 56.07415 & 474.2730 \\ 56.09792 & 474.2998 \\ 56.12170 & 474.3340 \\ 56.14547 & 474.3718 \\ 56.16925 & 474.4127 \\ 56.19302 & 474.4559 \\ 56.21680 & 474.5008 \\ 56.24058 & 474.5471 \\ 56.26435 & 474.5943 \\ 56.28813 & 474.6424 \\ 56.31190 & 474.6910 \\ 56.33568 & 474.7401 \\ 56.35946 & 474.7895 \\ 56.38324 & 474.8392 \\ 56.40701 & 474.8891 \\ 56.43079 & 474.9392 \\ 56.45456 & 474.9894 \\ 56.47834 & 475.0398 \\ 56.50211 & 475.0903 \\ 56.52589 & 475.1408 \\ 56.54967 & 475.1915 \\ 56.57344 & 475.2422 \\ 56.59722 & 475.2929 \\ 56.62099 & 475.3437 \\ 56.64477 & 475.3945 \\ 56.66855 & 475.4454 \\ 56.69233 & 475.4963 \\ 56.71610 & 475.5473 \\ 56.73988 & 475.5984 \\ 56.76365 & 475.6494 \\ 56.78743 & 475.7005 \\ 56.81121 & 475.7517 \\ 56.83498 & 475.8028 \\ 56.85876 & 475.8540 \\ 56.88253 & 475.9053 \\ 56.90631 & 475.9565 \\ 56.93008 & 476.0078 \\ 56.95386 & 476.0591 \\ 56.97764 & 476.1105 \\ 57.00142 & 476.1618 \\ 57.02519 & 476.2133 \\ 57.04897 & 476.2647 \\ 57.07274 & 476.3162 \\ 57.09652 & 476.3677 \\ 57.12030 & 476.4193 \\ 57.14407 & 476.4708 \\ 57.16785 & 476.5224 \\ 57.19162 & 476.5740 \\ 57.21540 & 476.6256 \\ 57.23918 & 476.6773 \\ 57.26295 & 476.7290 \\ 57.28673 & 476.7807 \\ 57.31050 & 476.8325 \\ 57.33428 & 476.8842 \\ 57.35806 & 476.9360 \\ 57.38183 & 476.9878 \\ 57.40561 & 477.0396 \\ 57.42939 & 477.0915\end{array}$




$\begin{array}{ll}57.45316 & 477.1433 \\ 57.47694 & 477.1952 \\ 57.50071 & 477.2471 \\ 57.52449 & 477.2990 \\ 57.54827 & 477.3509 \\ 57.57204 & 477.4029 \\ 57.59582 & 477.4548 \\ 57.61959 & 477.5068 \\ 57.64337 & 477.5589 \\ 57.66715 & 477.6109 \\ 57.69092 & 477.6629 \\ 57.71470 & 477.7150 \\ 57.73848 & 477.7671 \\ 57.76225 & 477.8191 \\ 57.78603 & 477.8712 \\ 57.80980 & 477.9233 \\ 57.83358 & 477.9755 \\ 57.85736 & 478.0276 \\ 57.88113 & 478.0798 \\ 57.90491 & 478.1320 \\ 57.92868 & 478.1841 \\ 57.95246 & 478.2363 \\ 57.97624 & 478.2886 \\ 58.00001 & 478.3408 \\ 58.02379 & 478.3931 \\ 58.04757 & 478.4453 \\ 58.07134 & 478.4975 \\ 58.09512 & 478.5498 \\ 58.11889 & 478.6021 \\ 58.14267 & 478.6544 \\ 58.16645 & 478.7067 \\ 58.19022 & 478.7590 \\ 58.21400 & 478.8113 \\ 58.23777 & 478.8636 \\ 58.26155 & 478.9160 \\ 58.28533 & 478.9684 \\ 58.30910 & 479.0207 \\ 58.33288 & 479.0731 \\ 58.35666 & 479.1255 \\ 58.38043 & 479.1779 \\ 58.40421 & 479.2303 \\ 58.42798 & 479.2827 \\ 58.45176 & 479.3351 \\ 58.45746 & 480.3622 \\ 58.46307 & 480.3237 \\ 58.46869 & 480.2871 \\ 58.47430 & 480.2523 \\ 58.47992 & 480.2193 \\ 58.48553 & 480.1878 \\ 58.49114 & 480.1580 \\ 58.49675 & 480.1297 \\ 58.50237 & 480.1027 \\ 58.50798 & 480.0771 \\ 58.51360 & 480.0528 \\ 58.51921 & 480.0296 \\ 58.52482 & 480.0077 \\ 58.53043 & 479.9867 \\ 58.53605 & 479.9669 \\ 58.54166 & 479.9480 \\ 58.54727 & 479.9300 \\ 58.55289 & 479.9129 \\ 58.55850 & 479.8966 \\ 58.56411 & 479.8812\end{array}$




$\begin{array}{ll}58.56973 & 479.8664 \\ 58.57534 & 479.8524 \\ 58.58095 & 479.8391 \\ 58.58656 & 479.8264 \\ 58.59218 & 479.8143 \\ 58.59779 & 479.8028 \\ 58.60340 & 479.7919 \\ 58.60902 & 479.7815 \\ 58.61463 & 479.7715 \\ 58.62024 & 479.7621 \\ 58.62586 & 479.7531 \\ 58.63147 & 479.7445 \\ 58.63708 & 479.7363 \\ 58.64270 & 479.7285 \\ 58.64831 & 479.7211 \\ 58.65392 & 479.7141 \\ 58.65953 & 479.7074 \\ 58.66515 & 479.7009 \\ 58.67076 & 479.6948 \\ 58.67638 & 479.6890 \\ 58.68199 & 479.6835 \\ 58.68760 & 479.6781 \\ 58.69321 & 479.6731 \\ 58.69883 & 479.6682 \\ 58.70444 & 479.6636 \\ 58.71006 & 479.6592 \\ 58.71567 & 479.6550 \\ 58.72128 & 479.6510 \\ 58.72689 & 479.6472 \\ 58.73251 & 479.6435 \\ 58.73812 & 479.6400 \\ 58.74373 & 479.6366 \\ 58.74935 & 479.6334 \\ 58.75496 & 479.6303 \\ 58.76057 & 479.6274 \\ 58.76619 & 479.6246 \\ 58.77180 & 479.6219 \\ 58.77741 & 479.6193 \\ 58.78302 & 479.6168 \\ 58.78864 & 479.6145 \\ 58.79425 & 479.6122 \\ 58.79987 & 479.6100 \\ 58.80548 & 479.6079 \\ 58.81109 & 479.6058 \\ 58.81670 & 479.6039 \\ 58.82232 & 479.6021 \\ 58.82793 & 479.6003 \\ 58.83355 & 479.5985 \\ 58.83916 & 479.5968 \\ 58.84477 & 479.5952 \\ 58.85038 & 479.5937 \\ 58.85600 & 479.5922 \\ 58.86161 & 479.5908 \\ 58.86722 & 479.5894 \\ 58.87284 & 479.5880 \\ 58.87845 & 479.5867 \\ 58.88406 & 479.5854 \\ 58.88968 & 479.5843 \\ 58.89529 & 479.5830 \\ 58.90090 & 479.5819 \\ 58.90652 & 479.5808 \\ 58.91213 & 479.5797 \\ 58.91774 & 479.5786\end{array}$




$\begin{array}{ll}58.92336 & 479.5776 \\ 58.92897 & 479.5766 \\ 58.93458 & 479.5756 \\ 58.94019 & 479.5747 \\ 58.94581 & 479.5738 \\ 58.95142 & 479.5729 \\ 58.95704 & 479.5720 \\ 58.96265 & 479.5712 \\ 58.96826 & 479.5703 \\ 58.97387 & 479.5695 \\ 58.97948 & 479.5688 \\ 58.98510 & 479.5680 \\ 58.99071 & 479.5672 \\ 58.99633 & 479.5665 \\ 59.00194 & 479.5657 \\ 59.00755 & 479.5650 \\ 59.01316 & 479.5643 \\ 59.01878 & 479.5636 \\ 59.02501 & 479.5627 \\ 59.03108 & 479.5645 \\ 59.03715 & 479.5675 \\ 59.04322 & 479.5716 \\ 59.04929 & 479.5768 \\ 59.05535 & 479.5830 \\ 59.06142 & 479.5902 \\ 59.06749 & 479.5983 \\ 59.07356 & 479.6073 \\ 59.07963 & 479.6172 \\ 59.08569 & 479.6278 \\ 59.09176 & 479.6393 \\ 59.09783 & 479.6514 \\ 59.10390 & 479.6642 \\ 59.10997 & 479.6776 \\ 59.11604 & 479.6917 \\ 59.12210 & 479.7065 \\ 59.12817 & 479.7217 \\ 59.13424 & 479.7376 \\ 59.14031 & 479.7539 \\ 59.14638 & 479.7707 \\ 59.15245 & 479.7879 \\ 59.15852 & 479.8056 \\ 59.16459 & 479.8238 \\ 59.17065 & 479.8423 \\ 59.17672 & 479.8612 \\ 59.18279 & 479.8804 \\ 59.18886 & 479.9001 \\ 59.19493 & 479.9200 \\ 59.20100 & 479.9403 \\ 59.20706 & 479.9608 \\ 59.21313 & 479.9816 \\ 59.21920 & 480.0027 \\ 59.22527 & 480.0240 \\ 59.23134 & 480.0456 \\ 59.23740 & 480.0674 \\ 59.24347 & 480.0894 \\ 59.2656168 & 480.1117 \\ 59.27382 & 480.1341 \\ 59.27988 & 480.1567 \\ 59.28595 & 480.1795 \\ 59202 & 480.2256 \\ 59 & 480.2489 \\ 590.2723\end{array}$




$\begin{array}{ll}59.29809 & 480.2958 \\ 59.30416 & 480.3195 \\ 59.31023 & 480.3432 \\ 59.31630 & 480.3671 \\ 59.32236 & 480.3911 \\ 59.32843 & 480.4153 \\ 59.33450 & 480.4395 \\ 59.34057 & 480.4638 \\ 59.34664 & 480.4882 \\ 59.35271 & 480.5127 \\ 59.35877 & 480.5373 \\ 59.36484 & 480.5619 \\ 59.37091 & 480.5866 \\ 59.37698 & 480.6114 \\ 59.38305 & 480.6363 \\ 59.38911 & 480.6613 \\ 59.39518 & 480.6862 \\ 59.40125 & 480.7113 \\ 59.40732 & 480.7364 \\ 59.41339 & 480.7615 \\ 59.41946 & 480.7868 \\ 59.42553 & 480.8120 \\ 59.43159 & 480.8372 \\ 59.43766 & 480.8626 \\ 59.44373 & 480.8879 \\ 59.44980 & 480.9134 \\ 59.45587 & 480.9388 \\ 59.46194 & 480.9643 \\ 59.46801 & 480.9898 \\ 59.47408 & 481.0154 \\ 59.48014 & 481.0410 \\ 59.48621 & 481.0666 \\ 59.49228 & 481.0922 \\ 59.49835 & 481.1179 \\ 59.50441 & 481.1436 \\ 59.51048 & 481.1693 \\ 59.51655 & 481.1951 \\ 59.52262 & 481.2208 \\ 59.52869 & 481.2466 \\ 59.53476 & 481.2724 \\ 59.54082 & 481.2982 \\ 59.54689 & 481.3241 \\ 59.55296 & 481.3500 \\ 59.55903 & 481.3759 \\ 59.56510 & 481.4018 \\ 59.57117 & 481.4277 \\ 59.57724 & 481.4536 \\ 59.58331 & 481.4796 \\ 59.58937 & 481.5056 \\ 59.59544 & 481.5315 \\ 59.60151 & 481.5575 \\ 59.60758 & 481.5836 \\ 59.61365 & 481.6096 \\ 59.61972 & 481.6356 \\ 59.62578 & 481.6617 \\ 59.63185 & 481.6877 \\ 59.64998 & 481.7537 \\ 59.66818 & 481.8122 \\ 59.68638 & 481.8614 \\ 59.70458 & 481.9035 \\ 59.72278 & 481.9397 \\ 59.74098 & 481.9714 \\ 59.75918 & 481.9993\end{array}$




$\begin{array}{ll}59.77738 & 482.0241 \\ 59.79558 & 482.0466 \\ 59.81378 & 482.0672 \\ 59.83198 & 482.0862 \\ 59.85018 & 482.1040 \\ 59.86839 & 482.1207 \\ 59.88659 & 482.1367 \\ 59.90479 & 482.1520 \\ 59.92299 & 482.1669 \\ 59.94118 & 482.1812 \\ 59.95938 & 482.1954 \\ 59.97759 & 482.2092 \\ 59.99579 & 482.2228 \\ 60.01399 & 482.2362 \\ 60.03219 & 482.2495 \\ 60.05039 & 482.2627 \\ 60.06859 & 482.2758 \\ 60.08679 & 482.2888 \\ 60.10499 & 482.3018 \\ 60.12319 & 482.3147 \\ 60.14139 & 482.3276 \\ 60.15959 & 482.3405 \\ 60.17779 & 482.3534 \\ 60.19600 & 482.3661 \\ 60.21420 & 482.3790 \\ 60.23240 & 482.3918 \\ 60.25060 & 482.4045 \\ 60.26880 & 482.4173 \\ 60.28699 & 482.4301 \\ 60.30520 & 482.4428 \\ 60.32340 & 482.4555 \\ 60.34160 & 482.4683 \\ 60.35980 & 482.4810 \\ 60.37800 & 482.4938 \\ 60.39620 & 482.5065 \\ 60.41440 & 482.5193 \\ 60.43260 & 482.5320 \\ 60.45080 & 482.5447 \\ 60.46900 & 482.5574 \\ 60.48720 & 482.5702 \\ 60.50540 & 482.5829 \\ 60.52360 & 482.5956 \\ 60.54181 & 482.6083 \\ 60.56001 & 482.6210 \\ 60.57821 & 482.6337 \\ 60.59641 & 482.6464 \\ 60.61460 & 482.6591 \\ 60.63280 & 482.6718 \\ 60.65101 & 482.6845 \\ 60.66921 & 482.6972 \\ 60.68741 & 482.7099 \\ 60.70561 & 482.7226 \\ 60.72381 & 482.7352 \\ 60.74201 & 482.7479 \\ 60.76021 & 482.7606 \\ 60.77841 & 482.7733 \\ 60.79661 & 482.7860 \\ 60.81481 & 482.7987 \\ 60.83301 & 482.8114 \\ 60.85121 & 482.8241 \\ 60.86942 & 482.8368 \\ 60.88762 & 482.8495 \\ 60.90582 & 482.8621\end{array}$




$\begin{array}{ll}60.92402 & 482.8748 \\ 60.94221 & 482.8875 \\ 60.96041 & 482.9001 \\ 60.97862 & 482.9128 \\ 60.99682 & 482.9255 \\ 61.01502 & 482.9382 \\ 61.03322 & 482.9508 \\ 61.05142 & 482.9635 \\ 61.06962 & 482.9761 \\ 61.08782 & 482.9887 \\ 61.10602 & 483.0014 \\ 61.12422 & 483.0141 \\ 61.14242 & 483.0267 \\ 61.16062 & 483.0393 \\ 61.17882 & 483.0520 \\ 61.19702 & 483.0646 \\ 61.21523 & 483.0772 \\ 61.23343 & 483.0899 \\ 61.25163 & 483.1026 \\ 61.26982 & 483.1152 \\ 61.28802 & 483.1278 \\ 61.30622 & 483.1405 \\ 61.32443 & 483.1531 \\ 61.34263 & 483.1657 \\ 61.36083 & 483.1784 \\ 61.37903 & 483.1910 \\ 61.39723 & 483.2036 \\ 61.41543 & 483.2163 \\ 61.43363 & 483.2289 \\ 61.45183 & 483.2415 \\ 61.47003 & 483.2542 \\ 61.47612 & 483.2700 \\ 61.48197 & 483.2700 \\ 61.48782 & 483.4413 \\ 61.49368 & 483.5986 \\ 61.49953 & 483.7451 \\ 61.50538 & 483.8813 \\ 61.51123 & 484.0080 \\ 61.51709 & 484.1259 \\ 61.52294 & 484.2355 \\ 61.52879 & 484.3376 \\ 61.53465 & 484.4324 \\ 61.54050 & 484.5207 \\ 61.54635 & 484.6028 \\ 61.55220 & 484.6792 \\ 61.55805 & 484.7504 \\ 61.56391 & 484.8166 \\ 61.56976 & 484.8783 \\ 61.57561 & 484.9358 \\ 61.58146 & 484.9893 \\ 61.58731 & 485.0392 \\ 61.59317 & 485.0858 \\ 61.59902 & 485.1293 \\ 61.60487 & 485.1699 \\ 61.61073 & 485.2079 \\ 61.61658 & 485.2434 \\ 61.62243 & 485.2766 \\ 61.62828 & 485.3076 \\ 61.63414 & 485.3368 \\ 61.63999 & 485.3640 \\ 61.64584 & 485.3897 \\ 61.65170 & 485.4138 \\ 61.65755 & 485.4365\end{array}$




$\begin{array}{ll}61.66340 & 485.4579 \\ 61.66925 & 485.4780 \\ 61.67511 & 485.4970 \\ 61.68096 & 485.5150 \\ 61.68681 & 485.5319 \\ 61.69266 & 485.5480 \\ 61.69851 & 485.5633 \\ 61.70437 & 485.5778 \\ 61.71022 & 485.5916 \\ 61.71607 & 485.6047 \\ 61.72192 & 485.6172 \\ 61.72778 & 485.6292 \\ 61.73363 & 485.6407 \\ 61.73948 & 485.6517 \\ 61.74533 & 485.6622 \\ 61.75119 & 485.6724 \\ 61.75704 & 485.6822 \\ 61.76289 & 485.6916 \\ 61.76875 & 485.7007 \\ 61.77460 & 485.7096 \\ 61.78045 & 485.7181 \\ 61.78630 & 485.7265 \\ 61.79216 & 485.7346 \\ 61.79801 & 485.7424 \\ 61.80386 & 485.7501 \\ 61.80971 & 485.7576 \\ 61.81556 & 485.7650 \\ 61.82142 & 485.7722 \\ 61.82727 & 485.7792 \\ 61.83312 & 485.7861 \\ 61.83897 & 485.7930 \\ 61.84483 & 485.7997 \\ 61.85068 & 485.8062 \\ 61.85653 & 485.8127 \\ 61.86238 & 485.8191 \\ 61.86824 & 485.8254 \\ 61.87409 & 485.8317 \\ 61.87994 & 485.8378 \\ 61.88580 & 485.8439 \\ 61.89165 & 485.8500 \\ 61.89750 & 485.8560 \\ 61.90335 & 485.8619 \\ 61.90921 & 485.8679 \\ 61.91506 & 485.8737 \\ 61.92091 & 485.8796 \\ 61.92676 & 485.8854 \\ 61.93261 & 485.8912 \\ 61.93847 & 485.8969 \\ 61.94432 & 485.9026 \\ 61.95017 & 485.9084 \\ 61.95602 & 485.9140 \\ 61.96188 & 485.9197 \\ 61.96773 & 485.9253 \\ 61.97358 & 485.9309 \\ 61.97943 & 485.9365 \\ 61.98529 & 485.9421 \\ 61.99114 & 485.9476 \\ 61.99699 & 485.9532 \\ 62.00285 & 485.9587 \\ 62.00870 & 485.9643 \\ 62.01455 & 485.9698 \\ 62.02040 & 485.9753 \\ 62.02626 & 485.9808\end{array}$




$\begin{array}{ll}62.03211 & 485.9863 \\ 62.03796 & 485.9918 \\ 62.04381 & 485.9973 \\ 62.04967 & 486.0027 \\ 62.05552 & 486.0082 \\ 62.06137 & 486.0137 \\ 62.08459 & 486.0366 \\ 62.10780 & 486.0734 \\ 62.13102 & 486.1157 \\ 62.15423 & 486.1586 \\ 62.17744 & 486.2027 \\ 62.20065 & 486.2474 \\ 62.22387 & 486.2926 \\ 62.24708 & 486.3382 \\ 62.27029 & 486.3839 \\ 62.29351 & 486.4298 \\ 62.31672 & 486.4759 \\ 62.33993 & 486.5219 \\ 62.36314 & 486.5681 \\ 62.38636 & 486.6144 \\ 62.40957 & 486.6606 \\ 62.43278 & 486.7069 \\ 62.45599 & 486.7532 \\ 62.47921 & 486.7996 \\ 62.50242 & 486.8459 \\ 62.52563 & 486.8922 \\ 62.54885 & 486.9386 \\ 62.57206 & 486.9850 \\ 62.59527 & 487.0313 \\ 62.61848 & 487.0777 \\ 62.64170 & 487.1241 \\ 62.66491 & 487.1705 \\ 62.68813 & 487.2169 \\ 62.71134 & 487.2634 \\ 62.73455 & 487.3098 \\ 62.75776 & 487.3562 \\ 62.78098 & 487.4026 \\ 62.80419 & 487.4490 \\ 62.82740 & 487.4955 \\ 62.85062 & 487.5420 \\ 62.87383 & 487.5884 \\ 62.89704 & 487.6349 \\ 62.92025 & 487.6813 \\ 62.94347 & 487.7278 \\ 62.96668 & 487.7743 \\ 62.98989 & 487.8208 \\ 63.01311 & 487.8672 \\ 63.03632 & 487.9137 \\ 63.05953 & 487.9602 \\ 63.08274 & 488.0067 \\ 63.10596 & 488.0532 \\ 63.12917 & 488.0996 \\ 63.15239 & 488.1462 \\ 63.17560 & 488.1927 \\ 63.19881 & 488.2392 \\ 63.22202 & 488.2857 \\ 63.24524 & 488.3322 \\ 63.26845 & 488.3788 \\ 63.29166 & 488.4253 \\ 63.31488 & 488.4717 \\ 63.33809 & 488.5183 \\ 63.36130 & 488.5648 \\ 63.38451 & 488.6113\end{array}$




$\begin{array}{ll}63.40773 & 488.6579 \\ 63.43094 & 488.7044 \\ 63.45415 & 488.7509 \\ 63.47737 & 488.7975 \\ 63.50058 & 488.8440 \\ 63.52379 & 488.8905 \\ 63.54700 & 488.9370 \\ 63.57022 & 488.9836 \\ 63.59343 & 489.0301 \\ 63.61664 & 489.0766 \\ 63.63986 & 489.1231 \\ 63.66307 & 489.1696 \\ 63.68628 & 489.2161 \\ 63.70950 & 489.2627 \\ 63.73271 & 489.3092 \\ 63.75592 & 489.3557 \\ 63.77913 & 489.4022 \\ 63.80235 & 489.4487 \\ 63.82556 & 489.4952 \\ 63.84877 & 489.5417 \\ 63.87199 & 489.5882 \\ 63.89520 & 489.6348 \\ 63.91841 & 489.6813 \\ 63.94162 & 489.7278 \\ 63.96484 & 489.7743 \\ 63.98805 & 489.8208 \\ 64.01126 & 489.8673 \\ 64.03448 & 489.9138 \\ 64.05769 & 489.9603 \\ 64.08090 & 490.0068 \\ 64.10412 & 490.0533 \\ 64.12733 & 490.0997 \\ 64.15054 & 490.1462 \\ 64.17375 & 490.1927 \\ 64.19697 & 490.2392 \\ 64.22018 & 490.2856 \\ 64.24339 & 490.3321 \\ 64.26661 & 490.3786 \\ 64.28982 & 490.4251 \\ 64.31303 & 490.4716 \\ 64.33624 & 490.5180 \\ 64.35946 & 490.5645 \\ 64.38267 & 490.6109 \\ 64.40588 & 490.6574 \\ 64.41123 & 491.4302 \\ 64.41669 & 491.3915 \\ 64.42213 & 491.3554 \\ 64.42758 & 491.3219 \\ 64.43303 & 491.2906 \\ 64.43848 & 491.2616 \\ 64.44393 & 491.2345 \\ 64.44938 & 491.2093 \\ 64.45483 & 491.1859 \\ 64.46027 & 491.1642 \\ 64.46572 & 491.1440 \\ 64.47118 & 491.1253 \\ 64.47662 & 491.1079 \\ 64.48207 & 491.0918 \\ 64.48752 & 491.0769 \\ 64.49297 & 491.0631 \\ 64.49841 & 491.0504 \\ 64.50387 & 491.0385 \\ 64.50932 & 491.0277\end{array}$




$\begin{array}{ll}64.51476 & 491.0176 \\ 64.52021 & 491.0084 \\ 64.52566 & 490.9999 \\ 64.53111 & 490.9921 \\ 64.53656 & 490.9850 \\ 64.54201 & 490.9785 \\ 64.54745 & 490.9725 \\ 64.55290 & 490.9671 \\ 64.55836 & 490.9622 \\ 64.56380 & 490.9577 \\ 64.56925 & 490.9537 \\ 64.57470 & 490.9502 \\ 64.58015 & 490.9470 \\ 64.58560 & 490.9441 \\ 64.59105 & 490.9416 \\ 64.59650 & 490.9394 \\ 64.60194 & 490.9375 \\ 64.60739 & 490.9359 \\ 64.61285 & 490.9346 \\ 64.61829 & 490.9335 \\ 64.62374 & 490.9326 \\ 64.62919 & 490.9319 \\ 64.63464 & 490.9314 \\ 64.64009 & 490.9312 \\ 64.64554 & 490.9311 \\ 64.65099 & 490.9311 \\ 64.65643 & 490.9313 \\ 64.66188 & 490.9316 \\ 64.66734 & 490.9322 \\ 64.67278 & 490.9327 \\ 64.67823 & 490.9335 \\ 64.68368 & 490.9343 \\ 64.68913 & 490.9352 \\ 64.69458 & 490.9363 \\ 64.70003 & 490.9374 \\ 64.70547 & 490.9386 \\ 64.71092 & 490.9398 \\ 64.71637 & 490.9412 \\ 64.72182 & 490.9426 \\ 64.72727 & 490.9441 \\ 64.73272 & 490.9456 \\ 64.73817 & 490.9473 \\ 64.74361 & 490.9489 \\ 64.74907 & 490.9506 \\ 64.75452 & 490.9524 \\ 64.75996 & 490.9542 \\ 64.76541 & 490.9560 \\ 64.77086 & 490.9579 \\ 64.77631 & 490.9598 \\ 64.78176 & 490.9617 \\ 64.78721 & 490.9637 \\ 64.79266 & 490.9657 \\ 64.79810 & 490.9677 \\ 64.80356 & 490.9697 \\ 64.80901 & 490.9718 \\ 64.81445 & 490.9739 \\ 64.81990 & 490.9760 \\ 64.82535 & 490.9782 \\ 64.83080 & 490.9803 \\ 64.83625 & 490.9825 \\ 64.84170 & 490.9847 \\ 64.84715 & 490.9869 \\ 64.85259 & 490.9891\end{array}$




$\begin{array}{ll}64.85805 & 490.9914 \\ 64.86349 & 490.9936 \\ 64.86894 & 490.9959 \\ 64.87439 & 490.9981 \\ 64.87984 & 491.0004 \\ 64.88529 & 491.0027 \\ 64.89074 & 491.0050 \\ 64.89619 & 491.0074 \\ 64.90163 & 491.0096 \\ 64.90708 & 491.0120 \\ 64.91254 & 491.0143 \\ 64.91798 & 491.0166 \\ 64.92343 & 491.0189 \\ 64.92888 & 491.0212 \\ 64.93433 & 491.0236 \\ 64.93978 & 491.0260 \\ 64.94523 & 491.0283 \\ 64.95068 & 491.0307 \\ 64.95612 & 491.0331 \\ 64.96181 & 491.0350 \\ 64.96768 & 491.0396 \\ 64.97356 & 491.0451 \\ 64.97942 & 491.0515 \\ 64.98530 & 491.0585 \\ 64.99117 & 491.0663 \\ 64.99704 & 491.0748 \\ 65.00291 & 491.0839 \\ 65.00878 & 491.0937 \\ 65.01466 & 491.1039 \\ 65.02052 & 491.1147 \\ 65.02640 & 491.1259 \\ 65.03226 & 491.1376 \\ 65.03814 & 491.1498 \\ 65.04401 & 491.1623 \\ 65.04988 & 491.1752 \\ 65.05576 & 491.1884 \\ 65.06162 & 491.2020 \\ 65.06750 & 491.2159 \\ 65.07336 & 491.2300 \\ 65.07924 & 491.2444 \\ 65.08511 & 491.2590 \\ 65.09098 & 491.2739 \\ 65.09686 & 491.2890 \\ 65.10272 & 491.3043 \\ 65.10860 & 491.3197 \\ 65.11446 & 491.3354 \\ 65.12034 & 491.3512 \\ 65.12621 & 491.3672 \\ 65.13208 & 491.3832 \\ 65.13795 & 491.3994 \\ 65.14382 & 491.4158 \\ 65.14970 & 491.4323 \\ 65.15556 & 491.4489 \\ 65.16144 & 491.4656 \\ 65.16730 & 491.4823 \\ 65.17318 & 491.4992 \\ 65.17905 & 491.5162 \\ 65.18492 & 491.5332 \\ 65.19080 & 491.5503 \\ 65.19666 & 491.5675 \\ 65.20254 & 491.5847 \\ 65.20840 & 491.6021 \\ 65.21428 & 491.6194\end{array}$




$\begin{array}{ll}65.22015 & 491.6368 \\ 65.22602 & 491.6543 \\ 65.23190 & 491.6699 \\ 65.23776 & 491.6699 \\ 65.24364 & 491.6699 \\ 65.24950 & 491.6699 \\ 65.25538 & 491.6699 \\ 65.26125 & 491.6699 \\ 65.26712 & 491.6699 \\ 65.27299 & 491.6699 \\ 65.27886 & 491.6699 \\ 65.28474 & 491.6699 \\ 65.29060 & 491.6699 \\ 65.29648 & 491.6699 \\ 65.30235 & 491.6699 \\ 65.30822 & 491.6699 \\ 65.31409 & 491.6699 \\ 65.31996 & 491.6699 \\ 65.32584 & 491.6699 \\ 65.33170 & 491.6699 \\ 65.33758 & 491.6699 \\ 65.34344 & 491.6699 \\ 65.34932 & 491.6699 \\ 65.35519 & 491.6699 \\ 65.36106 & 491.6699 \\ 65.36693 & 491.6699 \\ 65.37280 & 491.6699 \\ 65.37868 & 491.6699 \\ 65.38454 & 491.6699 \\ 65.39042 & 491.6699 \\ 65.39629 & 491.6699 \\ 65.40216 & 491.6699 \\ 65.40803 & 491.6699 \\ 65.41390 & 491.6699 \\ 65.41978 & 491.6699 \\ 65.42564 & 491.6699 \\ 65.43152 & 491.6699 \\ 65.43739 & 491.6699 \\ 65.44326 & 491.6699 \\ 65.44913 & 491.6699 \\ 65.45500 & 491.6699 \\ 65.46087 & 491.6699 \\ 65.46674 & 491.6699 \\ 65.47262 & 491.6699 \\ 65.47849 & 491.6699 \\ 65.48436 & 491.6699 \\ 65.49023 & 491.6699 \\ 65.49610 & 491.6699 \\ 65.50197 & 491.6699 \\ 65.50784 & 491.6699 \\ 65.51372 & 491.6699 \\ 65.51958 & 491.6699 \\ 65.52546 & 491.6699 \\ 65.53133 & 491.6699 \\ 65.53720 & 491.6699 \\ 65.54307 & 491.6699 \\ 65.54894 & 491.6699 \\ 65.56672 & 491.6699 \\ 65.58434 & 491.6699 \\ 65.60195 & 491.6699 \\ 65.61957 & 491.6699 \\ 65.63719 & 491.6699 \\ 65.65481 & 491.6699\end{array}$




$\begin{array}{ll}65.67242 & 491.6699 \\ 65.69005 & 491.6699 \\ 65.70766 & 491.6699 \\ 65.72528 & 491.6699 \\ 65.74290 & 491.6699 \\ 65.76052 & 491.6699 \\ 65.77814 & 491.6699 \\ 65.79575 & 491.6699 \\ 65.81338 & 491.6699 \\ 65.83099 & 491.6699 \\ 65.84861 & 491.6699 \\ 65.86623 & 491.6699 \\ 65.88385 & 491.6699 \\ 65.90147 & 491.6699 \\ 65.91909 & 491.6699 \\ 65.93671 & 491.6699 \\ 65.95432 & 491.6699 \\ 65.97194 & 491.6699 \\ 65.98956 & 491.6699 \\ 66.00718 & 491.6699 \\ 66.02480 & 491.6699 \\ 66.04242 & 491.6699 \\ 66.06004 & 491.6699 \\ 66.07765 & 491.6699 \\ 66.09528 & 491.6699 \\ 66.11289 & 491.6699 \\ 66.13051 & 491.6699 \\ 66.14812 & 491.6699 \\ 66.16575 & 491.6699 \\ 66.18336 & 491.6699 \\ 66.20098 & 491.6699 \\ 66.21861 & 491.6699 \\ 66.23622 & 491.6699 \\ 66.25384 & 491.6699 \\ 66.27146 & 491.6699 \\ 66.28908 & 491.6699 \\ 66.30669 & 491.6699 \\ 66.32431 & 491.6699 \\ 66.34193 & 491.6699 \\ 66.35955 & 491.6699 \\ 66.37717 & 491.6699 \\ 66.39479 & 491.6699 \\ 66.41241 & 491.6699 \\ 66.43002 & 491.6699 \\ 66.44765 & 491.6699 \\ 66.46526 & 491.6699 \\ 66.48288 & 491.6699 \\ 66.50050 & 491.6699 \\ 66.51812 & 491.6699 \\ 66.53574 & 491.6699 \\ 66.55335 & 491.6699 \\ 66.57098 & 491.6699 \\ 66.58859 & 491.6699 \\ 66.60621 & 491.6699 \\ 66.62383 & 491.6699 \\ 66.64145 & 491.6699 \\ 66.65907 & 491.6699 \\ 66.67668 & 491.6699 \\ 66.69431 & 491.6699 \\ 66.71192 & 491.6699 \\ 66.72954 & 491.6699 \\ 66.74716 & 491.6699 \\ 66.76478 & 491.6699\end{array}$




\begin{tabular}{|c|c|}
\hline 66.78239 & 491.6699 \\
\hline 66.80002 & 491.6699 \\
\hline 66.81763 & 491.6699 \\
\hline 66.83525 & 491.6699 \\
\hline 66.85287 & 491.6699 \\
\hline 66.87049 & 491.6699 \\
\hline 66.88811 & 491.6699 \\
\hline 66.90572 & 491.6699 \\
\hline 66.92335 & 491.6699 \\
\hline 66.94096 & 491.6699 \\
\hline 66.95858 & 491.6699 \\
\hline 66.97620 & 491.6699 \\
\hline 66.99382 & 491.6699 \\
\hline 67.01144 & 491.6699 \\
\hline 67.02906 & 491.6699 \\
\hline 67.04668 & 491.6699 \\
\hline 67.06429 & 491.6699 \\
\hline 67.08191 & 491.6699 \\
\hline 67.09953 & 491.6699 \\
\hline 67.11715 & 491.6699 \\
\hline 67.13477 & 491.6699 \\
\hline 67.15239 & 491.6699 \\
\hline 67.17001 & 491.6699 \\
\hline 67.18762 & 491.6699 \\
\hline 67.20525 & 491.6699 \\
\hline 67.22286 & 491.6699 \\
\hline 67.24048 & 491.6699 \\
\hline 67.25809 & 491.6699 \\
\hline 67.27572 & 491.6699 \\
\hline 67.29333 & 491.6699 \\
\hline 67.31095 & 491.6699 \\
\hline 67.32858 & 491.6699 \\
\hline 67.33422 & 491.6699 \\
\hline 67.33987 & 491.6699 \\
\hline 67.34552 & 491.6699 \\
\hline 67.35117 & 491.6699 \\
\hline 67.35682 & 491.6699 \\
\hline 67.36247 & 491.6699 \\
\hline 67.36813 & 491.6699 \\
\hline 67.37377 & 491.6699 \\
\hline 67.37943 & 491.6699 \\
\hline 67.38508 & 491.6699 \\
\hline 67.39072 & 491.6699 \\
\hline 67.39638 & 491.6699 \\
\hline 67.40202 & 491.6699 \\
\hline 67.40768 & 491.6699 \\
\hline 67.41333 & 491.6699 \\
\hline 67.41898 & 491.6699 \\
\hline 67.42463 & 491.6699 \\
\hline 67.43028 & 491.6699 \\
\hline 67.43593 & 491.6699 \\
\hline 67.44158 & 491.6699 \\
\hline 67.44724 & 491.6699 \\
\hline 67.45288 & 491.6699 \\
\hline 67.45853 & 491.6699 \\
\hline 67.46419 & 491.6699 \\
\hline 67.46983 & 491.6699 \\
\hline 67.47549 & 491.6699 \\
\hline 67.48114 & 491.6699 \\
\hline 67.48679 & 491.6699 \\
\hline 67.49244 & 491.6699 \\
\hline 67.49809 & 491.6699 \\
\hline 67.50374 & 491.6699 \\
\hline
\end{tabular}




\begin{tabular}{|c|c|}
\hline 67.50939 & 491.6699 \\
\hline 67.51504 & 491.6699 \\
\hline 67.52069 & 491.6699 \\
\hline 67.52634 & 491.6699 \\
\hline 67.53199 & 491.6699 \\
\hline 67.53764 & 491.6699 \\
\hline 67.54330 & 491.6699 \\
\hline 67.54894 & 491.6699 \\
\hline 67.55460 & 491.6699 \\
\hline 67.56025 & 491.6699 \\
\hline 67.56590 & 491.6699 \\
\hline 67.57155 & 491.6699 \\
\hline 67.57720 & 491.6699 \\
\hline 67.58285 & 491.6699 \\
\hline 67.58850 & 491.6699 \\
\hline 67.59415 & 491.6699 \\
\hline 67.59980 & 491.6699 \\
\hline 67.60545 & 491.6699 \\
\hline 67.61111 & 491.6699 \\
\hline 67.61675 & 491.6699 \\
\hline 67.62241 & 491.6699 \\
\hline 67.62805 & 491.6699 \\
\hline 67.63371 & 491.6699 \\
\hline 67.63936 & 491.6699 \\
\hline 67.64500 & 491.6699 \\
\hline 67.65066 & 491.6699 \\
\hline 67.65631 & 491.6699 \\
\hline 67.66196 & 491.6699 \\
\hline 67.66761 & 491.6699 \\
\hline 67.67326 & 491.6699 \\
\hline 67.67891 & 491.6699 \\
\hline 67.68456 & 491.6699 \\
\hline 67.69022 & 491.6699 \\
\hline 67.69586 & 491.6699 \\
\hline 67.70152 & 491.6699 \\
\hline 67.70717 & 491.6699 \\
\hline 67.71281 & 491.6699 \\
\hline 67.71847 & 491.6699 \\
\hline 67.72412 & 491.6699 \\
\hline 67.72977 & 491.6699 \\
\hline 67.73542 & 491.6699 \\
\hline 67.74107 & 491.6699 \\
\hline 67.74672 & 491.6699 \\
\hline 67.75237 & 491.6699 \\
\hline 67.75802 & 491.6699 \\
\hline 67.76367 & 491.6699 \\
\hline 67.76933 & 491.6699 \\
\hline 67.77497 & 491.6699 \\
\hline 67.78062 & 491.6699 \\
\hline 67.78628 & 491.6699 \\
\hline 67.79192 & 491.6699 \\
\hline 67.79758 & 491.6699 \\
\hline 67.80323 & 491.6699 \\
\hline 67.80888 & 491.6699 \\
\hline 67.81453 & 491.6699 \\
\hline 67.82018 & 491.6699 \\
\hline 67.82583 & 491.6699 \\
\hline 67.83148 & 491.6699 \\
\hline 67.83713 & 491.6699 \\
\hline 67.84278 & 491.6699 \\
\hline 67.84843 & 491.6699 \\
\hline 67.85408 & 491.6699 \\
\hline 67.85973 & 491.6699 \\
\hline
\end{tabular}




\begin{tabular}{|c|c|}
\hline 67.86539 & 491.6699 \\
\hline 67.87103 & 491.6699 \\
\hline 67.87669 & 491.6699 \\
\hline 67.88234 & 491.6699 \\
\hline 67.88799 & 491.6699 \\
\hline 67.89364 & 491.6699 \\
\hline 67.89929 & 491.6699 \\
\hline 67.92163 & 491.6699 \\
\hline 67.94381 & 491.6699 \\
\hline 67.96599 & 491.6699 \\
\hline 67.98816 & 491.6699 \\
\hline 68.01034 & 491.6699 \\
\hline 68.03252 & 491.6699 \\
\hline 68.05469 & 491.6699 \\
\hline 68.07687 & 491.6699 \\
\hline 68.09904 & 491.6699 \\
\hline 68.12122 & 491.6699 \\
\hline 68.14339 & 491.6699 \\
\hline 68.16557 & 491.6699 \\
\hline 68.18774 & 491.6699 \\
\hline 68.20992 & 491.6699 \\
\hline 68.23209 & 491.6699 \\
\hline 68.25427 & 491.6699 \\
\hline 68.27644 & 491.6699 \\
\hline 68.29862 & 491.6699 \\
\hline 68.32080 & 491.6699 \\
\hline 68.34297 & 491.6699 \\
\hline 68.36515 & 491.6699 \\
\hline 68.38732 & 491.6699 \\
\hline 68.40950 & 491.6699 \\
\hline 68.43168 & 491.6699 \\
\hline 68.45385 & 491.6699 \\
\hline 68.47603 & 491.6699 \\
\hline 68.49821 & 491.6699 \\
\hline 68.52038 & 491.6699 \\
\hline 68.54256 & 491.6699 \\
\hline 68.56473 & 491.6699 \\
\hline 68.58691 & 491.6699 \\
\hline 68.60909 & 491.6699 \\
\hline 68.63126 & 491.6699 \\
\hline 68.65343 & 491.6699 \\
\hline 68.67561 & 491.6699 \\
\hline 68.69778 & 491.6699 \\
\hline 68.71996 & 491.6699 \\
\hline 68.74213 & 491.6699 \\
\hline 68.76431 & 491.6699 \\
\hline 68.78649 & 491.6699 \\
\hline 68.80866 & 491.6699 \\
\hline 68.83084 & 491.6699 \\
\hline 68.85301 & 491.6699 \\
\hline 68.87519 & 491.6699 \\
\hline 68.89737 & 491.6699 \\
\hline 68.91954 & 491.6699 \\
\hline 68.94172 & 491.6699 \\
\hline 68.96390 & 491.6699 \\
\hline 68.98607 & 491.6699 \\
\hline 69.00825 & 491.6699 \\
\hline 69.03042 & 491.6699 \\
\hline 69.05260 & 491.6699 \\
\hline 69.07478 & 491.6699 \\
\hline 69.09695 & 491.6699 \\
\hline 69.11913 & 491.6699 \\
\hline 69.14130 & 491.6699 \\
\hline
\end{tabular}




$\begin{array}{ll}69.16348 & 491.6699 \\ 69.18565 & 491.6699 \\ 69.20782 & 491.6699 \\ 69.23000 & 491.6699 \\ 69.25218 & 491.6699 \\ 69.27435 & 491.6699 \\ 69.29653 & 491.6699 \\ 69.31870 & 491.6699 \\ 69.34088 & 491.6699 \\ 69.36306 & 491.6699 \\ 69.38523 & 491.6699 \\ 69.40741 & 491.6699 \\ 69.42959 & 491.6699 \\ 69.45176 & 491.6699 \\ 69.47394 & 491.6699 \\ 69.49611 & 491.6699 \\ 69.51829 & 491.6699 \\ 69.54047 & 491.6699 \\ 69.56264 & 491.6699 \\ 69.58482 & 491.6699 \\ 69.60699 & 491.6699 \\ 69.62917 & 491.6699 \\ 69.65134 & 491.6699 \\ 69.67352 & 491.6699 \\ 69.69569 & 491.6699 \\ 69.71787 & 491.6699 \\ 69.74004 & 491.6699 \\ 69.76222 & 491.6699 \\ 69.78439 & 491.6699 \\ 69.80657 & 491.6699 \\ 69.82875 & 491.6699 \\ 69.85092 & 491.6699 \\ 69.87310 & 491.6699 \\ 69.89528 & 491.6699 \\ 69.91745 & 491.6699 \\ 69.93963 & 491.6699 \\ 69.96180 & 491.6699 \\ 69.98398 & 491.6699 \\ 70.00616 & 491.6699 \\ 70.02833 & 491.6699 \\ 70.05051 & 491.6699 \\ 70.07268 & 491.6699 \\ 70.09486 & 491.6699 \\ 70.11703 & 491.6699 \\ 70.13921 & 491.6699 \\ 70.14417 & 491.6699 \\ 70.14937 & 491.6699 \\ 70.15456 & 491.6699 \\ 70.15974 & 491.6699 \\ 70.16494 & 491.6699 \\ 70.17013 & 491.6699 \\ 70.17532 & 491.6699 \\ 70.18051 & 491.6699 \\ 70.18570 & 491.6699 \\ 70.19089 & 491.6699 \\ 70.19608 & 491.6699 \\ 70.20127 & 491.6699 \\ 70.20647 & 491.6699 \\ 70.21165 & 491.6699 \\ 70.21684 & 491.6699 \\ 70.22204 & 491.6699 \\ 70.22723 & 491.6699 \\ 70.23241 & 491.6699\end{array}$




$\begin{array}{ll}70.23761 & 491.6699 \\ 70.24280 & 491.6699 \\ 70.24799 & 491.6699 \\ 70.25318 & 491.6699 \\ 70.25837 & 491.6699 \\ 70.26357 & 491.6699 \\ 70.26875 & 491.6699 \\ 70.27394 & 491.6699 \\ 70.27914 & 491.6699 \\ 70.28432 & 491.6699 \\ 70.28951 & 491.6699 \\ 70.29471 & 491.6699 \\ 70.29990 & 491.6699 \\ 70.30508 & 491.6699 \\ 70.31028 & 491.6699 \\ 70.31547 & 491.6699 \\ 70.32066 & 491.6699 \\ 70.32585 & 491.6699 \\ 70.33104 & 491.6699 \\ 70.33624 & 491.6699 \\ 70.34142 & 491.6699 \\ 70.34661 & 491.6699 \\ 70.35181 & 491.6699 \\ 70.35699 & 491.6699 \\ 70.36218 & 491.6699 \\ 70.36738 & 491.6699 \\ 70.37257 & 491.6699 \\ 70.37776 & 491.6699 \\ 70.38295 & 491.6699 \\ 70.38814 & 491.6699 \\ 70.39333 & 491.6699 \\ 70.39852 & 491.6699 \\ 70.40371 & 491.6699 \\ 70.40891 & 491.6699 \\ 70.41409 & 491.6699 \\ 70.41928 & 491.6699 \\ 70.42448 & 491.6699 \\ 70.42966 & 491.6699 \\ 70.43486 & 491.6699 \\ 70.44005 & 491.6699 \\ 70.44524 & 491.6699 \\ 70.45043 & 491.6699 \\ 70.45562 & 491.6699 \\ 70.46081 & 491.6699 \\ 70.46600 & 491.6699 \\ 70.47119 & 491.6699 \\ 70.47638 & 491.6699 \\ 70.48158 & 491.6699 \\ 70.48676 & 491.6699 \\ 70.49196 & 491.6699 \\ 70.49715 & 491.6699 \\ 70.50233 & 491.6699 \\ 70.50753 & 491.6699 \\ 70.51272 & 491.6699 \\ 70.5231791 & 491.6699 \\ 70.53869 & 491.6699 \\ 70.54386 & 491.6699 \\ 70.54906 & 491.6699 \\ 70.55424 & 491.6699 \\ 70.55943 & 491.6699 \\ 70 & 491.6699\end{array}$




$\begin{array}{ll}70.56463 & 491.6699 \\ 70.56982 & 491.6699 \\ 70.57500 & 491.6699 \\ 70.58020 & 491.6699 \\ 70.58539 & 491.6699 \\ 70.59058 & 491.6699 \\ 70.59577 & 491.6699 \\ 70.60096 & 491.6699 \\ 70.60616 & 491.6699 \\ 70.61134 & 491.6699 \\ 70.61653 & 491.6699 \\ 70.62173 & 491.6699 \\ 70.62691 & 491.6699 \\ 70.63210 & 491.6699 \\ 70.63730 & 491.6699 \\ 70.64249 & 491.6699 \\ 70.64768 & 491.6699 \\ 70.65287 & 491.6699 \\ 70.65806 & 491.6699 \\ 70.66325 & 491.6699 \\ 70.66892 & 491.6699 \\ 70.67455 & 491.6699 \\ 70.68016 & 491.6699 \\ 70.68578 & 491.6699 \\ 70.69140 & 491.6699 \\ 70.69701 & 491.6699 \\ 70.70263 & 491.6699 \\ 70.70825 & 491.6699 \\ 70.71387 & 491.6699 \\ 70.71948 & 491.6699 \\ 70.72511 & 491.6699 \\ 70.73072 & 491.6699 \\ 70.73634 & 491.6699 \\ 70.74196 & 491.6699 \\ 70.74757 & 491.6699 \\ 70.75319 & 491.6699 \\ 70.75881 & 491.6699 \\ 70.76443 & 491.6699 \\ 70.77005 & 491.6699 \\ 70.77567 & 491.6699 \\ 70.78128 & 491.6699 \\ 70.78690 & 491.6699 \\ 70.79252 & 491.6699 \\ 70.79813 & 491.6699 \\ 70.80376 & 491.6699 \\ 70.80937 & 491.6699 \\ 70.81499 & 491.6699 \\ 70.82061 & 491.6699 \\ 70.82623 & 491.6699 \\ 70.83184 & 491.6699 \\ 70.83746 & 491.6699 \\ 70.84308 & 491.6699 \\ 70.84869 & 491.6699 \\ 70.85432 & 491.6699 \\ 70.876953 & 491.66999 \\ 70.88240 & 491.66999 \\ 70.88802 & 491.6699 \\ 70.89364 & 491.6699 \\ 70.89925 & 491.6699 \\ 70.90488 & 491.6699 \\ 706 & \end{array}$




\begin{tabular}{|c|c|}
\hline 70.91049 & 491.6699 \\
\hline 70.91611 & 491.6699 \\
\hline 70.92173 & 491.6699 \\
\hline 70.92735 & 491.6699 \\
\hline 70.93296 & 491.6699 \\
\hline 70.93858 & 491.6699 \\
\hline 70.94420 & 491.6699 \\
\hline 70.94982 & 491.6699 \\
\hline 70.95544 & 491.6699 \\
\hline 70.96105 & 491.6699 \\
\hline 70.96667 & 491.6699 \\
\hline 70.97229 & 491.6699 \\
\hline 70.97791 & 491.6699 \\
\hline 70.98353 & 491.6699 \\
\hline 70.98914 & 491.6699 \\
\hline 70.99476 & 491.6699 \\
\hline 71.00038 & 491.6699 \\
\hline 71.00600 & 491.6699 \\
\hline 71.01161 & 491.6699 \\
\hline 71.01723 & 491.6699 \\
\hline 71.02285 & 491.6699 \\
\hline 71.02847 & 491.6699 \\
\hline 71.03409 & 491.6699 \\
\hline 71.03970 & 491.6699 \\
\hline 71.04532 & 491.6699 \\
\hline 71.05094 & 491.6699 \\
\hline 71.05656 & 491.6699 \\
\hline 71.06217 & 491.6699 \\
\hline 71.06779 & 491.6699 \\
\hline 71.07341 & 491.6699 \\
\hline 71.07903 & 491.6699 \\
\hline 71.08465 & 491.6699 \\
\hline 71.09026 & 491.6699 \\
\hline 71.09588 & 491.6699 \\
\hline 71.10150 & 491.6699 \\
\hline 71.10712 & 491.6699 \\
\hline 71.11273 & 491.6699 \\
\hline 71.11835 & 491.6699 \\
\hline 71.12397 & 491.6699 \\
\hline 71.12959 & 491.6699 \\
\hline 71.13521 & 491.6699 \\
\hline 71.14082 & 491.6699 \\
\hline 71.14645 & 491.6699 \\
\hline 71.15206 & 491.6699 \\
\hline 71.15768 & 491.6699 \\
\hline 71.16330 & 491.6699 \\
\hline 71.16891 & 491.6699 \\
\hline 71.17453 & 491.6699 \\
\hline 71.18015 & 491.6699 \\
\hline 71.18577 & 491.6699 \\
\hline 71.19138 & 491.6699 \\
\hline 71.19701 & 491.6699 \\
\hline 71.20262 & 491.6699 \\
\hline 71.20824 & 491.6699 \\
\hline 71.21386 & 491.6699 \\
\hline 71.21947 & 491.6699 \\
\hline 71.22509 & 491.6699 \\
\hline 71.23071 & 491.6699 \\
\hline 71.24777 & 491.6699 \\
\hline 71.26463 & 491.6699 \\
\hline 71.28149 & 491.6699 \\
\hline 71.29835 & 491.6699 \\
\hline 71.31521 & 491.6699 \\
\hline
\end{tabular}




\begin{tabular}{|c|c|}
\hline 71.33207 & 491.6699 \\
\hline 71.34892 & 491.6699 \\
\hline 71.36578 & 491.6699 \\
\hline 71.38264 & 491.6699 \\
\hline 71.39950 & 491.6699 \\
\hline 71.41636 & 491.6699 \\
\hline 71.43322 & 491.6699 \\
\hline 71.45007 & 491.6699 \\
\hline 71.46693 & 491.6699 \\
\hline 71.48379 & 491.6699 \\
\hline 71.50065 & 491.6699 \\
\hline 71.51751 & 491.6699 \\
\hline 71.53436 & 491.6699 \\
\hline 71.55122 & 491.6699 \\
\hline 71.56808 & 491.6699 \\
\hline 71.58494 & 491.6699 \\
\hline 71.60180 & 491.6699 \\
\hline 71.61866 & 491.6699 \\
\hline 71.63551 & 491.6699 \\
\hline 71.65237 & 491.6699 \\
\hline 71.66924 & 491.6699 \\
\hline 71.68609 & 491.6699 \\
\hline 71.70295 & 491.6699 \\
\hline 71.71980 & 491.6699 \\
\hline 71.73666 & 491.6699 \\
\hline 71.75352 & 491.6699 \\
\hline 71.77038 & 491.6699 \\
\hline 71.78724 & 491.6699 \\
\hline 71.80410 & 491.6699 \\
\hline 71.82095 & 491.6699 \\
\hline 71.83781 & 491.6699 \\
\hline 71.85468 & 491.6699 \\
\hline 71.87153 & 491.6699 \\
\hline 71.88839 & 491.6699 \\
\hline 71.90525 & 491.6699 \\
\hline 71.92210 & 491.6699 \\
\hline 71.93896 & 491.6699 \\
\hline 71.95583 & 491.6699 \\
\hline 71.97268 & 491.6699 \\
\hline 71.98954 & 491.6699 \\
\hline 72.00639 & 491.6699 \\
\hline 72.02325 & 491.6699 \\
\hline 72.04012 & 491.6699 \\
\hline 72.05697 & 491.6699 \\
\hline 72.07383 & 491.6699 \\
\hline 72.09069 & 491.6699 \\
\hline 72.10754 & 491.6699 \\
\hline 72.12440 & 491.6699 \\
\hline 72.14127 & 491.6699 \\
\hline 72.15812 & 491.6699 \\
\hline 72.17498 & 491.6699 \\
\hline 72.19184 & 491.6699 \\
\hline 72.20869 & 491.6699 \\
\hline 72.22556 & 491.6699 \\
\hline 72.24242 & 491.6699 \\
\hline 72.25927 & 491.6699 \\
\hline 72.27613 & 491.6699 \\
\hline 72.29298 & 491.6699 \\
\hline 72.30984 & 491.6699 \\
\hline 72.32671 & 491.6699 \\
\hline 72.34356 & 491.6699 \\
\hline 72.36042 & 491.6699 \\
\hline 72.37728 & 491.6699 \\
\hline
\end{tabular}




$\begin{array}{ll}72.39413 & 491.6699 \\ 72.41100 & 491.6699 \\ 72.42786 & 491.6699 \\ 72.44471 & 491.6699 \\ 72.46157 & 491.6699 \\ 72.47843 & 491.7829 \\ 72.49529 & 492.6719 \\ 72.51215 & 493.4771 \\ 72.52900 & 494.2052 \\ 72.54586 & 494.8625 \\ 72.56272 & 495.4550 \\ 72.57957 & 495.9886 \\ 72.59644 & 496.4685 \\ 72.61330 & 496.8998 \\ 72.63015 & 497.2871 \\ 72.64701 & 497.6347 \\ 72.66387 & 497.9467 \\ 72.68073 & 498.2264 \\ 72.69759 & 498.4773 \\ 72.71445 & 498.7023 \\ 72.73130 & 498.9043 \\ 72.74816 & 499.0855 \\ 72.76502 & 499.2484 \\ 72.78188 & 499.3947 \\ 72.79874 & 499.5264 \\ 72.81559 & 499.6451 \\ 72.83245 & 499.7521 \\ 72.84931 & 499.8489 \\ 72.86617 & 499.9366 \\ 72.88303 & 500.0161 \\ 72.89989 & 500.0884 \\ 72.91674 & 500.1544 \\ 72.93360 & 500.2147 \\ 72.93916 & 500.2484 \\ 72.94455 & 500.3764 \\ 72.94995 & 500.5193 \\ 72.95535 & 500.6556 \\ 72.96074 & 500.7869 \\ 72.96614 & 500.9130 \\ 72.97153 & 501.0342 \\ 72.97694 & 501.1508 \\ 72.98233 & 501.2628 \\ 72.98773 & 501.3705 \\ 72.99313 & 501.4739 \\ 72.99852 & 501.5733 \\ 73.00392 & 501.6688 \\ 73.00932 & 501.7604 \\ 73.01472 & 501.8485 \\ 73.02011 & 501.9331 \\ 73.02551 & 502.0143 \\ 73.03091 & 502.0923 \\ 73.03630 & 502.1673 \\ 73.04170 & 502.2392 \\ 73.04710 & 502.3082 \\ 73.05250 & 502.3745 \\ 73.05789 & 502.4381 \\ 73.06329 & 502.4992 \\ 73.06869 & 502.5578 \\ 73.07408 & 502.6141 \\ 73.07948 & 502.6681 \\ 73.08488 & 502.7199 \\ 73.09028 & 502.7696 \\ 73.09567 & 502.8173\end{array}$




$\begin{array}{ll}73.10107 & 502.8631 \\ 73.10647 & 502.9070 \\ 73.11187 & 502.9492 \\ 73.11726 & 502.9896 \\ 73.12266 & 503.0284 \\ 73.12806 & 503.0656 \\ 73.13345 & 503.1013 \\ 73.13885 & 503.1356 \\ 73.14425 & 503.1685 \\ 73.14965 & 503.2000 \\ 73.15504 & 503.2303 \\ 73.16044 & 503.2594 \\ 73.16584 & 503.2872 \\ 73.17123 & 503.3140 \\ 73.17664 & 503.3396 \\ 73.18203 & 503.3642 \\ 73.18743 & 503.3878 \\ 73.19283 & 503.4105 \\ 73.19822 & 503.4323 \\ 73.20362 & 503.4532 \\ 73.20901 & 503.4732 \\ 73.21442 & 503.4924 \\ 73.21981 & 503.5109 \\ 73.22521 & 503.5286 \\ 73.23061 & 503.5456 \\ 73.23600 & 503.5619 \\ 73.24140 & 503.5775 \\ 73.24680 & 503.5926 \\ 73.25220 & 503.6070 \\ 73.25759 & 503.6208 \\ 73.26299 & 503.6342 \\ 73.26839 & 503.6469 \\ 73.27378 & 503.6592 \\ 73.27918 & 503.6710 \\ 73.28458 & 503.6823 \\ 73.28998 & 503.6932 \\ 73.29537 & 503.7036 \\ 73.30077 & 503.7137 \\ 73.30617 & 503.7234 \\ 73.31156 & 503.7326 \\ 73.31696 & 503.7416 \\ 73.32236 & 503.7502 \\ 73.32776 & 503.7584 \\ 73.33315 & 503.7664 \\ 73.33855 & 503.7740 \\ 73.34395 & 503.7814 \\ 73.34934 & 503.7885 \\ 73.35474 & 503.7953 \\ 73.36014 & 503.8018 \\ 73.36554 & 503.8082 \\ 73.37093 & 503.8143 \\ 73.37634 & 503.8202 \\ 73.38173 & 503.8258 \\ 73.38712 & 503.8313 \\ 73.40871 & 503.8366 \\ 73.41951 & 503.8466 \\ 73.42491 & 503.8513 \\ 73.43031 & 503.8559 \\ 73.43570 & 503.8603 \\ 732 & 503.8687 \\ 7303.8727\end{array}$




$\begin{array}{ll}73.44110 & 503.8766 \\ 73.44650 & 503.8803 \\ 73.45190 & 503.8839 \\ 73.45729 & 503.8875 \\ 73.46269 & 503.8908 \\ 73.46809 & 503.8941 \\ 73.47348 & 503.8973 \\ 73.47888 & 503.9004 \\ 73.49987 & 503.9085 \\ 73.52092 & 503.9265 \\ 73.54196 & 503.9482 \\ 73.56301 & 503.9716 \\ 73.58405 & 503.9968 \\ 73.60509 & 504.0232 \\ 73.62614 & 504.0508 \\ 73.64719 & 504.0793 \\ 73.66823 & 504.1085 \\ 73.68928 & 504.1383 \\ 73.71032 & 504.1686 \\ 73.73136 & 504.1994 \\ 73.75241 & 504.2305 \\ 73.77345 & 504.2619 \\ 73.79450 & 504.2935 \\ 73.81554 & 504.3252 \\ 73.83659 & 504.3571 \\ 73.85764 & 504.3891 \\ 73.87868 & 504.4213 \\ 73.89972 & 504.4535 \\ 73.92077 & 504.4857 \\ 73.94181 & 504.5181 \\ 73.96285 & 504.5505 \\ 73.98390 & 504.5829 \\ 74.00494 & 504.6153 \\ 74.02599 & 504.6477 \\ 74.04704 & 504.6801 \\ 74.06808 & 504.7125 \\ 74.08913 & 504.7449 \\ 74.11017 & 504.7773 \\ 74.13121 & 504.8098 \\ 74.15226 & 504.8422 \\ 74.17330 & 504.8746 \\ 74.19435 & 504.9070 \\ 74.21539 & 504.9393 \\ 74.23643 & 504.9717 \\ 74.25748 & 505.0040 \\ 74.27853 & 505.0363 \\ 74.29957 & 505.0686 \\ 74.32062 & 505.1010 \\ 74.34166 & 505.1332 \\ 74.36270 & 505.1654 \\ 74.38375 & 505.1977 \\ 74.40479 & 505.2299 \\ 74.42584 & 505.2621 \\ 74.44688 & 505.2943 \\ 74.46793 & 505.3265 \\ 74.53106 & 505.3586 \\ 74.55211 & 505.3907 \\ 74.57315 & 505.4229 \\ 74.59419 & 505.4871 \\ 74.61524 & 505.5192 \\ 74.63628 & 505.5512 \\ 705.5833\end{array}$




$\begin{array}{ll}74.65733 & 505.6153 \\ 74.67838 & 505.6473 \\ 74.69942 & 505.6793 \\ 74.72047 & 505.7112 \\ 74.74151 & 505.7432 \\ 74.76255 & 505.7751 \\ 74.78360 & 505.8070 \\ 74.80464 & 505.8390 \\ 74.82569 & 505.8708 \\ 74.84673 & 505.9027 \\ 74.86777 & 505.9345 \\ 74.88882 & 505.9664 \\ 74.90987 & 505.9982 \\ 74.93091 & 506.0300 \\ 74.95196 & 506.0618 \\ 74.97300 & 506.0936 \\ 74.99404 & 506.1253 \\ 75.01509 & 506.1570 \\ 75.03613 & 506.1888 \\ 75.05718 & 506.2205 \\ 75.07822 & 506.2521 \\ 75.09927 & 506.2838 \\ 75.12032 & 506.3155 \\ 75.14136 & 506.3471 \\ 75.16240 & 506.3788 \\ 75.18345 & 506.4103 \\ 75.20449 & 506.4419 \\ 75.22553 & 506.4735 \\ 75.24658 & 506.5051 \\ 75.26762 & 506.5366 \\ 75.28867 & 506.5682 \\ 75.30972 & 506.5997 \\ 75.33076 & 506.6312 \\ 75.35181 & 506.6628 \\ 75.37285 & 506.6942 \\ 75.39389 & 506.7257 \\ 75.41494 & 506.7571 \\ 75.43598 & 506.7885 \\ 75.45703 & 506.8200 \\ 75.47807 & 506.8514 \\ 75.49911 & 506.8828 \\ 75.52016 & 506.9142 \\ 75.54121 & 506.9456 \\ 75.56225 & 506.9769 \\ 75.58330 & 507.0083 \\ 75.60434 & 507.0396 \\ 75.60910 & 507.6951 \\ 75.61401 & 507.6783 \\ 75.61890 & 507.6619 \\ 75.62380 & 507.6459 \\ 75.62870 & 507.6303 \\ 75.63361 & 507.6151 \\ 75.63850 & 507.6003 \\ 75.66790 & 507.5858 \\ 75.67280 & 507.5570 \\ 75.67770 & 507.5310 \\ 75.68260 & 507.5180 \\ 75.68750 & 507.5053 \\ 750 & 507.4689 \\ 750 & \end{array}$




$\begin{array}{ll}75.69240 & 507.4572 \\ 75.69730 & 507.4458 \\ 75.70220 & 507.4346 \\ 75.70710 & 507.4236 \\ 75.71200 & 507.4128 \\ 75.71690 & 507.4023 \\ 75.72180 & 507.3919 \\ 75.72670 & 507.3817 \\ 75.73160 & 507.3716 \\ 75.73650 & 507.3618 \\ 75.74140 & 507.3521 \\ 75.74630 & 507.3425 \\ 75.75120 & 507.3331 \\ 75.75610 & 507.3239 \\ 75.76099 & 507.3148 \\ 75.76590 & 507.3058 \\ 75.77080 & 507.2970 \\ 75.77570 & 507.2883 \\ 75.78059 & 507.2798 \\ 75.78550 & 507.2713 \\ 75.79040 & 507.2629 \\ 75.79530 & 507.2547 \\ 75.80019 & 507.2466 \\ 75.80509 & 507.2386 \\ 75.81000 & 507.2307 \\ 75.81490 & 507.2228 \\ 75.81979 & 507.2150 \\ 75.82469 & 507.2073 \\ 75.82960 & 507.1998 \\ 75.83450 & 507.1922 \\ 75.83939 & 507.1848 \\ 75.84429 & 507.1775 \\ 75.84919 & 507.1702 \\ 75.85410 & 507.1630 \\ 75.85899 & 507.1558 \\ 75.86389 & 507.1487 \\ 75.86879 & 507.1417 \\ 75.87370 & 507.1347 \\ 75.87859 & 507.1278 \\ 75.88349 & 507.1209 \\ 75.88839 & 507.1141 \\ 75.89329 & 507.1074 \\ 75.89819 & 507.1007 \\ 75.90309 & 507.0940 \\ 75.90799 & 507.0874 \\ 75.91289 & 507.0808 \\ 75.91779 & 507.0743 \\ 75.92269 & 507.0678 \\ 75.92759 & 507.0614 \\ 75.93249 & 507.0550 \\ 75.93739 & 507.0486 \\ 75.94229 & 507.0422 \\ 75.94719 & 507.0359 \\ 75.95209 & 507.0296 \\ 75.95699 & 507.0234 \\ 75.96189 & 507.0172 \\ 75.96679 & 507.0110 \\ 75.97659 & 507.0048 \\ 75.98149 & 506.9986 \\ 75.98639 & 506.9925 \\ 75.99129 & 506.9864 \\ 75.99619 & 506.9803 \\ 706.9742\end{array}$




$\begin{array}{ll}76.00108 & 506.9682 \\ 76.00599 & 506.9621 \\ 76.01089 & 506.9561 \\ 76.01579 & 506.9502 \\ 76.02068 & 506.9442 \\ 76.02559 & 506.9382 \\ 76.03049 & 506.9323 \\ 76.03539 & 506.9263 \\ 76.04028 & 506.9204 \\ 76.04518 & 506.9145 \\ 76.05009 & 506.9086 \\ 76.05499 & 506.9027 \\ 76.05988 & 506.8968 \\ 76.06478 & 506.8909 \\ 76.06969 & 506.8851 \\ 76.07458 & 506.8792 \\ 76.07948 & 506.8734 \\ 76.08438 & 506.8676 \\ 76.08928 & 506.8618 \\ 76.09418 & 506.8560 \\ 76.09908 & 506.8501 \\ 76.10417 & 506.8448 \\ 76.10944 & 506.8402 \\ 76.11472 & 506.8359 \\ 76.12000 & 506.8318 \\ 76.12527 & 506.8279 \\ 76.13055 & 506.8243 \\ 76.13582 & 506.8209 \\ 76.14110 & 506.8177 \\ 76.14637 & 506.8148 \\ 76.15165 & 506.8120 \\ 76.15692 & 506.8094 \\ 76.16220 & 506.8069 \\ 76.16747 & 506.8047 \\ 76.17274 & 506.8027 \\ 76.17802 & 506.8008 \\ 76.18330 & 506.7991 \\ 76.18858 & 506.7975 \\ 76.19385 & 506.7961 \\ 76.19913 & 506.7948 \\ 76.20440 & 506.7937 \\ 76.20967 & 506.7926 \\ 76.21495 & 506.7917 \\ 76.22022 & 506.7910 \\ 76.22550 & 506.7903 \\ 76.23077 & 506.7898 \\ 76.23605 & 506.7893 \\ 76.24133 & 506.7890 \\ 76.24660 & 506.7888 \\ 76.25188 & 506.7887 \\ 76.25715 & 506.7887 \\ 76.26243 & 506.7887 \\ 76.26770 & 506.7889 \\ 76.27298 & 506.7891 \\ 76.27825 & 506.7894 \\ 76.28880 & 506.7898 \\ 76.29408 & 506.7909 \\ 76.29935 & 506.7915 \\ 76.30463 & 506.7921 \\ 76.30991 & 506.7929 \\ 76.32046 & 506.7937 \\ 706 & 506.7946\end{array}$




$\begin{array}{ll}76.32573 & 506.7955 \\ 76.33101 & 506.7965 \\ 76.33628 & 506.7975 \\ 76.34155 & 506.7986 \\ 76.34683 & 506.7998 \\ 76.35210 & 506.8010 \\ 76.35738 & 506.8022 \\ 76.36266 & 506.8034 \\ 76.36794 & 506.8047 \\ 76.37321 & 506.8061 \\ 76.37848 & 506.8075 \\ 76.38376 & 506.8089 \\ 76.38903 & 506.8104 \\ 76.39431 & 506.8119 \\ 76.39958 & 506.8134 \\ 76.40486 & 506.8150 \\ 76.41013 & 506.8166 \\ 76.41541 & 506.8183 \\ 76.42068 & 506.8199 \\ 76.42596 & 506.8216 \\ 76.43124 & 506.8233 \\ 76.43651 & 506.8251 \\ 76.44179 & 506.8268 \\ 76.44706 & 506.8286 \\ 76.45234 & 506.8305 \\ 76.45761 & 506.8323 \\ 76.46288 & 506.8342 \\ 76.46816 & 506.8361 \\ 76.47343 & 506.8380 \\ 76.47871 & 506.8399 \\ 76.48399 & 506.8419 \\ 76.48927 & 506.8439 \\ 76.49454 & 506.8459 \\ 76.49982 & 506.8479 \\ 76.50509 & 506.8499 \\ 76.51036 & 506.8519 \\ 76.51564 & 506.8540 \\ 76.52091 & 506.8560 \\ 76.52619 & 506.8582 \\ 76.53146 & 506.8602 \\ 76.53674 & 506.8624 \\ 76.54202 & 506.8645 \\ 76.54729 & 506.8667 \\ 76.55257 & 506.8688 \\ 76.55784 & 506.8709 \\ 76.56312 & 506.8731 \\ 76.56839 & 506.8753 \\ 76.57367 & 506.8775 \\ 76.57894 & 506.8797 \\ 76.58422 & 506.8819 \\ 76.58949 & 506.8842 \\ 76.59476 & 506.8864 \\ 76.60004 & 506.8887 \\ 76.60532 & 506.8909 \\ 76.61060 & 506.8954 \\ 76.63169 & 506.8978 \\ 76.64760 & 506.9024 \\ 76.66342 & 506.9069 \\ 76.67924 & 506.9112 \\ 76.69508 & 506.9160 \\ 706.9217\end{array}$




$\begin{array}{ll}76.71090 & 506.9280 \\ 76.72672 & 506.9348 \\ 76.74255 & 506.9421 \\ 76.75838 & 506.9498 \\ 76.77420 & 506.9579 \\ 76.79002 & 506.9662 \\ 76.80585 & 506.9749 \\ 76.82168 & 506.9839 \\ 76.83750 & 506.9931 \\ 76.85333 & 507.0024 \\ 76.86916 & 507.0119 \\ 76.88498 & 507.0216 \\ 76.90081 & 507.0313 \\ 76.91663 & 507.0412 \\ 76.93246 & 507.0512 \\ 76.94829 & 507.0613 \\ 76.96411 & 507.0714 \\ 76.97993 & 507.0815 \\ 76.99576 & 507.0918 \\ 77.01159 & 507.1021 \\ 77.02741 & 507.1124 \\ 77.04324 & 507.1227 \\ 77.05907 & 507.1331 \\ 77.07489 & 507.1435 \\ 77.09071 & 507.1538 \\ 77.10654 & 507.1642 \\ 77.12237 & 507.1747 \\ 77.13819 & 507.1851 \\ 77.15402 & 507.1955 \\ 77.16985 & 507.2060 \\ 77.18567 & 507.2164 \\ 77.20149 & 507.2268 \\ 77.21732 & 507.2373 \\ 77.23315 & 507.2477 \\ 77.24897 & 507.2581 \\ 77.26480 & 507.2686 \\ 77.28062 & 507.2789 \\ 77.29645 & 507.2893 \\ 77.31228 & 507.2997 \\ 77.32810 & 507.3101 \\ 77.34393 & 507.3205 \\ 77.35976 & 507.3308 \\ 77.37558 & 507.3412 \\ 77.39140 & 507.3515 \\ 77.40723 & 507.3618 \\ 77.42306 & 507.3721 \\ 77.43888 & 507.3825 \\ 77.45470 & 507.3928 \\ 77.47054 & 507.4031 \\ 77.48636 & 507.4133 \\ 77.50218 & 507.4236 \\ 77.51801 & 507.4339 \\ 77.53384 & 507.4442 \\ 77.54966 & 507.4544 \\ 77.56549 & 507.4646 \\ 77.58131 & 507.4748 \\ 77.59714 & 507.4850 \\ 77.61297 & 507.5054 \\ 77.62879 & 507.5156 \\ 77.64462 & 507.5257 \\ 77.66045 & 507.5359 \\ 77.67627 & 507.5460 \\ 77.69209 & \end{array}$




\begin{tabular}{|c|c|}
\hline 77.70792 & 507.5561 \\
\hline 77.72375 & 507.5662 \\
\hline 77.73957 & 507.5763 \\
\hline 77.75539 & 507.5864 \\
\hline 77.77122 & 507.5964 \\
\hline 77.78705 & 507.6065 \\
\hline 77.80287 & 507.6165 \\
\hline 77.81870 & 507.6266 \\
\hline 77.83453 & 507.6366 \\
\hline 77.85035 & 507.6466 \\
\hline 77.86618 & 507.6566 \\
\hline 77.88200 & 507.6666 \\
\hline 77.89783 & 507.6766 \\
\hline 77.91365 & 507.6865 \\
\hline 77.92948 & 507.6965 \\
\hline 77.94530 & 507.7064 \\
\hline 77.96113 & 507.7163 \\
\hline 77.97696 & 507.7262 \\
\hline 77.99278 & 507.7361 \\
\hline 78.00861 & 507.7460 \\
\hline 78.02444 & 507.7559 \\
\hline 78.04026 & 507.7657 \\
\hline 78.05608 & 507.7756 \\
\hline 78.07191 & 507.7854 \\
\hline 78.08774 & 507.7952 \\
\hline 78.10356 & 507.8051 \\
\hline 78.11939 & 507.8148 \\
\hline 78.13522 & 507.8246 \\
\hline 78.15104 & 507.8344 \\
\hline 78.16686 & 507.8442 \\
\hline 78.18269 & 507.8539 \\
\hline 78.19852 & 507.8637 \\
\hline 78.21434 & 507.8734 \\
\hline 78.23017 & 507.8831 \\
\hline \multicolumn{2}{|c|}{ ARIABLES $=" x "$} \\
\hline DNE T=" Sta & c Air Temp \\
\hline 0.00000 & 416.9700 \\
\hline 40.07360 & 458.9067 \\
\hline 47.45212 & 467.8723 \\
\hline 49.25944 & 462.0252 \\
\hline 50.66056 & 473.7310 \\
\hline 54.75445 & 475.5438 \\
\hline 56.07415 & 476.0240 \\
\hline 58.45746 & 485.5237 \\
\hline 59.02501 & 485.0961 \\
\hline 59.64998 & 489.7584 \\
\hline 61.47612 & 490.6278 \\
\hline 62.08459 & 491.3479 \\
\hline 64.41123 & 500.0971 \\
\hline 64.96181 & 500.1538 \\
\hline 65.56672 & 503.5971 \\
\hline 67.33422 & 504.7017 \\
\hline 67.92163 & 505.4839 \\
\hline 70.14417 & 513.0594 \\
\hline 70.66892 & 513.6744 \\
\hline 71.24777 & 515.8846 \\
\hline 72.93916 & 516.7426 \\
\hline 73.49987 & 515.5519 \\
\hline 75.60910 & 521.0090 \\
\hline 76.10417 & 517.9590 \\
\hline 76.64760 & 519.1533 \\
\hline 78.23017 & 517.4634 \\
\hline
\end{tabular}


VARIABLES $=" x ", " y "$

ZONE $T="$ Location of Fan Leading Edge", $I=2.0$ $40.07360 \quad 400.0$

$40.07360 \quad 465.0$ 
Appendix G: NPSS cycle analysis for the altitude cruise conditions at 35000 feet

$5 \mu \mathrm{m}, \mathrm{ISA}+18 \mathrm{R}$

Date:05/10/13 Time:10:57:24 Model: Turbofan Engine - COMDES ON CONverge = 1 CASE: O

Version:NPSS 1.6.5 - Rev: -> Gas Package: Janaf iter/pass/Jacb/Broy= 25/39/1/23 Run by: Philip C Jonvergon 12 PC: 100

Temperature Stator 1 inlet: 449.58 Stator 1 exit: 468.32 Stator 2 inlet: 490.14 Stator 2 exit: 497.71

Stator 3 inlet: $526.40 \quad$ Stator 3 exit: 534.65 Stator 4 Unlet: 561.45 Stator 4 exit: 569.32
Stator 5 inlet: $590.12 \quad$ Stator 5 exit: $597.80 \quad$ Percent Blockage: 0.00

Ambient Relative Humidity

Fan Face Relative Humidity $\quad 5.12$

Fan Bypass Relative Humidity $\quad 0.16$

LPC Inlet Relative Humidity $\quad 2.30$

LPC Exit Relative Humidity $\quad 0.02$

HPC Relative Humidity $\quad 0.01$

Ambient Flow Velocity

$\begin{aligned} .0000050 & \text { Inlet Length } \\ 776.30 & \text { Fan/LPC Inlet Flow Velocity }\end{aligned}$

Ambient Flow Velocity

Ambient Static Temperature

Additional Water at IPC Exit

3.46 Fan/LPC Inlet Static Pressure $\quad 4.45$

411.85 Fan/LPC Inlet Static Temperature 442.76

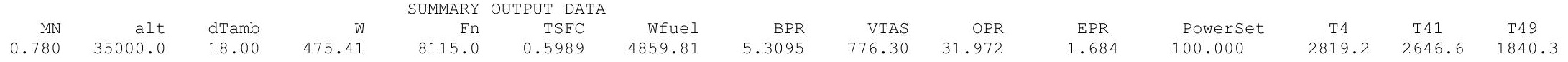

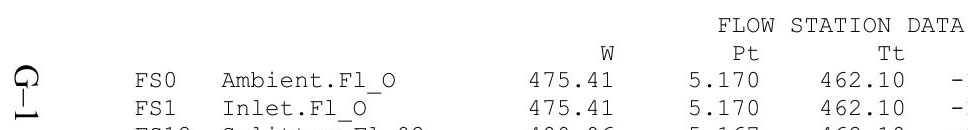

FS12 Splitter.F1_02

FS2 Splitter.Fl_

FS14 Fan.Fl_

ES24 VaporIN.F1

ES25 Bleed2.FI

FS3 HPC.Fl_O

FS36 Bleed3.F1 O

FS4 Burner.Fl-O

FS45 HPT.FI_O

FS49 LPT.FI-O

FS5 TEGV.FI_O

FS8 Core_Nozz.FIO

ES17 FanDuctLkg.Fi_O

FS171 Bleed15.F1_

FS172 FanDuct.Fl_O

TURBOMACHINERY PERFORMANCE DATA

\begin{tabular}{|c|c|c|c|c|c|c|c|c|c|}
\hline \\
\hline Fan & $\begin{array}{r}\text { WC } \\
1073.94\end{array}$ & $\begin{array}{r}P R \\
693\end{array}$ & $\begin{array}{r}\text { eff } \\
0.9109\end{array}$ & $\begin{array}{r}\text { NC } \\
4121.004\end{array}$ & $\begin{array}{r}\text { TR } \\
1.1783\end{array}$ & $\begin{array}{l}\text { efPoly } \\
0.9173\end{array}$ & $\begin{array}{r}\text { pwr } \\
-11181.7\end{array}$ & $\begin{array}{r}\text { SMN } \\
38.37\end{array}$ & $\begin{array}{r}\text { SMW } \\
23.45\end{array}$ \\
\hline LPC & 202.27 & $\begin{array}{l}1.693 \\
2.475\end{array}$ & 0.8306 & 4121.004 & 1.3557 & 0.8506 & -4206.4 & 2.96 & $\begin{array}{r}23.45 \\
2.22\end{array}$ \\
\hline HPC & 95.62 & 12.924 & 0.8588 & 10318.107 & 2.1966 & 0.8979 & -19637.7 & 26.74 & 25.93 \\
\hline $\mathrm{HPT}$ & 12.10 & 4.278 & 0.9118 & 214.076 & 1.3463 & 0.8911 & 19637.7 & & \\
\hline LPT & 55.87 & 4.346 & .915 & 90.456 & 1.3915 & 0.8986 & 15388.5 & & \\
\hline & NERY M & DATA & & & & & & & \\
\hline & WcMap & PRmap & effMap & NcMap & R/Parm & S_WcDes & S_PRdes & S_effDes & S_NcDes \\
\hline n & 1247.81 & 1.677 & & 4081.711 & & $\overline{0} .8607$ & $\overline{1} .0235$ & 0.9980 & $\overline{0} .9905$ \\
\hline C & 165.14 & 2.728 & 0.8329 & 1.088 & 0.0000 & $1.224 \xi$ & 0.8538 & 0.9972 & 0.0003 \\
\hline C & 86.96 & 12.363 & 0.8677 & 10043.034 & 13.4277 & & 1.0494 & 0.9898 & 0.97 \\
\hline TT & 0.96 & 4.187 & 0.911 & 1.287 & 4.1869 & 12.6299 & 0.9723 & 1.0000 & \\
\hline $\mathrm{T}$ & 0.86 & 3.516 & 0.9224 & 1.018 & 3.5163 & $65.259 c$ & 0.7521 & 0.9926 & 0.00 \\
\hline
\end{tabular}

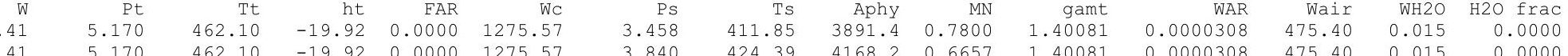

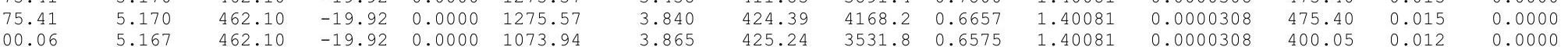

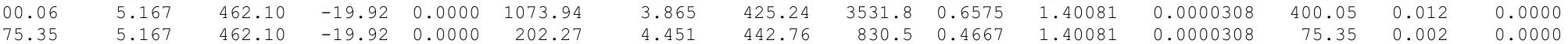

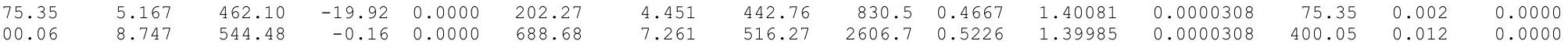

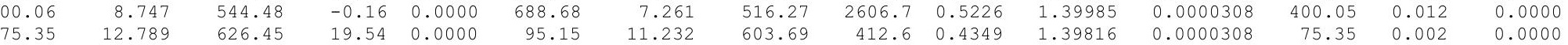

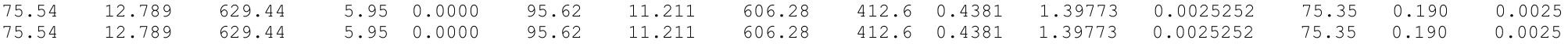

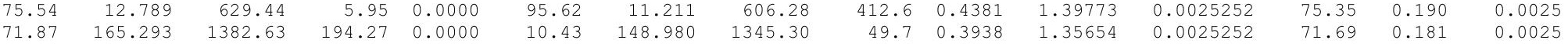

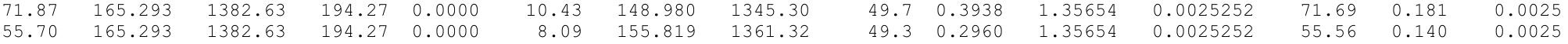

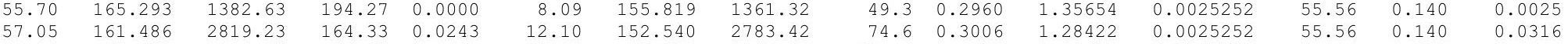

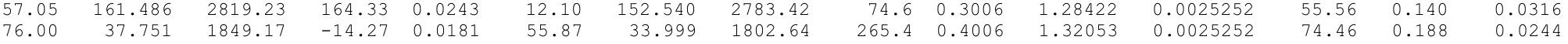
$\begin{array}{lllllllllllllll}76.89 & 8.687 & 1325.66 & -154.42 & 0.0179 & 207.94 & 7.493 & 1275.91 & 860.2 & 0.4724 & 1.34763 & 0.0025252 & 75.35 & 0.190 & 0.0241 \\ 76.89 & 8.687 & 1325.79 & -154.42 & 0.019 & 207.05 & 4.63 & 0.190 & 0.0241\end{array}$ $\begin{array}{lllllllllllllll}76.89 & 8.687 & 1325.79 & -154.42 & 0.0179 & 207.95 & 4.646 & 1125.68 & 613.4 & 1.0000 & 1.34762 & 0.0025252 & 75.35 & 0.190 & 0.0241\end{array}$ $\begin{array}{llllll}700.06 & 8.747 & 544.48 & -0.16 & 0.0000 & 688.68\end{array}$ $\begin{array}{llllll}400.06 & 8.747 & 544.48 & -0.16 & 0.0000 & 688.68\end{array}$ $\begin{array}{llllll}8.747 & 544.48 & -0.16 & 0.0000 & 688.68\end{array}$ $\begin{array}{lllll}8.747 & 544.48 & -0.16 & 0.0000 & 688.68\end{array}$

$\begin{array}{lllll}7.261 & 516.27 & 2606.7 & 0.5226 & 1.39985\end{array}$

$\begin{array}{lllll}7.056 & 512.06 & 2481.9 & 0.5625 & 1.39985 \\ 7.056 & 512.06 & 2481.9 & 0.5625 & 1.39985\end{array}$ $\begin{array}{lllll}7.056 & 512.06 & 2481.9 & 0.5625 & 1.39985 \\ 4.619 & 453.63 & 2006.9 & 1.0000 & 1.39985\end{array}$

$\begin{array}{lll}0.0000308 & 400.05 & 0.012\end{array}$

$\begin{array}{lll}0.0000308 & 400.05 & 0.012\end{array}$

$\begin{array}{lll}0.0000308 & 400.05 & 0.012\end{array}$

$\begin{array}{lll}400.05 & 0.012 & 0.0000 \\ 400.05 & 0.012 & 0.0000 \\ 400.05 & 0.012 & 0.0000\end{array}$ 0.0000 


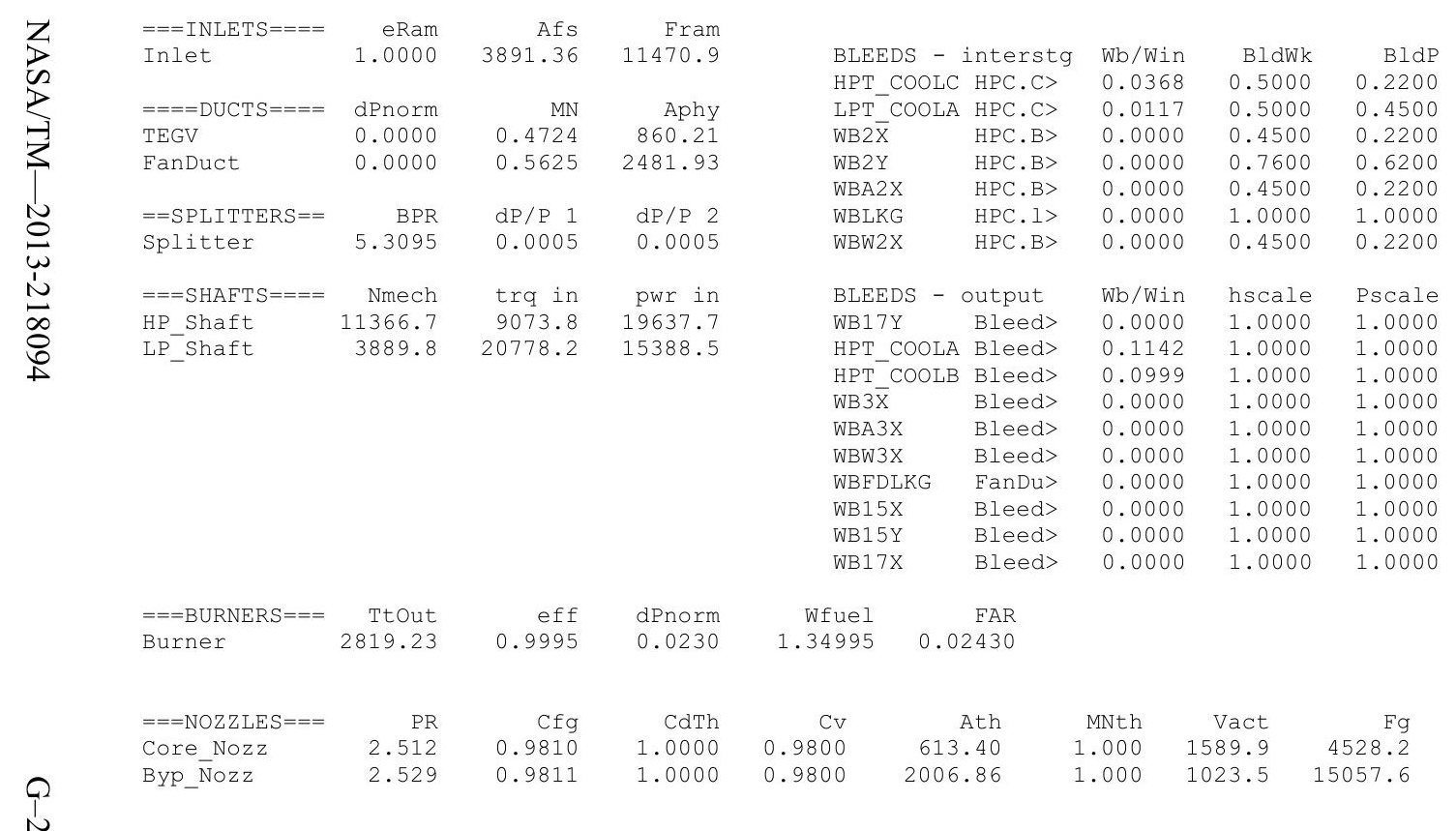


Date: $05 / 10 / 13$ Time: $10: 57: 57$ Model:

Turbofan Engine - COMDES ON COnverge $=1$ CASE: 0

Version:NPSS1.6.5 - Rev: -> Gas Package: Janaf iter/pass/Jacb/Broy=19/33/1/17 Run by: Philip C Jorgenson PC: 75

Temperature Stator 1 inlet: 459.18 Stator 1 exit: 475.50 Stator 2 inlet: 495.41 Stator 2 exit: 503.84 Stator 3 inlet: $527.44 \quad$ Stator 3 exit: 535.81 Stator 4 inlet: 558.04 Stator 4 exit: 565.66
Stator 5 inlet: $583.45 \quad$ Stator 5 exit: 590.65

Ambient Relative Humidity $\quad 10.00$

Fan Face Relative Humidity 3.59

Fan Bypass Relative Humidity 0.24

LPC Inlet Relative Humidity $\quad 1.84$

LPC Exit Relative Humidity $\quad 0.02$

HPC Relative Humidity

Drop Diameter

0.0000050

776.30

Inlet Length

40.00

Ambient

Ambient Static Temperature

3.46 Fan/LPC Inlet Static Pressure $\quad 412.05$

Additional Water at LPC Exit 0.0031004

$\begin{array}{lr}\text { Fan/LPC Inlet Static Pressure } & 4.64 \\ \text { Fan/LPC Inlet Static Temperature } & 447.94\end{array}$

$\begin{array}{rrrr}\text { MN } & \text { alt } & \text { dTamb } & \\ 0.780 & 35000.0 & 18.00 & 439.10\end{array}$

SUMMARY OUTPUT DATA

Fn TSFC

Wfuel

BPR
5.6163

VTAS

$\mathrm{OPR}$
26.730

EPR

Powerset

$\begin{array}{ccc}\text { T4 } & \text { T41 } & \text { T49 } \\ 2550.2 & 2398.0 & 1660.9\end{array}$

FSO Ambient.FI_O

FS1 Inlet.Fl_o-

$\begin{array}{ll}\text { FS12 } & \text { Splitter.F1_02 } \\ \text { FS2 } & \text { Splitter.F1_01 }\end{array}$

FS14 Fan.F1_O

$\begin{array}{ll}\text { FS23 } & \text { LPC.FI_O } \\ \text { FS24 } & \text { VaporIN.F1_O }\end{array}$

$\begin{array}{ll}\text { FS24 } & \text { VaporIN.F1- } \\ \text { FS25 } & \text { Bleed2.F1_O }\end{array}$

FS3 HPC.FI_O

FS36 Bleed3.F1_O

FS4 Burner.FI_O

FS49 LPT.FI-

FS5 TEGV.F̄

FS8 Core_Nozz.Fl_O

FS17 FanDuctLkg.Fì_O

FS172 FanDuct. F1-

FS173 Byp_Nozz.Fì_o

W
439.10
439.10
372.74
66.37
372.74
66.37
66.57
66.57
63.34
49.09
50.10
66.80
67.58
67.58
67.58
372.74
372.74
372.74
372.74

\section{FLOW STATION DATA}

$\begin{array}{rrrr}\text { Pt } & \text { Tt } & \text { ht } & \text { FAR } \\ 5.170 & 462.10 & -19.92 & 0.0000 \\ 5.170 & 462.10 & -19.92 & 0.0000 \\ 5.167 & 462.10 & -19.92 & 0.0000 \\ 5.167 & 462.10 & -19.92 & 0.0000 \\ 8.047 & 530.89 & -3.43 & 0.0000 \\ 12.409 & 613.63 & 16.45 & 0.0000 \\ 12.409 & 617.41 & -0.42 & 0.0000 \\ 12.409 & 617.41 & -0.42 & 0.0000 \\ 138.194 & 1299.37 & 169.25 & 0.0000 \\ 38.194 & 1299.37 & 169.25 & 0.0000 \\ 35.011 & 2550.20 & 144.50 & 0.0206 \\ 31.395 & 1668.42 & -17.41 & 0.0153 \\ 7.216 & 1189.32 & -142.92 & 0.0152 \\ 7.216 & 1189.39 & -142.92 & 0.0152 \\ 7.216 & 1189.52 & -142.92 & 0.0152 \\ 8.047 & 530.89 & -3.43 & 0.0000 \\ 8.047 & 530.89 & -3.43 & 0.0000 \\ 8.047 & 530.89 & -3.43 & 0.0000 \\ 8.047 & 530.89 & -3.43 & 0.0000\end{array}$

WC
178.15
178.15
1000.58
178.16
688.70
85.49
86.02
86.02
10.66
8.26
12.09
56.08
208.42
208.42
208.43
688.70
688.70
688.70
688.70

Ps
3.458

3.458
4.119
4.110

4.110
4.635

4.635
6.680

6.680
11.228
11.208

11.208

11.208
123.862
129.894

123.862
129.894
127.512

129.894
127.512

28.245
6.220

$6.220 \quad 1143.51$

$6.220 \quad 1143.58$

$\begin{array}{rr}3.848 & 1005.77 \\ 6.680 & 503.37\end{array}$

$\begin{array}{ll}6.680 & 503.37 \\ 6.491 & 499.26\end{array}$

$\begin{array}{ll}6.491 & 499.26 \\ 4.250 & 442.28\end{array}$

Ts
411.85
433.00
432.79
447.94
503.37
96.38
599.78
99.78
62.06
1278.16
2516.99
25.05
43.51
43.58
105.77
503.37
99.26
499.26
442.28

$\begin{array}{rr}\text { Aphy } & \text { MN } \\ 3594.2 & 0.7800 \\ 4168.2 & 0.5790 \\ 3531.8 & 0.5812 \\ 830.5 & 0.3970 \\ 2606.7 & 0.5226 \\ 412.6 & 0.3809 \\ 412.6 & 0.3842 \\ 412.6 & 0.3842 \\ 49.7 & 0.4037 \\ 49.3 & 0.3028 \\ 74.6 & 0.2992 \\ 265.4 & 0.4010 \\ 860.2 & 0.4719 \\ 860.2 & 0.4719 \\ 613.4 & 1.0000 \\ 2606.7 & 0.5226 \\ 2481.9 & 0.5625 \\ 2481.9 & 0.5625 \\ 2006.9 & 1.0000\end{array}$

gamt
1.40081
1.40081
1.40081
1.40081
1.40006
1.39848
1.39795
1.39795
1.36141
1.36141
1.29428
1.33048
1.35789
1.35789
1.35788
1.40006
1.40006
1.40006
1.40006

WAR
0.0000308
0.0000308
0.0000308
0.0000308
0.0000308
0.0000308
0.0031313
0.0031313
0.0031313
0.0031313
0.0031313
0.0031313
0.0031313
0.0031313
0.0031313
0.0000308
0.0000308
0.0000308
0.0000308

Wair

439.09 WH2

WH2O H2O frac

TURBOMACHINERY PERFORMANCE DATA

\begin{tabular}{|c|c|c|c|c|c|c|c|c|c|}
\hline & WC & $P R$ & eff & $\mathrm{NC}$ & $T R$ & efpoly & pwr & SMN & SMW \\
\hline Fan & 1000.58 & 1.557 & 0.9070 & 3844.865 & 1.1489 & 0.9127 & -8698.1 & 62.23 & 29.30 \\
\hline LPC & 178.16 & 2.401 & 0.8671 & 3844.865 & 1.3279 & 0.8823 & -3415.4 & 3.99 & 3.15 \\
\hline HPC & 86.02 & 11.137 & 0.8615 & 10038.885 & 2.1045 & 0.8982 & -15593.4 & 33.66 & 32.34 \\
\hline HPT & 12.09 & 4.300 & 0.9079 & 216.890 & 1.3559 & 0.8883 & 15593.4 & & \\
\hline LPT & 56.08 & 4.351 & 0.9115 & 88.848 & 1.4001 & 0.8942 & 12113.5 & & \\
\hline \multicolumn{10}{|c|}{ TURBOMACHINERY MAP DATA } \\
\hline & WcMap & PRmap & effMap & NcMap & R/Parm & S_WCDes & S_PRdes & s_effDes & S_NCDes \\
\hline Fan & 1162.58 & 1.544 & 0.9089 & 3808.205 & 0.0589 & $\overline{0} .8607$ & 1.0235 & 0.9980 & 0.9905 \\
\hline LPC & 143.26 & 2.433 & 0.8208 & 1.016 & 0.0000 & 1.2436 & 0.9782 & 1.0564 & 0.0003 \\
\hline HPC & 78.24 & 10.659 & 0.8704 & 9771.255 & 12.8223 & 1.0995 & 1.0494 & 0.9898 & 0.9733 \\
\hline HPT & 0.96 & 4.209 & 0.9079 & 1.303 & 4.2089 & 12.6299 & 0.9723 & 1.0000 & 0.0003 \\
\hline LPT & 0.86 & 3.520 & 0.9182 & 0.999 & 3.5203 & 65.2590 & 0.7521 & 0.9926 & 0.0005 \\
\hline
\end{tabular}




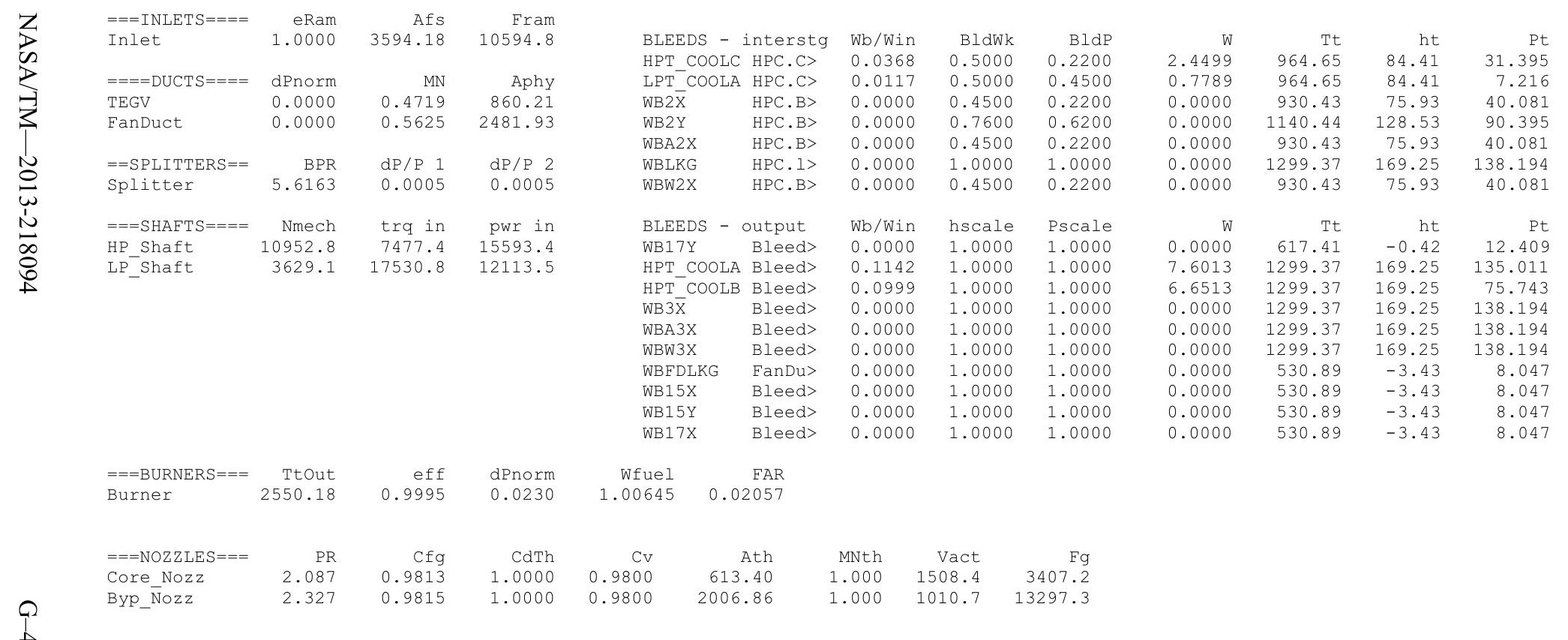


Ambient Relative Humidity $\quad 10.00$

Fan Face Relative Humidity 3.47

Fan Bypass Relative Humidity 0.25

LPC Inlet Relative Humidity $\quad 1.80$

LPC Exit Relative Humidity $\quad 0.02$

HPC Relative Humidity $\quad 0.01$

Drop Diameter

0.0000050

776.30
3.46

Inlet Length

40.00

Ambient Flow Velocity

Fan/LPC Inlet Flow Velocity $\quad 403.58$

Ambient Static Temperature

Additional Water at LPC Exit

411.85

Fan/LPC Inlet Static Temperature 448.52

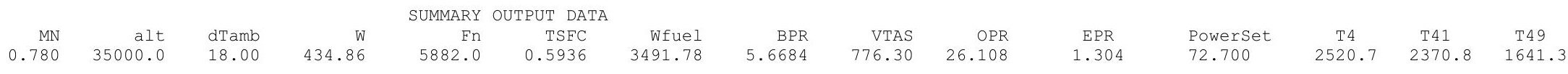

\section{FSO Ambient.FI_O $\quad 434.86$}

FS1 Inlet.Fl_O $\quad 434.86$

FS12 Splitter.F1_02

FS14 Fan.F1_OF__

FS23 2 IPC.

FS24 VaporIN.FI_O

FS25 Bleed2.FI_

HPC.FI_O

FS36 Bleed3.FI_O

FS45 Burner.F1-

$\begin{array}{ll}\text { FS45 } & \text { HPT.FI-O } \\ \text { FS49 } & \text { LPT.FI-O }\end{array}$

FS5 TEGV.FI

FS8 Core_Nozz.FI_O

FS17 FanDüctLkg.Fì_O

FS171 Bleed15.Fl_O

FS172 FanDuct.FI_O

TURBOMACHINERY PERFORMANCE DATA

\begin{tabular}{|c|c|c|c|c|c|c|c|c|c|c|}
\hline & & & & FLOW & STATION DA? & & & & & \\
\hline & & & W & Pt & $\mathrm{Tt}$ & ht & FAR & Wo & & Ps \\
\hline FSO & Ambient.Fl_o & & 434.86 & 5.170 & 462.10 & -19.92 & 0.0000 & $\begin{array}{ll}0 & 1166.77\end{array}$ & 3.45 & 411. \\
\hline FS1 & Inlet.Fl $0^{-}$ & & 434.86 & 5.170 & 462.10 & -19.92 & 0.0000 & $0 \quad 1166.77$ & 4.12 & 433. \\
\hline FS12 & Splitter.Fl_ & & 369.65 & 5.167 & 462.10 & -19.92 & 0.0000 & 992.29 & 4.13 & 433. \\
\hline FS2 & Splitter.FI- & & 65.21 & 5.167 & 462.10 & -19.92 & 0.0000 & 175.06 & 4.65 & 448. \\
\hline FS14 & Fan.Fl_o & & 369.65 & 7.968 & 529.26 & -3.82 & 0.0000 & 688.70 & 6.61 & 501. \\
\hline FS23 & LPC.FI_O & & 65.21 & 12.337 & 612.22 & 16.11 & 0.0000 & 84.40 & $11.1 \mathrm{~s}$ & 595 \\
\hline FS24 & VaporIN. Fl_o & & 65.42 & 12.337 & 616.12 & -1.31 & 0.0000 & 84.94 & 11.1 & \\
\hline FS25 & Bleed2.FI_ō & & 65.42 & 12.337 & 616.12 & -1.31 & 0.0000 & 84.94 & 11.17 & 599 \\
\hline FS3 & HPC.FI_O & & 62.25 & 134.976 & 1290.20 & 166.31 & 0.0000 & 10.69 & 120.89 & 94 \\
\hline FS36 & Bleed3.FI_O & & 48.24 & 134.976 & 1290.20 & 166.31 & 0.0000 & 8.28 & 126.82 & 23 \\
\hline FS4 & Burner.FI-o & & 49.21 & 131.868 & 2520.75 & 142.11 & 0.0202 & 12.09 & 124.54 & 2487. \\
\hline FS45 & HPT.FI_O & & 65.63 & 30.645 & 1648.68 & -17.96 & 0.0151 & 56.11 & 27.56 & 1605 \\
\hline FS 49 & LPT.FI_O & & 66.39 & 7.043 & 1174.70 & -141.85 & 0.0149 & 208.47 & 6.07 & 1129 \\
\hline FS5 & TEGV.FI O & & 66.39 & 7.043 & 1174.77 & -141.85 & 0.0149 & 208.48 & 6.07 & 1129 \\
\hline FS 8 & Core_Nozz.FI & & 66.39 & 7.043 & 1174.90 & -141.85 & 0.0149 & 208.49 & 3.75 & 992 \\
\hline FS17 & FanDūctLkg. F & & 369.65 & 7.968 & 529.26 & -3.82 & 0.0000 & 688.70 & 6.61 & 501 \\
\hline FS171 & Bleed15.FI & & 369.65 & 7.968 & 529.26 & -3.82 & 0.0000 & 688.70 & 6.42 & 497 \\
\hline FS172 & FanDuct.FI-O & & 369.65 & 7.968 & 529.26 & -3.82 & 0.0000 & 688.70 & 6.42 & 497 \\
\hline FS173 & Byp_Nozz.FI'_ & & 369.65 & 7.968 & 529.26 & -3.82 & 0.0000 & 688.70 & 4.20 & \\
\hline JRBC & MACHINERY PERI & ORMANCE & DATA & & & & & & & \\
\hline & & $\mathrm{PR}$ & eff & NC & TR & efPoly & & pwr & SMN & SMW \\
\hline Fan & 992.29 & 1.542 & 0.9070 & 3813.867 & 1.1453 & 0.9125 & -84 & 421.9 & 67.00 & 30.02 \\
\hline LPC & 175.06 & 2.387 & 0.8688 & 3813.867 & 1.3249 & 0.8837 & -33 & 324.5 & 4.27 & 3.36 \\
\hline HPC & 84.94 & 10.941 & 0.8615 & 10008.285 & 2.0941 & 0.8981 & -151 & 138.7 & 34.50 & 33.12 \\
\hline HPT & 12.09 & 4.303 & 0.9073 & 217.262 & 1.3571 & 0.8881 & 151 & 138.7 & & \\
\hline LPT & 56.11 & 4.351 & 0.9108 & 88.658 & 1.4008 & 0.8933 & 117 & 746.4 & & \\
\hline TURBC & MACHINERY MAP & DATA & & & & & & & & \\
\hline & WcMap & PRma & effMap & NcMap & R/Parm & S_WC & Des & S_PRdes & S_effDes & S_NcDes \\
\hline Fan & 1152.95 & 1.530 & 0.9089 & 3777.502 & 0.0584 & 0.8 & 3607 & 1.0235 & 0.9980 & 0.9905 \\
\hline LPC & 141.26 & 2.401 & 0.8202 & 1.007 & 0.0000 & 1.2 & 391 & 0.9905 & 1.0592 & 0.0003 \\
\hline HPC & 77.25 & 10.472 & 0.8704 & 9741.471 & 12.7535 & 1.0 & 995 & 1.0494 & 0.9898 & 0.9733 \\
\hline HPT & 0.96 & 4.212 & 0.9073 & 1.306 & 4.2116 & 12.6 & 299 & 0.9723 & 1.0000 & 0.0003 \\
\hline LPT & 0.86 & 3.520 & 0.9176 & 0.997 & 3.5203 & 65.2 & 590 & 0.7521 & 0.9926 & 0.0005 \\
\hline
\end{tabular}

$\begin{array}{rrrrrrr}\text { Aphy } & \text { MN } & \text { gamt } & \text { WAR } & \text { Wair } & \text { WH2O } & \text { H2O frac } \\ 3559.4 & 0.7800 & 1.40081 & 0.0000308 & 434.85 & 0.013 & 0.0000 \\ 4168.2 & 0.5701 & 1.40081 & 0.0000308 & 434.85 & 0.013 & 0.0000 \\ 3531.8 & 0.5735 & 1.40081 & 0.0000308 & 369.64 & 0.011 & 0.0000 \\ 830.5 & 0.3886 & 1.40081 & 0.0000308 & 65.21 & 0.002 & 0.0000 \\ 2606.7 & 0.5226 & 1.40008 & 0.0000308 & 369.64 & 0.011 & 0.0000 \\ 412.6 & 0.3750 & 1.39852 & 0.0000308 & 65.21 & 0.002 & 0.0000 \\ 412.6 & 0.3784 & 1.39796 & 0.0032329 & 65.21 & 0.211 & 0.0032 \\ 412.6 & 0.3784 & 1.39796 & 0.0032329 & 65.21 & 0.211 & 0.0032 \\ 49.7 & 0.4049 & 1.36195 & 0.0032329 & 62.05 & 0.201 & 0.0032 \\ 49.3 & 0.3036 & 1.36195 & 0.0032329 & 48.09 & 0.155 & 0.0032 \\ 74.6 & 0.2991 & 1.29540 & 0.0032329 & 48.09 & 0.155 & 0.0275 \\ 265.4 & 0.4011 & 1.33163 & 0.0032329 & 64.45 & 0.208 & 0.0214 \\ 860.2 & 0.4719 & 1.35901 & 0.0032329 & 65.21 & 0.211 & 0.0212 \\ 860.2 & 0.4719 & 1.35900 & 0.0032329 & 65.21 & 0.211 & 0.0212 \\ 613.4 & 1.0000 & 1.35899 & 0.0032329 & 65.21 & 0.211 & 0.0212 \\ 2606.7 & 0.5226 & 1.40008 & 0.0000308 & 369.64 & 0.011 & 0.0000 \\ 2481.9 & 0.5625 & 1.40008 & 0.0000308 & 369.64 & 0.011 & 0.0000 \\ 2481.9 & 0.5625 & 1.40008 & 0.0000308 & 369.64 & 0.011 & 0.0000 \\ 2006.9 & 1.0000 & 1.40008 & 0.0000308 & 369.64 & 0.011 & 0.0000\end{array}$

1.40008 


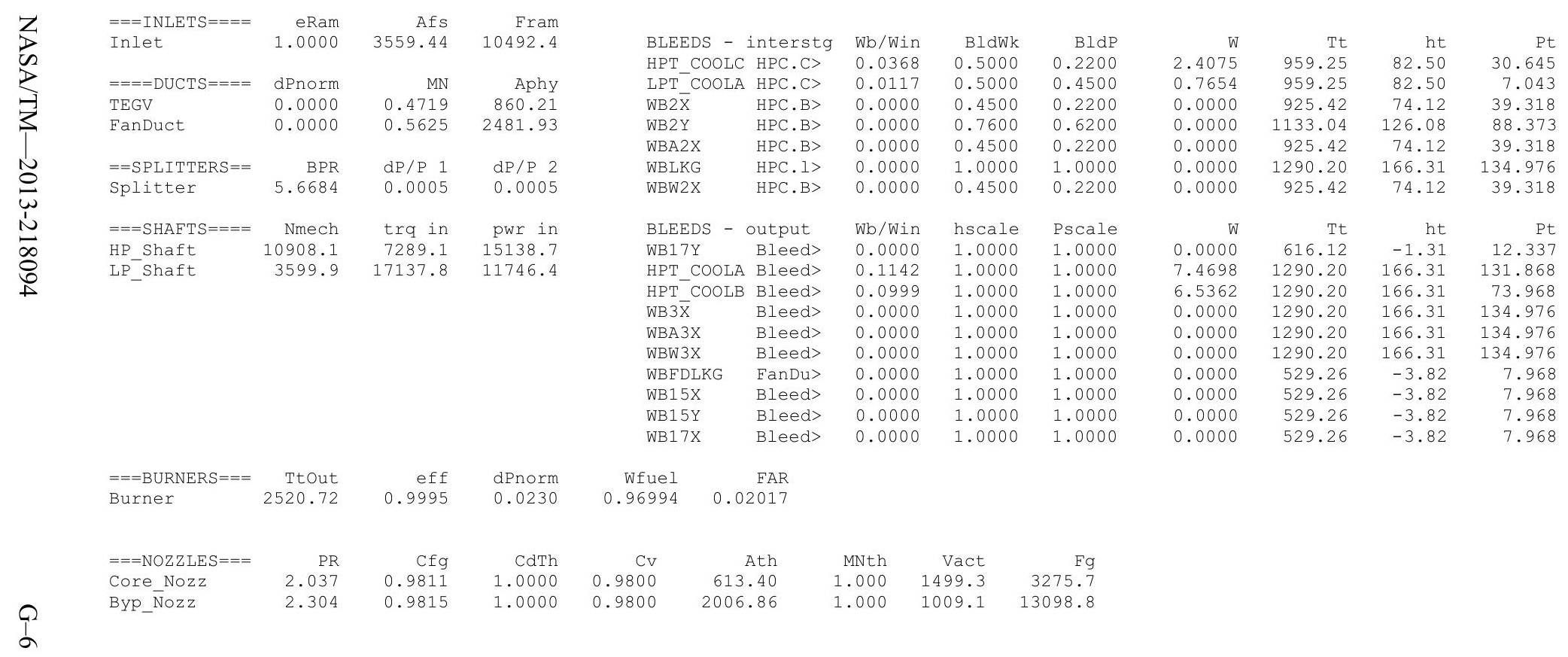


Date:05/10/13 Time:10:59:04 Model: Turbofan Engine - COMDES ON Converge $=1$ CASE: 0

Version:NPSS_1.6.5 - Rev: -> Gas Package: Janaf iter/pass/Jacb/Broy=20/34/1/18 Run by: Philip C Jorgenson PC: 69

Temperature Stator 1 inlet: $460.95 \quad$ Stator 1 exit: $476.77 \quad$ Stator 2 inlet: 496.25 $\quad$ Stator 2 exit: 504.70 $\begin{array}{llll}\text { Stator } 3 \text { inlet: } 527.37 & \text { Stator } 3 \text { exit: } 535.69 & \text { Stator } 4 \text { inlet: } 557.03 \text { Stator } 4 \text { exit: } 564.57 \\ \text { Stator } 5 \text { inlet: } 581.74 & \text { Stator } 5 \text { exit: } 588.81 & & \text { Unblocked Percent Blockage: } 0.00\end{array}$

Ambient Relative Humidity $\quad 10.00$

Fan Face Relative Humidity $\quad 3.37$

Fan Bypass Relative Humidity 0.26

LPC Inlet Relative Humidity $\quad 1.76$

LPC Exit Relative Humidity 0.02

HPC Relative Humidity $\quad 0.01$

Ambient Flow Velocity

Ambient Static Pressure

Additional Water at LPC Exit

$\begin{array}{rrrrr}\text { MN alt dTamb } & \text { W } & \text { SUMMARY OUTPUT DATA } \\ 0.780 & \text { Fn } & \text { TSFC }\end{array}$

$\begin{array}{llll}\text { Fn TSFC Wfuel BPR VTAS } & \\ 5 & \end{array}$

$\begin{array}{ccclccc}\text { VTAS } & \text { OPR } & \text { EPR } & \text { PowerSet } & \text { T4 } & \text { T41 } & \text { T49 } \\ 6.30 & 25.501 & 1.267 & 69.900 & 2492.3 & 2344.6 & 1622.6\end{array}$

FSO Ambient.FI_O

FS1 Inlet.Fl_O

$\begin{array}{ll}\text { FS12 } & \text { Splitter.F1_02 } \\ \text { FS2 } & \text { Splitter.FI_01 }\end{array}$

FS14 Fan.FI_O

$\begin{array}{ll}\text { FS23 } & \text { LPC.F1_O } \\ \text { FS24 } & \text { VaporIN.F1 }\end{array}$

$\begin{array}{ll}\text { FS24 } & \text { VaporIN.FI_O } \\ \text { FS25 } & \text { Bleed2.FI_O }\end{array}$

$\begin{array}{ll}\text { FS25 } & \text { Bleed2.F1 } \\ \text { FS3 } & \text { HPC.F1 O }\end{array}$

FS36 Bleed3.FI_O

FS4 Burner.FI_O

FS45 HPT.FI_

FS5 5 TEGV . FI

FS5 Core Nozz.Fl

$\begin{array}{ll}\text { FS8 } & \text { Core_Nozz.F1_O } \\ \text { FS17 } & \text { FanDuctLkg.FI_O }\end{array}$

$\begin{array}{ll}\text { FS17 } & \text { FanDuctLkg.FI } \\ \text { FS171 } & \text { Bleed15.FI_O }\end{array}$

FS172 FanDuct.Fl-O

TURBOMACHINERY PERFORMANCE DATA

$\begin{array}{lrrr} & \text { WC } & \text { PR } & \text { eff } \\ \text { Fan } & 984.46 & 1.528 & 0.9073 \\ \text { LPC } & 171.98 & 2.371 & 0.8701 \\ \text { HPC } & 83.92 & 10.759 & 0.8615 \\ \text { HPT } & 12.09 & 4.306 & 0.9067 \\ \text { LPT } & 56.14 & 4.351 & 0.9101\end{array}$

TURBOMACHINERY MAP DATA

$\begin{array}{lrrr} & \text { WCMap } & \text { PRmap } & \text { effMap } \\ \text { Fan } & 1143.84 & 1.515 & 0.9092 \\ \text { LPC } & 139.25 & 2.369 & 0.8196 \\ \text { HPC } & 76.33 & 10.300 & 0.8704 \\ \text { HPT } & 0.96 & 4.214 & 0.9067 \\ \text { LPT } & 0.86 & 3.520 & 0.9169\end{array}$

\section{FLOW STATION DATA}

\begin{tabular}{|c|c|c|c|}
\hline $\mathrm{Pt}$ & $\mathrm{Tt}$ & ht & FAR \\
\hline .170 & 462.10 & -19.92 & 0.0000 \\
\hline .170 & 462.10 & -19.92 & 0.0000 \\
\hline 5.167 & 462.10 & -19.92 & 0000 \\
\hline 5.167 & 462.10 & -19.92 & 0.0000 \\
\hline 7.893 & 527.69 & -4.19 & 0.0000 \\
\hline 12.253 & 610.66 & 15.74 & \\
\hline 12.253 & 614.68 & -2.14 & 0.0000 \\
\hline 12.253 & 614.68 & -2.14 & 0.0000 \\
\hline 31.839 & 1281.18 & 163.50 & 0.0000 \\
\hline 31.83 & & 163. & \\
\hline 28.803 & 2492.34 & 139.82 & 0.0198 \\
\hline 29.914 & 629.81 & -18.47 & \\
\hline 6.876 & 1160.77 & -140.80 & 0.0146 \\
\hline 6.876 & 1160.84 & -140.80 & 0.0146 \\
\hline 6.876 & 1160.97 & -140.80 & 0.0146 \\
\hline 7.893 & 527.69 & -4.19 & 0.0000 \\
\hline 7.893 & 527.69 & -4.19 & 0.0000 \\
\hline 7.893 & 527.69 & -4.19 & 0.0000 \\
\hline 7.893 & 527.69 & -4.19 & 0.0000 \\
\hline
\end{tabular}

BPR
5.7242

WC
1155.86 1155.86
1155.86 1155.86
984.46 171.98

688.70 83.37
83.92

83.92
83.92

83.92
10.71

8.30
12.09

12.09
56.14

56.14
208.52

208.52
208.52

208.52
208.53

688.70

688.70 688.70

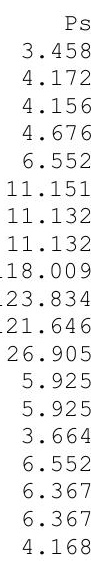

PS
.458

Ts
411.85
434.60

Aphy MN $\begin{array}{ll}3526.2 & 0.7800 \\ 4168.2 & 0.5618 \\ 3531.8 & 0.5664\end{array}$ $\begin{array}{rrr}434.18 & 3531.8 & 0.5664 \\ 449.07 & 830.5 & 0.38004\end{array}$ $\begin{array}{lrl}500.34 & 2606.7 & 0.5226\end{array}$ $\begin{array}{rrr}594.47 & 412.6 & 0.3696\end{array}$ $\begin{array}{lll}598.11 & 412.6 & 0.3730\end{array}$ $\begin{array}{lll}598.11 & 412.6 & 0.3730\end{array}$ $\begin{array}{rrr}1243.87 & 49.7 & 0.4060\end{array}$ $\begin{array}{lll}1259.98 & 49.3 & 0.3043\end{array}$

$\begin{array}{rrr}1259.98 & 74.6 & 0.2989 \\ 1587.15 & 265.4 & 0.4012\end{array}$ 1587.15

1587.15
1115.83

1115.89

1115.89
980.78

500.34

496.25

496.25

439.61

$\begin{array}{ll}265.4 & 0.4012 \\ 860.2 & 0.4718\end{array}$

860.20 .4718

$613.4 \quad 1.0000$

$2481.9 \quad 0.5625$

$2481.9 \quad 0.5625$

gamt
1.40081
1.40081
1.40081
1.40081
1.40010
1.39855
1.39799
1.39799
1.36250
1.36250
1.29650
1.33274
1.36007
1.36007
1.36006
1.40010
1.40010
1.40010
1.40010

WAR
0.0000308

Wair

$430.78-0.013$ H2O frac $\begin{array}{lll}430.78 & 0.013 & 0.0000\end{array}$ $\begin{array}{llll}0.0000308 & 366.72 & 0.011 & 0.0000\end{array}$ $\begin{array}{llll}0.0000308 & 64.06 & 0.011 & 0.0000 \\ 0.0000308 & 366.72 & 0.011 & 0.0000\end{array}$ $\begin{array}{llll}0.0000308 & 64.06 & 0.002 & 0.0000 \\ 0.0000308 & 64.06 & 0.011 & 0.0000\end{array}$ $\begin{array}{llll}0.0000308 & 366.72 & 0.011 & 0.0000 \\ 0.0033183 & 64.06 & 0.002 & 0.0000\end{array}$ $\begin{array}{llll}0.0033183 & 64.06 & 0.002 & 0.0000 \\ 0.0033183 & 64.06 & 0.213 & 0.0033\end{array}$ $\begin{array}{llll}0.0033183 & 64.06 & 0.213 & 0.0033 \\ 0.0033183 & 60.96 & 0.202 & 0.0033\end{array}$ $\begin{array}{llll}0.0033183 & 47.24 & 0.157 & 0.0033\end{array}$ $\begin{array}{llll}0.0033183 & 47.24 & 0.157 & 0.0033 \\ 0.0033183 & 47.24 & 0.157 & 0.0271\end{array}$ $\begin{array}{llll}0.0033183 & 63.32 & 0.210 & 0.0212\end{array}$ $\begin{array}{llll}0.0033183 & 63.32 & 0.210 & 0.0212 \\ 0.0033183 & 64.06 & 0.213 & 0.0210\end{array}$ $\begin{array}{llll}0.0033183 & 64.06 & 0.213 & 0.0210\end{array}$ $\begin{array}{llll}0.0033183 & 64.06 & 0.213 & 0.0210 \\ 0.0000308 & 366.72 & 0.011 & 0.0210\end{array}$ $\begin{array}{llll}0.0000308 & 366.72 & 0.011 & 0.0210\end{array}$ $\begin{array}{llll}0.0000308 & 366.72 & 0.011 & 0.0000\end{array}$ $\begin{array}{llll}0.0000308 & 366.72 & 0.011 & 0.0000\end{array}$ $\begin{array}{rllll}\text { NC } & T \text { TR efPoly } & \text { pwr } & \text { SMN } & \text { SMW }\end{array}$ $\begin{array}{rrrrrr}3782.622 & 1.1419 & 0.9127 & -8159.8 & 73.33 & 30.72\end{array}$ $\begin{array}{rrrrrr}3782.622 & 1.3215 & 0.8848 & -3232.3 & 4.55 & 3.56\end{array}$ $\begin{array}{rrrr}9979.760 & 2.0843 & 0.8979 & -14699.2 \\ 217.619 & 1.3581 & 0.8875 & 14699.2\end{array}$ $88.439 \quad 1.4015 \quad 0.8925 \quad 11392.4$

$\begin{array}{rrrrrr}\text { NcMap } & \text { R/Parm } & \text { S_WcDes } & \text { S_PRdes } & \text { S_effDes } & \text { S_NcDes } \\ 3746.555 & 0.0578 & 0.8607 & 1.0235 & 0.9980 & 0.9905 \\ 0.999 & 0.0000 & 1.2351 & 1.0017 & 1.0617 & 0.0003 \\ 9713.706 & 12.6887 & 1.0995 & 1.0494 & 0.9898 & 0.9733 \\ 1.308 & 4.2142 & 12.6299 & 0.9723 & 1.0000 & 0.0003 \\ 0.995 & 3.5201 & 65.2590 & 0.7521 & 0.9926 & 0.0005\end{array}$




\begin{tabular}{|c|c|c|c|c|c|c|c|c|c|c|c|c|}
\hline$===\operatorname{INLETS}====$ & eRam & Afs & Fram & & & & & & & & & \\
\hline Inlet & 1.0000 & 3526.18 & 10394.4 & BLEEDS - & interstg & $\mathrm{Wb} / \mathrm{Win}$ & BldWk & $\mathrm{BldP}$ & W & $\mathrm{Tt}$ & ht & Pt \\
\hline & & & & HPT COOLC & $C \mathrm{HPC} . \mathrm{C}>$ & 0.0368 & 0.5000 & 0.2200 & 2.3654 & 953.86 & 80.68 & 29.914 \\
\hline$====$ DUCTS $====$ & dPnorm & $\mathrm{MN}$ & Aphy & LPT COOLA & A HPC.C> & 0.0117 & 0.5000 & 0.4500 & 0.7520 & 953.86 & 80.68 & 6.876 \\
\hline TEGV & 0.0000 & 0.4718 & 860.21 & WB2 $\bar{X}$ & HPC.B> & 0.0000 & 0.4500 & 0.2200 & 0.0000 & 920.41 & 72.40 & 38.562 \\
\hline FanDuct & 0.0000 & 0.5625 & 2481.93 & WB2Y & HPC.B> & 0.0000 & 0.7600 & 0.6200 & 0.0000 & 1125.71 & 123.75 & 86.396 \\
\hline & & & & WBA $2 X$ & HPC.B> & 0.0000 & 0.4500 & 0.2200 & 0.0000 & 920.41 & 72.40 & 38.562 \\
\hline$==$ SPLITTERS $==$ & BPR & $\mathrm{dP} / \mathrm{P} 1$ & $\mathrm{dP} / \mathrm{P} 2$ & WBLKG & HPC. $1>$ & 0.0000 & 1.0000 & 1.0000 & 0.0000 & 1281.18 & 163.50 & 131.839 \\
\hline Splitter & 5.7242 & 0.0005 & 0.0005 & WBW2X & HPC.B> & 0.0000 & 0.4500 & 0.2200 & 0.0000 & 920.41 & 72.40 & 38.562 \\
\hline$===\mathrm{SHAFTS}====$ & Nmech & trg in & pwr in & BLEEDS - & output & Wb/Win & hscale & Pscale & W & Tt & ht & Pt \\
\hline HP_Shaft & 10864.3 & 7106.0 & 14699.2 & WB17Y & Bleed> & 0.0000 & 1.0000 & 1.0000 & 0.0000 & 614.68 & -2.14 & 12.253 \\
\hline LP_Shaft & 3570.4 & 16758.5 & 11392.4 & HPT_COOLA & A Bleed> & 0.1142 & 1.0000 & 1.0000 & 7.3392 & 1281.18 & 163.50 & 128.803 \\
\hline & & & & HPT COOLB & Bleed> & 0.0999 & 1.0000 & 1.0000 & 6.4220 & 1281.18 & 163.50 & 72.238 \\
\hline & & & & WB $3 \overline{\mathrm{X}}$ & Bleed> & 0.0000 & 1.0000 & 1.0000 & 0.0000 & 1281.18 & 163.50 & 131.839 \\
\hline & & & & WBA3X & Bleed> & 0.0000 & 1.0000 & 1.0000 & 0.0000 & 1281.18 & 163.50 & 131.839 \\
\hline & & & & WBW3X & Bleed> & 0.0000 & 1.0000 & 1.0000 & 0.0000 & 1281.18 & 163.50 & 131.839 \\
\hline & & & & WBFDLKG & FanDu> & 0.0000 & 1.0000 & 1.0000 & 0.0000 & 527.69 & -4.19 & 7.893 \\
\hline & & & & WB15X & Bleed> & 0.0000 & 1.0000 & 1.0000 & 0.0000 & 527.69 & -4.19 & 7.893 \\
\hline & & & & WB15Y & Bleed> & 0.0000 & 1.0000 & 1.0000 & 0.0000 & 527.69 & -4.19 & 7.893 \\
\hline & & & & WB17X & Bleed> & 0.0000 & 1.0000 & 1.0000 & 0.0000 & 527.69 & -4.19 & 7.893 \\
\hline$===$ BURNERS $===$ & Ttout & eff & dPnorm & Wfuel & FAR & & & & & & & \\
\hline Burner & 2492.31 & 0.9995 & 0.0230 & .93512 & 01979 & & & & & & & \\
\hline$===$ NOZZLES $===$ & PR & $\mathrm{Cfg}$ & CdTh & $\mathrm{Cv}$ & Ath & MNth & Vact & $\mathrm{Fg}$ & & & & \\
\hline Core Nozz & 1.988 & 0.9808 & 1.0000 & 0.9800 & 3.40 & 1.000 & 1490.6 & 3147.7 & & & & \\
\hline Byp_Nozz & 2.283 & 0.9815 & 1.0000 & 0.9800 & 6.86 & 1.000 & 1007.6 & 12911.0 & & & & \\
\hline
\end{tabular}




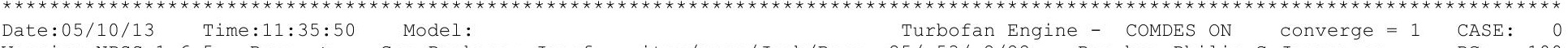

ersionsus 1.6.5 - Rev: -> Gas Package: Janaf iter/pass/Jacb/Broy $=25 / 53 / 2$

Temperature Stator 1 inlet: 469.84 Stator 1 exit: 489.48 Stator 2 inlet: 512.47 Stator 2 exit: 520.65

Stator 5 inlet: $617.26 \quad$ Stator 5 exit: $625.38 \quad$ Stator 4 Unblocked Percent Blockage: 0.00

Ambient Relative Humidity

Fan Face Relative Humidity

Fan Bypass Relative Humidity $\quad 0.21$

LPC Inlet Relative Humidity $\quad 2.51$

PC Exit Relative Humidity

Drop Diameter

0.01

Inlet Length $\quad 40.00$

Ambient Flow Velocity

Ambient Static Pressure

Ambient Static Temperature

Additional Water at IPC Exit

Fan/LPC Inlet Flow Velocity $\quad 490.02$

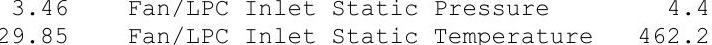

0.0048230 Fan/LPC Inlet Static Temperature 462.27

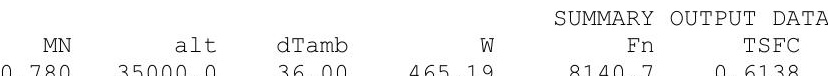
W
19
Wfuel
BPR
3274
VTAS
OPR
EPR
1.687
Powerset
$\begin{array}{rr}\text { T4 } & \text { T41 } \\ 2926.9 & 2748.9\end{array}$
T49

\section{FLOW STATION DATA}

\begin{tabular}{|c|c|c|c|c|c|c|c|c|c|c|c|}
\hline & & & & FL & & & FAR & & & & \\
\hline FSO & Ambient.Fl_O & & 465.19 & 5.170 & 482.28 & -15.41 & 0.0000 & 1275.1 & 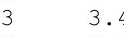 & & 429.8 \\
\hline FS1 & Inlet.Fl_o- & & 465.19 & 5.170 & 482.28 & -15.41 & 0.0000 & 1275.1 & 3.8 & & 442.9 \\
\hline FS12 & Splitter.Fl_0 & & 391.67 & 5.167 & 482.28 & -15.41 & 0.0000 & 1074.1 & 3.8 & & 443. \\
\hline FS2 & Splitter.Fl_0 & & 73.52 & 5.167 & 482.28 & -15.41 & .0000 & 201.6 & 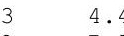 & & 462 \\
\hline FS14 & Fan.Fl_O & & 391.67 & 8.749 & 568.25 & 5.22 & 0.0000 & 688.6 & 7.2 & & 538 \\
\hline FS23 & LPC.FI_O & & 73.52 & 12.916 & 655.07 & 26.11 & 0.0000 & 94.0 & 11.3 & & 632 \\
\hline FS24 & 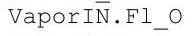 & & 73.88 & 12.916 & 660.53 & -0.14 & 0.0000 & 94.8 & 11.3 & & 63 \\
\hline 525 & Bleed2.Fl_o & & 73.88 & 12.916 & 660.53 & -0.14 & 0.0000 & 94.8 & 11.3 & & 63 \\
\hline 3 & HPC.FI_O & & 70.29 & 164.951 & 1439.80 & 196.08 & 0.0000 & 10.4 & 148.6 & & 1401 \\
\hline$\$ 36$ & Bleed3.Fl_O & & 54.48 & 64.951 & 1439.80 & 196.08 & .0000 & 8.0 & 155.4 & & 1417 \\
\hline 54 & Burner.Fl_o & & 55 & 161.152 & 2926.92 & 164.50 & 0.0256 & 12.1 & 152.2 & & 890 \\
\hline S45 & HPT.FI_O ${ }^{-}$ & & 74 & 37.752 & 1927.83 & -21.33 & 0 & 55.8 & 33.9 & & 879 \\
\hline FS49 & LPT.FI_O & & 75.26 & .712 & 1386.35 & -167.87 & 0189 & 207.5 & 7.5 & & 133 \\
\hline FS5 & TEGV.F̄__O & & 75.26 & 8.712 & 1386.41 & -167.87 & 0.0189 & 207.5 & 7.5 & & 13 \\
\hline 58 & Core_Nozz.FI & & 75.26 & 8.712 & 1386.54 & -167.87 & 0.0189 & 207.5 & 4.6 & & \\
\hline$\$ 17$ & FanDūctLkg.Fi & & 39 & 49 & 568.25 & 5.22 & 0 & 688.6 & 7.2 & & $53 \varepsilon$ \\
\hline S171 & Bleed15.Fl_o & & 57 & 8.749 & 568.25 & 5.22 & 0. & 688.6 & 7.0 & & \\
\hline FS172 & FanDuct.Fl_O & & 91.67 & 8.749 & 568.25 & 5.22 & 0 . & 688.6 & 7.0 & & 53 \\
\hline FS173 & Byp_Nozz.FI_O & & 391.67 & 8.749 & 568.25 & 5.22 & 0.0000 & 688.6 & 4.6 & & $41-$ \\
\hline $\mathrm{RBOI}$ & MACHINERY PERE & ANCE & DATA & & & & & & & & \\
\hline & & & eff & NC & TR & efPoly & & pwr & SMN & & $\mathrm{W}$ \\
\hline Fan & 1074.15 & 1.693 & 0.9110 & 4121.876 & 1.1783 & 0.9173 & -114 & 31.7 & 38.28 & 23. & 43 \\
\hline PC & 201.63 & 2.5 & 0.8340 & 1.876 & 1.3583 & 0.8538 & -4 & 18.8 & 2.96 & & 22 \\
\hline $\mathrm{PC}$ & 94.85 & 12.771 & 0.8590 & 10286.780 & 2.1798 & 0.8977 & -200 & 11.4 & 27.32 & 26. & 49 \\
\hline HPT & 12.10 & 4.269 & 0.9122 & 214.572 & 1.3415 & 0.8903 & 200 & 11.4 & & & \\
\hline LPT & 55.84 & 4.333 & 0.9169 & 90.524 & 1.3871 & 0.9007 & 157 & 50.5 & & & \\
\hline RB & MACH INERY & DA & & & & & & & & & \\
\hline & WcMa & PRmap & ffMap & NcMap & R/Parm & & & 1tics & effDes & & $\operatorname{NoD}$ \\
\hline Fan & 1248. & & & 82.574 & 0636 & & & $\overline{1} .0235$ & 0.9980 & & \\
\hline LPC & 165.2 & & & 1.089 & 0.0000 & & & 0.8676 & 1.0013 & & \\
\hline HPC & 86.27 & 12.216 & 0.8679 & 10012.541 & 13.3720 & & 0995 & 1.0494 & 0.9898 & & \\
\hline НРТ & & 4.17 & 1 & 1.290 & 4.1781 & & & 0.9723 & 1.0000 & & \\
\hline LPT & 0.86 & 3.507 & 0.9237 & 1.018 & 3.5069 & $65.2 \cdot x \cdot x$ & & 0.7521 & 0.9926 & & 0.0 \\
\hline
\end{tabular}




\begin{tabular}{|c|c|c|c|c|c|c|c|c|c|c|c|c|}
\hline$===$ INLET $S====$ & eRam & Afs & Fram & \multirow{2}{*}{\multicolumn{2}{|c|}{ BLEEDS - interstg }} & & & & & & & \\
\hline Inlet & 1.0000 & 3890.21 & 11466.7 & & & $g$ Wb/Win & BldWk & BldP & W & Tt & ht & Pt \\
\hline us & & & & HPT COOLC & C HPC.C> & 0.0368 & 0.5000 & 0.2200 & 2.7186 & 1058.91 & 97.97 & 37.752 \\
\hline$====$ DUCTS $====$ & dPnorm & MN & Aphy & LPT COOLZ & A HPC.C> & 0.0117 & 0.5000 & 0.4500 & 0.8643 & 1058.91 & 97.97 & 8.712 \\
\hline TEGV & 0.0000 & 0.4726 & 860.21 & WB2 $\overline{\mathrm{X}}$ & HPC. B> & 0.0000 & 0.4500 & 0.2200 & 0.0000 & 1019.81 & 88.16 & 46.364 \\
\hline FanDuct & 0.0000 & 0.5626 & 2481.93 & WB2Y & HPC. B> & 0.0000 & 0.7600 & 0.6200 & 0.0000 & 1259.22 & 148.98 & 107.178 \\
\hline & & & & WBA2X & HPC.B> & 0.0000 & 0.4500 & 0.2200 & 0.0000 & 1019.81 & 88.16 & 46.364 \\
\hline$=$ SPLITTERS $==$ & BPR & $\mathrm{dP} / \mathrm{P} 1$ & $\mathrm{dP} / \mathrm{P} 2$ & WBLKG & HPC. $1>$ & 0.0000 & 1.0000 & 1.0000 & 0.0000 & 1439.80 & 196.08 & 164.951 \\
\hline Splitter & 5.3274 & 0.0005 & 0.0005 & WBW2X & HPC.B> & 0.0000 & 0.4500 & 0.2200 & 0.0000 & 1019.81 & 88.16 & 46.364 \\
\hline$===$ SHAFTS $====$ & Nmech & $\operatorname{trg}$ in & pwr in & BLEEDS - & output & Wb/Win & hscale & Pscale & $\mathrm{W}$ & $\mathrm{Tt}$ & ht & Pt \\
\hline HP Shaft & 11608.6 & 9053.8 & 20011.4 & WB17Y & Bleed> & 0.0000 & 1.0000 & 1.0000 & 0.0000 & 660.53 & -0.14 & 12.916 \\
\hline LP_Shaft & 3974.6 & 20812.8 & 15750.5 & HPT_COOLZ & A Bleed> & 0.1142 & 1.0000 & 1.0000 & 8.4350 & 1439.80 & 196.08 & 161.152 \\
\hline & & & & $\mathrm{HPT}$ COOLH & B Bleed> & 0.0999 & 1.0000 & 1.0000 & 7.3809 & 1439.80 & 196.08 & 90.567 \\
\hline & & & & WB3 $\overline{\mathrm{X}}$ & Bleed> & 0.0000 & 1.0000 & 1.0000 & 0.0000 & 1439.80 & 196.08 & 164.951 \\
\hline & & & & WBA $3 \mathrm{X}$ & Bleed> & 0.0000 & 1.0000 & 1.0000 & 0.0000 & 1439.80 & 196.08 & 164.951 \\
\hline & & & & WBW3X & Bleed> & 0.0000 & 1.0000 & 1.0000 & 0.0000 & 1439.80 & 196.08 & 164.951 \\
\hline & & & & WBFDLKG & FanDu> & 0.0000 & 1.0000 & 1.0000 & 0.0000 & 568.25 & 5.22 & 8.749 \\
\hline & & & & WB15X & Bleed> & 0.0000 & 1.0000 & 1.0000 & 0.0000 & 568.25 & 5.22 & 8.749 \\
\hline & & & & WB15Y & Bleed> & 0.0000 & 1.0000 & 1.0000 & 0.0000 & 568.25 & 5.22 & 8.749 \\
\hline & & & & WB17X & Bleed> & 0.0000 & 1.0000 & 1.0000 & 0.0000 & 568.25 & 5.22 & 8.749 \\
\hline$===$ BURNERS $===$ & Ttout & eff & dPnorm & Wfuel & FAR & & & & & & & \\
\hline Burner & 2926.92 & 0.9995 & 0.0230 & .38793 & 02560 & & & & & & & \\
\hline$===$ NOZZLES $===$ & $\mathrm{PR}$ & $\mathrm{Cfg}$ & CdTh & $\mathrm{Cv}$ & Ath & MNth & Vact & $\mathrm{Fg}$ & & & & \\
\hline Core Nozz & 2.519 & 0.9810 & 1.0000 & 0.9800 & 3.40 & 1.000 & 1626.0 & 4544.8 & & & & \\
\hline Byp_Nozz & 2.530 & 0.9811 & 1.0000 & 0.9800 & 6.86 & 1.000 & 1045.7 & 15062.6 & & & & \\
\hline
\end{tabular}


Date:05/10/13 Time:11:36:21 Model: Turbofan Engine - COMDES ON COnverge = 1 CASE: 0

Version:NPSS 1.6.5 - Rev: -> Gas Package: Janaf iter/pass/Jacb/Broy=17/31/1/15 Run by: Philip C Jorgenson PC: 75

Temperature Stator 1 inlet: 479.71 Stator 1 exit: 496.80 Stator 2 inlet: $517.72 \quad$ Stator 2 exit: 526.64 $\begin{array}{llll}\text { Stator } 3 \text { inlet: } 551.23 & \text { Stator } 3 \text { exit: } 560.06 & \text { Stator } 4 \text { inlet: } 583.21 \text { Stator } 4 \text { exit: } 591.31 \\ \text { Stator } 5 \text { inlet: } 609.85 & \text { Stator } 5 \text { exit: } 617.42 & & \text { Unblocked Percent Blockage: } 0.00\end{array}$

Ambient Relative Humidity $\quad 10.00$

Fan Face Relative Humidity $\quad 3.84$

Fan Bypass Relative Humidity $\quad 0.30$

$\begin{array}{ll}\text { LPC Inlet Relative Humidity } & 2.04 \\ \text { LPC } & 0.03\end{array}$

$\begin{array}{ll}\text { LPC Exit Relative Humidity } & 0.03 \\ \text { HPC Relative Humidity } & 0.01\end{array}$

HPC Relative Humidity

Drop Diameter

0.0000050

Inlet

Ambient Static Pressure

Additional Water at LPC Exit

$\begin{array}{lr} & 40.00 \\ & \end{array}$

3.46 Fan/LPC Inlet Static Pressure 4.64

429.85

Fan/LPC Inlet Static Temperature 467.67

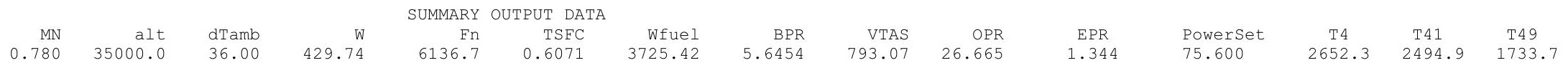

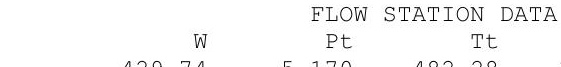 \\ FS0 Ambient.F1_O}

FS12 Splitter.F1_02

FS2 Splitter.FI-01

FS14 Fan.F1_O

$\begin{array}{ll}\text { FS23 } & \text { LPC.FI-O } \\ \text { FS24 } & \text { VaporIN.FI_O }\end{array}$

FS25 Bleed2.Fl_o

FS3 HPC.FIO

FS36 Bleed3.FI_O

FS4 Burner.Fl

FS45 HPT.F1_-

FS49 LPT.FI_O

$\begin{array}{ll}\text { FS5 } & \text { TEGV.FI_O } \\ \text { FS8 } & \text { Core Nozz.Fl }\end{array}$

FS17 FanDuctLkg.Fi_O

FS17 FanDuctLkg.F1-
FS171 Bleed15.FI_O

FS171
FS172 FanDuct.Fl_O

FS173 Byp_Nozz.FI_O

W
429.74
429.74
365.07
64.67
365.07
64.67
65.04
65.04
61.88
47.96
48.99
65.31
66.07
66.07
66.07
365.07
365.07
365.07
365.07

$\begin{array}{rrrrr}\text { Pt } & \text { Tt } & \text { ht } & \text { FAR } & \text { WC } \\ 5.170 & 482.28 & -15.41 & 0.0000 & 1177.95 \\ 5.170 & 482.28 & -15.41 & 0.0000 & 1177.95\end{array}$

$\begin{array}{lllll}5.170 & 482.28 & -15.41 & 0.0000 & 1177.95 \\ 5.167 & 482.28 & -15.41 & 0.0000 & 1001.99\end{array}$

$\begin{array}{lllll}5.167 & 482.28 & -15.41 & 0.0000 & 1001.19 \\ 5.167 & 482.28 & -15.41 & 0.0000 & 177.35\end{array}$

$\begin{array}{lrrrr}5.167 & 482.28 & -15.41 & 0.0000 & 1001.19 \\ 8.167 & 482.28 & -15.41 & 0.0000 & 177.35 \\ 8.053 & 554.16 & 1.84 & 0.0000 & 688.65\end{array}$

$\begin{array}{rrrrr}8.053 & 554.16 & 1.84 & 0.0000 & 688.65 \\ 12.480 & 641.48 & 22.83 & 0.0000 & 84.69\end{array}$

$\begin{array}{lllll}12.480 & 641.48 & 22.83 & 0.0000 & 84.69 \\ 12.480 & 648.09 & -8.26 & 0.0000 & 85.61 \\ 12.480 & 648.09 & -8.26 & 0.0000 & 85.61\end{array}$

$\begin{array}{llll}13 . .851 & 648.09 & -8.26 & 0.0000\end{array}$

$\begin{array}{llll}137.851 & 1355.53 & 169.01 & 0.0000\end{array}$

$\begin{array}{llll}134.677 & 2652.25 & 142.95 & 0.0217\end{array}$

$31.373-1741.54$

$\begin{array}{ll}7.232 & 1245.33 \\ 7.232 & 1245.40\end{array}$

$\begin{array}{llll}7.232 & 1245.33 & -157.52 & 0.0160\end{array}$

$\begin{array}{llll}7.232 & 1245.33 & -157.52 & 0.0160 \\ 7.232 & 1245.40 & -157.52 & 0.0160\end{array}$

$\begin{array}{rrrr}8.053 & 554.16 & 1.84 & 0.0000\end{array}$

$\begin{array}{llll}8.053 & 554.16 & 1.84 & 0.0000\end{array}$

$\begin{array}{llll}8.053 & 554.16 & 1.84 & 0.0000\end{array}$

85.61

10.67
8.27
12.09

8.27
12.09
56.06

12.09
56.06
208.04

208.04
208.04

208.04
208.06

688.65

688.65

$\begin{array}{lll}1.84 & 0.0000 & 688.65\end{array}$

$\begin{array}{rr}\text { Ps } & \text { TS } \\ 3.458 & 429.85 \\ 4.119 & 451.93 \\ 4.108 & 451.64 \\ 4.641 & 467.67 \\ 6.685 & 525.46 \\ 11.317 & 623.86 \\ 11.283 & 629.79 \\ 11.283 & 629.79 \\ 123.512 & 1316.79 \\ 129.550 & 1333.52 \\ 127.197 & 2618.07 \\ 28.223 & 1696.70 \\ 6.236 & 1197.89 \\ 6.236 & 1197.95 \\ 3.861 & 1055.02 \\ 6.685 & 525.46 \\ 6.496 & 521.17 \\ 6.496 & 521.17 \\ 4.253 & 461.71\end{array}$

$\begin{array}{rr}\text { Aphy } & \text { MN } \\ 3593.7 & 0.7800 \\ 4168.2 & 0.5789 \\ 3531.8 & 0.5818 \\ 830.5 & 0.3948 \\ 2606.7 & 0.5226 \\ 412.6 & 0.3767 \\ 412.6 & 0.3825 \\ 412.6 & 0.3825 \\ 49.7 & 0.4048 \\ 49.3 & 0.3036 \\ 74.6 & 0.2999 \\ 265.4 & 0.4019 \\ 860.2 & 0.4721 \\ 860.2 & 0.4721 \\ 613.4 & 1.0000 \\ 2606.7 & 0.5226 \\ 2481.9 & 0.5626 \\ 2481.9 & 0.5626 \\ 2006.9 & 1.0000\end{array}$

gamt
1.40064
1.40064
1.40064
1.40064
1.39969
1.39774
1.39675
1.39675
1.35759
1.35759
1.29008
1.32609
1.35345
1.35345
1.35344
1.39969
1.39969
1.39969
1.39969

WAR
0.0000871

0.0000871

0.0000871

0.0000871

0.0000871

0.0058084

0.0058084

0.0058084

0.0058084

0.0058084

0.0058084

0.0058084

0.00000871
0.0000871

0.00000871
0.0000871

0.0000871

$\begin{array}{rrr}\text { Wair } & \text { WH2O } & \text { H2O frac } \\ 429.70 & 0.037 & 0.0001 \\ 429.70 & 0.037 & 0.0001 \\ 365.04 & 0.032 & 0.0001 \\ 64.66 & 0.006 & 0.0001 \\ 365.04 & 0.032 & 0.0001 \\ 64.66 & 0.006 & 0.0001 \\ 64.66 & 0.376 & 0.0058 \\ 64.66 & 0.376 & 0.0058 \\ 61.53 & 0.357 & 0.0058 \\ 47.68 & 0.277 & 0.0058 \\ 47.68 & 0.277 & 0.0317 \\ 63.90 & 0.371 & 0.0252 \\ 64.66 & 0.376 & 0.0250 \\ 64.66 & 0.376 & 0.0250 \\ 64.66 & 0.376 & 0.0250 \\ 365.04 & 0.032 & 0.0001 \\ 365.04 & 0.032 & 0.0001 \\ 365.04 & 0.032 & 0.0001 \\ 365.04 & 0.032 & 0.0001\end{array}$

TURBOMACHINERY PERFORMANCE DATA

\begin{tabular}{|c|c|c|c|c|c|c|c|c|c|}
\hline & Wc & $\mathrm{PR}$ & eff & $\mathrm{NC}$ & $\mathrm{TR}$ & efpoly & pwr & SMN & SMW \\
\hline Fan & 1001.19 & 1.558 & 0.9071 & 3847.235 & 1.1491 & 0.9127 & -8907.1 & 62.06 & 29.24 \\
\hline LPC & 177.35 & 2.415 & 0.8671 & 3847.235 & 1.3301 & 0.8824 & -3499.0 & 3.97 & 3.14 \\
\hline HPC & 85.61 & 11.046 & 0.8615 & 10026.648 & 2.0916 & 0.8980 & -15916.1 & 34.17 & 32.82 \\
\hline HPT & 12.09 & 4.293 & 0.9089 & 217.630 & 1.3518 & 0.8892 & 15916.2 & & \\
\hline LPT & 56.06 & 4.338 & 0.9132 & 88.896 & 1.3957 & 0.8964 & 12406.1 & & \\
\hline \multicolumn{10}{|c|}{ TURBOMACHINERY MAP DE } \\
\hline & WcMap & PRmap & effMap & NcMap & R/Parm & s WCDes & s PRdes & $s$ effDes & s NcDes \\
\hline Fan & 1163.29 & 1.546 & 0.9089 & 3810.552 & 0.0590 & 0.8607 & 1.0235 & 0.9980 & 0.9905 \\
\hline LPC & 143.42 & 2.435 & 0.8209 & 1.016 & 0.0000 & 1.2365 & 0.9861 & 1.0563 & 0.0003 \\
\hline 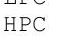 & 77.86 & 10.573 & 0.8704 & 9759.344 & 12.7792 & 1.0995 & 1.0494 & 0.9898 & 0.9733 \\
\hline HPT & 0.96 & 4.202 & 0.9089 & 1.308 & 4.2016 & 12.6299 & 0.9723 & 1.0000 & 0.0003 \\
\hline LPT & 0.86 & 3.511 & 0.9200 & 1.000 & 3.5106 & 65.2590 & 0.7521 & 0.9926 & 0.0005 \\
\hline
\end{tabular}




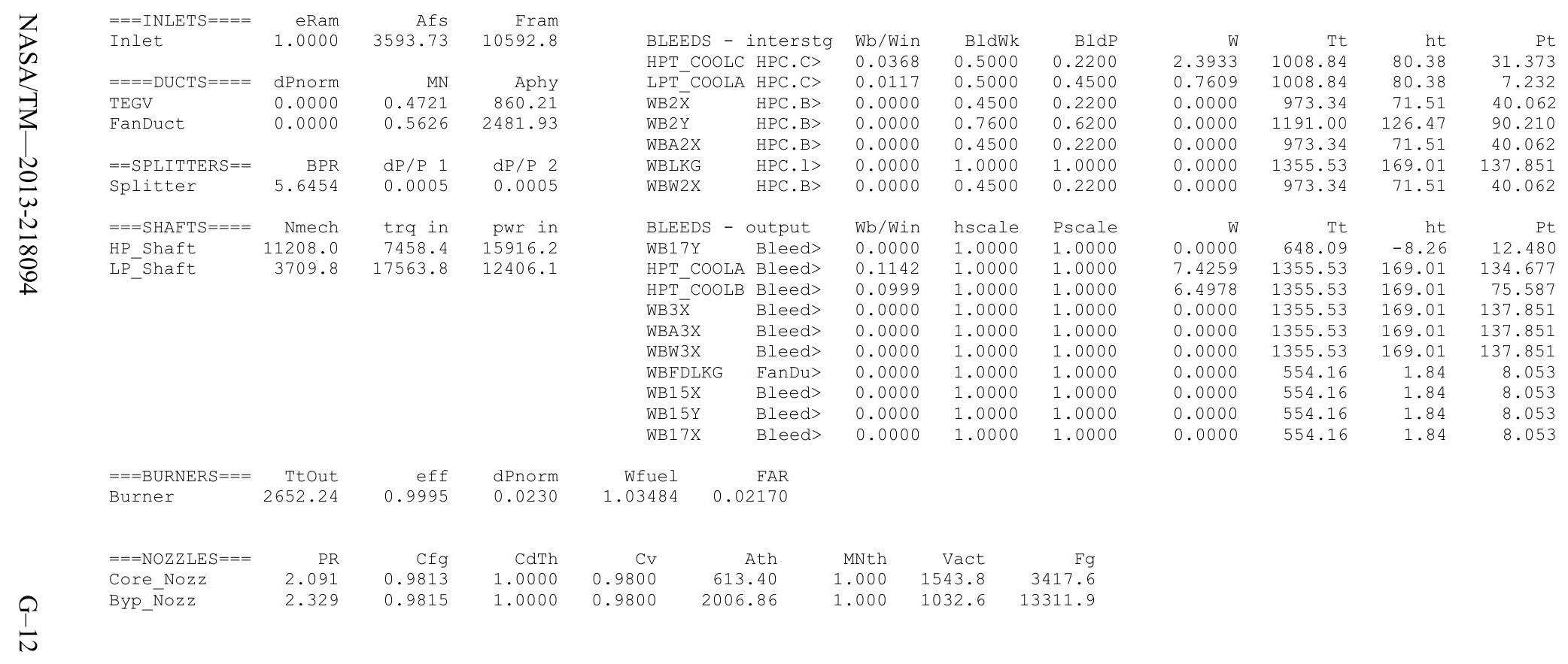


Date:05/10/13 Time:11:36:52 Model: Turbofan Engine - COMDES ON CONverge $=1$ CASE: 0

Version:NPSS_1.6.5 - Rev: -> Gas Package: Janaf iter/pass/Jacb/Broy=17/31/1/15 Run by: Philip C Jorgenson PC: 72

Temperature Stator 1 inlet: 480.66 Stator 1 exit: 497.49 Stator 2 inlet: 518.18 Stator 2 exit: 527.12

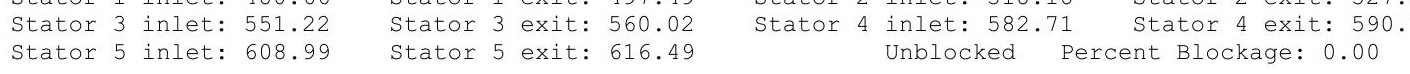

Ambient Relative Humidity $\quad 10.00$

Fan Face Relative Humidity $\quad 3.72$

Fan Bypass Relative Humidity 0.31

LPC Inlet Relative Humidity $\quad 1.99$

LPC Exit Relative Humidity $\quad 0.03$

HPC Relative Humidity

Drop Diameter
Ambient Flow Velocity

0.01

0.0000050
793.07
3.46

Ambient static Pressure

Additional Water at LPC Exit

Inlet Iength

$\begin{array}{rlr}000050 & \text { Inlet Length } & 40.00 \\ 93.07 & \text { Fan/LPC Inlet Flow Velocity } & 410.02 \\ 3.46 & \text { Fan/LPC Inlet Static Pressure } & 4.66\end{array}$

$\begin{array}{rrr}3.46 & \text { Fan/LPC Inlet Static Pressure r r } & 4.66 \\ 429.85 & \text { Fan/LPC Inlet Static Temperature } & 468.27\end{array}$

0.0058490

\section{MN alt dTamb W SUMMARY OUTPUT DATA}

$\begin{array}{rrrrrr}0.780 & 35000.0 & 36.00 & 425.58 & 5909.0 & 0.6075\end{array}$

$\begin{array}{rr}\text { Wfuel } & \text { BPR } \\ 3589.93 & 5.6990\end{array}$

$\begin{array}{rr}\text { VTAS } & \text { OPR } \\ 793.07 & 26.043\end{array}$

EPR Powerset

72.700

$\begin{array}{ccc}\text { T4 } & \text { T41 } & \text { T49 } \\ 2622.0 & 2466.9 & 1713.6\end{array}$

FS0 Ambient.F1_O

$\begin{array}{ll}\text { FS1 } & \text { Inlet.F1_O } \\ \text { FS12 } & \text { Splitter.Fl } 02\end{array}$

$\stackrel{9}{1}$

Splitter.Fl-01

FS14 Fan.Fl_O

FS24 VaporIN.F1_O

FS25 Bleed2.F1_o

FS3 HPC.F1_O

FS4 Burner.FI_O

FS45 HPT.FI_O

FS49 LPT.FI-O

FS5 TEGV.FI-O

FS17 FanDuctLkg.Fí_O

FS171 Bleed15.FI_O

FS172 FanDuct.Fl-O

$W$
425.58
425.58
362.05
63.53
362.05
63.53
63.90
63.90
60.80
47.12
48.12
64.15
64.90
64.90
64.90
362.05
362.05
362.05
362.05

FLOW STATION DATA

\begin{tabular}{rrrr}
\multicolumn{1}{c}{ Pt } & $\mathrm{Tt}$ & $\mathrm{ht}$ & FAR \\
5.170 & 482.28 & -15.41 & 0.0000 \\
5.170 & 482.28 & -15.41 & 0.0000 \\
5.167 & 482.28 & -15.41 & 0.0000 \\
5.167 & 482.28 & -15.41 & 0.0000 \\
7.974 & 552.47 & 1.43 & 0.0000 \\
12.404 & 639.98 & 22.47 & 0.0000 \\
12.404 & 646.75 & -9.31 & 0.0000 \\
12.404 & 646.75 & -9.31 & 0.0000 \\
134.635 & 1346.14 & 165.85 & 0.0000 \\
134.635 & 1346.14 & 165.85 & 0.0000 \\
131.534 & 2622.01 & 140.37 & 0.0213 \\
30.622 & 1721.32 & -26.72 & 0.0159 \\
7.059 & 1230.27 & -156.54 & 0.0157 \\
7.059 & 1230.34 & -156.54 & 0.0157 \\
7.059 & 1230.47 & -156.54 & 0.0157 \\
7.974 & 552.47 & 1.43 & 0.0000 \\
7.974 & 552.47 & 1.43 & 0.0000 \\
7.974 & 552.47 & 1.43 & 0.0000 \\
7.974 & 552.47 & 1.43 & 0.0000
\end{tabular}

WC
1166.55 1166.55
1166.55 992.91

688.66

688.66
83.61

84.54

84.54
10.69

10.69
8.29
12.09

12.09
56.08
208.08

208.08

208.09
208.10

208.10
688.66

688.66

688.66

688.66

PS
3.458
4.147

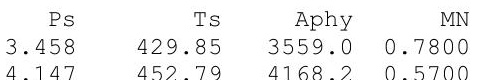

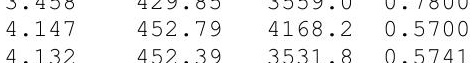

$\begin{array}{llll}4.661 & 468.27 & 351.8 & 0.5741 \\ 6.619 & 523.86 & 830.5 & 0.3864\end{array}$

$\begin{array}{llll}6.619 & 523.86 & 2606.7 & 0.5226\end{array}$

$\begin{array}{llll}11.281 & 622.92 & 412.6 & 0.3709\end{array}$

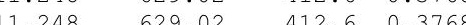

$\begin{array}{llll}120.549 & 1307.39 & 49.7 & 0.4060\end{array}$

$\begin{array}{llll}126.482 & 1324.13 & 49.3 & 0.3044\end{array}$

$\begin{array}{llll}126.482 & 1324.13 & 49.3 & 0.3044 \\ 27.542 & 1676.78 & 74.6 & 0.299\end{array}$

$\begin{array}{llll}6.542 & 1676.78 & 265.4 & 0.4020 \\ 6.086 & 1183.27 & 860.2 & 0.4721\end{array}$

$\begin{array}{llll}6.086 & 1183.34 & 860.2 & 0.4721\end{array}$

$\begin{array}{rrrr}6.0868 & 1041.79 & 613.4 & 1.0000 \\ 6.619 & 523.86 & 2606.7 & 0.5226\end{array}$

$\begin{array}{llll}6.432 & 519.59 & 2481.9 & 0.5626\end{array}$

$6.432-519.59$

$\begin{array}{ll}6.432 & 519.59 \\ 4.211 & 460.30\end{array}$

$\begin{array}{ll}2481.9 & 0.5626 \\ 2481.9 & 0.5626\end{array}$

$2006.9 \quad 1.0000$ gamt
1.40064
1.40064
1.40064
1.40064
1.39972
1.39779
1.39677
1.39677
1.35813
1.35813
1.29121
1.32721
1.35458
1.35458
1.35457
1.39972
1.39972
1.39972
1.39972

WAR
0.0000871
0.0000871
0.0000871
0.0000871
0.0000871
0.0000871
0.0059361
0.0059361
0.0059361
0.0059361
0.0059361
0.0059361
0.0059361
0.0059361
0.0059361
0.0000871
0.0000871
0.0000871
0.0000871

$\begin{array}{rrr}\text { Wair } & \text { WH2O } & \text { H2O frac } \\ 425.54 & 0.037 & 0.0001\end{array}$ $\begin{array}{lll}425.54 & 0.037 & 0.0001\end{array}$ $\begin{array}{lll}362.02 & 0.032=0.0001\end{array}$ $63.52-0.032-0.0001$ $362.02=0.032=0.0001$ $63.52-0.006=0.0001$ $\begin{array}{lll}63.52 & 0.0067 & 0.0001\end{array}$ $\begin{array}{lll}63.52 & 0.377 & 0.0059\end{array}$ $\begin{array}{lll}60.44 & 0.359 & 0.0059\end{array}$ $\begin{array}{lll}60.84 & 0.278 & 0.0059\end{array}$ $\begin{array}{lll}46.84 & 0.278 & 0.0313\end{array}$ $\begin{array}{lll}62.78 & 0.373=0.0250\end{array}$ $\begin{array}{lll}63.52 & 0.377 & 0.0248\end{array}$ $\begin{array}{lll}63.52 & 0.377 & 0.0248\end{array}$ $\begin{array}{lll}63.52 & 0.377 & 0.0248\end{array}$ $\begin{array}{lll}362.02 & 0.032 & 0.0001\end{array}$ $\begin{array}{lll}362.02 & 0.032 & 0.0001\end{array}$ $\begin{array}{lll}362.02 & 0.032 & 0.0001\end{array}$ 0.0001

\begin{tabular}{|c|c|c|c|c|c|c|c|c|c|}
\hline & Wc & PR & eff & $\mathrm{NC}$ & TR & efPoly & pwr & SMN & SMW \\
\hline Fan & 992.91 & 1.543 & 0.9070 & 3816.307 & 1.1456 & 0.9125 & -8626.0 & 66.53 & 29.96 \\
\hline LPC & 174.23 & 2.400 & 0.8685 & 3816.307 & 1.3270 & 0.8835 & -3405.0 & 4.24 & 3.34 \\
\hline HPC & 84.54 & 10.854 & 0.8615 & 9996.637 & 2.0814 & 0.8978 & -15452.3 & 35.00 & 33.60 \\
\hline HPT & 12.09 & 4.295 & 0.9084 & 218.001 & 1.3529 & 0.8888 & 15452.3 & & \\
\hline LPT & 56.08 & 4.338 & 0.9125 & 88.698 & 1.3965 & 0.8956 & 12031.0 & & \\
\hline TURE & INERY MAP & DATA & & & & & & & \\
\hline & WcMap & PRmap & effMap & NcMap & R/Parm & s WcDes & S PRdes & $s$ effDes & s NcDes \\
\hline Fan & 1153.67 & 1.531 & 0.9088 & 3779.919 & 0.0584 & $\overline{0} .8607$ & 1.0235 & 0.9980 & 0.9905 \\
\hline LPC & 141.42 & 2.403 & 0.8202 & 1.008 & 0.0000 & 1.2319 & 0.9980 & 1.0588 & 0.0003 \\
\hline HPC & 76.89 & 10.390 & 0.8705 & 9730.134 & 12.7112 & 1.0995 & 1.0494 & 0.9898 & 0.9733 \\
\hline HPT & 0.96 & 4.204 & 0.9084 & 1.310 & 4.2041 & 12.6299 & 0.9723 & 1.0000 & 0.0003 \\
\hline LPT & 0.86 & 3.511 & 0.9193 & 0.998 & 3.5106 & 65.2590 & 0.7521 & 0.9926 & 0.0005 \\
\hline
\end{tabular}




\begin{tabular}{|c|c|c|c|c|c|c|c|c|c|c|c|c|}
\hline$===$ INLET $S====$ & eRam & Afs & Fram & \multirow{2}{*}{\multicolumn{2}{|c|}{ BLEEDS - interstg }} & & & & & & & \\
\hline Inlet & 1.0000 & 3558.95 & 10490.3 & & & g Wb/Win & BldWk & BldP & w & $\mathrm{Tt}$ & ht & Pt \\
\hline s & & & & HPT_COOLC & C HPC.C> & 0.0368 & 0.5000 & 0.2200 & 2.3515 & 1003.29 & 78.27 & 30.622 \\
\hline$====$ DUCTS $====$ & dPnorm & MN & Aphy & LPT_COOLA & A HPC.C> & 0.0117 & 0.5000 & 0.4500 & 0.7476 & 1003.29 & 78.27 & 7.059 \\
\hline TEGV & 0.0000 & 0.4721 & 860.21 & WB2 $\bar{X}$ & HPC.B> & 0.0000 & 0.4500 & 0.2200 & 0.0000 & 968.20 & 69.52 & 39.295 \\
\hline FanDuct & 0.0000 & 0.5626 & 2481.93 & WB2Y & HPC. B> & 0.0000 & 0.7600 & 0.6200 & 0.0000 & 1183.41 & 123.82 & 88.187 \\
\hline & & & & WBA2X & HPC.B> & 0.0000 & 0.4500 & 0.2200 & 0.0000 & 968.20 & 69.52 & 39.295 \\
\hline$==$ SPLITTERS $==$ & BPR & $\mathrm{dP} / \mathrm{P} 1$ & $\mathrm{dP} / \mathrm{P} 2$ & WBLKG & HPC.I> & 0.0000 & 1.0000 & 1.0000 & 0.0000 & 1346.14 & 165.85 & 134.635 \\
\hline Splitter & 5.6990 & 0.0005 & 0.0005 & WBW2X & $\mathrm{HPC} \cdot \mathrm{B}>$ & 0.0000 & 0.4500 & 0.2200 & 0.0000 & 968.20 & 69.52 & 39.295 \\
\hline$===$ SHAFTS $====$ & Nmech & trq in & pwr in & BLEEDS - & - output & $\mathrm{Wb} / \mathrm{Win}$ & hscale & Pscale & W & $\mathrm{Tt}$ & ht & $\mathrm{Pt}$ \\
\hline HP_Shaft & 11162.9 & 7270.2 & 15452.3 & WB17Y & Bleed> & 0.0000 & 1.0000 & 1.0000 & 0.0000 & 646.75 & -9.31 & 12.404 \\
\hline LP_Shaft & 3680.0 & 17170.8 & 12031.0 & HPT_COOLA & A Bleed> & 0.1142 & 1.0000 & 1.0000 & 7.2962 & 1346.14 & 165.85 & 131.534 \\
\hline & & & & HPT COOLB & B Bleed> & 0.0999 & 1.0000 & 1.0000 & 6.3843 & 1346.14 & 165.85 & 73.813 \\
\hline & & & & WB $3 \overline{\mathrm{X}}$ & Bleed> & 0.0000 & 1.0000 & 1.0000 & 0.0000 & 1346.14 & 165.85 & 134.635 \\
\hline & & & & WBA3X & Bleed> & 0.0000 & 1.0000 & 1.0000 & 0.0000 & 1346.14 & 165.85 & 134.635 \\
\hline & & & & WBW3X & Bleed> & 0.0000 & 1.0000 & 1.0000 & 0.0000 & 1346.14 & 165.85 & 134.635 \\
\hline & & & & WBFDLKG & FanDu> & 0.0000 & 1.0000 & 1.0000 & 0.0000 & 552.47 & 1.43 & 7.974 \\
\hline & & & & WB15X & Bleed> & 0.0000 & 1.0000 & 1.0000 & 0.0000 & 552.47 & 1.43 & 7.974 \\
\hline & & & & WB15Y & Bleed> & 0.0000 & 1.0000 & 1.0000 & 0.0000 & 552.47 & 1.43 & 7.974 \\
\hline & & & & WB17X & Bleed> & 0.0000 & 1.0000 & 1.0000 & 0.0000 & 552.47 & 1.43 & 7.974 \\
\hline$===$ BURNERS $===$ & TtOut & eff & dPnorm & Wfuel & FAR & & & & & & & \\
\hline Burner & 2621.99 & 0.9995 & 0.0230 & 0.99720 & 02129 & & & & & & & \\
\hline$===$ NOZZLES $===$ & PR & Cfg & CdTh & $\mathrm{Cv}$ & Ath & MNth & Vact & $\mathrm{Fg}$ & & & & \\
\hline Core Nozz & 2.041 & 0.9811 & 1.0000 & 0.9800 & 3.40 & 1.000 & 1534.7 & 3285.5 & & & & \\
\hline Byp__̄ozz & 2.306 & 0.9815 & 1.0000 & 0.9800 & 6.86 & 1.000 & 1031.0 & 13113.7 & & & & \\
\hline
\end{tabular}


Date:05/10/13 Time:11:37:23 Model: Turbofan Engine - COMDES ON Converge = 1 CASE: 0

Version:NPSS 1.6.5 - Rev: -> Gas Package: Janaf iter/pass/Jacb/Broy=18/32/1/16 Run by: Philip C Jorgenson PC: 69

Temperature Stator 1 inlet: $481.53 \quad$ Stator 1 exit: $498.10 \quad$ Stator 2 inlet: 518.55 Stator 2 exit: 527.48 $\begin{array}{ccc}\text { Stator } 3 \text { inlet: } 551.10 & \text { Stator } 3 \text { exit: } 559.86 & \text { Stator } 4 \text { inlet: } 582.10 \text { Stator } 4 \text { exit: } 590.10 \\ \text { Stator } 5 \text { inlet: } 607.99 & \text { Stator } 5 \text { exit: } 615.43 & \text { Unblocked Percent Blockage: } 0.00\end{array}$

Ambient Relative Humidity $\quad 10.00$

Fan Face Relative Humidity $\quad 3.62$

Fan Bypass Relative Humidity $\quad 0.33$

LPC Inlet Relative Humidity $\quad 1.95$

$\begin{array}{ll}\text { LPC Exit Relative Humidity } & 0.03 \\ \text { HPC Relative Humidity } & 0.01\end{array}$

Drop Diameter

0.0000050

Inlet

Amblent Flow Velocity

Ambient Static Pressure

Additional Water at LPC Exit

$\begin{array}{lr} & 40.00 \\ \text { Fan/LPC Inlet Flow Velocity } & 401.52\end{array}$

$3.46 \quad$ Fan/LPC Inlet Static Pressure $\quad 4.68$
0.0059723

0.0059723 Fan/LPC Inlet Static Temperature 468.84

\begin{tabular}{|c|c|c|c|c|c|c|c|c|c|c|c|c|c|c|}
\hline & & & & SUMMARY & TPUT DA? & & & & & & & & & \\
\hline $\begin{array}{l}\text { MN } \\
780\end{array}$ & alt & dTamb & W & $\mathrm{Fn}$ & TSFC & Wfuel & BPR & VTAS & OPR & EPR & Powerset & T4 & T41 & $\begin{array}{r}\text { T49 } \\
1694.2\end{array}$ \\
\hline 780 & 35000.0 & 36.00 & 421.59 & 5690.4 & 0.6082 & 3460.64 & 5.7560 & 793.07 & 25.434 & 1.269 & 69.900 & 2592.8 & 2439.9 & 1694.2 \\
\hline
\end{tabular}

\begin{tabular}{|c|c|c|c|c|c|c|c|c|c|c|c|}
\hline & & & $\mathrm{W}$ & $\begin{array}{l}\text { FLOW } S \\
\text { Pt }\end{array}$ & STATION DAT & TA & & Wo & & PS & \\
\hline FSO & Ambient.Fl $O$ & & 421.59 & 5.170 & 482.28 & -15.41 & 0.0000 & 1155.62 & 3.45 & & 429. \\
\hline FS1 & Inlet.Fl O & & 421.59 & 5.170 & 482.28 & -15.41 & 0.0000 & 1155.62 & 4.17 & & 453 \\
\hline FS12 & Splitter.Fl 0 & & 359.19 & 5.167 & 482.28 & -15.41 & 0.0000 & 985.07 & 4.15 & & 453 \\
\hline FS2 & Splitter.Fl_o & & 62.40 & 5.167 & 482.28 & -15.41 & 0.0000 & 171.14 & 4.68 & & 468. \\
\hline FS14 & Fan.Fl_O & & 359.19 & 7.899 & 550.83 & 1.04 & 0.0000 & 688.66 & 6.55 & & 522. \\
\hline FS23 & LPC.FI_O & & 62.40 & 12.317 & 638.37 & 22.09 & 0.0000 & 82.60 & 11.23 & & 621 \\
\hline FS24 & VaporIN̄.FI_O & & 62.77 & 12.317 & 645.30 & -10.36 & 0.0000 & 83.55 & 11.19 & & 628 \\
\hline FS25 & Bleed2.Fl $\overline{0}$ & & 62.77 & 12.317 & 645.30 & -10.36 & 0.0000 & 83.55 & 11.19 & & 628 \\
\hline FS3 & HPC.FI_O & & 59.73 & 131.488 & 1336.96 & 162.78 & 0.0000 & 10.72 & 117.65 & & 1298 \\
\hline FS36 & Bleed3.FI_O & & 46.29 & 131.488 & 1336.96 & 162.78 & 0.0000 & 8.31 & 123.48 & & 1314 \\
\hline FS4 & Burner.Fl_o & & 47.25 & 128.460 & 2592.80 & 137.84 & 0.0209 & 12.09 & 121.32 & & 2559 \\
\hline FS45 & HPT.Fl_O & & 63.00 & 29.887 & 1701.78 & -27.43 & 0.0156 & 56.11 & 26.87 & & 1657 \\
\hline FS 49 & LPT.FI_O & & 63.74 & 6.890 & 1215.78 & -155.62 & 0.0154 & 208.13 & 5.94 & & 1169 \\
\hline FS5 & TEGV.F̄ & & 63.74 & 6.890 & 1215.85 & -155.62 & 0.0154 & 208.14 & 5.94 & & 1169 \\
\hline FS8 & Core_Nozz.Fl_ & & 63.74 & 6.890 & 1215.98 & -155.62 & 0.0154 & 208.15 & 3.67 & & 1029 \\
\hline FS17 & FanDuctLkg.Fi & & 359.19 & 7.899 & 550.83 & 1.04 & 0.0000 & 688.66 & 6.55 & & 522 \\
\hline FS171 & Bleed15.FI_O & & 359.19 & 7.899 & 550.83 & 1.04 & 0.0000 & 688.66 & 6.37 & & \\
\hline FS172 & FanDuct.FI_O & & 359.19 & 7.899 & 550.83 & 1.04 & 0.0000 & 688.66 & 6.37 & & 518 \\
\hline FS173 & Byp_Nozz.Fin_C & & 359.19 & 7.899 & 550.83 & 1.04 & 0.0000 & 688.66 & 4.17 & & 458 \\
\hline TURBON & MACHINERY PERE & ORMANCE & DATA & & & & & & & & \\
\hline & & $\mathrm{PR}$ & eff & $\mathrm{NC}$ & $\mathrm{TR}$ & efPoly & & pwr & SMN & & MW \\
\hline Fan & 985.07 & 1.529 & 0.9073 & 3785.224 & 1.1422 & 0.9127 & -8357 & $\$ 7.7$ & 72.77 & 30.6 & 66 \\
\hline LPC & 171.14 & 2.384 & 0.8695 & 3785.224 & 1.3237 & 0.8843 & -3310 & 0.5 & 4.53 & & 55 \\
\hline HPC & 83.55 & 10.676 & 0.8616 & 9968.416 & 2.0718 & 0.8977 & -15004 & 4.3 & 35.81 & 34.3 & 34 \\
\hline HPT & 12.09 & 4.298 & 0.9079 & 218.363 & 1.3539 & 0.8884 & 15004 & 4.3 & & & \\
\hline LPT & 56.11 & 4.338 & 0.9119 & 88.479 & 1.3972 & 0.8947 & 11668 & 8.2 & & & \\
\hline TURBON & MACHINERY MAP & DATA & & & & & & & & & \\
\hline & WcMap & PRmap & effMap & NcMap & R/Parm & s_WCI & Des & _PRdes & S_effDes & & NcDes \\
\hline Fan & 1144.55 & 1.517 & 0.9091 & 3749.132 & 0.0579 & $\overline{0} .8$ & 3607 & $\overline{1} .0235$ & 0.9980 & & $\overline{0} .9905$ \\
\hline LPC & 139.41 & 2.371 & 0.8196 & 1.000 & 0.0000 & 1.22 & 2275 & 1.0090 & 1.0608 & & 0.0003 \\
\hline HPC & 75.98 & 10.220 & 0.8705 & 9702.665 & 12.6468 & 1.0 & 0995 & 1.0494 & 0.9898 & & 0.9733 \\
\hline HPT & 0.96 & 4.207 & 0.9078 & 1.312 & 4.2068 & 12.62 & 5299 & 0.9723 & 1.0000 & & 0.0003 \\
\hline LPT & 0.86 & 3.510 & 0.9186 & 0.995 & 3.5104 & 65.25 & 2590 & 0.7521 & 0.9926 & & 0.0005 \\
\hline
\end{tabular}




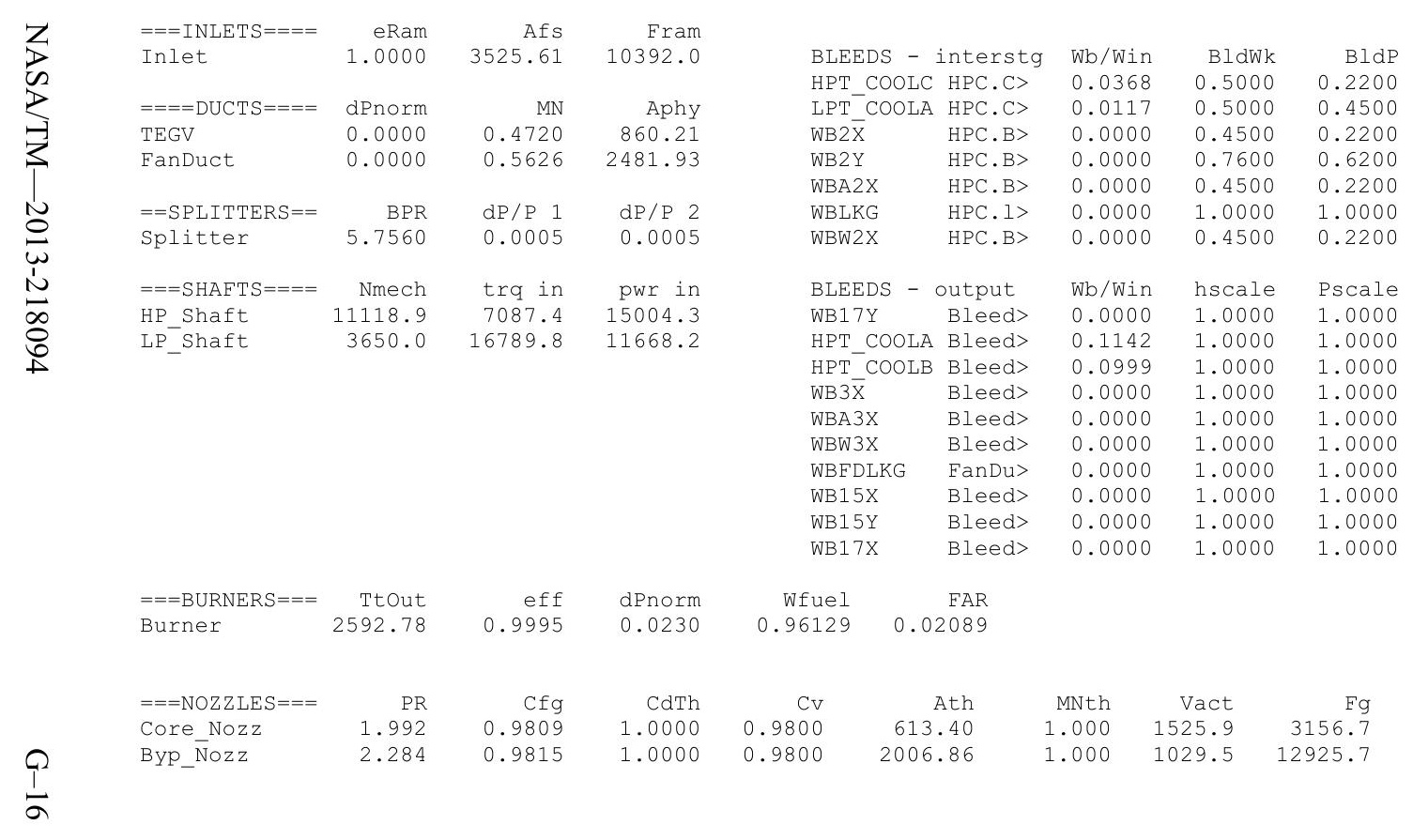


Date:05/13/13 Time:07:00:59 Model: Turbofan Engine - COMDES ON COnverge = 1 CASE: O

Gas Package: Janaf,iter/pass/Jacb/Broy= 26/40/1/24 Run by: Philip C Jorger

Stator 2 inlet: 489.45 Stator 2 exit: 496.80

Stator 3 inlet: 525.56 Stator 5 exit: 533.65 Stator 4 inlet: 560.59 Stator 4 exit: 568.37

Ambient Relative Humidity $\quad 10.00$

Fan Face Relative Humidity $\quad 5.11$

Fan Bypass Relative Humidity $\quad 0.16$

LPC Inlet Relative Humidity $\quad 2.31$

LPC Exit Relative Humidity $\quad 0.02$

HPC Relative Humidity $\quad 0.00$

Ambient Flow Velocity

.0000100

3.46
411.85

Inlet Length $\quad 40.00$

Ambient Static Pressure

Ambient Static Temperature

0.0005921

Fan/LPC Inlet Static Pressure $\quad 4.45$

SUMMARY OUTPUT DATA

MN alt dTamb W $\quad$ Fn TSFC Wfuel BPR

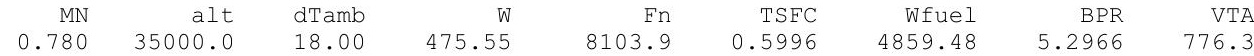

.2966
OPR
31.984

1.684
PowerSet
100.000 $\begin{array}{ccr}\text { T4 } & \text { T41 } & \text { T49 } \\ 2818.7 & 2645.8 & 1838 .\end{array}$

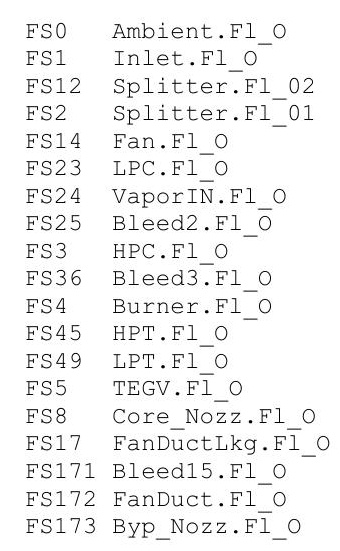

$W$
475.55
475.55
400.02
75.52
400.02
75.52
75.57
75.57
71.90
55.73
57.08
76.03
76.92
76.92
76.92
400.02
400.02
400.02
400.02

FLOW STATION DATA

\begin{tabular}{rrrrr}
\multicolumn{1}{c}{ Pt } & Tt & ht & FAR & WC \\
5.170 & 462.10 & -19.92 & 0.0000 & 1275.94 \\
5.170 & 462.10 & -19.92 & 0.0000 & 1275.94 \\
5.167 & 462.10 & -19.92 & 0.0000 & 1073.83 \\
5.167 & 462.10 & -19.92 & 0.0000 & 202.74 \\
8.746 & 544.46 & -0.17 & 0.0000 & 688.68 \\
12.696 & 625.54 & 19.32 & 0.0000 & 96.01 \\
12.696 & 626.30 & 16.10 & 0.0000 & 96.12 \\
12.696 & 626.30 & 16.10 & 0.0000 & 96.12 \\
165.353 & 1379.91 & 204.14 & 0.0000 & 10.42 \\
165.353 & 1379.91 & 204.14 & 0.0000 & 8.08 \\
161.545 & 2818.73 & 173.93 & 0.0242 & 12.10 \\
37.754 & 1847.79 & -4.33 & 0.0181 & 55.86 \\
8.680 & 1323.99 & -144.26 & 0.0179 & 208.07 \\
8.680 & 1324.05 & -144.26 & 0.0179 & 208.07 \\
8.680 & 1324.18 & -144.26 & 0.0179 & 208.08 \\
8.746 & 544.46 & -0.17 & 0.0000 & 688.68 \\
8.746 & 544.46 & -0.17 & 0.0000 & 688.68 \\
8.746 & 544.46 & -0.17 & 0.0000 & 688.68 \\
8.746 & 544.46 & -0.17 & 0.0000 & 688.68
\end{tabular}

Ps
3.458
3.839
3.865
4.446
7.260
11.117
11.112
11.112
149.090
155.906
152.607
34.006
7.487
7.487
4.642
7.260
7.055
7.055
4.619

Ts
411.85
424.35
425.25
442.65
516.25
602.32
602.96
602.96
1342.75
1358.69
2782.95
1801.32
1274.25
1274.32
1124.14
516.25
512.04
512.04
453.61

$\begin{array}{rr}\text { Aphy } & \text { MN } \\ 3892.5 & 0.7800 \\ 4168.2 & 0.6661 \\ 3531.8 & 0.6574 \\ 830.5 & 0.4682 \\ 2606.7 & 0.5226 \\ 412.6 & 0.4399 \\ 412.6 & 0.4407 \\ 412.6 & 0.4407 \\ 49.7 & 0.3930 \\ 49.3 & 0.2955 \\ 74.6 & 0.3004 \\ 265.4 & 0.4003 \\ 860.2 & 0.4723 \\ 860.2 & 0.4723 \\ 613.4 & 1.0000 \\ 2606.7 & 0.5226 \\ 2481.9 & 0.5625 \\ 2481.9 & 0.5625 \\ 2006.9 & 1.0000\end{array}$

gamt
1.40081
1.40081
1.40081
1.40081
1.39985
1.39818
1.39808
1.39808
1.35701
1.35701
1.28459
1.32089
1.34801
1.34800
1.34799
1.39985
1.39985
1.39985
1.39985

WAR

Wair WH2O H2O frac $\begin{array}{llll}0.0000308 & 475.53 & 0.015 & 0.0000\end{array}$ $\begin{array}{llll}0.0000308 & 475.53 & 0.015 & 0.0000 \\ 0.0000308 & 475.53 & 0.015 & 0.0000 \\ 0.0000308 & 400.01 & 0.012 & 0.0000\end{array}$ $\begin{array}{rrrr}0.0000308 & 475.53 & 0.015 & 0.0000 \\ 0.000308 & 400.01 & 0.012 & 0.0000 \\ 0.000308 & 75.52 & 0.002 & 0.0000\end{array}$ $\begin{array}{llll}0.0000308 & 400.01 & 0.012 & 0.0000\end{array}$ $\begin{array}{llll}0.0000308 & 75.52 & 0.002 & 0.0000\end{array}$ $\begin{array}{llll}0.0006230 & 75.52 & 0.047 & 0.0006\end{array}$ $\begin{array}{llll}0.0006230 & 75.52 & 0.047 & 0.0006\end{array}$ $\begin{array}{llll}0.0006230 & 71.86 & 0.045 & 0.0006\end{array}$ $\begin{array}{llll}0.0006230 & 55.69 & 0.035 & 0.0006\end{array}$ $\begin{array}{llll}0.0006230 & 55.69 & 0.035 & 0.0297\end{array}$ $\begin{array}{llll}0.0006230 & 74.64 & 0.046 & 0.0225\end{array}$ $\begin{array}{llll}0.0006230 & 75.52 & 0.047 & 0.0223\end{array}$ $\begin{array}{llll}0.0006230 & 75.52 & 0.047 & 0.0223\end{array}$ $\begin{array}{llll}0.0006230 & 75.52 & 0.047 & 0.0223 \\ 0.0000308 & 400.01 & 0.012 & 0.0000\end{array}$ $\begin{array}{llll}0.0000308 & 400.01 & 0.012 & 0.0223\end{array}$ $\begin{array}{llll}0.0000308 & 400.01 & 0.012 & 0.0000\end{array}$ $\begin{array}{lll}0.0000308 & 400.01 & 0.012 \\ 0.0000308 & 400.01 & 0.012 \\ & 400.01 & 0.012\end{array}$

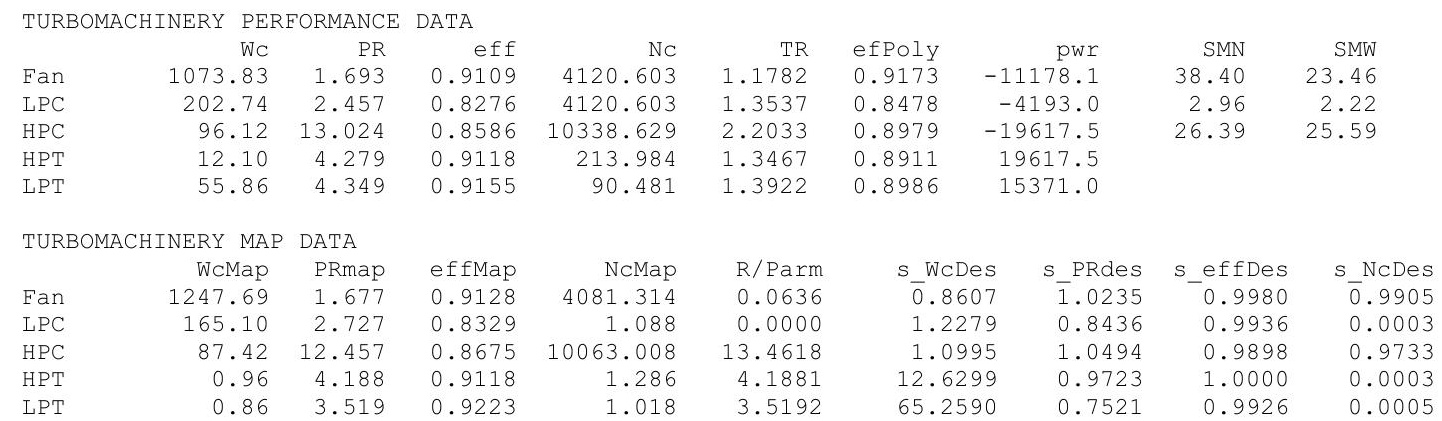




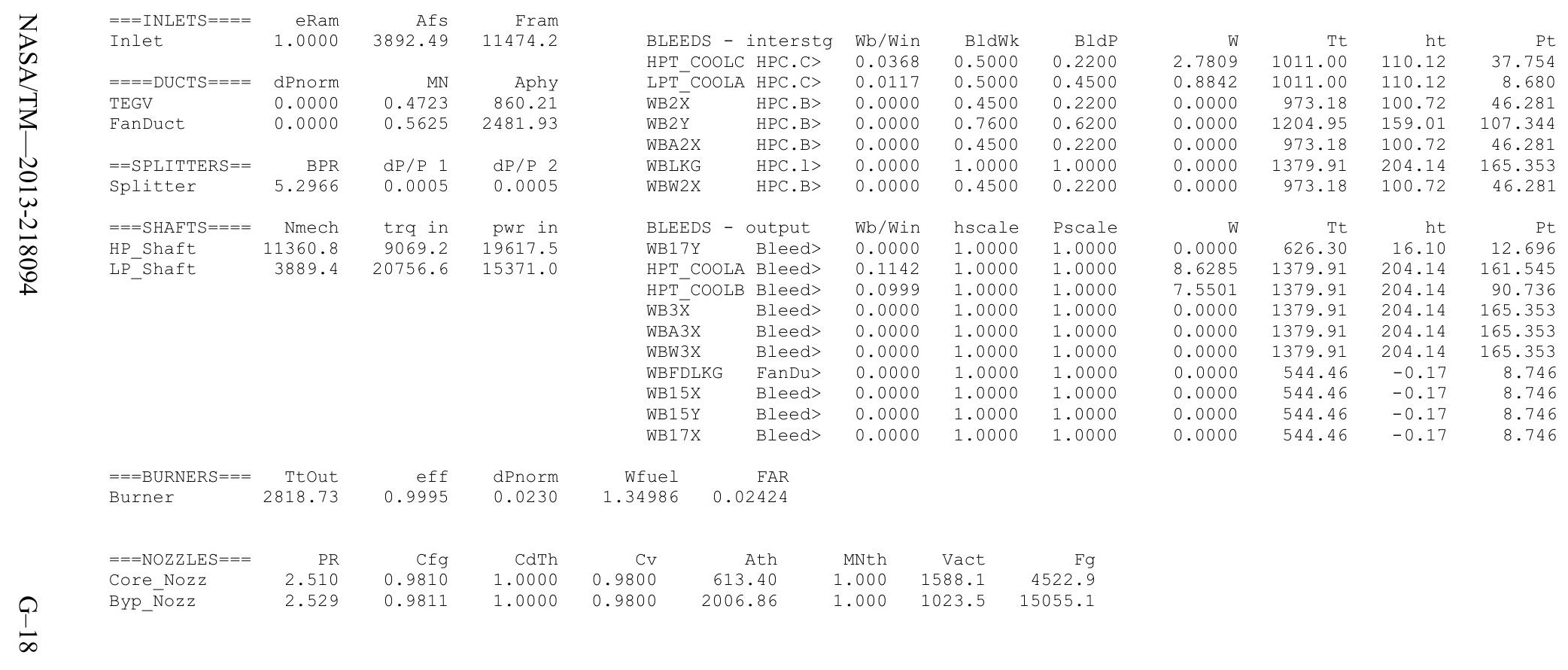


Turbofan Engine - COMDES ON Converge = 1 CASE:

Version:NPSS 1.6.5 - Rev: -> Gas Package: Janaf iter/pass/Jacb/Broy= 20/34/1/18 Run by: Philip C Jorgenson PC: 75

Temperature Stator 1 inlet: 458.76 Stator 1 exit: 475.02 Stator 2 inlet: 494.78 Stator 2 exit: 503.08 $\begin{array}{llll}\text { Stator } 3 \text { inlet: } 526.68 & \text { Stator } 3 \text { exit: } 534.96 & \text { Stator } 4 \text { inlet: } 557.27 & \text { Stator } 4 \text { exit: } 564.84 \\ \text { Stator } 5 \text { inlet: } 582.66 & \text { Stator } 5 \text { exit: } 589.79 & & \text { Unblocked Percent Blockage: } 0.00\end{array}$

Ambient Relative Humidity $\quad 10.00$

Fan Face Relative Humidity 3.58

Fan Bypass Relative Humidity $\quad 0.24$

LPC Inlet Relative Humidity 1.85

$\begin{array}{ll}\text { LPC Exit Relative Humidity } & 0.02 \\ \text { HPC Relative Humidity } & 0.00\end{array}$

0.0000100

776.30
3.46

Inlet Length

Ambient Flow Velocity

Ambient Static Pressure

411.85

Fan/LPC Inlet Static Pressure $\quad 4.63$

Additional Water at LPC Exit 0.0007012

an/LPC Inlet Static Temperature 447.81

\begin{tabular}{|c|c|c|c|c|c|c|c|c|c|c|c|c|c|c|}
\hline & & & & SUMMARY & TPUT DA & & & & & & & & & \\
\hline $\begin{array}{r}\text { MN } \\
0.780\end{array}$ & $\begin{array}{r}\text { alt } \\
35000.0\end{array}$ & $\begin{array}{l}\text { dTamb } \\
18.00\end{array}$ & $\begin{array}{r}W \\
439.16\end{array}$ & $\begin{array}{r}\text { Fn } \\
6089.2\end{array}$ & $\begin{array}{r}\text { TSFC } \\
0.5939\end{array}$ & $\begin{array}{r}\text { Wfuel } \\
3616.23\end{array}$ & $\begin{array}{r}\text { BPR } \\
5.5902\end{array}$ & $\begin{array}{r}\text { VTAS } \\
776.30\end{array}$ & $\begin{array}{r}\text { OPR } \\
26.743\end{array}$ & $\begin{array}{c}\text { EPR } \\
1.342\end{array}$ & $\begin{array}{l}\text { Powerset } \\
75.600\end{array}$ & $\begin{array}{c}T 4 \\
2544.9\end{array}$ & $\begin{array}{c}\text { T41 } \\
2393.3\end{array}$ & $\begin{array}{c}\text { T49 } \\
1656.2\end{array}$ \\
\hline
\end{tabular}

\begin{tabular}{|c|c|c|c|}
\hline & FSO & Ambient.Fl_O & 439.16 \\
\hline & FS1 & Inlet.Fl_o & 439.16 \\
\hline & FS12 & Splitter.Fl_02 & 372.52 \\
\hline & FS2 & Splitter.FI_01 & 66.64 \\
\hline 6 & FS14 & Fan.Fl o & 372.52 \\
\hline & FS23 & LPC.FI_O & 66.64 \\
\hline & FS24 & VaporIN.FI_O & 66.68 \\
\hline & FS25 & Bleed2.Fl 0 & 66.68 \\
\hline & FS 3 & HPC.Fl O & 63.45 \\
\hline & FS36 & Bleed3.Fl O & 49.17 \\
\hline & FS4 & Burner.Fl_o & 50.18 \\
\hline & FS45 & HPT.FI O & 66.91 \\
\hline & FS49 & LPT.FI_O & 67.69 \\
\hline & FS5 & TEGV.F̄ $O$ & 67.69 \\
\hline & FS 8 & Core Nozz.Fl O & 67.69 \\
\hline & FS17 & FanDüctLkg.Fi $O$ & 372.52 \\
\hline & FS171 & Bleed15.FI O & 372.52 \\
\hline & FS172 & FanDuct. FI & 372.52 \\
\hline & FS173 & Byp Nozz.Fì & 372.52 \\
\hline
\end{tabular}

\section{FLOW STATION DATA}

\begin{tabular}{|c|c|c|c|c|}
\hline Pt & Tt & ht & FAR & Wc \\
\hline 5.170 & 462.10 & -19.92 & 0.0000 & 1178.30 \\
\hline 5.170 & 462.10 & -19.92 & 0.0000 & 1178.30 \\
\hline 5.167 & 462.10 & -19.92 & 0.0000 & 1000.00 \\
\hline 5.167 & 462.10 & -19.92 & 0.0000 & 178.88 \\
\hline 8.041 & 530.78 & -3.45 & 0.0000 & 688.70 \\
\hline 12.339 & 612.64 & 16.22 & 0.0000 & 86.26 \\
\hline 12.339 & 613.54 & 12.40 & 0.0000 & 86.38 \\
\hline 12.339 & 613.54 & 12.40 & 0.0000 & 86.38 \\
\hline 38.259 & 1294.64 & 181.41 & 0.0000 & 10.66 \\
\hline 38.259 & 1294.64 & 181.41 & 0.0000 & 8.2 \\
\hline 35.075 & 2544.87 & 156.49 & 0.0204 & 12.09 \\
\hline 31.402 & 1663.71 & -4.74 & 0.0153 & 56.08 \\
\hline 7.208 & 1185.12 & -129.76 & 0.0151 & 208.6 \\
\hline 7.208 & 1185.18 & -129.76 & 0.0151 & 208.60 \\
\hline 7.208 & 1185.32 & -129.76 & 0.0151 & 208.62 \\
\hline 8.041 & 530.78 & -3.45 & 0.0000 & 688.70 \\
\hline 8.041 & 530.78 & -3.45 & 0.0000 & 688.70 \\
\hline 8.041 & 530.78 & -3.45 & 0.0000 & 688.70 \\
\hline 8.041 & 530.78 & -3.45 & 0.0000 & 688.70 \\
\hline
\end{tabular}

PS

3.458
4.118

4.118
4.112

4.630

6.675
11.141
11.136

11.136
11.136

11.136
123.964

129.978

127.582

28.257
6.213
6.213

$6.213-1139.46$

$3.843-1001.94$

6.675
6.487

$\begin{array}{ll}6.675 & 503.27 \\ 6.487 & 499.16\end{array}$

6.487
$4.247 \quad 442.19$

$\begin{array}{lll}503.27 & 2606.7 & 0.5226 \\ 442.19 & 2481.9 & 0.5625\end{array}$

$\begin{array}{ll}2481.9 & 0.5625 \\ 2006.9 & 1.0000\end{array}$

WAR
0.0000308
0.0000308
0.0000308
0.0000308
0.0000308
0.0000308
0.0007320
0.0007320
0.0007320
0.0007320
0.0007320
0.0007320
0.0007320
0.0007320
0.0007320
0.0000308
0.0000308
0.0000308
0.0000308

WAR

Wai 439.15
439.15
372.51 439.15
372.51 66.6
372.51

672.51

$\begin{array}{ll}66.64 & 0.049\end{array}$

$\begin{array}{ll}66.64 & 0.049\end{array}$

$\begin{array}{ll}63.40 & 0.046 \\ 49.14 & 0.036\end{array}$

$\begin{array}{ll}49.14 & 0.036 \\ 49.14 & 0.036\end{array}$

$\begin{array}{ll}49.14 & 0.03 \\ 65.86 & 0.04\end{array}$

$\begin{array}{ll}65.86 & 0.048 \\ 66.64 & 0.049\end{array}$

$66.64-0.049$

$\begin{array}{lll}66.64 & 0.049\end{array}$

$\begin{array}{ll}372.51 & 0.011\end{array}$

$372.51 \quad 0.011$

$372.51 \quad 0.011$

$\begin{array}{ll}372.51 & 0.011\end{array}$
H2O frac 0.0000 0.0000 0.0000 0.0007 0.0007 0.0007 0.0254 0.0192 0.0190 0.0190
0.0190
0.0000 0.0190
0.0000 0.0000 0.0000 0.0000

\begin{tabular}{|c|c|c|c|c|c|c|c|c|c|}
\hline \multicolumn{3}{|c|}{ TURBOMACHINERY PERFORMANCE } & \multicolumn{7}{|l|}{ DATA } \\
\hline & Wc & $P R$ & eff & $\mathrm{NC}$ & TR & efPoly & pwr & SMN & SMW \\
\hline Fan & 1000.00 & 1.556 & 0.9070 & 3842.664 & 1.1486 & 0.9126 & -8679.2 & 62.39 & 29.35 \\
\hline LPC & 178.88 & 2.388 & 0.8665 & 3842.664 & 1.3258 & 0.8817 & -3406.9 & 4.01 & 3.17 \\
\hline HPC & 86.38 & 11.205 & 0.8614 & 10048.977 & 2.1101 & 0.8983 & -15559.2 & 33.35 & 32.05 \\
\hline HPT & 12.09 & 4.301 & 0.9079 & 216.653 & 1.3566 & 0.8882 & 15559.2 & & \\
\hline LPT & 56.08 & 4.356 & 0.9114 & 88.923 & 1.4011 & 0.8940 & 12086.1 & & \\
\hline TURB & NERY MAP & DATA & & & & & & & \\
\hline & WcMap & PRmap & effMap & NcMap & R/Parm & s WCDes & s PRdes & $s$ effDes & s NcDes \\
\hline Fan & 1161.90 & 1.543 & 0.9088 & 3806.024 & 0.0589 & $\overline{0} .8607$ & $\overline{1} .0235$ & 0.9980 & $\overline{0} .9905$ \\
\hline LPC & 143.12 & 2.430 & 0.8208 & 1.015 & 0.0000 & 1.2499 & 0.9703 & 1.0557 & 0.0003 \\
\hline HPC & 78.56 & 10.724 & 0.8704 & 9781.078 & 12.8486 & 1.0995 & 1.0494 & 0.9898 & 0.9733 \\
\hline HPT & 0.96 & 4.210 & 0.9079 & 1.302 & 4.2099 & 12.6299 & 0.9723 & 1.0000 & 0.0003 \\
\hline LPT & 0.86 & 3.524 & 0.9182 & 1.000 & 3.5244 & 65.2590 & 0.7521 & 0.9926 & 0.0005 \\
\hline
\end{tabular}




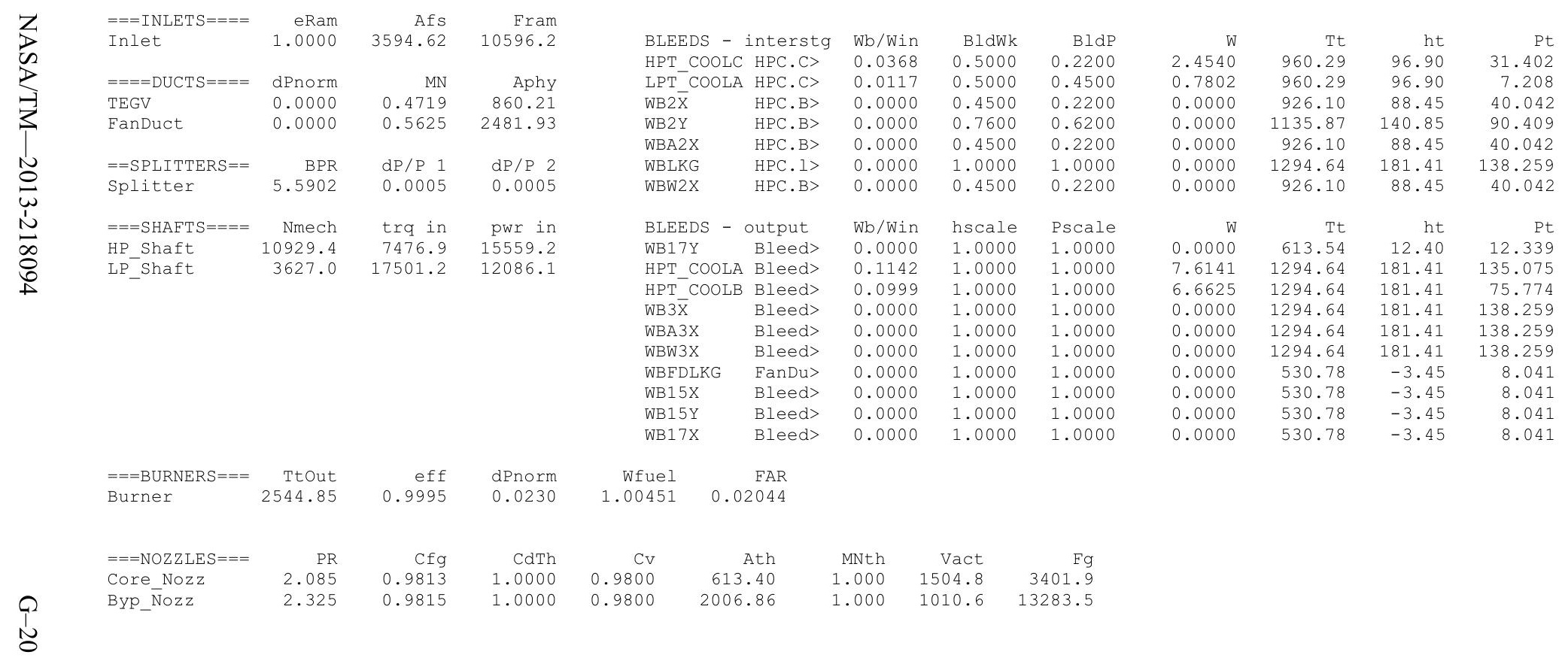




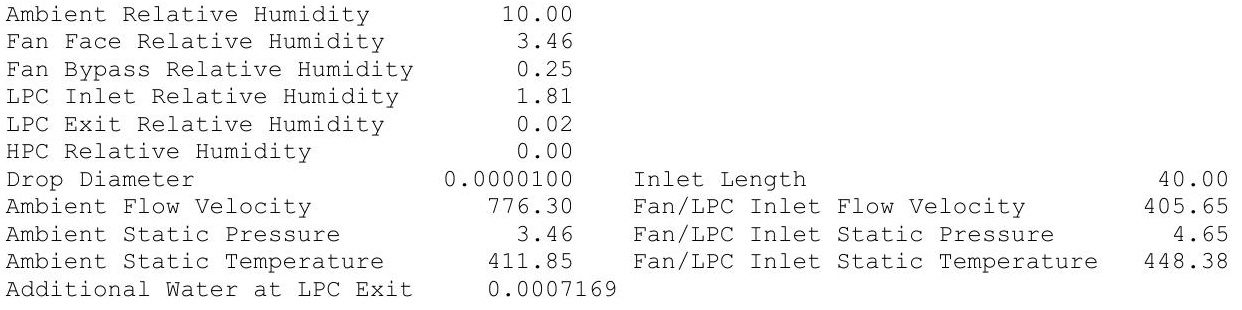

\begin{tabular}{|c|c|c|c|c|c|c|c|c|c|c|c|c|c|}
\hline $\begin{array}{r}\mathrm{MN} \\
.780\end{array}$ & $\begin{array}{r}\text { alt } \\
35000.0\end{array}$ & $\begin{array}{l}\text { dTamb } \\
18.00\end{array}$ & $\begin{array}{r}W \\
434.92\end{array}$ & $\begin{array}{r}\mathrm{Fn} \\
5860.9\end{array}$ & $\begin{array}{r}\text { TSFC } \\
0.5944\end{array}$ & $\begin{array}{r}\text { Wfuel } \\
3483.94\end{array}$ & $\begin{array}{r}\text { BPR } \\
5.6404\end{array}$ & $\begin{array}{r}\text { VTAS } \\
776.30\end{array}$ & $\begin{array}{r}\text { OPR } \\
26.121\end{array}$ & $\begin{array}{c}\text { EPR } \\
1.304\end{array}$ & $\begin{array}{l}\text { Powerset } \\
72.700\end{array}$ & $\begin{array}{c}\text { T4 } \\
2514.7\end{array}$ & $\begin{array}{c}\text { T41 } \\
2364.8\end{array}$ \\
\hline
\end{tabular}

\begin{tabular}{rrrrr}
\multicolumn{7}{c}{ FLOW } & \multicolumn{1}{l}{ STATION DATA } \\
W & Pt & Tt & ht & FAR \\
434.92 & 5.170 & 462.10 & -19.92 & 0.0000 \\
434.92 & 5.170 & 462.10 & -19.92 & 0.0000 \\
369.42 & 5.167 & 462.10 & -19.92 & 0.0000 \\
65.50 & 5.167 & 462.10 & -19.92 & 0.0000 \\
369.42 & 7.962 & 529.14 & -3.85 & 0.0000 \\
65.50 & 12.273 & 611.23 & 15.88 & 0.0000 \\
65.54 & 12.273 & 612.15 & 11.97 & 0.0000 \\
65.54 & 12.273 & 612.15 & 11.97 & 0.0000 \\
62.36 & 135.046 & 1285.16 & 178.88 & 0.0000 \\
48.33 & 135.046 & 1285.16 & 178.88 & 0.0000 \\
49.30 & 131.936 & 2514.71 & 154.51 & 0.0200 \\
65.74 & 30.655 & 1643.61 & -4.82 & 0.0150 \\
66.51 & 7.036 & 1170.20 & -128.19 & 0.0148 \\
66.51 & 7.036 & 1170.27 & -128.19 & 0.0148 \\
66.51 & 7.036 & 1170.40 & -128.19 & 0.0148 \\
369.42 & 7.962 & 529.14 & -3.85 & 0.0000 \\
369.42 & 7.962 & 529.14 & -3.85 & 0.0000 \\
369.42 & 7.962 & 529.14 & -3.85 & 0.0000 \\
369.42 & 7.962 & 529.14 & -3.85 & 0.0000
\end{tabular}

Wc
1166.92
1166.92
991.69
175.82
688.70
85.14
85.26
85.26
10.68
8.28
12.09
56.11
208.66
208.66
208.67
688.70
688.70
688.70
688.70

Ts
411.85
433.82
433.57
448.38
501.71
594.22
595.04
595.04
1248.06
1264.06
2481.86
1600.75
1124.92
1124.99
988.88
501.71
497.62
497.62
440.82

gamt
1.40081
1.40081
1.40081
1.40081
1.40008
1.39854
1.39842
1.39842
1.36267
1.36267
1.29608
1.33230
1.35969
1.35969
1.35968
1.40008
1.40008
1.40008
1.40008

WAR
0.0000308
0.0000308
0.0000308
0.0000308
0.0000308
0.0000308
0.0007477
0.0007477
0.0007477
0.0007477
0.0007477
0.0007477
0.0007477
0.0007477
0.0007477
0.0000308
0.0000308
0.0000308
0.0000308

Wair 434.90 434.90 0.013 $\begin{array}{ll}65.41 & 0.011\end{array}$ $\begin{array}{rr}65.49 & 0.002 \\ 369.41 & 0.01\end{array}$ $\begin{array}{ll}65.49 & 0.002\end{array}$ $\begin{array}{ll}65.49 & 0.002 \\ 65.49 & 0.049\end{array}$ $\begin{array}{ll}65.49 & 0.049 \\ 65.49 & 0.049\end{array}$ 62.320 .049 $\begin{array}{ll}62.32 & 0.04 \\ 48.30 & 0.03\end{array}$ $\begin{array}{ll}48.30 & 0.03 \\ 48.30 & 0.03\end{array}$ $\begin{array}{ll}64.73 & 0.048\end{array}$ $\begin{array}{ll}64.73 & 0.04 \\ 65.49 & 0.04\end{array}$ $65.49 \quad 0.049$ $65.49 \quad 0.049$ $369.41 \quad 0.011$ $369.41 \quad 0.01$ $\begin{array}{ll}369.41 & 0.011\end{array}$ $369.41 \quad 0.011$
H2O frac 0.0000 0.0000 0.0000 0.0000 0.0007 0.0007 0.0007 0.0007 0.0249 0.0189 0.0187 0.0187
0.0187 0.0187
0.0000 0.0000 0.0000

TURBOMACHINERY PERFORMANCE DATA

\begin{tabular}{|c|c|c|c|c|c|c|c|c|c|}
\hline & Wc & PR & eff & $\mathrm{NC}$ & $\mathrm{TR}$ & efPoly & pwr & SMN & SMW \\
\hline Fan & 991.69 & 1.541 & 0.9070 & 3811.543 & 1.1451 & 0.9125 & -8401.7 & 67.45 & 30.07 \\
\hline LPC & 175.82 & 2.375 & 0.8686 & 3811.543 & 1.3227 & 0.8835 & -3317.0 & 4.29 & 3.37 \\
\hline HPC & 85.26 & 11.004 & 0.8615 & 10017.646 & 2.0994 & 0.8982 & -15101.9 & 34.20 & 32.84 \\
\hline HPT & 12.09 & 4.304 & 0.9073 & 217.022 & 1.3577 & 0.8878 & 15101.9 & & \\
\hline LPT & 56.11 & 4.357 & .9107 & 88.741 & 1.4019 & 0.8932 & 11718.7 & & \\
\hline \multicolumn{10}{|c|}{ NERY MAP DATA } \\
\hline & WCMap & PRmap & effMap & NcMap & R/Parm & S_WcDes & S_PRdes & S_effDes & S_NcDes \\
\hline Fan & 1152.24 & 1.528 & 0.9089 & 3775.200 & 0.0583 & 0.8607 & $\overline{1} .0235$ & 0.9980 & $\overline{0} .99$ \\
\hline LPC & 141.11 & 2.398 & .8201 & 1.007 & 0.0000 & 1.2459 & 0.9834 & 1.0591 & 0.0003 \\
\hline HPC & 77.55 & 10.532 & .8704 & 9750.582 & 12.7785 & 1.0995 & 1.0494 & 0.9898 & 07 \\
\hline HPT & 0.96 & 4.212 & .9073 & 1.304 & 4.2124 & 12.6299 & 0.9723 & 1.0000 & 0.00 \\
\hline LPT & 0.86 & 3.525 & .9175 & 0.998 & 3.5247 & 65.2590 & 0.7521 & 0.9926 & 0.0 \\
\hline
\end{tabular}




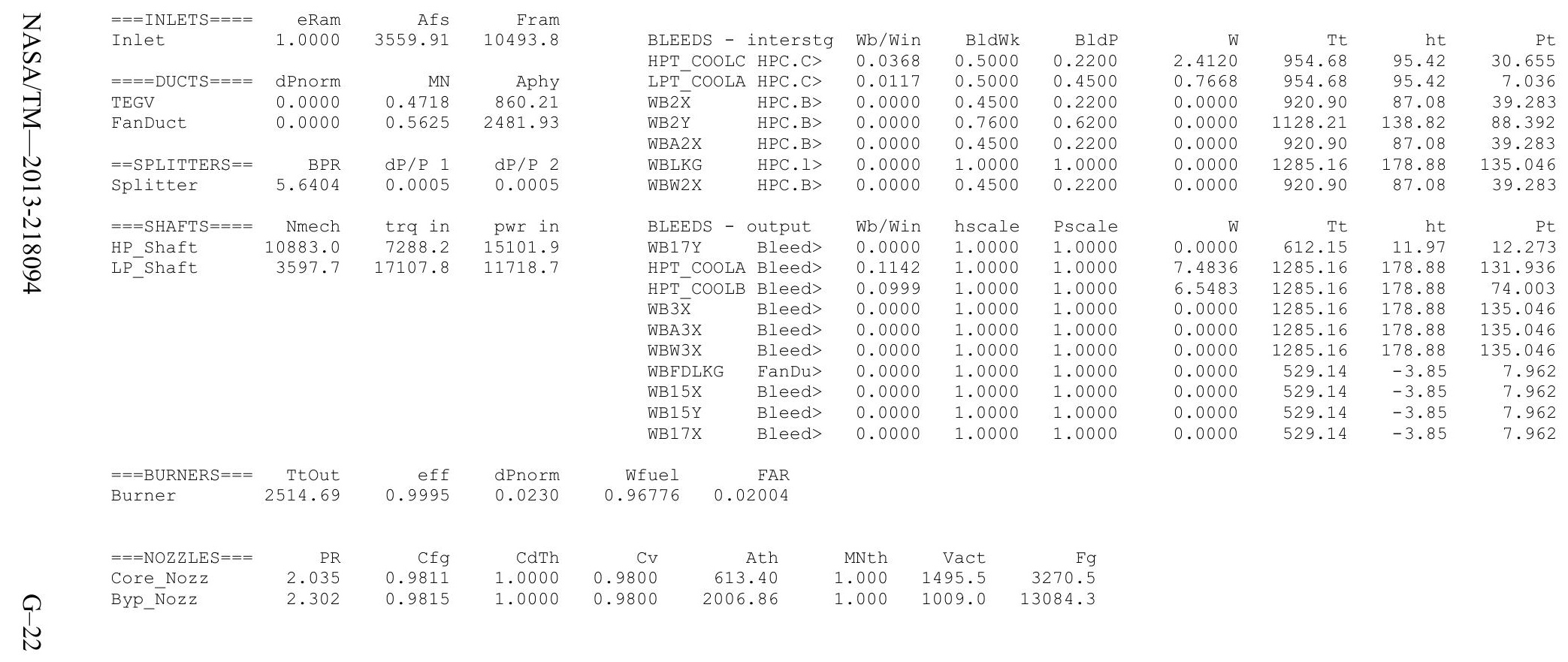


Date:05/13/13 Time:07:03:22 Model: Turbofan Engine - COMDES ON COnverge $=1$ CASE: 0

Version:NPSS 1.6.5 - Rev: -> Gas Package: Janaf iter/pass/Jacb/Broy= 38/52/1/36 Run by: Philip C Jorgenson PC: 69

Temperature Stator 1 inlet: 460.53 Stator 1 exit: 476.29 Stator 2 inlet: 495.63 Stator 2 exit: 503.96 $\begin{array}{llll}\text { Stator } 3 \text { inlet: } 526.63 & \text { Stator } 3 \text { exit: } 534.86 & \text { Stator } 4 \text { inlet: } 556.28 \text { Stator } 4 \text { exit: } 563.77 \\ \text { Stator } 5 \text { inlet: } 580.96 & \text { Stator } 5 \text { exit: } 587.97 & \text { Unblocked Percent Blockage: } 0.00\end{array}$

$\begin{array}{lclll}\text { Ambient Relative Humidity } & 10.00 & & & \\ \text { Fan Face Relative Humidity } & 3.36 & & & \\ \text { Fan Bypass Relative Humidity } & 0.26 & & & \\ \text { LPC Inlet Relative Humidity } & 1.77 & & & \\ \text { LPC Exit Relative Humidity } & 0.02 & & & \\ \text { HPC Relative Humidity } & 0.00 & & & \\ \text { Drop Diameter } & 0.0000100 & \text { Inlet Length } & & \\ \text { Ambient Flow Velocity } & 776.30 & \text { Fan/LPC Inlet Flow Velocity } & 397.39 \\ \text { Ambient Static Pressure } & 3.46 & \text { Fan/LPC Inlet Static Pressure } & 4.67 \\ \text { Ambient Static Temperature } & 411.85 & \text { Fan/LPC Inlet Static Temperature } & 448.93 \\ \text { Additional Water at LPC Exit } & 0.0007329 & & & \end{array}$

\begin{tabular}{|c|c|c|c|c|c|c|c|c|c|c|c|c|c|c|}
\hline & & & & SUMMARY & OUTPUT DATA & & & & & & & & & \\
\hline $\begin{array}{r}\text { MN } \\
0.780\end{array}$ & $\begin{array}{r}\text { alt } \\
35000.0\end{array}$ & $\begin{array}{l}\text { dTamb } \\
18.00\end{array}$ & $\begin{array}{r}W \\
430.85\end{array}$ & $\begin{array}{r}\text { Fn } \\
5642.7\end{array}$ & $\begin{array}{r}\text { TSFC } \\
0.5952\end{array}$ & $\begin{array}{r}\text { Wfuel } \\
3358.54\end{array}$ & $\begin{array}{r}\text { BPR } \\
5.6945\end{array}$ & $\begin{array}{r}\text { VTAS } \\
776.30\end{array}$ & $\begin{array}{r}\text { OPR } \\
25.516\end{array}$ & $\begin{array}{c}\text { EPR } \\
1.267\end{array}$ & $\begin{array}{l}\text { Powerset } \\
69.900\end{array}$ & $\begin{array}{c}T 4 \\
2486.0\end{array}$ & $\begin{array}{c}\text { T41 } \\
2338.4\end{array}$ & $\begin{array}{c}\text { T49 } \\
1617.1\end{array}$ \\
\hline
\end{tabular}

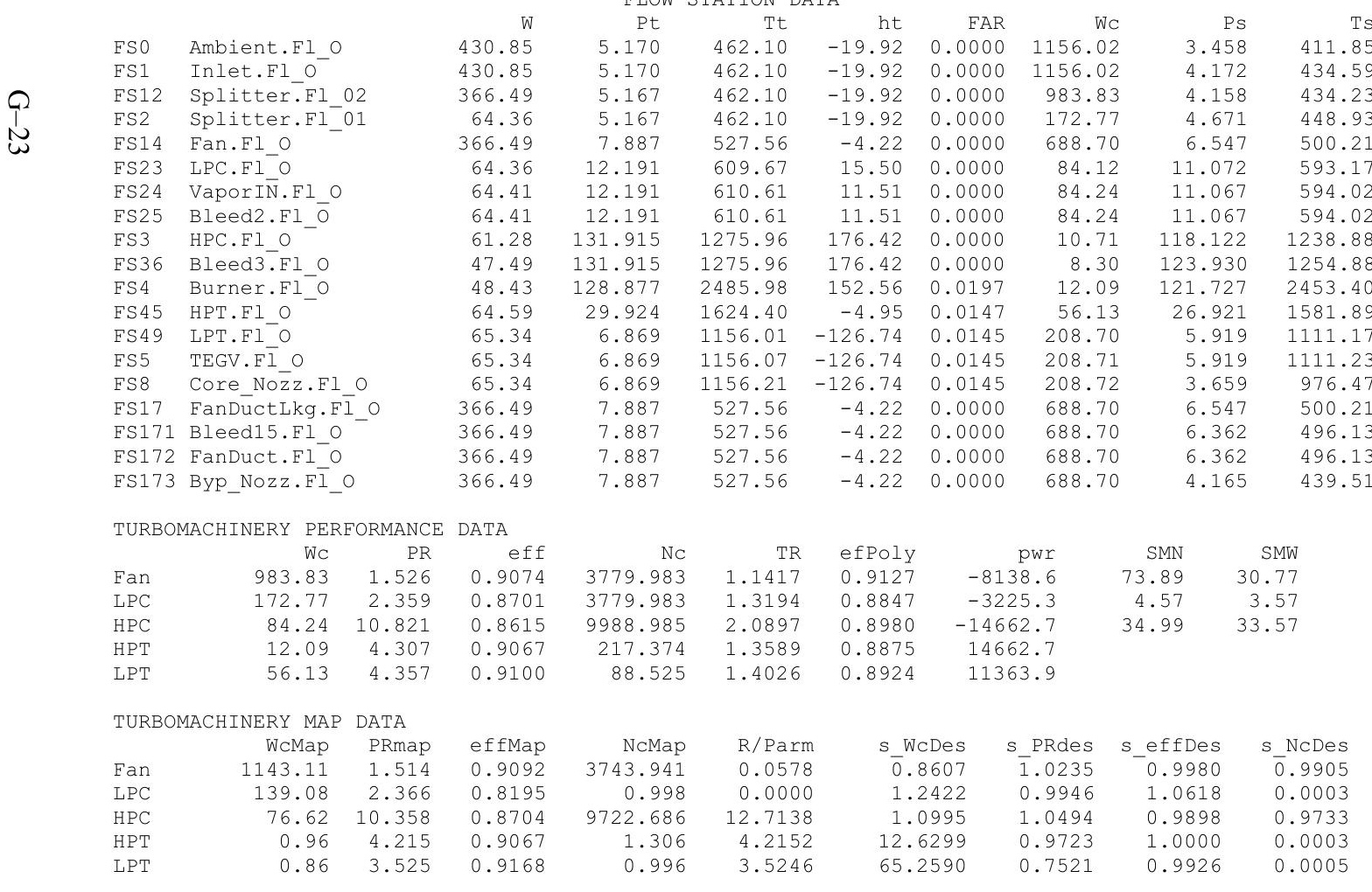




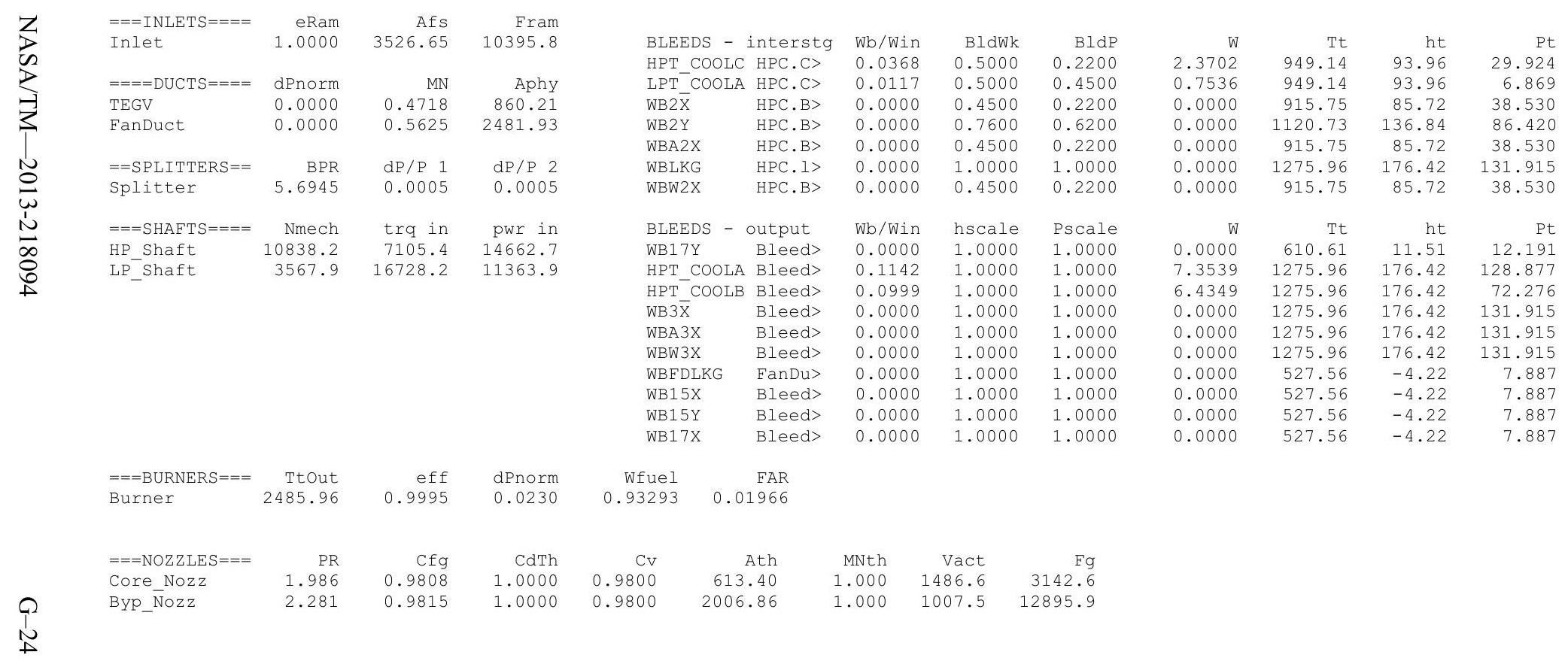


$\begin{array}{lll}\text { Date:05/13/13 Time:07:21:31 Model: } & \text { Turbofan Engine - COMDES ON COnverge }=1 \text { CASE: O }\end{array}$

Version:NPSS 1.6.5 - Rev: -> Gas Package: Janaf iter/pass/Jacb/Broy= 26/40/1/24 Run by: Philip C Jorgenson PC: 100

Stator 1 inlet: 468.91 Stator 1 exit: 488.41 Stator 2 inlet: 511.07 Stator 2 exit: 518.82 Stator 3 inlet: 548.81 Stator 3 exit: 557.31 stator lnlet: 585.38 Stator 4 exit: 593.55

Ambient Relative Humidity

Fan Face Relative Humidity 0

Fan Bypass Relative Humidity $\quad 0.21$

IPC Inlet Relative Humidity $\quad 2.53$

LPC Exit Relative Humidity $\quad 0.03$

Drop Diameter

Ambient Flow Velocity

.0000100

793.07 Fan/LPC Inlet

Fan/LPC Inlet Flow Velocity $\quad 40.00$

Ambient Static Pressure

Ambient Static Temperature

429.85 Fan/LPC Inlet Static Temperature 462.02

Additional Water at IPC Exit

0.0008426

SUMMARY OUTPUT DATA
$\begin{array}{rrr}\text { MN } & \text { alt } & \text { dTamb } \\ 0.780 & 35000.0 & 36.00\end{array}$
W
465.46
$\begin{array}{rr}\text { Fn } & \text { TSFC } \\ 8117.3 & 0.6152\end{array}$
Wfuel
4994.10
BPR
VTAS
OPR
$\mathrm{EPR}$
1.68
Powerset
T4 4
$\begin{array}{lr}\text { T41 } & \text { T49 } \\ 2747.5 & 1915.9\end{array}$

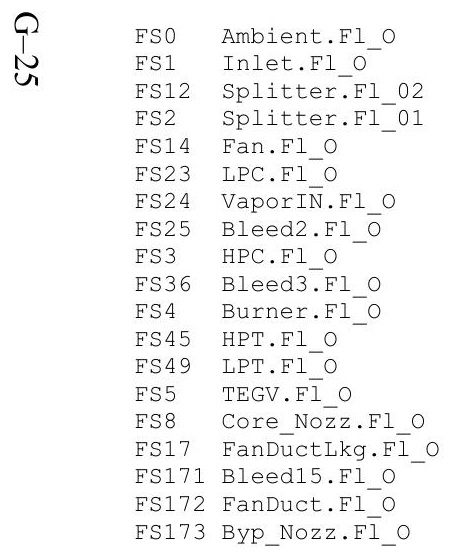

\begin{tabular}{|c|c|c|c|c|c|}
\hline W & $\mathrm{Pt}$ & $\mathrm{Tt}$ & ht & $R_{2}$ & Whe \\
\hline 5.46 & 5.170 & 482.28 & -15.41 & 0.0000 & 1275. \\
\hline 5.46 & 5.170 & 482.28 & -15.41 & 0.0000 & 1275.86 \\
\hline 1.59 & 5.167 & 482.28 & -15.41 & 0.0000 & 1073.92 \\
\hline 73.87 & 5.167 & 482.28 & -15.41 & 0.0000 & 202.58 \\
\hline 91.59 & 8.747 & 568.21 & 5.21 & 0.0000 & 688.62 \\
\hline 3.87 & 12.732 & 653.0 & 25.63 & 0.0 & 95.68 \\
\hline .93 & 12.732 & 654. & 21.04 & 0. & 95.83 \\
\hline & & & 21.04 & & \\
\hline .35 & 65.042 & 1434.36 & 216.67 & 0.0000 & 10.42 \\
\hline .52 & 5.042 & 434 & 216.67 & 0. & 8.07 \\
\hline & 161.241 & 924 & 184.53 & 0. & 12.1 \\
\hline 74.45 & 37.751 & 925 & -0.59 & 0.0 & 55.84 \\
\hline 7532 & 8.6 & 1383 & -146.66 & 0.0 & 207.83 \\
\hline 75.32 & 8.697 & 1383.21 & -146.66 & 0.0188 & 207.83 \\
\hline & 8.697 & 138 & -146.66 & 0.0188 & 207.84 \\
\hline 391 & 8.747 & 568.21 & 5.21 & 0.0 & 688.62 \\
\hline & 8.747 & 568.21 & 5.21 & 0.0000 & 688.62 \\
\hline & 8.747 & 568.21 & 5.21 & 0.0 & 688.62 \\
\hline 59 & 8.747 & 568.21 & 5.21 & 0.0000 & 688.62 \\
\hline
\end{tabular}

Ps
3.458
3.839
3.865
4.448
7.261
11.161
11.154
11.154
148.825
155.622
152.342
34.008
7.504
7.504
4.657
7.261
7.056
7.056
4.620

Ts
429.85
442.90
443.81
462.02
538.79
629.06
629.93
629.93
1396.01
1412.46
2889.33
1877.19
1331.76
1331.82
1176.42
538.79
534.40
534.40
473.45

$\begin{array}{rr}\text { Aphy } & \text { MN } \\ 3892.4 & 0.7800 \\ 4168.2 & 0.6661 \\ 3531.8 & 0.6576 \\ 830.5 & 0.4677 \\ 2606.7 & 0.5227 \\ 412.6 & 0.4381 \\ 412.6 & 0.4392 \\ 412.6 & 0.4392 \\ 49.7 & 0.3932 \\ 49.3 & 0.2957 \\ 74.6 & 0.3008 \\ 265.4 & 0.4008 \\ 860.2 & 0.4725 \\ 860.2 & 0.4725 \\ 613.4 & 1.0000 \\ 2606.7 & 0.5227 \\ 2481.9 & 0.5626 \\ 2481.9 & 0.5626 \\ 2006.9 & 1.0000\end{array}$

gamt
1.40064
1.40064
1.40064
1.40064
1.39944
1.39741
1.39726
1.39726
1.35386
1.35386
1.28075
1.31704
1.34383
1.34382
1.34381
1.39944
1.39944
1.39944
1.39944

WAR

Wair WH2O H2O frac $\begin{array}{llll}0.0000871 & 465.42 & 0.041 & 0.0001\end{array}$ $\begin{array}{llll}0.0000871 & 465.42 & 0.041 & 0.0001 \\ 0.0000871 & 391.55 & 0.034 & 0.0001\end{array}$ $\begin{array}{rrrr}0.0000871 & 391.55 & 0.034 & 0.0001 \\ 0.0000871 & 73.86 & 0.006 & 0.0001\end{array}$ $\begin{array}{llll}0.0000871 & 391.55 & 0.034 & 0.0001\end{array}$ $\begin{array}{llll}0.0000871 & 73.86 & 0.006 & 0.0001\end{array}$ $\begin{array}{llll}0.0009297 & 73.86 & 0.069 & 0.0009\end{array}$ $\begin{array}{llll}0.0009297 & 73.86 & 0.069 & 0.0009\end{array}$ $\begin{array}{llll}0.0009297 & 70.28 & 0.065 & 0.0009\end{array}$ $\begin{array}{llll}0.0009297 & 54.47 & 0.051 & 0.0009\end{array}$ $\begin{array}{llll}0.0009297 & 54.47 & 0.051 & 0.0315\end{array}$ $\begin{array}{llll}0.0009297 & 73.00 & 0.068 & 0.0239\end{array}$ $\begin{array}{llll}0.0009297 & 73.86 & 0.069 & 0.0236\end{array}$ $\begin{array}{llll}0.0009297 & 73.86 & 0.069 & 0.0236\end{array}$ $\begin{array}{llll}0.0009297 & 73.86 & 0.069 & 0.0236\end{array}$ $\begin{array}{llll}0.0000871 & 391.55 & 0.034 & 0.0001\end{array}$ $\begin{array}{llll}0.0000871 & 391.55 & 0.034 & 0.0001\end{array}$ $\begin{array}{llll}0.0000871 & 391.55 & 0.034 & 0.0001\end{array}$ TURBOMACHINERY PERFORMANCE DATA

\begin{tabular}{|c|c|c|c|c|c|c|c|c|c|}
\hline & WC & $\mathrm{PR}$ & \\
\hline Fan & 1073.92 & 1.693 & 0.9109 & 4120.978 & 1.1782 & 0.9173 & 423.5 & 38.36 & 23.45 \\
\hline LPC & 202.58 & 2.464 & 0.8288 & 4120.978 & 1.3542 & 0.8489 & 289.1 & 2.96 & 2.22 \\
\hline HPC & 95.83 & 12.963 & 0.8587 & 10326.703 & 2.1929 & 0.8978 & 967.3 & 26.63 & 25.82 \\
\hline HPT & 12.10 & 4.271 & 0.9122 & 214.382 & 1.3424 & 0.8901 & 967.3 & & \\
\hline LPT & 55.84 & 4.341 & 0.9168 & 90.567 & 1.3884 & 0.9006 & 712.3 & & \\
\hline \multicolumn{10}{|c|}{ TURBOMACHINERY MAP DATA } \\
\hline & WcMap & PRmap & effMap & NcMap & R/Parm & s_WcDes & S_PRdes & s_effDes & S_NCDes \\
\hline Fan & 1247.78 & 1.677 & 0.9128 & 4081.685 & 0.0636 & $\overline{0} .8607$ & $\overline{1} .0235$ & 0.9980 & $\overline{0} .9905$ \\
\hline LPC & 165.14 & 2.728 & 0.8329 & 1.088 & 0.0000 & 1.2268 & 0.8474 & 0.9950 & 0.0003 \\
\hline HPC & 87.16 & 12.399 & 0.8676 & 10051.400 & 13.4384 & 1.0995 & 1.0494 & 0.9898 & 0.9733 \\
\hline HРT & 0.96 & 4.181 & 0.9122 & 1.288 & 4.1805 & 12.6299 & 0.9723 & 1.0000 & 0.0003 \\
\hline LPT & 0.86 & 3.513 & 0.9236 & 1.019 & 3.5126 & 65.2590 & 0.7521 & 0.9926 & 0.0005 \\
\hline
\end{tabular}




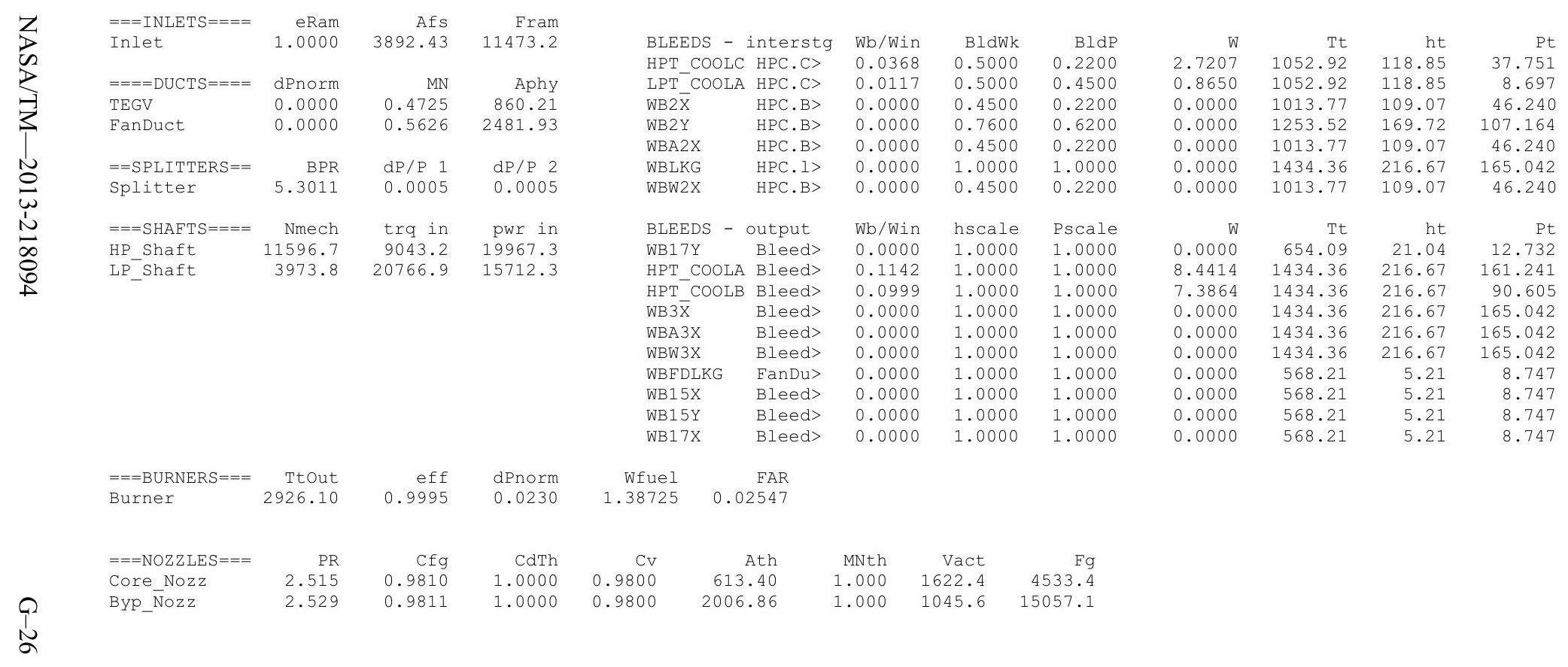


Date:05/13/13 Time:07:22:04 Model: Turbofan Engine - COMDES ON COnverge $=1$ CASE: 0

Version:NPSS 1.6.5 - Rev: -> Gas Package: Janaf iter/pass/Jacb/Broy=19/33/1/17 Run by: Philip C Jorgenson PC: 75

Temperature Stator 1 inlet: 478.88 Stator 1 exit: 495.86 Stator 2 inlet: 516.51 Stator 2 exit: 525.19 $\begin{array}{llll}\text { Stator } 3 \text { inlet: } 549.81 & \text { Stator } 3 \text { exit: } 558.46 & \text { Stator } 4 \text { inlet: } 581.72 \text { Stator } 4 \text { exit: } 589.63 \\ \text { Stator } 5 \text { inlet: } 608.21 & \text { Stator } 5 \text { exit: } 615.66 & \text { Unblocked Percent Blockage: } 0.00\end{array}$

\begin{abstract}
Ambient Relative Humidity $\quad 10.00$
Fan Face Relative Humidity $\quad 3.82$

Fan Bypass Relative Humidity $\quad 0.30$

LPC Inlet Relative Humidity $\quad 2.06$

LPC Exit Relative Humidity $\quad 0.03$

HPC Relative Humidity $\quad 0.00$

$\begin{array}{lr}\text { Drop Diameter } & 0.0000100 \\ \text { Ambient Flow Velocity } & 793.07\end{array}$

Ambient Static Pressure

Additional Water at LPC Exit

Inlet Length

3.46 Fan/LPC Inlet Flow Velocity $\quad 422.59$

429.85 Fan/LPC Inlet Static Pressure 4.63 0.0009991 Fan/LPC Inlet Static Temperature 467.40
\end{abstract}

\section{$\begin{array}{rlrr}\text { MN alt dTamb } & \text { W } & \text { SUMMAR OUTPUT DATA } \\ 0.780 & \text { Fn } & \text { TSFC }\end{array}$}

$\begin{array}{rrrr}0.780 & 35000.0 & 36.00 & 429.85\end{array}$
BPR
5.5955
VTAS
793.07
OPR
26.691

$\begin{array}{ccccc}\text { EPR } & \text { Powerset } & \text { T4 } & \text { T41 } & \text { T49 } \\ 1.344 & 75.600 & 2642.5 & 2485.1 & 1724.8\end{array}$

WC
1178.26
1178.26
1000.11
178.73
688.65
86.09
86.26
86.26
10.65
8.26
12.09
56.06
208.38
208.39
208.40
688.65
688.65
688.65
688.65

$\begin{array}{rr}\text { Ps } & \text { TS } \\ 3.458 & 429.85 \\ 4.118 & 451.90 \\ 4.111 & 451.74 \\ 4.631 & 467.40 \\ 6.676 & 525.26 \\ 11.158 & 621.22 \\ 11.152 & 622.30 \\ 11.152 & 622.30 \\ 23.721 & 1308.35 \\ 29.724 & 1324.88 \\ 127.343 & 2608.38 \\ 28.246 & 1688.06 \\ 6.223 & 1190.06 \\ 6.223 & 1190.12 \\ 3.852 & 1047.70 \\ 6.676 & 525.26 \\ 6.488 & 520.97 \\ 6.488 & 520.97 \\ 4.248 & 461.54\end{array}$$$
\text { A }
$$

Aphy MN

\section{$\begin{array}{ll}4168.2 & 0.7800 \\ 3531.8 & 0.5791\end{array}$}

$830.5 \quad 0.3986$

$2606.7 \quad 0.5226$

$412.6 \quad 0.3842$

$\begin{array}{ll}412.6 & 0.3853\end{array}$

$\begin{array}{rr}412.6 & 0.3853 \\ 49.7 & 0.4033\end{array}$

$\begin{array}{ll}49.7 & 0.4033 \\ 49.3 & 0.3026\end{array}$

$\begin{array}{ll}49.3 & 0.3026 \\ 74.6 & 0.2993\end{array}$

$\begin{array}{cc}74.6 & 0.2993 \\ 265.4 & 0.4010 \\ 860.2 & 0.4721\end{array}$

$\begin{array}{ll}860.2 & 0.4721 \\ 860.2 & 0.4721\end{array}$

$\begin{array}{ll}860.2 & 0.4721 \\ 613.4 & 1.0000\end{array}$

$\begin{array}{ll}2606.7 & 0.5226 \\ 2481.9 & 0.5626\end{array}$

$\begin{array}{ll}2481.9 & 0.5626 \\ 2481.9 & 0.5626\end{array}$

2006.91 .0000

gamt
1.40064
1.40064
1.40064
1.40064
1.39969
1.39780
1.39762
1.39762
1.35889
1.35889
1.29130
1.32729
1.35470
1.35469
1.35468
1.39969
1.39969
1.39969
1.39969

1.39969
1.39969

WAR
0.0000871
0.0000871
0.0000871
0.0000871
0.0000871
0.0000871
0.0010862
0.0010862
0.0010862
0.0010862
0.0010862
0.0010862
0.0010862
0.0010862
0.0010862
0.0000871
0.0000871
0.0000871
0.0000871

Wair
429.81
429.81
364.64
65.17
364.64
65.17
65.17
65.17
62.01
48.05
48.05
64.40
65.17
65.17
65.17
364.64
364.64
364.64
364.64
WH2O H2O frac $\begin{array}{ll}0.037 & 0.0001 \\ 0.037 & 0.0001\end{array}$ $\begin{array}{ll}0.037 & 0.0001 \\ 0.032 & 0.0001\end{array}$ $0.006 \quad 0.0001$ $0.032 \quad 0.0001$ $0.006 \quad 0.0001$ $\begin{array}{ll}0.032 & 0.0001 \\ 0.071 & 0.0011\end{array}$ $0.071 \quad 0.0011$ $0.070 \quad 0.0205$ $0.071 \quad 0.0203$ $0.071 \quad 0.0203$ $0.032 \quad 0.0001$ $\begin{array}{ll}0.032 & 0.0001 \\ 0.032 & 0.0001\end{array}$ 0.0001

TURBOMACHINERY PERFORMANCE DATA

\begin{tabular}{|c|c|c|c|c|c|c|c|c|c|}
\hline & WC & PR & eff & $\mathrm{Nc}$ & $\mathrm{TR}$ & efPoly & wr & SMN & SMW \\
\hline Fan & 1000.11 & 1.556 & 0.9070 & 3843.155 & 1.1486 & 0.9126 & -8871.2 & 62.35 & 29.33 \\
\hline LPC & 178.73 & 2.391 & 0.8667 & 3843.155 & 1.3259 & 0.8819 & -3481.8 & 4.00 & 3.16 \\
\hline HPC & 86.26 & 11.170 & 0.8615 & 10045.180 & 2.1020 & 0.8982 & -15851.7 & 33.59 & 32.28 \\
\hline HPT & 12.09 & 4.295 & 0.9089 & 217.184 & 1.3532 & 0.8892 & 15851.8 & & \\
\hline LPT & 56.06 & 4.348 & 0.9130 & 89.029 & 1.3976 & 0.8960 & 12352.9 & & \\
\hline \multicolumn{10}{|c|}{ TURBOMACHINERY MAP DATA } \\
\hline & WcMap & PRmap & effMap & NcMap & R/Parm & s WcDes & s PRdes & $s$ effDes & s NcDes \\
\hline Fan & 1162.03 & 1.544 & 0.9088 & 3806.511 & 0.0589 & $\overline{0} .8607$ & $\overline{1} .0235$ & 0.9980 & $\overline{0} .9905$ \\
\hline LPC & 143.15 & 2.431 & 0.8208 & 1.015 & 0.0000 & 1.2485 & 0.9719 & 1.0559 & 0.0003 \\
\hline HPC & 78.45 & 10.691 & 0.8704 & 9777.382 & 12.8276 & 1.0995 & 1.0494 & 0.9898 & 0.9733 \\
\hline HPT & 0.96 & 4.204 & 0.9089 & 1.305 & 4.2036 & 12.6299 & 0.9723 & 1.0000 & 0.0003 \\
\hline LPT & 0.86 & 3.518 & 0.9197 & 1.001 & 3.5184 & 65.2590 & 0.7521 & 0.9926 & 0.0005 \\
\hline
\end{tabular}


$\begin{array}{lrrr}===\text { INLETS }==== & \text { eRam } & \text { Afs } & \text { Fram } \\ \text { Inlet } & 1.0000 & 3594.66 & 10595.5\end{array}$

$====$ DUCTS $====$ dPnorm

$\begin{array}{ll} & \\ \text { TEGV } & 0.0000\end{array}$

FanDuct

0.0000

$\begin{array}{lr}==\text { SPLITTERS }== & \text { BPR } \\ \text { Splitter } & 5.5955\end{array}$

$===$ SHAFT $S====\quad$ Nmech

$\begin{array}{lr}\text { HP_Shaft } & 11164.3 \\ \text { LP_Shaft } & 3705.9\end{array}$

$=$ BURNERS $===\quad$ TtOut

Burner 2642.44

eff
0.9995

dPnorm

$===$ NOZZLES $===$

Core Nozz

$\mathrm{PR}$
2.087
2.326

Cfg
0.9813

CdTh

$\begin{array}{ll}0.9813 & 1.0000 \\ 0.9815 & 1.0000\end{array}$

$1.0000 \quad 0.9800$

0.9800
0.9800

$\begin{array}{llrr}\text { BLEEDS - interstg } & \text { Wb/Win } & \text { BldWk } & \text { BldP }\end{array}$

$\begin{array}{llll}\text { HPT_COOLC HPC.C> } & 0.0368 & 0.5000 & 0.2200 \\ \text { LPTCCOOLA HPC.C> } & 0.0117 & 0.5000 & 0.4500\end{array}$

$\begin{array}{lllll}\text { LPT COOLA HPC.C> } & 0.0117 & 0.5000 & 0.4500 \\ \text { WB2 } 2 \text { X } & \text { HPC.B> } & 0.0000 & 0.4500 & 0.2200\end{array}$

$\begin{array}{llllllllllllll}\text { WB2Y HPC.B } & 0.0000 & 0.7600 & 0.6200\end{array}$

$\begin{array}{lllll}\text { WBA2X HPC.B } & 0.0000 & 0.4500 & 0.2200\end{array}$

WBLKG HPC.1> $0.0000 \quad 1.0000 \quad 1.0000$

$\begin{array}{lllll}\text { WBW2X HPC.B> } & 0.0000 & 0.4500 & 0.2200\end{array}$

BLEEDS - output Wb/Win hscale Pscale

WB17Y Bleed> $0.0000 \quad 1.0000 \quad 1.0000$

HPT_COOLA Bleed> $0.1142 \quad 1.0000 \quad 1.0000$

$\begin{array}{lllll}\text { HPT_COOLB Bleed> } & 0.0999 & 1.0000 & 1.0000\end{array}$

$\begin{array}{lllll}\text { WB3X } & \text { Bleed> } & 0.0000 & 1.0000 & 1.0000 \\ \text { WBA3X } & \text { Bleed }> & 0.0000 & 1.0000 & 1.0000\end{array}$

$\begin{array}{lllll}\text { WBA3X } & \text { Bleed> } & 0.0000 & 1.0000 & 1.0000 \\ \text { WBW3X } & \text { Bleed }> & 0.0000 & 1.0000 & 1.0000\end{array}$

$\begin{array}{lllll}\text { WBW3X } & \text { Bleed> } & 0.0000 & 1.0000 & 1.0000 \\ \text { WBFDLKG } & \text { FanDu> } & 0.0000 & 1.0000 & 1.0000\end{array}$

$\begin{array}{llll}\text { WB15X Bleed> } & 0.0000 & 1.0000 & 1.0000\end{array}$

WB15Y Bleed> $0.0000 \quad 1.0000 \quad 1.0000$

$\begin{array}{lll}0.0000 & 1.0000 & 1.0000\end{array}$

$\begin{array}{rrrr}\text { W } & \text { Tt } & \text { ht } & \text { Pt } \\ 2.4008 & 1000.61 & 104.91 & 31.389 \\ 0.7633 & 1000.61 & 104.91 & 7.218 \\ 0.0000 & 965.18 & 96.11 & 39.993 \\ 0.0000 & 1182.44 & 150.67 & 90.246 \\ 0.0000 & 965.18 & 96.11 & 39.993 \\ 0.0000 & 1346.69 & 192.91 & 137.988 \\ 0.0000 & 965.18 & 96.11 & 39.993 \\ \text { W } & \text { Tt } & \text { ht } & \text { Pt } \\ 0.0000 & 640.68 & 16.91 & 12.353 \\ 7.4489 & 1346.69 & 192.91 & 134.810 \\ 6.5179 & 1346.69 & 192.91 & 75.653 \\ 0.0000 & 1346.69 & 192.91 & 137.988 \\ 0.0000 & 1346.69 & 192.91 & 137.988 \\ 0.0000 & 1346.69 & 192.91 & 137.988 \\ 0.0000 & 553.95 & 1.78 & 8.043 \\ 0.0000 & 553.95 & 1.78 & 8.043 \\ 0.0000 & 553.95 & 1.78 & 8.043 \\ 0.0000 & 553.95 & 1.78 & 8.043\end{array}$

$\underset{\infty}{\infty}$$$
0.02145
$$$$
\text { cis }
$$

5


Date:05/13/13 Time:07:22:36 Model: Turbofan Engine - COMDES ON COnverge = 1 CASE:

Version:NPSS 1.6.5 - Rev: -> Gas Package: Janaf iter/pass/Jacb/Broy=18/32/1/16 Run by: Philip C Jorgenson PC: 72

Temperature Stator 1 inlet: 479.85 Stator 1 exit: 496.57 Stator 2 inlet: 517.01 Stator 2 exit: 525.73 $\begin{array}{lll}\text { Stator } 3 \text { inlet: } 549.86 & \text { Stator } 3 \text { exit: } 558.50 & \text { Stator } 4 \text { inlet: } 581.29 \text { Stator } 4 \text { exit: } 589.17 \\ \text { Stator } 5 \text { inlet: } 607.42 & \text { Stator } 5 \text { exit: } 614.82 & \text { Unblocked Percent Blockage: } 0.00\end{array}$

\begin{abstract}
Ambient Relative Humidity $\quad 10.00$
$\begin{array}{ll}\text { Fan Face Relative Humidity } & 3.71 \\ \text { Fan Bypass Relative Humidity } & 0.31\end{array}$

$\begin{array}{ll}\text { Fan Bypass Relative Humidity } & 0.31 \\ \text { LPC } & 2.01\end{array}$

$\begin{array}{ll}\text { LPC Inlet Relative Humidity } & 2.01 \\ \text { LPC Exit Relative Humidity } & 0.03\end{array}$

$\begin{array}{ll}\text { LPC Exit Relative Humidity } & 0.03 \\ \text { HPC Relative Humidity } & 0.00\end{array}$

0.0000100

Fan/LPC Inlet Flow Velocity $\quad 414.01$

3.46 Fan/LPC Inlet Static Pressure 4.65
0.0010212 0.0010212

Ambient Static Temperatur

Additional Water at LPC Exit
\end{abstract}

SUMMARY OUTPUT DATA

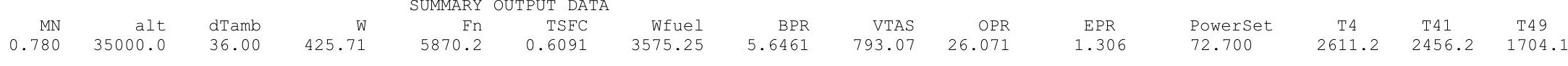

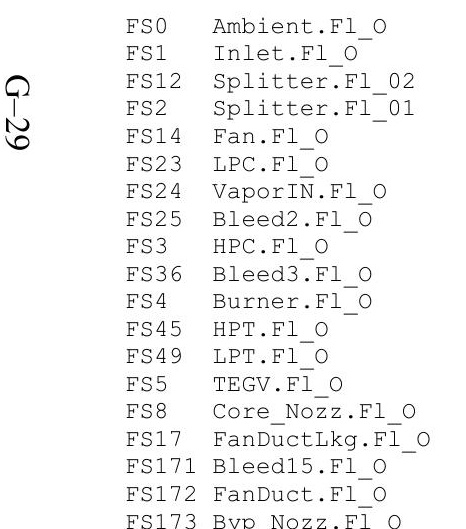

\begin{tabular}{rrrrrr}
\multicolumn{7}{c}{ FLOW } & \multicolumn{1}{c}{ STATION DATA } \\
$W$ & $\mathrm{Pt}$ & $\mathrm{Tt}$ & $\mathrm{ht}$ & FAR & WC \\
425.71 & 5.170 & 482.28 & -15.41 & 0.0000 & 1166.93 \\
425.71 & 5.170 & 482.28 & -15.41 & 0.0000 & 1166.93 \\
361.66 & 5.167 & 482.28 & -15.41 & 0.0000 & 991.84 \\
64.05 & 5.167 & 482.28 & -15.41 & 0.0000 & 175.67 \\
361.66 & 7.964 & 552.25 & 1.38 & 0.0000 & 688.66 \\
64.05 & 12.286 & 638.01 & 22.00 & 0.0000 & 84.98 \\
64.12 & 12.286 & 639.25 & 16.43 & 0.0000 & 85.15 \\
64.12 & 12.286 & 639.25 & 16.43 & 0.0000 & 85.15 \\
61.01 & 134.780 & 1336.94 & 190.25 & 0.0000 & 10.68 \\
47.28 & 134.780 & 1336.94 & 190.25 & 0.0000 & 8.28 \\
48.28 & 131.676 & 2611.22 & 164.41 & 0.0210 & 12.09 \\
64.36 & 30.641 & 1711.79 & -1.28 & 0.0157 & 56.08 \\
65.11 & 7.045 & 1221.69 & -130.11 & 0.0155 & 208.45 \\
65.11 & 7.045 & 1221.75 & -130.11 & 0.0155 & 208.45 \\
65.11 & 7.045 & 1221.89 & -130.11 & 0.0155 & 208.46 \\
361.66 & 7.964 & 552.25 & 1.38 & 0.0000 & 688.66 \\
361.66 & 7.964 & 552.25 & 1.38 & 0.0000 & 688.66 \\
361.66 & 7.964 & 552.25 & 1.38 & 0.0000 & 688.66 \\
361.66 & 7.964 & 552.25 & 1.38 & 0.0000 & 688.66
\end{tabular}

$\begin{array}{rr}\text { PS } & \text { Ts } \\ 3.458 & 429.85 \\ 4.146 & 452.77 \\ 4.135 & 452.49 \\ 4.652 & 467.99 \\ 6.611 & 523.65 \\ 11.132 & 620.35 \\ 11.126 & 621.46 \\ 11.126 & 621.46 \\ 120.762 & 1298.59 \\ 126.663 & 1315.13 \\ 124.383 & 2577.43 \\ 27.570 & 1667.55 \\ 6.074 & 1174.86 \\ 6.074 & 1174.93 \\ 3.759 & 1033.95 \\ 6.611 & 523.65 \\ 6.424 & 519.38 \\ 6.424 & 519.38 \\ 4.206 & 460.12\end{array}$

$\begin{array}{rr}\text { Aphy } & \text { MN } \\ 3560.1 & 0.7800 \\ 4168.2 & 0.5703 \\ 3531.8 & 0.5731 \\ 830.5 & 0.3903 \\ 2606.7 & 0.5226 \\ 412.6 & 0.3782 \\ 412.6 & 0.3793 \\ 412.6 & 0.3793 \\ 49.7 & 0.4045 \\ 49.3 & 0.3034 \\ 74.6 & 0.2991 \\ 265.4 & 0.4010 \\ 860.2 & 0.4720 \\ 860.2 & 0.4720 \\ 613.4 & 1.0000 \\ 2606.7 & 0.5226 \\ 2481.9 & 0.5626 \\ 2481.9 & 0.5626 \\ 2006.9 & 1.0000\end{array}$

gamt
1.40064
1.40064
1.40064
1.40064
1.39972
1.39784
1.39766
1.39766
1.35946
1.35946
1.29249
1.32848
1.35589
1.35588
1.35587
1.39972
1.39972
1.39972
1.39972

WAR
0.0000871
0.0000871
0.0000871
0.0000871
0.0000871
0.0000871
0.0011083
0.0011083
0.0011083
0.0011083
0.0011083
0.0011083
0.0011083
0.0011083
0.0011083
0.0000871
0.0000871
0.0000871
0.0000871

Wair
425.68
425.68
361.63
64.05
361.63
64.05
64.05
64.05
60.94
47.23
47.23
63.30
64.05
64.05
64.05
361.63
361.63
361.63
361.63

H2O frac $0.037-0.0001$ $0.006 \quad 0.0001$ 0.0520 .0011 $0.070 \quad 0.0201$ $0.071 \quad 0.0199$ 0.071 0.071 0.031 0.031 TURBOMACHINERY PERFORMANCE DATA

\begin{tabular}{|c|c|c|c|c|c|c|c|c|c|}
\hline & Wc & PR & eff & $\mathrm{NC}$ & TR & efPoly & pwr & SMN & SMW \\
\hline Fan & 991.84 & 1.541 & 0.9070 & 3812.224 & 1.1451 & 0.9125 & -8589.4 & 67.31 & 30.05 \\
\hline LPC & 175.67 & 2.378 & 0.8687 & 3812.224 & 1.3229 & 0.8836 & -3390.2 & 4.28 & 3.37 \\
\hline HPC & 85.15 & 10.970 & 0.8615 & 10013.931 & 2.0914 & 0.8980 & -15386.6 & 34.44 & 33.07 \\
\hline HPT & 12.09 & 4.297 & 0.9083 & 217.556 & 1.3543 & 0.8888 & 15386.6 & & \\
\hline LPT & 56.08 & 4.349 & 0.9124 & 88.850 & 1.3985 & 0.8954 & 11979.6 & & \\
\hline \multicolumn{10}{|c|}{ TURBOMACHINERY MAP DATA } \\
\hline & WcMap & PRmap & effMap & NcMap & R/Parm & S_WcDes & s_PRdes & S_effDes & S_NCDes \\
\hline Fan & 1152.42 & 1.529 & 0.9089 & 3775.875 & 0.0583 & $\overline{0} .8607$ & $\overline{1} .0235$ & 0.9980 & $\overline{0} .9905$ \\
\hline LPC & 141.16 & 2.399 & 0.8202 & 1.007 & 0.0000 & 1.2444 & 0.9848 & 1.0592 & 0.0003 \\
\hline HPC & 77.44 & 10.500 & 0.8704 & 9746.966 & 12.7574 & 1.0995 & 1.0494 & 0.9898 & 0.9733 \\
\hline HPT & 0.96 & 4.206 & 0.9083 & 1.307 & 4.2060 & 12.6299 & 0.9723 & 1.0000 & 0.0003 \\
\hline LPT & 0.86 & 3.519 & 0.9192 & 0.999 & 3.5189 & 65.2590 & 0.7521 & 0.9926 & 0.0005 \\
\hline
\end{tabular}




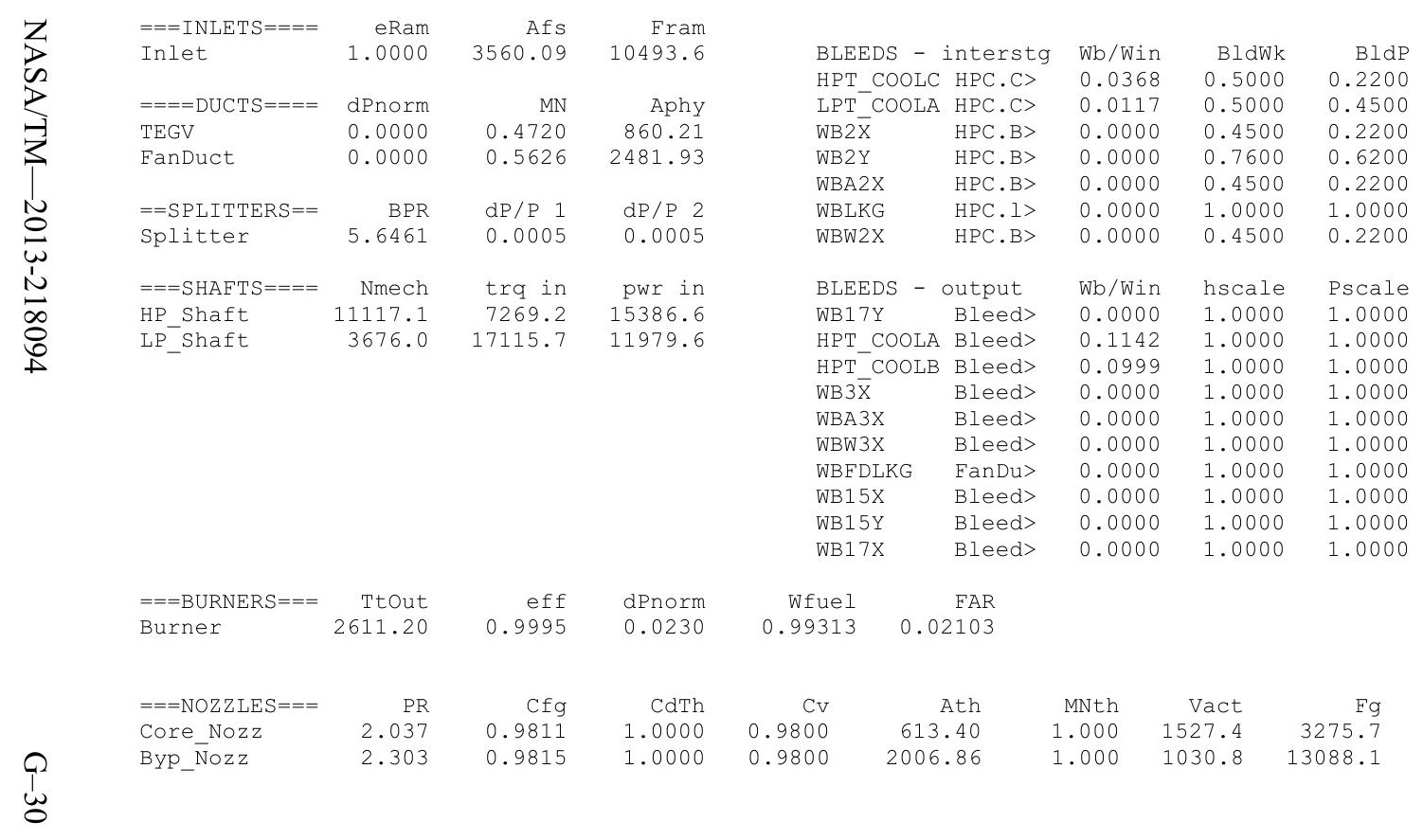




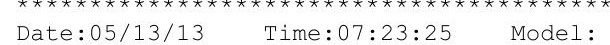

Turbofan Engine - COMDES ON COnverge $=1$ CASE: 0

Version:NPSS1.6.5 - Rev: -> Gas Package: Janaf iter/pass/Jacb/Broy= 37/51/1/35 Run by: Philip C Jorgenson PC: 69

Temperature Stator 1 inlet: 480.73 Stator 1 exit: 497.19 Stator 2 inlet: 517.39 Stator 2 exit: 526.11 Stator 3 inlet: 549.75 Stator 3 exit: 558.36 Stator 4 inlet: 580.69 Stator 4 exit: 588.52
Stator 5 inlet: $606.44 \quad$ Stator 5 exit: 613.76

an Face Relative Humidity $\quad 3.60$

Fan Bypass Relative Humidity 0.33

LPC Inlet Relative Humidity $\quad 1.97$

LPC Exit Relative Humidity

Drop Diameterumidity

0.00

Ambient Flow Velocity

Ambient Static Tressure

0.0000100

40.00

Additional Water at LPC Exit

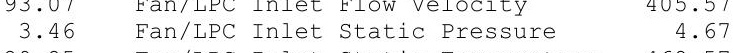
0.0010436

Fan/LPC Inlet Static Temperature $\quad 468.57$

$\begin{array}{rrr}\text { MN } & \text { alt } & \text { dTamb } \\ 0.780 & 35000.0 & 36.00\end{array}$

\section{SUMMARY OUTPUT DATA}

$0.780 \quad 35000.0 \quad 36.00$

W
421.73

FSO Ambient.Fl 0

W
421.73
421.73
358.79
62.94
358.79
62.94
63.01
63.01
59.95
46.46
47.42
63.23
63.96
63.96
63.96
358.79
358.79
358.79
358.79

\section{FLOW STATION DATA}

FS1 Ambient.FI-

FS12 Splitter.F1_02

FS2 Splitter.F1_01

$\begin{array}{ll}\text { FS14 } & \text { Fan.F1- } \\ \text { FS23 } & \text { LPC.F1- }\end{array}$

FS24 VaporIN.F1_O

FS25 Bleed2.F1_

FS3 HPC.FI_O

FS4 Bleed3.Fl_O

$\begin{array}{ll}\text { FS4 } & \text { Burner.F1 } \\ \text { FS45 } & \text { HPT.Fl }\end{array}$

FS49 LPT.FI-O

FS8 Core Nozz.Fl

FS17 FanDuctLkg. Fì

FS171 Bleed15.F1_O

FS173 Byp_Nozz.FI O

$\begin{array}{rrrr}\text { Pt } & \text { Tt } & \text { ht } & \text { FAR } \\ 5.170 & 482.28 & -15.41 & 0.0000 \\ 5.170 & 482.28 & -15.41 & 0.0000 \\ 5.167 & 482.28 & -15.41 & 0.0000 \\ 5.167 & 482.28 & -15.41 & 0.0000 \\ 7.889 & 550.60 & 0.98 & 0.0000 \\ 12.204 & 636.38 & 21.61 & 0.0000 \\ 12.204 & 637.64 & 15.92 & 0.0000 \\ 12.204 & 637.64 & 15.92 & 0.0000 \\ 31.643 & 1327.40 & 187.65 & 0.0000 \\ 31.643 & 1327.40 & 187.65 & 0.0000 \\ 28.611 & 2581.28 & 162.37 & 0.0206 \\ 29.909 & 1691.79 & -1.45 & 0.0154 \\ 6.877 & 1206.84 & -128.62 & 0.0152 \\ 6.877 & 1206.90 & -128.62 & 0.0152 \\ 6.877 & 1207.04 & -128.62 & 0.0152 \\ 7.889 & 550.60 & 0.98 & 0.0000 \\ 7.889 & 550.60 & 0.98 & 0.0000 \\ 7.889 & 550.60 & 0.98 & 0.0000 \\ 7.889 & 550.60 & 0.98 & 0.0000\end{array}$

$\mathrm{BPR}$
5.7004

VTAS

OPR

EPR

Powerset

$\begin{array}{ccc}\text { T4 } & \text { T41 } & \text { T49 } \\ 2581.3 & 2428.6 & 1684.2\end{array}$

TURBOMACHINERY PERFORMANCE DATA

\begin{tabular}{|c|c|c|c|c|c|c|c|c|c|}
\hline & Wc & PR & eff & Nc & TR & efPoly & pwr & SMN & SMW \\
\hline Fan & 983.98 & 1.527 & 0.9073 & 3780.705 & 1.1417 & 0.9127 & -8320.5 & 73.73 & 30.75 \\
\hline LPC & 172.61 & 2.362 & 0.8702 & 3780.705 & 1.3195 & 0.8848 & -3296.2 & 4.57 & 3.57 \\
\hline HPC & 84.13 & 10.787 & 0.8615 & 9985.183 & 2.0817 & 0.8978 & -14938.2 & 35.25 & 33.82 \\
\hline HPT & 12.09 & 4.300 & 0.9078 & 217.912 & 1.3554 & 0.8883 & 14938.2 & & \\
\hline LPT & 56.11 & 4.349 & 0.9117 & 88.634 & 1.3992 & 0.8945 & 11616.7 & & \\
\hline \multicolumn{10}{|c|}{ JRBOMACHINERY MAP DATA } \\
\hline & WcMap & PRmap & effMap & NcMap & R/Parm & S_WCDes & S_PRdes & S_effDes & S_NCDes \\
\hline Fan & 1143.28 & 1.515 & 0.9092 & 3744.657 & 0.0578 & $\overline{0} .8607$ & $\overline{1} .0235$ & 0.9980 & $\overline{0} .9905$ \\
\hline LPC & 139.13 & 2.367 & 0.8195 & 0.999 & 0.0000 & 1.2407 & 0.9961 & 1.0619 & 0.0003 \\
\hline HPC & 76.51 & 10.326 & 0.8704 & 9718.985 & 12.6923 & 1.0995 & 1.0494 & 0.9898 & 0.9733 \\
\hline HPT & 0.96 & 4.209 & 0.9078 & 1.310 & 4.2087 & 12.6299 & 0.9723 & 1.0000 & 0.0003 \\
\hline LPT & 0.86 & 3.519 & 0.9185 & 0.997 & 3.5189 & 65.2590 & 0.7521 & 0.9926 & 0.0005 \\
\hline
\end{tabular}




\begin{tabular}{|c|c|c|c|}
\hline$===$ INLET $S===$ & eRam & Afs & Fram \\
\hline Inlet & 1.0000 & 3526.80 & 10395.5 \\
\hline$====$ DUCTS $====$ & dPnorm & MN & Aphy \\
\hline TEGV & 0.0000 & 0.4720 & 860.21 \\
\hline FanDuct & 0.0000 & 0.5626 & 2481.93 \\
\hline$==$ SPLITTERS $==$ & BPR & $\mathrm{dP} / \mathrm{P} 1$ & $\mathrm{dP} / \mathrm{P} 2$ \\
\hline Splitter & 5.7004 & 0.0005 & 0.0005 \\
\hline$===$ SHAFTS $====$ & Nmech & $\operatorname{trg}$ in & pwr in \\
\hline HP Shaft & 11071.3 & 7086.5 & 14938.2 \\
\hline LP ${ }^{-}$Shaft & 3645.6 & 16735.7 & 11616.7 \\
\hline
\end{tabular}

BLEEDS - interstg Wb/Win BldWk BldP $\begin{array}{llll}\text { HPT_COOLC HPC.C }> & 0.0368 & 0.5000 & 0.2200\end{array}$ $\begin{array}{lllll}\text { LPT COOLA HPC.C> } & 0.0117 & 0.5000 & 0.4500 \\ \text { WB } 2 \bar{X} & \text { HPC.B> } & 0.0000 & 0.4500 & 0.2200\end{array}$ $\begin{array}{lllll}\text { WB2X } & \text { HPC.B> } & 0.0000 & 0.4500 & 0.2200 \\ \text { WB2Y } & \text { HPC. B> } & 0.0000 & 0.7600 & 0.6200\end{array}$ $\begin{array}{lllll}\text { WB2Y } & \text { HPC.B> } & 0.0000 & 0.7600 & 0.6200\end{array}$ $\begin{array}{lllll}\text { WBA2X HPC.B } & 0.0000 & 0.4500 & 0.2200\end{array}$ $\begin{array}{lllll}\text { WBLKG HPC.I> } & 0.0000 & 1.0000 & 1.0000\end{array}$ $\begin{array}{lllll}\text { WBW2X HPC.B> } & 0.0000 & 0.4500 & 0.2200\end{array}$

BLEEDS - output Wb/Win hscale Pscale $\begin{array}{llll}\text { WB17Y Bleed> } 0.0000 & 1.0000 & 1.0000\end{array}$ $\begin{array}{llll}\text { HPT_COOLA Bleed> } & 0.1142 & 1.0000 & 1.000\end{array}$ $\begin{array}{lllll}\text { HPT_COOLB } & \text { Bleed> } & 0.0999 & 1.0000 & 1.0000 \\ \text { WB } 3 \bar{X} & \text { Bleed> } & 0.0000 & 1.0000 & 1.0000\end{array}$ $\begin{array}{lllll}\text { WB3X } & \text { Bleed> } & 0.0000 & 1.0000 & 1.0000 \\ \text { WBA3X } & \text { Bleed }> & 0.0000 & 1.0000 & 1.0000\end{array}$ $\begin{array}{lllll}\text { WBA3X } & \text { Bleed> } & 0.0000 & 1.0000 & 1.0000 \\ \text { WBW3X } & \text { Bleed> } & 0.0000 & 1.0000 & 1.0000\end{array}$ $\begin{array}{lllll}\text { WBW3X } & \text { Bleed> } & 0.0000 & 1.0000 & 1.0000 \\ \text { WBFDLKG } & \text { FanDu> } & 0.0000 & 1.0000 & 1.0000\end{array}$ $\begin{array}{lllll}\text { WBFDLKG } & \text { FanDu> } & 0.0000 & 1.0000 & 1.0000 \\ \text { WB15X } & \text { Bleed }> & 0.0000 & 1.0000 & 1.0000 \\ \text { WB15Y } & \text { Bleed }> & 0.0000 & 1.0000 & 1.0000\end{array}$ $\begin{array}{lllll}\text { WB15X } & \text { Bleed }> & 0.0000 & 1.0000 & 1.0000 \\ \text { WB15Y } & \text { Bleed }> & 0.0000 & 1.0000 & 1.0000\end{array}$ WB17X Bleed> $0.0000 \quad 1.0000 \quad 1.0000$

$\begin{array}{rrrr}\text { W } & \text { Tt } & \text { ht } & \text { Pt } \\ 2.3187 & 989.07 & 101.79 & 29.909 \\ 0.7372 & 989.07 & 101.79 & 6.877 \\ 0.0000 & 954.46 & 93.20 & 38.480 \\ 0.0000 & 1166.77 & 146.44 & 86.256 \\ 0.0000 & 954.46 & 93.20 & 38.480 \\ 0.0000 & 1327.40 & 187.65 & 131.643 \\ 0.0000 & 954.46 & 93.20 & 38.480 \\ \text { W } & & & \\ 0.0000 & 637.64 & 15.92 & 12.204 \\ 7.1941 & 1327.40 & 187.65 & 128.611 \\ 6.2950 & 1327.40 & 187.65 & 72.153 \\ 0.0000 & 1327.40 & 187.65 & 131.643 \\ 0.0000 & 1327.40 & 187.65 & 131.643 \\ 0.0000 & 1327.40 & 187.65 & 131.643 \\ 0.0000 & 550.60 & 0.98 & 7.889 \\ 0.0000 & 550.60 & 0.98 & 7.889 \\ 0.0000 & 550.60 & 0.98 & 7.889 \\ 0.0000 & 550.60 & 0.98 & 7.889\end{array}$

$===$ BURNERS $===\quad$ TtOut Burner

eff
0.9995

dPnorm

\section{FAR}

$0.95714 \quad 0.02062$

\begin{tabular}{|c|c|c|c|c|c|c|c|c|}
\hline$===$ NOZZLES $===$ & PR & Cfg & CdTh & $\mathrm{Cv}$ & Ath & MNth & Vact & $\mathrm{Fg}$ \\
\hline Core_Nozz & 1.989 & 0.9808 & 1.0000 & 0.9800 & 613.40 & 1.000 & 1518.4 & 3147.3 \\
\hline Byp & 2.281 & 0.9815 & 1.0000 & 0.9800 & 2006.86 & 1.000 & 1029.3 & 12899.6 \\
\hline
\end{tabular}


Date:05/13/13 Time:09:31:08 Model: Turbofan Engine - COMDES ON COnverge $=1$ CASE: 0

Version:NPSS 1.6.5 - Rev: -> Gas Package: Janaf iter/pass/Jacb/Broy= 27/55/2/24 Run by: Philip C Jorgenson PC: 100

Temperature Stator 1 inlet: 449.02 Stator 1 exit: 467.66 Stator 2 inlet: 489.26 Stator 2 exit: 496.56

Stator 5 inlet: 588.99 Stator 5 exit: 596.56 Stator 4 Unblocked Percent Blockage: 0.00

Ambient Relative Humidity 10.00

Fan Face Relative Humidity

Fan Bypass Relative Humidity $\quad 0.16$

PCC Inlet Relative Humidity 2.31

LPC Exit Relative Humidity

Drop Diameter

0.02

Ambient Flow Velocity

0.0000250

Ambient Flow Velocity

Ambient Static Temperatur

Additional Water at IPC Exit

776.30
3.46

Inlet Length

40.00

411.85 Fan/LPC Inlet Static Temperature 442.62

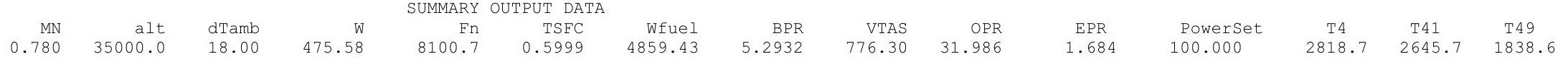

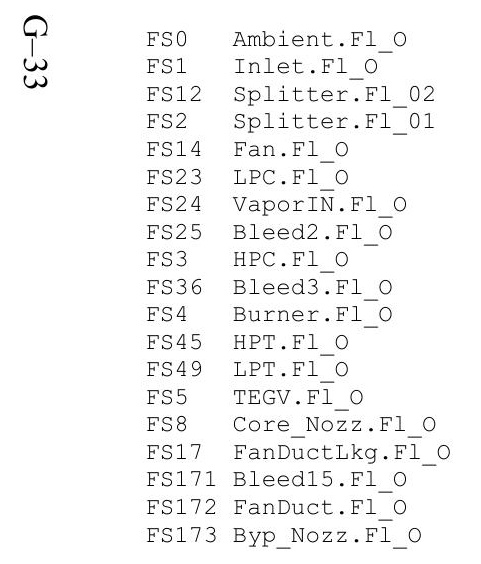

$W$
475.58
475.58
400.01
75.57
400.01
75.57
75.58
75.58
71.91
55.73
57.08
76.04
76.93
76.93
76.93
400.01
400.01
400.01
400.01

$$
\begin{array}{crl}
\text { FLOW } & \text { STATION DATA } \\
\text { Pt } & \text { Tt }
\end{array}
$$

$\begin{array}{rrrrr}\text { FLOW } & \text { STATION DATA } & \text { TI } & \text { FAR } & \text { WC } \\ \text { Pt } & \text { Tt } & \text { ht } & 0.0000 & 1276.02 \\ 5.170 & 462.10 & -19.92 & 0.0000 \\ 5.170 & 462.10 & -19.92 & 0.0000 & 1276.02 \\ 5.167 & 462.10 & -19.92 & 0.0000 & 1073.80 \\ 5.167 & 462.10 & -19.92 & 0.0000 & 202.86 \\ 8.745 & 544.45 & -0.17 & 0.0000 & 688.68 \\ 12.670 & 625.31 & 19.26 & 0.0000 & 96.24 \\ 12.670 & 625.46 & 18.86 & 0.0000 & 96.26 \\ 12.670 & 625.46 & 18.86 & 0.0000 & 96.26 \\ 165.364 & 1379.21 & 206.83 & 0.0000 & 10.42 \\ 165.364 & 1379.21 & 206.83 & 0.0000 & 8.08 \\ 161.555 & 2818.68 & 176.55 & 0.0242 & 12.10 \\ 37.753 & 1847.47 & -1.63 & 0.0181 & 55.87 \\ 8.678 & 1323.60 & -141.49 & 0.0179 & 208.11 \\ 8.678 & 1323.66 & -141.49 & 0.0179 & 208.11 \\ 8.678 & 1323.79 & -141.49 & 0.0179 & 208.12 \\ 8.745 & 544.45 & -0.17 & 0.0000 & 688.68 \\ 8.745 & 544.45 & -0.17 & 0.0000 & 688.68 \\ 8.745 & 544.45 & -0.17 & 0.0000 & 688.68 \\ 8.745 & 544.45 & -0.17 & 0.0000 & 688.68\end{array}$

Ps
3.458
3.838
3.865
4.445
7.260
11.085
11.084
11.084
149.114
155.924
152.619
34.007
7.485
7.485
4.641
7.260
7.055
7.055
4.619

Ts
411.85
424.34
425.25
442.62
516.24
601.95
602.07
602.07
1342.09
1358.01
2782.89
1801.01
1273.86
1273.93
1123.76
516.24
512.03
512.03
453.60

$\begin{array}{rr}\text { Aphy } & \text { MN } \\ 3892.8 & 0.7800 \\ 4168.2 & 0.6662 \\ 3531.8 & 0.6574 \\ 830.5 & 0.4685 \\ 2606.7 & 0.5226 \\ 412.6 & 0.4413 \\ 412.6 & 0.4415 \\ 412.6 & 0.4415 \\ 49.7 & 0.3928 \\ 49.3 & 0.2953 \\ 74.6 & 0.3003 \\ 265.4 & 0.4002 \\ 860.2 & 0.4724 \\ 860.2 & 0.4724 \\ 613.4 & 1.0000 \\ 2606.7 & 0.5226 \\ 2481.9 & 0.5625 \\ 2481.9 & 0.5625 \\ 2006.9 & 1.0000\end{array}$

gamt
1.40081
1.40081
1.40081
1.40081
1.39985
1.39819
1.39817
1.39817
1.35714
1.35714
1.28468
1.32099
1.34811
1.34810
1.34809
1.39985
1.39985
1.39985
1.39985 0.0000308 0.0000308 0.0000308 0.0000308 0.0000308 0.0001078 0.0001078 0.0001078 0.0001078 0.0001078 0.0001078 0.0001078 0.0000308 0.0000308 0.0000308 $\begin{array}{rr}\text { WAR } & \text { Wair } \\ 0.0000308 & 475.57\end{array}$ 475.57 400.00
75.5 $\begin{array}{ll}75.57 & 0.002 \\ 75.57 & 0.008\end{array}$ $\begin{array}{ll}75.57 & 0.008\end{array}$ $\begin{array}{ll}71.90 & 0.008\end{array}$ $\begin{array}{ll}55.73 & 0.006\end{array}$ $\begin{array}{ll}55.73 & 0.006\end{array}$ $\begin{array}{ll}74.68 & 0.008\end{array}$ $\begin{array}{ll}75.57 & 0.008\end{array}$ $\begin{array}{ll}75.57 & 0.008\end{array}$ $\begin{array}{ll}75.57 & 0.008\end{array}$ $400.00 \quad 0.012$ $400.00 \quad 0.012$ $400.00 \quad 0.012$ TURBOMACHINERY PERFORMANCE DATA

\begin{tabular}{|c|c|c|c|c|c|c|c|c|c|}
\hline & Wc & PR & eff & $\mathrm{NC}$ & TR & efPoly & pwr & SMN & SMW \\
\hline Fan & 1073.80 & 1.692 & 0.9109 & 4120.456 & 1.1782 & 0.9173 & 176.8 & 38.42 & 23.46 \\
\hline LPC & 202.86 & 2.452 & 0.8266 & 4120.456 & 1.3532 & 0.8469 & 189.6 & 2.96 & 2.22 \\
\hline HPC & 96.26 & 13.052 & 0.8586 & 10344.363 & 2.2051 & 0.8979 & 612.3 & 26.29 & 25.49 \\
\hline $\mathrm{HPT}$ & 12.10 & 4.279 & 0.9118 & 213.961 & 1.3469 & 0.8911 & 612.3 & & \\
\hline LPT & 55.87 & 4.350 & 0.9155 & 90.485 & 1.3923 & 0.8986 & 366.4 & & \\
\hline \multicolumn{10}{|c|}{ INERY MAP DF } \\
\hline & WcMap & PRmap & effmap & NcMap & R/Parm & s_WcDes & S_PRdes & S_effDes & S_NCDes \\
\hline Fan & 1247.65 & 1.677 & 0.9128 & 4081.168 & 0.0636 & 0.8607 & $\overline{1} .0235$ & 0.9980 & 0.9905 \\
\hline LPC & 165.09 & 2.727 & 0.8329 & 1.088 & 0.0000 & 1.2288 & 0.8407 & 0.9925 & 0.0003 \\
\hline HPC & 87.55 & 12.484 & 0.8674 & 10068.589 & 13.4710 & 1.0995 & 1.0494 & 0.9898 & 0.9733 \\
\hline HPT & 0.96 & 4.188 & 0.9118 & 1.286 & 4.1884 & 12.6299 & 0.9723 & 1.0000 & 0.0003 \\
\hline LPT & 0.86 & 3.520 & 0.9223 & 1.018 & 3.5199 & 65.2590 & 0.7521 & 0.9926 & 0.0005 \\
\hline
\end{tabular}




\begin{tabular}{|c|c|c|c|c|c|c|c|c|c|}
\hline Z & $===$ INLETS $====$ & eRam & Afs & Fram & \multirow{2}{*}{\multicolumn{2}{|c|}{ BLEEDS - interstg }} & & & \\
\hline D & Inlet & 1.0000 & 3892.75 & 11475.0 & & & $\begin{array}{l}\text { Wb/Win } \\
0.0368\end{array}$ & $\begin{array}{r}\text { BldWk } \\
0.5000\end{array}$ & $\begin{array}{r}\text { BldP } \\
0.2200\end{array}$ \\
\hline & $====$ DUCTS $====$ & dPnorm & MN & Aphy & \multicolumn{2}{|c|}{$\begin{array}{l}\text { HPT_COOLC HPC.C> } \\
\text { LPT_COOLA HPC.C> }\end{array}$} & 0.0117 & 0.5000 & 0.4500 \\
\hline & TEGV & 0.0000 & 0.4724 & 860.21 & WB $2 \bar{x}$ & HPC.B $>$ & 0.0000 & 0.4500 & 0.2200 \\
\hline & FanDuct & 0.0000 & 0.5625 & 2481.93 & WB2Y & HPC. B> & 0.0000 & 0.7600 & 0.6200 \\
\hline & & & & & \multirow{2}{*}{$\begin{array}{l}\text { WBA2X } \\
\text { WBLKG }\end{array}$} & $\mathrm{HPC} . \mathrm{B}>$ & 0.0000 & 0.4500 & 0.2200 \\
\hline & $==$ SPLITTERS $==$ & BPR & $\mathrm{dP} / \mathrm{P} 1$ & $\mathrm{dP} / \mathrm{P} 2$ & & HPC. $1>$ & 0.0000 & 1.0000 & 1.0000 \\
\hline & Splitter & 5.2932 & 0.0005 & 0.0005 & WBW2X & HPC. B> & 0.0000 & 0.4500 & 0.2200 \\
\hline & $===$ SHAFTS $====$ & Nmech & trq in & pwr in & \multicolumn{2}{|c|}{ BLEEDS - output } & Wb/Win & hscale & Pscale \\
\hline & HP Shaft & 11359.4 & 9067.9 & 19612.3 & WB17Y & Bleed> & 0.0000 & 1.0000 & 1.0000 \\
\hline & LP_Shaft & 3889.2 & 20751.0 & 15366.4 & HPT COOLA & A Bleed> & 0.1142 & 1.0000 & 1.0000 \\
\hline & & & & & $\begin{array}{l}\mathrm{HPT}^{-} \mathrm{COOLB} \\
\mathrm{NB} 3 \overline{\mathrm{x}}\end{array}$ & 3 Bleed> & 0.0999 & 1.0000 & 1.0000 \\
\hline & & & & & WB $3 \overline{\mathrm{X}}$ & Bleed> & 0.0000 & 1.0000 & 1.0000 \\
\hline & & & & & \multirow{2}{*}{$\begin{array}{l}\text { WBA3X } \\
\text { WBW3X }\end{array}$} & Bleed> & 0.0000 & 1.0000 & 1.0000 \\
\hline & & & & & & Bleed> & 0.0000 & 1.0000 & 1.0000 \\
\hline & & & & & & FanDu> & 0.0000 & 1.0000 & 1.0000 \\
\hline & & & & & $\begin{array}{l}\text { WBED DLKG } \\
\text { WB15X }\end{array}$ & Bleed> & 0.0000 & 1.0000 & 1.0000 \\
\hline & & & & & WB15Y & Bleed> & 0.0000 & 1.0000 & 1.0000 \\
\hline & & & & & WB17X & Bleed> & 0.0000 & 1.0000 & 1.0000 \\
\hline & $===$ BURNERS $===$ & Ttout & eff & dPnorm & & & & \\
\hline & Burner & 2818.67 & 0.9995 & 0.0230 & \multicolumn{2}{|c|}{$\begin{array}{rr}\text { Wfuel } & \text { FAR } \\
1.34984 & 0.02422\end{array}$} & & & \\
\hline & $===\operatorname{NOZZLES}===$ & PR & $\mathrm{Cfg}$ & CdTh & \multirow{2}{*}{$\begin{array}{r}\mathrm{CV} \\
0.9800\end{array}$} & \multirow{2}{*}{$\begin{array}{r}\text { Ath } \\
613.40\end{array}$} & MNth & Vact & $\mathrm{Fg}$ \\
\hline & Core_Nozz & 2.509 & 0.9810 & 1.0000 & & & 1.000 & \multirow{2}{*}{$\begin{array}{l}1587.7 \\
1023.5\end{array}$} & 4521.4 \\
\hline & Byp__̄ozz & 2.529 & 0.9811 & 1.0000 & \multicolumn{2}{|c|}{2006.86} & 1.000 & & 15054.2 \\
\hline
\end{tabular}


Date:05/13/13 Time:09:31:58 Model: Turbofan Engine - COMDES ON COnverge $=1$ CASE: 0

Version:NPSS 1.6.5 - Rev: -> Gas Package: Janaf iter/pass/Jacb/Broy= 21/49/2/18 Run by: Philip C Jorgenson PC: 75

Temperature Stator 1 inlet: 458.64 Stator 1 exit: 474.89 Stator 2 inlet: 494.62 Stator 2 exit: 502.88

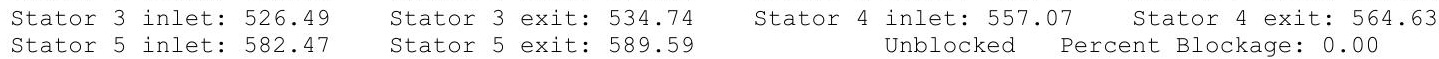

\begin{abstract}
Ambient Relative Humidity $\quad 10.00$
Fan Face Relative Humidity $\quad 3.58$

Fan Bypass Relative Humidity 0.24

LPC Inlet Relative Humidity $\quad 1.85$

LPC Exit Relative Humidity $\quad 0.02$

HPC Relative Humidity 0.00

Ambient Flow Velocity

Ambient Static Pressure

Additional Water at LPC Exit

0.00
0 0.0000967

Fan/LPC Inlet Static Temperature $\quad 447.77$
\end{abstract}

\section{$\begin{array}{crrr} & \text { SUMMARY OUTPUT DATA } \\ \text { W } & \text { Fn } & \text { TSFC }\end{array}$}

$\begin{array}{rrr}\text { MN } & \text { alt } & \text { dTamb } \\ 0.780 & 35000.0 & 18.00\end{array}$
439.16

BPR
5.5833

VTAS
776.30
OPR
26.748 $\begin{array}{cl}\text { EPR } & \text { PowerSet } \\ 1.342 & 75.600\end{array}$ $\begin{array}{ccc}\text { T4 } & \text { T41 } & \text { T49 } \\ 2543.7 & 2391.6 & 1654.9\end{array}$

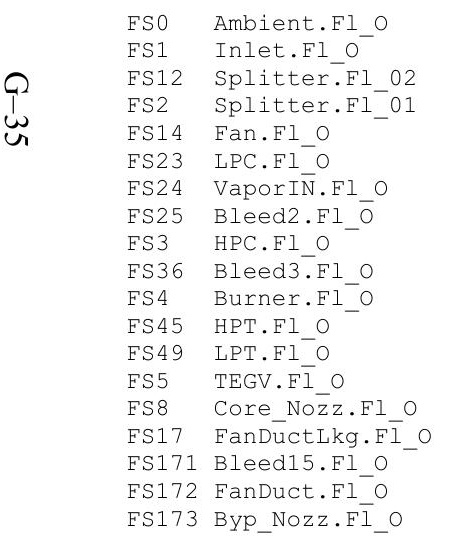

$W$
439.16
439.16
372.46
66.71
372.46
66.71
66.71
66.71
63.48
49.20
50.20
66.94
67.72
67.72
67.72
372.46
372.46
372.46
372.46

Wc
1178.31
1178.31
999.83
179.07
688.70
86.46
86.48
86.48
10.65
8.26
12.09
56.08
208.65
208.65
208.66
688.70
688.70
688.70
688.70

Ps
3.458
4.118
4.112
4.629
6.674
11.117
11.117
11.117
123.998
130.007
127.608
28.260
6.211
6.211
3.842
6.674
6.485
6.485
4.246

Ts
411.85
432.98
432.86
447.77
503.23
594.73
594.88
594.88
1256.46
1272.43
2510.54
1619.23
1138.28
1138.35
100.91
503.23
499.13
499.13
442.16

$\begin{array}{rr}\text { Aphy } & \text { MN } \\ 3594.7 & 0.7800 \\ 4168.2 & 0.5791 \\ 3531.8 & 0.5805 \\ 830.5 & 0.3995 \\ 2606.7 & 0.5226 \\ 412.6 & 0.3861 \\ 412.6 & 0.3862 \\ 412.6 & 0.3862 \\ 49.7 & 0.4027 \\ 49.3 & 0.3021 \\ 74.6 & 0.2988 \\ 265.4 & 0.4004 \\ 860.2 & 0.4719 \\ 860.2 & 0.4719 \\ 613.4 & 1.0000 \\ 2606.7 & 0.5226 \\ 2481.9 & 0.5625 \\ 2481.9 & 0.5625 \\ 2006.9 & 1.0000\end{array}$

gamt
1.40081
1.40081
1.40081
1.40081
1.40006
1.39851
1.39849
1.39849
1.36225
1.36225
1.29507
1.33128
1.35871
1.35871
1.35870
1.40006
1.40006
1.40006
1.40006

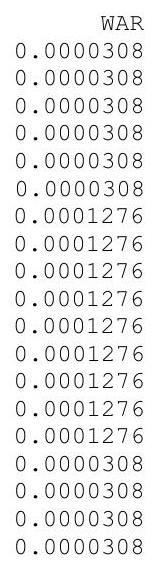

Wair
439.15
439.15
372.44
66.71
372.44
66.71
66.71
66.71
63.47
49.19
49.19
65.93
66.71
66.71
66.71
372.44
372.44
372.44
372.44

WH2O H2O frac $0.014 \quad 0.0000$ $\begin{array}{ll}0.011 & 0.0000 \\ 0.002 & 0.0000\end{array}$ $\begin{array}{ll}0.011 & 0.0000 \\ 0.002 & 0.0000 \\ 0.011 & 0.0000 \\ 0.002 & 0.0000\end{array}$ $\begin{array}{ll}0.002 & 0.0000 \\ 0.002 & 0.0000 \\ 0.009 & 0.0000\end{array}$ $\begin{array}{ll}0.011 & 0.0000 \\ 0.009 & 0.0001\end{array}$ $0.009 \quad 0.0001$ $\begin{array}{ll}0.009 & 0.0001 \\ 0.008 & 0.0001\end{array}$ $0.006 \quad 0.0001$ $0.006 \quad 0.0248$ $0.008 \quad 0.0186$ $0.009 \quad 0.0184$ $0.009 \quad 0.0184$ $0.011 \quad 0.0000$

\begin{tabular}{|c|c|c|c|c|c|c|c|c|c|}
\hline TURB & NERY PER & ORMANCE & DATA & & & & & & \\
\hline & Wc & PR & eff & Nc & TR & efPoly & pwr & SMN & SMW \\
\hline Fan & 999.83 & 1.556 & 0.9070 & 3842.004 & 1.1486 & 0.9126 & -8673.5 & 62.44 & 29.36 \\
\hline LPC & 179.07 & 2.384 & 0.8662 & 3842.004 & 1.3253 & 0.8814 & -3405.0 & 4.01 & 3.17 \\
\hline HPC & 86.48 & 11.224 & 0.8614 & 10051.885 & 2.1116 & 0.8984 & -15552.6 & 33.26 & 31.97 \\
\hline $\mathrm{HPT}$ & 12.09 & 4.302 & 0.9079 & 216.594 & 1.3570 & 0.8887 & 15552.6 & & \\
\hline LPT & 56.08 & 4.358 & 0.9114 & 88.942 & 1.4013 & 0.8940 & 12078.5 & & \\
\hline TURB & NERY MAP & DATA & & & & & & & \\
\hline & WcMap & PRmap & effMap & NcMap & R/Parm & s_WcDes & S_PRdes & s_effDes & S_NcDes \\
\hline Fan & 1161.70 & 1.543 & 0.9088 & 3805.371 & 0.0589 & $\overline{0} .8607$ & $\overline{1} .0235$ & 0.9980 & $\overline{0} .9905$ \\
\hline LPC & 143.08 & 2.430 & 0.8208 & 1.015 & 0.0000 & 1.2516 & 0.9682 & 1.0554 & 0.0003 \\
\hline HPC & 78.66 & 10.742 & 0.8703 & 9783.908 & 12.8557 & 1.0995 & 1.0494 & 0.9898 & 0.9733 \\
\hline $\mathrm{HPT}$ & 0.96 & 4.210 & 0.9079 & 1.302 & 4.2105 & 12.6299 & 0.9723 & 1.0000 & 0.0003 \\
\hline LPT & 0.86 & 3.525 & 0.9181 & 1.001 & 3.5255 & 65.2590 & 0.7521 & 0.9926 & 0.0005 \\
\hline
\end{tabular}




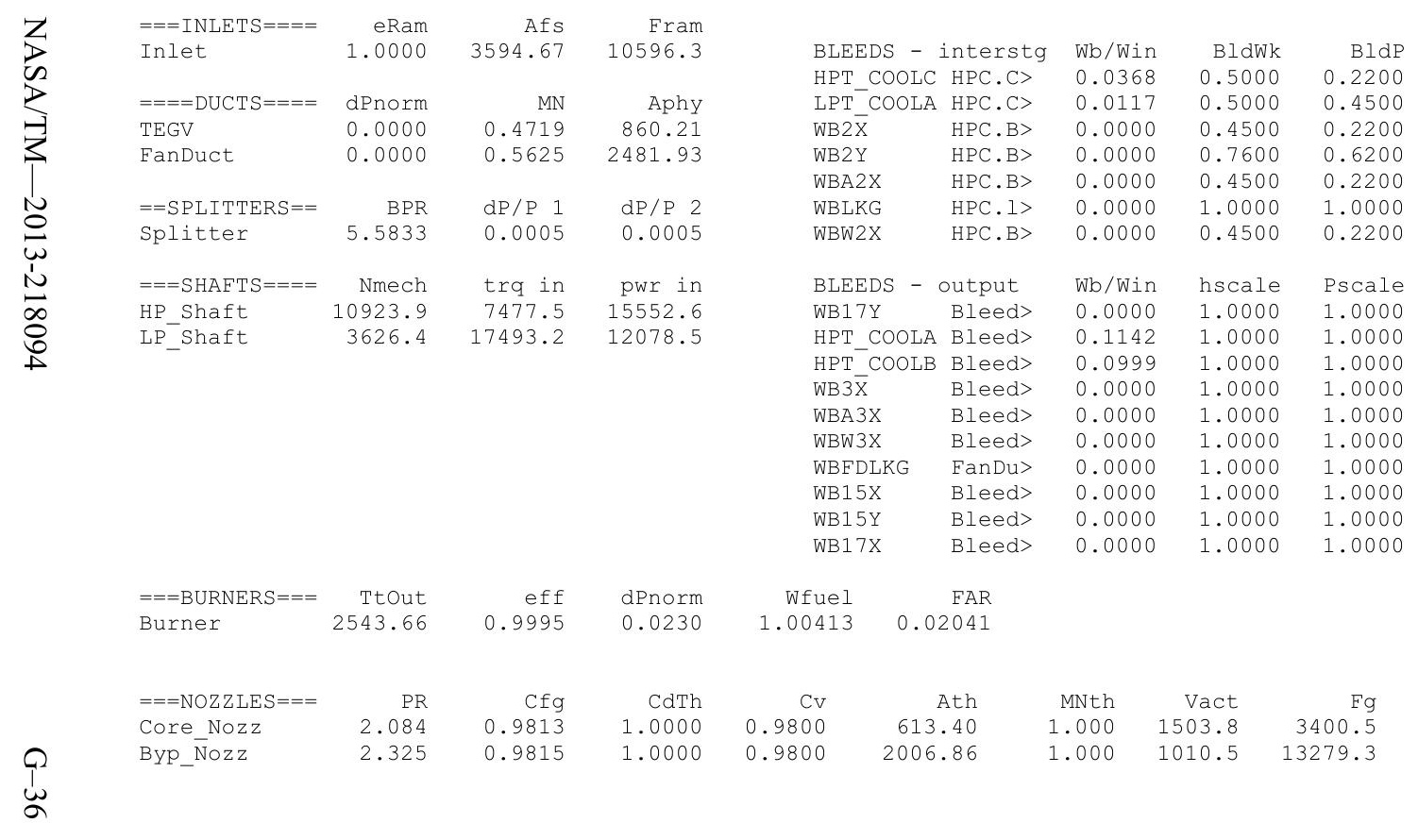


Date:05/13/13 Time:09:32:31 Model: Turbofan Engine - COMDES ON CONverge = 1 CASE: ON

Version:NPSS_1.6.5 - Rev: -> Gas Package: Janaf iter/pass/Jacb/Broy=18/ 32/ 1/16 Run by: Philip C Jorgenson PC: 72

Temperature Stator 1 inlet: 459.59 Stator 1 exit: 475.58 Stator 2 inlet: 495.12 Stator 2 exit: 503.42

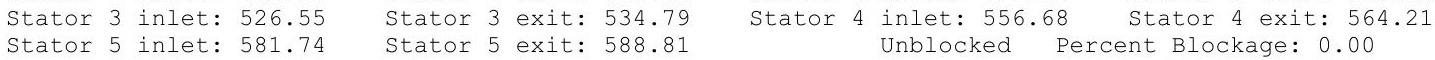

Ambient Relative Humidity $\quad 10.00$

$\begin{array}{ll}\text { Fan Face Relative Humidity } & 3.46 \\ \text { Fan Bypass Relative Humidity } & 0.25\end{array}$

$\begin{array}{ll}\text { Fan Bypass Relative Humidity } & 0.25 \\ \text { LPC Inlet Relative Humidity } & 1.81\end{array}$

LPC Exit Relative Humidity $\quad 0.02$

HPC Relative Humidity

0.00

Drop Diameter

0.0000250

Inlet Length $\quad 40.00$

Ambient rlow velocity

Ambient Static Temperature

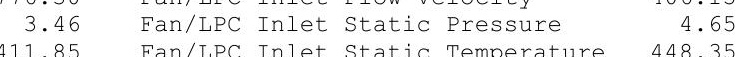

Additional Water at LPC Exit 0.0000997

\section{SUMMARY OUTPUT DATA}

alt dTamb
434.93

$5855.8 \quad 0.5946$
Wfuel

\section{FLOW STATION DATA}

FSO Ambient.Fl_O

FS1 Inlet.FI_O

FS12 Splitter.F1_02

FS14 Splitter.Fl_01

FS14 Fan.F1_9

FS24 VaporIN.FI_O

FS25 Bleed2.F1_O

FS3 HPC.FI_O

FS36 Bleed3.Fl_O

FS45 HPT.FI

FS49 LPT.FI_O

FS5 TEGV.F̄̄O

FS8 Core_Nozz.Fl_O

FS171 Bleed15.F1 O

FS172 FanDuct.Fl

FS173 Byp_Nozz.F̄' O

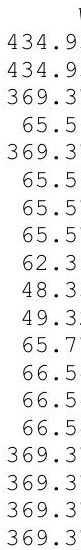

FLOW
Pt

$\begin{array}{rrr}\text { Tt } & \text { ht } & \text { FAR } \\ 462.10 & -19.92 & 0.0000 \\ 462.10 & -19.92 & 0.0000 \\ 462.10 & -19.92 & 0.0000 \\ 462.10 & -19.92 & 0.0000 \\ 529.11 & -3.85 & 0.0000 \\ 611.02 & 15.82 & 0.0000 \\ 611.19 & 15.29 & 0.0000 \\ 611.19 & 15.29 & 0.0000 \\ 1283.94 & 182.02 & 0.0000 \\ 1283.94 & 182.02 & 0.0000 \\ 2513.27 & 157.61 & 0.0200 \\ 1642.57 & -1.53 & 0.0149 \\ 1169.25 & -124.80 & 0.0148 \\ 1169.32 & -124.80 & 0.0148 \\ 1169.45 & -124.80 & 0.0148 \\ 529.11 & -3.85 & 0.0000 \\ 529.11 & -3.85 & 0.0000 \\ 529.11 & -3.85 & 0.0000 \\ 529.11 & -3.85 & 0.0000\end{array}$

5.170
5.167

5.167
5.167

7.961

12.25
12.25

12.257

135.057

135.057

131.946

30.656

7.034

7.034

7.034

7.961

7.961

529.11

TURBOMACHINERY PERFORMANCE DATA

\begin{tabular}{|c|c|c|c|c|c|c|c|c|c|}
\hline & Wc & PR & eff & $\mathrm{NC}$ & $T R$ & efPoly & pwr & SMN & SMW \\
\hline Fan & 991.53 & 1.541 & 0.9070 & 3810.950 & 1.1450 & 0.9125 & -8396.5 & 67.56 & 30.09 \\
\hline LPC & 176.00 & 2.372 & 0.8684 & 3810.950 & 1.3223 & 0.8833 & -3315.8 & 4.29 & 3.38 \\
\hline HPC & 85.34 & 11.019 & .8615 & 10019.853 & 2.1007 & 0.8982 & -15092.4 & 34.12 & 32.77 \\
\hline HPT & 12.09 & 4.304 & .9073 & 216.963 & 1.3577 & 0.8874 & 15092.4 & & \\
\hline $\mathrm{HT}$ & 56.11 & 4.358 & . 9107 & 88.755 & 1.4021 & 0.8932 & 11712.9 & & \\
\hline \multicolumn{10}{|c|}{ INERY MAP D } \\
\hline & WcMap & PRmap & effMap & NcMap & R/Parm & S_WcDes & S_PRdes & S_effDes & S_NcDes \\
\hline an & 1152.06 & 1.528 & & 3774.613 & 0.0583 & $\overline{0} .8607$ & $\overline{1} .0235$ & 0.9980 & $\overline{0} .9905$ \\
\hline $\mathrm{HPC}$ & 141.07 & 2.398 & 0.8201 & 1.007 & 0.0000 & 1.2475 & 0.9816 & 1.0588 & 0.0003 \\
\hline PC & 77.62 & 10.547 & 0. & 9752.731 & 12.7846 & 1.0995 & 1.0494 & 0.9898 & 0.9733 \\
\hline IPT & 0.96 & 4.213 & 0.9073 & 1.304 & 4.2125 & 12.6299 & 0.9723 & 1.0000 & 0.0003 \\
\hline LPT & 0.86 & 3.526 & 0.9175 & 0.998 & 3.5257 & 65.2590 & 0.7521 & 0.9926 & 0.0005 \\
\hline
\end{tabular}

$\begin{array}{rrrlccc}\text { VTAS } & \text { OPR } & \text { EPR } & \text { PowerSet } & \text { T4 } & \text { T41 } & \text { T49 } \\ 776.30 & 26.124 & 1.304 & 72.700 & 2513.3 & 2363.4 & 1635.2\end{array}$




\begin{tabular}{|c|c|c|c|}
\hline$===$ INLET $S===$ & eRam & Afs & Fram \\
\hline Inlet & 1.0000 & 3560.00 & 10494.1 \\
\hline$====$ DUCTS $====$ & dPnorm & MN & Aphy \\
\hline TEGV & 0.0000 & 0.4718 & 860.21 \\
\hline FanDuct & 0.0000 & 0.5625 & 2481.93 \\
\hline$==$ SPLITTERS $==$ & BPR & $\mathrm{dP} / \mathrm{P} 1$ & $\mathrm{dP} / \mathrm{P} 2$ \\
\hline Splitter & 5.6337 & 0.0005 & 0.0005 \\
\hline$===$ SHAFTS $====$ & Nmech & trq in & pwr in \\
\hline HP Shaft & 10876.9 & 7287.7 & 15092.4 \\
\hline LP ${ }^{-}$Shaft & 3597.1 & 17101.9 & 11712.9 \\
\hline
\end{tabular}

BLEEDS - interstg Wb/Win BldWk BldP $\begin{array}{llll}\text { HPT_COOLC HPC.C }> & 0.0368 & 0.5000 & 0.2200\end{array}$ $\begin{array}{lllll}\text { LPT COOLA HPC.C> } & 0.0117 & 0.5000 & 0.4500 \\ \text { WB } 2 \bar{X} & \text { HPC B } & 0.0000 & 0.4500 & 0.2200\end{array}$ $\begin{array}{lllll}\text { WB2X } & \text { HPC.B> } & 0.0000 & 0.4500 & 0.2200 \\ \text { WB2Y } & \text { HPC. B> } & 0.0000 & 0.7600 & 0.6200\end{array}$ $\begin{array}{lllll}\text { WB2Y } & \text { HPC.B> } & 0.0000 & 0.7600 & 0.6200\end{array}$ $\begin{array}{lllll}\text { WBA2X HPC.B } & 0.0000 & 0.4500 & 0.2200\end{array}$ $\begin{array}{lllll}\text { WBLKG HPC.1> } & 0.0000 & 1.0000 & 1.0000\end{array}$ $\begin{array}{lllll}\text { WBW2X HPC.B> } & 0.0000 & 0.4500 & 0.2200\end{array}$

BLEEDS - output Wb/Win hscale Pscale WB17Y Bleed> $0.0000 \quad 1.0000 \quad 1.0000$ $\begin{array}{lllll}\text { HPT_COOLA Bleed> } & 0.1142 & 1.0000 & 1.000\end{array}$ $\begin{array}{lllll}\text { HPT_COOLB } & \text { Bleed> } & 0.0999 & 1.0000 & 1.0000\end{array}$ $\begin{array}{lllll}\text { WB3X } & \text { Bleed> } & 0.0000 & 1.0000 & 1.0000 \\ \text { WBA3X } & \text { Bleed }> & 0.0000 & 1.0000 & 1.0000\end{array}$ $\begin{array}{lllll}\text { WBA3X } & \text { Bleed> } & 0.0000 & 1.0000 & 1.0000 \\ \text { WBW3X } & \text { Bleed> } & 0.0000 & 1.0000 & 1.0000\end{array}$ $\begin{array}{lllll}\text { WBW3X } & \text { Bleed> } & 0.0000 & 1.0000 & 1.0000 \\ \text { WBFDLKG } & \text { FanDu> } & 0.0000 & 1.0000 & 1.0000\end{array}$ $\begin{array}{lllll}\text { WBFDLKG } & \text { FanDu> } & 0.0000 & 1.0000 & 1.0000 \\ \text { WB15X } & \text { Bleed }> & 0.0000 & 1.0000 & 1.0000\end{array}$ $\begin{array}{lllll}\text { WB15X } & \text { Bleed }> & 0.0000 & 1.0000 & 1.0000 \\ \text { WB15Y } & \text { Bleed }> & 0.0000 & 1.0000 & 1.0000\end{array}$ WB17X Bleed> $0.0000 \quad 1.0000 \quad 1.0000$ $===$ BURNERS $===\quad$ TtOut eff
0.9995 dPnorm

$\begin{array}{rrrr}\text { W } & \text { Tt } & \text { ht } & \text { Pt } \\ 2.4130 & 953.58 & 98.66 & 30.656 \\ 0.7672 & 953.58 & 98.66 & 7.034 \\ 0.0000 & 919.81 & 90.32 & 39.273 \\ 0.0000 & 1127.04 & 142.00 & 88.393 \\ 0.0000 & 919.81 & 90.32 & 39.273 \\ 0.0000 & 1283.94 & 182.02 & 135.057 \\ 0.0000 & 919.81 & 90.32 & 39.273 \\ \text { W } & & & \\ 0.0000 & 611.19 & 15.29 & 12.257 \\ 7.4868 & 1283.94 & 182.02 & 131.946 \\ 6.5511 & 1283.94 & 182.02 & 74.008 \\ 0.0000 & 1283.94 & 182.02 & 135.057 \\ 0.0000 & 1283.94 & 182.02 & 135.057 \\ 0.0000 & 1283.94 & 182.02 & 135.057 \\ 0.0000 & 529.11 & -3.85 & 7.961 \\ 0.0000 & 529.11 & -3.85 & 7.961 \\ 0.0000 & 529.11 & -3.85 & 7.961 \\ 0.0000 & 529.11 & -3.85 & 7.961\end{array}$

$\mathrm{PR}$
2.034


Date:05/13/13 Time:09:33:03 Model: Turbofan Engine - COMDES ON COnverge $=1$ CASE: 0

Version:NPSS 1.6.5 - Rev: -> Gas Package: Janaf iter/pass/Jacb/Broy=18/32/1/16 Run by: Philip C Jorgenson PC: 69

Temperature Stator 1 inlet: 460.43 Stator 1 exit: 476.17 Stator 2 inlet: 495.48 Stator 2 exit: 503.78 $\begin{array}{lll}\text { Stator } 3 \text { inlet: } 526.45 & \text { Stator } 3 \text { exit: } 534.66 & \text { Stator } 4 \text { inlet: } 556.09 \text { Stator } 4 \text { exit: } 563.58 \\ \text { Stator } 5 \text { inlet: } 580.79 & \text { Stator } 5 \text { exit: } 587.80 & \text { Unblocked Percent Blockage: } 0.00\end{array}$

$\begin{array}{lclll}\text { Ambient Relative Humidity } & 10.00 & & & \\ \text { Fan Face Relative Humidity } & 3.36 & & & \\ \text { Fan Bypass Relative Humidity } & 0.26 & & & \\ \text { LPC Inlet Relative Humidity } & 1.77 & & & \\ \text { LPC Exit Relative Humidity } & 0.02 & & & \\ \text { HPC Relative Humidity } & 0.00 & & & \\ \text { Drop Diameter } & 0.0000250 & \text { Inlet Length } & & \\ \text { Ambient Flow Velocity } & 776.30 & \text { Fan/LPC Inlet Flow Velocity } & 397.91 \\ \text { Ambient Static Pressure } & 3.46 & \text { Fan/LPC Inlet Static Pressure } & 4.67 \\ \text { Ambient Static Temperature } & 411.85 & \text { Fan/LPC Inlet Static Temperature } & 448.90 \\ \text { Additional Water at LPC Exit } & 0.0001025 & & & \end{array}$

\begin{tabular}{|c|c|c|c|c|c|c|c|c|c|c|c|c|c|c|}
\hline & & dTa & & SUMMARY & DUTPUT DATA & Wf & & & & & & & & \\
\hline $\begin{array}{r}\mathrm{MN} \\
0.780\end{array}$ & $\begin{array}{r}\text { alt } \\
35000.0\end{array}$ & 18.00 & $430.86^{W}$ & $\begin{array}{r}\text { Fn } \\
5637.1\end{array}$ & $\begin{array}{r}\text { TSFC } \\
0.5955\end{array}$ & 3356.62 & 5.6872 & 776.30 & $\begin{array}{r}\text { OPR } \\
25.519\end{array}$ & 1.267 & 69.900 & 2484.5 & $\begin{array}{r}\text { T41 } \\
2336.9\end{array}$ & $\begin{array}{c}T 49 \\
1615.8\end{array}$ \\
\hline
\end{tabular}

\begin{tabular}{|c|c|c|c|c|c|c|c|c|c|c|}
\hline & & & & & & & & & & \\
\hline & & & $w$ & Pt & Tt & ht & FAR & Wo & & $\begin{array}{l}\text { Ps } \\
58\end{array}$ \\
\hline $\begin{array}{l}\text { FS0 } \\
\text { FS1 }\end{array}$ & Ambient.Fl_O & & 430.86 & 5.170 & 462.10 & $-19 \cdot 92$ & 0.0000 & 1156.0 & 3.45 & $\begin{array}{l}411.8 \\
434.5\end{array}$ \\
\hline $\begin{array}{l}\text { FS1 } \\
\text { FS12 }\end{array}$ & Inlet.Fl_o & & 430.86 & 5.170 & 462.10 & $-19 \cdot 92$ & 0.0000 & 1156.0 & 4.17 & $\begin{array}{l}434 . \\
434 .\end{array}$ \\
\hline FS2 & Splitter.FI- & & $\begin{array}{r}300.43 \\
64.43\end{array}$ & 5.167 & 462.10 & $\begin{array}{l}-19.92 \\
-19.92\end{array}$ & 0.0000 & $\begin{array}{l}983.6 \\
172.9\end{array}$ & $\begin{array}{l}4.13 \\
4.67\end{array}$ & $\begin{array}{l}454 . \\
448 .\end{array}$ \\
\hline FS14 & Fan.Fl_o & & 366.43 & 7.886 & 527.53 & -4.23 & 0.0000 & 688.76 & 6.54 & 500 \\
\hline FS23 & LPC. Fl O & & 64.43 & 12.175 & 609.45 & 15.45 & 0.0000 & 84.31 & 11.05 & 592 \\
\hline FS24 & 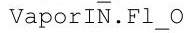 & & 64.44 & 12.175 & 609.62 & 14.90 & 0.0000 & 84.33 & 11.05 & 593 \\
\hline FS25 & Bleed2.Fl_ō & & 64.44 & 12.175 & 609.62 & 14.90 & 0.0000 & 84.33 & 11.05 & 593 \\
\hline FS3 & HPC.FI_O- & & 61.31 & 131.931 & 1274.71 & 179.63 & 0.0000 & 10.77 & 118.14 & 1237. \\
\hline FS36 & Bleed3.F1_o & & 47.52 & 131.931 & 1274.71 & 179.63 & 0.0000 & 8.36 & 123.95 & 1253 \\
\hline FS4 & Burner.Fl_o & & 48.45 & 128.892 & 2484.47 & 155.73 & 0.0196 & 12.09 & 121.74 & 2451. \\
\hline FS45 & HPT.Fl_O & & 64.62 & 29.926 & 1623.10 & -1.60 & 0.0146 & 56.13 & 26.92 & 1580 \\
\hline FS49 & LPT.FI_O & & 65.37 & 6.867 & 1154.86 & -123.25 & 0.0145 & 208.7 & 5.91 & 1110 \\
\hline FS5 & TEGV.F̄__O & & 65.37 & 6.867 & 1154.93 & -123.25 & 0.0145 & 208.7 & 5.91 & 1110 \\
\hline FS8 & Core_Noz̄z.Fl & & 65.37 & 6.867 & 1155.07 & -123.25 & 0.0145 & 208.78 & 3.65 & \\
\hline FS17 & FanDūctLkg. F & I_o & 366.43 & 7.886 & 527.53 & -4.23 & 0.0000 & 688.76 & 6.54 & 500 \\
\hline FS171 & Bleed15.FI_O & & 43 & 7.886 & 527.53 & -4.23 & 0.0000 & 688.76 & 6.36 & 496 \\
\hline FS172 & FanDuct.FI_O & & 366.43 & 7.886 & 527.53 & -4.23 & 0.0000 & $688.7 \mathrm{C}=\mathrm{x}$ & 6.36 & 496. \\
\hline FS173 & Byp_Nozz.FI__ & & 366.43 & 7.886 & 527.53 & -4.23 & 0.0000 & $688.7 \mathrm{C}-\mathrm{r}$ & 4.16 & 439. \\
\hline TURB & MACHINERY PER & EORMANCE & DATA & & & & & & & \\
\hline & & $P R$ & eff & $\mathrm{NC}$ & TR & efPoly & & pwr & SMN & SMW \\
\hline Fan & 983.66 & 1.526 & 0.9074 & 3779.269 & 1.1416 & 0.9127 & -81 & 32.9 & 74.05 & 30.79 \\
\hline LPC & 172.96 & 2.356 & 0.8699 & 3779.269 & 1.3189 & 0.8846 & -32 & 24.0 & 4.58 & 3.57 \\
\hline HPC & 84.33 & 10.836 & 0.8615 & 9991.297 & 2.0910 & 0.8980 & -146 & 53.9 & 34.92 & 33.50 \\
\hline HPT & 12.09 & 4.307 & 0.9067 & 217.315 & 1.3590 & 0.8875 & 146 & 53.9 & & \\
\hline LPT & 56.13 & 4.358 & 0.9100 & 88.543 & 1.4029 & 0.8923 & 113 & 56.9 & & \\
\hline TURBC & MACHINERY & $D A$ & & & & & & & & \\
\hline & WcMap & PRmap & effMap & NcMap & R/Parm & S_WC & cDes & S_PRdes & S_effDes & S_NcDes \\
\hline Fan & 1142.91 & 1.514 & 0.9092 & 3743.234 & 0.0578 & 0.8 & 8607 & $\overline{1} .0235$ & 0.9980 & $\overline{0} .9905$ \\
\hline LPC & 139.04 & 2.366 & 0.8195 & 0.998 & 0.0000 & & 2440 & 0.9929 & 1.0616 & 0.0003 \\
\hline HPC & 76.69 & 10.373 & 0.8704 & 9724.936 & 12.7199 & 1.0 & 0995 & 1.0494 & 0.9898 & 0.9733 \\
\hline $\mathrm{HPT}$ & 0.96 & 4.215 & 0.9067 & 1.306 & 4.2154 & 12.6 & 6299 & 0.9723 & 1.0000 & 0.0003 \\
\hline LPT & 0.86 & 3.526 & 0.9168 & 0.996 & 3.5257 & 65.2 & 2590 & 0.7521 & 0.9926 & 0.0005 \\
\hline
\end{tabular}




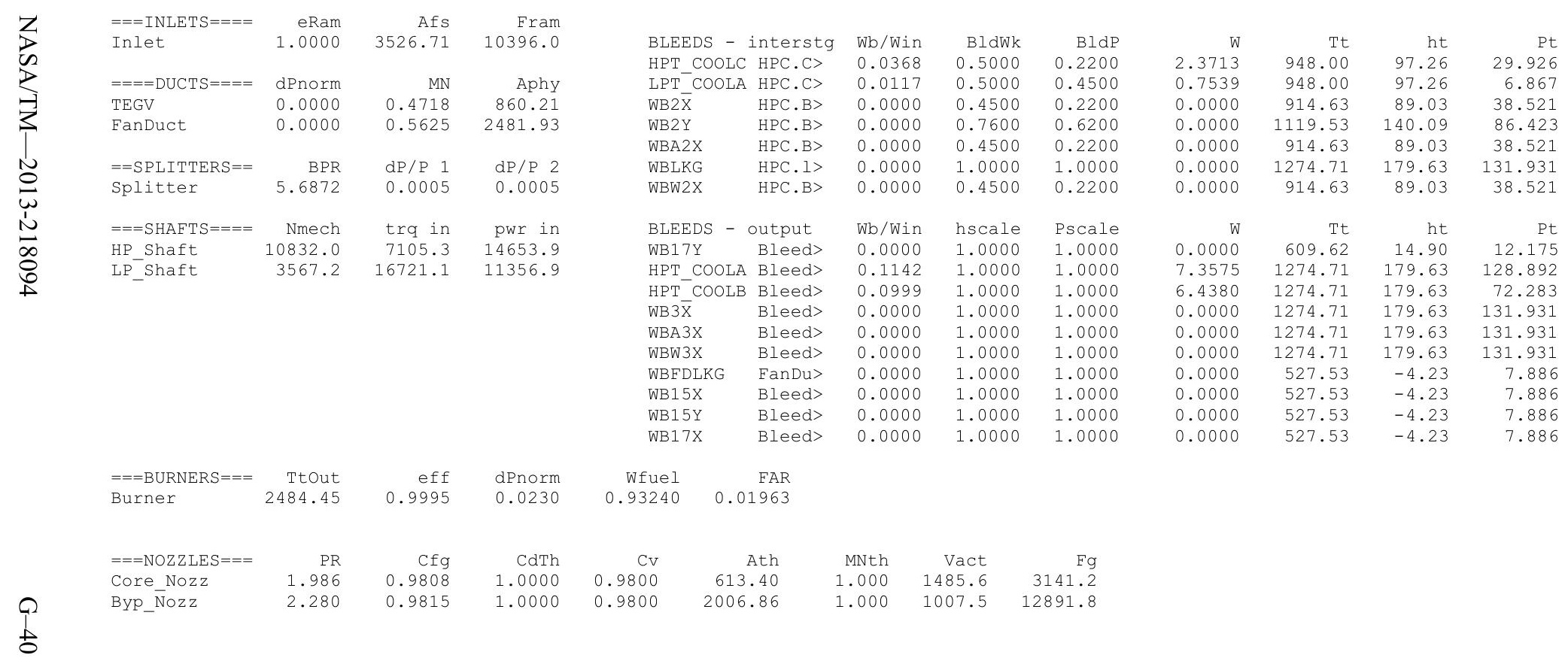


Date:05/13/13 Time:10:00:09 Model: Turbofan Engine - COMDES ON CONverge = 1 CASE: O

Version:NPSS 1.6.5 - Rev: -> Gas Package: Janaf iter/pass/Jacb/Broy=27/41/1/25 Run by: Philip C Jorgenson PC: 100

Ttator 1 inlet: 468.75 Stator 1 exit: 488.22 Stator 2 inlet: 510.83 Stator 2 exit: 518.50

Stator 3 inlet: 548.53 Stator 3 exit: 556.97 Stator 4 inlet: 585.11 Stator 4 exit: 593.25

Ambient Relative Humidity $\quad 10.00$

Ran Face Relative Hunidity

Fan Bypass Relative Humidity $\quad 0.21$

PCC Inlet Relative Humidity 2.54

LPC Exit Relative Humidity $\quad 0.03$

Ambient Flow Velocity

0.0000250

793.07 Inlet Length $\quad 40.00$

Ambient Static Pressure

Ambient Static Temperature

3.46

Fan/LPC Inlet Flow Velocity $\quad 493.51$

Additional Water at IPC Exit

429.85

Fan/LPC Inlet Static Temperature 461.98
$\begin{array}{rrrrrr} & & & & \text { SUMMARY } & \text { OUTPUT DATA } \\ \text { MN } & \text { alt } & \text { dTamb } & \text { W } & \text { Fn } & \text { TSFC } \\ 0.780 & 35000.0 & 36.00 & 465.49 & 8112.6 & 0.6156\end{array}$
465.49
$\begin{array}{lr}8112.6 & 0.6156\end{array}$
Wfuel
4993.72
$B P R$
5.2964
VTAS $\quad$ OPR
EPR
Powerset
T4
$\begin{array}{rr}T 41 & T 49 \\ 2747.3 & 1915.5\end{array}$

\begin{tabular}{|c|c|c|}
\hline FSO & Ambient.Fl O & 465.49 \\
\hline FS1 & Inlet.FI $0^{-}$ & 465.49 \\
\hline FS12 & Splitter.Fl 02 & 391.56 \\
\hline FS2 & Splitter.Fl_01 & 73.93 \\
\hline FS14 & Fan.Fl 0 & 391.56 \\
\hline FS23 & LPC.Fl- & 73.93 \\
\hline FS24 & VaporIN̄.FI_O & 73.94 \\
\hline FS25 & Bleed2.F1 $\overline{0}$ & 73.94 \\
\hline FS3 & HPC.FI O & 70.35 \\
\hline FS36 & Bleed3.F1 O & 54.52 \\
\hline FS4 & Burner.Fl-o & 55.91 \\
\hline FS45 & HPT.FI O- & 74.46 \\
\hline FS49 & LPT.Fl- & 75.33 \\
\hline FS5 & TEGV.FI $O$ & 75.33 \\
\hline FS8 & Core Nozz.El O & 75.33 \\
\hline FS17 & FanDuctLkg.Fì & 391.56 \\
\hline FS171 & Bleed15.FI_o & 391.56 \\
\hline FS172 & FanDuct.FI- & 391.56 \\
\hline FS173 & Byp Nozz.Fīo & 391.56 \\
\hline
\end{tabular}

FLOW STATION DATA

$\begin{array}{rrrrrrrrr}\text { Pt } & \text { Tt } & \text { ht } & \text { FAR } & \text { Wc } & \text { Ps } & \text { Ts } & \text { Aphy } & \text { MN } \\ 5.170 & 482.28 & -15.41 & 0.0000 & 1275.96 & 3.458 & 429.85 & 3892.7 & 0.7800 \\ 5.170 & 482.28 & -15.41 & 0.0000 & 1275.96 & 3.838 & 442.89 & 4168.2 & 0.6662 \\ 5.167 & 482.28 & -15.41 & 0.0000 & 1073.85 & 3.865 & 443.82 & 3531.8 & 0.6575 \\ 5.167 & 482.28 & -15.41 & 0.0000 & 202.75 & 4.446 & 461.98 & 830.5 & 0.4682 \\ 8.746 & 568.20 & 5.21 & 0.0000 & 688.62 & 7.261 & 538.78 & 2606.7 & 0.5227 \\ 12.698 & 652.75 & 25.55 & 0.0000 & 95.99 & 11.119 & 628.55 & 412.6 & 0.4400 \\ 12.698 & 652.96 & 24.89 & 0.0000 & 96.02 & 11.118 & 628.73 & 412.6 & 0.4401 \\ 12.698 & 652.96 & 24.89 & 0.0000 & 96.02 & 11.118 & 628.73 & 412.6 & 0.4401 \\ 125.047 & 1433.44 & 220.42 & 0.0000 & 10.41 & 148.847 & 1395.15 & 49.7 & 0.3930 \\ 65.047 & 1433.44 & 220.42 & 0.0000 & 8.07 & 155.636 & 1411.58 & 49.3 & 0.2955 \\ 61.246 & 2926.06 & 188.18 & 0.0254 & 12.10 & 152.349 & 2889.29 & 74.6 & 0.3007 \\ 37.748 & 1924.78 & 3.18 & 0.0190 & 55.84 & 34.006 & 1876.83 & 265.4 & 0.4007 \\ 8.694 & 1382.66 & -142.81 & 0.0188 & 207.89 & 7.501 & 1331.26 & 860.2 & 0.4726 \\ 8.694 & 1382.73 & -142.81 & 0.0188 & 207.90 & 7.501 & 1331.33 & 860.2 & 0.4726 \\ 8.694 & 1382.85 & -142.81 & 0.0188 & 207.91 & 4.655 & 1175.94 & 613.4 & 1.0000 \\ 8.746 & 568.20 & 5.21 & 0.0000 & 688.62 & 7.261 & 538.78 & 2606.7 & 0.5227 \\ 8.746 & 568.20 & 5.21 & 0.0000 & 688.62 & 7.056 & 534.39 & 2481.9 & 0.5626 \\ 8.746 & 568.20 & 5.21 & 0.0000 & 688.62 & 7.056 & 534.39 & 2481.9 & 0.5626 \\ 8.746 & 568.20 & 5.21 & 0.0000 & 688.62 & 4.619 & 473.44 & 2006.9 & 1.0000\end{array}$

gamt
1.40064
1.40064
1.40064
1.40064
1.39944
1.39742
1.39740
1.39740
1.35403
1.35403
1.28088
1.31717
1.34396
1.34396
1.34395
1.39944
1.39944
1.39944
1.39944

WAR

Wair WH2O H2O frac $\begin{array}{llll}0.0000871 & 465.45 & 0.041 & 0.0001\end{array}$ $\begin{array}{llll}0.0000871 & 391.53 & 0.034 & 0.0001\end{array}$ $\begin{array}{llll}0.0000871 & 73.92 & 0.006 & 0.0001\end{array}$ $\begin{array}{llll}0.0000871 & 391.53 & 0.034 & 0.0001\end{array}$ $\begin{array}{llll}0.0000871 & 73.92 & 0.006 & 0.0001\end{array}$ $\begin{array}{llll}0.0000871 & 73.92 & 0.006 & 0.0001 \\ 0.0002119 & 73.92 & 0.016 & 0.0002 \\ 0.0002119 & 73.92 & 0.016 & 0.0002\end{array}$ $\begin{array}{llll}0.0002119 & 70.34 & 0.015 & 0.0002\end{array}$ $\begin{array}{llll}0.0002119 & 54.51 & 0.012 & 0.0002\end{array}$ $\begin{array}{llll}0.0002119 & 54.51 & 0.012 & 0.0308\end{array}$ $\begin{array}{llll}0.0002119 & 73.06 & 0.015 & 0.0232\end{array}$ $\begin{array}{llll}0.0002119 & 73.92 & 0.016 & 0.0229\end{array}$ $\begin{array}{llll}0.0002119 & 73.92 & 0.016 & 0.0229\end{array}$ $\begin{array}{llll}0.0002119 & 73.92 & 0.016 & 0.0229\end{array}$ $\begin{array}{llll}0.0000871 & 391.53 & 0.034 & 0.0001\end{array}$ $\begin{array}{llll}0.0000871 & 391.53 & 0.034 & 0.0001\end{array}$ TURBOMACHINERY PERFORMANCE DATA

\begin{tabular}{|c|c|c|c|c|c|c|c|c|c|}
\hline & Wc & PR & eff & $\mathrm{NC}$ & TR & efPoly & pwr & SMN & SMW \\
\hline Fan & 1073.85 & 1.693 & 0.9109 & 4120.705 & 1.1782 & 0.9173 & -11421.0 & 38.39 & 23.45 \\
\hline LPC & 202.75 & 2.457 & 0.8276 & 4120.705 & 1.3535 & 0.8477 & -4284.4 & 2.96 & 2.22 \\
\hline HPC & 96.02 & 12.998 & 0.8587 & 10334.072 & 2.1953 & 0.8978 & -19959.6 & 26.51 & 25.70 \\
\hline HPT & 12.10 & 4.272 & 0.9122 & 214.352 & 1.3425 & 0.8900 & 19959.6 & & \\
\hline LPT & 55.84 & 4.342 & 0.9168 & 90.570 & 1.3886 & 0.9005 & 15705.8 & & \\
\hline \multicolumn{10}{|c|}{ JRBOMACHINERY MAP DATA } \\
\hline & WcMap & PRmap & effMap & NcMap & R/Parm & s WcDes & S_PRdes & s_effDes & S_NCDes \\
\hline Fan & 1247.70 & 1.677 & 0.9128 & 4081.415 & 0.0636 & $\overline{0} .8607$ & $\overline{1} .0235$ & 0.9980 & 0.9905 \\
\hline LPC & 165.11 & 2.727 & 0.8329 & 1.088 & 0.0000 & 1.2280 & 0.8437 & 0.9936 & 0.0003 \\
\hline HPC & 87.32 & 12.433 & 0.8676 & 10058.573 & 13.4500 & 1.0995 & 1.0494 & 0.9898 & 0.9733 \\
\hline HPT & 0.96 & 4.181 & 0.9122 & 1.288 & 4.1810 & 12.6299 & 0.9723 & 1.0000 & 0.0003 \\
\hline LPT & 0.86 & 3.514 & 0.9236 & 1.019 & 3.5135 & 65.2590 & 0.7521 & 0.9926 & 0.0005 \\
\hline
\end{tabular}




\begin{tabular}{|c|c|c|c|}
\hline $\begin{array}{l}===\text { INLETS }==== \\
\text { Inlet }\end{array}$ & $\begin{array}{r}\text { eRam } \\
1.0000\end{array}$ & $\begin{array}{r}\text { Afs } \\
3892.72\end{array}$ & $\begin{array}{r}\text { Fram } \\
11474.1\end{array}$ \\
\hline$====$ DUCTS $====$ & dPnorm & MN & Aphy \\
\hline TEGV & 0.0000 & 0.4726 & 860.21 \\
\hline FanDuct & 0.0000 & 0.5626 & 2481.93 \\
\hline$==$ SPLITTERS $==$ & BPR & $\mathrm{dP} / \mathrm{P} 1$ & $\mathrm{dP} / \mathrm{P} 2$ \\
\hline Splitter & 5.2964 & 0.0005 & 0.0005 \\
\hline$===$ SHAFTS $====$ & Nmech & trq in & pwr in \\
\hline HP Shaft & 11595.0 & 9041.0 & 19959.6 \\
\hline LP Shaft & 3973.5 & 20759.7 & 15705.8 \\
\hline
\end{tabular}

BLEEDS - interstg Wb/Win BldWk BldP $\begin{array}{llll}\text { HPT_COOLC HPC.C> } & 0.0368 & 0.5000 & 0.2200 \\ \text { LPT COOLA HPC.C } & 0.0117 & 0.5000 & 0.4500\end{array}$ $\begin{array}{llllll} & \text { WB } 2 \bar{X} & \text { HPC. } B> & 0.0000 & 0.4500 & 0.2200\end{array}$ $\begin{array}{lllll}\text { WB2Y HPC.B } & 0.0000 & 0.7600 & 0.6200\end{array}$ WBA2X HPC.B> $0.0000 \quad 0.4500 \quad 0.2200$ WBLKG HPC.I> $0.0000 \quad 1.0000 \quad 1.0000$ $\begin{array}{llll}\text { WBW2X HPC.B> } & 0.0000 & 0.4500 & 0.2200\end{array}$ BLEEDS - output Wb/Win hscale Pscale $\begin{array}{llll}\text { WB17Y Bleed } & 0.0000 & 1.0000 & 1.0000\end{array}$ HPT_COOLA Bleed> $0.1142 \quad 1.0000 \quad 1.0000$ ${ }_{\mathrm{HPT}}$ COOLB Bleed> $0.0999 \quad 1.0000 \quad 1.0000$

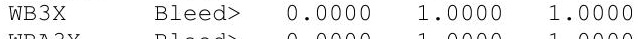
$\begin{array}{lllll}\text { WBA3X } & \text { Bleed> } & 0.0000 & 1.0000 & 1.0000 \\ \text { WBW3X } & \text { Bleed }> & 0.0000 & 1.0000 & 1.0000\end{array}$ $\begin{array}{lllll}\text { WBW3X } & \text { Bleed> } & 0.0000 & 1.0000 & 1.0000 \\ \text { WBFDLKG FanDu> } & 0.0000 & 1.0000 & 1.0000\end{array}$ $\begin{array}{lllll}\text { WBFDLKG } & \text { FanDu> } & 0.0000 & 1.0000 & 1.0000 \\ \text { WB15X } & \text { Bleed> } & 0.0000 & 1.0000 & 1.0000\end{array}$ $\begin{array}{lllll}\text { WB15X } & \text { Bleed> } & 0.0000 & 1.0000 & 1.0000 \\ \text { WB15Y } & \text { Bleed> } & 0.0000 & 1.0000 & 1.0000\end{array}$ WB17X Bleed> $0.0000 \quad 1.0000 \quad 1.0000$

$\begin{array}{rrrr}\text { W } & \text { Tt } & \text { ht } & \text { Pt } \\ 2.7209 & 1051.90 & 122.65 & 37.748 \\ 0.8651 & 1051.90 & 122.65 & 8.694 \\ 0.0000 & 1012.73 & 112.88 & 46.214 \\ 0.0000 & 1252.55 & 173.49 & 107.154 \\ 0.0000 & 1012.73 & 112.88 & 46.214 \\ 0.0000 & 1433.44 & 220.42 & 165.047 \\ 0.0000 & 1012.73 & 112.88 & 46.214 \\ \text { W } & & & \\ 0.0000 & 652.96 & 24.89 & 12.698 \\ 8.4423 & 1433.44 & 220.42 & 161.246 \\ 7.3872 & 1433.44 & 220.42 & 90.605 \\ 0.0000 & 1433.44 & 220.42 & 165.047 \\ 0.0000 & 1433.44 & 220.42 & 165.047 \\ 0.0000 & 1433.44 & 220.42 & 165.047 \\ 0.0000 & 568.20 & 5.21 & 8.746 \\ 0.0000 & 568.20 & 5.21 & 8.746 \\ 0.0000 & 568.20 & 5.21 & 8.746 \\ 0.0000 & 568.20 & 5.21 & 8.746\end{array}$

$===$ BURNERS $===\quad$ TtOut 2926.06

eff
0.9995

dPnorm

Wfuel Bar

$1.38714 \quad 0.02545$

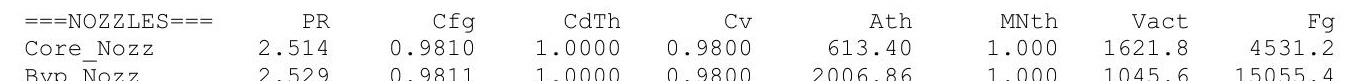


Date:05/13/13 Time:10:00:41 Model: Turbofan Engine - COMDES ON Converge = 1 CASE:

Version:NPSS 1.6.5 - Rev: -> Gas Package: Janaf iter/pass/Jacb/Broy=18/32/1/16 Run by: Philip C Jorgenson PC: 75

Temperature Stator 1 inlet: 478.73 Stator 1 exit: 495.69 Stator 2 inlet: 516.30 Stator 2 exit: 524.95 $\begin{array}{lll}\text { Stator } 3 \text { inlet: } 549.58 & \text { Stator } 3 \text { exit: } 558.21 & \text { Stator } 4 \text { inlet: } 581.50 \text { Stator } 4 \text { exit: } 589.41 \\ \text { Stator } 5 \text { inlet: } 608.02 & \text { Stator } 5 \text { exit: } 615.47 & \text { Unblocked Percent Blockage: } 0.00\end{array}$

\begin{abstract}
Ambient Relative Humidity $\quad 10.00$
Fan Face Relative Humidity $\quad 3.82$

Fan Bypass Relative Humidity $\quad 0.30$

LPC Inlet Relative Humidity $\quad 2.06$

LPC Exit Relative Humidity $\quad 0.03$

HPC Relative Humidity $\quad 0.00$

Drop Diameter $\quad 0.0000250$

Ambient Flow Velocity

Ambient Static Pressure

Additional Water at LPC Exit

40.00
Fan/LPC Inlet Flow Velocity $\quad 423.28$

3.46 Fan/LPC Inlet Static Pressure 4.63 0.000155
\end{abstract}

\section{MN SUMMARY OUTPUT DATA}

$\begin{array}{rrrrrr} & \text { alt } & \text { dTamb } & \text { W } & \text { Fn } & \text { TSFC } \\ 0.780 & 35000.0 & 36.00 & 429.86 & 6091.0 & 0.6090\end{array}$
Wfuel $\begin{array}{rr}3709.16 & \text { BPR } \\ & 5.5867\end{array}$
VTAS 793.07
OPR

\section{FLOW STATION DATA}

W
429.86
429.86
364.60
65.26
364.60
65.26
65.27
65.27
62.11
48.13
49.16
65.54
66.30
66.30
66.30
364.60
364.60
364.60
364.60

\begin{tabular}{rrrr}
\multicolumn{1}{c}{ Pt } & Tt & ht & FAR \\
5.170 & 482.28 & -15.41 & 0.0000 \\
5.170 & 482.28 & -15.41 & 0.0000 \\
5.167 & 482.28 & -15.41 & 0.0000 \\
5.167 & 482.28 & -15.41 & 0.0000 \\
8.040 & 553.91 & 1.77 & 0.0000 \\
12.330 & 639.17 & 22.28 & 0.0000 \\
12.330 & 639.41 & 21.44 & 0.0000 \\
12.330 & 639.41 & 21.44 & 0.0000 \\
138.011 & 1345.21 & 197.23 & 0.0000 \\
138.011 & 1345.21 & 197.23 & 0.0000 \\
134.833 & 2640.85 & 170.73 & 0.0214 \\
31.391 & 1731.20 & 3.30 & 0.0160 \\
7.216 & 1235.99 & -127.06 & 0.0158 \\
7.216 & 1236.05 & -127.06 & 0.0158 \\
7.216 & 1236.19 & -127.06 & 0.0158 \\
8.040 & 553.91 & 1.77 & 0.0000 \\
8.040 & 553.91 & 1.77 & 0.0000 \\
8.040 & 553.91 & 1.77 & 0.0000 \\
8.040 & 553.91 & 1.77 & 0.0000
\end{tabular}

WC
1178.28
1178.28
999.89
178.98
688.65
86.35
86.38
86.38
10.65
8.25
12.09
56.06
208.45
208.45
208.46
688.65
688.65
688.65
688.65

$\begin{array}{rr}\text { PS } & \text { TS } \\ 3.458 & 429.85 \\ 4.118 & 451.90 \\ 4.112 & 451.76 \\ 4.629 & 467.35 \\ 6.675 & 525.21 \\ 11.130 & 620.80 \\ 11.129 & 621.02 \\ 11.129 & 621.02 \\ 123.758 & 1306.93 \\ 129.754 & 1323.44 \\ 127.369 & 2606.79 \\ 28.250 & 1686.62 \\ 6.221 & 1188.74 \\ 6.221 & 1188.80 \\ 3.851 & 1046.46 \\ 6.675 & 525.21 \\ 6.486 & 520.93 \\ 6.486 & 520.93 \\ 4.246 & 461.50\end{array}$

$\begin{array}{rr}\text { Aphy } & \text { MN } \\ 3594.7 & 0.7800 \\ 4168.2 & 0.5792 \\ 3531.8 & 0.5806 \\ 830.5 & 0.3993 \\ 2606.7 & 0.5226 \\ 412.6 & 0.3856 \\ 412.6 & 0.3857 \\ 412.6 & 0.3857 \\ 49.7 & 0.4031 \\ 49.3 & 0.3024 \\ 74.6 & 0.2992 \\ 265.4 & 0.4009 \\ 860.2 & 0.4720 \\ 860.2 & 0.4721 \\ 613.4 & 1.0000 \\ 2606.7 & 0.5226 \\ 2481.9 & 0.5626 \\ 2481.9 & 0.5626 \\ 2006.9 & 1.0000\end{array}$

gamt
1.40064
1.40064
1.40064
1.40064
1.39969
1.39781
1.39778
1.39778
1.35911
1.35911
1.29152
1.32751
1.35492
1.35491
1.35490
1.39969
1.39969
1.39969
1.39969

WAR
0.0000871
0.0000871
0.0000871
0.0000871
0.0000871
0.0000871
0.0002430
0.0002430
0.0002430
0.0002430
0.0002430
0.0002430
0.0002430
0.0002430
0.0002430
0.0000871
0.0000871
0.0000871
0.0000871

Wair
429.82
429.82
364.56
65.26
364.56
65.26
65.26
65.26
62.09
48.12
48.12
64.49
65.26
65.26
65.26
364.56
364.56
364.56
364.56
$0.037 \quad 0.0001$ $\begin{array}{ll}0.037 & 0.0001 \\ 0.032 & 0.0001\end{array}$ $0.032 \quad 0.0001$ $0.006 \quad 0.0001$ $\begin{array}{ll}0.016 & 0.0196 \\ 0.016 & 0.0194\end{array}$ $0.016-0.0194$ $\begin{array}{ll}0.032 & 0.0001 \\ 0.032 & 0.0001\end{array}$ $\begin{array}{ll}0.032 & 0.0001 \\ 0.032 & 0.0001 \\ 0.032 & 0.0001\end{array}$ TURBOMACHINERY PERFORMANCE DATA

\begin{tabular}{|c|c|c|c|c|c|c|c|c|c|}
\hline & Wc & PR & eff & NC & TR & efPoly & pwr & SMN & SMW \\
\hline Fan & 999.89 & 1.556 & 0.9070 & 3842.318 & 1.1485 & 0.9126 & -8863.9 & 62.41 & 29.35 \\
\hline LPC & 178.98 & 2.386 & 0.8663 & 3842.318 & 1.3253 & 0.8815 & -3479.9 & 4.01 & 3.17 \\
\hline HPC & 86.38 & 11.193 & 0.8615 & 10048.502 & 2.1038 & 0.8982 & -15840.8 & 33.48 & 32.19 \\
\hline HPT & 12.09 & 4.295 & 0.9089 & 217.106 & 1.3534 & 0.8892 & 15840.8 & & \\
\hline LPT & 56.06 & 4.350 & 0.9129 & 89.048 & 1.3979 & 0.8960 & 12343.7 & & \\
\hline \multirow{2}{*}{\multicolumn{10}{|c|}{ TURBOMACHINERY MAP DATA }} \\
\hline & & & & & & & & & s NcDes \\
\hline Fan & 1161.78 & 1.543 & 0.9088 & 3805.682 & 0.0589 & $\overline{0} .8607$ & $\overline{1} .0235$ & 0.9980 & 0.9905 \\
\hline LPC & 143.10 & 2.430 & 0.8208 & 1.015 & 0.0000 & 1.2507 & 0.9694 & 1.0555 & 0.0003 \\
\hline HPC & 78.56 & 10.712 & 0.8704 & 9780.616 & 12.8362 & 1.0995 & 1.0494 & 0.9898 & 0.9733 \\
\hline HPT & 0.96 & 4.204 & 0.9089 & 1.305 & 4.2040 & 12.6299 & 0.9723 & 1.0000 & 0.0003 \\
\hline LPT & 0.86 & 3.520 & 0.9197 & 1.002 & 3.5198 & 65.2590 & 0.7521 & 0.9926 & 0.0005 \\
\hline
\end{tabular}




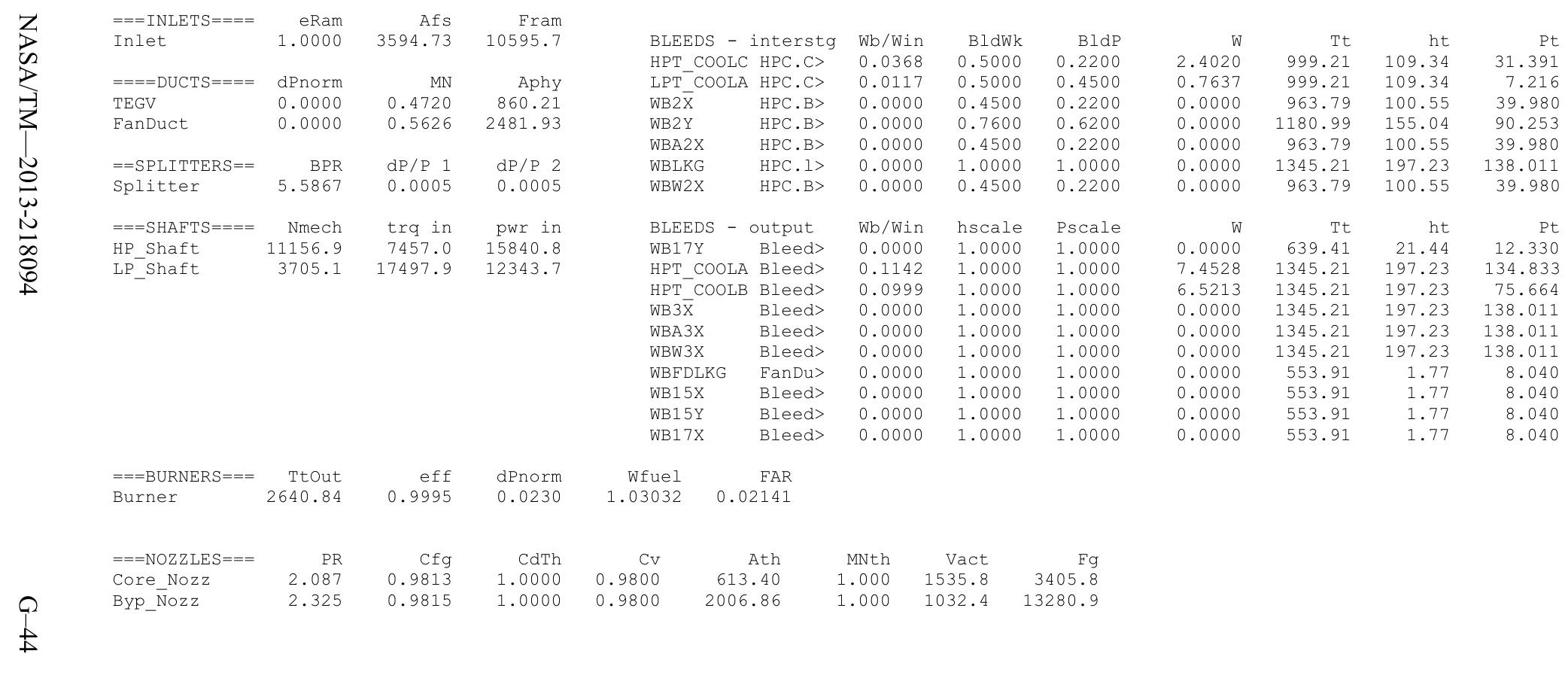




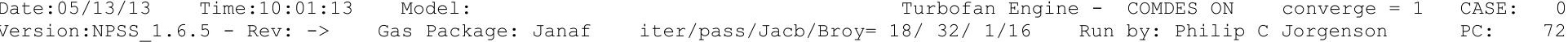

Temperature Stator 1 inlet: 479.70 Stator 1 exit: 496.41 Stator 2 inlet: 516.81 Stator 2 exit: 525.49 $\begin{array}{cccc}\text { Stator } 3 \text { inlet: } 549.63 & \text { Stator } 3 \text { exit: } 558.25 & \text { Stator } 4 \text { inlet: } 581.09 \text { Stator } 4 \text { exit: } 588.96 \\ \text { Stator } 5 \text { inlet: } 607.25 & \text { Stator } 5 \text { exit: } 614.64 & \text { Unblocked Percent Blockage: } 0.00\end{array}$

Ambient Relative Humidity $\quad 10.00$

Fan Face Relative Humidity $\quad 3.70$

Fan Bypass Relative Humidity 0.31

LPC Inlet Relative Humidity $\quad 2.02$

LPC Exit Relative Humidity 0.03

HPC Relative Humidity

0.00

Drop Diameter

0.0000250
793.07

Inlet Length

40.00

Ambient Flow Velocity

Ambient Static Temperature

.46 $\quad 414.72$

Additional Water at LPC Exit $\quad \begin{array}{ll}429.85 \\ 0.0001602\end{array}$ Fan/LPC Inlet Static Temperature 467.94

\begin{tabular}{|c|c|c|c|c|c|c|c|c|c|c|c|c|c|c|}
\hline MN & alt & dTamb & W & $\begin{array}{r}\text { UMMMARY } \\
\text { Fn }\end{array}$ & $\begin{array}{l}\text { TPUT DAT } \\
\text { TSEC }\end{array}$ & Wfuel & BPR & VTAS & OPR & EPR & Powerset & T4 & T41 & 99 \\
\hline 0.780 & 35000.0 & 36.00 & 425.73 & 5862.8 & 0.6094 & 3572.69 & 5.6367 & 793.07 & 26.076 & 1.306 & 72.700 & 2609.4 & 2454.5 & 1702.5 \\
\hline
\end{tabular}

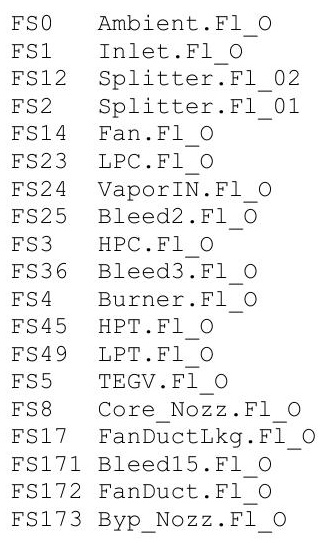

\begin{tabular}{|c|c|c|c|c|}
\hline W & Pt & Tt & ht & FAR \\
\hline 5.73 & 5.170 & 482.28 & -15.41 & 0.0000 \\
\hline 5.73 & 5.170 & 482.28 & -15.41 & 0.0000 \\
\hline 1.58 & 5.167 & 482.28 & -15.41 & 0.0000 \\
\hline .15 & 5.167 & 482.28 & -15.41 & 0.0000 \\
\hline 1.58 & 7.962 & 552.21 & 1.37 & 0.0 \\
\hline 64.15 & 12.266 & 637.72 & 21.93 & 0.0000 \\
\hline 4.16 & 12.266 & 637.97 & 21.07 & 0.0000 \\
\hline 64.16 & 12.266 & 637.97 & 21.07 & 0.0000 \\
\hline 61.05 & 34.805 & 1335.40 & 194.66 & 0.0000 \\
\hline 47.31 & 134.805 & 1335.40 & 194.66 & 0.0000 \\
\hline 48.30 & 131.701 & 2609.43 & 168.75 & 0.0210 \\
\hline 64.40 & 30.644 & 1710.19 & 3.30 & 0.0157 \\
\hline 65.15 & 7.043 & 1220.24 & -125.36 & 0.0155 \\
\hline 65.15 & 7.043 & 1220.30 & -125.36 & 0.0155 \\
\hline .15 & 7.043 & 1220.44 & -125.36 & 0.0155 \\
\hline 361.58 & 7.962 & 552.21 & 1.37 & 0.0000 \\
\hline & 7.962 & 552.21 & 1.37 & \\
\hline & 7.962 & 552.21 & 1.37 & 0. \\
\hline 58 & 7.962 & 552.21 & 1.37 & 0.000 \\
\hline
\end{tabular}

WC
1166.96
1166.96
991.62
175.92
688.66
85.22
85.25
85.25
10.68
8.28
12.09
56.08
208.51
208.52
208.53
688.66
688.66
688.66
688.66

Ps
3.458
4.146
4.136
4.650
6.609
11.106
11.105
11.105
120.800
126.695
124.411
27.575
6.072
6.071
3.757
6.609
6.423
6.423
4.205

Ts
429.85
452.76
452.51
467.94
523.60
619.95
620.17
620.17
1297.12
1313.62
2575.67
1666.00
1173.45
1173.51
1032.63
523.60
519.33
519.33
460.08

$\begin{array}{rr}\text { Aphy } & \text { MN } \\ 3560.2 & 0.7800 \\ 4168.2 & 0.5703 \\ 3531.8 & 0.5729 \\ 830.5 & 0.3910 \\ 2606.7 & 0.5226 \\ 412.6 & 0.3795 \\ 412.6 & 0.3797 \\ 412.6 & 0.3797 \\ 49.7 & 0.4043 \\ 49.3 & 0.3032 \\ 74.6 & 0.2990 \\ 265.4 & 0.4009 \\ 860.2 & 0.4720 \\ 860.2 & 0.4720 \\ 613.4 & 1.0000 \\ 2606.7 & 0.5226 \\ 2481.9 & 0.5626 \\ 2481.9 & 0.5626 \\ 2006.9 & 1.0000\end{array}$

gamt
1.40064
1.40064
1.40064
1.40064
1.39972
1.39785
1.39782
1.39782
1.35969
1.35969
1.29271
1.32870
1.35612
1.35611
1.35610
1.39972
1.39972
1.39972
1.39972

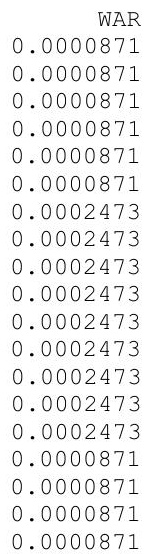

$\begin{array}{rr}\text { Wair } & \text { WH2O } \\ 425.69 & 0.037\end{array}$ $\begin{array}{ll}425.69 & 0.037\end{array}$

$64.14 \quad 0.031$

$\begin{array}{rr}64.14 & 0.031 \\ 361.55 & 0.031\end{array}$

$\begin{array}{ll}64.14 & 0.006\end{array}$

$\begin{array}{ll}64.14 & 0.016 \\ 64.14 & 0.016\end{array}$

$\begin{array}{lll}61.03 & 0.015\end{array}$

$\begin{array}{ll}47.30 & 0.012\end{array}$

\begin{tabular}{ll}
$47.30 \quad 0.012$ \\
\hline 3.39 & 0.016
\end{tabular}

$63.39 \quad 0.016$

$64.14 \quad 0.016$

$64.14 \quad 0.016$

$\begin{array}{ll}64.14 & 0.016\end{array}$

$\begin{array}{ll}361.55 & 0.031 \\ 361.55 & 0.031\end{array}$

$361.55 \quad 0.031$

0.031

TURBOMACHINERY PERFORMANCE DATA

\begin{tabular}{|c|c|c|c|c|c|c|c|c|c|}
\hline & Wc & $\mathrm{PR}$ & eff & $\mathrm{NC}$ & TR & efPoly & pwr & SMN & SMW \\
\hline Fan & 991.62 & 1.541 & 0.9070 & 3811.363 & 1.1450 & 0.9125 & -8581.8 & 67.48 & 30.07 \\
\hline LPC & 175.92 & 2.374 & 0.8684 & 3811.363 & 1.3223 & 0.8833 & -3388.8 & 4.29 & 3.38 \\
\hline HPC & 85.25 & 10.991 & 0.8615 & 10017.005 & 2.0932 & 0.8980 & -15375.4 & 34.34 & 32.98 \\
\hline $\mathrm{HPT}$ & 12.09 & 4.298 & 0.9083 & 217.479 & 1.3545 & 0.8888 & 15375.4 & & \\
\hline $\mathrm{PT}$ & 56.08 & 4.351 & .9124 & 88.871 & 1.3988 & 0.8953 & 11970.5 & & \\
\hline \multicolumn{10}{|c|}{ NE } \\
\hline & WcMap & PRmap & effmap & NcMap & R/Parm & S_WCDes & S_PRdes & S_effDes & S_NCDes \\
\hline an & 1152.16 & .528 & 0.9089 & 3775.022 & 0.0583 & $\overline{0} .8607$ & $\overline{1} .0235$ & 0.9980 & $\overline{0} .9905$ \\
\hline$\triangle \mathrm{PC}$ & 141.10 & 2.398 & 0.8201 & 1.007 & 0.0000 & 1.2467 & 0.9825 & 1.0588 & 0.0003 \\
\hline${ }^{\mathrm{C}}$ & 77.54 & 10.520 & 0.87 & 9749.958 & 12.7655 & 1.0995 & 1.0494 & 0.9898 & 0.9733 \\
\hline $\mathrm{APT}$ & 0.96 & 4.206 & 0.9083 & 1.307 & 4.2064 & 12.6299 & 0.9723 & 1.0000 & 0.0003 \\
\hline LPT & 0.86 & 3.520 & 0.9192 & 1.000 & 3.5204 & 65.2590 & 0.7521 & 0.9926 & 0.0005 \\
\hline
\end{tabular}




\begin{tabular}{|c|c|c|c|c|c|c|c|c|c|}
\hline Z & $===$ INLETS $====$ & eRam & Afs & Fram & \multirow{2}{*}{\multicolumn{2}{|c|}{ BLEEDS - interstg }} & & & \\
\hline Bs & Inlet & 1.0000 & 3560.18 & 10493.9 & & & $\begin{array}{l}\text { Wb/Win } \\
0.0368\end{array}$ & $\begin{array}{r}\text { BldWk } \\
0.5000\end{array}$ & $\begin{array}{r}\text { BldP } \\
0.2200\end{array}$ \\
\hline 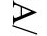 & $====$ DUCTS $====$ & dPnorm & MN & Aphy & \multicolumn{2}{|c|}{$\begin{array}{l}\text { HPT_COOLC HPC.C> } \\
\text { LPT COOLA HPC.C> }\end{array}$} & 0.0117 & 0.5000 & 0.4500 \\
\hline$\theta$ & TEGV & 0.0000 & 0.4720 & 860.21 & WB $2 \overline{\mathrm{x}}$ & HPC.B> & 0.0000 & 0.4500 & 0.2200 \\
\hline$z$ & FanDuct & 0.0000 & 0.5626 & 2481.93 & \multirow{2}{*}{$\begin{array}{l}\text { WB2Y } \\
\text { WBA2X }\end{array}$} & HPC.B> & 0.0000 & 0.7600 & 0.6200 \\
\hline & & & & & & HPC.B> & 0.0000 & 0.4500 & 0.2200 \\
\hline E & $==$ SPLITTERS $==$ & BPR & $\mathrm{dP} / \mathrm{P} 1$ & $\mathrm{dP} / \mathrm{P} 2$ & WBLKG & HPC.1> & 0.0000 & 1.0000 & 1.0000 \\
\hline & Splitter & 5.6367 & 0.0005 & 0.0005 & WBW2X & $\mathrm{HPC} . \mathrm{B}>$ & 0.0000 & 0.4500 & 0.2200 \\
\hline N & $===$ SHAFTS $====$ & Nmech & trg in & pwr in & \multicolumn{2}{|c|}{ BLEEDS - output } & Wb/Win & hscale & Pscale \\
\hline $\bar{\alpha}$ & HP Shaft & 11109.4 & 7268.9 & 15375.4 & \multirow{2}{*}{\multicolumn{2}{|c|}{$\begin{array}{ll}\text { WB17Y } & \text { Bleed }> \\
\text { HPT COOLA } & \text { Bleed }>\end{array}$}} & 0.0000 & 1.0000 & 1.0000 \\
\hline & LP_Shaft & 3675.2 & 17106.7 & 11970.5 & & & 0.1142 & 1.0000 & 1.0000 \\
\hline & & & & & $\begin{array}{l}\text { HPT } \\
\text { WB } 3 \overline{\mathrm{X}}-\mathrm{LO} B\end{array}$ & 3 Bleed> & 0.0999 & 1.0000 & 1.0000 \\
\hline & & & & & \multirow{2}{*}{$\begin{array}{l}\text { WB3 } 3 \overline{\mathrm{X}} \\
\text { WBA3X }\end{array}$} & Bleed> & 0.0000 & 1.0000 & 1.0000 \\
\hline & & & & & & Bleed> & 0.0000 & 1.0000 & 1.0000 \\
\hline & & & & & WBW3X & Bleed> & 0.0000 & 1.0000 & 1.0000 \\
\hline & & & & & WBFDLKG & FanDu> & 0.0000 & 1.0000 & 1.0000 \\
\hline & & & & & WB15X & Bleed> & 0.0000 & 1.0000 & 1.0000 \\
\hline & & & & & WB15Y & Bleed> & 0.0000 & 1.0000 & 1.0000 \\
\hline & & & & & WB17X & Bleed> & 0.0000 & 1.0000 & 1.0000 \\
\hline & $===$ BURNERS $===$ & TtOut & eff & dPnorm & & & & \\
\hline & Burner & 2609.41 & 0.9995 & 0.0230 & \multicolumn{2}{|c|}{$\begin{array}{rr}\text { Wfuel } & \text { FAR } \\
0.99241 & 0.02098\end{array}$} & & & \\
\hline & $===$ NOZZLES $===$ & $\mathrm{PR}$ & $\mathrm{Cfg}$ & CdTh & $\begin{array}{r}\mathrm{Cv} \\
9800\end{array}$ & Ath & MNth & Vact & $\mathrm{Fg}$ \\
\hline & Core_Nozz & 2.037 & 0.9811 & 1.0000 & \multirow{2}{*}{\multicolumn{2}{|c|}{$\begin{array}{l}0.9800 \\
0.9800\end{array}$}} & 1.000 & 1526.2 & 3274.0 \\
\hline$\Omega$ & Byp_Nōozz & 2.302 & 0.9815 & 1.0000 & & & 1.000 & 1030.8 & 13082.8 \\
\hline
\end{tabular}


Date:05/13/13 Time:10:01:44 Model: Turbofan Engine - COMDES ON Converge = 1 CASE: 0

Version:NPSS1.6.5 - Rev: -> Gas Package: Janaf iter/pass/Jacb/Broy=18/32/1/16 Run by: Philip C Jorgenson PC: 69

Temperature Stator 1 inlet: 480.59 Stator 1 exit: $497.03 \quad$ Stator 2 inlet: $517.20 \quad$ Stator 2 exit: 525.88 $\begin{array}{lll}\text { Stator } 3 \text { inlet: } 549.54 & \text { Stator } 3 \text { exit: } 558.12 & \text { Stator } 4 \text { inlet: } 580.49 \text { Stator } 4 \text { exit: } 588.3 \\ \text { Stator } 5 \text { inlet: } 606.27 & \text { Stator } 5 \text { exit: } 613.59 & \end{array}$

Ambient Relative Humidity $\quad 10.00$

Fan Bypass Relative Humidity $\quad 0.33$

LPC Inlet Relative Humidity $\quad 1.9$

$\begin{array}{ll}\text { LPC Exit Relative Humidity } & 0.03 \\ \text { HPC Relative Humidity } & 0.00\end{array}$

Drop Diameter

0.00
.0000250

Ambient Flow Velocity

0.0000250
793.07
3.46

Inlet Length

Ambient Static Pressure

Additional Water at LPC Exit

3.46
429.85

Fan/LPC Inlet Static $\quad 406.28$

0.0001646

$\begin{array}{rr} & 4.67 \\ \text { Fan/LPC Inlet Static Temperature } & 468.52\end{array}$

$\begin{array}{rrr}\text { MN } & \text { alt } & \text { dTamb } \\ 0.780 & 35000.0 & 36.00\end{array}$

$\begin{array}{lrr} & \text { SUMMARY OUTPUT DATA } \\ \text { W } & \text { Fn } & \text { TSFC }\end{array}$

Wfuel

VThS

$\begin{array}{ccclccc} & & & & & & \\ \text { TPR } & \text { EPR } & \text { PowerSet } & \text { T4 } & \text { T41 } & \text { T49 } \\ 793.07 & 25.469 & 1.269 & 69.900 & 2579.5 & 2426.8 & 1682.6\end{array}$

FSO Ambient.Fl_O

FS1 Inlet.Fl_O

$\begin{array}{lll}\text { FS12 } & \text { Splitter.F1_02 } \\ \text { FS2 } & \text { Splitter.Fl_01 }\end{array}$

FS14 Fan.FI_

FS23 LPC.FI-O

FS25 Bleed2.FI_O

$\begin{array}{ll}\text { FS25 } & \text { Bleed2.Fl } \\ \text { FS3 } & \text { HPC.F1 O }\end{array}$

FS36 Bleed3.F1_O

FS4 Burner.FI_O

FS45 HPT.FI_O

FS4 LPT.FI-

FS8 Core_Nozz.FI_O

FS17 FanDuctlkg.FI_O

FS171 Bleed15.F1_O

FS172 FanDuct. Fl-O

FS173 Byp_Nozz.Fí_

$W$
421.74
421.74
358.71
63.03
358.71
63.03
63.05
63.05
59.99
46.49
47.45
63.26
64.00
64.00
64.00
358.71
358.71
358.71
358.71

FLOW STATION DATA

\begin{tabular}{rrrrr}
\multicolumn{1}{c}{ Pt } & Tt & ht & FAR & Wc \\
5.170 & 482.28 & -15.41 & 0.0000 & 1156.04 \\
5.170 & 482.28 & -15.41 & 0.0000 & 1156.04 \\
5.167 & 482.28 & -15.41 & 0.0000 & 983.75 \\
5.167 & 482.28 & -15.41 & 0.0000 & 172.87 \\
7.887 & 550.56 & 0.97 & 0.0000 & 688.66 \\
12.183 & 636.10 & 21.54 & 0.0000 & 84.20 \\
12.183 & 636.35 & 20.65 & 0.0000 & 84.23 \\
12.183 & 636.35 & 20.65 & 0.0000 & 84.23 \\
131.669 & 1325.83 & 192.16 & 0.0000 & 10.70 \\
131.669 & 1325.83 & 192.16 & 0.0000 & 8.30 \\
128.637 & 2579.48 & 166.81 & 0.0206 & 12.09 \\
29.912 & 1690.18 & 3.23 & 0.0154 & 56.11 \\
6.875 & 1205.38 & -123.77 & 0.0152 & 208.57 \\
6.875 & 1205.45 & -123.77 & 0.0152 & 208.57 \\
6.875 & 1205.58 & -123.77 & 0.0152 & 208.59 \\
7.887 & 550.56 & 0.97 & 0.0000 & 688.66 \\
7.887 & 550.56 & 0.97 & 0.0000 & 688.66 \\
7.887 & 550.56 & 0.97 & 0.0000 & 688.66 \\
7.887 & 550.56 & 0.97 & 0.0000 & 688.66
\end{tabular}

PS
3.458
4.172
4.158
4.670
6.547
11.062
11.061
11.061
117.915
123.706
121.513
26.913
5.926
5.926
3.666
6.547
6.362
6.362
4.165

Ts
429.85
453.57
453.20
468.52
522.04
618.86
619.08
619.08
1287.56
1304.07
2546.01
1646.33
1159.02
1159.09
1019.59
522.04
517.78
517.78
458.70

$\begin{array}{rr}\text { Aphy } & \text { MN } \\ 3526.9 & 0.7800\end{array}$

$\begin{array}{ll}4168.2 & 0.5620 \\ 3531.8 & 0.5658\end{array}$

$\begin{array}{rr}3531.8 & 0.5658 \\ 830.5 & 0.3828\end{array}$

$\begin{array}{rr}2606.7 & 0.5226\end{array}$

$\begin{array}{ll}412.6 & 0.3741 \\ 412.6 & 0.3743\end{array}$

$\begin{array}{ll}412.6 & 0.3743 \\ 412.6 & 0.3743\end{array}$

$\begin{array}{ll}49.7 & 0.4054 \\ 49.3 & 0.3040\end{array}$

$\begin{array}{ll}49.3 & 0.3040 \\ 74.6 & 0.2989\end{array}$

$\begin{array}{ll}265.4 & 0.4009\end{array}$

$\begin{array}{ll}265.4 & 0.4009 \\ 860.2 & 0.4720\end{array}$

$\begin{array}{ll}860.2 & 0.4720 \\ 613.4 & 1.0000\end{array}$

$2606.7 \quad 0.5226$

$2481.9 \quad 0.5626$

$\begin{array}{ll}2481.9 & 0.5626\end{array}$

2006.91 .0000
WAR
0.0000871
0.0000871
0.0000871
0.0000871
0.0000871
0.0000871
0.0002517
0.0002517
0.0002517
0.0002517
0.0002517
0.0002517
0.0002517
0.0002517
0.0002517
0.0000871
0.0000871
0.0000871
0.0000871

Wair
421.71

$\begin{array}{lll}421.71 & 0.037 & 0.0001\end{array}$ $\begin{array}{lll}421.71 & 0.037 & 0.0001\end{array}$ $\begin{array}{lll}358.68 & 0.031 & 0.0001\end{array}$ $\begin{array}{lll}358.68 & 0.031 & 0.0001\end{array}$ $\begin{array}{lll}63.03 & 0.005 & 0.0001\end{array}$ $\begin{array}{lll}63.03 & 0.016-0.0003\end{array}$ $63.03=0.016-0.0003$ $\begin{array}{lll}59.97 & 0.015 & 0.0003\end{array}$ $\begin{array}{lll}46.48 & 0.012=0.0003\end{array}$ $\begin{array}{lll}46.48 & 0.012 & 0.0251\end{array}$

$\begin{array}{lll}62.29 & 0.016 & 0.0189\end{array}$

$\begin{array}{lll}62.29 & 0.016 & 0.0189 \\ 63.03 & 0.016 & 0.0187\end{array}$

$\begin{array}{lll}63.03 & 0.016 & 0.0187\end{array}$

$\begin{array}{lll}63.03 & 0.016 & 0.0187\end{array}$

$\begin{array}{lll}358.68 & 0.031 & 0.0001\end{array}$ $\begin{array}{lll}358.68 & 0.031 & 0.0001\end{array}$ $\begin{array}{lll}358.68 & 0.031 & 0.0001\end{array}$ 0.0001

\begin{tabular}{|c|c|c|c|c|c|c|c|c|c|}
\hline an & Wc & $P R$ & eff & Nc & TR & efPoly & pwr & SMN & SMW \\
\hline LPC & 983.75 & 1.526 & 0.9074 & 3779.750 & 1.1416 & 0.9127 & -8312.7 & 73.94 & 30.77 \\
\hline $\mathrm{PC}$ & 172.87 & 2.358 & 0.8699 & 3779.750 & 1.3189 & 0.8845 & -3295.1 & 4.57 & 3.57 \\
\hline $\mathrm{HPT}$ & .23 & 10.807 & 8615 & 9988.260 & 2.0835 & 0.8979 & -14927.3 & 35.15 & 33.72 \\
\hline $\mathrm{LPT}$ & $\begin{array}{l}12.09 \\
56.11\end{array}$ & $\begin{array}{l}4.30 \\
4.35\end{array}$ & .9078 & $\begin{array}{r}217.834 \\
88.654\end{array}$ & $\begin{array}{l}1.3556 \\
1.3996\end{array}$ & 0.8883 & 14927.3 & & \\
\hline$R B$ & JERY & DATA & & & & & & & \\
\hline & WcMap & PRmap & effMap & NcMap & R/Parm & s WcDes & S_PRdes & S effDes & S_NCDes \\
\hline an & 1143.02 & 1.514 & 000 & 3743.710 & 0.0578 & $\overline{0} .8607$ & $\overline{1} .0235$ & 0.9980 & $\overline{0} .9905$ \\
\hline PC & 139.07 & 2.366 & 0.8195 & 0.998 & 0.0000 & 1.2430 & 0.9938 & 1.0 & 0.0003 \\
\hline HPC & 76.61 & 10.345 & .8704 & 9721.979 & 12.7004 & 1.0995 & 1.0494 & 0.9 & 0.9733 \\
\hline $\mathrm{T}$ & & & .9078 & & & 12. 424 & 0.9723 & 1.0000 & 0.00 \\
\hline $\mathrm{PT}$ & 0.86 & 3.520 & 0.9185 & 0.997 & 3.5204 & 65.2590 & 0.7521 & 0.9926 & 0.0005 \\
\hline
\end{tabular}




\begin{tabular}{|c|c|c|c|}
\hline$===$ INLET $S===$ & eRam & Afs & Fram \\
\hline Inlet & 1.0000 & 3526.88 & 10395.7 \\
\hline$====$ DUCTS $====$ & dPnorm & MN & Aphy \\
\hline TEGV & 0.0000 & 0.4720 & 860.21 \\
\hline FanDuct & 0.0000 & 0.5626 & 2481.93 \\
\hline$==$ SPLITTERS $==$ & BPR & $\mathrm{dP} / \mathrm{P} 1$ & $\mathrm{dP} / \mathrm{P} 2$ \\
\hline Splitter & 5.6907 & 0.0005 & 0.0005 \\
\hline$===$ SHAFT $\mathrm{S}===$ & Nmech & trg in & pwr in \\
\hline HP Shaft & 11063.5 & 7086.4 & 14927.3 \\
\hline LP Shaft & 3644.7 & 16727.0 & 11607.8 \\
\hline
\end{tabular}

BLEEDS - interstg Wb/Win BldWk BldP $\begin{array}{llll}\text { HPT_COOLC HPC.C> } & 0.0368 & 0.5000 & 0.2200 \\ \text { LPT COOLA HPC.C } & 0.0117 & 0.5000 & 0.4500\end{array}$ $\begin{array}{lllll}\text { WB } 2 \overline{\mathrm{X}} & \text { HPC. } B> & 0.0000 & 0.4500 & 0.2200\end{array}$ $\begin{array}{lllll}\text { WB2Y HPC.B } & 0.0000 & 0.7600 & 0.6200\end{array}$ $\begin{array}{lllll}\text { WBA2X HPC.B> } & 0.0000 & 0.4500 & 0.2200\end{array}$ WBLKG HPC.I> $0.0000 \quad 1.0000 \quad 1.0000$ $\begin{array}{llll}\text { WBW2X HPC.B> } & 0.0000 & 0.4500 & 0.2200\end{array}$ BLEEDS - output Wb/Win hscale Pscale WB17Y Bleed> $0.0000 \quad 1.0000 \quad 1.0000$ HPT_COOLA Bleed> $0.1142 \quad 1.0000 \quad 1.0000$ $\begin{array}{lllll}\text { HPT COOLB } & \text { Bleed> } & 0.0999 & 1.0000 & 1.0000 \\ \text { WB } 3 \bar{X} & \text { Bleed> } & 0.0000 & 1.0000 & 1.0000\end{array}$ $\begin{array}{lllll}\text { WB3X } & \text { Bleed> } & 0.0000 & 1.0000 & 1.0000 \\ \text { WBA3X } & \text { Bleed }> & 0.0000 & 1.0000 & 1.0000\end{array}$ $\begin{array}{lllll}\text { WBA3X } & \text { Bleed }> & 0.0000 & 1.0000 & 1.0000 \\ \text { WBW3X } & \text { Bleed }> & 0.0000 & 1.0000 & 1.0000\end{array}$ $\begin{array}{lllll}\text { WBW3X } & \text { Bleed> } & 0.0000 & 1.0000 & 1.0000 \\ \text { WBFDLKG FanDu> } & 0.0000 & 1.0000 & 1.0000\end{array}$ $\begin{array}{lllll}\text { WBFDLKG } & \text { FanDu> } & 0.0000 & 1.0000 & 1.0000 \\ \text { WB15X } & \text { Bleed> } & 0.0000 & 1.0000 & 1.0000\end{array}$ $\begin{array}{lllll}\text { WB15X } & \text { Bleed> } & 0.0000 & 1.0000 & 1.0000 \\ \text { WB15Y } & \text { Bleed> } & 0.0000 & 1.0000 & 1.0000\end{array}$ Bleed> $0.0000 \quad 1.0000 \quad 1.0000$

$\begin{array}{rrrr}\text { W } & \text { Tt } & \text { ht } & \text { Pt } \\ 2.3201 & 987.62 & 106.41 & 29.912 \\ 0.7376 & 987.62 & 106.41 & 6.875 \\ 0.0000 & 953.02 & 97.83 & 38.470 \\ 0.0000 & 1165.26 & 151.00 & 86.264 \\ 0.0000 & 953.02 & 97.83 & 38.470 \\ 0.0000 & 1325.83 & 192.16 & 131.669 \\ 0.0000 & 953.02 & 97.83 & 38.470 \\ & & & \\ \text { W } & \text { Tt } & \text { ht } & \text { Pt } \\ 0.0000 & 636.35 & 20.65 & 12.183 \\ 7.1985 & 1325.83 & 192.16 & 128.637 \\ 6.2988 & 1325.83 & 192.16 & 72.166 \\ 0.0000 & 1325.83 & 192.16 & 131.669 \\ 0.0000 & 1325.83 & 192.16 & 131.669 \\ 0.0000 & 1325.83 & 192.16 & 131.669 \\ 0.0000 & 550.56 & 0.97 & 7.887 \\ 0.0000 & 550.56 & 0.97 & 7.887 \\ 0.0000 & 550.56 & 0.97 & 7.887 \\ 0.0000 & 550.56 & 0.97 & 7.887\end{array}$
$===$ BURNERS $===\quad$ TtOut eff
0.9995

dPnorm

Wfuel FAR

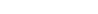

0.0230

0.95645

$$
.02058
$$

$\mathrm{PR}$
1.988

$\mathrm{Cfg}$
0.9808

CdTh
1.0000

$\mathrm{CV}$
0.9800

Ath
613.40

MNth Vact

$\mathrm{Fg}$
3145.6 
Date:07/05/13 Time:09:22:01 Model: $\quad$ Turbofan Engine - COMDES ON Converge $=1$ CASE: 0
Version:NPSS 1.6.5 - Rev: $\rightarrow \quad$ Gas Package: Janaf iter/pass/Jacb/Broy=29/57/2/26 Run by: Philip C Jorgenson

$\begin{array}{lllll} & \\ \text { Temperature Stator } 1 \text { inlet: } 449.01 & \text { Stator } 1 \text { exit: } 467.65 & \text { Stator } 2 \text { inlet: } 489.24 & \text { Stator } 2 \text { exit: } 496.53\end{array}$

Stator 3 inlet: 525.31 Stator 3 exit: 533.35 Stator anlet: 560.32 Stator 4 exit: 568.08

Ambient Relative Humidity $\quad 10.00$

Fan Face Relative Humidity $\quad 5.11$

E 0.16

LPC Exit Relative Humidity $\quad 0.02$

Drop Diameter

Ambient Flow Velocity

0.0000500

Inlet Length

Ambient Static Pressure

Ambient Static Temperature

Additional Water at IPC Exit

Fan/LPC Inlet

Flow Velocity $\quad 483.44$

$411.85 \quad$ Fan/LPC Inlet Static Temperature 442.61

$\begin{array}{lll}\text { MN alt dTamb SUMMARY OUTPUT DATA } & \\ 0.780 & \text { W } & \text { Fn }\end{array}$

$\begin{array}{llllll}0.780 & 35000.0 & 18.00 & 475.58 & 8100.1 & 0.5999\end{array}$

$\begin{array}{rr}\text { Wfuel } & \text { BPR } \\ 4859.43 & 5.2928\end{array}$

VTAS
776.30

$\mathrm{OPR}$

EPR
1.684

Powerset

$\begin{array}{ccr}\text { T4 } & \text { T41 } & \text { T49 } \\ 2818.7 & 2645.7 & 1838.6\end{array}$

\begin{tabular}{|c|c|}
\hline FSO & Ambient.Fl_O \\
\hline FS1 & Inlet.Fl_O \\
\hline S12 & Splitter.Fl_02 \\
\hline FS2 & Splitter.Fl_01 \\
\hline FS14 & Fan.Fl_O \\
\hline FS23 & LPC. $\mathrm{FI}_{-}^{-} \mathrm{O}$ \\
\hline FS24 & 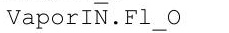 \\
\hline FS25 & Bleed2.FI_-̄ \\
\hline & HPC.Fl_O \\
\hline FS36 & Bleed3.FI_O \\
\hline FS4 & Burner.FI_O \\
\hline FS45 & HPT.FI_O \\
\hline FS 49 & LPT.FI_O \\
\hline FS5 & TEGV.F̄ \\
\hline FS\& & Core_Nozzz.Fl_O \\
\hline ES17 & FanDūctLkg.F̄̄ \\
\hline 17 & Bleed15.Fl_o \\
\hline S17 & FanDuct.FI_O \\
\hline & Byp_Nozz.Fi _ \\
\hline
\end{tabular}

W
475.58
475.58
400.01
75.58
400.01
75.58
75.58
75.58
71.91
55.73
57.08
76.04
76.93
76.93
76.93
400.01
400.01
400.01
400.01

FLOW STATION DATA

$\begin{array}{rrrrr}r \text { Pt } & \text { Tt } & \text { ht } & \text { FAR } & \text { Wc } \\ 5.170 & 462.10 & -19.92 & 0.0000 & 1276.02 \\ 5.170 & 462.10 & -19.92 & 0.0000 & 1276.02 \\ 5.167 & 462.10 & -19.92 & 0.0000 & 1073.78 \\ 5.167 & 462.10 & -19.92 & 0.0000 & 202.88 \\ 8.745 & 544.45 & -0.17 & 0.0000 & 688.68 \\ 12.667 & 625.29 & 19.26 & 0.0000 & 96.27 \\ 12.667 & 625.36 & 19.20 & 0.0000 & 96.28 \\ 12.667 & 625.36 & 19.20 & 0.0000 & 96.28 \\ 65.364 & 1379.14 & 207.17 & 0.0000 & 10.42 \\ 165.364 & 1379.14 & 207.17 & 0.0000 & 8.08 \\ 61.556 & 2818.71 & 176.88 & 0.0242 & 12.10 \\ 37.752 & 1847.45 & -1.29 & 0.0181 & 55.87 \\ 8.678 & 1323.57 & -141.15 & 0.0179 & 208.11 \\ 8.678 & 1323.64 & -141.15 & 0.0179 & 208.12 \\ 8.678 & 1323.77 & -141.15 & 0.0179 & 208.13 \\ 8.745 & 544.45 & -0.17 & 0.0000 & 688.68 \\ 8.745 & 544.45 & -0.17 & 0.0000 & 688.68 \\ 8.745 & 544.45 & -0.17 & 0.0000 & 688.68 \\ 8.745 & 544.45 & -0.17 & 0.0000 & 688.68\end{array}$

PS
3.458
3.838
3.865
4.445
7.260
11.081
11.081
11.081
149.116
155.925
152.620
34.006
7.485
7.485
4.641
7.260
7.055
7.055
4.619

Ts
411.85
424.34
425.25
442.61
516.24
601.91
601.98
601.98
1342.03
1357.95
2782.93
1801.00
1273.84
1273.90
1123.74
516.24
512.03
512.03
453.60

$\begin{array}{rr}\text { Aphy } & \text { MN } \\ 3892.7 & 0.7800 \\ 4168.2 & 0.6662 \\ 3531.8 & 0.6574 \\ 830.5 & 0.4686 \\ 2606.7 & 0.5226 \\ 412.6 & 0.4415 \\ 412.6 & 0.4415 \\ 412.6 & 0.4415 \\ 49.7 & 0.3928 \\ 49.3 & 0.2953 \\ 74.6 & 0.3003 \\ 265.4 & 0.4002 \\ 860.2 & 0.4724 \\ 860.2 & 0.4724 \\ 613.4 & 1.0000 \\ 2606.7 & 0.5226 \\ 2481.9 & 0.5625 \\ 2481.9 & 0.5625 \\ 2006.9 & 1.0000\end{array}$
1.39985
1.39819 1.39818 1.32100
1.34812 1.34811
1.34810 1.34810
1.39985 1.39985
1.39985 TURBOMACHINERY PERFORMANCE DATA

\begin{tabular}{|c|c|c|c|c|c|c|c|c|c|}
\hline \\
\hline & $\begin{array}{r}\text { WC } \\
\end{array}$ & $\mathrm{PR}$ & eff & Nc & TR & efPoly & pwr & SMN & SMW \\
\hline Fan & 1073.78 & 1.692 & 0.9109 & 4120.393 & 1.1782 & 0.9173 & -11176.3 & 38.42 & 23.46 \\
\hline LPC & 202.88 & 2.451 & 0.8264 & 4120.393 & 1.3532 & 0.8467 & -4189.4 & 2.96 & 2.22 \\
\hline $\mathrm{HPC}$ & 96.28 & 13.055 & 0.8586 & 10345.054 & 2.2053 & .8979 & -19611.7 & 26.28 & 25.48 \\
\hline $\mathrm{HPT}$ & 12.10 & 4.279 & 0.9118 & 213.958 & 1.3469 & 0.8911 & 19611.7 & & \\
\hline LPT & 55.87 & 4.350 & . 9155 & 90.484 & 1.3923 & 0.8986 & 15365.7 & & \\
\hline $\mathrm{TRI}$ & NER & $\mathrm{DA}$ & & & & & & & \\
\hline & WcMap & PRmap & effmap & NcMap & R/Parm & S_WcDes & S_PRdes & S_effDes & S_NCDes \\
\hline an & 1247.63 & 1.677 & 0.9128 & 4081.106 & 0.0636 & $\overline{0} .8607$ & $\overline{1} .0235$ & 0.9980 & $\overline{0} .9905$ \\
\hline PC & 165.08 & 2.727 & 0.8329 & 1.088 & 0.0000 & 1.2289 & 0.8404 & 0.9923 & 0.0003 \\
\hline${ }^{\mathrm{C}} \mathrm{C}$ & 87.57 & 12.487 & 0.8674 & 10069.262 & 13.4722 & 1.0995 & 1.0494 & 0.9898 & 0.9733 \\
\hline $\mathrm{PT}$ & 0.96 & 4.188 & 0.9118 & 1.286 & 4.1885 & 12.6299 & 0.9723 & 1.0000 & 0.0003 \\
\hline LPT & 0.86 & 3.520 & 0.9223 & 1.018 & 3.5200 & 65.2590 & 0.7521 & 0.9926 & 0.0005 \\
\hline
\end{tabular}
1.40081
1.40081 1.40081 1.40081 1.39818
1.35715 1.35715
1.35715 1.35715
1.28469 1.28469
1.32100

WAR Wair WH2O H2O frac $\begin{array}{llll}0.0000308 & 475.57 & 0.015 & 0.0000\end{array}$ $\begin{array}{llll}0.0000308 & 475.57 & 0.015 & 0.0000 \\ 0.0000308 & 399.99 & 0.012 & 0.0000\end{array}$ $\begin{array}{llll}0.0000308 & 75.57 & 0.002 & 0.0000\end{array}$ $\begin{array}{llll}0.0000308 & 399.99 & 0.012 & 0.0000\end{array}$ $\begin{array}{llll}0.0000308 & 75.57 & 0.002 & 0.0000\end{array}$ $\begin{array}{llll}0.0000435 & 75.57 & 0.003 & 0.0000\end{array}$ $\begin{array}{llll}0.0000435 & 75.57 & 0.003 & 0.0000\end{array}$ $\begin{array}{llll}0.0000435 & 71.91 & 0.003 & 0.0000\end{array}$ $\begin{array}{llll}0.0000435 & 55.73 & 0.002 & 0.0000\end{array}$ $\begin{array}{llll}0.0000435 & 55.73 & 0.002 & 0.0000\end{array}$ $\begin{array}{llll}0.0000435 & 74.69 & 0.003 & 0.0219\end{array}$ $\begin{array}{llll}0.0000435 & 75.57 & 0.003 & 0.0217\end{array}$ $\begin{array}{llll}0.0000435 & 75.57 & 0.003 & 0.0217 \\ 0.0000435 & 75.57 & 0.003 & 0.0217 \\ 0.000435 & 75.57 & 0.003 & 0.0217\end{array}$ $\begin{array}{llll}0.0000308 & 399.99 & 0.012 & 0.0000\end{array}$ $\begin{array}{llll}0.0000308 & 399.99 & 0.012 & 0.0000\end{array}$ $\begin{array}{llll}0.0000308 & 399.99 & 0.012 & 0.0000\end{array}$ $\begin{array}{lllll}1.39985 & 0.0000308 & 399.99 & 0.012 & 0.0000\end{array}$ 


\begin{tabular}{|c|c|c|c|}
\hline$===$ INLETS $====$ & eRam & Afs & Fram \\
\hline Inlet & 1.0000 & 3892.74 & 11474.9 \\
\hline$====$ DUCT $S====$ & dPnorm & MN & Aphy \\
\hline TEGV & 0.0000 & 0.4724 & 860.21 \\
\hline FanDuct & 0.0000 & 0.5625 & 2481.93 \\
\hline$==$ SPLITTERS $==$ & BPR & $\mathrm{dP} / \mathrm{P} 1$ & $\mathrm{dP} / \mathrm{P} 2$ \\
\hline Splitter & 5.2928 & 0.0005 & 0.0005 \\
\hline$===$ SHAFTS $====$ & Nmech & trq in & pwr in \\
\hline HP_Shaft & 11359.4 & 9067.7 & 19611.7 \\
\hline LP_Shaft & 3889.2 & 20750.4 & 15365.7 \\
\hline
\end{tabular}

BLEEDS - interstg Wb/Win BldWk BldP $\begin{array}{llll}\text { HPT_COOLC HPC.C }> & 0.0368 & 0.5000 & 0.2200\end{array}$ $\begin{array}{lllll}\text { LPT COOLA HPC.C> } & 0.0117 & 0.5000 & 0.4500 \\ \text { WB } 2 \bar{X} & \text { HPC. }> & 0.0000 & 0.4500 & 0.2200\end{array}$ $\begin{array}{lllll}\text { WB2X } & \text { HPC.B> } & 0.0000 & 0.4500 & 0.2200 \\ \text { WB2Y } & \text { HPC.B> } & 0.0000 & 0.7600 & 0.6200\end{array}$ $\begin{array}{lllll}\text { WB2Y } & \text { HPC.B } & 0.0000 & 0.7600 & 0.6200 \\ \text { WBA2X } & \text { HPC.B> } & 0.0000 & 0.4500 & 0.2200\end{array}$ $\begin{array}{lllll}\text { WBA2X HPC.B> } & 0.0000 & 0.4500 & 0.2200\end{array}$ $\begin{array}{lllll}\text { WBLKG } & \text { HPC.I> } & 0.0000 & 1.0000 & 1.0000 \\ \text { WBW2X } & \text { HPC.B } & 0.0000 & 0.4500 & 0.2200\end{array}$ BLEEDS - output Wb/Win hscale Pscale $\begin{array}{llll}\text { WB17Y Bleed> } 0.0000 & 1.0000 & 1.0000\end{array}$ HPT_COOLA Bleed> $0.1142 \quad 1.0000 \quad 1.0000$ $\begin{array}{lllll}\text { HPT COOLB } & \text { Bleed> } & 0.0999 & 1.0000 & 1.0000 \\ \text { WB 3 } 3 \text { X } & \text { Bleed> } & 0.0000 & 1.0000 & 1.0000\end{array}$ $\begin{array}{lllll}\text { WB3X } & \text { Bleed> } & 0.0000 & 1.0000 & 1.0000 \\ \text { WBA3X } & \text { Bleed> } & 0.0000 & 1.0000 & 1.0000\end{array}$ $\begin{array}{lllll}\text { WBA3X } & \text { Bleed> } & 0.0000 & 1.0000 & 1.0000 \\ \text { WBW3X } & \text { Bleed> } & 0.0000 & 1.0000 & 1.0000\end{array}$ $\begin{array}{lllll}\text { WBW3X } & \text { Bleed> } & 0.0000 & 1.0000 & 1.0000 \\ \text { WBFDLKG } & \text { FanDu> } & 0.0000 & 1.0000 & 1.0000\end{array}$ $\begin{array}{lllll}\text { WBFDLKG } & \text { FanDu> } & 0.0000 & 1.0000 & 1.0000 \\ \text { WB15X } & \text { Bleed }> & 0.0000 & 1.0000 & 1.0000 \\ \text { WB15Y } & \text { Bleed }> & 0.0000 & 1.0000 & 1.0000\end{array}$ $\begin{array}{lllll}\text { WB15X } & \text { Bleed }> & 0.0000 & 1.0000 & 1.0000 \\ \text { WB15Y } & \text { Bleed }> & 0.0000 & 1.0000 & 1.0000\end{array}$ WB17X Bleed> $0.0000 \quad 1.0000 \quad 1.0000$

$\begin{array}{rrrr}\text { W } & \text { Tt } & \text { ht } & \text { Pt } \\ 2.7812 & 1010.15 & 113.19 & 37.752 \\ 0.8842 & 1010.15 & 113.19 & 8.678 \\ 0.0000 & 972.31 & 103.79 & 46.260 \\ 0.0000 & 1204.14 & 162.06 & 107.339 \\ 0.0000 & 972.31 & 103.79 & 46.260 \\ 0.0000 & 1379.14 & 207.17 & 165.364 \\ 0.0000 & 972.31 & 103.79 & 46.260 \\ \text { W } & & & \\ 0.0000 & 625.36 & 19.20 & 12.667 \\ 8.6293 & 1379.14 & 207.17 & 161.556 \\ 7.5509 & 1379.14 & 207.17 & 90.740 \\ 0.0000 & 1379.14 & 207.17 & 165.364 \\ 0.0000 & 1379.14 & 207.17 & 165.364 \\ 0.0000 & 1379.14 & 207.17 & 165.364 \\ 0.0000 & 544.45 & -0.17 & 8.745 \\ 0.0000 & 544.45 & -0.17 & 8.745 \\ 0.0000 & 544.45 & -0.17 & 8.745 \\ 0.0000 & 544.45 & -0.17 & 8.745\end{array}$

$===$ BURNERS $===\quad$ TtOut Burner 2818.71

$\begin{array}{rr}\text { eff } & \text { dPnorm } \\ 0.9995 & 0.0230\end{array}$

Wfuel
1.34984 $\begin{array}{ll}44.45 & -0.17\end{array}$

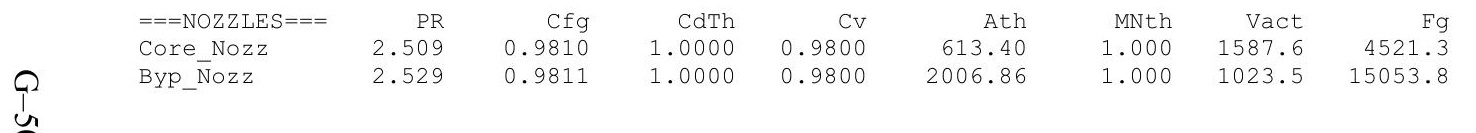


Date:07/05/13 Time:09:22:34 Model:_ Turbofan Engine - COMDES ON COnverge = 1 CASE: 0

Version:NPSS 1.6.5 - Rev: -> Gas Package: Janaf iter/pass/Jacb/Broy= 18/ 32/1/16 Run by: Philip C Jorgenson PC: 75

Temperature Stator 1 inlet: 458.63 Stator 1 exit: 474.87 Stator 2 inlet: 494.60 Stator 2 exit: 502.86 $\begin{array}{llll}\text { Stator } 3 \text { inlet: } 526.46 & \text { Stator } 3 \text { exit: } 534.71 & \text { Stator } 4 \text { inlet: } 557.04 \text { Stator } 4 \text { exit: } 564.60 \\ \text { Stator } 5 \text { inlet: } 582.44 & \text { Stator } 5 \text { exit: } 589.57 & \text { Unblocked Percent Blockage: } 0.00\end{array}$

Ambient Relative Humidity $\quad 10.00$

Fan Face Relative Humidity $\quad 3.58$

Fan Bypass Relative Humidity 0.24

LPC Init Relative Humidity $\quad 0.86$

HPC Exit Relative Humidity

0.00

0.0000500

776.30

Inlet Length

40.00

Ambient Static Pressur

Ambient Static Temperature
Additional Water at LPC Exit

31.46 0.0000162

Fan/LPC Inlet Static Temperature 447.77

SUMMARY OUTPUT DATA

$\begin{array}{rrr}\text { MN } & \text { alt } & \text { dTamb } \\ 0.780 & 35000.0 & 18.00\end{array}$

W39.17

Fn
6082.8

TSFC
0.5942

Wfuel
3614.58

BPR
5.5824

VTAS
776.30

OPR
26.748

EPR
1.342

Powerset

$\begin{array}{ccc}\text { T4 } & \text { T41 } & \text { T49 } \\ 2543.5 & 2391.4 & 1654.7\end{array}$

FSO Ambient.Fl_O

FS1 Inlet.Fl_O

$\begin{array}{ll}\text { FS12 } & \text { Splitter.Fl_02 } \\ \text { FS2 Splitter.Fl_01 }\end{array}$

FS14 Fan.Fl_O

FS23 LPC.FI_O

FS25 Bleed2.Fl-

FS3 HPC.Fl O

FS36 Bleed3.F1_O

FS4 Burner.Fl_

FS45 HPT.FI_

$\begin{array}{ll}\text { FS49 } & \text { LPT.FI- } \\ \text { FS5 } & \text { TEGV.FI }\end{array}$

FS8 Core_Nozz.FI_O

FS17 FanDuctLkg.Fi_o

FS171 Bleed15.FI_O

FS172 FanDuct. FI_O

FS173 Byp Nozz. Fi

$W$
439.17
439.17
372.45
66.72
372.45
66.72
66.72
66.72
63.48
49.20
50.20
66.94
67.72
67.72
67.72
372.45
372.45
372.45
372.45

FLOW STATION DATA

TURBOMACHINERY PERFORMANCE DATA

$\begin{array}{lrrrrrrrrr} & \text { Wc } & \text { PR } & \text { eff } & \text { NC } & \text { TR } & \text { efPoly } & \text { pwr } & \text { SMN } & \text { SMW } \\ \text { Fan } & 999.81 & 1.556 & 0.9070 & 3841.927 & 1.1486 & 0.9126 & -8672.9 & 62.44 & 29.36 \\ \text { LPC } & 179.10 & 2.384 & 0.8662 & 3841.927 & 1.3252 & 0.8814 & -3404.6 & 4.01 & 3.17 \\ \text { HPC } & 86.49 & 11.226 & 0.8614 & 10052.195 & 2.1118 & 0.8984 & -15551.3 & 33.25 & 31.96 \\ \text { HPT } & 12.09 & 4.302 & 0.9079 & 216.586 & 1.3570 & 0.8887 & 15551.3 & & \\ \text { LPT } & 56.08 & 4.358 & 0.9114 & 88.946 & 1.4014 & 0.8940 & 12077.5 & \end{array}$

TURBOMACHINERY MAP DATA

\begin{tabular}{|c|c|c|c|c|c|c|c|c|c|}
\hline & WcMa & PRmap & effmap & NcMap & R/Parm & $\mathrm{s}$ WCDes & s PRdes & $s$ effDes & S_NCDes \\
\hline an & 1161.68 & 1.543 & 0.9088 & 3805.294 & 0.0589 & $\overline{0} .8607$ & $\overline{1} .0235$ & 0.9980 & $\overline{0} .9905$ \\
\hline & 143.07 & 2.4 & 0.8207 & & & & & 1.0554 & \\
\hline PC & 78.67 & 10.744 & 0.8703 & 784.210 & 12.8566 & 1.0995 & 1.0494 & 0.9898 & 0.9733 \\
\hline דו ד & 0.96 & & & & & & & & \\
\hline $\mathrm{PT}$ & 0.86 & 3.526 & 0.9181 & 1.001 & 3.5256 & 65.2590 & 0.7521 & 0.9926 & 0.0005 \\
\hline
\end{tabular}




$\begin{array}{lrrr}===\text { INLETS }==== & \begin{array}{r}\text { eRam } \\ 1.0000\end{array} & \begin{array}{r}\text { Afs } \\ \text { Inlet }\end{array} & \begin{array}{r}\text { Fram } \\ 1594.69\end{array} \\ \begin{array}{l}10596.3 \\ ===\text { DUCTS }==== \\ \text { dPnorm }\end{array} & \text { MN } & \text { Aphy } \\ \text { TEGV } & 0.0000 & 0.4719 & 860.21 \\ \text { FanDuct } & 0.0000 & 0.5625 & 2481.93 \\ & & & \\ ==\text { SPLITTERS }== & \text { BPR } & \mathrm{dP} / \mathrm{P} \mathrm{1} & \mathrm{dP} / \mathrm{P} \mathrm{2} \\ \text { Splitter } & 5.5824 & 0.0005 & 0.0005 \\ & & & \\ ===\text { SHAFTS }==== & \text { Nmech } & \text { trq in } & \text { pwrin } \\ \text { HP_Shaft } & 10923.1 & 7477.5 & 15551.3 \\ \text { LP_Shaft } & 3626.3 & 17492.0 & 12077.5\end{array}$

BLEEDS - interstg Wb/Win BldWk BldP $\begin{array}{llll}\text { HPT_COOLC HPC.C> } & 0.0368 & 0.5000 & 0.2200 \\ \text { LPT_COOLA HPC.C> } & 0.0117 & 0.5000 & 0.4500\end{array}$ $\begin{array}{lllll}\text { LPT COOLA HPC.C> } & 0.0117 & 0.5000 & 0.4500 \\ \text { WB } 2 \overline{\mathrm{X}} & \text { HPC. B> } & 0.0000 & 0.4500 & 0.2200\end{array}$ $\begin{array}{lllll}\text { WB2Y HPC.B> } & 0.0000 & 0.7600 & 0.6200\end{array}$ $\begin{array}{lllll}\text { WBA2X HPC.B } & 0.0000 & 0.4500 & 0.2200\end{array}$

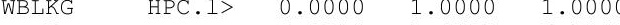
$\begin{array}{llll}\text { WBW2X HPC.B> } & 0.0000 & 0.4500 & 0.2200\end{array}$ BLEEDS - output Wb/Win hscale Pscale WB17Y Bleed> $0.0000 \quad 1.0000 \quad 1.0000$ HPT_COOLA Bleed> $0.1142 \quad 1.0000 \quad 1.0000$ $\begin{array}{lllll}\text { HPT COOLB } & \text { Bleed> } & 0.0999 & 1.0000 & 1.0000 \\ \text { WB } 3 \bar{X} & \text { Bleed } & 0.0000 & 1.0000 & 1.0000\end{array}$

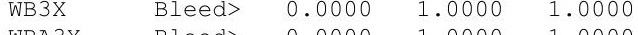
$\begin{array}{lllll}\text { WBA3X } & \text { Bleed> } & 0.0000 & 1.0000 & 1.0000 \\ \text { WBW3X } & \text { Bleed }> & 0.0000 & 1.0000 & 1.0000\end{array}$ $\begin{array}{lllll}\text { WBW3X } & \text { Bleed } & 0.0000 & 1.0000 & 1.0000 \\ \text { WBFDLKG FanDu> } & 0.0000 & 1.0000 & 1.0000\end{array}$ $\begin{array}{lllll}\text { WBFDLKG } & \text { FanDu> } & 0.0000 & 1.0000 & 1.0000 \\ \text { WB15X } & \text { Bleed }> & 0.0000 & 1.0000 & 1.0000\end{array}$ $\begin{array}{lllll}\text { WB15X } & \text { Bleed> } & 0.0000 & 1.0000 & 1.0000 \\ \text { WB15Y } & \text { Bleed> } & 0.0000 & 1.0000 & 1.0000\end{array}$ WB17X Bleed> $0.0000 \quad 1.0000 \quad 1.0000$

$\begin{array}{rrrr}\text { W } & \text { Tt } & \text { ht } & \text { Pt } \\ 2.4553 & 959.07 & 100.49 & 31.404 \\ 0.7806 & 959.07 & 100.49 & 7.206 \\ 0.0000 & 924.89 & 92.05 & 40.031 \\ 0.0000 & 1134.62 & 144.39 & 90.417 \\ 0.0000 & 924.89 & 92.05 & 40.031 \\ 0.0000 & 1293.34 & 184.91 & 138.285 \\ 0.0000 & 924.89 & 92.05 & 40.031 \\ & & & \\ \text { W } & \mathrm{Tt} & \mathrm{ht} & \mathrm{Pt} \\ 0.0000 & 612.43 & 16.07 & 12.318 \\ 7.6180 & 1293.34 & 184.91 & 135.100 \\ 6.6659 & 1293.34 & 184.91 & 75.786 \\ 0.0000 & 1293.34 & 184.91 & 138.285 \\ 0.0000 & 1293.34 & 184.91 & 138.285 \\ 0.0000 & 1293.34 & 184.91 & 138.285 \\ 0.0000 & 530.74 & -3.46 & 8.040 \\ 0.0000 & 530.74 & -3.46 & 8.040 \\ 0.0000 & 530.74 & -3.46 & 8.040 \\ 0.0000 & 530.74 & -3.46 & 8.040\end{array}$

$===$ BURNERS $===\quad$ TtOut eff
0.9995

dPnorm

Wfuel FAR

Burner 2543.45

0.90 
Date:07/05/13 Time:09:23:08 Model: Turbofan Engine - COMDES ON COnverge = 1 CASE: O

Version:NPSS_1.6.5 - Rev: -> Gas Package: Janaf iter/pass/Jacb/Broy=19/33/1/17 Run by: Philip C Jorgenson PC: 72

Temperature Stator 1 inlet: 459.57 Stator 1 exit: 475.57 Stator 2 inlet: 495.09 Stator 2 exit: 503.39

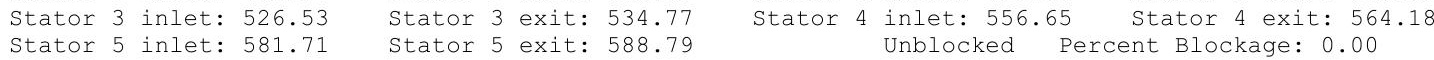

Ambient Relative Humidity $\quad 10.00$

$\begin{array}{ll}\text { Fan Face Relative Humidity } & 3.46 \\ \text { Fan Bypass Relative Humidity } & 0.25\end{array}$

$\begin{array}{ll}\text { Fan Bypass Relative Humidity } & 0.25 \\ \text { LPC Inlet Relative Humidity } & 1.81\end{array}$

LPC Exit Relative Humidity $\quad 0.02$

HPC Relative Hunidity

Drop Diame Humidity

0.0000500

Ambient Flow Velocity

Ambient Static Pressure

Additional Water at LPC Exit

776.30

Inlet Length

3.46 Fan/LPC Inlet Static Pressure $\quad 4.65$ 0.0000168

\section{SUMMARY OUTPUT DATA}

$\begin{array}{rrr}\text { MN } & \text { alt } & \text { dTamb } \\ 0.780 & 35000.0 & 18.00\end{array}$
434.93
5854. TSFC Wfuel

\section{FLOW STATION DATA}

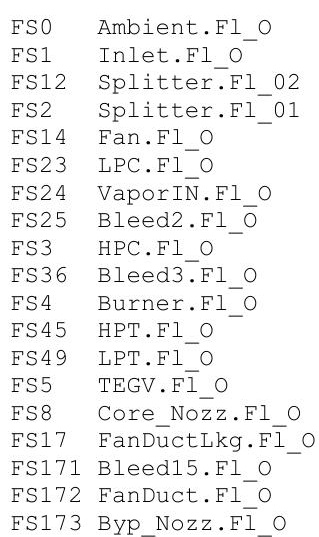

$W$
434.93
434.93
369.36
65.57
369.36
65.57
65.58
65.58
62.40
48.36
49.32
65.78
66.54
66.54
66.54
369.36
369.36
369.36
369.36

W
3

$$
\begin{array}{cc}
\text { Pt } & \text { Tt } \\
5.170 & 462.10
\end{array}
$$$$
5.170
$$

5.170
5.167

5.167
5.167

5.167
7.960
12.254

12.254

12.254
12.254

135.064

135.064
135.064

131.953

30.657
7.034

7.034
7.034
7.034

7.034
7.960

7.960

7.960
7.960

462.10
462.10
462.10

$\begin{array}{llll}462.10 & -19.92 & 0.0000 & 991.51\end{array}$

$\begin{array}{rrrr}462.10 & -19.92 & 0.0000 & 176.03 \\ 529.11 & -3.85 & 0.0000 & 688.70\end{array}$

610.98

611.04

611.04
1283.78

1283.78

2513.10

1642.24
1168.97

$-124.330 .0147$

$\begin{array}{lll}169.04 & -124.33 & 0.0147 \\ 529.18 & -124.33 & 0.0147\end{array}$

$\begin{array}{lrr}529.11 & -3.85 & 0.0000\end{array}$

$\begin{array}{lll}529.11 & -3.85 & 0.0000 \\ 529.11 & -3.85 & 0.0000\end{array}$

$\begin{array}{llll}529.11 & -3.85 & 0.0000\end{array}$

$\begin{array}{lll}-3.85 & 0.0000 & 688.7\end{array}$

\begin{tabular}{|c|c|c|c|c|c|c|c|c|c|}
\hline Fan & $\begin{array}{r}\text { Wc } \\
991.51\end{array}$ & $\begin{array}{r}\mathrm{PR} \\
1.541\end{array}$ & $\begin{array}{r}\text { eff } \\
0.9070\end{array}$ & $\begin{array}{r}\text { NC } \\
3810.868\end{array}$ & $\begin{array}{r}\mathrm{TR} \\
1.1450\end{array}$ & $\begin{array}{l}\text { efPoly } \\
0.9125\end{array}$ & $\begin{array}{r}\text { pwr } \\
-8395.8\end{array}$ & $\begin{array}{r}\text { SMN } \\
67.58\end{array}$ & $\begin{array}{r}\text { SMW } \\
30.09\end{array}$ \\
\hline LPC & 176.03 & 2.371 & 0.8684 & 3810.868 & 1.3222 & 0.8833 & -3315.3 & 4.29 & 3.38 \\
\hline HPC & 85.36 & 11.022 & .8615 & 10020.329 & 2.1010 & 0.8982 & -15091.9 & 34.11 & 32.76 \\
\hline HPT & 12.09 & 4.3 & & 216.954 & 1.3579 & 0.8878 & 15091.9 & & \\
\hline LPT & 56.1 & 4.358 & 107 & 88.762 & 1.4022 & 0.8932 & 11711.1 & & \\
\hline $\mathrm{UR}$ & INERY I & DATA & & & & & & & \\
\hline & WcMap & PRmap & effMap & NcMap & R/Parm & S_WcDes & S_PRdes & S_effDes & S_NcDes \\
\hline an & 1152.04 & 1.528 & & 3774.531 & 583 & $\overline{0} .8607$ & $\overline{1} .0235$ & 0.9980 & $\overline{0} .9905$ \\
\hline LPC & 141.07 & 2.398 & .8201 & 1.007 & 0.0000 & 1.2478 & 0.9813 & 1.0588 & 0.0003 \\
\hline HPC & 77.63 & 10.550 & 0.8704 & 9753.193 & 12.7857 & 1.0995 & 1.0494 & 0.9898 & 0.9733 \\
\hline PT & & 4.213 & 0.9073 & 1.304 & 4.2126 & 12.6290 & 0.9723 & 1.0000 & 0.0003 \\
\hline$\angle P T$ & 0.86 & 3.526 & 0.9175 & 0.998 & 3.5259 & 65.2590 & 0.7521 & 0.9926 & 0.0005 \\
\hline
\end{tabular}




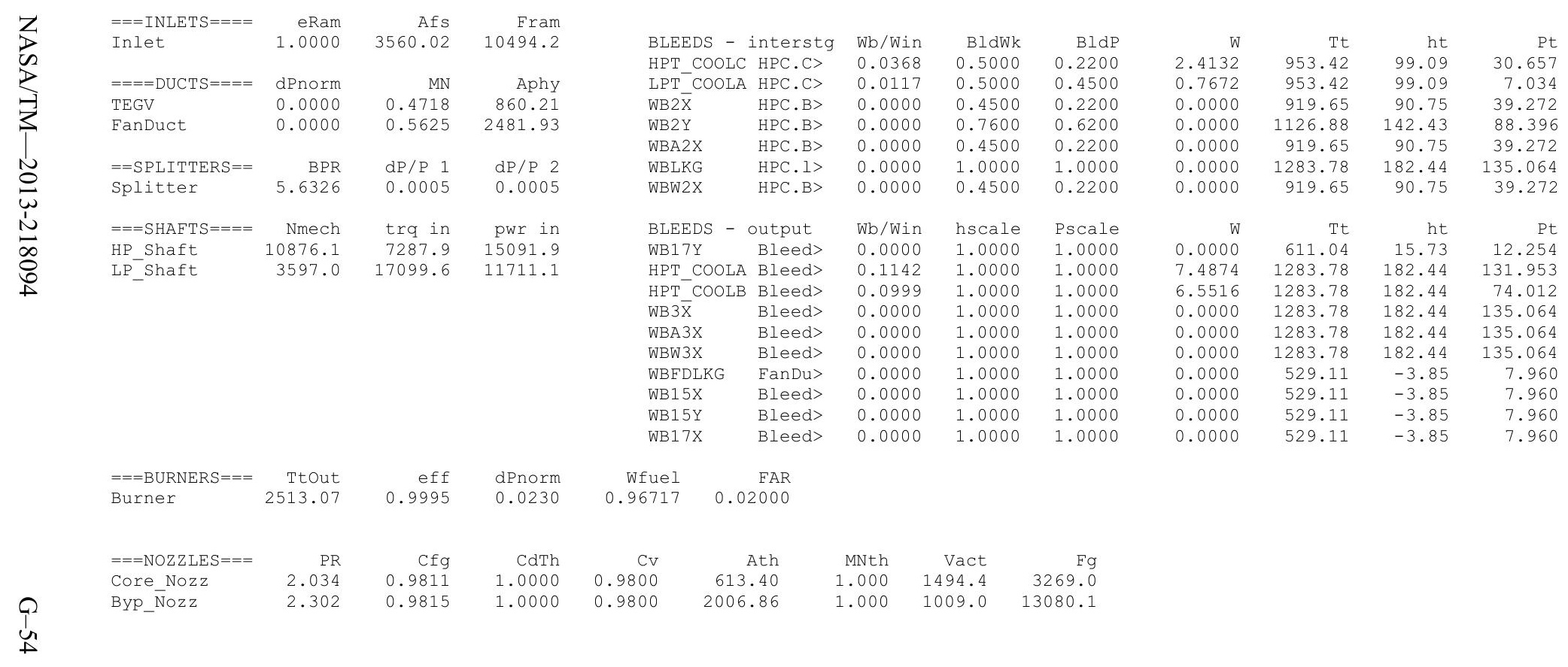


Date: 07/05/13 Time:09:23:40 Model: COMDES ON Converge = 1 CASE: 0

Version:NPSS_1.6.5 - Rev: -> Gas Package: Janaf iter/pass/Jacb/Broy=18/32/1/16 Run by: Philip C Jorgenson PC: 69

Temperature Stator 1 inlet: 460.42 Stator 1 exit: 476.16 Stator 2 inlet: 495.46 Stator 2 exit: 503.76

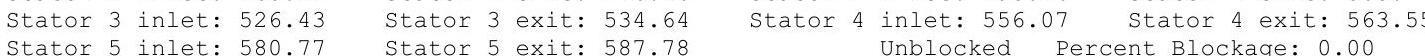

Ambient Relative Humidity $\quad 10.00$

Fan Face Relative Humidity $\quad 3.36$

Fan Bypass Relative Humidity 0.26

LPC Inlet Relative Humidity $\quad 1.77$

LPC Exit Relative Humidity $\quad 0.02$

HPC Relative Humidity $\quad 0.00$

Ambient Flow Velocity

Ambient Static Pressure

Additional Water at LPC Exit

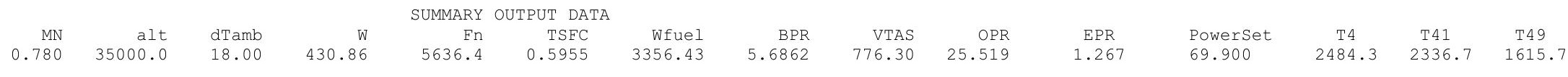

FS0 Ambient.F1_O

$\begin{array}{ll}\text { FS1 Inlet.FI_O } & 430.86 \\ \text { FS12 } & 430.86\end{array}$

FS12 Splitter.F1_02 366.42

FS2 Splitter.F1_0

FS14 Fan.F1_O

$\begin{array}{ll}\text { FS23 } & \text { LPC.FI } \\ \text { FS24 VaporIN.FI }\end{array}$

FS25 Bleed2.FI_O

FS3 HPC.FI_O

FS36 Bleed3.F1_O

FS4 Burner.Fl-

$\begin{array}{lll}\text { FS45 } & \text { HPT.F1_O } \\ \text { FS49 } & \text { LPT.FI- }\end{array}$

FS5 TEGV.FI_O

FS8 Core_Nozz.Fl_O

FS17 FanDüctLkg.Fì_O

FS171 Bleed15.F1_O

FS172 FanDuct.Fl-O
FS173 Byp Nozz. FI

TURBOMACHINERY PERFORMANCE DATA

$\begin{array}{lrrr} & \text { WC } & \text { PR } & \text { eff } \\ \text { Fan } & 983.64 & 1.526 & 0.9074 \\ \text { LPC } & 172.99 & 2.356 & 0.8699 \\ \text { HPC } & 84.34 & 10.839 & 0.8615 \\ \text { HPT } & 12.09 & 4.307 & 0.9067 \\ \text { LPT } & 56.13 & 4.358 & 0.9100\end{array}$

FLOW STATION DATA

$\begin{array}{rrrr}\text { FLOW } & \text { STATION DATA } & \text { ht } \\ \text { Pt } & \text { Tt } & \text { ht } & \text { FAR } \\ 5.170 & 462.10 & -19.92 & 0.0000 \\ 5.170 & 462.10 & -19.92 & 0.0000 \\ 5.167 & 462.10 & -19.92 & 0.0000 \\ 5.167 & 462.10 & -19.92 & 0.0000 \\ 7.885 & 527.52 & -4.23 & 0.0000 \\ 12.172 & 609.42 & 15.44 & 0.0000 \\ 12.172 & 609.49 & 15.36 & 0.0000 \\ 12.172 & 609.49 & 15.36 & 0.0000 \\ 31.932 & 1274.55 & 180.06 & 0.0000 \\ 31.932 & 1274.55 & 180.06 & 0.0000 \\ 18.894 & 2484.30 & 156.16 & 0.0196 \\ 29.926 & 1622.95 & -1.15 & 0.0146 \\ 6.867 & 1154.72 & -122.78 & 0.0145 \\ 6.867 & 1154.79 & -122.78 & 0.0145 \\ 6.867 & 1154.93 & -122.78 & 0.0145 \\ 7.885 & 527.52 & -4.23 & 0.0000 \\ 7.885 & 527.52 & -4.23 & 0.0000 \\ 7.885 & 527.52 & -4.23 & 0.0000 \\ 7.885 & 527.52 & -4.23 & 0.0000\end{array}$

Wc
1156.04
1156.04
983.64
172.99
688.70
84.33
84.34
84.34
10.71
8.30
12.09
56.13
208.77
208.77
208.78
688.70
688.70
688.70
688.70

Ps
3.458
4.172
4.158
4.670
6.546
11.049
11.048
11.048
118.149
123.952
121.744
26.924
5.917
5.917
3.658
6.546
6.361
6.361
4.164

TS
411.85
434.59
434.24
448.89
500.18
592.82
592.89
592.89
1237.52
1253.50
2451.73
1580.48
1109.91
1109.97
975.31
500.18
496.10
496.10
439.47

$\begin{array}{rr}\text { Aphy } & \text { MN } \\ 3526.7 & 0.7800 \\ 4168.2 & 0.5619 \\ 3531.8 & 0.5656 \\ 830.5 & 0.3830 \\ 2606.7 & 0.5226 \\ 412.6 & 0.3747 \\ 412.6 & 0.3747 \\ 412.6 & 0.3747 \\ 49.7 & 0.4049 \\ 49.3 & 0.3036 \\ 74.6 & 0.2985 \\ 265.4 & 0.4005 \\ 860.2 & 0.4718 \\ 860.2 & 0.4718 \\ 613.4 & 1.0000 \\ 2606.7 & 0.5226 \\ 2481.9 & 0.5625 \\ 2481.9 & 0.5625 \\ 2006.9 & 1.0000\end{array}$

gamt
1.40081
1.40081
1.40081
1.40081
1.40011
1.39858
1.39858
1.39858
1.36344
1.36344
1.29739
1.33363
1.36099
1.36098
1.36097
1.40011
1.40011
1.40011
1.40011

WAR
0.0000308 0.0000308
0.0000308 0.0000308

0.0000308

0.0000308

0.0000482

0.0000482

0.0000482

0.0000482

0.0000482

0.0000482

0.0000482

0.0000482
0.0000482
0.0000308

0.0000482
0.0000308
0.0000308

0.0000308

0.0000308

0.0000308
H2O frac 0.0000 0.0000 0.0000 0.0000
0.0000 0.0000 0.0000 0.0000
0.00000 0.0000 0.0000
0.0238 0.0178 0.0178 0.0176 0.0177 0.0000 0.0000 0.0000 0.0000

TURBOMACHINERY MAP DATA

\begin{tabular}{|c|c|c|c|c|c|c|c|c|c|}
\hline & WcMap & PRmap & effMap & NcMap & $\mathrm{R} /$ Parm & s WcDes & S PRdes & $s$ effDes & S NCDes \\
\hline Fan & 1142.89 & 1.514 & 0.9092 & 3743.156 & 0.0578 & $\overline{0} .8607$ & $\overline{1} .0235$ & 0.9980 & 0.9905 \\
\hline LPC & 139.03 & 2.366 & 0.8195 & 0.998 & 0.0000 & 1.2442 & 0.9926 & 1.0615 & 0.0003 \\
\hline HPC & 76.71 & 10.375 & 0.8704 & 9725.294 & 12.7209 & 1.0995 & 1.0494 & 0.9898 & 0.9733 \\
\hline HPT & 0.96 & 4.215 & 0.9067 & 1.306 & 4.2155 & 12.6299 & 0.9723 & 1.0000 & .0003 \\
\hline LPT & 0.86 & 3.526 & 0.9168 & 0.996 & 3.5258 & 65.2590 & 0.7521 & 0.9926 & 0.0005 \\
\hline
\end{tabular}




\begin{tabular}{|c|c|c|c|}
\hline$===$ INLETS $====$ & eRam & Afs & Fram \\
\hline Inlet & 1.0000 & 3526.73 & 10396.0 \\
\hline$====$ DUCTS $====$ & dPnorm & MN & Aphy \\
\hline TEGV & 0.0000 & 0.4718 & 860.21 \\
\hline FanDuct & 0.0000 & 0.5625 & 2481.93 \\
\hline$==$ SPLITTERS $==$ & BPR & $\mathrm{dP} / \mathrm{P} 1$ & $\mathrm{dP} / \mathrm{P} 2$ \\
\hline Splitter & 5.6862 & 0.0005 & 0.0005 \\
\hline$===$ SHAFTS $====$ & Nmech & trq in & pwr in \\
\hline HP_Shaft & 10831.1 & 7105.3 & 14652.9 \\
\hline LP_Shaft & 3567.1 & 16720.3 & 11356.1 \\
\hline
\end{tabular}

BLEEDS - interstg Wb/Win BldWk BldP $\begin{array}{llll}\text { HPT_COOLC HPC.C> } & 0.0368 & 0.5000 & 0.2200 \\ \text { LPT COOLA HPC.C } & 0.0117 & 0.5000 & 0.4500\end{array}$ $\begin{array}{llllll}\text { WB } 2 \bar{X} & \text { HPC. B }> & 0.0000 & 0.4500 & 0.2200\end{array}$ $\begin{array}{llllll}\text { WB2Y HPC.B } & 0.0000 & 0.7600 & 0.6200\end{array}$ $\begin{array}{llll}\text { WBA2X HPC.B> } & 0.0000 & 0.4500 & 0.2200\end{array}$ WBLKG HPC.I> $0.0000 \quad 1.0000 \quad 1.0000$ $\begin{array}{lllll}\text { WBW2X HPC.B> } & 0.0000 & 0.4500 & 0.2200\end{array}$ BLEEDS - output Wb/Win hscale Pscale WB17Y Bleed> $0.0000 \quad 1.0000 \quad 1.0000$ HPT_COOLA Bleed> $0.1142 \quad 1.0000 \quad 1.0000$ $\begin{array}{lllll}\text { HPT COOLB } & \text { Bleed> } & 0.0999 & 1.0000 & 1.0000 \\ \text { WB } 3 \bar{X} & \text { Bleed }> & 0.0000 & 1.0000 & 1.0000\end{array}$ $\begin{array}{llll}\text { WB3X } & \text { Bleed }>0.0000 & 1.0000 & 1.0000\end{array}$ $\begin{array}{lllll}\text { WBA3X } & \text { Bleed> } & 0.0000 & 1.0000 & 1.0000 \\ \text { WBW3X } & \text { Bleed }> & 0.0000 & 1.0000 & 1.0000\end{array}$ $\begin{array}{lllll}\text { WBW3X } & \text { Bleed> } & 0.0000 & 1.0000 & 1.0000 \\ \text { WBFDLKG } & \text { FanDu> } & 0.0000 & 1.0000 & 1.0000\end{array}$ $\begin{array}{lllll}\text { WBFDLKG } & \text { FanDu> } & 0.0000 & 1.0000 & 1.0000 \\ \text { WB15X } & \text { Bleed }> & 0.0000 & 1.0000 & 1.0000\end{array}$ $\begin{array}{lllll}\text { WB15X } & \text { Bleed> } & 0.0000 & 1.0000 & 1.0000 \\ \text { WB15Y } & \text { Bleed> } & 0.0000 & 1.0000 & 1.0000\end{array}$ WB17X Bleed> $0.0000 \quad 1.0000 \quad 1.0000$

$\begin{array}{rrrr}\text { W } & \text { Tt } & \text { ht } & \text { Pt } \\ 2.3714 & 947.85 & 97.71 & 29.926 \\ 0.7540 & 947.85 & 97.71 & 6.867 \\ 0.0000 & 914.48 & 89.47 & 38.519 \\ 0.0000 & 1119.37 & 140.53 & 86.423 \\ 0.0000 & 914.48 & 89.47 & 38.519 \\ 0.0000 & 1274.55 & 180.06 & 131.932 \\ 0.0000 & 914.48 & 89.47 & 38.519 \\ & & & \\ \text { W } & T t & h t & \text { Pt } \\ 0.0000 & 609.49 & 15.36 & 12.172 \\ 7.3579 & 1274.55 & 180.06 & 128.894 \\ 6.4384 & 1274.55 & 180.06 & 72.284 \\ 0.0000 & 1274.55 & 180.06 & 131.932 \\ 0.0000 & 1274.55 & 180.06 & 131.932 \\ 0.0000 & 1274.55 & 180.06 & 131.932 \\ 0.0000 & 527.52 & -4.23 & 7.885 \\ 0.0000 & 527.52 & -4.23 & 7.885 \\ 0.0000 & 527.52 & -4.23 & 7.885 \\ 0.0000 & 527.52 & -4.23 & 7.885\end{array}$
$===$ BURNERS $===\quad$ TtOut Burner 2484.27

eff
0.9995

dPnorm FAR

$0.93234 \quad 0.01962$

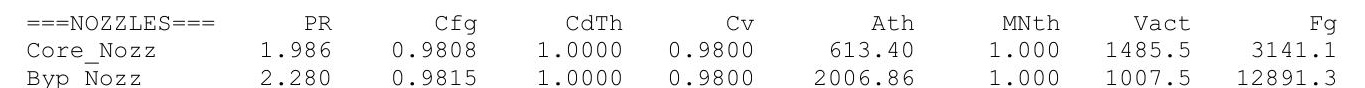


$\begin{array}{lll}\text { Date:07/05/13 Time:09:33:02 Model: } & \text { Turbofan Engine - COMDES ON COnverge = 1 CASE: O }\end{array}$

Version:NPSS1.6.5 - Rev: -> Gas Package: Janaf iter/pass/Jacb/Broy=28/56/2/25 Run by: Philip C Jorgenson PC: 100

Temperature Stator 1 inlet: 468.73 Stator 1 exit: 488.20 Stator 2 inlet: 510.80 Stator 2 exit: 518.47

Stator 3 inlet: 548.50 Stator 3 exit: 556.94 Stator inlet: 585.08 Stator 4 exit: 593.22

Ambient Relative Humidity $\quad 10.00$

Fan Face Relative Humidity

Fan Bypass Relative Humidity $\quad 0.21$

2.54

LPC Exit Relative Humidity $\quad 0.03$

HPC Relative Humidity 0.000050

Ambient Flow Velocity

0.0000500

$\begin{aligned} 793.07 & \text { Fan/LPC Inlet Flow Velocity } \\ 3.46 & \text { Fan/LPC Inlet Static Pressure }\end{aligned}$

Inlet Length

40.00

Ambient Static Pressure

Ambient Static Temperature

429.85 Fan/LPC Inlet Static Temperature 461.98

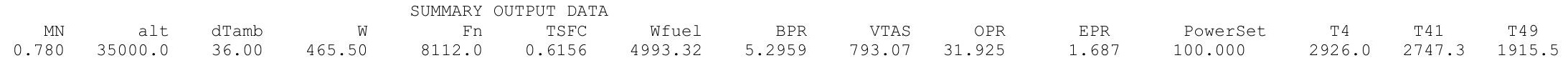

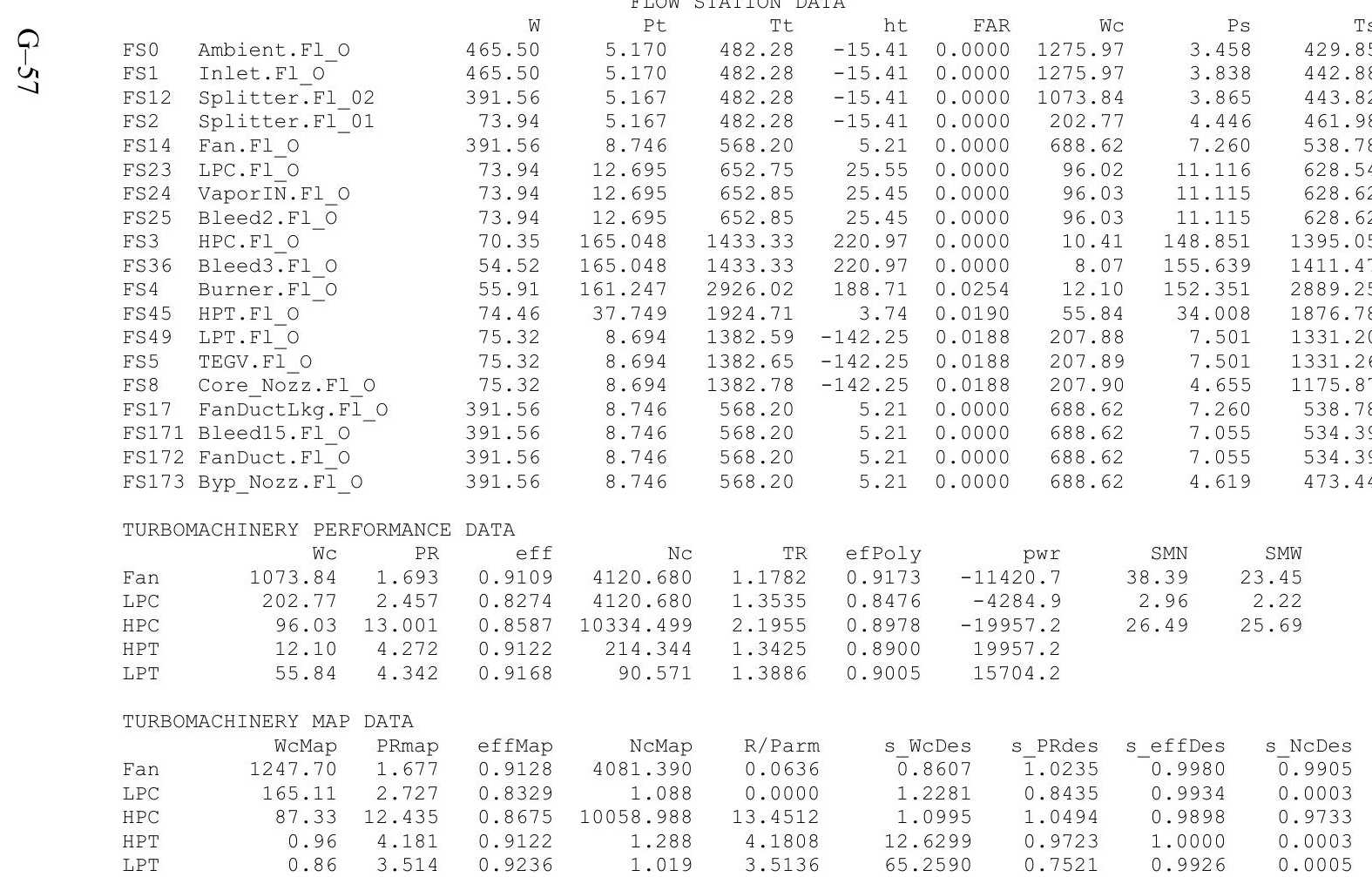




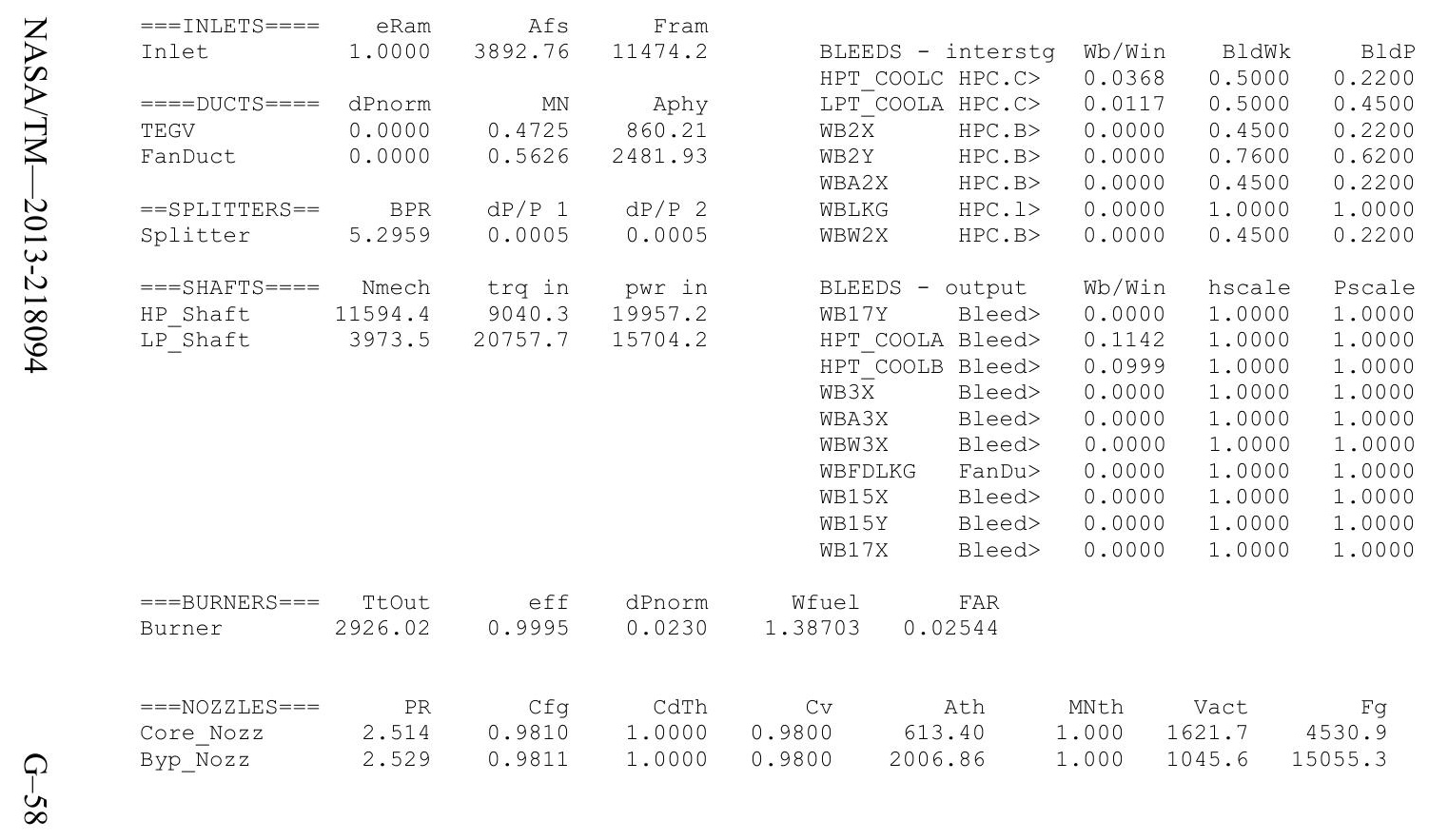


Date:07/05/13 Time:09:33:34 Model: Turbofan Engine - COMDES ON COnverge $=1$ CASE: 0

Version:NPSS 1.6.5 - Rev: -> Gas Package: Janaf iter/pass/Jacb/Broy=18/32/1/16 Run by: Philip C Jorgenson PC: 75

Temperature Stator 1 inlet: 478.71 Stator 1 exit: 495.67 Stator 2 inlet: 516.27 Stator 2 exit: 524.91 $\begin{array}{ll}\text { Stator } 3 \text { inlet: } 549.55 & \text { Stator } 3 \text { exit: } 558.17 \\ \text { Stator } 5 \text { inlet: } 607.99 & \text { Stator } 5 \text { exit: } 615.44\end{array} \quad$ Unblocked Percent Blockage: 0.00 .37

\begin{abstract}
Ambient Relative Humidity $\quad 10.00$
Fan Face Relative Humidity $\quad 3.82$

Fan Bypass Relative Humidity $\quad 0.30$

LPC Inlet Relative Humidity $\quad 2.07$

LPC Exit Relative Humidity $\quad 0.03$

HPC Relative Humidity $\quad 0.00$

Drop Diameter $\quad 0.0000500$

Ambient Flow Velocity

Ambient Static Pressure

Additional Water at LPC Exit

Inlet Length

$\begin{array}{rr} & 40.00 \\ \text { Fan/LPC Inlet Flow Velocity } \quad 423.38\end{array}$

$429.85 \quad$ Fan/LPC Inlet Static Pressure 4.63 0.0000290 Fan/LPC Inlet Static Temperature 467.34
\end{abstract}

\section{$\begin{array}{rrrrrr}\text { MN } & \text { alt } & \text { dTamb } & & \text { SUMMARY } & \text { OUTPUT DATA } \\ 0.780 & 35000.0 & 36.00 & 429.86 & 6089.9 & 0.6090\end{array}$}

$0.780 \quad 35000.0 \quad 36.00 \quad 429.8$

FSO Ambient.Fl 0

FS1 Inlet.FI_O

Splitter.FI_01

FS14 Fan.F1_

FLOW STATION DATA

FS23 LPC.FI_O

FS25 Bleed2.F1_O

S3 HPC.Fl_O

$\begin{array}{ll}\text { FS36 } & \text { Bleed3.F1_O } \\ \text { FS4 } & \text { Burner.FI_O }\end{array}$

FS45 HPT.FI_O

$\begin{array}{ll}\text { FS49 } & \text { LPT.FI-O } \\ \text { FS5 } & \text { TEGV.FI'O }\end{array}$

FS5 TEGV.FI_O

FS17 FanDuctlkg.Fi_o

FS171 Bleed15.FI_O

FS172 FanDuct.Fl_O

$W$
429.86
429.86
364.58
65.27
364.58
65.27
65.28
65.28
62.11
48.14
49.17
65.54
66.31
66.31
66.31
364.58
364.58
364.58
364.58

TURBOMACHINERY PERFORMANCE DATA

\begin{tabular}{|c|c|c|c|c|c|c|c|c|c|}
\hline & Wc & PR & eff & NC & TR & efPoly & pwr & SMN & SMW \\
\hline Fan & 999.86 & 1.556 & 0.9070 & 3842.192 & 1.1485 & 0.9126 & -8862.8 & 62.42 & 29.35 \\
\hline LPC & 179.01 & 2.386 & 0.8662 & 3842.192 & 1.3252 & 0.8814 & -3479.7 & 4.01 & 3.17 \\
\hline HPC & 86.39 & 11.196 & 0.8615 & 10049.022 & 2.1041 & 0.8982 & -15839.3 & 33.47 & 32.17 \\
\hline HPT & 12.09 & 4.295 & 0.9089 & 217.095 & 1.3535 & 0.8892 & 15839.3 & & \\
\hline LPT & 56.06 & 4.351 & 0.9129 & 89.050 & 1.3979 & 0.8960 & 12342.4 & & \\
\hline \multicolumn{10}{|c|}{ TURBOMACHINERY MAP DATA } \\
\hline & WcMap & PRmap & effMap & NcMap & R/Parm & S_WcDes & s_PRdes & S_effDes & S_NCDes \\
\hline Fan & 1161.74 & 1.543 & 0.9088 & 3805.558 & 0.0589 & $\overline{0} .8607$ & $\overline{1} .0235$ & 0.9980 & 0.9905 \\
\hline LPC & 143.09 & 2.430 & 0.8208 & 1.015 & 0.0000 & 1.2510 & 0.9690 & 1.0554 & 0.0003 \\
\hline HPC & 78.57 & 10.716 & 0.8704 & 9781.122 & 12.8375 & 1.0995 & 1.0494 & 0.9898 & 0.9733 \\
\hline HPT & 0.96 & 4.204 & 0.9089 & 1.305 & 4.2040 & 12.6299 & 0.9723 & 1.0000 & 0.0003 \\
\hline LPT & 0.86 & 3.520 & 0.9197 & 1.002 & 3.5200 & 65.2590 & 0.7521 & 0.9926 & 0.0005 \\
\hline
\end{tabular}




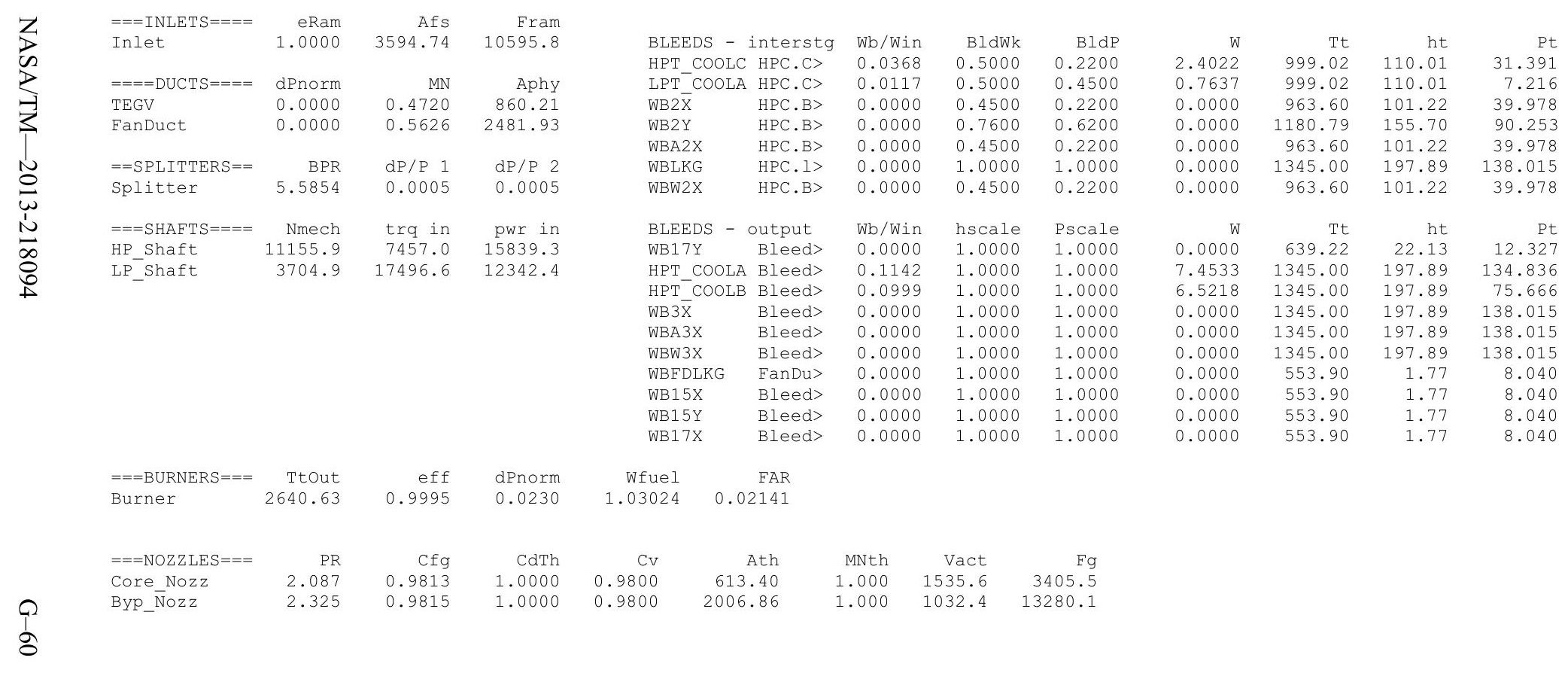


Time:09:34:07 Model: Turbofan Engine - COMDES ON Converge $=1 \quad$ CASE: 0 Stator 3 inlet: 549.60 Stator 3 exit: 558.22 Stator 4 inlet: 581.05 Stator 4 exit: 588.92 Stator 5 inlet: 607.22 Stator 5 exit: 614.61 Unblocked Percent Blockage: 0.00

\begin{abstract}
Ambient Relative Humidity $\quad 10.00$
Fan Face Relative Humidity $\quad 3.70$

Fan Bypass Relative Humidity $\quad 0.31$

LPC Inlet Relative Humidity $\quad 2.02$

LPC Exit Relative Humidity $\quad 0.03$

HPC Relative Humidity $\quad 0.00$

Drop Diameter 0.0000500

Ambient Flow Velocity

Ambient Static Pressure

Additional Water at LPC Exit

3.46 Fan/LPC Inlet Static Pressure $\quad 4.65$

429.85 Fan/LPC Inlet Static Temperature 467.94
\end{abstract}

$\begin{array}{rrrrrr} & & & & \text { SUMMARY } & \text { OUTPUT DATA } \\ \text { MN } & \text { alt } & \text { dTamb } & \text { W } & \text { Fn } & \text { TSFC } \\ 0.780 & 35000.0 & 36.00 & 425.73 & 5861.8 & 0.6094\end{array}$
Wfuel
3572.28 $\begin{array}{rr}\text { BPR } \\ 3572.28 & 5.6353\end{array}$
VTAS 793.07
OPR
26.076

\section{FLOW STATION DATA}

$W$
425.73
425.73
361.57
64.16
361.57
64.16
64.16
64.16
61.05
47.31
48.31
64.40
65.16
65.16
65.16
361.57
361.57
361.57
361.57

$\begin{array}{rrr}\text { ht } & \text { FAR } & \text { WC } \\ -15.41 & 0.0000 & 1166.96\end{array}$ $\begin{array}{lll}-15.41 & 0.0000 & 1166.96\end{array}$ $\begin{array}{lll}-15.41 & 0.0000 & 991.5 \\ -15.41 & 0.0000 & 175.96\end{array}$ $\begin{array}{rll}15.41 & 0.0000 & 175.96\end{array}$ $\begin{array}{rrr}1.36 & 0.0000 & 688.66\end{array}$ $\begin{array}{lll}1.36 & 0.0000 & 688.66 \\ 21.77 & 0.0000-85.26\end{array}$ $\begin{array}{lll}21.77 & 0.00000 & 85.27\end{array}$

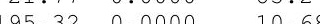
$\begin{array}{lll}195.32 & 0.0000 & -8.68\end{array}$ $\begin{array}{lll}169.41 & 0.0210-12.09\end{array}$ $\begin{array}{lll}3.99 & 0.0157 & 56.08\end{array}$ $\begin{array}{lll}-124.64 & 0.0155 & 208.52\end{array}$ $\begin{array}{lll}-124.64 & 0.0155 & 208.52\end{array}$ $\begin{array}{lll}-124.64 & 0.0155 & 208.53\end{array}$ $\begin{array}{lll}1.36 & 0.0000 & 688.66\end{array}$ $\begin{array}{lll}1.36 & 0.0000 & 688.66\end{array}$ $\begin{array}{lll}1.36 & 0.0000 & 688.66\end{array}$

$\begin{array}{rr}\text { PS } & \text { TS } \\ 3.458 & 429.85 \\ 4.146 & 452.76 \\ 4.136 & 452.51 \\ 4.650 & 467.94 \\ 6.609 & 523.60 \\ 11.102 & 619.88 \\ 11.102 & 619.97 \\ 11.102 & 619.97 \\ 120.807 & 1296.89 \\ 126.701 & 1313.38 \\ 124.416 & 2575.38 \\ 27.576 & 1665.75 \\ 6.071 & 1173.22 \\ 6.071 & 1173.28 \\ 3.757 & 1032.42 \\ 6.609 & 523.60 \\ 6.422 & 519.33 \\ 6.422 & 519.33 \\ 4.205 & 460.08\end{array}$

\section{Aphy MN} $4168.2=0.5700$ $3531.8 \quad 0.5729$ $2606.7 \quad 0.5226$ $\begin{array}{lll}412.6 & 0.3797\end{array}$ $\begin{array}{lll}412.6 & 0.3797 \\ 412.6 & 0.3798\end{array}$ $\begin{array}{lll}412.6 & 0.3798\end{array}$ $\begin{array}{rr}49.7 & 0.4042 \\ 49.3 & 0.3032\end{array}$ $\begin{array}{ll}49.3 & 0.3032\end{array}$ $\begin{array}{ll}74.6 & 0.2990\end{array}$ $265.4 \quad 0.4009$ $\begin{array}{ll}265.4 & 0.4720\end{array}$ $860.2 \quad 0.4720$ $613.4 \quad 1.0000$ $2606.7 \quad 0.5226$ $2481.9 \quad 0.5626$ $2481.9 \quad 0.5626$ $\begin{array}{lccc} & & & \\ \text { PowerSet } & T 4 & T 41 & T 49 \\ 72.700 & 2609.1 & 2454.2 & 1702.3\end{array}$

TURBOMACHINERY PERFORMANCE DATA

\begin{tabular}{|c|c|c|c|c|c|c|c|c|}
\hline WC & PR & eff & $\mathrm{NC}$ & TR & efPoly & pwr & SMN & SMW \\
\hline 991.59 & 1.541 & 0.9070 & 3811.247 & 1.1450 & 0.9125 & -8580.7 & 67.50 & 30.08 \\
\hline 175.96 & 2.373 & 0.8684 & 3811.247 & 1.3222 & 0.8833 & -3388.4 & 4.29 & 3.38 \\
\hline 85.27 & 10.994 & 0.8615 & 10017.451 & 2.0935 & 0.8980 & -15373.6 & 34.33 & 32.97 \\
\hline 12.09 & 4.298 & .9083 & 217.467 & 1.3546 & 0.8888 & 15373.6 & & \\
\hline 56.08 & 4.351 & 0.9124 & 88.875 & 1.3989 & 0.8953 & 11969.1 & & \\
\hline INERY MAP & DATA & & & & & & & \\
\hline WcMap & PRmap & effMap & NcMap & R/Parm & s WcDes & s PRdes & $s$ effDes & s NcDes \\
\hline 1152.13 & 1.528 & 0.9089 & 3774.907 & 0.0583 & $\overline{0} .8607$ & $\overline{1} .0235$ & 0.9980 & $\overline{0} .9905$ \\
\hline 141.09 & 2.398 & 0.8201 & 1.007 & 0.0000 & 1.2471 & 0.9822 & 1.0589 & 0.0003 \\
\hline 77.55 & 10.523 & 0.8704 & 9750.393 & 12.7668 & 1.0995 & 1.0494 & 0.9898 & 0.9733 \\
\hline 0.96 & 4.206 & 0.9083 & 1.307 & 4.2064 & 12.6299 & 0.9723 & 1.0000 & 0.0003 \\
\hline 0.86 & 3.521 & 0.9192 & 1.000 & 3.5206 & 65.2590 & 0.7521 & 0.9926 & 0.0005 \\
\hline
\end{tabular}


$\begin{array}{lrrr}\begin{array}{l}==- \text { INLETS }==== \\ \text { Inlet }\end{array} & \text { eRam } & \text { AfS } & \text { Fram } \\ 1.0000 & 3560.21 & 10494.0\end{array}$

$====$ DUCTS $====$ dPnorm MN Aphy

BLEEDS - interstg wb/Win Bldwk BldP $\begin{array}{llll}\text { HPT_COOLC HPC.C> } & 0.0368 & 0.5000 & 0.2200 \\ \text { LPT COOLA HPC.C } & 0.0117 & 0.5000 & 0.4500\end{array}$ $\begin{array}{lllr}\text { TEGV } & 0.0000 & 0.4720 & 860.21 \\ \text { FanDuct } & 0.0000 & 0.5626 & 2481.93\end{array}$ $\begin{array}{lllll}\text { WB } 2 \bar{X} & \text { HPC.B } & 0.0000 & 0.4500 & 0.2200 \\ \text { WB } & & 0.45 & \end{array}$ $\begin{array}{lrll}==\text { SPLITTERS }== & \mathrm{BPR} & \mathrm{dP} / \mathrm{P}_{1} & \mathrm{dP} / \mathrm{P}_{2} \\ \text { Splitter } & 5.6353 & 0.0005 & 0.0005\end{array}$ $\begin{array}{lllll}\text { WB2Y } & \text { HPC.B } & 0.0000 & 0.7600 & 0.6200 \\ \text { WBA2X HPC. B> } & 0.0000 & 0.4500 & 0.2200\end{array}$ WBLKG HPC $>0.0000 \quad 1.0000 \quad 1.0000$ WBW2X HPC.B> $0.0000 \quad 0.4500 \quad 0.2200$

BLEEDS - output Wb/Win hscale Pscale $\begin{array}{llll}\text { WB17Y Bleed> } & 0.0000 & 1.0000 & 1.0000\end{array}$ HPT_COOLA Bleed> $0.1142 \quad 1.0000 \quad 1.0000$ $\begin{array}{lllll}\text { HPT COOLB } & \text { Bleed> } & 0.0999 & 1.0000 & 1.0000 \\ \text { WB } 3 \bar{X} & \text { Bleed }> & 0.0000 & 1.0000 & 1.0000\end{array}$ $\begin{array}{lllll}\text { WB3X } & \text { Bleed }> & 0.0000 & 1.0000 & 1.0000 \\ \text { WBA3X } & \text { Bleed }> & 0.0000 & 1.0000 & 1.0000\end{array}$ $\begin{array}{lllll}\text { WBA3X } & \text { Bleed }> & 0.0000 & 1.0000 & 1.0000 \\ \text { WBW3X } & \text { Bleed }> & 0.0000 & 1.0000 & 1.0000\end{array}$ $\begin{array}{lllll}\text { WBW3X } & \text { Bleed> } & 0.0000 & 1.0000 & 1.0000 \\ \text { WBFDLKG FanDu> } & 0.0000 & 1.0000 & 1.0000\end{array}$ $\begin{array}{lllll}\text { WBFDLKG } & \text { FanDu> } & 0.0000 & 1.0000 & 1.0000 \\ \text { WB15X } & \text { Bleed> } & 0.0000 & 1.0000 & 1.0000\end{array}$ $\begin{array}{lllll}\text { WB15X } & \text { Bleed> } & 0.0000 & 1.0000 & 1.0000 \\ \text { WB15Y } & \text { Bleed> } & 0.0000 & 1.0000 & 1.0000\end{array}$ Bleed $>0.0000 \quad 1.0000 \quad 1.0000$

$\begin{array}{rrrr}\text { W } & \text { Tt } & \text { ht } & \text { Pt } \\ 2.3612 & 993.17 & 108.55 & 30.645 \\ 0.7507 & 993.17 & 108.55 & 7.043 \\ 0.0000 & 958.17 & 99.87 & 39.223 \\ 0.0000 & 1172.81 & 153.67 & 88.242 \\ 0.0000 & 958.17 & 99.87 & 39.223 \\ 0.0000 & 1335.15 & 195.32 & 134.810 \\ 0.0000 & 958.17 & 99.87 & 39.223 \\ \text { W } & & & \\ 0.0000 & 637 & \mathrm{ht} & \mathrm{Pt} \\ 7.3261 & 1335.15 & 21.77 & 12.263 \\ 6.4105 & 1335.15 & 195.32 & 131.706 \\ 0.0000 & 1335.15 & 195.32 & 134.899 \\ 0.0000 & 1335.15 & 195.32 & 134.810 \\ 0.0000 & 1335.15 & 195.32 & 134.810 \\ 0.0000 & 552.20 & 1.36 & 7.961 \\ 0.0000 & 552.20 & 1.36 & 7.961 \\ 0.0000 & 552.20 & 1.36 & 7.961 \\ 0.0000 & 552.20 & 1.36 & 7.961\end{array}$
$===$ BURNERS $===\quad$ TtOut eff
0.9995

dPnorm

Wfuel FAR 2609.12 0.99230 .02097

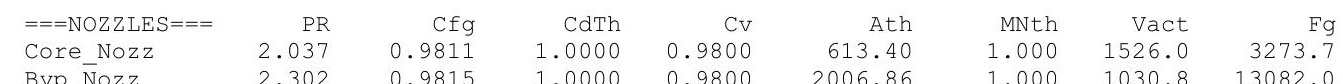


Date:07/05/13 Time:09:34:37 Model: Turbofan Engine - COMDES ON CONverge = 1 CASE: 0

Version:NPSS 1.6.5 - Rev: -> Gas Package: Janaf iter/pass/Jacb/Broy=17/31/1/15 Run by: Philip C Jorgenson PC: 69

Temperature Stator 1 inlet: 480.57 Stator 1 exit: 497.00 Stator 2 inlet: 517.17 Stator 2 exit: 525.85 $\begin{array}{ll}\text { Stator } 3 \text { inlet: } 549.50 & \text { Stator } 3 \text { exit: } 558.08 \text { Stator } 4 \text { inlet: } 580.46 \text { Stator } 4 \text { exit: } 588.27 \\ \text { Stator } 5 \text { inlet: } 606.24 & \text { Stator } 5 \text { exit: } 613.56\end{array}$

$\begin{array}{lclll}\text { Ambient Relative Humidity } & 10.00 & & & \\ \text { Fan Face Relative Humidity } & 3.60 & & & \\ \text { Fan Bypass Relative Humidity } & 0.33 & & & \\ \text { LPC Inlet Relative Humidity } & 1.98 & & & \\ \text { LPC Exit Relative Humidity } & 0.03 & & & \\ \text { HPC Relative Humidity } & 0.00 & & & \\ \text { Drop Diameter } & 0.0000500 & \text { Inlet Length } & & \\ \text { Ambient Flow Velocity } & 793.07 & \text { Fan/LPC Inlet Flow Velocity } & 406.39 \\ \text { Ambient Static Pressure } & 3.46 & \text { Fan/LPC Inlet Static Pressure } & 4.67 \\ \text { Ambient Static Temperature } & 429.85 & \text { Fan/LPC Inlet Static Temperature } & 468.51 \\ \text { Additional Water at LPC Exit } & 0.0000311 & & & \\ \end{array}$

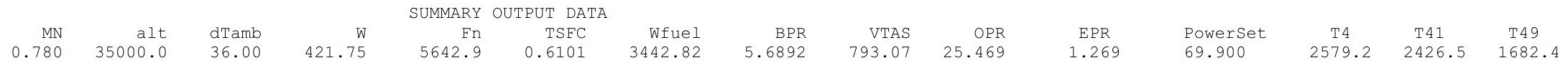

\begin{tabular}{|c|c|c|c|c|c|c|c|c|c|c|}
\hline & & & W & Pt & $\mathrm{Tt}$ & ht & FAR & Wo & & Ps \\
\hline FSO & Ambient.FI_O & & 421.75 & 5.170 & 482.28 & -15.41 & 0.0000 & 1156.05 & 3.45 & 429 \\
\hline FS1 & Inlet.Fl o & & 421.75 & 5.170 & 482.28 & -15.41 & 0.0000 & 1156.05 & 4.17 & 453. \\
\hline FS12 & Splitter.Fl_o & & 358.70 & 5.167 & 482.28 & -15.41 & 0.0000 & $983.72=0$ & 4.15 & 453 \\
\hline FS2 & Splitter.Fl_o & & 63.05 & 5.167 & 482.28 & -15.41 & 0.0000 & 172.91 & 4.67 & 468. \\
\hline FS14 & Fan.Fl_O & & 358.70 & 7.886 & 550.55 & 0.97 & 0.0000 & 688.66 & 6.54 & 522. \\
\hline FS23 & LPC.FI_O & & 63.05 & 12.180 & 636.05 & 21.53 & 0.0000 & 84.24 & 11.05 & 618. \\
\hline FS24 & VaporIN .FI_O & & 63.05 & 12.180 & 636.14 & 21.37 & 0.0000 & 84.25 & 11.05 & 618. \\
\hline FS25 & Bleed2.Fl_o & & 63.05 & 12.180 & 636.14 & 21.37 & 0.0000 & 84.25 & 11.05 & 618. \\
\hline FS3 & HPC.FI_O & & 59.99 & 131.672 & 1325.58 & 192.84 & 0.0000 & $10.7 \mathrm{C}$ & 117.92 & 1287. \\
\hline FS36 & Bleed3.FI_O & & 46.49 & 131.672 & 1325.58 & 192.84 & 0.0000 & 8.30 & 123.71 & 1303. \\
\hline FS4 & Burner.Fl-o & & 47.45 & 128.640 & 2579.17 & 167.48 & 0.0206 & $12.0 \mathrm{~s}$ & 121.51 & 17 \\
\hline FS45 & HPT.Fl O & & 63.27 & 29.912 & 1689.91 & 3.94 & 0.0153 & 56.11 & 26.91 & 1646 \\
\hline FS 49 & LPT.FI_O & & 64.01 & 6.874 & 1205.14 & -123.03 & 0.0152 & 208.58 & 5.92 & 1158 \\
\hline FS5 & TEGV.FI_O & & 64.01 & 6.874 & 1205.21 & -123.03 & 0.0152 & 208.5 & 5.92 & 1158 \\
\hline FS8 & Core_Nozz.Fl & & 64.01 & 6.874 & 1205.34 & -123.03 & 0.0152 & 208.60 & 3.66 & 1019 \\
\hline FS17 & FanDuctLkg.Fi & & 358.70 & 7.886 & 550.55 & 0.97 & 0.0000 & 688.66 & 6.54 & 522 \\
\hline FS171 & Bleed15.FI_o & & 358.70 & 7.886 & 550.55 & 0.97 & 0.0000 & 688.66 & 6.36 & 517 \\
\hline FS172 & FanDuct.FI- 0 & & 358.70 & 7.886 & 550.55 & 0.97 & 0.0000 & 688.66 & 6.36 & 517 \\
\hline FS173 & Byp_Nozz.FI_o & & 358.70 & 7.886 & 550.55 & 0.97 & 0.0000 & 688.66 & 4.16 & \\
\hline TURBON & MACHINERY PERE & PORMANCE & DATA & & & & & & & \\
\hline & Wc & PR & eff & $\mathrm{NC}$ & TR & efPoly & & pwr & SMN & SMW \\
\hline Fan & 983.72 & 1.526 & 0.9074 & 3779.613 & 1.1416 & 0.9127 & & 311.6 & 73.97 & 30.78 \\
\hline LPC & 172.91 & 2.357 & 0.8699 & 3779.613 & 1.3188 & 0.8845 & -32 & 294.8 & 4.57 & 3.57 \\
\hline $\begin{array}{l}\mathrm{HPC} \\
\end{array}$ & 84.25 & 10.810 & 0.8615 & 9988.697 & 2.0838 & 0.8979 & -149 & 325.5 & 35.13 & 33.71 \\
\hline HPT & 12.09 & 4.301 & 0.9078 & 217.822 & 1.3557 & 0.8883 & 149 & 25.5 & & \\
\hline $\mathrm{LPT}$ & 56.11 & 4.351 & 0.9117 & 88.658 & 1.3996 & 0.8945 & 116 & 506.4 & & \\
\hline TURB & MACH INERY MAP & DATA & & & & & & & & \\
\hline & WcMap & PRmap & effMap & NcMap & R/Parm & S WC & cDes & S PRdes & $s$ effDes & S NCDes \\
\hline Fan & 1142.98 & 1.514 & 0.9092 & 3743.575 & 0.0578 & 0.8 & 8607 & 1.0235 & 0.9980 & 0.9905 \\
\hline LPC & 139.06 & 2.366 & 0.8195 & 0.998 & 0.0000 & & 2433 & 0.9935 & 1.0615 & 0.0003 \\
\hline HPC & 76.62 & 10.348 & 0.8704 & 9722.405 & 12.7017 & & 0995 & 1.0494 & 0.9898 & 0.9733 \\
\hline HPT & 0.96 & 4.209 & 0.9078 & 1.309 & 4.2092 & 12.6 & 6299 & 0.9723 & 1.0000 & 0.0003 \\
\hline LPT & 0.86 & 3.521 & 0.9185 & 0.997 & 3.5206 & 65.2 & 2590 & 0.7521 & 0.9926 & 0.0005 \\
\hline
\end{tabular}




\begin{tabular}{|c|c|c|c|}
\hline$===$ INLET $S===$ & eRam & Afs & Fram \\
\hline Inlet & 1.0000 & 3526.90 & 10395.8 \\
\hline$====$ DUCTS $====$ & dPnorm & MN & Aphy \\
\hline TEGV & 0.0000 & 0.4720 & 860.21 \\
\hline FanDuct & 0.0000 & 0.5626 & 2481.93 \\
\hline$==$ SPLITTERS $==$ & BPR & $\mathrm{dP} / \mathrm{P} 1$ & $\mathrm{dP} / \mathrm{P} 2$ \\
\hline Splitter & 5.6892 & 0.0005 & 0.0005 \\
\hline$===$ SHAFTS $====$ & Nmech & $\operatorname{trg}$ in & pwr in \\
\hline HP Shaft & 11062.2 & 7086.3 & 14925.5 \\
\hline LP ${ }^{-}$Shaft & 3644.6 & 16725.6 & 11606.4 \\
\hline
\end{tabular}

BLEEDS - interstg Wb/Win BldWk BldP HPT_COOLC HPC.C> $0.0368 \quad 0.5000 \quad 0.2200$ $\begin{array}{lllll}\text { LPT COOLA HPC.C> } & 0.0117 & 0.5000 & 0.4500 \\ \text { WB } 2 \bar{X} & \text { HPC.B> } & 0.0000 & 0.4500 & 0.2200\end{array}$ $\begin{array}{lllll}\text { WB2X } & \text { HPC.B> } & 0.0000 & 0.4500 & 0.2200 \\ \text { WB2Y } & \text { HPC. B> } & 0.0000 & 0.7600 & 0.6200\end{array}$ $\begin{array}{lllll}\text { WB2Y } & \text { HPC.B> } & 0.0000 & 0.7600 & 0.6200\end{array}$ $\begin{array}{lllll}\text { WBA2X HPC.B } & 0.0000 & 0.4500 & 0.2200\end{array}$ $\begin{array}{lllll}\text { WBLKG HPC.I> } & 0.0000 & 1.0000 & 1.0000\end{array}$ $\begin{array}{lllll}\text { WBW2X HPC.B> } & 0.0000 & 0.4500 & 0.2200\end{array}$

BLEEDS - output Wb/Win hscale Pscale $\begin{array}{lllll}\text { WB17Y Bleed> } & 0.0000 & 1.0000 & 1.0000\end{array}$ $\begin{array}{llll}\text { HPT_COOLA Bleed> } & 0.1142 & 1.0000 & 1.0000\end{array}$ $\begin{array}{lllll}\text { HPT COOLB } & \text { Bleed> } & 0.0999 & 1.0000 & 1.0000 \\ \text { WB 3 } & \text { Bleed> } & 0.0000 & 1.0000 & 1.0000\end{array}$ $\begin{array}{lllll}\text { WB3X } & \text { Bleed> } & 0.0000 & 1.0000 & 1.0000 \\ \text { WBA3X } & \text { Bleed> } & 0.0000 & 1.0000 & 1.0000\end{array}$ $\begin{array}{lllll}\text { WBA3X } & \text { Bleed> } & 0.0000 & 1.0000 & 1.0000 \\ \text { WBW3X } & \text { Bleed> } & 0.0000 & 1.0000 & 1.0000\end{array}$ $\begin{array}{lllll}\text { WBW3X } & \text { Bleed> } & 0.0000 & 1.0000 & 1.0000 \\ \text { WBFDLKG } & \text { FanDu> } & 0.0000 & 1.0000 & 1.0000\end{array}$ $\begin{array}{lllll}\text { WBFDLKG } & \text { FanDu> } & 0.0000 & 1.0000 & 1.0000 \\ \text { WB15X } & \text { Bleed }> & 0.0000 & 1.0000 & 1.0000 \\ \text { WB15Y } & \text { Bleed }> & 0.0000 & 1.0000 & 1.0000\end{array}$ $\begin{array}{lllll}\text { WB15X } & \text { Bleed }> & 0.0000 & 1.0000 & 1.0000 \\ \text { WB15Y } & \text { Bleed }> & 0.0000 & 1.0000 & 1.0000\end{array}$ WB17X Bleed> $0.0000 \quad 1.0000 \quad 1.0000$

$\begin{array}{rrrr}\text { W } & \text { Tt } & \text { ht } & \text { Pt } \\ 2.3203 & 987.39 & 107.11 & 29.912 \\ 0.7377 & 987.39 & 107.11 & 6.874 \\ 0.0000 & 952.79 & 98.53 & 38.468 \\ 0.0000 & 1165.02 & 151.69 & 86.265 \\ 0.0000 & 952.79 & 98.53 & 38.468 \\ 0.0000 & 1325.58 & 192.84 & 131.672 \\ 0.0000 & 952.79 & 98.53 & 38.468 \\ \text { W } & & & \\ 0.0000 & 636.14 & 21.37 & 12.180 \\ 7.1992 & 1325.58 & 192.84 & 128.640 \\ 6.2994 & 1325.58 & 192.84 & 72.167 \\ 0.0000 & 1325.58 & 192.84 & 131.672 \\ 0.0000 & 1325.58 & 192.84 & 131.672 \\ 0.0000 & 1325.58 & 192.84 & 131.672 \\ 0.0000 & 550.55 & 0.97 & 7.886 \\ 0.0000 & 550.55 & 0.97 & 7.886 \\ 0.0000 & 550.55 & 0.97 & 7.886 \\ 0.0000 & 550.55 & 0.97 & 7.886\end{array}$

$===$ BURNERS $===\quad$ TtOut Burner 2579.15 eff
0.9995

dPnorm

Wfuel

0.0000

50.55

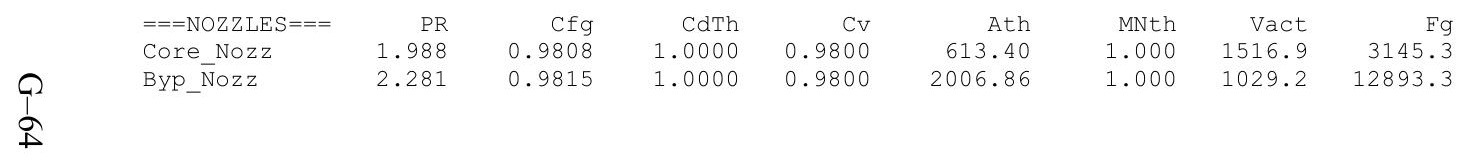


$\begin{array}{lll}\text { Date:07/05/13 Time:09:45:29 Model: } & \text { Turbofan Engine - COMDES ON Converge }=1 \text { CASE: Co }\end{array}$

Version:NPSS 1.6.5 - Rev: -> Gas Package: Janaf iter/pass/Jacb/Broy=27/41/1/25 Run by: Philip C Jorgenson PC: 100

emperature Stator 1 inlet: 449.01 Stator 1 exit: 467.64 Stator 2 inlet: 489.24 Stator 2 exit: 496.53

Stator 3 inlet: 525.31 Stator 3 exit: 533.35 Stator lnlet: 560.32 Stator 4 exit: 568.08

Ambient Relative Humidity $\quad 10.00$

Fan Face Relative Humidity $\quad 5.11$

Fan Bypass Relative Humidity $\quad 0.16$

LPC Inlet Relative Humidity 2.31

LPC Exit Relative Humidity

Drop Diameter

0.00

$\begin{array}{lr}\text { Drop Diameter } & 0.0001000 \\ \text { Ambient Flow Velocity } & 776.30\end{array}$

Ambient Static Pressure

3.46
411.85

Inlet Length

Fan/LPC Inlet Flow Velocity $\quad 40.00$

Ambient Static Temperature

Additional Water at LPC Exit

0.0000058

Inlet Static Pressure $\quad 4.45$

SUMMARY OUTPUT DATA

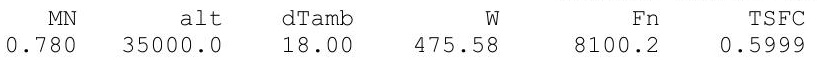

\begin{tabular}{|c|c|}
\hline FSO & Ambient.Fl_O \\
\hline FS1 & Inlet.Fl_O \\
\hline FS12 & Splitter.Fl_02 \\
\hline FS2 & Splitter.Fl_-01 \\
\hline FS14 & Fan.Fl_O \\
\hline FS23 & $\mathrm{LPC} . \mathrm{Fl} \mathrm{O}^{-}$ \\
\hline FS24 & VaporIN̄.FI_O \\
\hline FS25 & Bleed2.Fl $\bar{O}$ \\
\hline FS3 & HPC.FI_O \\
\hline FS36 & Bleed3.FI_O \\
\hline FS4 & Burner.Fl_o \\
\hline FS45 & HPT.FI_O \\
\hline FS49 & LPT.FI_O \\
\hline FS5 & TEGV.F̄ $O$ \\
\hline FS8 & Core_Nozz.Fl_O \\
\hline FS17 & FanDuctLkg.FìO \\
\hline FS171 & Bleed15.Fl O \\
\hline FS172 & FanDuct.Fl- ${ }^{-}$ \\
\hline FS173 & Byp_Nozz.F̄ \\
\hline
\end{tabular}

$W$
475.58
475.58
400.01
75.58
400.01
75.58
75.58
75.58
71.91
55.73
57.08
76.04
76.93
76.93
76.93
400.01
400.01
400.01
400.01

TURBOMACHINERY PERFORMANCE DATA

\begin{tabular}{|c|c|c|c|c|c|c|c|c|c|c|}
\hline & & & W & $\begin{array}{l}\text { FLOW } s \\
\text { Pt }\end{array}$ & $\begin{array}{c}\text { STATION DA } \\
\text { Tt }\end{array}$ & $\mathrm{ATA}$ & FAR & Wo & & Ps \\
\hline FSO & Ambient.Fl O & & 475.58 & 5.170 & 462.10 & -19.92 & 0.0000 & 1276.02 & 3.45 & 411. \\
\hline FS1 & Inlet.Fl $0^{-}$ & & 475.58 & 5.170 & 462.10 & -19.92 & 0.0000 & 1276.02 & 3.83 & 424. \\
\hline FS12 & Splitter.Fl & & 400.01 & 5.167 & 462.10 & -19.92 & 0.0000 & 1073.78 & 3.86 & 425 \\
\hline FS2 & Splitter.FI_C & & 75.58 & 5.167 & 462.10 & -19.92 & 0.0000 & 202.88 & 4.44 & 442 \\
\hline FS14 & Fan.Fl 0 & & 400.01 & 8.745 & 544.45 & -0.17 & 0.0000 & 688.68 & 7.26 & 516 \\
\hline FS23 & LPC.FI_-O & & 75.58 & 12.667 & 625.30 & 19.26 & 0.0000 & $96.28-1$ & 11.08 & 601 \\
\hline FS24 & VaporIN̄.FI_O & & 75.58 & 12.667 & 625.36 & 19.24 & 0.0000 & 96.28 & 11.08 & 601 \\
\hline FS25 & Bleed2.Fl_ō & & 75.58 & 12.667 & 625.36 & 19.24 & 0.0000 & $96.28-1$ & 11.08 & 601. \\
\hline FS3 & HPC.FI_O & & 71.91 & 165.367 & 1379.15 & 207.21 & 0.0000 & 10.42 & 149.11 & 1342 \\
\hline FS36 & Bleed3.Fl_o & & 55.73 & 165.367 & 1379.15 & 207.21 & 0.0000 & 8.08 & 155.92 & 1357 \\
\hline FS4 & Burner.Fl_o & & 57.08 & 161.558 & 2818.73 & 176.92 & 0.0242 & 12.10 & 152.62 & 2782 \\
\hline FS45 & $\begin{array}{l}\text { HPT.FI O } \\
\text { H. }\end{array}$ & & 76.04 & 37.753 & 1847.47 & -1.25 & 0.0181 & 55.8 & 34.00 & 180 \\
\hline FS49 & LPT.FI_O & & 76.93 & 8.678 & 1323.58 & -141.11 & 0.0179 & 208.11 & 7.48 & 127 \\
\hline FS5 & TEGV.FI_O & & 76.93 & 8.678 & 1323.65 & -141.11 & 0.0179 & 208.12 & 7.48 & 127 \\
\hline FS8 & Core_Nozz.Fl_ & & 76.93 & 8.678 & 1323.78 & -141.11 & 0.0179 & 208.13 & 4.64 & 112 \\
\hline FS17 & FanDúctLkg.F. & & 400.01 & 8.745 & 544.45 & -0.17 & 0.0000 & 688.68 & 7.26 & \\
\hline FS171 & Bleed15.FI_o & & 400 & 8.745 & 544.45 & -0.17 & 0.0000 & $688.68-1-2$ & 7.05 & \\
\hline FS172 & FanDuct.Fl- 0 & & 400.01 & 8.745 & 544.45 & -0.17 & 0.0000 & 688.68 & 7.05 & \\
\hline FS173 & Byp_Nozz.Fin_C & & 400.01 & 8.745 & 544.45 & -0.17 & 0.0000 & 688.68 & 4.61 & \\
\hline URBON & MACHINERY PERE & ORMANCE & DATA & & & & & & & \\
\hline & & PR & eff & $\mathrm{Nc}$ & TR & efPoly & & pwr & SMN & SMW \\
\hline Fan & 1073.78 & 1.692 & 0.9109 & 4120.398 & 1.1782 & 0.9173 & -111 & 176.3 & 38.42 & 23.46 \\
\hline LPC & 202.88 & 2.451 & 0.8264 & 4120.398 & 1.3532 & 0.8467 & -41 & 189.7 & 2.96 & 2.22 \\
\hline HPC & 96.28 & 13.055 & 0.8586 & 10345.089 & 2.2054 & 0.8979 & -196 & 511.9 & 26.28 & 25.48 \\
\hline HPT & 12.10 & 4.279 & 0.9118 & 213.958 & 1.3469 & 0.8911 & 196 & 511.9 & & \\
\hline LPT & 55.87 & 4.351 & 0.9155 & 90.484 & 1.3923 & 0.8986 & 153 & 365.9 & & \\
\hline URBON & MACHINERY MAP & DATA & & & & & & & & \\
\hline & WcMap & PRmap & effMap & NcMap & R/Parm & S_WC & cDes & S_PRdes & S_effDes & S_NCDes \\
\hline Fan & 1247.63 & 1.677 & 0.9128 & 4081.111 & 0.0636 & 0.8 & 8607 & $\overline{1} .0235$ & 0.9980 & 0.9905 \\
\hline LPC & 165.08 & 2.727 & 0.8329 & 1.088 & 0.0000 & & 2290 & 0.8404 & 0.9922 & 0.0003 \\
\hline HPC & 87.57 & 12.487 & 0.8674 & 10069.296 & 13.4723 & 1.0 & 0995 & 1.0494 & 0.9898 & 0.9733 \\
\hline HPT & 0.96 & 4.188 & 0.9118 & 1.286 & 4.1885 & 12.6 & 6299 & 0.9723 & 1.0000 & 0.0 \\
\hline LPT & 0.86 & 3.520 & 0.9223 & 1.018 & 3.5200 & 65.2 & 2590 & 0.7521 & 0.9926 & 0.0005 \\
\hline
\end{tabular}


$===$ INLETS $====$ eRam AfS Fram

Inlet

$====$ DUCTS $====\quad$ dPnorm
TEGV

TEGV 0.0000

FanDuct

0.0000

$\begin{array}{lr}==\text { SPLITTERS }== & \text { BPR } \\ \text { Splitter } & 5.2927\end{array}$

$===$ SHAFTS $====\quad$ Nmech

$\begin{array}{lr}\text { HP_Shaft } & 11359.4 \\ \text { LP_Shaft } & 3889.2\end{array}$

$=$ BURNERS $===\quad$ TtOut

Burner 2818.73

ef
0.9995

dPnorm

$\begin{array}{rl}\text { Cfg CdTh } \\ 0.9810 & 1.0000\end{array}$

CdTh
1.0000
BLEEDS - interstg Wb/Win BldWk BldP $\begin{array}{llll}\text { HPT_COOLC HPC.C> } & 0.0368 & 0.5000 & 0.2200 \\ \text { IPT_COOC }\end{array}$ $\begin{array}{lllll}\text { LPT_COOLA HPC.C> } & 0.0117 & 0.5000 & 0.4500 \\ \text { WB } 2 \bar{X} & \text { HPC.B> } & 0.0000 & 0.4500 & 0.2200\end{array}$ $\begin{array}{lllll}\text { WB2X } & \text { HPC.B> } & 0.0000 & 0.4500 & 0.2200 \\ \text { WB2Y } & \text { HPC.B> } & 0.0000 & 0.7600 & 0.6200\end{array}$

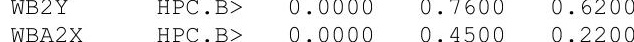
$\begin{array}{lllll}\text { WBA2X HPC.B } & 0.0000 & 0.4500 & 0.2200\end{array}$ $\begin{array}{lllll}\text { WBLKG } & \text { HPC.I> } & 0.0000 & 1.0000 & 1.0000 \\ \text { WBW2X } & \text { HPC.B } & 0.0000 & 0.4500 & 0.2200\end{array}$

BLEEDS - output Wb/Win hscale Pscale WB17Y Bleed> $0.0000 \quad 1.0000 \quad 1.0000$ HPT_COOLA Bleed> $0.1142-1.0000 \quad 1.000$ HPT_COOLB Bleed> $0.0999 \quad 1.0000 \quad 1.0000$ $\begin{array}{lllll}\text { WB3X } & \text { Bleed> } & 0.0000 & 1.0000 & 1.0000 \\ \text { WBA3X } & \text { Bleed> } & 0.0000 & 1.0000 & 1.0000\end{array}$ $\begin{array}{lllll}\text { WBA3X } & \text { Bleed> } & 0.0000 & 1.0000 & 1.0000 \\ \text { WBW3X } & \text { Bleed> } & 0.0000 & 1.0000 & 1.0000\end{array}$ $\begin{array}{lllll}\text { WBW3X } & \text { Bleed> } & 0.0000 & 1.0000 & 1.0000 \\ \text { WBFDLKG } & \text { FanDu> } & 0.0000 & 1.0000 & 1.0000\end{array}$ $\begin{array}{lllll}\text { WBFDLKG } & \text { FanDu> } & 0.0000 & 1.0000 & 1.0000 \\ \text { WB15X } & \text { Bleed }> & 0.0000 & 1.0000 & 1.0000\end{array}$ $\begin{array}{lllll}\text { WB15X } & \text { Bleed> } & 0.0000 & 1.0000 & 1.0000 \\ \text { WB15Y } & \text { Bleed> } & 0.0000 & 1.0000 & 1.0000\end{array}$ WB17X Bleed> $0.0000 \quad 1.0000 \quad 1.0000$

$\begin{array}{rrrr}\text { W } & \text { Tt } & \text { ht } & \text { Pt } \\ 2.7812 & 1010.15 & 113.23 & 37.753 \\ 0.8843 & 1010.15 & 113.23 & 8.678 \\ 0.0000 & 972.32 & 103.83 & 46.261 \\ 0.0000 & 1204.15 & 162.10 & 107.341 \\ 0.0000 & 972.32 & 103.83 & 46.261 \\ 0.0000 & 1379.15 & 207.21 & 165.367 \\ 0.0000 & 972.32 & 103.83 & 46.261 \\ \text { W } & & & \\ 0.0000 & 625.36 & 19.24 & 12.667 \\ 8.6294 & 1379.15 & 207.21 & 161.558 \\ 7.5509 & 1379.15 & 207.21 & 90.742 \\ 0.0000 & 1379.15 & 207.21 & 165.367 \\ 0.0000 & 1379.15 & 207.21 & 165.367 \\ 0.0000 & 1379.15 & 207.21 & 165.367 \\ 0.0000 & 544.45 & -0.17 & 8.745 \\ 0.0000 & 544.45 & -0.17 & 8.745 \\ 0.0000 & 544.45 & -0.17 & 8.745 \\ 0.0000 & 544.45 & -0.17 & 8.745\end{array}$

Wfuel 0.0000

8.745 
Date:07/05/13 Time:09:46:02 Model: Turbofan Engine - COMDES ON CONverge = 1 CASE: 0

Version:NPSS 1.6.5 - Rev: -> Gas Package: Janaf iter/pass/Jacb/Broy=18/32/1/16 Run by: Philip C Jorgenson PC: 75

Temperature Stator 1 inlet: 458.63 Stator 1 exit: 474.88 Stator 2 inlet: 494.60 Stator 2 exit: 502.86 $\begin{array}{llll}\text { Stator } 3 \text { inlet: } 526.47 & \text { Stator } 3 \text { exit: } 534.71 & \text { Stator } 4 \text { inlet: } 557.05 & \text { Stator } 4 \text { exit: } 564.61 \\ \text { Stator } 5 \text { inlet: } 582.45 & \text { Stator } 5 \text { exit: } 589.57 & \text { Unblocked Percent Blockage: } 0.00\end{array}$

\begin{abstract}
Ambient Relative Humidity $\quad 10.00$
Fan Face Relative Humidity $\quad 3.58$

Fan Bypass Relative Humidity $\quad 0.24$

LPC Inlet Relative Humidity $\quad 1.86$

LPC Exit Relative Humidity $\quad 0.02$

HPC Relative Humidity

Drop Diameter

0.0001000

776.30

3.46
411.85

0.0000060

Fan/IPC Inlet Flow Velocity $\quad 40.00$

Fan/LPC Inlet Static Pressure $\quad 4.63$

Ambient Static Pressure

Additional Water at LPC Exit
\end{abstract}

SUMMARY OUTPUT DATA

MN alt dTamb

W 439.17

$\begin{array}{rr}\text { Fn } & \text { TSFC } \\ 6082.8 & 0.5942\end{array}$

Wfuel

BPR
5.5824

VTAS
776.30

OPR
26.748

$\begin{array}{cl}\text { EPR } & \text { Powerset } \\ 1.342 & 75.600\end{array}$

$\begin{array}{ccc}\text { T4 } & \text { T41 } & \text { T49 } \\ 2543.5 & 2391.4 & 1654.7\end{array}$

Wc
1178.32
1178.32
999.81
179.10
688.70
86.49
86.50
86.50
10.65
8.26
12.09
56.08
208.65
208.66
208.67
688.70
688.70
688.70
688.70

$\begin{array}{rr}\text { PS } & \text { TS } \\ 3.458 & 411.85 \\ 4.118 & 432.98 \\ 4.112 & 432.86 \\ 4.629 & 447.77 \\ 6.674 & 503.23 \\ 11.114 & 594.67 \\ 11.114 & 594.73 \\ 11.114 & 594.73 \\ 124.002 & 1256.31 \\ 130.011 & 1272.27 \\ 127.611 & 2510.39 \\ 28.260 & 1619.08 \\ 6.211 & 1138.15 \\ 6.211 & 1138.21 \\ 3.842 & 1000.78 \\ 6.674 & 503.23 \\ 6.485 & 499.13 \\ 6.485 & 499.13 \\ 4.246 & 442.16\end{array}$

$\begin{array}{rr}\text { Aphy } & \text { MN } \\ 3594.7 & 0.7800 \\ 4168.2 & 0.5791 \\ 3531.8 & 0.5805 \\ 830.5 & 0.3996 \\ 2606.7 & 0.5226 \\ 412.6 & 0.3863 \\ 412.6 & 0.3863 \\ 412.6 & 0.3863 \\ 49.7 & 0.4027 \\ 49.3 & 0.3021 \\ 74.6 & 0.2988 \\ 265.4 & 0.4004 \\ 860.2 & 0.4719 \\ 860.2 & 0.4719 \\ 613.4 & 1.0000 \\ 2606.7 & 0.5226 \\ 2481.9 & 0.5625 \\ 2481.9 & 0.5625 \\ 2006.9 & 1.0000\end{array}$

gamt
1.40081
1.40081
1.40081
1.40081
1.40006
1.39851
1.39851
1.39851
1.36228
1.36228
1.29509
1.33130
1.35873
1.35873
1.35872
1.40006
1.40006
1.40006
1.40006

WAR
0.0000308
0.0000308
0.0000308
0.0000308
0.0000308
0.0000308
0.0000368
0.0000368
0.0000368
0.0000368
0.0000368
0.0000368
0.0000368
0.0000368
0.0000368
0.0000308
0.0000308
0.0000308
0.0000308

Wair 439.15
439.15

FSO Ambient.FI_O

FS12 Splitter.F1_02

FS14 Fan.Fl_o

FS23 LPC.FI-O

FS25 Bleed2.F1_-

FS3 HPC.FI_O

FS4 Burner. Fl-

FS45 HPT.FI_O

FS8 Core_Nozz.FI_O

FS171 Bleed15.F1_O

FS172 FanDuct.Fl-0

8.040

$\begin{array}{lll}-3.46 & 0.0000\end{array}$

\begin{tabular}{|c|c|c|c|c|c|c|c|c|c|}
\hline \multicolumn{2}{|c|}{ TURBOMACHINERY P } & $\begin{array}{l}\text { PERFORMANCE } \\
\text { WC PR }\end{array}$ & \multicolumn{2}{|l|}{ DATA } & & \multirow[b]{2}{*}{ efPoly } & \multirow[b]{2}{*}{ pwr } & \multirow[b]{2}{*}{ SMN } & \multirow[b]{2}{*}{ SMW } \\
\hline & Wc & PR & eff & $\mathrm{NC}$ & TR & & & & \\
\hline Fan & 999.81 & 1.556 & 0.9070 & 3841.924 & 1.1486 & 0.9126 & 672.8 & 62.44 & 29.36 \\
\hline LPC & 179.10 & 2.384 & 0.8661 & 3841.924 & 1.3252 & 0.8813 & 404.7 & 4.01 & 3.17 \\
\hline HPC & 86.50 & 11.227 & 0.8614 & 10052.335 & 2.1119 & 0.8984 & 551.5 & 33.25 & 31.96 \\
\hline HPT & 12.09 & 4.302 & 0.9079 & 216.585 & 1.3570 & 0.8887 & 551.5 & & \\
\hline LPT & 56.08 & 4.358 & 0.9114 & 88.945 & 1.4014 & 0.8940 & 077.5 & & \\
\hline TURI & NERY MAP & DATA & & & & & & & \\
\hline & WcMap & PRmap & effMap & NcMap & R/Parm & s WcDes & S PRdes & $s$ effDes & s NCDes \\
\hline Fan & 1161.68 & 1.543 & 0.9088 & 3805.291 & 0.0589 & $\overline{0} .8607$ & $\overline{1} .0235$ & 0.9980 & $\overline{0} .9905$ \\
\hline LPC & 143.07 & 2.430 & 0.8207 & 1.015 & 0.0000 & 1.2519 & 0.9679 & 1.0553 & 0.0003 \\
\hline HPC & 78.67 & 10.745 & 0.8703 & 9784.346 & 12.8567 & 1.0995 & 1.0494 & 0.9898 & 0.9733 \\
\hline HPT & 0.96 & 4.210 & 0.9079 & 1.302 & 4.2105 & 12.6299 & 0.9723 & 1.0000 & 0.0003 \\
\hline LPT & & 3.526 & 0.9181 & 1.001 & 3.5256 & & 0.7521 & 0.9926 & 0.0005 \\
\hline
\end{tabular}




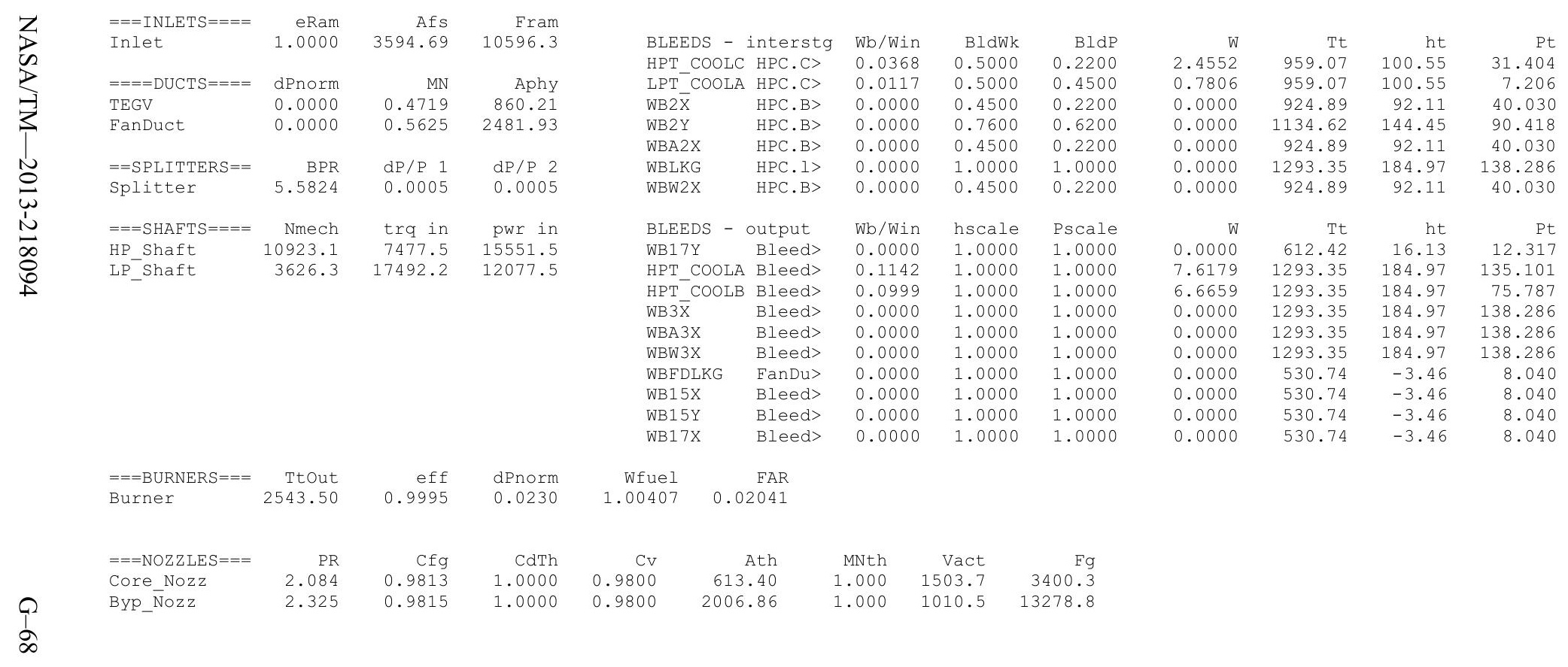


Turbofan Engine - COMDES ON Converge = 1 CASE: Stator 3 inlet: $526.52 \quad$ Stator 3 exit: 534.76 Stator 4 inlet: 556.65 Stator 4 exit: 564.18 Stator 5 inlet: 581.71 Stator 5 exit: 588.79 Unblocked Percent Blockage: 0.00

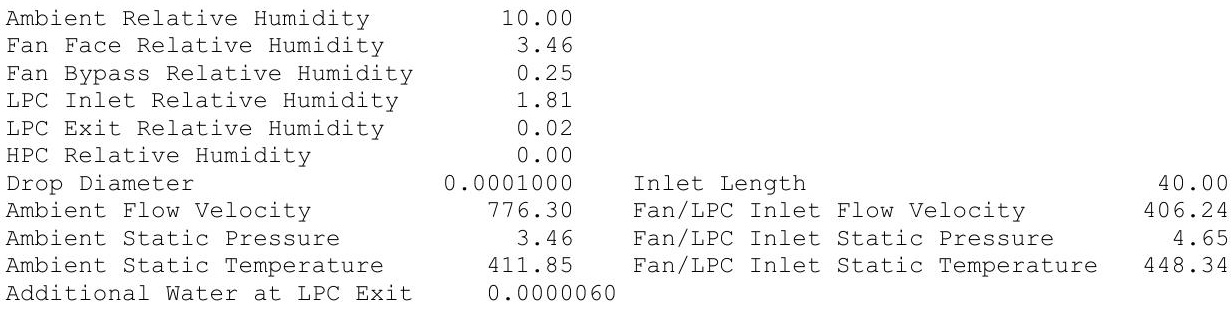

\begin{tabular}{|c|c|c|c|c|c|c|c|c|c|c|c|c|c|c|}
\hline & alt & dTamb & W & $\begin{array}{r}\text { SUMMARY } \\
\text { Fn }\end{array}$ & $\begin{array}{r}\text { TPUT DA } \\
\text { TSFC }\end{array}$ & Wfuel & BPR & VTAS & OPR & EPR & Powerset & T4 & T41 & T49 \\
\hline 0.780 & 35000.0 & 18.00 & 434.93 & 5854.8 & 0.5947 & 3481.75 & 5.6325 & 776.30 & 26.125 & 1.304 & 72.700 & 2513.1 & 2363.2 & 1634.8 \\
\hline
\end{tabular}

FSO Ambient.Fl

FS1 Inlet.Fi_O

FS12 Splitter.Fl_02

FS14 Fan. Fl

FS23 LPC.F1-

FS24 VaporIN.F1

FS25 Bleed2.F1_O

HPC.FI_O

FS36 Bleed3.FI_O

FS4 Burner.Fl_O

FS45 4 HPT.F1-

ES5 TEGV.FI

FS8 Core_Nozz.Fl_O

FS17 FanDuctLkg.Fi__

FS171 Bleed15.FI_O

FS172 FanDuct.FI-O

\begin{tabular}{rrrrrr}
\multicolumn{7}{c}{ FLOW } & \multicolumn{1}{l}{ STATION DATA } \\
W & Pt & Tt & ht & FAR & Wc \\
434.93 & 5.170 & 462.10 & -19.92 & 0.0000 & 1166.95 \\
434.93 & 5.170 & 462.10 & -19.92 & 0.0000 & 1166.95 \\
369.35 & 5.167 & 462.10 & -19.92 & 0.0000 & 991.50 \\
65.58 & 5.167 & 462.10 & -19.92 & 0.0000 & 176.03 \\
369.35 & 7.960 & 529.10 & -3.85 & 0.0000 & 688.70 \\
65.58 & 12.254 & 610.98 & 15.81 & 0.0000 & 85.35 \\
65.58 & 12.254 & 611.03 & 15.79 & 0.0000 & 85.36 \\
65.58 & 12.254 & 611.03 & 15.79 & 0.0000 & 85.36 \\
62.40 & 135.065 & 1283.76 & 182.50 & 0.0000 & 10.68 \\
48.36 & 135.065 & 1283.76 & 182.50 & 0.0000 & 8.28 \\
49.32 & 131.955 & 2513.06 & 158.08 & 0.0200 & 12.09 \\
65.78 & 30.657 & 1642.20 & -1.04 & 0.0149 & 56.11 \\
66.54 & 7.034 & 1168.95 & -124.27 & 0.0147 & 208.71 \\
66.54 & 7.034 & 1169.02 & -124.27 & 0.0147 & 208.72 \\
66.54 & 7.034 & 1169.15 & -124.27 & 0.0147 & 208.73 \\
369.35 & 7.960 & 529.10 & -3.85 & 0.0000 & 688.70 \\
369.35 & 7.960 & 529.10 & -3.85 & 0.0000 & 688.70 \\
369.35 & 7.960 & 529.10 & -3.85 & 0.0000 & 688.70 \\
369.35 & 7.960 & 529.10 & -3.85 & 0.0000 & 688.70
\end{tabular}

$\begin{array}{rr}\text { Ps } & \text { Ts } \\ 3.458 & 411.85 \\ 4.146 & 433.81 \\ 4.136 & 433.58 \\ 4.649 & 448.34 \\ 6.608 & 501.68 \\ 11.092 & 593.87 \\ 11.092 & 593.92 \\ 11.092 & 593.92 \\ 121.032 & 1246.72 \\ 126.938 & 1262.69 \\ 124.637 & 2480.22 \\ 27.585 & 1599.39 \\ 6.062 & 1123.70 \\ 6.062 & 1123.77 \\ 3.749 & 987.74 \\ 6.608 & 501.68 \\ 6.421 & 497.59 \\ 6.421 & 497.59 \\ 4.204 & 440.79\end{array}$

$\begin{array}{rr}\text { Aphy } & \text { MN } \\ 3560.0 & 0.7800 \\ 4168.2 & 0.5703 \\ 3531.8 & 0.5728 \\ 830.5 & 0.3912 \\ 2606.7 & 0.5226 \\ 412.6 & 0.3801 \\ 412.6 & 0.3801 \\ 412.6 & 0.3801 \\ 49.7 & 0.4038 \\ 49.3 & 0.3029 \\ 74.6 & 0.2987 \\ 265.4 & 0.4004 \\ 860.2 & 0.4718 \\ 860.2 & 0.4718 \\ 613.4 & 1.0000 \\ 2606.7 & 0.5226 \\ 2481.9 & 0.5625 \\ 2481.9 & 0.5625 \\ 2006.9 & 1.0000\end{array}$

gamt
1.40081
1.40081
1.40081
1.40081
1.40008
1.39855
1.39854
1.39854
1.36287
1.36287
1.29627
1.33249
1.35988
1.35988
1.35987
1.40008
1.40008
1.40008
1.40008

WAR
0.0000308
0.0000308
0.0000308
0.0000308
0.0000308
0.0000308
0.0000368
0.0000368
0.0000368
0.0000368
0.0000368
0.0000368
0.0000368
0.0000368
0.0000368
0.0000308
0.0000308
0.0000308
0.0000308

\begin{tabular}{|c|c|c|c|c|c|c|c|c|c|}
\hline \multicolumn{2}{|c|}{ TURBOMACH INERY } & PERFORMANCE & \multicolumn{2}{|l|}{ DATA } & \multirow[b]{2}{*}{ TR } & \multirow[b]{2}{*}{ efPoly } & \multirow[b]{2}{*}{ pwr } & \multirow[b]{2}{*}{ SMN } & \multirow[b]{2}{*}{ SMW } \\
\hline & Wc & PR & eff & Nc & & & & & \\
\hline Fan & 991.50 & 1.541 & 0.9070 & 3810.842 & 1.1450 & 0.9125 & -8395.6 & 67.58 & 30.09 \\
\hline LPC & 176.03 & 2.372 & 0.8684 & 3810.842 & 1.3222 & 0.8833 & -3315.4 & 4.29 & 3.38 \\
\hline HPC & 85.36 & 11.022 & 0.8615 & 10020.330 & 2.1010 & 0.8982 & -15091.7 & 34.11 & 32.76 \\
\hline HPT & 12.09 & 4.304 & 0.9073 & 216.954 & 1.3579 & 0.8878 & 15091.7 & & \\
\hline LPT & 56.11 & 4.358 & 0.9107 & 88.762 & 1.4022 & 0.8932 & 11710.9 & & \\
\hline \multicolumn{10}{|c|}{ TURBOMACHINERY MAP DATA } \\
\hline & WcMap & PRmap & effmap & NcMap & R/Parm & s_WcDes & s_PRdes & s_effDes & S_NcDes \\
\hline Fan & 1152.03 & 1.528 & 0.9089 & 3774.506 & 0.0583 & $\overline{0} .8607$ & $\overline{1} .0235$ & 0.9980 & $\overline{0} .9905$ \\
\hline LPC & 141.07 & 2.398 & 0.8201 & 1.007 & 0.0000 & 1.2479 & 0.9813 & 1.0589 & 0.0003 \\
\hline HPC & 77.63 & 10.550 & 0.8704 & 9753.195 & 12.7857 & 1.0995 & 1.0494 & 0.9898 & 0.9733 \\
\hline HPT & 0.96 & 4.213 & 0.9073 & 1.304 & 4.2126 & 12.6299 & 0.9723 & 1.0000 & 0.0003 \\
\hline LPT & 0.86 & 3.526 & 0.9175 & 0.998 & 3.5259 & 65.2590 & 0.7521 & 0.9926 & 0.0005 \\
\hline
\end{tabular}




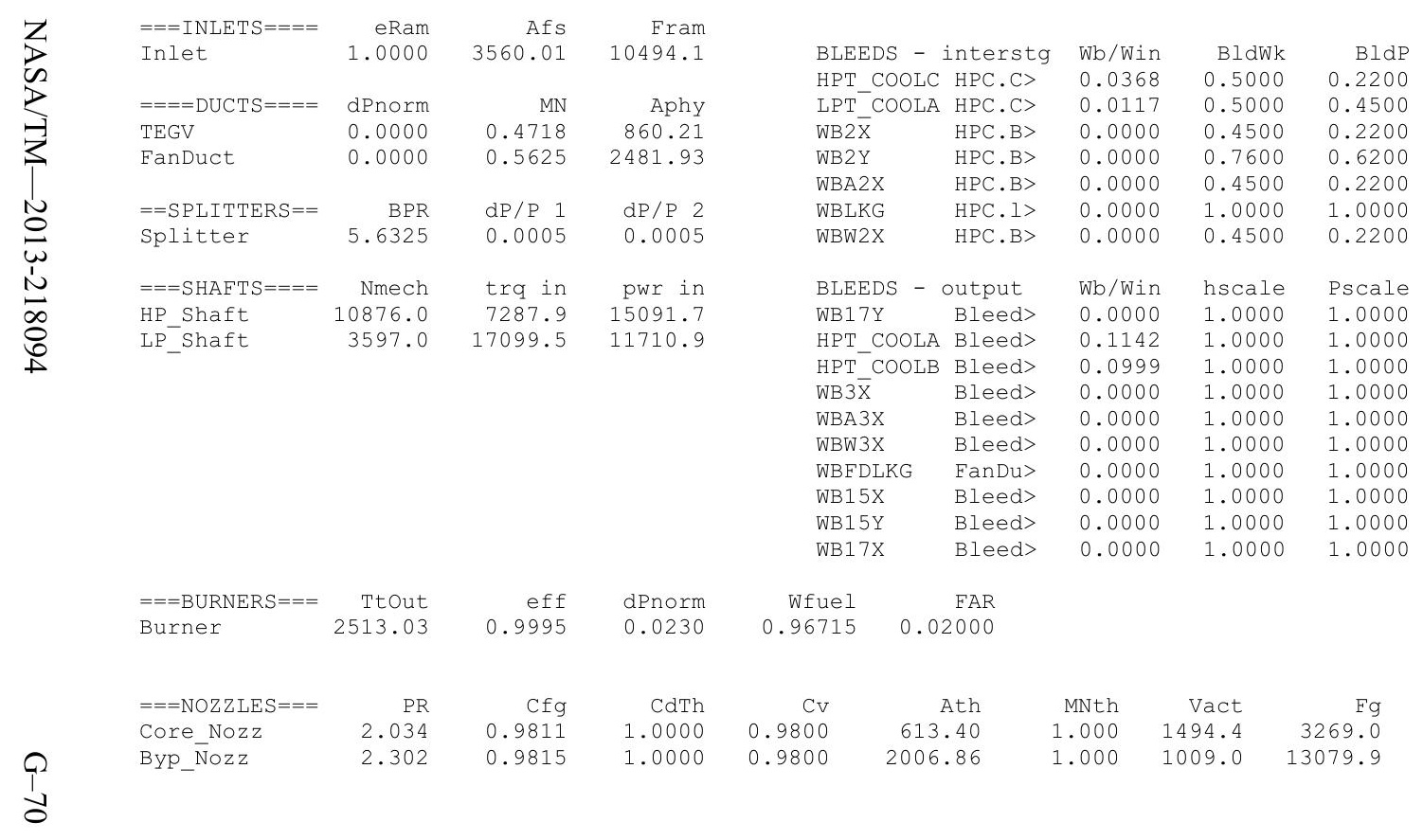


Date:07/05/13 Time:09:47:07 Model: Turbofan Engine - COMDES ON CONverge = 1 CASE:

Version:NPSS 1.6.5 - Rev: -> Gas Package: Janaf iter/pass/Jacb/Broy=18/32/1/16 Run by: Philip C Jorgenson PC: 69

Temperature Stator 1 inlet: 460.42 Stator 1 exit: 476.16 Stator 2 inlet: 495.46 Stator 2 exit: 503.76 $\begin{array}{llll}\text { Stator } 3 \text { inlet: } 526.43 & \text { Stator } 3 \text { exit: } 534.63 & \text { Stator } 4 \text { inlet: } 556.07 \text { Stator } 4 \text { exit: } 563.55 \\ \text { Stator } 5 \text { inlet: } 580.77 & \text { Stator } 5 \text { exit: } 587.78 & \text { Unblocked Percent Blockage: } 0.00\end{array}$

$\begin{array}{lclll}\text { Ambient Relative Humidity } & 10.00 & & & \\ \text { Fan Face Relative Humidity } & 3.36 & & & \\ \text { Fan Bypass Relative Humidity } & 0.26 & & & \\ \text { LPC Inlet Relative Humidity } & 1.77 & & & \\ \text { LPC Exit Relative Humidity } & 0.02 & & & \\ \text { HPC Relative Humidity } & 0.00 & & & \\ \text { Drop Diameter } & 0.0001000 & \text { Inlet Length } & & \\ \text { Ambient Flow Velocity } & 776.30 & \text { Fan/LPC Inlet Flow Velocity } & 397.99 \\ \text { Ambient Static Pressure } & 3.46 & \text { Fan/LPC Inlet Static Pressure } & 4.67 \\ \text { Ambient Static Temperature } & 411.85 & \text { Fan/LPC Inlet Static Temperature } & 448.89 \\ \text { Additional Water at LPC Exit } & 0.0000060 & & & \end{array}$

\begin{tabular}{|c|c|c|c|c|c|c|c|c|c|c|c|c|c|c|}
\hline & & & & SUMMARY & UTPUT DA & Wfuel & & VTAS & & $E P$ & Power Set & $T 2$ & T41 & 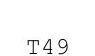 \\
\hline 0.780 & 35000.0 & 18.00 & 430.86 & 5636.2 & 0.5955 & 3356.39 & 5.6861 & 776.30 & 25.519 & 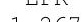 & FUWLDEL & 14 & 23367 & . \\
\hline
\end{tabular}

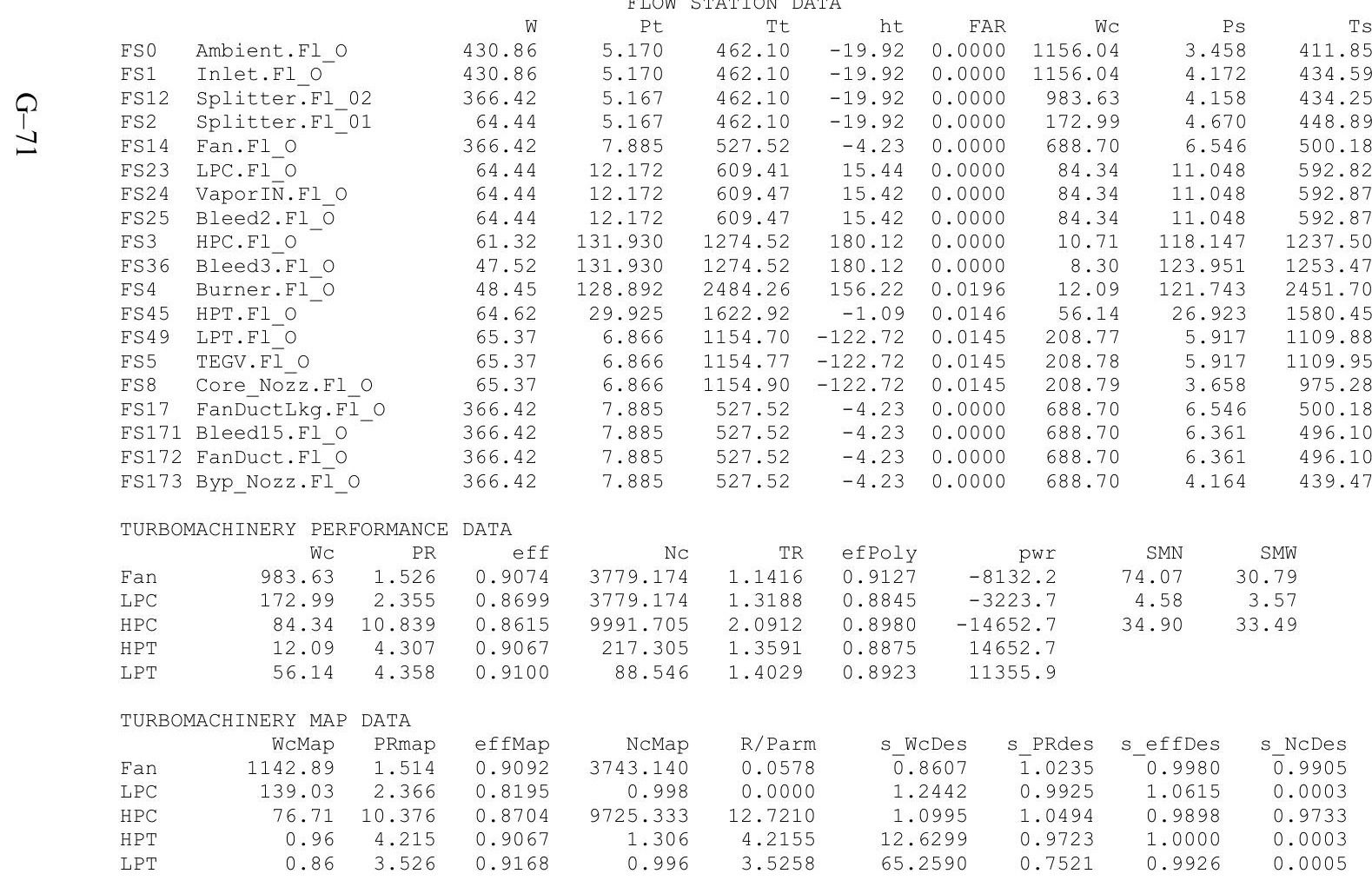




\begin{tabular}{|c|c|c|c|}
\hline$===$ INLETS $====$ & eRam & Afs & Fram \\
\hline Inlet & 1.0000 & 3526.73 & 10396.0 \\
\hline$====$ DUCTS $====$ & dPnorm & MN & Aphy \\
\hline TEGV & 0.0000 & 0.4718 & 860.21 \\
\hline FanDuct & 0.0000 & 0.5625 & 2481.93 \\
\hline$==$ SPLITTERS $==$ & BPR & $\mathrm{dP} / \mathrm{P} 1$ & $\mathrm{dP} / \mathrm{P} 2$ \\
\hline Splitter & 5.6861 & 0.0005 & 0.0005 \\
\hline$===$ SHAFTS $====$ & Nmech & $\operatorname{trg}$ in & pwr in \\
\hline HP Shaft & 10831.0 & 7105.3 & 14652.7 \\
\hline LP Shaft & 3567.1 & 16720.1 & 11355.9 \\
\hline
\end{tabular}

BLEEDS - interstg Wb/Win BldWk BldP HPT_COOLC HPC.C> $0.0368 \quad 0.5000 \quad 0.2200$ $\begin{array}{lllll}\text { LPT COOLA HPC.C> } & 0.0117 & 0.5000 & 0.4500 \\ \text { WB } 2 \bar{X} & \text { HPC. }> & 0.0000 & 0.4500 & 0.2200\end{array}$ $\begin{array}{lllll}\text { WB2X } & \text { HPC.B } & 0.0000 & 0.4500 & 0.2200 \\ \text { WB2Y } & \text { HPC. B> } & 0.0000 & 0.7600 & 0.6200\end{array}$ $\begin{array}{lllll}\text { WB2Y } & \text { HPC.B> } & 0.0000 & 0.7600 & 0.6200\end{array}$ $\begin{array}{llll}\text { WBA2X HPC.B> } & 0.0000 & 0.4500 & 0.2200\end{array}$ $\begin{array}{lllll}\text { WBLKG HPC.I> } & 0.0000 & 1.0000 & 1.0000\end{array}$ $\begin{array}{lllll}\text { WBW2X HPC.B> } & 0.0000 & 0.4500 & 0.2200\end{array}$

BLEEDS - output Wb/Win hscale Pscale WB17Y Bleed> $0.0000 \quad 1.0000 \quad 1.0000$ HPT_COOLA Bleed> $0.1142 \quad 1.0000 \quad 1.0000$ $\begin{array}{lllll}\text { HPT_COOLB } & \text { Bleed> } & 0.0999 & 1.0000 & 1.0000 \\ \text { WB } 3 \bar{X} & \text { Bleed> } & 0.0000 & 1.0000 & 1.0000\end{array}$ $\begin{array}{lllll}\text { WB3X } & \text { Bleed> } & 0.0000 & 1.0000 & 1.0000 \\ \text { WBA3X } & \text { Bleed }> & 0.0000 & 1.0000 & 1.0000\end{array}$ $\begin{array}{lllll}\text { WBA3X } & \text { Bleed> } & 0.0000 & 1.0000 & 1.0000 \\ \text { WBW3X } & \text { Bleed> } & 0.0000 & 1.0000 & 1.0000\end{array}$ $\begin{array}{lllll}\text { WBW3X } & \text { Bleed> } & 0.0000 & 1.0000 & 1.0000 \\ \text { WBFDLKG } & \text { FanDu> } & 0.0000 & 1.0000 & 1.0000\end{array}$ $\begin{array}{lllll}\text { WBFDLKG } & \text { FanDu> } & 0.0000 & 1.0000 & 1.0000 \\ \text { WB15X } & \text { Bleed }> & 0.0000 & 1.0000 & 1.0000 \\ \text { WB15Y } & \text { Bleed }> & 0.0000 & 1.0000 & 1.0000\end{array}$ $\begin{array}{lllll}\text { WB15X } & \text { Bleed }> & 0.0000 & 1.0000 & 1.0000 \\ \text { WB15Y } & \text { Bleed }> & 0.0000 & 1.0000 & 1.0000\end{array}$ WB17X Bleed> $0.0000 \quad 1.0000 \quad 1.0000$

$\begin{array}{rrrr}\text { W } & \text { Tt } & \text { ht } & \text { Pt } \\ 2.3715 & 947.83 & 97.77 & 29.925 \\ 0.7540 & 947.83 & 97.77 & 6.866 \\ 0.0000 & 914.45 & 89.53 & 38.519 \\ 0.0000 & 1119.35 & 140.59 & 86.422 \\ 0.0000 & 914.45 & 89.53 & 38.519 \\ 0.0000 & 1274.52 & 180.12 & 131.930 \\ 0.0000 & 914.45 & 89.53 & 38.519 \\ \text { W } & \text { Tt } & \text { ht } & \text { Pt } \\ 0.0000 & 609.47 & 15.42 & 12.172 \\ 7.3580 & 1274.52 & 180.12 & 128.892 \\ 6.4384 & 1274.52 & 180.12 & 72.283 \\ 0.0000 & 1274.52 & 180.12 & 131.930 \\ 0.0000 & 1274.52 & 180.12 & 131.930 \\ 0.0000 & 1274.52 & 180.12 & 131.930 \\ 0.0000 & 527.52 & -4.23 & 7.885 \\ 0.0000 & 527.52 & -4.23 & 7.885 \\ 0.0000 & 527.52 & -4.23 & 7.885 \\ 0.0000 & 527.52 & -4.23 & 7.885\end{array}$

$===$ BURNERS $===\quad$ TtOut Burner 2484.24

eff
0.9995

dPnorm

0.93233

0.01962

$\mathrm{PR}$
1.986
2.280

\section{Cfg
0.9808}

CdTh

$\mathrm{CV}$
0.9800

Ath

Core_Nozz

0.9815

$0.9800 \quad 2006.86$

MNth Vact

$\begin{array}{lrr}1.000 & 1485.5 & \text { Fg } \\ 1.000 & 1007.5 & 12891.0\end{array}$ 
Date:07/05/13 Time:09:57:39 Model: Turbofan Engine - COMDES ON COnverge = 1 CASE: O

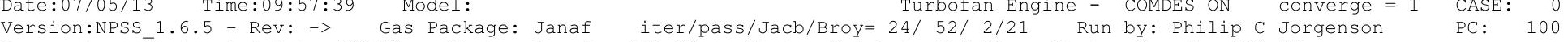

$\begin{array}{llll}\text { Version:NPSS 1.6.5 - Rev: -> Gas Package: Janaf iter/pass/Jacb/Broy= 24/ } 52 / 2 / 21 & \text { Run by: Philip C Jorge } \\ \text { Temperature Stator } 1 \text { inlet: } 468.73 \quad \text { Stator } 1 \text { exit: } 488.20 \quad \text { Stator } 2 \text { inlet: } 510.79 \text { Stator } 2 \text { exit: } 518.46\end{array}$

$\begin{array}{rllll}\text { Temperature } & \text { Stator } 1 \text { inlet: } 468.73 & \text { Stator } 1 \text { exit: } 488.20 & \text { Stator } 2 \text { inlet: } 510.79 & \text { Stator } 2 \text { exit: } 518.46 \\ \text { Stator } 3 \text { inlet: } 548.49 & \text { Stator } 3 \text { exit: 556.93 } & \text { Stator } 4 \text { inlet: 585.07 } & \text { Stator } 4 \text { exit: } 593.21\end{array}$

Stator 5 inlet: 615.01 Stator 5 exit: $622.94 \quad$ Unblocked Percent Blockage: 0.00

Ambient Relative Humidity

Fan Face Relative Humidity $\quad 5.34$

Fan Bypass Relative Humidity $\quad 0.21$

LPC Inlet Relative Humidity $\quad 2.54$

LPC Exit Relative Humidity $\quad 0.03$

Drop Diameter

Ambient Flow Velocity

0001000
793.07

3.46
429.85

Inlet Length

Ambient Static Pressure

Additional Water at LPC Exit

0.0000034

Inlet Flow Velocity $\quad 493.59$

SUMMARY OUTPUT DATA

$\begin{array}{rrrrrr}\text { MN } & \text { alt } & \text { dTamb } & \text { W } & \text { Fn } & \text { TSFC } \\ 0.780 & 35000.0 & 36.00 & 465.50 & 8111.9 & 0.6156\end{array}$

BPR
5.2957

VTAS

OPR
1.926

$E P R$
1.687

PowerSet

$\begin{array}{ccr}\text { T4 } & \text { T41 } & \text { T49 } \\ 2926.0 & 2747.3 & 1915.5\end{array}$

\begin{tabular}{|c|c|}
\hline FSO & Ambient.Fl_O \\
\hline FS1 & Inlet.Fl_O \\
\hline FS12 & Splitter.Fl_02 \\
\hline FS2 & Splitter.Fl_01 \\
\hline FS14 & Fan.Fl_O - \\
\hline FS23 & LPC.Fl_o \\
\hline FS24 & VaporIN. Fl O \\
\hline FS25 & Bleed2.F1_o \\
\hline FS3 & HPC.Fl O \\
\hline FS36 & Bleed3.F1_o \\
\hline FS4 & Burner.FI_O \\
\hline FS45 & HPT.FI_O \\
\hline FS49 & LPT.FI_O \\
\hline FS5 & TEGV.FI_O \\
\hline FS8 & Core Nozz.Fl \\
\hline FS17 & FanDüctLkg.Fì_o \\
\hline FS171 & Bleed15.FI_O \\
\hline FS172 & FanDuct.F \\
\hline$\$ 173$ & Byp_Nozz \\
\hline
\end{tabular}

$W$
465.50
465.50
391.56
73.94
391.56
73.94
73.94
73.94
70.35
54.52
55.91
74.46
75.33
75.33
75.33
391.56
391.56
391.56
391.56
DATA

FLOW STATION DATA

TURBOMACHINERY PERFORMANCE DATA

\begin{tabular}{|c|c|c|c|c|c|c|c|c|c|}
\hline & Wc & PR & eff & $\mathrm{NC}$ & TR & efPoly & pwr & SMN & SMW \\
\hline Fan & 1073.84 & 1.693 & 0.9109 & 4120.682 & 1.1782 & 0.9173 & 20.7 & 38.39 & 23.45 \\
\hline LPC & 202.77 & 2.457 & 0.8274 & 4120.682 & 1.3534 & 0.8476 & 284.5 & 2.96 & 2.22 \\
\hline HPC & 96.03 & 13.002 & 0.8587 & 10334.793 & 2.1956 & 0.8978 & 57.3 & 26.49 & 25.69 \\
\hline HPT & 12.10 & 4.272 & 0.9122 & 214.343 & 1.3425 & 0.8900 & 57.3 & & \\
\hline LPT & 55.84 & 4.342 & 0.9168 & 90.571 & 1.3886 & 0.9005 & 104.1 & & \\
\hline \multicolumn{10}{|c|}{ TURBOMACHINERY MAP DATA } \\
\hline & WcMap & PRmap & effMap & NcMap & R/Parm & S_WcDes & S_PRdes & s_effDes & S_NCDes \\
\hline Fan & 1247.70 & 1.677 & 0.9128 & 4081.391 & 0.0636 & $\overline{0} .8607$ & $\overline{1} .0235$ & 0.9980 & $\overline{0} .9905$ \\
\hline LPC & 165.11 & 2.727 & 0.8329 & 1.088 & 0.0000 & 1.2281 & 0.8433 & 0.9934 & 0.0003 \\
\hline HPC & 87.34 & 12.437 & 0.8675 & 10059.274 & 13.4517 & 1.0995 & 1.0494 & 0.9898 & 0.9733 \\
\hline $\mathrm{HPT}$ & 0.96 & 4.181 & 0.9122 & 1.288 & 4.1809 & 12.6299 & 0.9723 & 1.0000 & 0.0003 \\
\hline LPT & 0.86 & 3.514 & 0.9236 & 1.019 & 3.5137 & 65.2590 & 0.7521 & 0.9926 & 0.0005 \\
\hline
\end{tabular}




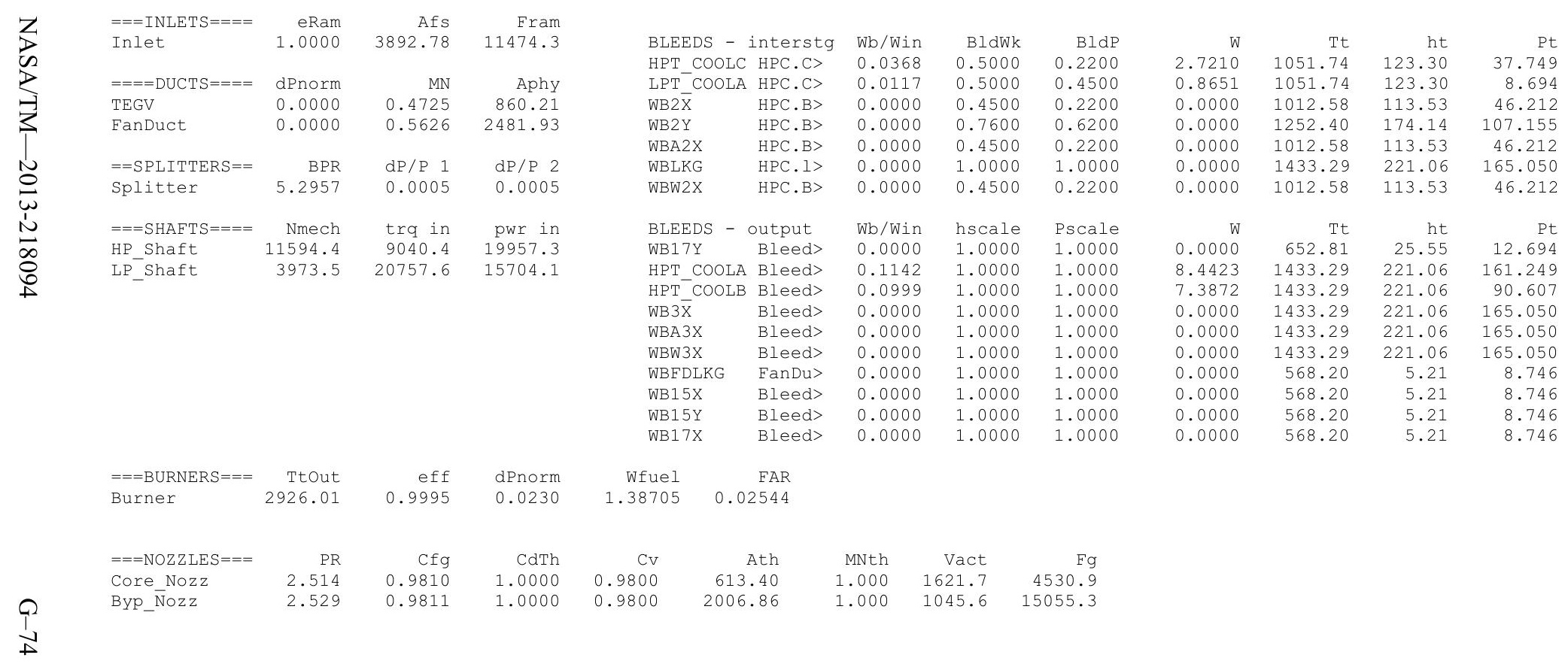


Turbofan Engine - COMDES ON COnverge $=1$ CASE: 0

Version:NPSS 1.6.5 - Rev: -> Gas Package: Janaf iter/pass/Jacb/Broy=18/32/1/16 Run by: Philip C Jorgenson PC: 75

Temperature Stator 1 inlet: 478.71 Stator 1 exit: 495.67 Stator 2 inlet: 516.27 Stator 2 exit: 524.91 $\begin{array}{cccc}\text { Stator } 3 \text { inlet: } 549.54 & \text { Stator } 3 \text { exit: } 558.17 & \text { Stator } 4 \text { inlet: } 581.47 \text { Stator } 4 \text { exit: } 589.37 \\ \text { Stator } 5 \text { inlet: } 607.99 & \text { Stator } 5 \text { exit: } 615.44 & \text { Unblocked Percent Blockage: } 0.00\end{array}$

Ambient Relative Humidity $\quad 10.00$

Fan Face Relative Humidity $\quad 3.82$

Fan Bypass Relative Humidity $\quad 0.30$

LPC Inlet Relative Humidity $\quad 2.07$

LPC Exit Relative Humidity $\quad 0.03$

HPC Relative Humidity 0.00

Ambient Flow Velocity 0.0001000

Fan/IPC Inlet Flow Velocity 40.00

Ambient Static Pressure

Additional Water at LPC Exit

3.46 Fan/LPC Inlet Static Pressure $\quad 4.63$ 0.0000042

Fan/LPC Inlet Static Temperature 467.34

\section{MN 2 alt}

alt dTamb $\begin{array}{ccc}\text { SUMMARY OUTPUT DATA } \\ \text { Fn } & \text { TSFC } \\ 6089.7 & 0.6090\end{array}$
TION DATA

FSO Ambient.Fl_O

FS1 Inlet.FI_O

FS12 Splitter.F1_02

ES2 Splitter.FI_01

FS14 Fan.FI_O

FS24 VaporIN.F1_O

FS25 Bleed2.F1_O

FS3 HPC.FI_O

FS4 Burner. Fl_

FS45 HPT.FI_O

FS49 LPT.FI O

FS8 Core Nozz.Fl

FS17 FanDúctLkg.Fì_o

FS171 Bleed15.FI_O

FS172 FanDuct.Fl_O
FS173 Byp Nozz.FİO

$W$
429.86
429.86
364.58
65.28
364.58
65.28
65.28
65.28
62.11
48.14
49.17
65.54
66.31
66.31
66.31
364.58
364.58
364.58
364.58

\section{$\begin{array}{cc}\text { Pt } & \text { Tt } \\ \text { Pt } & -15\end{array}$}

$\mathrm{Pt}$
5.170
5.170

5.170
5.167

5.167

5.167
8.040

12.326

12.326

138.014

138.014
138.014

134.836

482.28
482.28
482.28

482.28
482.28

553.90

639.11
639.18

639.18
639.18

1344.96

1344.96
2640.61
1730.96

$\begin{array}{llll}7.215 & 1235.77 & -126.23 & 0.0158 \\ 7.215 & 1235.83 & -126.23 & 0.0158\end{array}$

$\begin{array}{rrrr}7.215 & 1235.97 & -126.23 & 0.0158 \\ 8.040 & 553.90 & 1.77 & 0.0000\end{array}$

$\begin{array}{llll}8.040 & 553.90 & 1.77 & 0.0000\end{array}$

$\begin{array}{llll}8.040 & 553.90 & 1.77 & 0.0000 \\ 8.040 & 553.90 & 1.77 & 0.0000\end{array}$ $\begin{array}{rrrr} & \text { FAR } & \text { WC } \\ & & & \\ & & \end{array}$
Wfuel

BPR
5851

VTAS
793.07

OPR

\section{Ps} $\begin{array}{lll}-15.41 & 0.0000 & -179.8\end{array}$ $\begin{array}{rrr}1.77 & 0.0000 & 688.65 \\ 22.27 & 0.0000 & 86.39\end{array}$ $\begin{array}{lll}22.26 & 0.0000 & 86.39\end{array}$ $\begin{array}{lll}22.26 & 0.0000 & 86.40\end{array}$ $98.01 \quad 0.0000$ $\begin{array}{ll}98.01 & 0.0000\end{array}$ 10.65
8.25
12.09 12.09
56.06 208.46 208.47 208.48 688.65
688.65 688.65 688.65
688.65

$\begin{array}{rr}\text { Ps } & \text { Ts } \\ 3.458 & 429.85 \\ 4.118 & 451.90 \\ 4.112 & 451.76 \\ 4.629 & 467.34 \\ 6.674 & 525.21 \\ 11.124 & 620.72 \\ 11.124 & 620.78 \\ 11.124 & 620.78 \\ 123.763 & 1306.69 \\ 129.759 & 1323.19 \\ 127.372 & 2606.55 \\ 28.250 & 1686.39 \\ 6.221 & 1188.52 \\ 6.221 & 1188.58 \\ 3.851 & 1046.26 \\ 6.674 & 525.21 \\ 6.486 & 520.93 \\ 6.486 & 520.93 \\ 4.246 & 461.49\end{array}$

EPR
1.344
Powerset

$\begin{array}{ccc}\text { T4 } & \text { T41 } & \text { T49 } \\ 2640.6 & 2483.3 & 1723.1\end{array}$

TURBOMACHINERY PERFORMANCE DATA

\begin{tabular}{|c|c|c|c|c|c|c|c|c|c|}
\hline & Wc & $\mathrm{PR}$ & eff & NC & TR & efPoly & pwr & SMN & SMW \\
\hline Fan & 999.86 & 1.556 & 0.9070 & 3842.188 & 1.1485 & 0.9126 & 362.7 & 62.42 & 29.35 \\
\hline LPC & 179.02 & 2.385 & 0.8662 & 3842.188 & 1.3252 & 0.8814 & 779.5 & 4.01 & 3.17 \\
\hline HPC & 86.40 & 11.197 & 0.8615 & 10049.149 & 2.1042 & 0.8982 & 339.0 & 33.46 & 32.17 \\
\hline HPT & 12.09 & 4.295 & 0.9089 & 217.092 & 1.3535 & 0.8892 & 39.0 & & \\
\hline LPT & 56.06 & 4.351 & 0.9129 & 89.051 & 1.3979 & 0.8960 & 342.2 & & \\
\hline \multirow{2}{*}{\multicolumn{10}{|c|}{ TURBOMACHINERY MAP DATA }} \\
\hline & & & & & & & & & s NcDes \\
\hline Fan & 1161.74 & 1.543 & 0.9088 & 3805.553 & 0.0589 & $\overline{0} .8607$ & $\overline{1} .0235$ & 0.9980 & $\overline{0} .9905$ \\
\hline LPC & 143.09 & 2.430 & 0.8208 & 1.015 & 0.0000 & 1.2510 & 0.9689 & 1.0554 & 0.0003 \\
\hline HPC & 78.58 & 10.717 & 0.8704 & 9781.245 & 12.8379 & 1.0995 & 1.0494 & 0.9898 & 0.9733 \\
\hline HPT & 0.96 & 4.204 & 0.9089 & 1.305 & 4.2040 & 12.6299 & 0.9723 & 1.0000 & 0.0003 \\
\hline LPT & 0.86 & 3.520 & 0.9197 & 1.002 & 3.5201 & 65.2590 & 0.7521 & 0.9926 & 0.0005 \\
\hline
\end{tabular}

$\begin{array}{rrrrrrr}\text { Aphy } & \text { MN } & \text { gamt } & \text { WAR } & \text { Wair } & \text { WH2O } & \text { H2O frac } \\ 3594.8 & 0.7800 & 1.40064 & 0.0000871 & 429.82 & 0.037 & 0.0001 \\ 4168.2 & 0.5792 & 1.40064 & 0.0000871 & 429.82 & 0.037 & 0.0001 \\ 3531.8 & 0.5806 & 1.40064 & 0.0000871 & 364.55 & 0.032 & 0.0001 \\ 830.5 & 0.3994 & 1.40064 & 0.0000871 & 65.27 & 0.006 & 0.0001 \\ 2606.7 & 0.5226 & 1.39969 & 0.0000871 & 364.55 & 0.032 & 0.0001 \\ 412.6 & 0.3858 & 1.39781 & 0.0000871 & 65.27 & 0.006 & 0.0001 \\ 412.6 & 0.3858 & 1.39781 & 0.0000913 & 65.27 & 0.006 & 0.0001 \\ 412.6 & 0.3858 & 1.39781 & 0.0000913 & 65.27 & 0.006 & 0.0001 \\ 49.7 & 0.4030 & 1.35915 & 0.0000913 & 62.11 & 0.006 & 0.0001 \\ 49.3 & 0.3023 & 1.35915 & 0.0000913 & 48.13 & 0.004 & 0.0001 \\ 74.6 & 0.2992 & 1.29155 & 0.0000913 & 48.13 & 0.004 & 0.0259 \\ 265.4 & 0.4008 & 1.32754 & 0.0000913 & 64.51 & 0.006 & 0.0195 \\ 860.2 & 0.4721 & 1.35496 & 0.0000913 & 65.27 & 0.006 & 0.0193 \\ 860.2 & 0.4721 & 1.35495 & 0.0000913 & 65.27 & 0.006 & 0.0193 \\ 613.4 & 1.0000 & 1.35494 & 0.0000913 & 65.27 & 0.006 & 0.0193 \\ 2606.7 & 0.5226 & 1.39969 & 0.0000871 & 364.55 & 0.032 & 0.0001 \\ 2481.9 & 0.5626 & 1.39969 & 0.0000871 & 364.55 & 0.032 & 0.0001 \\ 2481.9 & 0.5626 & 1.39969 & 0.0000871 & 364.55 & 0.032 & 0.0001 \\ 2006.9 & 1.0000 & 1.39969 & 0.0000871 & 364.55 & 0.032 & 0.0001\end{array}$




\begin{tabular}{|c|c|c|c|c|c|c|c|c|c|c|c|c|}
\hline $\begin{array}{l}===\text { INLETS }==== \\
\text { Inlet }\end{array}$ & eRam & Afs & Fram & \multirow{2}{*}{\multicolumn{2}{|c|}{ BLEEDS - interstg }} & & & & & & & \\
\hline Inlet & 1.0000 & 3594.76 & 10595.8 & & & $9 \quad \mathrm{~Wb} / \mathrm{Win}$ & BldWk & BldP & $\begin{array}{r}W \\
2.4022\end{array}$ & $\begin{array}{r}\text { Tt } \\
998.97\end{array}$ & ht & $\begin{array}{r}\text { Pt } \\
31.391\end{array}$ \\
\hline$====$ DUCTS $====$ & dPnorm & MN & Aphy & \multicolumn{2}{|c|}{ HPT_COOLC HPC.C> } & 0.0117 & 0.5000 & 0.4500 & 0.7637 & 998.97 & 110.14 & 7.215 \\
\hline TEGV & 0.0000 & 0.4721 & 860.21 & \multicolumn{2}{|c|}{$\begin{array}{ll}\text { LPT }^{-} \text {COOLA } & \text { HPC.C } C> \\
\text { WB } 2 \bar{X} & \text { HPC.B }>\end{array}$} & 0.0000 & 0.4500 & 0.2200 & 0.0000 & 963.55 & 101.35 & 39.977 \\
\hline FanDuct & 0.0000 & 0.5626 & 2481.93 & WB2Y & HPC.B> & 0.0000 & 0.7600 & 0.6200 & 0.0000 & 1180.75 & 155.83 & 90.253 \\
\hline & & & & \multirow{2}{*}{$\begin{array}{l}\text { WBA2X } \\
\text { WBLKG }\end{array}$} & HPC.B> & 0.0000 & 0.4500 & 0.2200 & 0.0000 & 963.55 & 101.35 & 39.977 \\
\hline$==$ SPLITTERS $==$ & BPR & $\mathrm{dP} / \mathrm{P} 1$ & $\mathrm{dP} / \mathrm{P} 2$ & & HPC.I> & 0.0000 & 1.0000 & 1.0000 & 0.0000 & 1344.96 & 198.01 & 138.014 \\
\hline Splitter & 5.5851 & 0.0005 & 0.0005 & WBW2X & HPC.B> & 0.0000 & 0.4500 & 0.2200 & 0.0000 & 963.55 & 101.35 & 39.977 \\
\hline$===$ SHAFTS $====$ & Nmech & trq in & pwr in & \multicolumn{2}{|c|}{ BLEEDS - output } & Wb/Win & hscale & Pscale & W & $\mathrm{Tt}$ & ht & Pt \\
\hline HP_Shaft & 11155.7 & 7457.0 & 15839.0 & WB17Y & Bleed> & 0.0000 & 1.0000 & 1.0000 & 0.0000 & 639.18 & 22.26 & 12.326 \\
\hline LP_Shaft & 3704.9 & 17496.3 & 12342.2 & HPT_COOLA & A Bleed> & 0.1142 & 1.0000 & 1.0000 & 7.4534 & 1344.96 & 198.01 & 134.836 \\
\hline & & & & \multirow{2}{*}{$\mathrm{HPT}_{-}^{-} \mathrm{COOLB}$} & B Bleed> & 0.0999 & 1.0000 & 1.0000 & 6.5219 & 1344.96 & 198.01 & 75.665 \\
\hline & & & & & Bleed> & 0.0000 & 1.0000 & 1.0000 & 0.0000 & 1344.96 & 198.01 & 138.014 \\
\hline & & & & WBA3X & Bleed> & 0.0000 & 1.0000 & 1.0000 & 0.0000 & 1344.96 & 198.01 & 138.014 \\
\hline & & & & \multirow{2}{*}{$\begin{array}{l}\text { WBW3X } \\
\text { WBFDLKG }\end{array}$} & Bleed> & 0.0000 & 1.0000 & 1.0000 & 0.0000 & 1344.96 & 198.01 & 138.014 \\
\hline & & & & & FanDu> & 0.0000 & 1.0000 & 1.0000 & 0.0000 & 553.90 & 1.77 & 8.040 \\
\hline & & & & WB15X & Bleed> & 0.0000 & 1.0000 & 1.0000 & 0.0000 & 553.90 & 1.77 & 8.040 \\
\hline & & & & WB15Y & Bleed> & 0.0000 & 1.0000 & 1.0000 & 0.0000 & 553.90 & 1.77 & 8.040 \\
\hline & & & & WB17X & Bleed> & 0.0000 & 1.0000 & 1.0000 & 0.0000 & 553.90 & 1.77 & 8.040 \\
\hline$===$ BURNERS $===$ & TtOut & eff & dPnorm & \multicolumn{2}{|c|}{ Wfuel FAR } & & & & & & & \\
\hline Burner & 2640.59 & 0.9995 & 0.0230 & \multicolumn{2}{|c|}{$\begin{array}{rr}\text { Wfuel } & \text { FAR } \\
1.03023 & 0.02140\end{array}$} & & & & & & & \\
\hline$===$ NOZZLES $===$ & $\mathrm{PR}$ & $\mathrm{Cfg}$ & CdTh & $\mathrm{Cv}$ & & MNth & Vact & $\mathrm{Fg}$ & & & & \\
\hline Core_Nozz & 2.087 & 0.9813 & 1.0000 & $\begin{array}{r}\mathrm{CV} \\
0.9800\end{array}$ & $\begin{array}{r}\text { Ath } \\
613.40\end{array}$ & 1.000 & 1535.6 & \multirow{2}{*}{$\begin{array}{r}3405.4 \\
13280.1\end{array}$} & & & & \\
\hline Byp__Nozz & 2.325 & 0.9815 & 1.0000 & \multicolumn{2}{|c|}{2006.86} & 1.000 & 1032.4 & & & & & \\
\hline
\end{tabular}


Turbofan Engine - COMDES ON COnverge $=1$ CASE: 0

Version:NPSS 1.6.5 - Rev: -> Gas Package: Janaf iter/pass/Jacb/Broy=17/31/1/15 Run by: Philip C Jorgenson PC: 72

Temperature Stator 1 inlet: 479.68 Stator 1 exit: 496.38 Stator 2 inlet: 516.78 Stator 2 exit: 525.45

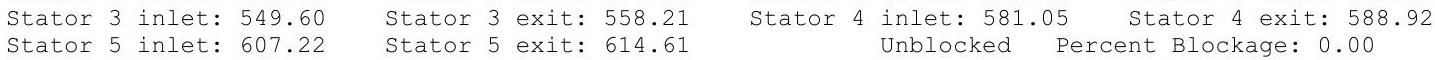

Ambient Relative Humidity $\quad 10.00$

Fan Face Relative Humidity $\quad 3.70$

Fan Bypass Relative Humidity $\quad 0.31$

LPC Inlet Relative Humidity $\quad 2.02$

LPC Exit Relative Humidity $\quad 0.03$

HPC Relative Humidity

0.00

Ambient Flow Velocity

0.0001000

Inlet Length $\quad 40.00$

Ambient Static Pressure

Additional Water at LPC Exit

3.46 Fan/LPC Inlet Static Pressure $\quad 4.65$

0.0000044

Fan/LPC Inlet Static Temperature 467.94

$\begin{array}{lll}\text { MN } & \text { alt dTamb } & \text { SUMMARY OUTPUT DATA } \\ 0.780 & \text { Fn } & \text { TSFC }\end{array}$

$\begin{array}{lrr}0.780 & 35000.0 & 36.00\end{array}$
W
425.73
Dith
FSO Ambient.Fl 0

FS1 Inlet.Fl_o
Wfuel 3572.18
BPR
5.6350

$(5.6350$

VTAS
793.07
OPR
6.077

$\begin{array}{rr}\text { Ps } & \text { Ts } \\ 3.458 & 429.85 \\ 4.146 & 452.76 \\ 4.136 & 452.51 \\ 4.650 & 467.94 \\ 6.609 & 523.59 \\ 11.102 & 619.87 \\ 11.102 & 619.94 \\ 11.102 & 619.94 \\ 120.809 & 1296.84 \\ 126.704 & 1313.33 \\ 124.418 & 2575.32 \\ 27.576 & 1665.70 \\ 6.071 & 1173.17 \\ 6.071 & 1173.24 \\ 3.757 & 1032.37 \\ 6.609 & 523.59 \\ 6.422 & 519.33 \\ 6.422 & 519.33 \\ 4.205 & 460.07\end{array}$

$\mathrm{EPR}$
1.306

\section{Aphy}

$\begin{array}{rr}\text { Aphy } & \text { MN } \\ 3560.2 & 0.7800 \\ 4168.2 & 0.5703 \\ 3531.8 & 0.5729 \\ 830.5 & 0.3911 \\ 2606.7 & 0.5226 \\ 412.6 & 0.3797 \\ 412.6 & 0.3798 \\ 412.6 & 0.3798 \\ 49.7 & 0.4042 \\ 49.3 & 0.3032 \\ 74.6 & 0.2990 \\ 265.4 & 0.4008 \\ 860.2 & 0.4720 \\ 860.2 & 0.4720 \\ 613.4 & 1.0000 \\ 2606.7 & 0.5226 \\ 2481.9 & 0.5626 \\ 2481.9 & 0.5626 \\ 2006.9 & 1.0000\end{array}$

Powerset 72.700

$\begin{array}{ccc}\text { T4 } & \text { T41 } & \text { T49 } \\ 2609.1 & 2454.1 & 1702.2\end{array}$

$\begin{array}{rrrrrr}\text { W } & \text { Pt } & \text { Tt } & \text { ht } & \text { FAR } & \text { WC } \\ 425.73 & 5.170 & 482.28 & -15.41 & 0.0000 & 1166.96 \\ 425.73 & 5.170 & 482.28 & -15.41 & 0.0000 & 1166.96 \\ 361.56 & 5.167 & 482.28 & -15.41 & 0.0000 & 991.58 \\ 64.16 & 5.167 & 482.28 & -15.41 & 0.0000 & 175.97 \\ 361.56 & 7.961 & 552.20 & 1.36 & 0.0000 & 688.66 \\ 64.16 & 12.262 & 637.67 & 21.92 & 0.0000 & 85.26 \\ 64.16 & 12.262 & 637.73 & 21.91 & 0.0000 & 85.27 \\ 64.16 & 12.262 & 637.73 & 21.91 & 0.0000 & 85.27 \\ 61.05 & 134.813 & 1335.11 & 195.46 & 0.0000 & 10.68 \\ 47.32 & 134.813 & 1335.11 & 195.46 & 0.0000 & 8.28 \\ 48.31 & 131.708 & 2609.08 & 169.53 & 0.0210 & 12.09 \\ 64.41 & 30.645 & 1709.88 & 4.13 & 0.0156 & 56.08 \\ 65.16 & 7.043 & 1219.96 & -124.50 & 0.0155 & 208.52 \\ 65.16 & 7.043 & 1220.02 & -124.50 & 0.0155 & 208.52 \\ 65.16 & 7.043 & 1220.16 & -124.50 & 0.0155 & 208.53 \\ 361.56 & 7.961 & 552.20 & 1.36 & 0.0000 & 688.66 \\ 361.56 & 7.961 & 552.20 & 1.36 & 0.0000 & 688.66 \\ 361.56 & 7.961 & 552.20 & 1.36 & 0.0000 & 688.66 \\ 361.56 & 7.961 & 552.20 & 1.36 & 0.0000 & 688.66\end{array}$

gamt
1.40064
1.40064
1.40064
1.40064
1.39972
1.39785
1.39785
1.39785
1.35974
1.35974
1.29275
1.32874
1.35616
1.35615
1.35614
1.39972
1.39972
1.39972
1.39972

WAR
0.0000871
0.0000871
0.0000871
0.0000871
0.0000871
0.0000871
0.0000915
0.0000915
0.0000915
0.0000915
0.0000915
0.0000915
0.0000915
0.0000915
0.0000915
0.0000871
0.0000871
0.0000871
0.0000871

Wair
425.69

WH2O H2O frac

$\begin{array}{lll}425.69 & 0.037 & 0.0001\end{array}$

$\begin{array}{lll}361.53 & 0.031 & 0.0001\end{array}$

$\begin{array}{lll}64.16 & 0.006 & 0.0001\end{array}$

$\begin{array}{lll}361.53 & 0.031 & 0.0001\end{array}$

$\begin{array}{lll}64.16 & 0.006 & 0.0001\end{array}$

$\begin{array}{lll}64.16 & 0.006 & 0.0001 \\ 64.16 & 0.006 & 0.0001\end{array}$

$\begin{array}{lll}61.05 & 0.006 & 0.0001\end{array}$

$\begin{array}{lll}47.31 & 0.004 & 0.0001\end{array}$

$\begin{array}{ll}47.31 & 0.004\end{array}$

$\begin{array}{ll}63.41 & 0.006\end{array}$ $\begin{array}{ll}64.16 & 0.006\end{array}$ $\begin{array}{ll}64.16 & 0.006\end{array}$ $361.53 \quad 0.031$ $361.53 \quad 0.031$ $361.53 \quad 0.031$ 0.0191
0.0189 0.0189 0.0189 0.0001 0.0001 0.0001 FS172 FanDuct.FI-O

\section{$\mathrm{NC}$}

$$
\begin{array}{rr}
\text { NERY } & \text { PERFORMANCE } \\
\text { WC } & \text { PR } \\
991.58 & 1.541 \\
175.97 & 2.373 \\
85.27 & 10.994 \\
12.09 & 4.298 \\
56.08 & 4.351
\end{array}
$$

\section{DATA}

\begin{tabular}{|c|c|c|c|c|c|c|c|c|c|}
\hline & Wc & PR & eff & $\mathrm{NC}$ & TR & efPoly & pwr & SMN & SMW \\
\hline Fan & 991.58 & 1.541 & 0.9070 & 3811.212 & 1.1450 & 0.9125 & -8580.4 & 67.51 & 30.08 \\
\hline LPC & 175.97 & 2.373 & 0.8684 & 3811.212 & 1.3222 & 0.8833 & -3388.5 & 4.29 & 3.38 \\
\hline HPC & 85.27 & 10.994 & 0.8615 & 10017.491 & 2.0935 & 0.8980 & -15373.2 & 34.33 & 32.96 \\
\hline HPT & 12.09 & 4.298 & 0.9083 & 217.465 & 1.3546 & 0.8888 & 15373.2 & & \\
\hline LPT & 56.08 & 4.351 & 0.9124 & 88.876 & 1.3989 & 0.8953 & 11968.8 & & \\
\hline \multirow{2}{*}{\multicolumn{10}{|c|}{ TURBOMACHINERY MAP DATA }} \\
\hline & & & & & & & & & s NcDes \\
\hline Fan & 1152.12 & 1.528 & 0.9089 & 3774.872 & 0.0583 & $\overline{0} .8607$ & $\overline{1} .0235$ & 0.9980 & $\overline{0} .9905$ \\
\hline LPC & 141.09 & 2.398 & 0.8201 & 1.007 & 0.0000 & 1.2471 & 0.9822 & 1.0589 & 0.0003 \\
\hline HPC & 77.55 & 10.523 & 0.8704 & 9750.431 & 12.7670 & 1.0995 & 1.0494 & 0.9898 & 0.9733 \\
\hline HPT & 0.96 & 4.206 & 0.9083 & 1.307 & 4.2065 & 12.6299 & 0.9723 & 1.0000 & 0.0003 \\
\hline LPT & 0.86 & 3.521 & 0.9192 & 1.000 & 3.5206 & 65.2590 & 0.7521 & 0.9926 & 0.0005 \\
\hline
\end{tabular}


$\begin{array}{lrrr}===\text { INLETS }==== & \text { eRam } & \text { Afs } & \text { Fram } \\ \text { Inlet } & 1.0000 & 3560.20 & 10494.0\end{array}$

$====$ DUCTS $====$ dPnorm

FanDuct

0.0000

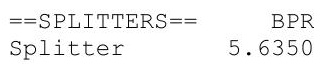

HP_Shaft 11107.9

LP_Shaft

3675.1

$===$ BURNERS $===\quad$ TtOut

Burner 2609.06

eff
0.9995

dPnorm

Core_Nozz

$P R$
2.037
2.302

$\mathrm{Cfg}$
0.9811

0.9815

CdTh

1.0000
1.0000
$\begin{array}{ll}E===\text { TEGT } & 0.0000\end{array}$

$===$ SHAFTS $====\quad$ Nmech

$===$ NOZZIFS $===$

0.9800

0.9800
0.9800

$\begin{array}{llrr}\text { BLEEDS - interstg Wb/Win } & \text { BldWk } & \text { BldP }\end{array}$

$\begin{array}{llll}\text { HPT_COOLC HPC.C> } & 0.0368 & 0.5000 & 0.2200 \\ \text { LPT COOIA HPC.C> } & 0.0117 & 0.5000 & 0.4500\end{array}$

$\begin{array}{lllll}\text { LPT COOLA HPC.C> } & 0.0117 & 0.5000 & 0.4500 \\ \text { WB } 2 \bar{X} & \text { HPC. B> } & 0.0000 & 0.4500 & 0.2200\end{array}$

WB2Y HPC.B> $0.0000 \quad 0.7600 \quad 0.6200$

$\begin{array}{lllll}\text { WBA2X HPC.B } & 0.0000 & 0.4500 & 0.2200\end{array}$

$\begin{array}{lllll}\text { WBLKG HPC.1> } & 0.0000 & 1.0000 & 1.0000\end{array}$

$\begin{array}{lllll}\text { WBW2X HPC.B> } & 0.0000 & 0.4500 & 0.2200\end{array}$

BLEEDS - output Wb/Win hscale Pscale

WB17Y Bleed> $0.0000 \quad 1.0000 \quad 1.0000$

HPT_COOLA Bleed> $0.1142 \quad 1.0000 \quad 1.0000$

$\begin{array}{llll}\text { HPT COOLB Bleed> } & 0.0999 & 1.0000 & 1.0000\end{array}$

$\begin{array}{lllll}\text { WB3X } & \text { Bleed> } & 0.0000 & 1.0000 & 1.0000 \\ \text { WBA3X } & \text { Bleed> } & 0.0000 & 1.0000 & 1.0000\end{array}$

$\begin{array}{lllll}\text { WBA3X } & \text { Bleed> } & 0.0000 & 1.0000 & 1.0000 \\ \text { WBW3X } & \text { Bleed }> & 0.0000 & 1.0000 & 1.0000\end{array}$

$\begin{array}{lllll}\text { WBW3X } & \text { Bleed> } & 0.0000 & 1.0000 & 1.0000 \\ \text { WBFDLKG } & \text { FanDu> } & 0.0000 & 1.0000 & 1.0000\end{array}$

$\begin{array}{llll}\text { WB15X Bleed> } & 0.0000 & 1.0000 & 1.0000\end{array}$

WB15Y Bleed> $0.0000 \quad 1.0000 \quad 1.0000$

$\begin{array}{lll}0.0000 & 1.0000 & 1.0000\end{array}$

$\begin{array}{rrrr}\text { W } & \text { Tt } & \text { ht } & \text { Pt } \\ 2.3612 & 993.13 & 108.68 & 30.645 \\ 0.7507 & 993.13 & 108.68 & 7.043\end{array}$

$\begin{array}{llll}0.0000 & 958.13 & 100.00 & 39.223\end{array}$

$\begin{array}{llll}0.0000 & 1172.77 & 153.80 & 88.243\end{array}$

$\begin{array}{lrrr}0.0000 & 958.13 & 100.00 & 39.223\end{array}$

$\begin{array}{llll}0.0000 & 1335.11 & 195.46 & 134.813\end{array}$

$\begin{array}{lrrr}0.0000 & 958.13 & 100.00 & 39.223\end{array}$

$\begin{array}{rrrr}\text { W } & \text { Tt } & \text { ht } & \text { Pt } \\ 0.0000 & 637.73 & 21.91 & 12.262\end{array}$

$\begin{array}{lllr}7.3263 & 1335.11 & 195.46 & 131.708\end{array}$

$\begin{array}{lllr}6.4106 & 1335.11 & 195.46 & 73.900\end{array}$

$\begin{array}{llll}0.0000 & 1335.11 & 195.46 & 134.813 \\ 0.0000 & 1335.11 & 195.46 & 134.813\end{array}$

$\begin{array}{llll}0.0000 & 1335.11 & 195.46 & 134.813 \\ 0.00000 & 1335.11 & 195.46 & 134.813\end{array}$

$\begin{array}{rrrr}0.0000 & 1335.11 & 195.46 & 134.813 \\ 0.00000 & 552.20 & 1.36 & 7.961\end{array}$

$\begin{array}{llll}0.0000 & 1335.11 & 195.46 & 134.813 \\ 0.0000 & 552.20 & 1.36 & 7.961\end{array}$

$\begin{array}{llll}0.0000 & 552.20 & 1.36 & 7.961\end{array}$

$\frac{1}{\infty}$ $\begin{array}{rr}\text { FAR } \\ 0.99227 & 0.02097\end{array}$
7.961 
Date:07/05/13 Time:09:59:12 Model:

Turbofan Engine - COMDES ON Converge = 1 CASE: 0

Version:NPSS 1.6.5 - Rev: -> Gas Package: Janaf iter/pass/Jacb/Broy=17/31/1/15 Run by: Philip C Jorgenson PC: 69

Temperature Stator 1 inlet: 480.57 Stator 1 exit: 497.00 Stator 2 inlet: 517.17 Stator 2 exit: 525.84 $\begin{array}{ll}\text { Stator } 3 \text { inlet: } 549.50 & \text { Stator } 3 \text { exit: } 558.08 \quad \text { Stator } 4 \text { inlet: } 580.45 \text { Stator } 4 \text { exit: } 588.27 \\ \text { Stator } 5 \text { inlet: } 606.24 & \text { Stator } 5 \text { exit: } 613.56\end{array}$

Ambient Relative Humidity $\quad 10.00$

Fan Face Relative Humidity $\quad 3.60$

Ean

1.98

PCC Relative He Humidity

Drop Diameter

mbient Flow Velocity

Ambient Static Pressure

Additional

$\begin{array}{clr}0.00 & & \\ 0.0001000 & \text { Inlet Length } & 40.00 \\ 793.07 & \text { Fan/LPC Inlet Flow Velocity } & 406.41 \\ 3.46 & \text { Fan/LPC Inlet Static Pressure } & 4.67 \\ 429.85 & \text { Fan/LPC Inlet Static Temperature } & 468.51 \\ 0.0000045 & & \end{array}$

0.0000045

$\begin{array}{rrrrr}\text { MN alt dTamb } & \text { SUMPY OUTPUT DATA } \\ 0.780 & \text { Fn } & \text { TSFC }\end{array}$

$\begin{array}{rrr}\text { MN alt dTamb } \\ 0.780 & 35000.0 & \end{array}$ W
421.75
Wfuel

BPR
5.6888

FLOW STATION DATA

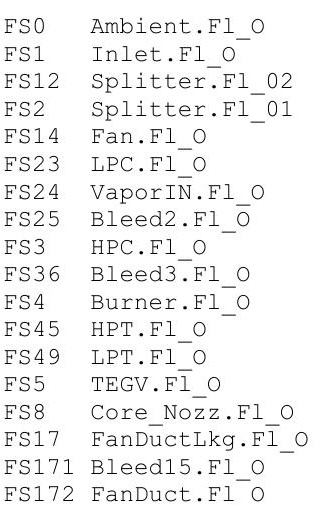

FS172 FanDuct.FI-O

FS173 Byp Nozz.FI O

$\begin{array}{rrrrrr}\text { W } & \mathrm{Pt} & \mathrm{Tt} & \mathrm{ht} & \mathrm{FAR} & \text { WC } \\ 421.75 & 5.170 & 482.28 & -15.41 & 0.0000 & 1156.05 \\ 421.75 & 5.170 & 482.28 & -15.41 & 0.0000 & 1156.05 \\ 358.69 & 5.167 & 482.28 & -15.41 & 0.0000 & 983.71 \\ 63.05 & 5.167 & 482.28 & -15.41 & 0.0000 & 172.92 \\ 358.69 & 7.886 & 550.55 & 0.97 & 0.0000 & 688.66 \\ 63.05 & 12.180 & 636.04 & 21.52 & 0.0000 & 84.25 \\ 63.05 & 12.180 & 636.11 & 21.51 & 0.0000 & 84.25 \\ 63.05 & 12.180 & 636.11 & 21.51 & 0.0000 & 84.25 \\ 59.99 & 131.676 & 1325.53 & 192.98 & 0.0000 & 10.70 \\ 46.50 & 131.676 & 1325.53 & 192.98 & 0.0000 & 8.30 \\ 47.45 & 128.643 & 2579.11 & 167.61 & 0.0206 & 12.09 \\ 63.27 & 29.913 & 1689.85 & 4.09 & 0.0153 & 56.11 \\ 64.01 & 6.874 & 1205.09 & -122.88 & 0.0152 & 208.58 \\ 64.01 & 6.874 & 1205.16 & -122.88 & 0.0152 & 208.58 \\ 64.01 & 6.874 & 1205.29 & -122.88 & 0.0152 & 208.60 \\ 358.69 & 7.886 & 550.55 & 0.97 & 0.0000 & 688.66 \\ 358.69 & 7.886 & 550.55 & 0.97 & 0.0000 & 688.66 \\ 358.69 & 7.886 & 550.55 & 0.97 & 0.0000 & 688.66 \\ 358.69 & 7.886 & 550.55 & 0.97 & 0.0000 & 688.66\end{array}$

Ps
3.458
4.172
4.158
4.670
6.547
11.058
11.058
11.058
117.924
123.714
121.520
26.914
5.926
5.926
3.666
6.547
6.362
6.362
4.165

TS
429.85
453.57
453.21
468.51
522.03
618.78
618.84
618.84
1287.27
1303.78
2545.64
1646.02
1158.74
1158.81
1019.33
522.03
517.77
517.77
458.70

$\begin{array}{rr}\text { Aphy } & \mathrm{MN} \\ 3526.9 & 0.7800 \\ 4168.2 & 0.5620 \\ 3531.8 & 0.5657 \\ 830.5 & 0.3829 \\ 2606.7 & 0.5226 \\ 412.6 & 0.3743 \\ 412.6 & 0.3743 \\ 412.6 & 0.3743 \\ 49.7 & 0.4053 \\ 49.3 & 0.3039 \\ 74.6 & 0.2988 \\ 265.4 & 0.4009 \\ 860.2 & 0.4719 \\ 860.2 & 0.4719 \\ 613.4 & 1.0000 \\ 2606.7 & 0.5226 \\ 2481.9 & 0.5626 \\ 2481.9 & 0.5626 \\ 2006.9 & 1.0000\end{array}$

gamt
1.40064
1.40064
1.40064
1.40064
1.39975
1.39789
1.39789
1.39789
1.36031
1.36031
1.29390
1.32989
1.35729
1.35729
1.35728
1.39975
1.39975
1.39975
1.39975

WAR
0.0000871
0.0000871
0.0000871
0.0000871
0.0000871
0.0000871
0.0000916
0.0000916
0.0000916
0.0000916
0.0000916
0.0000916
0.0000916
0.0000916
0.0000916
0.0000871
0.0000871
0.0000871
0.0000871

Wair

H2O frac

$\begin{array}{lll}421.71 & 0.037 & 0.0001 \\ 421.71 & 0.037 & 0.0001\end{array}$

$\begin{array}{lll}358.66 & 0.031 & 0.0001\end{array}$

$\begin{array}{lll}63.05 & 0.005 & 0.0001\end{array}$

$\begin{array}{lll}358.66 & 0.031 & 0.0001\end{array}$

$\begin{array}{lll}63.05 & 0.005 & 0.0001\end{array}$

$\begin{array}{lll}63.05 & 0.006 & 0.0001\end{array}$

$\begin{array}{lll}63.05 & 0.006 & 0.0001\end{array}$

$\begin{array}{lll}59.99 & 0.005 & 0.0001\end{array}$

$\begin{array}{lll}46.49 & 0.004 & 0.0001\end{array}$

$\begin{array}{lll}46.49 & 0.004 & 0.0249 \\ 62.31 & 0.006 & 0.0187\end{array}$

$\begin{array}{lll}63.05 & 0.006 & 0.0185\end{array}$

$\begin{array}{lll}63.05 & 0.006 & 0.0185\end{array}$

$\begin{array}{lll}63.05 & 0.006 & 0.0185\end{array}$

$\begin{array}{lll}358.66 & 0.031 & 0.0001\end{array}$

$\begin{array}{lll}358.66 & 0.031 & 0.0001 \\ 358.66 & 0.031 & 0.0001\end{array}$

0.0001

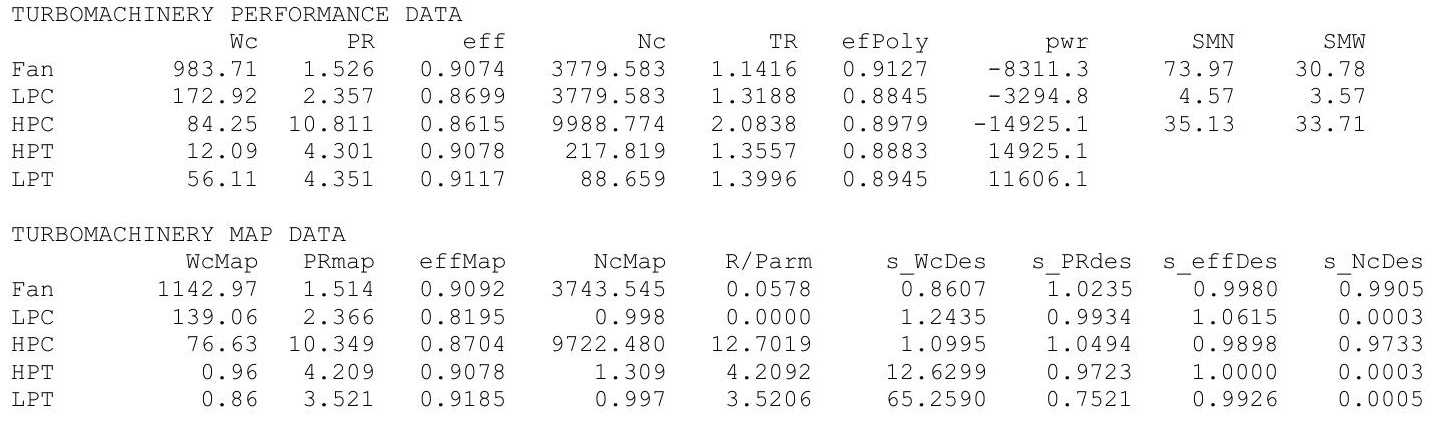




\begin{tabular}{|c|c|c|c|}
\hline$===$ INLET $S===$ & eRam & Afs & Fram \\
\hline Inlet & 1.0000 & 3526.91 & 10395.8 \\
\hline$====$ DUCTS $====$ & dPnorm & MN & Aphy \\
\hline TEGV & 0.0000 & 0.4719 & 860.21 \\
\hline FanDuct & 0.0000 & 0.5626 & 2481.93 \\
\hline$==$ SPLITTERS $==$ & BPR & $\mathrm{dP} / \mathrm{P} 1$ & $\mathrm{dP} / \mathrm{P} 2$ \\
\hline Splitter & 5.6888 & 0.0005 & 0.0005 \\
\hline$===$ SHAFT $\mathrm{S}===$ & Nmech & trg in & pwr in \\
\hline HP Shaft & 11061.9 & 7086.3 & 14925.1 \\
\hline LP Shaft & 3644.6 & 16725.3 & 11606.1 \\
\hline
\end{tabular}

BLEEDS - interstg Wb/Win BldWk BldP $\begin{array}{lllll}\text { HPT_COOLC HPC.C } & 0.0368 & 0.5000 & 0.2200\end{array}$ $\begin{array}{lllll}\text { LPT }^{-} \text {COOLA } & \text { HPC.C> } & 0.0117 & 0.5000 & 0.4500 \\ \text { WB2 } 2 \text { X } & \text { HPC.B> } & 0.0000 & 0.4500 & 0.2200\end{array}$ $\begin{array}{lllll}\text { WB2X } & \text { HPC.B } & 0.0000 & 0.4500 & 0.2200 \\ \text { WB2Y } & \text { HPC. B> } & 0.0000 & 0.7600 & 0.6200\end{array}$ $\begin{array}{lllll}\text { WB2Y } & \text { HPC.B> } & 0.0000 & 0.7600 & 0.6200 \\ \text { WBA2X } & \text { HPC.B> } & 0.0000 & 0.4500 & 0.2200\end{array}$ $\begin{array}{lllll}\text { WBA2X } & \text { HPC.B } & 0.0000 & 0.7600 & 0.6200 \\ \text { WBTKG } & 0.000 & 0.2200\end{array}$ $\begin{array}{lllll}\text { WBLKG } & \text { HPC.I> } & 0.0000 & 1.0000 & 1.0000 \\ \text { WBW2X } & \text { HPC.B } & 0.0000 & 0.4500 & 0.2200\end{array}$

BLEEDS - output Wb/Win hscale Pscale $\begin{array}{llll}\text { WB17Y Bleed> } & 0.0000 & 1.0000 & 1.0000\end{array}$ HPT_COOLA Bleed> $0.1142 \quad 1.0000 \quad 1.0000$ $\begin{array}{lllll}\text { HPT COOLB } & \text { Bleed> } & 0.0999 & 1.0000 & 1.0000 \\ \text { WB } 3 \bar{X} & \text { Bleed> } & 0.0000 & 1.0000 & 1.0000\end{array}$ $\begin{array}{lllll}\text { WB3X } & \text { Bleed }> & 0.0000 & 1.0000 & 1.0000 \\ \text { WBA3X } & \text { Bleed }> & 0.0000 & 1.0000 & 1.0000\end{array}$ $\begin{array}{lllll}\text { WBA3X } & \text { Bleed }> & 0.0000 & 1.0000 & 1.0000 \\ \text { WBW3X } & \text { Bleed }> & 0.0000 & 1.0000 & 1.0000\end{array}$ WBFDLKG FanDu> $0.0000 \quad 1.0000 \quad 1.0000$ $\begin{array}{lllll}\text { WBFDLKG } & \text { FanDu> } & 0.0000 & 1.0000 & 1.0000 \\ \text { WB15X } & \text { Bleed> } & 0.0000 & 1.0000 & 1.0000\end{array}$ $\begin{array}{lllll}\text { WB15X } & \text { Bleed> } & 0.0000 & 1.0000 & 1.0000 \\ \text { WB15Y } & \text { Bleed> } & 0.0000 & 1.0000 & 1.0000\end{array}$ WB17X Bleed> $0.0000 \quad 1.0000 \quad 1.0000$

$\begin{array}{rrrr}\text { W } & \text { Tt } & \text { ht } & \text { Pt } \\ 2.3203 & 987.34 & 107.24 & 29.913 \\ 0.7377 & 987.34 & 107.24 & 6.874 \\ 0.0000 & 952.75 & 98.67 & 38.469 \\ 0.0000 & 1164.97 & 151.82 & 86.267 \\ 0.0000 & 952.75 & 98.67 & 38.469 \\ 0.0000 & 1325.53 & 192.98 & 131.676 \\ 0.0000 & 952.75 & 98.67 & 38.469 \\ \text { W } & & & \\ 0.0000 & 636.11 & 21.51 & 12.180 \\ 7.1993 & 1325.53 & 192.98 & 128.643 \\ 6.2996 & 1325.53 & 192.98 & 72.169 \\ 0.0000 & 1325.53 & 192.98 & 131.676 \\ 0.0000 & 1325.53 & 192.98 & 131.676 \\ 0.0000 & 1325.53 & 192.98 & 131.676 \\ 0.0000 & 550.55 & 0.97 & 7.886 \\ 0.0000 & 550.55 & 0.97 & 7.886 \\ 0.0000 & 550.55 & 0.97 & 7.886 \\ 0.0000 & 550.55 & 0.97 & 7.886\end{array}$
$===$ BURNERS $===\quad$ TtOut eff
0.9995

dPnorm

Wfuel FAR

.

.02057

$\begin{array}{lrrrrrrrr}===\text { NOZZLES }=== & \text { PR } & \text { Cfg } & \text { CdTh } & \text { CV } & \text { Ath } & \text { MNth } & \text { Vact } & \text { Fg } \\ \text { Core_Nozz } & 1.988 & 0.9808 & 1.0000 & 0.9800 & 613.40 & 1.000 & 1516.9 & 3145.3 \\ \text { Byp_Nozz } & 2.281 & 0.9815 & 1.0000 & 0.9800 & 2006.86 & 1.000 & 1029.2 & 12893.2\end{array}$


Appendix H: Compressor code analysis for the altitude cruise conditions at 35000 feet

$5 \mu \mathrm{m}, \mathrm{ISA}+18 \mathrm{R}$

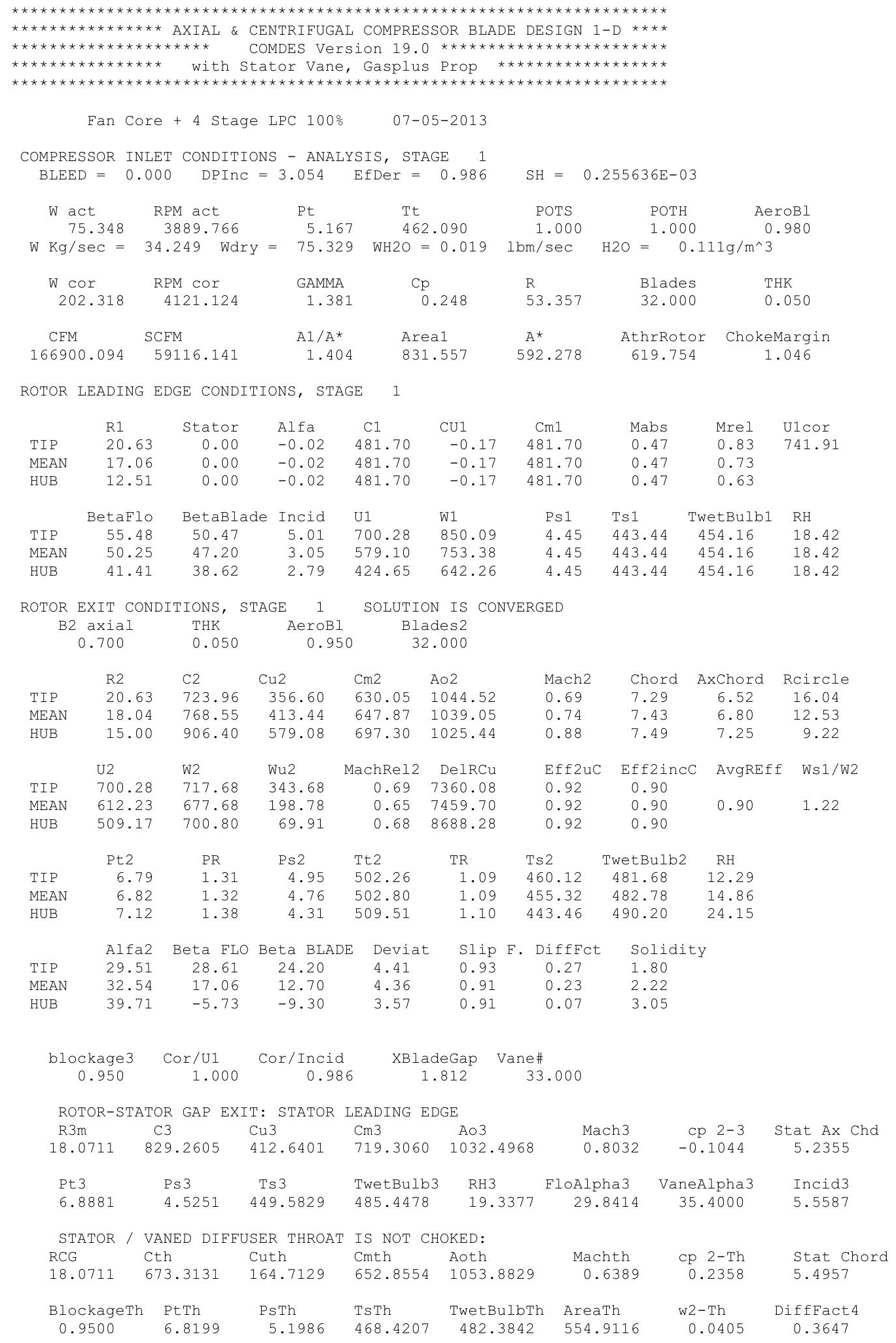




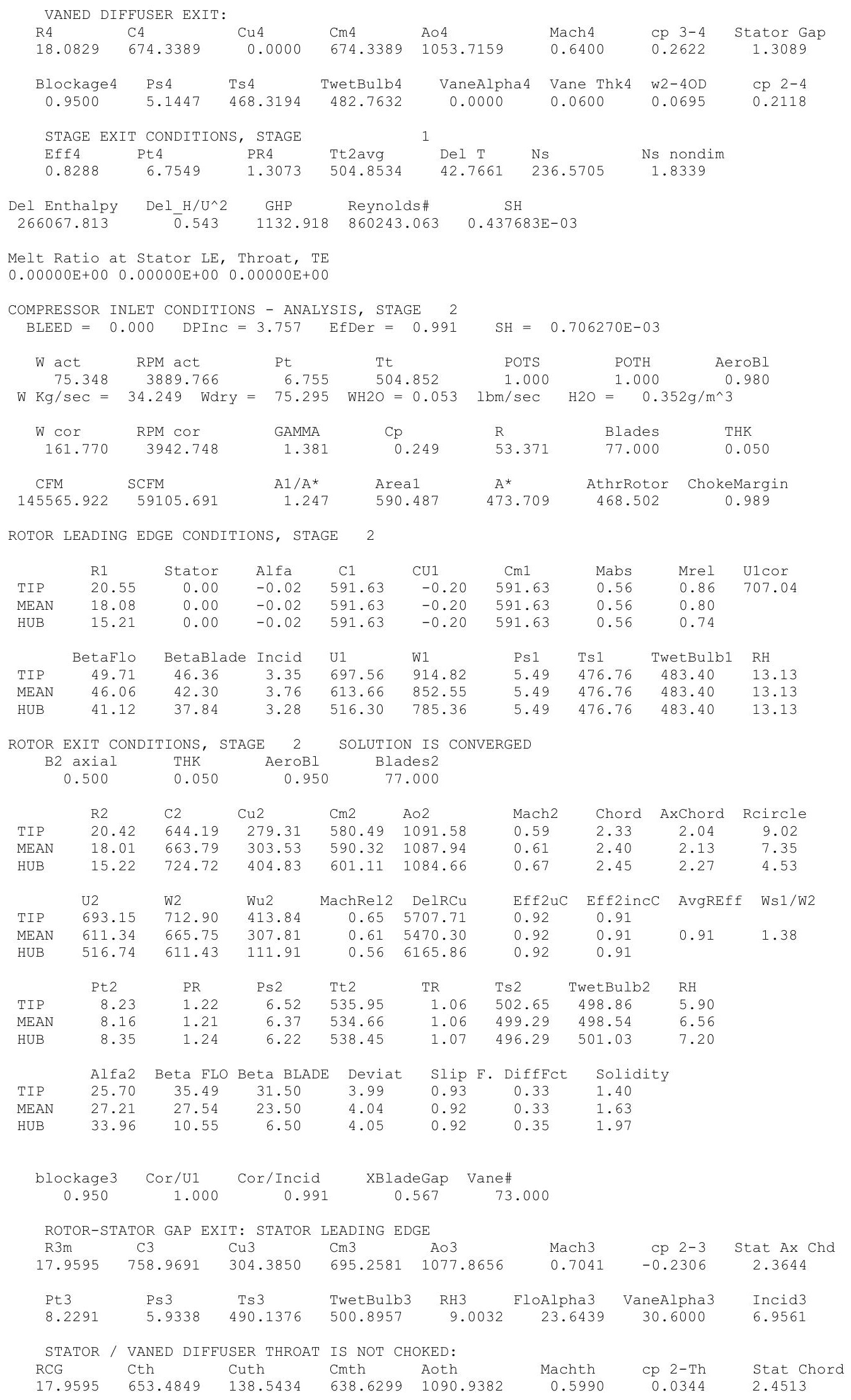




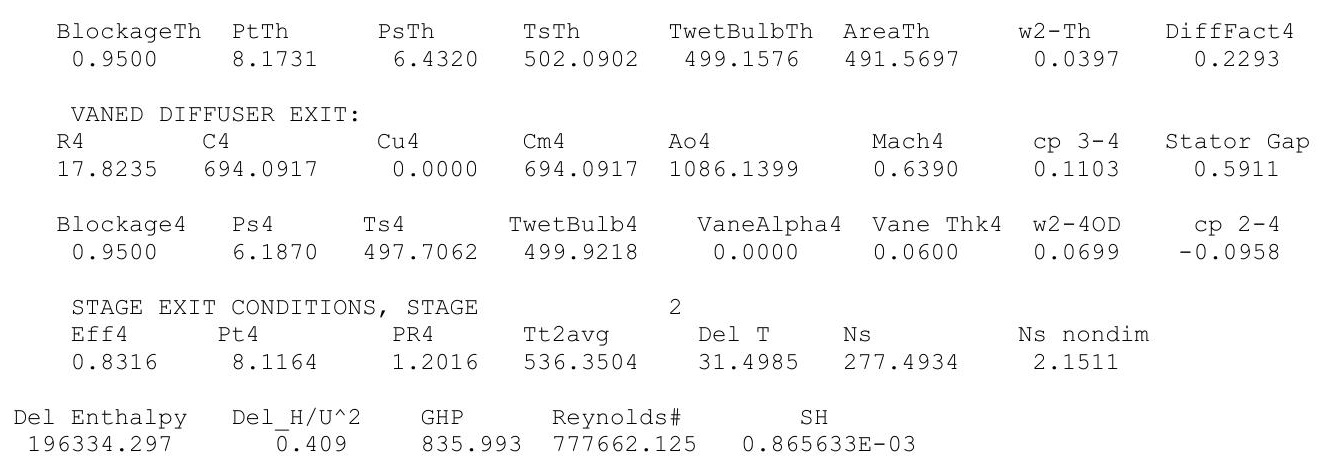

Melt Ratio at Stator LE, Throat, TE

$0.00000 \mathrm{E}+000.00000 \mathrm{E}+000.00000 \mathrm{E}+00$

COMPRESSOR INLET CONDITIONS - ANALYSIS, STAGE 3 $\mathrm{BLEED}=0.000 \quad$ DPInC $=4.173 \quad$ EfDer $=0.993$

$\begin{array}{llllll}W \text { act } & \text { RPM act } & \text { Pt } & \text { POTS } & \text { POTH } & \text { AeroBI }\end{array}$

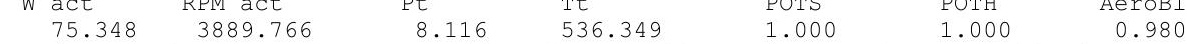

$\mathrm{W} \mathrm{Kg} / \mathrm{sec}=34.249$ Wdry $=75.266$ WH2O $=0.083 \mathrm{lbm} / \mathrm{sec} \quad \mathrm{H} 2 \mathrm{O}=0.637 \mathrm{~g} / \mathrm{m}^{\wedge} 3$

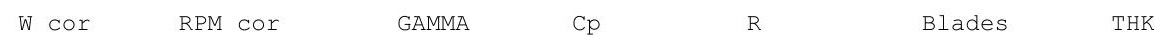

$\begin{array}{cccclll}138.770 & 3825.228 & \text { GAMMA } & \text { Cp } & \text { R } & \text { Blades } & \text { THK } \\ & 1.380 & 0.249 & 53.384 & 77.000 & 0.050\end{array}$

$\begin{array}{lllcl}\text { CFM } & \text { SCFM } & \text { A1/A* } & \text { Area1 } & \text { A* AthrRotor ChokeMargin }\end{array}$

$\begin{array}{lllllll}125123.148 & 59096.594 & 1.342 & 545.479 & 406.477 & 424.468 & 1.044\end{array}$

ROTOR LEADING EDGE CONDITIONS, STAGE 3

$\begin{array}{lccccccccc} & \text { R1 } & \text { Stator } & \text { Alfa } & \text { C1 } & \text { CU1 } & \text { Cm1 } & \text { Mabs } & \text { Mrel } & \text { U1cor } \\ \text { TIP } & 20.07 & 0.00 & -0.02 & 550.50 & -0.19 & 550.50 & 0.50 & 0.80 & 669.95 \\ \text { MEAN } & 17.74 & 0.00 & -0.02 & 550.50 & -0.19 & 550.50 & 0.50 & 0.74 & \\ \text { HUB } & 15.05 & 0.00 & -0.02 & 550.50 & -0.19 & 550.50 & 0.50 & 0.68 & \\ & & & & & & & & & \\ & \text { BetaFlo } & \text { BetaBlade } & \text { Incid } & \text { U1 } & \text { W1 } & \text { Ps } 1 & \text { Ts1 } & \text { TwetBulb1 } & \text { RH } \\ \text { TIP } & 51.07 & 46.36 & 4.71 & 681.27 & 876.04 & 6.86 & 512.06 & 499.06 & 6.23 \\ \text { MEAN } & 47.57 & 43.40 & 4.17 & 602.13 & 815.99 & 6.86 & 512.06 & 499.06 & 6.23 \\ \text { HUB } & 42.87 & 38.84 & 4.03 & 510.87 & 751.15 & 6.86 & 512.06 & 499.06 & 6.23\end{array}$

ROTOR EXIT CONDITIONS, STAGE 3 SOLUTION IS CONVERGED

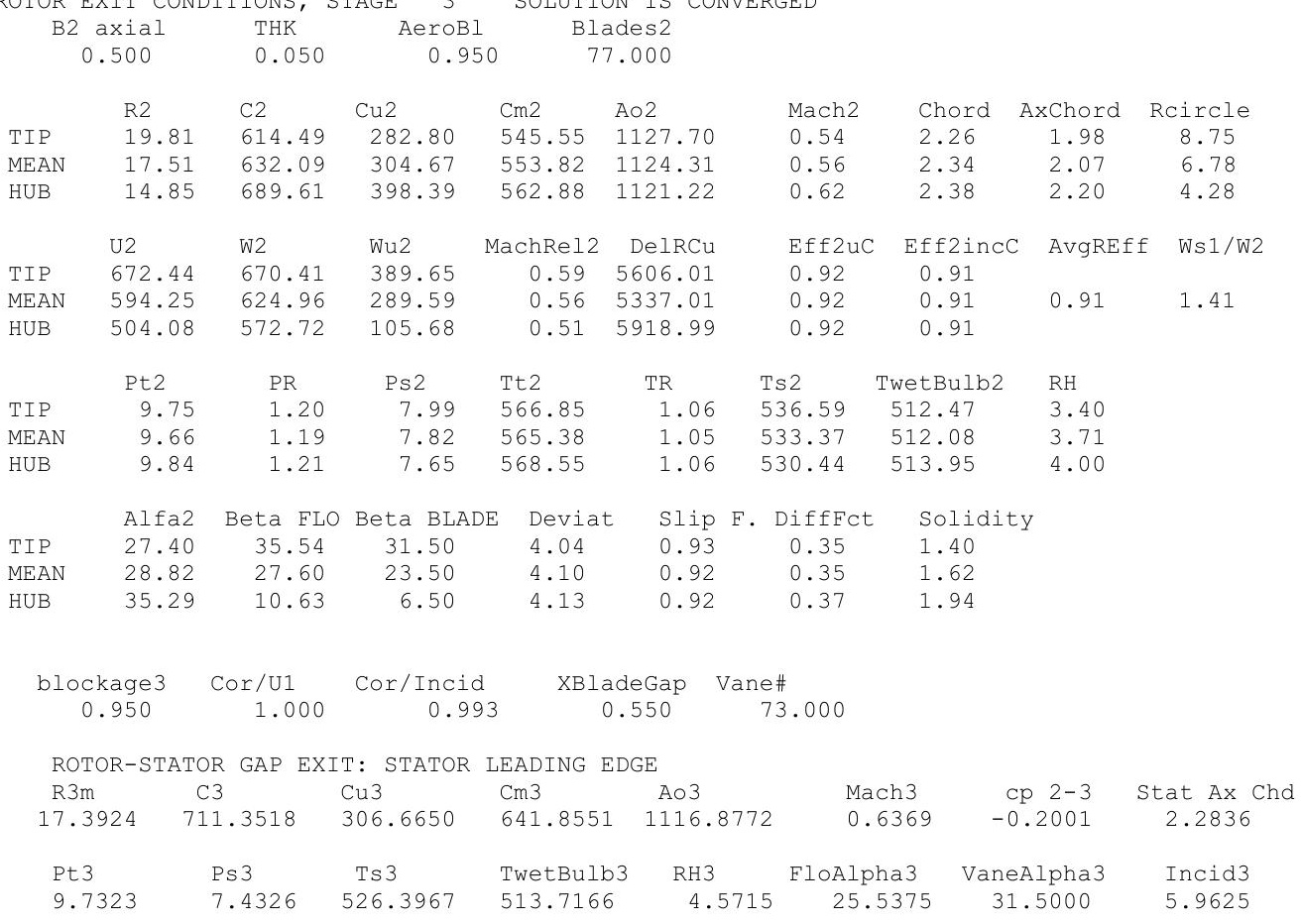




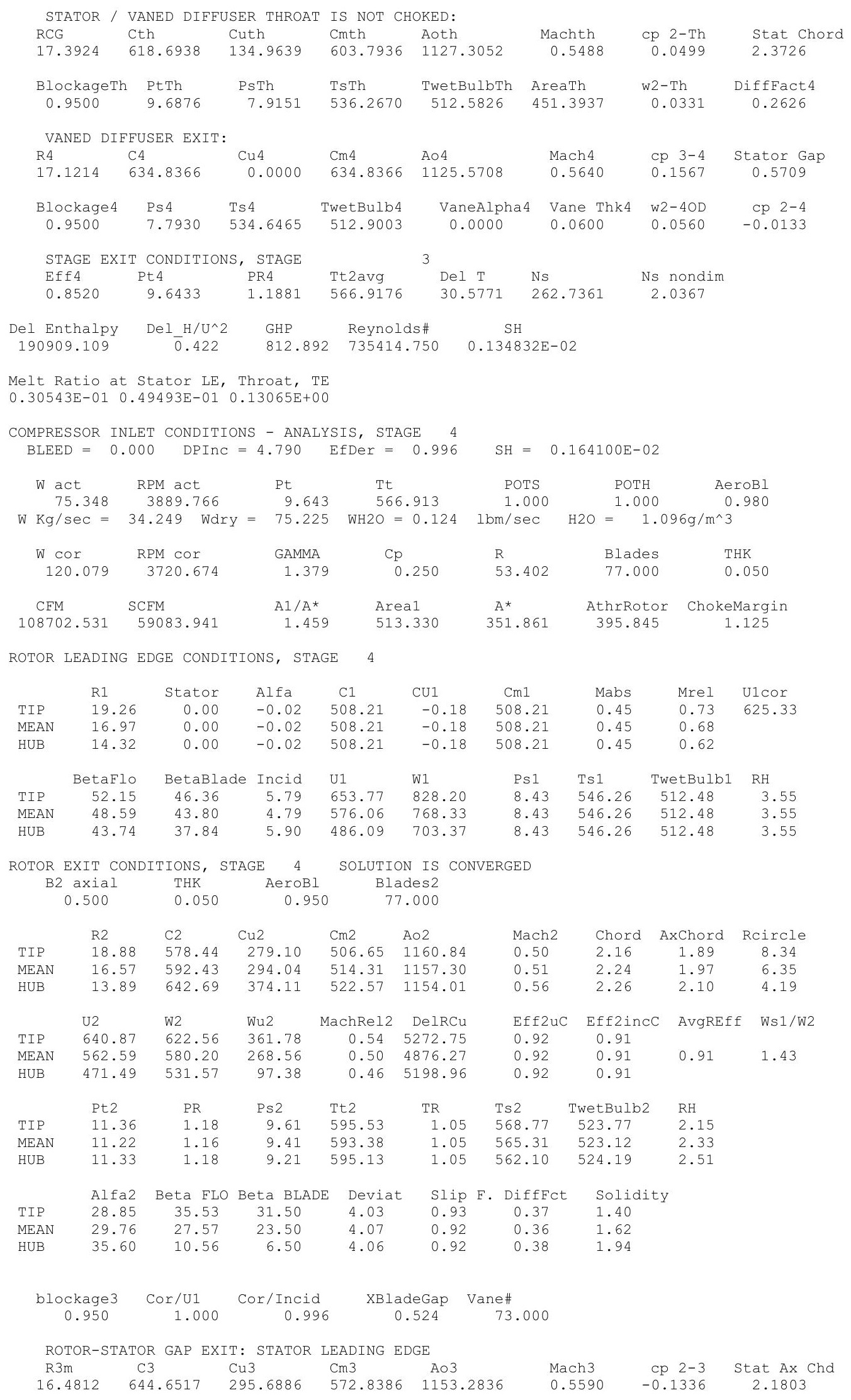




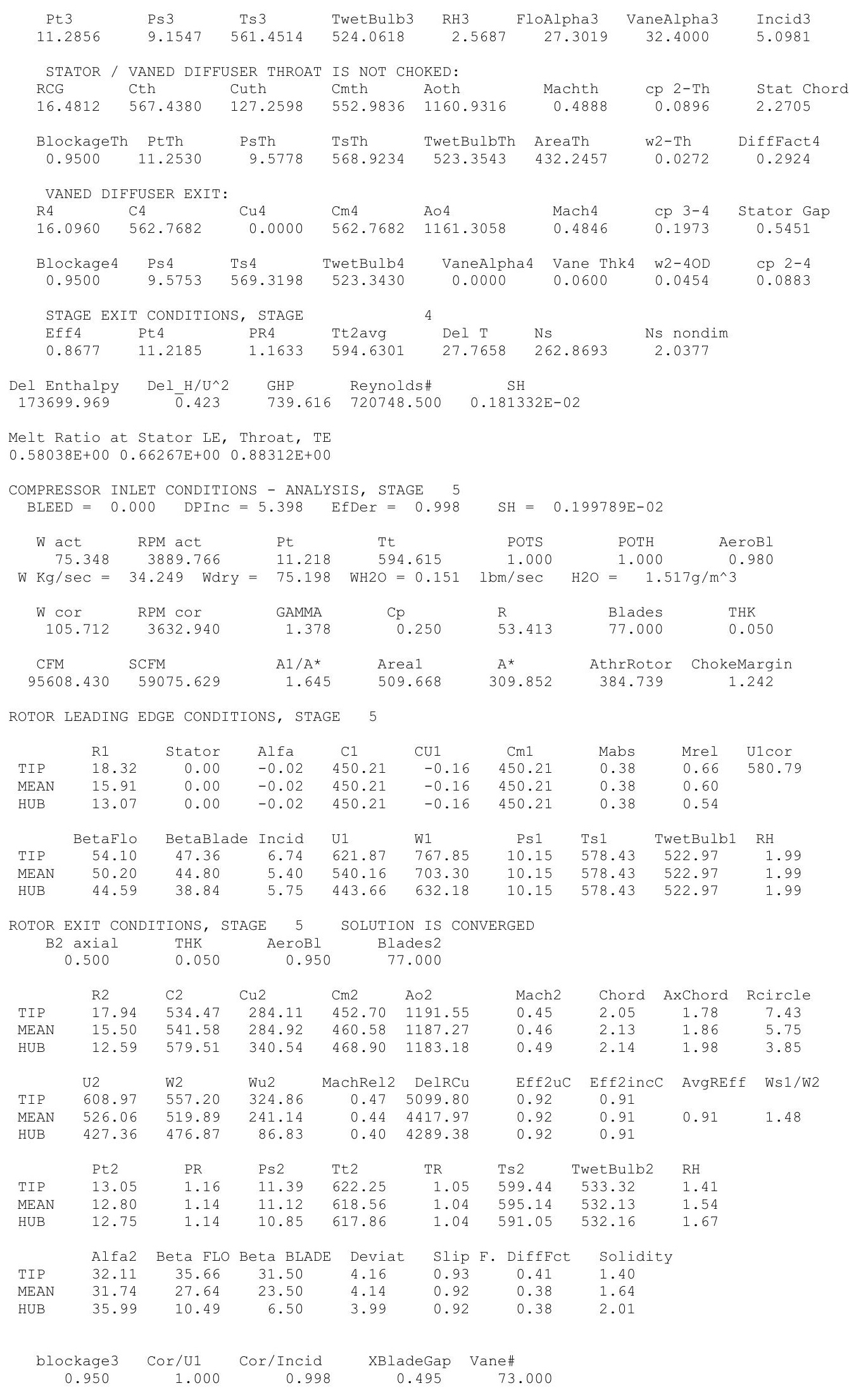




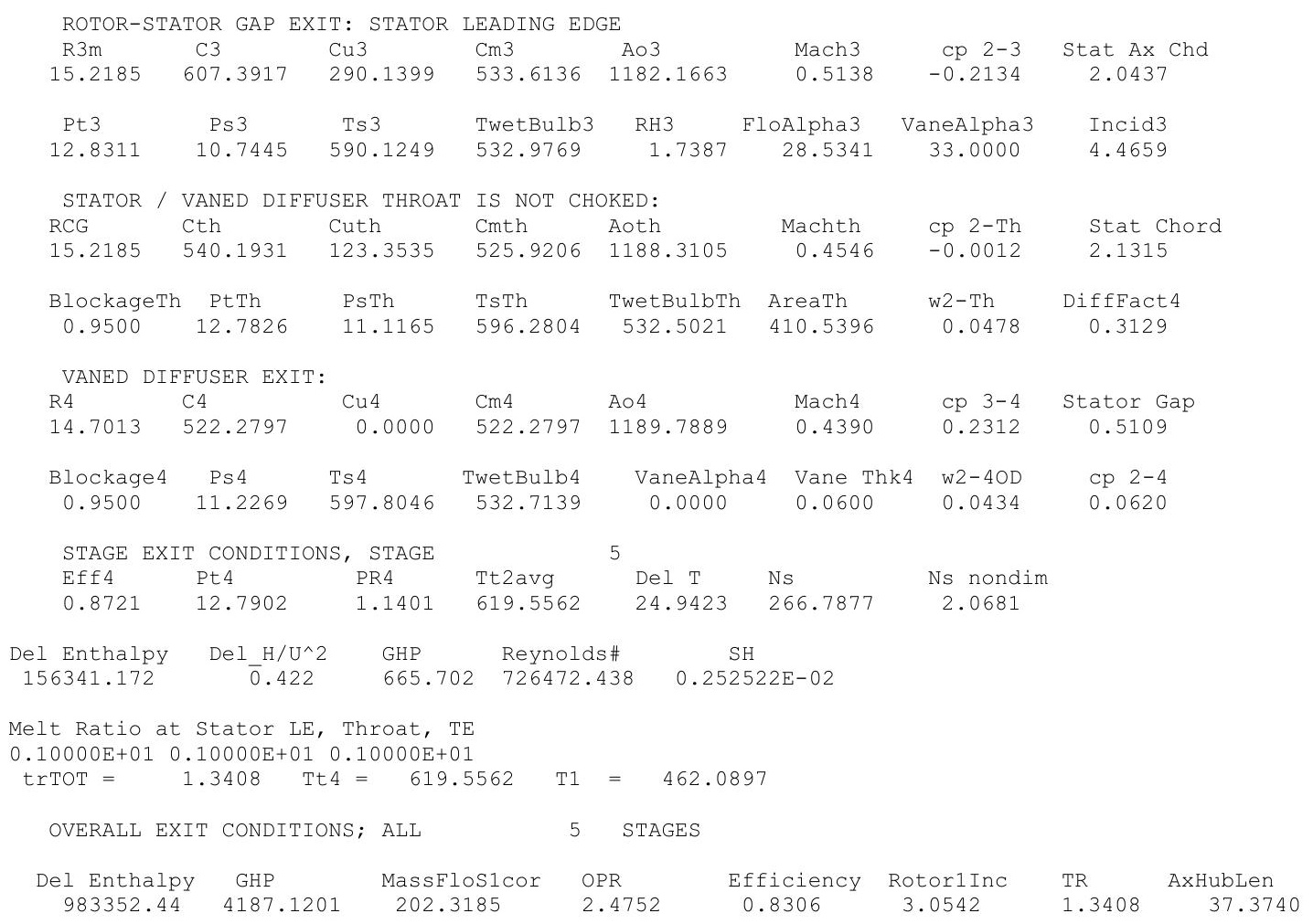




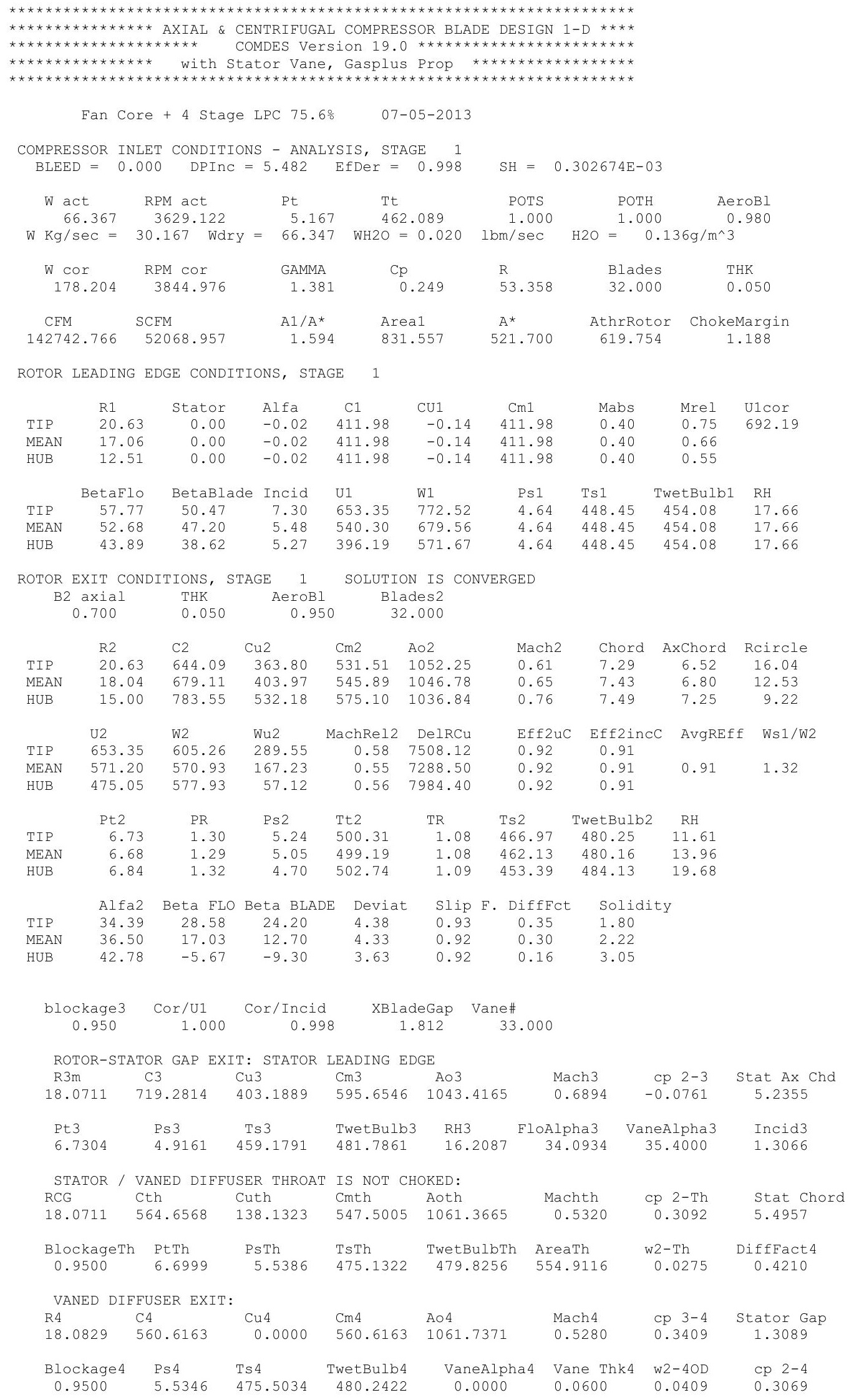




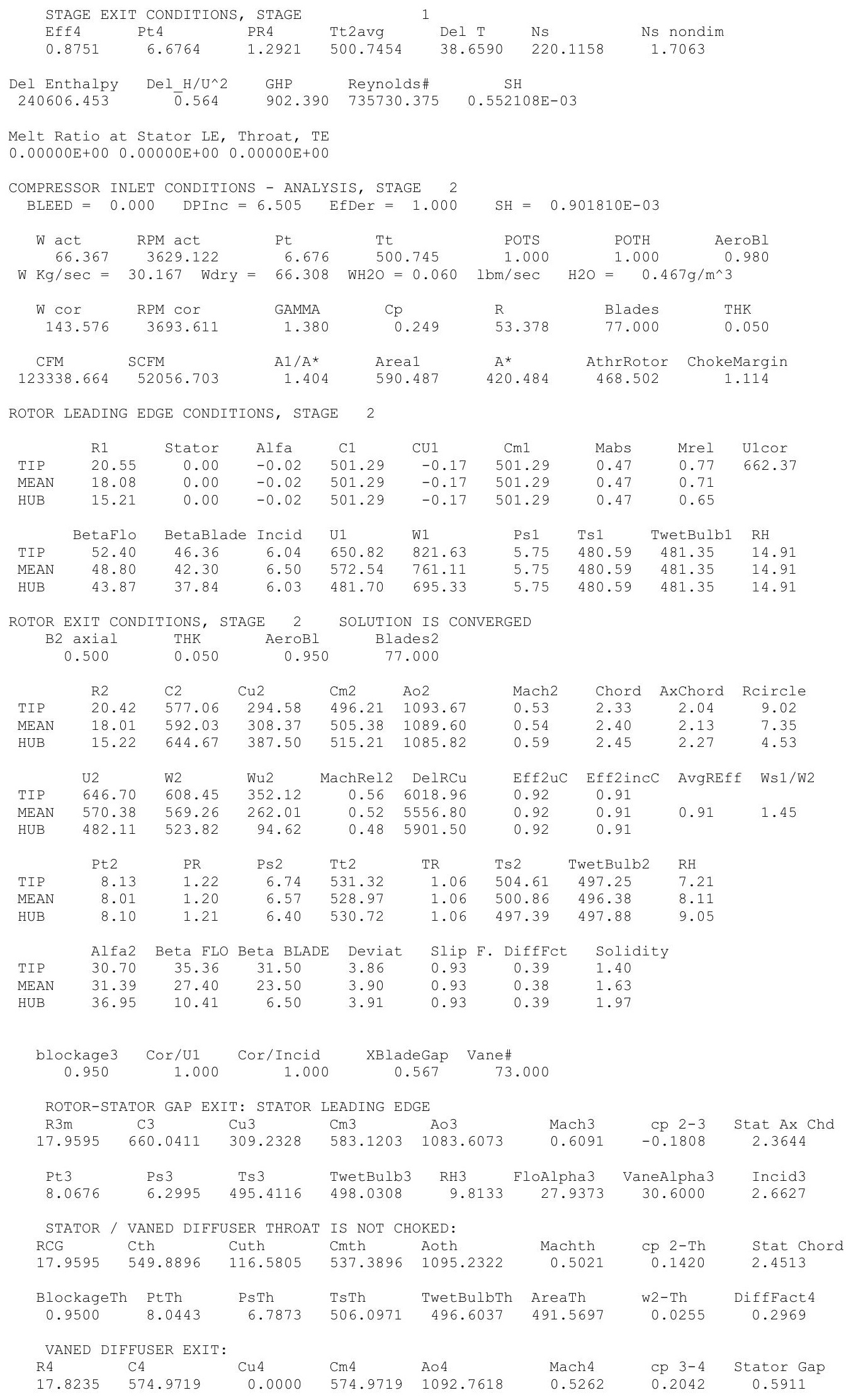




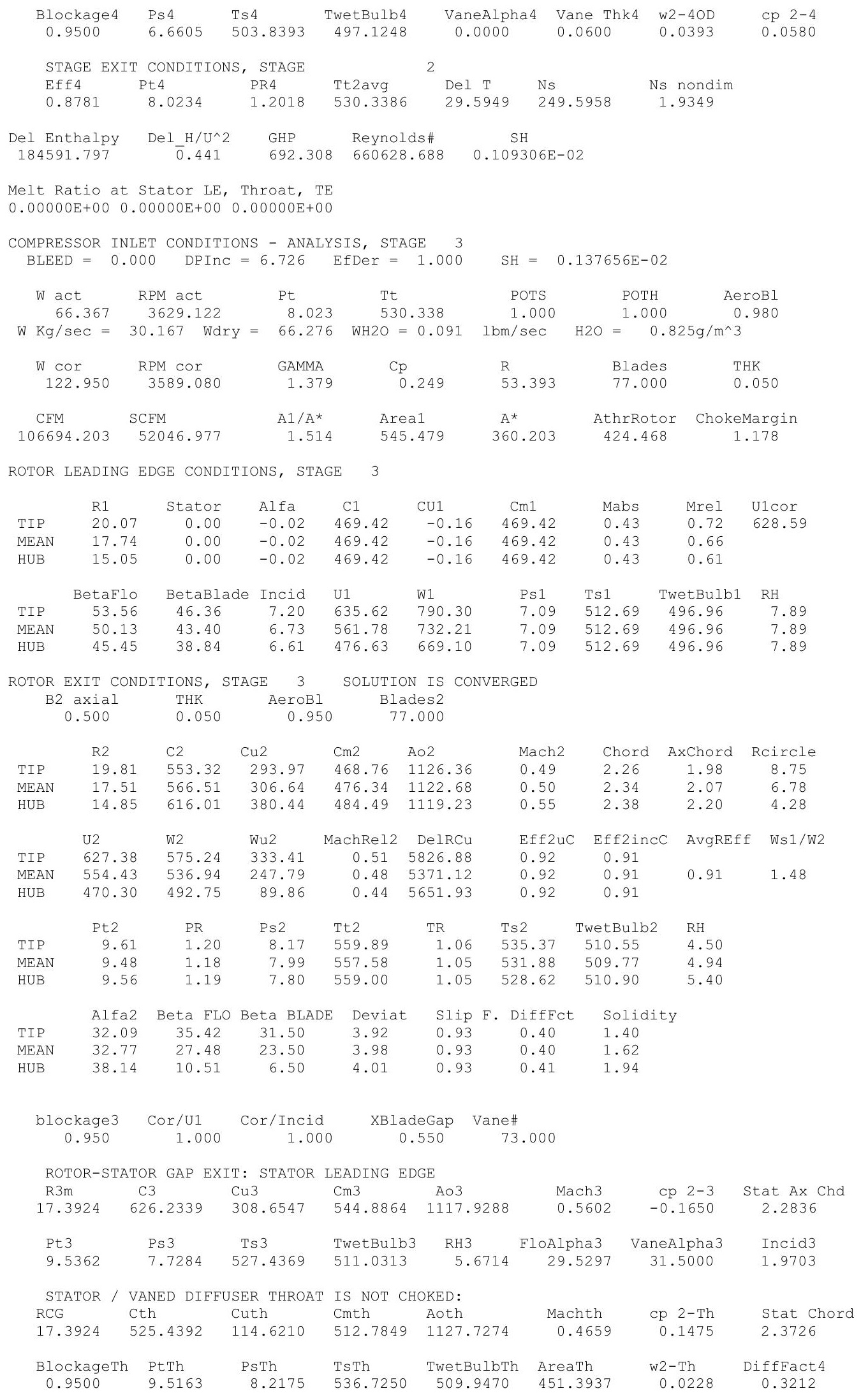




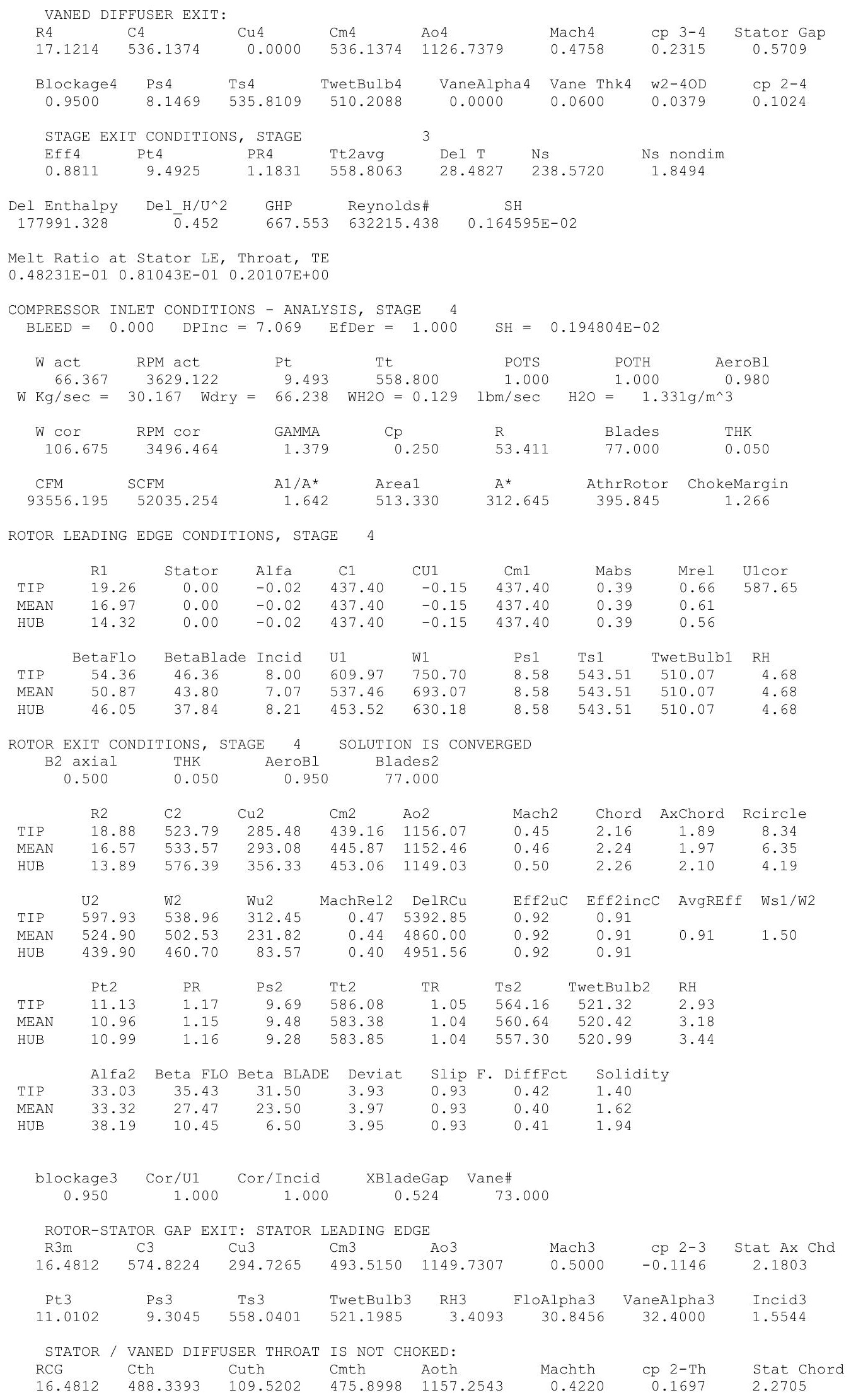




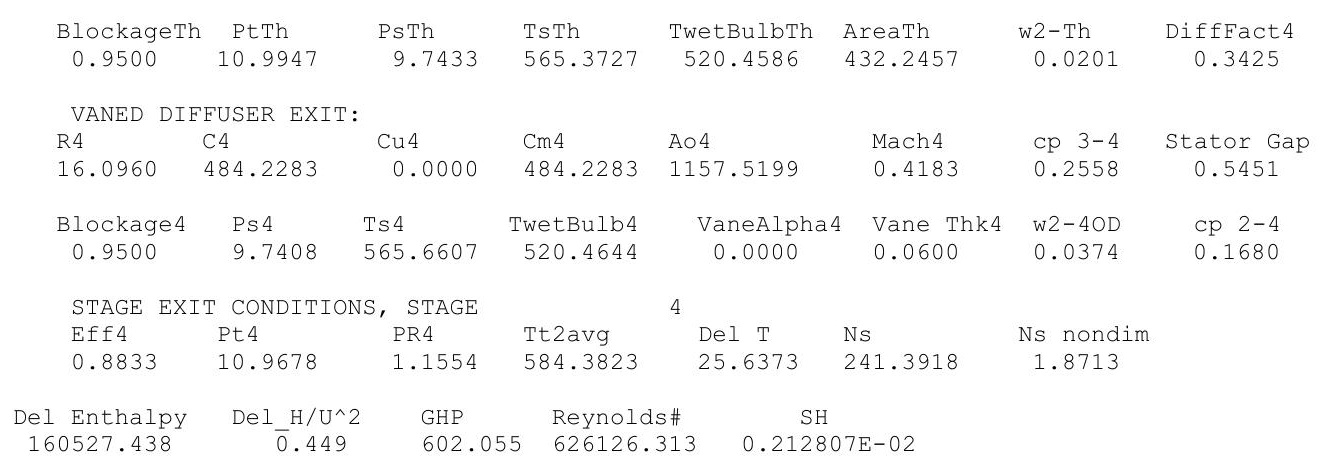

Melt Ratio at Stator LE, Throat, TE

$0.67647 \mathrm{E}+00 \quad 0.76553 \mathrm{E}+00 \quad 0.10000 \mathrm{E}+01$

COMPRESSOR INLET CONDITIONS - ANALYSIS, STAGE 5 $\mathrm{BLEED}=0.000 \quad \mathrm{DPInC}=7.324 \quad$ EfDer $=0.999$

$\mathrm{SH}=0.251636 \mathrm{E}-02$

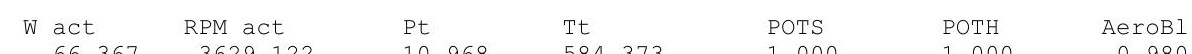
$\mathrm{W} \mathrm{Kg} / \mathrm{sec}=30.167$ Wdry $=66.200 \quad$ WH2O $=0.167 \quad 1 \mathrm{bm} / \mathrm{sec} \quad \mathrm{H} 2 \mathrm{O}=\begin{gathered}1.000 \\ 1.932 \mathrm{~g} / \mathrm{m}^{\wedge} 3\end{gathered}$

$\begin{array}{ccccccc}\text { W Cor } & \text { RPM cor } & \text { GAMMA } & \text { Cp } & \text { R } & \text { Blades } & \text { THK } \\ 94.415 & 3419.095 & 1.378 & 0.251 & 53.430 & 77.000 & 0.050 \\ & & & & & & \\ \text { CFM } & \text { SCFM } & \text { A1/A* } & \text { Area1 } & \text { A* } & \text { AthrRotor } & \text { ChokeMargin } \\ 83267.594 & 52023.574 & 1.841 & 509.668 & 276.823 & 384.739 & 1.390\end{array}$

ROTOR LEADING EDGE CONDITIONS, STAGE 5

$\begin{array}{lccccccccc} & \text { R1 } & \text { Stator } & \text { Alfa } & \text { C1 } & \text { CU1 } & \text { Cm1 } & \text { Mabs } & \text { Mrel } & \text { U1cor } \\ \text { TIP } & 18.32 & 0.00 & -0.02 & 392.09 & -0.14 & 392.09 & 0.34 & 0.60 & 546.60 \\ \text { MEAN } & 15.91 & 0.00 & -0.02 & 392.09 & -0.14 & 392.09 & 0.34 & 0.55 & \\ \text { HUB } & 13.07 & 0.00 & -0.02 & 392.09 & -0.14 & 392.09 & 0.34 & 0.49 & \\ & & & & & & & & & \\ \text { TIP } & \text { BetaFlo } & \text { BetaBlade } & \text { Incid } & \text { U1 } & \text { W1 } & \text { Ps1 } & \text { Ts1 } & \text { TwetBulb1 } & \text { RH } \\ \text { MEAN } & 55.96 & 47.36 & 8.60 & 580.20 & 700.37 & 10.15 & 572.12 & 520.55 & 3.00 \\ \text { HUB } & 46.56 & 38.84 & 7.72 & 413.93 & 570.25 & 10.15 & 572.12 & 520.55 & 3.00\end{array}$

ROTOR EXIT CONDITIONS, STAGE 5 SOLUTION IS CONVERGED

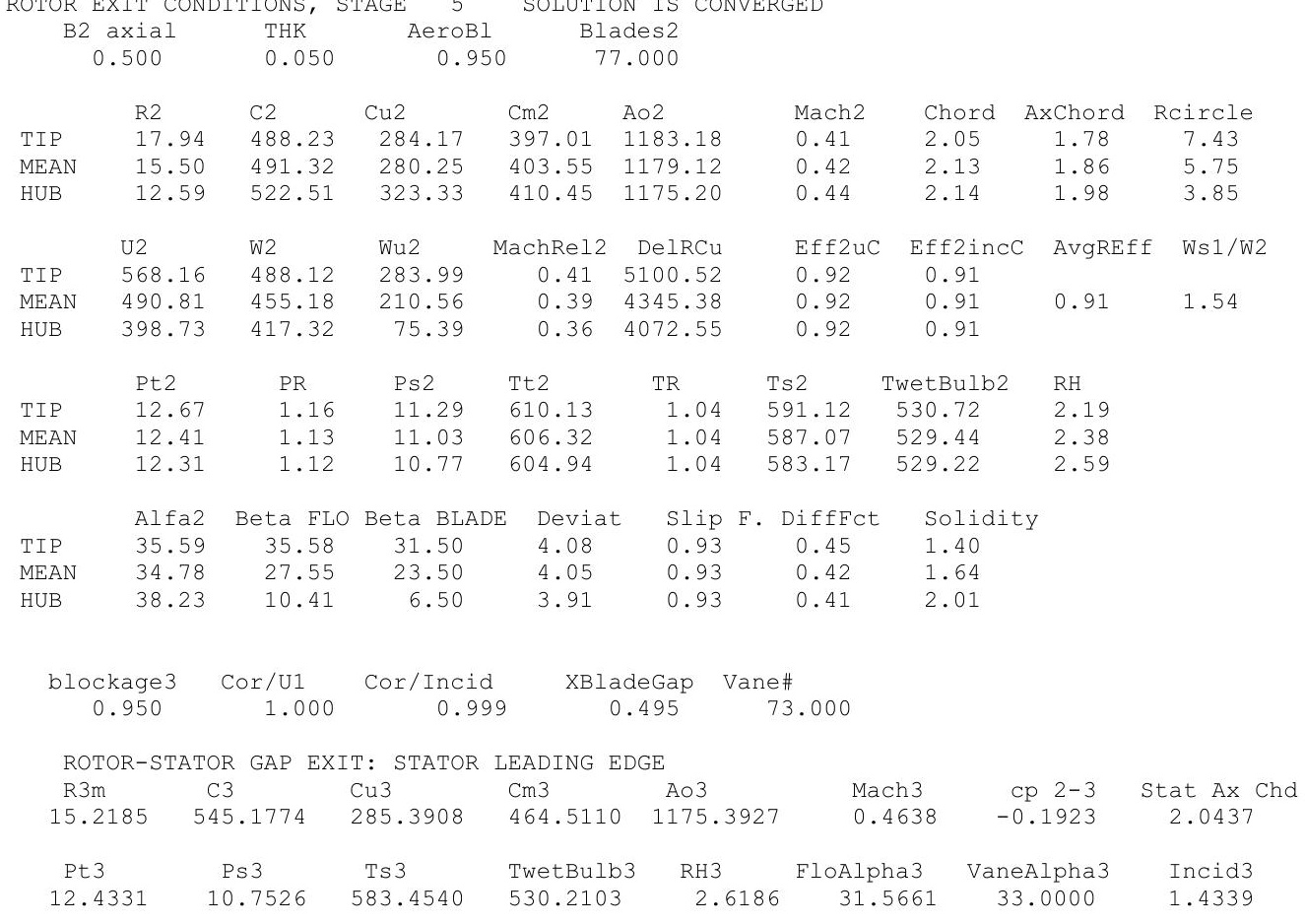




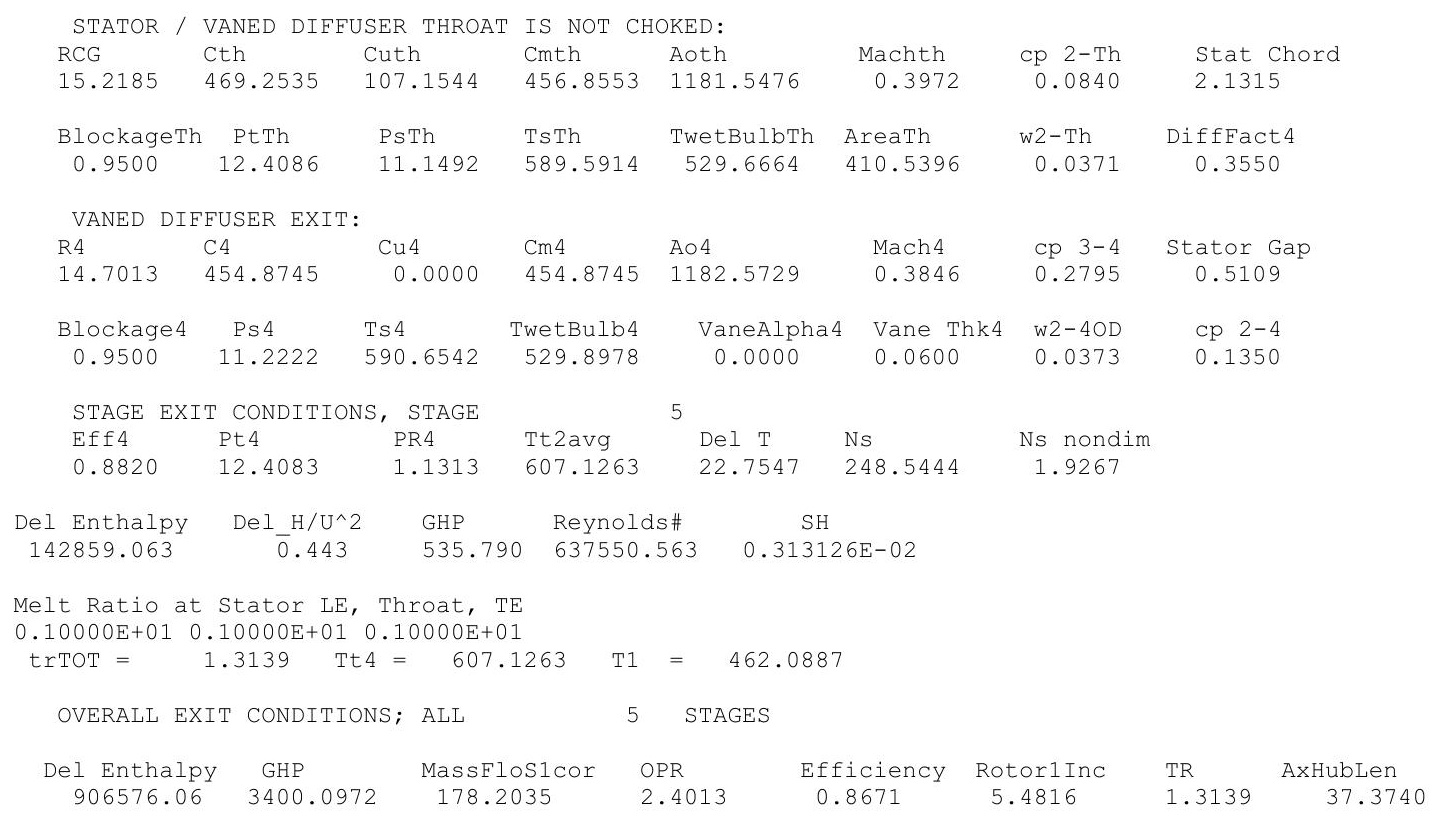




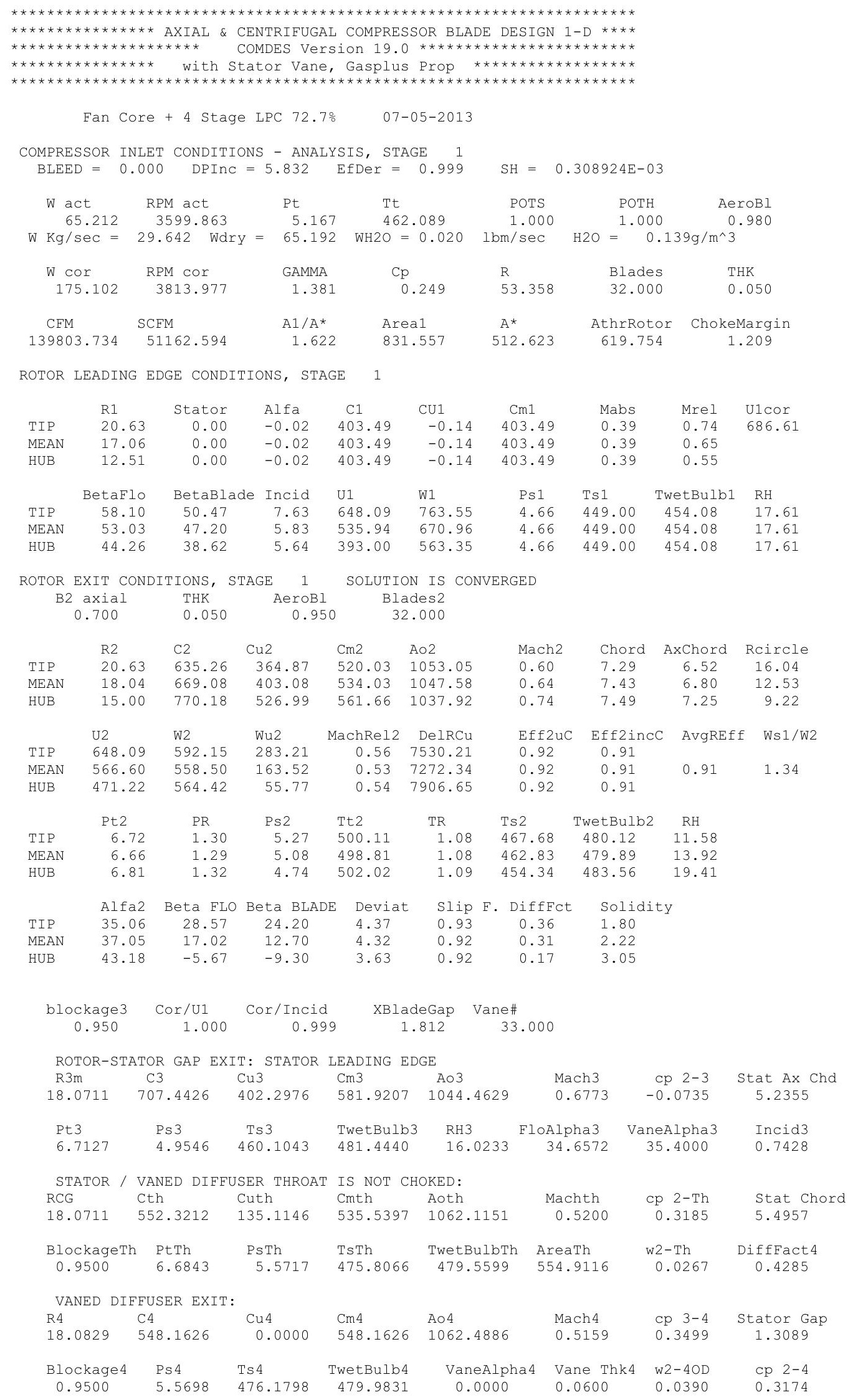




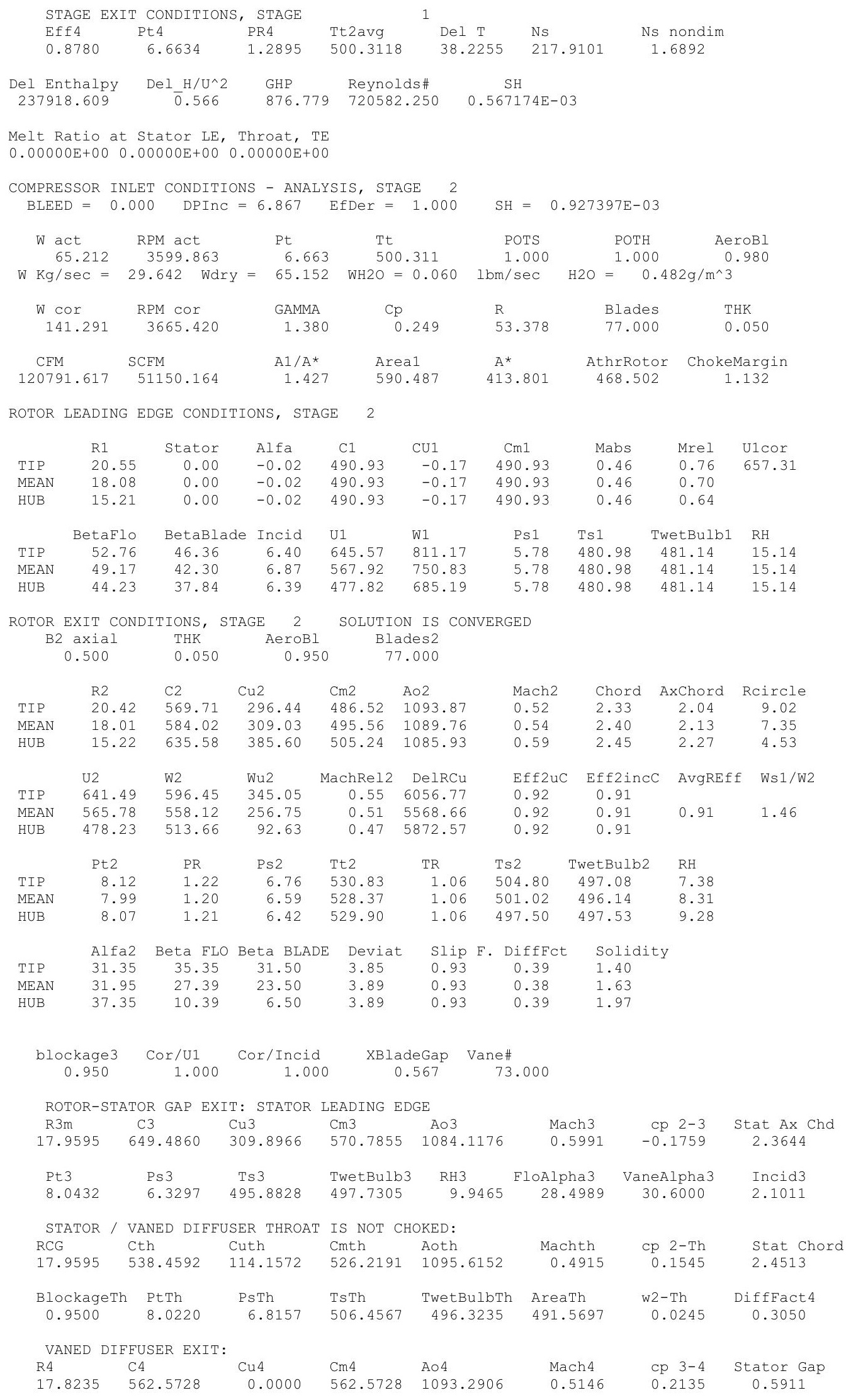




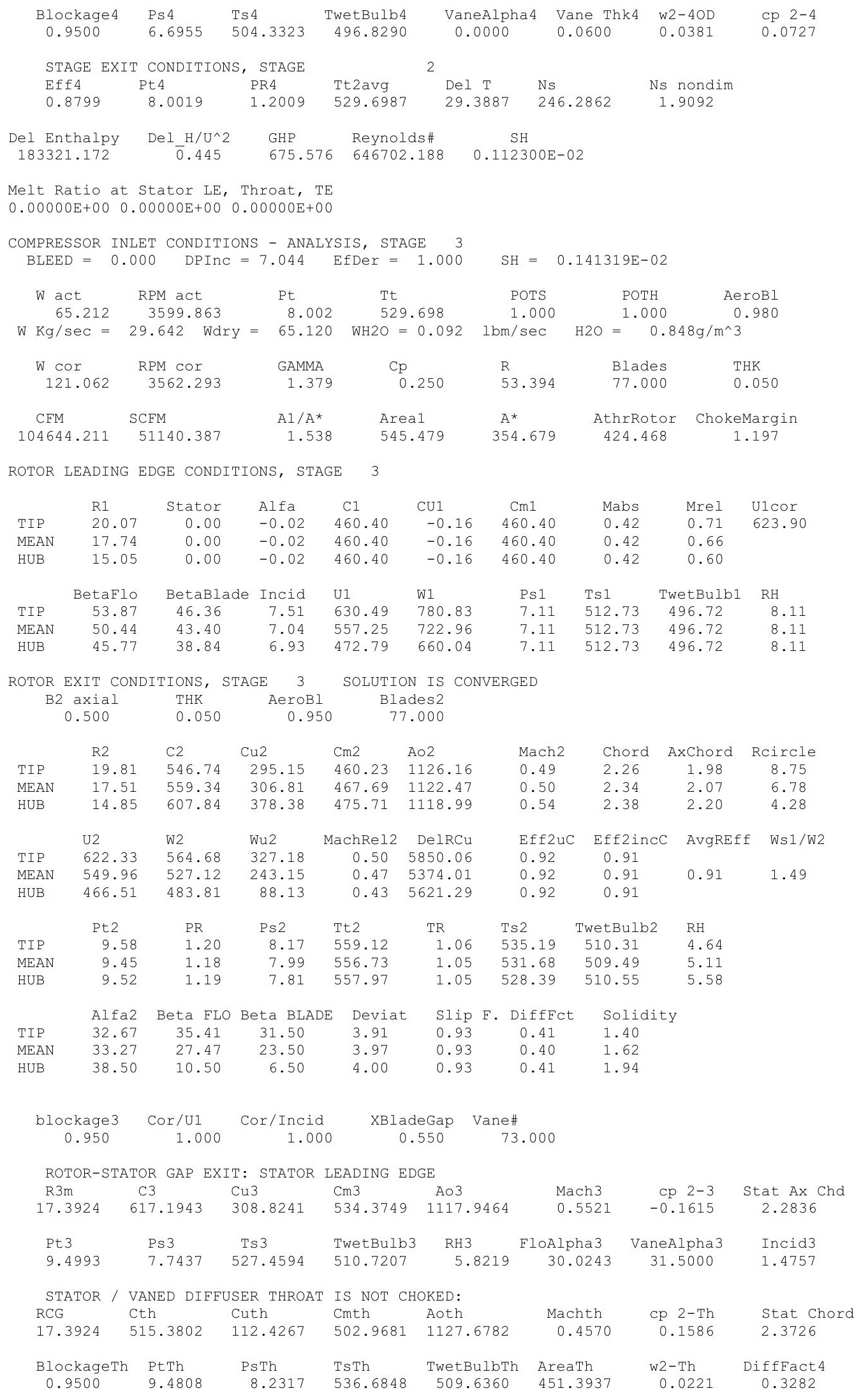




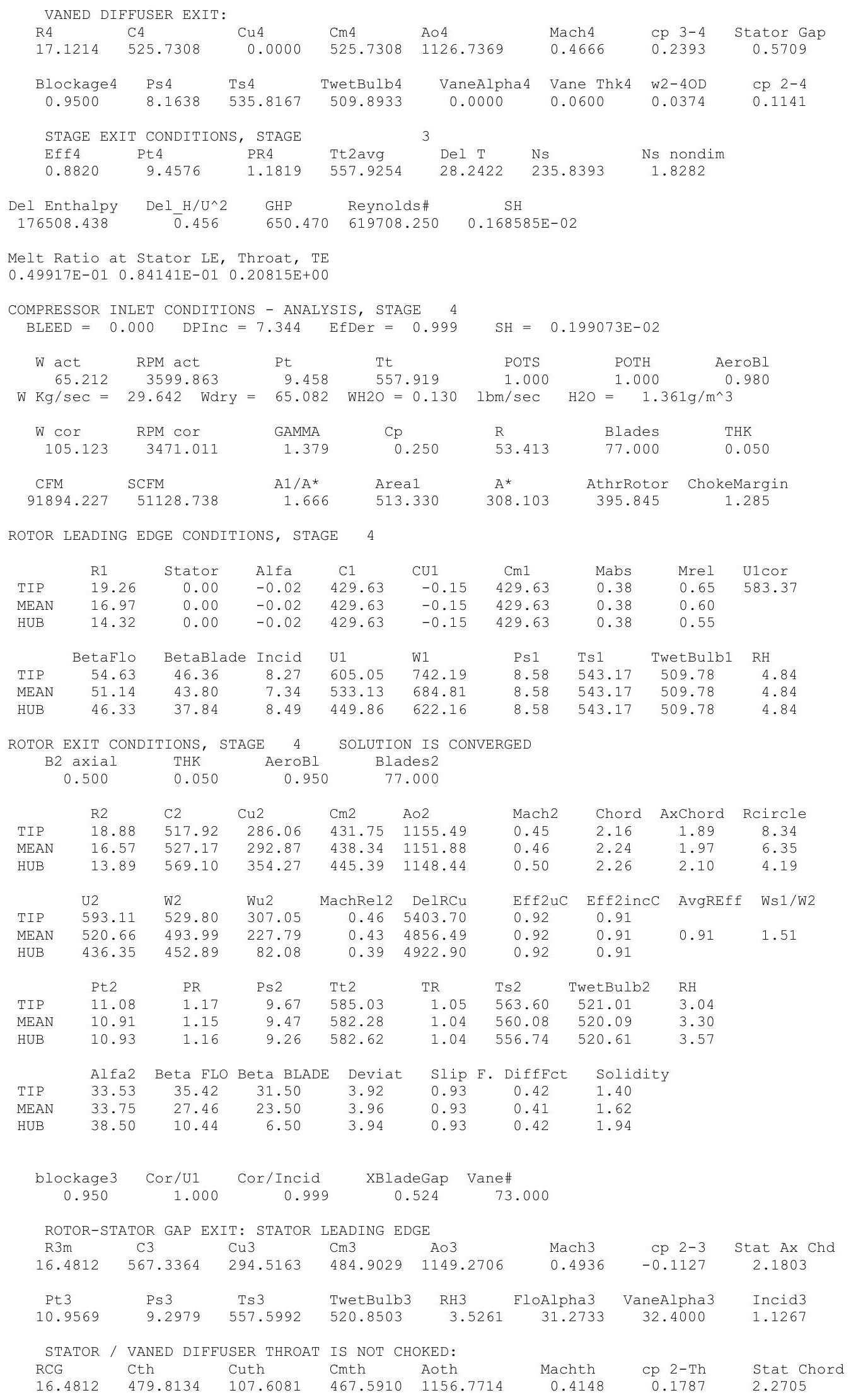




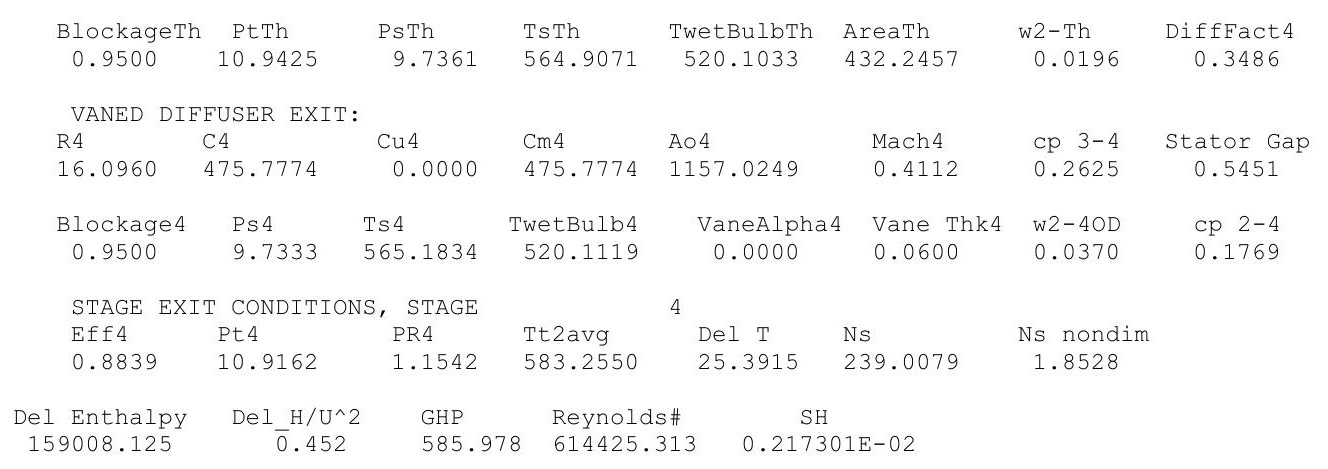

Melt Ratio at Stator LE, Throat, TE

$0.68643 \mathrm{E}+00 \quad 0.77626 \mathrm{E}+00 \quad 0.10000 \mathrm{E}+01$

COMPRESSOR INLET CONDITIONS - ANALYSIS, STAGE 5 $\mathrm{BLEED}=0.000 \quad$ DPInC $=7.556 \quad$ EfDer $=0.999$

65.212 RPM act Pt Tt

$\mathrm{W} \mathrm{Kg} / \mathrm{sec}=29.642$ Wdry $=65.042$ WH2O $=0.170$

$\mathrm{SH}=0.260876 \mathrm{E}-02$

\begin{tabular}{|c|c|c|c|c|c|c|}
\hline $\begin{array}{ll}W & \text { cor } \\
& 93.121\end{array}$ & $\begin{array}{l}\text { RPM cor } \\
3394.805\end{array}$ & $\begin{array}{l}\text { GAMMA } \\
1.378\end{array}$ & $\begin{array}{l}\mathrm{Cp} \\
0.251\end{array}$ & $\begin{array}{l}R \\
53.433\end{array}$ & $\begin{array}{l}\text { Blades } \\
77.000\end{array}$ & $\begin{array}{l}\text { THK } \\
0.050\end{array}$ \\
\hline $\begin{array}{l}\text { CFM } \\
81909.859\end{array}$ & $\begin{array}{l}\text { SCFM } \\
51116.258\end{array}$ & $\begin{array}{l}\mathrm{A} 1 / \mathrm{A}^{\star} \\
1.867\end{array}$ & $\begin{array}{l}\text { Area1 } \\
509.668\end{array}$ & $\begin{array}{c}A^{*} \\
273.042\end{array}$ & $\begin{array}{c}\text { AthrRotor } \\
384.739\end{array}$ & $\begin{array}{c}\text { ChokeMargin } \\
1.409\end{array}$ \\
\hline
\end{tabular}

ROTOR LEADING EDGE CONDITIONS, STAGE 5

$\begin{array}{lrrrrrrrrr} & \text { R1 } & \text { Stator } & \text { Alfa } & \text { C1 } & \text { CU1 } & \text { Cm1 } & \text { Mabs } & \text { Mrel } & \text { U1cor } \\ \text { TIP } & 18.32 & 0.00 & -0.02 & 385.69 & -0.13 & 385.69 & 0.33 & 0.60 & 542.72 \\ \text { MEAN } & 15.91 & 0.00 & -0.02 & 385.69 & -0.13 & 385.69 & 0.33 & 0.54 & \\ \text { HUB } & 13.07 & 0.00 & -0.02 & 385.69 & -0.13 & 385.69 & 0.33 & 0.48 & \\ & & & & & & & & & \\ & \text { BetaFlo } & \text { BetaBlade } & \text { Incid } & \text { U1 } & \text { W1 } & \text { Ps1 } & \text { Ts1 } & \text { TwetBulb1 } & \text { RH } \\ \text { TIP } & 56.18 & 47.36 & 8.82 & 575.52 & 692.92 & 10.13 & 571.39 & 520.29 & 3.17 \\ \text { MEAN } & 52.36 & 44.80 & 7.56 & 499.90 & 631.50 & 10.13 & 571.39 & 520.29 & 3.17 \\ \text { HUB } & 46.80 & 38.84 & 7.96 & 410.59 & 563.43 & 10.13 & 571.39 & 520.29 & 3.17\end{array}$

ROTOR EXIT CONDITIONS, STAGE 5 SOLUTION IS CONVERGED

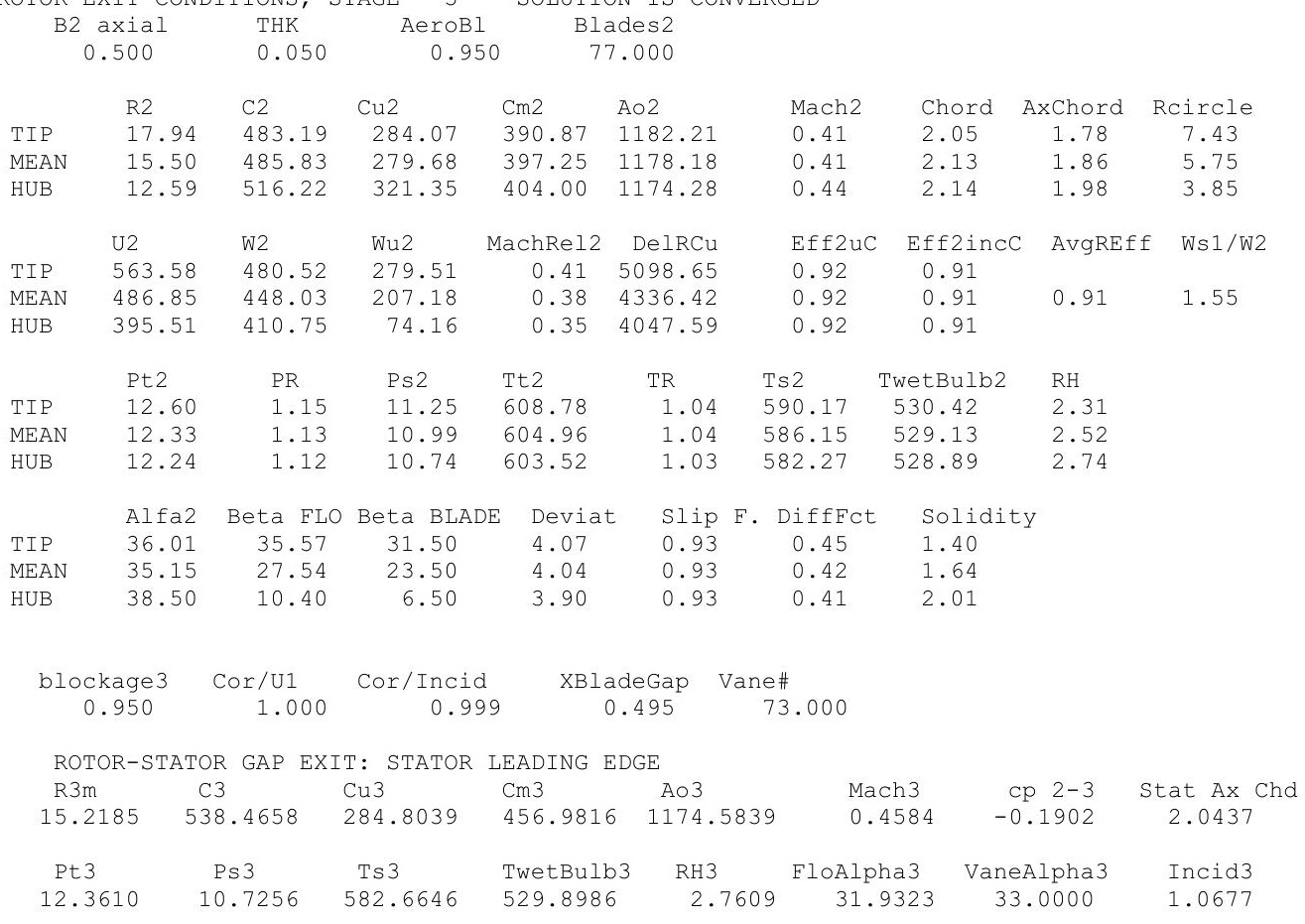




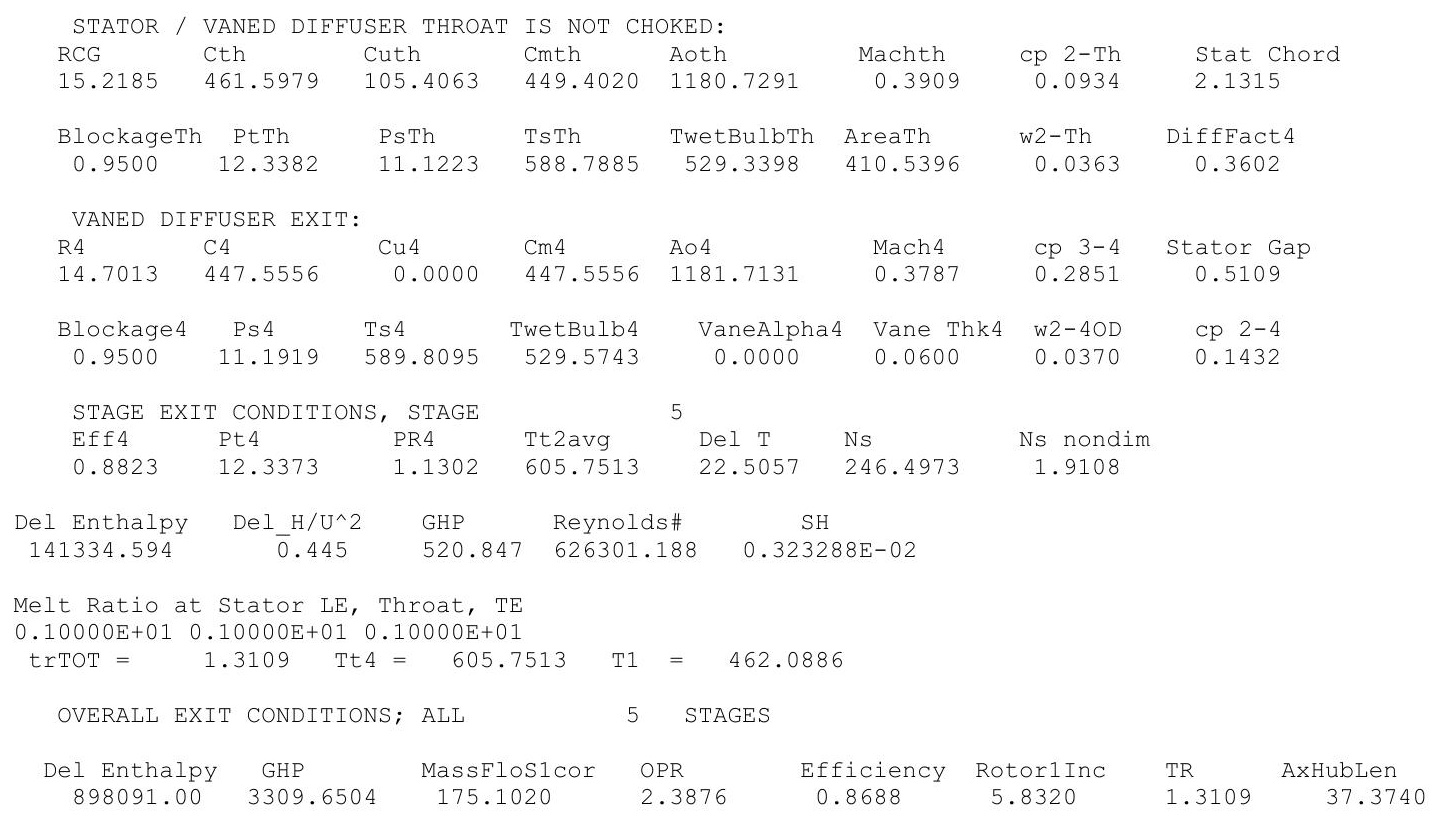




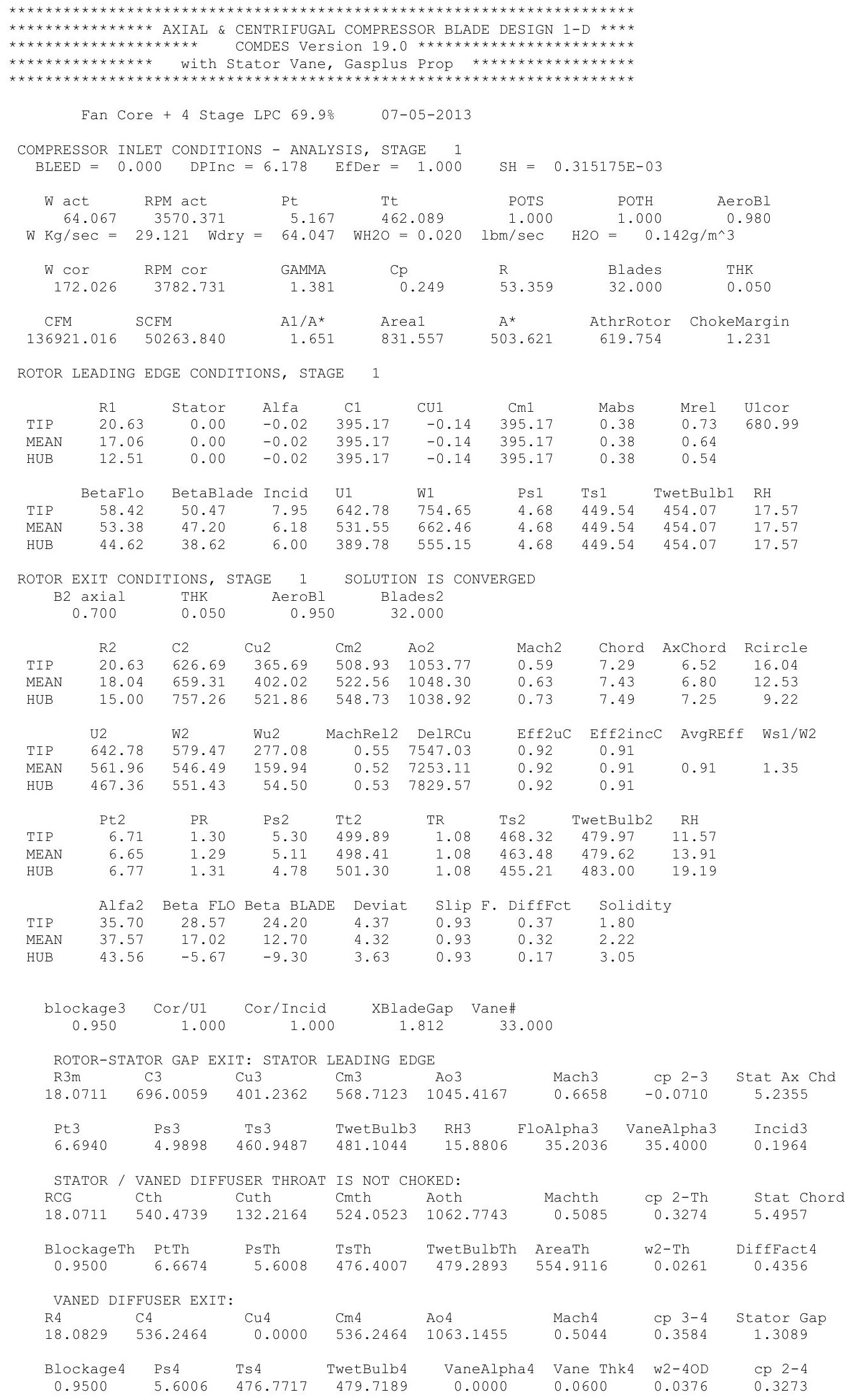




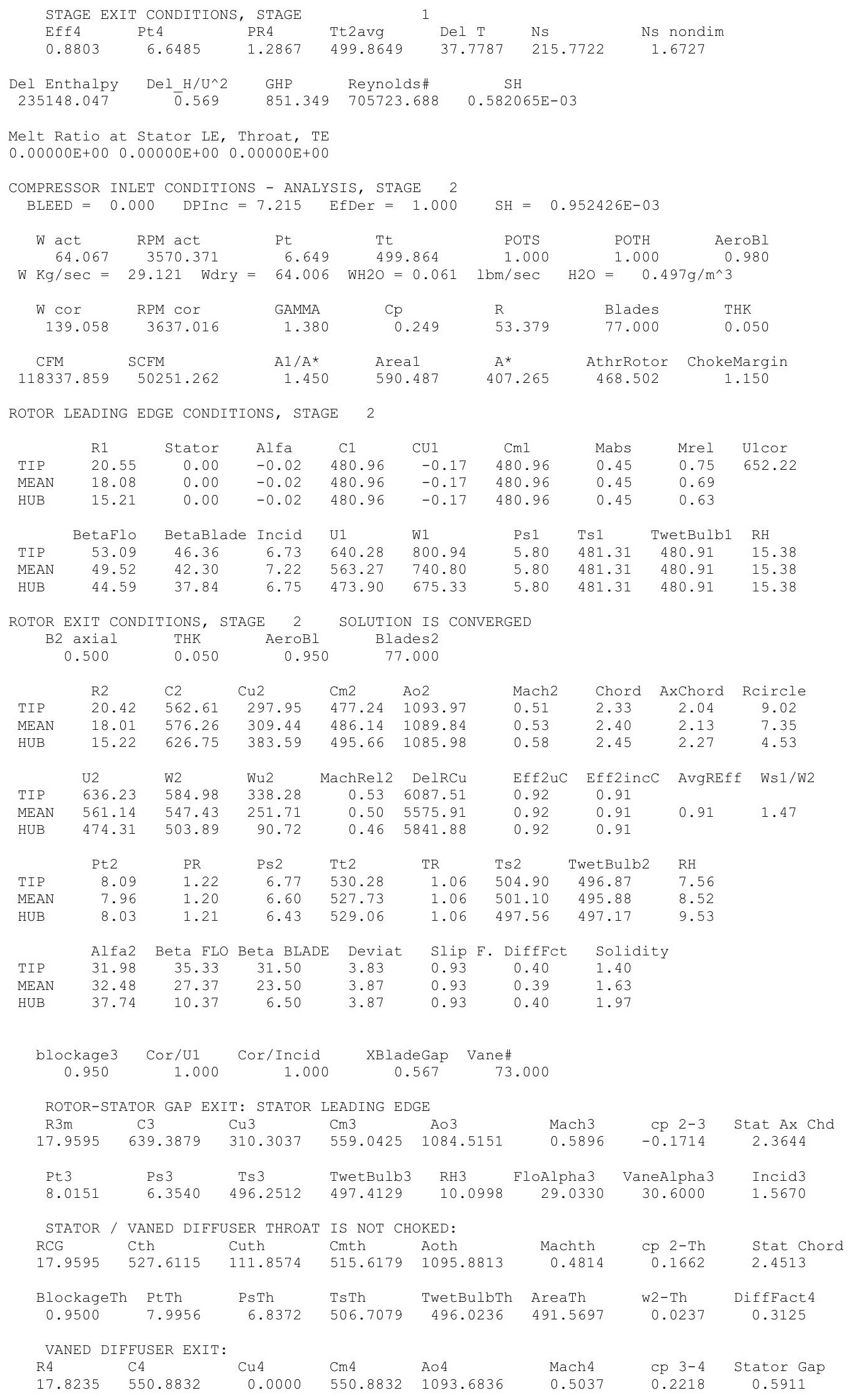




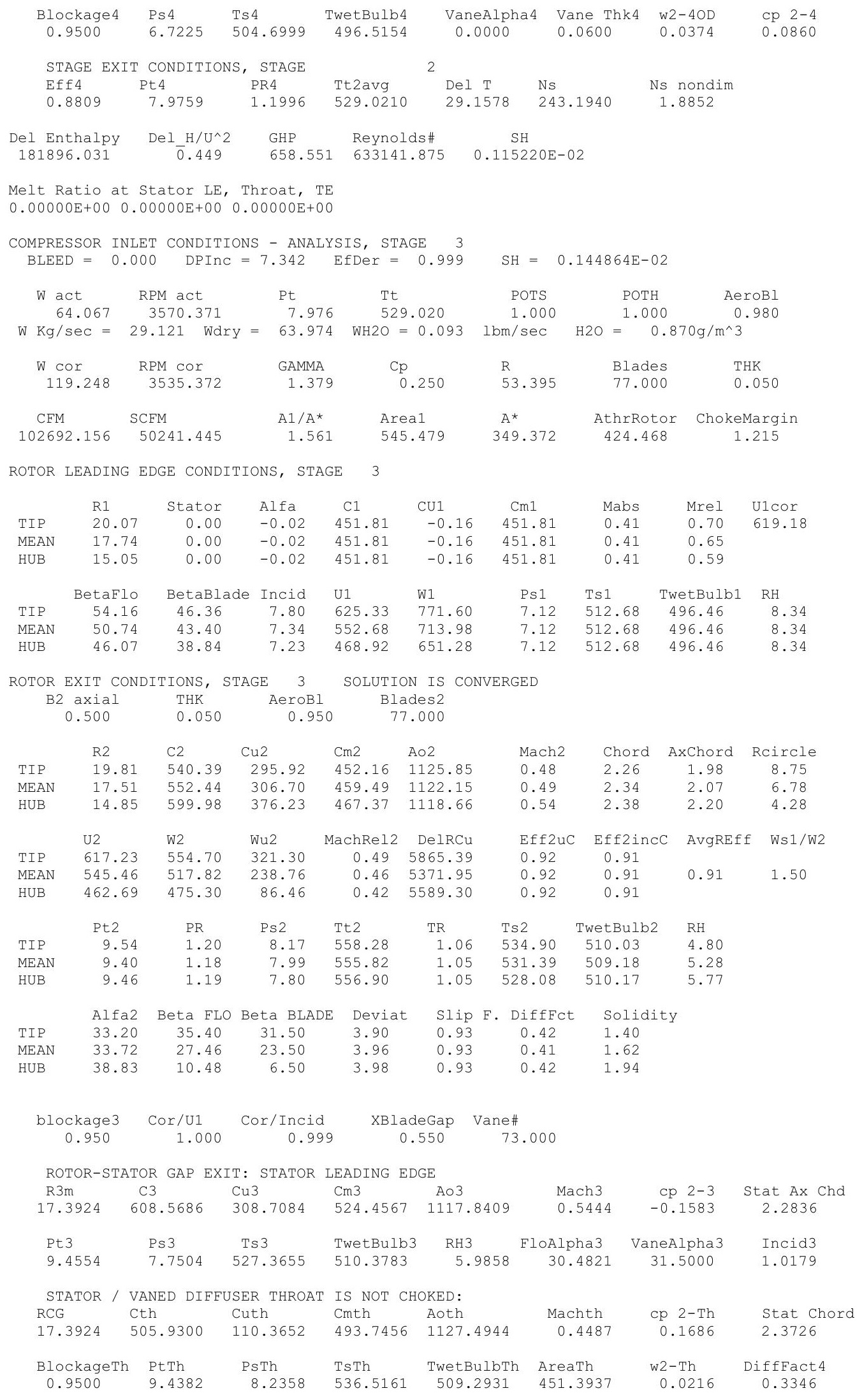




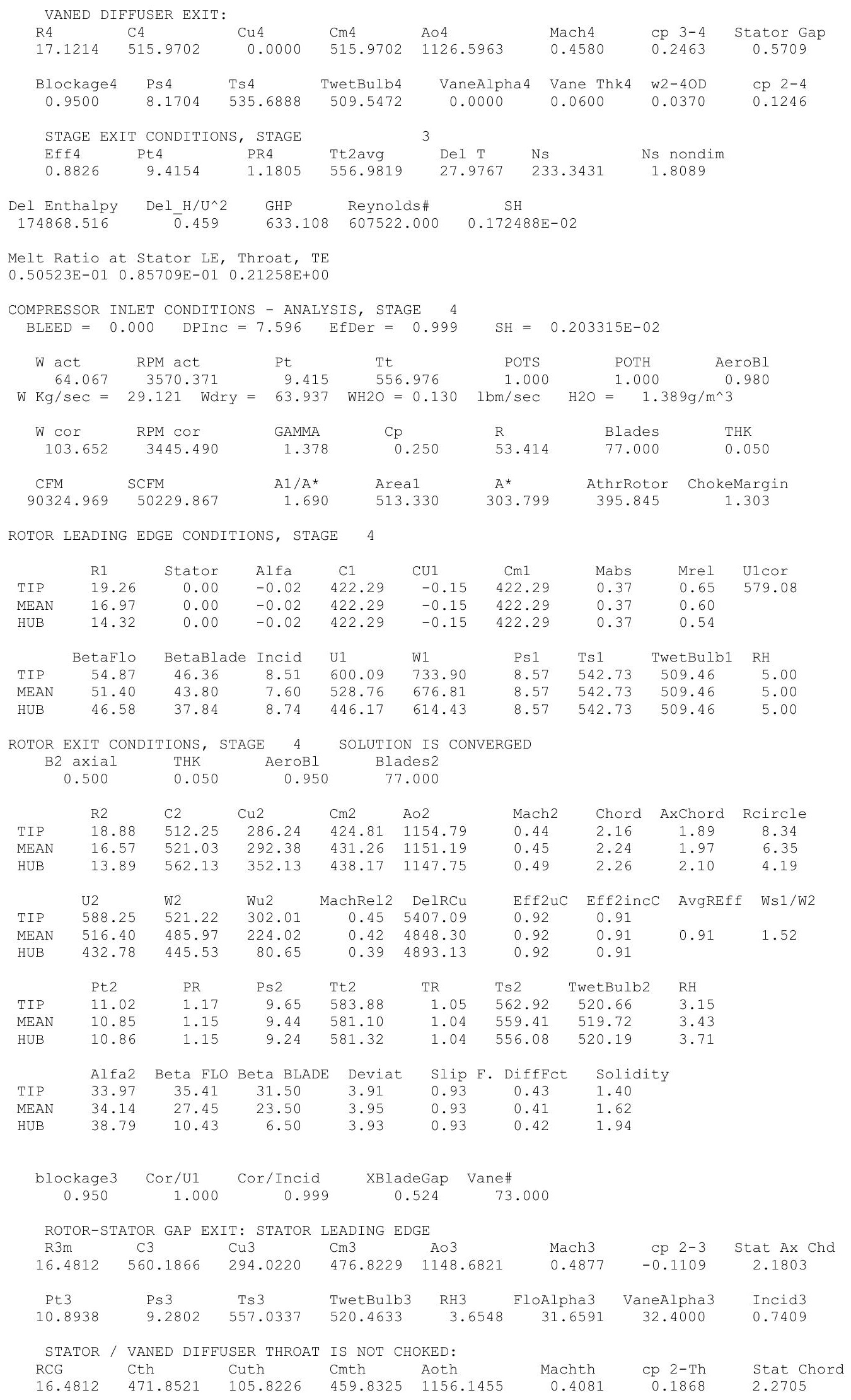




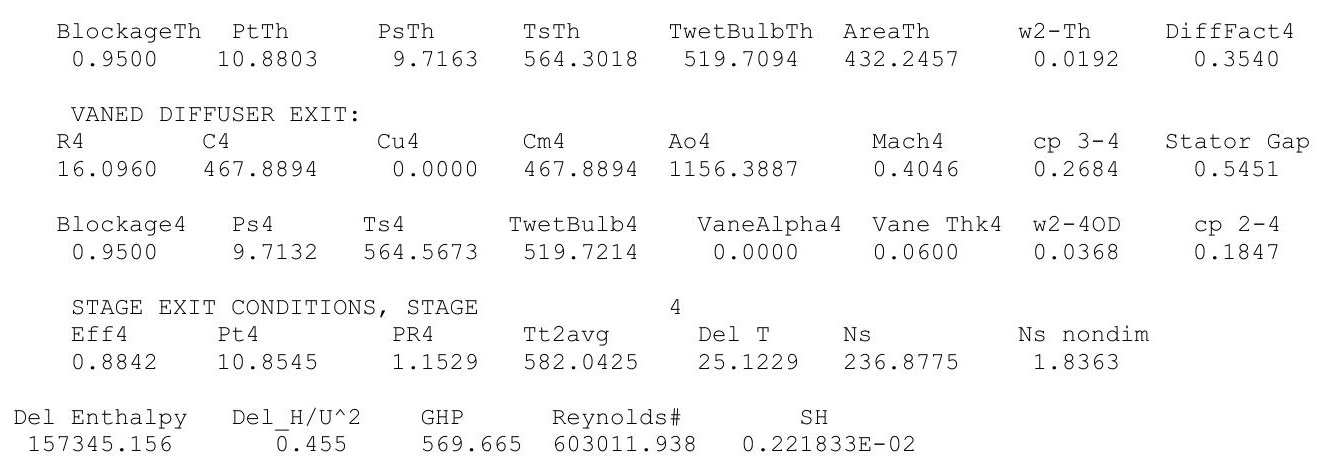

Melt Ratio at Stator LE, Throat, TE

$0.69261 \mathrm{E}+000.78303 \mathrm{E}+00 \quad 0.10000 \mathrm{E}+01$

COMPRESSOR INLET CONDITIONS - ANALYSIS, STAGE 5 $\mathrm{BLEED}=0.000 \quad$ DPInC $=7.762 \quad$ EfDer $=0.999$

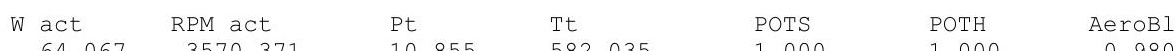
$\mathrm{W} \mathrm{Kg} / \mathrm{sec}=29.121$ Wdry $=63.895 \mathrm{WH} 2 \mathrm{O}=0.172 \quad \mathrm{lbm} / \mathrm{sec} \quad \mathrm{H} 2 \mathrm{O}=2.056 \mathrm{~g} / \mathrm{m}^{\wedge} 3$

$\begin{array}{ccccccc}\text { W Cor } & \text { RPM cor } & \text { GAMMA } & \text { Cp } & \text { R } & \text { Blades } & \text { THK } \\ 91.909 & 3370.499 & 1.377 & 0.251 & 53.435 & 77.000 & 0.050 \\ & & & & & & \\ \text { CFM } & \text { SCFM } & \text { A1/A* } & \text { Area1 } & A^{*} & \text { AthrRotor } & \text { ChokeMargin } \\ 80636.117 & 50216.895 & 1.891 & 509.668 & 269.502 & 384.739 & 1.428\end{array}$

ROTOR LEADING EDGE CONDITIONS, STAGE 5

$\begin{array}{lccccccccc} & \text { R1 } & \text { Stator } & \text { Alfa } & \text { C1 } & \text { CU1 } & \text { Cm1 } & \text { Mabs } & \text { Mrel } & \text { U1cor } \\ \text { TIP } & 18.32 & 0.00 & -0.02 & 379.70 & -0.13 & 379.69 & 0.33 & 0.59 & 538.83 \\ \text { MEAN } & 15.91 & 0.00 & -0.02 & 379.70 & -0.13 & 379.69 & 0.33 & 0.54 & \\ \text { HUB } & 13.07 & 0.00 & -0.02 & 379.70 & -0.13 & 379.69 & 0.33 & 0.48 & \\ & & & & & & & & & \\ \text { TIP } & \text { BetaFlo } & \text { BetaBlade } & \text { Incid } & \text { U1 } & \text { W1 } & \text { Ps1 } & \text { Ts1 } & \text { TwetBulb1 } & \text { RH } \\ \text { MEAN } & 56.37 & 47.36 & 9.01 & 570.80 & 685.66 & 10.09 & 570.55 & 519.96 & 3.33 \\ \text { HUB } & 47.01 & 34.80 & 7.76 & 495.81 & 624.60 & 10.09 & 570.55 & 519.96 & 3.33 \\ & 4.84 & 8.17 & 407.23 & 556.87 & 10.09 & 570.55 & 519.96 & 3.33\end{array}$

ROTOR EXIT CONDITIONS, STAGE 5 SOLUTION IS CONVERGED

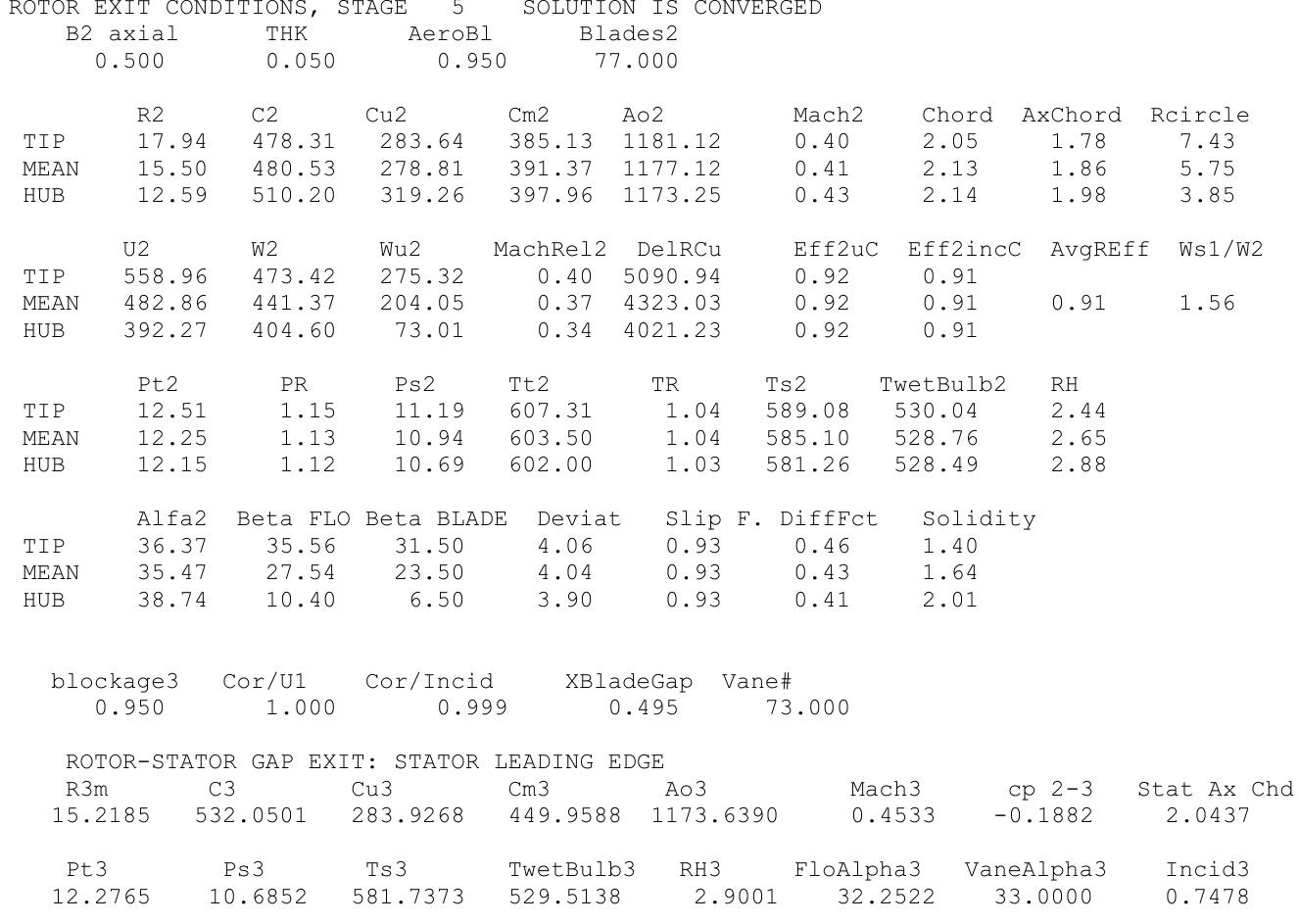




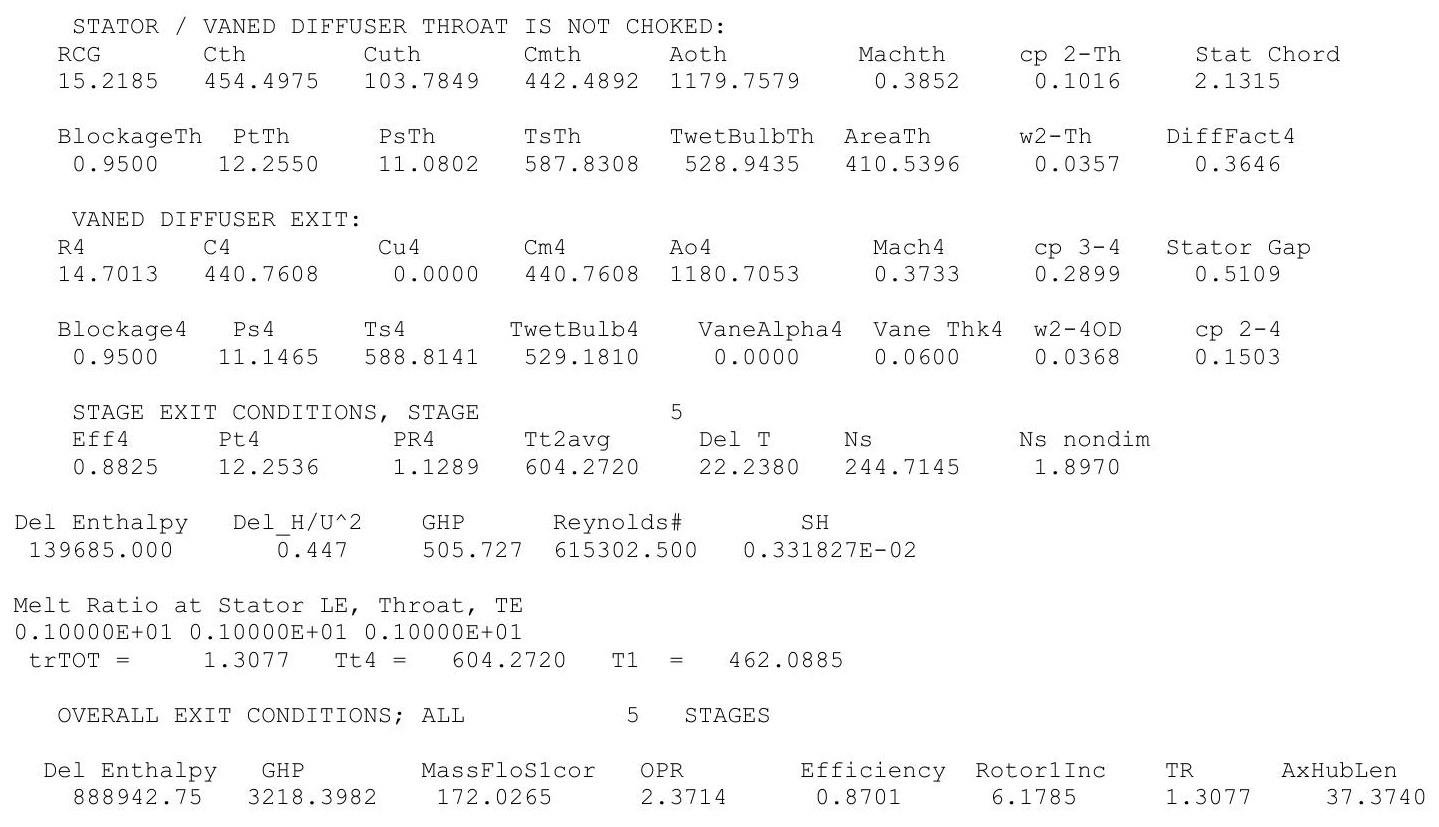


$5 \mu \mathrm{m}, \mathrm{ISA}+36 \mathrm{R}$

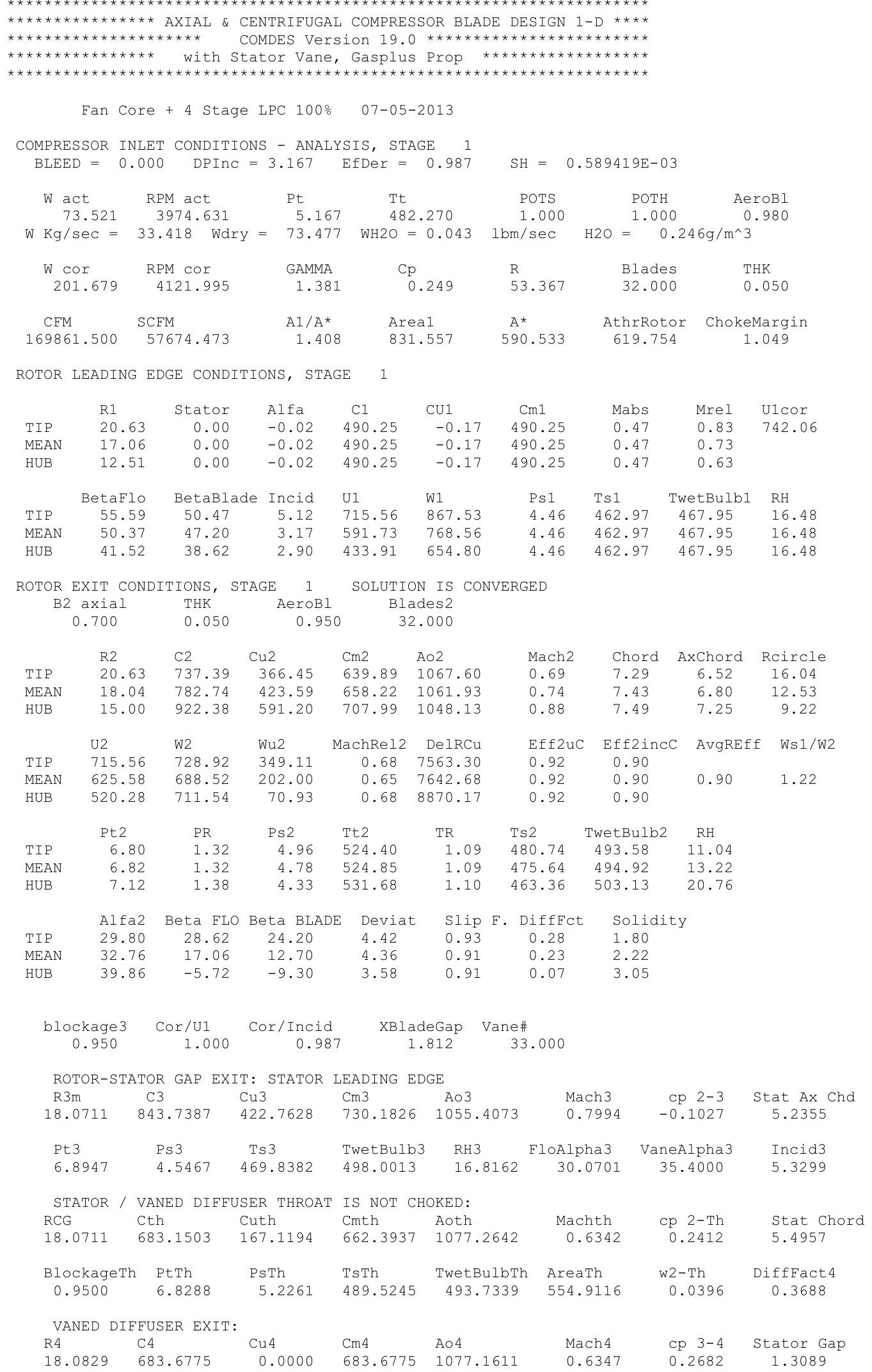




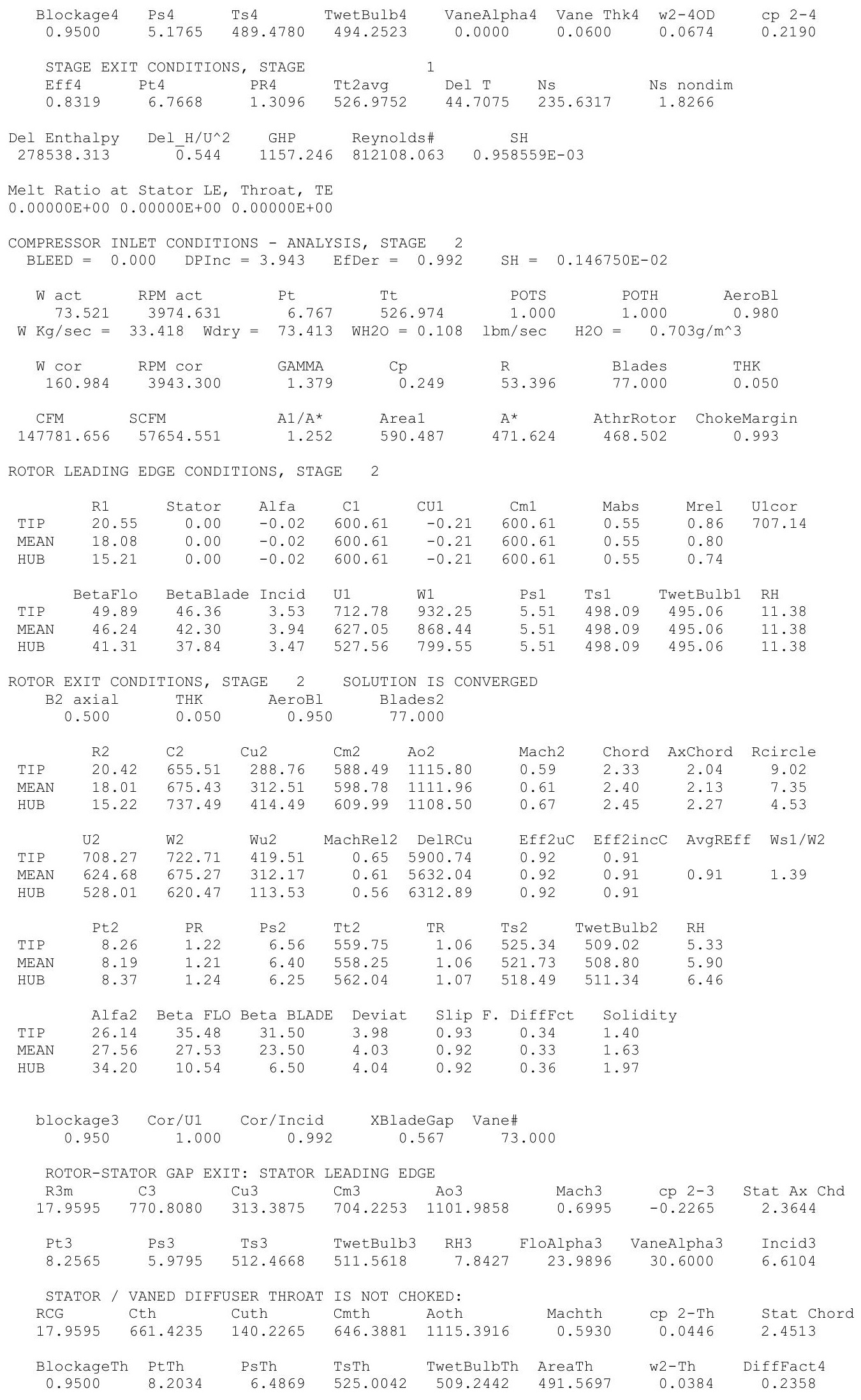




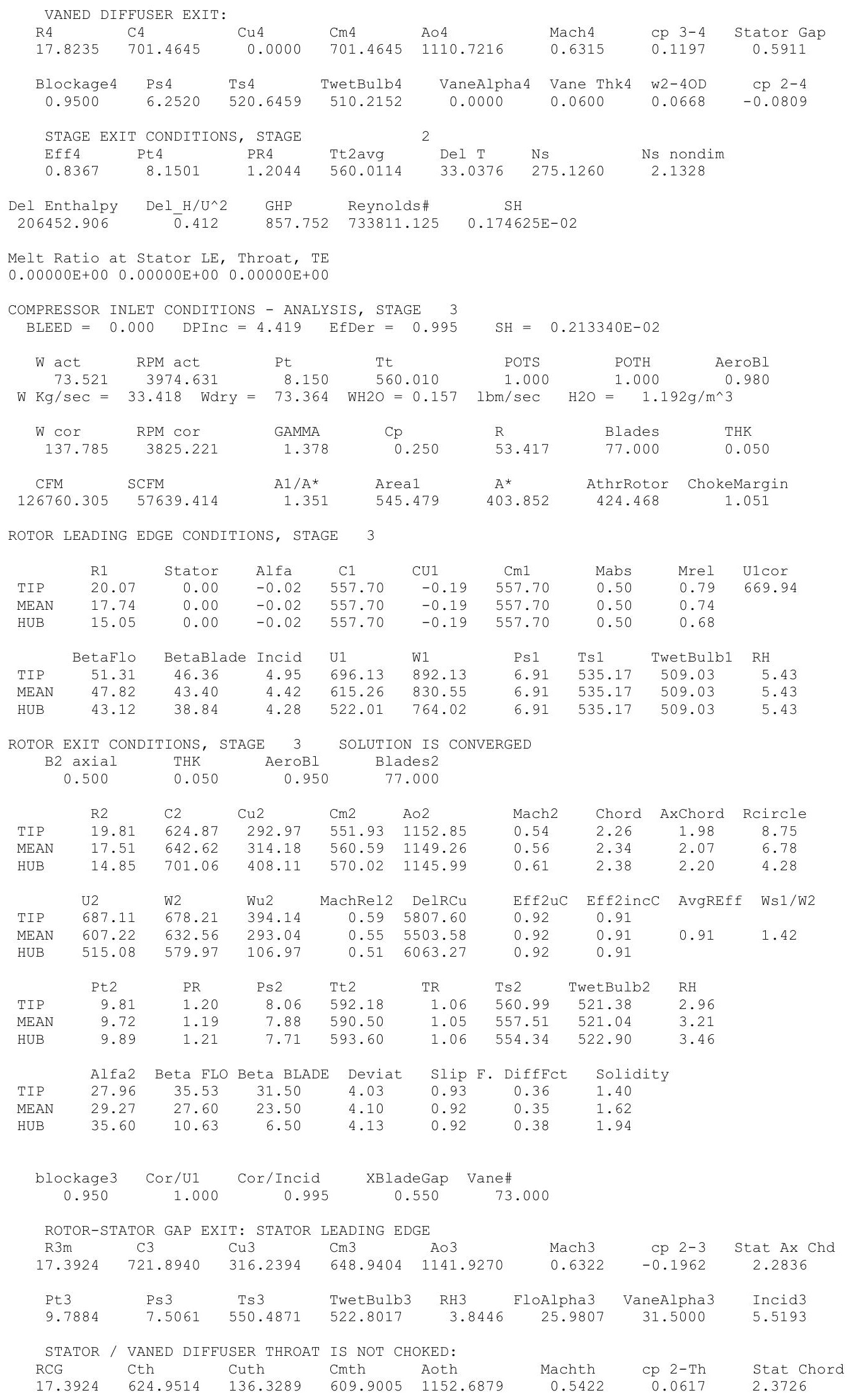




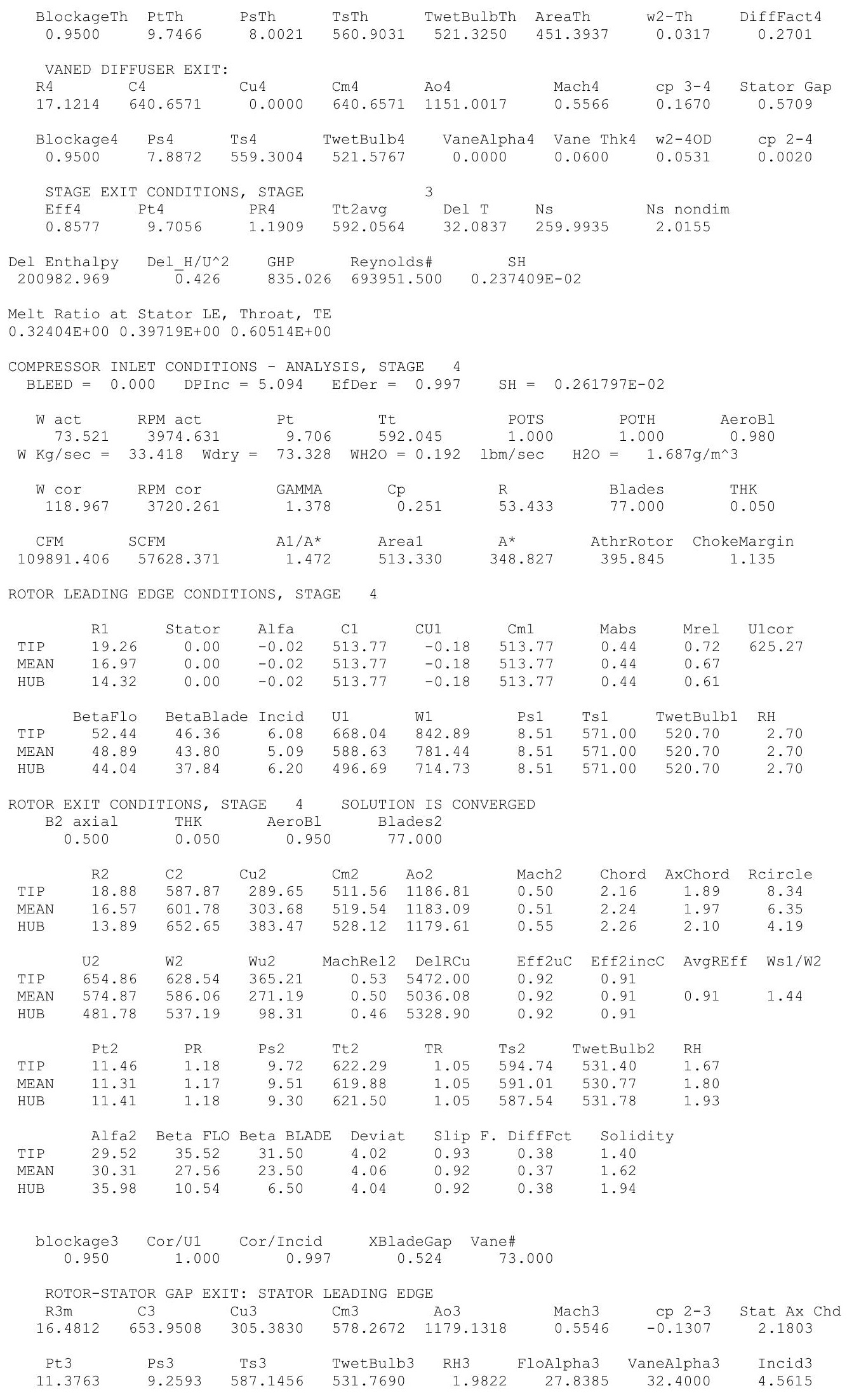




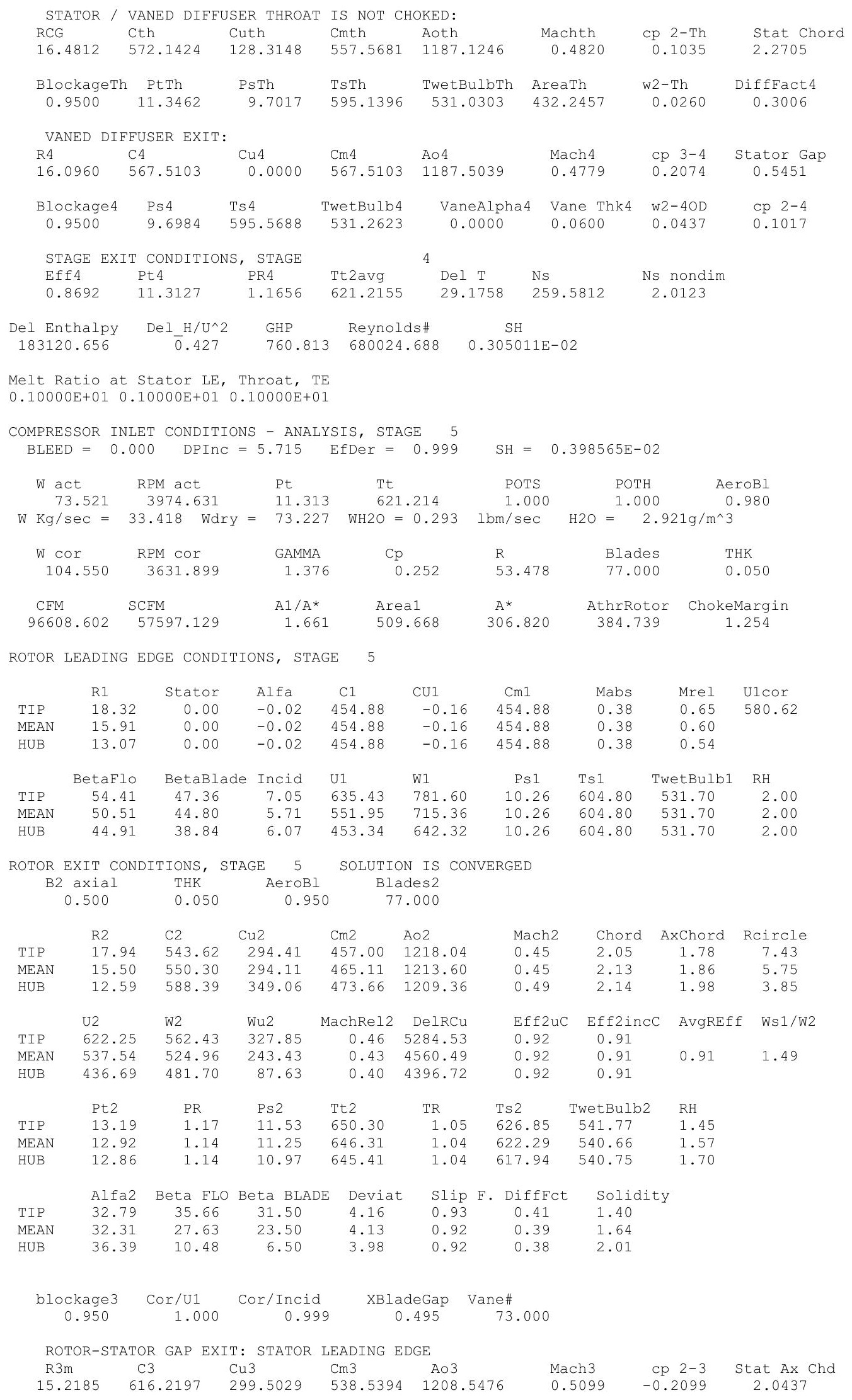




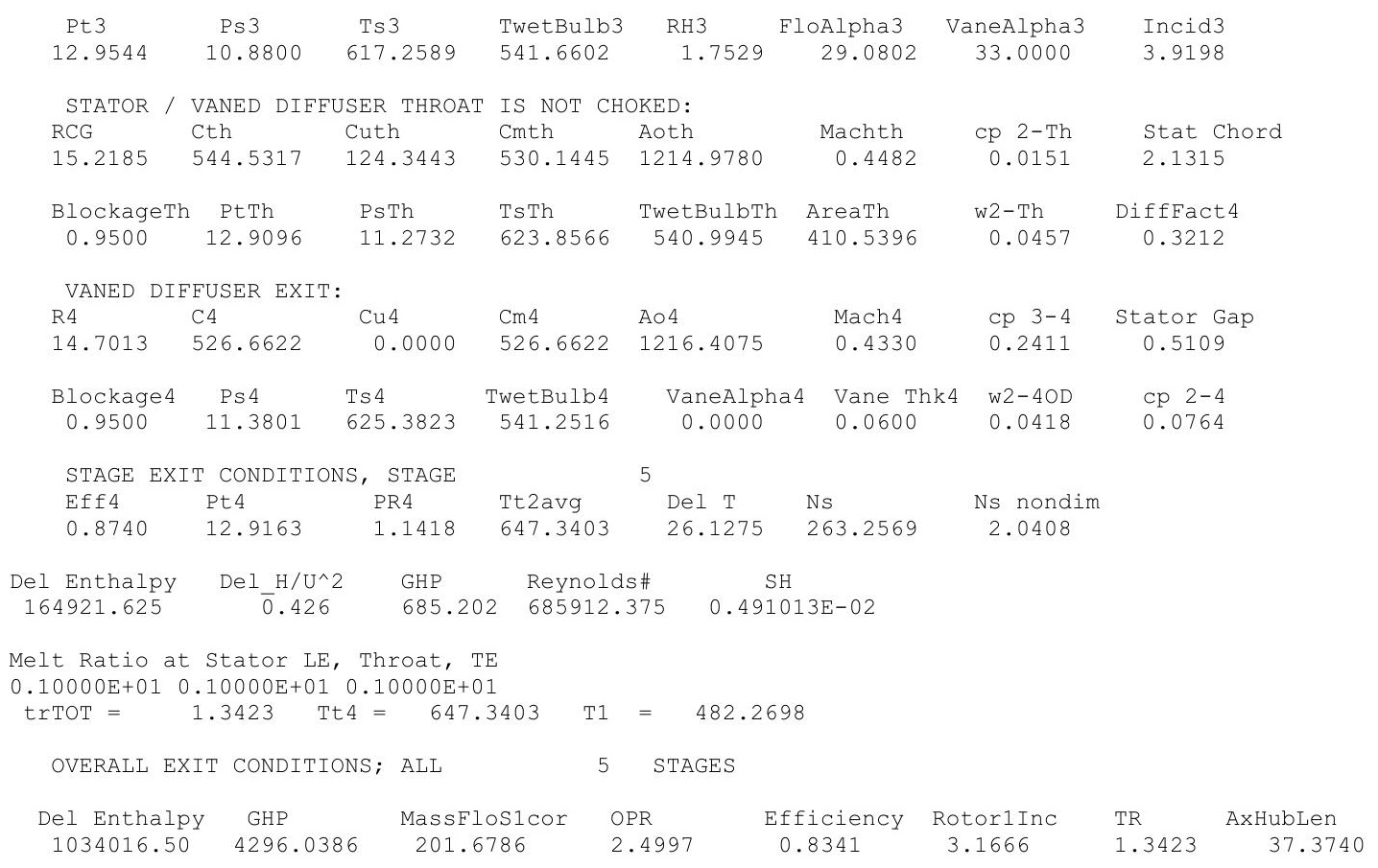




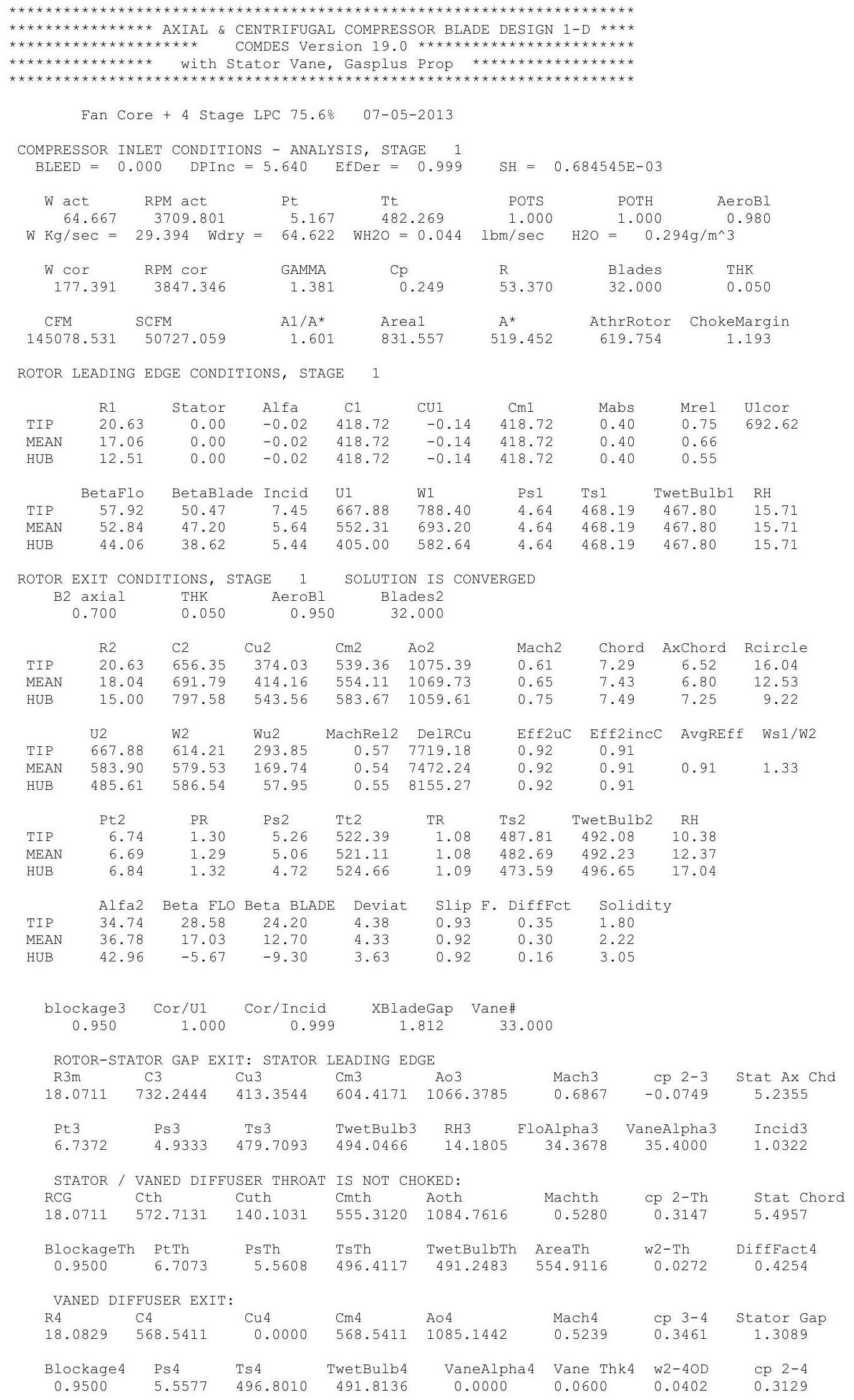




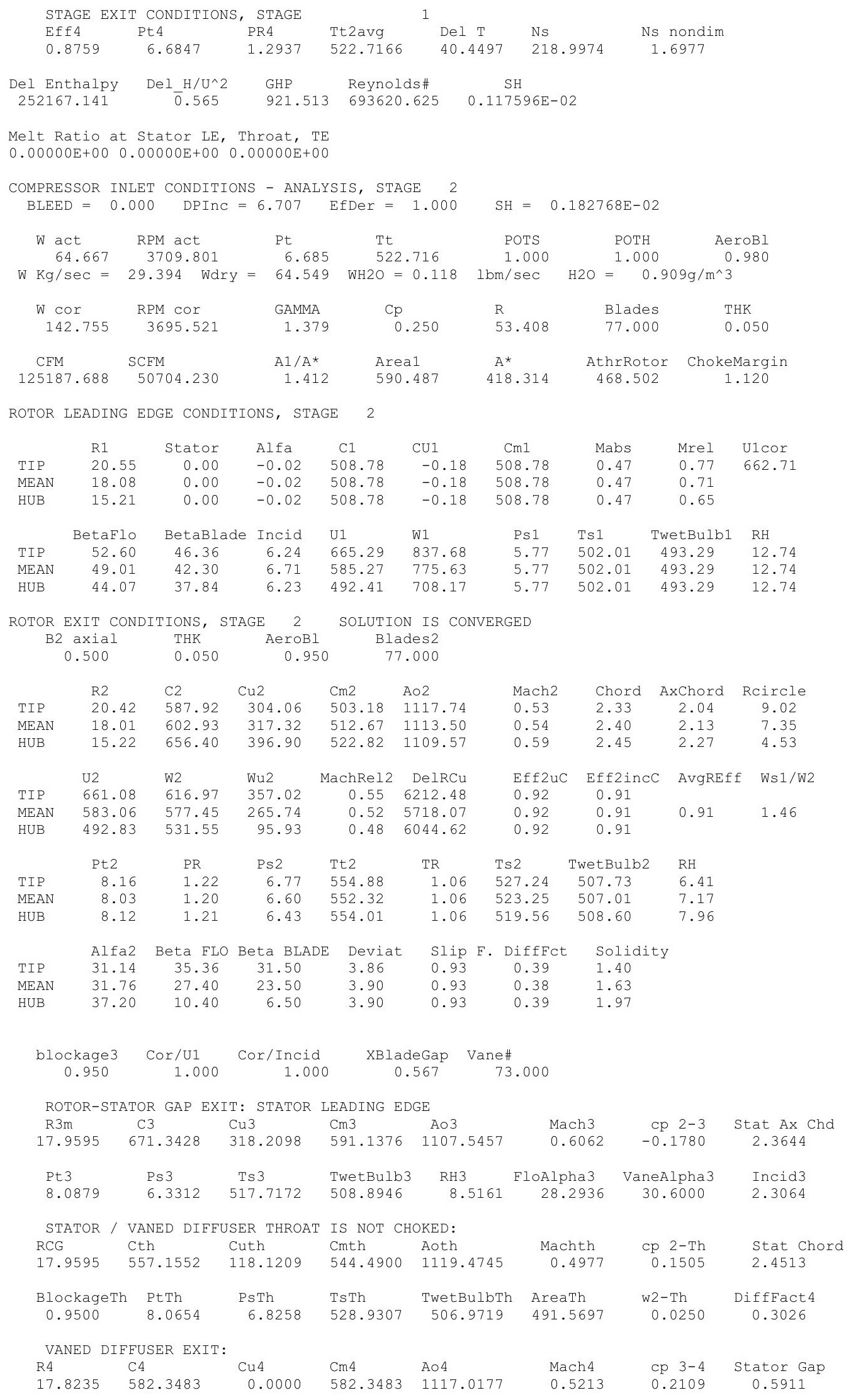




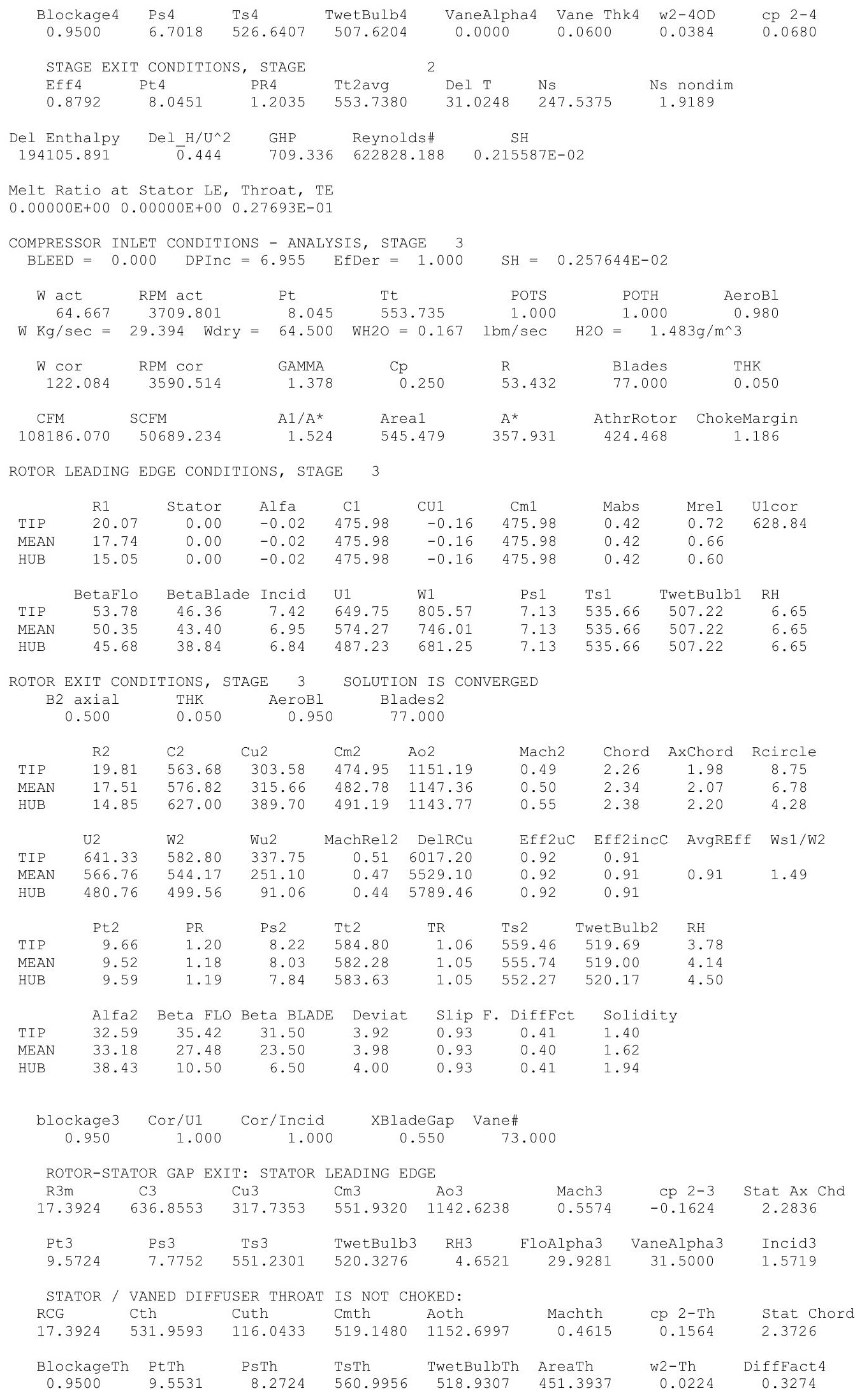




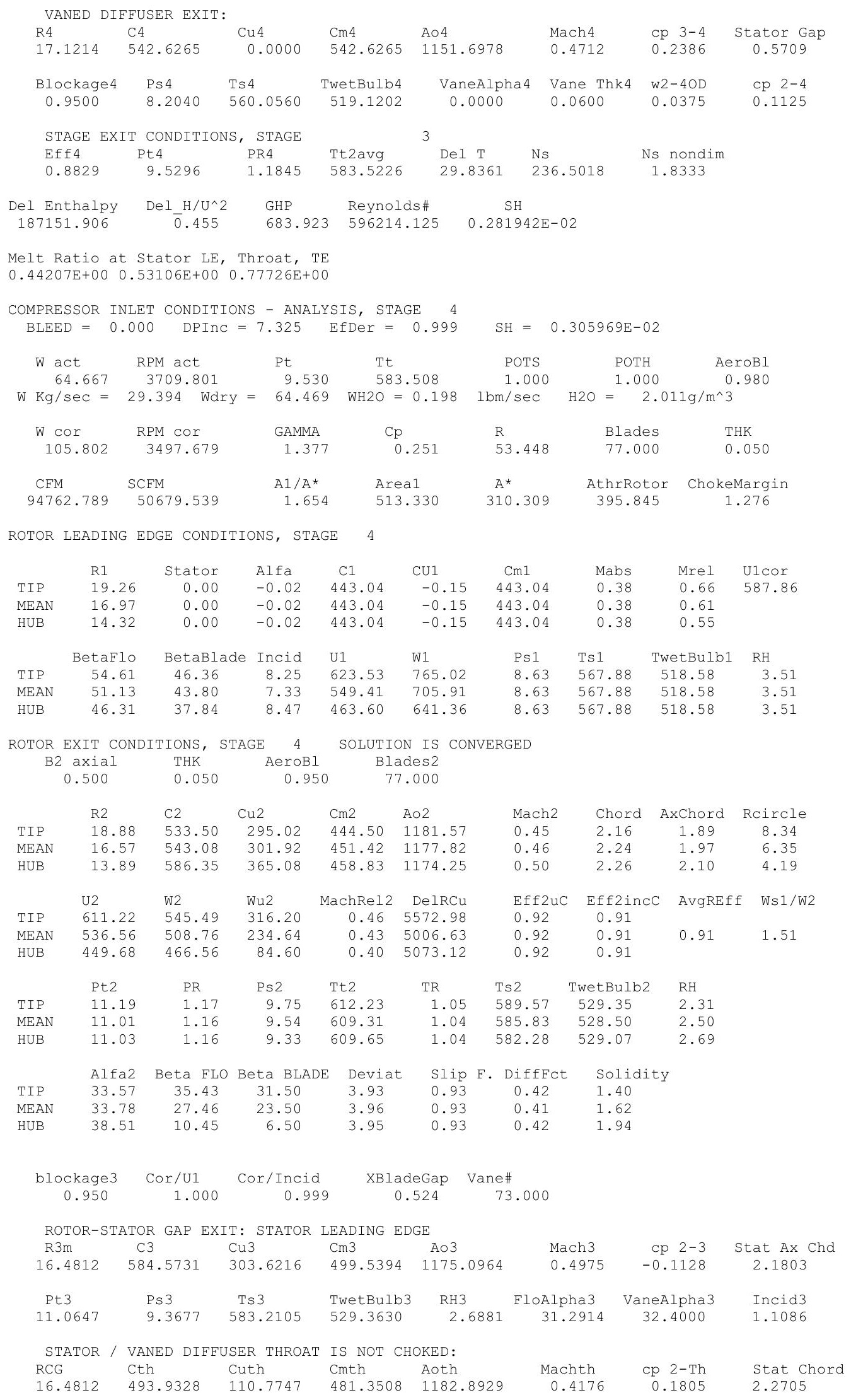




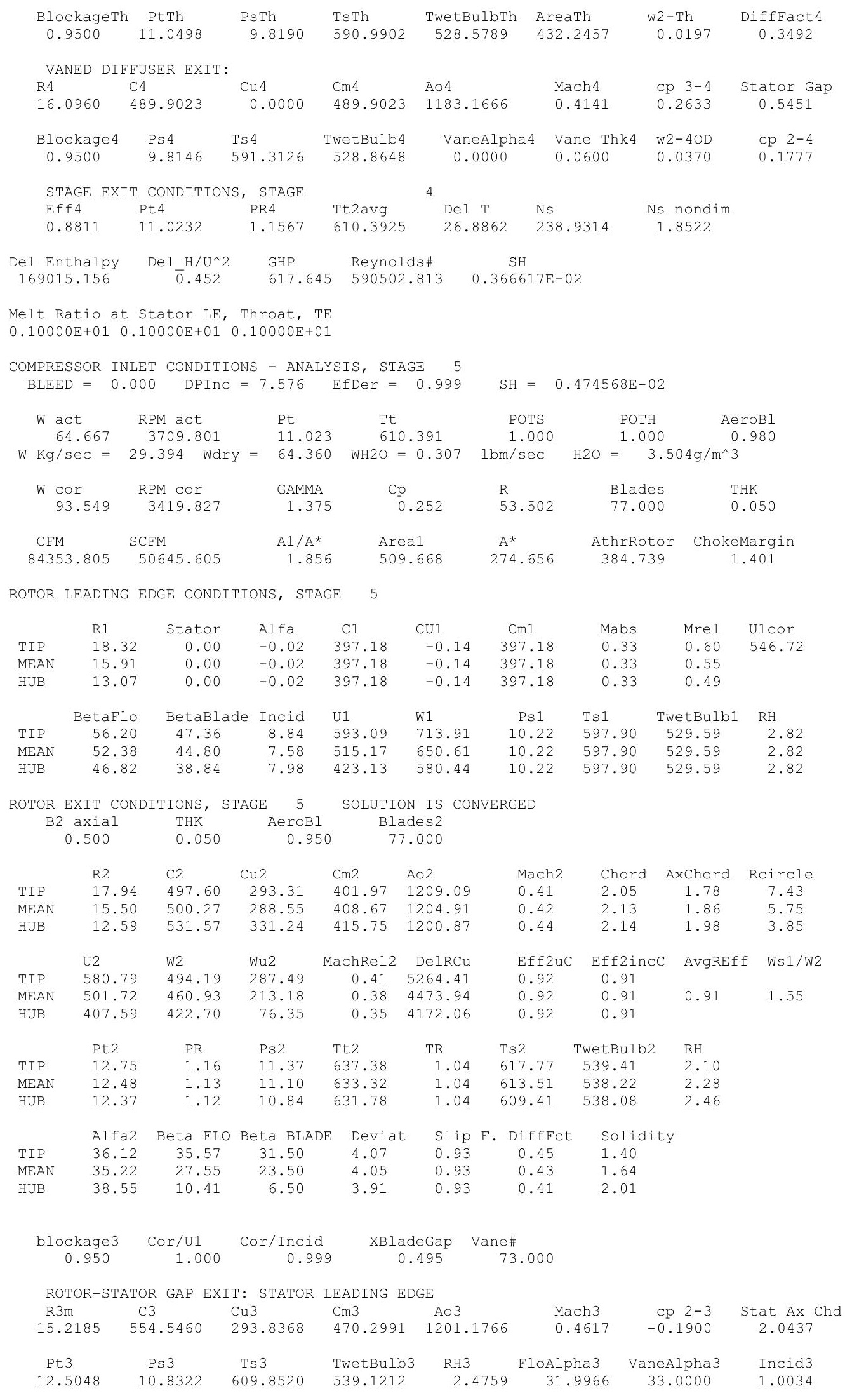




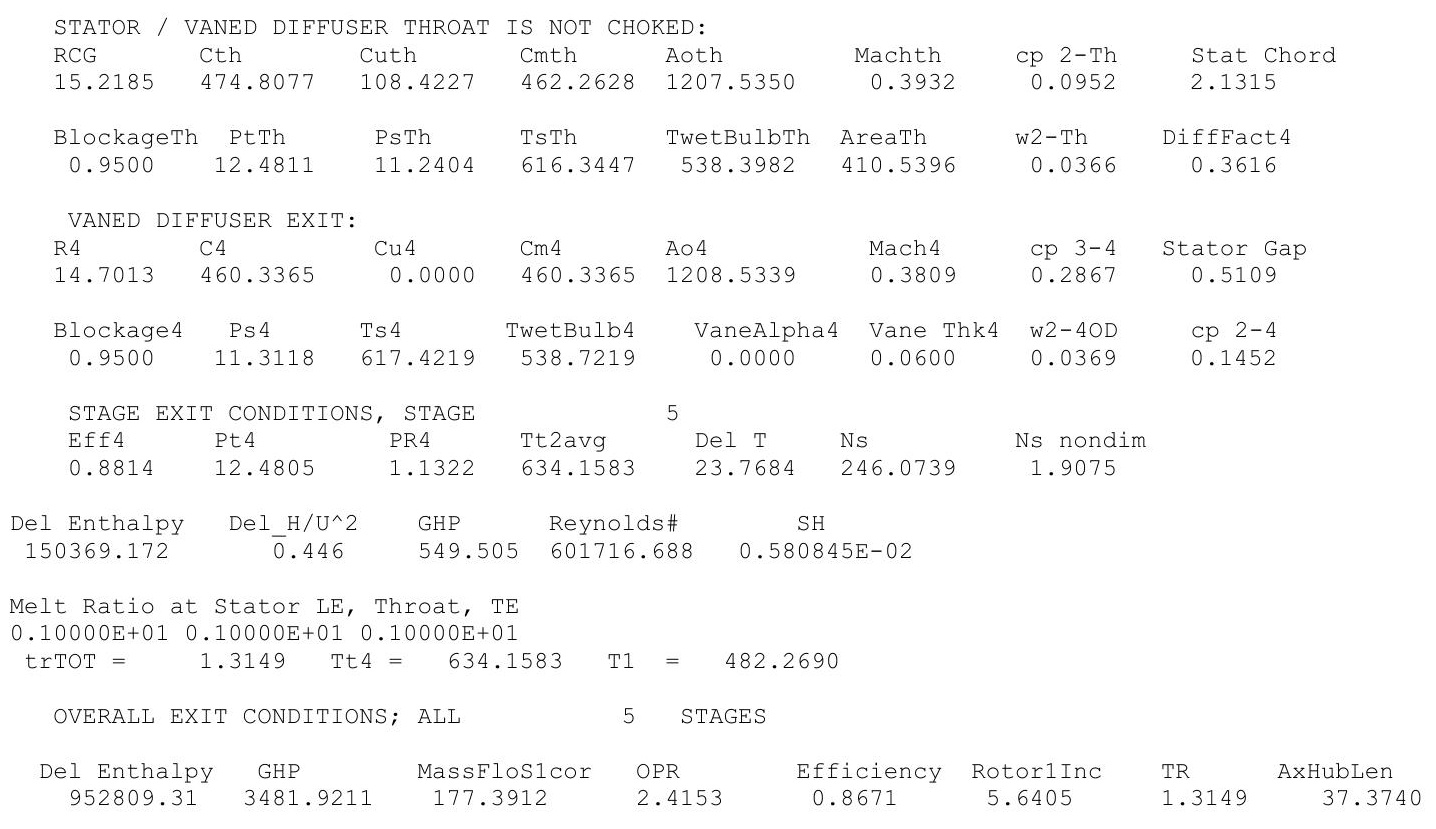




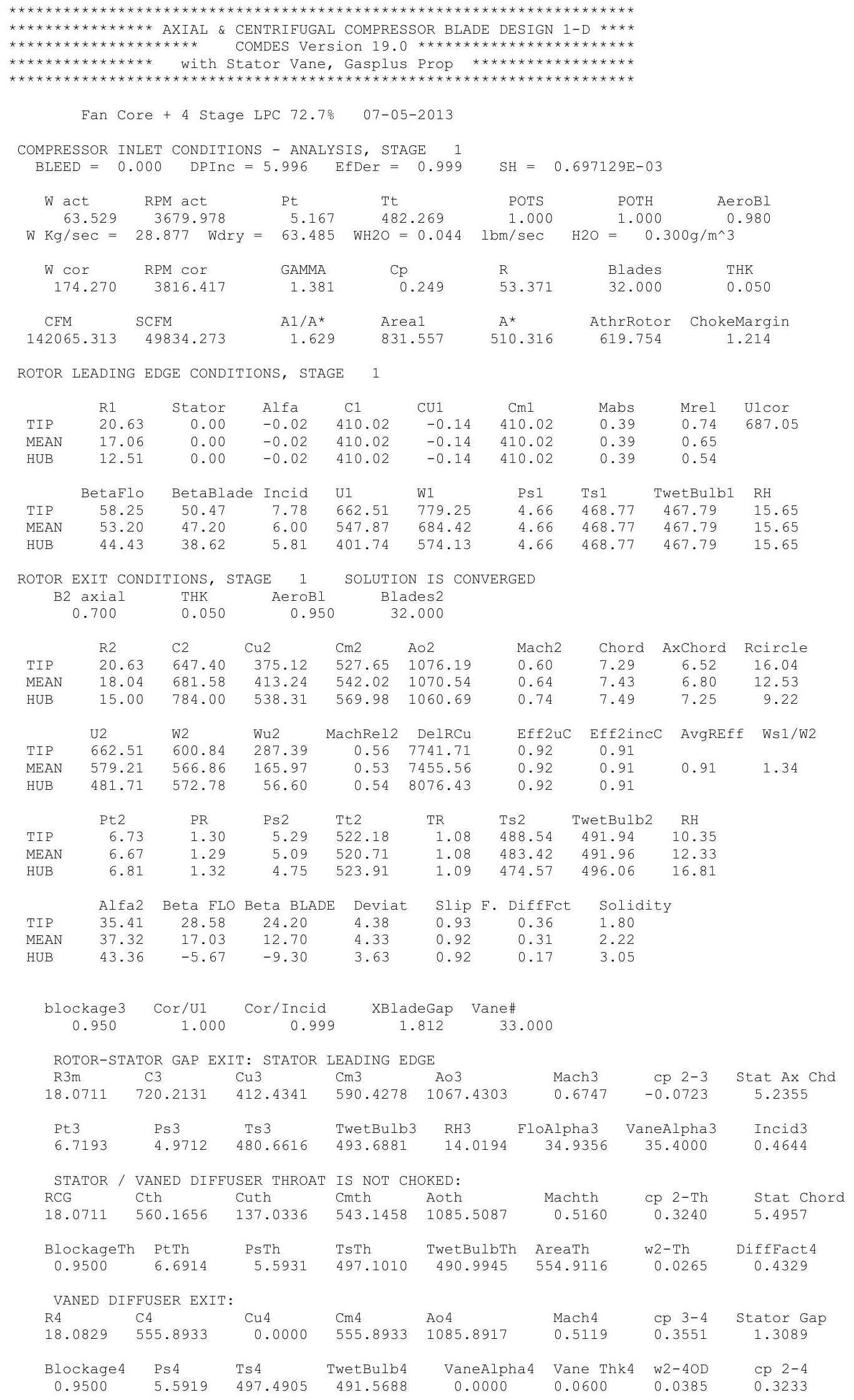




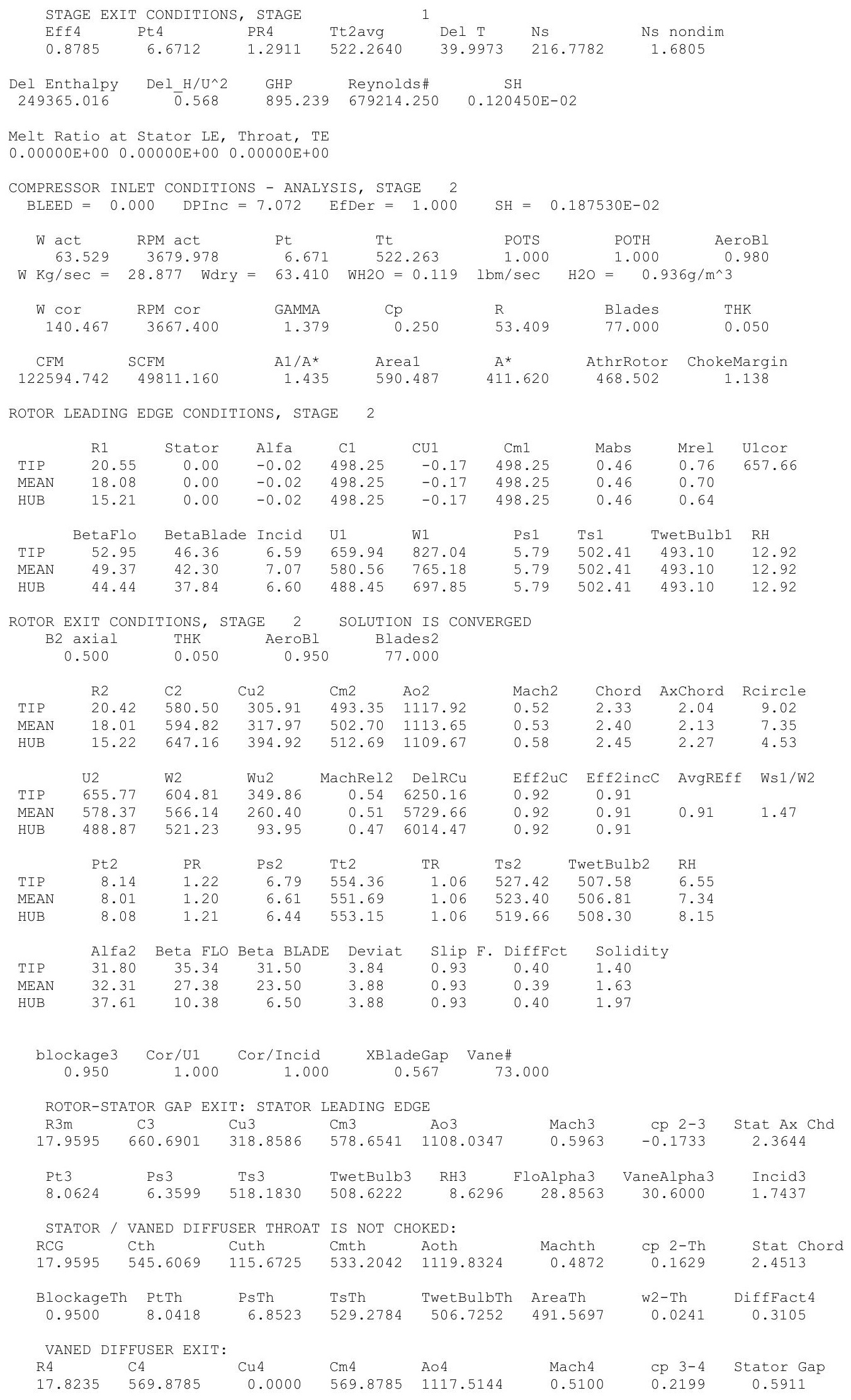




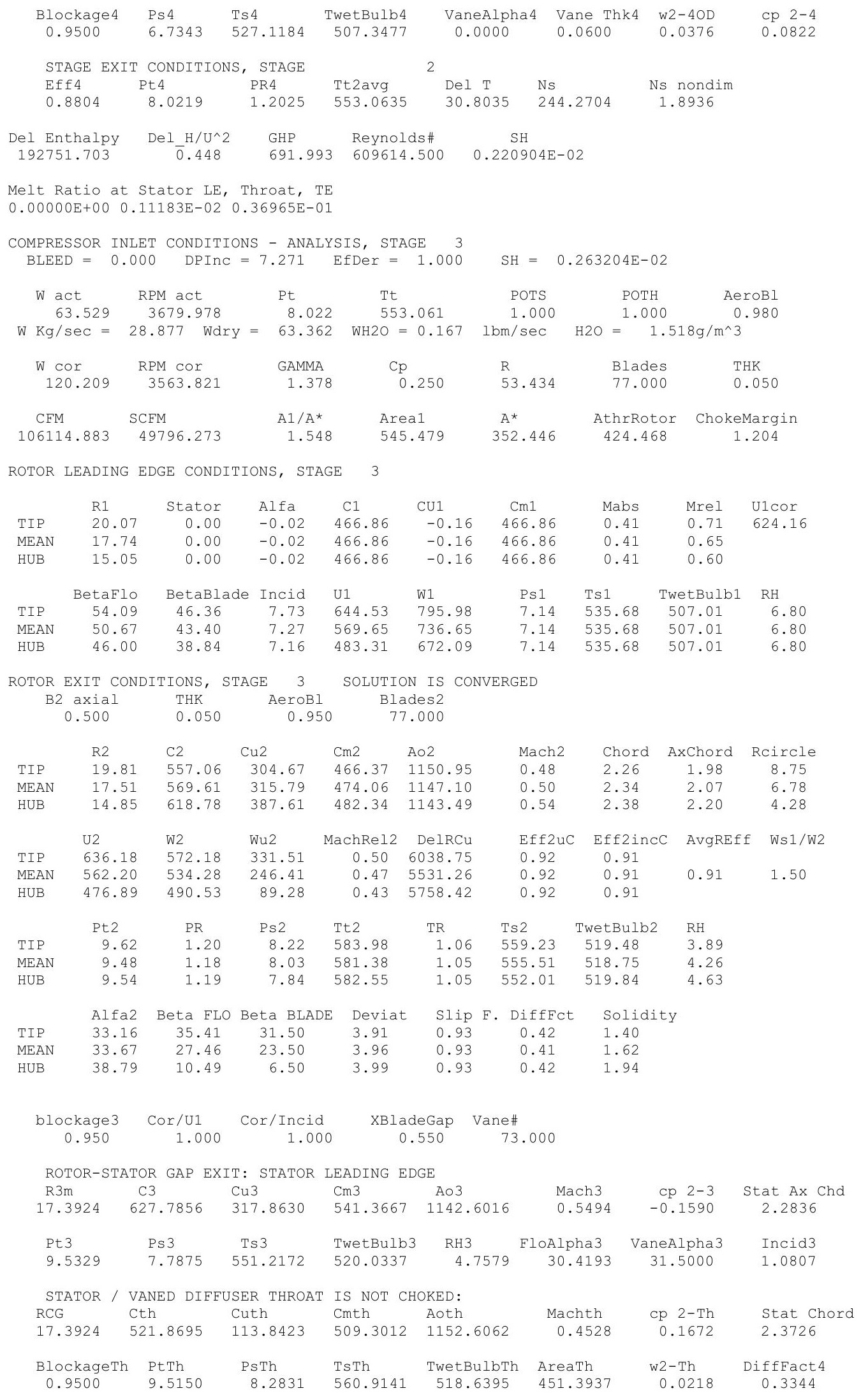




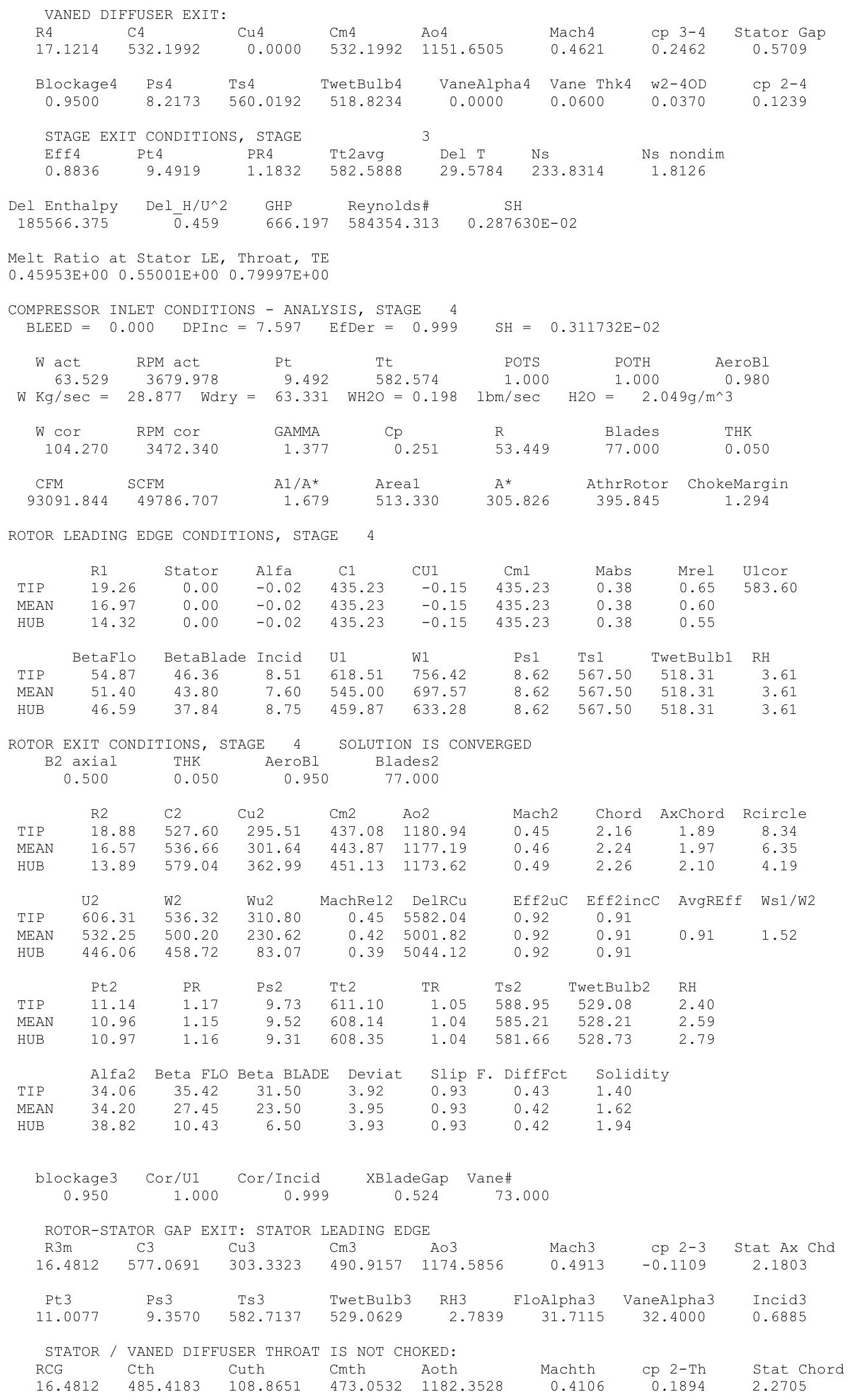




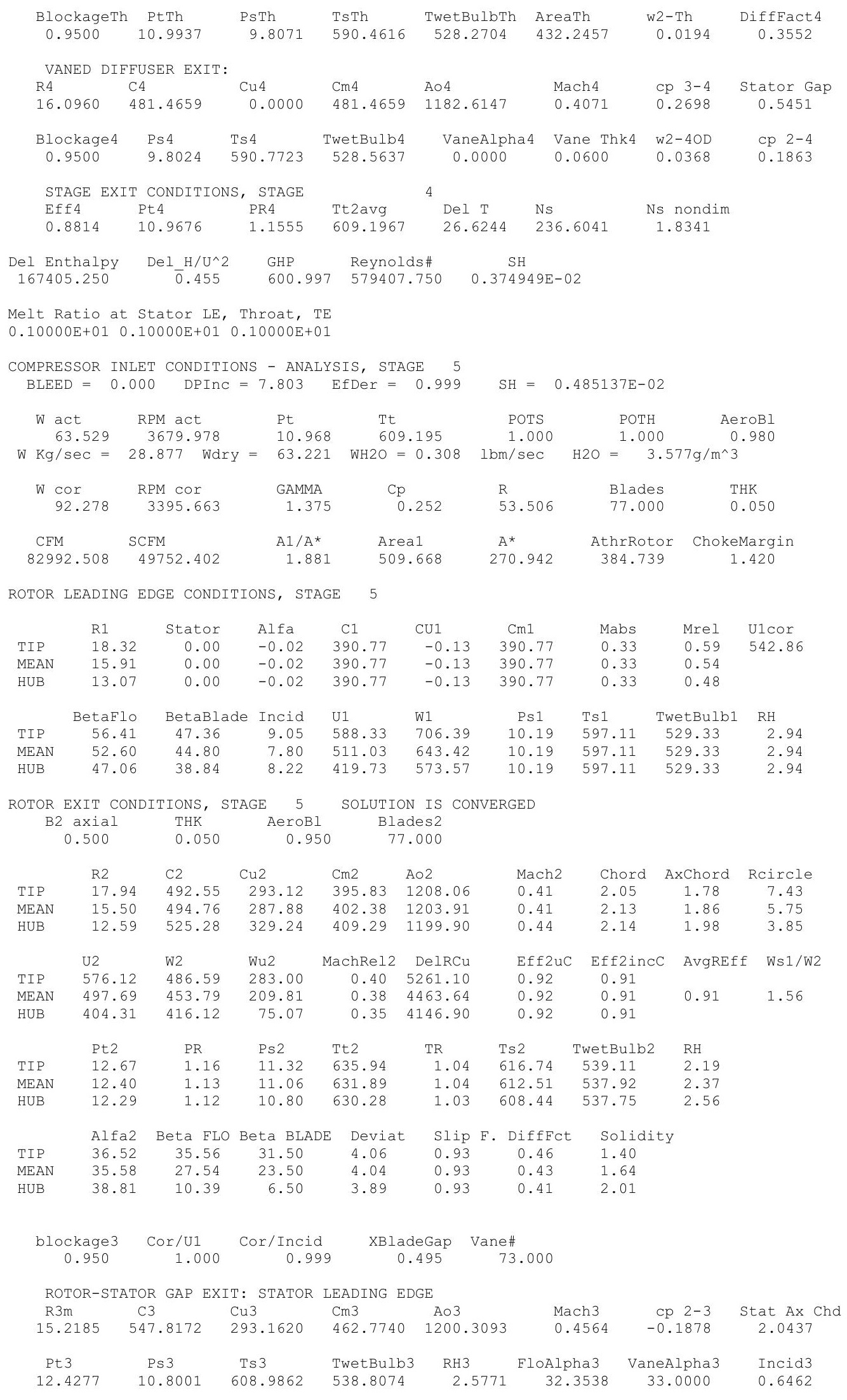




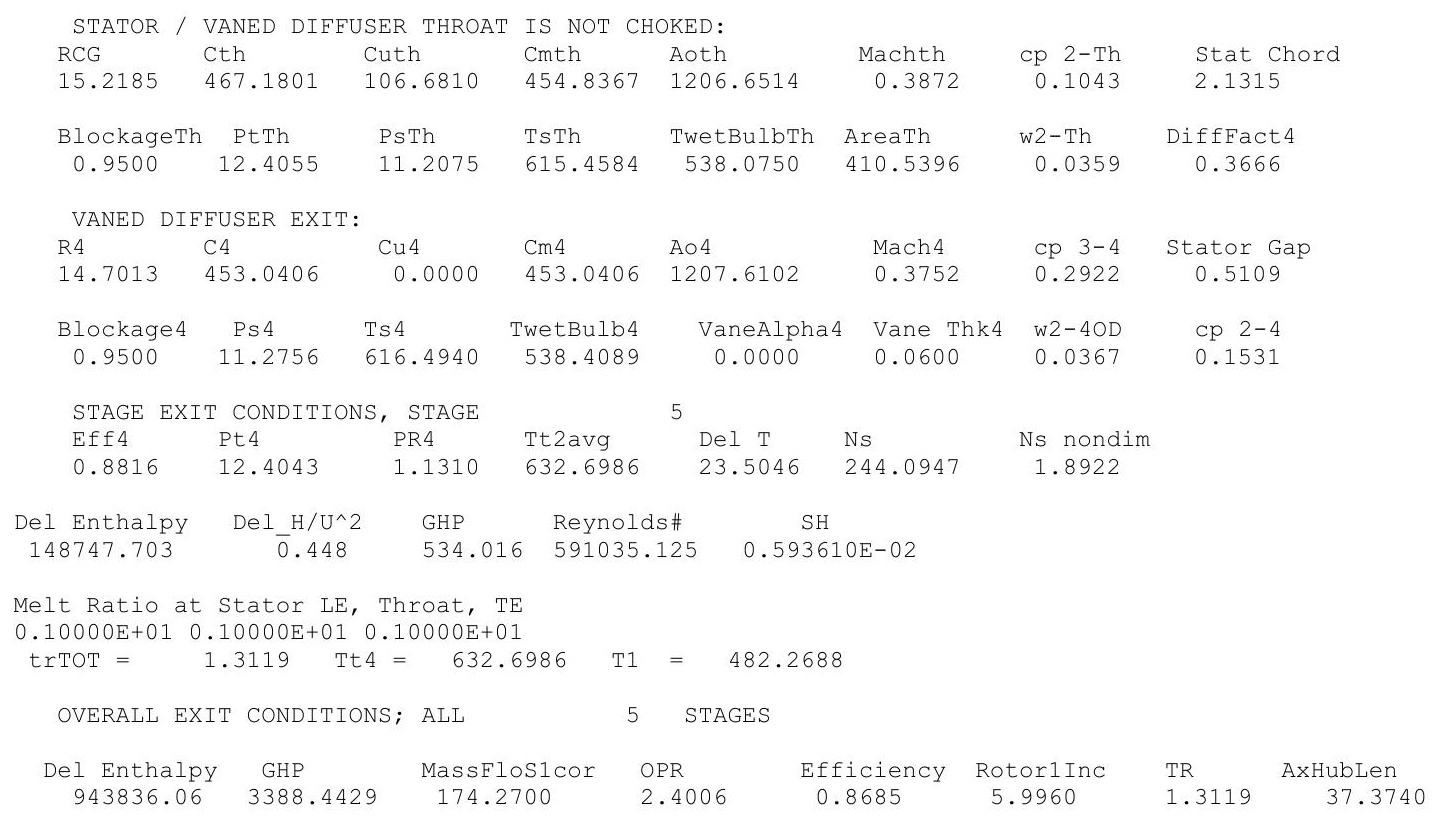




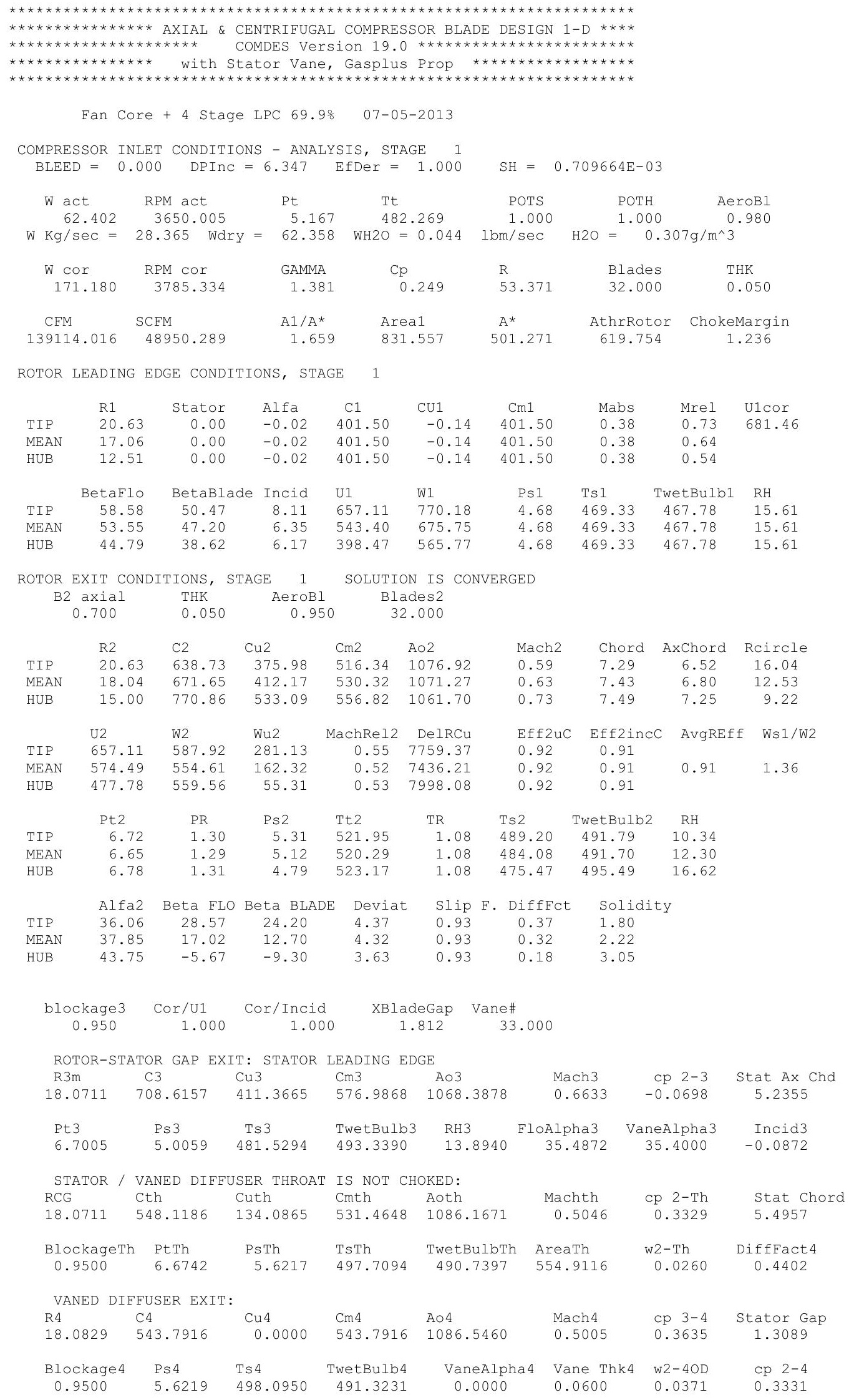




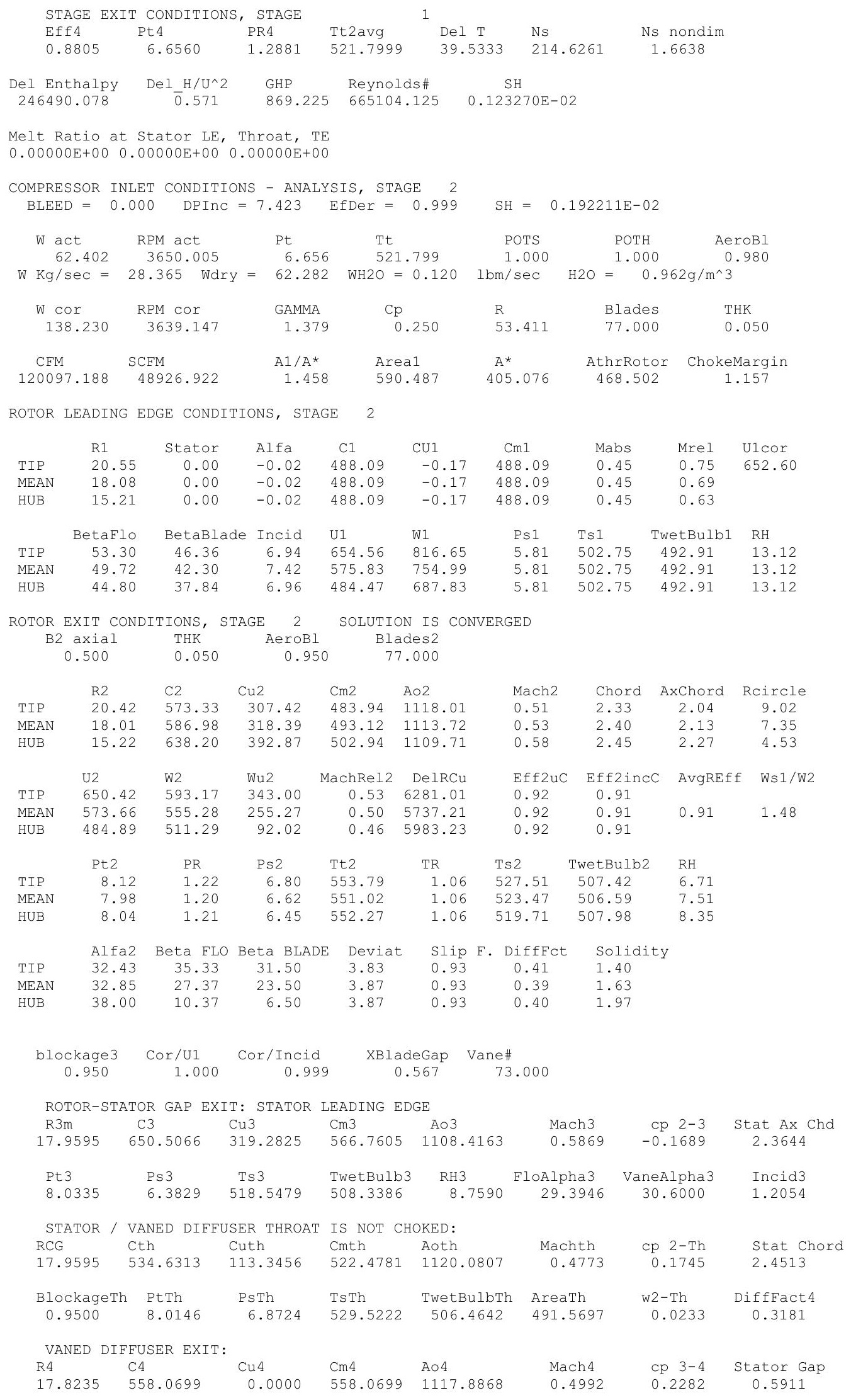




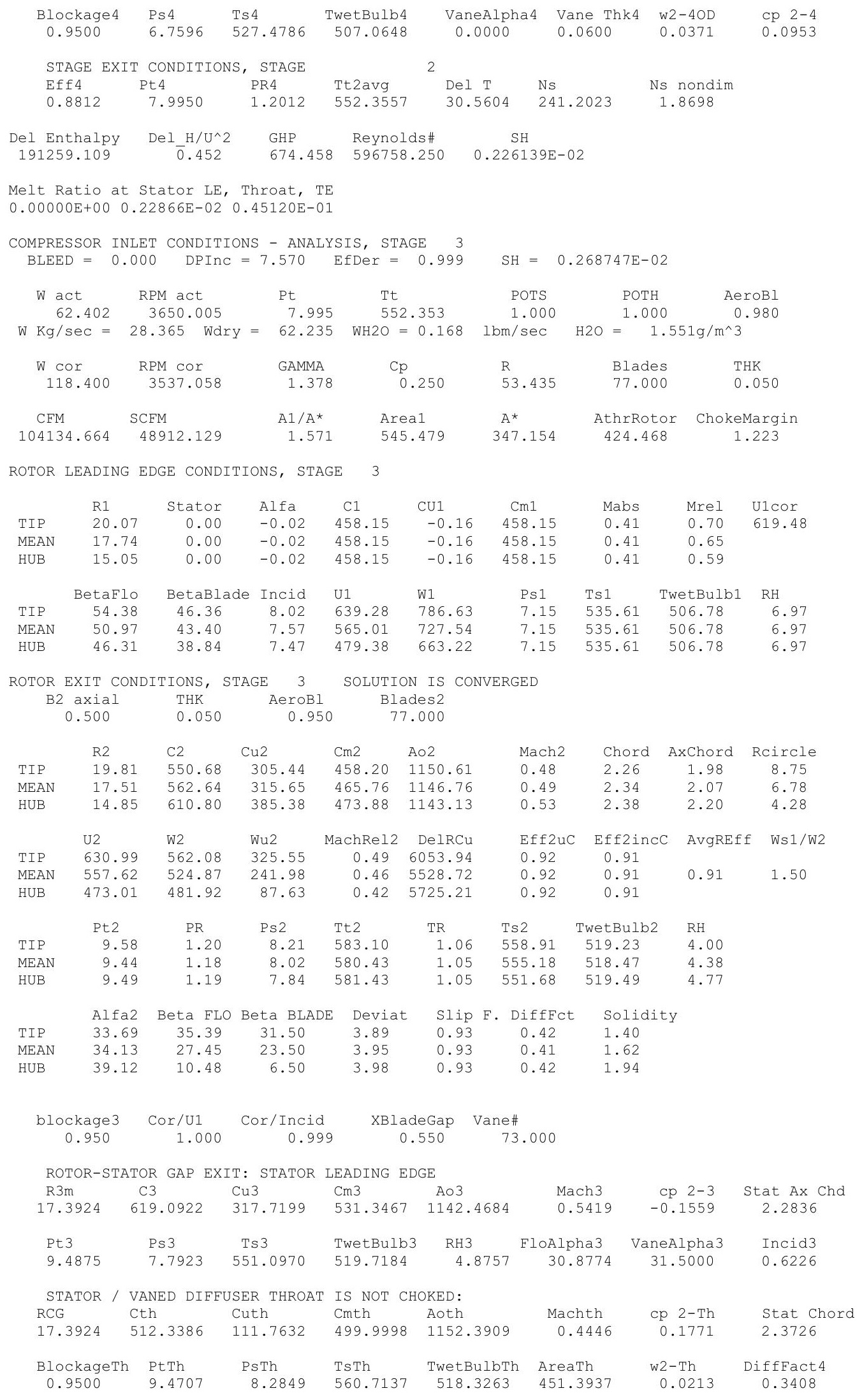




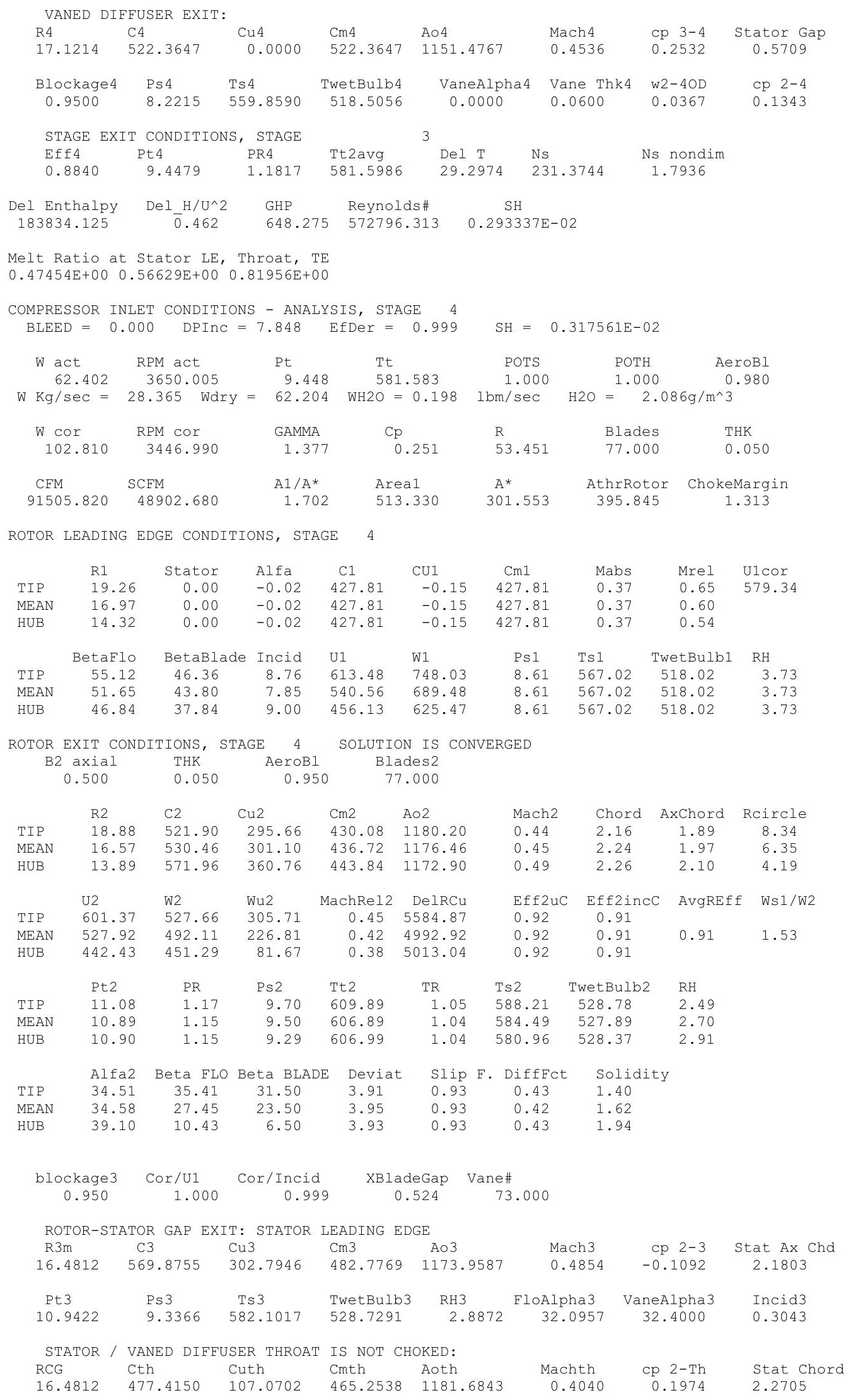




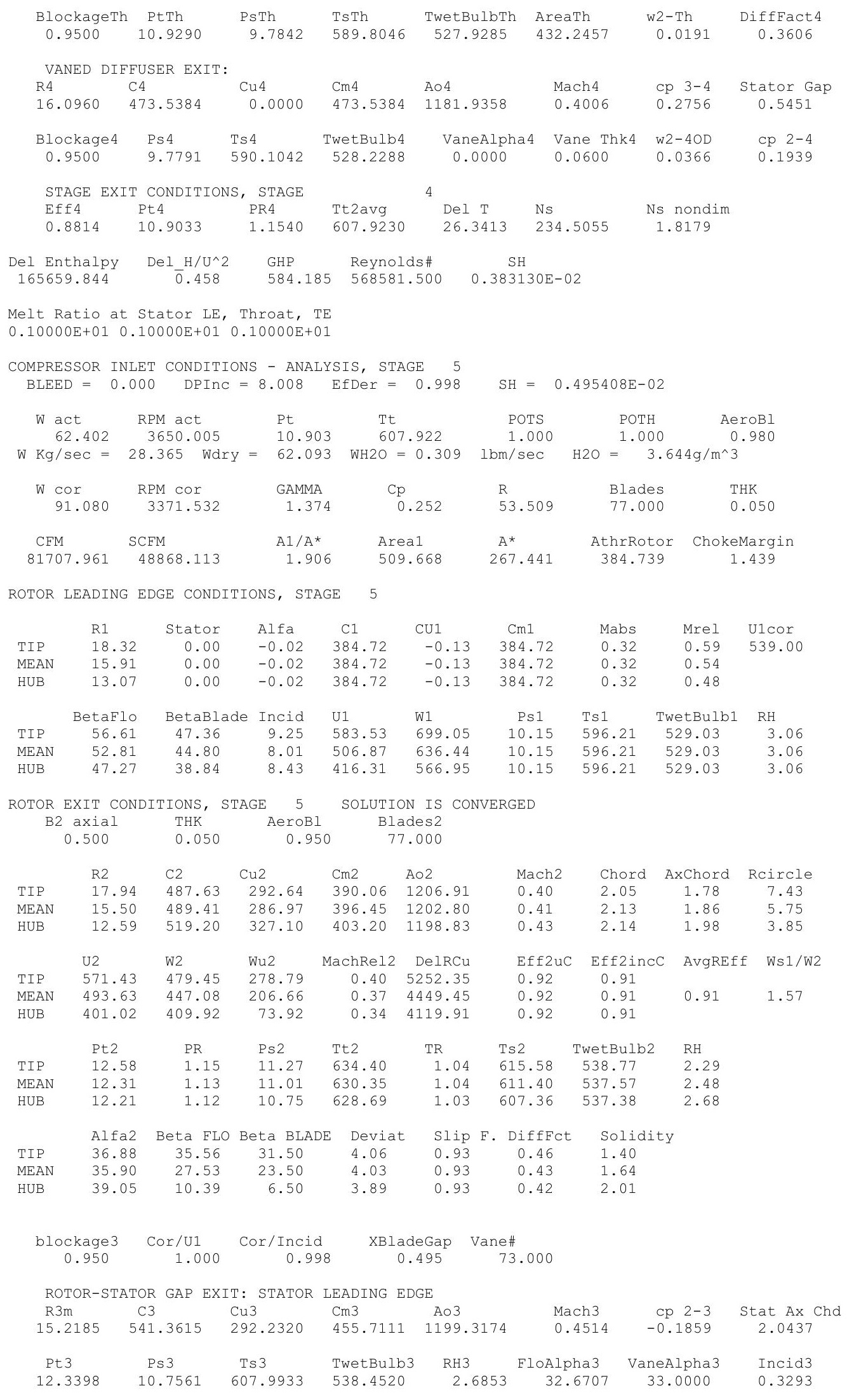




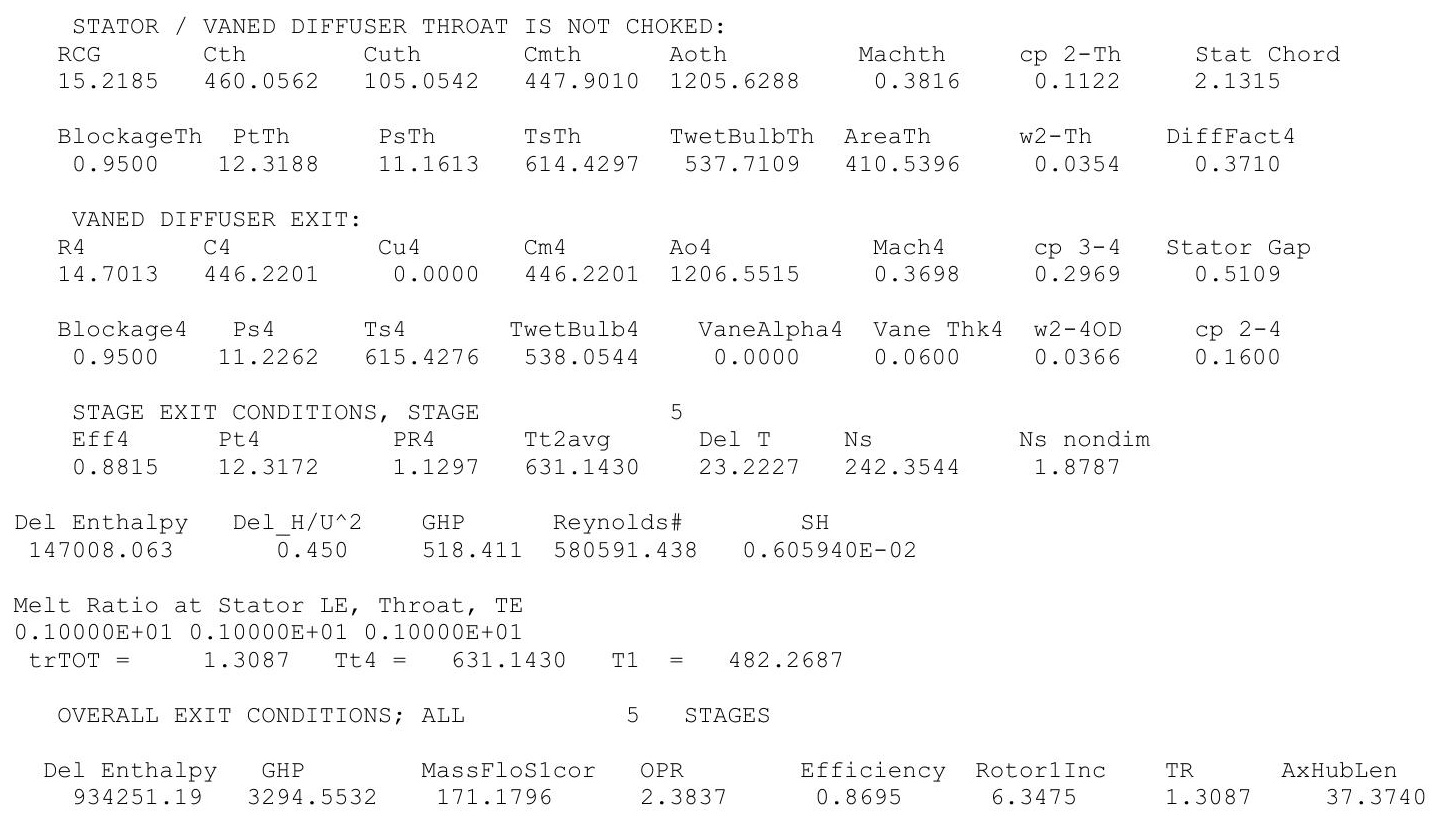




\section{$10 \mu \mathrm{m}$, ISA $+18 \mathrm{R}$}

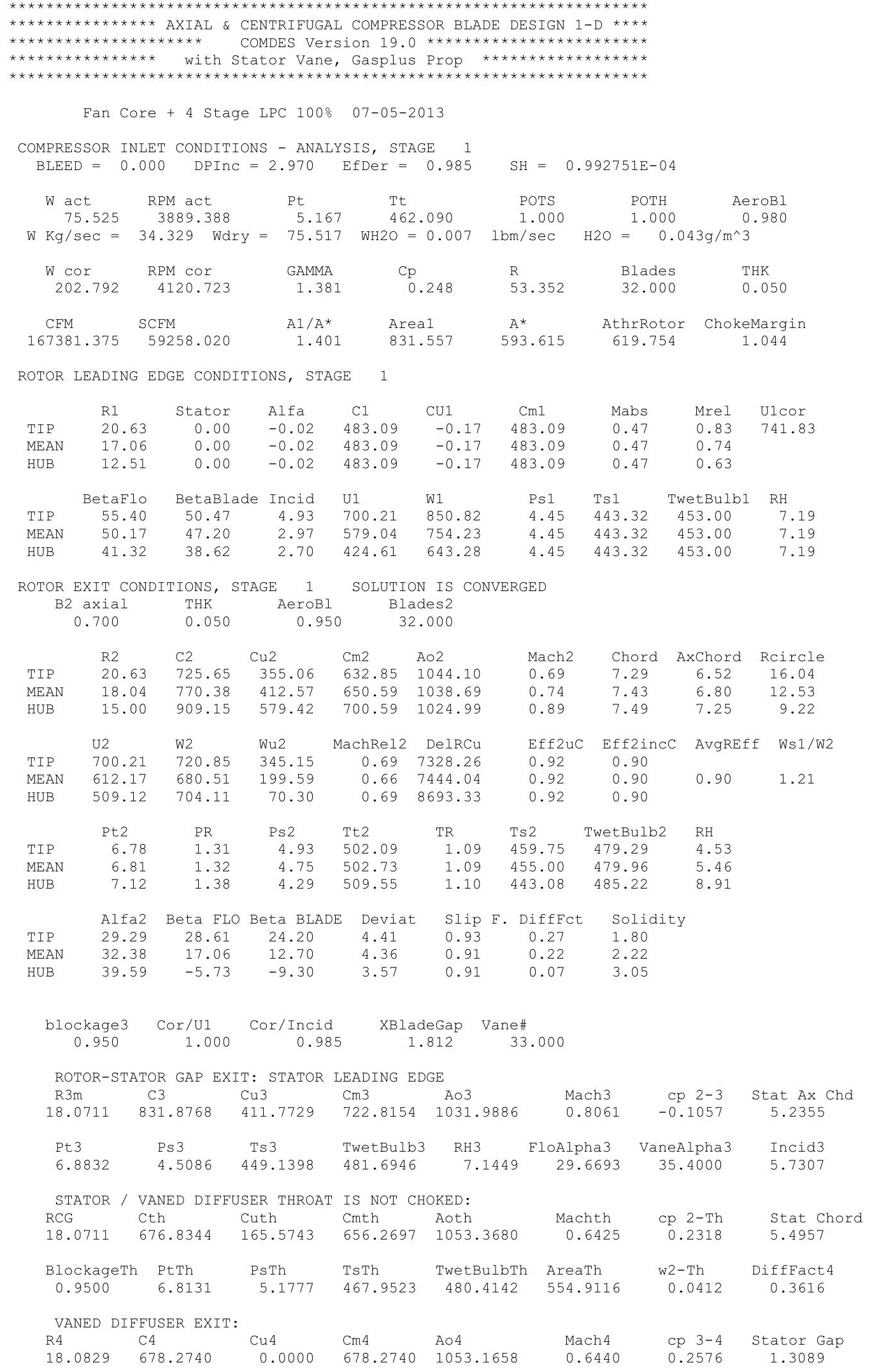




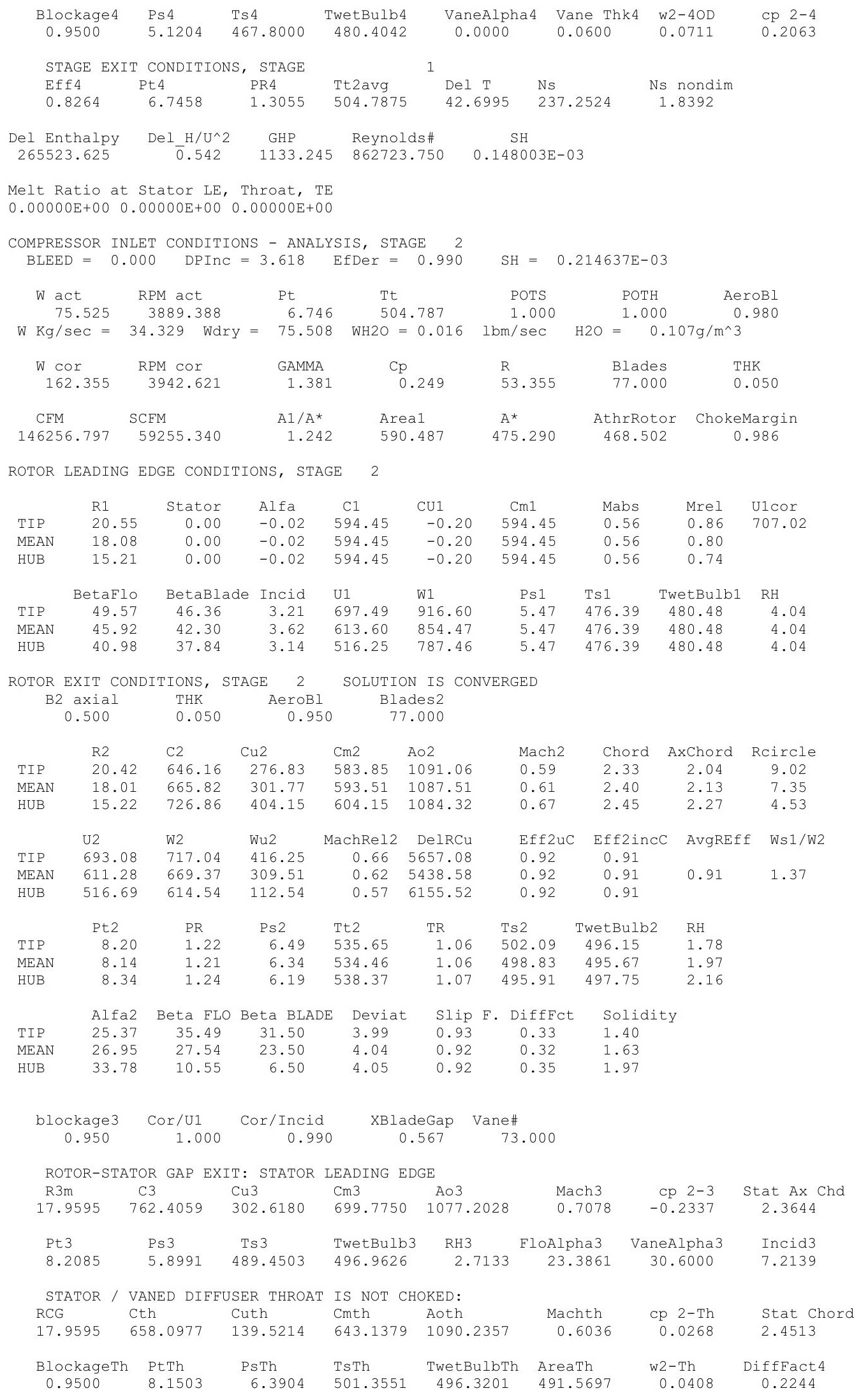




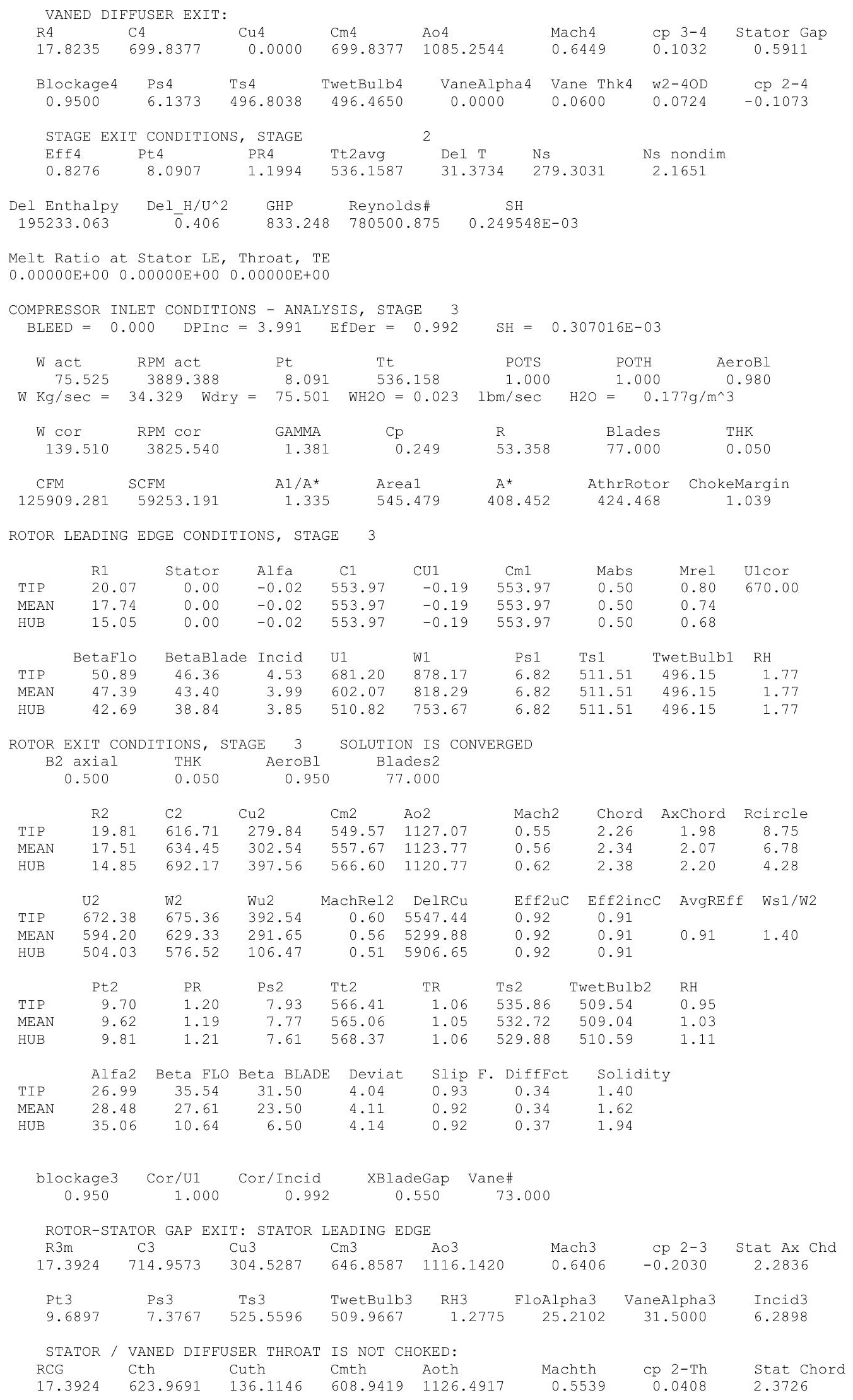




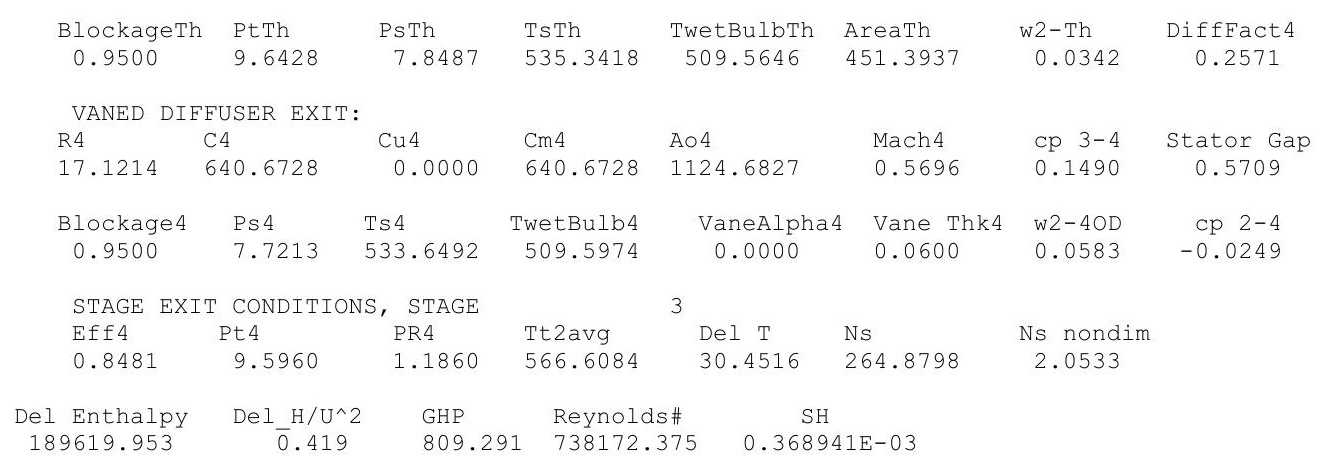

Melt Ratio at Stator LE, Throat, TE

$0.00000 \mathrm{E}+000.00000 \mathrm{E}+000.70166 \mathrm{E}-02$

COMPRESSOR INLET CONDITIONS - ANALYSIS, STAGE 4 $\mathrm{BLEED}=0.000 \quad$ DPInC $=4.572 \quad$ EfDer $=0.995$

$\mathrm{SH}=0.459095 \mathrm{E}-03$

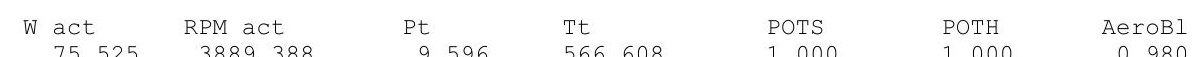
$\mathrm{W} \mathrm{Kg} / \mathrm{sec}=34.329$ Wdry $=75.490 \quad \mathrm{WH} 2 \mathrm{O}=0.035 \quad \mathrm{lbm} / \mathrm{sec} \quad \mathrm{H} 2 \mathrm{O}=0.305 \mathrm{~g} / \mathrm{m}^{\wedge} 3$

$\begin{array}{ccccccc}\text { W cor } & \text { RPM cor } & \text { GAMMA } & \text { Cp } & \text { R } & \text { Blades } & \text { THK } \\ 120.921 & 3721.328 & 1.380 & 0.249 & 53.363 & 77.000 & 0.050 \\ \text { CFM } & \text { SCFM } & \text { A1/A* } & \text { Area1 } & \text { A* } & \text { AthrRotor } & \text { ChokeMargin } \\ 109526.742 & 59249.656 & 1.450 & 513.330 & 354.077 & 395.845 & 1.118\end{array}$

ROTOR LEADING EDGE CONDITIONS, STAGE 4

$\begin{array}{lccccccccc} & \text { R1 } & \text { Stator } & \text { Alfa } & \text { C1 } & \text { CU1 } & \text { Cm1 } & \text { Mabs } & \text { Mrel } & \text { U1cor } \\ \text { TIP } & 19.26 & 0.00 & -0.02 & 512.07 & -0.18 & 512.07 & 0.45 & 0.73 & 625.44 \\ \text { MEAN } & 16.97 & 0.00 & -0.02 & 512.07 & -0.18 & 512.07 & 0.45 & 0.68 & \\ \text { HUB } & 14.32 & 0.00 & -0.02 & 512.07 & -0.18 & 512.07 & 0.45 & 0.62 & \\ & & & & & & & & & \\ & \text { BetaFlo } & \text { BetaBlade } & \text { Incid } & \text { U1 } & \text { W1 } & \text { Ps1 } & \text { Ts1 } & \text { TwetBulb1 } & \text { RH } \\ \text { TIP } & 51.93 & 46.36 & 5.57 & 653.71 & 830.53 & 8.36 & 545.56 & 509.49 & 1.01 \\ \text { MEAN } & 48.37 & 43.80 & 4.57 & 576.01 & 770.85 & 8.36 & 545.56 & 509.49 & 1.01 \\ \text { HUB } & 43.52 & 37.84 & 5.68 & 486.04 & 706.13 & 8.36 & 545.56 & 509.49 & 1.01\end{array}$

ROTOR EXIT CONDITIONS, STAGE 4 SOLUTION IS CONVERGED

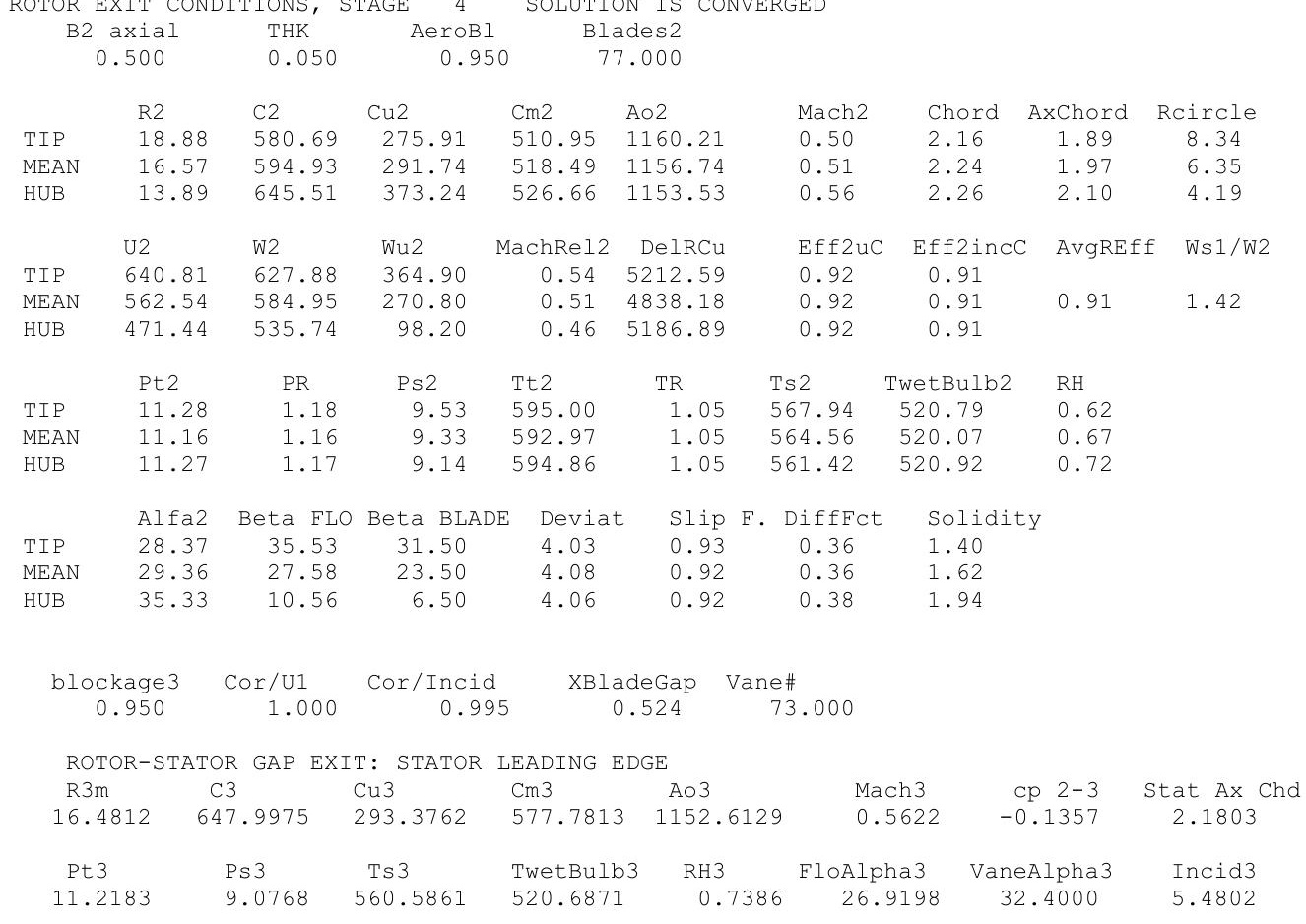




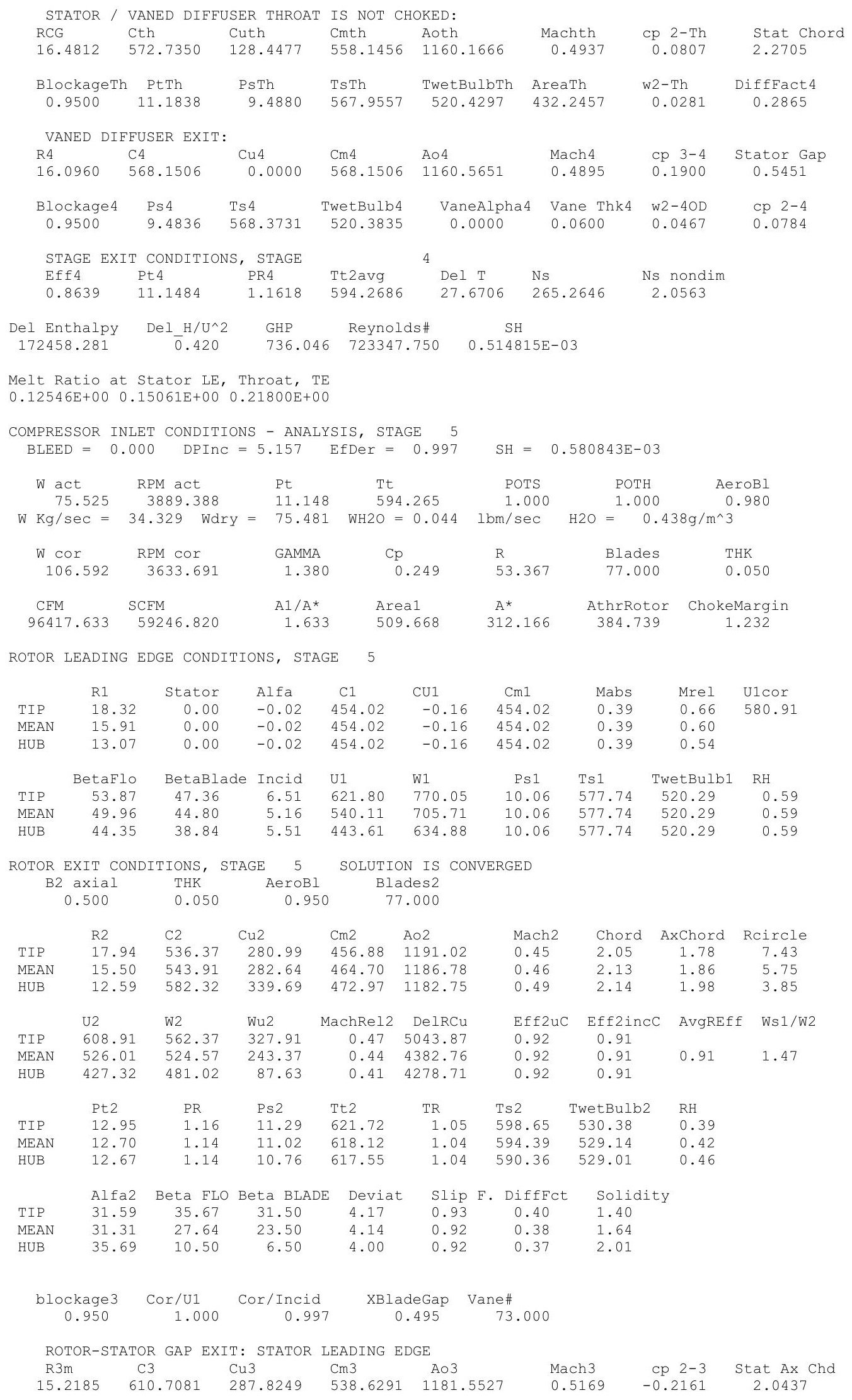




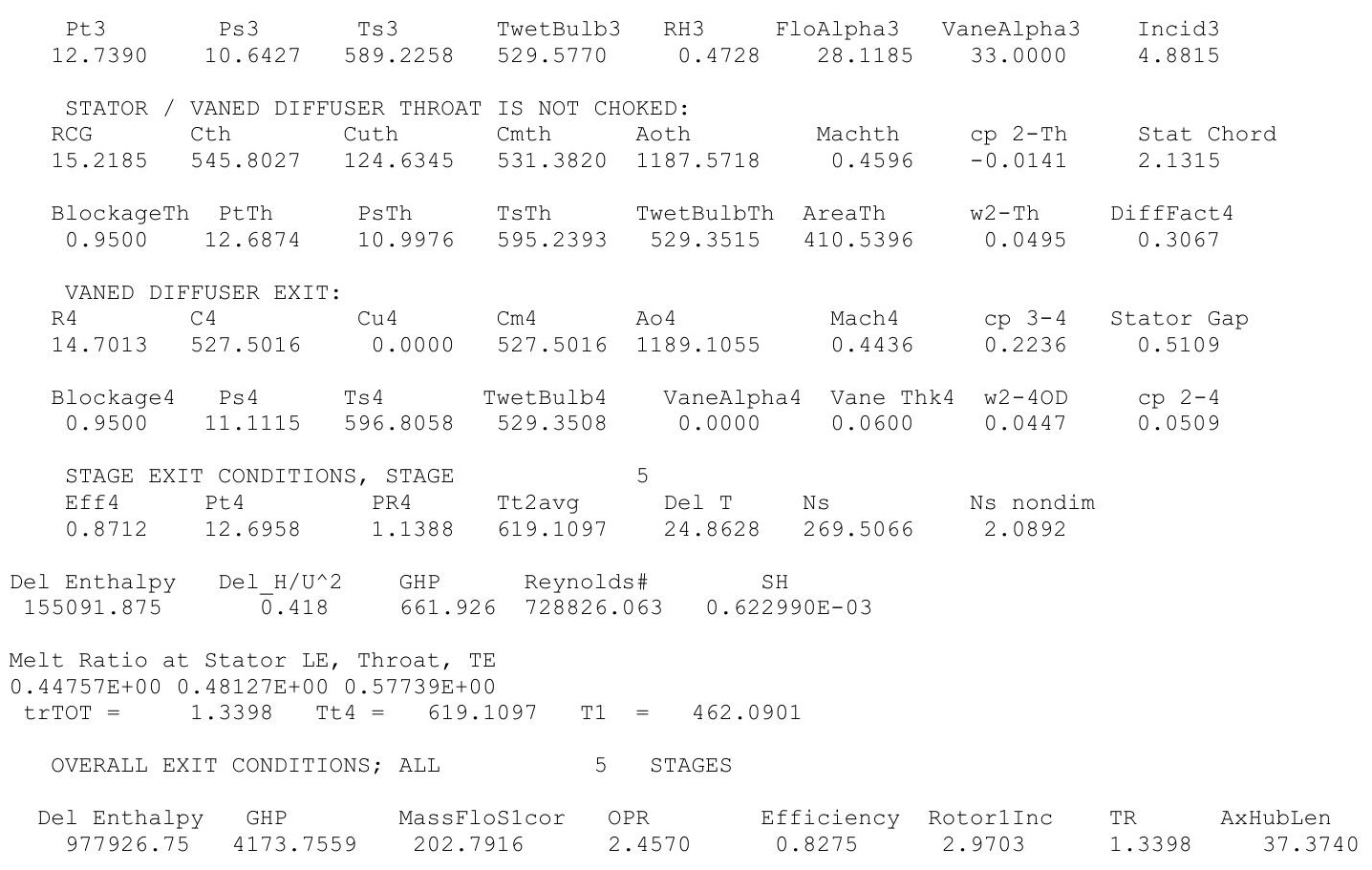




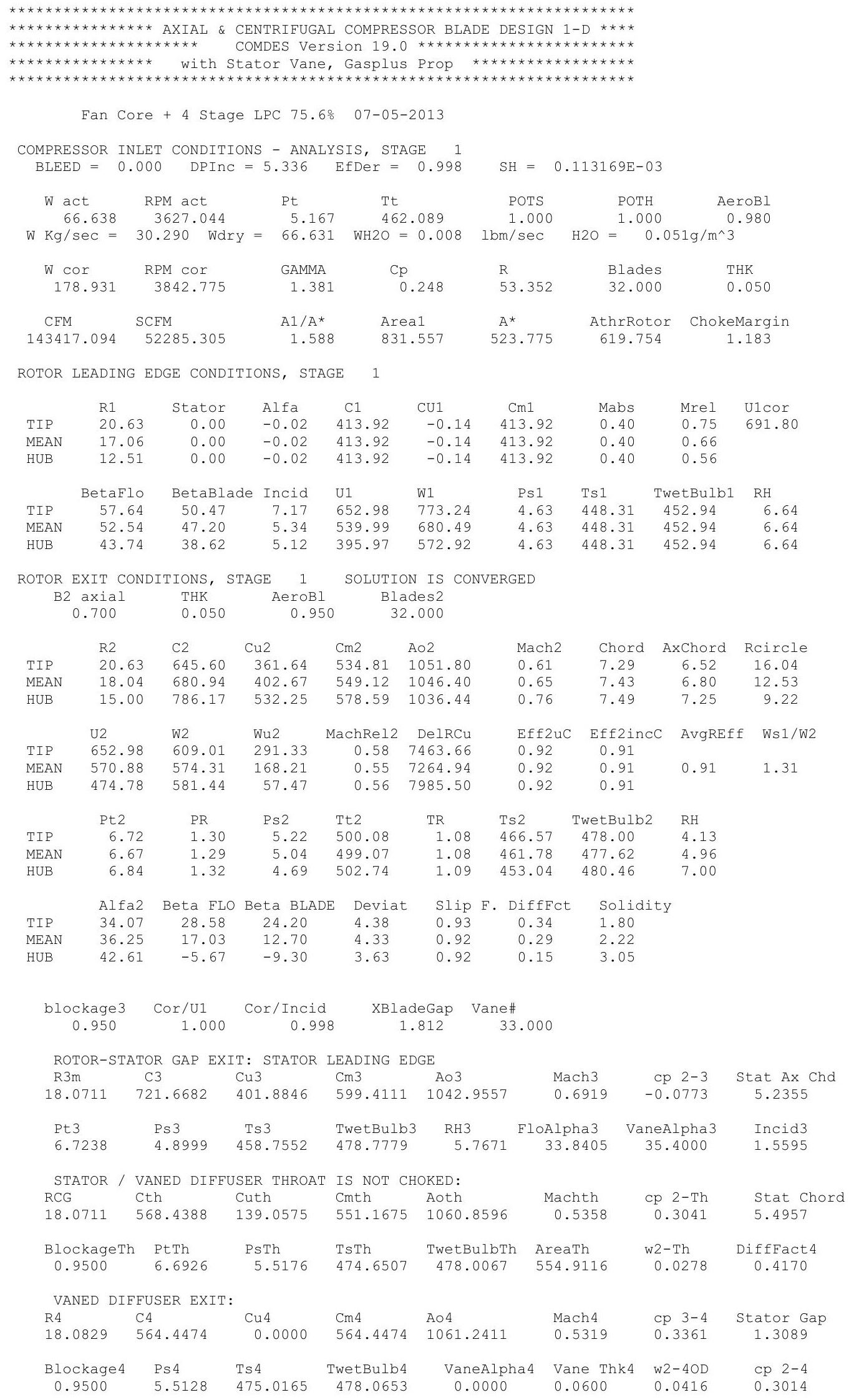




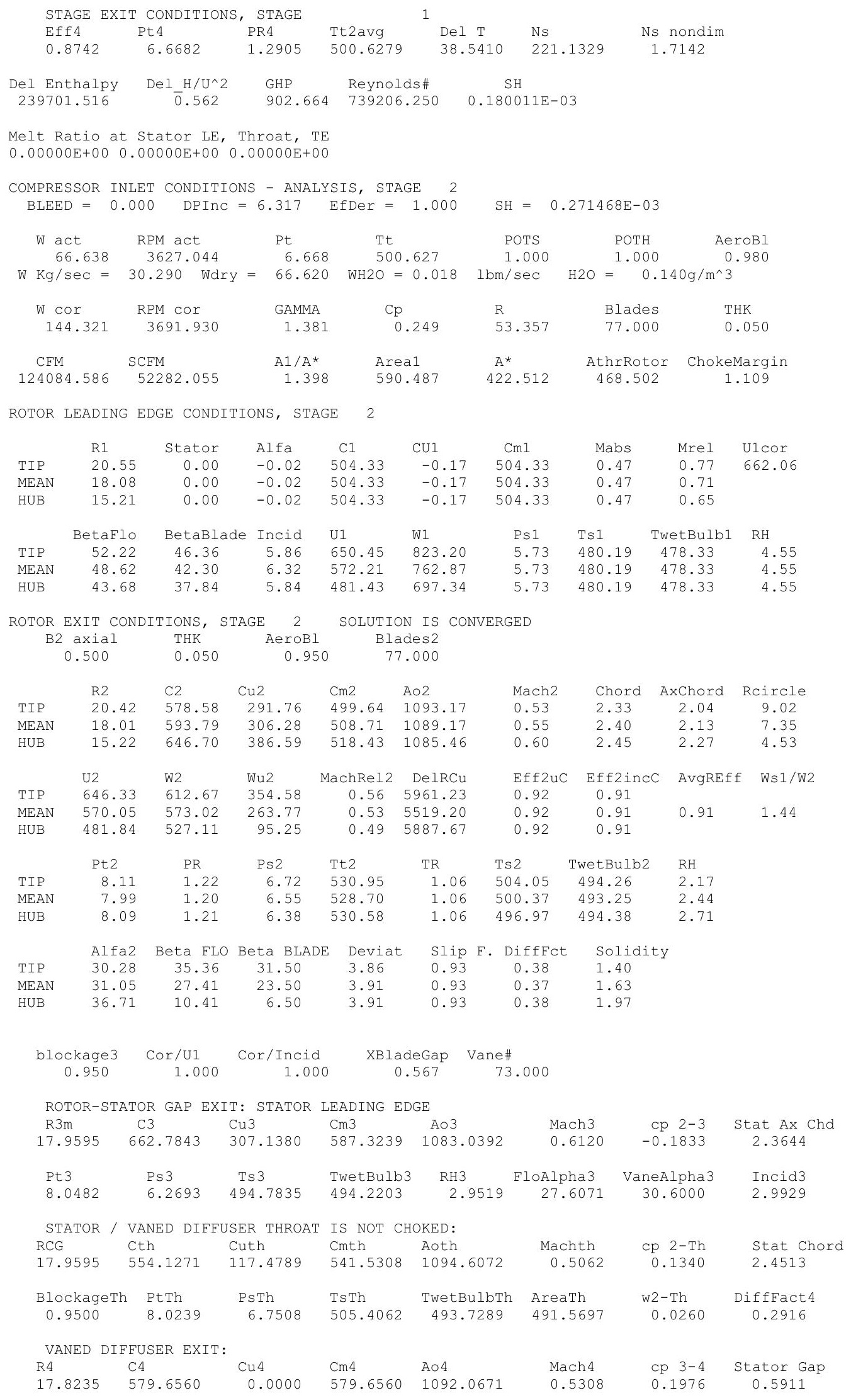




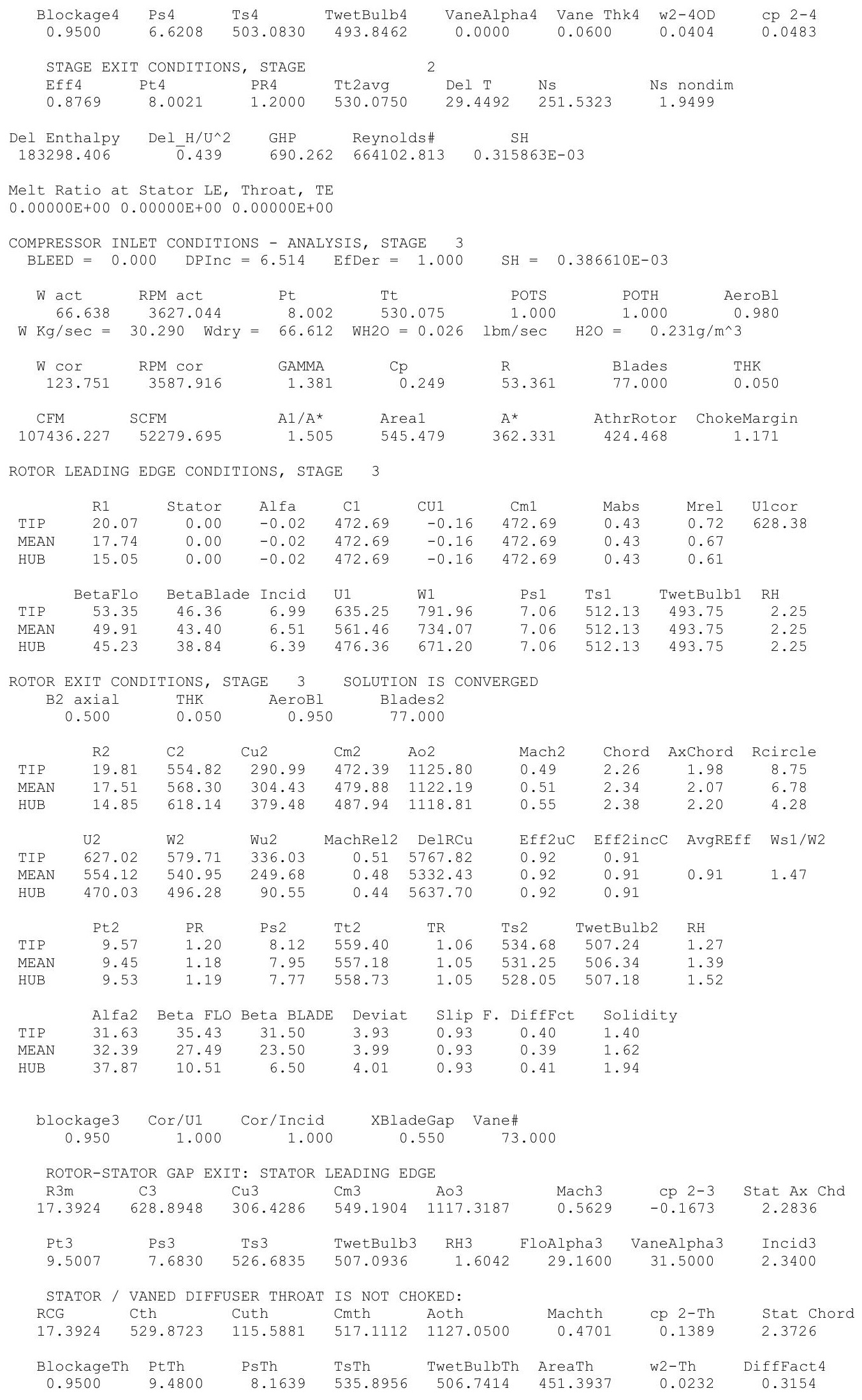




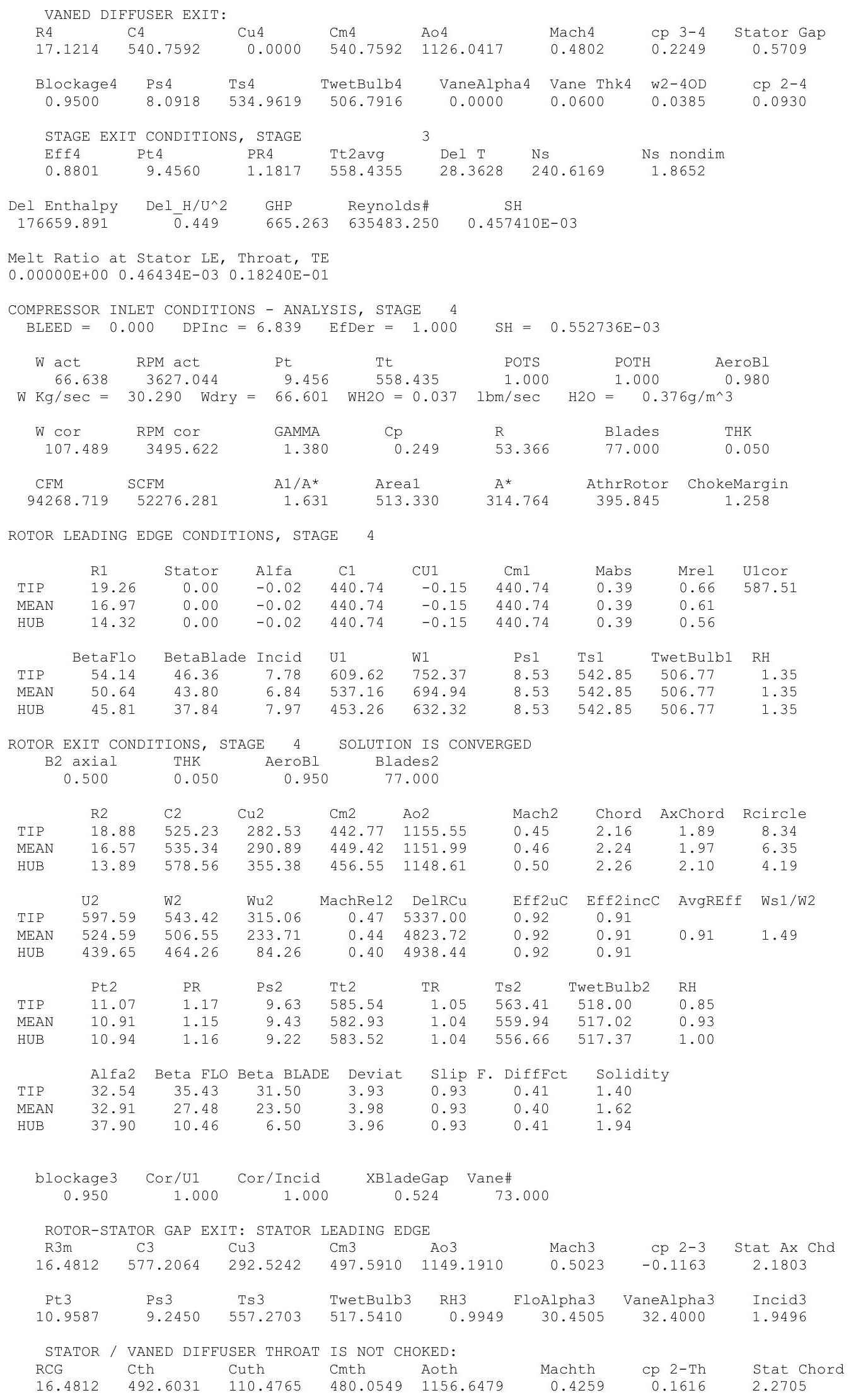




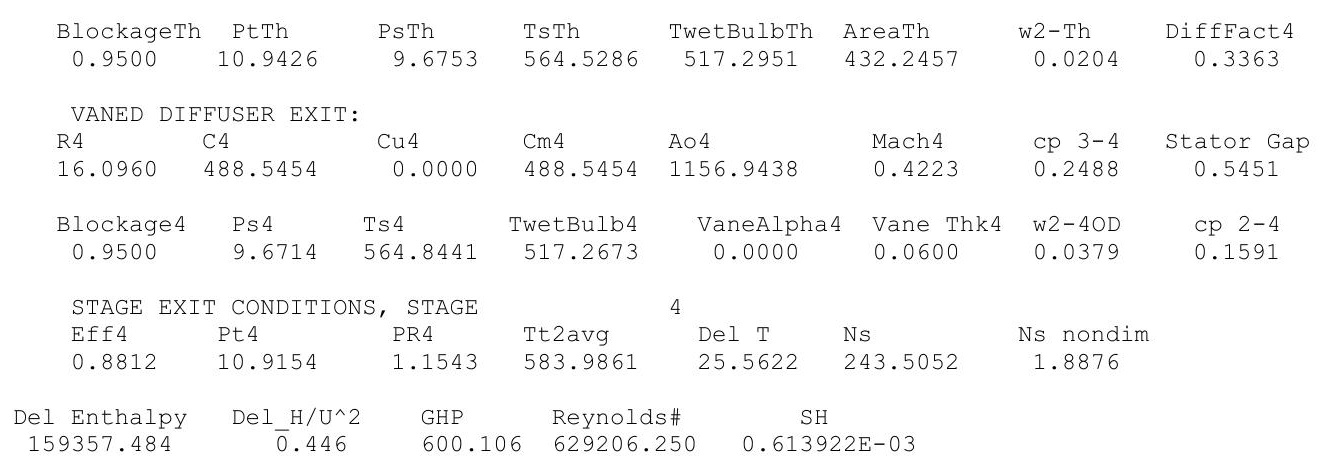

Melt Ratio at Stator LE, Throat, TE

$0.14301 \mathrm{E}+00 \quad 0.17047 \mathrm{E}+00 \quad 0.24355 \mathrm{E}+00$

COMPRESSOR INLET CONDITIONS - ANALYSIS, STAGE 5 $\mathrm{BLEED}=0.000 \quad$ DPInC $=7.085 \quad$ EfDer $=1.000$

$\mathrm{SH}=0.685380 \mathrm{E}-03$

$\begin{array}{llllll}\text { W act } & \text { RPM act } & \text { Pt } & \text { POTS } & \text { POTH } & \text { AeroBI }\end{array}$ $\mathrm{W} \mathrm{Kg} / \mathrm{sec}=30.290$ Wdry $=66.593 \quad \mathrm{WH} 2 \mathrm{O}=0.046 \mathrm{lbm} / \mathrm{sec} \quad \mathrm{H} 2 \mathrm{O}=0.524 \mathrm{~g} / \mathrm{m}^{\wedge} 3$

$\begin{array}{ccccccc}\text { W Cor } & \text { RPM cor } & \text { GAMMA } & \text { Cp } & \text { R } & \text { Blades } & \text { THK } \\ 95.223 & 3418.296 & 1.380 & 0.249 & 53.371 & 77.000 & 0.050 \\ & & & & & & \\ \text { CFM } & \text { SCFM } & \text { A1/A* } & \text { Area1 } & \text { A* } & \text { AthrRotor } & \text { ChokeMargin } \\ 83937.172 & 52273.559 & 1.828 & 509.668 & 278.886 & 384.739 & 1.380\end{array}$

ROTOR LEADING EDGE CONDITIONS, STAGE 5

$\begin{array}{lccccccccc} & \text { R1 } & \text { Stator } & \text { Alfa } & \text { C1 } & \text { CU1 } & \text { Cm1 } & \text { Mabs } & \text { Mrel } & \text { U1cor } \\ \text { TIP } & 18.32 & 0.00 & -0.02 & 395.25 & -0.14 & 395.25 & 0.34 & 0.60 & 546.47 \\ \text { MEAN } & 15.91 & 0.00 & -0.02 & 395.25 & -0.14 & 395.25 & 0.34 & 0.55 & \\ \text { HUB } & 13.07 & 0.00 & -0.02 & 395.25 & -0.14 & 395.25 & 0.34 & 0.49 & \\ & & & & & & & & & \\ \text { BIP } & \text { BetaFlo } & \text { BetaBlade } & \text { Incid } & \text { U1 } & \text { W1 } & \text { Ps1 } & \text { Ts1 } & \text { TwetBulb1 } & \text { RH } \\ \text { MEAN } & 55.73 & 47.36 & 8.37 & 579.86 & 701.87 & 10.09 & 571.46 & 517.22 & 0.83 \\ \text { HUB } & 46.32 & 44.80 & 7.08 & 503.68 & 640.35 & 10.09 & 571.46 & 517.22 & 0.83 \\ & & 38.84 & 7.48 & 413.69 & 572.26 & 10.09 & 571.46 & 517.22 & 0.83\end{array}$

ROTOR EXIT CONDITIONS, STAGE 5 SOLUTION IS CONVERGED

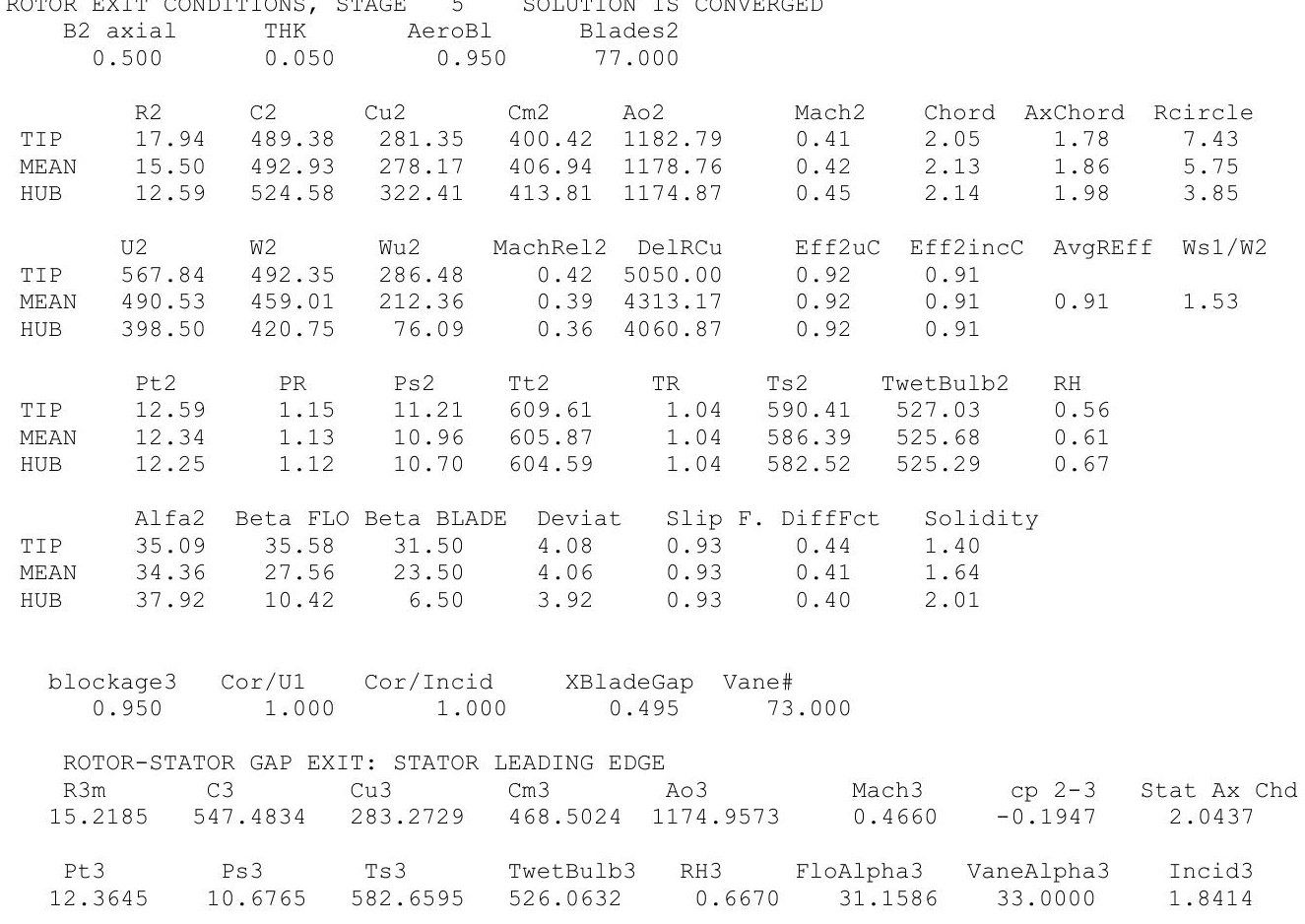




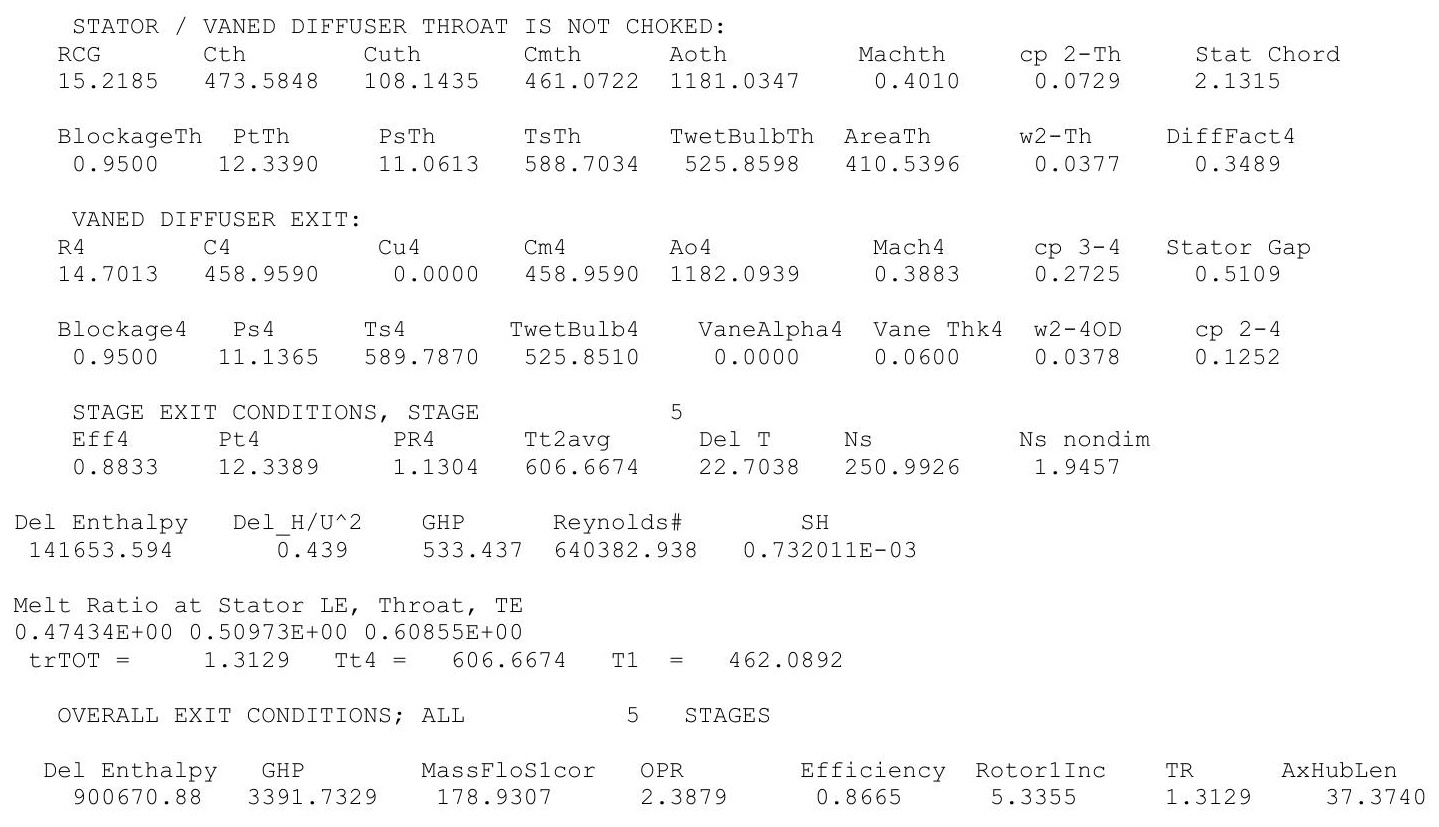




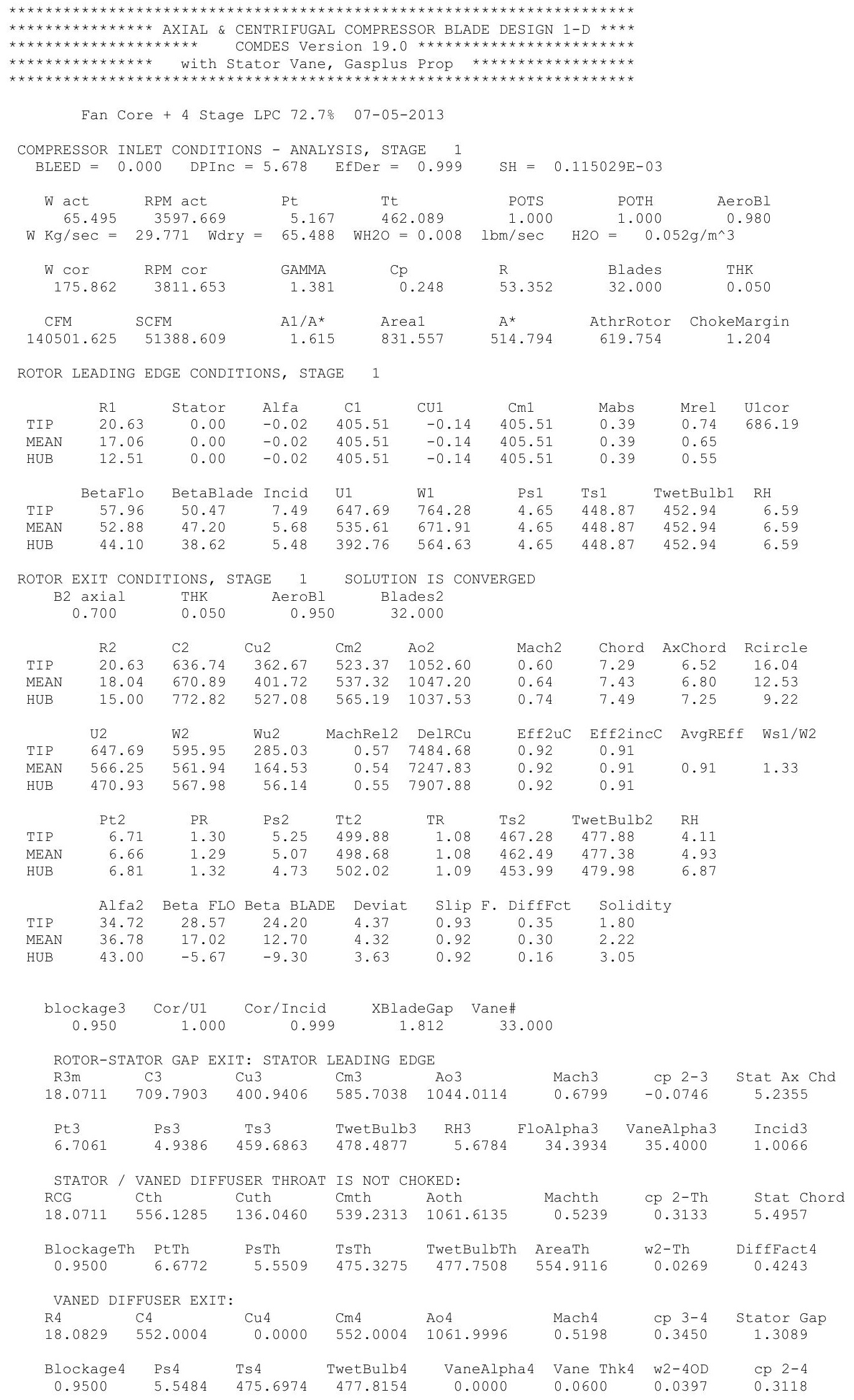




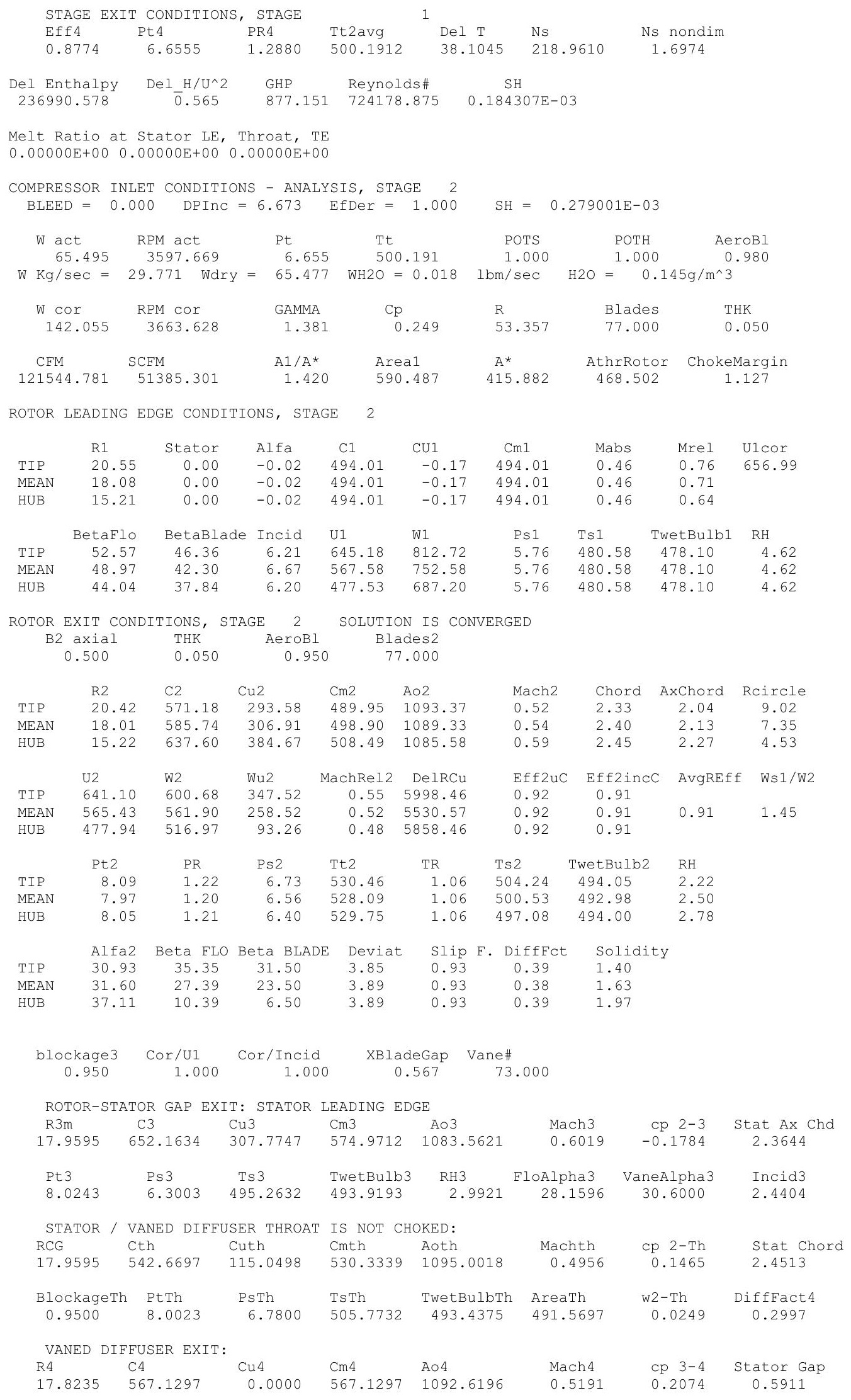




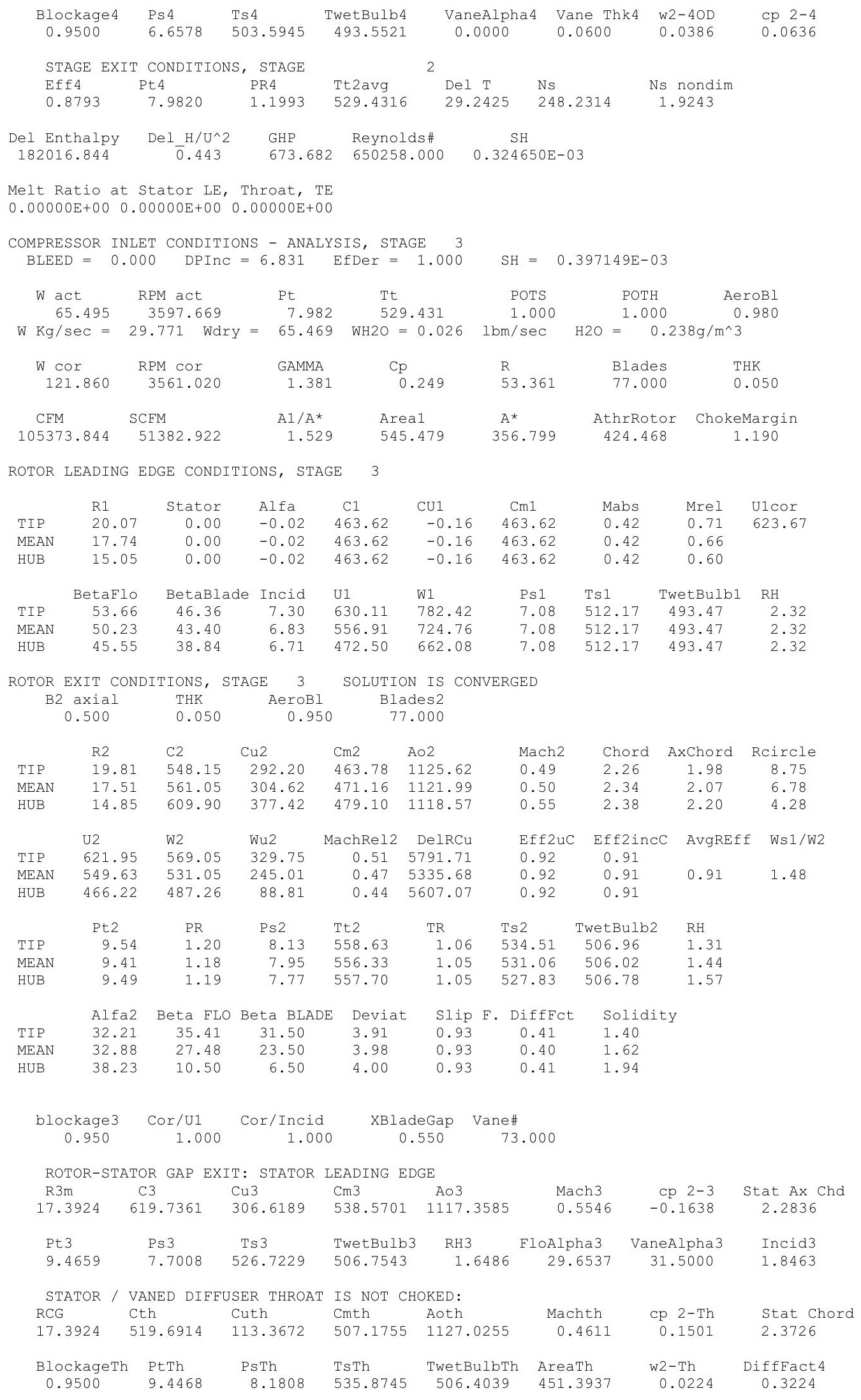




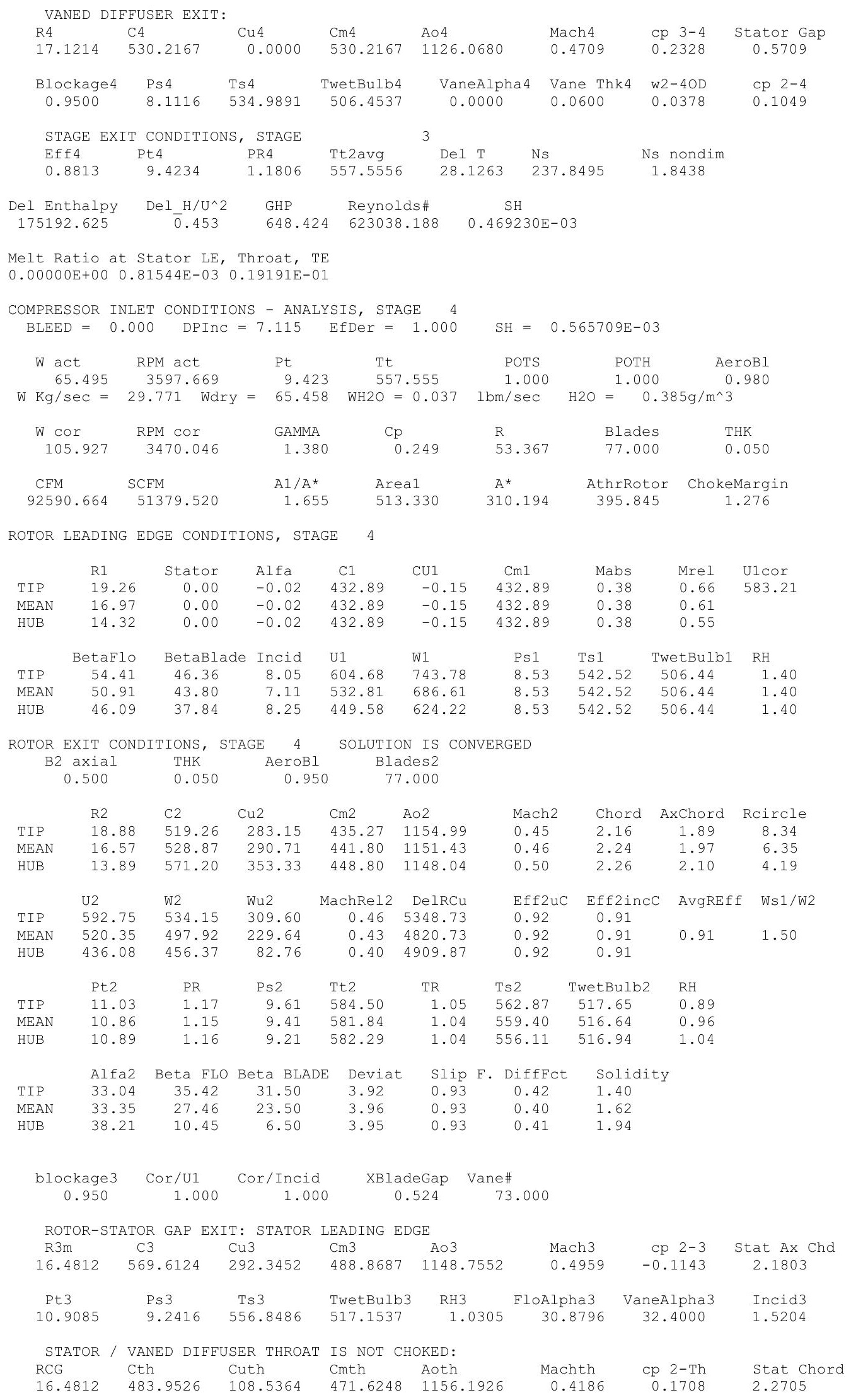




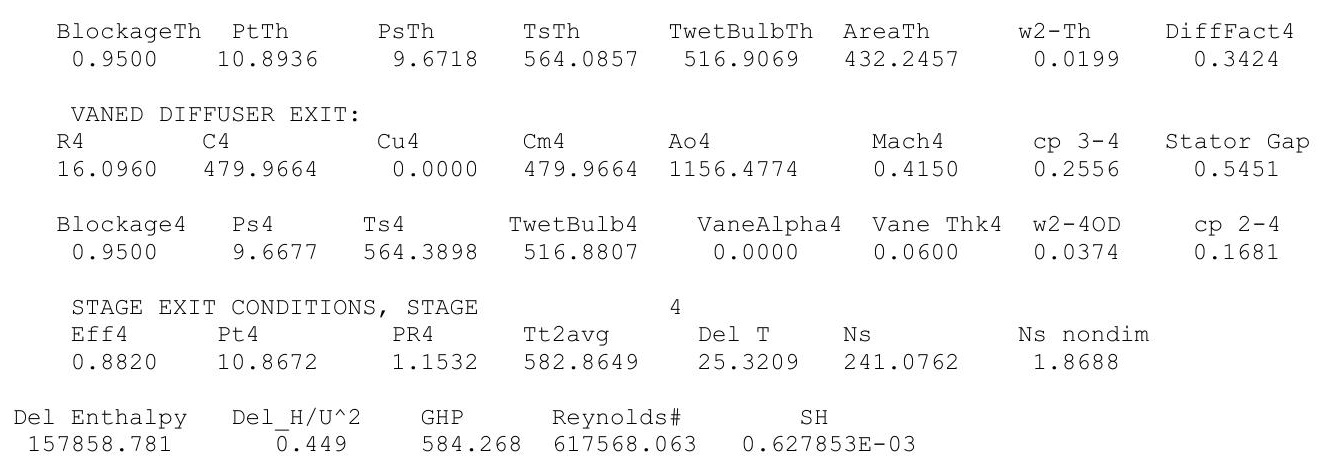

Melt Ratio at Stator LE, Throat, TE

$0.14435 \mathrm{E}+00 \quad 0.17204 \mathrm{E}+00 \quad 0.24568 \mathrm{E}+00$

COMPRESSOR INLET CONDITIONS - ANALYSIS, STAGE 5 BLEED $=0.000 \quad$ DPInC $=7.319 \quad$ EfDer $=0.999$

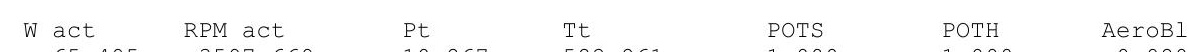

ROTOR LEADING EDGE CONDITIONS, STAGE 5

$\begin{array}{lccccccccc} & \text { R1 } & \text { Stator } & \text { Alfa } & \text { C1 } & \text { CU1 } & \text { Cm1 } & \text { Mabs } & \text { Mrel } & \text { U1cor } \\ \text { TIP } & 18.32 & 0.00 & -0.02 & 388.77 & -0.13 & 388.77 & 0.33 & 0.60 & 542.57 \\ \text { MEAN } & 15.91 & 0.00 & -0.02 & 388.77 & -0.13 & 388.77 & 0.33 & 0.54 & \\ \text { HUB } & 13.07 & 0.00 & -0.02 & 388.77 & -0.13 & 388.77 & 0.33 & 0.49 & \\ & & & & & & & & & \\ & & & & & & & & \\ \text { TIP } & 55.95 & 47.36 & 8.59 & 575.17 & 694.34 & 10.07 & 570.74 & 516.83 & 0.86 \\ \text { MEAN } & 52.12 & 44.80 & 7.32 & 499.60 & 633.14 & 10.07 & 570.75 & 516.83 & 0.86 \\ \text { HUB } & 46.56 & 38.84 & 7.72 & 410.34 & 565.36 & 10.07 & 570.74 & 516.83 & 0.86\end{array}$

ROTOR EXIT CONDITIONS, STAGE 5 SOLUTION IS CONVERGED

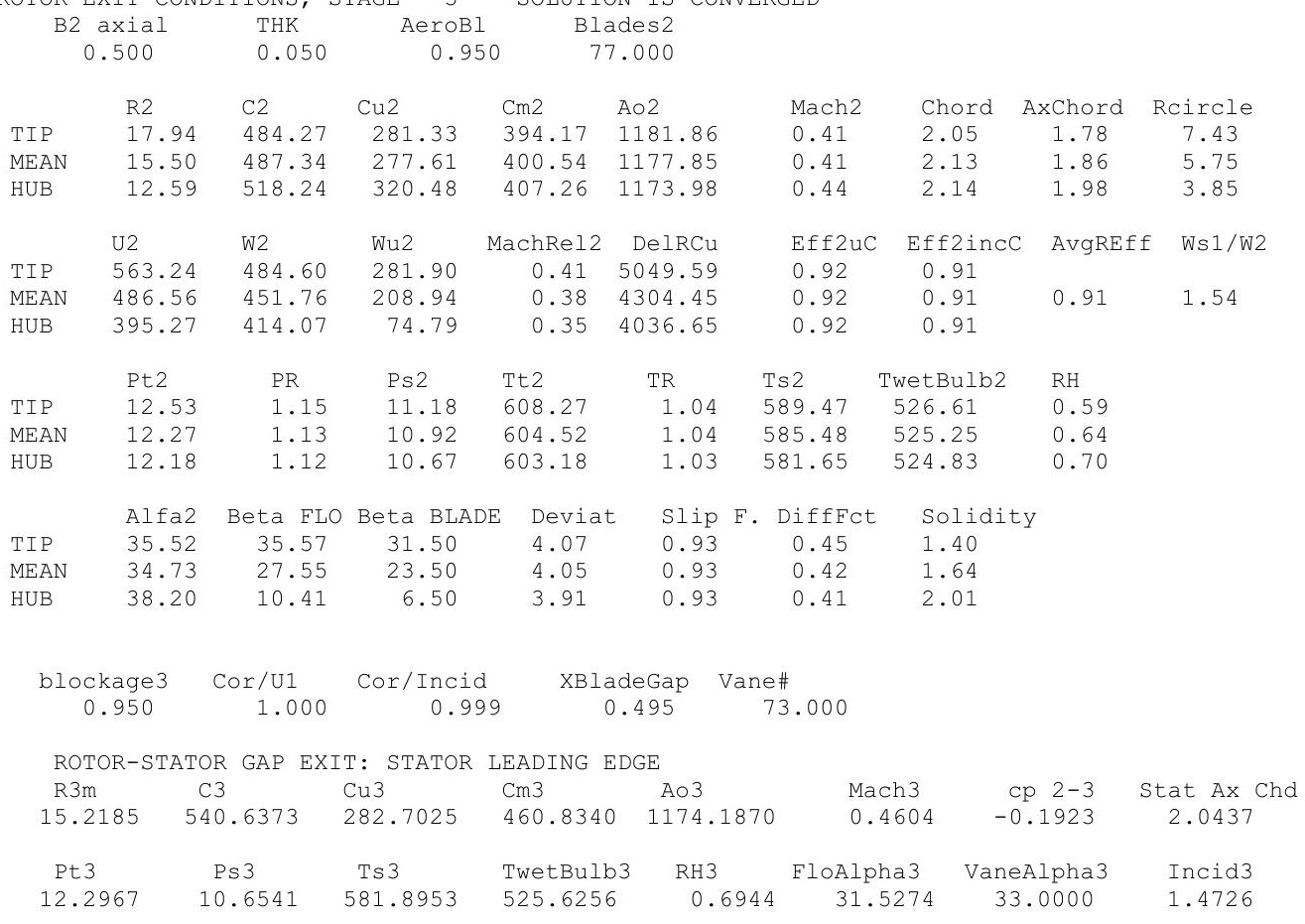




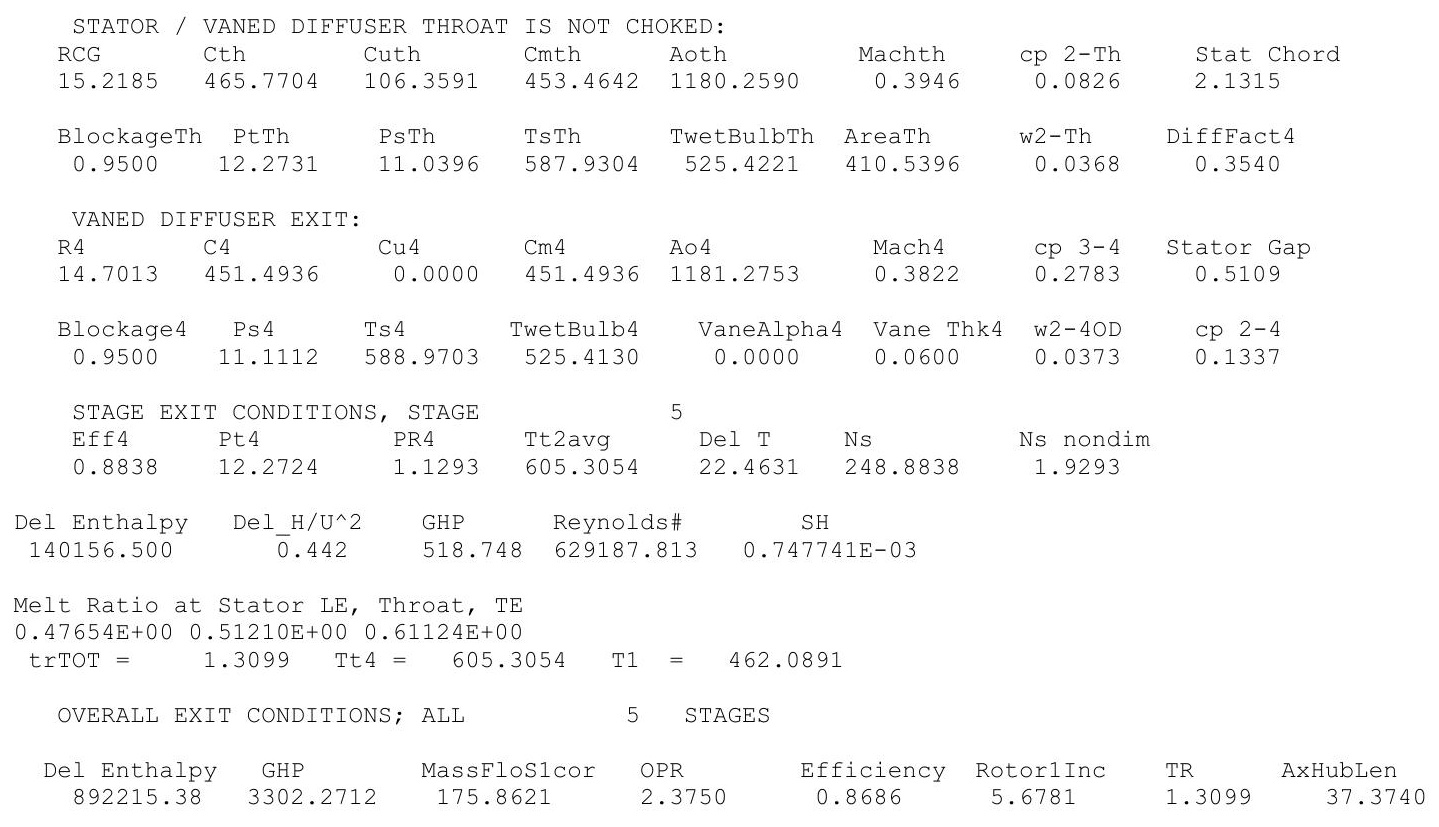




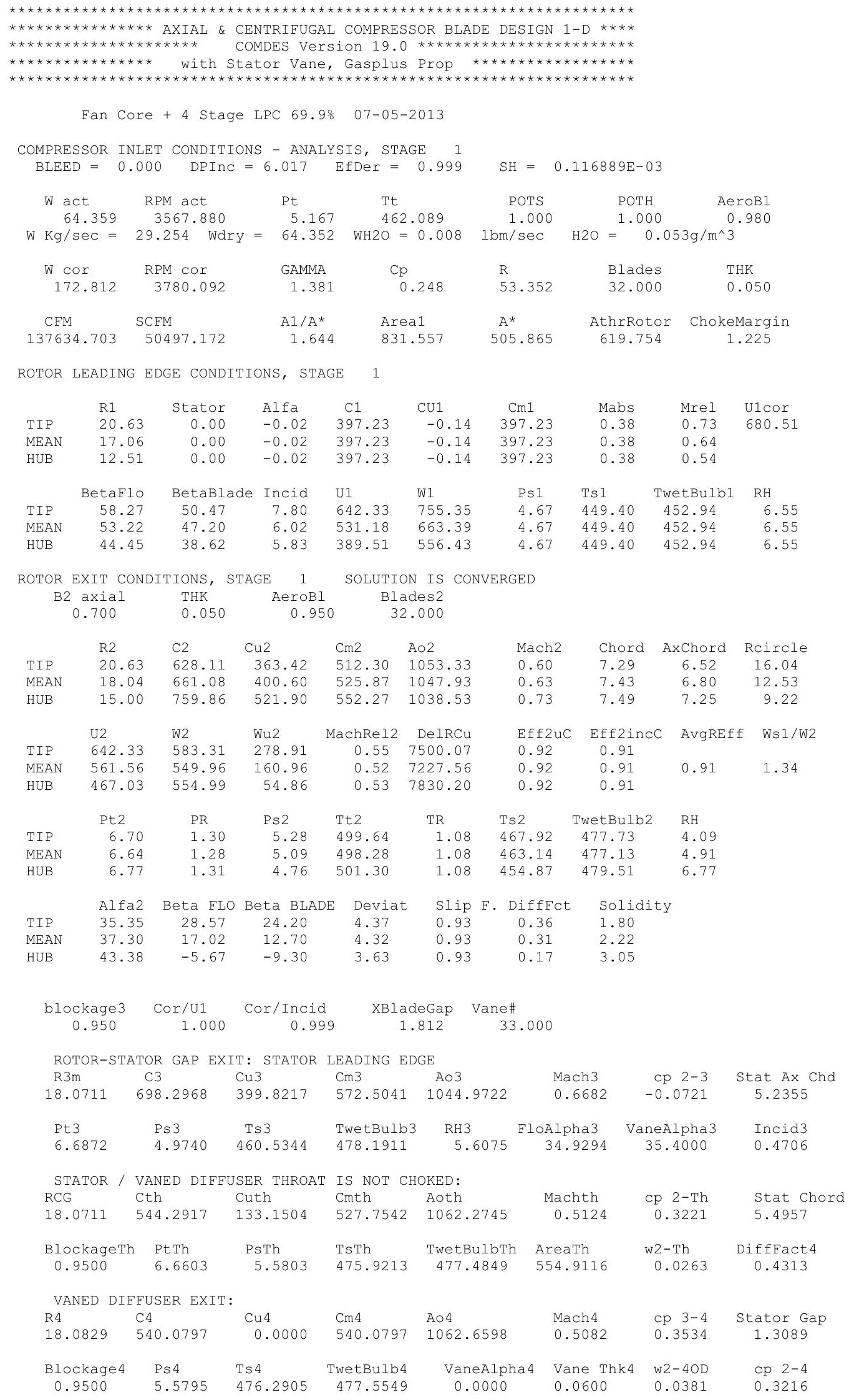




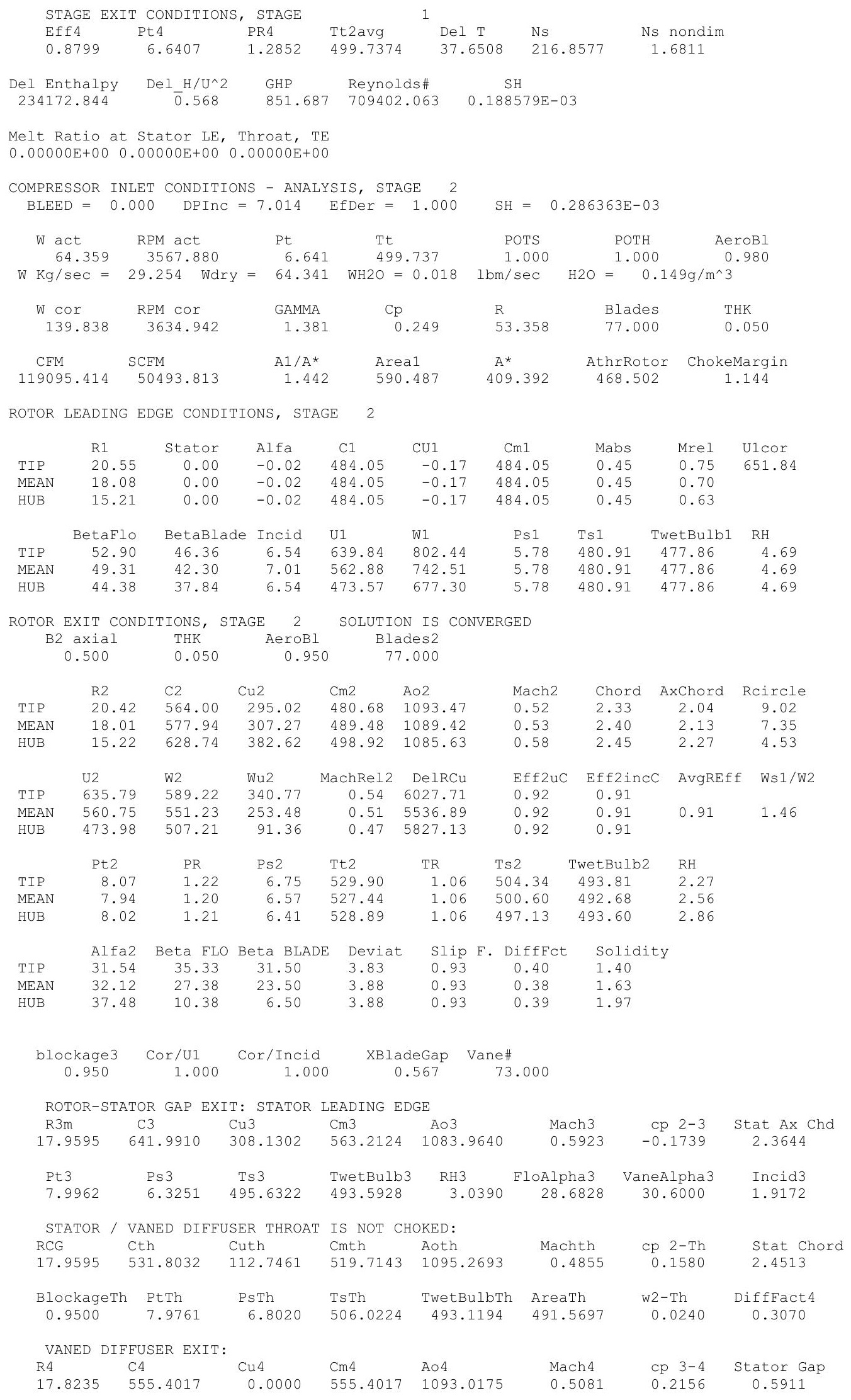




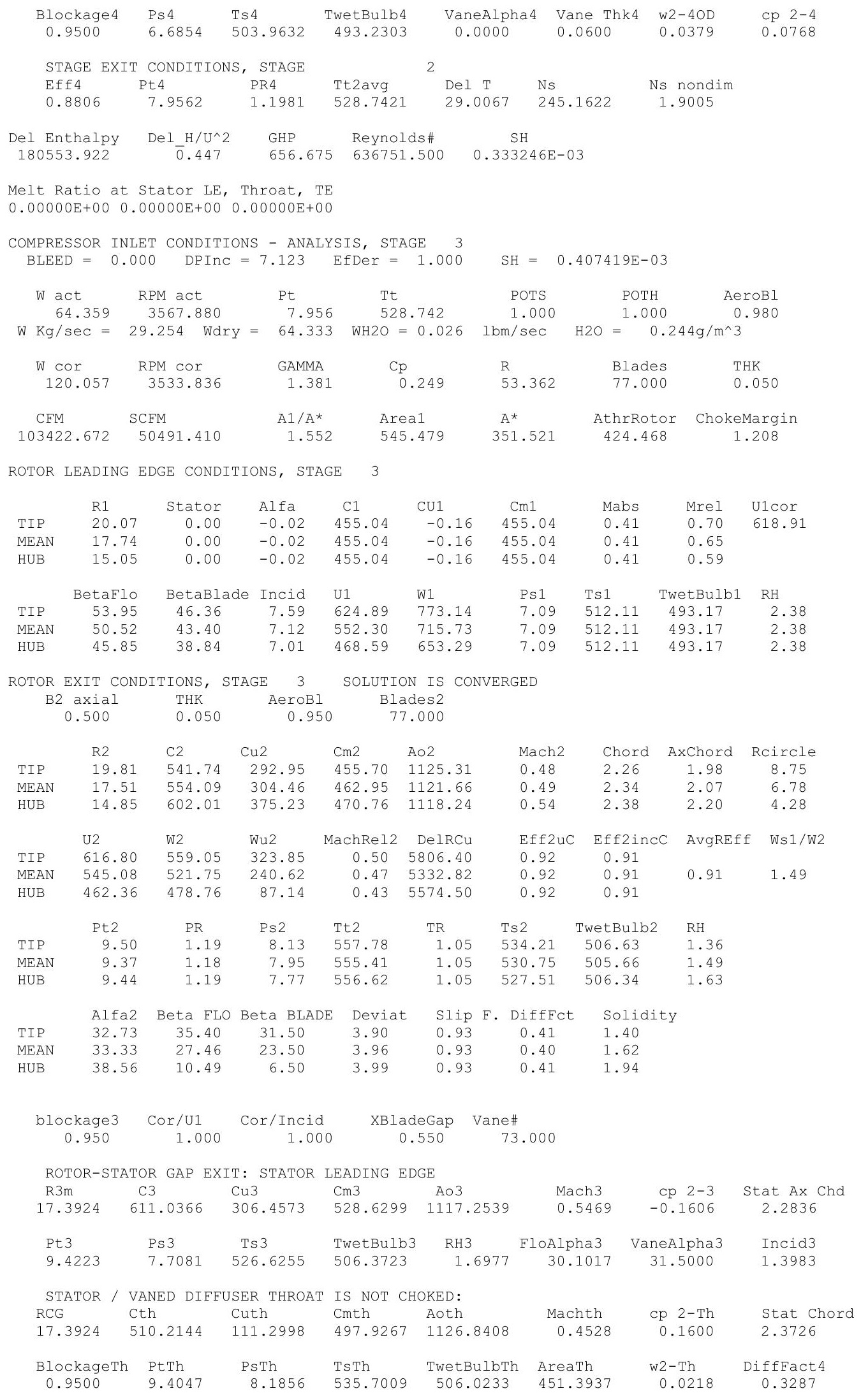




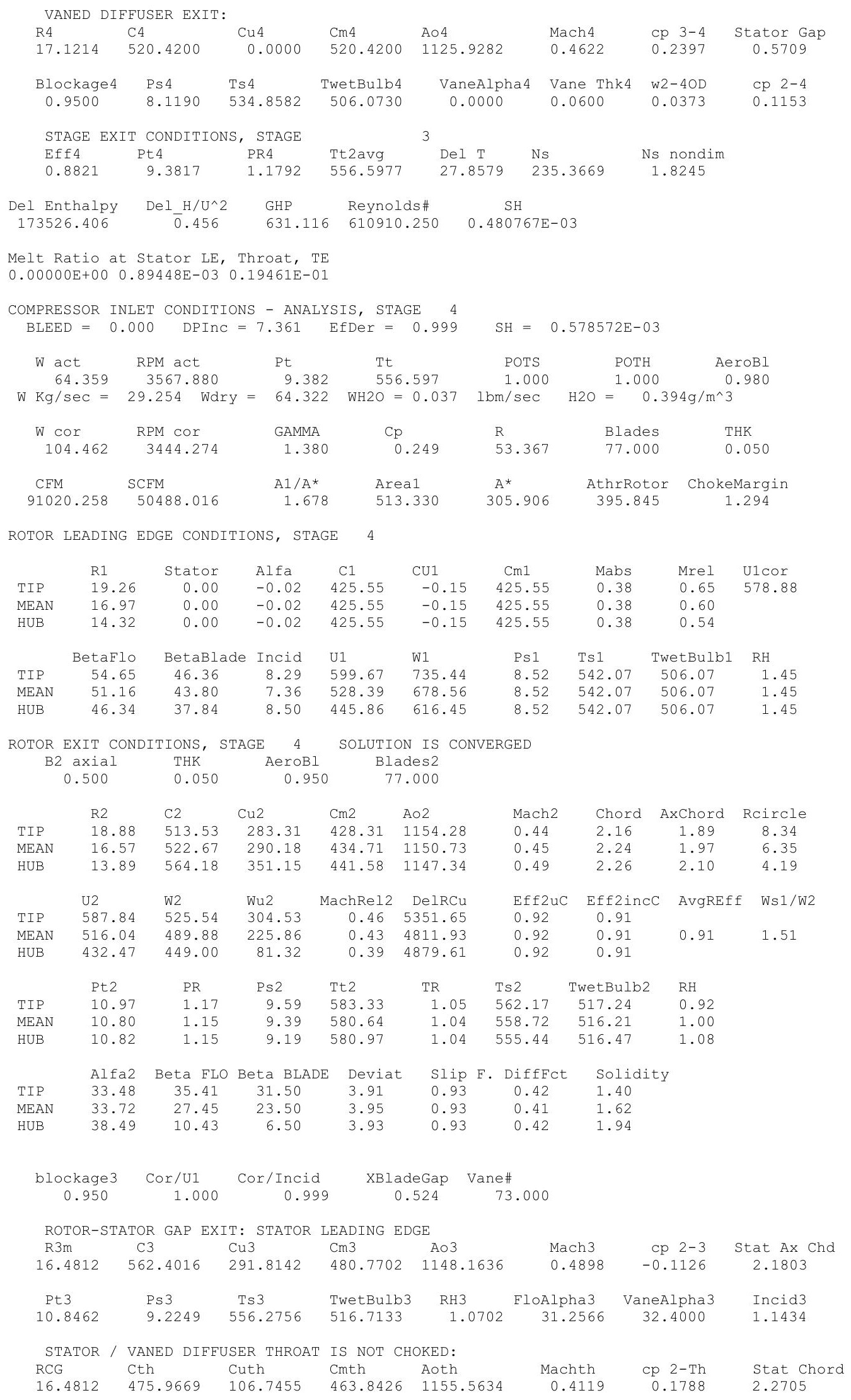




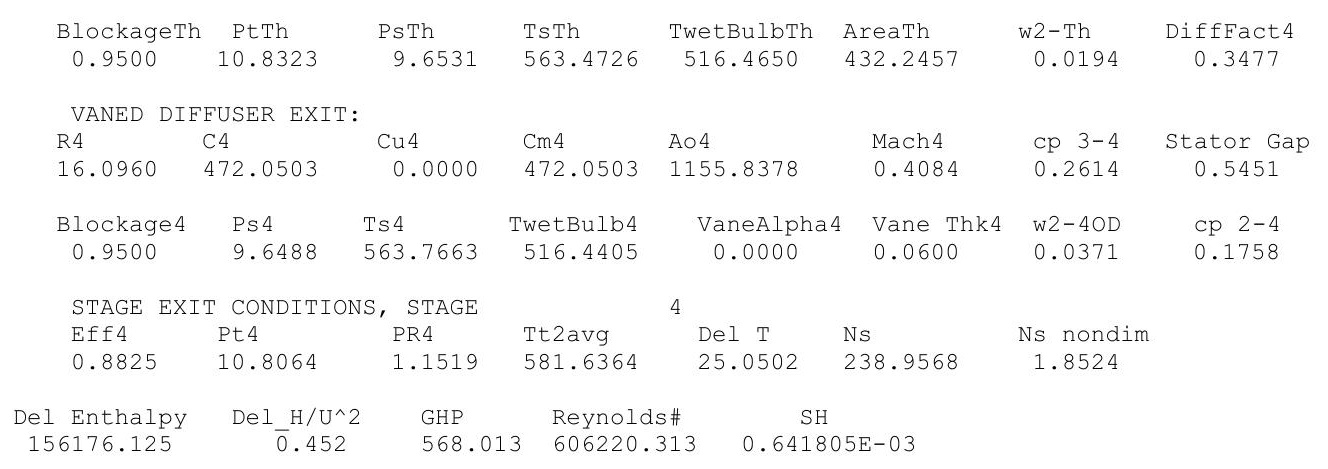

Melt Ratio at Stator LE, Throat, TE

$0.14418 \mathrm{E}+00 \quad 0.17194 \mathrm{E}+00 \quad 0.24582 \mathrm{E}+00$

COMPRESSOR INLET CONDITIONS - ANALYSIS, STAGE 5 BLEED $=0.000 \quad$ DPInC $=7.521 \quad$ EfDer $=0.999$

$\mathrm{SH}=0.715508 \mathrm{E}-03$

$\begin{array}{llllll}\text { W act } & \text { RPM act } & \text { Pt } & \text { POTS } & \text { POTH } & \text { AeroBI }\end{array}$ W Kg/sec $=29.254$ Wdry $=64.313$ WH2O $=0.046 \quad 1 \mathrm{bm} / \mathrm{sec} \quad \mathrm{H} 2 \mathrm{O}=\begin{gathered}1.000 \\ 0.546 \mathrm{~g} / \mathrm{m}^{\wedge} 3\end{gathered}$

$\begin{array}{ccccccc}\text { W Cor } & \text { RPM cor } & \text { GAMMA } & \text { CP } & \text { R } & \text { Blades } & \text { THK } \\ 92.708 & 3369.323 & 1.380 & 0.249 & 53.372 & 77.000 & 0.050 \\ & & & & & & \\ \text { CFM } & \text { SCFM } & \text { A1/A* } & \text { Areal } & A^{*} & \text { AthrRotor } & \text { ChokeMargin } \\ 81282.375 & 50485.305 & 1.877 & 509.668 & 271.523 & 384.739 & 1.417\end{array}$

ROTOR LEADING EDGE CONDITIONS, STAGE 5

\begin{tabular}{|c|c|c|c|c|c|c|c|c|c|}
\hline & R1 & Stator & Alfa & $\mathrm{C} 1$ & CU1 & $\mathrm{Cm} 1$ & Mabs & Mrel & U1cor \\
\hline TIP & 18.32 & 0.00 & -0.02 & 382.75 & -0.13 & 382.75 & 0.33 & 0.59 & 538.64 \\
\hline MEAN & 15.91 & 0.00 & -0.02 & 382.75 & -0.13 & 382.75 & 0.33 & 0.54 & \\
\hline HUB & 13.07 & 0.00 & -0.02 & 382.75 & -0.13 & 382.75 & 0.33 & 0.48 & \\
\hline & BetaFlo & BetaBlade & Incid & U1 & W1 & Ps 1 & Ts1 & TwetBulb1 & $\mathrm{RH}$ \\
\hline TIP & 56.14 & 47.36 & 8.78 & 570.40 & 687.03 & 10.03 & 569.89 & 516.40 & 0.90 \\
\hline MEAN & 52.32 & 44.80 & 7.52 & 495.46 & 626.19 & 10.03 & 569.89 & 516.40 & 0.90 \\
\hline HUB & 46.76 & 38.84 & 7.92 & 406.94 & 558.76 & 10.03 & 569.89 & 516.40 & 0.90 \\
\hline
\end{tabular}

ROTOR EXIT CONDITIONS, STAGE 5 SOLUTION IS CONVERGED

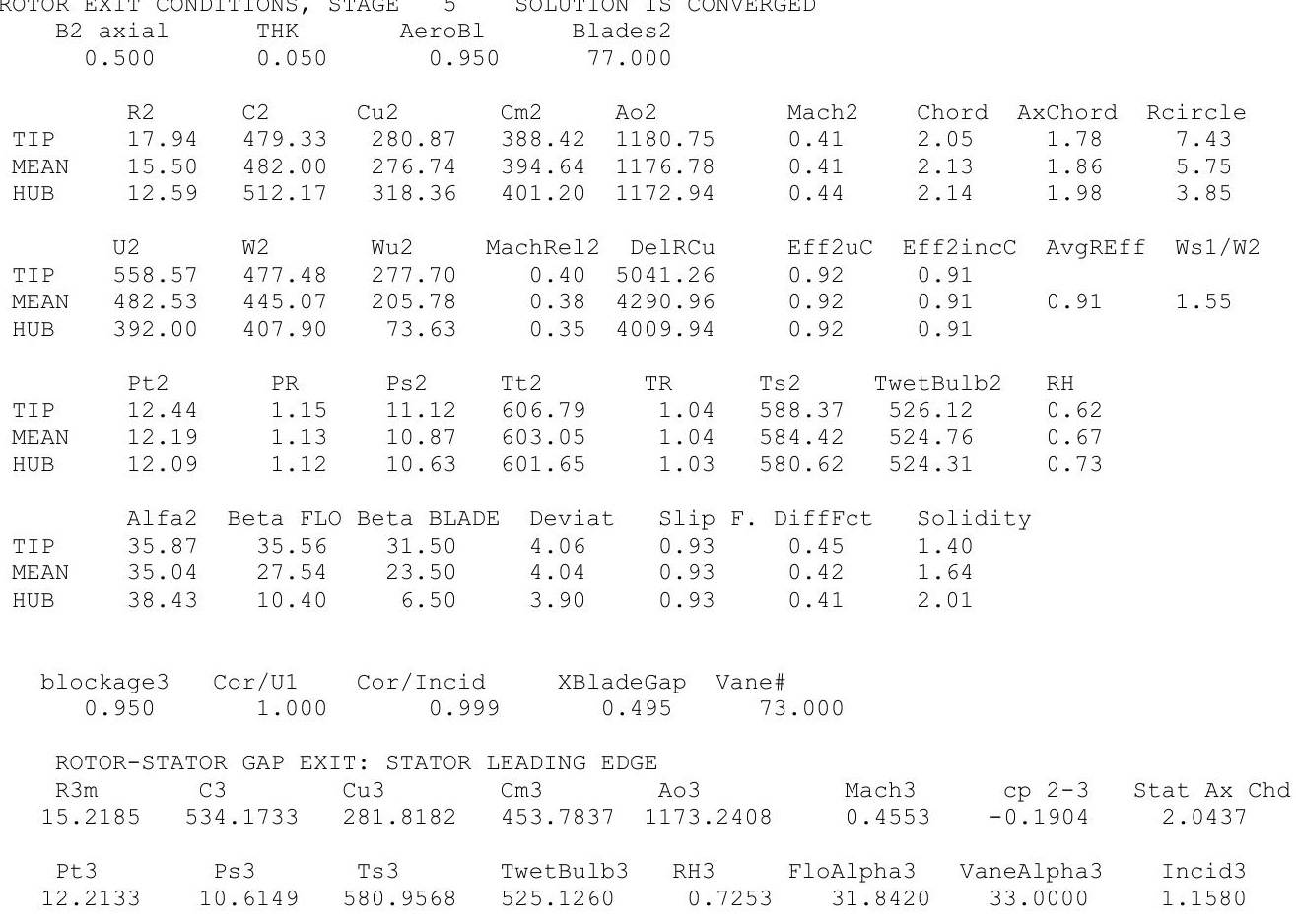




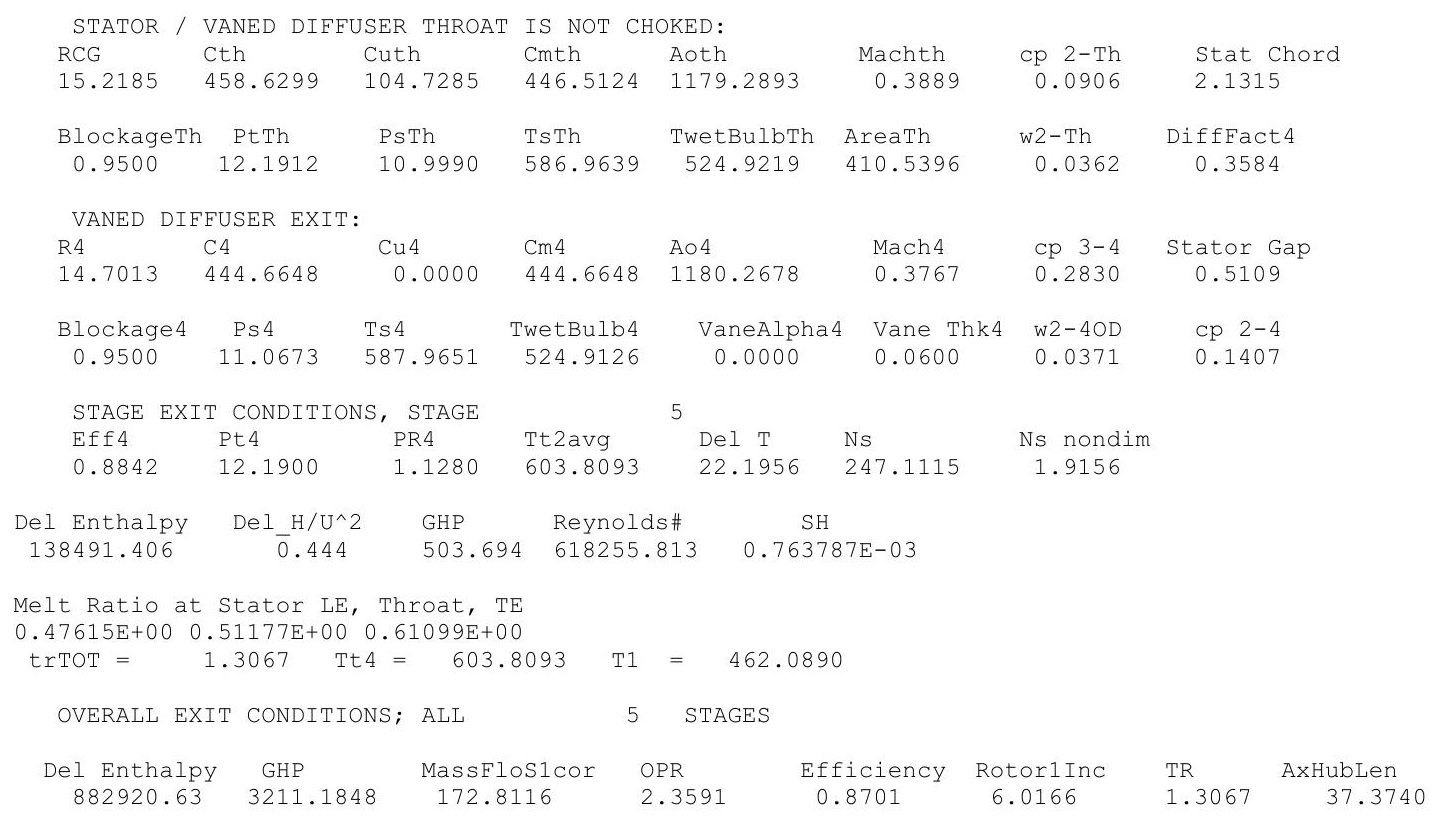


$10 \mu \mathrm{m}, \mathrm{ISA}+36 \mathrm{R}$

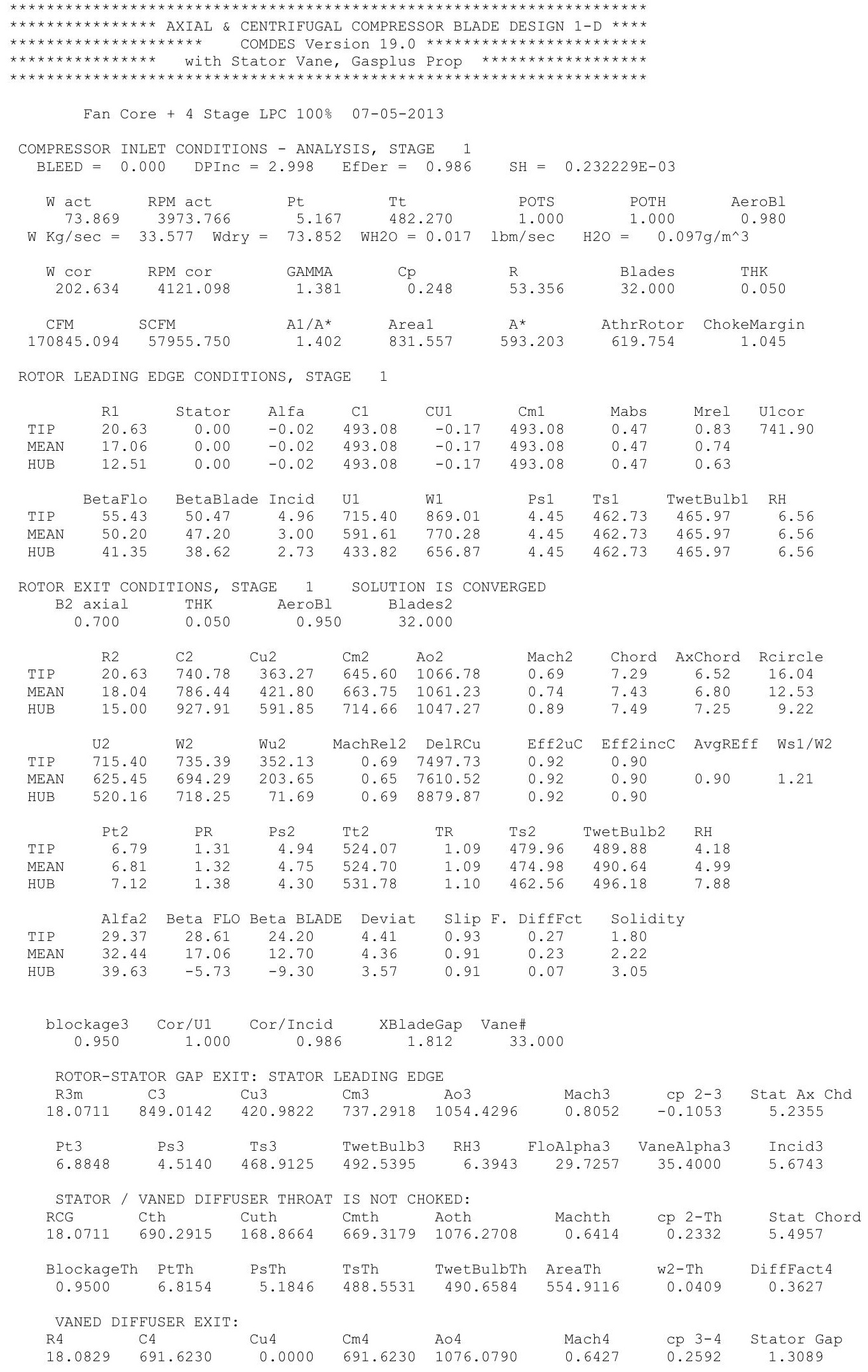




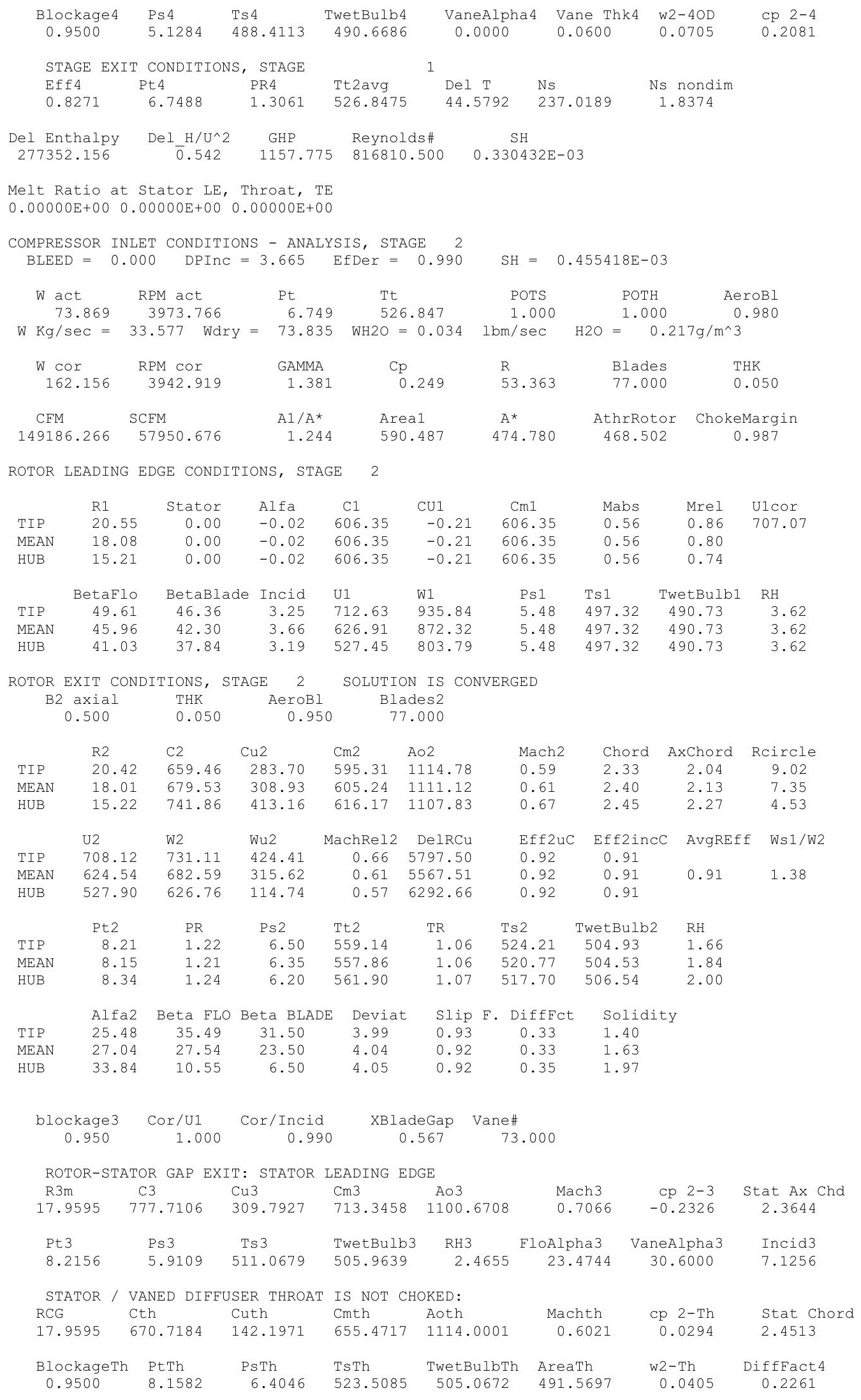




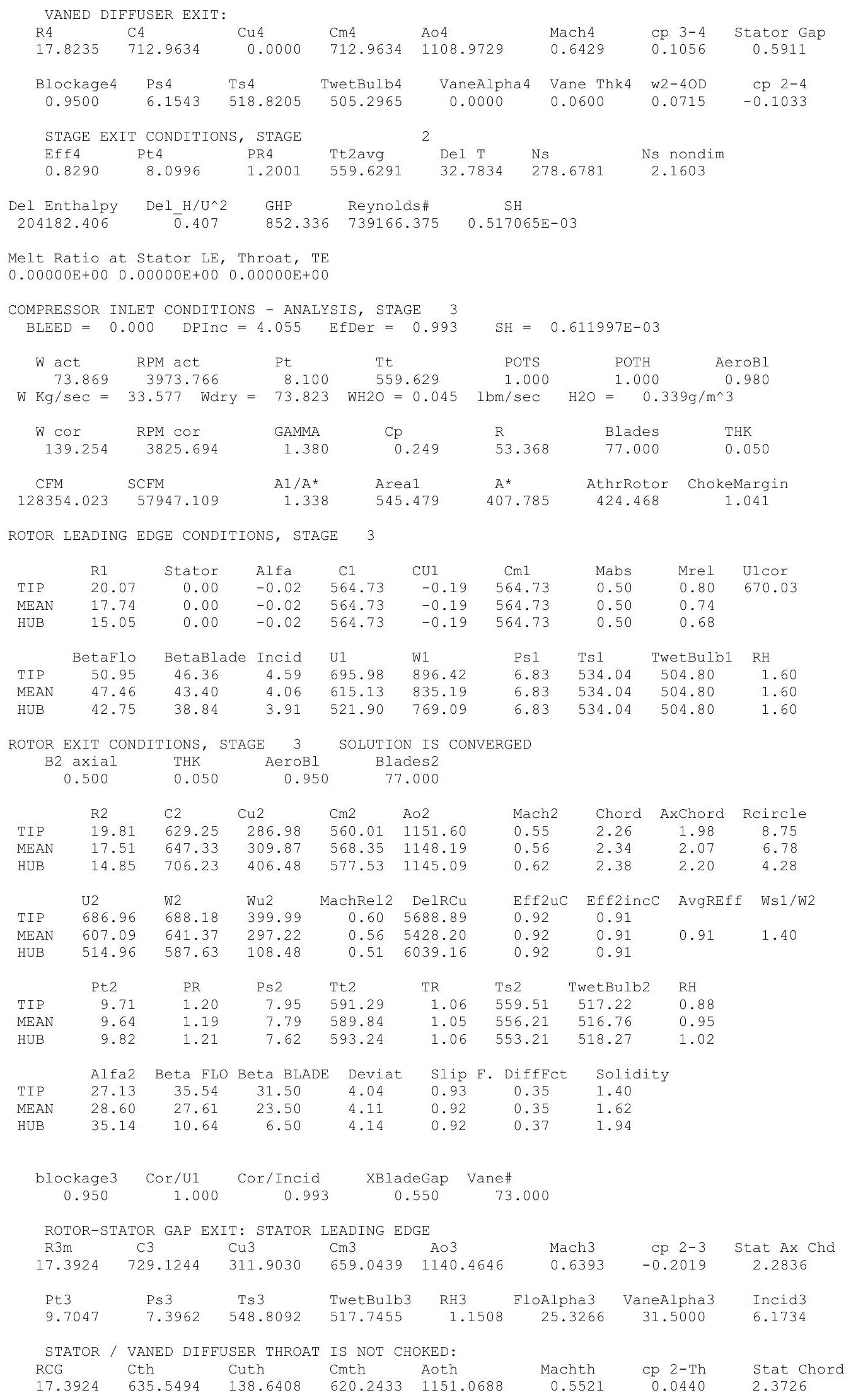




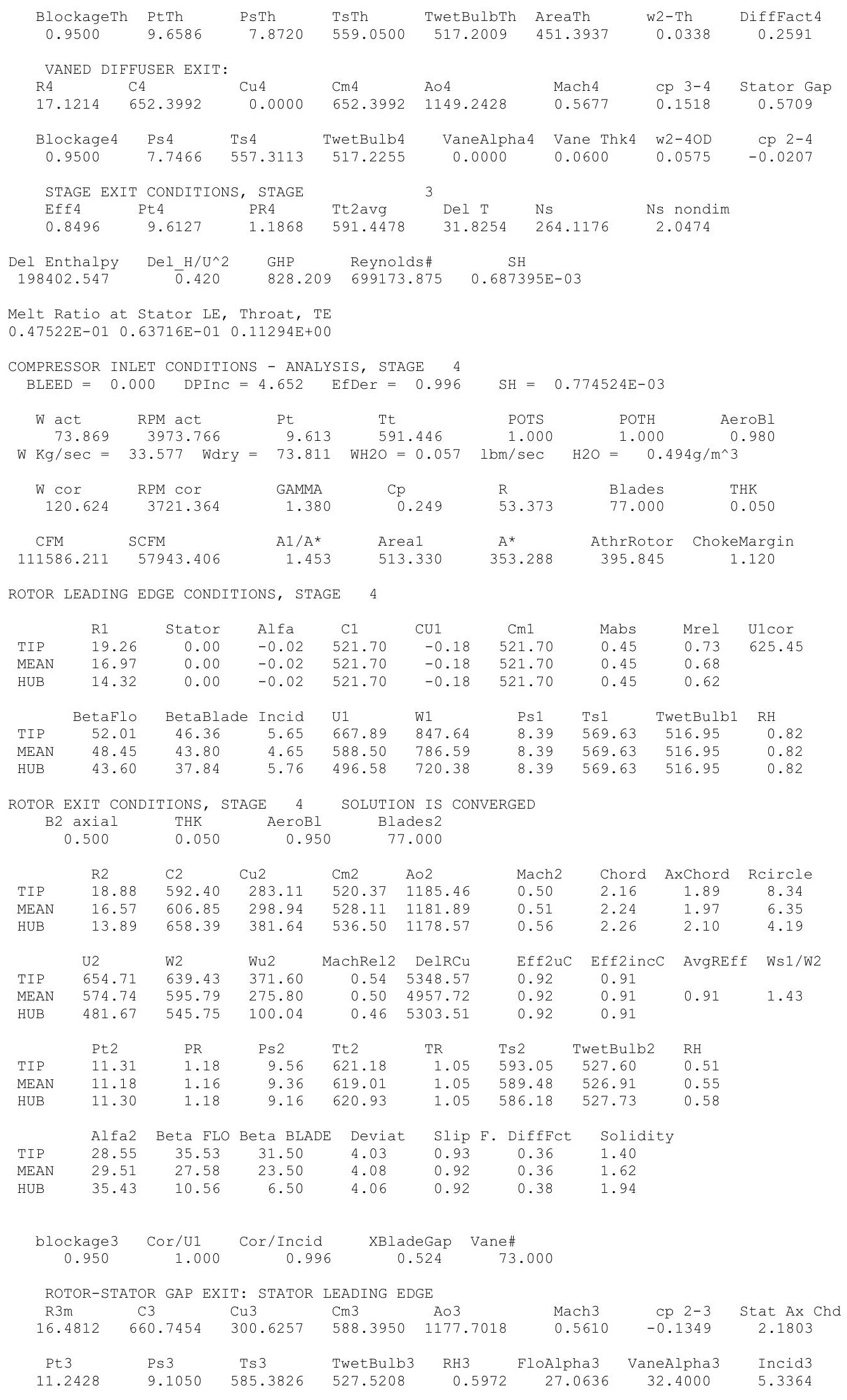




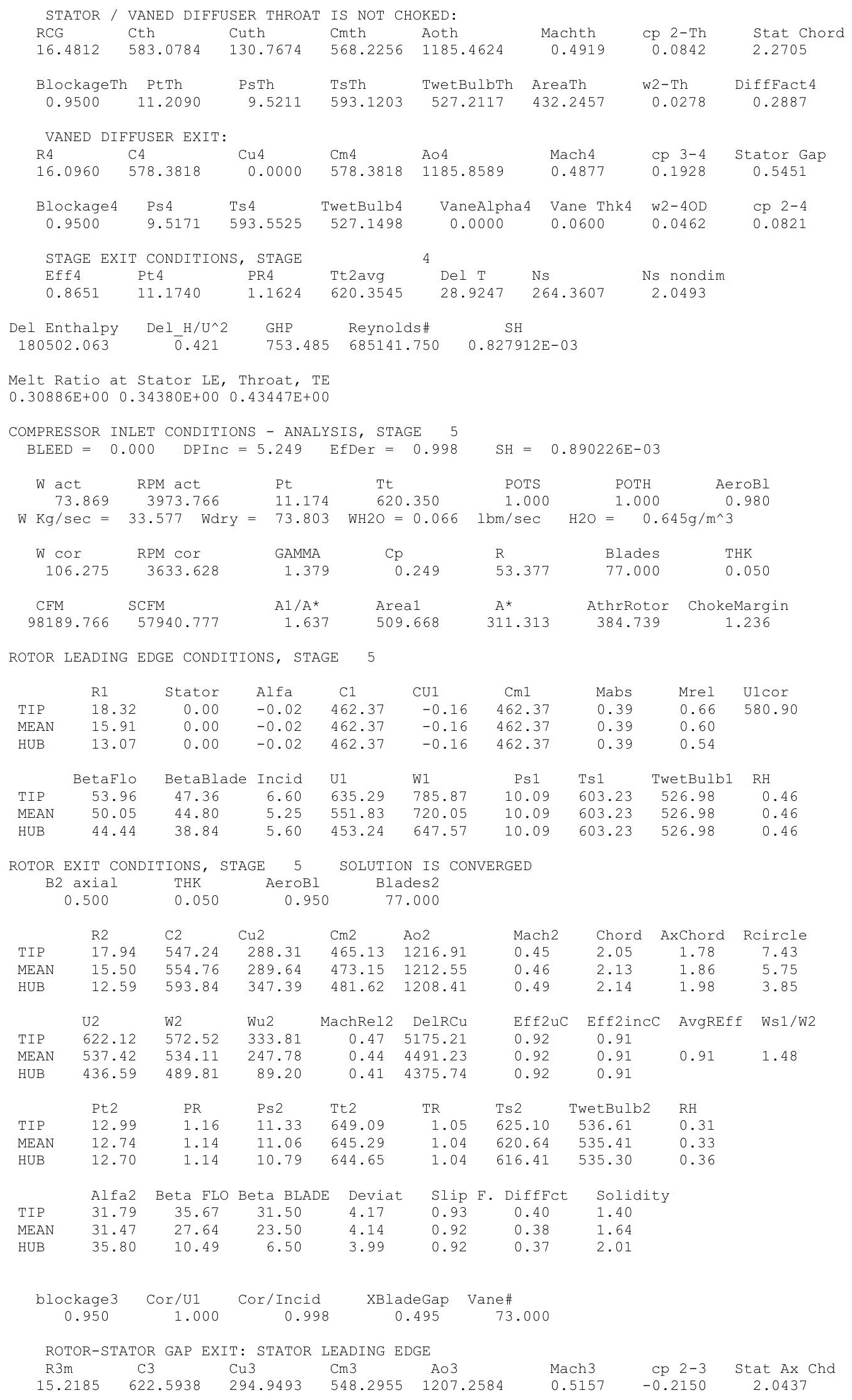




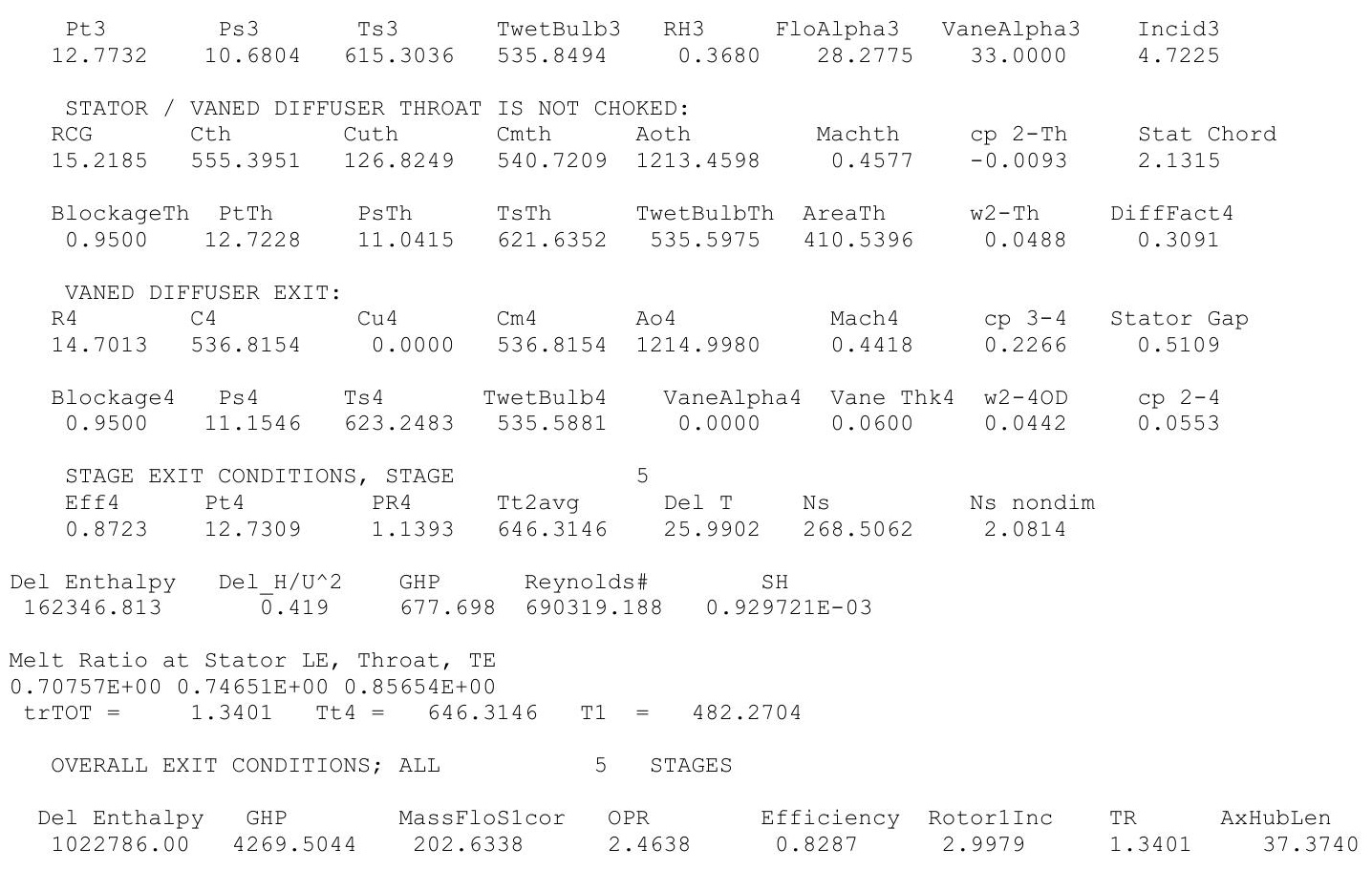




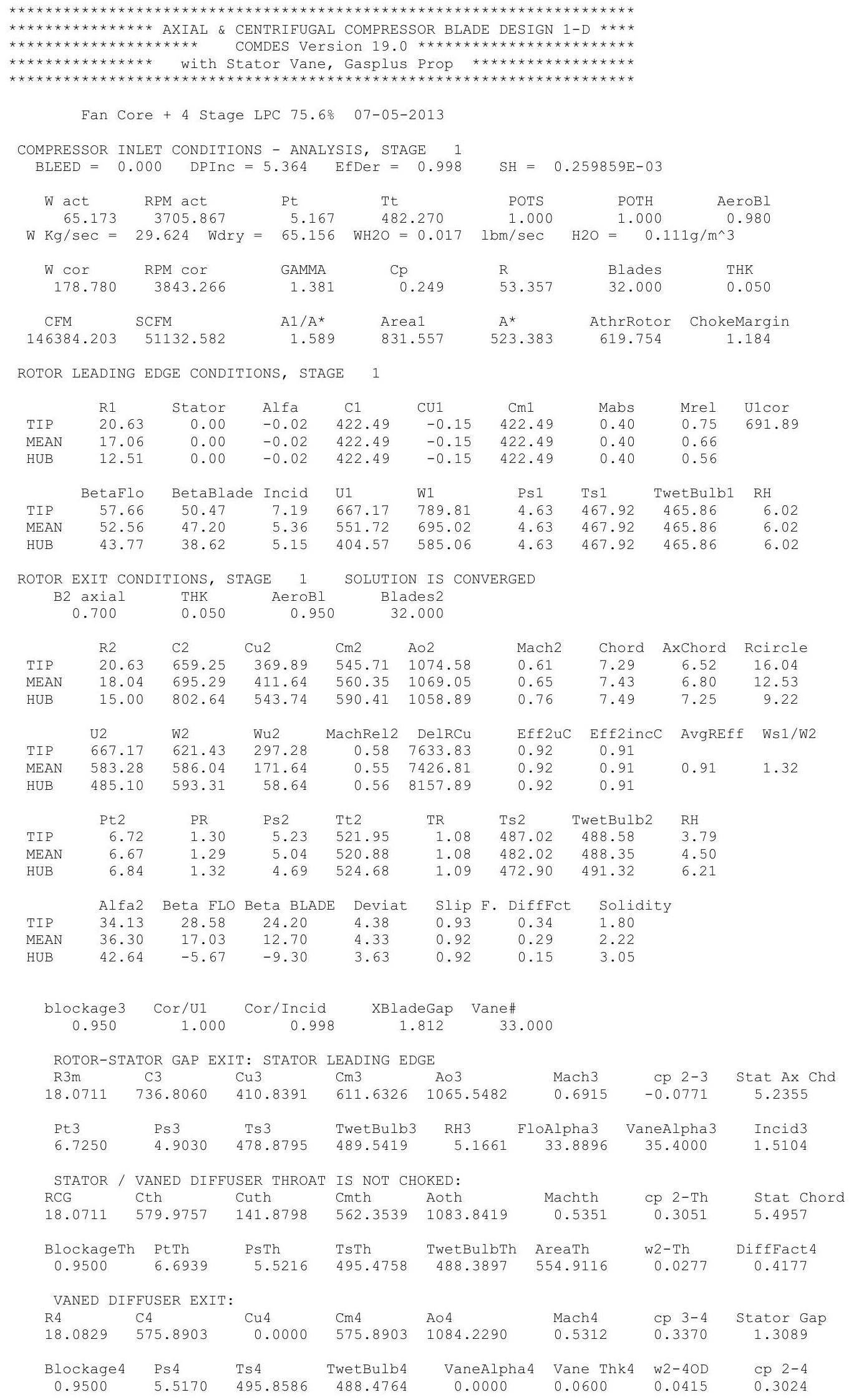




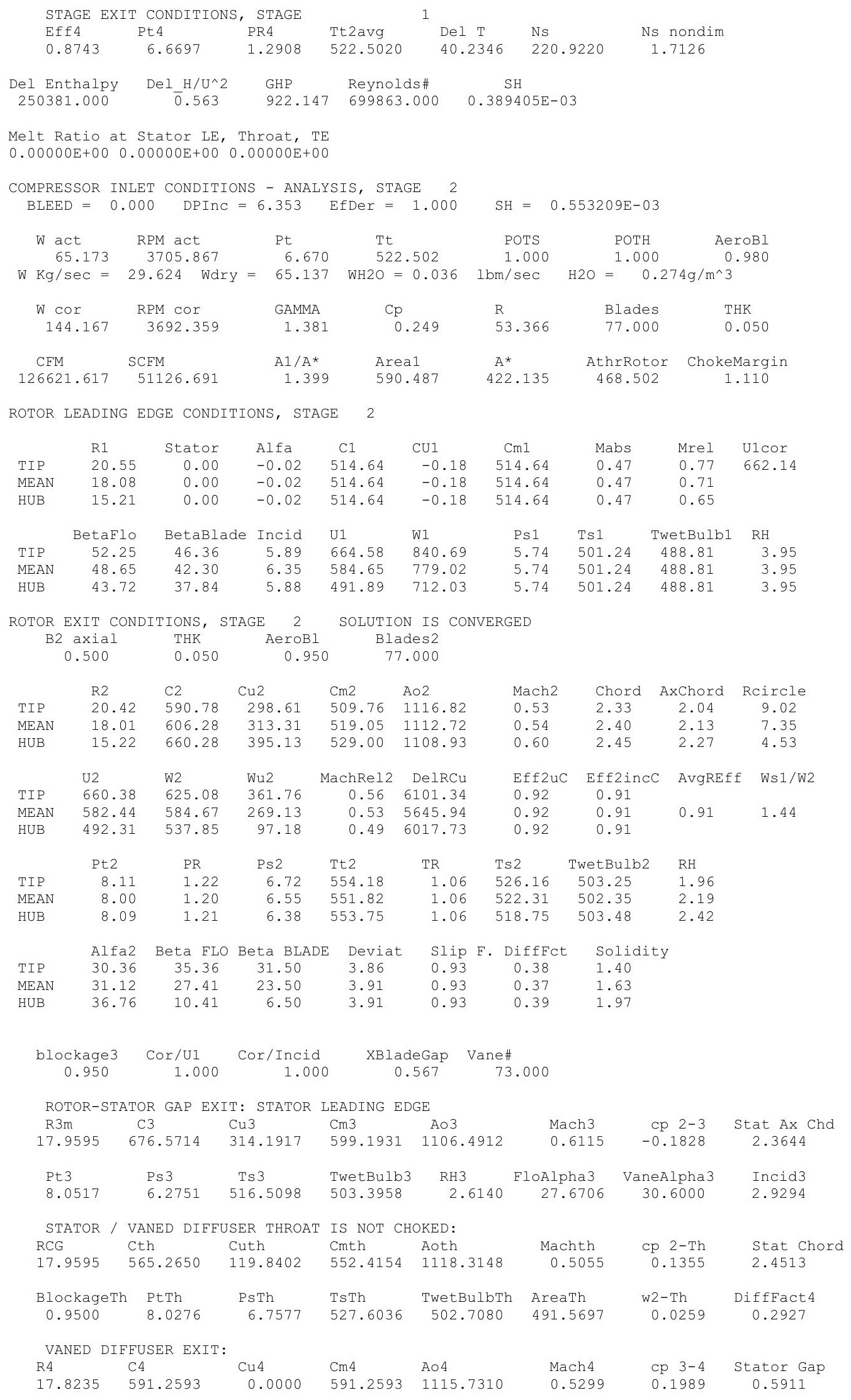




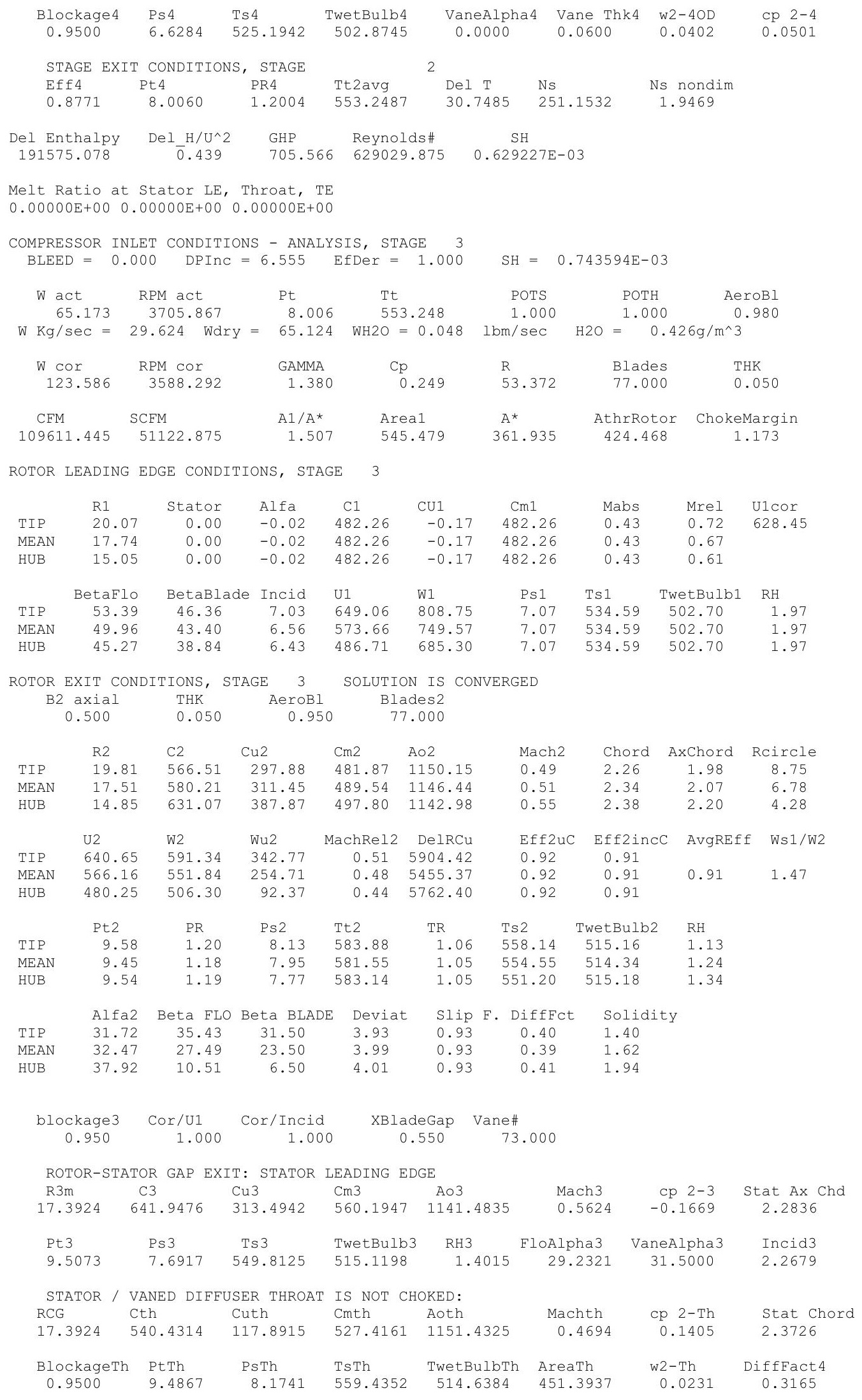




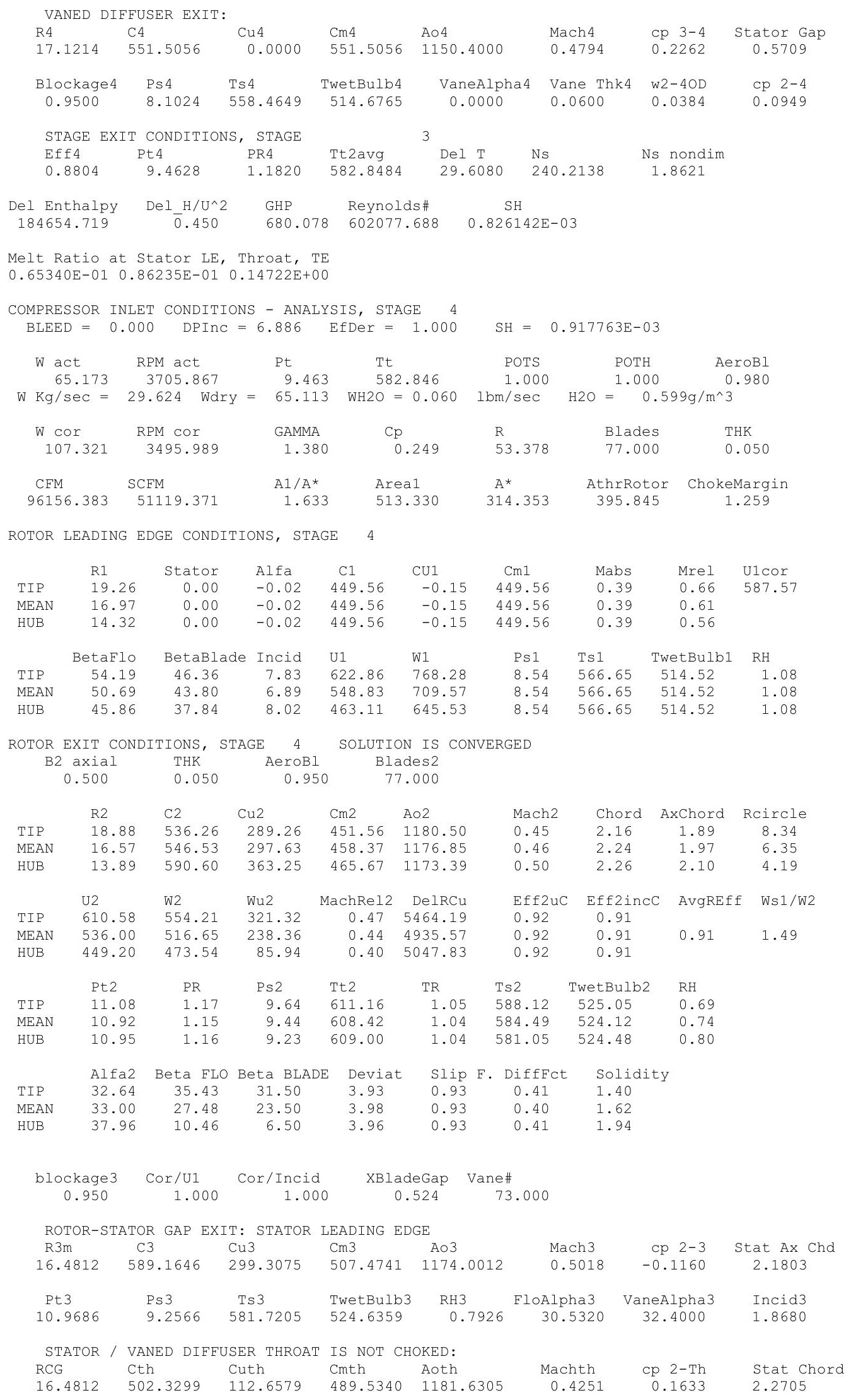




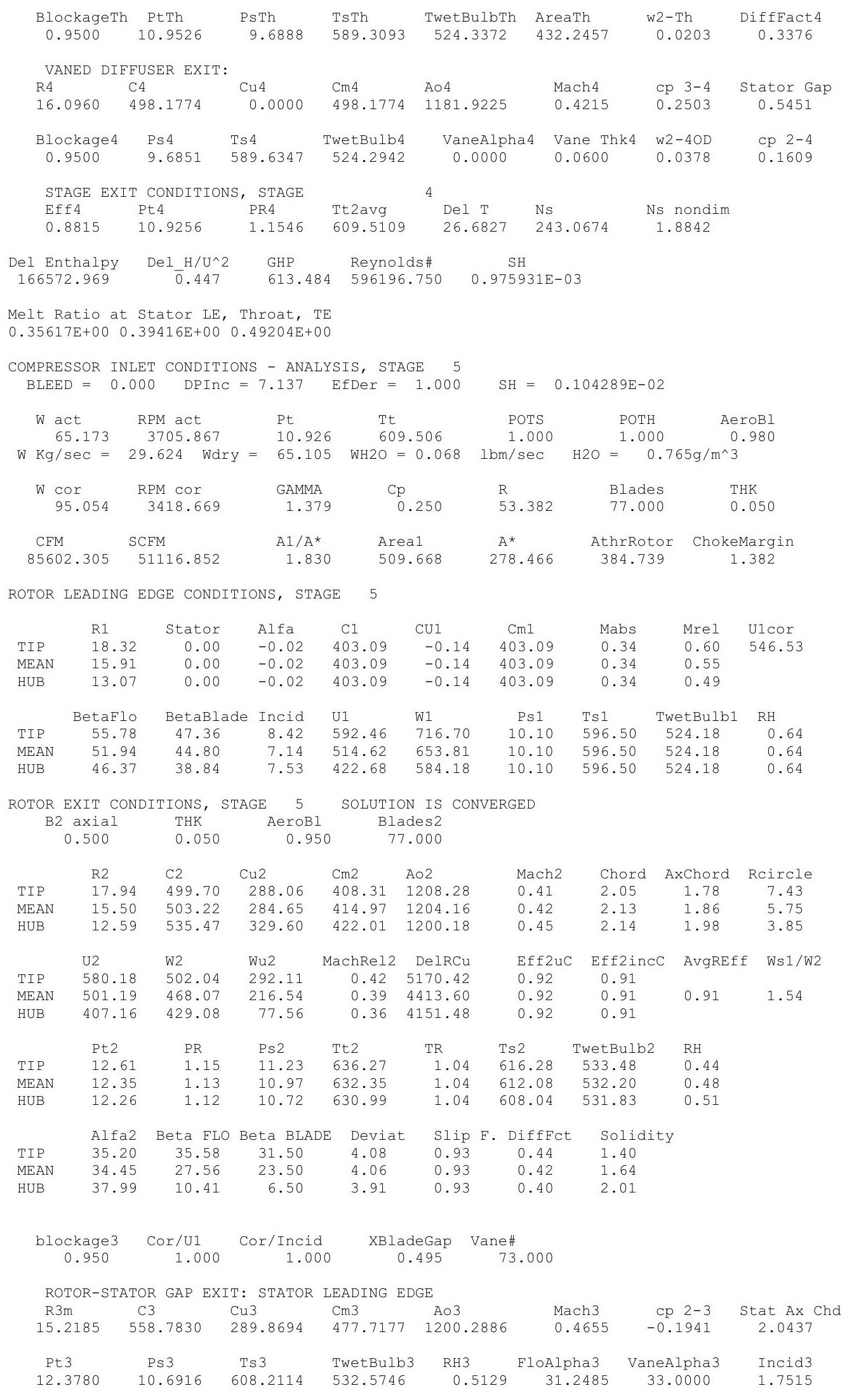




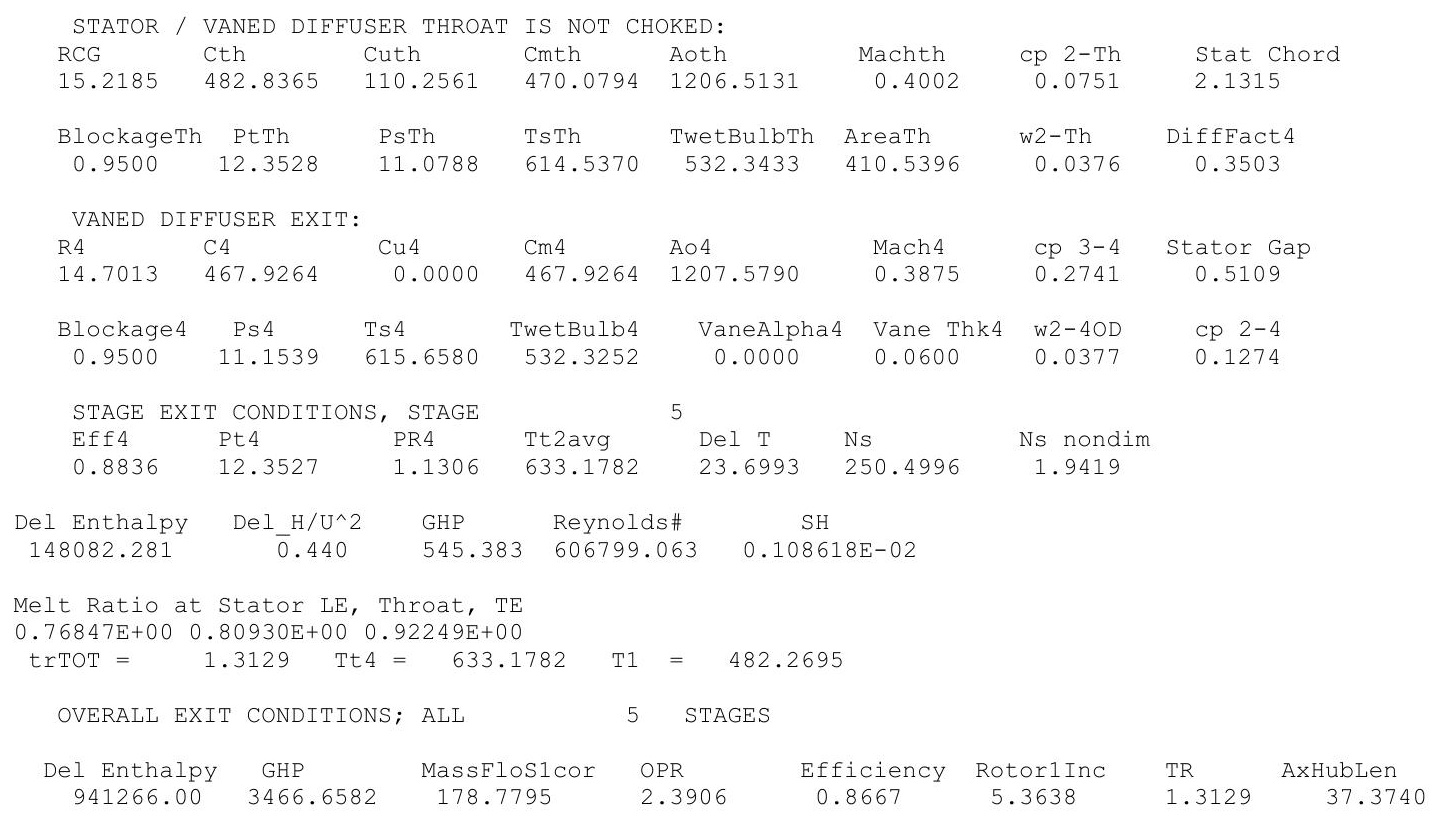




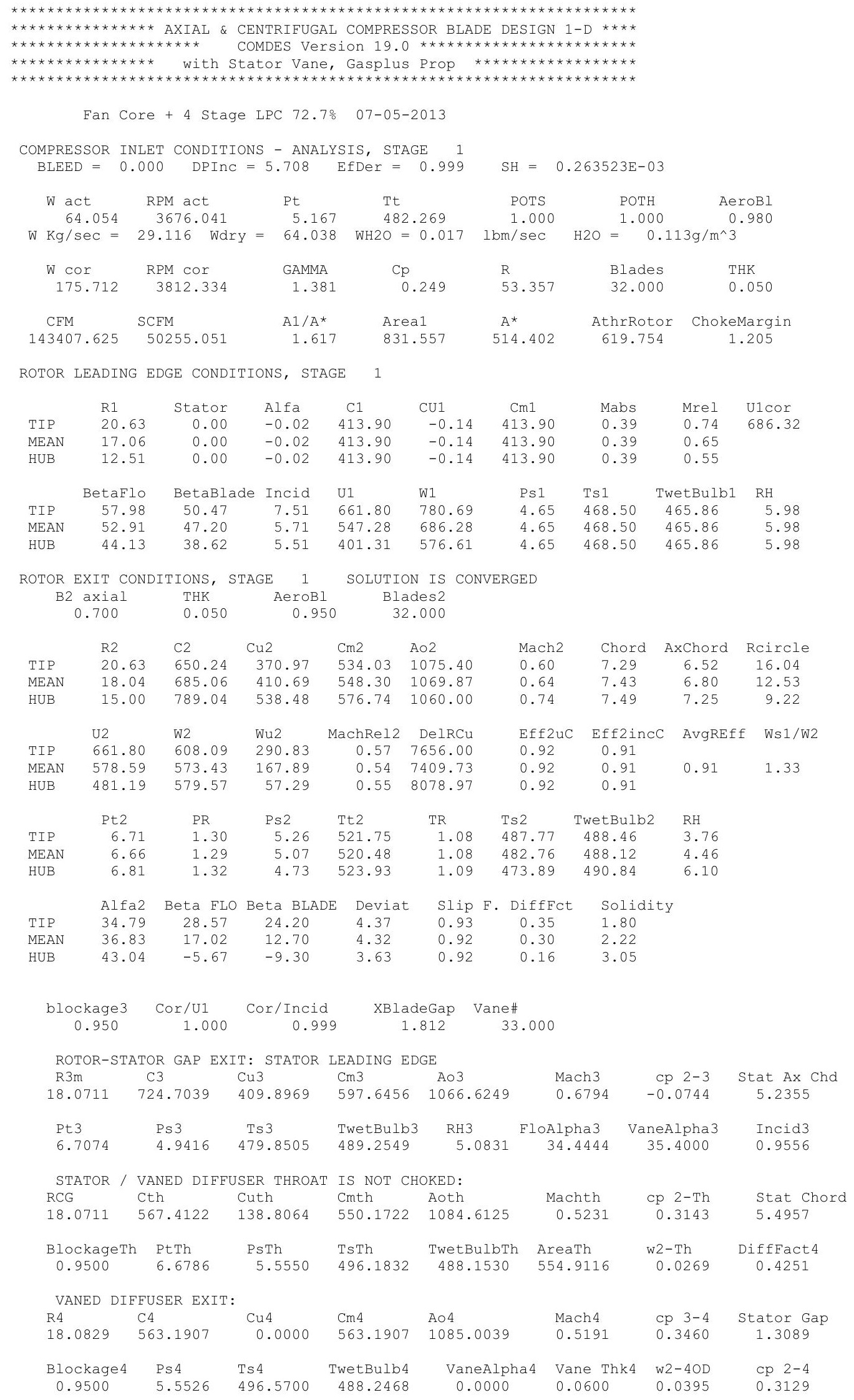




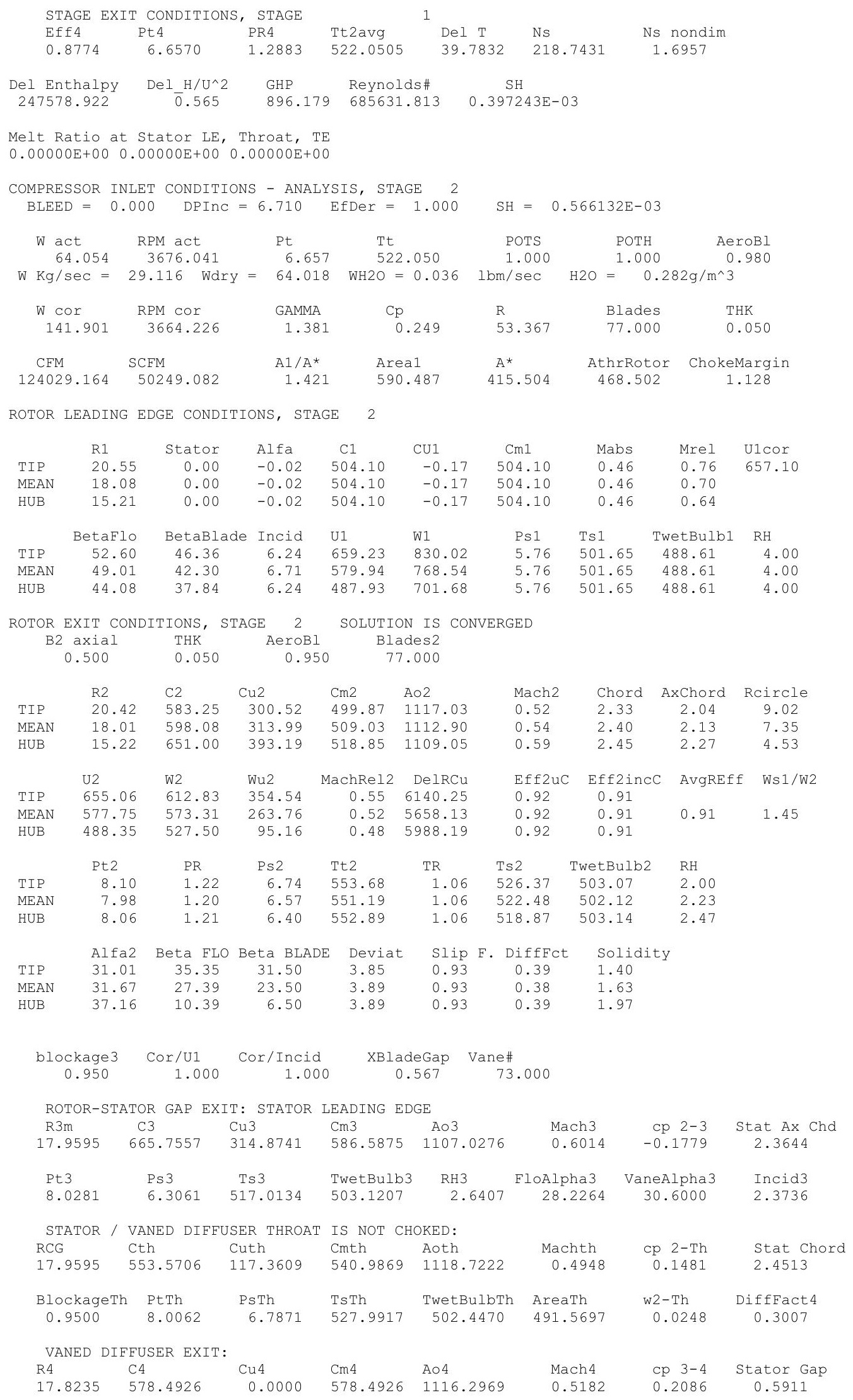




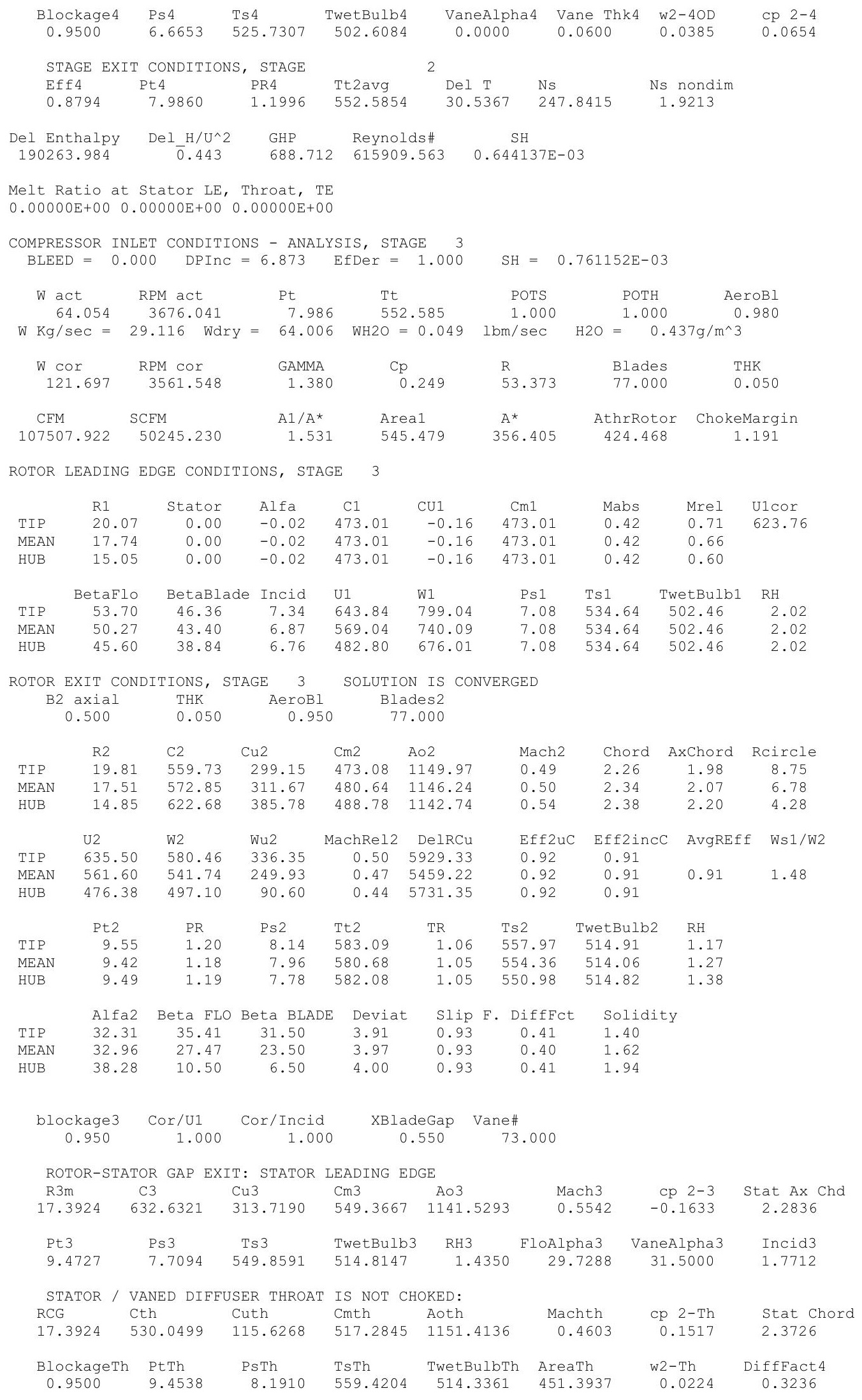




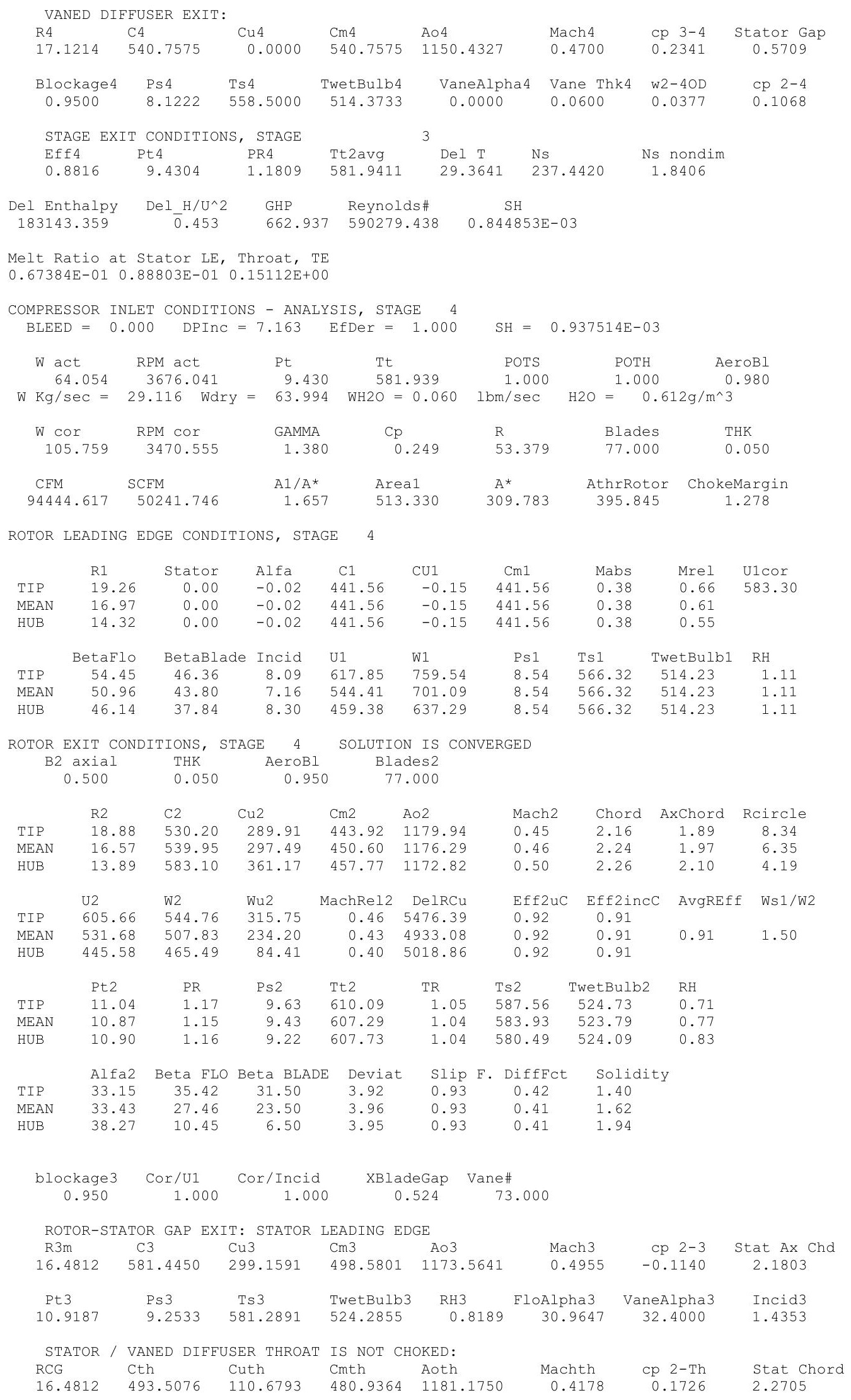




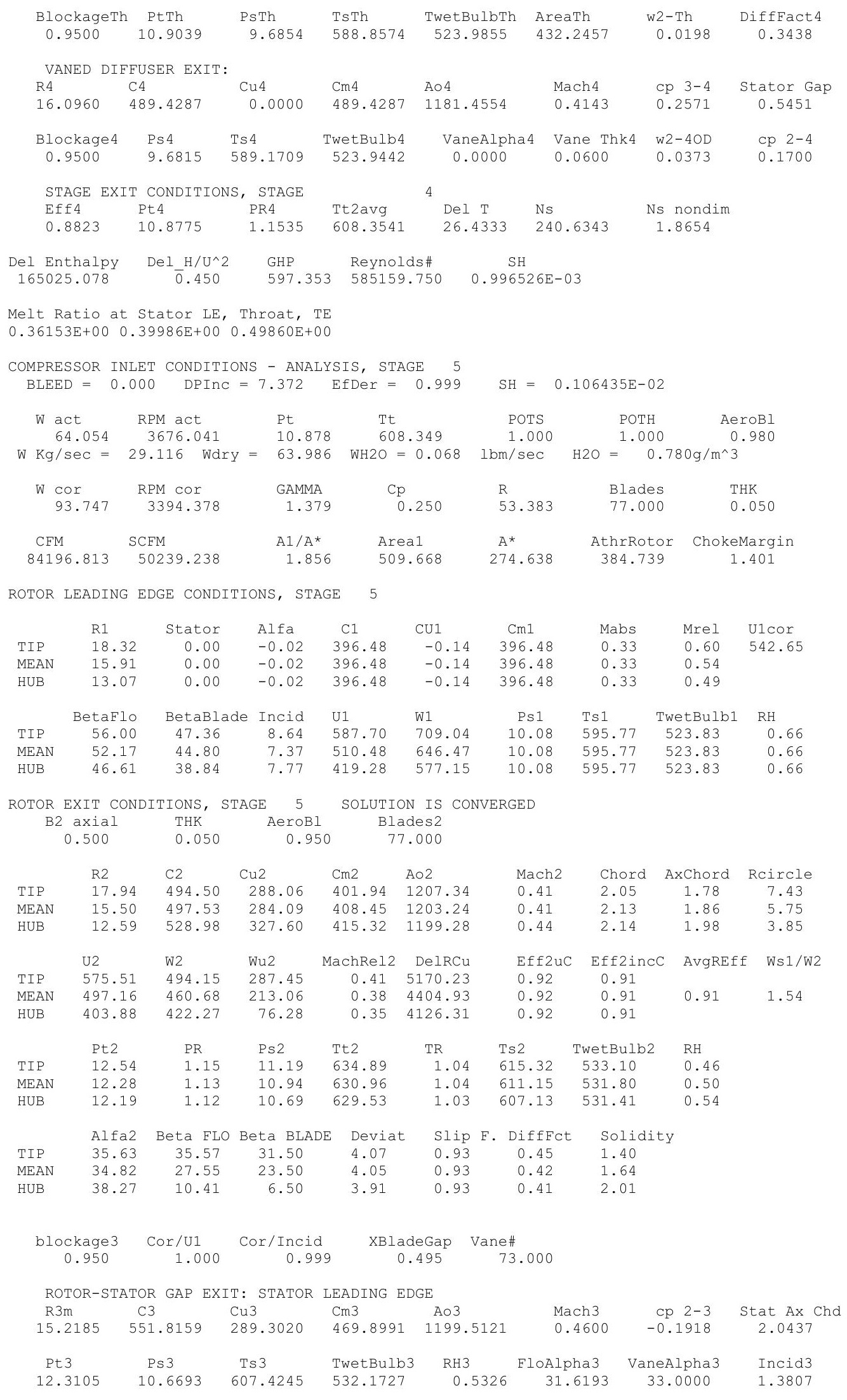




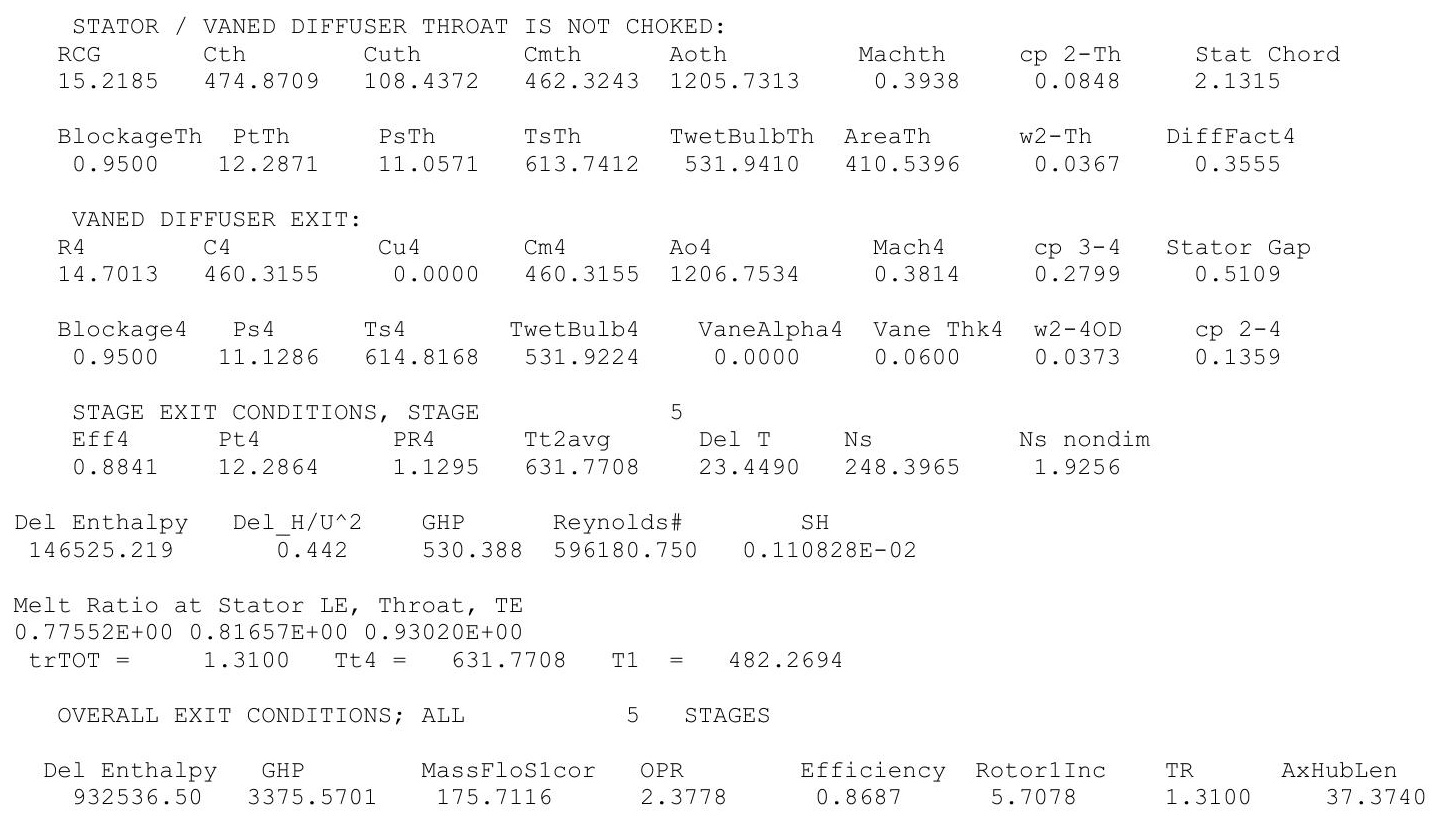




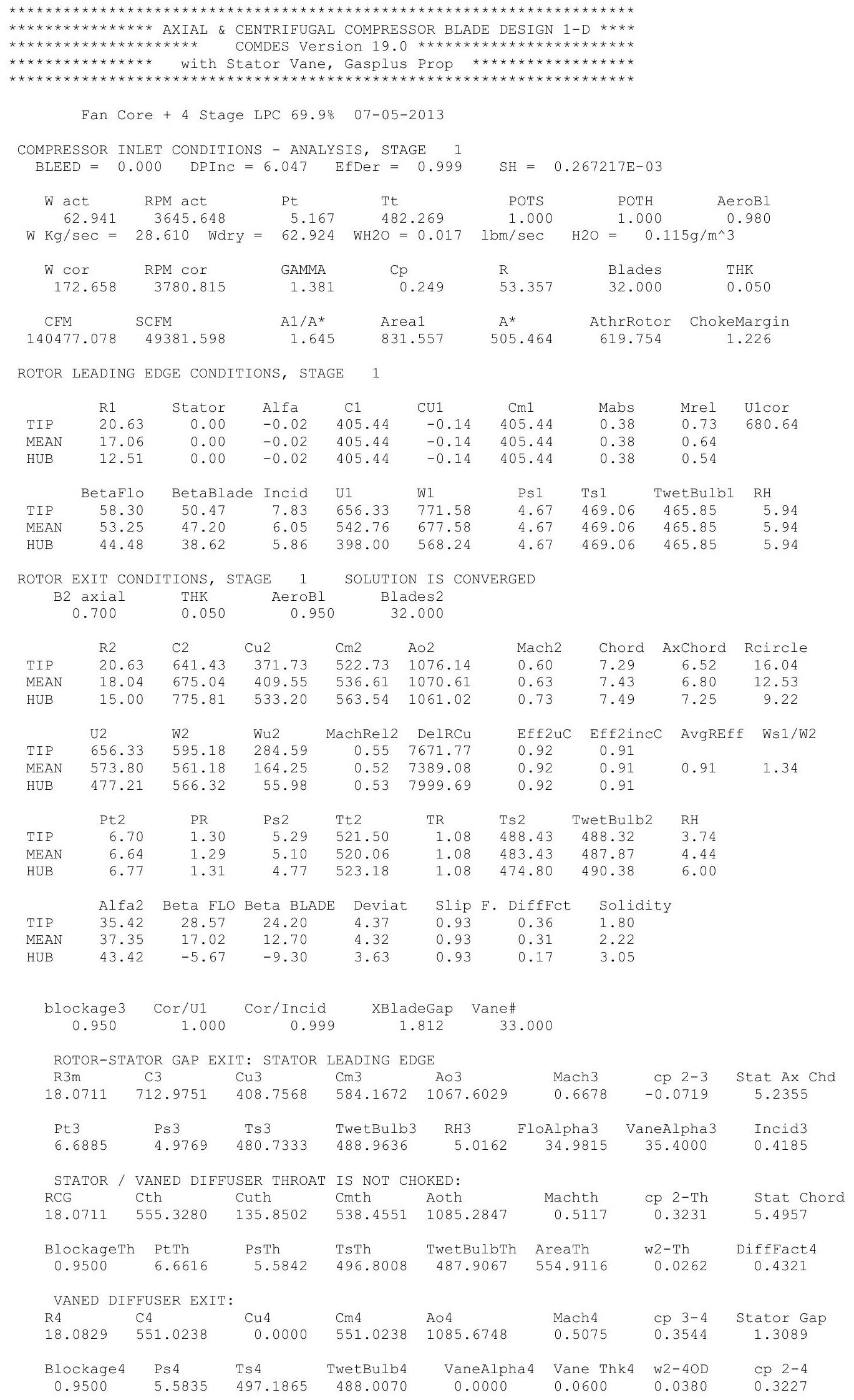




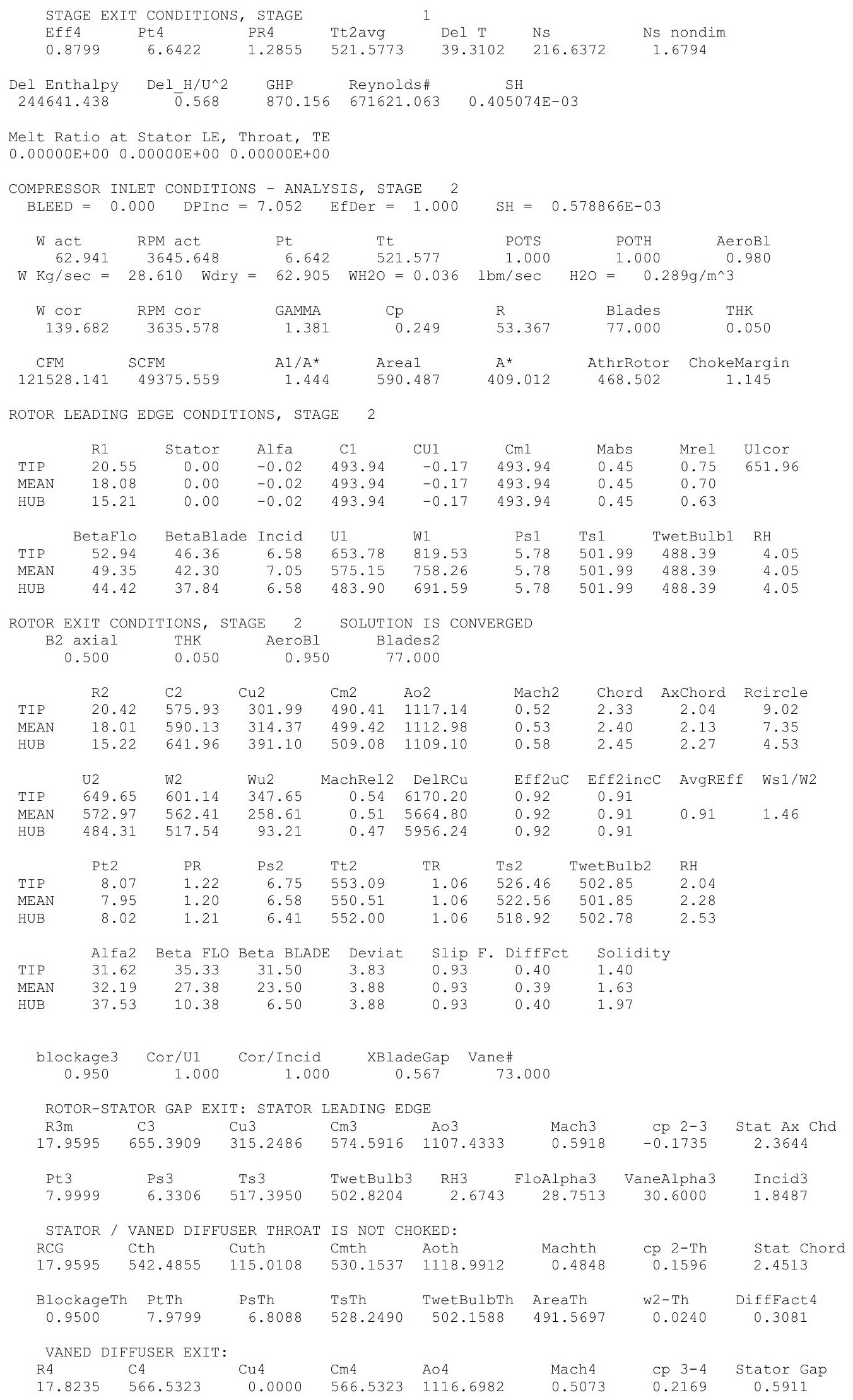




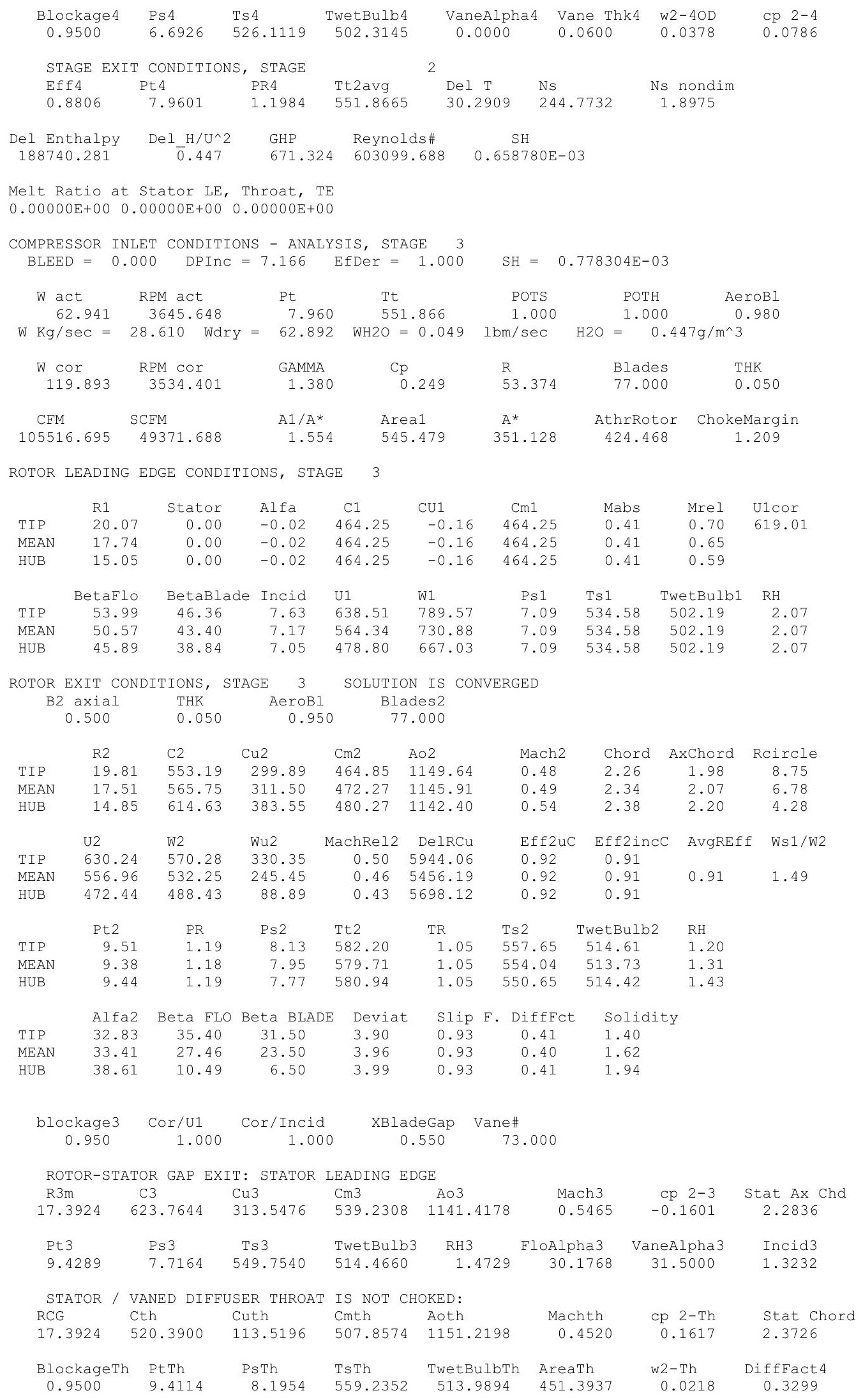




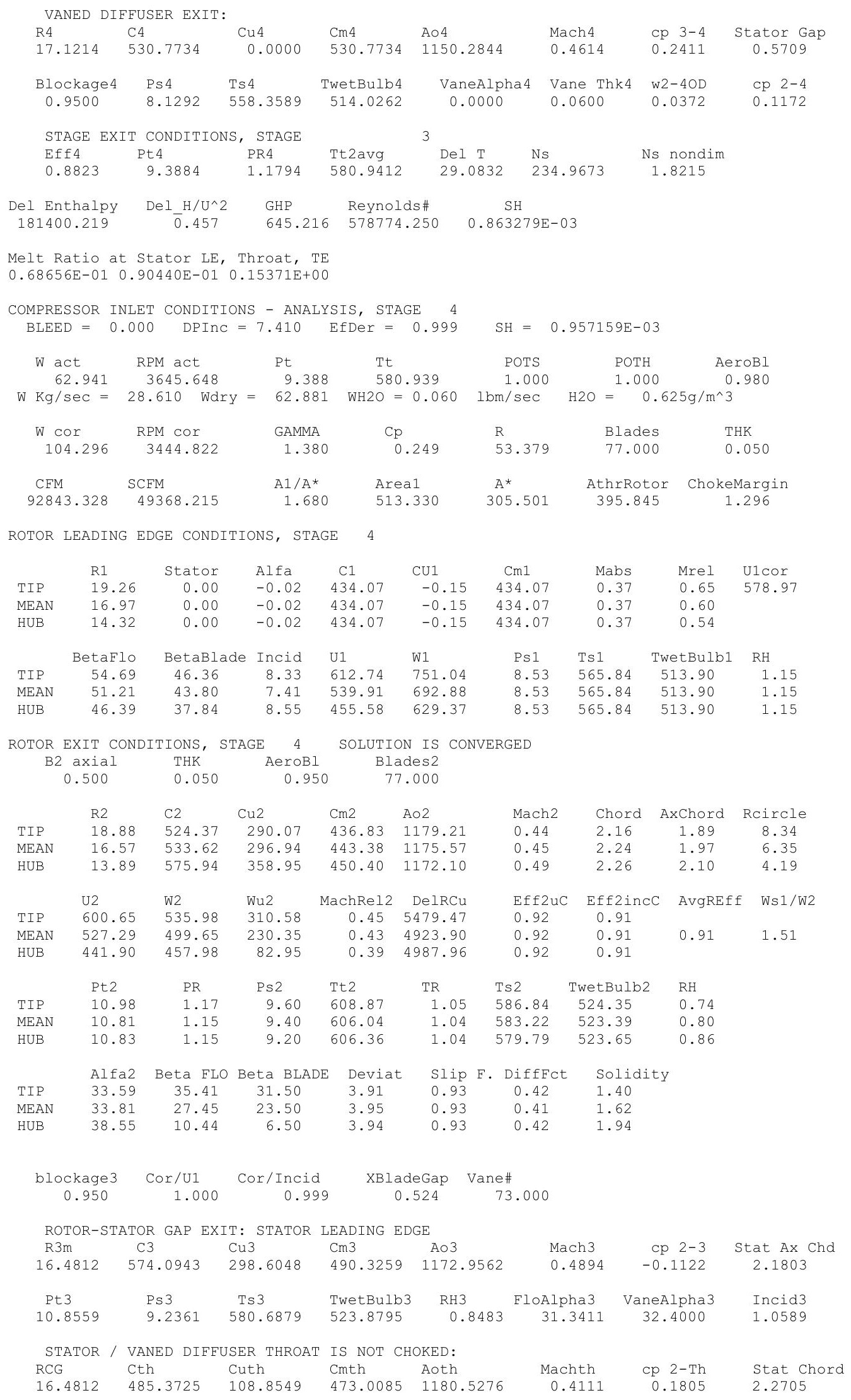




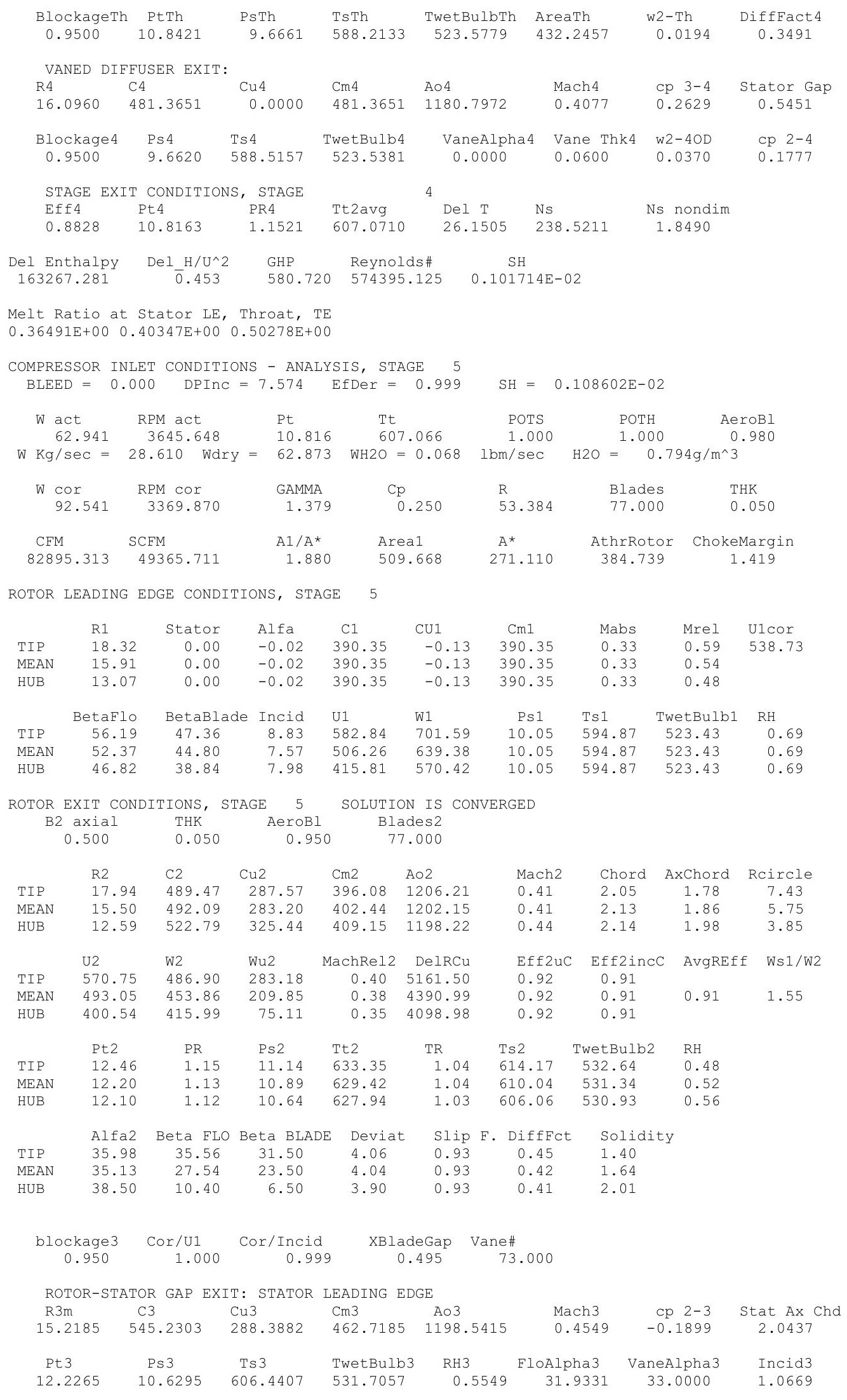




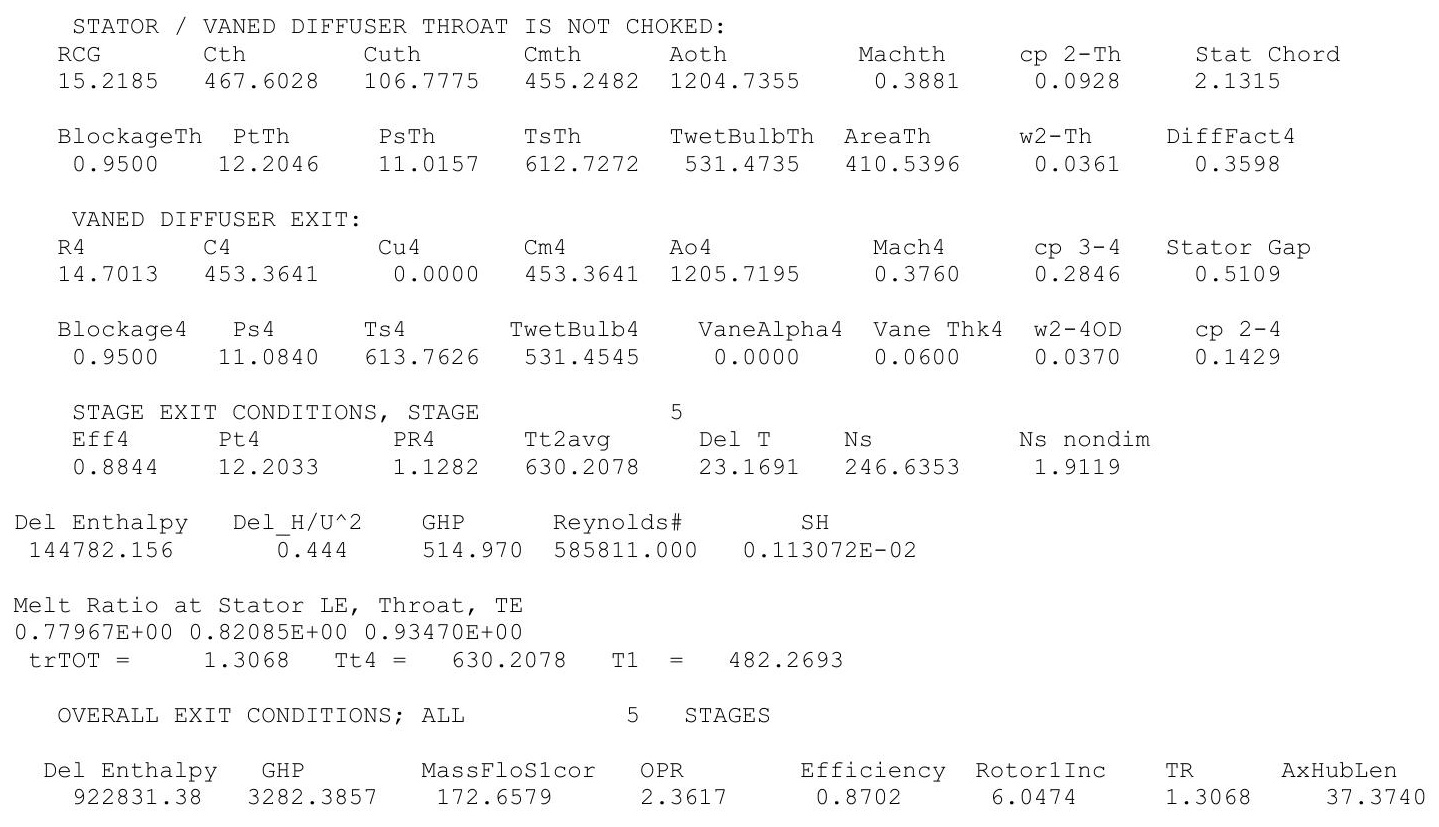




\section{$25 \mu \mathrm{m}, \mathrm{ISA}+18 \mathrm{R}$}

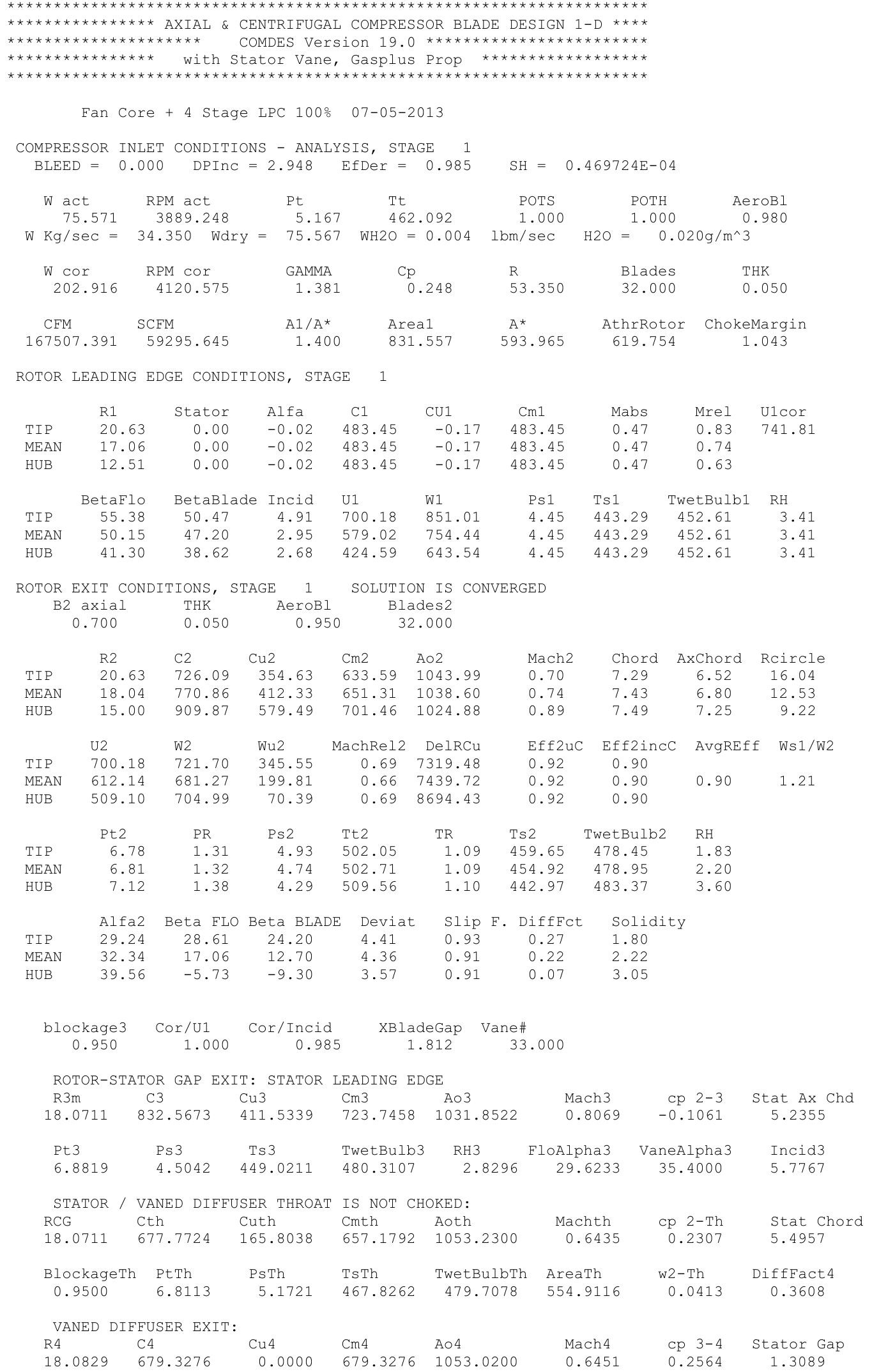




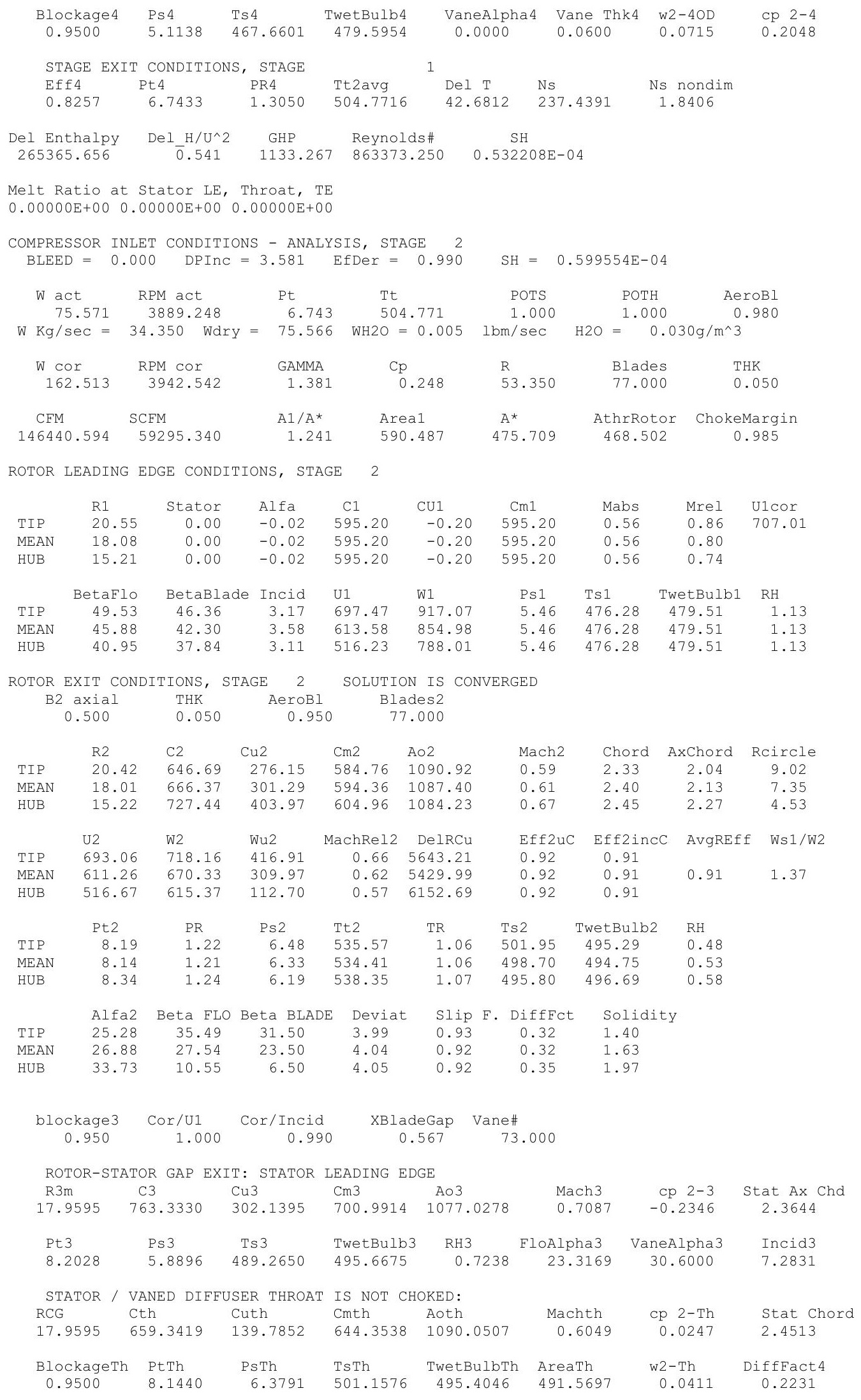




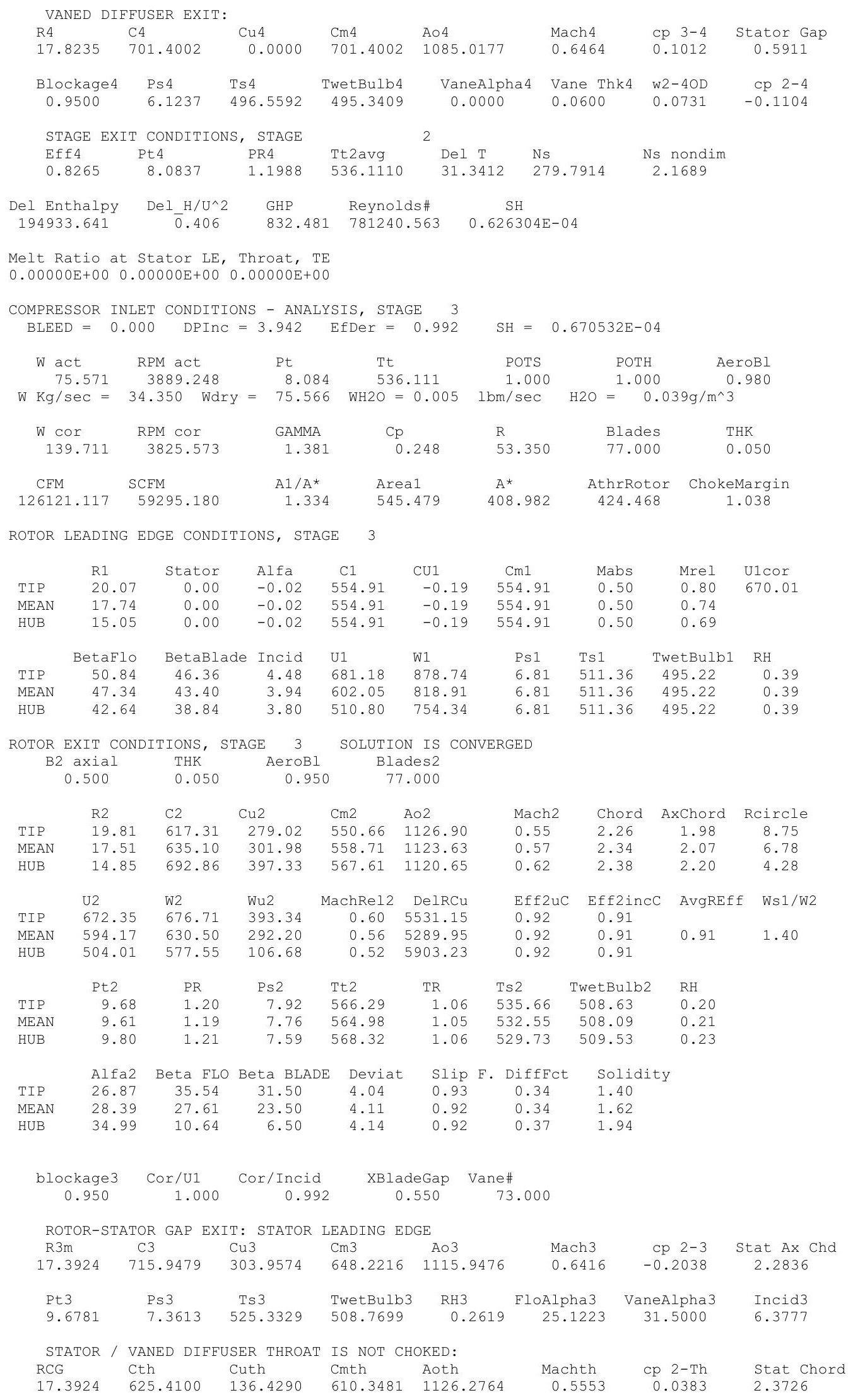




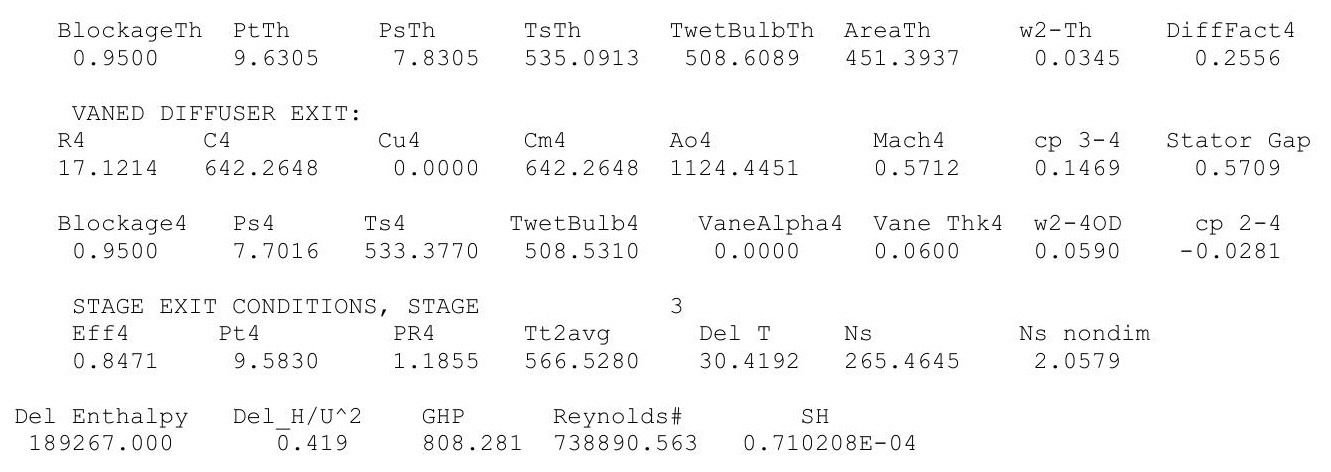

Melt Ratio at Stator LE, Throat, TE

$0.00000 \mathrm{E}+00 \quad 0.00000 \mathrm{E}+00 \quad 0.00000 \mathrm{E}+00$

COMPRESSOR INLET CONDITIONS - ANALYSIS, STAGE 4 $\mathrm{BLEED}=0.000 \quad$ DPInC $=4.513 \quad$ EfDer $=0.995$

$\mathrm{SH}=0.784810 \mathrm{E}-04$

$\begin{array}{llllll}\text { W act } & \text { RPM act } & \text { Pt } & \text { POTS } & \text { POTH } & \text { AeroBI }\end{array}$ $\mathrm{W} \mathrm{Kg} / \mathrm{sec}=34.350$ Wdry $=75.565 \quad \mathrm{WH} 2 \mathrm{O}=0.006 \mathrm{lbm} / \mathrm{sec} \quad \mathrm{H} 2 \mathrm{O}=0.052 \mathrm{~g} / \mathrm{m}^{\wedge} 3$

$\begin{array}{ccccccc}\text { W cor } & \text { RPM cor } & \text { GAMMA } & \text { CP } & \text { R } & \text { Blades } & \text { THK } \\ 121.151 & 3721.458 & 1.381 & 0.249 & 53.351 & 77.000 & 0.050 \\ & & & & & & \\ \text { CFM } & \text { SCFM } & \text { A1/A* } & \text { Area1 } & A^{*} & \text { AthrRotor } & \text { ChokeMargin } \\ 109747.586 & 59294.914 & 1.447 & 513.330 & 354.670 & 395.845 & 1.116\end{array}$

ROTOR LEADING EDGE CONDITIONS, STAGE 4

$\begin{array}{lccccccccc} & \text { R1 } & \text { Stator } & \text { Alfa } & \text { C1 } & \text { CU1 } & \text { Cm1 } & \text { Mabs } & \text { Mrel } & \text { U1cor } \\ \text { TIP } & 19.26 & 0.00 & -0.02 & 513.11 & -0.18 & 513.11 & 0.45 & 0.73 & 625.47 \\ \text { MEAN } & 16.97 & 0.00 & -0.02 & 513.11 & -0.18 & 513.11 & 0.45 & 0.68 & \\ \text { HUB } & 14.32 & 0.00 & -0.02 & 513.11 & -0.18 & 513.11 & 0.45 & 0.62 & \\ & & & & & & & & & \\ & \text { BetaFlo } & \text { BetaBlade } & \text { Incid } & \text { U1 } & \text { W1 } & \text { Ps1 } & \text { Ts1 } & \text { TwetBulb1 } & \text { RH } \\ \text { TIP } & 51.88 & 46.36 & 5.52 & 653.69 & 831.15 & 8.35 & 545.37 & 508.48 & 0.17 \\ \text { MEAN } & 48.31 & 43.80 & 4.51 & 575.99 & 771.52 & 8.35 & 545.37 & 508.48 & 0.17 \\ \text { HUB } & 43.46 & 37.84 & 5.62 & 486.02 & 706.87 & 8.35 & 545.37 & 508.48 & 0.17\end{array}$

ROTOR EXIT CONDITIONS, STAGE 4 SOLUTION IS CONVERGED

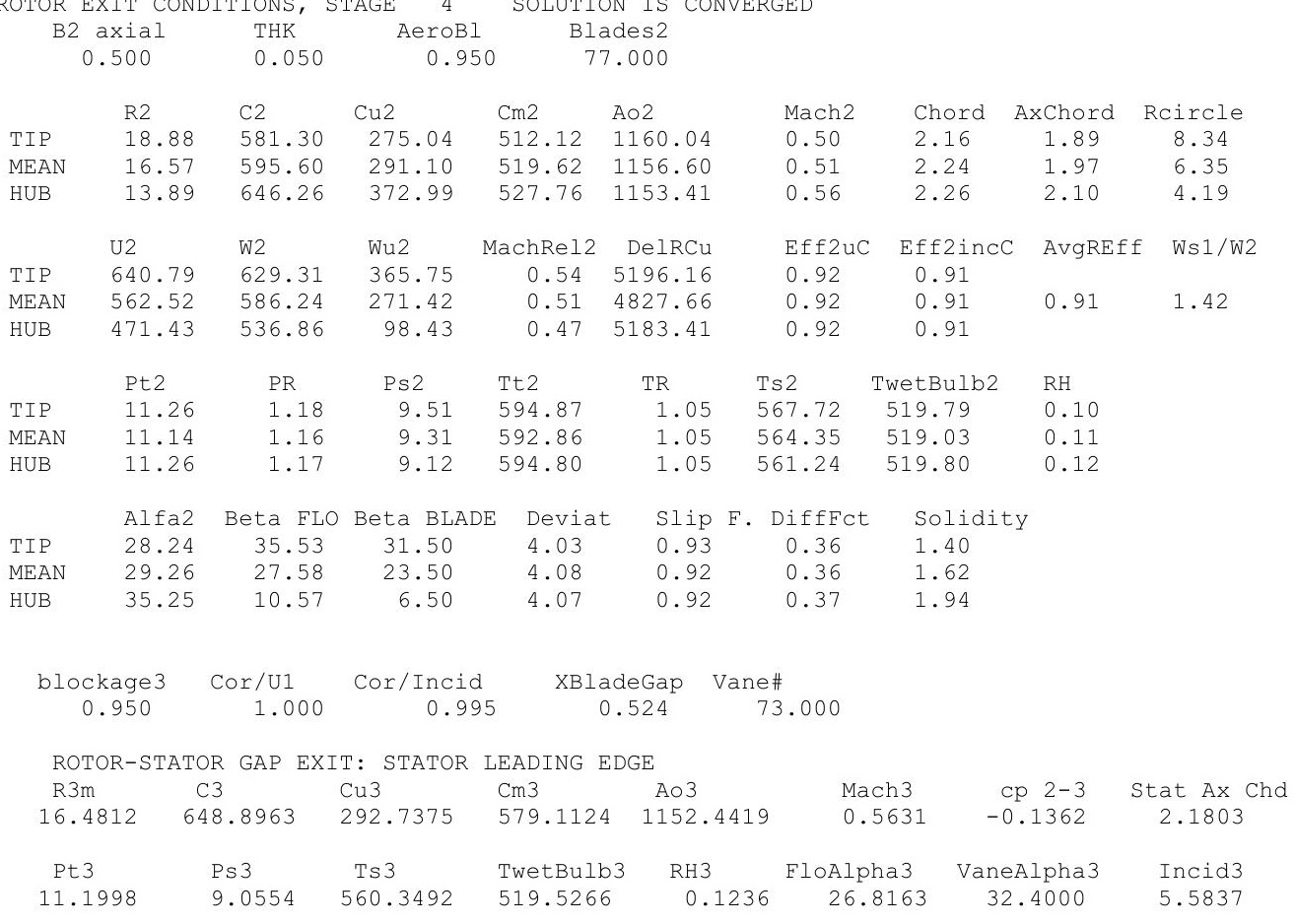




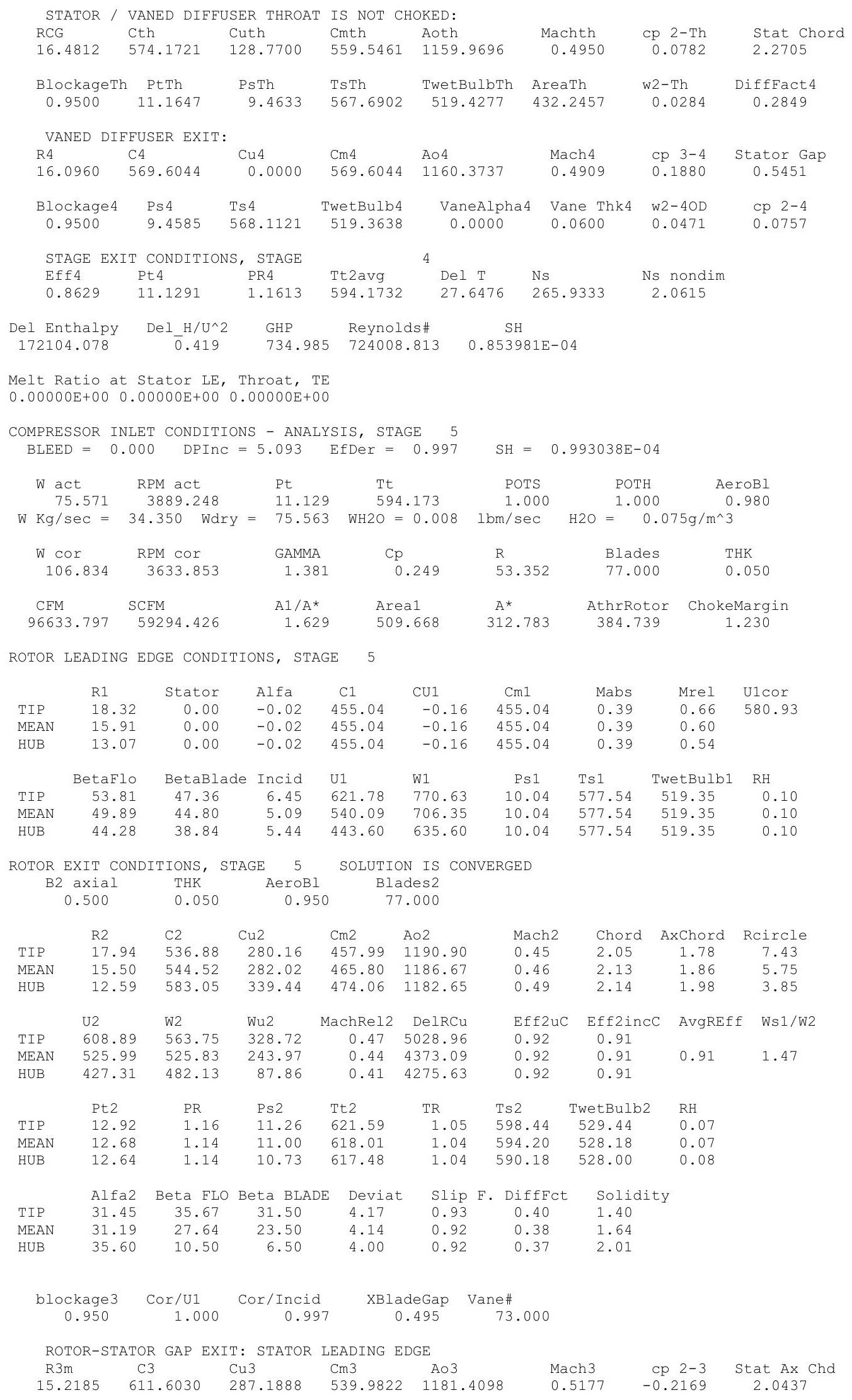




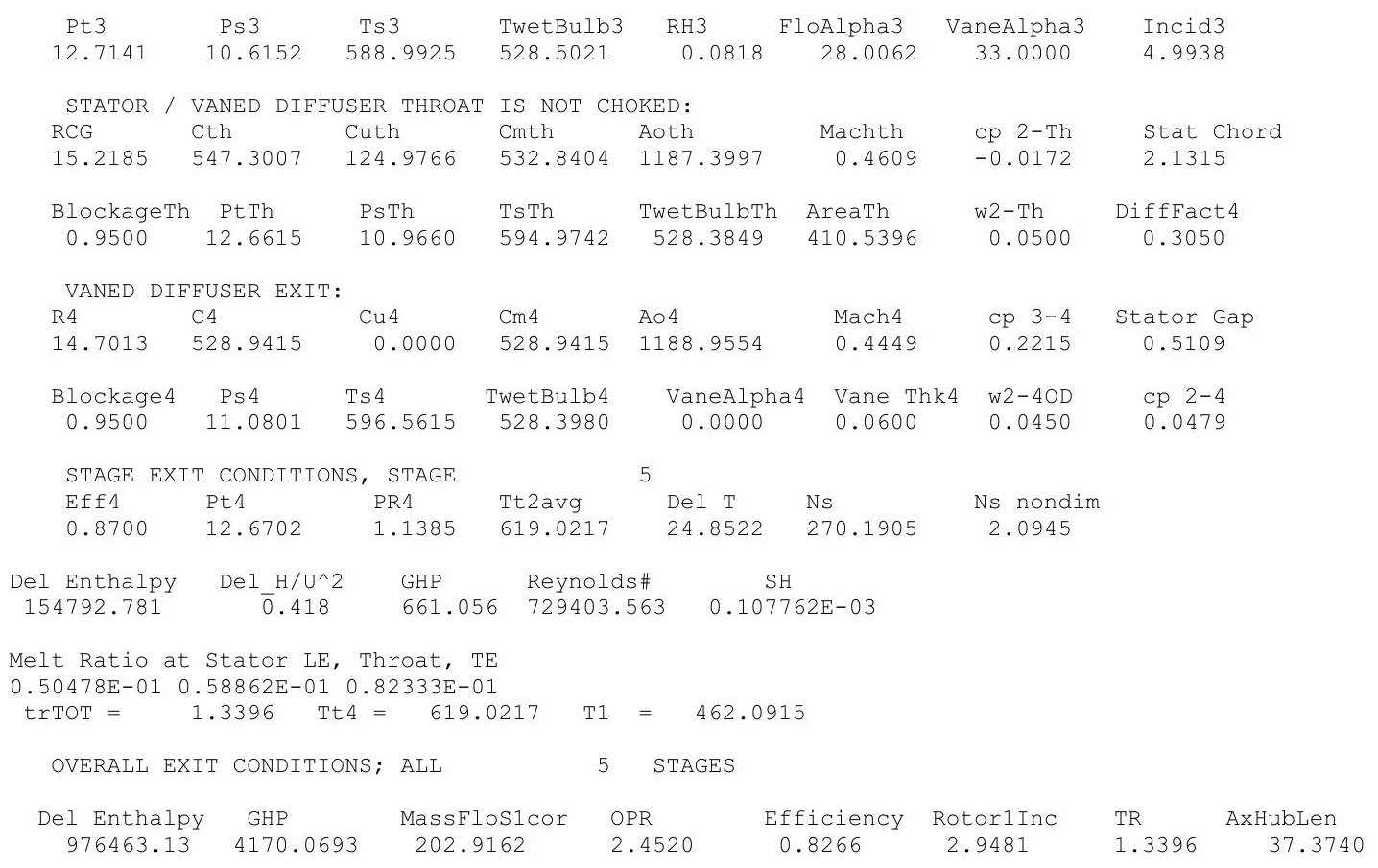




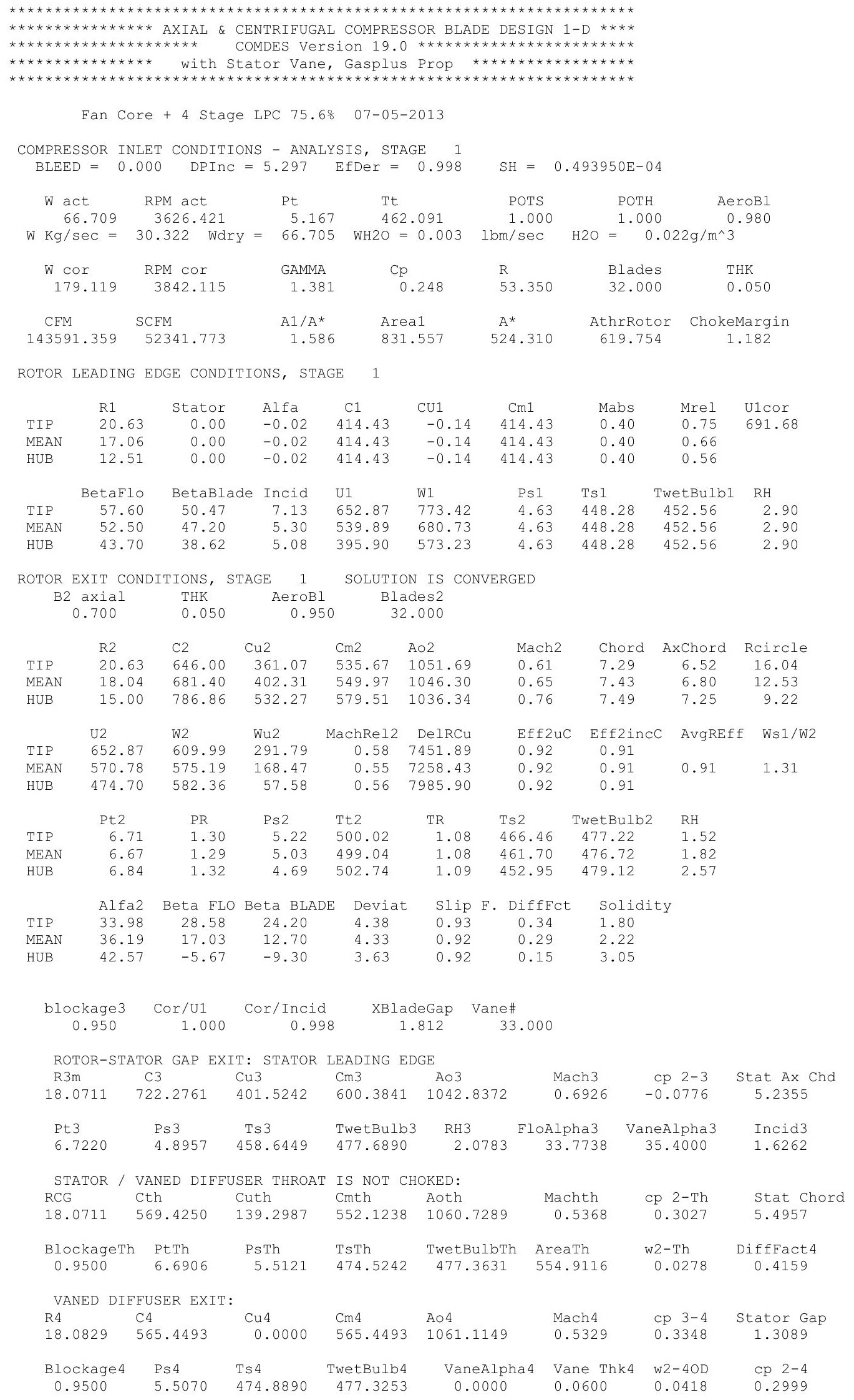




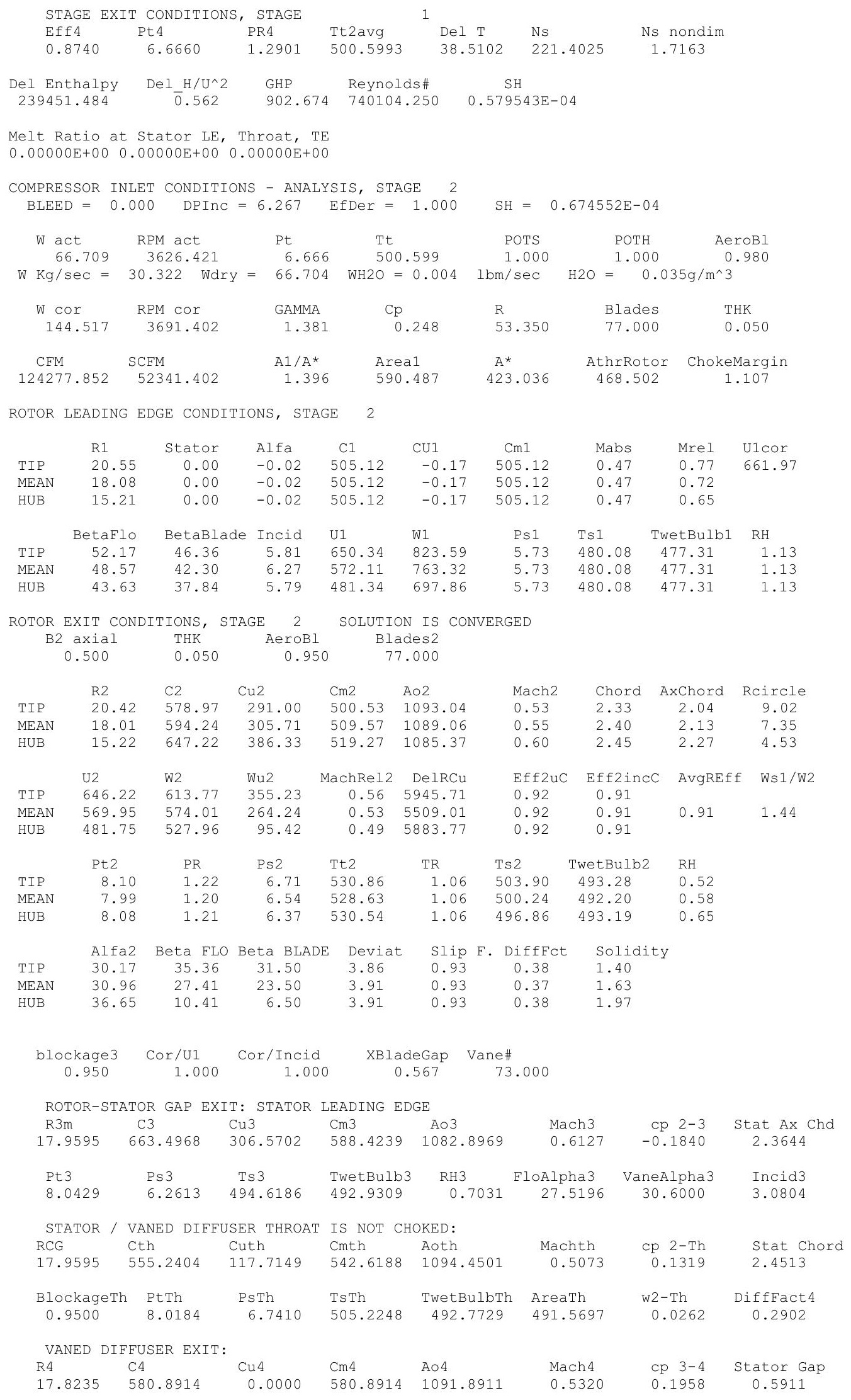




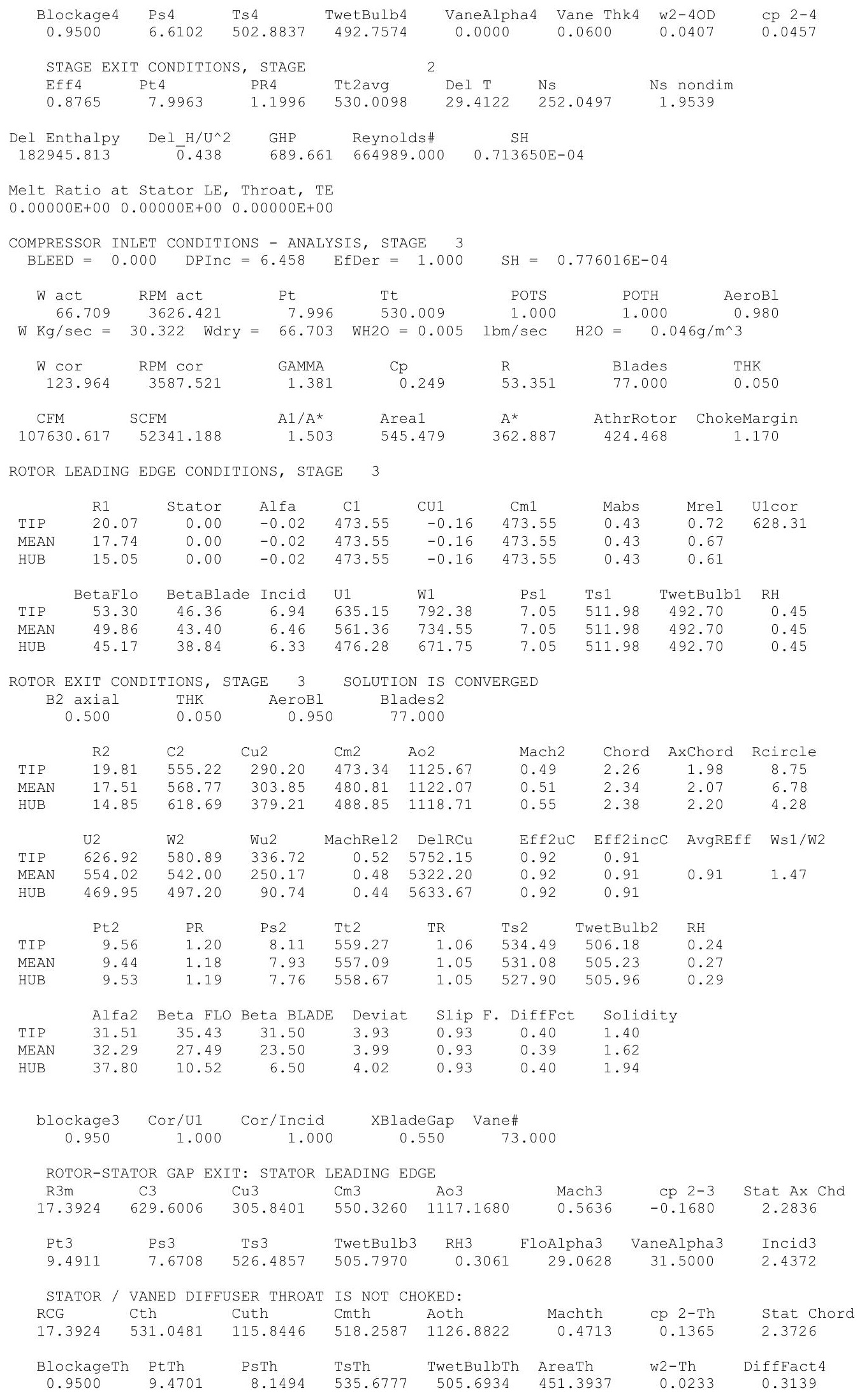




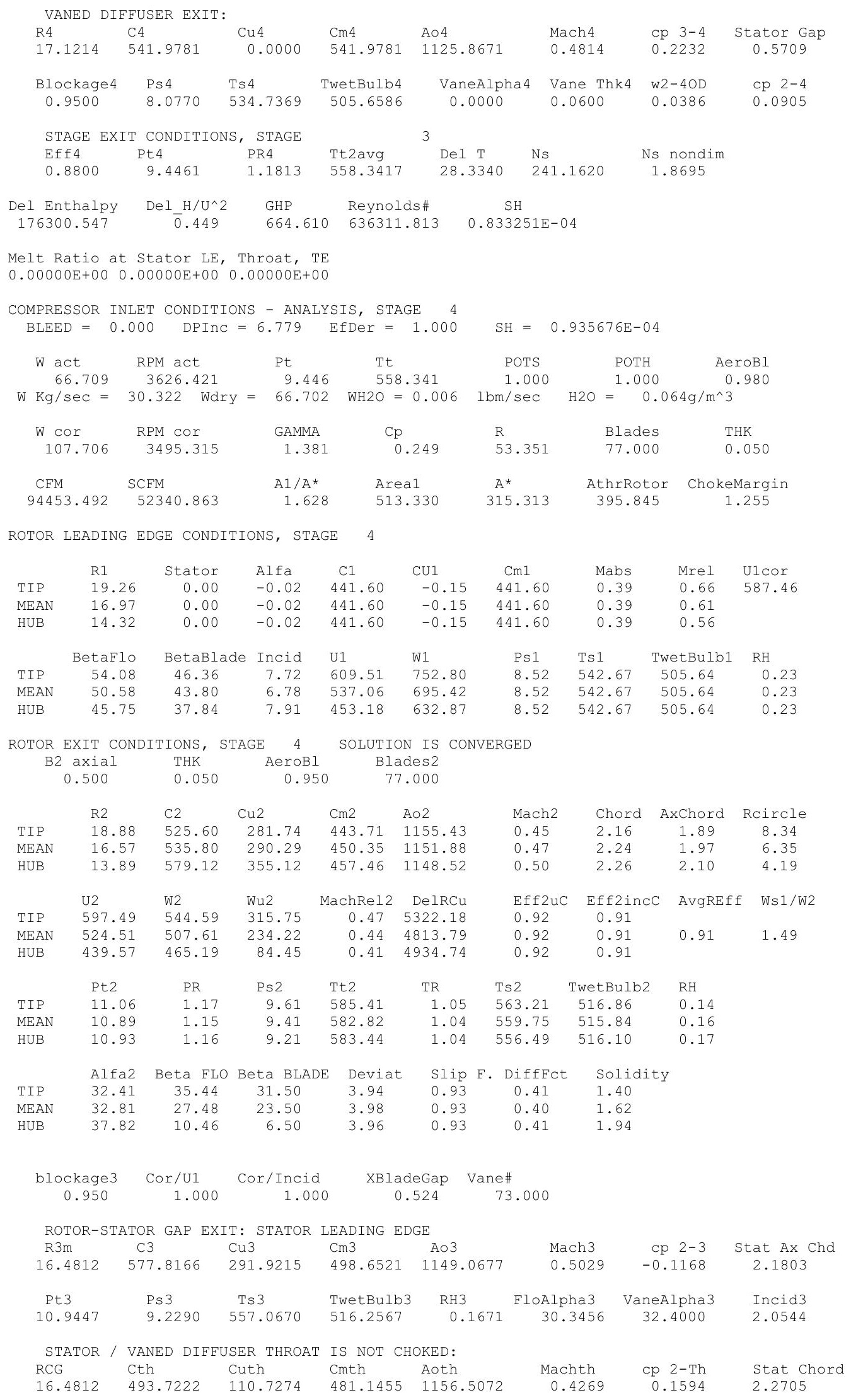




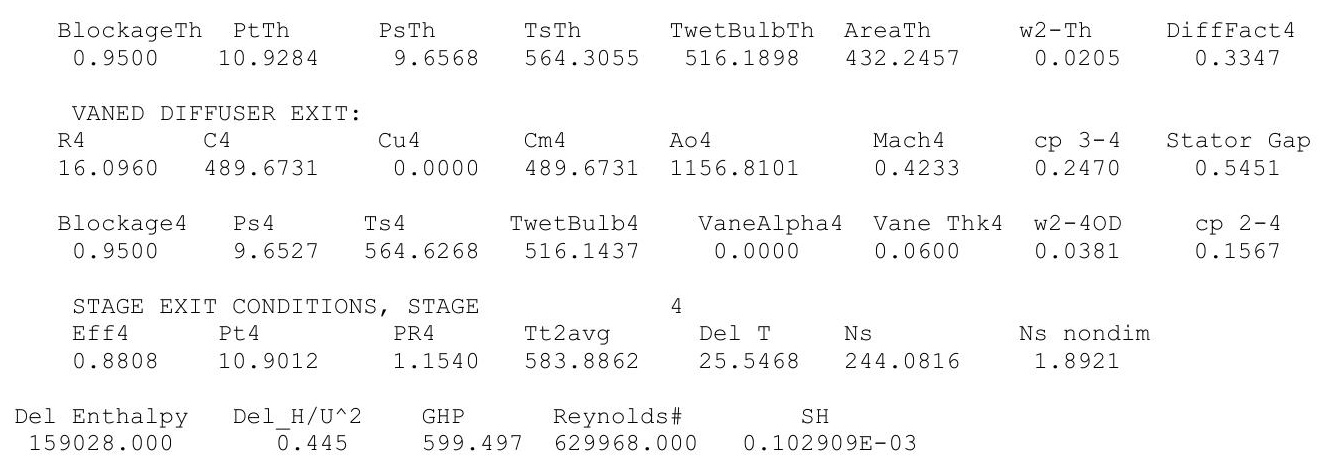

Melt Ratio at Stator LE, Throat, TE

$0.00000 \mathrm{E}+00 \quad 0.00000 \mathrm{E}+00 \quad 0.92996 \mathrm{E}-02$

COMPRESSOR INLET CONDITIONS - ANALYSIS, STAGE 5 $\mathrm{BLEED}=0.000 \quad$ DPInC $=7.022 \quad$ EfDer $=1.000$

66.709 RPM act Pt Tt

$\mathrm{SH}=0.118128 \mathrm{E}-03$ $\mathrm{W} \mathrm{Kg} / \mathrm{sec}=30.322 \mathrm{Wdry}=66.701 \quad \mathrm{WH} 2 \mathrm{O}=0.008 \quad \mathrm{lbm} / \mathrm{sec} \quad \mathrm{H} 2 \mathrm{O}=0.090 \mathrm{~g} / \mathrm{m}^{\wedge} 3$

$\begin{array}{ccccccc}\text { W Cor } & \text { RPM cor } & \text { GAMMA } & \text { Cp } & \text { R } & \text { Blades } & \text { THK } \\ 95.440 & 3418.001 & 1.381 & 0.249 & 53.352 & 77.000 & 0.050 \\ & & & & & & \\ \text { CFM } & \text { SCFM } & \text { A1/A* } & \text { Area1 } & A^{*} & \text { AthrRotor } & \text { ChokeMargin } \\ 84113.250 & 52340.363 & 1.824 & 509.668 & 279.425 & 384.739 & 1.377\end{array}$

ROTOR LEADING EDGE CONDITIONS, STAGE 5

$\begin{array}{lccccccccc} & \text { R1 } & \text { Stator } & \text { Alfa } & \text { C1 } & \text { CU1 } & \text { Cm1 } & \text { Mabs } & \text { Mrel } & \text { U1cor } \\ \text { TIP } & 18.32 & 0.00 & -0.02 & 396.08 & -0.14 & 396.08 & 0.34 & 0.60 & 546.43 \\ \text { MEAN } & 15.91 & 0.00 & -0.02 & 396.08 & -0.14 & 396.08 & 0.34 & 0.55 & \\ \text { HUB } & 13.07 & 0.00 & -0.02 & 396.08 & -0.14 & 396.08 & 0.34 & 0.49 & \\ & & & & & & & & & \\ & \text { BetaFlo } & \text { BetaBlade } & \text { Incid } & \text { U1 } & \text { W1 } & \text { Ps1 } & \text { Ts1 } & \text { TwetBulb1 } & \text { RH } \\ \text { TIP } & 55.67 & 47.36 & 8.31 & 579.76 & 702.26 & 10.07 & 571.29 & 516.14 & 0.14 \\ \text { MEAN } & 51.82 & 44.80 & 7.02 & 503.59 & 640.80 & 10.07 & 571.29 & 516.14 & 0.14 \\ \text { HUB } & 46.25 & 38.84 & 7.41 & 413.62 & 572.78 & 10.07 & 571.29 & 516.14 & 0.14\end{array}$

ROTOR EXIT CONDITIONS, STAGE 5 SOLUTION IS CONVERGED

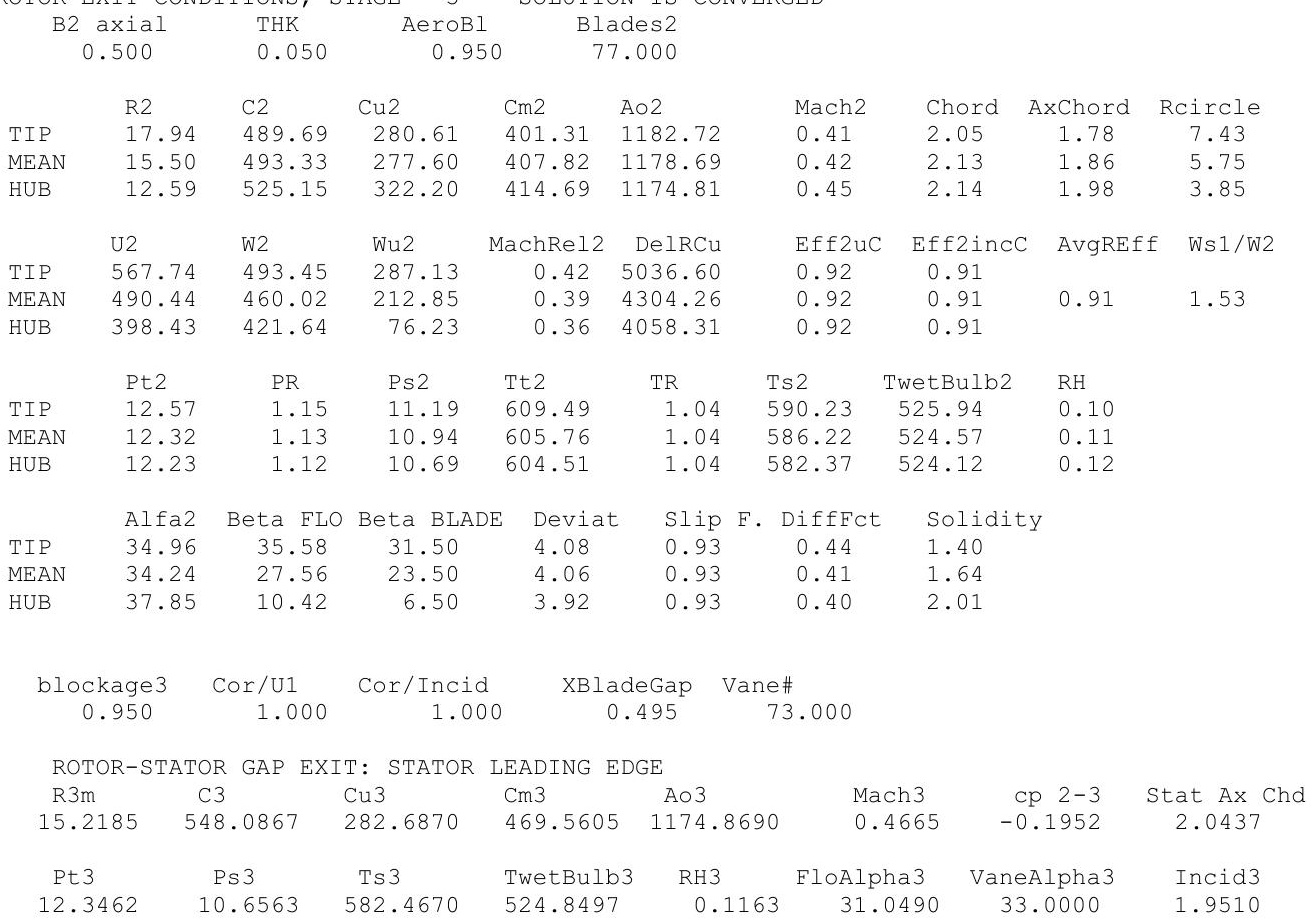




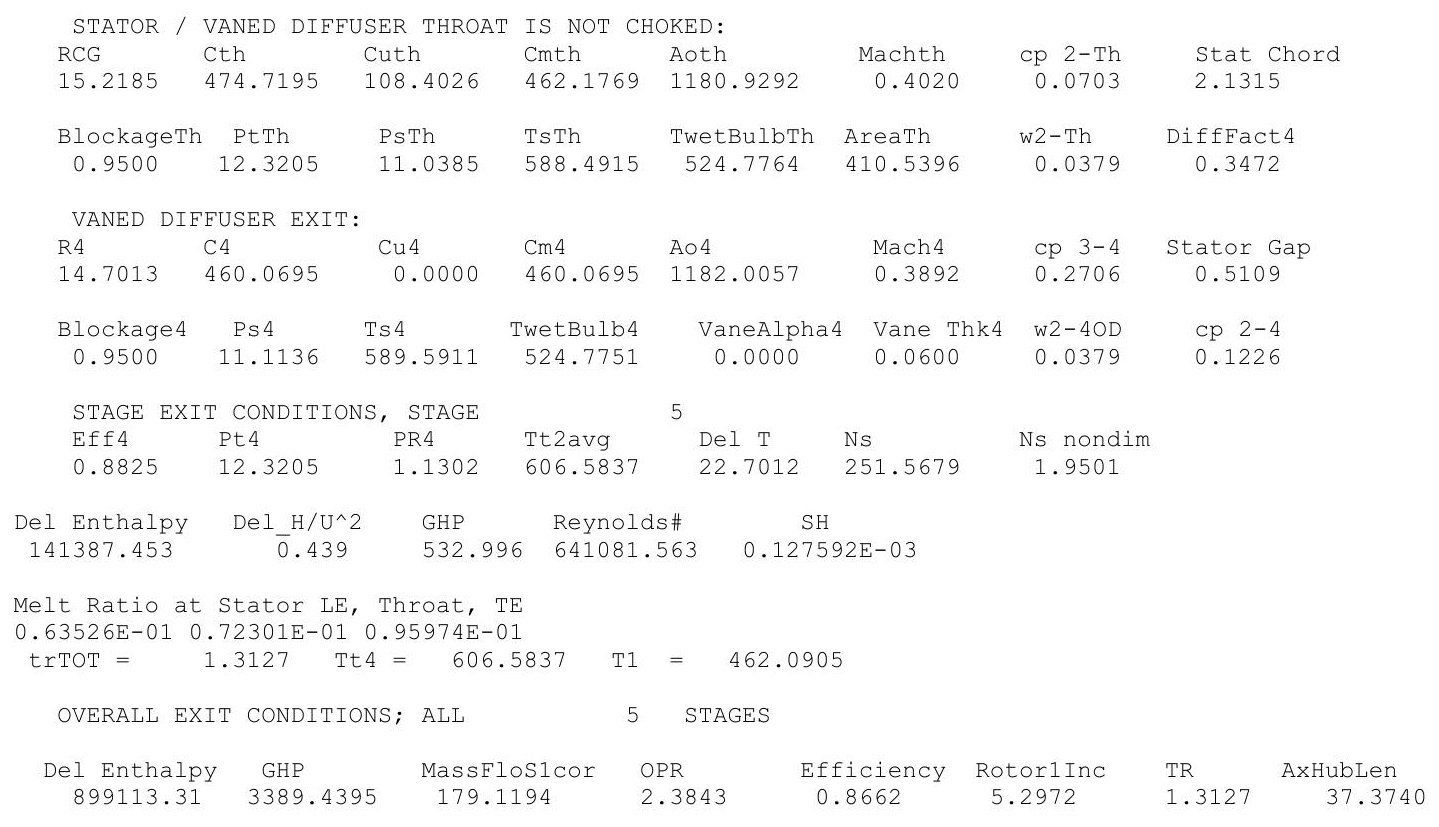




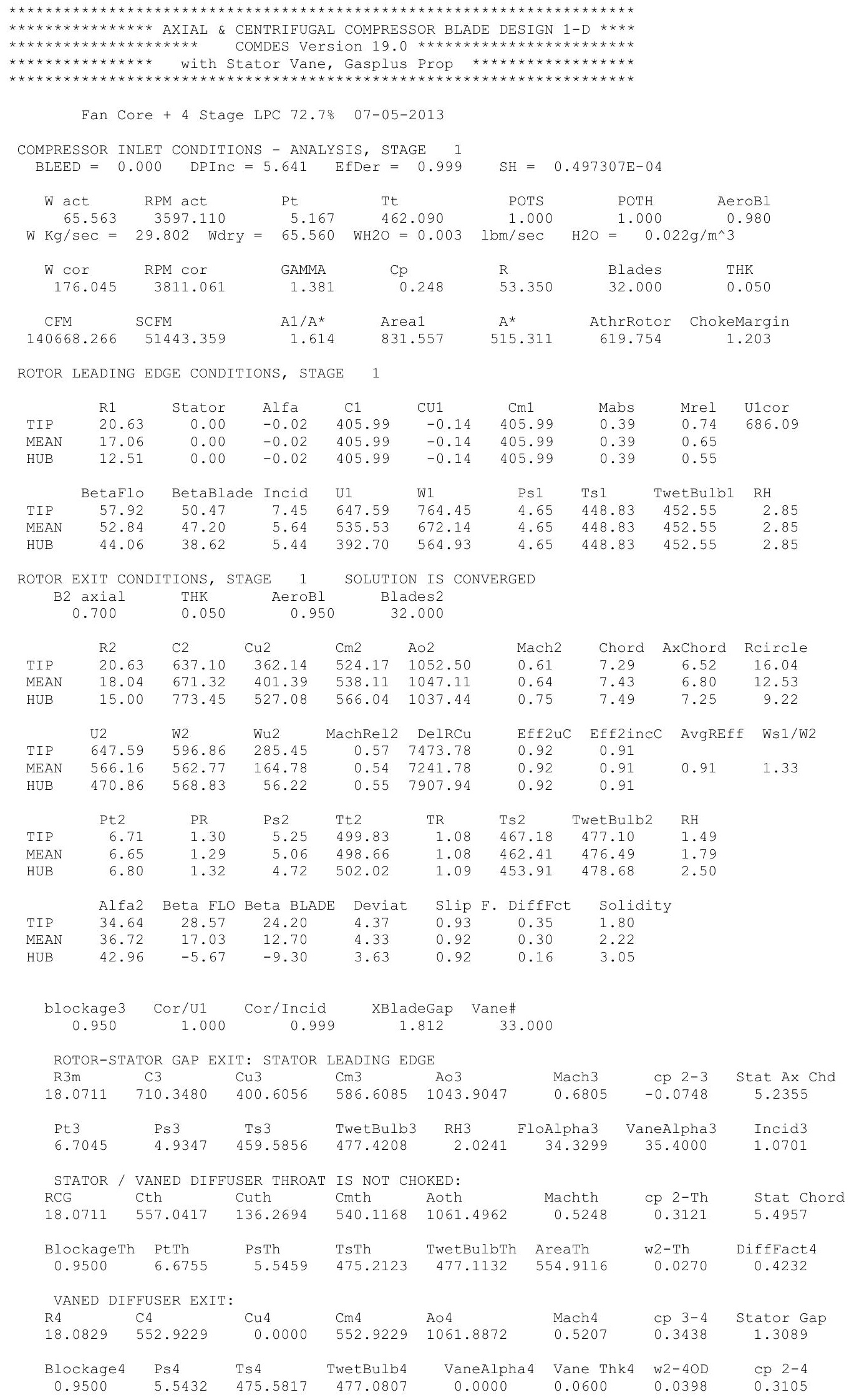




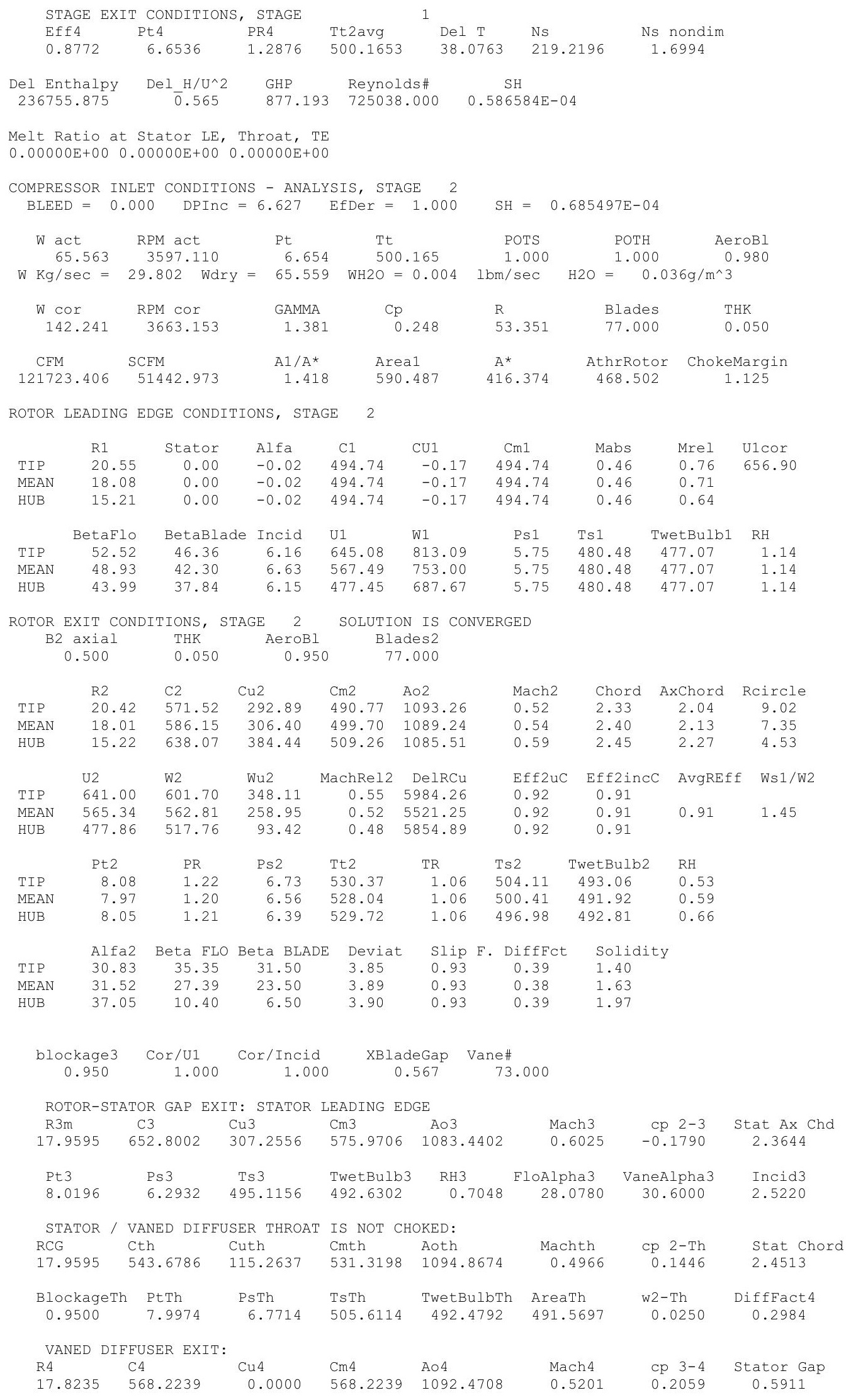




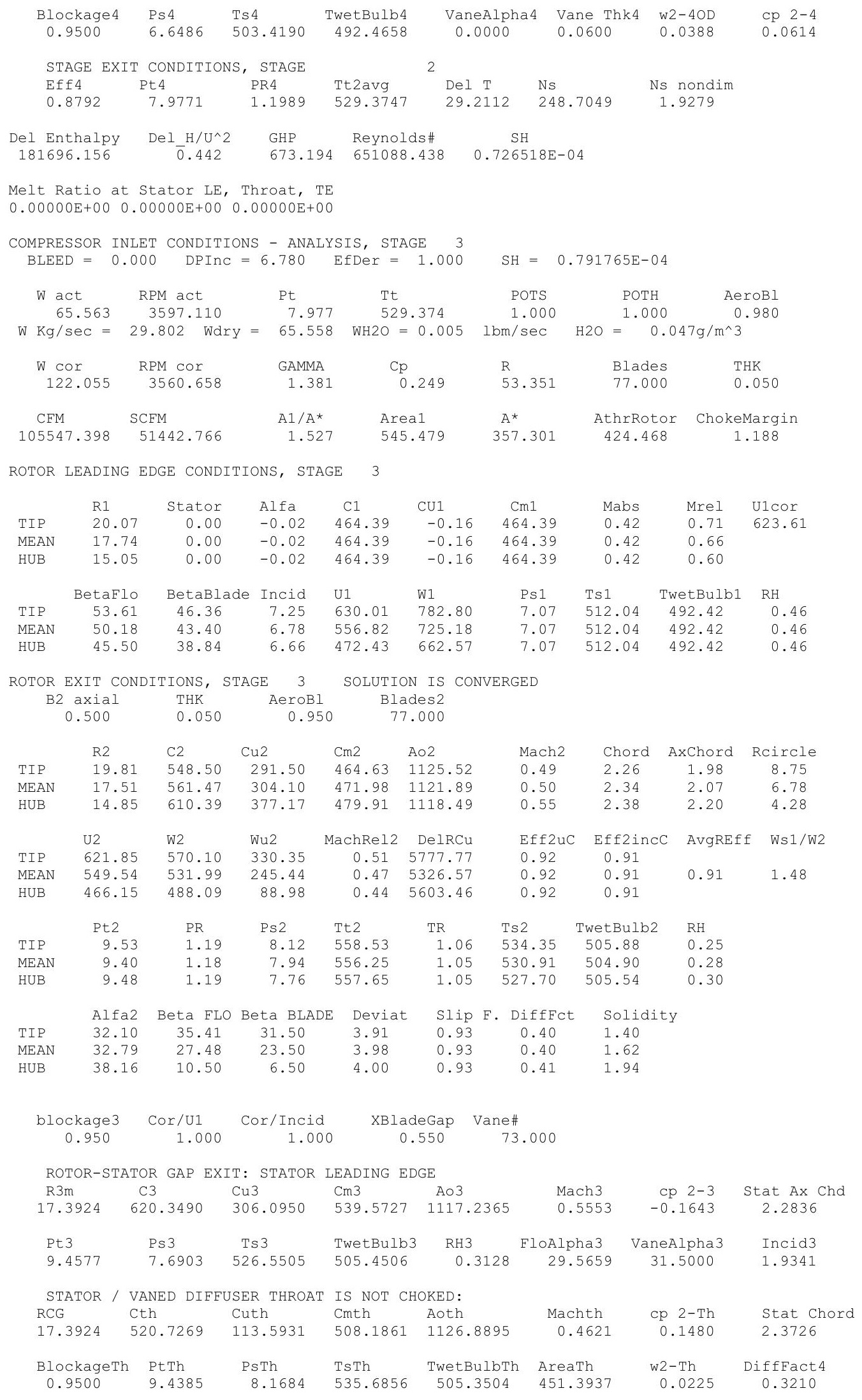




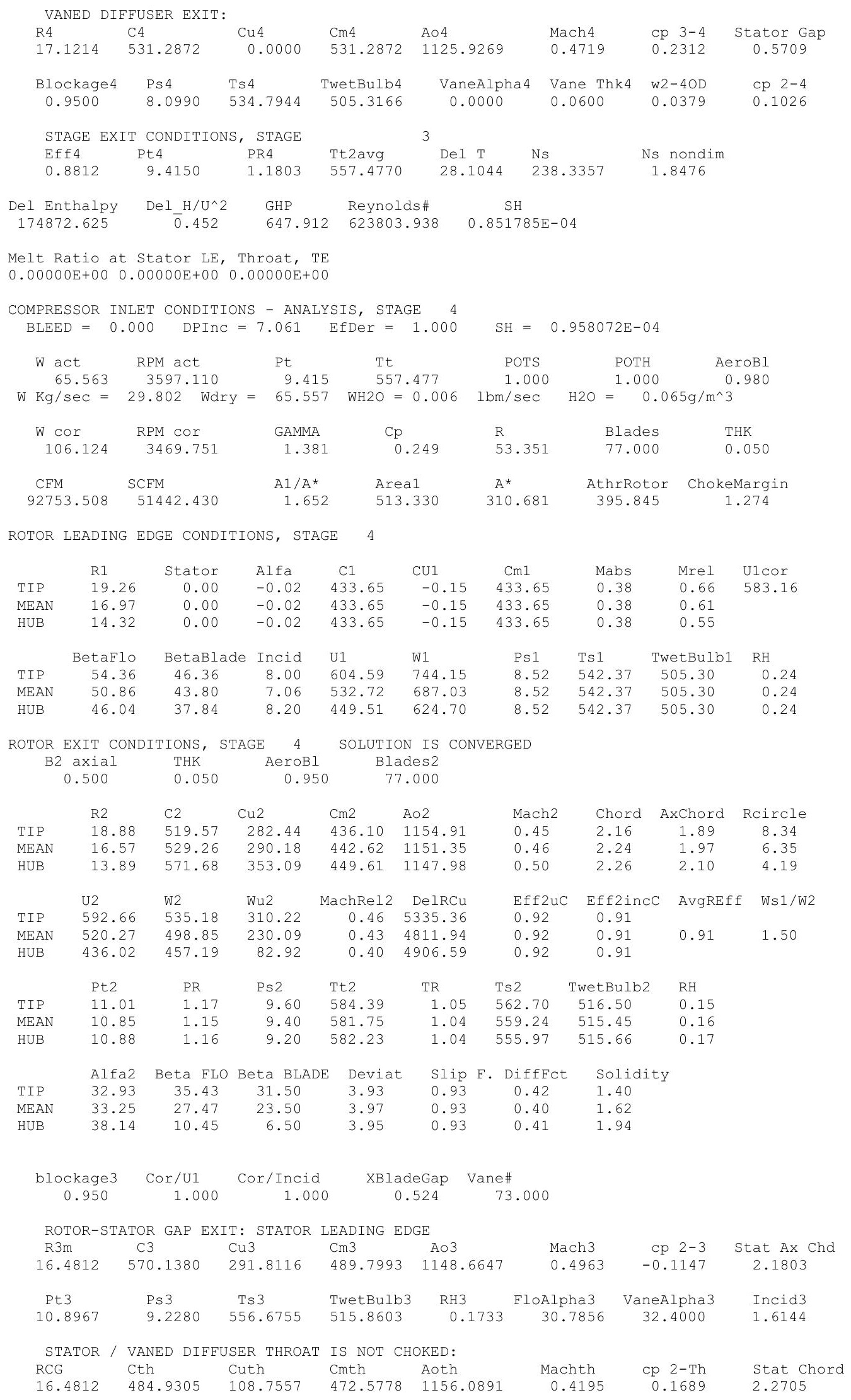




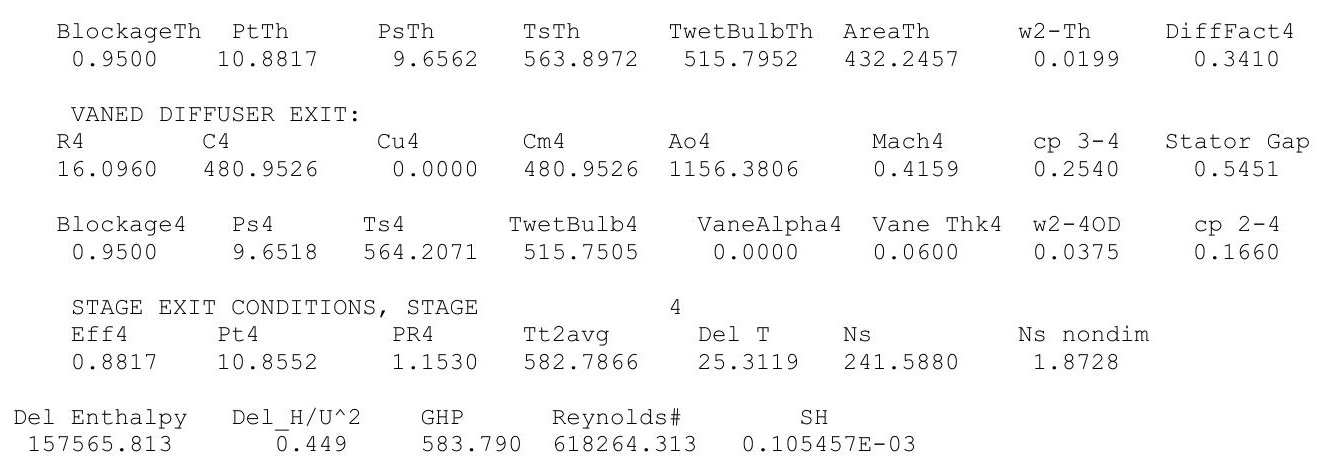

Melt Ratio at Stator LE, Throat, TE

$0.00000 \mathrm{E}+000.00000 \mathrm{E}+000.10646 \mathrm{E}-01$

COMPRESSOR INLET CONDITIONS - ANALYSIS, STAGE 5 $\mathrm{BLEED}=0.000 \quad$ DPInC $=7.263 \quad$ EfDer $=1.000$

ROTOR LEADING EDGE CONDITIONS, STAGE 5

$\begin{array}{lccccccccc} & \text { R1 } & \text { Stator } & \text { Alfa } & \text { C1 } & \text { CU1 } & \text { Cm1 } & \text { Mabs } & \text { Mrel } & \text { U1cor } \\ \text { TIP } & 18.32 & 0.00 & -0.02 & 389.49 & -0.13 & 389.49 & 0.33 & 0.60 & 542.52 \\ \text { MEAN } & 15.91 & 0.00 & -0.02 & 389.49 & -0.13 & 389.49 & 0.33 & 0.54 & \\ \text { HUB } & 13.07 & 0.00 & -0.02 & 389.49 & -0.13 & 389.49 & 0.33 & 0.49 & \\ & & & & & & & & & \\ & \text { BetaFlo } & \text { BetaBlade } & \text { Incid } & \text { U1 } & \text { W1 } & \text { Ps1 } & \text { Ts1 } & \text { TwetBulb1 } & \text { RH } \\ \text { TIP } & 55.90 & 47.36 & 8.54 & 575.08 & 694.68 & 10.05 & 570.60 & 515.75 & 0.15 \\ \text { MEAN } & 52.06 & 44.80 & 7.26 & 499.52 & 633.53 & 10.05 & 570.60 & 515.75 & 0.15 \\ \text { HUB } & 46.50 & 38.84 & 7.66 & 410.28 & 565.81 & 10.05 & 570.60 & 515.75 & 0.15\end{array}$

ROTOR EXIT CONDITIONS, STAGE 5 SOLUTION IS CONVERGED

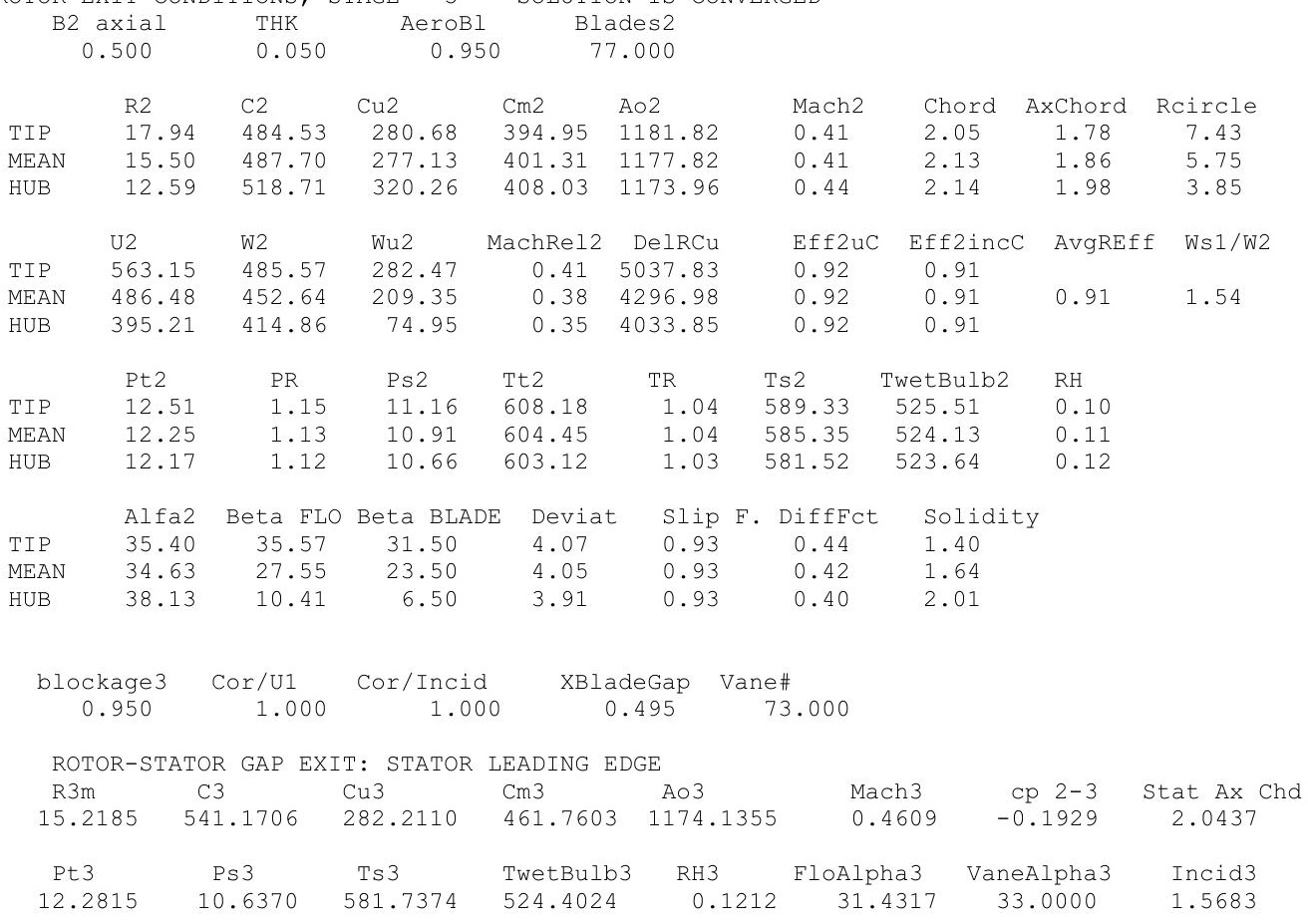




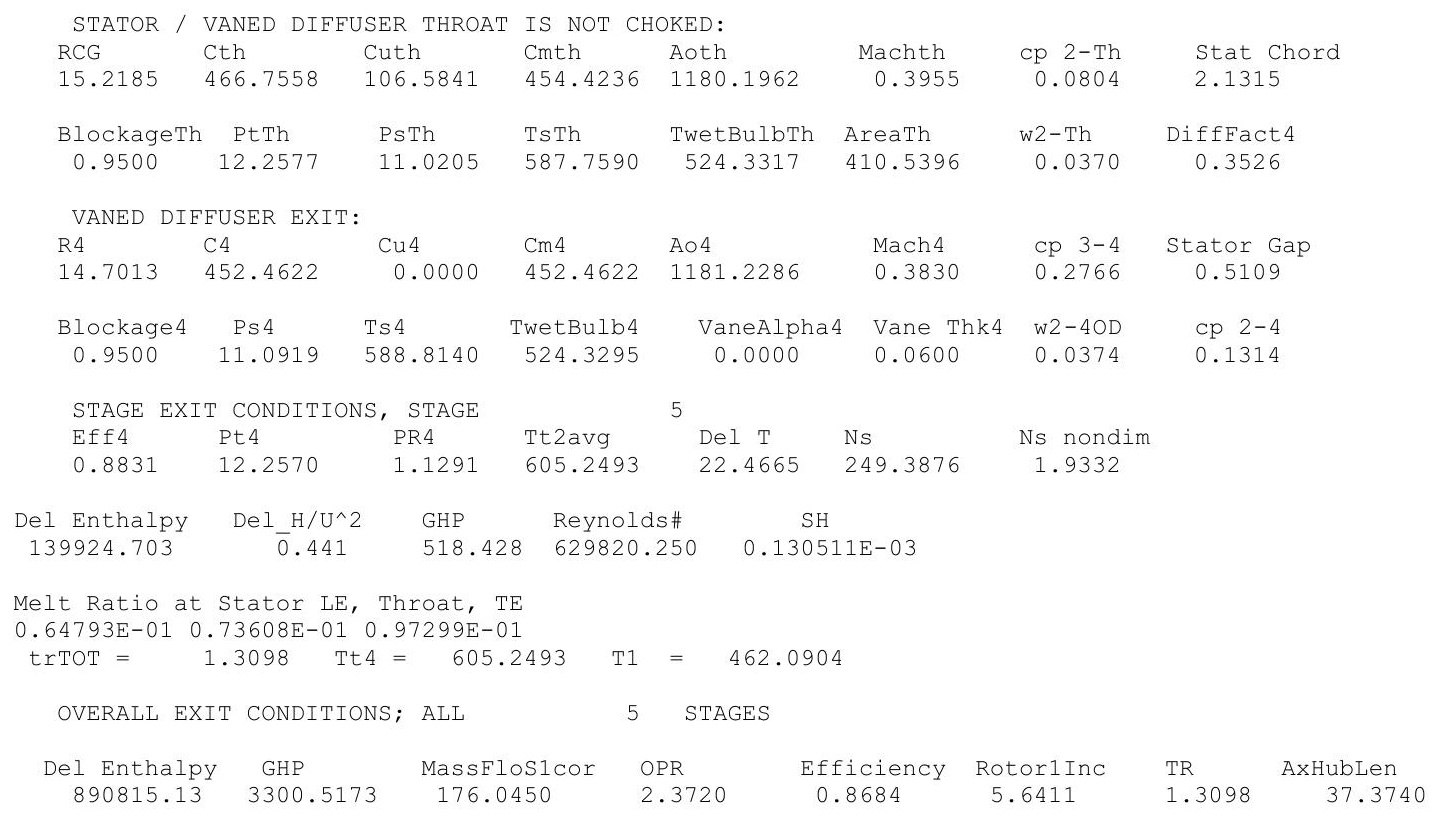




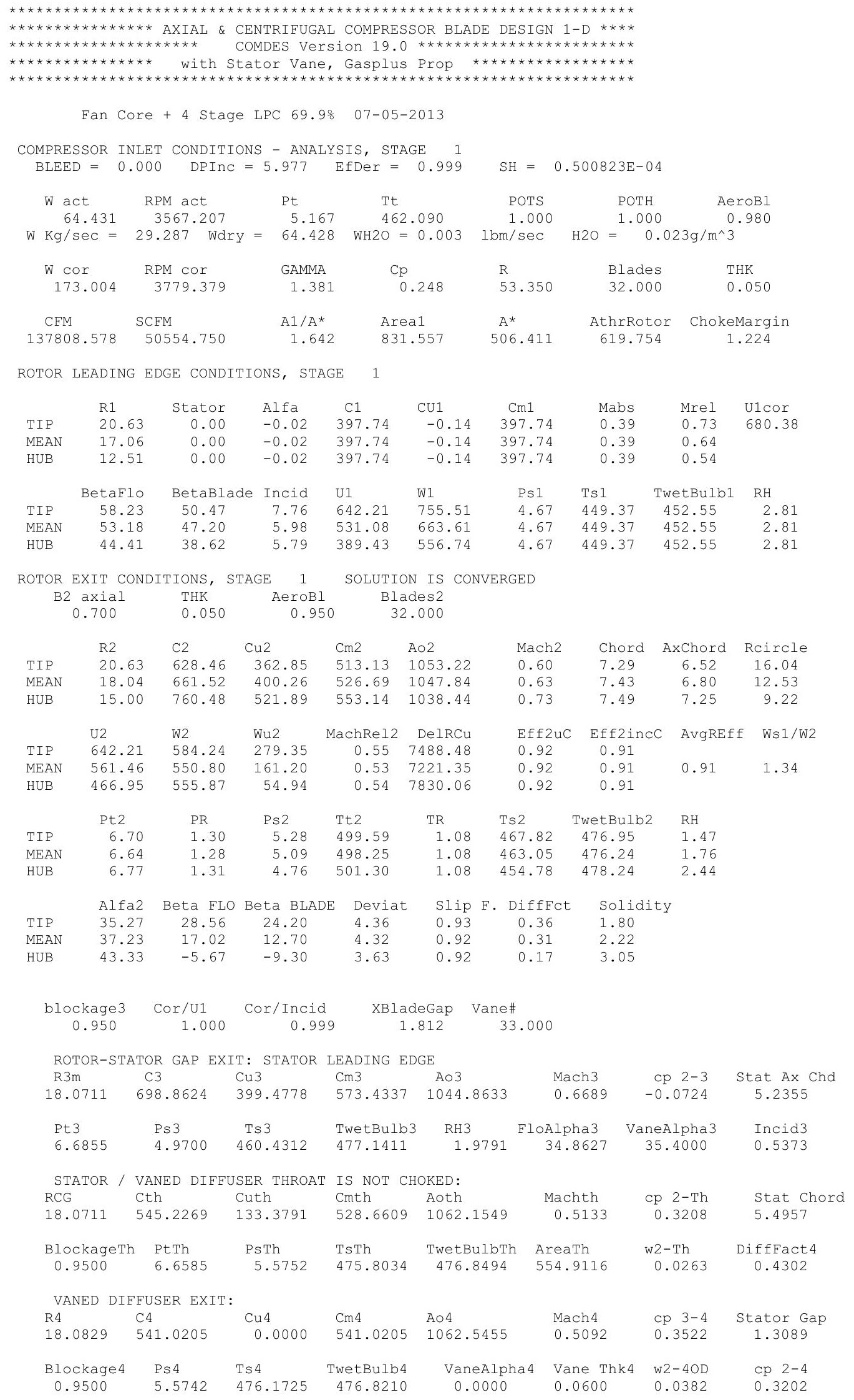




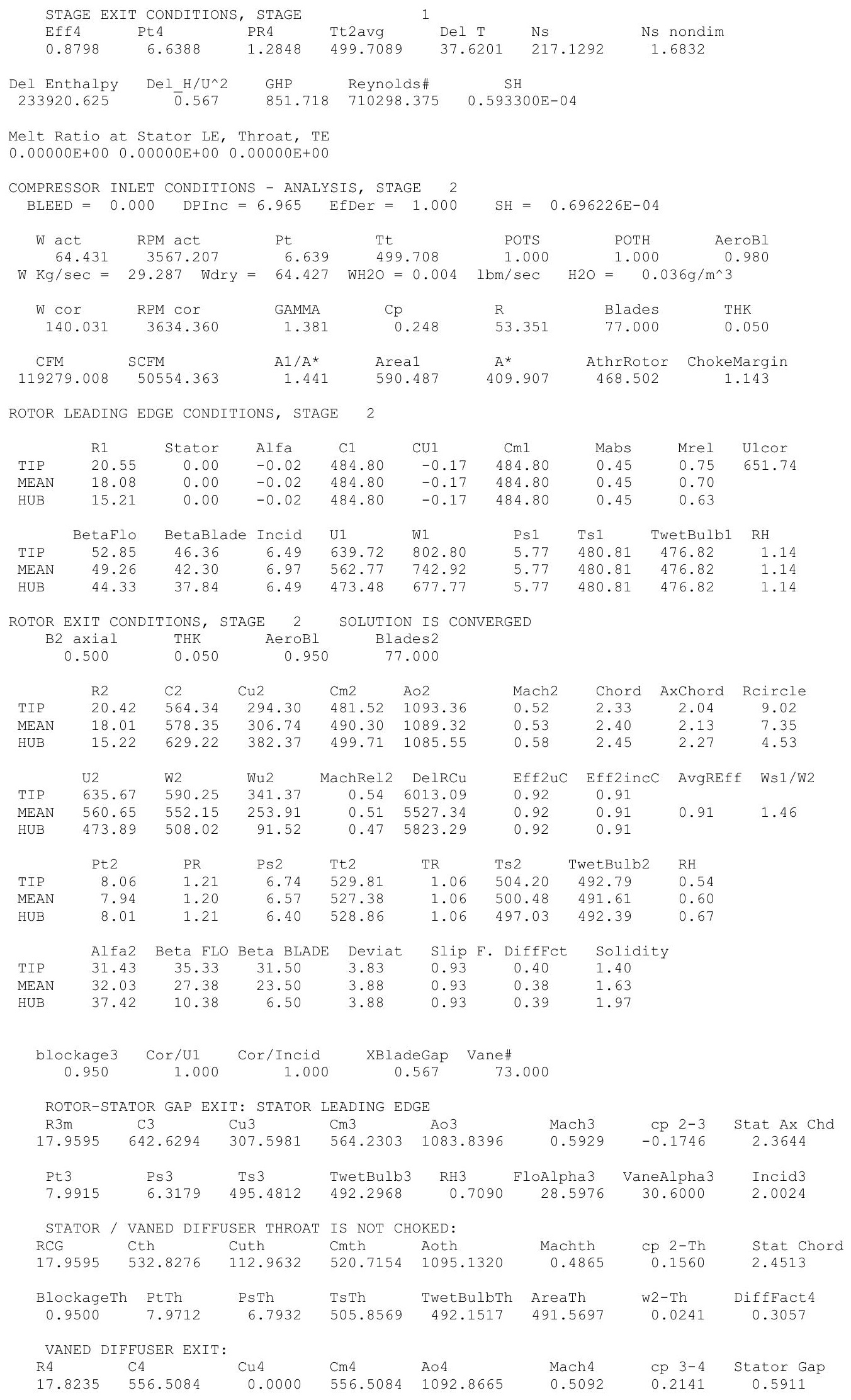




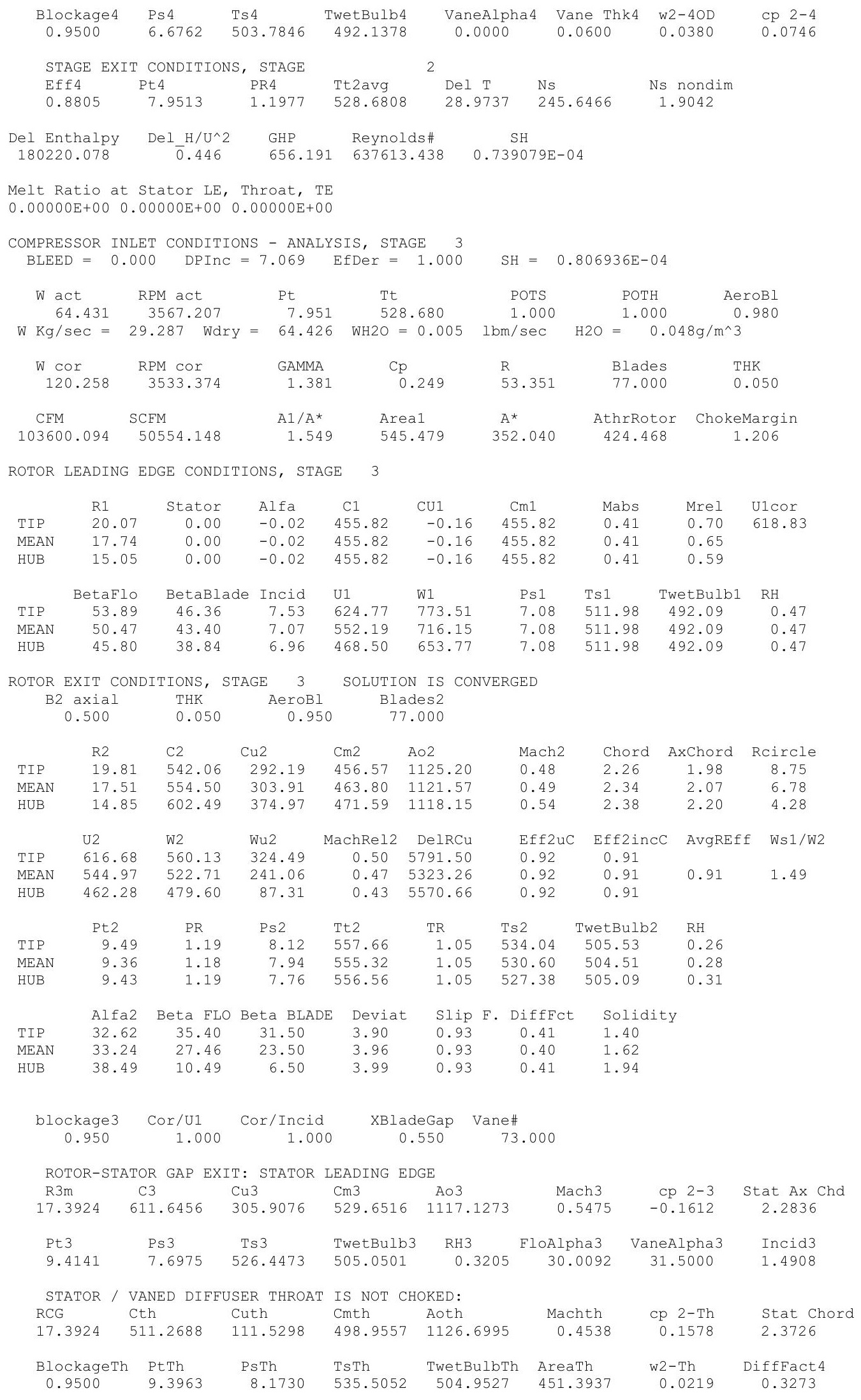




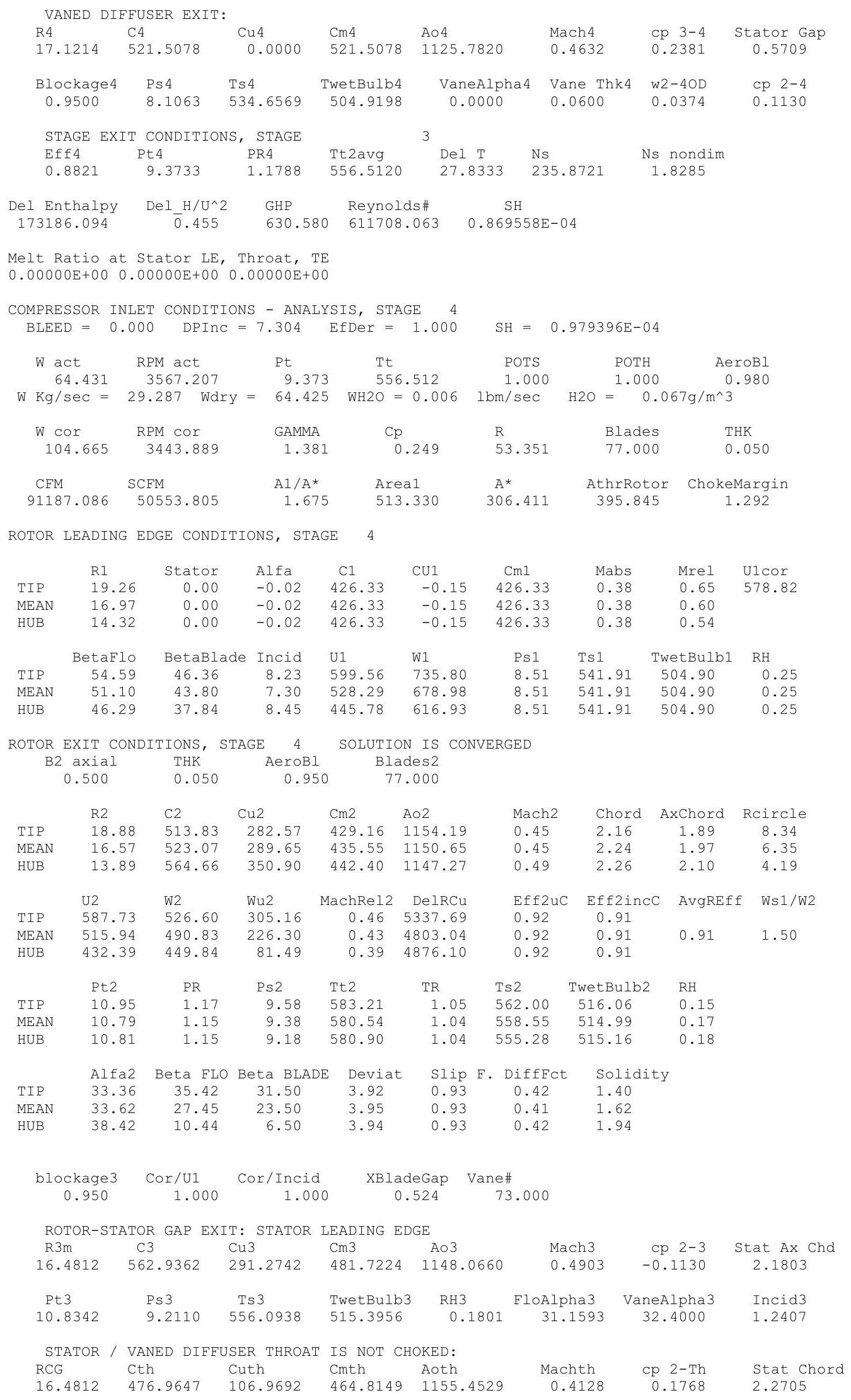




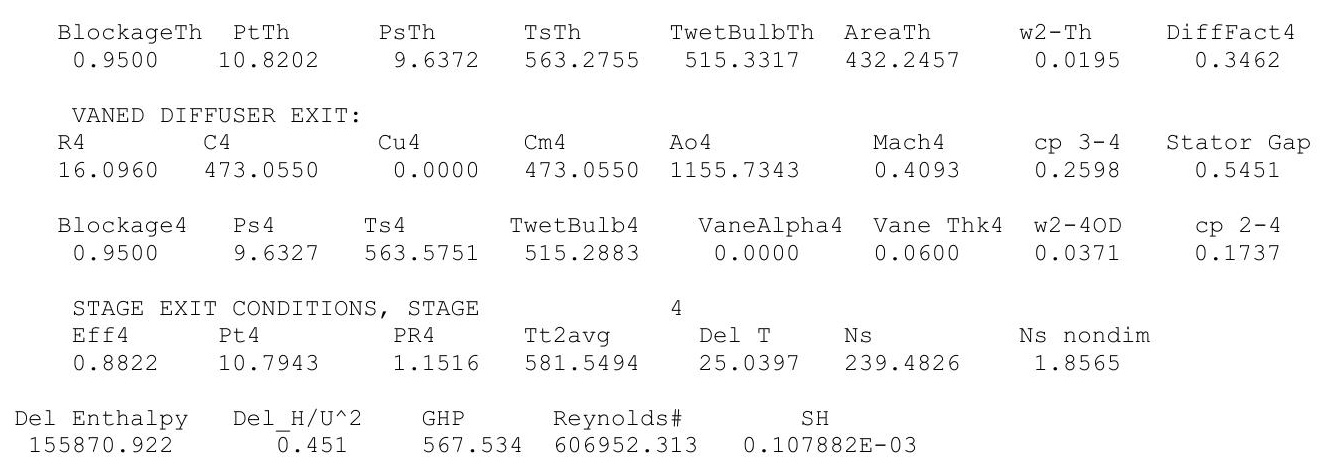

Melt Ratio at Stator LE, Throat, TE

$0.00000 \mathrm{E}+00 \quad 0.00000 \mathrm{E}+00 \quad 0.11460 \mathrm{E}-01$

COMPRESSOR INLET CONDITIONS - ANALYSIS, STAGE 5 $\mathrm{BLEED}=0.000 \quad$ DPInC $=7.462 \quad$ EfDer $=0.999$

$\begin{array}{llllllr}\text { W act } & \text { RPM act } & \text { Pt } & \text { Tt } & \text { POTS } & \text { POTH } & \text { AeroBI } \\ 64.431 & 3567.207 & 10.794 & 581.549 & 1.000 & 1.000 & 0.980\end{array}$ $\mathrm{W} \mathrm{Kg} / \mathrm{sec}=29.287 \mathrm{WdrY}=64.423 \mathrm{WH} 2 \mathrm{O}=0.008 \mathrm{lbm} / \mathrm{sec} \quad \mathrm{H} 2 \mathrm{O}=0.094 \mathrm{~g} / \mathrm{m}^{\wedge} 3$

$\begin{array}{llllll}W & \text { Cor } & \text { RPM cor } & \text { GAMMA } & \text { Blades } & \text { THK }\end{array}$

$\begin{array}{lccclll}\text { Cor } & \text { RPM Cor } & \text { GAMMA } & \text { Cp } & \text { R } & \text { Blades } & \text { THK } \\ 92.908 & 3368.938 & 1.381 & 0.249 & 53.352 & 77.000 & 0.050\end{array}$

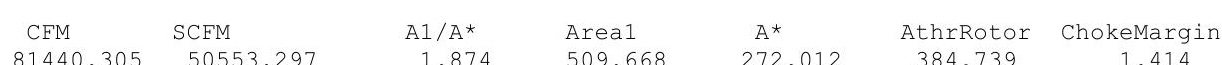

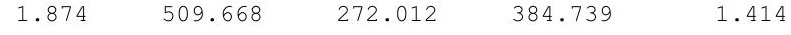

ROTOR LEADING EDGE CONDITIONS, STAGE 5

$\begin{array}{lccccccccc} & \text { R1 } & \text { Stator } & \text { Alfa } & \text { C1 } & \text { CU1 } & \text { Cm1 } & \text { Mabs } & \text { Mrel } & \text { U1cor } \\ \text { TIP } & 18.32 & 0.00 & -0.02 & 383.50 & -0.13 & 383.50 & 0.33 & 0.59 & 538.58 \\ \text { MEAN } & 15.91 & 0.00 & -0.02 & 383.50 & -0.13 & 383.50 & 0.33 & 0.54 & \\ \text { HUB } & 13.07 & 0.00 & -0.02 & 383.50 & -0.13 & 383.50 & 0.33 & 0.48 & \\ & & & & & & & & & \\ & \text { BetaFlo } & \text { BetaBlade } & \text { Incid } & \text { U1 } & \text { W1 } & \text { Ps1 } & \text { Ts1 } & \text { TwetBulb1 } & \text { RH } \\ \text { TIP } & 56.09 & 47.36 & 8.73 & 570.30 & 687.36 & 10.02 & 569.74 & 515.29 & 0.16 \\ \text { MEAN } & 52.26 & 44.80 & 7.46 & 495.37 & 626.57 & 10.02 & 569.74 & 515.29 & 0.16 \\ \text { HUB } & 46.70 & 38.84 & 7.86 & 406.87 & 559.21 & 10.02 & 569.74 & 515.29 & 0.16\end{array}$

ROTOR EXIT CONDITIONS, STAGE 5 SOLUTION IS CONVERGED

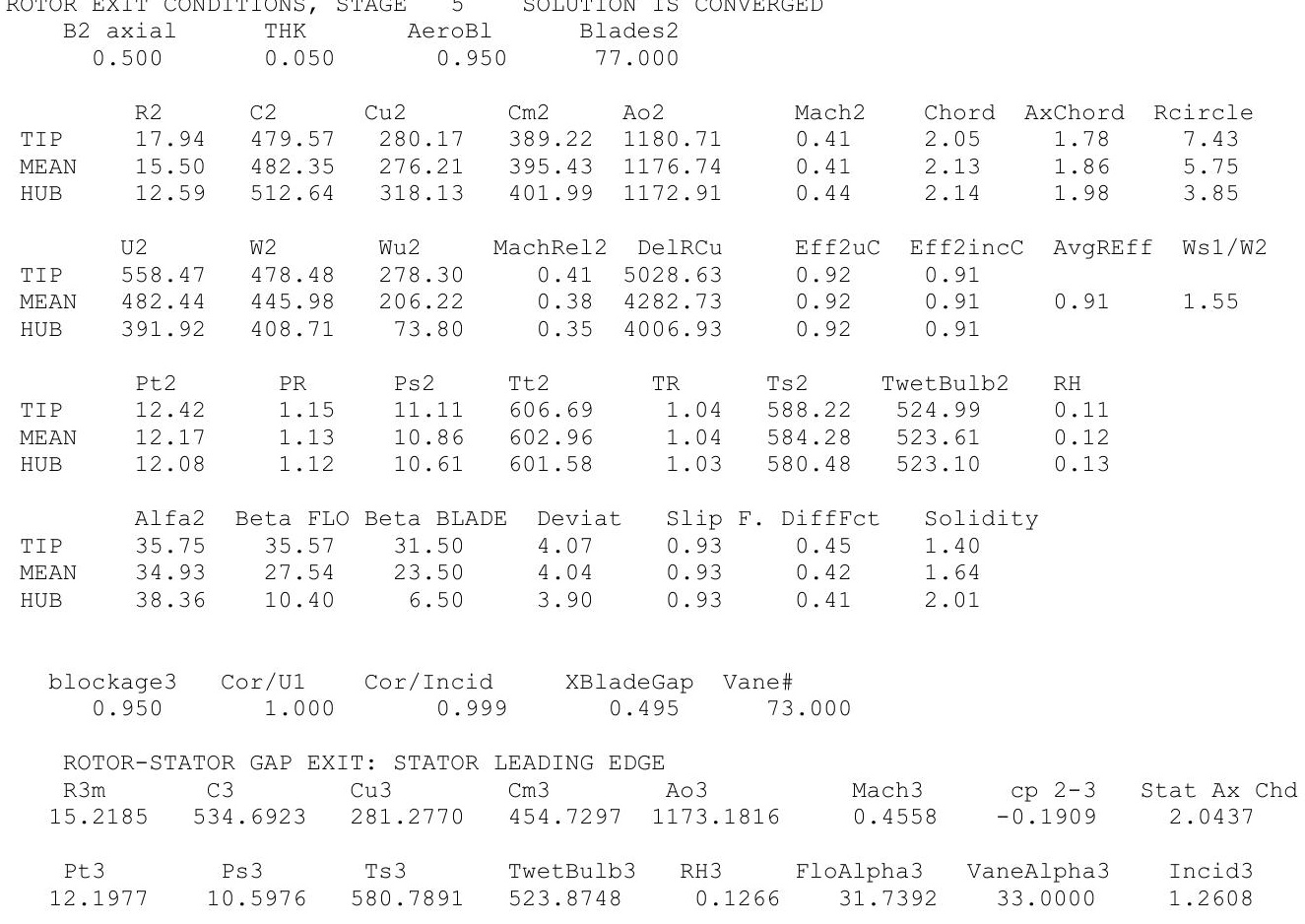




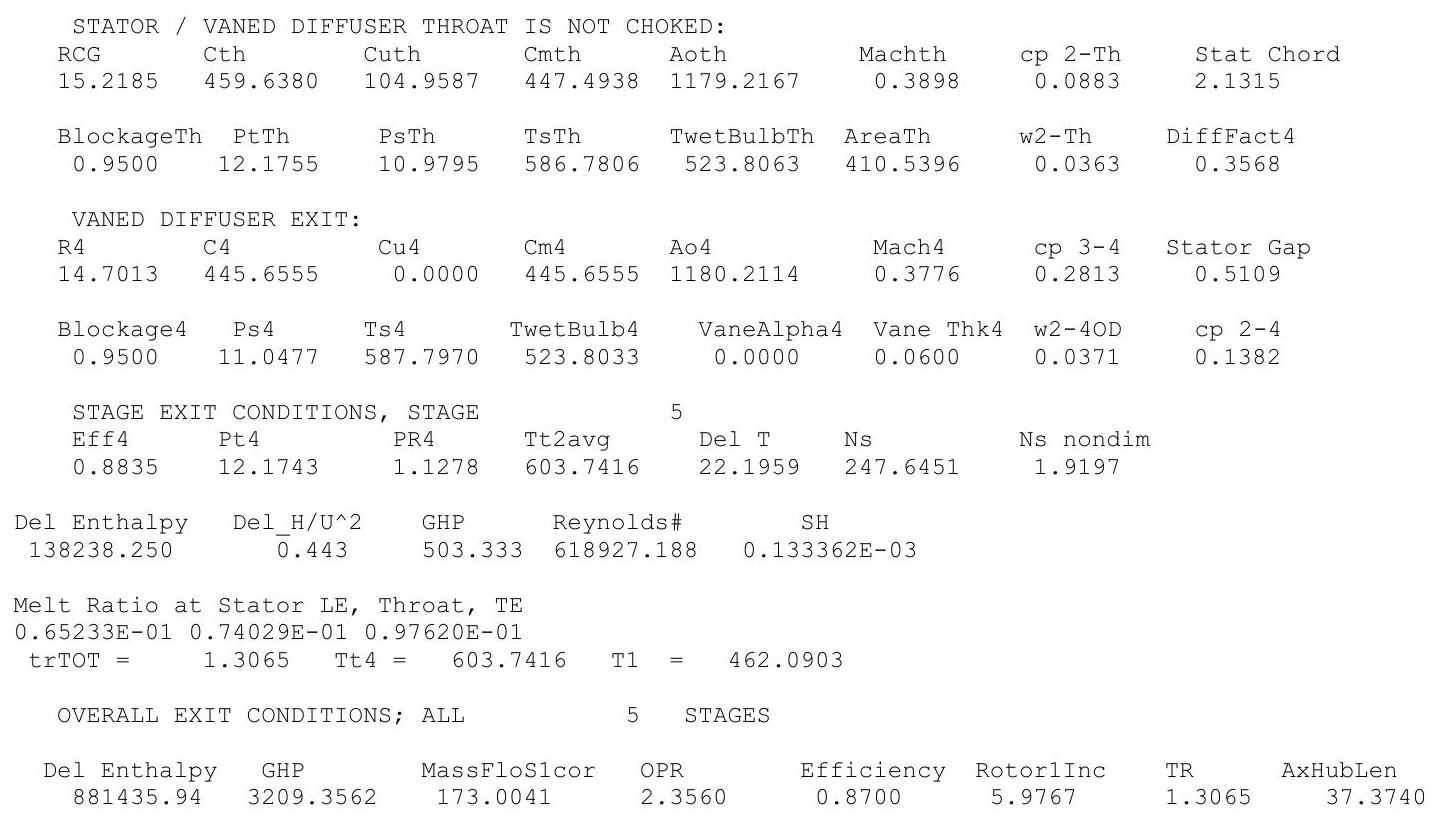




\section{$25 \mu \mathrm{m}, \mathrm{ISA}+36 \mathrm{R}$}

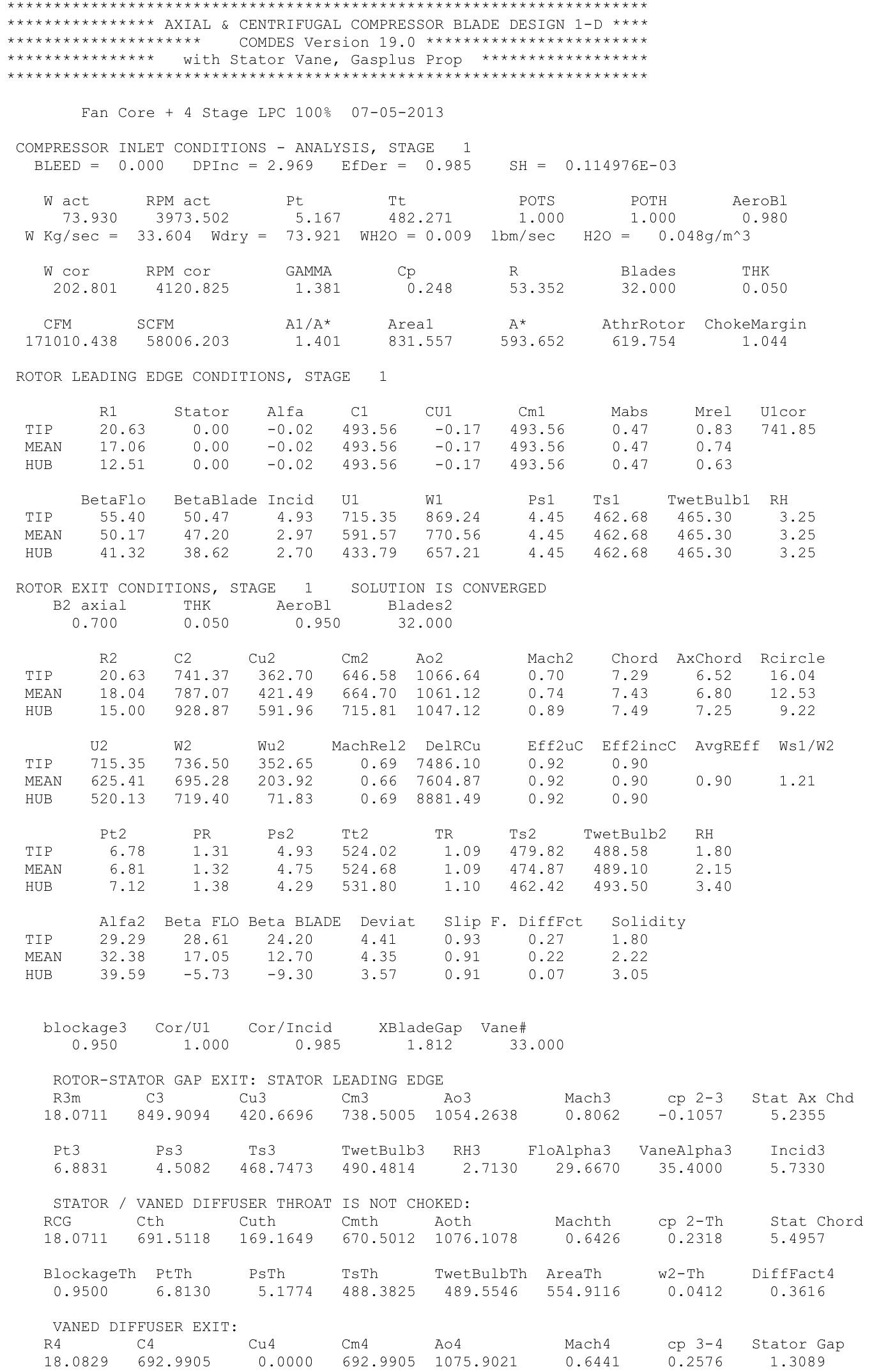

$\begin{array}{lccccccccc} & \text { R1 } & \text { Stator } & \text { Alfa } & \text { C1 } & \text { CU1 } & \text { Cm1 } & \text { Mabs } & \text { Mrel } & \text { U1cor } \\ \text { TIP } & 20.63 & 0.00 & -0.02 & 493.56 & -0.17 & 493.56 & 0.47 & 0.83 & 741.85 \\ \text { MEAN } & 17.06 & 0.00 & -0.02 & 493.56 & -0.17 & 493.56 & 0.47 & 0.74 & \\ \text { HUB } & 12.51 & 0.00 & -0.02 & 493.56 & -0.17 & 493.56 & 0.47 & 0.63 & \\ & & & & & & & & & \\ & \text { BetaFlo } & \text { BetaBlade } & \text { Incid } & \text { U1 } & \text { W1 } & \text { Ts 1 } & \text { TwetBulb1 } & \text { RH } \\ \text { TIP } & 55.40 & 50.47 & 4.93 & 715.35 & 869.24 & 4.45 & 462.68 & 465.30 & 3.25 \\ \text { MEAN } & 50.17 & 47.20 & 2.97 & 591.57 & 770.56 & 4.45 & 462.68 & 465.30 & 3.25 \\ \text { HUB } & 41.32 & 38.62 & 2.70 & 433.79 & 657.21 & 4.45 & 462.68 & 465.30 & 3.25\end{array}$

ROTOR EXIT CONDITIONS, STAGE 1 SOLUTION IS CONVERGED

\begin{tabular}{|c|c|c|c|c|c|c|c|c|c|}
\hline & $\begin{array}{l}\text { axial } \\
700\end{array}$ & $\begin{array}{l}\text { THK } \\
0.050\end{array}$ & $\begin{array}{l}\text { AeroBl } \\
0.950\end{array}$ & $\begin{array}{r}\text { Bla } \\
32\end{array}$ & $\begin{array}{l}\text { ades } 2 \\
2.000\end{array}$ & & & & \\
\hline & $\mathrm{R} 2$ & $\mathrm{C} 2$ & $\mathrm{Cu} 2$ & $\mathrm{Cm} 2$ & $\mathrm{~A} \circ 2$ & Mach2 & Chord & AxChord & Rcircle \\
\hline TIP & 20.63 & 741.37 & 362.70 & 646.58 & 1066.64 & 0.70 & 7.29 & 6.52 & 16.04 \\
\hline MEAN & 18.04 & 787.07 & 421.49 & 664.70 & 1061.12 & 0.74 & 7.43 & 6.80 & 12.53 \\
\hline HUB & 15.00 & 928.87 & 591.96 & 715.81 & 1047.12 & 0.89 & 7.49 & 7.25 & 9.22 \\
\hline & $\mathrm{U} 2$ & W2 & Wu2 & MachRel2 & DelRCu & Eff $2 u C$ & Eff 2 incC & AvgREff & Ws $1 /$ W2 \\
\hline TIP & 715.35 & 736.50 & 352.65 & 0.69 & 7486.10 & 0.92 & 0.90 & & \\
\hline MEAN & 625.41 & 695.28 & 203.92 & 0.66 & 7604.87 & 0.92 & 0.90 & 0.90 & 1.21 \\
\hline HUB & 520.13 & 719.40 & 71.83 & 0.69 & 8881.49 & 0.92 & 0.90 & & \\
\hline & Pt2 & PR & Ps2 & Tt2 & $\mathrm{TR}$ & Ts 2 & TwetBulb2 & $\mathrm{RH}$ & \\
\hline TIP & 6.78 & 1.31 & 4.93 & 524.02 & 1.09 & 479.82 & 488.58 & 1.80 & \\
\hline MEAN & 6.81 & 1.32 & 4.75 & 524.68 & 1.09 & 474.87 & 489.10 & 2.15 & \\
\hline UB & 7.12 & 1.38 & 4.29 & 531.80 & 1.10 & 462.42 & 493.50 & 3.40 & \\
\hline & Alfa2 & Beta FLO & Beta BLADE & Deviat & Slip & F. DiffFct & Solidity & & \\
\hline IP & 29.29 & 28.61 & 24.20 & 4.41 & 0.93 & 0.27 & 1.80 & & \\
\hline GAN & 32.38 & 17.05 & 12.70 & 4.35 & 0.91 & 0.22 & 2.22 & & \\
\hline JB & 39.59 & -5.73 & -9.30 & 3.57 & 0.91 & 0.07 & 3.05 & & \\
\hline
\end{tabular}




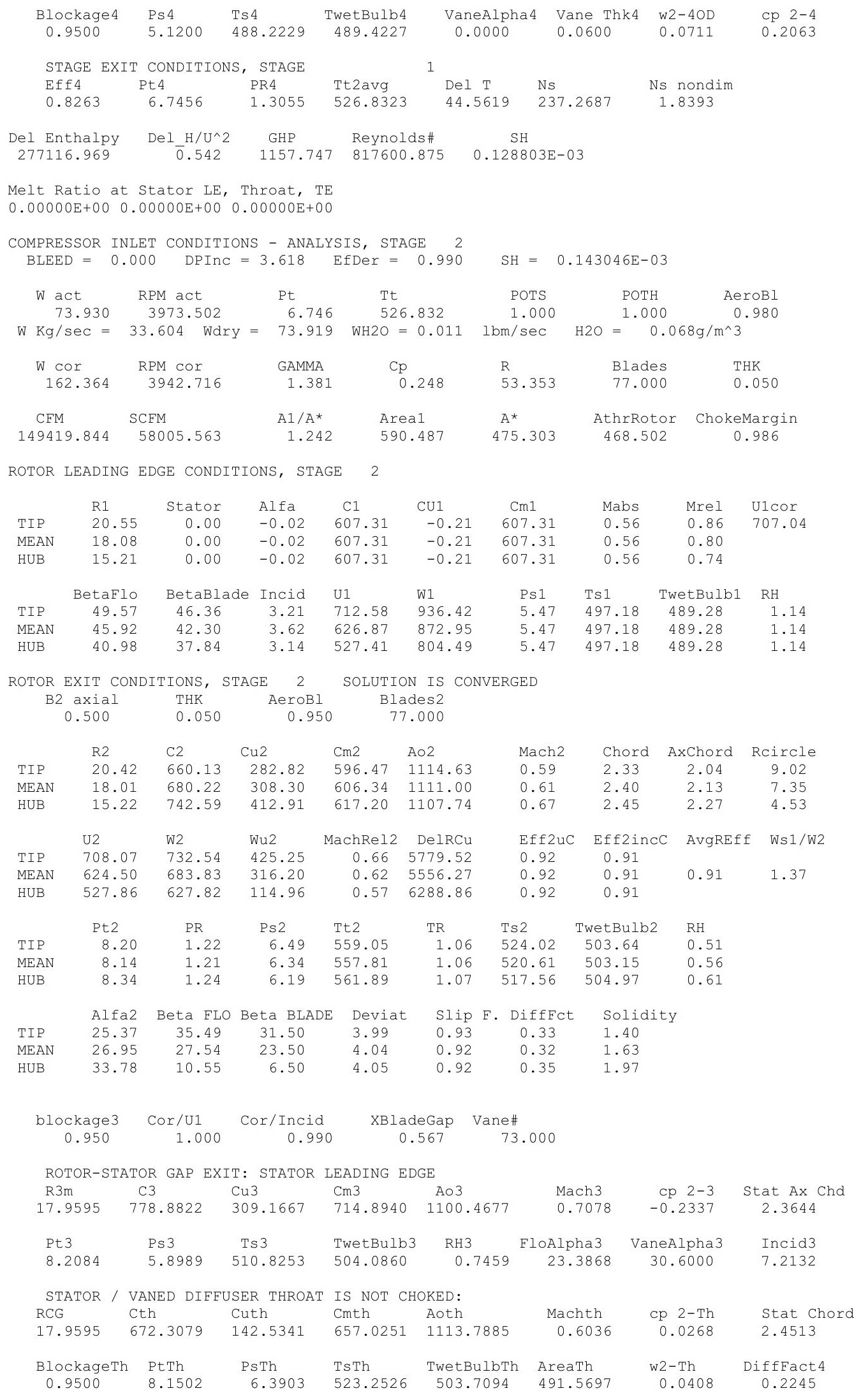




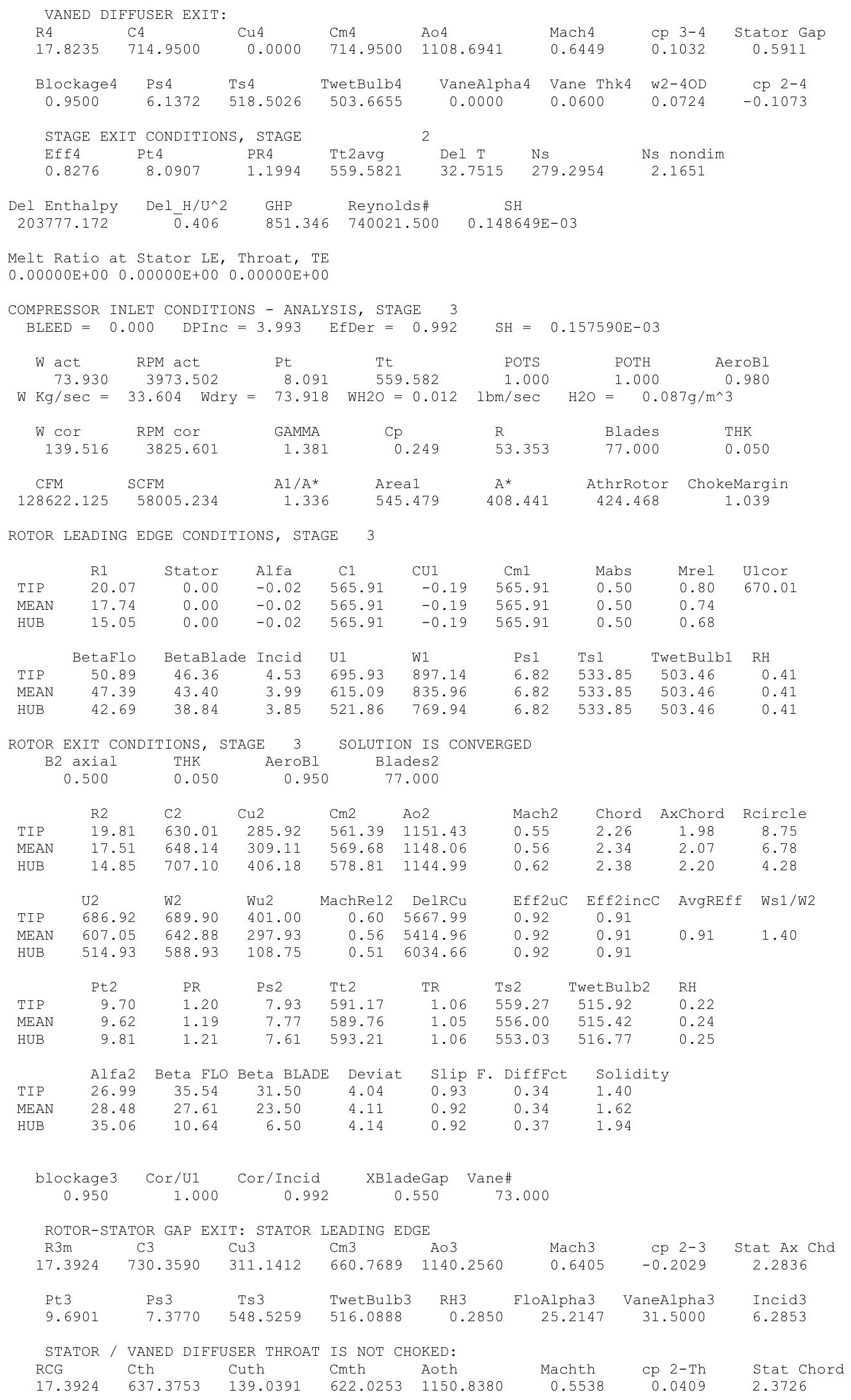




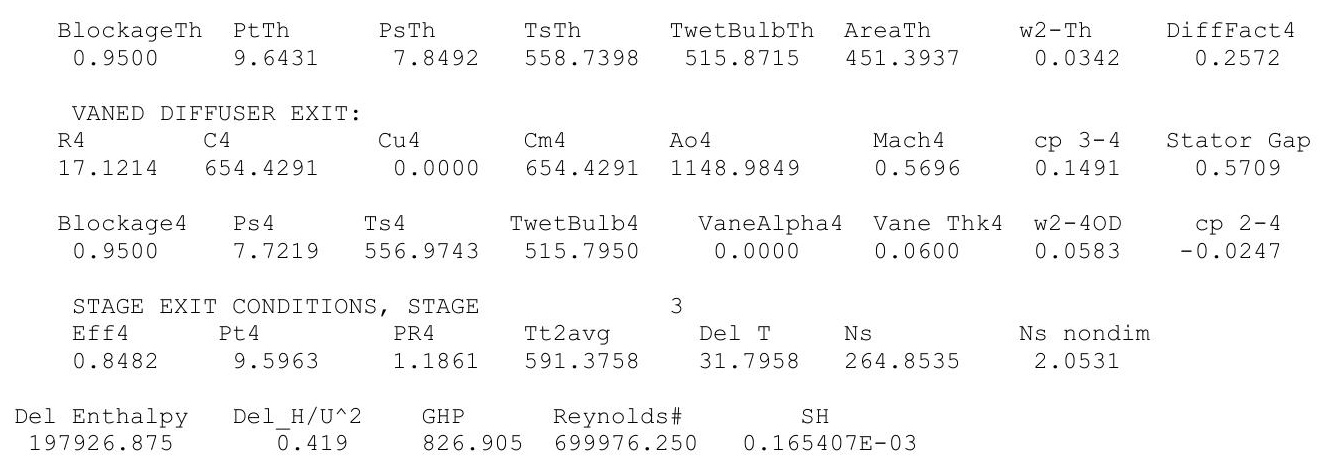

Melt Ratio at Stator LE, Throat, TE

$0.00000 \mathrm{E}+00 \quad 0.00000 \mathrm{E}+00 \quad 0.00000 \mathrm{E}+00$

COMPRESSOR INLET CONDITIONS - ANALYSIS, STAGE 4 $\mathrm{BLEED}=0.000 \quad$ DPInC $=4.577 \quad$ EfDer $=0.995$

ROTOR LEADING EDGE CONDITIONS, STAGE 4

$\begin{array}{lccccccccc} & \text { R1 } & \text { Stator } & \text { Alfa } & \text { C1 } & \text { CU1 } & \text { Cm1 } & \text { Mabs } & \text { Mrel } & \text { U1cor } \\ \text { TIP } & 19.26 & 0.00 & -0.02 & 523.04 & -0.18 & 523.04 & 0.45 & 0.73 & 625.45 \\ \text { MEAN } & 16.97 & 0.00 & -0.02 & 523.04 & -0.18 & 523.04 & 0.45 & 0.68 & \\ \text { HUB } & 14.32 & 0.00 & -0.02 & 523.04 & -0.18 & 523.04 & 0.45 & 0.62 & \\ & & & & & & & & & \\ & \text { BetaFlo } & \text { BetaBlade } & \text { Incid } & \text { U1 } & \text { W1 } & \text { Ps1 } & \text { Ts1 } & \text { TwetBulb1 } & \text { RH } \\ \text { TIP } & 51.94 & 46.36 & 5.58 & 667.85 & 848.43 & 8.37 & 569.41 & 515.71 & 0.19 \\ \text { MEAN } & 48.38 & 43.80 & 4.58 & 588.47 & 787.45 & 8.37 & 569.41 & 515.71 & 0.19 \\ \text { HUB } & 43.52 & 37.84 & 5.68 & 496.55 & 721.33 & 8.37 & 569.41 & 515.71 & 0.19\end{array}$

ROTOR EXIT CONDITIONS, STAGE 4 SOLUTION IS CONVERGED

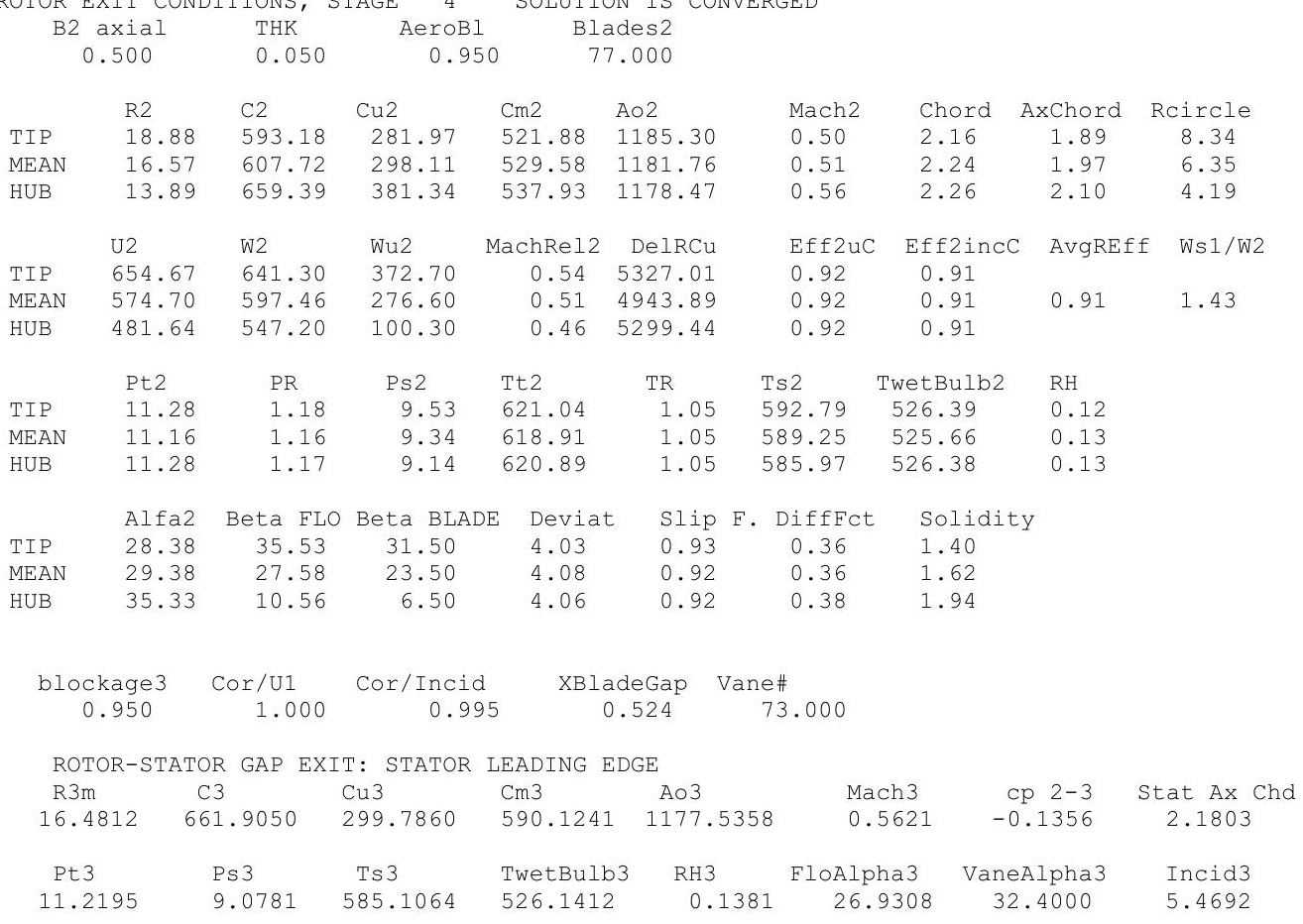




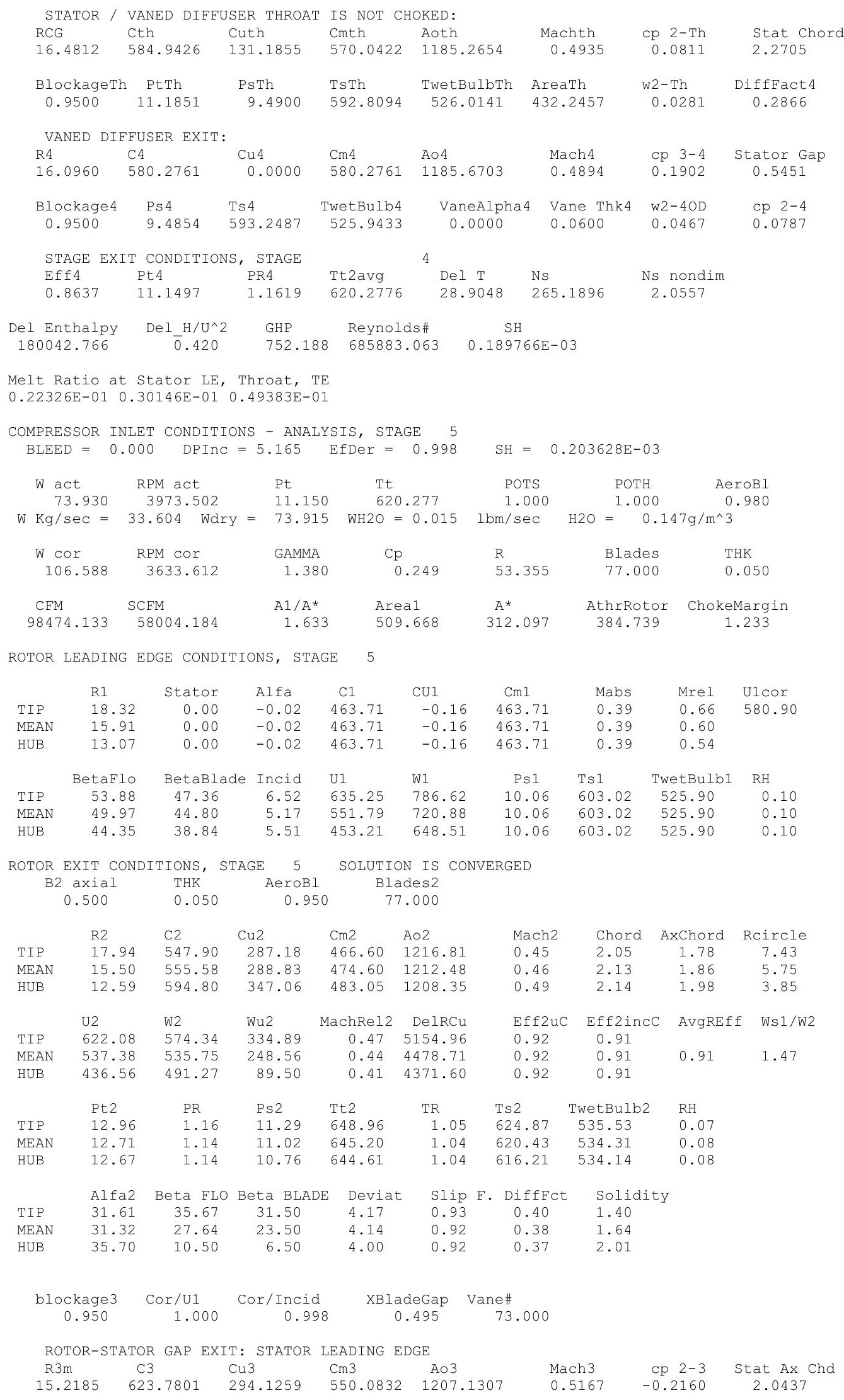




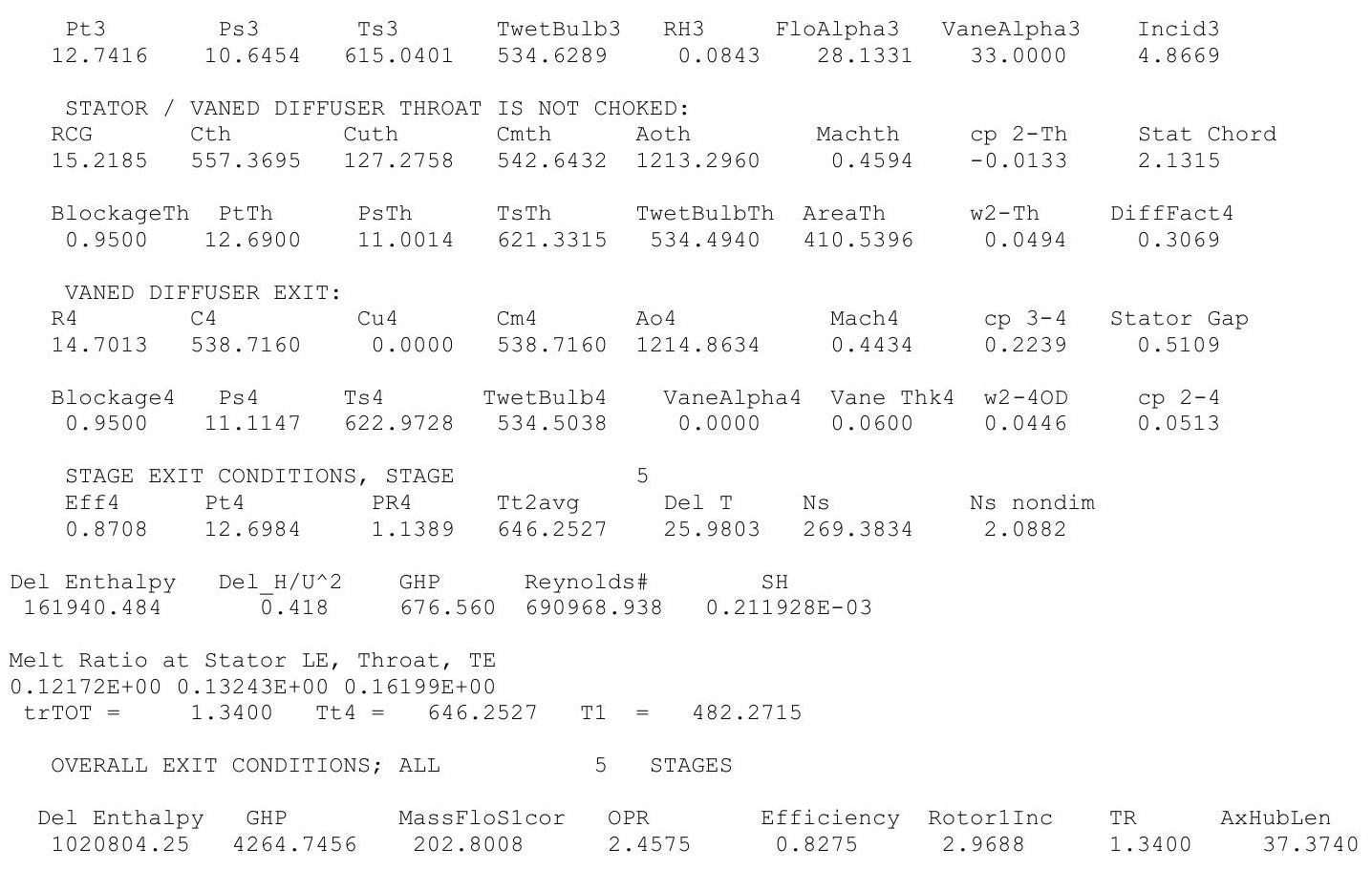




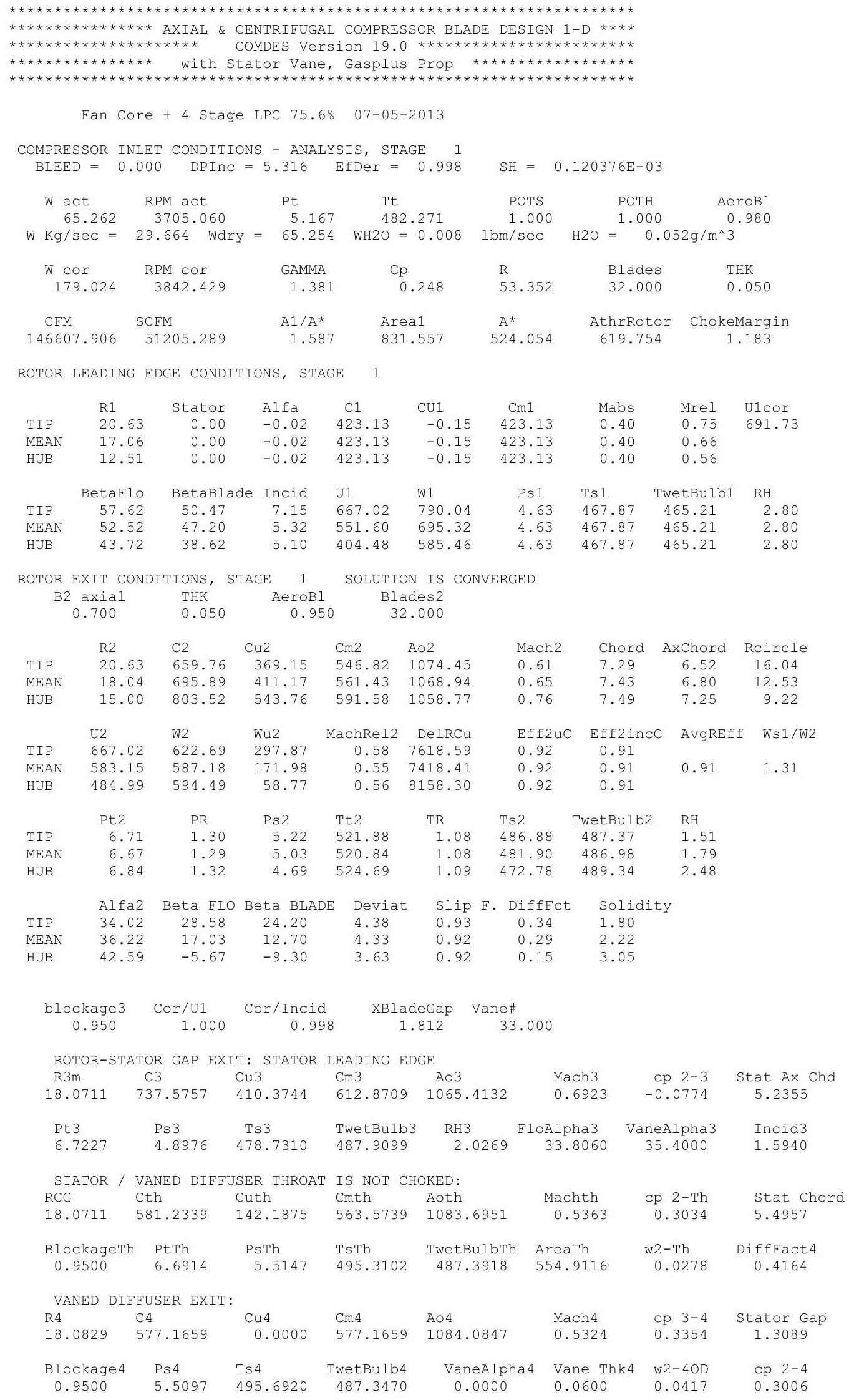




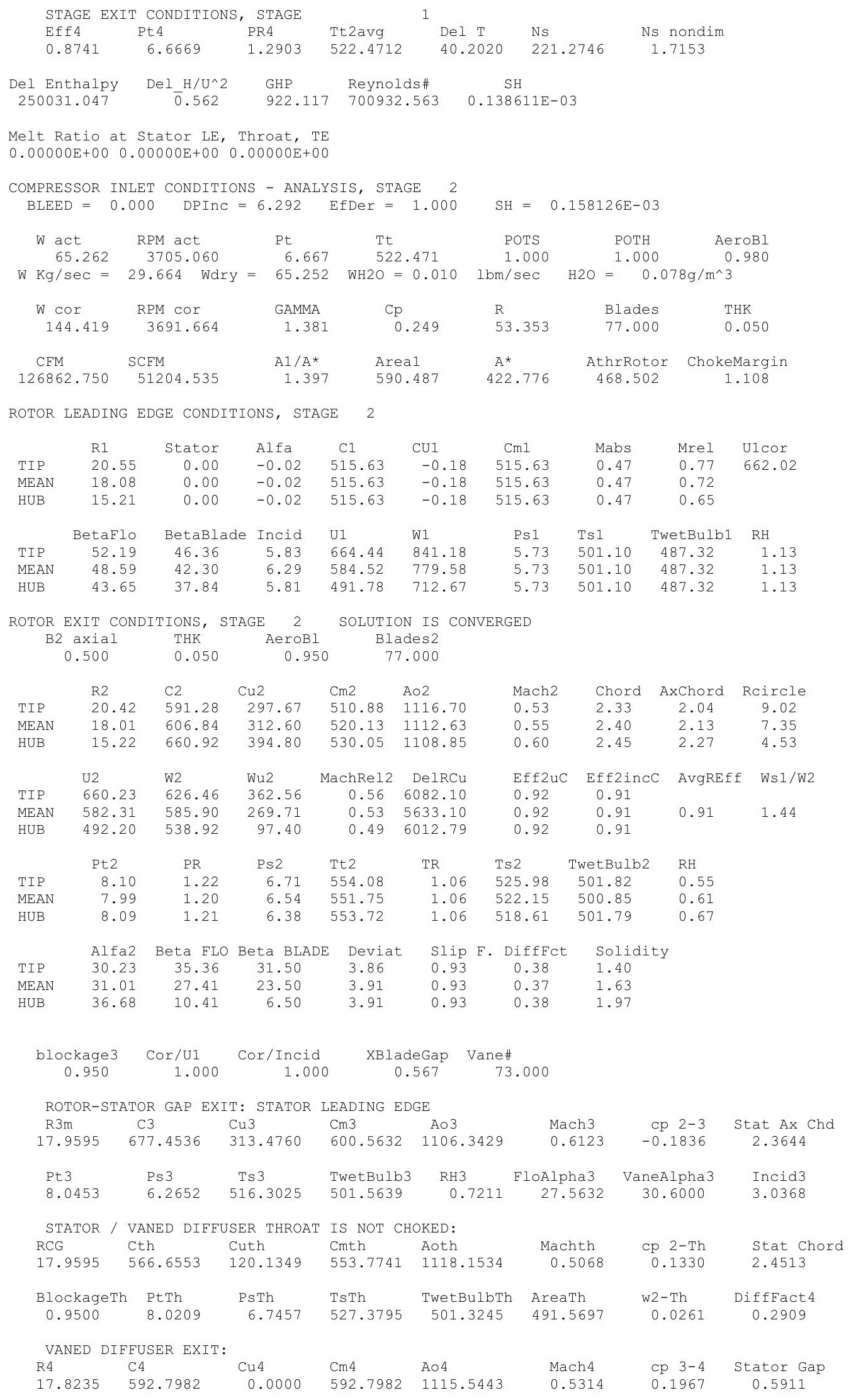




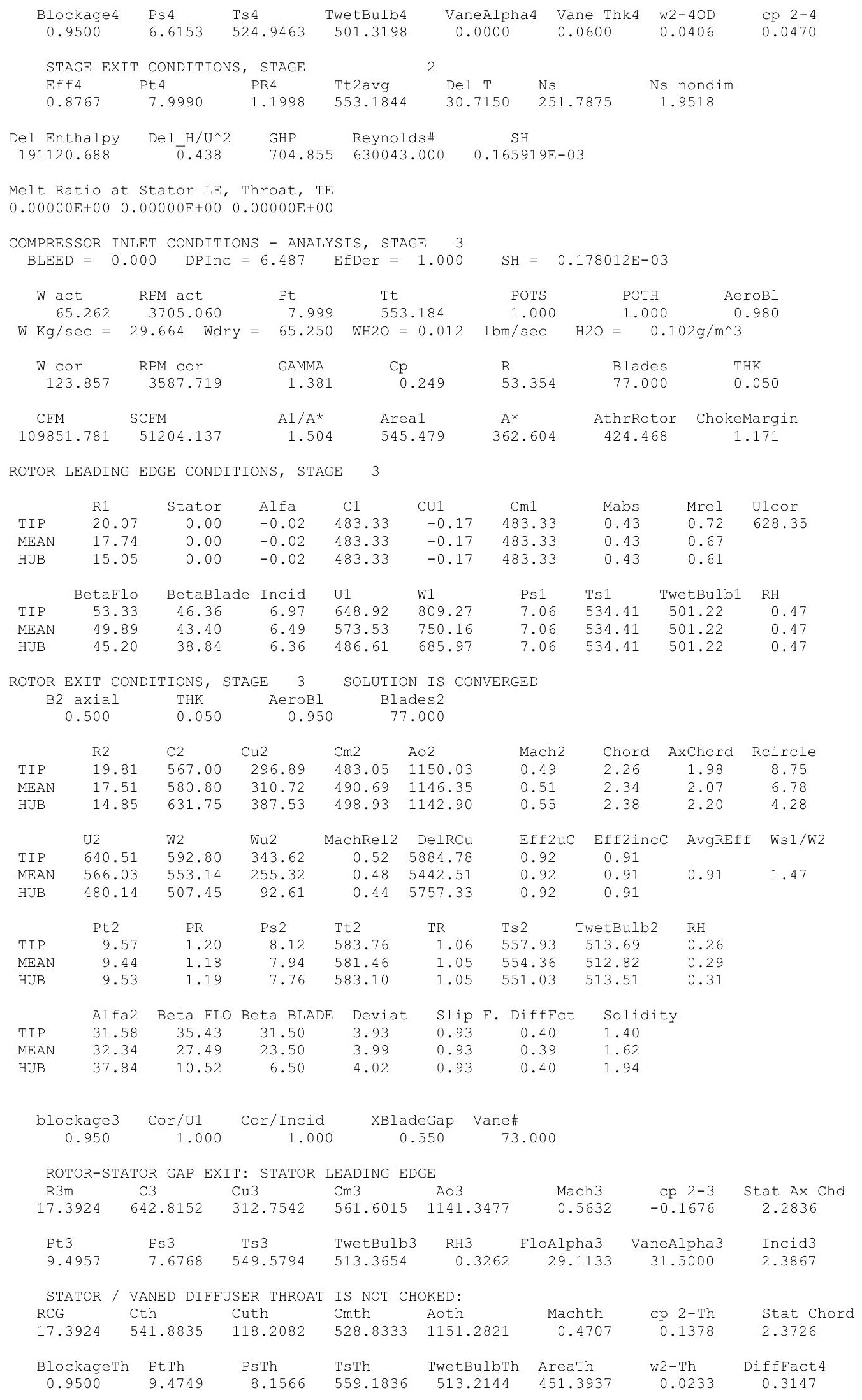




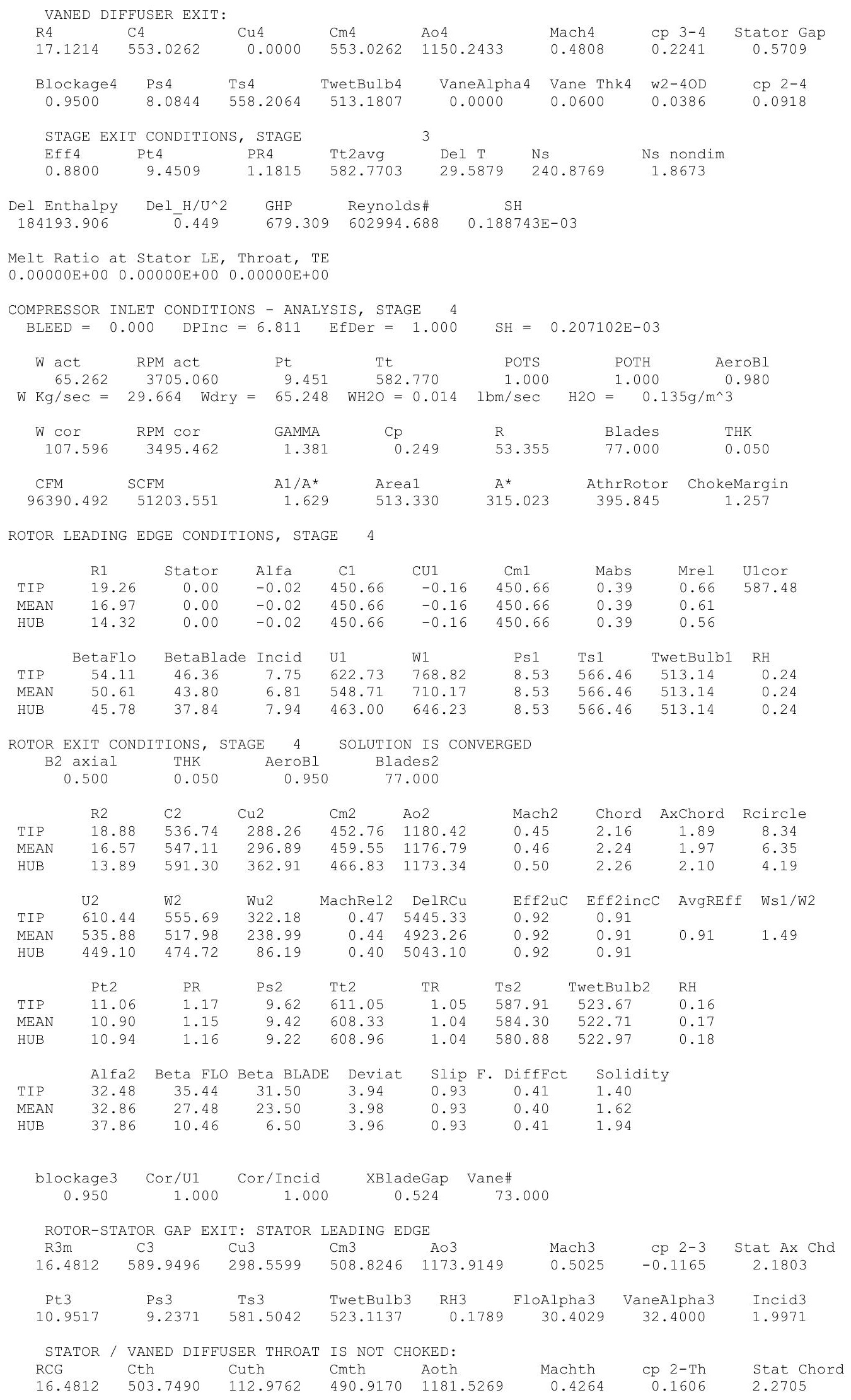




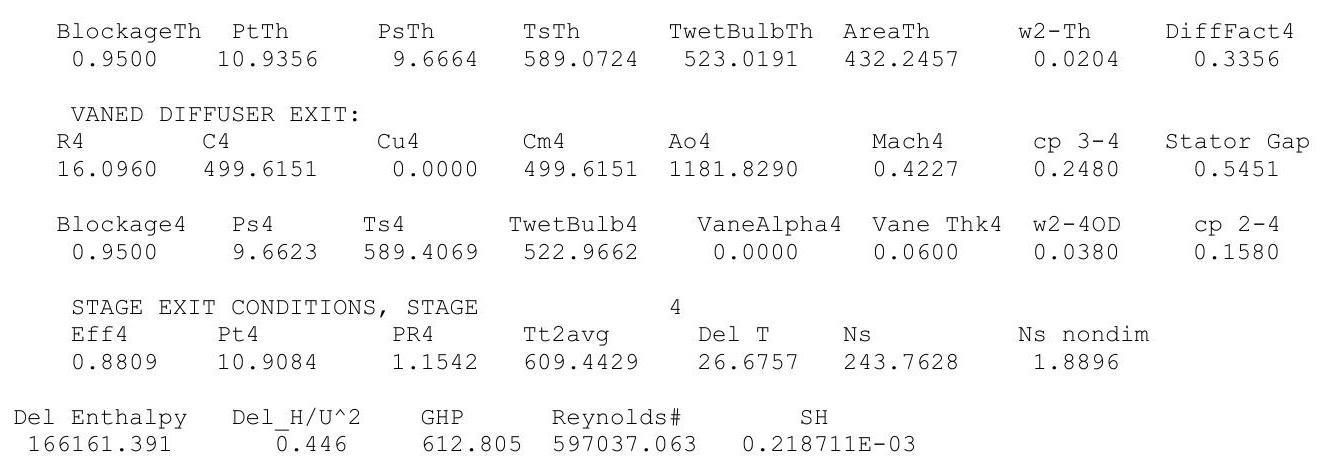

Melt Ratio at Stator LE, Throat, TE

$0.38448 \mathrm{E}-01 \quad 0.47042 \mathrm{E}-01 \quad 0.67898 \mathrm{E}-01$

COMPRESSOR INLET CONDITIONS - ANALYSIS, STAGE 5 $\mathrm{BLEED}=0.000 \quad$ DPInC $=7.057 \quad$ EfDer $=1.000$

$\begin{array}{llllll}W \text { act } & \text { RPM act } & \text { Pt } & \text { POTS } & \text { POTH } & \text { AeroBI }\end{array}$

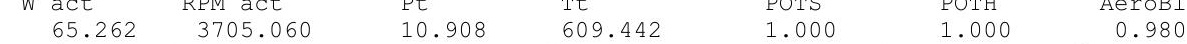
$\mathrm{W} \mathrm{Kg} / \mathrm{sec}=29.664$ Wdry $=65.247$ WH2O $=0.015 \quad \mathrm{lbm} / \mathrm{sec} \quad \mathrm{H} 2 \mathrm{O}=0.171 \mathrm{~g} / \mathrm{m}^{\wedge} 3$

$\begin{array}{ccccccc}\text { W Cor } & \text { RPM cor } & \text { GAMMA } & \text { Cp } & \text { R } & \text { Blades } & \text { THK } \\ 95.329 & 3418.116 & 1.380 & 0.249 & 53.356 & 77.000 & 0.050 \\ & & & & & & \\ \text { CFM } & \text { SCFM } & \text { A1/A* } & \text { Area1 } & \text { A* } & \text { AthrRotor } & \text { ChokeMargin } \\ 85828.719 & 51203.020 & 1.826 & 509.668 & 279.132 & 384.739 & 1.378\end{array}$

ROTOR LEADING EDGE CONDITIONS, STAGE 5

$\begin{array}{lccccccccc} & \text { R1 } & \text { Stator } & \text { Alfa } & \text { C1 } & \text { CU1 } & \text { Cm1 } & \text { Mabs } & \text { Mrel } & \text { U1cor } \\ \text { TIP } & 18.32 & 0.00 & -0.02 & 404.16 & -0.14 & 404.16 & 0.34 & 0.60 & 546.45 \\ \text { MEAN } & 15.91 & 0.00 & -0.02 & 404.16 & -0.14 & 404.16 & 0.34 & 0.55 & \\ \text { HUB } & 13.07 & 0.00 & -0.02 & 404.16 & -0.14 & 404.16 & 0.34 & 0.49 & \\ & & & & & & & & & \\ \text { TIP } & \text { BetaFlo } & \text { BetaBlade } & \text { Incid } & \text { U1 } & \text { W1 } & \text { Ps1 } & \text { Ts1 } & \text { TwetBulb1 } & \text { RH } \\ \text { MEAN } & 55.70 & 47.36 & 8.34 & 592.34 & 717.20 & 10.08 & 596.33 & 522.94 & 0.14 \\ \text { HUB } & 46.29 & 44.80 & 7.06 & 514.51 & 654.38 & 10.08 & 596.33 & 522.94 & 0.14 \\ & & 38.84 & 7.45 & 422.59 & 584.85 & 10.08 & 596.33 & 522.94 & 0.14\end{array}$

ROTOR EXIT CONDITIONS, STAGE 5 SOLUTION IS CONVERGED

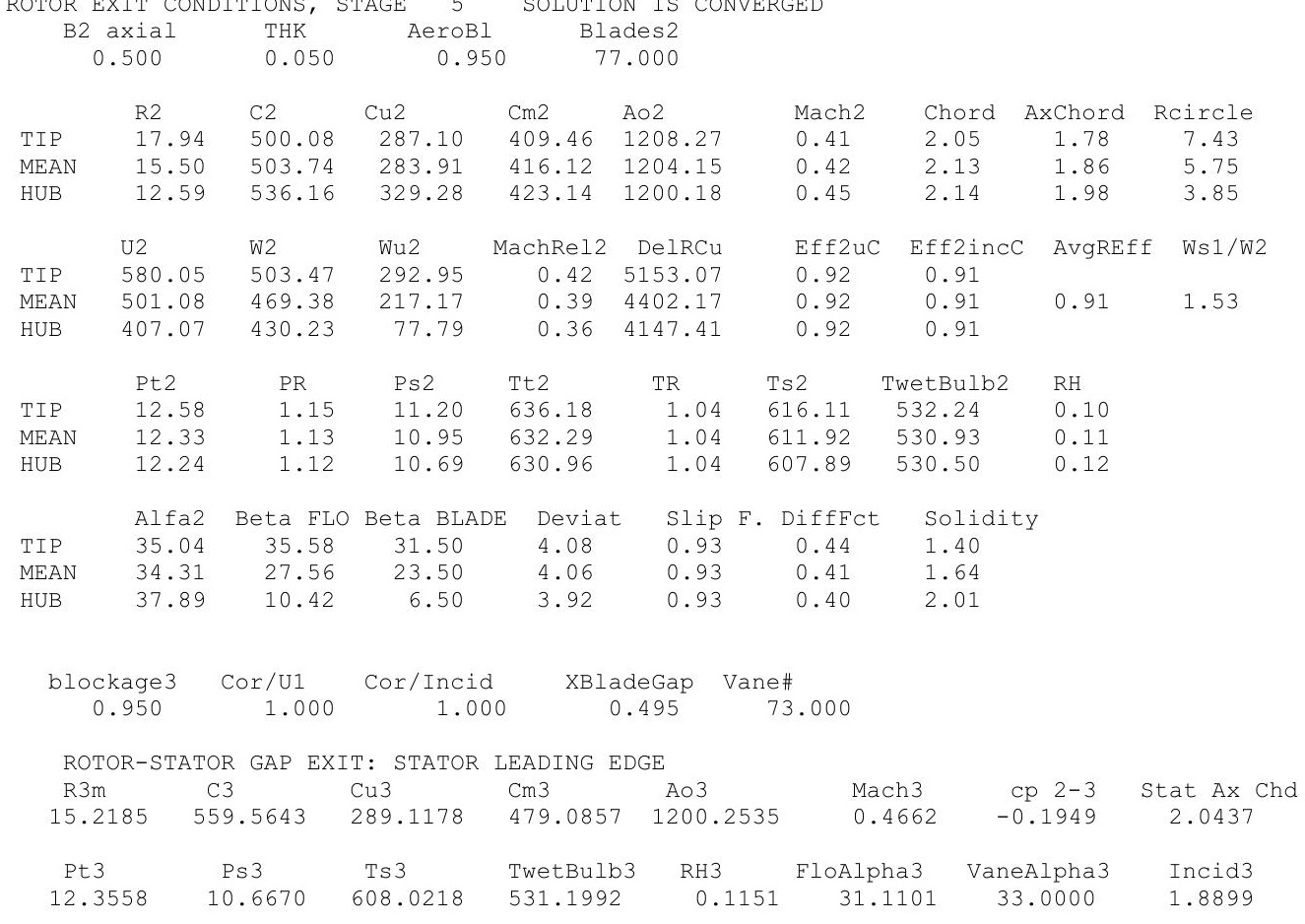




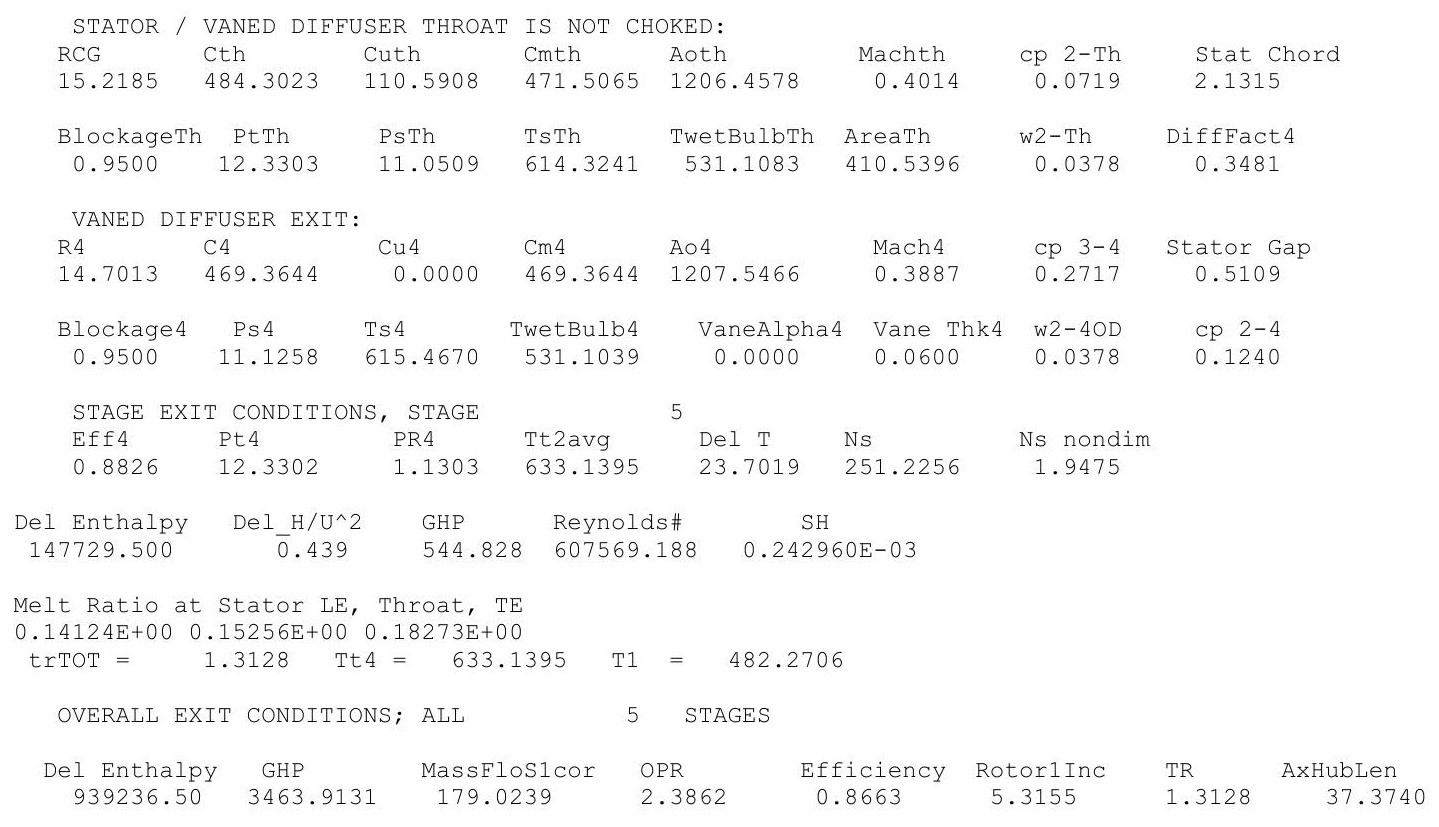




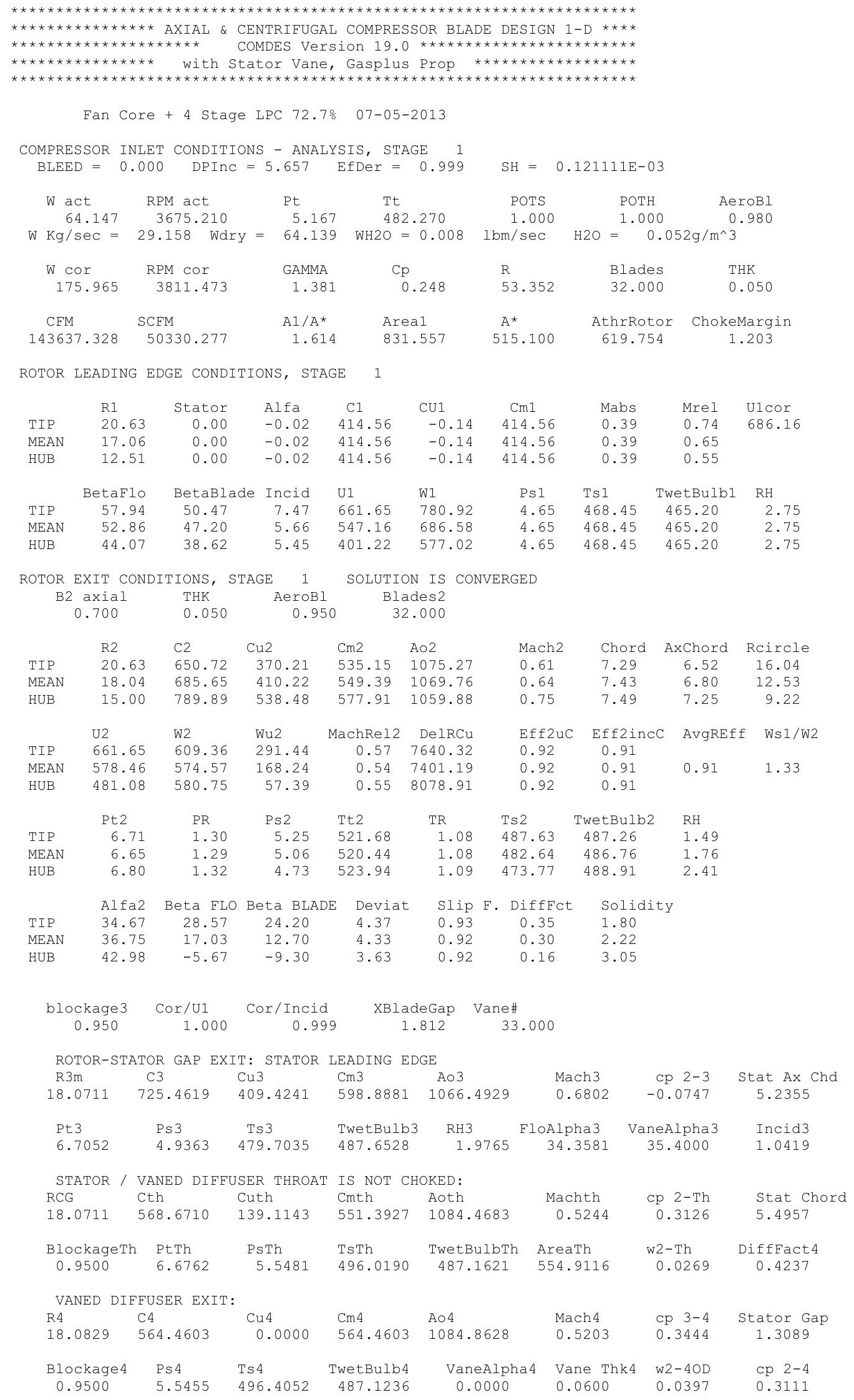




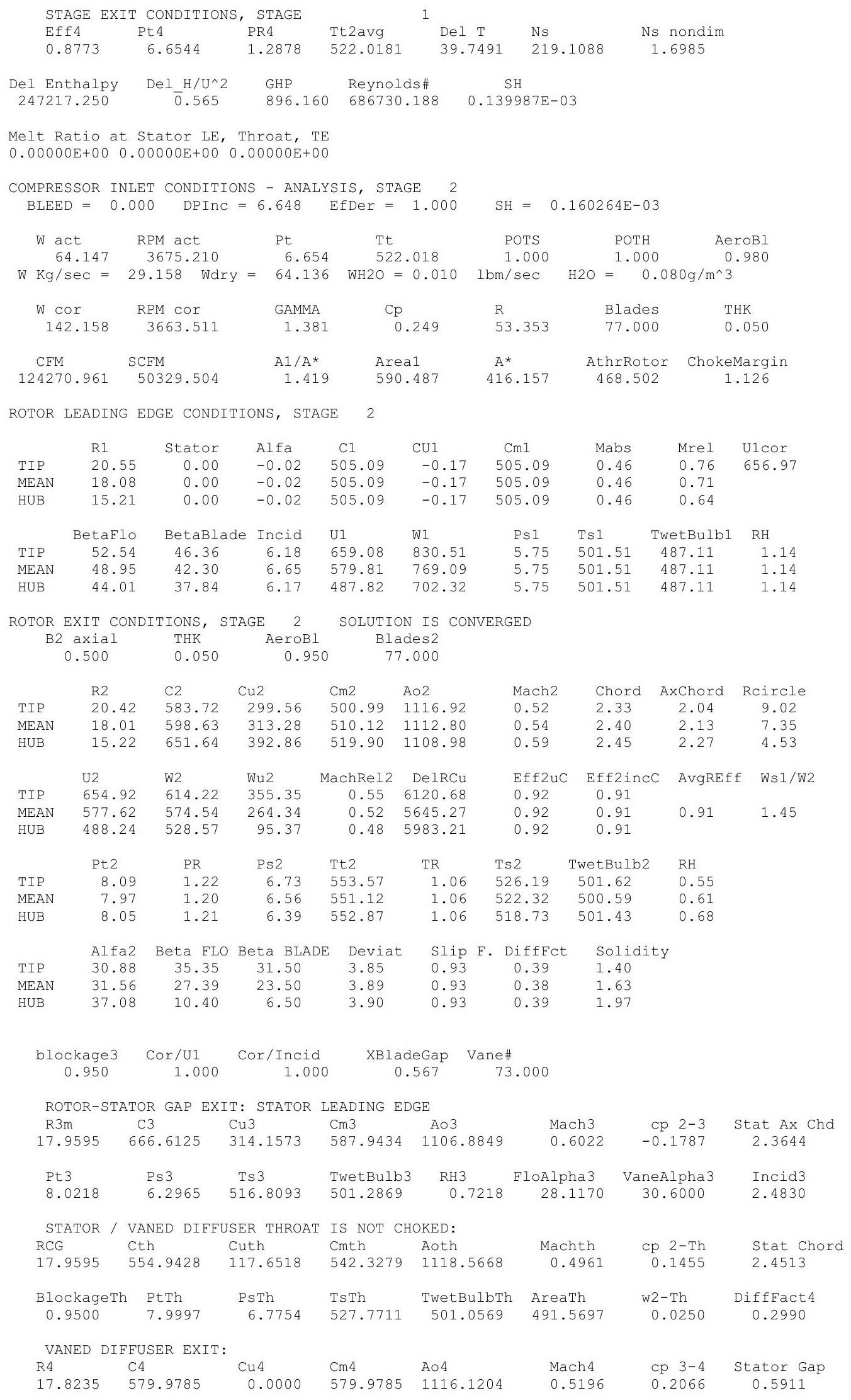




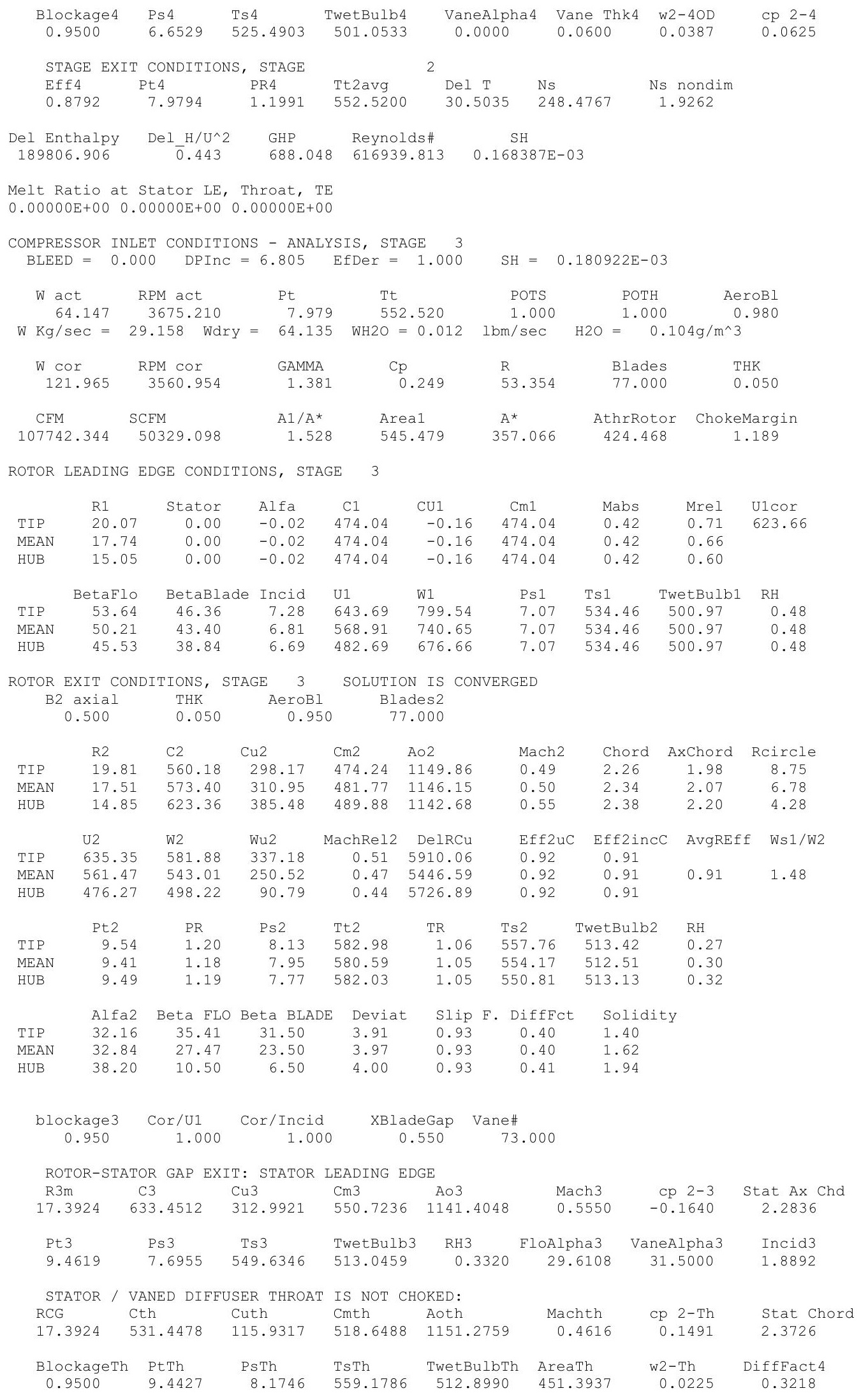




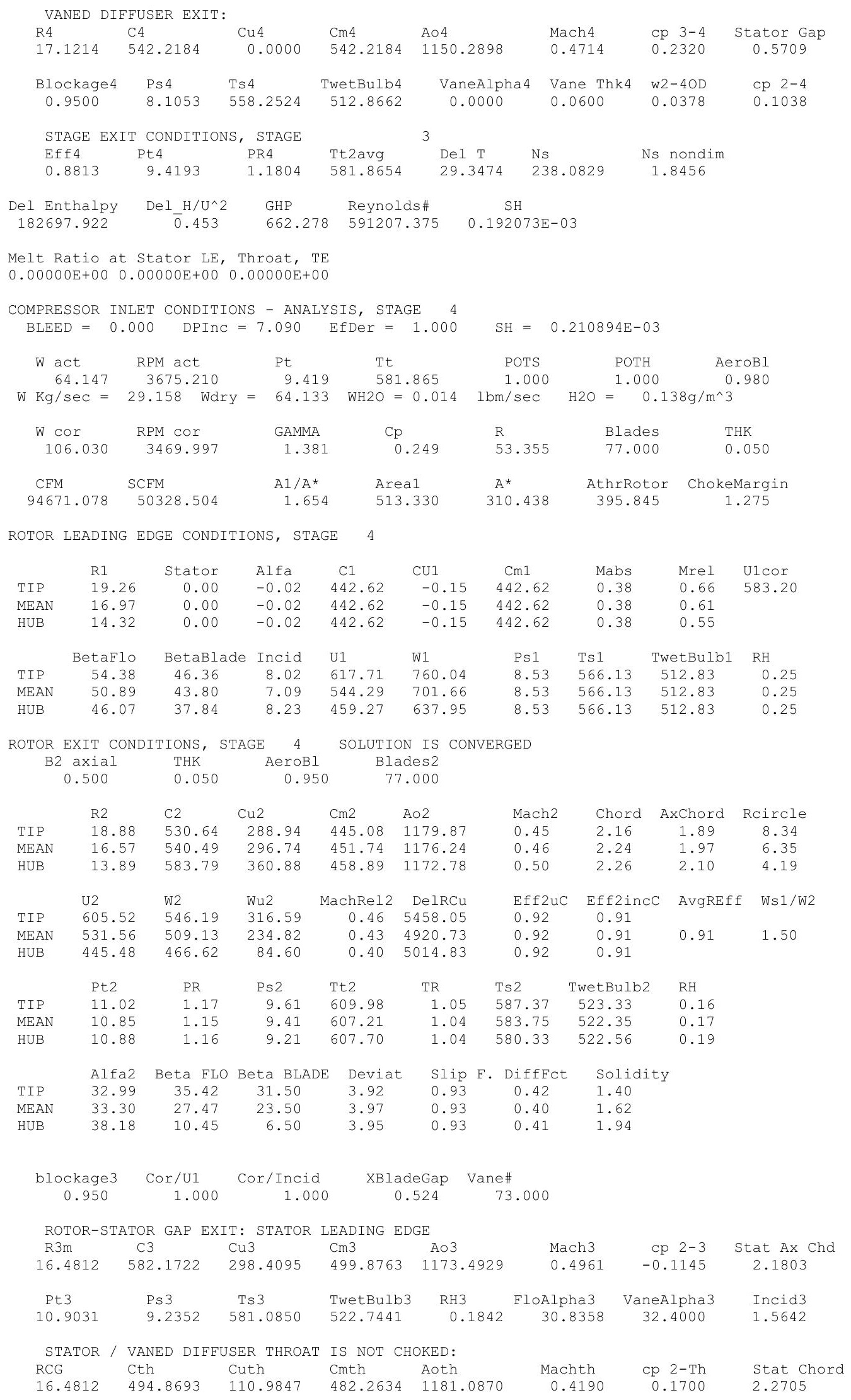




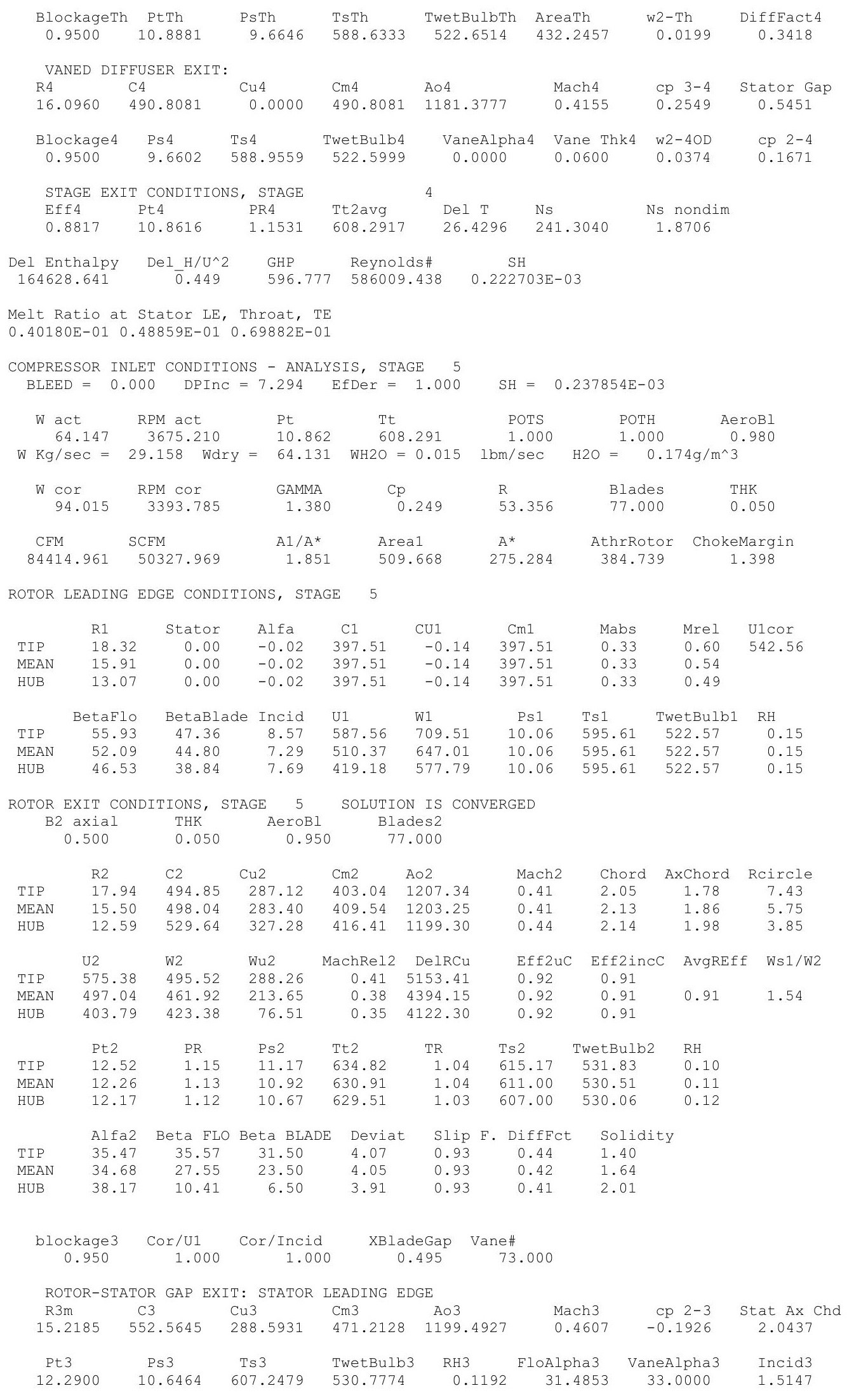




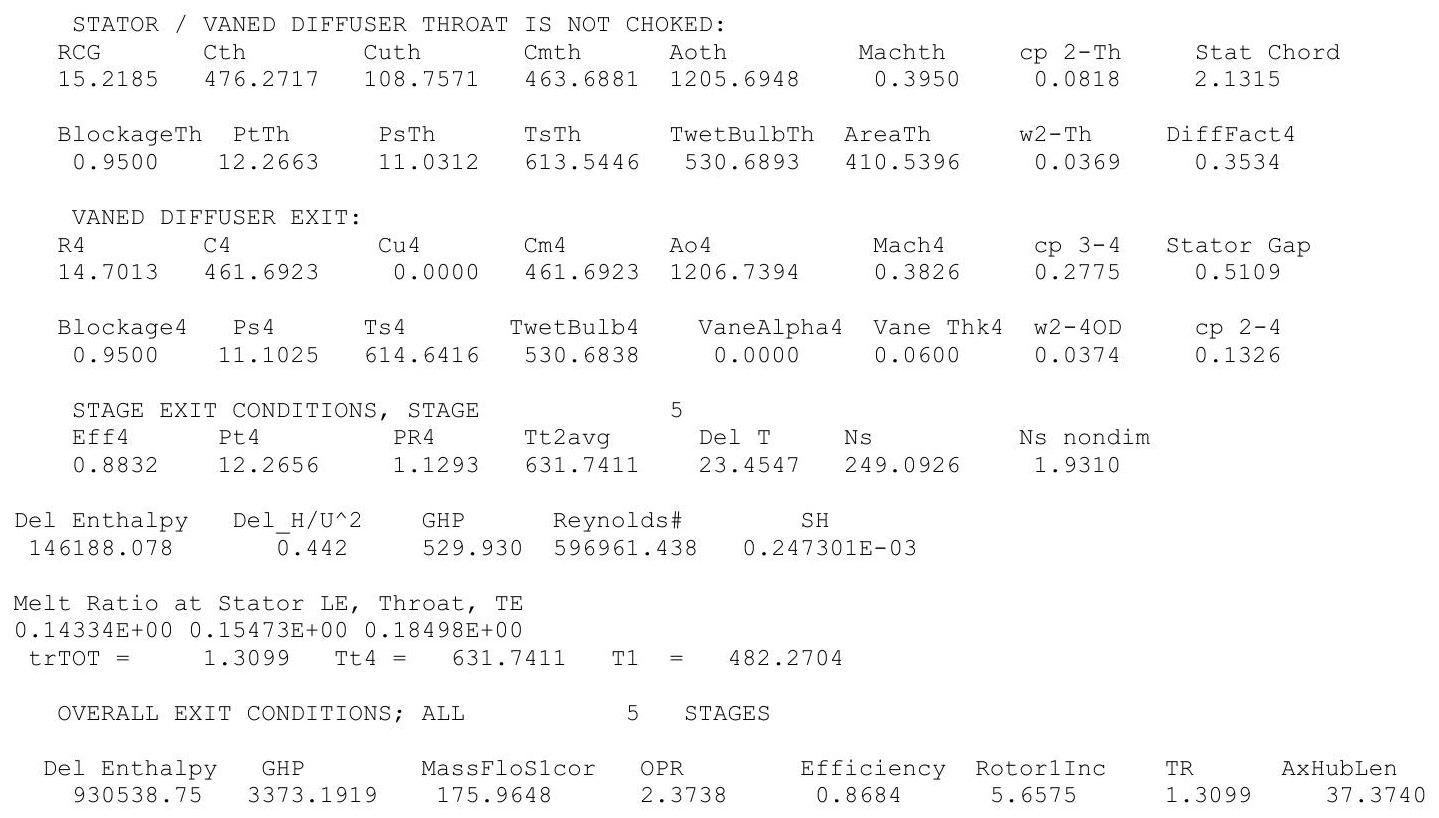




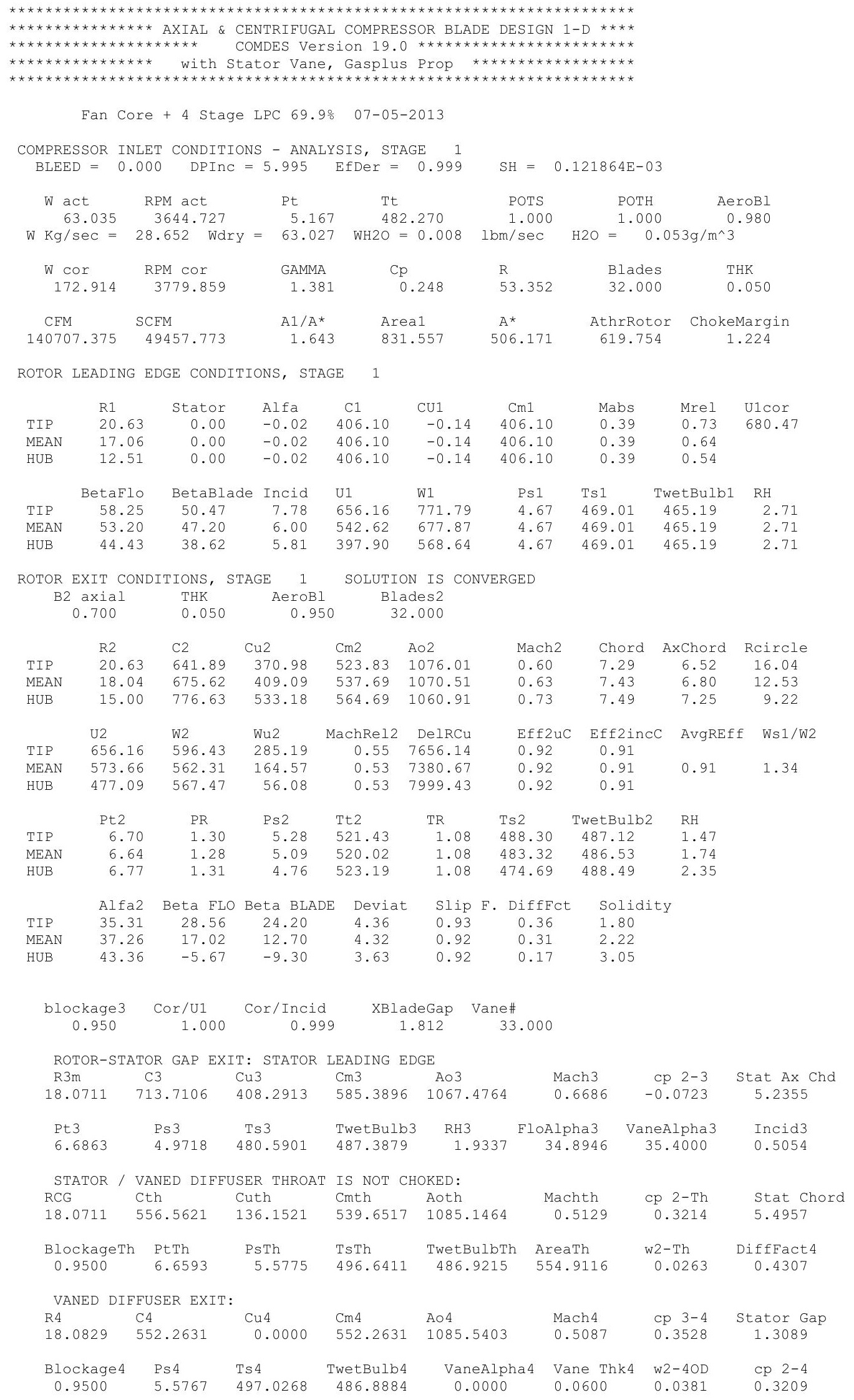




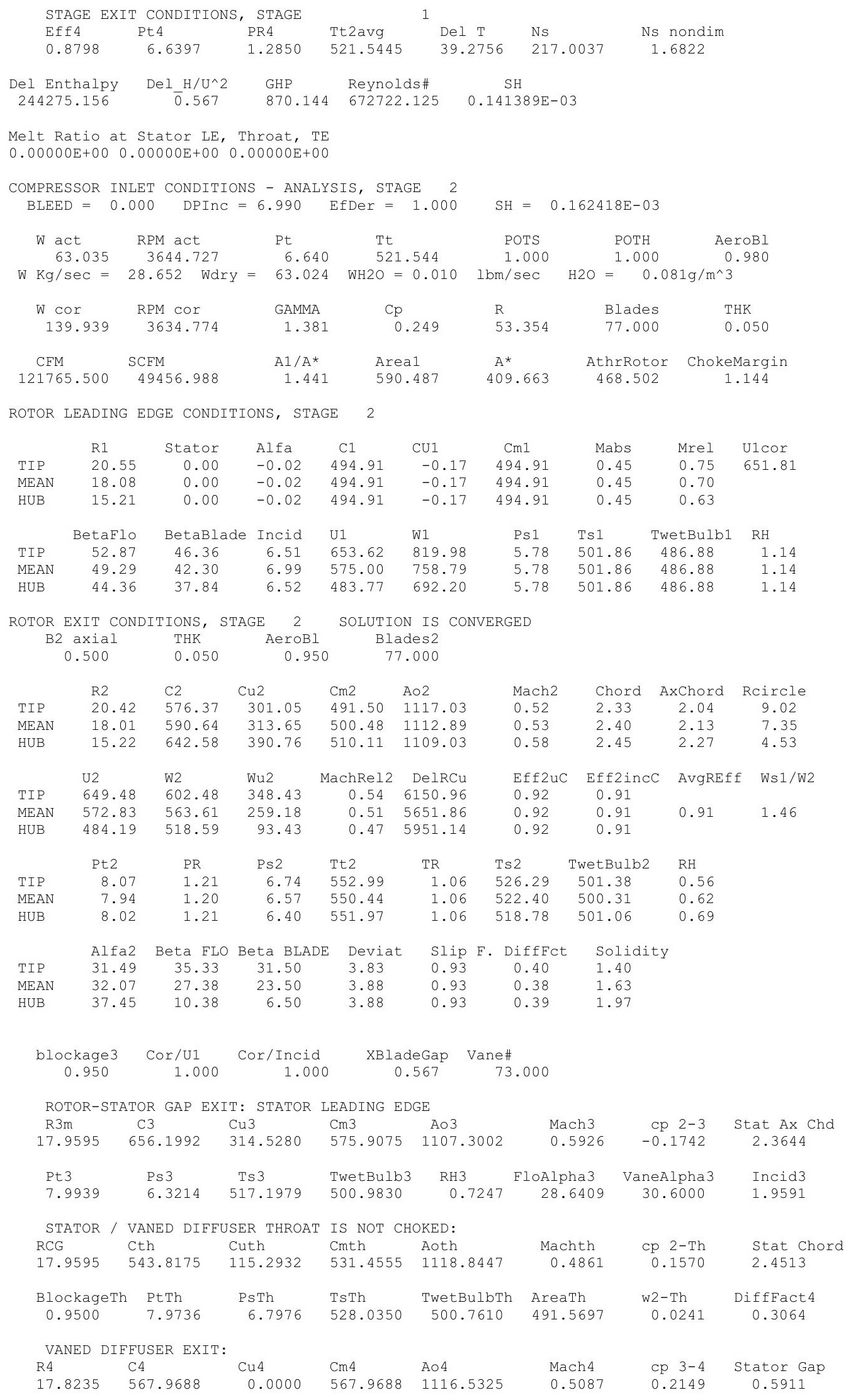




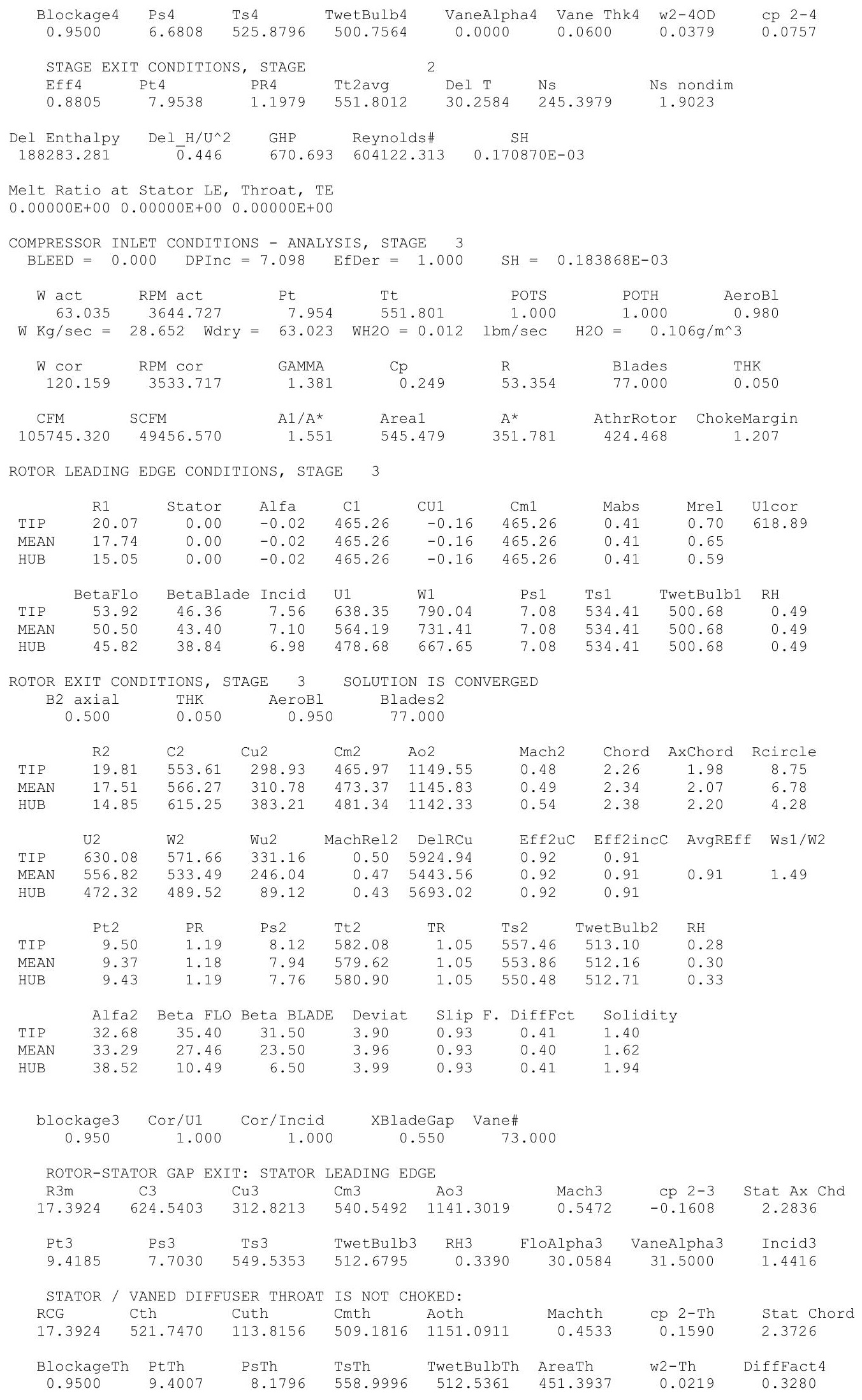




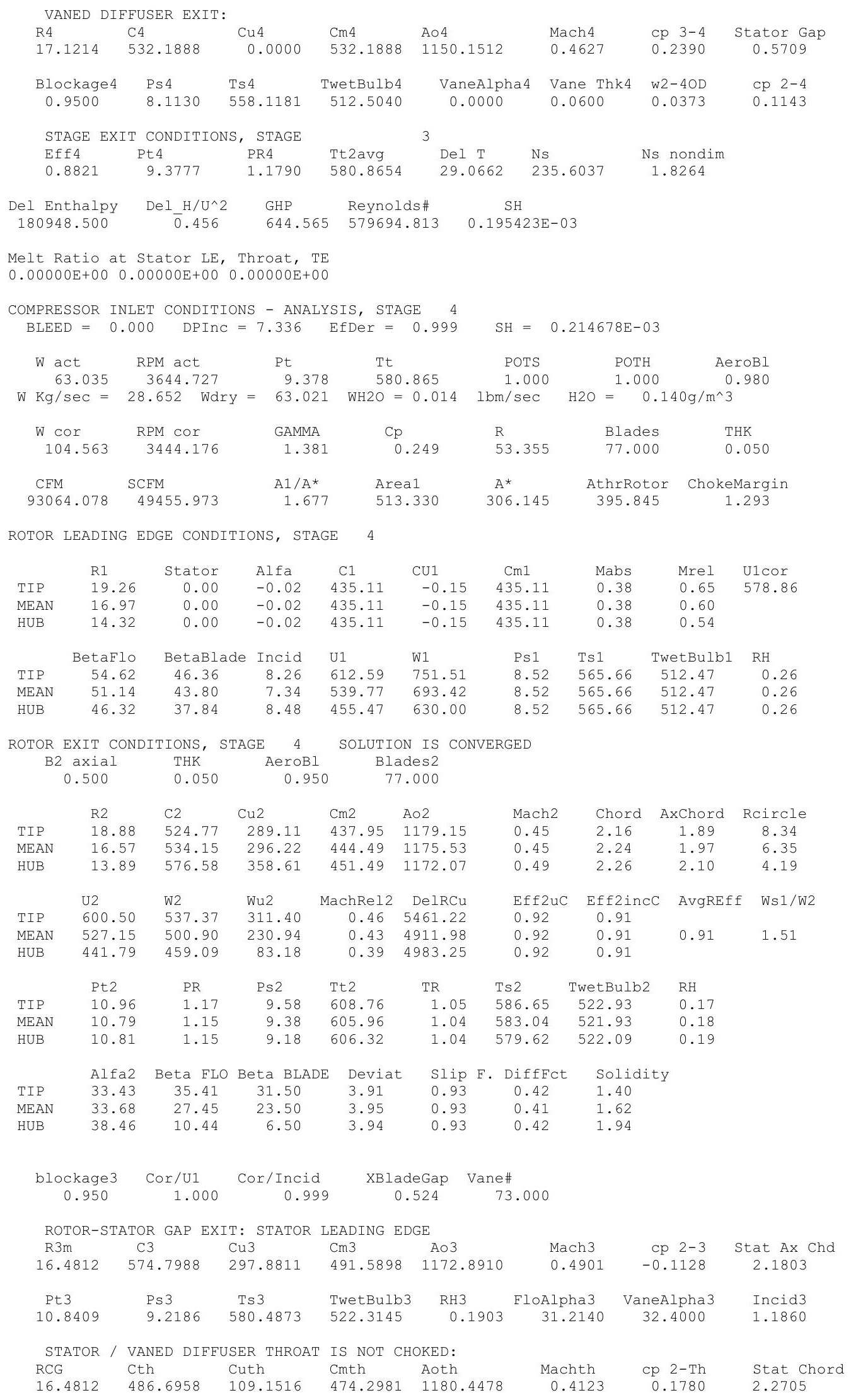




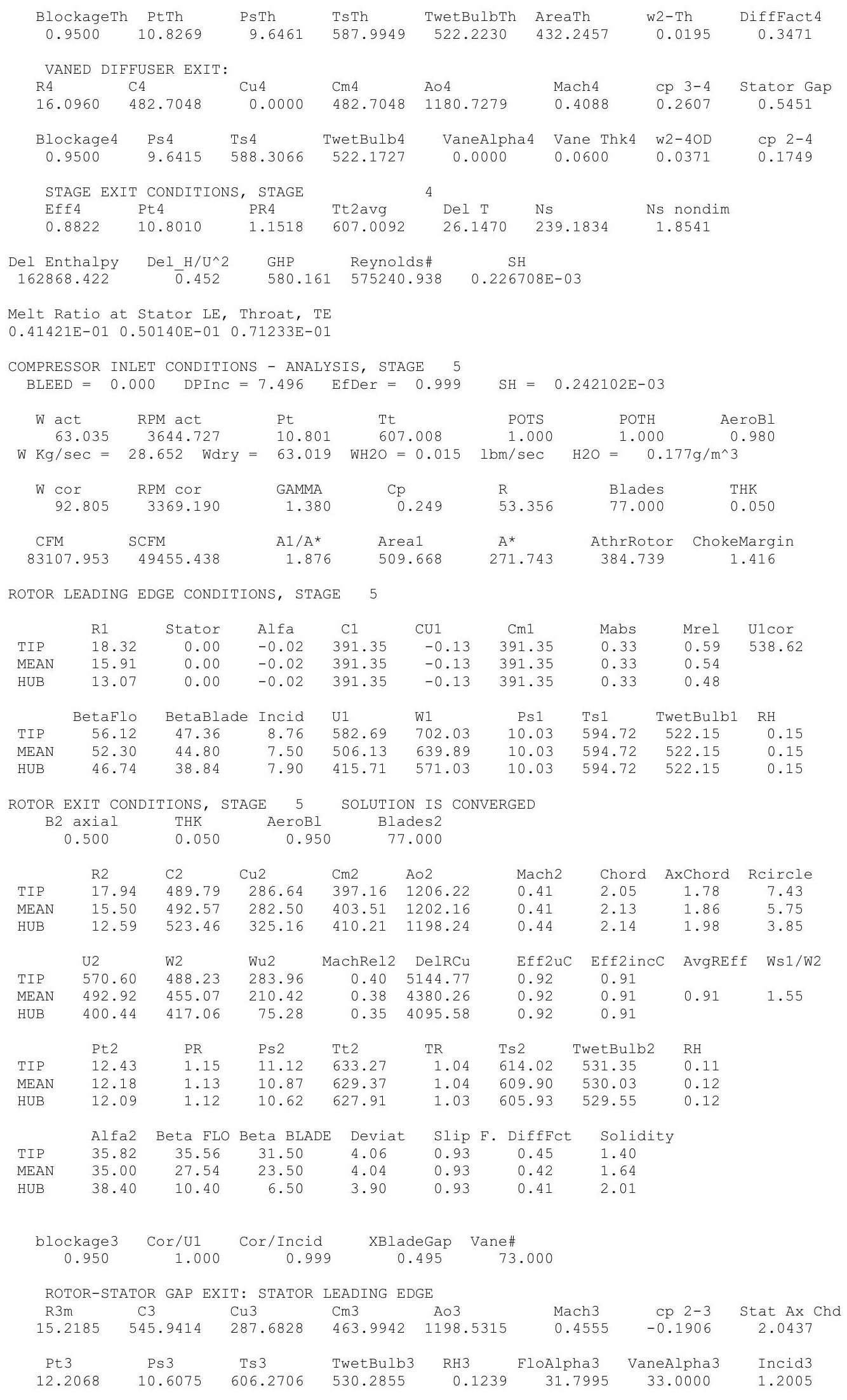




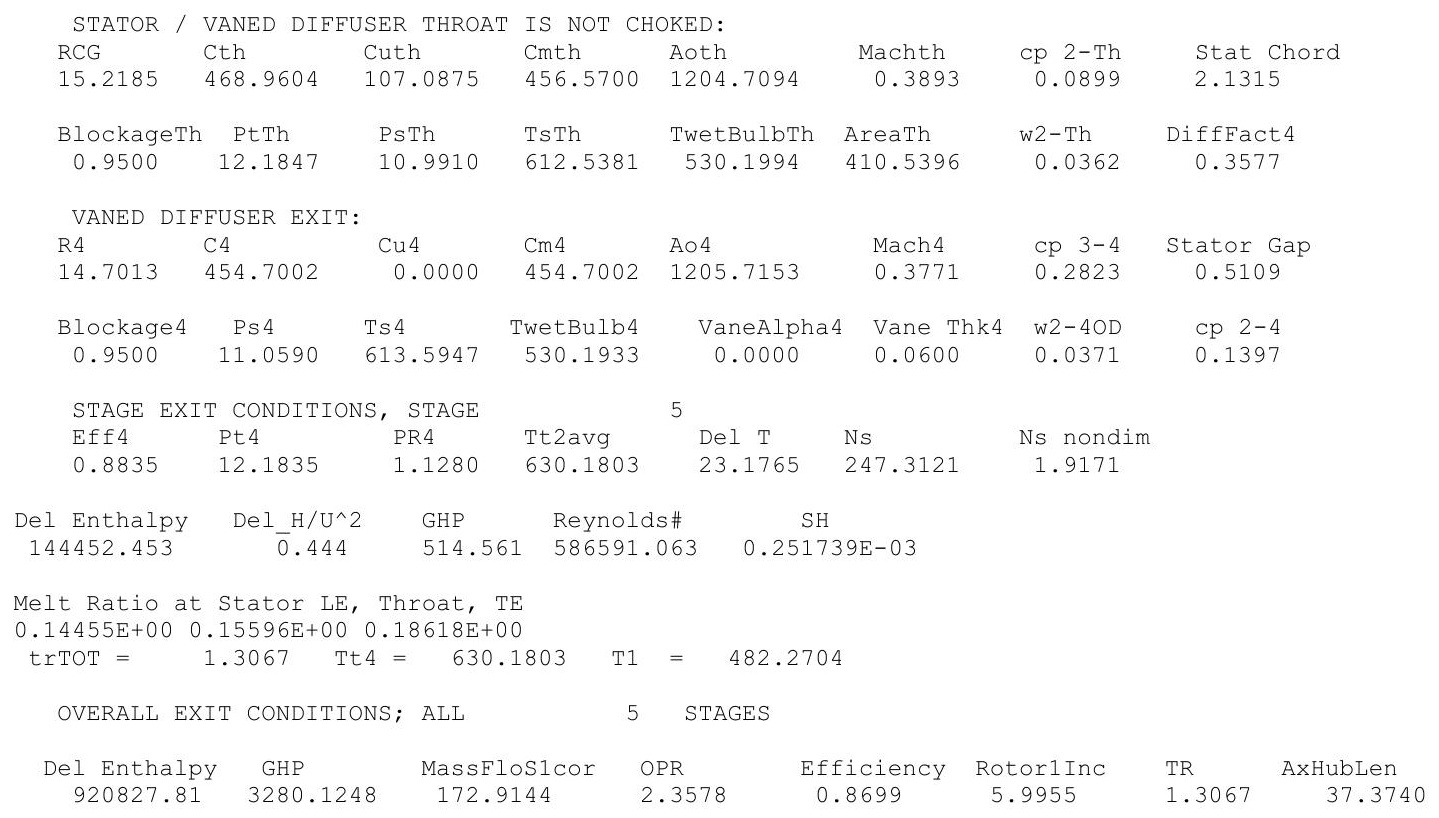




\section{$50 \mu \mathrm{m}, \mathrm{ISA}+18 \mathrm{R}$}

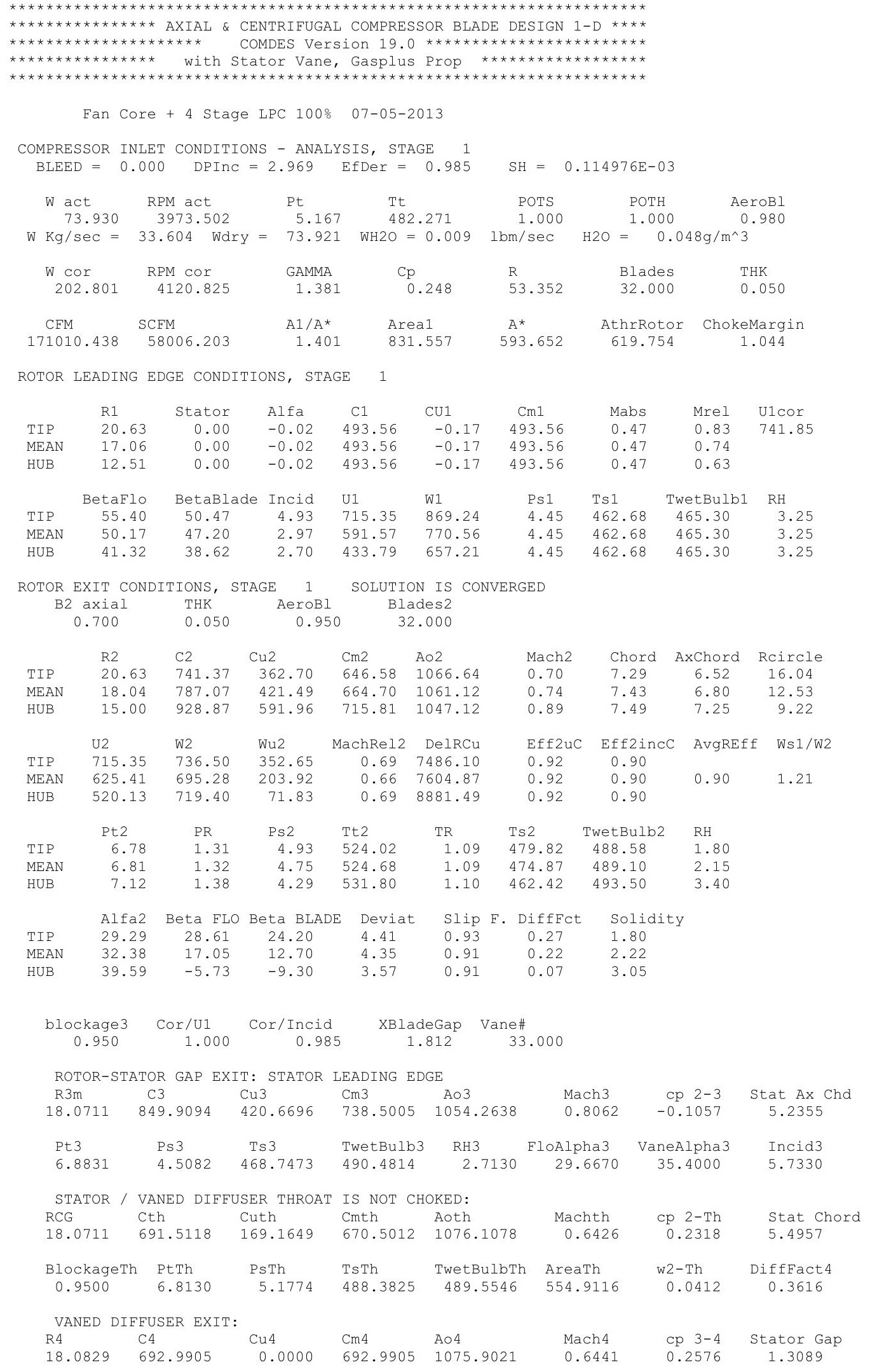




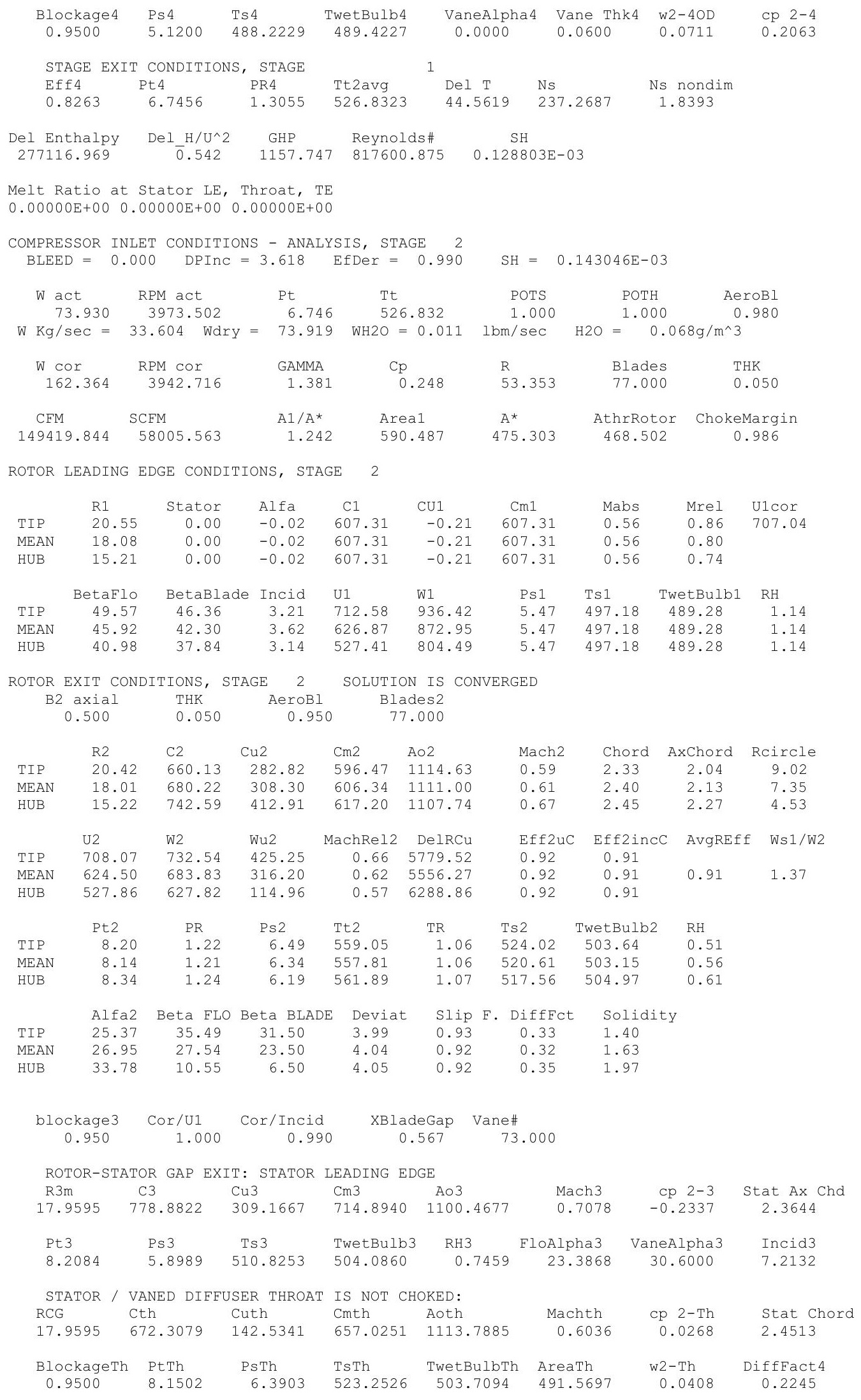




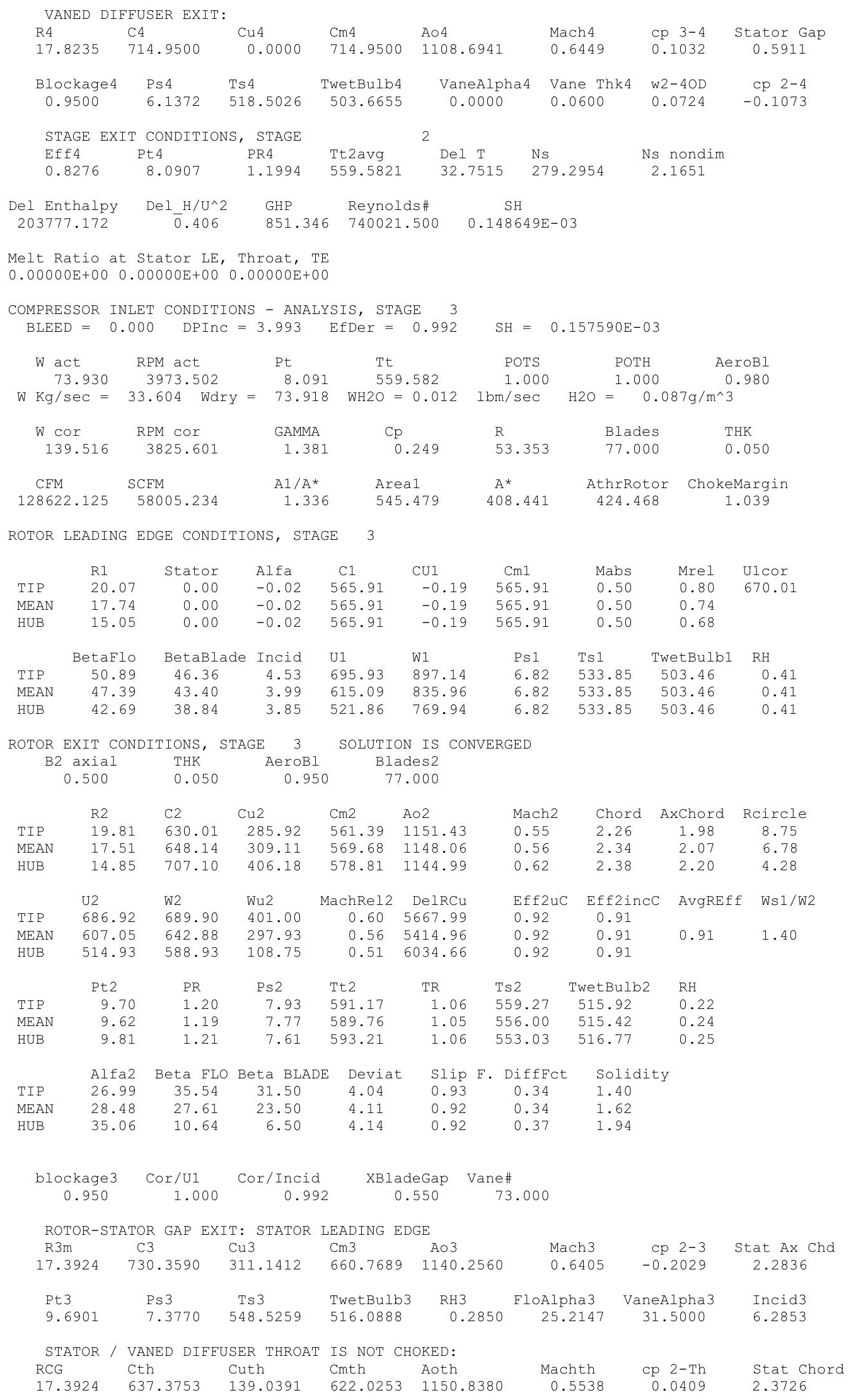




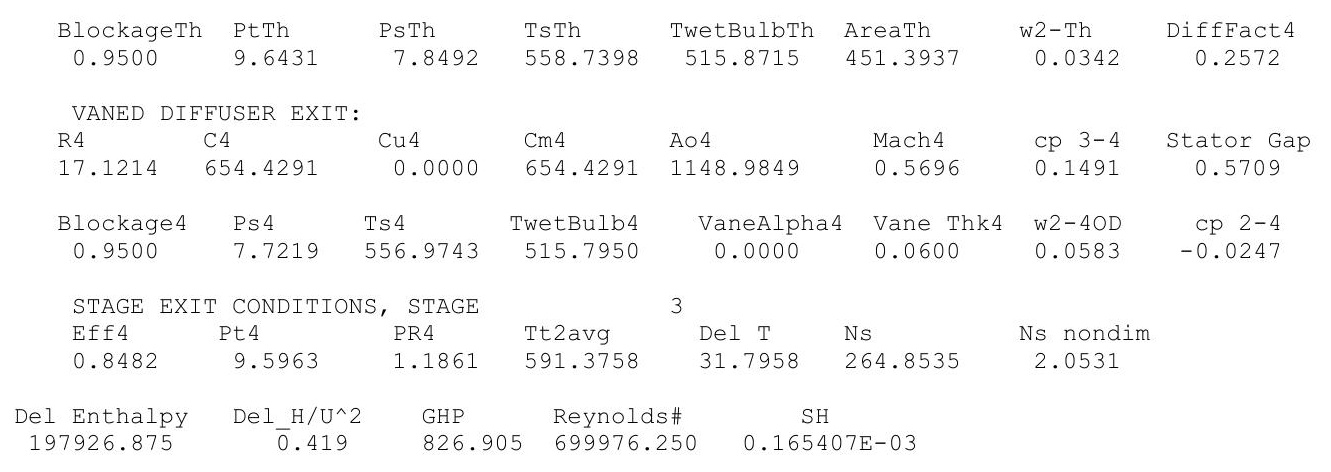

Melt Ratio at Stator LE, Throat, TE

$0.00000 \mathrm{E}+00 \quad 0.00000 \mathrm{E}+00 \quad 0.00000 \mathrm{E}+00$

COMPRESSOR INLET CONDITIONS - ANALYSIS, STAGE 4 $\mathrm{BLEED}=0.000 \quad$ DPInC $=4.577 \quad$ EfDer $=0.995$

ROTOR LEADING EDGE CONDITIONS, STAGE 4

$\begin{array}{lccccccccc} & \text { R1 } & \text { Stator } & \text { Alfa } & \text { C1 } & \text { CU1 } & \text { Cm1 } & \text { Mabs } & \text { Mrel } & \text { U1cor } \\ \text { TIP } & 19.26 & 0.00 & -0.02 & 523.04 & -0.18 & 523.04 & 0.45 & 0.73 & 625.45 \\ \text { MEAN } & 16.97 & 0.00 & -0.02 & 523.04 & -0.18 & 523.04 & 0.45 & 0.68 & \\ \text { HUB } & 14.32 & 0.00 & -0.02 & 523.04 & -0.18 & 523.04 & 0.45 & 0.62 & \\ & & & & & & & & & \\ & \text { BetaFlo } & \text { BetaBlade } & \text { Incid } & \text { U1 } & \text { W1 } & \text { Ps1 } & \text { Ts1 } & \text { TwetBulb1 } & \text { RH } \\ \text { TIP } & 51.94 & 46.36 & 5.58 & 667.85 & 848.43 & 8.37 & 569.41 & 515.71 & 0.19 \\ \text { MEAN } & 48.38 & 43.80 & 4.58 & 588.47 & 787.45 & 8.37 & 569.41 & 515.71 & 0.19 \\ \text { HUB } & 43.52 & 37.84 & 5.68 & 496.55 & 721.33 & 8.37 & 569.41 & 515.71 & 0.19\end{array}$

ROTOR EXIT CONDITIONS, STAGE 4 SOLUTION IS CONVERGED

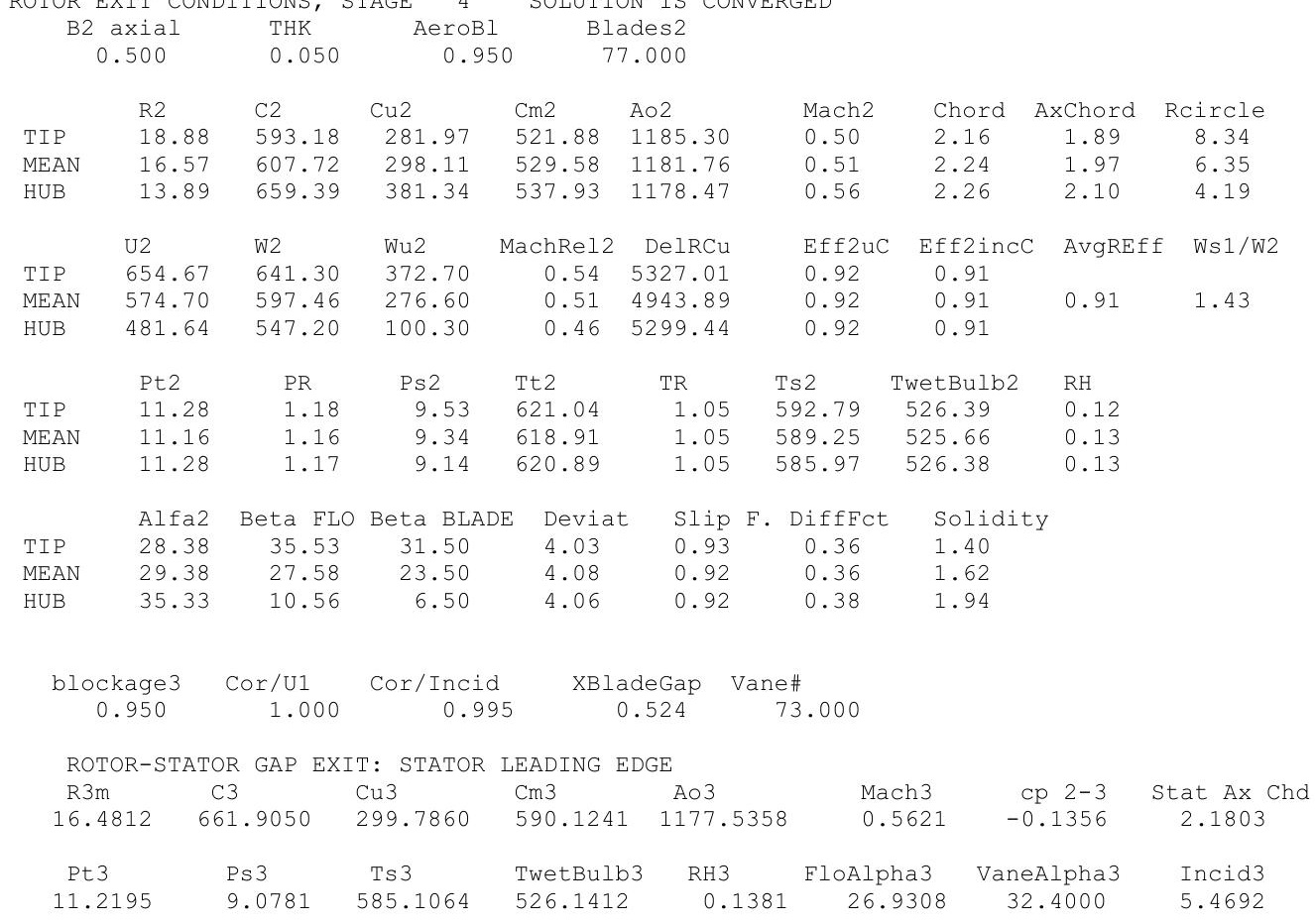




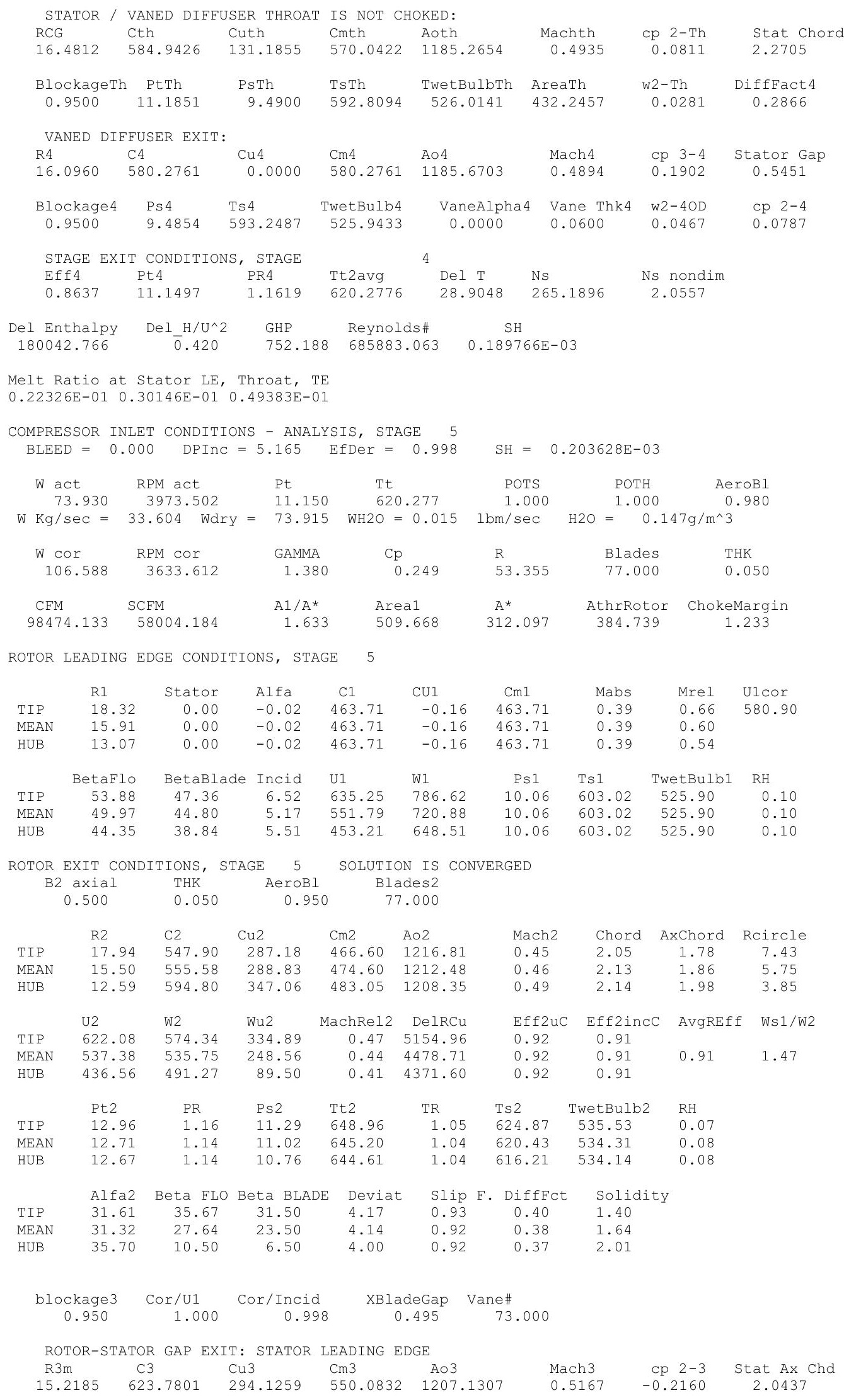




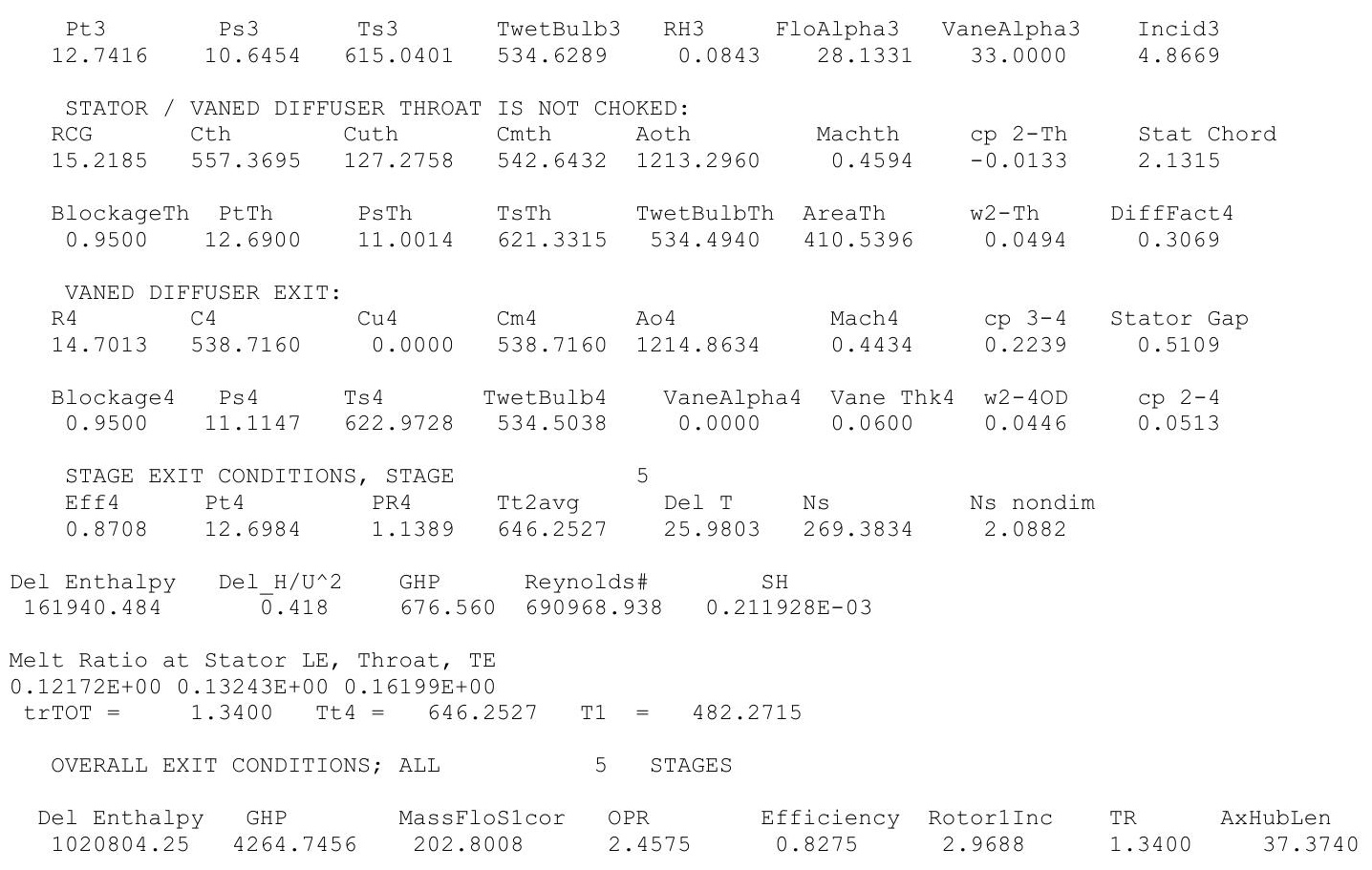




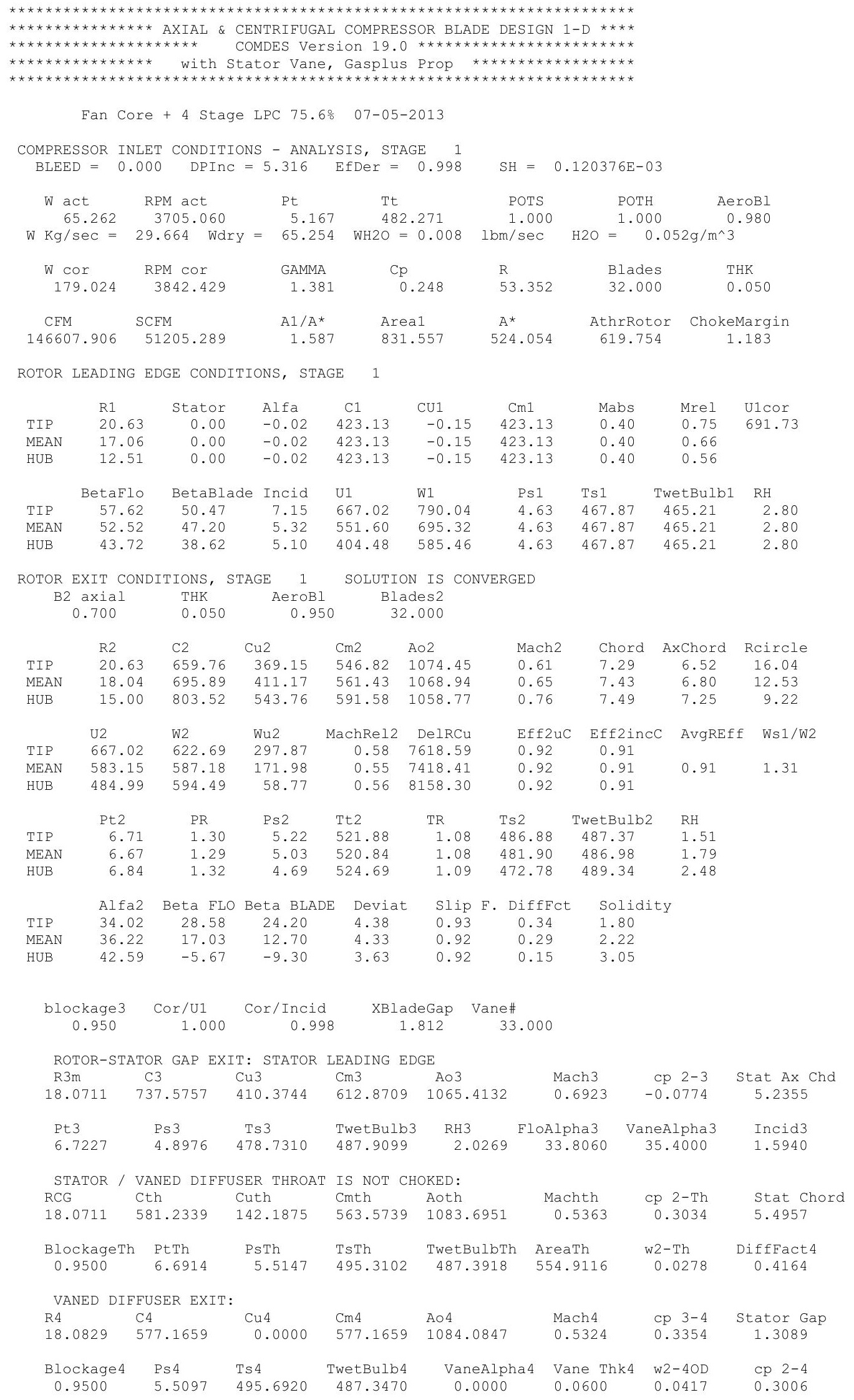




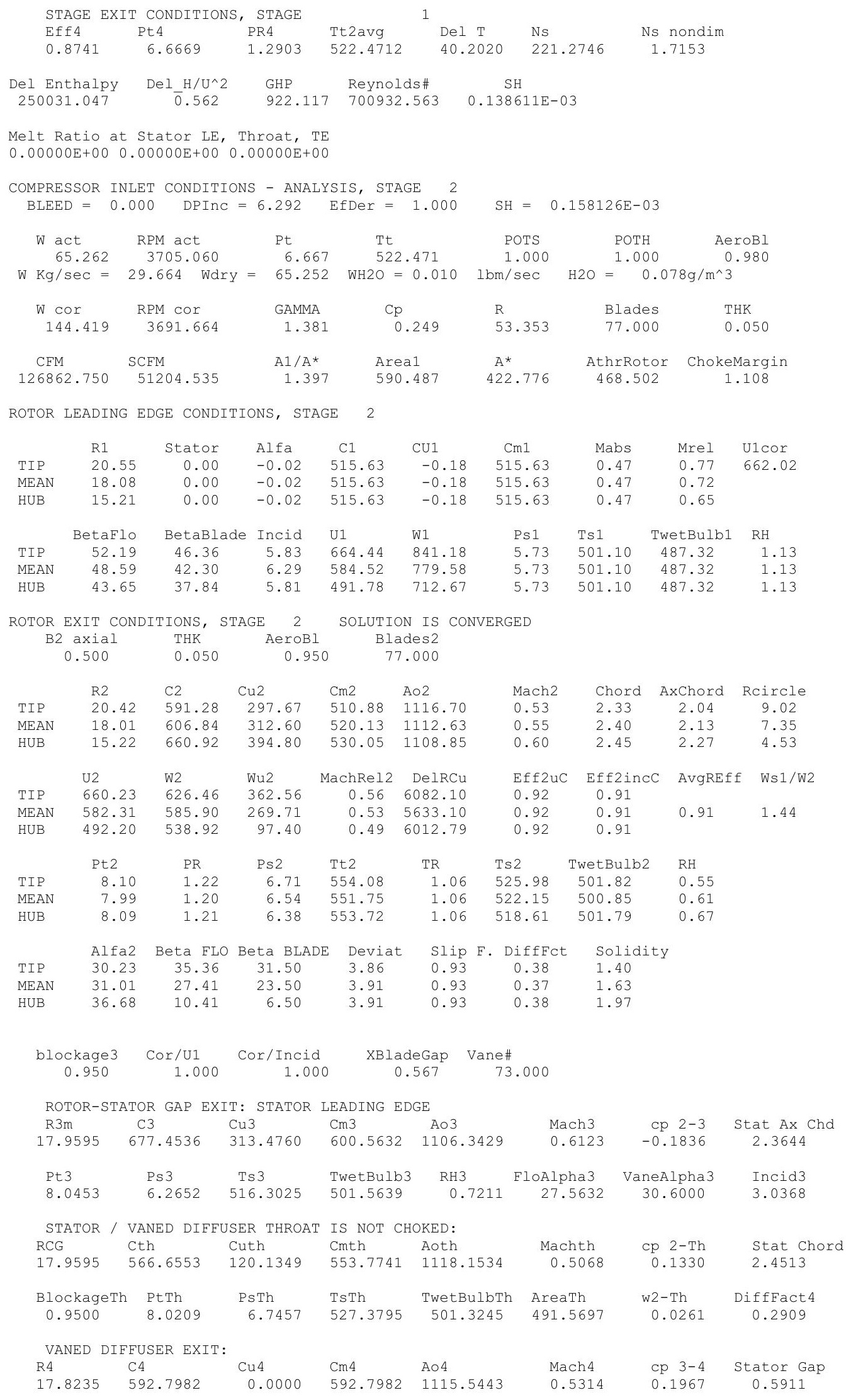




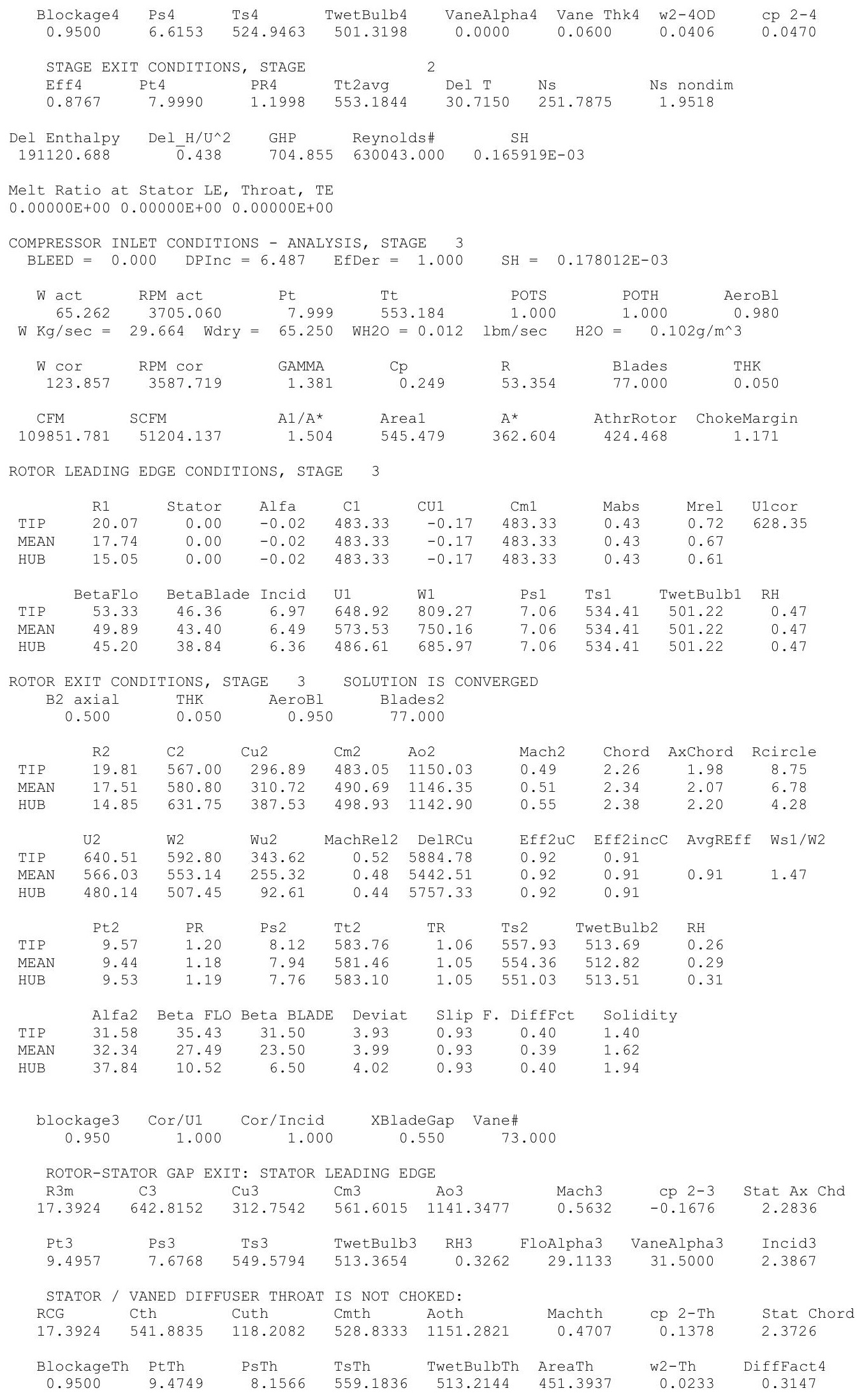




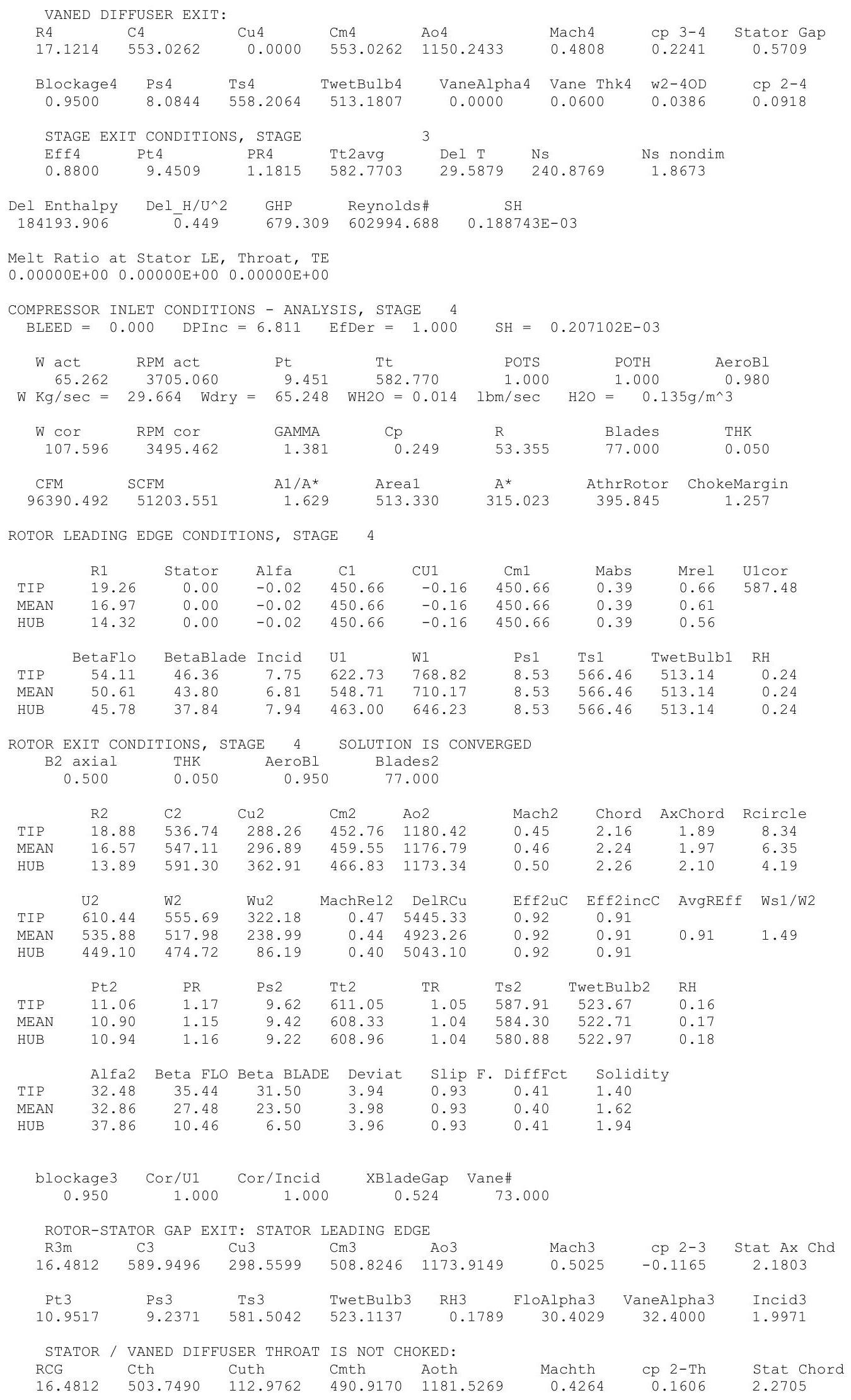




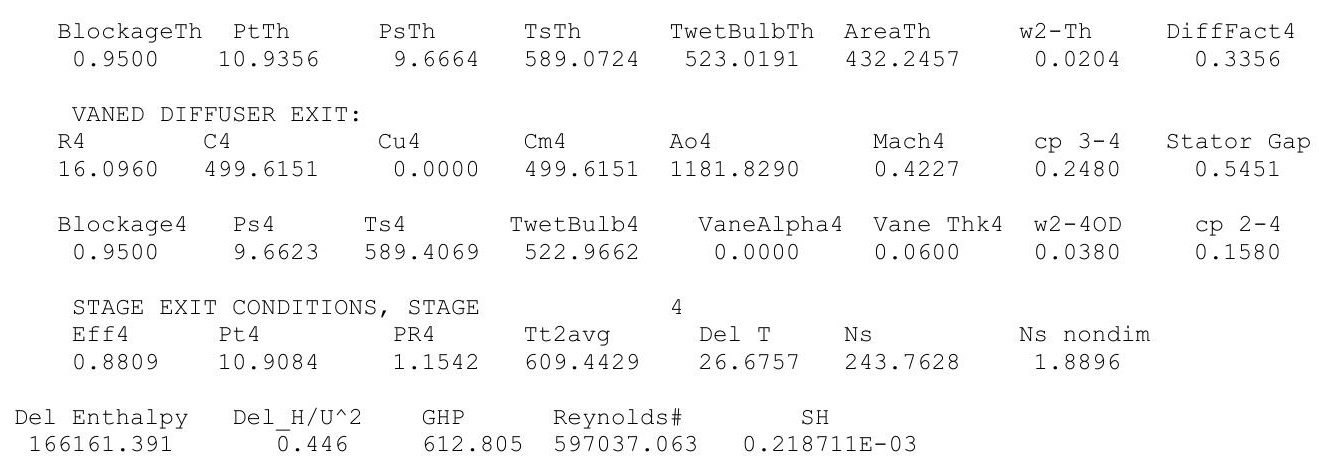

Melt Ratio at Stator LE, Throat, TE

$0.38448 \mathrm{E}-01 \quad 0.47042 \mathrm{E}-01 \quad 0.67898 \mathrm{E}-01$

COMPRESSOR INLET CONDITIONS - ANALYSIS, STAGE 5 $\mathrm{BLEED}=0.000 \quad$ DPInC $=7.057 \quad$ EfDer $=1.000$

$\begin{array}{llllll}W & \text { Ret } & \text { Pt } & \text { POTS } & \text { POTH } & \text { AeroBl }\end{array}$ $\mathrm{W} \mathrm{Kg} / \mathrm{sec}=29.664$ Wdry $=65.247 \quad \mathrm{WH} 2 \mathrm{O}=0.015 \quad \mathrm{lbm} / \mathrm{sec} \quad \mathrm{H} 2 \mathrm{O}=0.171 \mathrm{~g} / \mathrm{m}^{\wedge} 3$

$\begin{array}{ccccccc}\begin{array}{c}\text { W Cor } \\ 95.329\end{array} & \text { RPM cor } & \text { GAMMA } & \text { Cp } & \text { R } & \text { Blades } & \text { THK } \\ & 3418.116 & 1.380 & 0.249 & 53.356 & 77.000 & 0.050 \\ \text { CFM } & \text { SCFM } & \text { A1/A* } & \text { Area1 } & A^{*} & \text { AthrRotor } & \text { ChokeMargin } \\ 85828.719 & 51203.020 & 1.826 & 509.668 & 279.132 & 384.739 & 1.378\end{array}$

ROTOR LEADING EDGE CONDITIONS, STAGE 5

$\begin{array}{lccccccccc} & \text { R1 } & \text { Stator } & \text { Alfa } & \text { C1 } & \text { CU1 } & \text { Cm1 } & \text { Mabs } & \text { Mrel } & \text { U1cor } \\ \text { TIP } & 18.32 & 0.00 & -0.02 & 404.16 & -0.14 & 404.16 & 0.34 & 0.60 & 546.45 \\ \text { MEAN } & 15.91 & 0.00 & -0.02 & 404.16 & -0.14 & 404.16 & 0.34 & 0.55 & \\ \text { HUB } & 13.07 & 0.00 & -0.02 & 404.16 & -0.14 & 404.16 & 0.34 & 0.49 & \\ & & & & & & & & & \\ \text { TIP } & \text { BetaFlo } & \text { BetaBlade } & \text { Incid } & \text { U1 } & \text { W1 } & \text { Ps1 } & \text { Ts1 } & \text { TwetBulb1 } & \text { RH } \\ \text { MEAN } & 55.70 & 47.36 & 8.34 & 592.34 & 717.20 & 10.08 & 596.33 & 522.94 & 0.14 \\ \text { HUB } & 46.29 & 44.80 & 7.06 & 514.51 & 654.38 & 10.08 & 596.33 & 522.94 & 0.14 \\ & & 38.84 & 7.45 & 422.59 & 584.85 & 10.08 & 596.33 & 522.94 & 0.14\end{array}$

ROTOR EXIT CONDITIONS, STAGE 5 SOLUTION IS CONVERGED

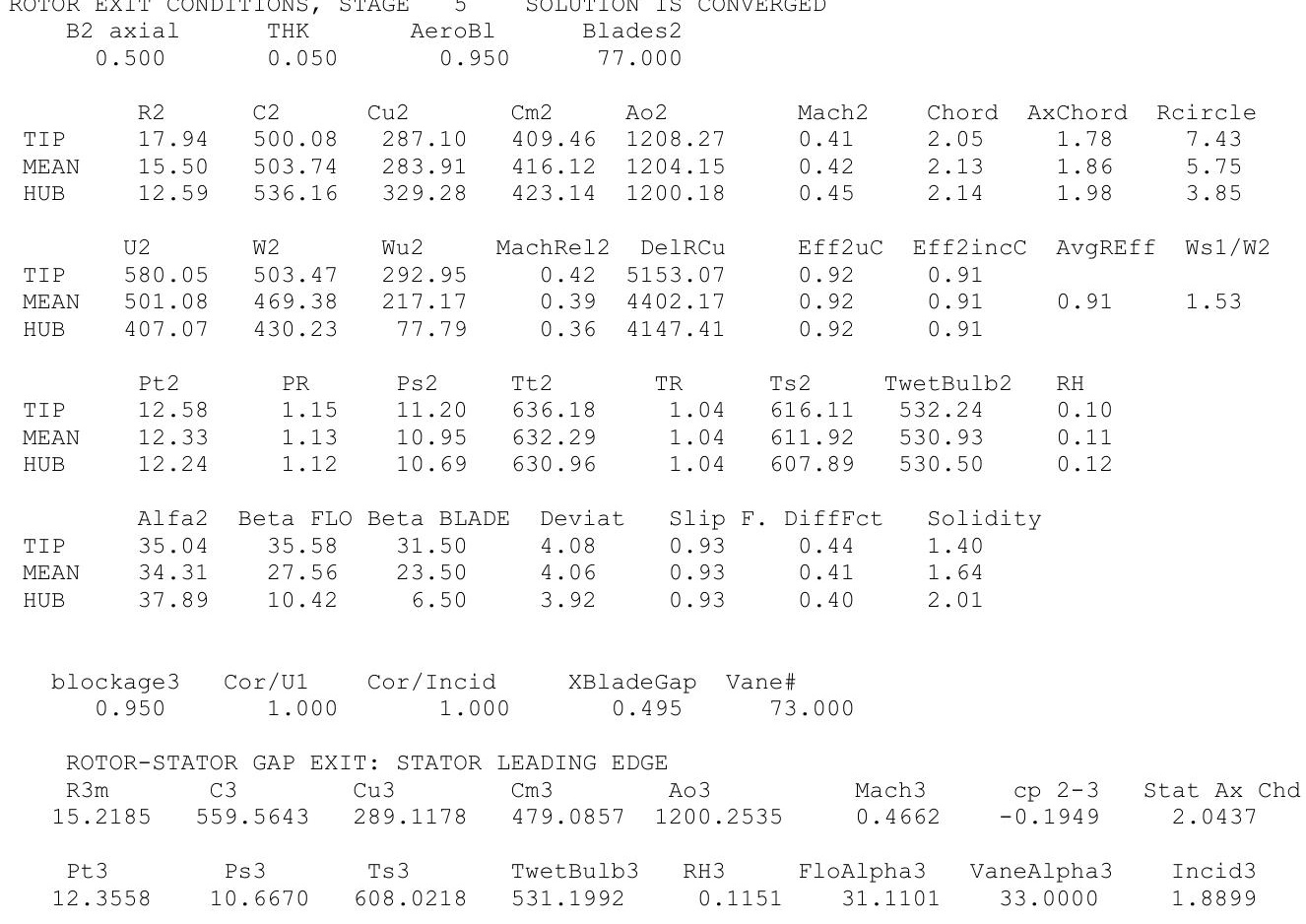




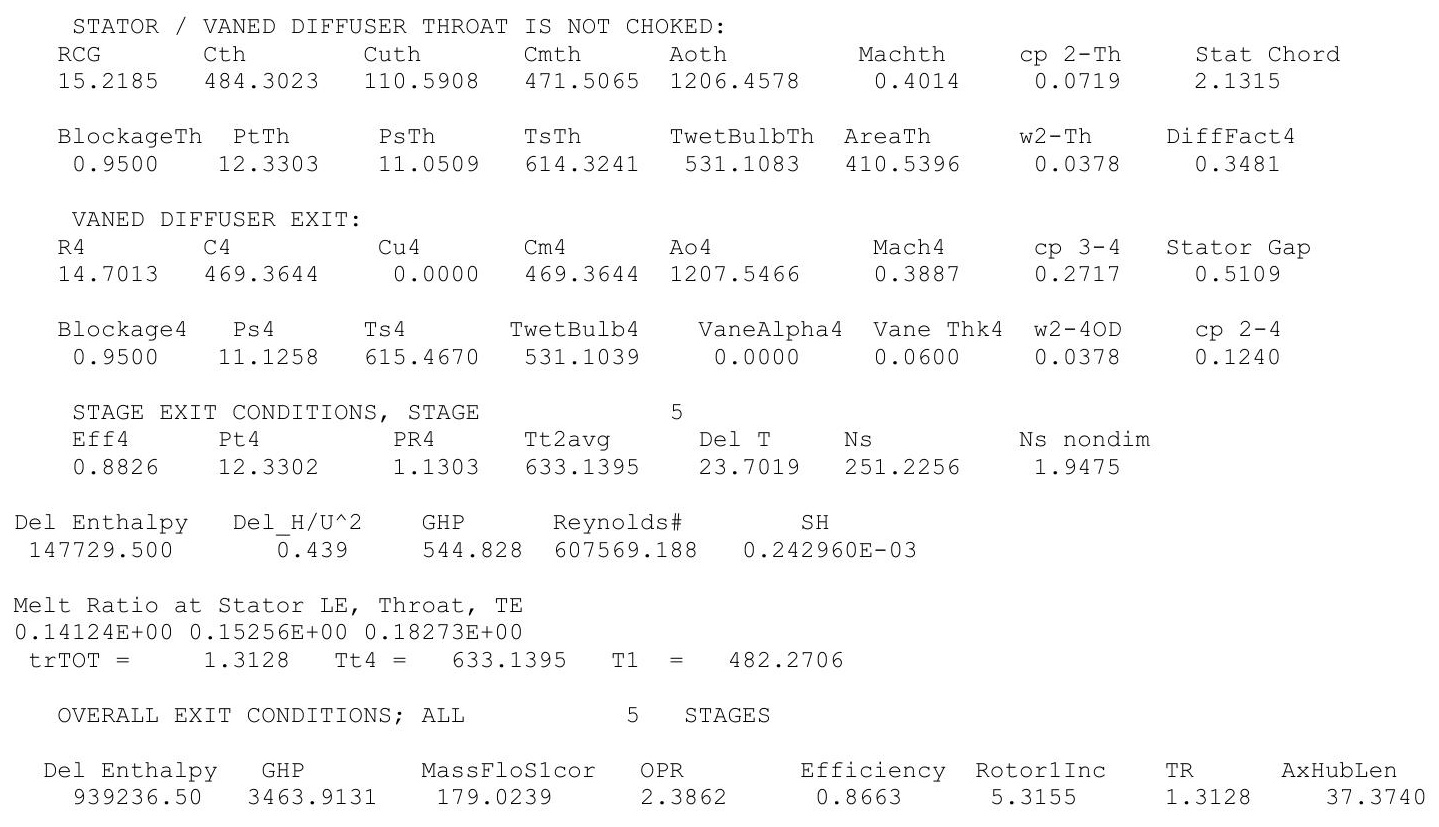




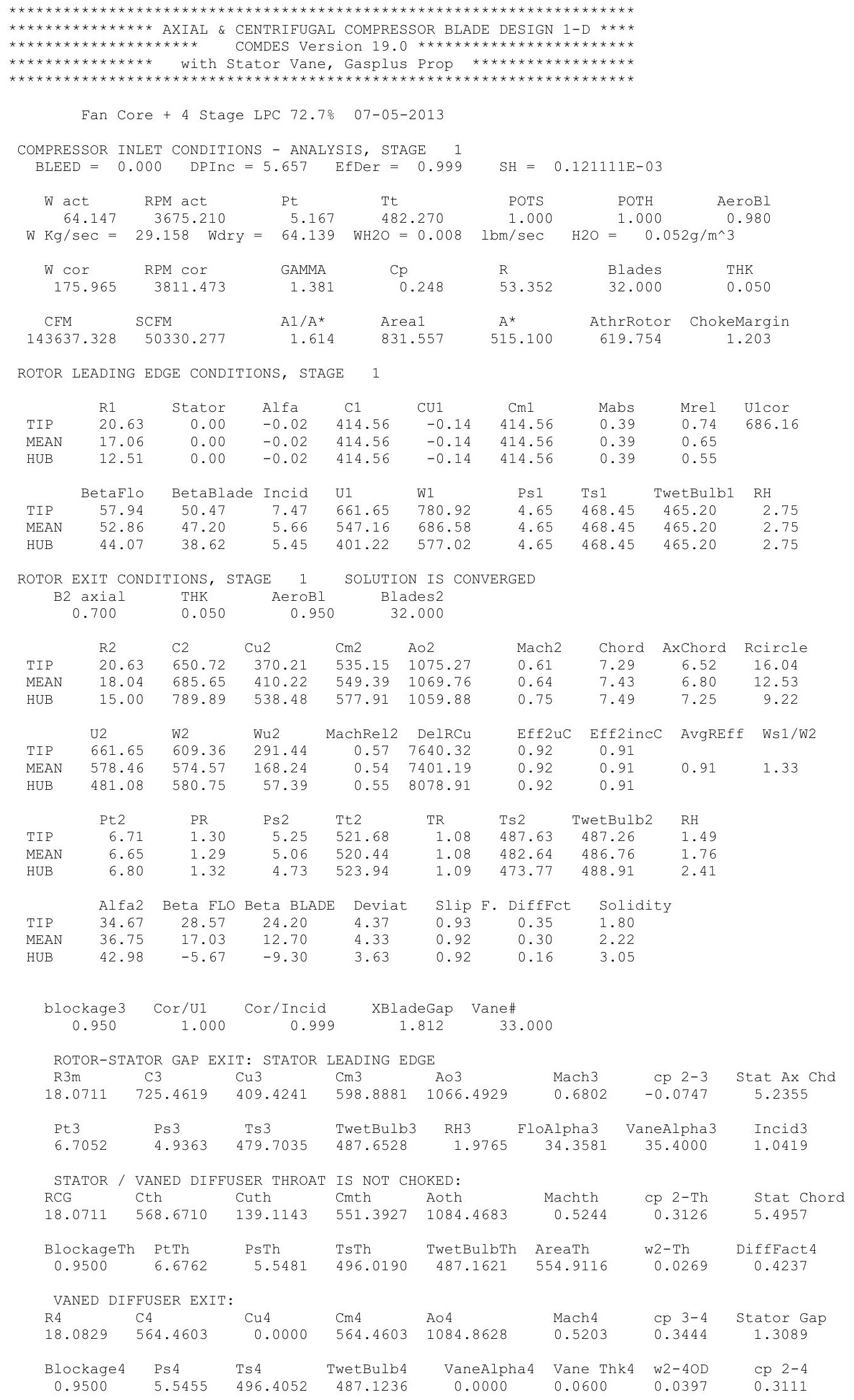




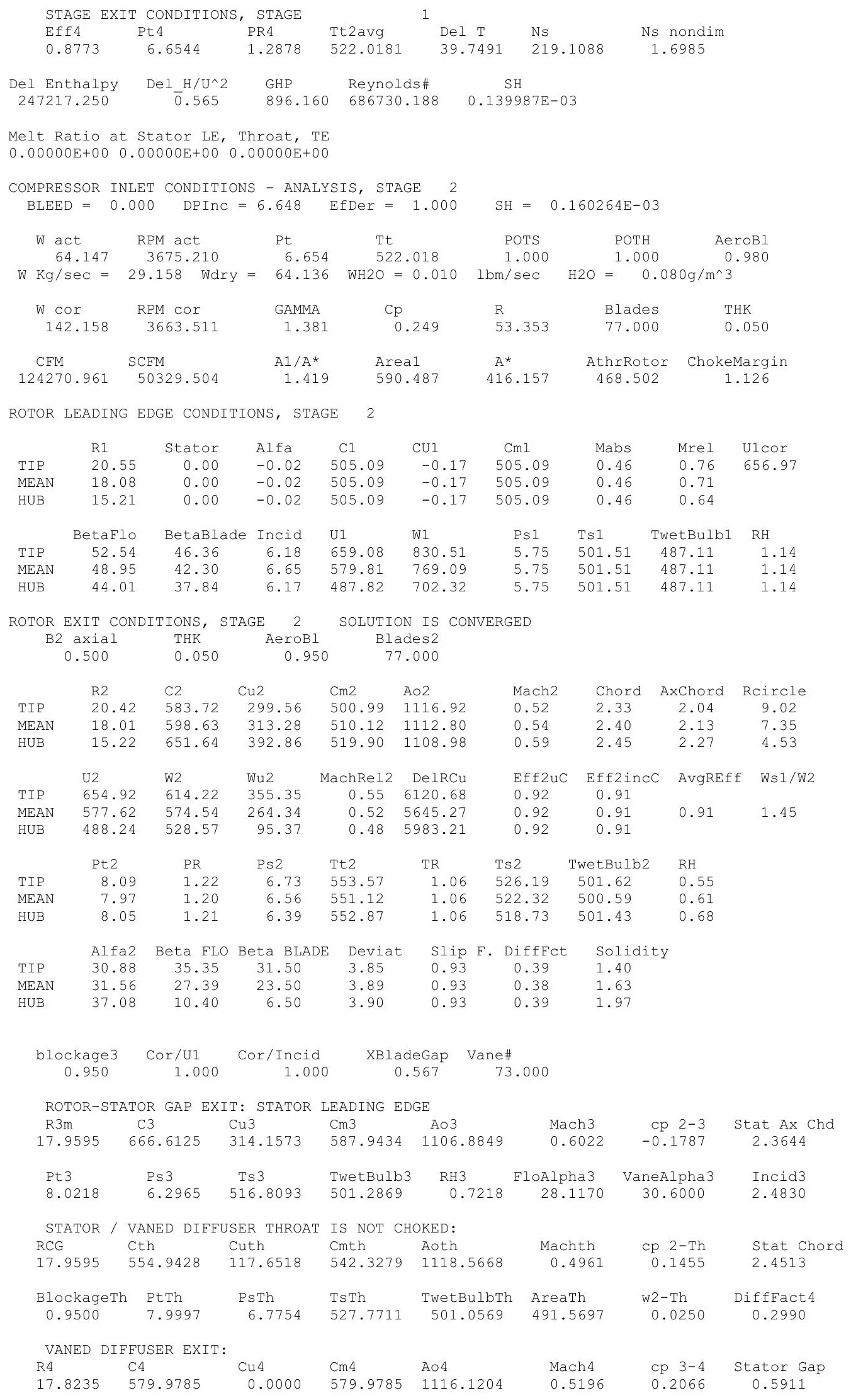




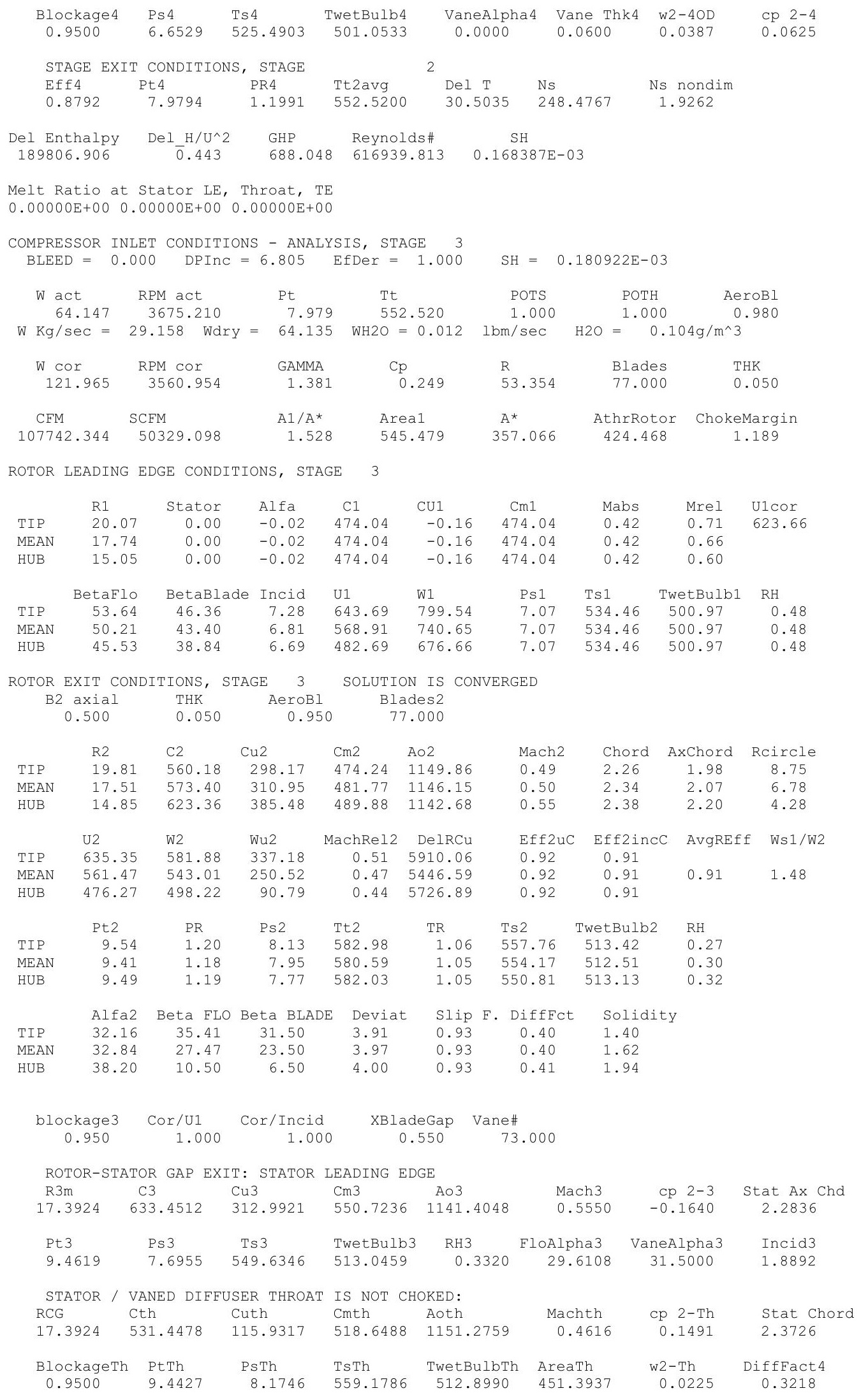




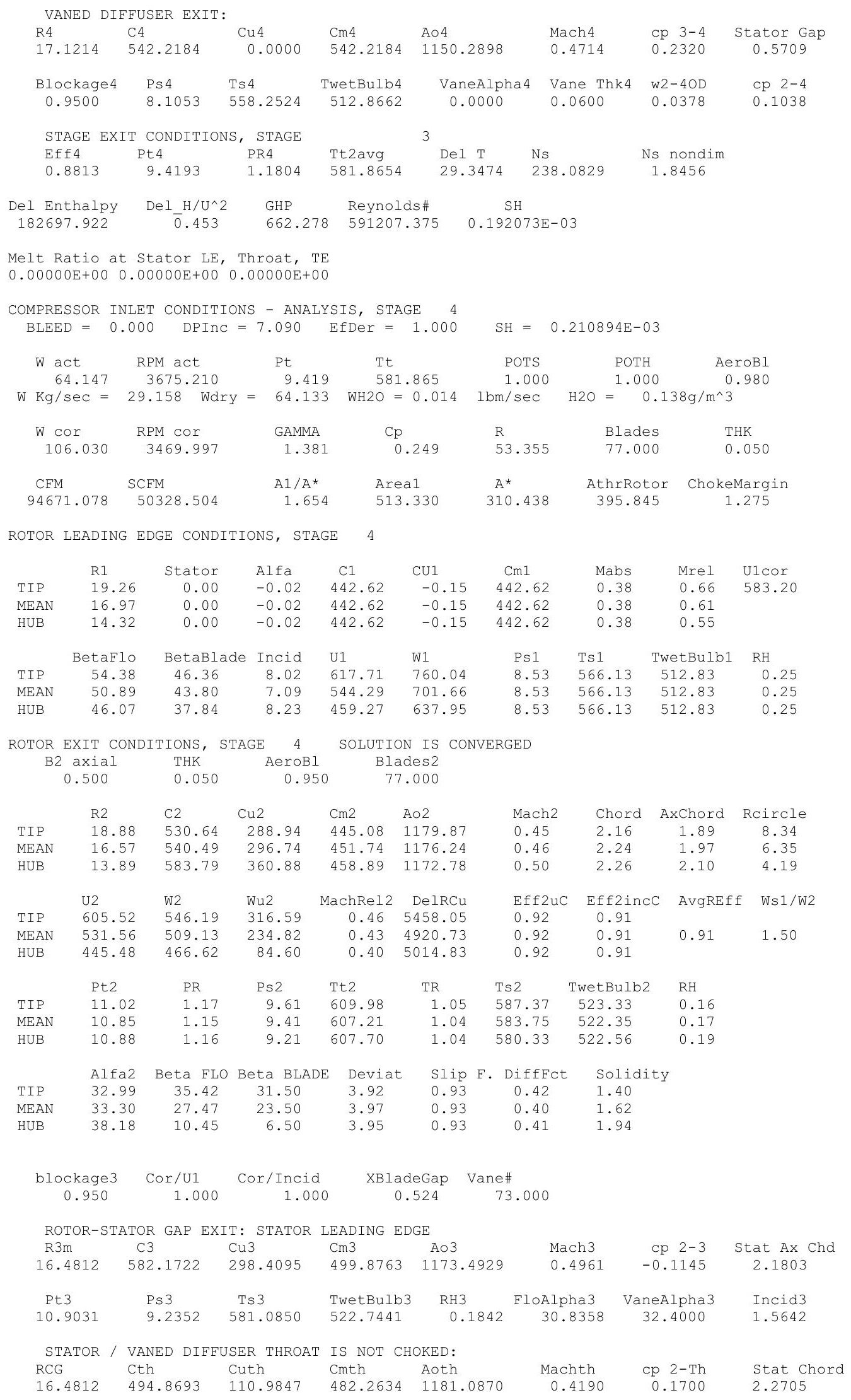




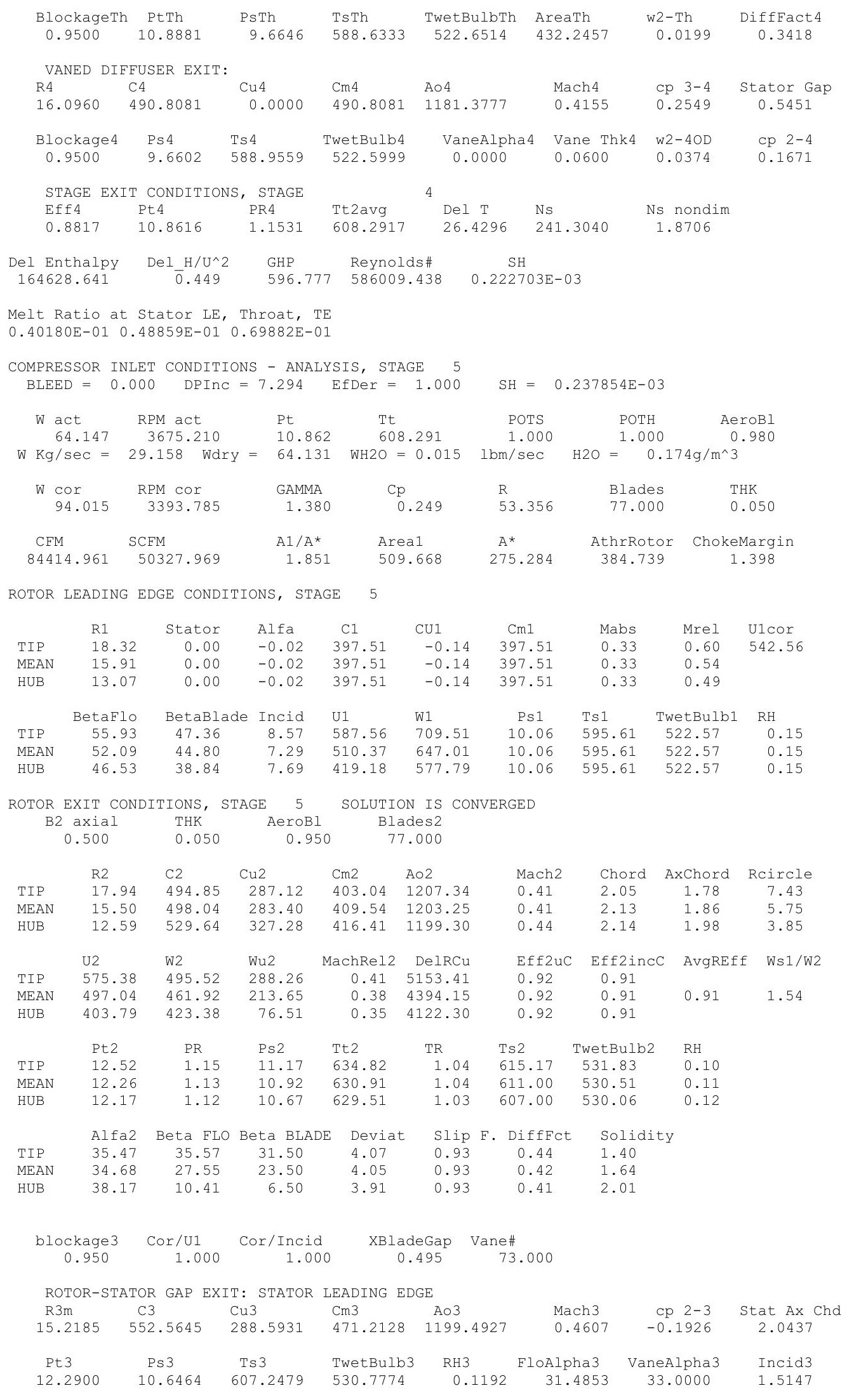




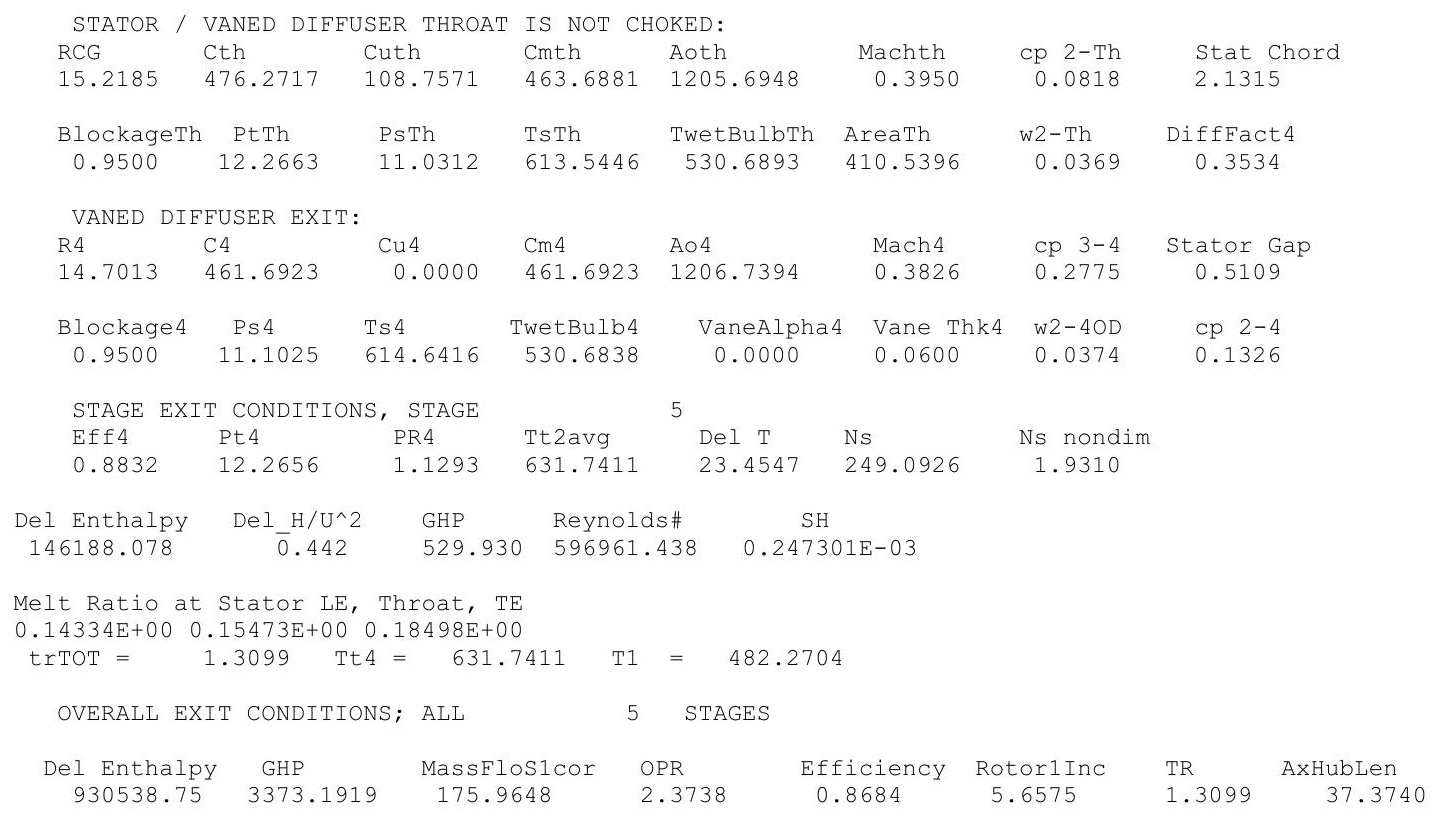




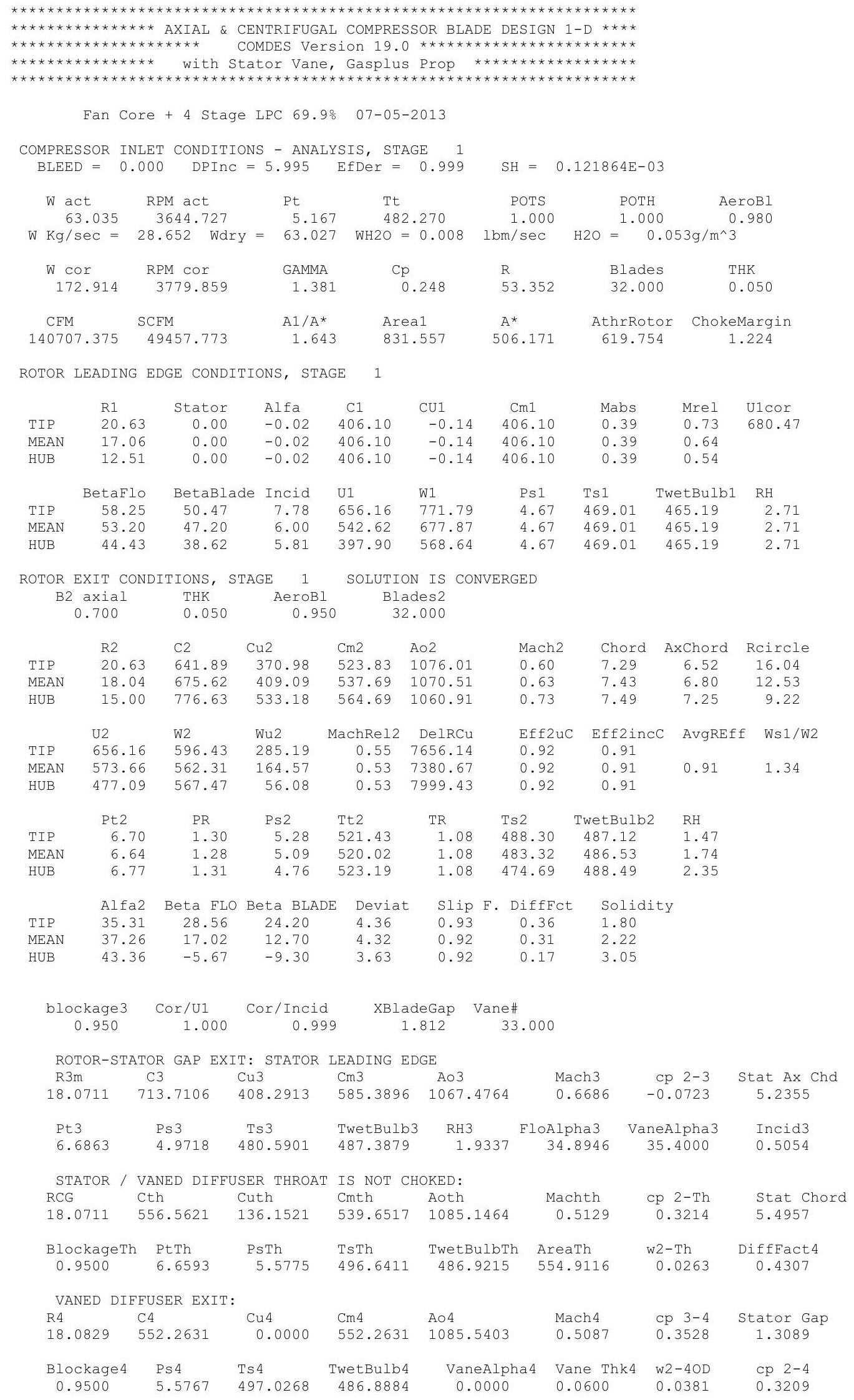




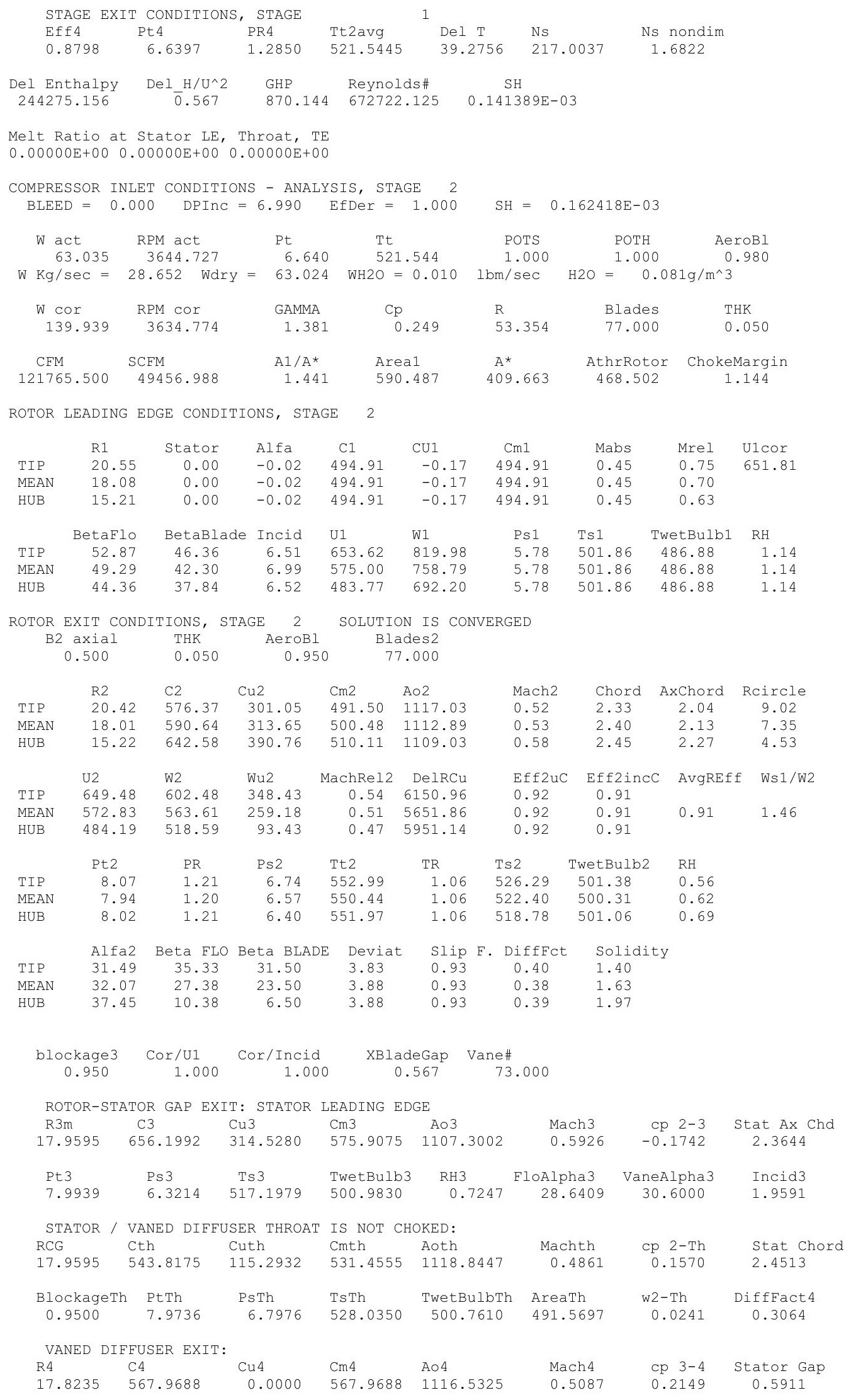




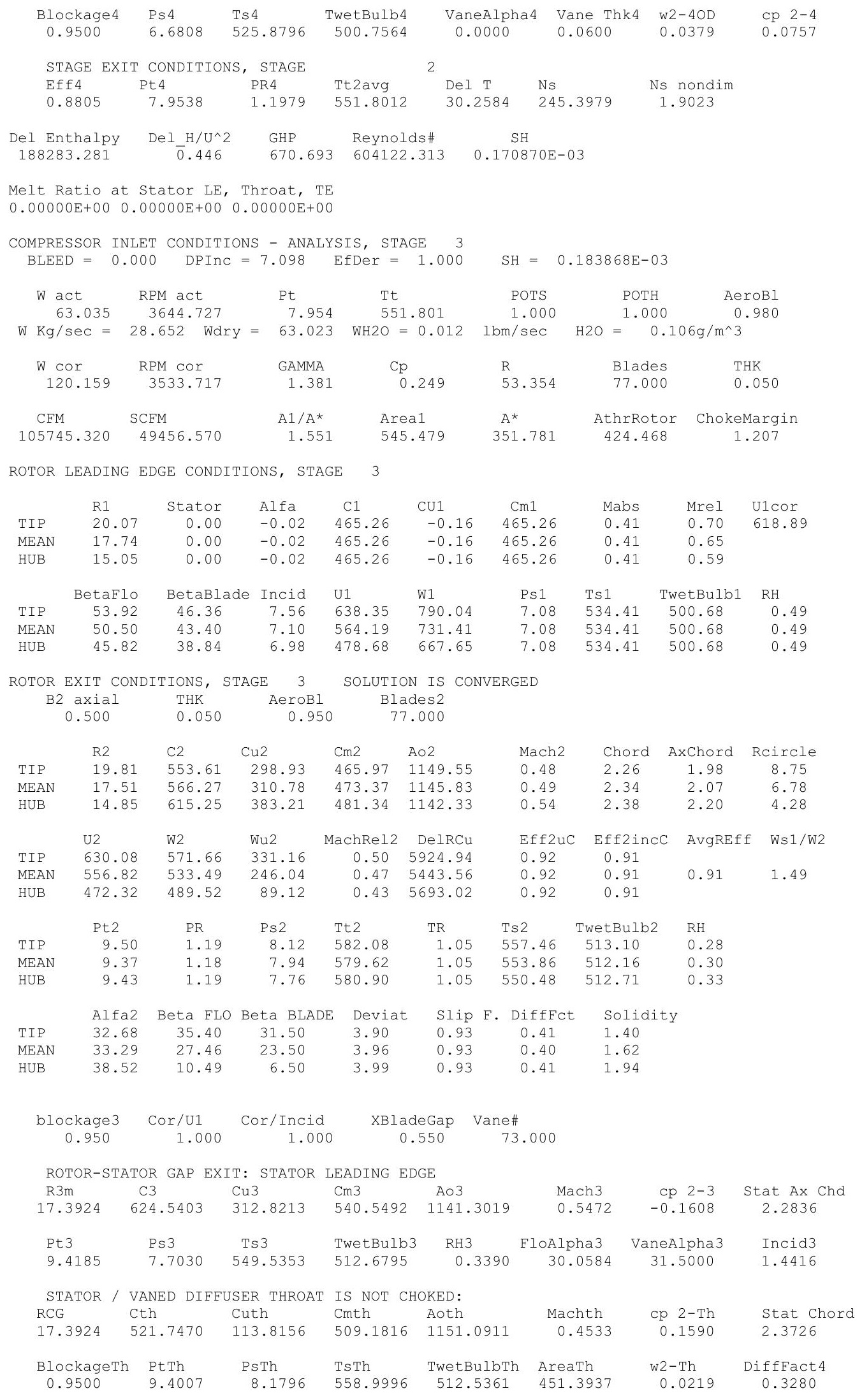




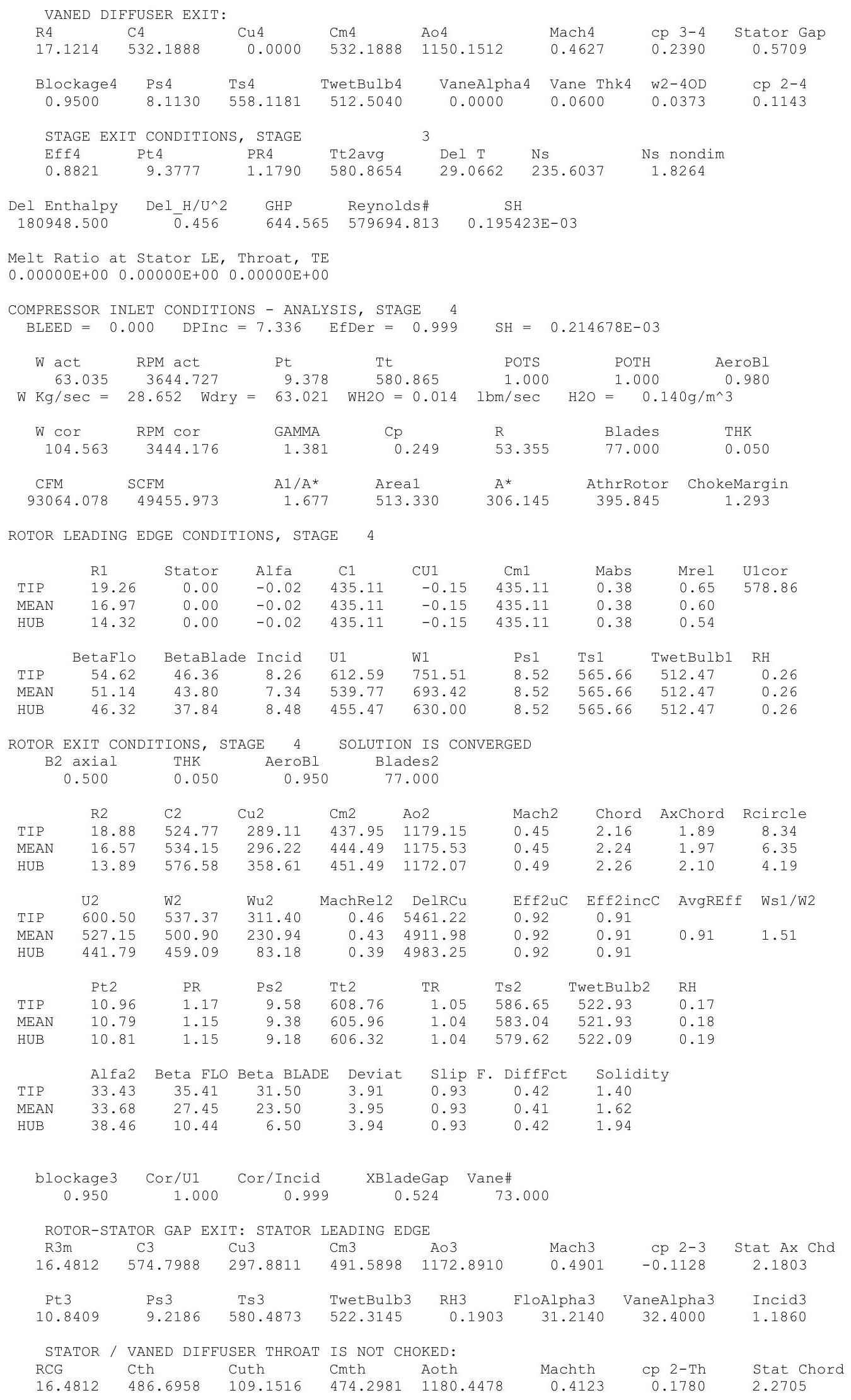




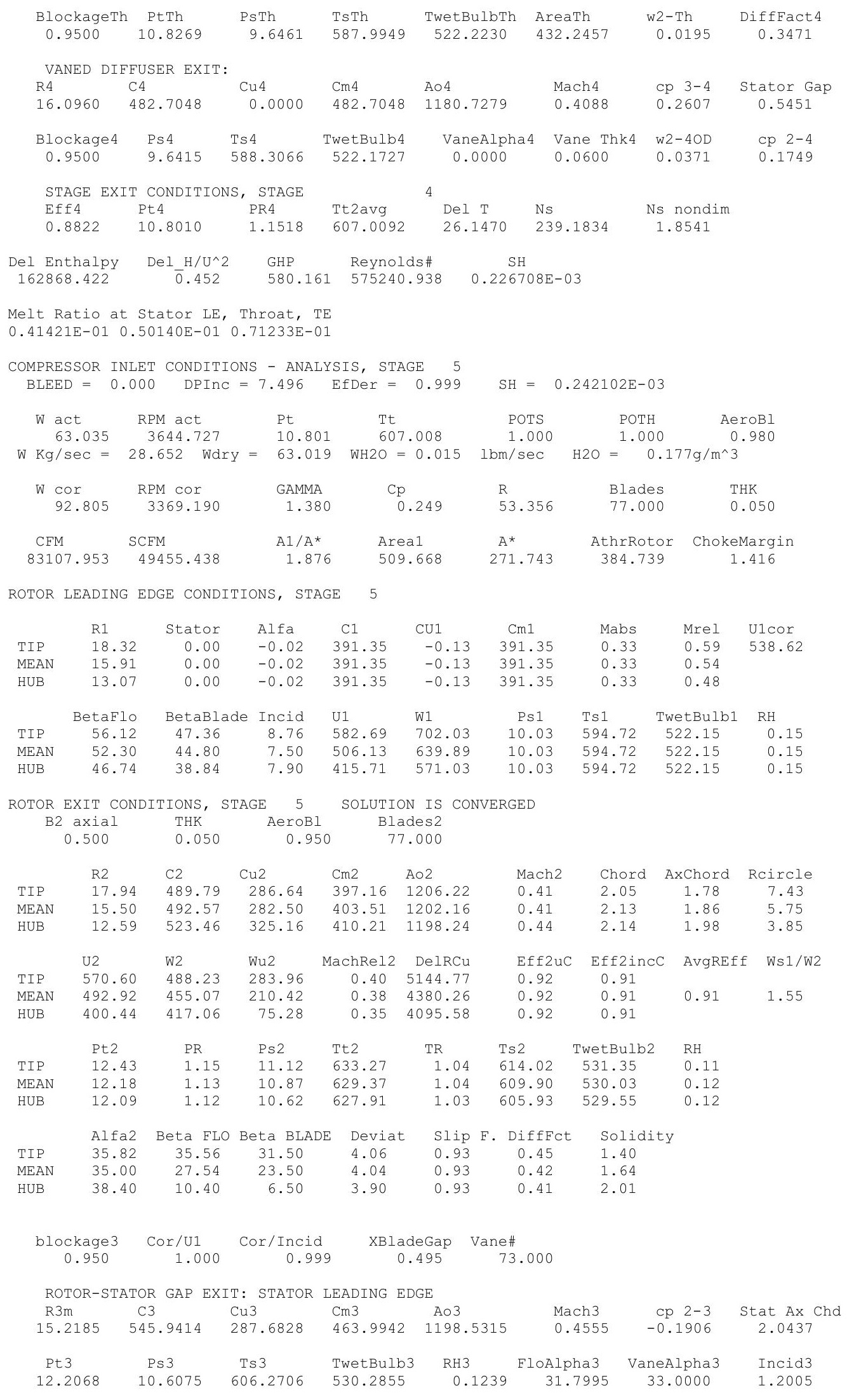




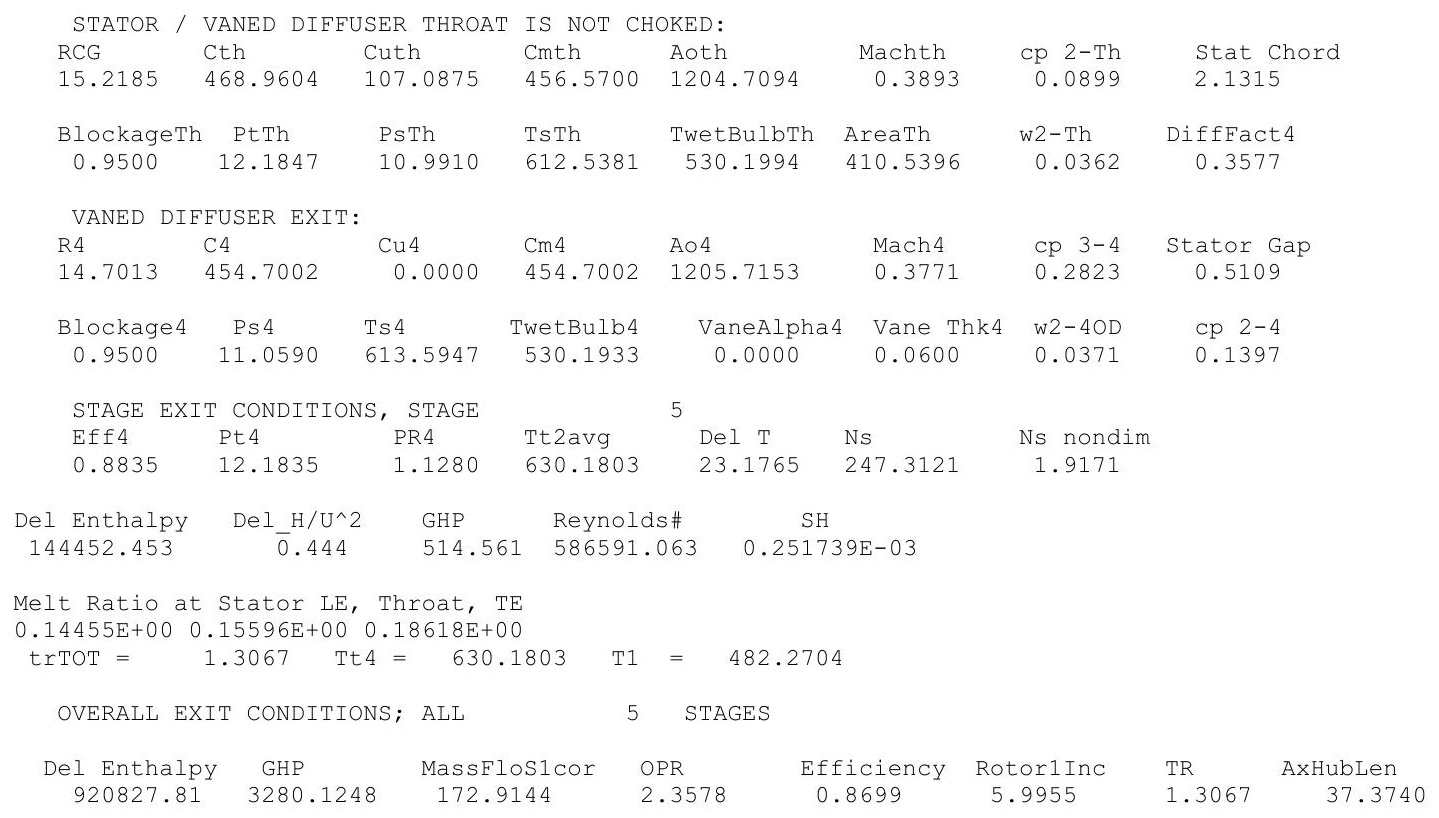


$50 \mu \mathrm{m}$, ISA $+36 \mathrm{R}$

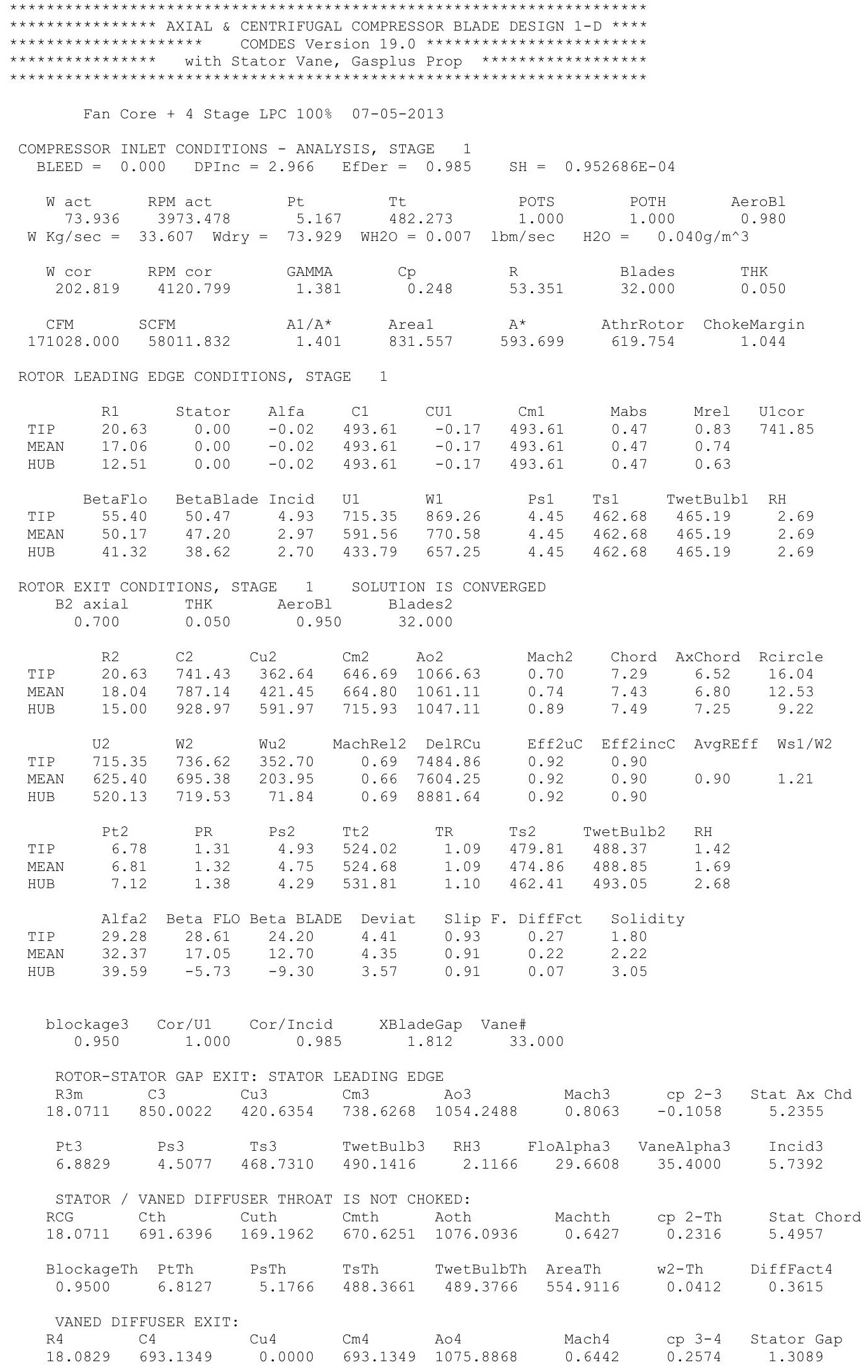




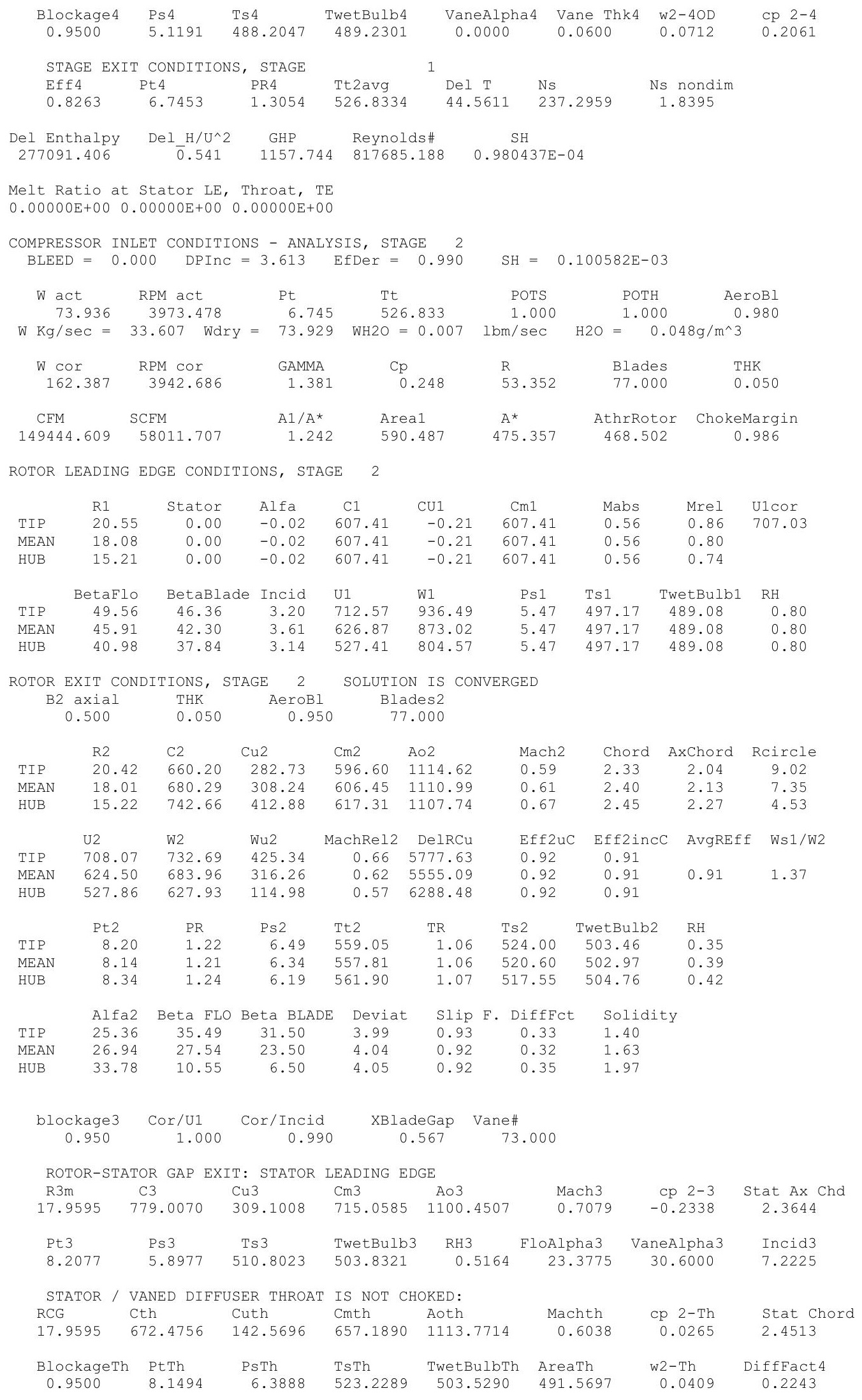




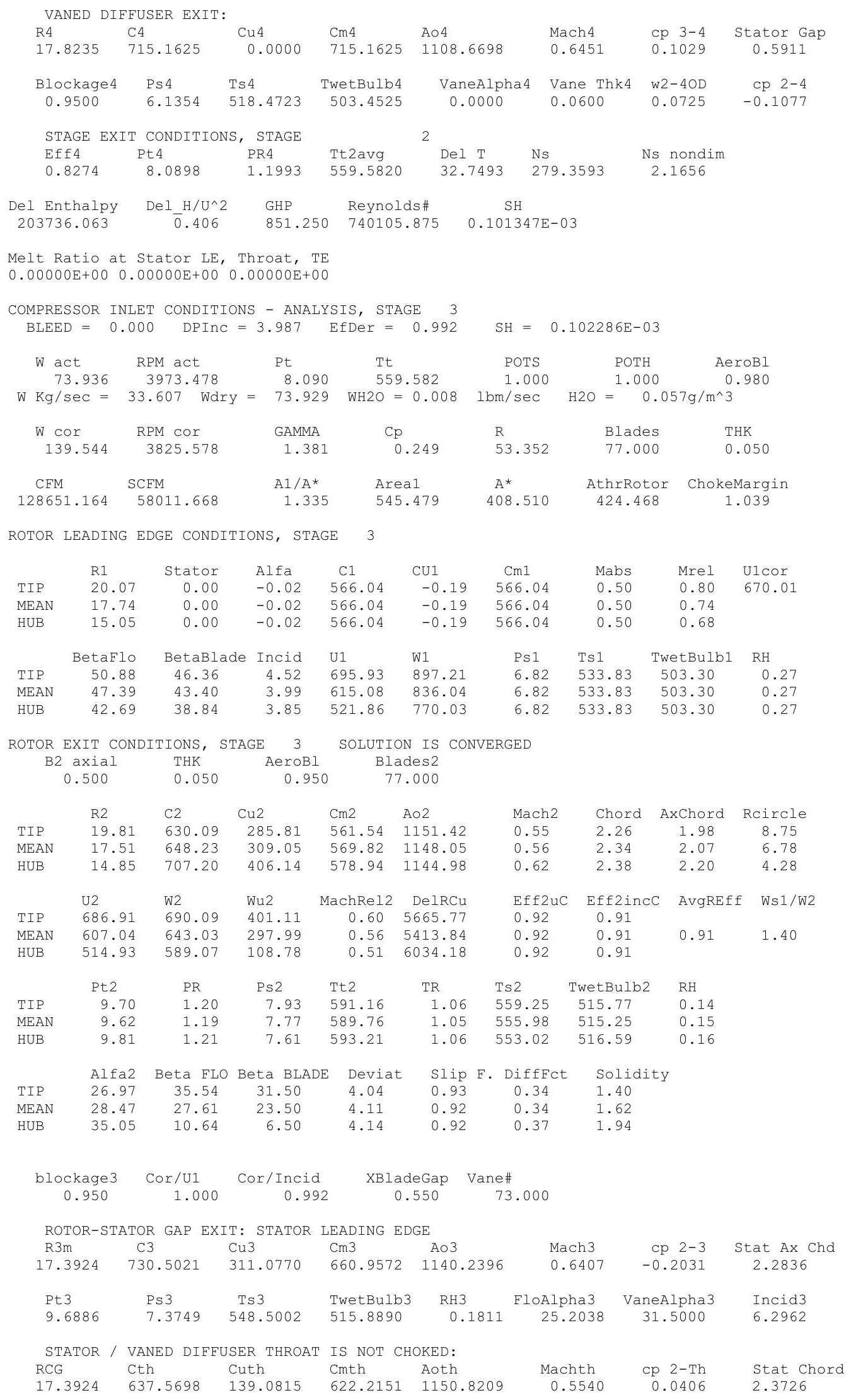




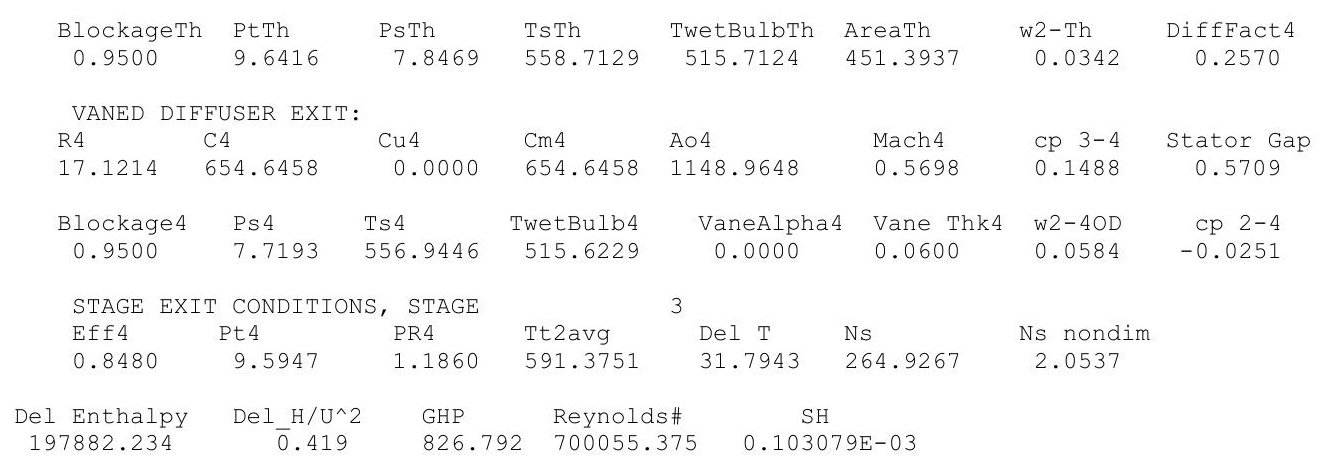

Melt Ratio at Stator LE, Throat, TE

$0.00000 \mathrm{E}+00 \quad 0.00000 \mathrm{E}+00 \quad 0.00000 \mathrm{E}+00$

COMPRESSOR INLET CONDITIONS - ANALYSIS, STAGE 4 BLEED $=0.000 \quad$ DPInC $=4.569 \quad$ EfDer $=0.995$

$\mathrm{SH}=0.104302 \mathrm{E}-03$

$\begin{array}{llllll}\text { W act } & \text { RPM act } & \text { Pt } & \text { POTS } & \text { POTH } & \text { AeroBI }\end{array}$ $\mathrm{W} \mathrm{Kg} / \mathrm{sec}=33.607$ Wdry $=73.928 \quad$ WH2O $=0.008 \quad \mathrm{lbm} / \mathrm{sec} \quad \mathrm{H} 2 \mathrm{O}=\begin{gathered}1.000 \\ 0.066 \mathrm{~g} / \mathrm{m}^{\wedge} 3\end{gathered}$

$\begin{array}{ccccccc}\text { W cor } & \text { RPM cor } & \text { GAMMA } & \text { Cp } & \text { R } & \text { Blades } & \text { THK } \\ 120.953 & 3721.323 & 1.381 & 0.249 & 53.352 & 77.000 & 0.050 \\ \text { CFM } & \text { SCFM } & \text { A1/A* } & \text { Area1 } & A^{*} & \text { AthrRotor } & \text { ChokeMargin } \\ 111902.813 & 58011.621 & 1.450 & 513.330 & 354.110 & 395.845 & 1.118\end{array}$

ROTOR LEADING EDGE CONDITIONS, STAGE 4

\begin{tabular}{|c|c|c|c|c|c|c|c|c|c|}
\hline & R1 & Stator & Alfa & $\mathrm{C} 1$ & CU1 & $\mathrm{Cm} 1$ & Mabs & Mrel & U1cor \\
\hline TIP & 19.26 & 0.00 & -0.02 & 523.19 & -0.18 & 523.19 & 0.45 & 0.73 & 625.44 \\
\hline MEAN & 16.97 & 0.00 & -0.02 & 523.19 & -0.18 & 523.19 & 0.45 & 0.68 & \\
\hline HUB & 14.32 & 0.00 & -0.02 & 523.19 & -0.18 & 523.19 & 0.45 & 0.62 & \\
\hline & BetaFlo & BetaBlade & Incid & U1 & W1 & Ps 1 & Ts1 & TwetBulb1 & $\mathrm{RH}$ \\
\hline TIP & 51.93 & 46.36 & 5.57 & 667.84 & 848.52 & 8.36 & 569.39 & 515.56 & 0.11 \\
\hline MEAN & 48.37 & 43.80 & 4.57 & 588.46 & 787.54 & 8.36 & 569.39 & 515.56 & 0.11 \\
\hline HUB & 43.51 & 37.84 & 5.67 & 496.55 & 721.43 & 8.36 & 569.39 & 515.56 & 0.11 \\
\hline
\end{tabular}

ROTOR EXIT CONDITIONS, STAGE 4 SOLUTION IS CONVERGED

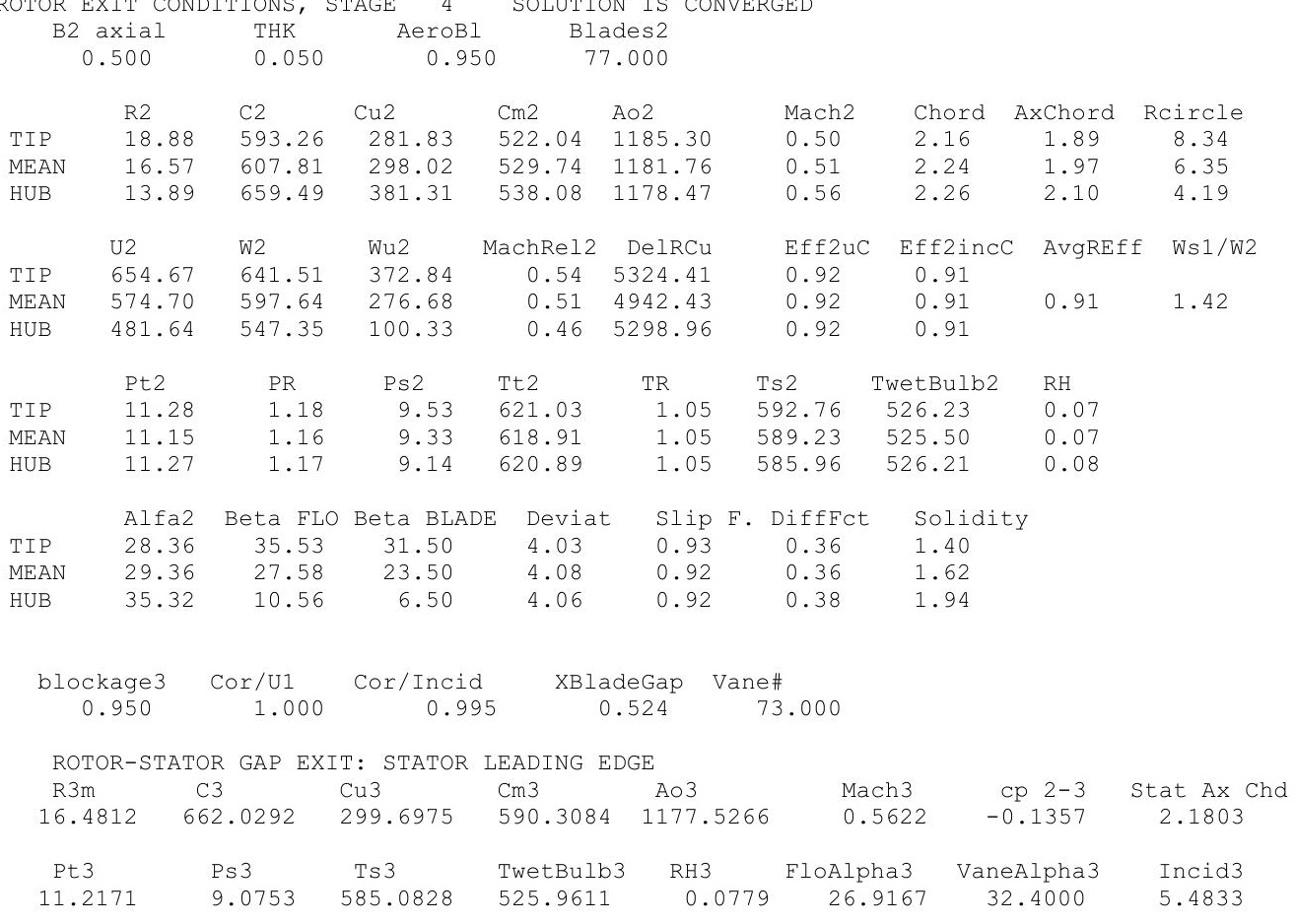




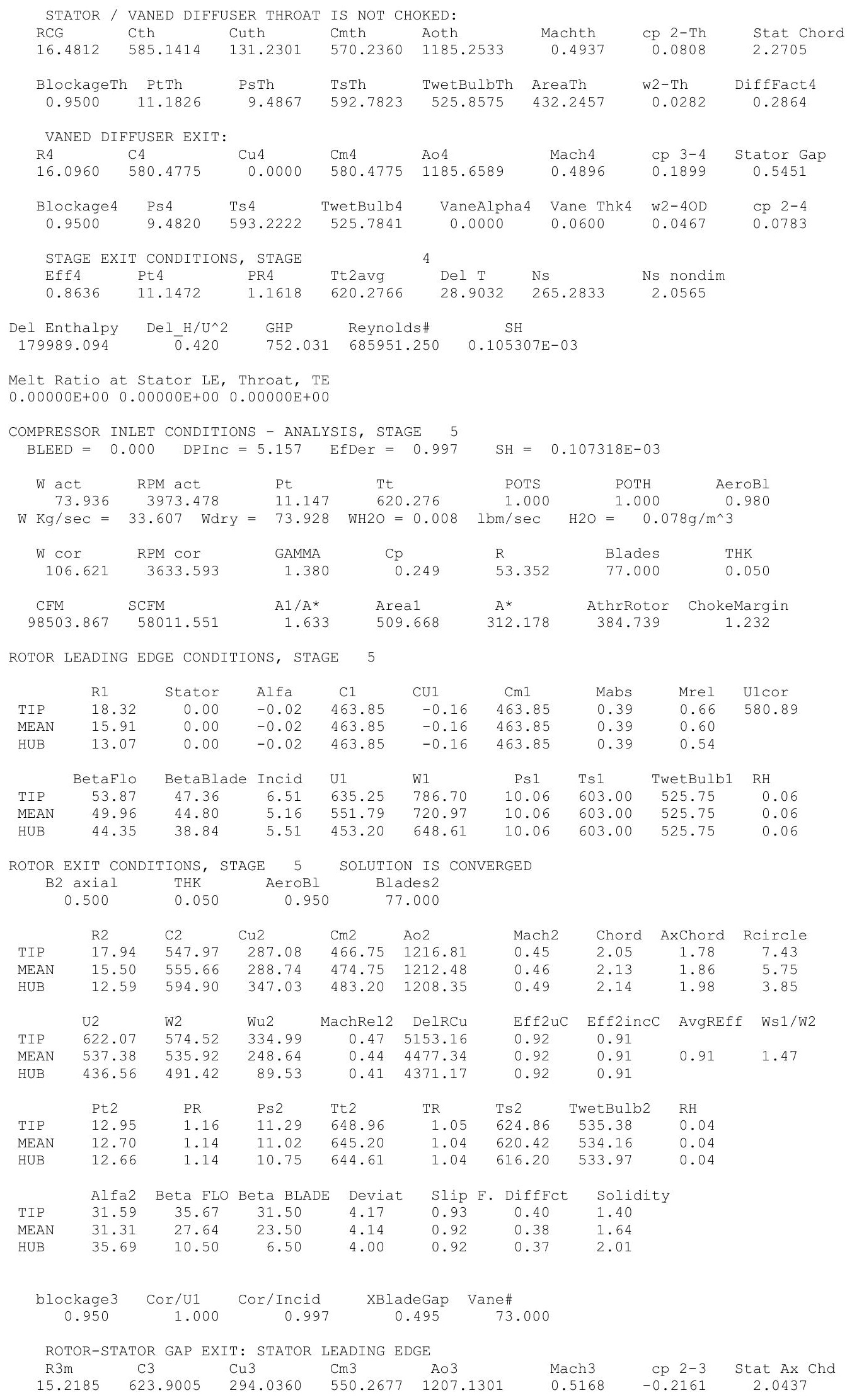




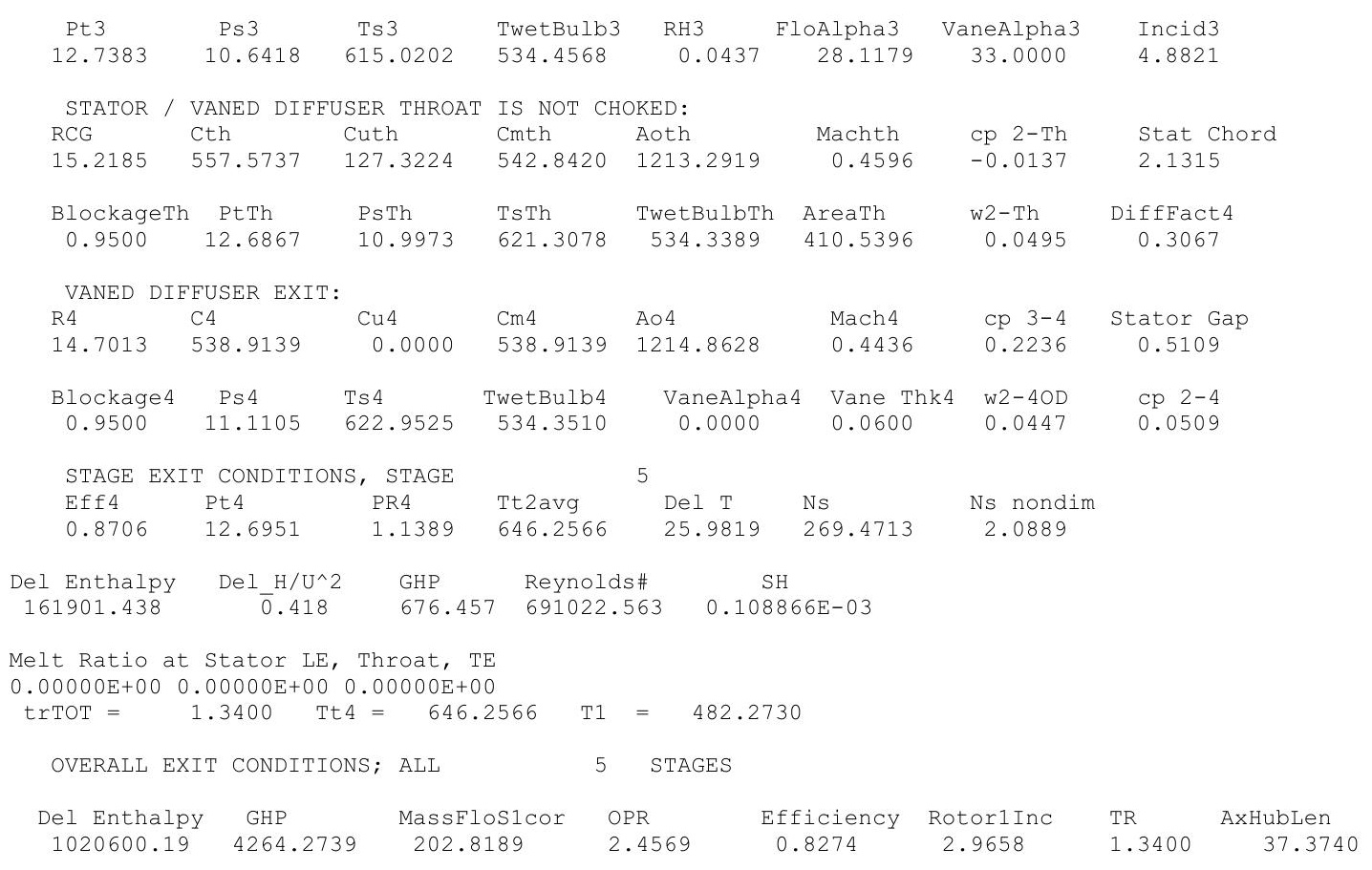




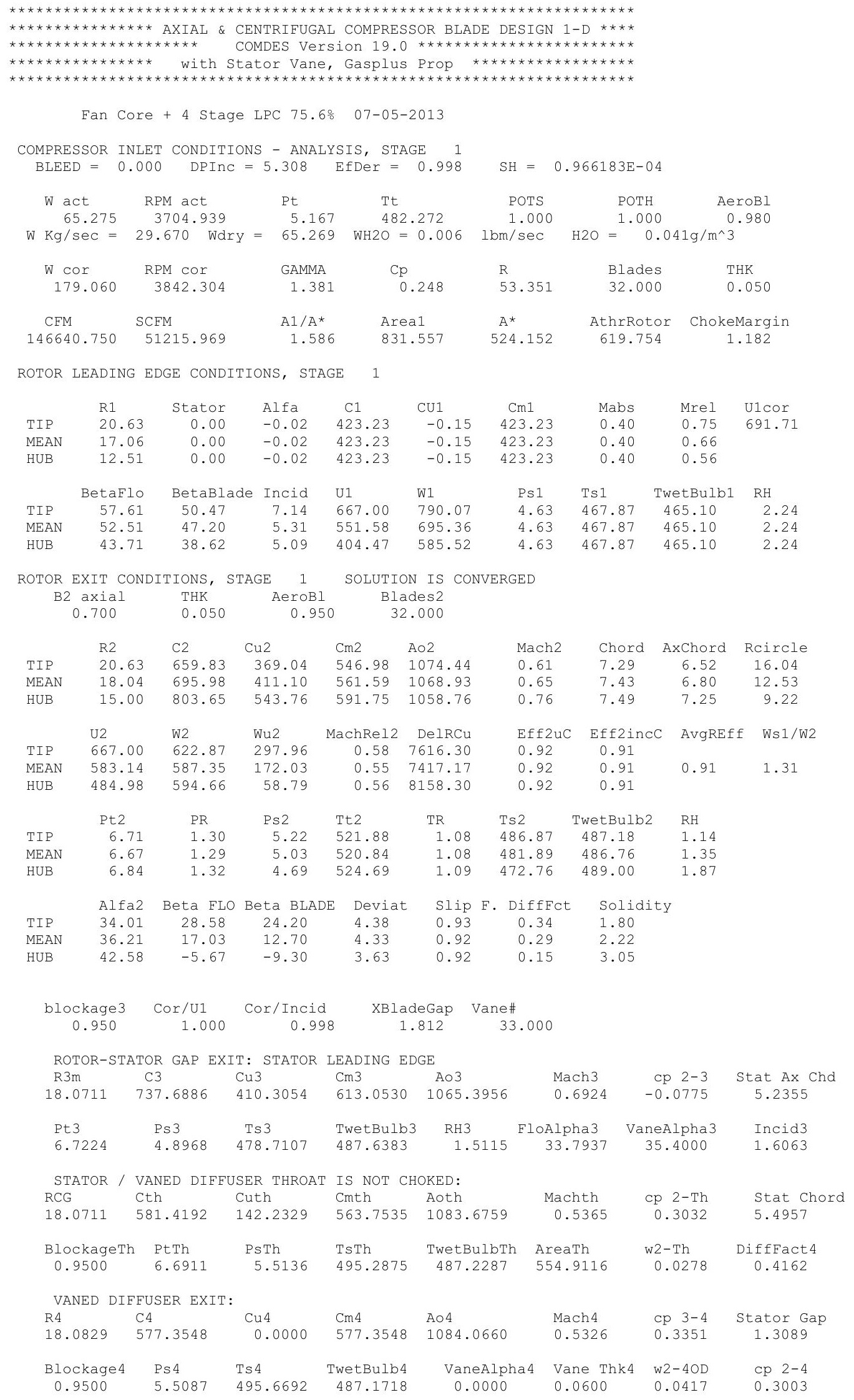




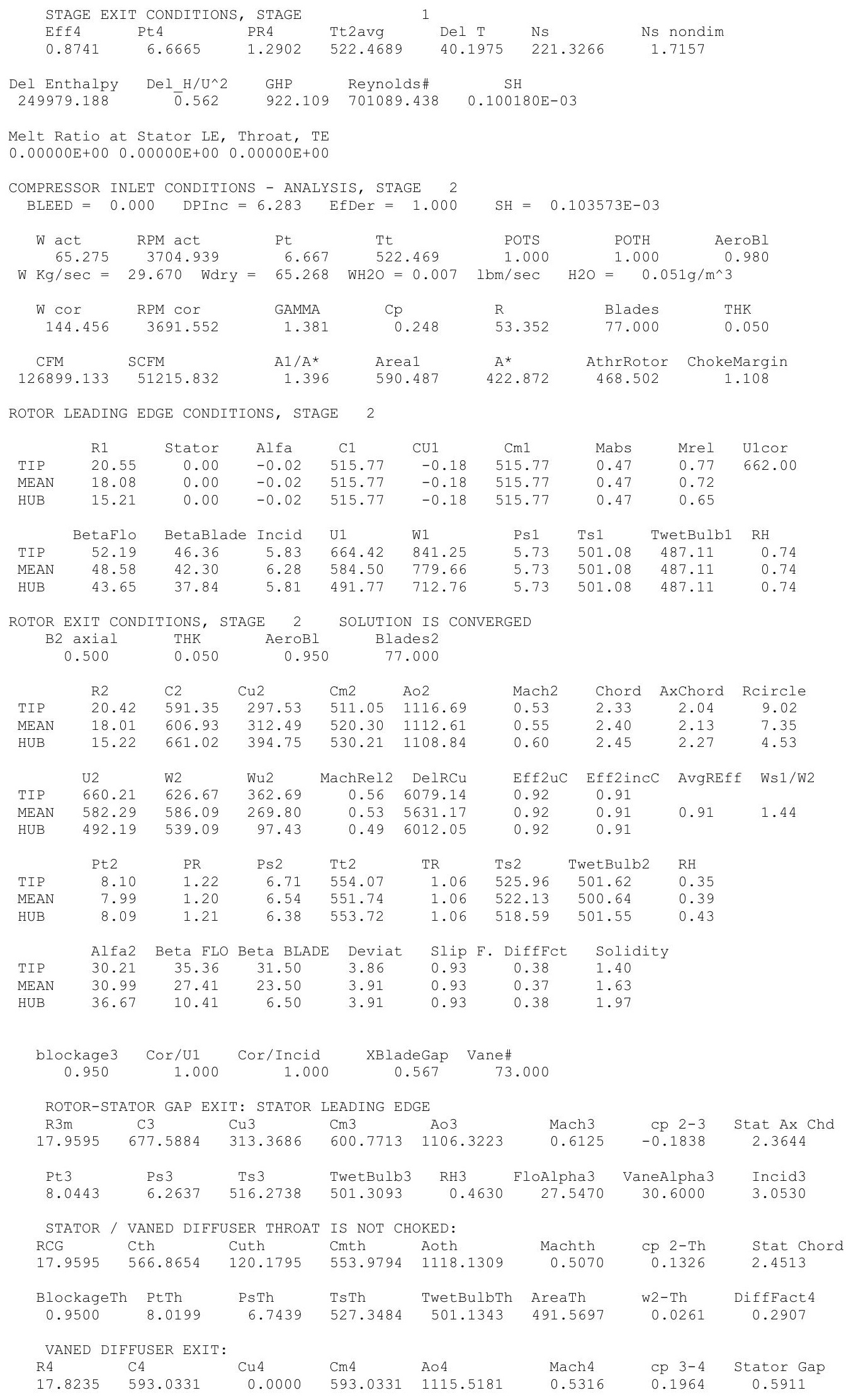




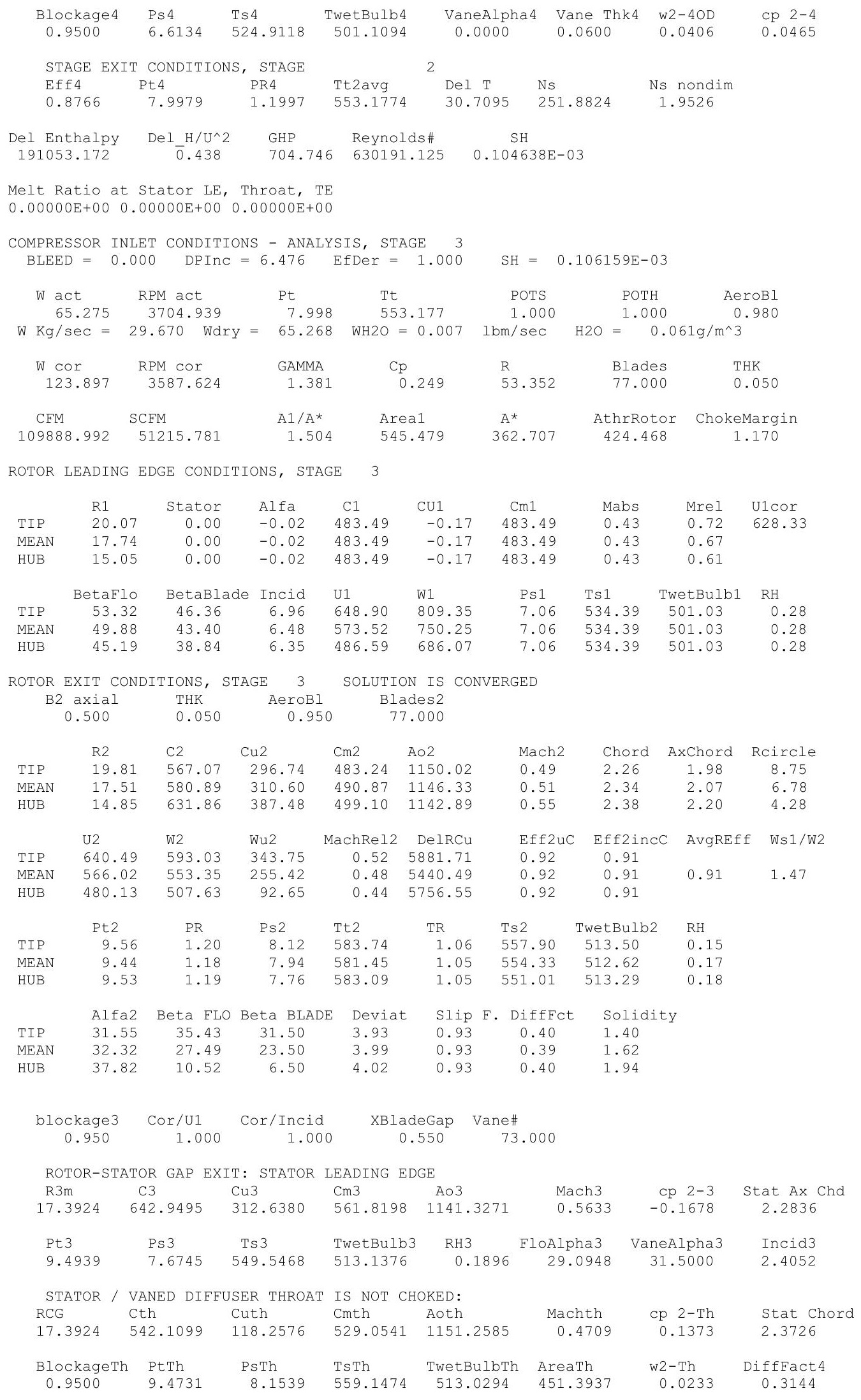




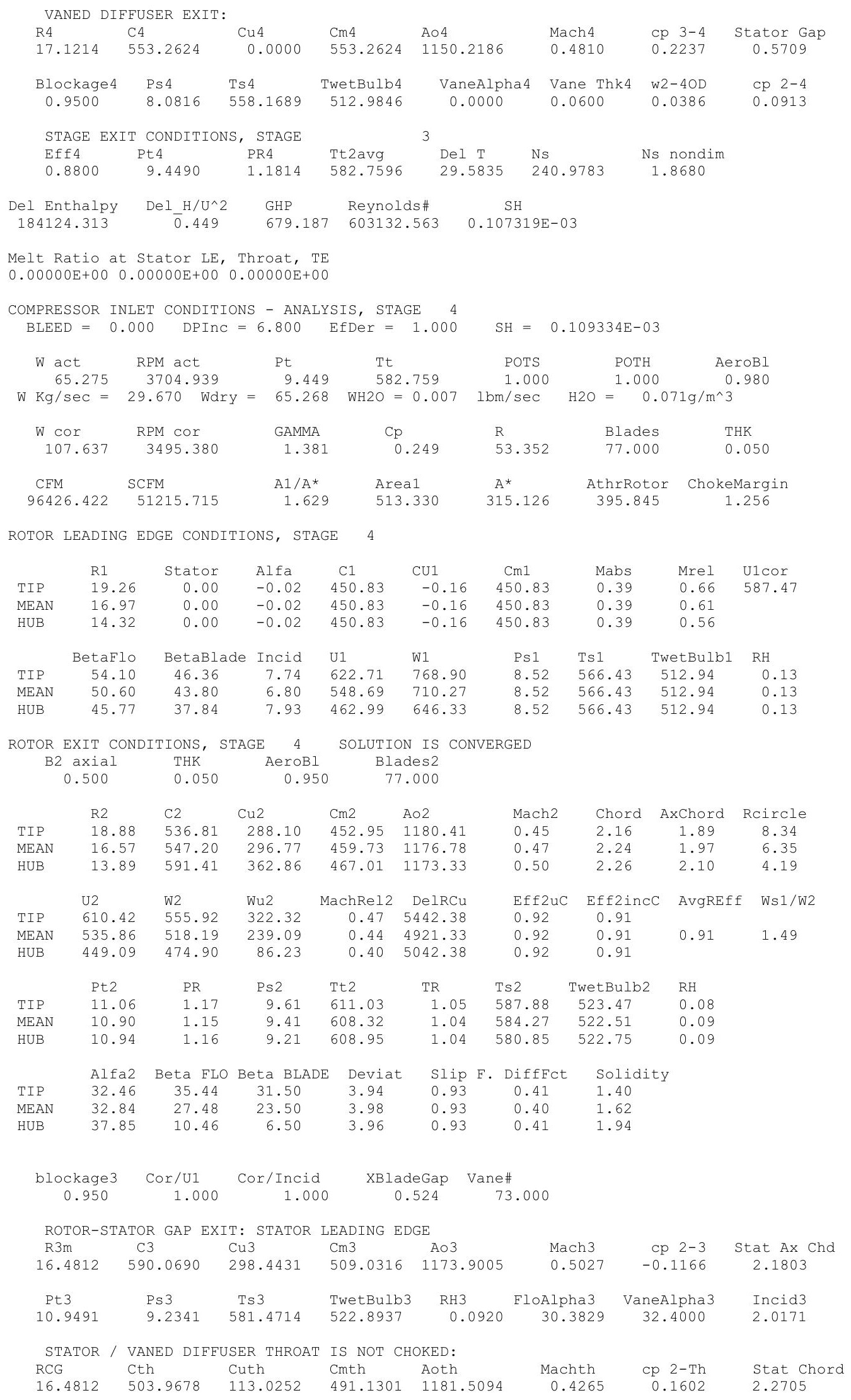




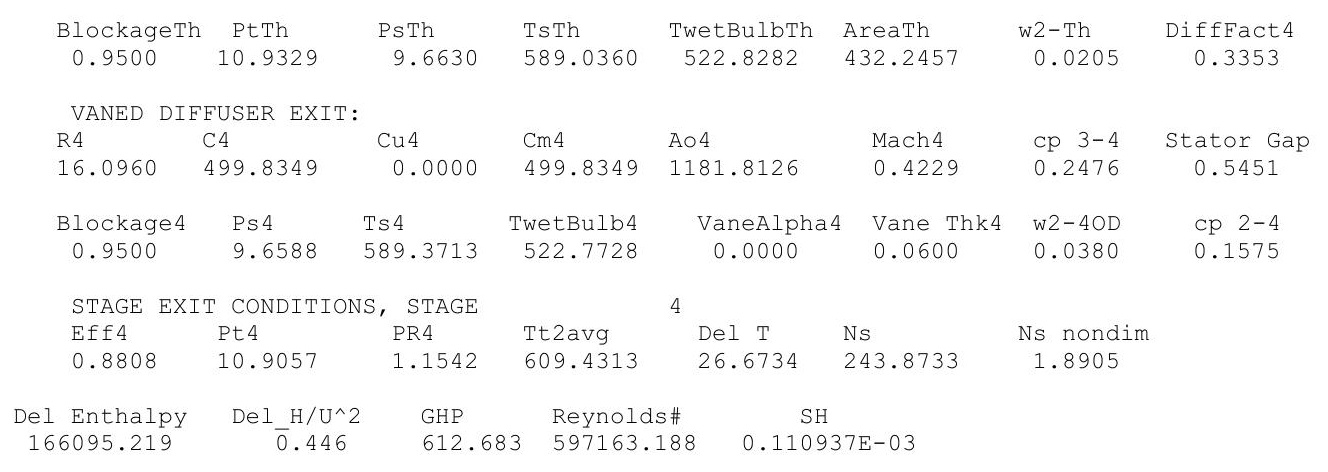

Melt Ratio at Stator LE, Throat, TE

$0.00000 \mathrm{E}+00 \quad 0.00000 \mathrm{E}+00 \quad 0.00000 \mathrm{E}+00$

COMPRESSOR INLET CONDITIONS - ANALYSIS, STAGE 5 $\mathrm{BLEED}=0.000 \quad$ DPInC $=7.045 \quad$ EfDer $=1.000$

$\mathrm{SH}=0.113783 \mathrm{E}-03$

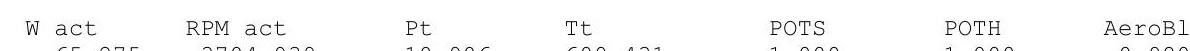

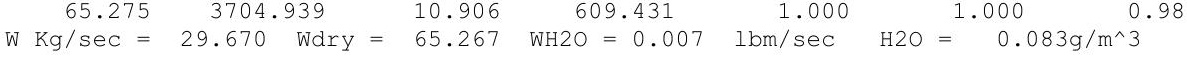

$\begin{array}{ccccccc}\text { W Cor } & \text { RPM cor } & \text { GAMMA } & \text { Cp } & \text { R } & \text { Blades } & \text { THK } \\ 95.370 & 3418.037 & 1.380 & 0.249 & 53.352 & 77.000 & 0.050 \\ & & & & & & \\ \text { CFM } & \text { SCFM } & \text { A1/A* } & \text { Area1 } & \text { A* } & \text { AthrRotor } & \text { ChokeMargin } \\ 85863.016 & 51215.629 & 1.825 & 509.668 & 279.233 & 384.739 & 1.378\end{array}$

ROTOR LEADING EDGE CONDITIONS, STAGE 5

\begin{tabular}{|c|c|c|c|c|c|c|c|c|c|}
\hline & R1 & Stator & Alfa & $\mathrm{C} 1$ & CU1 & $\mathrm{Cm} 1$ & Mabs & Mrel & U1cor \\
\hline TIP & 18.32 & 0.00 & -0.02 & 404.32 & -0.14 & 404.32 & 0.34 & 0.60 & 546.43 \\
\hline MEAN & 15.91 & 0.00 & -0.02 & 404.32 & -0.14 & 404.32 & 0.34 & 0.55 & \\
\hline HUB & 13.07 & 0.00 & -0.02 & 404.32 & -0.14 & 404.32 & 0.34 & 0.49 & \\
\hline & BetaFlo & BetaBlade & Incid & U1 & W1 & Ps 1 & Ts1 & TwetBulb1 & $\mathrm{RH}$ \\
\hline TIP & 55.69 & 47.36 & 8.33 & 592.32 & 717.27 & 10.08 & 596.31 & 522.75 & 0.07 \\
\hline MEAN & 51.84 & 44.80 & 7.04 & 514.49 & 654.47 & 10.08 & 596.31 & 522.75 & 0.07 \\
\hline HUB & 46.27 & 38.84 & 7.43 & 422.58 & 584.95 & 10.08 & 596.31 & 522.75 & 0.07 \\
\hline
\end{tabular}

ROTOR EXIT CONDITIONS, STAGE 5 SOLUTION IS CONVERGED

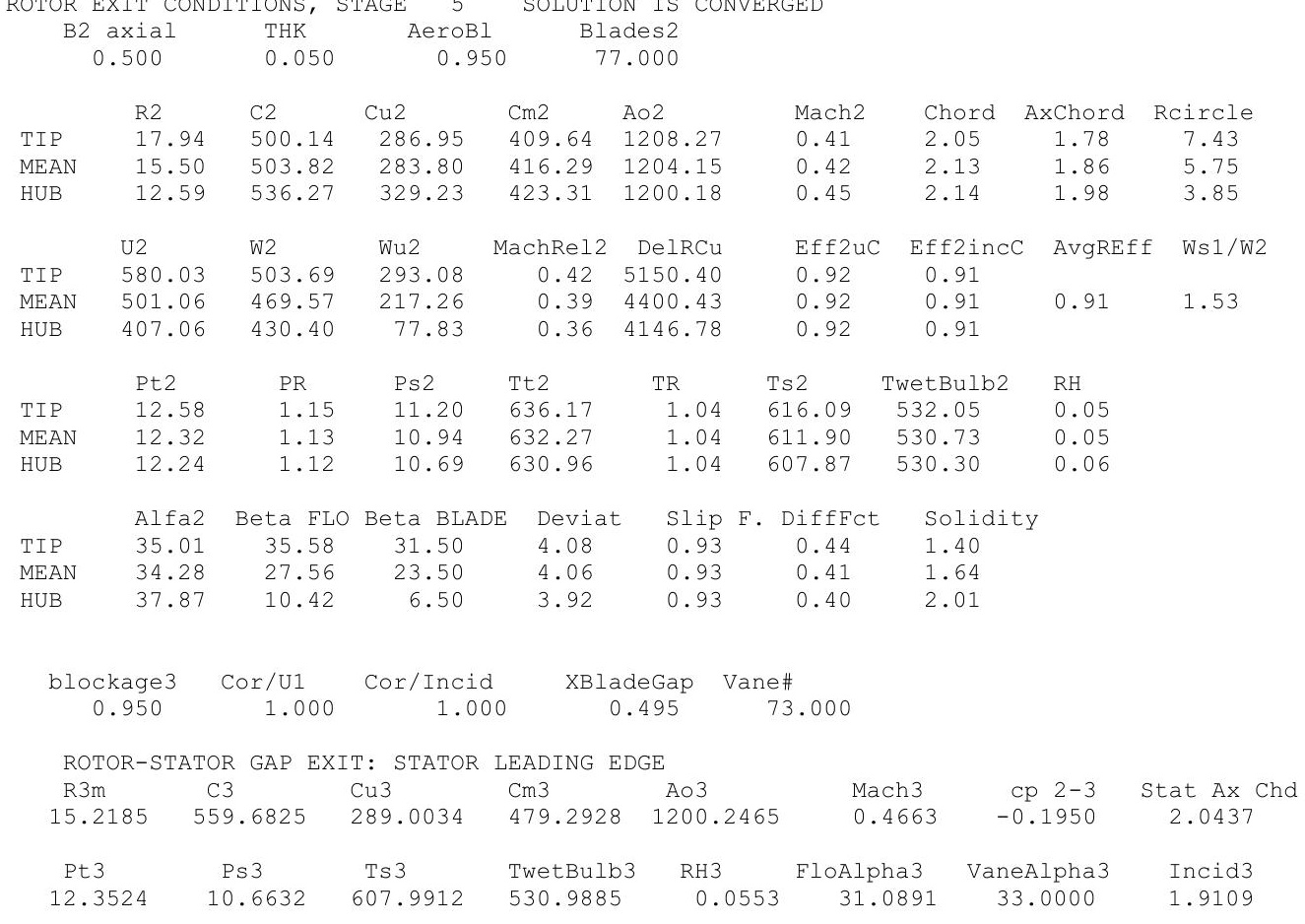




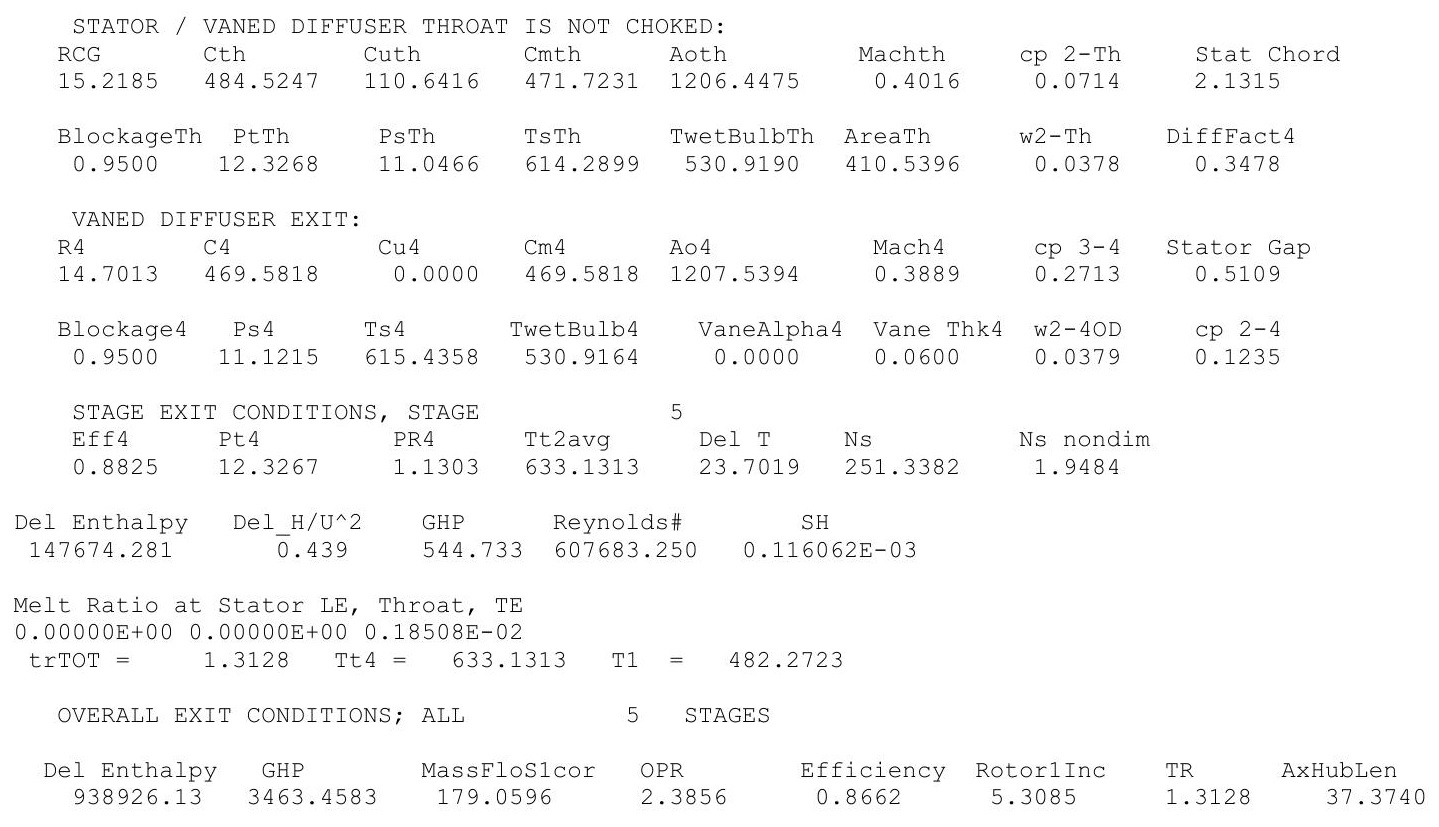




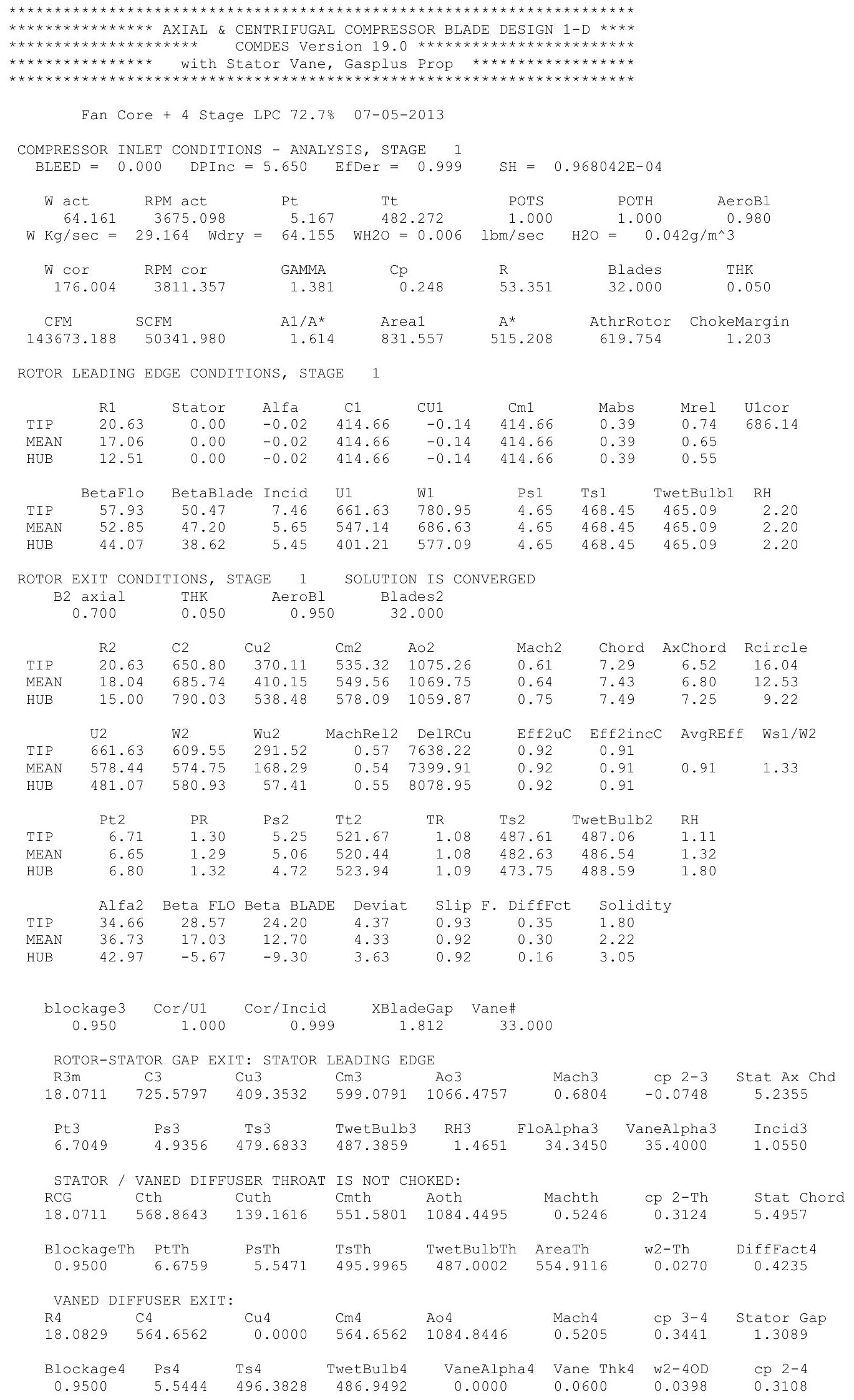




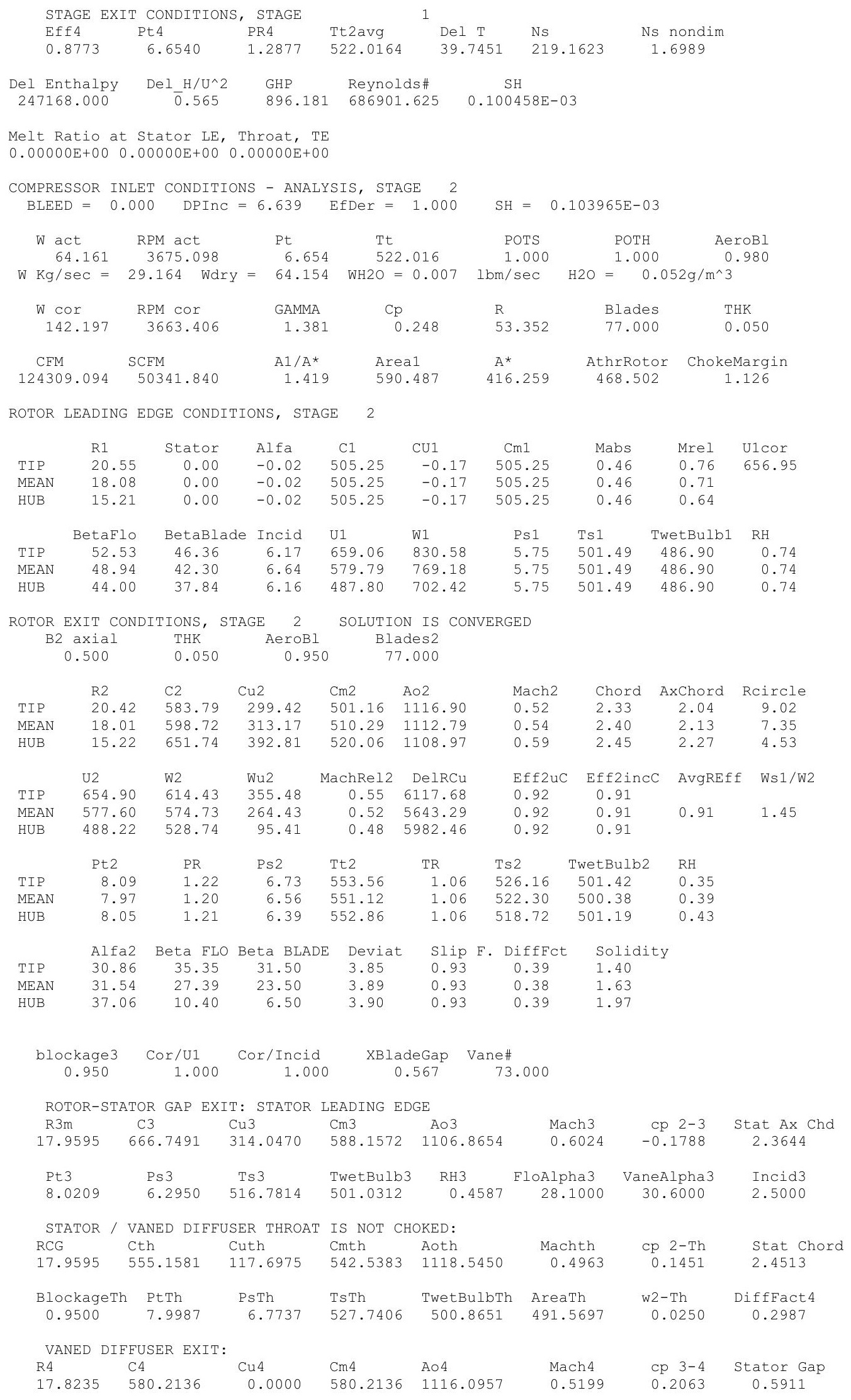




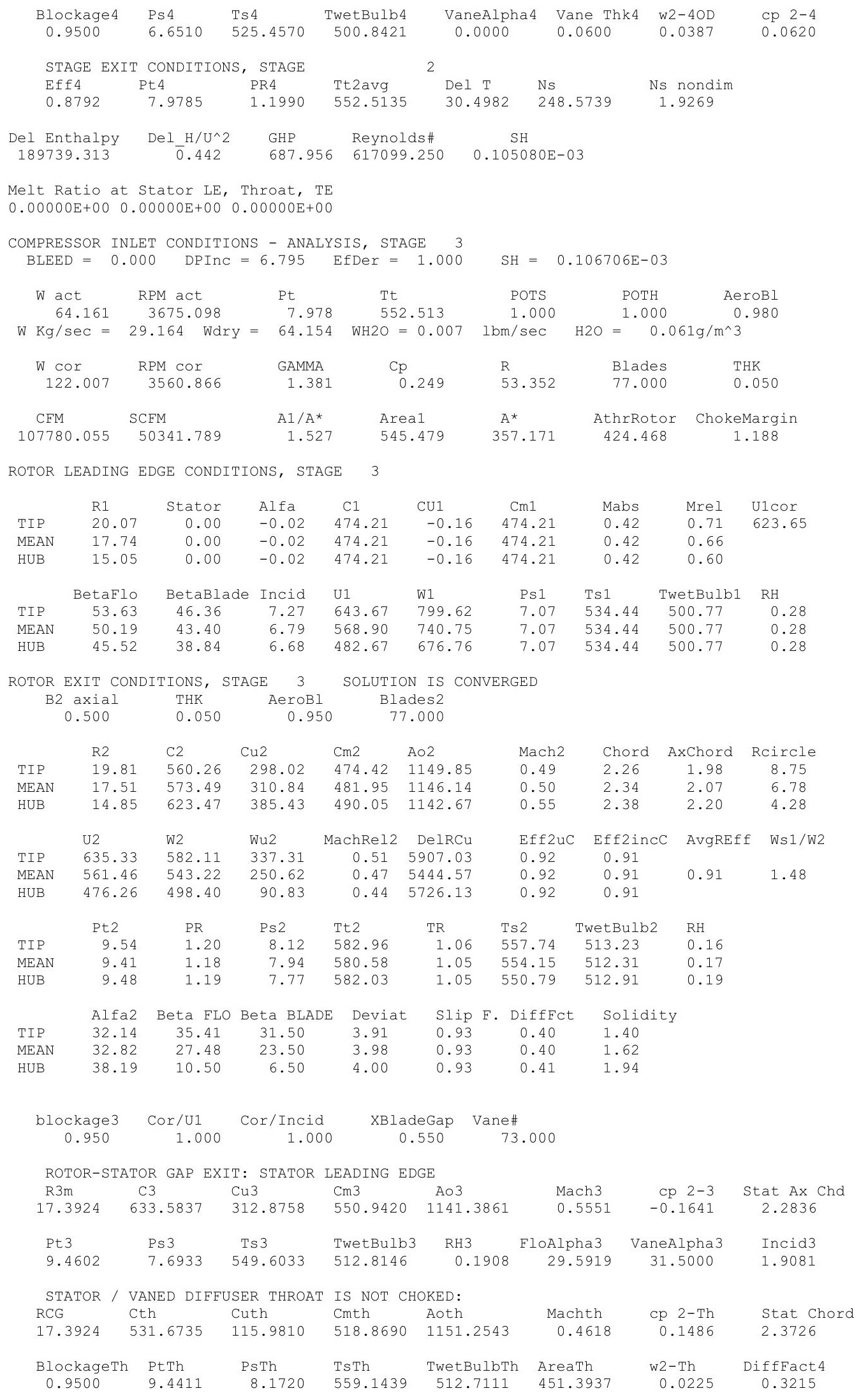




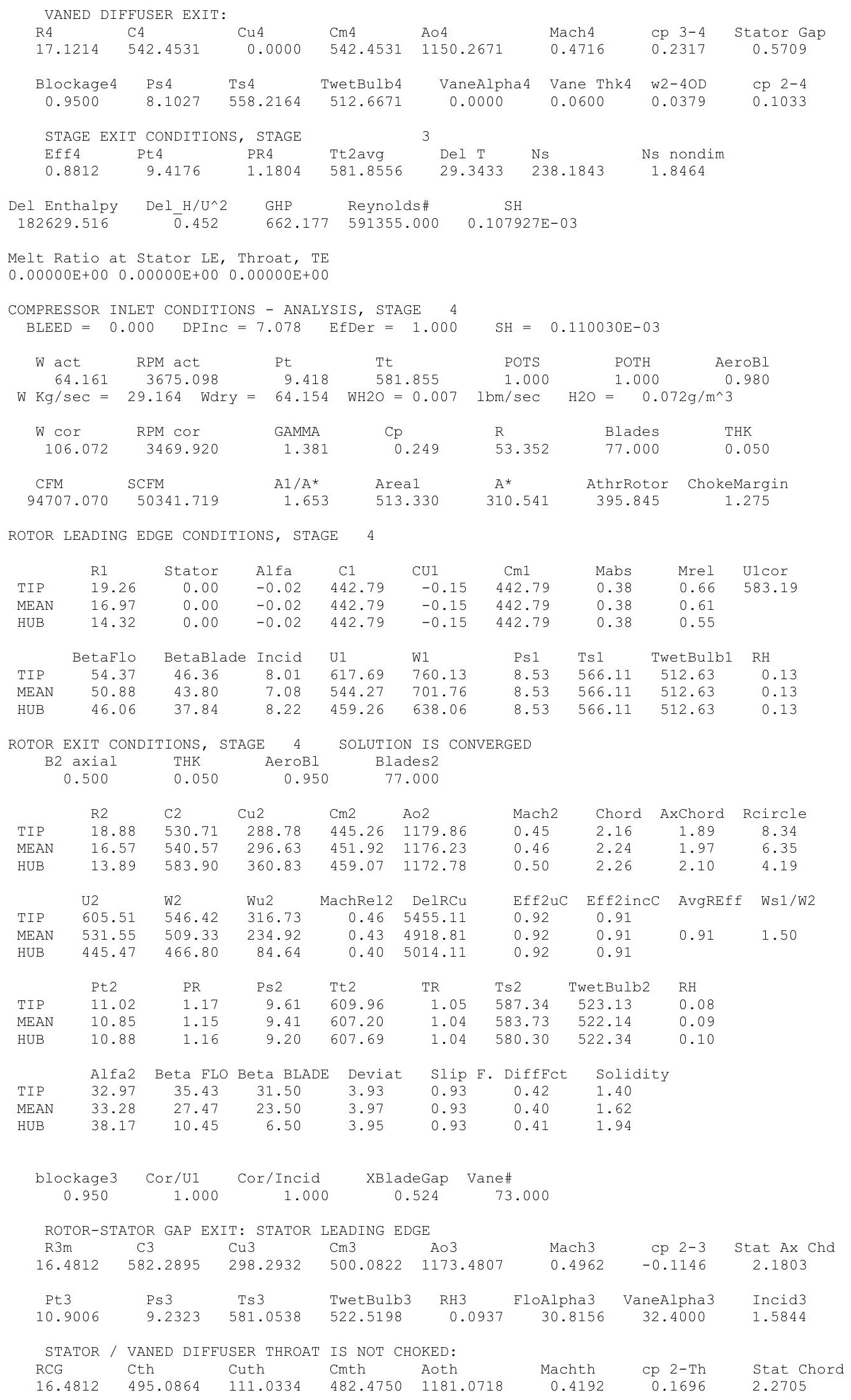




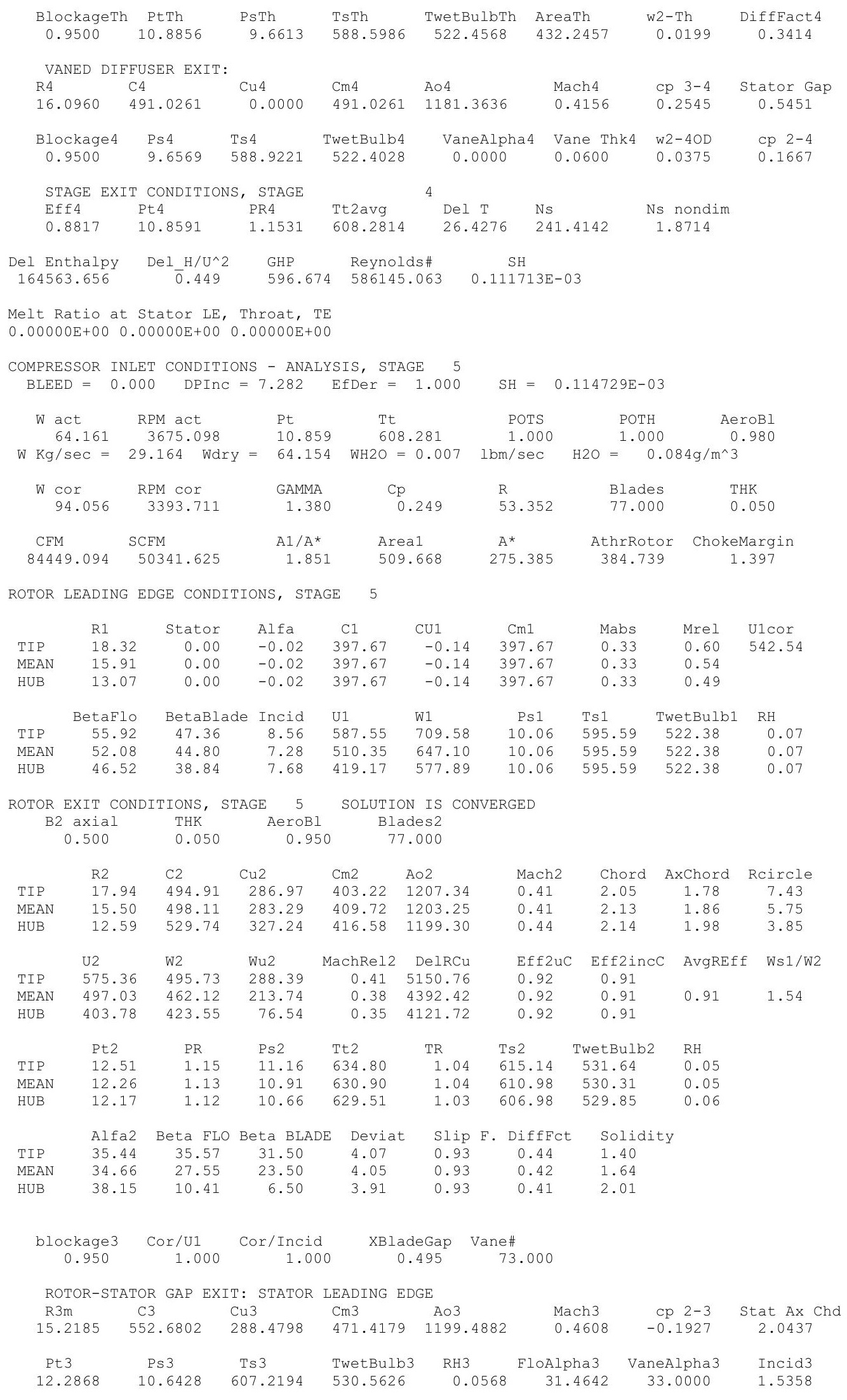




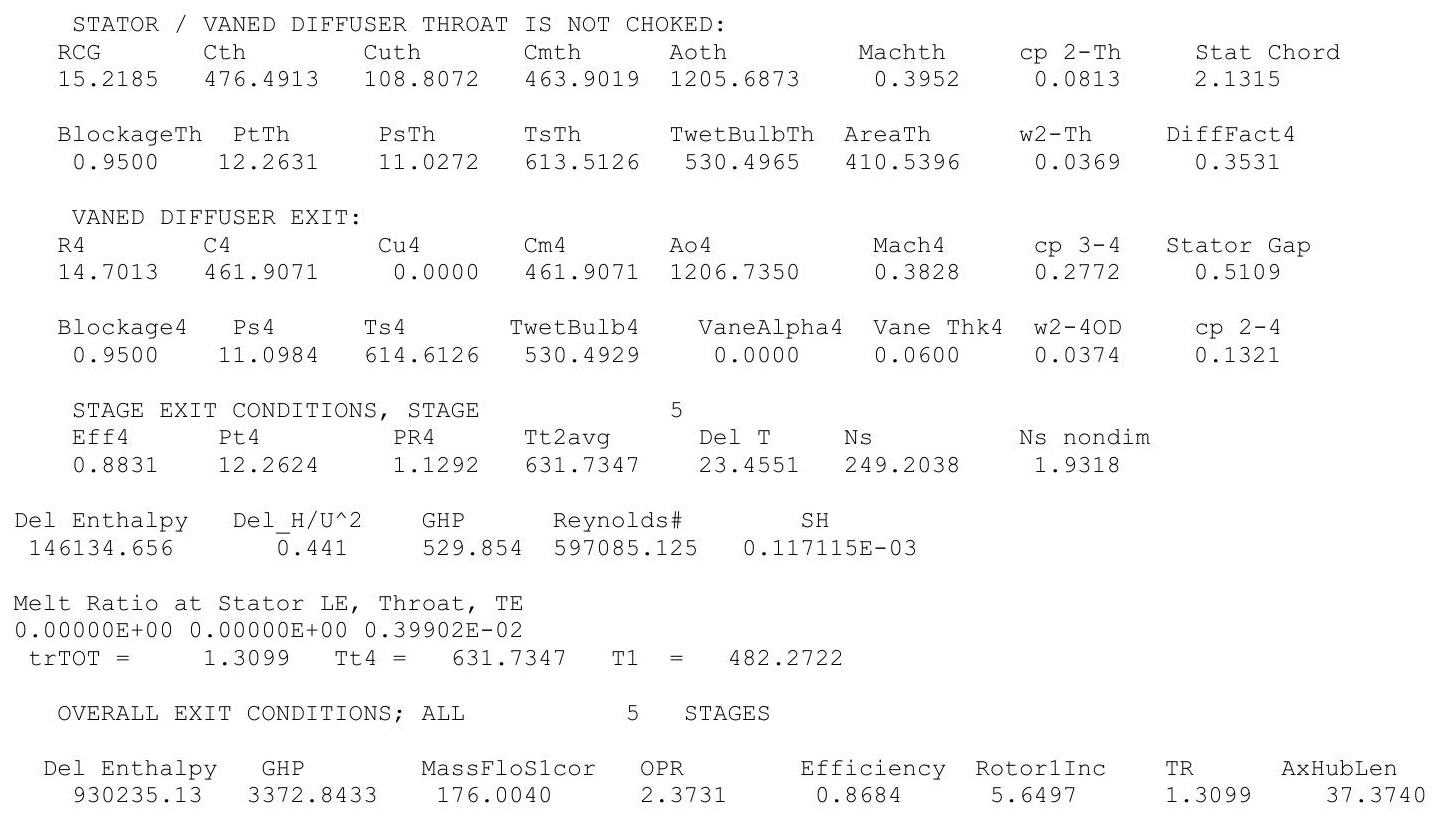




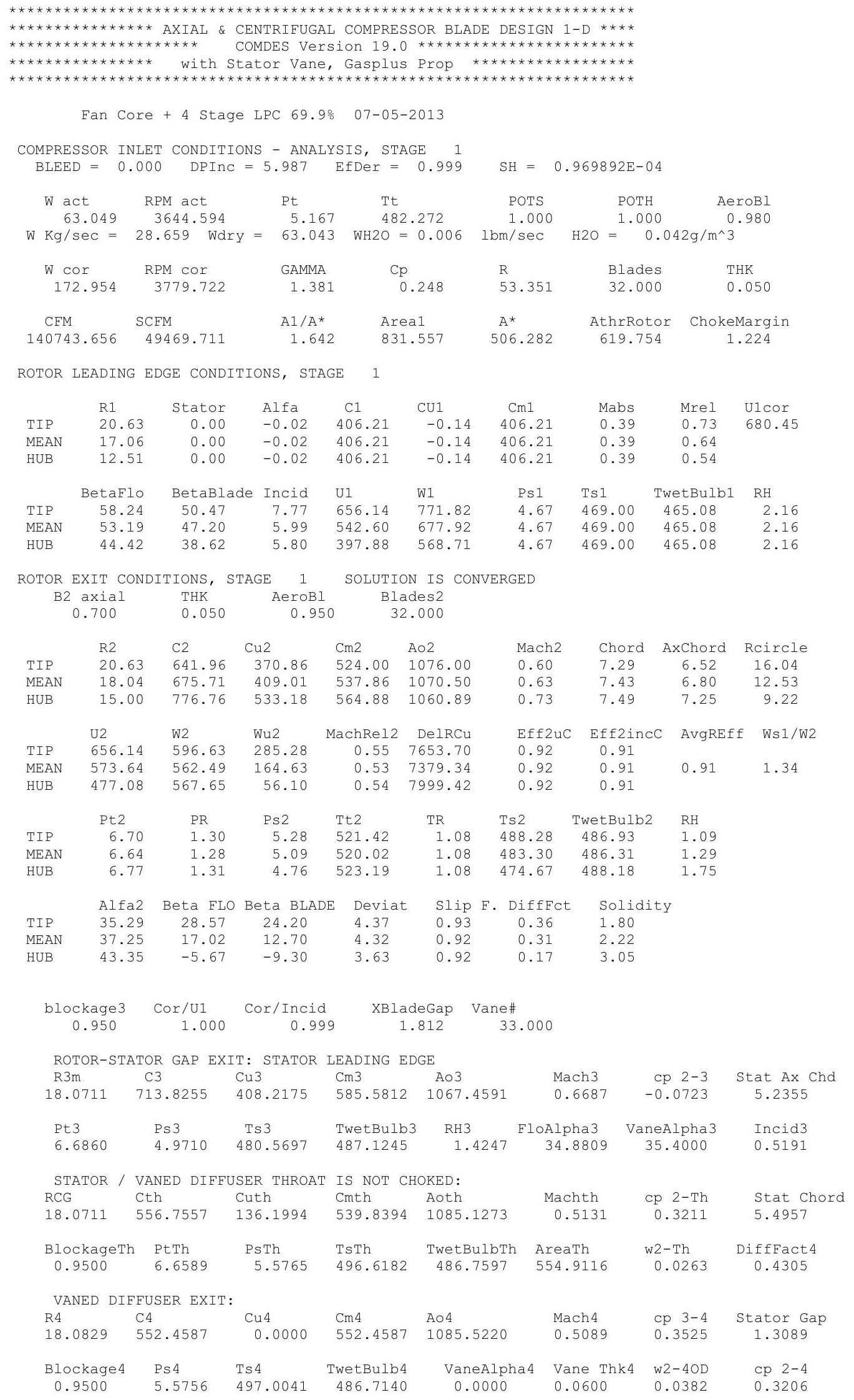




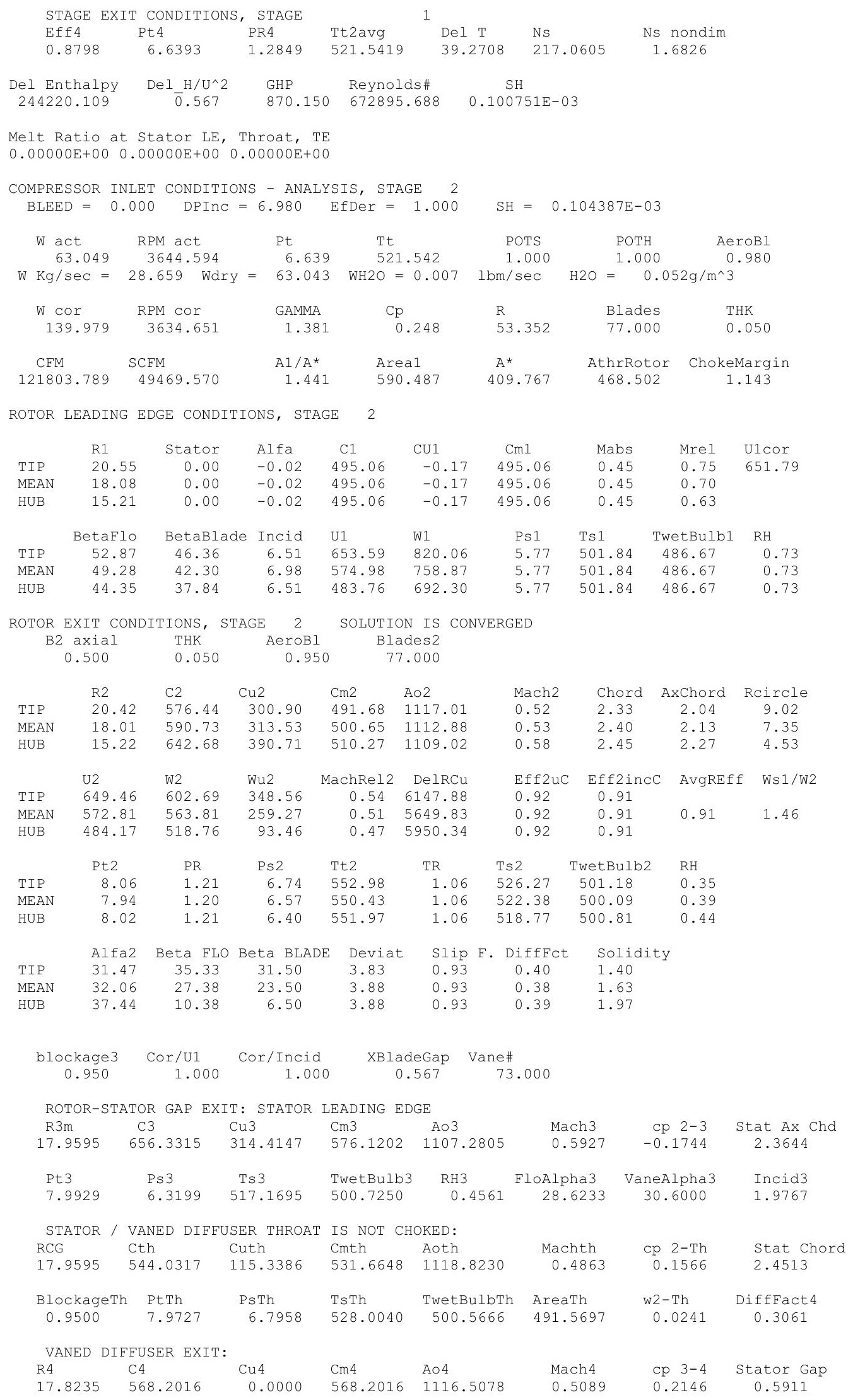




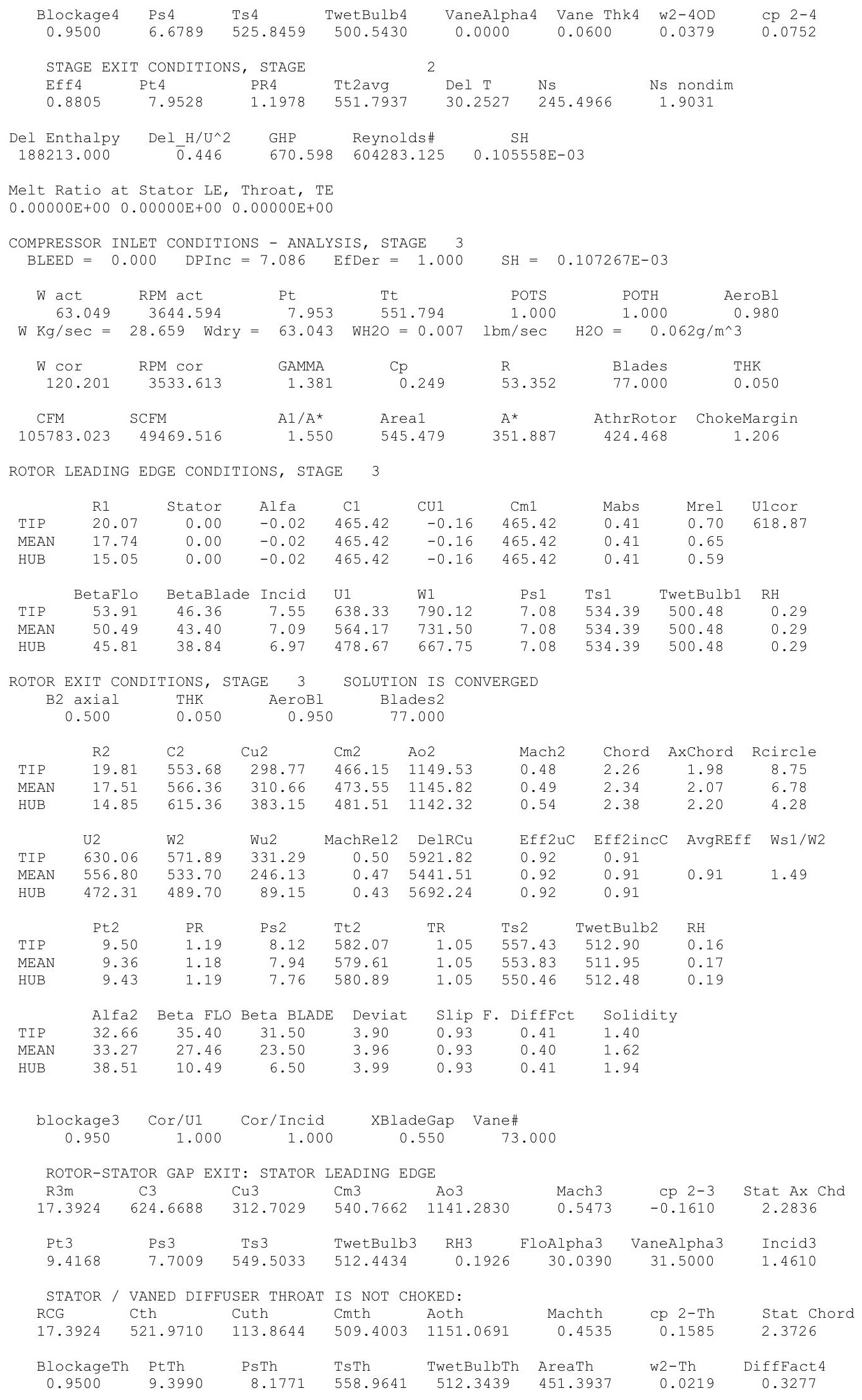




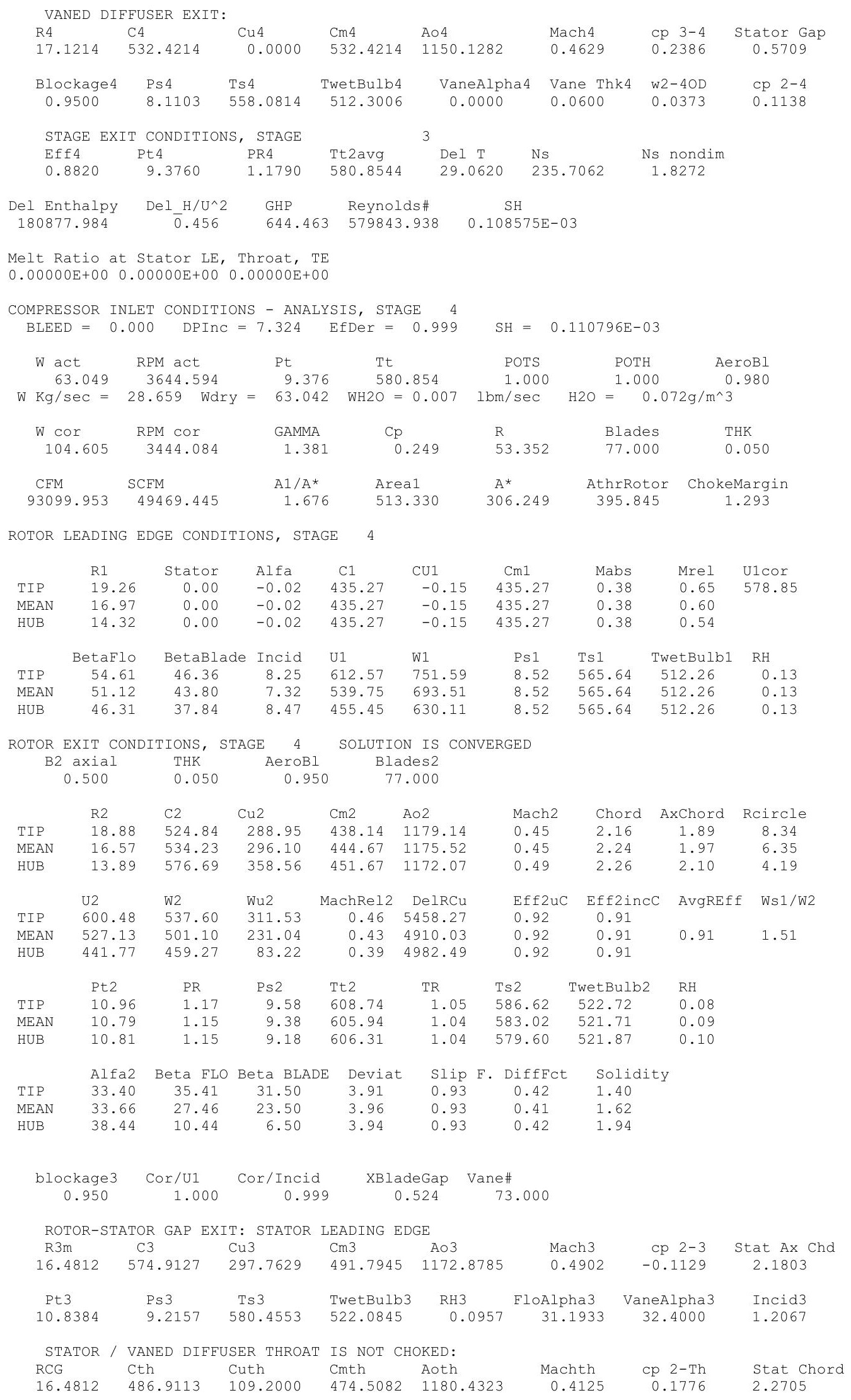




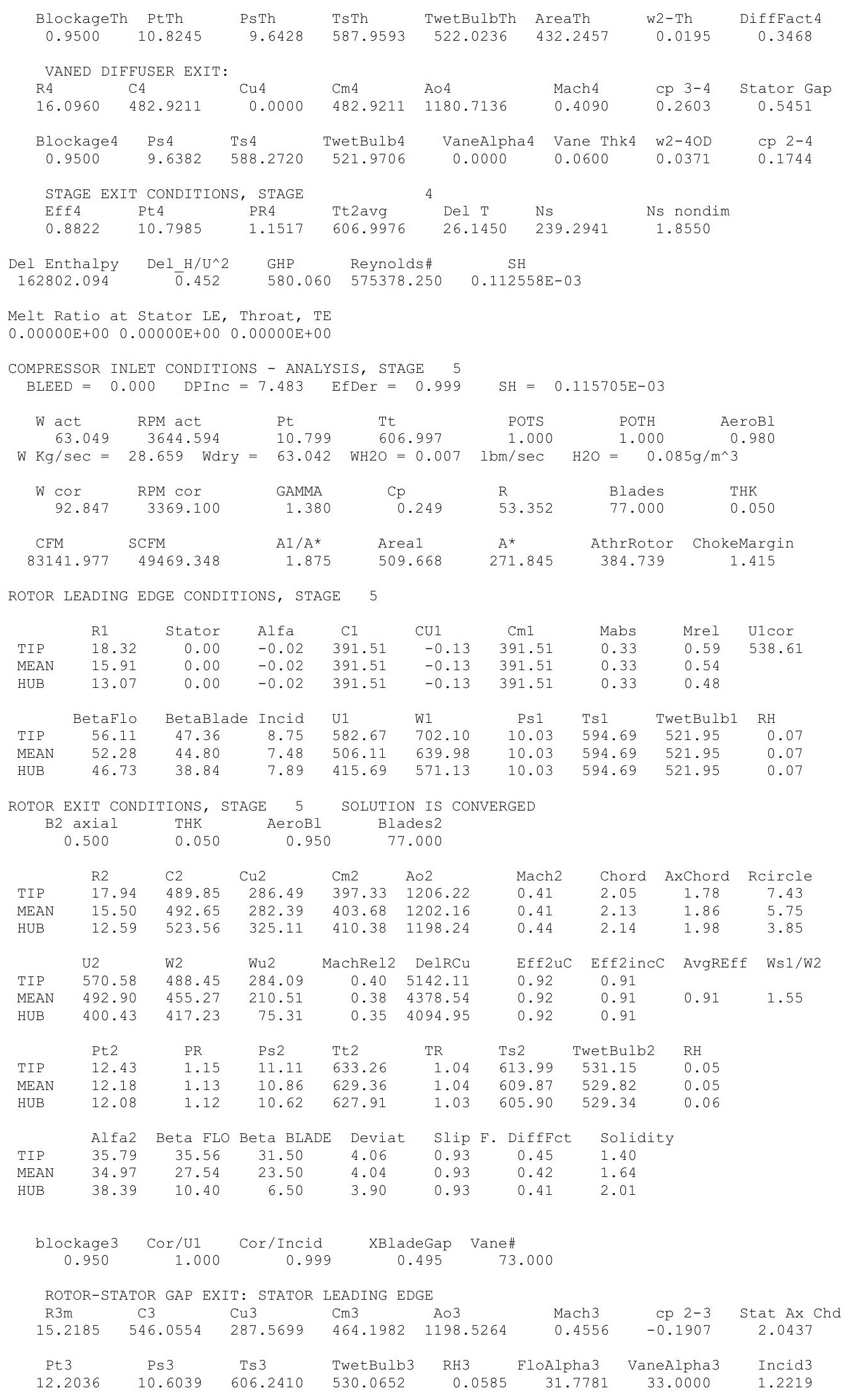




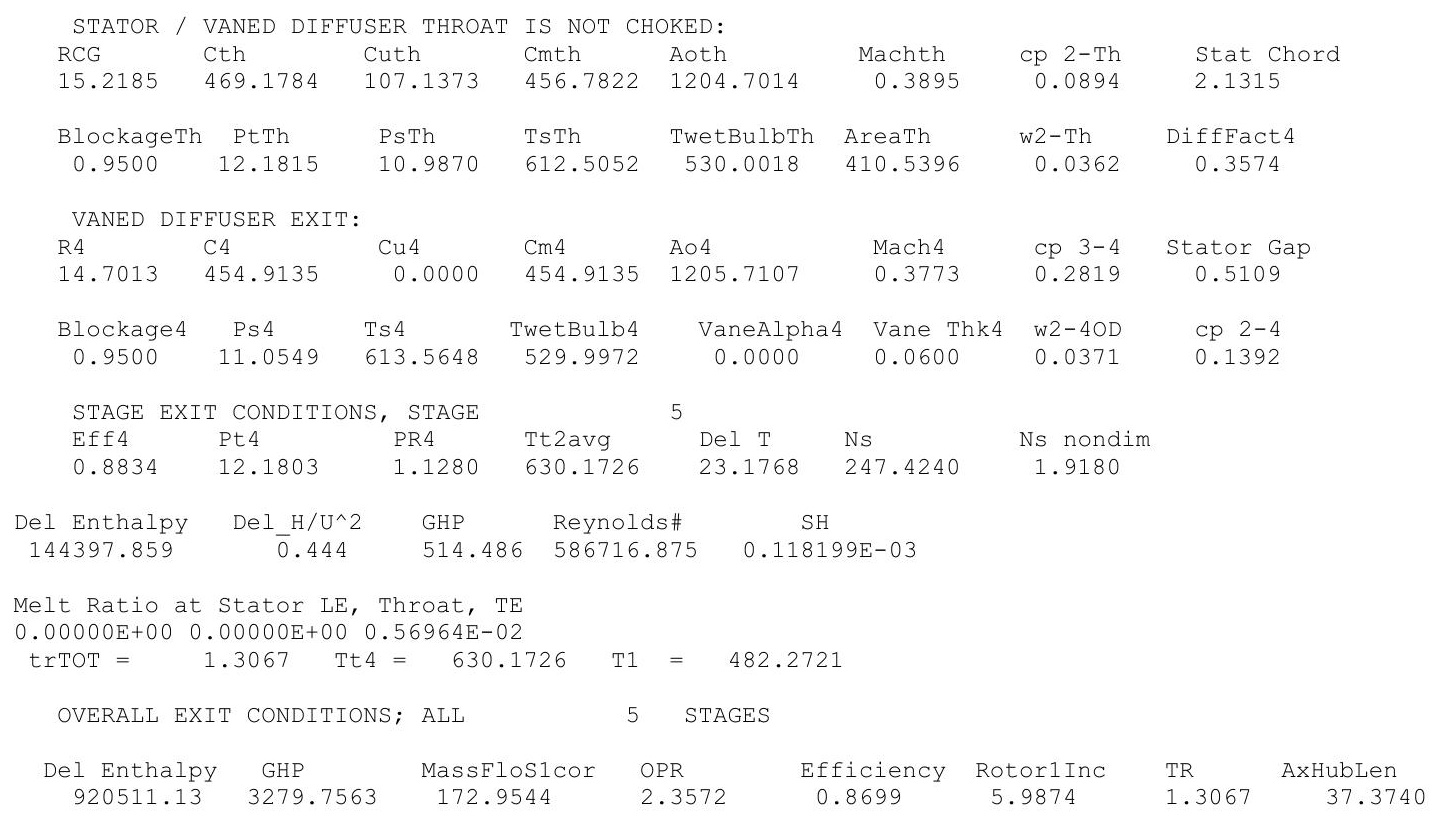




\section{$100 \mu \mathrm{m}$, ISA $+18 \mathrm{R}$}

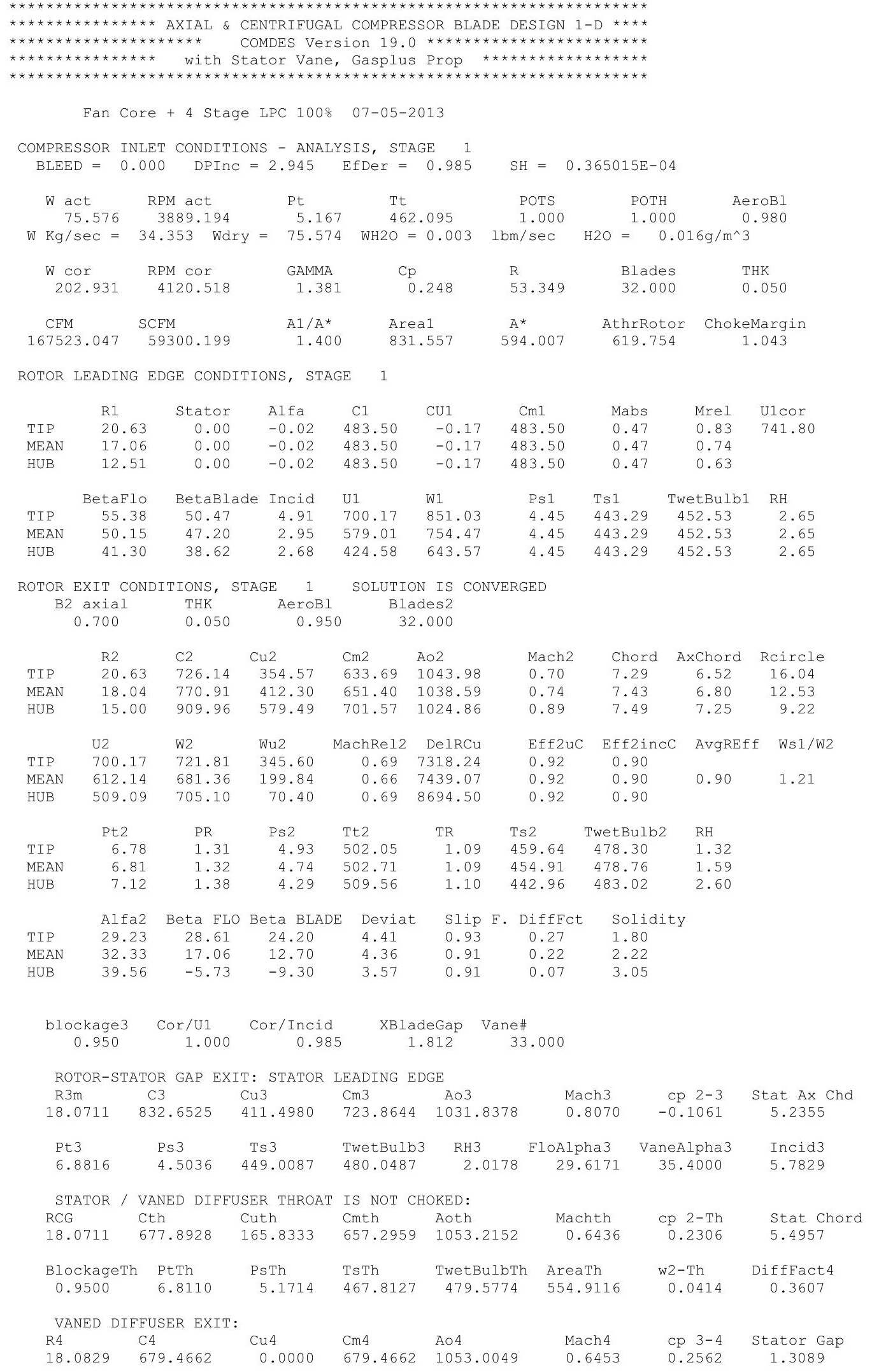




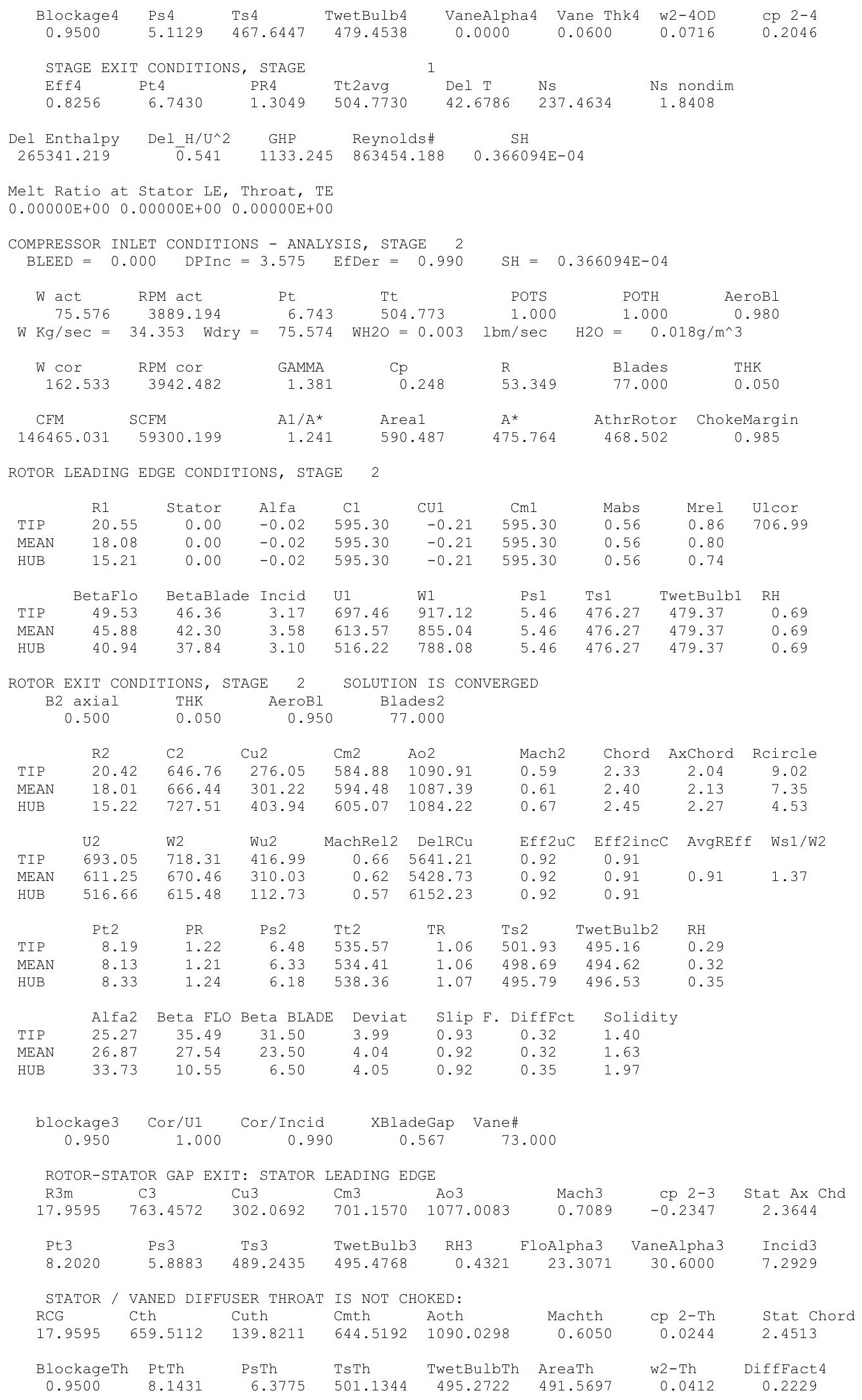




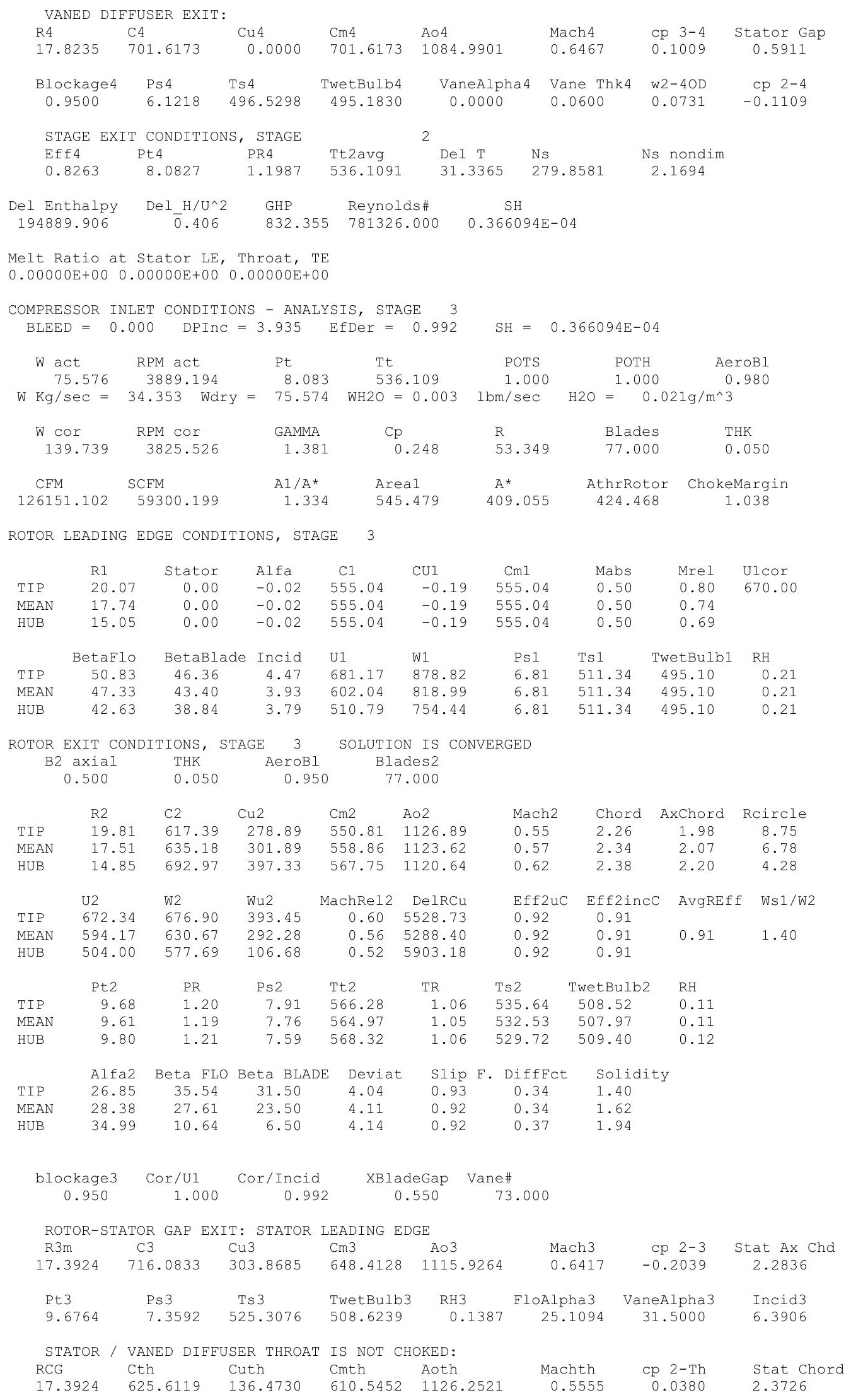




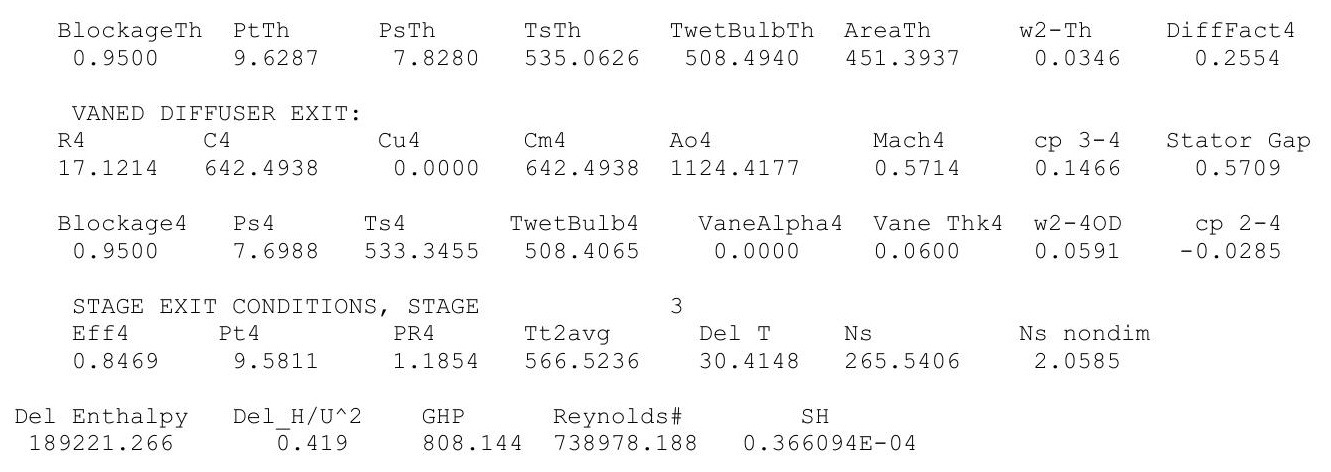

Melt Ratio at Stator LE, Throat, TE

$0.00000 \mathrm{E}+00 \quad 0.00000 \mathrm{E}+00 \quad 0.00000 \mathrm{E}+00$

COMPRESSOR INLET CONDITIONS - ANALYSIS, STAGE 4 BLEED $=0.000 \quad$ DPInC $=4.504 \quad$ EfDer $=0.995$

$\mathrm{SH}=0.366094 \mathrm{E}-04$

$\begin{array}{llllll}\text { W act } & \text { RPM act } & \text { Pt } & \text { POTS } & \text { POTH } & \text { AeroBI }\end{array}$ $\mathrm{W} \mathrm{Kg} / \mathrm{sec}=34.353$ Wdry $=75.574 \quad \mathrm{WH} 2 \mathrm{O}=0.003 \quad \mathrm{lbm} / \mathrm{sec} \quad \mathrm{H} 2 \mathrm{O}=0.024 \mathrm{~g} / \mathrm{m}^{\wedge} 3$

$\begin{array}{ccccccc}\text { W cor } & \text { RPM cor } & \text { GAMMA } & \text { Cp } & \text { R } & \text { Blades } & \text { THK } \\ 121.183 & 3721.421 & 1.381 & 0.249 & 53.349 & 77.000 & 0.050 \\ \text { CFM } & \text { SCFM } & \text { A1/A* } & \text { Area1 } & \text { A* } & \text { AthrRotor } & \text { ChokeMargin } \\ 109780.039 & 59300.199 & 1.447 & 513.330 & 354.755 & 395.845 & 1.116\end{array}$

ROTOR LEADING EDGE CONDITIONS, STAGE 4

\begin{tabular}{|c|c|c|c|c|c|c|c|c|c|}
\hline & R1 & Stator & Alfa & $\mathrm{C} 1$ & CU1 & $\mathrm{Cm} 1$ & Mabs & Mrel & U1cor \\
\hline TIP & 19.26 & 0.00 & -0.02 & 513.26 & -0.18 & 513.26 & 0.45 & 0.73 & 625.46 \\
\hline MEAN & 16.97 & 0.00 & -0.02 & 513.26 & -0.18 & 513.26 & 0.45 & 0.68 & \\
\hline HUB & 14.32 & 0.00 & -0.02 & 513.26 & -0.18 & 513.26 & 0.45 & 0.62 & \\
\hline & BetaFlo & BetaBlade & Incid & U1 & W1 & Ps 1 & Ts1 & TwetBulb1 & $\mathrm{RH}$ \\
\hline TIP & 51.87 & 46.36 & 5.51 & 653.68 & 831.24 & 8.35 & 545.35 & 508.37 & 0.08 \\
\hline MEAN & 48.30 & 43.80 & 4.50 & 575.98 & 771.62 & 8.35 & 545.35 & 508.37 & 0.08 \\
\hline HUB & 43.45 & 37.84 & 5.61 & 486.02 & 706.98 & 8.35 & 545.35 & 508.37 & 0.08 \\
\hline
\end{tabular}

ROTOR EXIT CONDITIONS, STAGE 4 SOLUTION IS CONVERGED

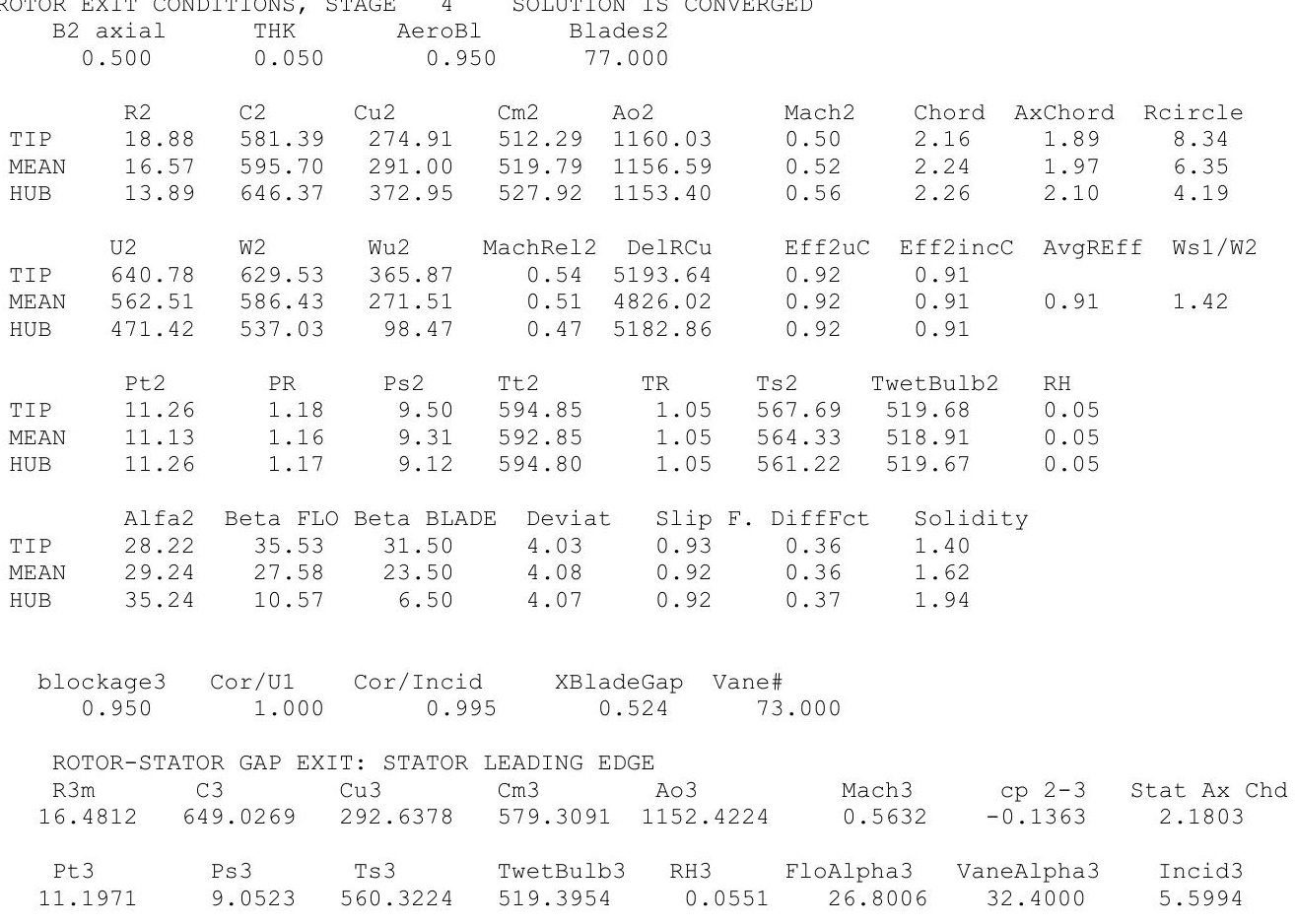




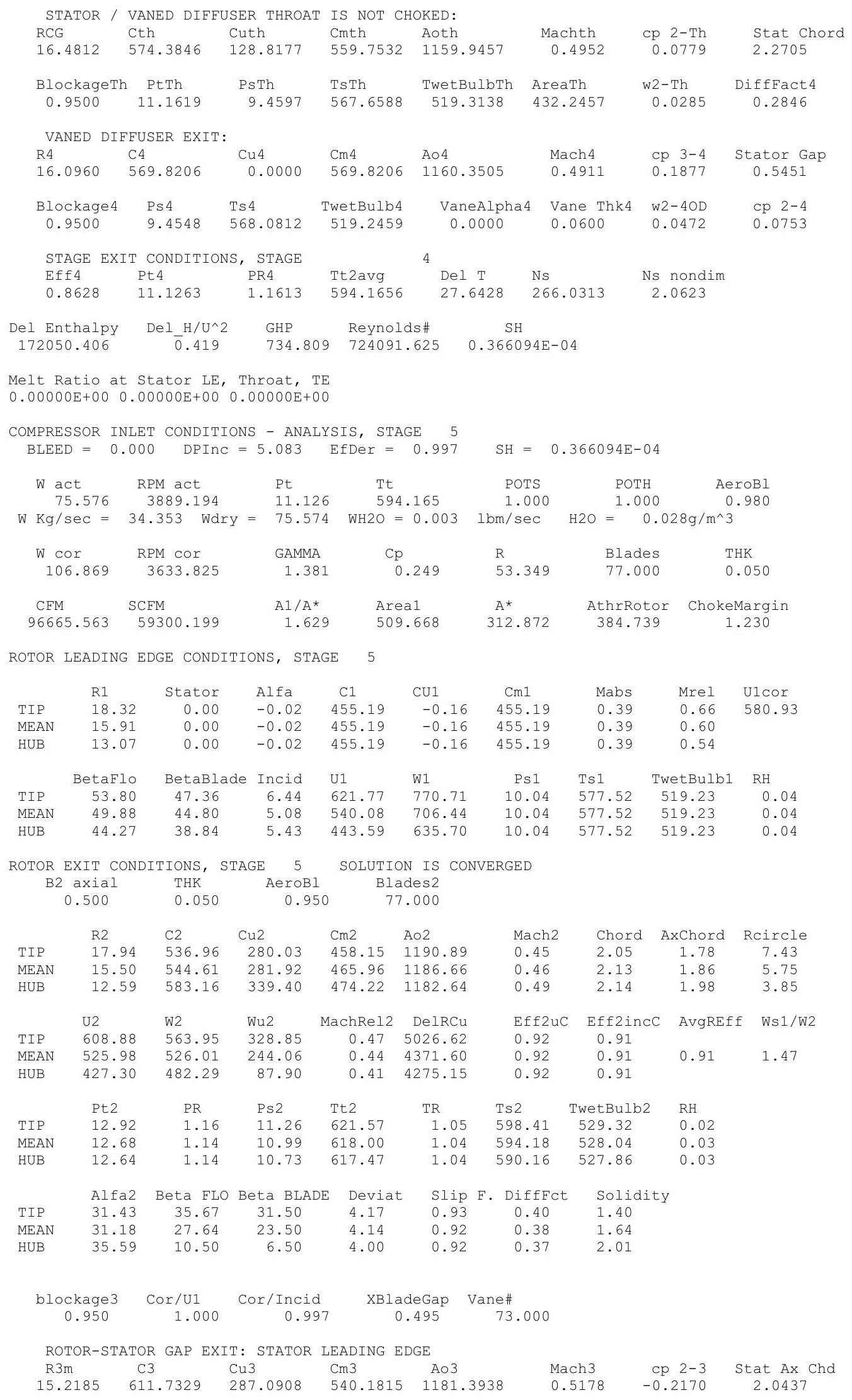




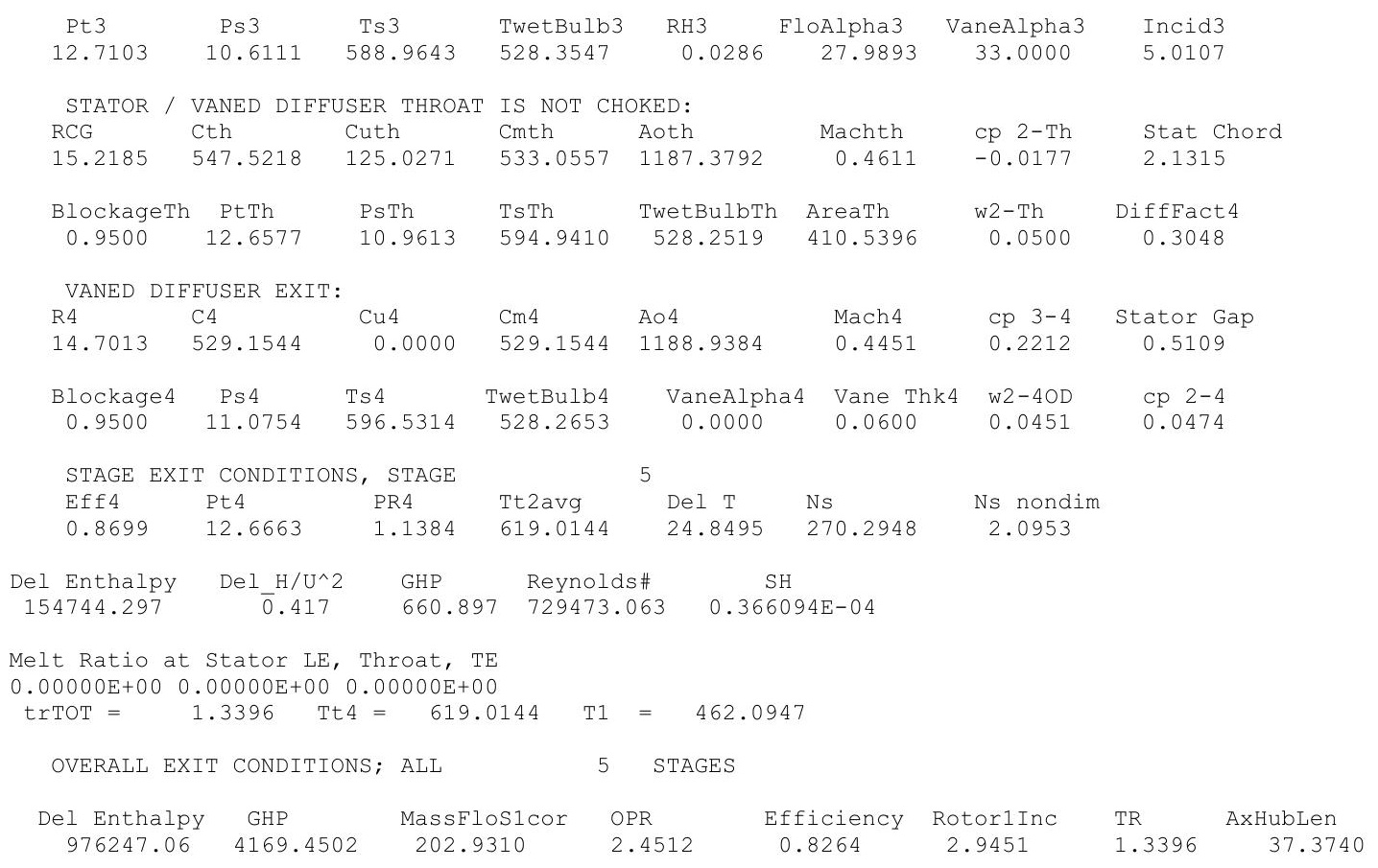




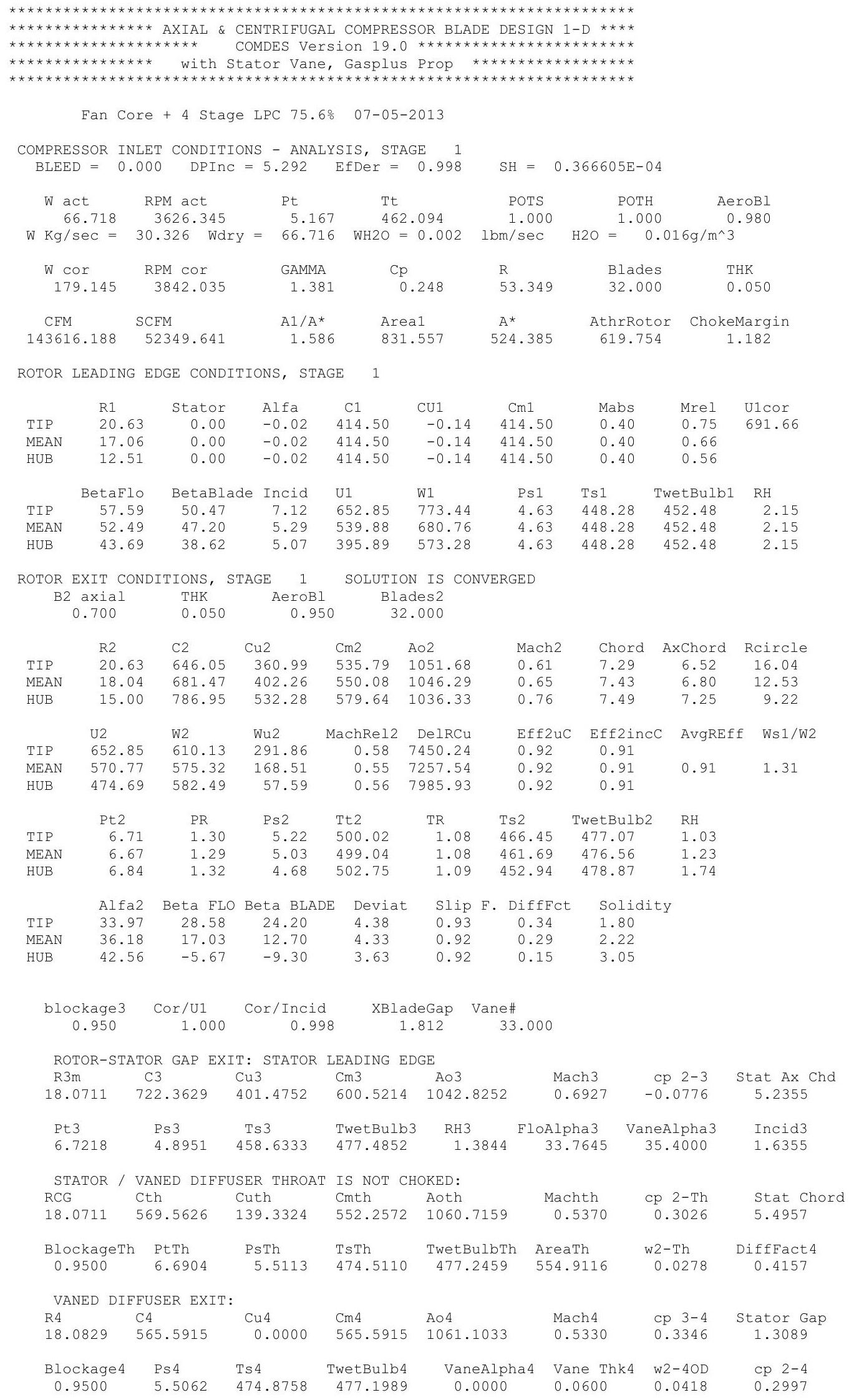




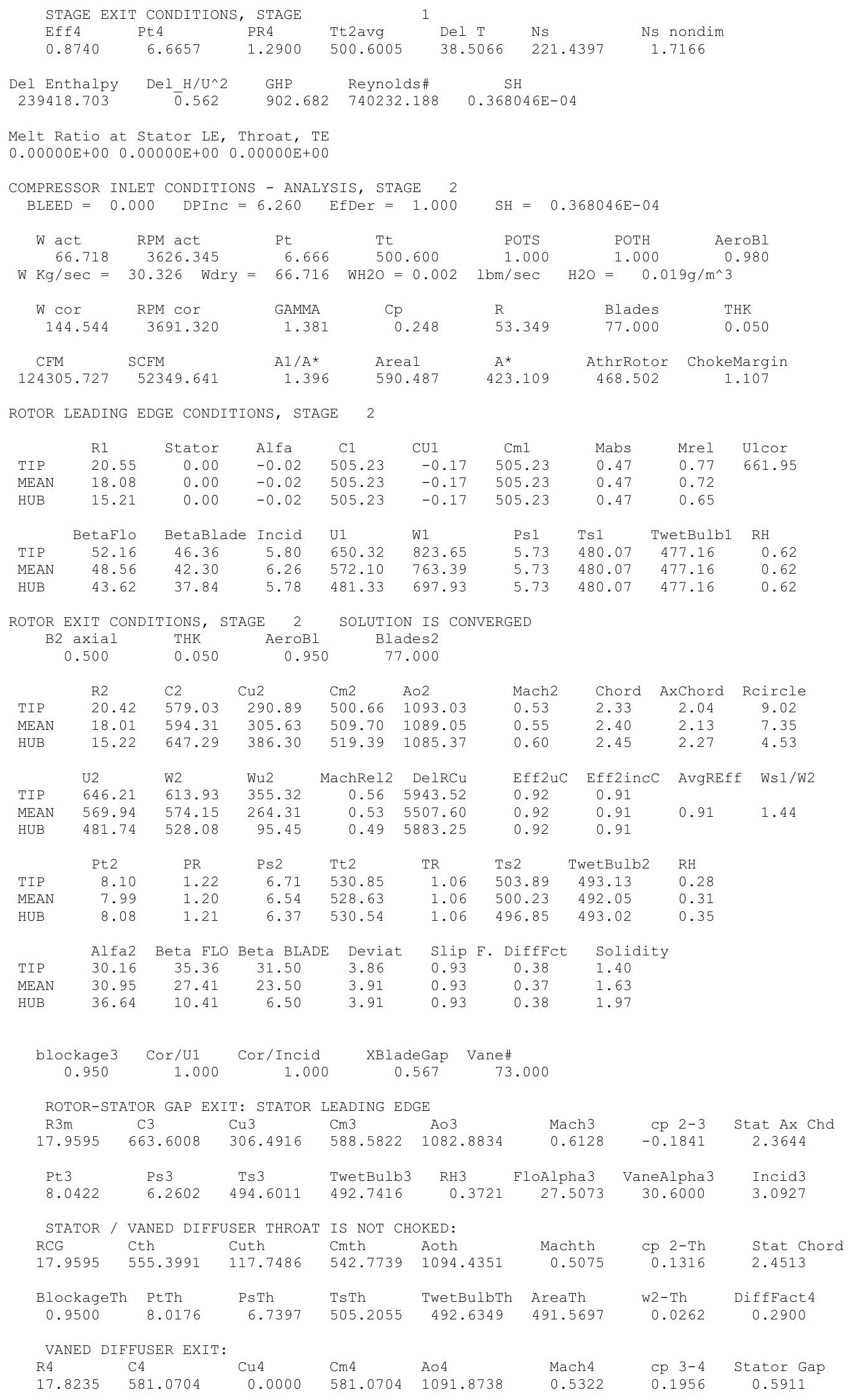




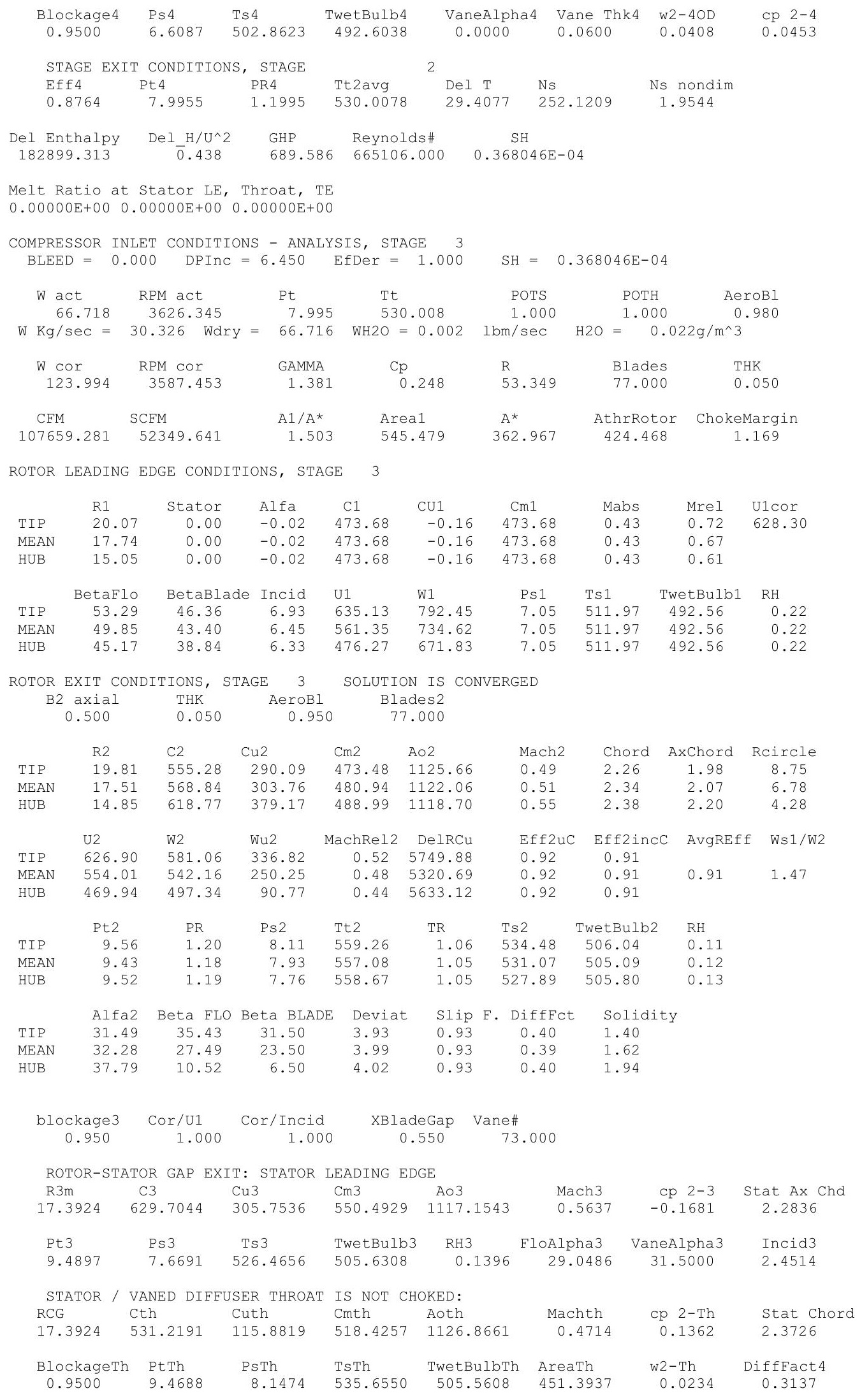




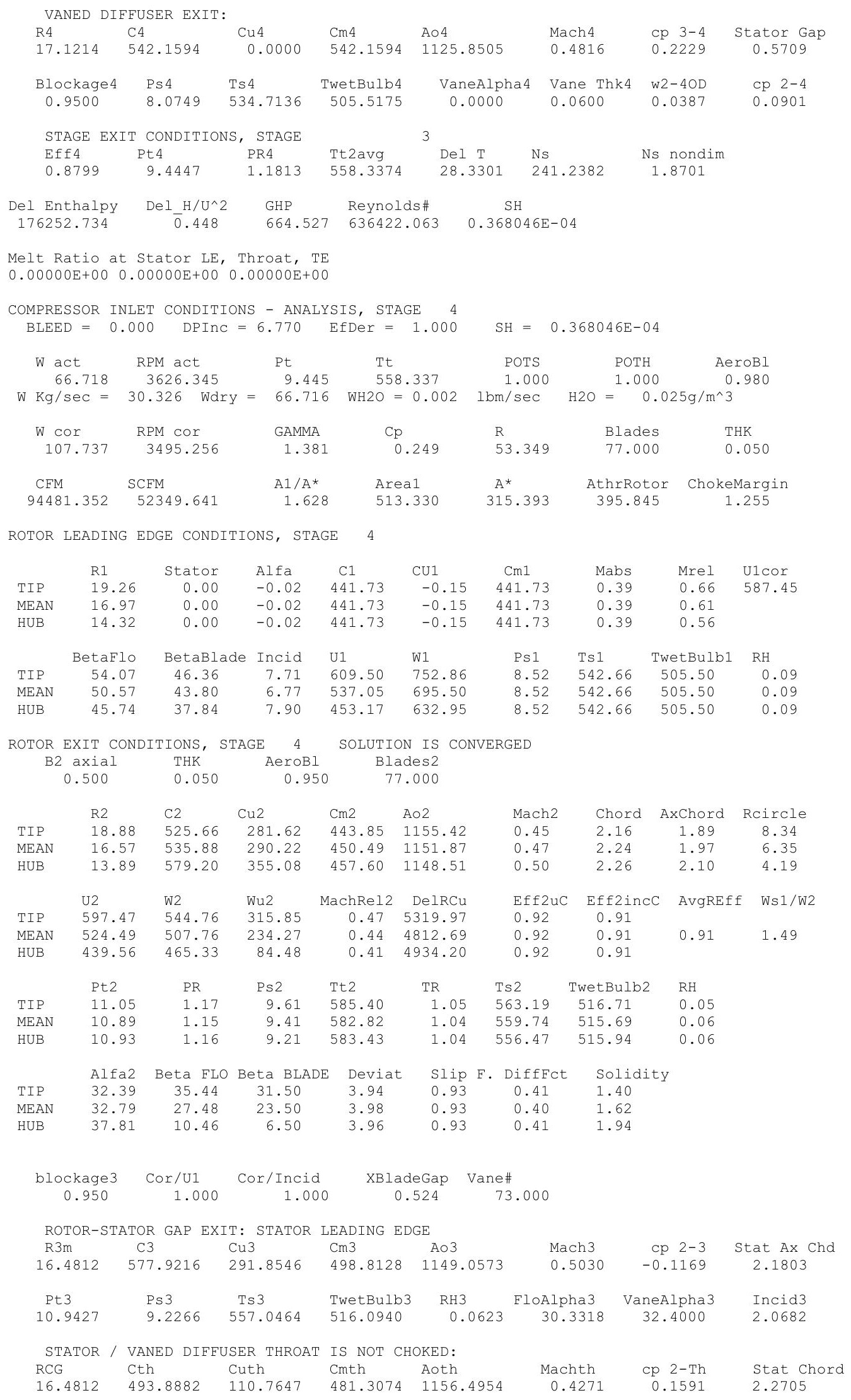




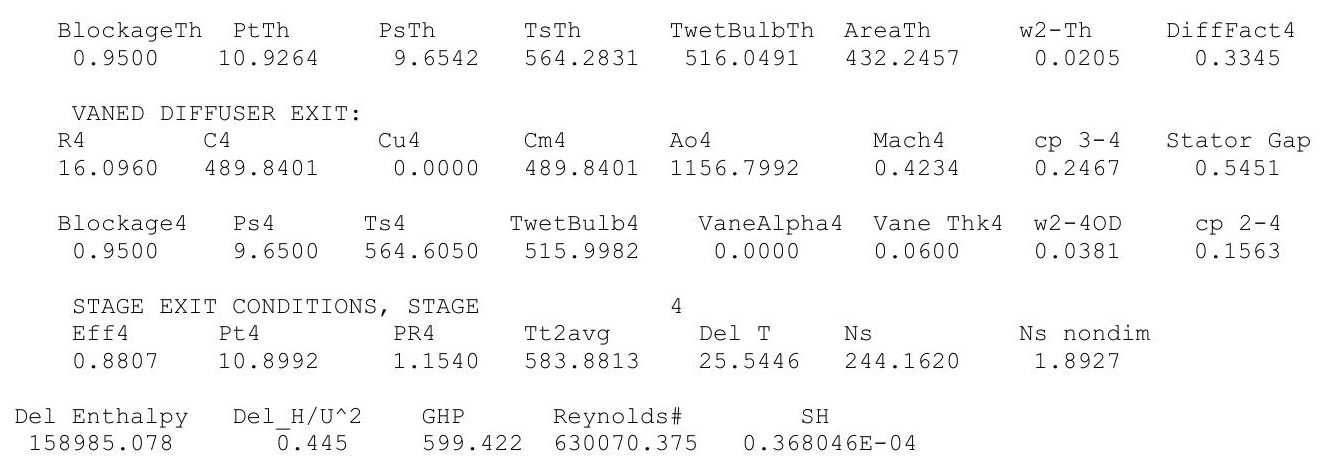

Melt Ratio at Stator LE, Throat, TE

$0.00000 \mathrm{E}+00 \quad 0.00000 \mathrm{E}+00 \quad 0.00000 \mathrm{E}+00$

COMPRESSOR INLET CONDITIONS - ANALYSIS, STAGE 5 BLEED $=0.000 \quad$ DPInC $=7.013 \quad$ EfDer $=1.000$

$\begin{array}{llllll}\text { W act } & \text { RPM act } & \text { Pt } & \text { POTS } & \text { POTH } & \text { AeroBI }\end{array}$

ROTOR LEADING EDGE CONDITIONS, STAGE 5

$\begin{array}{lccccccccc} & \text { R1 } & \text { Stator } & \text { Alfa } & \text { C1 } & \text { CU1 } & \text { Cm1 } & \text { Mabs } & \text { Mrel } & \text { U1cor } \\ \text { TIP } & 18.32 & 0.00 & -0.02 & 396.21 & -0.14 & 396.21 & 0.34 & 0.60 & 546.42 \\ \text { MEAN } & 15.91 & 0.00 & -0.02 & 396.21 & -0.14 & 396.21 & 0.34 & 0.55 & \\ \text { HUB } & 13.07 & 0.00 & -0.02 & 396.21 & -0.14 & 396.21 & 0.34 & 0.49 & \\ & & & & & & & & & \\ & \text { BetaFlo } & \text { BetaBlade } & \text { Incid } & \text { U1 } & \text { W1 } & \text { Ps1 } & \text { Ts 1 } & \text { TwetBulb1 } & \text { RH } \\ \text { TIP } & 55.66 & 47.36 & 8.30 & 579.75 & 702.32 & 10.07 & 571.27 & 515.99 & 0.04 \\ \text { MEAN } & 51.81 & 44.80 & 7.01 & 503.58 & 640.87 & 10.07 & 571.27 & 515.99 & 0.04 \\ \text { HUB } & 46.24 & 38.84 & 7.40 & 413.61 & 572.86 & 10.07 & 571.27 & 515.99 & 0.04\end{array}$

ROTOR EXIT CONDITIONS, STAGE 5 SOLUTION IS CONVERGED

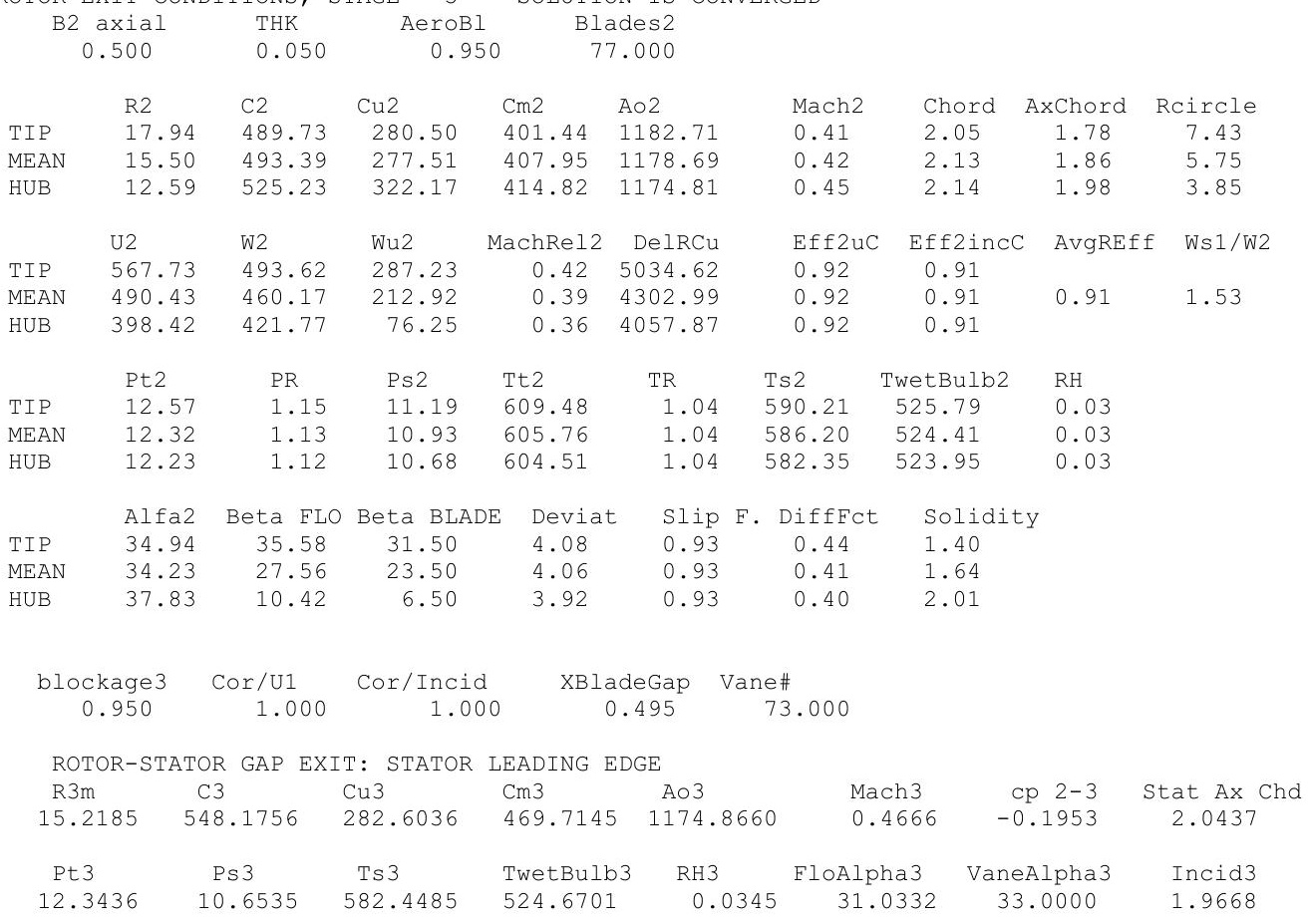




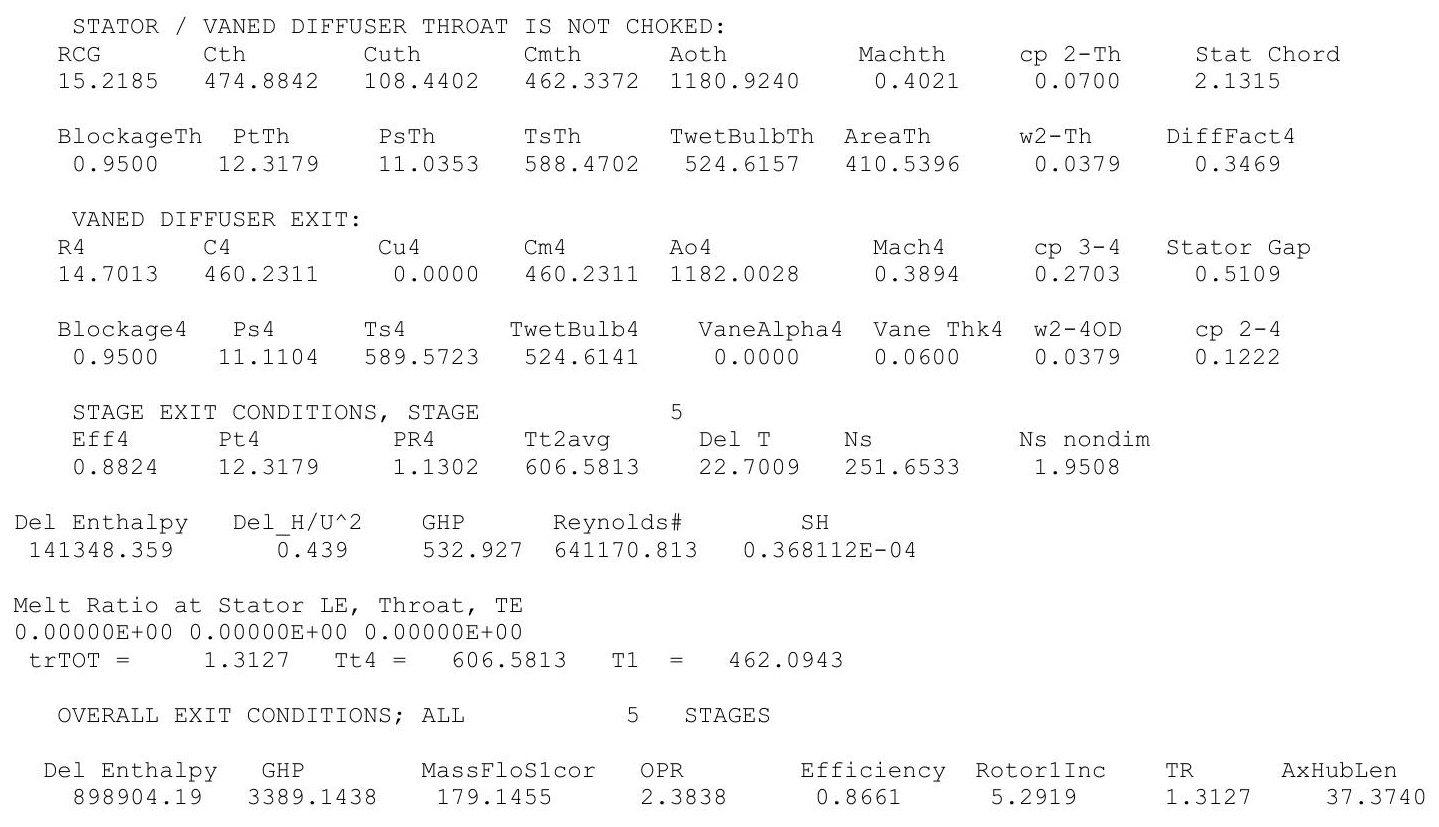




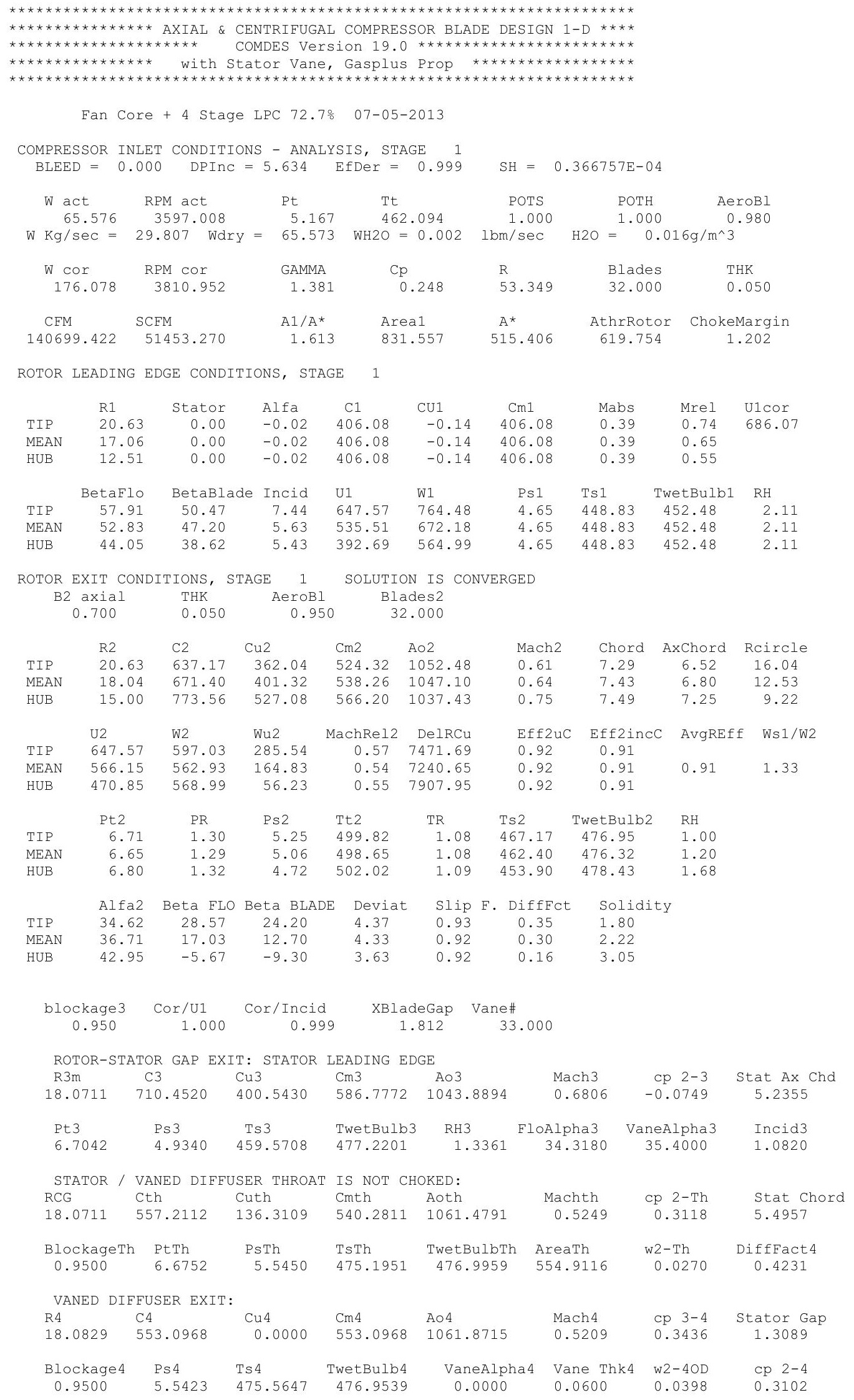




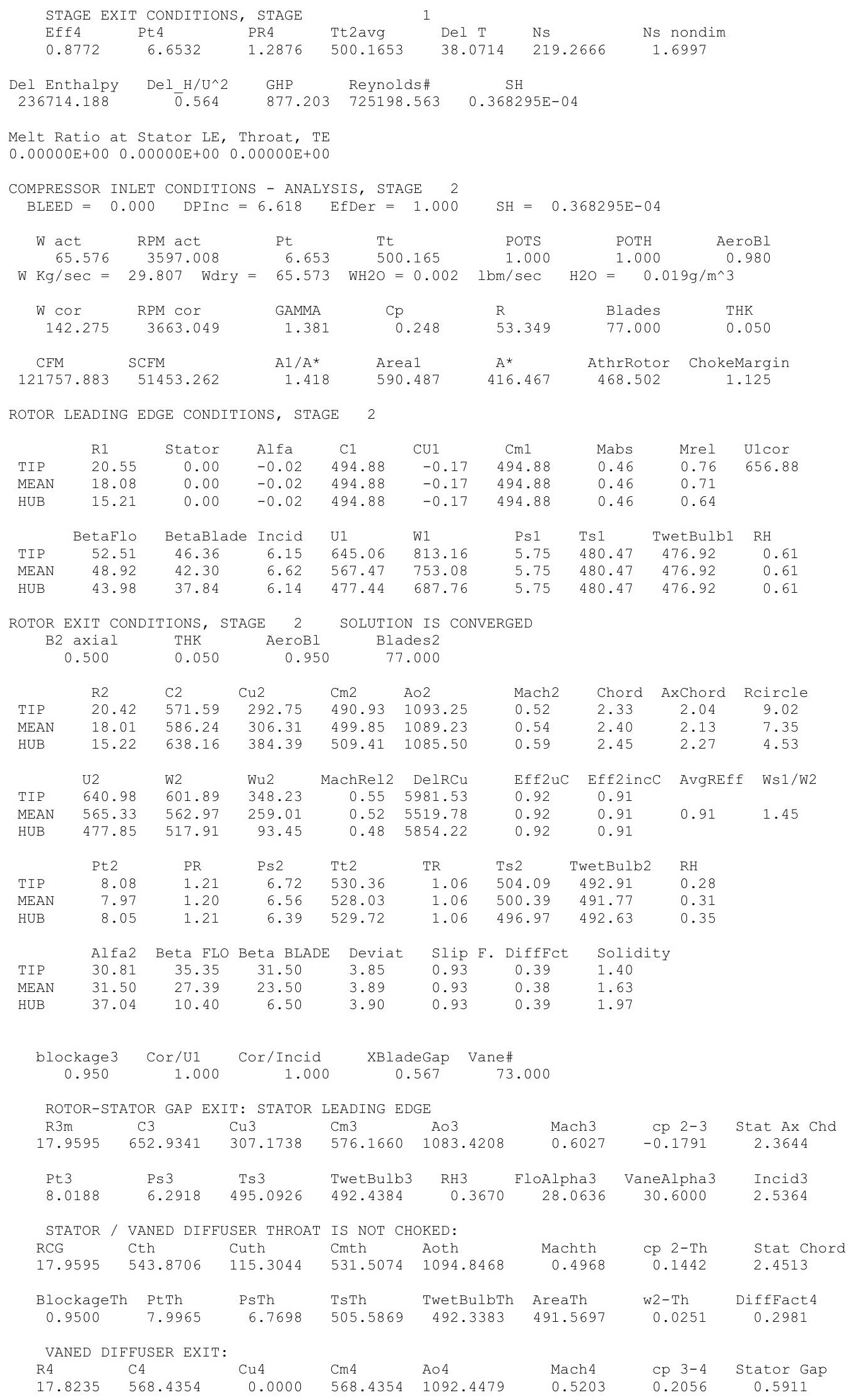




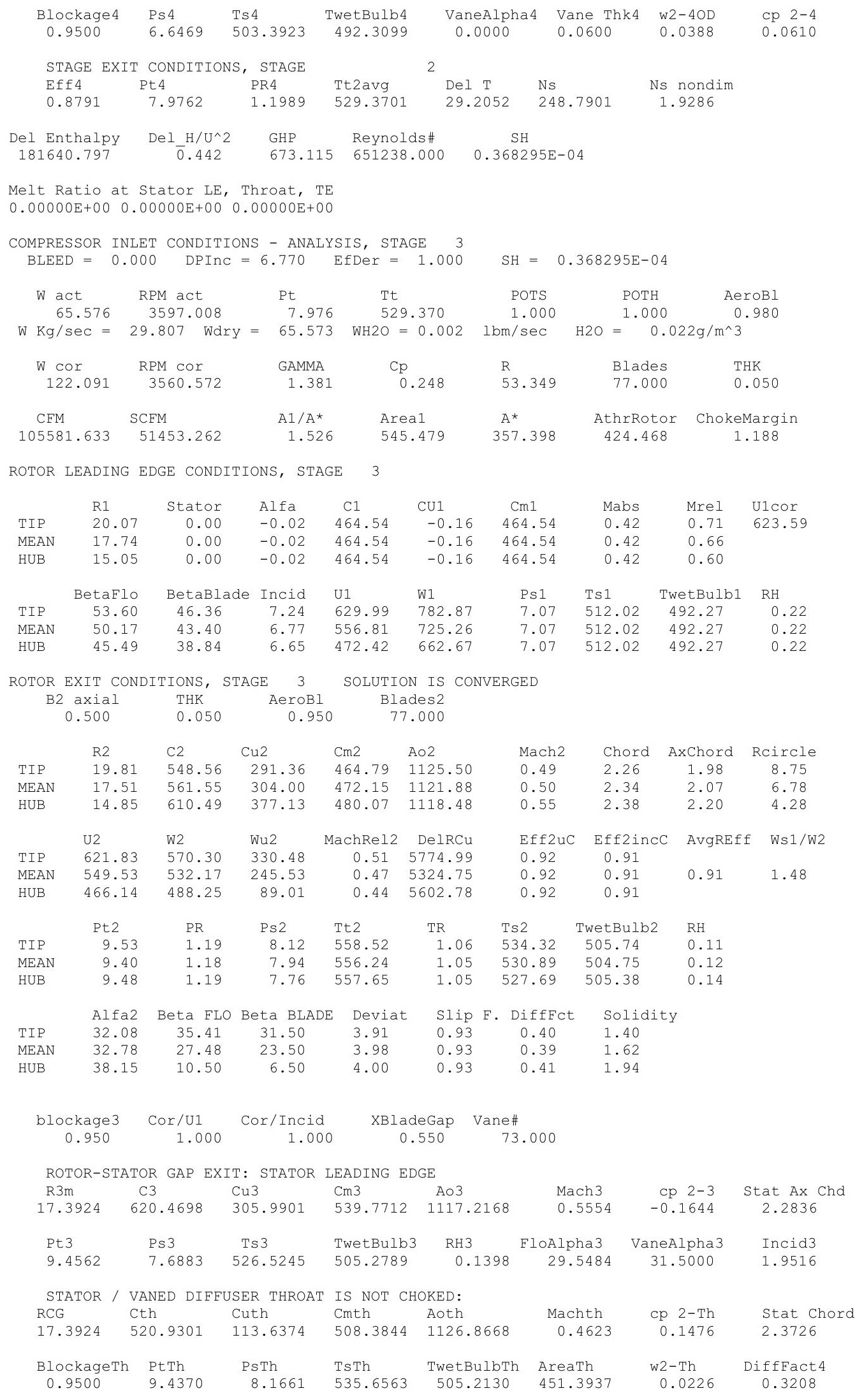




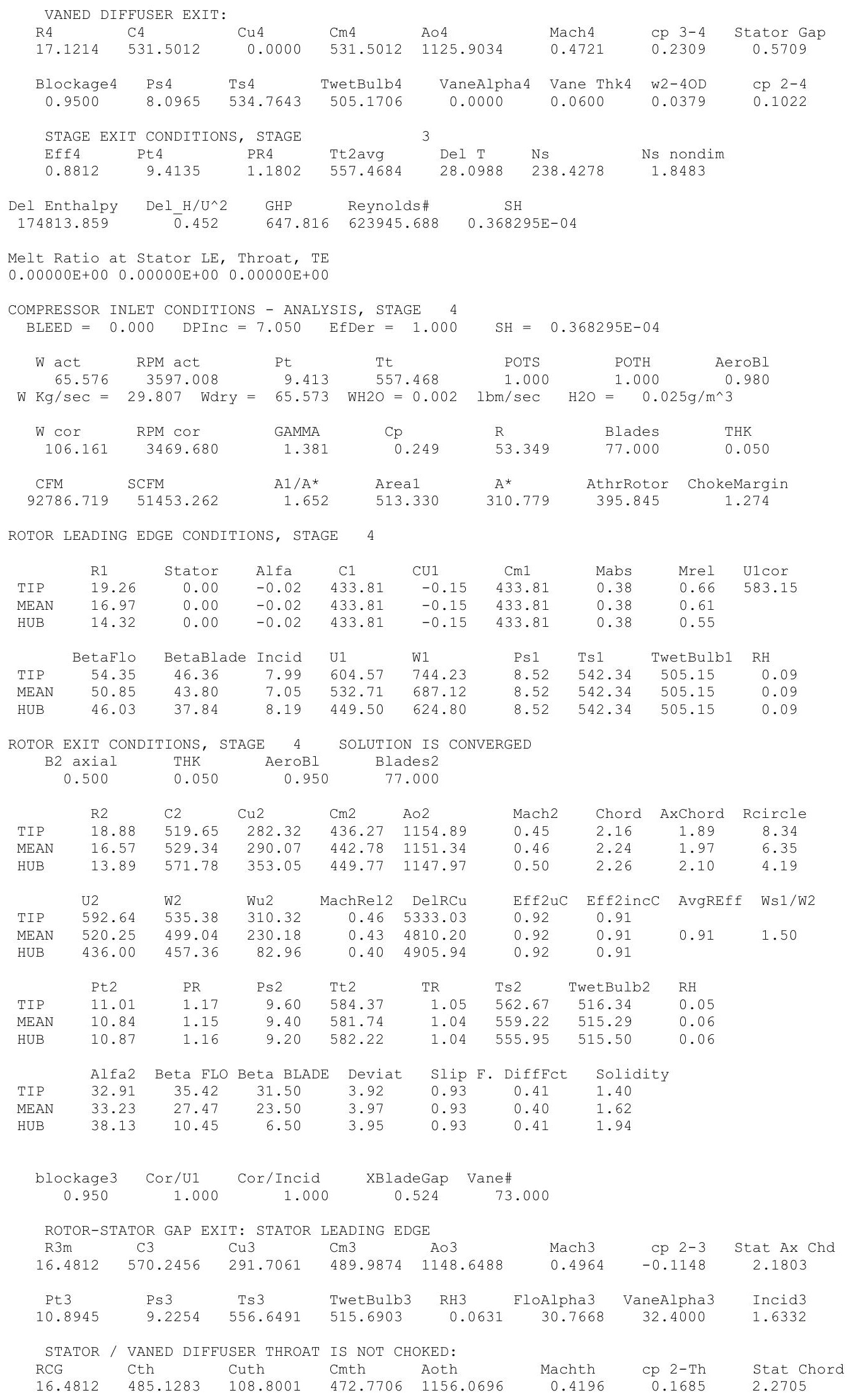




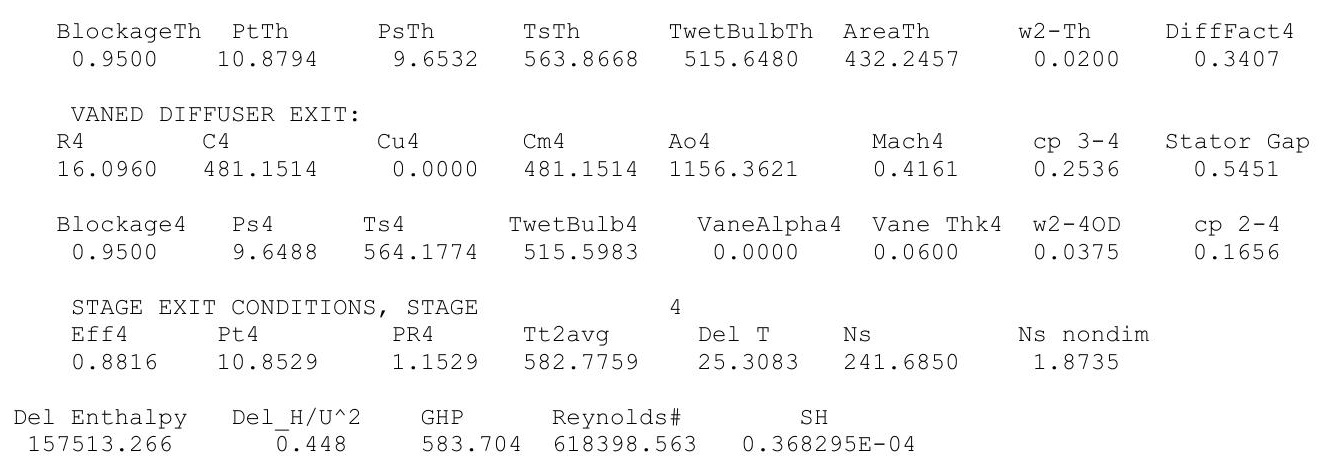

Melt Ratio at Stator LE, Throat, TE

$0.00000 \mathrm{E}+00 \quad 0.00000 \mathrm{E}+00 \quad 0.00000 \mathrm{E}+00$

COMPRESSOR INLET CONDITIONS - ANALYSIS, STAGE 5 $\mathrm{BLEED}=0.000 \quad$ DPInC $=7.251 \quad$ EfDer $=1.000$ $\begin{array}{llllllr}W \text { act } & \text { RPM act } & \text { Pt } & \text { Tt } & \text { POTS } & \text { POTH } & \text { AeroBI } \\ 65.576 & 3597.008 & 10.853 & 582.776 & 1.000 & 1.000 & 0.980\end{array}$ $\mathrm{W} \mathrm{Kg} / \mathrm{sec}=29.807$ Wdry $=65.573 \mathrm{WH} 2 \mathrm{O}=0.002 \mathrm{lbm} / \mathrm{sec} \quad \mathrm{H} 2 \mathrm{O}=0.028 \mathrm{~g} / \mathrm{m}^{\wedge} 3$

$\begin{array}{ccccccc}\text { W Cor } & \text { RPM cor } & \text { GAMMA } & \text { CP } & \text { R } & \text { Blades } & \text { THK } \\ 94.147 & 3393.506 & 1.381 & 0.249 & 53.349 & 77.000 & 0.050 \\ & & & & & & \\ \text { CFM } & \text { SCFM } & \text { A1/A* } & \text { Areal } & A^{*} & \text { AthrRotor } & \text { ChokeMargin } \\ 82744.711 & 51453.262 & 1.849 & 509.668 & 275.626 & 384.739 & 1.396\end{array}$

ROTOR LEADING EDGE CONDITIONS, STAGE 5

$\begin{array}{lccccccccc} & \text { R1 } & \text { Stator } & \text { Alfa } & \text { C1 } & \text { CU1 } & \text { Cm1 } & \text { Mabs } & \text { Mrel } & \text { U1cor } \\ \text { TIP } & 18.32 & 0.00 & -0.02 & 389.64 & -0.13 & 389.64 & 0.34 & 0.60 & 542.51 \\ \text { MEAN } & 15.91 & 0.00 & -0.02 & 389.64 & -0.13 & 389.64 & 0.34 & 0.54 & \\ \text { HUB } & 13.07 & 0.00 & -0.02 & 389.64 & -0.13 & 389.64 & 0.34 & 0.49 & \\ & & & & & & & & & \\ \text { TIP } & \text { BetaFlo } & \text { BetaBlade } & \text { Incid } & \text { U1 } & \text { W1 } & \text { Ps1 } & \text { Ts1 } & \text { TwetBulb1 } & \text { RH } \\ \text { MEAN } & 55.89 & 47.36 & 8.53 & 575.06 & 694.74 & 10.05 & 570.58 & 515.59 & 0.05 \\ \text { HUB } & 46.49 & 38.84 & 7.65 & 410.26 & 565.90 & 10.05 & 570.58 & 515.59 & 0.05\end{array}$

ROTOR EXIT CONDITIONS, STAGE 5 SOLUTION IS CONVERGED

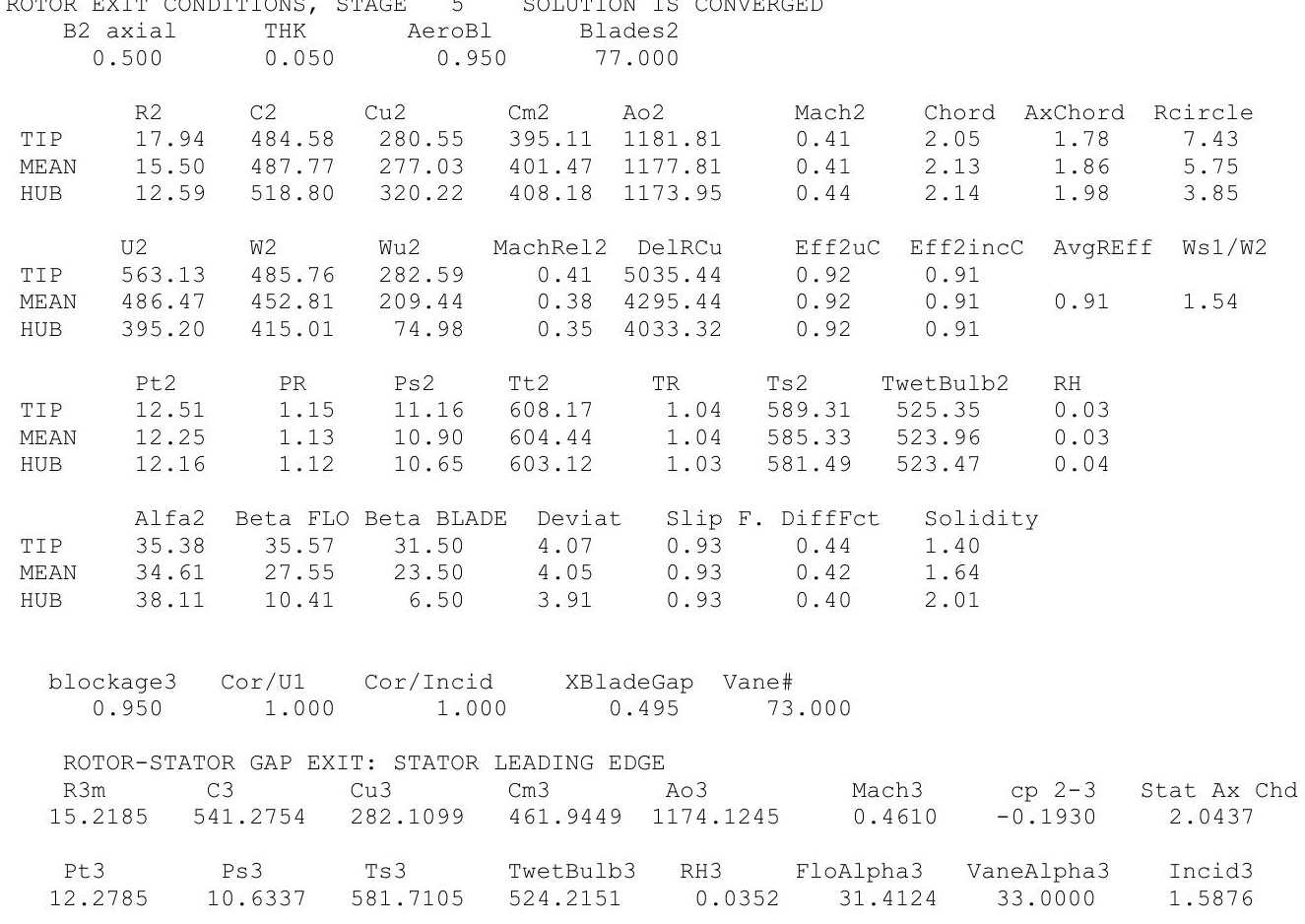




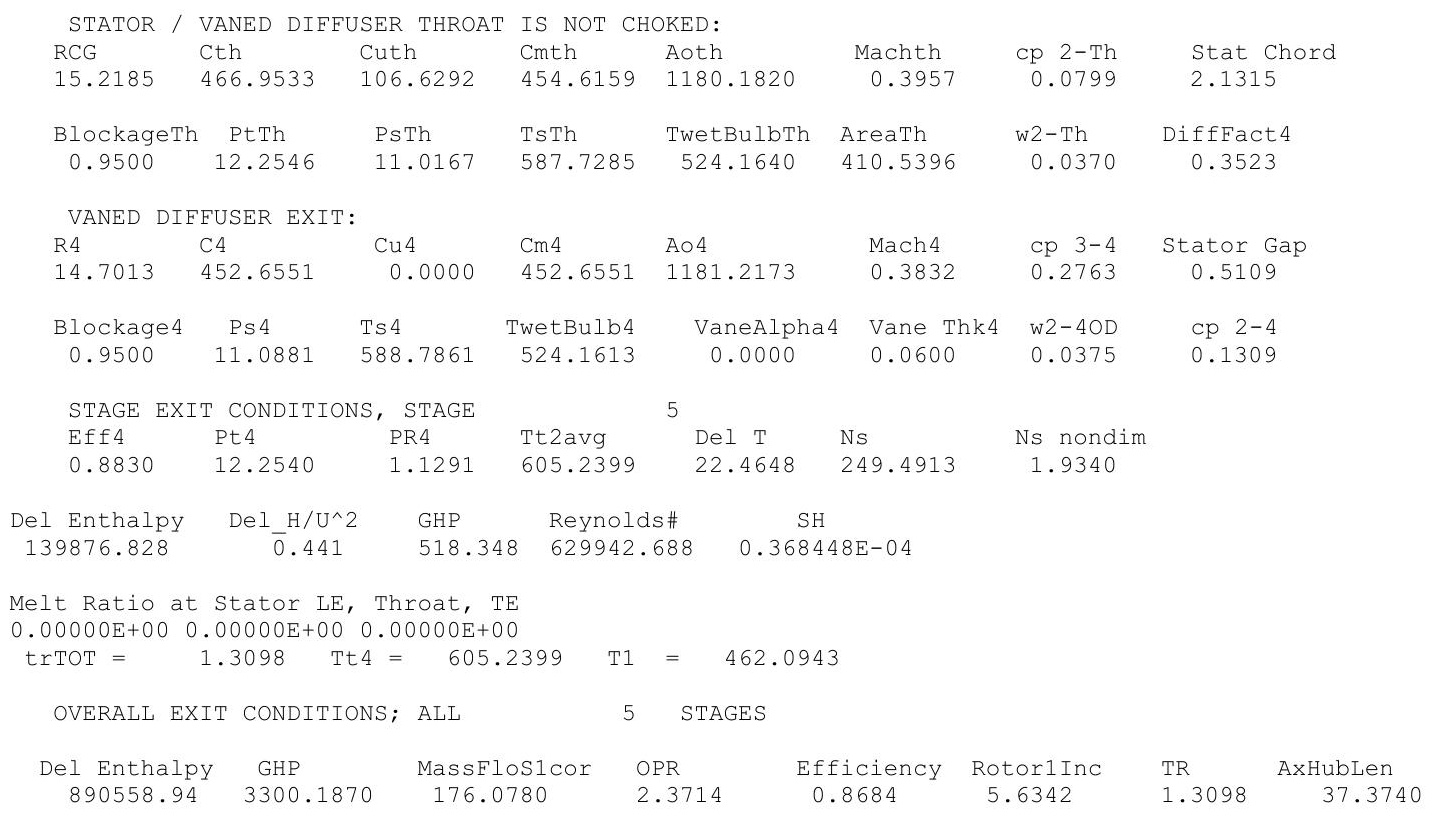




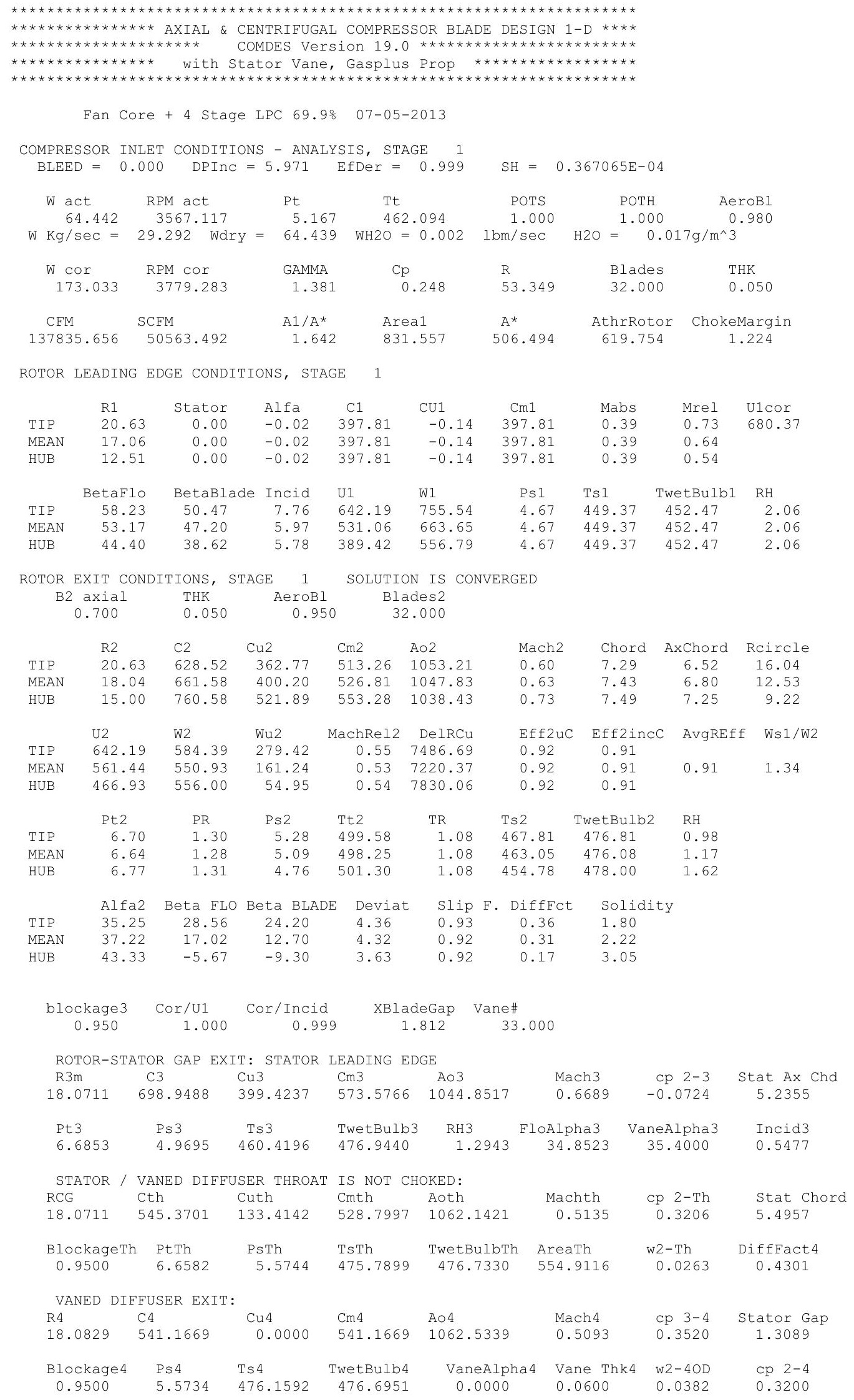




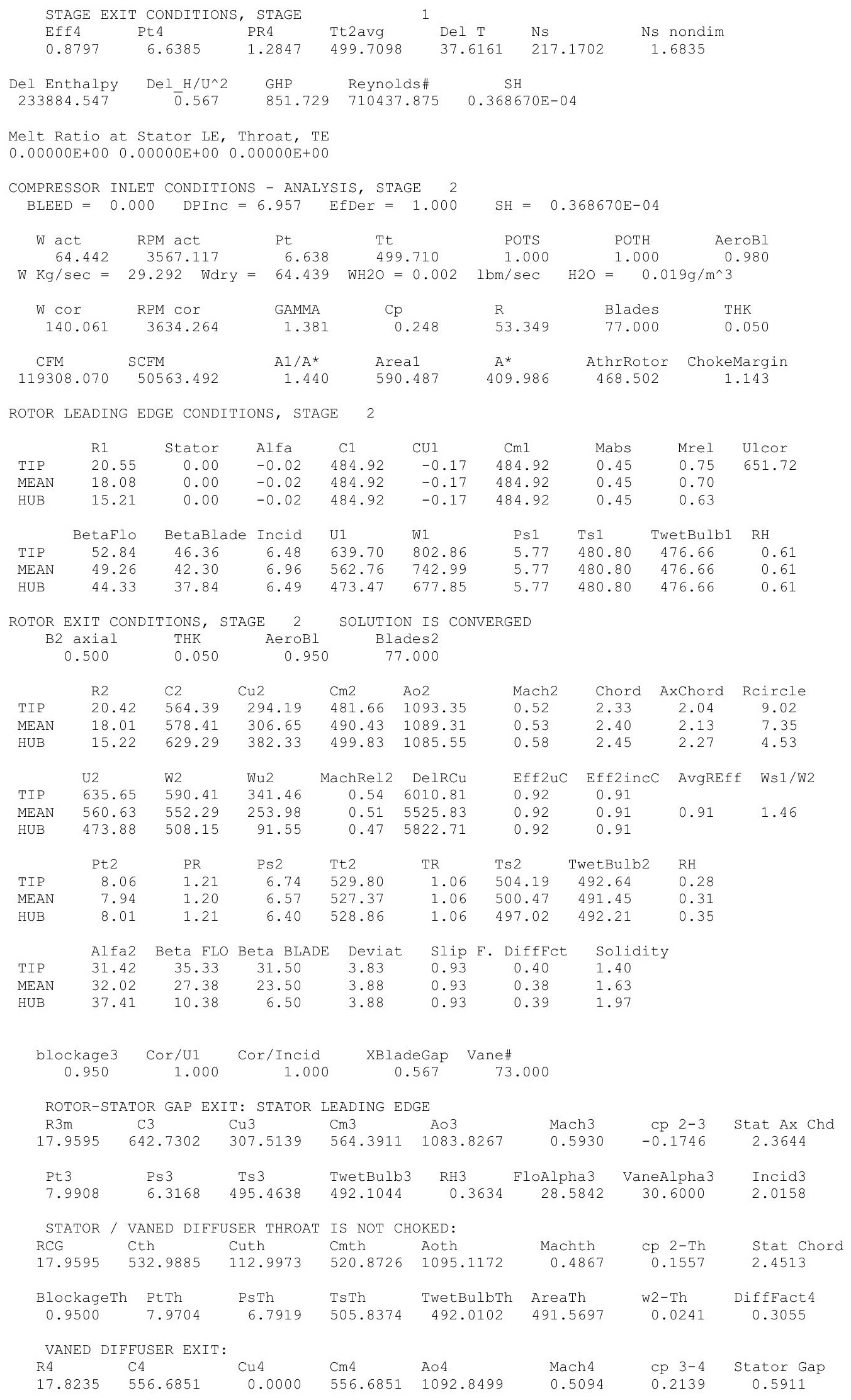




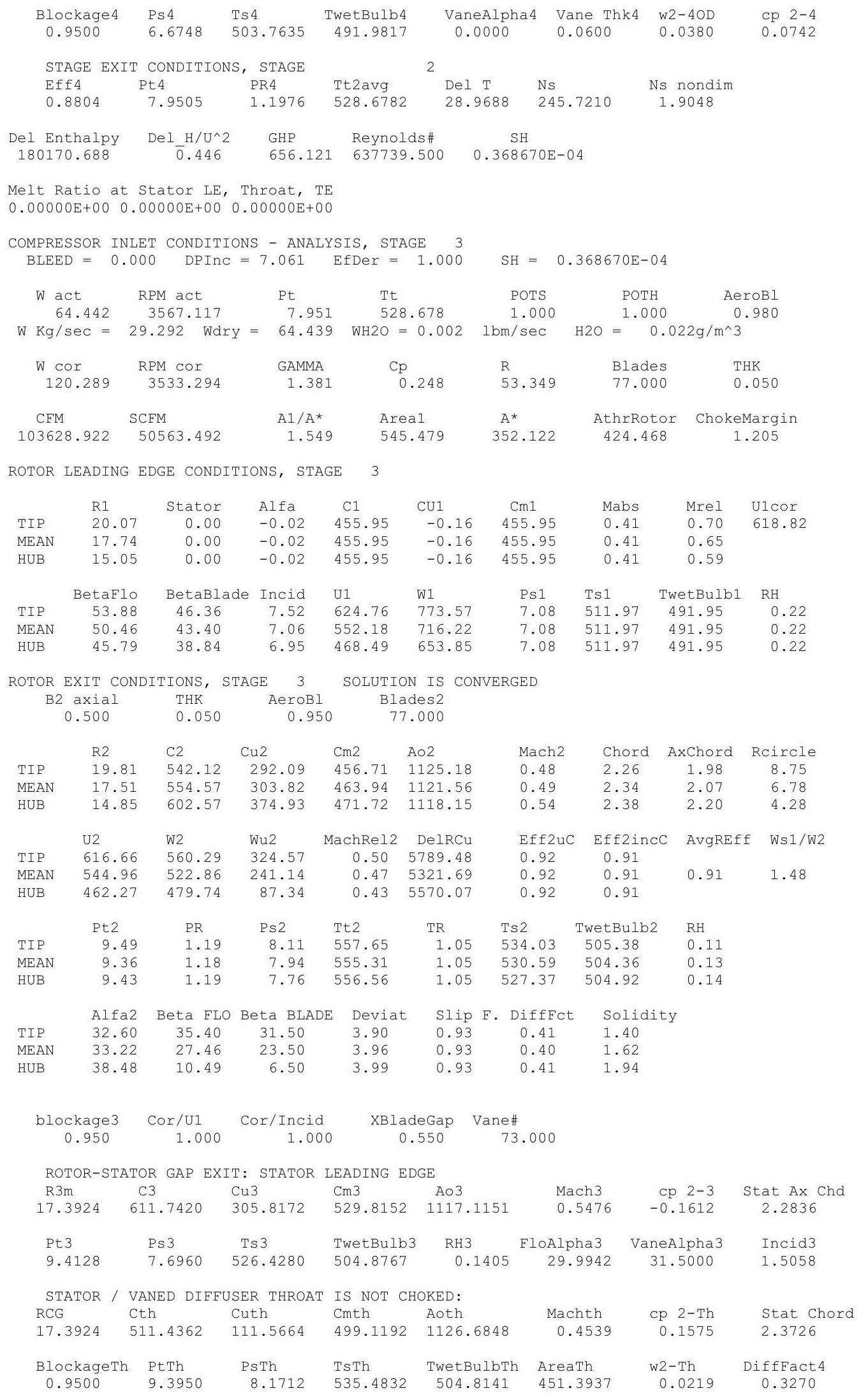




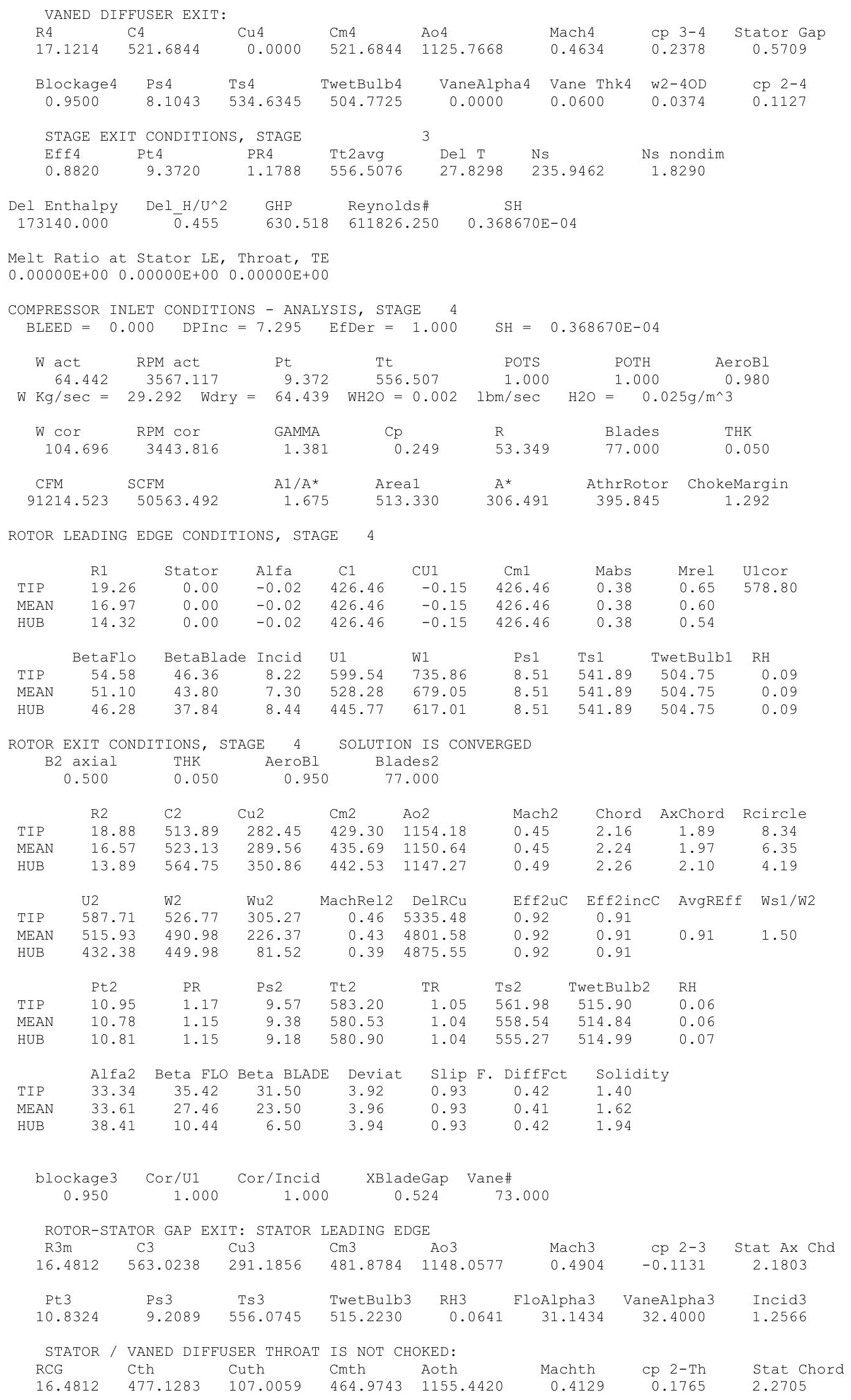




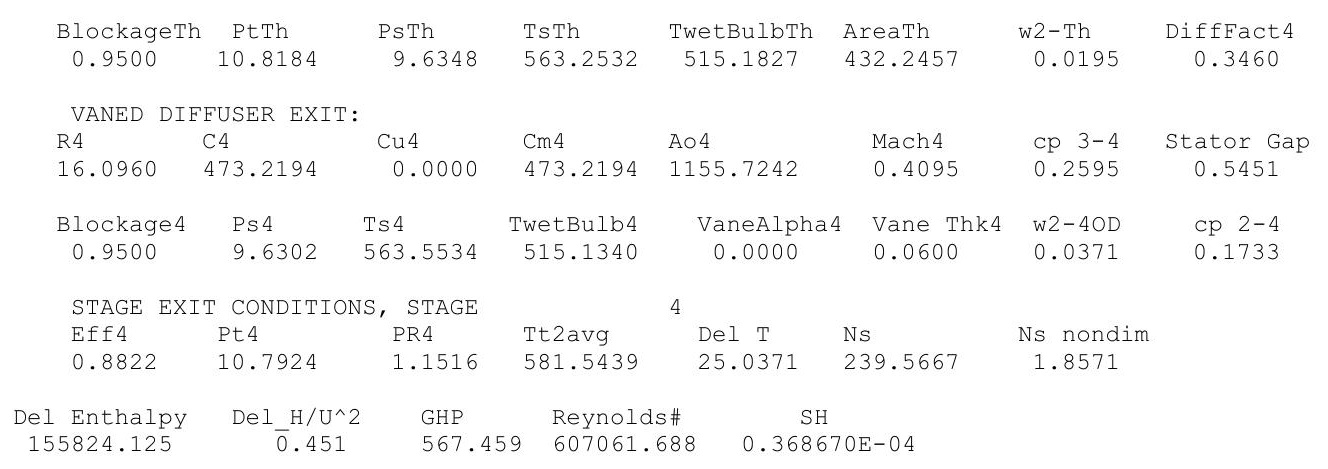

Melt Ratio at Stator LE, Throat, TE

$0.00000 \mathrm{E}+00 \quad 0.00000 \mathrm{E}+00 \quad 0.00000 \mathrm{E}+00$

COMPRESSOR INLET CONDITIONS - ANALYSIS, STAGE 5 $\mathrm{BLEED}=0.000 \quad$ DPInC $=7.452 \quad$ EfDer $=0.999$

RPM act

Pt $\quad$ Tt

$\mathrm{SH}=0.368670 \mathrm{E}-04$

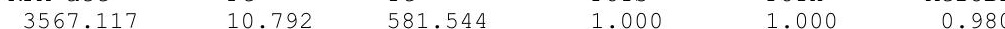

$\mathrm{W} \mathrm{Kg} / \mathrm{sec}=29.292$ Wdry $=64.439 \mathrm{WH} 2 \mathrm{O}=0.002 \mathrm{lbm} / \mathrm{sec} \quad \mathrm{H} 2 \mathrm{O}=0.028 \mathrm{~g} / \mathrm{m}^{\wedge} 3$

$\begin{array}{llllll}\text { W } & \text { RPM cor } & \text { GAMMA } & \text { Cp } & \text { Blades } & \text { THK }\end{array}$

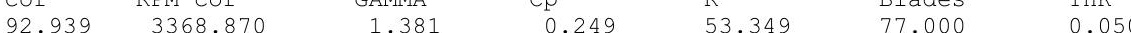

CFM SCFM A1/A* Area1 A A* AthrRotor ChokeMargin

$\begin{array}{lllllll}81465.781 & 50563.492 & 1.873 & 509.668 & 272.089 & 384.739 & 1.414\end{array}$

ROTOR LEADING EDGE CONDITIONS, STAGE 5

$\begin{array}{lrrrrrrrrr} & \text { R1 } & \text { Stator } & \text { Alfa } & \text { C1 } & \text { CU1 } & \text { Cm1 } & \text { Mabs } & \text { Mrel } & \text { U1cor } \\ \text { TIP } & 18.32 & 0.00 & -0.02 & 383.62 & -0.13 & 383.62 & 0.33 & 0.59 & 538.57 \\ \text { MEAN } & 15.91 & 0.00 & -0.02 & 383.62 & -0.13 & 383.62 & 0.33 & 0.54 & \\ \text { HUB } & 13.07 & 0.00 & -0.02 & 383.62 & -0.13 & 383.62 & 0.33 & 0.48 & \\ & & & & & & & & & \\ & \text { BetaFlo } & \text { BetaBlade } & \text { Incid } & \text { U1 } & \text { W1 } & \text { Ps1 } & \text { Ts } 1 & \text { TwetBulb1 } & \text { RH } \\ \text { TIP } & 56.08 & 47.36 & 8.72 & 570.28 & 687.41 & 10.02 & 569.72 & 515.12 & 0.05 \\ \text { MEAN } & 52.25 & 44.80 & 7.45 & 495.36 & 626.63 & 10.02 & 569.72 & 515.12 & 0.05 \\ \text { HUB } & 46.69 & 38.84 & 7.85 & 406.86 & 559.29 & 10.02 & 569.72 & 515.12 & 0.05\end{array}$

ROTOR EXIT CONDITIONS, STAGE 5 SOLUTION IS CONVERGED

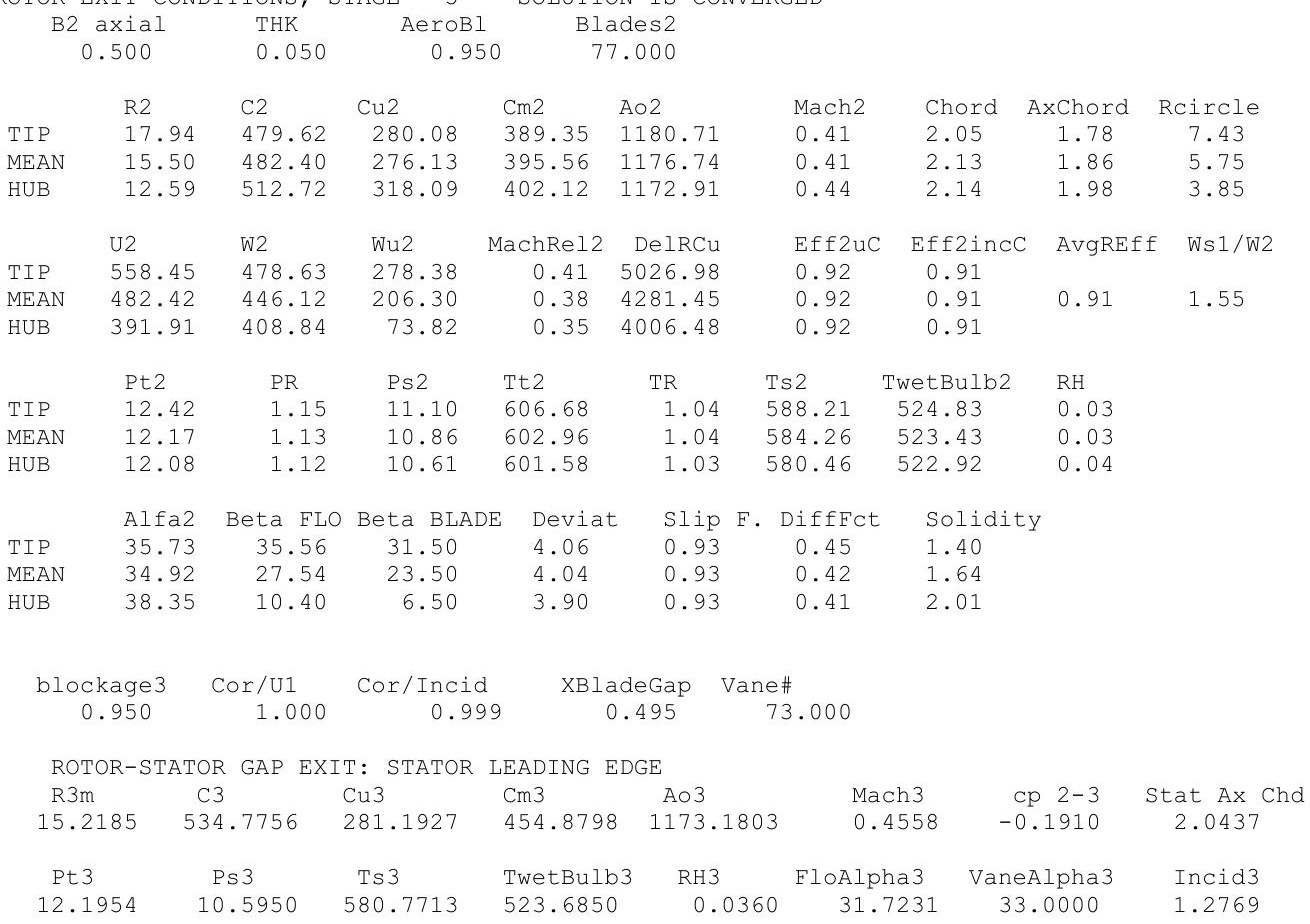




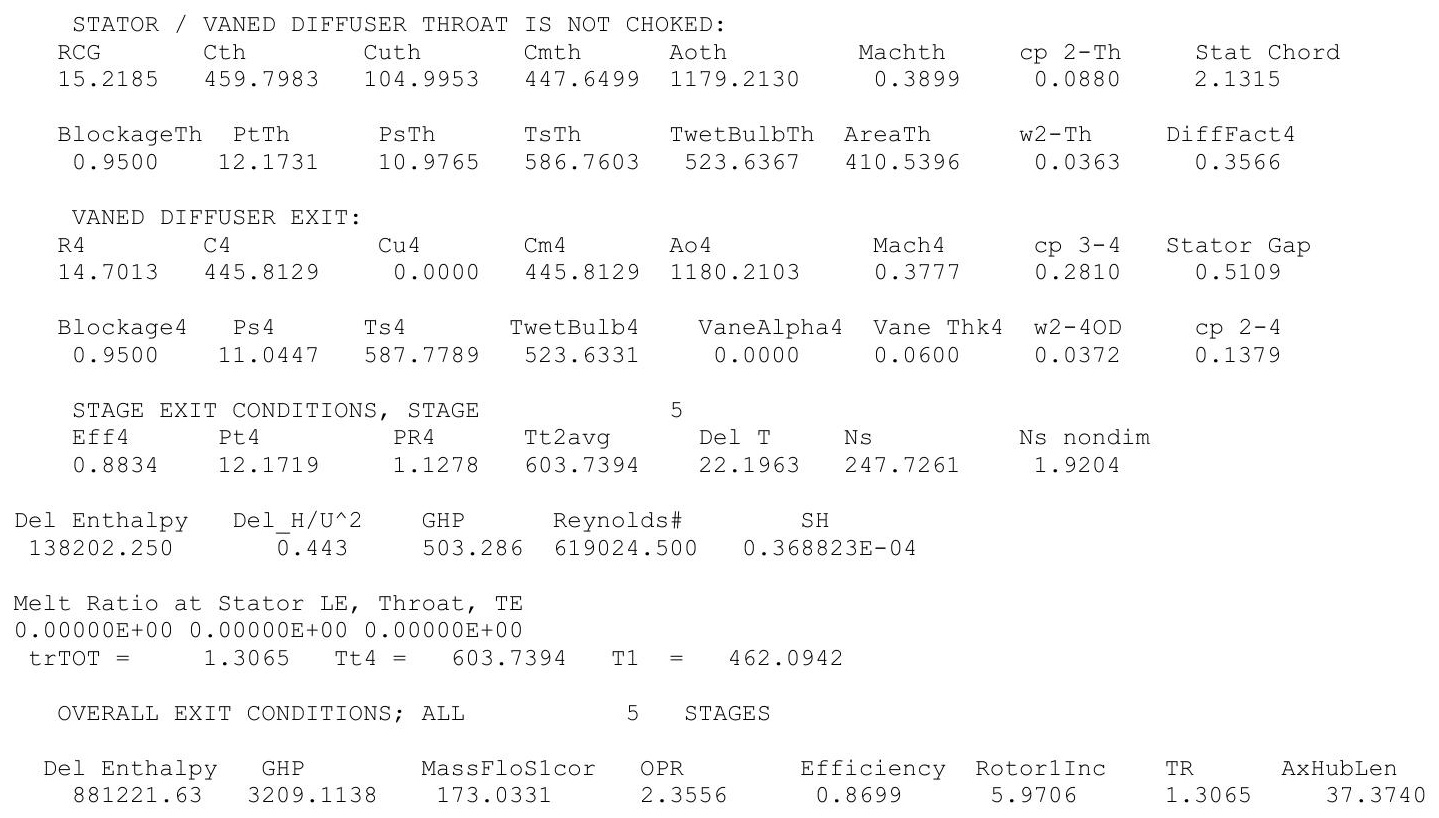


$100 \mu \mathrm{m}, \mathrm{ISA}+36 \mathrm{R}$

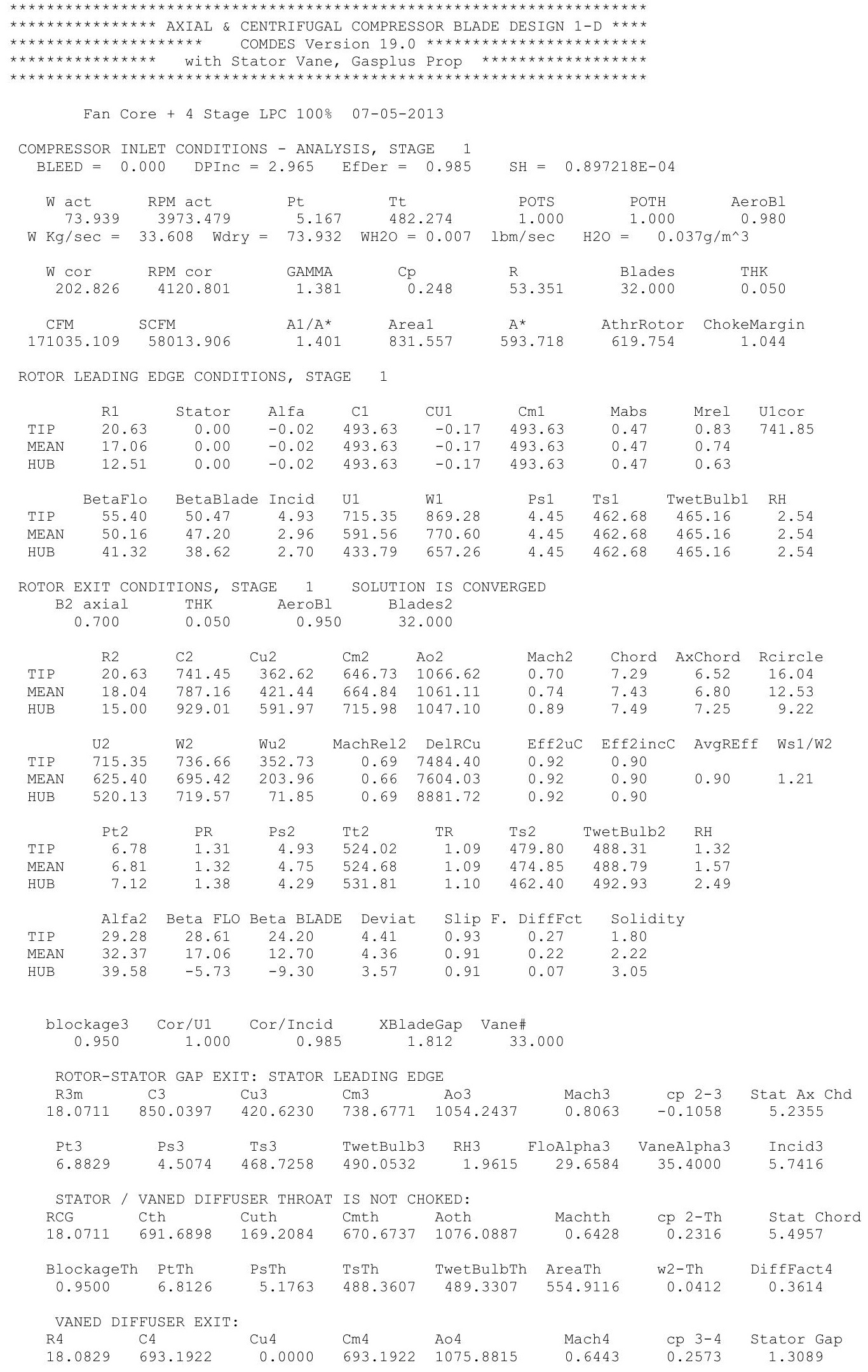




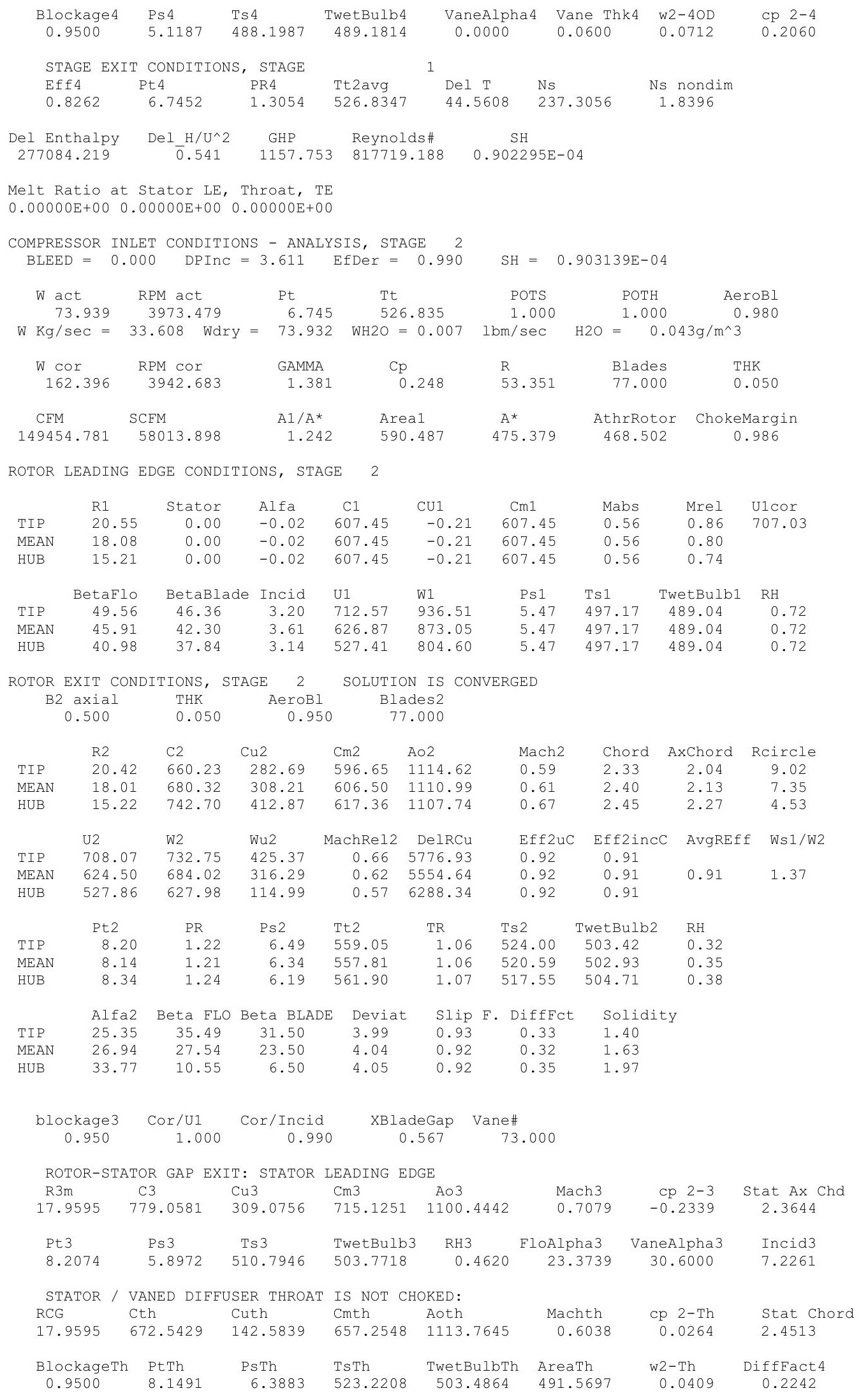




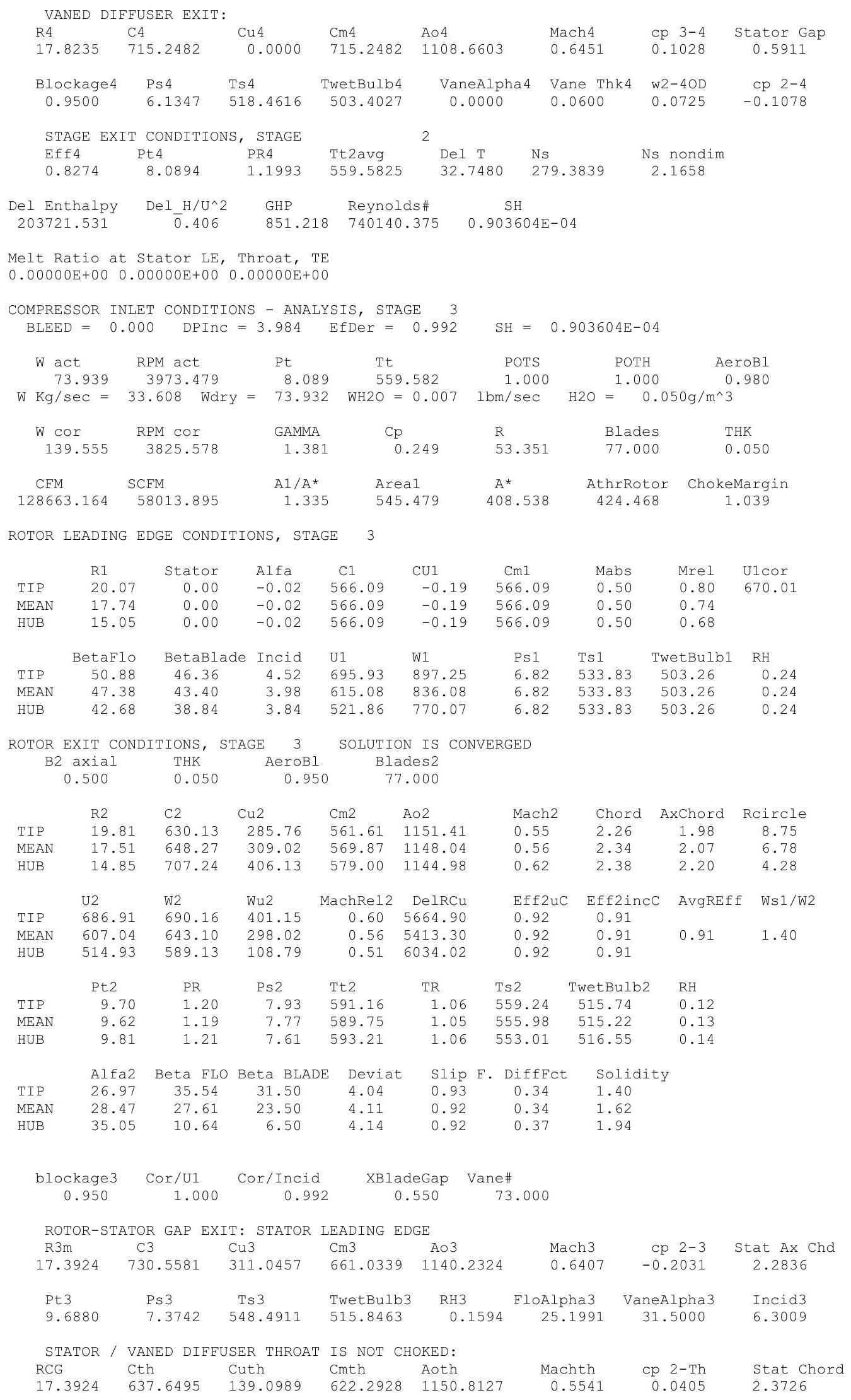




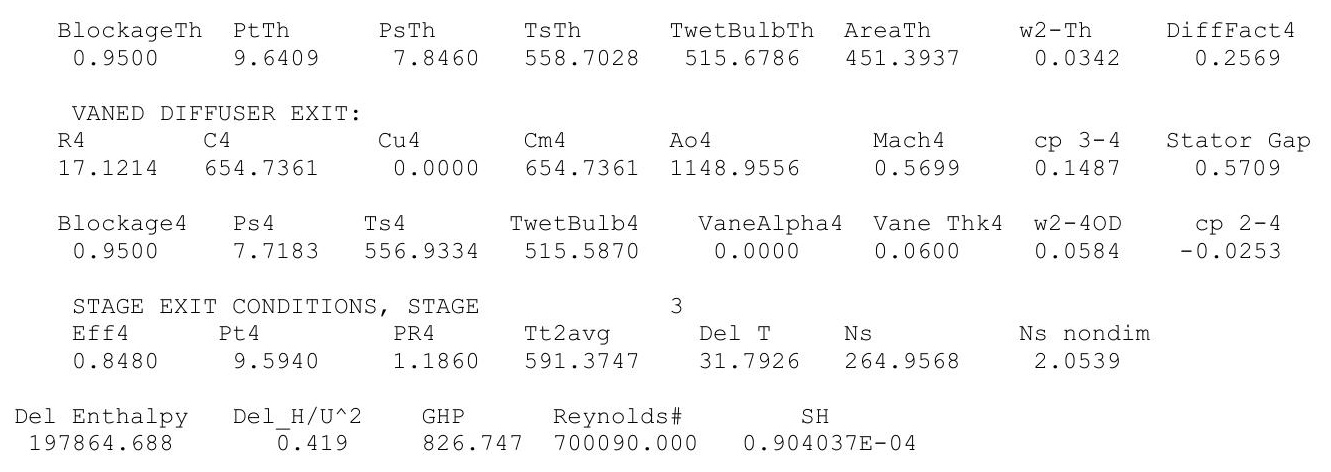

Melt Ratio at Stator LE, Throat, TE

$0.00000 \mathrm{E}+00 \quad 0.00000 \mathrm{E}+00 \quad 0.00000 \mathrm{E}+00$

COMPRESSOR INLET CONDITIONS - ANALYSIS, STAGE 4 $\mathrm{BLEED}=0.000 \quad$ DPInC $=4.566 \quad$ EfDer $=0.995$

$\mathrm{SH}=0.904037 \mathrm{E}-04$

$\begin{array}{llllll}\text { W act } & \text { RPM act } & \text { Pt } & \text { POTS } & \text { POTH } & \text { AeroBI }\end{array}$

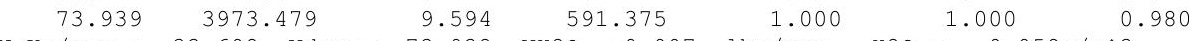
$\mathrm{WKg} / \mathrm{sec}=33.608$ Wdry $=73.932 \mathrm{WH} 2 \mathrm{O}=0.007 \quad \mathrm{lbm} / \mathrm{sec} \quad \mathrm{H} 2 \mathrm{O}=0.058 \mathrm{~g} / \mathrm{m}^{\wedge} 3$

$\begin{array}{ccccccc}\text { W Cor } & \text { RPM Cor } & \text { GAMMA } & \text { Cp } & \text { R } & \text { Blades } & \text { THK } \\ 120.966 & 3721.326 & 1.381 & 0.249 & 53.351 & 77.000 & 0.050 \\ & & & & & & \\ \text { CFM } & \text { SCFM } & \text { A1/A* } & \text { Area1 } & \text { A* } & \text { AthrRotor } & \text { ChokeMargin } \\ 111915.844 & 58013.898 & 1.450 & 513.330 & 354.143 & 395.845 & 1.118\end{array}$

ROTOR LEADING EDGE CONDITIONS, STAGE 4

$\begin{array}{lccccccccc} & \text { R1 } & \text { Stator } & \text { Alfa } & \text { C1 } & \text { CU1 } & \text { Cm1 } & \text { Mabs } & \text { Mrel } & \text { U1cor } \\ \text { TIP } & 19.26 & 0.00 & -0.02 & 523.25 & -0.18 & 523.25 & 0.45 & 0.73 & 625.44 \\ \text { MEAN } & 16.97 & 0.00 & -0.02 & 523.25 & -0.18 & 523.25 & 0.45 & 0.68 & \\ \text { HUB } & 14.32 & 0.00 & -0.02 & 523.25 & -0.18 & 523.25 & 0.45 & 0.62 & \\ & & & & & & & & & \\ & \text { BetaFlo } & \text { BetaBlade } & \text { Incid } & \text { U1 } & \text { W1 } & \text { Ps1 } & \text { Ts1 } & \text { TwetBulb1 } & \text { RH } \\ \text { TIP } & 51.93 & 46.36 & 5.57 & 667.84 & 848.55 & 8.36 & 569.38 & 515.53 & 0.10 \\ \text { MEAN } & 48.37 & 43.80 & 4.57 & 588.46 & 787.58 & 8.36 & 569.38 & 515.53 & 0.10 \\ \text { HUB } & 43.51 & 37.84 & 5.67 & 496.55 & 721.47 & 8.36 & 569.38 & 515.53 & 0.10\end{array}$

ROTOR EXIT CONDITIONS, STAGE 4 SOLUTION IS CONVERGED

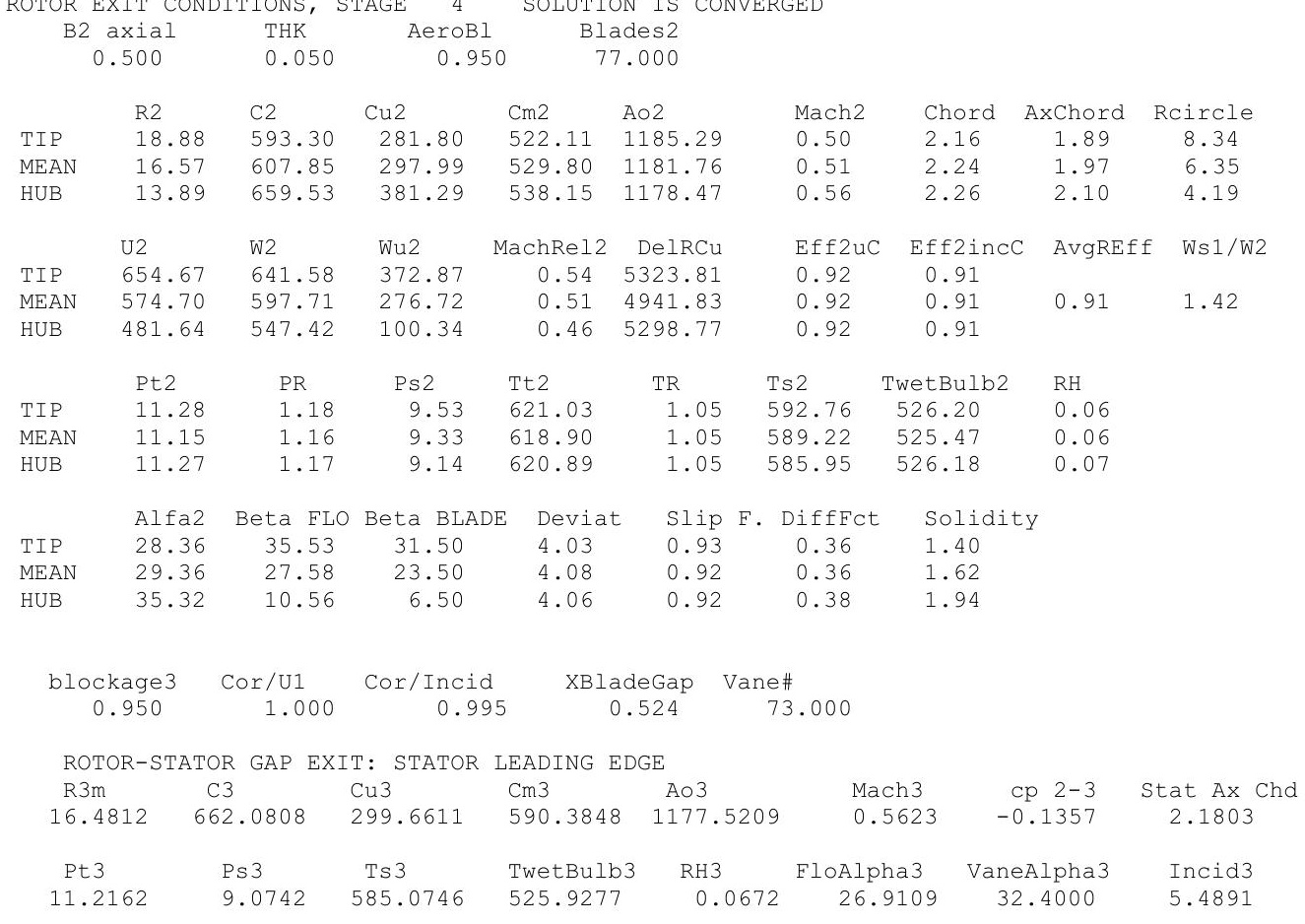




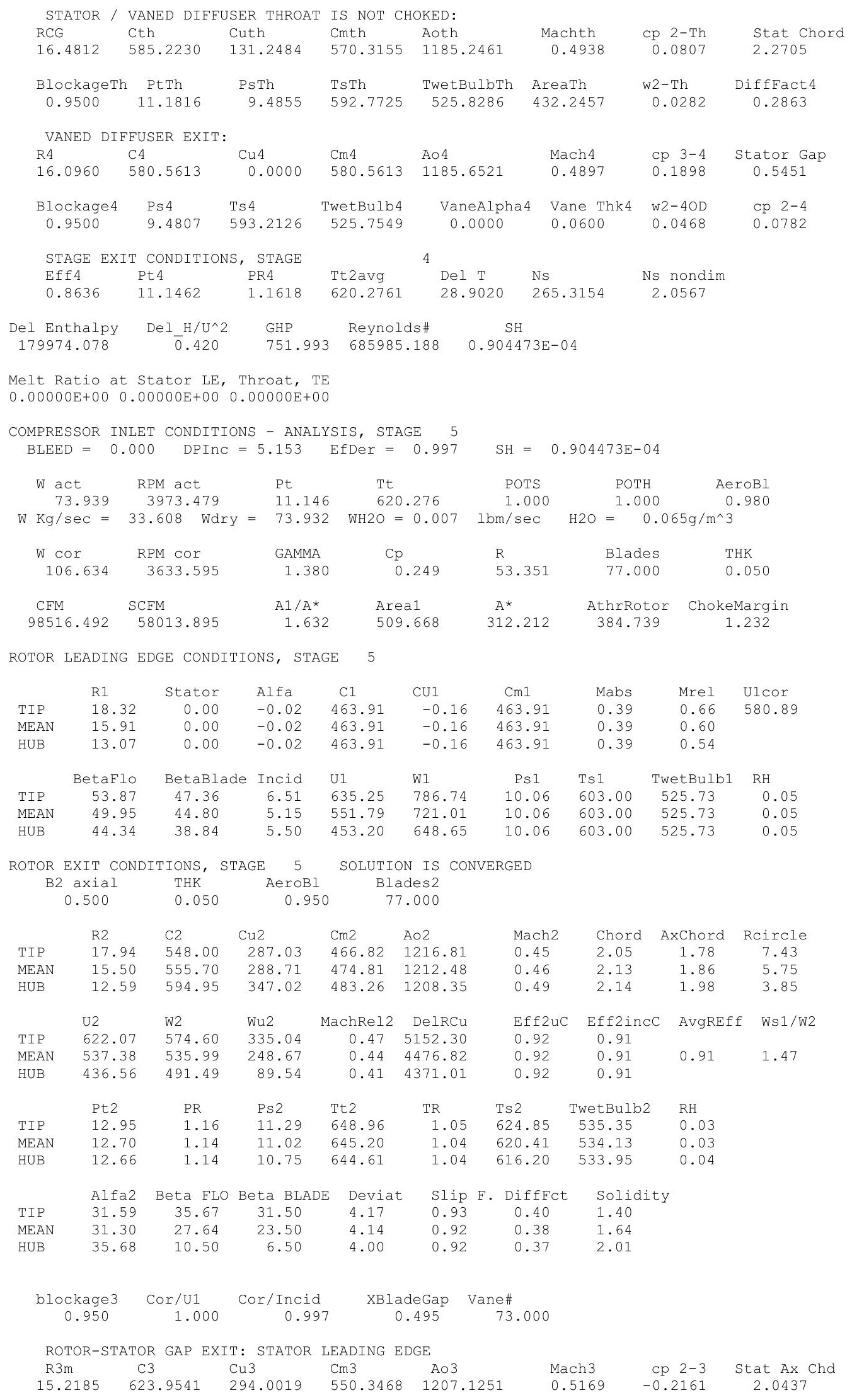




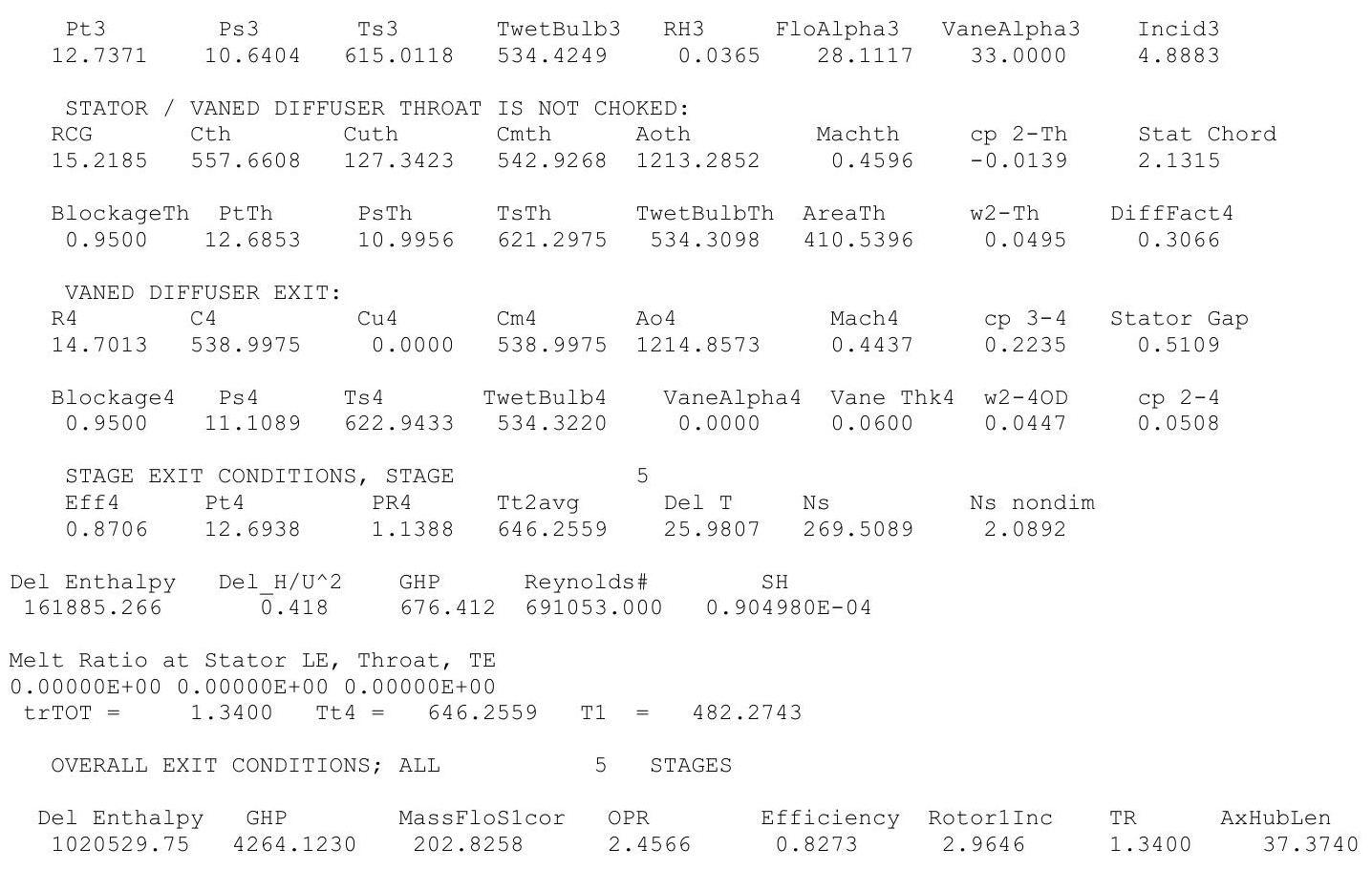




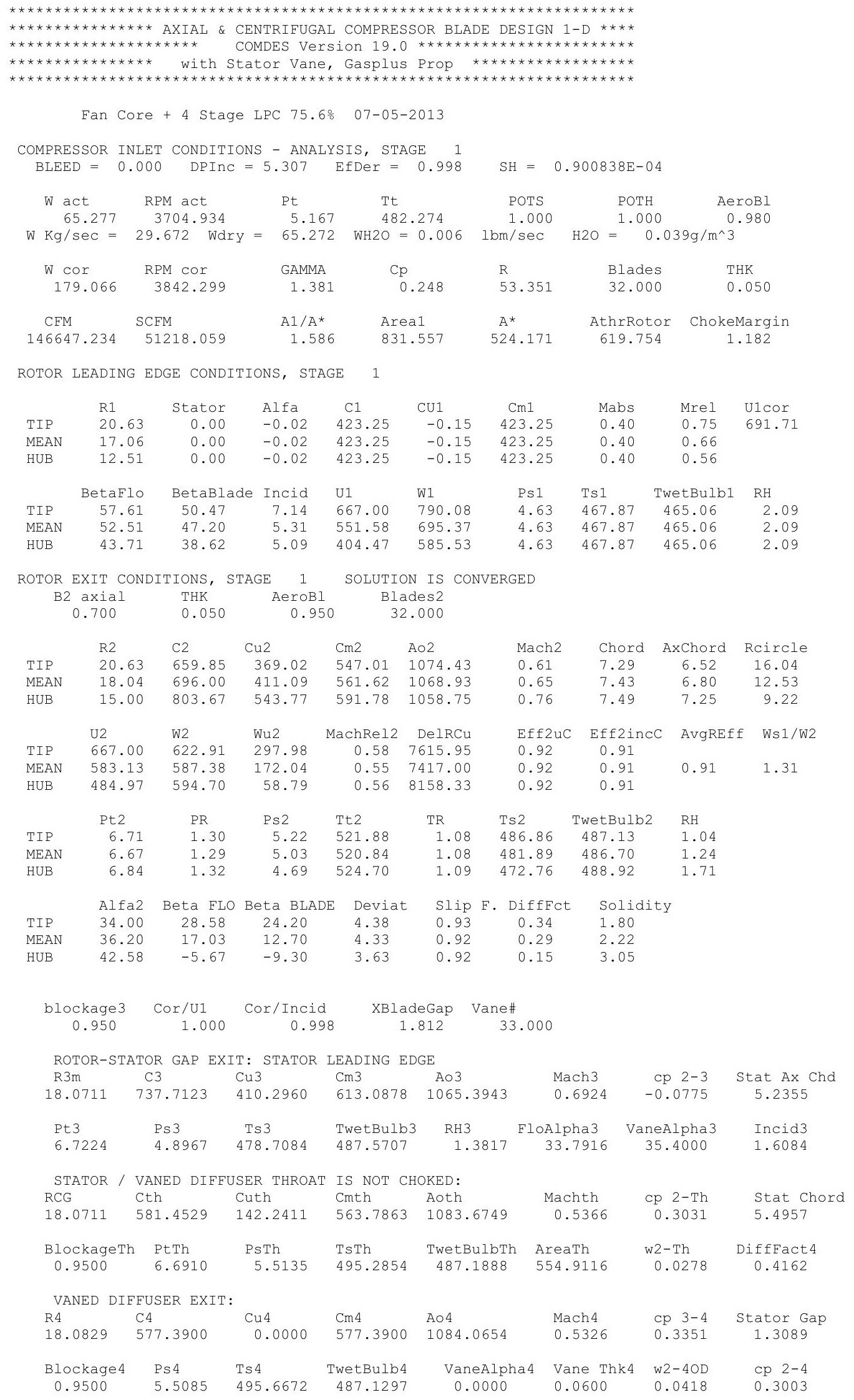




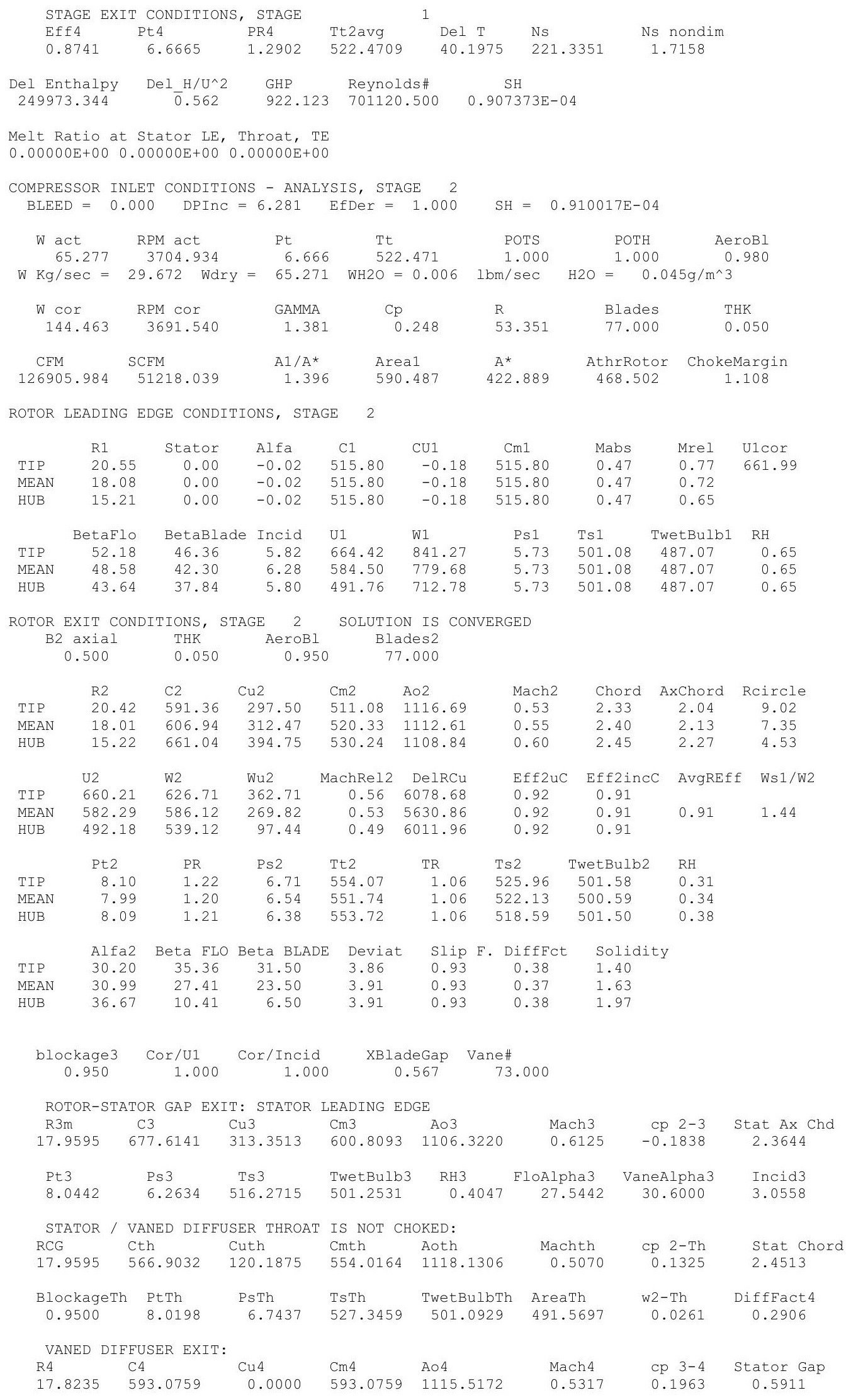




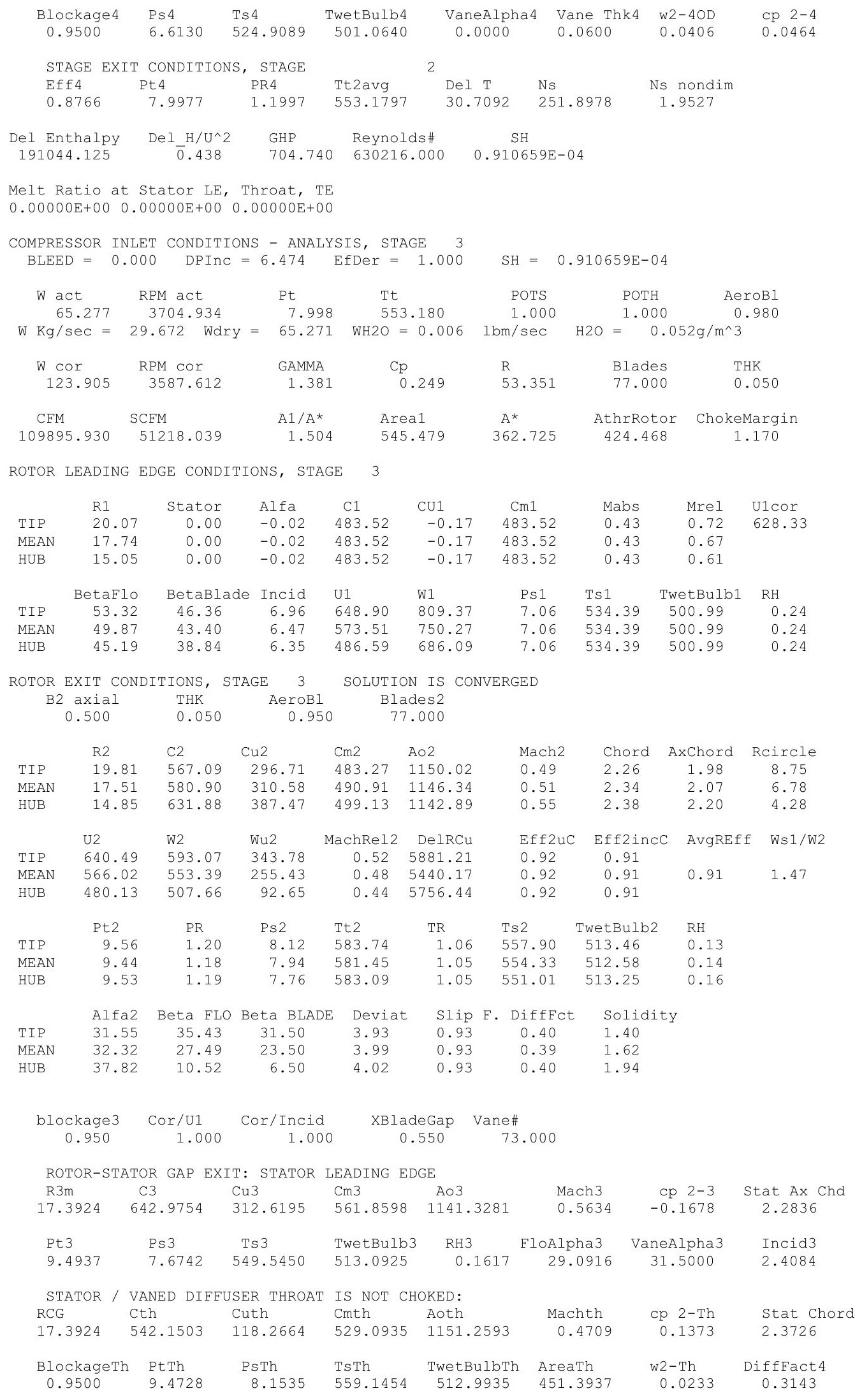




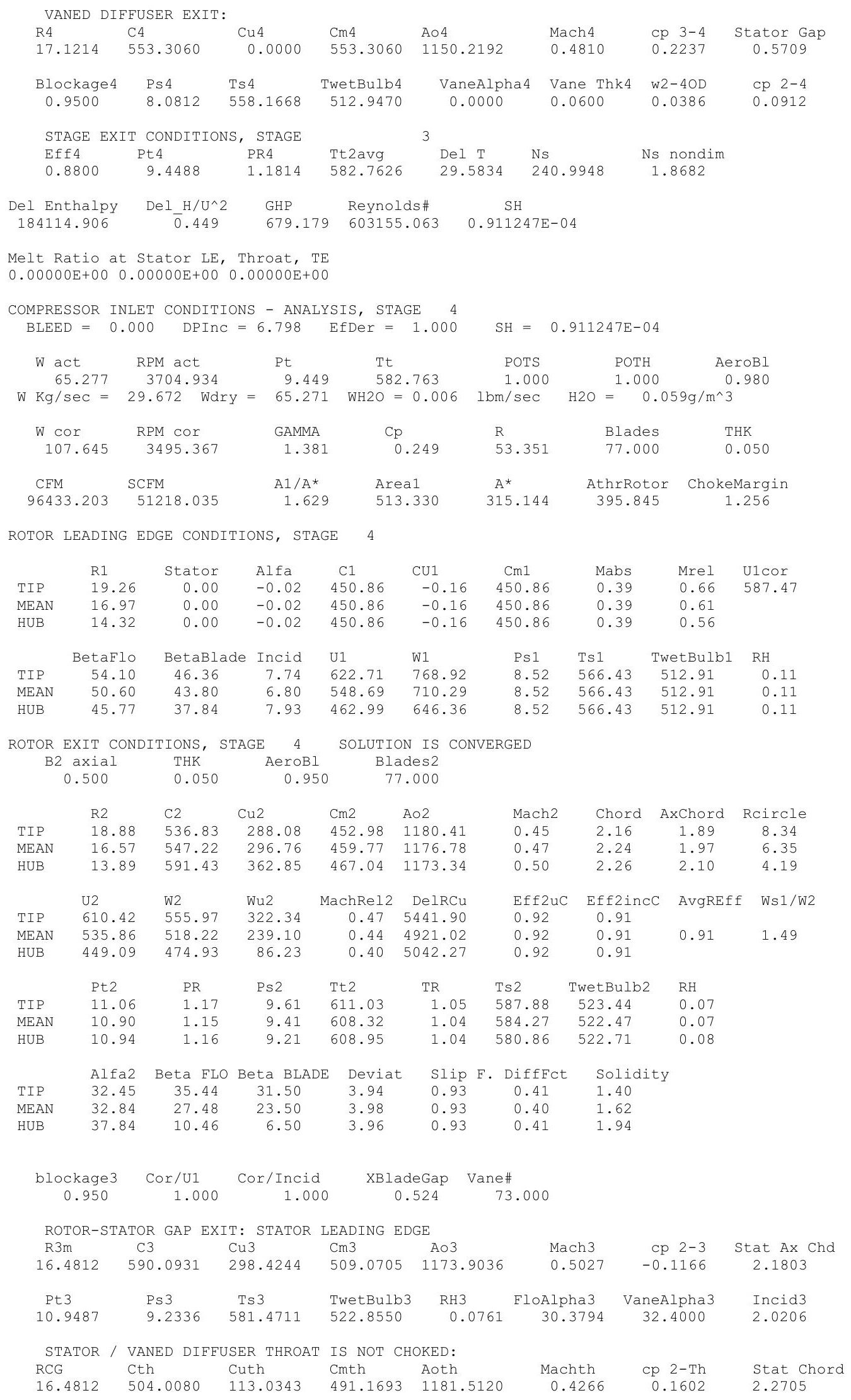




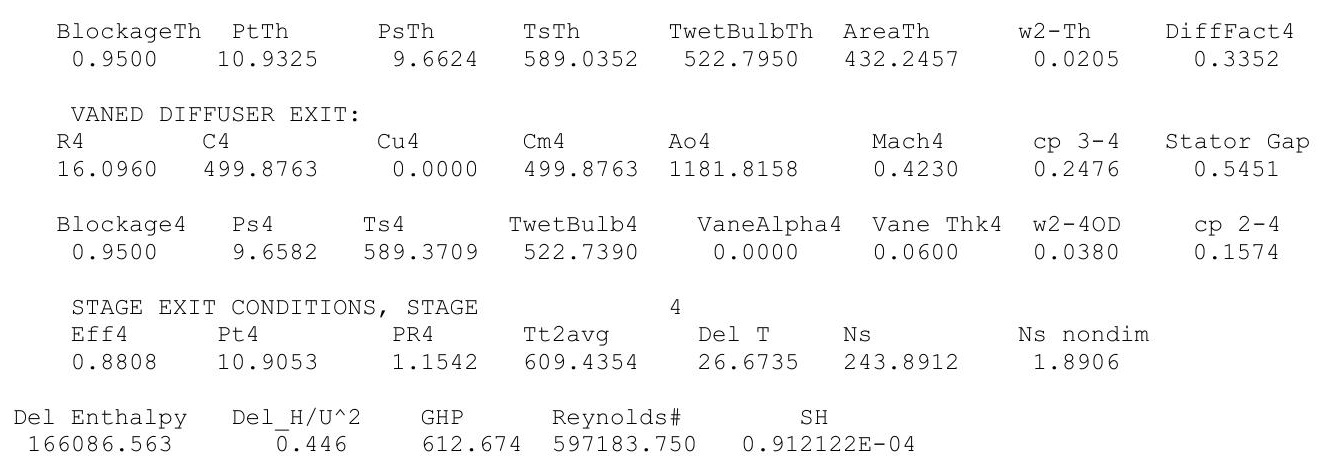

Melt Ratio at Stator LE, Throat, TE

$0.00000 \mathrm{E}+000.00000 \mathrm{E}+000.00000 \mathrm{E}+00$

COMPRESSOR INLET CONDITIONS - ANALYSIS, STAGE 5 $\mathrm{BLEED}=0.000 \quad$ DPInC $=7.043 \quad$ EfDer $=1.000$

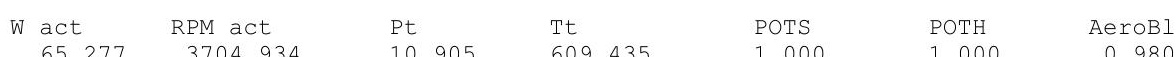
$\mathrm{W} \mathrm{Kg} / \mathrm{sec}=29.672$ Wdry $=65.271 \quad \mathrm{WH} 2 \mathrm{O}=0.006 \mathrm{lbm} / \mathrm{sec} \quad \mathrm{H} 2 \mathrm{O}=0.067 \mathrm{~g} / \mathrm{m}^{\wedge} 3$

$\begin{array}{ccccccc}\text { W cor } & \text { RPM cor } & \text { GAMMA } & \text { Cp } & \text { R } & \text { Blades } & \text { THK } \\ 95.378 & 3418.021 & 1.381 & 0.249 & 53.351 & 77.000 & 0.050 \\ & & & & & & \\ \text { CFM } & \text { SCFM } & \text { A1/A* } & \text { Area1 } & \text { A* } & \text { AthrRotor } & \text { ChokeMargin } \\ 85869.484 & 51218.043 & 1.825 & 509.668 & 279.251 & 384.739 & 1.378\end{array}$

ROTOR LEADING EDGE CONDITIONS, STAGE 5

\begin{tabular}{|c|c|c|c|c|c|c|c|c|c|}
\hline & R1 & Stator & Alfa & $\mathrm{C} 1$ & CU1 & $\mathrm{Cm} 1$ & Mabs & Mrel & U1cor \\
\hline TIP & 18.32 & 0.00 & -0.02 & 404.35 & -0.14 & 404.35 & 0.34 & 0.60 & 546.43 \\
\hline MEAN & 15.91 & 0.00 & -0.02 & 404.35 & -0.14 & 404.35 & 0.34 & 0.55 & \\
\hline HUB & 13.07 & 0.00 & -0.02 & 404.35 & -0.14 & 404.35 & 0.34 & 0.49 & \\
\hline & BetaFlo & BetaBlade & Incid & U1 & W1 & Ps 1 & Ts1 & TwetBulb1 & $\mathrm{RH}$ \\
\hline TIP & 55.69 & 47.36 & 8.33 & 592.32 & 717.29 & 10.08 & 596.31 & 522.72 & 0.06 \\
\hline MEAN & 51.84 & 44.80 & 7.04 & 514.49 & 654.48 & 10.08 & 596.31 & 522.72 & 0.06 \\
\hline HUB & 46.27 & 38.84 & 7.43 & 422.57 & 584.97 & 10.08 & 596.31 & 522.72 & 0.06 \\
\hline
\end{tabular}

ROTOR EXIT CONDITIONS, STAGE 5 SOLUTION IS CONVERGED

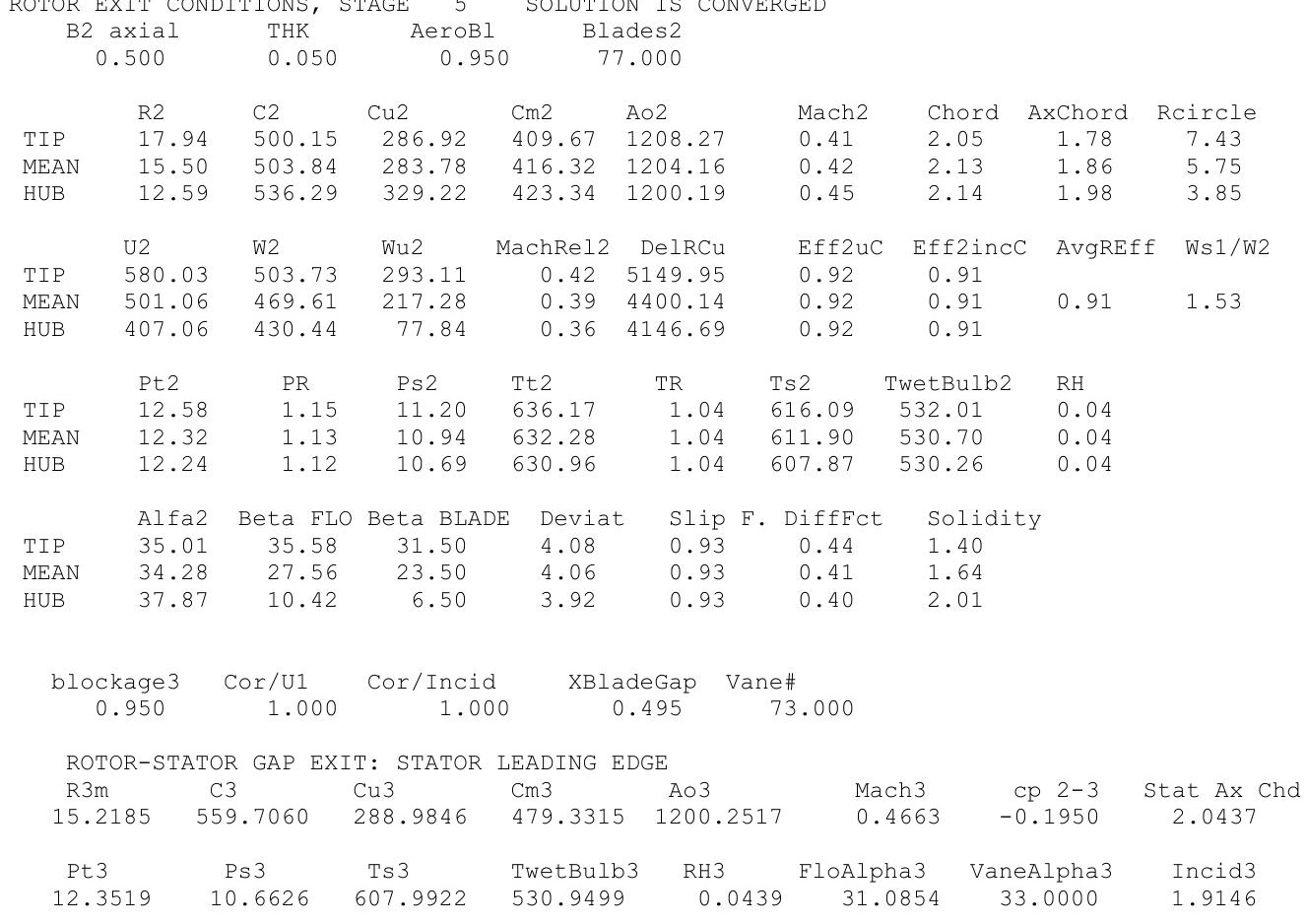




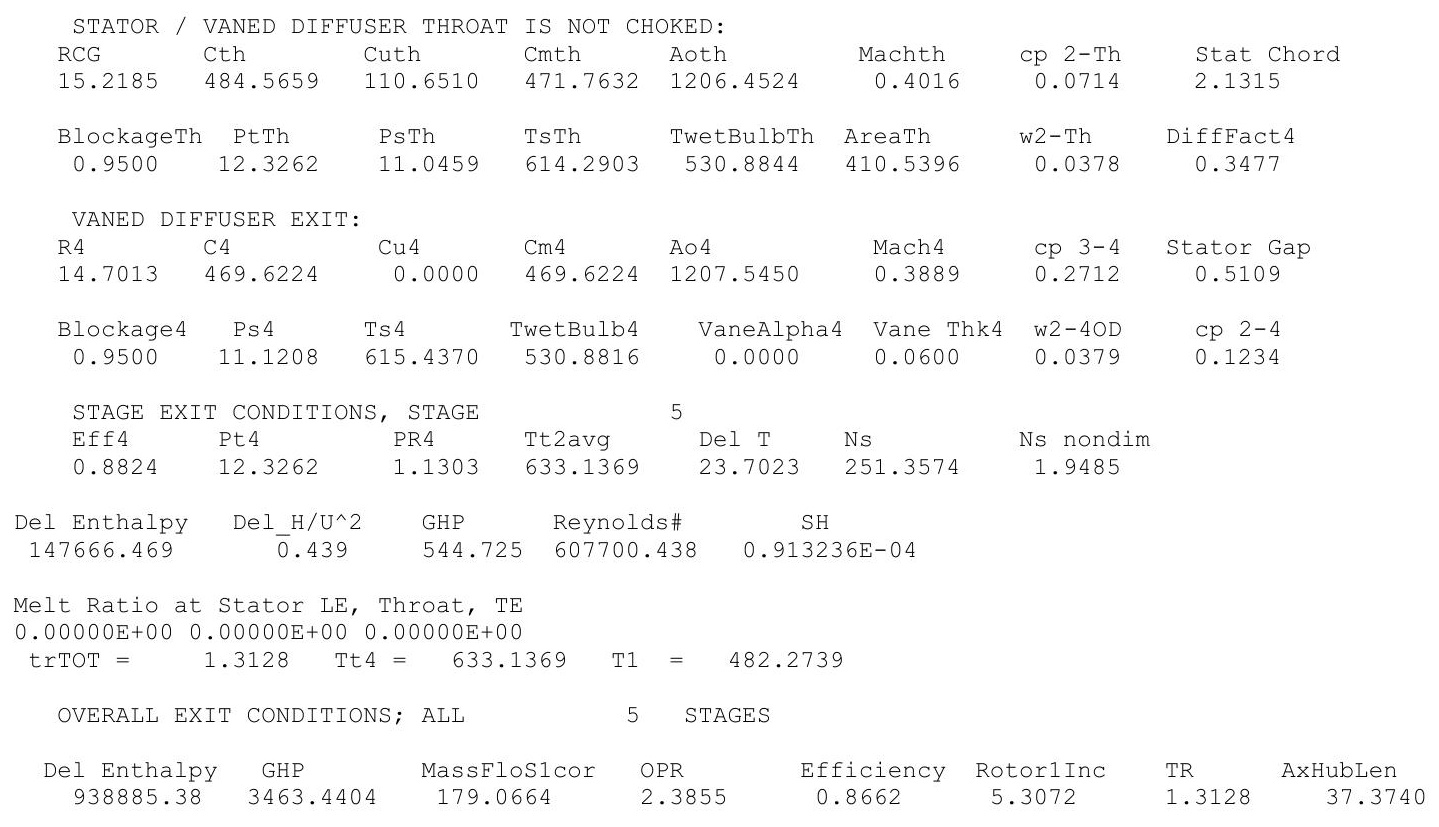




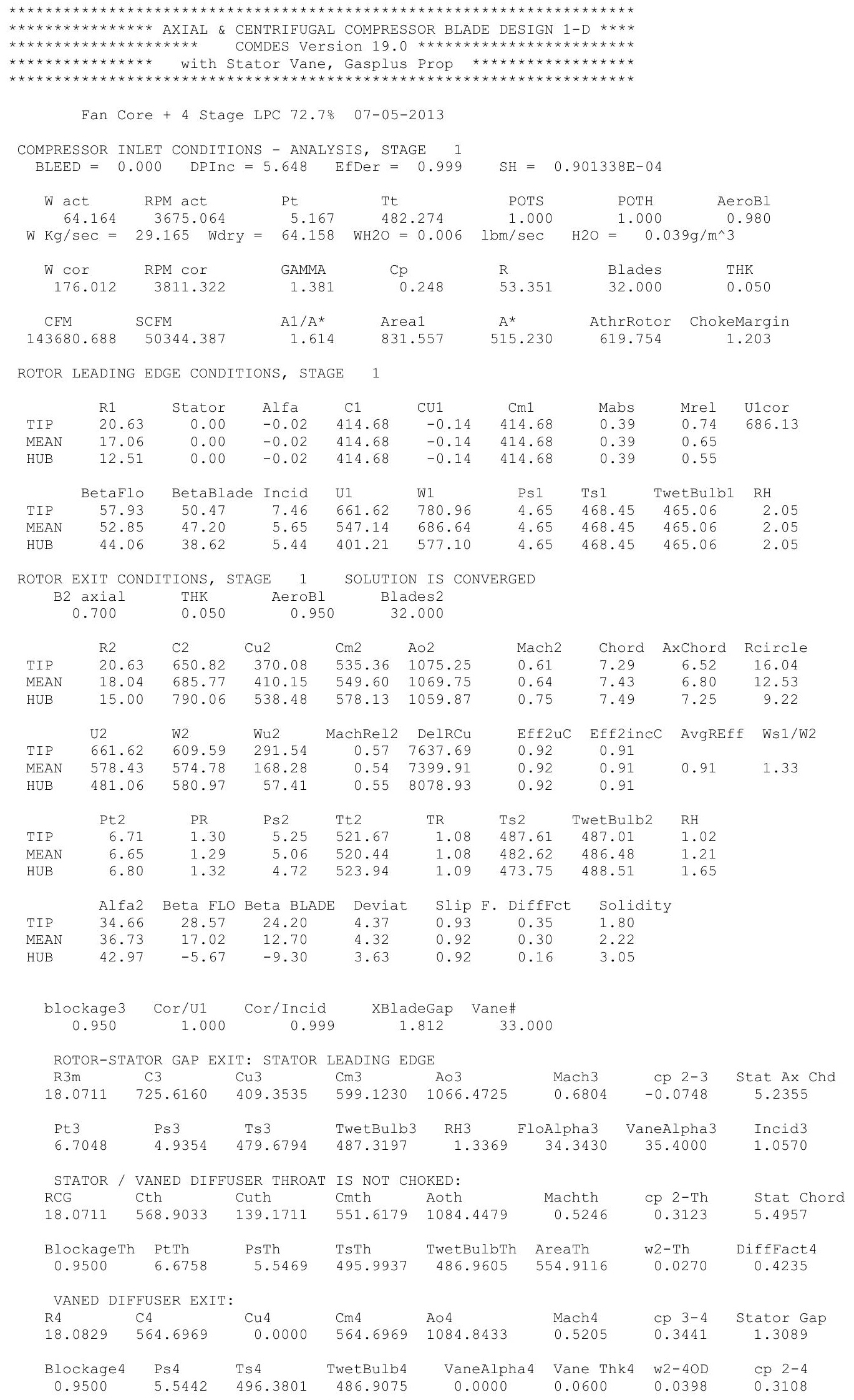




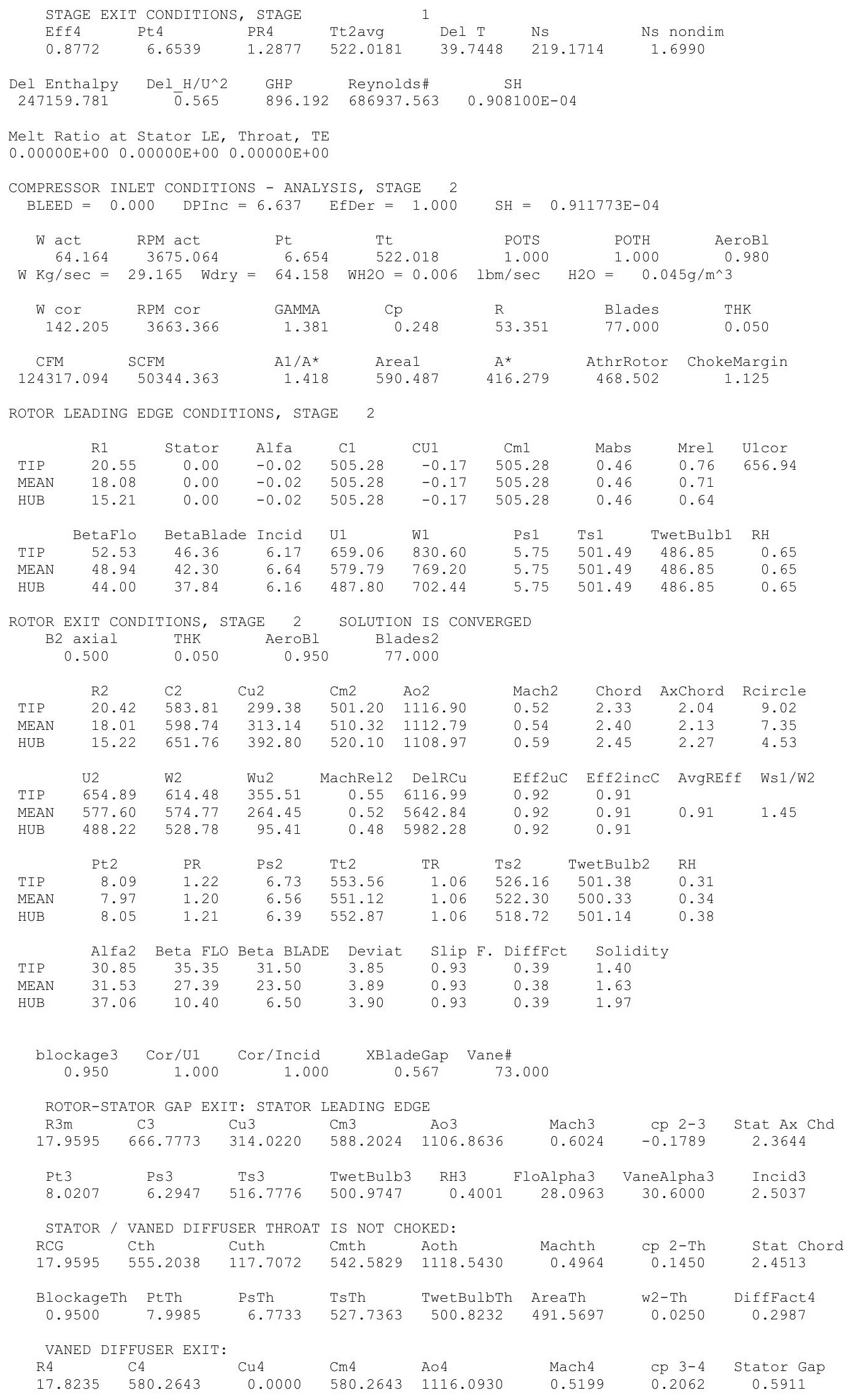




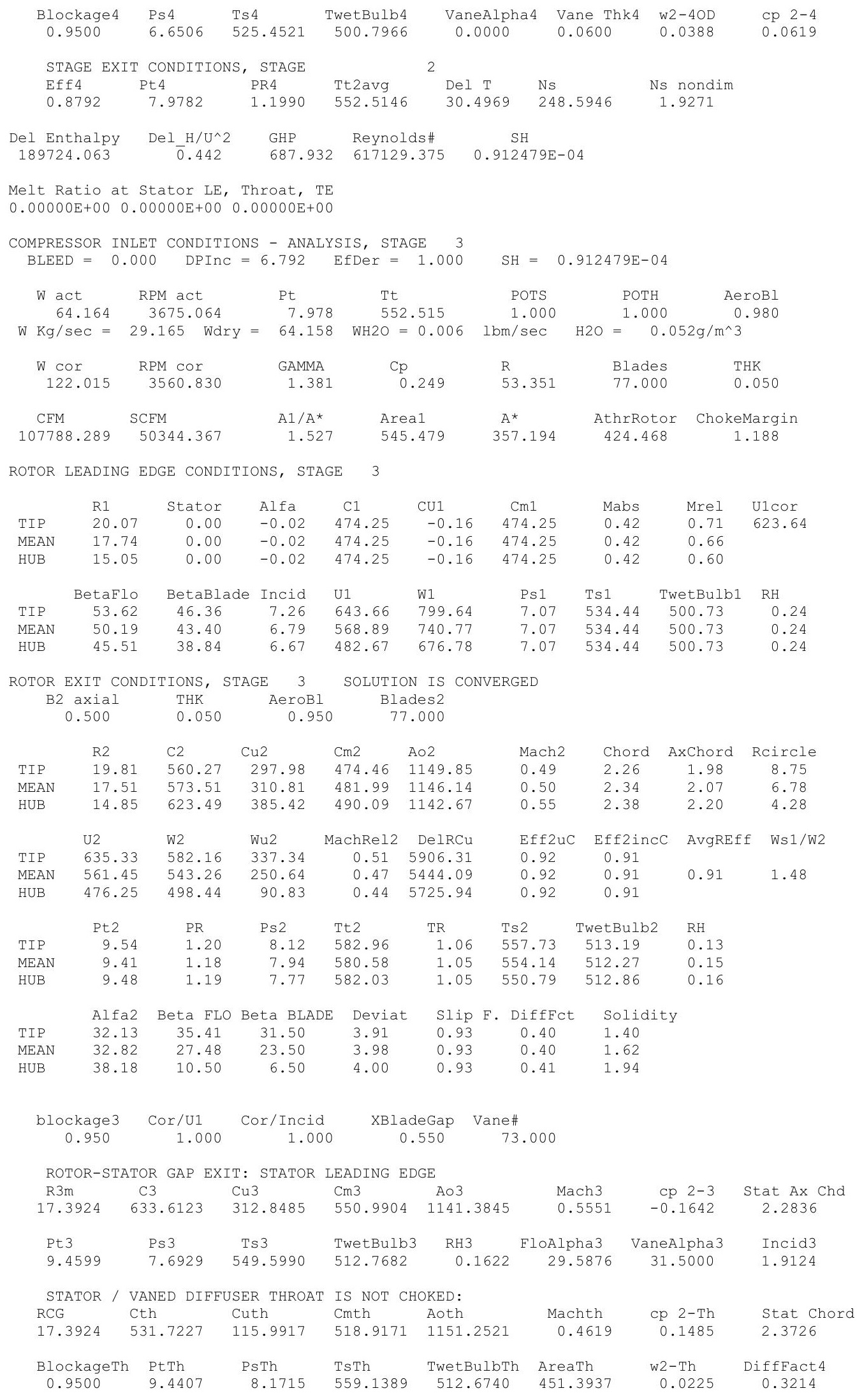




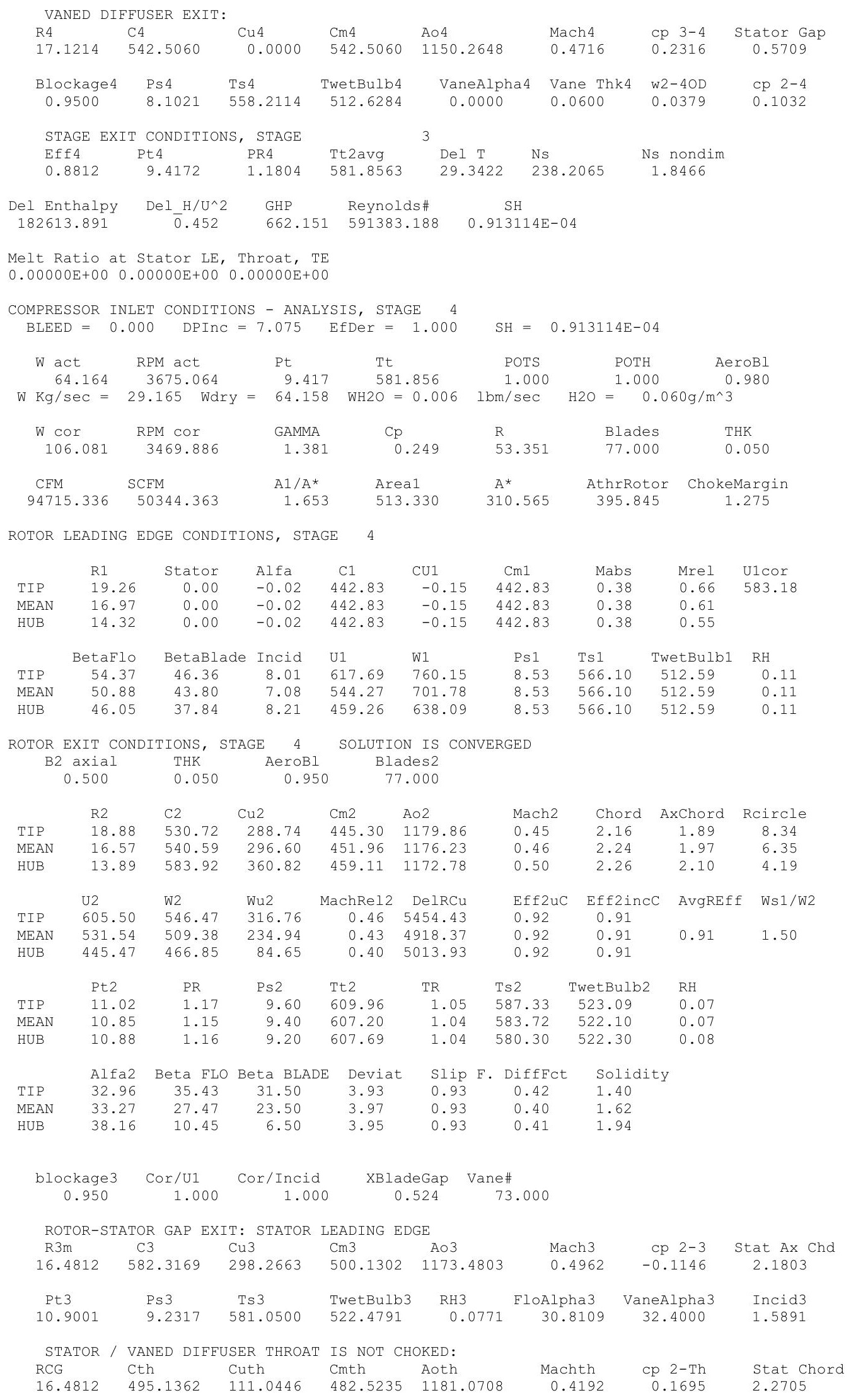




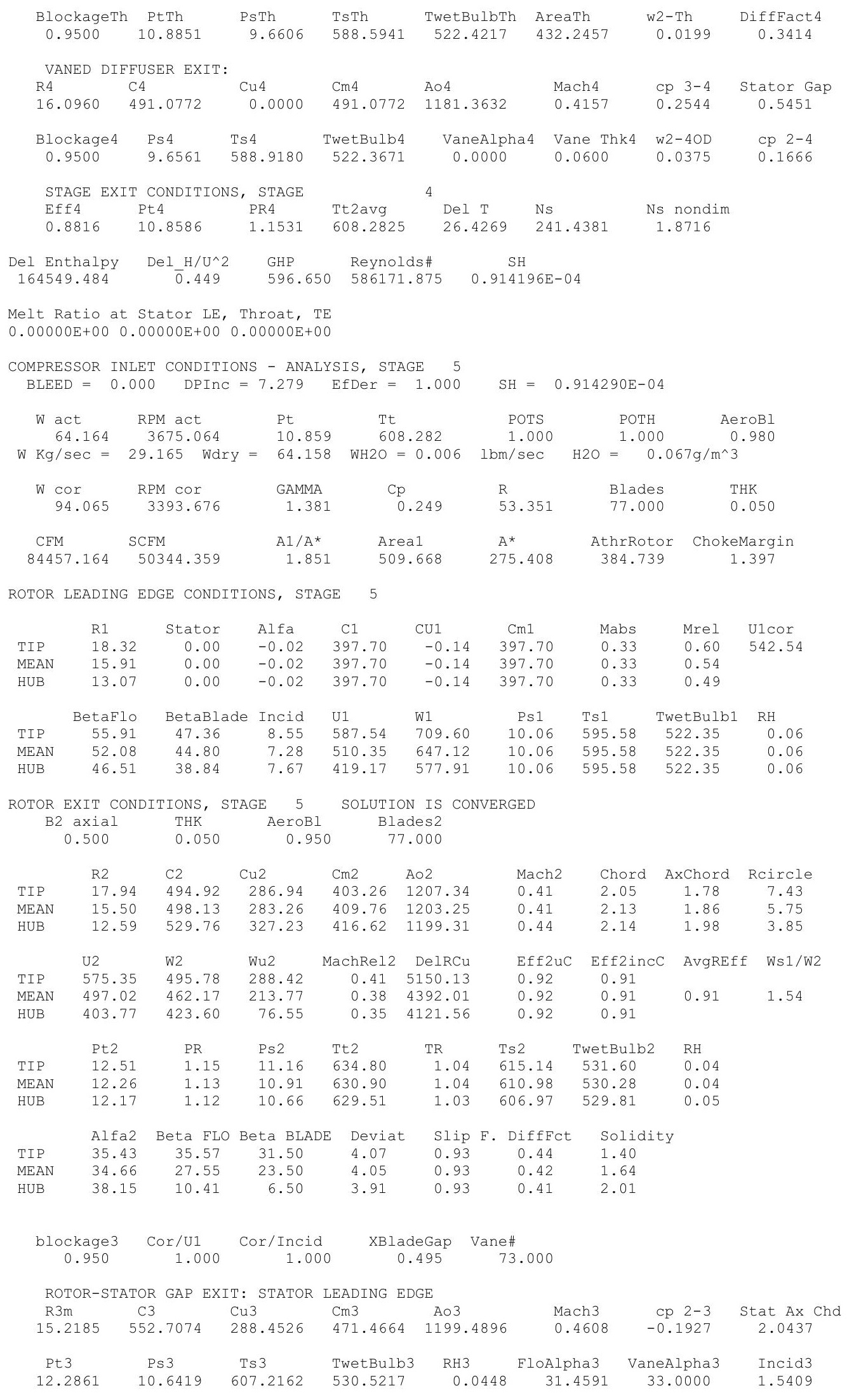




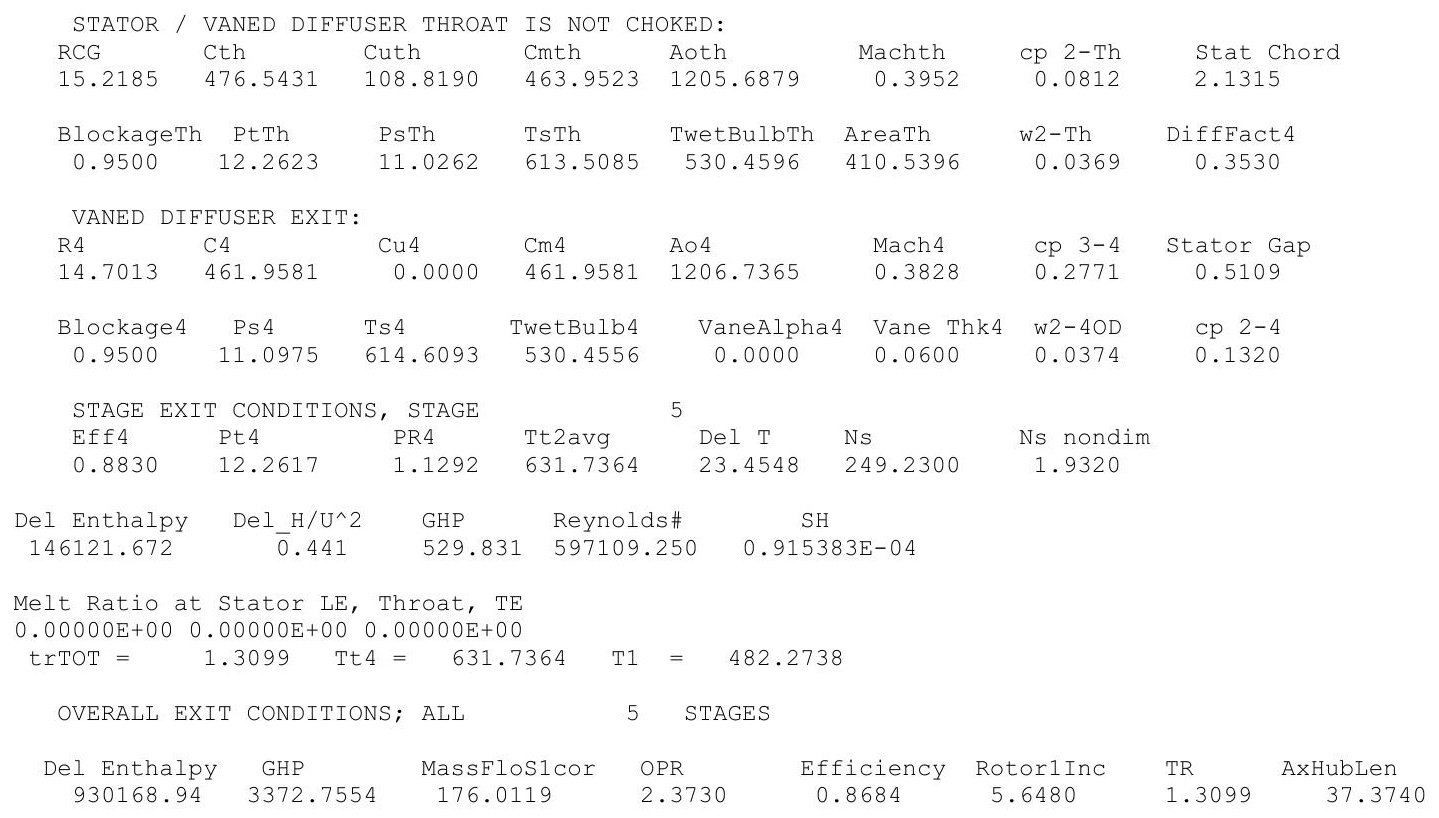




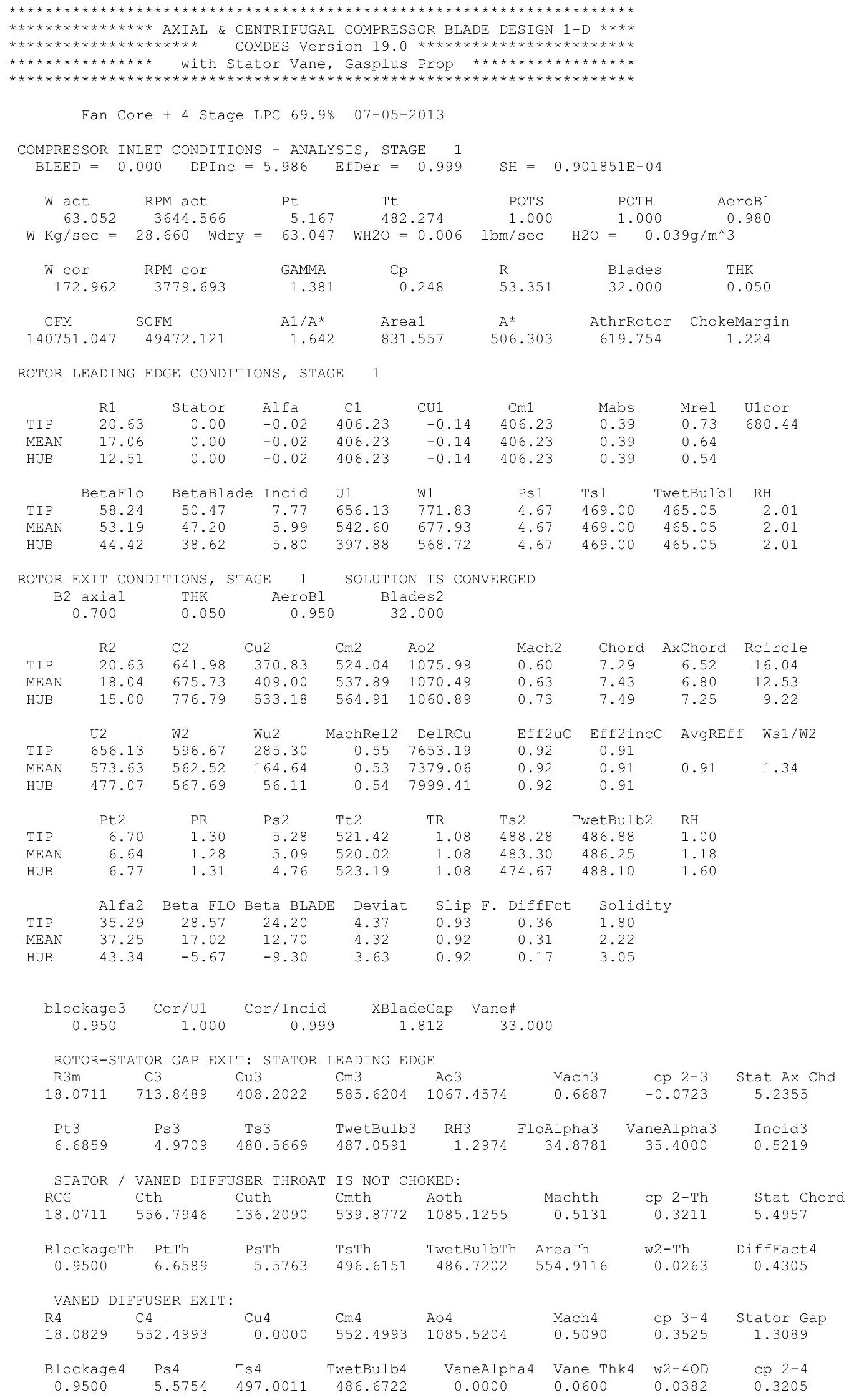




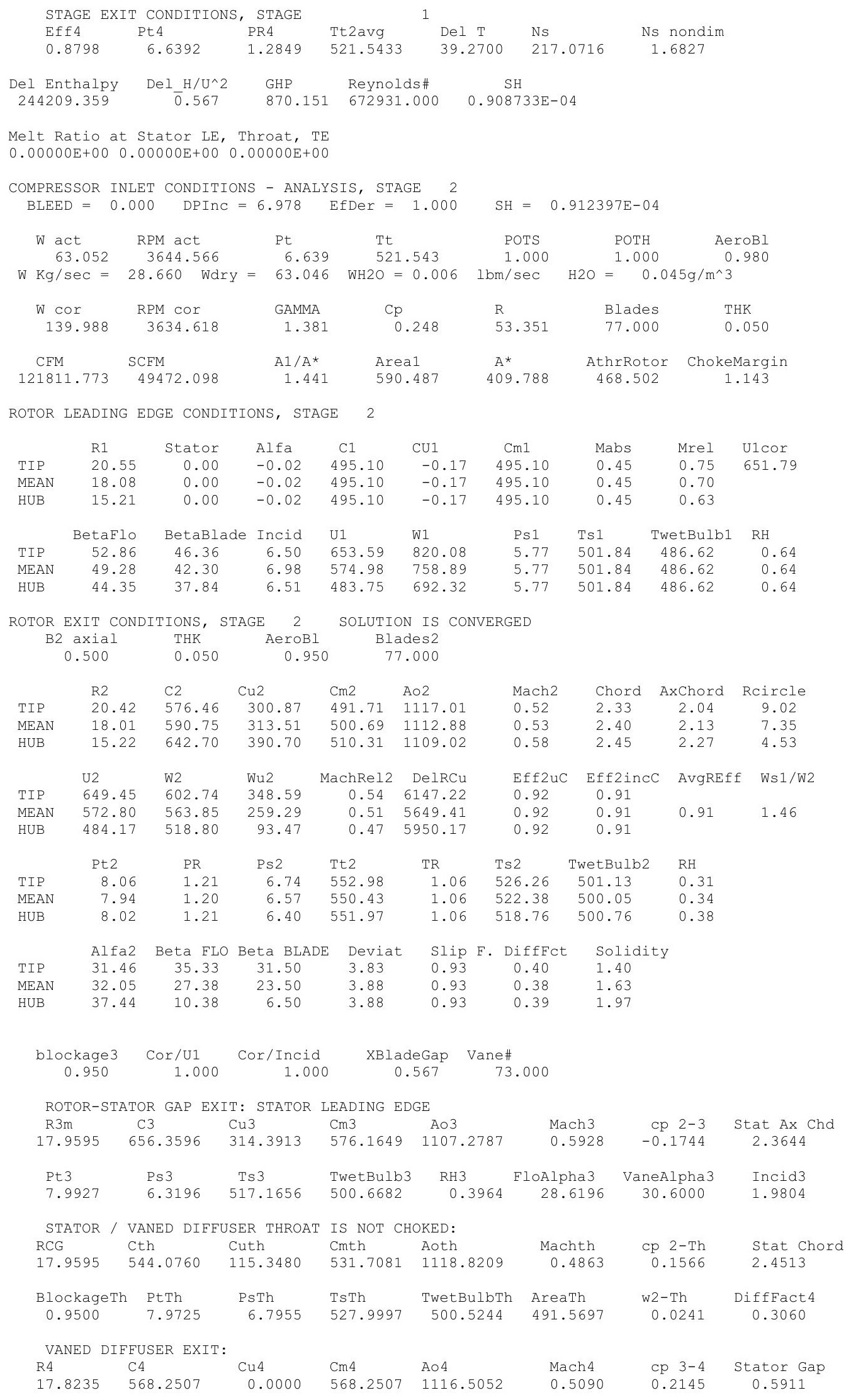




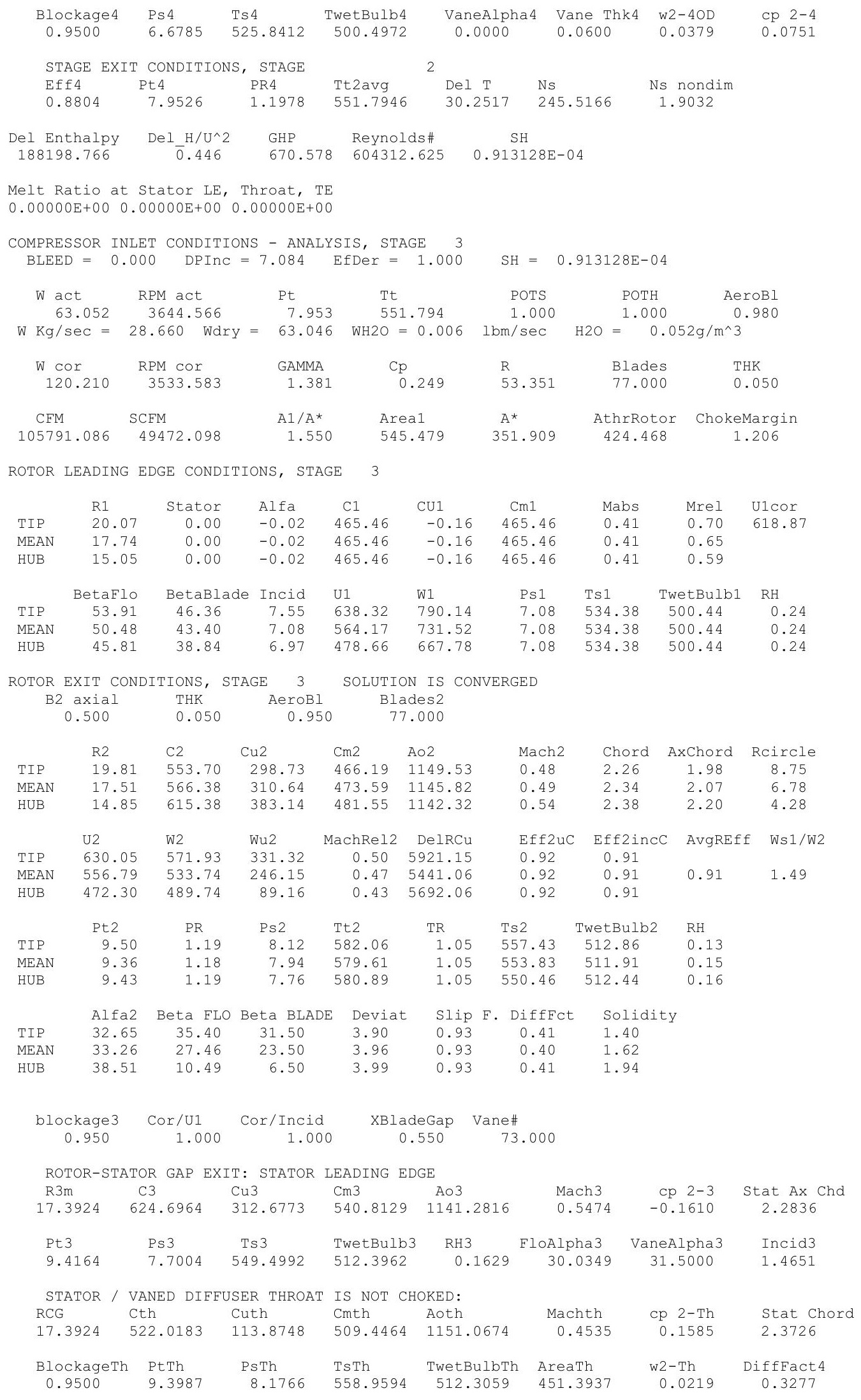




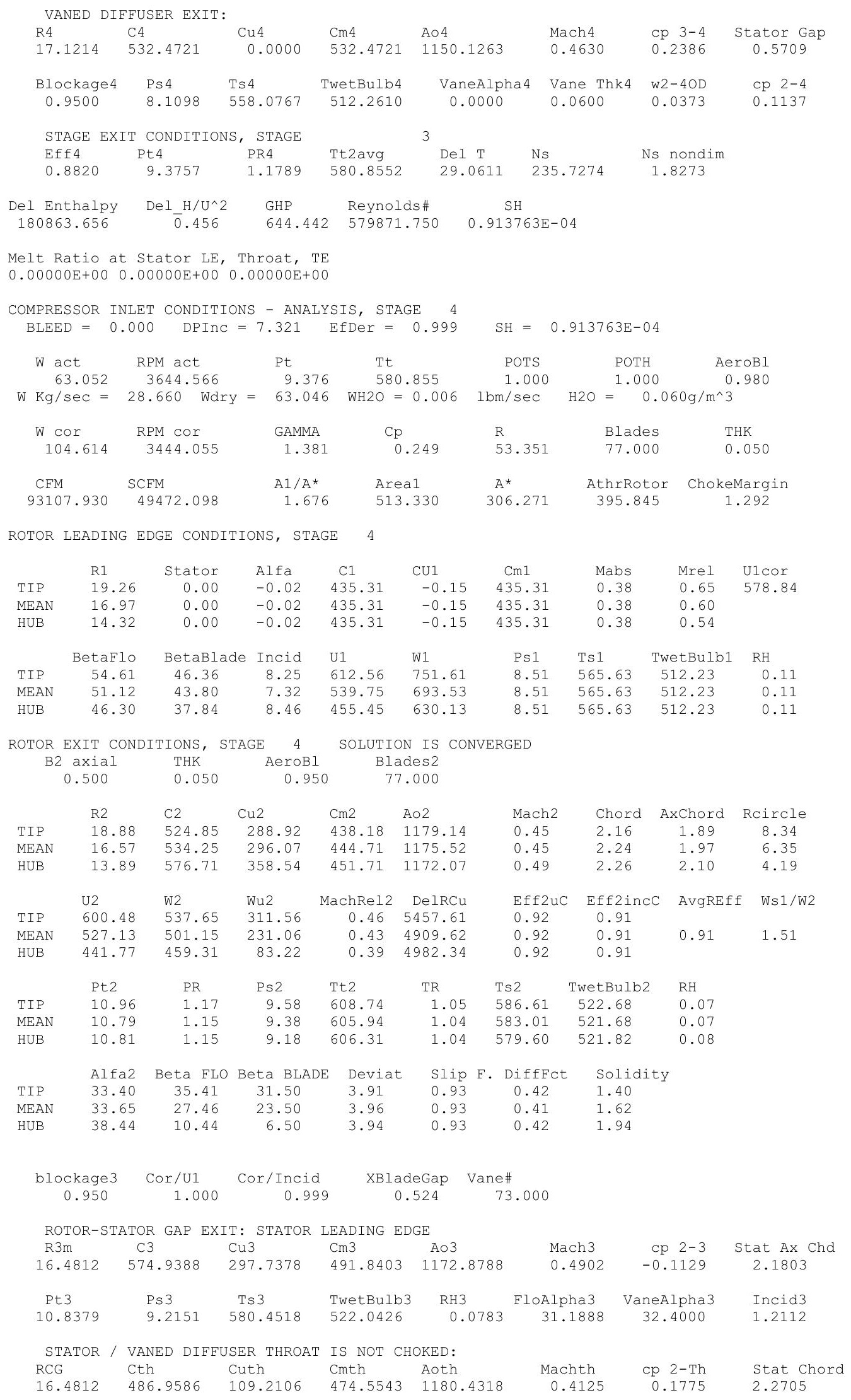




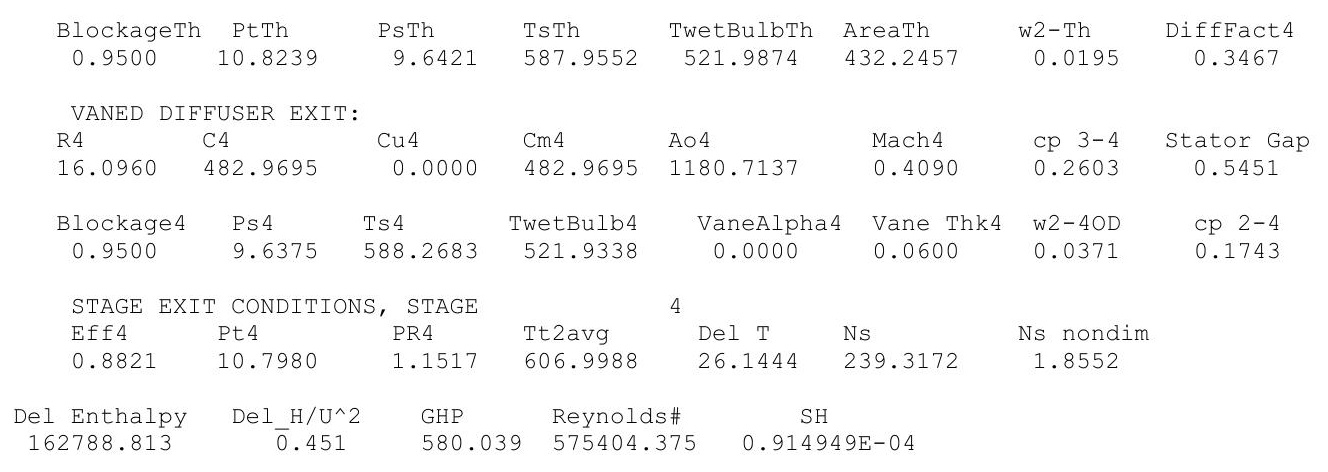

Melt Ratio at Stator LE, Throat, TE

$0.00000 \mathrm{E}+00 \quad 0.00000 \mathrm{E}+00 \quad 0.00000 \mathrm{E}+00$

COMPRESSOR INLET CONDITIONS - ANALYSIS, STAGE 5 $\mathrm{BLEED}=0.000 \quad$ DPInC $=7.480 \quad$ EfDer $=0.999$

$\begin{array}{llllll}\text { W act } & \text { RPM act } & \text { POTS } & \text { POTH } & \text { AeroBI }\end{array}$

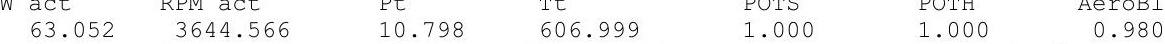

$\mathrm{W} \mathrm{Kg} / \mathrm{sec}=28.660$ Wdry $=63.046 \mathrm{WH} 2 \mathrm{O}=0.006 \mathrm{lbm} / \mathrm{sec} \quad \mathrm{H} 2 \mathrm{O}=0.067 \mathrm{~g} / \mathrm{m}^{\wedge} 3$

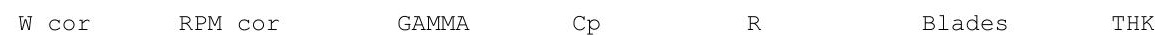

$\begin{array}{ccccccc}92.856 & 3369.070 & 1.381 & 0.249 & 53.351 & 77.000 & 0.050\end{array}$

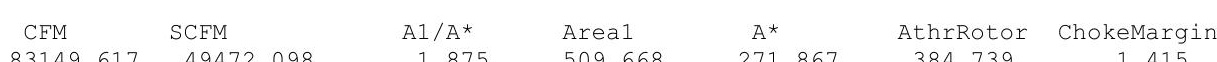

$\begin{array}{lllllll}83149.617 & 49472.098 & 1.875 & 509.668 & 271.867 & 384.739 & 1.415\end{array}$

ROTOR LEADING EDGE CONDITIONS, STAGE 5

$\begin{array}{lccccccccc} & \text { R1 } & \text { Stator } & \text { Alfa } & \text { C1 } & \text { CU1 } & \text { Cm1 } & \text { Mabs } & \text { Mrel } & \text { U1cor } \\ \text { TIP } & 18.32 & 0.00 & -0.02 & 391.55 & -0.13 & 391.55 & 0.33 & 0.59 & 538.60 \\ \text { MEAN } & 15.91 & 0.00 & -0.02 & 391.55 & -0.13 & 391.55 & 0.33 & 0.54 & \\ \text { HUB } & 13.07 & 0.00 & -0.02 & 391.55 & -0.13 & 391.55 & 0.33 & 0.48 & \\ & & & & & & & & & \\ \text { TIP } & \text { BetaFlo } & \text { BetaBlade } & \text { Incid } & \text { U1 } & \text { W1 } & \text { Ps1 } & \text { Ts1 } & \text { TwetBulb1 } & \text { RH } \\ \text { MEAN } & 56.11 & 47.36 & 8.75 & 582.66 & 702.11 & 10.02 & 594.69 & 521.91 & 0.06 \\ \text { HUB } & 46.72 & 44.80 & 7.48 & 506.11 & 639.99 & 10.02 & 594.69 & 521.91 & 0.06 \\ & 48.84 & 7.88 & 415.69 & 571.16 & 10.02 & 594.69 & 521.91 & 0.06\end{array}$

ROTOR EXIT CONDITIONS, STAGE 5 SOLUTION IS CONVERGED

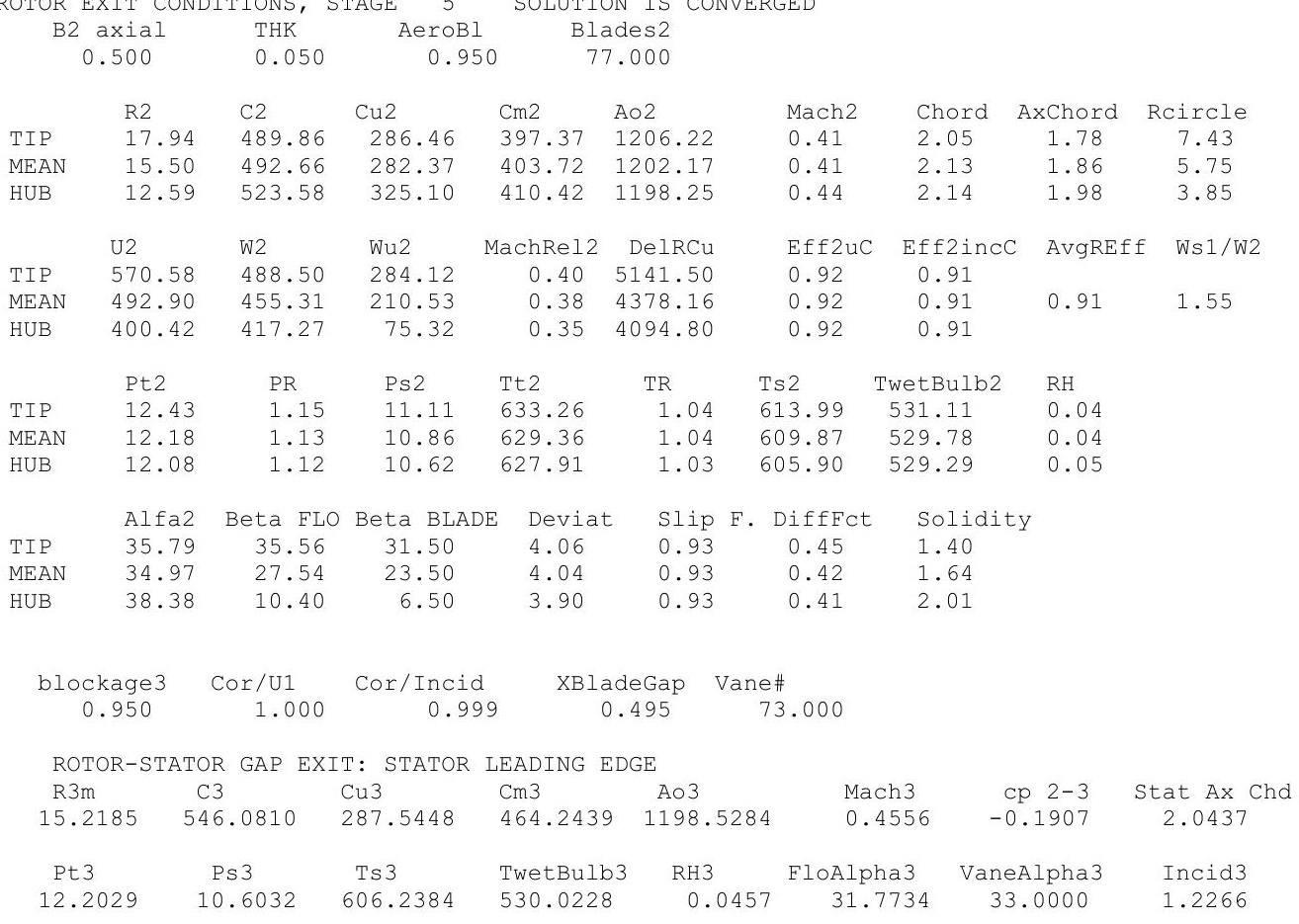




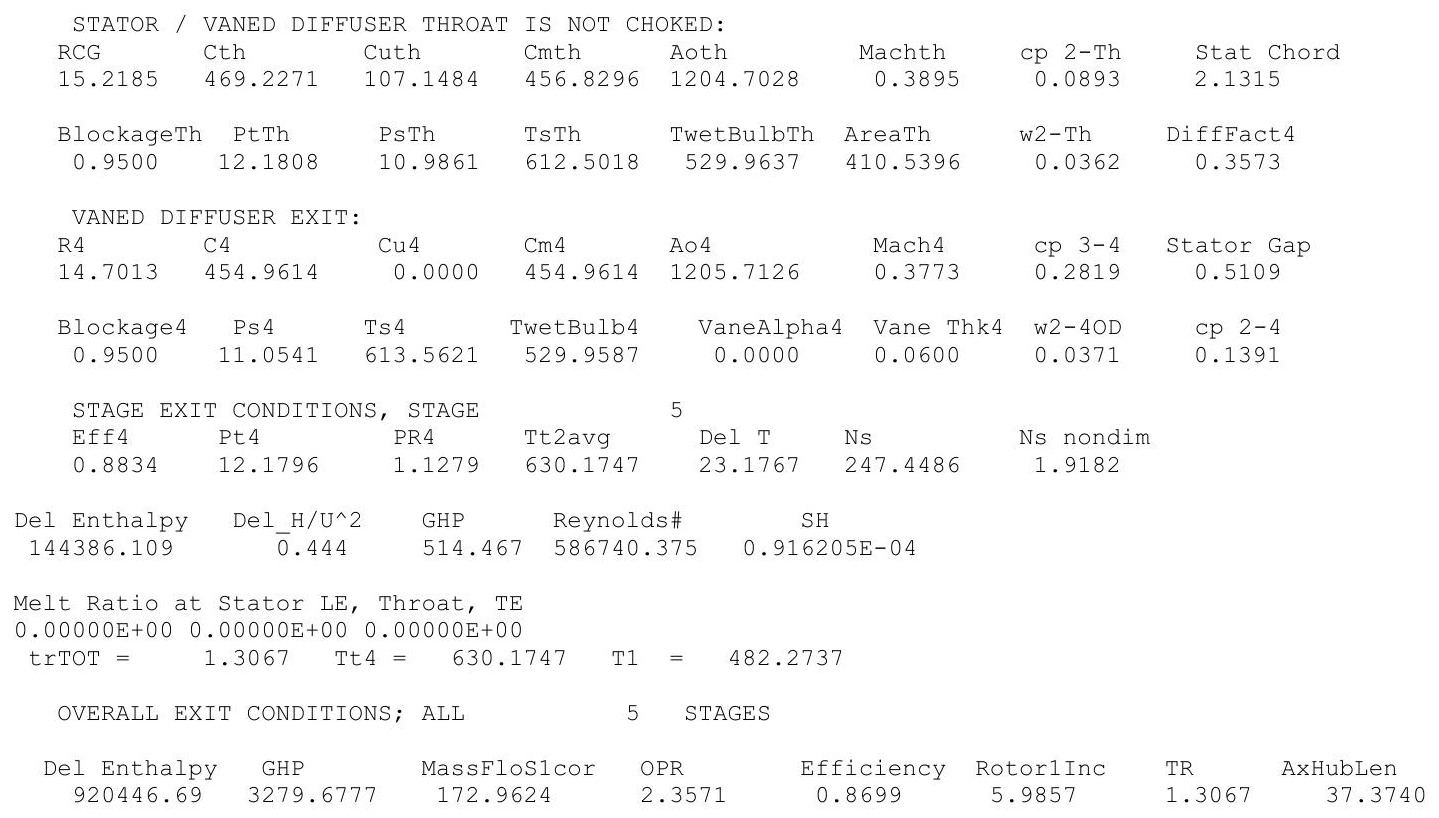




\section{Appendix I: NPSS cycle analysis for the altitude cruise conditions at 39000 feet}

\section{$5 \mu \mathrm{m}, \mathrm{ISA}+18 \mathrm{R}$}

Date:05/10/13 Time:11:00:06 Model: Turbofan Engine - COMDES ON CONverge $=1$ CASE: 0

Temperature Stator 1 inlet: 444.75 Stator 1 exit: 463.49 Stator 2 inlet: 485.28 Stator 2 exit: 492.72 Stator 3 inlet: 521.55 Stator 3 exit: 529.73 Stator 4 lnlet: 556.62 Stator 4 exit: 564.45

Ambient Relative Humidity $\quad 10.00$

Fan Face Relative Humidity $\quad 5.21$

Fan Bypass Relative Humidity 0.15

LPC Exit Relative Humidity

HPC Relative Humidity

Drop Diameter

0.02

Ambient Flow Velocity

0.0000050

et Length

$\begin{array}{lll}\text { Ambient Static Temperature } & 407.97 \\ \text { Additional Water at LPC Exit } & 0.0029812\end{array}$ Fan/LPC Inlet Static Temperature 438.28

483.11

407.97 Fan/IPC Inlet Static Temperature 438.28

MN alt dTamb W SUMMARY OUTPUT DAT

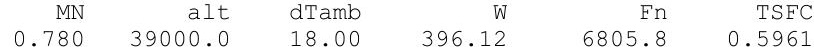

Wfuel

Wfuel
4057.03
BPR
5.3007
VTAS
$\mathrm{OPR}$
32.312
Powerset

100.000 $\begin{array}{ccr}\text { T4 } & \text { T41 } & \text { T49 } \\ 2814.3 & 2641.6 & 1836 .\end{array}$

\begin{tabular}{rrrrrr}
\multicolumn{7}{c}{ FLOW } & \multicolumn{1}{l}{ STATION DATA } & & Wt \\
W & Pt & Tt & ht & FAR & Wc \\
396.12 & 4.267 & 457.74 & -20.96 & 0.0000 & 1281.69 \\
396.12 & 4.267 & 457.74 & -20.96 & 0.0000 & 1281.69 \\
333.25 & 4.265 & 457.74 & -20.96 & 0.0000 & 1078.81 \\
62.87 & 4.265 & 457.74 & -20.96 & 0.0000 & 203.52 \\
333.25 & 7.257 & 540.20 & -1.18 & 0.0000 & 688.69 \\
62.87 & 10.583 & 621.71 & 18.40 & 0.0000 & 95.58 \\
63.06 & 10.583 & 625.30 & 2.18 & 0.0000 & 96.14 \\
63.06 & 10.583 & 625.30 & 2.18 & 0.0000 & 96.14 \\
60.00 & 137.870 & 1377.26 & 190.17 & 0.0000 & 10.42 \\
46.50 & 137.870 & 1377.26 & 190.17 & 0.0000 & 8.08 \\
47.62 & 134.694 & 2814.25 & 160.34 & 0.0243 & 12.10 \\
63.45 & 31.488 & 1845.31 & -17.97 & 0.0181 & 55.85 \\
64.18 & 7.245 & 1322.51 & -157.86 & 0.0179 & 207.90 \\
64.18 & 7.245 & 1322.57 & -157.86 & 0.0179 & 207.91 \\
64.18 & 7.245 & 1322.70 & -157.86 & 0.0179 & 207.92 \\
333.25 & 7.257 & 540.20 & -1.18 & 0.0000 & 688.69 \\
333.25 & 7.257 & 540.20 & -1.18 & 0.0000 & 688.69 \\
333.25 & 7.257 & 540.20 & -1.18 & 0.0000 & 688.69 \\
333.25 & 7.257 & 540.20 & -1.18 & 0.0000 & 688.69 \\
& & & & &
\end{tabular}

$\begin{array}{rr}\text { PS } & \text { TS } \\ 2.854 & 407.97 \\ 3.152 & 419.74 \\ 3.174 & 420.64 \\ 3.664 & 438.28 \\ 6.024 & 512.21 \\ 9.281 & 598.87 \\ 9.260 & 601.96 \\ 9.260 & 601.96 \\ 124.294 & 1340.14 \\ 129.984 & 1356.06 \\ 127.230 & 2778.51 \\ 28.359 & 1798.88 \\ 6.249 & 1272.87 \\ 6.249 & 1272.93 \\ 3.875 & 1123.01 \\ 6.024 & 512.21 \\ 5.854 & 508.03 \\ 5.854 & 508.03 \\ 3.833 & 450.06\end{array}$

$\begin{array}{rrr}\text { Aphy } & \text { MN } & \text { gamt } \\ 3910.0 & 0.7800 & 1.40084 \\ 4168.2 & 0.6720 & 1.40084 \\ 3531.8 & 0.6633 & 1.40084 \\ 830.5 & 0.4706 & 1.40084 \\ 2606.7 & 0.5226 & 1.39992 \\ 412.6 & 0.4374 & 1.39828 \\ 412.6 & 0.4413 & 1.39776 \\ 412.6 & 0.4413 & 1.39776 \\ 49.7 & 0.3933 & 1.35677 \\ 49.3 & 0.2957 & 1.35677 \\ 74.6 & 0.3007 & 1.28425 \\ 265.4 & 0.4006 & 1.32062 \\ 860.2 & 0.4723 & 1.34775 \\ 860.2 & 0.4723 & 1.34775 \\ 613.4 & 1.0000 & 1.34774 \\ 2606.7 & 0.5226 & 1.39992 \\ 2481.9 & 0.5625 & 1.39992 \\ 2481.9 & 0.5625 & 1.39992 \\ 2006.9 & 1.0000 & 1.39992\end{array}$

WAR 0.0000295 0.0000295 0.0000295 0.0000295 0.0000295 0.0030106 0.0030106 0.0030106 0.0030106 0.0030106 0.0030106 0.0030106 0.0030106 0.0030106 0.0000295
0.0000295 0.0000295 0.0000295 0.0000295

TURBOMACHINERY PERFORMANCE DATA

\begin{tabular}{|c|c|c|c|c|c|c|c|c|c|}
\hline & WC & PR & & N & TD & 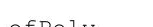 & nwr & SMNT & SMTT \\
\hline Fan & 1078.81 & 1.702 & $\begin{array}{l}\text { eII } \\
0.9114\end{array}$ & $\begin{array}{r}\text { NC } \\
4139.469\end{array}$ & $\begin{array}{r}1.1801 \\
1 R\end{array}$ & 0.9178 & $\begin{array}{r}\text { pWr } \\
-9322.3\end{array}$ & 36.69 & 23.09 \\
\hline 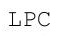 & 203.52 & 2.482 & 0.8276 & 4139.469 & 1.3582 & 0.8480 & -3501.0 & 2.97 & 2.24 \\
\hline HPC & 96.14 & 13.027 & 0.8586 & 10339.379 & 2.2026 & 0.8979 & -16365.1 & 26.38 & 25.58 \\
\hline HPT & 12.10 & 4.278 & 0.9118 & 213.999 & 1.3463 & 0.8912 & 16364.9 & & \\
\hline LPT & 55.85 & 4.346 & 0.9155 & 90.526 & 1.3918 & 0.8989 & 12823.3 & & \\
\hline & INERY & DATA & & & & & & & \\
\hline & WcMap & PRmap & effMap & NcMap & R/Parm & S_WCDes & S_PRdes & S_effDes & S_NCDes \\
\hline $\operatorname{Tan}$ & 1253.47 & 1.686 & 0.9132 & 4100.000 & 0.0639 & $\overline{0} .8607$ & $\overline{1} .0235$ & 0.9980 & $\overline{0} .9905$ \\
\hline PC & 166.84 & 2.748 & 0.8341 & 1.093 & 0.0000 & 1.2198 & 0.8477 & 0.9922 & 0.0003 \\
\hline${ }^{\mathrm{C}} \mathrm{C}$ & 87.44 & 12.460 & 0.8675 & 10063.738 & 13.4623 & & 1.0494 & 0.9898 & 0.9733 \\
\hline & & & 118 & 1.286 & 4.1868 & 12.6299 & 0.9723 & 1.0000 & 0.0003 \\
\hline PT & 0.86 & 3.517 & 0.9223 & 1.018 & 3.5170 & 65.2590 & 0.7521 & 0.9926 & 0.0005 \\
\hline
\end{tabular}




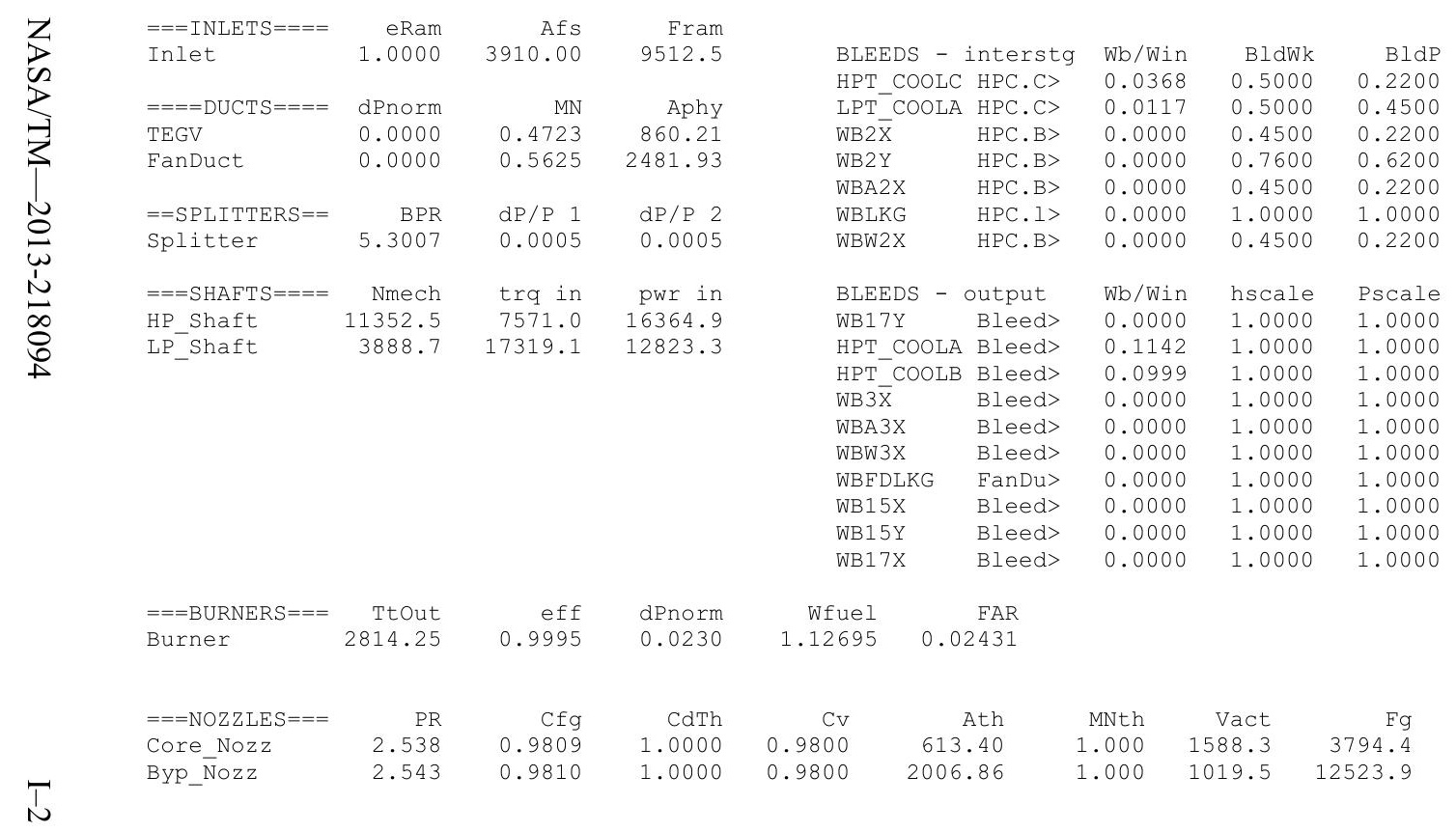


Date:05/10/13 Time:11:00:41 Model: Turbofan Engine - COMDES ON Converge = 1 CASE: 0

Version:NPSS 1.6.5 - Rev: -> Gas Package: Janaf iter/pass/Jacb/Broy=18/32/1/16 Run by: Philip C Jorgenson PC: 84

Temperature Stator 1 inlet: 450.83 Stator 1 exit: 468.07 Stator 2 inlet: 488.64 Stator 2 exit: 496.76 $\begin{array}{lll}\text { Stator } 3 \text { inlet: } 522.20 & \text { Stator } 3 \text { exit: } 530.54 & \text { Stator } 4 \text { inlet: } 554.47 \text { Stator } 4 \text { exit: } 562.16 \\ \text { Stator } 5 \text { inlet: } 581.05 & \text { Stator } 5 \text { exit: } 588.40 & \text { Unblocked Percent Blockage: } 0.00\end{array}$

Ambient Relative Humidity $\quad 10.00$

Fan Face Relative Humidity $\quad 4.13$

Fan Bypass Relative Humidity 0.18

LPC Inlet Relative Humidity $\quad 1.99$

LPC Exit Relative Humidity $\quad 0.02$

HPC Relative Humidity

0.01

Ambient Flow Vecity 0.0000050

Ambient Static Pressu

Ambient Static Pressure

$\begin{array}{rrr}772.64 & \text { Fan/LPC Inlet Flow Velocity } & 443.02 \\ 2.85 & \text { Fan/LPC Inlet Static Pressure } & 3.76\end{array}$

Inlet Length

40.00

Additional Water at LPC Exit 0.0032403

$\begin{array}{rr}\text { Fan/LPC Inlet Static Pressure } & 3.76 \\ \text { Fan/LPC Inlet Static Temperature } 441.38\end{array}$

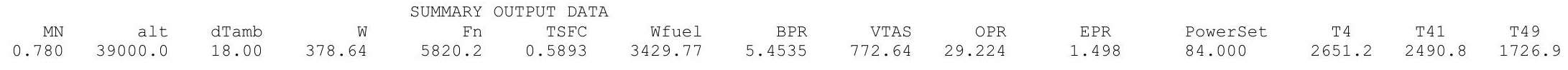

\begin{tabular}{|c|c|}
\hline FSO & Ambient.Fl_O \\
\hline FS1 & Inlet.Fl_o- \\
\hline & Splitter.Fl 02 \\
\hline FS2 & Splitter.FI_01 \\
\hline FS14 & Fan.Fl_O \\
\hline$\$ 23$ & LPC.Fl-o \\
\hline FS24 & 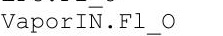 \\
\hline S25 & Bleed2.Fl_ō \\
\hline Fs 3 & HPC.FI_O \\
\hline 36 & Bleed3.F1 O \\
\hline 4 & Burner.Fl_O \\
\hline S45 & HPT.FI_O \\
\hline FS49 & LPT.FI_O \\
\hline & TEGV.FI__O \\
\hline 58 & Core_Nozz.FI_ \\
\hline FS17 & FanDuctLkg.FI__o \\
\hline ES171 & Bleed15.Fl_o \\
\hline & FanDuct.Fl- \\
\hline & Byp_Nozz.Fi \\
\hline
\end{tabular}

TURBOMACHINERY PERFORMANCE DATA

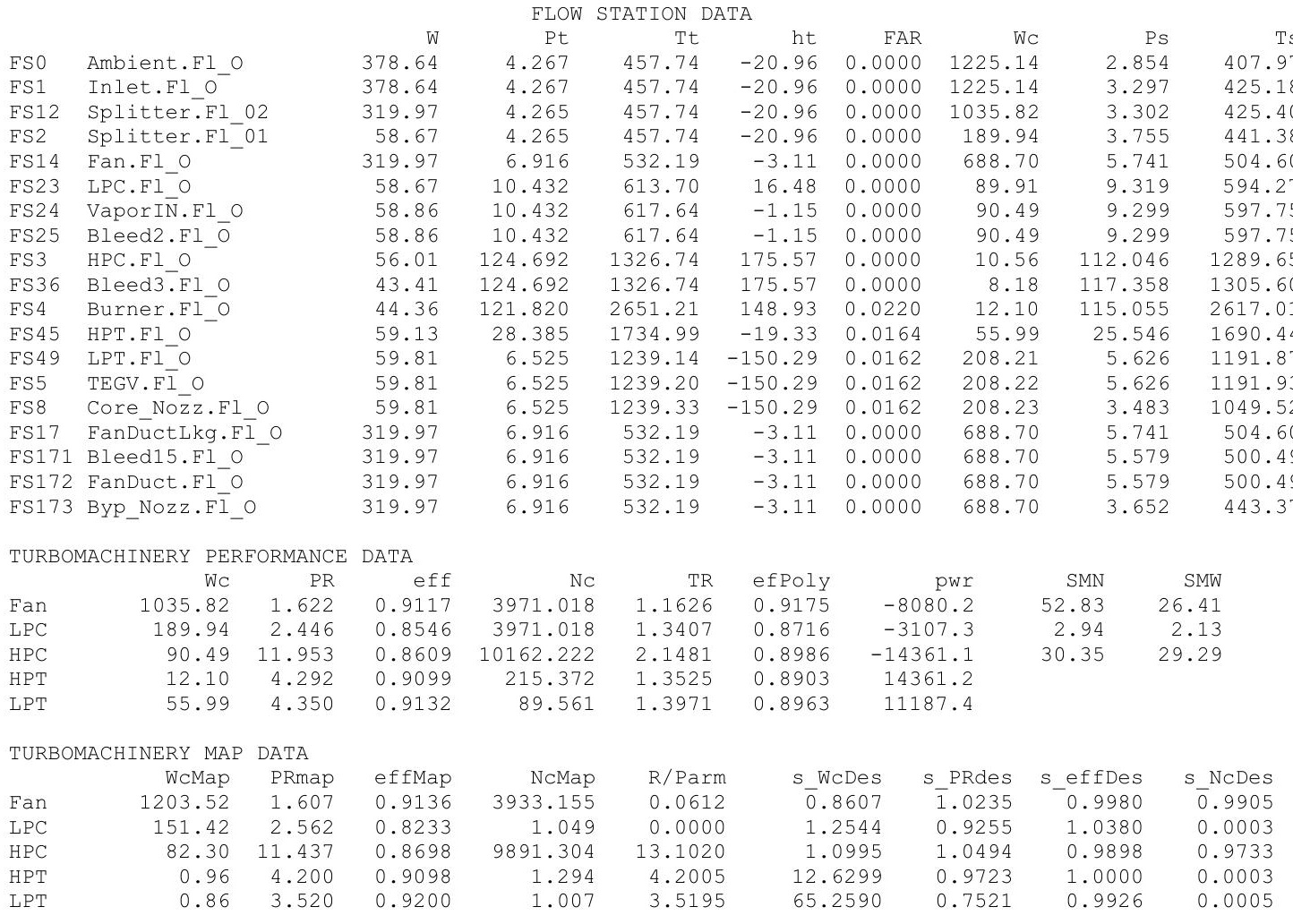

$\begin{array}{rrrrrrr}\text { Aphy } & \text { MN } & \text { gamt } & \text { WAR } & \text { Wair } & \text { WH2O } & \text { H2O frac } \\ 3737.5 & 0.7800 & 1.40084 & 0.0000295 & 378.63 & 0.011 & 0.0000 \\ 4168.2 & 0.6181 & 1.40084 & 0.0000295 & 378.63 & 0.011 & 0.0000 \\ 3531.8 & 0.6157 & 1.40084 & 0.0000295 & 319.96 & 0.009 & 0.0000 \\ 830.5 & 0.4300 & 1.40084 & 0.0000295 & 58.67 & 0.002 & 0.0000 \\ 2606.7 & 0.5226 & 1.40004 & 0.0000295 & 319.96 & 0.009 & 0.0000 \\ 412.6 & 0.4050 & 1.39848 & 0.0000295 & 58.67 & 0.002 & 0.0000 \\ 412.6 & 0.4088 & 1.39792 & 0.0032697 & 58.67 & 0.192 & 0.0033 \\ 412.6 & 0.4088 & 1.39792 & 0.0032697 & 58.67 & 0.192 & 0.0033 \\ 49.7 & 0.3991 & 1.35972 & 0.0032697 & 55.82 & 0.183 & 0.0033 \\ 49.3 & 0.2997 & 1.35972 & 0.0032697 & 43.26 & 0.141 & 0.0033 \\ 74.6 & 0.2999 & 1.29033 & 0.0032697 & 43.26 & 0.141 & 0.0297 \\ 265.4 & 0.4009 & 1.32659 & 0.0032697 & 57.98 & 0.190 & 0.0231 \\ 860.2 & 0.4721 & 1.35402 & 0.0032697 & 58.67 & 0.192 & 0.0229 \\ 860.2 & 0.4721 & 1.35402 & 0.0032697 & 58.67 & 0.192 & 0.0229 \\ 613.4 & 1.0000 & 1.35401 & 0.0032697 & 58.67 & 0.192 & 0.0229 \\ 2606.7 & 0.5226 & 1.40004 & 0.0000295 & 319.96 & 0.009 & 0.0000 \\ 2481.9 & 0.5625 & 1.40004 & 0.0000295 & 319.96 & 0.009 & 0.0000 \\ 2481.9 & 0.5625 & 1.40004 & 0.0000295 & 319.96 & 0.009 & 0.0000 \\ 2006.9 & 1.0000 & 1.40004 & 0.0000295 & 319.96 & 0.009 & 0.0000\end{array}$




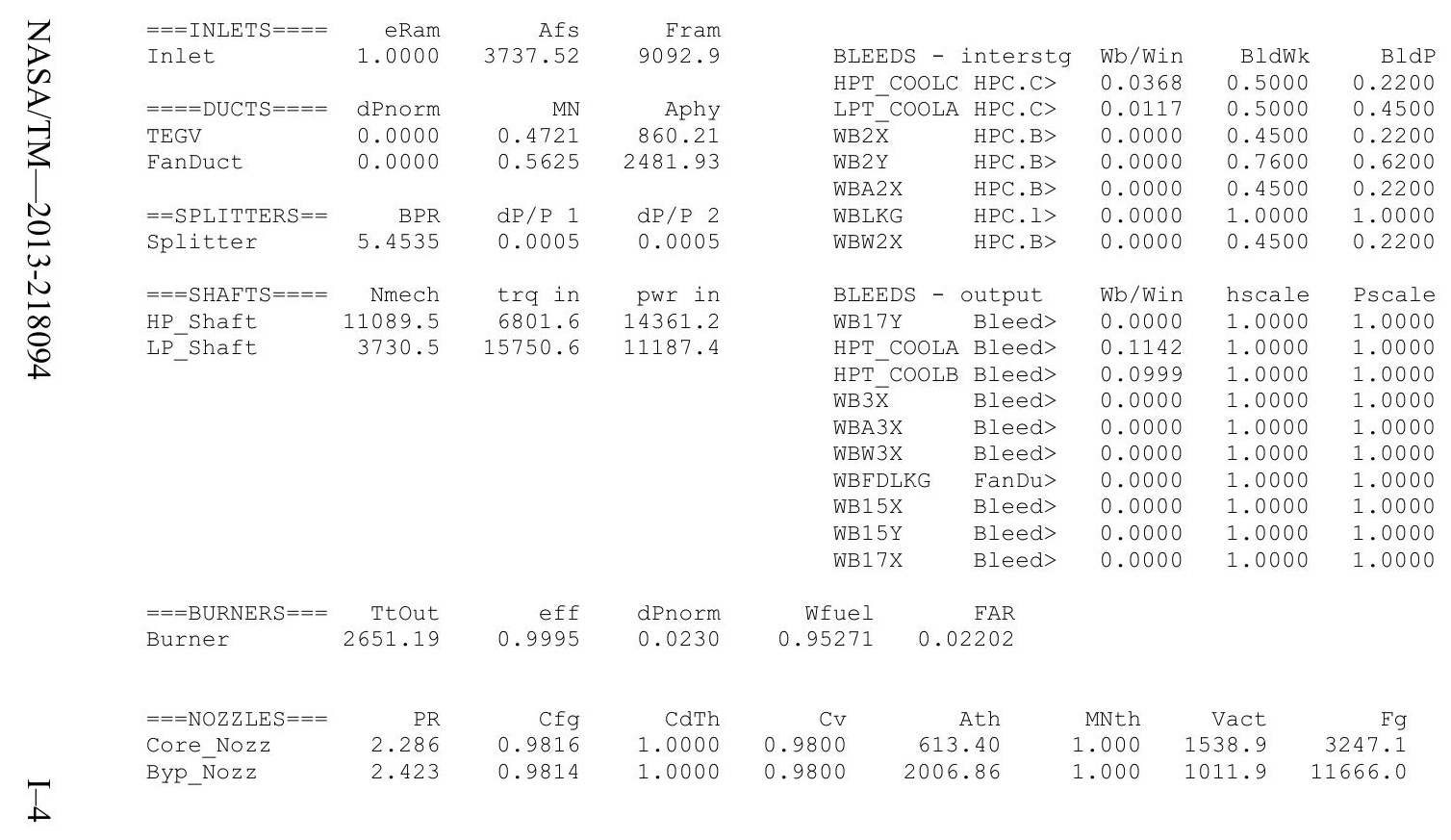


Date:05/10/13 Time:11:01:31 Model: Turbofan Engine - COMDES ON Converge $=1$ CASE: 0

Version:NPSS_1.6.5 - Rev: -> Gas Package: Janaf iter/pass/Jacb/Broy=19/47/2/16 Run by: Philip C Jorgenson PC: 79

Temperature Stator 1 inlet: 452.44 Stator 1 exit: 469.27 Stator 2 inlet: 489.52 Stator 2 exit: 497.76 $\begin{array}{lll}\text { Stator } 3 \text { inlet: } 522.38 & \text { Stator } 3 \text { exit: } 530.72 & \text { Stator } 4 \text { inlet: } 553.90 \text { Stator } 4 \text { exit: } 561.54 \\ \text { Stator } 5 \text { inlet: } 579.94 & \text { Stator } 5 \text { exit: } 587.21 & \text { Unblocked Percent Blockage: } 0.00\end{array}$

Ambient Relative Humidity $\quad 10.00$

Fan Face Relative Humidity $\quad 3.89$

Fan Bypass Relative Humidity 0.20

LPC Inlet Relative Humidity 1.91

LPC Exit Relative Humidity $\quad 0.02$

HPC Relative Humidity

0.01

Ambient Flow 0.0000050

Ambient Static velty

Ambient Static Temperature

7.64
2.85
407.97

Inlet Length

40.00

Fan/LPC Inlet Static Pressure $\quad 3.78$ Additional Water at LPC Exit 0.0033318 Fan/LPC Inlet Static Temperature 442.28

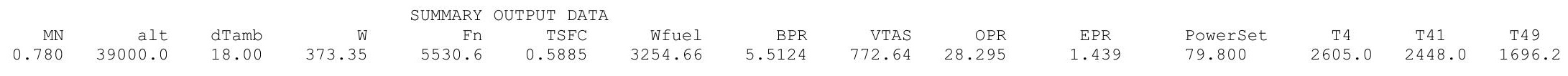

\begin{tabular}{|c|c|}
\hline FSO & Ambient.FI_O \\
\hline FS1 & Inlet.Fl_O \\
\hline FS12 & Splitter.F1_02 \\
\hline FS2 & Splitter.Fl_01 \\
\hline FS14 & Fan.Fl_O - \\
\hline FS23 & LPC.FI_O \\
\hline FS24 & 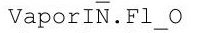 \\
\hline FS25 & Bleed2.F1_o \\
\hline FS3 & HPC.FI_O ${ }^{-}$ \\
\hline FS36 & Bleed3.Fl_O \\
\hline & Burner.Fl_o \\
\hline FS 45 & HPT.FI_O \\
\hline FS 49 & LPT.FI_O \\
\hline FS5 & TEGV.FI__O \\
\hline FS $\varepsilon$ & Core_Nozz.FI_O \\
\hline FS17 & FanDüctLkg.F̄__o \\
\hline 17. & Bleed15.Fl_O \\
\hline & FanDuct.FI_O \\
\hline & Byp_Nozz.F̄ \\
\hline
\end{tabular}

TURBOMACHINERY PERFORMANCE DATA

\begin{tabular}{|c|c|c|c|c|c|c|c|c|c|c|c|}
\hline & & & & $\begin{array}{l}\text { FLOW } \\
\text { Pt }\end{array}$ & STATION DA & & $\mathrm{FAl}$ & Wo & & Ps & \\
\hline FSO & Ambient.Fl 0 & & 373.35 & 4.267 & 457.74 & $\begin{array}{r}h t \\
-20.96\end{array}$ & 0.000 & 1208.02 & 2.8 & & $40^{\circ}$ \\
\hline FS1 & Inlet.Fl_o- & & 373.35 & 4.267 & 457.74 & -20.96 & 0.000 & 1208.02 & 3.3 & & 426. \\
\hline FS12 & Splitter.Fl_ & & 316.02 & 4.265 & 457.74 & -20.96 & 0.000 & 1023.04 & 3.3 & & 426 \\
\hline FS2 & Splitter.FI- & & 57.33 & 4.265 & 457.74 & -20.96 & 0.000 & 185.59 & 3.78 & & 442 \\
\hline FS14 & Fan.Fl_O & & 316.02 & 6.815 & 529.79 & -3.68 & 0.000 & 688.70 & 5.6 & & 502 \\
\hline ES23 & LPC.FI_O & & 57.33 & 10.371 & 611.54 & 15.96 & 0.000 & 88.21 & 9.3. & & 593 \\
\hline ES24 & VaporIN̄.Fl O & & 57.52 & 10.371 & 615.61 & -2.17 & 0.000 & 88.80 & 9.2 & & \\
\hline s25 & Bleed2.Fl_o & & 57.52 & 10.371 & 615.61 & -2.17 & 0.000 & 88.80 & 9.2 & & \\
\hline 53 & HPC.Fl_O & & 54.73 & 120.730 & 1312.35 & 171.33 & 0.000 & 10.60 & 108.38 & & 127 \\
\hline ES36 & Bleed3.FI_O & & 42.42 & 120.730 & 1312.35 & 171.33 & 0.000 & 8.21 & $113.5^{\prime}$ & & \\
\hline S4 & Burner.FI_O & & 43.32 & 117.950 & 2604.98 & 145.56 & 0.021 & 12.10 & 111.3 & & 571 \\
\hline s45 & HPT.FI_O & & 57.75 & 27.458 & 1704.05 & -19.80 & 0.016 & 56.02 & 24.7 & & 66 \\
\hline ES49 & LPT.FI_O & & 58.42 & 6.311 & 1215.93 & -148.25 & 0.015 & 208.29 & 5.4 & & 169 \\
\hline ES5 & TEGV.F̄ O & & 58.42 & 6.311 & 1215.99 & -148.25 & 0.015 & 208.30 & 5.4 & & 1169 \\
\hline FS 8 & Core_Nozz.Fl & & 58.42 & 6.311 & 1216.12 & -148.25 & 0.015 & 208.31 & 3.3 & & 1029 \\
\hline FS17 & FanDüctLkg. F & & 316.02 & 6.815 & 529.79 & -3.68 & 0.000 & 688.70 & 5.6 & & \\
\hline FS171 & Bleed15.F1 O & & 316.02 & 6.815 & 529.79 & -3.68 & 0.000 & 688.70 & 5.49 & & \\
\hline FS172 & FanDuct.FI_o & & 316.02 & 6.815 & 529.79 & -3.68 & 0.000 & 688.70 & 5.49 & & 498 \\
\hline FS173 & Byp_Nozz.Fin_ & & 316.02 & 6.815 & 529.79 & -3.68 & 0.000 & 688.70 & 3.59 & & \\
\hline URBOI & MACHINERY P & ORMANCE & DATA & & & & & & & & \\
\hline & & $\mathrm{PR}$ & eff & NC & TR & efPoly & & pwr & SMN & & MW \\
\hline Tan & 1023.04 & 1.598 & 0.9114 & 3923.364 & 1.1574 & 0.9171 & & 23.8 & 56.99 & 27. & \\
\hline LPC & 185.59 & 2.432 & 0.8601 & 3923.364 & 1.3360 & 0.8764 & -2 & 994.1 & 3.32 & 2. & 60 \\
\hline HPC & 88.80 & 11.642 & 0.8614 & 10115.453 & 2.1318 & 0.8987 & -13 & 777.4 & 31.59 & 30. & \\
\hline HPT & 12.10 & 4.296 & 0.9090 & 215.918 & 1.3541 & 0.8897 & 13 & 777.4 & & & \\
\hline LPT & 56.02 & 4.351 & 0.9125 & 89.286 & 1.3985 & 0.8954 & 10 & 17.9 & & & \\
\hline & NERY & $\mathrm{DA}$ & & & & & & & & & \\
\hline & WCMa & PRmap & effMap & NcMap & R/Parm & S_Wc & Des & S_PRdes & S_effDes & & NcDes \\
\hline Tan & 1188.67 & 1.584 & 0.9133 & 3885.955 & 0.0604 & 0.8 & 3607 & 1.0235 & 0.9980 & & $\overline{0} .9905$ \\
\hline PC & 148.34 & 2.513 & 0.8224 & 1.036 & 0.0000 & & 11 & 0.9460 & 1.0459 & & 0.0003 \\
\hline PC & 80.76 & 11.140 & 0.8703 & 9845.782 & 12.9962 & 1.0 & 0995 & 1.0494 & 0.9898 & & 0.9733 \\
\hline HPT & 0.96 & 4.204 & 0.9090 & 1.298 & 4.2043 & 12.6 & 5299 & 0.9723 & 1.0000 & & 0.0003 \\
\hline LPT & 0.86 & 3.520 & 0.9192 & 1.004 & 3.5201 & 65.2 & 2590 & 0.7521 & 0.9926 & & 0.0005 \\
\hline
\end{tabular}

$\begin{array}{rrrrrrr}\text { Aphy } & \text { MN } & \text { gamt } & \text { WAR } & \text { Wair } & \text { WH2O } & \text { H2O frac } \\ 3685.3 & 0.7800 & 1.40084 & 0.0000295 & 373.34 & 0.011 & 0.0000 \\ 4168.2 & 0.6033 & 1.40084 & 0.0000295 & 373.34 & 0.011 & 0.0000 \\ 3531.8 & 0.6028 & 1.40084 & 0.0000295 & 316.01 & 0.009 & 0.0000 \\ 830.5 & 0.4176 & 1.40084 & 0.0000295 & 57.33 & 0.002 & 0.0000 \\ 2606.7 & 0.5226 & 1.40007 & 0.0000295 & 316.01 & 0.009 & 0.0000 \\ 412.6 & 0.3956 & 1.39853 & 0.0000295 & 57.33 & 0.002 & 0.0000 \\ 412.6 & 0.3994 & 1.39796 & 0.0033613 & 57.33 & 0.193 & 0.0034 \\ 412.6 & 0.3994 & 1.39796 & 0.0033613 & 57.33 & 0.193 & 0.0034 \\ 49.7 & 0.4008 & 1.36058 & 0.0033613 & 54.55 & 0.183 & 0.0034 \\ 49.3 & 0.3008 & 1.36058 & 0.0033613 & 42.27 & 0.142 & 0.0034 \\ 74.6 & 0.2996 & 1.29207 & 0.0033613 & 42.27 & 0.142 & 0.0290 \\ 265.4 & 0.4009 & 1.32834 & 0.0033613 & 56.66 & 0.190 & 0.0226 \\ 860.2 & 0.4720 & 1.35578 & 0.0033613 & 57.33 & 0.193 & 0.0224 \\ 860.2 & 0.4720 & 1.35577 & 0.0033613 & 57.33 & 0.193 & 0.0224 \\ 613.4 & 1.0000 & 1.35576 & 0.0033613 & 57.33 & 0.193 & 0.0224 \\ 2606.7 & 0.5226 & 1.40007 & 0.0000295 & 316.01 & 0.009 & 0.0000 \\ 2481.9 & 0.5625 & 1.40007 & 0.0000295 & 316.01 & 0.009 & 0.0000 \\ 2481.9 & 0.5625 & 1.40007 & 0.0000295 & 316.01 & 0.009 & 0.0000 \\ 2006.9 & 1.0000 & 1.40007 & 0.0000295 & 316.01 & 0.009 & 0.0000\end{array}$




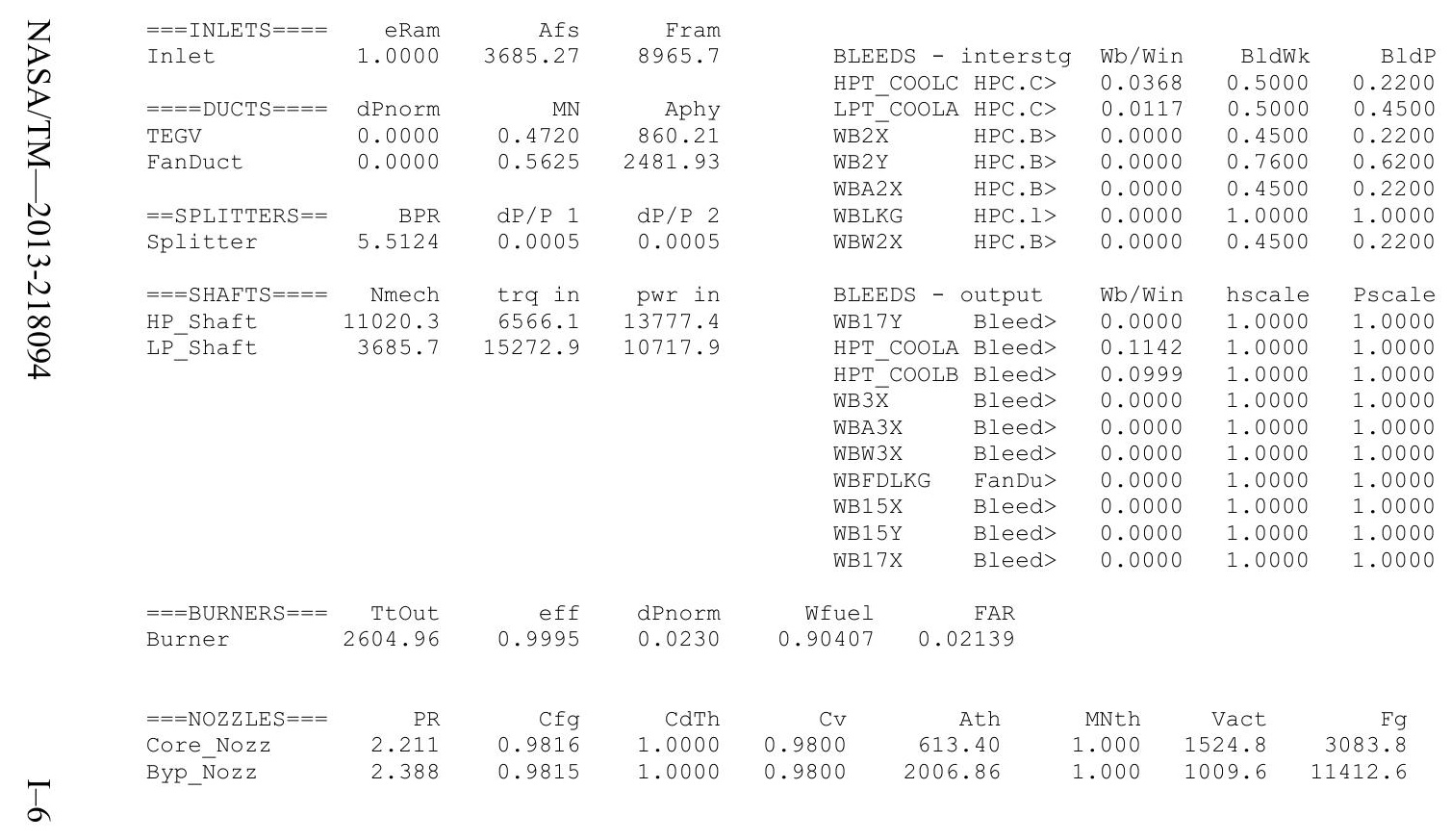


Date:05/10/13 Time:11:02:07 Model: Turbofan Engine - COMDES ON Converge = 1 CASE: 0

Version:NPSS_1.6.5 - Rev: -> Gas Package: Janaf iter/pass/Jacb/Broy= 20/34/1/18 Run by: Philip C Jorgenson PC: 76

Temperature Stator 1 inlet: 453.52 Stator 1 exit: 470.08 Stator 2 inlet: 490.14 Stator 2 exit: 498.45 $\begin{array}{lll}\text { Stator } 3 \text { inlet: } 522.55 & \text { Stator } 3 \text { exit: } 530.89 & \text { Stator } 4 \text { inlet: } 553.58 \text { Stator } 4 \text { exit: } 561.19 \\ \text { Stator } 5 \text { inlet: } 579.25 & \text { Stator } 5 \text { exit: } 586.48 & \text { Unblocked Percent Blockage: } 0.00\end{array}$

Ambient Relative Humidity $\quad 10.00$

Fan Face Relative Humidity $\quad 3.73$

Fan Bypass Relative Humidity $\quad 0.21$

LPC Inlet Relative Humidity 1.86

LPC Exit Relative Humidity $\quad 0.02$

HPC Relative Humidity

0.01

Ambient Flow Velocity 0.0000050

Ambient Static

Ambient Static Pressure

$\begin{array}{rlr}772.64 & \text { Fan/LPC Inlet Flow Velocity } & 421.98 \\ 2.85 & \text { Fan/LPC Inlet Static Pressure } & 3.80\end{array}$

Inlet Length

Additional Water at IPC Exit 0.0034016 Fan/LPC Inlet Static Temperature 442.90

\begin{tabular}{|c|c|c|c|c|c|c|c|c|c|c|c|c|c|c|}
\hline & & & & SUMMARY & JTPUT DA & & & & & & & & & \\
\hline MN & alt & dTamb & W & $\mathrm{Fn}$ & TSFC & Wfuel & $\begin{array}{r}\text { BPR } \\
\quad 5548\end{array}$ & VTAS & $\begin{array}{r}\mathrm{OPR} \\
\quad 645\end{array}$ & EPR & Powerset & $\begin{array}{c}\text { T4 } \\
2573\end{array}$ & T41 & $\begin{array}{r}\text { T49 } \\
1675\end{array}$ \\
\hline
\end{tabular}

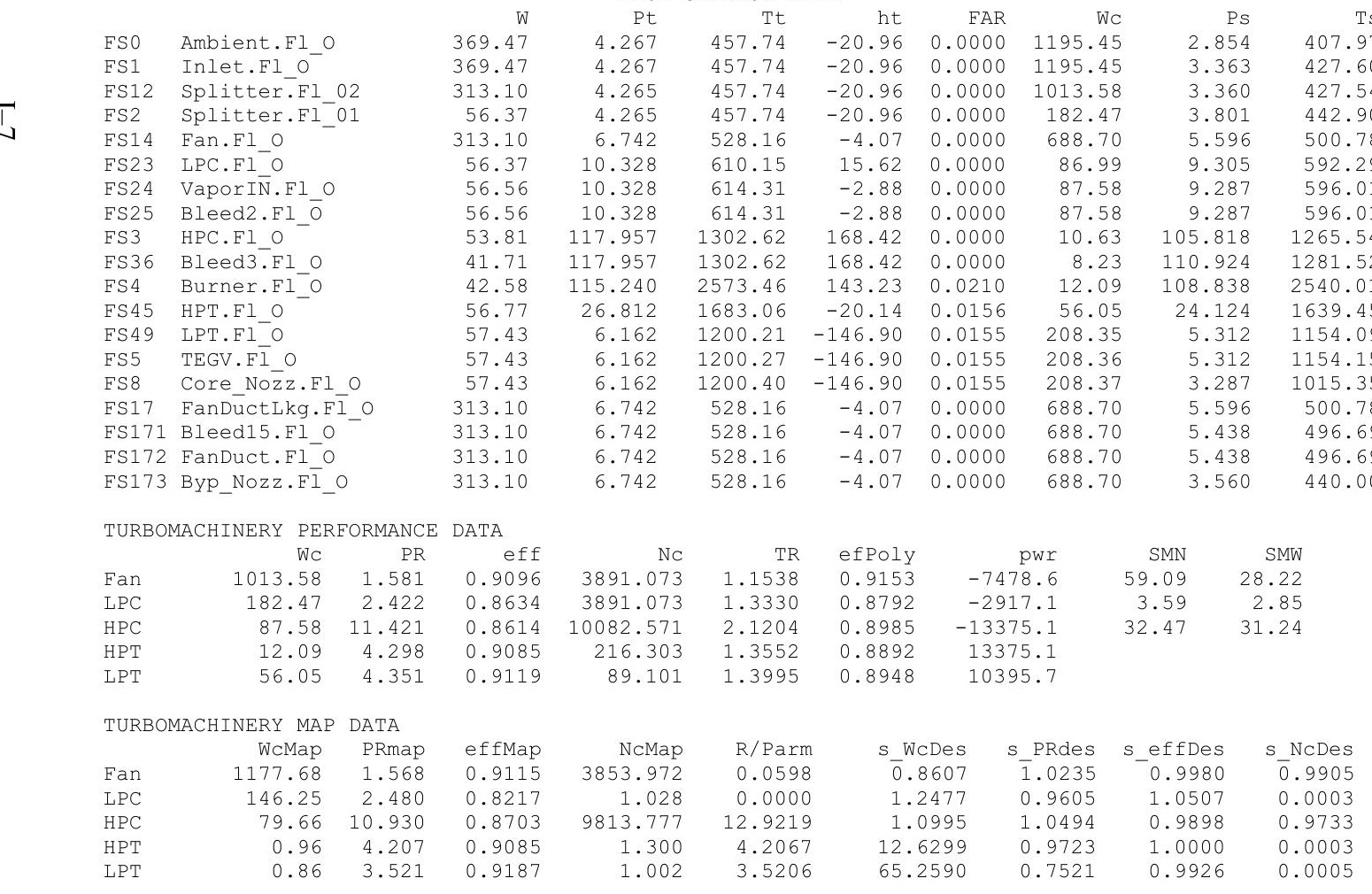




\begin{tabular}{|c|c|c|c|c|c|c|c|c|}
\hline $\begin{array}{l}===\text { INLETS }==== \\
\text { Inlet }\end{array}$ & $\begin{array}{r}\text { eRam } \\
1.0000\end{array}$ & $\begin{array}{r}\text { Afs } \\
3646.93\end{array}$ & $\begin{array}{r}\text { Fram } \\
8872.5\end{array}$ & \multicolumn{2}{|c|}{ BLEEDS - interstg } & $\begin{array}{l}\text { Wb/Win } \\
0.0368\end{array}$ & $\begin{array}{r}\text { BldWk } \\
0.5000\end{array}$ & $\begin{array}{r}\mathrm{Bld} \text { L } \\
0.220 \mathrm{C}\end{array}$ \\
\hline$====$ DUCTS $====$ & dPnorm & MN & Aphy & \multirow{2}{*}{$\begin{array}{l}\text { LPT_COOL } z \\
\text { WB2 } 2\end{array}$} & A HPC.C> & 0.0117 & 0.5000 & 0.4500 \\
\hline TEGV & 0.0000 & 0.4719 & 860.21 & & HPC.B $>$ & 0.0000 & 0.4500 & 0.2200 \\
\hline FanDuct & 0.0000 & 0.5625 & 2481.93 & WB2Y & HPC.B> & 0.0000 & 0.7600 & 0.62 \\
\hline & & & & \multirow{2}{*}{$\begin{array}{l}\text { WBA2X } \\
\text { WBLKG }\end{array}$} & HPC.B> & 0.0000 & 0.4500 & 0.2200 \\
\hline$==$ SPLITTERS $==$ & BPR & $\mathrm{dP} / \mathrm{P} 1$ & $\mathrm{dP} / \mathrm{P} 2$ & & HPC.1> & 0.0000 & 1.0000 & 1.00 \\
\hline Splitter & 5.5548 & 0.0005 & 0.0005 & WBW2X & HPC.B> & 0.0000 & 0.4500 & 0.22 \\
\hline$===$ SHAFT $S====$ & Nmech & trq in & pwr in & BLEEDS - & output & Wb/Win & hscale & Psc \\
\hline HP_Shaft & 10972.9 & 6401.9 & 13375.1 & WB17Y & Bleed> & 0.0000 & 1.0000 & 1.00 \\
\hline LP ${ }^{-}$Shaft & 3655.4 & 14936.8 & 10395.7 & HPT_COOLA & A Bleed> & 0.1142 & 1.0000 & \\
\hline & & & & \multirow{2}{*}{$\begin{array}{l}\mathrm{HPT}^{-} \mathrm{COOLB} \\
\text { WB } 3 \overline{\mathrm{X}}\end{array}$} & 3 Bleed> & 0.0999 & 1.0000 & 1.0 \\
\hline & & & & & Bleed> & 0.0000 & 1.0000 & 1.04 \\
\hline & & & & WBA3X & Bleed> & 0.0000 & 1.0000 & 1.0 \\
\hline & & & & WBW3X & Bleed> & 0.0000 & 1.0000 & 1.00 \\
\hline & & & & $\begin{array}{l}\text { WBEDLKG } \\
\text { WB1 } 5 \mathrm{X}\end{array}$ & FanDu> & 0.0000 & 1.0000 & 1.0 \\
\hline & & & & \multirow{2}{*}{$\begin{array}{l}\text { WB15X } \\
\text { WB15Y }\end{array}$} & Bleed> & 0.0000 & 1.0000 & 1. \\
\hline & & & & & Bleed> & 0.0000 & 1.0000 & 1.00 \\
\hline & & & & WB17X & Bleed> & 0.0000 & 1.0000 & 1.0 \\
\hline$===$ BURNERS $===$ & Ttout & eff & dPnorm & \multirow{2}{*}{\multicolumn{2}{|c|}{$\begin{array}{rr}\text { Wfuel } & \text { FAR } \\
0.87100 & 0.02096\end{array}$}} & & & \\
\hline Burner & 2573.44 & 0.9995 & 0.0230 & & & & & \\
\hline$===$ NOZZLES $S===$ & PR & $\mathrm{Cfg}$ & CdTh & & Ath & & \multirow{2}{*}{$\begin{array}{r}\text { Vact } \\
1515.2\end{array}$} & $\mathrm{Fo}$ \\
\hline Core_Nozz & 2.159 & 0.9815 & 1.0000 & & 613.40 & \multirow{2}{*}{$\begin{array}{l}\text { Minth } \\
1.000 \\
1.000\end{array}$} & & 2969.8 \\
\hline Byp $\bar{N} \circ z z$ & 2.362 & 0.9815 & 1.0000 & \multicolumn{2}{|c|}{$\begin{array}{lr}0.9800 & 613.40 \\
0.9800 & 2006.86\end{array}$} & & 1008.1 & 11227.9 \\
\hline
\end{tabular}




\section{$5 \mu \mathrm{m}, \mathrm{ISA}+36 \mathrm{R}$}

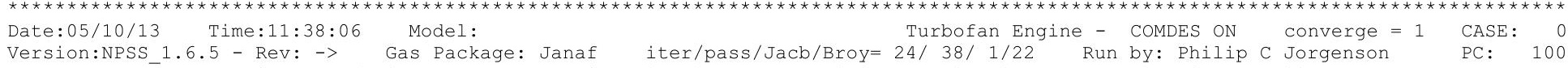

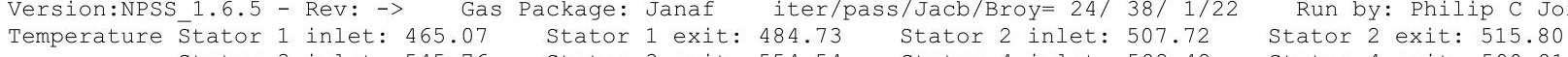

Stator 3 inlet: 545.76 Stator 3 exit: 554.54 stator 4 inlet: 582.42 Stator 2 exit: 590.81

$$
\text { Stator } 5 \text { inlet: } 612.50 \text { Stator } 5 \text { exit: } 620.61 \text { Unblocked Percent Blockage: } 0.00
$$

Ambient Relative Humidity

Fan Bypass Relative Humidity $\quad 5.44$

LPC Inlet Relative Humidity

LPC Exit Relative Humidity

HPC Relative Humidity

Drop Diameter

0.19

0.02

Velocity

0.0000050

Ambient Static Pressure

Ambient Static Temperature

89.49

425.97

Inlet Length $\quad 40.00$

Additional Water temperature

0.0055918

Fan/LPC Inlet Flow Velocity $\quad 491.33$

Fan/LPC Inlet Static Temperature 457.80

MN alt dTamb SUMMARY OUTPUT DATA
$0.780 \quad 39000.0 \quad 36.00$
W
387.42
Fn TSFC
Wfuel
4168.86
BPR
3204
VTAS
$\mathrm{OPR}$
2.228
EPR
1.708
Powerset
T4 T41
T 49

FLOW STATION DATA

\begin{tabular}{|c|c|}
\hline ESO & Ambient.Fl_o \\
\hline FS1 & Inlet.Fl_O \\
\hline FS12 & Splitter.Fl_02 \\
\hline FS2 & Splitter.FI_01 \\
\hline FS14 & Fan.FI_O \\
\hline FS23 & LPC.FI- O \\
\hline FS24 & VaporIN .FI_O \\
\hline FS25 & Bleed2.Fl $\bar{O}$ \\
\hline FS 3 & HPC.FI_O \\
\hline FS36 & Bleed3.Flo \\
\hline FS4 & Burner.Fl-O \\
\hline FS 45 & HPT.Fl_O \\
\hline FS49 & LPT.FI_O \\
\hline FS5 & TEGV.FI O \\
\hline FS 8 & Core Nozzz.F \\
\hline FS17 & FanDuctIkg.FI__o \\
\hline FS171 & Bleed15.Fl_o \\
\hline FS172 & FanDuct.FI_O \\
\hline FS173 & Byp Nozz.F̄ \\
\hline
\end{tabular}

$W$
387.42
387.42
326.13
61.30
326.13
61.30
61.64
61.64
58.65
45.45
46.61
62.08
62.80
62.80
62.80
326.13
326.13
326.13
326.13

$\begin{array}{rrrrrr}\text { W } & \text { Pt } & \text { Tt } & \text { ht } & \text { FAR } & \text { WC } \\ 387.42 & 4.267 & 477.92 & -16.44 & 0.0000 & 1280.92\end{array}$ $\begin{array}{llllll}387.42 & 4.267 & 477.92 & -16.44 & 0.0000 & 1280.92\end{array}$ $\begin{array}{lllll}4.265 & 477.92 & -16.44 & 0.0000 & 1078.79\end{array}$ $\begin{array}{lllll}4.265 & 477.92 & -16.44 & 0.0000 & 202.77\end{array}$ $\begin{array}{lllll}7.257 & 563.97 & 4.20 & 0.0000 & 688.63\end{array}$ $\begin{array}{lllll}10.700 & 650.42 & 25.00 & 0.0000 & 94.28 \\ 10.700 & 656.78 & -5.40 & 0.0000 & 95.27\end{array}$ $\begin{array}{lllll}10.700 & 656.78 & -5.40 & 0.0000 & 95.27 \\ 10.700 & 656.78 & -5.40 & 0.0000 & 95.27\end{array}$ $\begin{array}{llll}10.700 & 656.78 & -5.40 & 0.0000\end{array}$ $\begin{array}{llll}656.78 & -5.40 & 0.0000 & 95.27\end{array}$ $\begin{array}{rrrrr}137.506 & 1434.67 & 190.50 & 0.0000 & 10.43 \\ 137.506 & 1434.67 & 190.50 & 0.0000 & 8.08\end{array}$

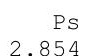
3.154 3.669 6.025 9.425
9.388 9.388 123.924 $\begin{array}{rrrrr}134.340 & 2921.68 & 159.09 & 0.0256 & 12.10 \\ 31.473 & 1923.93 & -26.47 & 0.0191 & 55.83\end{array}$ $\begin{array}{rrrrr}31.473 & 1923.93 & -26.47 & 0.0191 & 55.83 \\ 7.264 & 1383.50 & -172.77 & 0.0189 & 207.51\end{array}$ $\begin{array}{lllll}7.264 & 1383.50 & -172.77 & 0.0189 & 207.51 \\ 7.264 & 1383.57 & -172.77 & 0.0189 & 207.51\end{array}$ $\begin{array}{lllll}7.264 & 1383.57 & -172.77 & 0.0189 & 207.51 \\ 7.257 & 563.69 & -172.77 & 0.0189 & 207.52\end{array}$ $\begin{array}{rrrrr}7.257 & 563.97 & 4.20 & 0.0000 & 688.63\end{array}$ $\begin{array}{lllll}7.257 & 563.97 & 4.20 & 0.0000 & 688.63\end{array}$ $\begin{array}{lllll}7.257 & 563.97 & 4.20 & 0.0000 & 688.63\end{array}$ $\begin{array}{lllll}7.257 & 563.97 & 4.20 & 0.0000 & 688.63\end{array}$

TURBOMACHINERY PERFORMANCE DATA

$\begin{array}{rrr} & \text { WC } & \text { PR } \\ \text { Fan } & 1078.79 & 1.702 \\ \text { LPC } & 202.77 & 2.509 \\ \text { HPC } & 95.27 & 12.851 \\ \text { HPT } & 12.10 & 4.268 \\ \text { LPT } & 55.83 & 4.333\end{array}$

$\begin{array}{rr}\text { eff } & \text { NC } \\ 0.9114 & 4139.469 \\ 0.8319 & 4139.469 \\ 0.8589 & 10303.595 \\ 0.9122 & 214.505 \\ 0.9168 & 90.590\end{array}$

TR
1.1801
1.3609
2.1844
1.3415
1.3872


R/Parm
0.0639
0.0000
13.3993
4.1778
3.5068

ef
0.917
0.852
0.897
0.890

TURBOMACHINERY MAP DATA

$\begin{array}{lrrrrrrrrr} & \text { WcMap } & \text { PRmap } & \text { effMap } & \text { NcMap } & \text { R/Parm } & \text { S_WcDes } & \text { S_PRdes } & \text { S_effDes } & \text { S_NcDes } \\ \text { Fan } & 1253.45 & 1.686 & 0.9132 & 4100.000 & 0.0639 & 0.8607 & \overline{1} .0235 & 0.9980 & 0.9905 \\ \text { LPC } & 166.84 & 2.748 & 0.8341 & 1.093 & 0.0000 & 1.2153 & 0.8633 & 0.9974 & 0.0003 \\ \text { HPC } & 86.64 & 12.293 & 0.8678 & 10028.908 & 13.3993 & 1.0995 & 1.0494 & 0.9898 & 0.9733 \\ \text { HPT } & 0.96 & 4.178 & 0.9121 & 1.289 & 4.1778 & 12.6299 & 0.9723 & 1.0000 & 0.0003 \\ \text { LPT } & 0.86 & 3.507 & 0.9236 & 1.019 & 3.5068 & 65.2590 & 0.7521 & 0.9926 & 0.0005\end{array}$

Ts
425.97
438.33
439.19
457.80
534.77
627.35
632.82
632.82
1396.17
1412.70
2884.92
1875.95
1332.19
1332.26
1177.08
534.77
530.41
530.41
469.91
$\begin{array}{ll}4168.2 & 0.6713 \\ 3531.8 & 0.6634\end{array}$ $830.5 \quad 0.4683$ $\begin{array}{ll}8606.7 & 0.5227\end{array}$ $\begin{array}{lll}412.6 & 0.4299\end{array}$ $\begin{array}{lll}412.6 & 0.4368\end{array}$ $\begin{array}{lll}412.6 & 0.4368\end{array}$ $\begin{array}{lll}49.7 & 0.3944\end{array}$ $\begin{array}{ll}49.3 & 0.2965 \\ 74.6 & 0.3014\end{array}$ $\begin{array}{cc}74.6 & 0.3014 \\ 265.4 & 0.4014\end{array}$ $\begin{array}{ll}860.2 & 0.4726\end{array}$ $860.2 \quad 0.4726$ $\begin{array}{rr}613.4 & 1.0000 \\ 2606.7 & 0.5227\end{array}$ $\begin{array}{ll}2606.7 & 0.5227 \\ 2481.9 & 0.5626\end{array}$ $\begin{array}{ll}2481.9 & 0.5626 \\ 2481.9 & 0.5626\end{array}$ 2006.91 .0000

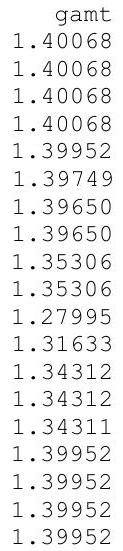

1.40068
1.40068
1.40068
1.40068
1.39952
1.39749
1.39650
1.39650
1.35306
1.35306
1.27995
1.31633
1.34312
1.34312
1.34311
1.39952
1.39952
1.39952
1.39952
WAR 0.0000851 0.0000851 0.0000851 0.0000851 0.0000851 0.0000851 0.0056769 0.0056769 0.0056769 0.0056769 0.0056769 0.0056769 0.0056769 0.0056769 0.0000851 0.0000851 0.0000851 0.0000851
Wair WH2O H2O frac $\begin{array}{lll}387.39 & 0.033 & 0.0001 \\ 387.39 & 0.033 & 0.0001\end{array}$ $\begin{array}{lll}387.39 & 0.033 & 0.0001 \\ 326.10 & 0.028 & 0.0001\end{array}$ $\begin{array}{lll}38.10 & 0.028 & 0.0001\end{array}$ $\begin{array}{lll}32.29 & 0.005 & 0.0001 \\ 26.10 & 0.028 & 0.0001\end{array}$ $\begin{array}{lll}61.29 & 0.005 & 0.0001\end{array}$ $\begin{array}{lll}61.29 & 0.348 & 0.0056\end{array}$ $\begin{array}{lll}61.29 & 0.348 & 0.0056\end{array}$ $\begin{array}{lll}58.32 & 0.331 & 0.0056\end{array}$ $\begin{array}{lll}58.32 & 0.331 & 0.0056\end{array}$ $\begin{array}{lll}45.20 & 0.257 & 0.0361\end{array}$ $\begin{array}{lll}60.58 & 0.344 & 0.0285\end{array}$ $\begin{array}{lll}61.29 & 0.348 & 0.0283\end{array}$ $\begin{array}{lll}61.29 & 0.348 & 0.0283 \\ 61.29 & 0.348 & 0.0283\end{array}$ $\begin{array}{lll}61.29 & 0.348 & 0.0283\end{array}$ $\begin{array}{lll}326.10 & 0.348 & 0.028-0.0283\end{array}$ $\begin{array}{lll}326.10 & 0.028 & 0.0001\end{array}$ $326.10=0.028-0.00001$ 
$\begin{array}{lrrr}===\text { INLETS }==== & \text { eRam } & \text { Afs } & \text { Fram } \\ \text { Inlet } & 1.0000 & 3907.84 & 9506.6\end{array}$

$====$ DUCTS $====$ dPnorm MN Aphy

$\begin{array}{llrr}\text { TEGV } & 0.0000 & 0.4726 & 860.21 \\ \text { FanDuct } & 0.0000 & 0.5626 & 2481.93\end{array}$

BPR
5.3204

$\mathrm{dP} / \mathrm{P} 1$

$\mathrm{dP} / \mathrm{P} 2$

Spler

Nmech

$=-$ SHAF

LP_Shaft

11594.5

trq in pwr

$7551.7 \quad 16671.1$

13120.2

$===$ BURNERS $===\begin{array}{r}\text { TtOut } \\ \text { Burner }\end{array}$
2921.68

eff

dPnorm

$$
\text { Burner }
$$$$
0.9995
$$$$
0.0230
$$

$\begin{array}{ll}\text { BLEEDS - interstg } & \text { Wb/Win } \\ \text { HPT_COOLC HPC.C }> & 0.0368 \\ \text { LPT COOLA HPC.C }> & 0.0117\end{array}$

$\begin{array}{lll}0.0368 & 0.5000 & 0.2200\end{array}$

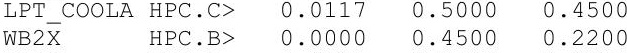

$\begin{array}{lllll}\text { WB2Y HPC.B } & 0.0000 & 0.7600 & 0.6200\end{array}$

$\begin{array}{lllll}\text { WBA2X } & \text { HPC.B } & 0.0000 & 0.4500 & 0.2200 \\ \text { WBLKG } & \text { HPC. } 1> & 0.0000 & 1.0000 & 1.0000\end{array}$

WBW2X HPC.B> $0.0000 \quad 0.4500 \quad 0.2200$

$\begin{array}{lcccc}\text { BLEEDS - output } & \text { Wb/Win } & \text { hscale } & \text { Pscale } \\ \text { WB17Y } & \text { Bleed }> & 0.0000 & 1.0000 & 1.0000\end{array}$

HPT_COOLA Bleed> $0.1142 \quad 1.0000 \quad 1.0000$

$\begin{array}{lllll}\text { HPT }^{-} \text {COOLB } & \text { Bleed> } & 0.0999 & 1.0000 & 1.0000 \\ \text { WB } 3 \bar{X} & \text { Bleed> } & 0.0000 & 1.0000 & 1.0000\end{array}$

$\begin{array}{lllll}\text { WB3X } & \text { Bleed }> & 0.0000 & 1.0000 & 1.0000 \\ \text { WBA3X } & \text { Bleed }> & 0.0000 & 1.0000 & 1.0000\end{array}$

WBW3X Bleed> $0.0000 \quad 1.0000 \quad 1.0000$

$\begin{array}{lllll}\text { WBFDLKG } & \text { FanDu> } & 0.0000 & 1.0000 & 1.0000 \\ \text { WB15X } & \text { Bleed }> & 0.0000 & 1.0000 & 1.0000\end{array}$

WB15X Bleed> $0.0000 \quad 1.0000 \quad 1.0000$

$\begin{array}{lllll}\text { WB15Y } & \text { Bleed }> & 0.0000 & 1.0000 & 1.0000 \\ \text { WB17X } & \text { Bleed }> & 0.0000 & 1.0000 & 1.0000\end{array}$

$===$ NOZZLES $===$

Core Nozz

$P R$
2.545
2.543

Cfg
0.9809
0.9810

CdTh
1.0000

1.0000
1.0000

Wfuel

.

\section{$\begin{array}{rl}\mathrm{CV} & \text { Ath } \\ 0.9800 & 613.40\end{array}$} 0.9800

613.40 $\begin{array}{rrr}\text { MNth } & \text { Vact } & \text { Fg } \\ 1.000 & 1624.7 & 3806.9\end{array}$

$\begin{array}{rrr}1.000 & 1624.7 & 3806.9 \\ 1.000 & 1041.7 & 12523.7\end{array}$

$\begin{array}{rrrr}\text { W } & \text { Tt } & \text { ht } & \text { Pt } \\ 2.2684 & 1054.43 & 92.55 & 31.473 \\ 0.7212 & 1054.43 & 92.55 & 7.264 \\ 0.0000 & 1015.39 & 82.75 & 38.597 \\ 0.0000 & 1254.40 & 143.48 & 89.320 \\ 0.0000 & 1015.39 & 82.75 & 38.597 \\ 0.0000 & 1434.67 & 190.50 & 137.506 \\ 0.0000 & 1015.39 & 82.75 & 38.597 \\ \text { W } & \text { Tt } & \text { ht } & \text { Pt } \\ 0.0000 & 656.78 & -5.40 & 10.700 \\ 7.0381 & 1434.67 & 190.50 & 134.340 \\ 6.1585 & 1434.67 & 190.50 & 75.500 \\ 0.0000 & 1434.67 & 190.50 & 137.506 \\ 0.0000 & 1434.67 & 190.50 & 137.506 \\ 0.0000 & 1434.67 & 190.50 & 137.506 \\ 0.0000 & 563.97 & 4.20 & 7.257 \\ 0.0000 & 563.97 & 4.20 & 7.257 \\ 0.0000 & 563.97 & 4.20 & 7.257 \\ 0.0000 & 563.97 & 4.20 & 7.257\end{array}$

Pt

7.264
38.597 3.597
38.506 8.597 340 137.506 137.506 7.257 7.257 
Date:05/10/13 Time:11:38:42 Model: Turbofan Engine - COMDES ON Converge = 1 CASE: 0

Version:NPSS 1.6.5 - Rev: -> Gas Package: Janaf iter/pass/Jacb/Broy= 20/34/1/18 Run by: Philip C Jorgenson PC: 84

Temperature Stator 1 inlet: 470.84 Stator 1 exit: 489.03 Stator 2 inlet: 510.79 Stator 2 exit: 519.44 $\begin{array}{lll}\text { Stator } 3 \text { inlet: } 546.19 & \text { Stator } 3 \text { exit: } 555.04 & \text { Stator } 4 \text { inlet: } 580.14 \text { Stator } 4 \text { exit: } 588.35 \\ \text { Stator } 5 \text { inlet: } 608.21 & \text { Stator } 5 \text { exit: } 616.00 & \text { Unblocked Percent Blockage: } 0.00\end{array}$

Ambient Relative Humidity $\quad 10.00$

Fan Face Relative Humidity $\quad 4.46$

Fan Bypass Relative Humidity 0.23

LPC Inlet Relative Humidity 2.21

LPC Exit Relative Humidity $\quad 0.03$

HPC Relative Humidity

0.01

Drop Diameter

0.0000050

000050 Inlet Length

40.00

Ambient Static Pressure

Ambient Static Temperature
Additional Water at LPC Exit

2.85 Fan/LPC Inlet Flow Velocity 453.72

$\begin{array}{lrr}42.85 & \text { Fan/LPC Inlet Static Pressure } & 3.75 \\ 425.97 & \text { Fan/LPC Inlet Static Temperature } & 460.77\end{array}$ 
$\begin{array}{lrrr}===\text { INLETS }==== & \text { eRam } & \text { Afs } & \text { Fram } \\ \text { Inlet } & 1.0000 & 3751.73 & 9126.8\end{array}$

$====$ DUCTS $====$ dPnorm MN Aphy

$\begin{array}{llrr}\text { TEGV } & 0.0000 & 0.4723 & 860.21 \\ \text { FanDuct } & 0.0000 & 0.5626 & 2481.93\end{array}$

Splitter

BPR
5.4660

LP_Shaft

11368.0

$\mathrm{dP} / \mathrm{P} 1$

$\mathrm{dP} / \mathrm{P} 2$

$0.0005-0.0005$

trq in pwr in

$6847.7 \quad 14821.5$

11596.0

$===$ BURNERS $===\begin{array}{r}\text { TtOut } \\ \text { Burner }\end{array}$
2769.97

eff

dPnor

$===$ NOZZLES $===$

Core Nozz

PR
2.314
2.435

Cfg
0.9816
0.9814

CdTh

1.0000

0.9800
0.9800

BLEEDS - interstg Wb/Win BldWk

HPT COOLC HPC.C $>0.0368$

$\begin{array}{lllll}\text { LPT_COOLA HPC.C> } & 0.0117 & 0.5000 & 0.4500 \\ \text { WB } 2 \overline{\mathrm{X}} & 0.000 & 0.4200\end{array}$

$\begin{array}{lllll}\text { WB2X } & \text { HPC.B } & 0.0000 & 0.4500 & 0.2200 \\ \text { WB2Y } & \text { HPC.B } & 0.0000 & 0.7600 & 0.6200\end{array}$

$\begin{array}{lllll}\text { WBA2X } & \text { HPC.B> } & 0.0000 & 0.4500 & 0.2200 \\ \text { WBLKG } & \text { HPC. I> } & 0.0000 & 1.0000 & 1.0000\end{array}$

$\begin{array}{lllll}\text { WBW2X HPC.B> } & 0.0000 & 0.4500 & 0.2200\end{array}$

BLEEDS - output Wb/Win hscale Pscale

WB17Y Bleed> $0.0000 \quad 1.0000 \quad 1.0000$

HPT_COOLA Bleed> $0.1142 \quad 1.0000 \quad 1.0000$

$\begin{array}{lllll}\text { HPT COOLB } & \text { Bleed> } & 0.0999 & 1.0000 & 1.0000 \\ \text { WB } 3 \bar{X} & \text { Bleed> } & 0.0000 & 1.0000 & 1.0000\end{array}$

$\begin{array}{lllll}\text { WB3X } & \text { Bleed> } & 0.0000 & 1.0000 & 1.0000 \\ \text { WBA3X } & \text { Bleed> } & 0.0000 & 1.0000 & 1.0000\end{array}$

$\begin{array}{lllll}\text { WBA3X } & \text { Bleed> } & 0.0000 & 1.0000 & 1.0000 \\ \text { WBW3X } & \text { Bleed> } & 0.0000 & 1.0000 & 1.0000\end{array}$

$\begin{array}{lllll}\text { WBW3X } & \text { Bleed> } & 0.0000 & 1.0000 & 1.0000 \\ \text { WBFDLKG } & \text { FanDu> } & 0.0000 & 1.0000 & 1.0000\end{array}$

$\begin{array}{lllll}\text { WBFDLKG } & \text { FanDu> } & 0.0000 & 1.0000 & 1.0000 \\ \text { WB15X } & \text { Bleed }> & 0.0000 & 1.0000 & 1.0000\end{array}$

WB17X Bleed> $0.0000 \quad 1.0000 \quad 1.0000$

Wfuel FAR

$.99416 \quad 0.02344$

$\begin{array}{rrrr}\text { W } & \text { Tt } & \text { ht } & \text { Pt } \\ 2.1298 & 1026.69 & 82.75 & 28.636 \\ 0.6771 & 1026.69 & 82.75 & 6.605 \\ 0.000 & 989.63 & 73.47 & 35.832 \\ 0.0000 & 1216.73 & 130.98 & 81.822 \\ 0.0000 & 989.63 & 73.47 & 35.832 \\ 0.0000 & 1388.23 & 175.50 & 125.513 \\ 0.0000 & 989.63 & 73.47 & 35.832 \\ & & & \\ W & \text { Tt } & \text { ht } & \mathrm{Pt} \\ 0.000 & 649.67 & -10.01 & 10.537 \\ 6.0081 & 1388.23 & 175.50 & 122.622 \\ 5.7822 & 1388.23 & 175.50 & 68.862 \\ 0.0000 & 1388.23 & 175.50 & 125.513 \\ 0.0000 & 1388.23 & 175.50 & 125.513 \\ 0.0000 & 1388.23 & 175.50 & 125.513 \\ 0.0000 & 556.40 & 2.39 & 6.950 \\ 0.0000 & 556.40 & 2.39 & 6.950 \\ 0.0000 & 556.40 & 2.39 & 6.950 \\ 0.0000 & 556.40 & 2.39 & 6.950\end{array}$

1.822

.513

0.537

8. 862

125.513

6.950

6.950

6.950 
Date:05/10/13 Time:11:39:17 Model: Turbofan Engine - COMDES ON COnverge = 1 CASE: 0

Version:NPSS 1.6.5 - Rev: -> Gas Package: Janaf iter/pass/Jacb/Broy= 20/34/1/18 Run by: Philip C Jorgen

Temperature Stator 1 inlet: 472.55 Stator 1 exit: 490.30 Stator 2 inlet: 511.72 Stator 2 exit: 520.48 $\begin{array}{llll}\text { Stator } 3 \text { inlet: } 546.36 & \text { Stator } 3 \text { exit: } 555.19 & \text { Stator } 4 \text { inlet: } 579.49 & \text { Stator } 4 \text { exit: } 587.65 \\ \text { Stator } 5 \text { inlet: } 606.98 & \text { Stator } 5 \text { exit: } 614.67 & \text { Unblocked Percent Blockage: } 0.00\end{array}$

Ambient Relative Humidity $\quad 10.00$

Fan Face Relative Humidity $\quad 4.21$

Fan Bypass Relative Humidity 0.25

LPC Inlet Relative Humidity 2.13

LPC Exit Relative Humidity 0.03

HPC Relative Humidity

Drop Diameter

0.0000050

Inlet Ienth

40.00

Ambient Flow Velocity

Ambient Static Pressure

\begin{tabular}{rrr}
2.85 & Fan/LPC Inlet Flow Velocity & 440.91 \\
\hline & Fan/LPC Inlet Static Pressure & 3.78
\end{tabular}

Ambient Static Temperature $\quad 425.97 \quad$ Fan/LPC Inlet Static Temperature 461.72

\section{SUMMARY OUTPUT DATA}

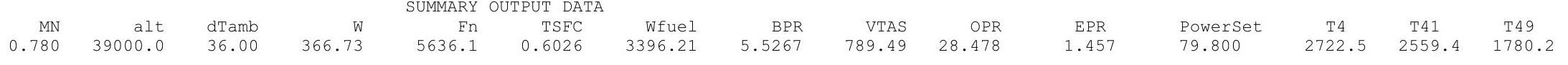

\begin{tabular}{|c|c|}
\hline FSO & Ambient.FI_O \\
\hline 51 & Inlet.FI_- \\
\hline FS12 & Splitter.Fl_02 \\
\hline FS2 & Splitter.Fl_01 \\
\hline FS14 & Fan.Fl_o \\
\hline FS23 & LPC.FI_O \\
\hline FS24 & VaporIN.FI_O \\
\hline FS25 & Bleed2.FI_-̄ \\
\hline FS3 & HPC.FI_O \\
\hline FS36 & Bleed3.FI_O \\
\hline ES4 & Burner.FI_O \\
\hline ES45 & HPT.FI_O \\
\hline FS 49 & LPT.FI_O \\
\hline FS5 & TEGV.F̄ \\
\hline FS8 & Core_Nozz.Fl_ \\
\hline FS17 & FanDūctLkg.Fì \\
\hline FS171 & Bleed15.FI_o \\
\hline FS172 & FanDuct.Fl- \\
\hline ES173 & Byp_Nozz.FI__ \\
\hline
\end{tabular}

\begin{tabular}{|c|c|c|c|c|c|}
\hline W & $\begin{array}{c}\text { FLOW } \\
\text { Pt }\end{array}$ & $\begin{array}{l}\text { TATION } \\
\text { Tt }\end{array}$ & ATA & FAR & \\
\hline 66.73 & 4.267 & 477.92 & -16.44 & 0.0000 & 21 \\
\hline 66.73 & 4.267 & 477.92 & -16.44 & 0.0000 & 1212. \\
\hline 310.54 & 4.265 & 477.92 & -16.44 & 0.0000 & 1027.2 \\
\hline 56.19 & 4.265 & 477.92 & -16.44 & 0.0000 & 185. \\
\hline 310.54 & 6.848 & 553.91 & 1.79 & 0.0000 & 688 \\
\hline 56.19 & 10.470 & 640.36 & 22.58 & 0.0000 & 87.6 \\
\hline 54 & 10.4 & 647. & -11.63 & 0.0 & \\
\hline 56.54 & 10.470 & 647.64 & -11.63 & 0.0 & 88.6 \\
\hline 53.80 & 121.507 & 1373.71 & 170.60 & 0.0 & 10.5 \\
\hline 41.70 & 121.507 & 1373.71 & 170.60 & 0.0000 & 8.2 \\
\hline 42.64 & 118.709 & 2722.47 & 143.23 & 0.0228 & 12.0 \\
\hline 56.82 & 27.696 & 1788.41 & -30.20 & 0.0 & 55.9 \\
\hline 57.49 & 6.387 & 1280.76 & -165.54 & 0.0168 & 207.8 \\
\hline 57.49 & 6 & 1280.82 & -165.54 & 0.0168 & 07 \\
\hline 57.49 & 6.387 & 1280.95 & -165.54 & 0.0168 & 207.8 \\
\hline 310.54 & 6.848 & 553.91 & 1.79 & 0.0000 & \\
\hline 4 & 6.848 & 553.91 & 1.79 & 0.0000 & 688 \\
\hline 310.54 & 6.848 & 553.91 & 1.79 & 0.0000 & \\
\hline 310.54 & 6.848 & 553.91 & 1.79 & 0.0000 & 688. \\
\hline
\end{tabular}

$\begin{array}{rr}\text { Ps } & \text { Ts } \\ 2.854 & 425.97 \\ 3.326 & 445.04 \\ 3.324 & 445.05 \\ 3.780 & 461.72 \\ 5.685 & 525.22 \\ 9.416 & 621.30 \\ 9.382 & 627.76 \\ 9.382 & 627.76 \\ 109.067 & 1335.19 \\ 114.299 & 1351.77 \\ 112.117 & 2687.59 \\ 24.922 & 1742.75 \\ 5.508 & 1232.31 \\ 5.508 & 1232.37 \\ 3.413 & 1086.26 \\ 5.685 & 525.22 \\ 5.525 & 520.94 \\ 5.525 & 520.94 \\ 3.617 & 461.51\end{array}$

$\begin{array}{rr}\text { Aphy } & \text { MN } \\ 3699.1 & 0.7800 \\ 4168.2 & 0.6072 \\ 3531.8 & 0.6071 \\ 830.5 & 0.4184 \\ 2606.7 & 0.5226 \\ 412.6 & 0.3925 \\ 412.6 & 0.3994 \\ 412.6 & 0.3994 \\ 49.7 & 0.4016 \\ 49.3 & 0.3014 \\ 74.6 & 0.3004 \\ 265.4 & 0.4018 \\ 860.2 & 0.4722 \\ 860.2 & 0.4722 \\ 613.4 & 1.0000 \\ 2606.7 & 0.5226 \\ 2481.9 & 0.5626 \\ 2481.9 & 0.5626 \\ 2006.9 & 1.0000\end{array}$

gamt
1.40068
1.40068
1.40068
1.40068
1.39969
1.39778
1.39668
1.39668
1.35643
1.35643
1.28726
1.32336
1.35064
1.35063
1.35062
1.39969
1.39969
1.39969
1.39969

WAR
0.0000851
0.0000851
0.0000851
0.0000851
0.0000851
0.0000851
0.0063824
0.0063824
0.0063824
0.0063824
0.0063824
0.0063824
0.0063824
0.0063824
0.0063824
0.0000851
0.0000851
0.0000851
0.0000851

$\begin{array}{rrr}\text { Wair } & \text { WH2O } & \text { H2O frac } \\ 366.70 & 0.031 & 0.0001 \\ 366.70 & 0.031 & 0.0001 \\ 310.51 & 0.026 & 0.0001 \\ 56.18 & 0.005 & 0.0001 \\ 310.51 & 0.026 & 0.0001 \\ 56.18 & 0.005 & 0.0001 \\ 56.18 & 0.359 & 0.0063 \\ 56.18 & 0.359 & 0.0063 \\ 53.46 & 0.341 & 0.0063 \\ 41.43 & 0.264 & 0.0063 \\ 41.43 & 0.264 & 0.0335 \\ 55.53 & 0.354 & 0.0267 \\ 56.18 & 0.359 & 0.0265 \\ 56.18 & 0.359 & 0.0265 \\ 56.18 & 0.359 & 0.0265 \\ 310.51 & 0.026 & 0.0001 \\ 310.51 & 0.026 & 0.0001 \\ 310.51 & 0.026 & 0.0001 \\ 310.51 & 0.026 & 0.0001\end{array}$

TURBOMACHINERY PERFORMANCE DATA

\begin{tabular}{|c|c|c|c|c|c|c|c|c|c|}
\hline & Wc & PR & eff & $\mathrm{NC}$ & $\mathrm{TR}$ & efPoly & pwr & SMN & SMW \\
\hline Fan & 1027.24 & 1.606 & 0.9118 & 3938.129 & 1.1590 & 0.9175 & $\begin{array}{r}\text { pwr } \\
3009.1\end{array}$ & 56.02 & 27.11 \\
\hline LPC & 185.87 & 2.455 & 0.8600 & 3938.129 & 1.3399 & 0.8764 & -3101.8 & 3.20 & 2.45 \\
\hline HPC & 88.68 & 11.605 & 0.8614 & 10111.890 & 2.1211 & 0.8985 & $\begin{array}{r}-314224.8 \\
-1422.3\end{array}$ & 31.85 & 30.67 \\
\hline HPT & 12.09 & 4.286 & 0.9102 & 216.556 & 1.3491 & 0.8899 & 142243 & & \\
\hline LPT & 55.99 & 4.336 & 0.9143 & 89.390 & 1.3934 & 0.8977 & 11110.9 & & \\
\hline \multirow{2}{*}{\multicolumn{3}{|c|}{ TURBOMACHINERY MAP DF }} & & & & \multirow{3}{*}{ s_WcDes } & & & \\
\hline & & & effMap & NcMap & R/Parm & & s PRdes & $s$ effDes & s NCDes \\
\hline Fan & 1193.55 & 1.592 & 0.9137 & 3900.579 & 0.0606 & & $\overline{1} .0235$ & 0.9980 & $\overline{0} .9905$ \\
\hline LPC & 149.29 & 2.529 & 0.8227 & 1.040 & 0.0000 & $\overline{0} .8607$ & 0.9519 & 1.0454 & 0.0003 \\
\hline HPC & 80.65 & 11.105 & 0.8703 & 9842.314 & 12.9732 & 1.0995 & 1.0494 & 0.9898 & 0.9733 \\
\hline $\mathrm{HPT}$ & 0.96 & 4.195 & 0.9102 & 1.301 & 4.1951 & & 0.9723 & 1.0000 & 0.0003 \\
\hline LPT & 0.86 & 3.509 & 0.9211 & 1.006 & 3.5093 & $\begin{array}{l}12.6299 \\
65.2590\end{array}$ & 0.7521 & 0.9926 & 0.0005 \\
\hline
\end{tabular}




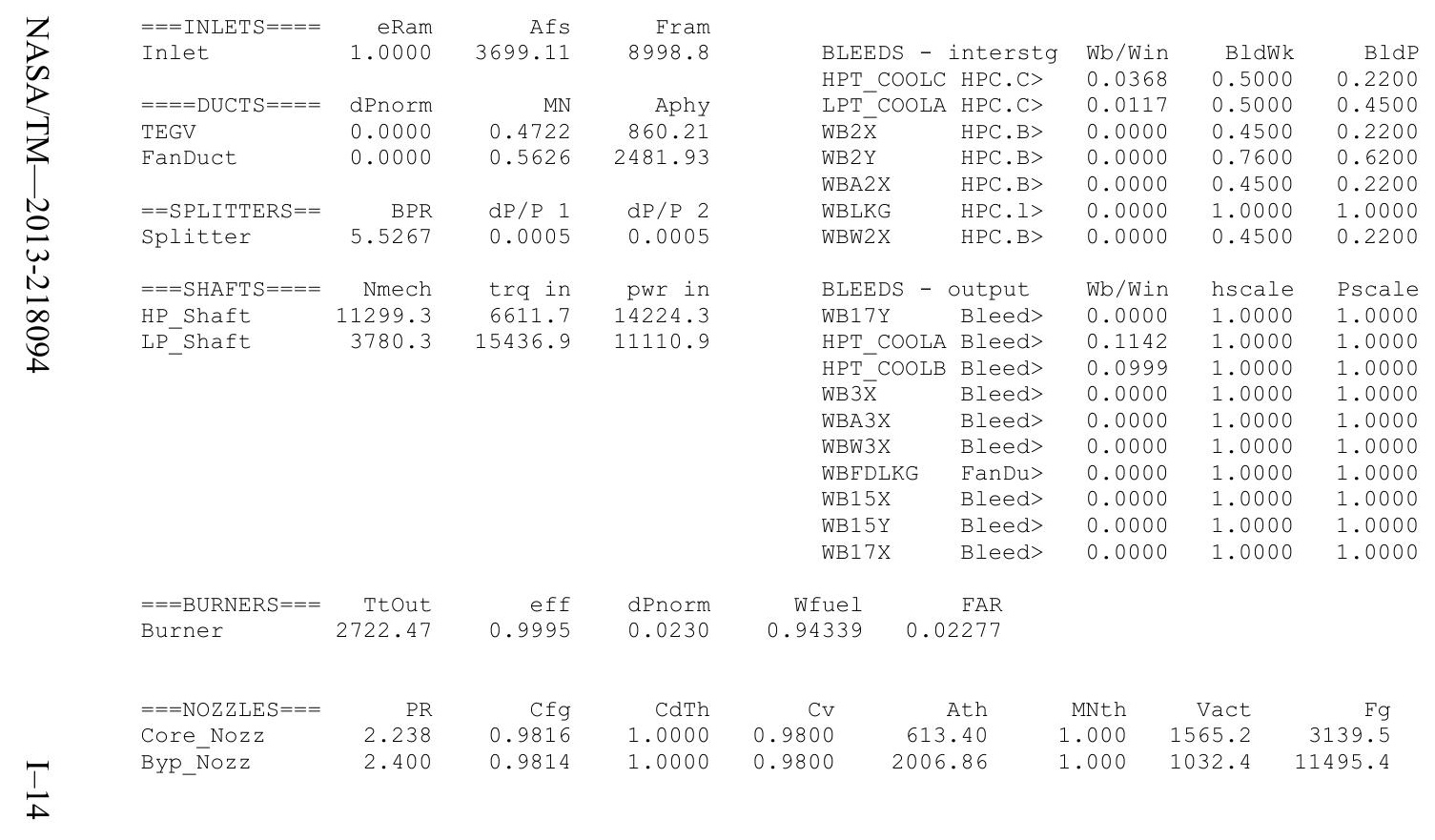


Date:05/10/13 Time:11:39:52 Model:

Turbofan Engine - COMDES ON converge $=1$ CASE: 0

Version:NPSS 1.6.5 - Rev: -> Gas Package: Janaf iter/pass/Jacb/Broy= 19/33/1/17 Run by: Philip C Jorgenson PC: 76

Temperature Stator 1 inlet: 473.71 Stator 1 exit: 491.17 Stator 2 inlet: 512.37 Stator 2 exit: 521.20

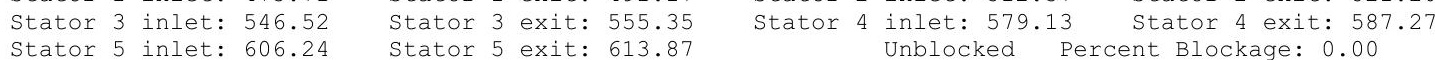

Ambient Relative Humidity $\quad 10.00$

Fan Face Relative Humidity $\quad 4.05$

Fan Bypass Relative Humidity $\quad 0.26$

$\begin{array}{ll}\text { LPC Inlet Relative Humidity } & 2.08 \\ \text { LPC Exit Relative Humidity } & 0.03\end{array}$

HPC Exit Relative Humidity

Drop Dative Humidity

0.01

0.0000050

Ambient Flow Velocity

Ambient static Pressure

Additional Water at LPC Exit

Inlet Length

40.00

2.85 Fan/LPC Inlet Flow Velocity 431.80 0.0064518

$\begin{array}{lr}\text { Fan/LPC Inlet Static Pressure } & 3.80 \\ \text { Fan/LPC Inlet Static Temperature } & 462.38\end{array}$

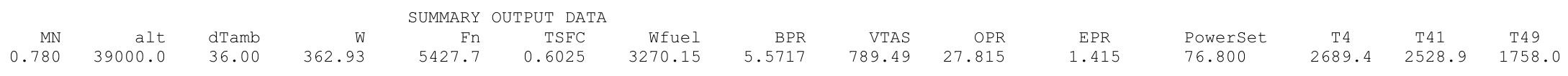

W FLOW STATION DATA

FS0 Ambient.Fl_

FS1 Inlet.Fl_O

FS14 Fan.FI_

FS24 VaporIñ.FI

FS25 Bleed2.FI_O

FS3 HPC.FI_O

FS36 Bleed3.FI_O

FS4 Burner.FI

FS49 4 TPT. F1-

FS5 5 LPT.FI-O

FS8 Core_Nozz.Fl_O

FS17 FanDuctLkg.Fi_O

FS171 Bleed15.FI_O

FS172 FanDuct.Fl_o

FS173 Byp Nozz.FI

\subsection{3}

$\begin{array}{ccc}\text { Pt } & \text { Tt } \\ 4.267 & 477.92 & -16 . \\ 4.267 & 477.92 & -16 .\end{array}$

(5)

$\begin{array}{rrrrrr}307.70 & 4.265 & 477.92 & -16.44 & 0.0000 & 1017.85 \\ 55.23 & 4.265 & 477.92 & -16.44 & 0.0000 & 182.68\end{array}$

$\begin{array}{rrrrrr}55.23 & 4.265 & 477.92 & -16.44 & 0.0000 & 1017.85 \\ 307.70 & 6.775 & 552.16 & 1.37 & 0.0000 & 182.68\end{array}$

$\begin{array}{rrrrrr}55.23 & 10.425 & 638.89 & 22.22 & 0.0000 & 86.40\end{array}$

$\begin{array}{rrrrrr}55.23 & 10.425 & 638.89 & 22.22 & 0.0000 & 86.40 \\ 55.58 & 10.425 & 646.36 & -12.81 & 0.0000 & 87.47\end{array}$

$\begin{array}{llllll}55.58 & 10.425 & 646.36 & -12.81 & 0.0000 & 87.47 \\ 55.58 & 10.425 & 646.36 & -12.81 & 0.0000 & 87.47\end{array}$

$\begin{array}{llllll}52.89 & 118.679 & 1363.70 & 167.12 & 0.0000\end{array}$

$\begin{array}{lllll}40.99 & 118.679 & 1363.70 & 167.12 & 0.0000\end{array}$

$\begin{array}{rrrrr}41.89 & 115.946 & 2689.43 & 140.40 & 0.0223\end{array}$

56.49

56.49

56.49

56.49

307.70
307.70
307.70

307.70

6.233
6.233

$\begin{array}{llll}6.233 & 1263.92 & -164.52 & 0.0164\end{array}$

$\begin{array}{llll}6.233 & 1263.99 & -164.52 & 0.0164 \\ 6.233 & 1264.12 & -164.52 & 0.0164\end{array}$

$\begin{array}{rrrr}6.233 & 1264.12 & -164.52 & 0.0164 \\ 6.775 & 552.16 & 1.37 & 0.0000\end{array}$

$\begin{array}{llll}6.775 & 552.16 & 1.37 & 0.0000 \\ 6.775 & 552.16 & 1.37 & 0.0000\end{array}$

$\begin{array}{llll}6.775 & 552.16 & 1.37 & 0.0000\end{array}$

$1.37 \quad 0.0000$

9.375
-9.16

$\begin{array}{lll}10.62 & 106.450 & 1325.14\end{array}$

$\begin{array}{rrr}8.23 & 111.596 & 1341.76 \\ 12.09 & 109.507 & 2654.85 \\ 56.01 & 24.323 & 1720.82\end{array}$

$\begin{array}{rrr}56.01 & 24.323 & 1720.82\end{array}$

207.91

$\begin{array}{ll}5.375 & 1215.96 \\ 5.375 & 1216.02\end{array}$

$\begin{array}{rr}5.330 & 1071.44 \\ 5.624 & 523.55\end{array}$

688.6

$\begin{array}{ll}5.624 & 523.55 \\ 5.465 & 519.29\end{array}$

$\begin{array}{ll}5.465 & 519.29\end{array}$

688.66

3.578

460.04

$\begin{array}{rr}\text { Aphy } & \text { MN } \\ 3660.7 & 0.7800 \\ 4168.2 & 0.5966 \\ 3531.8 & 0.5978 \\ 830.5 & 0.4095 \\ 2606.7 & 0.5226 \\ 412.6 & 0.3859 \\ 412.6 & 0.3927 \\ 412.6 & 0.3927 \\ 49.7 & 0.4029 \\ 49.3 & 0.3023 \\ 74.6 & 0.3002 \\ 265.4 & 0.4019 \\ 860.2 & 0.4722 \\ 860.2 & 0.4722 \\ 613.4 & 1.0000 \\ 2606.7 & 0.5226 \\ 2481.9 & 0.5626 \\ 2481.9 & 0.5626 \\ 2006.9 & 1.0000\end{array}$

gamt
1.40068
1.40068
1.40068
1.40068
1.39972
1.39782
1.39669
1.39669
1.35699
1.35699
1.28848
1.32457
1.35191
1.35190
1.35189
1.39972
1.39972
1.39972
1.39972

WAR
0.0000851

Wair $\begin{array}{ll}0.0000851 & 362.90 \\ 0.0000851 & 307.67\end{array}$ 0.0000851 307.67
55.22 $\begin{array}{lll}0.0000851 & 55.22 & 0.005\end{array}$

0.0065369 55.22
55.22

$\begin{array}{lll}0.0065369 & 55.22 & 0.361 \\ 0.0065369-52.54 & 0.361\end{array}$

0.0065369

52.54

$\begin{array}{ll}40.72 & 0.266\end{array}$

$\begin{array}{ll}54.57 & 0.357\end{array}$

$\begin{array}{lll}55.22 & 0.361\end{array}$

065369

0.0065369

0.0065369

55.22
55.22

0.361
0.361

TURBOMACHINERY PERFORMANCE DATA

$\begin{array}{lrrrrrrrrr} & \text { Wc } & \text { PR } & \text { eff } & \text { NC } & \text { TR } & \text { efPoly } & \text { pwr } & \text { SMN } & \text { SMW } \\ \text { Fan } & 1017.85 & 1.589 & 0.9108 & 3905.459 & 1.1553 & 0.9164 & -7752.1 & 58.14 & 27.87 \\ \text { LPC } & 182.68 & 2.445 & 0.8631 & 3905.459 & 1.3368 & 0.8791 & -3021.0 & 3.47 & 2.76 \\ \text { HPC } & 87.47 & 11.384 & 0.8614 & 10078.845 & 2.1098 & 0.8983 & -13806.9 & 32.74 & 31.50 \\ \text { HPT } & 12.09 & 4.289 & 0.9097 & 216.957 & 1.3504 & 0.8898 & 13806.9 & & \\ \text { LPT } & 56.01 & 4.337 & 0.9138 & 89.207 & 1.3944 & 0.8971 & 10773.1 & \end{array}$

TURBOMACHINERY MAP DATA

\begin{tabular}{|c|c|c|c|c|c|c|c|c|c|}
\hline & WcMar & PRmap & effMap & NcMap & R/Parm & s WCDes & s PRdes & $s$ effDes & S NcDes \\
\hline an & 1182.64 & 1.575 & 0.9126 & 3868.221 & 0.0600 & $\overline{0} .8607$ & $\overline{1} .0235$ & 0.9980 & $\overline{0} .9905$ \\
\hline & 147.18 & 2.495 & 0.8220 & 1.032 & & 1. & 0.9662 & 1.0500 & \\
\hline${ }^{\mathrm{C}} \mathrm{C}$ & 79.55 & 10.895 & 0.8703 & 9810.149 & 12.8977 & 1.0995 & 1.0494 & 0.9898 & 0.9733 \\
\hline & 0.96 & & & & & & 3 & & \\
\hline PT & 0.86 & 3.510 & 0.9206 & 1.003 & 3.5098 & 652590 & 0.7521 & 0.9926 & 0.0005 \\
\hline
\end{tabular}




\begin{tabular}{|c|c|c|c|c|c|c|c|c|}
\hline \multirow{2}{*}{$\begin{array}{ll}Z & ===\text { INLETS }==== \\
& \text { Inlet }\end{array}$} & eRam & Afs & Fram & \multirow{2}{*}{\multicolumn{2}{|c|}{ BLEEDS - interstg }} & \multirow[b]{2}{*}{ Wb/Win } & \multirow[b]{2}{*}{ BldWk } & \multirow[b]{2}{*}{$\begin{array}{r}\text { BldP } \\
0.2200\end{array}$} \\
\hline & 1.0000 & 3660.74 & 8905.5 & & & & & \\
\hline$====$ DUCTS $====$ & dPnorm & MN & Aphy & LPT $\mathrm{COOLA}$ & A HPC.C> & 0.0117 & 0.5000 & 0.4500 \\
\hline TEGV & 0.0000 & 0.4722 & 860.21 & WB2 $2 \bar{x}$ & HPC.B> & 0.0000 & 0.4500 & 0.2200 \\
\hline FanDuct & 0.0000 & 0.5626 & 2481.93 & WB2Y & HPC.B> & 0.0000 & 0.7600 & 0.6200 \\
\hline & & & & WBA2X & $\mathrm{HPC} \cdot \mathrm{B}>$ & 0.0000 & 0.4500 & 0.2200 \\
\hline$==$ SPLITTERS $==$ & BPR & $\mathrm{dP} / \mathrm{P} 1$ & $\mathrm{dP} / \mathrm{P} 2$ & WBLKG & HPC.1> & 0.0000 & 1.0000 & 1.0000 \\
\hline Splitter & 5.5717 & 0.0005 & 0.0005 & WBW2X & $\mathrm{HPC} \cdot \mathrm{B}>$ & 0.0000 & 0.4500 & 0.2200 \\
\hline$===\mathrm{SHAFTS}====$ & Nmech & trg in & pwr in & BLEEDS - & output & $\mathrm{Wb} / \mathrm{Win}$ & hscale & Psca \\
\hline HP Shaft & 11251.3 & 6445.0 & 13806.9 & WB17Y & Bleed> & 0.0000 & 1.0000 & 1.000 \\
\hline LP_Shaft & 3748.9 & 15092.9 & 10773.1 & HPT_COOLI & A Bleed> & 0.1142 & 1.0000 & 1.000 \\
\hline & & & & $\mathrm{HPT}_{-}^{-} \mathrm{COOLE}$ & B Bleed> & 0.0999 & 1.0000 & 1.000 \\
\hline & & & & WB $3 \bar{x}$ & Bleed $>$ & 0.0000 & 1.0000 & 1.000 \\
\hline & & & & WBA3X & Bleed> & 0.0000 & 1.0000 & 1.000 \\
\hline & & & & WBW3X & Bleed> & 0.0000 & 1.0000 & 1.000 \\
\hline & & & & WBFDLKG & FanDu> & 0.0000 & 1.0000 & 1.000 \\
\hline & & & & WB15X & Bleed> & 0.0000 & 1.0000 & 1.000 \\
\hline & & & & WB15Y & Bleed> & 0.0000 & 1.0000 & 1.000 \\
\hline & & & & WB17X & Bleed> & 0.0000 & 1.0000 & 1.000 \\
\hline$===$ BURNERS $===$ & Ttout & eff & dPnorm & Wfuel & FAR & & & \\
\hline Burner & 2689.42 & 0.9995 & 0.0230 & .90837 & 02231 & & & \\
\hline$===$ NOZZLES $===$ & & Cfg & CdTh & $\mathrm{Cv}$ & Ath & MNth & Vact & $\mathrm{Fg}$ \\
\hline Core_Nozz & 2.184 & 0.9816 & 1.0000 & 0.9800 & 3.40 & 1.000 & 1555.2 & 3022.4 \\
\hline Byp_Nozz & 2.374 & 0.9815 & 1.0000 & 0.9800 & & 1.000 & 1030.7 & 11310.8 \\
\hline
\end{tabular}




\section{$10 \mu \mathrm{m}, \mathrm{ISA}+18 \mathrm{R}$}

Date:05/13/13 Time:07:04:22 Model:

Version:NPSS 1.6.5 - Rev: -> Gas Package: Janaf iter/pass/Jacb/Broy=23/51/2/20 Run by: Philip C Jorgenson PC: 100

Temperature Stator 1 inlet: 444.20 Stator 1 exit: $462.85 \quad$ Stator 2 inlet: 484.44 Stator 2 exit: 491.60

Stator 3 inlet: 520.52 Stator 3 exit: 528.49 Stator 4 inlet: 555.54 Stator 4 exit: 563.26

Ambient Relative Humidity $\quad 10.00$

Fan Face Relative Humidity $\quad 5.21$

FPC Inlet Relative Humidity 0.15

LPC Exit Relative Humidity 2.29

Drop Diameter $\quad 0.0000100$

0.00

mient

Ambient Static Temperature

Additional

7000100

2.85
407.97

Inlet Length

40.00

407.97 Fan/LPC Inlet Static Temperature 438.14

$\begin{array}{rrrrr} & & & \text { SUMMARY OUTPUT DATA } \\ \text { MN alt dTamb } & \text { W } & \text { Fn } & \text { TSFC }\end{array}$

\section{FLOW STATION DATA}

\begin{tabular}{rrrrrr}
\multicolumn{7}{c}{ FLOW } & STATION DATA \\
W & Pt & Tt & ht & FAR & WC \\
396.30 & 4.267 & 457.74 & -20.96 & 0.0000 & 1282.27 \\
396.30 & 4.267 & 457.74 & -20.96 & 0.0000 & 1282.27 \\
333.25 & 4.265 & 457.74 & -20.96 & 0.0000 & 1078.81 \\
63.05 & 4.265 & 457.74 & -20.96 & 0.0000 & 204.10 \\
333.25 & 7.257 & 540.20 & -1.18 & 0.0000 & 688.69 \\
63.05 & 10.488 & 620.60 & 18.14 & 0.0000 & 96.64 \\
63.10 & 10.488 & 621.54 & 14.10 & 0.0000 & 96.78 \\
63.10 & 10.488 & 621.54 & 14.10 & 0.0000 & 96.78 \\
60.03 & 137.939 & 1374.13 & 201.80 & 0.0000 & 10.41 \\
46.53 & 137.939 & 1374.13 & 201.80 & 0.0000 & 8.07 \\
47.65 & 134.763 & 2814.20 & 171.65 & 0.0242 & 12.10 \\
63.48 & 31.493 & 1844.04 & -6.29 & 0.0181 & 55.86 \\
64.22 & 7.239 & 1320.92 & -145.95 & 0.0179 & 208.07 \\
64.22 & 7.239 & 1320.99 & -145.95 & 0.0179 & 208.08 \\
64.22 & 7.239 & 1321.11 & -145.95 & 0.0179 & 208.09 \\
333.25 & 7.257 & 540.20 & -1.18 & 0.0000 & 688.69 \\
333.25 & 7.257 & 540.20 & -1.18 & 0.0000 & 688.69 \\
333.25 & 7.257 & 540.20 & -1.18 & 0.0000 & 688.69 \\
333.25 & 7.257 & 540.20 & -1.18 & 0.0000 & 688.69
\end{tabular}

VTAS
OPR
32.328

$\begin{array}{rr}\text { PS } & \text { TS } \\ 2.854 & 407.97\end{array}$

$\begin{array}{rrrrr}2.854 & 407.97 & \text { Aphy } & \text { MN } & \text { gamt }\end{array}$

$\begin{array}{lllll}3.150 & 419.67 & 4168.2 & 0.6726 & 1.40084\end{array}$

$\begin{array}{rrrrr}3.174 & 420.64 & 3531.8 & 0.6633 & 1.40084 \\ 3.660 & 438.14 & 830.5 & 0.4724 & 1.40084\end{array}$

$\begin{array}{rrrrr}3.660 & 438.14 & 830.5 & 0.4724 & 1.40084 \\ 6.024 & 512.21 & 2606.7 & 0.5226 & 1.39992\end{array}$

$\begin{array}{lllll}9.163 & 597.17 & 412.6 & 0.4436 & 1.39831\end{array}$

$\begin{array}{lllll}9.158 & 597.98 & 412.6 & 0.4447 & 1.39818 \\ 9.158 & 597.98 & 412.6 & 0.4447 & 1.39818\end{array}$

$\begin{array}{lllll}9.158 & 597.98 & 412.6 & 0.4447 & 1.39818\end{array}$

$\begin{array}{lllll}124.410 & 1337.20 & 49.7 & 0.3924 & 1.35732 \\ 130.079 & 1353.03 & 49.3 & 0.2951 & 1.35732\end{array}$

$\begin{array}{rrrrr}30.079 & 1353.03 & 49.3 & 0.2951 & 1.35732 \\ 27.304 & 2778.46 & 74.6 & 0.3004 & 1.28466\end{array}$

$\begin{array}{lll}265.4 & 0.4002 & 1.28466\end{array}$

$\begin{array}{lll}860.2 & 0.4724 & 1.34817\end{array}$

$\begin{array}{lll}860.2 & 0.4724 & 1.34817\end{array}$

$\begin{array}{rrr}613.4 & 1.0000 & 1.34816 \\ 2606.7 & 0.5226 & 1.39992\end{array}$

$\begin{array}{lll}2606.7 & 0.5226 & 1.39992 \\ 2481.9 & 0.5625 & 1.39992\end{array}$

$\begin{array}{lll}2481.9 & 0.5625 & 1.39992 \\ 2481.9 & 0.5625 & 1.39992\end{array}$

$2481.9 \quad 0.562$

$2006.9 \quad 1.0000 \quad 1.39992$ $\begin{array}{ccr}\text { T4 } & \text { T41 } & \text { T49 } \\ 2814.2 & 2641.1 & 1835.1\end{array}$

TURBOMACHINERY PERFORMANCE DATA

\begin{tabular}{|c|c|c|c|c|c|c|c|c|c|}
\hline \multirow{2}{*}{ 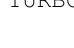 } & WT & $\mathrm{PR}$ & eff & NT & Rת & $f \mathrm{Pat}$ & nw & & SMW \\
\hline & 1078.81 & $\begin{array}{l}1.702 \\
1.702\end{array}$ & $\begin{array}{l}\text { eII } \\
0.9114\end{array}$ & $\begin{array}{r}\text { NC } \\
4139.469\end{array}$ & $\begin{array}{r}1.1801 \\
1 R\end{array}$ & $\begin{array}{l}\text { eIfo1y } \\
0.9178\end{array}$ & $\begin{array}{r}\text { PWr } \\
-9322.3\end{array}$ & $\begin{array}{r}\text { SMIN } \\
36.69\end{array}$ & 23.09 \\
\hline LPC & 204.10 & 2.459 & 0.8238 & 4139.469 & 1.3558 & 0.8444 & -3487.4 & 2.97 & 2.24 \\
\hline HPC & 96.78 & 13.153 & 0.8584 & 10365.299 & 2.2109 & 0.8979 & -16349.9 & 25.94 & 25.16 \\
\hline HPT & 12.10 & 4.279 & 0.9118 & 213.891 & 1.3468 & 0.8911 & 16349.9 & & \\
\hline LPT & 55.86 & 4.351 & 0.9155 & 90.557 & 1.3925 & 0.8988 & 12809.7 & & \\
\hline & INERY M $z$ & DATA & & & & & & & \\
\hline & WcMap & PRmap & effMap & NcMap & R/Parm & S_WcDes & S_PRdes & S_effDes & S_NCDes \\
\hline & 1253.47 & 1.686 & 0.9132 & 4100.000 & 0.0639 & $\overline{0} .8607$ & $\overline{1} .0235$ & 0.9980 & $\overline{0} .9905$ \\
\hline${ }^{C} \mathrm{C}$ & 166.84 & 2.748 & 0.8341 & 1.093 & 0.0000 & 1.2233 & 0.8348 & 0.9876 & 0.0003 \\
\hline $\mathrm{PC}$ & 88.02 & 12.580 & 0.8673 & 10088.967 & 13.5045 & 1.0995 & 1.0494 & 0.9898 & 0.9733 \\
\hline & 0.96 & 4.188 & 0.9118 & 1.285 & 4.1882 & 12.6299 & 0.9723 & 1.0000 & 0.0003 \\
\hline PT & 0.86 & 3.520 & 0.9223 & 1.019 & 3.5201 & 65.2590 & 0.7521 & 0.9926 & 0.0005 \\
\hline
\end{tabular}

WAR Wair WH2O H2O frac $\begin{array}{llll}0.0000295 & 396.29 & 0.012 & 0.0000\end{array}$ $\begin{array}{llll}0.00000295 & 333.24 & 0.010 & 0.0000\end{array}$ $\begin{array}{lrrr}0.0000295 & 333.24 & 0.010 & 0.0000 \\ 0.0000295 & 63.05 & 0.002 & 0.0000 \\ 0.0000295 & 333.24 & 0.010 & 0.0000\end{array}$ $\begin{array}{llll}0.0000295 & 63.05 & 0.002 & 0.0000\end{array}$ $\begin{array}{llll}0.0007710 & 63.05 & 0.049 & 0.0008\end{array}$ $\begin{array}{llll}0.0007710 & 63.05 & 0.049 & 0.0008\end{array}$ $\begin{array}{llll}0.0007710 & 59.99 & 0.046 & 0.0008\end{array}$ $\begin{array}{llll}0.0007710 & 46.49 & 0.036 & 0.0008\end{array}$ $\begin{array}{llll}0.0007710 & 46.49 & 0.036 & 0.0008 \\ 0.0007710 & 46.49 & 0.036 & 0.0299 \\ 0.0007710 & 62.31 & 0.048 & 0.0227\end{array}$ $\begin{array}{llll}0.0007710 & 63.05 & 0.049 & 0.0224\end{array}$ $\begin{array}{llll}0.0007710 & 63.05 & 0.049 & 0.0224\end{array}$ $\begin{array}{llll}0.0007710 & 63.05 & 0.049 & 0.0224\end{array}$ $\begin{array}{llll}0.0000295 & 333.24 & 0.010 & 0.0000\end{array}$ $\begin{array}{llll}0.0000295 & 333.24 & 0.010 & 0.0000\end{array}$ $\begin{array}{llll}0.0000295 & 333.24 & 0.010 & 0.0000\end{array}$ $\begin{array}{llll}0.0000295 & 333.24 & 0.010 & 0.0000\end{array}$ 


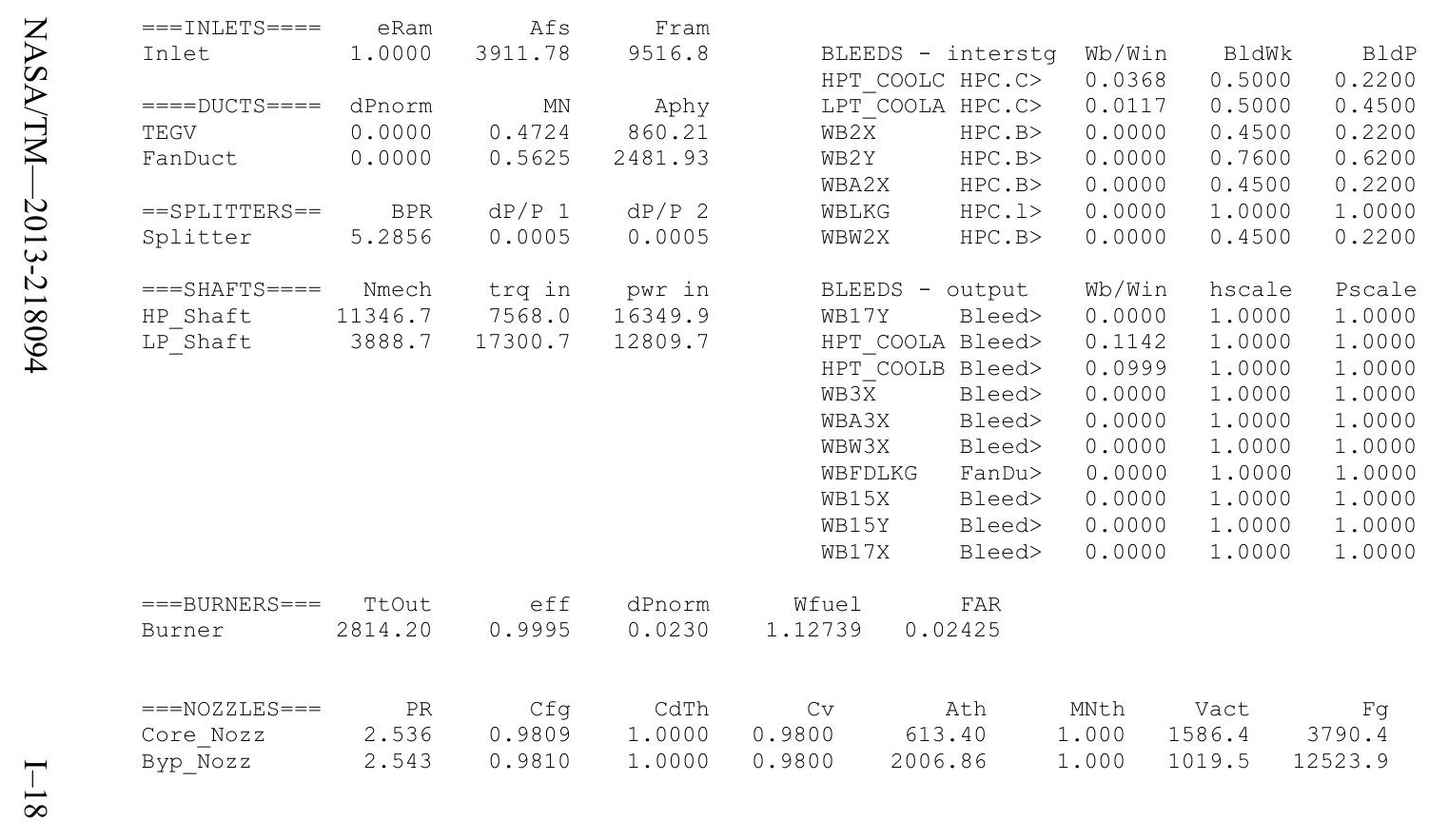


Date: 05/13/13 Time: 07:05:00 Model: Temperature Stator 1 inlet: 450.34 Stator 1 exit: 467.50 Stator 2 inlet: 487.90 Stator 2 exit: 495.85 $\begin{array}{llll}\text { Stator } 3 & \text { inlet: } 521.31 & \text { Stator } 3 \text { exit: } 529.50 & \text { Stator } 4 \text { inlet: } 553.54 \quad \text { Stator } 4 \text { exit: } 561.15 \\ \text { Stator } 5 \text { inlet: } 580.11 & \text { Stator } 5 \text { exit: } 587.37 & \text { Unblocked Percent Blockage: } 0.00\end{array}$

Ambient Relative Humidity $\quad 10.00$

Fan Face Relative Humidity 4.13

Fan Bypass Relative Humidity $\quad 0.18$

LPC Inlet Relative Humidity

LPC Exit Relative Humidity

HPC Relative Humidity

0.00

Ambient Flow Velocity

0.0000100

772.64

Inlet Length

2.85 Fan/LPC Inlet Flow Velocity $\quad 444.99$

Ambient Static Pressure

Ambient Static Temperature

3.75

Additional Water at LPC Exit 0.0008168

Fan/LPC Inlet Static Temperature 441.23

SUMMARY OUTPUT DATA

$\begin{array}{rrrrrr}\text { MN } & \text { alt } & \text { dTamb } & \text { W } & \text { Fn } & \text { TSFC } \\ 0.780 & 39000.0 & 18.00 & 378.72 & 5804.3 & 0.5902\end{array}$

$\begin{array}{rrr} & \text { Wfuel } \\ 5804.3 & 0.5902 & 3425.49\end{array}$

\section{FLOW STATION DATA}

$\begin{array}{ll}\text { FS0 } & \text { Ambient.FI- } \\ \text { FS1 } & \text { Inlet.FI_O }\end{array}$

$\begin{array}{ll}\text { FS12 } & \text { Splitter.F1_02 } \\ \text { FS2 } & \text { Splitter.Fl_01 }\end{array}$

FS14 Fan.Fl_o

$\begin{array}{ll}\text { FS23 } & \text { LPC.FI-O } \\ \text { FS24 } & \text { VaporIN.FI }\end{array}$

FS25 Bleed2.FI_o

FS3 HPC.FIO

FS36 Bleed3.F1

FS4 Burner.FI-O

FS45 HPT.FI

FS49 LPT.FI_O

FS8 8 COre.

FS17 FanDuctLkg.Fi_O

FS171 Bleed15.FI_O

FS172 FanDuct.

FS173 Byp Nozz.Fì

$W$
378.72
378.72
319.83
58.88
319.83
58.88
58.93
58.93
56.07
43.46
44.41
59.19
59.88
59.88
59.88
319.83
319.83
319.83
319.83

$\begin{array}{rrrrr}\text { Pt } & \text { Tt } & \text { ht } & \text { FAR } & \text { WC } \\ 4.267 & 457.74 & -20.96 & 0.0000 & 1225.39\end{array}$

$\begin{array}{lllll}4.267 & 457.74 & -20.96 & 0.0000 & 1225.39\end{array}$

$\begin{array}{lllll}4.265 & 457.74 & -20.96 & 0.0000 & 1035.38 \\ 4.265 & 457.74 & -20.96 & 0.0000 & 190.62\end{array}$

$\begin{array}{rrrrr}4.265 & 457.74 & -20.96 & 0.0000 & 1035.38 \\ 6.913 & 532.10 & -3.13 & 0.0000 & 688.70 \\ 10.356 & 612.59 & 16.21 & 0.0000 & 90.81\end{array}$

$\begin{array}{rrrrr}6.913 & 532.10 & -3.13 & 0.0000 & 688.70 \\ 10.356 & 612.59 & 16.21 & 0.0000 & 90.81\end{array}$

$\begin{array}{lllll}10.356 & 612.59 & 16.21 & 0.0000 & 90.81 \\ 10.356 & 613.62 & 11.76 & 0.0000 & 90.96\end{array}$

$\begin{array}{lllll}10.356 & 613.62 & 11.76 & 0.0000 & 90.96\end{array}$

$\begin{array}{llll}124.756 & 1322.38 & 187.96 & 0.0000\end{array}$

$\begin{array}{llll}124.7362 & 1322.38 & 187.94 & 0.0000\end{array}$

$\begin{array}{lllll}28.387 & 1731.31 & -6.59 & 0.0164\end{array}$

$\begin{array}{llll}28.387 & 1731.31 & -6.59 & 0.0164\end{array}$

.00 .965

115.103

$25.553-1613.42$

$\begin{array}{llll}1731.31 & -6.59 & 0.0164 & 55.99\end{array}$

$5.620 \quad 1188.48$

$\begin{array}{ll}5.620 & 1188.55 \\ 3.479 & 1046.35\end{array}$

$\begin{array}{lllll}6.518 & 1235.88 & -137.13 & 0.0162 & 208.4\end{array}$

$\begin{array}{lllll}6.913 & 532.10 & -3.13 & 0.0000 & 688.70 \\ 6.913 & 532.10 & -3.13 & 0.0000 & 688.70 \\ 6.913 & 532.10 & -3.13 & 0.0000 & 688.70\end{array}$

$\begin{array}{lllll}6.913 & 532.10 & -3.13 & 0.0000 & 688.70\end{array}$

$\begin{array}{lllll}6.913 & 532.10 & -3.13 & 0.0000 & 688.70\end{array}$

$5.738 \quad 504.53$

$\begin{array}{ll}.576 & 500.41\end{array}$

$3.651 \quad 443.30$

TURBOMACHINERY PERFORMANCE DATA

$\begin{array}{lrr} & \text { WC } & \text { PR } \\ \text { Fan } & 1035.38 & 1.621 \\ \text { LPC } & 190.62 & 2.428 \\ \text { HPC } & 90.96 & 12.045 \\ \text { HPT } & 12.10 & 4.293 \\ \text { LPT } & 55.99 & 4.355\end{array}$

eff

NC
3969.398
3969.398

EPR
1.498

PowerSet
84.000
T4
2647. $\begin{array}{cc}\text { T41 } & \text { T49 } \\ 2487.0 & 1723.2\end{array}$

TURBOMACHINERY MAP DATA

$\begin{array}{lrrrrrrrrr} & \text { WcMap } & \text { PRmap } & \text { effMap } & \text { NcMap } & \text { R/Parm } & \text { S_WcDes } & \text { S_PRdes } & \text { S_effDes } & \text { S_NcDes } \\ \text { Fan } & 1203.01 & 1.607 & 0.9135 & 3931.550 & 0.0611 & 0.8607 & 1.0235 & 0.9980 & 0.9905 \\ \text { LPC } & 151.31 & 2.561 & 0.8233 & 1.048 & 0.0000 & 1.2598 & 0.9151 & 1.0358 & 0.0003 \\ \text { HPC } & 82.73 & 11.524 & 0.8696 & 9904.302 & 13.1355 & 1.0995 & 1.0494 & 0.9898 & 0.9733 \\ \text { HPT } & 0.96 & 4.202 & 0.9099 & 1.293 & 4.2016 & 12.6299 & 0.9723 & 1.0000 & 0.0003 \\ \text { LPT } & 0.86 & 3.523 & 0.9199 & 1.008 & 3.5235 & 65.2590 & 0.7521 & 0.9926 & 0.0005\end{array}$




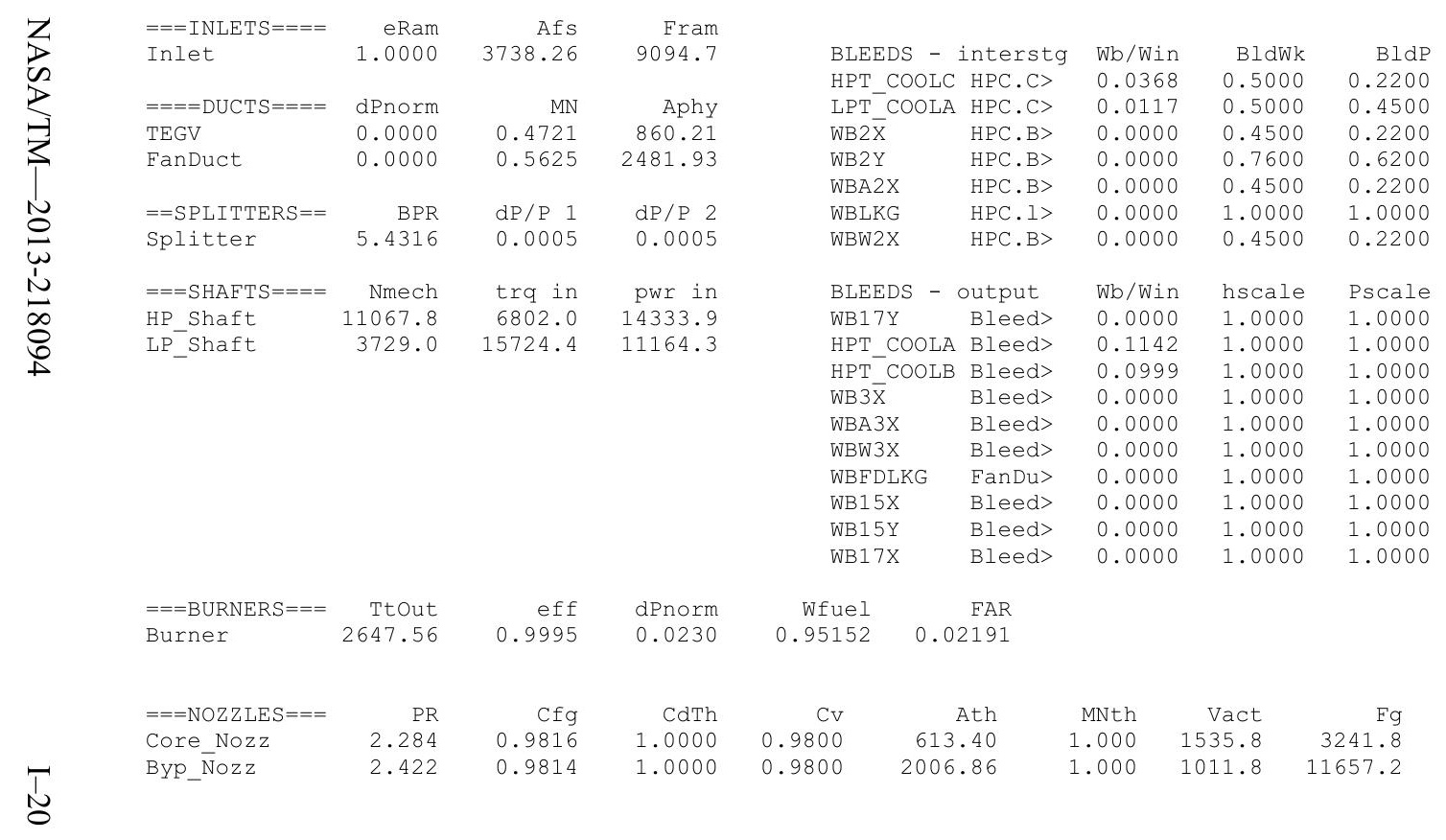


Date: 05/13/13 Time:07:05:36 Model:_ Turbofan Engine - COMDES ON COnverge = 1 CASE: 0

Version:NPSS 1.6.5 - Rev: -> Gas Package: Janaf iter/pass/Jacb/Broy= 20/34/1/18 Run by: Philip C Jorgenson PC: 79

Temperature Stator 1 inlet: 451.95 Stator 1 exit: 468.71 Stator 2 inlet: 488.79 Stator 2 exit: 496.87

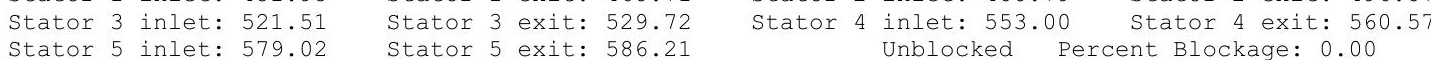

Ambient Relative Humidity $\quad 10.00$

Fan Face Relative Humidity $\quad 3.88$

Fan Bypass Relative Humidity $\quad 0.20$

LPC Inlet Relative Humidity $\quad 1.92$

LPC Exit Relative Humidity

0.00

Ambient Flow Velocity

772.64

2.85

Inlet Length

40.00

Ambient Static Pressure

Additional Water at LPC Exit 0.0008427

$\begin{array}{lr} & 40.00 \\ \text { Fan/LPC Inlet Static Pressure } & 3.78\end{array}$

$\begin{array}{rr}\text { Fan/LPC Inlet Static Temperature } & 442.78 \\ & \end{array}$

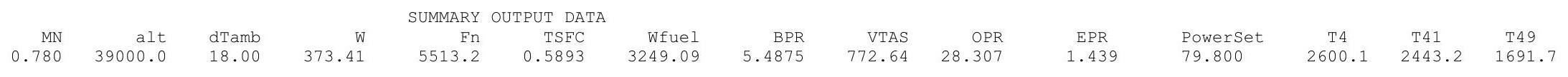

FLOW STATION DATA

FSO Ambient.FI_O

FS1 Inlet.Fl_O

FS14 Fan.Fl_O

ES23 LPC.FIO

FS25 VaporIN. FI_O

FS3 3 HPC.FI_O

FS36 Bleed3.F1_O

FS4 Burner.FI_O

FS45 HPT.FI-

FS49 LPT.F1_O

FS8 Core Nozz.FIO

FS17 FanDuctLkg.Fi_O

FS171 Bleed15.FI_O

FS172 FanDuct. Fl-0

FS173 Byp_Nozz.Fì_O

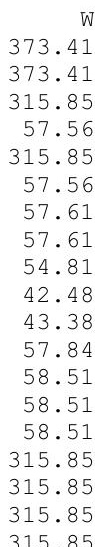

$\begin{array}{rrrrr}\text { Pt } & \text { Tt } & \text { ht } & \text { FAR } & \text { WC } \\ 4.267 & 457.74 & -20.96 & 0.0000 & 1208.21\end{array}$ $\begin{array}{lllll}4.267 & 457.74 & -20.96 & 0.0000 & 1208.21 \\ 4.267 & 457.74 & -20.96 & 0.0000 & 1208.21\end{array}$ $\begin{array}{lllll}4.265 & 457.74 & -20.96 & 0.0000 & 1022.48\end{array}$ $\begin{array}{lllll}4.265 & 457.74 & -20.96 & 0.0000 & 1022.48 \\ 4.265 & 457.74 & -20.96 & 0.0000 & 186.33\end{array}$ $\begin{array}{rrrrr}4.265 & 457.74 & -20.96 & 0.0000 & 186.33 \\ 10.300 & 610.43 & -3.70 & 0.0000 & 688.7\end{array}$ $\begin{array}{lllll}10.300 & 529.69 & -3.70 & 0.0000 & 688.70\end{array}$

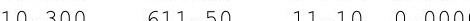
$\begin{array}{lllll}120.781 & 1307.56 & 183.97 & 0.0000\end{array}$ $\begin{array}{llll}120.781 & 1307.56 & 183.97 & 0.0000\end{array}$ $\begin{array}{llll}120.781 & 1307.56 & 183.97 & 0.0000 \\ 117.999 & 2600.14 & 157.99 & 0.0000\end{array}$ $\begin{array}{lllll}27.463 & 1699.57 & -6.69 & 0.0159\end{array}$ $\begin{array}{lllll}6.304 & 1211.86 & -134.67 & 0.0157\end{array}$ $\begin{array}{lllll}6.304 & 1211.93 & -134.67 & 0.0157\end{array}$ $\begin{array}{lllll}6.304 & 1212.06 & -134.67 & 0.0157\end{array}$ $\begin{array}{llll}6.811 & 529.69 & -3.70 & 0.0000\end{array}$ $\begin{array}{llll}6.811 & 529.69 & -3.70 & 0.0000\end{array}$ $\begin{array}{llll}6.811 & 529.69 & -3.70 & 0.0000\end{array}$

$-3.70 \quad 0.0000$

$\begin{array}{rr}\text { PS } & \text { TS } \\ 2.854 & 407.97 \\ 3.336 & 426.59 \\ 3.337 & 426.71 \\ 3.778 & 442.13 \\ 5.654 & 502.24 \\ 9.224 & 591.52 \\ 9.219 & 592.47 \\ 9.219 & 592.47 \\ 108.469 & 1270.71 \\ 113.643 & 1286.58 \\ 111.454 & 2566.43 \\ 24.718 & 1655.72 \\ 5.434 & 1165.36 \\ 5.434 & 1165.42 \\ 3.363 & 1025.43 \\ 5.654 & 502.24 \\ 5.494 & 498.14 \\ 5.494 & 498.14 \\ 3.597 & 441.29\end{array}$

$\begin{array}{rr}\text { Aphy } & \text { MN } \\ 3685.8 & 0.7800 \\ 4168.2 & 0.6035 \\ 3531.8 & 0.6023 \\ 830.5 & 0.4197 \\ 2606.7 & 0.5226 \\ 412.6 & 0.4004 \\ 412.6 & 0.4014 \\ 412.6 & 0.4014 \\ 49.7 & 0.4000 \\ 49.3 & 0.3002 \\ 74.6 & 0.2993 \\ 265.4 & 0.4004 \\ 860.2 & 0.4720 \\ 860.2 & 0.4720 \\ 613.4 & 1.0000 \\ 2606.7 & 0.5226 \\ 2481.9 & 0.5625 \\ 2481.9 & 0.5625 \\ 2006.9 & 1.0000\end{array}$

WAR
0.0000295
0.0000295
0.0000295
0.0000295
0.0000295
0.0000295
0.0008721
0.0008721
0.0008721
0.0008721
0.0008721
0.0008721
0.0008721
0.0008721
0.0008721
0.0000295
0.0000295
0.0000295
0.0000295

Wair
373.40
373.40
315.84
57.56
315.84
57.56
57.56
57.56
54.77
42.44
42.44
56.88
57.56
57.56
57.56
315.84
315.84
315.84
315.84

WH2O H2O frac $0.011 \quad 0.0000$ $0.009 \quad 0.0000$ $0.002=0.0000$ 0.0020 .0000 $\begin{array}{ll}0.050 & 0.0009 \\ 0.008 & 0.0009\end{array}$ $0.058 \quad 0.0009$ $0.037 \quad 0.0009$ $0.037 \quad 0.0265$ $0.050 \quad 0.0201$ $0.050=0.0199$ $\begin{array}{lll}0.050 & 0.0199\end{array}$ 0.0000

\begin{tabular}{|c|c|c|c|c|c|c|c|c|c|}
\hline TURI & ENERY PER & ORMANCE & DATA & & & & & & \\
\hline & Wc & PR & eff & $\mathrm{NC}$ & TR & efPoly & pwr & SMN & SMW \\
\hline Fan & 1022.48 & 1.597 & 0.9114 & 3921.443 & 1.1572 & 0.9170 & -7709.0 & 57.11 & 27.50 \\
\hline LPC & 186.33 & 2.415 & 0.8589 & 3921.443 & 1.3336 & 0.8752 & -2984.3 & 3.33 & 2.62 \\
\hline HPC & 89.24 & 11.726 & 0.8613 & 10127.580 & 2.1383 & 0.8988 & -13747.6 & 31.23 & 30.09 \\
\hline HPT & 12.10 & 4.297 & & 215.655 & 1.35 & 0.8897 & 13747.6 & & \\
\hline LPT & 56.02 & 4.356 & .9124 & 89.359 & 1.3995 & 0.8952 & 10693.3 & & \\
\hline $\mathrm{JRF}$ & [NE! & DATA & & & & & & & \\
\hline & WcMap & PRmap & effMap & NcMap & R/Parm & s WcDes & S_PRdes & s_effDes & S_NCDes \\
\hline an & 1188.02 & 1.583 & 0.9132 & 3884.052 & 0.0603 & 0.8607 & 1.0235 & 0.9980 & 0.9905 \\
\hline $\mathrm{APC}$ & 148.21 & 2.511 & 0.8223 & 1.036 & 0.0000 & 1.2572 & 0.9364 & 1.0445 & 0.0003 \\
\hline PC & 81.17 & 11.221 & 0. & 9857.585 & 13.0271 & 1.0995 & 1.04 & 0.9898 & 0.9733 \\
\hline $\mathrm{PT}$ & 0.96 & 4.205 & 0.9090 & 1.296 & 4.2053 & 12.6299 & 0.9723 & 1.0000 & 0.0003 \\
\hline $\mathrm{T}$ & 0.86 & 3.524 & .9191 & 1.005 & 3.5244 & 65.2590 & 0.7521 & 0.9926 & 0.00 \\
\hline
\end{tabular}


$\begin{array}{lrrr}===\text { INLETS }==== & \text { eRam } & \text { Afs } & \text { Fram } \\ \text { Inlet } & 1.0000 & 3685.85 & 8967.1\end{array}$

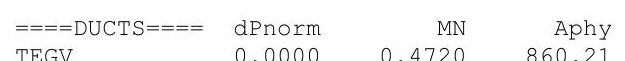

$\begin{array}{llrr}\text { TEGV } & 0.0000 & 0.4720 & 860.21 \\ \text { FanDuct } & 0.0000 & 0.5625 & 2481.93\end{array}$

nouct

Splitter

5.4875

$\mathrm{dP} / \mathrm{P}$

0.0005

$\mathrm{dP} / \mathrm{P} 2$

$===$ SHAFTS $====$

5.4875

$\begin{array}{lrrr}===\text { SHAFTS }==== & \text { Nmech } & \text { trq in } & \text { pwr in } \\ \text { HP_Shaft } & 10996.6 & 6566.1 & 13747.6 \\ 3683.9 & 15245.3 & 10693.3\end{array}$

13747.6
10693.3

$===$ BURNERS $===\begin{array}{r}\text { TtOut } \\ 2600.12\end{array}$
Burner

eff
0.9995

dPnorm

0.0230

$===$ NOZZLES $===$

Core Nozz

PR
2.209
2.387

Cfg
0.9816
0.9815

CdTh
1.0000

$\begin{array}{ll}1.0000 & 0.9800\end{array}$

BLEEDS - interstg Wb/Win BldWk

HPT COOLC HPC.C $>0.0368$

$\begin{array}{lllll}\text { LPT }^{-} \text {COOLA HPC.C> } & 0.0117 & 0.5000 & 0.4500 \\ \text { WB2 } & & 0.0170\end{array}$

$\begin{array}{lllll}\text { WB2X } & \text { HPC.B } & 0.0000 & 0.4500 & 0.2200 \\ \text { WB2Y } & \text { HPC.B } & 0.0000 & 0.7600 & 0.6200\end{array}$

$\begin{array}{llllll}\text { WBA2X HPC.B } & 0.0000 & 0.4500 & 0.2200\end{array}$

WBLKG HPC.I> $0.0000 \quad 1.0000 \quad 1.0000$

$\begin{array}{lllll}\text { WBW2X HPC.B> } & 0.0000 & 0.4500 & 0.2200\end{array}$

BLEEDS - output Wb/Win hscale Pscale

$\begin{array}{lllll}\text { WB17Y Bleed> } 0.0000 & 1.0000 & 1.0000\end{array}$

HPT_COOLA Bleed> $0.1142 \quad 1.0000 \quad 1.0000$

$\begin{array}{lllll}\text { HPT COOLB } & \text { Bleed }> & 0.0999 & 1.0000 & 1.0000 \\ \text { WB } 3 \bar{X} & \text { Bleed }> & 0.0000 & 1.0000 & 1.0000\end{array}$

$\begin{array}{lllll}\text { WB3X } & \text { Bleed> } & 0.0000 & 1.0000 & 1.0000 \\ \text { WBA3X } & \text { Bleed }> & 0.0000 & 1.0000 & 1.0000\end{array}$

$\begin{array}{lllll}\text { WBA3X } & \text { Bleed> } & 0.0000 & 1.0000 & 1.0000 \\ \text { WBW3X } & \text { Bleed> } & 0.0000 & 1.0000 & 1.0000\end{array}$

$\begin{array}{lllll}\text { WBW3X } & \text { Bleed> } & 0.0000 & 1.0000 & 1.0000 \\ \text { WBFDIKG } & \text { FanDu> } & 0.0000 & 1.0000 & 1.0000\end{array}$

$\begin{array}{lllll}\text { WBFDLKG } & \text { FanDu> } & 0.0000 & 1.0000 & 1.0000 \\ \text { WB15X } & \text { Bleed }> & 0.0000 & 1.0000 & 1.0000\end{array}$

WB15X 1.0000

WB17X Bleed> $0.0000 \quad 1.0000$

1.0000
1.0000

1.0000
1.0000

$\begin{array}{rr}\text { Wfuel } & \text { FAR } \\ 0.90252 & 0.02126\end{array}$

$.90252 \quad 0.02126$

$\begin{array}{rrrrr}\text { CV } & \text { Ath } & \text { MNth } & \text { Vact } & \text { Fg } \\ 0.9800 & 613.40 & 1.000 & 1521.3 & 3078.7 \\ 0.9800 & 2006.86 & 1.000 & 1009.5 & 11401.7\end{array}$

$\begin{array}{rrrr}W & \text { Tt } & \text { ht } & \text { Pt } \\ 2.1199 & 966.04 & 97.54 & 27.463 \\ 0.6740 & 966.04 & 97.54 & 6.304 \\ 0.0000 & 931.10 & 88.89 & 34.606 \\ 0.0000 & 1145.44 & 142.48 & 78.798 \\ 0.0000 & 931.10 & 88.89 & 34.606 \\ 0.0000 & 1307.56 & 183.97 & 120.781 \\ 0.0000 & 931.10 & 88.89 & 34.606 \\ W & T t & h t & \mathrm{Pt} \\ 0.000 & 611.50 & 11.10 & 10.300 \\ 6.5775 & 1307.56 & 183.97 & 117.999 \\ 5.7555 & 1307.56 & 183.97 & 66.212 \\ 0.0000 & 1307.56 & 183.97 & 120.781 \\ 0.0000 & 1307.56 & 183.97 & 120.781 \\ 0.0000 & 1307.56 & 183.97 & 120.781 \\ 0.0000 & 529.69 & -3.70 & 6.811 \\ 0.0000 & 529.69 & -3.70 & 6.811 \\ 0.0000 & 529.69 & -3.70 & 6.811 \\ 0.0000 & 529.69 & -3.70 & 6.811\end{array}$


Date: 05/13/13 Time:07:06:12 Model: Turbofan Engine - COMDES ON Converge = 1 CASE: 0

Version:NPSS1.6.5 - Rev: -> Gas Package: Janaf iter/pass/Jacb/Broy= 20/34/1/18 Run by: Philip C Jorgenson PC: 76

Temperature Stator 1 inlet: $453.04 \quad$ Stator 1 exit: $469.53 \quad$ Stator 2 inlet: 489.43 Stator 2 exit: 497.59 Stator 3 inlet: $521.69 \quad$ Stator 3 exit: $529.91 \quad$ Stator 4 inlet: 552.69 Stator 4 exit: 560.2
Stator 5 inlet: 578.36
Stator 5 exit: 585.50

Ambient Relative Humidity $\quad 10.00$

Fan Face Relative Humidity $\quad 3.72$

Fan Bypass Relative Humidity 0.21

LPC Inlet Relative Humidity

LPC Exit Relative Humidity $\quad 0.02$

HPC Relative Humidity

Drop Diameter

0.00
.000100

Ambient Flow Velocity

0.0000100
772.64

7.64
2.85

Inlet Length

40.00

Ambient Static Pressure

Additional Water at LPC Exit 0.0008620

Fan/LPC Inlet Static Pressure $\quad 3.80$

Fan/LPC Inlet Static Temperature $\quad \begin{array}{r}4.80 \\ \end{array}$

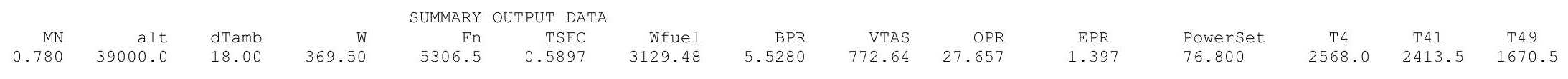

FLOW STATION DATA

FS0 Ambient.F1_O

FS1 Inlet.Fl_O

$\begin{array}{ll}\text { FS12 } & \text { Splitter.F1_02 } \\ \text { FS2 } & \text { Splitter.Fl-01 }\end{array}$

FS14 Fan.Fl_

FS23 LPC.FI_O

FS25 Bleed2.FI_

$\begin{array}{ll}\text { FS25 } & \text { Bleed2.FI_ } \\ \text { FS3 HPC.F1_O }\end{array}$

FS36 Bleed3.F1_O

FS4 Burner.FI_O

FS45 HPT.FI_O

FS49 LPT.FI_O

$\begin{array}{ll}\text { FS5 } & \text { TEGV.FI_O } \\ \text { FS8 } & \text { Core Nozz.FI }\end{array}$

FS17 FanDuctlkg.FI_O

FS171 Bleed15.FI_O

FS172 FanDuct. F1

FS173 Byp_Nozz.FI_O

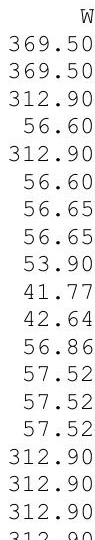

$\begin{array}{ll}\text { Pt } & \text { Tt } \\ 4.267 & 457.74\end{array}$

$(5.280$

(7).

TURBOMACHINERY PERFORMANCE DATA

\begin{tabular}{|c|c|c|c|c|c|c|c|c|c|}
\hline & WC & PR & eff & NC & $\mathrm{TR}$ & efPoly & pwr & SMN & SMW \\
\hline Fan & 1012.92 & 1.580 & 0.9094 & 3888.842 & 1.1536 & 0.9151 & -7462.2 & 59.24 & 28.27 \\
\hline LPC & 183.23 & 2.406 & 0.8625 & 3888.842 & 1.3305 & 0.8783 & -2908.0 & 3.61 & 2.87 \\
\hline HPC & 88.00 & 11.501 & .8614 & 10094.302 & 2.1268 & 0.8986 & -13344.0 & 32.12 & 30.91 \\
\hline $\mathrm{HPT}$ & 12.10 & 4.299 & 0.9085 & 216.045 & 1.3560 & 0.8892 & 13344.1 & & \\
\hline $\mathrm{HPT}$ & 56.05 & 4.357 & .9118 & 89.179 & 1.4005 & 0.8946 & 10370.2 & & \\
\hline \multirow{2}{*}{\multicolumn{10}{|c|}{ TURBOMACHINERY MAP DATA }} \\
\hline & & & & & & & S_PRdes & s_effDes & S_NcDes \\
\hline an & 1176.91 & 1.566 & 0.9113 & 3851.762 & 0.0597 & $\overline{0} .8607$ & 1.0235 & 0.9980 & $\overline{0} .9905$ \\
\hline$\triangle P C$ & 146.11 & 2.478 & 0.8217 & 1.027 & 0.0000 & 1.2541 & 0.9513 & 1.0497 & 0.0003 \\
\hline C & 80.04 & 11.0 & 0. & 9825.195 & 12.9518 & 1.0995 & 1.0494 & 0.9898 & 0.9733 \\
\hline T & 0.9 & 4.208 & 0.9085 & 1.298 & 4.2078 & 12.6299 & 0.9723 & 1.0000 & 0.0003 \\
\hline $\mathrm{PT}$ & 0.86 & 3.525 & .9186 & 1.003 & 3.5249 & 65.2590 & 0.7521 & 0.9926 & 0.0005 \\
\hline
\end{tabular}




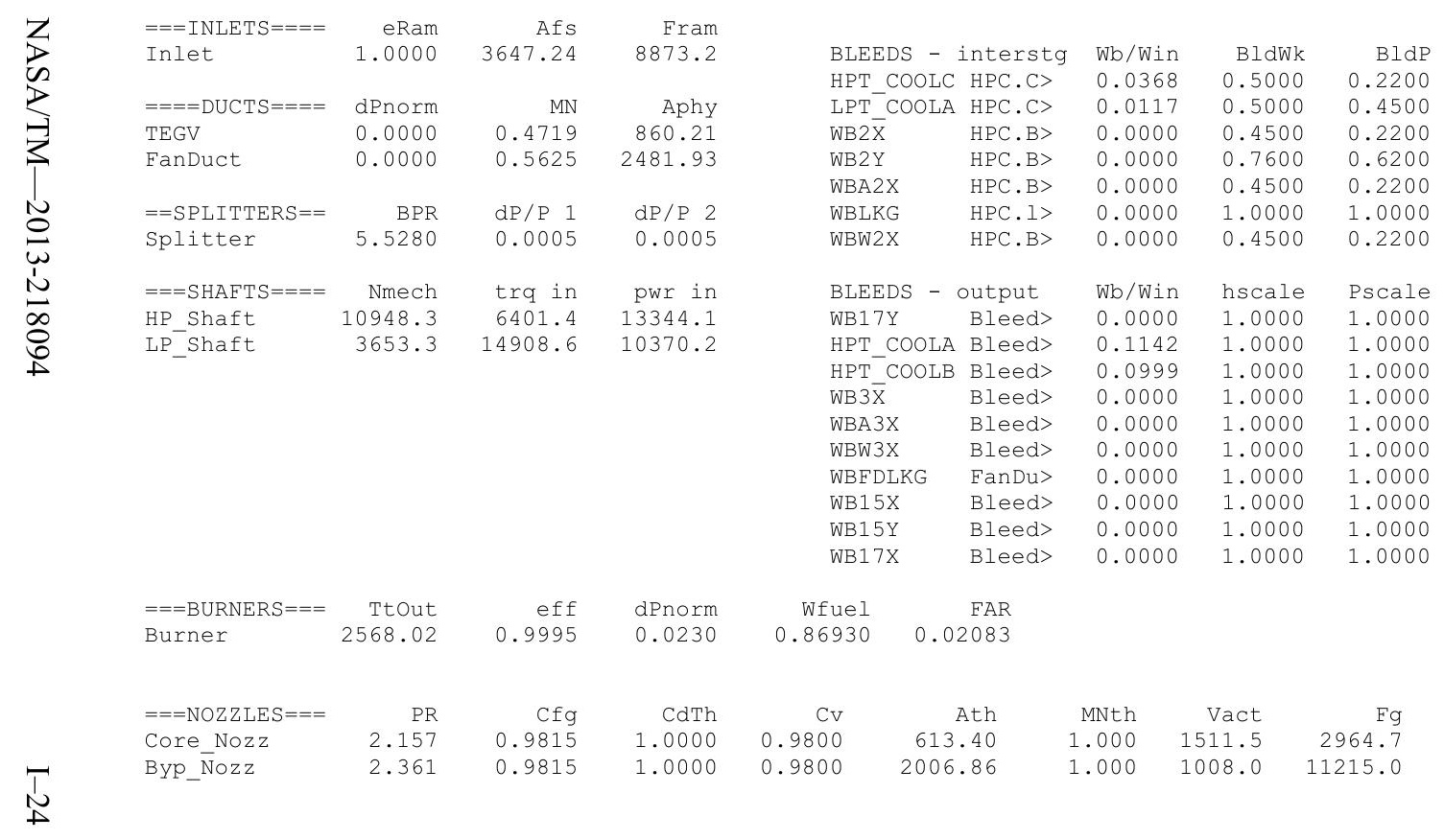




\section{$10 \mu \mathrm{m}, \mathrm{ISA}+36 \mathrm{R}$}

Date:05/13/13 Time:07:24:08 Model: Turbofan Engine -

Version:NPSS 1.6.5 - Rev: -> Gas Package: Janaf iter/pass/Jacb/Broy=24/38/1/22 Run by: Philip C Jorgenson PC: 100

Temperature Stator 1 inlet: 463.97 Stator 1 exit: 483.46 Stator 2 inlet: 506.07 Stator 2 exit: 513.64

Stator 3 inlet: 543.80 Stator 3 exit: 552.19 Stator 4 inlet:580.37 stator 4 exit: 588.48

Ambient Relative Humidity $\quad 10.00$

Fan Face Relative Humidity $\quad 5.44$

IPC Inlet Relative Humidity 0.19

LPC Exit Relative Humidity 2.52

Drop Diameter

0.00

Drop Diameter

0.0000100

789.49

Inlet Length

40.00

Ambient Static Pressure

Ambient Static Temperature

2.85

Fan/LPC Inlet Flow Velocity $\quad 494.89$

Ambient Static Temperature

0.0010505

Fan/LPC Inlet Static Temperature 457.51

SUMMARY OUTPUT DATA

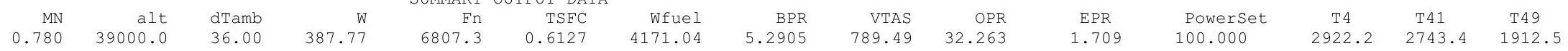

\begin{tabular}{|c|c|}
\hline So & Ambient.Fl_O \\
\hline FS1 & Inlet.Fl_o \\
\hline FS12 & Splitter.Fl_02 \\
\hline FS2 & Splitter.FI_01 \\
\hline FS14 & Fan.Fl O \\
\hline FS23 & LPC.FI- O \\
\hline FS24 & VaporIN̄.FI_O \\
\hline FS25 & Bleed2.FI_o \\
\hline FS3 & HPC.FI O \\
\hline FS36 & Bleed3.FI_O \\
\hline FS4 & Burner.Fl-O \\
\hline FS 45 & HPT.FI_O \\
\hline FS49 & LPT.EI_O \\
\hline FS5 & TEGV.FI \\
\hline S8 & Core Nozzz.Fl \\
\hline S17 & FanDuctLkg.Fİ_o \\
\hline FS171 & Bleed15.FI O \\
\hline 5172 & FanDuct.Fl 0 \\
\hline S173 & Byp Nozz.Fī \\
\hline
\end{tabular}

\begin{tabular}{rrrrrr}
\multicolumn{7}{c}{ FLOW } & \multicolumn{1}{l}{ STATION DATA } \\
W & Pt & Tt & ht & FAR & WC \\
387.77 & 4.267 & 477.92 & -16.44 & 0.0000 & 1282.06 \\
387.77 & 4.267 & 477.92 & -16.44 & 0.0000 & 1282.06 \\
326.13 & 4.265 & 477.92 & -16.44 & 0.0000 & 1078.79 \\
61.64 & 4.265 & 477.92 & -16.44 & 0.0000 & 203.91 \\
326.13 & 7.257 & 563.97 & 4.20 & 0.0000 & 688.63 \\
61.64 & 10.520 & 648.20 & 24.47 & 0.0000 & 96.27 \\
61.71 & 10.520 & 649.45 & 18.74 & 0.0000 & 96.47 \\
61.71 & 10.520 & 649.45 & 18.74 & 0.0000 & 96.47 \\
58.72 & 137.658 & 1428.86 & 214.09 & 0.0000 & 10.40 \\
45.50 & 137.658 & 1428.86 & 214.09 & 0.0000 & 8.06 \\
46.66 & 134.488 & 2922.17 & 182.01 & 0.0255 & 12.10 \\
62.14 & 31.487 & 1921.74 & -2.84 & 0.0190 & 55.83 \\
62.87 & 7.253 & 1380.52 & -148.68 & 0.0188 & 207.83 \\
62.87 & 7.253 & 1380.58 & -148.68 & 0.0188 & 207.83 \\
62.87 & 7.253 & 1380.71 & -148.68 & 0.0188 & 207.84 \\
326.13 & 7.257 & 563.97 & 4.20 & 0.0000 & 688.63 \\
326.13 & 7.257 & 563.97 & 4.20 & 0.0000 & 688.63 \\
326.13 & 7.257 & 563.97 & 4.20 & 0.0000 & 688.63 \\
326.13 & 7.257 & 563.97 & 4.20 & 0.0000 & 688.63 \\
& & & & &
\end{tabular}

$\begin{array}{rr}\text { PS } & \text { Ts } \\ 2.854 & 425.97 \\ 3.151 & 438.20 \\ 3.174 & 439.19 \\ 3.661 & 457.51 \\ 6.025 & 534.77 \\ 9.203 & 623.99 \\ 9.195 & 625.06 \\ 9.195 & 625.06 \\ 124.168 & 1390.75 \\ 129.821 & 1407.11 \\ 127.064 & 2885.43 \\ 28.365 & 1873.87 \\ 6.258 & 1329.20 \\ 6.258 & 1329.26 \\ 3.883 & 1174.12 \\ 6.025 & 534.77 \\ 5.854 & 530.41 \\ 5.854 & 530.41 \\ 3.833 & 469.91\end{array}$

$\begin{array}{rrr}\text { Aphy } & \text { MN } & \text { gamt } \\ 3911.3 & 0.7800 & 1.400068 \\ 4168.2 & 0.6725 & 1.40068 \\ 3531.8 & 0.6634 & 1.40068 \\ 830.5 & 0.4718 & 1.40068 \\ 2606.7 & 0.5227 & 1.39952 \\ 412.6 & 0.4416 & 1.39756 \\ 412.6 & 0.4430 & 1.39737 \\ 412.6 & 0.4430 & 1.39737 \\ 49.7 & 0.3926 & 1.35413 \\ 49.3 & 0.2952 & 1.35413 \\ 74.6 & 0.3008 & 1.28077 \\ 265.4 & 0.4007 & 1.31714 \\ 860.2 & 0.4726 & 1.34395 \\ 860.2 & 0.4726 & 1.34394 \\ 613.4 & 1.0000 & 1.34393 \\ 2606.7 & 0.5227 & 1.39952 \\ 2481.9 & 0.5626 & 1.39952 \\ 2481.9 & 0.5626 & 1.39952 \\ 2006.9 & 1.0000 & 1.39952\end{array}$

WAR 0.0000851 0.0000851 0.0000851 0.0000851 0.0000851 0.0011356 0.0011356 0.0011356 0.0011356 0.0011356 0.0011356 0.0011356 0.0011356 0.0011356 0.0000851 0.0000851 0.0000851 0.0000851

TURBOMACHINERY PERFORMANCE DATA

\begin{tabular}{|c|c|c|c|c|c|c|c|c|c|}
\hline & WC & PR & eff & NC & TR & efPoly & pwr & SMN & SMW \\
\hline Fan & 1078.79 & 1.702 & 0.9114 & 4139.469 & 1.1801 & 0.9178 & -9525.9 & 36.68 & 23.09 \\
\hline LPC & 203.91 & 2.467 & 0.8251 & 4139.469 & 1.3563 & 0.8457 & -3567.8 & 2.97 & 2.24 \\
\hline PC & 96.47 & 13.086 & 0.8585 & 10352.276 & 2.2001 & 0.8978 & -16641.9 & 26.20 & 25.41 \\
\hline HPT & 12.10 & 4.271 & 0.9122 & 214.295 & 1.3425 & 0.8903 & 16641.9 & & \\
\hline LPT & 55.83 & 4.341 & 0.9167 & 90.642 & 1.3885 & 0.9005 & 13093.6 & & \\
\hline & INERY MZ & DATA & & & & & & & \\
\hline & WcMap & PRmap & effMap & NcMap & R/Parm & s WcDes & PRdes & $s$ effDes & S NCDes \\
\hline an & 1253.45 & 1.686 & 0.9132 & 4100.000 & 0.0639 & $\overline{0} .8607$ & 1.0235 & 0.9980 & 0.9905 \\
\hline & 166.84 & 2.748 & 41 & 1.093 & 0.0000 & 1.2221 & 0.8391 & 0.9892 & 0.0003 \\
\hline PC & 87.73 & 12.517 & 0.8674 & 10076.291 & 13.4793 & 1.0995 & 1.0494 & 0.9898 & 0.9733 \\
\hline & 0.96 & 4.181 & 0.9122 & 1.288 & 4.1806 & 12.6299 & 0.9723 & 1.0000 & 0.0003 \\
\hline & 0.86 & 3.513 & 0.9235 & 1.020 & 3.5132 & 65.2590 & 0.7521 & 0.9926 & 0.0005 \\
\hline
\end{tabular}




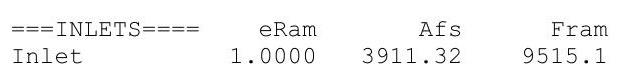

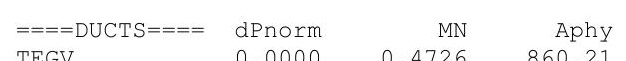

$\begin{array}{llrr} & & \text { Aphy } \\ \text { TEGV } & 0.0000 & 0.4726 & 860.21 \\ \text { FanDuct } & 0.0000 & 0.5626 & 2481.93\end{array}$

$==$ SPLITTERS $==\quad \mathrm{BPR} \quad \mathrm{dP} / \mathrm{P} 1 \mathrm{dP} / \mathrm{P}_{2}$

$\begin{array}{llll}\text { Splitter } & 5.2905 & 0.0005 & 0.0005\end{array}$

$===$ SHAFTS $====$ Nmech trq in pwr in

$\begin{array}{lrrr}\text { HP_Shaft } & 11584.2 & \text { trq in } & \text { pwr in } \\ \text { HP Shaft } & 3973.5 & 16641.9\end{array}$

$\begin{array}{lrrr}\text { LP_Shaft } & 11584.2 & 7545.2 & 16641.9 \\ & 3973.5 & 17306.7 & 13093.6\end{array}$

$===$ BURNERS $===\quad$ TtOut

Burner 2922.17

0.9995

dPnorm $===$ NOZZLES $===$ Core_Nozz PR
2.541
2.543 Cfg
0.9809
0.9810

\section{CdTh} $0.9810-1.0000$ $1.0000 \quad 0.9800$

$\begin{array}{rrrr}\text { W } & \text { Tt } & \text { ht } & \text { Pt } \\ 2.2709 & 1047.82 & 116.41 & 31.487 \\ 0.7220 & 1047.82 & 116.41 & 7.253 \\ 0.0000 & 1008.71 & 106.65 & 38.490 \\ 0.0000 & 1248.21 & 167.20 & 89.346 \\ 0.0000 & 1008.71 & 106.65 & 38.490 \\ 0.0000 & 1428.86 & 214.09 & 137.658 \\ 0.0000 & 1008.71 & 106.65 & 38.490 \\ \text { W } & \text { Tt } & \text { ht } & \text { Pt } \\ 0.0000 & 649.45 & 18.74 & 10.520 \\ 7.0458 & 1428.86 & 214.09 & 134.488 \\ 6.1652 & 1428.86 & 214.09 & 75.571 \\ 0.0000 & 1428.86 & 214.09 & 137.658 \\ 0.0000 & 1428.86 & 214.09 & 137.658 \\ 0.0000 & 1428.86 & 214.09 & 137.658 \\ 0.0000 & 563.97 & 4.20 & 7.257 \\ 0.0000 & 563.97 & 4.20 & 7.257 \\ 0.0000 & 563.97 & 4.20 & 7.257 \\ 0.0000 & 563.97 & 4.20 & 7.257\end{array}$

\begin{tabular}{|c|c|c|c|c|}
\hline BLEED, & terstg & Wb/Win & $\mathrm{BldWk}$ & $\mathrm{B} 1$ \\
\hline OLC & HPC.C> & 0.0368 & 0.5000 & 23 \\
\hline LPT_COOLA & HPC.C> & 0.0117 & 0.5000 & 0.4 \\
\hline WB2 $2 \bar{x}$ & HPC. B> & 0.0000 & 0.4500 & 0.22 \\
\hline WB2Y & HPC.B> & 0.0000 & 0.7600 & \\
\hline WBA2X & HPC. B > & .0000 & 0.4500 & \\
\hline WBLKG & HPC. $1>$ & 0 & 1.0 & \\
\hline WBW2X & HPC.B> & .0000 & 0.4500 & \\
\hline BLEEDS & output & 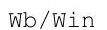 & hscale & \\
\hline WB17Y & Blee & .0000 & & \\
\hline HPT COOLA & Bleed> & 0.1142 & 1.0000 & \\
\hline HPT COOLB & Bleed> & 99 & 1.0 & \\
\hline WB $3 \bar{x}$ & Bleed> & 0000 & 1.0 & \\
\hline WBA $3 \mathrm{X}$ & Bleed> & 0000 & 1.0 & \\
\hline WBW3X & Bleed> & .0 & 1.0 & \\
\hline WBFDLKG & FanDu> & 0 & 1.0000 & \\
\hline WB15X & Blee & 0 & 1.0 & \\
\hline WB15Y & Bleed> & .0000 & 1.0000 & \\
\hline WB17X & Bleed> & 0.0000 & 1.0000 & 10 \\
\hline
\end{tabular}

Wfuel

FAR$$
\mathrm{CV} \quad \text { Ath }
$$

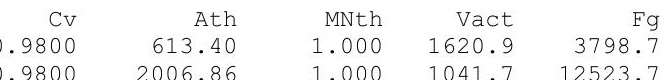


Model:

Turbofan Engine - COMDES ON

converge $=1$ CASE: 0

Version:NPSS 1.6.5 - Rev: -> Gas Package: Janaf iter/pass/Jacb/Broy= 20/34/1/18 Run by: Philip C Jorgenson PC: 84

Temperature Stator 1 inlet: 469.82 Stator 1 exit: 487.88 Stator 2 inlet: 509.30 Stator 2 exit: 517.60 $\begin{array}{lll}\text { Stator } 3 \text { inlet: } 544.42 & \text { Stator } 3 \text { exit: } 553.00 & \text { Stator } 4 \text { inlet: } 578.30 \text { Stator 4 exit: } 586.27 \\ \text { Stator } 5 \text { inlet: } 606.20 & \text { Stator } 5 \text { exit: } 613.82 & \text { Unblocked Percent Blockage: } 0.00\end{array}$

Ambient Relative Humidity $\quad 10.00$

Fan Face Relative Humidity $\quad 4.45$

Fan Bypass Relative Humidity 0.23

LPC Inlet Relative Humidity $\quad 2.24$

LPC Exit Relative Humidity

0.00

0.0000100

Amblent Flow velocity

Ambient Static Pressure

Additional Water at LPC Exit

789.49

2.85

Inlet Length

40.00

Fan/LPC Inlet Flow Velocity $\quad 457.71$ 0.001143

Fan/LPC Inlet Static Temperature $\quad 460.46$

SUMMARY OUTPUT DATA

MN alt dTamb W $\quad$ Fn TSFC Wfuel

\section{FLOW STATION DATA}

FSO Ambient.Fl_O

FS1 Inlet.Fl_O

$\begin{array}{ll}\text { FS12 } & \text { Splitter.F1_02 } \\ \text { FS2 } & \text { Splitter.Fl-01 }\end{array}$

FS14 Fan.Fl_O

LPC.FI_O

FS25 Bleed2.FI-

FS25 Bleed2.F1

FS36 BPC.F1-

FS4 Burner. F1-O

FS4 Burner.FI

FS49 4 HPT. FI-

ES5 5 TEGV FI

FS8 Core Nozz.Fl

FS17 FanDuctLkg.Fi_o

FS171 Bleed15.FI_O

FS172 FanDuct. F1-0

FS173 Byp Nozz. Fi-

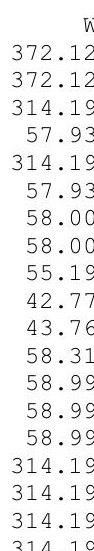

$\begin{array}{rr}\mathrm{Pt} & \mathrm{Tt} \\ 4.267 & 477.92 \\ 4.267 & 47.92\end{array}$

3571.07

BPR
5.4231

789.49

OPR
29.440

ht FAR WC

$\begin{array}{lllll}4.267 & 477.92 & -16.44 & 0.0000 & 1230.32\end{array}$

$\begin{array}{rrrrr}4.265 & 477.92 & -16.44 & 0.0000 & 1039.29 \\ 4.265 & 477.92 & -16.44 & 0.0000 & 191.64\end{array}$

$\begin{array}{rrrrr}4.265 & 477.92 & -16.44 & 0.0000 & 1039.29 \\ 6.943 & 556.26 & 2.35 & 0.0000 & 191.64\end{array}$

$\begin{array}{rrrrr}4.265 & 477.92 & -16.44 & 0.0000 & 191.64 \\ 10.389 & 540.26 & 2.35 & 0.0000 & 688.65\end{array}$

$\begin{array}{lllll}10.389 & 640.38 & 22.58 & 0.0000 & 91.06 \\ 10.389 & 641.75 & 16.35 & 0.0000 & 91.26\end{array}$

$10.389-641.75$

$125.614-1380.13$

91.26
10.53

10.53
8.16

$200.82 \quad 0.00$

$\begin{array}{llll}125.614 & 1380.13 & 200.82 & 0.0000\end{array}$

$\begin{array}{lllll}28.642 & 1813.72 & -3.22 & 0.0173\end{array}$

$\begin{array}{llll}6.591 & 1298.42 & -140.33 & 0.0171\end{array}$

$\begin{array}{lllll}6.591 & 1298.49 & -140.33 & 0.0171\end{array}$

$\begin{array}{llll}6.591 & 1298.62 & -140.33 & 0.0171\end{array}$

$\begin{array}{llll}6.943 & 556.26 & 2.35 & 0.0000\end{array}$

$\begin{array}{llll}6.943 & 556.26 & 2.35 & 0.0000\end{array}$

$\begin{array}{llll}6.943 & 556.26 & 2.35 & 0.0000\end{array}$

$6.943-556.26 \quad 2.35 \quad 0.0000$

12.10

208.13

208.13

208.14

688.65
688.65

688.65

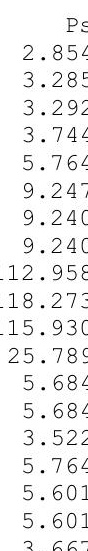

(1)

$\begin{array}{clccc}\text { EPR } & \text { Powerset } & \text { T4 } & \text { T41 } & \text { T49 } \\ 1.518 & 84.000 & 2764.0 & 2597.0 & 1805.3\end{array}$

TURBOMACHINERY PERFORMANCE DATA

\begin{tabular}{|c|c|c|c|c|c|c|c|c|c|}
\hline & Wc & PR & eff & NC & TR & efPoly & pwr & SMN & SMW \\
\hline Fan & 1039.29 & 1.628 & 0.9121 & 3983.460 & 1.1639 & 0.9179 & -8353.4 & 51.66 & 26.14 \\
\hline LPC & 191.64 & 2.436 & 0.8514 & 3983.460 & 1.3399 & 0.8687 & -3198.7 & 2.91 & 2.10 \\
\hline HPC & 91.26 & 12.091 & .8607 & 10183.487 & 2.1506 & 0.8985 & -14771.2 & 29.85 & 28.84 \\
\hline $\mathrm{HPT}$ & 12.10 & 4.285 & 0.9109 & 215.460 & 1.3487 & 0.8905 & 14771.3 & & \\
\hline $\mathrm{HPT}$ & 55.95 & 4.346 & .9148 & 89.786 & 1.3937 & 0.8982 & 11551.9 & & \\
\hline \multicolumn{10}{|c|}{ ACHINERY MAP DATA } \\
\hline & WcMap & PRmap & effMap & NcMap & R/Parm & s_WcDes & S_PRdes & s_effDes & S_NcDes \\
\hline an & 1207.56 & 1.614 & 0.9139 & 3945.478 & 0.0614 & $\overline{0} .8607$ & 1.0235 & 0.9980 & $\overline{0} .9905$ \\
\hline $\mathrm{PC}$ & 152.44 & 2.576 & 0.8239 & 1.052 & 0.0000 & 1.2571 & 0.9113 & 1.0334 & 0.0003 \\
\hline ?C & 83.00 & 11.5 & 0. & 9912.002 & 13.1441 & 1.0995 & 1.0494 & 0.9898 & 0.9733 \\
\hline PT & 0.96 & 4.194 & 0.9109 & 1.295 & 4.1936 & 12.6299 & 0.9723 & 1.0000 & 0.0003 \\
\hline $\mathrm{PT}$ & 0.86 & 3.517 & .9216 & 1.010 & 3.5166 & 65.2590 & 0.7521 & 0.9926 & 0.0005 \\
\hline
\end{tabular}

$\begin{array}{rrrrrrr}\text { Aphy } & \text { MN } & \text { gamt } & \text { WAR } & \text { Wair } & \text { WH2O } & \text { H2O frac } \\ 3753.5 & 0.7800 & 1.40068 & 0.0000851 & 372.09 & 0.032 & 0.0001 \\ 4168.2 & 0.6227 & 1.40068 & 0.0000851 & 372.09 & 0.032 & 0.0001 \\ 3531.8 & 0.6194 & 1.40068 & 0.0000851 & 314.16 & 0.027 & 0.0001 \\ 830.5 & 0.4350 & 1.40068 & 0.0000851 & 57.93 & 0.005 & 0.0001 \\ 2606.7 & 0.5226 & 1.39966 & 0.0000851 & 314.16 & 0.027 & 0.0001 \\ 412.6 & 0.4115 & 1.39778 & 0.0000851 & 57.93 & 0.005 & 0.0001 \\ 412.6 & 0.4129 & 1.39757 & 0.0012282 & 57.93 & 0.071 & 0.0012 \\ 412.6 & 0.4129 & 1.39757 & 0.0012282 & 57.93 & 0.071 & 0.0012 \\ 49.7 & 0.3981 & 1.35690 & 0.0012282 & 55.12 & 0.068 & 0.0012 \\ 49.3 & 0.2990 & 1.35690 & 0.0012282 & 42.72 & 0.052 & 0.0012 \\ 74.6 & 0.3000 & 1.28663 & 0.0012282 & 42.72 & 0.052 & 0.0291 \\ 265.4 & 0.4008 & 1.32277 & 0.0012282 & 57.25 & 0.070 & 0.0222 \\ 860.2 & 0.4722 & 1.34999 & 0.0012282 & 57.93 & 0.071 & 0.0219 \\ 860.2 & 0.4722 & 1.34999 & 0.0012282 & 57.93 & 0.071 & 0.0220 \\ 613.4 & 1.0000 & 1.34998 & 0.0012282 & 57.93 & 0.071 & 0.0220 \\ 2606.7 & 0.5226 & 1.39966 & 0.0000851 & 314.16 & 0.027 & 0.0001 \\ 2481.9 & 0.5626 & 1.39966 & 0.0000851 & 314.16 & 0.027 & 0.0001 \\ 2481.9 & 0.5626 & 1.39966 & 0.0000851 & 314.16 & 0.027 & 0.0001 \\ 2006.9 & 1.0000 & 1.39966 & 0.0000851 & 314.16 & 0.027 & 0.0001\end{array}$




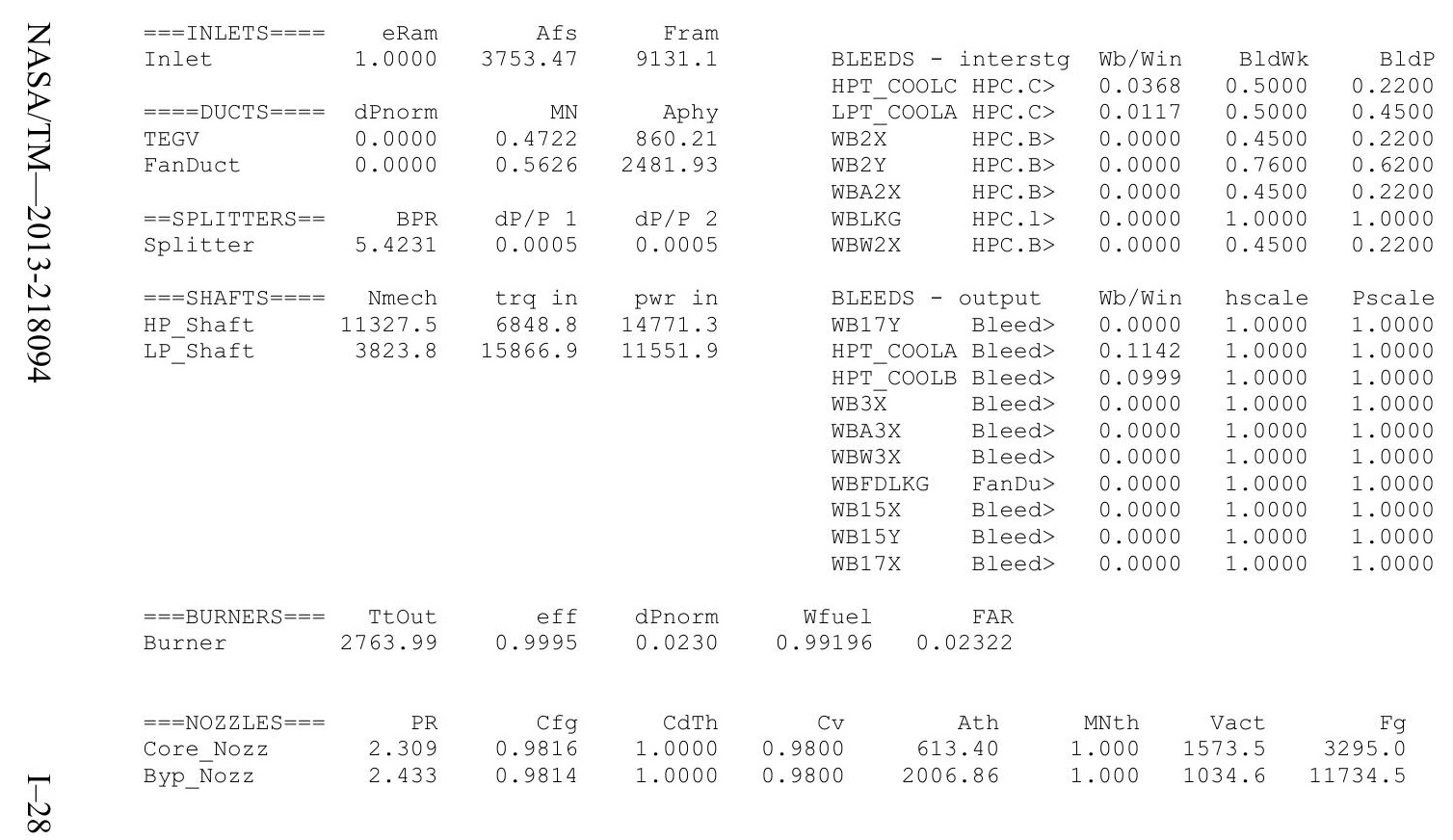


Date:05/13/13 Time:07:25:20 Model: Turbofan Engine - COMDES ON COnverge = 1 CASE: 0

Version:NPSS_1.6.5 - Rev: -> Gas Package: Janaf iter/pass/Jacb/Broy= 21/ 35/1/19 Run by: Philip C Jorgenson

Temperature Stator 1 inlet: 471.54 Stator 1 exit: $489.16 \quad$ Stator 2 inlet: $510.24 \quad$ Stator 2 exit: 518.67 $\begin{array}{llll}\text { Stator } 3 \text { inlet: } 544.60 & \text { Stator } 3 \text { exit: } 553.19 & \text { Stator } 4 \text { inlet: } 577.67 \text { Stator 4 exit: } 585.60 \\ \text { Stator } 5 \text { inlet: } 604.98 & \text { Stator } 5 \text { exit: } 612.52 & \text { Unblocked Percent Blockage: } 0.00\end{array}$

Ambient Relative Humidity $\quad 10.00$

Fan Face Relative Humidity $\quad 4.20$

Fan Bypass Relative Humidity $\quad 0.25$

$\begin{array}{ll}\text { LPC Exit Relative Humidity } & 0.16 \\ & 0.03\end{array}$

HPC Relative Humidity

Drop Diameter

0.00

Ambient $\quad 0.0000100$

Ambient Static Pressure

Ambient Static Temperature

789.49

2.85

Inlet Length

40.00

Additional Water at LPC Exit

Fan/LPC Inlet Flow Velocity $\quad 445.14$ 0.001178

$\begin{array}{lr}\text { Fan/LPC Inlet Static Temperature } & 461.41\end{array}$

MN SUMMARY OUTPUT DATA

$\begin{array}{rrrrrr}\text { MN } & \text { alt } & \text { dTamb } & \text { W } & \text { Fn } & \text { TSFC } \\ 0.780 & 39000.0 & 36.00 & 366.87 & 5602.3 & 0.6042\end{array}$

FSO Ambient.FI_O

FS1 Inlet.Fl_O

$\begin{array}{ll}\text { FS12 } & \text { Splitter.Fl_02 } \\ \text { FS2 } & \text { Splitter.Fl_01 }\end{array}$

FS14 Fan.FI_O

FS23 LPC.FIO

VIN.FI_O

$\begin{array}{ll}\text { FS25 } & \text { Bleed2.FI_ } \\ \text { FS3 } & \text { HPC.FI_O }\end{array}$

FS36 Bleed3.FI_O

FS4 Burner.FI_O

FS45 HPT.F1_O

FS49 LPT.FI_O

$\begin{array}{ll}\text { FS5 } & \text { TEGV.FI_O } \\ \text { FS8 } & \text { Core Nozzz.Fl }\end{array}$

FS17 FanDuctLkg.Fi_o

FS171 Bleed15.FI_O

FS172 FanDuct.Fl-

FS173 Byp_Nozz.FI_O

$W$
366.87
366.87
310.23
56.63
310.23
56.63
56.70
56.70
53.95
41.81
42.75
56.98
57.64
57.64
57.64
310.23
310.23
310.23
310.23

FLOW STATION DATA

$\begin{array}{crrrr}\text { Pt } & \text { Tt } & \text { ht } & \text { FAR } & \text { WC } \\ 4.267 & 477.92 & -16.44 & 0.0000 & 1212.95\end{array}$

$\begin{array}{lllll}4.267 & 477.92 & -16.44 & 0.0000 & 1212.95 \\ 4.267 & 477.92 & -16.44 & 0.0000 & 1212.95\end{array}$

$\begin{array}{lllll}4.265 & 477.92 & -16.44 & 0.0000 & 1026.23\end{array}$

$\begin{array}{lllll}4.265 & 477.92 & -16.44 & 0.0000 & 187.34\end{array}$

$\begin{array}{lllll}6.840 & 553.72 & 1.74 & 0.0000 & 688.65\end{array}$

$\begin{array}{lllll}10.331 & 638.03 & 22.02 & 0.0000 & 89.35\end{array}$

$\begin{array}{lllll}10.331 & 639.45 & 15.59 & 0.0000 & 89.5 \\ 10.331 & 639.45 & 15.59 & 0.0000 & 89.56\end{array}$

$\begin{array}{rrrrr}121.614 & 1364.61 & 196.58 & 0.0000 & 10.57\end{array}$

$\begin{array}{rrrrr}121.614 & 1364.61 & 196.58 & 0.0000 & 8.20 \\ 118.813 & 2713.83 & 168.74 & 0.0225 & 12.10\end{array}$

$\begin{array}{rrrrr}118.813 & 2713.83 & 168.74 & 0.0225 & 12.10 \\ 27.704 & 1779.91 & -3.36 & 0.0168 & 55.99\end{array}$

$\begin{array}{rrrrr}27.704 & 1779.91 & -3.36 & 0.0168 & 55.99 \\ 6.373 & 1272.88 & -137.75 & 0.0166 & 208.23\end{array}$

$\begin{array}{llllll}6.373 & 1272.94 & -137.75 & 0.0166 & 208.24\end{array}$

$\begin{array}{llllll}6.373 & 1273.08 & -137.74 & 0.0166 & 208.24 \\ 6.840 & 553.72 & 1.74 & 0.0000 & 688.65\end{array}$

$\begin{array}{lrrrr}6.840 & 553.72 & 1.74 & 0.0000 & 688.65\end{array}$

$\begin{array}{lllll}6.840 & 553.72 & 1.74 & 0.0000 & 688.65 \\ 6.840 & 553.72 & 1.74 & 0.0000 & 688.65\end{array}$

$\begin{array}{lllll}6.840 & 553.72 & 1.74 & 0.0000 & 688.65 \\ 6.840 & 553.72 & 1.74 & 0.0000 & 688.65\end{array}$

$\begin{array}{lllll}6.840 & 553.72 & 1.74 & 0.0000 & 688.65\end{array}$

TURBOMACHINERY PERFORMANCE DATA

\begin{tabular}{lrrrrrrrrr}
\multicolumn{1}{c}{ Wan } & Wc & PR & eff & NC & TR & efPoly & pwr & SMN & SMW \\
Fan & 1026.23 & 1.604 & 0.9118 & 3934.513 & 1.1586 & 0.9175 & -7980.7 & 56.27 & 27.19 \\
LPC & 187.34 & 2.422 & 0.8578 & 3934.513 & 1.3350 & 0.8742 & -3081.4 & 3.23 & 2.49 \\
HPC & 89.56 & 11.772 & 0.8613 & 10135.831 & 2.1340 & 0.8987 & -14166.9 & 31.13 & 30.01 \\
HPT & 12.10 & 4.289 & 0.9102 & 216.036 & 1.3505 & 0.8900 & 14167.0 & & \\
LPT & 55.99 & 4.347 & 0.9141 & 89.521 & 1.3953 & 0.8974 & 11062.1 & &
\end{tabular}

TURBOMACHINERY MAP DATA

$\begin{array}{lrrrrrrrrr} & \text { WcMap } & \text { PRmap } & \text { effMap } & \text { NcMap } & \text { R/Parm } & \text { S_WcDes } & \text { S_PRdes } & \text { S_effDes } & \text { S_NcDes } \\ \text { Fan } & 1192.37 & 1.590 & 0.9136 & 3896.998 & 0.0606 & 0.8607 & \overline{1} .0235 & 0.9980 & 0.9905 \\ \text { LPC } & 149.06 & 2.525 & 0.8226 & 1.039 & 0.0000 & 1.2568 & 0.9328 & 1.0428 & 0.0003 \\ \text { HPC } & 81.45 & 11.265 & 0.8702 & 9865.617 & 13.0344 & 1.0995 & 1.0494 & 0.9898 & 0.9733 \\ \text { HPT } & 0.96 & 4.197 & 0.9102 & 1.298 & 4.1975 & 12.6299 & 0.9723 & 1.0000 & 0.0003 \\ \text { LPT } & 0.86 & 3.518 & 0.9208 & 1.007 & 3.5176 & 65.2590 & 0.7521 & 0.9926 & 0.0005\end{array}$

\begin{tabular}{|c|c|}
\hline & \\
\hline 2.8 & 42 \\
\hline 3.3 & 445 \\
\hline 3.3 & 445 \\
\hline 3.7 & 461 \\
\hline 5. & 525 \\
\hline 9.2 & 618 \\
\hline 9.2 & 61 \\
\hline 9.2 & 7 \\
\hline 109.2 & 1326 \\
\hline 114. & 1342 \\
\hline 112.2 & 2679 \\
\hline 24. & 1734 \\
\hline 5. & 1224 \\
\hline 5.2 & 1224 \\
\hline 3.2 & 1079 \\
\hline 5.6 & 525 \\
\hline 5.5 & 520 \\
\hline 5.5 & 520 \\
\hline 3. & 461 \\
\hline & SMW \\
\hline & 27.19 \\
\hline & 2.49 \\
\hline & 30.01 \\
\hline es & S_NCDes \\
\hline & $\overline{0} .9905$ \\
\hline 0428 & 0.00 \\
\hline & 0.973 \\
\hline & .000 \\
\hline & 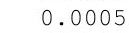 \\
\hline
\end{tabular}

$\begin{array}{clccc}\text { EPR } & \text { PowerSet } & \text { T4 } & \text { T41 } & \text { T49 } \\ 1.457 & 79.800 & 2713.8 & 2550.6 & 1771.7\end{array}$

$\begin{array}{rrr}\text { Aphy } & \text { MN } & \text { gamt } \\ 3700.5 & 0.7800 & 1.40068 \\ 4168.2 & 0.6076 & 1.40068 \\ 3531.8 & 0.6061 & 1.40068 \\ 830.5 & 0.4226 & 1.40068 \\ 2606.7 & 0.5226 & 1.39970 \\ 412.6 & 0.4020 & 1.39784 \\ 412.6 & 0.4033 & 1.39763 \\ 412.6 & 0.4033 & 1.39763 \\ 49.7 & 0.3999 & 1.35780 \\ 49.3 & 0.3002 & 1.35780 \\ 74.6 & 0.2998 & 1.28851 \\ 265.4 & 0.4009 & 1.32460 \\ 860.2 & 0.4722 & 1.35193 \\ 860.2 & 0.4722 & 1.35192 \\ 613.4 & 1.0000 & 1.35191 \\ 2606.7 & 0.5226 & 1.39970 \\ 2481.9 & 0.5626 & 1.39970 \\ 2481.9 & 0.5626 & 1.39970 \\ 2006.9 & 1.0000 & 1.39970\end{array}$

WAR
0.0000851
0.0000851
0.0000851
0.0000851
0.0000851
0.0000851
0.0012632
0.0012632
0.0012632
0.0012632
0.0012632
0.0012632
0.0012632
0.0012632
0.0012632
0.0000851
0.0000851
0.0000851
0.0000851

Wair
366.84 366.84 366.84
310.21 310.21 310.21
56.63

56.63

56.63
53.88

$\begin{array}{ll}53.88 & 0.068 \\ 41.76 & 0.053\end{array}$

$\begin{array}{ll}53.88 & 0.068 \\ 41.76 & 0.053 \\ 55.97 & 0.053\end{array}$

$\begin{array}{lll}45.97 & 0.071\end{array}$

$\begin{array}{ll}55.97 & 0.071 \\ 56.63 & 0.072\end{array}$

$\begin{array}{ll}56.63 & 0.072 \\ 56.63 & 0.072\end{array}$

$\begin{array}{rr}56.63 & 0.072 \\ 310.21 & 0.026 \\ 310.21 & 0.026\end{array}$

$\begin{array}{ll}310.21 & 0.026 \\ 310.21 & 0.026\end{array}$

$\begin{array}{ll}310.21 & 0.026 \\ 310.21 & 0.026\end{array}$

$310.21 \quad 0.026$
H2O frac 0.0001 0.00001
0.0001 0.0001 0.0001 0.0013 0.0013 0.0013 0.0216 0.0216 0.0214 0.0214 0.0001 0.0001 0.0001 


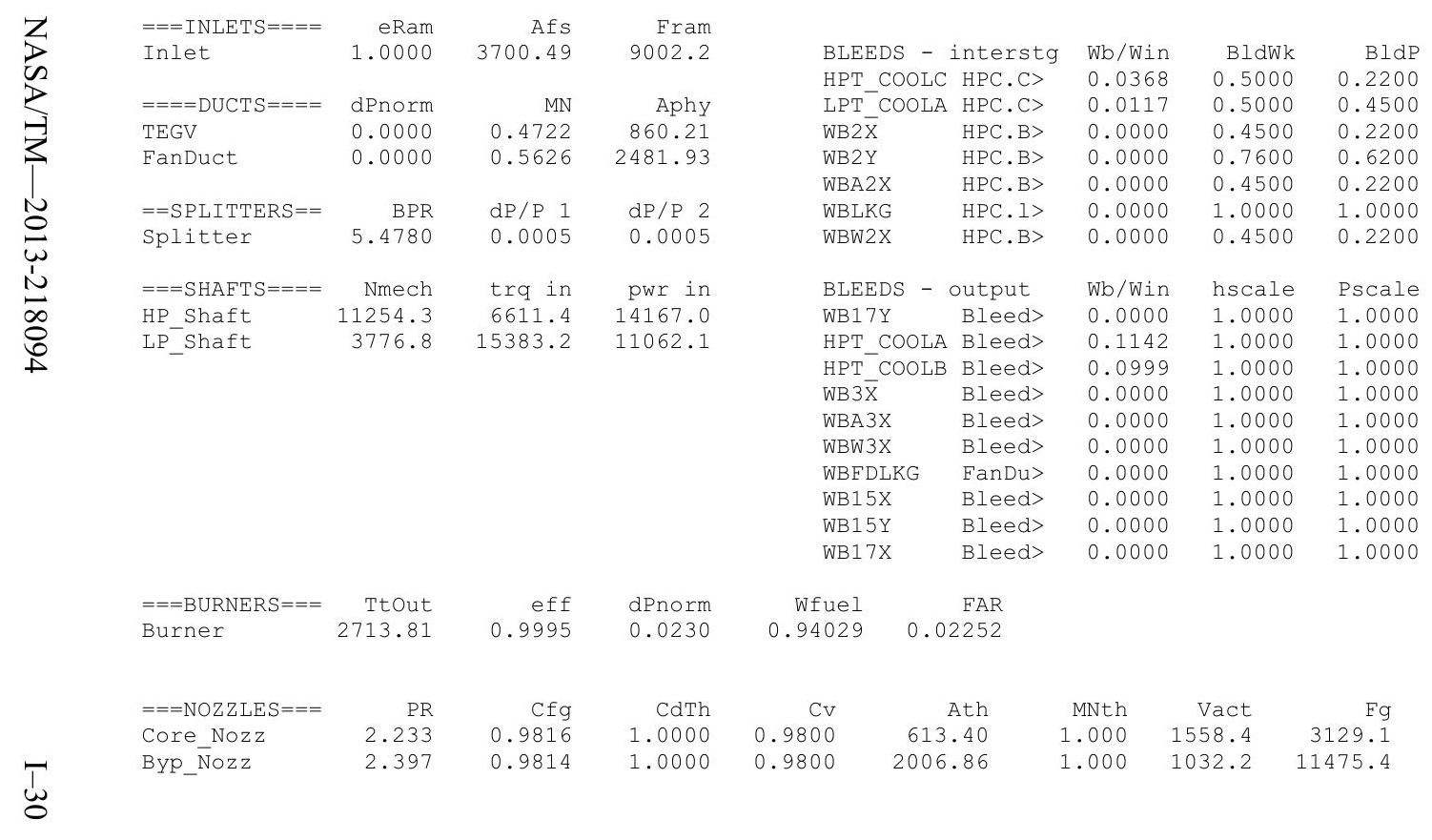


Date:05/13/13 Time:07:25:54 Model: Turbofan Engine - COMDES ON COnverge $=1$ CASE: 0

Version:NPSS 1.6.5 - Rev: -> Gas Package: Janaf iter/pass/Jacb/Broy=19/ 33/1/17 Run by: Philip C Jorgenson

Temperature Stator 1 inlet: 472.72 Stator 1 exit: $490.05 \quad$ Stator 2 inlet: 510.93 Stator 2 exit: 519.45 $\begin{array}{lll}\text { Stator } 3 \text { inlet: } 544.81 & \text { Stator } 3 \text { exit: } 553.41 & \text { Stator } 4 \text { inlet: } 577.36 \quad \text { Stator } 4 \text { exit: } 585.27 \\ \text { Stator } 5 \text { inlet: } 604.30 & \text { Stator } 5 \text { exit: } 611.78 & \text { Unblocked Percent Blockage: } 0.00\end{array}$

Fan Face Relative Humidity $\quad 10.00$

Fan Bypass Relative Humidity $\quad 0.26$

LPC Inlet Relative Humidity $\quad 2.10$

$\begin{array}{ll}\text { LPC Exit Relative Humidity } & 0.03 \\ \text { HPC Relative Humidity } & 0.00\end{array}$

Drop Diameter

0.00
000100

Ambient Flow Velocity

Ambient Static Pressure

Ambient Static Temperature
Additional Water at LPC Exit

789.49

2.85 0.0012042

$\begin{array}{lr}436.14 \\ \text { an/LPC Inlet Static Pressure } & 3.79\end{array}$

Ean/LPC Inlet Static Temperature 462.07

\begin{tabular}{|c|c|c|c|c|c|c|c|c|c|c|c|c|c|c|}
\hline & & & & SUMMARY & TPUT DAT & & & & & & & & & \\
\hline MN & alt & dTamb & $3633^{W}$ & $\mathrm{Fn}$ & TSFC & $\begin{array}{l}\text { Wfuel } \\
\end{array}$ & $\begin{array}{r}\text { BPR } \\
5 \text {. }\end{array}$ & VTAS & OPR & EPR & Powerset & T4 & T41 & T49 \\
\hline 0.780 & 39000.0 & 36.00 & 363.04 & 5392.0 & 0.6042 & 3257.78 & 5.5193 & 789.49 & 27.840 & 1.415 & 76.800 & 2679.5 & 2518.9 & 1748.8 \\
\hline
\end{tabular}

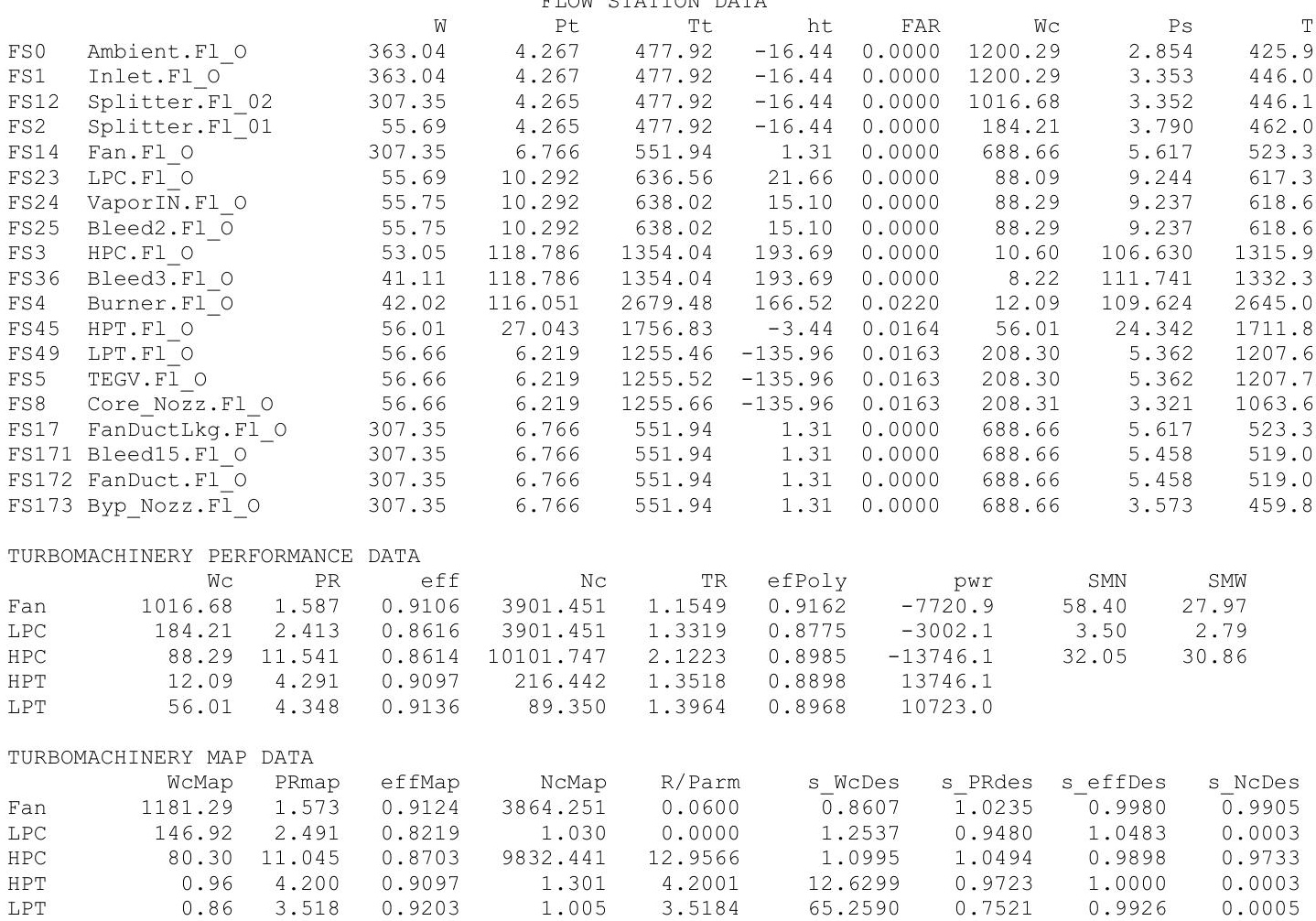


$\begin{array}{lrrr}===\text { INLETS }==== & \text { eRam } & \text { AfS } & \text { Fram } \\ \text { Inlet } & 1.0000 & 3661.85 & 8908.2\end{array}$

$====$ DUCTS $====$ dPnorm MN Aphy

$\begin{array}{llrr}\text { TEGV } & 0.0000 & 0.4721 & 860.21 \\ \text { FanDuct } & 0.0000 & 0.5626 & 2481.93\end{array}$

.0000

Splitter

5.5193

Nmech

11203.8

$\mathrm{dP} / \mathrm{P} 1$

$\mathrm{dP} / \mathrm{P} 2$

$0.0005-0.0005$

$6443.9 \quad 13746.1$

LP_Shaft
$===$ BURNERS $===\quad$ TtOut

Burner 2679.46

$===$ NOZZLES $===$ Core Nozz $P R$
2.179
2.371 eff

dPnorm
BLEEDS - interstg Wb/Win BldWk BldP $\begin{array}{lllll}\text { LPT COOLA HPC.C> } & 0.0117 & 0.5000 & 0.4500\end{array}$ $\begin{array}{lllll}\text { WB2X } & \text { HPC.B> } & 0.0000 & 0.4500 & 0.2200 \\ \text { WB2Y } & \text { HPC.B> } & 0.0000 & 0.7600 & 0.6200\end{array}$ WBA2X HPC.B> $0.0000 \quad 0.4500 \quad 0.2200$ WBLKG HPC.I> $0.0000 \quad 1.0000 \quad 1.0000$ $\begin{array}{lllll}\text { WBW2X HPC.B> } & 0.0000 & 0.4500 & 0.2200\end{array}$

$\begin{array}{llll}\text { BLEEDS - output Wb/Win hscale } & \text { Pscale } \\ \text { WB17Y Bleed> } & 0.0000 & 1.0000 & 1.0000\end{array}$ WB17Y Bleed> $0.0000 \quad 1.0000 \quad 1.0000$ HPT_COOLA Bleed> $0.1142 \quad 1.0000 \quad 1.0000$ HPT COOLB Bleed> $0.0999 \quad 1.0000 \quad 1.0000$

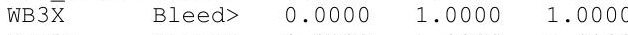
$\begin{array}{lllll}\text { WBA3X } & \text { Bleed> } & 0.0000 & 1.0000 & 1.0000 \\ \text { WBW3X } & \text { Bleed> } & 0.0000 & 1.0000 & 1.0000\end{array}$ $\begin{array}{lllll}\text { WBW3X } & \text { Bleed> } & 0.0000 & 1.0000 & 1.0000 \\ \text { WBFDIKG } & \text { FanDu> } & 0.0000 & 1.0000 & 1.0000\end{array}$ $\begin{array}{lllll}\text { WBFDLKG } & \text { FanDu> } & 0.0000 & 1.0000 & 1.0000 \\ \text { WB15X } & \text { Bleed }> & 0.0000 & 1.0000 & 1.0000\end{array}$ WB15X Bleed> $0.0000-1.0000-1.0000$ $\begin{array}{lllll}\text { WB15Y } & \text { Bleed> } & 0.0000 & 1.0000 & 1.0000 \\ \text { WB17X } & \text { Bleed> } & 0.0000 & 1.0000 & 1.0000\end{array}$

\begin{tabular}{|c|c|c|c|}
\hline W & Tt & ht & Pt \\
\hline .0517 & 1003.16 & 104.39 & 27.043 \\
\hline 0.6523 & 1003.16 & 104.39 & 6.219 \\
\hline 0.0000 & 967.22 & 95.46 & 34.161 \\
\hline 0.0000 & 1187.54 & 150.83 & 77.559 \\
\hline 0.0000 & 967.22 & 95.46 & 34.161 \\
\hline 0.0000 & 1354.04 & 193.69 & 118.786 \\
\hline 0.0000 & 967.22 & 95.46 & 34.161 \\
\hline $\mathrm{W}$ & $\mathrm{Tt}$ & $h t$ & $\mathrm{Pt}$ \\
\hline 0.0000 & 638.02 & 15.10 & 10.292 \\
\hline 6.3659 & 1354.04 & 193.69 & 116.051 \\
\hline 5.5703 & 1354.04 & 193.69 & 65.139 \\
\hline 0.0000 & 1354.04 & 193.69 & 118.786 \\
\hline 0.0000 & 1354.04 & 193.69 & 118.786 \\
\hline 0.0000 & 1354.04 & 193.69 & 118.786 \\
\hline 0.0000 & 551.94 & 1.31 & 6.766 \\
\hline 0.0000 & 551.94 & 1.31 & 6.766 \\
\hline 0.0000 & 551.94 & 1.31 & 6.766 \\
\hline 0.0000 & 551.94 & 1.31 & 6.766 \\
\hline
\end{tabular}

$\begin{array}{lr}\text { Wfuel } & \text { FAR } \\ .90494 & 0.02204\end{array}$ 
Date: 05/13/13 Time:09:33:46 Model:

Version:NPSS 1.6.5 - Rev: -> Gas Package: Janaf iter/pass/Jacb/Broy=22/36/1/20 Run by: Philip C Jorgenson PC: 100

Temperature Stator 1 inlet: 444.04 Stator 1 exit: 462.66 Stator 2 inlet: 484.20 Stator 2 exit: 491.28

Stator 3 inlet: 520.22 Stator 3 exit: 528.14 Stator lnlet: 555.22 stator 4 exit: 562.91

Ambient Relative Humidity $\quad 10.00$

Fan Face Relative Humidity $\quad 5.21$

LPC Inl Relative Humidity

LPC Exit Relative Humidity $\quad 0.02$

0.0000250

Ambient Flow Velocit

772.64

2.85
407.97

Fan/LPC Inlet Flow Velocity $\quad 485.39$

Ambient Static Pressure

Ambient Static Temperature

407.97
0.0000959

Fan/LPC Inlet Static Temperature 438.10

Additional Water at LPC Exit

SUMMARY OUTPUT DATA

$0.780-39000.0 \quad 18.00-396.35$

$\begin{array}{rrr}\text { W } & \text { Fn } & \text { TSFC } \\ 396.35 & 6795.2 & 0.5973\end{array}$
Wfuel
4059.07
BPR
5.2814

FLOW STATION DATA $\begin{array}{rrr}\text { ht } & \text { FAR } & \text { WC } \\ 20.96 & 0.0000 & \end{array}$

\begin{tabular}{|c|c|}
\hline FSO & Ambient.Fl_O \\
\hline FS1 & Inlet.FI_O- \\
\hline FS12 & Splitter.Fl_02 \\
\hline FS2 & Splitter.FI_01 \\
\hline FS14 & Fan.Fl_O \\
\hline FS23 & LPC. $F 1$ \\
\hline FS24 & 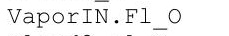 \\
\hline FS25 & Bleed2.Fl_o \\
\hline FS3 & HPC.FI_O \\
\hline FS36 & Bleed3.FI_O \\
\hline FS 4 & Burner.Fl_O \\
\hline FS45 & HPT.FI_O ${ }^{-}$ \\
\hline FS49 & LPT.FI_O \\
\hline FS5 & TEGV.F̄̄_O \\
\hline FS8 & Core_Nozz.Fl_C \\
\hline FS17 & FanDūctLkg.F̄i_o \\
\hline FS171 & Bleed15.Fl_o \\
\hline S172 & FanDuct.Fl_o \\
\hline S173 & Byp_Nozz.Fi_ \\
\hline
\end{tabular}

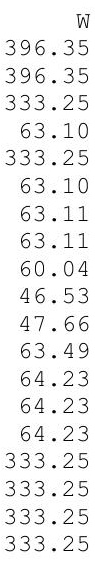

TURBOMACHINERY PERFORMANCE DATA

$\begin{array}{rrr} & \text { WC } & \text { PR } \\ \text { Fan } & 1078.81 & 1.702 \\ \text { LPC } & 204.27 & 2.453 \\ \text { HPC } & 96.96 & 13.189 \\ \text { HPT } & 12.10 & 4.280 \\ \text { LPT } & 55.86 & 4.352\end{array}$

$$
\begin{array}{rr}
\text { eff } & \text { NC } \\
0.9114 & 4139.469 \\
0.8226 & 4139.469 \\
0.8584 & 10372.775 \\
0.9118 & 213.860
\end{array}
$$

TURBOMACHINERY MAP DATA

$\begin{array}{lrrrrr} & \text { WcMap } & \text { PRmap } & \text { effMap } & \text { NcMap } & \text { R/Parm } \\ \text { Fan } & 1253.47 & 1.686 & 0.9132 & 4100.000 & 0.0639 \\ \text { LPC } & 166.84 & 2.748 & 0.8341 & 1.093 & 0.0000 \\ \text { HPC } & 88.19 & 12.615 & 0.8672 & 10096.243 & 13.5168 \\ \text { HPT } & 0.96 & 4.189 & 0.9118 & 1.285 & 4.1886 \\ \text { LPT } & 0.86 & 3.521 & 0.9223 & 1.019 & 3.5211\end{array}$
31.497 7.237$$
7.257
$$$$
7.257
$$$$
7.257
$$$$
7.257
$$
$\begin{array}{lllll}4.267 & 457.74 & -20.96 & 0.0000 & 1282.43 \\ 4.267 & 457.74 & -20.96 & 0.0000 & 1282.43 \\ 4.265 & 457.74 & -20.96 & 0.0000 & 1078.81\end{array}$ $\begin{array}{llllr}4.265 & 457.74 & -20.96 & 0.0000 & 1078.81 \\ 4.265 & 457.74 & -20.96 & 0.0000 & 204.27 \\ 7.257 & 540.20 & -1.18 & 0.0000 & 688.69\end{array}$ $\begin{array}{lllll}7.257 & 540.20 & -1.18 & 0.0000 & 688.69\end{array}$ $\begin{array}{lllll}10.461 & 620.31 & 18.07 & 0.0000 & 96.94\end{array}$ $\begin{array}{lllll}10.461 & 620.48 & 17.56 & 0.0000 & 96.96 \\ 10.461 & 620.48 & 17.56 & 0.0000 & 96.96\end{array}$ $\begin{array}{rrrrr}137.969 & 1373.28 & 205.18 & 0.0000 & 10.41\end{array}$ $\begin{array}{llll}137.969 & 1373.28 & 205.18 & 0.0000\end{array}$ $\begin{array}{llll}134.792 & 2814.27 & 174.94 & 0.0242\end{array}$ 


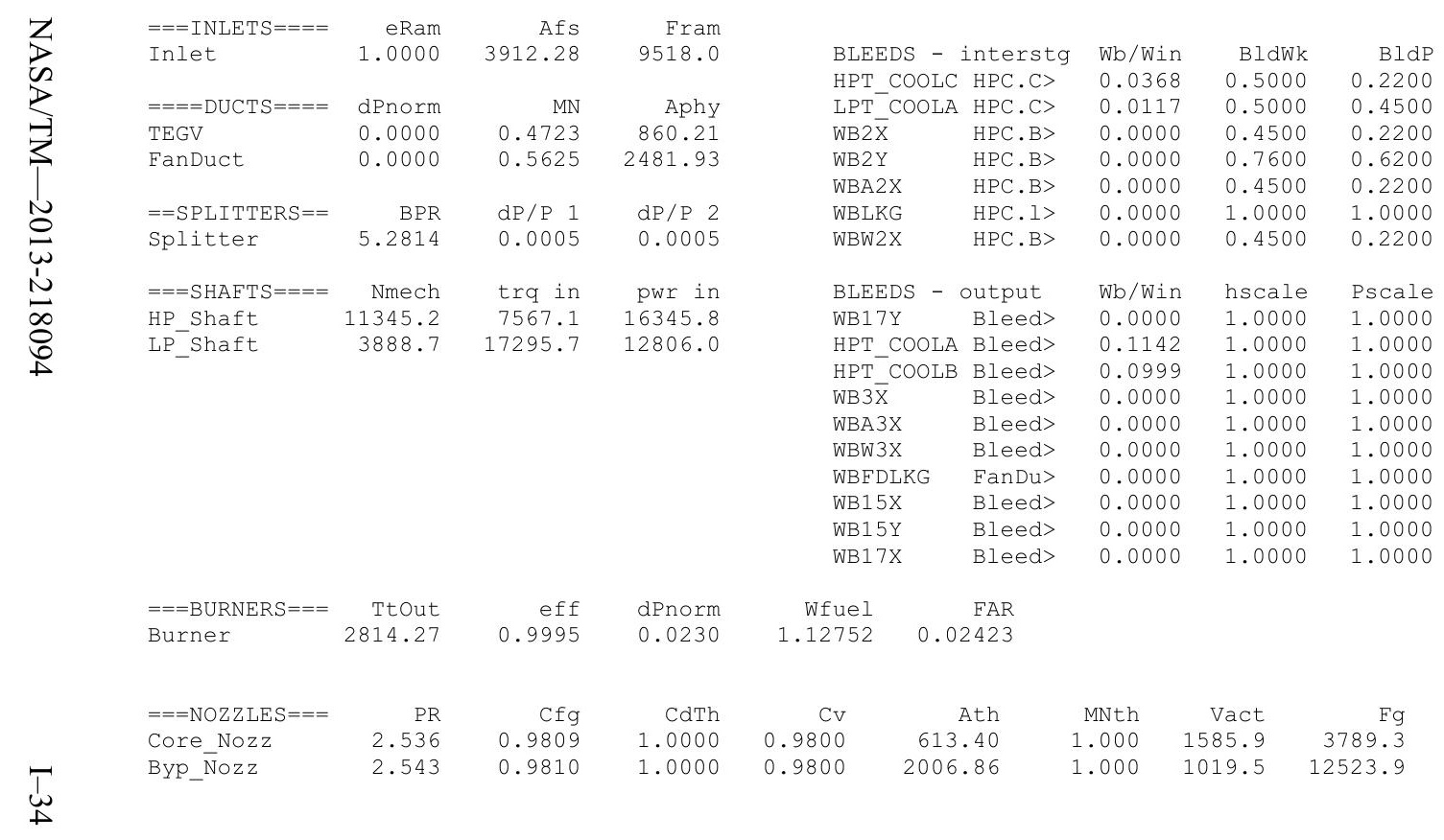


Date:05/13/13 Time:09:34:22 Model:

Turbofan Engine - COMDES ON converge $=1$ CASE: 0

Version:NPSS1.6.5 - Rev: -> Gas Package: Janaf iter/pass/Jacb/Broy=19/33/1/17 Run by: Philip C Jorgenson PC: 84

Temperature Stator 1 inlet: 450.19 Stator 1 exit: 467.34 Stator 2 inlet: 487.68 Stator 2 exit: 495.58 $\begin{array}{lll}\text { Stator } 3 \text { inlet: } 521.05 & \text { Stator } 3 \text { exit: } 529.20 & \text { Stator } 4 \text { inlet: } 553.27 \text { Stator } 4 \text { exit: } 560.86 \\ \text { Stator } 5 \text { inlet: } 579.84 & \text { Stator } 5 \text { exit: } 587.09 & \text { Unblocked Percent Blockage: } 0.00\end{array}$

Ambient Relative Humidity $\quad 10.00$

Fan Face Relative Humidity $\quad 4.12$

Fan Bypass Relative Humidity $\quad 0.18$

LPC Inlet Relative Humidity $\quad 2.00$

HPC Exit Relative Humidity

0.00

0.0000250

Amblent Flow velocity

Ambient Static Pressure

Additional Water at LPC Exit

772.64

2.85

Inlet Length

40.00

Fan/LPC Inlet Flow Velocity $\quad 445.56$ 0.000110

Fan/LPC Inlet Static Temperature $\quad \begin{array}{rr}3.75 \\ \end{array}$

SUMMARY OUTPUT DATA

$\begin{array}{rrrrrr}\text { MN } & \text { alt } & \text { dTamb } & \text { W } & \text { Fn } & \text { TSFC } \\ 0.780 & 39000.0 & 18.00 & 378.74 & 5799.6 & 0.5904\end{array}$

FSO Ambient.Fl_O

FS1 Inlet.FI_O

$\begin{array}{ll}\text { FS12 } & \text { Splitter.F1_02 } \\ \text { FS2 } & \text { Splitter.Fl-01 }\end{array}$

FS14 Fan.FI_O

FS23 LPC.F1 0

FS25 BPorIN.F1_O

$\begin{array}{ll}\text { FS25 } & \text { Bleed2.FI_ } \\ \text { FS3 HPC.FI_O }\end{array}$

FS36 Bleed3.F1_O

FS4 Burner.FI_O

FS45 HPT.FI

FS49 LPT.F1_O

FS8 Core_Nozz.FI_O

FS17 FanDuctLkg.Fì_O

FS171 Bleed15.F1_O

FS173 Byp Nozz.FI?

TURBOMACHINERY PERFORMANCE DATA

\begin{tabular}{|c|c|c|c|c|c|c|c|c|c|c|}
\hline & & & & FLOW & STATION DA & & & & & \\
\hline FSO & Ambient.Fl O & & $\begin{array}{r}\text { W } \\
378.74\end{array}$ & $\begin{array}{r}P t \\
4.267\end{array}$ & $\begin{array}{r}\mathrm{Tt} \\
457.74\end{array}$ & $\begin{array}{r}h t \\
-20.96\end{array}$ & $\begin{array}{r}\text { FAI } \\
0.000\end{array}$ & $\begin{array}{r}\text { WC } \\
1225.4\end{array}$ & $\begin{array}{r}P \\
2.85\end{array}$ & $\begin{array}{l}\text { Ps } \\
54\end{array}$ \\
\hline 1 & Inlet.Fl $0^{-}$ & & 378.74 & 4.267 & 457.74 & -20.96 & 0.000 & 1225.4 & 3.29 & 425. \\
\hline 12 & Splitter.Fl_o & & 319.79 & 4.265 & 457.74 & -20.96 & 0.000 & 1035.2 & 3.30 & 425 \\
\hline 2 & Splitter.Fl_o & & 58.94 & 4.265 & 457.74 & -20.96 & 0.000 & 190.8 & 3.75 & \\
\hline 14 & Fan.Fl_O & & 319.79 & 6.912 & 532.08 & -3.13 & 0.000 & 688.7 & 5.73 & 504 \\
\hline 23 & LPC.FI-O & & 58.94 & 10.333 & 612.28 & 16.14 & 0.0 & 91.08 & 9.19 & 592 \\
\hline 24 & VaporIN.FI_O & & 5 & 10.333 & 612.46 & 15.55 & 0.0 & 91.11 & 9.19 & \\
\hline 25 & Bleed2.F1_o & & 95 & 10.333 & 612.46 & 15.55 & 0.000 & 91.11 & 9.19 & \\
\hline & HPC.Fl_O & & 9 & 124.744 & 1321.16 & 191.58 & & 10.5 & 112.15 & \\
\hline 36 & Bleed3.Fl O & & 7 & 4.744 & 1321.16 & 191.58 & 0.0 & 8.1 & 117.43 & 38 \\
\hline & Burr & & & 1 & .63 & 164.64 & & 12.10 & 115. & \\
\hline 45 & HPT. & & & 28.387 & 1 & -2.86 & & 55.9 & 25.55 & \\
\hline 49 & LPT.FI_O & & & 6.516 & 1234.74 & -133.29 & 0 & 208.4 & 5.61 & \\
\hline 5 & TEGV.FI__O & & & 6.516 & 1234.81 & -133.29 & & 208.4 & 5.61 & \\
\hline 8 & Core_Nozz.FI & & & 6.516 & 1234.94 & -133.29 & & 208.4 & 3.47 & 77 \\
\hline 17 & Fant & & & 6.912 & 532.08 & -3.13 & & 688.7 & 5.73 & \\
\hline 171 & Bleed15.FI_o & & & 6.912 & 532.08 & -3.13 & & 688.7 & 5.57 & \\
\hline 172 & FanDuct.FI_O & & & 6.912 & 08 & -3.13 & & 688.7 & 5.57 & \\
\hline S173 & Byp_Nozz.Fì_o & & 319.79 & 6.912 & 532.08 & -3.13 & 0.000 & 688.7 & 3.65 & \\
\hline $\mathrm{R}$ & INERY PERE & NCE & DATA & & & & & & & \\
\hline & & D & ef & $\mathrm{NC}$ & $\mathrm{Tt}$ & & & r & & SMW \\
\hline an & 1035.24 & 1.62 & 9117 & 3968.903 & 1624 & 75 & -8 & 64.3 & 53.0 & 26.46 \\
\hline & 190.82 & & & .903 & & & -3 & 93.4 & 2. & \\
\hline ec & 91.11 & 12.0 & & 10179.543 & 571 & 0.8986 & -14 & 26.3 & 29.85 & 28.83 \\
\hline 17 & 12.10 & 4.2 & & 215.019 & 3534 & 0.8903 & & 26.3 & & \\
\hline PT & 55.99 & 4.357 & 0.9131 & 89.634 & 1.3983 & 0.8961 & 11 & 57.7 & & \\
\hline & NERY & DA & & & & & & & & \\
\hline & WcMap & PRmap & $\mathrm{ap}$ & NcMap & R/Parm & s_W & Des & S_PRdes & S_effDes & S_NCDes \\
\hline & 120 & & & 31. & & & & & & \\
\hline & 151.28 & 2 & & 1.048 & 0.0000 & & 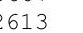 & 0.9120 & 1.0350 & \\
\hline & 82.86 & 11.550 & 0.8696 & 9908.163 & 13.1455 & 1.0 & 995 & 1.0494 & 0.9898 & 0.973 \\
\hline & 0.96 & 4.202 & 0.9099 & 1.292 & 4.2020 & & & 0.9723 & 1.0000 & 0.00 \\
\hline & & & .91 & & & & & & & \\
\hline
\end{tabular}

TURBOMACHINERY MAP DATA

\begin{tabular}{|c|c|c|c|c|c|c|c|c|c|c|}
\hline & & & & FLOW $S$ & STATION DA & & & & & Ps \\
\hline ESO & Ambient.Fl 0 & & $\begin{array}{r}W \\
378.74\end{array}$ & $\begin{array}{r}P t \\
4.267\end{array}$ & $\begin{array}{r}\text { Tt } \\
457.74\end{array}$ & $\begin{array}{r}h t \\
-20.96\end{array}$ & $\begin{array}{r}\text { FAR } \\
0.0000\end{array}$ & $\begin{array}{r}W \\
1225.4\end{array}$ & 2.8 & $\begin{array}{l}\text { PS } \\
54\end{array}$ \\
\hline S1 & Inlet.Fl $0^{-}$ & & 378.74 & 4.267 & 457.74 & -20.96 & 0.0000 & 1225.4 & 3.2 & 425 \\
\hline S12 & Splitter.Fl_o & & 319.79 & 4.265 & 457.74 & -20.96 & .0000 & 1035.2 & 3.3 & 03 \\
\hline s2 & Splitter.Fl_o & & 58.94 & 4.265 & 457.74 & -20.96 & .0000 & 190.8 & 3.73 & \\
\hline$\$ 14$ & Fan.Fl_O & & 319.79 & 6.912 & 532.08 & -3.13 & 0.0000 & 688.7 & 5.7 & 504 \\
\hline S23 & LPC.FI-O & & 58.94 & 10.333 & 612.28 & 16.14 & 0.0000 & 91.0 & 9.1 & \\
\hline 24 & VaporIN.FI_O & & 5 & 10.333 & 612.46 & 15.55 & 0.0000 & 91.1 & 9.1 & 96 \\
\hline 525 & Bleed2.FI_o & & 5 & 10.333 & 612.46 & 15.55 & 0.0000 & 91.1 & 9.1 & \\
\hline 3 & HPC.FI_O & & 56 & 24. & 1321.16 & 191.58 & 0 & 10.5 & 112.1 & \\
\hline 36 & Bleed3.FI_O & & & 4 & 1321.16 & 191.58 & 0 & 8.1 & 117.4 & 38 \\
\hline & $\mathrm{Bu}$ & & & & & 164 & & 12 & 115 & \\
\hline 35 & $\mathrm{HPT}$ & & & & 31 & -2.86 & & & 25. & \\
\hline$\$ 49$ & LPT.FI_O & & & & 1234.74 & -133.29 & .0161 & 208 & 6 & 17 \\
\hline 55 & TEGV.FI_O & & 5 & & 1234.81 & -133.29 & 0.0 & 208.4 & 5.6 & \\
\hline 8 & Core_Nożz.Fl_ & & & & 1234.94 & -133.29 & 0 & 208.4 & 3.4 & 77 \\
\hline 517 & Fan & & & & 532.08 & -3.13 & & 68 & 0 & \\
\hline S171 & Blee & & & 6.912 & 532.08 & -3.13 & 0. & 688.7 & 5.57 & \\
\hline$\$ 172$ & Fand & & & & 532.08 & -3.13 & & 688 & 5.57 & 75 \\
\hline FS173 & Byp_Nozz.FI_O & & 319.79 & 6.912 & 532.08 & -3.13 & 0.0000 & 688.7 & 3.65 & \\
\hline JRB & INERY PERE & 0 & $\mathrm{D}$ & & & & & & & \\
\hline & & & 1 & & $\mathrm{~T}$ & & & & & \\
\hline an & 1035.24 & 62 & 9117 & 8.903 & 1624 & & -80 & 64.3 & 53.03 & 26.46 \\
\hline PC & 190.82 & & & .903 & 6 & & -3 & & & 2.15 \\
\hline ec & 91.11 & 12.0 & 7 & 101 & 2.1571 & 0.8986 & -143 & 26.3 & 29.85 & 28.83 \\
\hline IPT & 12.10 & & & 21 & 3534 & 0.8903 & 143 & 26.3 & & \\
\hline $\mathrm{HT}$ & 55.99 & 4.357 & 0.9131 & 89.634 & 1.3983 & 0.8961 & 111 & 57.7 & & \\
\hline JR & ER & & & & & & & & & \\
\hline & WCM & & & NcMap & /Parm & s_h & s & $S_{S} \mathrm{PF}$ & effDes & S_NCDes \\
\hline & & & & & & & & & & \\
\hline$P C$ & & & & & & & & 0.9120 & & \\
\hline HPC & 82.86 & 11.550 & 0.8696 & 9908.163 & 13.1455 & & 995 & 1.0494 & 0.9898 & 0.9733 \\
\hline HPT & 0.96 & 4.202 & 0.9099 & 1.292 & 4.2020 & & & 0.9723 & 1.0000 & 0 \\
\hline & & & ) 919 & & & & & & & \\
\hline
\end{tabular}

gamt
1.40084
1.40084
1.40084
1.40084
1.40004
1.39852
1.39850
1.39850
1.36057
1.36057
1.29107
1.32733
1.35478
1.35478
1.35477
1.40004
1.40004
1.40004
1.40004

WAR 0.0000295 0.0000295 0.0000295 0.0000295 0.0001396 0.0001396 0.0001396 0.0001396 0.0001396 0.0001396 0.0001396 0.0000295 0.0000295 0.0000295 0.0000295

$\begin{array}{rrr}\text { Wair } & \text { WH2O } & \text { H2O frac } \\ 378.73 & 0.011 & 0.0000 \\ 378.73 & 0.011 & 0.0000 \\ 319.78 & 0.009 & 0.0000 \\ 58.94 & 0.002 & 0.0000 \\ 319.78 & 0.009 & 0.0000 \\ 58.94 & 0.002 & 0.0000 \\ 58.94 & 0.008 & 0.0001 \\ 58.94 & 0.008 & 0.0001 \\ 56.08 & 0.008 & 0.0001 \\ 43.47 & 0.006 & 0.0001 \\ 43.47 & 0.006 & 0.0265 \\ 58.25 & 0.008 & 0.0199 \\ 58.94 & 0.008 & 0.0197 \\ 58.94 & 0.008 & 0.0197 \\ 58.94 & 0.008 & 0.0197 \\ 319.78 & 0.009 & 0.0000 \\ 319.78 & 0.009 & 0.0000 \\ 319.78 & 0.009 & 0.0000 \\ 319.78 & 0.009 & 0.0000\end{array}$

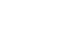

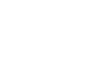




\begin{tabular}{|c|c|c|c|c|c|c|c|c|c|c|c|c|}
\hline$===$ INLETS $====$ & eRam & Afs & Fram & \multirow{2}{*}{\multicolumn{2}{|c|}{ BLEEDS - interstg }} & & & & & & & \\
\hline Inlet & 1.0000 & 3738.44 & 9095.1 & & & $\begin{array}{l}\text { Wb } / \text { Win } \\
0.0368\end{array}$ & $\begin{array}{r}\text { BldWk } \\
0.5000\end{array}$ & $\begin{array}{r}\text { BldP } \\
0.2200\end{array}$ & $\begin{array}{r}\text { W } \\
2.1694\end{array}$ & $\begin{array}{r}\mathrm{Tt} \\
973.60\end{array}$ & $\begin{array}{r}\text { ht } \\
103.56\end{array}$ & $\begin{array}{r}P t \\
28.387\end{array}$ \\
\hline$====$ DUCTS $====$ & dPnorm & MN & Aphy & \multicolumn{2}{|c|}{ LPT COOLA HPC.C> } & 0.0117 & 0.5000 & 0.4500 & 0.6897 & 973.60 & 103.56 & 6.516 \\
\hline TEGV & 0.0000 & 0.4721 & 860.21 & WB $2 \overline{\mathrm{X}}$ & HPC. B > & 0.0000 & 0.4500 & 0.2200 & 0.0000 & 938.03 & 94.76 & 35.504 \\
\hline FanDuct & 0.0000 & 0.5625 & 2481.93 & WB2Y & HPC.B> & 0.0000 & 0.7600 & 0.6200 & 0.0000 & 1156.20 & 149.33 & 81.268 \\
\hline$==$ SPLITTERS $==$ & BPR & $d P / P 1$ & $d P / P 2$ & $\begin{array}{l}\text { WBA2X } \\
\text { WBLKG }\end{array}$ & HPC.B> & 0.0000 & 0.4500 & 0.2200 & 0.0000 & $\begin{array}{r}938.03 \\
1321.16\end{array}$ & $\begin{array}{r}94.76 \\
91.58\end{array}$ & $\begin{array}{r}35.504 \\
124.744\end{array}$ \\
\hline $\begin{array}{l}==\text { SPLITTERS== } \\
\text { Splitter }\end{array}$ & 5.4253 & 0.0005 & 0.0005 & $\begin{array}{l}\text { WBLKG } \\
\text { WBW2X }\end{array}$ & HPC.B> & 0.0000 & 0.4500 & 0.2200 & 0.0000 & 938.03 & 94.76 & 35.504 \\
\hline$===$ SHAFTS $====$ & Nmech & trq in & pwr in & BLEEDS - & output & Wb/Win & hscale & Pscale & w & Tt & ht & Pt \\
\hline HP Shaft & 11061.7 & 6802.1 & 14326.3 & $\begin{array}{l}\text { WB17Y } \\
\text { HPT }\end{array}$ & Bleed> & 0.0000 & 1.0000 & 1.0000 & 0.0000 & 612.46 & 15.55 & 10.333 \\
\hline $\mathrm{LP}^{-}$Shaft & 3728.5 & 15717.2 & 11157.7 & & Bleed> & 0.1142 & 1.0000 & 1.0000 & 6.7311 & 1321.16 & 191.58 & 121.871 \\
\hline & & & & $\begin{array}{l}\text { HPT_COOLA } \\
\text { HPT_COOLB }\end{array}$ & Bleed> & 0.0999 & 1.0000 & 1.0000 & 5.8898 & 1321.16 & 191.58 & 68.398 \\
\hline & & & & WB $3 \overline{\mathrm{X}}$ & Bleed> & 0.0000 & 1.0000 & 1.0000 & 0.0000 & 1321.16 & 191.58 & 124.744 \\
\hline & & & & WBA3X & Bleed> & 0.0000 & 1.0000 & 1.0000 & 0.0000 & 1321.16 & 191.58 & 124.744 \\
\hline & & & & \multirow{2}{*}{$\begin{array}{l}\text { WBW3X } \\
\text { WBFDLKG }\end{array}$} & Bleed> & 0.0000 & 1.0000 & 1.0000 & 0.0000 & 1321.16 & 191.58 & 124.744 \\
\hline & & & & & FanDu> & 0.0000 & 1.0000 & 1.0000 & 0.0000 & 532.08 & -3.13 & 6.912 \\
\hline & & & & WB15X & Bleed> & 0.0000 & 1.0000 & 1.0000 & 0.0000 & 532.08 & -3.13 & 6.912 \\
\hline & & & & \multirow{2}{*}{$\begin{array}{l}\text { WB15Y } \\
\text { WB17X }\end{array}$} & Bleed> & 0.0000 & 1.0000 & 1.0000 & 0.0000 & 532.08 & -3.13 & 6.912 \\
\hline & & & & & Bleed> & 0.0000 & 1.0000 & 1.0000 & 0.0000 & 532.08 & -3.13 & 6.912 \\
\hline$===$ BURNERS $===$ & Ttout & eff & dPnorm & \multirow{2}{*}{\multicolumn{2}{|c|}{$\begin{array}{rr}\text { Wfuel } & \text { FAR } \\
0.95121 & 0.02188\end{array}$}} & & & & & & & \\
\hline Burner & 2646.61 & 0.9995 & 0.0230 & & & & & & & & & \\
\hline$===$ NOZZLES $===$ & PR & $\mathrm{Cfg}$ & CdTh & & Ath & MNth & Vact & \multirow{2}{*}{$\begin{array}{r}\mathrm{Fg} \\
3240.2\end{array}$} & & & & \\
\hline Core_Nozz & 2.283 & 0.9816 & 1.0000 & \multirow{2}{*}{\multicolumn{2}{|c|}{$\begin{array}{l}0.9800 \\
0.9800\end{array}$}} & 1.000 & \multirow{2}{*}{$\begin{array}{r}\text { Vact } \\
1534.9 \\
1011.8\end{array}$} & & & & & \\
\hline Byp_Nozz & 2.422 & 0.9814 & 1.0000 & & & 1.000 & & 11654.5 & & & & \\
\hline
\end{tabular}


Model:

Turbofan Engine - COMDES ON converge $=1$ CASE: 0

Version:NPSS 1.6.5 - Rev: -> Gas Package: Janaf iter/pass/Jacb/Broy= 19/33/1/17 Run by: Philip C Jorgenson PC: 79

Temperature Stator 1 inlet: 451.81 Stator 1 exit: 468.55 Stator 2 inlet: 488.59 Stator 2 exit: 496.62

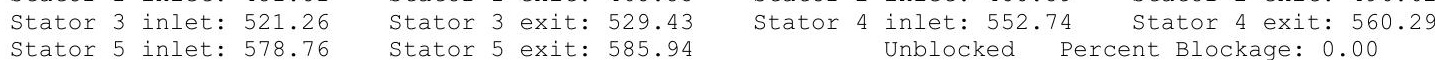

Ambient Relative Humidity $\quad 10.00$

Fan Face Relative Humidity $\quad 3.88$

Fan Bypass Relative Humidity $\quad 0.20$

LPC Inlet Relative Humidity $\quad 1.93$

LPC Exit Relative Humidity

Dro Relative Humidity

0.00

0.0000250

Amblent Flow velocity

Ambient Static Pressure

Additional Water at LPC Exit

772.64

2.85

Inlet Length

40.00

Fan/LPC Inlet Flow Velocity $\quad 433.38$ 0.0001148

$\begin{array}{rr} & 3.78 \\ \text { Fan/LPC Inlet Static Temperature } & 442.09\end{array}$

MN SUMMARY OUTPUT DATA

$\begin{array}{rrrrrr}\text { MN } & \text { alt } & \text { dTamb } & \text { W } & \text { Fn } & \text { TSFC } \\ 0.780 & 39000.0 & 18.00 & 373.42 & 5508.1 & 0.5896\end{array}$ 3247.58 BPR
5.4804
OPR
28.311 1.439
Powerset 79.800 $\begin{array}{ccc}\text { T4 } & \text { T41 } & \text { T49 } \\ 2598.9 & 2441.9 & 1690.5\end{array}$
FSO Ambient.FI_O

FS1 Inlet.Fl_O-

$\begin{array}{ll}\text { FS12 } & \text { Splitter.Fl_02 } \\ \text { FS2 } & \text { Splitter.Fl-01 }\end{array}$

FS14 Fan.Fl_

LPC.FI_O

FS24 VaporIN.FI

FS25 Bleed2.F1

FS36 Ble.FI_O

FS4 Burner.F1_-

FS45 HPT.FI_O

FS49 LPT.FI_O

FS5 TEGV.FI_O

FS8 Core_Nozz.Fl_O

FS17 FanDüctLkg.Fì_O

FS171 Bleed15.FI

FS173 Byp Nozz. FI

TURBOMACHINERY PERFORMANCE DATA

\begin{tabular}{|c|c|c|c|c|c|c|c|c|c|c|c|}
\hline & & & W & $\begin{array}{c}\text { FLOW } \\
\text { Pt }\end{array}$ & $\begin{array}{c}\text { STATION DA } \\
\text { Tt }\end{array}$ & TA & $\mathrm{FA}$ & & & Ps & \\
\hline FSO & Ambient.Fl O & & 373.42 & 4.267 & 457.74 & -20.96 & 0.000 & 1208.2 & 2.8 & & 407 \\
\hline s1 & Inlet.FI_O- & & 373.42 & 4.267 & 457.74 & -20.96 & 0.000 & 1208.2 & 3.3 & & \\
\hline ES12 & Splitter.Fl_o & & 315.80 & 4.265 & 457.74 & -20.96 & 0.000 & 1022.3 & 3.3 & & 426 \\
\hline 52 & Splitter.Fl_o & & 57.62 & 4.265 & 457.74 & -20.96 & 0.000 & 186.5 & 3.7 & & \\
\hline$\$ 14$ & Fan.Fl o & & 315.80 & 6.810 & 529.66 & -3.71 & 0.000 & 688.7 & 5.6 & & 502 \\
\hline 23 & LPC.Fl_O & & 57.62 & 10.280 & 610.14 & 15.62 & 0.000 & 89.3 & 9.1 & & 591 \\
\hline 24 & VaporIN.FI_O & & 57.63 & 10.280 & 610.33 & 15.01 & 0.000 & 89.3 & 9.1 & & \\
\hline 25 & Bleed2.Fl_o & & 57.63 & 10.280 & 610.33 & 15.01 & 0.000 & 89.3 & 9.1 & & 591 \\
\hline & HPC.Fl_O & & 54.83 & 120.797 & 1306.23 & 187.69 & 0.000 & 10.5 & 108.4 & & 126 \\
\hline 36 & Bleed3.Fl O & & 42.50 & 120.797 & 1306.23 & 187.69 & 0.000 & 8.2 & 113.6 & & 12 \\
\hline & Burner.Fl_o & & 43.40 & 118.015 & 2598.87 & 161.65 & 0.021 & 12.1 & 111.4 & & \\
\hline$\$ 5$ & HPT.FI_O & & 57.86 & 27.464 & 1698.37 & -2.84 & 0.015 & 56.0 & 24.7 & & 165 \\
\hline 39 & LPT.FI-O & & 58.53 & 6.302 & 1210.77 & -130.68 & 0.015 & 208.5 & 5.4 & & 116 \\
\hline 5 & TEGV.F̄ $O$ & & 58.53 & 6.302 & 1210.83 & -130.68 & 0.015 & 208.5 & 5.4 & & 116 \\
\hline 8 & Core_Nozz.FI & & 58.53 & 6.302 & 1210.97 & -130.68 & 0.015 & 208.5 & 3.3 & & 102 \\
\hline 17 & FanDūctLkg.Fi & & 315.80 & 6.810 & 529.66 & -3.71 & 0.000 & 688.7 & 5.6 & & 502 \\
\hline FS171 & Bleed15.FI_o & & & 6.810 & 529.66 & -3.71 & 0.000 & 688.7 & 5.4 & & \\
\hline FS172 & FanDuct.FI_o & & 315.80 & 6.810 & 529.66 & -3.71 & 0.000 & 688.7 & 5.4 & & \\
\hline FS173 & Byp_Nozz.Fin_C & & 315.80 & 6.810 & 529.66 & -3.71 & 0.000 & 688.7 & 3.5 & & 44 \\
\hline TURBOM & MACHINERY PERE & ORMANCE & DATA & & & & & & & & \\
\hline & & PR & eff & $\mathrm{NC}$ & TR & efPoly & & pwr & SMN & & SMW \\
\hline an & 1022.31 & 1.597 & 0.9114 & 3920.858 & 1.1571 & 0.9170 & & 704.5 & 57.15 & 27. & \\
\hline OC & 186.54 & 2.411 & 0.8584 & 3920.858 & 1.3329 & 0.8747 & & 982.0 & 3.34 & & .63 \\
\hline${ }_{C} \mathrm{C}$ & 89.37 & 11.750 & 0.8613 & 10131.152 & 2.1402 & 0.8988 & -13 & 739.2 & 31.12 & 29. & .99 \\
\hline HPT & 12.10 & 4.297 & 0.9091 & 215.577 & 1.3551 & 0.8897 & & 39.2 & & & \\
\hline LPT & 56.02 & 4.358 & 0.9123 & 89.378 & 1.3998 & 0.8952 & & 586.4 & & & \\
\hline 101 & MACHINERY & DATA & & & & & & & & & \\
\hline & WcMap & PRmap & effMap & NcMap & R/Parm & S_W & cDes & S_PRdes & S_effDes & & S_NcDes \\
\hline & 1187.83 & 1.583 & 0.9132 & 3883.473 & 0.0603 & $\overline{0} .8$ & 8607 & $\overline{1} .0235$ & 0.9980 & & $\overline{0} .9905$ \\
\hline & 148.17 & 2.511 & 0.8223 & 1.036 & 0.0000 & & 2589 & 0.9336 & 1.0439 & & 0.0003 \\
\hline $\mathrm{PC}$ & 81.28 & 11.244 & 0.8702 & 9861.062 & 13.0364 & 1.0 & 0995 & 1.0494 & 0.9898 & & 0.9733 \\
\hline PT & 0.96 & 4.206 & 0.9090 & 1.296 & 4.2057 & & 6299 & 0.9723 & 1.0000 & & 0.0003 \\
\hline LPT & 0.86 & 3.526 & 0.9191 & 1.005 & 3.5256 & 65.2 & 2590 & 0.7521 & 0.9926 & & 0.0005 \\
\hline
\end{tabular}

TURBOMACHINERY MAP DATA

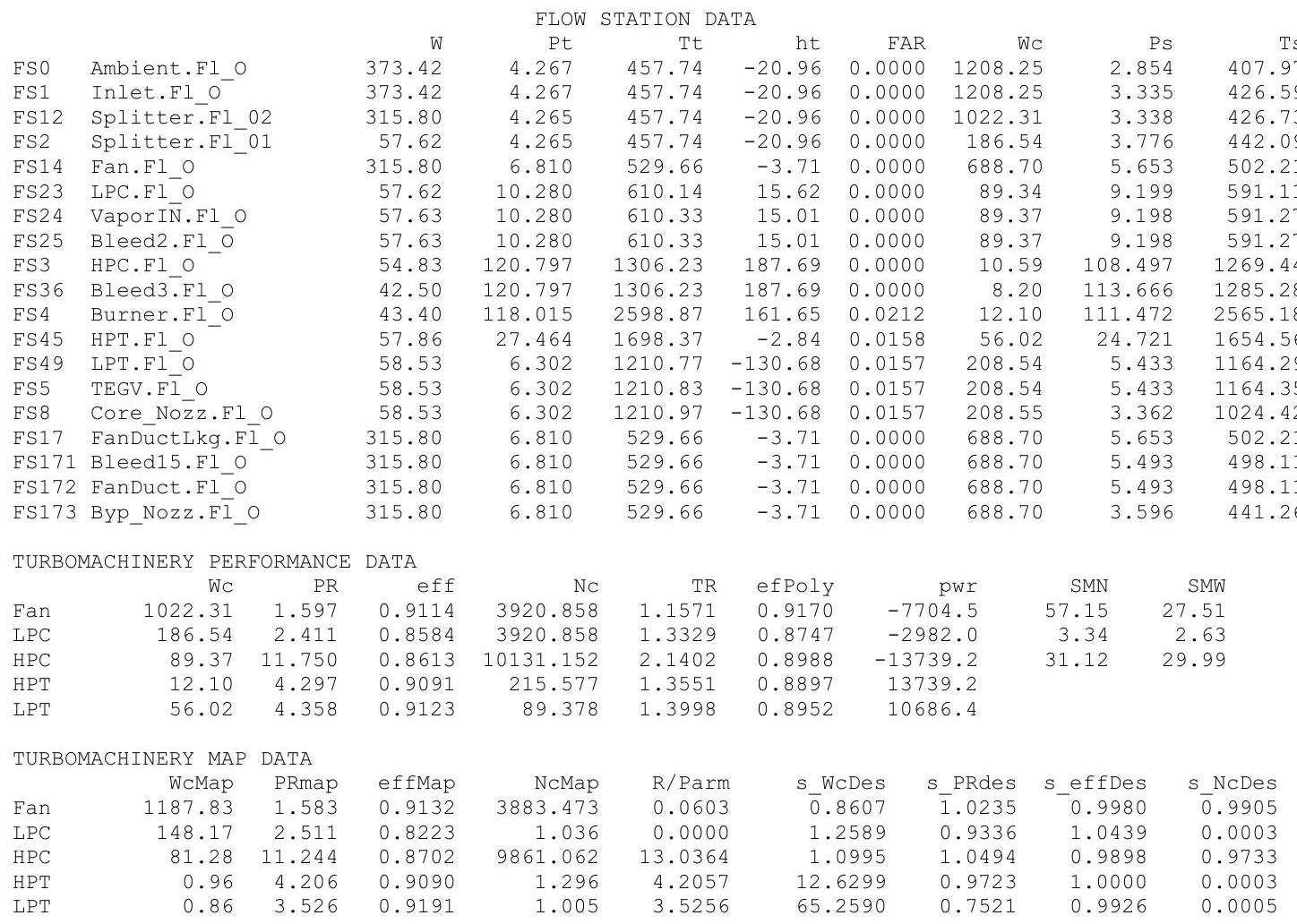

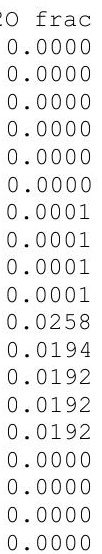


$\begin{array}{lrrr}===\text { INLETS }==== & \text { eRam } & \text { Afs } & \text { Fram } \\ \text { Inlet } & 1.0000 & 3685.97 & 8967.5\end{array}$

$====$ DUCTS $====$ dPnorm MN Aphy

$\begin{array}{llrr}\text { TEGV } & 0.0000 & 0.4720 & 860.21 \\ \text { FanDuct } & 0.0000 & 0.5625 & 2481.93\end{array}$

BPR
5.4804

$\mathrm{dP} / \mathrm{P} 2$

$0.0005 \quad 0.0005$

$===$ SHAFTS $====\quad$ Nmech

HP_Shaft

10989.9

trq in pwr in

$6566.0 \quad 13739.2$

10686.4

$===$ BURNERS $===\quad$ TtOut

Burner 2598.85

eff

dPnor

$===$ NOZZLES $===$

Core Nozz

$P R$
2.208
2.386

Cfg
0.9816

0.9815
BLEEDS - interstg Wb/Win BldWk

$\mathrm{BldP}$
0.2200
0.4500

LPT COOLA HPC.C> $0.0117 \quad 0.5000 \quad 0.4500$

$\begin{array}{lllll}\text { WB2X } & \text { HPC.B> } & 0.0000 & 0.4500 & 0.2200 \\ \text { WB2Y } & \text { HPC.B> } & 0.0000 & 0.7600 & 0.6200\end{array}$

$\begin{array}{lllll}\text { WBA2X HPC.B> } & 0.0000 & 0.4500 & 0.2200\end{array}$

WBLKG HPC.I> $0.0000 \quad 1.0000 \quad 1.0000$

$\begin{array}{lllll}\text { WBW2X HPC.B } & 0.0000 & 0.4500 & 0.2200\end{array}$

BLEEDS - output Wb/Win hscale Pscale

WB17Y Bleed> $0.0000 \quad 1.0000 \quad 1.0000$

HPT_COOLA Bleed> $0.1142 \quad 1.0000 \quad 1.0000$

$\begin{array}{lllll}\text { HPT COOLB } & \text { Bleed> } & 0.0999 & 1.0000 & 1.0000 \\ \text { WB } 3 \bar{X} & \text { Bleed> } & 0.0000 & 1.0000 & 1.0000\end{array}$

$\begin{array}{lllll}\text { WB3X } & \text { Bleed> } & 0.0000 & 1.0000 & 1.0000 \\ \text { WBA3X } & \text { Bleed> } & 0.0000 & 1.0000 & 1.0000\end{array}$

$\begin{array}{lllll}\text { WBA3X } & \text { Bleed> } & 0.0000 & 1.0000 & 1.0000 \\ \text { WBW3X } & \text { Bleed }> & 0.0000 & 1.0000 & 1.0000\end{array}$

$\begin{array}{lllll}\text { WBW3X } & \text { Bleed> } & 0.0000 & 1.0000 & 1.0000 \\ \text { WBFDLKG } & \text { FanDu> } & 0.0000 & 1.0000 & 1.0000\end{array}$

$\begin{array}{lllll}\text { WBFDLKG } & \text { FanDu> } & 0.0000 & 1.0000 & 1.0000 \\ \text { WB15X } & \text { Bleed }> & 0.0000 & 1.0000 & 1.0000\end{array}$

WB15Y Bleed> $0.0000 \quad 1.0000 \quad 1.0000$

WB17X Bleed> $0.0000 \quad 1.0000 \quad 1.0000$

$\begin{array}{rr}\text { Wfuel } & \text { FAR } \\ 0.90211 & 0.02123\end{array}$

$\begin{array}{rrrr}\text { W } & \text { Tt } & \text { ht } & \text { Pt } \\ 2.1208 & 964.77 & 101.35 & 27.464 \\ 0.6743 & 964.77 & 101.35 & 6.302 \\ 0.0000 & 929.84 & 92.72 & 34.594 \\ 0.0000 & 1144.14 & 146.25 & 78.801 \\ 0.0000 & 929.84 & 92.72 & 34.594 \\ 0.0000 & 1306.23 & 187.69 & 120.797 \\ 0.0000 & 929.84 & 92.72 & 34.594 \\ \text { W } & \text { Tt } & \text { ht } & \text { Pt } \\ 0.0000 & 610.33 & 15.01 & 10.280 \\ 6.5802 & 1306.23 & 187.69 & 118.015 \\ 5.7578 & 1306.23 & 187.69 & 66.220 \\ 0.0000 & 1306.23 & 187.69 & 120.797 \\ 0.0000 & 1306.23 & 187.69 & 120.797 \\ 0.0000 & 1306.23 & 187.69 & 120.797 \\ 0.0000 & 529.66 & -3.71 & 6.810 \\ 0.0000 & 529.66 & -3.71 & 6.810 \\ 0.0000 & 529.66 & -3.71 & 6.810 \\ 0.0000 & 529.66 & -3.71 & 6.810\end{array}$

$\begin{array}{rrrrr}\text { CV } & \text { Ath } & \text { MNth } & \text { Vact } & \text { Fg } \\ .9800 & 613.40 & 1.000 & 1520.3 & 3077.2 \\ .9800 & 2006.86 & 1.000 & 1009.5 & 11398.4\end{array}$


Date:05/13/13 Time:09:35:32 Model: Turbofan Engine - COMDES ON Converge = 1 CASE: 0

Version:NPSS_1.6.5 - Rev: -> Gas Package: Janaf iter/pass/Jacb/Broy=18/32/1/16 Run by: Philip C Jorgenson PC: 76

Temperature Stator 1 inlet: 452.90 Stator 1 exit: 469.37 Stator 2 inlet: 489.22 Stator 2 exit: 497.33 $\begin{array}{lll}\text { Stator } 3 \text { inlet: } 521.44 & \text { Stator } 3 \text { exit: } 529.62 & \text { Stator } 4 \text { inlet: } 552.42 \text { Stator } 4 \text { exit: } 559.96 \\ \text { Stator } 5 \text { inlet: } 578.10 & \text { Stator } 5 \text { exit: } 585.24 & \text { Unblocked Percent Blockage: } 0.00\end{array}$

Ambient Relative Humidity $\quad 10.00$

Fan Face Relative Humidity $\quad 3.72$

Fan Bypass Relative Humidity 0.21

LPC Inlet Relative Humidity 1.88

LPC Exit Relative Humidity $\quad 0.02$

HPC Relative Humidity

0.00

Ambient Flow Velocity $\quad 0.0000250$

Ambient Static Pressu

Ambient Static Temperature

$\begin{array}{rrr}772.64 & \text { Fan/LPC Inlet Flow Velocity } & 424.73 \\ 2.85 & \text { Fan/LPC Inlet Static Pressure } & 3.79\end{array}$

Inlet Length

40.00

Additional Water at LPC Exit 0.0001183

Fan/LPC Inlet Static Pressure $\quad 3.79$

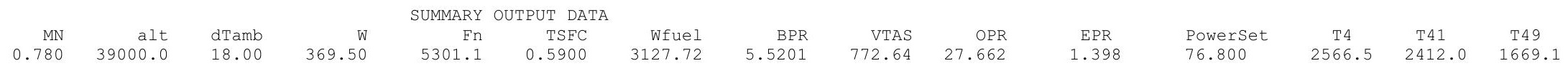

\begin{tabular}{|c|c|}
\hline SO & Ambient.Fl O \\
\hline FS1 & Inlet.Fl o \\
\hline FS12 & Splitter.Fl 02 \\
\hline FS2 & Splitter.FI-01 \\
\hline FS14 & Fan.Fl 0 \\
\hline FS23 & LPC.FI- $\mathrm{O}$ \\
\hline FS24 & VaporIÑ.FI O \\
\hline FS25 & Bleed2.F1_o \\
\hline FS3 & HPC.FI O \\
\hline S36 & Bleed3.FI O \\
\hline FS4 & Burner.FI_O \\
\hline FS45 & $\begin{array}{l}\text { HPT.FI O } \\
\text { HPT }\end{array}$ \\
\hline FS49 & LPT.FI_O \\
\hline FS5 & TEGV.FI__O \\
\hline 58 & Core Nozzz.Fl O \\
\hline FS17 & FanDuctLkg.Fì \\
\hline FS171 & Bleed15.FI O \\
\hline FS172 & FanDuct.Fl \\
\hline S173 & Byp Nozz.Fì \\
\hline
\end{tabular}

TURBOMACHINERY PERFORMANCE DATA

$\begin{array}{lrrrrrrrrr} & \text { Wc } & \text { PR } & \text { eff } & \text { NC } & \text { TR } & \text { efPoly } & \text { pwr } & \text { SMN } & \text { SMW } \\ \text { Fan } & 1012.72 & 1.579 & 0.9094 & 3888.172 & 1.1535 & 0.9150 & -7457.2 & 59.28 & 28.29 \\ \text { LPC } & 183.46 & 2.401 & 0.8622 & 3888.172 & 1.3299 & 0.8780 & -2905.6 & 3.61 & 2.87 \\ \text { HPC } & 88.12 & 11.525 & 0.8614 & 10097.708 & 2.1286 & 0.8986 & -13335.0 & 32.02 & 30.82 \\ \text { HPT } & 12.10 & 4.300 & 0.9085 & 215.968 & 1.3562 & 0.8892 & 13335.1 & & \\ \text { LPT } & 56.05 & 4.359 & 0.9118 & 89.201 & 1.4008 & 0.8945 & 10362.8 & \end{array}$

TURBOMACHINERY MAP DATA

$\begin{array}{lrrrrrrrrr} & \text { WcMap } & \text { PRmap } & \text { effMap } & \text { NcMap } & \text { R/Parm } & \text { S_WcDes } & \text { S_PRdes } & \text { S_effDes } & \text { S_NcDes } \\ \text { Fan } & 1176.68 & 1.566 & 0.9112 & 3851.098 & 0.0597 & 0.8607 & \overline{1} .0235 & 0.9980 & 0.9905 \\ \text { LPC } & 146.06 & 2.477 & 0.8217 & 1.027 & 0.0000 & 1.2561 & 0.9487 & 1.0493 & 0.0003 \\ \text { HPC } & 80.15 & 11.029 & 0.8703 & 9828.510 & 12.9606 & 1.0995 & 1.0494 & 0.9898 & 0.9733 \\ \text { HPT } & 0.96 & 4.208 & 0.9085 & 1.298 & 4.2081 & 12.6299 & 0.9723 & 1.0000 & 0.0003 \\ \text { LPT } & 0.86 & 3.526 & 0.9186 & 1.003 & 3.5261 & 65.2590 & 0.7521 & 0.9926 & 0.0005\end{array}$

\begin{tabular}{|c|c|c|c|c|c|c|c|c|c|c|c|c|c|c|}
\hline W & $\mathrm{Pt}$ & $\mathrm{Tt}$ & ht & FAR & Wc & Ps & Ts & Aphy & MN & gamt & WAR & Wair & WH2O & frac \\
\hline 9.50 & 4.267 & 457.74 & -20.96 & 0.0000 & 1195.58 & 2.854 & 407.97 & 3647.3 & 0.7800 & 1.40084 & 0.0000295 & 369.49 & 0.011 & 0.0000 \\
\hline 59.50 & 4.267 & 457.74 & -20.96 & 0.0000 & 1195.58 & 3.363 & 427.60 & 4168.2 & 0.5930 & 1.40084 & 0.0000295 & 369.49 & 0.011 & 0.0000 \\
\hline 312.83 & 4.265 & 457.74 & -20.96 & 0.0000 & 1012.72 & 3.362 & 427.62 & 3531.8 & 0.5927 & 1.40084 & 0.0000295 & 312.82 & 0.009 & 0.0000 \\
\hline 56.67 & 4.265 & 457.74 & -20.96 & 0.0000 & 183.46 & 3.795 & 442.70 & 830.5 & 0.4116 & 1.40084 & 0.0000295 & 56.67 & 0.002 & 0.0000 \\
\hline 312.83 & 6.735 & 528.02 & -4.11 & 0.0000 & 688.70 & 5.591 & 500.65 & 2606.7 & 0.5226 & 1.40010 & 0.0000295 & 312.82 & 0.009 & 0.0000 \\
\hline 56.67 & 10.241 & 608.73 & 15.28 & 0.0000 & 88.10 & 9.198 & 590.37 & 412.6 & 0.3950 & 1.39860 & 0.0000295 & 56.67 & 0.002 & 0.0000 \\
\hline 56.68 & 10.241 & 608.93 & 14.65 & 0.0000 & 88.12 & 9.197 & 590.54 & 412.6 & 0.3951 & 1.39858 & 0.0001478 & 56.67 & 0.008 & 0.0001 \\
\hline 56.68 & 10.241 & 608.93 & 14.65 & 0.0000 & 88.12 & 9.197 & 590.54 & 412.6 & 0.3951 & 1.39858 & 0.0001478 & 56.67 & & 0.0001 \\
\hline 53.93 & 118.028 & 1296.17 & 185.07 & 0.0000 & 10.62 & 105.936 & 1259.37 & 49.7 & 0.4009 & 1.36 & & 53.92 & & 0.0001 \\
\hline 41.79 & 118.028 & 1296.17 & 185.07 & 0.0000 & 8.23 & 111.020 & 1275.22 & 49.3 & 0.3009 & 1.36209 & 0.0001478 & 41.79 & 0.006 & 0.0001 \\
\hline 42.66 & 115.310 & 2566.49 & 159.63 & 0.0208 & 12.10 & 108.915 & 2533.12 & 74.6 & 0.2990 & 1.29413 & 478 & 41 & & 0.0252 \\
\hline 56.88 & 26.819 & 1676.79 & -2.82 & 0.0155 & 56.05 & 24.137 & 1633.37 & 265.4 & 0.4003 & 1.33040 & 0.0001478 & 56.01 & & 0.0190 \\
\hline 57.55 & 6.153 & 1194.61 & -128.91 & 0.0153 & 208.59 & 5.304 & 1148.61 & 860.2 & 0.4719 & 1.35785 & 0.0001478 & 56.67 & 108 & 0.0188 \\
\hline 57.55 & 153 & 1194.67 & -128.91 & 0.0153 & 208.60 & 5.304 & 3.67 & 860.2 & 0.4719 & 1.3 & & & & 0.0188 \\
\hline 57.55 & 6.153 & 1194.81 & -128.91 & 0.0153 & 208.61 & 3.281 & 1010.25 & 613.4 & 1.0000 & 1.35783 & 0.0001478 & 56.67 & 0.008 & 0.0188 \\
\hline .83 & 6.735 & 528.02 & -4.11 & 0.0000 & 688. & 5.591 & 500.65 & 2606.7 & 0.5226 & 1.40 & 295 & .82 & & 0.0000 \\
\hline 2.83 & 6.735 & 528.02 & -4.11 & 0.0000 & 688.70 & 5.433 & 496.56 & 2481.9 & 0.5625 & 1.40010 & 0.0000295 & 312.82 & 0.009 & 0.0000 \\
\hline 312.83 & 6.735 & 528.02 & -4.11 & 0.0000 & 688.70 & 5.433 & 496.56 & 2481.9 & 0.5625 & 1.40010 & 0.0000295 & 312.82 & 0.009 & 0.0000 \\
\hline 312.83 & 6.735 & 528.02 & -4.11 & 0.0000 & 688.70 & 3.557 & 439.89 & 2006.9 & 1.0000 & 1.40010 & 0.0000295 & 312.82 & 0.009 & 0.0000 \\
\hline
\end{tabular}




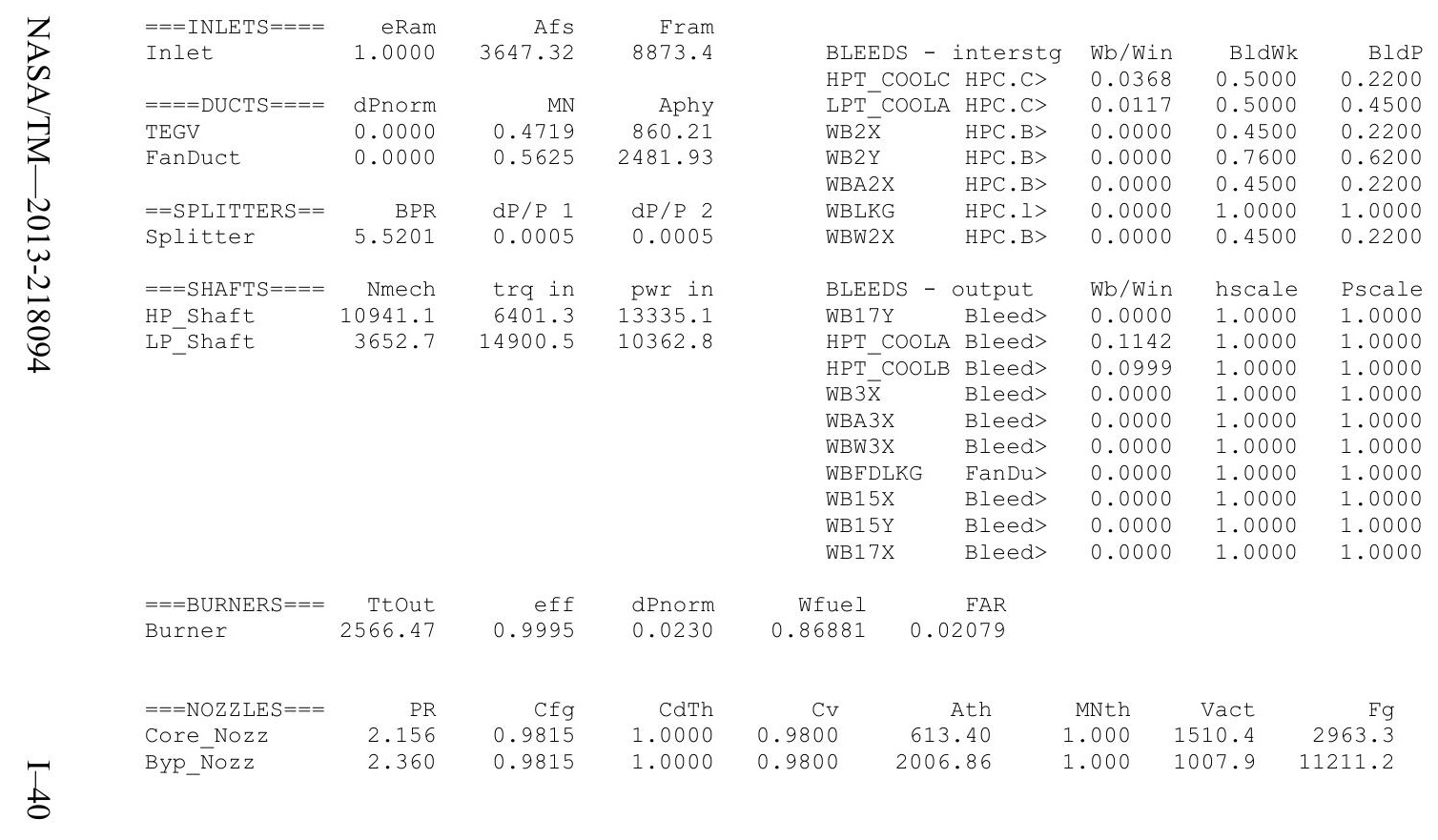




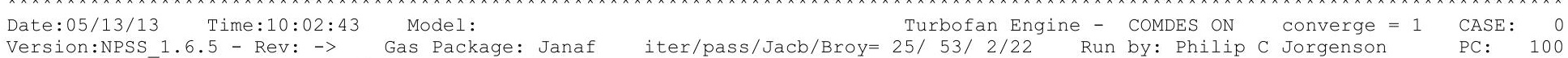
$\begin{array}{llllll}\text { Version:NPSS 1.6.5 - Rev: -> Gas Package: Janaf lter/pass/Jacb/Broy= } 25 / 53 / 2 / 22 & \text { Run by: Philip C Jorge } \\ \text { Temperature Stator } 1 \text { inlet: } 463.74 \quad \text { Stator } 1 \text { exit: } 483.21 \quad \text { Stator } 2 \text { inlet: } 505.74 \text { Stator } 2 \text { exit: } 513.20\end{array}$ Stator 3 inlet: 543.41 Stator 3 exit: 551.73 Stator 4 inlet: 579.99 stator 4 exit: 588.05

Ambient Relative Humidity $\quad 10.00$

Fan Face Relative Humidity $\quad 5.44$

IPC Inlet Relative Humidity $\quad 0.19$

LPC Exit Relative Humidity 2.52

Drop Diameter

0.00

0.0000250

789.49

Ambient Static Pressure

Ambient Static Temperature

Additional

2.85

Inlet Length

\begin{tabular}{l}
40.00 \\
\hline
\end{tabular}

Fan/IPC Inlet Static Pressure $\quad 495.61$

425.97 Fan/LPC Inlet Static Temperature 457.45

$\begin{array}{rrrrr}\text { MN alt dTamb } & \text { W } & \text { SUMMARY OUTPUT DATA } \\ \text { Mn } & \text { TSFC }\end{array}$

\section{FLOW STATION DATA}

\begin{tabular}{rrrrrr}
\multicolumn{7}{c}{ FLOW } & STATION DATA \\
W & Pt & Tt & $\mathrm{ht}$ & FAR & WC \\
387.84 & 4.267 & 477.92 & -16.44 & 0.0000 & 1282.29 \\
387.84 & 4.267 & 477.92 & -16.44 & 0.0000 & 1282.29 \\
326.13 & 4.265 & 477.92 & -16.44 & 0.0000 & 1078.79 \\
61.71 & 4.265 & 477.92 & -16.44 & 0.0000 & 204.14 \\
326.13 & 7.257 & 563.97 & 4.20 & 0.0000 & 688.63 \\
61.71 & 10.484 & 647.79 & 24.37 & 0.0000 & 96.67 \\
61.72 & 10.484 & 648.03 & 23.57 & 0.0000 & 96.70 \\
61.72 & 10.484 & 648.03 & 23.57 & 0.0000 & 96.70 \\
58.73 & 137.687 & 1427.73 & 218.81 & 0.0000 & 10.40 \\
45.51 & 137.687 & 1427.73 & 218.81 & 0.0000 & 8.06 \\
46.67 & 134.516 & 2922.28 & 186.59 & 0.0255 & 12.10 \\
62.16 & 31.489 & 1921.32 & 1.89 & 0.0190 & 55.83 \\
62.88 & 7.250 & 1379.92 & -143.86 & 0.0188 & 207.91 \\
62.88 & 7.250 & 1379.98 & -143.86 & 0.0188 & 207.91 \\
62.88 & 7.250 & 1380.11 & -143.86 & 0.0188 & 207.92 \\
326.13 & 7.257 & 563.97 & 4.20 & 0.0000 & 688.63 \\
326.13 & 7.257 & 563.97 & 4.20 & 0.0000 & 688.63 \\
326.13 & 7.257 & 563.97 & 4.20 & 0.0000 & 688.63 \\
326.13 & 7.257 & 563.97 & 4.20 & 0.0000 & 688.63
\end{tabular}

\section{DATA}

TURBOMACHINERY PERFORMANCE DATA

\section{NC}

\section{$\begin{array}{r}4139.469 \\ 4139.469 \\ \hline 10361.858\end{array}$}

0.8236

$0.8585 \quad 10361.858$

0.9167
TR
1.1801
1.3554
2.2032
1.3427
1.3888

efPoly
0.9178
0.8443
0.8978
0.8903

0.8903
0.9005

\section{pwr
-9525.9}

-9525.9
-3563.2

$-16636.2$

16636.3
13088.9
VTAS
$\mathrm{OPR}$

270
EPR
T4

$T 41$
2743.4

49

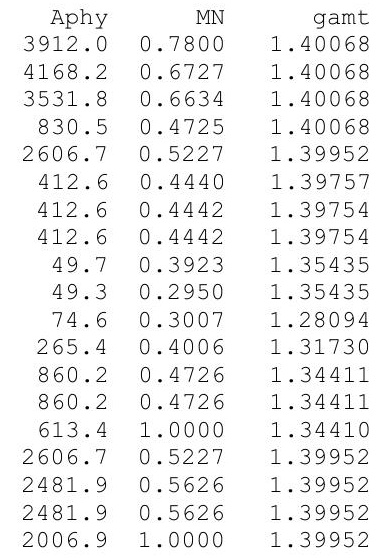

WAR WAR
0.0000851 0.0000851 0.0000851 0.0000851 0.0000851 0.0000851 0.0002334 0.0002334 0.0002334 0.0002334 0.0002334 0.0002334 0.0002334 0.0002334 0.0002334 0.0000851 0.0000851 0.0000851 0.0000851
Wair WH2O H2O frac $\begin{array}{lll}387.81 & 0.033 & 0.0001\end{array}$ $\begin{array}{lll}387.81 & 0.033 & 0.0001\end{array}$ $\begin{array}{lll}326.10 & 0.028 & 0.0001\end{array}$ $\begin{array}{lll}326.10 & 0.028 & 0.0001 \\ 326.10 & 0.005 & 0.0001 \\ 61.71 & 0.028 & 0.0001\end{array}$ $\begin{array}{lll}61.71 & 0.005 & 0.0001\end{array}$ $\begin{array}{lll}61.71 & 0.014 & 0.0002\end{array}$ $\begin{array}{lll}61.71 & 0.014 & 0.0002 \\ 61.71 & 0.014 & 0.0002\end{array}$ $\begin{array}{lll}58.71 & 0.014 & 0.0002\end{array}$ $\begin{array}{lll}58.71 & 0.014 & 0.0002 \\ 45.50 & 0.011 & 0.0002\end{array}$ $\begin{array}{lll}45.50 & 0.011 & 0.0002\end{array}$ $\begin{array}{lll}60.99 & 0.014 & 0.0232\end{array}$ $\begin{array}{lll}61.71 & 0.014 & 0.0230\end{array}$ $\begin{array}{lll}61.71 & 0.014 & 0.0230\end{array}$ $\begin{array}{lll}61.71 & 0.014 & 0.0230\end{array}$ $\begin{array}{lll}326.10 & 0.028 & 0.0001\end{array}$ $\begin{array}{lll}326.10 & 0.028 & 0.0001\end{array}$ $\begin{array}{lll}326.10 & 0.028 & 0.0001 \\ & & 0.0001\end{array}$

TURBOMACHINERY MAP DATA

$\begin{array}{lrrrrrrrrr} & \text { WcMap } & \text { PRmap } & \text { effMap } & \text { NcMap } & \text { R/Parm } & \text { S_WcDes } & \text { S_PRdes } & \text { S_effDes } & \text { S_NcDes } \\ \text { Fan } & 1253.45 & 1.686 & 0.9132 & 4100.000 & 0.0639 & 0.8607 & \overline{1} .0235 & 0.9980 & 0.9905 \\ \text { LPC } & 166.84 & 2.748 & 0.8341 & 1.093 & 0.0000 & 1.2236 & 0.8344 & 0.9874 & 0.0003 \\ \text { HPC } & 87.95 & 12.561 & 0.8673 & 10085.617 & 13.4951 & 1.0995 & 1.0494 & 0.9898 & 0.9733 \\ \text { HPT } & 0.96 & 4.181 & 0.9122 & 1.288 & 4.1812 & 12.6299 & 0.9723 & 1.0000 & 0.0003 \\ \text { LPT } & 0.86 & 3.515 & 0.9235 & 1.020 & 3.5146 & 65.2590 & 0.7521 & 0.9926 & 0.0005\end{array}$




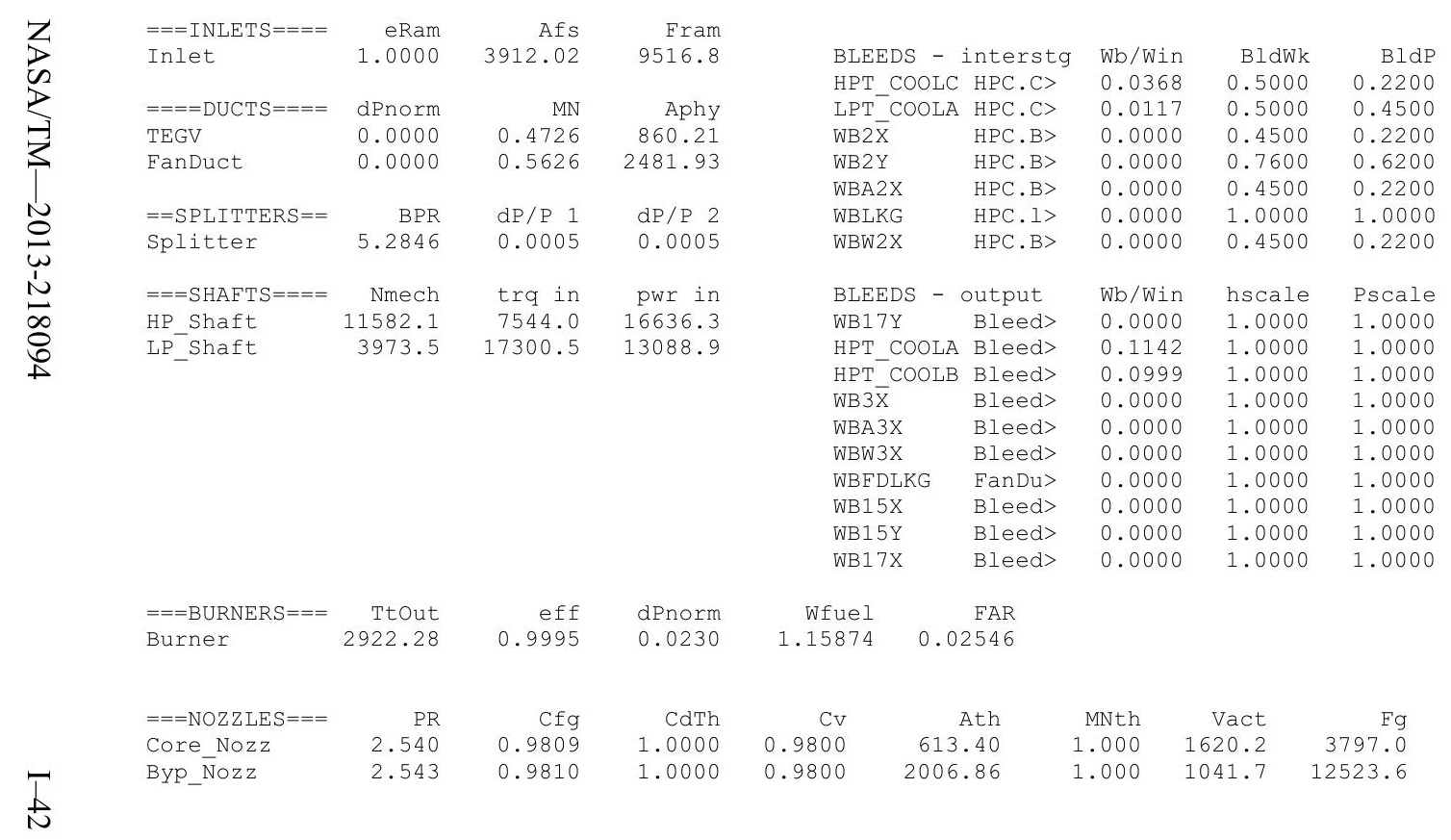


Date:05/13/13 Time:10:03:19 Model:

Turbofan Engine - COMDES ON converge $=1$ CASE: 0

Version:NPSS1.6.5 - Rev: -> Gas Package: Janaf iter/pass/Jacb/Broy= 20/34/1/18 Run by: Philip C Jorgenson PC: 84

Temperature Stator 1 inlet: 469.62 Stator 1 exit: 487.66 Stator 2 inlet: 509.01 Stator 2 exit: 517.25 $\begin{array}{lll}\text { Stator } 3 \text { inlet: } 544.09 & \text { Stator } 3 \text { exit: } 552.62 & \text { Stator } 4 \text { inlet: } 577.97 \text { Stator } 4 \text { exit: } 585.92 \\ \text { Stator } 5 \text { inlet: } 605.89 & \text { Stator } 5 \text { exit: } 613.49 & \text { Unblocked Percent Blockage: } 0.00\end{array}$

Ambient Relative Humidity $\quad 10.00$

Fan Face Relative Humidity $\quad 4.44$

Fan Bypass Relative Humidity $\quad 0.23$

LPC Inlet Relative Humidity $\quad 2.25$

LPC Exit Relative Humidity

0.00

0.0000250

Amblent rlow velocity

Ambient Static Pressure

Additional Water at LPC Exit

789.49

2.85

Inlet Length

40.00

Fan/LPC Inlet Flow Velocity $\quad 458.48$ 0.000166

$\begin{array}{lr}\text { Fan/LPC Inlet Static Temperature } & 460.40\end{array}$

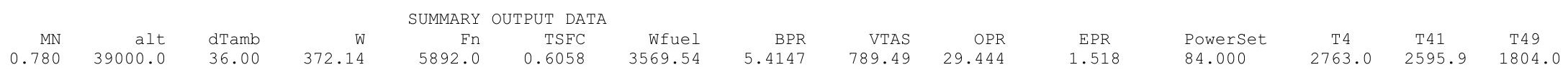

\begin{tabular}{|c|c|c|c|c|c|c|c|c|c|c|c|}
\hline & & \multicolumn{4}{|c|}{ FLOW STATION DATA } & TA & FAR & Wo & & Ps & \\
\hline FSO & \multicolumn{2}{|l|}{ Ambient.Fl 0} & 372.14 & 4.267 & 477.92 & -16.44 & 0.0000 & 1230.39 & 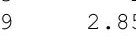 & & 425 \\
\hline FS1 & \multicolumn{2}{|l|}{ Inlet.Fl o } & 372.14 & 4.267 & 477.92 & -16.44 & 0.0000 & 1230.39 & $3.28-12-1$ & & 443 \\
\hline FS12 & \multicolumn{2}{|l|}{ Splitter.F1 02} & 314.13 & 4.265 & 477.92 & -16.44 & 0.0000 & 1039.10 & 3.25 & & 443. \\
\hline FS2 & \multicolumn{2}{|l|}{ Splitter.Fl- 01} & 58.01 & 4.265 & 477.92 & -16.44 & 0.0000 & 191.90 & 3.72 & & 460. \\
\hline FS14 & \multicolumn{2}{|l|}{ Fan.Fl 0} & 314.13 & 6.942 & 556.22 & 2.34 & 0.0000 & 688.65 & 5.7 & & 527. \\
\hline FS23 & \multicolumn{2}{|l|}{ LPC.FI-O } & 58.01 & 10.359 & 639.99 & 22.49 & 0.0000 & 91.42 & 9.21 & & 618. \\
\hline FS24 & \multicolumn{2}{|l|}{ VaporIN̄.FI_O } & 58.02 & 10.359 & 640.24 & 21.59 & 0.0000 & 91.45 & 9.26 & & \\
\hline FS25 & \multicolumn{2}{|l|}{ Bleed2.FI_ō } & 58.02 & 10.359 & 640.24 & 21.59 & 0.0000 & 91.45 & 9.20 & & 619 \\
\hline FS3 & \multicolumn{2}{|l|}{ HPC.FI O } & 55.21 & 125.630 & 1378.62 & 205.87 & 0.0000 & 10.53 & 112.99 & & 340 \\
\hline FS36 & \multicolumn{2}{|l|}{ Bleed3.F1 O } & 42.79 & 125.630 & 1378.62 & 205.87 & 0.0000 & 8.16 & 118.29 & & $356 \mathrm{~F}-2 \times$ \\
\hline FS4 & \multicolumn{2}{|l|}{ Burner.FI_O } & 43.78 & 122.737 & 2762.97 & 176.94 & 0.0232 & 12.10 & 115.94 & & 2727 \\
\hline FS45 & \multicolumn{2}{|l|}{$\begin{array}{ll}\text { HPT.FI } & -\end{array}$} & 58.34 & 28.643 & 1812.51 & 1.94 & 0.0173 & 55.95 & 25.79 & & 76 \\
\hline FS49 & \multicolumn{2}{|l|}{ LPT.FI- } & 59.01 & 6.588 & 1297.24 & -135.02 & 0.0171 & 208.21 & 5.68 & & 248 \\
\hline FS5 & \multicolumn{2}{|l|}{ TEGV.FI_O } & 59.01 & 6.588 & 1297.30 & -135.02 & 0.0171 & 208.21 & 5.68 & & 1248 \\
\hline FS8 & & 59.01 & 6.588 & 1297.43 & -135.02 & 0.0171 & 208.22 & 3.52 & & 1100 \\
\hline FS17 & \multicolumn{2}{|c|}{$\begin{array}{l}\text { Core_Nozz.F'_o } \\
\text { FanDuctLkg.FÍ }\end{array}$} & 314.13 & 6.942 & 556.22 & 2.34 & 0.0000 & $688.65 \mathrm{rat} \cdot \mathrm{r} \cdot \mathrm{r} \cdot \mathrm{s}$ & 5.76 & & 527 \\
\hline FS171 & \multicolumn{2}{|c|}{$\begin{array}{l}\text { FanDuctLkg.Fin_O } \\
\text { Bleed15.F1 O }\end{array}$} & 314.13 & 6.942 & 556.22 & 2.34 & 0.0000 & 688.65 & 5.60 & & \\
\hline FS172 & \multicolumn{2}{|l|}{$\begin{array}{l}\text { Bleed15.Fl_O } \\
\text { FanDuct.FI-O }\end{array}$} & 314.13 & 6.942 & 556.22 & 2.34 & 0.0000 & 688.65 & 5.60 & & 523 \\
\hline FS173 & \multicolumn{2}{|l|}{ Byp_Nozz.F̄'_O } & 314.13 & 6.942 & 556.22 & 2.34 & 0.0000 & 688.65 & 3.66 & & 463 \\
\hline & \multicolumn{11}{|c|}{ TURBOMACHINERY PERFORMANCE D } \\
\hline & & PR & eff & NC & TR & efPoly & & \multicolumn{3}{|c|}{ SMW } \\
\hline Fan & 1039.10 & 1.628 & 0.9121 & 3982.795 & 1.1638 & 0.9179 & & & 51.72 & 26. & \\
\hline LPC & 191.90 & 2.429 & 0.8504 & 3982.795 & 1.3391 & 0.8677 & \multicolumn{2}{|c|}{$\begin{array}{l}-8348.2 \\
-3195.2\end{array}$} & 2.91 & & 10 \\
\hline HPC & 91.45 & 12.127 & 0.8606 & 10188.703 & 2.1533 & 0.8985 & -147 & 61.5 & 29.71 & 28. & \\
\hline HPT & 12.10 & 4.285 & 0.9109 & 215.356 & 1.3490 & 0.8905 & 147 & 61.6 & & & \\
\hline LPT & 55.95 & 4.348 & 0.9147 & 89.801 & 1.3941 & 0.8982 & 115 & 43.3 & & & \\
\hline TURBON & MACHINERY & DAT: & & & & & & & & & \\
\hline & WcMap & PRmap & effMap & NcMap & R/Parm & s Wc & Des & S_PRdes & S effDes & & NCDes \\
\hline Fan & 1207.34 & 1.613 & 0.9139 & 3944.819 & 0.0614 & 0.8 & 3607 & 1.0235 & 0.9980 & & 0.9905 \\
\hline LPC & 152.38 & 2.575 & 0.8239 & 1.052 & 0.0000 & 1.2 & 593 & 0.9073 & 1.0322 & & 0.0003 \\
\hline HPC & 83.17 & 11.603 & 0.8695 & 9917.079 & 13.1570 & & 995 & 1.0494 & 0.9898 & & 0.9733 \\
\hline HPT & 0.96 & 4.194 & 0.9109 & 1.294 & 4.1941 & 12.6 & 5299 & 0.9723 & 1.0000 & & 0.0003 \\
\hline LPT & & 3.518 & 0.9215 & 1.010 & & & & .7521 & & & .0005 \\
\hline
\end{tabular}




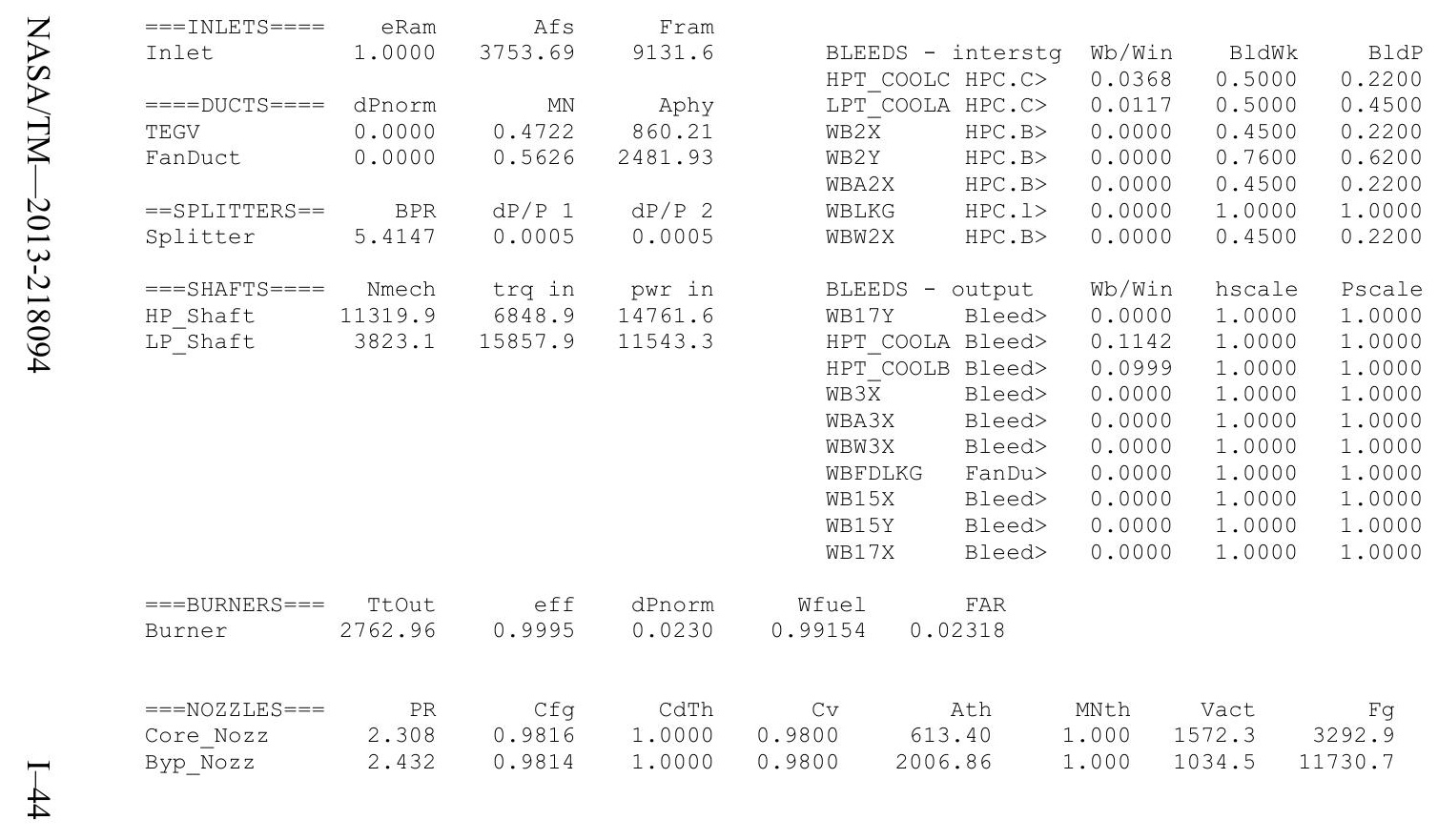


$\begin{array}{ll}\text { Date:05/13/13 Time:10:03:55 Model: } & \text { Turbofan Engine }- \text { COMDES ON CONverge }=1 \text { CASE: } 0 \\ \text { Version:NPSS 1.6.5 - Rev: -> Gas Package: Janaf iter/pass/Jacb/Broy= 20/34/1/18 Run by: Philip C Jorgenson PC: }\end{array}$

Temperature Stator 1 inlet: 471.34 Stator 1 exit: 488.94 Stator 2 inlet: 509.96 Stator 2 exit: 518.33 $\begin{array}{llll}\text { Stator } 3 \text { inlet: } 544.28 & \text { Stator } 3 \text { exit: } 552.83 & \text { Stator } 4 \text { inlet: } 577.35 \text { Stator } 4 \text { exit: } 585.26 \\ \text { Stator } 5 \text { inlet: } 604.69 & \text { Stator } 5 \text { exit: } 612.21 & \text { Unblocked Percent Blockage: } 0.00\end{array}$

Ambient Relative Humidity $\quad 10.00$

Fan Face Relative Humidity $\quad 4.19$

Fan Bypass Relative Humidity $\quad 0.25$

$\begin{array}{ll}\text { LPC Inlet Relative Humidity } & 2.16 \\ \text { LPC Exit Relative Humidity } & 0.03\end{array}$

HPC Relative Humidity

Drop Diameter

0.00

Ambient Flow Velocity $\quad 0.0000250$

low velocity

Ambient Static Pressure

Additional Water at LPC Exit

789.49

Inlet Length

40.00

2.85 Fan/LPC Inlet Flow Velocity 445.95

0.0001730

3.77

Fan/LPC Inlet Static Temperature 461.35

$\begin{array}{rrrrrc} & & & & \text { SUMMARY OUTPUT DATA } \\ \text { MN } & \text { alt } & \text { dTamb } & \text { W } & \text { Fn } & \text { TSFC } \\ 0.780 & 39000.0 & 36.00 & 366.89 & 5595.6 & 0.6046\end{array}$

VTAS
EPR Powerset 79.800 $\begin{array}{ccc}\text { T4 } & \text { T41 } & \text { T49 } \\ 2712.4 & 2549.2 & 1770.2\end{array}$
FSO Ambient.FI_O

FS1 Inlet.Fl_O

$\begin{array}{ll}\text { FS12 } & \text { Splitter.Fl_02 } \\ \text { FS2 } & \text { Splitter.Fl_01 }\end{array}$

FS14 Fan.Fl_

TS23 LPC.FI-O

FS25 BaporIN.FI_O

FS25 Bleed2.F1

FS36 Ble.FI_O

FS4 Burner.F1_-

FS4 Burner.F1-

FS44 HPT.FI_

FS5 5 TPGV FI

FS8 Core_Nozz.FI_O

FS17 FanDuctLkg.F̄_o

FS171 Bleed15.F1.F

FS172 FanDuct.

FS173 Byp Noz7. Fi

TURBOMACHINERY PERFORMANCE DATA

\begin{tabular}{|c|c|c|c|c|c|c|c|c|c|c|}
\hline \multicolumn{11}{|c|}{ DATA } \\
\hline & & $\begin{array}{r}\text { W } \\
366-89\end{array}$ & $\mathrm{Pt}$ & $\mathrm{Tt}$ & $\mathrm{ht}$ & FA & W & C & $\begin{array}{l}\text { Ps } \\
54\end{array}$ \\
\hline $\begin{array}{l}\text { ES0 } \\
\text { ES1 }\end{array}$ & \multicolumn{2}{|l|}{ Ambient.FI_O } & $\begin{array}{l}366.89 \\
366.89\end{array}$ & $\begin{array}{l}4.267 \\
4.267\end{array}$ & $\begin{array}{l}477.92 \\
477.92\end{array}$ & $\begin{array}{l}-16.44 \\
-16.44\end{array}$ & 0.000 & $\begin{array}{l}0 \\
0\end{array}$ & $\begin{array}{l}2.8 \\
3.3\end{array}$ & $\begin{array}{l}425 \\
444\end{array}$ \\
\hline S12 & \multicolumn{2}{|c|}{ Splitter.F1 02} & 310.17 & 4.265 & 477.92 & -16.44 & 0.000 & D 1026.0 & $\begin{array}{l}3.02 \\
3.32 \\
3\end{array}$ & 445 \\
\hline 52 & \multicolumn{2}{|l|}{ Splitter.FI_01 } & 56.72 & 4.265 & 477.92 & -16.44 & 0.000 & $0 \quad 187.6$ & 3.7 & \\
\hline 514 & \multicolumn{2}{|l|}{ Fan.Fl_O - } & 310.17 & 6.839 & 553.68 & 1.73 & 0.000 & 688.6 & 5.6 & 77 \\
\hline 523 & \multicolumn{2}{|l|}{ LPC.Fl-O } & 56.72 & 10.303 & 637.63 & 21.92 & 0.000 & 89.7 & 9.2 & \\
\hline$\$ 24$ & \multicolumn{2}{|l|}{ VaporIN̄.Fl O } & 73 & 10.303 & 637.89 & 0.99 & .000 & 89.7 & & \\
\hline 25 & \multicolumn{2}{|l|}{ Bleed2.Fl $\overline{0}$} & 56.73 & 10.303 & 637.89 & 20.99 & .000 & 89.7 & 9.2 & 617 \\
\hline 3 & \multicolumn{2}{|l|}{ HPC.FIO } & 5 & 121.635 & 1362.94 & 201.75 & 0.000 & 10.5 & 109.28 & 84 \\
\hline 536 & \multicolumn{2}{|l|}{ Bleed3.FI_O } & $47-1$ & 21.635 & 362.94 & 201.75 & 0 & 8. & 114. & \\
\hline 54 & \multicolumn{2}{|l|}{ Burner.Fl-o } & 42. & 118.833 & 2712.39 & 173.82 & 0.022 & 12.1 & 112.2 & \\
\hline S45 & \multicolumn{2}{|l|}{$\mathrm{HF}$} & 57. & 27.706 & 778.43 & 1.96 & 0.0 & 55.9 & 24.9 & \\
\hline$\$ 49$ & \multirow{2}{*}{\multicolumn{2}{|c|}{$\begin{array}{l}\text { LPT.FI_- } \\
\text { TEGV.FI'O }\end{array}$}} & 57. & & 271.48 & 132.25 & 016 & 208.3 & 0 & 93 \\
\hline 5 & & 5 & & .54 & -132.25 & 0 & 20 & 1 & 93 \\
\hline S8 & & & 57.67 & 6.370 & 1271.67 & -132.25 & 0 & 208 & 2 & 107 \\
\hline 517 & \multicolumn{2}{|c|}{ FanDuctLkg.Fi $O$} & 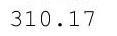 & & .68 & 1.73 & 0 & 68 & 5 & \\
\hline S171 & \multicolumn{2}{|c|}{ Bleed15.F1 O - } & & & & 1.73 & & & & \\
\hline S172 & \multicolumn{2}{|l|}{ FanDuct.Fl_o } & 7 & 6.839 & 553.68 & 1.73 & 0. & 688 & 5.51 & 520 \\
\hline S173 & \multicolumn{2}{|l|}{ Byp_Nozz.Fì_o } & 310.17 & 6.839 & 553.68 & 1.73 & 0.000 & 688.6 & 3.61 & \\
\hline \multicolumn{3}{|c|}{ URBOMACH INERY } & D & \multirow{2}{*}{\multicolumn{2}{|c|}{$\mathrm{TR}$}} & & \multirow{2}{*}{\multicolumn{2}{|c|}{ pwr }} & \multirow{2}{*}{\multicolumn{2}{|c|}{ SN $>>$}} \\
\hline & & & ff & & & efPoly & & & & \\
\hline an & 1026.01 & & 17 & .75 & 585 & & & & 27.2 \\
\hline $\mathrm{PC}$ & 187.62 & & & $3,75>-15$ & 842 & 5 & & & & 2.5 \\
\hline $\mathrm{PC}$ & 89.73 & 11.8 & 0.8 & 10140.735 & 2.1366 & 0.8 & \multicolumn{2}{|c|}{$\begin{array}{r}-3078.3 \\
-14156.4\end{array}$} & 0.99 & 29.88 \\
\hline PT & 12.10 & & & .93 & 508 & 0.8 & & 156.4 & & \\
\hline $\mathrm{PT}$ & 55.99 & 4.349 & 0.9140 & 89.541 & 1.3957 & 0.8973 & & 553.0 & & \\
\hline JRB & ER: & DA & & & & & & & & \\
\hline & WCM & & effmap & NcMap & $\mathrm{R} / \mathrm{Pa}$ & & cDes & S_PRdes & effDes & S_NCDe \\
\hline 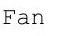 & & & & 396.249 & . & & & & & $\overline{0} .990$ \\
\hline PC & 14 & & & 1.039 & 0.0000 & & 91 & 0.9291 & 419 & 0.000 \\
\hline $\mathrm{APC}$ & 81.61 & 11.296 & 0.8702 & 9870.389 & 13.0466 & & 0995 & 1.0494 & 0.9898 & 0.9733 \\
\hline IDT & 0.9 & 4.198 & 02 & 1.298 & 4.1980 & 12.6 & 6299 & 0.9723 & 1.0000 & 0.0003 \\
\hline & & 3.5 & 9208 & 1007 & & & & 7521 & .9926 & \\
\hline
\end{tabular}

TURBOMACHINERY MAP DATA

\begin{tabular}{|c|c|c|c|c|c|c|c|c|c|c|}
\hline \multicolumn{11}{|c|}{ DATA } \\
\hline & & $\begin{array}{r}\text { W } \\
366-89\end{array}$ & $\mathrm{Pt}$ & $\mathrm{Tt}$ & $\mathrm{ht}$ & FA & W & C & $\begin{array}{l}\text { Ps } \\
54\end{array}$ \\
\hline $\begin{array}{l}\text { ES0 } \\
\text { ES1 }\end{array}$ & \multicolumn{2}{|l|}{ Ambient.FI_O } & $\begin{array}{l}366.89 \\
366.89\end{array}$ & $\begin{array}{l}4.267 \\
4.267\end{array}$ & $\begin{array}{l}477.92 \\
477.92\end{array}$ & $\begin{array}{l}-16.44 \\
-16.44\end{array}$ & 0.000 & $\begin{array}{l}0 \\
0\end{array}$ & $\begin{array}{l}2.8 \\
3.3\end{array}$ & $\begin{array}{l}425 \\
444\end{array}$ \\
\hline S12 & \multicolumn{2}{|c|}{ Splitter.F1 02} & 310.17 & 4.265 & 477.92 & -16.44 & 0.000 & D 1026.0 & $\begin{array}{l}3.02 \\
3.32 \\
3\end{array}$ & 445 \\
\hline 52 & \multicolumn{2}{|l|}{ Splitter.FI_01 } & 56.72 & 4.265 & 477.92 & -16.44 & 0.000 & $0 \quad 187.6$ & 3.7 & \\
\hline 514 & \multicolumn{2}{|l|}{ Fan.Fl_O - } & 310.17 & 6.839 & 553.68 & 1.73 & 0.000 & 688.6 & 5.6 & 77 \\
\hline 523 & \multicolumn{2}{|l|}{ LPC.Fl-O } & 56.72 & 10.303 & 637.63 & 21.92 & 0.000 & 89.7 & 9.2 & \\
\hline$\$ 24$ & \multicolumn{2}{|l|}{ VaporIN̄.Fl O } & 73 & 10.303 & 637.89 & 0.99 & .000 & 89.7 & & \\
\hline 25 & \multicolumn{2}{|l|}{ Bleed2.Fl $\overline{0}$} & 56.73 & 10.303 & 637.89 & 20.99 & .000 & 89.7 & 9.2 & 617 \\
\hline 3 & \multicolumn{2}{|l|}{ HPC.FIO } & 5 & 121.635 & 1362.94 & 201.75 & 0.000 & 10.5 & 109.28 & 84 \\
\hline 536 & \multicolumn{2}{|l|}{ Bleed3.FI_O } & $47-1$ & 21.635 & 362.94 & 201.75 & 0 & 8. & 114. & \\
\hline 54 & \multicolumn{2}{|l|}{ Burner.Fl-o } & 42. & 118.833 & 2712.39 & 173.82 & 0.022 & 12.1 & 112.2 & \\
\hline S45 & \multicolumn{2}{|l|}{$\mathrm{HF}$} & 57. & 27.706 & 778.43 & 1.96 & 0.0 & 55.9 & 24.9 & \\
\hline$\$ 49$ & \multirow{2}{*}{\multicolumn{2}{|c|}{$\begin{array}{l}\text { LPT.FI_- } \\
\text { TEGV.FI'O }\end{array}$}} & 57. & & 271.48 & 132.25 & 016 & 208.3 & 0 & 93 \\
\hline 5 & & 5 & & .54 & -132.25 & 0 & 20 & 1 & 93 \\
\hline S8 & & & 57.67 & 6.370 & 1271.67 & -132.25 & 0 & 208 & 2 & 107 \\
\hline 517 & \multicolumn{2}{|c|}{ FanDuctLkg.Fi $O$} & 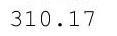 & & .68 & 1.73 & 0 & 68 & 5 & \\
\hline S171 & \multicolumn{2}{|c|}{ Bleed15.F1 O - } & & & & 1.73 & & & & \\
\hline S172 & \multicolumn{2}{|l|}{ FanDuct.Fl_o } & 7 & 6.839 & 553.68 & 1.73 & 0. & 688 & 5.51 & 520 \\
\hline S173 & \multicolumn{2}{|l|}{ Byp_Nozz.Fì_o } & 310.17 & 6.839 & 553.68 & 1.73 & 0.000 & 688.6 & 3.61 & \\
\hline \multicolumn{3}{|c|}{ URBOMACH INERY } & D & \multirow{2}{*}{\multicolumn{2}{|c|}{$\mathrm{TR}$}} & & \multirow{2}{*}{\multicolumn{2}{|c|}{ pwr }} & \multirow{2}{*}{\multicolumn{2}{|c|}{ SN $>>$}} \\
\hline & & & ff & & & efPoly & & & & \\
\hline an & 1026.01 & & 17 & .75 & 585 & & & & 27.2 \\
\hline $\mathrm{PC}$ & 187.62 & & & $3,75>-15$ & 842 & 5 & & & & 2.5 \\
\hline $\mathrm{PC}$ & 89.73 & 11.8 & 0.8 & 10140.735 & 2.1366 & 0.8 & \multicolumn{2}{|c|}{$\begin{array}{r}-3078.3 \\
-14156.4\end{array}$} & 0.99 & 29.88 \\
\hline PT & 12.10 & & & .93 & 508 & 0.8 & & 156.4 & & \\
\hline $\mathrm{PT}$ & 55.99 & 4.349 & 0.9140 & 89.541 & 1.3957 & 0.8973 & & 553.0 & & \\
\hline JRB & ER: & DA & & & & & & & & \\
\hline & WCM & & effmap & NcMap & $\mathrm{R} / \mathrm{Pa}$ & & cDes & S_PRdes & effDes & S_NCDe \\
\hline 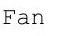 & & & & 396.249 & . & & & & & $\overline{0} .990$ \\
\hline PC & 14 & & & 1.039 & 0.0000 & & 91 & 0.9291 & 419 & 0.000 \\
\hline $\mathrm{APC}$ & 81.61 & 11.296 & 0.8702 & 9870.389 & 13.0466 & & 0995 & 1.0494 & 0.9898 & 0.9733 \\
\hline IDT & 0.9 & 4.198 & 02 & 1.298 & 4.1980 & 12.6 & 6299 & 0.9723 & 1.0000 & 0.0003 \\
\hline & & 3.5 & 9208 & 1007 & & & & 7521 & .9926 & \\
\hline
\end{tabular}

$\begin{array}{rrrrrrr}\text { Aphy } & \text { MN } & \text { gamt } & \text { WAR } & \text { Wair } & \text { WH2O } & \text { H2O frac } \\ 3700.7 & 0.7800 & 1.400068 & 0.0000851 & 366.85 & 0.031 & 0.0001 \\ 4168.2 & 0.6076 & 1.40068 & 0.0000851 & 366.85 & 0.031 & 0.0001 \\ 3531.8 & 0.6058 & 1.40068 & 0.0000851 & 310.14 & 0.026 & 0.0001 \\ 830.5 & 0.4234 & 1.40068 & 0.0000851 & 56.71 & 0.005 & 0.0001 \\ 2606.7 & 0.5226 & 1.39970 & 0.0000851 & 310.14 & 0.026 & 0.0001 \\ 412.6 & 0.4039 & 1.39785 & 0.0000851 & 56.71 & 0.005 & 0.0001 \\ 412.6 & 0.4041 & 1.39782 & 0.0002581 & 56.71 & 0.015 & 0.0003 \\ 412.6 & 0.4041 & 1.39782 & 0.0002581 & 56.71 & 0.015 & 0.0003 \\ 49.7 & 0.3996 & 1.35806 & 0.0002581 & 53.96 & 0.014 & 0.0003 \\ 49.3 & 0.3000 & 1.35806 & 0.0002581 & 41.82 & 0.011 & 0.0003 \\ 74.6 & 0.2996 & 1.28875 & 0.0002581 & 41.82 & 0.011 & 0.0273 \\ 265.4 & 0.4007 & 1.32483 & 0.0002581 & 56.05 & 0.014 & 0.0206 \\ 860.2 & 0.4722 & 1.35217 & 0.0002581 & 56.71 & 0.015 & 0.0204 \\ 860.2 & 0.4722 & 1.35217 & 0.0002581 & 56.71 & 0.015 & 0.0204 \\ 613.4 & 1.0000 & 1.35216 & 0.0002581 & 56.71 & 0.015 & 0.0204 \\ 2606.7 & 0.5226 & 1.39970 & 0.0000851 & 310.14 & 0.026 & 0.0001 \\ 2481.9 & 0.5626 & 1.39970 & 0.0000851 & 310.14 & 0.026 & 0.0001 \\ 2481.9 & 0.5626 & 1.39970 & 0.0000851 & 310.14 & 0.026 & 0.0001 \\ 2006.9 & 1.0000 & 1.39970 & 0.0000851 & 310.14 & 0.026 & 0.0001\end{array}$


$\begin{array}{lrrr}===\text { INLETS }==== & \text { eRam } & \text { Afs } & \text { Fram } \\ \text { Inlet } & 1.0000 & 3700.68 & 9002.6\end{array}$

$\begin{array}{lrrr}====\text { DUCTS }==== & \text { dPnorm } & \text { MN } & \text { Aphy } \\ \text { TEGV } & 0.0000 & 0.4722 & 860.21\end{array}$

$==$ SPLITTERS $==$

Splitter

$===$ SHAFTS $===$

$===$ SHAFTS
HP_Shaft

LP_Shaft

5.4687

$\begin{array}{ll}\mathrm{dP} / \mathrm{P}_{1} 1 & \mathrm{dP} / \mathrm{P}^{2} \\ 0.0005 & 0.0005\end{array}$

$\mathrm{dP} / \mathrm{P} 2$

trq in pwr in

$6611.3 \quad 14156.4$

11246.0

$\begin{array}{ll}15373.6 & 11053.0\end{array}$

$===$ BURNERS $===\begin{array}{r}\text { TtOut } \\ \text { Burner }\end{array}$
2712.37

0.9995

dPnorm

$===$ NOZZLES $===$

Core_Nozz

$P R$
2.232
2.396

Cfg
0.9816
0.9814

CdTh

1.0000
1.0000

$\mathrm{CV}$
0.9800
0.9800 $\begin{array}{lllr}\text { FanDuct } & 0.0000 & 0.4722 & 860.21 \\ & 0.0000 & 0.5626 & 2481.93\end{array}$

BLEEDS - interstg Wb/Win BldWk BldP $\begin{array}{llll}\text { HPT_COOLC HPC.C> } & 0.0368 & 0.5000 & 0.2200 \\ \text { IPT_COOD } & 0.500 & 0.4500\end{array}$ $\begin{array}{lllll}\text { LPT_COOLA } & \text { HPC.C }> & 0.0117 & 0.5000 & 0.4500 \\ \text { WB2 } & \text { HPC.B } & 0.0000 & 0.4500 & 0.2200 \\ \text { WB2Y } & \text { HPC.B } & 0.0000 & 0.7600 & 0.6200\end{array}$ $\begin{array}{lllll}\text { WB2Y HPC.B } & 0.0000 & 0.7600 & 0.6200\end{array}$ $\begin{array}{lllll}\text { WBA2X HPC.B } & 0.0000 & 0.4500 & 0.2200\end{array}$ $\begin{array}{lllll}\text { WBLKG } & \text { HPC.I> } & 0.0000 & 1.0000 & 1.0000 \\ \text { WBW2X } & \text { HPC.B> } & 0.0000 & 0.4500 & 0.2200\end{array}$

BLEEDS - output Wb/Win hscale Pscale WB17Y Bleed> $0.0000 \quad 1.0000 \quad 1.0000$ HPT_COOLA Bleed> $0.1142 \quad 1.0000 \quad 1.0000$ $\begin{array}{lllll}\text { HPT COOLB } & \text { Bleed }> & 0.0999 & 1.0000 & 1.0000 \\ \text { WB } 3 \bar{X} & \text { Bleed }> & 0.0000 & 1.0000 & 1.0000\end{array}$ $\begin{array}{lllll}\text { WB3X } & \text { Bleed }> & 0.0000 & 1.0000 & 1.0000 \\ \text { WBA3X } & \text { Bleed }> & 0.0000 & 1.0000 & 1.0000\end{array}$ $\begin{array}{lllll}\text { WBA3X } & \text { Bleed> } & 0.0000 & 1.0000 & 1.0000 \\ \text { WBW3X } & \text { Bleed> } & 0.0000 & 1.0000 & 1.0000\end{array}$ $\begin{array}{lllll}\text { WBW3X } & \text { Bleed> } & 0.0000 & 1.0000 & 1.0000 \\ \text { WBFDIKG } & \text { FanDu> } & 0.0000 & 1.0000 & 1.0000\end{array}$ $\begin{array}{lllll}\text { WBFDLKG } & \text { FanDu> } & 0.0000 & 1.0000 & 1.0000 \\ \text { WB15X } & \text { Bleed> } & 0.0000 & 1.0000 & 1.0000\end{array}$ WB15X Bleed> $0.0000 \quad 1.0000-1.0000$ $\begin{array}{lllll}\text { WB15Y } & \text { Bleed }> & 0.0000 & 1.0000 & 1.0000 \\ \text { WB17X } & \text { Bleed }> & 0.0000 & 1.0000 & 1.0000\end{array}$

Wfuel FAR $0.93976 \quad 0.02247$

$\begin{array}{rrrr}\text { W } & \text { Tt } & \text { ht } & \text { Pt } \\ 2.0876 & 1007.74 & 111.37 & 27.706 \\ 0.6637 & 1007.74 & 111.37 & 6.370 \\ 0.0000 & 971.35 & 102.33 & 34.796 \\ 0.0000 & 1194.40 & 158.37 & 79.329 \\ 0.0000 & 971.35 & 102.33 & 34.796 \\ 0.0000 & 1362.94 & 201.75 & 121.635 \\ 0.0000 & 971.35 & 102.33 & 34.796 \\ & & \mathrm{Tt} & \mathrm{Pt} \\ \mathrm{W} & \mathrm{Tt} & \mathrm{ht} & 10.303 \\ 0.0000 & 637.89 & 20.99 & \mathbf{9} \\ 6.4771 & 1362.94 & 201.75 & 118.833 \\ 5.6676 & 1362.94 & 201.75 & 66.708 \\ 0.0000 & 1362.94 & 201.75 & 121.635 \\ 0.0000 & 1362.94 & 201.75 & 121.635 \\ 0.0000 & 1362.94 & 201.75 & 121.635 \\ 0.0000 & 553.68 & 1.73 & 6.839 \\ 0.0000 & 553.68 & 1.73 & 6.839 \\ 0.0000 & 553.68 & 1.73 & 6.839 \\ 0.0000 & 553.68 & 1.73 & 6.839\end{array}$


Date:05/13/13 Time:10:04:30 Model: Turbofan Engine - COMDES ON Converge $=1$ CASE: 0

Temperature Stator 1 inlet: $472.53 \quad$ Stator 1 exit: $489.84 \quad$ Stator 2 inlet: 510.66 Stator 2 exit: 519.13 Stator 5 inlet: 604.02 Stator 5 exit: 611.50 Unblocked Percent Blockage: 0.00

Ambient Relative Humidity $\quad 10.00$

Fan Face Relative Humidity $\quad 4.03$

an Bypass Relative Humidity

Inlet Relative Humidity

HPC Relative Humidity

Drop Diameter

0.00

0.0000250

Inlet Length

Fan/LPC Inlet Flow Velocity $\quad \begin{aligned} 40.00 \\ \text { Fan/LPC Inlet }\end{aligned}$

Ambient Flow Velocity

Ambient Static Pressure

Additional Water at LPC Exit

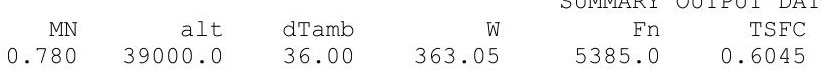

$\begin{array}{rrr}5385.0 & 0.6045 & \text { Wfuel } \\ & & \end{array}$

BPR
5.5094

VTAS
789.49

$O P R$
27.844

$\begin{array}{cl}\text { EPR } & \text { PowerSet } \\ 1.415 & 76.800\end{array}$

$\begin{array}{ccc}\text { T4 } & \text { T41 } & \text { T49 } \\ 2677.6 & 2517.0 & 1747.3\end{array}$

FSO Ambient

FS1 Inlet.FI_O

FS12 Splitter.F1_02

FS2 Splitter.Fl_01

FS14 Fan.F1_-

FS23 LPC.FI_O

FS25 Bleed2.FI_O

FS3 HPC.Fl_O

FS36 Bleed3.F1_O

FS4 Burner.FI_O

FS45 HPT.FI

FS5 TEGV.FI O

FS8 Core Nozz.FI

FS17 FanDuctLkg.Fì_O

FS171 Bleed15.FI

FS172 FanDuct.FI_O

FLOW STATION DATA

\begin{tabular}{rrrrrr} 
W & \multicolumn{2}{c}{ FLOW } & \multicolumn{1}{l}{ STATION DATA } \\
Pt & Tt & ht & FAR & WC \\
363.05 & 4.267 & 477.92 & -16.44 & 0.0000 & 1200.33 \\
363.05 & 4.267 & 477.92 & -16.44 & 0.0000 & 1200.33 \\
307.28 & 4.265 & 477.92 & -16.44 & 0.0000 & 1016.44 \\
55.77 & 4.265 & 477.92 & -16.44 & 0.0000 & 184.49 \\
307.28 & 6.764 & 551.90 & 1.30 & 0.0000 & 688.66 \\
55.77 & 10.268 & 636.19 & 21.57 & 0.0000 & 88.41 \\
55.78 & 10.268 & 636.45 & 20.61 & 0.0000 & 88.44 \\
55.78 & 10.268 & 636.45 & 20.61 & 0.0000 & 88.44 \\
53.08 & 118.804 & 1352.21 & 198.94 & 0.0000 & 10.60 \\
41.14 & 118.804 & 1352.21 & 198.94 & 0.0000 & 8.22 \\
42.04 & 116.068 & 2677.57 & 171.67 & 0.0220 & 12.09 \\
56.03 & 27.046 & 1755.32 & 2.00 & 0.0164 & 56.01 \\
56.69 & 6.217 & 1254.04 & -130.35 & 0.0162 & 208.37 \\
56.69 & 6.217 & 1254.11 & -130.35 & 0.0162 & 208.38 \\
56.69 & 6.217 & 1254.24 & -130.35 & 0.0162 & 208.39 \\
307.28 & 6.764 & 551.90 & 1.30 & 0.0000 & 688.66 \\
307.28 & 6.764 & 551.90 & 1.30 & 0.0000 & 688.66 \\
307.28 & 6.764 & 551.90 & 1.30 & 0.0000 & 688.66 \\
307.28 & 6.764 & 551.90 & 1.30 & 0.0000 & 688.66 \\
& & & & &
\end{tabular}

Ps
2.854
3.353
3.352
3.789
5.615
9.213
9.212
9.212
106.663
111.766
109.644
24.346
5.360
5.360
3.319
5.615
5.456
5.456
3.572

Ts
425.97
446.06
446.12
462.01
523.31
616.85
617.09
617.09
1314.18
1330.57
2643.14
1710.38
1206.27
1206.33
1062.36
523.31
519.04
519.04
459.82$$
\text { . }
$$

$\begin{array}{rrr}\text { Aphy MN gamt } & \end{array}$ $\begin{array}{lll}4168.2 & 0.7800 & 1.40068 \\ 3531.8 & 0.5970 & 1.40068\end{array}$ $\begin{array}{lll}3531.8 & 0.5964 & 1.40068\end{array}$ $\begin{array}{lll}830.5 & 0.4146 & 1.40068\end{array}$ $\begin{array}{lll}8306.7 & 0.4146 & 1.40068 \\ 412.6 & 0.3968 & 1.39973\end{array}$ $\begin{array}{lll}412.6 & 0.3970 & 1.39786\end{array}$ $\begin{array}{rrr}412.6 & 0.3970 & 1.39786 \\ 49.7 & 0.4009 & 1.35869\end{array}$ $\begin{array}{llll}49.3 & 0.3009 & 1.35869\end{array}$ $\begin{array}{llll}74.6 & 0.3009 & 1.35869\end{array}$ $\begin{array}{lll}74.6 & 0.2994 & 1.29006\end{array}$ $\begin{array}{lll}265.4 & 0.4007 & 1.32611 \\ 860.2 & 0.4721 & 1.35350\end{array}$ $860.20 .4721 \quad 1.35350$ $\begin{array}{rrr}613.4 & 1.0000 & 1.35349\end{array}$ $\begin{array}{lll}2606.7 & 0.5226 & 1.39973 \\ 2481.9 & 0.5626 & 1.39973\end{array}$ $2481.9 \quad 0.5626 \quad 1.39973$ $2006.9 \quad 1.0000$

$\begin{array}{rrrr}\text { WAR } & \text { Wair } & \text { WH2O } & \text { H2O frac } \\ 0.0000851 & 363.02 & 0.031 & 0.0001 \\ 0.0000851 & 363.02 & 0.031 & 0.0001 \\ 0.0000851 & 307.25 & 0.026 & 0.0001 \\ 0.0000851 & 55.77 & 0.005 & 0.0001 \\ 0.0000851 & 307.25 & 0.026 & 0.0001 \\ 0.0000851 & 55.77 & 0.005 & 0.0001 \\ 0.0002632 & 55.77 & 0.015 & 0.0003 \\ 0.0002632 & 55.77 & 0.015 & 0.0003 \\ 0.0002632 & 53.06 & 0.014 & 0.0003 \\ 0.0002632 & 41.12 & 0.011 & 0.0003 \\ 0.0002632 & 41.12 & 0.011 & 0.0268 \\ 0.0002632 & 55.12 & 0.015 & 0.0202 \\ 0.0002632 & 55.77 & 0.015 & 0.0199 \\ 0.0002632 & 55.77 & 0.015 & 0.0199 \\ 0.0002632 & 55.77 & 0.015 & 0.0199 \\ 0.0000851 & 307.25 & 0.026 & 0.0001 \\ 0.0000851 & 307.25 & 0.026 & 0.0001 \\ 0.0000851 & 307.25 & 0.026 & 0.0001 \\ 0.0000851 & 307.25 & 0.026 & 0.0001\end{array}$

0.0001
0.0001

TURBOMACHINERY PERFORMANCE DATA

\begin{tabular}{|c|c|c|c|c|c|c|c|c|c|}
\hline & Wc & PR & eff & $\mathrm{NC}$ & TR & efPoly & pwr & SMN & SMW \\
\hline Fan & 1016.44 & 1.586 & 0.9105 & 3900.605 & 1.1548 & 0.9162 & -7714.3 & 58.46 & 27.99 \\
\hline LPC & 184.49 & 2.408 & 0.8610 & 3900.605 & 1.3312 & 0.8770 & -2999.6 & 3.51 & 2.79 \\
\hline HPC & 88.44 & 11.570 & 0.8614 & 10105.824 & 2.1246 & 0.8985 & -13733.0 & 31.92 & 30.74 \\
\hline HPT & 12.09 & 4.291 & 0.9097 & 216.341 & 1.3518 & 0.8892 & 13733.0 & & \\
\hline LPT & 56.01 & 4.351 & 0.9135 & 89.369 & 1.3968 & 0.8967 & 10714.2 & & \\
\hline \multicolumn{10}{|c|}{ TURBOMACH INER } \\
\hline & WcMap & PRmap & effMap & NcMap & R/Parm & S_WCDes & S_PRdes & s_effDes & S_NcDes \\
\hline Fan & 1181.00 & 1.573 & 0.9124 & 3863.413 & 0.0599 & $\overline{0} .8607$ & $\overline{1} .0235$ & 0.9980 & $\overline{0} .9905$ \\
\hline LPC & 146.87 & 2.490 & 0.8219 & 1.030 & 0.0000 & 1.2562 & 0.9448 & 1.0476 & 0.0003 \\
\hline HPC & 80.44 & 11.072 & 0.8703 & 9836.410 & 12.9676 & 1.0995 & 1.0494 & 0.9898 & 0.9733 \\
\hline HPT & 0.96 & 4.200 & 0.9097 & 1.300 & 4.2003 & 12.6299 & 0.9723 & 1.0000 & 0.0003 \\
\hline LPT & 0.86 & 3.520 & 0.9203 & 1.005 & 3.5201 & 65.2590 & 0.7521 & 0.9926 & 0.0005 \\
\hline
\end{tabular}




\begin{tabular}{|c|c|c|c|c|c|c|c|c|c|c|c|c|}
\hline \multirow{2}{*}{$\begin{array}{ll}Z & ===\text { INLETS }==== \\
& \text { Inlet }\end{array}$} & eRam & Afs & Fram & \multirow{2}{*}{\multicolumn{2}{|c|}{ BIFEDS - intersta }} & \multirow[b]{2}{*}{ Wb/Win } & & & & \multirow[b]{2}{*}{$\mathrm{Tt}$} & \multirow[b]{2}{*}{$h t$} & \multirow[b]{2}{*}{$\begin{array}{r}P t \\
27.046\end{array}$} \\
\hline & 1.0000 & 3661.98 & 8908.5 & $\begin{array}{l}\text { BLEEDS - } \\
\text { HPT COOLC }\end{array}$ & & & $\begin{array}{r}\text { BldWk } \\
0.5000\end{array}$ & $\begin{array}{r}\text { BldP } \\
0.2200\end{array}$ & $\begin{array}{r}\text { W } \\
2.0528\end{array}$ & & & \\
\hline$====$ DUCTS $====$ & dPnorm & MN & Aphy & LPT COOLA & A HPC.C> & 0.0117 & 0.5000 & 0.4500 & 0.6527 & 1001.43 & 109.77 & 6.217 \\
\hline TEGV & 0.0000 & 0.4721 & 860.21 & WB2 $\overline{\mathrm{X}}$ & HPC. B> & 0.0000 & 0.4500 & 0.2200 & 0.0000 & 965.51 & 100.86 & 34.146 \\
\hline FanDuct & 0.0000 & 0.5626 & 2481.93 & $\begin{array}{l}\text { WB2Y } \\
\text { WBA2X }\end{array}$ & HPC.B> & 0.0000 & 0.7600 & 0.6200 & 0.0000 & 1185.75 & 156.14 & 77.560 \\
\hline$==$ SPLITTERS $==$ & $B P R$ & $d P / P 1$ & $\mathrm{dP} / \mathrm{P} 2$ & $\begin{array}{l}\text { WBA2X } \\
\text { WBLKG }\end{array}$ & $\begin{array}{l}\text { HPC.B }> \\
\text { HPC. } 1>\end{array}$ & 0.0000 & 0.4500 & 0.2200 & 0.0000 & $\begin{array}{r}965.51 \\
1352.21\end{array}$ & 100.86 & $\begin{array}{r}34.146 \\
118.804\end{array}$ \\
\hline $\begin{array}{l}==\text { SPLITTERS== } \\
\text { Splitter }\end{array}$ & 5.5094 & 0.0005 & 0.0005 & $\begin{array}{l}\text { WBLAG } \\
\text { WBW2X }\end{array}$ & HPC.B> & 0.0000 & 0.4500 & 0.2200 & 0.0000 & 965.51 & 100.86 & $\begin{array}{r}118.804 \\
34.146\end{array}$ \\
\hline$===$ SHAFTS $====$ & Nmech & trq in & pwr in & BLEEDS - & output & Wb/Win & hscale & Pscale & W & Tt & ht & Pt \\
\hline HP Shaft & 11194.6 & 6443.1 & 13733.0 & WB17Y & Bleed> & 0.0000 & 1.0000 & 1.0000 & 0.0000 & 636.45 & 20.61 & 10.268 \\
\hline LP' Shaft & 3744.2 & 15029.0 & 10714.2 & HPT COOLA & A Bleed> & 0.1142 & 1.0000 & 1.0000 & 6.3693 & 1352.21 & 198.94 & 116.068 \\
\hline & & & & HPT COOLB & B Bleed> & 0.0999 & 1.0000 & 1.0000 & 5.5733 & 1352.21 & 198.94 & 65.147 \\
\hline & & & & WB3 $3 \bar{x}$ & Bleed> & 0.0000 & 1.0000 & 1.0000 & 0.0000 & 1352.21 & 198.94 & 118.804 \\
\hline & & & & WBA3X & Bleed> & 0.0000 & 1.0000 & 1.0000 & 0.0000 & 1352.21 & 198.94 & 118.804 \\
\hline & & & & WBW3X & Bleed> & 0.0000 & 1.0000 & 1.0000 & 0.0000 & 1352.21 & 198.94 & 118.804 \\
\hline & & & & WBFDLKG & FanDu> & 0.0000 & 1.0000 & 1.0000 & 0.0000 & 551.90 & 1.30 & 6.764 \\
\hline & & & & WB15X & Bleed> & 0.0000 & 1.0000 & 1.0000 & 0.0000 & 551.90 & 1.30 & 6.764 \\
\hline & & & & WB15Y & Bleed> & 0.0000 & 1.0000 & 1.0000 & 0.0000 & 551.90 & 1.30 & 6.764 \\
\hline & & & & WB17X & Bleed> & 0.0000 & 1.0000 & 1.0000 & 0.0000 & 551.90 & 1.30 & 6.764 \\
\hline$===$ BURNERS $===$ & TtOut & eff & dPnorm & Wfuel & FAR & & & & & & & \\
\hline Burner & 2677.55 & 0.9995 & 0.0230 & 0.90419 & 02199 & & & & & & & \\
\hline$===$ NOZZLES $===$ & $\mathrm{PR}$ & $\mathrm{Cfg}$ & CdTh & $\mathrm{CV}$ & Ath & MNth & Vact & $\mathrm{Fg}$ & & & & \\
\hline Core_Nozz & 2.178 & 0.9815 & 1.0000 & 0.9800 & 3.40 & 1.000 & 1546.6 & 3010.3 & & & & \\
\hline Byp_Nozz & 2.370 & 0.9815 & 1.0000 & 0.9800 & 6.86 & 1.000 & 1030.5 & 11283.2 & & & & \\
\hline
\end{tabular}




\section{$50 \mu \mathrm{m}, \mathrm{ISA}+18 \mathrm{R}$}

Date:07/05/13 Time:09:24:24 Model: Turbofan Engine -

Version:NPSS 1.6.5 - Rev: -> Gas Package: Janaf iter/pass/Jacb/Broy=24/38/1/22 Run by: Philip C Jorgenson PC: 100

Temperature Stator 1 inlet: 444.03 Stator 1 exit: 462.65 Stator 2 inlet: 484.18 Stator 2 exit: 491.25

Stator 3 inlet: 520.20 Stator 3 exit: 528.10 Stator 4 inlet: 555.20 Stator 4 exit: 562.88

Ambient Relative Humidity $\quad 10.00$

Fan Face Relative Humidity $\quad 5.21$

LPC Inlet Relative Humidity 0.15

LPC Exit Relative Humidity 2.30

Cepitative Hunidity

Drop Diameter

0.00

$0.0000500 \quad$ Inlet Length 40.00

Ambient Flow Velocity

Ambient Static Pressure

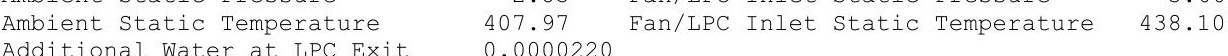

Fan/LPC Inlet Flow Velocity $\quad 485.44$

SUMMARY OUTPUT DATA

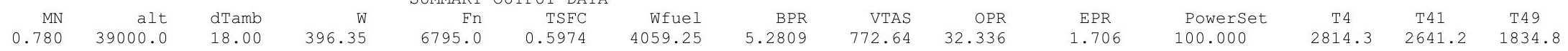

\begin{tabular}{|c|c|}
\hline FSO & Ambient.FI_O \\
\hline & Inlet.FI_O \\
\hline FS12 & Splitter.Fl_02 \\
\hline FS2 & Splitter.FI_01 \\
\hline FS14 & Fan.Fl O \\
\hline FS23 & LPC.FI- \\
\hline FS24 & VaporIN.FI_O \\
\hline 525 & Bleed2.Fl_o \\
\hline FS3 & HPC.Fl O \\
\hline S36 & Bleed3.FI_O \\
\hline F 4 & Burner.Fl_o \\
\hline$S 45$ & HPT.FI_O \\
\hline S49 & LPT.FI_O \\
\hline 55 & TEGV.F̄ $O$ \\
\hline & Core Nozz.Fl \\
\hline 517 & FanDúctLkg.Fì_o \\
\hline S171 & Bleed15.FI O \\
\hline & FanDuct.Fl \\
\hline & Byp Nozz.Fì \\
\hline
\end{tabular}

\begin{tabular}{|c|c|c|c|c|c|}
\hline W & Pt & $\mathrm{Tt}$ & ht & FAR & \\
\hline 96.35 & 4.267 & 457.74 & -20.96 & 0.0000 & 1282. \\
\hline 6.35 & 4.267 & 457.74 & -20.96 & 0.0000 & 12 \\
\hline 3.25 & 4.265 & 457.74 & -20.96 & 0.0000 & 107 \\
\hline 63.10 & 4.265 & 457.74 & -20.96 & 0.0000 & \\
\hline 33.25 & 7.257 & 540.20 & -1.18 & 0.0000 & \\
\hline 63.10 & 10.457 & 620.27 & 18.06 & 0.0 & \\
\hline 63.11 & 10.457 & 620.35 & 17.95 & 0.0 & \\
\hline 63.11 & 10.457 & 620.35 & 17.95 & 0.0 & \\
\hline 60.04 & 137.970 & 1373.20 & 205.57 & & \\
\hline 46.53 & 37.970 & 1373.20 & 205.57 & 0.0 & \\
\hline 47.66 & 134.793 & 2814.34 & 175.32 & 0.0242 & \\
\hline 63.49 & 31.497 & 1843.74 & -2.52 & 0.0 & \\
\hline 64.23 & 7.238 & 1320.50 & -142.11 & 0.0 & \\
\hline 64.23 & 7.238 & 1320.57 & -142.11 & 0.0 & \\
\hline 64.23 & 7.238 & 1320.70 & -142.11 & 0.0 & \\
\hline 333.25 & 7.257 & 540.20 & -1.18 & 0.0000 & \\
\hline 333.25 & 7.257 & 540.20 & -1.18 & 0.0000 & \\
\hline & 7.2 & & -1.18 & & \\
\hline 3 & 7.257 & 540.20 & -1.18 & 0.0000 & \\
\hline
\end{tabular}

$\begin{array}{rr}\text { PS } & \text { Ts } \\ 2.854 & 407.97 \\ 3.150 & 419.66 \\ 3.174 & 420.64 \\ 3.659 & 438.10 \\ 6.024 & 512.21 \\ 9.125 & 596.65 \\ 9.125 & 596.72 \\ 9.125 & 596.72 \\ 24.457 & 1336.34 \\ -30.119 & 1352.13 \\ 27.337 & 2778.61 \\ 28.373 & 1797.37 \\ 6.243 & 1270.87 \\ 6.243 & 1270.93 \\ 3.870 & 1121.05 \\ 6.024 & 512.21 \\ 5.854 & 508.03 \\ 5.854 & 508.03 \\ 3.833 & 450.06\end{array}$

$\begin{array}{rrr}\text { Aphy } & \text { MN } & \text { gamt } \\ 3912.3 & 0.7800 & 1.40084 \\ 4168.2 & 0.6728 & 1.40084 \\ 3531.8 & 0.6633 & 1.40084 \\ 830.5 & 0.4729 & 1.40084 \\ 2606.7 & 0.5226 & 1.39992 \\ 412.6 & 0.4457 & 1.39831 \\ 412.6 & 0.4457 & 1.39831 \\ 412.6 & 0.4457 & 1.39831 \\ 49.7 & 0.3921 & 1.35749 \\ 49.3 & 0.2948 & 1.35749 \\ 74.6 & 0.3003 & 1.28479 \\ 265.4 & 0.4001 & 1.32115 \\ 860.2 & 0.4723 & 1.34830 \\ 860.2 & 0.4723 & 1.34830 \\ 613.4 & 1.0000 & 1.34829 \\ 2606.7 & 0.5226 & 1.39992 \\ 2481.9 & 0.5625 & 1.39992 \\ 2481.9 & 0.5625 & 1.39992 \\ 2006.9 & 1.0000 & 1.39992\end{array}$

WAR 0.0000295 0.0000295 0.0000295 0.0000295 0.0000295 0.0000515 0.0000515 0.0000515 0.0000515 0.0000515 0.0000515 0.0000515 0.0000515 0.0000515 0.0000295 0.0000295
0.0000295 0.0000295
Wair WH2O H2O frac $\begin{array}{lll}396.34 & 0.012 & 0.0000\end{array}$ $\begin{array}{lll}396.34 & 0.012 & 0.0000\end{array}$ $\begin{array}{rrr}396.34 & 0.012 & 0.0000 \\ 63.10 & 0.010 & 0.0000 \\ 333.24 & 0.010 & 0.0000\end{array}$ $\begin{array}{lll}333.24 & 0.010 & 0.0000\end{array}$ $\begin{array}{lll}63.10 & 0.002 & 0.0000\end{array}$ $\begin{array}{lll}63.10 & 0.002 & 0.0000\end{array}$ $\begin{array}{lll}63.10 & 0.003 & 0.0001\end{array}$ $\begin{array}{lll}63.10 & 0.003 & 0.0001 \\ 60.04 & 0.003 & 0.0001\end{array}$ $\begin{array}{lll}60.04 & 0.003 & 0.0001\end{array}$ $\begin{array}{lll}46.53 & 0.002 & 0.0001 \\ 66.53 & 0.002 & 0.0292\end{array}$ $\begin{array}{lll}62.36 & 0.003 & 0.0219\end{array}$ $\begin{array}{lll}62.36 & 0.003 & 0.0219\end{array}$ $\begin{array}{lll}63.10 & 0.003 & 0.0217\end{array}$ $\begin{array}{lll}63.10 & 0.003 & 0.0217\end{array}$ $\begin{array}{lll}633.10 & 0.003 & 0.0240\end{array}$ $\begin{array}{lll}333.24 & 0.010 & 0.0000\end{array}$ $333.24 \quad 0.010 \quad 0.0000$ 0.0000

TURBOMACHINERY PERFORMANCE DATA

\begin{tabular}{|c|c|c|c|c|c|c|c|c|c|}
\hline & WC & $\mathrm{PR}$ & eff & $\mathrm{NC}$ & $T R$ & efPoly & pwr & SMN & SMW \\
\hline Fan & 1078.81 & 1.702 & 0.9114 & 4139.469 & 1.1801 & 0.9178 & -9322.3 & 36.69 & 23.09 \\
\hline LPC & 204.28 & 2.452 & 0.8224 & 4139.469 & 1.3551 & 0.8432 & -3483.4 & 2.97 & 2.24 \\
\hline HPC & 96.99 & 13.194 & 0.8584 & 10373.799 & 2.2136 & 0.8979 & -16345.6 & 25.80 & 25.03 \\
\hline HPT & 12.10 & 4.280 & 0.9118 & 213.857 & 1.3470 & 0.8911 & 16345.5 & & \\
\hline LPT & 55.86 & 4.352 & 0.9155 & 90.565 & 1.3927 & 0.8988 & 12805.4 & & \\
\hline URI & ENERY M & DATA & & & & & & & \\
\hline & WcMap & PRmap & effMap & NcMap & R/Parm & S_WcDes & S_PRd & S_effDes & $S_{S} N_{N} \subset D$ \\
\hline an & 1253.47 & 1.686 & 0.9132 & 4100.000 & 0.0639 & $\overline{0} .8607$ & 1.0235 & 0.9980 & 0.9905 \\
\hline PC & 166.84 & 2.748 & 0. & 1.093 & 00 & 1 . & 0.8307 & 0.9860 & 0.0003 \\
\hline HPC & 88.21 & 12.619 & 0.8672 & 10097.240 & 13.5185 & 1.0995 & 1.0494 & 0.9898 & 0.9733 \\
\hline HPT & 0.96 & 4.189 & 0.9118 & 1.285 & 4.1887 & 12.6299 & 0.9723 & 1.0000 & 0.0003 \\
\hline LPT & 0.86 & 3.521 & 0.9223 & 1.019 & 3.5210 & 65.2590 & 0.7521 & 0.9926 & 0.0005 \\
\hline
\end{tabular}


$===$ INLETS $====\begin{array}{rrr}\text { eRam } & \text { Afs } & \text { Fram } \\ 1.0000 & 3912.33 & \end{array}$

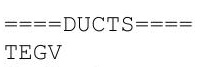

FanDu

apnorm

0.0000

$==$ SPLITTERS $=$

splitter

BPR

5.2809

$===\mathrm{SHAFTS}=$

HP_Shaf

LP_Shaft

Nhech

11345.2
3888.7

$=$ BURNERS $==$

Ttout

Burner 2814.3

ef

dPnorm $===$ NOZZLES $S===$ Core_Nozz $\mathrm{PR}$
2.536
2.543 Cfg
0.9809
0.9810 CdTh 1.0000
1.0000 $1.0000-0.9800$

$\begin{array}{rrrr}\text { W } & \text { Tt } & \text { ht } & \text { Pt } \\ 2.3223 & 1004.61 & 111.76 & 31.497 \\ 0.7383 & 1004.61 & 111.76 & 7.238 \\ 0.0000 & 966.82 & 102.38 & 38.510 \\ 0.0000 & 1198.38 & 160.54 & 89.515 \\ 0.0000 & 966.82 & 102.38 & 38.510 \\ 0.0000 & 1373.20 & 205.57 & 137.970 \\ 0.0000 & 966.82 & 102.38 & 38.510 \\ \text { W } & & & \\ 0.0000 & 620.35 & \text { ht } & \text { Pt } \\ 7.2054 & 1373.20 & 205.55 & 10.457 \\ 6.3049 & 1373.20 & 205.57 & 134.793 \\ 0.0000 & 1373.20 & 205.57 & 137.970 \\ 0.0000 & 1373.20 & 205.57 & 137.970 \\ 0.0000 & 1373.20 & 205.57 & 137.970 \\ 0.0000 & 540.20 & -1.18 & 7.257 \\ 0.0000 & 540.20 & -1.18 & 7.257 \\ 0.0000 & 540.20 & -1.18 & 7.257 \\ 0.0000 & 540.20 & -1.18 & 7.257\end{array}$

\begin{tabular}{|c|c|c|c|c|}
\hline & & $\mathrm{Wb} / \mathrm{Win}$ & BldWk & BI \\
\hline & $\mathrm{C} \mathrm{HE}$ & 0.0368 & 0.5000 & .2200 \\
\hline $\mathrm{LPT}^{-} \mathrm{COOLA}$ & A HPC.C> & 0.0117 & 0.5000 & \\
\hline WB $2 \bar{x}$ & HPC.B $>$ & 0.0000 & .4500 & \\
\hline $32 Y$ & $\mathrm{HPC} \cdot \mathrm{B}>$ & & .7600 & \\
\hline$x$ & HPC , B> & & & \\
\hline $\mathrm{C}$ & HPC. & & 0 & \\
\hline WBW2 & HPC. B> & & . & \\
\hline LEEDS & & & & \\
\hline WB1 & & & & \\
\hline HPT_COOLA & A Bleed> & & & \\
\hline HPT COOLB & & & & \\
\hline WB3 $\bar{X}$ & Blee & & . & \\
\hline WBA & & & & \\
\hline WBW & leed> & & 1.0 & \\
\hline WBF & the & & 1.0 & \\
\hline WB1 & & & & \\
\hline WB1 & $d>$ & & 1. & \\
\hline & &. & & \\
\hline
\end{tabular}

$\begin{array}{rr}\text { Wfuel } & \text { FAR } \\ 1.12757 & 0.02423\end{array}$

$\begin{array}{rrrrr}\text { CV } & \text { Ath } & \text { MNth } & \text { Vact } & \text { Fg } \\ 9800 & 613.40 & 1.000 & 1585.8 & 3789.3\end{array}$


Gackage: Janaf iter/pass/Jacb/Broy= 20/34/1/18 Run by: Philip C Jorgenson PC: 84

Temperature Stator 1 inlet: 450.18 Stator 1 exit: 467.32 Stator 2 inlet: 487.66 Stator 2 exit: 495.55 $\begin{array}{llll}\text { Stator } 3 \text { inlet: } 521.02 & \text { Stator } 3 \text { exit: } 529.17 & \text { Stator } 4 \text { inlet: } 553.24 \text { Stator } 4 \text { exit: } 560.82 \\ \text { Stator } 5 \text { inlet: } 579.81 & \text { Stator } 5 \text { exit: } 587.06 & & \text { Unblocked Percent Blockage: } 0.00\end{array}$

Ambient Relative Humidity $\quad 10.00$

Fan Face Relative Humidity $\quad 4.12$

Fan Bypass Relative Humidity $\quad 0.18$

LPC Inlet Relative Humidity $\quad 0.02$

HPC Exit Relative Humidity

Dre Relative Humidity

0.00

0.0000500

Ambient Flow Velocity

Ambient Static Pressure

Additional Water at LPC Exit

772.64

2.85

Inlet Length

40.00

Fan/LPC Inlet Flow Velocity $\quad 445.62$

0.000024

Fan/LPC Inlet Static Temperature $\quad \begin{array}{r}3.75 \\ \end{array}$

MN SUMMARY OUTPUT DATA

$\begin{array}{rrrrr}\text { MN alt dTamb } & \text { W } & \text { Fn } & \text { TSFC }\end{array}$

FSO Ambient.FI_O

ES1 Inlet.Fl_O

$\begin{array}{ll}\text { FS12 } & \text { Splitter.F1_02 } \\ \text { FS2 } & \text { Splitter.F1-01 }\end{array}$

FS14 Fan.Fl_O

FS23 LPC.FI-O

FS25 25 Bleed2.FI_O

FS25 Bleed2.F1

FS36 BPC.F1-

FS4 4 Burner. F1-O

FS4 Burner.F1-

FS49 4 ST FI-

FS5 5 TEGV Fi-O

FS8 Core_Nozz.Fl_O

FS17 FanDuctLkg. Fì_O

FS171 Bleed15.F1

FS172 FanDuct.

FS173 Byp Nozz. Fi

TURBOMACHINERY PERFORMANCE DATA

\begin{tabular}{|c|c|c|c|c|c|c|c|c|c|c|}
\hline \multicolumn{11}{|c|}{ LOW $S$} \\
\hline & & Ambient.Fl_O & $\begin{array}{r}W \\
378.74\end{array}$ & Pt & $\mathrm{Tt}$ & ht & FAR & W & \multicolumn{2}{|c|}{ Ps } \\
\hline $\begin{array}{l}\text { FS0 } \\
\text { FS1 }\end{array}$ & \multicolumn{2}{|l|}{ Inlet.FI O } & $\begin{array}{l}378.74 \\
378.74\end{array}$ & $\begin{array}{l}4.267 \\
4.267\end{array}$ & $\begin{array}{l}457.74 \\
457.74\end{array}$ & $\begin{array}{l}-20.96 \\
-20.96\end{array}$ & $\begin{array}{l}0.0000 \\
0.0000\end{array}$ & $\begin{array}{l}1225.4 \\
1225.4\end{array}$ & $\begin{array}{l}2.85 \\
3.29\end{array}$ & $\begin{array}{l}407 \\
425\end{array}$ \\
\hline 12 & \multicolumn{2}{|c|}{ Splitter.F1 02} & 319.79 & 4.265 & 457.74 & -20.96 & 0.0000 & 1035.2 & 3.30 & \\
\hline & \multicolumn{2}{|l|}{ Splitter.FI_01 } & 58.95 & 4.265 & 457.74 & -20.96 & 0.0000 & 190.8 & 3.75 & \\
\hline 14 & \multicolumn{2}{|l|}{ Fan.Fl_o } & 319.79 & 6.911 & 532.08 & -3.13 & 0.0000 & 688.7 & 5.73 & \\
\hline 23 & \multicolumn{2}{|l|}{ LPC.FI-O } & 58.95 & 10.331 & 612.25 & 16.13 & 0.0000 & 91.1 & 9.19 & \\
\hline 24 & \multicolumn{2}{|l|}{ VaporIN̄.FIO } & 95 & 10.331 & 612.33 & 16.01 & 0.0000 & 91.1 & 9.19 & \\
\hline 25 & \multicolumn{2}{|l|}{ Bleed2.FI $\overline{0}$} & 8.95 & 10.331 & 612.33 & 16.01 & 0.0000 & 91.1 & 9.19 & 94 \\
\hline 3 & \multicolumn{2}{|l|}{ HPC.FI_O } & 9 & 124.751 & 1321.02 & 192.02 & 0.0 & 10.5 & 112.16 & \\
\hline 36 & \multicolumn{2}{|l|}{ Bleed3.FI_O } & & 4.751 & 1321.02 & 192.02 & & 8.1 & 17.44 & \\
\hline 4 & \multicolumn{2}{|l|}{ Burner.Fl-o } & & 121.878 & 2646.52 & 165.07 & 0. & 12.1 & 115.12 & \\
\hline 45 & \multicolumn{2}{|l|}{ HPT.FI_O } & & 28.388 & 1730.19 & -2.41 & & 55.9 & 25.55 & \\
\hline 49 & \multirow{2}{*}{\multicolumn{2}{|c|}{ LPT.FI_O }} & & 16 & 123 & -132.82 & & 208.4 & 5.61 & \\
\hline 5 & & TEGV.FĪ O & & 6.516 & 123 & -132.82 & & 208 & 5. & \\
\hline & \multicolumn{2}{|c|}{ Core_Nozz.FI_O } & 59 & 6.516 & 1234.83 & -132.82 & 0.0 & 208.4 & 3.47 & \\
\hline 17 & \multicolumn{2}{|c|}{ FanDuctLkg.F̄_o } & & 6.911 & .08 & -3.13 & & 688.7 & 5.73 & \\
\hline 171 & \multicolumn{2}{|c|}{ Bleed15.FI O - } & & 6.911 & & -3.13 & & & & \\
\hline 172 & \multicolumn{2}{|l|}{ FanDuct.Fl_o } & & 6.911 & 532.08 & -3.13 & 0. & 688.7 & 5.57 & \\
\hline 173 & \multicolumn{2}{|l|}{ Byp_Nozz.Fİ_O } & 319.79 & 6.911 & 532.08 & -3.13 & 0.0000 & 688.7 & 3.65 & \\
\hline \multirow{2}{*}{\multicolumn{3}{|c|}{ TURBOMACH INERY }} & $\mathrm{DA}$ & & \multirow[b]{2}{*}{$T R$} & & & & & \\
\hline & & & eff & & & efPoly & & pwr & SMN & SMW \\
\hline & 1035.22 & 1 & 9117 & .836 & 624 & 0.9175 & -8063 & 3.8 & & \\
\hline & 190.84 & & & 3.836 & 76 & 1 & $-309 ?$ & 3.1 & 2. & 2 \\
\hline & 91.12 & 12.0 & 7 & 10180.008 & 2.1 & 86 & -14325 & 5.4 & 29.83 & 28.82 \\
\hline & 12.10 & 4.2 & & .009 & & & 14325 & 5.4 & & \\
\hline & 55.99 & 4.357 & 0.9131 & 89.635 & 1.3983 & 0.8961 & 1115 & 6.9 & & \\
\hline & NERY & $\mathrm{DA}$ & & & & & & & & \\
\hline & WcMap & & ef & $\mathrm{N} \subset \mathrm{Ma}$ & R/Parm & s_t & cDes & PRdes & S_effDes & S_NCDes \\
\hline & 1202.83 & 1 & & 3930.9 & 0.0611 & & & & 980 & $\overline{0}$. \\
\hline & 151.27 & 2.560 & & 1.0 & 0.0000 & & 2616 & 0.9117 & 1.0349 & 0.0003 \\
\hline & 82.87 & 11.553 & 696 & 9908.615 & 13.1467 & & 0995 & 1.0494 & 0.9898 & 0.9733 \\
\hline & 0.96 & 4202 & 9099 & 1.292 & 4.2020 & & 6299 & 0.9723 & 1.0000 & 0 \\
\hline & $0.8, \gamma-8$ & 3.525 & 199 & & & & & 521 & 0.9926 & 0 \\
\hline
\end{tabular}

TURBOMACHINERY MAP DATA

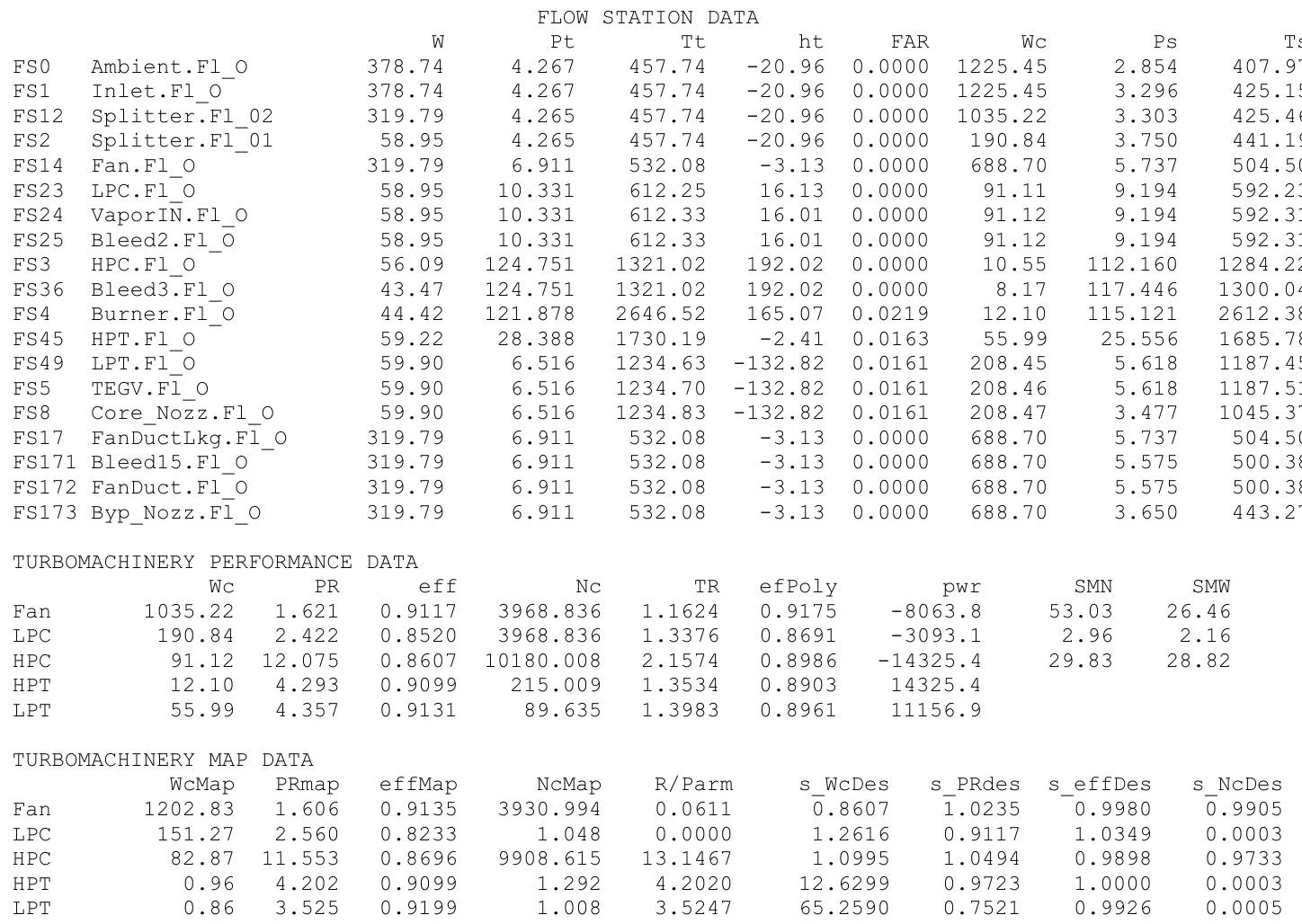

LP

gamt
1.40084
1.40084
1.40084
1.40084
1.40004
1.39852
1.39851
1.39851
1.36059
1.36059
1.29109
1.32734
1.35480
1.35480
1.35479
1.40004
1.40004
1.40004
1.40004

WAR
0.0000295
0.0000295
0.0000295
0.0000295
0.0000295
0.0000295
0.0000535
0.0000535
0.0000535
0.0000535
0.0000535
0.0000535
0.0000535
0.0000535
0.0000535
0.0000295
0.0000295
0.0000295
0.0000295

Wair
378.73
378.73 378.73
378.73 319.78 58.95
319.78 319.78
58.95 $\begin{array}{ll}58.95 & 0.002 \\ 58.95 & 0.003\end{array}$ $\begin{array}{ll}58.95 & 0.003 \\ 56.09 & 0.003\end{array}$ $\begin{array}{ll}43.47 & 0.002 \\ 43.47 & 0.002\end{array}$ $\begin{array}{ll}43.47 & 0.002 \\ 58.26 & 0.003\end{array}$ $\begin{array}{ll}58.26 & 0.00 \\ 58.95 & 0.003\end{array}$ $\begin{array}{ll}58.95 & 0.003\end{array}$ $\begin{array}{ll}58.95 & 0.003\end{array}$ $\begin{array}{ll}319.78 & 0.00\end{array}$ $\begin{array}{ll}319.78 & 0.009\end{array}$ $\begin{array}{ll}319.78 & 0.009\end{array}$ $\begin{array}{ll}319.78 & 0.009\end{array}$

$\begin{array}{rr}20 & \text { H2O frac } \\ 011 & 0.0000 \\ 011 & 0.0000 \\ 09 & 0.0000 \\ 02 & 0.0000 \\ 09 & 0.0000 \\ 02 & 0.0000 \\ 03 & 0.0001 \\ 03 & 0.0001 \\ 03 & 0.0001 \\ 02 & 0.0001 \\ 02 & 0.0264 \\ 03 & 0.0199 \\ 03 & 0.0196 \\ 03 & 0.0196 \\ 03 & 0.0196 \\ 09 & 0.0000 \\ 09 & 0.0000 \\ 09 & 0.0000 \\ 09 & 0.0000\end{array}$

.




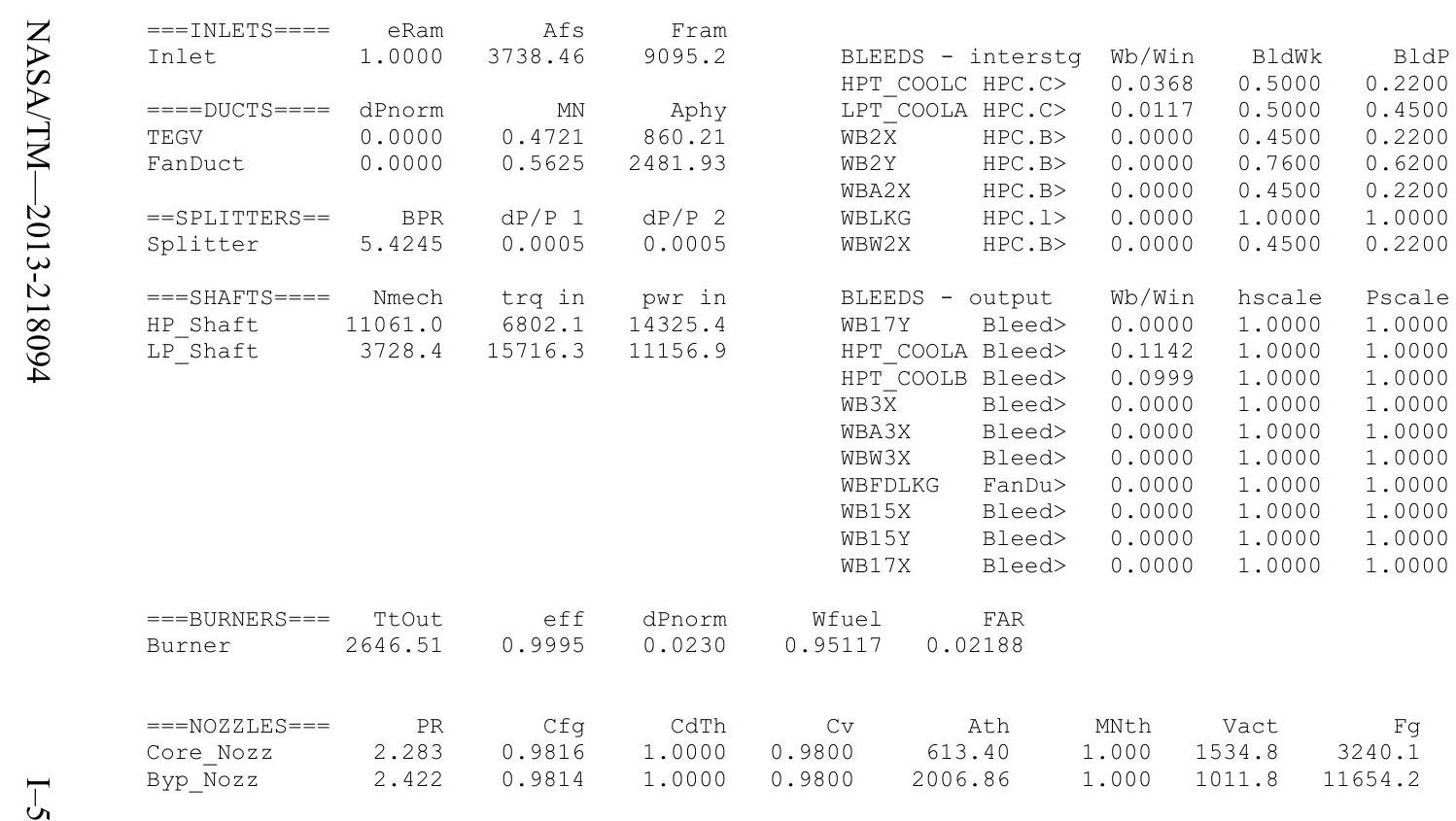


Date:07/05/13 Time:09:25:38 Model: Turbofan Engine - COMDES ON Converge = 1 CASE: 0

Version:NPSS_1.6.5 - Rev: -> Gas Package: Janaf iter/pass/Jacb/Broy=19/33/ 1/17 Run by: Philip C Jorgenson PC: 79

Temperature Stator 1 inlet: 451.80 Stator 1 exit: 468.53 Stator 2 inlet: 488.56 Stator 2 exit: 496.59

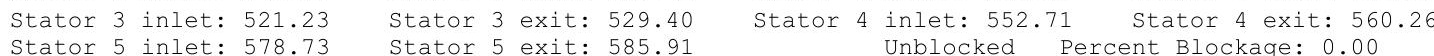

Ambient Relative Humidity $\quad 10.00$

Fan Face Relative Humidity 3.88

Fan Bypass Relative Humidity $\quad 0.20$

LPC Inlet Relative Humidity $\quad 1.93$

LPC Exit Relative Humidity 0.02

HPC Relative Humidity

Drop Diameter

0.00

0.0000500

772.64
2.85

Inlet Length

Ambient Static Pressure

Ambient Static Temperature

Fan/LPC Inlet Static Pressure $\quad 433.45$

Additional Water at LPC Exit 0.0000248

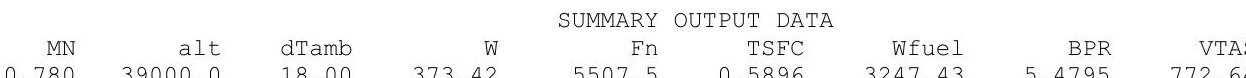

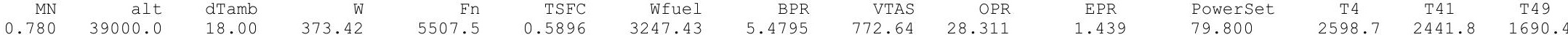

FSO Ambient.Fl_O

FS1 Inlet.Fl_o

$\begin{array}{ll}\text { FS12 } & \text { Splitter.Fl_02 } \\ \text { FS2 } & \text { Splitter.Fl_01 }\end{array}$

FS14 Fan.Fl_O

FS23 LPC.F1-O

FS25 Bleed2.FI-

FS25 Bleed2.F1

FS36 Bleed3.F1

FS36 Bleed3.F1_O

FS45 HPT.FI_O

FS49 LPT.FI-O

FS5 TEGV.FI_O

S8 Core_Nozz.Fl_O

FS17 FanDuctLkg.FI_O

FS172 Fleed15. F1-

FS173 Byp Nozz. FI

TURBOMACHINERY PERFORMANCE DATA

\begin{tabular}{|c|c|c|c|c|c|c|c|c|c|c|c|}
\hline & & & & FLOW & STATION DA & & & & & & \\
\hline FSO & Ambient.Fl O & & $\begin{array}{r}W \\
373.42\end{array}$ & $\begin{array}{r}P t \\
4.267\end{array}$ & $\begin{array}{r}\mathrm{Tt} \\
457.74\end{array}$ & $\begin{array}{r}h t \\
-20.96\end{array}$ & $\begin{array}{r}\text { FAR } \\
0.0000\end{array}$ & $\begin{array}{rr}W c \\
1208.25\end{array}$ & 2.8 & $\begin{array}{l}\text { Ps } \\
54\end{array}$ & $407.9^{7}-3$ \\
\hline FS1 & Inlet.Fl o- & & 373.42 & 4.267 & 457.74 & -20.96 & 0.0000 & 1208.25 & 3.3 & & \\
\hline FS12 & Splitter.Fl_0 & & 315.79 & 4.265 & 457.74 & -20.96 & 0.0000 & 1022.29 & 3.3 & & 426 \\
\hline s2 & Splitter.Fl_o & & 57.63 & 4.265 & 457.74 & -20.96 & 0.0000 & 186.5 & 3.7 & & 442 \\
\hline$\$ 14$ & Fan.Fl_O & & 315.79 & 6.810 & 529.66 & -3.71 & 0.0000 & 688.70 & 5.6 & & 502 \\
\hline 523 & LPC.FI O & & 57.63 & 10.278 & 610.11 & 15.61 & 0.0000 & 89.37 & 9.1 & & 591 \\
\hline 524 & VaporIN.FI_O & & 57.63 & 10.278 & 610.19 & 15.49 & 0.0000 & 89.38 & 9.1 & & 591 \\
\hline 525 & Bleed2.FI_o & & 57.63 & 10.278 & 610.19 & 15.49 & 0.0000 & 89.38 & 9.1 & & 591 \\
\hline 3 & HPC.FI_O & & 54.84 & 120.799 & 1306.08 & 188.16 & 0.0000 & 10.59 & 108.5 & & 1269 \\
\hline S36 & Bleed3.Fl O & & 42.50 & 120.799 & 1306.08 & 188.16 & 0.0000 & 8.20 & 113.6 & & 285 \\
\hline 54 & Burner.Fl_o & & 43.40 & 118.017 & 2598.74 & 162.11 & 0.0212 & 12.10 & 111.4 & & \\
\hline S45 & HPT.Fl_O & & 57.86 & 27.465 & 1698.24 & -2.37 & 0.0158 & 56.02 & 24.7 & & 654. \\
\hline S49 & LPT.FI_O & & 58 & 6.302 & 1210.65 & -130.19 & 0.0157 & 208.54 & 5.4 & & 164 \\
\hline S5 & TEGV.FI $O$ & & 58 & 6.302 & 1210.71 & -130.19 & 0.0157 & 208.55 & 5.4 & & \\
\hline 58 & Core_Nozz.FI_ & & 58.53 & 6.302 & 1210.85 & -130.19 & 0.0157 & 208.56 & 3.3 & & 1024 \\
\hline S17 & FanDúctLkg. Fi & & 315.79 & 6.810 & 529.66 & -3.71 & 0.0000 & 688.70 & 5.6 & & 502 \\
\hline S171 & Bleed15.Fl O & & 315.79 & 6.810 & 529.66 & -3.71 & 0.0000 & 688.70 & 5.4 & & 498 \\
\hline FS172 & FanDuct.FI_O & & 79 & 6.810 & 529.66 & -3.71 & 0.0 & 688.70 & 5.4 & & 49 \\
\hline FS173 & Byp_Nozz.Fin_o & & 315.79 & 6.810 & 529.66 & -3.71 & 0.0000 & 688.70 & 3.5 & & \\
\hline $\mathrm{RBC}$ & MACHINERY PERF & ORMANCE & DATA & & & & & & & & \\
\hline & & $\mathrm{PB}$ & eff & $\mathrm{NC}$ & TR & efPoly & & pwr & SMN & & W \\
\hline an & 1022.29 & 1.597 & 0.9114 & 3920.781 & 1.1571 & 0.9170 & -77 & 103.9 & 57.15 & 27. & \\
\hline $\mathrm{PC}^{2} \mathrm{r}-\mathrm{a}$ & 186.57 & 2.4 & 85 & 3920.781 & 1.3329 & 0.8746 & -29 & 881.8 & 3.34 & & .63 \\
\hline PC & 89.38 & 11.753 & .8613 & 10131.613 & 2.1405 & 0.8988 & -137 & 738.3 & 31.11 & 29. & .98 \\
\hline $\mathrm{PT}$ & 12.10 & 4.297 & 0.9091 & 215.567 & 1.3551 & 0.8897 & 137 & 38.3 & & & \\
\hline $\mathrm{PT}$ & 56.02 & 4.358 & 0.9123 & 89.379 & 1.3998 & 0.8952 & 106 & 585.6 & & & \\
\hline $\mathrm{RB}$ & ACHINERY M & $\mathrm{DA}$ & & & & & & & & & \\
\hline & WcMap & PRmap & effMap & NcMap & R/Parm & $s_{s}$ W & cDes & PRdes & effDes & & 3 NoDes \\
\hline an & 1187.80 & 1.583 & 0.9132 & 3883.396 & 0.0603 & & 8607 & 1.0235 & 0.9980 & & $\overline{0} .9905$ \\
\hline PC & 148.17 & 2.511 & 0.8223 & 1.036 & 0.0000 & & 2591 & 0.9333 & 1.0438 & & 0.0003 \\
\hline PC & 81.29 & 11.247 & 0.8702 & 9861.511 & 13.0375 & & 0995 & 1.0494 & 0.9898 & & 0.9733 \\
\hline PT & 0.96 & 4.20 & & 1.296 & 4.2057 & 12. & 6299 & 0.9723 & 1.0000 & & 0.0003 \\
\hline PT & 0.86 & 3.526 & 0.9191 & 1.005 & 3.5257 & 65.2 & 2590 & 0.7521 & 0.9926 & & 0.0005 \\
\hline
\end{tabular}

TURBOMACHINERY MAP DATA

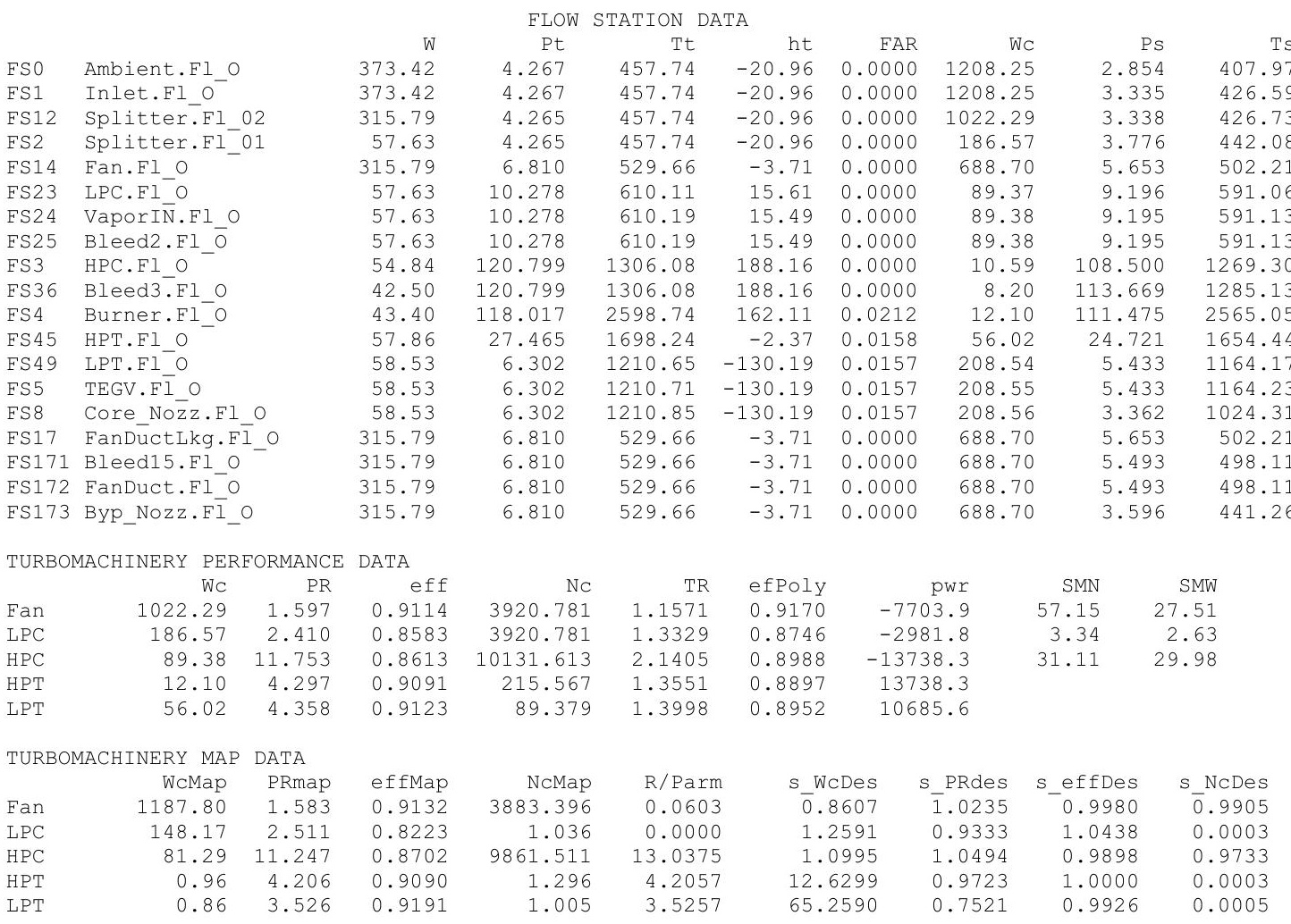

$0.86 \quad 3.526$
20 frac
0.0000
0.0000
0.0000
0.0000
0.0000
0.0000
0.0001
0.0001
0.0001
0.0001
0.0257
0.0193
0.0191
0.0191
0.0191
0.0000
0.0000
0.0000
0.0000

\begin{abstract}
(1.0.
\end{abstract}


$\begin{array}{lrrr}===\text { INLETS }==== & \text { eRam } & \text { Afs } & \text { Fram } \\ \text { Inlet } & 1.0000 & 3685.98 & 8967.5\end{array}$

$\begin{array}{lrrr}====\text { DUCTS }==== & \text { dPnorm } & \text { MN } & \text { Aphy } \\ \text { TEGV } & 0.0000 & 0.4720 & 860.21 \\ & 0.0000 & 0.5625 & 2481.93\end{array}$

$\begin{array}{llrr} & 0.0000 & 0.4720 & 860.21 \\ \text { FanDuct } & 0.0000 & 0.5625 & 2481.93\end{array}$

$==$ SPLITTERS $==$

Splitter

$===$ SHAFTS $====\quad$ Nmech

HP_Shaft

10989.2

$\mathrm{dP} / \mathrm{P}$

$0.0005 \quad 0.0005$

trq in pwr in

$6566.0 \quad 13738.3$

$===$ BURNERS $===\quad$ TtOut

Burner 2598.72

eff

dPnor

$===$ NOZZLES $S===$

Core Nozz

$P R$
2.208
2.386

Cfg
0.9816

0.9815
$15236.9 \quad 10685.6$
BLEEDS - interstg Wb/Win BldWk

0.2200

$\begin{array}{lllll}\text { LPT COOLA HPC.C> } & 0.0117 & 0.5000 & 0.4500 \\ \text { WB } 2 \bar{X} & 0.000 & 0.4500 & 0.2200\end{array}$

$\begin{array}{lllll}\text { WB2X } & \text { HPC.B> } & 0.0000 & 0.4500 & 0.2200 \\ \text { WB2Y } & \text { HPC.B> } & 0.0000 & 0.7600 & 0.6200\end{array}$

$\begin{array}{lllll}\text { WBA2X HPC.B> } & 0.0000 & 0.4500 & 0.2200\end{array}$

$\begin{array}{lllll}\text { WBLKG } & \text { HPC.I> } & 0.0000 & 1.0000 & 1.0000 \\ \text { WBW2X } & \text { HPC.B } & 0.0000 & 0.4500 & 0.2200\end{array}$

BLEEDS - output Wb/Win hscale Pscale

WB17Y Bleed> $0.0000 \quad 1.0000 \quad 1.0000$

HPT_COOLA Bleed> $0.1142 \quad 1.0000 \quad 1.0000$

$\begin{array}{lllll}\text { HPT COOLB } & \text { Bleed> } & 0.0999 & 1.0000 & 1.0000 \\ \text { WB 3X } & \text { Bleed> } & 0.0000 & 1.0000 & 1.0000\end{array}$

$\begin{array}{lllll}\text { WB3X } & \text { Bleed> } & 0.0000 & 1.0000 & 1.0000 \\ \text { WBA3X } & \text { Bleed }> & 0.0000 & 1.0000 & 1.0000\end{array}$

$\begin{array}{lllll}\text { WBA3X } & \text { Bleed> } & 0.0000 & 1.0000 & 1.0000 \\ \text { WBW3X } & \text { Bleed }> & 0.0000 & 1.0000 & 1.0000\end{array}$

$\begin{array}{lllll}\text { WBW3X } & \text { Bleed> } & 0.0000 & 1.0000 & 1.0000 \\ \text { WBFDLKG } & \text { FanDu> } & 0.0000 & 1.0000 & 1.0000\end{array}$

$\begin{array}{lllll}\text { WB15X Bleed> } & 0.0000 & 1.0000 & 1.0000\end{array}$

WB15Y Bleed> $0.0000 \quad 1.0000 \quad 1.0000$

WB17X Bleed> $0.0000 \quad 1.0000 \quad 1.0000$

$\begin{array}{rr}\text { Wfuel } & \text { FAR } \\ 0.90206 & 0.02123\end{array}$

$\begin{array}{rrrr}\text { W } & \text { Tt } & \text { ht } & \text { Pt } \\ 2.1209 & 964.62 & 101.82 & 27.465 \\ 0.6743 & 964.62 & 101.82 & 6.302 \\ 0.0000 & 929.70 & 93.19 & 34.593 \\ 0.0000 & 1143.99 & 146.72 & 78.801 \\ 0.0000 & 929.70 & 93.19 & 34.593 \\ 0.0000 & 1306.08 & 188.16 & 120.799 \\ 0.0000 & 929.70 & 93.19 & 34.593 \\ \text { W } & \text { Tt } & \text { ht } & \text { Pt } \\ 0.0000 & 610.19 & 15.49 & 10.278 \\ 6.5805 & 1306.08 & 188.16 & 118.017 \\ 5.7581 & 1306.08 & 188.16 & 66.221 \\ 0.0000 & 1306.08 & 188.16 & 120.799 \\ 0.0000 & 1306.08 & 188.16 & 120.799 \\ 0.0000 & 1306.08 & 188.16 & 120.799 \\ 0.0000 & 529.66 & -3.71 & 6.810 \\ 0.0000 & 529.66 & -3.71 & 6.810 \\ 0.0000 & 529.66 & -3.71 & 6.810 \\ 0.0000 & 529.66 & -3.71 & 6.810\end{array}$

$\begin{array}{rrrrr}\text { CV } & \text { Ath } & \text { MNth } & \text { Vact } & \text { Fg } \\ .9800 & 613.40 & 1.000 & 1520.2 & 3077.0 \\ .9800 & 2006.86 & 1.000 & 1009.5 & 11397.9\end{array}$


COMDES ON COnverge $=1$ CASE:

Date:07/05/13 Time:09:26:12 Model:

Temperature Stator 1 inlet: $452.89 \quad$ Stator 1 exit: $469.36 \quad$ Stator 2 inlet: $489.20 \quad$ Stator 2 exit: 497.31 Stator 3 inlet: 521.41 Stator 3 exit: 529.59 Stator 4 inlet: 552.40 Stator 4 exit: 559.93 Stator 5 inlet: 578.07 Stator 5 exit: $585.21 \quad$ Unblocked Percent Blockage: 0.00

Ambient Relative Humidity $\quad 10.00$

Fan Face Relative Humidity $\quad 3.72$

Fan Bypass Relative Humidity $\quad 0.21$

LPC Inlet Relative Humidity

HPC Exit Relative Humidity

Drop Diameter

0.02
0.00

0.0000500

772.64

Inlet Length

0.00

Ambient Flow Velocity

2.85

Fan/LPC Inle

424.80

Ambient Static Temperature

Additional Water at LPC Exit

0.0000254

$\begin{array}{rr} & 3.79 \\ \text { an/LPC Inlet Static Temperature } & 442.70\end{array}$

SUMMARY OUTPUT DATA

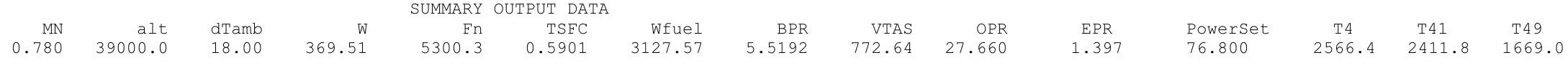

\begin{tabular}{|c|c|}
\hline FSO & Ambient.FI_O \\
\hline FS1 & Inlet.Fl_o- \\
\hline FS12 & Splitter.Fl_02 \\
\hline FS2 & Splitter.Fl_01 \\
\hline FS14 & Fan.Fl O \\
\hline FS23 & LPC.FI_O \\
\hline FS24 & 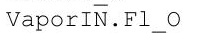 \\
\hline FS25 & Bleed2.F1_o \\
\hline FS3 & HPC.FI_O \\
\hline FS36 & Bleed3.FI_O \\
\hline FS4 & Burner.Fl_o \\
\hline FS45 & HPT.FI_O \\
\hline FS49 & LPT.FI_O \\
\hline FS5 & TEGV.F̄_OO \\
\hline FS8 & Core_Nozz.Fl_O \\
\hline FS17 & FanDúctLkg.Fí_o \\
\hline FS171 & Bleed15.FI_O \\
\hline P172 & FanDuct.FI_O \\
\hline & Byp Nozz.FI \\
\hline
\end{tabular}

\begin{tabular}{|c|c|c|c|c|c|}
\hline & FLOW & TATION & ATA & & \\
\hline W & Pt & Tt & ht & FAR & Wo \\
\hline 69.51 & 4.267 & 457.74 & -20.96 & 0.0000 & 1195.58 \\
\hline 59.51 & 4.267 & 457.74 & -20.96 & 0.0000 & 1195.58 \\
\hline 2.83 & 4.265 & 457.74 & -20.96 & 0.0000 & 1012. \\
\hline 56.68 & 4.265 & 457.74 & -20.96 & 0.0000 & 183. \\
\hline 12.83 & 6.735 & 528.01 & -4.11 & 0.0000 & 688.70 \\
\hline 56.68 & 10.238 & 608.70 & 15.27 & 0.0000 & 88.14 \\
\hline 56.68 & 10.238 & 608.78 & 15.15 & 0.0000 & 88.1 \\
\hline 56.68 & 10.238 & 608.78 & 15.15 & 0.0 & 88.15 \\
\hline 53.93 & 118.020 & 1296.01 & 185.55 & 0.0000 & 10.62 \\
\hline 41.80 & 8.020 & 1296.01 & 185.55 & 0.0000 & 8.23 \\
\hline 42.67 & 115.302 & 2566.36 & 160.10 & 0.0 & 12.10 \\
\hline 56.89 & 26.817 & 1676.65 & -2.33 & 0.0155 & 56.05 \\
\hline 57.55 & 22 & 1194.48 & -128.41 & 0. & 208.62 \\
\hline .55 & .152 & 1194.55 & -128.41 & 0.0153 & 208.62 \\
\hline 57.55 & .152 & 1194.68 & -128.40 & 0.0153 & 208.6 \\
\hline & 6.7 & .01 & -4.11 & & \\
\hline & 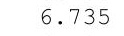 & 528.01 & -4.11 & 0 & ( \\
\hline 2.83 & 6.735 & 528.01 & -4.11 & 0.0000 & 8.7 \\
\hline & & & & & 588 \\
\hline
\end{tabular}

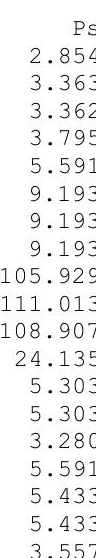

$\begin{array}{rrr}\text { Ts } & \text { Aphy } & \text { MN } \\ 407.97 & 3647.3 & 0.7800 \\ 427.59 & 4168.2 & 0.5930 \\ 427.62 & 3531.8 & 0.5927 \\ 442.70 & 830.5 & 0.4117 \\ 500.64 & 2606.7 & 0.5226 \\ 590.31 & 412.6 & 0.3952 \\ 590.38 & 412.6 & 0.3953 \\ 590.38 & 412.6 & 0.3953 \\ 1259.22 & 49.7 & 0.4009 \\ 1275.06 & 49.3 & 0.3009 \\ 2532.99 & 74.6 & 0.2990 \\ 1633.23 & 265.4 & 0.4003 \\ 1148.48 & 860.2 & 0.4719 \\ 1148.54 & 860.2 & 0.4719 \\ 1010.13 & 613.4 & 1.0000 \\ 500.64 & 2606.7 & 0.5226 \\ 496.56 & 2481.9 & 0.5625 \\ 496.56 & 2481.9 & 0.5625 \\ 439.88 & 2006.9 & 1.0000\end{array}$

gamt
1.40084
1.40084
1.40084
1.40084
1.40010
1.39860
1.39859
1.39859
1.36211
1.36211
1.29415
1.33042
1.35787
1.35786
1.35785
1.40010
1.40010
1.40010
1.40010

WAR
0.0000295
0.0000295
0.0000295
0.0000295
0.0000295
0.0000295
0.0000549
0.0000549
0.0000549
0.0000549
0.0000549
0.0000549
0.0000549
0.0000549
0.0000549
0.0000295
0.0000295
0.0000295
0.0000295

AR
95
95
95
95
95
95
95
95
49
49
49
49
49
49
49
49
49
95
95
95
95

\begin{tabular}{|c|c|c|c|c|c|c|c|c|c|}
\hline & WC & $\mathrm{PR}$ & & & (TP & 17 & nur & SMNT & SMVT \\
\hline Fan & 1012.69 & $\begin{array}{r}\text { PR } \\
1.579\end{array}$ & $\begin{array}{l}\text { eII } \\
0.9094\end{array}$ & $\begin{array}{l}3888.090 \\
\text { NC }\end{array}$ & $\begin{array}{r}\mathrm{TR} \\
1.1535\end{array}$ & $\begin{array}{l}\text { expo1y } \\
0.9150\end{array}$ & $\begin{array}{r}\text { pWr } \\
-7456.6\end{array}$ & $\begin{array}{r}\text { SMN } \\
59.29\end{array}$ & 28.29 \\
\hline LPC & 183.48 & 2.401 & 0.8620 & 3888.090 & 1.3298 & 0.8778 & -2905.3 & 3.61 & 2.87 \\
\hline $\mathrm{HPC}$ & 88.15 & 11.5 & 86 & 10098.210 & 2.1289 & 0.8986 & -13334.1 & 32.00 & 30.80 \\
\hline HPT & 12.10 & 4.300 & .9085 & 215.958 & 1.3562 & 0.8892 & 13334.1 & & \\
\hline LPT & 56.05 & 4.359 & .9118 & 89.203 & 1.4008 & 0.8945 & 10362.0 & & \\
\hline $\mathrm{JRI}$ & INERY MAP & DATA & & & & & & & \\
\hline & WcMap & PRmap & effMap & NcMap & R/Parm & S_WcDes & S_PRdes & s_effDes & S_NCDes \\
\hline an & 1176.65 & 1.566 & 0.9112 & 3851.017 & 0.0597 & $\overline{0} .8607$ & $\overline{1} .0235$ & 0.9980 & $\overline{0.9905}$ \\
\hline PC & 146.06 & 2.477 & .8217 & 1.027 & 0.0000 & 1.2562 & 0.9482 & 1.0491 & 0.0003 \\
\hline HPC & 80.17 & 11.032 & 0.8703 & 9828.998 & 12.9618 & 1.0995 & 1.0494 & 0.9898 & 0.9733 \\
\hline TT & 0.9 & 4.2 & .9085 & 1.298 & 4.2082 & 12.6299 & 0.9723 & 1.0000 & 0.0 \\
\hline $\mathrm{LPT}$ & 0.86 & 3.526 & 0.9185 & 1.003 & 3.5263 & 65.2590 & 0.7521 & 0.9926 & 0.0005 \\
\hline
\end{tabular}


$\begin{array}{lrr}==\text { INLETS }^{\prime}=== & \text { eRam } & \text { AfS } \\ 1.0000 & 3647.33 & \text { Fram }\end{array}$

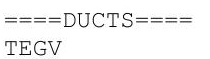

$0.5625 \quad 2481.93$

Splitter

5.5192

$\mathrm{dP} / \mathrm{P}$

0.0005

$\mathrm{dP} / \mathrm{P} 2$
0.0005

Nmech
10940.3

$\begin{array}{lr}\text { trq in } & \text { pwr in } \\ 6401.3 & 13334.1\end{array}$

LP_Shaft

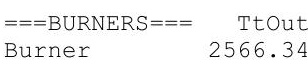

ef

dPnorm

$===$ NOZZLES $===$

Core_Nozz

PR
2.156
2.360

Cfg
0.9815
0.9815

CdTh
1.0000

$1.0000 \quad 0.9800$

0.9800
- interstg Wb/Win Blawk BldP

$\begin{array}{llll}\text { HPICCOOLC HPC.C }> & 0.0368 & 0.5000 & 0.2200\end{array}$

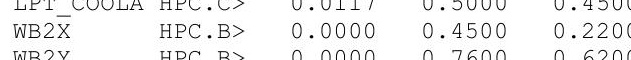

$\begin{array}{lllll}\text { WB2Y HPC.B } & 0.0000 & 0.7600 & 0.6200\end{array}$

$\begin{array}{lllll}\text { WBA2X } & \text { HPC.B } & 0.0000 & 0.7600 & 0.6200 \\ \text { WBLKG } & \text { HPC. } 1> & 0.0000 & 1.4500 & 0.2200\end{array}$

$\begin{array}{llll}\text { WBW2X HPC.B> } & 0.0000 & 0.4500 & 0.2200\end{array}$

BLEEDS - output Wb/Win hscale Pscale

WB17Y Bleed> $0.0000 \quad 1.0000 \quad 1.0000$

HPT_COOLA Bleed> $0.1142 \quad 1.0000 \quad 1.0000$

$\begin{array}{lllll}\text { HPT COOLB } & \text { Bleed }> & 0.0999 & 1.0000 & 1.0000 \\ \text { WB } 3 \bar{X} & \text { Bleed }> & 0.0000 & 1.0000 & 1.0000\end{array}$

$\begin{array}{lllll}\text { WB3X } & \text { Bleed> } & 0.0000 & 1.0000 & 1.0000 \\ \text { WBA3X } & \text { Bleed }> & 0.0000 & 1.0000 & 1.0000\end{array}$

$\begin{array}{lllll}\text { WBA3X } & \text { Bleed> } & 0.0000 & 1.0000 & 1.0000 \\ \text { WBW3X } & \text { Bleed> } & 0.0000 & 1.0000 & 1.0000\end{array}$

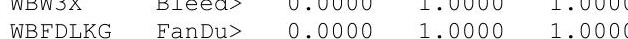

$\begin{array}{lllll}\text { WBFDLKG } & \text { FanDu> } & 0.0000 & 1.0000 & 1.0000 \\ \text { WB15X } & \text { Bleed }> & 0.0000 & 1.0000 & 1.0000\end{array}$

WB17X Bleed> $0.0000 \quad 1.0000 \quad 1.0000$

Wfuel FAR 
$\begin{array}{ll}\text { Date:07/05/13 Time:09:35:17 Model: } & \text { Turbofan Engine - COMDES ON CONverge }=1 \text { CASE: } 0 \\ \text { Version:NPSS 1.6.5 - Rev: -> Gas Package: Janaf iter/pass/Jacb/Broy=22/36/1/20 Run by: Philip C Jorgenson PC: } 100\end{array}$

Version:NPSS1.6.5 - Rev: -> Gas Package: Janaf 1ter/pass/Jacb/Broy= 22/ $36 / 1 / 20$ Run by: Philip C Jorge
Temperature Stator 1 inlet: $463.71 \quad$ Stator 1 exit: 483.18 Stator 2 inlet: 505.70 Stator 2 exit: 513.15

$\begin{array}{ccc}\text { Stator } 3 \text { inlet: } 543.36 & \text { Stator } 3 \text { exit: } 551.67 & \text { Stator } 4 \text { inlet: } 579.94 \quad \text { Stator } 4 \text { exit: } 588.00 \\ \text { Stator } 5 \text { inlet: } 609.83 & \text { Stator } 5 \text { exit: } 617.71 & \text { Unblocked Percent Blockage: } 0.00\end{array}$

Ambient Relative Humidity $\quad 10.00$

Fan Face Relative Humidity $\quad 5.44$

IPC Inl Relative Humidity 0.19

LPC Exit Relative Humidity $\quad 0.02$

HPC Relative Humidity

0.0000500

Inlet Length $\quad 40.00$

Ambient Flow Velocity

789.49
2.85

495.70

Ambient Static Pressure

Ambient Static Temperature

425.97
0.0000239

$\begin{array}{rr}\text { Fan/LPC Inlet Static Pressure } & 3.66 \\ \text { Fan/LPC Inlet Static Temperature } & 457.44\end{array}$

SUMMARY OUTPUT DATA

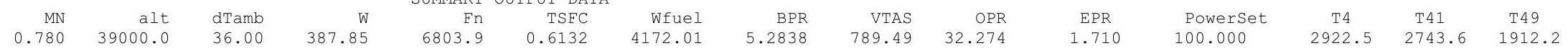

\begin{tabular}{|c|c|}
\hline & ent.Fl \\
\hline FS1 & Inlet.FI 0 \\
\hline FS12 & Splitter.Fl_02 \\
\hline FS2 & Splitter.Fl-01 \\
\hline FS14 & Fan.Fl_o \\
\hline FS23 & LPC.FI_O \\
\hline FS24 & VaporIÑ.FIO \\
\hline FS25 & Bleed2.FI_o \\
\hline FS3 & HPC.FIO \\
\hline FS36 & Bleed3.FI O \\
\hline FS4 & Burner.FI-O \\
\hline FS45 & HPT.FI_O \\
\hline FS49 & LPT.FI_O \\
\hline FS5 & TEGV.FI \\
\hline FS8 & Core_Nozz. \\
\hline FS17 & FanDúctLkg.Fì \\
\hline FS171 & Bleed15.F1 O \\
\hline FS172 & FanDuct.Fl- \\
\hline FS173 & Byp Nozz.Fi \\
\hline
\end{tabular}

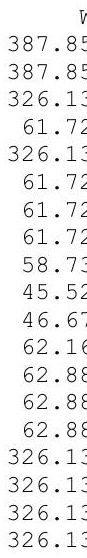

TURBOMACHINERY PERFORMANCE DATA

$\begin{array}{lrr} & \text { WC } & \text { PR } \\ \text { Fan } & 1078.79 & 1.702 \\ \text { LPC } & 204.17 & 2.457 \\ \text { HPC } & 96.74 & 13.140 \\ \text { HPT } & 12.10 & 4.272 \\ \text { LPT } & 55.83 & 4.343\end{array}$

$$
\begin{array}{r}
\text { eff } \\
0.9114 \\
0.8233 \\
0.8585 \\
0.9122 \\
0.9167
\end{array}
$$

TURBOMACHINERY MAP DATA

$\begin{array}{lrr} & \text { WcMap } & \text { PRmap } \\ \text { Fan } & 1253.45 & 1.686 \\ \text { LPC } & 166.84 & 2.748 \\ \text { HPC } & 87.99 & 12.568 \\ \text { HPT } & 0.96 & 4.181 \\ \text { LPT } & 0.86 & 3.515\end{array}$

FLOW STATION DATA

$\begin{array}{rrrrr}\mathrm{Pt} & \mathrm{Tt} & \mathrm{ht} & \text { FAR } & \text { Wc } \\ 4.267 & 477.92 & -16.44 & 0.0000 & 1282.32 \\ 4.267 & 477.92 & -16.44 & 0.0000 & 1282.32 \\ 4.265 & 477.92 & -16.44 & 0.0000 & 1078.79 \\ 4.265 & 477.92 & -16.44 & 0.0000 & 204.17 \\ 7.257 & 563.97 & 4.20 & 0.0000 & 688.63 \\ 10.479 & 647.76 & 24.36 & 0.0000 & 96.73 \\ 10.479 & 647.85 & 24.25 & 0.0000 & 96.74 \\ 10.479 & 647.85 & 24.25 & 0.0000 & 96.74 \\ 137.704 & 1427.67 & 219.49 & 0.0000 & 10.40 \\ 137.704 & 1427.67 & 219.49 & 0.0000 & 8.06 \\ 134.533 & 2922.53 & 187.25 & 0.0255 & 12.10 \\ 31.492 & 1921.42 & 2.55 & 0.0190 & 55.83 \\ 7.251 & 1379.99 & -143.20 & 0.0188 & 207.89 \\ 7.251 & 1380.05 & -143.20 & 0.0188 & 207.90 \\ 7.251 & 1380.18 & -143.20 & 0.0188 & 207.91 \\ 7.257 & 563.97 & 4.20 & 0.0000 & 688.63 \\ 7.257 & 563.97 & 4.20 & 0.0000 & 688.63 \\ 7.257 & 563.97 & 4.20 & 0.0000 & 688.63 \\ 7.257 & 563.97 & 4.20 & 0.0000 & 688.63\end{array}$

PS
2.85
3.15
3.17
3.65
6.025
9.15
9.152
9.152
124.235
129.878
127.111
28.372
6.25
6.25
3.882
6.025
5.85
5.85
3.83

Ts
425.97
438.17
439.19
457.44
534.77
623.27
623.36
623.36
1389.65
1405.97
2885.79
1873.58
1328.67
1328.73
1173.58
534.77
530.41
530.41
469.91

$\begin{array}{rr}\text { Aphy } & \text { MN } \\ 3912.1 & 0.7800 \\ 4168.2 & 0.6727 \\ 3531.8 & 0.6634 \\ 830.5 & 0.4726 \\ 2606.7 & 0.5227 \\ 412.6 & 0.4443 \\ 412.6 & 0.4444 \\ 412.6 & 0.4444 \\ 49.7 & 0.3922 \\ 49.3 & 0.2950 \\ 74.6 & 0.3007 \\ 265.4 & 0.4005 \\ 860.2 & 0.4725 \\ 860.2 & 0.4725 \\ 613.4 & 1.0000 \\ 2606.7 & 0.5227 \\ 2481.9 & 0.5626 \\ 2481.9 & 0.5626 \\ 2006.9 & 1.0000\end{array}$

gamt
1.40068
1.40068
1.40068
1.40068
1.39952
1.39757
1.39757
1.39757
1.35437
1.35437
1.28095
1.31732
1.34412
1.34412
1.34411
1.39952
1.39952
1.39952
1.39952

0.0000851 0.0000851 0.0000851 0.0000851 0.0000851 0.00000851
0.0001090 0.0001090 0.0001090 0.0001090 0.0001090 0.0001090 0.0001090 0.0001090 0.0001090 0.0000851 0.0000851 0.0000851

\begin{tabular}{|c|c|c|c|c|c|}
\hline NC & TR & efPoly & pwr & SMN & SMW \\
\hline 4139.469 & 1.1801 & 0.9178 & -9525.9 & 36.68 & 23.09 \\
\hline 4139.469 & 1.3554 & 0.8440 & -3563.0 & 2.97 & 2.24 \\
\hline 10363.492 & 2.2037 & 0.8978 & -16636.9 & 26.01 & 25.23 \\
\hline 214.250 & 1.3428 & 0.8903 & 16636.9 & & \\
\hline 90.650 & 1.3888 & 0.9005 & 13088.7 & & \\
\hline NcMap & R/Parm & S_WCDes & S_PRdes & s_effDes & S_NcDes \\
\hline 4100.000 & 0.0639 & $\overline{0} .8607$ & $\overline{1} .0235$ & 0.9980 & $\overline{0} .9905$ \\
\hline 1.093 & 0.0000 & 1.2237 & 0.8337 & 0.9870 & 0.0003 \\
\hline 10087.208 & 13.4977 & 1.0995 & 1.0494 & 0.9898 & 0.9733 \\
\hline 1.288 & 4.1813 & 12.6299 & 0.9723 & 1.0000 & 0.0003 \\
\hline 1.020 & 3.5146 & 65.2590 & 0.7521 & 0.9926 & 0.0005 \\
\hline
\end{tabular}
0.0000851

$\begin{array}{rrr}\text { Wair } & \text { WH2O } & \text { H2O frac } \\ 387.82 & 0.033 & 0.0001 \\ 387.82 & 0.033 & 0.0001 \\ 326.10 & 0.028 & 0.0001 \\ 61.72 & 0.005 & 0.0001 \\ 326.10 & 0.028 & 0.0001 \\ 61.72 & 0.005 & 0.0001 \\ 61.72 & 0.007 & 0.0001 \\ 61.72 & 0.007 & 0.0001 \\ 58.72 & 0.006 & 0.0001 \\ 45.51 & 0.005 & 0.0001 \\ 45.51 & 0.005 & 0.0307 \\ 60.99 & 0.007 & 0.0231 \\ 61.72 & 0.007 & 0.0228 \\ 61.72 & 0.007 & 0.0228 \\ 61.72 & 0.007 & 0.0228 \\ 326.10 & 0.028 & 0.0001 \\ 326.10 & 0.028 & 0.0001 \\ 326.10 & 0.028 & 0.0001 \\ 326.10 & 0.028 & 0.0001\end{array}$


$\begin{array}{lrr}===\text { INLETS }==== & \text { eRam } & \text { AfS } \\ \text { Inlet } & \text { Fram }\end{array}$

0.0000

0.0000

$==$ SPLITTERS $==$

Splitter

BPR

5.2838

- Shats

LP_Shaf

11582.4

3973.5

in pwr in

$7544.1 \quad 16636.9$

$\begin{array}{ll}7300.3 & 13088.7\end{array}$

$===$ BURNERS $===\quad$ TtOut

Burner 2922.53

ef

dPnorm

$===$ NOZZLES $===$

Core Nozz

$\mathrm{PR}$
2.541
2.543

CdTh

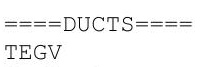

$\begin{array}{rrrrrrr}\text { Cfg } & \text { CdTh } & \text { CV } & \text { Ath } & \text { MNth } & \text { Vact } & \text { Fg } \\ 0.9809 & 1.0000 & 0.9800 & 613.40 & 1.000 & 1620.2 & 3797.3 \\ 0.9810 & 1.0000 & 0.9800 & 2006.86 & 1.000 & 1041.7 & 12523.7\end{array}$

$\begin{array}{rrrrrrr}\text { Cfg } & \text { CdTh } & \text { Cv } & \text { Ath } & \text { MNth } & \text { Vact } & \text { Fg } \\ 0.9809 & 1.0000 & 0.9800 & 613.40 & 1.000 & 1620.2 & 3797.3 \\ 0.9810 & 1.0000 & 0.9800 & 2006.86 & 1.000 & 1041.7 & 12523.7\end{array}$

$\begin{array}{rrrrrrr}\text { Cfg } & \text { CdTh } & \text { Cv } & \text { Ath } & \text { MNth } & \text { Vact } & \text { Fg } \\ 0.9809 & 1.0000 & 0.9800 & 613.40 & 1.000 & 1620.2 & 3797.3 \\ 0.9810 & 1.0000 & 0.9800 & 2006.86 & 1.000 & 1041.7 & 12523.7\end{array}$

BLEEDS - interstg Wb/Win BldWk BldP

HPI_COOLC HPC.C> 0.0368

$\begin{array}{llll}\text { LPT COOLA HPC.C> } & 0.0117 & 0.5000 & 0.4500\end{array}$

$\begin{array}{llll}\text { WB2Y HPC.B } & 0.0000 & 0.7600 & 0.6200\end{array}$

$\begin{array}{lllll}\text { WBA2X HPC.B } & 0.0000 & 0.4500 & 0.2200\end{array}$

$\begin{array}{llllll}\text { WBLKG HPC.1> } & 0.0000 & 1.0000 & 1.0000\end{array}$

$\begin{array}{lllll}\text { WBW2X HPC.B } & 0.0000 & 0.4500 & 0.2200\end{array}$

BLEEDS - output Wb/Win hscale Pscale

$\begin{array}{lllll}\text { WB17Y Bleed } & 0.0000 & 1.0000 & 1.0000\end{array}$

HPT_COOLA Bleed> $0.1142 \quad 1.0000 \quad 1.0000$

HPT_COOLB Bleed> $0.0999 \quad 1.0000 \quad 1.0000$

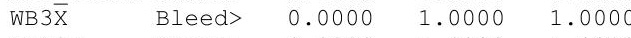

$\begin{array}{lllll}\text { WBA3X Bleed } & 0.0000 & 1.0000 & 1.0000\end{array}$

WBW3X Bleed> $0.0000-1.0000-1.0000$

WBFDLKG FanDu> $0.0000 \quad 1.0000 \quad 1.0000$

WB15X

WB15Y Bleed $0.0000-1.0000-1.00000$

$0.0000 \quad 1.0000 \quad 1.0000$

$\begin{array}{rr}\text { Wfuel } & \text { FAR } \\ 1.15889 & 0.02546\end{array}$

$\begin{array}{rrrr}W & \text { Tt } & \text { ht } & \text { Pt } \\ 2.2714 & 1046.42 & 121.87 & 31.492 \\ 0.7222 & 1046.42 & 121.87 & 7.251 \\ 0.0000 & 1007.29 & 112.10 & 38.469 \\ 0.0000 & 1246.92 & 172.63 & 89.359 \\ 0.0000 & 1007.29 & 112.10 & 38.469 \\ 0.0000 & 1427.67 & 219.49 & 137.704 \\ 0.0000 & 1007.29 & 112.10 & 38.469 \\ W & T t & \text { ht } & \text { Pt } \\ \text { W } & \text { Tt } & 24.25 & 10.479 \\ 0.000 & 647.85 & 219.49 & 134.533 \\ 7.0476 & 1427.67 & 2199.49 \\ 6.1668 & 1427.67 & 219.49 & 75.594 \\ 0.0000 & 1427.67 & 219.49 & 137.704 \\ 0.0000 & 1427.67 & 219.49 & 137.704 \\ 0.0000 & 1427.67 & 219.49 & 137.704 \\ 0.0000 & 563.97 & 4.20 & 7.257 \\ 0.0000 & 563.97 & 4.20 & 7.257 \\ 0.0000 & 563.97 & 4.20 & 7.257 \\ 0.0000 & 563.97 & 4.20 & 7.257\end{array}$

469 .469 8.469 $\mathrm{Pt}$
0.479 13.533 137.704 17.704 257 257 


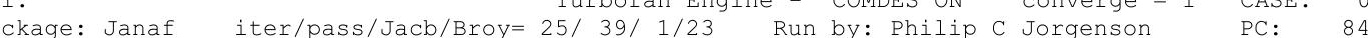

Temperature Stator 1 inlet: 469.60 Stator 1 exit: 487.63 Stator 2 inlet: 508.98 Stator 2 exit: 517.20 $\begin{array}{llll}\text { Stator } 3 \text { inlet: } 544.05 & \text { Stator } 3 \text { exit: } 552.57 & \text { Stator } 4 \text { inlet: } 577.92 \text { Stator } 4 \text { exit: } 585.87 \\ \text { Stator } 5 \text { inlet: } 605.84 & \text { Stator } 5 \text { exit: } 613.45 & \text { Unblocked Percent Blockage: } 0.00\end{array}$

$\begin{array}{lclll}\text { Ambient Relative Humidity } & 10.00 & & & \\ \text { Fan Face Relative Humidity } & 4.44 & & & \\ \text { Fan Bypass Relative Humidity } & 0.23 & & & \\ \text { LPC Inlet Relative Humidity } & 2.25 & & & \\ \text { LPC Exit Relative Humidity } & 0.03 & & & \\ \text { HPC Relative Humidity } & 0.00 & & & \\ \text { Drop Diameter } & 0.000500 & \text { Inlet Length } & & \\ \text { Ambient Flow Velocity } & 789.49 & \text { Fan/LPC Inlet Flow Velocity } & 458.59 \\ \text { Ambient Static Pressure } & 2.85 & \text { Fan/LPC Inlet Static Pressure } & 3.74 \\ \text { Ambient Static Temperature } & 425.97 & \text { Fan/LPC Inlet Static Temperature } & 460.40 \\ \text { Additional Water at LPC Exit } & 0.0000276 & & & \end{array}$

\begin{tabular}{|c|c|c|c|c|c|c|c|c|c|c|c|c|c|c|}
\hline & & & & SUMMARY & TPUT DAT & & & & & & & & & \\
\hline $\begin{array}{r}\mathrm{MN} \\
0.780\end{array}$ & $\begin{array}{r}\text { alt } \\
39000.0\end{array}$ & $\begin{array}{l}\text { dTamb } \\
36.00\end{array}$ & $\begin{array}{r}W \\
372.15\end{array}$ & $\begin{array}{r}\text { Fn } \\
5891.2\end{array}$ & $\begin{array}{r}\text { TSFC } \\
0.6059\end{array}$ & $\begin{array}{r}\text { Wfuel } \\
3569.34\end{array}$ & $\begin{array}{r}\text { BPR } \\
5.4136\end{array}$ & $\begin{array}{r}\text { VTAS } \\
789.49\end{array}$ & $\begin{array}{r}\text { OPR } \\
29.445\end{array}$ & $\begin{array}{c}\text { EPR } \\
1.518\end{array}$ & $\begin{array}{l}\text { Powerset } \\
84.000\end{array}$ & $\begin{array}{c}\text { T4 } \\
2762.8\end{array}$ & $\begin{array}{c}\text { T41 } \\
2595.8\end{array}$ & $\begin{array}{c}\text { T49 } \\
1803.9\end{array}$ \\
\hline
\end{tabular}

\begin{tabular}{|c|c|c|c|c|c|c|c|c|c|c|}
\hline & & & & & & & & & & \\
\hline & & & $\mathrm{W}$ & $\mathrm{Pt}$ & Tt & ht & FAR & Wo & & Ps \\
\hline FSO & Ambient.FI_O & & 372.15 & 4.267 & 477.92 & -16.44 & 0.0000 & 1230.47 & 2.8 & 425.9 \\
\hline FS1 & Inlet.Fl o- & & 372.15 & 4.267 & 477.92 & -16.44 & 0.0000 & 1230.41 & 3.28 & 443. \\
\hline FS12 & Splitter.Fl 0 & & 314.12 & 4.265 & 477.92 & -16.44 & 0.0000 & 1039.08 & 3.2 & 443.8 \\
\hline FS2 & Splitter.Fl-0 & & 58.02 & 4.265 & 477.92 & -16.44 & 0.0000 & 191.94 & 3.7. & 460. \\
\hline FS14 & Fan.Fl_o & & 314.12 & 6.942 & 556.22 & 2.34 & 0.0000 & 688.65 & 5.7 & 527.4 \\
\hline FS23 & LPC.FI- & & 58.02 & 10.355 & 639.92 & 22.47 & 0.0000 & 91.47 & 9.2 & 618.8 \\
\hline FS24 & VaporIÑ.FIO & & 58.03 & 10.355 & 640.02 & 22.34 & 0.0000 & 91.48 & 9.2 & 618.9 \\
\hline FS25 & Bleed2.FI $\overline{-}$ & & 58.03 & 10.355 & 640.02 & 22.34 & 0.0000 & 91.48 & 9.28 ( & 618.9 \\
\hline $\begin{array}{l}\text { FS3 } \\
\text { FS }\end{array}$ & HPC.FI O- & & 55.21 & 125.632 & 1378.40 & 206.59 & 0.0000 & 10.53 & 112.99 & 1340. \\
\hline FS36 & Bleed3.F1 O & & 42.79 & 125.632 & 1378.40 & 206.59 & 0.000 & 8.16 & 118.3 & 1356.7 \\
\hline FS4 & Burner.FI_o & & 43.78 & 122.738 & 2762.82 & 177.64 & 0.0232 & 12.10 & 115.9 & 2727 \\
\hline FS45 & HPT.FI O & & 58.34 & 28.643 & 1812.34 & 2.67 & 0.0173 & 55.95 & 25.78 & 1766. \\
\hline FS49 & LPT.FI- & & 59.02 & 6.587 & 1297.07 & -134.27 & 0.0171 & 208.22 & 5.68 & 1248. \\
\hline FS5 & TEGV.F̄ $O$ & & 59.02 & 6.587 & 1297.13 & -134.27 & 0.0171 & 208.22 & 5.68 & 1248 . \\
\hline FS8 & Core Nozz.Fl & & 59.02 & 6.587 & 1297.26 & -134.27 & 0.0171 & 208.23 & 3.5 & 1100. \\
\hline FS17 & FanDuctLkg.FI & & 314.12 & 6.942 & 556.22 & 2.34 & 0.0000 & 688.65 & 5.7 & 527. \\
\hline FS171 & Bleed15.FI O & & 314.12 & 6.942 & 556.22 & 2.34 & 0.0000 & 688.65 & 5.6 & 523. \\
\hline FS172 & FanDuct.Fl-o & & 314.12 & 6.942 & 556.22 & 2.34 & 0.0000 & 688.65 & 5.6 & 523. \\
\hline FS173 & Byp_Nozz.FI'_o & & 314.12 & 6.942 & 556.22 & 2.34 & 0.0000 & 688.65 & 3.6 & 463. \\
\hline$J$ & NERY PERE & ORMANCE & DATA & & & & & & & \\
\hline & & $\mathrm{PR}$ & eff & NC & $\mathrm{TR}$ & efPoly & & pwr & SMN & SMW \\
\hline Fan & 1039.08 & 1.62 & 0.9120 & 3982.719 & 1.1638 & 0.9179 & $-83 \cdot \ln (x)$ & 347.6 & 51.73 & 26.15 \\
\hline LPC & 191.94 & 2.428 & 0.8503 & 3982.719 & 1.3390 & 0.8676 & -31 & 94.6 & 2.91 & 2.10 \\
\hline HPC & 91.48 & 12.132 & 0.8606 & 10189.485 & 2.1537 & 0.8985 & -147 & 60.1 & 29.69 & 28.69 \\
\hline HPT & 12.10 & $\begin{array}{r}4.285 \\
4.285\end{array}$ & 0.9109 & 215.341 & 1.3490 & 0.8905 & 147 & 60.1 & & \\
\hline LPT & 55.95 & 4.348 & 0.9147 & 89.803 & 1.3941 & 0.8982 & 115 & 52.1 & & \\
\hline $\mathrm{UR}$ & MACHINERY & $\mathrm{DA}$ & & & & & & & & \\
\hline & WcMap & PRmap & effMap & NcMap & R/Parm & $s$ Wo & cDes & s PRdes & $s$ effDes & s NCDes \\
\hline Fan & 1207.31 & 1.613 & 0.9139 & 3944.744 & 0.0614 & 0.8 & 8607 & 1.0235 & 0.9980 & 0.9905 \\
\hline LPC & 152.37 & 2.575 & 0.8239 & 1.052 & 0.0000 & & 2597 & 0.9067 & 1.0320 & 0.0003 \\
\hline $\begin{array}{l}\text { HPC } \\
\text { HPC }\end{array}$ & 83.20 & 11.608 & 0.8695 & 9917.840 & 13.1589 & 1.0 & 0995 & 1.0494 & 0.9898 & 0.9733 \\
\hline $\begin{array}{l}\text { HPT } \\
\text { HPT }\end{array}$ & 0.96 & 4.194 & 0.9109 & 1.294 & 4.1941 & 12.6 & 6299 & 0.9723 & 1.0000 & 0.0003 \\
\hline LPT & 0.86 & 3.518 & 0.9215 & 1.010 & 3.5183 & 65.2 & 2590 & 0.7521 & 0.9926 & 0.0005 \\
\hline
\end{tabular}


$===$ INLETS $====$ eRam Afs Fram

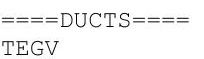

3753.73

9131.7

FanDuct

Aphy
860.21

$\begin{array}{lr}0.5626 & 860.21 \\ & \end{array}$

$==\mathrm{SPLITTERS}==$

$\mathrm{dP} / \mathrm{P}$

$\mathrm{dP} / \mathrm{P} 2$

splitter

5.4136

$=-$ SHAFIS

Nmech

0.0005

LP_Shaft

3823.1

14760.1
11542.1

$===$ BURNERS $===\quad$ TtOut

Burner 2762.81

ef

dPnorm $===$ NOZZLES $===$ Core Nozz PR
2.308
2.432 Cfg
0.9816
0.9814 CdTh $0.9814 \quad 1.0000$ 2.432
$6848.9 \quad 14760.1$

BLEEDS - interstg Wb/Win BldWk LPT COOLA HPC.C> $0.0117 \quad 0.5000 \quad 0.4500$

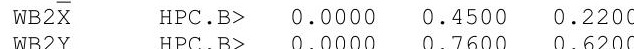
WBA2X HPC.B> $0.0000 \quad 0.4500 \quad 0.2200$ WBLKG HPC.1> $0.0000 \quad 1.0000 \quad 1.0000$ $\begin{array}{lllll}\text { WBW2X HPC.B> } & 0.0000 & 0.4500 & 0.2200\end{array}$

BLEEDS - output Wb/Win hscale Pscale
WB17Y Bleed> $0.0000 \quad 1.0000 \quad 1.0000$ HPT_COOLA Bleed> $0.1142 \quad 1.0000 \quad 1.0000$ HPT_COOLB Bleed> $0.0999 \quad 1.0000 \quad 1.0000$ WB3X $\quad$ Bleed> $0.0000 \quad 1.0000 \quad 1.0000$ $\begin{array}{lllll}\text { WBA3X } & \text { Bleed } & 0.0000 & 1.0000 & 1.0000\end{array}$ WBW3X Bleed> $0.0000-1.0000-1.0000$ WBFDLKG FanDu> $0.0000 \quad 1.0000 \quad 1.0000$ WB15X Bleed> $0.0000-1.0000-1.0000$ $\begin{array}{lllll}\text { WB15Y } & \text { Bleed }> & 0.0000 & 1.0000 & 1.0000 \\ \text { WB17X } & \text { Bleed }> & 0.0000 & 1.0000 & 1.0000\end{array}$ FAR

$\begin{array}{rr}\text { Wfuel } & \text { FAR } \\ 0.99148 & 0.02317\end{array}$ 0.9800 Ath
613.40 MNth Vact 3292.6 $\begin{array}{llrr}0.9800-2006.86 & 1.000 & 1034.5 & 11730.3\end{array}$

$\begin{array}{rrrr}W & \text { Tt } & \text { ht } & \text { Pt } \\ 2.1354 & 1016.84 & 114.46 & 28.643 \\ 0.6789 & 1016.84 & 114.46 & 6.587 \\ 0.0000 & 979.79 & 105.25 & 35.716 \\ 0.0000 & 1206.89 & 162.37 & 81.826 \\ 0.0000 & 979.79 & 105.25 & 35.716 \\ 0.0000 & 1378.40 & 206.59 & 125.632 \\ 0.0000 & 979.79 & 105.25 & 35.716 \\ W & T t & \text { ht } & \text { Pt } \\ \text { W } & \text { Tt } & 22.34 & 10.355 \\ 0.000 & 640.02 & 20.59 & 122.738 \\ 6.6254 & 1378.40 & 206.59 \\ 5.7974 & 1378.40 & 206.59 & 68.916 \\ 0.0000 & 1378.40 & 206.59 & 125.632 \\ 0.0000 & 1378.40 & 206.59 & 125.632 \\ 0.0000 & 1378.40 & 206.59 & 125.632 \\ 0.0000 & 556.22 & 2.34 & 6.942 \\ 0.0000 & 556.22 & 2.34 & 6.942 \\ 0.0000 & 556.22 & 2.34 & 6.942 \\ 0.0000 & 556.22 & 2.34 & 6.942\end{array}$

\section{3} 6.587 81.826 .632 Pt 122.738 68.916 15.632 6.942 6.942 $0.0000-556.22$ 
Date: $07 / 05 / 13$ Time: 09:36:33 Model:

COAnverge $=1$ CASE:

Date:07/05/13 Time:09:36:33 Model:

Temperature Stator 1 inlet: 471.32 Stator 1 exit: 488.91 Stator 2 inlet: 509.92 Stator 2 exit: 518.28

Stator 3 inlet: $544.23 \quad$ Stator 3 exit: 552.77 Stator 4 inlet: 577.31

Ambient Relative Humidity $\quad 10.00$

Fan Face Relative Humidity $\quad 4.19$

Fan Bypass Relative Humidity $\quad 0.25$

LPC Inlet Relative Humidity

LPC Exit Relative Humidity

HPC Relative Humidity

2.16

Ambient Flow Velocity

Ambient Static Pressur

Ambient Static Temperature

789.49
2.85

Inlet Length

40.00

5 Fan/LPC Inlet Flow Velocity 446.07

Additional Water at LPC Exit 0.0000291

Fan/LPC Inlet Static Temperature $\quad \begin{array}{r}3.77 \\ \end{array}$

SUMMARY OUTPUT DATA
$\begin{array}{rrr}\text { MN } & \text { alt } & \text { dTamb } \\ 0.780 & 39000.0 & 36.00\end{array}$
W
66.89
Fn
5594.6
TSFC
0.6047
Wfuel
3382.81
BPR
5.4673
VTAS
789.49
OPR
28.509
EPR
1.457
PowerSet
79.800
$T 4$
2712.2
$\begin{array}{cc}\text { T41 } & \text { T49 } \\ 2548.9 & 1770.0\end{array}$

FSO Ambient.FI

FS1 Inlet.Fl_o

$\begin{array}{ll}\text { FS12 } & \text { Splitter.FI_02 } \\ \text { FS2 } & \text { Splitter.Fl_01 }\end{array}$

FS14 Fan.F1_O

FS23 LPC.FI_O

FS25 Bleed2.FI_O

FS3 HPC.FI_O

FS36 Bleed3.F1_

FS4 Burner.FI_-

FS45 HPT.F1-

FS5 TEGV.FĪ

FS8 Core_Nozz.Fl

FS17 FanDuctLkg.FI_O

FS171 Bleed15.FI_O

ES172 FanDuct.FI-O

3
366.89
366.89
310.16
56.73
310.16
56.73
56.73
56.73
53.98
41.83
42.77
57.01
57.67
57.67
57.67
310.16
310.16
310.16
310.16

FLOW STATION DATA

$\begin{array}{rrrrr}\text { Pt } & \text { Tt } & \text { ht } & \text { FAR } & \text { WC } \\ 4.267 & 477.92 & -16.44 & 0.0000 & 1213.02\end{array}$ $\begin{array}{lllll}4.267 & 477.92 & -16.44 & 0.0000 & 1213.02 \\ 4.267 & 477.92 & -16.44 & 0.0000 & 1213.02\end{array}$ $\begin{array}{lllll}4.265 & 477.92 & -16.44 & 0.0000 & 1025.97\end{array}$ $\begin{array}{lllll}4.265 & 477.92 & -16.44 & 0.0000 & 1025.97 \\ 4.835 & 47.92 & -16.44 & 0.0000 & 187.66\end{array}$ $\begin{array}{lllll}4.2638 & 577.92 & -16.44 & 0.0000 & 187.66 \\ 10.300 & 637.58 & 1.73 & 0.0000 & 688.65\end{array}$ $\begin{array}{lllll}10.300 & 637.58 & 21.91 & 0.0000 & 89.74 \\ 10.300 & 637.68 & 21.76 & 0.0000 & 89.75\end{array}$ $\begin{array}{lllll}10.300 & 637.58 & 21.91 & 0.0000 & 89.74 \\ 10.300 & 637.68 & 21.76 & 0.0000 & 89.75\end{array}$ $\begin{array}{lllll}10.300 & 637.68 & 21.76 & 0.0000 & 89.75 \\ 121.641 & 1362.71 & 202.50 & 0.0000 & 89.75\end{array}$ $\begin{array}{lllll}121.641 & 1362.71 & 202.50 & 0.0000 & 10.57\end{array}$ $\begin{array}{llll}121.641 & 1362.71 & 202.50 & 0.0000\end{array}$ $\begin{array}{llll}27.707 & 1778.22 & 2.72 & 0.0168\end{array}$ 9.205
109.293 $\begin{array}{rrrr}\text { PS } & \text { Ts } & \text { Aphy } & \text { MN } \\ 2.854 & 425.97 & 3700.7 & 0.7800 \\ 3.325 & 444.99 & 4168.2 & 0.6076\end{array}$ $\begin{array}{llll}2.8545 & 44.99 & 4168.2 & 0.7800\end{array}$ 3.328 $\begin{array}{rrr}524.99 & 2606.7 & 0.5226\end{array}$ $\begin{array}{llll}9.205 & 617.51 & 412.6 & 0.4041 \\ 9.205 & 617.59 & 412.6 & 0.4042\end{array}$ $\begin{array}{llll}9.205 & 617.59 & 412.6 & 0.4042\end{array}$ $\begin{array}{rrr}617.59 & 412.6 & 0.4042\end{array}$ $49.7 \quad 0.3995$ $\begin{array}{llll}114.481 & 1341.05 & 49.3 & 0.3000 \\ 112.266 & 2677.40 & 74.6 & 0.2996\end{array}$ 24.944 .1732 .88 $\begin{array}{lll}74.6 & 0.2996\end{array}$ $\begin{array}{lllll}6.370 & 1271.28 & -131.47 & 0.0166 & 208.31\end{array}$ $\begin{array}{lllll}6.370 & 1271.34 & -131.47 & 0.0166 & 208.31 \\ 6.370 & 1271.47 & -131.47 & 0.0166 & 208.33\end{array}$

$\begin{array}{rrrrr}6.370 & 1271.47 & -131.47 & 0.0166 & 208.31 \\ 6.838 & 553.67 & 1.73 & 0.0166 & 208.33 \\ 6.838 & 553.67 & 1.73 & 0.0000 & 688.65\end{array}$

$\begin{array}{lllll}6.838 & 553.67 & 1.73 & 0.0166 & 208.33 \\ 6.838 & 553.67 & 1.73 & 0.0000 & 688.65 \\ 6.838 & 553.67 & 1.73 & 0.0000 & 688.65\end{array}$

$\begin{array}{lllll}6.838 & 553.67 & 1.73 & 0.0000 & 688.65\end{array}$

$\begin{array}{lllll}6.838 & 553.67 & 1.73 & 0.0000 & 688.65 \\ 6.838 & 553.67 & 1.73 & 0.0000 & 688.65\end{array}$

$\begin{array}{ll}765.4 & 0.4006 \\ 860.2 & 0.4721\end{array}$

$\begin{array}{llll}5.493 & 1223.07 & 860.2 & 0.4722\end{array}$

$5.677 \quad 524.99$

$613.4 \quad 1.0000$

$\begin{array}{llll}.516 & 520.71 & 2481.9 & 0.5626\end{array}$

$\begin{array}{lll}520.71 & 2481.9 & 0.5626\end{array}$

$461.30 \quad 2006.9 \quad 1.0000$

$\begin{array}{rrrrr}\text { gamt } & \text { WAR } & \text { Wair } & \text { WH2O } & \text { H2O frac } \\ 1.40068 & 0.0000851 & 366.86 & 0.031 & 0.0001 \\ 1.40068 & 0.0000851 & 366.86 & 0.031 & 0.0001 \\ 1.40068 & 0.0000851 & 310.13 & 0.026 & 0.0001 \\ 1.40068 & 0.0000851 & 56.72 & 0.005 & 0.0001 \\ 1.39970 & 0.0000851 & 310.13 & 0.026 & 0.0001 \\ 1.39785 & 0.0000851 & 56.72 & 0.005 & 0.0001 \\ 1.39785 & 0.0001142 & 56.72 & 0.006 & 0.0001 \\ 1.39785 & 0.0001142 & 56.72 & 0.006 & 0.0001 \\ 1.35810 & 0.0001142 & 53.97 & 0.006 & 0.0001 \\ 1.35810 & 0.0001142 & 41.83 & 0.005 & 0.0001 \\ 1.28878 & 0.0001142 & 41.83 & 0.005 & 0.0272 \\ 1.32487 & 0.0001142 & 56.06 & 0.006 & 0.0204 \\ 1.35221 & 0.0001142 & 56.72 & 0.006 & 0.0202 \\ 1.35220 & 0.0001142 & 56.72 & 0.006 & 0.0202 \\ 1.35219 & 0.0001142 & 56.72 & 0.006 & 0.0202 \\ 1.39970 & 0.0000851 & 310.13 & 0.026 & 0.0001 \\ 1.39970 & 0.0000851 & 310.13 & 0.026 & 0.0001 \\ 1.39970 & 0.0000851 & 310.13 & 0.026 & 0.0001 \\ 1.39970 & 0.0000851 & 310.13 & 0.026 & 0.0001\end{array}$

TURBOMACHINERY PERFORMANCE DATA

$\begin{array}{lrrrrrrrrr} & \text { WC } & \text { PR } & \text { eff } & \text { NC } & \text { TR } & \text { efPoly } & \text { pwr } & \text { SMN } & \text { SMW } \\ \text { Fan } & 1025.97 & 1.604 & 0.9117 & 3933.633 & 1.1585 & 0.9174 & -7973.7 & 56.33 & 27.21 \\ \text { LPC } & 187.66 & 2.415 & 0.8569 & 3933.633 & 1.3341 & 0.8734 & -3078.1 & 3.24 & 2.50 \\ \text { HPC } & 89.75 & 11.810 & 0.8612 & 10141.348 & 2.1370 & 0.8987 & -14154.9 & 30.97 & 29.86 \\ \text { HPT } & 12.10 & 4.289 & 0.9102 & 215.920 & 1.3509 & 0.8900 & 14154.9 & & \\ \text { LPT } & 55.99 & 4.350 & 0.9140 & 89.543 & 1.3957 & 0.8973 & 11051.7 & \end{array}$

TURBOMACHINERY MAP DATA

$\begin{array}{lrrrrrrrrr} & \text { WcMap } & \text { PRmap } & \text { effMap } & \text { NcMap } & \text { R/Parm } & \text { S_WcDes } & \text { S_PRdes } & \text { S_effDes } & \text { S_NcDes } \\ \text { Fan } & 1192.08 & 1.590 & 0.9136 & 3896.126 & 0.0605 & 0.8607 & 1.0235 & 0.9980 & 0.9905 \\ \text { LPC } & 149.00 & 2.524 & 0.8226 & 1.039 & 0.0000 & 1.2595 & 0.9287 & 1.0418 & 0.0003 \\ \text { HPC } & 81.63 & 11.300 & 0.8701 & 9870.986 & 13.0483 & 1.0995 & 1.0494 & 0.9898 & 0.9733 \\ \text { HPT } & 0.96 & 4.198 & 0.9102 & 1.298 & 4.1981 & 12.6299 & 0.9723 & 1.0000 & 0.0003 \\ \text { LPT } & 0.86 & 3.519 & 0.9208 & 1.007 & 3.5195 & 65.2590 & 0.7521 & 0.9926 & 0.0005\end{array}$




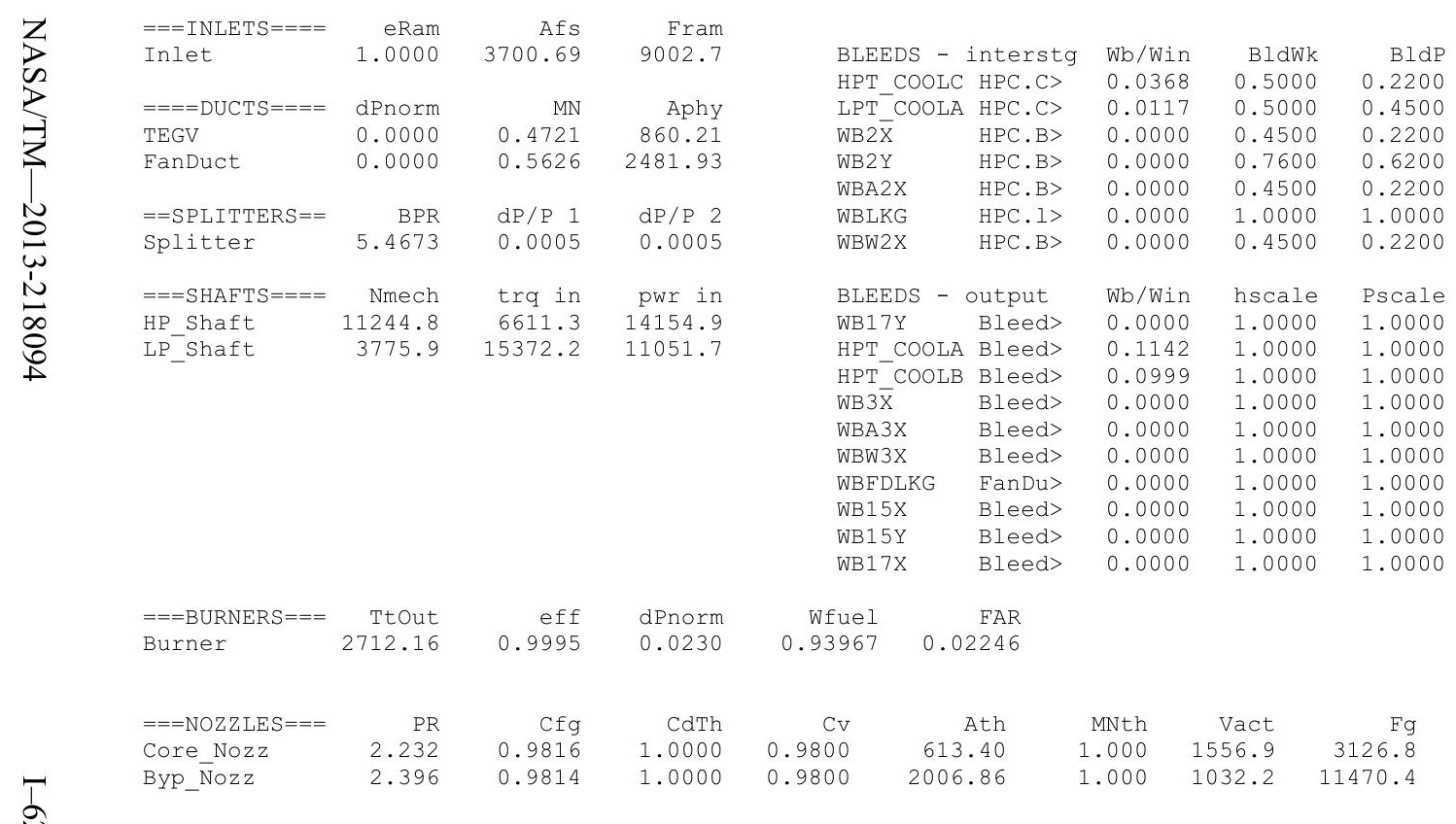


Temperature Stator 1 inlet: 472.50 Stator 1 exit: 489.81 Stator 2 inlet: 510.62 Stator 2 exit: 519.08 $\begin{array}{llll}\text { Stator } 3 \text { inlet: } 544.45 & \text { Stator } 3 \text { exit: } 553.01 & \text { Stator } 4 \text { inlet: } 577.01 \text { Stator } 4 \text { exit: } 584.90 \\ \text { Stator } 5 \text { inlet: } 603.97 & \text { Stator } 5 \text { exit: } 611.45 & \text { Unblocked Percent Blockage: } 0.00\end{array}$

Ambient Relative Humidity $\quad 10.00$

Fan Face Relative Humidity $\quad 4.03$

Fan Bypass Relative Humidity 0.26

LPC Exit Relative Humidity $\quad 0.03$

HPC Exit Relative Humidity

Dre Relative Humidity

0.00

0.0000500

Ambient Flow Velocity

Ambient Static Pressure

Additional Water at LPC Exit

789.49

2.85

Inlet Length

40.00

Fan/LPC Inlet Flow Velocity $\quad 437.10$ 0.0000303

Fan/LPC Inlet Static Temperature $\quad \begin{array}{r}3.79 \\ \end{array}$

MN SUMMARY OUTPUT DATA

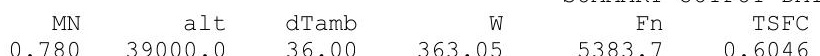

FLOW STATION DATA

FSO Ambient.Fl_O

FS1 Inlet.FI_O

$\begin{array}{ll}\text { FS12 } & \text { Splitter.FI_02 } \\ \text { FS2 } & \text { Splitter.FI_01 }\end{array}$

FS14 Fan.FI_O

FS23 LPC.FI-O

FS25 BaporIN.FI_O

FS25 Bleed2.FI

FS36 Ble.Fi_

FS4 Burner. F1_O

FS4 Burner.FI

FS49 4 STT. FI-O

FS5 5 TEGV FI

FS8 Core_Nozz.Fl_O

FS17 FanDuctLkg. FI_O

FS172 FanDuct. Fl-0

FS173 Byp Nozz. FI

363.05

$\begin{array}{llllll}363.05 & 4.267 & 477.92 & -16.44 & 0.0000 & 1200.34 \\ 363.05 & 4.267 & 477.92 & -16.44 & 0.0000 & 1200.34 \\ 307.26 & 4.265 & 477.92 & -16.44 & 0.0000 & 1016.40\end{array}$

$\begin{array}{cc}\text { Pt } & \text { Tt } \\ 4.267 & 477.92\end{array}$

$\begin{array}{llllll}363.05 & 4.267 & 477.92 & -16.44 & 0.0000 & 1200.34 \\ 55.79 & 4.265 & 477.92 & -16.44 & 0.0000 & 1016.40\end{array}$

$-16.440 .0000 \quad 184.54$ $.0000 \quad 184.5$

55.79

55.79

10.263

10.263

636.11

$1.30 \quad 0.0000$

$\begin{array}{ll} & \end{array}$

$41.14 \quad 118.805-1351.96$

56.04

27.045

2677.35
1754.90

21.400 .0000

$199.70 \quad 0.0000$

56.69

56.69

307.26

307.26

6.216

$\begin{array}{lll}.67 & -129.52 & 0.0162\end{array}$

$\begin{array}{llll}6.764 & 551.89 & 1.30 & 0.0000\end{array}$

$\begin{array}{llll}6.764 & 551.89 & 1.30 & 0.0000 \\ 6.764 & 551.89 & 1.30 & 0.0000\end{array}$

$\begin{array}{llll}6.764 & 551.89 & 1.30 & 0.0000 \\ 6.764 & 551.89 & 1.30 & 0.0000\end{array}$

688.66
88.47

88.47
88.48

88.48
10.60

8.22
12.09

12.09
56.01

208.39

208.39
208.40

208.40
688.66

688.66
688.66

688.66

VTA

789.49
$\mathrm{OPR}$
27.845 $\begin{array}{rr}\text { Ps } & \text { Ts } \\ 2.854 & 425.97\end{array}$

3.353

3.353

5.615

9.207

9.207

106.666

111.768

09.646
24.346

$\begin{array}{ll}5.615 & 523.30 \\ 5.456 & 519.03\end{array}$

5.456
3.572 519.03

$3.572 \quad 459.82$ $\begin{array}{ccccc}\text { EPR } & \text { Powerset } & \text { T4 } & \text { T41 } & \text { T49 } \\ 1.415 & 76.800 & 2677.4 & 2516.8 & 1746.8\end{array}$

TURBOMACHINERY PERFORMANCE DATA

\begin{tabular}{|c|c|c|c|c|c|c|c|c|c|}
\hline & Wc & PR & eff & NC & TR & efPoly & pwr & SMN & SMW \\
\hline Fan & 1016.40 & 1.586 & 0.9105 & 3900.457 & 1.1548 & 0.9162 & -7713.2 & 58.47 & 27.99 \\
\hline LPC & 184.54 & 2.406 & 0.8609 & 3900.457 & 1.3310 & 0.8769 & -2998.9 & 3.51 & 2.79 \\
\hline HPC & 88.48 & 11.576 & 0.8614 & 10106.712 & 2.1250 & 0.8986 & -13732.3 & 31.90 & 30.71 \\
\hline HPT & 12.09 & 4.292 & 0.9097 & 216.327 & 1.3521 & 0.8896 & 13732.3 & & \\
\hline LPT & 56.01 & 4.351 & 0.9135 & 89.376 & 1.3969 & 0.8967 & 10712.0 & & \\
\hline \multirow{2}{*}{\multicolumn{10}{|c|}{ TURBOMACHINERY MAP DE }} \\
\hline & WcMap & PRmap & effMap & NcMap & R/Parm & s_WCDes & s_PRdes & & s NcDes \\
\hline an & 1180.95 & 1.573 & 0.9124 & 363.266 & 0.0599 & $\overline{0} .8607$ & $\overline{1} .0235$ & 0.9980 & $\overline{0} .9905$ \\
\hline $\mathrm{PC}$ & 146.86 & 2.490 & .8219 & 1.030 & 0.0000 & 1.2566 & 0.9440 & 1.0474 & 0.0003 \\
\hline HPC & 80.47 & 11.078 & 0.8703 & 9837.273 & 12.9696 & 1.0995 & 1.0494 & 0.9898 & 0.9733 \\
\hline $\mathrm{HPT}$ & 0.96 & 4.201 & 0.9097 & 1.300 & 4.2005 & 12.6299 & 0.9723 & 1.0000 & 0.0003 \\
\hline LPT & 0.86 & 3.520 & 0.9203 & 1.005 & 3.5203 & 65.2590 & 0.7521 & 0.9926 & 0.0005 \\
\hline
\end{tabular}

$\begin{array}{rrr}\text { Aphy } & \text { MN } & \text { gamt } \\ 3662.0 & 0.7800 & 1.40068 \\ 4168.2 & 0.5970 & 1.40068 \\ 3531.8 & 0.5963 & 1.40068 \\ 830.5 & 0.4147 & 1.40068 \\ 2606.7 & 0.5226 & 1.39973 \\ 412.6 & 0.3971 & 1.39789 \\ 412.6 & 0.3972 & 1.39789 \\ 412.6 & 0.3972 & 1.39789 \\ 49.7 & 0.4008 & 1.35873 \\ 49.3 & 0.3009 & 1.35873 \\ 74.6 & 0.2994 & 1.29010 \\ 265.4 & 0.4007 & 1.32616 \\ 860.2 & 0.4721 & 1.35355 \\ 860.2 & 0.4721 & 1.35355 \\ 613.4 & 1.0000 & 1.35354 \\ 2606.7 & 0.5226 & 1.39973 \\ 2481.9 & 0.5626 & 1.39973 \\ 2481.9 & 0.5626 & 1.39973 \\ 2006.9 & 1.0000 & 1.39973\end{array}$

WAR
0.0000851
0.0000851
0.0000851
0.0000851
0.0000851
0.0000851
0.0001153
0.0001153
0.0001153
0.0001153
0.0001153
0.0001153
0.0001153
0.0001153
0.0001153
0.0000851
0.0000851
0.0000851
0.0000851

WAR

Wair 363.02
363.02 307.24 $307.24 \quad 0.026$ $\begin{array}{ll}55.78 & 0.005 \\ 55.78 & 0.006\end{array}$ $\begin{array}{ll}55.78 & 0.005 \\ 55.78 & 0.006\end{array}$ $\begin{array}{ll}55.78 & 0.006 \\ 53.08 & 0.006\end{array}$

$\begin{array}{lll}43.08 & 0.006\end{array}$

$\begin{array}{ll}41.13 & 0.005 \\ 41.13 & 0.005\end{array}$

$\begin{array}{ll}41.13 & 0.005 \\ 55.13 & 0.006\end{array}$ $\begin{array}{ll}55.78 & 0.006\end{array}$ $\begin{array}{ll}55.78 & 0.006 \\ 55.78 & 0.006\end{array}$ $307.24 \quad 0.026$ $307.24 \quad 0.026$ $307.24 \quad 0.026$ $307.24 \quad 0.026$
H2O frac 0.0001 0.0001 0.0001 0.0001 0.0001 0.0001 0.0001 0.0260 0.0198 0.0198 0.0198 0.0001 0.0001 0.0001 $\begin{array}{lll}0.86 & 3.520 & 0.9203\end{array}$

1.005

65.2590

.7521

0.0005 


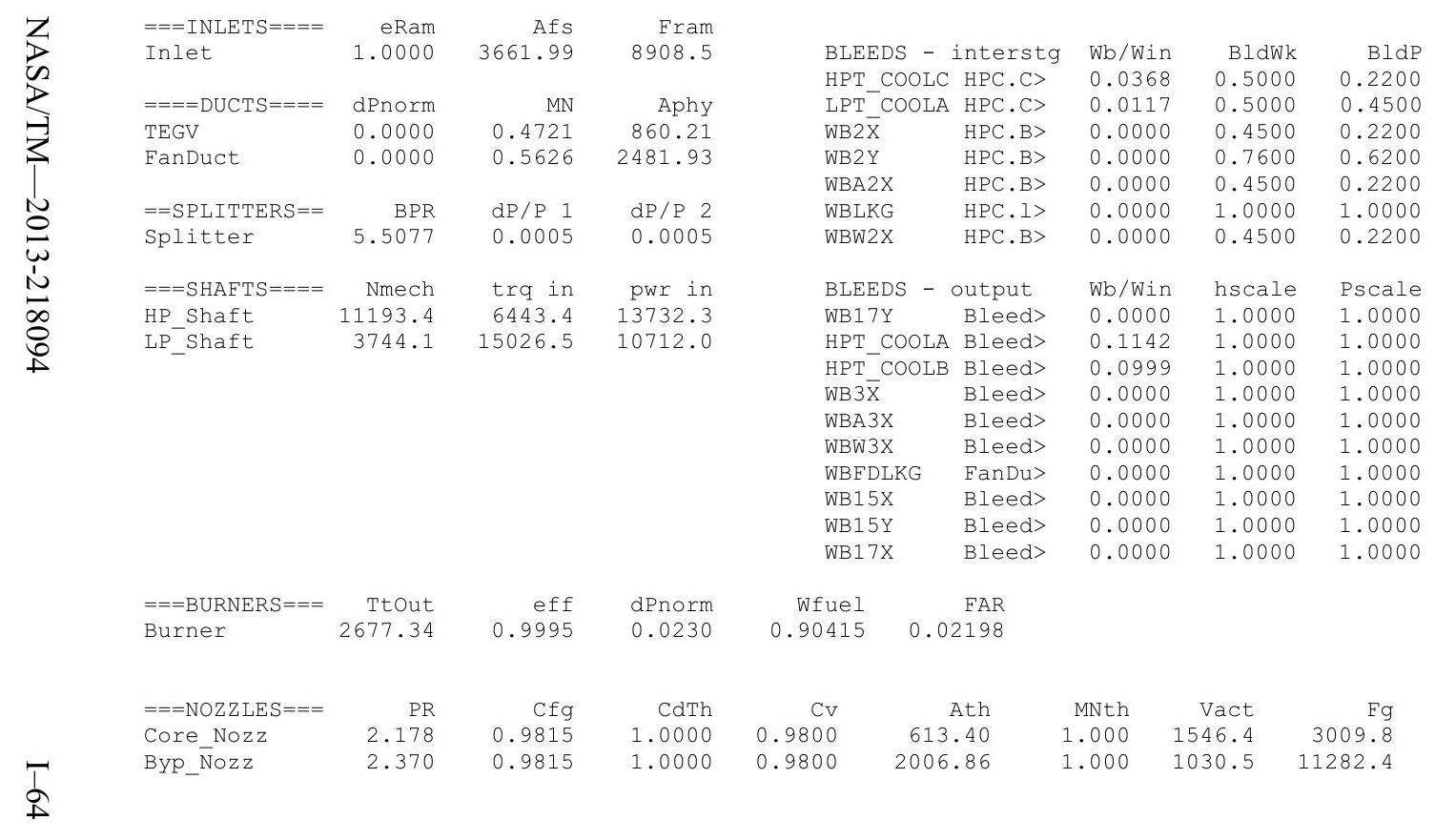


Date:07/05/13 Time:09:47:54 Model: Turbofan Engine

Version:NPSS 1.6.5 - Rev: -> Gas Package: Janaf iter/pass/Jacb/Broy=24/38/1/22 Run by: Philip C Jorgenson PC: 100

Temperature Stator 1 inlet: 444.03 Stator 1 exit: 462.65 Stator 2 inlet: 484.17 Stator 2 exit: 491.24

Stator 3 inlet: 520.19 Stator 3 exit: 528.10 Stator 4 inlet: 555.19 Stator 4 exit: 562.88

Ambient Relative Humidity $\quad 10.00$

Fan Face Relative Humidity $\quad 5.21$

FPC Inlet Relative Humidity 0.15

LPC Exit Relative Humidity 2.30

Drop Diameter

0.00

Drop Dianer

0.0001000

772.64

Ambient Static Pressure

Ambient Static Temperature

2.85

Inlet Length

40.00

$\begin{array}{ll} & 40.00 \\ & \end{array}$

407.97 Fan/LPC Inlet Static Temperature 438.10

SUMMARY OUTPUT DATA

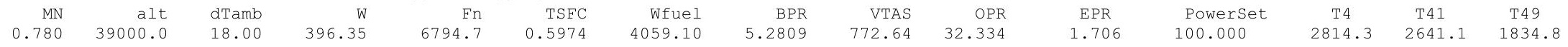

\begin{tabular}{|c|c|}
\hline SO & Ambient.FI_O \\
\hline FS1 & Inlet.Fl_o- \\
\hline FS12 & Splitter.Fl_02 \\
\hline FS2 & Splitter.Fl_01 \\
\hline FS14 & Fan.Fl_O \\
\hline FS23 & LPC.Fl_o \\
\hline FS24 & VaporIN̄.FI_O \\
\hline FS25 & Bleed2.Fl_o \\
\hline FS3 & HPC.FI_O \\
\hline FS36 & Bleed3.FI_O \\
\hline FS 4 & Burner.FI_O \\
\hline FS45 & HPT.FI_O \\
\hline FS49 & LPT.FI_O \\
\hline FS5 & TEGV.F̄'_O \\
\hline FS8 & Core Nozz.Fl \\
\hline FS17 & FanDuctLkg.Fì_o \\
\hline FS171 & Bleed15.FI_o \\
\hline FS172 & FanDuct.Fl- \\
\hline S173 & Byp_Nozz.Fi \\
\hline
\end{tabular}

\begin{tabular}{rrrrrr}
\multicolumn{7}{c}{ FLOW } & STATION DATA \\
W & Pt & Tt & ht & FAR & WC \\
396.35 & 4.267 & 457.74 & -20.96 & 0.0000 & 1282.45 \\
396.35 & 4.267 & 457.74 & -20.96 & 0.0000 & 1282.45 \\
333.25 & 4.265 & 457.74 & -20.96 & 0.0000 & 1078.81 \\
63.11 & 4.265 & 457.74 & -20.96 & 0.0000 & 204.29 \\
333.25 & 7.257 & 540.20 & -1.18 & 0.0000 & 688.69 \\
63.11 & 10.456 & 620.26 & 18.06 & 0.0000 & 96.99 \\
63.11 & 10.456 & 620.33 & 17.99 & 0.0000 & 97.00 \\
63.11 & 10.456 & 620.33 & 17.99 & 0.0000 & 97.00 \\
60.05 & 137.963 & 1373.15 & 205.60 & 0.0000 & 10.41 \\
46.54 & 137.963 & 1373.15 & 205.60 & 0.0000 & 8.07 \\
47.66 & 134.786 & 2814.26 & 175.35 & 0.0242 & 12.11 \\
63.50 & 31.495 & 1843.67 & -2.48 & 0.0181 & 55.86 \\
64.23 & 7.237 & 1320.43 & -142.07 & 0.0179 & 208.13 \\
64.23 & 7.237 & 1320.50 & -142.07 & 0.0179 & 208.13 \\
64.23 & 7.237 & 1320.63 & -142.07 & 0.0179 & 208.14 \\
333.25 & 7.257 & 540.20 & -1.18 & 0.0000 & 688.69 \\
333.25 & 7.257 & 540.20 & -1.18 & 0.0000 & 688.69 \\
333.25 & 7.257 & 540.20 & -1.18 & 0.0000 & 688.69 \\
333.25 & 7.257 & 540.20 & -1.18 & 0.0000 & 688.69
\end{tabular}

$\begin{array}{rr}\text { Ps } & \text { Ts } \\ 2.854 & 407.97 \\ 3.150 & 419.65 \\ 3.174 & 420.64 \\ 3.659 & 438.10 \\ 6.024 & 512.21 \\ 9.124 & 596.63 \\ 9.124 & 596.69 \\ 9.124 & 596.69 \\ 4.449 & 1336.29 \\ 0.111 & 1352.09 \\ 7.330 & 2778.52 \\ 8.371 & 1797.31 \\ 6.242 & 1270.79 \\ 6.242 & 1270.85 \\ 3.870 & 1120.99 \\ 6.024 & 512.21 \\ 5.854 & 508.03 \\ 5.854 & 508.03 \\ 3.833 & 450.06\end{array}$

$\begin{array}{rrr}\text { Aphy } & \text { MN } & \text { gamt } \\ 3912.3 & 0.7800 & 1.40084 \\ 4168.2 & 0.6728 & 1.40084 \\ 3531.8 & 0.6633 & 1.40084 \\ 830.5 & 0.4730 & 1.40084 \\ 2606.7 & 0.5226 & 1.39992 \\ 412.6 & 0.4457 & 1.39831 \\ 412.6 & 0.4458 & 1.39831 \\ 412.6 & 0.4458 & 1.39831 \\ 49.7 & 0.3921 & 1.35750 \\ 49.3 & 0.2948 & 1.35750 \\ 74.6 & 0.3003 & 1.28479 \\ 265.4 & 0.4001 & 1.32115 \\ 860.2 & 0.4724 & 1.34831 \\ 860.2 & 0.4724 & 1.34830 \\ 613.4 & 1.0000 & 1.34829 \\ 2606.7 & 0.5226 & 1.39992 \\ 2481.9 & 0.5625 & 1.39992 \\ 2481.9 & 0.5625 & 1.39992 \\ 2006.9 & 1.0000 & 1.39992\end{array}$

WAR

Wair TURBOMACHINERY PERFORMANCE DATA

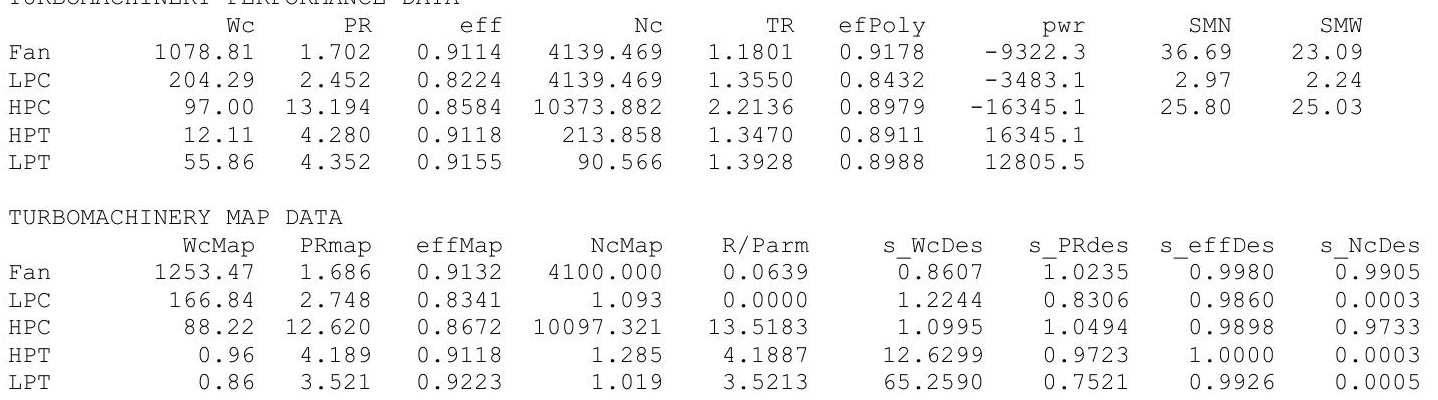




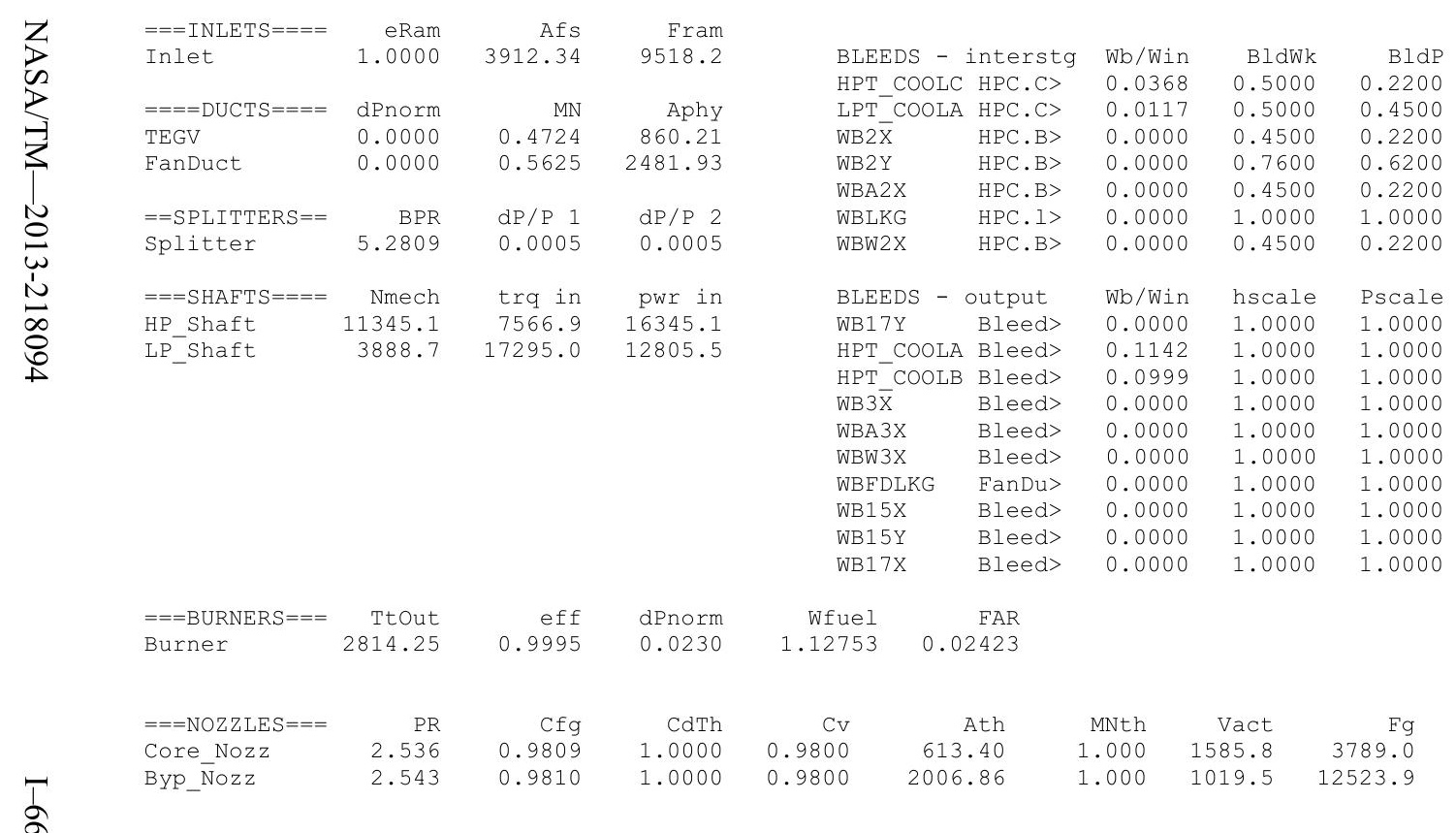


Date: $07 / 05 / 13$ Time: $09: 48: 31$ Model:

onverge $=1 \quad$ CASE: 0

Version:NPSS 1.6.5 - Rev: -> Gas Package: Janaf iter/pass/Jacb/Broy=19/33/1/17 Run by: Philip C Jorgenson PC: 84

Temperature Stator 1 inlet: 450.18 Stator 1 exit: 467.32 Stator 2 inlet: 487.66 Stator 2 exit: 495.55 $\begin{array}{llll}\text { Stator } 3 \text { inlet: } 521.02 & \text { Stator } 3 \text { exit: } 529.17 & \text { Stator } 4 \text { inlet: } 553.24 \quad \text { Stator } 4 \text { exit: } 560.82 \\ \text { Stator } 5 \text { inlet: } 579.81 & \text { Stator } 5 \text { exit: } 587.06 & \end{array}$

Fan Face Relative Humidity

Fan Bypass Relative Humidity

LPC Inlet Relative Humidity

LPC Exit Relative Humidity

Drop Diameter

Ambient Flow velocity

Ambient static Pressure

Additional Water at LPC Exit

10.00

4.12
0.18
2.01

2.01

0.02

0.0001000

0.0001000

2.64
2.85
0.007

Inlet Length

40.00

Fan/LPC Inlet Flow velocity

3.75

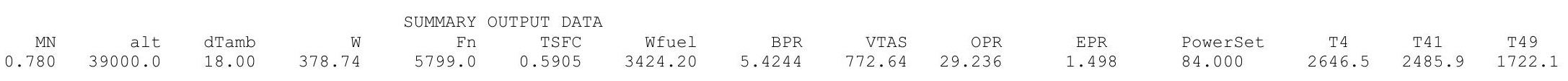

Ambient.F1_

Inlet.ri_o

FS2 Splitter.FI-01

FS14 Fan.Fl

(t)

SS25 Vaporin. F1-O

FS3 3 HPC.F1.0

FS36 Bleed3.Fl

FS4 Burner.FI-

FS45 HPT.FI_O

FS49 LPT.FI_O

FS5 TEGV.F̄i_O

FS17 Fanducts.

FS171 Bleed15.F1.FI-

FS171 17 FanDuct. F1

FS173 Byp Nozz. FI

$W$
378.74
378.74
319.79
58.95
319.79
58.95
58.95
58.95
56.09
43.47
44.42
59.22
59.91
59.91
59.91
319.79
319.79
319.79
319.79

FLOW STATION DATA

$\begin{array}{crrrr}\text { Pt } & \text { Tt } & \text { ht } & \text { FAR } & \text { WC } \\ 4.267 & 457.74 & -20.96 & 0.0000 & 1225.46\end{array}$

$\begin{array}{lllll}4.267 & 457.74 & -20.96 & 0.0000 & 1225.46 \\ 4.267 & 457.74 & -20.96 & 0.0000 & 1225.46\end{array}$

$\begin{array}{lllll}4.265 & 457.74 & -20.96 & 0.0000 & 1035.23\end{array}$

$\begin{array}{lllll}4.265 & 457.74 & -20.96 & 0.0000 & 190.85\end{array}$

$\begin{array}{rrrrr}6.911 & 532.08 & -3.13 & 0.0000 & 688.70 \\ 10.330 & 612.23 & 16.12 & 0.0000 & 91.12\end{array}$

$\begin{array}{lllll}10.330 & 612.23 & 16.12 & 0.0000 & 91.12 \\ 10.330 & 612.30 & 16.05 & 0.0000 & 91.13\end{array}$

$\begin{array}{llll}10.330 & 612.30 & 16.05 & 0.0000\end{array}$

$\begin{array}{llll}124.744 & 1320.99 & 192.07 & 0.0000\end{array}$

$\begin{array}{llll}124.744 & 1320.99 & 192.07 & 0.0000\end{array}$

$\begin{array}{llll}121.871 & 2646.49 & 165.11 & 0.0219\end{array}$

$\begin{array}{llll}28.387 & 1730.17 & -2.36 & 0.0163\end{array}$

$\begin{array}{llll}6.515 & 1234.61 & -132.77 & 0.0161\end{array}$

$\begin{array}{llll}6.515 & 1234.67 & -132.77 & 0.0161\end{array}$

$\begin{array}{rrrr}6.515 & 1234.81 & -132.77 & 0.0161 \\ 6.911 & 532.08 & -3.13 & 0.0000\end{array}$

$\begin{array}{llll}6.911 & 532.08 & -3.13 & 0.0000 \\ 6.911 & 532.08 & -3.13 & 0.0000\end{array}$

$\begin{array}{llll}6.911 & 532.08 & -3.13 & 0.0000 \\ 6.911 & 532.08 & -3.13 & 0.0000\end{array}$

91.13

$\begin{array}{ll}10.55 & 112.152 \\ 8.17 & 117.439\end{array}$

$\begin{array}{rr}8.17 & 117.439 \\ 12.10 & 115.114\end{array}$

55.99

208.47

208.47
208.48

688.70
688.70

532.08

688.70

Ps
2.854
3.296
3.303
3.750
5.737
9.193
9.193
9.193
112.152
117.439
115.114
25.554
5.617
5.617
3.477
5.737
5.575
5.575
3.650

Ts
407.97
425.15
425.46
441.19
504.50
592.21
592.28
592.28
1284.18
1300.01
2612.35
1685.75
1187.42
1187.48
1045.35
504.50
500.38
500.38
443.27

$\begin{array}{rr}\text { Aphy } & \text { MN } \\ 3738.5 & 0.7800 \\ 4168.2 & 0.6183\end{array}$ $\begin{array}{ll}4168.2 & 0.6183 \\ 3531.8 & 0.6151\end{array}$ $\begin{array}{rr}3531.8 & 0.6151 \\ 830.5 & 0.4326\end{array}$

$2606.7 \quad 0.5226$

$\begin{array}{ll}412.6 & 0.4118\end{array}$

$\begin{array}{ll}412.6 & 0.4118 \\ 412.6 & 0.4118\end{array}$

$\begin{array}{lll}49.7 & 0.3980\end{array}$

$\begin{array}{ll}49.3 & 0.2989 \\ 74.6 & 0.2995\end{array}$

$\begin{array}{rr}74.6 & 0.2995 \\ 265.4 & 0.4003\end{array}$

860.20 .4721

$\begin{array}{lll}860.2 & 0.4721\end{array}$

613.41 .0000

$2606.7 \quad 0.5226$

$2481.9 \quad 0.5625$

$\begin{array}{ll}2481.9 & 0.5625 \\ 2481.9 & 0.5625\end{array}$

2006.91 .0000

\begin{tabular}{|c|c|c|c|c|c|c|c|c|c|}
\hline & WT & & & & & & & & \\
\hline Fan & 1035.23 & $\begin{array}{r}\mathrm{PR} \\
1.621\end{array}$ & $\begin{array}{r}\text { ett } \\
0.9117\end{array}$ & 3968.841 & 1.1624 & 0.9175 & $\begin{array}{r}\text { pWr } \\
-8063.8\end{array}$ & 53.03 & $\begin{array}{r}\text { SMW } \\
26.46\end{array}$ \\
\hline LPC & 190.85 & 2.422 & 0.8520 & 3968.841 & 1.3375 & 0.8691 & -3092.8 & 2.96 & 2.16 \\
\hline HPC & 91.13 & 12.076 & 0.8607 & 10180.126 & 2.1574 & 0.8986 & -14325.3 & 29.83 & 28.82 \\
\hline $\mathrm{APT}$ & 12.10 & 4.293 & .9099 & 215.008 & 1.3534 & 0.8903 & 14325.2 & & \\
\hline LPT & 55.99 & 4.357 & 0.9131 & 89.636 & 1.3983 & 0.8961 & 11156.8 & & \\
\hline RD & INERY & DATA & & & & & & & \\
\hline & WcMap & PRmap & effmap & NcMap & R/Parm & S_WCDes & S_PRdes & S_effDes & S_NcDe \\
\hline an & 1202.83 & 1.606 & 0.9135 & 3930.998 & 0.0611 & $\overline{0} .8607$ & 1.0235 & 0.9980 & 0.9905 \\
\hline PC & 151.27 & 2.560 & .02 & 1.048 & 0.0000 & 1.2615 & 0.91 & 19 & 0. \\
\hline PC & 82.88 & 11.554 & 0.8696 & 9908.731 & 13.1468 & 1.0995 & 1.0494 & 0.9898 & 0.973 \\
\hline${ }_{T}$ & 0.96 & 4.202 & .9099 & 1.292 & 4.2020 & 12.6299 & 0.9723 & 1.0000 & 0.0003 \\
\hline $\mathrm{PT}$ & 0.86 & 3.525 & 0.9199 & 1.008 & 3.5247 & 65.2590 & 0.7521 & 0.9926 & 0.0005 \\
\hline
\end{tabular}




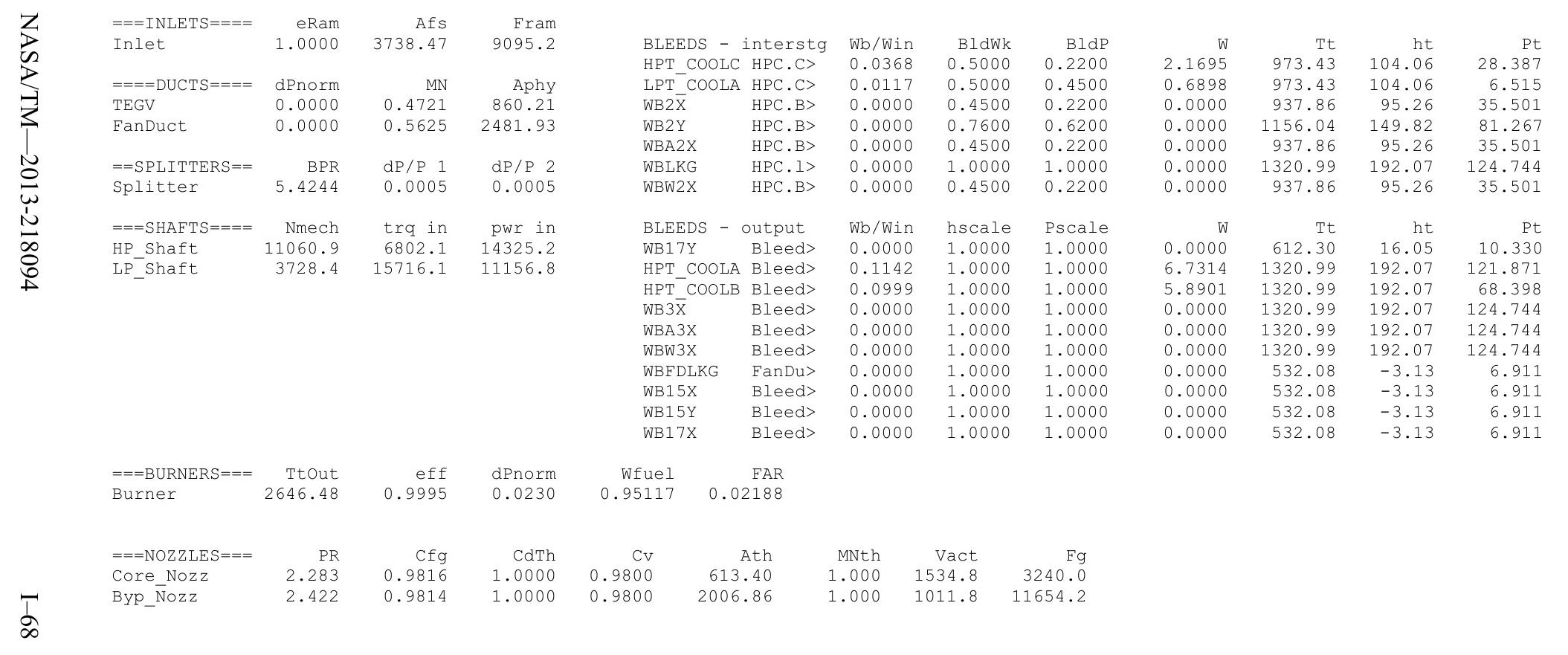


Date:07/05/13 Time:09:49:07 Model: Turbofan Engine - COMDES ON COnverge = 1 CASE: 0

Version:NPSS_1.6.5 - Rev: -> Gas Package: Janaf iter/pass/Jacb/Broy=19/ 33/1/17 Run by: Philip C Jorgenson

Temperature Stator 1 inlet: 451.80 Stator 1 exit: 468.53 Stator 2 inlet: 488.56 Stator 2 exit: 496.59 $\begin{array}{lll}\text { Stator } 3 \text { inlet: } 521.23 & \text { Stator } 3 \text { exit: } 529.40 & \text { Stator } 4 \text { inlet: } 552.71 \text { Stator } 4 \text { exit: } 560.26 \\ \text { Stator } 5 \text { inlet: } 578.74 & \text { Stator } 5 \text { exit: } 585.91 & \text { Unblocked Percent Blockage: } 0.00\end{array}$

Ambient Relative Humidity $\quad 10.00$

Fan Face Relative Humidity $\quad 3.88$

Fan Bypass Relative Humidity $\quad 0.20$

LPC Inlet Relative Humidity 1.93

LPC Exit Relative Humidity

Drop Diameter

0.0001000

Drblent rlow Velocity

Ambient static Pressure

Additional Water at LPC Exit

0.00

772.64

Inlet Iength

2.85 0.0000152 \begin{tabular}{lr} 
Fan/LPC Inlet Static Pressure & 433.45 \\
\hline
\end{tabular}

Fan/LPC Inlet Static Temperature 442.08
MN

$\begin{array}{rrrrrr}\text { MN } & \text { alt } & \text { dTamb } & \text { W } & \text { Fn } & \text { TSFC } \\ 0.780 & 39000.0 & 18.00 & 373.42 & 5507.4 & 0.5896\end{array}$

\section{FLOW STATION DATA}

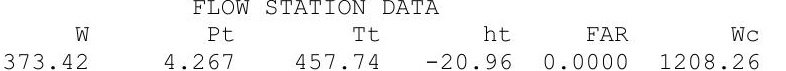

FSO Ambient.FI_O

FS1 Inlet.FI_O

$\begin{array}{ll}\text { FS12 } & \text { Splitter.Fl_02 } \\ \text { FS2 } & \text { Splitter.Fl-01 }\end{array}$

FS14 Fan.Fl_o

FS23 LPC.FI_O

FS24 VaporIN.F1_-

$\begin{array}{ll}\text { FS25 } & \text { Bleed2.FI_ } \\ \text { FS3 HPC.F1_O }\end{array}$

FS36 Bleed3.FI_O

FS4 Burner.FI_

FS45 HPT.FI

FS49 LPT.FI-O

FS8 Core_Nozz.Fl_O

FS17 FanDuctlkg.Fi_O

FS171 Bleed15.FI_O

FS171 Bleed15. F1_O

FS173 Byp_Nozz.FI_O

$\begin{array}{llllll}373.42 & 4.267 & 457.74 & -20.96 & 0.0000 & 1208.26 \\ 373.42 & 4.267 & 457.74 & -20.96 & 0.0000 & 1208.26\end{array}$

$\begin{array}{lllllll}315.79 & 4.265 & 457.74 & -20.96 & 0.0000 & 1208.26\end{array}$

$\begin{array}{llllll}57.63 & 4.265 & 457.74 & -20.96 & 0.0000 & 1022.29 \\ 315.79 & 6.810 & 529.66 & -3.76 & 0.0000 & 186.57\end{array}$

315.79

57.63

57.63

57.63

54.84

42.50
43.40

57.86

58.53

58.53

58.53

315.79
315.79

315.79

315.79

TURBOMACHINERY PERFORMANCE DATA

\begin{tabular}{lrrrrrrrrr}
\multicolumn{1}{c}{ W } & Wc & PR & eff & NC & TR & efPoly & pwr & SMN & SMW \\
Fan & 1022.29 & 1.597 & 0.9114 & 3920.781 & 1.1571 & 0.9170 & -7703.9 & 57.15 & 27.51 \\
LPC & 186.57 & 2.410 & 0.8583 & 3920.781 & 1.3329 & 0.8746 & -2981.7 & 3.34 & 2.63 \\
HPC & 89.39 & 11.754 & 0.8613 & 10131.674 & 2.1405 & 0.8988 & -13738.2 & 31.10 & 29.98 \\
HPT & 12.10 & 4.297 & 0.9091 & 215.566 & 1.3551 & 0.8897 & 13738.2 & & \\
LPT & 56.02 & 4.358 & 0.9123 & 89.380 & 1.3998 & 0.8952 & 10685.5 & &
\end{tabular}

TURBOMACHINERY MAP DATA

\begin{tabular}{|c|c|c|c|c|c|c|c|c|c|}
\hline & WcMa & PRmap & effMap & NcMap & R/Parm & s WCDes & s PRdes & $s$ effDes & S NcDes \\
\hline $\operatorname{Tan}$ & 1187.80 & 1.583 & 0.9132 & 3883.397 & 0.0603 & $\overline{0} .8607$ & $\overline{1} .0235$ & 0.9980 & $\overline{0} .9905$ \\
\hline & 148.17 & 2.511 & & 1.036 & & & 332 & & \\
\hline${ }^{\mathrm{C}} \mathrm{C}$ & 81.30 & 11.247 & 0.8702 & 9861.570 & 13.0377 & 1.0995 & 1.0494 & 0.9898 & 0.9733 \\
\hline & 0.9 & & & & & & & & \\
\hline PT & 0.86 & 3.526 & 0.9191 & 1.005 & 3.5257 & 652590 & 0.7521 & 0.9926 & 0.0005 \\
\hline
\end{tabular}

\begin{tabular}{|c|c|}
\hline & \\
\hline 2.8 & 407 \\
\hline 3.3 & 426 \\
\hline 3.3 & 426 \\
\hline 3.7 & 442 \\
\hline 5. & 502 \\
\hline 9.1 & \\
\hline 9.1 & 59 \\
\hline 9.1 & 59 \\
\hline 108. & 1269 \\
\hline 113.6 & 1285 \\
\hline 111. & 2565 \\
\hline 24. & 1654 \\
\hline 5. & 1164 \\
\hline 5.2 & 1164 \\
\hline 3.3 & 1024 \\
\hline 5. & 502 \\
\hline 5. & 498 \\
\hline 5.2 & 498 \\
\hline 3.5 & 44 \\
\hline & SMW \\
\hline & 27.51 \\
\hline & 2.63 \\
\hline & 29.98 \\
\hline es & S_NCDes \\
\hline & $\overline{0} .9905$ \\
\hline .0438 & 0.000 \\
\hline 98 & 0.973 \\
\hline & .000 \\
\hline & 0 \\
\hline
\end{tabular}

$\begin{array}{clccc}\text { EPR } & \text { PowerSet } & \text { T4 } & \text { T41 } & \text { T49 } \\ 1.439 & 79.800 & 2598.7 & 2441.7 & 1690.4\end{array}$

gamt
1.40084
1.40084
1.40084
1.40084
1.40008
1.39857
1.39856
1.39856
1.36150
1.36150
1.29291
1.32917
1.35663
1.35663
1.35662
1.40008
1.40008
1.40008
1.40008

Wair 373.41 373.41
315.78 315.78
57.63 57.63
315.78 $\begin{array}{ll}57.63 & 0.002 \\ 57.63 & 0.003\end{array}$ $\begin{array}{ll}57.63 & 0.003 \\ 57.63 & 0.003\end{array}$ $\begin{array}{ll}57.63 & 0.003 \\ 54.84 & 0.002\end{array}$ $\begin{array}{ll}54.84 & 0.002 \\ 42.50 & 0.002\end{array}$ $\begin{array}{ll}42.50 & 0.002 \\ 42.50 & 0.002\end{array}$ $\begin{array}{ll}56.96 & 0.003\end{array}$ $\begin{array}{ll}57.63 & 0.003\end{array}$ $\begin{array}{ll}57.63 & 0.003\end{array}$ $\begin{array}{ll}57.63 & 0.003\end{array}$ $\begin{array}{ll}315.78 & 0.009\end{array}$ $\begin{array}{ll}315.78 & 0.009\end{array}$ $\begin{array}{ll}315.78 & 0.009\end{array}$ $\begin{array}{ll}315.78 & 0.009\end{array}$

H2O frac
0.0000
0.0000
0.0000
0.0000
0.0000
0.0000
0.0000
0.0000
0.0000
0.0000
0.0257
0.0193
0.0191
0.0191
0.0191
0.0000
0.0000
0.0000
0.0000




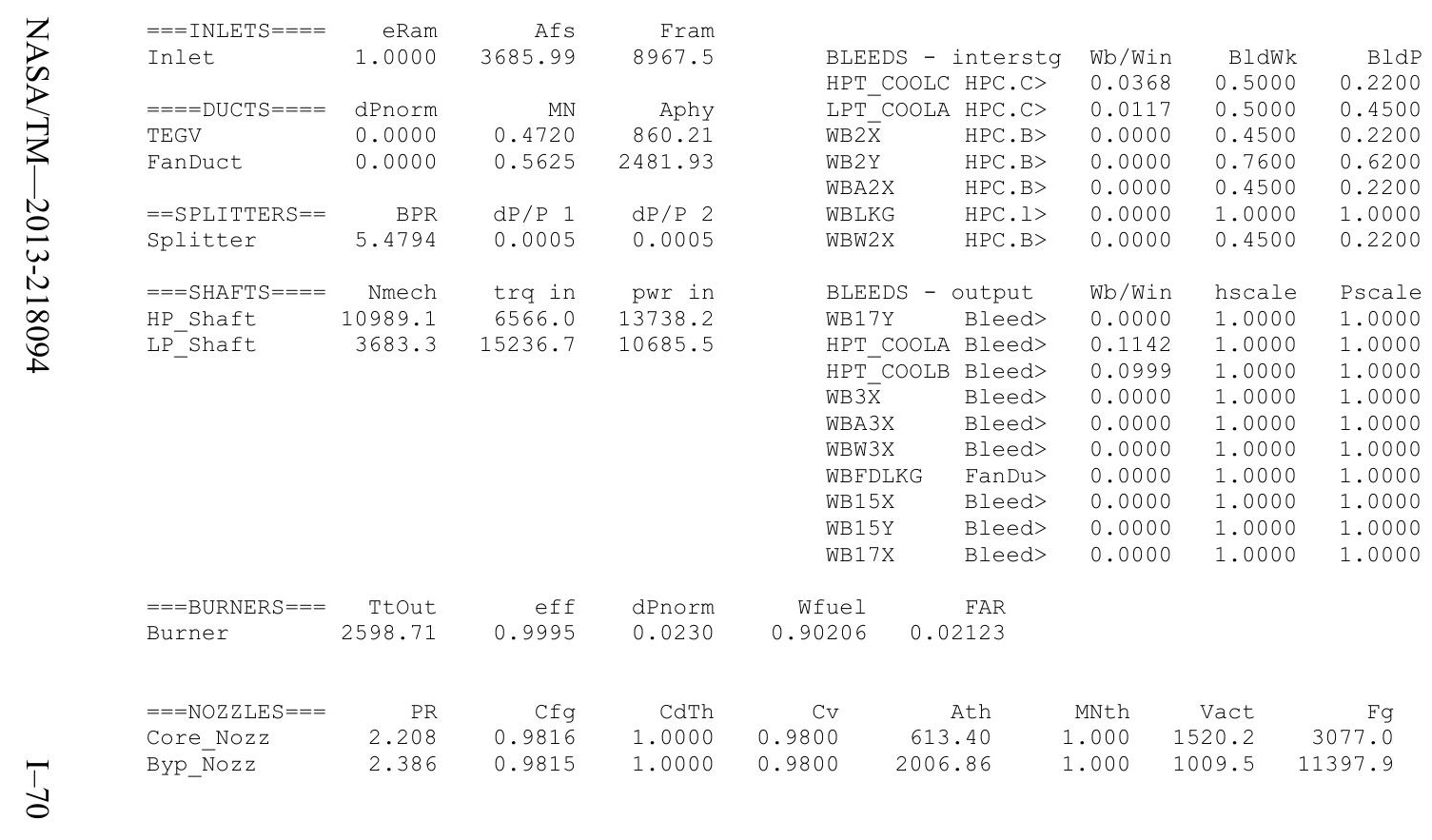


Date: 07/05/13 Time:09:49:42 Model:

Turbofan Engine

converge $=1 \quad$ CASE: $\quad 0$

(1.6.5 - Rev: -> Gas Package: Janaf iter/pass/Jacb/Broy= 18/ 32/ 1/16 Run by: Philip C Jorgenson $\begin{array}{llll}\text { Stator } 1 \text { inlet: } 452.89 & \text { Stator } 1 \text { exit: } 469.36 & \text { Stator } 2 \text { inlet: } 489.19 & \text { Stator } 2 \text { exit: } 497.30 \\ \text { Stator } 3 \text { inlet: 521.41 } & \text { Stator } 3 \text { exit: } 529.59 & \text { Stator } 4 \text { inlet: } 552.40 \quad \text { Stator } 4 \text { exit: 559.9 }\end{array}$ Stator 5 inlet: 578.07 Stator 5 exit: 585.21 Unblocked Percent Blockage: 0.00

Ambient Relative Humidity $\quad 10.00$

Fan Face Relative Humidity $\quad 3.72$

Fan Bypass Relative Humidity 0.21

LPC Inlet Relative Humidity

LPC Exit Relative Humidity

Delative Humidity

$\begin{array}{lr}\text { Drop Diameter } & 0.0001000 \\ \text { Ambient Flow Velocity } & 772.64\end{array}$

0.00

Drop Diant flow velocity

Ambient Static Pressure

772.64

2.85

Inlet Length

40.00

Additional Water at LPC Exit 0.0000152

(3) $\quad 424.81$

Fan/LPC Inlet Static Pressure $\quad 3.79$

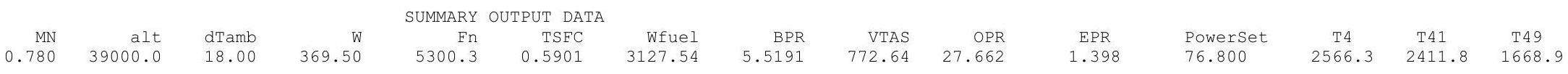

FSO Ambient.FI_O

FS1 Inlet.F1_

FS14 Fan.F1_O

FS23 LPC.FIO

FS24 VaporIN̄.FI_O

FS25 Bleed2.F1_

FS36 Bleed3.FI

FS4 Burner.FI-

FS45 HPT.FIO

FS49 LPT.FI-O

FS5 TEGV.F̄_O

FS17 Fandúctio

FS171 Bleed15.Fl_- 1

FS172 FanDuct. Fl-

FS173 Byp NozZ F1

$W$
369.50
369.50
312.82
56.68
312.82
56.68
56.68
56.68
53.93
41.80
42.67
56.89
57.55
57.55
57.55
312.82
312.82
312.82
312.82

FLOW STATION DATA

TURBOMACHINERY PERFORMANCE DATA

\begin{tabular}{lrrrrrrrrr}
\multicolumn{1}{c}{ W } & WC & PR & eff & NC & TR & efPoly & pwr & SMN & SMW \\
Fan & 1012.69 & 1.579 & 0.9094 & 3888.072 & 1.1535 & 0.9150 & -7456.5 & 59.29 & 28.29 \\
LPC & 183.49 & 2.401 & 0.8620 & 3888.072 & 1.3298 & 0.8778 & -2905.4 & 3.61 & 2.87 \\
HPC & 88.15 & 11.528 & 0.8614 & 10098.219 & 2.1289 & 0.8986 & -13334.0 & 32.00 & 30.80 \\
HPT & 12.10 & 4.300 & 0.9085 & 215.957 & 1.3562 & 0.8892 & 13334.0 & & \\
LPT & 56.05 & 4.359 & 0.9118 & 89.203 & 1.4008 & 0.8945 & 10361.9 & &
\end{tabular}

TURBOMACHINERY MAP DATA

$\begin{array}{lrrrrrrrrr} & \text { WcMap } & \text { PRmap } & \text { effMap } & \text { NcMap } & \text { R/Parm } & \text { S_WcDes } & \text { S_PRdes } & \text { S_effDes } & \text { S_NcDes } \\ \text { Fan } & 1176.64 & 1.566 & 0.9112 & 3850.999 & 0.0597 & 0.8607 & 1.0235 & 0.9980 & 0.9905 \\ \text { LPC } & 146.06 & 2.477 & 0.8217 & 1.027 & 0.0000 & 1.2562 & 0.9482 & 1.0491 & 0.0003 \\ \text { HPC } & 80.17 & 11.032 & 0.8703 & 9829.008 & 12.9619 & 1.0995 & 1.0494 & 0.9898 & 0.9733 \\ \text { HPT } & 0.96 & 4.208 & 0.9085 & 1.298 & 4.2082 & 12.6299 & 0.9723 & 1.0000 & 0.0003 \\ \text { LPT } & 0.86 & 3.526 & 0.9185 & 1.003 & 3.5263 & 65.2590 & 0.7521 & 0.9926 & 0.0005\end{array}$




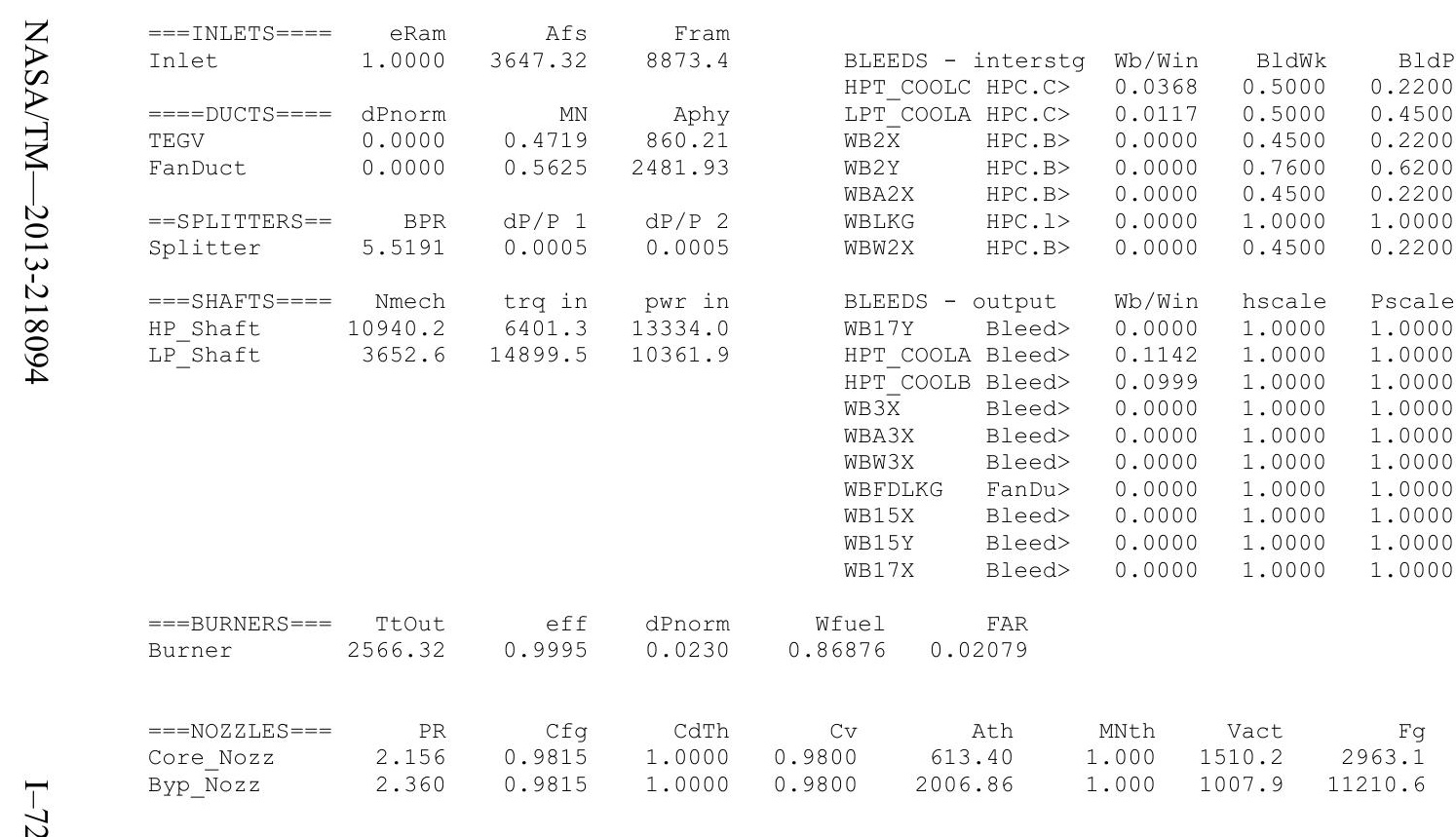


Date:07/05/13 Time:09:59:54 Model:

Version:NPSS 1.6.5 - Rev: -> Gas Package: Janaf iter/pass/Jacb/Broy=24/38/1/22 Run by: Philip C Jorgenson PC: 100

Temperature Stator 1 inlet: 463.71 Stator 1 exit: 483.17 Stator 2 inlet: 505.69 Stator 2 exit: 513.14

Stator 3 inlet: 543.36 Stator 3 exit: 551.66 stator 4 inlet: 579.93 stator 4 exit: 587.99

Ambient Relative Humidity $\quad 10.00$

Fan Face Relative Humidity $\quad 5.44$

LPC Inl Relative Humidity $\quad 2.52$

LPC Exit Relative Humidity 2.52

Drop Diameter

Dropient

0.02
0.00

Ambient Stow Velocity

0.0001000

789.49

Inlet Length

0.00

Ambient Static Pressure

Additional

425.97 Fan/LPC Inlet Static Temperature 457.44

Fan/LPC Inlet Flow Velocity $\quad 495.72$

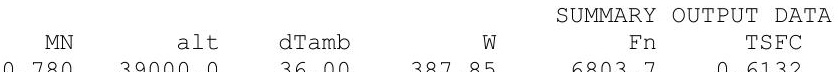

$\begin{array}{lrrrrr}0.780 & 39000.0 & 36.00 & 387.85 & 6803.7 & 0.6132\end{array}$

FLOW STATION DATA

\begin{tabular}{|c|c|}
\hline & ent.Fl \\
\hline FS1 & Inlet.FI 0 \\
\hline FS12 & Splitter.Fl_02 \\
\hline FS2 & Splitter.Fl-01 \\
\hline FS14 & Fan.Fl_o \\
\hline FS23 & LPC.FI_O \\
\hline FS24 & VaporIN. FI O \\
\hline FS25 & Bleed2.FI_o \\
\hline FS3 & HPC.FI O \\
\hline FS36 & Bleed3.FI O \\
\hline FS4 & Burner.FI-O \\
\hline FS45 & HPT.FI_O \\
\hline FS49 & LPT.FI_O \\
\hline FS5 & TEGV.FI \\
\hline FS8 & Core_Nozz. \\
\hline FS17 & FanDúctLkg.Fì \\
\hline FS171 & Bleed15.F1 O \\
\hline FS172 & FanDuct.FI- \\
\hline FS173 & Byp Nozz.F $\bar{l}$ \\
\hline
\end{tabular}

TURBOMACHINERY PERFORMANCE DATA

$\begin{array}{rrr} & \text { WC } & \text { PR } \\ \text { Fan } & 1078.79 & 1.702 \\ \text { LPC } & 204.18 & 2.457 \\ \text { HPC } & 96.75 & 13.142 \\ \text { HPT } & 12.10 & 4.272 \\ \text { LPT } & 55.83 & 4.343\end{array}$

$\begin{array}{rrr}\text { W } & \text { Pt } \\ 387.85 & 4.267 \\ 387.85 & 4.267 \\ 326.13 & 4.265 \\ 61.72 & 4.265 \\ 326.13 & 7.257 \\ 61.72 & 10.478 \\ 61.72 & 10.478 \\ 61.72 & 10.478 & \\ 58.73 & 137.698 & 1 \\ 45.52 & 137.698 & 1 \\ 46.67 & 134.527 & 2 \\ 62.16 & 31.490 & 1 \\ 62.88 & 7.250 & 1 \\ 62.88 & 7.250 & 1 \\ 62.88 & 7.250 & 138 \\ 326.13 & 7.257 & \\ 326.13 & 7.257 & \\ 326.13 & 7.257 & \\ 326.13 & 7.257 & \\ & & \end{array}$

$\begin{array}{rrrr}\text { Tt } & \text { ht } & \text { FAR } & \text { Wc } \\ 477.92 & -16.44 & 0.0000 & 1282.33 \\ 477.92 & -16.44 & 0.0000 & 1282.33 \\ 477.92 & -16.44 & 0.0000 & 1078.79 \\ 477.92 & -16.44 & 0.0000 & 204.18 \\ 563.97 & 4.20 & 0.0000 & 688.63 \\ 647.73 & 24.35 & 0.0000 & 96.75 \\ 647.80 & 24.35 & 0.0000 & 96.75 \\ 647.80 & 24.35 & 0.0000 & 96.75 \\ 427.61 & 219.58 & 0.0000 & 10.40 \\ 427.61 & 219.58 & 0.0000 & 8.06 \\ 922.48 & 187.34 & 0.0255 & 12.10 \\ 921.38 & 2.65 & 0.0190 & 55.83 \\ 379.95 & -143.09 & 0.0188 & 207.90 \\ 380.01 & -143.09 & 0.0188 & 207.91 \\ 380.14 & -143.09 & 0.0188 & 207.92 \\ 563.97 & 4.20 & 0.0000 & 688.63 \\ 563.97 & 4.20 & 0.0000 & 688.63 \\ 563.97 & 4.20 & 0.0000 & 688.63 \\ 563.97 & 4.20 & 0.0000 & 688.63\end{array}$

$\begin{array}{rr}\text { Wfuel } & \text { BPR } \\ 4171.96 & 5.2836\end{array}$

$\mathrm{OPR}$
32.273

$\begin{array}{cc}\text { T4 } & \text { T41 } \\ 2922.5 & 2743.5\end{array}$

T49

$\begin{array}{rr}\text { PS } & \text { TS } \\ 2.854 & 425.97 \\ 3.150 & 438.17 \\ 3.174 & 439.19 \\ 3.659 & 457.44 \\ 6.025 & 534.77 \\ 9.151 & 623.23 \\ 9.150 & 623.29 \\ 9.150 & 623.29 \\ 124.228 & 1389.59 \\ 129.872 & 1405.91 \\ 127.105 & 2885.75 \\ 28.370 & 1873.53 \\ 6.256 & 1328.62 \\ 6.256 & 1328.69 \\ 3.882 & 1173.55 \\ 6.025 & 534.77 \\ 5.854 & 530.41 \\ 5.854 & 530.41 \\ 3.833 & 469.91\end{array}$

$\begin{array}{rr}\text { Aphy } & \text { MN } \\ 3912.1 & 0.7800 \\ 4168.2 & 0.6727 \\ 3531.8 & 0.6634 \\ 830.5 & 0.4726 \\ 2606.7 & 0.5227 \\ 412.6 & 0.4444 \\ 412.6 & 0.4444 \\ 412.6 & 0.4444 \\ 49.7 & 0.3922 \\ 49.3 & 0.2950 \\ 74.6 & 0.3007 \\ 265.4 & 0.4005 \\ 860.2 & 0.4725 \\ 860.2 & 0.4725 \\ 613.4 & 1.0000 \\ 2606.7 & 0.5227 \\ 2481.9 & 0.5626 \\ 2481.9 & 0.5626 \\ 2006.9 & 1.0000\end{array}$

gamt
1.40068
1.40068
1.40068
1.40068
1.39952
1.39757
1.39757
1.39757
1.35438
1.35438
1.28096
1.31732
1.34413
1.34413
1.34412
1.39952
1.39952
1.39952
1.39952

WAR
0.0000851 0.0000851 0.0000851 0.0000851 0.0000851 0.0000851 0.0000888 0.0000888 0.0000888 0.0000888 0.0000888 0.0000888 0.0000888 0.0000888 0.0000888 0.0000851 0.0000851 0.0000851 0.0000851

$\begin{array}{rrr}\text { Wair } & \text { WH2O } & \text { H2O frac } \\ 387.82 & 0.033 & 0.0001 \\ 387.82 & 0.033 & 0.0001 \\ 326.10 & 0.028 & 0.0001 \\ 61.72 & 0.005 & 0.0001 \\ 326.10 & 0.028 & 0.0001 \\ 61.72 & 0.005 & 0.0001 \\ 61.72 & 0.005 & 0.0001 \\ 61.72 & 0.005 & 0.0001 \\ 58.73 & 0.005 & 0.0001 \\ 45.51 & 0.004 & 0.0001 \\ 45.51 & 0.004 & 0.0307 \\ 61.00 & 0.005 & 0.0231 \\ 61.72 & 0.005 & 0.0228 \\ 61.72 & 0.005 & 0.0228 \\ 61.72 & 0.005 & 0.0228 \\ 326.10 & 0.028 & 0.0001 \\ 326.10 & 0.028 & 0.0001 \\ 326.10 & 0.028 & 0.0001 \\ 326.10 & 0.028 & 0.0001\end{array}$

TURBOMACHINERY MAP DATA

$\begin{array}{lrrrrrrrrr} & \text { WcMap } & \text { PRmap } & \text { effMap } & \text { NcMap } & \text { R/Parm } & \text { S_WcDes } & \text { S_PRdes } & \text { s_effDes } & \text { S_NcDes } \\ \text { Fan } & 1253.45 & 1.686 & 0.9132 & 4100.000 & 0.0639 & 0.8607 & 1.0235 & 0.9980 & 0.9905 \\ \text { LPC } & 166.84 & 2.748 & 0.8341 & 1.093 & 0.0000 & 1.2238 & 0.8335 & 0.9870 & 0.0003 \\ \text { HPC } & 88.00 & 12.570 & 0.8673 & 10087.563 & 13.4980 & 1.0995 & 1.0494 & 0.9898 & 0.9733 \\ \text { HPT } & 0.96 & 4.181 & 0.9122 & 1.288 & 4.1813 & 12.6299 & 0.9723 & 1.0000 & 0.0003 \\ \text { LPT } & 0.86 & 3.515 & 0.9235 & 1.020 & 3.5146 & 65.2590 & 0.7521 & 0.9926 & 0.0005\end{array}$




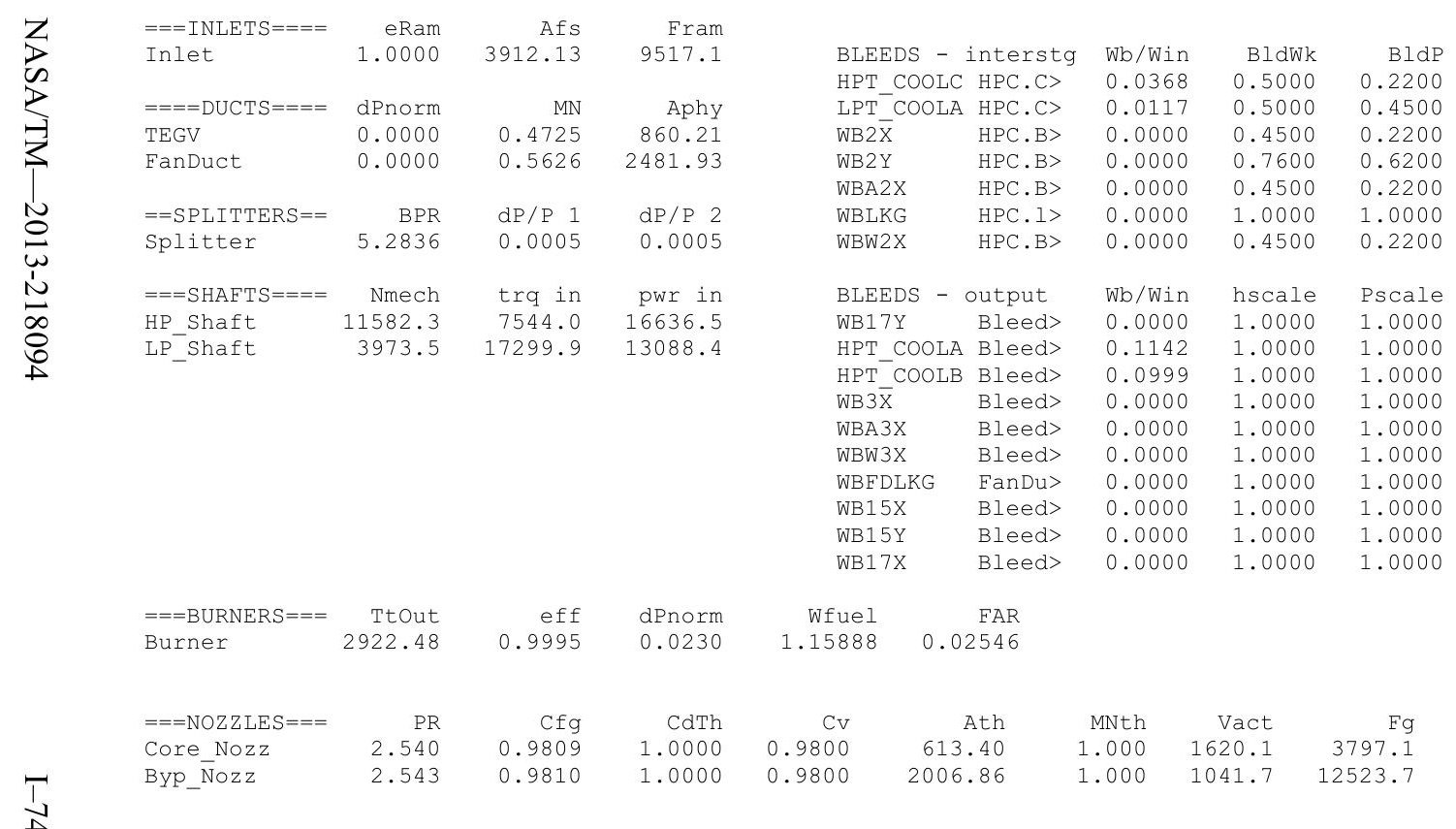


Date: 07/05/13 Time: 10:00:50 Model:

Turbofan Engine

converge $=1 \quad$ CASE: $\quad 0$

Version:NPSS 1.6.5 - Rev: -> Gas Package: Janaf iter/pass/Jacb/Broy= 25/53/2/22 Run by: Philip C Jorgenson PC: 84 Stator 3 inle: 54.54 Stator 1 exit: 487.62 Stat 2 inlet: 508.97 Stator 4 exit: 517.196 Stator 5 inlet: 605.83 Stator 5 exit: 613.44 Unblocked Percent Blockage: 0.00

Ambient Relative Humidity 10.00

Fan Face Relative Humidity

Fan Bypass Relative Humidity

LPC Inlet Relative Humidity

LPC Exit Relative Humidity

Drop Diameter

4.44

0.23

0.03 0.00

Ambient Static Pressure

Additional Water at LPC Exit

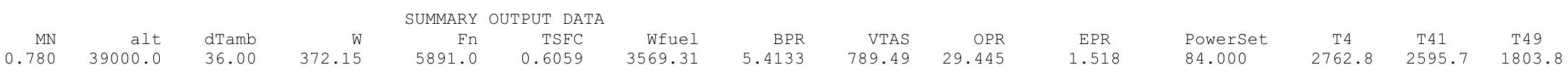

FS0 Ambient.F1_O

ES1 Inlet.FI_O

FS12 Splitter.F1_02

FS14 Fan.FI_O

(t)

FS24 VaporIN. FI_O

FS25 Bleed2. F1

FS36 Bleed3.Fl

FS4 Burner.F1-O

FS45 HPT.FIO

FS49 LPT.FIO

FS8 Core_Nozz.FI_O

FS17 FanDüctLkg.F̄__o

FS171 Bleed15.FI_O

FS173 FanDuct. FI-O

TURBOMACHINERY PERFORMANCE DATA

\begin{tabular}{|c|c|c|c|c|c|c|c|c|c|c|}
\hline \multicolumn{11}{|c|}{ ATION DATE } \\
\hline & & $\begin{array}{r}\text { W } \\
372.15\end{array}$ & Pt & $\mathrm{Tt}$ & ht & FA & W & & $\begin{array}{l}\text { Ps } \\
54\end{array}$ \\
\hline $\begin{array}{l}\text { FS0 } \\
\text { FS1 }\end{array}$ & \multicolumn{2}{|l|}{ Ambient.FI_O } & $\begin{array}{l}372.15 \\
372.15\end{array}$ & $\begin{array}{l}4.267 \\
4.267\end{array}$ & $\begin{array}{l}477.92 \\
477.92\end{array}$ & $\begin{array}{l}-16.44 \\
-16.44\end{array}$ & 0.000 & $\begin{array}{l}1230.4 \\
1230.4\end{array}$ & $\begin{array}{l}2.8 \\
3.2\end{array}$ & $\begin{array}{l}425 \\
443\end{array}$ \\
\hline 12 & \multicolumn{2}{|c|}{ Splitter.F1 02} & 314.12 & 4.265 & 477.92 & -16.44 & 0.000 & 1039.0 & 3.2 & \\
\hline 2 & \multicolumn{2}{|c|}{ Splitter.FI_01 } & 58.03 & 4.265 & 477.92 & -16.44 & 0.000 & 191.9 & 3.7 & \\
\hline 14 & \multicolumn{2}{|c|}{ Fan.Fl 0} & 314.12 & 6.942 & 556.22 & 2.34 & 0.000 & 688.6 & 5.7 & \\
\hline 23 & \multicolumn{2}{|l|}{ LPC.FI-O } & 8.03 & 10.354 & 639.92 & 22.47 & 0.000 & 91.4 & 9.2 & \\
\hline 24 & \multicolumn{2}{|l|}{ VaporIN..$F 10$} & 3 & 10.354 & 639.98 & 22.46 & 0.0 & 91. & 9.2 & \\
\hline 825 & \multicolumn{2}{|l|}{ Bleed2.Fl $\bar{O}$} & 58.03 & 10.354 & 639.98 & 22.46 & 0.000 & 91.4 & 9.2 & 04 \\
\hline 3 & \multicolumn{2}{|l|}{ HPC.FIO } & & 125.632 & 378.37 & 206.71 & & 10. & 12.9 & \\
\hline 36 & \multicolumn{2}{|l|}{ Bleed3.Fl_o } & & 25.632 & 378.37 & 206.71 & & 8. & 18.3 & \\
\hline 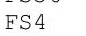 & \multicolumn{2}{|l|}{ Burner.Fl-o } & & 122.739 & .79 & 177.76 & 0. & 12.1 & 115.9 & \\
\hline 545 & \multicolumn{2}{|l|}{ HPT.FI_O } & 4 & 28.642 & 31 & 2.80 & & 55.9 & 25.7 & \\
\hline 49 & \multirow{2}{*}{\multicolumn{2}{|c|}{$\begin{array}{l}\text { LPT.FI-O } \\
\text { TEGV.FIOO }\end{array}$}} & & 6.587 & .04 & 134.14 & r & 208.2 & 5.6 & \\
\hline 5 & & & 6.587 & 129 & -134.14 & & 20 & 3 & \\
\hline 8 & & & & 6.587 & 1297.23 & $-134 \cdot 14$ & & 208 & 3. & \\
\hline 17 & \multicolumn{2}{|c|}{ FanDuctLkg.Fí } & & 6.942 & & 2.34 & 0 . & D & 5.7 & \\
\hline 171 & \multicolumn{2}{|c|}{ Bleed15.FI O- } & & 6.942 & & 2.34 & & & 5. & \\
\hline 172 & \multicolumn{2}{|l|}{ FanDuct.FI_o } & & 6.942 & 556.22 & 2.34 & 0.0 & 688 & 5.6 & \\
\hline$\$ 173$ & \multicolumn{2}{|l|}{ Byp_Nozz.FI_O } & 314.12 & 6.942 & 556.22 & 2.34 & 0.000 & 688.6 & 3.6 & \\
\hline \multirow{2}{*}{\multicolumn{3}{|c|}{ JRBOMACH INERY }} & D & & & & \multirow{2}{*}{\multicolumn{2}{|c|}{ pwr }} & \multirow{2}{*}{\multicolumn{2}{|c|}{ SMTH }} \\
\hline & & & $=f$ & NC & TR & efPoly & & & & \\
\hline$n$ & 1039.08 & 1.0 & 20 & 82.695 & 638 & 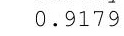 & \multirow{2}{*}{\multicolumn{2}{|c|}{-8347.4}} & & \\
\hline${ }_{\mathrm{C}}^{\mathrm{C}}$ & 191.95 & & & 3982.695 & 390 & 6 & & & & 0 \\
\hline$c_{0}$ & 91.48 & 12.133 & 6 & 10189.614 & 2.1538 & 0.8985 & \multicolumn{2}{|c|}{-14760.0} & 29.68 & 28.68 \\
\hline $\mathrm{T}$ & 12.10 & 4.2 & & 215.339 & & 0.8905 & 14 & 760.0 & & \\
\hline $\mathrm{T}$ & 55.95 & 4.348 & 0.9147 & 89.803 & 1.3941 & 0.8982 & 11 & 542.0 & & \\
\hline R & ACHINERY & DA & & & & & & & & \\
\hline & WcMap & & effMap & NcMap & R/Parm & s_t & Des & S_PRdes & S_effDes & S_NcDe \\
\hline & 1207.30 & 13 & & 3944.720 & & & 267078 & $\overline{1} .0$ & & \\
\hline & 152.37 & 2.575 & & 1.052 & 0.0000 & & 2597 & 0.9066 & 1.0320 & 0.0 \\
\hline${ }^{\mathrm{C}} \mathrm{C}$ & 83.20 & 11.609 & 0.8695 & 9917.965 & 13.1591 & & 0995 & 1.0494 & 0.9898 & \\
\hline & 0.96 & 4.194 & 9109 & 1.294 & 4.1942 & & 6000 & 0.9723 & 1.0000 & 0.0003 \\
\hline & $0.86 \mathrm{r}$ & 3.518 & .9215 & .010 & & & & .7521 & & \\
\hline
\end{tabular}

TURBOMACHINERY MAP DATA

\begin{tabular}{|c|c|c|c|c|c|c|c|c|c|c|}
\hline & & & & FLOW S & STATION DA & & & & & \\
\hline & & & $\begin{array}{r}W \\
372.15\end{array}$ & Pt & $\mathrm{Tt}$ & ht & FA & R Wc & & $\begin{array}{l}\text { Ps } \\
54\end{array}$ \\
\hline $\begin{array}{l}\text { FS0 } \\
\text { FS1 }\end{array}$ & $\begin{array}{l}\text { Ambient.Fl_o } \\
\text { Inlet.Fl }\end{array}$ & & $\begin{array}{l}372.15 \\
372.15\end{array}$ & $\begin{array}{l}4.267 \\
4.267\end{array}$ & $\begin{array}{l}477.92 \\
477.92\end{array}$ & $\begin{array}{l}-16.44 \\
-16.44\end{array}$ & 0.000 & $\begin{array}{ll}0 & 1230.4 \\
0 & 1230.4\end{array}$ & $\begin{array}{l}2.8 \\
3.2\end{array}$ & $\begin{array}{l}425 \\
443\end{array}$ \\
\hline$\$ 12$ & Splitter.FI_ & & 314.12 & 4.265 & 477.92 & -16.44 & 0.000 & 1039.08 & 3.2 & \\
\hline 32 & Splitter.FI- & & 58.03 & 4.265 & 477.92 & -16.44 & 0.000 & 191.9 & 3.7 & 460 \\
\hline 514 & Fan.Fl_o & & 314.12 & 6.942 & 556.22 & 2.34 & 0.000 & 688.65 & 5.7 & 63 \\
\hline 523 & LPC.FI_O & & 58.03 & 10.354 & 639.92 & 22.47 & 0.000 & $91.4 \varepsilon$ & 9.2 & \\
\hline S24 & VaporIñ.Fl_o & & 58.03 & 10.354 & 639.98 & 22.46 & 0.000 & $91.4 \varepsilon$ & 9.2 & \\
\hline S25 & Bleed2.FI_ō & & 58.03 & 10.354 & 639.98 & 22.46 & 0.000 & $91.4 \xi \quad 4$ & 9.2 & 618 \\
\hline FS3 & HPC.FI_O & & 55.21 & 125.632 & 1378.37 & 206.71 & 0.000 & 10.53 & 112.9 & $134 \mathrm{C}$ \\
\hline s36 & Bleed3.Fl_o & & 42.79 & 125.632 & 1378.37 & 206.71 & 0.000 & 8.16 & 118.3 & 135 \\
\hline S4 & Burner.FI-o & & 43.78 & 122.739 & 2762.79 & 177.76 & 0.023 & $12.1 \mathrm{c}$ & 115.9 & 2727 \\
\hline S45 & HPT.FI_O & & 58.34 & 28.642 & 1812.31 & 2.80 & 0.017 & 55.95 & 25.7 & \\
\hline$\$ 49$ & LPT.FI_O & & 59.02 & 6.587 & 1297.04 & $-134 \cdot 14$ & 0.017 & 208.22 & 5.6 & 81 \\
\hline 55 & TEGV.FI O & & 59.02 & 6.587 & 1297.10 & -134.14 & 0.017 & 208.2 & 5.6 & \\
\hline S8 & Core_Nozz.FI & & 59.02 & 6.587 & 1297.23 & -134.14 & 0.017 & 208.2 & 3.5 & 110 \\
\hline S17 & FanDüctLkg. F & & 314.12 & 6.942 & 556.22 & 2.34 & 0.000 & $688.65-5$ & 5.7 & \\
\hline$\$ 171$ & Bleed15.FI_O & & 3 & 6.942 & 556.22 & 2.34 & 0. & 688.65 & 5.6 & \\
\hline 5172 & FanDuct.FI_O & & 314.12 & 6.942 & 556.22 & 2.34 & 0.000 & $688.65-5$ & 5.6 & \\
\hline S173 & Byp_Nozz.Fi _ & & 314.12 & 6.942 & 556.22 & 2.34 & 0.000 & 688.65 & 3.6 & \\
\hline RD & CHINERY PER & ORMANCE & DATA & & & & & & & \\
\hline & & $\mathrm{PR}$ & eff & $\mathrm{NC}$ & TR & efPoly & & pwr & SMN & SMW \\
\hline an & 1039.08 & 1.628 & 0.9120 & 3982.695 & 1.1638 & 0.9179 & & 347.4 & 51.73 & 26.15 \\
\hline${ }_{\mathrm{C}}^{\mathrm{C}}$ & 191.95 & 2.428 & 0.8502 & 3982.695 & 1.3390 & 0.8676 & & 194.5 & 2.91 & 2.10 \\
\hline${ }^{\mathrm{C}} \mathrm{C}$ & 91.48 & 12.133 & 0.8606 & 10189.614 & 2.1538 & 0.8985 & -14 & 760.0 & 29.68 & 28.68 \\
\hline $\mathrm{eT}$ & 12.10 & 4.285 & 0.9109 & 215.339 & 1.3490 & 0.8905 & & 760.0 & & \\
\hline $\mathrm{PT}$ & 55.95 & 4.348 & 0.9147 & 89.803 & 1.3941 & 0.8982 & & 542.0 & & \\
\hline RB & MACHINERY MAP & DAT & & & & & & & & \\
\hline & WcMap & PRmap & effMap & NcMap & R/Parm & $s_{1}$ & cDes & S_PRdes & S_effDes & S_NCDe \\
\hline$x^{2}$ & 1207.30 & 1.613 & 0.9139 & 3944.720 & 0.0614 & & 3607 & $\overline{1} .0235$ & 0.9980 & $\overline{0} .9905$ \\
\hline C & 152.37 & 2.575 & 0.8239 & 1.052 & 0.0000 & & 2597 & 0.9066 & 1.0320 & 0.0003 \\
\hline PC & 83.20 & 11.609 & 0.8695 & 9917.965 & 13.1591 & & 0995 & 1.0494 & 0.9898 & 0.9733 \\
\hline PT & 0.96 & 4.194 & .9109 & 1.294 & 4.1942 & 12. & 6299 & 0.9723 & 1.0000 & 0.000 \\
\hline $\mathrm{PT}$ & 0.86 & 3.518 & 0.9215 & 1.010 & 3.5183 & 65. & 2590 & 0.7521 & 0.9926 & 0.0005 \\
\hline
\end{tabular}

gamt
1.40068
1.40068
1.40068
1.40068
1.39966
1.39779
1.39779
1.39779
1.35719
1.35719
1.28688
1.32302
1.35025
1.35025
1.35024
1.39966
1.39966
1.39966
1.39966

WAR
0.0000851 0.0000851 0.0000851 0.0000851 0.0000851 0.0000851 0.00081 0.000891 0.0000891 0.00000891
0.0000891 0.0000891 0.00000891 0.0000891 0.0000891 0.0000851 0.0000851 0.0000851 0.0000851

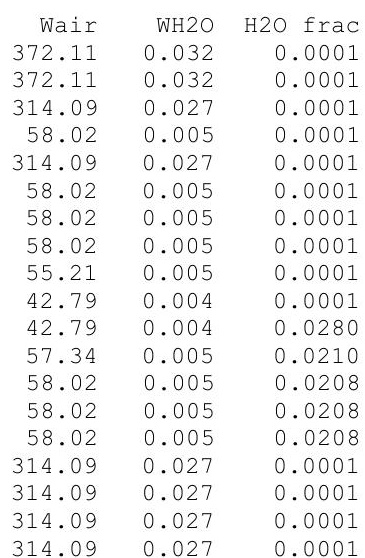

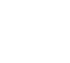




\begin{tabular}{|c|c|c|c|}
\hline$===$ INLETS $====$ & eRam & Afs & Fram \\
\hline Inlet & 1.0000 & 3753.73 & 9131.7 \\
\hline$====$ DUCTS $====$ & dPnorm & MN & Aphy \\
\hline TEGV & 0.0000 & 0.4723 & 860.21 \\
\hline FanDuct & 0.0000 & 0.5626 & 2481.93 \\
\hline$==$ SPLITTERS $==$ & BPR & $\mathrm{dP} / \mathrm{P} 1$ & $\mathrm{dP} / \mathrm{P} 2$ \\
\hline Splitter & 5.4133 & 0.0005 & 0.0005 \\
\hline$===$ SHAFTS $====$ & Nmech & trq in & pwr in \\
\hline HP Shaft & 11318.7 & 6848.9 & 14760.0 \\
\hline LP_Shaft & 3823.0 & 15856.4 & 11542.0 \\
\hline
\end{tabular}

\begin{tabular}{|c|c|c|c|c|}
\hline BLEEDS - & interstg & Wb/Win & BldWk & $\mathrm{BldP}$ \\
\hline HPT COOLC & HPC.C> & 0.0368 & 0.5000 & 0.2200 \\
\hline LPT_COOLA & HPC.C> & 0.0117 & 0.5000 & 0.4500 \\
\hline WB $2 \overline{\mathrm{X}}$ & HPC.B> & 0.0000 & 0.4500 & 0.2200 \\
\hline WB2Y & HPC.B> & 0.0000 & 0.7600 & 0.6200 \\
\hline WBA2X & HPC.B> & 0.0000 & 0.4500 & 0.2200 \\
\hline WBLKG & HPC. $1>$ & 0.0000 & 1.0000 & 1.0000 \\
\hline WBW2X & HPC.B> & 0.0000 & 0.4500 & 0.2200 \\
\hline BLEEDS - & output & Wb/Win & hscale & Pscale \\
\hline WB17Y & Bleed> & 0.0000 & 1.0000 & 1.0000 \\
\hline HPT_COOLA & Bleed> & 0.1142 & 1.0000 & 1.0000 \\
\hline $\mathrm{HPT}^{-} \mathrm{COOLB}$ & Bleed> & 0.0999 & 1.0000 & 1.0000 \\
\hline WB $3 \bar{x}$ & Bleed> & 0.0000 & 1.0000 & 1.0000 \\
\hline WBA3X & Bleed> & 0.0000 & 1.0000 & 1.0000 \\
\hline WBW3X & Bleed> & 0.0000 & 1.0000 & 1.0000 \\
\hline WBFDLKG & FanDu> & 0.0000 & 1.0000 & 1.0000 \\
\hline WB15X & Bleed> & 0.0000 & 1.0000 & 1.0000 \\
\hline WB15Y & Bleed> & 0.0000 & 1.0000 & 1.0000 \\
\hline WB17X & Bleed> & 0.0000 & 1.0000 & 1.0000 \\
\hline
\end{tabular}

$\begin{array}{rrrr}\text { W } & \text { Tt } & \text { ht } & \text { Pt } \\ 2.1354 & 1016.81 & 114.59 & 28.642 \\ 0.6789 & 1016.81 & 114.59 & 6.587 \\ 0.0000 & 979.75 & 105.37 & 35.715 \\ 0.0000 & 1206.85 & 162.49 & 81.826 \\ 0.0000 & 979.75 & 105.37 & 35.715 \\ 0.0000 & 1378.37 & 206.71 & 125.632 \\ 0.0000 & 979.75 & 105.37 & 35.715 \\ \text { W } & \text { Tt } & \text { ht } & \text { Pt } \\ 0.0000 & 639.98 & 22.46 & 10.354 \\ 6.6255 & 1378.37 & 206.71 & 122.739 \\ 5.7975 & 1378.37 & 206.71 & 68.916 \\ 0.0000 & 1378.37 & 206.71 & 125.632 \\ 0.0000 & 1378.37 & 206.71 & 125.632 \\ 0.0000 & 1378.37 & 206.71 & 125.632 \\ 0.0000 & 556.22 & 2.34 & 6.942 \\ 0.0000 & 556.22 & 2.34 & 6.942 \\ 0.0000 & 556.22 & 2.34 & 6.942 \\ 0.0000 & 556.22 & 2.34 & 6.942\end{array}$

$===$ BURNERS $===\begin{array}{r}\text { Ttout } \\ \text { Burner }\end{array}$
2762.79

$\begin{array}{rr}\text { eff } & \text { dPnorm } \\ 0.9995 & 0.0230\end{array}$

Wfuel

FAR

$===$ NOZZLES $S===$

PR
2.308
2.432

Cfg
0.9816
0.9814

CdTh
1.0000
1.0000

$\mathrm{CV}$
0.9800

Ath
613.40

$\begin{array}{rrr}\text { MNth } & \text { Vact } & \mathrm{Fg} \\ 1.000 & 1572.1 & 3292.5\end{array}$ 
Date: 07/05/13 Time:10:01:41 Model:

converge $=1$ CASE: 0

Version:NPSS 1.6.5 - Rev: -> Gas Package: Janaf iter/pass/Jacb/Broy=21/49/2/18 Run by: Philip C Jorgenson PC: 79

Temperature Stator 1 inlet: 471.31 Stator 1 exit: 488.90 Stator 2 inlet: 509.91 Stator 2 exit: 518.28 Stator 3 inlet: $544.23 \quad$ Stator 3 exit: 552.77 Stator 4 inlet: $577.30 \quad$ Stator 4 exit: 585.21

Ambient Relative Humidity $\quad 10.00$

Fan Face Relative Humidity 4.19

Fan Bypass Relative Humidity 0.25

LPC Inlet Relative Humidity

LPC Exit Relative Humidity

Delative Humidity

0.00

Ambient Flow Velocity

Ambient Static Pressure

Additional Water at LPC Exit

Inlet Length

40.00

2.85

446.09

0.0000042

Fan/LPC Inlet Static Pressure $\quad 3.77$

n/LPC Inlet Static Temperature 461.34

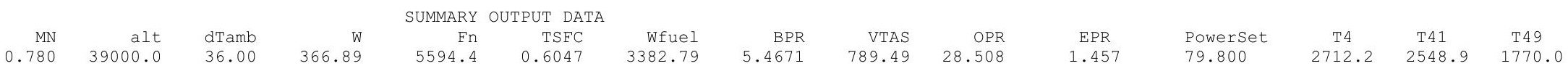

FS0 Ambient.F1_O

ES1 Inlet.F1_O

FS2 Splitter.F1-01

FS14 Fan.FI_O

(S25

FS25 Bleed2.F1-

536 Hleed3. 1

FS4 Burner.FI-

FS45 HPT.FI O

FS49 LPT.FIO

FS5 TEGV.Fì O

FS8 Core_Nozz.El_O

ES17 FanDüctLkg.F̄__o

FS171 Bleed15.Fl_O

FS173 Byp Nozz. Fi

TURBOMACHINERY PERFORMANCE DATA

\begin{tabular}{|c|c|c|c|c|c|c|c|c|c|c|}
\hline & & & W & $\begin{array}{l}\text { FLOW } \\
\text { Pt }\end{array}$ & $\begin{array}{c}\text { STATION DAT } \\
\text { Tt }\end{array}$ & TA ht & FA & Wo & & Ps \\
\hline so & Ambient.Fl_O & & 366.89 & 4.267 & 477.92 & -16.44 & 0.000 & 1213.03 & 2.85 & $425.9^{-}-x$ \\
\hline & Inlet.FI_o- & & 366.89 & 4.267 & 477.92 & -16.44 & 0.000 & 1213.03 & 3.32 & 444.9 \\
\hline$\$ 12$ & Splitter.Fl & & 310.16 & 4.265 & 477.92 & -16.44 & 0.000 & 1025.97 & 3.32 & 445.18 \\
\hline S2 & Splitter.Fl_o & & 56.73 & 4.265 & 477.92 & -16.44 & 0.000 & 187.66 & 3.76 & 461.3 \\
\hline 14 & Fan.Fl_O & & 310.16 & 6.838 & 553.67 & 1.73 & 0.000 & 688.65 & 5.67 & 524.9 \\
\hline 23 & LPC.Fl_O & & 56.73 & 10.299 & 637.57 & 21.91 & 0.000 & 89.75 & 9.20 & 617.4 \\
\hline 24 & VaporIN̄.FI_O & & 56.73 & 10.299 & 637.64 & 21.90 & 0.000 & 89.76 & 9.20 & 617. \\
\hline 25 & Bleed2.Fl_o & & 56.73 & 10.299 & 637.64 & 21.90 & 0.000 & 89.76 & 9.20 & 617 \\
\hline & HPC.Fl_O & & 53.98 & 121.637 & 1362.67 & 202.62 & 0.000 & 10.57 & 109.28 & 1324. \\
\hline 36 & Bleed3.Fl O & & 41.83 & 121.637 & 1362.67 & 202.62 & 0.000 & 8.19 & 114.47 & 1341. \\
\hline 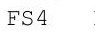 & Burner.Fl_o & & 42.77 & 118.836 & 2712.15 & 174.67 & 0.022 & 12.10 & 112.26 & 2677.3 \\
\hline 45 & HPT.FI_O & & 57.01 & 27.706 & 1778.19 & 2.85 & 0.016 & 55.99 & 24.94 & 1732. \\
\hline 49 & LPT.FI-O & & 57.67 & 6.369 & 1271.25 & -131.33 & 0.016 & 208.32 & 5.49 & 1222 \\
\hline 19 & TEGV.FI O & & 57.67 & 6.369 & 1271.31 & -131.33 & 0.016 & 208.32 & 5.49 & 1223. \\
\hline FS 8 & Core_Nozz.Fl & & 57.67 & 6.369 & 1271.44 & -131.33 & 0.016 & 208.34 & 3.40 & 1077. \\
\hline 17 & FanDūctLkg.F & & 310.16 & 6.838 & 553.67 & 1.73 & 0.0 & 688.65 & 5.67 & 524 \\
\hline & Bleed15.FI_o & & 310. & 6.838 & 553.67 & 1.73 & 0.000 & 688.65 & 5.51 & \\
\hline 172 & FanDuct.FI_O & & 310.16 & 6.838 & 553.67 & 1.73 & 0.000 & 688.65 & 5.51 & 520 \\
\hline 173 & Byp_Nozz.Fin_c & & 310.16 & 6.838 & 553.67 & 1.73 & 0.000 & 688.65 & 3.61 & 461 \\
\hline & MACHINERY PERE & ORMANCE & DATA & & & & & & & \\
\hline & & PR & eff & $\mathrm{NC}$ & $T R$ & efPoly & & pwr & SMN & SMW \\
\hline & 1025.97 & 1.604 & 0.9117 & 3933.623 & 1.1585 & 0.9174 & & 73.6 & 56.33 & 27.21 \\
\hline & 187.66 & 2.415 & 0.8569 & 3933.623 & 1.3341 & 0.8733 & & 77.9 & 3.24 & 2.50 \\
\hline & 89.76 & 11.811 & 0.8612 & 10141.517 & 2.1371 & 0.8987 & -14 & 54.7 & 30.96 & 29.86 \\
\hline IPT & 12.10 & 4.289 & 0.9102 & 215.917 & 1.3509 & 0.8900 & & 54.7 & & \\
\hline & 55.99 & 4.350 & 0.9140 & 89.544 & 1.3958 & 0.8973 & & 51.5 & & \\
\hline & MACHINERY & $\mathrm{DA}^{\mathrm{T}}$ & & & & & & & & \\
\hline & WCMap & PRmap & effMap & NcMap & R/Parm & S_WC & CDes & s_PRdes & S_effDes & S_NCDes \\
\hline & 1192.08 & 1.590 & 0.9136 & 3896.116 & 0.0605 & $\overline{0} .8$ & 8607 & $\overline{1} .0235$ & 0.9980 & $\overline{0} .9905$ \\
\hline & 149.00 & 2.524 & 0.8226 & 1.039 & 0.0000 & & 2595 & 0.9285 & 1.0417 & 0.0003 \\
\hline & 81.63 & 11.301 & 0.8701 & 9871.151 & 13.0487 & 1.0 & 0995 & 1.0494 & 0.9898 & 0.9733 \\
\hline & 0.96 & 4.198 & 0.9102 & 1.298 & 4.1981 & & 6299 & 0.9723 & 1.0000 & 0.0003 \\
\hline & 0.86 & 3.519 & 0.9208 & 1.007 & 3.5195 & 65.2 & 2590 & 0.7521 & 0.9926 & 0.0005 \\
\hline
\end{tabular}

TURBOMACHINERY MAP DATA

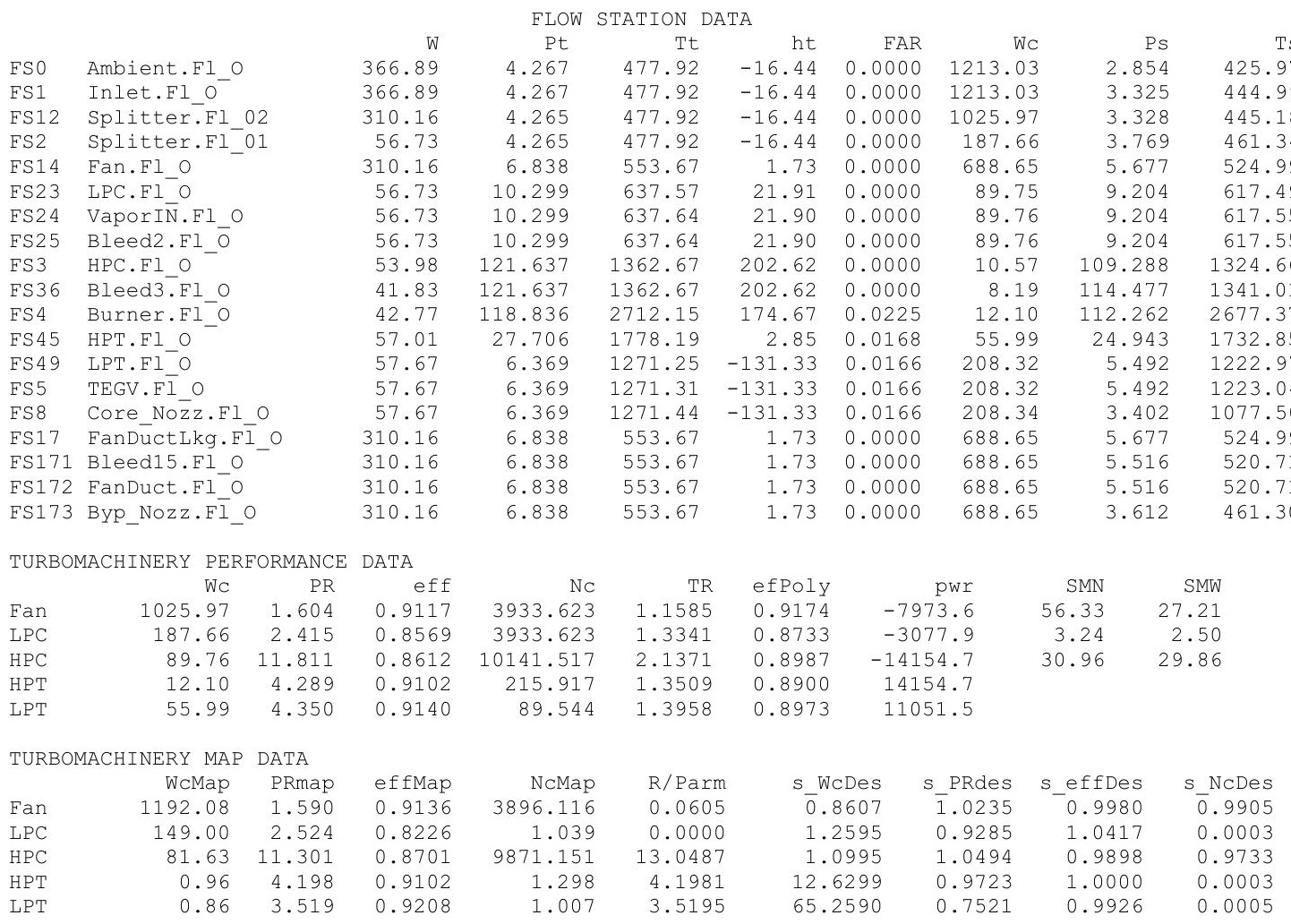

$\begin{array}{rrrrrrr}\text { Aphy } & \text { MN } & \text { gamt } & \text { WAR } & \text { Wair } & \text { WH2O } & \text { H2O frac } \\ 3700.7 & 0.7800 & 1.40068 & 0.0000851 & 366.86 & 0.031 & 0.0001 \\ 4168.2 & 0.6076 & 1.40068 & 0.0000851 & 366.86 & 0.031 & 0.0001 \\ 3531.8 & 0.6058 & 1.40068 & 0.0000851 & 310.13 & 0.026 & 0.0001 \\ 830.5 & 0.4235 & 1.40068 & 0.0000851 & 56.73 & 0.005 & 0.0001 \\ 2606.7 & 0.5226 & 1.39970 & 0.0000851 & 310.13 & 0.026 & 0.0001 \\ 412.6 & 0.4042 & 1.39785 & 0.0000851 & 56.73 & 0.005 & 0.0001 \\ 412.6 & 0.4042 & 1.39785 & 0.0000893 & 56.73 & 0.005 & 0.0001 \\ 412.6 & 0.4042 & 1.39785 & 0.0000893 & 56.73 & 0.005 & 0.0001 \\ 49.7 & 0.3995 & 1.35810 & 0.0000893 & 53.98 & 0.005 & 0.0001 \\ 49.3 & 0.3000 & 1.35810 & 0.0000893 & 41.83 & 0.004 & 0.0001 \\ 74.6 & 0.2996 & 1.28879 & 0.0000893 & 41.83 & 0.004 & 0.0272 \\ 265.4 & 0.4007 & 1.32487 & 0.0000893 & 56.06 & 0.005 & 0.0204 \\ 860.2 & 0.4722 & 1.35221 & 0.0000893 & 56.73 & 0.005 & 0.0202 \\ 860.2 & 0.4722 & 1.35221 & 0.0000893 & 56.73 & 0.005 & 0.0202 \\ 613.4 & 1.0000 & 1.35220 & 0.0000893 & 56.73 & 0.005 & 0.0202 \\ 2606.7 & 0.5226 & 1.39970 & 0.0000851 & 310.13 & 0.026 & 0.0001 \\ 2481.9 & 0.5626 & 1.39970 & 0.0000851 & 310.13 & 0.026 & 0.0001 \\ 2481.9 & 0.5626 & 1.39970 & 0.0000851 & 310.13 & 0.026 & 0.0001 \\ 2006.9 & 1.0000 & 1.39970 & 0.0000851 & 310.13 & 0.026 & 0.0001\end{array}$


$\begin{array}{lrrr}===\text { INLETS }==== & \text { eRam } & \text { Afs } & \text { Fram } \\ \text { Inlet } & 1.0000 & 3700.70 & 9002.7\end{array}$

$\begin{array}{lrrr}====\text { DUCTS }==== & \text { dPnorm } & \text { MN } & \text { Aphy } \\ \text { TEGV } & 0.0000 & 0.4722 & 860.21\end{array}$

$\begin{array}{lllr} & 0.0000 & 0.4722 & 860.21 \\ \text { FanDuct } & 0.0000 & 0.5626 & 2481.93\end{array}$

$==$ SPLITTERS $==$

Splitter

$===$ SHAFTS $===$

HP_Shaft
LP_Shaft

Nmech

$\mathrm{dP} / \mathrm{P}$

$\mathrm{dP} / \mathrm{P} 2$

$0.0005 \quad 0.0005$

trq in pwr in

$6611.3 \quad 14154.7$ $15372.0 \quad 11051.5$ Burner 2712.14

eff
0.9995

dPnorm

$===$ NOZZLES $===$

Core $\mathrm{Nozz}$

PR
2.232
2.396

Cfg
0.9816

0.9814
$===$ BURNERS $===\quad$ TtOut

BLEEDS - interstg Wb/Win Bldwk HPT COOLC HPC.C> 0.0368 $\begin{array}{lllll}\text { LPT_COOLA HPC.C> } & 0.0117 & 0.5000 & 0.4500\end{array}$ $\begin{array}{lllll}\text { WB2X } & \text { HPC.B } & 0.0000 & 0.4500 & 0.2200 \\ \text { WB2Y } & \text { HPC.B } & 0.0000 & 0.7600 & 0.6200\end{array}$ $\begin{array}{lllll}\text { WBA2X HPC.B> } & 0.0000 & 0.4500 & 0.2200\end{array}$ WBLKG HPC.1> $0.0000-1.0000-1.0000$ $\begin{array}{lllll}\text { WBW2X HPC.B> } & 0.0000 & 0.4500 & 0.2200\end{array}$

BLEEDS - output Wb/Win hscale Pscale
WB17Y Bleed> $0.0000 \quad 1.0000 \quad 1.0000$ HPT_COOLA Bleed> $0.1142 \quad 1.0000 \quad 1.0000$ HPT COOLB Bleed> $0.0999 \quad 1.0000 \quad 1.0000$

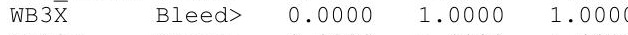
$\begin{array}{lllll}\text { WBA3X } & \text { Bleed> } & 0.0000 & 1.0000 & 1.0000 \\ \text { WBW3X } & \text { Bleed }> & 0.0000 & 1.0000 & 1.0000\end{array}$

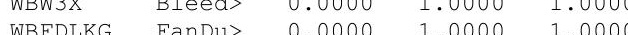
$\begin{array}{lllll}\text { WBFDLKG } & \text { FanDu> } & 0.0000 & 1.0000 & 1.00000 \\ \text { WB15X } & \text { Bleed } & 0.0000 & 1.0000 & 1.0000\end{array}$ WB15X Bleed> $0.0000-1.0000-1.0000$ $\begin{array}{lllll}\text { WB15Y } & \text { Bleed }> & 0.0000 & 1.0000 & 1.0000 \\ \text { WB17X } & \text { Bleed }> & 0.0000 & 1.0000 & 1.0000\end{array}$

Wfuel FAR $0.93966 \quad 0.02246$

$\begin{array}{rrrrrr}\text { CdTh } & \text { CV } & \text { Ath } & \text { MNth } & \text { Vact } & \text { Fg } \\ 1.0000 & 0.9800 & 613.40 & 1.000 & 1556.9 & 3126.7 \\ 1.0000 & 0.9800 & 2006.86 & 1.000 & 1032.2 & 11470.4\end{array}$

$\begin{array}{rrrr}W & \text { Tt } & \text { ht } & \text { Pt } \\ 2.0877 & 1007.47 & 112.26 & 27.706 \\ 0.6638 & 1007.47 & 112.26 & 6.369 \\ 0.0000 & 971.08 & 103.22 & 34.793 \\ 0.0000 & 1194.13 & 159.25 & 79.329 \\ 0.0000 & 971.08 & 103.22 & 34.793 \\ 0.0000 & 1362.67 & 202.62 & 121.637 \\ 0.0000 & 971.08 & 103.22 & 34.793 \\ W & T t & h t & \mathrm{Pt} \\ \text { W } & \mathrm{Tt} & \mathrm{ht} & 10.299 \\ 0.0000 & 637.64 & 21.90 & 10.836 \\ 6.4776 & 1362.67 & 202.62 & 118.836 \\ 5.6681 & 1362.67 & 202.62 & 66.709 \\ 0.0000 & 1362.67 & 202.62 & 121.637 \\ 0.0000 & 1362.67 & 202.62 & 121.637 \\ 0.0000 & 1362.67 & 202.62 & 121.637 \\ 0.0000 & 553.67 & 1.73 & 6.838 \\ 0.0000 & 553.67 & 1.73 & 6.838 \\ 0.0000 & 553.67 & 1.73 & 6.838 \\ 0.0000 & 553.67 & 1.73 & 6.838\end{array}$


Date:07/05/13 Time:10:02:15 Model: Turbofan Engine - COMDES ON Converge $=1$ CASE: 0

Version:NPSS 1.6.5 - Rev: -> Gas Package: Janaf iter/pass/Jacb/Broy=19/ 33/1/17 Run by: Philip C Jorgenson

Temperature Stator 1 inlet: $472.50 \quad$ Stator 1 exit: $489.80 \quad$ Stator 2 inlet: 510.61 Stator 2 exit: 519.07 $\begin{array}{ll}\text { Stator } 3 \text { inlet: } 544.45 & \text { Stator } 3 \text { exit: } 553.00 \quad \text { Stator } 4 \text { inlet: } 577.00 \text { Stator } 4 \text { exit: } 584.89 \\ \text { Stator } 5 \text { inlet: } 603.96 & \text { Stator } 5 \text { exit: } 611.44\end{array}$

$\begin{array}{lclll}\text { Ambient Relative Humidity } & 10.00 & & & \\ \text { Fan Face Relative Humidity } & 4.03 & & & \\ \text { Fan Bypass Relative Humidity } & 0.26 & & & \\ \text { LPC Inlet Relative Humidity } & 2.11 & & & \\ \text { LPC Exit Relative Humidity } & 0.03 & & & \\ \text { HPC Relative Humidity } & 0.00 & & & \\ \text { Drop Diameter } & 0.0001000 & \text { Inlet Length } & & \\ \text { Ambient Flow Velocity } & 789.49 & \text { Fan/LPC Inlet Flow Velocity } & 437.12 \\ \text { Ambient Static Pressure } & 2.85 & \text { Fan/LPC Inlet Static Pressure } & 3.79 \\ \text { Ambient Static Temperature } & 425.97 & \text { Fan/LPC Inlet Static Temperature } & 462.00 \\ \text { Additional Water at LPC Exit } & 0.0000043 & & & \end{array}$
$\begin{array}{rrr}\text { MN } & \text { alt } & \text { dTamb } \\ 0.780 & 39000.0 & 36.00\end{array}$
W SUMMARY OUTPUT DATA
W
Wfuel
3254.95
BPR
5.5075
VTAS
789.49
$\mathrm{OPR}$
27.846
$\begin{array}{cl}\text { EPR } & \text { PowerSet } \\ 1.415 & 76.800\end{array}$
$\begin{array}{ccc}\text { T4 } & \text { T41 } & \text { T49 } \\ 2677.4 & 2516.8 & 1746.9\end{array}$

FSO Ambient.FI_O

FS1 Inlet.Fl_O

$\begin{array}{ll}\text { FS12 } & \text { Splitter.F1_02 } \\ \text { FS2 } & \text { Splitter.F1_01 }\end{array}$

FS14 Fan.FI_O

FS23 LPC.F1-O

ES25 Ble

$\begin{array}{ll}\text { FS25 } & \text { Bleed2.F1_- } \\ \text { FS3 } & \text { HPC.F1_O }\end{array}$

FS36 Bleed3.F1_O

FS4 Burner.FI-

FS45 HPT.FI_O

FS49 LPT.FI-O

FS8 Core_Nozz.FI_O

FS17 FanDuctLkg.Fi_o

FS171 Bleed15.FI_O

FS172 FanDuct.FI-O

$W$
363.05
363.05
307.26
55.79
307.26
55.79
55.79
55.79
53.08
41.14
42.04
56.04
56.69
56.69
56.69
307.26
307.26
307.26
307.26

FLOW STATION DATA

$\begin{array}{rrrrr}\text { Pt } & \text { Tt } & \text { ht } & \text { FAR } & \text { WC } \\ 4.267 & 477.92 & -16.44 & 0.0000 & 1200.33\end{array}$

$\begin{array}{lllll}4.267 & 477.92 & -16.44 & 0.0000 & 1200.33\end{array}$

$\begin{array}{lllll}4.265 & 477.92 & -16.44 & 0.0000 & 1016.39 \\ 4.265 & 477.92 & -16.44 & 0.0000 & 184.55\end{array}$

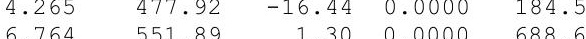

$\begin{array}{rrrrr}6.764 & 551.89 & 1.30 & 0.0000 & 688.66 \\ 10.262 & 636.11 & 21.55 & 0.0000 & 88.48\end{array}$

$\begin{array}{lllll}10.262 & 636.11 & 21.55 & 0.0000 & 88.48 \\ 10.262 & 636.18 & 21.54 & 0.0000 & 88.48\end{array}$

$\begin{array}{llll}10.262 & 636.18 & 21.54 & 0.0000\end{array}$

$\begin{array}{llll}118.262 & 636.18 & 21.54 & 0.0000\end{array}$

$\begin{array}{llll}118.809 & 1351.95 & 199.84 & 0.0000\end{array}$

$\begin{array}{llll}116.073 & 2677.38 & 172.56 & 0.0220\end{array}$

$\begin{array}{rrrr}27.046 & 1754.98 & 2.92 & 0.0164 \\ 6.216 & 1253.73 & -129.39 & 0.0162\end{array}$

$\begin{array}{lllll}6.216 & 1253.79 & -129.39 & 0.0162\end{array}$

$\begin{array}{llll}6.216 & 1253.93 & -129.39 & 0.0162\end{array}$

$\begin{array}{rrrr}6.764 & 551.89 & 1.30 & 0.0000\end{array}$

$\begin{array}{llll}6.764 & 551.89 & 1.30 & 0.0000\end{array}$

$\begin{array}{llll}6.764 & 551.89 & 1.30 & 0.0000\end{array}$

88.48

10.60
8.22
12.09

8.22
12.09
56.01

56.01
208.39

208.39
208.40
208.41

208.41
688.66

688.66

688.66
688.66

PS
2.854
3.353
3.353
3.788
5.615
9.206
9.206
9.206
106.670
111.773
109.650
24.346
5.359
5.359
3.319
5.615
5.456
5.456
3.572

Ts
425.97
446.06
446.13
462.00
523.30
616.74
616.80
616.80
1313.93
1330.32
2642.95
1710.04
1205.96
1206.02
1062.08
523.30
519.03
519.03
459.81

$\begin{array}{rr}\text { Aphy } & \text { MN } \\ 3662.0 & 0.7800\end{array}$ $\begin{array}{ll}4168.2 & 0.5970 \\ 3531.8 & 0.5963\end{array}$ $\begin{array}{rr}3531.8 & 0.5963 \\ 830.5 & 0.4147 \\ 2606.7 & 0.5226\end{array}$

$2606.7 \quad 0.5226$

$\begin{array}{ll}412.6 & 0.3972 \\ 412.6 & 0.3972\end{array}$

$\begin{array}{ll}412.6 & 0.3972 \\ 412.6 & 0.3972\end{array}$

$\begin{array}{ll}49.7 & 0.4008 \\ 49.3 & 0.3008\end{array}$

$\begin{array}{ll}49.3 & 0.3008 \\ 74.6 & 0.2994\end{array}$

$\begin{array}{lll}765.4 & 0.4007\end{array}$

$\begin{array}{ll}265.4 & 0.4007 \\ 860.2 & 0.4721\end{array}$

$\begin{array}{ll}860.2 & 0.4721\end{array}$

$\begin{array}{rr}613.4 & 1.0000 \\ 2606.7 & 0.5226\end{array}$

$\begin{array}{ll}2606.7 & 0.5226 \\ 2481.9 & 0.5626\end{array}$

$\begin{array}{ll}2481.9 & 0.5626 \\ 2481.9 & 0.5626\end{array}$

2006.91 .0000

gamt
1.40068
1.40068
1.40068
1.40068
1.39973
1.39789
1.39789
1.39789
1.35874
1.35874
1.29010
1.32616
1.35555
1.35355
1.35354
1.39973
1.39973
1.39973
1.39973

WAR
0.0000851
0.0000851
0.0000851
0.0000851
0.0000851
0.0000851
0.0000894
0.0000894
0.0000894
0.0000894
0.0000894
0.0000894
0.0000894
0.0000894
0.0000894
0.0000851
0.0000851
0.0000851
0.0000851

Wair
363.02
363.02
307.23
55.78
307.23
55.78
55.78
55.78
53.08
41.14
41.14
55.13
55.78
55.78
55.78
307.23
307.23
307.23
307.23

WH2O H2O frac $\begin{array}{ll}0.031 & 0.0001 \\ 0.031 & 0.0001\end{array}$ $0.026 \quad 0.0001$ $0.005 \quad 0.0001$ $0.026 \quad 0.0001$ $0.005 \quad 0.0001$

$0.005 \quad 0.0001$

$0.005 \quad 0.0001$

$0.004 \quad 0.0001$

$0.004 \quad 0.0266$

$\begin{array}{ll}0.004 & 0.0266 \\ 0.005 & 0.0200 \\ 0.005 & 0.0198\end{array}$

$0.005-0.0198$

0.005

0.026

0.026

0.026

0.0198

0.0001

0.0001

0.0001
0.0001

\begin{tabular}{|c|c|c|c|c|c|c|c|c|c|}
\hline TURB & INERY PER & ORMANCE & DATA & & & & & & \\
\hline & Wc & PR & eff & $\mathrm{NC}$ & $T R$ & efPoly & pwr & SMN & SMW \\
\hline Fan & 1016.39 & 1.586 & 0.9105 & 3900.420 & 1.1548 & 0.9162 & -7712.9 & 58.47 & 27.99 \\
\hline LPC & 184.55 & 2.406 & 0.8608 & 3900.420 & 1.3310 & 0.8768 & -2999.0 & 3.51 & 2.79 \\
\hline HPC & 88.48 & 11.578 & 0.8614 & 10106.860 & 2.1251 & 0.8986 & -13732.3 & 31.89 & 30.71 \\
\hline HPT & 12.09 & 4.292 & 0.9097 & 216.324 & 1.3520 & 0.8895 & 13732.3 & & \\
\hline LPT & 56.01 & 4.351 & 0.9135 & 89.373 & 1.3969 & 0.8967 & 10712.4 & & \\
\hline TURB & INERY MAP & DATA & & & & & & & \\
\hline & WcMap & PRmap & effMap & NcMap & R/Parm & S_WCDes & S_PRdes & S_effDes & S_NcDes \\
\hline Fan & 1180.94 & 1.573 & 0.9124 & 3863.230 & 0.0599 & $\overline{0} .8607$ & $\overline{1} .0235$ & 0.9980 & $\overline{0} .9905$ \\
\hline LPC & 146.85 & 2.490 & 0.8219 & 1.030 & 0.0000 & 1.2567 & 0.9440 & 1.0473 & 0.0003 \\
\hline HPC & 80.48 & 11.079 & 0.8703 & 9837.418 & 12.9699 & 1.0995 & 1.0494 & 0.9898 & 0.9733 \\
\hline HPT & 0.96 & 4.201 & 0.9097 & 1.300 & 4.2005 & 12.6299 & 0.9723 & 1.0000 & 0.0003 \\
\hline LPT & 0.86 & 3.520 & 0.9203 & 1.005 & 3.5204 & 65.2590 & 0.7521 & 0.9926 & 0.0005 \\
\hline
\end{tabular}




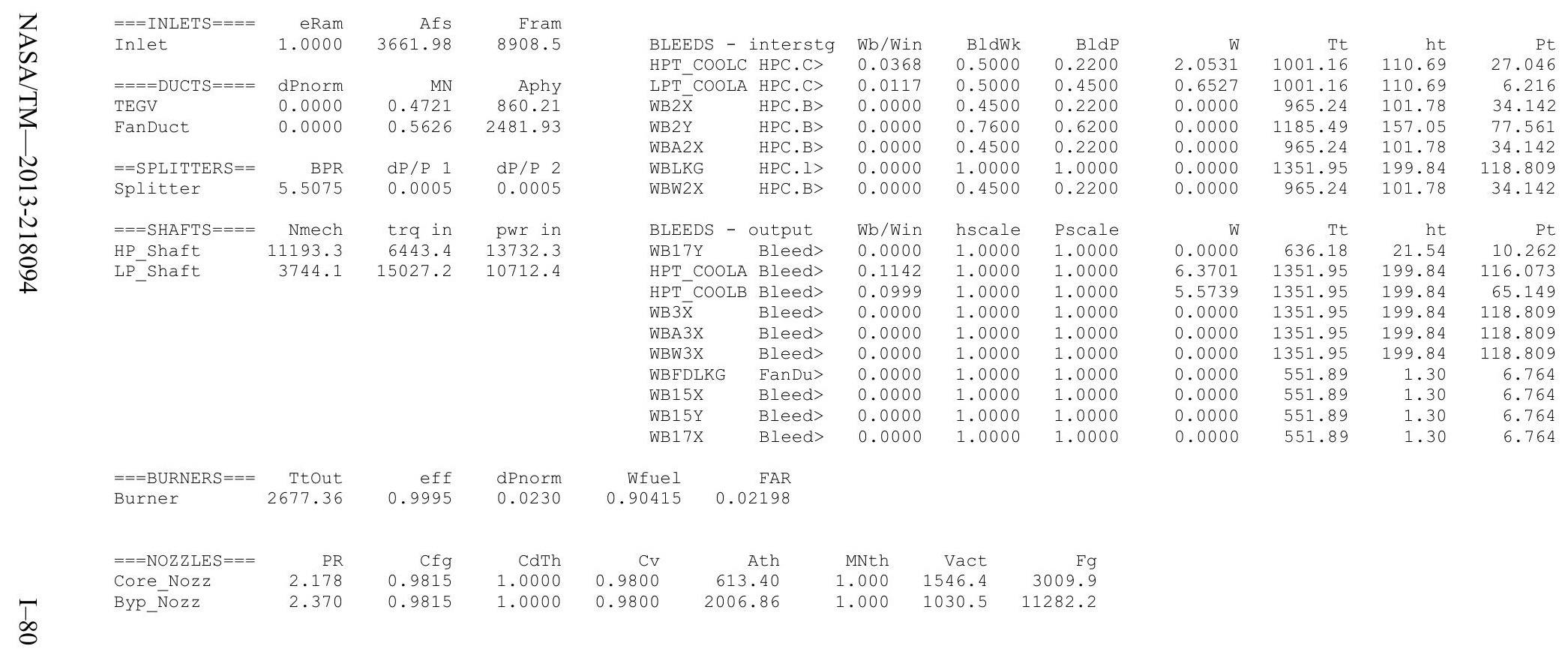


Appendix J: Compressor code analysis for the altitude cruise conditions at 39000 feet

$5 \mu \mathrm{m}, \mathrm{ISA}+18 \mathrm{R}$

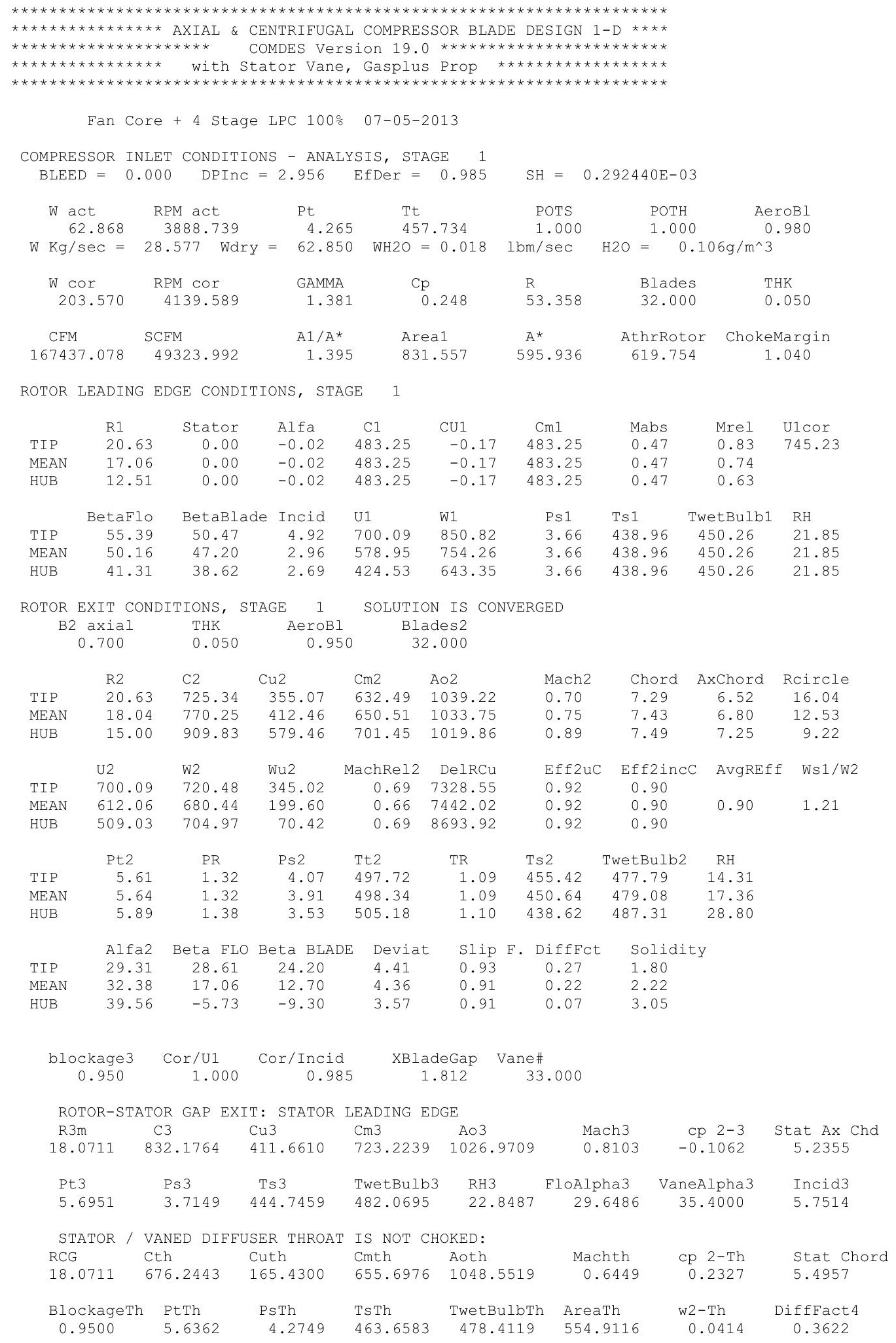




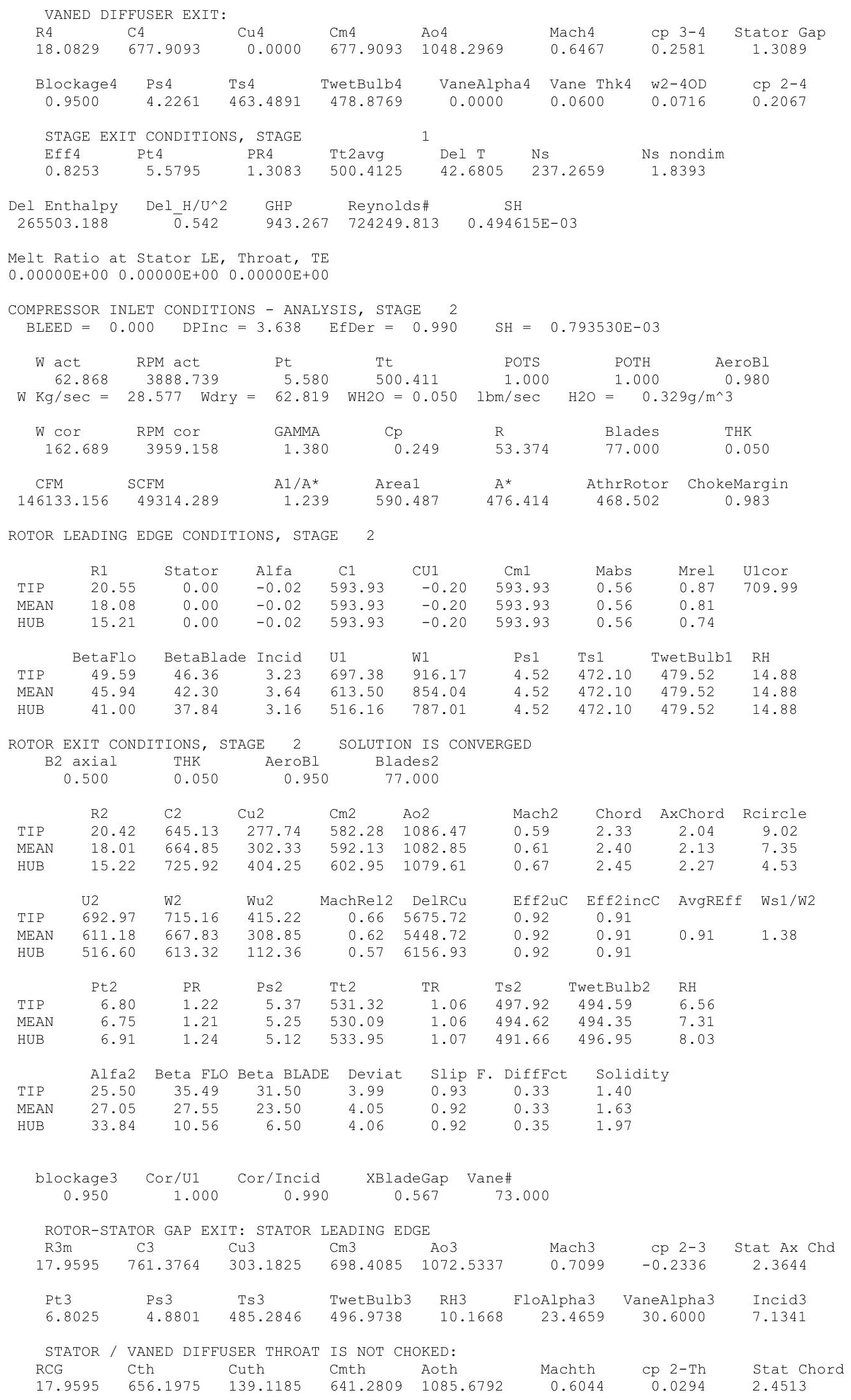




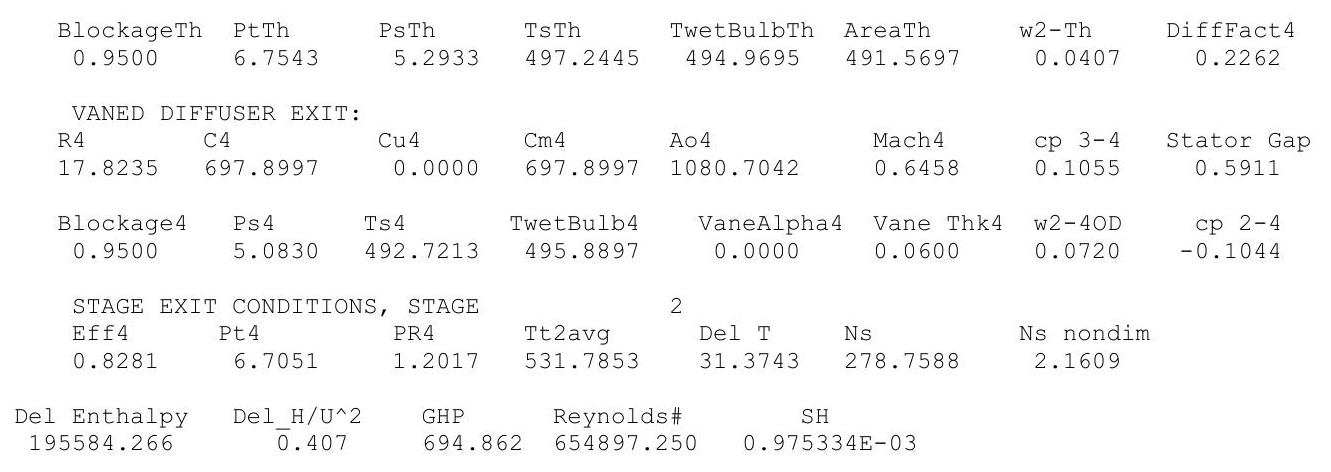

Melt Ratio at Stator LE, Throat, TE

$0.00000 \mathrm{E}+00 \quad 0.00000 \mathrm{E}+00 \quad 0.00000 \mathrm{E}+00$

COMPRESSOR INLET CONDITIONS - ANALYSIS, STAGE 3 BLEED $=0.000 \quad$ DPInC $=4.069 \quad$ EfDer $=0.993$

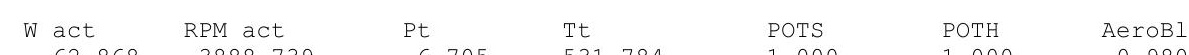

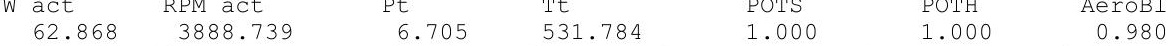

$\mathrm{W} \mathrm{Kg} / \mathrm{sec}=28.577$ Wdry $=62.791 \quad \mathrm{WH} 2 \mathrm{O}=0.078 \quad \mathrm{lbm} / \mathrm{sec} \quad \mathrm{H} 2 \mathrm{O}=0.597 \mathrm{~g} / \mathrm{m}^{\wedge} 3$

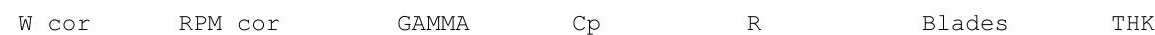

$\begin{array}{ccccccc}139.559 & 3840.597 & 1.380 & 0.249 & 53.388 & 77.000 & 0.050\end{array}$

$\begin{array}{lllcl}\text { CFM } & \text { SCFM } & \text { A1/A* } & \text { Area1 } & \text { A* AthrRotor ChokeMargin }\end{array}$

$\begin{array}{lllllll}125550.727 & 49305.676 & 1.334 & 545.479 & 408.813 & 424.468 & 1.038\end{array}$

ROTOR LEADING EDGE CONDITIONS, STAGE 3

$\begin{array}{lccccccccc} & \text { R1 } & \text { Stator } & \text { Alfa } & \text { C1 } & \text { CU1 } & \text { Cm1 } & \text { Mabs } & \text { Mrel } & \text { U1cor } \\ \text { TIP } & 20.07 & 0.00 & -0.02 & 552.38 & -0.19 & 552.38 & 0.50 & 0.80 & 672.64 \\ \text { MEAN } & 17.74 & 0.00 & -0.02 & 552.38 & -0.19 & 552.38 & 0.50 & 0.75 & \\ \text { HUB } & 15.05 & 0.00 & -0.02 & 552.38 & -0.19 & 552.38 & 0.50 & 0.69 & \\ & & & & & & & & & \\ & & & & & & & & & \\ \text { TIP } & 50.96 & 46.36 & 4.60 & 681.09 & 877.08 & 5.65 & 507.34 & 494.83 & 6.90 \\ \text { MEAN } & 47.47 & 43.40 & 4.07 & 601.97 & 817.14 & 5.65 & 507.34 & 494.83 & 6.90 \\ \text { HUB } & 42.77 & 38.84 & 3.93 & 510.73 & 752.44 & 5.65 & 507.34 & 494.83 & 6.90\end{array}$

ROTOR EXIT CONDITIONS, STAGE 3 SOLUTION IS CONVERGED

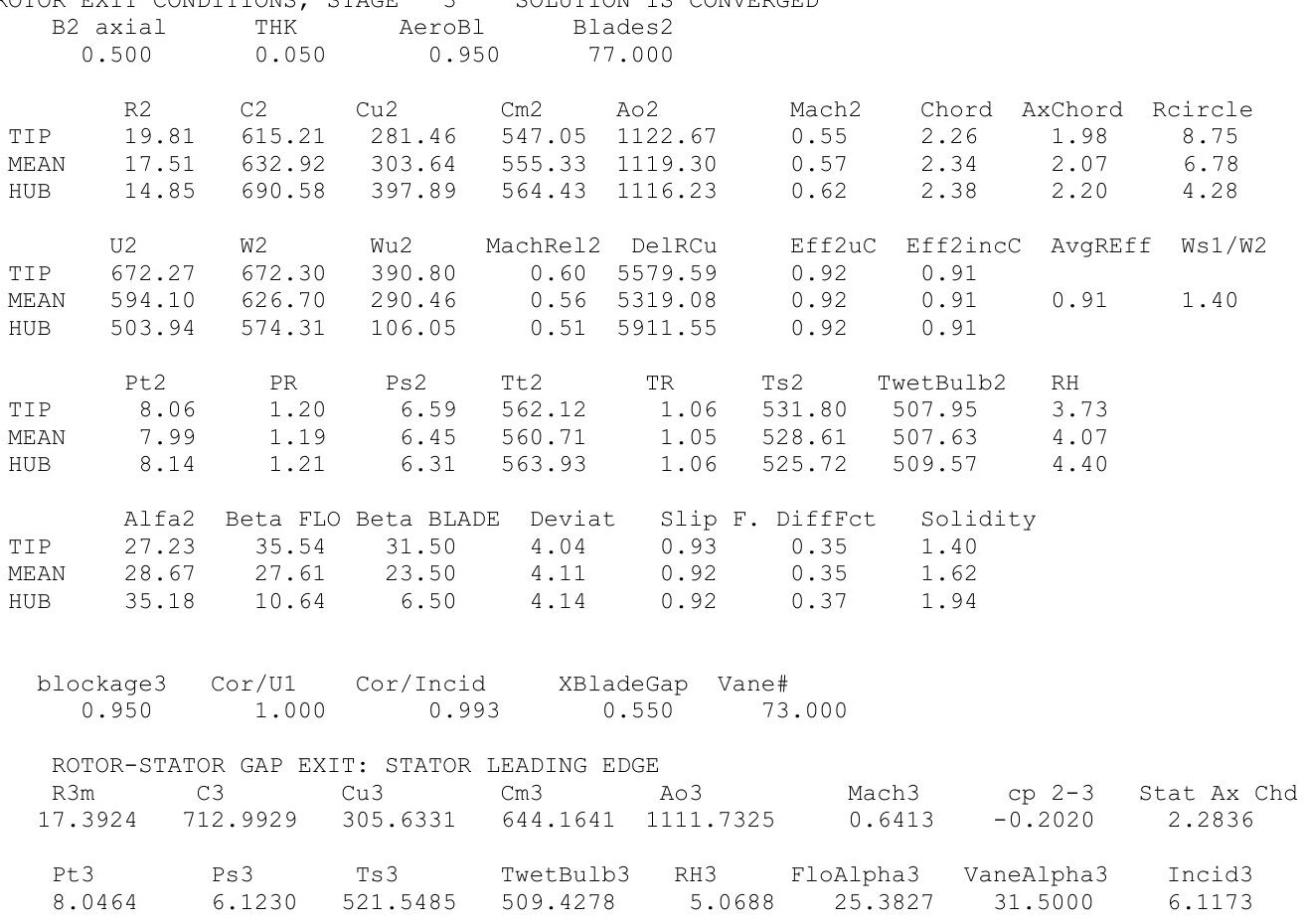




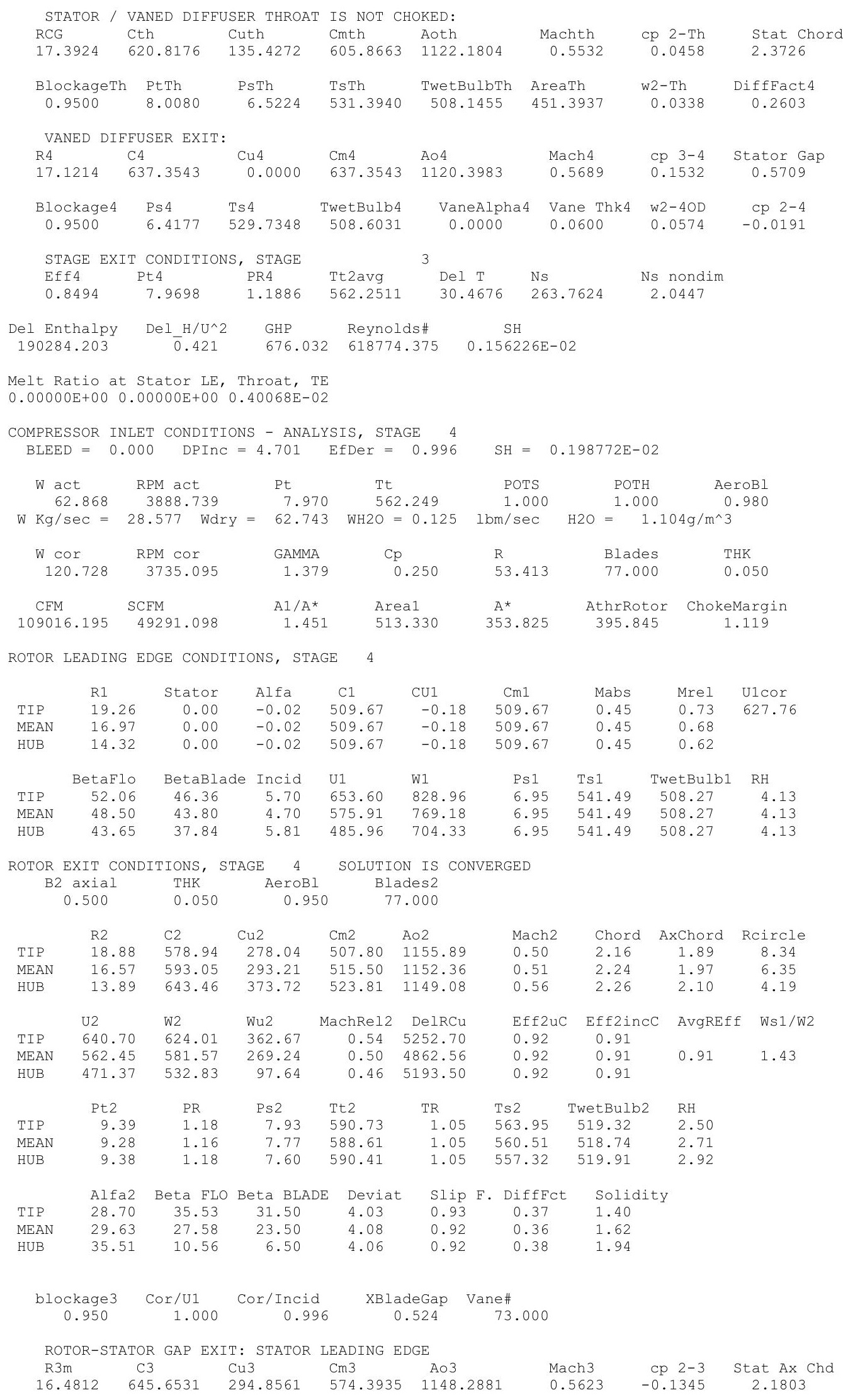




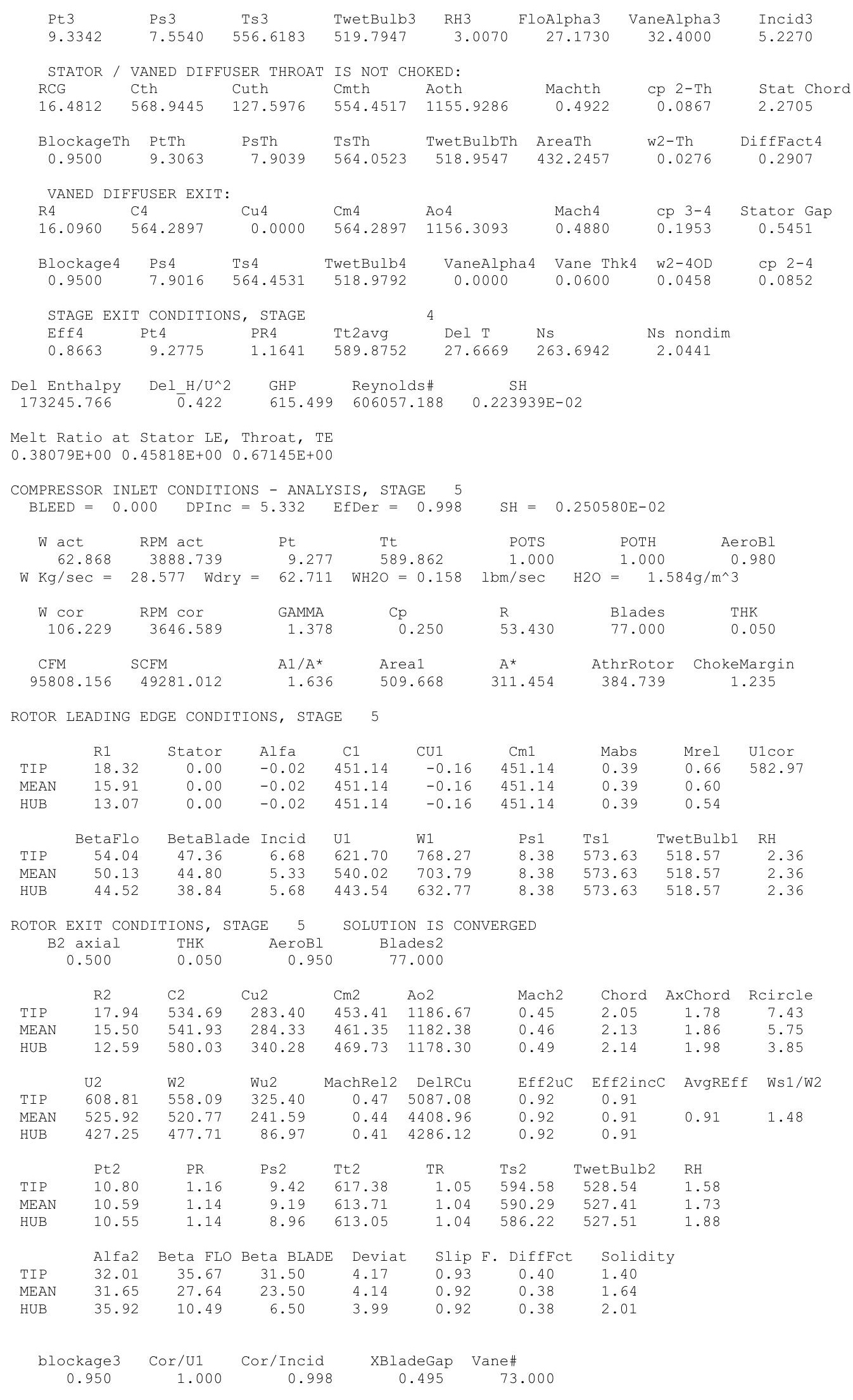




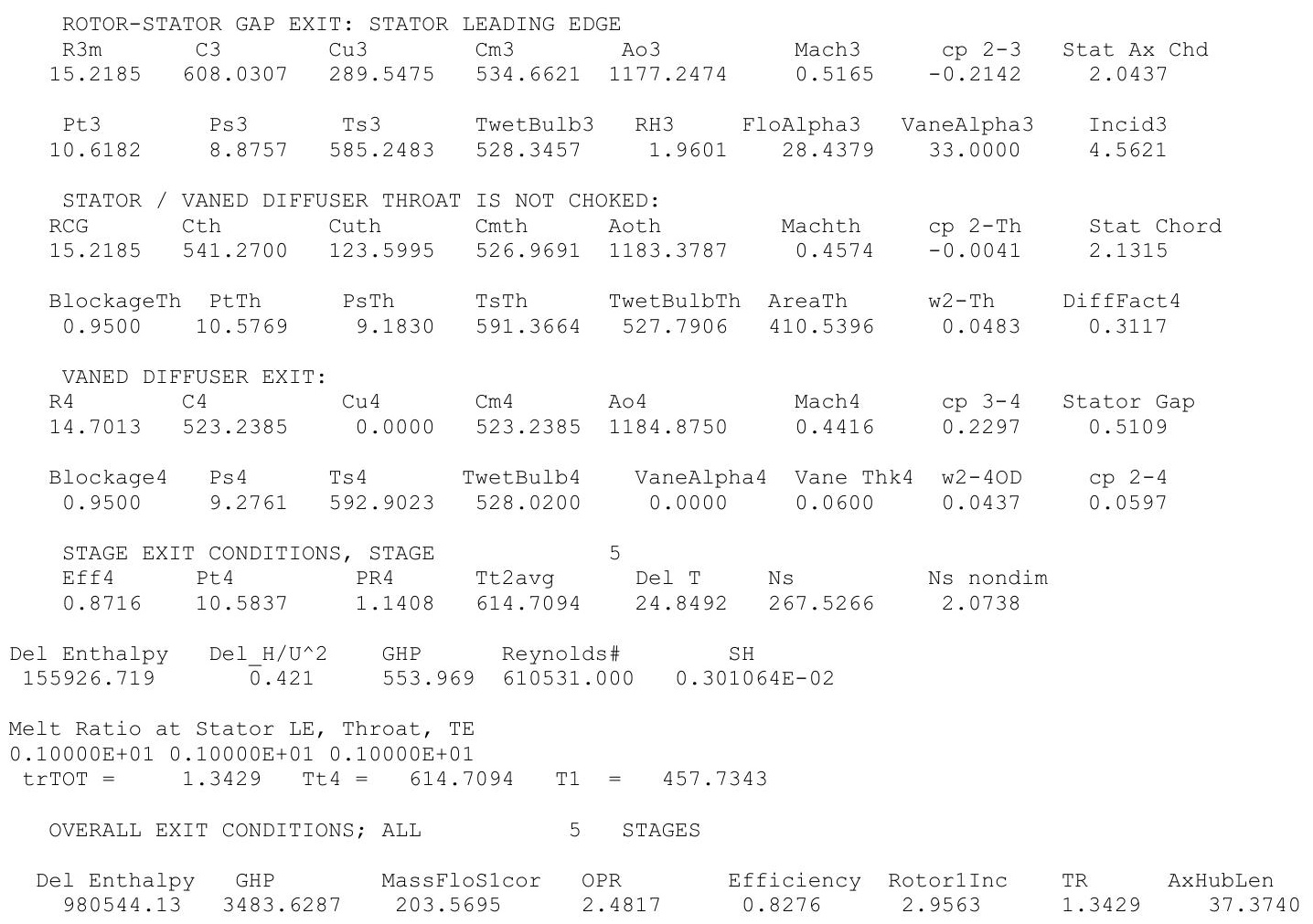




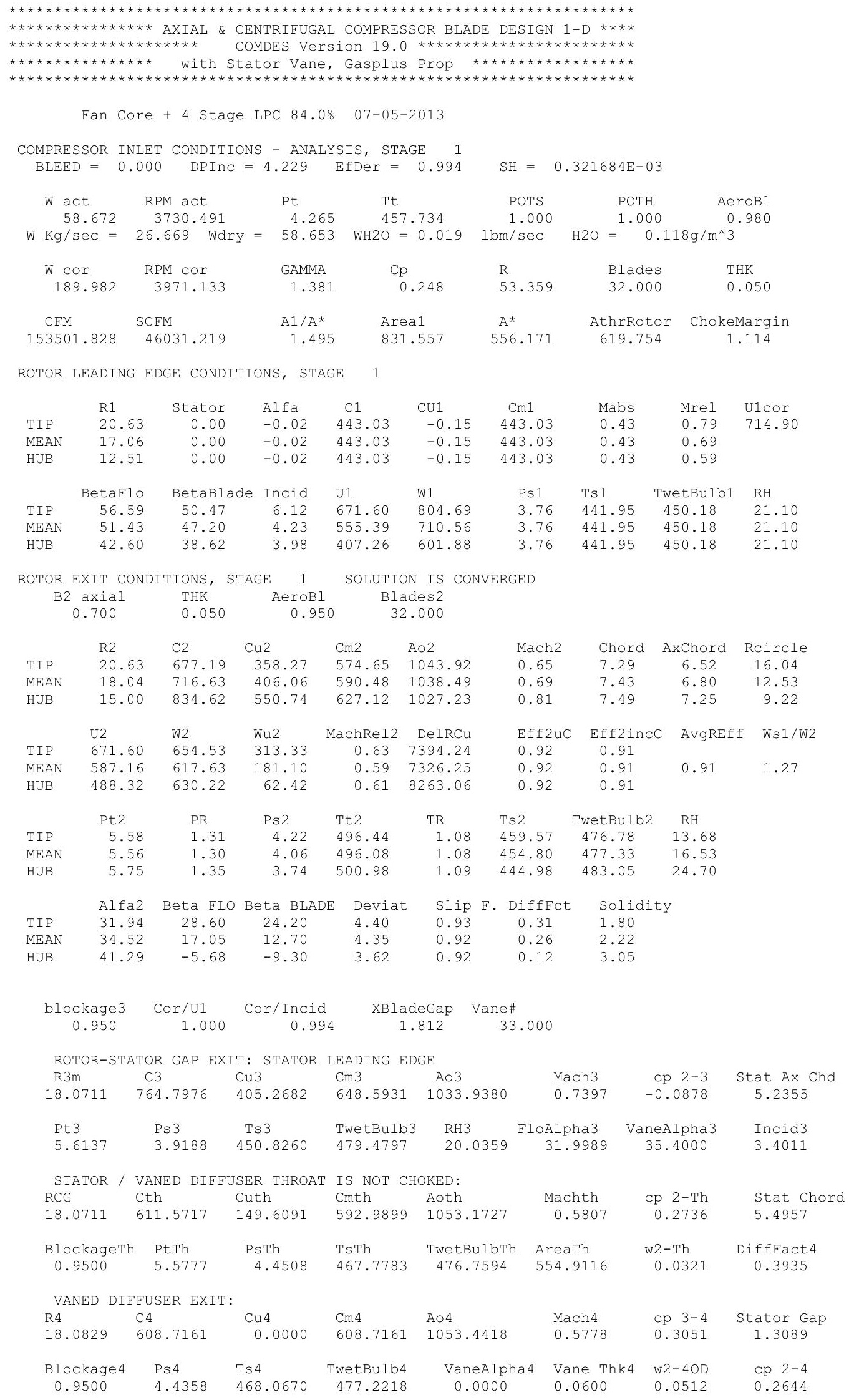




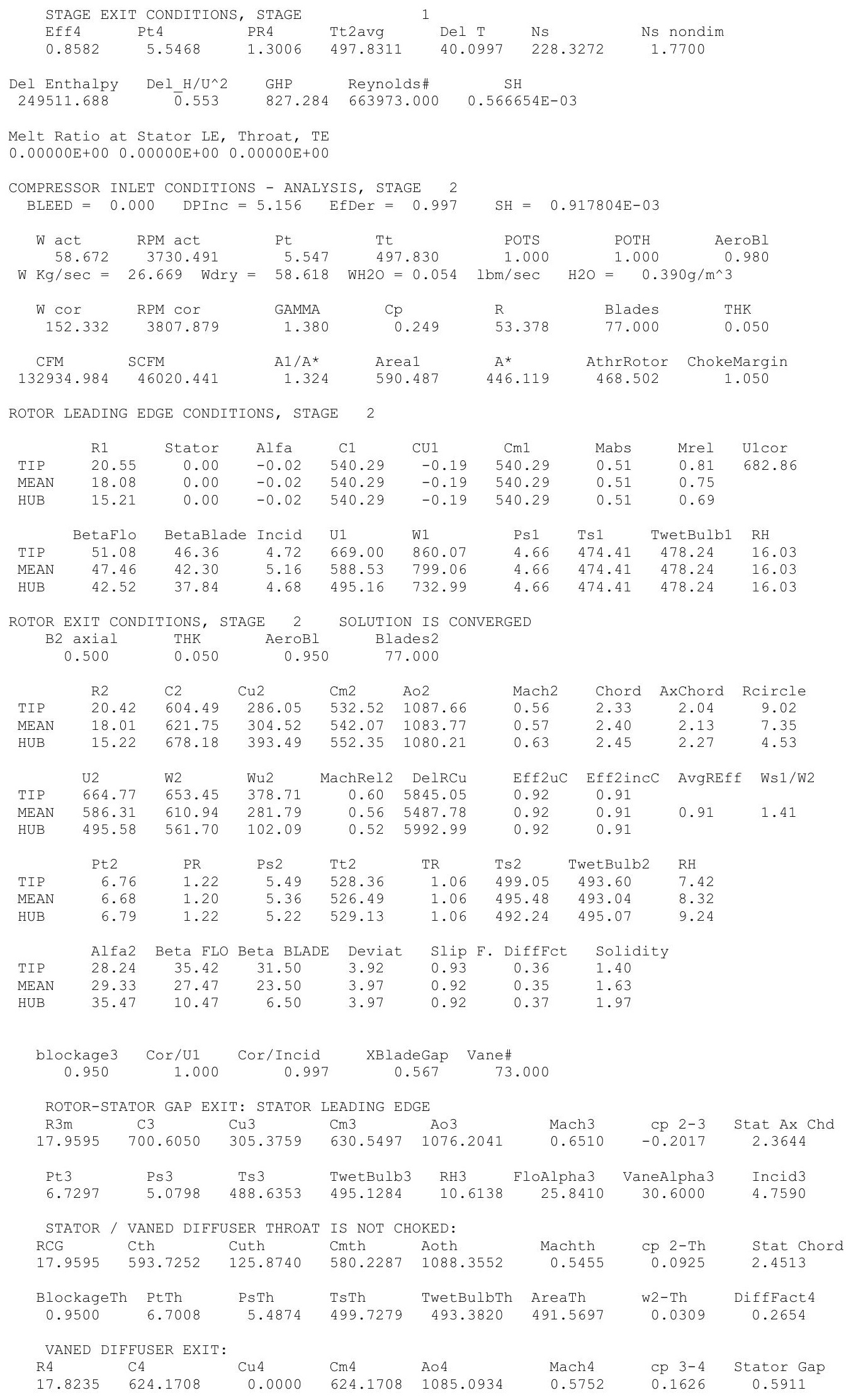




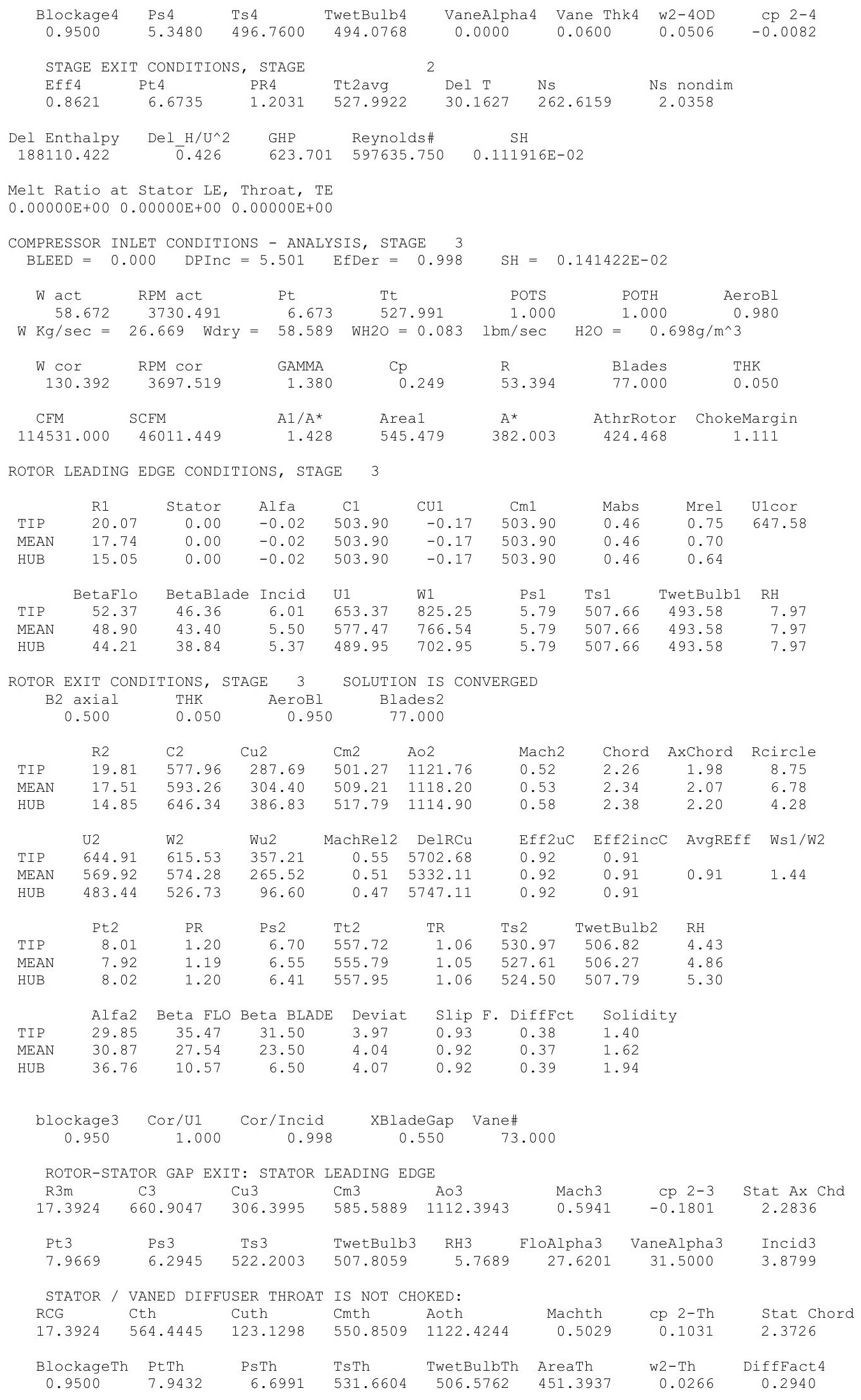




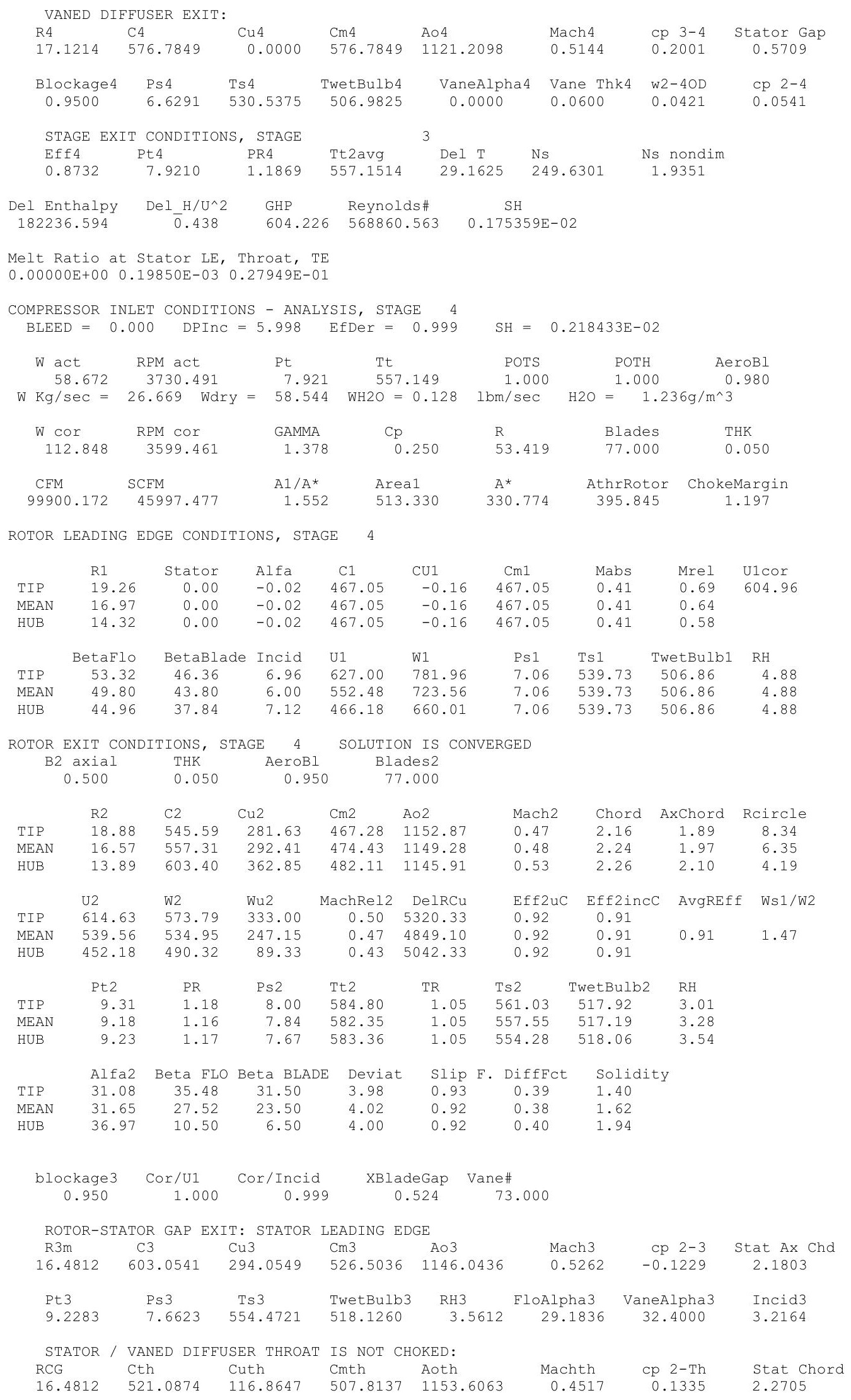




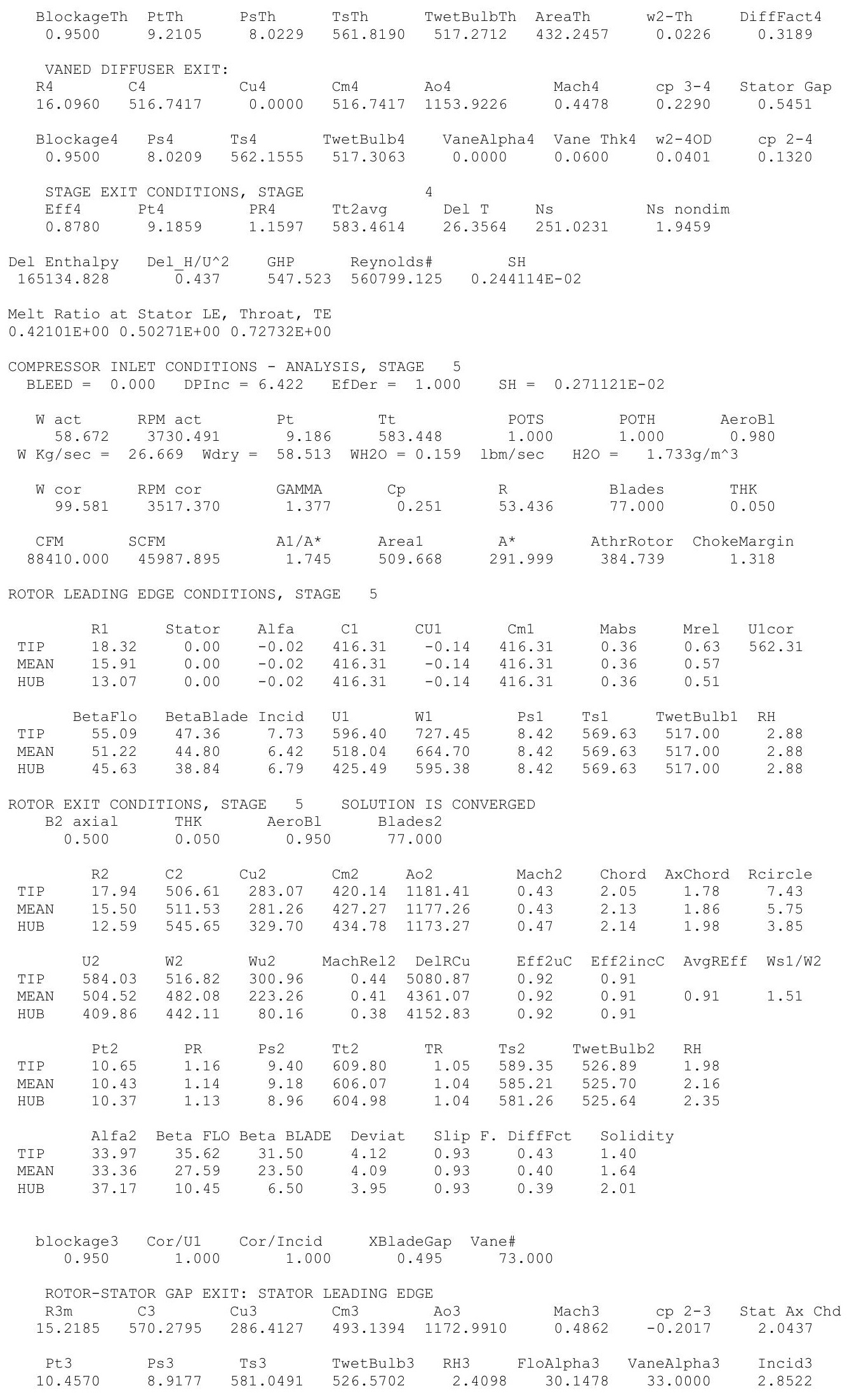




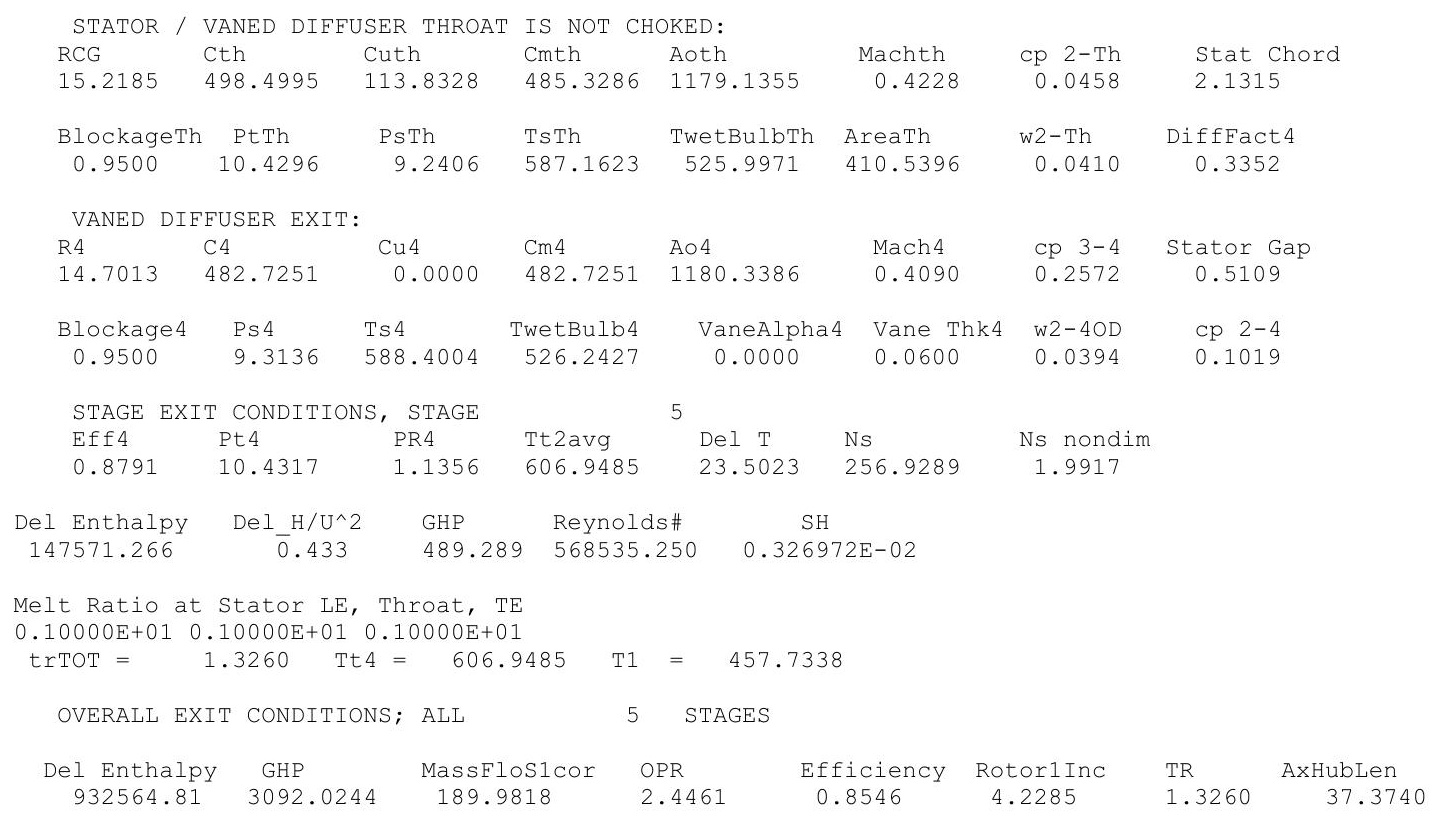




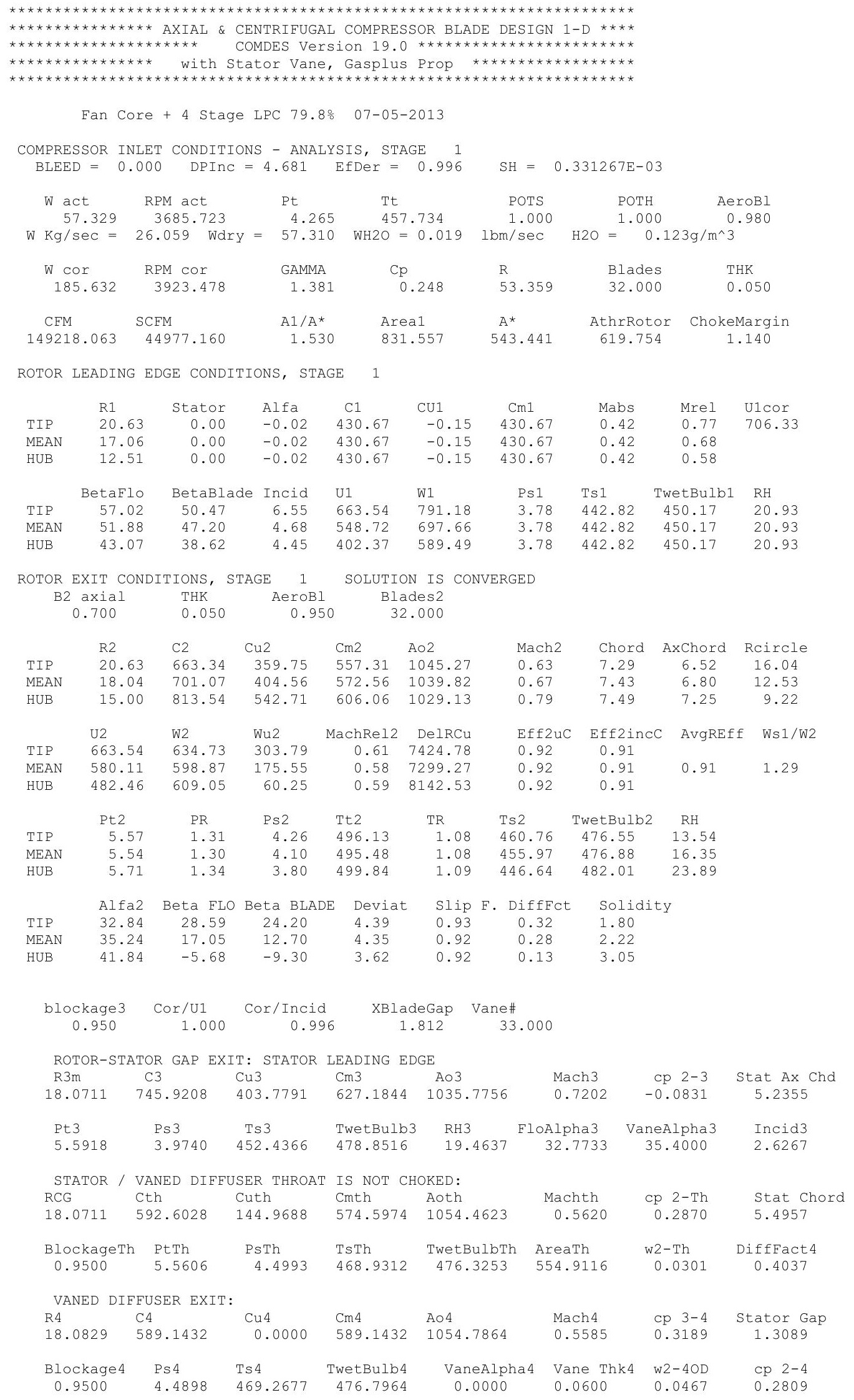




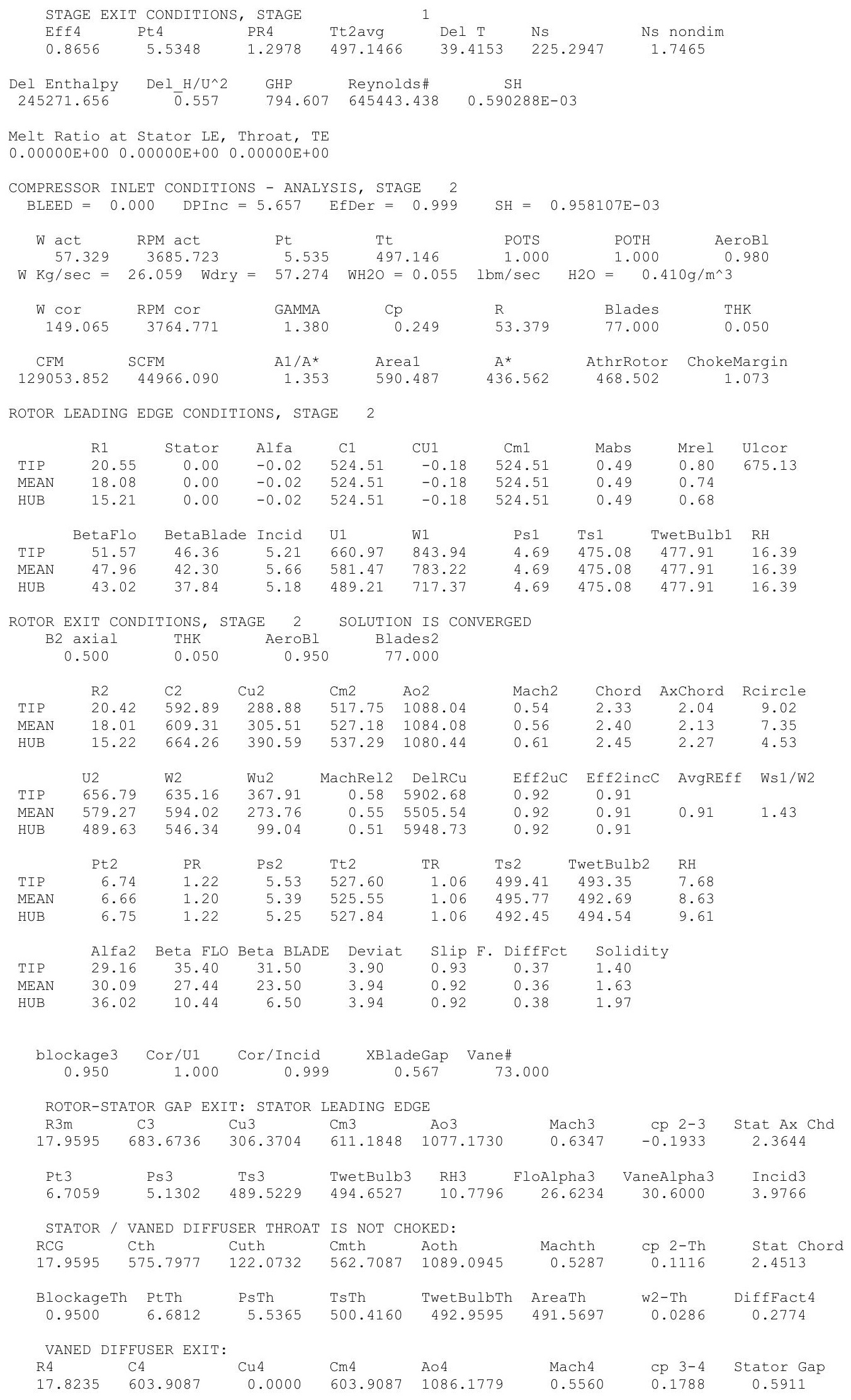




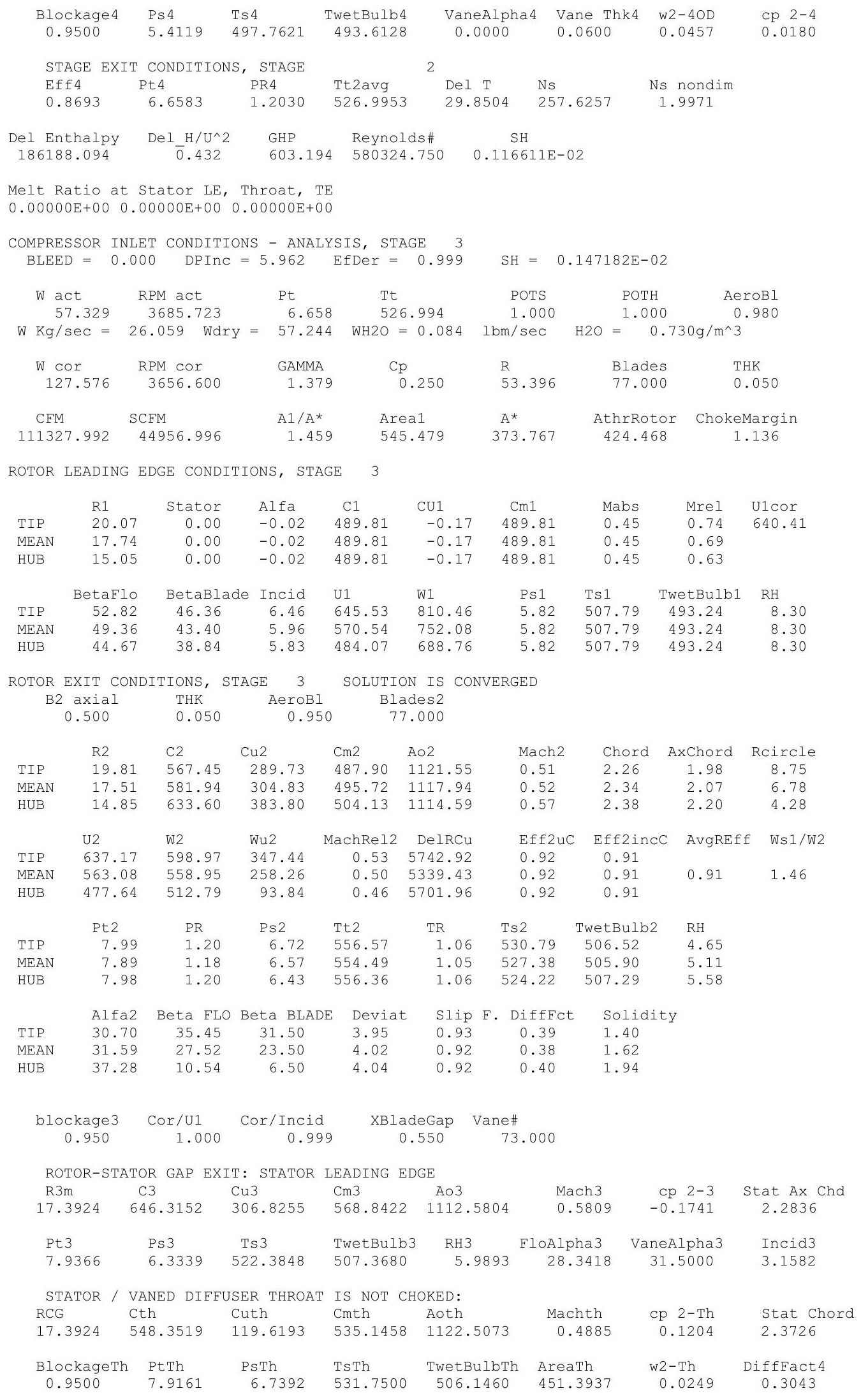




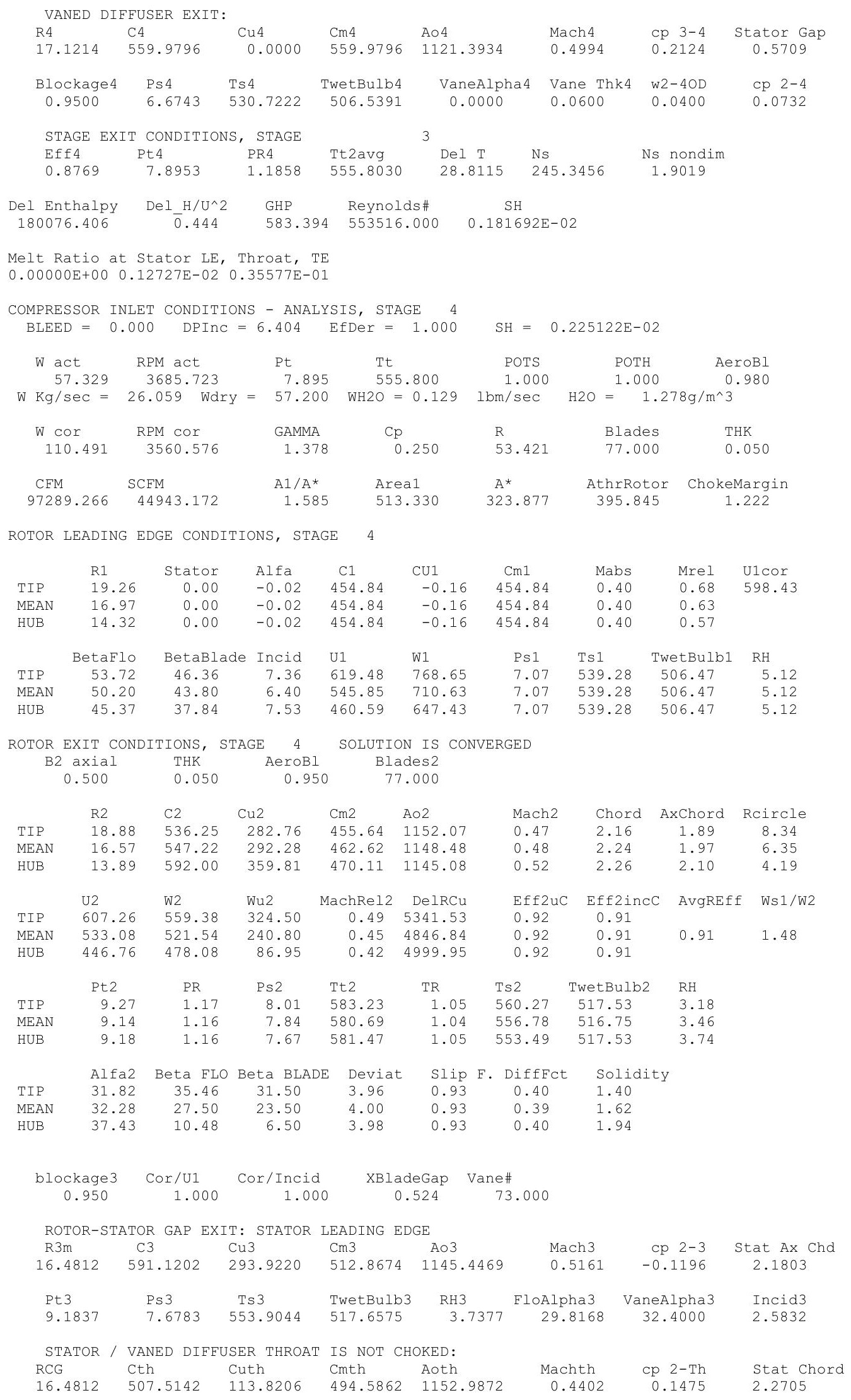




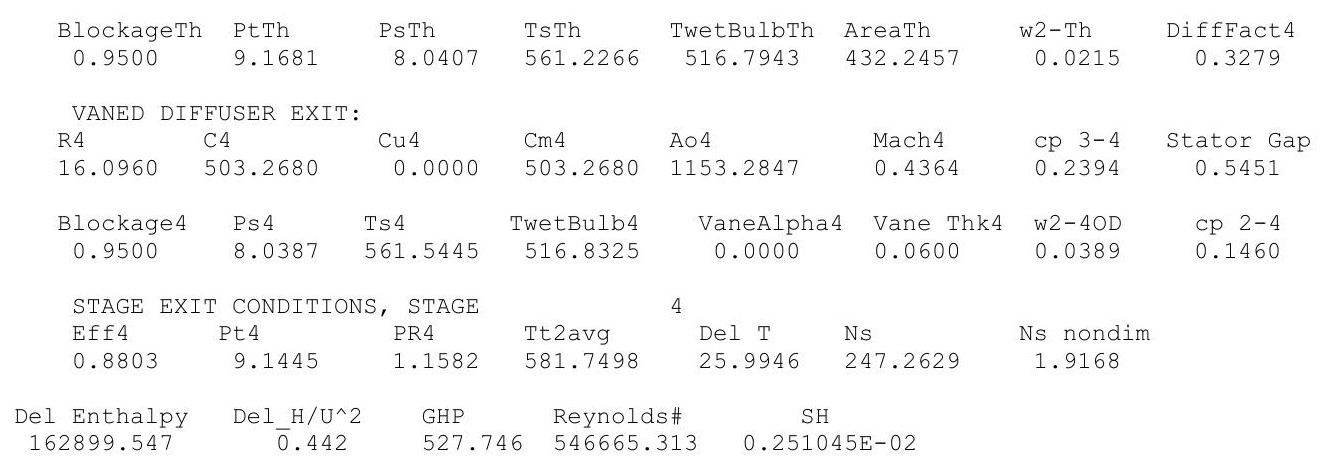

Melt Ratio at Stator LE, Throat, TE

$0.43423 \mathrm{E}+00 \quad 0.51730 \mathrm{E}+00 \quad 0.74559 \mathrm{E}+00$

COMPRESSOR INLET CONDITIONS - ANALYSIS, STAGE 5 $\mathrm{BLEED}=0.000 \quad$ DPInC $=6.767 \quad$ EfDer $=1.000$

$\mathrm{SH}=0.278245 \mathrm{E}-02$

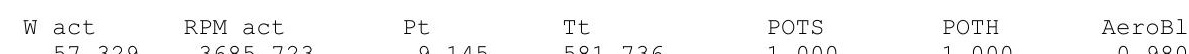
$\mathrm{W} \mathrm{Kg} / \mathrm{sec}=26.059$ Wdry $=57.169 \quad \mathrm{WH} 2 \mathrm{O}=0.160 \quad \mathrm{lbm} / \mathrm{sec} \quad \mathrm{H} 2 \mathrm{O}=\begin{gathered}1.000 \\ 1.781 \mathrm{~g} / \mathrm{m}^{\wedge} 3\end{gathered}$

$\begin{array}{ccccccc}\text { W Cor } & \text { RPM cor } & \text { GAMMA } & \text { Cp } & \text { R } & \text { Blades } & \text { THK } \\ 97.598 & 3480.268 & 1.377 & 0.251 & 53.439 & 77.000 & 0.050 \\ & & & & & & \\ \text { CFM } & \text { SCFM } & \text { A1/A* } & \text { Areal } & A^{*} & \text { AthrRotor } & \text { ChokeMargin } \\ 86276.836 & 44933.730 & 1.781 & 509.668 & 286.195 & 384.739 & 1.344\end{array}$

ROTOR LEADING EDGE CONDITIONS, STAGE 5

\begin{tabular}{lccccccccc} 
& R1 & Stator & Alfa & C1 & \multicolumn{1}{l}{ CU1 } & Cm1 & Mabs & Mrel & U1cor \\
TIP & 18.32 & 0.00 & -0.02 & 406.26 & -0.14 & 406.26 & 0.35 & 0.62 & 556.38 \\
MEAN & 15.91 & 0.00 & -0.02 & 406.26 & -0.14 & 406.26 & 0.35 & 0.56 & \\
HUB & 13.07 & 0.00 & -0.02 & 406.26 & -0.14 & 406.26 & 0.35 & 0.50 & \\
& & & & & & & & & \\
TIP & BetaFlo & BetaBlade & Incid & U1 & W1 & Ts1 & TwetBulb1 & RH \\
MEAN & 51.42 & 47.36 & 8.06 & 589.24 & 715.84 & 8.41 & 568.58 & 516.55 & 3.05 \\
HUB & 45.99 & 38.84 & 7.15 & 420.38 & 584.71 & 8.41 & 568.58 & 516.55 & 3.05
\end{tabular}

ROTOR EXIT CONDITIONS, STAGE 5 SOLUTION IS CONVERGED

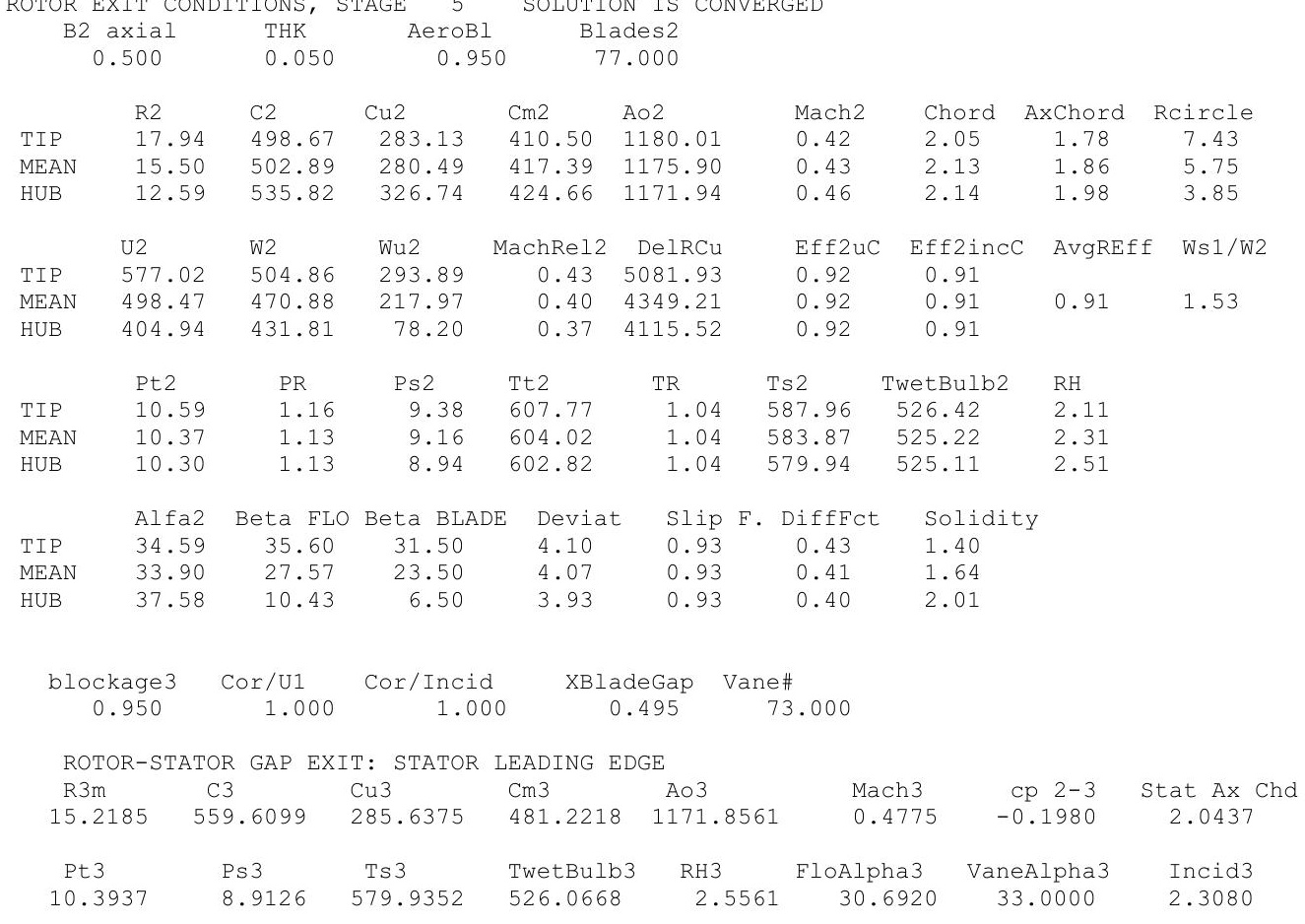




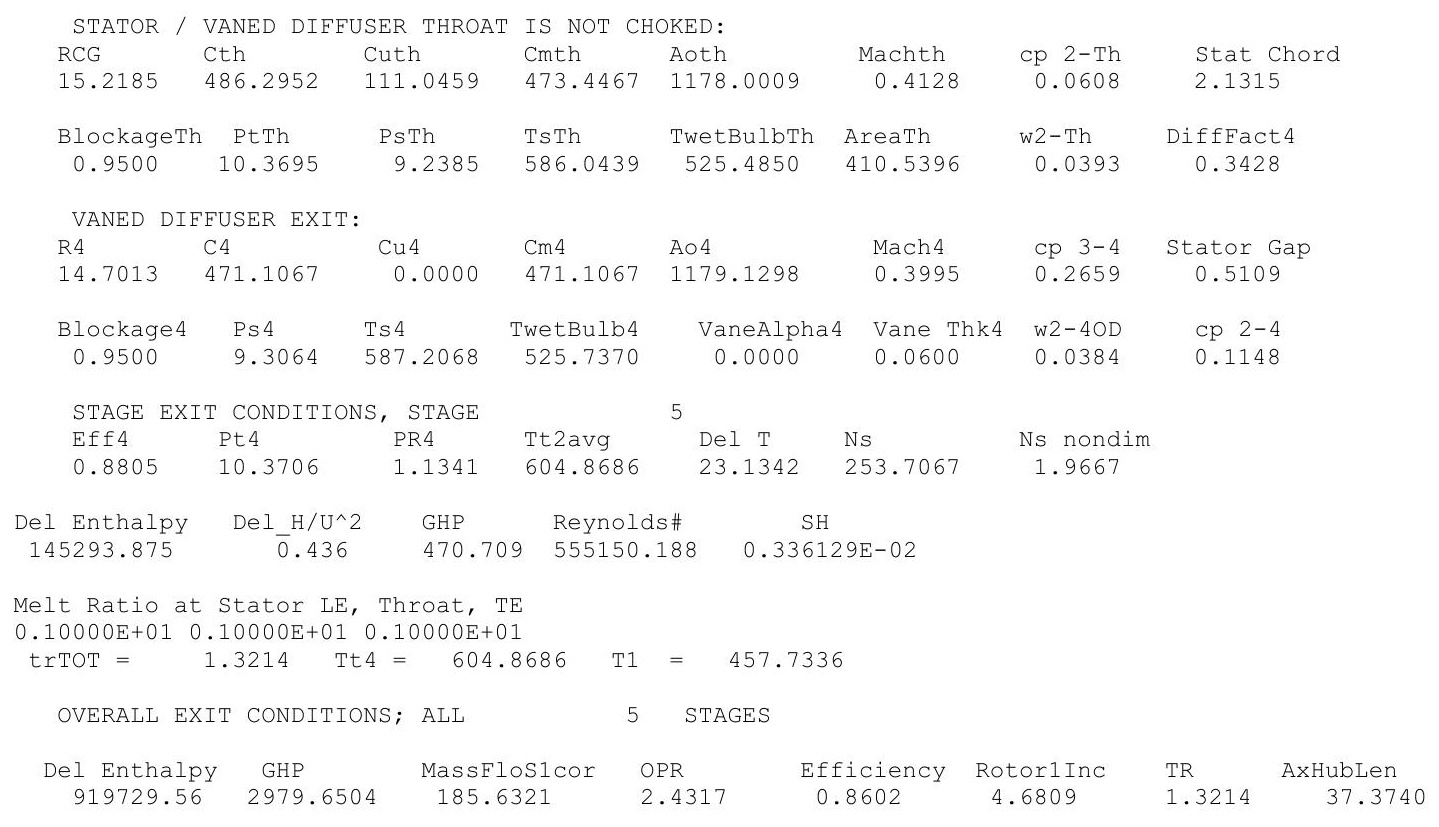




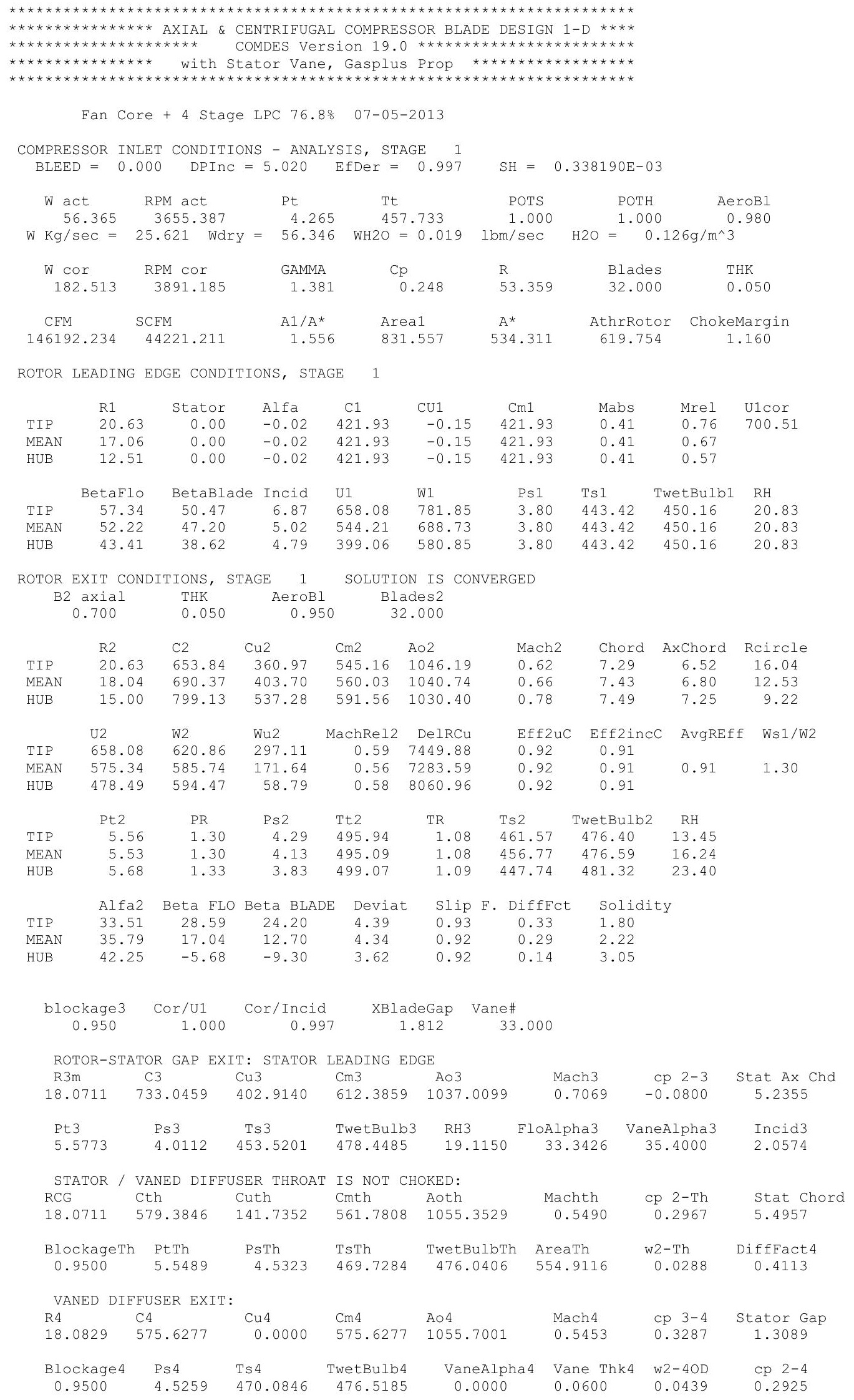




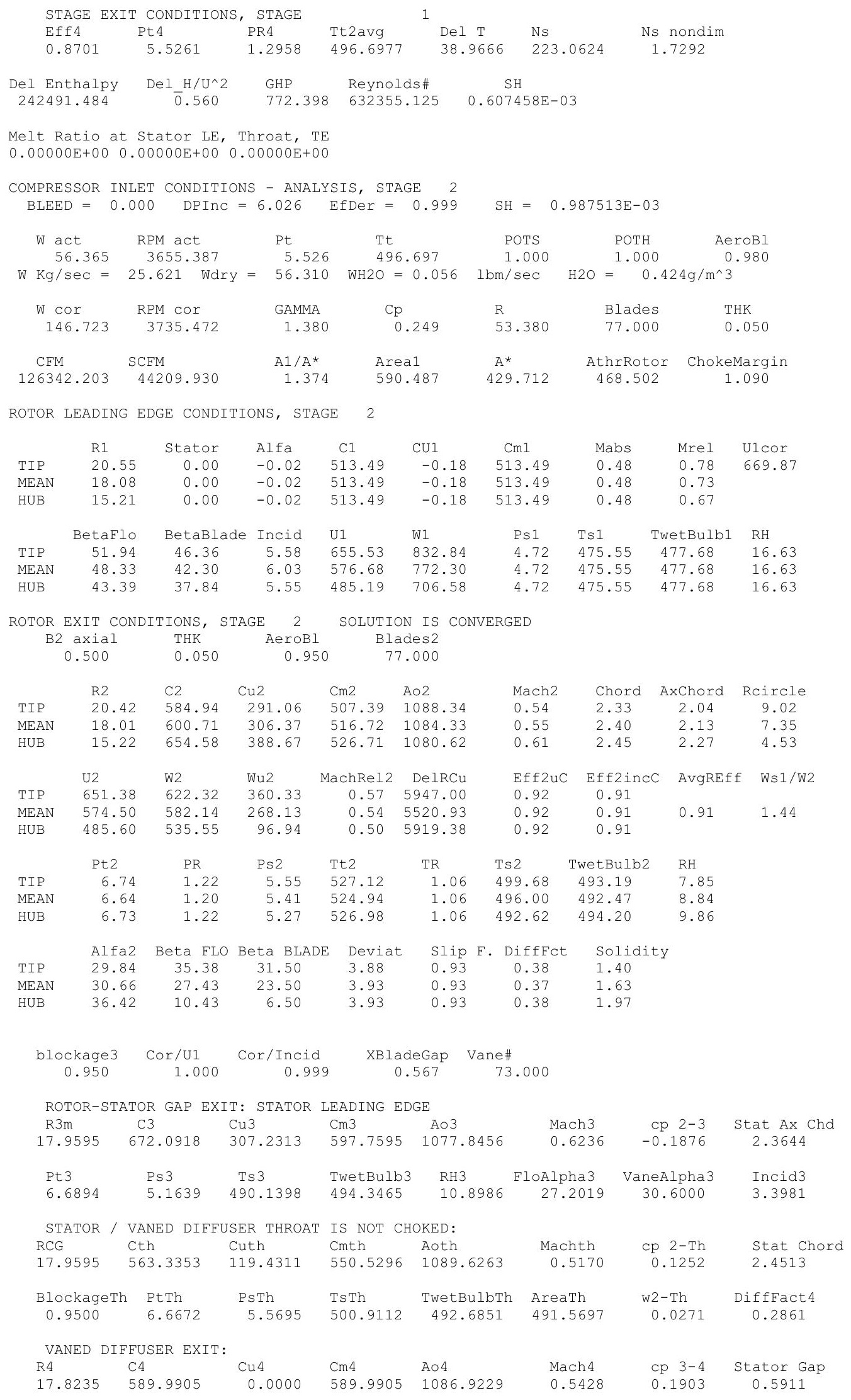




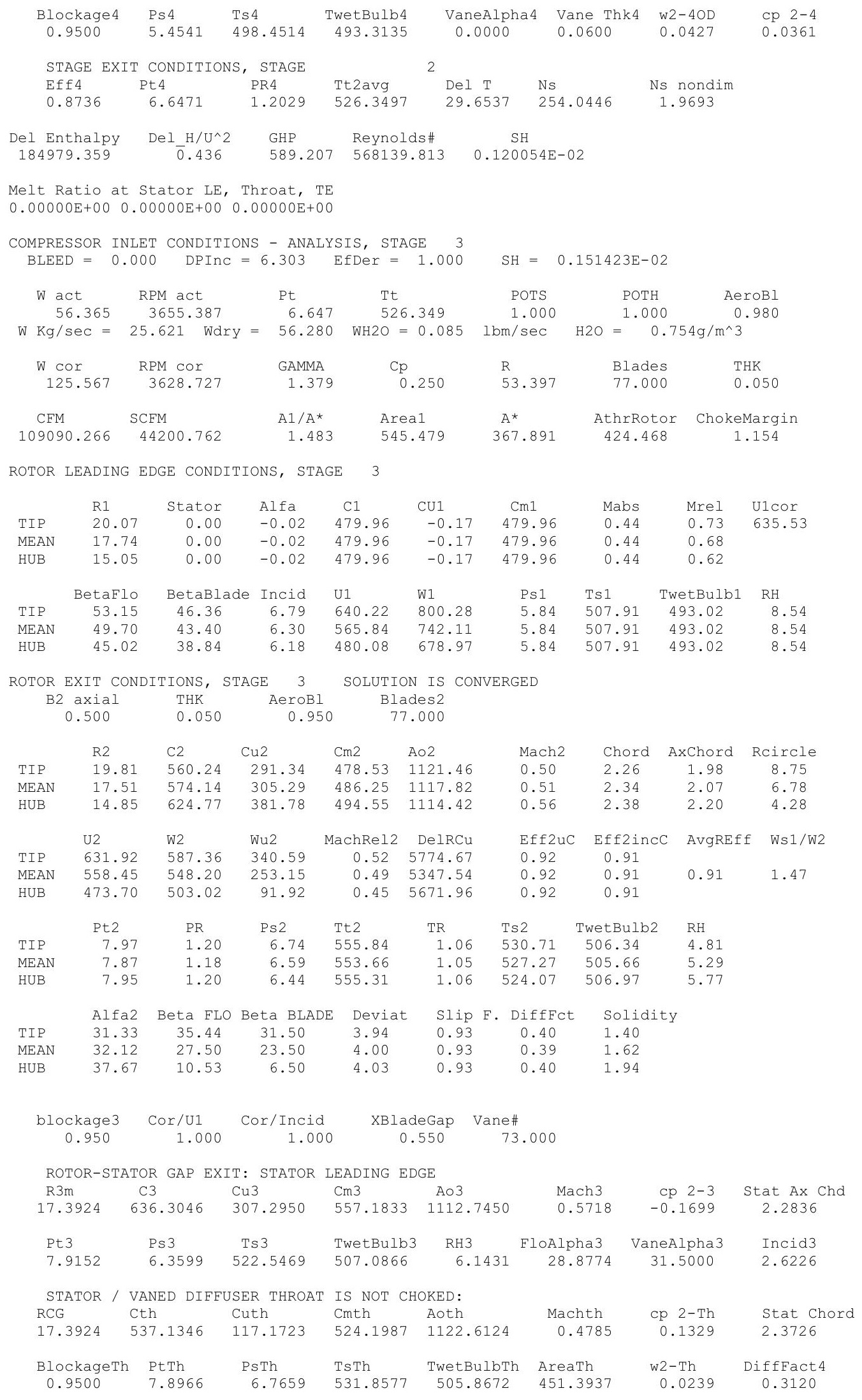




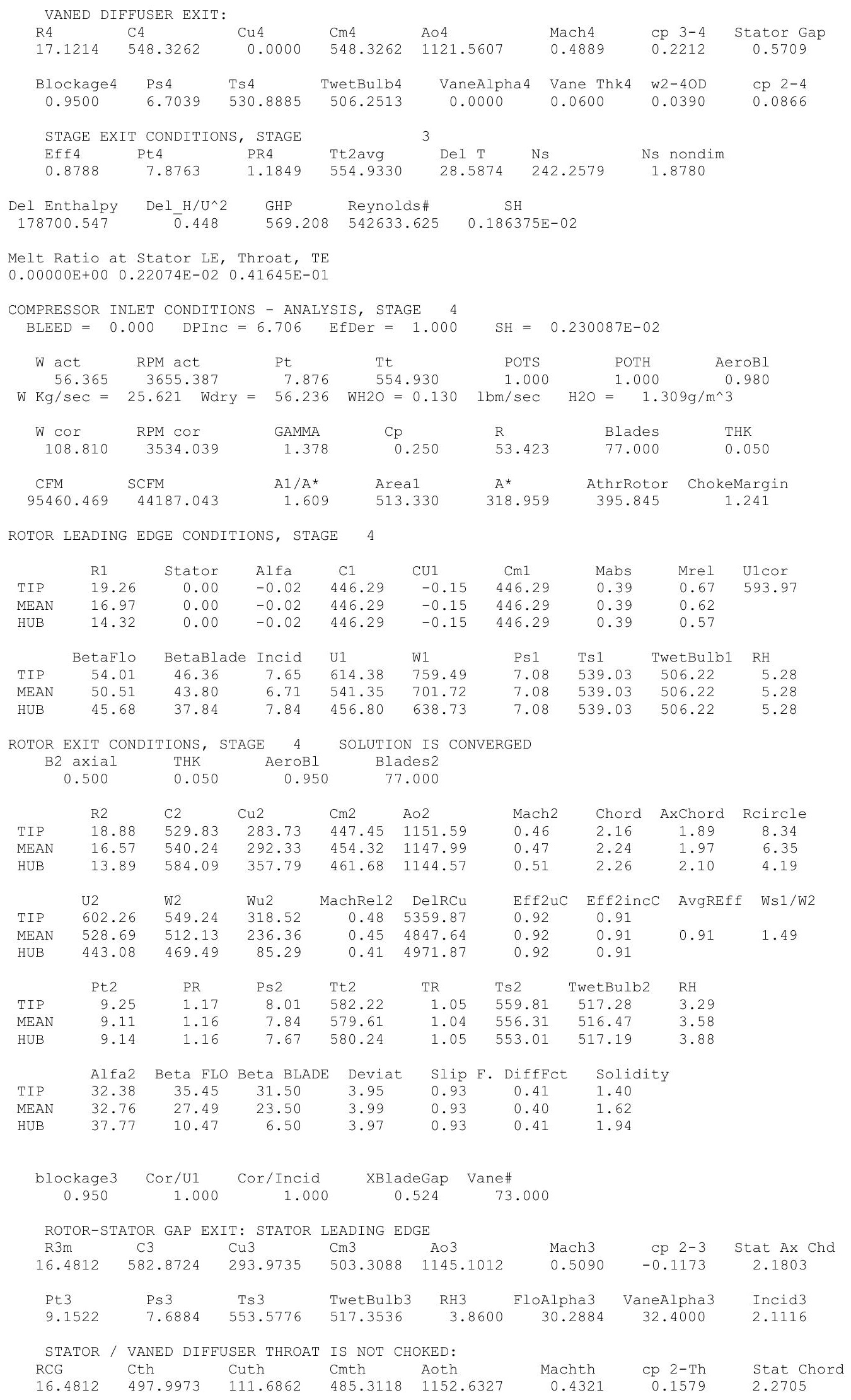




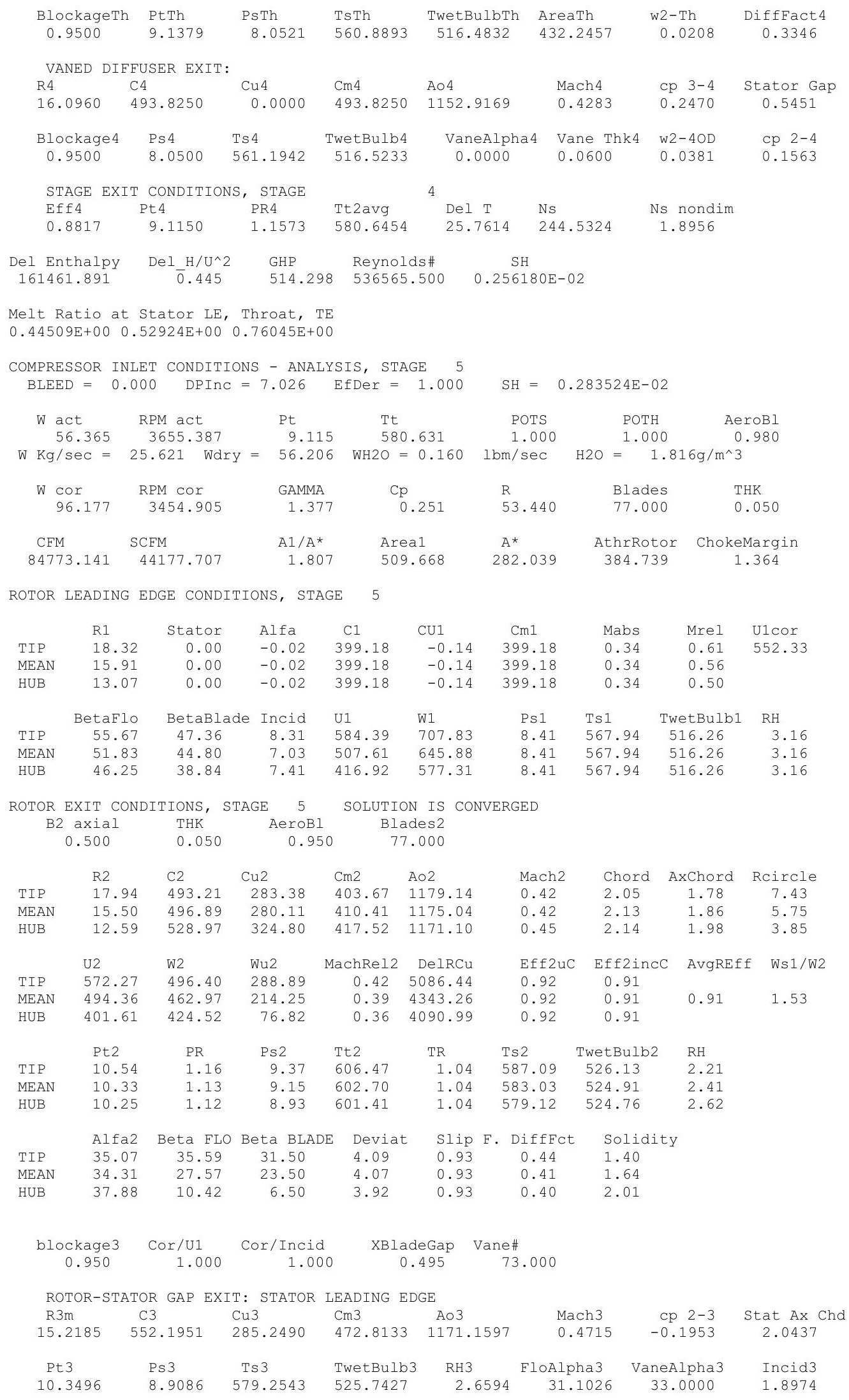




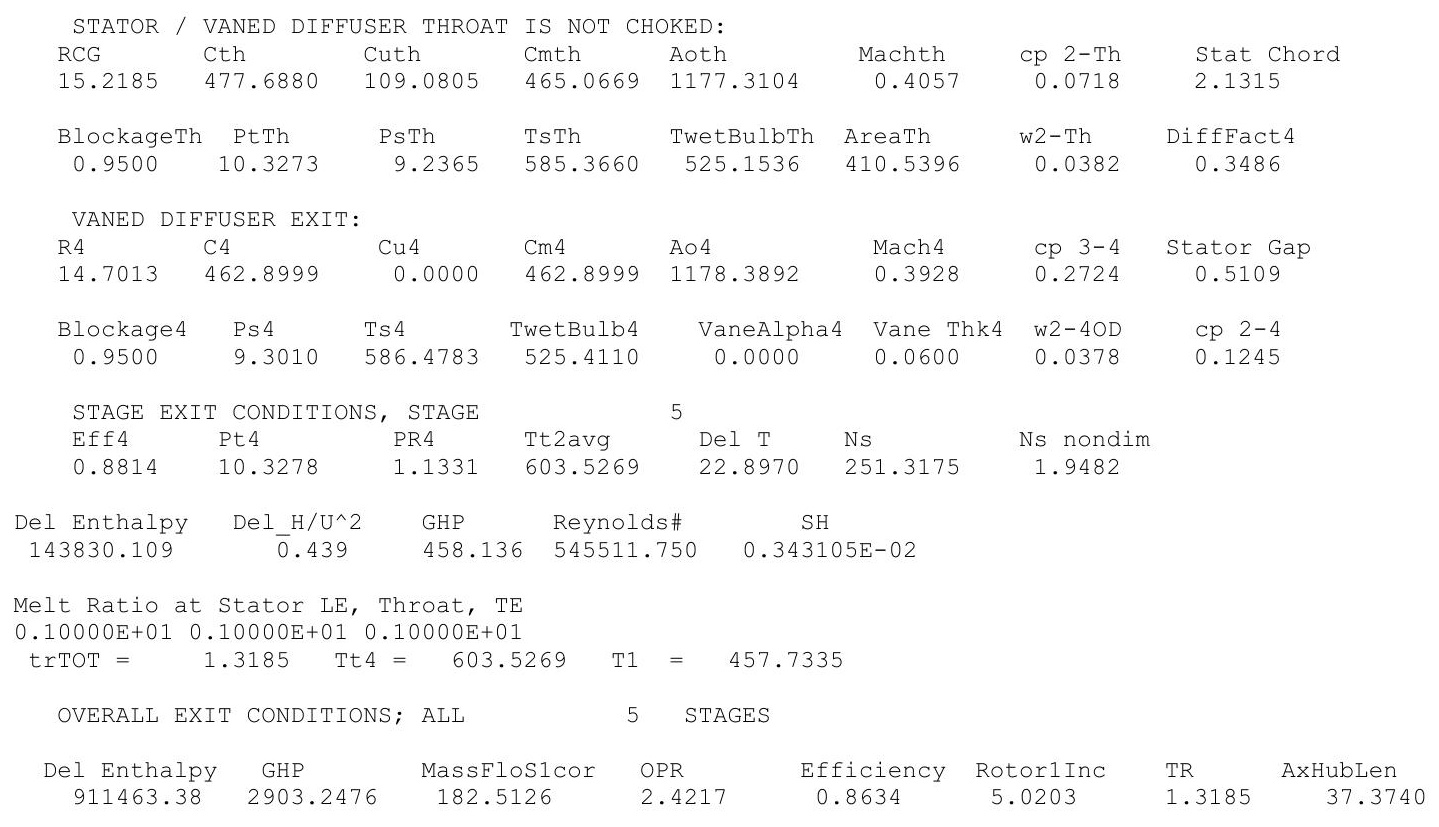


$5 \mu \mathrm{m}, \mathrm{ISA}+36 \mathrm{R}$

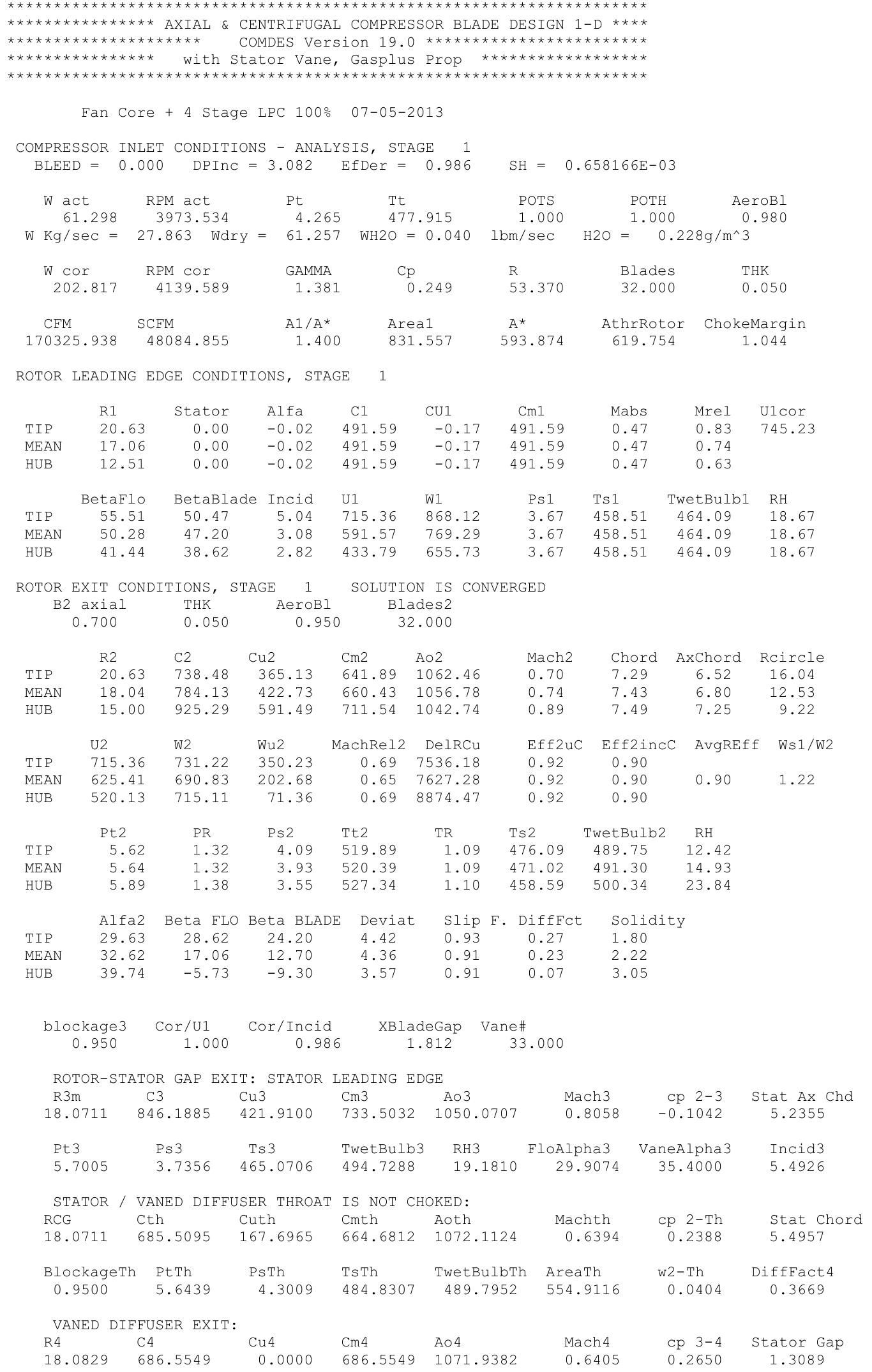




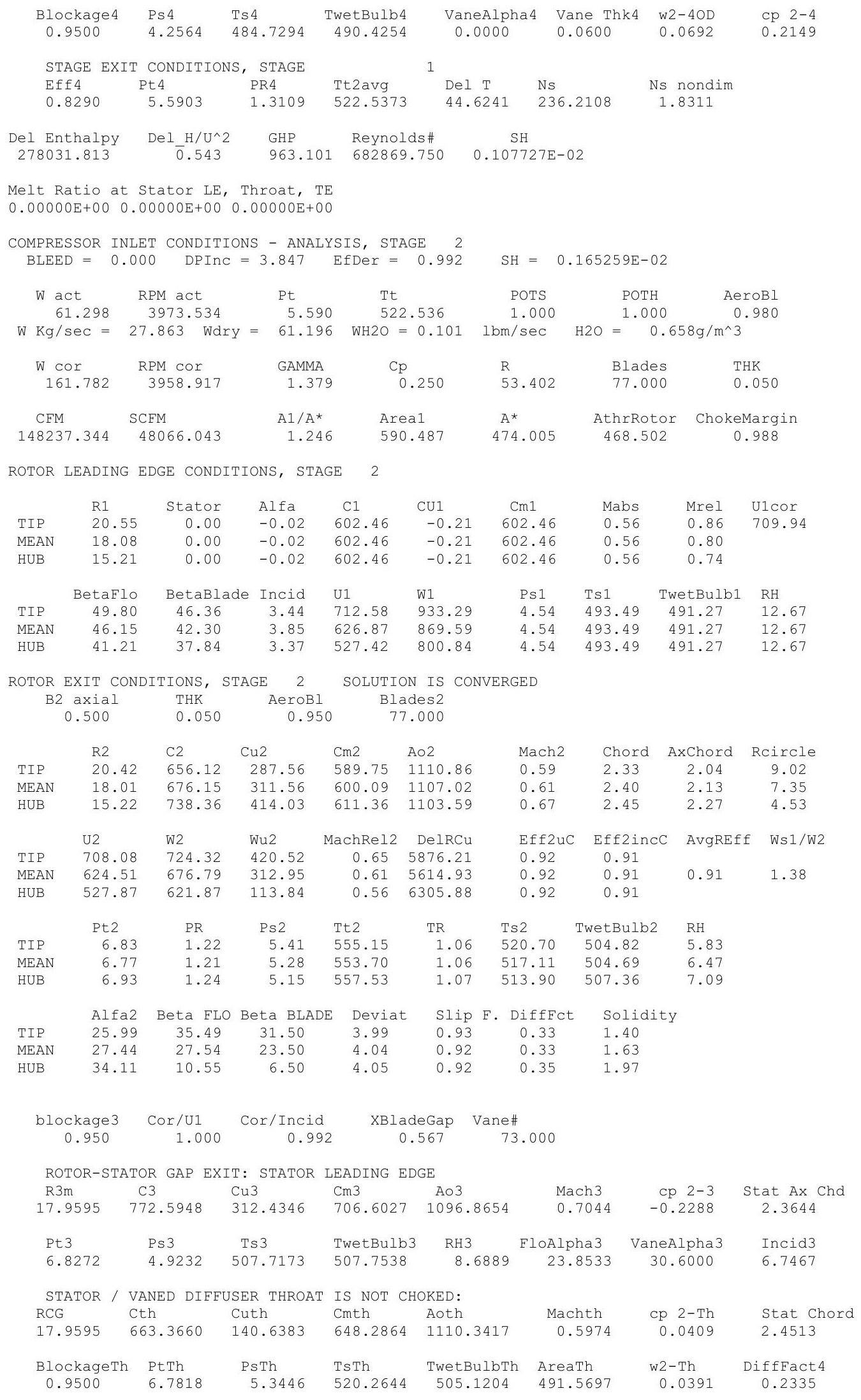




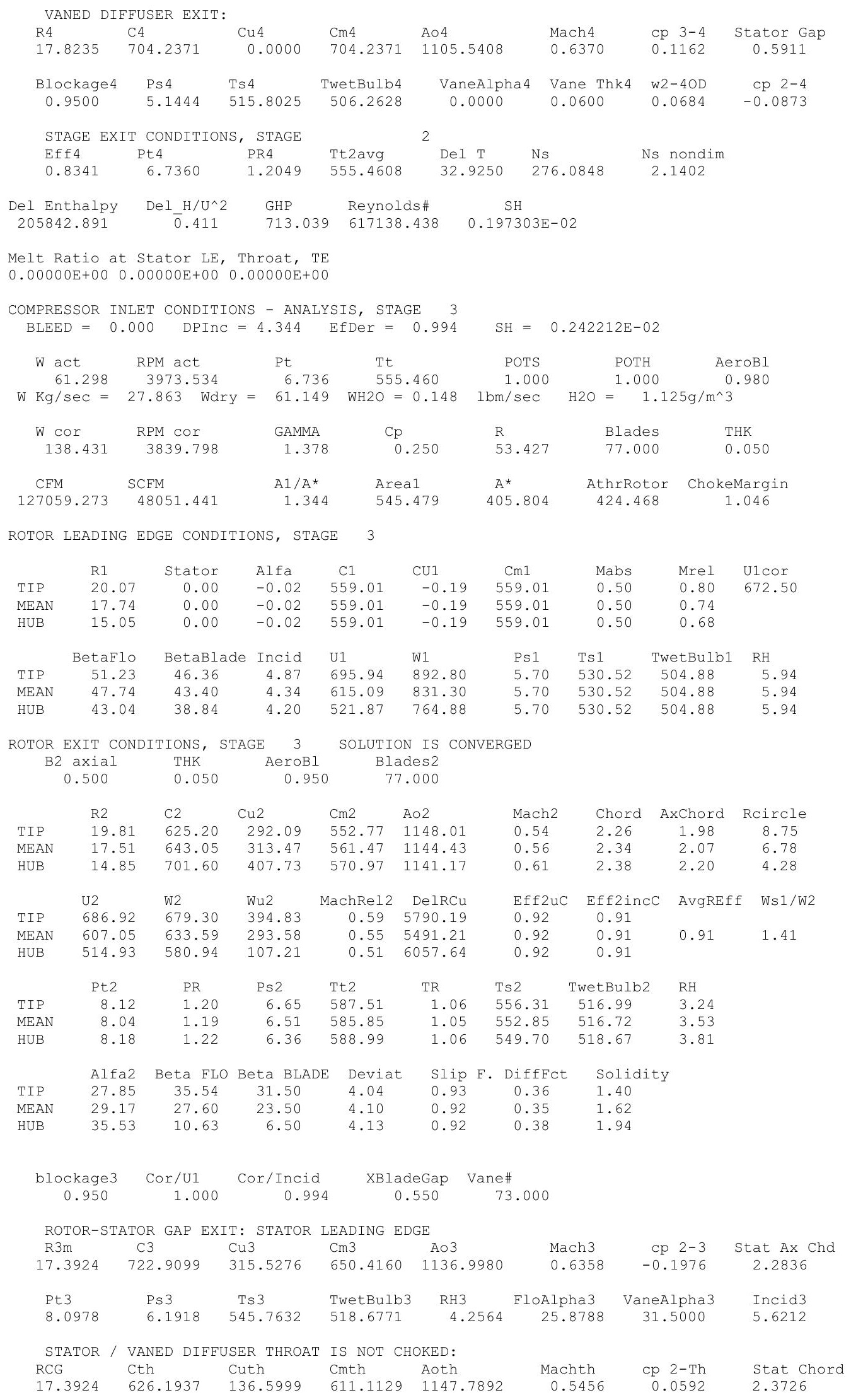




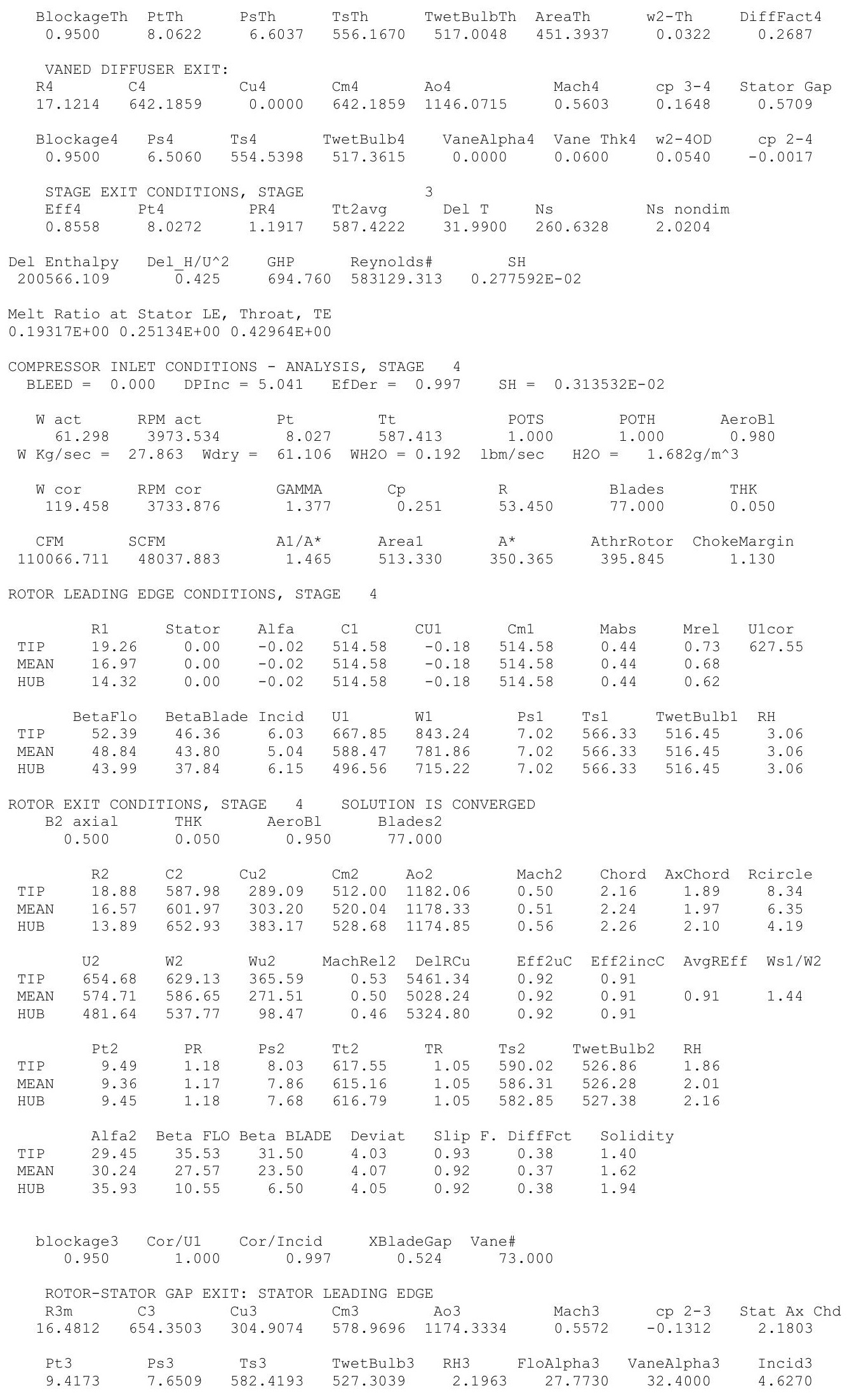




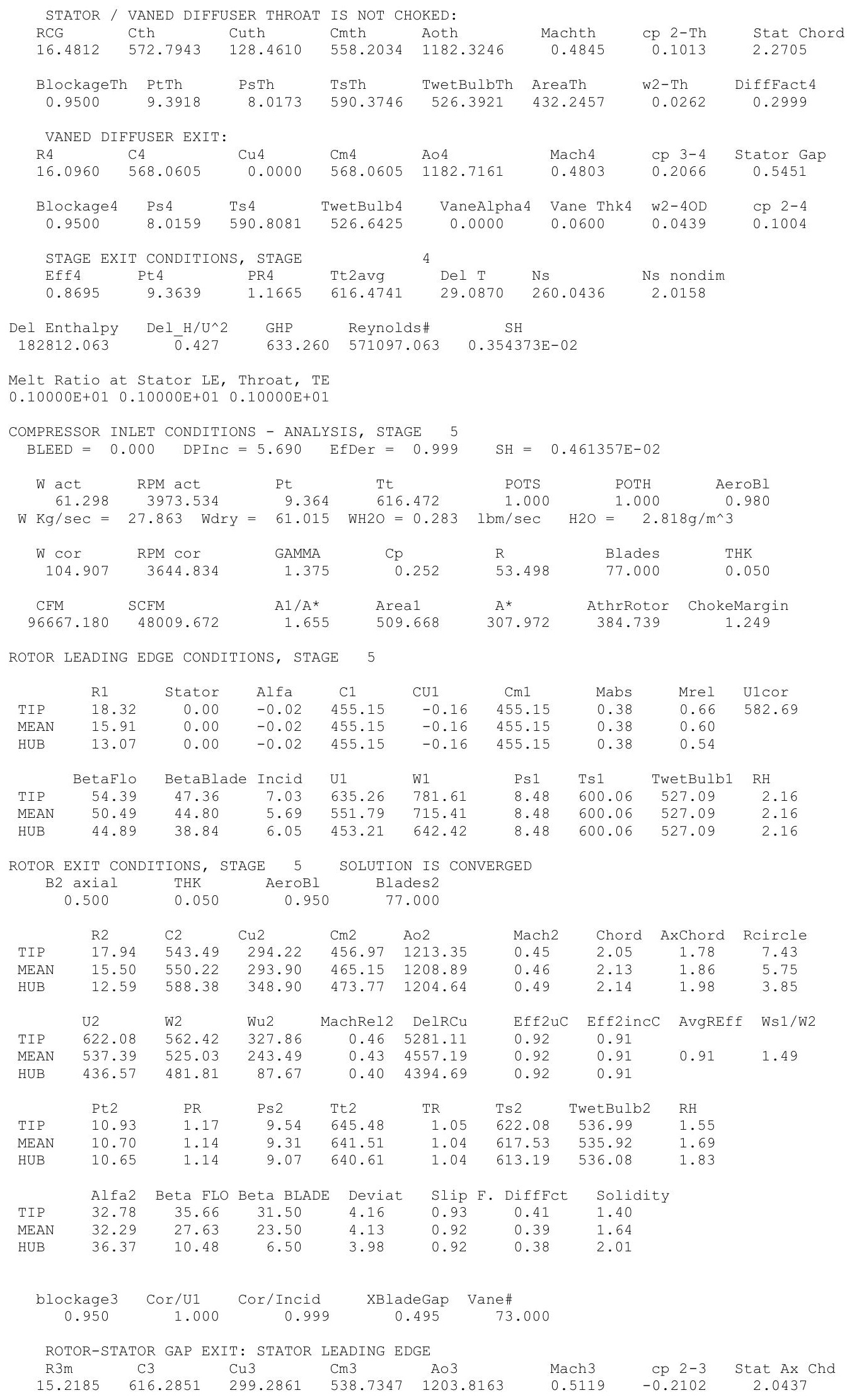




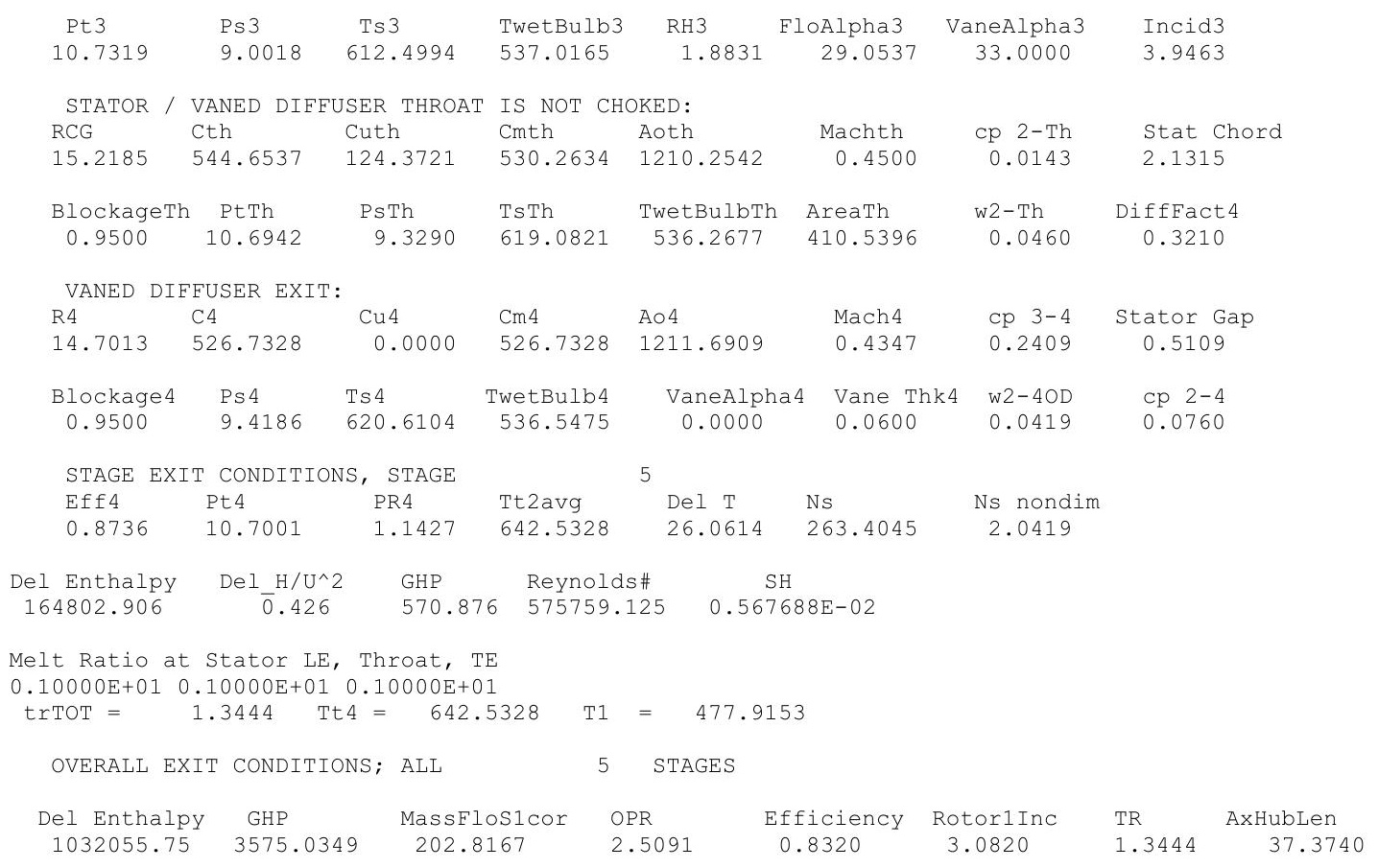




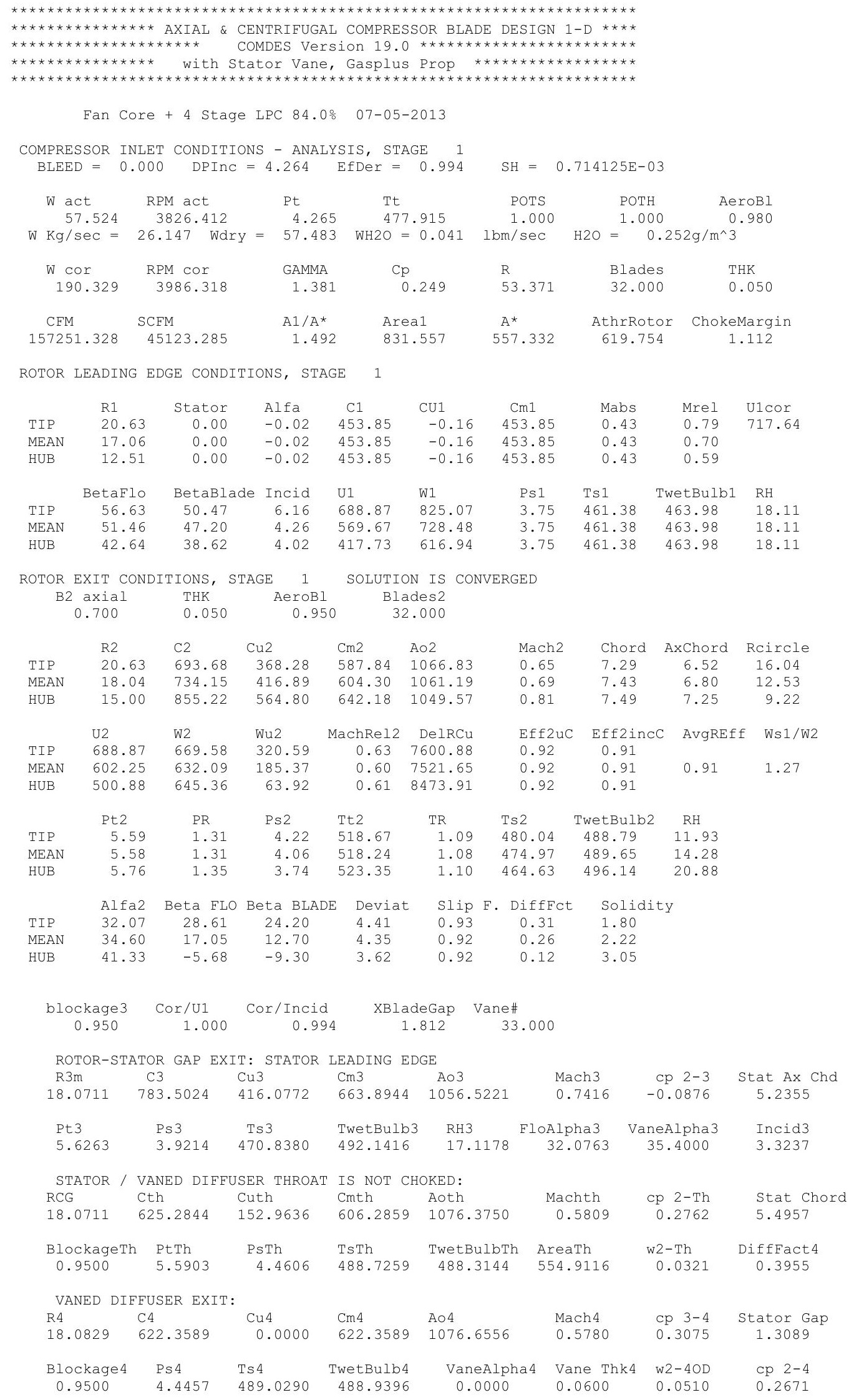




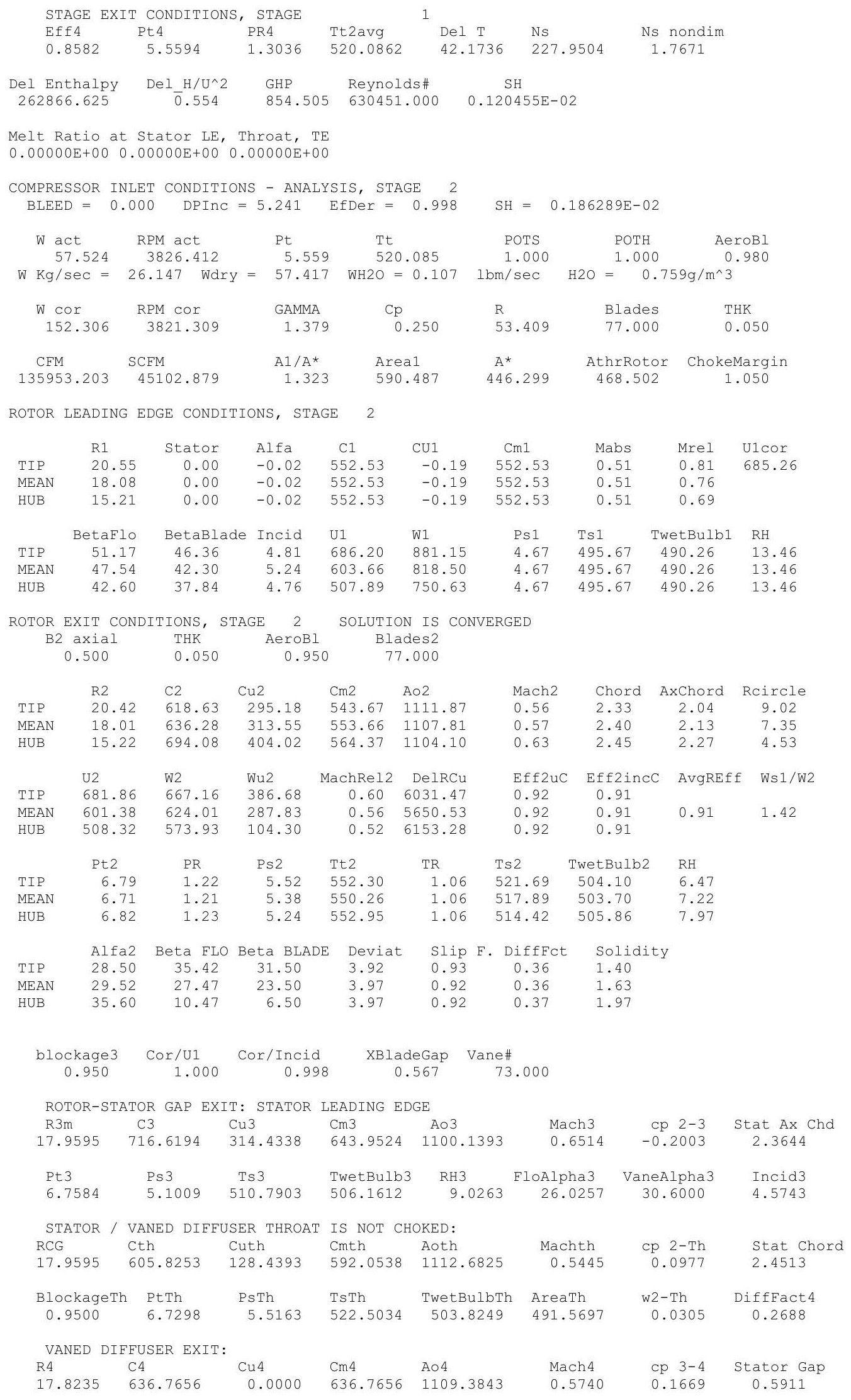




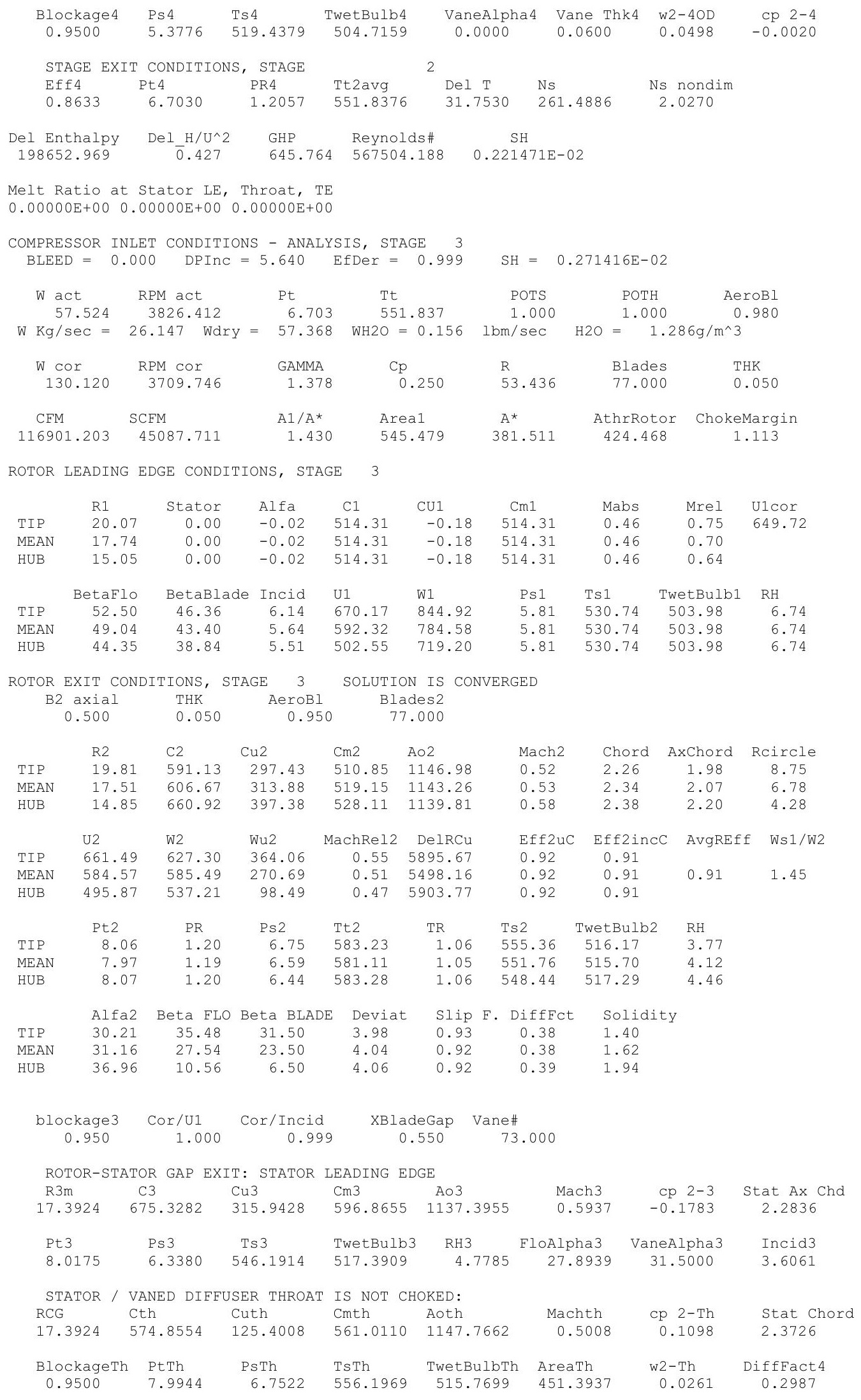




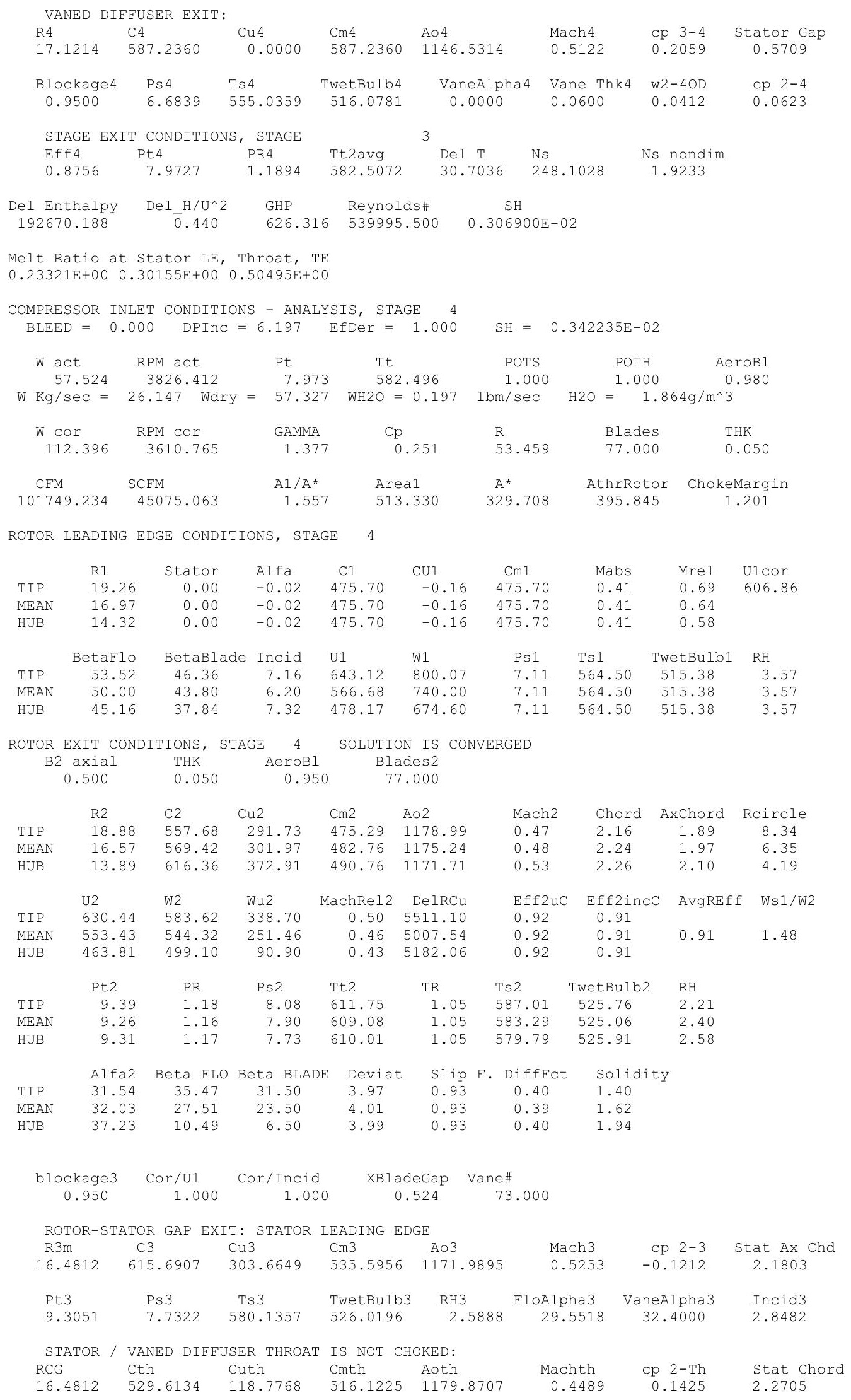




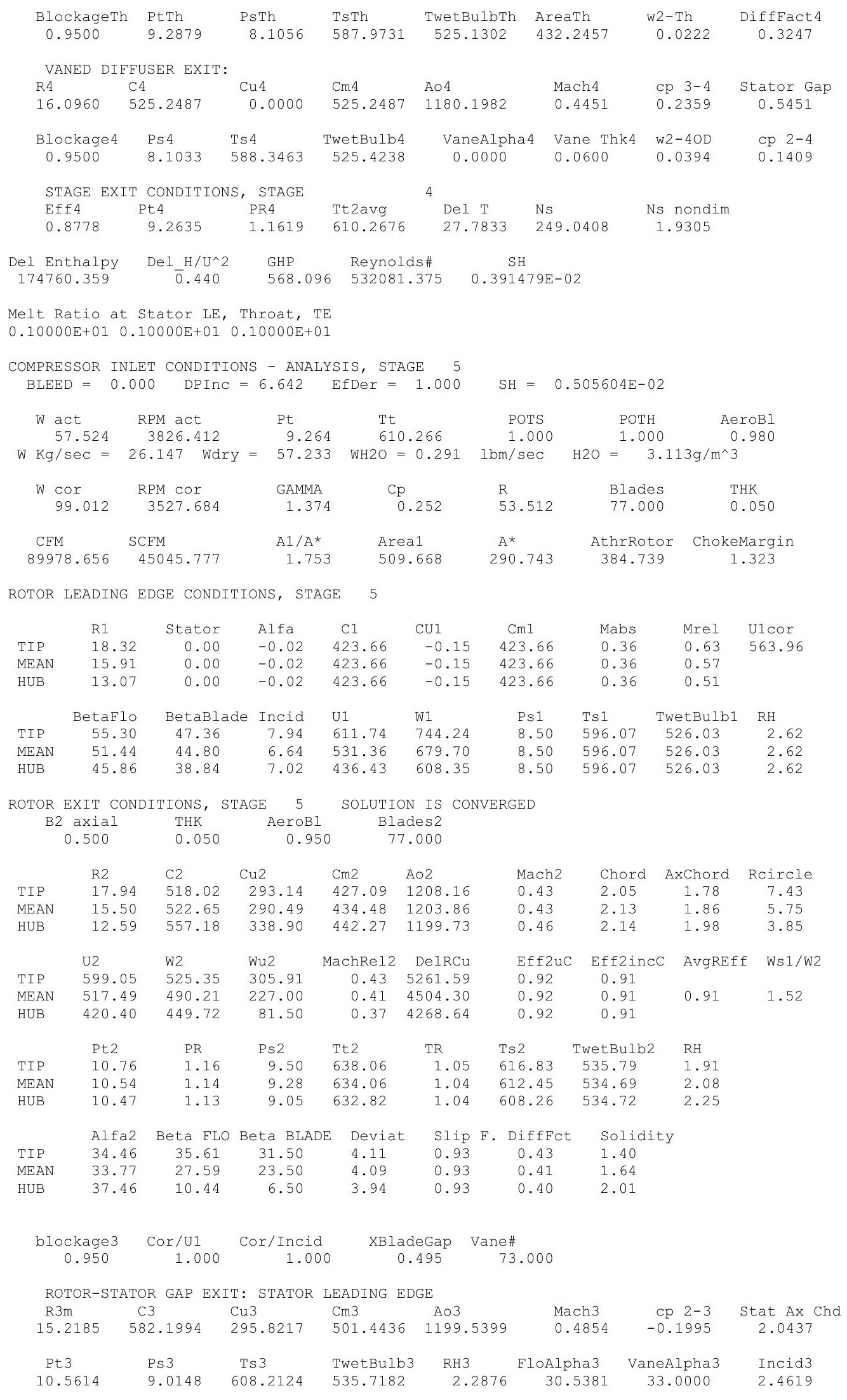




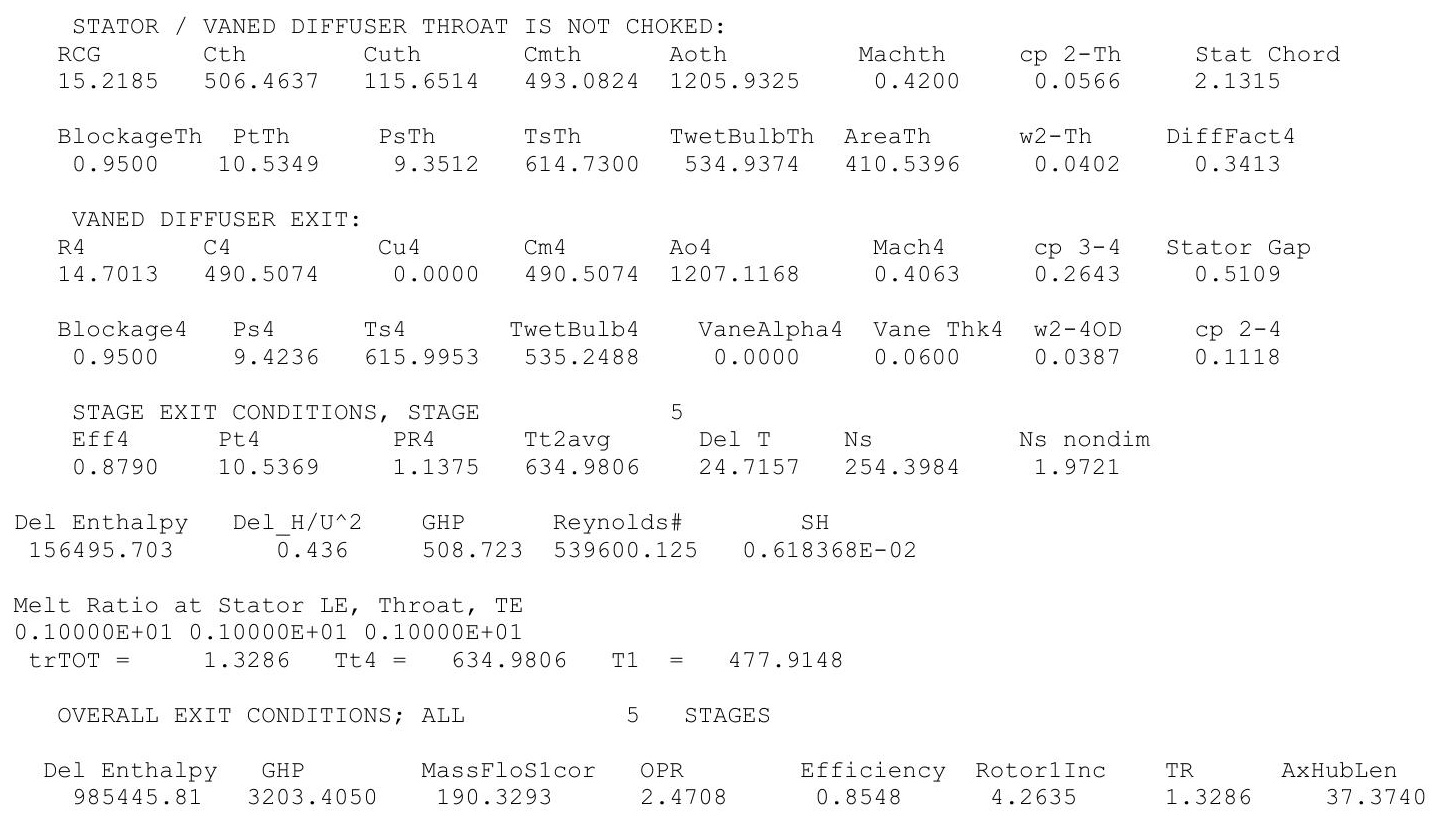




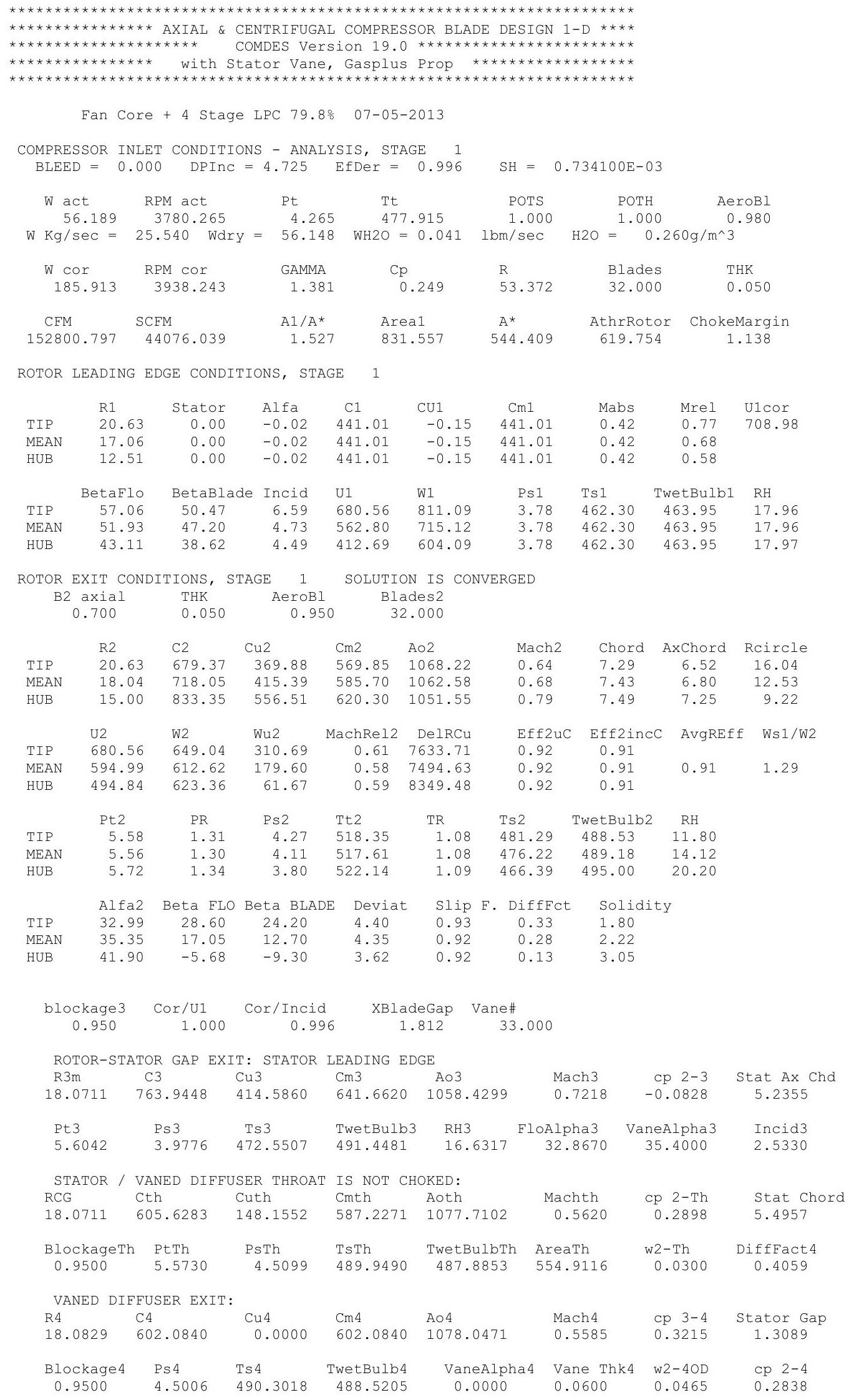




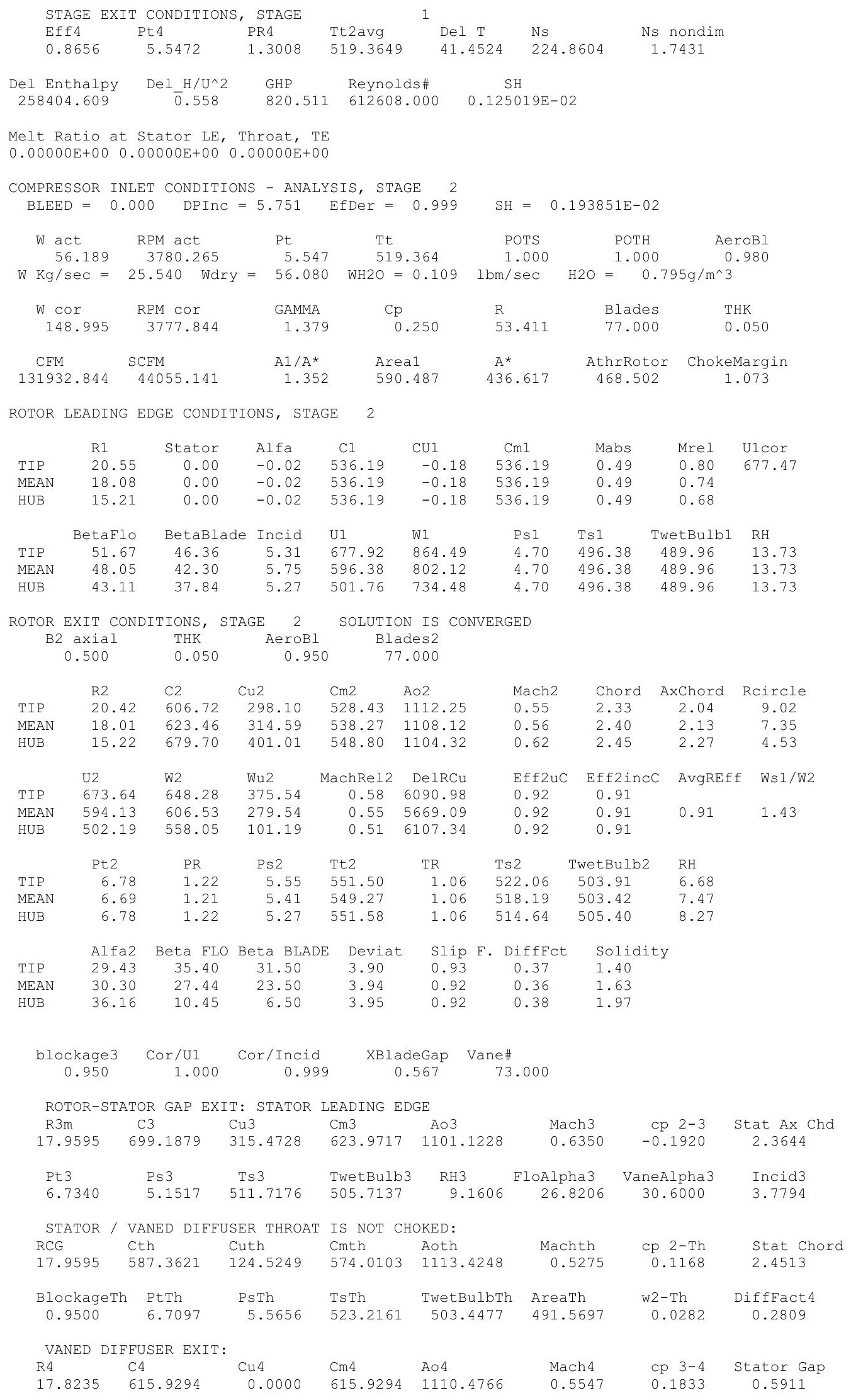




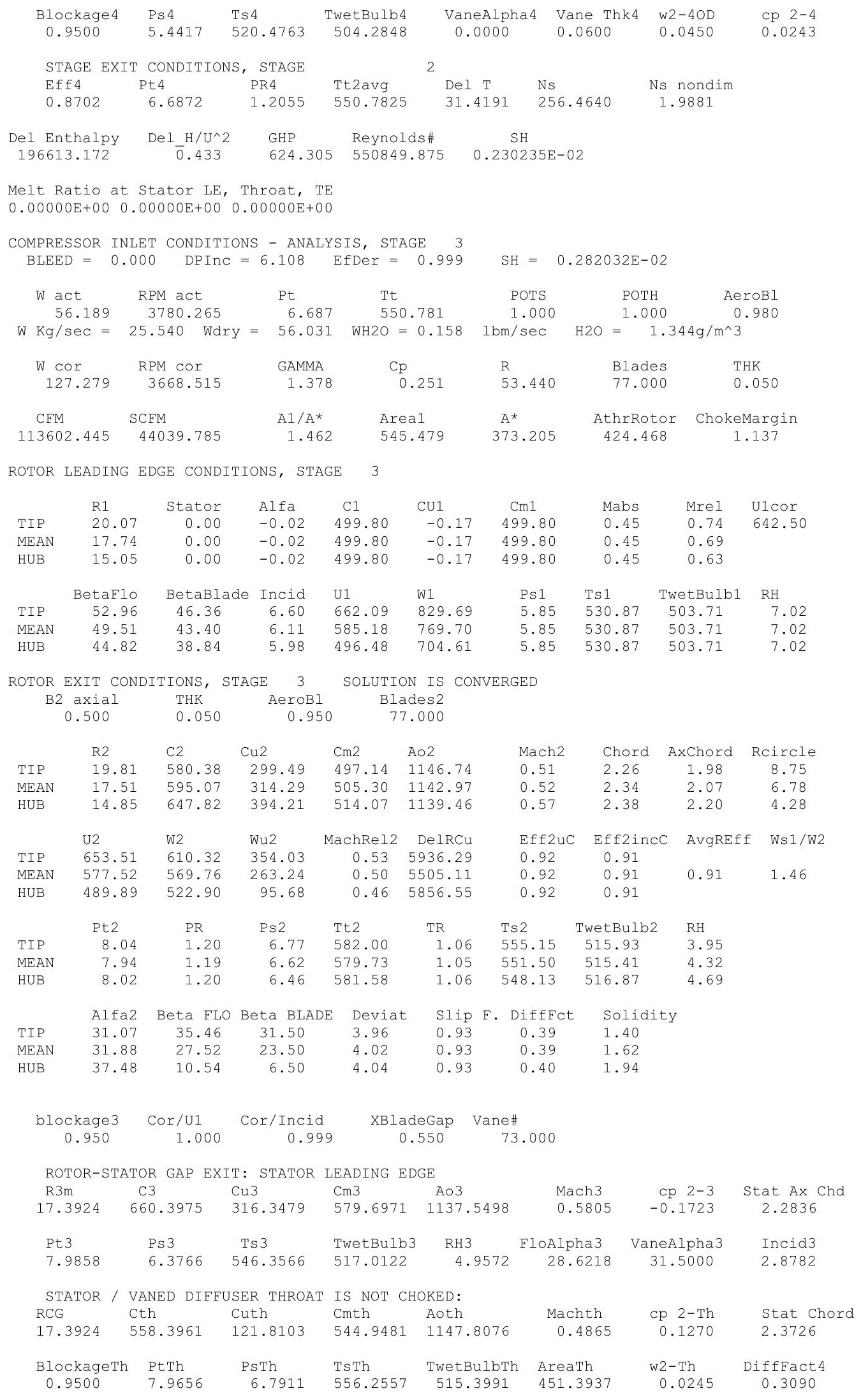




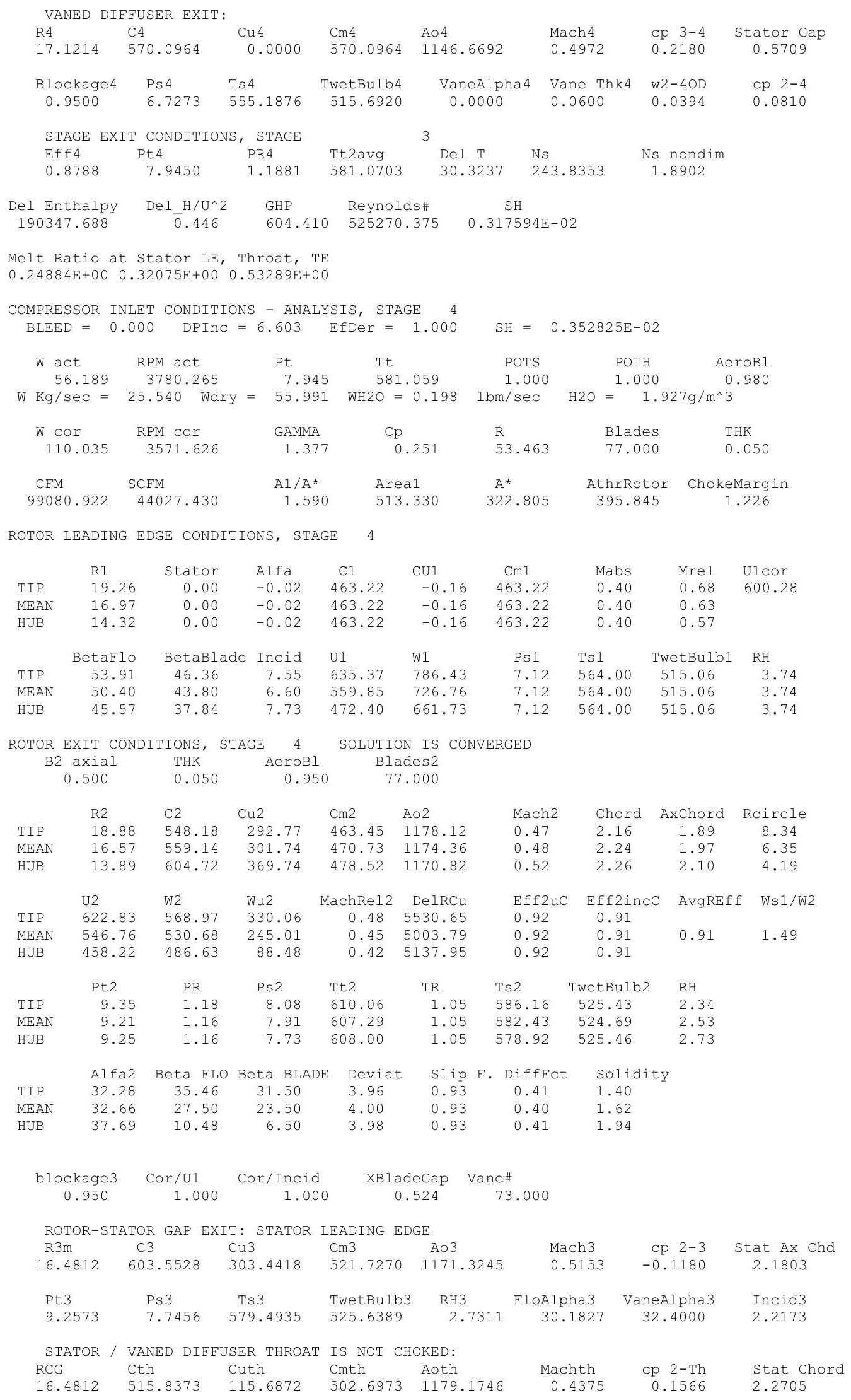




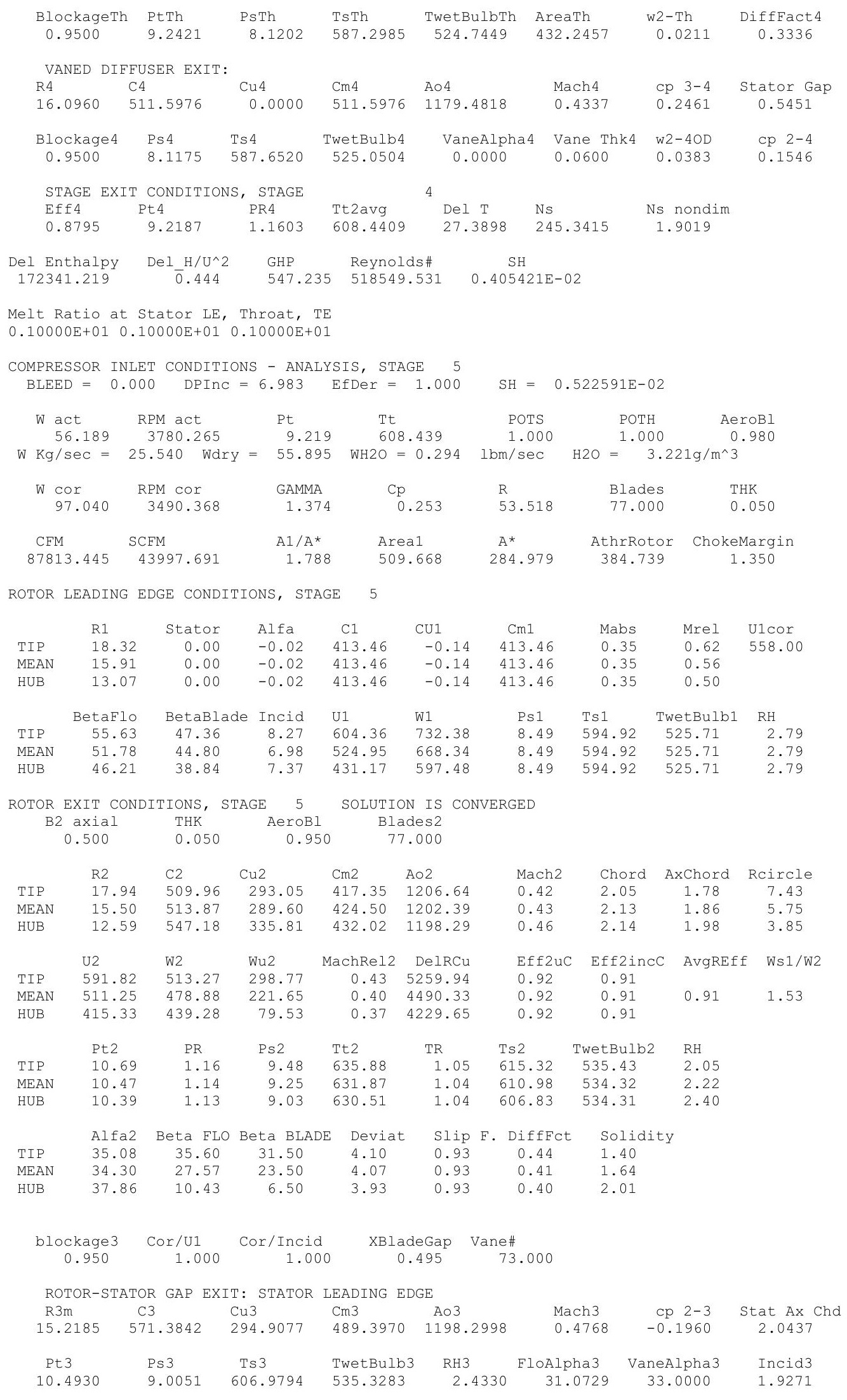




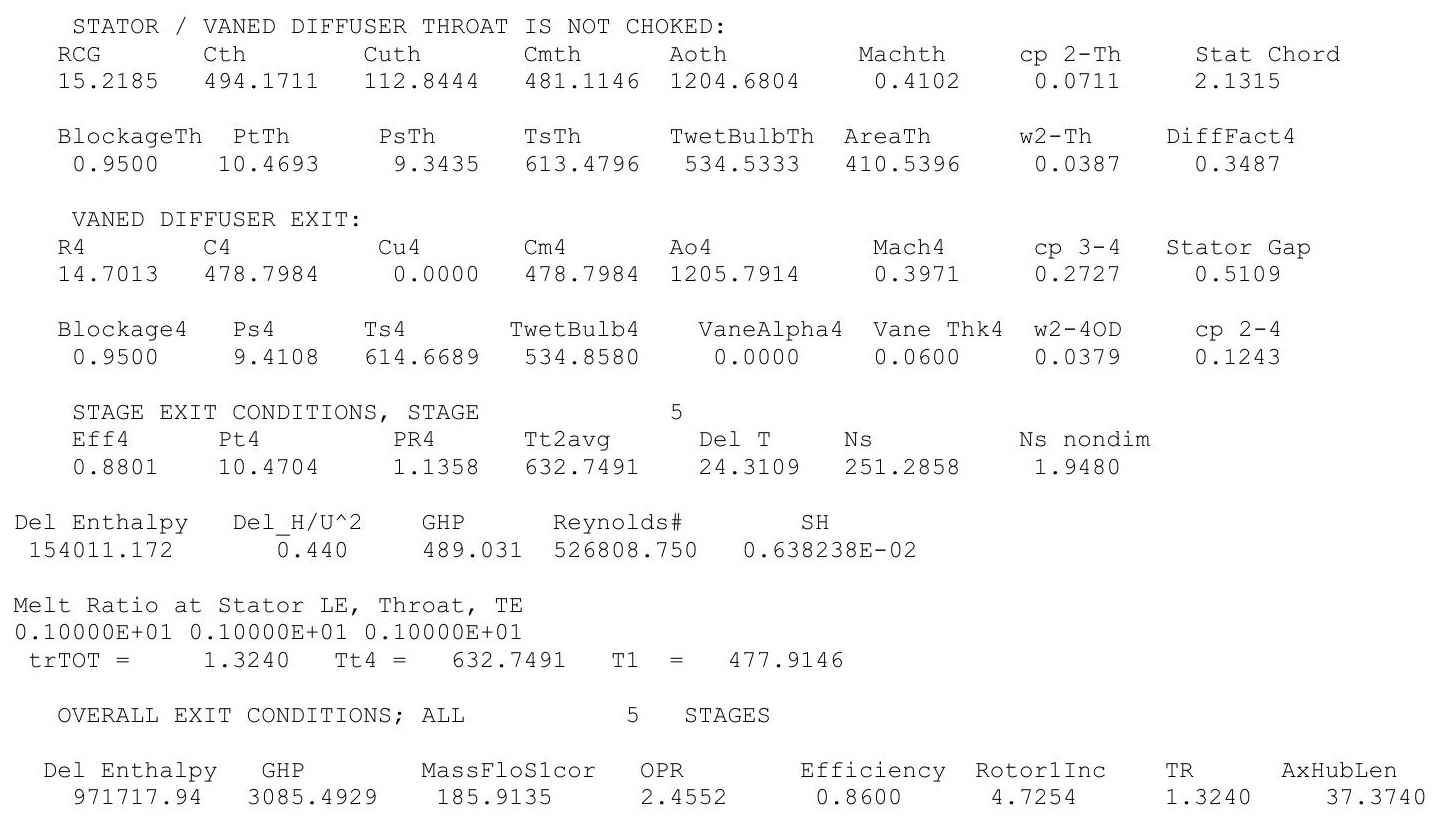




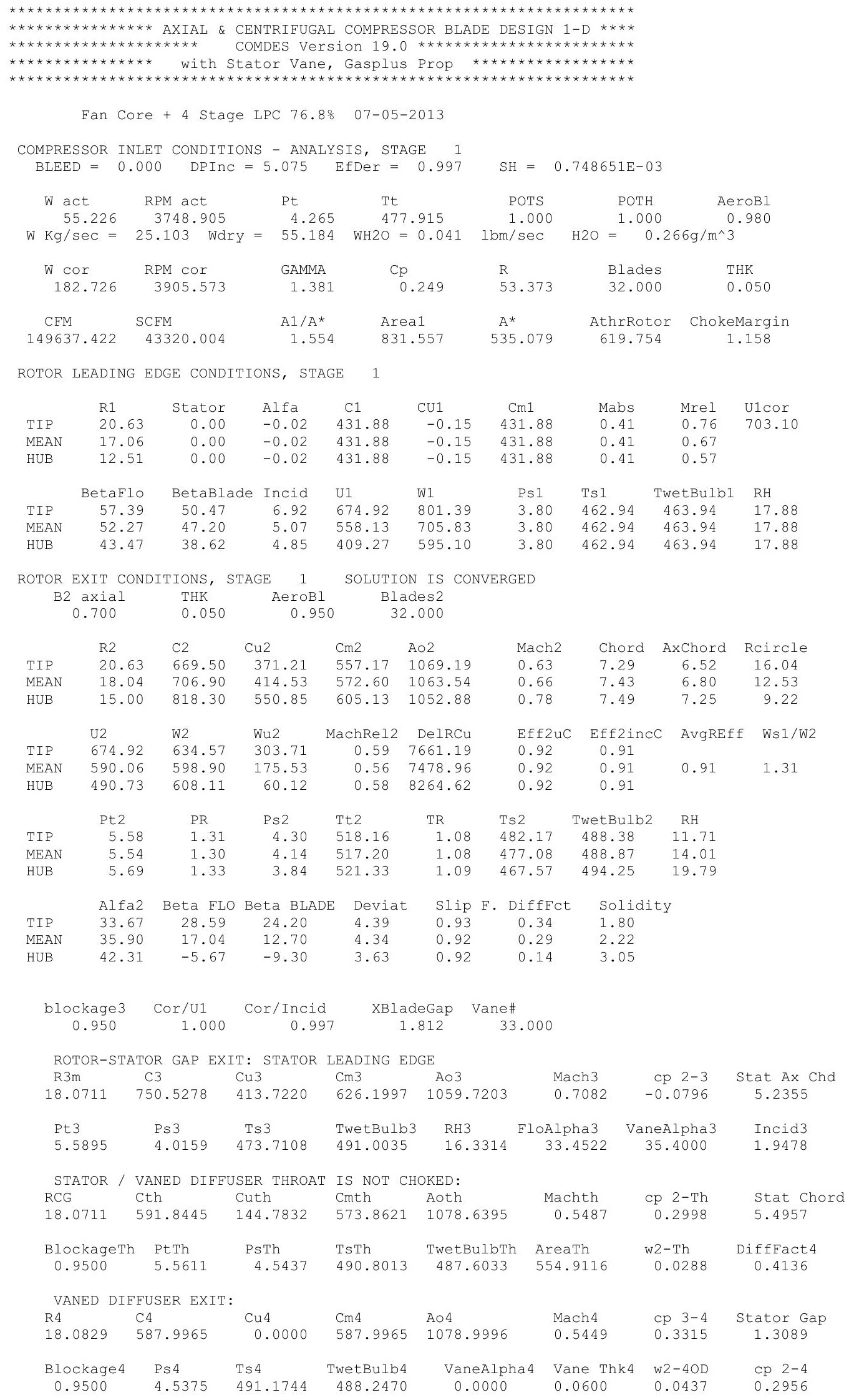




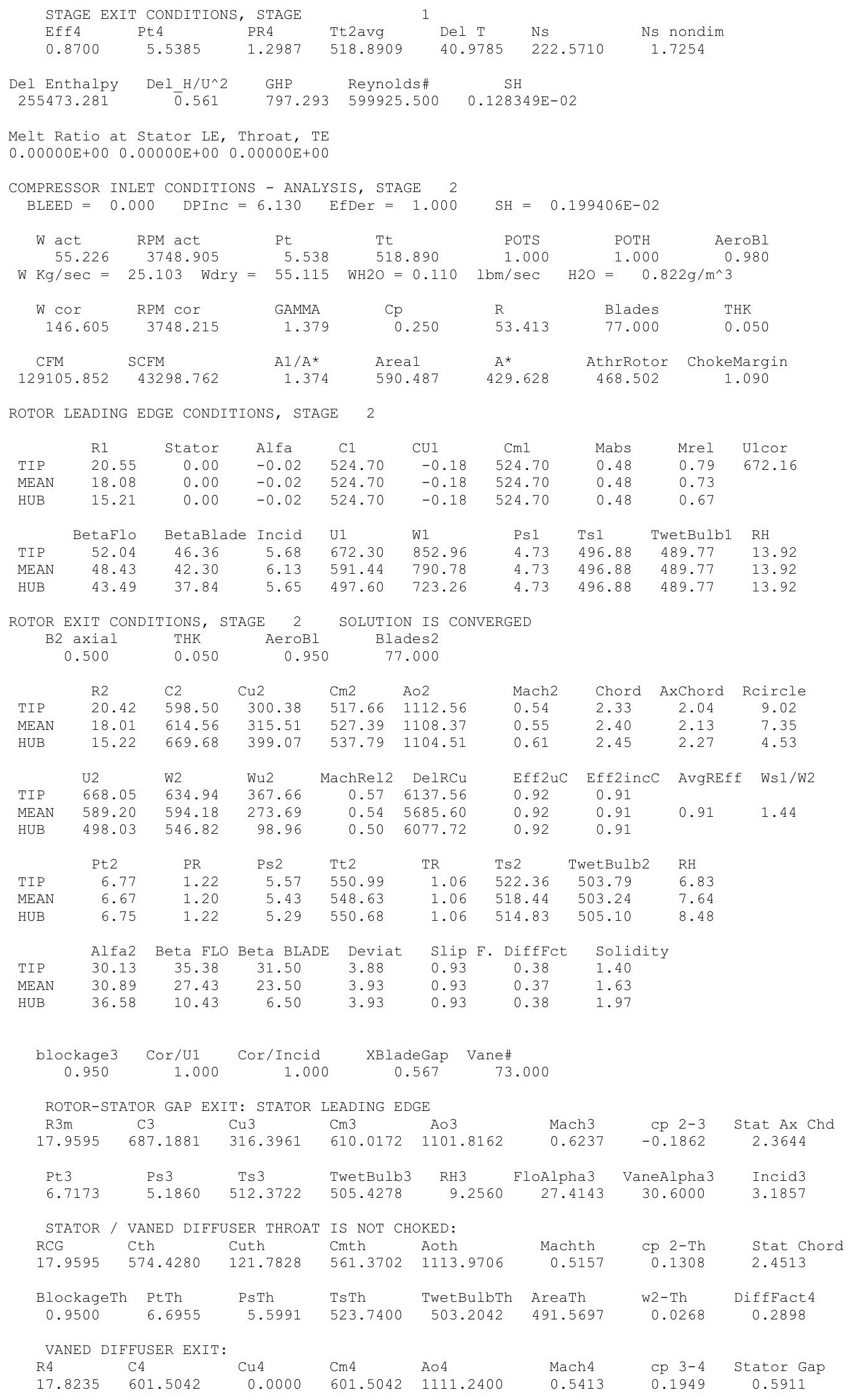




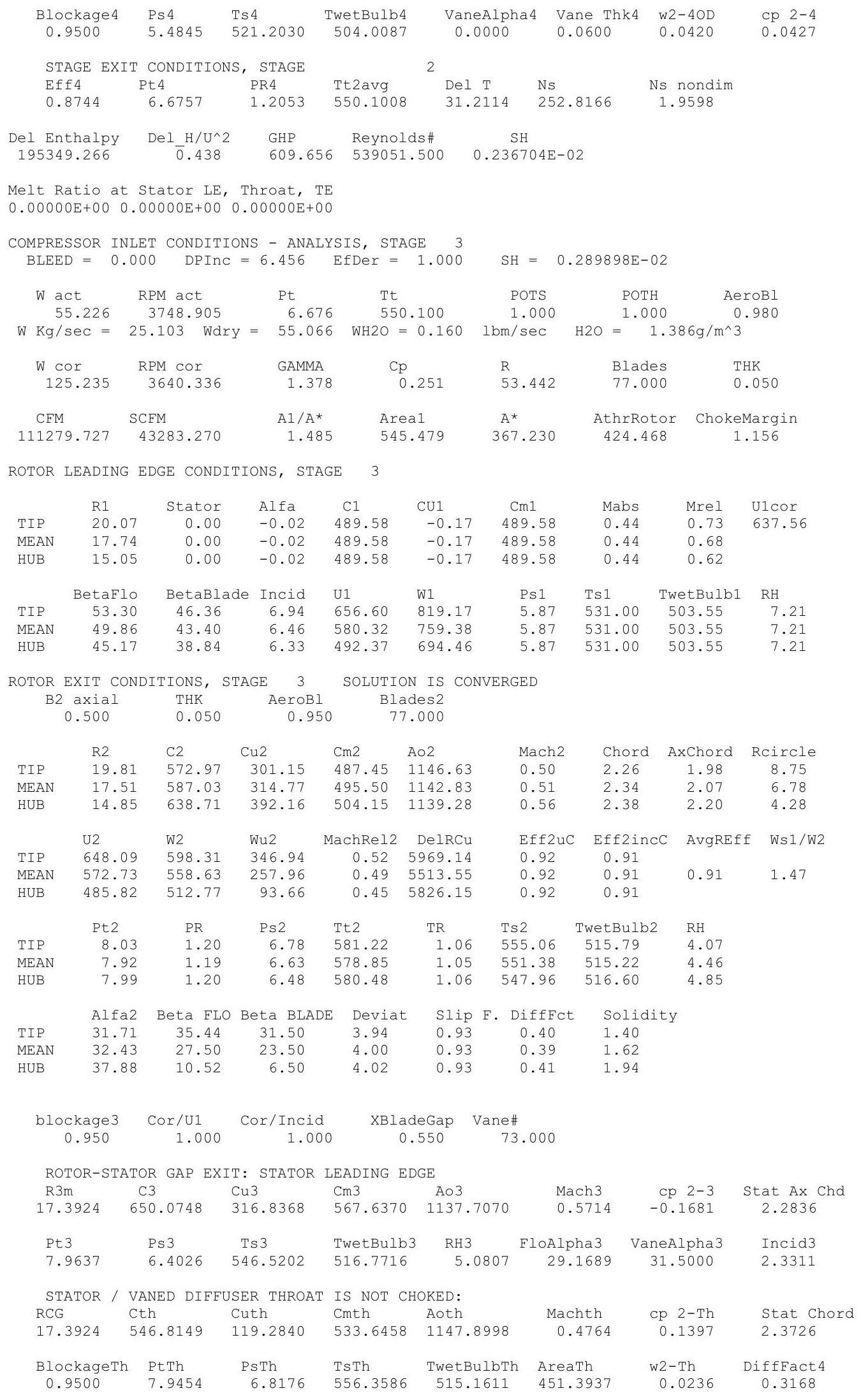




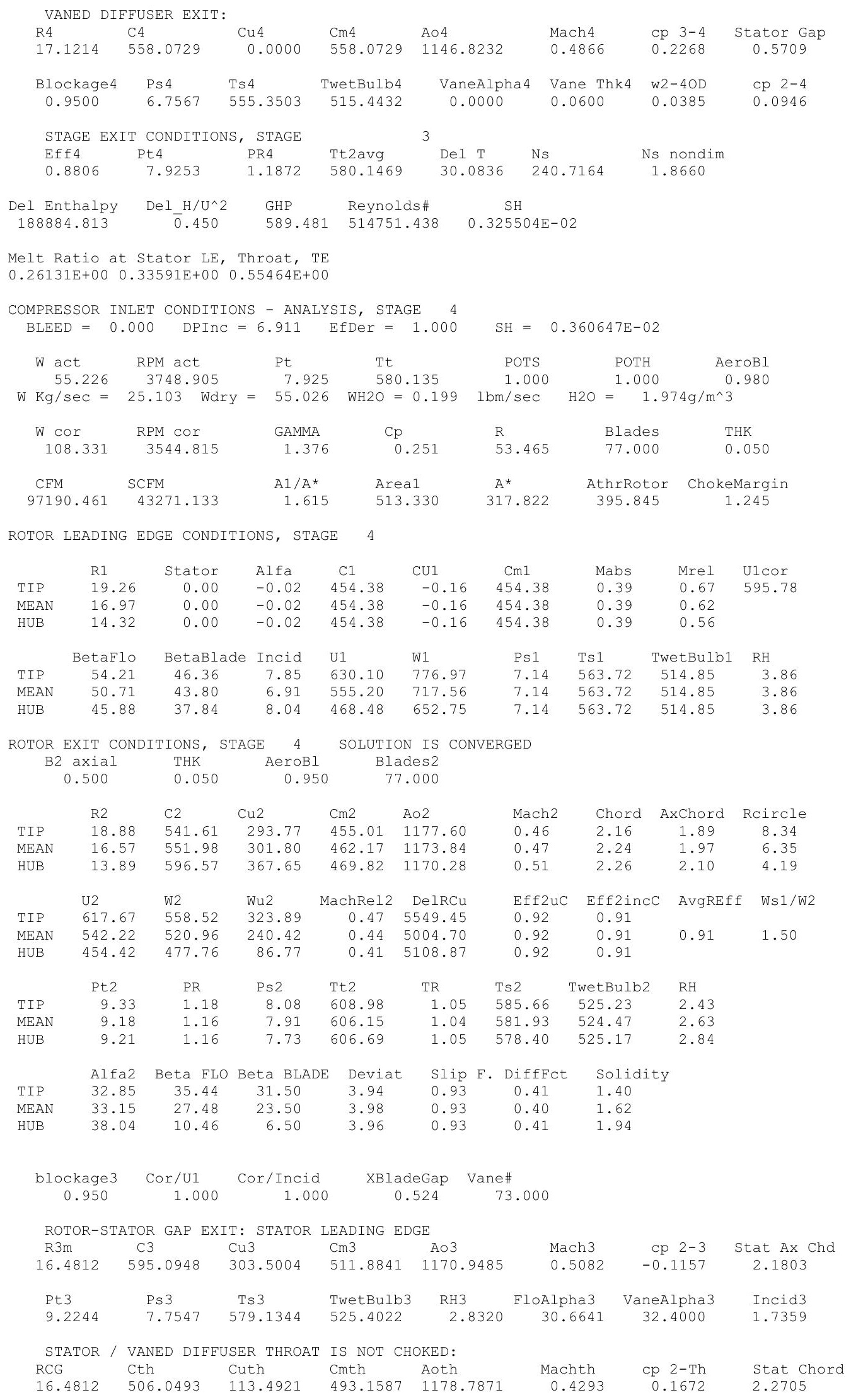




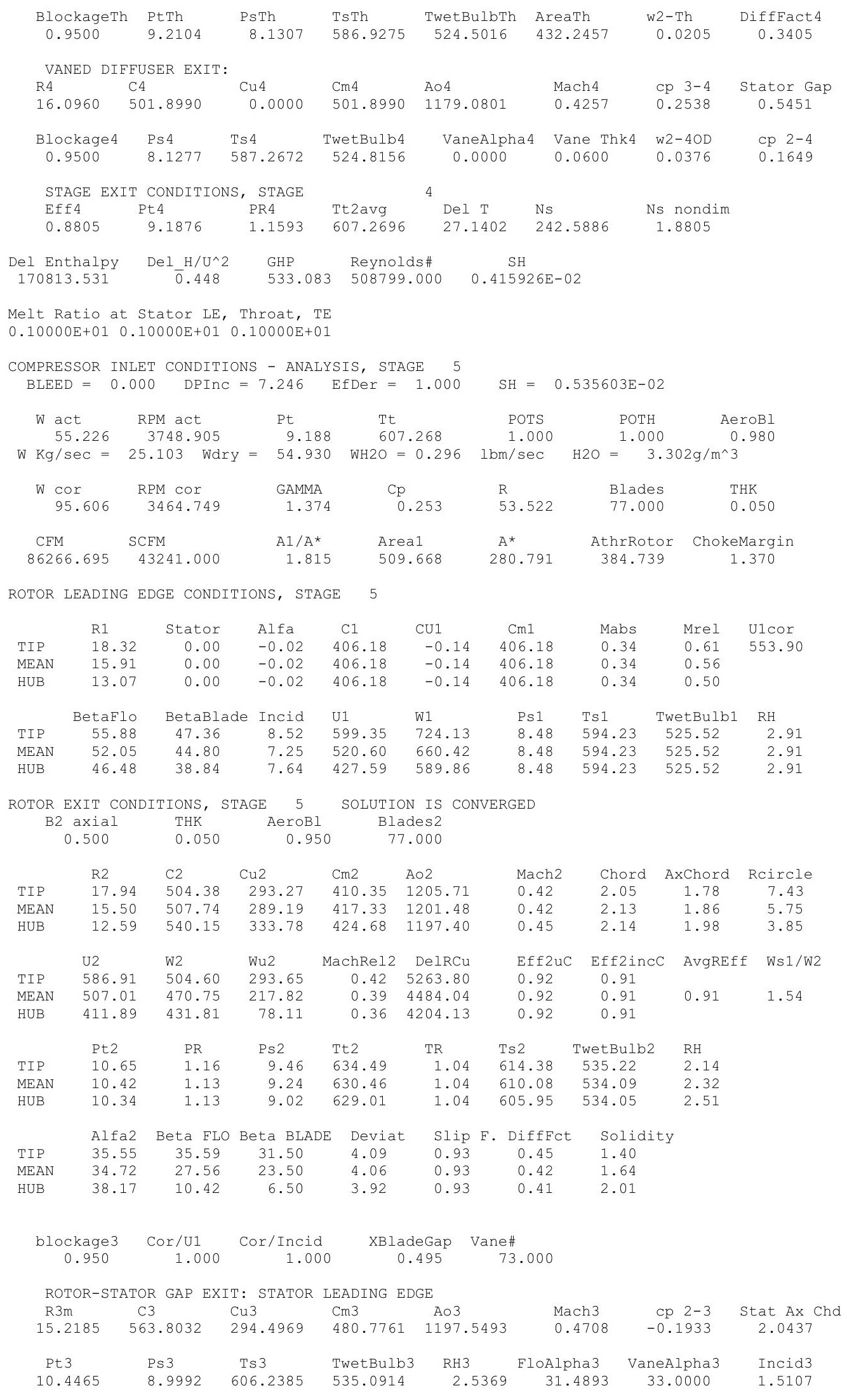




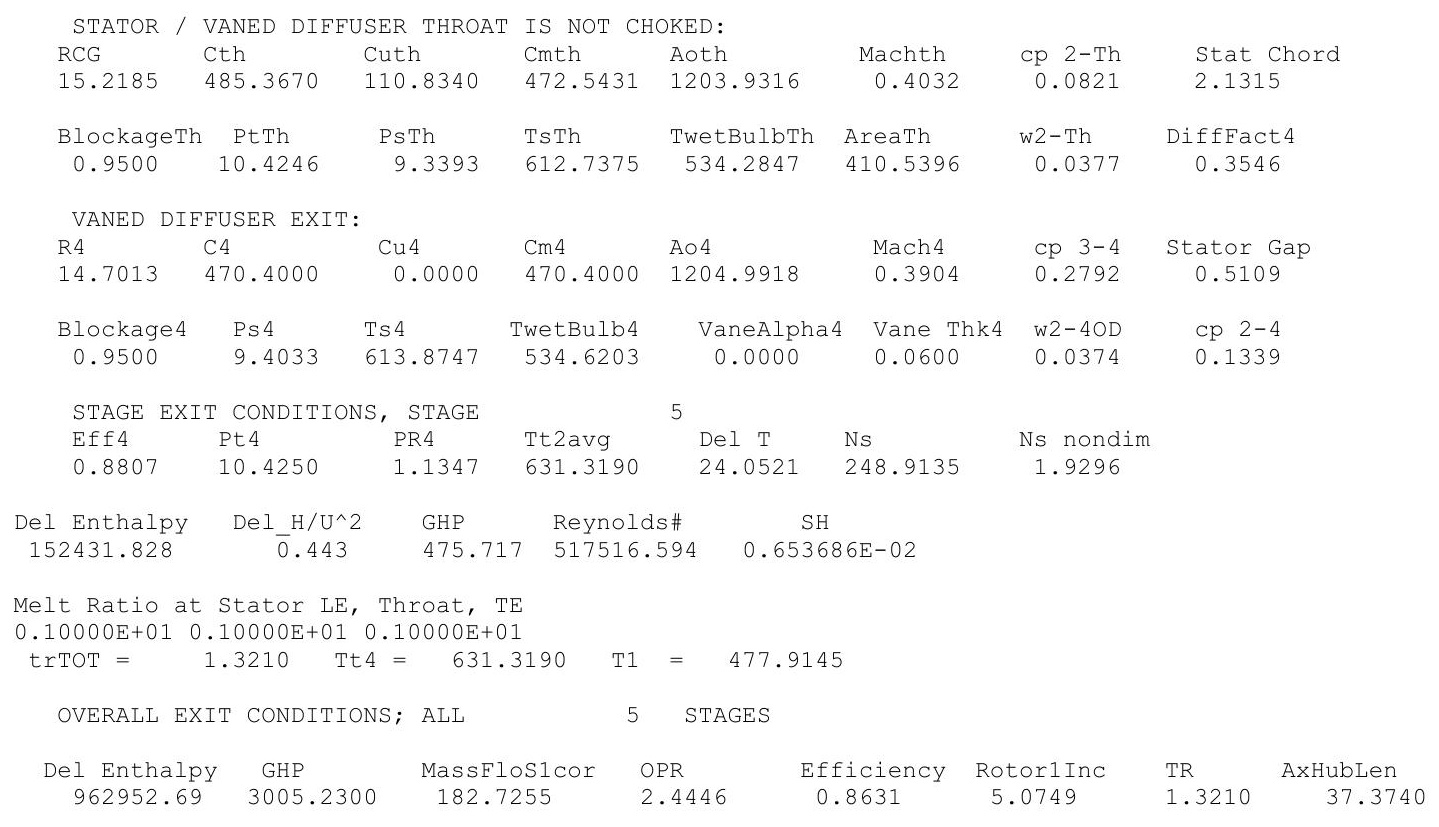




\section{$10 \mu \mathrm{m}$, ISA $+18 \mathrm{R}$}

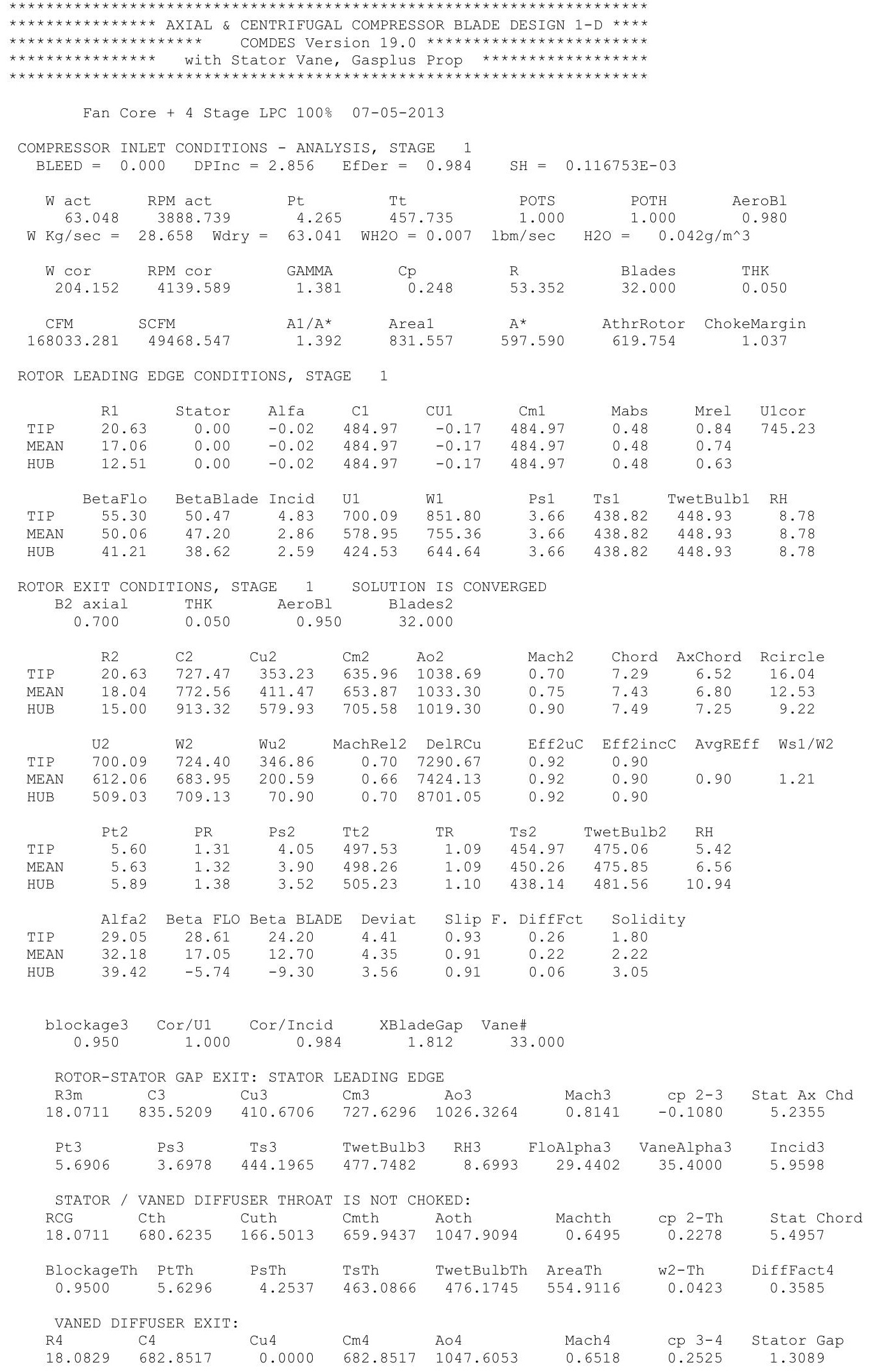




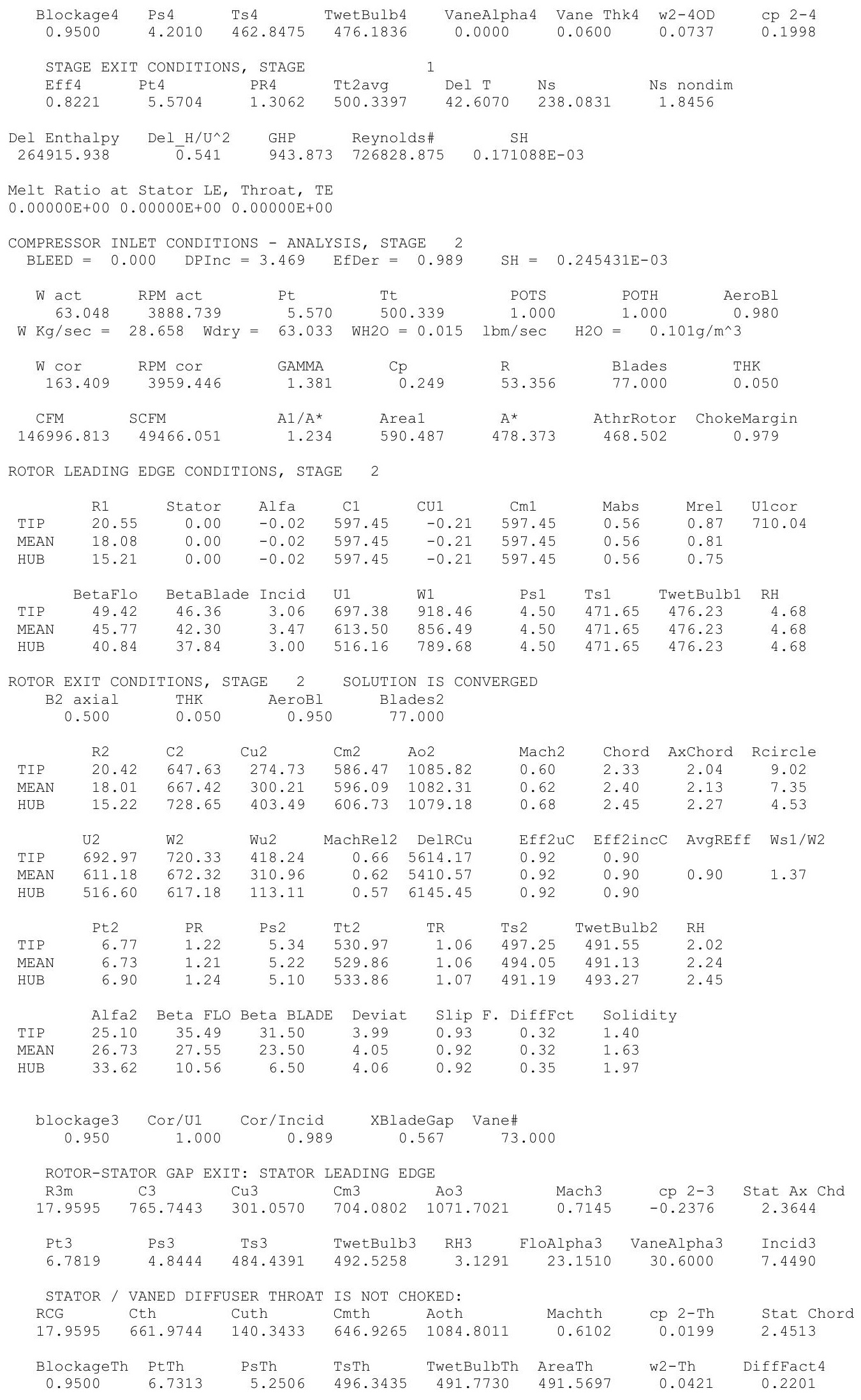




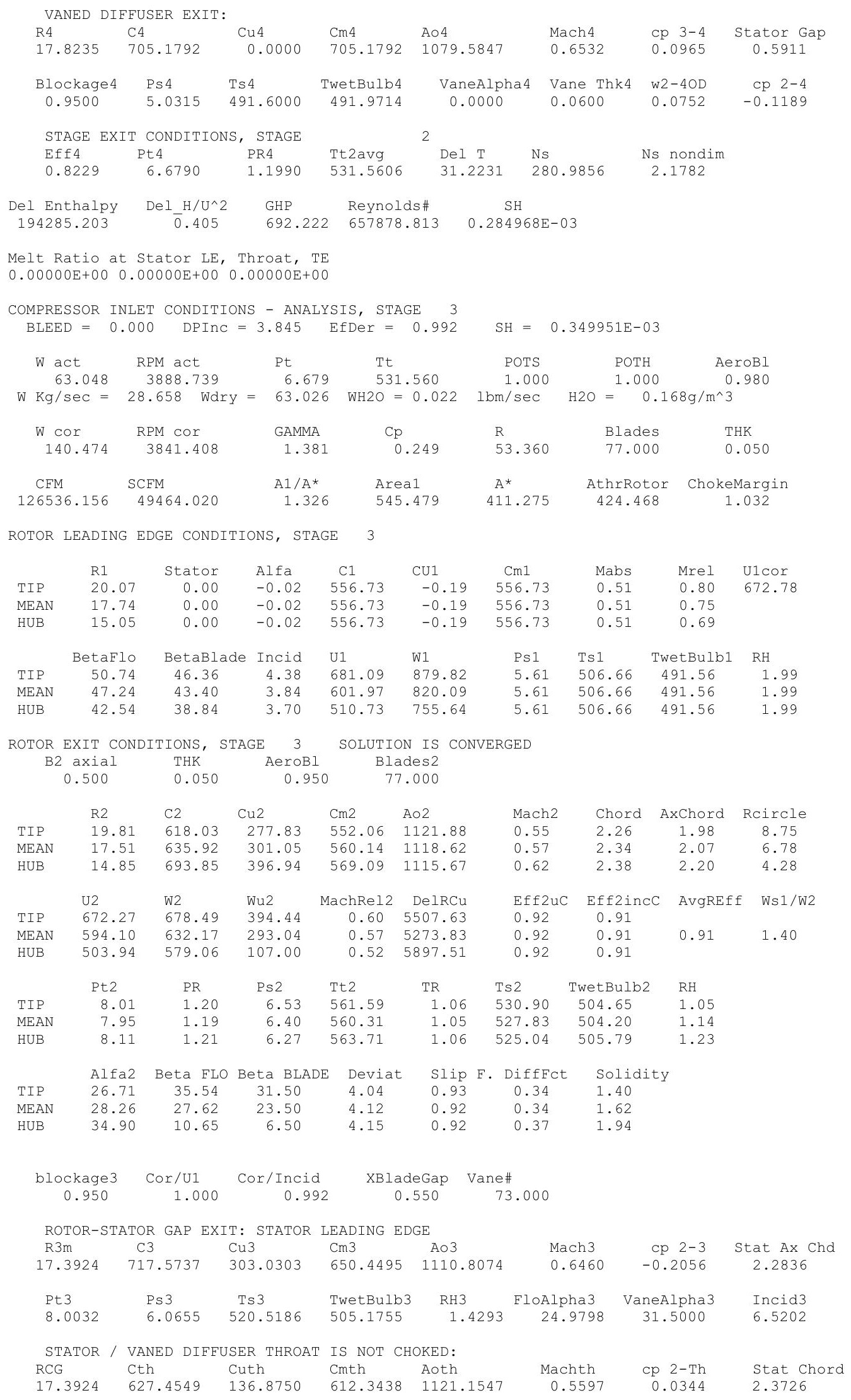




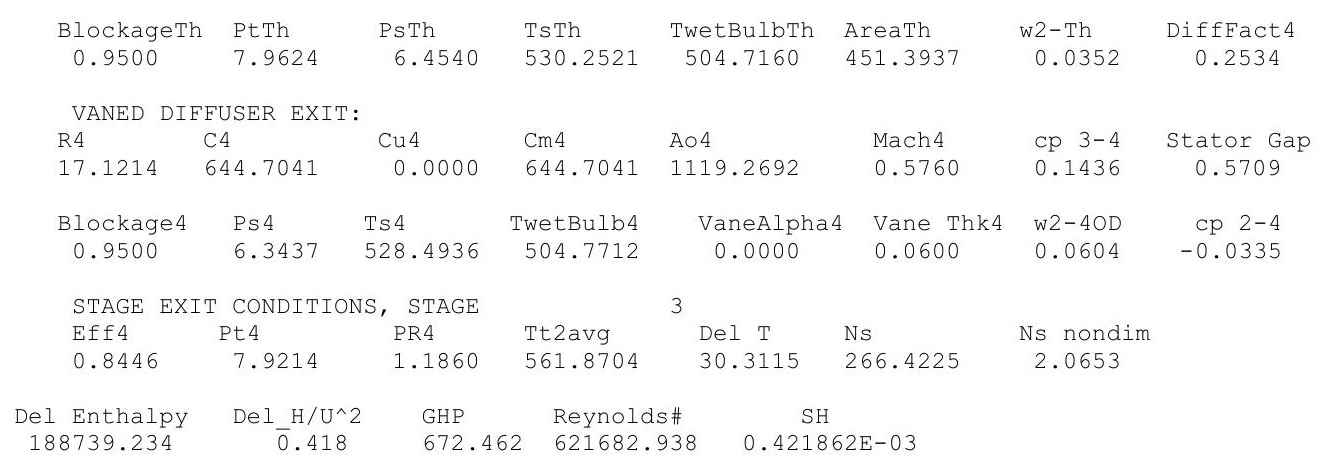

Melt Ratio at Stator LE, Throat, TE

$0.00000 \mathrm{E}+00 \quad 0.00000 \mathrm{E}+00 \quad 0.00000 \mathrm{E}+00$

COMPRESSOR INLET CONDITIONS - ANALYSIS, STAGE 4 $\mathrm{BLEED}=0.000 \quad$ DPInC $=4.434 \quad$ EfDer $=0.995$

$\mathrm{SH}=0.535832 \mathrm{E}-03$

$\begin{array}{llllll}\text { W act } & \text { RPM act } & \text { Pt } & \text { POTS } & \text { POTH } & \text { AeroBI }\end{array}$ $\mathrm{W} \mathrm{Kg} / \mathrm{sec}=28.658$ Wdry $=63.015$ WH2O $=0.034 \quad \mathrm{lbm} / \mathrm{sec} \quad \mathrm{H} 2 \mathrm{O}=\begin{gathered}1.000 \\ 0.296 \mathrm{~g} / \mathrm{m}^{\wedge} 3\end{gathered}$

$\begin{array}{ccccccc}\text { W cor } & \text { RPM cor } & \text { GAMMA } & \text { Cp } & \text { R } & \text { Blades } & \text { THK } \\ 121.772 & 3736.361 & 1.380 & 0.249 & 53.366 & 77.000 & 0.050 \\ \text { CFM } & \text { SCFM } & \text { A1/A* } & \text { Area1 } & \text { A* } & \text { AthrRotor } & \text { ChokeMargin } \\ 110039.742 & 49460.410 & 1.440 & 513.330 & 356.576 & 395.845 & 1.110\end{array}$

ROTOR LEADING EDGE CONDITIONS, STAGE 4

\begin{tabular}{|c|c|c|c|c|c|c|c|c|c|}
\hline & R1 & Stator & Alfa & $\mathrm{C} 1$ & CU1 & $\mathrm{Cm} 1$ & Mabs & Mrel & U1cor \\
\hline TIP & 19.26 & 0.00 & -0.02 & 514.47 & -0.18 & 514.47 & 0.45 & 0.73 & 627.97 \\
\hline MEAN & 16.97 & 0.00 & -0.02 & 514.47 & -0.18 & 514.47 & 0.45 & 0.68 & \\
\hline HUB & 14.32 & 0.00 & -0.02 & 514.47 & -0.18 & 514.47 & 0.45 & 0.63 & \\
\hline & BetaFlo & BetaBlade & Incid & U1 & W1 & Ps 1 & Ts 1 & TwetBulb1 & $\mathrm{RH}$ \\
\hline TIP & 51.80 & 46.36 & 5.44 & 653.60 & 831.93 & 6.89 & 540.63 & 504.65 & 1.14 \\
\hline MEAN & 48.23 & 43.80 & 4.43 & 575.91 & 772.37 & 6.89 & 540.63 & 504.65 & 1.14 \\
\hline HUB & 43.38 & 37.84 & 5.54 & 485.96 & 707.82 & 6.89 & 540.63 & 504.65 & 1.14 \\
\hline
\end{tabular}

ROTOR EXIT CONDITIONS, STAGE 4 SOLUTION IS CONVERGED

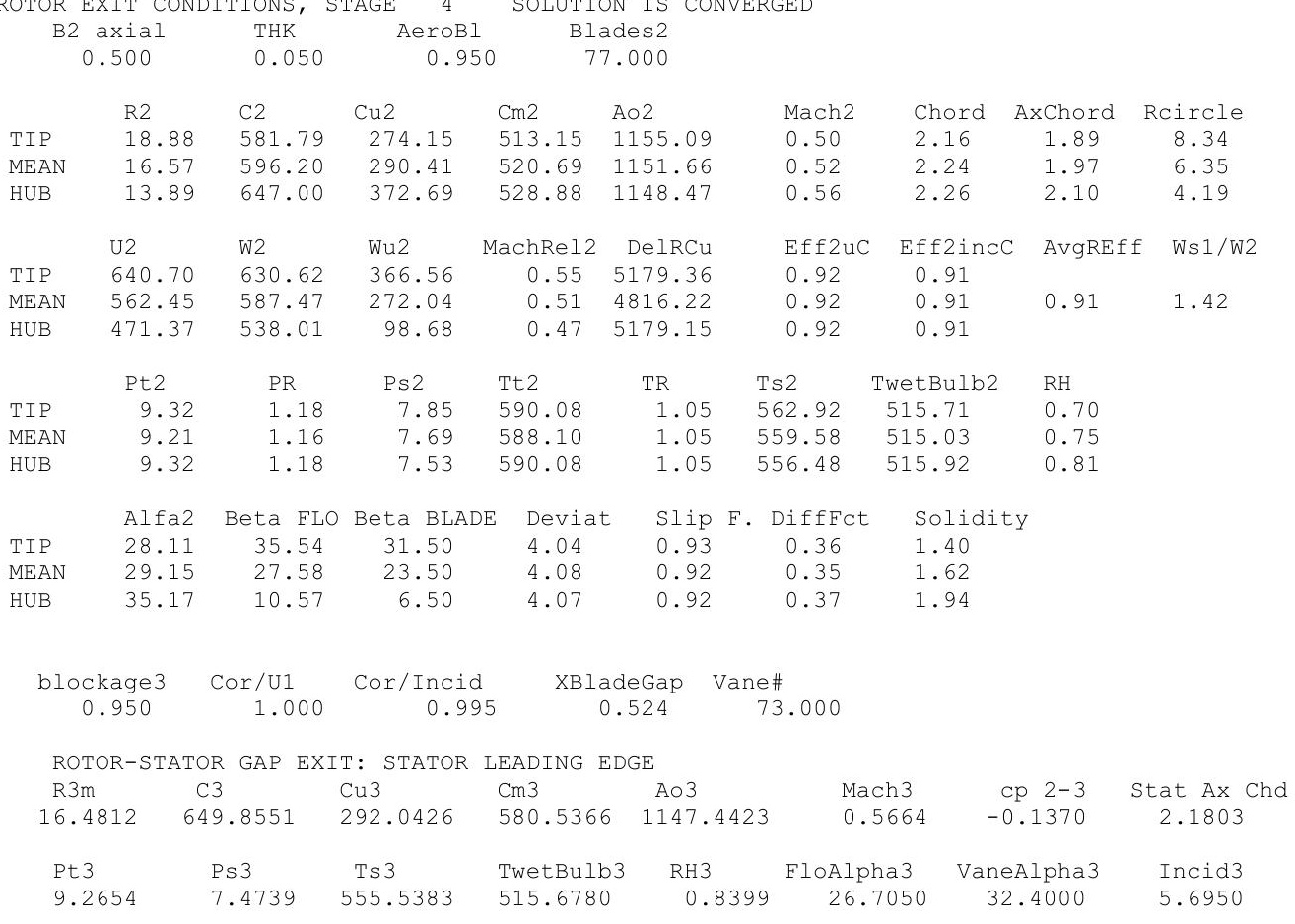




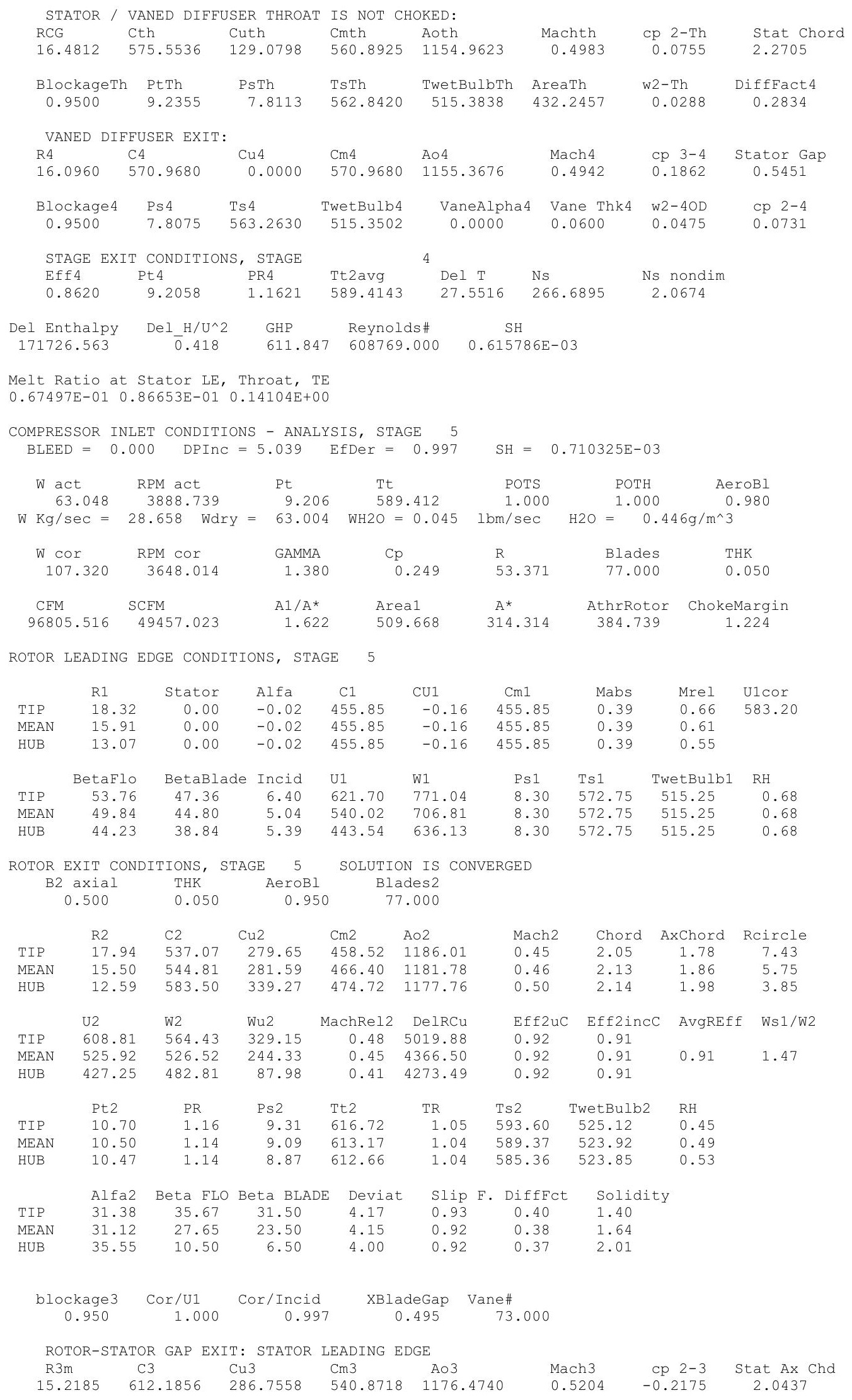




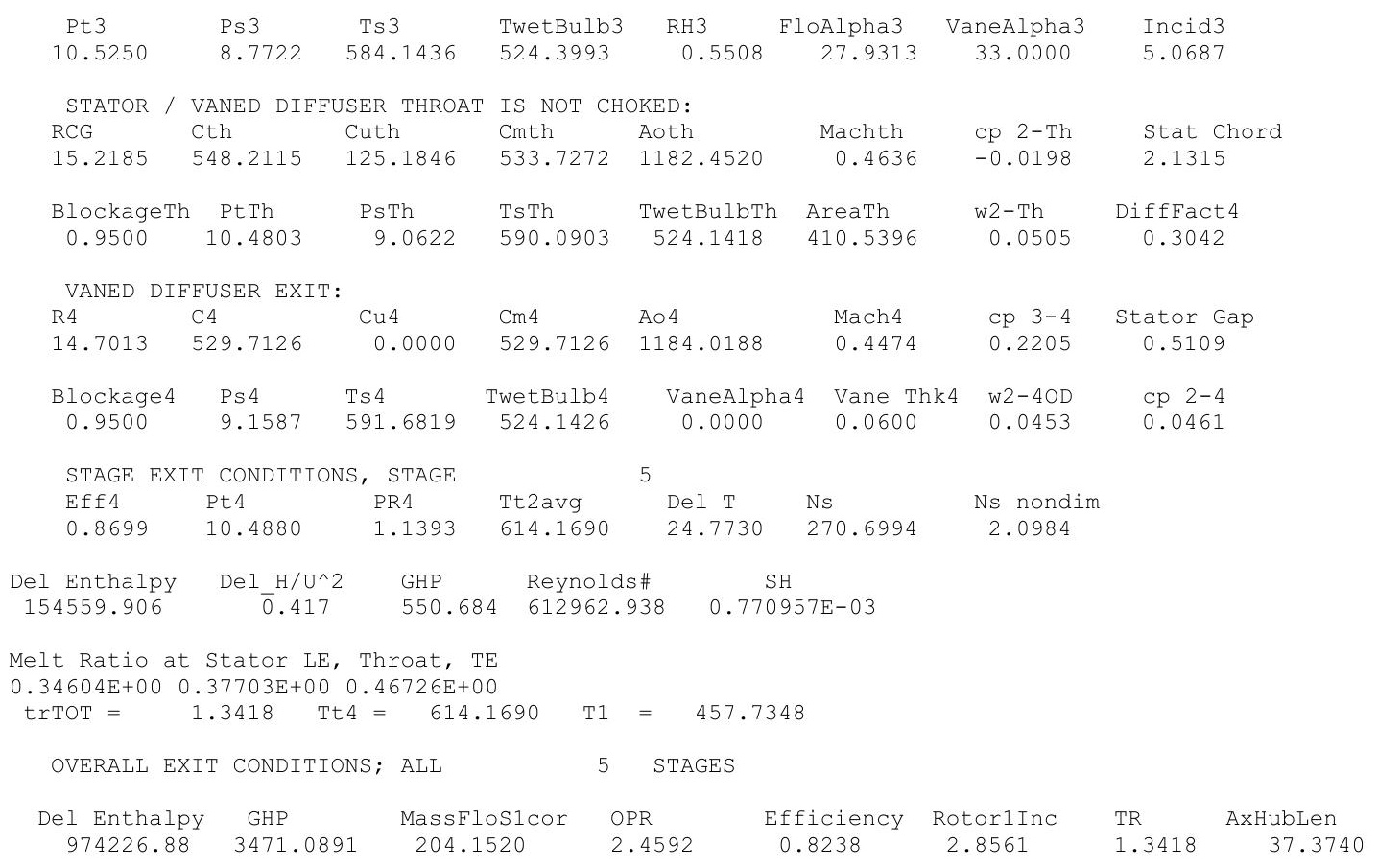




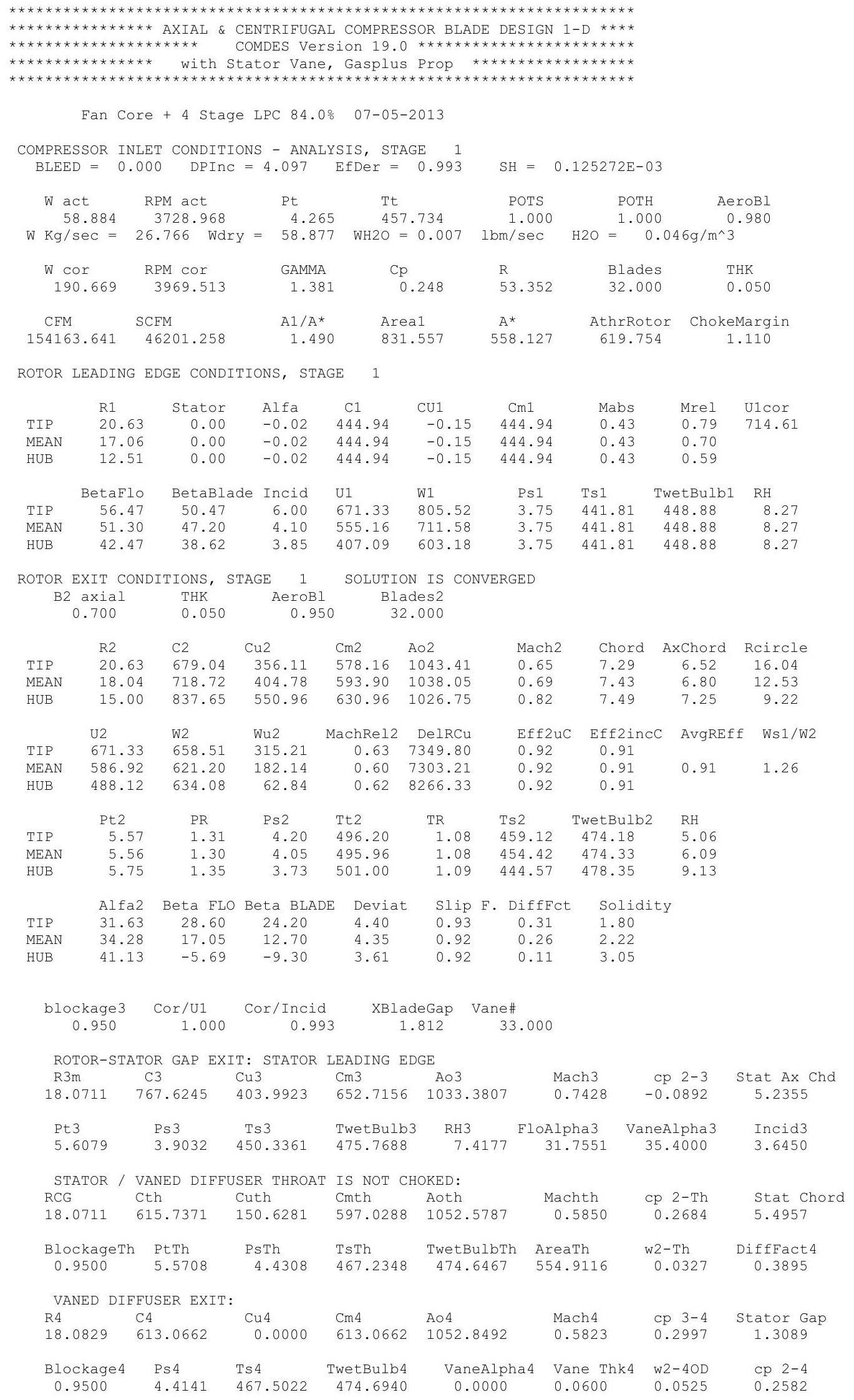




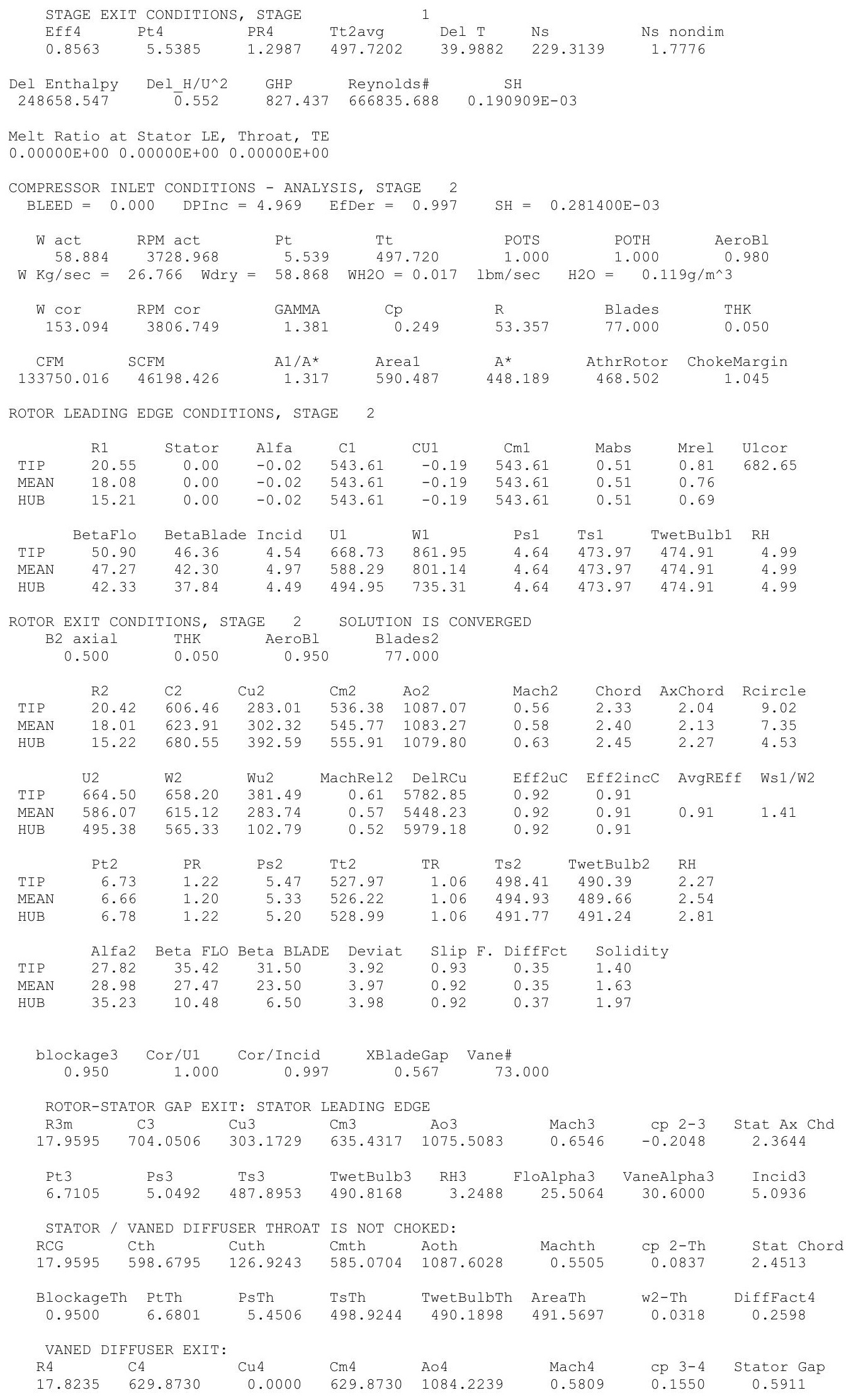




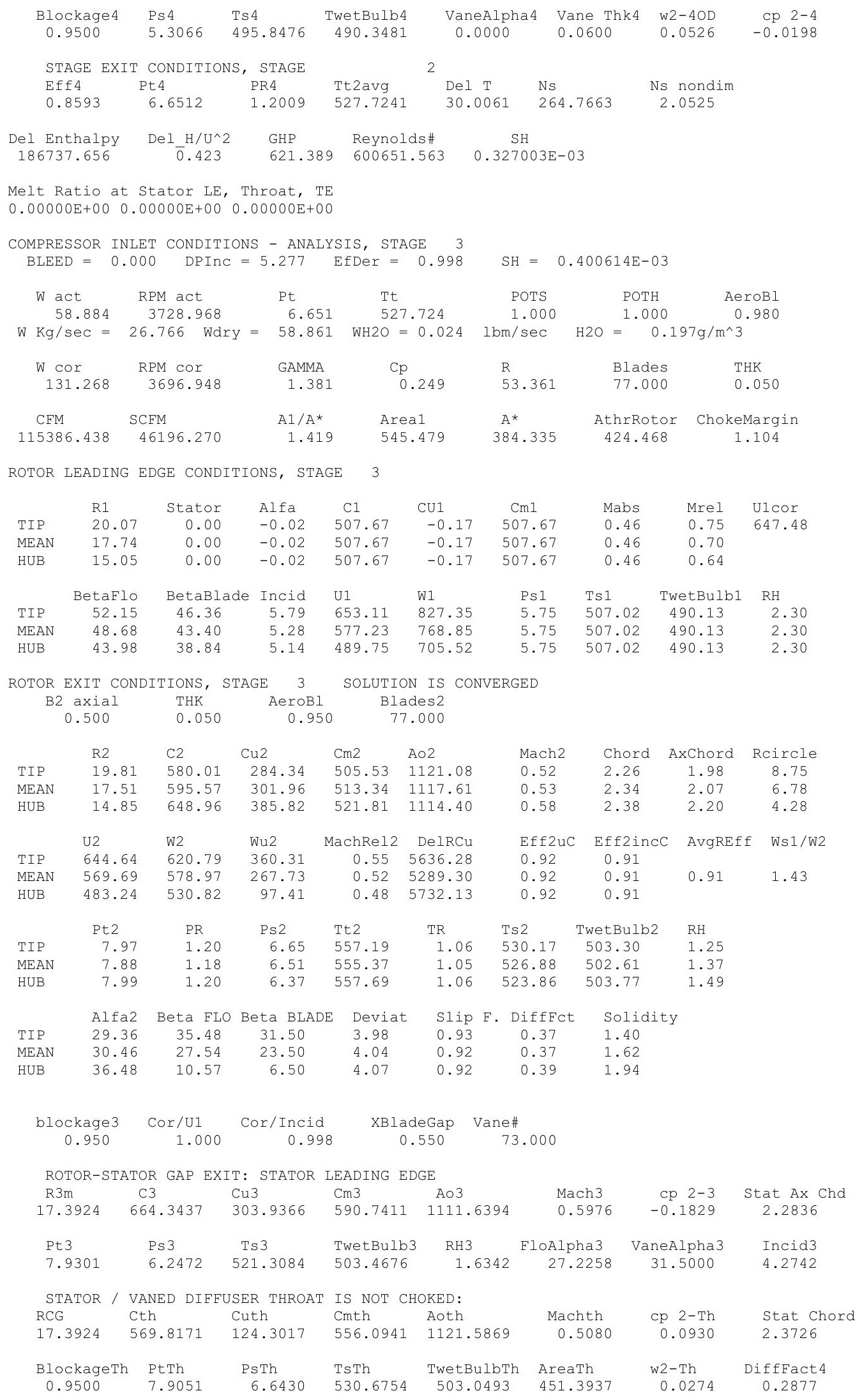




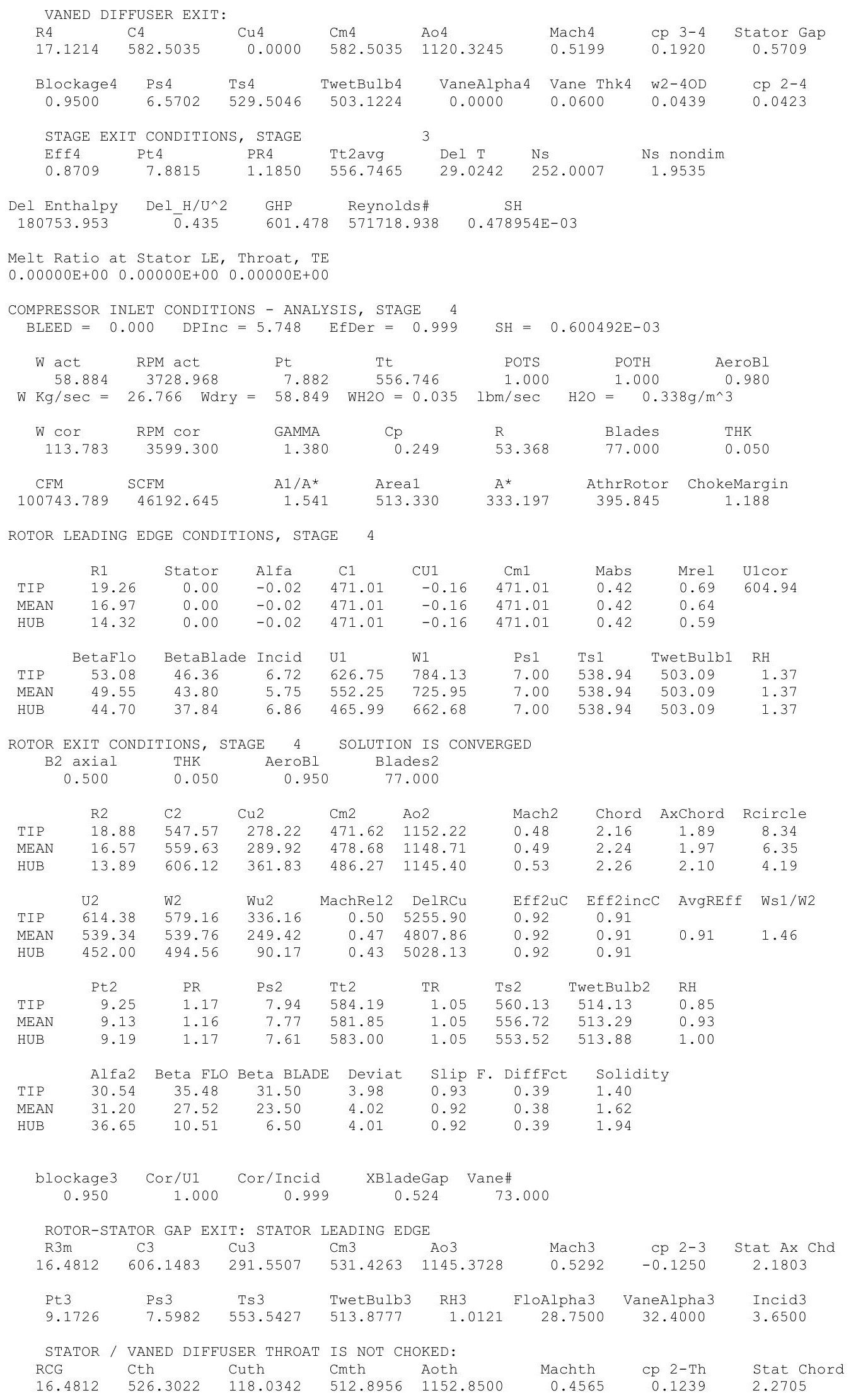




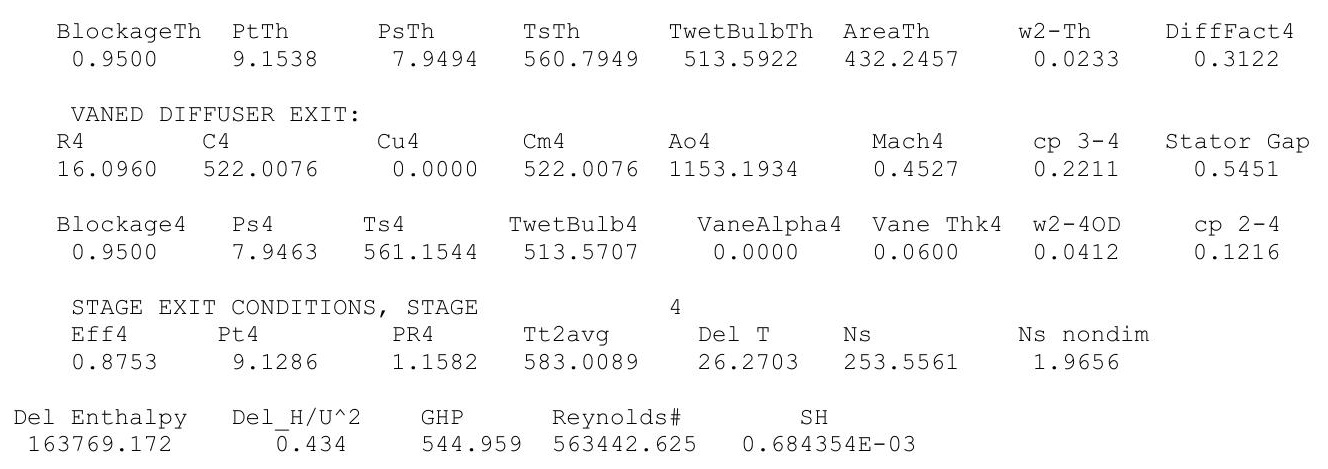

Melt Ratio at Stator LE, Throat, TE

$0.70622 \mathrm{E}-01 \quad 0.90963 \mathrm{E}-01 \quad 0.14848 \mathrm{E}+00$

COMPRESSOR INLET CONDITIONS - ANALYSIS, STAGE 5 $\mathrm{BLEED}=0.000 \quad$ DPInC $=6.154 \quad$ EfDer $=1.000$

$\begin{array}{llllll}W & \text { Ret } & \text { RPM act } & \text { Pt } & \text { POTS } & \text { POTH }\end{array}$ $\mathrm{W} \mathrm{Kg} / \mathrm{sec}=26.766$ Wdry $=58.838 \quad \mathrm{WH} 2 \mathrm{O}=0.046 \mathrm{lbm} / \mathrm{sec} \quad \mathrm{H} 2 \mathrm{O}=0.497 \mathrm{~g} / \mathrm{m}^{\wedge} 3$

$\begin{array}{ccccccc}\text { W cor } & \text { RPM cor } & \text { GAMMA } & \text { Cp } & \text { R } & \text { Blades } & \text { THK } \\ 100.529 & 3517.299 & 1.380 & 0.249 & 53.374 & 77.000 & 0.050 \\ \text { CFM } & \text { SCFM } & \text { A1/A* } & \text { Area1 } & A^{*} & \text { AthrRotor } & \text { ChokeMargin } \\ 89221.414 & 46189.336 & 1.731 & 509.668 & 294.434 & 384.739 & 1.307\end{array}$

ROTOR LEADING EDGE CONDITIONS, STAGE 5

$\begin{array}{lccccccccc} & \text { R1 } & \text { Stator } & \text { Alfa } & \text { C1 } & \text { CU1 } & \text { Cm1 } & \text { Mabs } & \text { Mrel } & \text { U1cor } \\ \text { TIP } & 18.32 & 0.00 & -0.02 & 420.13 & -0.14 & 420.13 & 0.36 & 0.63 & 562.30 \\ \text { MEAN } & 15.91 & 0.00 & -0.02 & 420.13 & -0.14 & 420.13 & 0.36 & 0.57 & \\ \text { HUB } & 13.07 & 0.00 & -0.02 & 420.13 & -0.14 & 420.13 & 0.36 & 0.52 & \\ & & & & & & & & & \\ & \text { BetaFlo } & \text { BetaBlade } & \text { Incid } & \text { U1 } & \text { W1 } & \text { Ps } 1 & \text { Ts1 } & \text { TwetBulb1 } & \text { RH } \\ \text { TIP } & 54.83 & 47.36 & 7.47 & 596.16 & 729.45 & 8.35 & 568.86 & 513.51 & 0.84 \\ \text { MEAN } & 50.95 & 44.80 & 6.15 & 517.83 & 666.94 & 8.35 & 568.86 & 513.51 & 0.84 \\ \text { HUB } & 45.36 & 38.84 & 6.52 & 425.32 & 597.94 & 8.35 & 568.86 & 513.51 & 0.84\end{array}$

ROTOR EXIT CONDITIONS, STAGE 5 SOLUTION IS CONVERGED

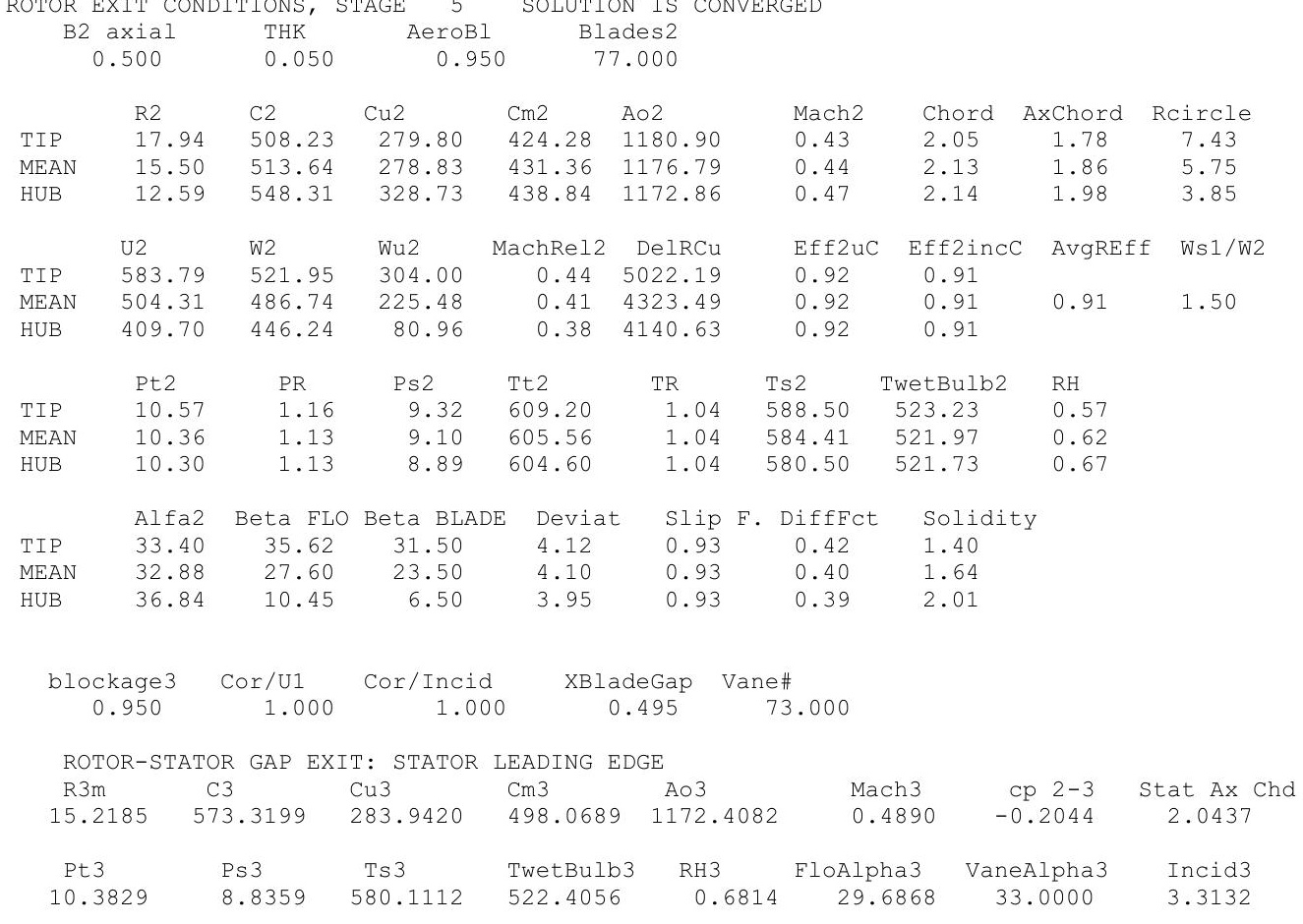




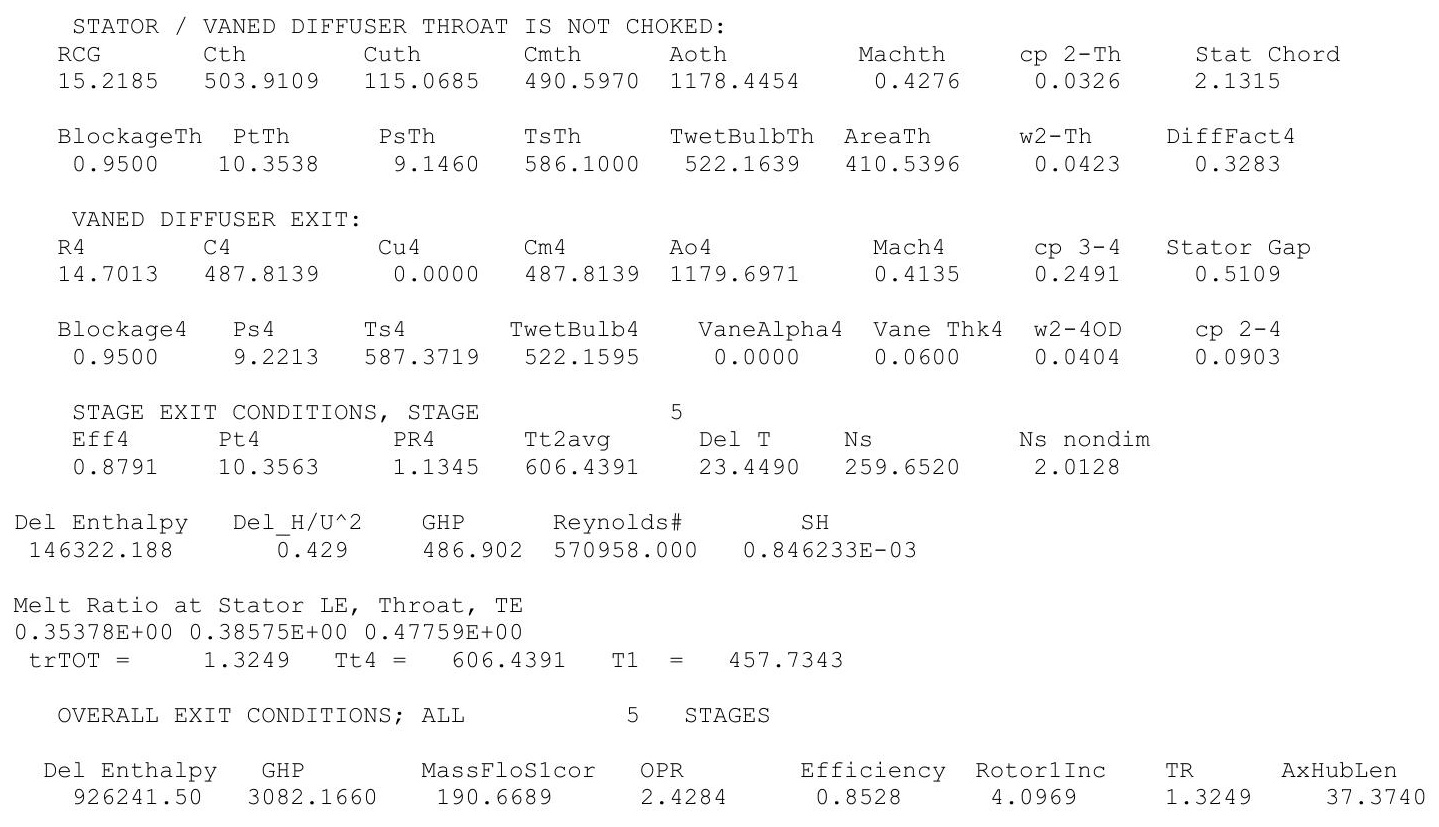




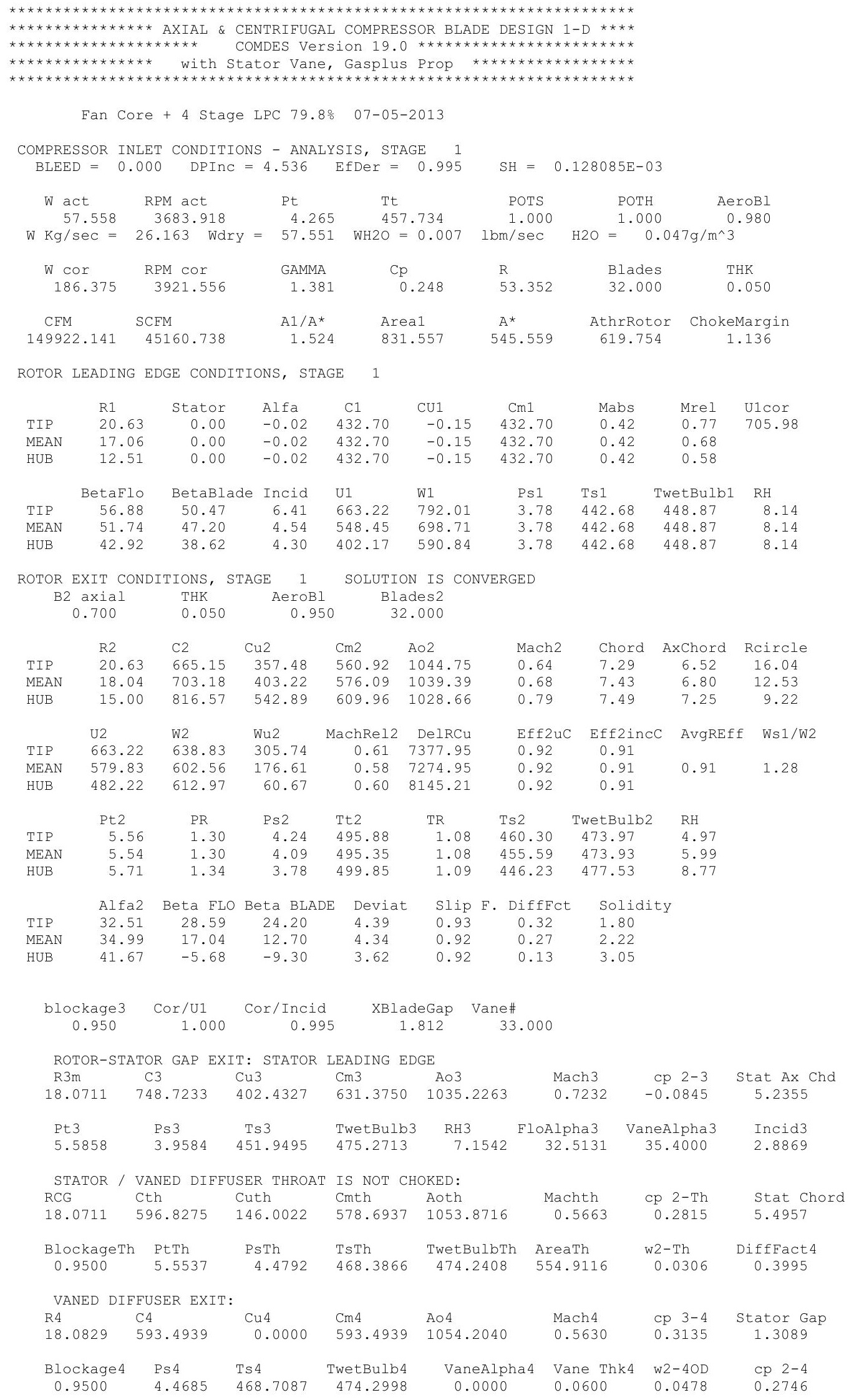




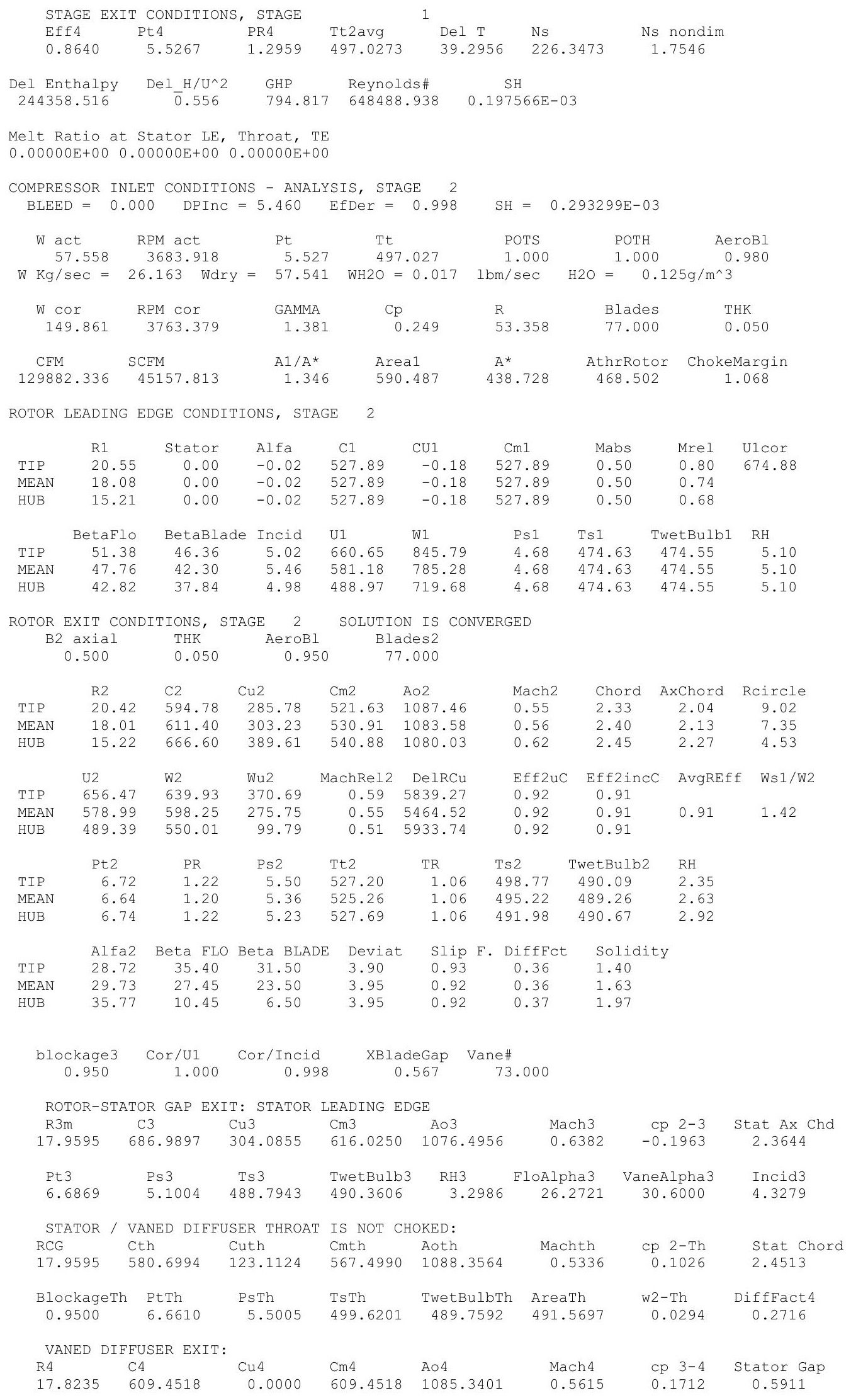




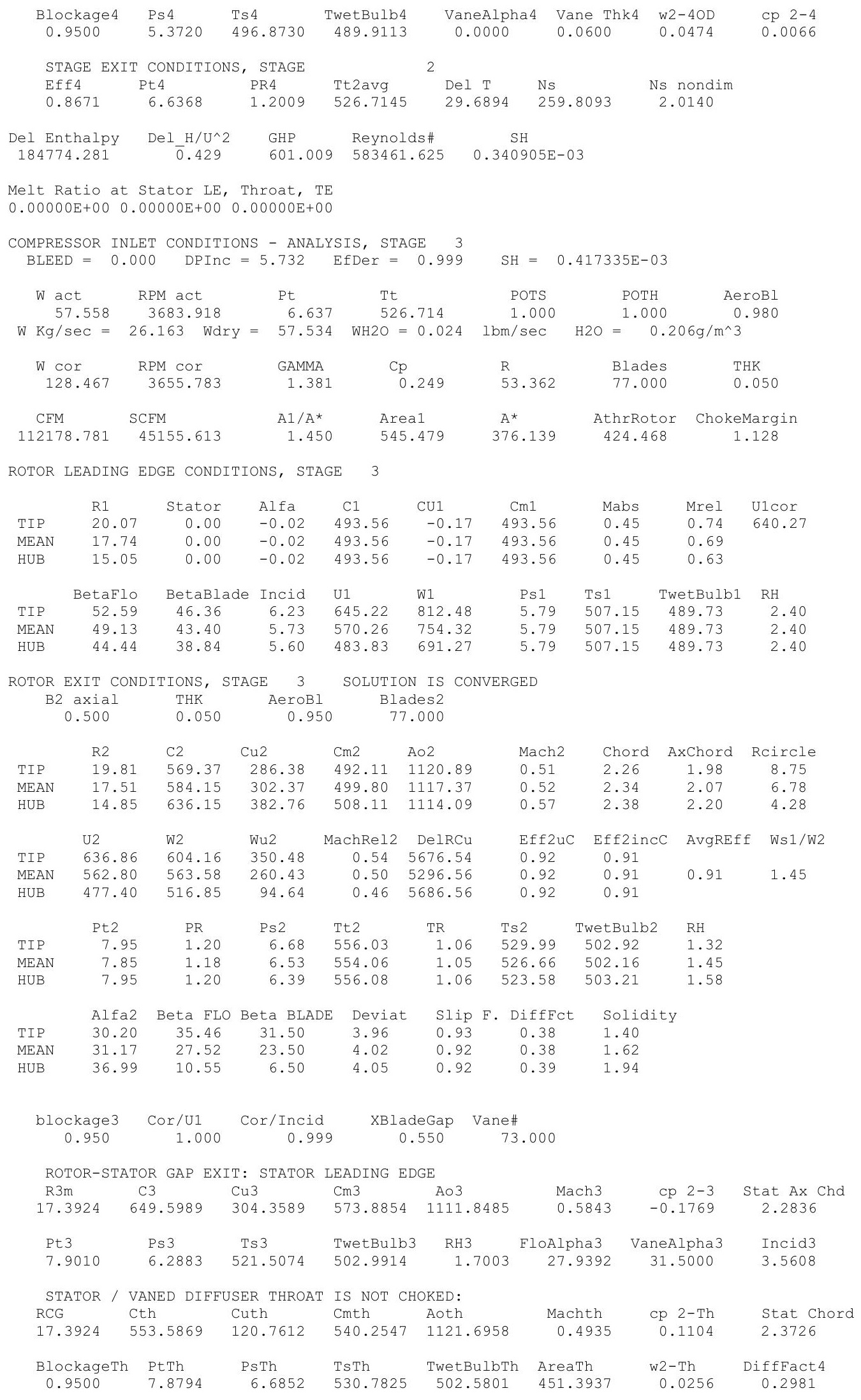




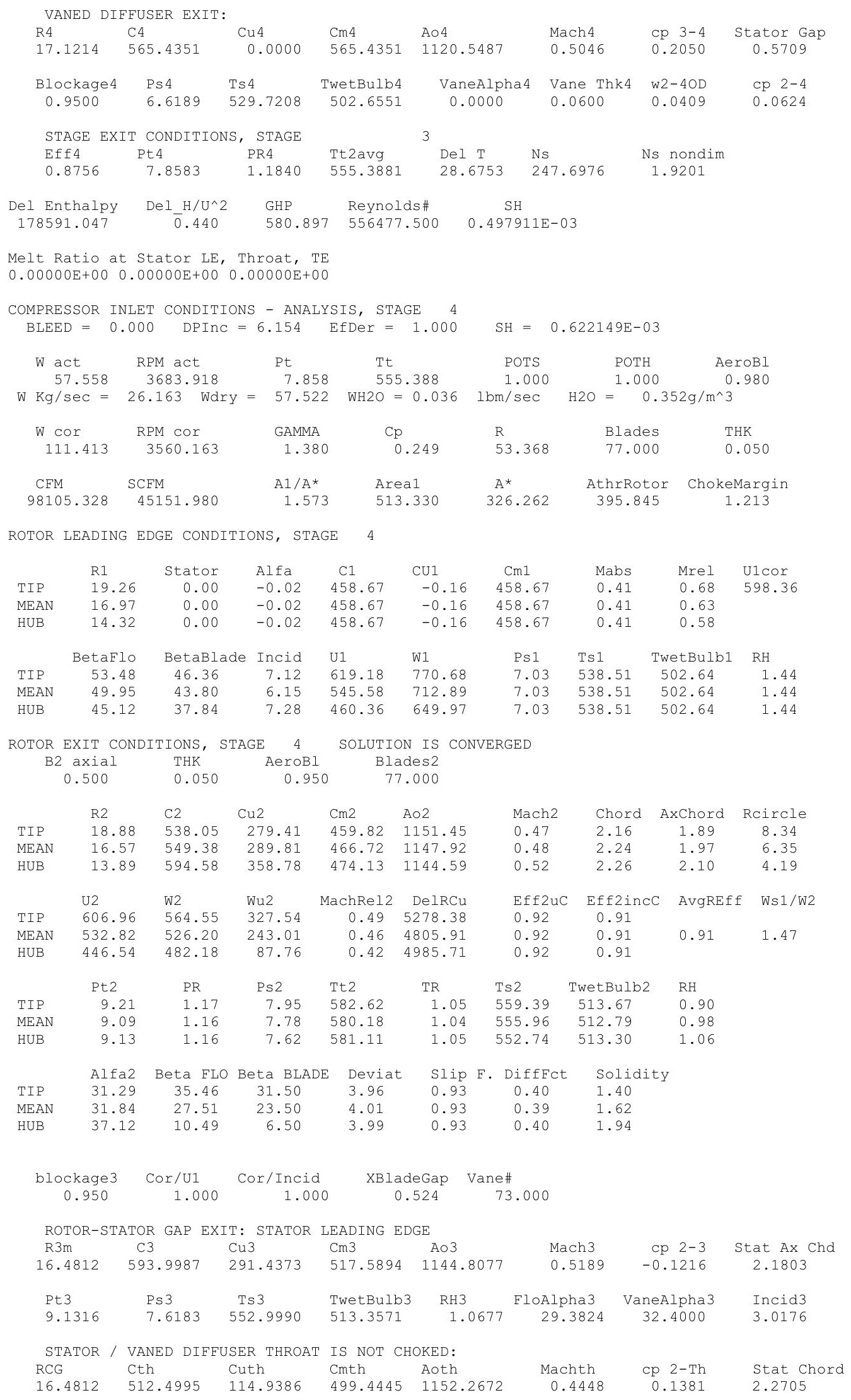




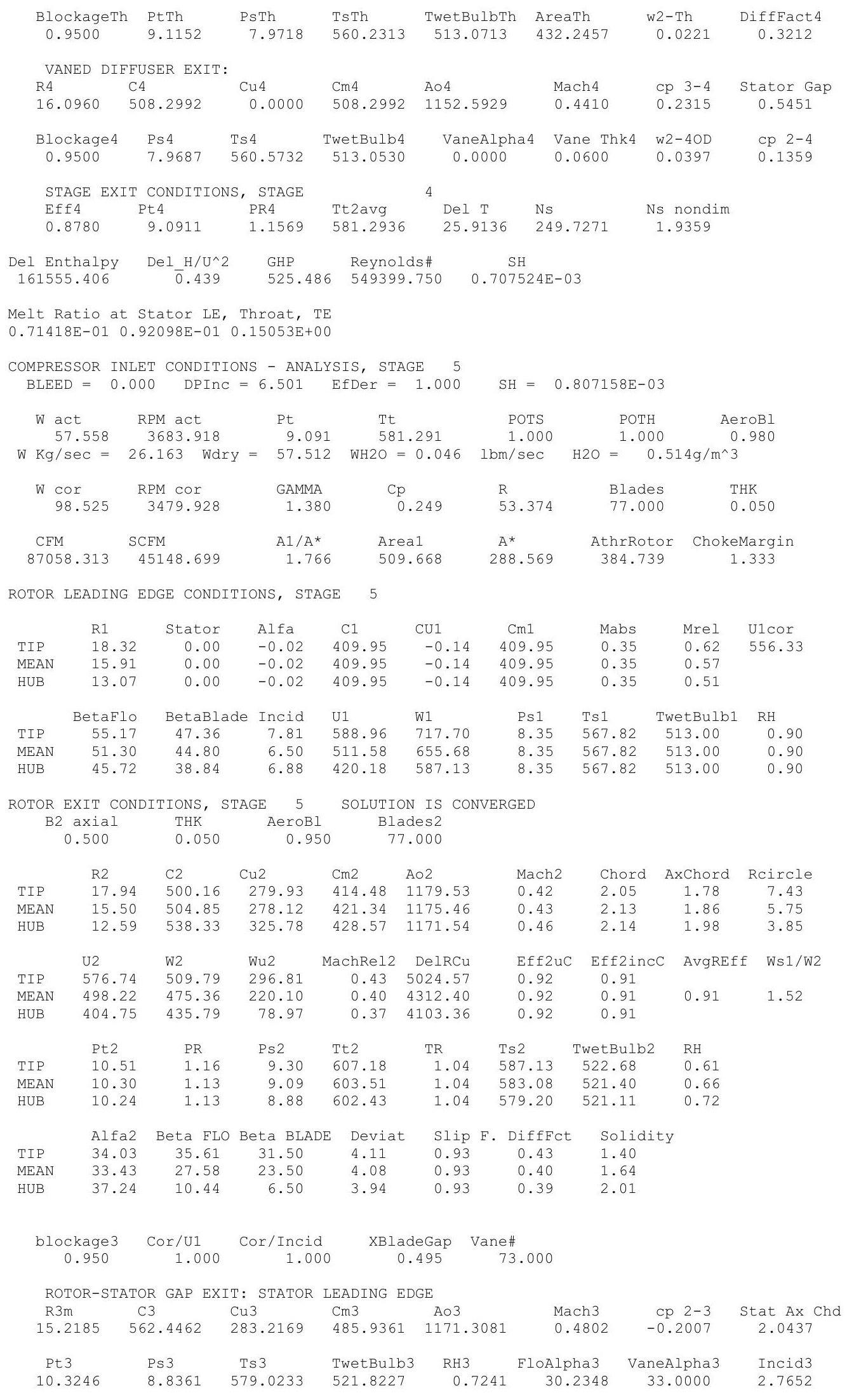




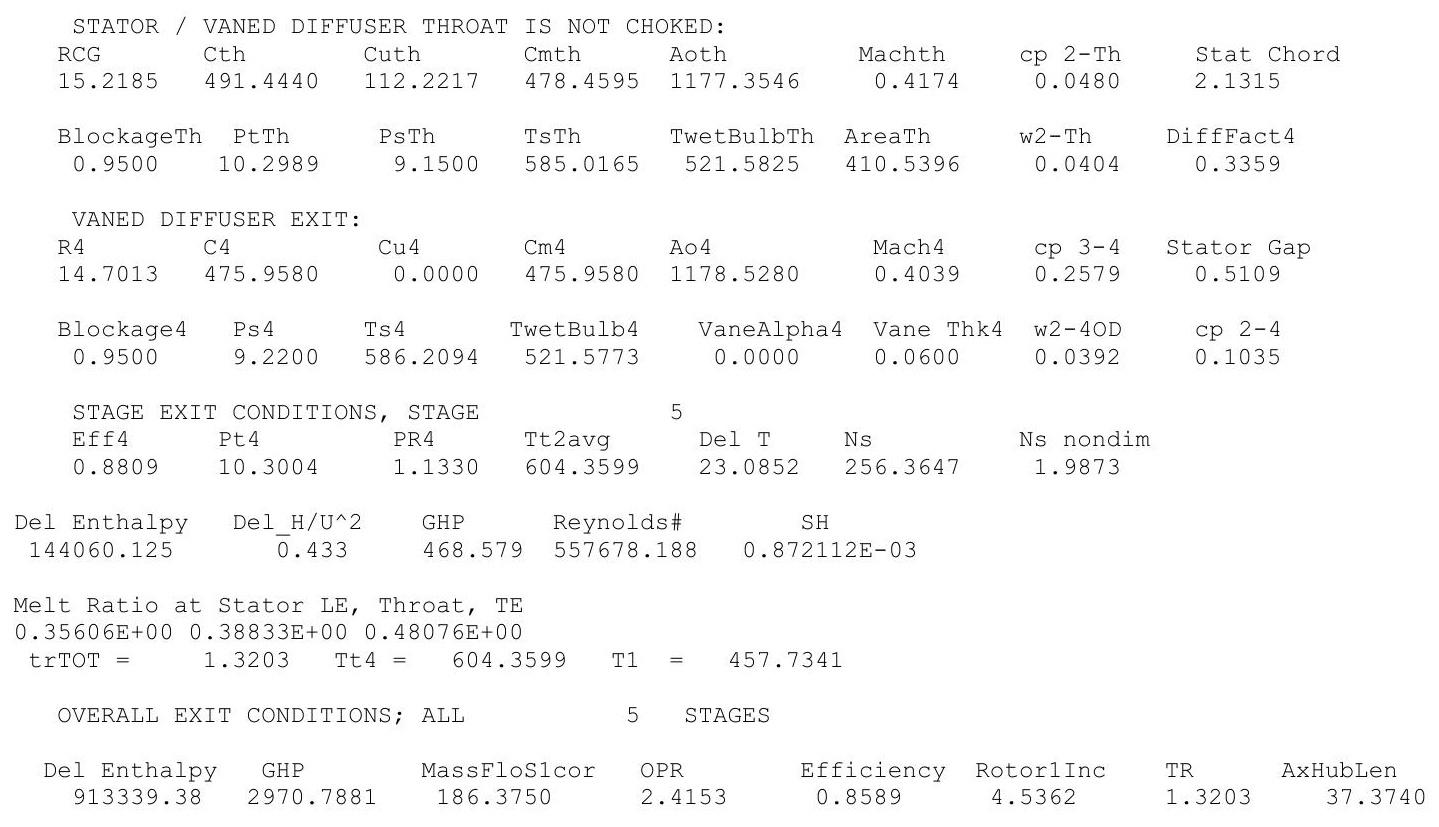




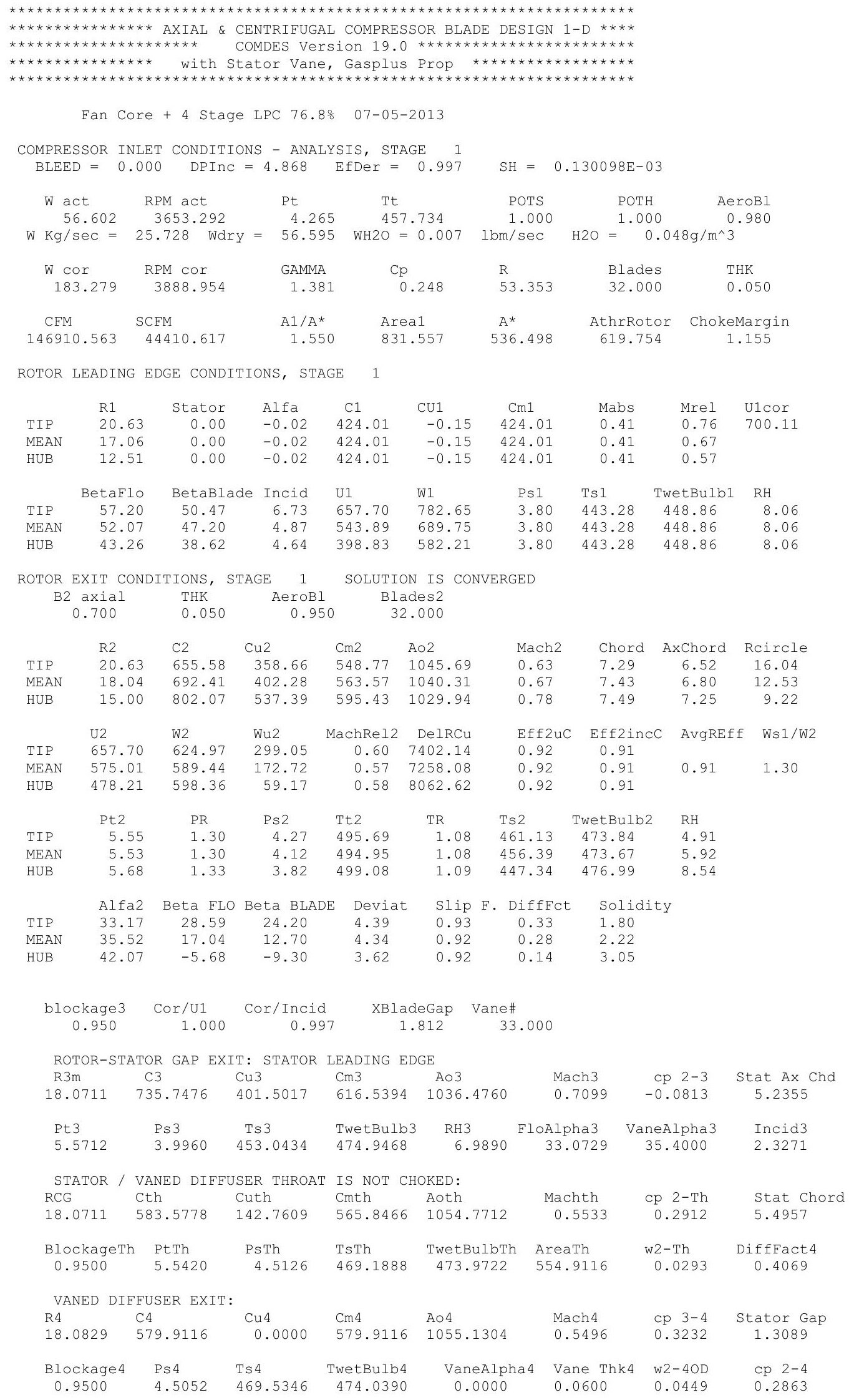




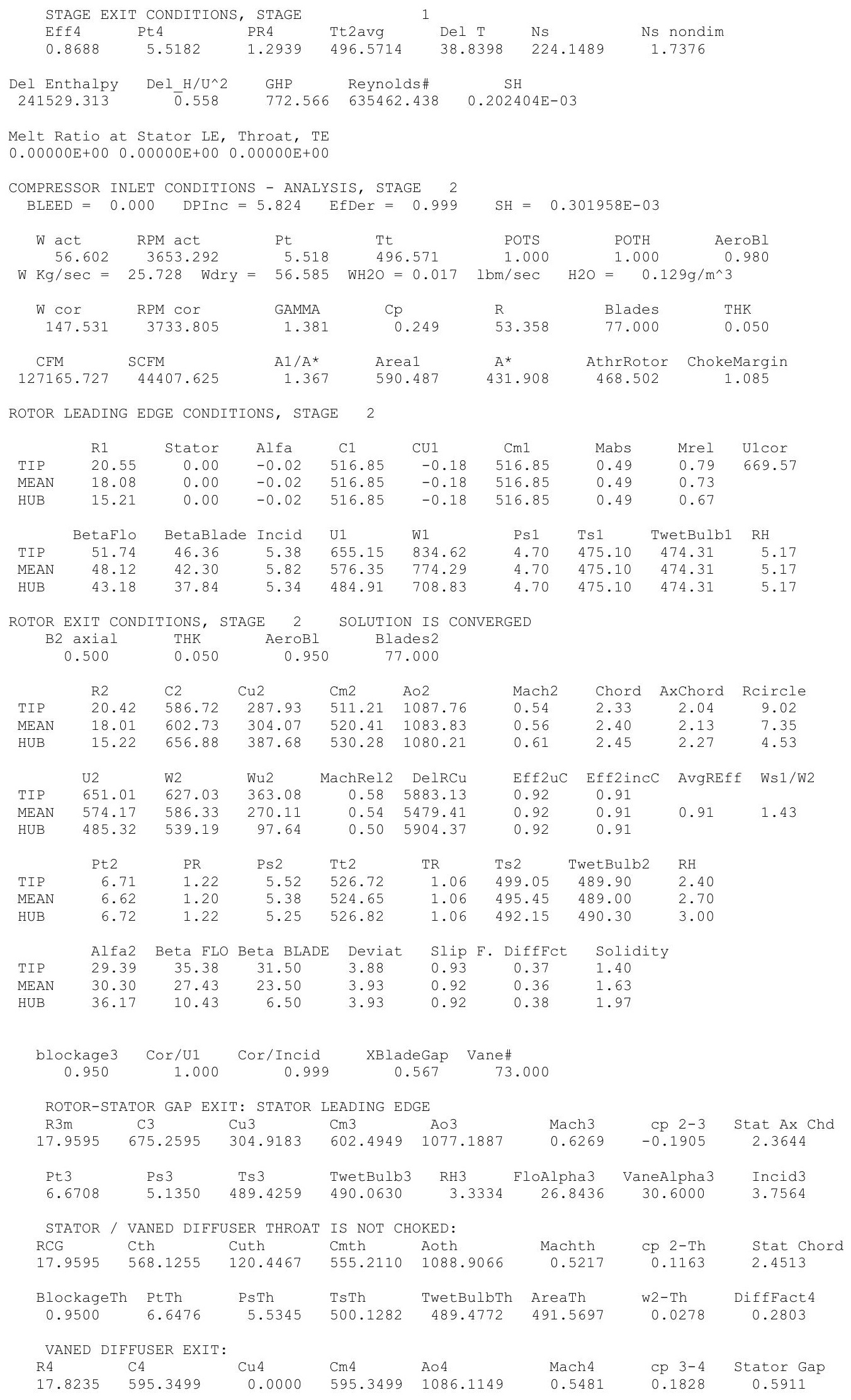




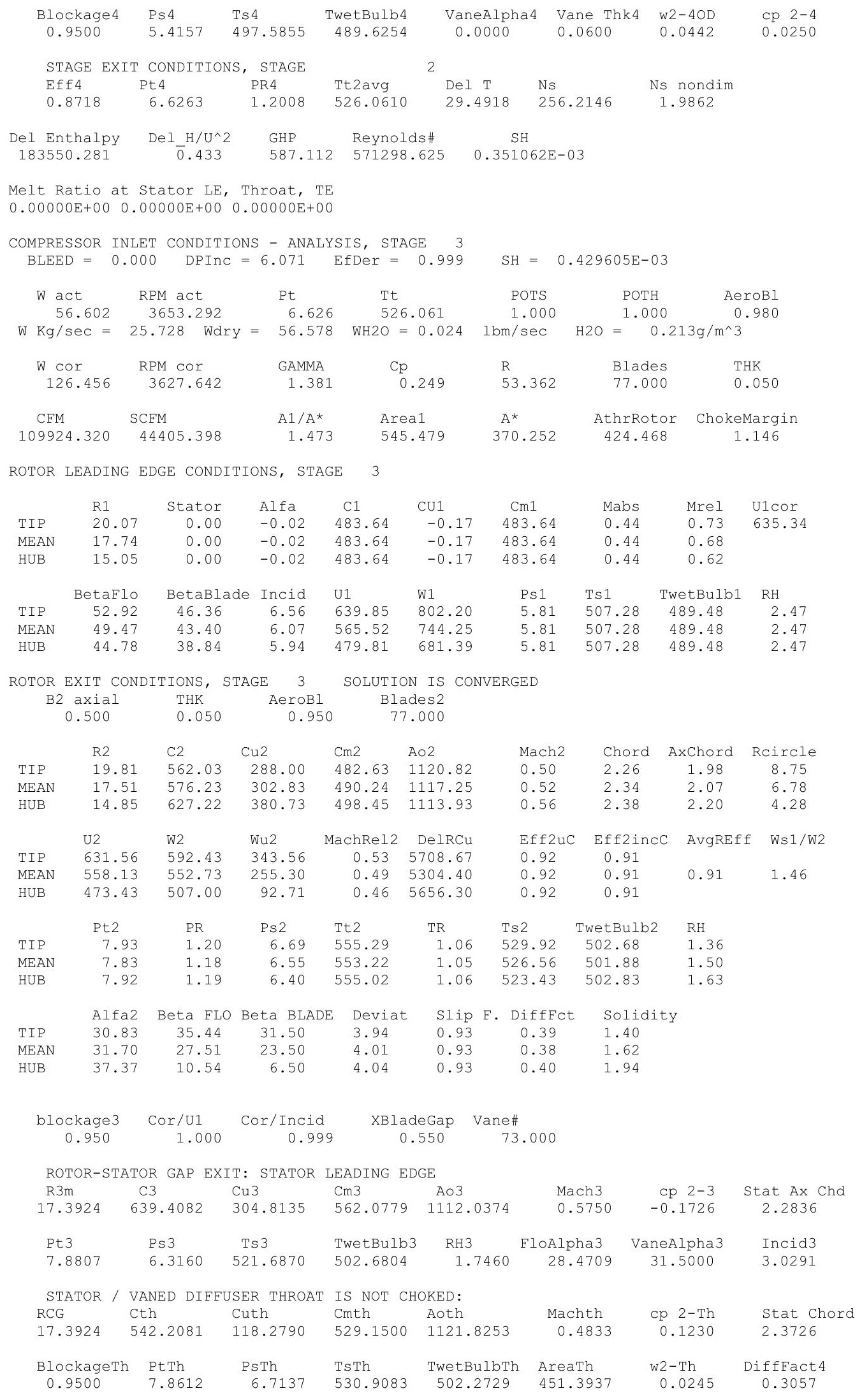




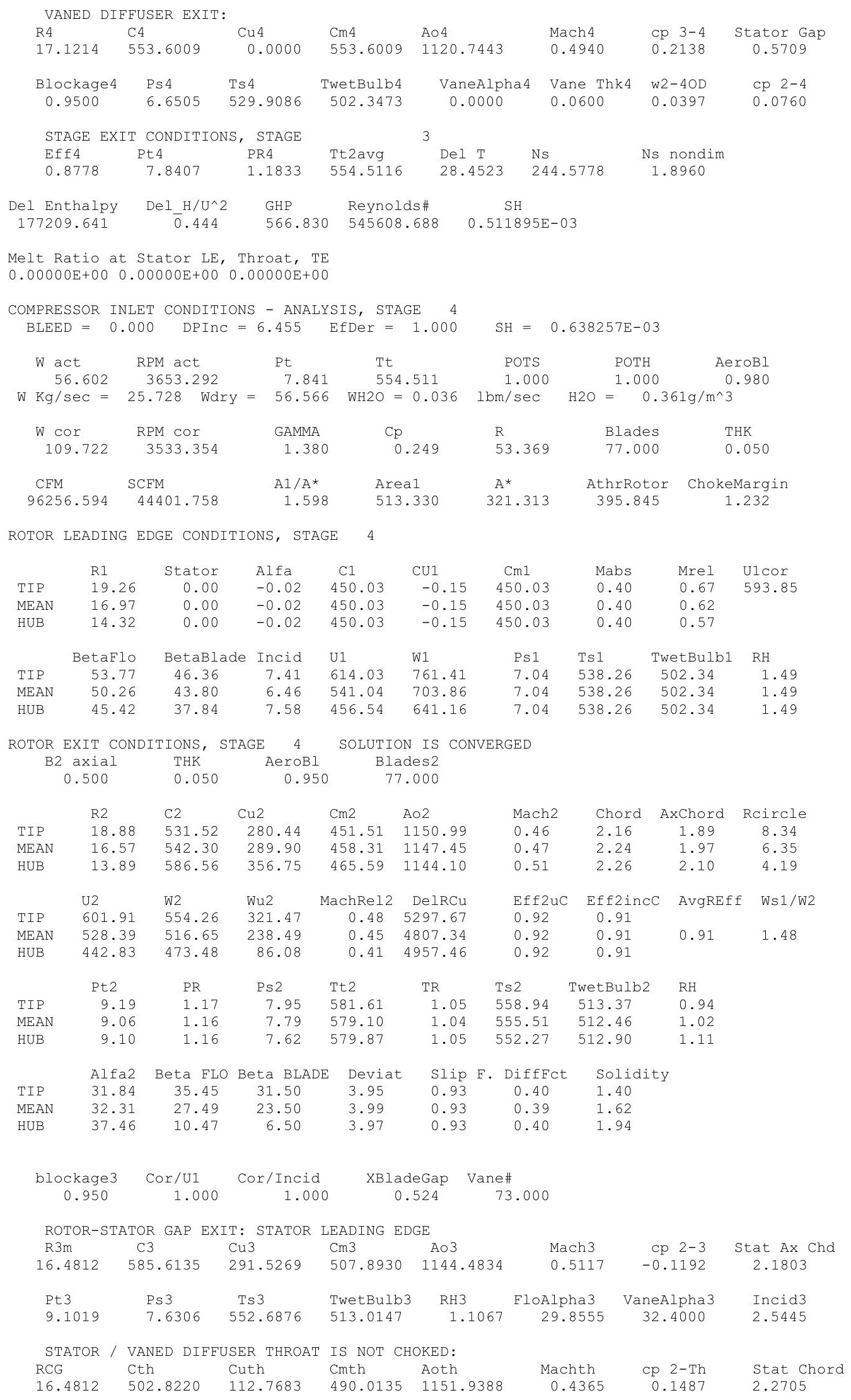




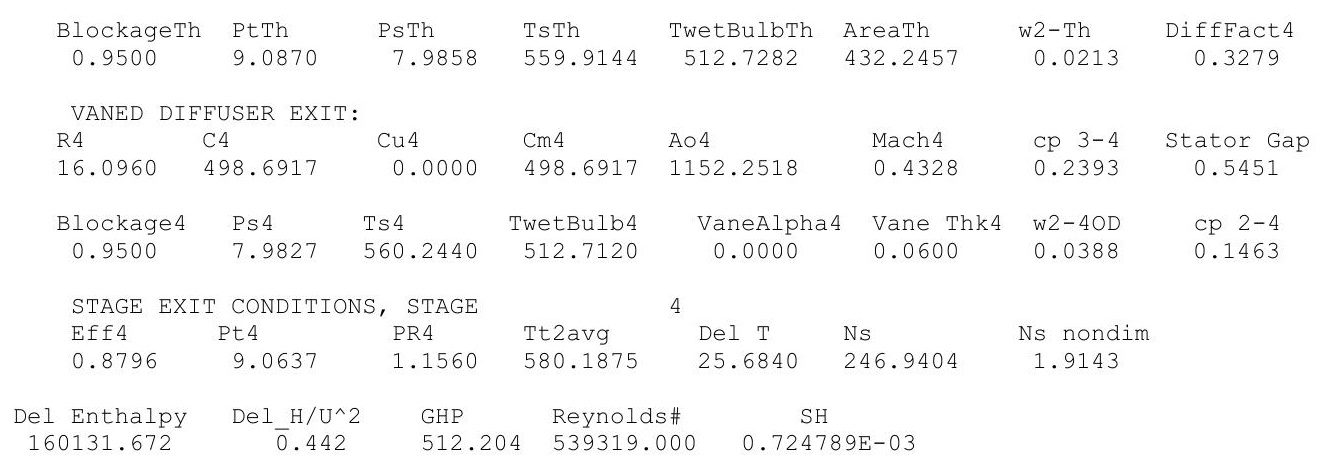

Melt Ratio at Stator LE, Throat, TE

$0.72270 \mathrm{E}-01 \quad 0.93243 \mathrm{E}-01 \quad 0.15245 \mathrm{E}+00$

COMPRESSOR INLET CONDITIONS - ANALYSIS, STAGE 5 $\mathrm{BLEED}=0.000 \quad$ DPInC $=6.761 \quad$ EfDer $=1.000$

$\mathrm{SH}=0.825575 \mathrm{E}-03$

$\begin{array}{llllll}\text { W act } & \text { RPM act } & \text { Pt } & \text { POTS } & \text { POTH } & \text { AeroBI }\end{array}$

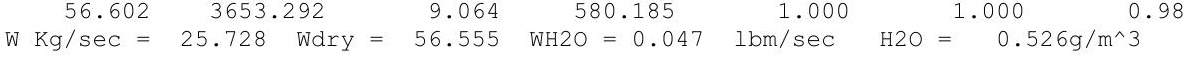

$\begin{array}{ccccccc}\text { W Cor } & \text { RPM cor } & \text { GAMMA } & \text { Cp } & \text { R } & \text { Blades } & \text { THK } \\ 97.089 & 3454.286 & 1.380 & 0.249 & 53.375 & 77.000 & 0.050 \\ & & & & & & \\ \text { CFM } & \text { SCFM } & \text { A1/A* } & \text { Area1 } & \text { A* } & \text { AthrRotor } & \text { ChokeMargin } \\ 85533.148 & 44398.488 & 1.792 & 509.668 & 284.367 & 384.739 & 1.353\end{array}$

ROTOR LEADING EDGE CONDITIONS, STAGE 5

\begin{tabular}{|c|c|c|c|c|c|c|c|c|c|}
\hline & R1 & Stator & Alfa & $\mathrm{C} 1$ & CU1 & $\mathrm{Cm} 1$ & Mabs & Mrel & U1cor \\
\hline TIP & 18.32 & 0.00 & -0.02 & 402.77 & -0.14 & 402.77 & 0.35 & 0.61 & 552.23 \\
\hline MEAN & 15.91 & 0.00 & -0.02 & 402.77 & -0.14 & 402.77 & 0.35 & 0.56 & \\
\hline HUB & 13.07 & 0.00 & -0.02 & 402.77 & -0.14 & 402.77 & 0.35 & 0.50 & \\
\hline & BetaFlo & BetaBlade & Incid & U1 & W1 & Ps 1 & Ts1 & TwetBulb1 & $\mathrm{RH}$ \\
\hline TIP & 55.42 & 47.36 & 8.06 & 584.06 & 709.58 & 8.35 & 567.18 & 512.67 & 0.93 \\
\hline MEAN & 51.56 & 44.80 & 6.76 & 507.32 & 647.87 & 8.35 & 567.18 & 512.67 & 0.93 \\
\hline HUB & 45.98 & 38.84 & 7.14 & 416.68 & 579.62 & 8.35 & 567.18 & 512.67 & 0.93 \\
\hline
\end{tabular}

ROTOR EXIT CONDITIONS, STAGE 5 SOLUTION IS CONVERGED

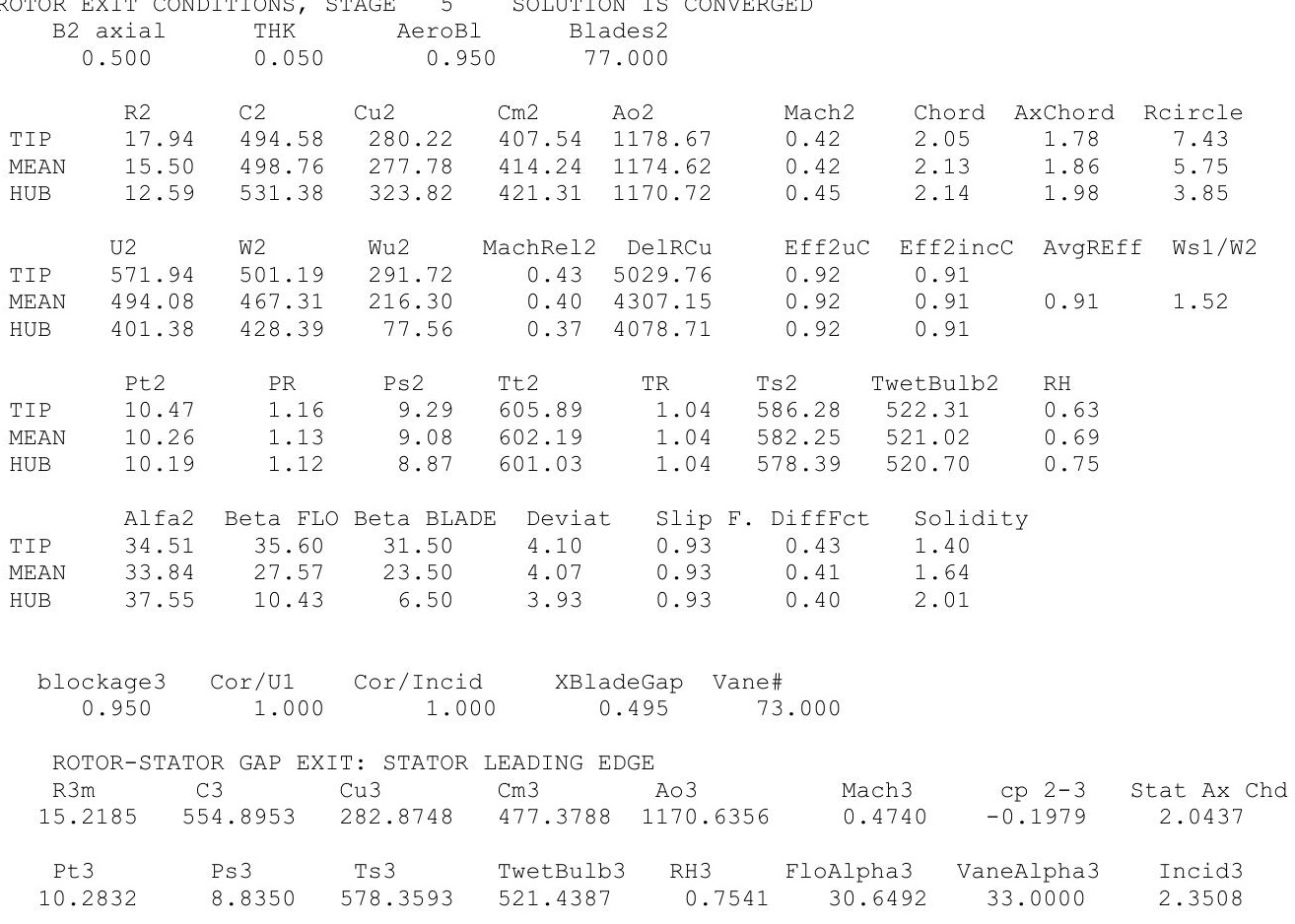




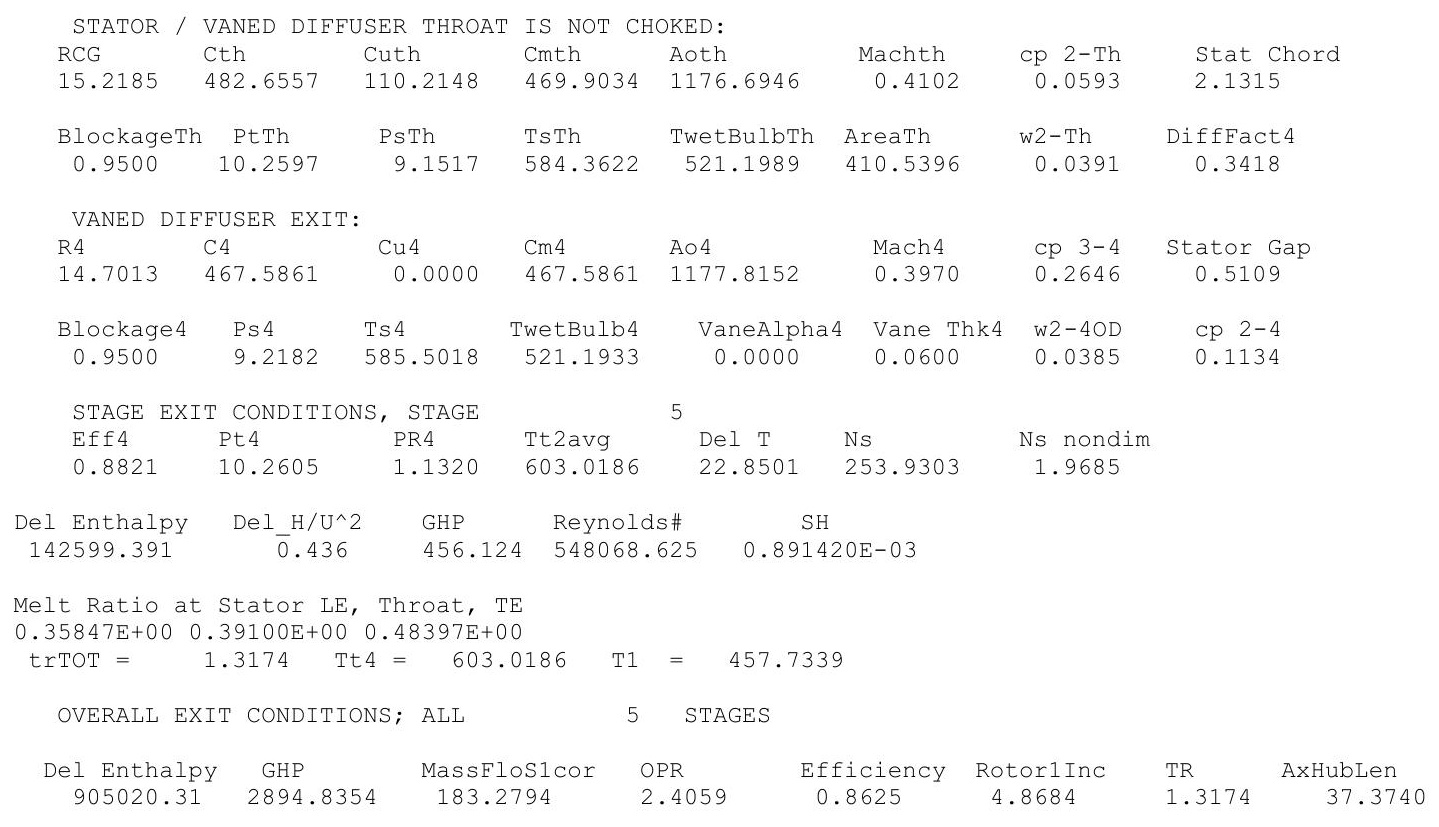


$10 \mu \mathrm{m}, \mathrm{ISA}+36 \mathrm{R}$

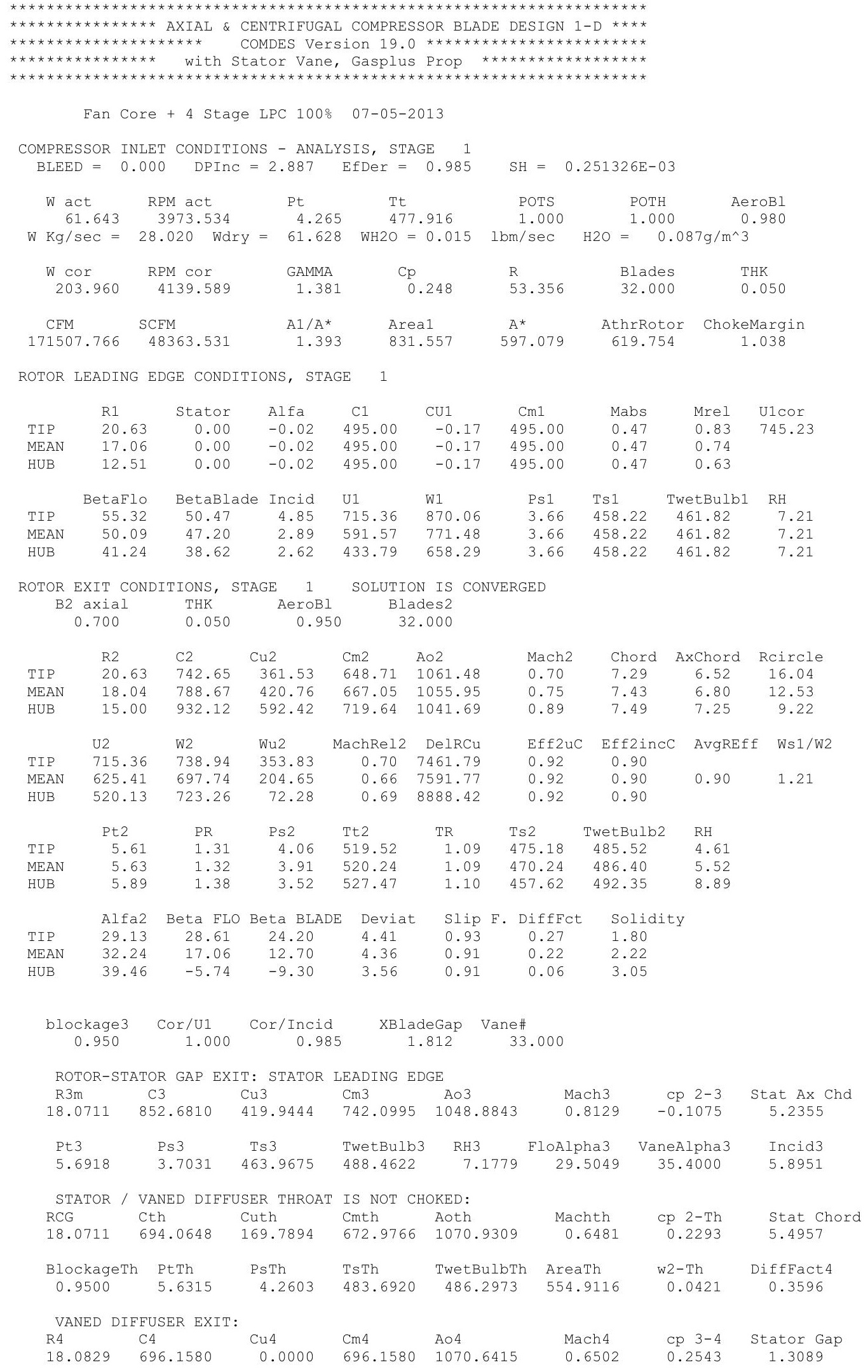




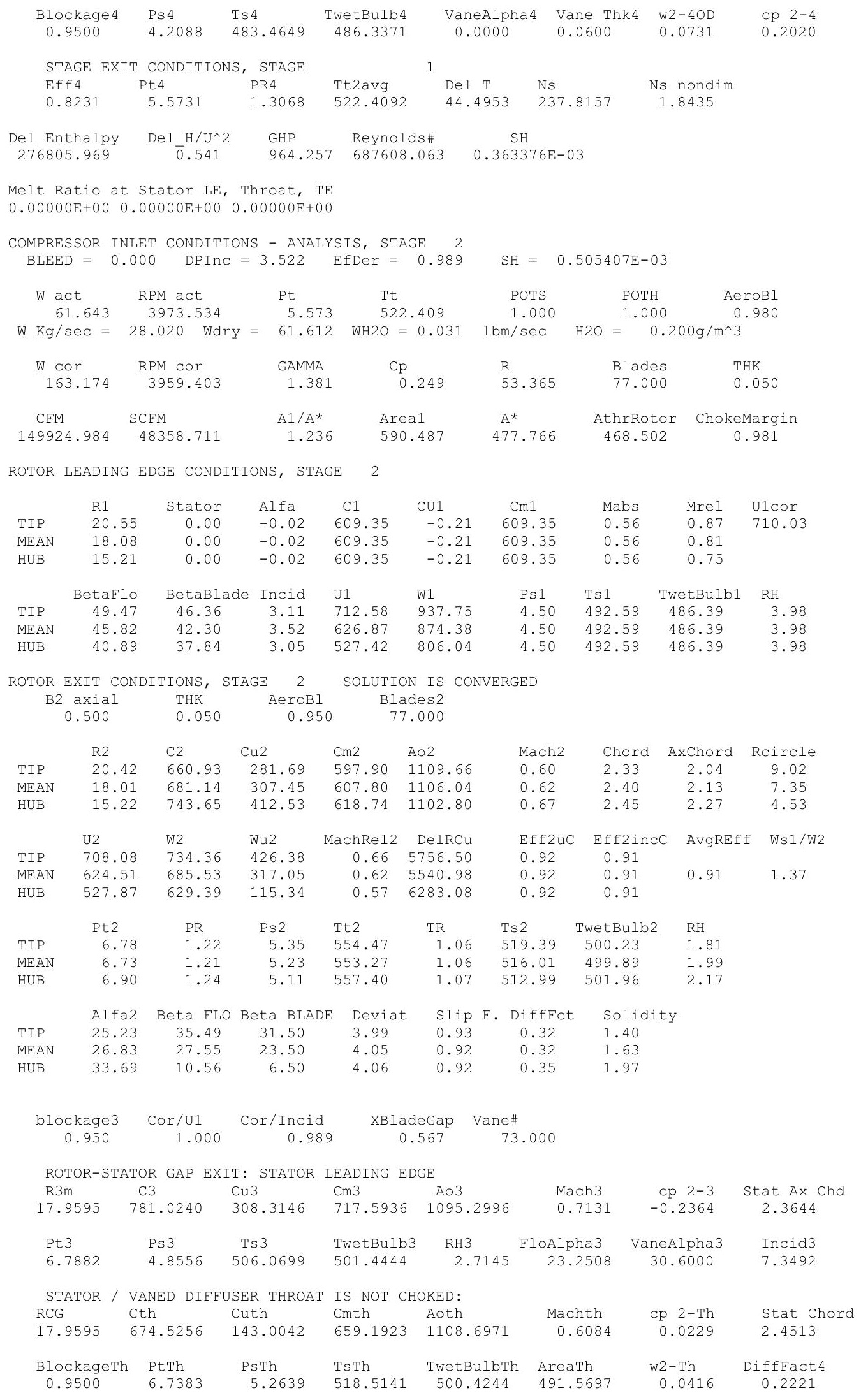




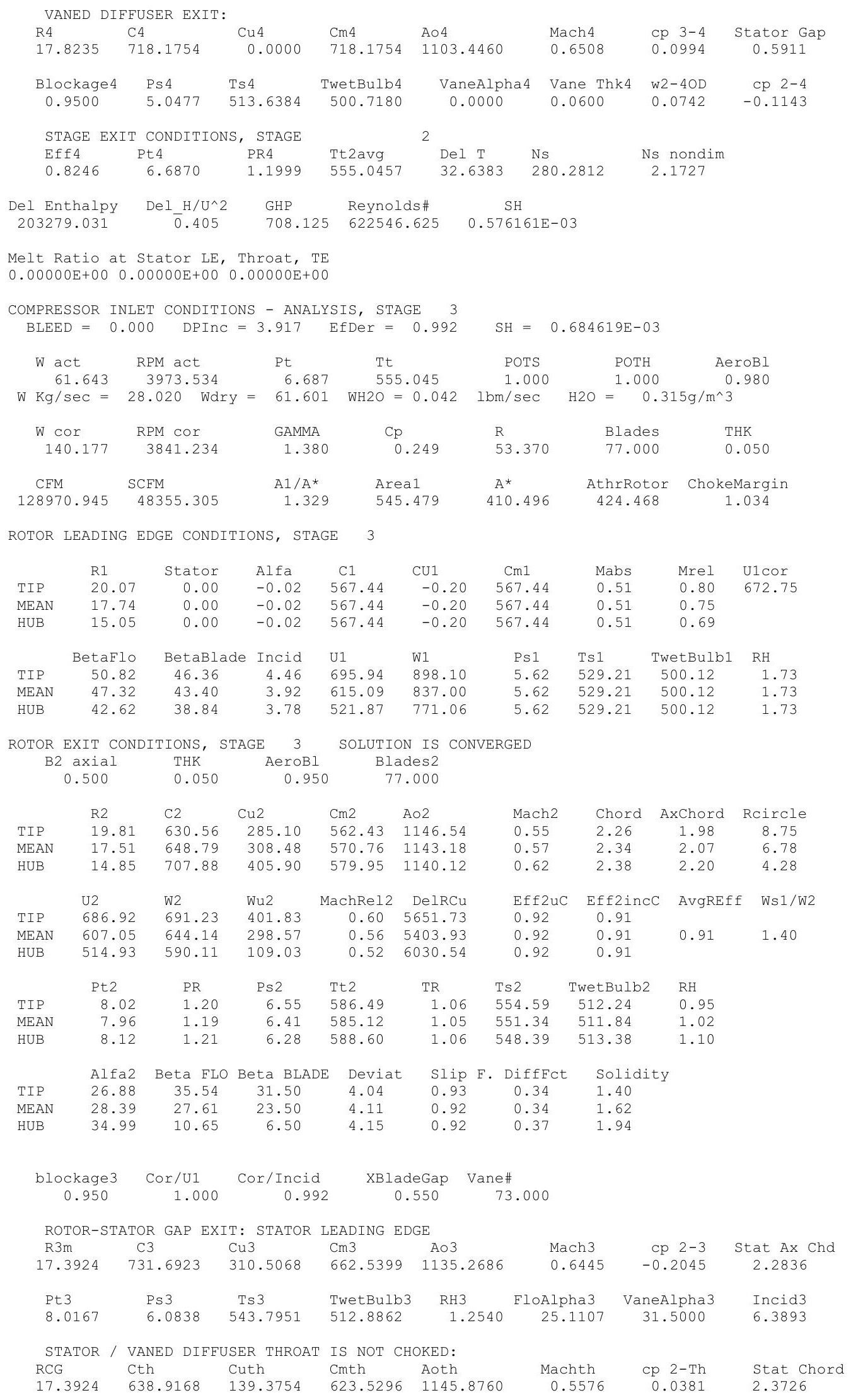




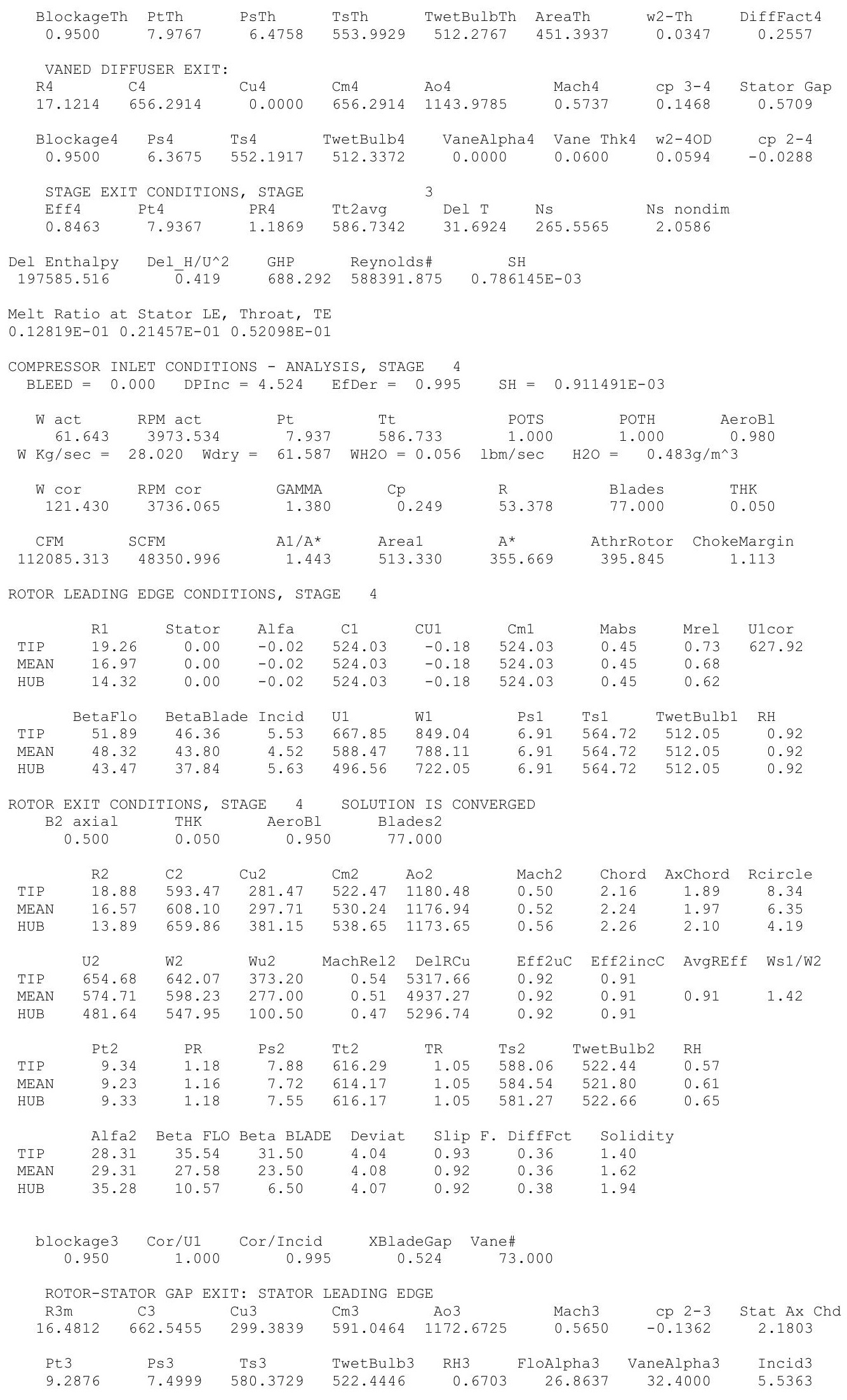




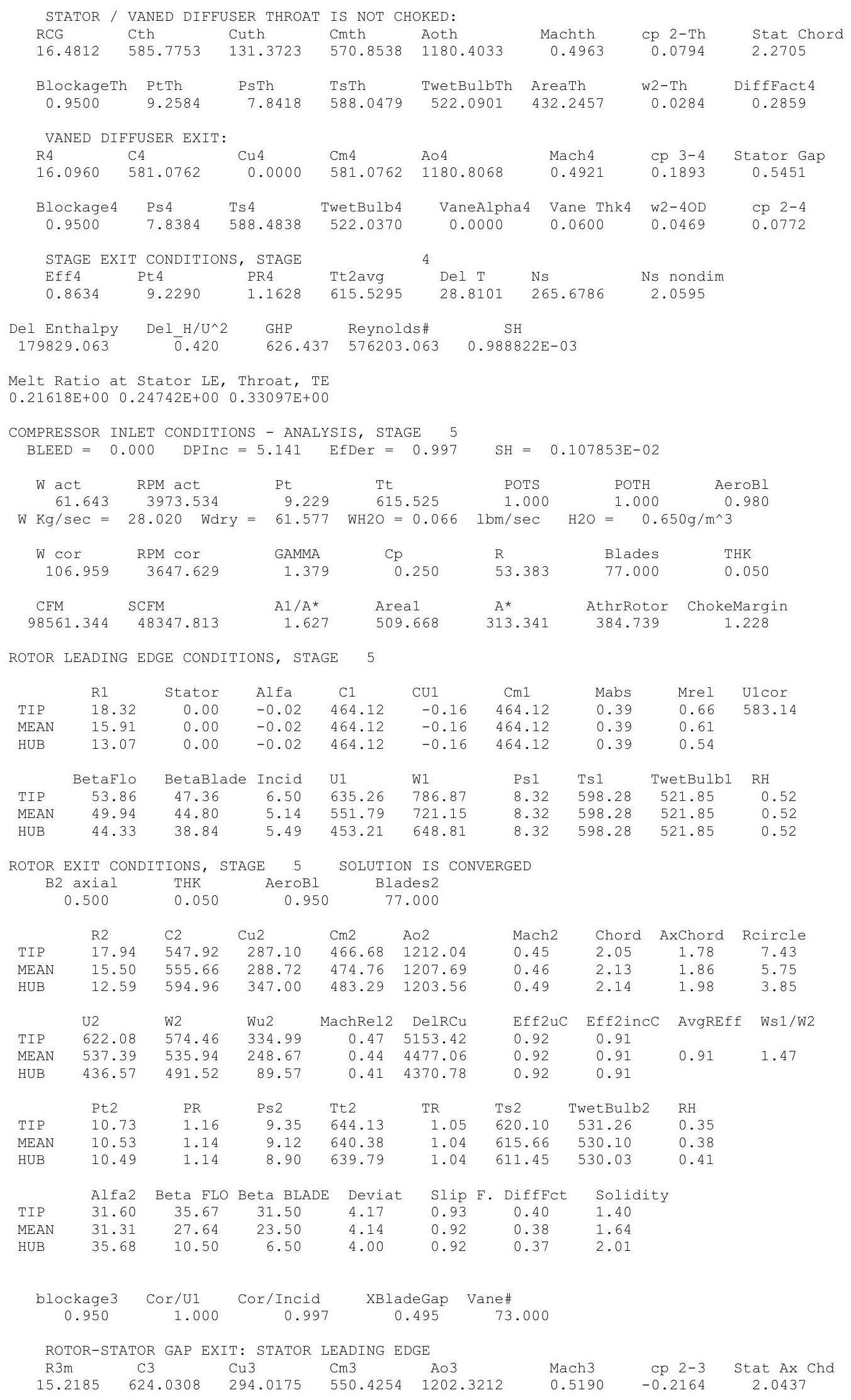




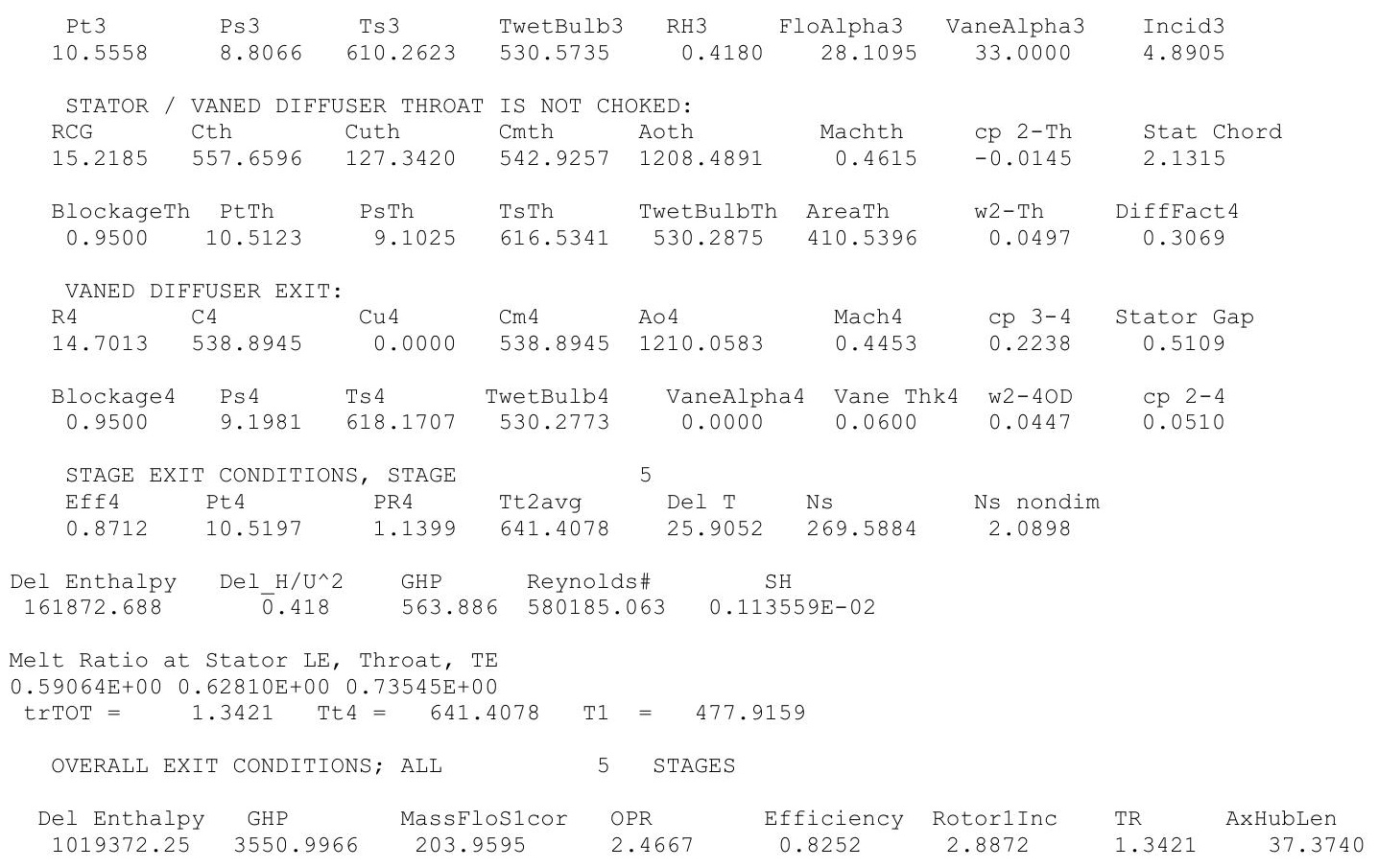




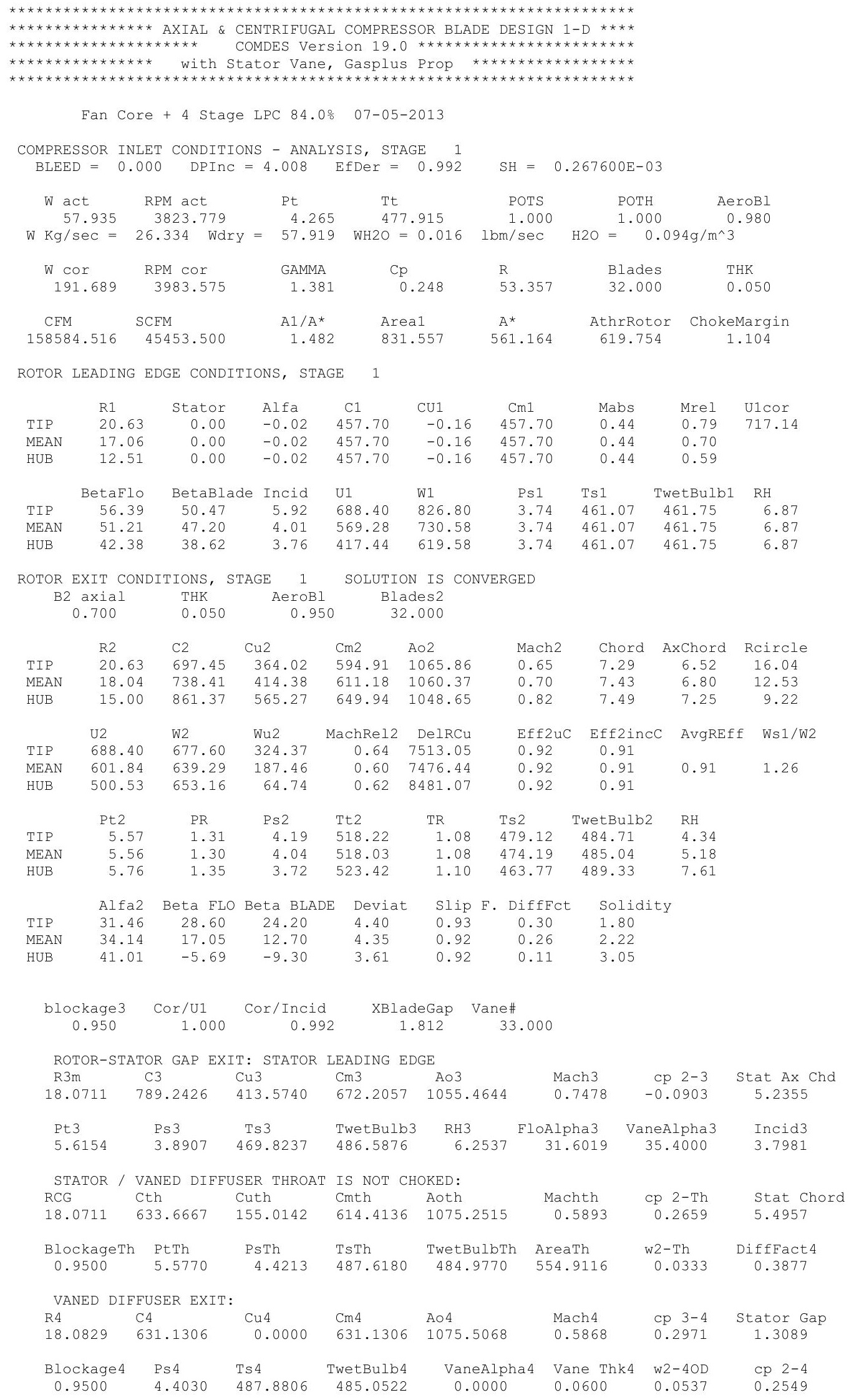




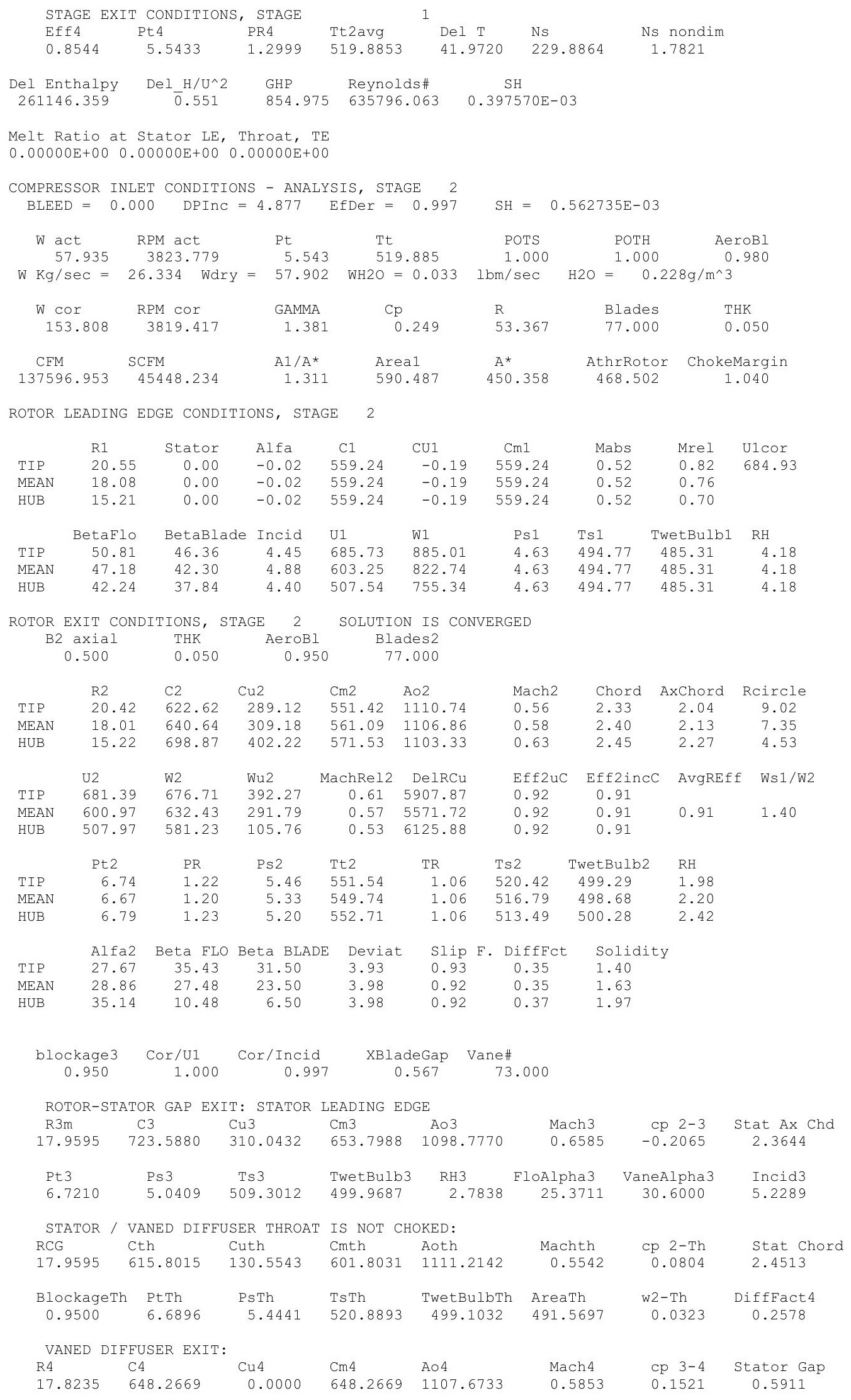




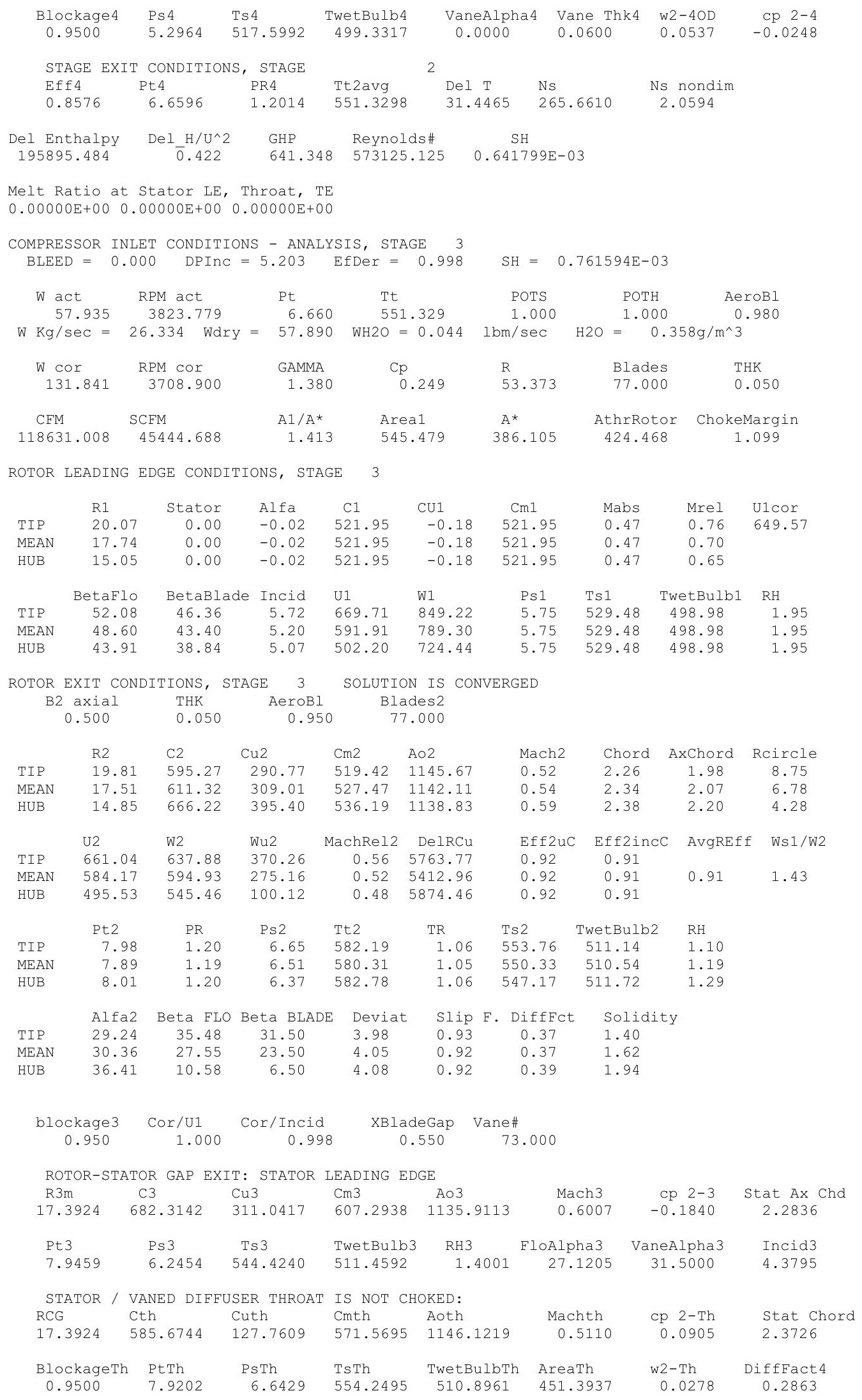




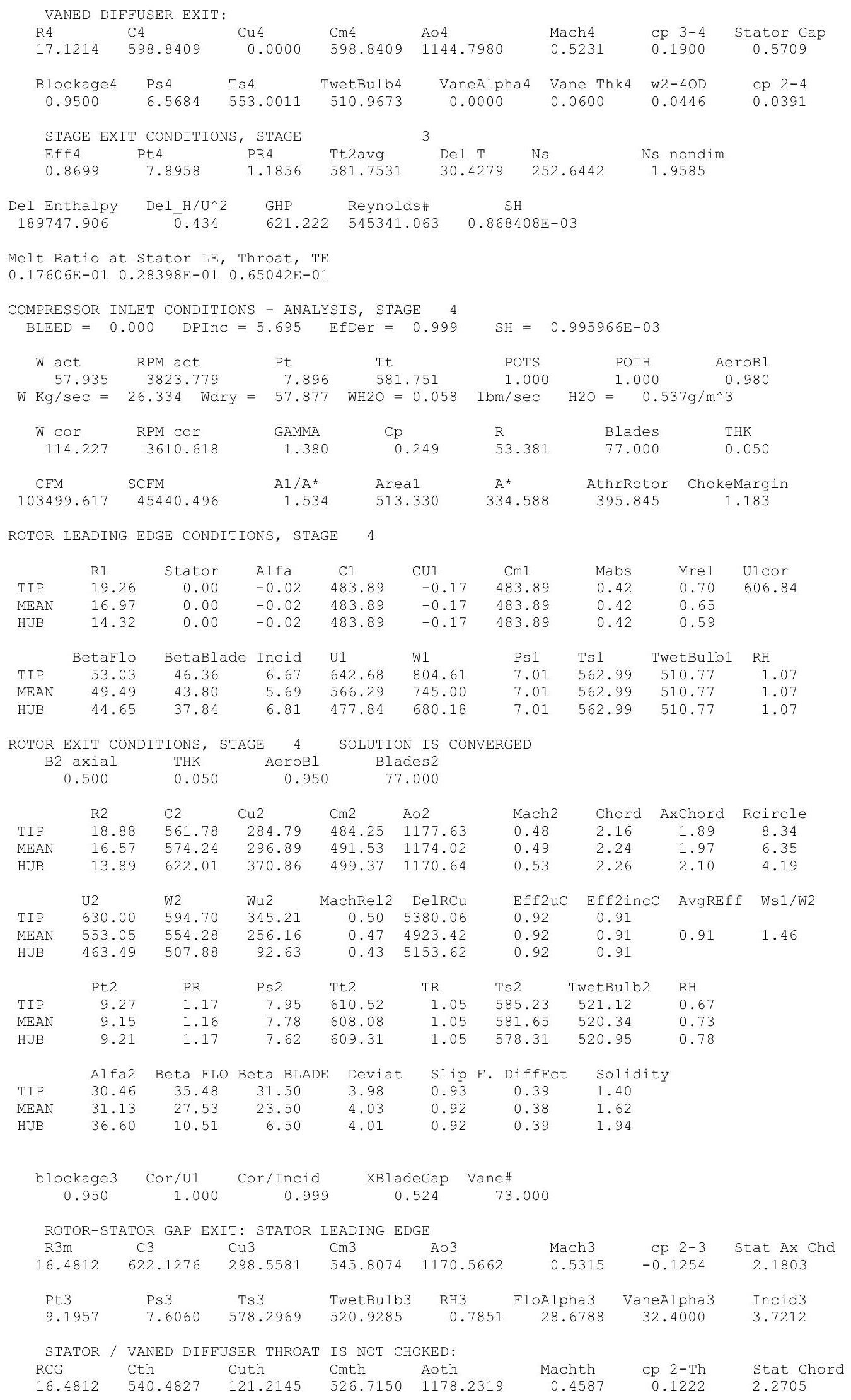




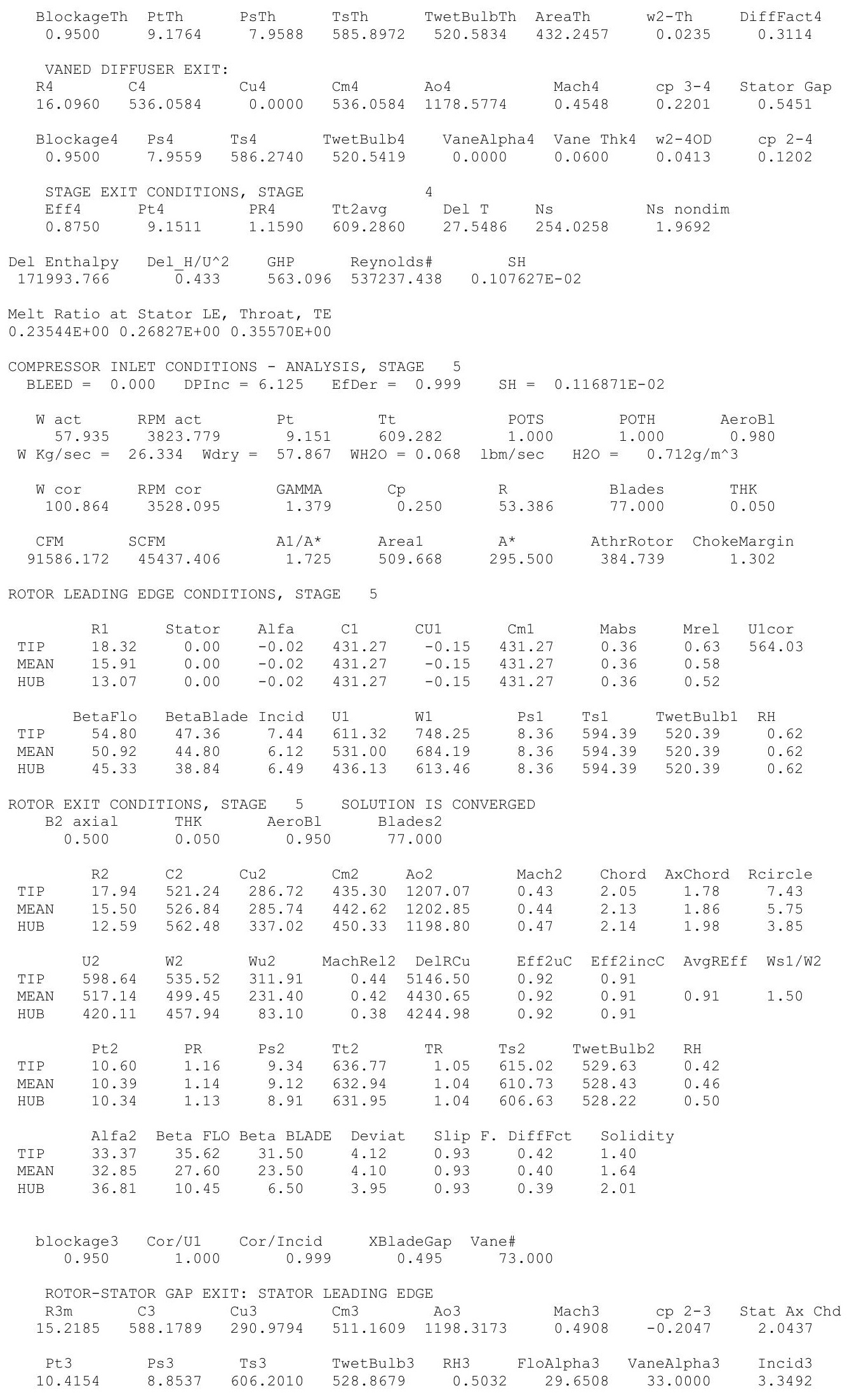




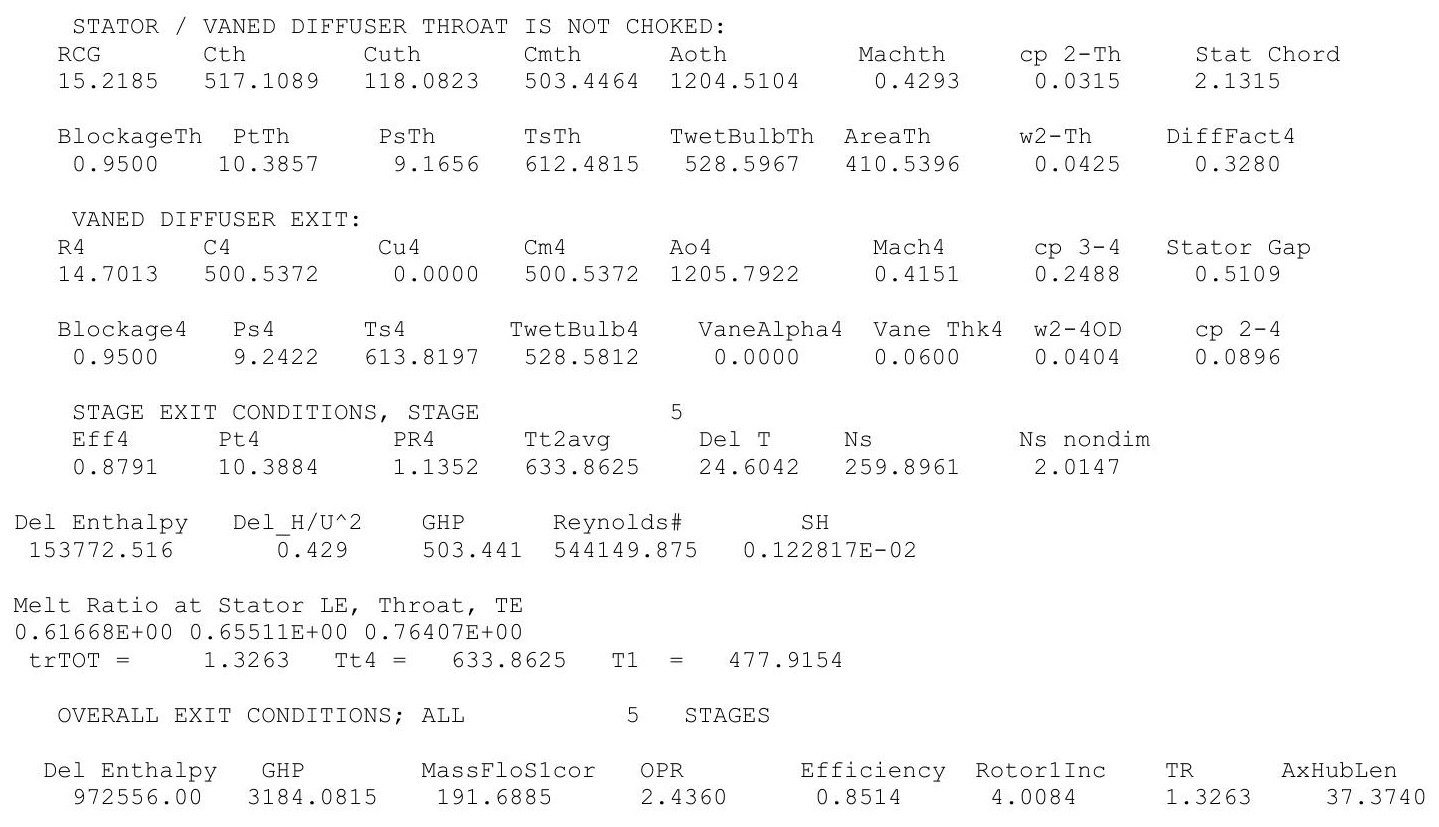




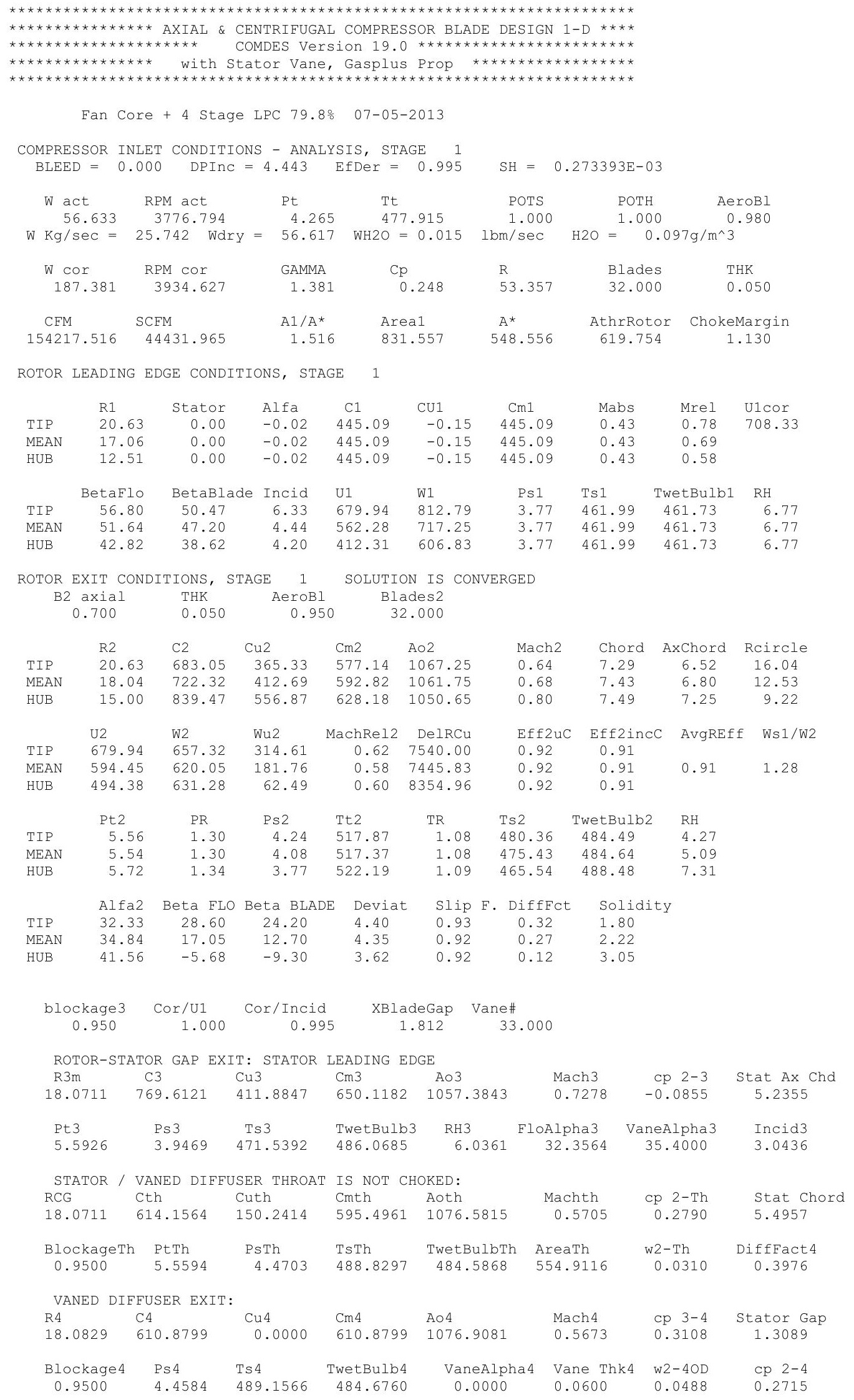




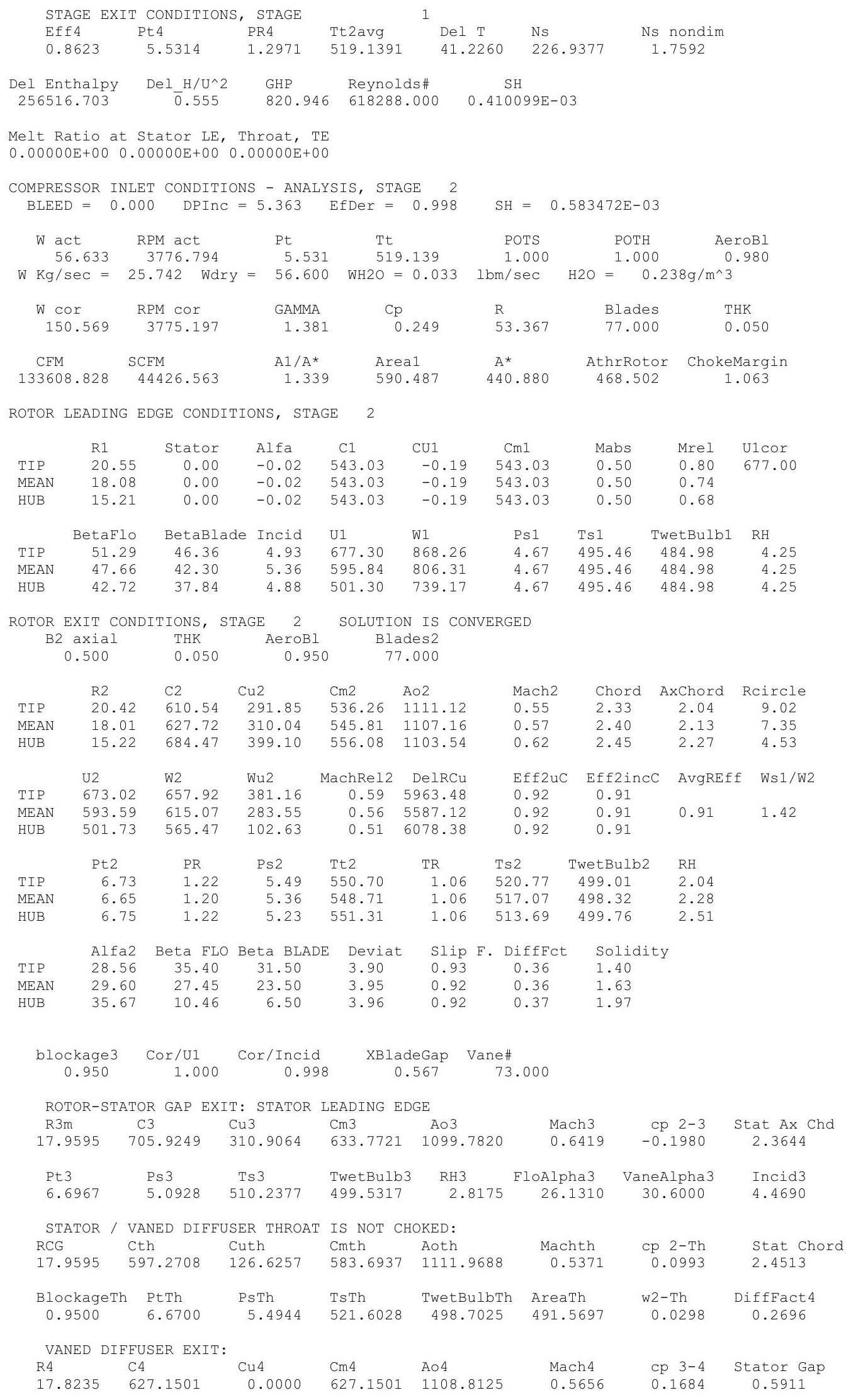




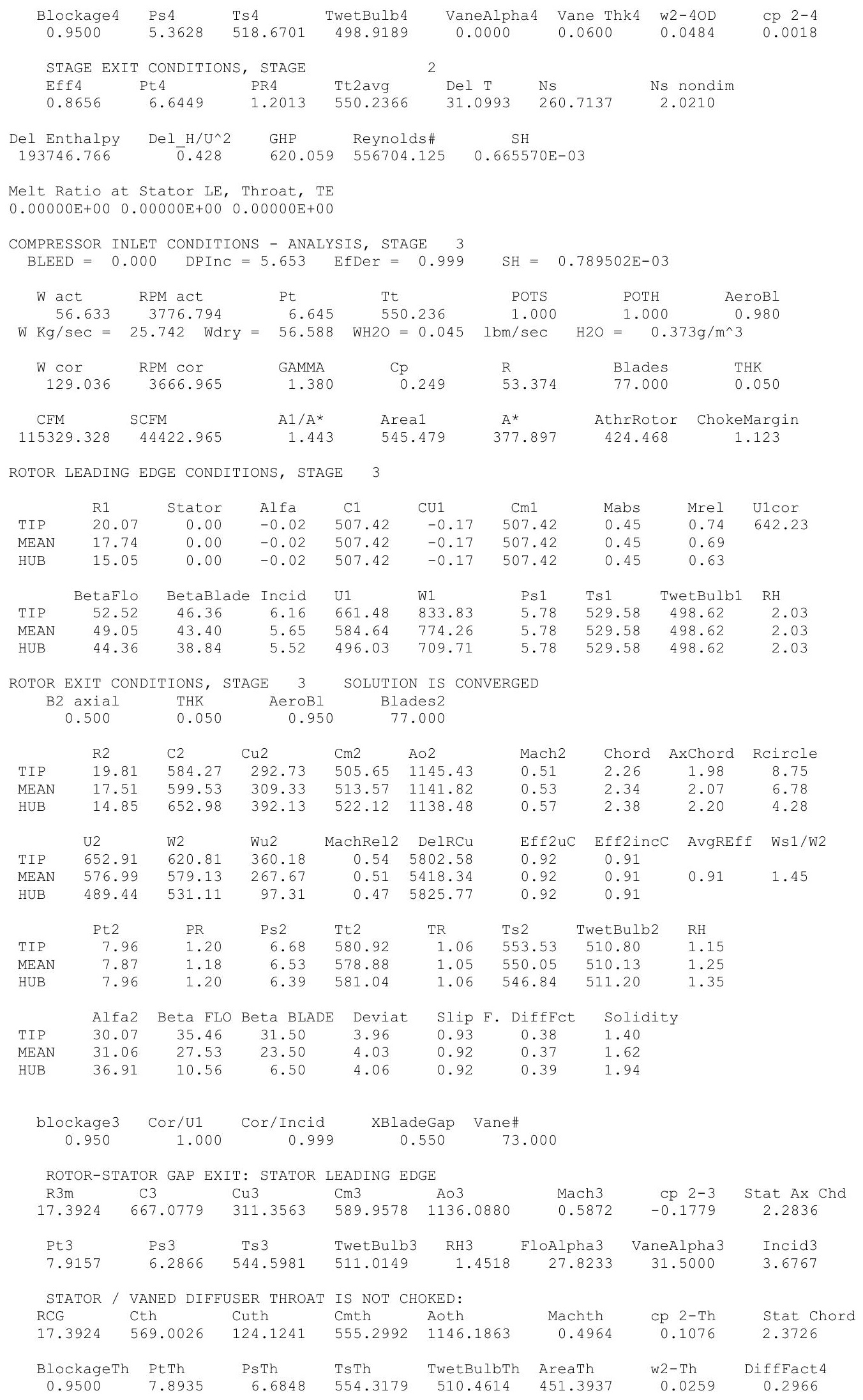




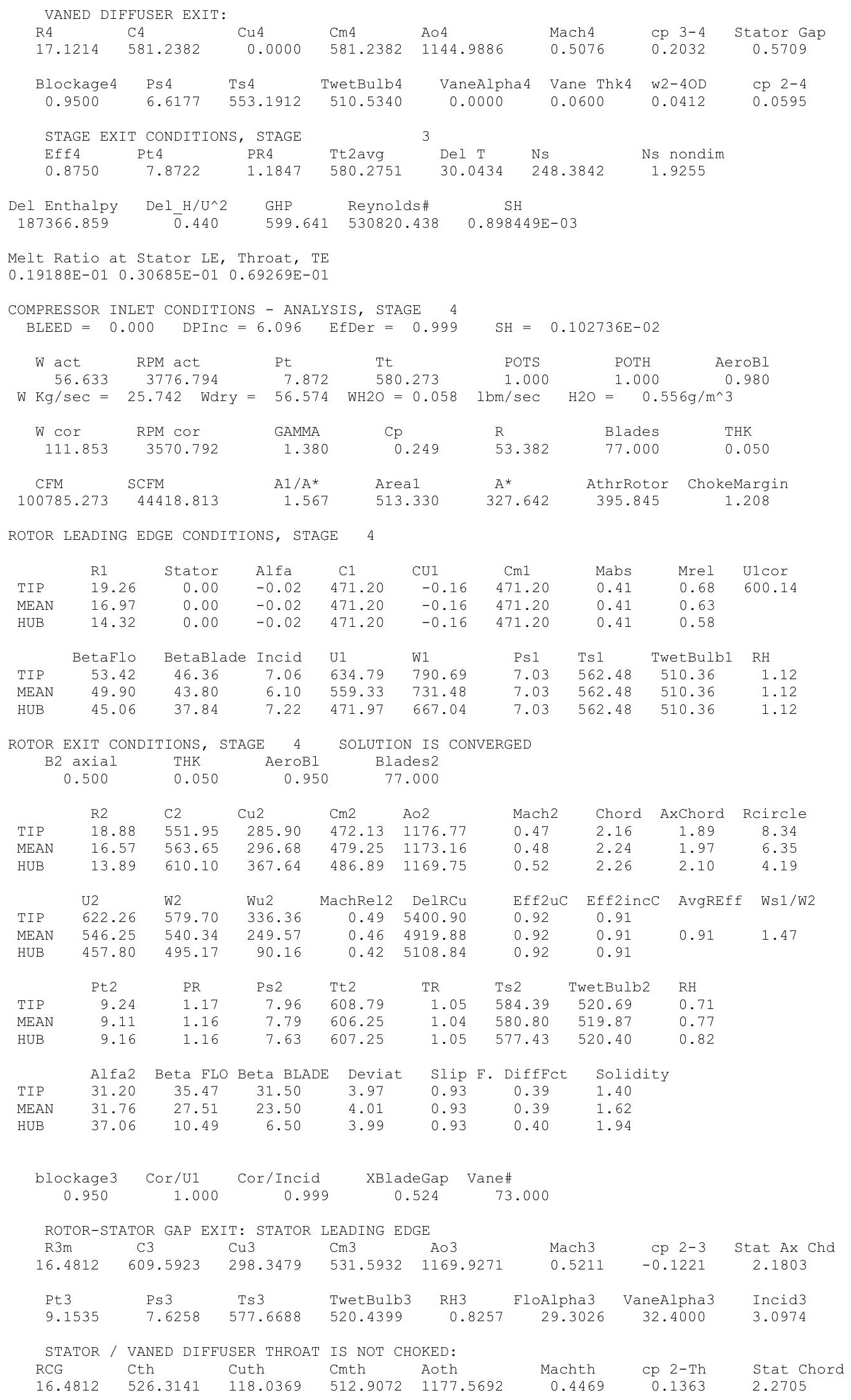




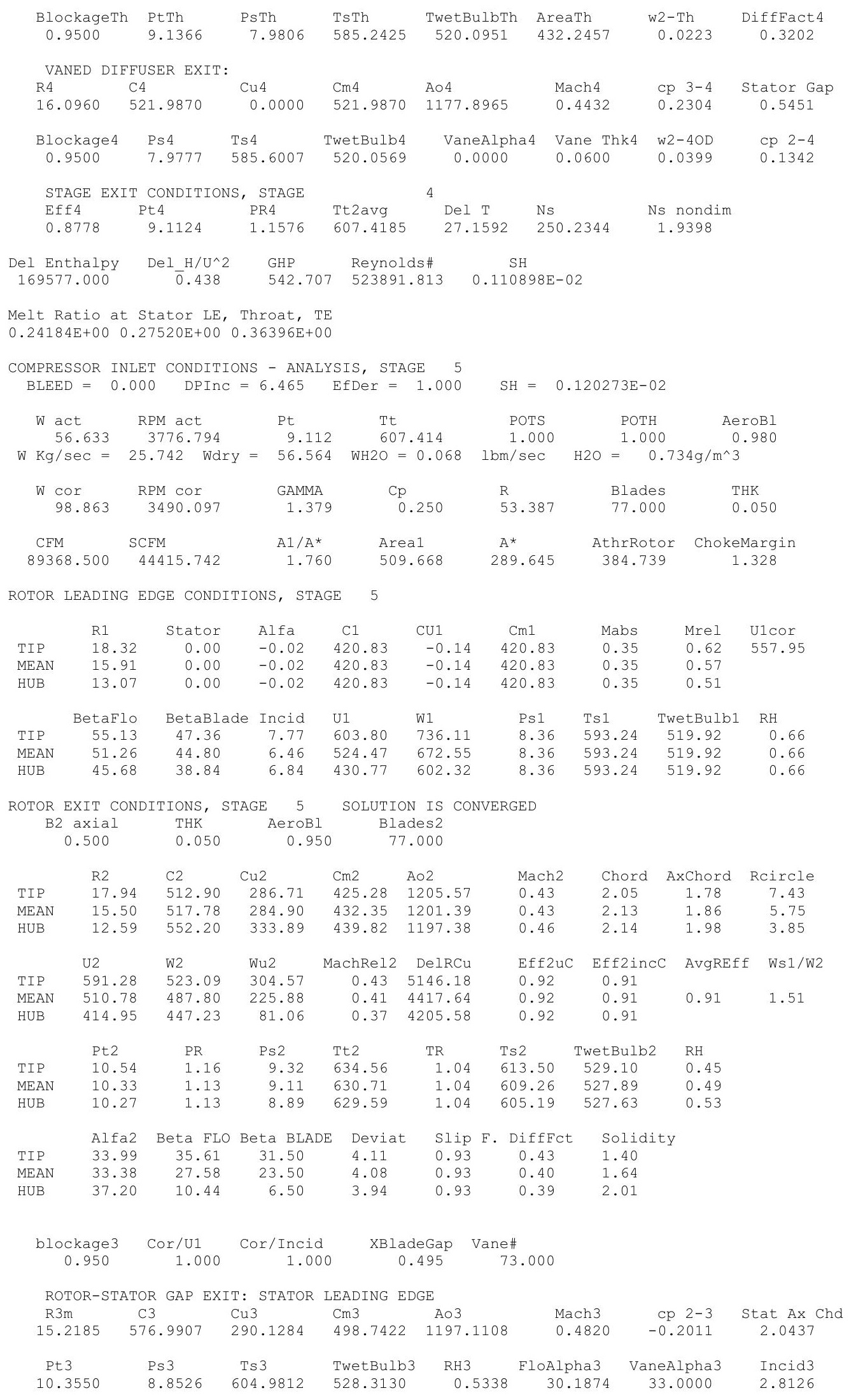




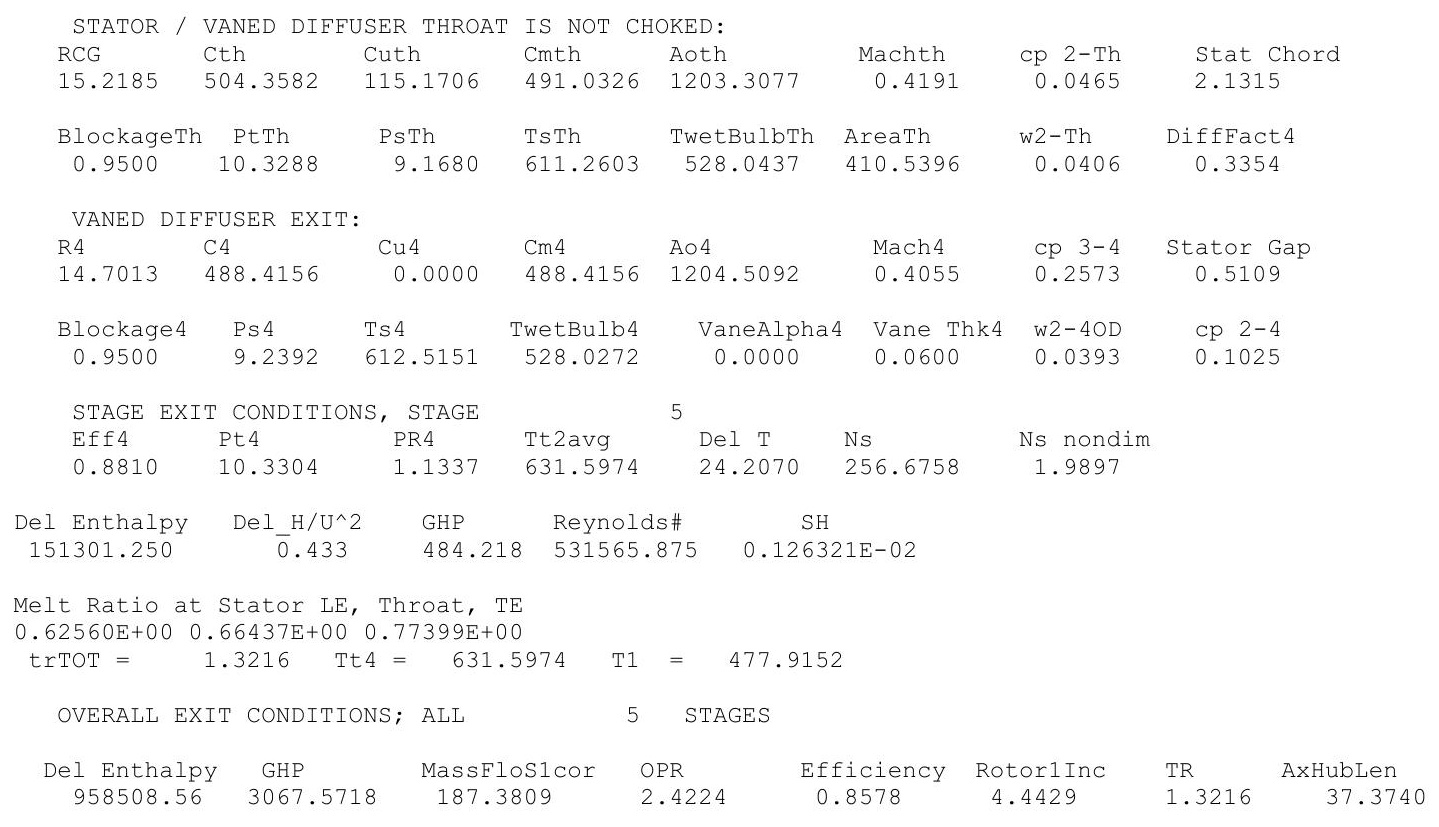




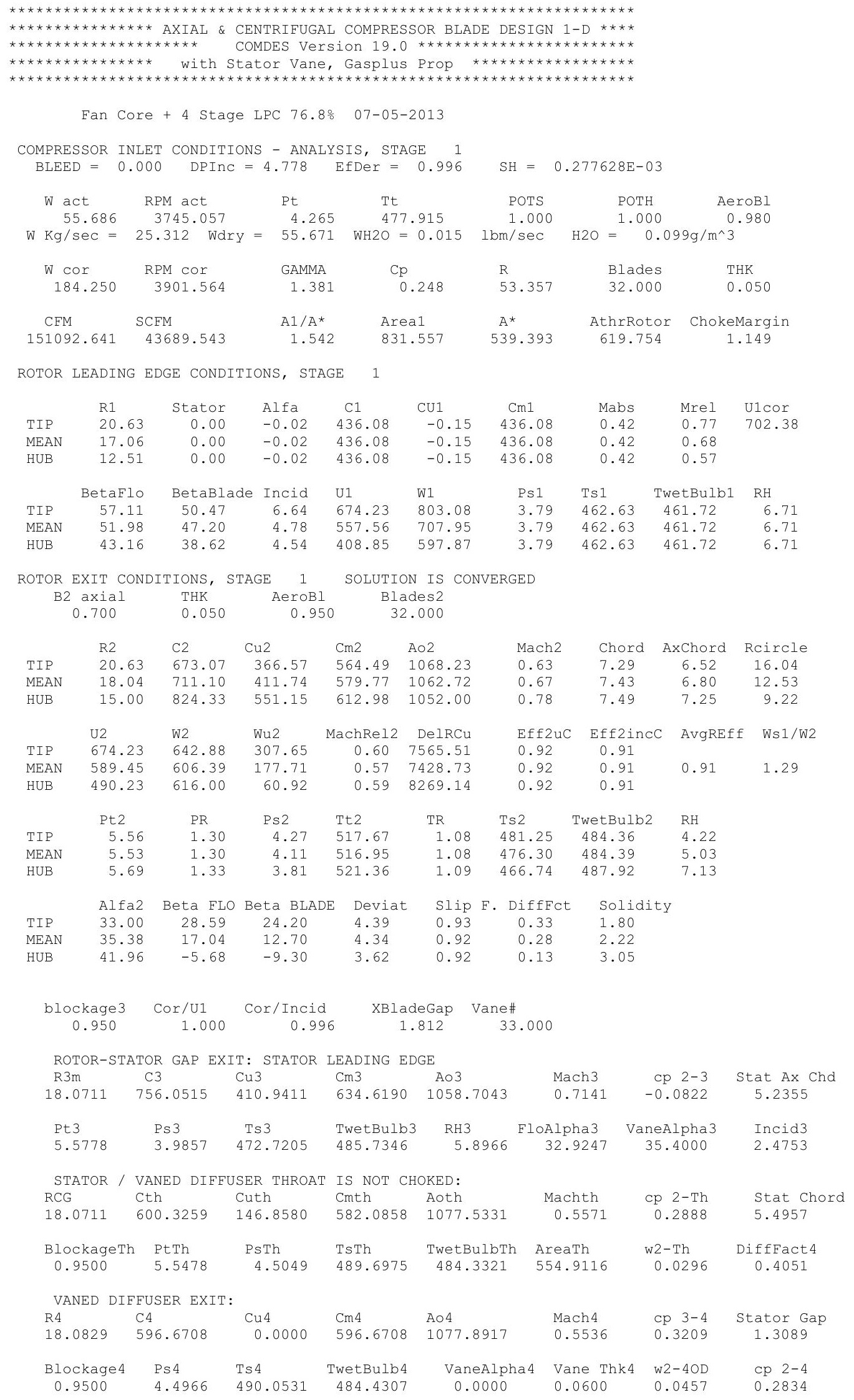




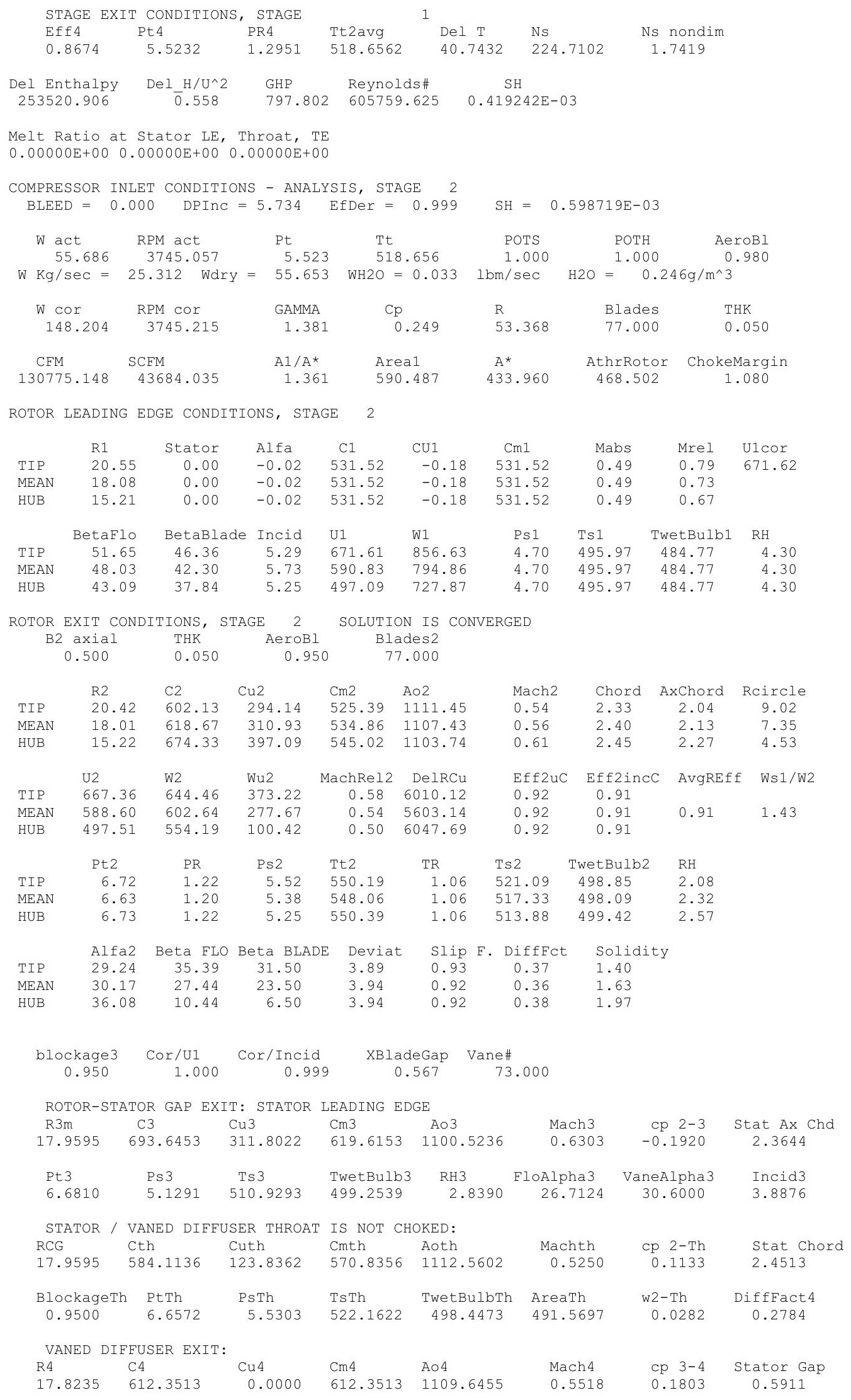




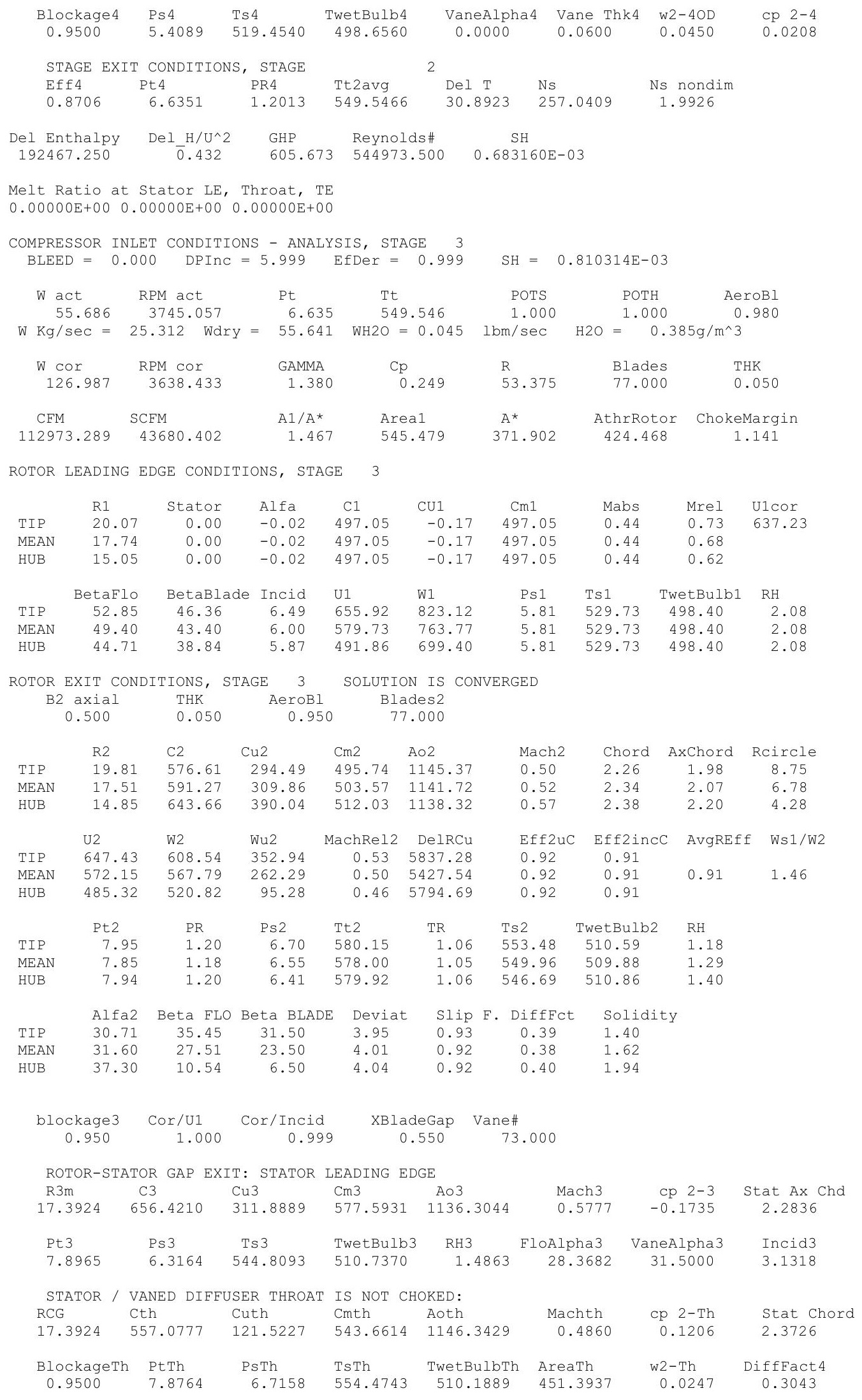




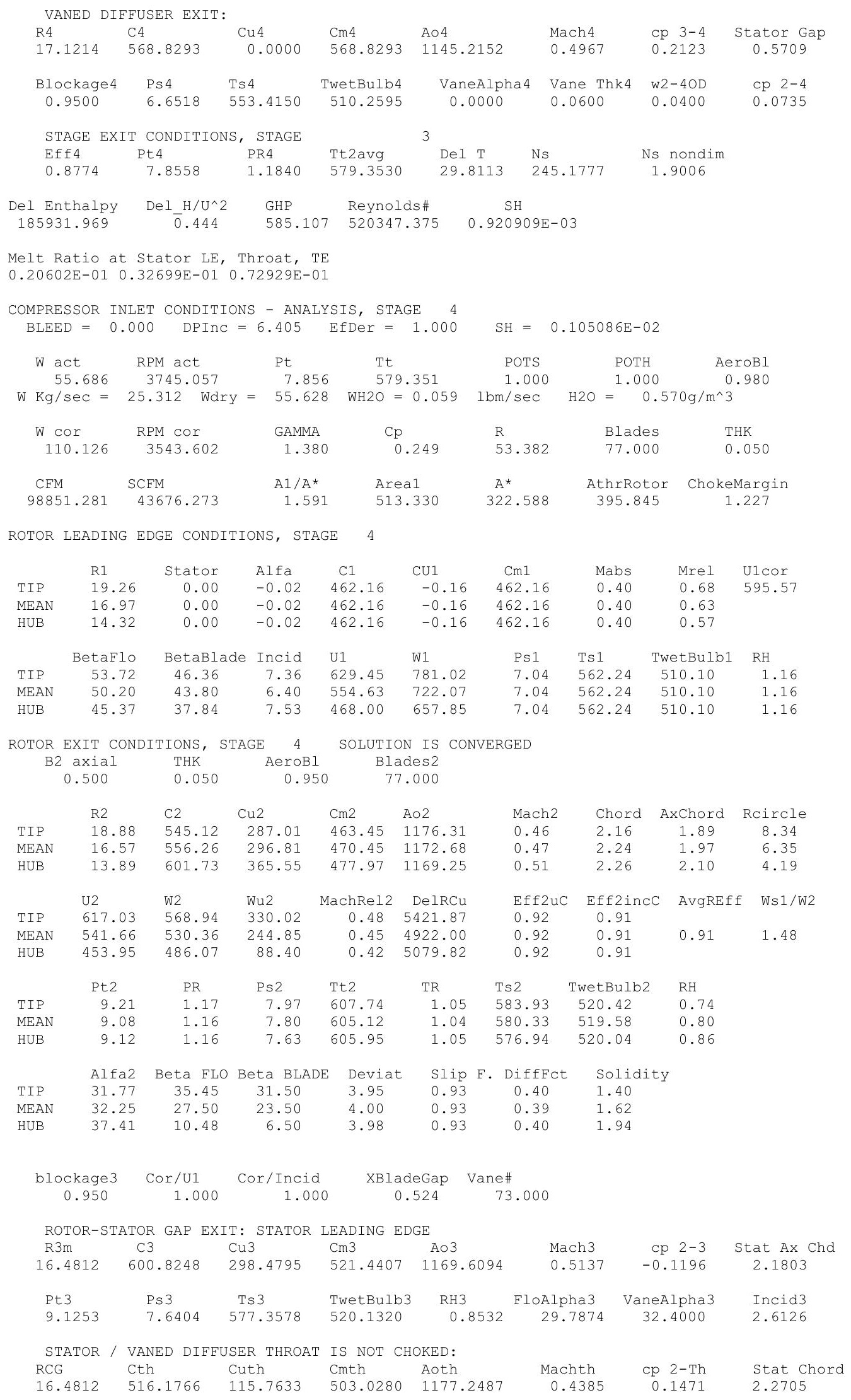




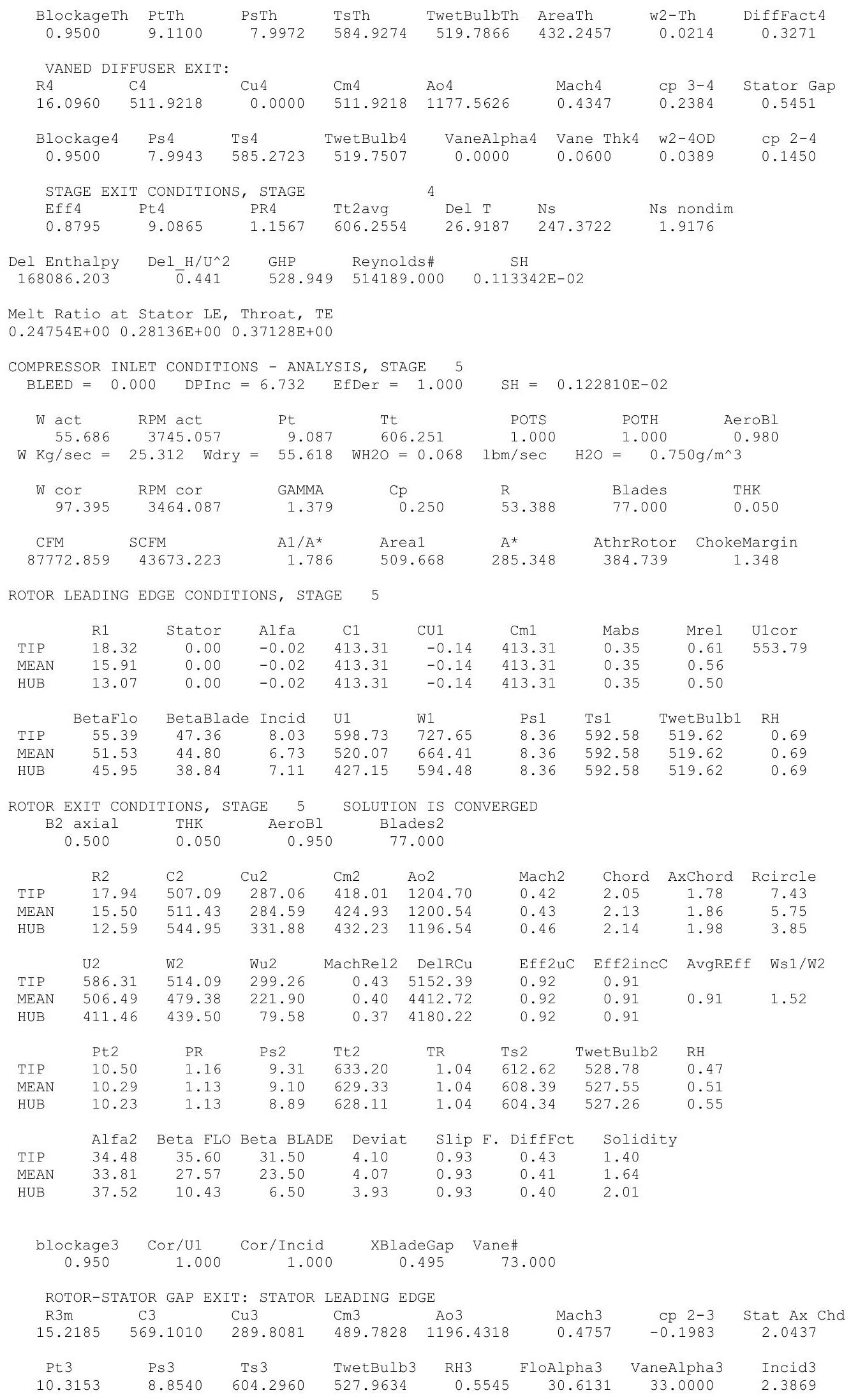




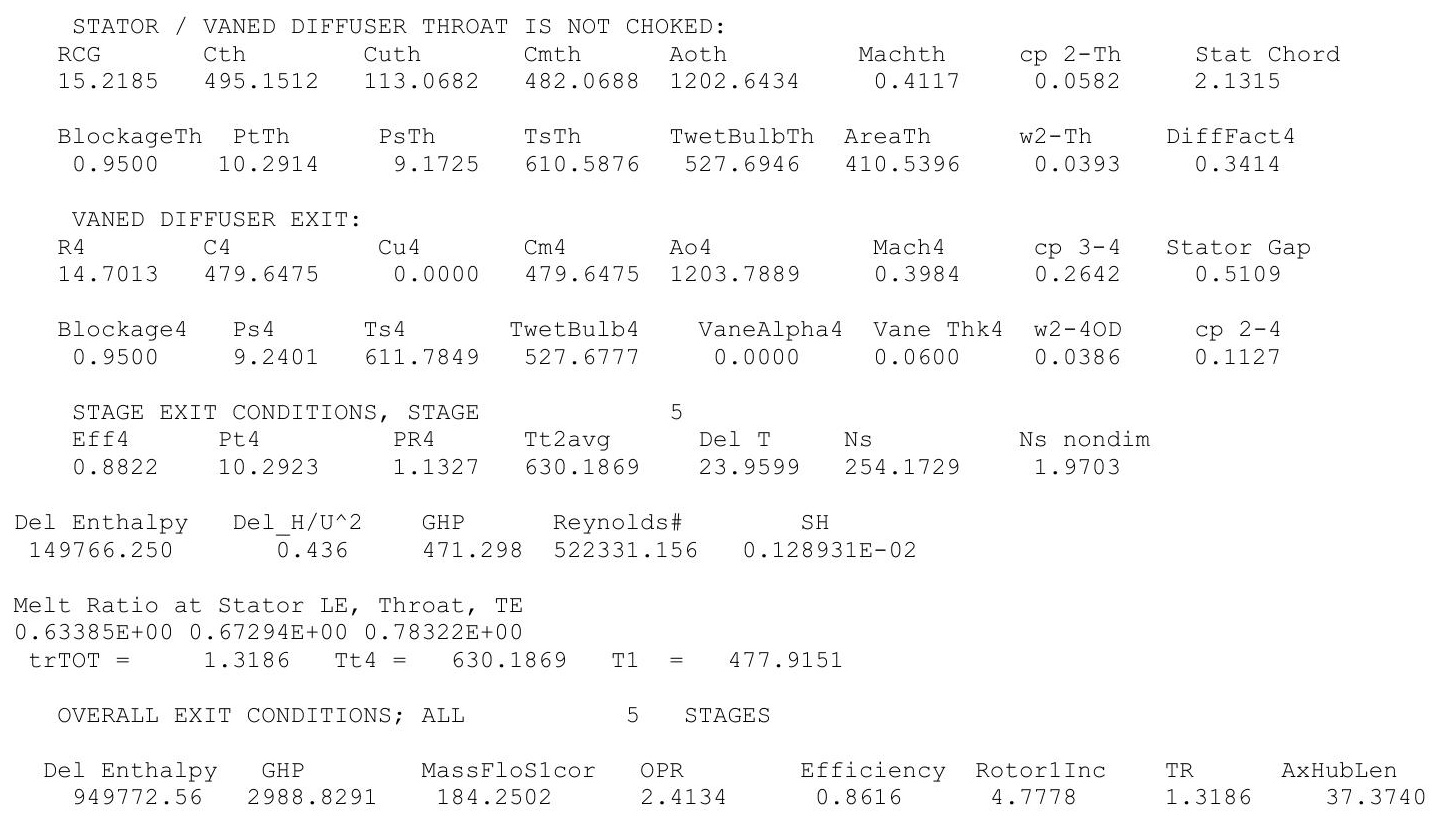




\section{$25 \mu \mathrm{m}, \mathrm{ISA}+18 \mathrm{R}$}

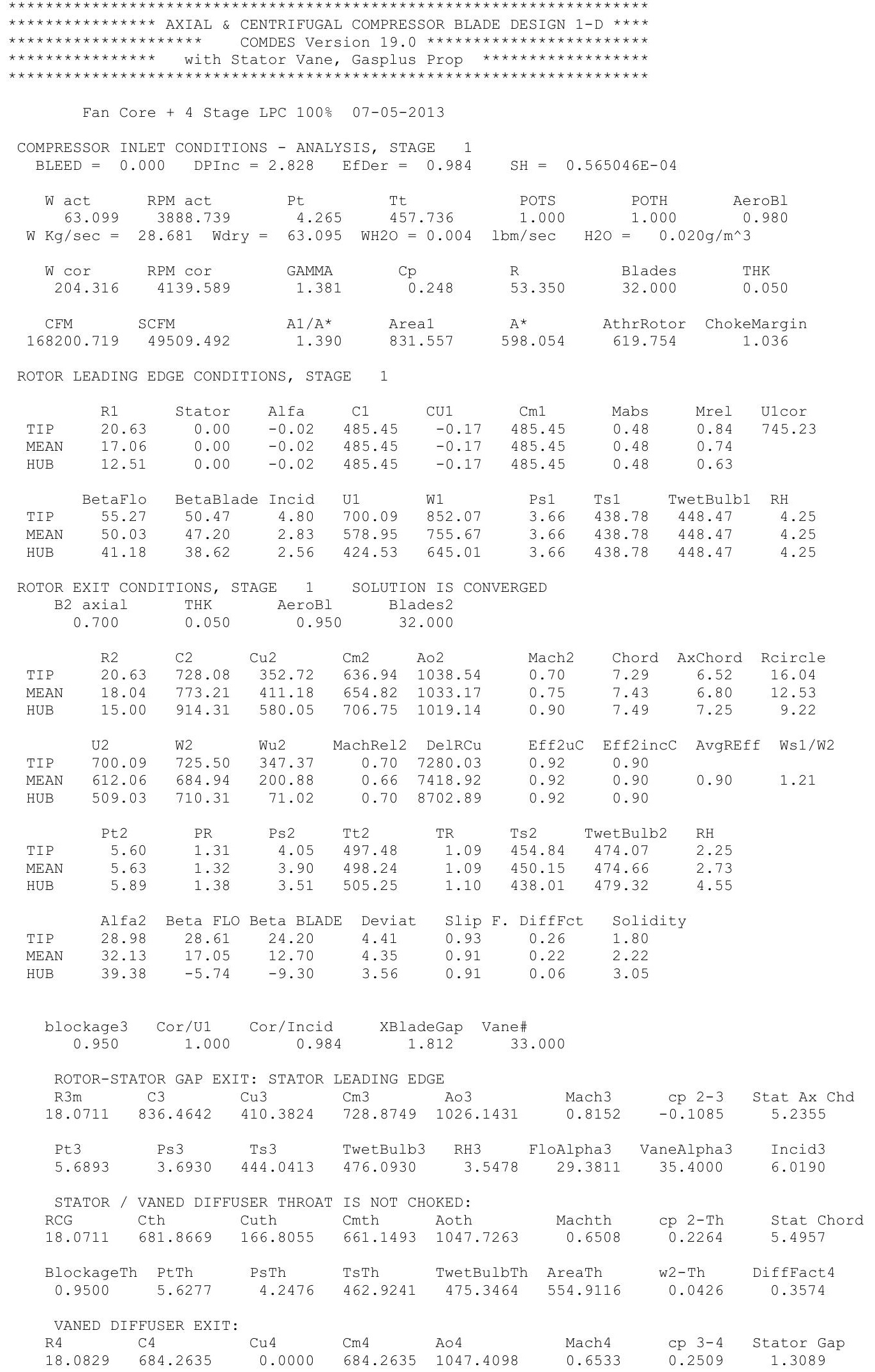




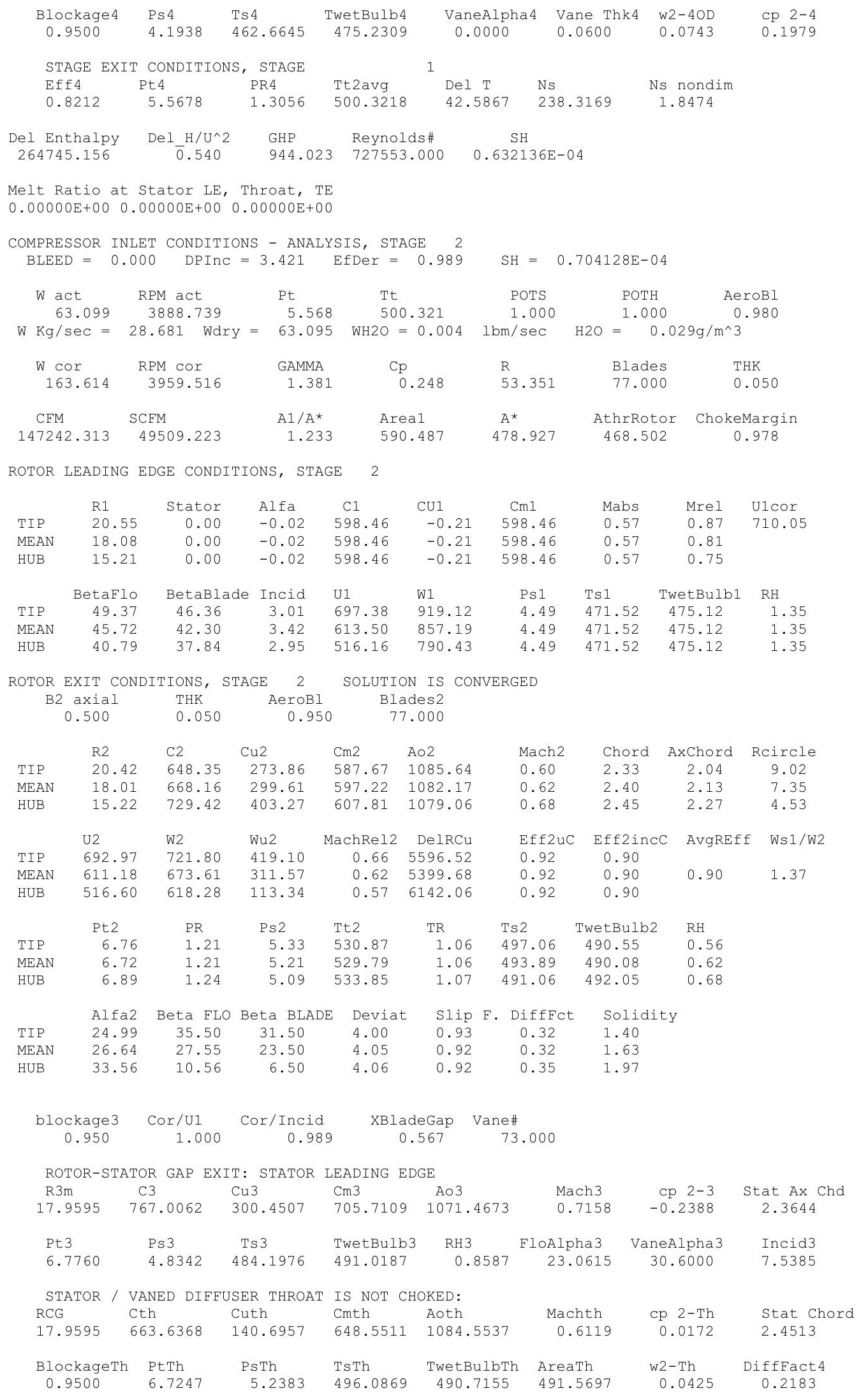




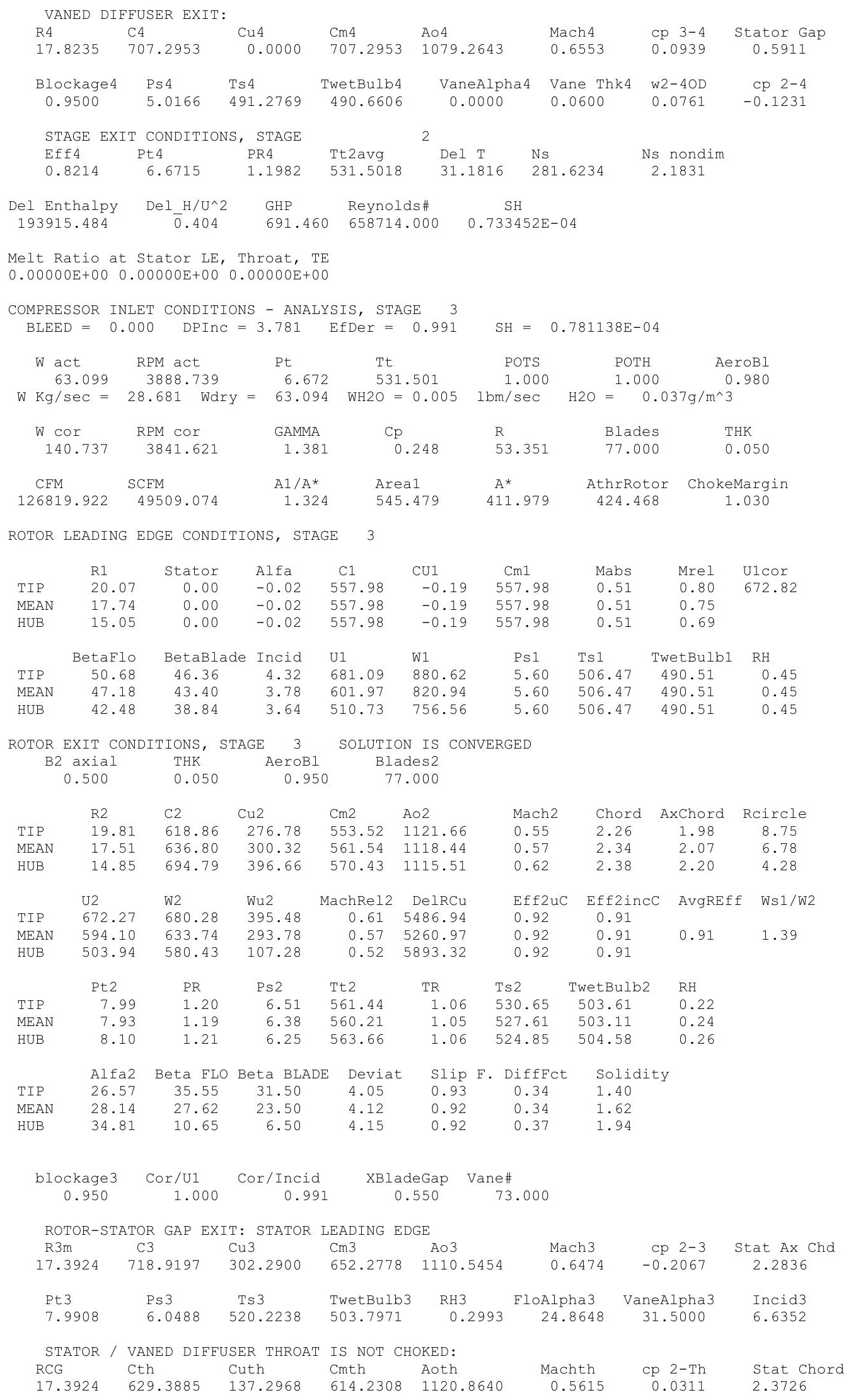




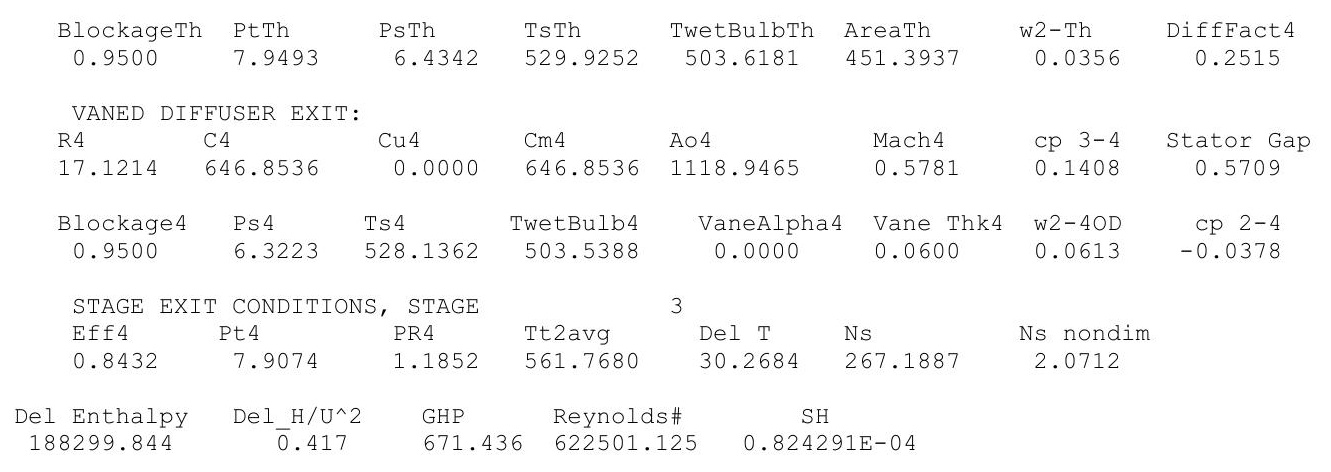

Melt Ratio at Stator LE, Throat, TE

$0.00000 \mathrm{E}+00 \quad 0.00000 \mathrm{E}+00 \quad 0.00000 \mathrm{E}+00$

COMPRESSOR INLET CONDITIONS - ANALYSIS, STAGE 4 $\mathrm{BLEED}=0.000 \quad$ DPInC $=4.357 \quad$ EfDer $=0.994$

63.099 RPM act Pt Tt

$\mathrm{SH}=0.905579 \mathrm{E}-04$ $\mathrm{W} \mathrm{Kg} / \mathrm{sec}=28.681$ Wdry $=63.093 \mathrm{WH} 2 \mathrm{O}=0.006 \mathrm{lbm} / \mathrm{sec} \quad \mathrm{H} 2 \mathrm{O}=0.050 \mathrm{~g} / \mathrm{m}^{\wedge} 3$

$\begin{array}{ccccccc}\text { W Cor } & \text { RPM Cor } & \text { GAMMA } & \text { Cp } & \text { R } & \text { Blades } & \text { THK } \\ 122.075 & 3736.701 & 1.381 & 0.249 & 53.351 & 77.000 & 0.050 \\ \text { CFM } & \text { SCFM } & \text { A1/A* } & \text { Area1 } & A^{*} & \text { AthrRotor } & \text { ChokeMargin } \\ 110335.977 & 49508.832 & 1.436 & 513.330 & 357.368 & 395.845 & 1.108\end{array}$

ROTOR LEADING EDGE CONDITIONS, STAGE 4

$\begin{array}{lrrrrrrrrr} & \text { R1 } & \text { Stator } & \text { Alfa } & \text { C1 } & \text { CU1 } & \text { Cm1 } & \text { Mabs } & \text { Mrel } & \text { U1cor } \\ \text { TIP } & 19.26 & 0.00 & -0.02 & 515.86 & -0.18 & 515.86 & 0.46 & 0.74 & 628.03 \\ \text { MEAN } & 16.97 & 0.00 & -0.02 & 515.86 & -0.18 & 515.86 & 0.46 & 0.68 & \\ \text { HUB } & 14.32 & 0.00 & -0.02 & 515.86 & -0.18 & 515.86 & 0.46 & 0.63 & \\ & & & & & & & & & \\ & \text { BetaFlo } & \text { BetaBlade } & \text { Incid } & \text { U1 } & \text { W1 } & \text { Ts1 } & \text { TwetBulb1 } & \text { RH } \\ \text { TIP } & 51.73 & 46.36 & 5.37 & 653.60 & 832.79 & 6.87 & 540.38 & 503.48 & 0.19 \\ \text { MEAN } & 48.16 & 43.80 & 4.36 & 575.91 & 773.30 & 6.87 & 540.38 & 503.48 & 0.19 \\ \text { HUB } & 43.30 & 37.84 & 5.46 & 485.96 & 708.83 & 6.87 & 540.38 & 503.48 & 0.19\end{array}$

ROTOR EXIT CONDITIONS, STAGE 4 SOLUTION IS CONVERGED

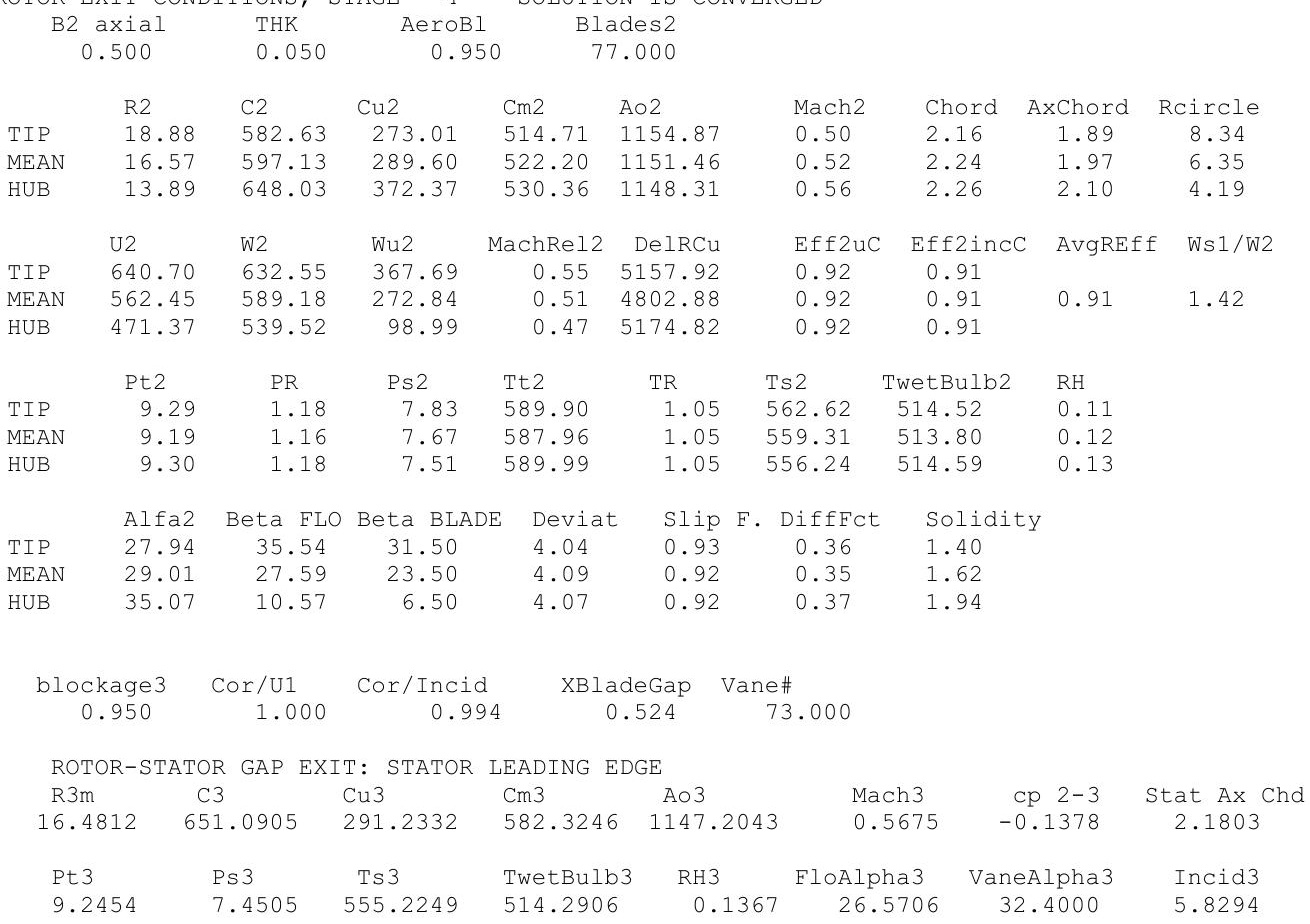




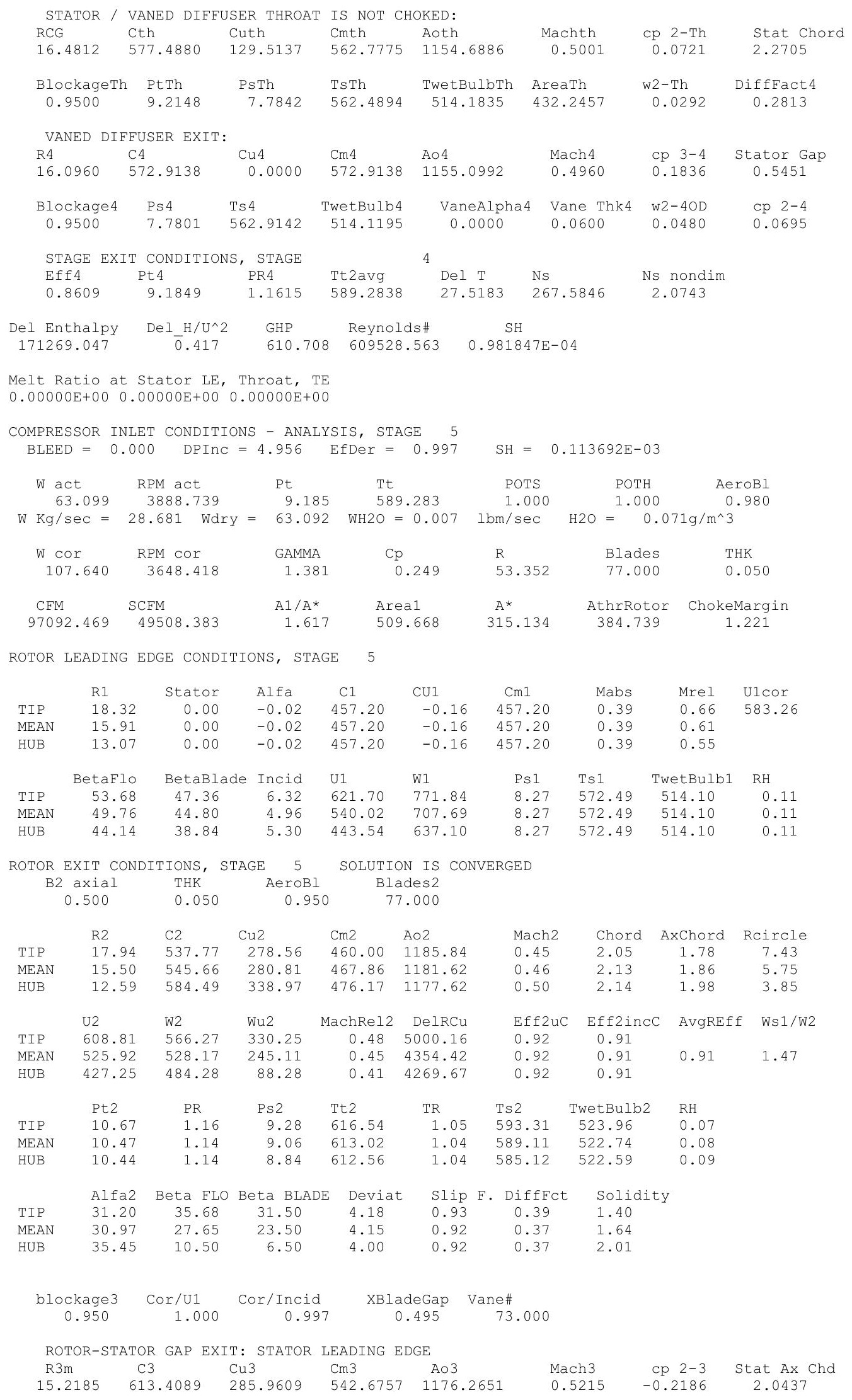




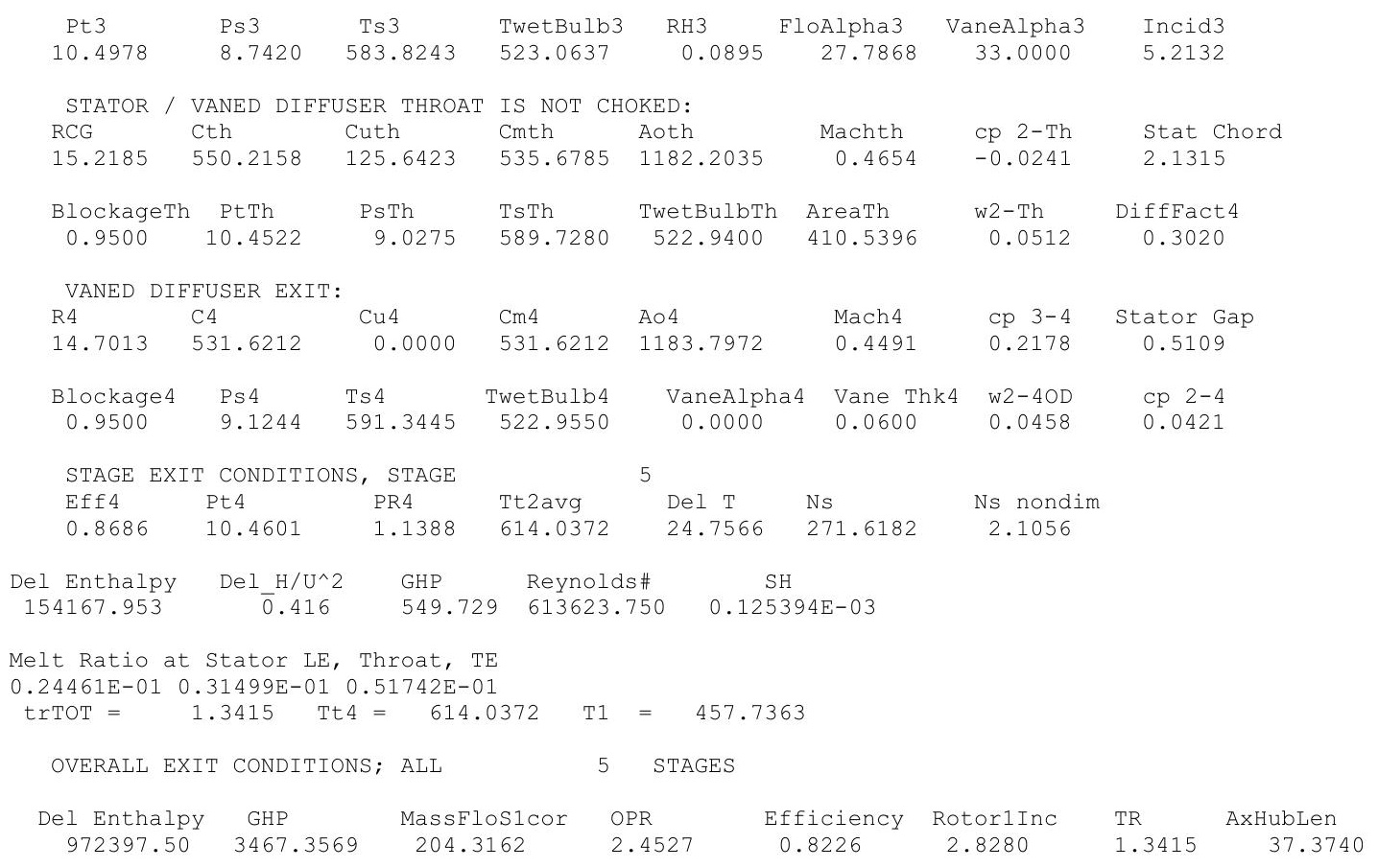




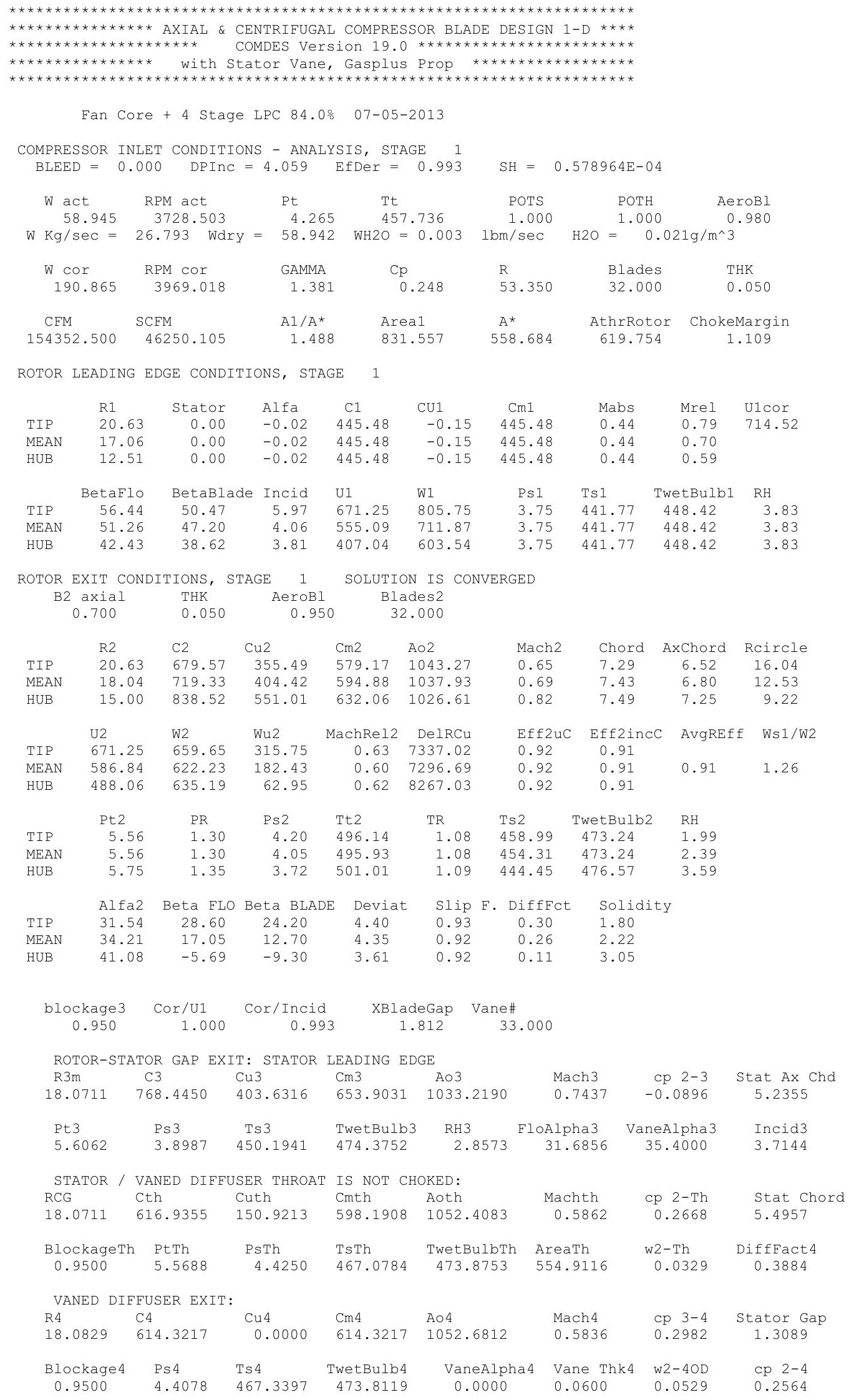




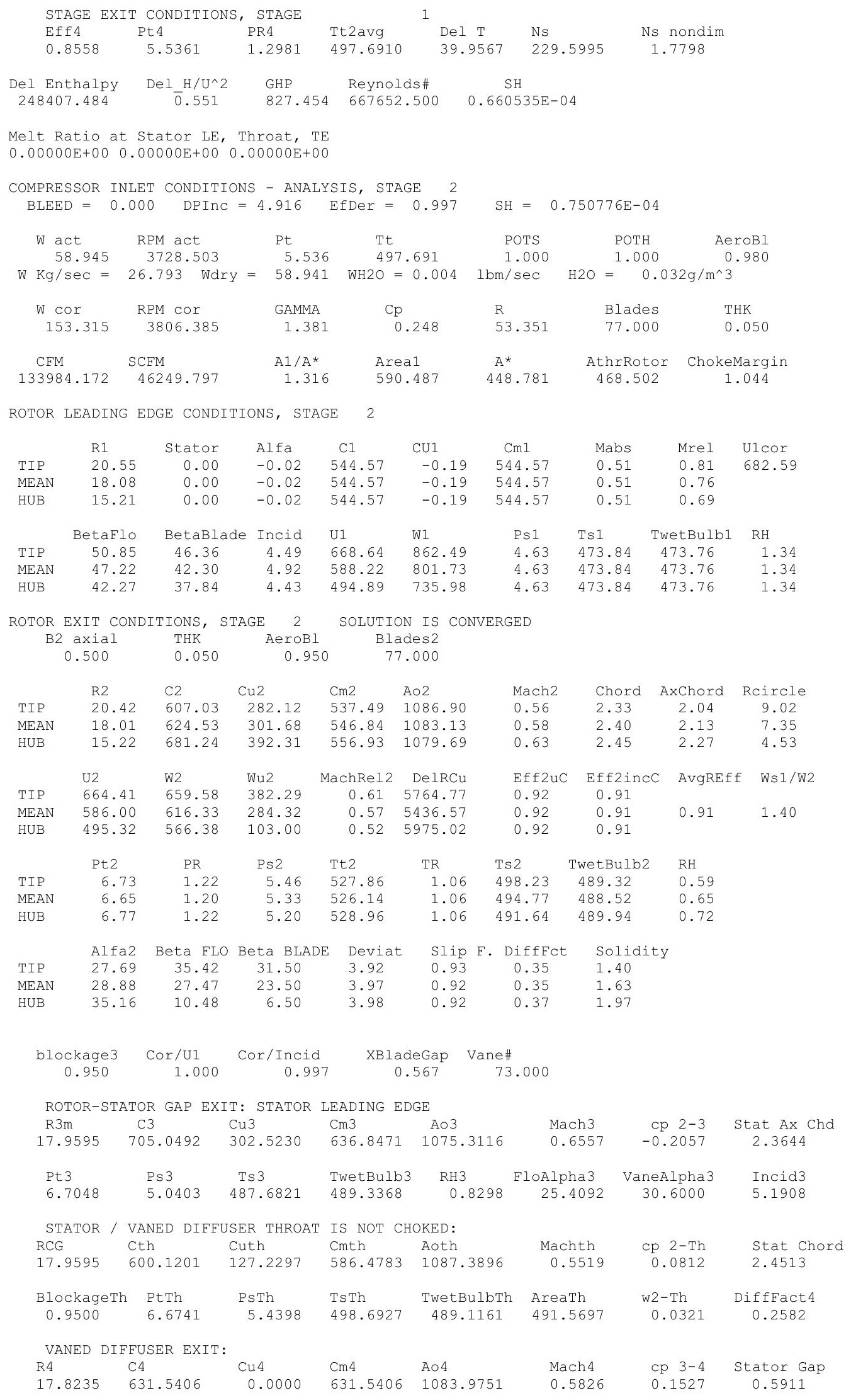




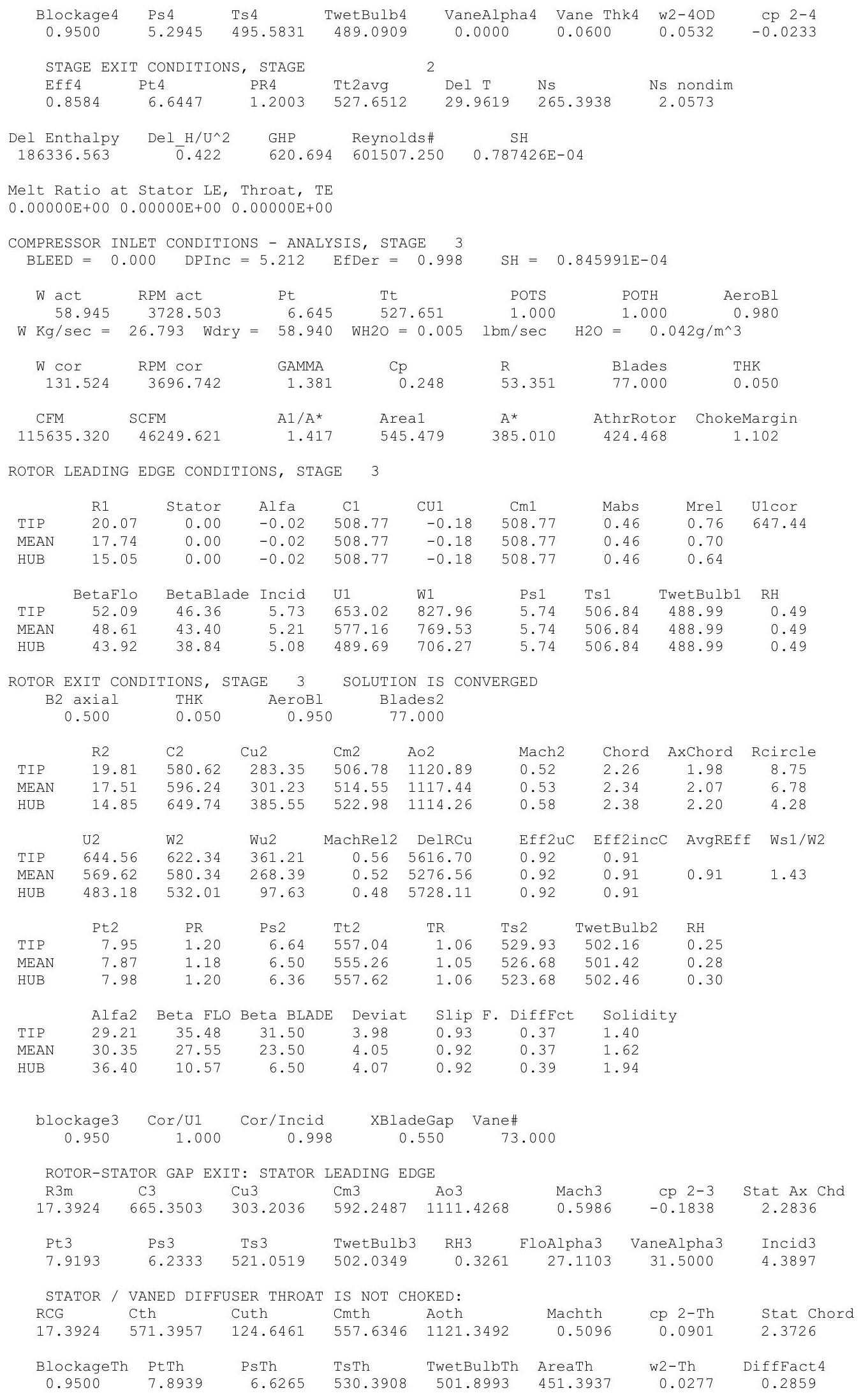




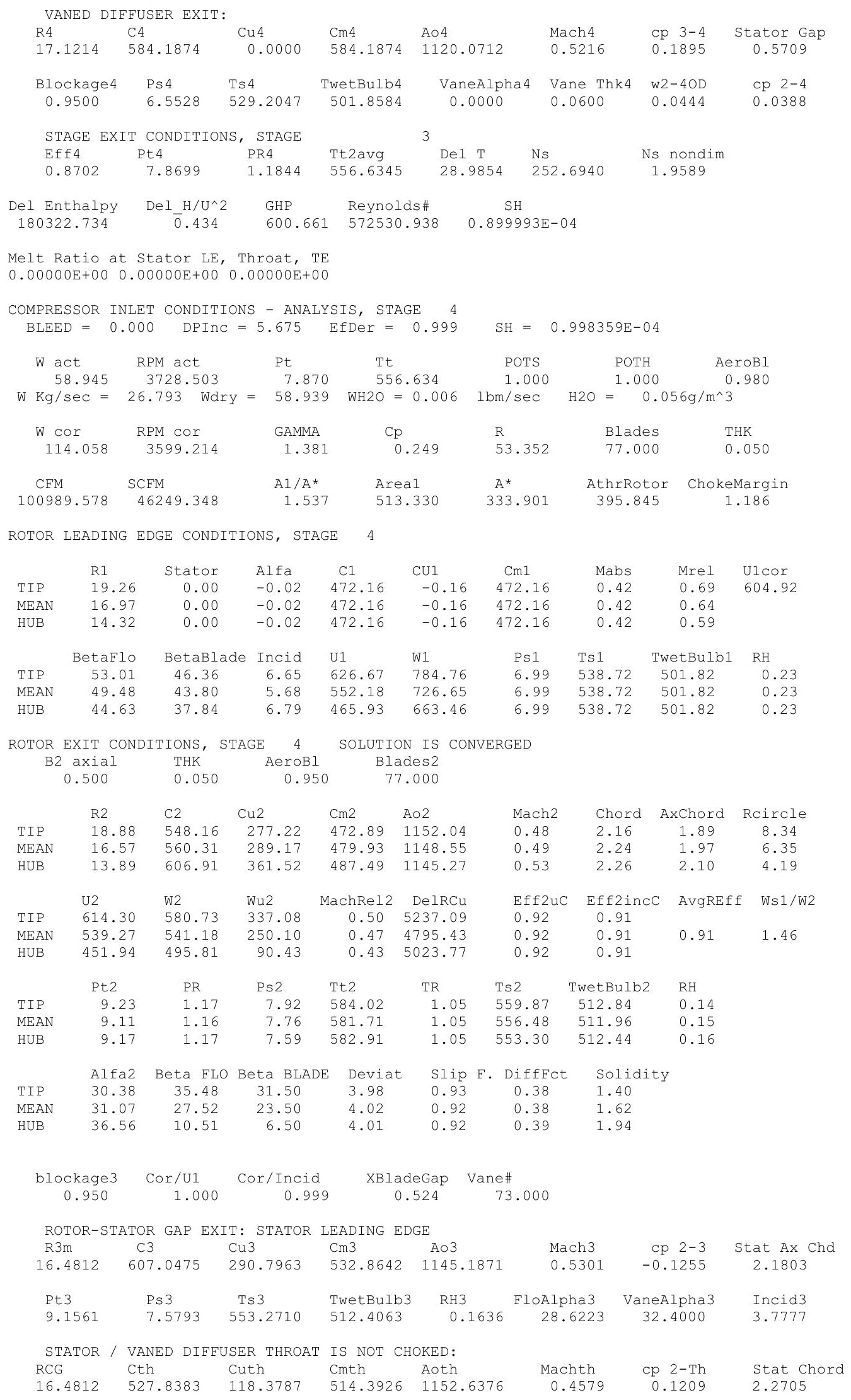




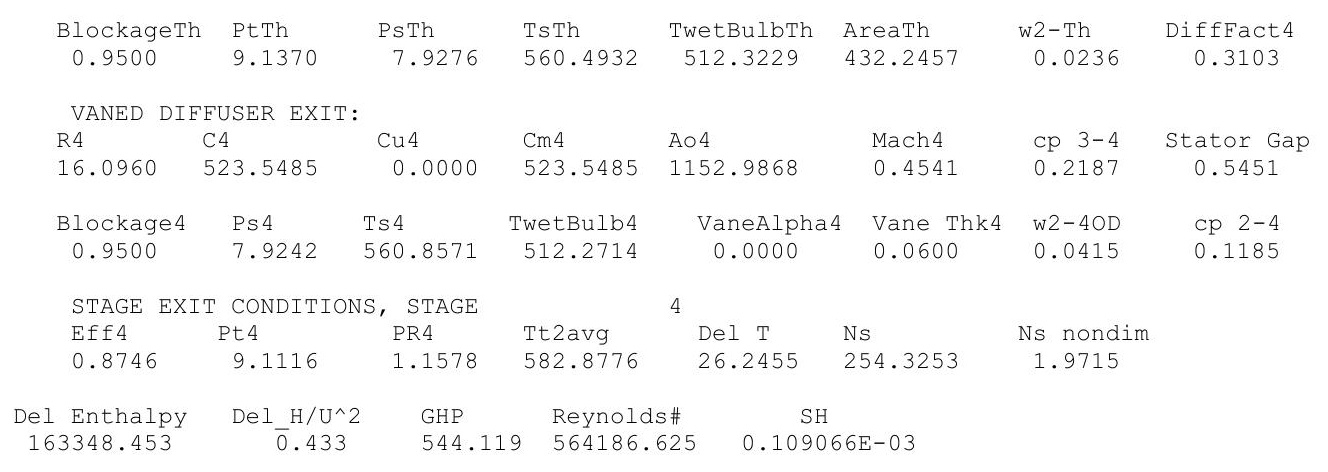

Melt Ratio at Stator LE, Throat, TE

$0.00000 \mathrm{E}+000.00000 \mathrm{E}+000.00000 \mathrm{E}+00$

COMPRESSOR INLET CONDITIONS - ANALYSIS, STAGE 5 $\mathrm{BLEED}=0.000 \quad$ DPInC $=6.077 \quad$ EfDer $=0.999$

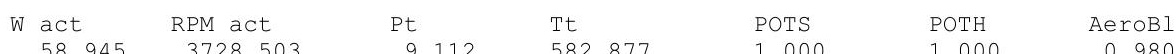
$\mathrm{W} \mathrm{Kg} / \mathrm{sec}=26.793$ Wdry $=58.938$ WH2O $=0.007 \quad \mathrm{lbm} / \mathrm{sec} \quad \mathrm{H} 2 \mathrm{O}=0.081 \mathrm{~g} / \mathrm{m}^{\wedge} 3$

$\begin{array}{ccccccc}\text { W cor } & \text { RPM Cor } & \text { GAMMA } & \text { Cp } & \text { R } & \text { Blades } & \text { THK } \\ 100.809 & 3517.256 & 1.381 & 0.249 & 53.352 & 77.000 & 0.050 \\ & & & & & & \\ \text { CFM } & \text { SCFM } & \text { A1/A* } & \text { Area1 } & \text { A* } & \text { AthrRotor } & \text { ChokeMargin } \\ 89455.836 & 46248.852 & 1.727 & 509.668 & 295.137 & 384.739 & 1.304\end{array}$

ROTOR LEADING EDGE CONDITIONS, STAGE 5

$\begin{array}{lccccccccc} & \text { R1 } & \text { Stator } & \text { Alfa } & \text { C1 } & \text { CU1 } & \text { Cm1 } & \text { Mabs } & \text { Mrel } & \text { U1cor } \\ \text { TIP } & 18.32 & 0.00 & -0.02 & 421.24 & -0.15 & 421.24 & 0.36 & 0.63 & 562.29 \\ \text { MEAN } & 15.91 & 0.00 & -0.02 & 421.24 & -0.15 & 421.24 & 0.36 & 0.58 & \\ \text { HUB } & 13.07 & 0.00 & -0.02 & 421.24 & -0.15 & 421.24 & 0.36 & 0.52 & \\ & & & & & & & & & \\ & \text { BetaFlo } & \text { BetaBlade } & \text { Incid } & \text { U1 } & \text { W1 } & \text { Ps1 } & \text { Ts1 } & \text { TwetBulb1 } & \text { RH } \\ \text { TIP } & 54.76 & 47.36 & 7.40 & 596.08 & 730.02 & 8.33 & 568.62 & 512.26 & 0.14 \\ \text { MEAN } & 50.88 & 44.80 & 6.08 & 517.77 & 667.59 & 8.33 & 568.62 & 512.26 & 0.14 \\ \text { HUB } & 45.28 & 38.84 & 6.44 & 425.26 & 598.68 & 8.33 & 568.62 & 512.26 & 0.14\end{array}$

ROTOR EXIT CONDITIONS, STAGE 5 SOLUTION IS CONVERGED

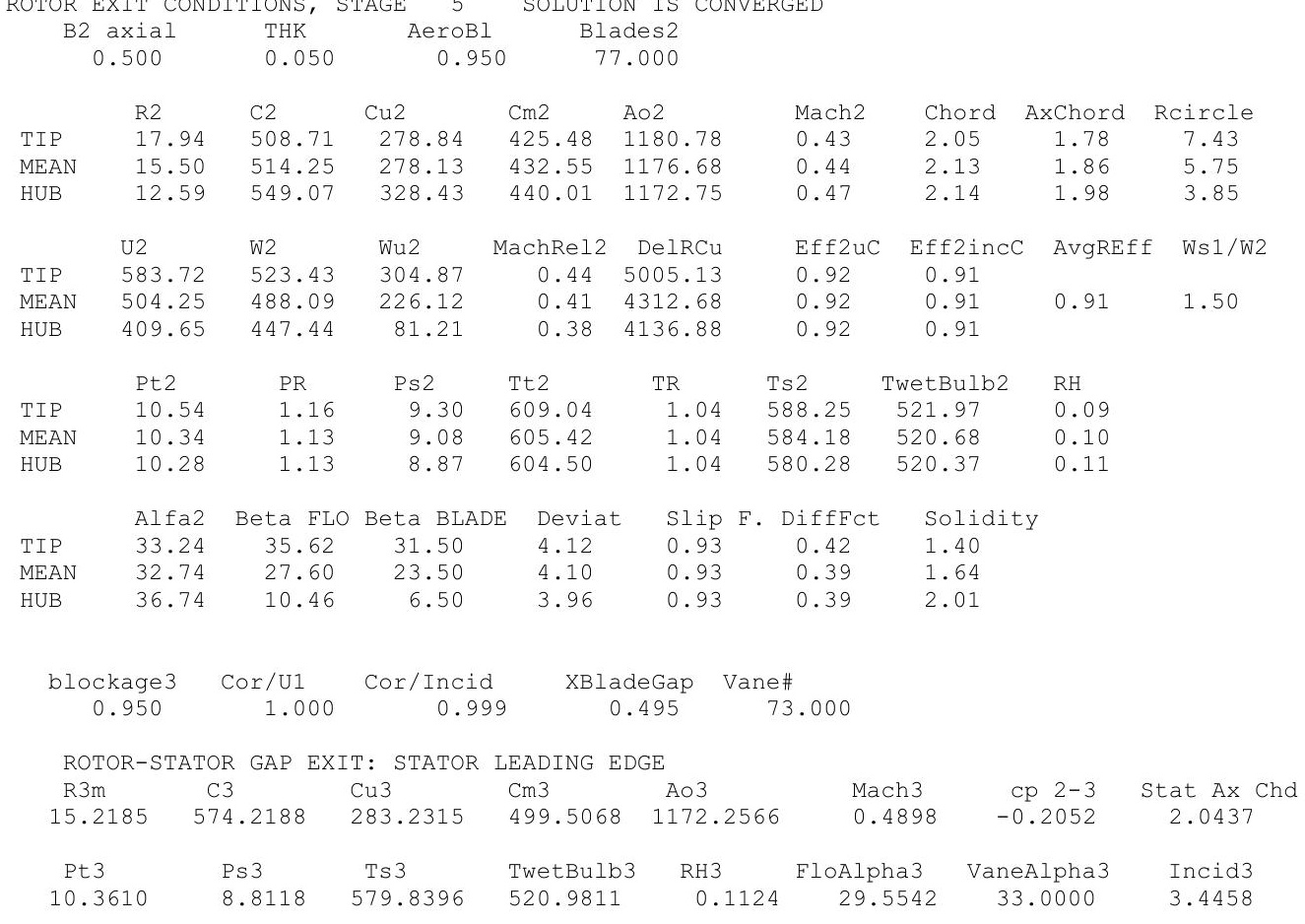




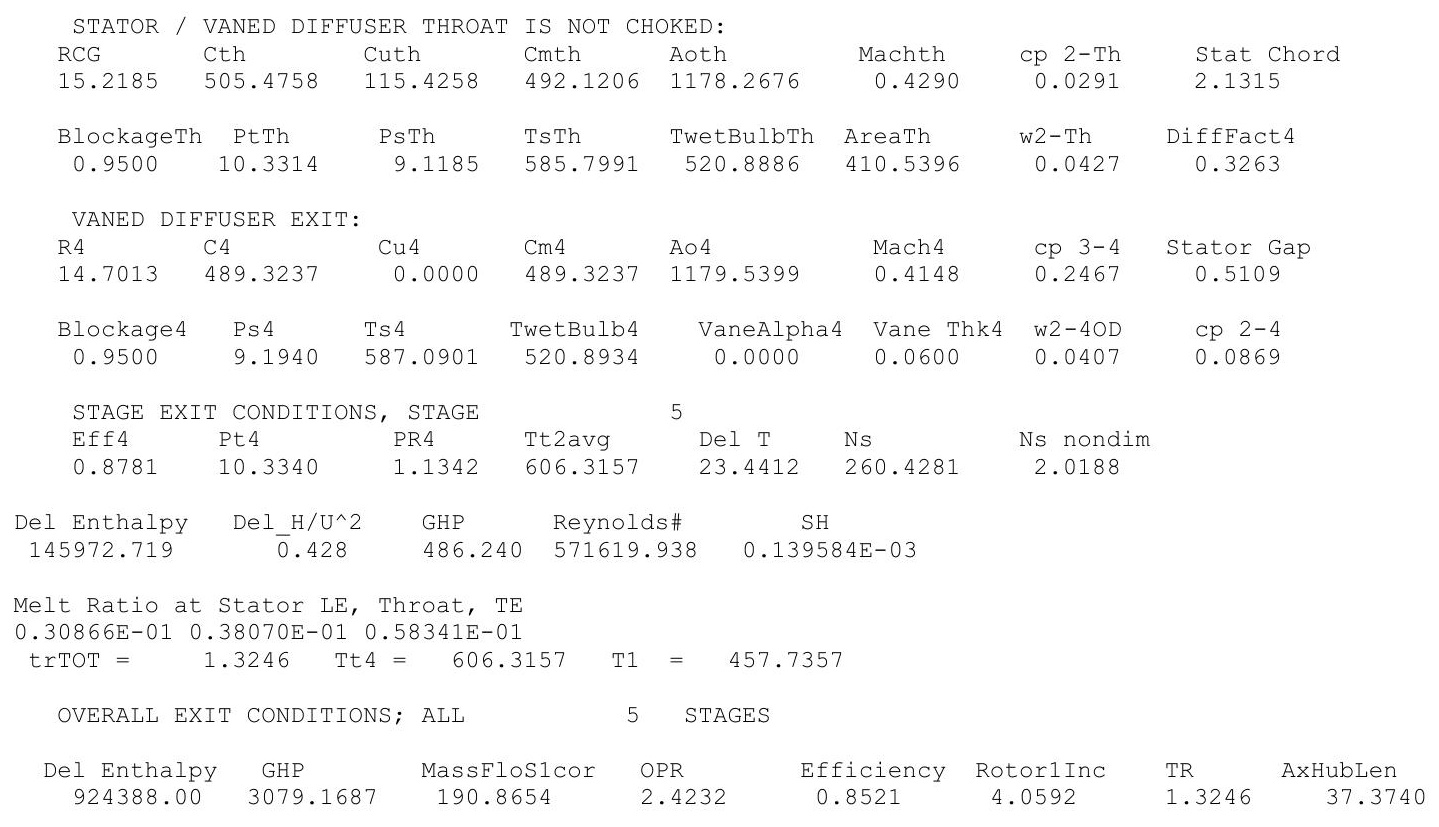




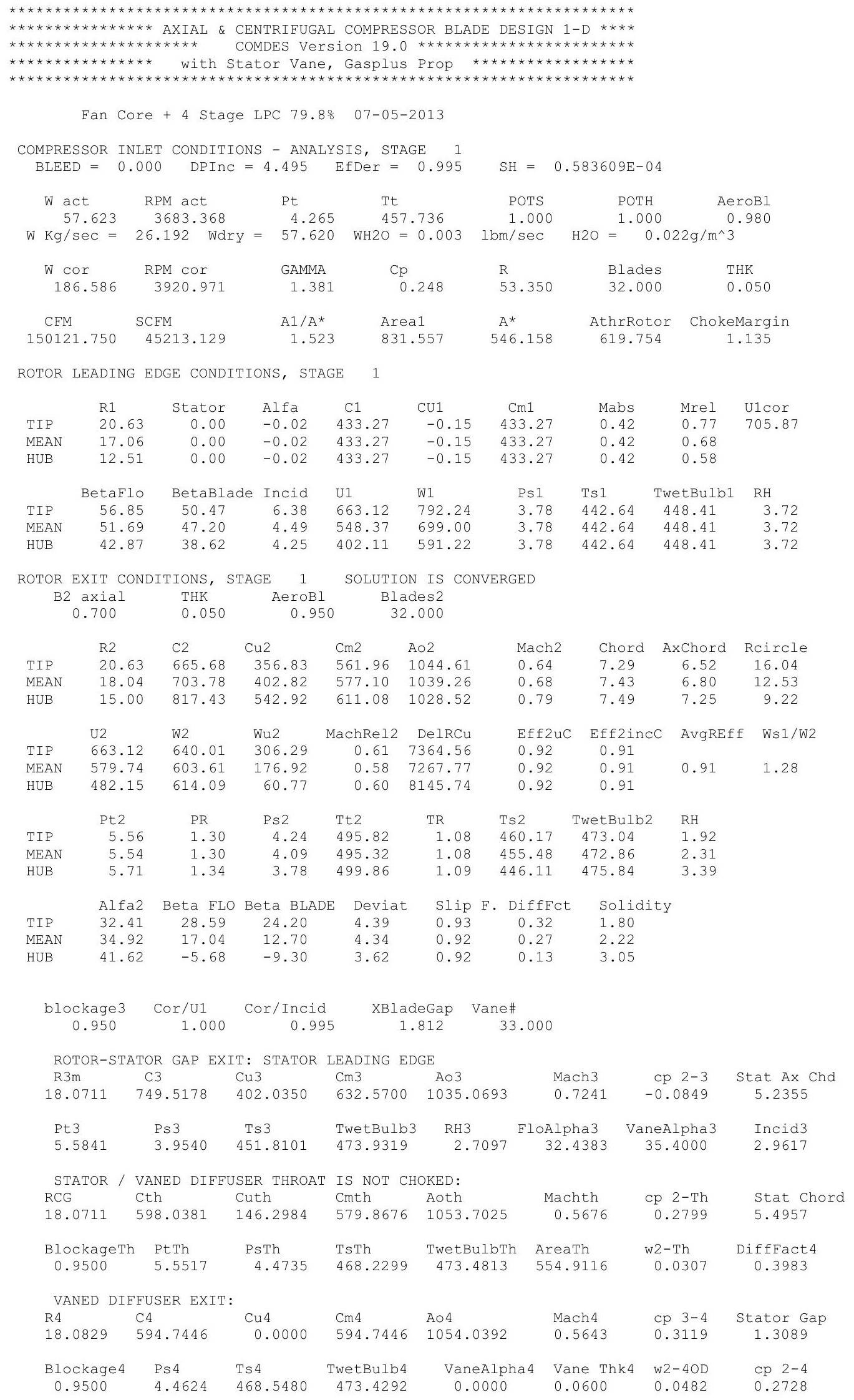




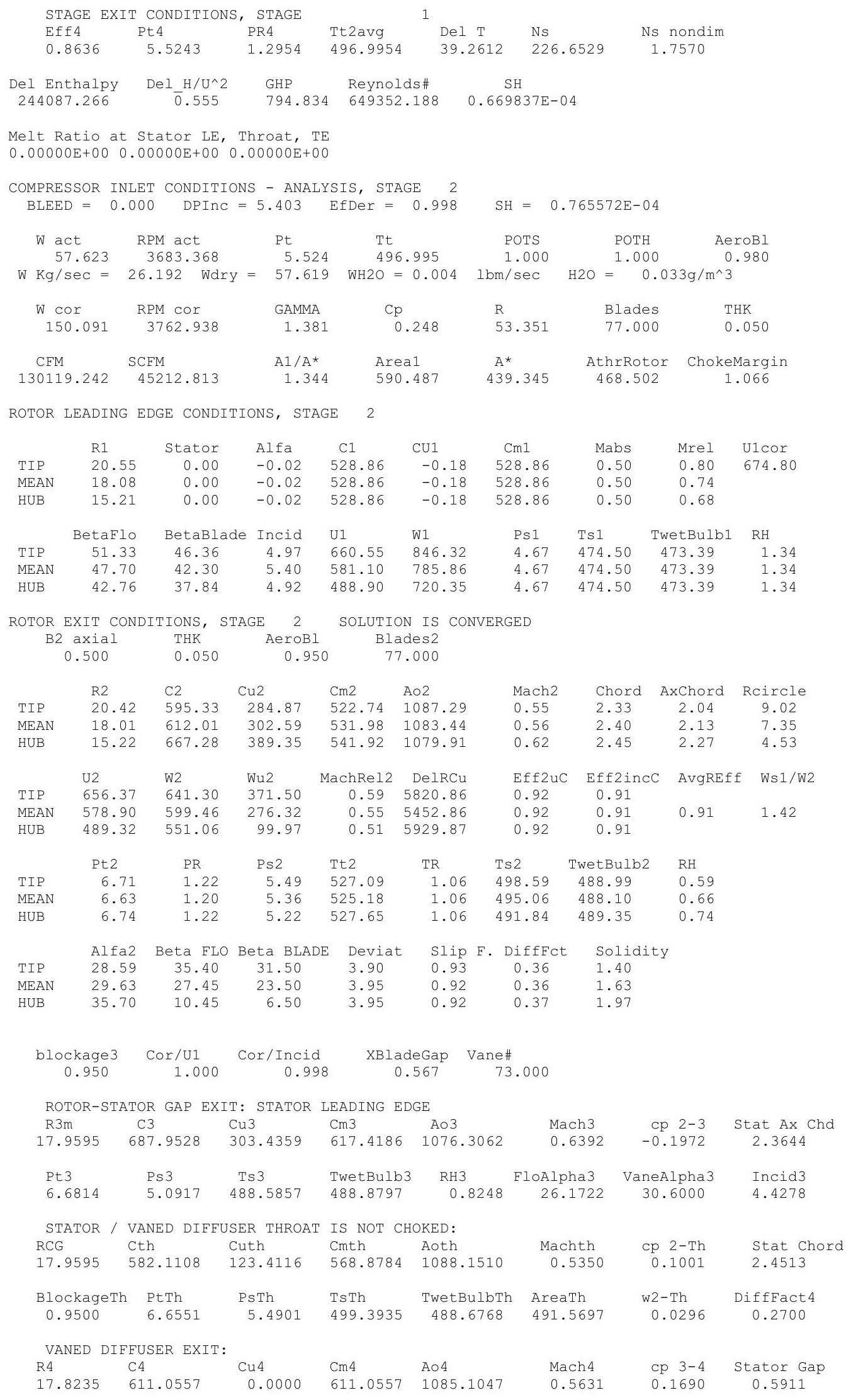




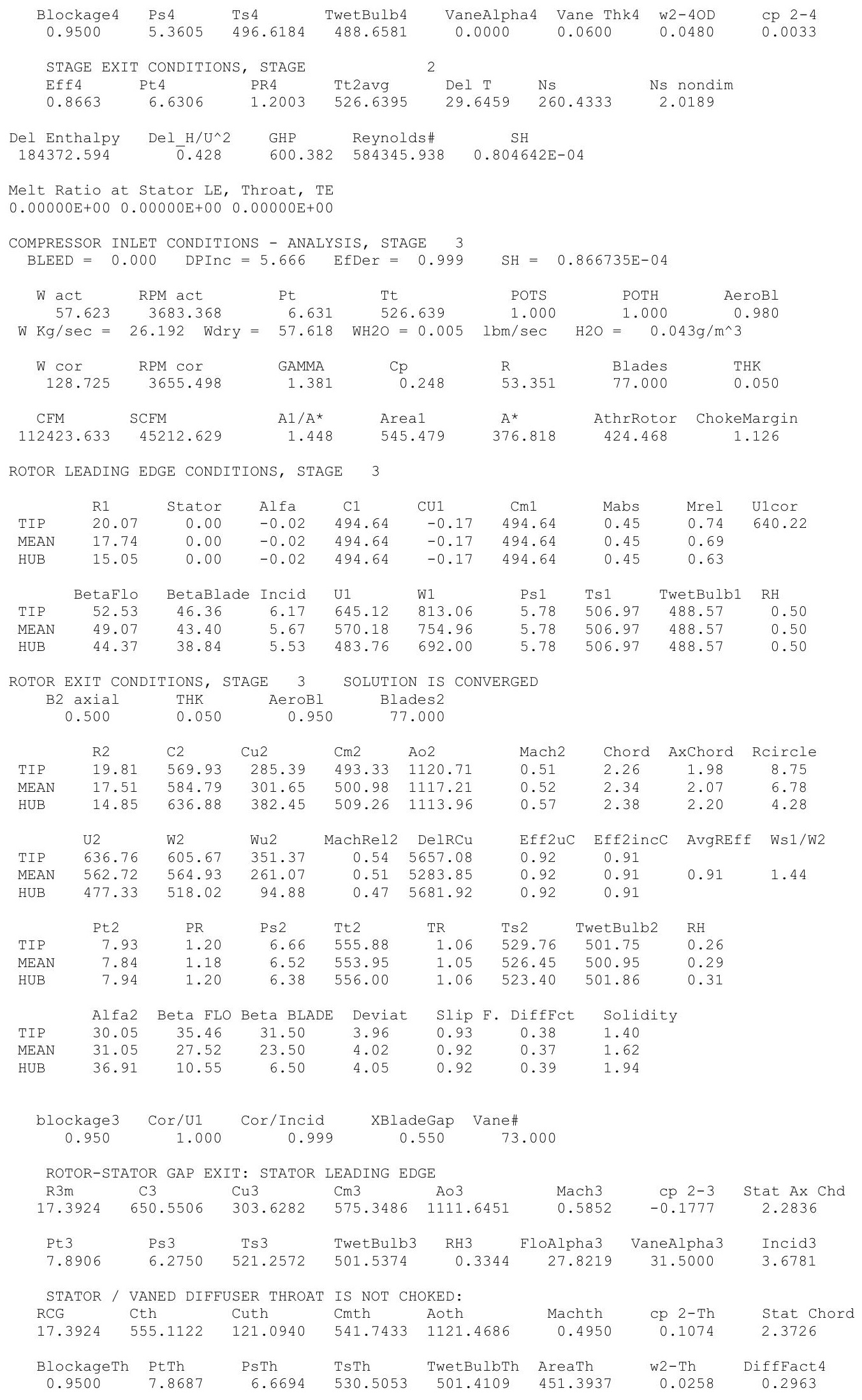




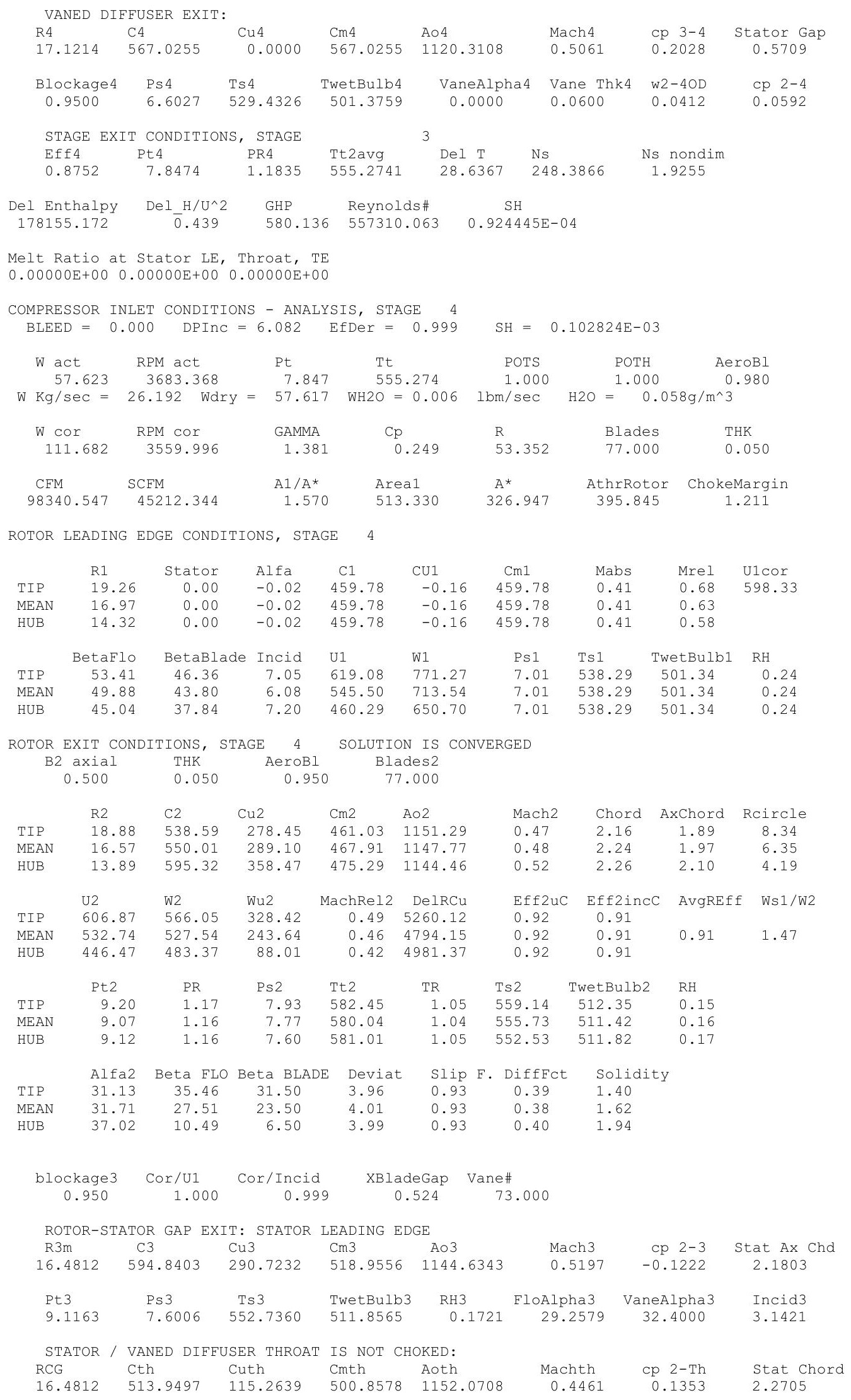




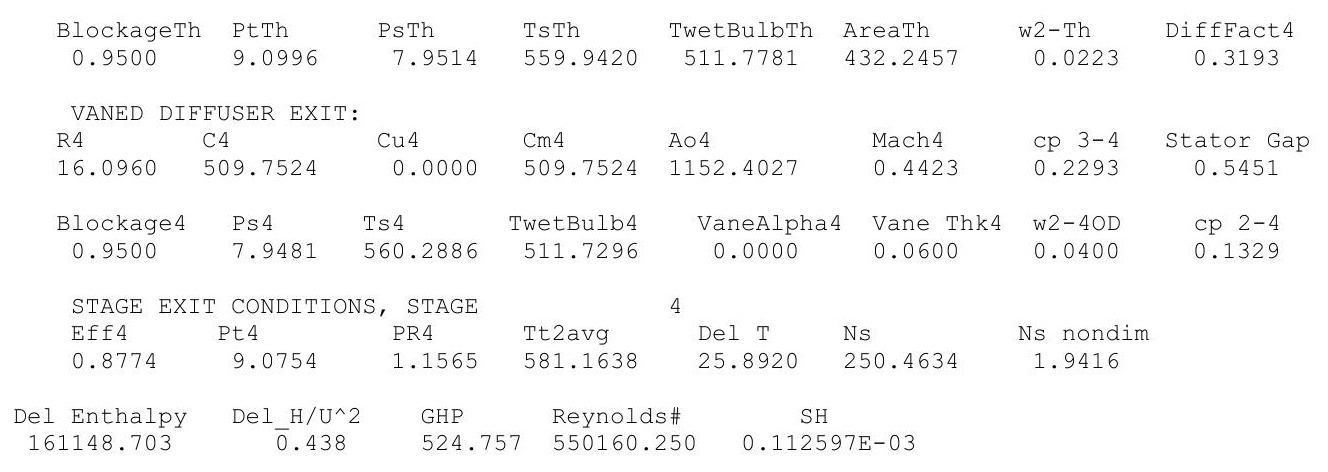

Melt Ratio at Stator LE, Throat, TE

$0.00000 \mathrm{E}+00 \quad 0.00000 \mathrm{E}+00 \quad 0.00000 \mathrm{E}+00$

COMPRESSOR INLET CONDITIONS - ANALYSIS, STAGE 5 BLEED $=0.000 \quad$ DPInC $=6.425 \quad$ EfDer $=1.000$

ROTOR LEADING EDGE CONDITIONS, STAGE 5

$\begin{array}{lccccccccc} & \text { R1 } & \text { Stator } & \text { Alfa } & \text { C1 } & \text { CU1 } & \text { Cm1 } & \text { Mabs } & \text { Mrel } & \text { U1cor } \\ \text { TIP } & 18.32 & 0.00 & -0.02 & 411.00 & -0.14 & 411.00 & 0.35 & 0.62 & 556.31 \\ \text { MEAN } & 15.91 & 0.00 & -0.02 & 411.00 & -0.14 & 411.00 & 0.35 & 0.57 & 0.51 \\ \text { HUB } & 13.07 & 0.00 & -0.02 & 411.00 & -0.14 & 411.00 & 0.35 & 0.73 \\ & & & & & & & & & \\ & \text { BetaFlo } & \text { BetaBlade } & \text { Incid } & \text { U1 } & \text { W1 } & \text { Ts1 } & \text { TwetBulb1 } & \text { RH } \\ \text { TIP } & 55.09 & 47.36 & 7.73 & 588.87 & 718.23 & 8.33 & 567.60 & 511.73 & 0.15 \\ \text { MEAN } & 51.23 & 44.80 & 6.43 & 511.50 & 656.28 & 8.33 & 567.60 & 511.73 & 0.15 \\ \text { HUB } & 45.64 & 38.84 & 6.80 & 420.11 & 587.82 & 8.33 & 567.60 & 511.73 & 0.15\end{array}$

ROTOR EXIT CONDITIONS, STAGE 5 SOLUTION IS CONVERGED

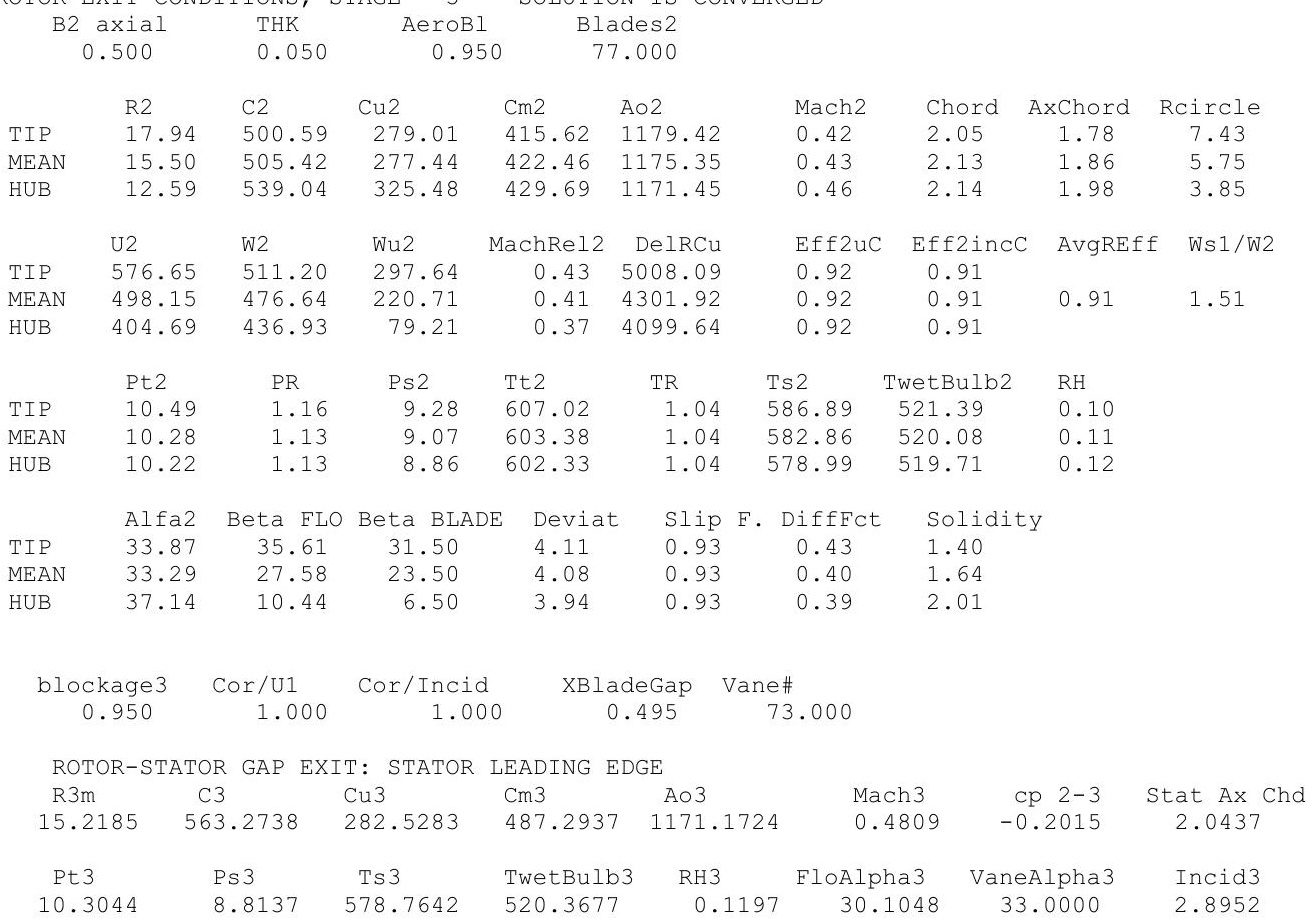




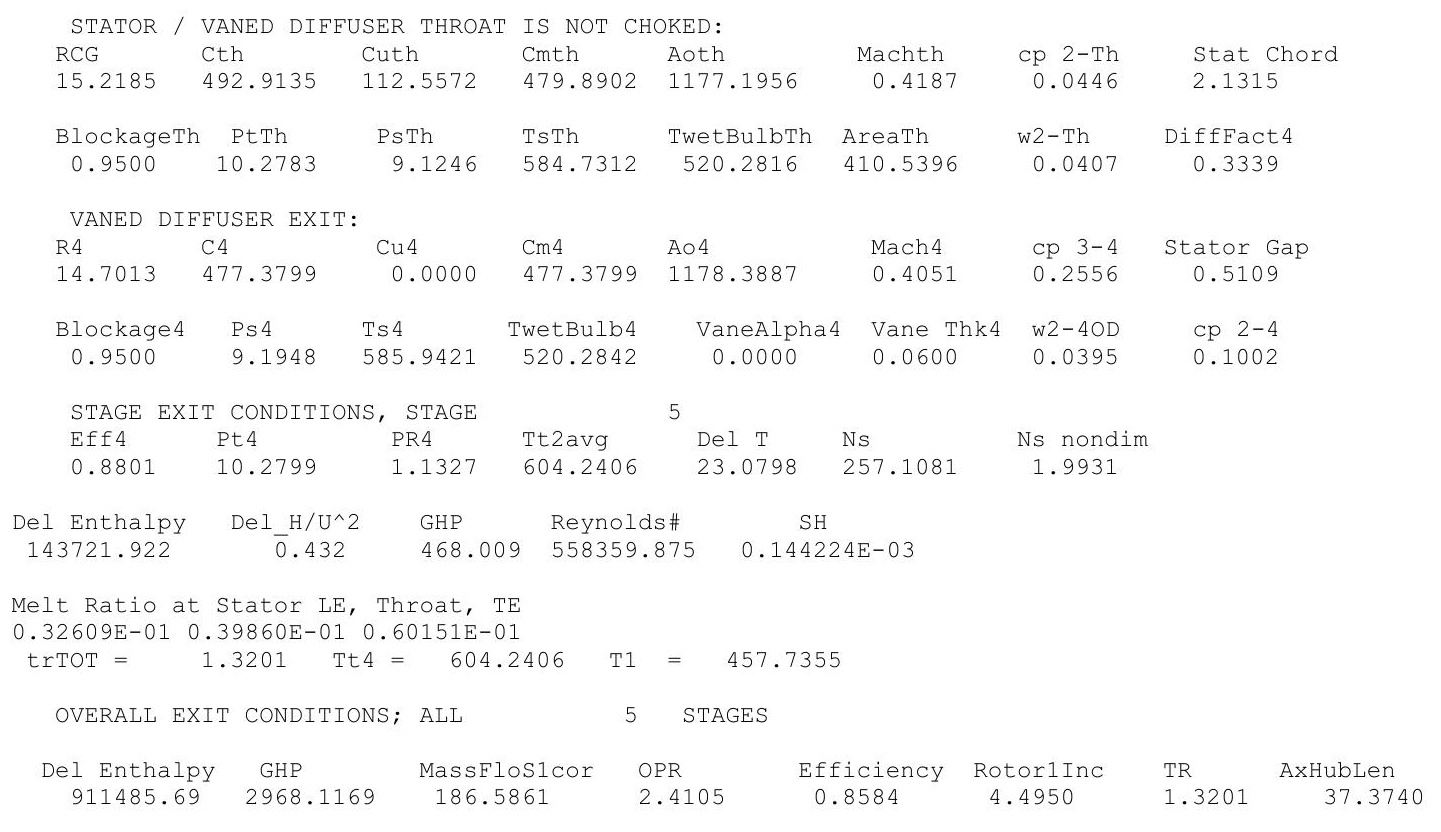




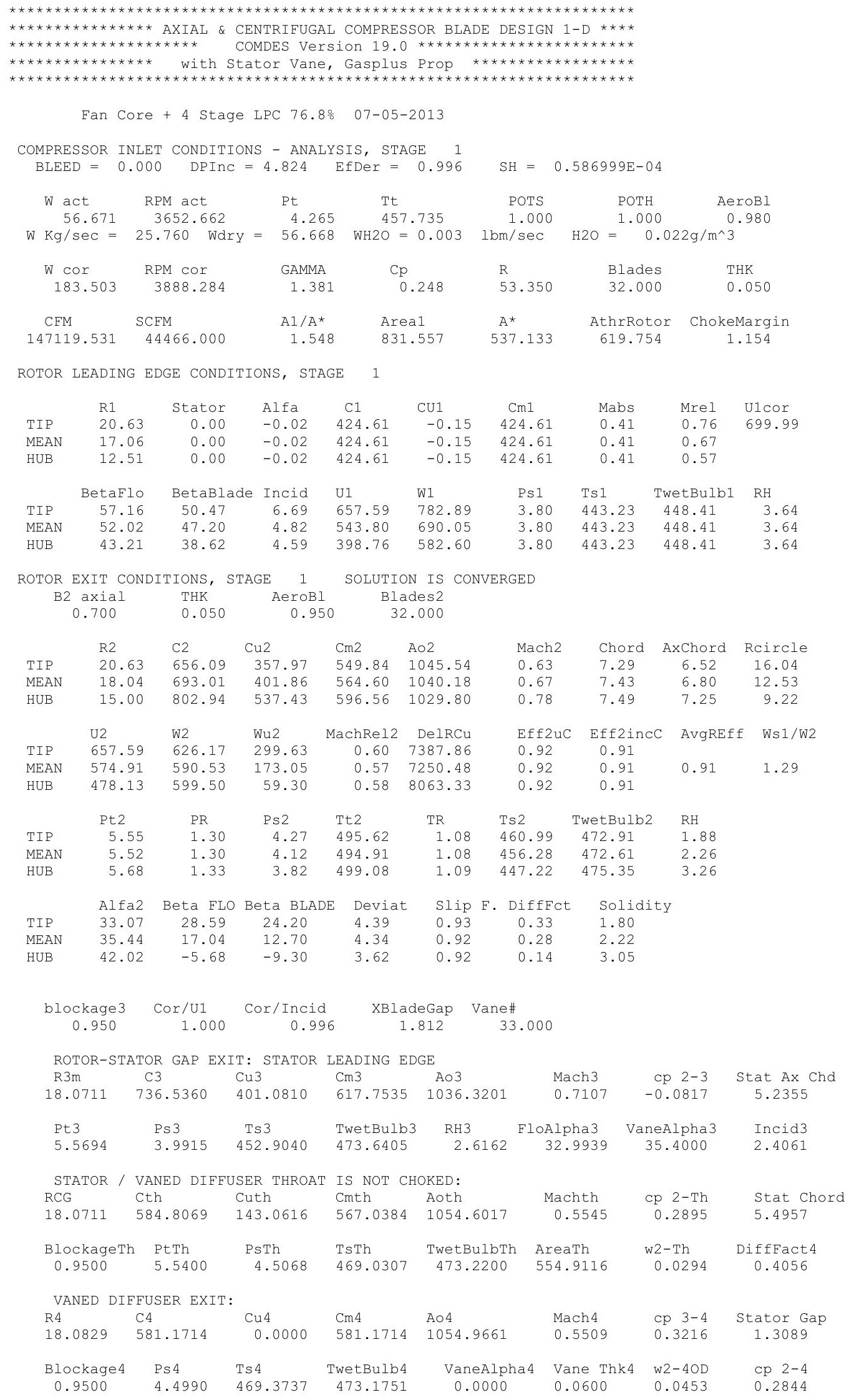




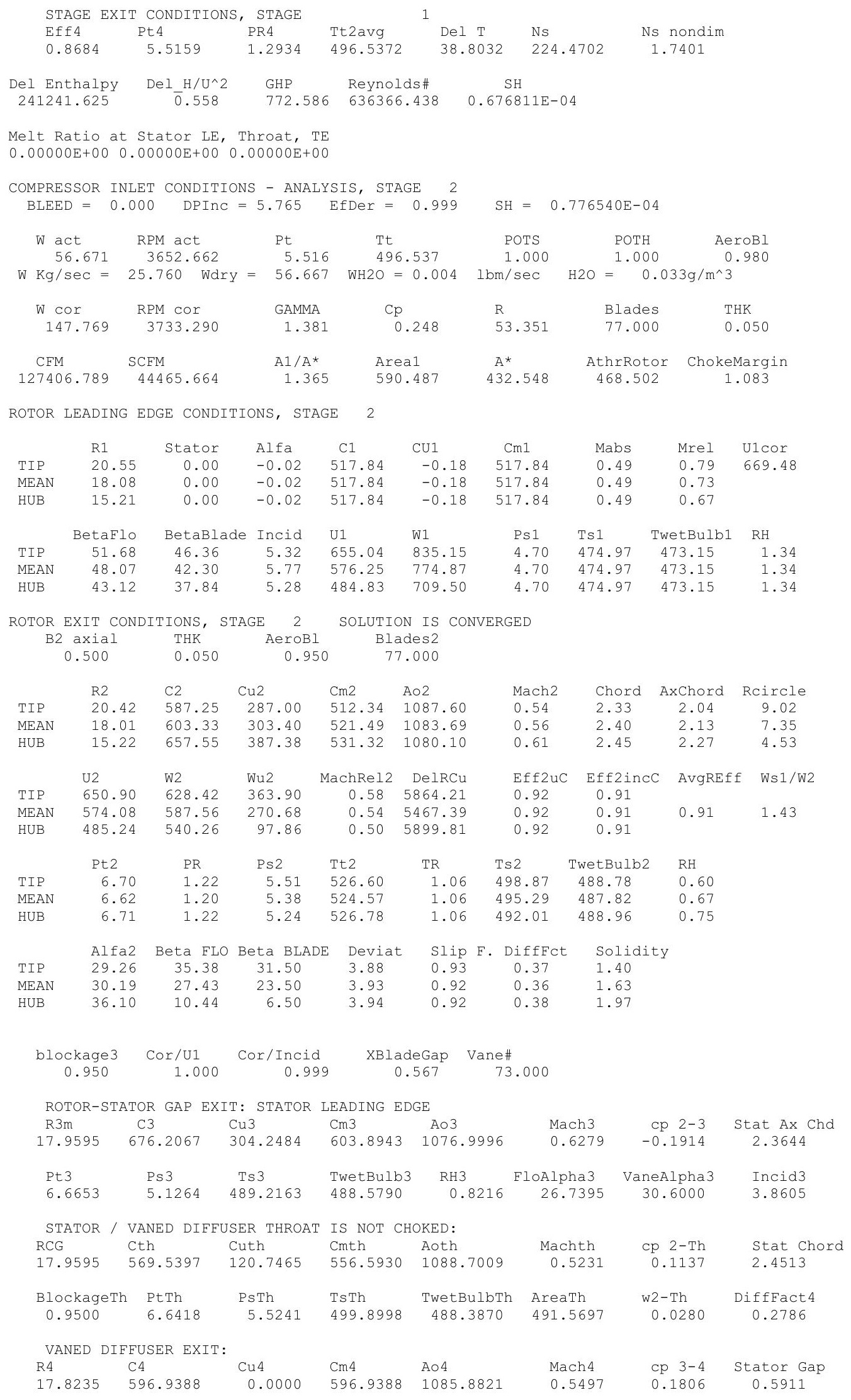




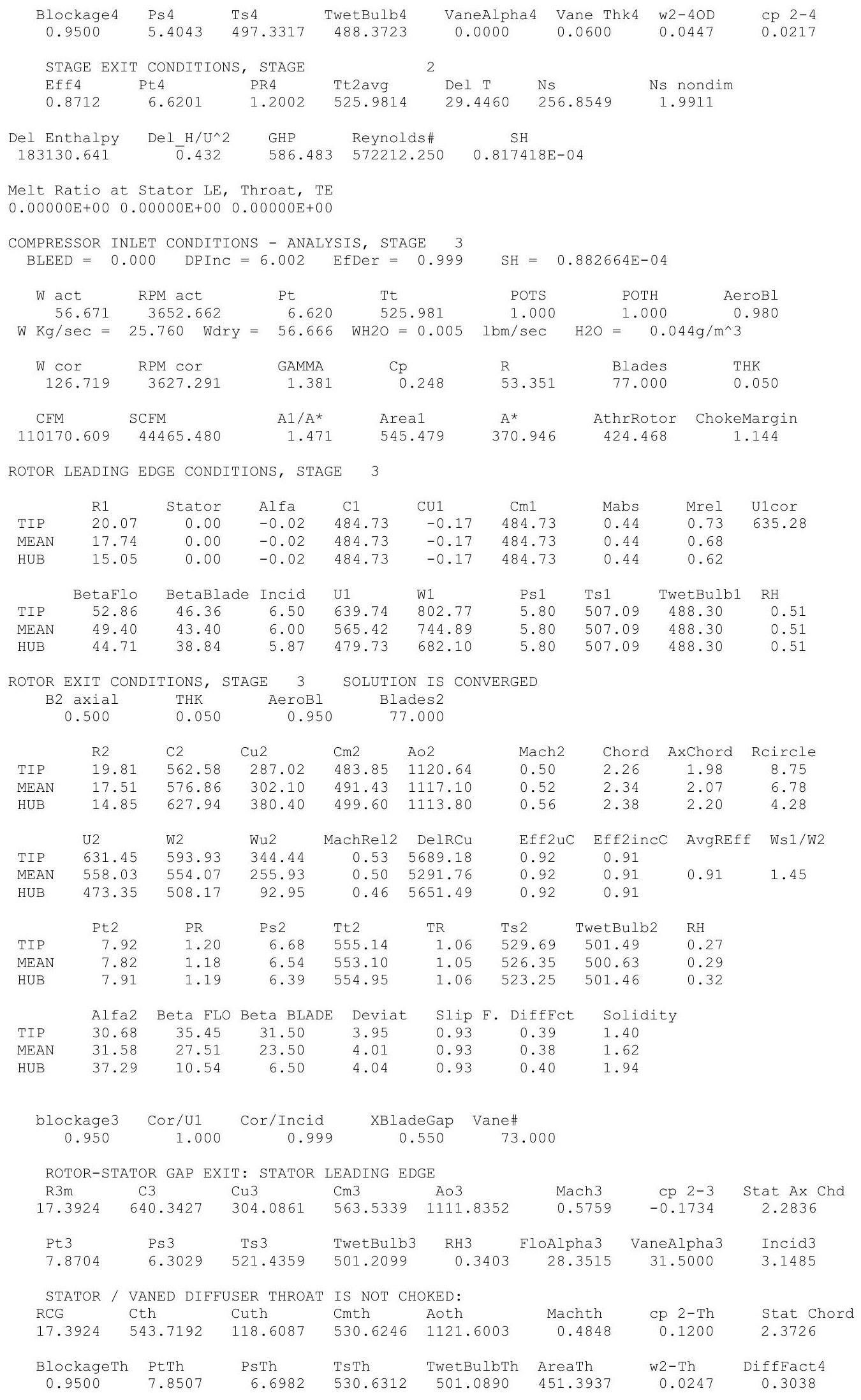




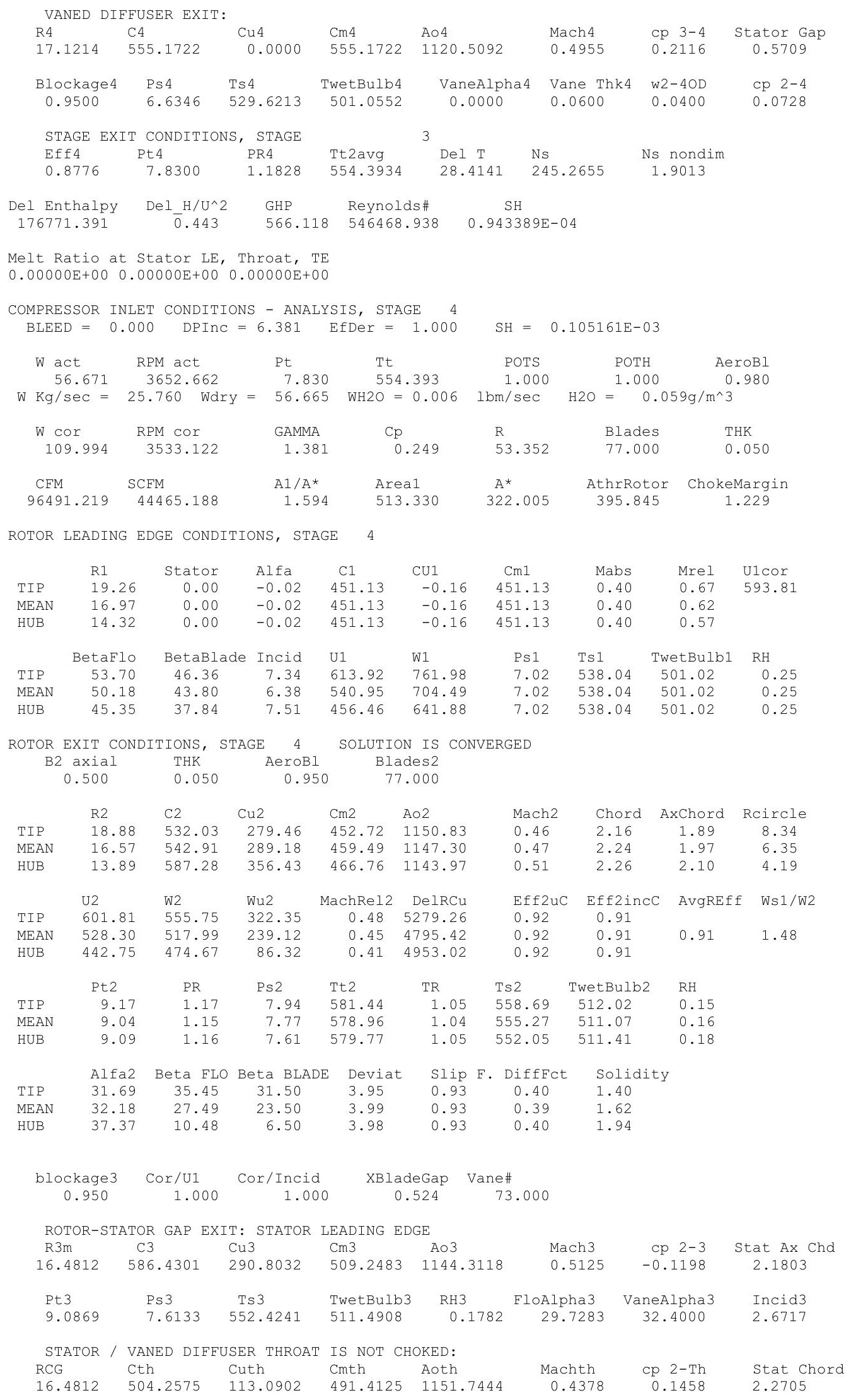




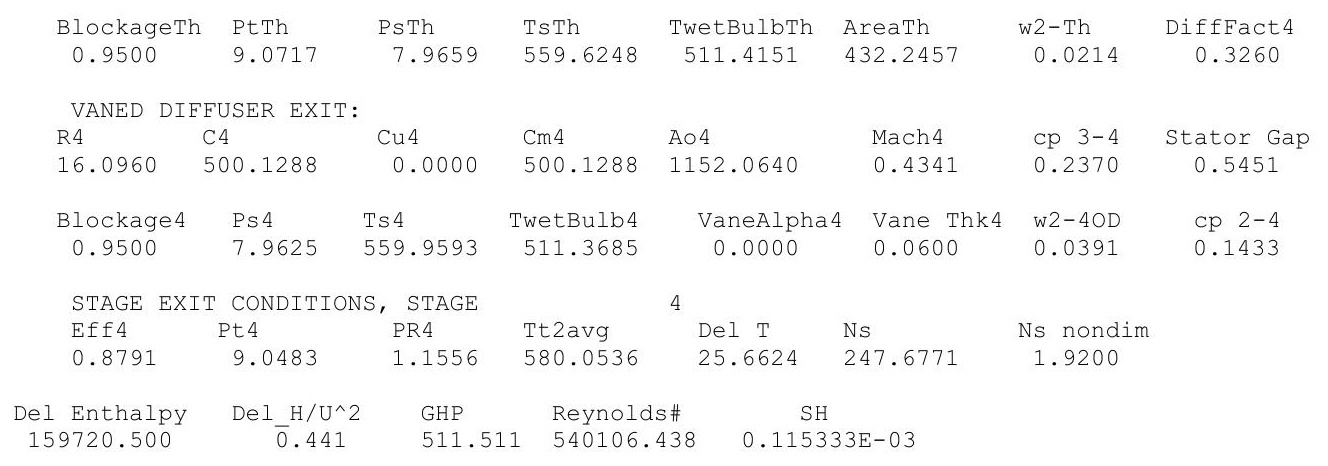

Melt Ratio at Stator LE, Throat, TE

$0.00000 \mathrm{E}+000.00000 \mathrm{E}+00 \quad 0.00000 \mathrm{E}+00$

COMPRESSOR INLET CONDITIONS - ANALYSIS, STAGE 5 $\mathrm{BLEED}=0.000 \quad$ DPInC $=6.684 \quad$ EfDer $=1.000$

$\mathrm{SH}=0.134651 \mathrm{E}-03$

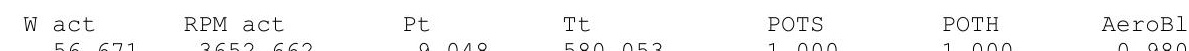
$\mathrm{W} \mathrm{Kg} / \mathrm{sec}=25.760$ Wdry $=56.664 \quad \mathrm{WH} 2 \mathrm{O}=0.008 \quad \mathrm{lbm} / \mathrm{sec} \quad \mathrm{H} 2 \mathrm{O}=0.086 \mathrm{~g} / \mathrm{m}^{\wedge} 3$

$\begin{array}{ccccccc}\text { W } \begin{array}{l}\text { Cor } \\ 97.362\end{array} & \text { RPM cor } & \text { GAMMA } & \text { Cp } & \text { R } & \text { Blades } & \text { THK } \\ & 3454.089 & 1.381 & 0.249 & 53.353 & 77.000 & 0.050 \\ \text { CFM } & \text { SCFM } & \text { A1/A* } & \text { Area1 } & \text { A* } & \text { AthrRotor } & \text { ChokeMargin } \\ 85754.742 & 44464.672 & 1.788 & 509.668 & 285.045 & 384.739 & 1.350\end{array}$

ROTOR LEADING EDGE CONDITIONS, STAGE 5

\begin{tabular}{|c|c|c|c|c|c|c|c|c|c|}
\hline & $\mathrm{R} 1$ & Stator & Alfa & $\mathrm{C} 1$ & CU1 & $\mathrm{Cm} 1$ & Mabs & Mrel & U1cor \\
\hline TIP & 18.32 & 0.00 & -0.02 & 403.81 & -0.14 & 403.81 & 0.35 & 0.61 & 552.20 \\
\hline MEAN & 15.91 & 0.00 & -0.02 & 403.81 & -0.14 & 403.81 & 0.35 & 0.56 & \\
\hline HUB & 13.07 & 0.00 & -0.02 & 403.81 & -0.14 & 403.81 & 0.35 & 0.50 & \\
\hline & Betaflo & BetaBlade & Incid & U1 & W1 & Ps 1 & Ts1 & TwetBulb1 & $\mathrm{RH}$ \\
\hline TIP & 55.34 & 47.36 & 7.98 & 583.96 & 710.10 & 8.33 & 566.96 & 511.37 & 0.15 \\
\hline MEAN & 51.48 & 44.80 & 6.68 & 507.23 & 648.46 & 8.33 & 566.96 & 511.37 & 0.15 \\
\hline HUB & 45.90 & 38.84 & 7.06 & 416.61 & 580.30 & 8.33 & 566.96 & 511.37 & 0.15 \\
\hline
\end{tabular}

ROTOR EXIT CONDITIONS, STAGE 5 SOLUTION IS CONVERGED

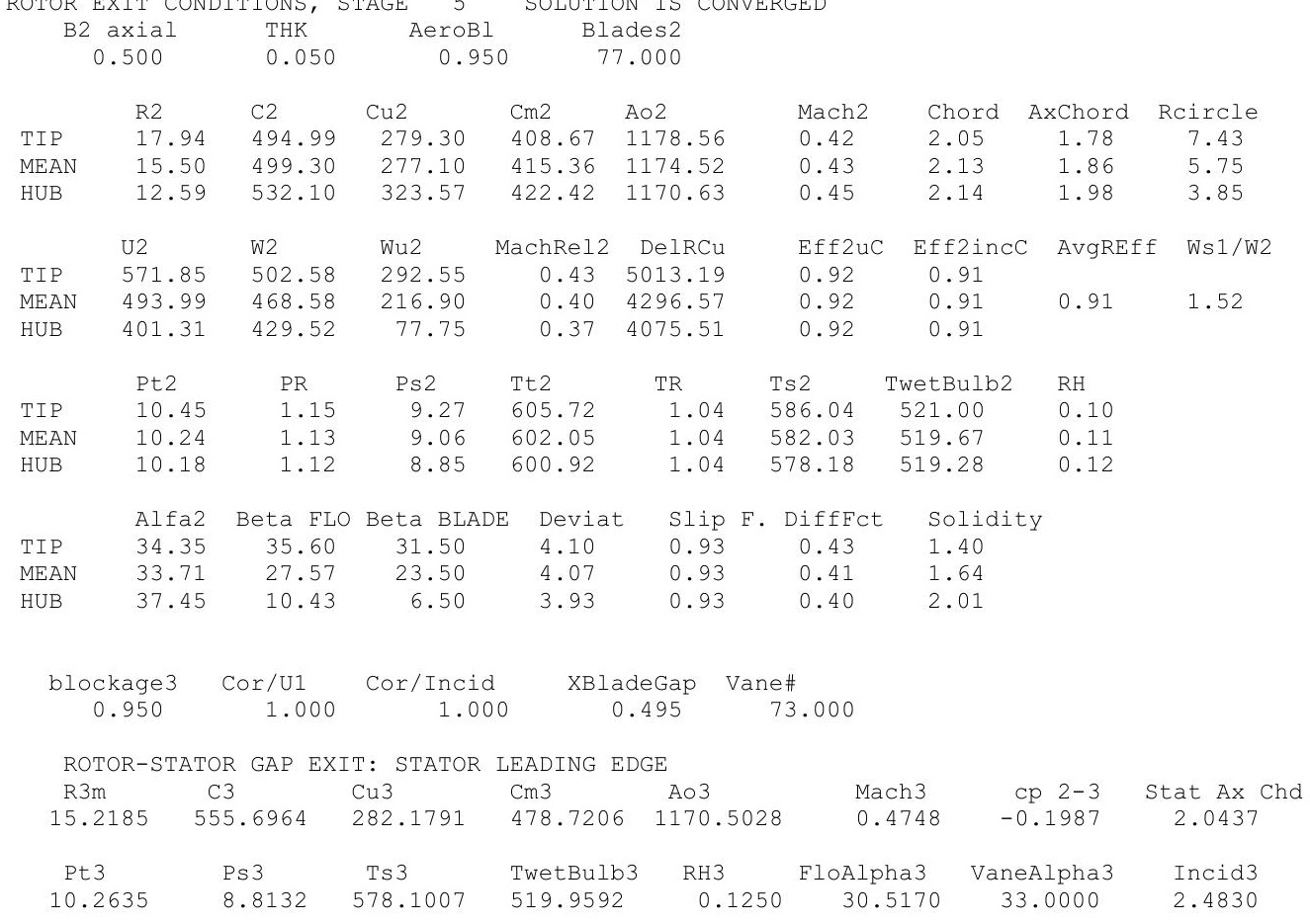




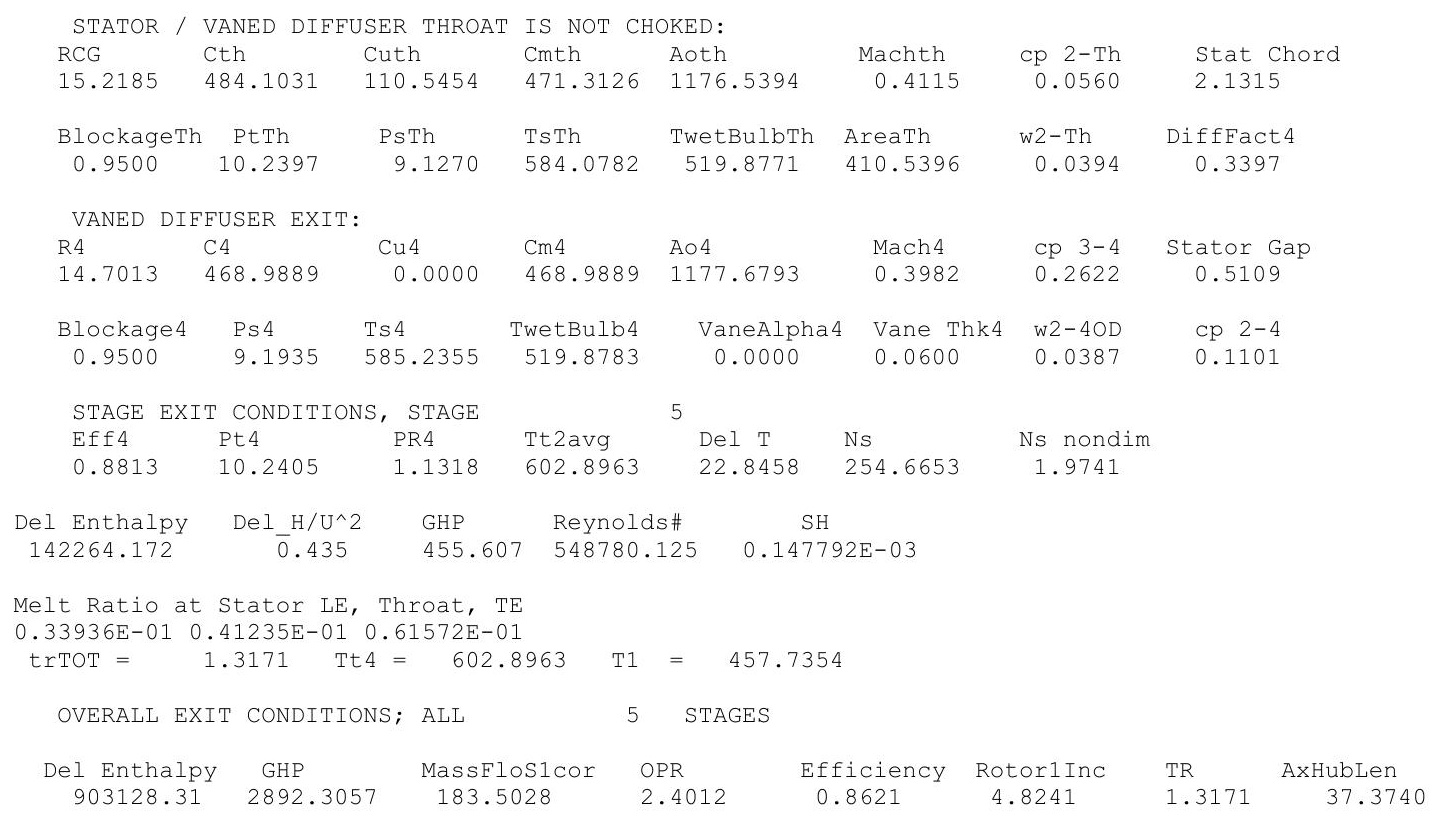




\section{$25 \mu \mathrm{m}, \mathrm{ISA}+36 \mathrm{R}$}

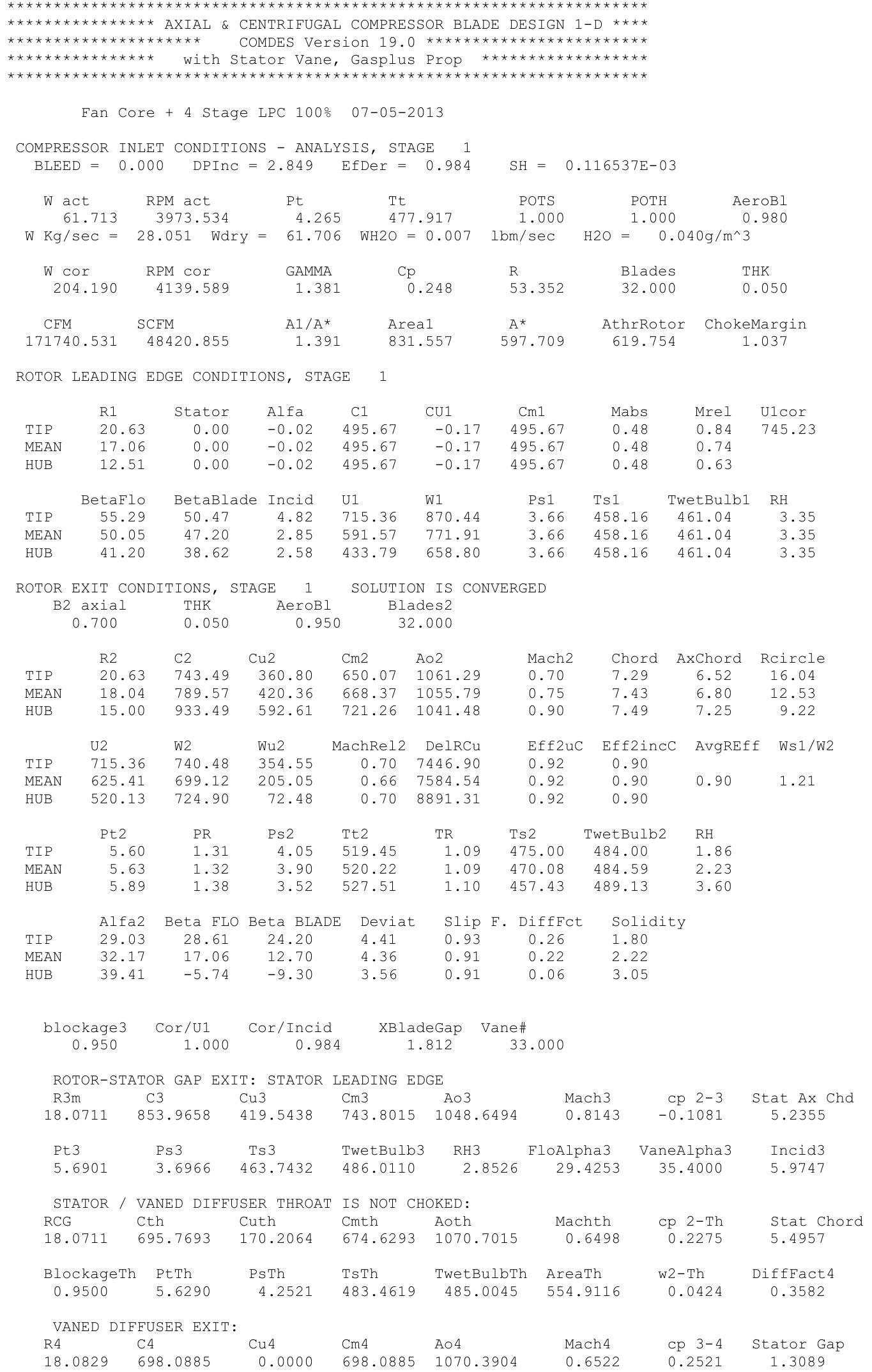

$\begin{array}{lrrrrrrrrr} & \text { R1 } & \text { Stator } & \text { Alfa } & \text { C1 } & \text { CU1 } & \text { Cm1 } & \text { Mabs } & \text { Mrel } & \text { U1cor } \\ \text { TIP } & 20.63 & 0.00 & -0.02 & 495.67 & -0.17 & 495.67 & 0.48 & 0.84 & 745.23 \\ \text { MEAN } & 17.06 & 0.00 & -0.02 & 495.67 & -0.17 & 495.67 & 0.48 & 0.74 & \\ \text { HUB } & 12.51 & 0.00 & -0.02 & 495.67 & -0.17 & 495.67 & 0.48 & 0.63 & \\ & & & & & & & & & \\ & \text { BetaFlo } & \text { BetaBlade } & \text { Incid } & \text { U1 } & \text { W1 } & \text { Ps1 } & \text { Ts1 } & \text { TwetBulb1 } & \text { RH } \\ \text { TIP } & 55.29 & 50.47 & 4.82 & 715.36 & 870.44 & 3.66 & 458.16 & 461.04 & 3.35 \\ \text { MEAN } & 50.05 & 47.20 & 2.85 & 591.57 & 771.91 & 3.66 & 458.16 & 461.04 & 3.35 \\ \text { HUB } & 41.20 & 38.62 & 2.58 & 433.79 & 658.80 & 3.66 & 458.16 & 461.04 & 3.35\end{array}$

ROTOR EXIT CONDITIONS, STAGE 1 SOLUTION IS CONVERGED

\begin{tabular}{|c|c|c|c|c|c|c|c|c|c|}
\hline & $\begin{array}{l}\text { axial } \\
700\end{array}$ & $\begin{array}{l}\text { THK } \\
0.050\end{array}$ & $\begin{array}{r}\text { AeroBl } \\
0.950\end{array}$ & $\begin{array}{r}\text { Bla } \\
32\end{array}$ & $\begin{array}{l}\text { ades } 2 \\
2.000\end{array}$ & & & & \\
\hline & $\mathrm{R} 2$ & $\mathrm{C} 2$ & $\mathrm{Cu} 2$ & $\mathrm{Cm} 2$ & $\mathrm{~A} \circ 2$ & Mach2 & Chord & AxChord & Rcircle \\
\hline TIP & 20.63 & 743.49 & 360.80 & 650.07 & 1061.29 & 0.70 & 7.29 & 6.52 & 16.04 \\
\hline MEAN & 18.04 & 789.57 & 420.36 & 668.37 & 1055.79 & 0.75 & 7.43 & 6.80 & 12.53 \\
\hline HUB & 15.00 & 933.49 & 592.61 & 721.26 & 1041.48 & 0.90 & 7.49 & 7.25 & 9.22 \\
\hline & $\mathrm{U} 2$ & W2 & Wu2 & MachRel2 & DelRCu & Eff $2 u C$ & Eff 2 incC & AvgREff & Ws $1 /$ W2 \\
\hline TIP & 715.36 & 740.48 & 354.55 & 0.70 & 7446.90 & 0.92 & 0.90 & & \\
\hline MEAN & 625.41 & 699.12 & 205.05 & 0.66 & 7584.54 & 0.92 & 0.90 & 0.90 & 1.21 \\
\hline HUB & 520.13 & 724.90 & 72.48 & 0.70 & 8891.31 & 0.92 & 0.90 & & \\
\hline & Pt2 & PR & Ps2 & Tt2 & TR & Ts 2 & TwetBulb2 & $\mathrm{RH}$ & \\
\hline TIP & 5.60 & 1.31 & 4.05 & 519.45 & 1.09 & 475.00 & 484.00 & 1.86 & \\
\hline MEAN & 5.63 & 1.32 & 3.90 & 520.22 & 1.09 & 470.08 & 484.59 & 2.23 & \\
\hline UB & 5.89 & 1.38 & 3.52 & 527.51 & 1.10 & 457.43 & 489.13 & 3.60 & \\
\hline & Alfa2 & Beta FLO & Beta BLADE & Deviat & Slip & F. DiffFct & Solidity & & \\
\hline IP & 29.03 & 28.61 & 24.20 & 4.41 & 0.93 & 0.26 & 1.80 & & \\
\hline GAN & 32.17 & 17.06 & 12.70 & 4.36 & 0.91 & 0.22 & 2.22 & & \\
\hline JB & 39.41 & -5.74 & -9.30 & 3.56 & 0.91 & 0.06 & 3.05 & & \\
\hline
\end{tabular}




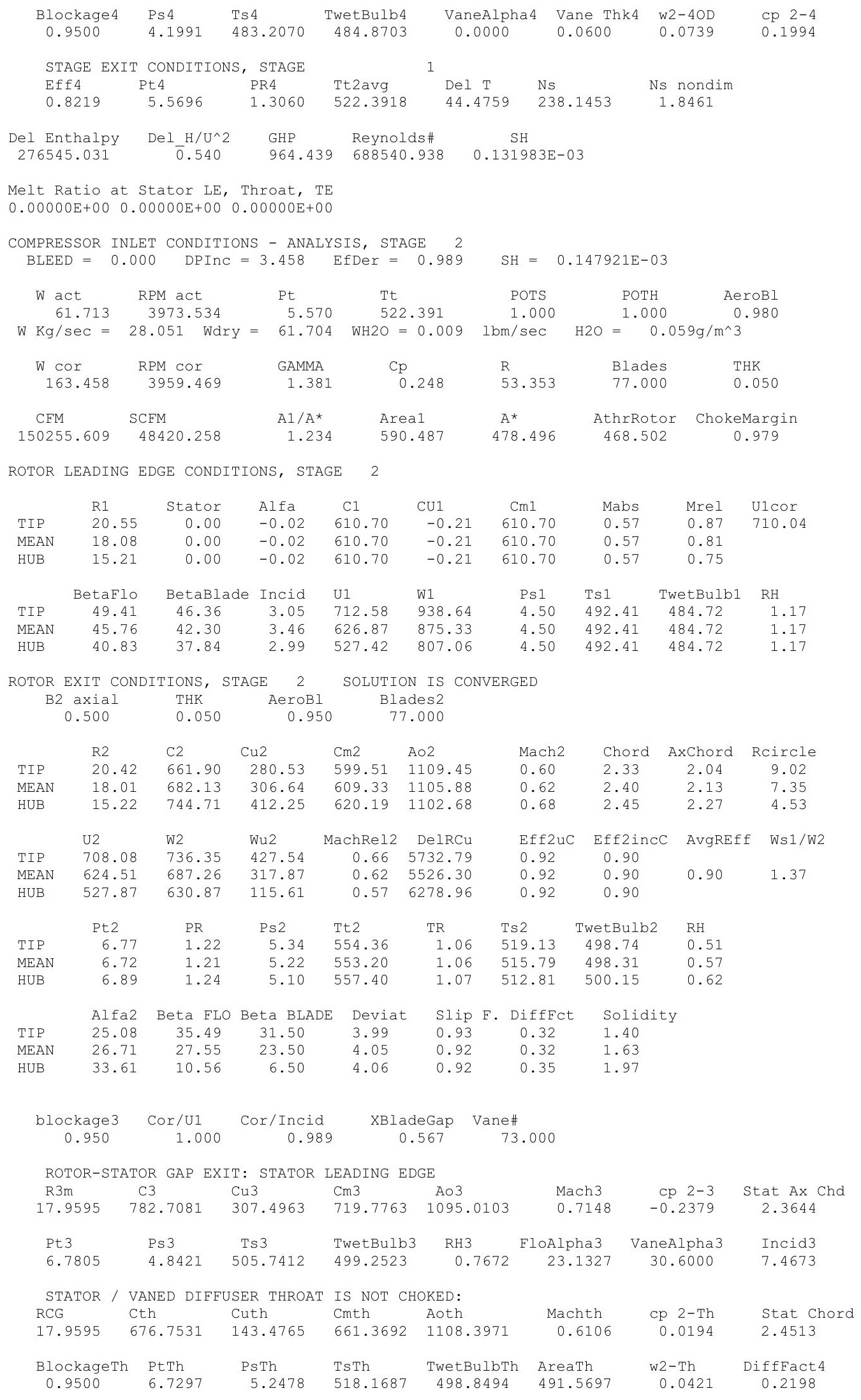




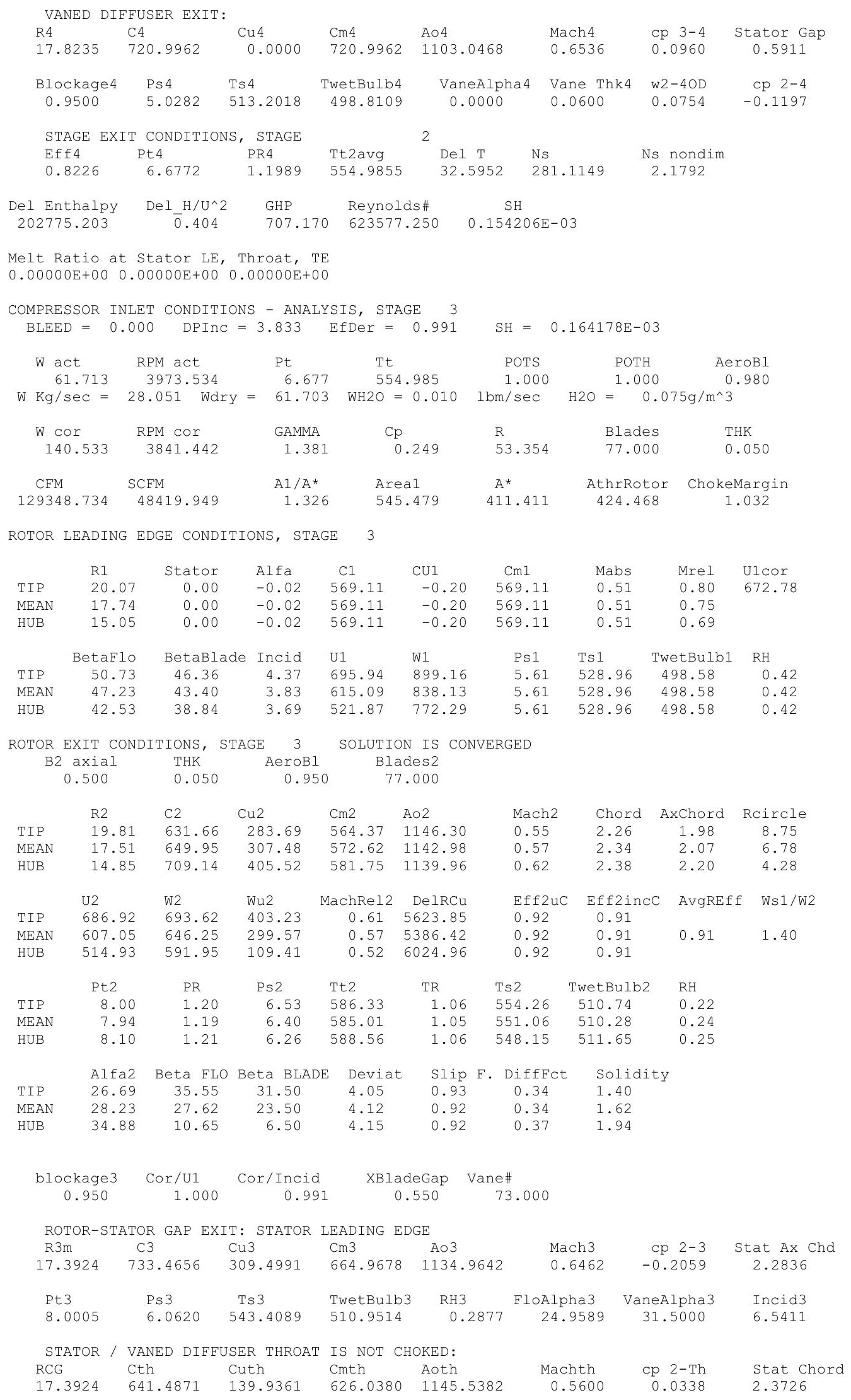




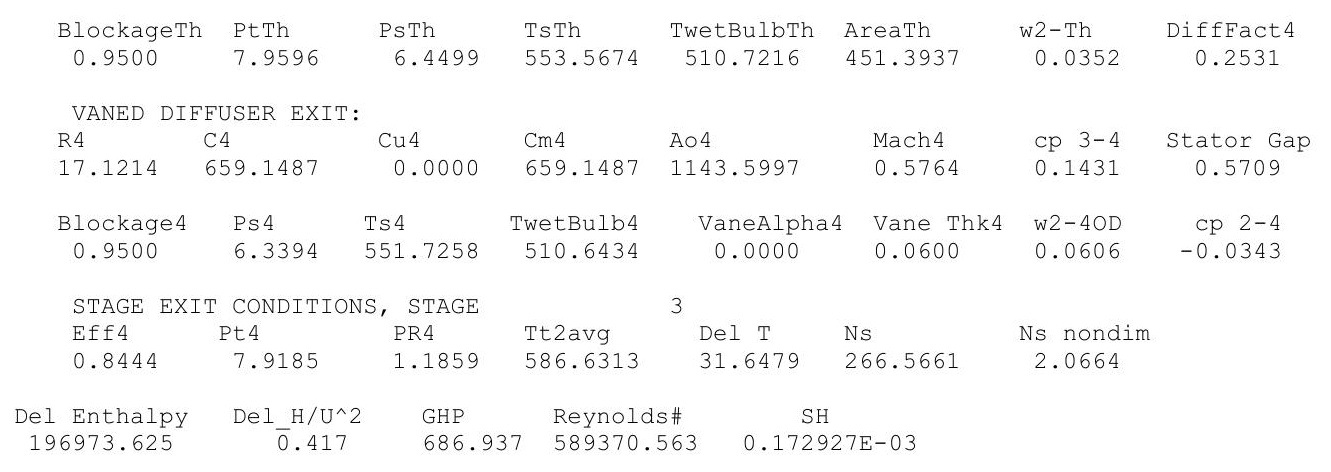

Melt Ratio at Stator LE, Throat, TE

$0.00000 \mathrm{E}+00 \quad 0.00000 \mathrm{E}+00 \quad 0.00000 \mathrm{E}+00$

COMPRESSOR INLET CONDITIONS - ANALYSIS, STAGE 4 BLEED $=0.000 \quad$ DPInC $=4.422 \quad$ EfDer $=0.995$

$\mathrm{SH}=0.188695 \mathrm{E}-03$

$\begin{array}{llllll}\text { W act } & \text { RPM act } & \text { Pt } & \text { POTS } & \text { POTH } & \text { AeroBI }\end{array}$ $\mathrm{W} \mathrm{Kg} / \mathrm{sec}=28.051$ Wdry $=61.701$ WH2O $=0.012 \quad \mathrm{lbm} / \mathrm{sec} \quad \mathrm{H} 2 \mathrm{O}=\begin{gathered}1.000 \\ 0.100 \mathrm{~g} / \mathrm{m}^{\wedge} 3\end{gathered}$

$\begin{array}{ccccccc}\text { W cor } & \text { RPM cor } & \text { GAMMA } & \text { Cp } & \text { R } & \text { Blades } & \text { THK } \\ 121.836 & 3736.392 & 1.381 & 0.249 & 53.354 & 77.000 & 0.050 \\ & & & & & & \\ \text { CFM } & \text { SCFM } & \text { A1/A* } & \text { Area1 } & \text { A* } & \text { AthrRotor } & \text { ChokeMargin } \\ 112483.953 & 48419.484 & 1.439 & 513.330 & 356.704 & 395.845 & 1.110\end{array}$

ROTOR LEADING EDGE CONDITIONS, STAGE 4

$\begin{array}{lccccccccc} & \text { R1 } & \text { Stator } & \text { Alfa } & \text { C1 } & \text { CU1 } & \text { Cm1 } & \text { Mabs } & \text { Mrel } & \text { U1cor } \\ \text { TIP } & 19.26 & 0.00 & -0.02 & 525.90 & -0.18 & 525.90 & 0.45 & 0.74 & 627.98 \\ \text { MEAN } & 16.97 & 0.00 & -0.02 & 525.90 & -0.18 & 525.90 & 0.45 & 0.68 & \\ \text { HUB } & 14.32 & 0.00 & -0.02 & 525.90 & -0.18 & 525.90 & 0.45 & 0.63 & \\ & & & & & & & & & \\ & \text { BetaFlo } & \text { BetaBlade } & \text { Incid } & \text { U1 } & \text { W1 } & \text { Ps1 } & \text { Ts1 } & \text { TwetBulb1 } & \text { RH } \\ \text { TIP } & 51.79 & 46.36 & 5.43 & 667.85 & 850.20 & 6.88 & 564.42 & 510.55 & 0.19 \\ \text { MEAN } & 48.22 & 43.80 & 4.42 & 588.47 & 789.36 & 6.88 & 564.42 & 510.55 & 0.19 \\ \text { HUB } & 43.37 & 37.84 & 5.53 & 496.56 & 723.41 & 6.88 & 564.42 & 510.55 & 0.19\end{array}$

ROTOR EXIT CONDITIONS, STAGE 4 SOLUTION IS CONVERGED

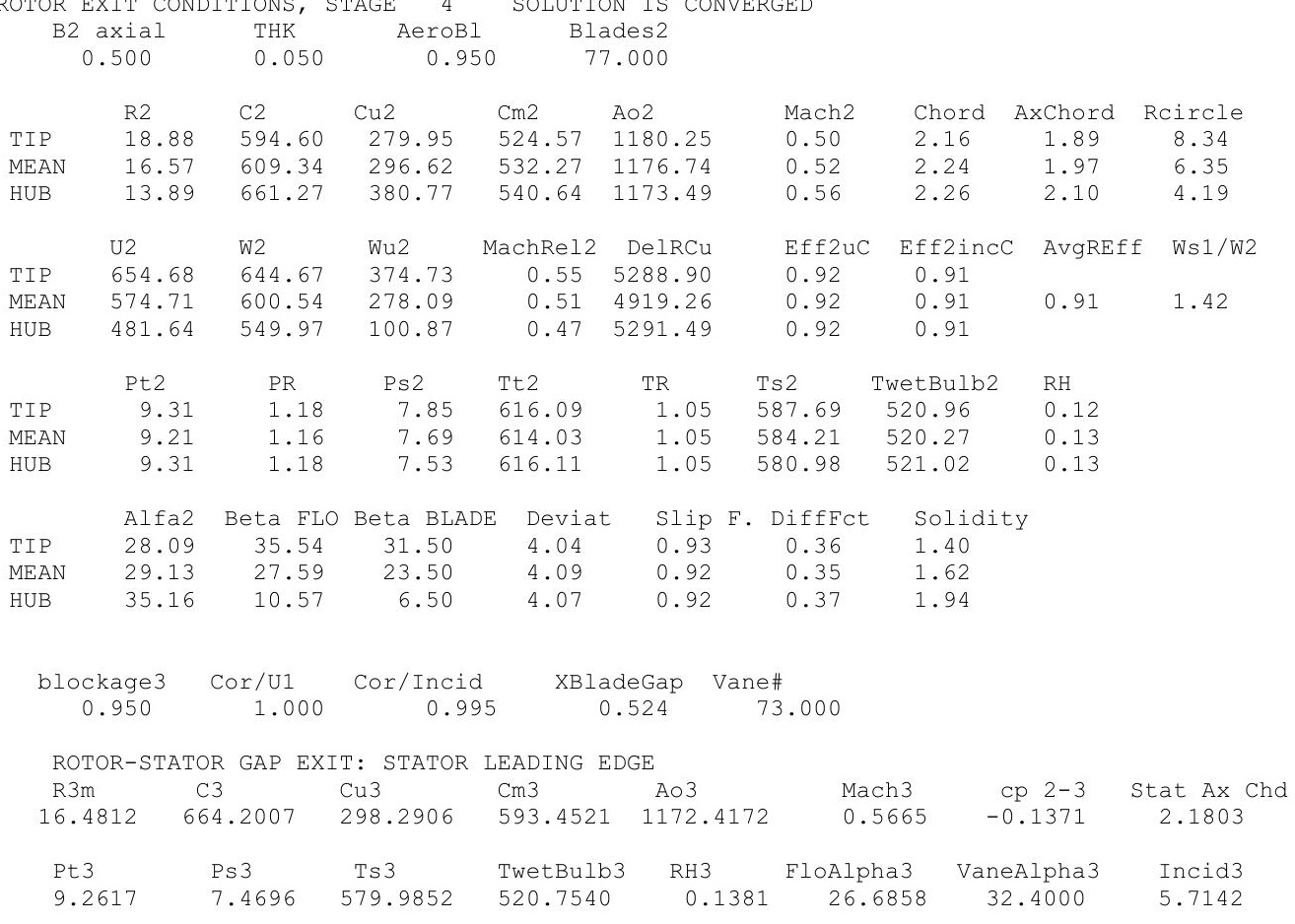




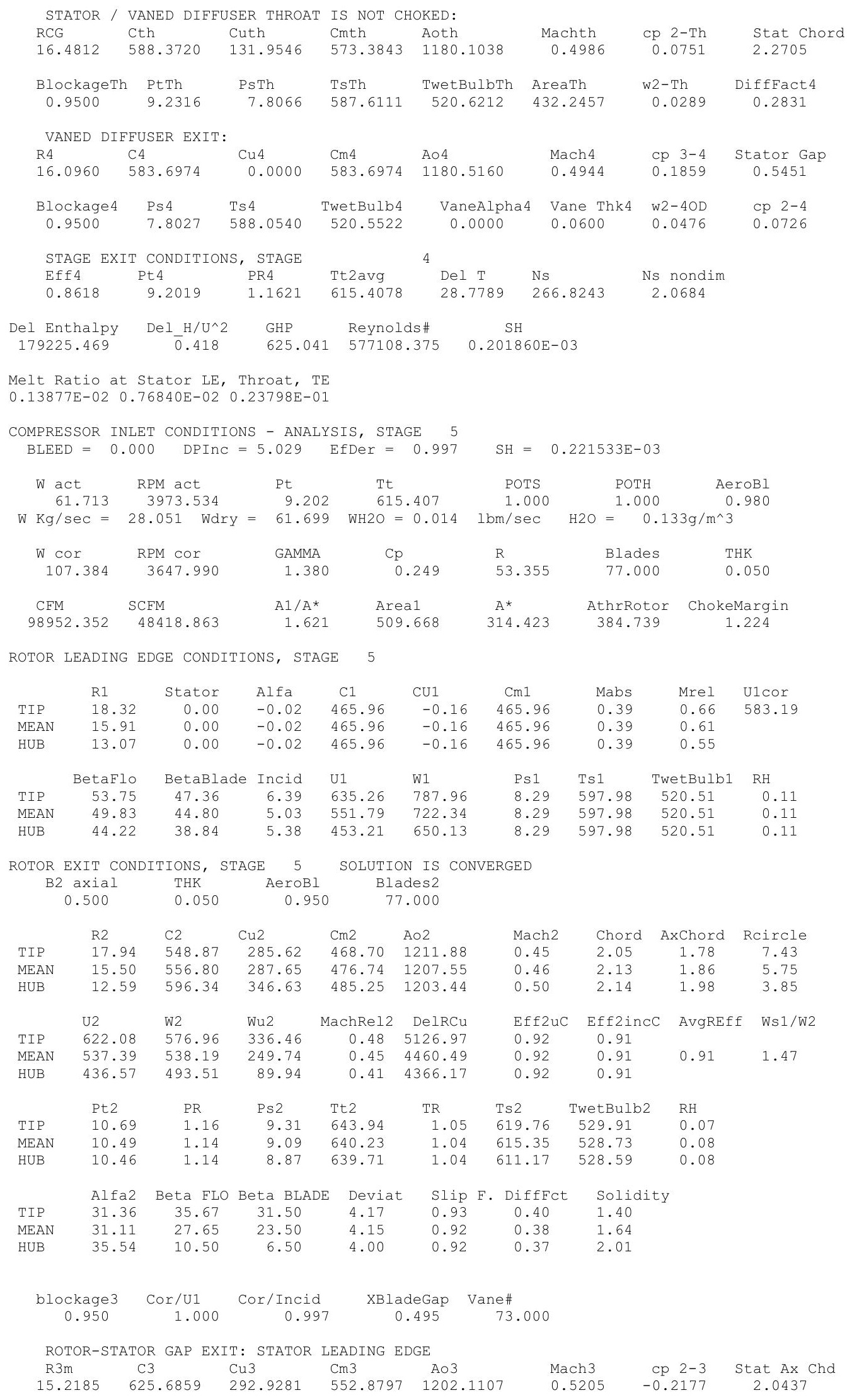




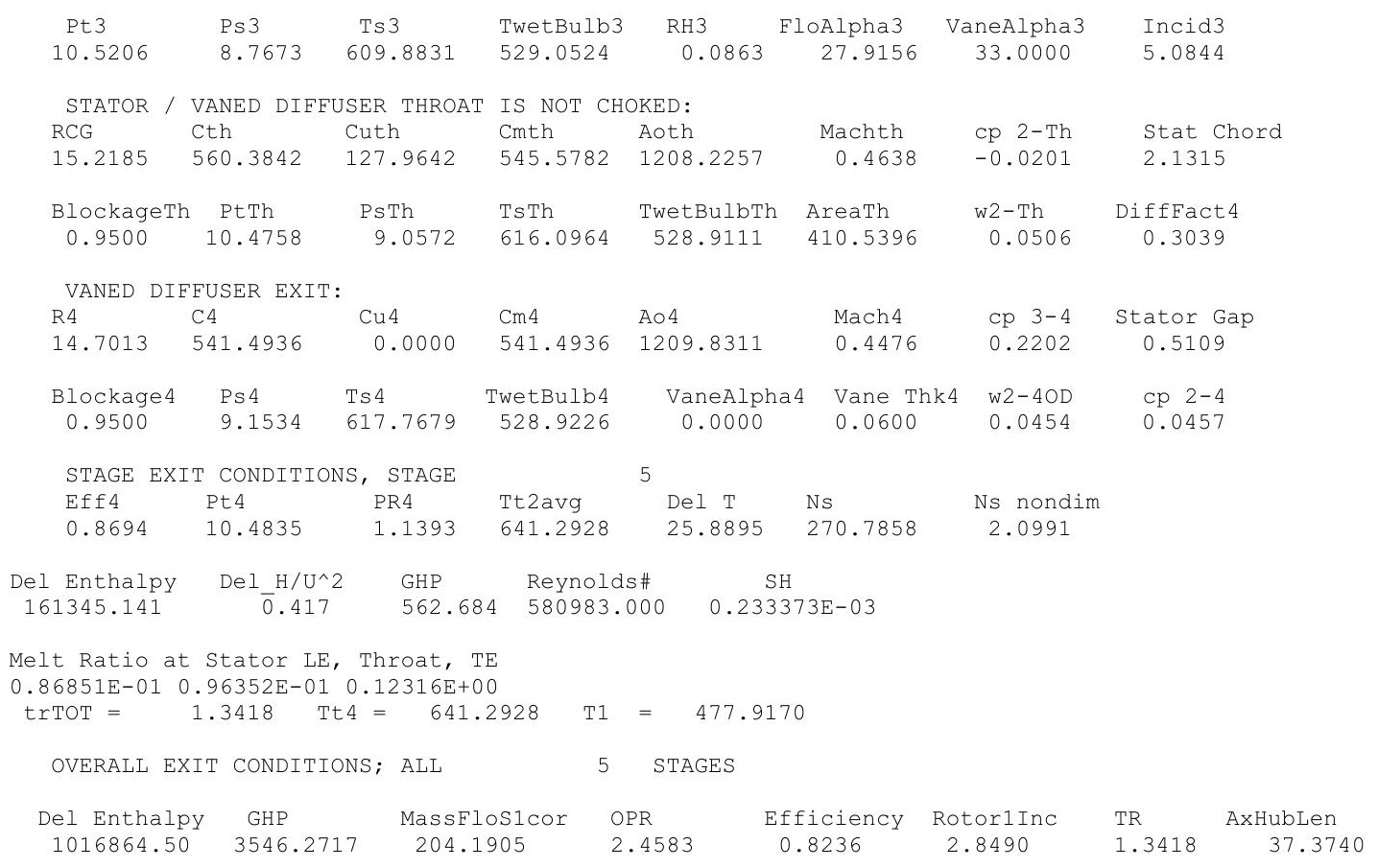




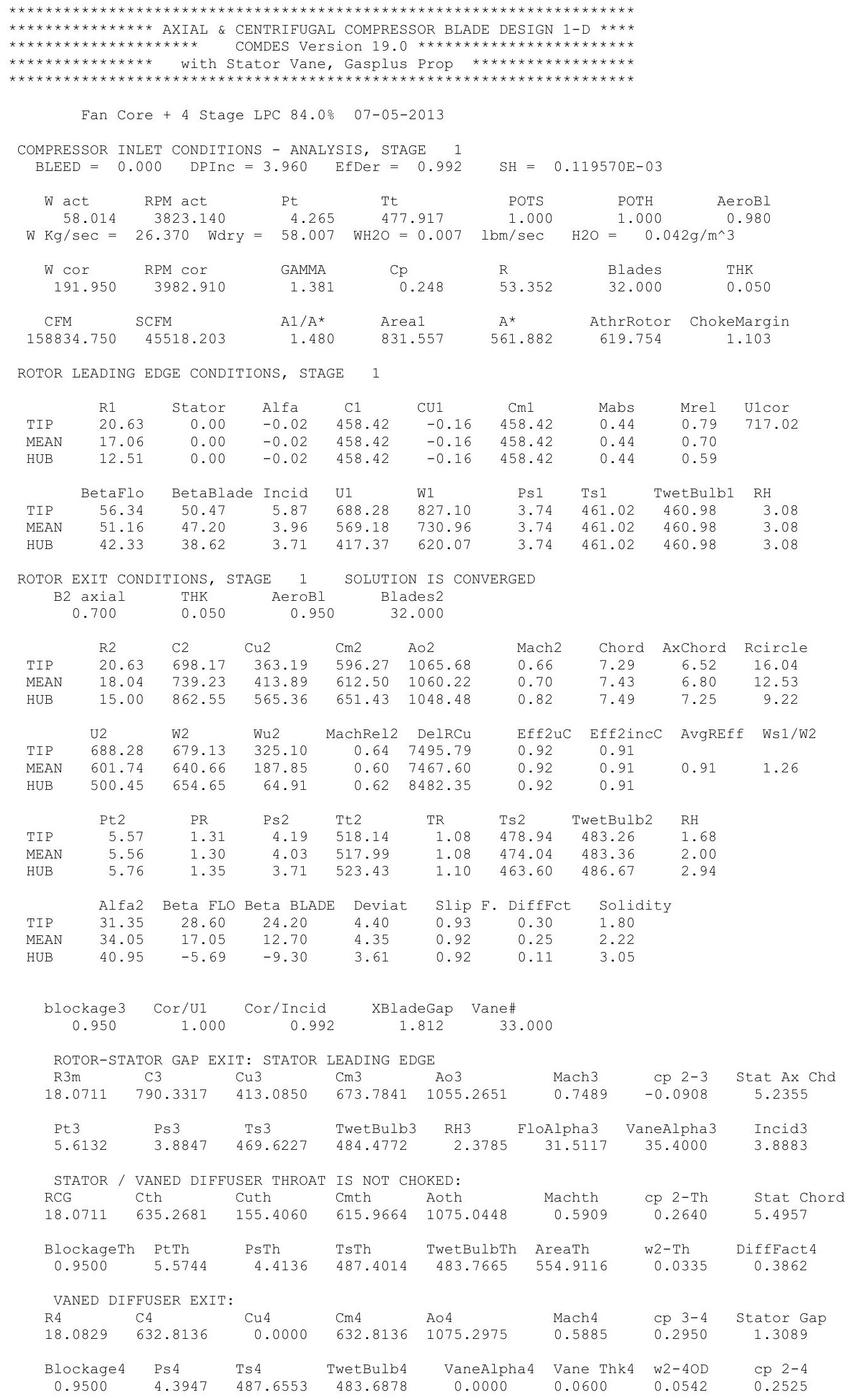




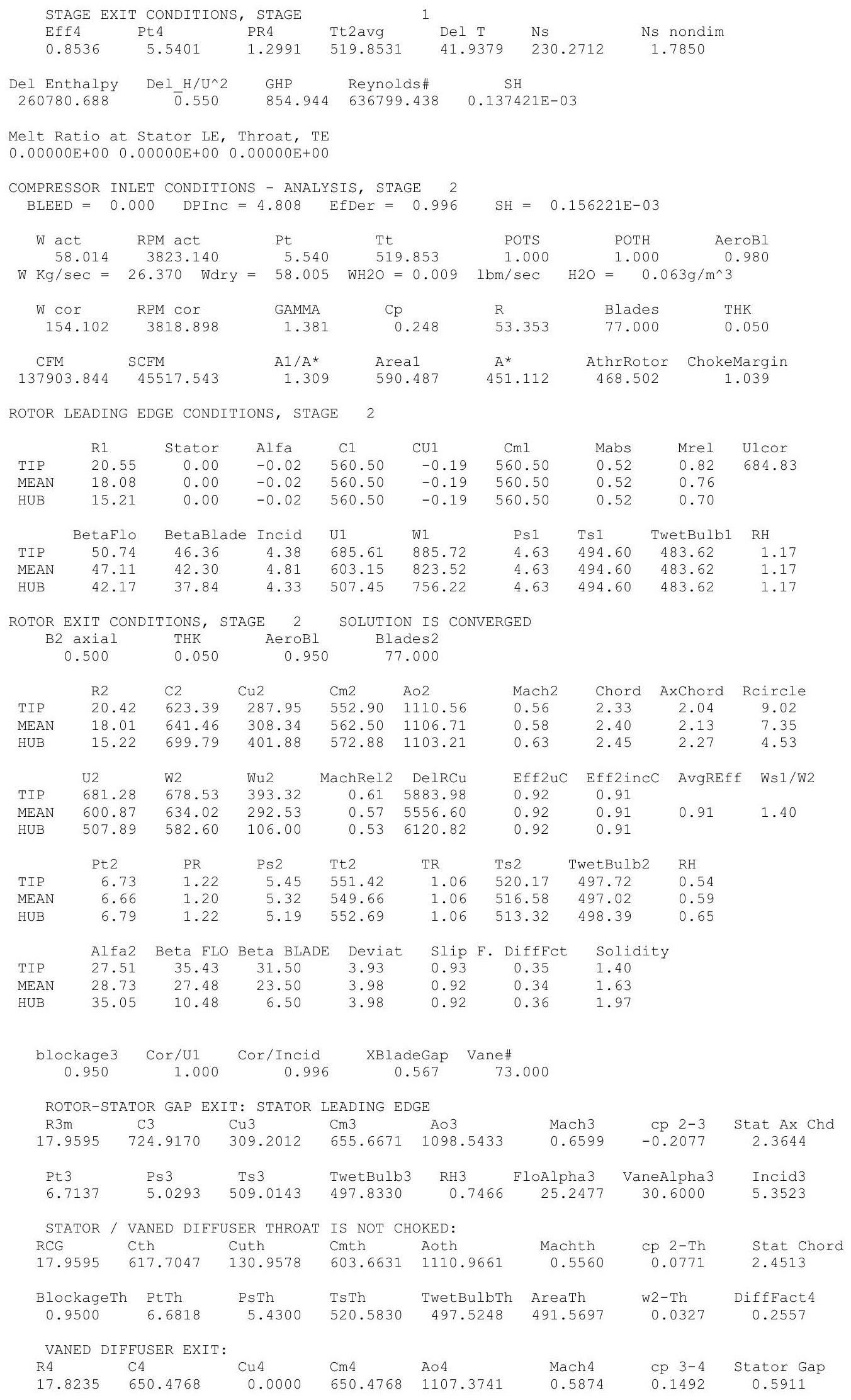




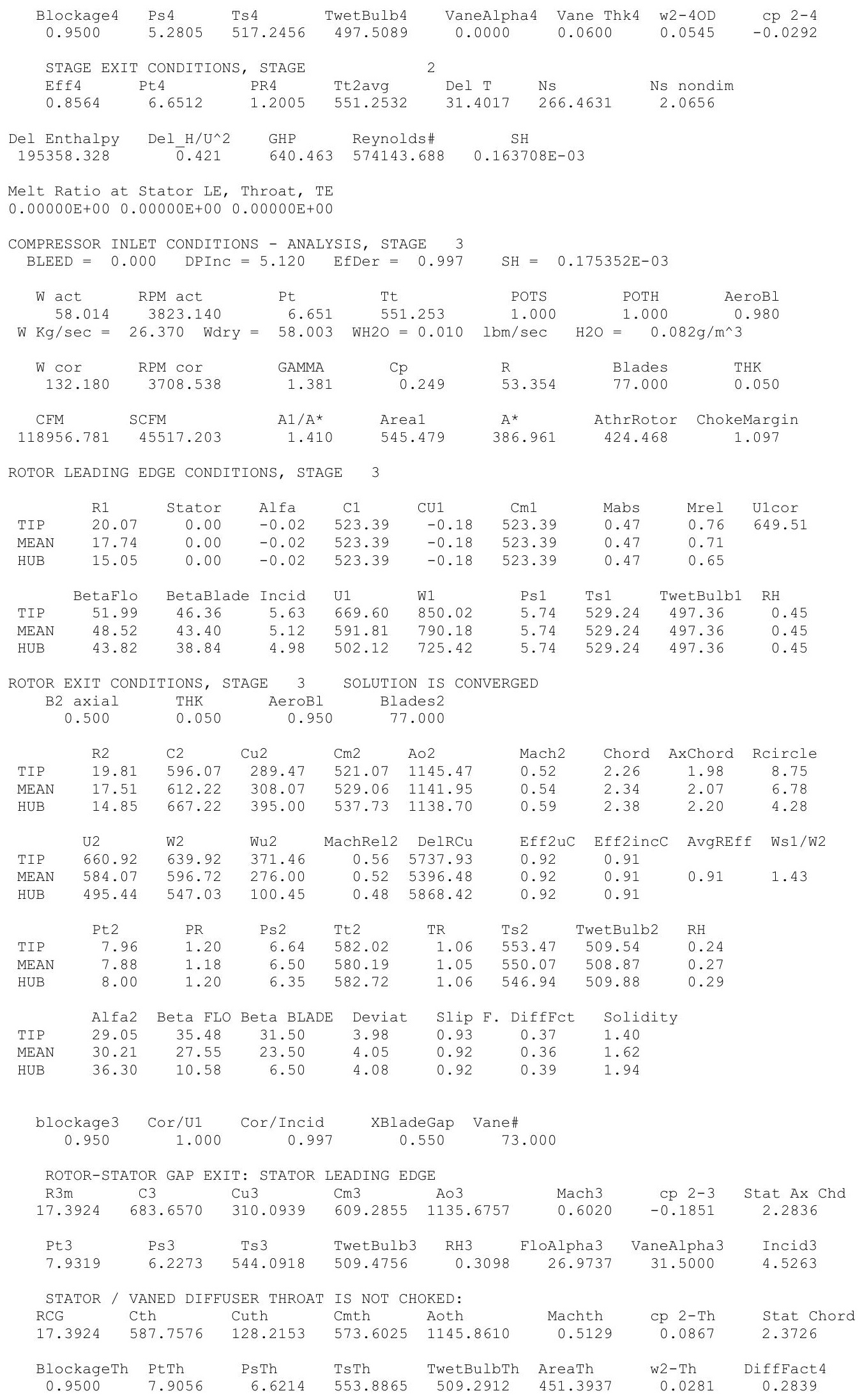




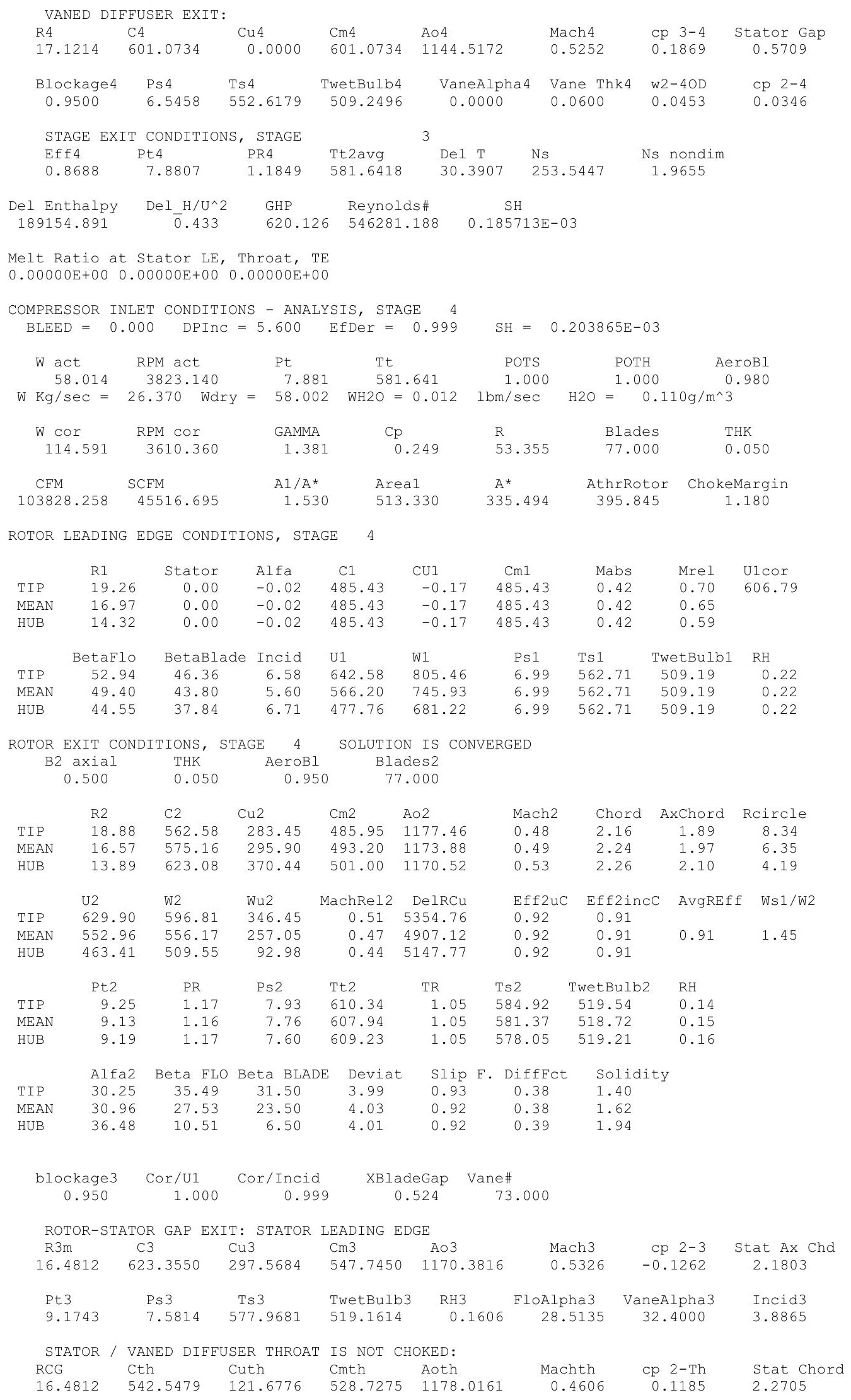




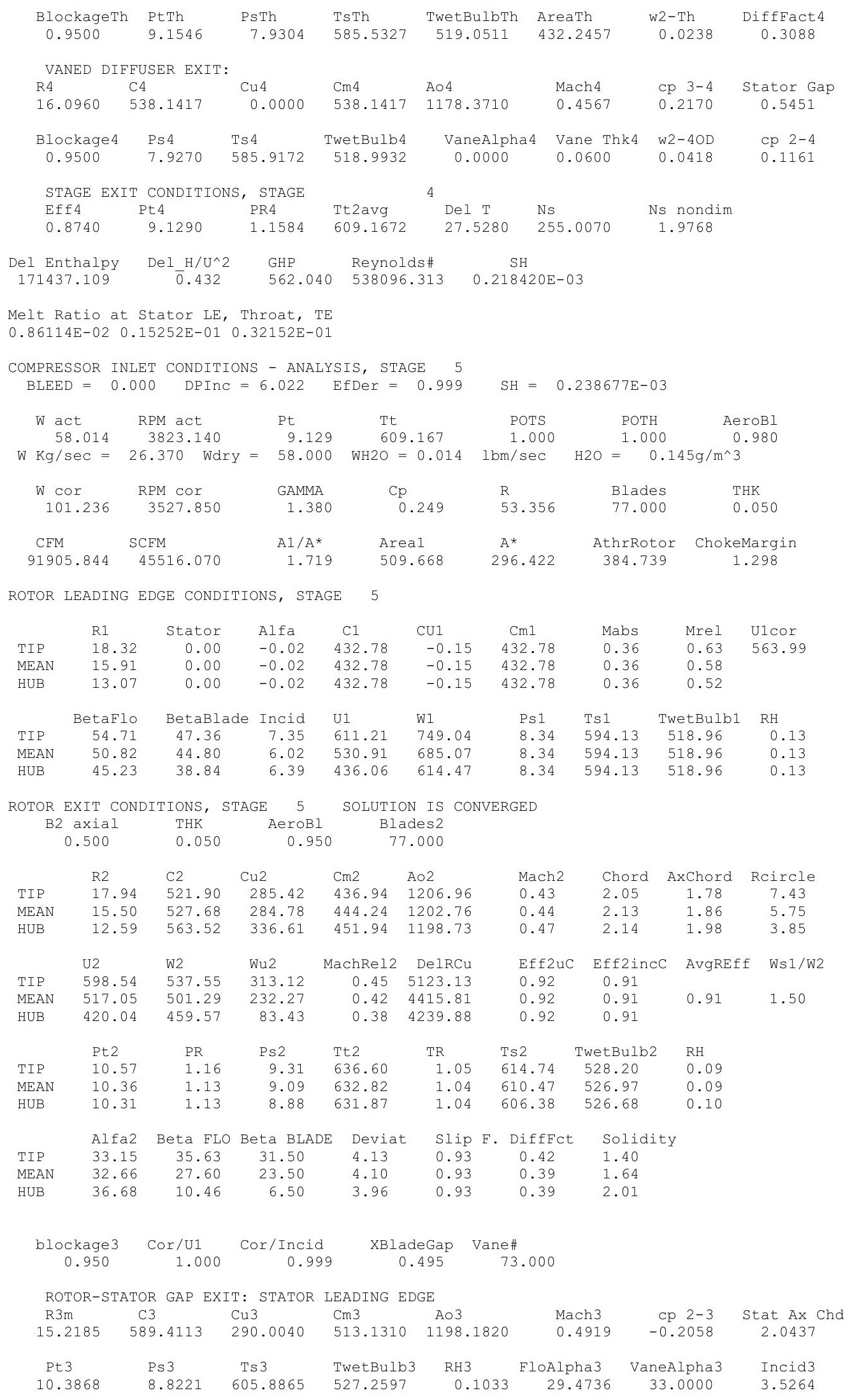




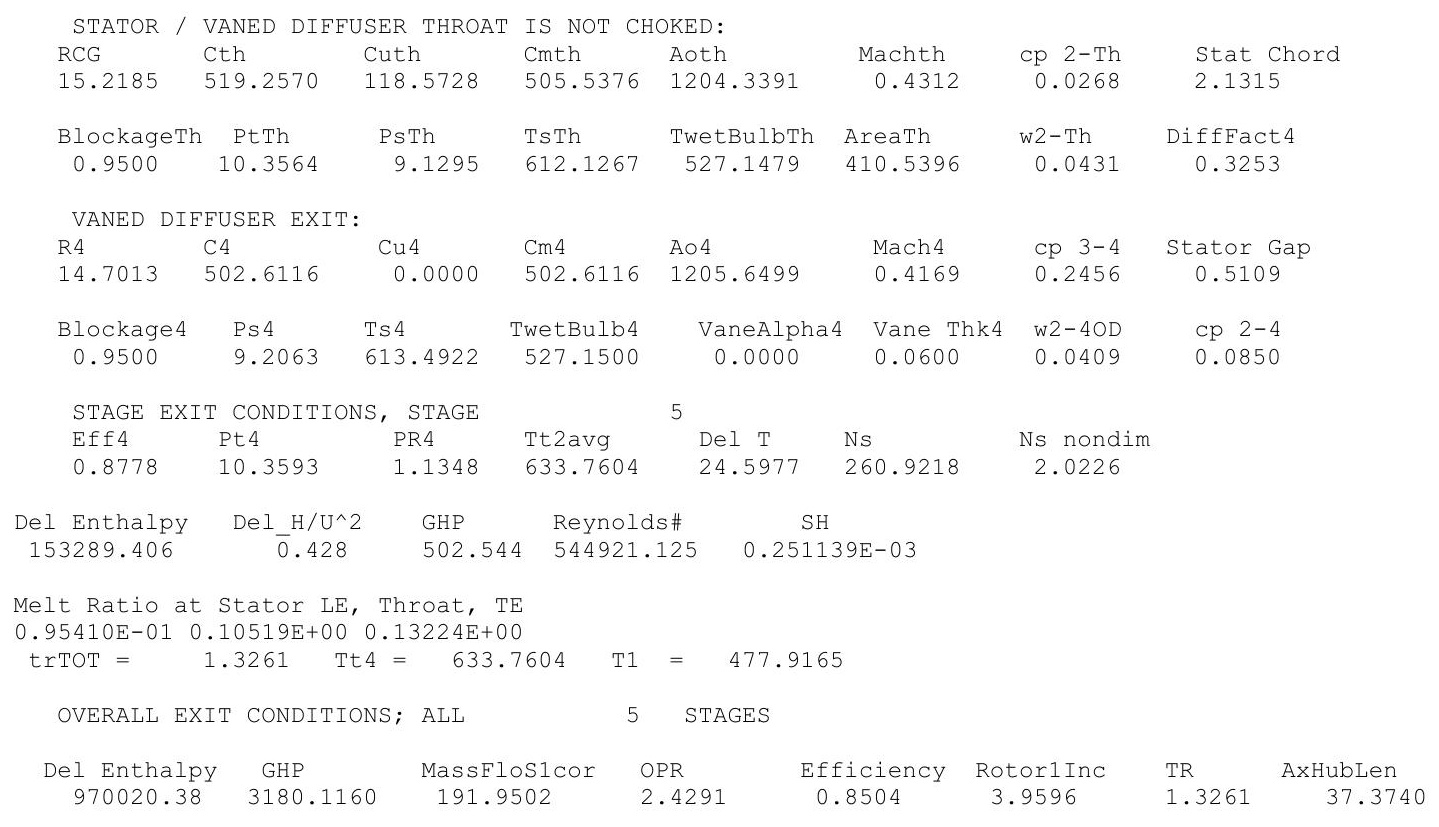




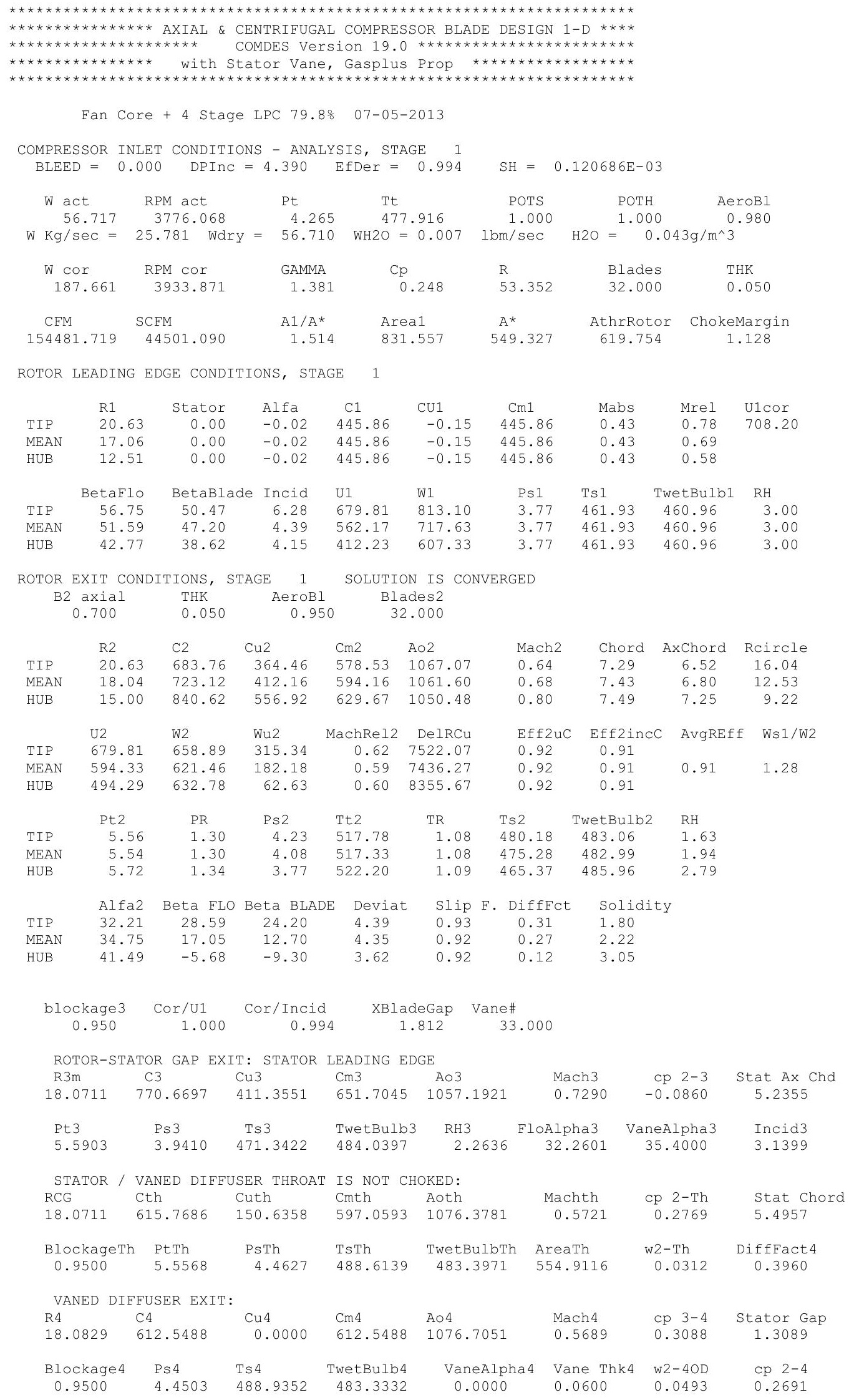




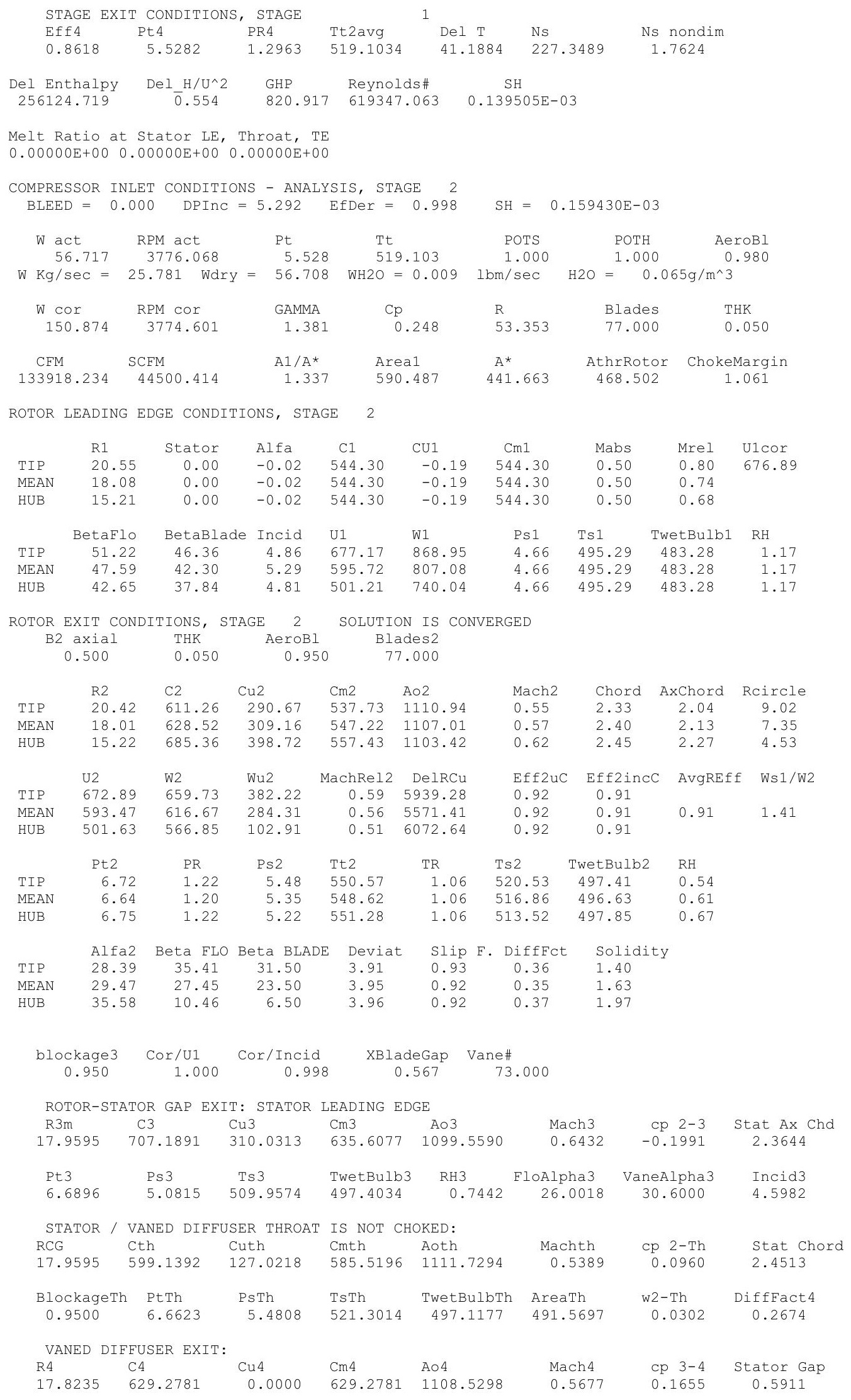




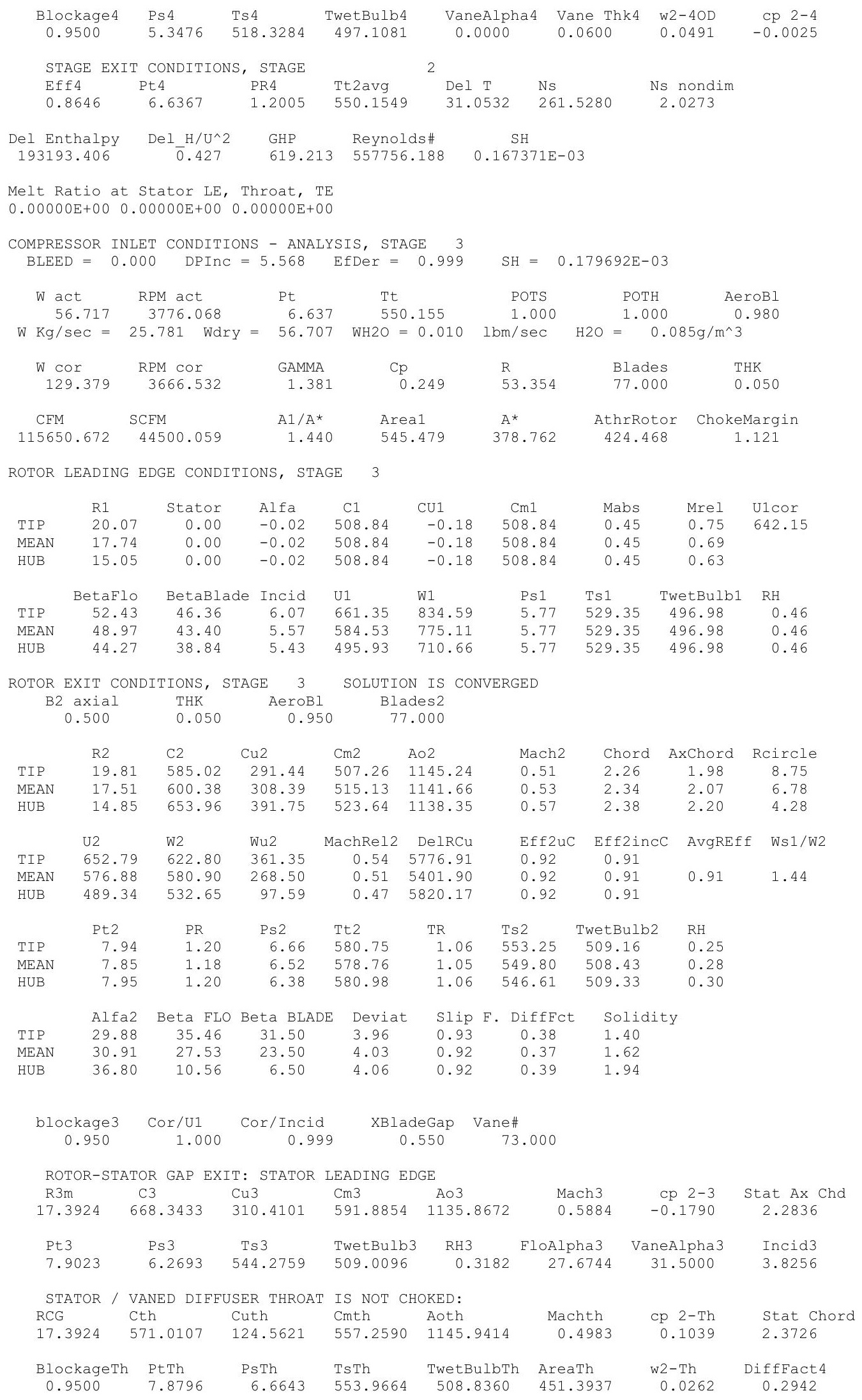




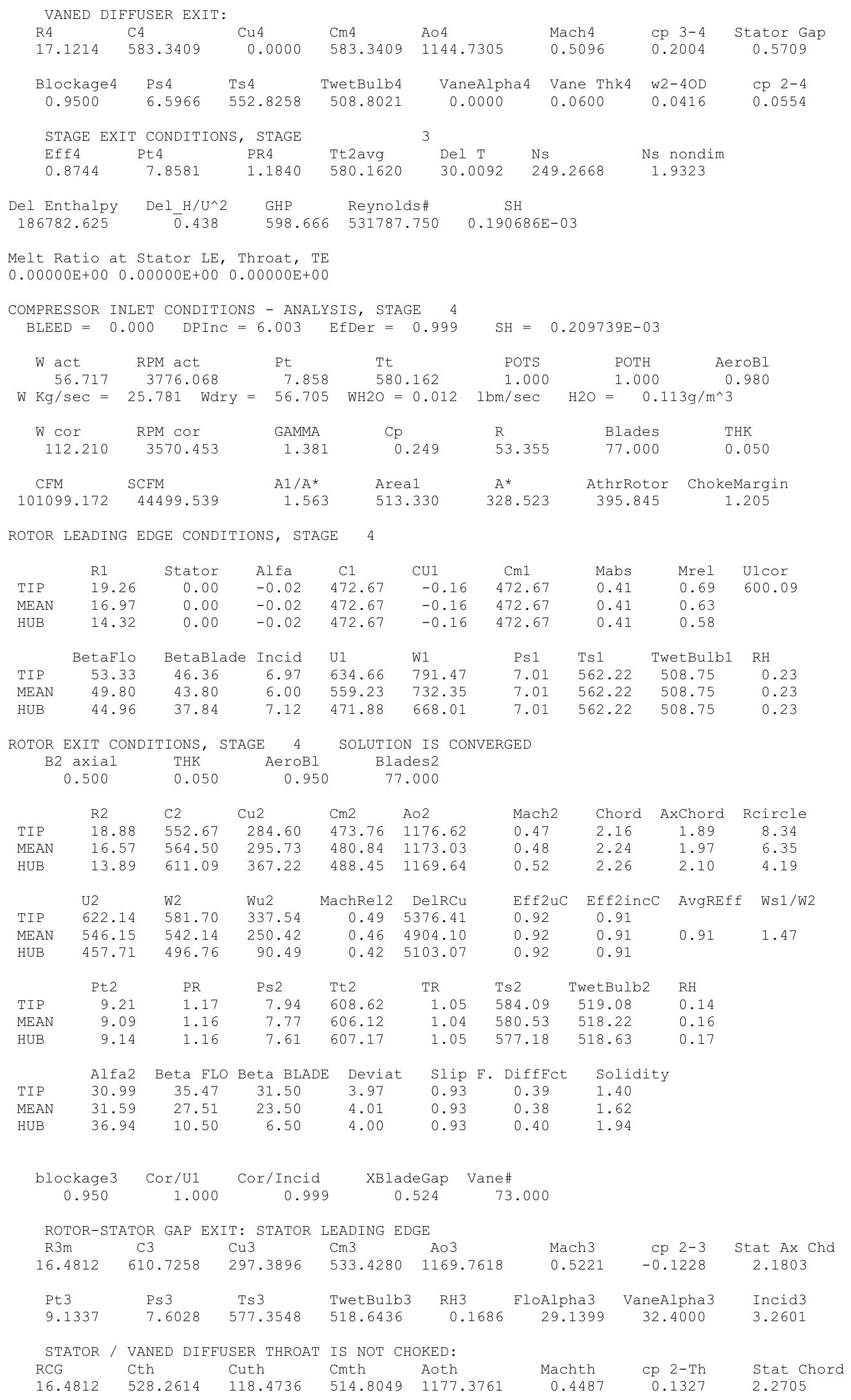




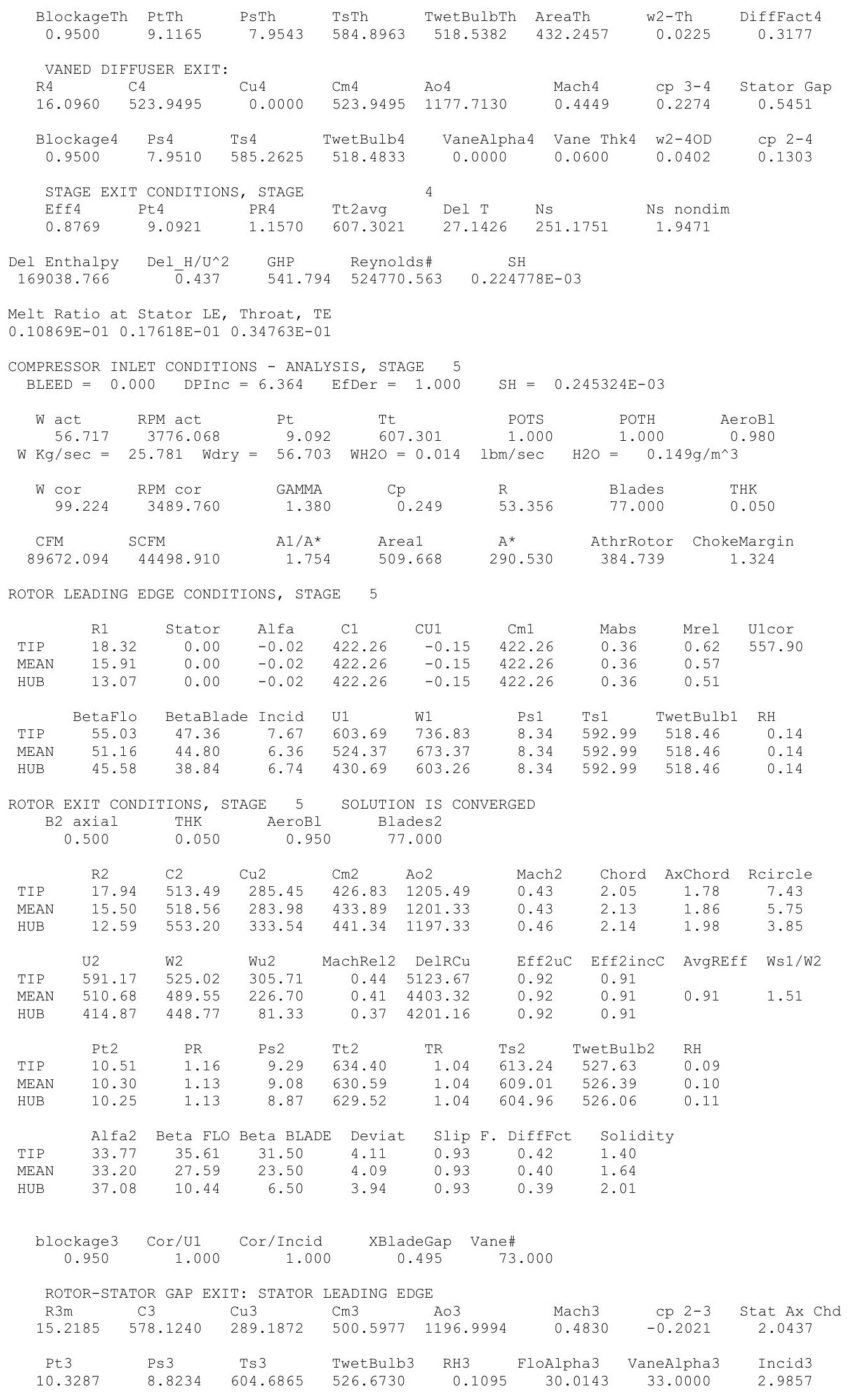




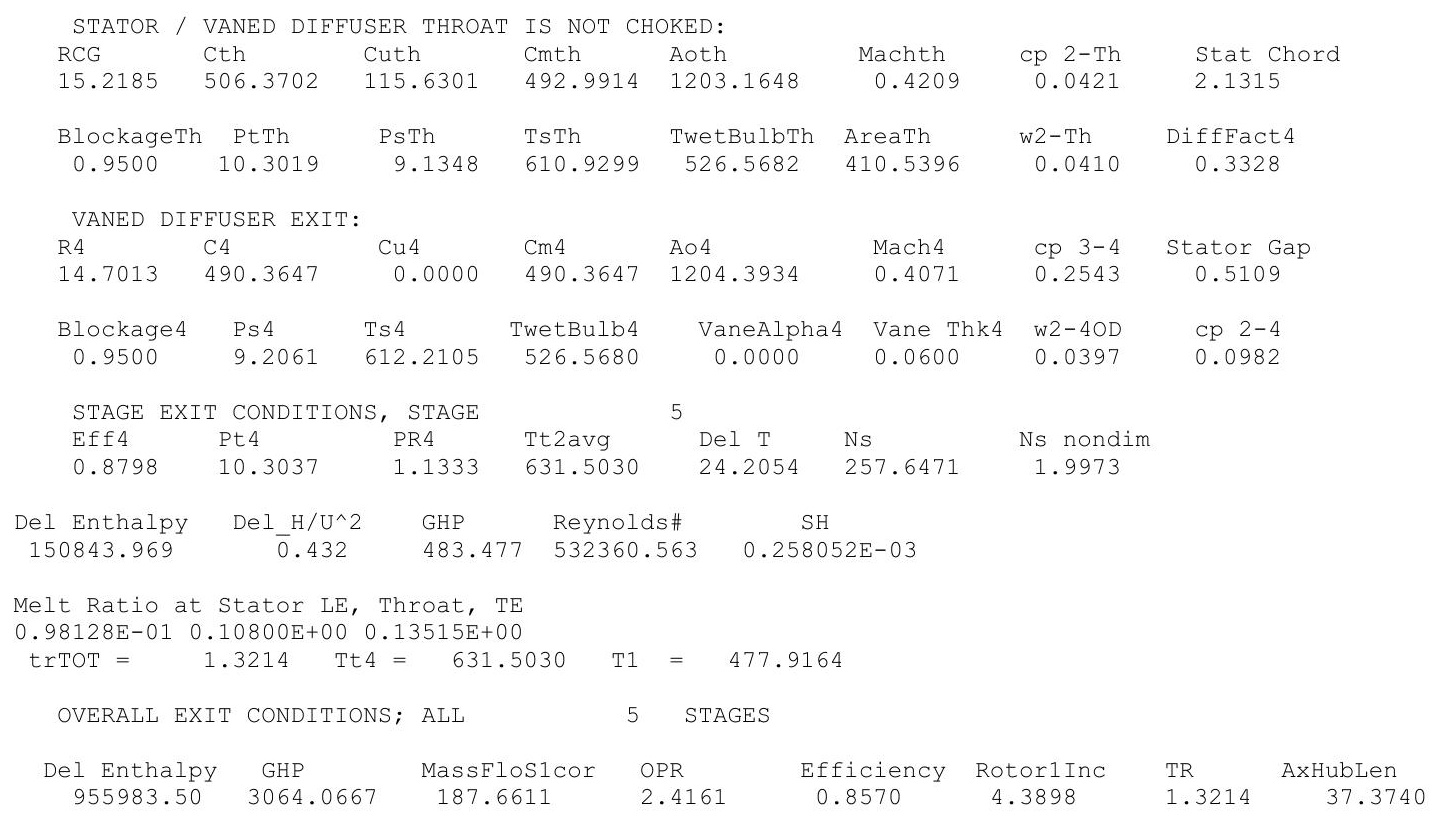




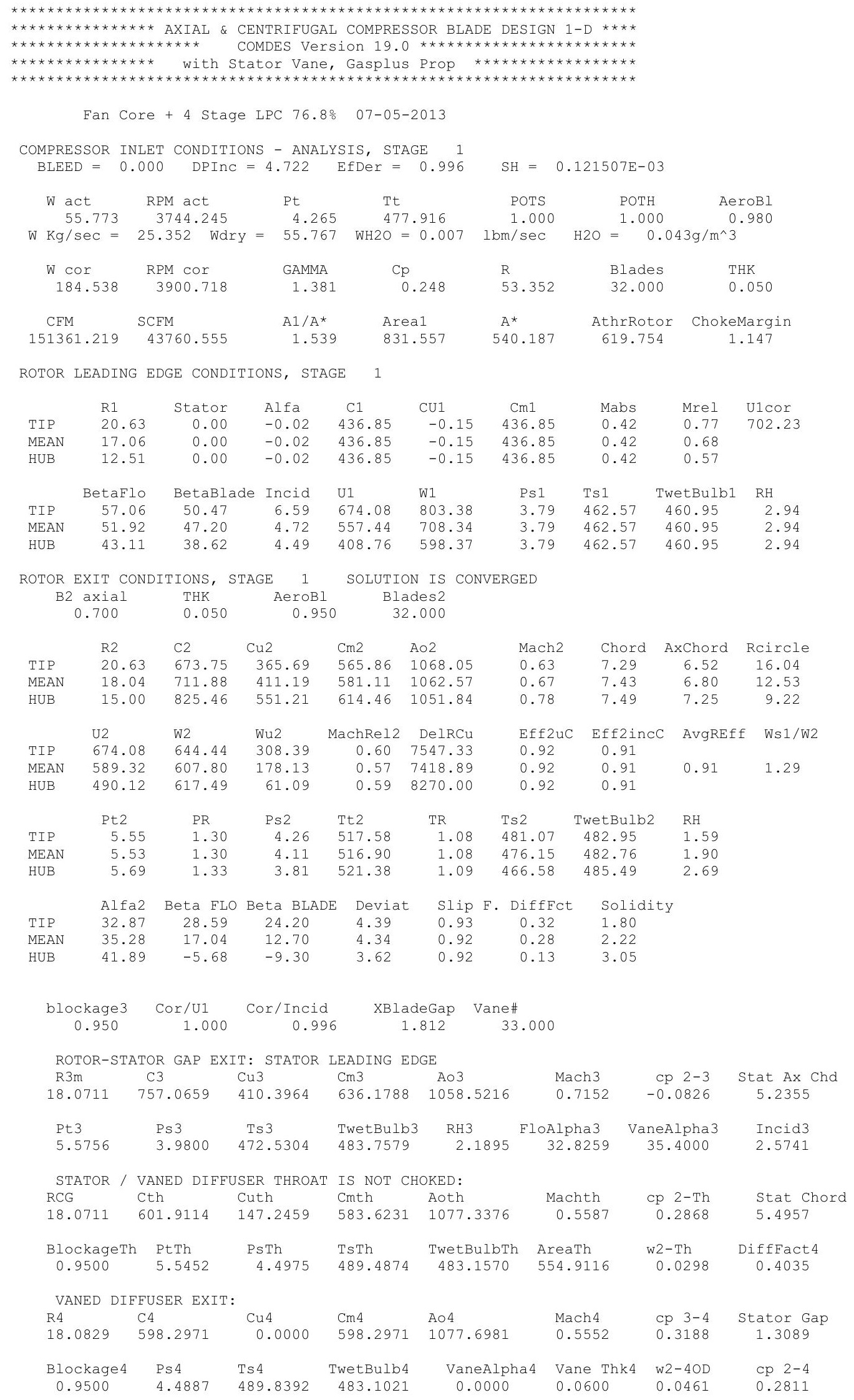




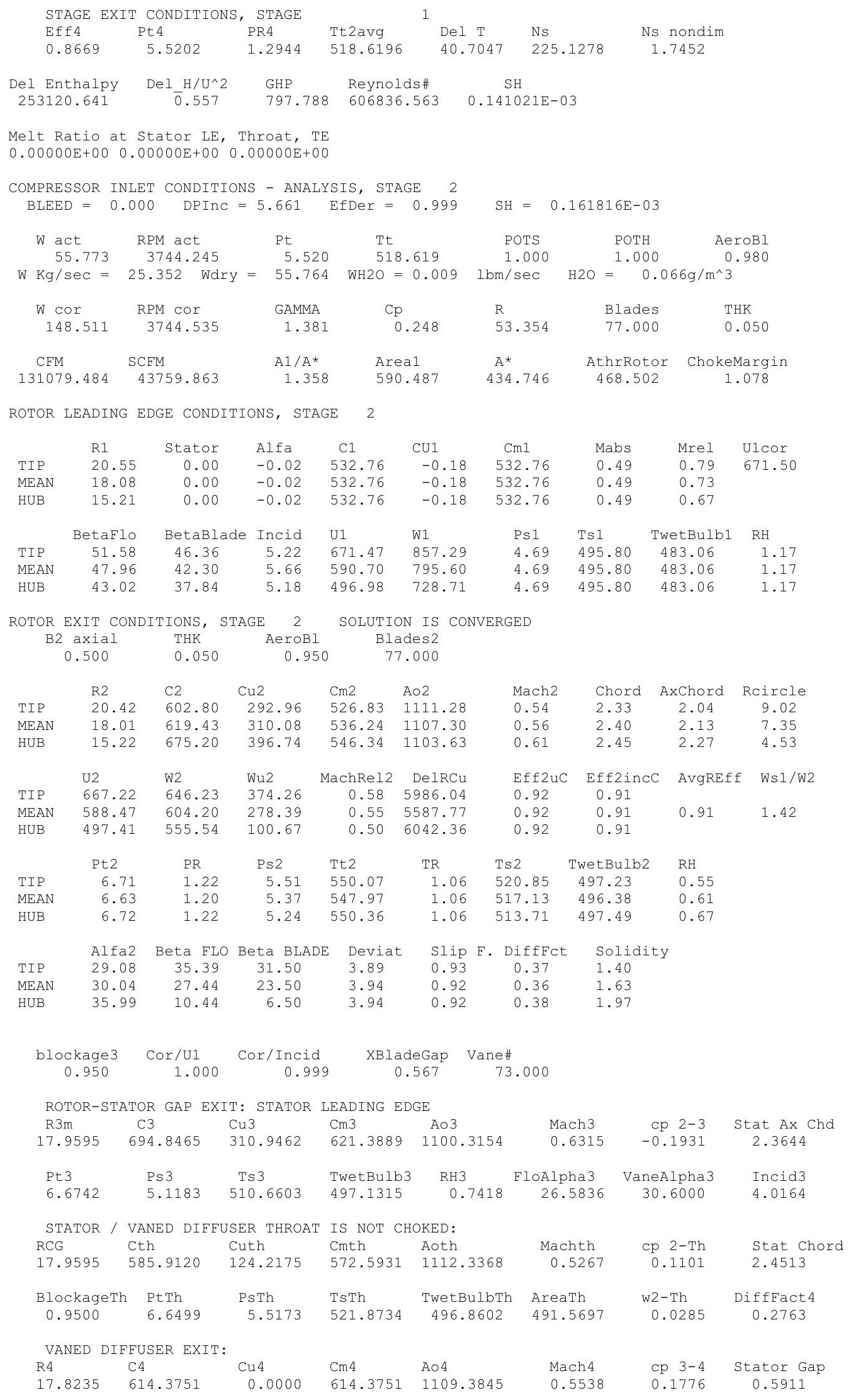




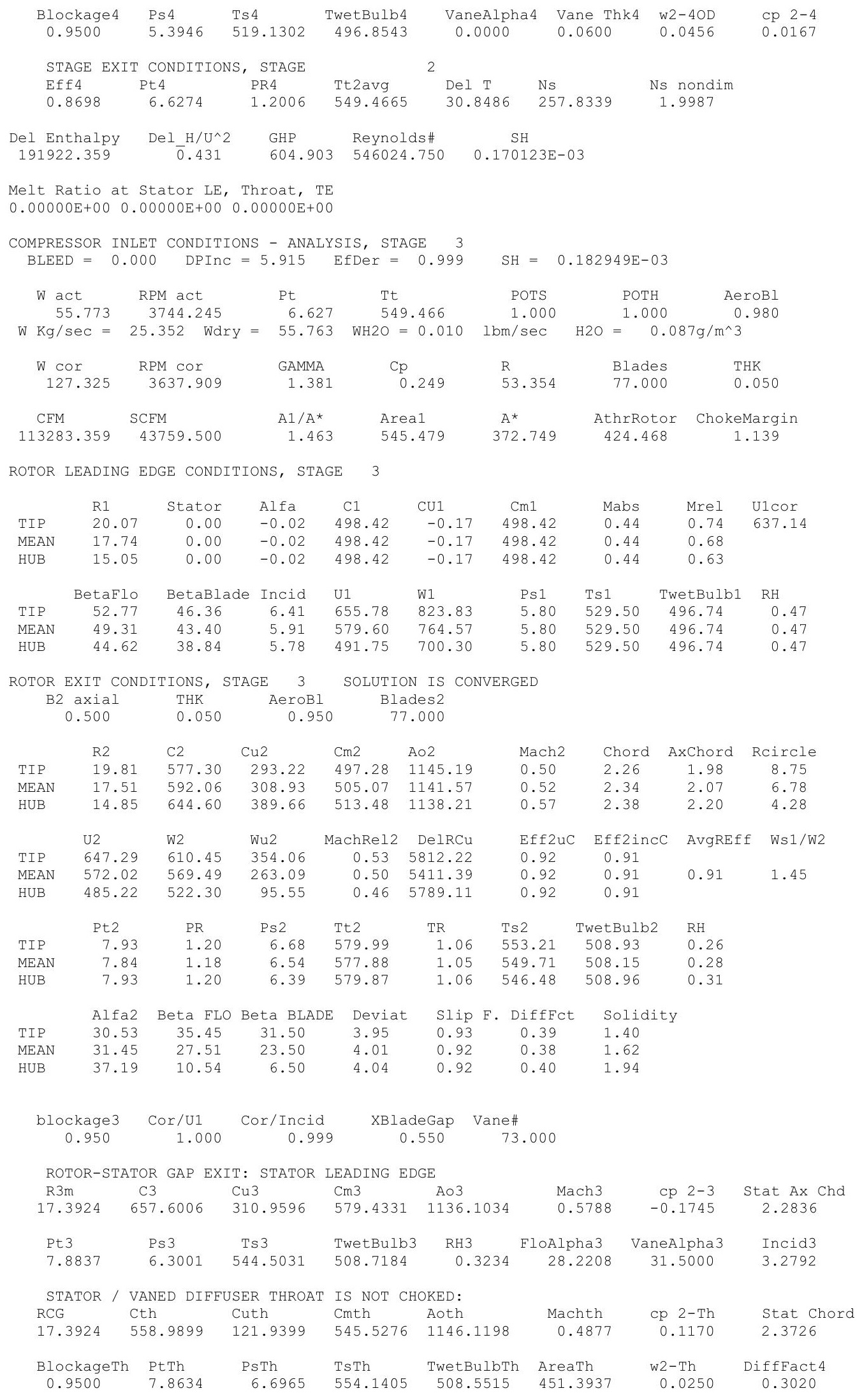




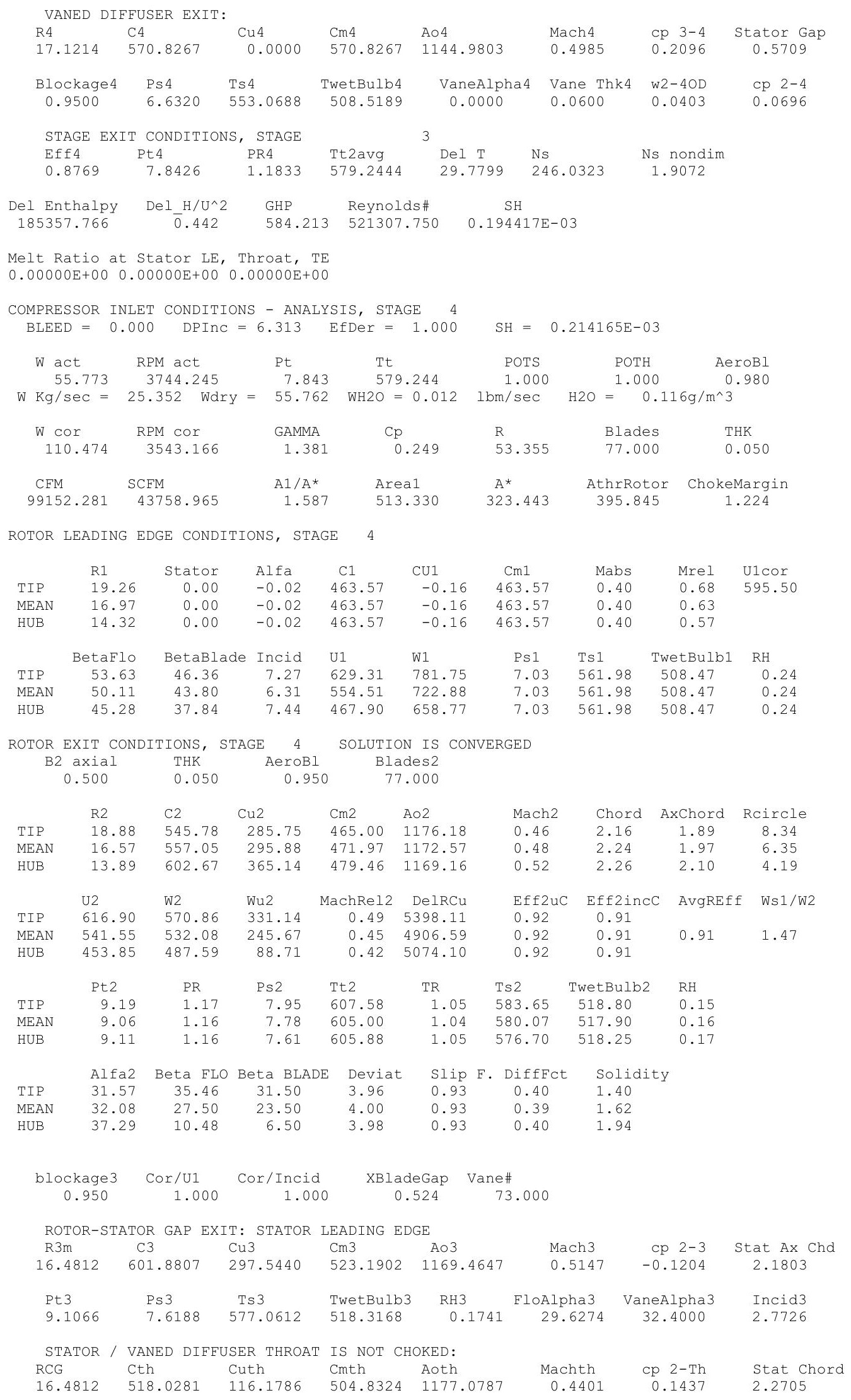




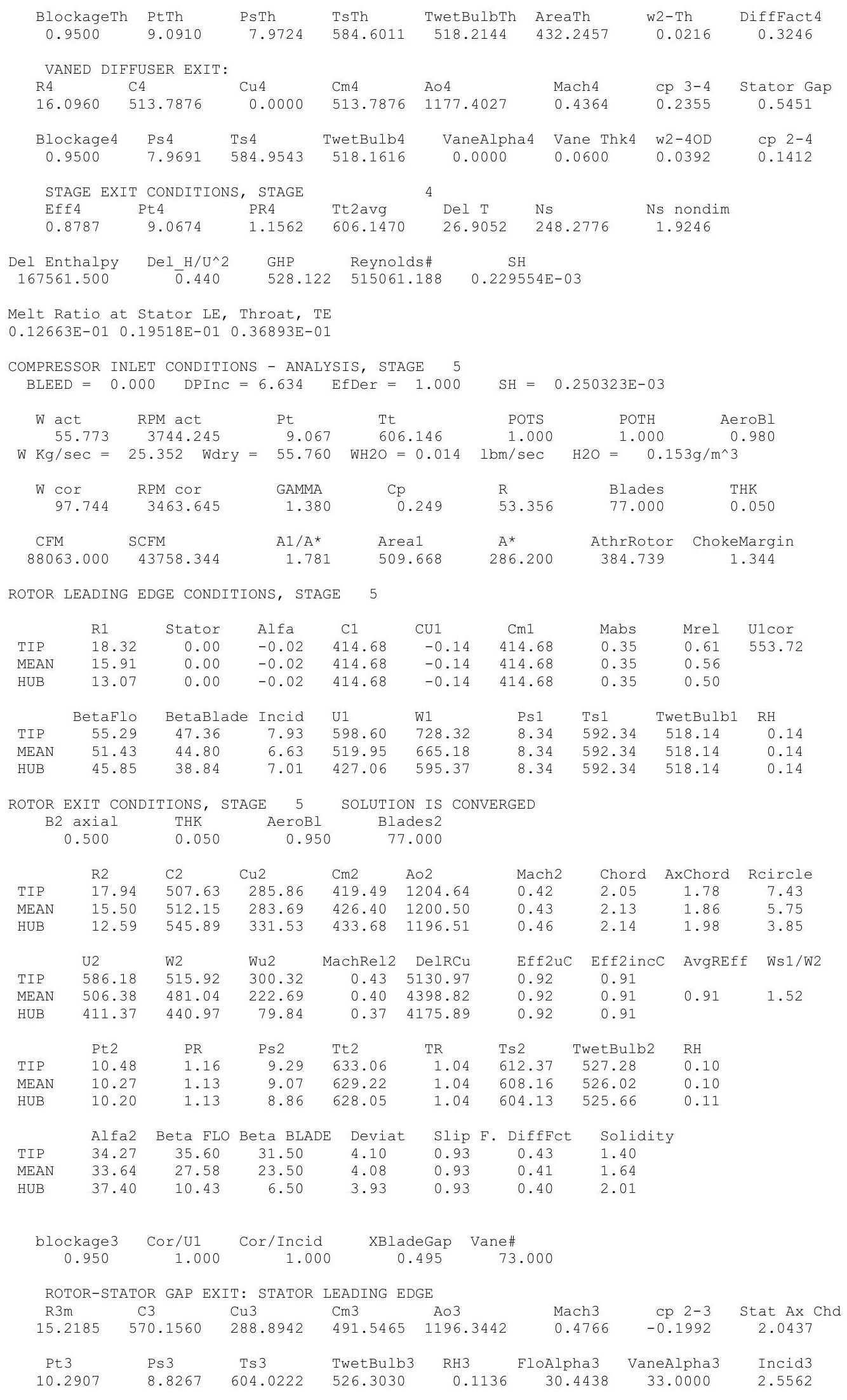




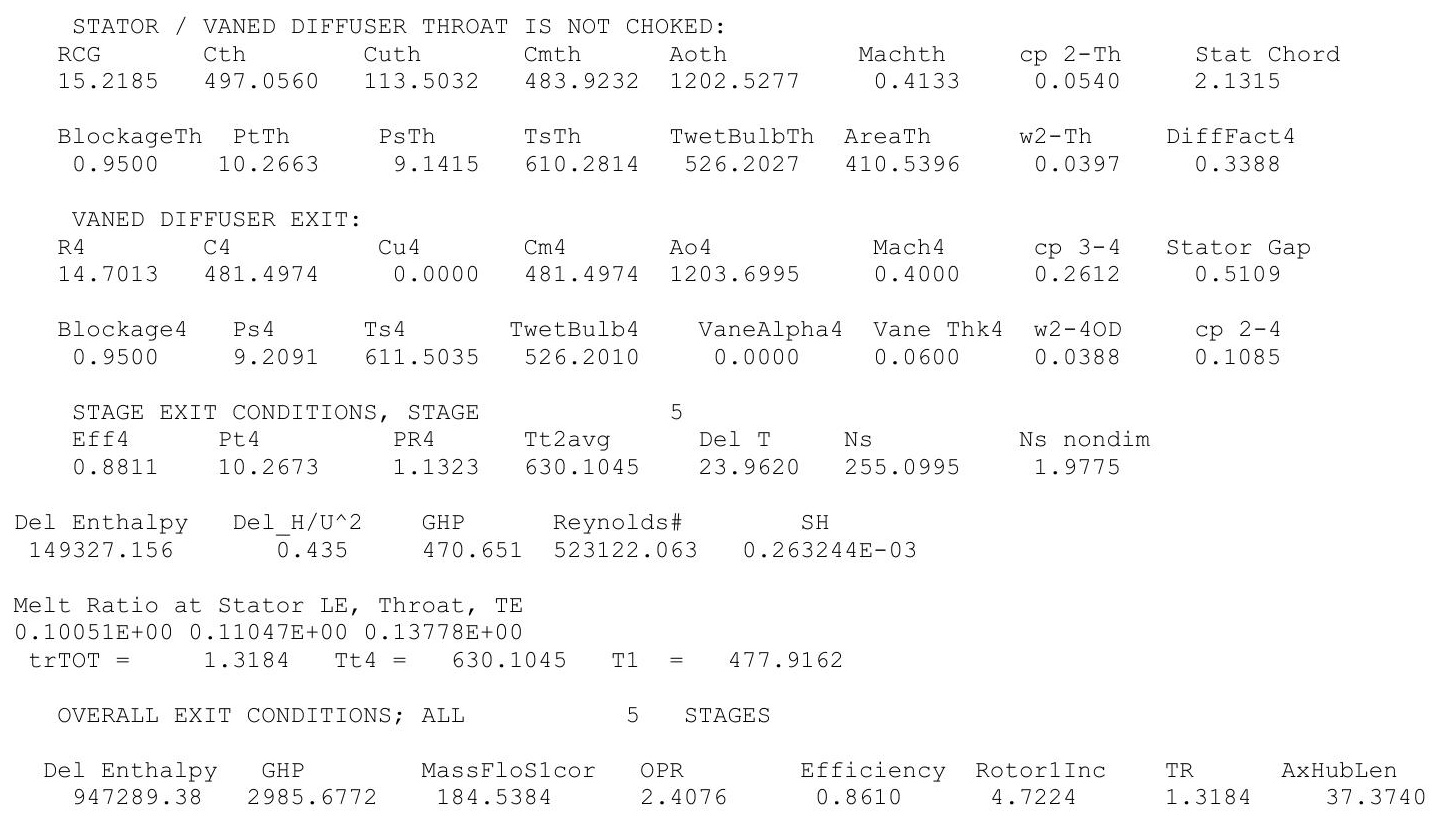




\section{$50 \mu \mathrm{m}, \mathrm{ISA}+18 \mathrm{R}$}

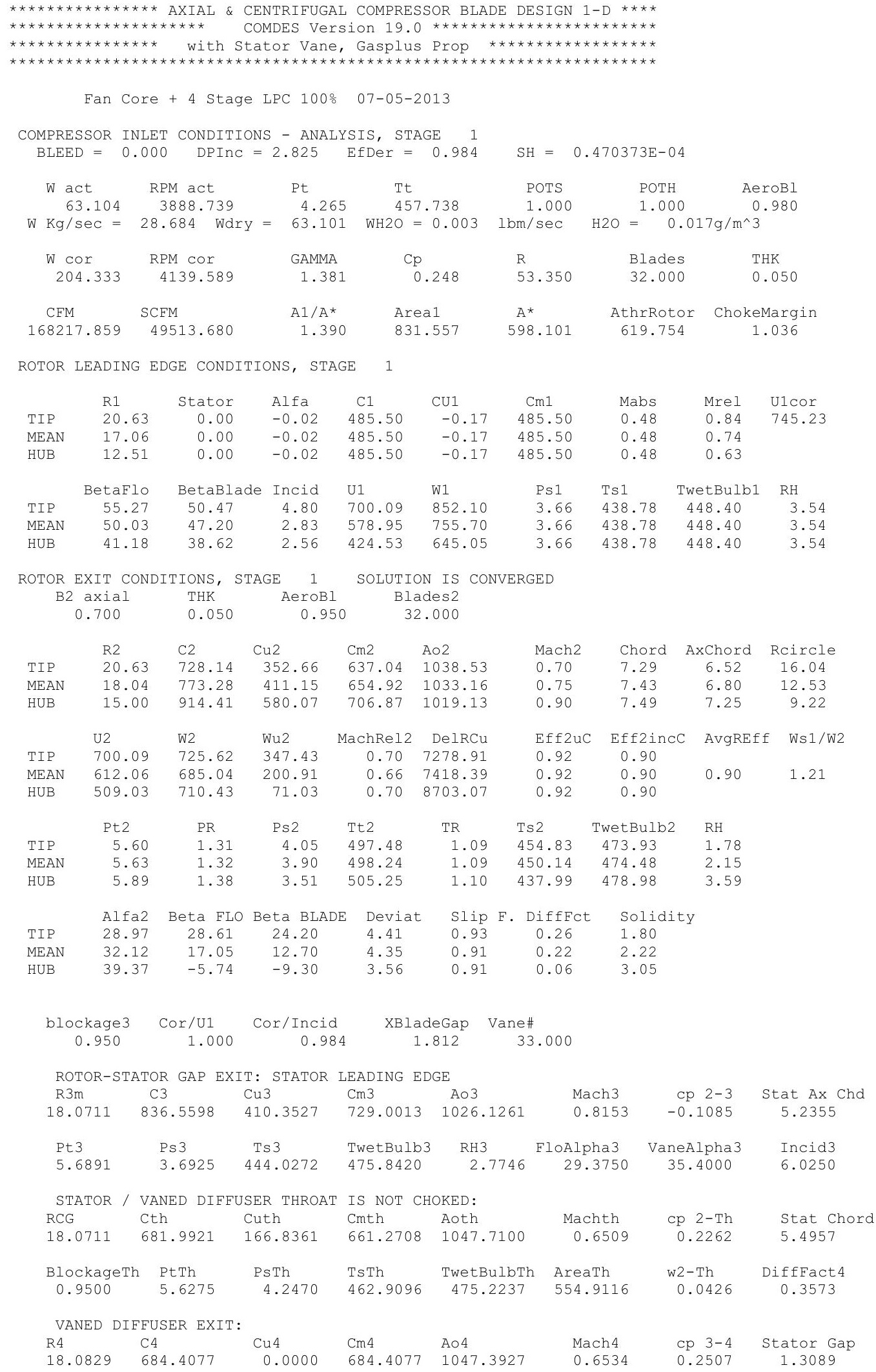




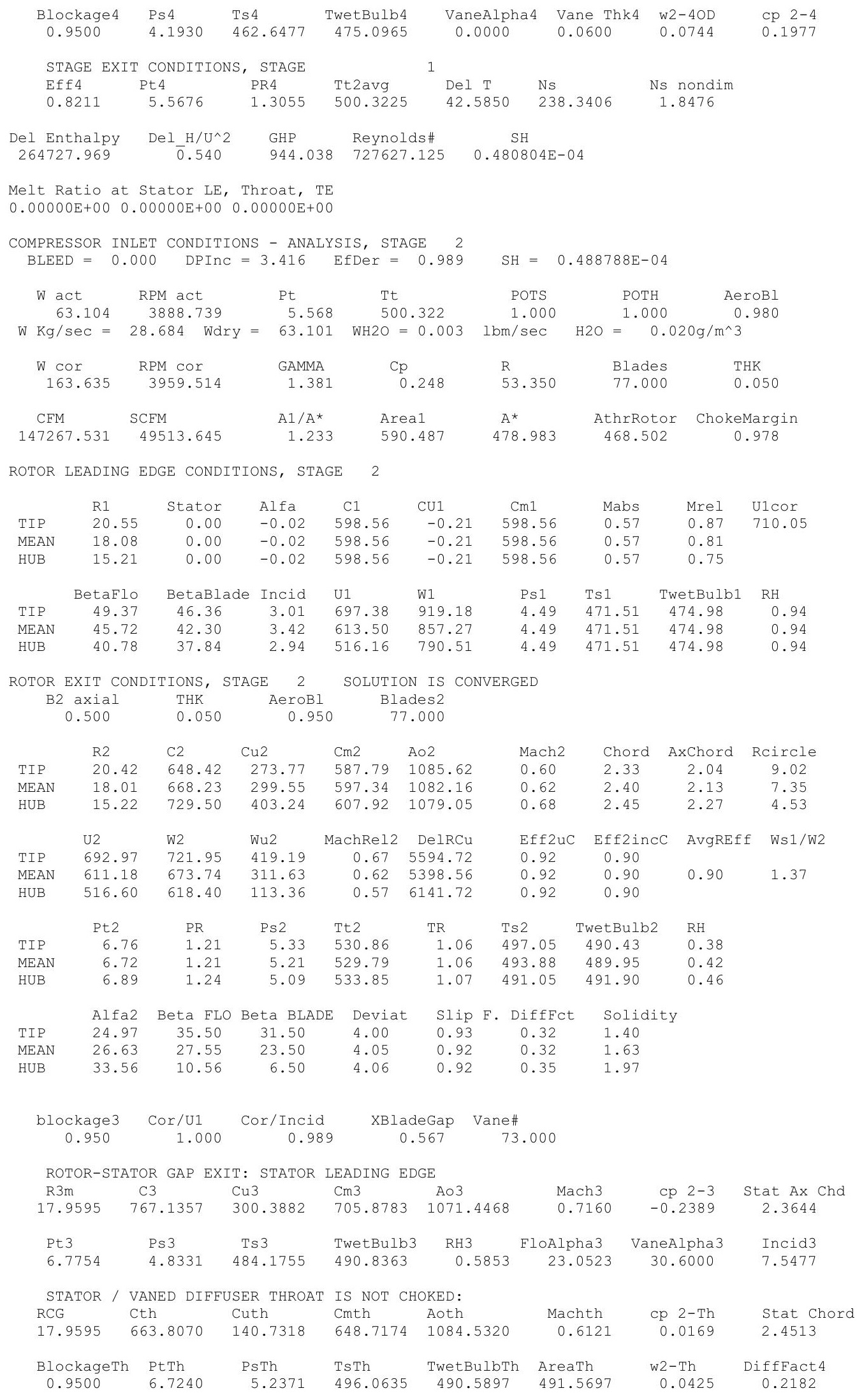




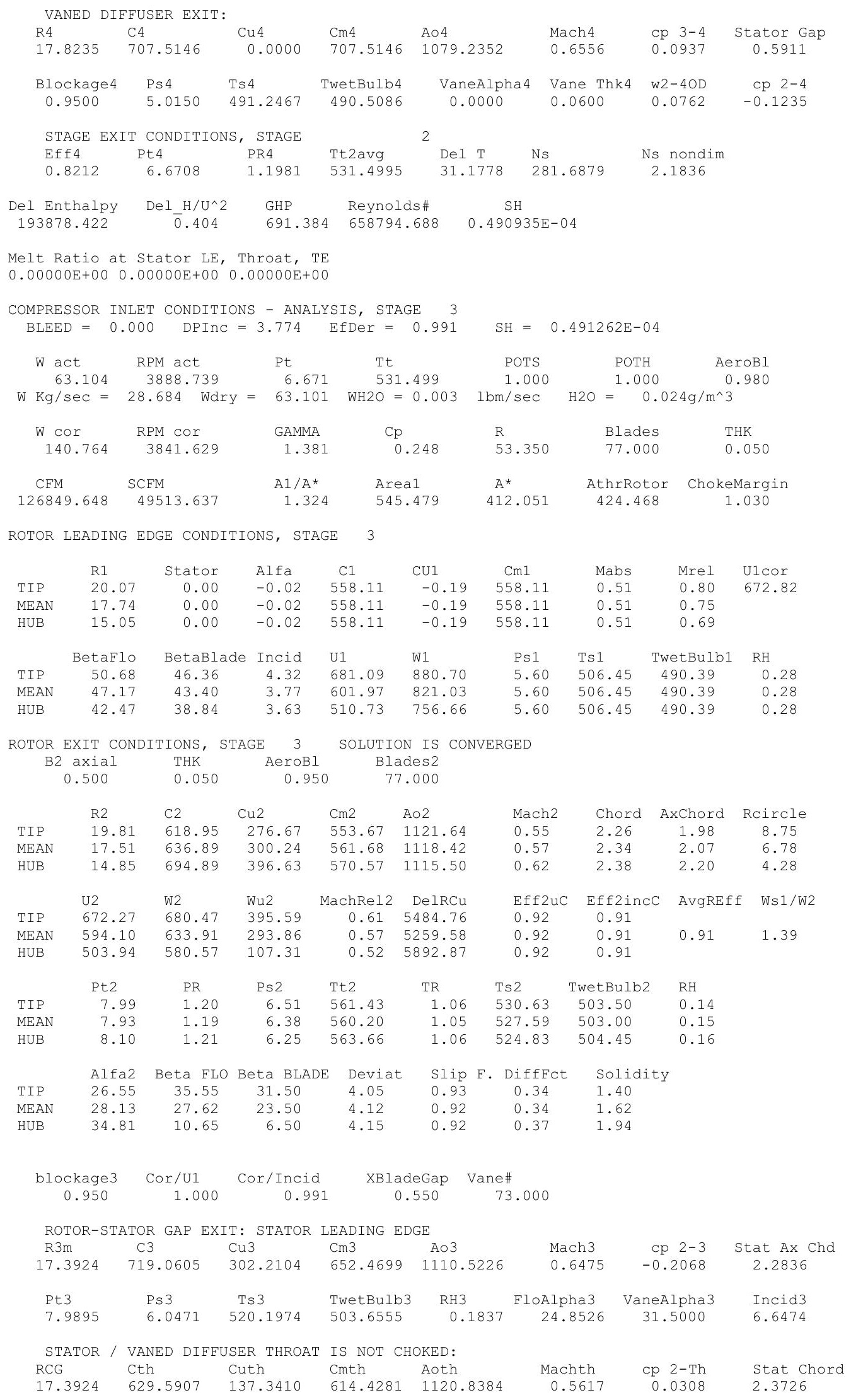




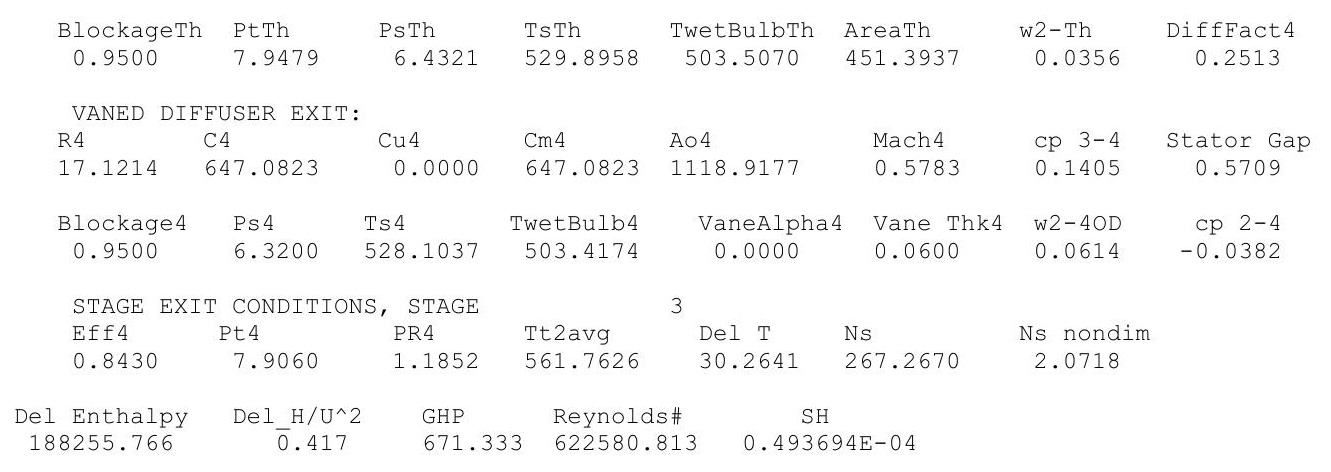

Melt Ratio at Stator LE, Throat, TE

$0.00000 \mathrm{E}+00 \quad 0.00000 \mathrm{E}+00 \quad 0.00000 \mathrm{E}+00$

COMPRESSOR INLET CONDITIONS - ANALYSIS, STAGE 4 BLEED $=0.000 \quad$ DPInC $=4.349 \quad$ EfDer $=0.994$

63.104 RPM act Pt Tt

$\mathrm{W} \mathrm{Kg} / \mathrm{sec}=28.684$ Wdry $=63.101$ WH2O $=0.003$

$\mathrm{SH}=0.496608 \mathrm{E}-04$

\begin{tabular}{|c|c|c|c|c|c|c|}
\hline $\begin{array}{l}\text { W cor } \\
122.106\end{array}$ & $\begin{array}{l}\text { RPM cor } \\
3736.719\end{array}$ & $\begin{array}{l}\text { GAMMA } \\
1.381\end{array}$ & $\begin{array}{l}\mathrm{Cp} \\
0.249\end{array}$ & $\begin{array}{l}R \\
53.350\end{array}$ & $\begin{array}{l}\text { Blades } \\
77.000\end{array}$ & $\begin{array}{l}\text { THK } \\
0.050\end{array}$ \\
\hline $\begin{array}{c}\text { CFM } \\
110367.977\end{array}$ & $\begin{array}{l}\mathrm{SCFM} \\
49513.625\end{array}$ & $\begin{array}{l}\mathrm{A} 1 / \mathrm{A}^{\star} \\
1.436\end{array}$ & $\begin{array}{l}\text { Area1 } \\
513.330\end{array}$ & $\begin{array}{l}A^{*} \\
357.452\end{array}$ & $\begin{array}{c}\text { AthrRotor } \\
395.845\end{array}$ & $\begin{array}{c}\text { ChokeMargin } \\
1.107\end{array}$ \\
\hline
\end{tabular}

ROTOR LEADING EDGE CONDITIONS, STAGE 4

$\begin{array}{lrrrrrrrrr} & \text { R1 } & \text { Stator } & \text { Alfa } & \text { C1 } & \text { CU1 } & \text { Cm1 } & \text { Mabs } & \text { Mrel } & \text { U1cor } \\ \text { TIP } & 19.26 & 0.00 & -0.02 & 516.01 & -0.18 & 516.01 & 0.46 & 0.74 & 628.03 \\ \text { MEAN } & 16.97 & 0.00 & -0.02 & 516.01 & -0.18 & 516.01 & 0.46 & 0.68 & \\ \text { HUB } & 14.32 & 0.00 & -0.02 & 516.01 & -0.18 & 516.01 & 0.46 & 0.63 & \\ & & & & & & & & & \\ & & & & & & & & & \\ \text { TIP } & 51.72 & 46.36 & 5.36 & 653.60 & 832.88 & 6.87 & 540.36 & 503.37 & 0.11 \\ \text { MEAN } & 48.15 & 43.80 & 4.35 & 575.91 & 773.40 & 6.87 & 540.36 & 503.37 & 0.11 \\ \text { HUB } & 43.29 & 37.84 & 5.45 & 485.96 & 708.94 & 6.87 & 540.36 & 503.37 & 0.11\end{array}$

ROTOR EXIT CONDITIONS, STAGE 4 SOLUTION IS CONVERGED

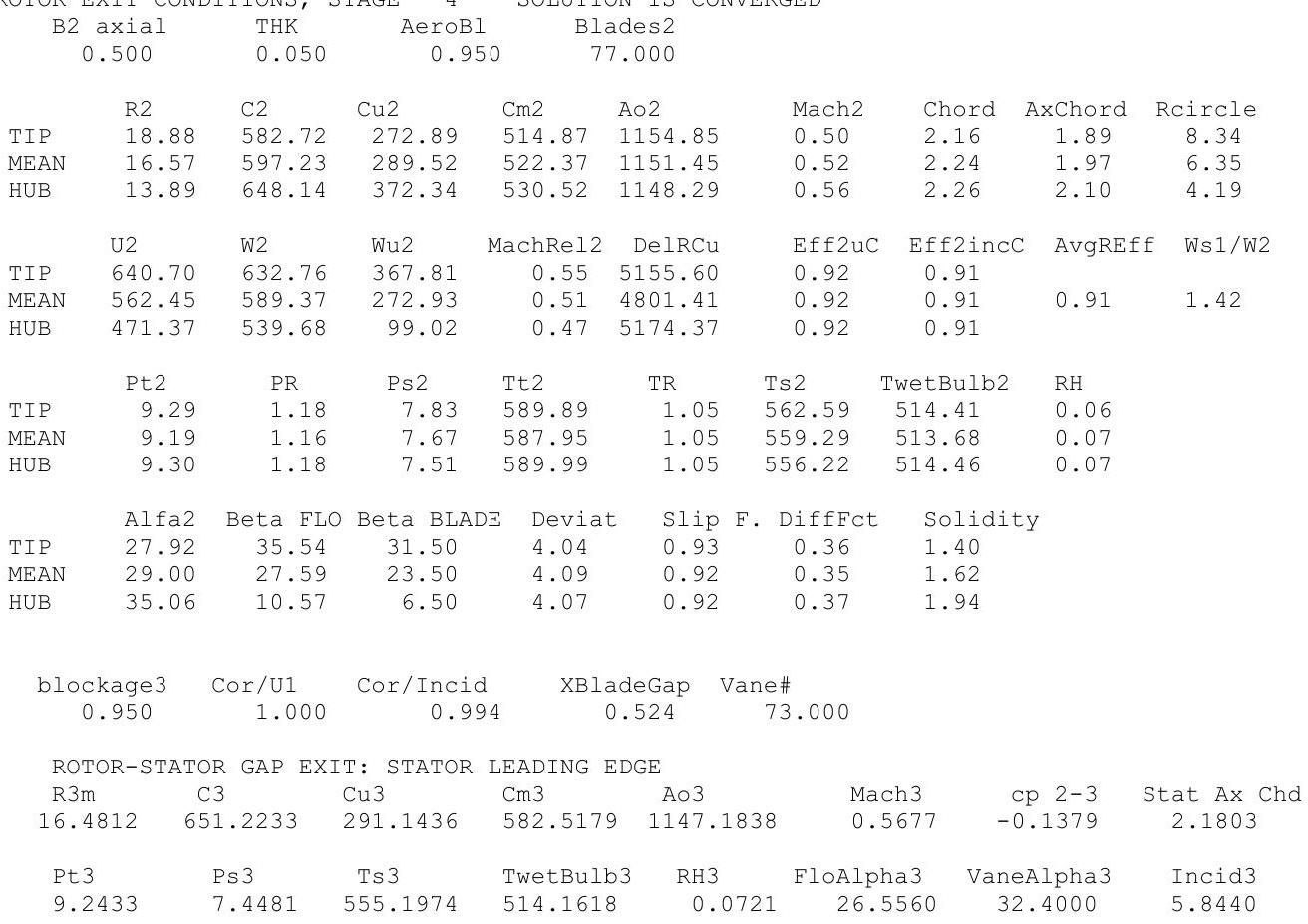




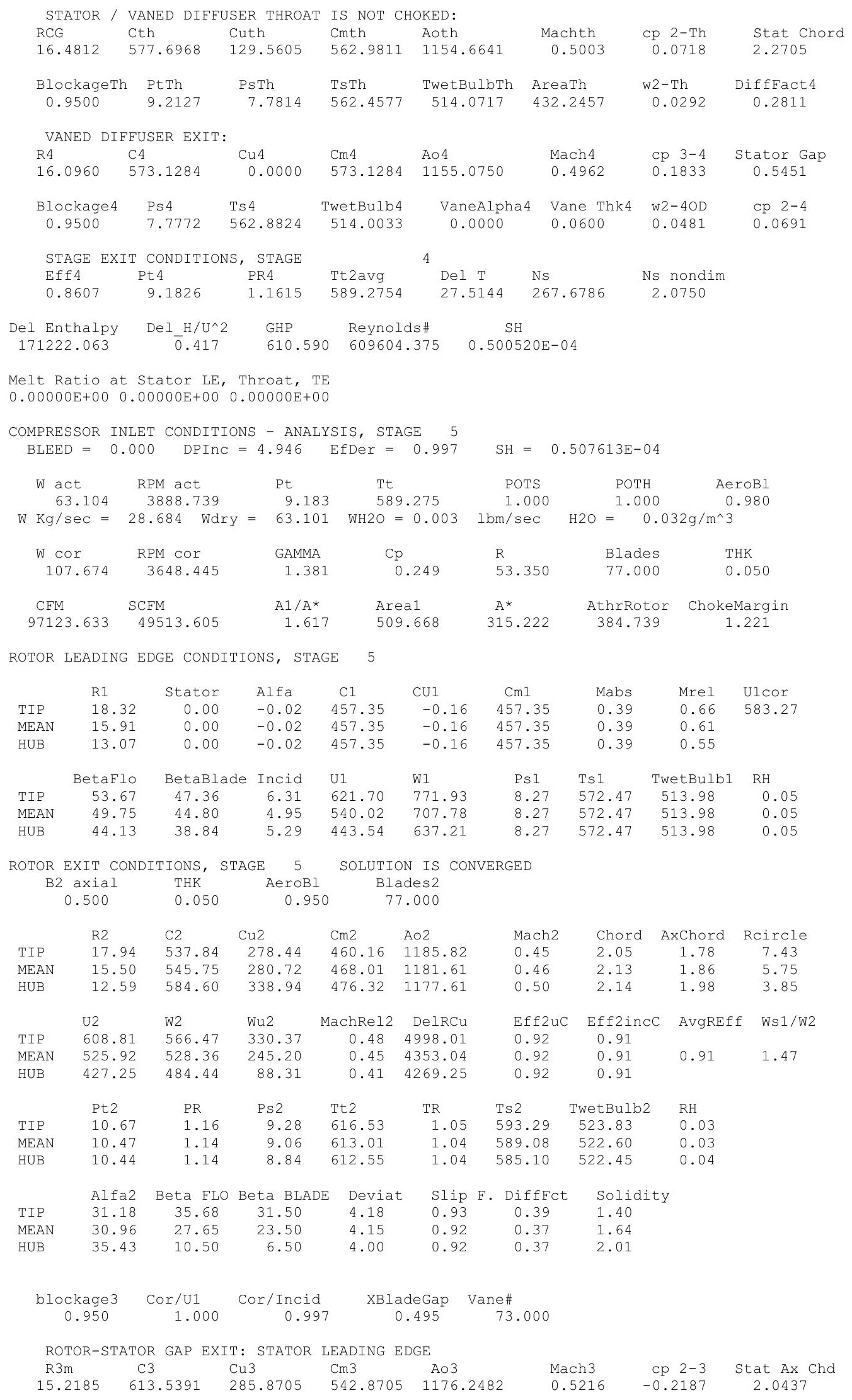




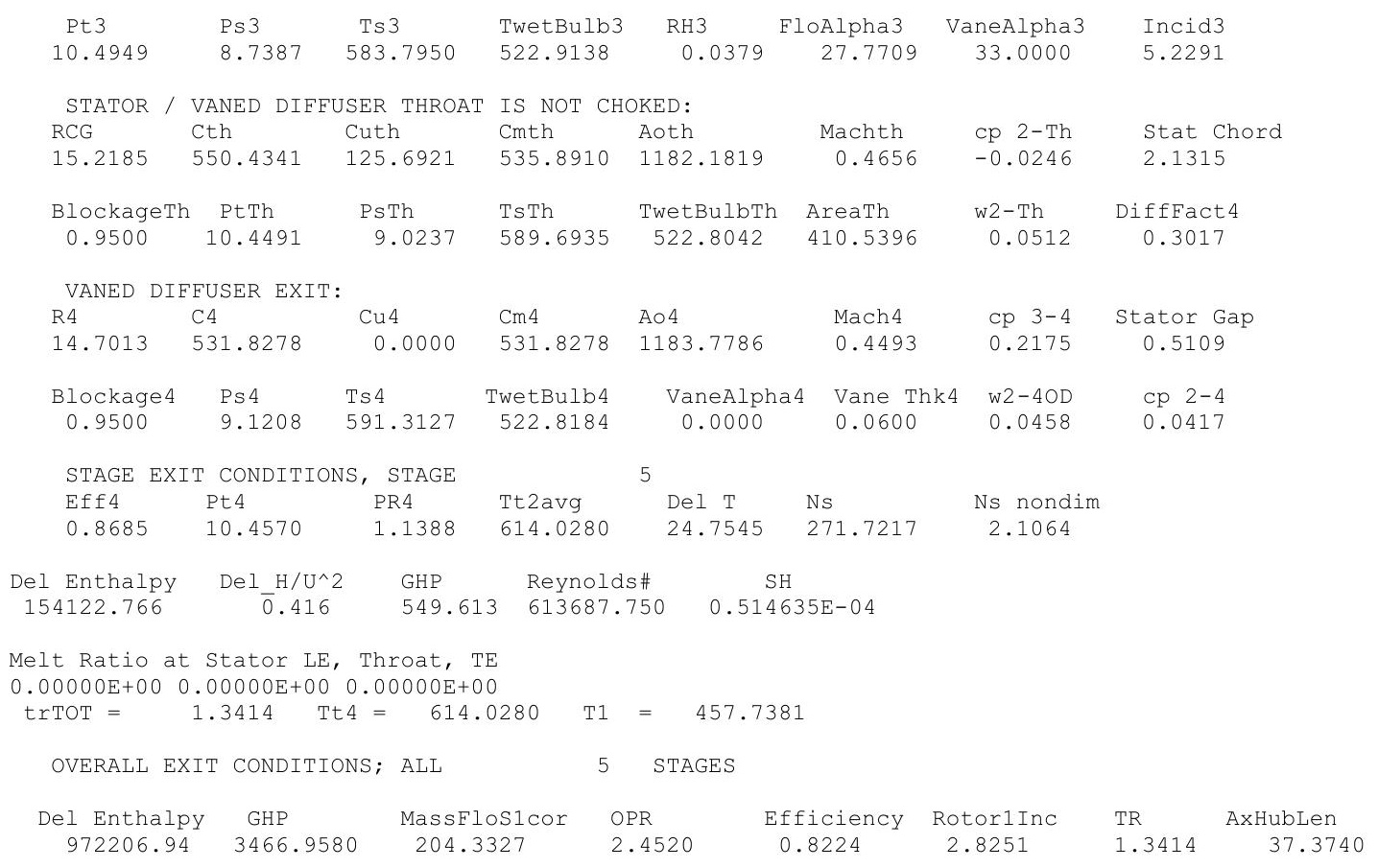




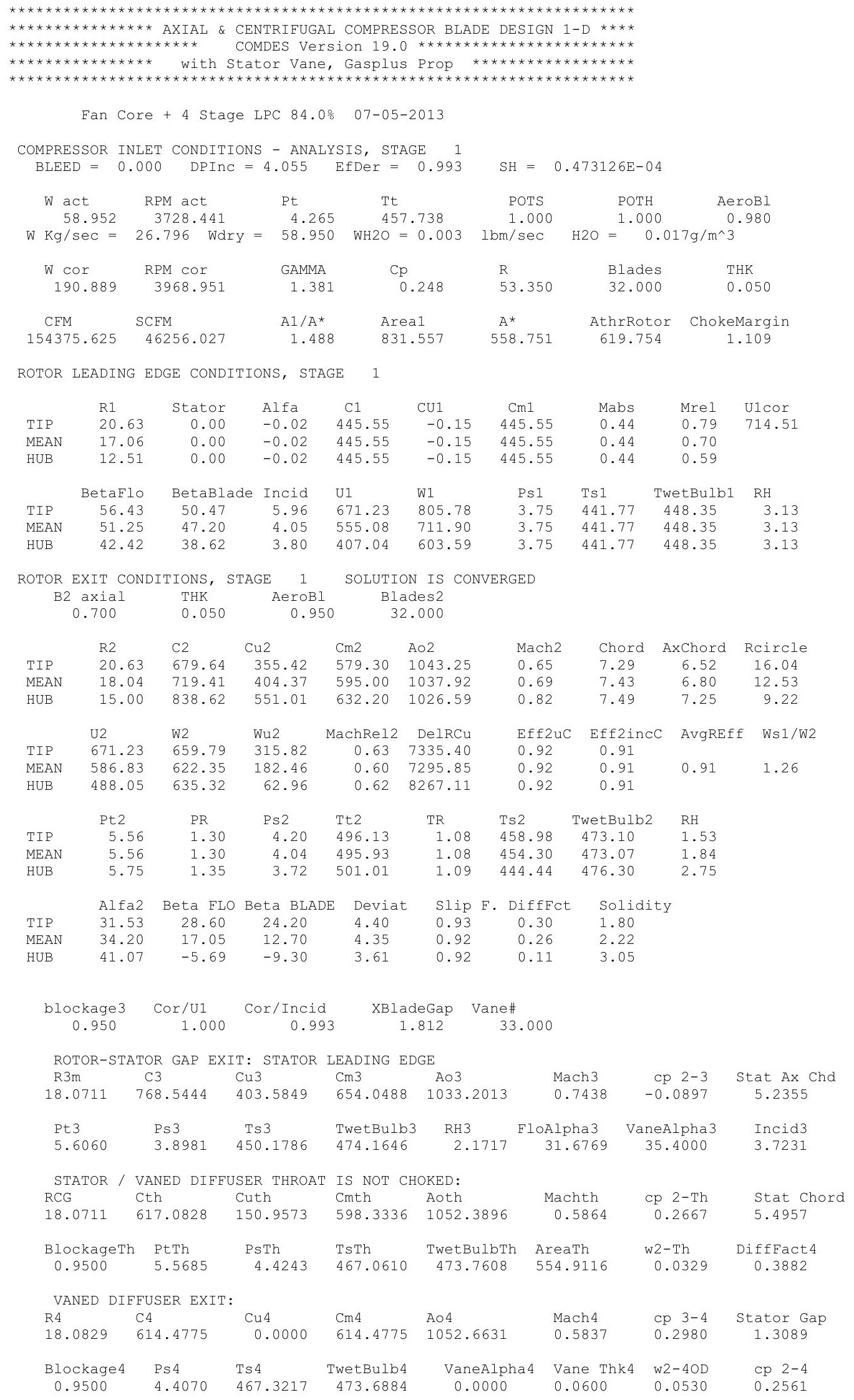




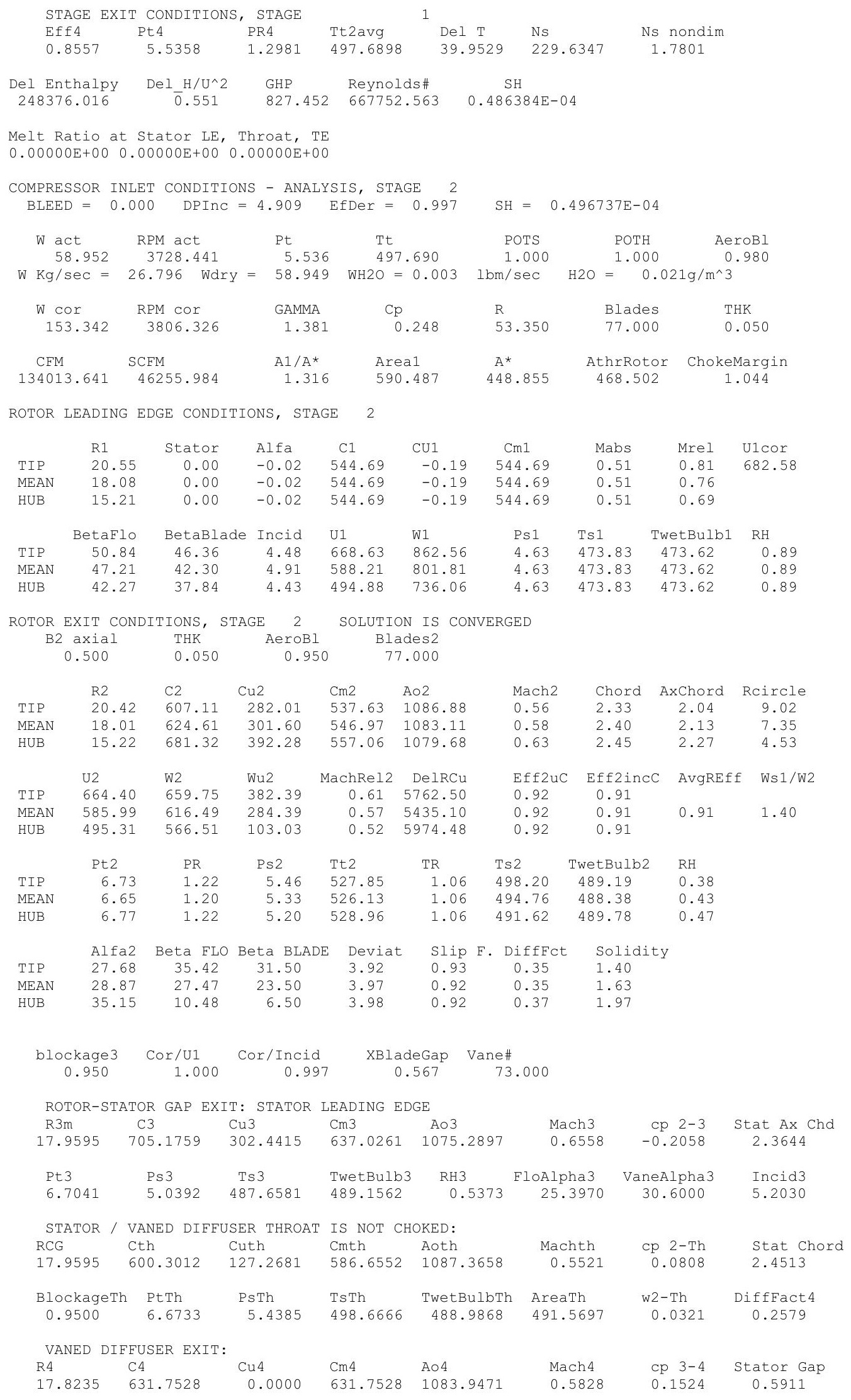




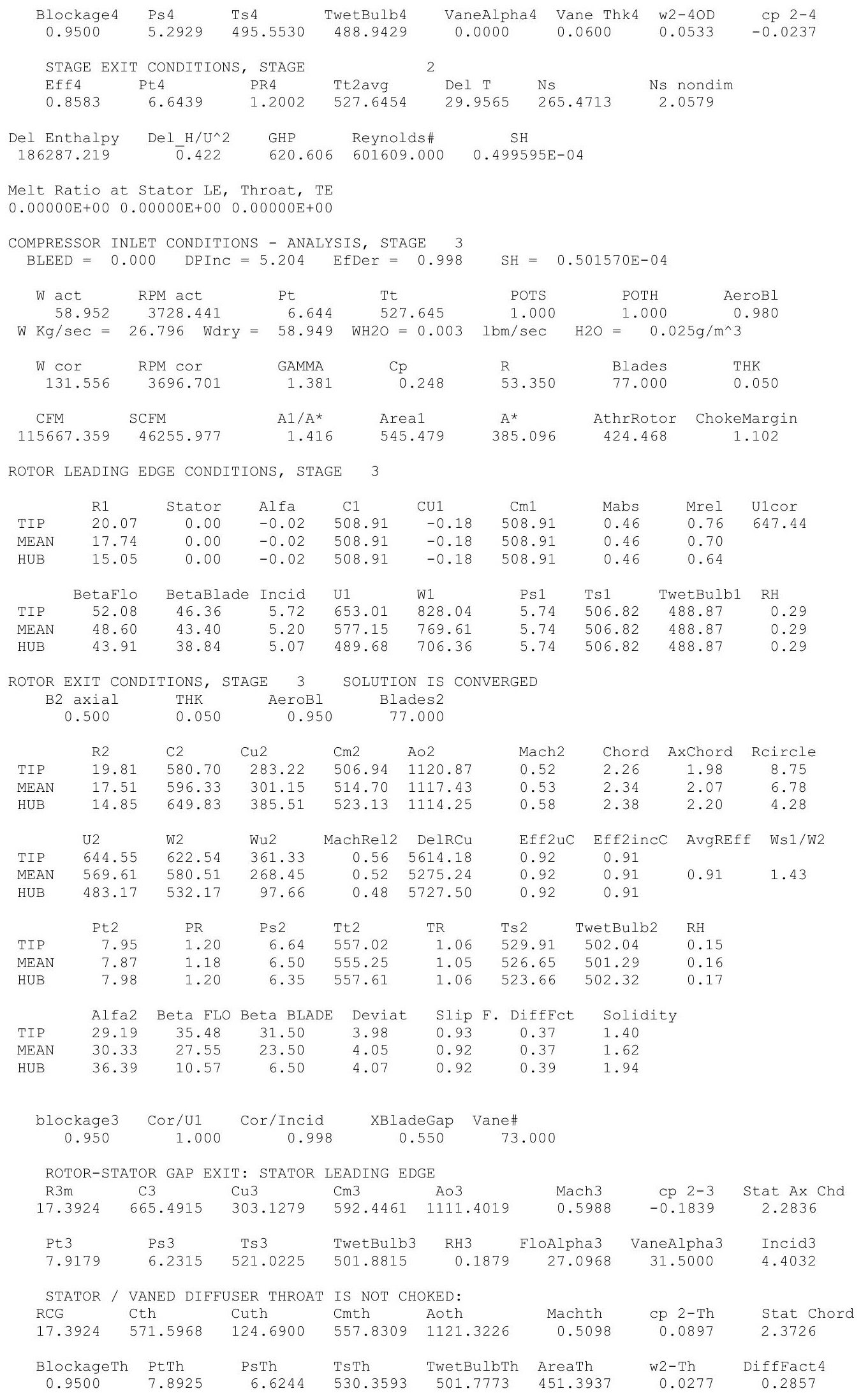




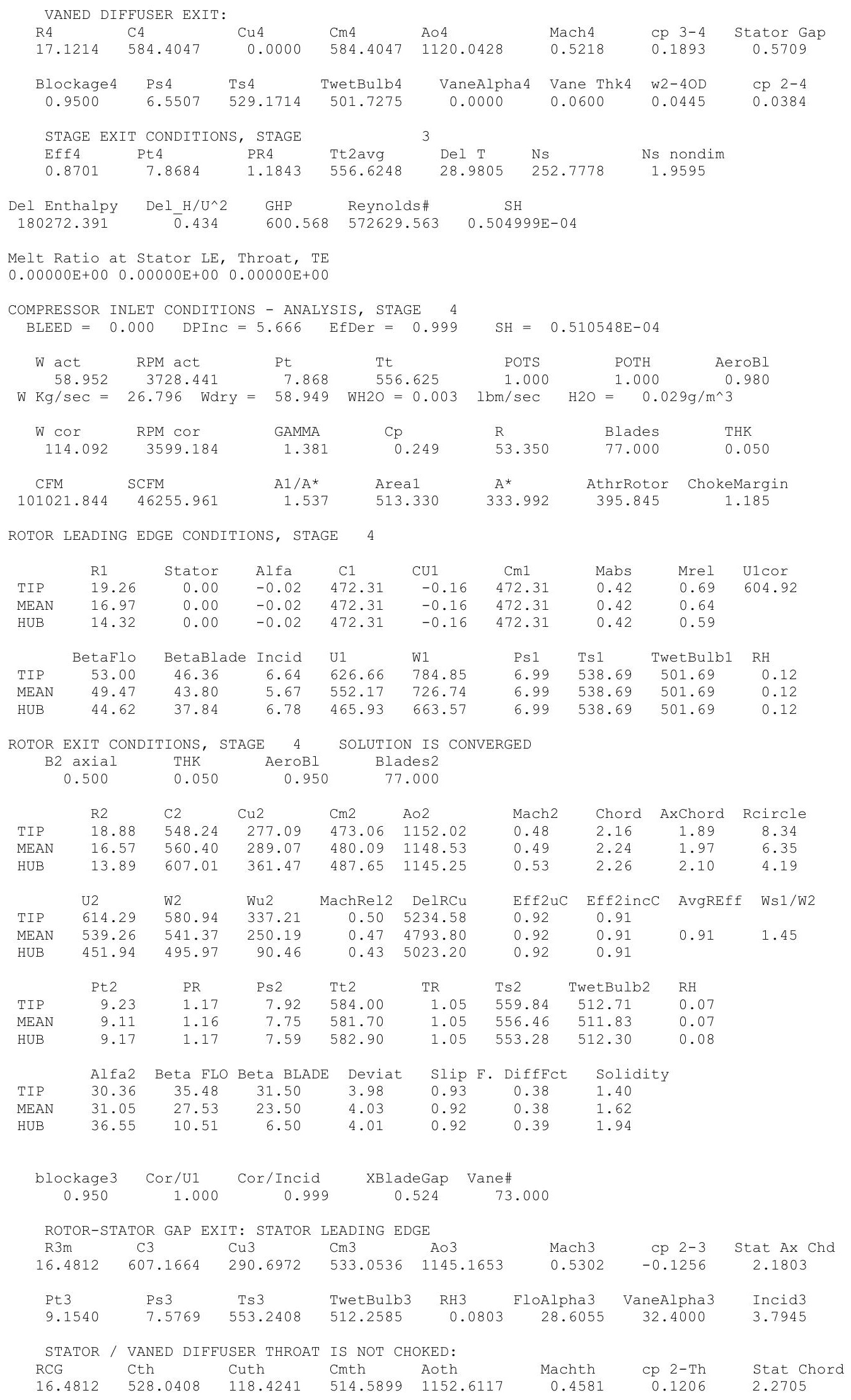




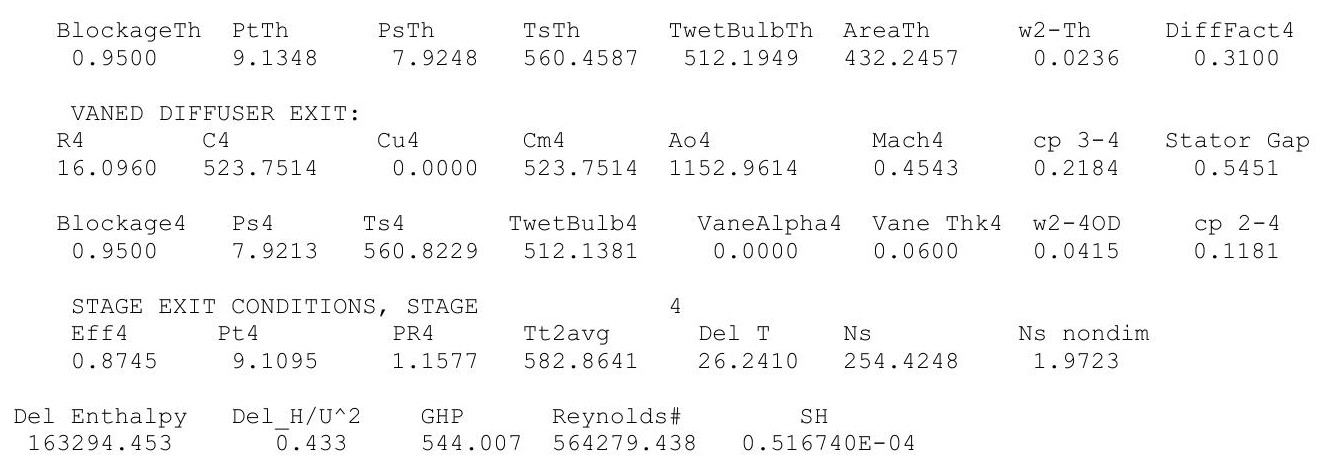

Melt Ratio at Stator LE, Throat, TE

$0.00000 \mathrm{E}+00 \quad 0.00000 \mathrm{E}+00 \quad 0.00000 \mathrm{E}+00$

COMPRESSOR INLET CONDITIONS - ANALYSIS, STAGE 5 $\mathrm{BLEED}=0.000 \quad$ DPInC $=6.067 \quad$ EfDer $=0.999$

$\begin{array}{llllll}W & \text { Ret } & \text { RPM act } & \text { Pt } & \text { POTS } & \text { POTH }\end{array}$ $\mathrm{W} \mathrm{Kg} / \mathrm{sec}=26.796$ Wdry $=58.949$ WH2O $=0.003 \quad \mathrm{lbm} / \mathrm{sec} \quad \mathrm{H} 2 \mathrm{O}=0.033 \mathrm{~g} / \mathrm{m}^{\wedge} 3$

$\begin{array}{ccccccc}\text { W cor } & \text { RPM cor } & \text { GAMMA } & \text { CP } & \text { R } & \text { Blades } & \text { THK } \\ 100.844 & 3517.238 & 1.381 & 0.249 & 53.350 & 77.000 & 0.050 \\ & & & & & & \\ \text { CFM } & \text { SCFM } & \text { A1/A* } & \text { Area1 } & \text { A* } & \text { AthrRotor } & \text { ChokeMargin } \\ 89486.250 & 46255.934 & 1.726 & 509.668 & 295.228 & 384.739 & 1.303\end{array}$

ROTOR LEADING EDGE CONDITIONS, STAGE 5

\begin{tabular}{|c|c|c|c|c|c|c|c|c|c|}
\hline & R1 & Stator & Alfa & $\mathrm{C} 1$ & CU1 & $\mathrm{Cm} 1$ & Mabs & Mrel & U1cor \\
\hline TIP & 18.32 & 0.00 & -0.02 & 421.39 & -0.15 & 421.39 & 0.36 & 0.63 & 562.29 \\
\hline MEAN & 15.91 & 0.00 & -0.02 & 421.39 & -0.15 & 421.39 & 0.36 & 0.58 & \\
\hline HUB & 13.07 & 0.00 & -0.02 & 421.39 & -0.15 & 421.39 & 0.36 & 0.52 & \\
\hline & BetaFlo & BetaBlade & Incid & U1 & W1 & Ps 1 & Ts1 & TwetBulb1 & $\mathrm{RH}$ \\
\hline TIP & 54.75 & 47.36 & 7.39 & 596.07 & 730.10 & 8.33 & 568.60 & 512.12 & 0.06 \\
\hline MEAN & 50.87 & 44.80 & 6.07 & 517.76 & 667.67 & 8.33 & 568.60 & 512.12 & 0.06 \\
\hline HUB & 45.27 & 38.84 & 6.43 & 425.26 & 598.78 & 8.33 & 568.60 & 512.12 & 0.06 \\
\hline
\end{tabular}

ROTOR EXIT CONDITIONS, STAGE 5 SOLUTION IS CONVERGED

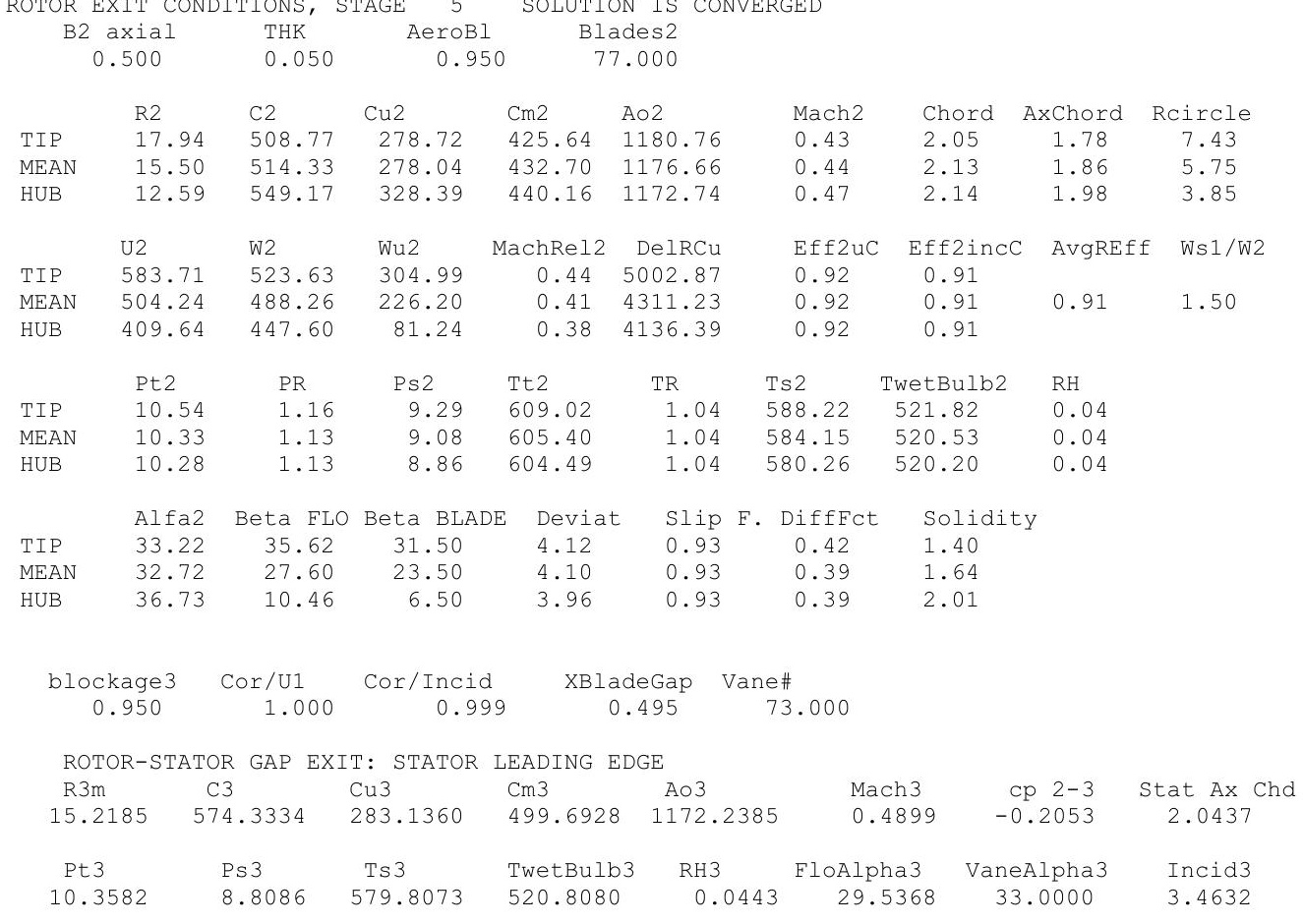




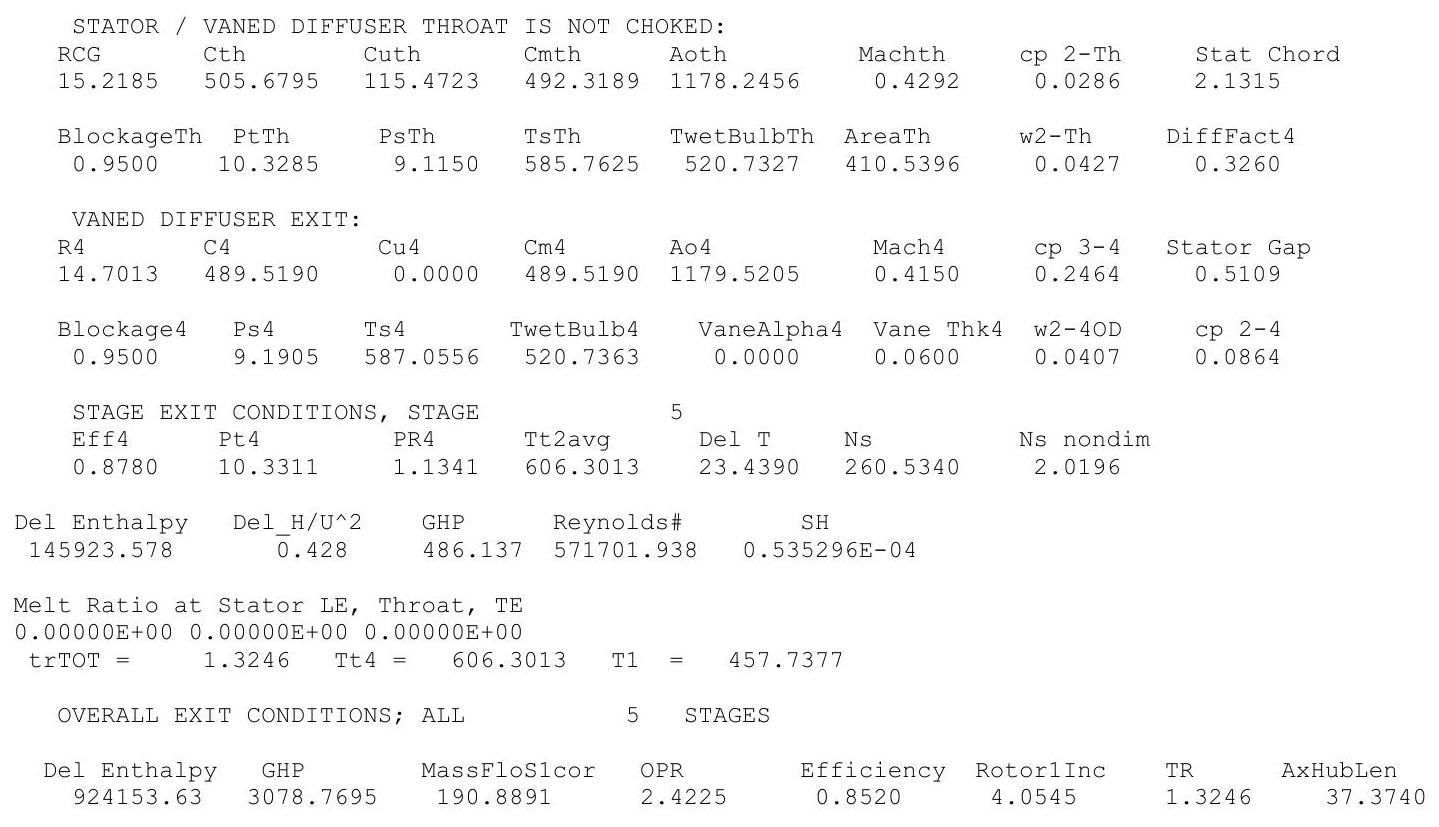




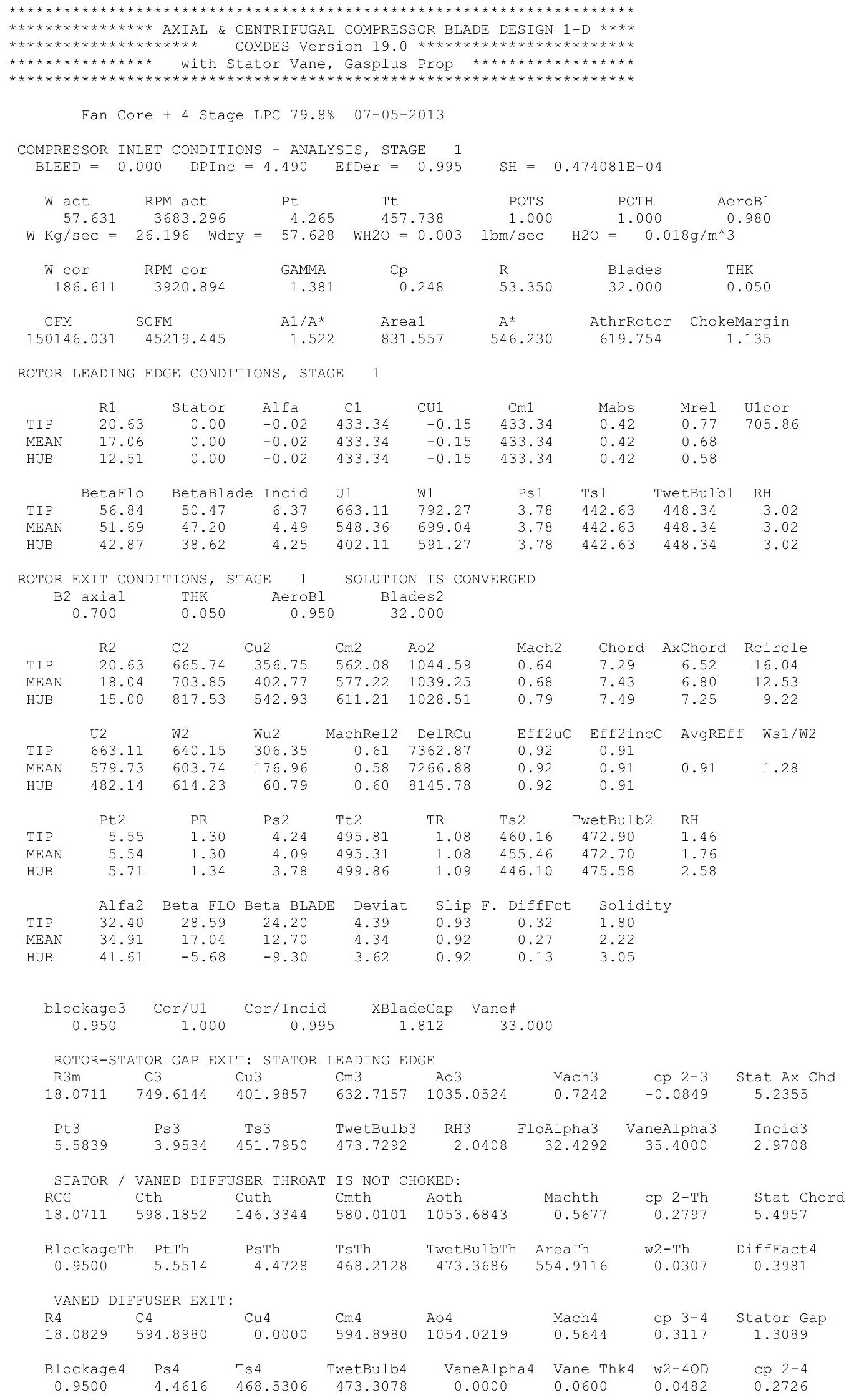




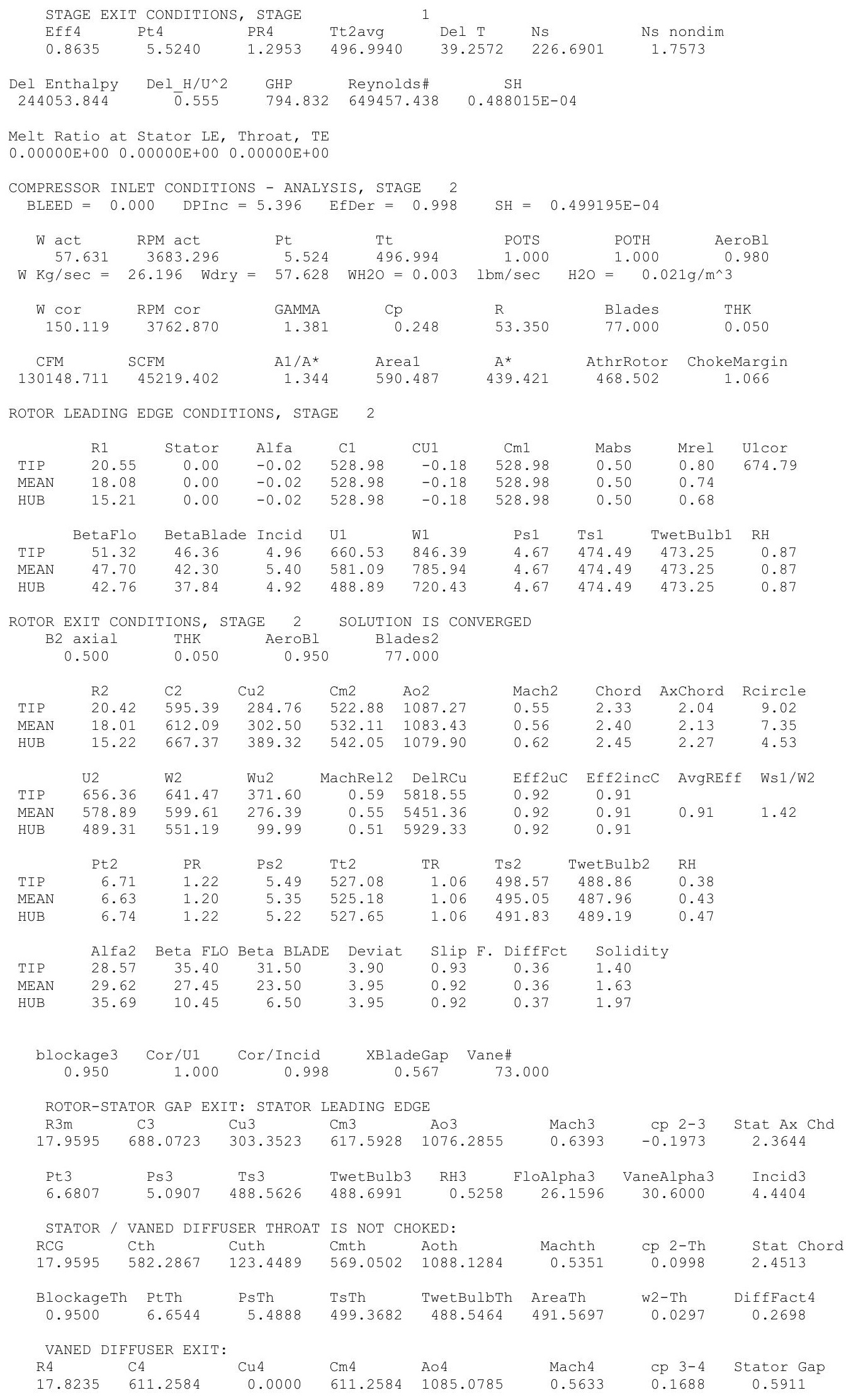




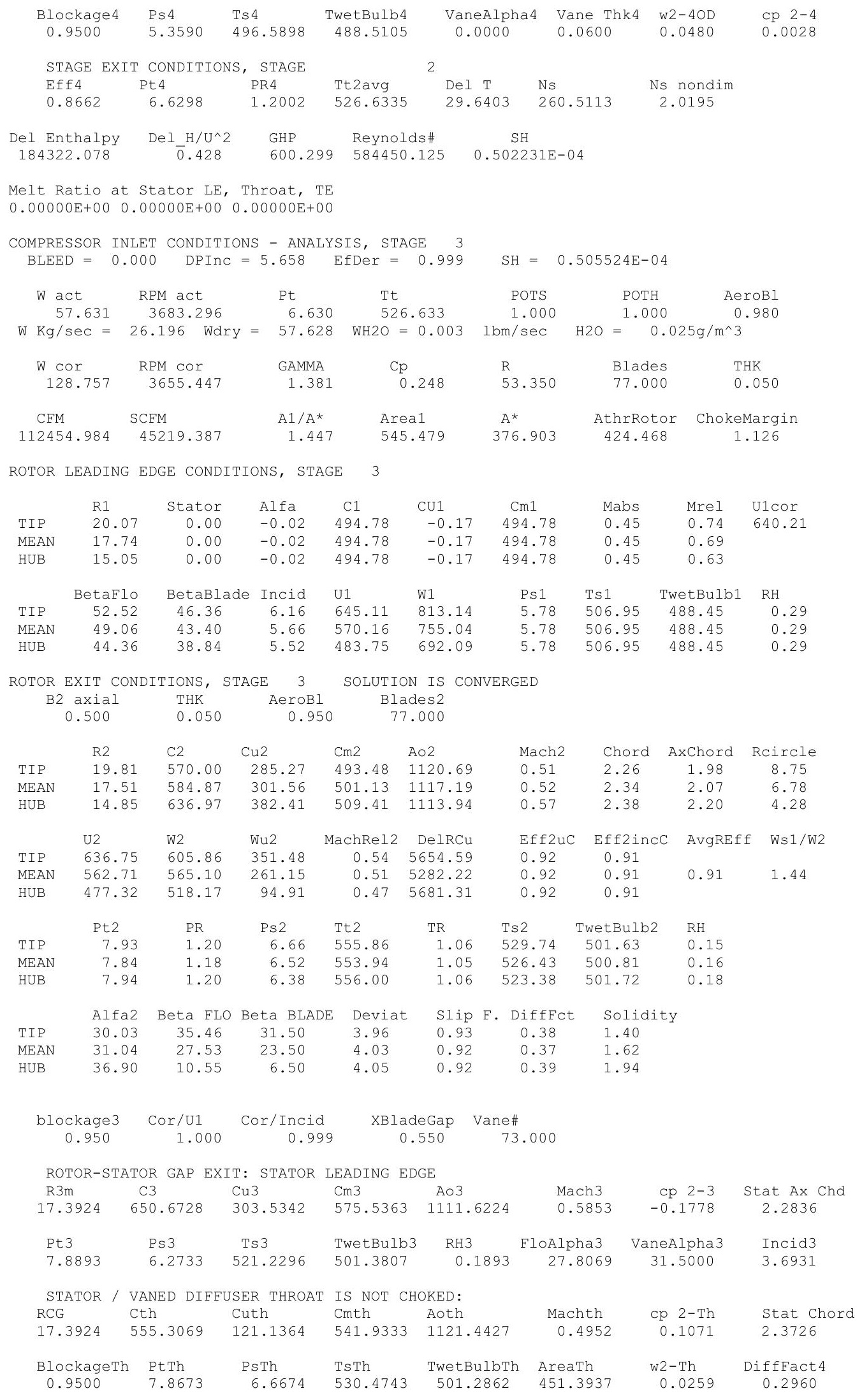




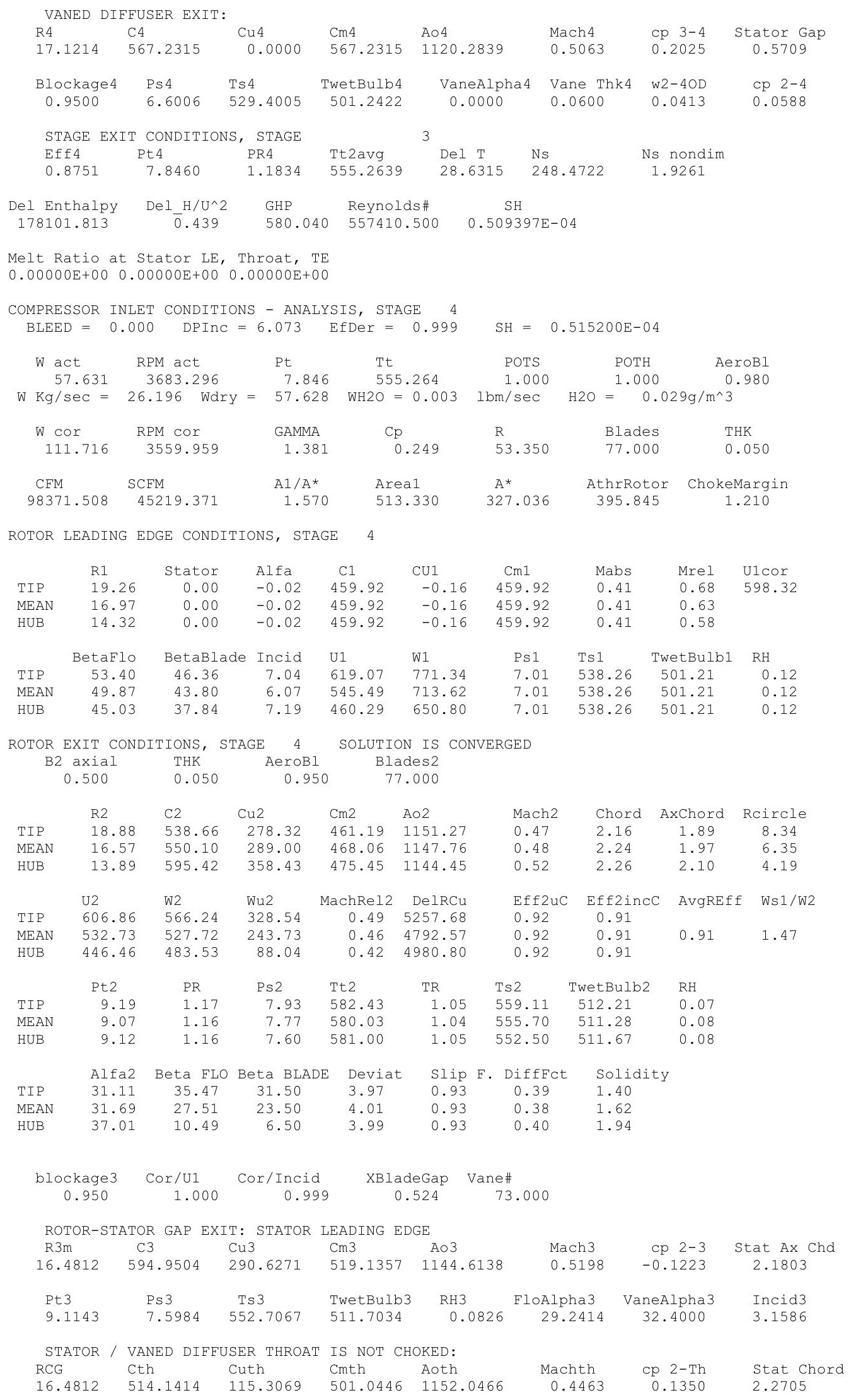




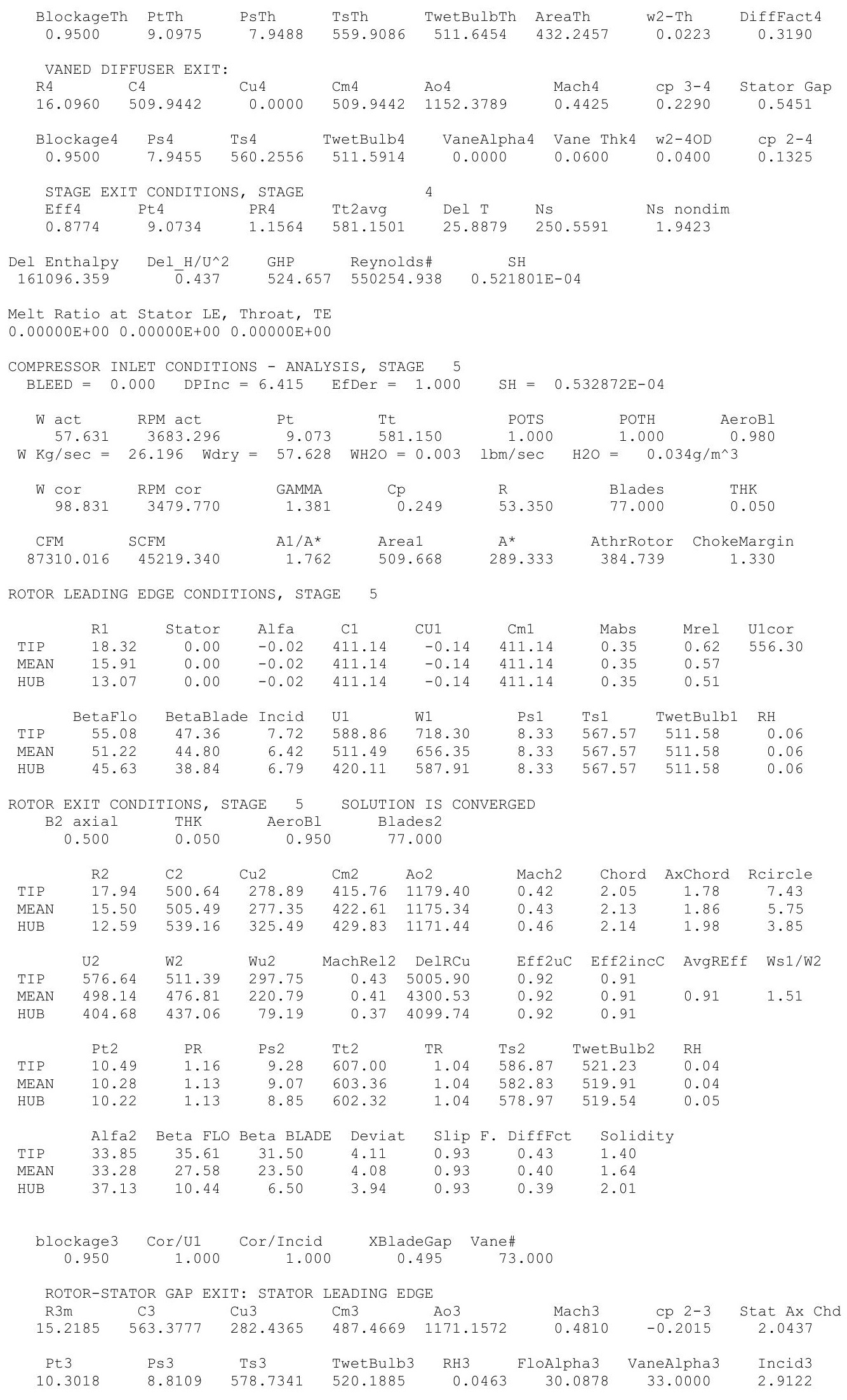




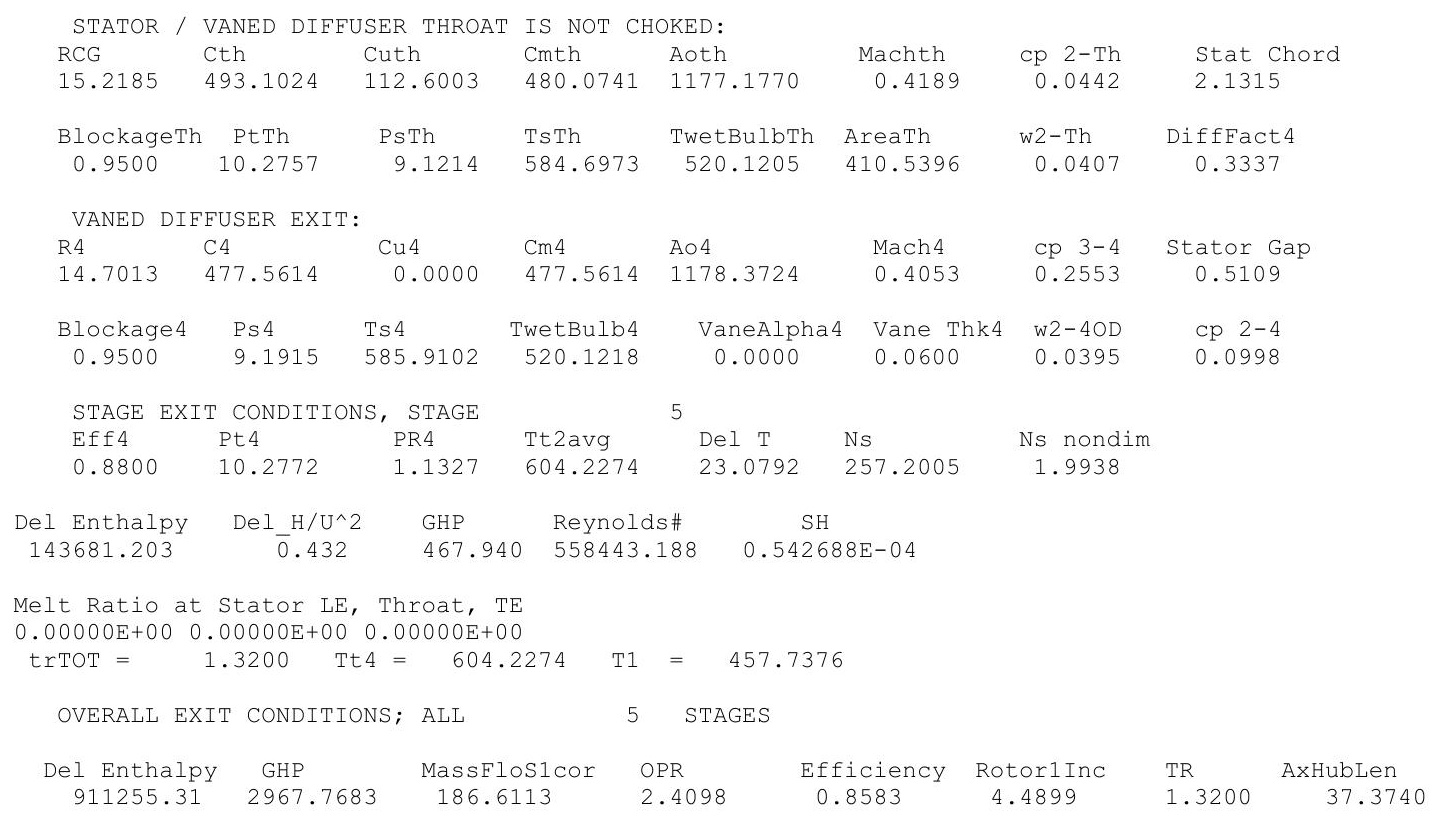




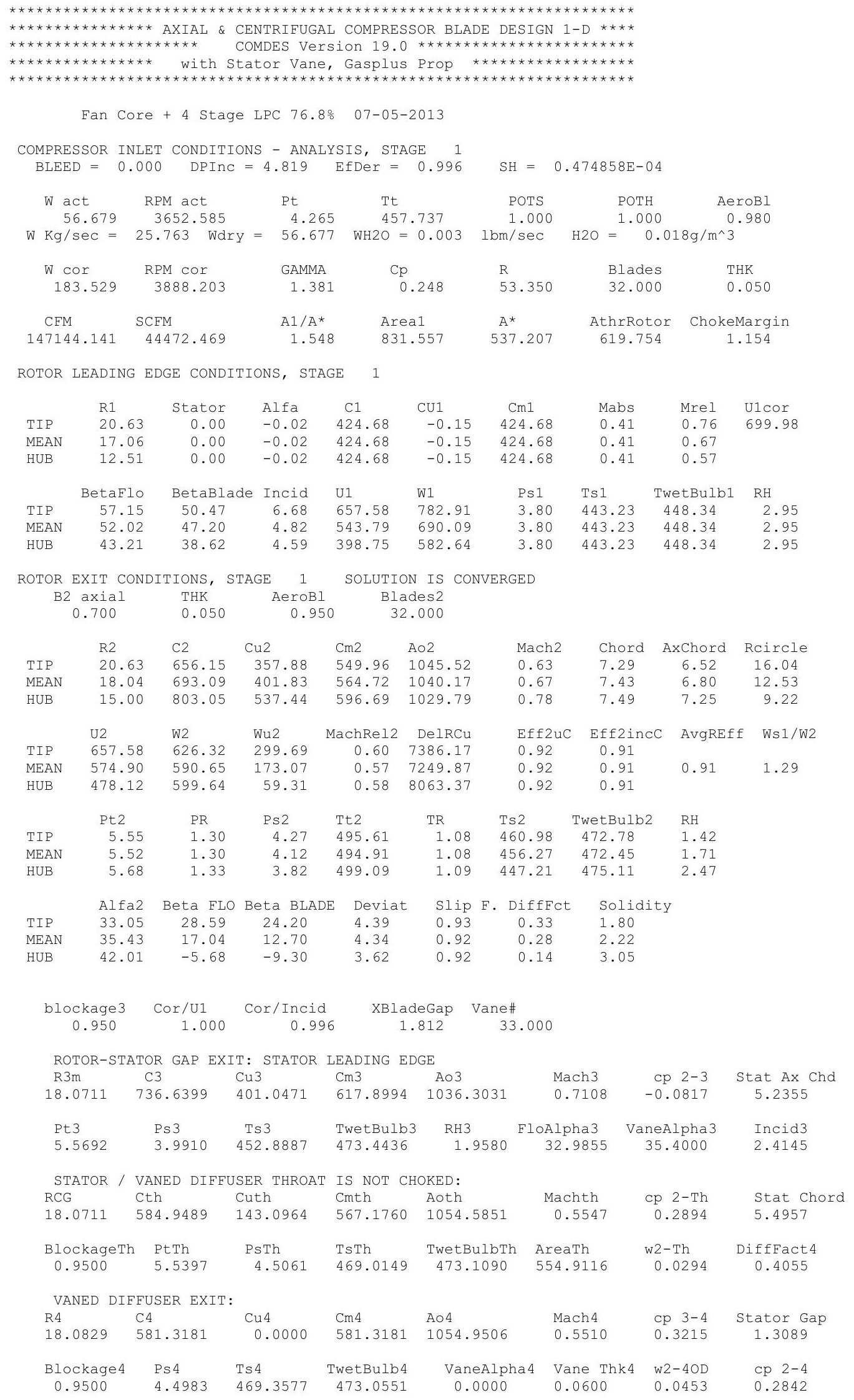




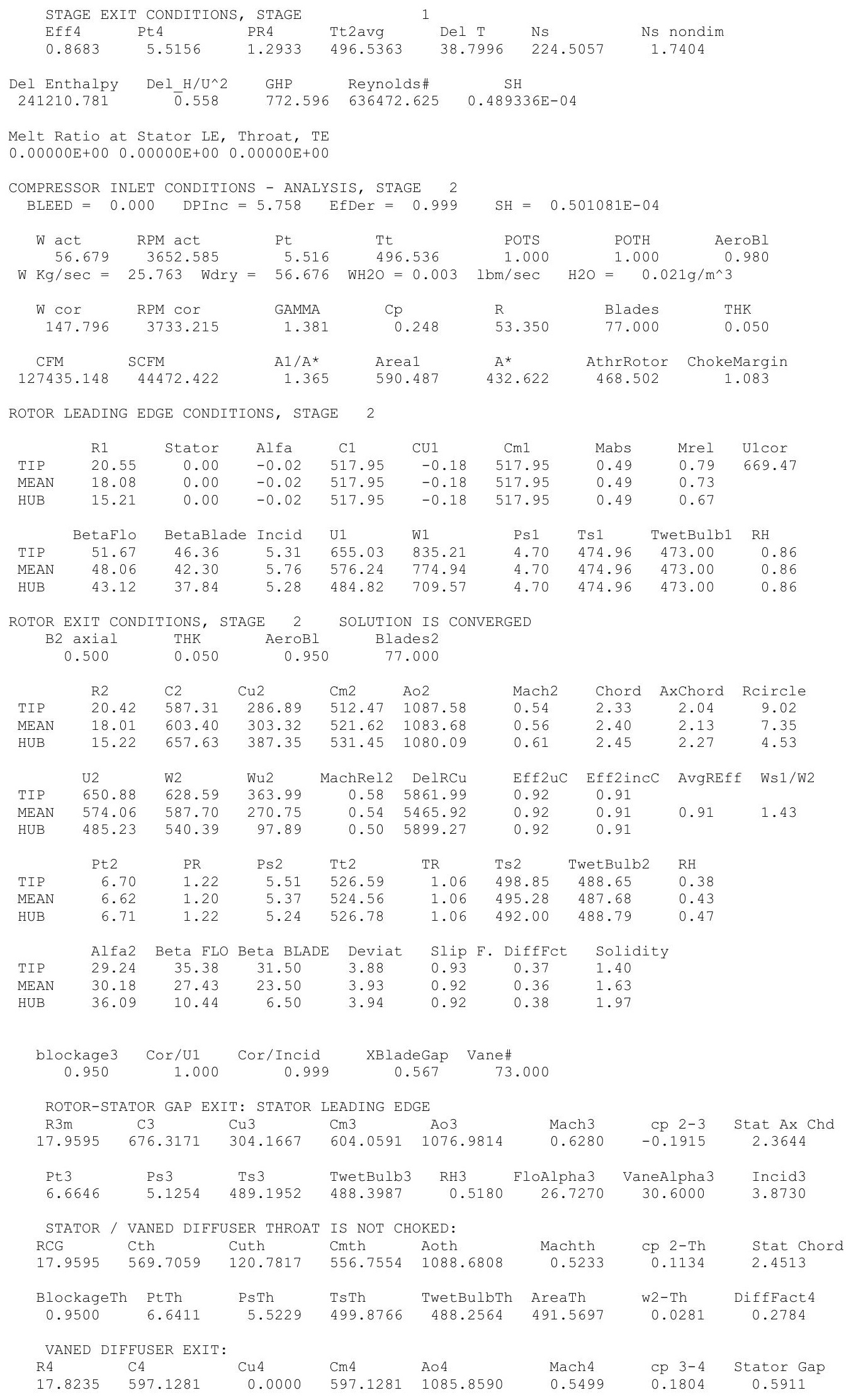




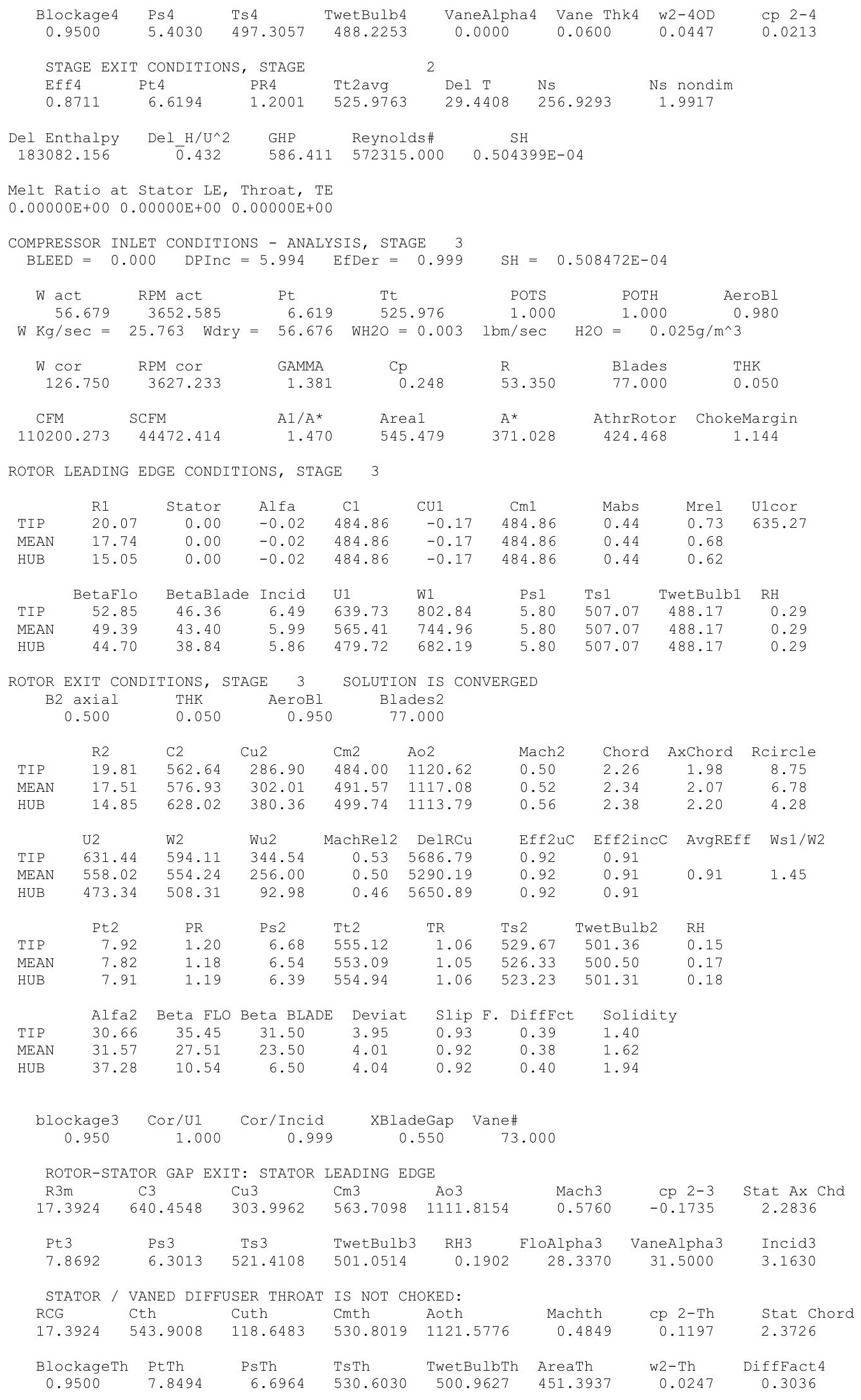




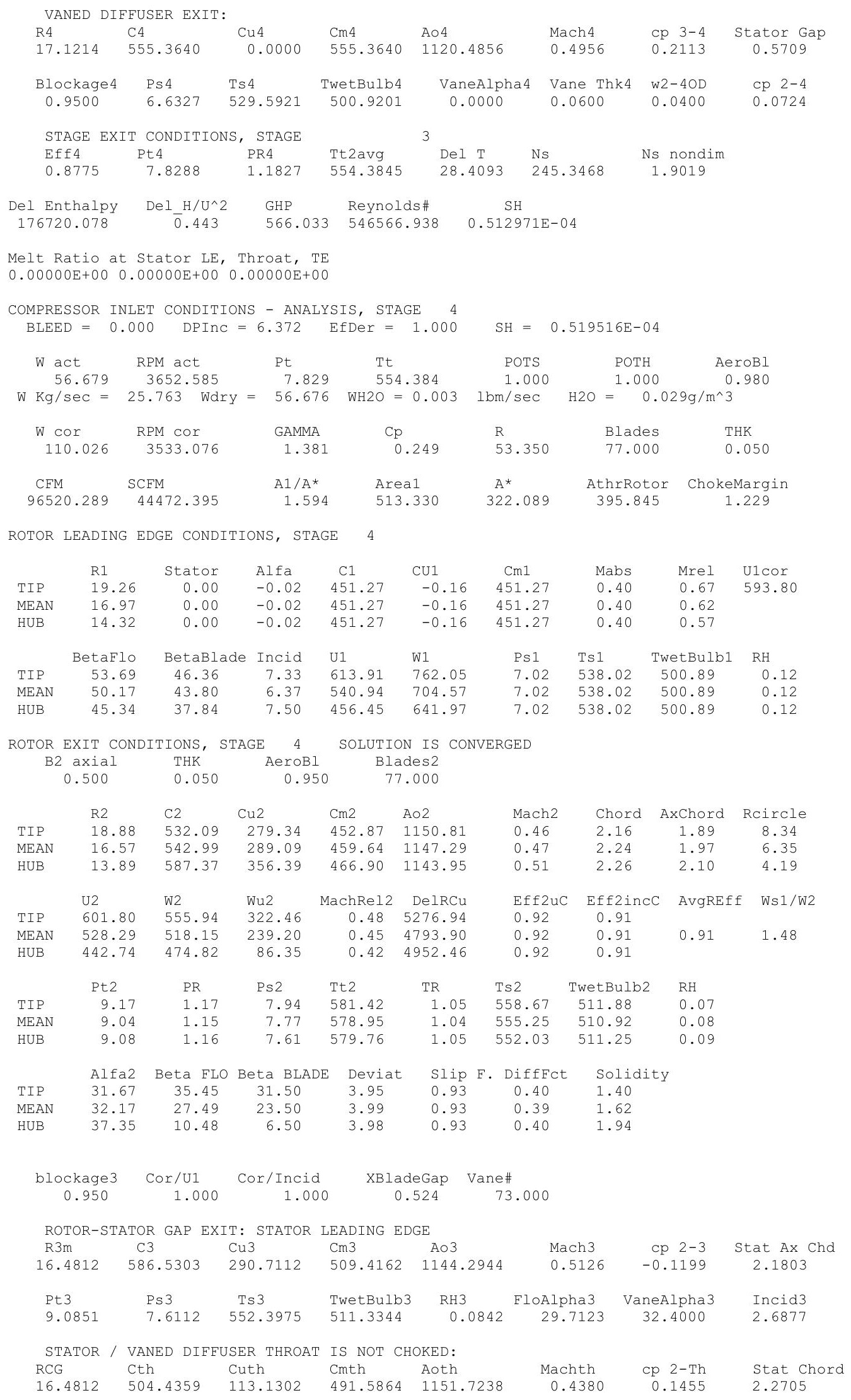




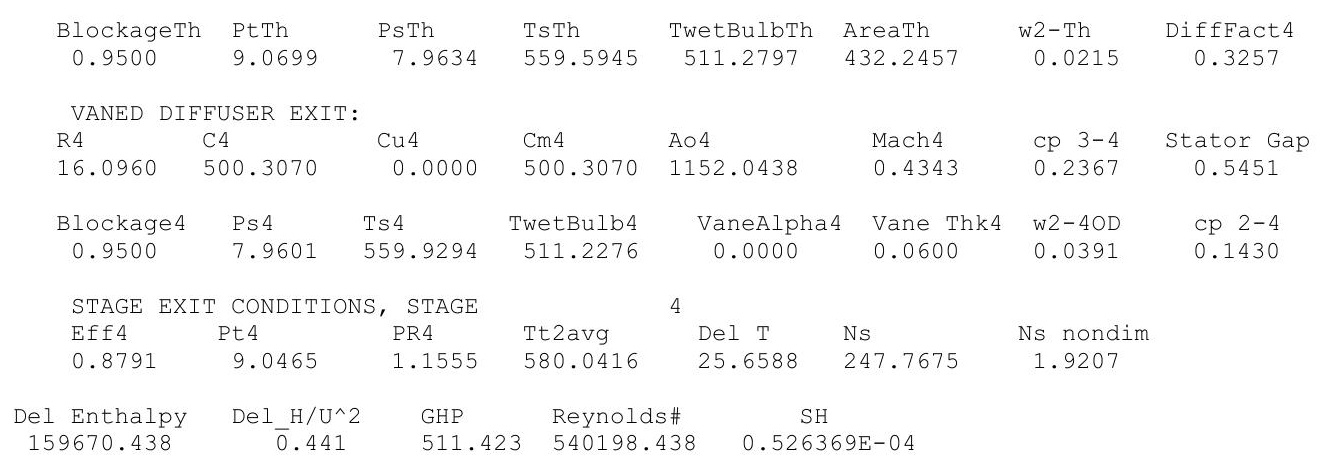

Melt Ratio at Stator LE, Throat, TE

$0.00000 \mathrm{E}+00 \quad 0.00000 \mathrm{E}+00 \quad 0.00000 \mathrm{E}+00$

COMPRESSOR INLET CONDITIONS - ANALYSIS, STAGE 5 BLEED $=0.000 \quad$ DPInC $=6.675 \quad$ EfDer $=1.000$

ROTOR LEADING EDGE CONDITIONS, STAGE 5

$\begin{array}{lccccccccc} & \text { R1 } & \text { Stator } & \text { Alfa } & \text { C1 } & \text { CU1 } & \text { Cm1 } & \text { Mabs } & \text { Mrel } & \text { U1cor } \\ \text { TIP } & 18.32 & 0.00 & -0.02 & 403.94 & -0.14 & 403.94 & 0.35 & 0.61 & 552.19 \\ \text { MEAN } & 15.91 & 0.00 & -0.02 & 403.94 & -0.14 & 403.94 & 0.35 & 0.56 & \\ \text { HUB } & 13.07 & 0.00 & -0.02 & 403.94 & -0.14 & 403.94 & 0.35 & 0.50 & \\ & & & & & & & & & \\ \text { TIP } & \text { BetaFlo } & \text { BetaBlade } & \text { Incid } & \text { U1 } & \text { W1 } & \text { Ps1 } & \text { Ts1 } & \text { TwetBulb1 } & \text { RH } \\ \text { MEAN } & 51.33 & 47.36 & 7.97 & 583.95 & 710.16 & 8.33 & 566.93 & 511.21 & 0.06 \\ \text { HUB } & 45.89 & 44.80 & 6.67 & 507.22 & 648.53 & 8.33 & 566.93 & 511.21 & 0.06 \\ & & 38.84 & 7.05 & 416.60 & 580.38 & 8.33 & 566.93 & 511.21 & 0.06\end{array}$

ROTOR EXIT CONDITIONS, STAGE 5 SOLUTION IS CONVERGED

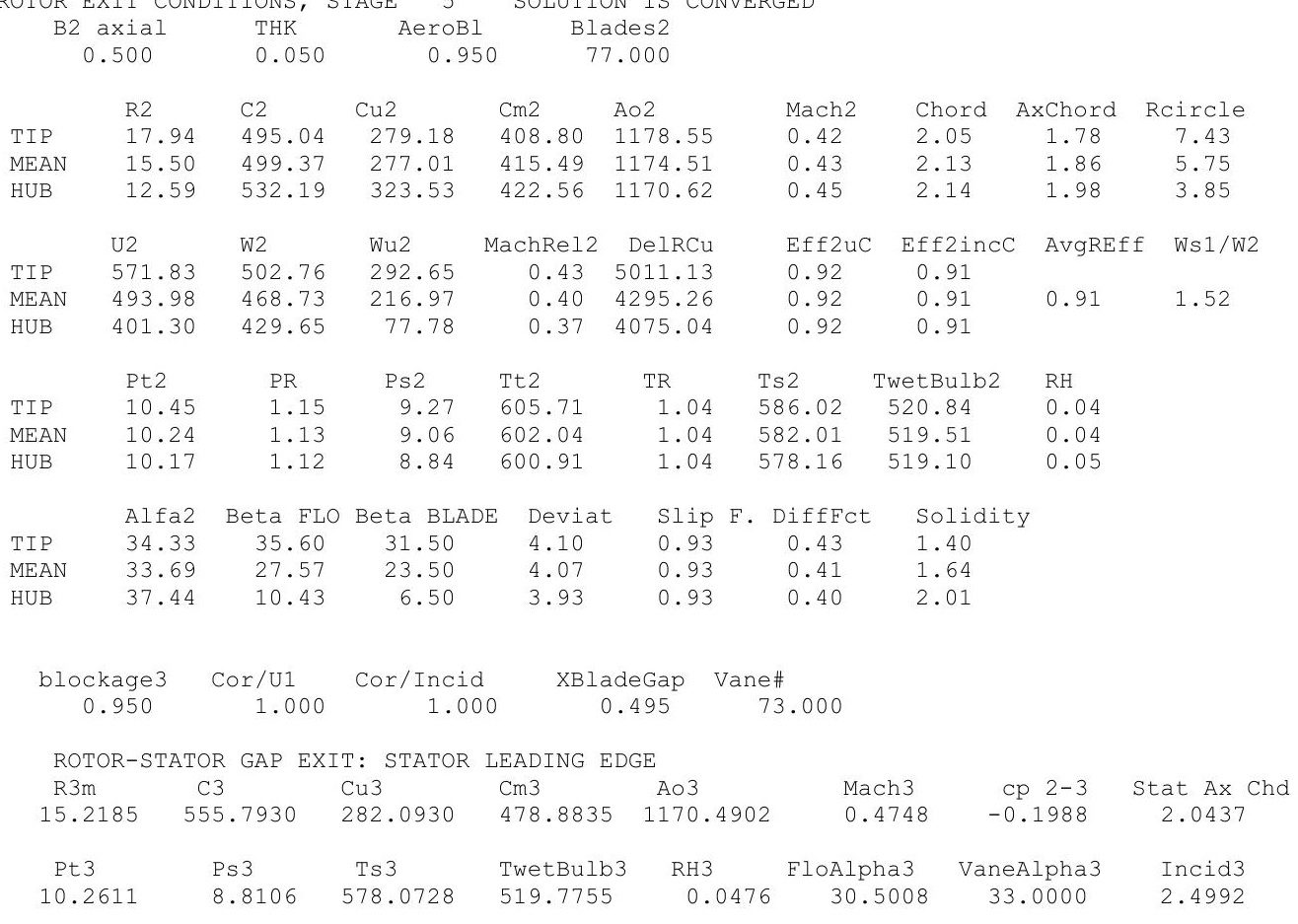




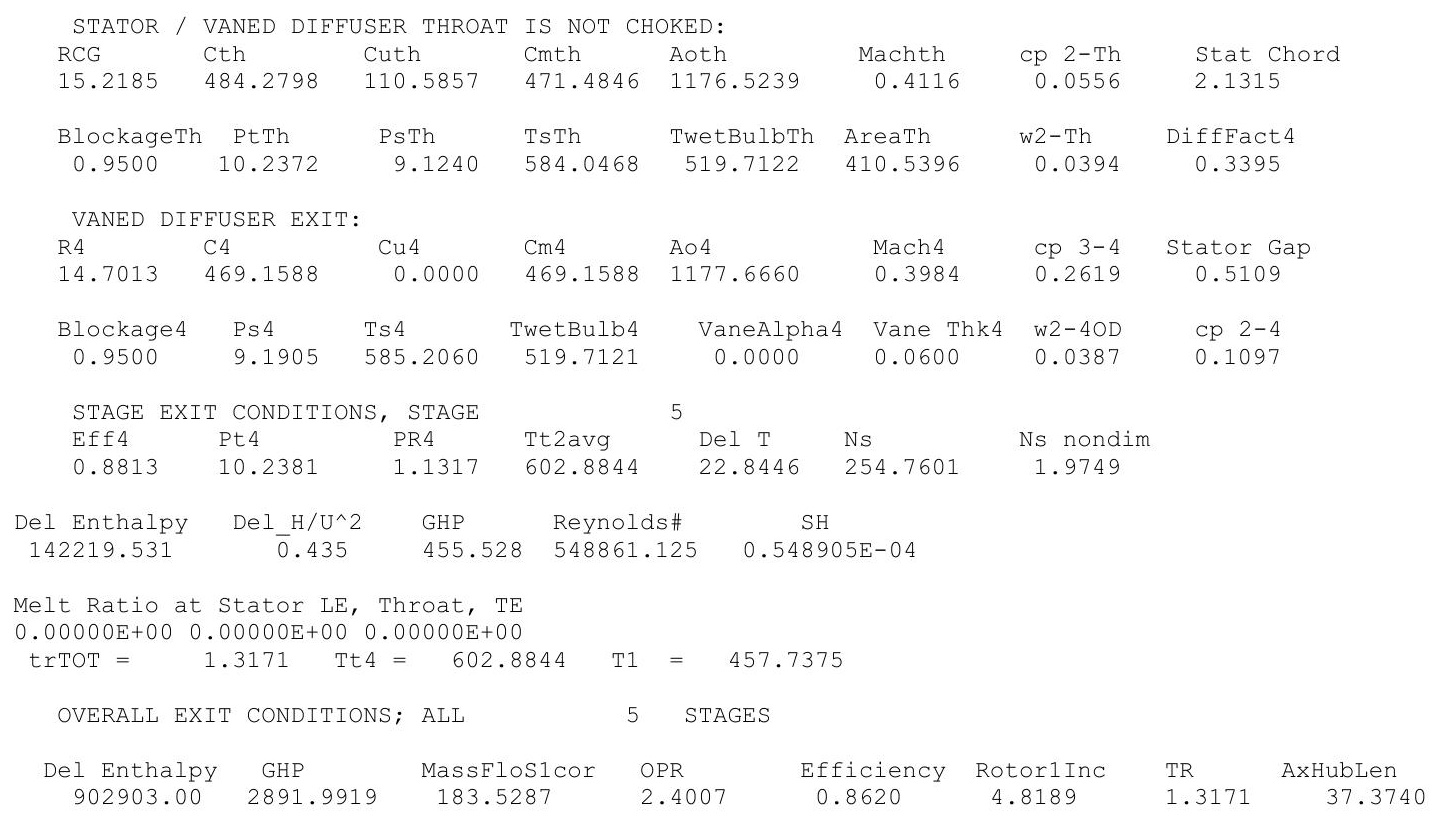


$50 \mu \mathrm{m}$, ISA $+36 \mathrm{R}$

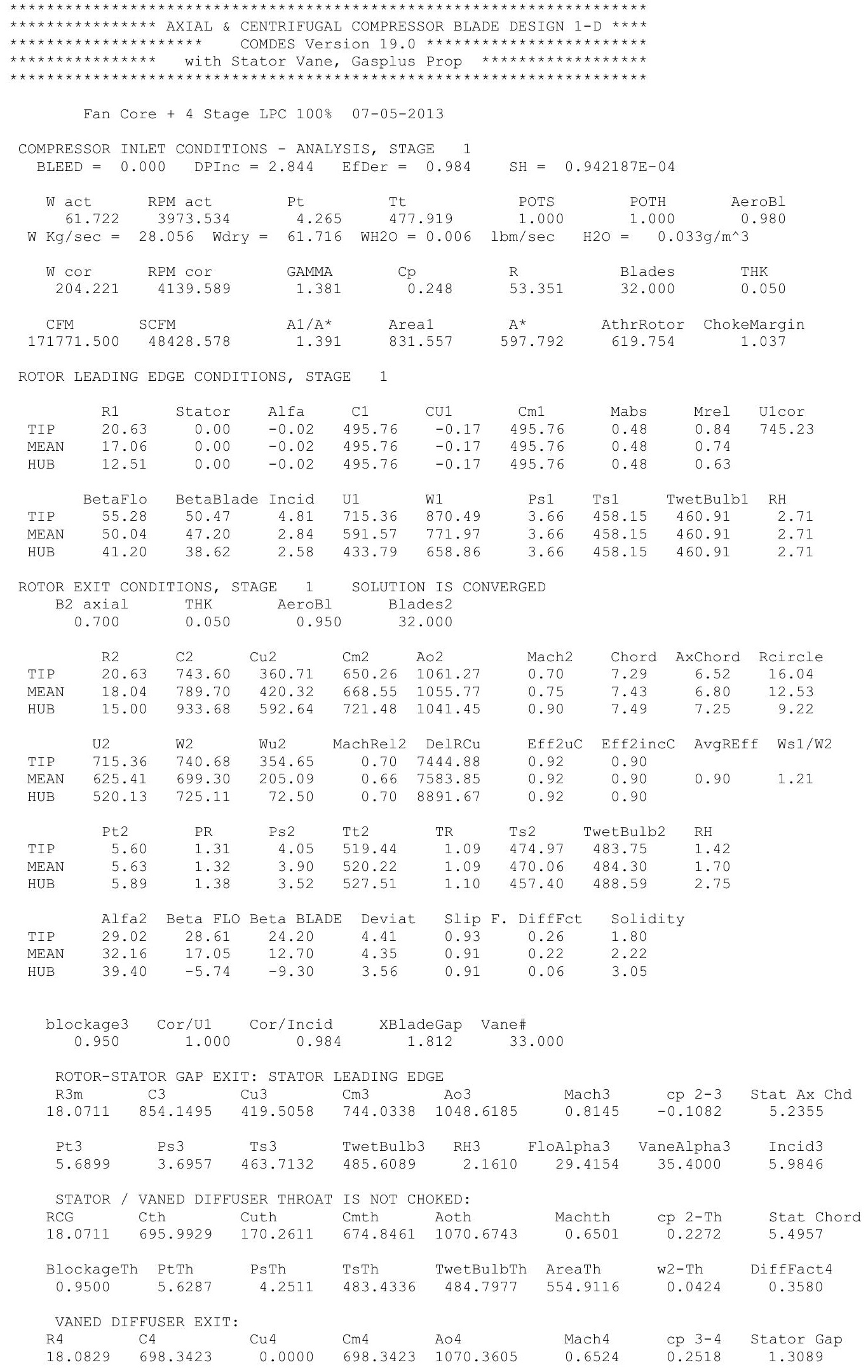




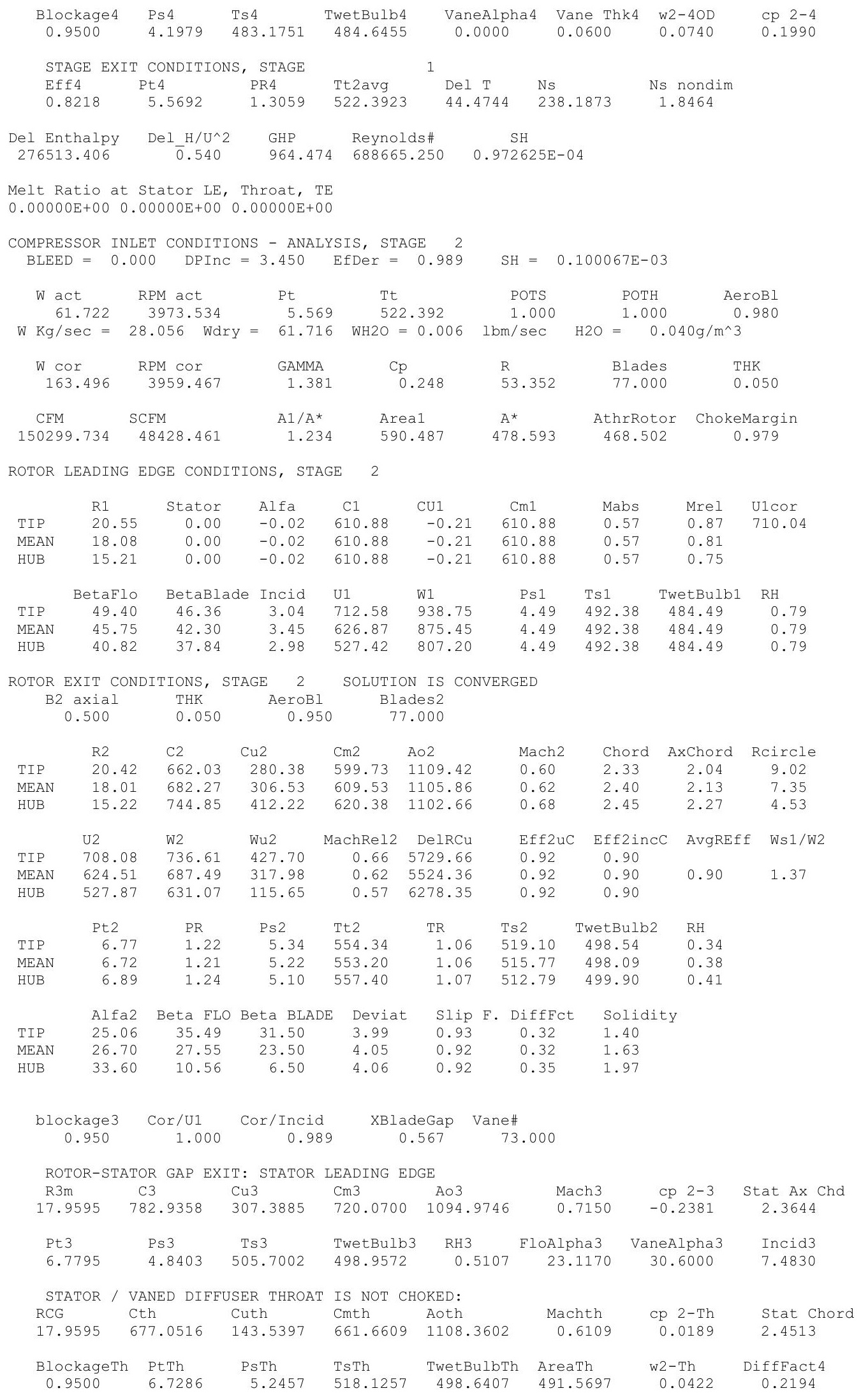




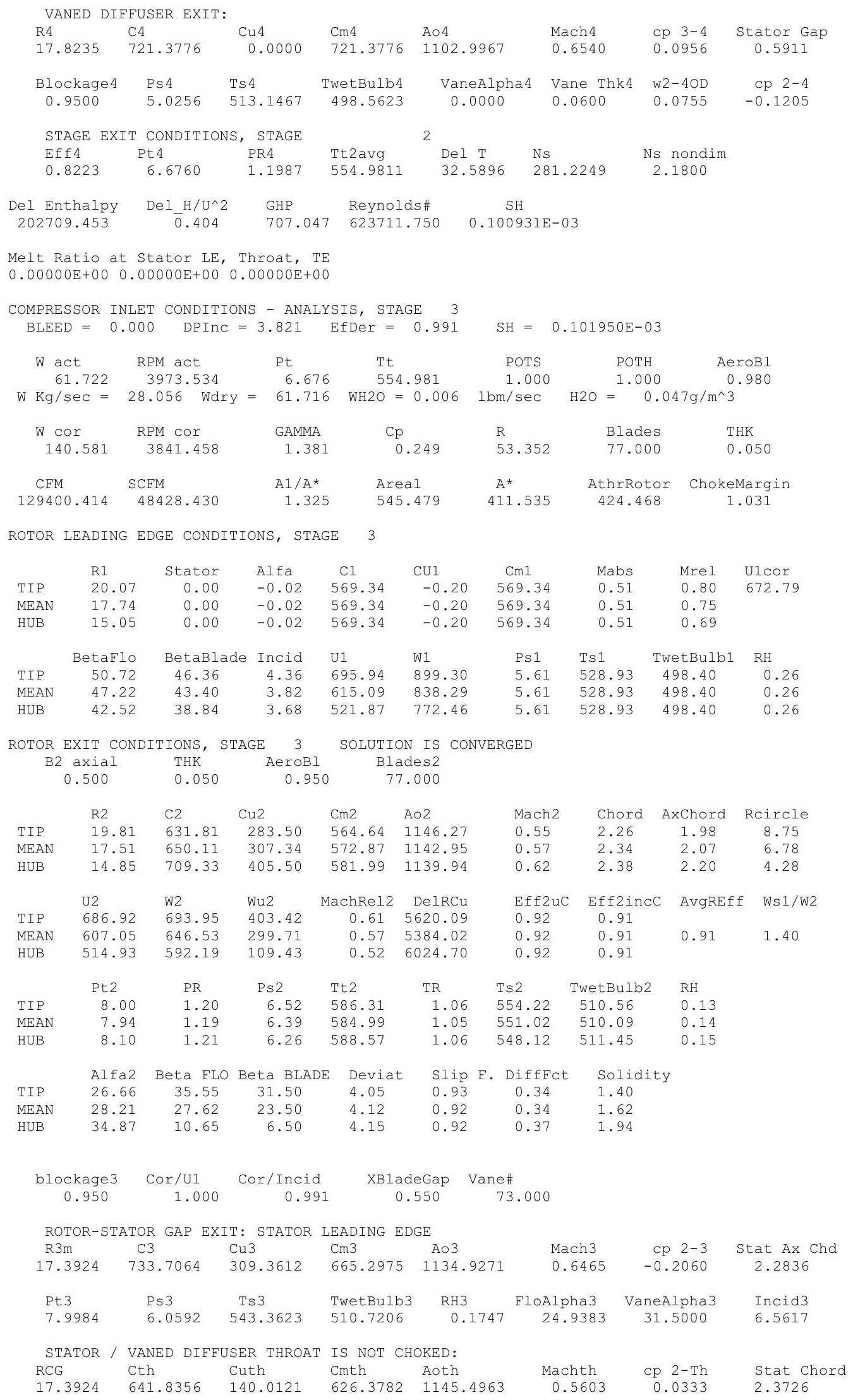




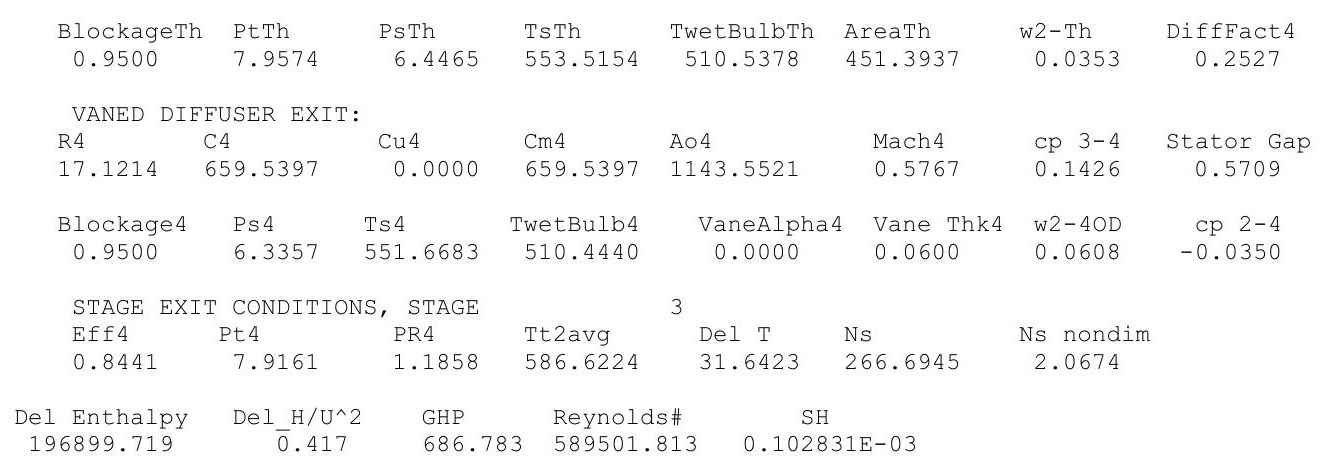

Melt Ratio at Stator LE, Throat, TE

$0.00000 \mathrm{E}+00 \quad 0.00000 \mathrm{E}+00 \quad 0.00000 \mathrm{E}+00$

COMPRESSOR INLET CONDITIONS - ANALYSIS, STAGE 4 BLEED $=0.000 \quad$ DPInC $=4.409 \quad$ EfDer $=0.995$

$\mathrm{SH}=0.104054 \mathrm{E}-03$

$\begin{array}{llllll}\text { W act } & \text { RPM act } & \text { Pt } & \text { POTS } & \text { POTH } & \text { AeroBI }\end{array}$

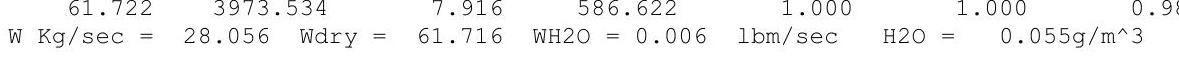

\begin{tabular}{|c|c|c|c|c|c|c|}
\hline $\begin{array}{l}\text { W cor } \\
121.891\end{array}$ & $\begin{array}{l}\text { RPM cor } \\
3736.421\end{array}$ & $\begin{array}{l}\text { GAMMA } \\
1.381\end{array}$ & $\begin{array}{l}\mathrm{Cp} \\
0.249\end{array}$ & $\begin{array}{l}R \\
53.352\end{array}$ & $\begin{array}{l}\text { Blades } \\
77.000\end{array}$ & $\begin{array}{l}\text { THK } \\
0.050\end{array}$ \\
\hline $\begin{array}{c}\text { CFM } \\
112538.602\end{array}$ & $\begin{array}{l}\text { SCFM } \\
48428.391\end{array}$ & $\begin{array}{l}\mathrm{A} 1 / \mathrm{A}^{\star} \\
1.439\end{array}$ & $\begin{array}{l}\text { Area1 } \\
513.330\end{array}$ & $\begin{array}{l}A^{\star} \\
356.845\end{array}$ & $\begin{array}{c}\text { AthrRotor } \\
395.845\end{array}$ & $\begin{array}{c}\text { ChokeMargin } \\
1.109\end{array}$ \\
\hline
\end{tabular}

ROTOR LEADING EDGE CONDITIONS, STAGE 4

\begin{tabular}{|c|c|c|c|c|c|c|c|c|c|}
\hline & R1 & Stator & Alfa & $\mathrm{C} 1$ & CU1 & $\mathrm{Cm} 1$ & Mabs & Mrel & U1cor \\
\hline TIP & 19.26 & 0.00 & -0.02 & 526.16 & -0.18 & 526.16 & 0.45 & 0.74 & 627.98 \\
\hline MEAN & 16.97 & 0.00 & -0.02 & 526.16 & -0.18 & 526.16 & 0.45 & 0.68 & \\
\hline HUB & 14.32 & 0.00 & -0.02 & 526.16 & -0.18 & 526.16 & 0.45 & 0.63 & \\
\hline & BetaFlo & BetaBlade & Incid & U1 & W1 & Ps 1 & Ts1 & TwetBulb1 & $\mathrm{RH}$ \\
\hline TIP & 51.78 & 46.36 & 5.42 & 667.85 & 850.36 & 6.88 & 564.38 & 510.37 & 0.11 \\
\hline MEAN & 48.21 & 43.80 & 4.41 & 588.47 & 789.53 & 6.88 & 564.38 & 510.37 & 0.11 \\
\hline HUB & 43.35 & 37.84 & 5.51 & 496.56 & 723.59 & 6.88 & 564.38 & 510.37 & 0.11 \\
\hline
\end{tabular}

ROTOR EXIT CONDITIONS, STAGE 4 SOLUTION IS CONVERGED

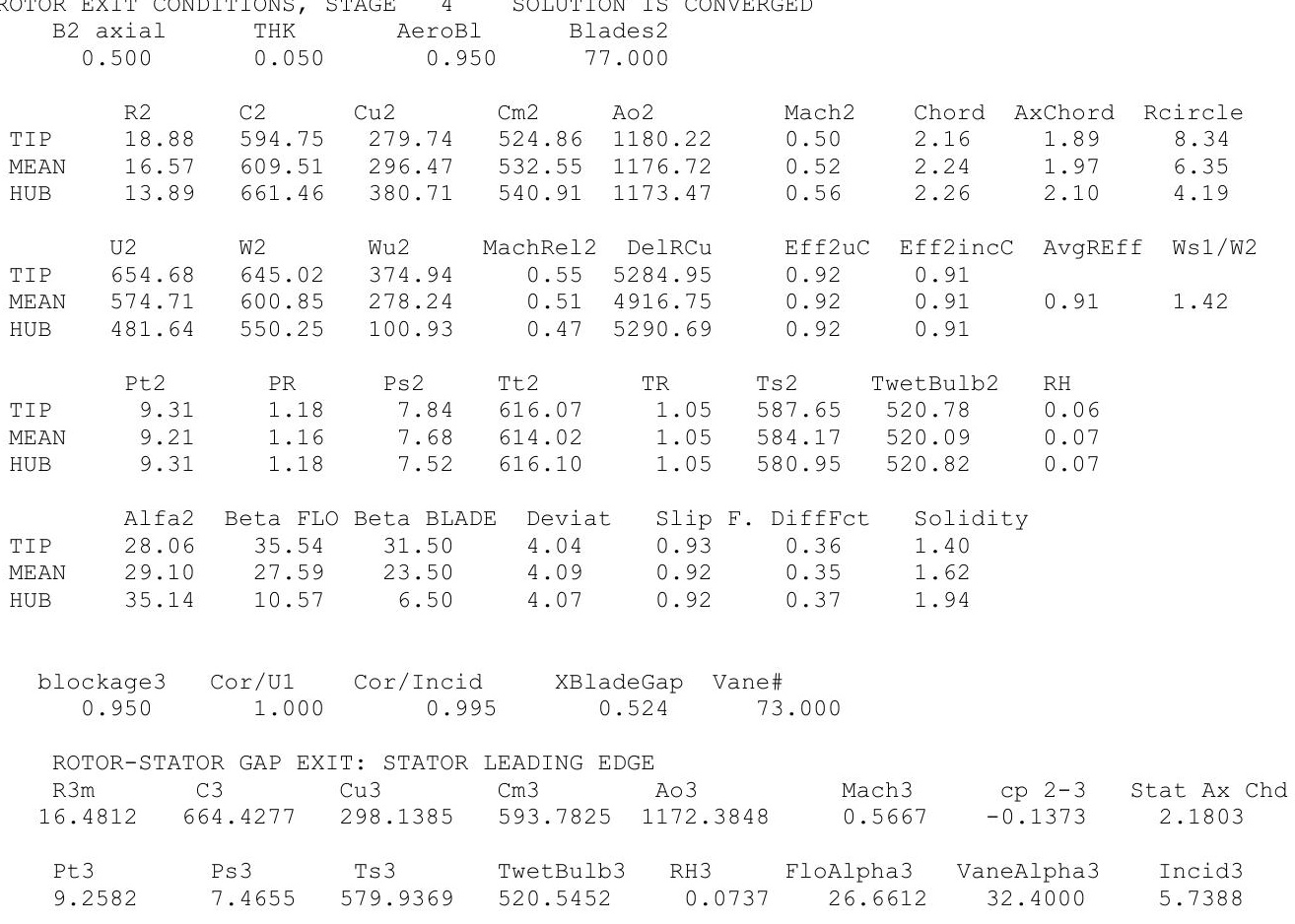




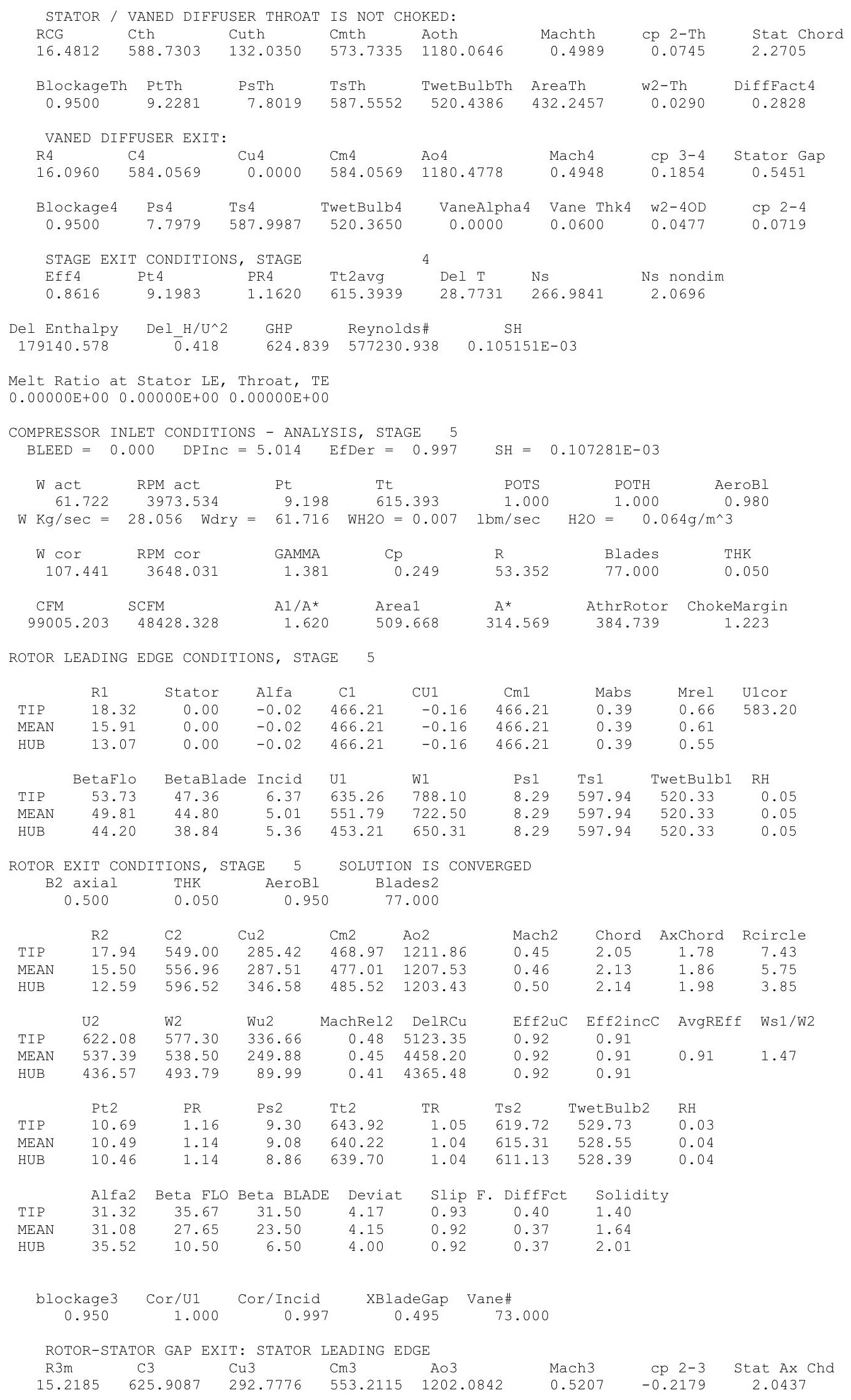




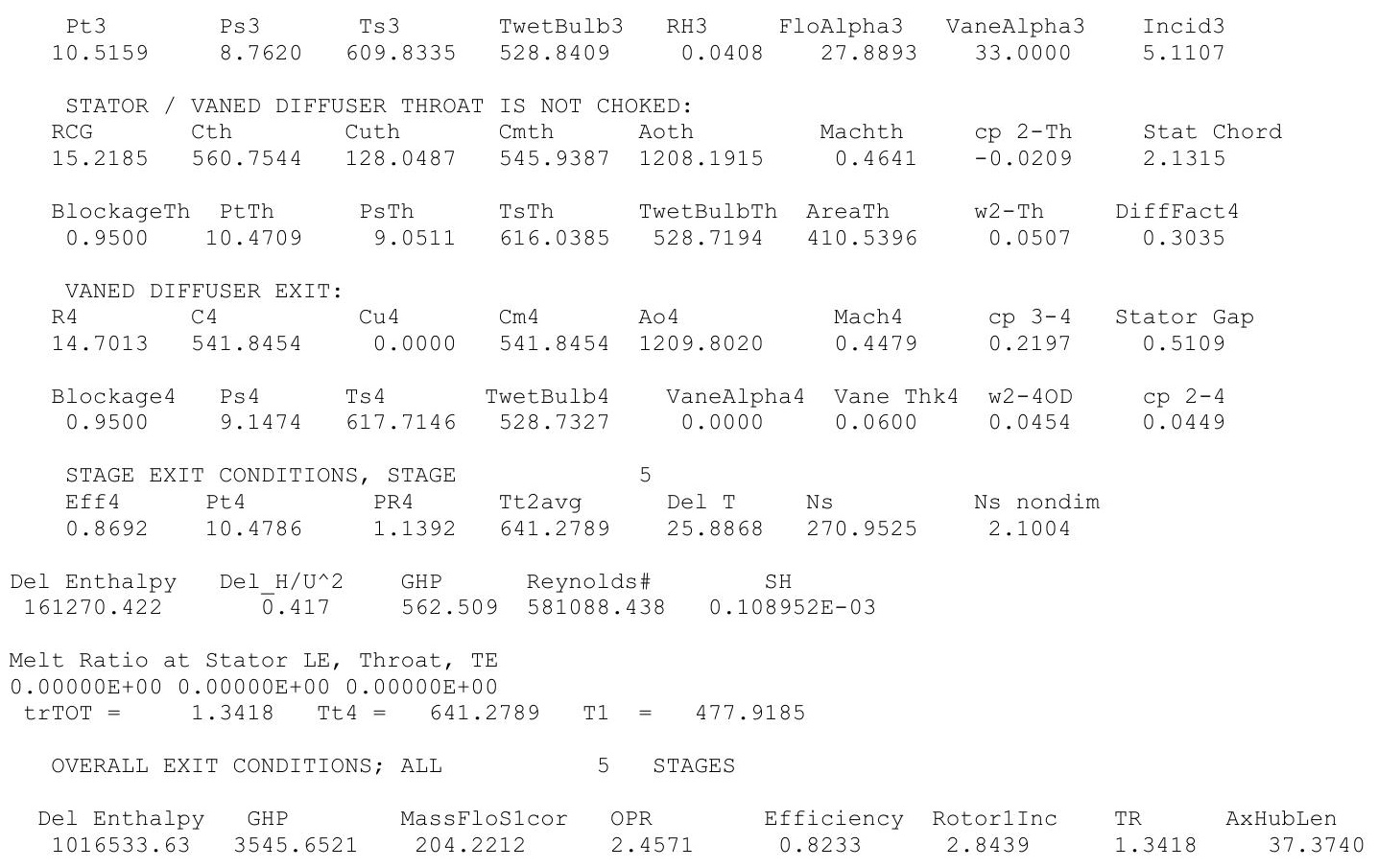




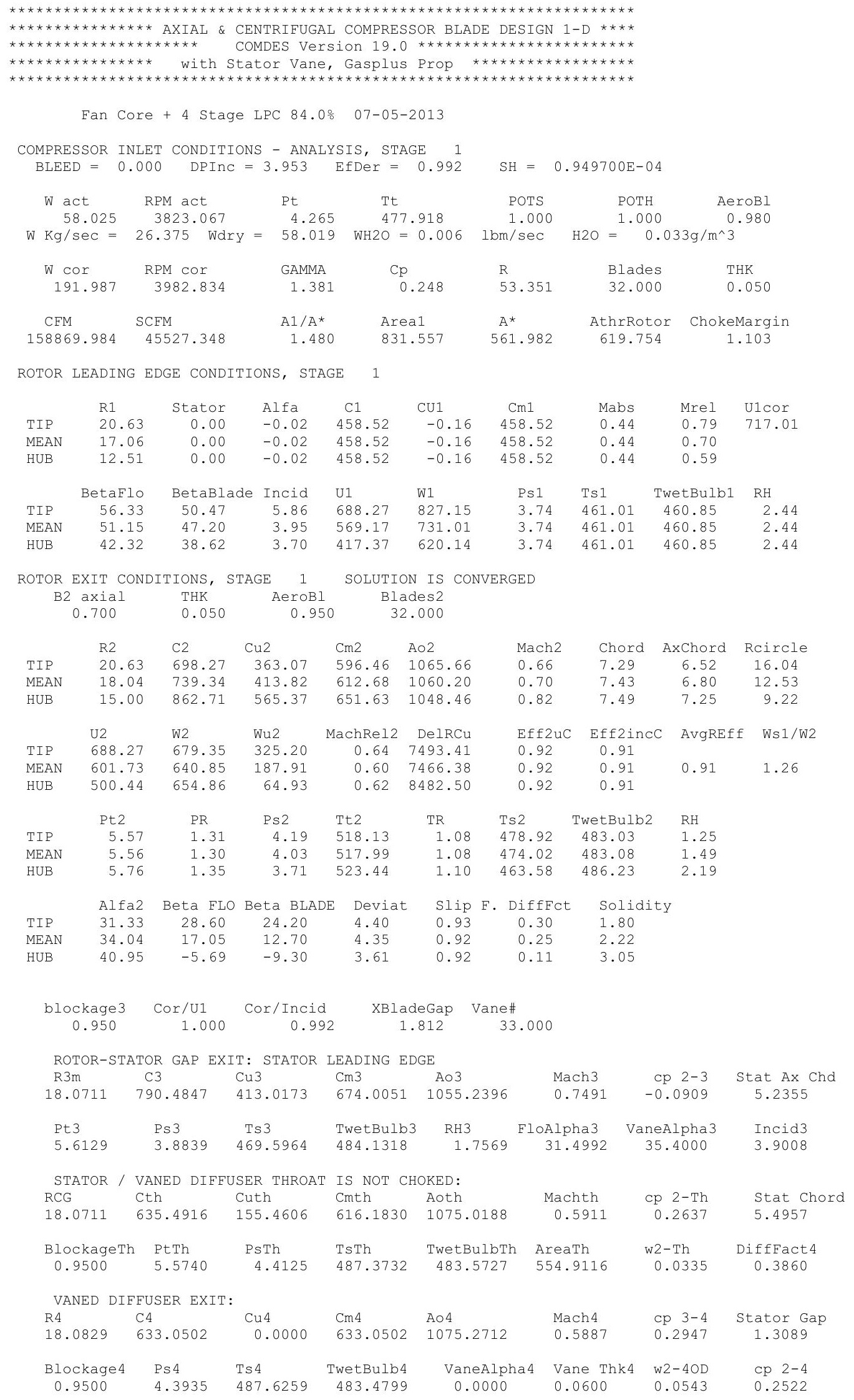




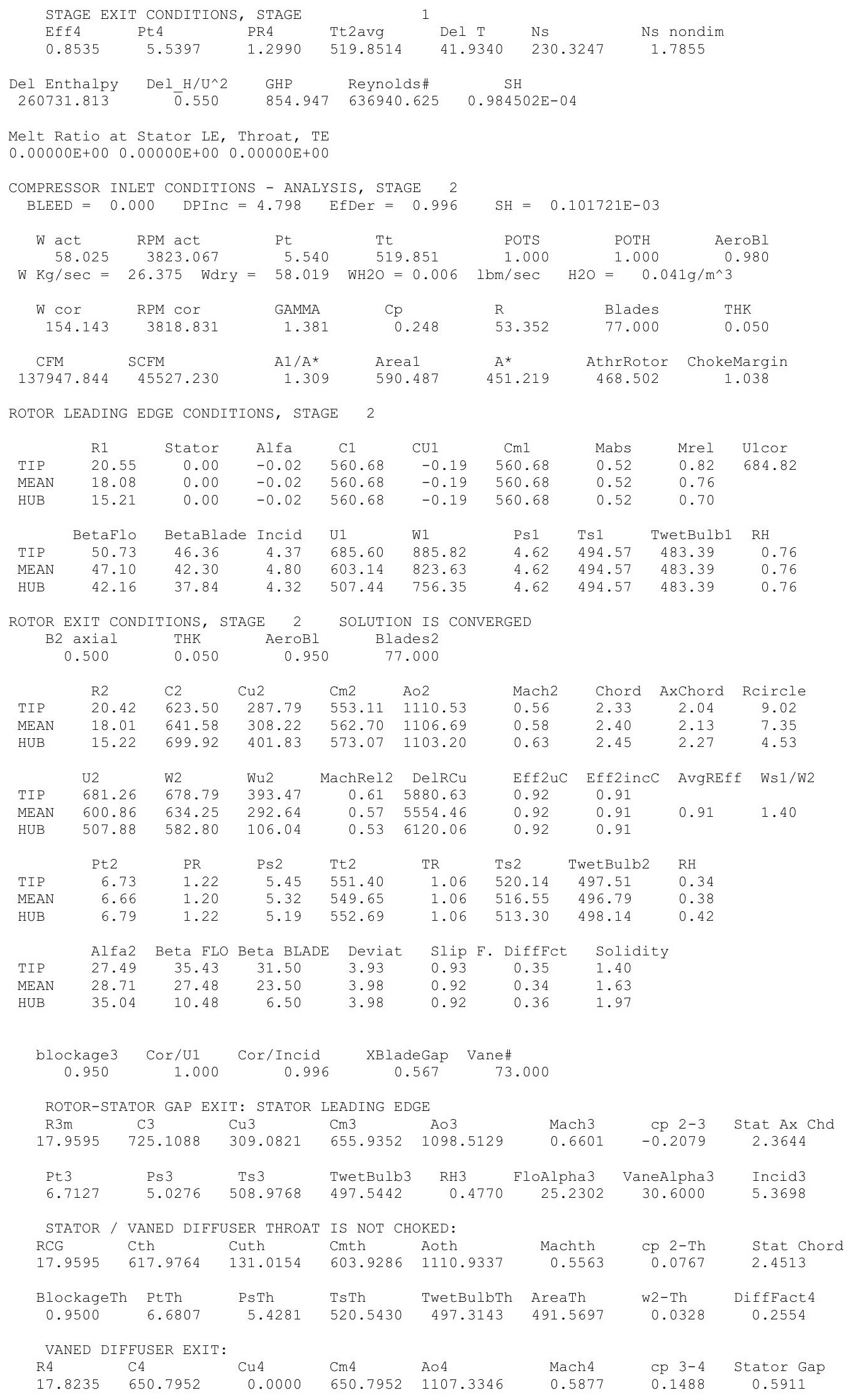




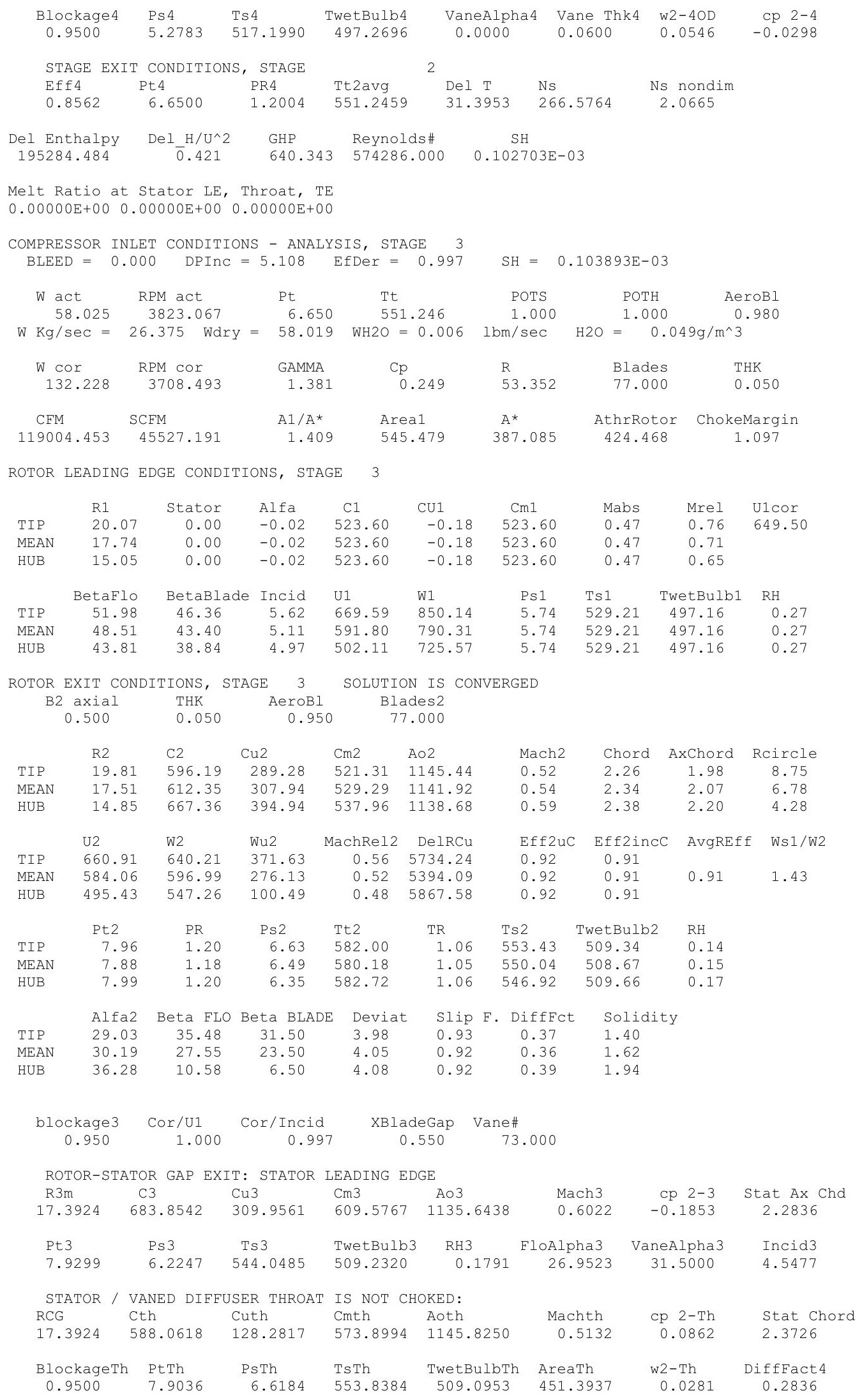




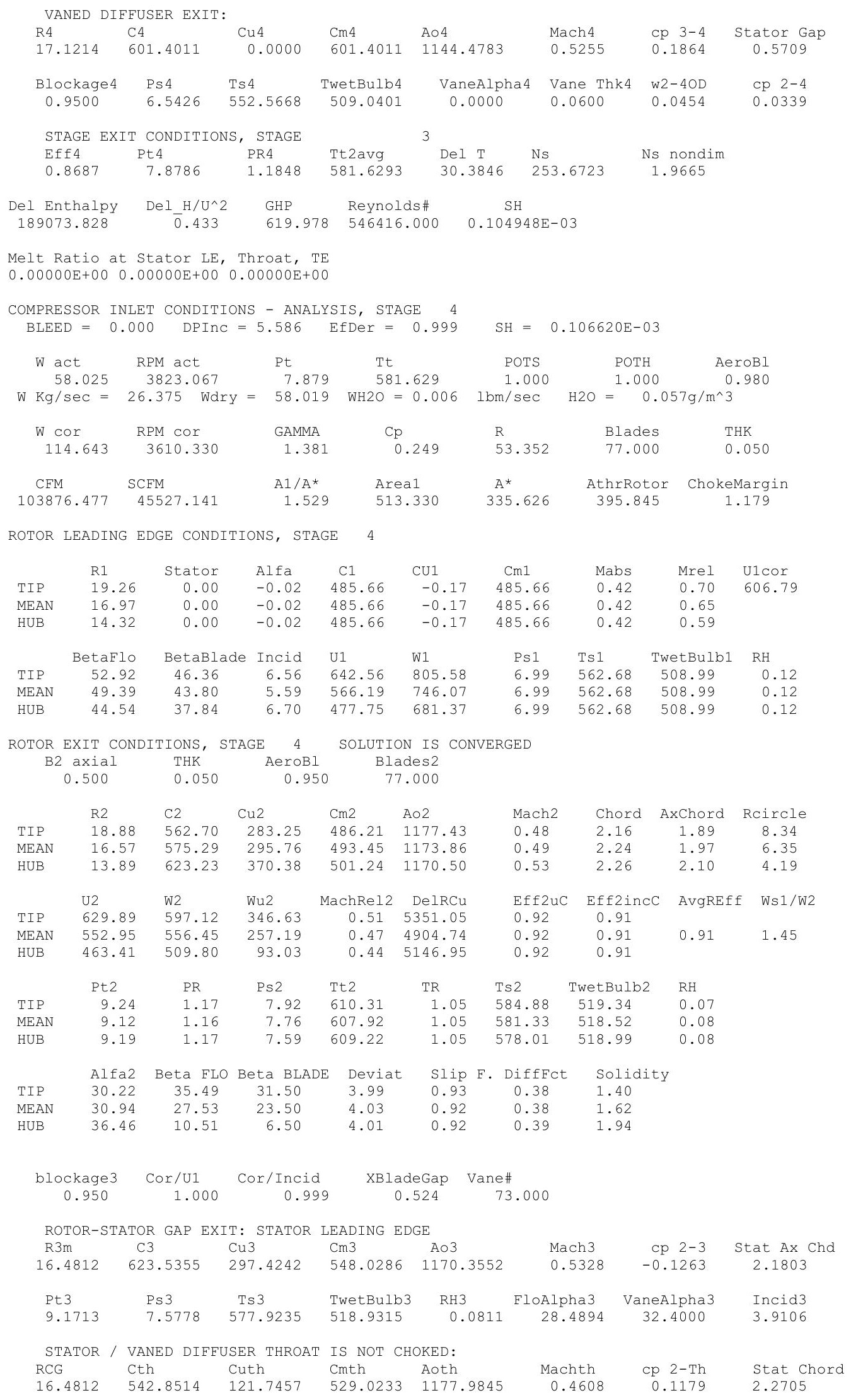




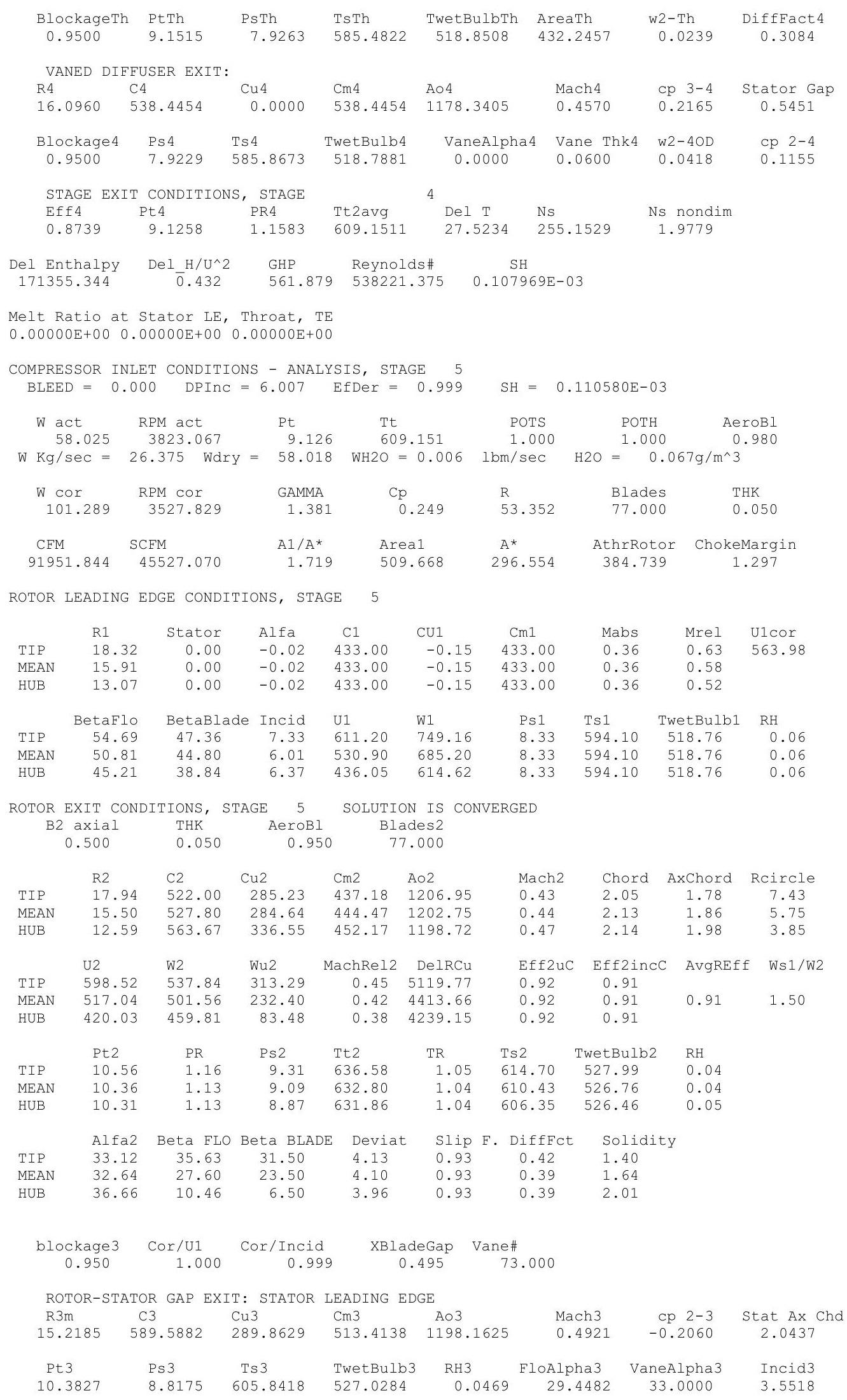




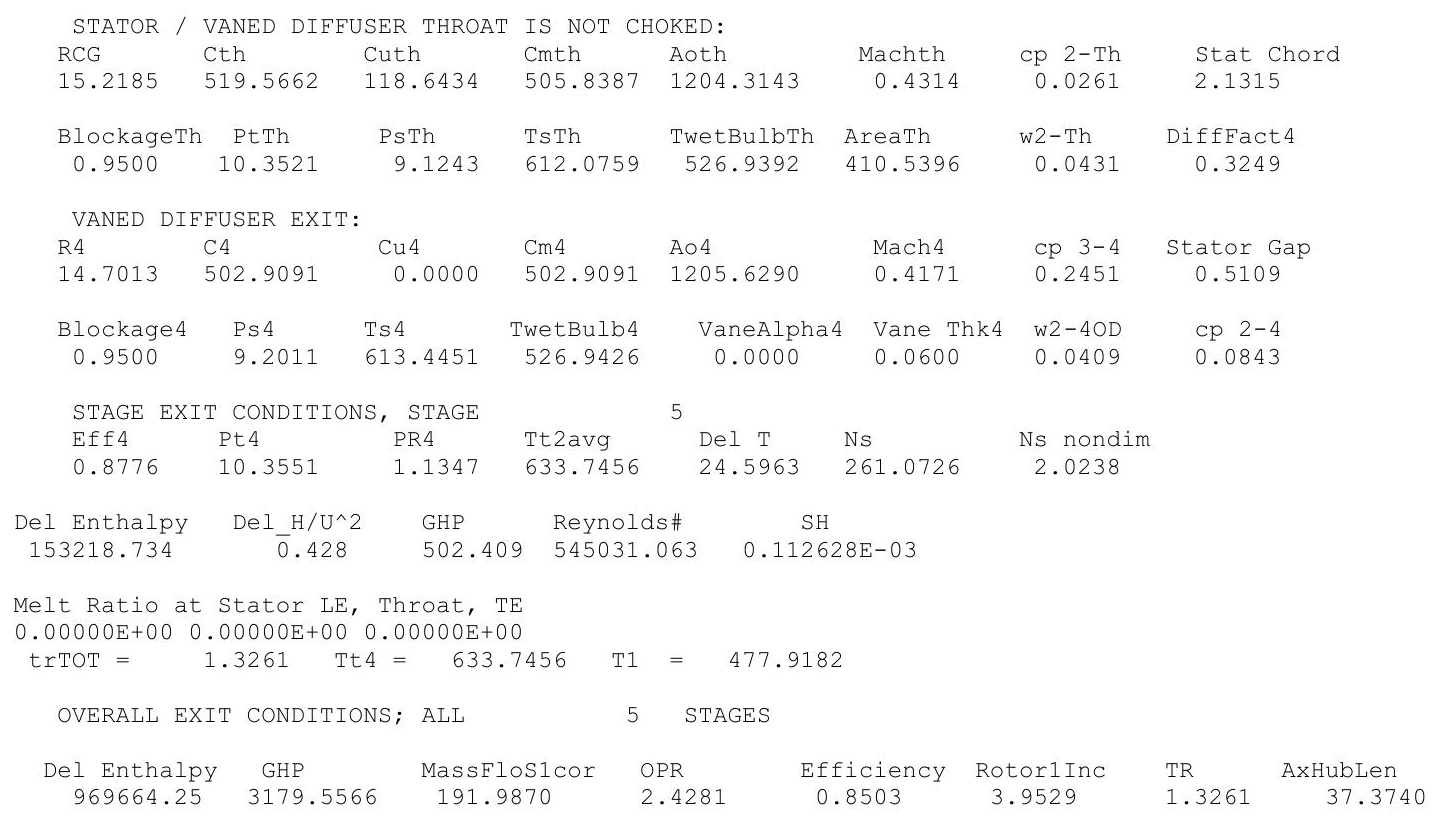




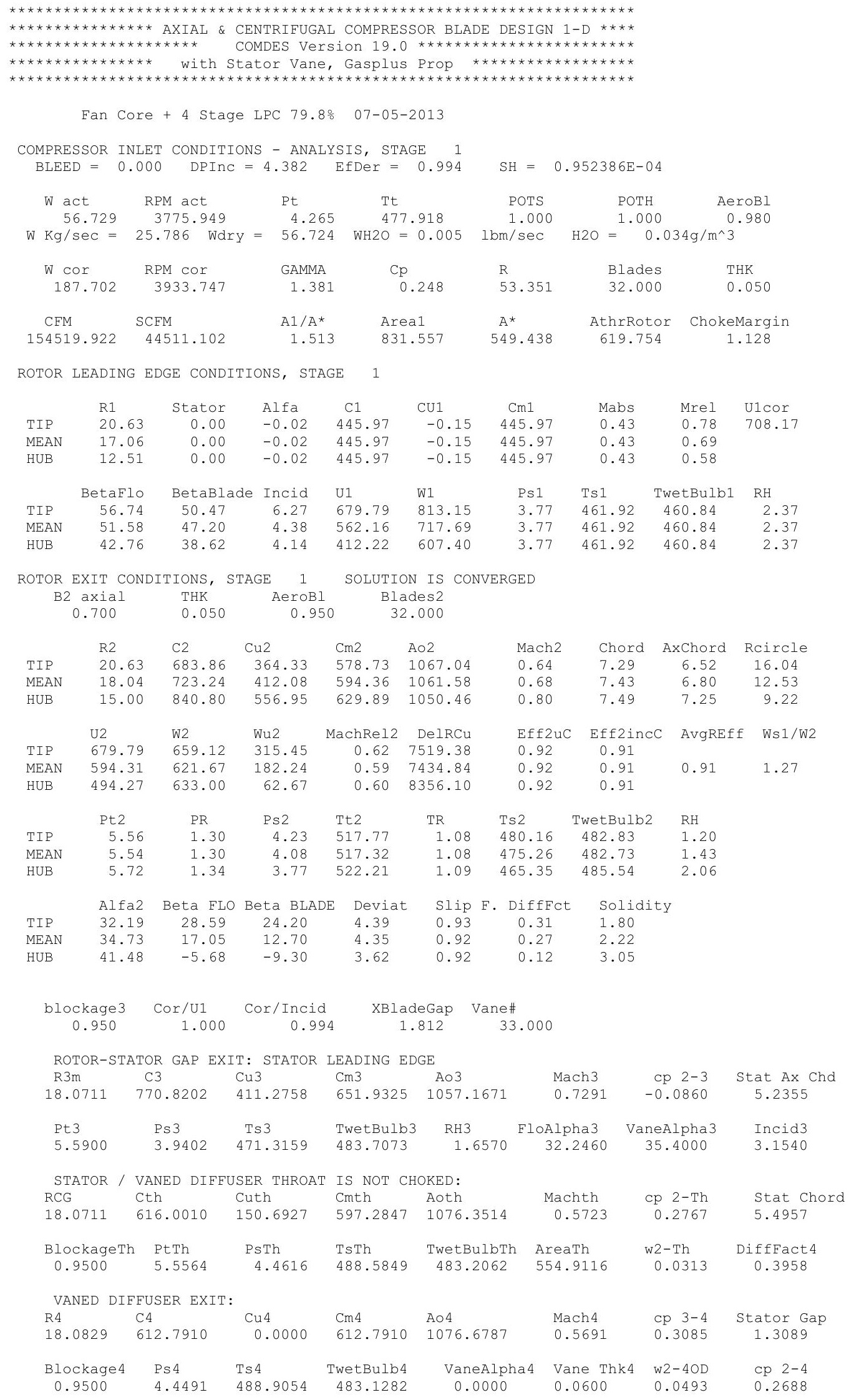




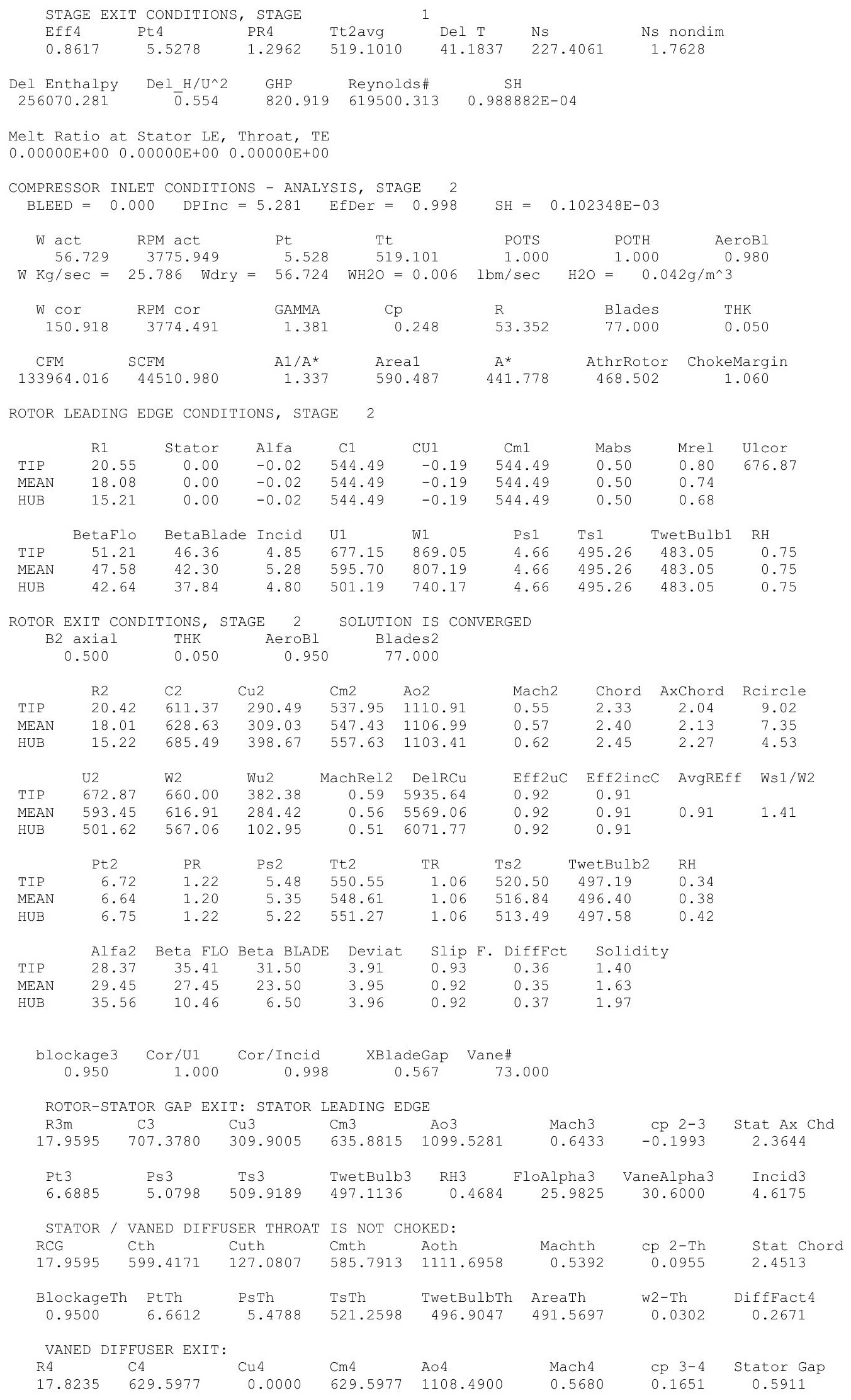




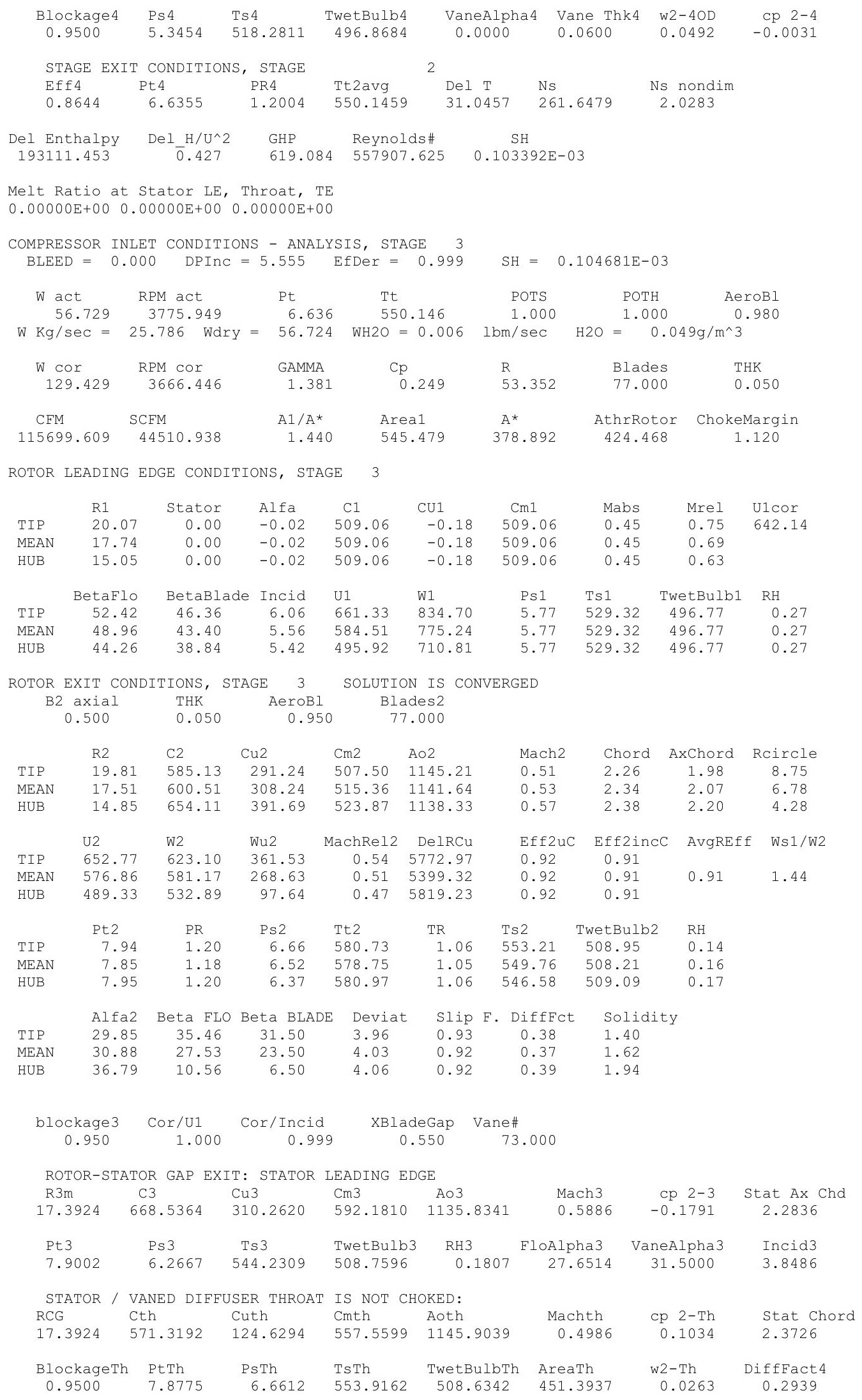




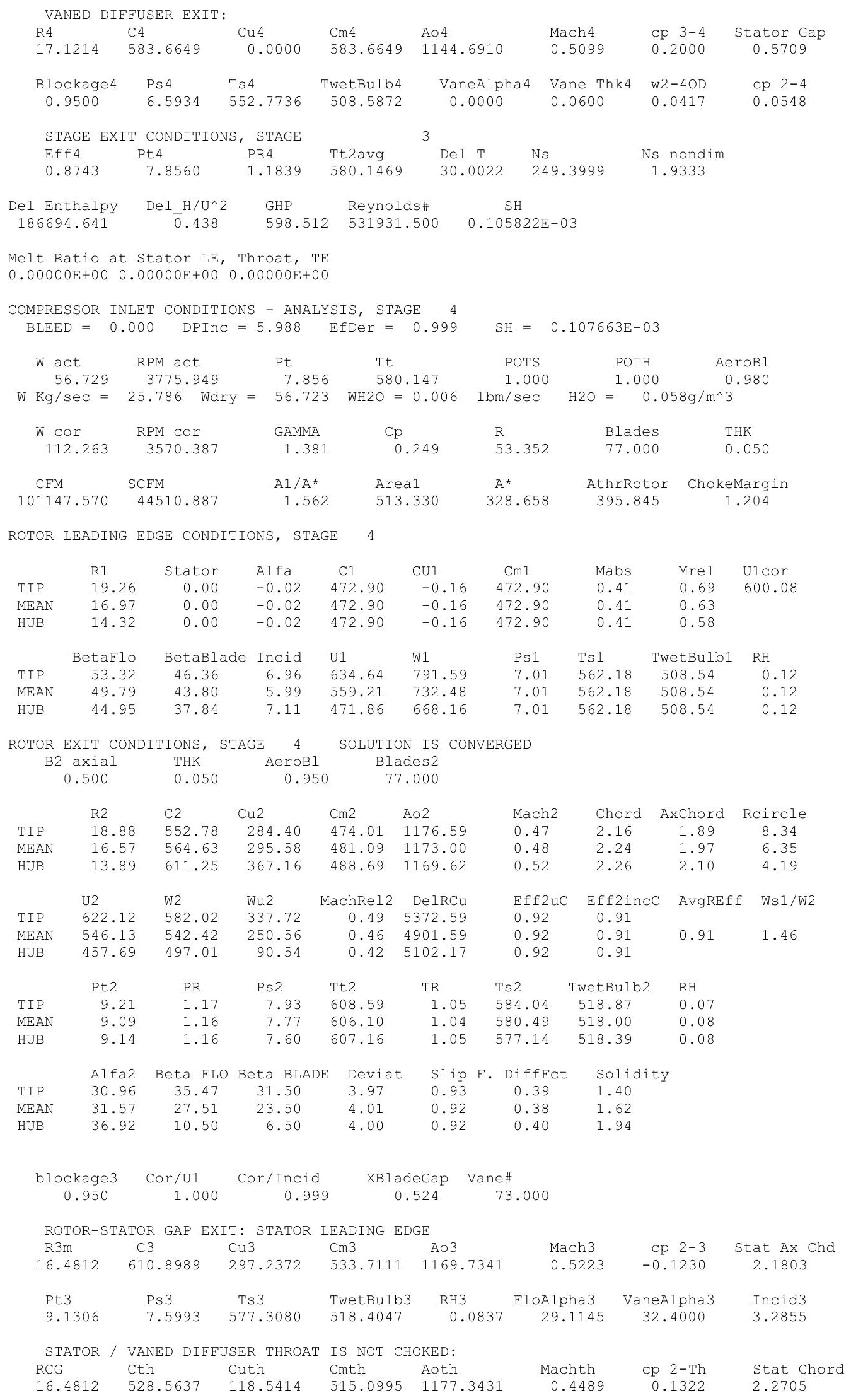




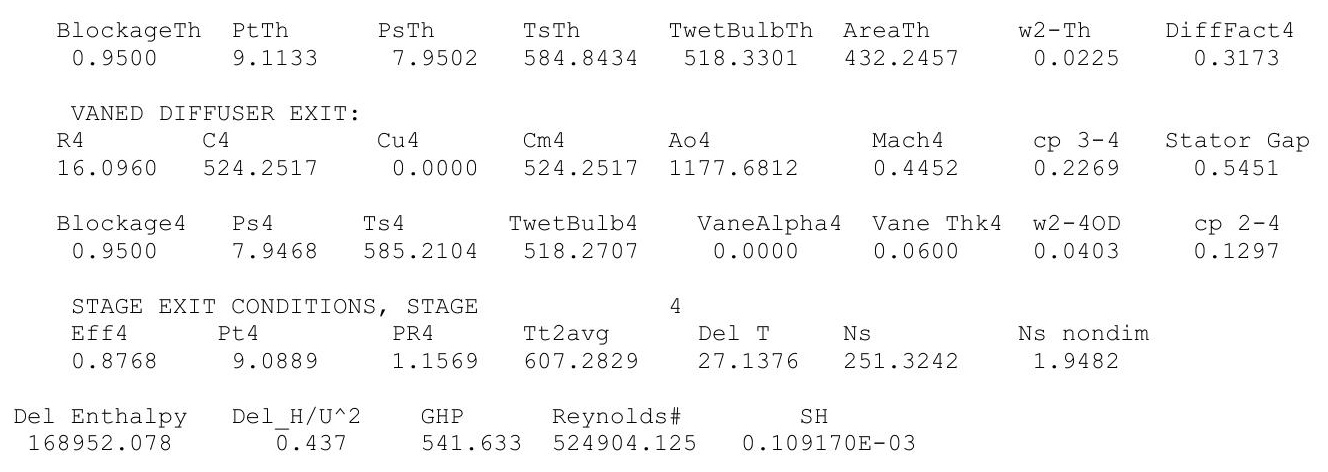

Melt Ratio at Stator LE, Throat, TE

$0.00000 \mathrm{E}+00 \quad 0.00000 \mathrm{E}+00 \quad 0.00000 \mathrm{E}+00$

COMPRESSOR INLET CONDITIONS - ANALYSIS, STAGE 5 $\mathrm{BLEED}=0.000 \quad$ DPInC $=6.349 \quad$ EfDer $=1.000$

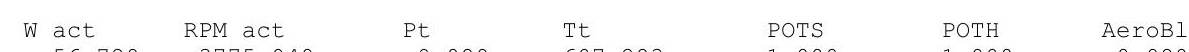

ROTOR LEADING EDGE CONDITIONS, STAGE 5

\begin{tabular}{|c|c|c|c|c|c|c|c|c|c|}
\hline & R1 & Stator & Alfa & $\mathrm{C} 1$ & CU1 & $\mathrm{Cm} 1$ & Mabs & Mrel & U1cor \\
\hline TIP & 18.32 & 0.00 & -0.02 & 422.48 & -0.15 & 422.48 & 0.36 & 0.62 & 557.89 \\
\hline MEAN & 15.91 & 0.00 & -0.02 & 422.48 & -0.15 & 422.48 & 0.36 & 0.57 & \\
\hline HUB & 13.07 & 0.00 & -0.02 & 422.48 & -0.15 & 422.48 & 0.36 & 0.51 & \\
\hline & BetaFlo & BetaBlade & Incid & U1 & W1 & Ps 1 & Ts1 & TwetBulb1 & $\mathrm{RH}$ \\
\hline TIP & 55.02 & 47.36 & 7.66 & 603.67 & 736.94 & 8.33 & 592.95 & 518.25 & 0.06 \\
\hline MEAN & 51.15 & 44.80 & 6.35 & 524.36 & 673.49 & 8.33 & 592.95 & 518.25 & 0.06 \\
\hline HUB & 45.56 & 38.84 & 6.72 & 430.67 & 603.40 & 8.33 & 592.95 & 518.25 & 0.06 \\
\hline
\end{tabular}

ROTOR EXIT CONDITIONS, STAGE 5 SOLUTION IS CONVERGED

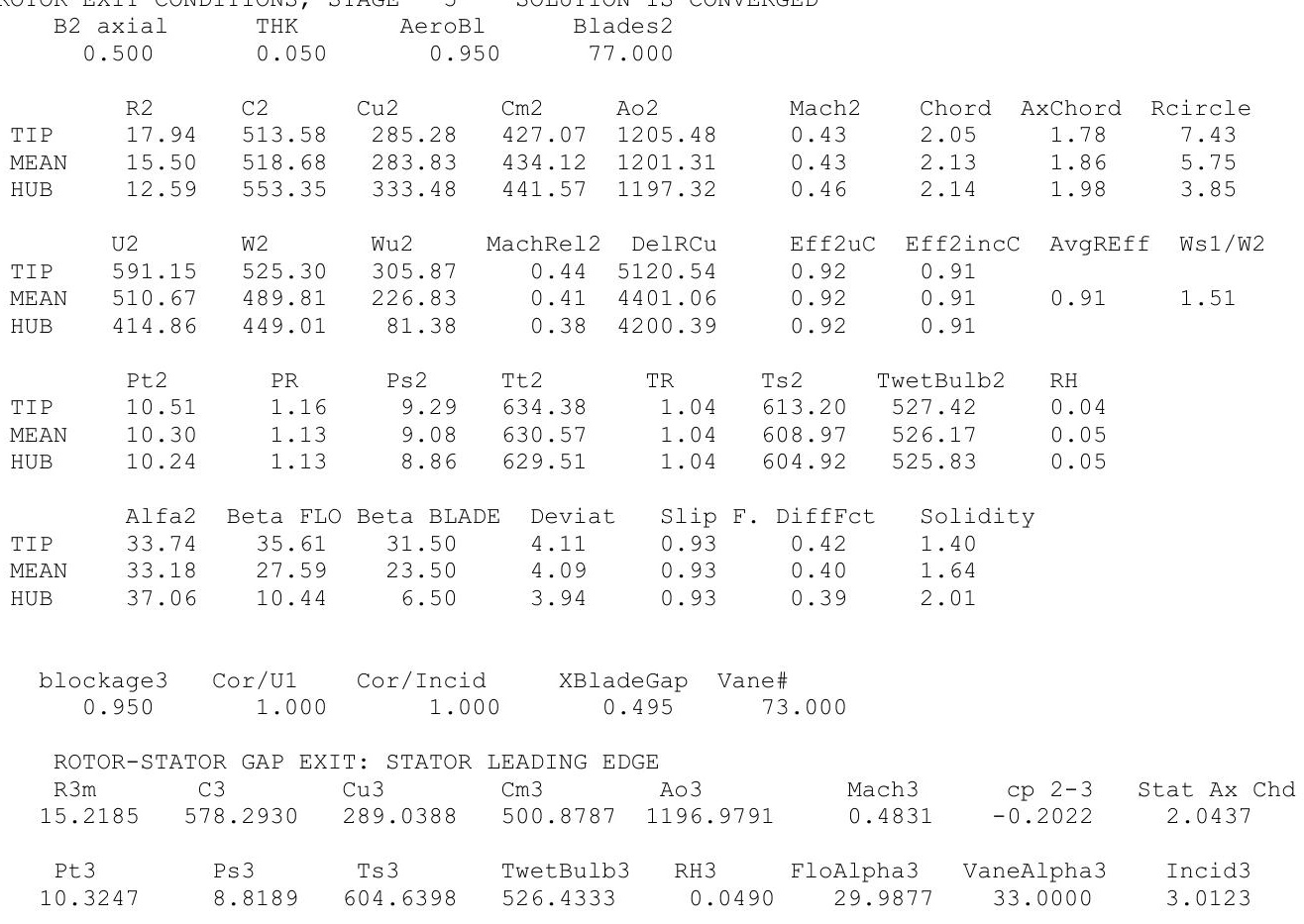




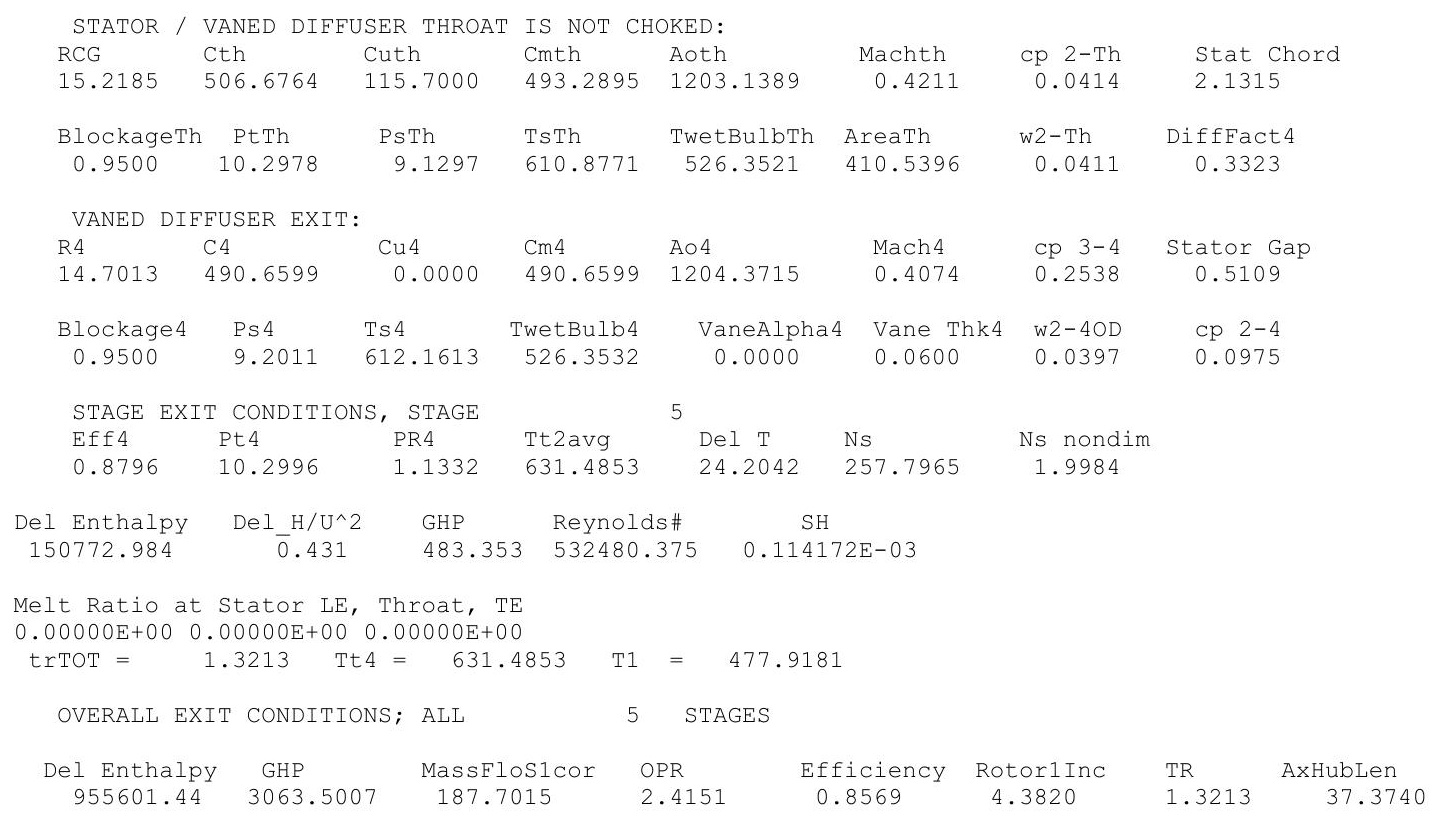




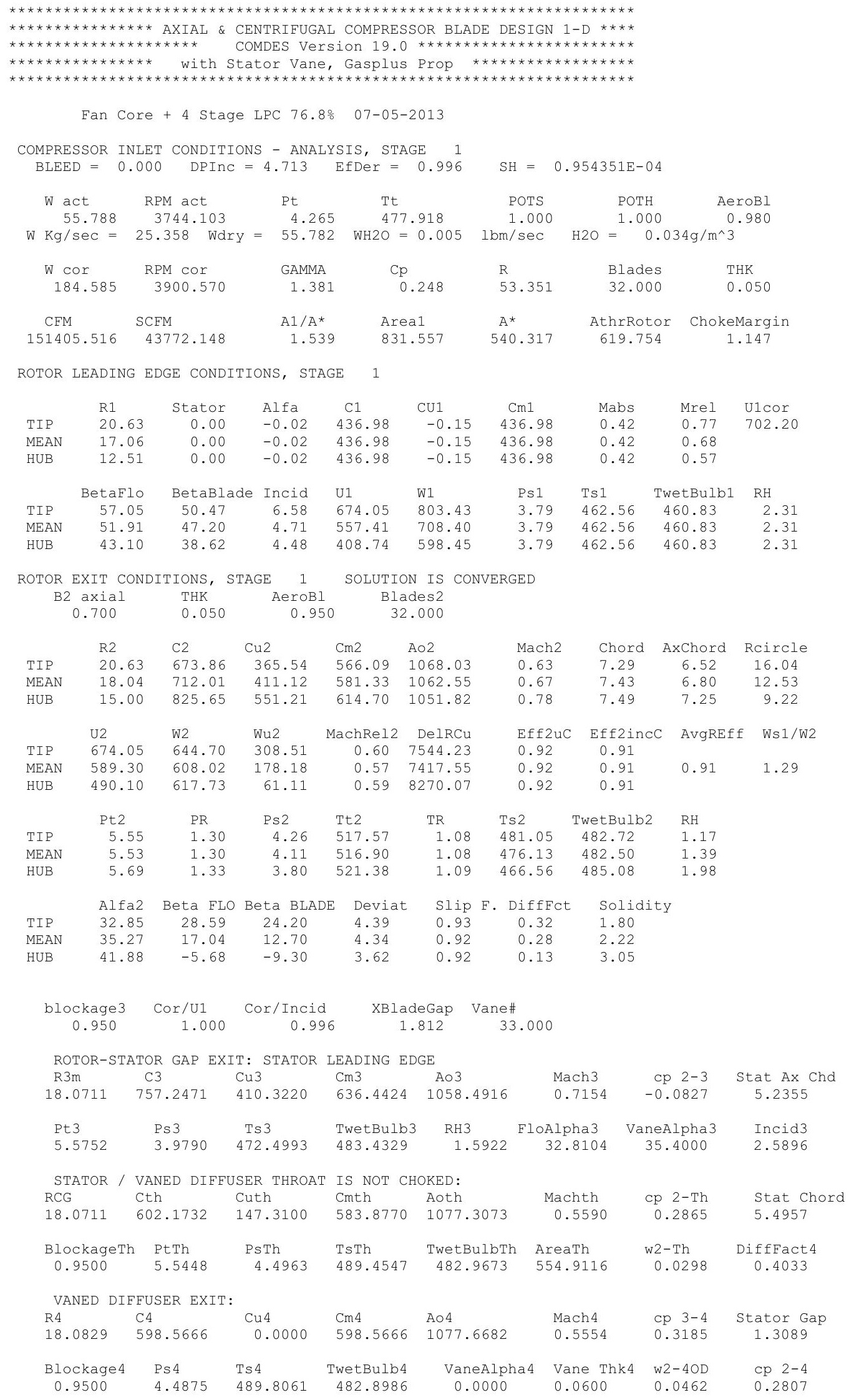




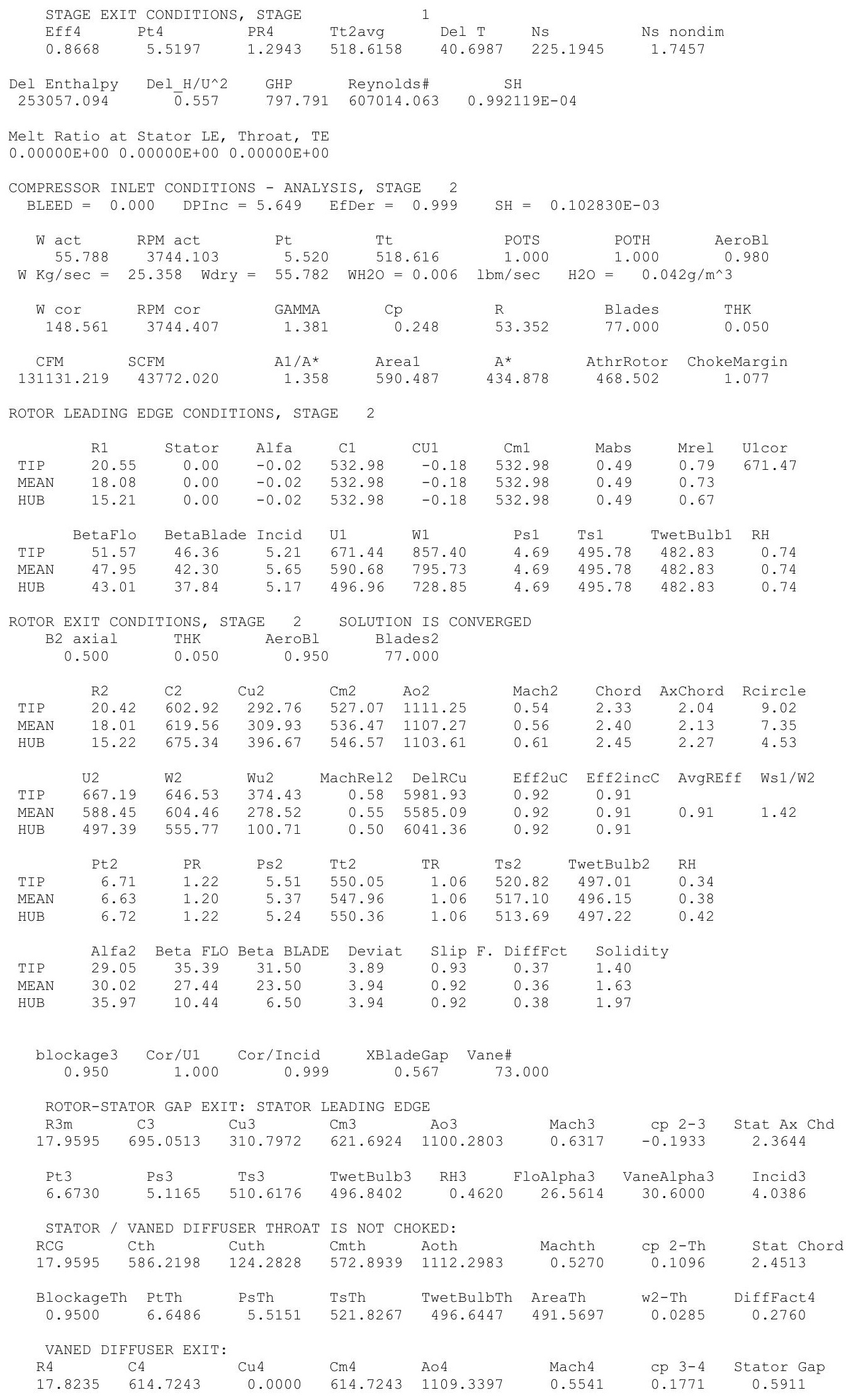




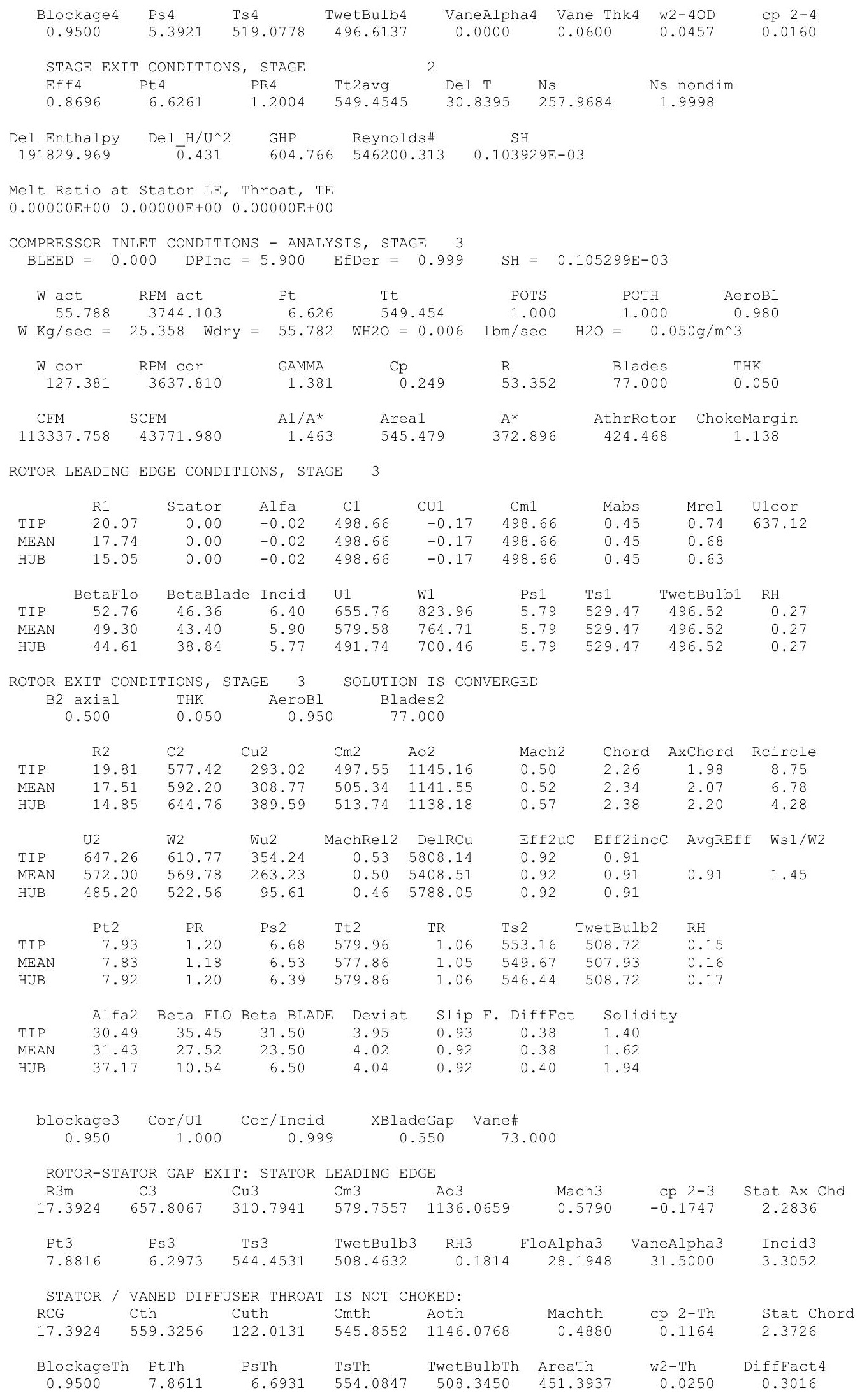




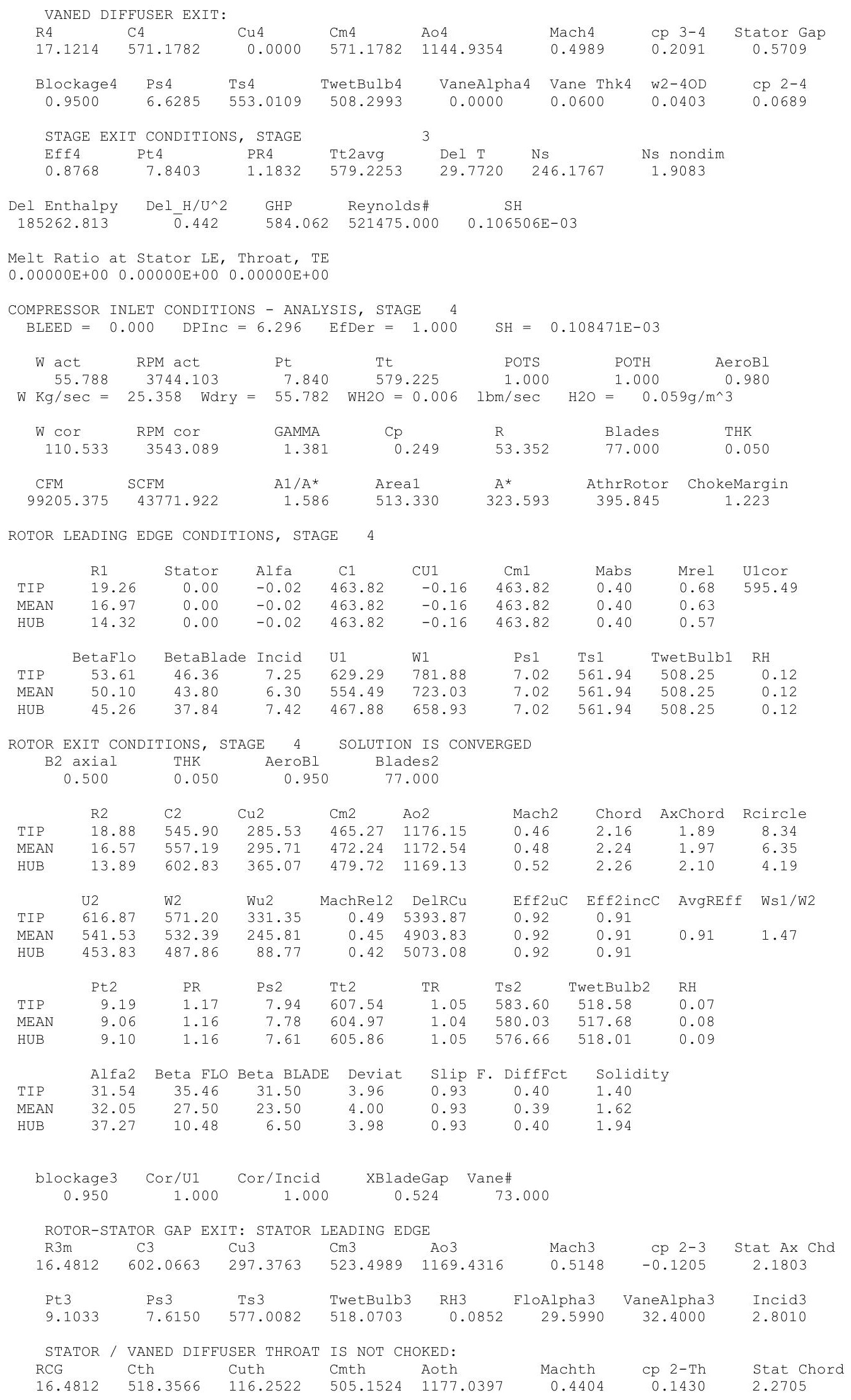




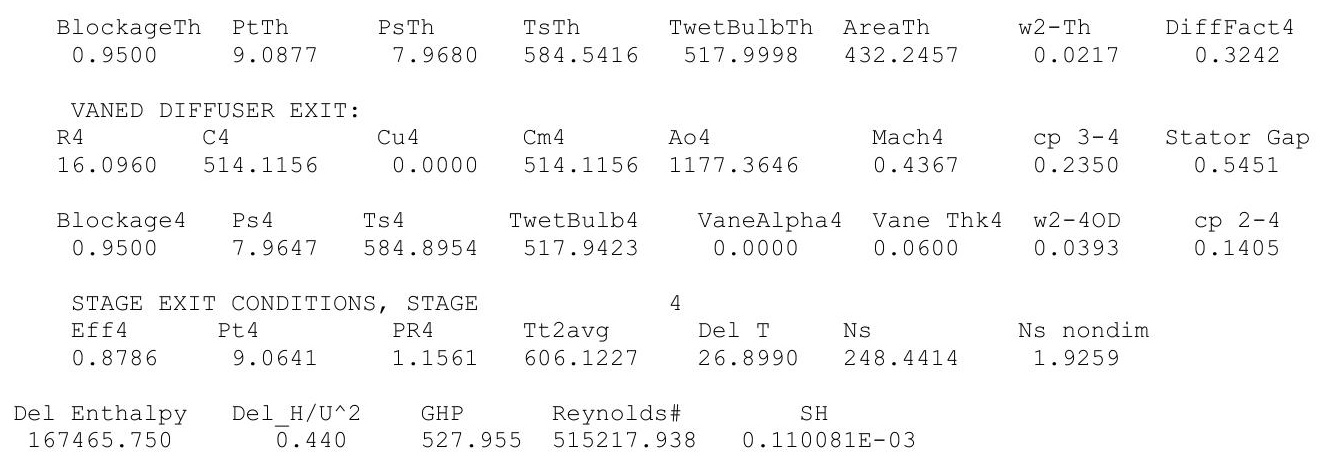

Melt Ratio at Stator LE, Throat, TE

$0.00000 \mathrm{E}+000.00000 \mathrm{E}+00 \quad 0.00000 \mathrm{E}+00$

COMPRESSOR INLET CONDITIONS - ANALYSIS, STAGE 5 $\mathrm{BLEED}=0.000 \quad$ DPInC $=6.617 \quad$ EfDer $=1.000$

$\begin{array}{llllll}W & \text { Ret } & \text { PPM act } & \text { POTS } & \text { POTH } & \text { AeroBI }\end{array}$

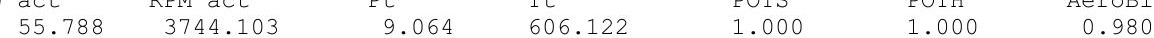

$\mathrm{W} \mathrm{Kg} / \mathrm{sec}=25.358$ Wdry $=55.781$ WH2O $=0.006 \mathrm{lbm} / \mathrm{sec} \quad \mathrm{H} 2 \mathrm{O}=0.069 \mathrm{~g} / \mathrm{m}^{\wedge} 3$

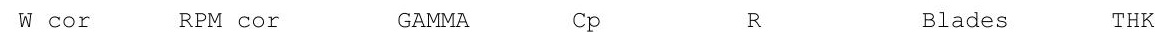

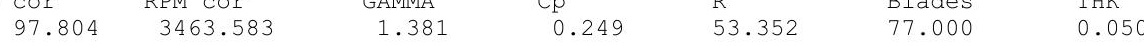

$\begin{array}{ccccc}\text { CFM } & \text { SCFM } & \text { A1/A* } & \text { Area1 } & \text { A* AthrRotor ChokeMargin }\end{array}$

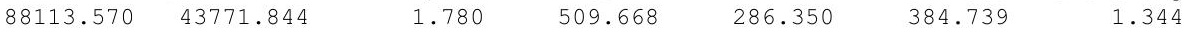

ROTOR LEADING EDGE CONDITIONS, STAGE 5

$\begin{array}{lccccccccc} & \text { R1 } & \text { Stator } & \text { Alfa } & \text { C1 } & \text { CU1 } & \text { Cm1 } & \text { Mabs } & \text { Mrel } & \text { U1cor } \\ \text { TIP } & 18.32 & 0.00 & -0.02 & 414.92 & -0.14 & 414.92 & 0.35 & 0.61 & 553.71 \\ \text { MEAN } & 15.91 & 0.00 & -0.02 & 414.92 & -0.14 & 414.92 & 0.35 & 0.56 & \\ \text { HUB } & 13.07 & 0.00 & -0.02 & 414.92 & -0.14 & 414.92 & 0.35 & 0.50 & \\ & & & & & & & & & \\ & & & & & & & & & \\ \text { TIP } & 55.28 & 47.36 & 7.92 & 598.58 & 728.44 & 8.34 & 592.30 & 517.92 & 0.06 \\ \text { MEAN } & 51.42 & 44.80 & 6.62 & 519.93 & 665.31 & 8.34 & 592.30 & 517.92 & 0.06 \\ \text { HUB } & 45.83 & 38.84 & 6.99 & 427.04 & 595.52 & 8.34 & 592.30 & 517.92 & 0.06\end{array}$

ROTOR EXIT CONDITIONS, STAGE 5 SOLUTION IS CONVERGED

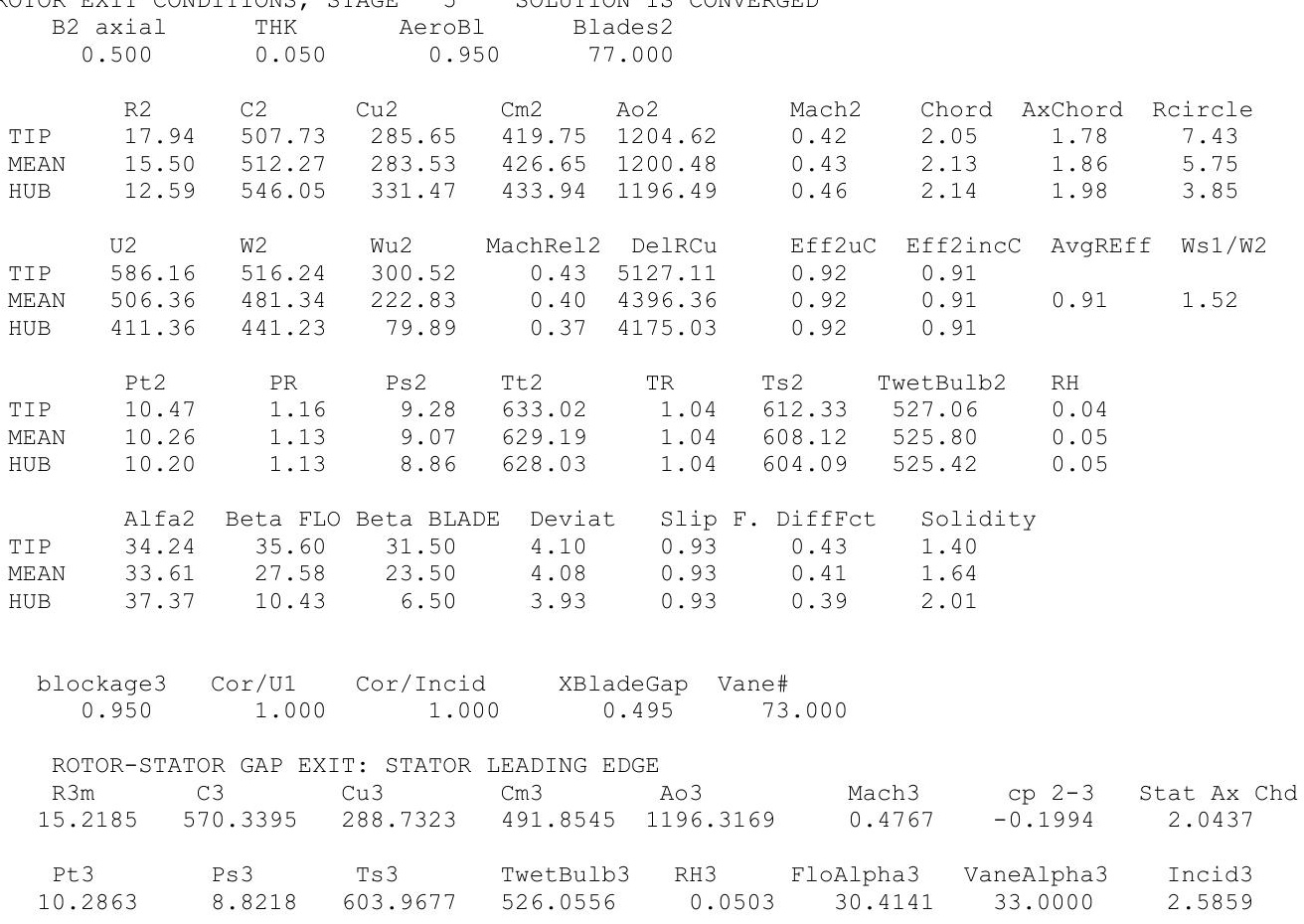




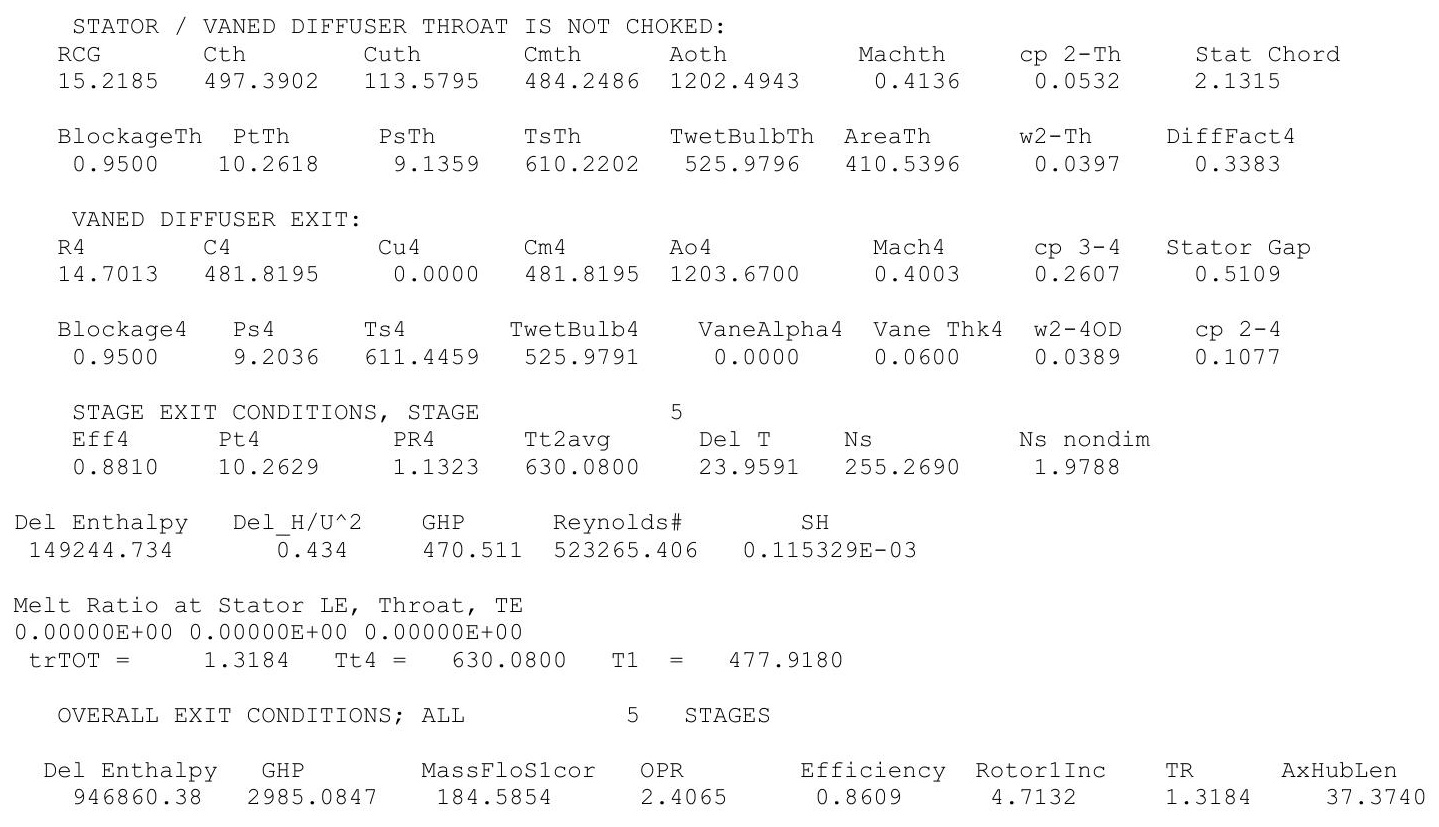




\section{$100 \mu \mathrm{m}$, ISA $+18 \mathrm{R}$}

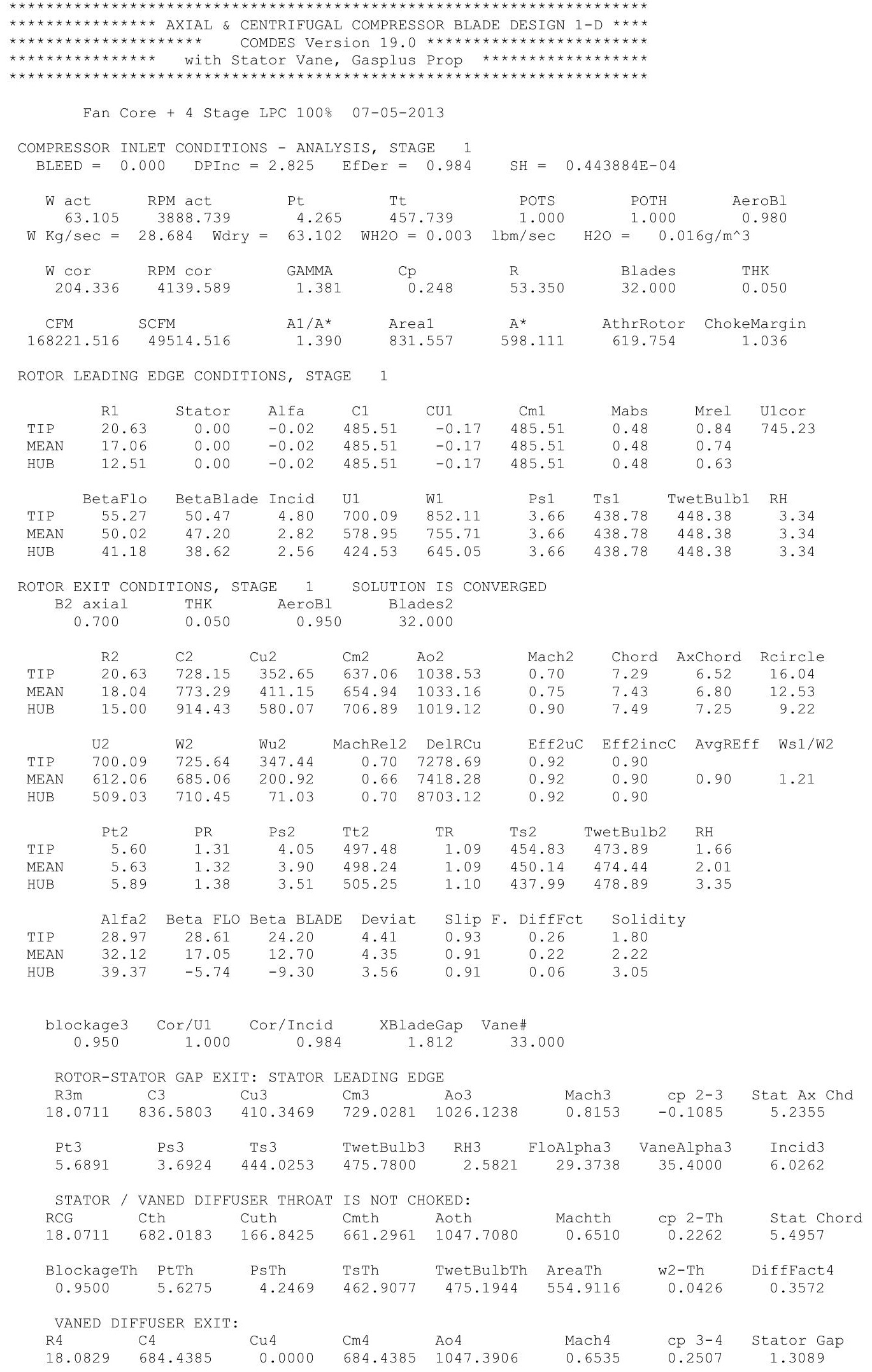




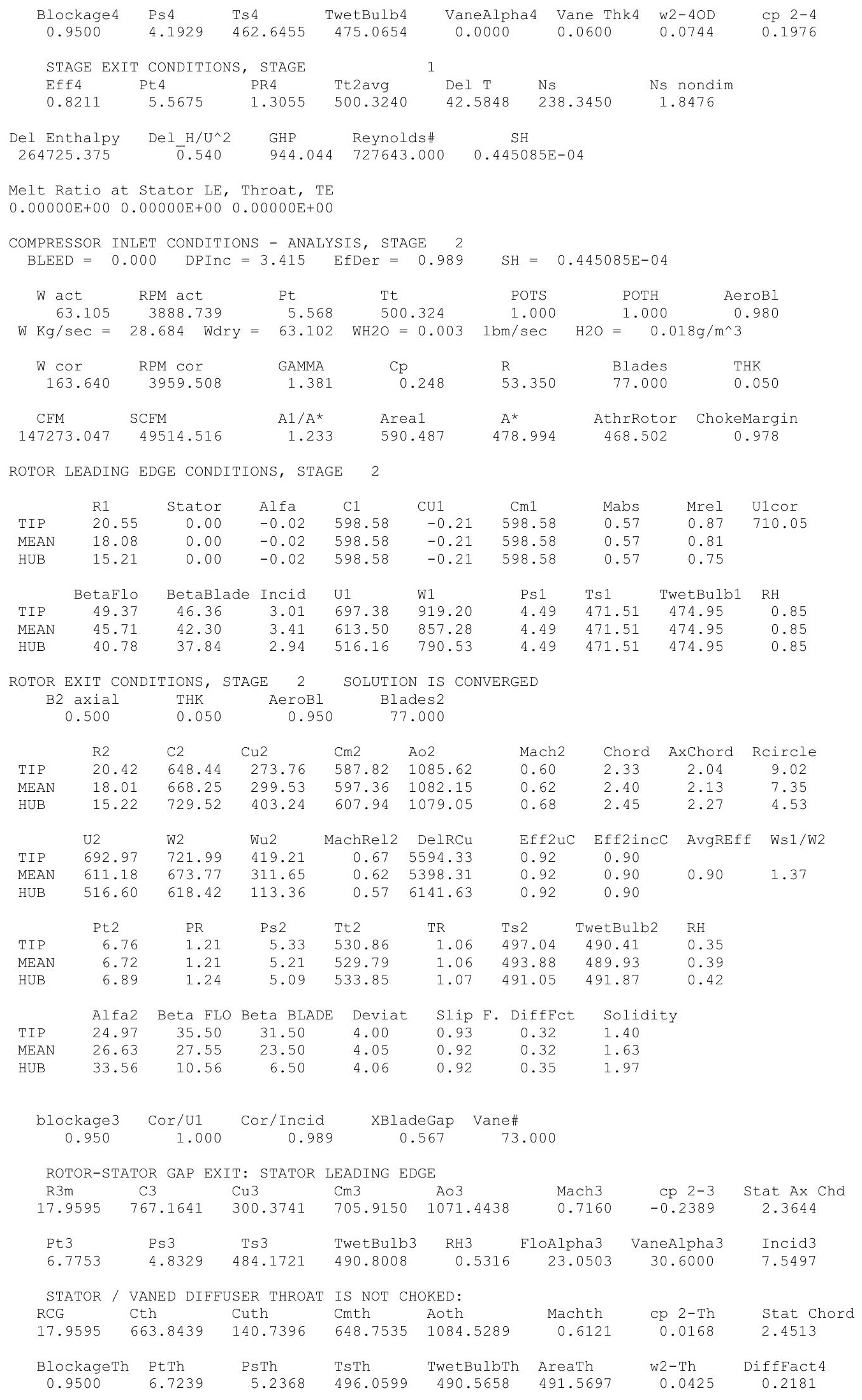




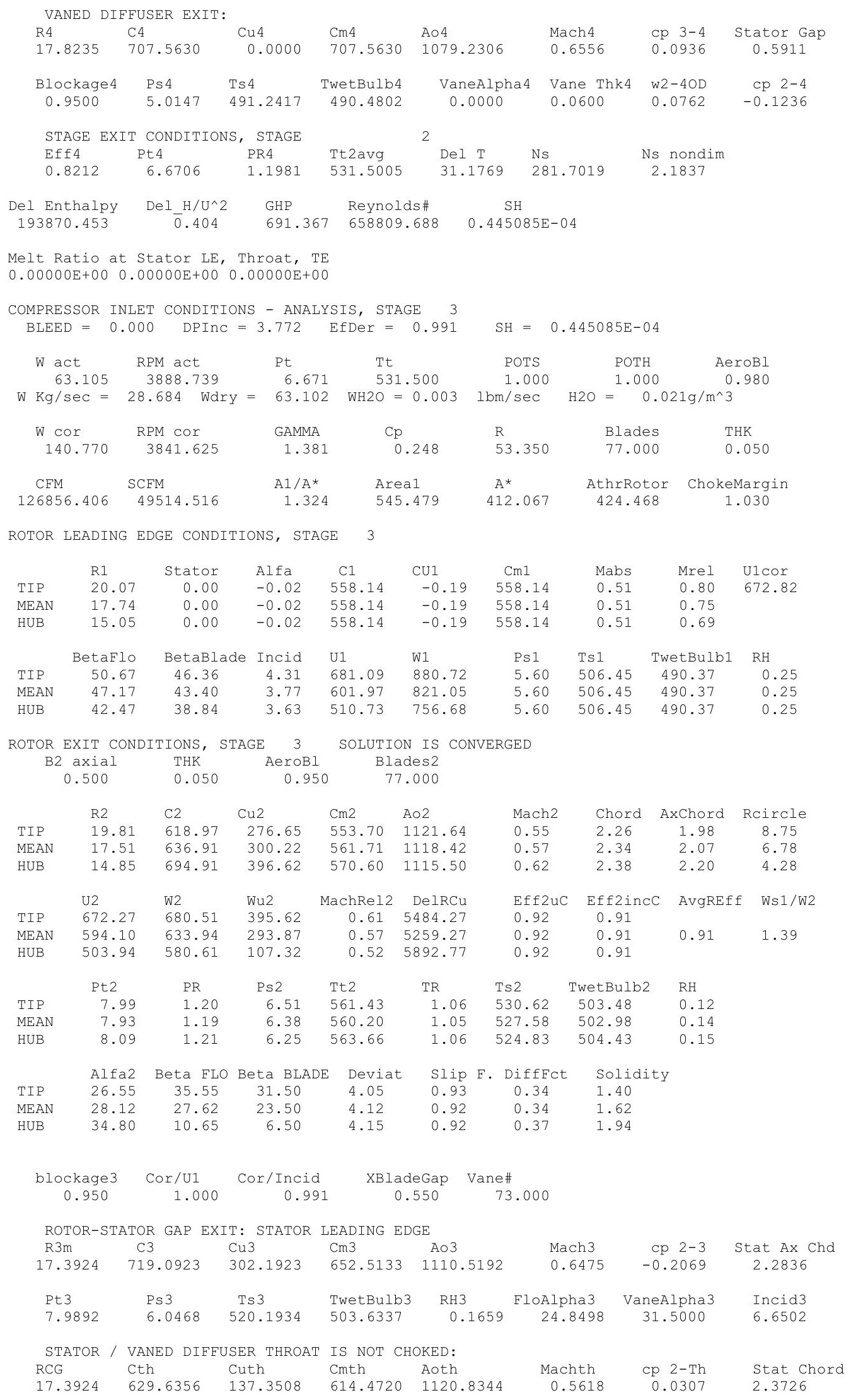




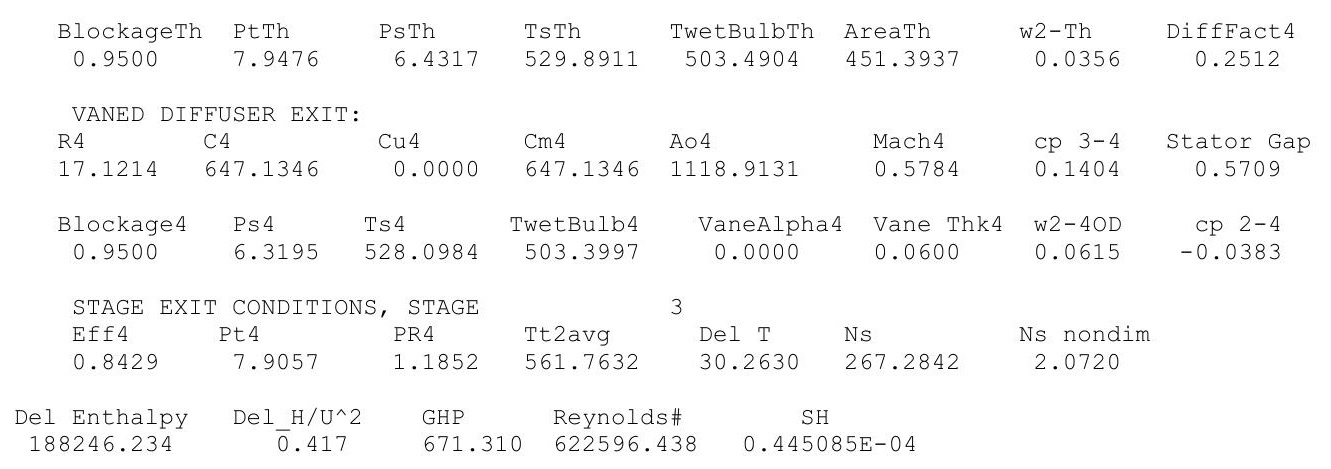

Melt Ratio at Stator LE, Throat, TE

$0.00000 \mathrm{E}+00 \quad 0.00000 \mathrm{E}+00 \quad 0.00000 \mathrm{E}+00$

COMPRESSOR INLET CONDITIONS - ANALYSIS, STAGE 4 $\mathrm{BLEED}=0.000 \quad$ DPInC $=4.347 \quad$ EfDer $=0.994$

$\begin{array}{lllllll}\text { W act } & \text { RPM act } & \text { Pt } & \text { POTS } & \text { POTH } & \text { AeroBI }\end{array}$

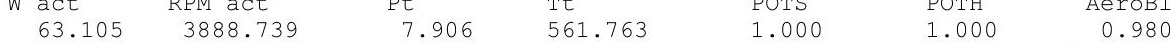
$\mathrm{W} \mathrm{Kg} / \mathrm{sec}=28.684$ WdrY $=63.102 \mathrm{WH} 2 \mathrm{O}=0.003 \mathrm{lbm} / \mathrm{sec} \quad \mathrm{H} 2 \mathrm{O}=0.025 \mathrm{~g} / \mathrm{m}^{\wedge} 3$

\begin{tabular}{|c|c|c|c|c|c|c|}
\hline $\begin{array}{l}W \text { cor } \\
122.113\end{array}$ & $\begin{array}{l}\text { RPM cor } \\
3736.718\end{array}$ & $\begin{array}{l}\text { GAMMA } \\
1.381\end{array}$ & $\begin{array}{l}\text { Cp } \\
0.249\end{array}$ & $\begin{array}{l}R \\
53.350\end{array}$ & $\begin{array}{l}\text { Blades } \\
77.000\end{array}$ & $\begin{array}{l}\text { THK } \\
0.050\end{array}$ \\
\hline $\begin{array}{l}\text { CFM } \\
10375.500\end{array}$ & $\begin{array}{l}\text { SCFM } \\
\quad 49514.516\end{array}$ & $\begin{array}{l}\mathrm{A} 1 / \mathrm{A}^{*} \\
1.436\end{array}$ & $\begin{array}{l}\text { Area1 } \\
513.330\end{array}$ & $\begin{array}{l}A^{*} \\
357.471\end{array}$ & $\begin{array}{c}\text { AthrRotor } \\
395.845\end{array}$ & $\begin{array}{c}\text { ChokeMargin } \\
1.107\end{array}$ \\
\hline
\end{tabular}

ROTOR LEADING EDGE CONDITIONS, STAGE 4

\begin{tabular}{|c|c|c|c|c|c|c|c|c|c|}
\hline & R1 & Stator & Alfa & $\mathrm{C} 1$ & $\mathrm{CU} 1$ & $\mathrm{Cm} 1$ & Mabs & Mrel & U1cor \\
\hline TIP & 19.26 & 0.00 & -0.02 & 516.04 & -0.18 & 516.04 & 0.46 & 0.74 & 628.03 \\
\hline MEAN & 16.97 & 0.00 & -0.02 & 516.04 & -0.18 & 516.04 & 0.46 & 0.68 & \\
\hline HUB & 14.32 & 0.00 & -0.02 & 516.04 & -0.18 & 516.04 & 0.46 & 0.63 & \\
\hline & Betaflo & BetaBlade & Incid & U1 & W1 & Ps 1 & Ts1 & TwetBulb1 & $\mathrm{RH}$ \\
\hline TIP & 51.72 & 46.36 & 5.36 & 653.60 & 832.90 & 6.87 & 540.36 & 503.36 & 0.09 \\
\hline MEAN & 48.15 & 43.80 & 4.35 & 575.91 & 773.42 & 6.87 & 540.36 & 503.36 & 0.09 \\
\hline HUB & 43.29 & 37.84 & 5.45 & 485.96 & 708.96 & 6.87 & 540.36 & 503.36 & 0.09 \\
\hline
\end{tabular}

ROTOR EXIT CONDITIONS, STAGE 4 SOLUTION IS CONVERGED

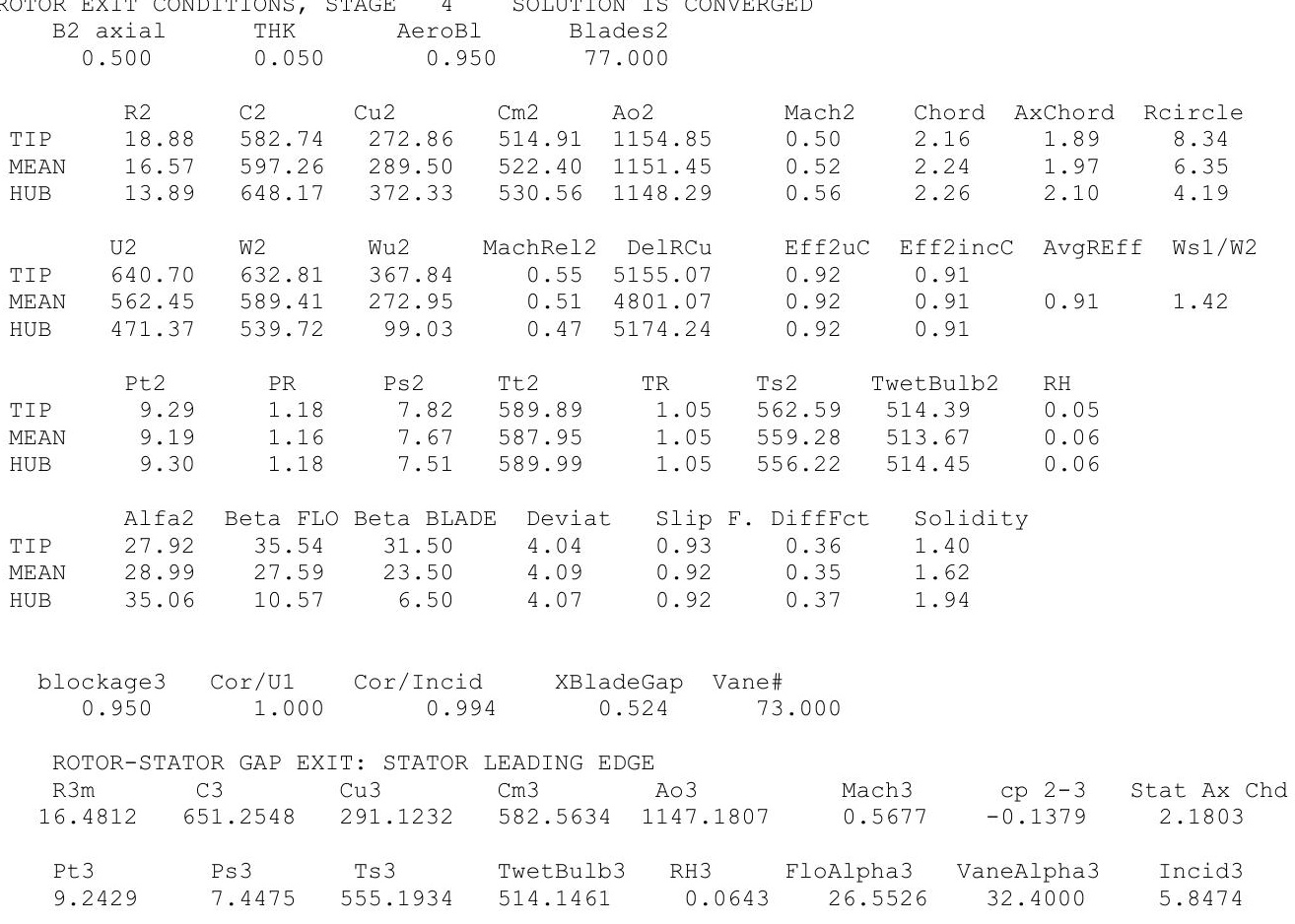




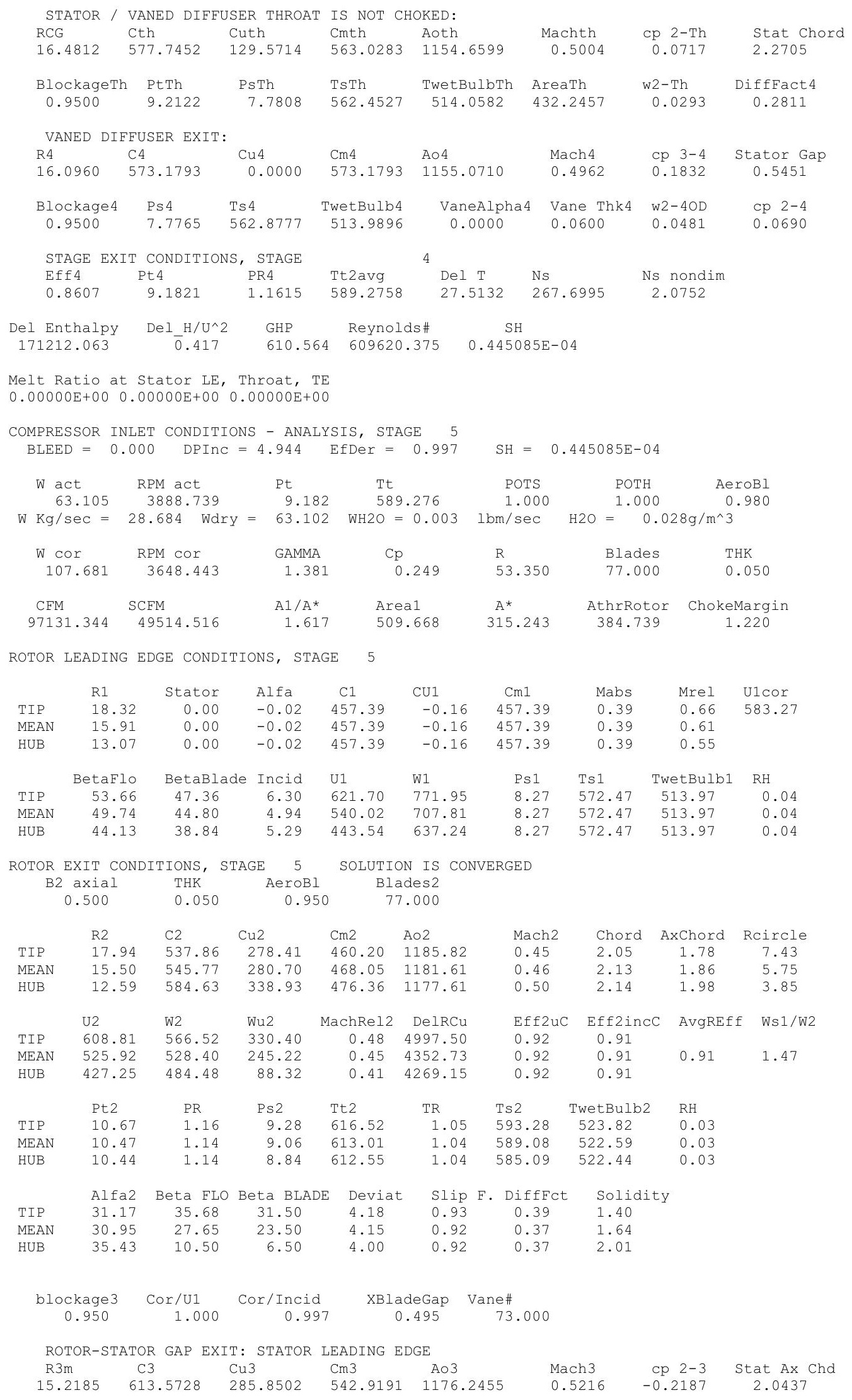




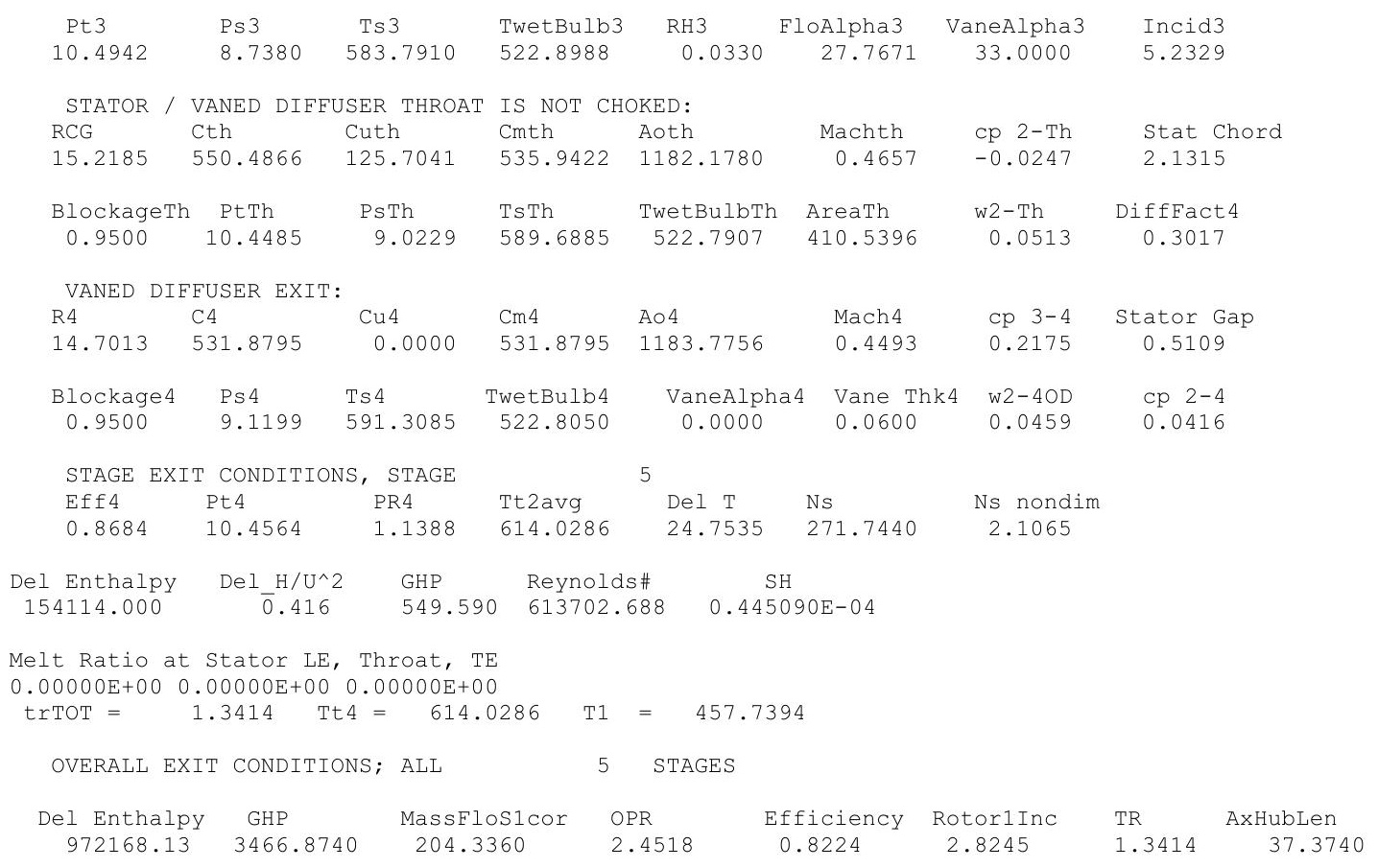




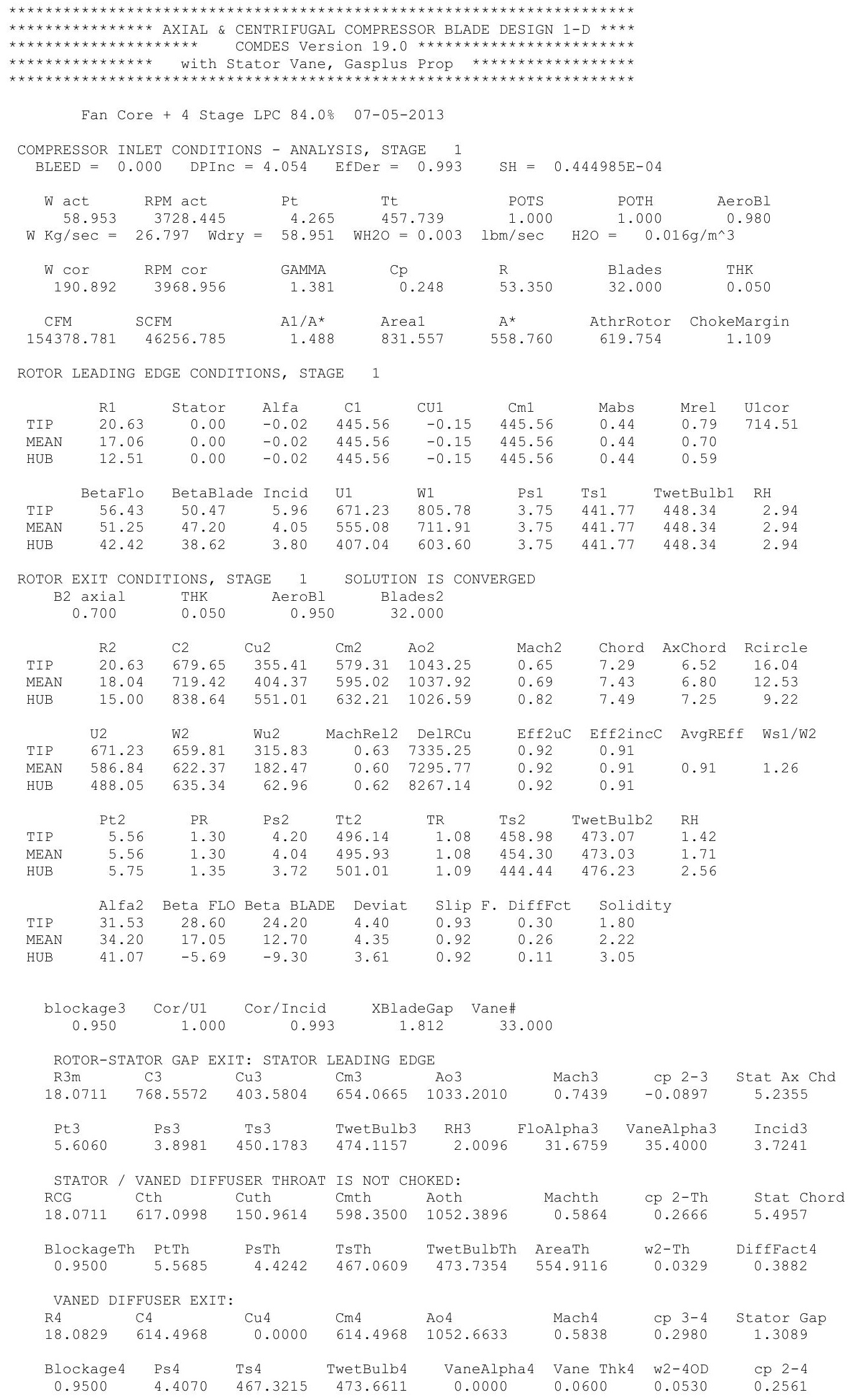




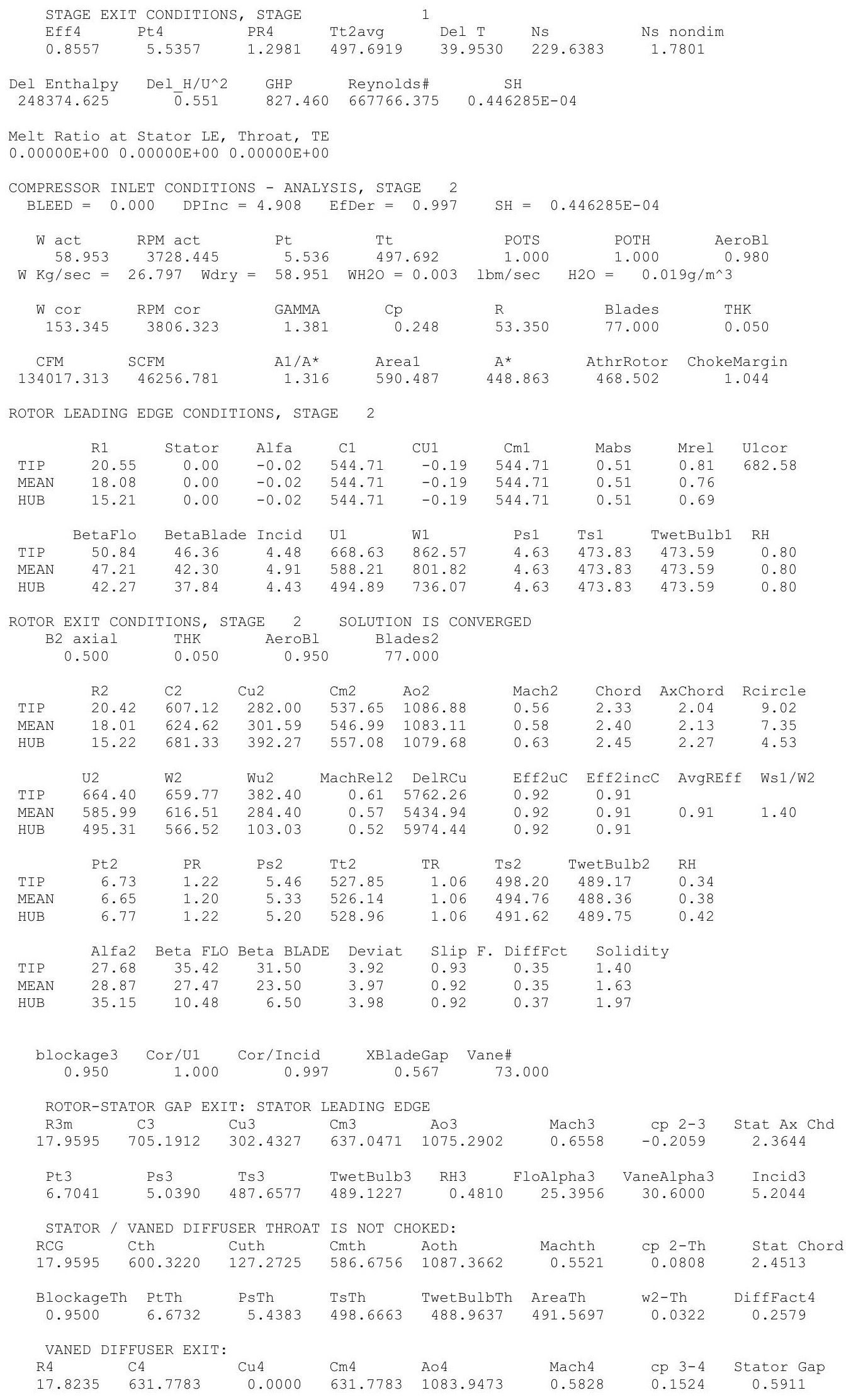




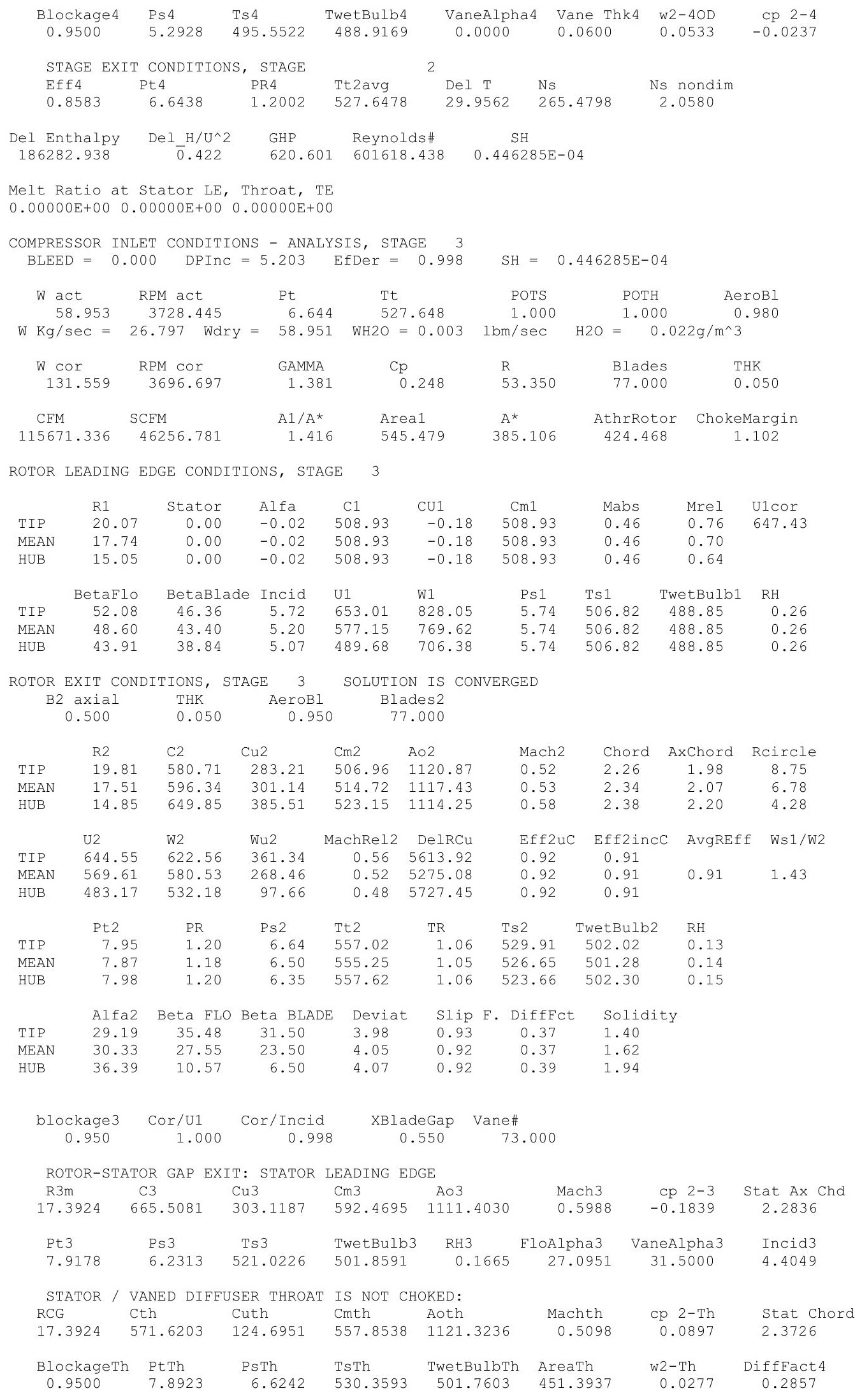




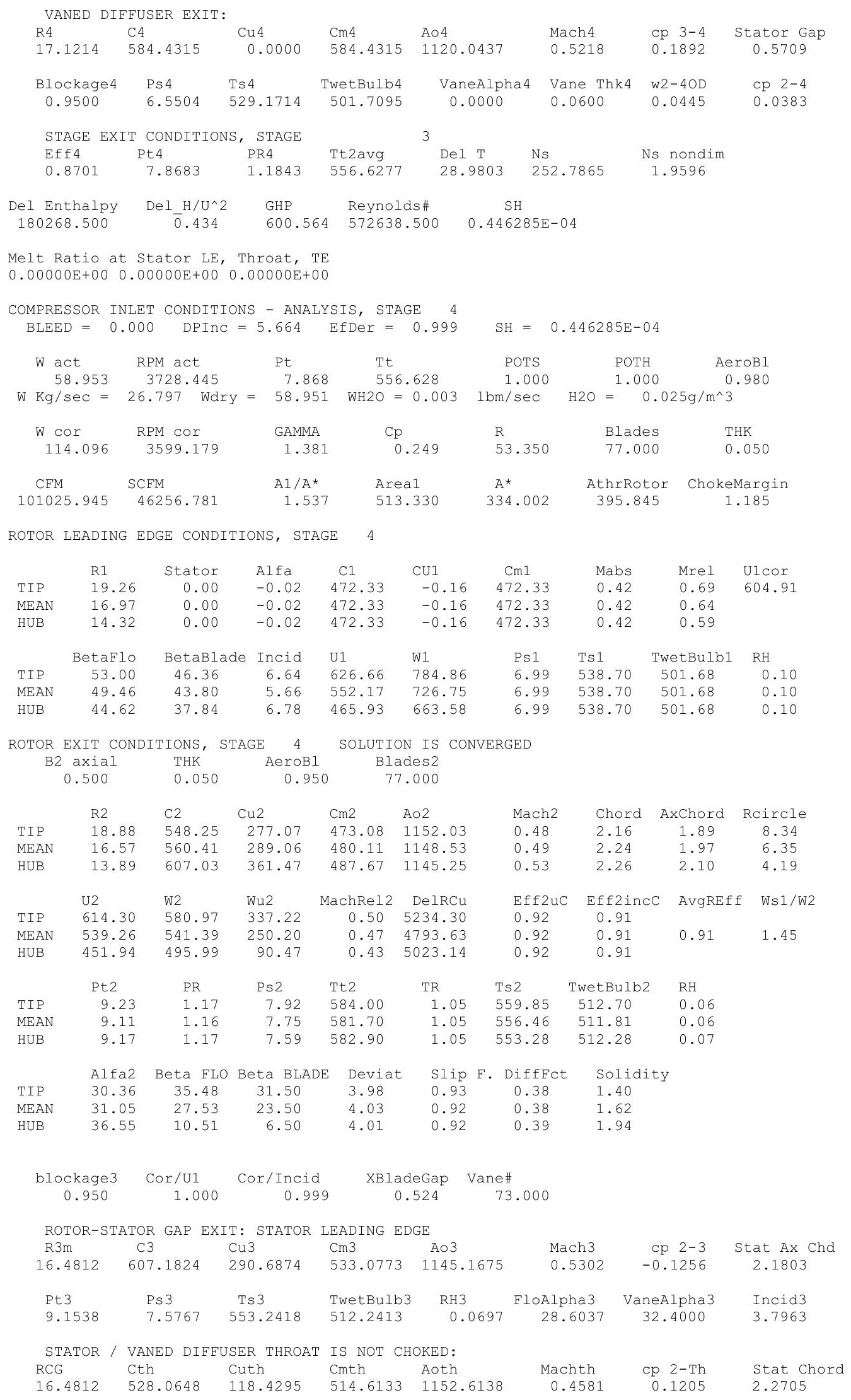




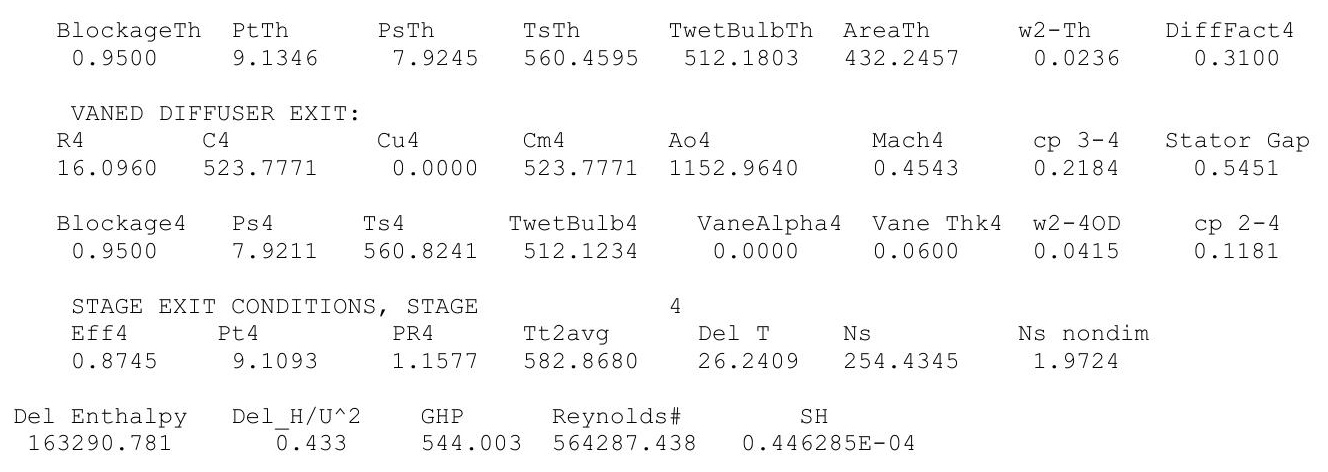

Melt Ratio at Stator LE, Throat, TE

$0.00000 \mathrm{E}+000.00000 \mathrm{E}+000.00000 \mathrm{E}+00$

COMPRESSOR INLET CONDITIONS - ANALYSIS, STAGE 5 $\mathrm{BLEED}=0.000 \quad$ DPInC $=6.066 \quad$ EfDer $=0.999$

$\begin{array}{llllll}W & \text { Ret } & \text { RPM act } & \text { POTS } & \text { POTH } & \text { AeroBI }\end{array}$ $\begin{array}{lll}1.000 & 1.000 & 0.980\end{array}$ $\mathrm{W} \mathrm{Kg} / \mathrm{sec}=26.797$ Wdry $=58.951$ WH2O $=0.003 \mathrm{lbm} / \mathrm{sec} \quad \mathrm{H} 20=0.028 \mathrm{~g} / \mathrm{m}^{\wedge} 3$

$\begin{array}{ccccccc}\text { W Cor } & \text { RPM cor } & \text { GAMMA } & \text { Cp } & \text { R } & \text { Blades } & \text { THK } \\ 100.848 & 3517.230 & 1.381 & 0.249 & 53.350 & 77.000 & 0.050 \\ \text { CFM } & \text { SCFM } & \text { A1/A* } & \text { Areal } & \text { A* } & \text { AthrRotor } & \text { ChokeMargin } \\ 89490.234 & 46256.781 & 1.726 & 509.668 & 295.238 & 384.739 & 1.303\end{array}$

ROTOR LEADING EDGE CONDITIONS, STAGE 5

$\begin{array}{lccccccccc} & \text { R1 } & \text { Stator } & \text { Alfa } & \text { C1 } & \text { CU1 } & \text { Cm1 } & \text { Mabs } & \text { Mrel } & \text { U1cor } \\ \text { TIP } & 18.32 & 0.00 & -0.02 & 421.40 & -0.15 & 421.40 & 0.36 & 0.63 & 562.29 \\ \text { MEAN } & 15.91 & 0.00 & -0.02 & 421.40 & -0.15 & 421.40 & 0.36 & 0.58 & \\ \text { HUB } & 13.07 & 0.00 & -0.02 & 421.40 & -0.15 & 421.40 & 0.36 & 0.52 & \\ & & & & & & & & & \\ \text { TIP } & \text { BetaFlo } & \text { BetaBlade } & \text { Incid } & \text { U1 } & \text { W1 } & \text { Ps } 1 & \text { Ts1 } & \text { TwetBulb1 } & \text { RH } \\ \text { MEAN } & 54.75 & 47.36 & 7.39 & 596.07 & 730.11 & 8.33 & 568.60 & 512.11 & 0.05 \\ \text { HUB } & 45.27 & 44.80 & 6.07 & 517.76 & 667.69 & 8.33 & 568.60 & 512.11 & 0.05 \\ & & 38.84 & 6.43 & 425.26 & 598.79 & 8.33 & 568.60 & 512.11 & 0.05\end{array}$

ROTOR EXIT CONDITIONS, STAGE 5 SOLUTION IS CONVERGED

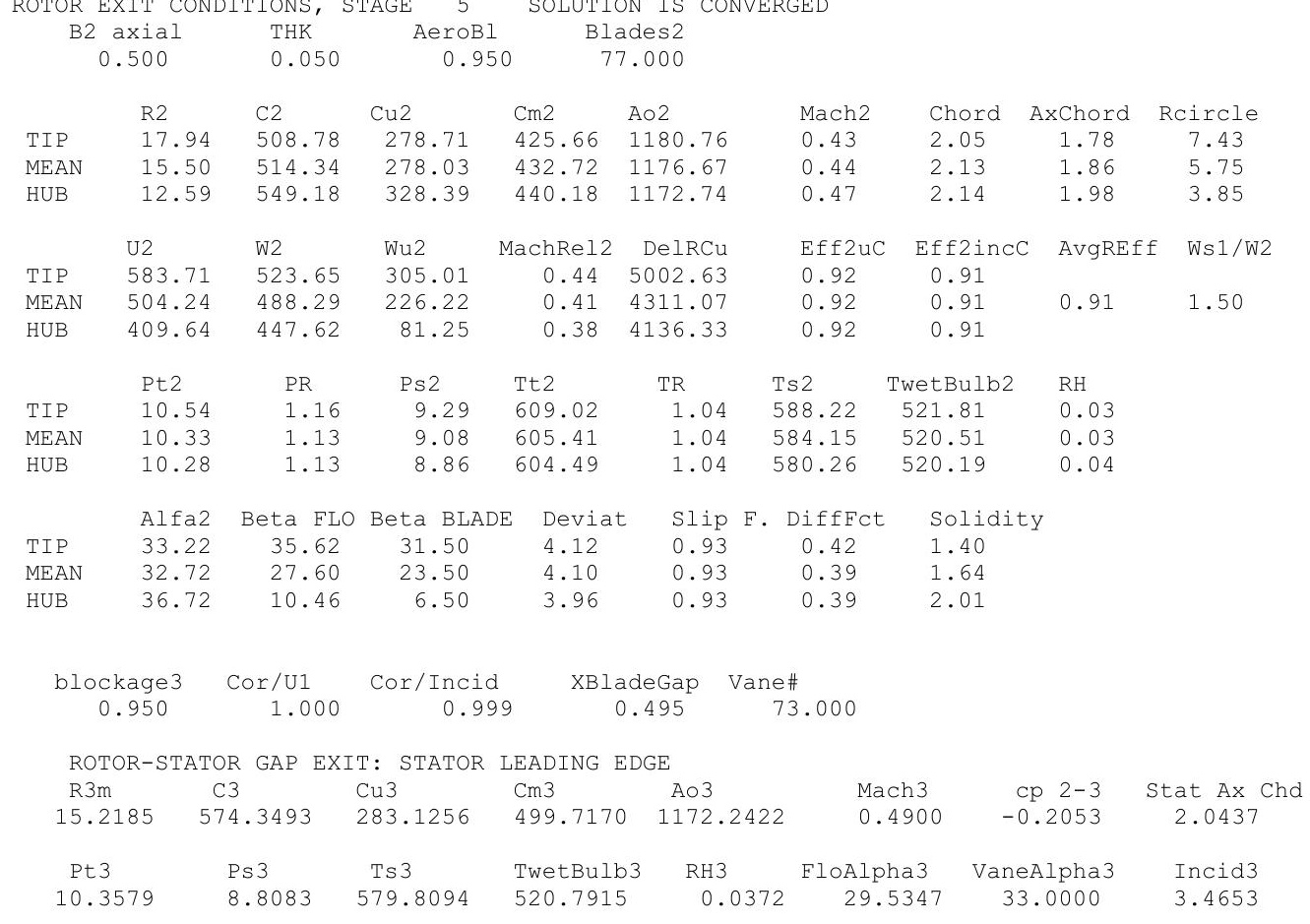




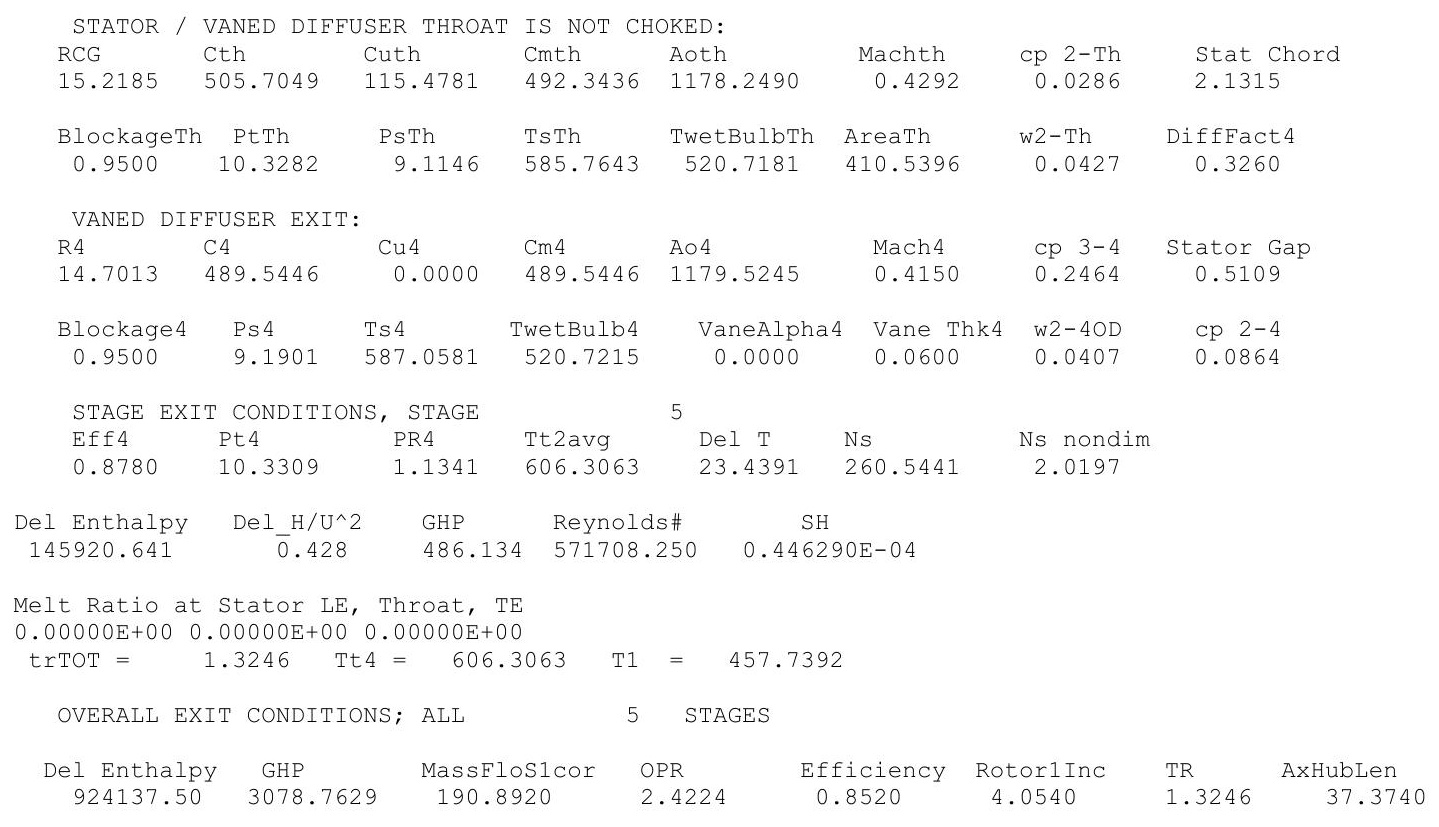




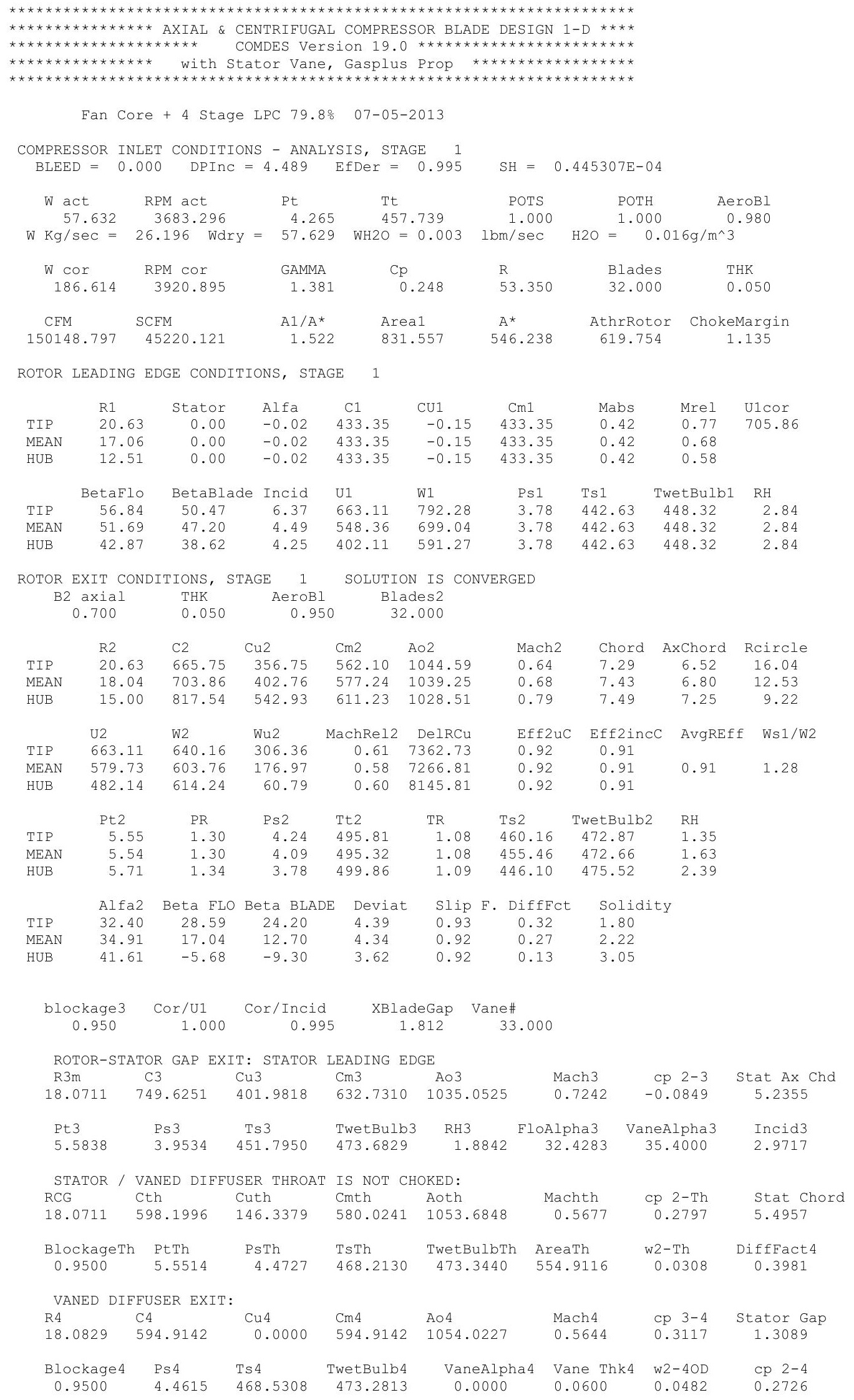




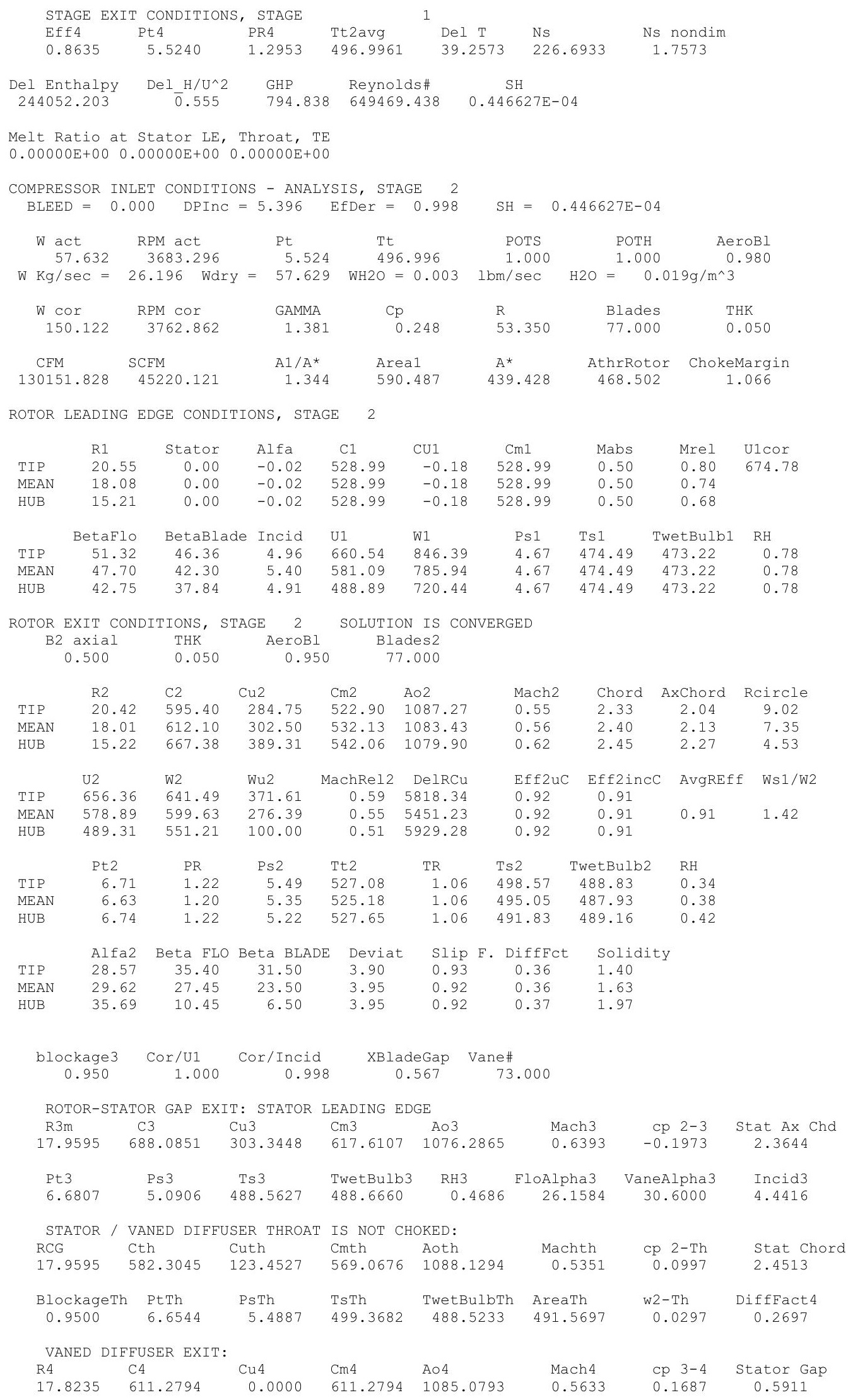




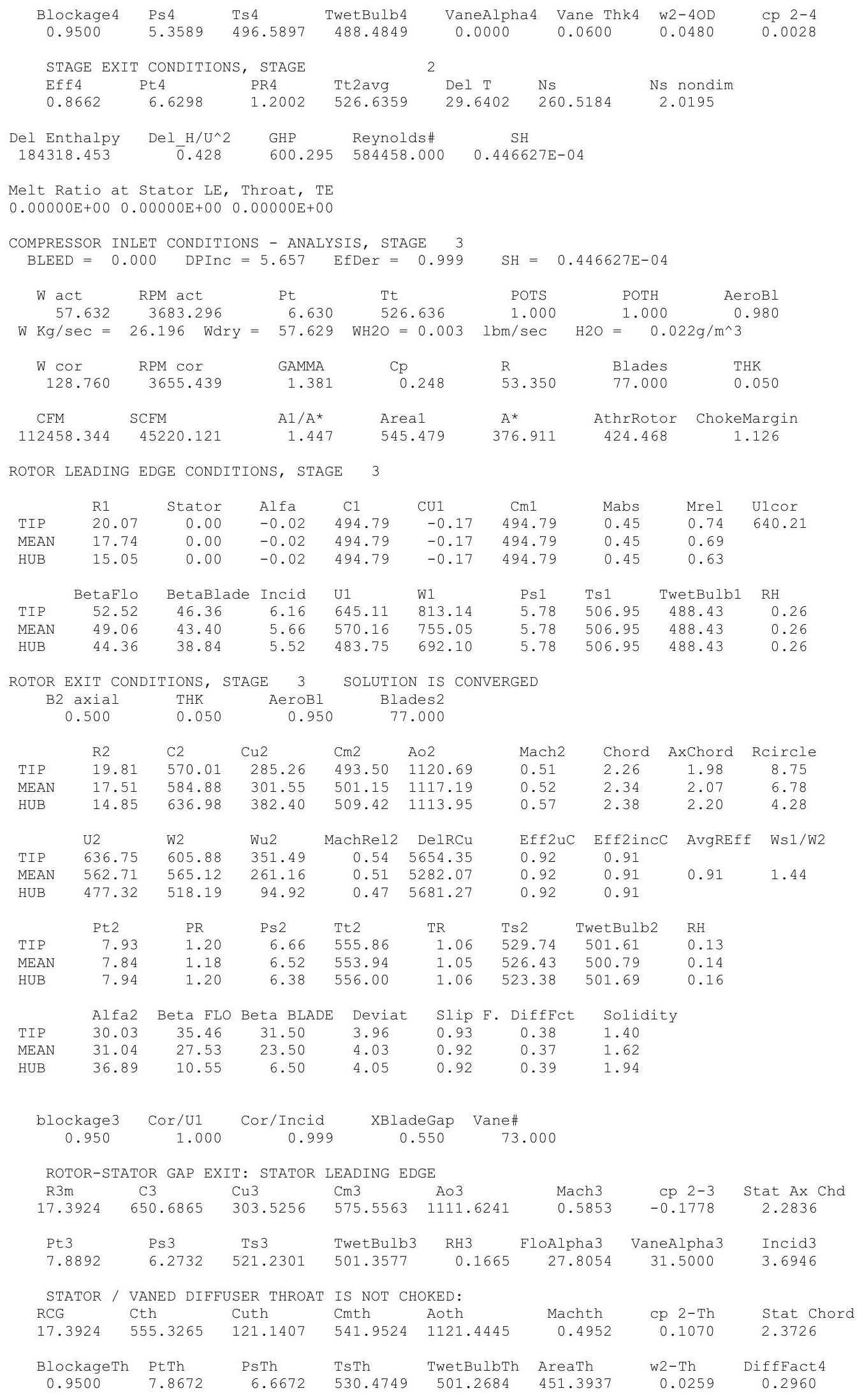




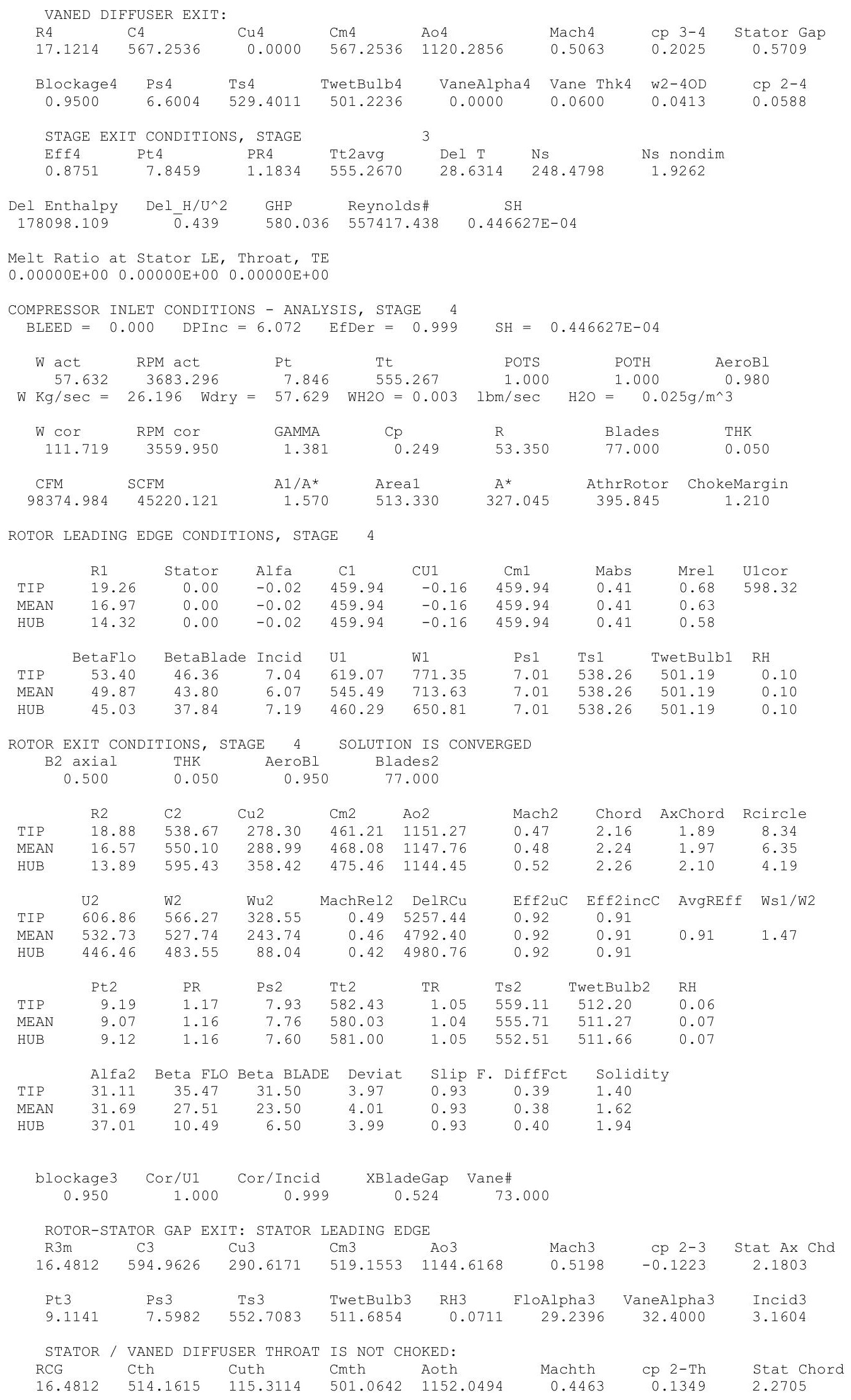




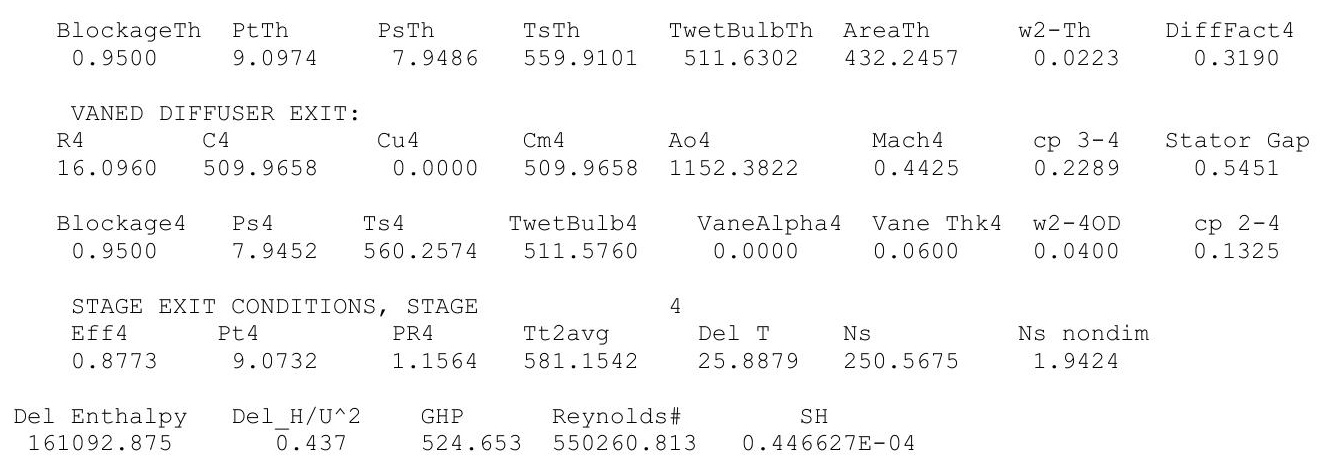

Melt Ratio at Stator LE, Throat, TE

$0.00000 \mathrm{E}+00 \quad 0.00000 \mathrm{E}+00 \quad 0.00000 \mathrm{E}+00$

COMPRESSOR INLET CONDITIONS - ANALYSIS, STAGE 5 BLEED $=0.000 \quad$ DPInC $=6.414 \quad$ EfDer $=1.000$

$\begin{array}{llllll}W & \text { Ret } & \text { RPM act } & \text { POTS } & \text { POTH } & \text { AeroBI }\end{array}$ $\begin{array}{llllll}57.632 & 3683.296 & 981.154 & 1.000 & 1.000 & 0.980\end{array}$ $\mathrm{W} \mathrm{Kg} / \mathrm{sec}=26.196$ Wdry $=57.629 \mathrm{WH} 2 \mathrm{O}=0.003 \mathrm{lbm} / \mathrm{sec} \quad \mathrm{H} 2 \mathrm{O}=0.028 \mathrm{~g} / \mathrm{m}^{\wedge} 3$

$\begin{array}{ccccccc}\text { W Cor } & \text { RPM cor } & \text { GAMMA } & \text { CP } & \text { R } & \text { Blades } & \text { THK } \\ 98.834 & 3479.759 & 1.381 & 0.249 & 53.350 & 77.000 & 0.050 \\ & & & & & & \\ \text { CFM } & \text { SCFM } & \text { A1/A* } & \text { Area1 } & A^{*} & \text { AthrRotor } & \text { ChokeMargin } \\ 87313.414 & 45220.121 & 1.761 & 509.668 & 289.342 & 384.739 & 1.330\end{array}$

ROTOR LEADING EDGE CONDITIONS, STAGE 5

$\begin{array}{lccccccccc} & \text { R1 } & \text { Stator } & \text { Alfa } & \text { C1 } & \text { CU1 } & \text { Cm1 } & \text { Mabs } & \text { Mrel } & \text { U1cor } \\ \text { TIP } & 18.32 & 0.00 & -0.02 & 411.15 & -0.14 & 411.15 & 0.35 & 0.62 & 556.30 \\ \text { MEAN } & 15.91 & 0.00 & -0.02 & 411.15 & -0.14 & 411.15 & 0.35 & 0.57 & \\ \text { HUB } & 13.07 & 0.00 & -0.02 & 411.15 & -0.14 & 411.15 & 0.35 & 0.51 & \\ & & & & & & & & & \\ \text { TIP } & \text { BetaFlo } & \text { BetaBlade } & \text { Incid } & \text { U1 } & \text { W1 } & \text { Ps1 } & \text { Ts1 } & \text { TwetBulb1 } & \text { RH } \\ \text { MEAN } & 51.08 & 47.36 & 7.72 & 588.86 & 718.31 & 8.33 & 567.57 & 511.56 & 0.05 \\ \text { HUB } & 45.63 & 38.84 & 6.79 & 420.11 & 587.93 & 8.33 & 567.57 & 511.56 & 0.05\end{array}$

ROTOR EXIT CONDITIONS, STAGE 5 SOLUTION IS CONVERGED

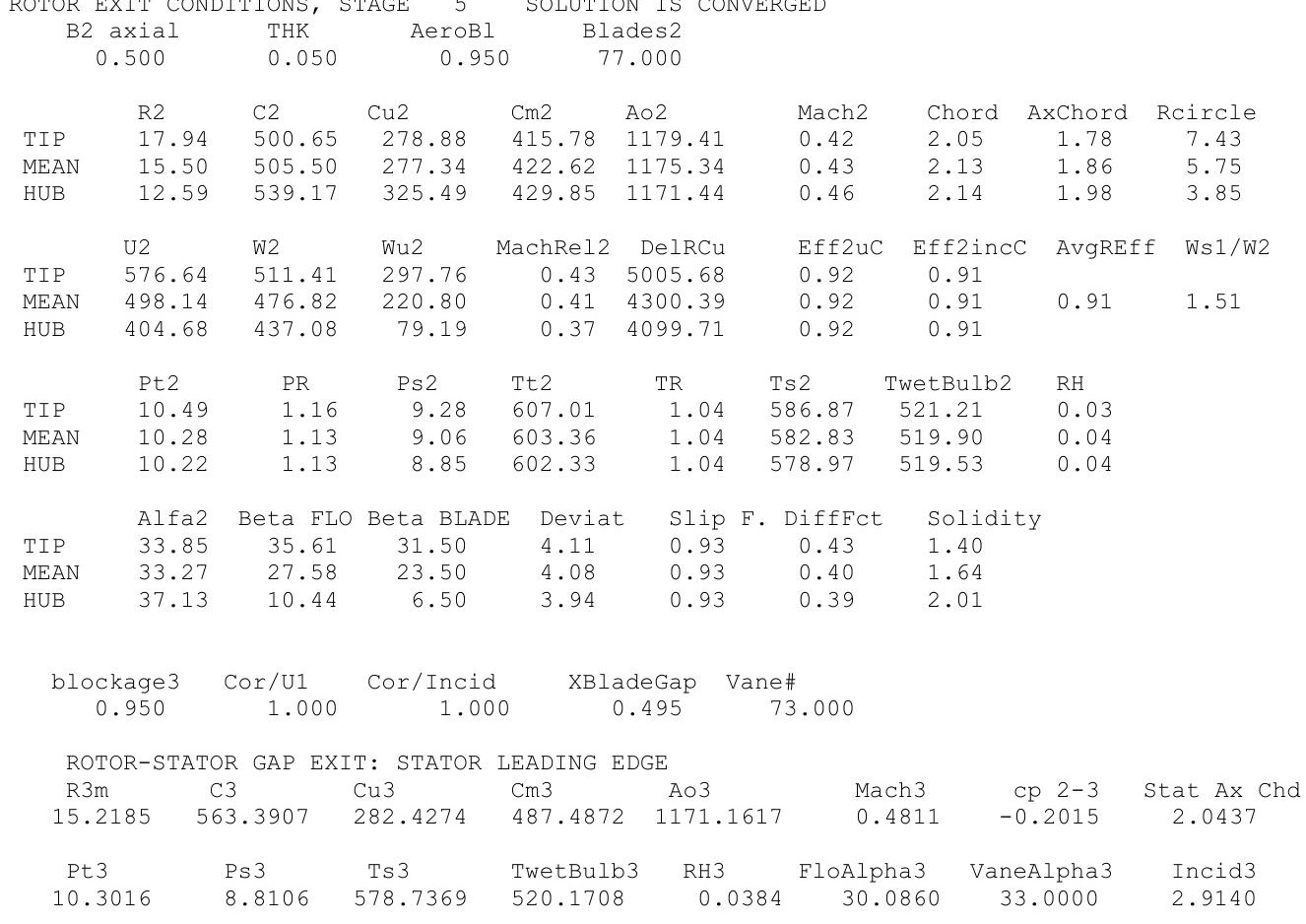




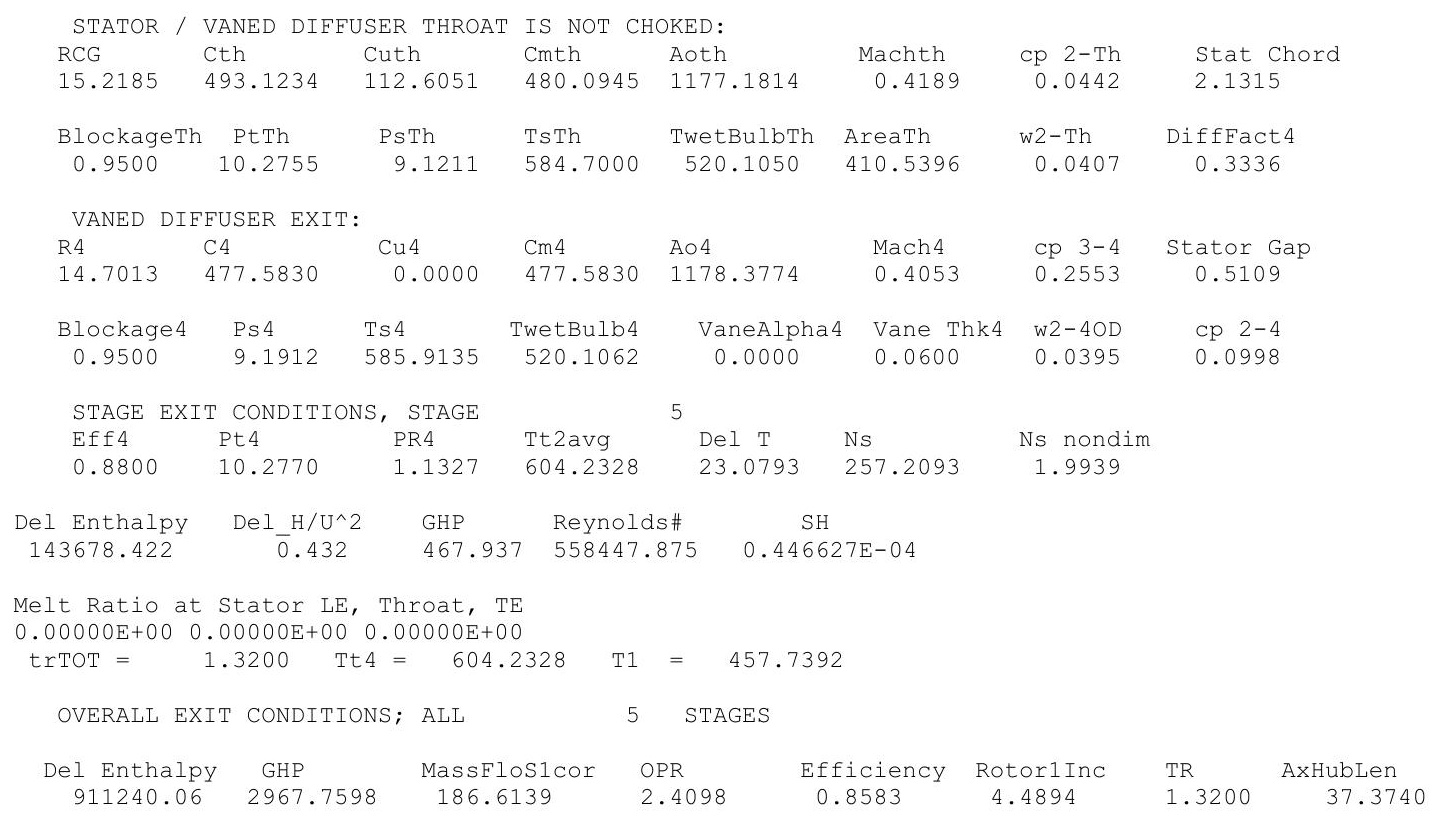




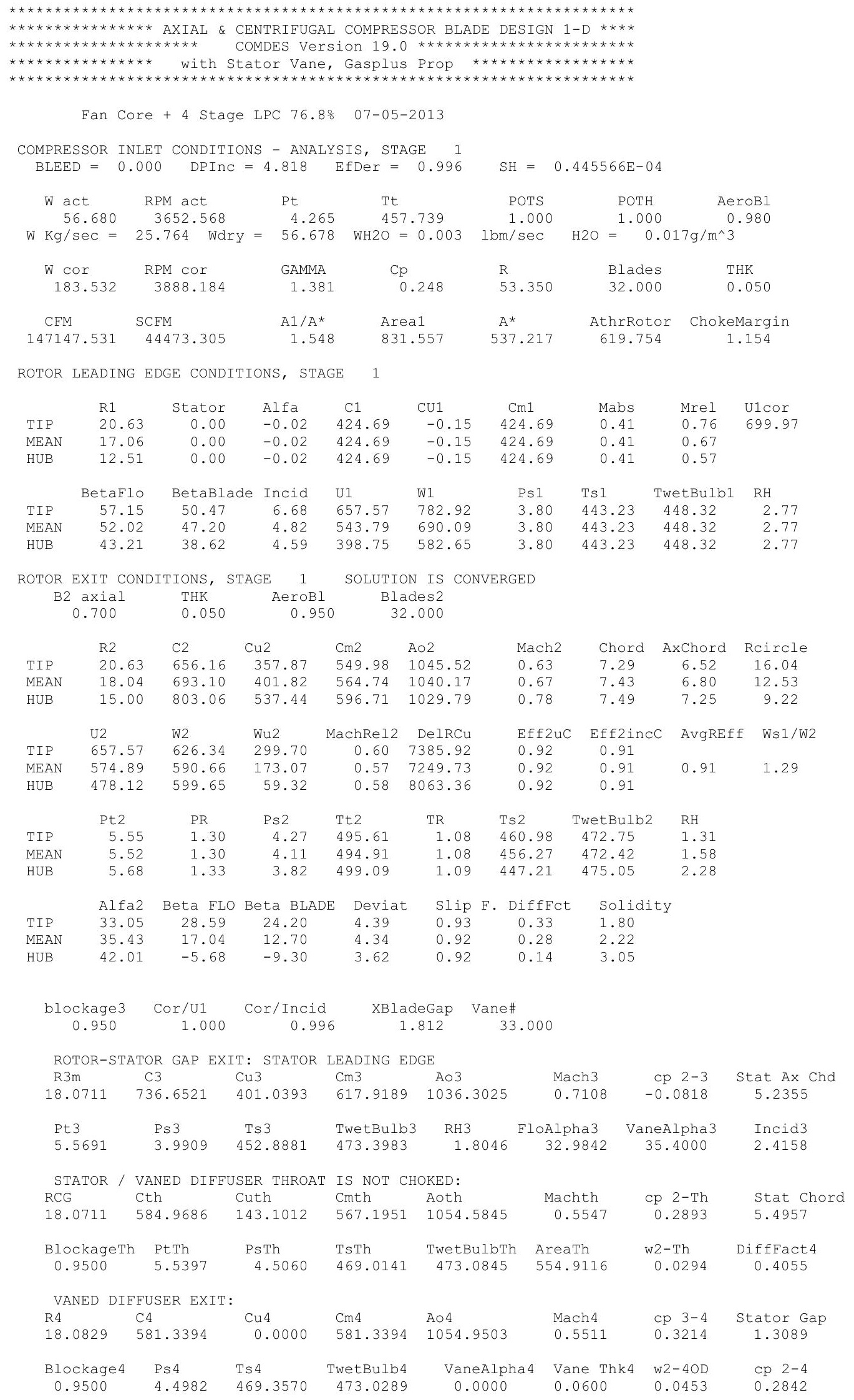




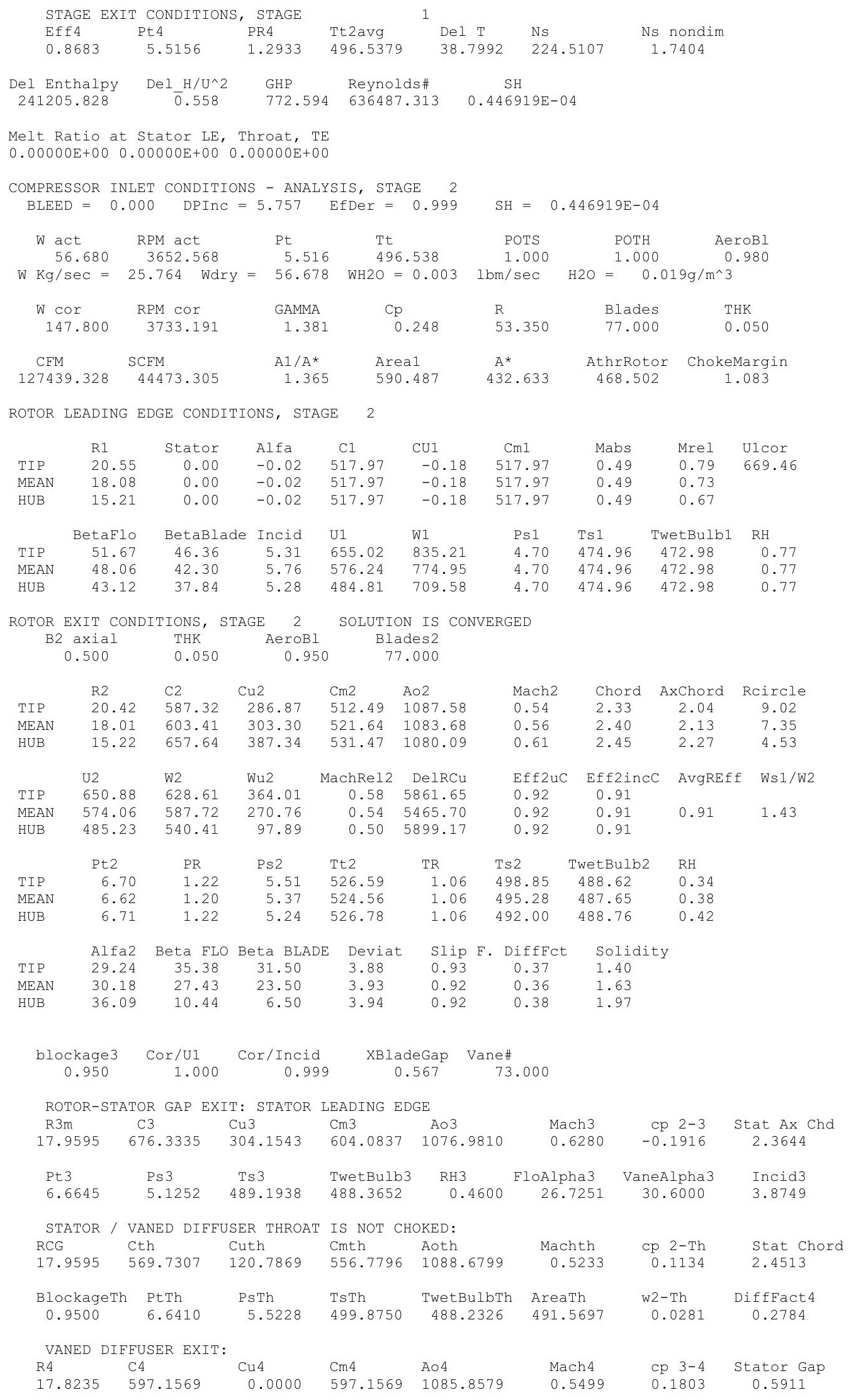




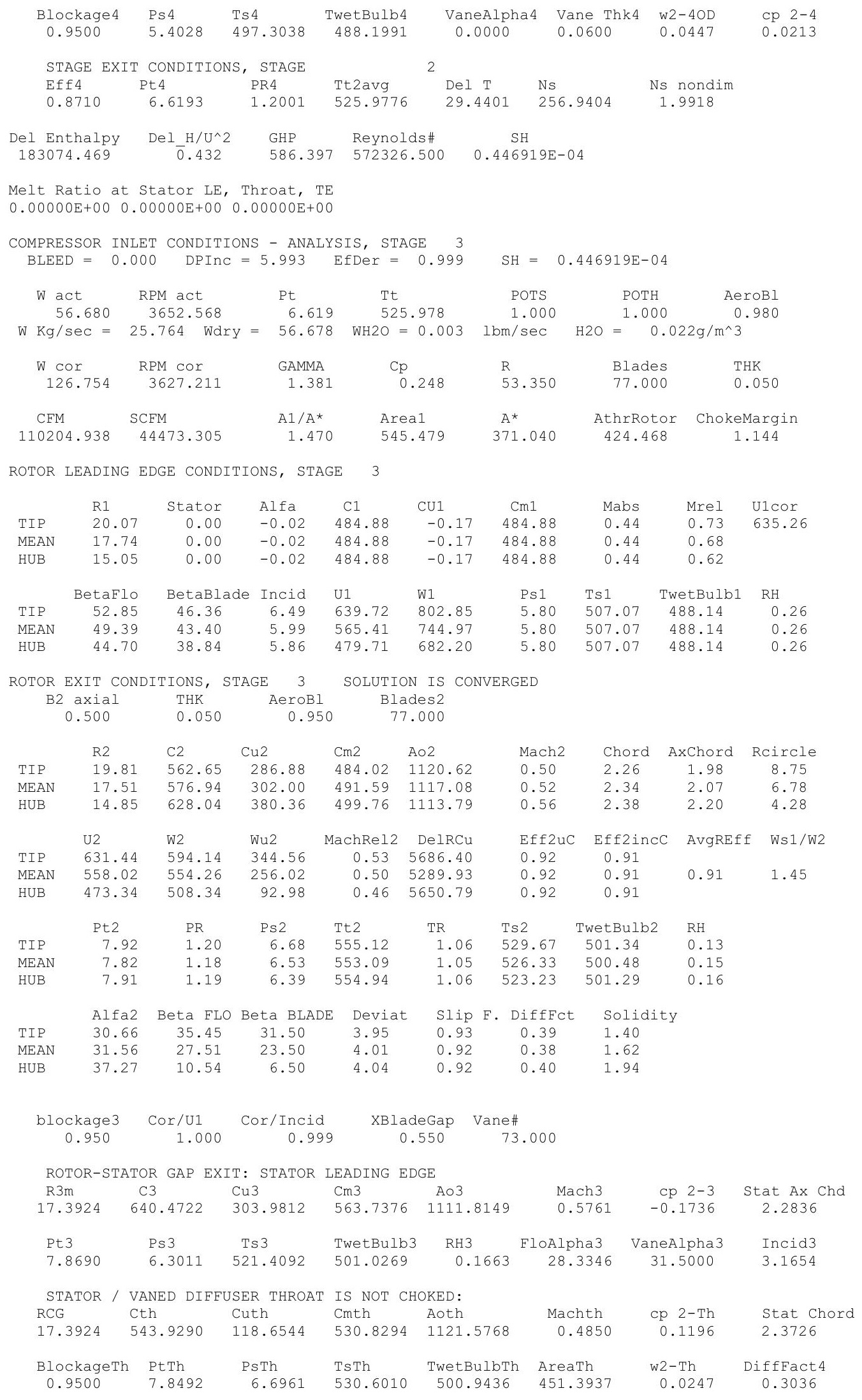




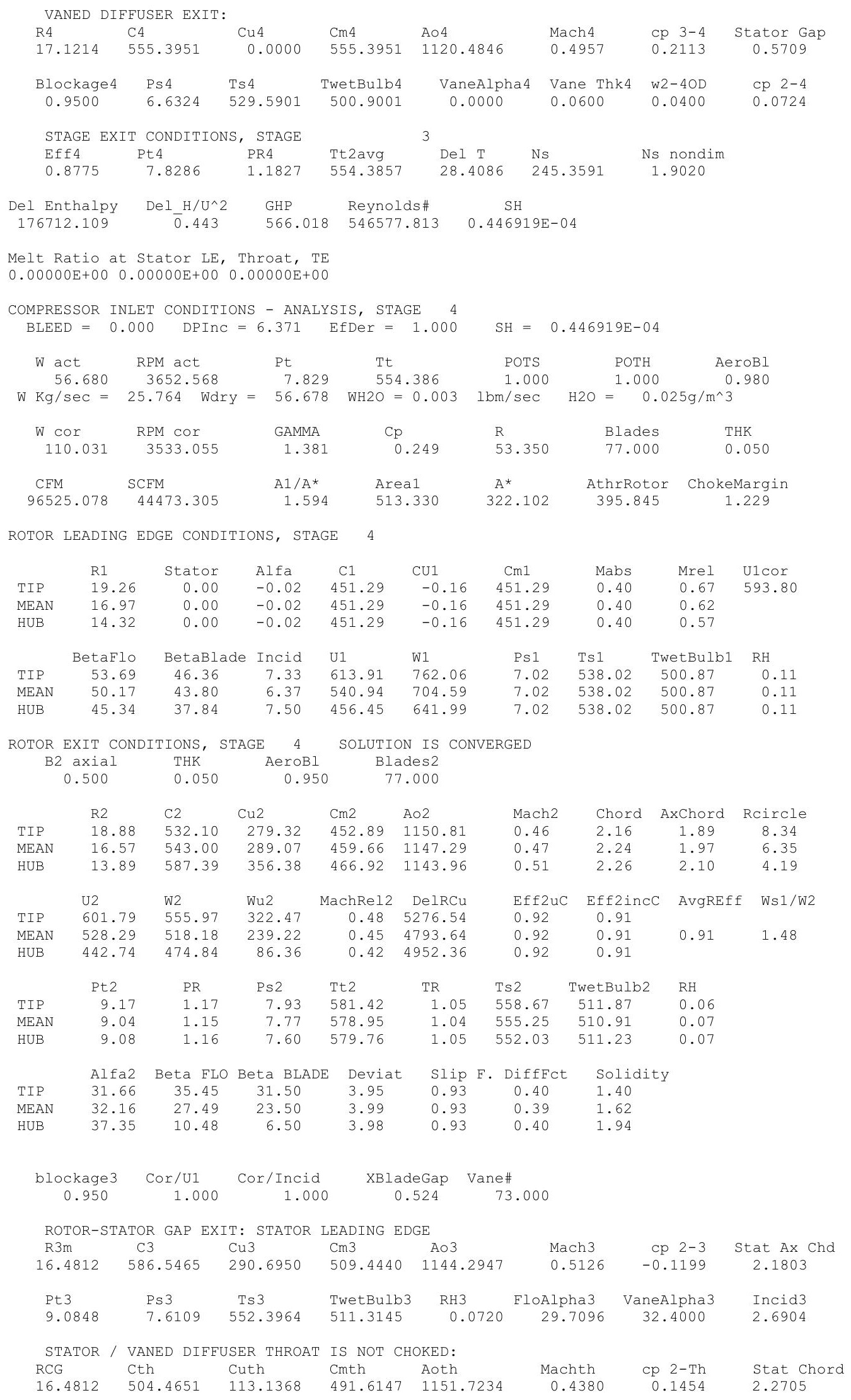




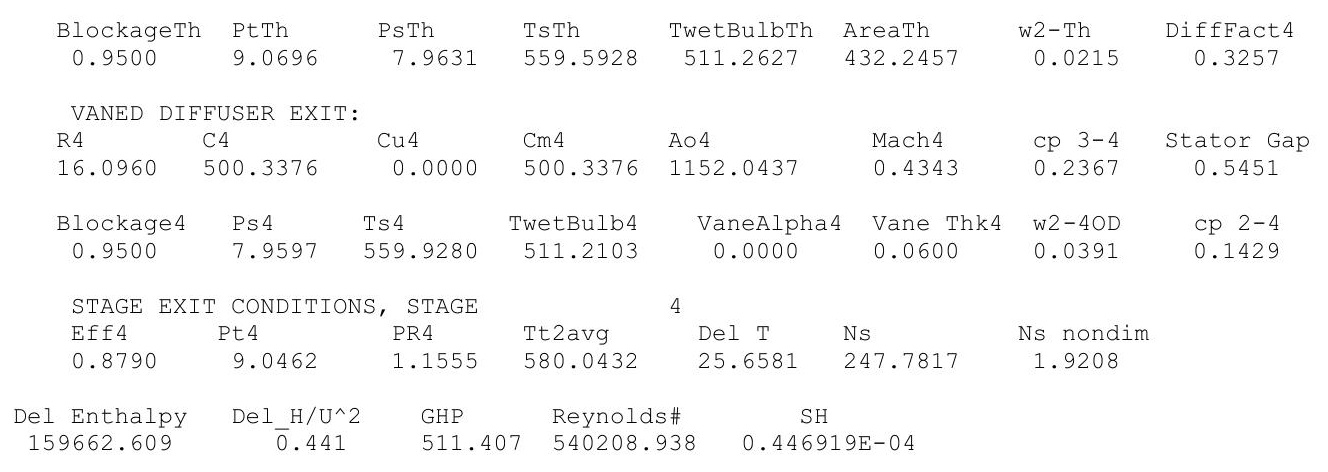

Melt Ratio at Stator LE, Throat, TE

$0.00000 \mathrm{E}+00 \quad 0.00000 \mathrm{E}+00 \quad 0.00000 \mathrm{E}+00$

COMPRESSOR INLET CONDITIONS - ANALYSIS, STAGE 5 $\mathrm{BLEED}=0.000 \quad$ DPInC $=6.673 \quad$ EfDer $=1.000$

$\begin{array}{llllll}W & \text { Ret } & \text { RPM act } & \text { POTS } & \text { POTH } & \text { AeroBI }\end{array}$ $\begin{array}{lllllll}56.680 & 3652.568 & 9.046 & 580.043 & 1.000 & 1.000 & 0.980\end{array}$ $\mathrm{W} \mathrm{Kg} / \mathrm{sec}=25.764$ Wdry $=56.678 \mathrm{WH} 2 \mathrm{O}=0.003 \mathrm{lbm} / \mathrm{sec} \quad \mathrm{H} 2 \mathrm{O}=0.028 \mathrm{~g} / \mathrm{m}^{\wedge} 3$

$\begin{array}{ccccccc}\text { W Cor } & \text { RPM cor } & \text { GAMMA } & \text { CP } & \text { R } & \text { Blades } & \text { THK } \\ 97.399 & 3454.032 & 1.381 & 0.249 & 53.350 & 77.000 & 0.050 \\ & & & & & & \\ \text { CFM } & \text { SCFM } & \text { A1/A* } & \text { Area1 } & A^{*} & \text { AthrRotor } & \text { ChokeMargin } \\ 85786.469 & 44473.305 & 1.787 & 509.668 & 285.141 & 384.739 & 1.349\end{array}$

ROTOR LEADING EDGE CONDITIONS, STAGE 5

\begin{tabular}{|c|c|c|c|c|c|c|c|c|c|}
\hline & R1 & Stator & Alfa & $\mathrm{C} 1$ & CU1 & $\mathrm{Cm} 1$ & Mabs & Mrel & U1cor \\
\hline TIP & 18.32 & 0.00 & -0.02 & 403.96 & -0.14 & 403.96 & 0.35 & 0.61 & 552.19 \\
\hline MEAN & 15.91 & 0.00 & -0.02 & 403.96 & -0.14 & 403.96 & 0.35 & 0.56 & \\
\hline HUB & 13.07 & 0.00 & -0.02 & 403.96 & -0.14 & 403.96 & 0.35 & 0.50 & \\
\hline & BetaFlo & BetaBlade & Incid & U1 & W1 & Ps 1 & Ts1 & TwetBulb1 & $\mathrm{RH}$ \\
\hline TIP & 55.33 & 47.36 & 7.97 & 583.94 & 710.17 & 8.33 & 566.93 & 511.20 & 0.05 \\
\hline MEAN & 51.47 & 44.80 & 6.67 & 507.22 & 648.54 & 8.33 & 566.93 & 511.20 & 0.05 \\
\hline HUB & 45.89 & 38.84 & 7.05 & 416.60 & 580.40 & 8.33 & 566.93 & 511.20 & 0.05 \\
\hline
\end{tabular}

ROTOR EXIT CONDITIONS, STAGE 5 SOLUTION IS CONVERGED

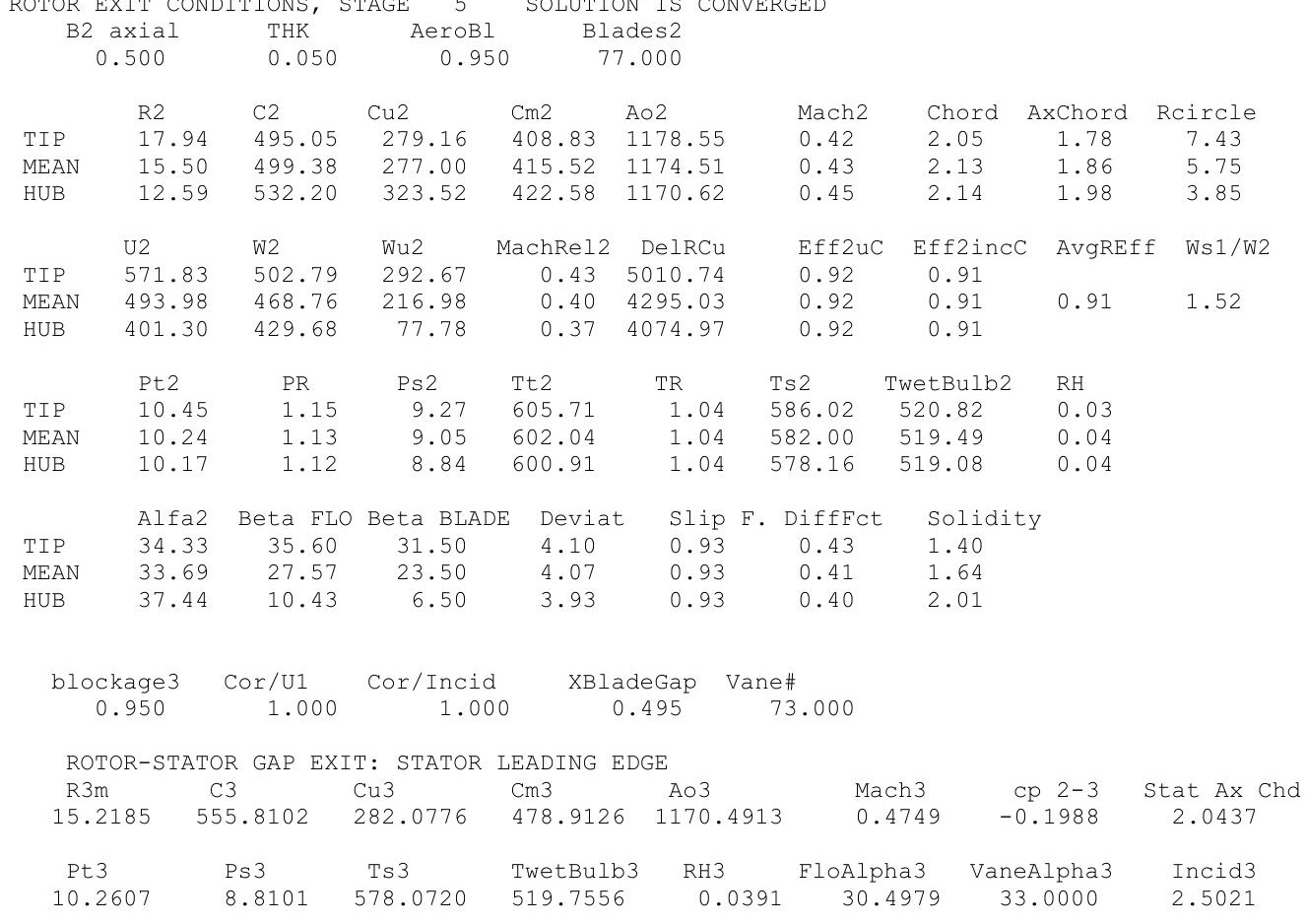




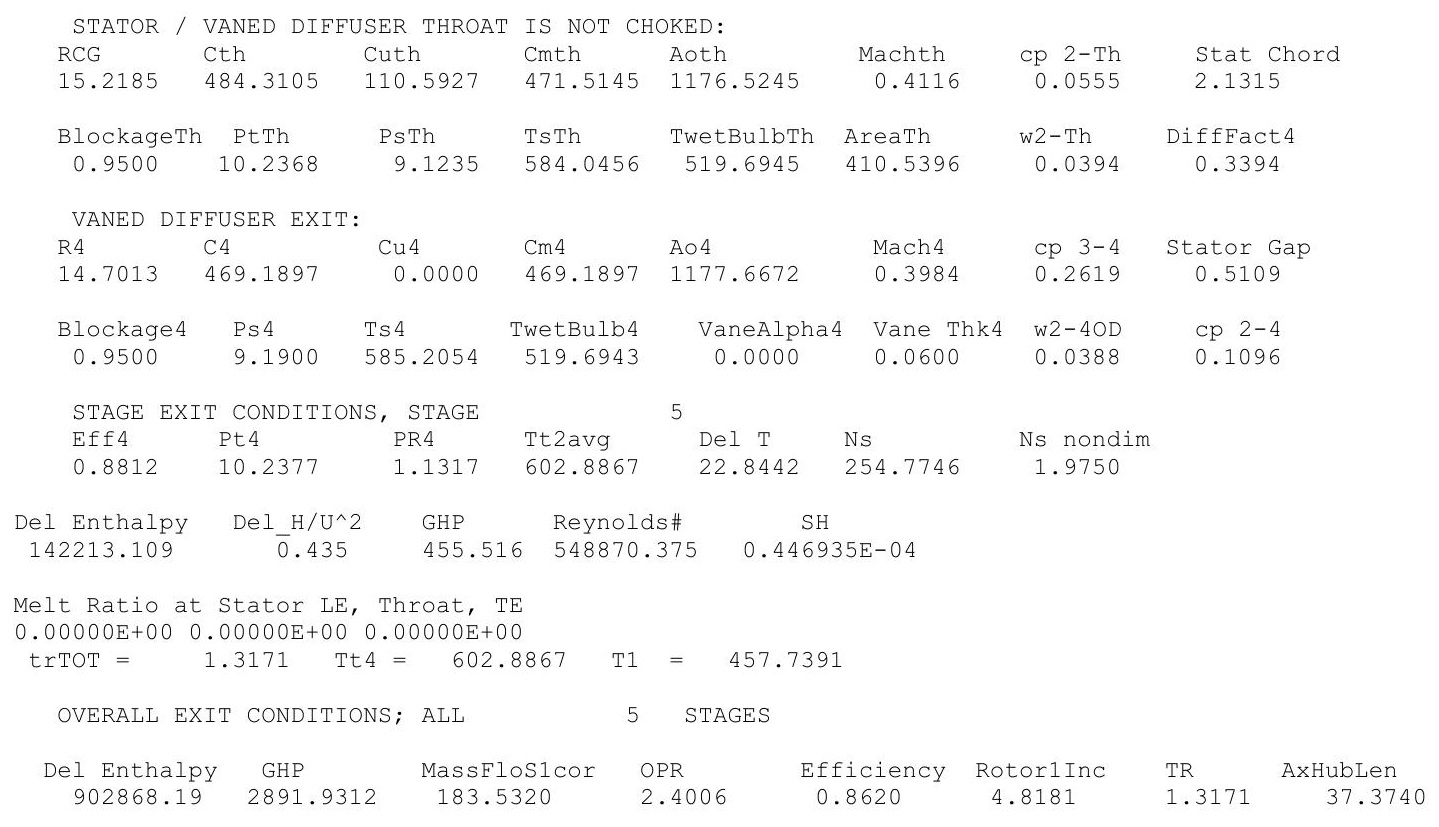


$100 \mu \mathrm{m}, \mathrm{ISA}+36 \mathrm{R}$

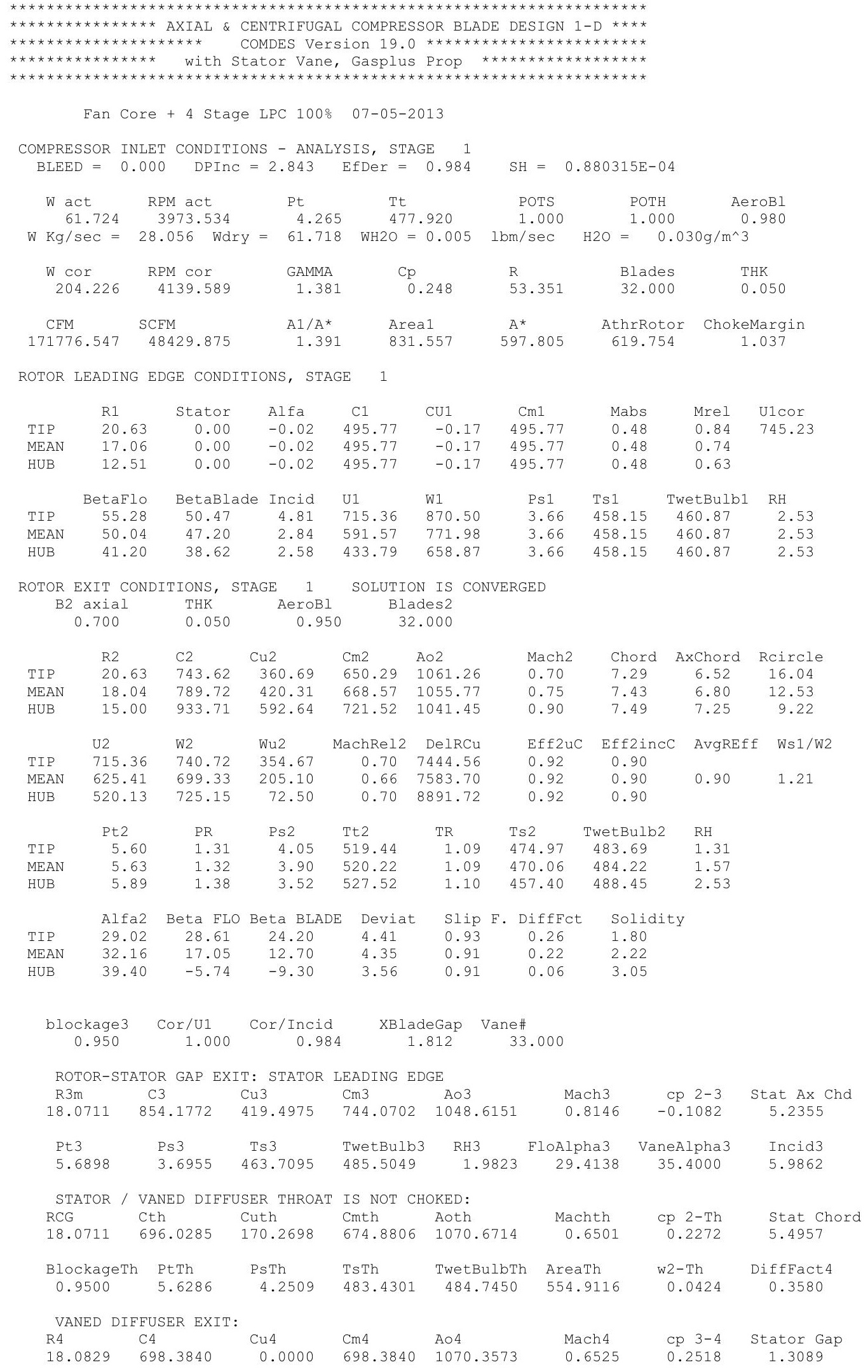




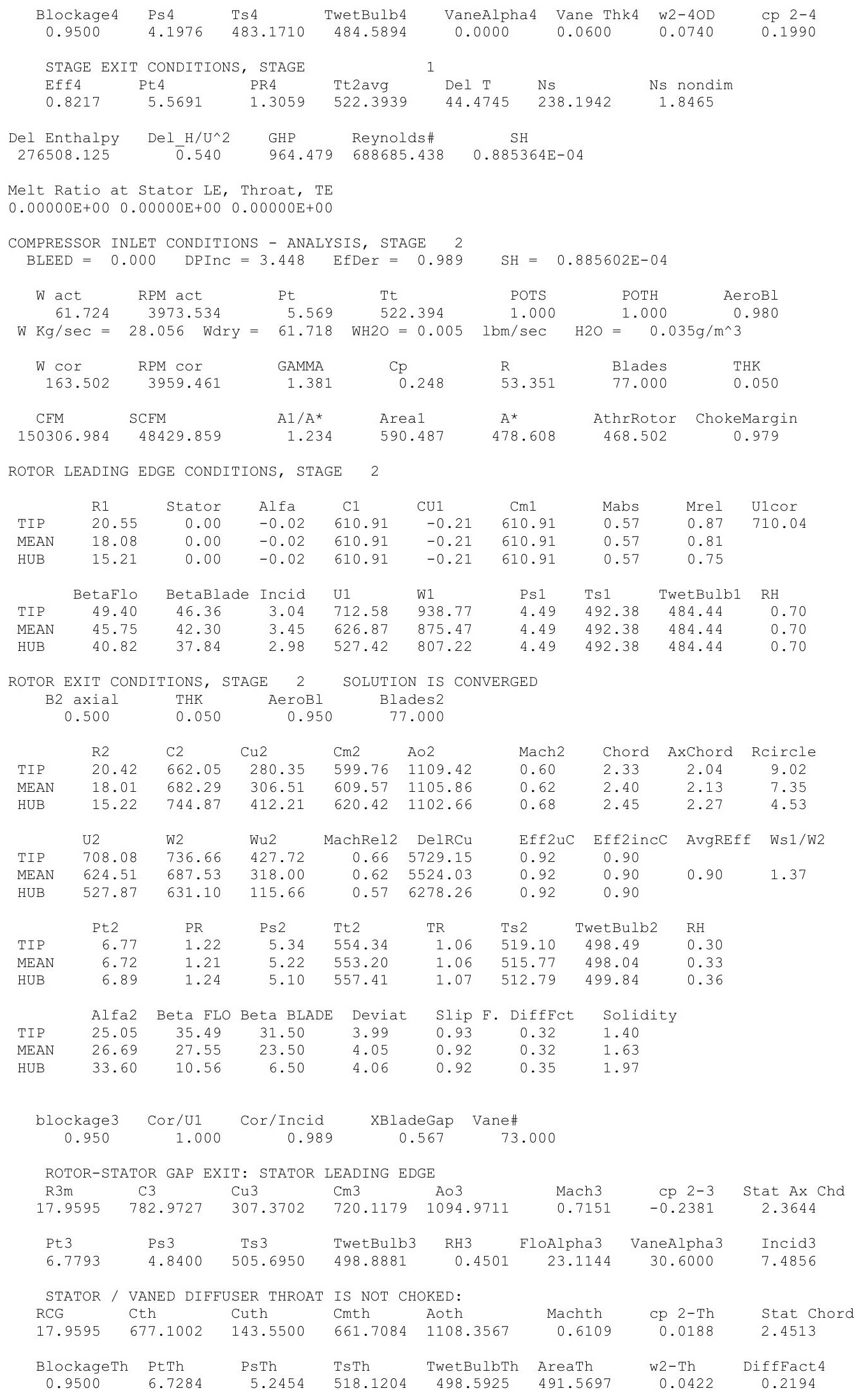




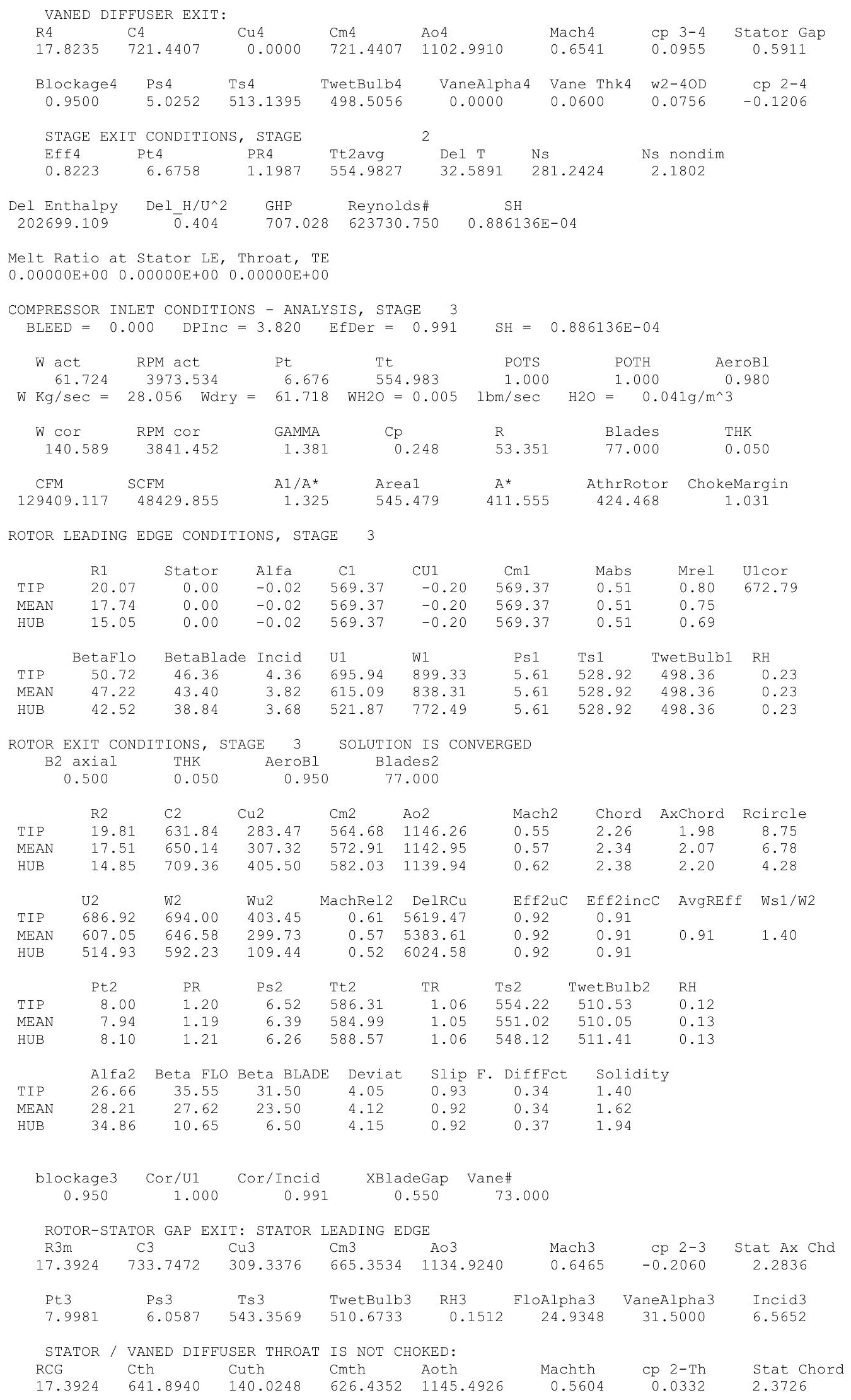




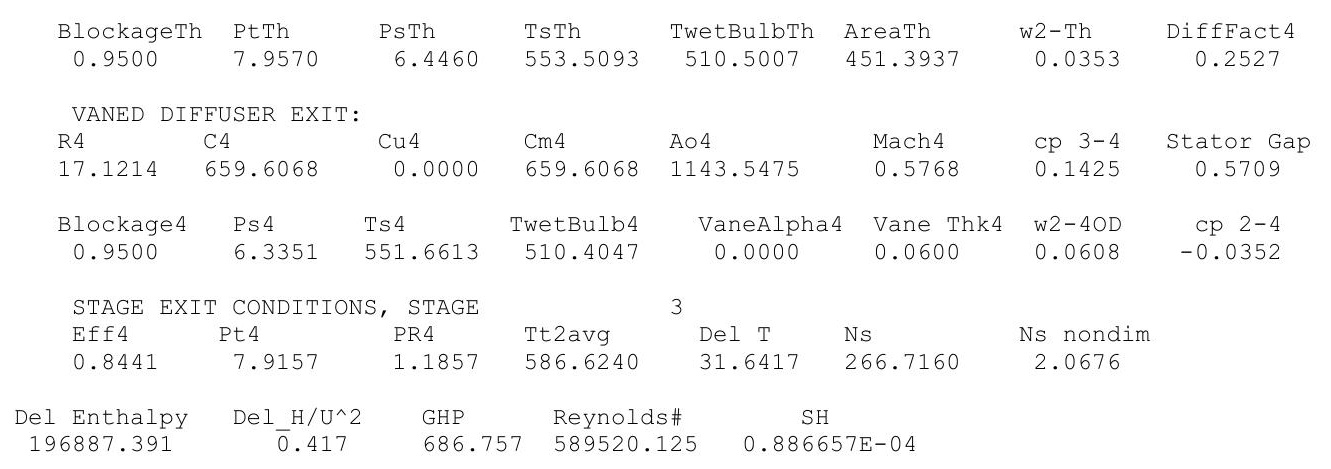

Melt Ratio at Stator LE, Throat, TE

$0.00000 \mathrm{E}+000.00000 \mathrm{E}+00 \quad 0.00000 \mathrm{E}+00$

COMPRESSOR INLET CONDITIONS - ANALYSIS, STAGE 4 $\mathrm{BLEED}=0.000 \quad$ DPInC $=4.406 \quad$ EfDer $=0.995$

61.724 RPM act Pt Tt

$\mathrm{W} \mathrm{Kg} / \mathrm{sec}=28.056$ Wdry $=61.718$ WH2O $=0.005$

$\mathrm{SH}=0.886657 \mathrm{E}-04$

$\begin{array}{ccccccc}\text { W cor } & \text { RPM cor } & \text { GAMMA } & \text { Cp } & \text { R } & \text { Blades } & \text { THK } \\ 121.900 & 3736.416 & 1.381 & 0.249 & 53.351 & 77.000 & 0.050 \\ & & & & & & \\ \text { CFM } & \text { SCFM } & \text { A1/A* } & \text { Area1 } & A^{*} & \text { AthrRotor } & \text { ChokeMargin } \\ 112548.188 & 48429.859 & 1.438 & 513.330 & 356.868 & 395.845 & 1.109\end{array}$

ROTOR LEADING EDGE CONDITIONS, STAGE 4

$\begin{array}{lrrrrrrrrr} & \text { R1 } & \text { Stator } & \text { Alfa } & \text { C1 } & \text { CU1 } & \text { Cm1 } & \text { Mabs } & \text { Mrel } & \text { U1cor } \\ \text { TIP } & 19.26 & 0.00 & -0.02 & 526.20 & -0.18 & 526.20 & 0.45 & 0.74 & 627.98 \\ \text { MEAN } & 16.97 & 0.00 & -0.02 & 526.20 & -0.18 & 526.20 & 0.45 & 0.68 & \\ \text { HUB } & 14.32 & 0.00 & -0.02 & 526.20 & -0.18 & 526.20 & 0.45 & 0.63 & \\ & & & & & & & & & \\ & \text { BetaFlo } & \text { BetaBlade } & \text { Incid } & \text { U1 } & \text { W1 } & \text { Ts1 } & \text { TwetBulb1 } & \text { RH } \\ \text { TIP } & 51.77 & 46.36 & 5.41 & 667.85 & 850.39 & 6.88 & 564.38 & 510.34 & 0.09 \\ \text { MEAN } & 48.21 & 43.80 & 4.41 & 588.47 & 789.56 & 6.88 & 564.38 & 510.34 & 0.09 \\ \text { HUB } & 43.35 & 37.84 & 5.51 & 496.56 & 723.63 & 6.88 & 564.38 & 510.34 & 0.09\end{array}$

ROTOR EXIT CONDITIONS, STAGE 4 SOLUTION IS CONVERGED

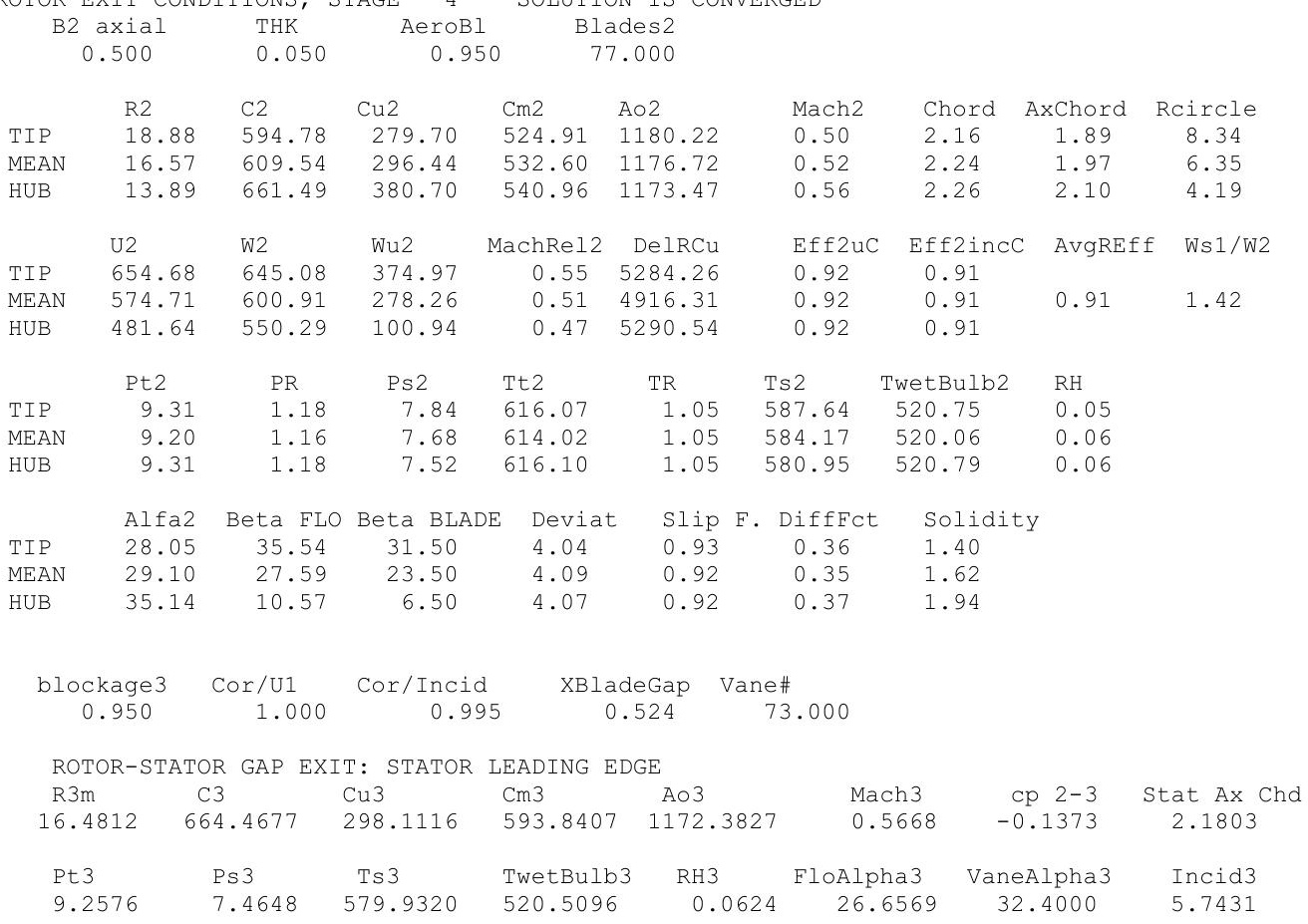




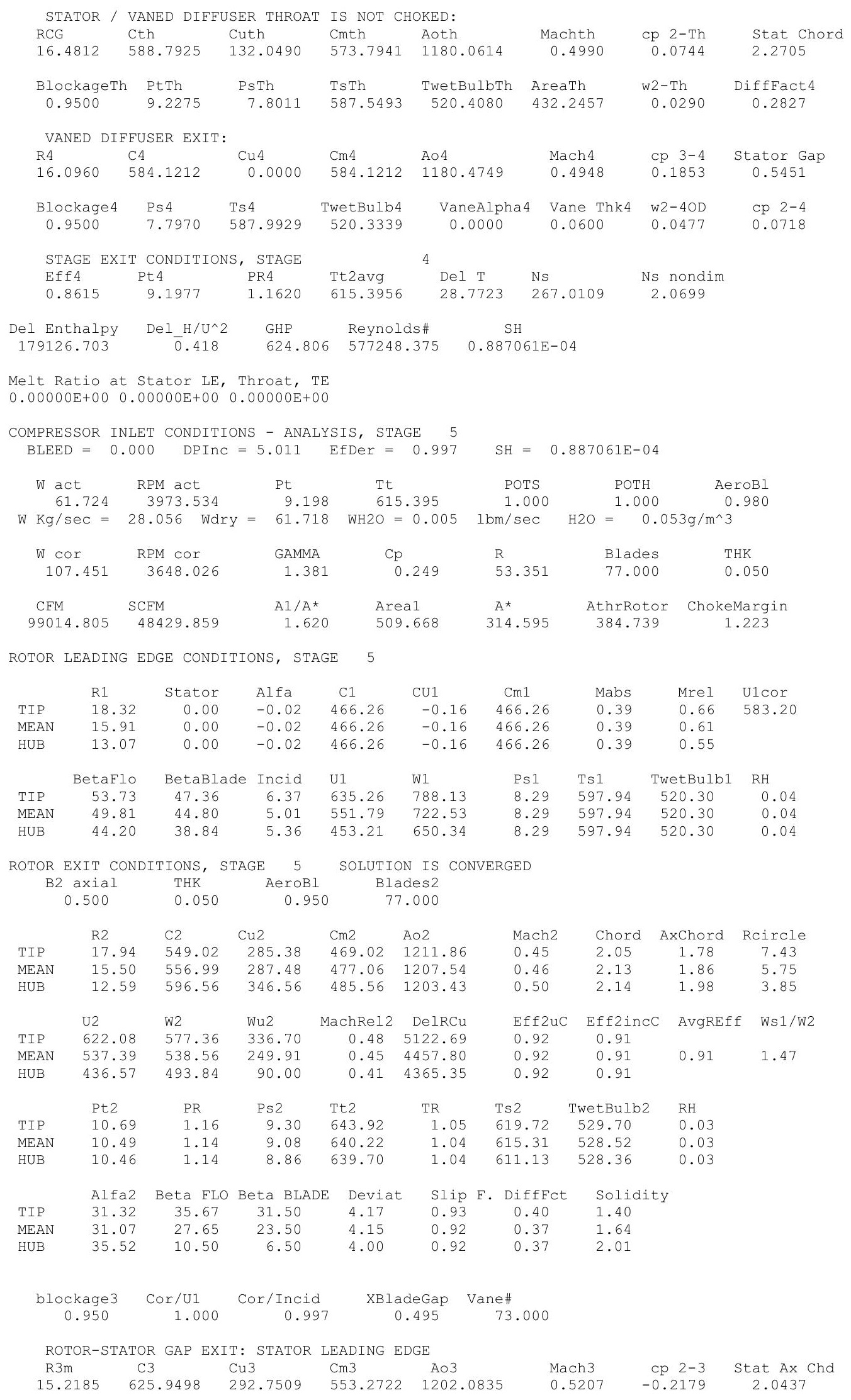




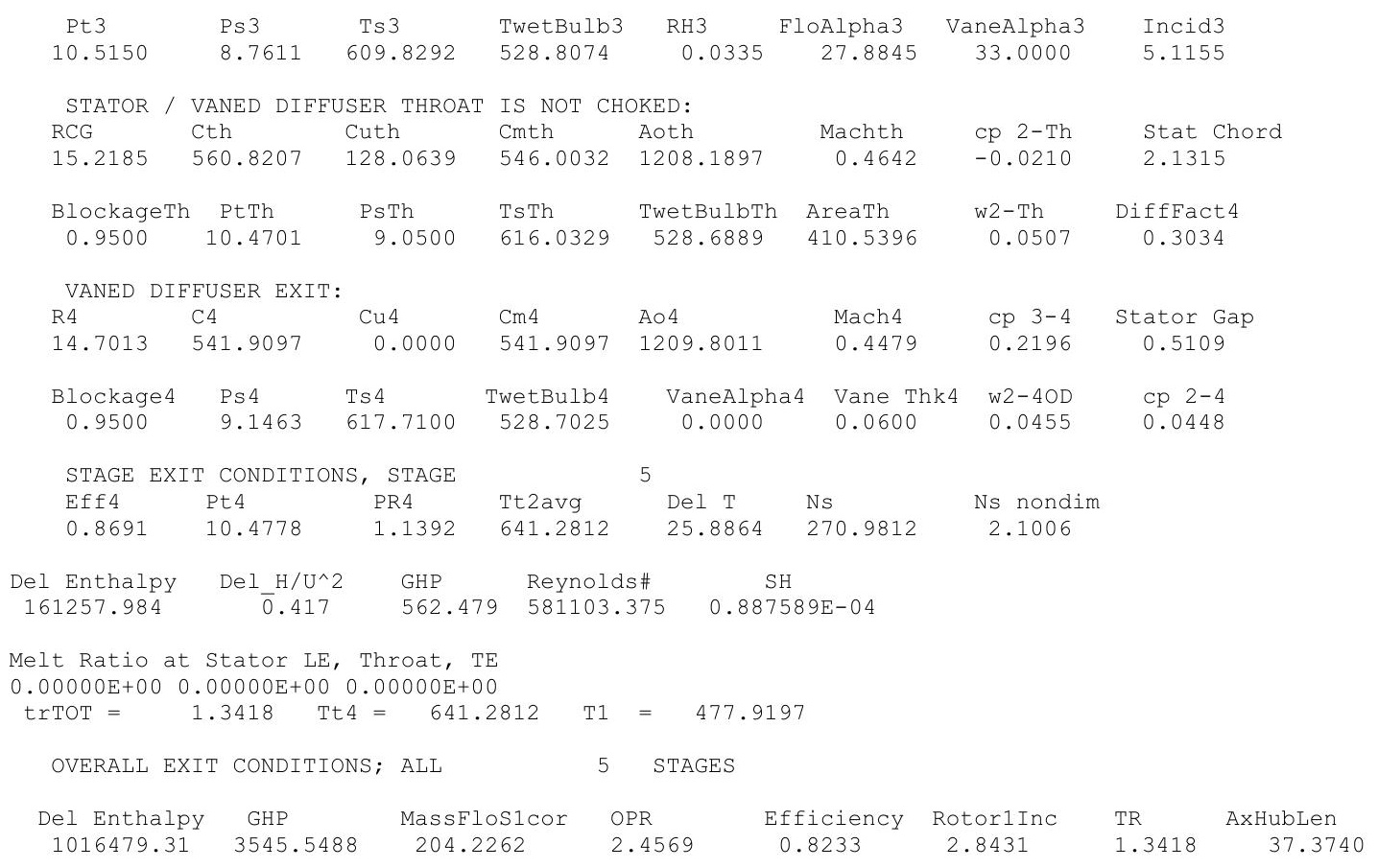




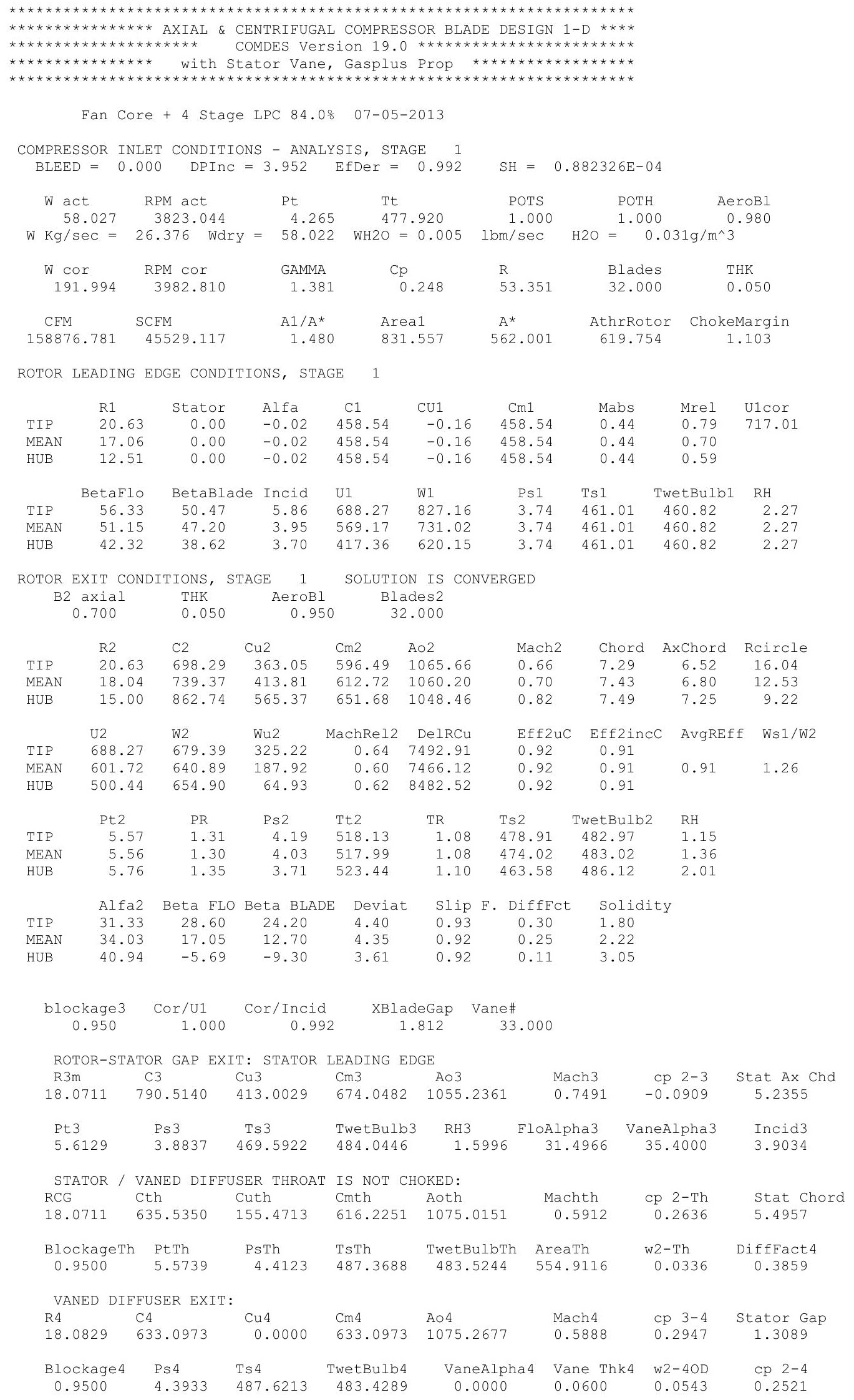




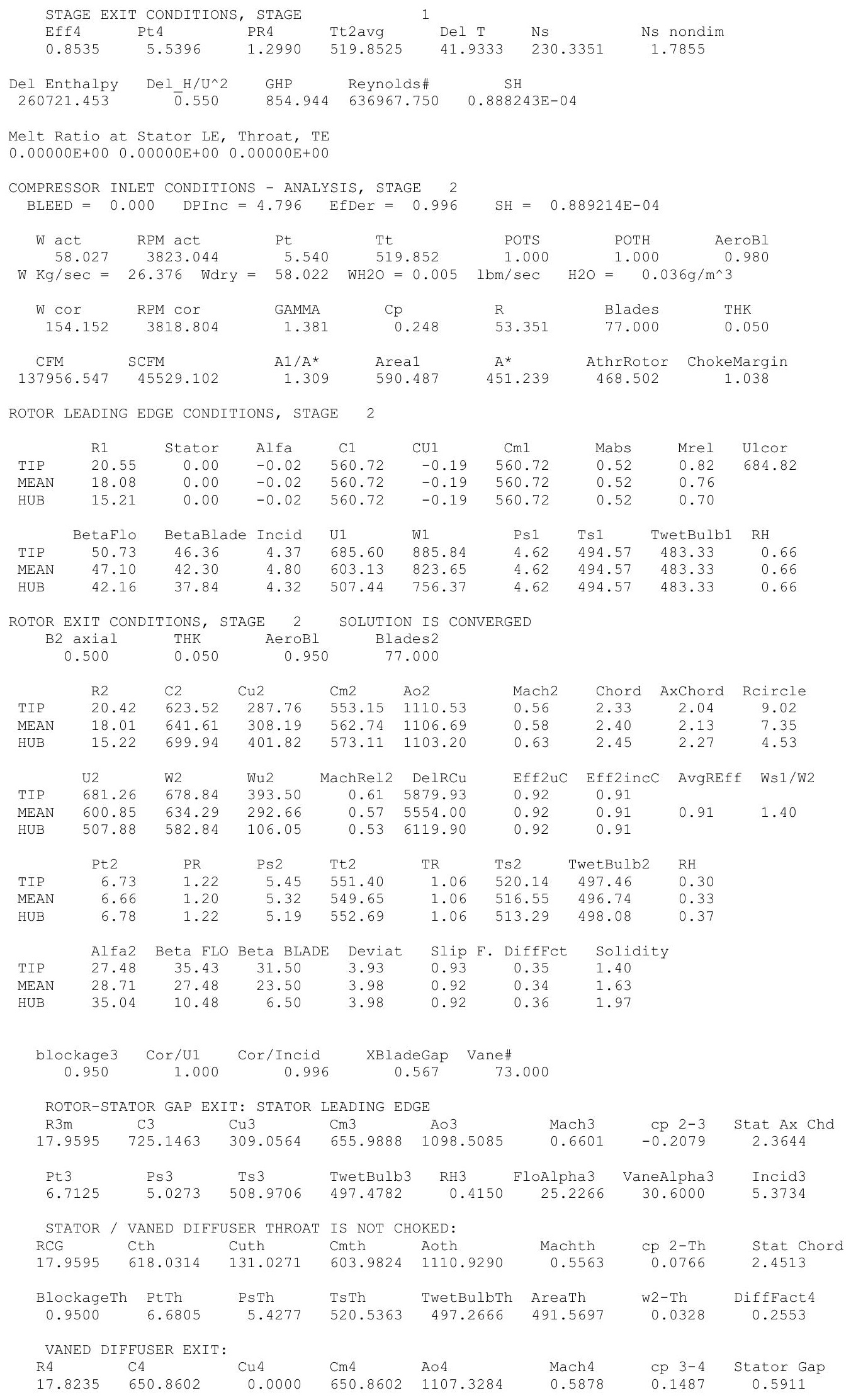




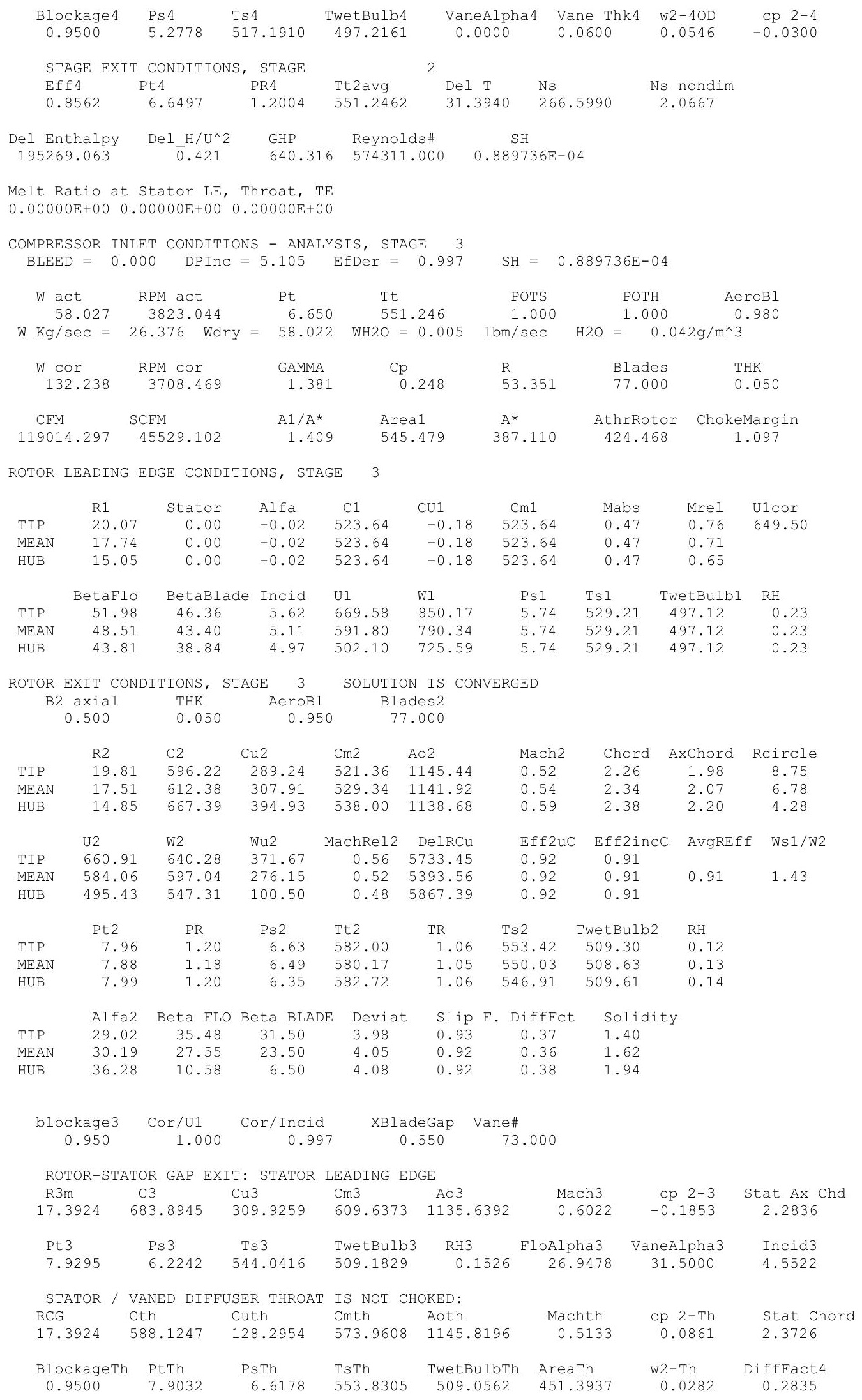




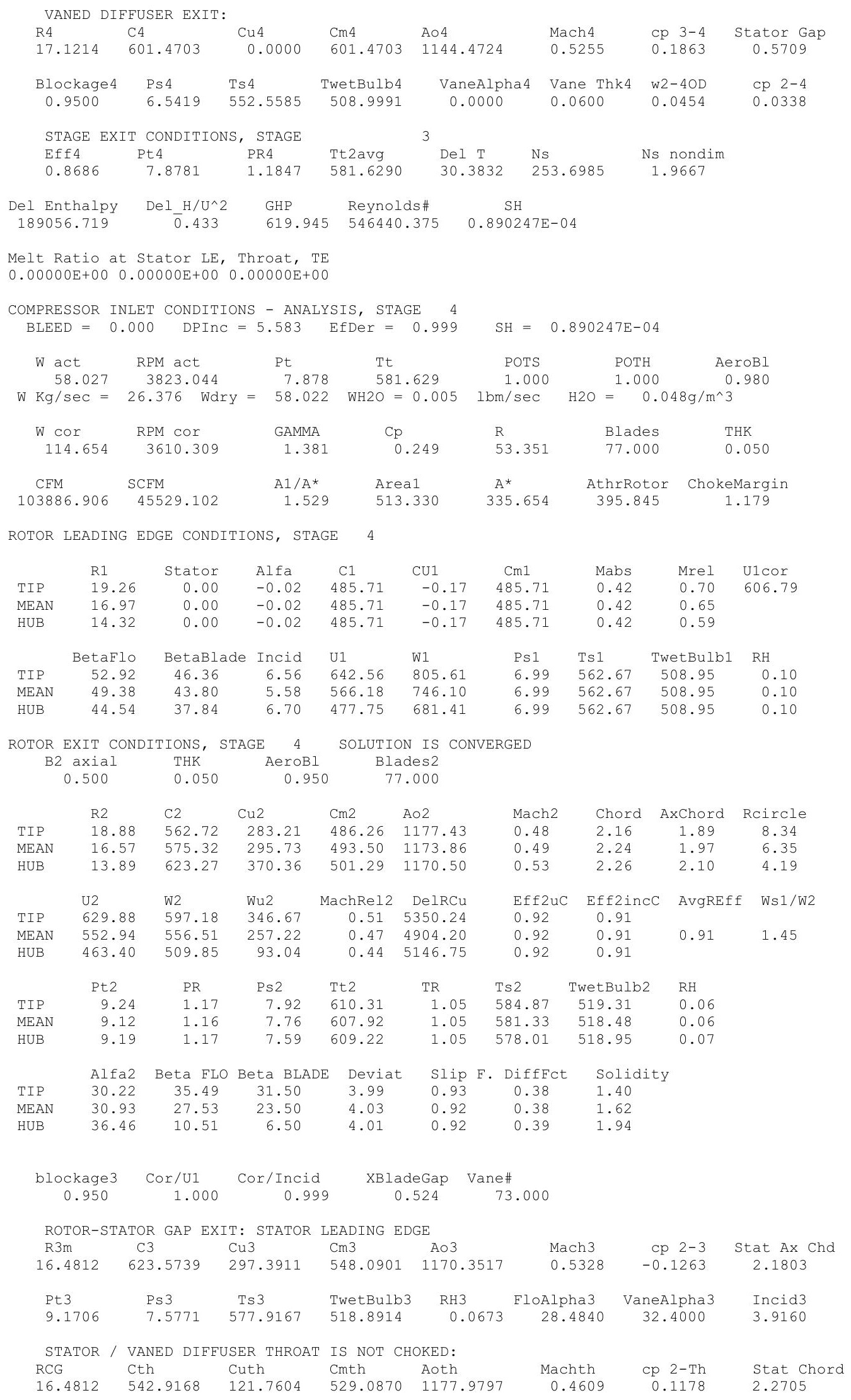




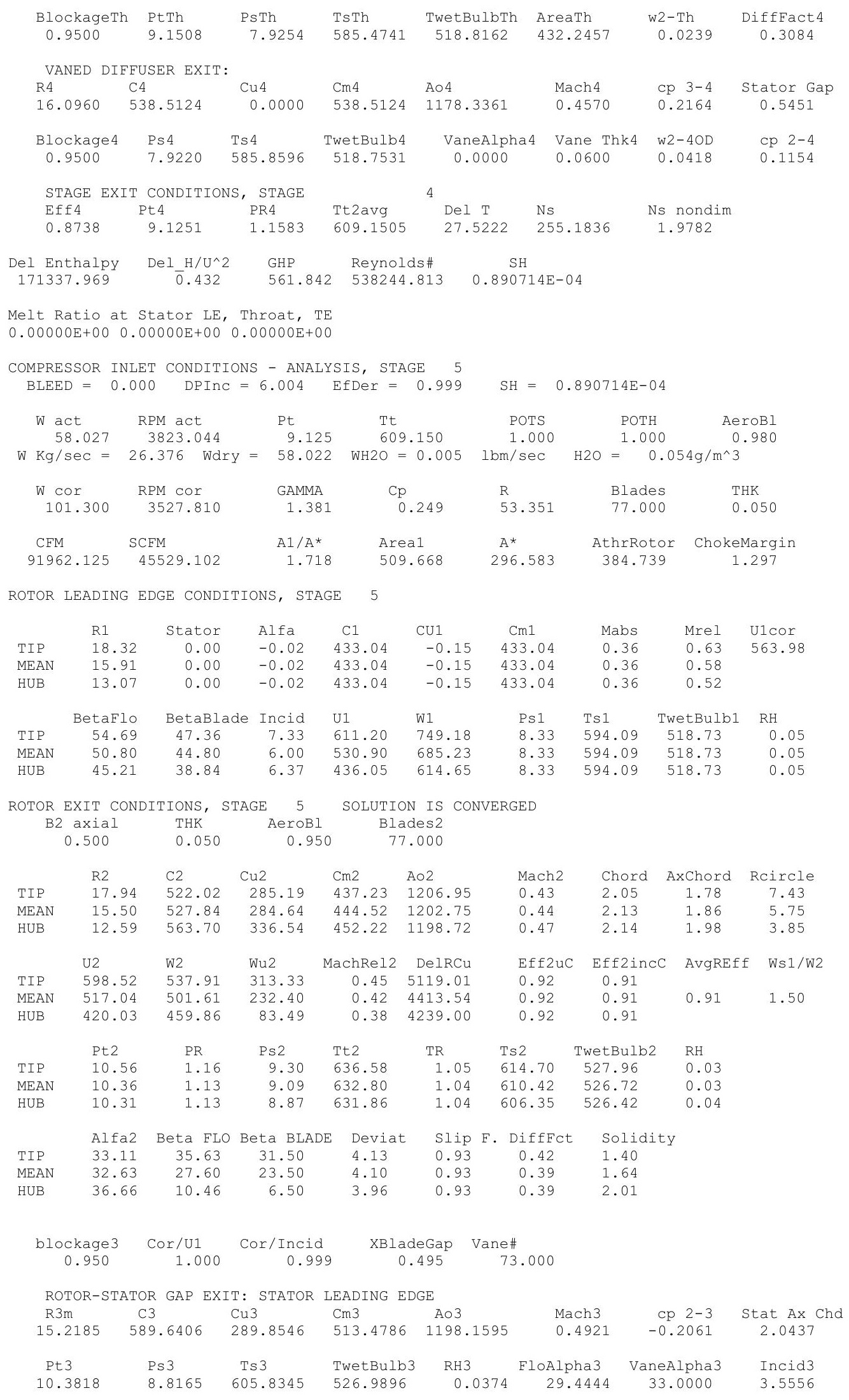




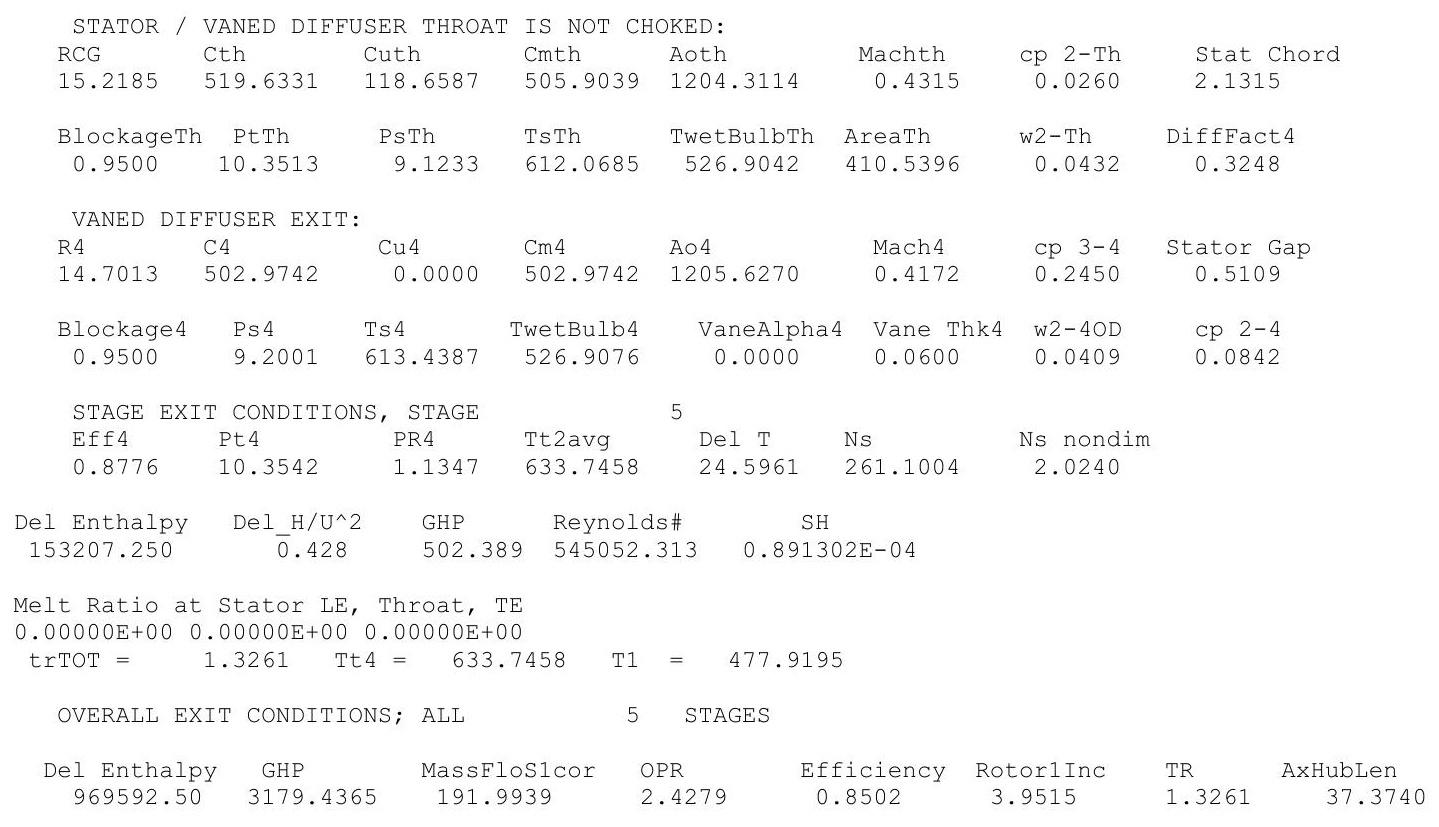




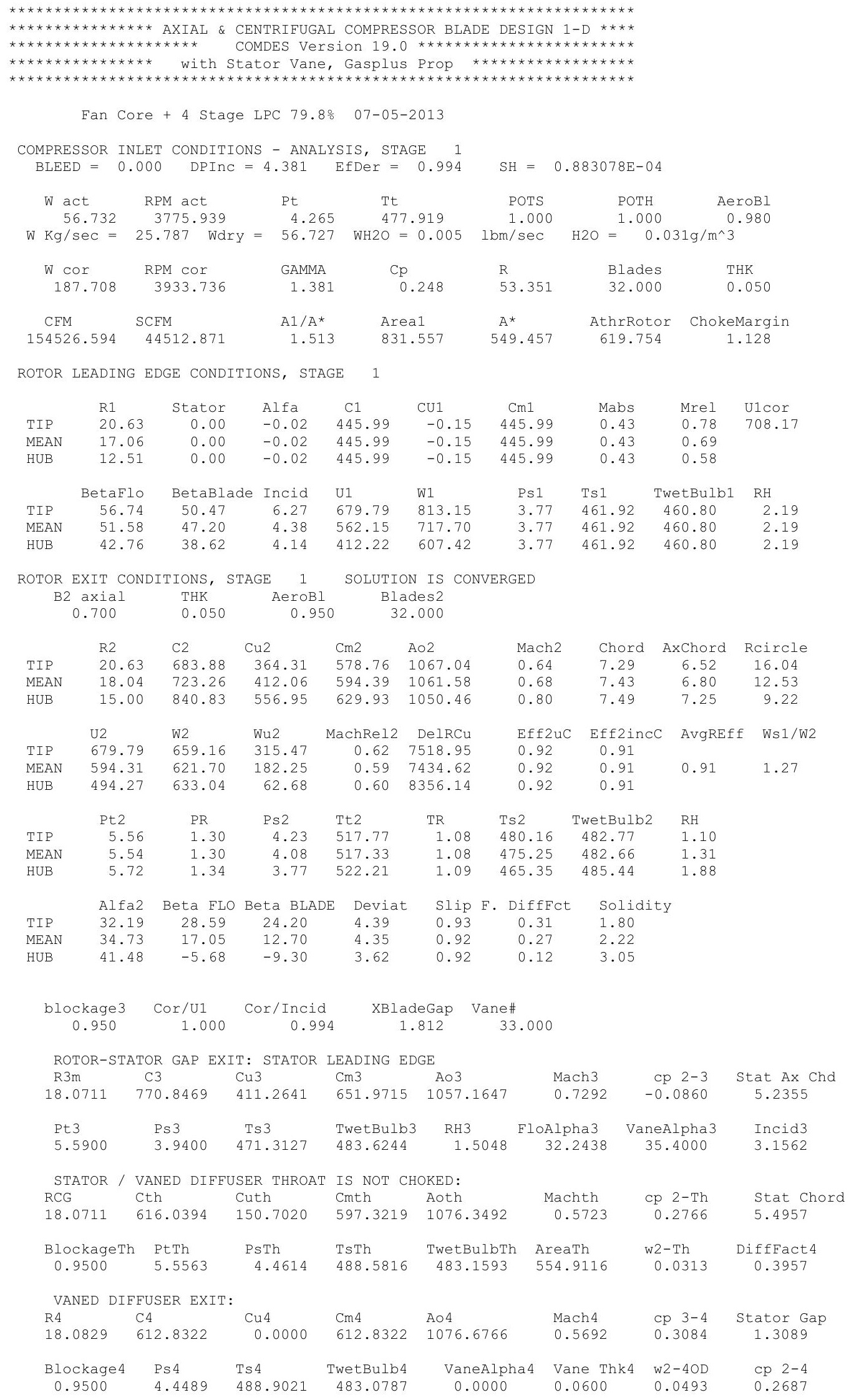




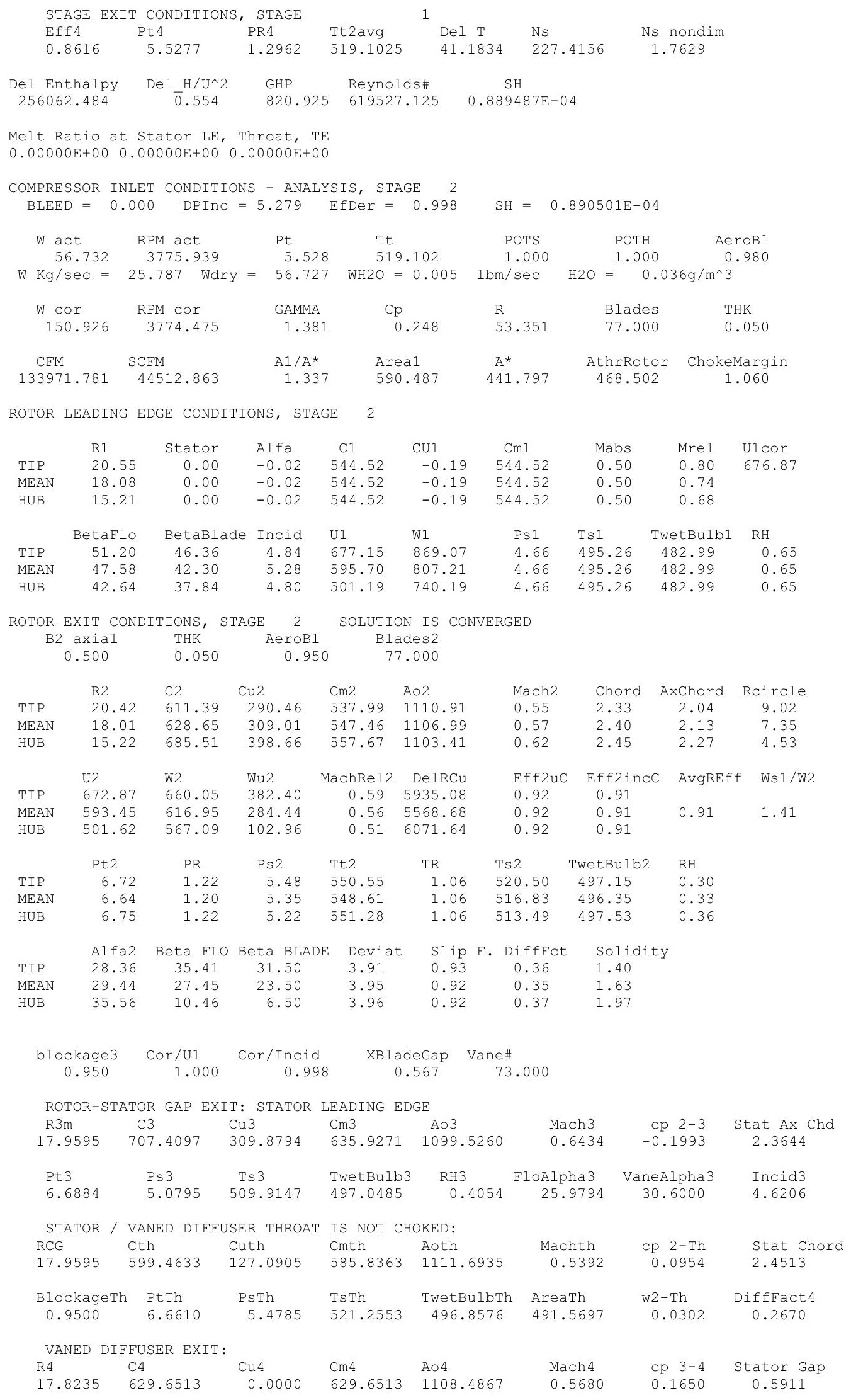




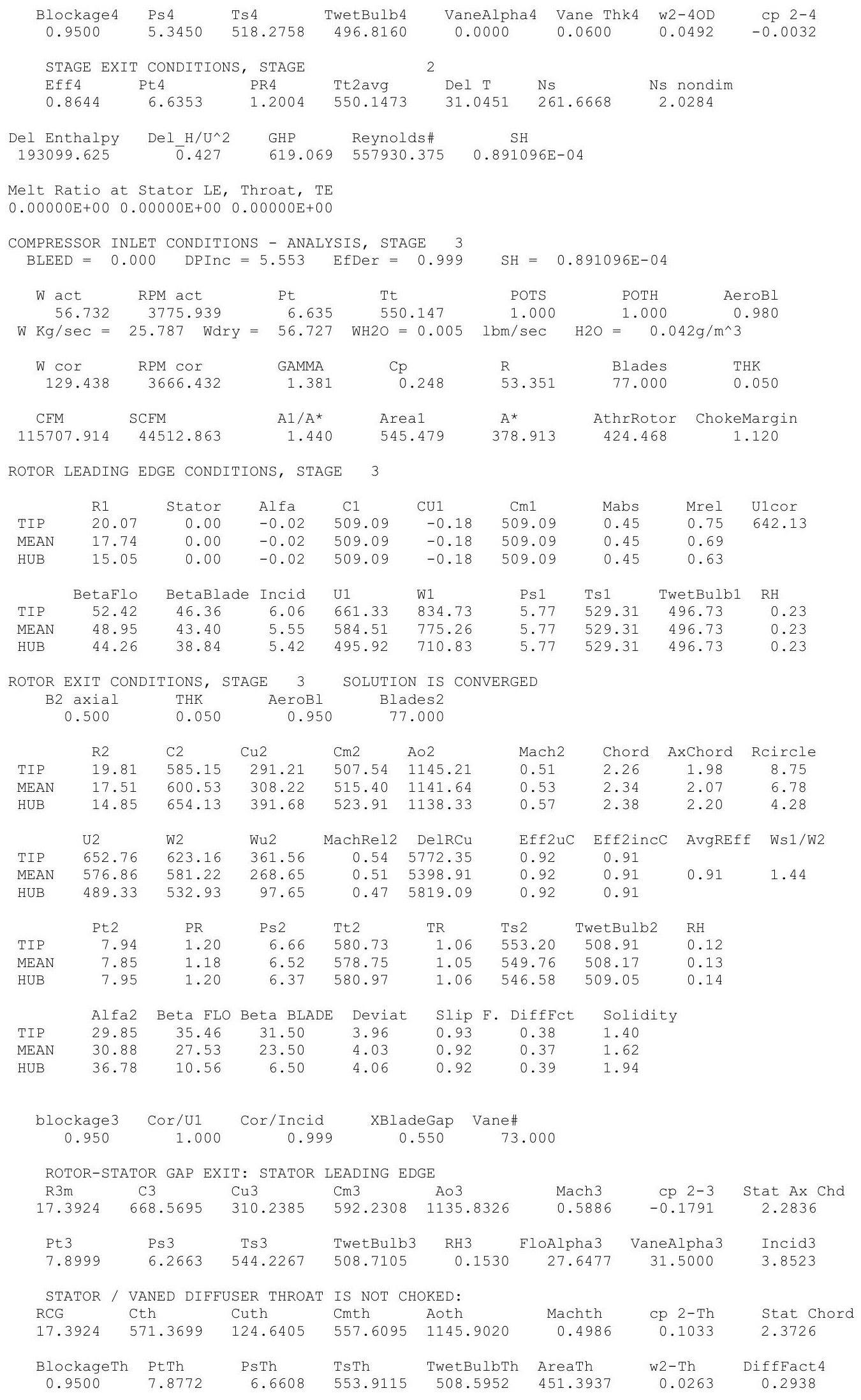




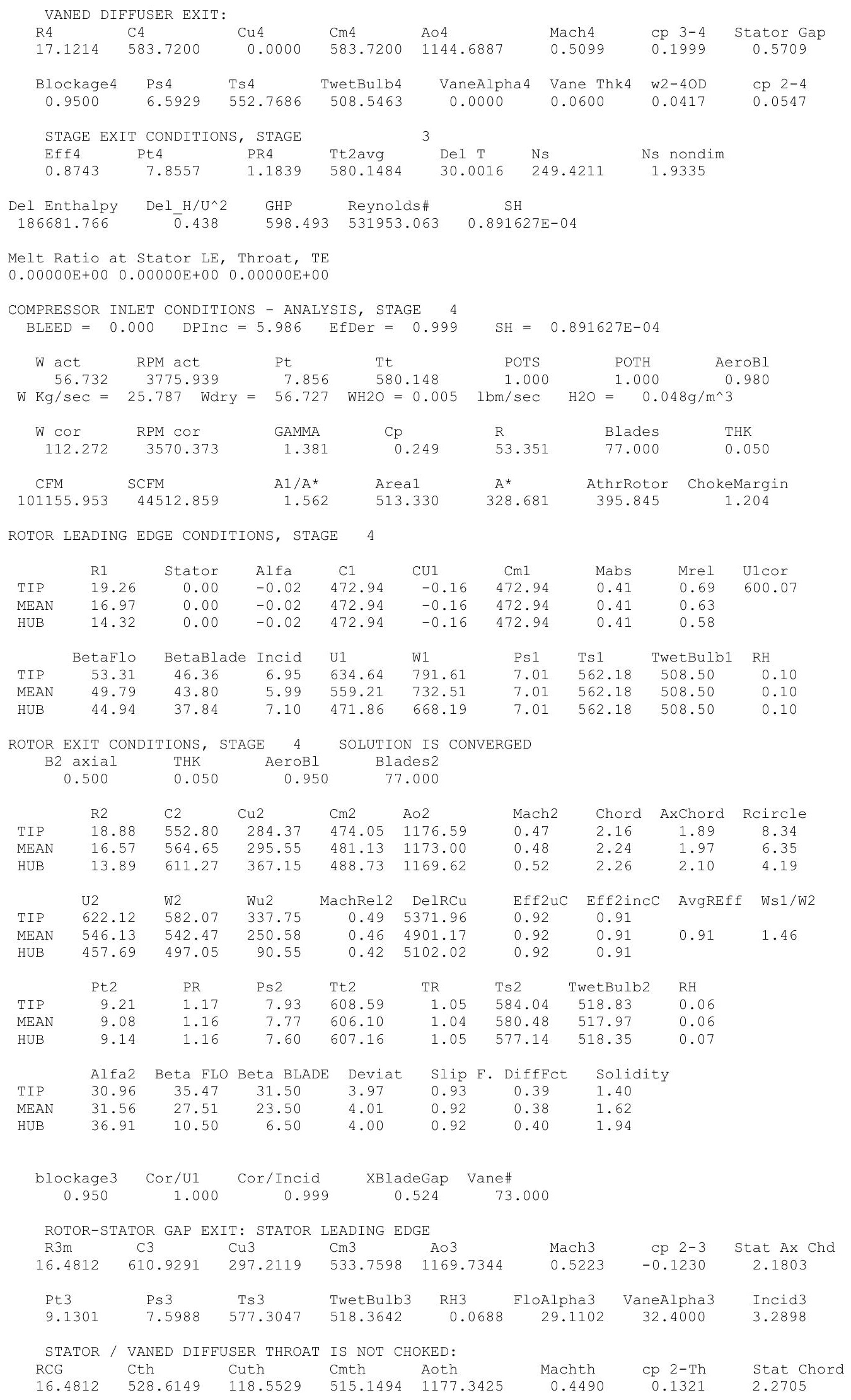




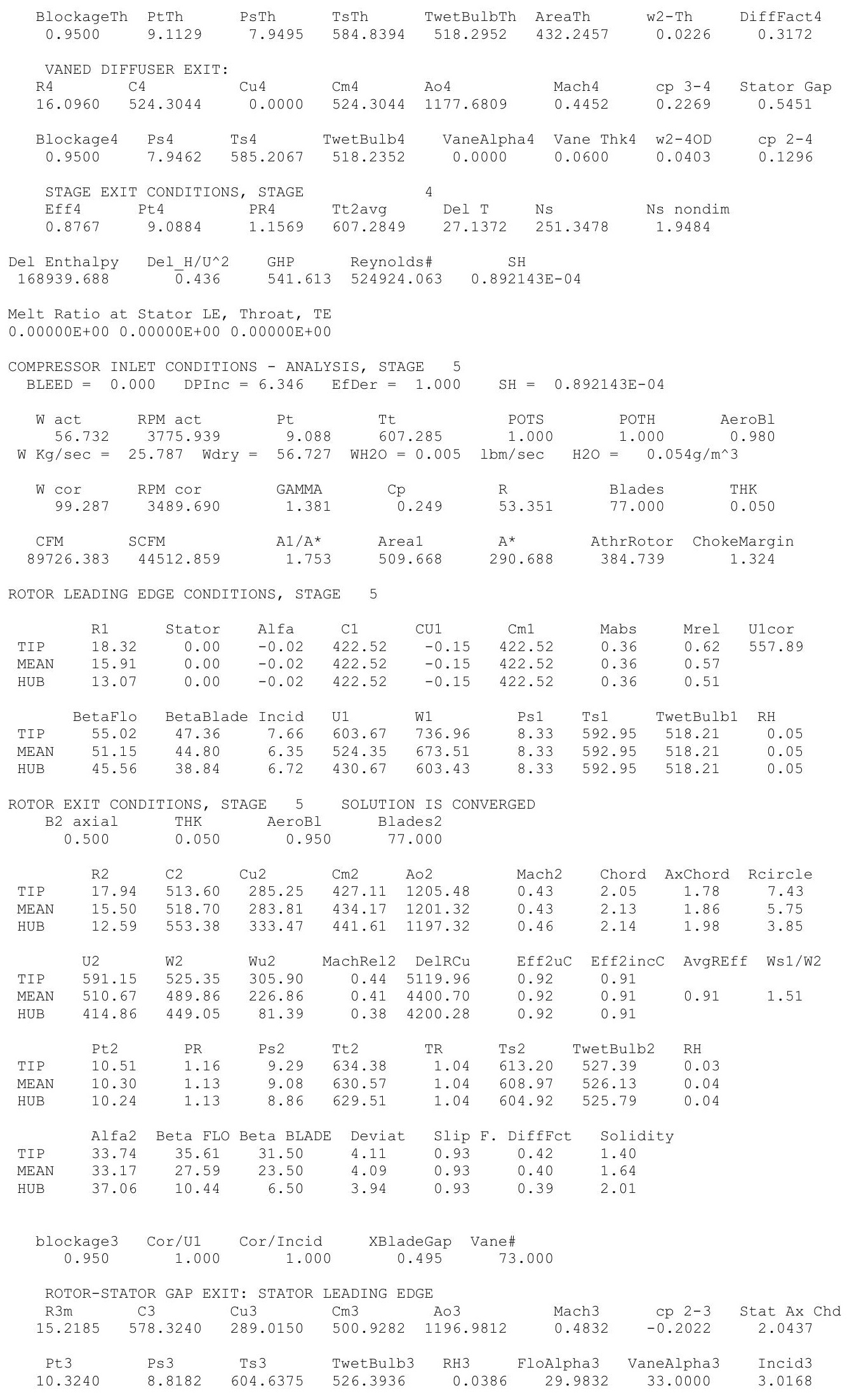




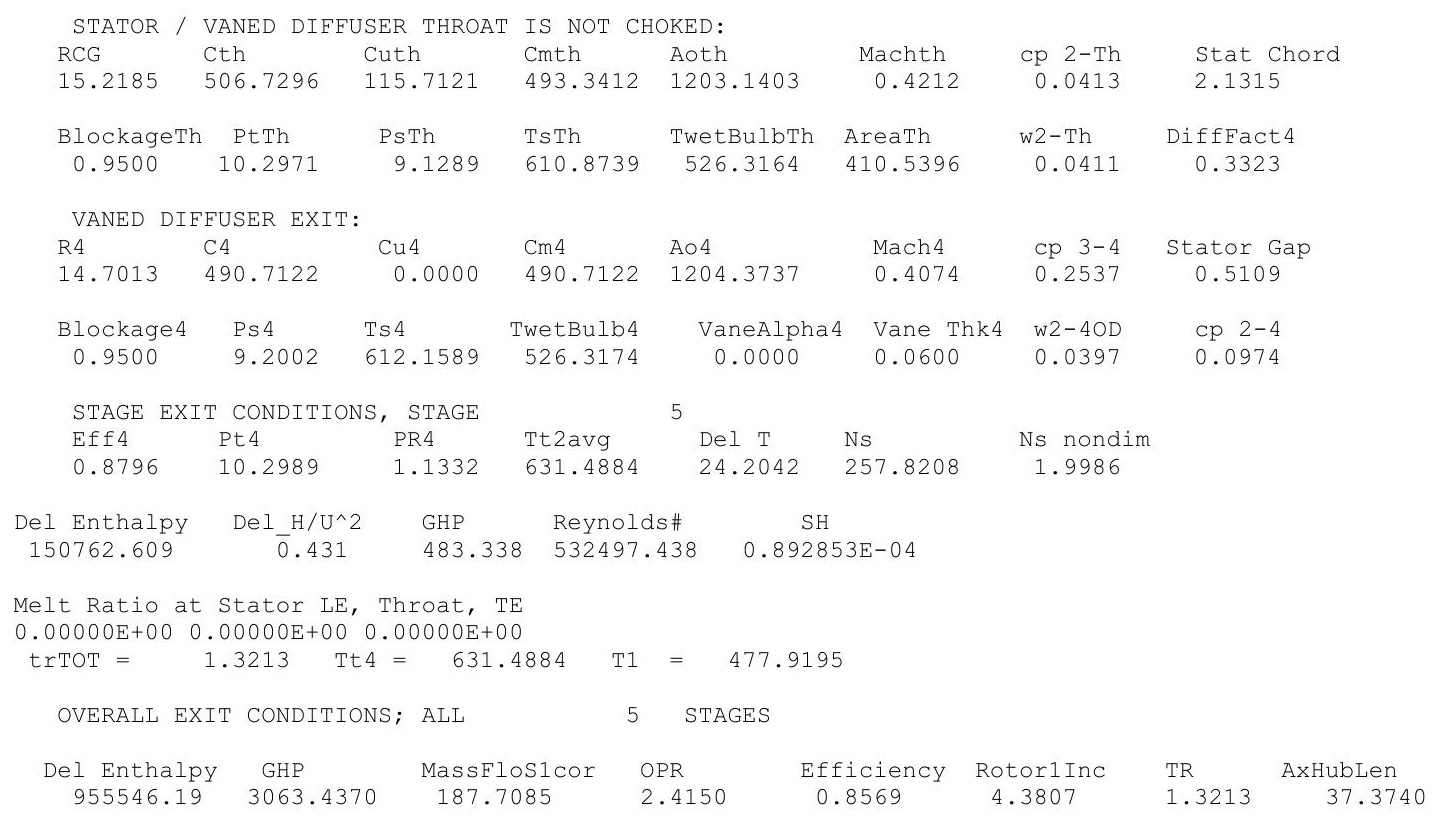




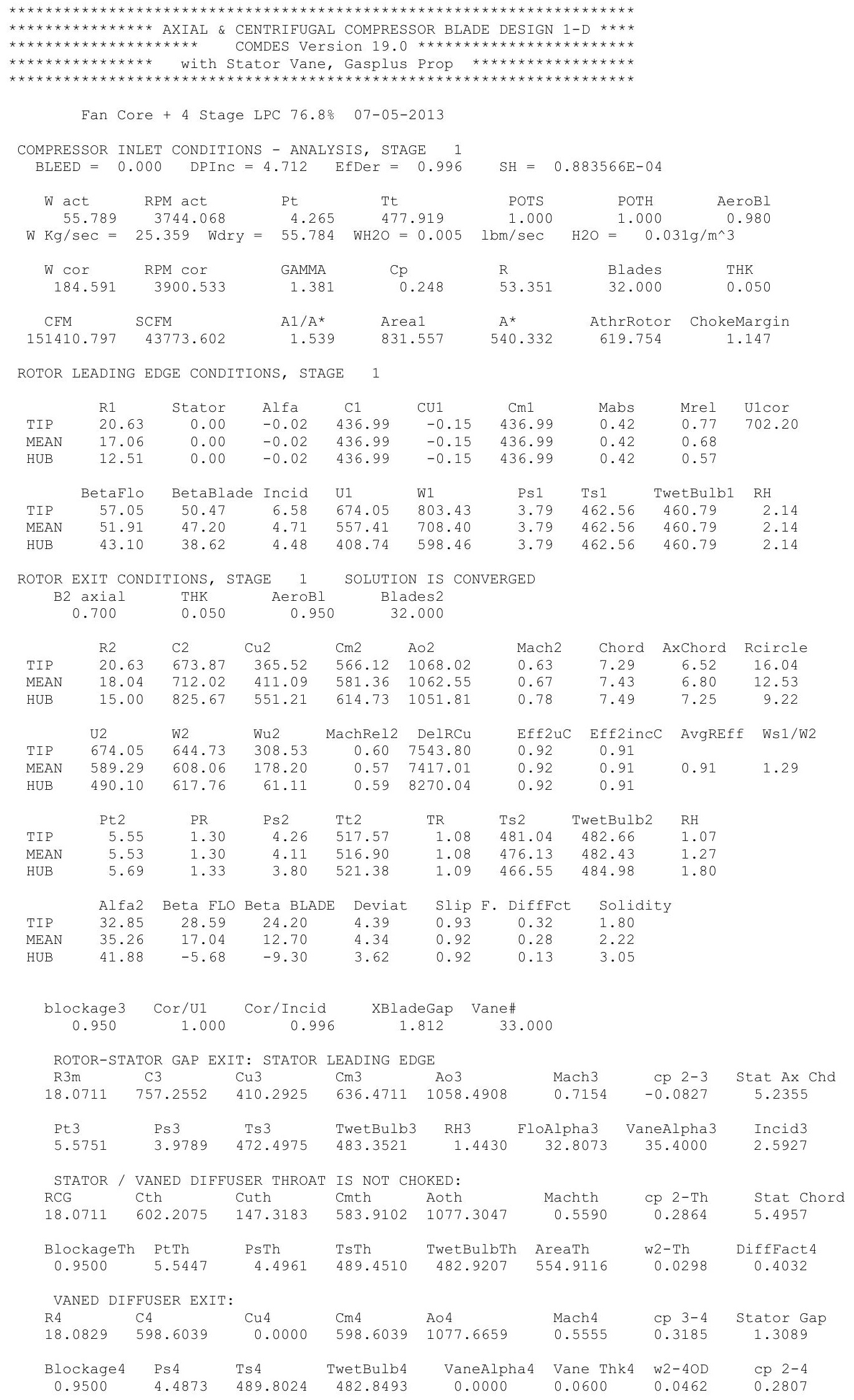




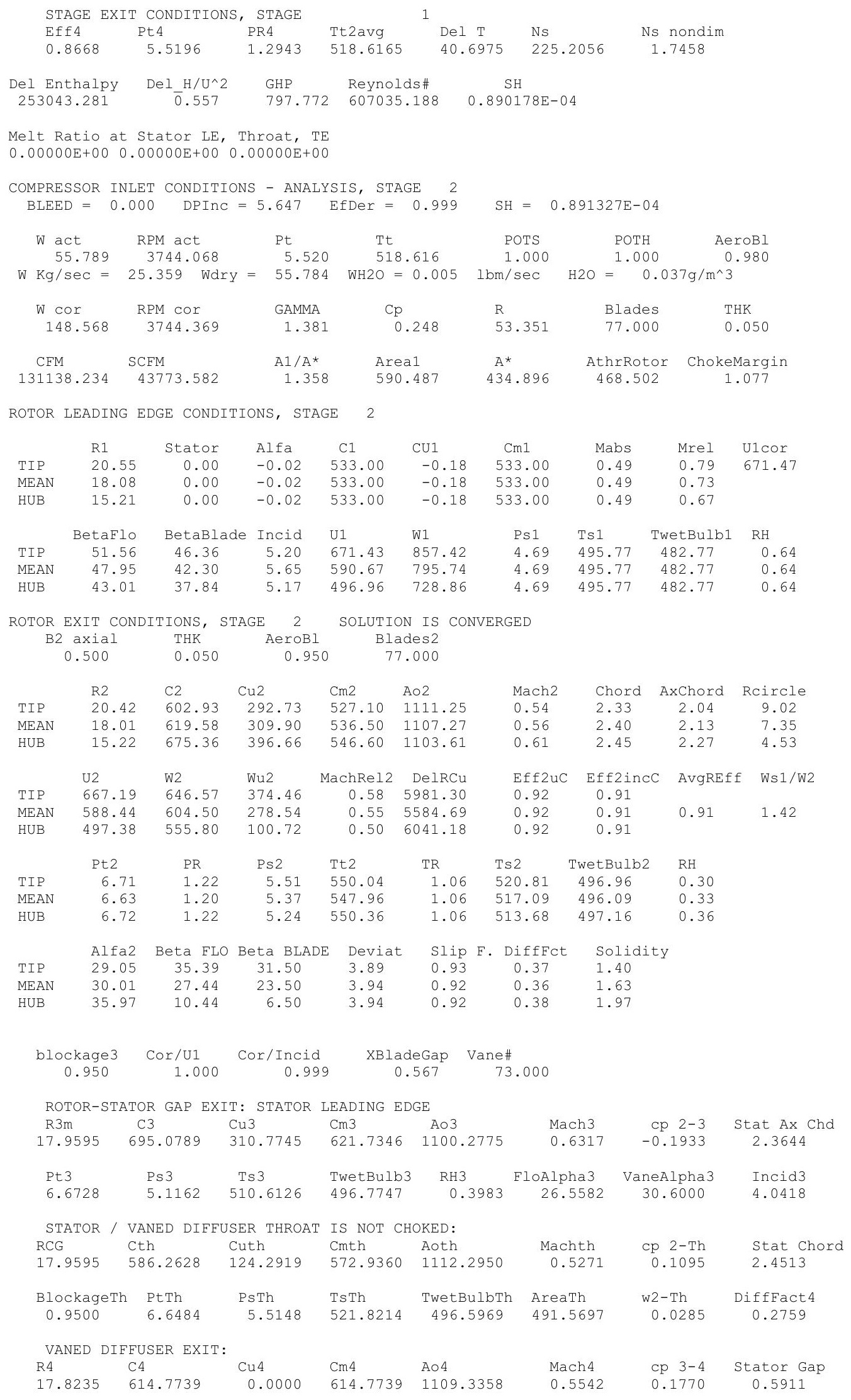




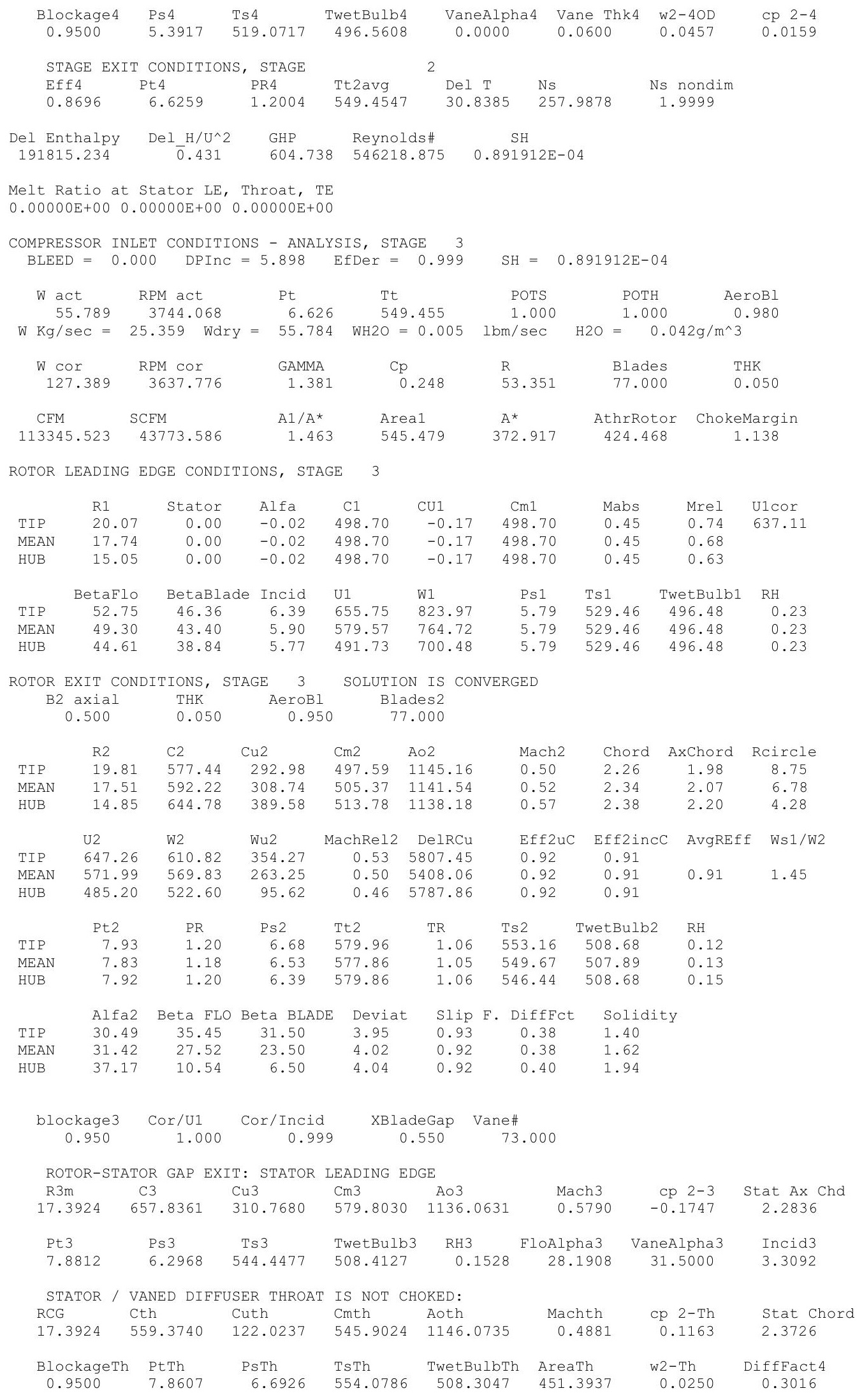




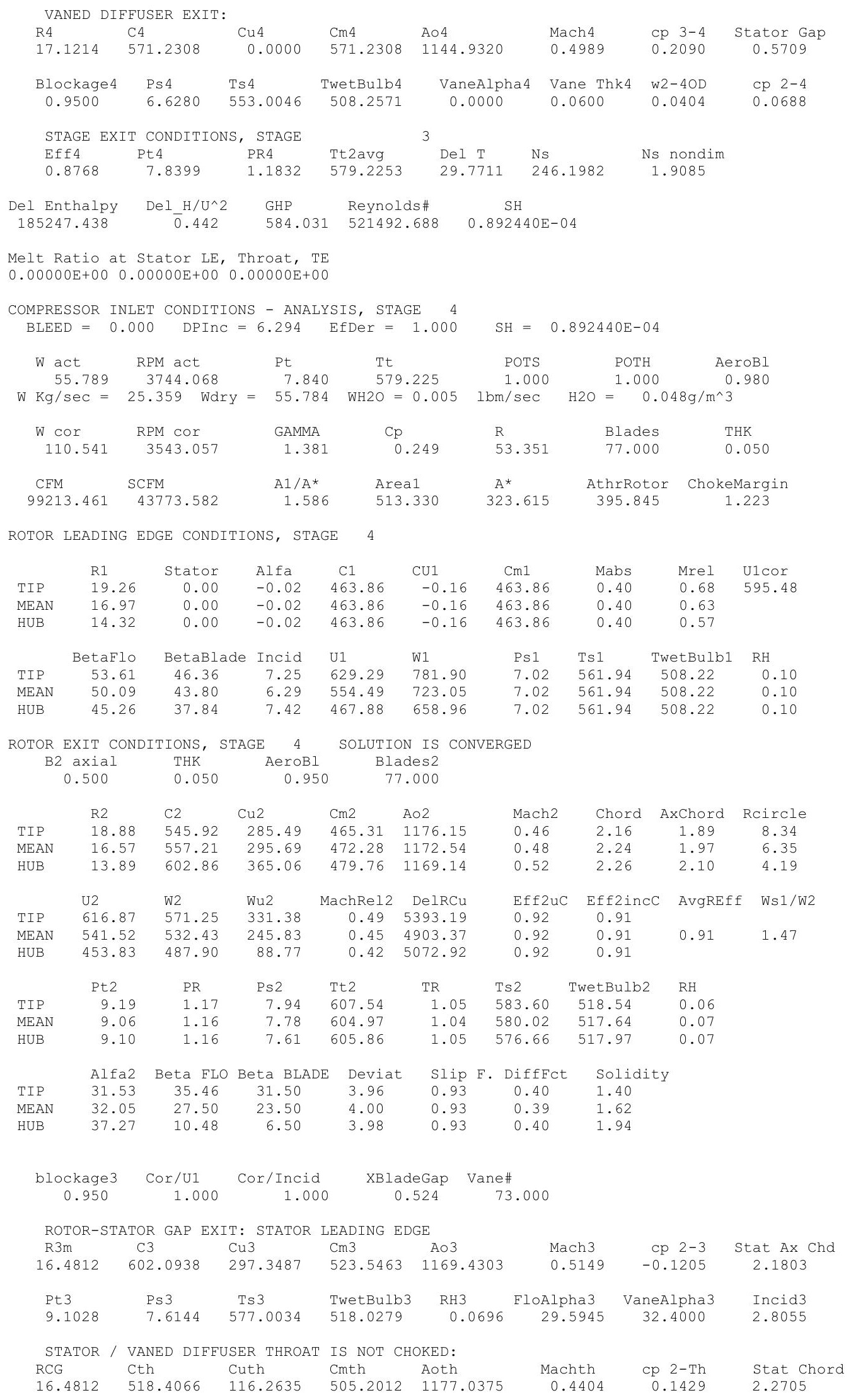




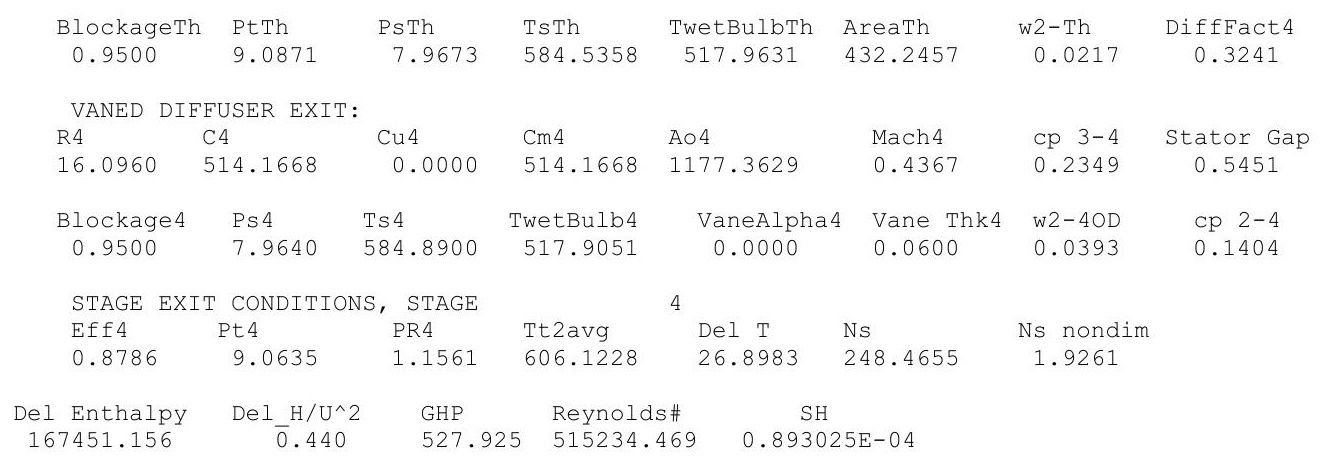

Melt Ratio at Stator LE, Throat, TE

$0.00000 \mathrm{E}+000.00000 \mathrm{E}+00 \quad 0.00000 \mathrm{E}+00$

COMPRESSOR INLET CONDITIONS - ANALYSIS, STAGE 5 $\mathrm{BLEED}=0.000 \quad$ DPInC $=6.614 \quad$ EfDer $=1.000$

$\begin{array}{lllllll}W \text { act } & \text { RPM act } & \text { Pt } & \text { POTS } & \text { POTH } & \text { AeroBI }\end{array}$ $\mathrm{W} \mathrm{Kg} / \mathrm{sec}=25.359$ Wary $=55.784 \quad \mathrm{WH} 2 \mathrm{O}=0.005 \quad \mathrm{bbm} / \mathrm{sec} \quad \mathrm{H} 2 \mathrm{O}=0.054 \mathrm{~g} / \mathrm{m}^{\wedge} 3$

$\begin{array}{ccccccc}\text { W Cor } & \text { RPM Cor } & \text { GAMMA } & \text { Cp } & \text { R } & \text { Blades } & \text { THK } \\ 97.813 & 3463.551 & 1.381 & 0.249 & 53.351 & 77.000 & 0.050 \\ & & & & & & \\ \text { CFM } & \text { SCFM } & \text { A1/A* } & \text { Area1 } & A^{*} & \text { AthrRotor } & \text { ChokeMargin } \\ 88121.492 & 43773.582 & 1.780 & 509.668 & 286.372 & 384.739 & 1.343\end{array}$

ROTOR LEADING EDGE CONDITIONS, STAGE 5

\begin{tabular}{|c|c|c|c|c|c|c|c|c|c|}
\hline & R1 & Stator & Alfa & $\mathrm{C} 1$ & CU1 & $\mathrm{Cm} 1$ & Mabs & Mrel & U1cor \\
\hline TIP & 18.32 & 0.00 & -0.02 & 414.96 & -0.14 & 414.96 & 0.35 & 0.61 & 553.71 \\
\hline MEAN & 15.91 & 0.00 & -0.02 & 414.96 & -0.14 & 414.96 & 0.35 & 0.56 & \\
\hline HUB & 13.07 & 0.00 & -0.02 & 414.96 & -0.14 & 414.96 & 0.35 & 0.50 & \\
\hline & BetaFlo & BetaBlade & Incid & U1 & W1 & Ps 1 & Ts1 & TwetBulb1 & $\mathrm{RH}$ \\
\hline TIP & 55.27 & 47.36 & 7.91 & 598.57 & 728.46 & 8.34 & 592.30 & 517.88 & 0.05 \\
\hline MEAN & 51.41 & 44.80 & 6.61 & 519.93 & 665.33 & 8.34 & 592.30 & 517.88 & 0.05 \\
\hline HUB & 45.83 & 38.84 & 6.99 & 427.04 & 595.55 & 8.34 & 592.30 & 517.88 & 0.05 \\
\hline
\end{tabular}

ROTOR EXIT CONDITIONS, STAGE 5 SOLUTION IS CONVERGED

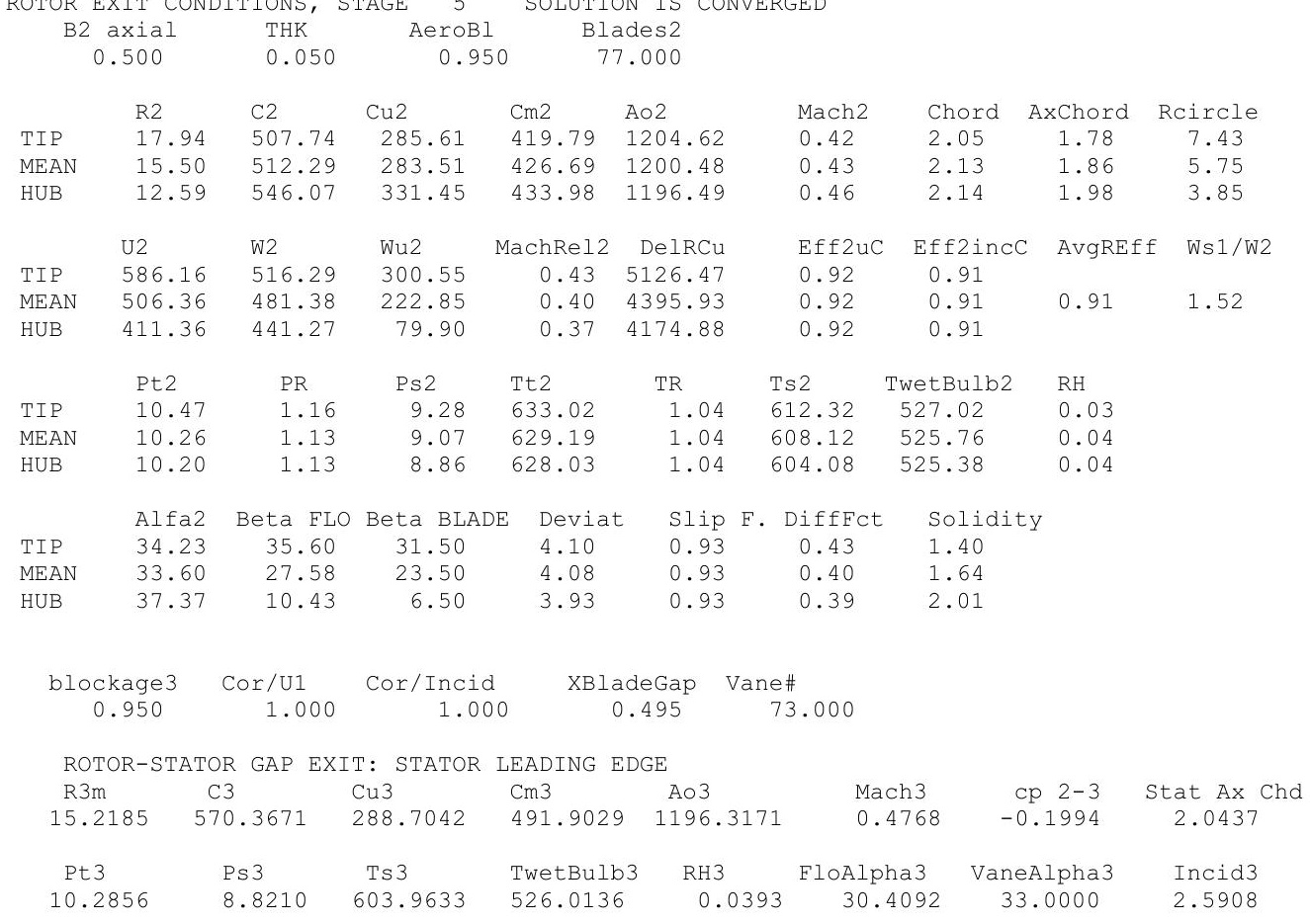




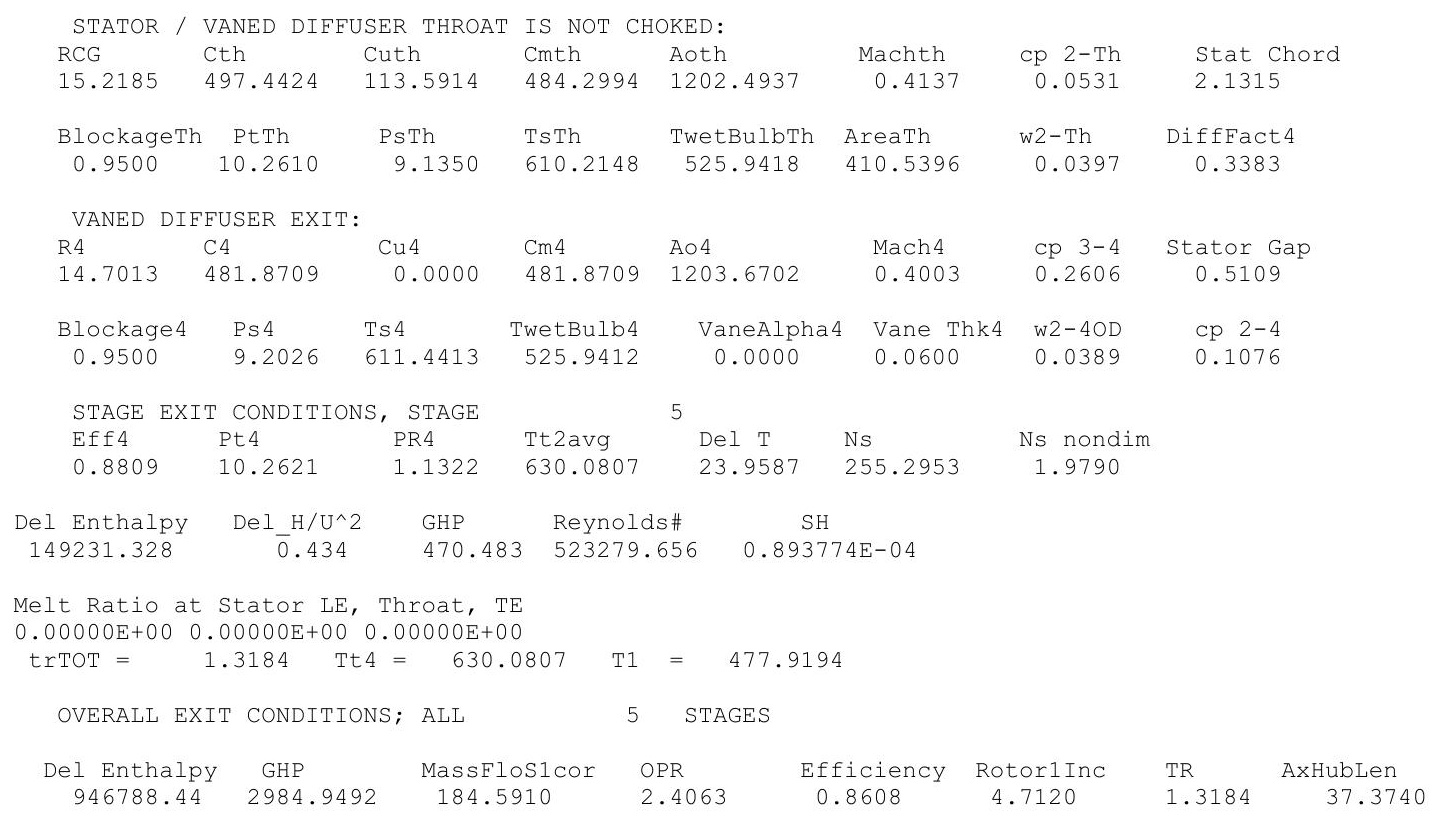


Appendix K: NPSS cycle analysis for the descent conditions

$5 \mu \mathrm{m}, 2 \mathrm{~g} / \mathrm{m}^{3}$, ISA $+18 \mathrm{R}$

Date:05/10/13 Time:11:02:24 Model:

Model:

Turbofan Engine - COMDES ON Converge $=1 \quad$ CASE: 0

Version:NPSS 1.6.5 - Rev: -> Gas Package: Janaf iter/pass/Jacb/Broy=12/ $26 / 1 / 10$

Temperature Stator 1 inlet: 463.46 Stator 1 exit: 470.96 Stator 2 inlet: 480.37 Stator 2 exit: 485.22

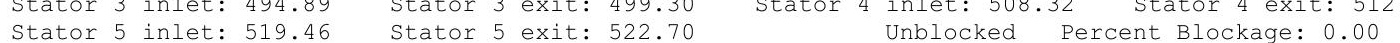

Ambient Relative Humidity

Fan Face Relative Humidity 10.00

Fan Bypass Relative Humidity 1.23

LPC Inlet Relative Humidity $\quad 1.13$

LPC Exit Relative Humidity $\quad 0.10$

HPC Relative Humidity $\quad 0.04$

Ambient Flow Velocity

0.0000050

$772.64 \quad 40.00$

Ambient Flow Velocity

Ambient Static Temperature

$2.85 \quad$ Fan/LPC Inlet Static Pressure

86.05

Additional Water at LPC Exit

Fan/LPC Inlet Static Temperature 454.86

SUMMARY OUTPUT DATA

$\begin{array}{rrrrrr}\text { MN } & \text { alt } & \text { dTamb } & \text { W } & \text { Fn } & \text { TSFC } \\ 0.780 & 39000.0 & 18.00 & 257.43 & 631.1 & 1.1046\end{array}$
Wfuel BPR VTAS

697.06
BPR
8.6926
VTAS
72.64

OPR
9.498$$
\text { EPR }
$$

Powerset

10.000

$\begin{array}{rr}T 4 & T 41 \\ 1711 & 1618\end{array}$

T49

FLOW STATION DATA

\begin{tabular}{|c|c|c|c|c|c|}
\hline W & Pt & Tt & ht & FAR & We \\
\hline 257.43 & 4.267 & 457.74 & -20.96 & 0.0000 & 832.95 \\
\hline 257.43 & 4.267 & 457.74 & -20.96 & 0.0000 & 832. \\
\hline 230.87 & 4.265 & 457.74 & -20.96 & 0.0000 & 747. \\
\hline 26.56 & 4.265 & 457.74 & -20.96 & 0.0000 & 85 \\
\hline 230.87 & 4.820 & 475.75 & -16.64 & 0.0000 & 674 \\
\hline 26.56 & 6.666 & 532.40 & -3.05 & 0.0000 & 59 \\
\hline .70 & 6.666 & 539.36 & -30.78 & 0.0000 & \\
\hline 24.03 & 6.666 & 539.36 & -30.78 & 0.0000 & 54 \\
\hline 22.86 & 40.527 & 976.91 & 76.09 & 0.0000 & 11. \\
\hline 17.72 & 40.527 & 976.91 & 76.09 & 0.0000 & \\
\hline 17.91 & 39.594 & 1711.35 & 63.90 & 0.0110 & \\
\hline 23.94 & 9.499 & 1130.07 & -39.67 & 0.0082 & \\
\hline 24.22 & 3.261 & 885.66 & -99.71 & 0.0081 & 142. \\
\hline 4.22 & 3.261 & 885.73 & -99.71 & 0.0081 & \\
\hline 24.22 & 3.261 & 885.80 & -99.71 & 0.0081 & 142. \\
\hline 230.87 & 4.820 & 475.75 & -16.64 & 0.0000 & 674 \\
\hline 33.54 & 4.820 & 476.48 & -16.80 & 0.0000 & 68 \\
\hline & 4.820 & 476.48 & -16.80 & 0.0000 & \\
\hline 3.54 & 4.820 & 476.48 & -16.80 & 0.0000 & \\
\hline
\end{tabular}

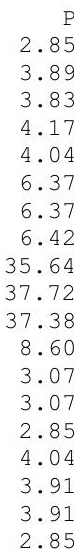

Ts
407.97
445.87
444.17
454.86
452.46
525.73
532.42
533.81
942.82
957.76
1687.04
1100.35
871.00
871.07
853.73
452.46
448.81
448.81
410.12

$\begin{array}{rr}\text { Aphy } & \text { MN } \\ 2541.1 & 0.7800 \\ 4168.2 & 0.3645 \\ 3531.8 & 0.3904 \\ 830.5 & 0.1779 \\ 2606.7 & 0.5068 \\ 412.6 & 0.2518 \\ 412.6 & 0.2555 \\ 412.6 & 0.2282 \\ 49.7 & 0.4347 \\ 49.3 & 0.3235 \\ 74.6 & 0.2947 \\ 265.4 & 0.3835 \\ 860.2 & 0.2969 \\ 860.2 & 0.2969 \\ 613.5 & 0.4432 \\ 2606.7 & 0.5068 \\ 2481.9 & 0.5546 \\ 2481.9 & 0.5546 \\ 2006.9 & 0.8984\end{array}$

gamt
1.40084
1.40084
1.40084
1.40084
1.40070
1.40004
1.39923
1.39923
1.38133
1.38133
1.33108
1.36604
1.38136
1.38136
1.38135
1.40070
1.40069
1.40069
1.40069

WAR
0.0000295
0.0000295
0.0000295
0.0000295
0.0000295
0.0000295
0.0051516
0.0051516
0.0051516
0.0051516
0.0051516
0.0051516
0.0051516
0.0051516
0.0051516
0.0000295
0.0000877
0.0000877
0.0000877

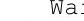

257.42 WH2O H2O frac $\begin{array}{lll}257.42 & 0.008 & 0.0000 \\ 237.42 & 0.008 & 0.0000\end{array}$ $\begin{array}{lll}230.86 & 0.007 & 0.0000\end{array}$ $\begin{array}{lll}26.56 & 0.001 & 0.0000\end{array}$ $\begin{array}{lll}230.86 & 0.007 & 0.0000\end{array}$ $\begin{array}{lll}26.56 & 0.001 & 0.0000\end{array}$ $\begin{array}{lll}26.56 & 0.137 & 0.0051\end{array}$ $\begin{array}{lll}23.90 & 0.123 & 0.0051\end{array}$ $\begin{array}{lll}22.74 & 0.117 & 0.0051\end{array}$ $\begin{array}{lll}17.63 & 0.091 & 0.0051\end{array}$ $\begin{array}{lll}17.63 & 0.091 & 0.0184\end{array}$ $\begin{array}{lll}17.63 & 0.091 & 0.0184\end{array}$ $\begin{array}{lll}23.90 & 0.123 & 0.0150\end{array}$

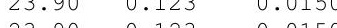
$\begin{array}{lll}23.90 & 0.123 & 0.0150\end{array}$ $\begin{array}{lll}230.86 & 0.007 & 0.0000\end{array}$ $\begin{array}{lll}233.52 & 0.020 & 0.0001\end{array}$ $\begin{array}{lll}233.52 & 0.020 & 0.0001\end{array}$

TURBOMACHINERY PERFORMANCE DATA

\begin{tabular}{|c|c|c|c|c|c|c|c|c|c|}
\hline \\
\hline Tan & $\begin{array}{r}\text { WC } \\
747.39\end{array}$ & $\begin{array}{r}P R \\
130\end{array}$ & $\begin{array}{r}\text { eff } \\
0.9056\end{array}$ & $\begin{array}{r}\text { NC } \\
2571.761\end{array}$ & $\begin{array}{r}\text { TR } \\
1.0393\end{array}$ & efPoly & $\begin{array}{r}\text { pwr } \\
-1409.8\end{array}$ & $\begin{array}{r}\text { SMN } \\
1585.81\end{array}$ & $\begin{array}{r}\text { SMW } \\
54.78\end{array}$ \\
\hline$\triangle P C$ & 85.98 & $\begin{array}{l}1.130 \\
1.563\end{array}$ & 0.8352 & 2571.761 & 1.1631 & 0.8452 & -672.7 & 6.98 & 3.75 \\
\hline HPC & 54.02 & 6.080 & 0.8183 & 8958.397 & 1.8112 & 0.8570 & -3544.8 & 61.41 & 58.06 \\
\hline $\mathrm{HPT}$ & 12.0 & 4.168 & 0.8749 & 220.829 & 1.3651 & 0.8525 & 3544.8 & & \\
\hline $\mathrm{EPT}$ & 54.6 & 2.913 & 0.8525 & 71.869 & 1.2742 & 0.8326 & 2082.4 & & \\
\hline JRB & NERY & DATA & & & & & & & \\
\hline & WcMap & PRmap & effMap & NcMap & R/Parm & S_WcDes & S_PRdes & s_effDes & S_NCDes \\
\hline an & 868.39 & 1.127 & 0.9075 & 2547.239 & 0.0414 & $\overline{0} .8607$ & $\overline{1} .0235$ & 0.9980 & $\overline{0} .9905$ \\
\hline PC & 74.06 & 1.524 & 0.7801 & 0.679 & 0.0000 & 1.1 & 1.0752 & 1.0706 & 0.0003 \\
\hline IPC & 49.13 & 5.841 & 0.8268 & 8719.572 & 10.9737 & 1.0 & 1.0494 & 0.9898 & 0.9733 \\
\hline $\mathrm{APT}$ & 0. & 4.081 & 0.8749 & 1.327 & 4.0806 & 12.6299 & 0.9723 & 1.0000 & 0 . \\
\hline PT & 0.84 & 2.439 & 0.8589 & 0.808 & 2.4386 & 65.2590 & 0.7521 & 0.9926 & 0.000 \\
\hline
\end{tabular}




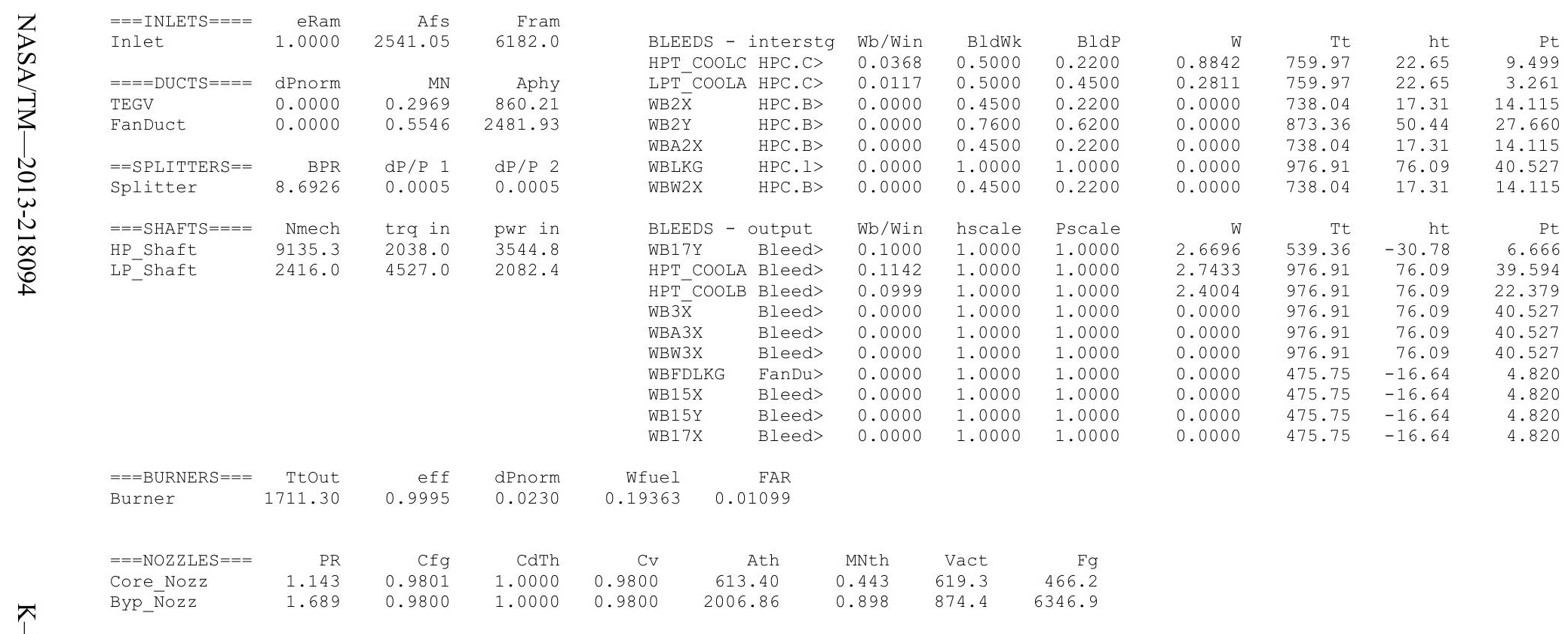


Date: 05/10/13 Time:11:03:04 Model:

Version:NPSS 1.6.5 - Rev: -> Gas Package: Janaf iter/pass/Jacb/Broy= 17/ 59/3/13 Run by: Philip C Jorgenson PC: 10

Temperature Stator 1 inlet: 456.93 Stator 1 exit: 463.94 Stator 2 inlet: 472.74 Stator 2 exit: 477.26

$\begin{array}{lll}\text { Stator } 3 \text { inlet: } 486.30 & \text { Stator } 3 \text { exit: } 490.39 & \text { Stator } 4 \text { inlet: } 498.82 \text { Stator } 4 \text { exit: } 502.29 \\ \text { Stator } 5 \text { inlet: } 509.20 & \text { Stator } 5 \text { exit: } 512.21 & \text { Unblocked Percent Blockage: } 0.00\end{array}$

Ambient Relative Humidity $\quad 10.00$

Fan Face Relative Humidity 2.24

Fan Bypass Relative Humidity $\quad 1.60$

$\begin{array}{ll}\text { LPC Inlet Relative Humidity } & 1.44 \\ \text { LPC Exit Relative Humidity } & 0.13\end{array}$

HPC Rel Relative Humidity

Drop Diative Humidity

0.04

0.0000050

723.11
2.95

Inlet Length

40.00

Drbient Flow Velocity

Fan/LPC Inlet Flow Velocity $\quad 179.78$

Ambient static Pressure

407.97

$\begin{array}{lr}\text { Fan/LPC Inlet Static Temperature } & 448.87\end{array}$

Additional Water at LPC Exit

$\begin{array}{rrrrrr} & & & & \text { SUMMARY } & \text { OUTPUT DAT } \\ \text { MN } & \text { alt } & \text { dTamb } & \text { W } & \text { Fn } & \text { TSFC } \\ 0.730 & 38334.0 & 18.00 & 250.47 & 617.3 & 1.0753\end{array}$

FLOW STATION DATA

FSO Ambient.Fl_O

FS1 Inlet.Fl_O

FS12 Splitter.F1_02

FS14 Fan.FI_O

S23 LPC.F1_O

FS24 VaporIN.FI_

FS3 HPC.Fl_O

FS36 Bleed3.FI_O

FS4 Burner.FI_O

FS45 HPT.FI_-

FS49 LPT.FI-O

FS8 Core_Nozz.FI_O

FS17 FanDuctLkg.Fi_o

FS171 Bleed15. F1

FS172 FanDuct. Fl-O

FS173 Byp Nozz.Fì

$W$
250.47
250.47
224.84
25.64
224.84
25.64
25.75
23.17
22.05
17.09
17.27
23.08
23.35
23.35
23.35
224.84
227.41
227.41
227.41

$\begin{array}{rr}\text { Pt } & \text { Tt } \\ 4.201 & 451.57 \quad-22\end{array}$

$\begin{array}{lrrrr}4.201 & 451.57 & -22.43 & 0.0000 & 817.49\end{array}$

$\begin{array}{lllll}4.201 & 451.57 & -22.43 & 0.0000 & 817.49 \\ 4.199 & 451.57 & -22.43 & 0.0000 & 734.18\end{array}$

$\begin{array}{lllll}4.199 & 451.57 & -22.43 & 0.0000 & 817.49 \\ 4.199 & 451.57 & -22.43 & 0.0000 & 734.18\end{array}$

$\begin{array}{lllll}4.199 & 451.57 & -22.43 & 0.0000 & 734.18 \\ 4.713 & 468.33 & -22.43 & 0.0000 & 83.72\end{array}$

$\begin{array}{lllll}6.423 & 521.36 & -5.70 & 0.0000 & 58.81\end{array}$

$\begin{array}{lllll}6.423 & 527.16 & -28.44 & 0.0000 & 59.38 \\ 6.423 & 527.16 & -28.44 & 0.0000 & 53.45\end{array}$

$\begin{array}{rrrrr}6.423 & 527.16 & -28.44 & 0.0000 & 53.45 \\ 38.732 & 953.52 & 75.46 & 0.0000 & 11.34\end{array}$

$\begin{array}{lllll}38.732 & 953.52 & 75.46 & 0.0000 & 8.79\end{array}$

$\begin{array}{rrrrr}37.840 & 1682.41 & 63.43 & 0.0108 & 12.08\end{array}$

$\begin{array}{lrrrr}9.192 & 1112.08 & -37.23 & 0.0081 & 54.04 \\ 3.311 & 881.94 & -93.56 & 0.0080 & 135.17\end{array}$

$\begin{array}{llllll}3.311 & 882.01 & -93.56 & 0.0080 & 135.17\end{array}$

$3.311 \quad 882.08$

$4.713 \quad 468.33$

$4.713 \quad 469.00$

$4.713 \quad 469.00$

$\begin{array}{lll}-93.56 & 0.0080 & 135.18\end{array}$

$\begin{array}{lll}-18.41 & 0.0000 & 666.15\end{array}$

$\begin{array}{lll}-18.53 & 0.0000 & 674.26\end{array}$

TURBOMACHINERY PERFORMANCE DATA

\begin{tabular}{|c|c|c|c|c|c|c|c|c|c|}
\hline & Wo & PR & eff & NC & $\mathrm{TR}$ & efpoly & pwr & SMN & SMW \\
\hline Fan & 734.18 & 1.122 & 0.9048 & 2506.966 & 1.0371 & 0.9064 & -1277.8 & 2157.02 & 54.66 \\
\hline LPC & 83.72 & 1.530 & 0.8363 & 2506.966 & 1.1546 & 0.8458 & -606.9 & 6.78 & 3.35 \\
\hline HPC & 53.45 & 6.030 & 0.8176 & 8940.025 & 1.8088 & 0.8563 & -3323.8 & 61.25 & 57.86 \\
\hline $\mathrm{HPT}$ & 12.08 & 4.117 & 0.8735 & 219.735 & & 0.8510 & 3323.8 & & \\
\hline LPT & 54.04 & 2.776 & 0.8468 & 70.145 & 1.2590 & 0.8271 & 1884.6 & & \\
\hline
\end{tabular}

TURBOMACHINERY MAP DATA

$\begin{array}{lrrrrrrrrr} & \text { WcMap } & \text { PRmap } & \text { effMap } & \text { NcMap } & \text { R/Parm } & \text { S_WCDes } & \text { S_PRdes } & \text { S_effDes } & \text { S_NCDes } \\ \text { Fan } & 853.04 & 1.120 & 0.9066 & 2483.062 & 0.0413 & 0.8607 & 1.0235 & 0.9980 & 0.9905 \\ \text { LPC } & 71.25 & 1.495 & 0.7770 & 0.662 & 0.0000 & 1.1749 & 1.0707 & 1.0762 & 0.0003 \\ \text { HPC } & 48.61 & 5.793 & 0.8261 & 8701.690 & 10.9899 & 1.0995 & 1.0494 & 0.9898 & 0.9733 \\ \text { HPT } & 0.96 & 4.030 & 0.8735 & 1.321 & 4.0303 & 12.6299 & 0.9723 & 1.0000 & 0.0003 \\ \text { LPT } & 0.83 & 2.336 & 0.8531 & 0.789 & 2.3358 & 65.2590 & 0.7521 & 0.9926 & 0.0005\end{array}$




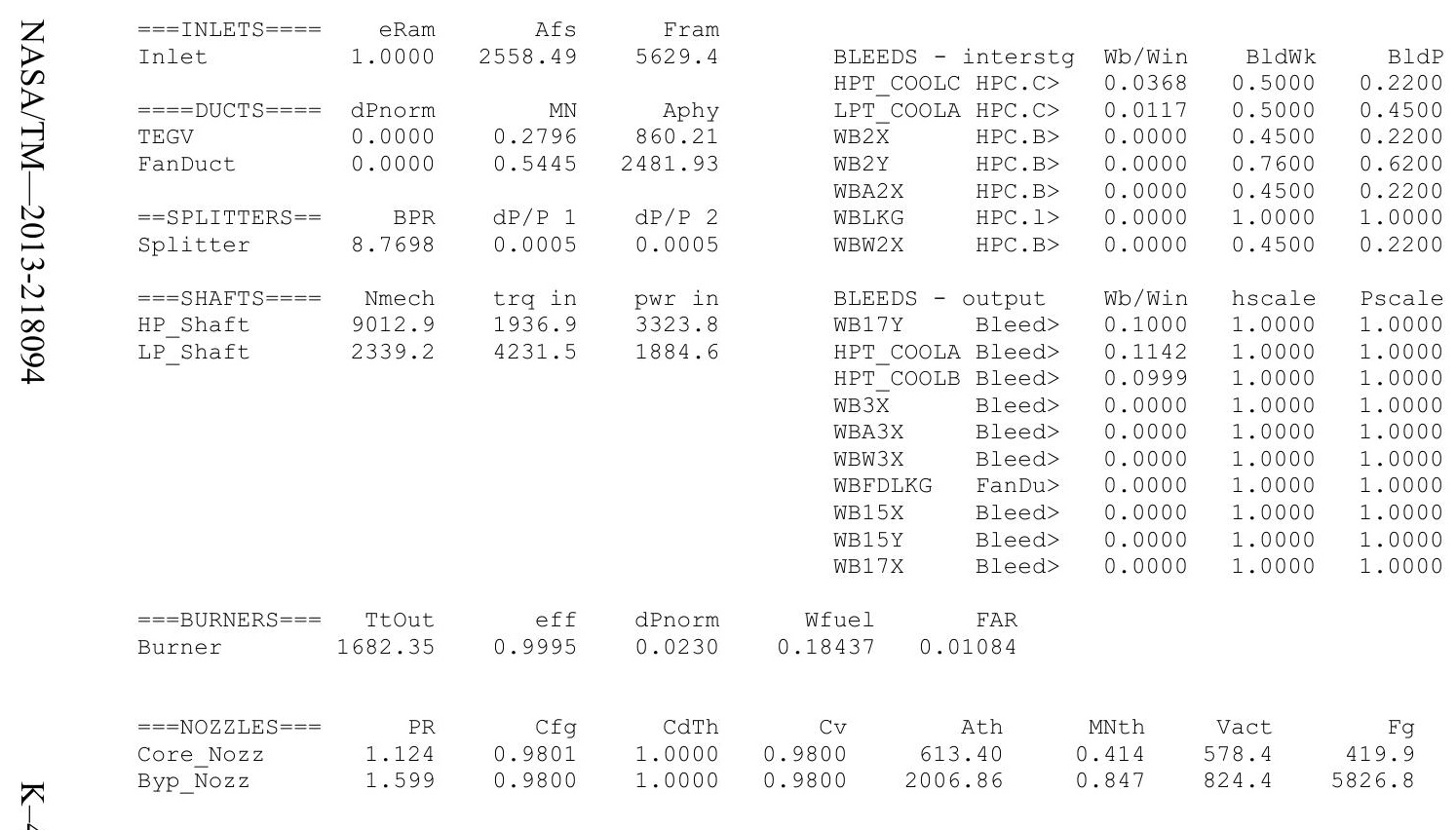


Turbofan Engine - COMDES ON converge $=1$ CASE: 0

Version:NPSS 1.6.5 - Rev: -> Gas Package: Janaf iter/pass/Jacb/Broy=12/26/1/10 Run by: Philip C Jorgenson PC: 10

Temperature Stator 1 inlet: 454.91 Stator 1 exit: $461.74 \quad$ Stator 2 inlet: $470.30 \quad$ Stator 2 exit: 474.69 $\begin{array}{cccc}\text { Stator } 3 \text { inlet: } 483.50 & \text { Stator } 3 \text { exit: } 487.47 & \text { Stator } 4 \text { inlet: } 495.68 \text { Stator } 4 \text { exit: } 499.04 \\ \text { Stator } 5 \text { inlet: } 505.77 & \text { Stator } 5 \text { exit: } 508.68 & \text { Unblocked Percent Blockage: } 0.00\end{array}$

$\begin{array}{lclll}\text { Ambient Relative Humidity } & 10.00 & & & \\ \text { Fan Face Relative Humidity } & 2.40 & & & \\ \text { Fan Bypass Relative Humidity } & 1.74 & & & \\ \text { LPC Inlet Relative Humidity } & 1.56 & & & \\ \text { LPC Exit Relative Humidity } & 0.15 & & & \\ \text { HPC Relative Humidity } & 0.04 & & & \\ \text { Drop Diameter } & 0.0000050 & \text { Inlet Length } & & \\ \text { Ambient Flow Velocity } & 707.26 & \text { Fan/LPC Inlet Flow Velocity } & 177.40 \\ \text { Ambient Static Pressure } & 3.09 & \text { Fan/LPC Inlet Static Pressure } & 4.25 \\ \text { Ambient Static Temperature } & 407.97 & \text { Fan/LPC Inlet Static Temperature } & 447.05 \\ \text { Additional Water at LPC Exit } & 0.0037136 & & & \\ \end{array}$
$\begin{array}{rrr}\text { MN } & \text { alt } & \text { dTamb } \\ 0.714 & 37357.0 & 18.00\end{array}$
257.21
SUMMARY OUTPUT DATA
$\begin{array}{rr}\text { Fn } & \text { TSFC } \\ 630.5 & 1.0705\end{array}$
Wfuel
675.00
BPR
8.7997
VTAS
707.26
OPR
9.100
$\begin{array}{ll}\text { EPR } & \text { PowerSet } \\ 0.772 & 10.000\end{array}$
$\begin{array}{ccc}\text { T4 } & \text { T41 } & \text { T49 } \\ 1670.8 & 1579.3 & 1100.7\end{array}$

0.00

FSO Ambient.FI_O

FS1 Inlet.Fl_O

$\begin{array}{ll}\text { FS12 } & \text { Splitter.Fl_02 } \\ \text { FS2 } & \text { Splitter.Fl_01 }\end{array}$

FS14 Fan.F1_O

$\begin{array}{ll}\text { FS23 } & \text { LPC.FI } \\ \text { FS24 } & \text { VaporIN.FI }\end{array}$

FS24 VaporIN.FI_-

FS3 HPC.FI_O

FS36 Bleed3.F1_O

FS4 Burner.FI_O

FS45 HPT.FI_O

FS49 LPT.FI-O

FS8 Core Nozz.Flo

FS17 FanDuctLkg.Fi_o

FS171 Bleed15.FI_O

FS172 FanDuct. F1_O

$W$
257.21
257.21
230.97
26.25
230.97
26.25
26.34
23.71
22.56
17.48
17.67
23.62
23.90
23.90
23.90
230.97
233.60
233.60
233.60

FLOW STATION DATA

$\begin{array}{rrrr}\text { Pt } & \text { Tt } & \text { ht } & \text { FAR } \\ 4.339 & 449.68 & -22.87 & 0.0000 \\ 4.339 & 449.68 & -22.87 & 0.0000 \\ 4.337 & 449.68 & -22.87 & 0.0000 \\ 4.337 & 449.68 & -22.87 & 0.0000 \\ 4.855 & 465.97 & -18.97 & 0.0000 \\ 6.577 & 517.63 & -6.59 & 0.0000 \\ 6.577 & 522.79 & -26.70 & 0.0000 \\ 6.577 & 522.79 & -26.70 & 0.0000 \\ 39.490 & 944.85 & 76.06 & 0.0000 \\ 39.490 & 944.85 & 76.06 & 0.0000 \\ 38.580 & 1670.79 & 64.10 & 0.0108 \\ 9.412 & 1104.81 & -35.44 & 0.0080 \\ 3.451 & 880.02 & -90.37 & 0.0079 \\ 3.451 & 880.09 & -90.37 & 0.0079 \\ 3.451 & 880.16 & -90.37 & 0.0079 \\ 4.855 & 465.97 & -18.97 & 0.0000 \\ 4.855 & 466.62 & -19.06 & 0.0000 \\ 4.855 & 466.62 & -19.06 & 0.0000 \\ 4.855 & 466.62 & -19.06 & 0.0000\end{array}$

Wc
811.10
811.10
728.70
82.81
662.71
58.59
59.10
53.19
11.33
8.78
12.08
53.83
132.54
132.55
132.55
662.71
670.74
670.74
670.74

PS
3.088
3.980
3.928
4.249
4.106
6.300
6.294
6.350
34.782
36.786
36.427
8.554
3.279
3.279
3.088
4.106
3.980
3.980
3.088

Ts
407.97
438.70
437.11
447.05
444.18
511.31
516.28
517.58
912.13
926.44
1646.92
1076.71
867.60
867.67
853.50
444.18
440.83
440.83
409.96

$\begin{array}{rr}\text { Aphy } & \text { MN } \\ 2563.0 & 0.7140 \\ 4168.2 & 0.3533 \\ 3531.8 & 0.3787 \\ 830.5 & 0.1711 \\ 2606.7 & 0.4947 \\ 412.6 & 0.2484 \\ 412.6 & 0.2511 \\ 412.6 & 0.2243 \\ 49.7 & 0.4319 \\ 49.3 & 0.3217 \\ 74.6 & 0.2945 \\ 265.4 & 0.3760 \\ 860.2 & 0.2736 \\ 860.2 & 0.2736 \\ 613.4 & 0.4039 \\ 2606.7 & 0.4947 \\ 2481.9 & 0.5402 \\ 2481.9 & 0.5402 \\ 2006.9 & 0.8302\end{array}$

gamt
1.40090
1.40090
1.40090
1.40090
1.40079
1.40024
1.39967
1.39967
1.38345
1.38345
1.33337
1.36800
1.38198
1.38198
1.38197
1.40079
1.40078
1.40078
1.40078

WAR
0.0000272
0.0000272
0.0000272
0.0000272
0.0000272
0.0000272
0.0037408
0.0037408
0.0037408
0.0037408
0.0037408
0.0037408
0.0037408
0.0037408
0.0037408
0.0000272
0.0000689
0.0000689
0.0000689

Wair
257.21 257.21
257.21 230.96 26.25
230.96 26.25
26.25 26.25
23.62

23.62
22.48
17.42

17.42
17.42

17.42
23.35

23.62
23.62

23.62
23.62
230.96 230.96
233.59 233.59
233.59
233.59 0.0000689 233.59

WH2O H2O frac $0.007 \quad 0.0000$ $0.001 \quad 0.0000$ $0.006 \quad 0.0000$ $0.065 \quad 0.0168$ $0.088-0.0134$ 0.088 0.006 0.016 0.016

\begin{tabular}{|c|c|c|c|c|c|c|c|c|c|}
\hline \multicolumn{2}{|c|}{ TURBOMACHINERY } & PERFORMANCE & \multicolumn{2}{|l|}{ DATA } & \multirow[b]{2}{*}{$\mathrm{TR}$} & \multirow[b]{2}{*}{ efPoly } & \multirow[b]{2}{*}{ pwr } & \multirow[b]{2}{*}{ SMN } & \multirow[b]{2}{*}{ SMW } \\
\hline & Wc & PR & eff & $\mathrm{NC}$ & & & & & \\
\hline Fan & 728.70 & 1.119 & 0.9044 & 2480.486 & 1.0362 & 0.9059 & -1276.0 & 2532.49 & 54.59 \\
\hline LPC & 82.81 & 1.516 & 0.8368 & 2480.486 & 1.1511 & 0.8461 & -604.9 & 6.70 & 3.28 \\
\hline HPC & 53.19 & 6.005 & 0.8172 & 8931.793 & 1.8073 & 0.8560 & -3363.9 & 61.28 & 57.86 \\
\hline HРT & 12.08 & 4.099 & 0.8729 & 219.379 & 1.3610 & 0.8503 & 3363.9 & & \\
\hline LPT & 53.83 & 2.727 & 0.8445 & 69.486 & 1.2534 & 0.8250 & 1880.9 & & \\
\hline$x=10$ & NERY & DATA & & & & & & & \\
\hline & WcMap & PRmap & effMap & NcMap & R/Parm & s_WcDes & S_PRdes & s_effDes & S_NCDes \\
\hline Fan & 846.67 & 1.117 & 0.9063 & 2456.835 & 0.0413 & $\overline{0} .8607$ & $\overline{1} .0235$ & 0.9980 & $\overline{0} .9905$ \\
\hline LPC & 70.11 & 1.483 & 0.7758 & 0.655 & 0.0000 & 1.1812 & 1.0695 & 1.0787 & 0.0003 \\
\hline $\mathrm{PPC}$ & 48.38 & 5.769 & 0.8256 & 8693.677 & 10.9915 & 1.0995 & 1.0494 & 0.9898 & 0.9733 \\
\hline HРT & 0.96 & 4.013 & 0.8728 & 1.318 & 4.0134 & 12.6299 & 0.9723 & 1.0000 & 0.0003 \\
\hline $\mathrm{PT}$ & 0.82 & 2.299 & 0.8508 & 0.782 & 2 & 65 & 21 & 0.9926 & 0.00 \\
\hline
\end{tabular}




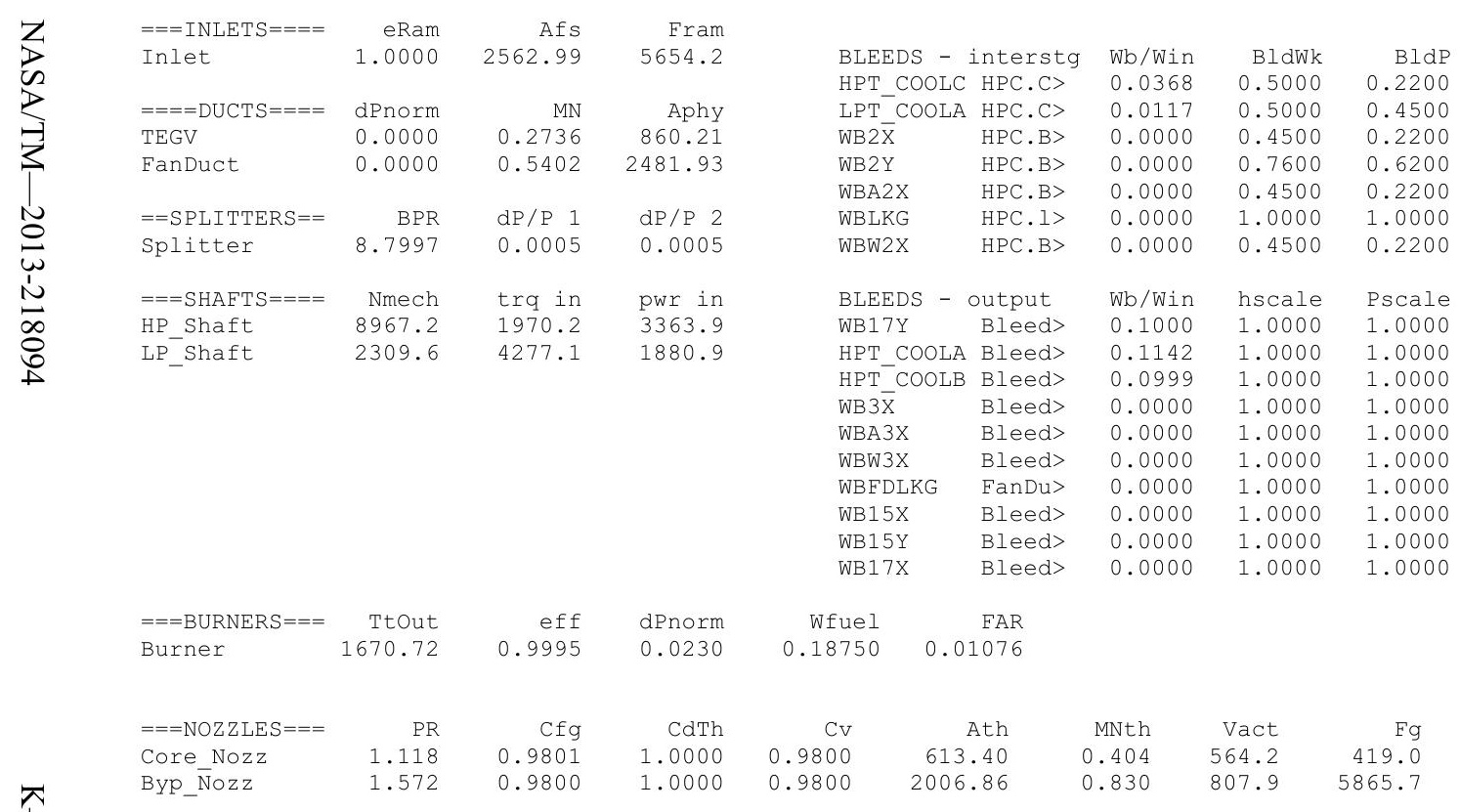


Date:05/10/13 Time:11:03:37 Model: Turbofan Engine - COMDES ON Converge = 1 CASE: 0

Version:NPSS 1.6.5 - Rev: -> Gas Package: Janaf iter/pass/Jacb/Broy=12/ 26/1/10 Run by: Philip C Jorgenson PC: 10

Temperature Stator 1 inlet: 456.54 Stator 1 exit: 462.95 Stator 2 inlet: 470.98 Stator 2 exit: 475.09

$\begin{array}{ccc}\text { Stator } 3 \text { inlet: } 483.34 & \text { Stator } 3 \text { exit: } 487.03 & \text { Stator } 4 \text { inlet: } 494.73 \text { Stator } 4 \text { exit: } 497.84 \\ \text { Stator } 5 \text { inlet: } 504.13 & \text { Stator } 5 \text { exit: } 506.82 & \text { Unblocked Percent Blockage: } 0.00\end{array}$

Ambient Relative Humidity $\quad 10.00$

Fan Face Relative Humidity $\quad 3.00$

Fan Bypass Relative Humidity 2.25

LPC Exit Relative Humidity 0.22

HPC Relative Humidity 0.04

0.0000050

667.90
3.58

Inlet Length

Ambient Flow Velocity

17.93
Inlet Static

Ambient static

414.42

Fan/LPC Inlet Static Temperature 449.15

Additional Water at LPC Exit

$\begin{array}{rrrrrr} & & & & \text { SUMMARY } & \text { OUTPUT DATA } \\ \text { MN } & \text { alt } & \text { dTamb } & \text { W } & \text { Fn } & \text { TSFC } \\ 0.669 & 34281.0 & 18.00 & 278.75 & 685.0 & 1.0571\end{array}$
BPR
8.8754

8.8754
VTAS
667.90
$O P R$
8.765
Powerset 10.000 $\begin{array}{ccc}\text { T4 } & \text { T41 } & \text { T49 } \\ 1662.7 & 1571.2 & 1097.2\end{array}$

\section{FLOW STATION DATA}

\begin{tabular}{rrrr}
\multicolumn{5}{c}{ FLOW } & STATION DATA \\
Pt & Tt & ht & FAR \\
4.831 & 451.61 & -22.45 & 0.0000 \\
4.831 & 451.61 & -22.45 & 0.0000 \\
4.829 & 451.61 & -22.45 & 0.0000 \\
4.829 & 451.61 & -22.45 & 0.0000 \\
5.366 & 466.93 & -18.78 & 0.0000 \\
7.140 & 515.39 & -7.17 & 0.0000 \\
7.140 & 520.06 & -25.28 & 0.0000 \\
7.140 & 520.06 & -25.28 & 0.0000 \\
42.344 & 937.10 & 76.19 & 0.0000 \\
42.344 & 937.10 & 76.19 & 0.0000 \\
41.369 & 1662.68 & 64.25 & 0.0107 \\
10.201 & 1101.30 & -34.01 & 0.0080 \\
3.943 & 888.52 & -85.91 & 0.0079 \\
3.943 & 888.59 & -85.91 & 0.0079 \\
3.943 & 888.66 & -85.91 & 0.0079 \\
5.366 & 466.93 & -18.78 & 0.0000 \\
5.366 & 467.53 & -18.86 & 0.0000 \\
5.366 & 467.53 & -18.86 & 0.0000 \\
5.366 & 467.53 & -18.86 & 0.0000
\end{tabular}

FSO Ambient.FI_

FS1 Inlet.Fl_O

FS2 Splitter.F1_02

FS14 Fan.F1_O

FS23 LPC.FI_O

FS24 Bleed2.F1-

FS3 HPC.FI_O

FS36 Bleed3.FI_O

FS4 Burner.Fl_o

FS45 HPT.FI-

FS49 LPT.FI-O

FS8 Core_Nozz.Fl_O

FS17 FanDüctLkg.Fì 0

FS171 Bleed15.F1_O

FS172 FanDuct.Fl_O

TURBOMACHINERY PERFORMANCE DATA

\begin{tabular}{|c|c|c|c|c|c|c|c|c|c|}
\hline & Wc & $P R$ & eff & $\mathrm{NC}$ & $\mathrm{TR}$ & efpoly & pwr & SMN & SMW \\
\hline Tan & 711.46 & 1.111 & 0.9036 & 2402.972 & 1.0339 & 0.9050 & -1301.2 & 5205.99 & 54.13 \\
\hline LPC & 80.16 & 1.479 & 0.8382 & 2402.972 & 1.1412 & 0.8469 & -610.6 & 6.43 & 3.07 \\
\hline HPC & 52.53 & 5.931 & 0.8159 & 8910.155 & 1.8019 & 0.8548 & -3570.6 & 61.54 & 58.05 \\
\hline $\mathrm{HPT}$ & 12.08 & 4.056 & 0.8723 & 218.806 & 1.3580 & 0.8499 & 3570.6 & & \\
\hline LPT & 53.30 & 2.587 & 0.8398 & 67.567 & 1.2372 & 0.8209 & 1911.7 & & \\
\hline \multicolumn{10}{|c|}{ TURBOMACHINERY MAP DATA } \\
\hline & WcMap & PRmap & effmap & NcMap & R/Parm & s_WcDes & S_PRdes & S_effDes & S_NcDes \\
\hline n & 826.64 & 1.109 & 0.9054 & 380.060 & 0.0413 & $\overline{0} .8607$ & $\overline{1} .0235$ & 0.9980 & $\overline{0} .9905$ \\
\hline PC & 66.75 & 1.448 & 0.7722 & 0.635 & 0.0000 & 1.2009 & 1.0681 & 1.0856 & 0.0003 \\
\hline $\mathrm{PC}$ & 47.78 & 5.698 & 0.82 & 8672.616 & 10.9836 & 1.0995 & 1.0494 & 0.9898 & 0.9733 \\
\hline ET & 0.96 & 3.971 & 0.8723 & 1.315 & 3.9709 & 12.6299 & 0.9723 & 1.0000 & 0.0003 \\
\hline pT & 0.82 & 2.194 & 0.8460 & 0.760 & 2.1936 & 65.2590 & 0.7521 & 0.9926 & 0.0005 \\
\hline
\end{tabular}

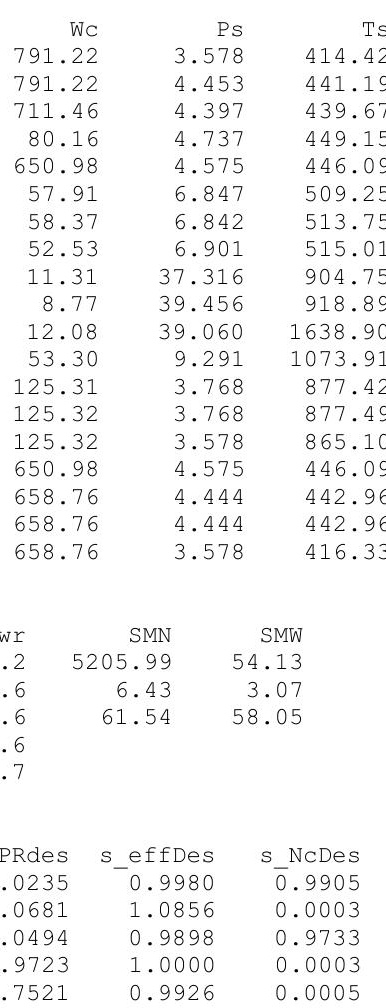

$\begin{array}{rrrrrrr}\text { Aphy } & \text { MN } & \text { gamt } & \text { WAR } & \text { Wair } & \text { WH2O } & \text { H2O frac } \\ 2579.0 & 0.6690 & 1.40088 & 0.0000348 & 278.74 & 0.010 & 0.0000 \\ 4168.2 & 0.3432 & 1.40088 & 0.0000348 & 278.74 & 0.010 & 0.0000 \\ 3531.8 & 0.3680 & 1.40088 & 0.0000348 & 250.51 & 0.009 & 0.0000 \\ 830.5 & 0.1654 & 1.40088 & 0.0000348 & 28.23 & 0.001 & 0.0000 \\ 2606.7 & 0.4827 & 1.40078 & 0.0000348 & 250.51 & 0.009 & 0.0000 \\ 412.6 & 0.2453 & 1.40027 & 0.0000348 & 28.23 & 0.001 & 0.0000 \\ 412.6 & 0.2477 & 1.39975 & 0.0033774 & 28.23 & 0.095 & 0.0034 \\ 412.6 & 0.2213 & 1.39975 & 0.0033774 & 25.40 & 0.086 & 0.0034 \\ 49.7 & 0.4309 & 1.38397 & 0.0033774 & 24.17 & 0.082 & 0.0034 \\ 49.3 & 0.3210 & 1.38397 & 0.0033774 & 18.73 & 0.063 & 0.0034 \\ 74.6 & 0.2944 & 1.33383 & 0.0033774 & 18.73 & 0.063 & 0.0164 \\ 265.4 & 0.3715 & 1.36830 & 0.0033774 & 25.11 & 0.085 & 0.0131 \\ 860.2 & 0.2574 & 1.38156 & 0.0033774 & 25.40 & 0.086 & 0.0130 \\ 860.2 & 0.2574 & 1.38156 & 0.0033774 & 25.40 & 0.086 & 0.0130 \\ 613.3 & 0.3774 & 1.38155 & 0.0033774 & 25.40 & 0.086 & 0.0130 \\ 2606.7 & 0.4827 & 1.40078 & 0.0000348 & 250.51 & 0.009 & 0.0000 \\ 2481.9 & 0.5260 & 1.40077 & 0.0000720 & 253.33 & 0.018 & 0.0001 \\ 2481.9 & 0.5260 & 1.40077 & 0.0000720 & 253.33 & 0.018 & 0.0001 \\ 2006.9 & 0.7832 & 1.40077 & 0.0000720 & 253.33 & 0.018 & 0.0001\end{array}$

1.40077 


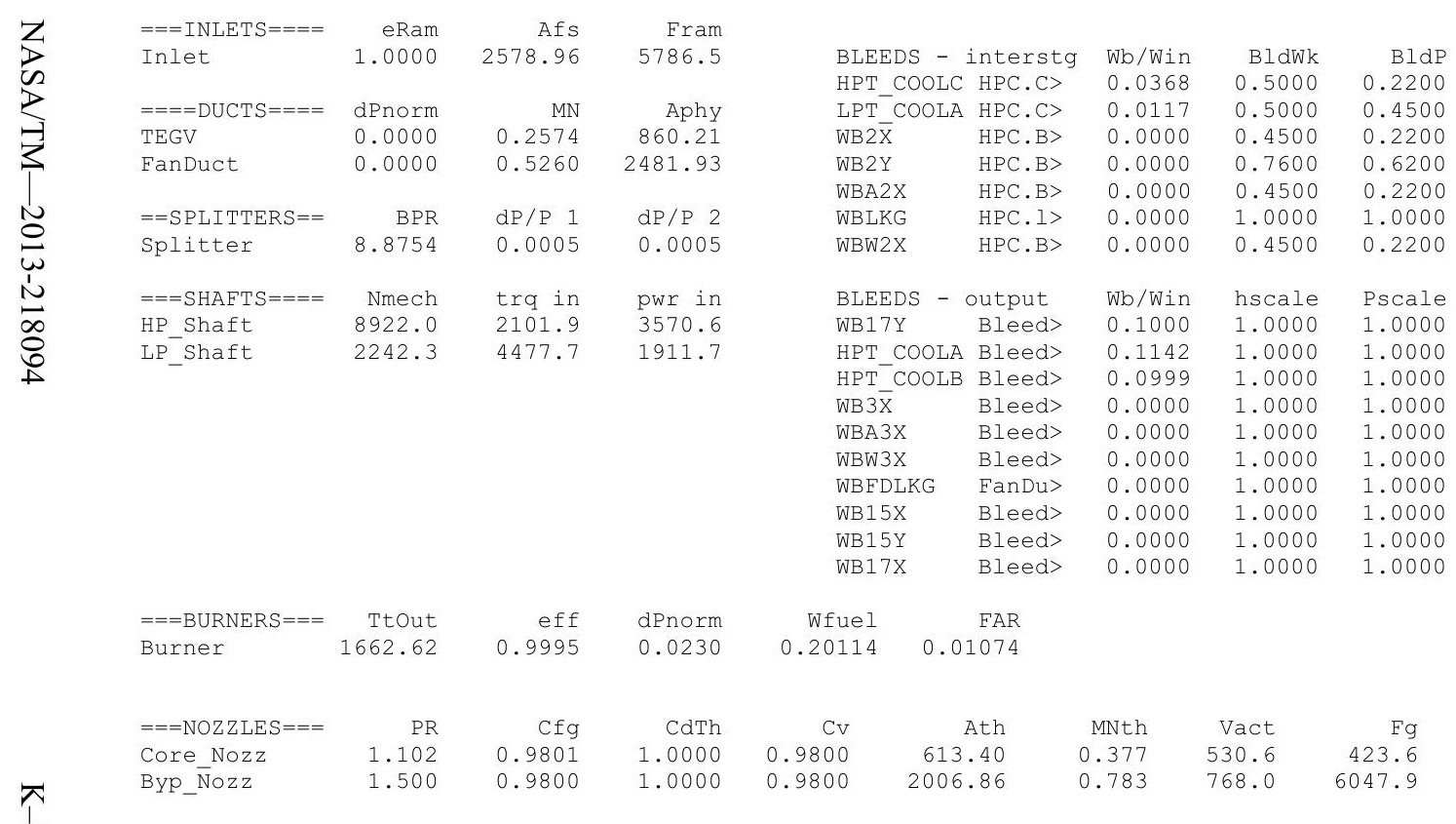


Date:05/10/13 Time:11:04:04 Model: Turbofan Engine - COMDES ON Converge = 1 CASE: 0

Version:NPSS 1.6.5 - Rev: -> Gas Package: Janaf iter/pass/Jacb/Broy=17/ 45/2/14 Run by: Philip C Jorgenson PC: 10

Temperature Stator 1 inlet: 465.17 Stator 1 exit: 470.74 Stator 2 inlet: 477.63 $\quad$ Stator 2 exit: 480.97 $\begin{array}{lll}\text { Stator } 3 \text { inlet: } 488.14 & \text { Stator } 3 \text { exit: } 491.08 & \text { Stator } 4 \text { inlet: 497.81 Stator 4 exit: } 500.22 \\ \text { Stator } 5 \text { inlet: } 505.66 & \text { Stator } 5 \text { exit: } 507.72 & \text { Unblocked Percent Blockage: } 0.00\end{array}$

$\begin{array}{lclll}\text { Ambient Relative Humidity } & 10.00 & & & \\ \text { Fan Face Relative Humidity } & 3.96 & & & \\ \text { Fan Bypass Relative Humidity } & 3.10 & & & \\ \text { LPC Inlet Relative Humidity } & 2.83 & & & \\ \text { LPC Exit Relative Humidity } & 0.46 & & & \\ \text { HPC Relative Humidity } & 0.05 & & & \\ \text { Drop Diameter } & 0.0000050 & \text { Inlet Length } & \\ \text { Ambient Flow Velocity } & 617.99 & \text { Fan/LPC Inlet Flow Velocity } & 173.93 \\ \text { Ambient Static Pressure } & 4.36 & \text { Fan/LPC Inlet Static Pressure } & 5.49 \\ \text { Ambient Static Temperature } & 429.58 & \text { Fan/LPC Inlet Static Temperature } & 458.90 \\ \text { Additional Water at LPC Exit } & 0.0034488 & & & \end{array}$

Additional Water at LPC Exit

SUMMARY OUTPUT DATA
MN alt dTamb

W
305.33 $\begin{array}{rc}\text { Fn } & \text { TSFC } \\ 746.5 & 1.0593\end{array}$

Wfuel
790.75

BPR
8.4334

VTAS
617.99
OPR
8.209
Powerset 10.000 $\begin{array}{ccc}\text { T4 } & \text { T41 } & \text { T49 } \\ 1667.2 & 1575.1 & 1102.9\end{array}$
FSO Ambient.Fl

FS1 Inlet.Fi_o

FS12 Splitter.F1_02

FS2 Splitter.

FS23 LPC.FI-

FS24 VaporIN. FI_O

FS25 Bleed2.F1_-

FS3 HPC.FI_O

FS36 Bleed3.FI_O

$\begin{array}{ll}\text { FS4 } & \text { Burner.FI } \\ \text { FS45 } & \text { HPT.FI O }\end{array}$

FS49 LPT.FI_O

FS5 TEGV.FI_O

FS8 Core_Nozz.Fl_O

FS171 RanDuctLkg.FI-

FS171 Bleed15.F1-

FS173 Byp Nozz.Fi'

TURBOMACHINERY PERFORMANCE DATA

\begin{tabular}{|c|c|c|c|c|c|c|c|c|c|c|c|}
\hline & & & & FLOW S & STATION DA: & & & & & PS & \\
\hline ESO & Ambient.Fl O & & 305.33 & $\begin{array}{r}\mathrm{Pt} \\
5.595\end{array}$ & $\begin{array}{r}\mathrm{Tt} \\
461.42\end{array}$ & $\begin{array}{r}h t \\
-20.30\end{array}$ & $\begin{array}{r}\text { FAR } \\
0.0000\end{array}$ & $\begin{array}{r}\text { Wc } \\
756.40\end{array}$ & 4.3 & & 429.5 \\
\hline FS1 & Inlet.Fl o- & & 305.33 & 5.595 & 461.42 & -20.30 & 0.0000 & 756.40 & 5. & & 451.8 \\
\hline FS12 & Splitter.Fl_0 & & 272.96 & 5.592 & 461.42 & -20.30 & 0.0000 & 676.55 & 5. & & 450 \\
\hline FS2 & Splitter.Fl_o & & 32.37 & 5.592 & 461.42 & -20.30 & 0.0000 & 80.22 & & & 458 \\
\hline S14 & Fan.Fl 0 & & 272.96 & 6.146 & 475.40 & -16.95 & 0.0000 & 624.92 & 5.3 & & 456 \\
\hline FS23 & LPC.FI_O & & 32.37 & 7.851 & 516.54 & -7.08 & 0.0000 & 60.46 & 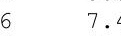 & & 509 \\
\hline FS24 & VaporIN.FI_O & & 32.48 & 7.851 & 521.34 & -25.77 & 0.0000 & 60.95 & 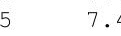 & & 51 \\
\hline S25 & Bleed2.Fl_o & & 27.61 & 7.851 & 521.34 & -25.77 & 0.0000 & 51.81 & 7.5 & & 51 \\
\hline s3 & HPC.FI_O & & 26.27 & 45.928 & 936.05 & 75.15 & 0.0000 & 11.29 & 40.2 & & \\
\hline S36 & Bleed3.Fl O & & 20.36 & 45.928 & 936.05 & 75.15 & 0.0000 & 8.75 & $42 . \varepsilon$ & & 91 \\
\hline 4 & Burner.Fl_o & & 20 & .870 & 1667.23 & 63.12 & & 12.08 & 42.3 & & \\
\hline 545 & HPT.FI_O & & 27.50 & 11.217 & 1107.02 & -34.56 & 81 & 52.64 & 10.2 & & 108 \\
\hline 549 & LPT.FI- O & & 27.83 & 4.719 & 911.84 & -82.11 & 0.0 & 114.91 & 4.5 & & 902 \\
\hline F 5 & TEGV.Fİ_O & & 27.83 & 4.719 & 911.92 & -82.11 & 0.0080 & 114.91 & 4.5 & & \\
\hline S8 & Core_Nozz.FI & & 27.83 & 4.719 & 911.99 & -82.11 & 0.0080 & 114.92 & 4.3 & & $892-2 \cdot \ln$ \\
\hline FS17 & FanDuctLkg.Fi & & 272 & 6.146 & 475.40 & -16.95 & 0. & 624.92 & 5.3 & & 45 \\
\hline FS171 & Bleed15.Fl O & & 277.83 & 6.146 & 476.21 & -17.10 & 0.0000 & 636.61 & 5.1 & & 453 \\
\hline FS172 & FanDuct.FI_O & & 277.83 & 6.146 & 476.21 & -17.10 & 0. & 636.61 & 5.1 & & 45 \\
\hline FS173 & Byp_Nozz.FI_o & & 277.83 & 6.146 & 476.21 & -17.10 & 0.0000 & 636.61 & 4.3 & & 431 \\
\hline JRBON & MACHINERY PERE & ORMANCE & DATA & & & & & & & & \\
\hline & & $\mathrm{PR}$ & eff & $\mathrm{NC}$ & $\mathrm{TR}$ & efPoly & & r & SMN & & SMW \\
\hline an & 676.55 & 1.099 & 0.9026 & 2257.238 & 1.0303 & 0.9039 & -12 & 94.1 & & & .66 \\
\hline$\lrcorner \mathrm{PC}$ & 80.22 & 1.40 & 0.8528 & 2257.238 & 1.1195 & 0.8597 & & 505.1 & 5.94 & & .65 \\
\hline PC & 51.81 & 5.850 & 0.8145 & 8886.483 & 1.7955 & 0.8534 & -38 & 346.1 & 1.83 & & .24 \\
\hline $\mathrm{HPT}$ & 12.08 & 4.000 & 0.8725 & 218.196 & 1.3538 & 0.8503 & & 346.1 & & & \\
\hline $\mathrm{EPT}$ & 52.64 & 2.377 & 0.8317 & 63.988 & 1.2115 & 0.8139 & & 99.2 & & & \\
\hline $\mathrm{RBB} C$ & MACHINERY MAP & $\mathrm{DA}:$ & & & & & & & & & \\
\hline & WcMap & PRmap & effMap & NcMap & R/Parm & $\mathrm{s}_{-} \mathrm{W}$ & cDes & s_PRdes & S_effDes & & S_NCDes \\
\hline Fan & 786.09 & 1.097 & & 2235.715 & 0.0417 & & 8607 & 1.0235 & 0.9980 & & 0.9905 \\
\hline LPC & 60.58 & 1.385 & 0.7657 & 0.596 & 0.0000 & & 3242 & 1.0502 & 1.1138 & & 0.0003 \\
\hline HPC & 47.12 & 5.622 & 0.8229 & 8649.575 & 10.9757 & & 0995 & 1.0494 & 0.9898 & & 0.9733 \\
\hline HPT & 0.96 & 3.91 & 0.87 & 1.311 & 3.9172 & 12. & 6299 & 0.9723 & 1.0000 & & 0.0003 \\
\hline LPT & 0.81 & 2.036 & 0.8379 & 0.720 & 2.0357 & 65.2 & 2590 & 0.7521 & 0.9926 & & 0.0005 \\
\hline
\end{tabular}

$\begin{array}{rrr}\text { Aphy } & \text { MN } & \text { gamt } \\ 2598.1 & 0.6080 & 1.40081 \\ 4168.2 & 0.3259 & 1.40081 \\ 3531.8 & 0.3469 & 1.40081 \\ 830.5 & 0.1656 & 1.40081 \\ 2606.7 & 0.4570 & 1.40070 \\ 412.6 & 0.2570 & 1.40025 \\ 412.6 & 0.2596 & 1.39972 \\ 412.6 & 0.2181 & 1.39972 \\ 49.7 & 0.4298 & 1.38401 \\ 49.3 & 0.3203 & 1.38401 \\ 74.6 & 0.2945 & 1.33353 \\ 265.4 & 0.3662 & 1.36786 \\ 860.2 & 0.2346 & 1.38014 \\ 860.2 & 0.2346 & 1.38014 \\ 613.4 & 0.3410 & 1.38014 \\ 2606.7 & 0.4570 & 1.40070 \\ 2481.9 & 0.5010 & 1.40069 \\ 2481.9 & 0.5010 & 1.40069 \\ 2006.9 & 0.7180 & 1.40069\end{array}$

WAR
0.0000681
0.0000681
0.0000681
0.0000681
0.0000681
0.0000681
0.0035169
0.0035169
0.0035169
0.0035169
0.0035169
0.0035169
0.0035169
0.0035169
0.0035169
0.0000681
0.0001284
0.0001284
0.0001284

Wair 305.31 305.31
272.94 32.36 32.36
32.36 $\begin{array}{ll}32.36 & 0.114\end{array}$ $\begin{array}{ll}27.51 & 0.097 \\ 26.18 & 0.092\end{array}$ $\begin{array}{ll}26.18 & 0.092 \\ 20.29 & 0.071\end{array}$ $20.29 \quad 0.07$ $\begin{array}{ll}27.29 & 0.071 \\ 27.19 & 0.096\end{array}$ $\begin{array}{ll}27.51 & 0.097\end{array}$ $\begin{array}{ll}27.51 & 0.097\end{array}$ $\begin{array}{ll}27.51 & 0.097\end{array}$ $\begin{array}{ll}272.94 & 0.019\end{array}$ $\begin{array}{ll}277.80 & 0.036\end{array}$ $\begin{array}{ll}277.80 & 0.036 \\ 277.80 & 0.036\end{array}$ $277.80 \quad 0.036$

$\begin{array}{rr}\text { H2O } & \text { H2O frac } \\ .021 & 0.0001 \\ .021 & 0.0001 \\ .019 & 0.0001 \\ 002 & 0.0001 \\ .019 & 0.0001 \\ 002 & 0.0001 \\ 14 & 0.0035 \\ 097 & 0.0035 \\ 092 & 0.0035 \\ .071 & 0.0035 \\ .071 & 0.0166 \\ .096 & 0.0133 \\ .097 & 0.0132 \\ 097 & 0.0132 \\ 097 & 0.0132 \\ .019 & 0.0001 \\ .036 & 0.0001 \\ .036 & 0.0001 \\ 036 & 0.0001\end{array}$

\begin{abstract}
(1)
\end{abstract}




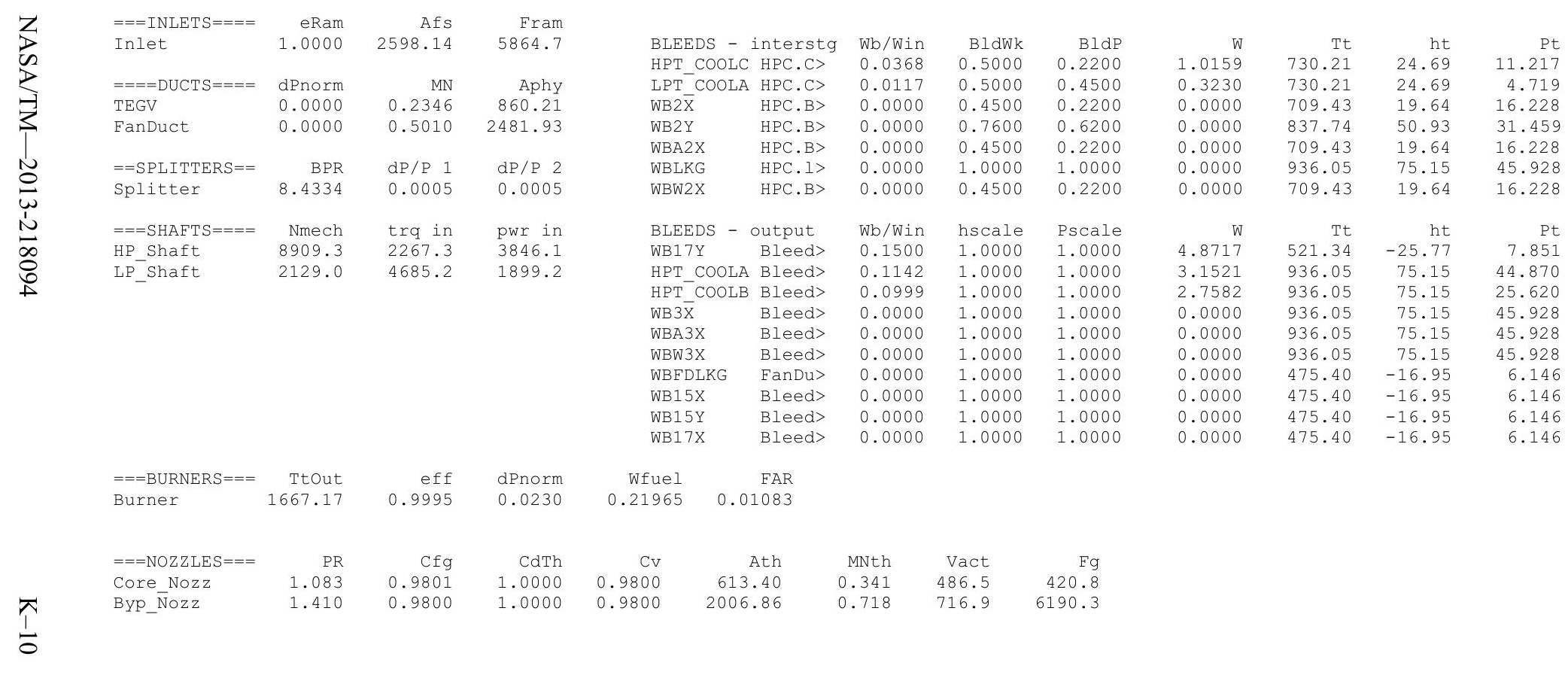


Date:05/10/13 Time:11:04:33 Model: Turbofan Engine - COMDES ON Converge = 1 CASE: 0

Version:NPSS 1.6.5 - Rev: -> Gas Package: Janaf iter/pass/Jacb/Broy= 20/48/2/17 Run by: Philip C Jorgenson PC: 10

Temperature Stator 1 inlet: 473.22 Stator 1 exit: 477.33 Stator 2 inlet: $482.00 \quad$ Stator 2 exit: 483.85 $\begin{array}{cccc}\text { Stator } 3 \text { inlet: } 489.04 & \text { Stator } 3 \text { exit: } 490.47 & \text { Stator } 4 \text { inlet: } 495.44 \text { Stator } 4 \text { exit: } 496.48 \\ \text { Stator } 5 \text { inlet: } 500.12 & \text { Stator } 5 \text { exit: } 500.87 & \text { Unblocked Percent Blockage: } 0.00\end{array}$

Ambient Relative Humidity $\quad 10.00$

Fan Face Relative Humidity $\quad 4.82$

Fan Bypass Relative Humidity $\quad 3.93$

LPC Inlet Relative Humidity

HPC Relative Humidity

Drop Diameter

0.05

Ambient Flow Velocity $\quad 0.0000050$

Ambient Static Pressure

Ambient Static Temperature
Additional Water at LPC Exit

571.13

Inlet Length

-40.00
-193.70

MN alt dTamb SUMMARY OUTPUT DATA $\begin{array}{rrrrrr} & \text { alt } & \text { dTamb } & \text { W } & \text { Fn } & \text { TSFC } \\ 0.552 & 25666.0 & 18.00 & 330.79 & 766.2 & 1.1014\end{array}$
Wfuel $\begin{array}{rr}\text { BPR } \\ 843.96 & 7.0896\end{array}$
VTAS 571.13
OPR
7.351

FSO Ambient.FI_O

FS1 Inlet.F1_- $0^{-}$

无

FLOW STATION DATA

$W$
330.79
330.79
289.90
40.89
289.90
40.89
41.02
28.71
27.32
21.17
21.41
28.61
28.95
28.95
28.95
289.90
302.20
302.20
302.20

$\begin{array}{rrrr}\text { Pt } & \text { Tt } & \text { ht } & \text { FAR } \\ 6.516 & 472.33 & -18.03 & 0.0000 \\ 6.516 & 472.33 & -18.03 & 0.0000 \\ 6.513 & 472.33 & -18.03 & 0.0000 \\ 6.513 & 472.33 & -18.03 & 0.0000 \\ 7.063 & 484.59 & -15.09 & 0.0000 \\ 8.237 & 512.12 & -8.48 & 0.0000 \\ 8.237 & 516.49 & -25.37 & 0.0000 \\ 8.237 & 516.49 & -25.37 & 0.0000 \\ 47.900 & 926.25 & 74.26 & 0.0000 \\ 47.900 & 926.25 & 74.26 & 0.0000 \\ 46.797 & 1676.50 & 61.93 & 0.0111 \\ 12.051 & 1117.30 & -34.37 & 0.0083 \\ 5.628 & 943.48 & -76.59 & 0.0082 \\ 5.628 & 943.55 & -76.59 & 0.0082 \\ 5.628 & 943.62 & -76.59 & 0.0082 \\ 7.063 & 484.59 & -15.09 & 0.0000 \\ 7.063 & 485.89 & -15.51 & 0.0000 \\ 7.063 & 485.89 & -15.51 & 0.0000 \\ 7.063 & 485.89 & -15.51 & 0.0000\end{array}$

Wc
711.91
711.91
624.22
88.05
583.04
72.49
73.03
51.12
11.20
8.68
12.09
51.21
101.94
101.95
101.95
583.04
608.61
608.61
608.61

Ts
445.14
463.72
463.04
469.20
468.19
502.20
506.31
511.76
895.01
908.63
1652.57
1092.03
935.92
935.99
928.03
468.19
465.21
465.21
447.50

Ts
5.14
3.72
3.04
9.20
8.19
2.20
6.31
1.76
5.01
8.63
2.57
2.03
5.92
5.99
8.03
8.19
5.21
5.21
7.50

$\begin{array}{rr}\text { Aphy } & \text { MN } \\ 2597.0 & 0.5520\end{array}$ $\begin{array}{ll}2597.0 & 0.5520 \\ 4168.2 & 0.3043\end{array}$ $\begin{array}{ll}3531.8 & 0.3163\end{array}$ 830.50 .1824 $\begin{array}{lll}412.6 & 0.3142\end{array}$ $\begin{array}{ll}412.6 & 0.3172\end{array}$ $\begin{array}{ll}412.6 & 0.2150\end{array}$ $\begin{array}{ll}49.7 & 0.4253\end{array}$ $\begin{array}{ll}49.3 & 0.3173\end{array}$ $\begin{array}{ll}74.6 & 0.2946\end{array}$ $\begin{array}{ll}265.4 & 0.3545\end{array}$ $\begin{array}{ll}860.2 & 0.2068\end{array}$ $\begin{array}{ll}860.2 & 0.2068\end{array}$ $\begin{array}{ll}613.4 & 0.2979\end{array}$ $2606.7 \quad 0.4181$ $2481.9 \quad 0.4710$ $2481.9 \quad 0.4710$ $2006.9 \quad 0.6543$
Powerset Powerse
10.000 $\begin{array}{ccc}\text { T4 } & \text { T41 } & \text { T49 } \\ 1676.5 & 1582.1 & 1112.9\end{array}$

TURBOMACHINERY PERFORMANCE DATA

\begin{tabular}{|c|c|c|c|c|c|c|c|c|c|}
\hline & Wc & $P R$ & eff & $\mathrm{NC}$ & $T R$ & efpoly & pwr & SMN & SMW \\
\hline Fan & 624.22 & 1.084 & 0.9036 & 2051.291 & 1.0260 & 0.9047 & $-1205.4=$ & -2829.56 & 49.84 \\
\hline LPC & 88.05 & 1.265 & 0.8244 & 2051.291 & 1.0843 & 0.8301 & -552.0 & 6.37 & 2.41 \\
\hline HPC & 51.12 & 5.815 & 0.8137 & 8864.996 & 1.7933 & 0.8527 & -3949.3 & 60.93 & 57.30 \\
\hline HPT & 12.09 & 3.883 & 0.8738 & 216.055 & 1.3452 & 0.8524 & 3949.3 & & \\
\hline LPT & 51.21 & 2.141 & 0.8223 & 58.562 & 1.1813 & 0.8061 & 1757.5 & & \\
\hline \multicolumn{10}{|c|}{ RURBOMACH INERY MAP D } \\
\hline & WcMap & PRmap & effmap & NcMap & R/Parm & s WCDes & s PRdes & $s s$ effDes & s NcDes \\
\hline an & 725.28 & 1.082 & 0.9055 & 31.732 & 0.0426 & $\overline{0} .8607$ & $\overline{1} .0235$ & 0.9980 & $\overline{0} .9905$ \\
\hline LPC & 53.69 & 1.313 & 0.7609 & 0.542 & 0.0000 & 1.6397 & 0.8445 & 1.0834 & 0.0003 \\
\hline $\mathrm{PC}$ & 46.49 & 5.588 & 0.8222 & 8628.661 & 11.0393 & 1.0995 & 1.0494 & 0.9898 & 0.9733 \\
\hline HPT & 0.96 & 3.803 & 0.8738 & 1.298 & 3.8034 & 12.6299 & 0.9723 & 1.0000 & 0.0003 \\
\hline LPT & 0.78 & 1.858 & 0.8284 & 0.659 & 1.8583 & 65.2590 & 0.7521 & 0.9926 & 0.0005 \\
\hline
\end{tabular}




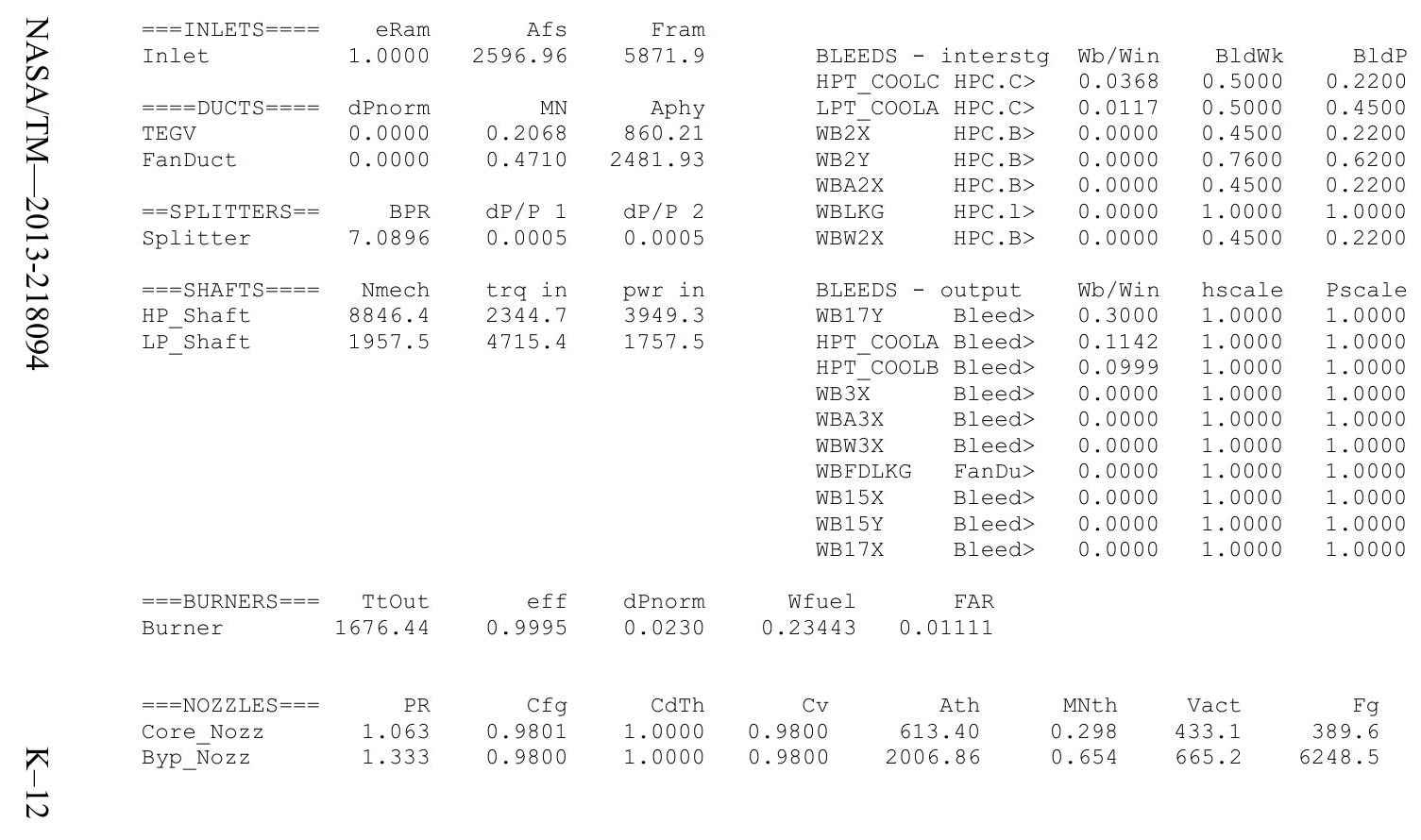


Date:05/10/13 Time:11:05:11 Model: Turbofan Engine - COMDES ON Converge = 1 CASE: 0

Version:NPSS 1.6.5 - Rev: -> Gas Package: Janaf iter/pass/Jacb/Broy= 35/63/2/32 Run by: Philip C Jorgenson PC: 10

Temperature Stator 1 inlet: $486.38 \quad$ Stator 1 exit: $489.37 \quad$ Stator 2 inlet: 492.19 Stator 2 exit: 492.99 Stator 5 inlet: 502.04 Stator 5 exit: 501.92 Unblocked Percent Blockage: 0.00

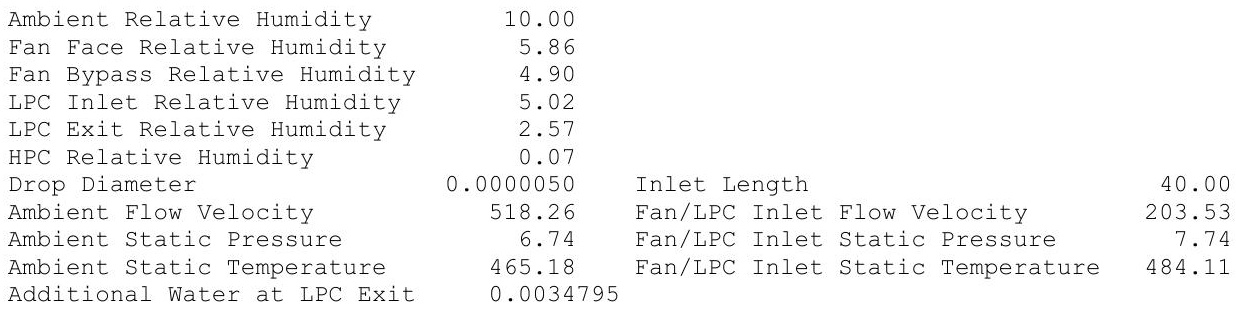
SUMMARY OUTPUT DAT

$\begin{array}{rrr}\text { MN } & \text { alt } & \text { dTamb } \\ 0.490 & 20047.0 & 18.00\end{array}$

$W$
368.47

$\begin{array}{rrr}\text { Fn } & \text { TSFC } & \text { Wfuel } \\ 853.8 & 1.1023 & \end{array}$ $\begin{array}{rr}\text { BPR } \\ 941.09 & 6.2711\end{array}$

VTAS
518.26 $\mathrm{OPR}$
6.475

Powerset 10.000
FSO Ambient.FI

FS1 Inlet.Fi_o

Splitter.Fl_01

FS14 Fan.F1_-

FS24 VaporIN.FI

FS25 Bleed2.F1_O

FS3 HPC.FI_O

S36 Bleed3.Fl_O

FS4 Burner.FI

$\begin{array}{ll}\text { FS45 } & \text { HPT.F1- } \\ \text { FS49 } & \text { LPT.FI- }\end{array}$

FS4 5 LPT.FI-O

FS8 Core_Nozz.FI_O

FS17 FanDüctLkg.Fi__o

FS171 Bleed15.FI_O

FS172 FanDuct.FI-O

W
368.47
368.47
317.79
50.68
317.79
50.68
50.85
30.51
29.03
22.50
22.76
30.42
30.77
30.77
30.77
317.79
338.13
338.13
338.13

FLOW STATION DATA

$\begin{array}{rrrr}\text { Pt } & \text { Tt } & \text { ht } & \text { FAR } \\ 7.943 & 487.56 & -15.16 & 0.0000 \\ 7.943 & 487.56 & -15.16 & 0.0000 \\ 7.939 & 487.56 & -15.16 & 0.0000 \\ 7.939 & 487.56 & -15.16 & 0.0000 \\ 8.519 & 498.52 & -12.53 & 0.0000 \\ 9.114 & 515.69 & -8.41 & 0.0000 \\ 9.114 & 520.54 & -27.25 & 0.0000 \\ 9.114 & 520.54 & -27.25 & 0.0000 \\ 51.433 & 926.42 & 71.49 & 0.0000 \\ 51.433 & 926.42 & 71.49 & 0.0000 \\ 50.248 & 1710.66 & 58.59 & 0.0117 \\ 13.583 & 1149.03 & -36.64 & 0.0087 \\ 7.050 & 994.22 & -74.21 & 0.0086 \\ 7.050 & 994.29 & -74.21 & 0.0086 \\ 7.050 & 994.36 & -74.21 & 0.0086 \\ 8.519 & 498.52 & -12.53 & 0.0000 \\ 8.519 & 499.85 & -13.42 & 0.0000 \\ 8.519 & 499.85 & -13.42 & 0.0000 \\ 8.519 & 499.85 & -13.42 & 0.0000\end{array}$

WC
660.94

660.94
570.33

570.33

90.94

81.48

81.48
82.15

49.29

11.09
8.59

12.09

48.98

88.81

88.82

88.82
537.45

572.61

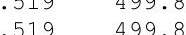

$\begin{array}{ll}-13.42 & 0.0000\end{array}$

572.61

$\begin{array}{rr}\text { PS } & \text { TS } \\ 6.740 & 465.18 \\ 7.522 & 480.01 \\ 7.501 & 479.70 \\ 7.745 & 484.11 \\ 7.717 & 484.62 \\ 8.335 & 502.69 \\ 8.319 & 507.15 \\ 8.846 & 516.13 \\ 45.607 & 895.95 \\ 48.075 & 909.19 \\ 47.438 & 1686.33 \\ 12.579 & 1125.65 \\ 6.896 & 988.27 \\ 6.896 & 988.34 \\ 6.740 & 982.25 \\ 7.717 & 484.62 \\ 7.480 & 481.60 \\ 7.480 & 481.60 \\ 6.740 & 467.46\end{array}$

$\begin{array}{rr}\text { Aphy } & \text { MN } \\ 2618.3 & 0.4900 \\ 4168.2 & 0.2802 \\ 3531.8 & 0.2859 \\ 830.5 & 0.1886 \\ 2606.7 & 0.3784 \\ 412.6 & 0.3595 \\ 412.6 & 0.3635 \\ 412.6 & 0.2069 \\ 49.7 & 0.4200 \\ 49.3 & 0.3137 \\ 74.6 & 0.2950 \\ 265.4 & 0.3371 \\ 860.2 & 0.1793 \\ 860.2 & 0.1793 \\ 613.4 & 0.2564 \\ 2606.7 & 0.3784 \\ 2481.9 & 0.4350 \\ 2481.9 & 0.4350 \\ 2006.9 & 0.5881\end{array}$

gamt
1.40057
1.40057
1.40057
1.40057
1.40045
1.40024
1.39970
1.39970
1.38453
1.38453
1.33086
1.36468
1.37471
1.37470
1.37470
1.40045
1.40040
1.40040
1.40040

WAR
0.0002627
0.0002627
0.0002627
0.0002627
0.0002627
0.0002627
0.0037423
0.0037423
0.0037423
0.0037423
0.0037423
0.0037423
0.0037423
0.0037423
0.0037423
0.0002627
0.0004714
0.0004714
0.0004714

WAR

Wair $\begin{array}{lll}368.37 & 0.097 & 0.0003\end{array}$ $\begin{array}{lll}368.37 & 0.097 & 0.0003 \\ 317.71 & 0.083 & 0.0003\end{array}$ $\begin{array}{lll}50.66 & 0.013-0.0003\end{array}$ $\begin{array}{lll}317.71 & 0.083 & 0.0003\end{array}$ $\begin{array}{lll}50.66 & 0.013 & 0.0003 \\ 50.66 & 0.190 & 0.0037\end{array}$ $\begin{array}{lll}30.40 & 0.114 & 0.0037\end{array}$ $\begin{array}{lll}28.92 & 0.108 & 0.0037\end{array}$ $\begin{array}{lll}28.92 & 0.108 & 0.0037\end{array}$ $\begin{array}{lll}22.42 & 0.084 & 0.0178\end{array}$ $\begin{array}{lll}22.42 & 0.084 & 0.0037 \\ 30.04 & 0.112 & 0.0178\end{array}$ $30.40-0.114 \quad 0.0142$ $\begin{array}{lll}30.40 & 0.114 & 0.0142\end{array}$ $\begin{array}{lll}30.40 & 0.114 & 0.0142\end{array}$ $\begin{array}{lll}317.71 & 0.083 & 0.0003\end{array}$ $\begin{array}{lll}337.97 & 0.159 & 0.0005\end{array}$ $\begin{array}{lll}317.71 & 0.083 & 0.0003 \\ 337.97 & 0.159 & 0.0005 \\ 337.97 & 0.159 & 0.0005\end{array}$ TURBOMACHINERY PERFORMANCE DATA

$\begin{array}{lrrrrrrrrr} & \text { WC } & \text { PR } & \text { eff } & \text { NC } & \text { TR } & \text { efPoly } & \text { pwr } & \text { SMN } & \text { SMW } \\ \text { Fan } & 570.33 & 1.073 & 0.9058 & 1856.126 & 1.0225 & 0.9067 & -1181.7 & -1892.99 & 46.38 \\ \text { LPC } & 90.94 & 1.148 & 0.6971 & 1856.126 & 1.0577 & 0.7030 & -483.8 & 6.94 & 2.22 \\ \text { HPC } & 49.29 & 5.643 & 0.8099 & 8805.853 & 1.7797 & 0.8490 & -4159.2 & 60.74 & 56.91 \\ \text { HPT } & 12.09 & 3.699 & 0.8761 & 213.291 & 1.3305 & 0.8559 & 4159.2 & & \\ \text { LPT } & 48.98 & 1.927 & 0.8155 & 53.090 & 1.1524 & 0.8012 & 1665.6 & & \end{array}$

TURBOMACHINERY MAP DATA

$\begin{array}{lrrrrrrrrr} & \text { WcMap } & \text { PRmap } & \text { effMap } & \text { NcMap } & \text { R/Parm } & \text { S_WcDes } & \text { S_PRdes } & \text { S_effDes } & \text { S_NcDes } \\ \text { Fan } & 662.66 & 1.071 & 0.9076 & 1838.428 & 0.0439 & 0.8607 & 1.0235 & 0.9980 & 0.9905 \\ \text { LPC } & 47.26 & 1.249 & 0.7560 & 0.490 & 0.0000 & 1.9242 & 0.5939 & 0.9220 & 0.0003 \\ \text { HPC } & 44.83 & 5.425 & 0.8182 & 8571.094 & 11.0764 & 1.0995 & 1.0494 & 0.9898 & 0.9733 \\ \text { HPT } & 0.96 & 3.625 & 0.8761 & 1.282 & 3.6246 & 12.6299 & 0.9723 & 1.0000 & 0.0003 \\ \text { LPT } & 0.75 & 1.697 & 0.8216 & 0.597 & 1.6970 & 65.2590 & 0.7521 & 0.9926 & 0.0005\end{array}$




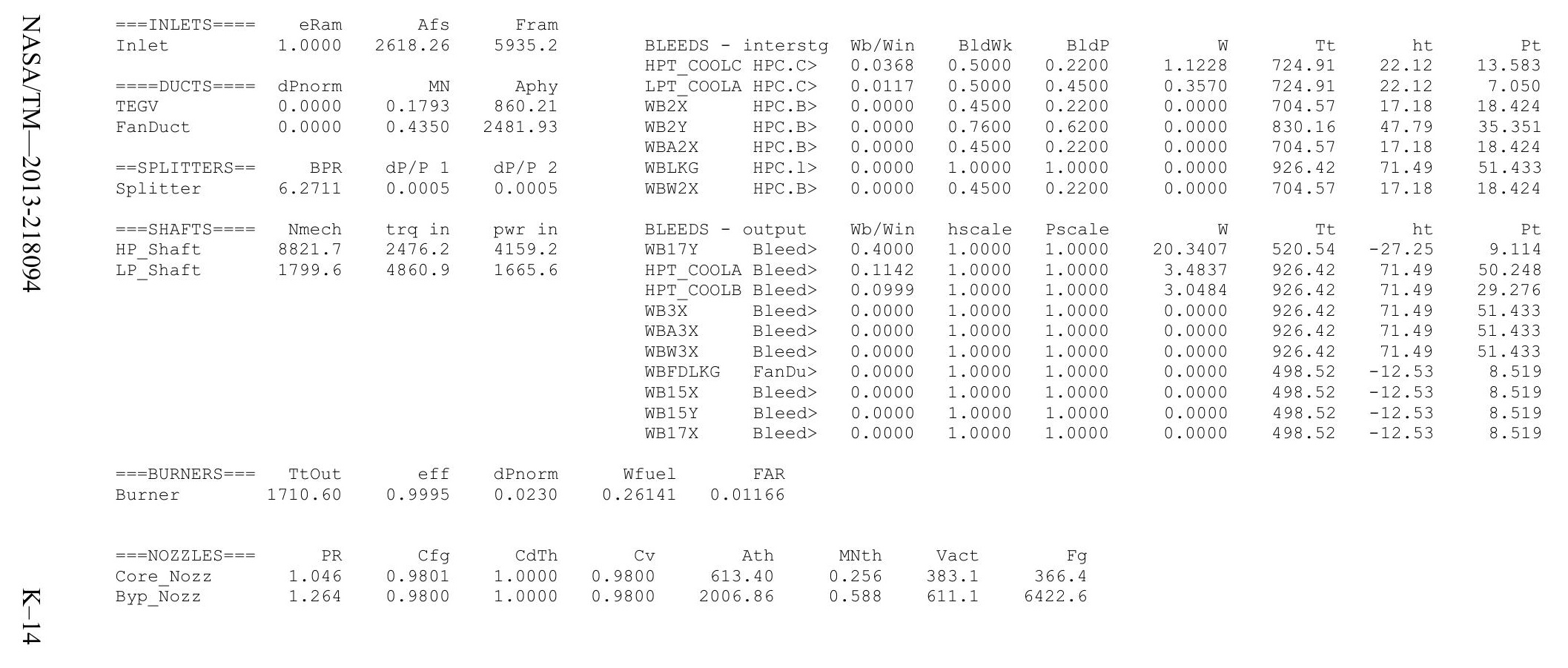


Turbofan Engine - COMDES ON Converge = 1 CASE: 0

Version:NPSS_1.6.5 - Rev: -> Gas Package: Janaf iter/pass/Jacb/Broy= 42/70/2/39 Run by: Philip C Jorgenson PC: 10

Temperature Stator 1 inlet: $500.38 \quad$ Stator 1 exit: $502.78 \quad$ Stator 2 inlet: 505.16 $\quad$ Stator 2 exit: 505.91 $\begin{array}{llcl}\text { Stator } 3 \text { inlet: } 508.66 & \text { Stator } 3 \text { exit: } 509.07 & \text { Stator } 4 \text { inlet: } 511.70 \text { Stator } 4 \text { exit: } 511.88 \\ \text { Stator } 5 \text { inlet: } 513.53 & \text { Stator } 5 \text { exit: } 513.57 & \text { Unblocked Percent Blockage: } 0.00\end{array}$

$\begin{array}{lclll}\text { Ambient Relative Humidity } & 10.00 & & & \\ \text { Fan Face Relative Humidity } & 6.54 & & & \\ \text { Fan Bypass Relative Humidity } & 5.94 & & & \\ \text { LPC Inlet Relative Humidity } & 5.71 & & & \\ \text { LPC Exit Relative Humidity } & 3.35 & & & \\ \text { HPC Relative Humidity } & 0.12 & & & \\ \text { Drop Diameter } & 0.000050 & \text { Inlet Length } & \\ \text { Ambient Flow Velocity } & 479.99 & \text { Fan/LPC Inlet Flow Velocity } & 172.62 \\ \text { Ambient Static Pressure } & 8.15 & \text { Fan/LPC Inlet Static Pressure } & 9.18 \\ \text { Ambient Static Temperature } & 481.63 & \text { Fan/LPC Inlet Static Temperature } & 498.33 \\ \text { Additional Water at LPC Exit } & 0.0046347 & & & \end{array}$

Additional Water at LPC Exit

SUMMARY OUTPUT DATA

\begin{tabular}{|c|c|c|c|c|c|c|c|c|c|c|c|c|c|c|}
\hline $\mathrm{MN}$ & alt & dTamb & W & $\begin{array}{r}\text { UMMARY } \\
\text { Fn }\end{array}$ & $\begin{array}{r}\text { TPUT DA } \\
\text { TSFC } \\
3373\end{array}$ & Wfuel & $\begin{array}{r}\text { BPR } \\
8286\end{array}$ & VTAS & $\begin{array}{l}\text { OPR } \\
5 \quad 312\end{array}$ & EPR & Powerset & $\begin{array}{r}\text { T4 } \\
1665\end{array}$ & $\begin{array}{r}\text { T41 } \\
1568 \text {. }\end{array}$ & $\begin{array}{l}\text { T49 } \\
1136.4\end{array}$ \\
\hline 0.446 & 15435.0 & 18.00 & 387.25 & 669.1 & 1.3373 & 894.73 & 6.8286 & 479.99 & 5.312 & 0.902 & 10.000 & 1665.1 & 1568.8 & 1136.4 \\
\hline
\end{tabular}

FSO Ambient.Fl O

FS1 Inlet.F1_O

衣

FS14 Fan.F1_

FS24 VaporIN. FI_O

FS25 Bleed2.F1_0

FS3 HPC.FI_O

FS36 Bleed3.F1_O

FS4 Burner.FI_

FS49 IPT.FI-

FS5 TEGV.F1

FS8 Core_Nozz.FI_O

FS17 FanDuctLkg.Fí

FS171 Bleed15.FI_O

FS172 FanDuct.FI-O

W
387.25
387.25
337.78
49.47
337.78
49.47
49.69
29.82
28.37
21.99
22.24
29.72
30.07
30.07
30.07
337.78
357.66
357.66
357.66

$\begin{array}{cc}\text { FLOW } & \text { STATION DATA } \\ \text { Pt } & \text { Tt }\end{array}$

$\begin{array}{rrrrr}\text { Pt } & \text { Tt } & \text { ht } & \text { FAR } & \text { WC } \\ 9.342 & 500.81 & -13.05 & 0.0000 & 598.60 \\ 9.342 & 500.81 & -13.05 & 0.0000 & 598.60 \\ 9.337 & 500.81 & -13.05 & 0.0000 & 522.40 \\ 9.337 & 500.81 & -13.05 & 0.0000 & 76.50 \\ 9.823 & 508.93 & -11.10 & 0.0000 & 500.60 \\ 10.469 & 523.60 & -7.58 & 0.0000 & 69.77 \\ 10.469 & 529.97 & -32.65 & 0.0000 & 70.52 \\ 10.469 & 529.97 & -32.65 & 0.0000 & 42.31 \\ 49.622 & 898.40 & 57.00 & 0.0000 & 11.06 \\ 49.622 & 898.40 & 57.00 & 0.0000 & 8.57 \\ 48.479 & 1665.12 & 44.60 & 0.0114 & 12.08 \\ 14.309 & 1141.07 & -41.71 & 0.0085 & 45.27 \\ 8.399 & 1015.41 & -71.97 & 0.0084 & 73.61 \\ 8.399 & 1015.48 & -71.97 & 0.0084 & 73.61 \\ 8.399 & 1015.55 & -71.97 & 0.0084 & 73.61 \\ 9.823 & 508.93 & -11.10 & 0.0000 & 500.60 \\ 9.823 & 510.11 & -12.30 & 0.0000 & 530.67 \\ 9.823 & 510.11 & -12.30 & 0.0000 & 530.67 \\ 9.823 & 510.11 & -12.30 & 0.0000 & 530.67\end{array}$

Ps
8.149
8.940
8.909
9.177
9.033
9.831
9.815
10.244
44.030
46.398
45.772
13.417
8.27
8.27
8.14
9.03
8.81
8.81
8.149

TS
481.63
494.55
494.14
498.33
496.89
514.28
520.29
526.69
868.94
881.74
1641.35
1121.56
1011.29
1011.36
1007.26
496.89
494.61
494.61
483.58

$\begin{array}{rr}\text { Aphy } & \text { MN } \\ 2544.6 & 0.4460\end{array}$ $\begin{array}{ll}2544.6 & 0.4460 \\ 4168.2 & 0.2515\end{array}$ $3531.8 \quad 0.259$ $\begin{array}{rr}830.5 & 0.1577 \\ 2606.7 & 0.3480\end{array}$ $\begin{array}{ll}412.6 & 0.3009\end{array}$ $\begin{array}{ll}412.6 & 0.3051\end{array}$ $\begin{array}{rrr}412.6 & 0.1765 \\ 49.7 & 0.4186\end{array}$ $\begin{array}{ll}49.7 & 0.4186 \\ 49.3 & 0.3127\end{array}$ $\begin{array}{ll}74.6 & 0.2945\end{array}$ $\begin{array}{ll}265.4 & 0.3083\end{array}$ 860.20 .1478 860.20 .1478 $\begin{array}{rr}613.4 & 0.2100\end{array}$ $2481.9 \quad 0.3956$ $\begin{array}{ll}2481.9 & 0.3956 \\ 2481.9 & 0.3956\end{array}$ $2006.9 \quad 0.5234$

gamt
1.40040
1.40040
1.40040
1.40040
1.40030
1.40011
1.39938
1.39938
1.38593
1.38593
1.33304
1.36515
1.37332
1.37331
1.37331
1.40030
1.40025
1.40025
1.40025

WAR
0.0004484
0.0004484
0.0004484
0.0004484
0.0004484
0.0004484
0.0050830
0.0050830
0.0050830
0.0050830
0.0050830
0.0050830
0.0050830
0.0050830
0.0050830
0.0004484
0.0007048
0.0007048
0.0007048

Wair

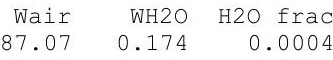
$\begin{array}{lll}387.07 & 0.174 & 0.0004\end{array}$ $\begin{array}{lll}49.44 & 0.022 & 0.0004\end{array}$ $\begin{array}{lll}337.63 & 0.151 & 0.0004\end{array}$ $\begin{array}{lll}49.44 & 0.022 & 0.0004\end{array}$ $\begin{array}{lll}49.44 & 0.251 & 0.0051 \\ 29.67 & 0.151 & 0.0051\end{array}$ $\begin{array}{lll}29.67 & 0.151 & 0.0051 \\ 28.23 & 0.143 & 0.0051\end{array}$ $\begin{array}{lll}21.88 & 0.111 & 0.0051\end{array}$ $\begin{array}{lll}21.88 & 0.111 & 0.0188\end{array}$ $\begin{array}{lll}29.32 & 0.149 & 0.0188\end{array}$ $\begin{array}{lll}29.67 & 0.151 & 0.0152\end{array}$ $\begin{array}{lll}29.67 & 0.151 & 0.0152\end{array}$ $\begin{array}{lll}29.67 & 0.151 & 0.0152\end{array}$ $\begin{array}{lll}337.63 & 0.151 & 0.0004\end{array}$ $\begin{array}{lll}357.41 & 0.252 & 0.0007\end{array}$ TURBOMACHINERY PERFORMANCE DATA

\begin{tabular}{lrrrrrrrrr}
\multicolumn{1}{c}{ WC } & PR & eff & NC & TR & efPoly & pwr & SMN & SMW \\
Fan & 522.40 & 1.052 & 0.8996 & 1618.857 & 1.0162 & 0.9004 & -931.1 & -1526.51 & 44.70 \\
LPC & 76.50 & 1.121 & 0.7304 & 1618.857 & 1.0455 & 0.7348 & -382.6 & 8.21 & 1.95 \\
HPC & 42.31 & 4.740 & 0.7956 & 8544.066 & 1.6952 & 0.8340 & -3690.6 & 68.43 & 63.22 \\
HPT & 12.08 & 3.388 & 0.8678 & 211.651 & 1.3031 & 0.8480 & 3690.5 & & \\
LPT & 45.27 & 1.704 & 0.8023 & 47.092 & 1.1202 & 0.7901 & 1313.8 & &
\end{tabular}

TURBOMACHINERY MAP DATA

$\begin{array}{lrrrrrrrrr} & \text { WcMap } & \text { PRmap } & \text { effMap } & \text { NcMap } & \text { R/Parm } & \text { S_WcDes } & \text { S_PRdes } & \text { S_effDes } & \text { S_NcDes } \\ \text { Fan } & 606.98 & 1.051 & 0.9015 & 1603.421 & 0.0442 & 0.8607 & 1.0235 & 0.9980 & 0.9905 \\ \text { LPC } & 39.96 & 1.187 & 0.7483 & 0.428 & 0.0000 & 1.9142 & 0.6495 & 0.9761 & 0.0003 \\ \text { HPC } & 38.48 & 4.564 & 0.8038 & 8316.287 & 10.7481 & 1.0995 & 1.0494 & 0.9898 & 0.9733 \\ \text { HPT } & 0.96 & 3.322 & 0.8678 & 1.272 & 3.3218 & 12.6299 & 0.9723 & 1.0000 & 0.0003 \\ \text { LPT } & 0.69 & 1.529 & 0.8083 & 0.530 & 1.5293 & 65.2590 & 0.7521 & 0.9926 & 0.0005\end{array}$




\begin{tabular}{|c|c|c|c|c|c|c|c|c|c|}
\hline Z & $===$ INLET $S====$ & eRam & Afs & Fram & \multirow{2}{*}{\multicolumn{2}{|c|}{ BLEEDS - interstg }} & & & \\
\hline D & Inlet & 1.0000 & 2544.56 & 5777.1 & & & $\begin{array}{l}\text { Wb/Win } \\
0.0368\end{array}$ & $\begin{array}{r}\text { BldWk } \\
0.5000\end{array}$ & $\begin{array}{r}\text { BldP } \\
0.2200\end{array}$ \\
\hline & $====$ DUCTS $====$ & dPnorm & MN & Aphy & \multicolumn{2}{|c|}{ LPT_COOLA HPC.C> } & 0.0117 & 0.5000 & 0.4500 \\
\hline & TEGV & 0.0000 & 0.1478 & 860.21 & WB2 $\overline{\mathrm{X}}$ & $\mathrm{HPC} . \mathrm{B}>$ & 0.0000 & 0.4500 & 0.2200 \\
\hline & FanDuct & 0.0000 & 0.3956 & 2481.93 & \multirow{2}{*}{$\begin{array}{l}\text { WB2Y } \\
\text { WBA2X }\end{array}$} & HPC. B> & 0.0000 & 0.7600 & 0.6200 \\
\hline & & & & & & HPC.B> & 0.0000 & 0.4500 & 0.2200 \\
\hline e & $==$ SPLITTERS $==$ & BPR & $\mathrm{dP} / \mathrm{P} 1$ & $\mathrm{dP} / \mathrm{P} 2$ & WBLKG & HPC.1> & 0.0000 & 1.0000 & 1.0000 \\
\hline & Splitter & 6.8286 & 0.0005 & 0.0005 & WBW2X & HPC. B> & 0.0000 & 0.4500 & 0.2200 \\
\hline 1 & $===$ SHAFTS $====$ & Nmech & trq in & pwr in & \multicolumn{2}{|c|}{ BLEEDS - output } & Wb/Win & hscale & Pscale \\
\hline & HP_Shaft & 8636.6 & 2244.3 & 3690.5 & WB17Y & Bleed> & 0.4000 & 1.0000 & 1.0000 \\
\hline & LP_Shaft & 1590.7 & 4337.7 & 1313.8 & HPT COOLA & A Bleed> & 0.1142 & 1.0000 & 1.0000 \\
\hline & & & & & $\begin{array}{l}\mathrm{HPT}^{-} \mathrm{COOLB} \\
\text { WB3 } 3 \mathrm{X}^{-}\end{array}$ & 3 Bleed> & 0.0999 & 1.0000 & 1.0000 \\
\hline & & & & & \multirow{2}{*}{$\begin{array}{l}\text { WB } 3 \bar{x} \\
\text { WBA3X }\end{array}$} & Bleed> & 0.0000 & 1.0000 & 1.0000 \\
\hline & & & & & & Bleed $>$ & 0.0000 & 1.0000 & 1.0000 \\
\hline & & & & & WBW3X & Bleed> & 0.0000 & 1.0000 & 1.0000 \\
\hline & & & & & WBFDLKG & FanDu> & 0.0000 & 1.0000 & 1.0000 \\
\hline & & & & & WB15X & Bleed> & 0.0000 & 1.0000 & 1.0000 \\
\hline & & & & & $\begin{array}{l}\text { WB15Y } \\
\text { WB17X }\end{array}$ & Bleed> & 0.0000 & 1.0000 & 1.0000 \\
\hline & & & & & WB17X & Bleed> & 0.0000 & 1.0000 & 1.0000 \\
\hline & $===$ BURNERS $===$ & Ttout & eff & dPnorm & & & & \\
\hline & Burner & 1665.06 & 0.9995 & 0.0230 & \multicolumn{2}{|c|}{$\begin{array}{rr}\text { Wfuel } & \text { FAR } \\
0.24854 & 0.01136\end{array}$} & & & \\
\hline & $===$ NOZZLES $===$ & $\mathrm{PR}$ & $\mathrm{Cfg}$ & CdTh & $\mathrm{Cv}$ & Ath & MNth & Vact & $\mathrm{Fg}$ \\
\hline & Core_Nozz & 1.031 & 0.9801 & 1.0000 & 0.9800 & 613.40 & \multirow{2}{*}{$\begin{array}{l}0.210 \\
0.523\end{array}$} & 317.7 & \multirow{2}{*}{$\begin{array}{r}296.9 \\
6149.3\end{array}$} \\
\hline & Byp_Nozzz & 1.205 & 0.9800 & 1.0000 & \multicolumn{2}{|c|}{2006.86} & & 553.2 & \\
\hline
\end{tabular}




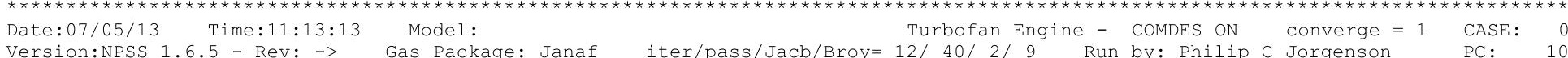

Temperature Stator 1 inlet: 463.54 Stator 1 exit: 471.07 Stator 2 inlet: 480.53 Stator 2 exit: 485.41

Stator 3 inlet: 495.10 Stator 3 exit: 499.53 Stator 4 inlet: 508.56 Stator 4 exit: 512.31

Stator 5 inlet: 519.67 Stator 5 exit: 522.89 Utator 4 Unblocked Percent Blockage: 0.00

Ambient Relative Humidity $\quad 10.00$

Fan Bypass Relative Humidity 1.76

Fan Bypass Relative Humidity 1.22

LPC Exit Relative Humidity 0.09

HPC Relative Humidity

Drop Diameter

Ambient Flow Velocity

0.0000050

Ambient Static Pressure

Ambient Static Temperature

772.64

Inlet Length

40.00

Additional

407.97

Fan/LPC Inlet Flow Velocity $\quad 185.27$

0.0072598

an/LPC Inlet Static Temperature 454.88

$\begin{array}{rrrrrr}\text { MN } & \text { alt } & \text { dTamb } & \text { W } & \text { Fn } & \text { TSFC } \\ 0.780 & 39000.0 & 18.00 & 257.50 & 640.6 & 1.0925\end{array}$

Wfuel
699.88

BPR
8.7350

0.736

T4

T41 $\quad$ T49

FLOW STATION DATA

$\begin{array}{rrrrr}\text { Pt } & \text { Tt } & \text { ht } & \text { FAR } \\ 4.267 & 457.74 & -20.96 & 0.0000\end{array}$
$\begin{array}{llll}4.267 & 457.74 & -20.96 & 0.0000 \\ 4.267 & 457.74 & -20.96 & 0.0000\end{array}$ $\begin{array}{rrr}457.74 & -20.96 & 0.0000 \\ -457.74 & -20.96 & 0.0000\end{array}$

$W$
257.50
257.50
231.05
26.45
231.05
26.45
26.64
23.98
22.82
17.68
17.88
23.89
24.17
24.17
24.17
231.05
233.72
233.72
233.72
$\begin{array}{llll}4.265 & 457.74 & -20.96 & 0.0000\end{array}$ 6.676 6.676 6.676
40.539 40.539 40.539
39.605 9.502 3.262 3.262 3.262

4.824

4.824

4.824

4.824 $\begin{array}{rrrr}75.86 & -16.61 & 0.0000 & 674.27 \\ 52.79 & -2.96 & 0.0000 & 59.02\end{array}$ $\begin{array}{llll}542.79 & -2.96 & 0.0000 & 59.02 \\ 542.60 & -42.18 & 0.0000 & 59.99\end{array}$ $\begin{array}{llll}542.60 & -42.18 & 0.0000 & 53.9\end{array}$ $\begin{array}{llll}981.98 & 65.37 & 0.0000 & 11.38\end{array}$ $\begin{array}{ll}65.37 & 0.0000\end{array}$ $\begin{array}{rr}53.22 & 0.011 \\ -51.03 & 0.0083\end{array}$

12.07
54.67 54.67
142.65
142.65 $889.88-111.50 \quad 0.0082$ $889.95-111.50 \quad 0.0082$ $\begin{array}{llll}775.86 & -16.61 & 0.0000 & 674.27\end{array}$ $\begin{array}{llll}475.63 & -16.90 & 0.0000 & 682.5\end{array}$ $\begin{array}{lll}476.63 & -16.90 & 0.0000\end{array}$ FS172 Fand TURBOMACHINERY PERFORMANCE DATA

$\begin{array}{lrrr} & \text { WC } & \text { PR } & \text { eff } \\ \text { Fan } & 747.97 & 1.131 & 0.9058 \\ \text { LPC } & 85.63 & 1.565 & 0.8338 \\ \text { HPC } & 53.99 & 6.073 & 0.8182 \\ \text { HPT } & 12.07 & 4.168 & 0.8753 \\ \text { LPT } & 54.67 & 2.913 & 0.8533\end{array}$

NC
2576.390
2576.390
8957.455
220.999
71.829

$\begin{array}{rr}\text { TR } & \text { efPoly } \\ 1.0396 & 0.9075 \\ 1.1640 & 0.8440 \\ 1.8098 & 0.8568 \\ 1.3647 & 0.8530 \\ 1.2741 & 0.8335\end{array}$

pwr
-1419.8
-673.4
-3560.4
3560.4
2093.2

682.59

PS
2.854
3.892
3.838
4.172
4.047
6.391
6.379
6.438
5.643
7.730
7.392
8.602
3.071
3.071
2.854
4.047
3.913
3.913
2.854

TS
407.97
445.86
444.14
454.88
452.56
526.19
535.62
537.02
947.67
962.71
1694.21
1105.53
875.14
875.21
857.76
452.56
448.94
448.94
410.16

4.

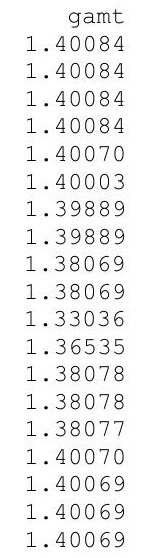

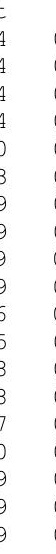

WAR Wair WH2O H2O frac $\begin{array}{llll}0.0000295 & 257.50 & 0.008 & 0.0000\end{array}$ $\begin{array}{llll}0.0000295 & 257.50 & 0.008 & 0.0000 \\ 0.0000295 & 231.05 & 0.007 & 0.0000\end{array}$ $\begin{array}{rrrr}0.0000295 & 231.05 & 0.007 & 0.0000 \\ 0.0000295 & 26.45 & 0.001 & 0.0000\end{array}$ $\begin{array}{llll}0.0000295 & 231.05 & 0.007 & 0.0000\end{array}$ $\begin{array}{llll}0.0000295 & 26.45 & 0.001 & 0.0000\end{array}$ $\begin{array}{llll}0.0072892 & 26.45 & 0.193 & 0.0072\end{array}$ $\begin{array}{llll}0.0072892 & 23.81 & 0.174 & 0.0072\end{array}$ $\begin{array}{llll}0.0072892 & 22.65 & 0.165 & 0.0072\end{array}$ $\begin{array}{llll}0.0072892 & 17.55 & 0.128 & 0.0072\end{array}$ $\begin{array}{llll}0.0072892 & 17.55 & 0.128 & 0.0072 \\ 0.0072892 & 17.55 & 0.128 & 0.0206\end{array}$ $\begin{array}{llll}0.0072892 & 17.55 & 0.128 & 0.0206 \\ 0.0072892 & 23.53 & 0.171 & 0.0172\end{array}$ $\begin{array}{llll}0.0072892 & 23.81 & 0.174 & 0.0171\end{array}$ $\begin{array}{llll}0.0072892 & 23.81 & 0.174 & 0.0171 \\ 0.0072892 & 23.81 & 0.174 & 0.0171\end{array}$ $\begin{array}{llll}0.0072892 & 23.81 & 0.174 & 0.0171 \\ 0.0072892 & 23.81 & 0.174 & 0.0171\end{array}$ $\begin{array}{llll}0.0000295 & 231.05 & 0.007 & 0.0000\end{array}$ $\begin{array}{llll}0.00001116 & 233.69 & 0.007 & 0.0000 \\ 0.0001116 & 233.69 & 0.026 & 0.0001\end{array}$ $\begin{array}{llll}0.0001116 & 233.69 & 0.026 & 0.0001\end{array}$

TURBOMACHINERY MAP DATA

$\begin{array}{lrrrrrrrrr} & \text { WCMap } & \text { PRmap } & \text { effMap } & \text { NCMap } & \text { R/Parm } & \text { S_WcDes } & \text { S_PRdes } & \text { S_effDes } & \text { S_NcDes } \\ \text { Fan } & 869.07 & 1.128 & 0.9077 & 2551.824 & 0.0414 & 0.8607 & \overline{1} .0235 & 0.9980 & 0.9905 \\ \text { LPC } & 74.26 & 1.526 & 0.7803 & 0.680 & 0.0000 & 1.1531 & 1.0755 & 1.0686 & 0.0003 \\ \text { HPC } & 49.11 & 5.834 & 0.8266 & 8718.656 & 10.9660 & 1.0995 & 1.0494 & 0.9898 & 0.9733 \\ \text { HPT } & 0.96 & 4.080 & 0.8753 & 1.328 & 4.0804 & 12.6299 & 0.9723 & 1.0000 & 0.0003 \\ \text { LPT } & 0.84 & 2.439 & 0.8596 & 0.808 & 2.4387 & 65.2590 & 0.7521 & 0.9926 & 0.0005\end{array}$




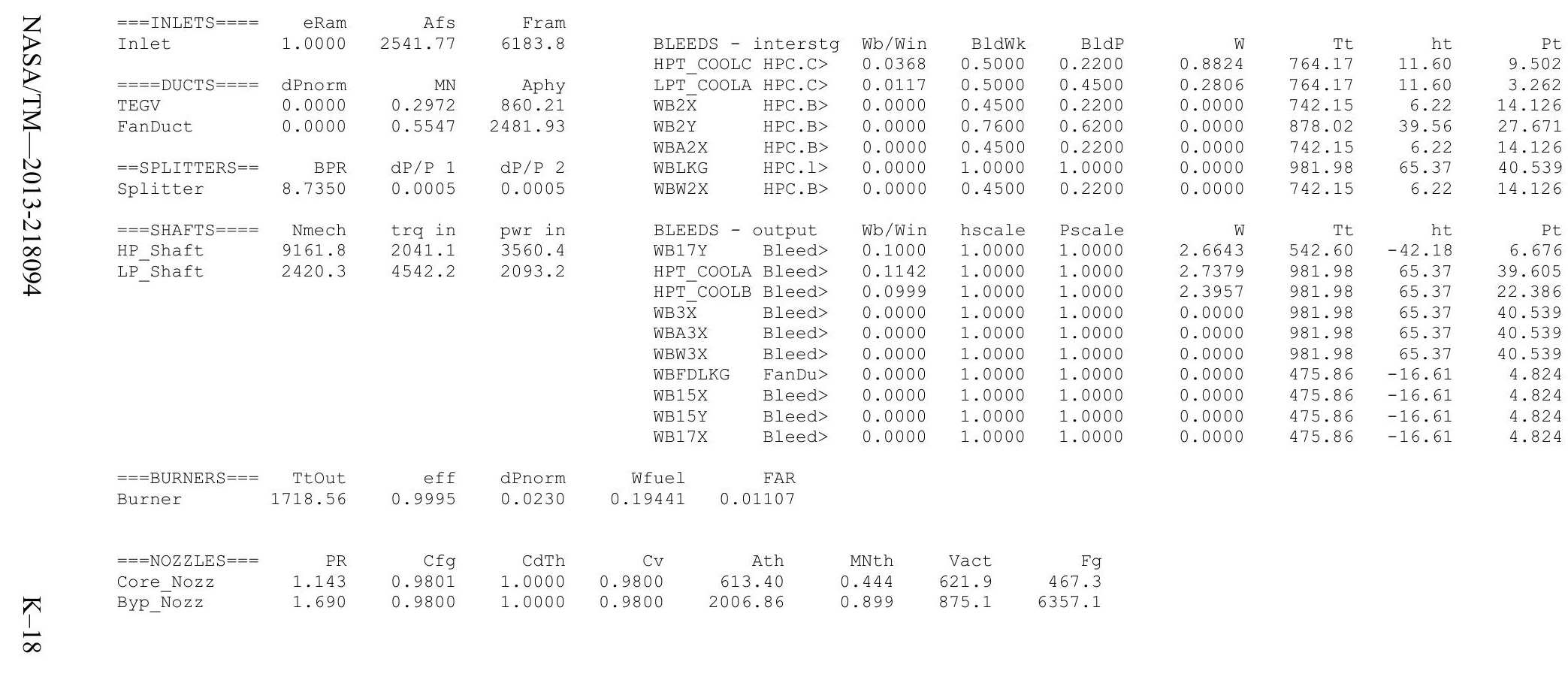


Date:07/05/13 Time:11:13:38 Model: Turbofan Engine - COMDES ON Converge = 1 CASE: 0

Version:NPSS 1.6.5 - Rev: -> Gas Package: Janaf iter/pass/Jacb/Broy=11/39/2/8 Run by: Philip C Jorgenson PC: 10

Temperature Stator 1 inlet: $457.01 \quad$ Stator 1 exit: $464.06 \quad$ Stator 2 inlet: $472.90 \quad$ Stator 2 exit: 477.45 Stator 5 inlet: 509.49 Stator 5 exit: 512.50 Unblocked Percent Blockage: 0.00

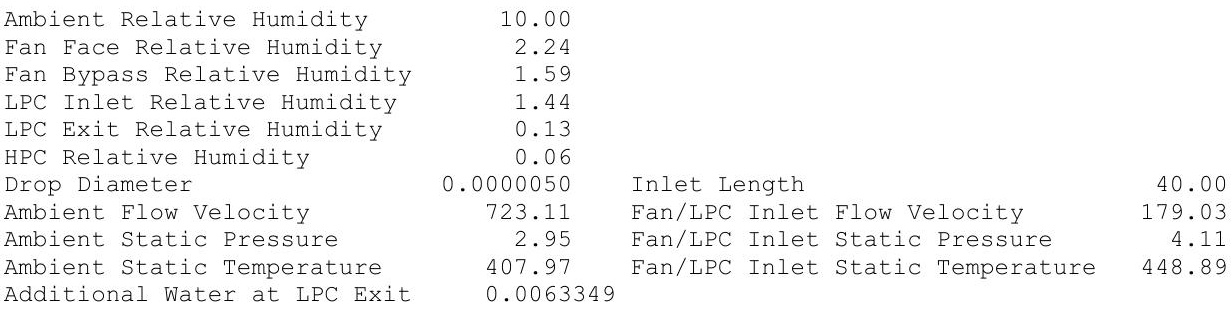

MN alt dTamb W SUMMARY OUTPUT DATA

$\begin{array}{rrrrrrrrrrrrrrr}\text { MN } & \text { alt } & \text { dTamb } & \text { W } & \text { Fn } & \text { TSFC } & \text { Wfuel } & \text { BPR } & \text { VTAS } & \text { OPR } & \text { EPR } & \text { PowerSet } & \text { T4 } & \text { T41 } & \text { T49 } \\ 0.730 & 38334.0 & 18.00 & 250.56 & 626.4 & 1.0643 & 666.61 & 8.8127 & 723.11 & 9.223 & 0.763 & 10.000 & 1690.0 & 1597.9 & 1113.5\end{array}$

FSO Ambient.Fl_o

FS1 Inlet.Fl_ 0

$W$
250.56
250.56
225.02
25.53
225.02
25.53
25.70
23.13
22.00
17.05
17.24
23.04
23.31
23.31
23.31
225.02
227.59
227.59
227.59

FLOW STATION DATA

\begin{tabular}{lrrr}
\multicolumn{1}{c}{ FLW } & Tt & ht & FAR \\
Pt & Tt & -22.43 & 0.0000 \\
4.201 & 451.57 & -22.43 & 0.0000 \\
4.201 & 451.57 & -22 \\
4.199 & 451.57 & -22.43 & 0.0000 \\
4.199 & 451.57 & -22.43 & 0.0000 \\
4.717 & 468.44 & -18.39 & 0.0000 \\
6.434 & 521.81 & -5.59 & 0.0000 \\
6.434 & 530.51 & -39.83 & 0.0000 \\
6.434 & 530.51 & -39.83 & 0.0000 \\
38.751 & 958.82 & 64.78 & 0.0000 \\
38.751 & 958.82 & 64.78 & 0.0000 \\
37.858 & 1690.05 & 52.79 & 0.0109 \\
9.196 & 1117.65 & -48.58 & 0.0082 \\
3.312 & 886.36 & -105.33 & 0.0081 \\
3.312 & 886.43 & -105.33 & 0.0081 \\
3.312 & 886.50 & -105.33 & 0.0081 \\
4.717 & 468.44 & -18.39 & 0.0000 \\
4.717 & 469.14 & -18.63 & 0.0000 \\
4.717 & 469.14 & -18.63 & 0.0000 \\
4.717 & 469.14 & -18.63 & 0.0000
\end{tabular}

WC
817.76
817.76
734.79
83.38
666.28
58.50
59.36
53.42
11.35
8.79
12.08
54.05
135.21
135.22
135.22
666.28
674.39
674.39
674.39

Ts
407.97
440.34
438.70
448.89
446.21
515.47
523.84
525.18
925.51
940.09
1665.94
1088.97
873.29
873.36
858.32
446.21
442.81
442.81
410.05

$\begin{array}{rrr}\text { Aphy } & \text { MN } & \text { gamt } \\ 2559.3 & 0.7300 & 1.40089 \\ 4168.2 & 0.3567 & 1.40089 \\ 3531.8 & 0.3825 & 1.40089 \\ 830.5 & 0.1723 & 1.40089 \\ 2606.7 & 0.4985 & 1.40077 \\ 412.6 & 0.2480 & 1.40019 \\ 412.6 & 0.2526 & 1.39920 \\ 412.6 & 0.2256 & 1.39920 \\ 49.7 & 0.4333 & 1.38222 \\ 49.3 & 0.3226 & 1.38222 \\ 74.6 & 0.2949 & 1.33194 \\ 265.4 & 0.3784 & 1.36670 \\ 860.2 & 0.2800 & 1.38118 \\ 860.2 & 0.2800 & 1.38117 \\ 613.4 & 0.4145 & 1.38117 \\ 2606.7 & 0.4985 & 1.40077 \\ 2481.9 & 0.5446 & 1.40075 \\ 2481.9 & 0.5446 & 1.40075 \\ 2006.9 & 0.8478 & 1.40075\end{array}$

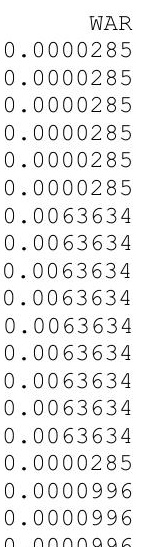

WAR

(22

$\begin{array}{rrr}\text { Wair } & \text { WH2O } & \text { H2O frac } \\ 250.55 & 0.007 & 0.0000 \\ 250.55 & 0.007 & 0.0000 \\ 225.02 & 0.006 & 0.0000 \\ 25.53 & 0.001 & 0.0000 \\ 225.02 & 0.006 & 0.0000 \\ 25.53 & 0.001 & 0.0000 \\ 25.53 & 0.162 & 0.0063 \\ 22.98 & 0.146 & 0.0063 \\ 21.87 & 0.139 & 0.0063 \\ 16.95 & 0.108 & 0.0063 \\ 16.95 & 0.108 & 0.0195 \\ 22.71 & 0.145 & 0.0162 \\ 22.98 & 0.146 & 0.0161 \\ 22.98 & 0.146 & 0.0161 \\ 22.98 & 0.146 & 0.0161 \\ 225.02 & 0.006 & 0.0000 \\ 227.57 & 0.023 & 0.0001 \\ 227.57 & 0.023 & 0.0001 \\ 227.57 & 0.023 & 0.0001\end{array}$

TURBOMACHINERY PERFORMANCE DATA

\begin{tabular}{|c|c|c|c|c|c|c|c|c|c|}
\hline & Wc & $\mathrm{PR}$ & eff & $\mathrm{NC}$ & $T R$ & efPoly & pwr & SMN & SMW \\
\hline Fan & 734.79 & 1.123 & 0.9050 & 2511.599 & 1.0374 & 0.9066 & -1287.0 & 2101.01 & 54.60 \\
\hline LPC & 83.38 & 1.532 & 0.8342 & 2511.599 & 1.1556 & 0.8439 & -608.3 & 6.80 & 3.37 \\
\hline PC & 53.42 & 6.023 & .8175 & 8939.174 & 1.8073 & 0.8562 & -3339.8 & 61.38 & 57.98 \\
\hline HPT & 12.08 & 4.117 & 0.8739 & 219.913 & 1.3616 & 0.8516 & 3339.8 & & \\
\hline LPT & 54.05 & 2.777 & 0.8476 & 70.099 & 1.2590 & 0.8280 & 1895.3 & & \\
\hline$J R B$ & IERY MAP & DATA & & & & & & & \\
\hline & WcMap & PRmap & effMap & NcMap & R/Parm & s_WCDes & S_PRdes & s_effDes & S_NCDes \\
\hline an & 853.75 & 1.120 & 0.9069 & 2487.652 & 0.0414 & 0.8607 & 1.0235 & 0.9980 & 0.9905 \\
\hline $\mathrm{PC}$ & 71.46 & 1.497 & & 0.663 & & 1.1 & 1.0712 & 1.0 & 0.0003 \\
\hline PC & 48.59 & 5.786 & 0.8259 & 8700.862 & 10.9822 & 1.0995 & 1.0494 & 0.9898 & 0.9733 \\
\hline IPT & 0.96 & 4.030 & 0.8739 & 1.322 & 4.0304 & 12.6299 & 0.9723 & 1.0000 & 0.0003 \\
\hline $\mathrm{PT}$ & 0.83 & 2.336 & 0.8539 & 0.789 & 2.3362 & 65.2590 & 0.7521 & 0.9926 & 0.0005 \\
\hline
\end{tabular}




\begin{tabular}{|c|c|c|c|c|c|c|c|c|c|}
\hline$Z$ & $===$ INLETS $====$ & eRam & Afs & Fram & \multirow{2}{*}{\multicolumn{2}{|c|}{ BLEEDS - interstg }} & & & \\
\hline D & Inlet & 1.0000 & 2559.33 & 5631.2 & & & $\begin{array}{l}\text { g Wb/Win } \\
0.0368\end{array}$ & $\begin{array}{r}\text { BldWk } \\
0.5000\end{array}$ & $\begin{array}{r}\text { BldP } \\
0.2200\end{array}$ \\
\hline & $====$ DUCTS $====$ & dPnorm & MN & Aphy & \multirow{2}{*}{\multicolumn{2}{|c|}{$\begin{array}{ll}\text { LPT COOLA } & \text { HPC.C> } \\
\text { WB } 2 \bar{X} & \text { HPC. B> }\end{array}$}} & 0.0117 & 0.5000 & 0.4500 \\
\hline & TEGV & 0.0000 & 0.2800 & 860.21 & & & 0.0000 & 0.4500 & 0.2200 \\
\hline & FanDuct & 0.0000 & 0.5446 & 2481.93 & WB2Y & HPC.B> & 0.0000 & 0.7600 & 0.6200 \\
\hline & & & & & \multirow{2}{*}{$\begin{array}{l}\text { WBA2X } \\
\text { WBLKG }\end{array}$} & $\mathrm{HPC} \cdot \mathrm{B}>$ & 0.0000 & 0.4500 & 0.2200 \\
\hline & $==$ SPLITTERS $==$ & BPR & $\mathrm{dP} / \mathrm{P} 1$ & $\mathrm{dP} / \mathrm{P} 2$ & & HPC.I> & 0.0000 & 1.0000 & 1.0000 \\
\hline & Splitter & 8.8127 & 0.0005 & 0.0005 & WBW2X & $\mathrm{HPC} \cdot \mathrm{B}>$ & 0.0000 & 0.4500 & 0.2200 \\
\hline & $===$ SHAFT $S====$ & Nmech & trq in & pwr in & \multicolumn{2}{|c|}{ BLEEDS - output } & Wb/Win & hscale & Pscale \\
\hline & HP_Shaft & 9040.7 & 1940.3 & 3339.8 & \multicolumn{2}{|c|}{ WB17Y Bleed } & 0.1000 & 1.0000 & 1.0000 \\
\hline & LP_Shaft & 2343.5 & 4247.6 & 1895.3 & \multirow{2}{*}{\multicolumn{2}{|c|}{$\begin{array}{l}\text { HPT_COOLA Bleed> } \\
\text { HPT COOLB Bleed> }\end{array}$}} & 0.1142 & 1.0000 & 1.0000 \\
\hline & & & & & & & 0.0999 & 1.0000 & 1.0000 \\
\hline & & & & & \multicolumn{2}{|c|}{ WB3 $\bar{x} \quad$ Bleed $>$} & 0.0000 & 1.0000 & 1.0000 \\
\hline & & & & & \multicolumn{2}{|c|}{ WBA3X Bleed> } & 0.0000 & 1.0000 & 1.0000 \\
\hline & & & & & \multicolumn{2}{|c|}{$\begin{array}{ll}\text { WBW3X } & \text { Bleed> } \\
\text { WBFDLKG } & \text { FanDu > }\end{array}$} & 0.0000 & 1.0000 & 1.0000 \\
\hline & & & & & \multirow{2}{*}{\multicolumn{2}{|c|}{$\begin{array}{ll}\text { WBFDLKG } & \text { FanDu> } \\
\text { WB15X } & \text { Bleed }>\end{array}$}} & 0.0000 & 1.0000 & 1.0000 \\
\hline & & & & & & & 0.0000 & 1.0000 & 1.0000 \\
\hline & & & & & WB15Y & Bleed> & 0.0000 & 1.0000 & 1.0000 \\
\hline & & & & & WB17X & Bleed> & 0.0000 & 1.0000 & 1.0000 \\
\hline & $===$ BURNERS $===$ & TtOut & eff & dPnorm & & & & \\
\hline & Burner & 1689.98 & 0.9995 & 0.0230 & \multicolumn{2}{|c|}{$\begin{array}{rr}\text { Wfuel } & \text { FAR } \\
0.18517 & 0.01093\end{array}$} & & & \\
\hline & $===\operatorname{NOZZLES}===$ & PR & Cfg & CdTh & $\mathrm{Cv}$ & Ath & MNth & Vact & $\mathrm{Fg}$ \\
\hline & Core_Nozz & 1.124 & 0.9801 & 1.0000 & \multirow{2}{*}{$\begin{array}{l}0.9800 \\
0.9800\end{array}$} & 613.40 & \multirow{2}{*}{$\begin{array}{l}0.415 \\
0.848\end{array}$} & \multirow{2}{*}{$\begin{array}{l}580.9 \\
825.1\end{array}$} & 420.9 \\
\hline & Byp_Nozzz & 1.601 & 0.9800 & 1.0000 & & 2006.86 & & & 5836.7 \\
\hline
\end{tabular}


Turbofan Engine - COMDES ON Converge = 1 CASE: 0

Version:NPSS 1.6.5 - Rev: -> Gas Package: Janaf iter/pass/Jacb/Broy=11/ 39/2/8 Run by: Philip C Jorgenson PC: 10

Temperature Stator 1 inlet: $454.98 \quad$ Stator 1 exit: $461.84 \quad$ Stator 2 inlet: $470.44 \quad$ Stator 2 exit: 474.87 $\begin{array}{llll}\text { Stator } 3 \text { inlet: } 483.70 & \text { Stator } 3 \text { exit: } 487.70 & \text { Stator } 4 \text { inlet: 495.92 } \text { Stator 4 exit: } 499.3 \\ \text { Stator } 5 \text { inlet: } 506.04 & \text { Stator } 5 \text { exit: } 508.97 & & \text { Unblocked Percent Blockage: } 0.00\end{array}$

$\begin{array}{lclll}\text { Ambient Relative Humidity } & 10.00 & & & \\ \text { Fan Face Relative Humidity } & 2.40 & & & \\ \text { Fan Bypass Relative Humidity } & 1.73 & & & \\ \text { LPC Inlet Relative Humidity } & 1.56 & & & \\ \text { LPC Exit Relative Humidity } & 0.14 & & & \\ \text { HPC Relative Humidity } & 0.06 & & \\ \text { Drop Diameter } & 0.0000050 & \text { Inlet Length } & \\ \text { Ambient Flow Velocity } & 707.26 & \text { Fan/LPC Inlet Flow Velocity } & 176.74 \\ \text { Ambient Static Pressure } & 3.09 & \text { Fan/LPC Inlet Static Pressure } & 4.25 \\ \text { Ambient Static Temperature } & 407.97 & \text { Fan/LPC Inlet Static Temperature } & 447.07 \\ \text { Additional Water at LPC Exit } & 0.0056534 & & & \end{array}$
W SUMMARY OUTPUT DATA
Fn
257.30

\section{FLOW STATION DATA}

FSO Ambient.Fl_O

FS1 Inlet.Fl_O

六

FS2 Splitter. Fl-01

$B P R$
8.8384

VTAS
707.26

OPR
9.105

$E P R$
0.772

Powerset

10.000

$\begin{array}{ccc}\text { T4 } & \text { T41 } & \text { T49 } \\ 1677.8 & 1586.0 & 1105.8\end{array}$

W
257.30
257.30
231.15
26.15
231.15
26.15
26.30
23.67
22.52
17.45
17.64
23.58
23.86
23.86
23.86
231.15
233.78
233.78
233.78

$\begin{array}{lrrr}\text { FLOW } & \text { STATION DATA } & \\ \text { Pt } & \text { Tt } & \text { ht } & \text { FAR } \\ 4.339 & 449.68 & -22.87 & 0.0000 \\ 4.339 & 449.68 & -22.87 & 0.0000 \\ 4.337 & 449.68 & -22.87 & 0.0000 \\ 4.337 & 449.68 & -22.87 & 0.0000 \\ 4.858 & 466.07 & -18.95 & 0.0000 \\ 6.587 & 518.04 & -6.49 & 0.0000 \\ 6.587 & 525.86 & -37.06 & 0.0000 \\ 6.587 & 525.86 & -37.06 & 0.0000 \\ 39.511 & 949.74 & 66.36 & 0.0000 \\ 39.511 & 949.74 & 66.36 & 0.0000 \\ 38.601 & 1677.81 & 54.42 & 0.0108 \\ 9.416 & 1109.91 & -45.77 & 0.0081 \\ 3.452 & 884.08 & -101.08 & 0.0080 \\ 3.452 & 884.15 & -101.08 & 0.0080 \\ 3.452 & 884.22 & -101.08 & 0.0080 \\ 4.858 & 466.07 & -18.95 & 0.0000 \\ 4.858 & 466.75 & -19.15 & 0.0000 \\ 4.858 & 466.75 & -19.15 & 0.0000 \\ 4.858 & 466.75 & -19.15 & 0.0000\end{array}$

FS14 Fan.F1_O

FS24 VaporIN. Fl O

FS25 Bleed2.F1_O

FS3 HPC.FI_O

FS36 Bleed3.F1_O

FS45 HPT.FI_O

FS49 LPT.FI_O

FS8 Core 8 TOzz.FI

FS17 FanDuctLkg.Fì

FS171 Bleed15.F1_

FS172 FanDuct.Fl_O

TURBOMACHINERY PERFORMANCE DATA

\begin{tabular}{|c|c|c|c|c|c|c|c|c|c|}
\hline & Wc & PR & eff & NC & $T R$ & efPoly & pwr & SMN & SMW \\
\hline Fan & 729.27 & 1.120 & 0.9046 & 2484.788 & 1.0365 & 0.9062 & -1284.6 & 2460.98 & 54.53 \\
\hline LPC & 82.51 & 1.519 & 0.8349 & 2484.788 & 1.1520 & 0.8444 & -606.4 & 6.71 & 3.29 \\
\hline HPC & 53.18 & 5.999 & 0.8171 & 8931.188 & 1.8061 & 0.8558 & -3379.4 & 61.40 & 57.97 \\
\hline HPT & 12.08 & 4.100 & 0.8733 & 219.546 & 1.3607 & 0.8508 & 3379.4 & & \\
\hline LPT & 53.84 & 2.727 & 0.8453 & 69.446 & 1.2534 & 0.8258 & 1891.0 & & \\
\hline \multirow{2}{*}{\multicolumn{10}{|c|}{$\begin{array}{c}\text { TURBOMACHINERY MAP DATA } \\
\text { WCMap } \\
\text { PRmap }\end{array}$}} \\
\hline & WcMap & PRmap & effMap & NcMap & R/Parm & s WcDes & s PRdes & $s$ effDes & s NcDes \\
\hline in & 847.34 & 1.117 & 0.9065 & 2461.096 & 0.0413 & $\overline{0} .8607$ & $\overline{1} .0235$ & 0.9980 & $\overline{0.9905}$ \\
\hline $\mathrm{PC}$ & 70.29 & 1.485 & 0.7760 & 0.656 & 0.0000 & 1.1738 & 1.0700 & 1.0760 & 0.0003 \\
\hline${ }^{\mathrm{C}} \mathrm{C}$ & 48.36 & 5.763 & 0.8255 & 8693.089 & 10.9845 & 1.0995 & 1.0494 & 0.9898 & 0.9733 \\
\hline T & 0.96 & 4.014 & 0.8732 & 1.319 & 4.0136 & 12.6299 & 0.9723 & 1.0000 & 0.0003 \\
\hline & 0.83 & 2.299 & 0.8515 & 0.781 & 2.2992 & 65.2590 & 0.7521 & 0.9926 & 0.0005 \\
\hline
\end{tabular}

$\begin{array}{lll}0.83 & 2.299 & 0.8515\end{array}$

$\begin{array}{rrrrrrrr}\text { Ts } & \text { Aphy } & \text { MN } & \text { gamt } & \text { WAR } & \text { Wair } & \text { WH2O } & \text { H2O frac } \\ 407.97 & 2563.9 & 0.7140 & 1.40090 & 0.0000272 & 257.29 & 0.007 & 0.0000 \\ 438.69 & 4168.2 & 0.3534 & 1.40090 & 0.0000272 & 257.29 & 0.007 & 0.0000 \\ 437.09 & 3531.8 & 0.3790 & 1.40090 & 0.0000272 & 231.14 & 0.006 & 0.0000 \\ 447.07 & 830.5 & 0.1705 & 1.40090 & 0.0000272 & 26.15 & 0.001 & 0.0000 \\ 444.26 & 2606.7 & 0.4949 & 1.40079 & 0.0000272 & 231.14 & 0.006 & 0.0000 \\ 511.78 & 412.6 & 0.2472 & 1.40024 & 0.0000272 & 26.15 & 0.001 & 0.0000 \\ 519.31 & 412.6 & 0.2512 & 1.39936 & 0.0056806 & 26.15 & 0.149 & 0.0056 \\ 520.62 & 412.6 & 0.2244 & 1.39936 & 0.0056806 & 23.54 & 0.134 & 0.0056 \\ 916.80 & 49.7 & 0.4325 & 1.38287 & 0.0056806 & 22.40 & 0.127 & 0.0056 \\ 931.21 & 49.3 & 0.3221 & 1.38287 & 0.0056806 & 17.36 & 0.099 & 0.0056 \\ 1653.86 & 74.6 & 0.2947 & 1.33269 & 0.0056806 & 17.36 & 0.099 & 0.0187 \\ 1081.67 & 265.4 & 0.3764 & 1.36735 & 0.0056806 & 23.26 & 0.132 & 0.0155 \\ 871.58 & 860.2 & 0.2740 & 1.38144 & 0.0056806 & 23.54 & 0.134 & 0.0153 \\ 871.65 & 860.2 & 0.2740 & 1.38144 & 0.0056806 & 23.54 & 0.134 & 0.0154 \\ 857.40 & 613.4 & 0.4045 & 1.38143 & 0.0056806 & 23.54 & 0.134 & 0.0154 \\ 444.26 & 2606.7 & 0.4949 & 1.40079 & 0.0000272 & 231.14 & 0.006 & 0.0000 \\ 440.94 & 2481.9 & 0.5404 & 1.40077 & 0.0000905 & 233.76 & 0.021 & 0.0001 \\ 440.94 & 2481.9 & 0.5404 & 1.40077 & 0.0000905 & 233.76 & 0.021 & 0.0001 \\ 410.00 & 2006.9 & 0.8309 & 1.40077 & 0.0000905 & 233.76 & 0.021 & 0.0001\end{array}$

$W c$
811.37
811.37
729.27
82.51
662.85
58.32
59.09
53.18
11.34
8.79
12.08
53.84
132.60
132.60
132.61
662.85
670.88
670.88
670.88

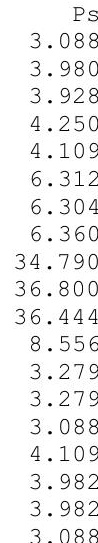

$2006.9 \quad 0.8309$ 


$\begin{array}{lrrr}===\text { INLETS }==== & \text { eRam } & \text { AfS } & \text { Fram } \\ \text { Inlet } & 1.0000 & 2563.86 & 5656.1 \\ ====\text { DUCTS }==== & \text { dPnorm } & \text { MN } & \text { Aphy } \\ \text { TEGV } & 0.0000 & 0.2740 & 860.21 \\ \text { FanDuct } & 0.0000 & 0.5404 & 2481.93 \\ & & & \\ ==\text { SPLITTERS }== & \text { BPR } & \text { dP } / \text { P 1 } & \text { dP } / \text { P 2 } \\ \text { Splitter } & 8.8384 & 0.0005 & 0.0005 \\ & & & \\ ===\text { SHAFTS }==== & \text { Nmech } & \text { trq in } & \text { pwr in } \\ \text { HP_Shaft } & 8992.9 & 1973.7 & 3379.4 \\ \text { LP_Shaft } & 2313.6 & 4292.6 & 1891.0\end{array}$

$\begin{array}{lllrr}\text { BLEEDS - interstg } & \text { Wb/Win } & \text { BldWk } & \text { BldP } \\ \text { HPT_COOLC HPC.C> } & 0.0368 & 0.5000 & 0.2200 \\ \text { LPT_COOLA HPC.C> } & 0.0117 & 0.5000 & 0.4500 \\ \text { WB2X } & \text { HPC.B> } & 0.0000 & 0.4500 & 0.2200 \\ \text { WB2Y } & \text { HPC.B } & 0.0000 & 0.7600 & 0.6200 \\ \text { WBA2X } & \text { HPC.B } & 0.0000 & 0.4500 & 0.2200 \\ \text { WBLKG } & \text { HPC.I> } & 0.0000 & 1.0000 & 1.0000 \\ \text { WBW2X } & \text { HPC.B } & 0.0000 & 0.4500 & 0.2200 \\ \text { BLEEDS - output } & \text { Wb/Win } & \text { hscale } & \text { Pscale }\end{array}$

$\begin{array}{rrrr}\text { W } & \text { Tt } & \text { ht } & \text { Pt } \\ 0.8711 & 739.43 & 14.65 & 9.416 \\ 0.2769 & 739.43 & 14.65 & 3.452 \\ 0.0000 & 718.18 & 9.48 & 13.830 \\ 0.0000 & 849.31 & 41.54 & 27.000 \\ 0.0000 & 718.18 & 9.48 & 13.830\end{array}$

$\begin{array}{rrrr}0.0000 & 718.18 & 9.48 & 13.830\end{array}$

$\begin{array}{rrrr}0.0000 & 949.74 & 66.36 & 39.511 \\ 0.0000 & 718.18 & 9.48 & 13.830\end{array}$

BLEEDS - output Wb/Win hscale Pscale $\begin{array}{llll}\text { WB17Y Bleed> } 0.1000 & 1.0000 & 1.0000\end{array}$ $\begin{array}{llll}\text { HPT_COOLA Bleed> } & 0.1142 & 1.0000 & 1.0000\end{array}$ $\begin{array}{lllll}\text { HPT_COOLB Bleed> } & 0.0999 & 1.0000 & 1.0000\end{array}$ $\begin{array}{lllll}\text { WB3X } & \text { Bleed }> & 0.0000 & 1.0000 & 1.0000\end{array}$ $\begin{array}{lllll}\text { WBA3X } & \text { Bleed } & 0.0000 & 1.0000 & 1.0000\end{array}$ $\begin{array}{llll}\text { WBW3X Bleed } & 0.0000 & 1.0000 & 1.0000\end{array}$ WBFDLKG FanDu> $0.0000 \quad 1.0000 \quad 1.0000$ WB15X Mleed> $0.0000-1.0000 \quad 1.000$ $\begin{array}{lllll}\text { WB15Y Bleed } & 0.0000 & 1.0000 & 1.0000\end{array}$ $\begin{array}{rrrr}\text { W } & \text { Tt } & \text { ht } & \text { Pt } \\ 2.6301 & 525.86 & -37.06 & 6.587\end{array}$ $\begin{array}{llll}2.7027 & 949.74 & 66.36 & 38.601\end{array}$ $\begin{array}{llll}2.3649 & 949.74 & 66.36 & 21.907\end{array}$ $\begin{array}{llll}0.0000 & 949.74 & 66.36 & 39.511\end{array}$ $\begin{array}{llll}0.0000 & 949.74 & 66.36 & 39.511\end{array}$ $\begin{array}{llll}0.0000 & 949.74 & 66.36 & 39.511\end{array}$ $\begin{array}{llll}0.0000 & 466.07 & -18.95 & 4.858\end{array}$ $\begin{array}{llll}0.0000 & 466.07 & -18.95 & 4.858\end{array}$ $\begin{array}{llll}0.0000 & 466.07 & -18.95 & 4.858\end{array}$

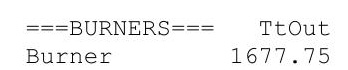
eff
0.9995

dPnorm Bleed>

4.858

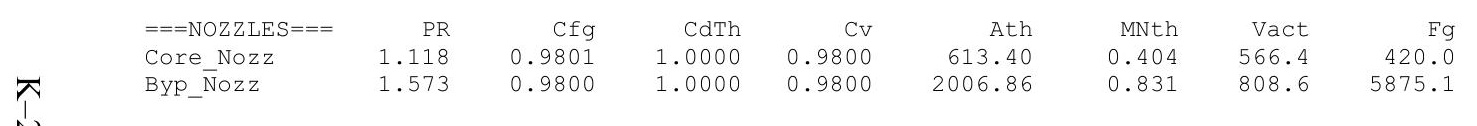


Date:07/05/13 Time:11:14:19 Model: Turbofan Engine - COMDES ON Converge = 1 CASE: 0

Version:NPSS 1.6.5 - Rev: -> Gas Package: Janaf iter/pass/Jacb/Broy= $12 / 26 / 1 / 10$ Run by: Philip C Jorgenson PC: 10

Temperature Stator 1 inlet: $456.59 \quad$ Stator 1 exit: $463.04 \quad$ Stator 2 inlet: $471.10 \quad$ Stator 2 exit: 475.24

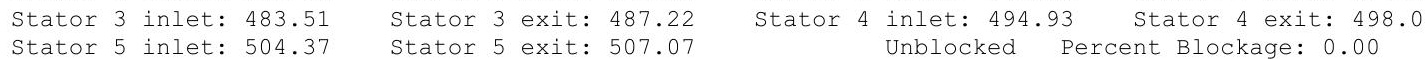

$\begin{array}{lclll}\text { Ambient Relative Humidity } & 10.00 & & & \\ \text { Fan Face Relative Humidity } & 3.01 & & & \\ \text { Fan Bypass Relative Humidity } & 2.24 & & & \\ \text { LPC Inlet Relative Humidity } & 2.00 & & & \\ \text { LPC Exit Relative Humidity } & 0.21 & & & \\ \text { HPC Relative Humidity } & 0.06 & & & \\ \text { Drop Diameter } & 0.0000050 & \text { Inlet Length } & \\ \text { Ambient Flow Velocity } & 667.90 & \text { Fan/LPC Inlet Flow Velocity } & 171.39 \\ \text { Ambient Static Pressure } & 3.58 & \text { Fan/LPC Inlet Static Pressure } & 4.74 \\ \text { Ambient Static Temperature } & 414.42 & \text { Fan/LPC Inlet Static Temperature } & 449.16 \\ \text { Additional Water at LPC Exit } & 0.0050695 & & & \end{array}$

Additional Water at LPC Exit

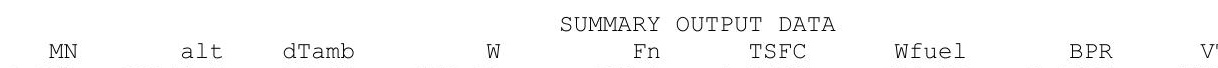

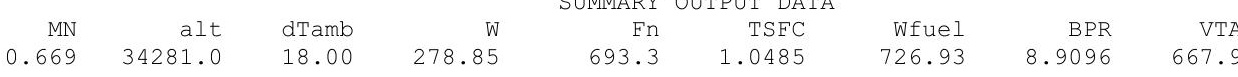

\begin{tabular}{|c|c|}
\hline FSO & Ambient.Fl O \\
\hline FS1 & Inlet.Fl $0^{-}$ \\
\hline FS12 & Splitter.Fl_02 \\
\hline FS2 & Splitter.FI 01 \\
\hline FS14 & Fan.Fl O \\
\hline FS23 & LPC.Fl_o \\
\hline FS24 & VaporI $\bar{N} \cdot$ FI_O \\
\hline FS25 & Bleed2.Fl $\overline{0}$ \\
\hline FS3 & HPC.Fl_O \\
\hline FS36 & Bleed3.Fl O \\
\hline FS4 & Burner.Fl- \\
\hline FS45 & HPT.FI_O \\
\hline FS49 & LPT.FI_O \\
\hline FS5 & TEGV.FI O \\
\hline FS8 & Core Nozz.FIO \\
\hline FS17 & FanDuctLkg.Fì_o \\
\hline FS171 & Bleed15.FI \\
\hline FS172 & FanDuct.FI- \\
\hline FS173 & Byp_Nozz.FI_C \\
\hline
\end{tabular}

\begin{tabular}{rrrrr}
\multicolumn{7}{c}{ FLOW } & \multicolumn{2}{l}{ STATION DATA } \\
W & Pt & Tt & ht & FAR \\
278.85 & 4.831 & 451.61 & -22.45 & 0.0000 \\
278.85 & 4.831 & 451.61 & -22.45 & 0.0000 \\
250.71 & 4.829 & 451.61 & -22.45 & 0.0000 \\
28.14 & 4.829 & 451.61 & -22.45 & 0.0000 \\
250.71 & 5.369 & 467.01 & -18.76 & 0.0000 \\
28.14 & 7.150 & 515.75 & -7.08 & 0.0000 \\
28.28 & 7.150 & 522.79 & -34.51 & 0.0000 \\
25.45 & 7.150 & 522.79 & -34.51 & 0.0000 \\
24.22 & 42.371 & 941.47 & 67.54 & 0.0000 \\
18.77 & 42.371 & 941.47 & 67.54 & 0.0000 \\
18.97 & 41.395 & 1669.03 & 55.63 & 0.0108 \\
25.36 & 10.206 & 1105.90 & -43.21 & 0.0081 \\
25.66 & 3.944 & 892.20 & -95.45 & 0.0080 \\
25.66 & 3.944 & 892.27 & -95.45 & 0.0080 \\
25.66 & 3.944 & 892.34 & -95.45 & 0.0080 \\
250.71 & 5.369 & 467.01 & -18.76 & 0.0000 \\
253.54 & 5.369 & 467.64 & -18.94 & 0.0000 \\
253.54 & 5.369 & 467.64 & -18.94 & 0.0000 \\
253.54 & 5.369 & 467.64 & -18.94 & 0.0000
\end{tabular}

Wc
791.52
791.52
712.00
79.91
651.15
57.68
58.36
52.53
11.32
8.77
12.08
53.32
125.38
125.39
125.39
651.15
658.93
658.93
658.93

Ps
3.578
4.452
4.396
4.738
4.577
6.859
6.851
6.910
37.330
39.475
39.082
9.295
3.768
3.768
3.578
4.577
4.446
4.446
3.578

Ts
414.42
441.18
439.65
449.16
446.16
509.66
516.45
517.71
908.93
923.16
1645.17
1078.39
881.04
881.11
868.66
446.16
443.05
443.05
416.36

$\begin{array}{rr}\text { Aphy } & \text { MN } \\ 2579.9 & 0.6690 \\ 4168.2 & 0.3434 \\ 3531.8 & 0.3683 \\ 830.5 & 0.1649 \\ 2606.7 & 0.4829 \\ 412.6 & 0.2442 \\ 412.6 & 0.2479 \\ 412.6 & 0.2214 \\ 49.7 & 0.4314 \\ 49.3 & 0.3214 \\ 74.6 & 0.2947 \\ 265.4 & 0.3719 \\ 860.2 & 0.2578 \\ 860.2 & 0.2578 \\ 613.4 & 0.3779 \\ 2606.7 & 0.4829 \\ 2481.9 & 0.5262 \\ 2481.9 & 0.5262 \\ 2006.9 & 0.7838\end{array}$

gamt
1.40088
1.40088
1.40088
1.40088
1.40078
1.40027
1.39948
1.39948
1.38344
1.38344
1.33322
1.36771
1.38107
1.38107
1.38106
1.40078
1.40077
1.40077
1.40077

WAR
0.0000348
0.0000348
0.0000348
0.0000348
0.0000348
0.0000348
0.0051043
0.0051043
0.0051043
0.0051043
0.0051043
0.0051043
0.0051043
0.0051043
0.0051043
0.0000348
0.0000911
0.0000911
0.0000911

$\begin{array}{rrr}\text { Wair } & \text { WH2O } & \text { H2O frac } \\ 78.84 & 0.010 & 0.0000 \\ 78.84 & 0.010 & 0.0000 \\ 50.70 & 0.009 & 0.0000 \\ 28.14 & 0.001 & 0.0000 \\ 50.70 & 0.009 & 0.0000 \\ 28.14 & 0.001 & 0.0000 \\ 28.14 & 0.144 & 0.0051 \\ 25.32 & 0.129 & 0.0051 \\ 24.10 & 0.123 & 0.0051 \\ 18.67 & 0.095 & 0.0051 \\ 18.67 & 0.095 & 0.0181 \\ 25.03 & 0.128 & 0.0149 \\ 25.32 & 0.129 & 0.0148 \\ 25.32 & 0.129 & 0.0148 \\ 25.32 & 0.129 & 0.0148 \\ 50.70 & 0.009 & 0.0000 \\ 55.52 & 0.023 & 0.0001 \\ 53.52 & 0.023 & 0.0001 \\ 253.52 & 0.023 & 0.0001\end{array}$

TURBOMACHINERY PERFORMANCE DATA

\begin{tabular}{|c|c|c|c|c|c|c|c|c|c|}
\hline & Wc & PR & eff & $\mathrm{NC}$ & $T R$ & efpoly & pwr & SMN & SMW \\
\hline Fan & 712.00 & 1.112 & 0.9038 & 2406.933 & 1.0341 & 0.9052 & -1309.4 & 4933.57 & 54.09 \\
\hline LPC & 79.91 & 1.481 & 0.8366 & 2406.933 & 1.1420 & 0.8454 & -612.1 & 6.45 & 3.08 \\
\hline HPC & 52.53 & 5.926 & 0.8159 & 8909.850 & 1.8009 & 0.8546 & -3586.1 & 61.64 & 58.15 \\
\hline HPT & 12.08 & 4.056 & 0.8727 & 218.955 & 1.3577 & 0.8503 & 3586.1 & & \\
\hline LPT & 53.32 & 2.588 & 0.8405 & 67.537 & 1.2373 & 0.8217 & 1921.4 & & \\
\hline TURB & NERY MAP & DATA & & & & & & & \\
\hline & WcMap & PRmap & effMap & NcMap & R/Parm & s WcDes & s PRdes & s effDes & s NCDes \\
\hline Fan & 827.28 & 1.109 & 0.9056 & 2383.983 & 0.0413 & 0.8607 & $\overline{1} .0235$ & 0.9980 & 0.9905 \\
\hline LPC & 66.92 & 1.450 & 0.7723 & 0.636 & 0.0000 & 1.1940 & 1.0684 & 1.0832 & 0.0003 \\
\hline HPC & 47.77 & 5.694 & 0.8243 & 8672.320 & 10.9775 & 1.0995 & 1.0494 & 0.9898 & 0.9733 \\
\hline HPT & 0.96 & 3.971 & 0.8727 & 1.316 & 3.9712 & 12.6299 & 0.9723 & 1.0000 & 0.0003 \\
\hline LPT & 0.82 & 2.194 & 0.8467 & 0.760 & 2.1942 & 65.2590 & 0.7521 & 0.9926 & 0.0005 \\
\hline
\end{tabular}




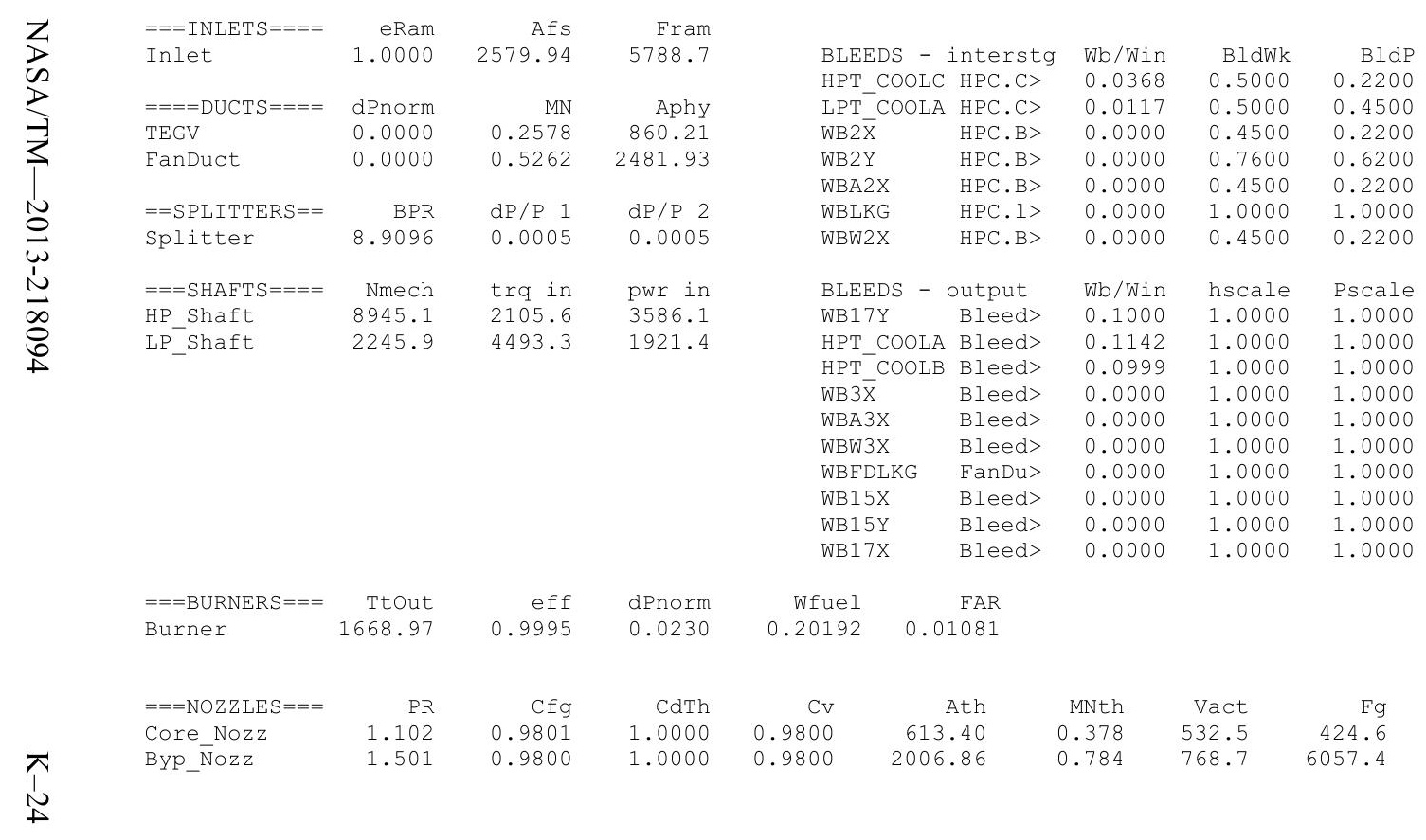




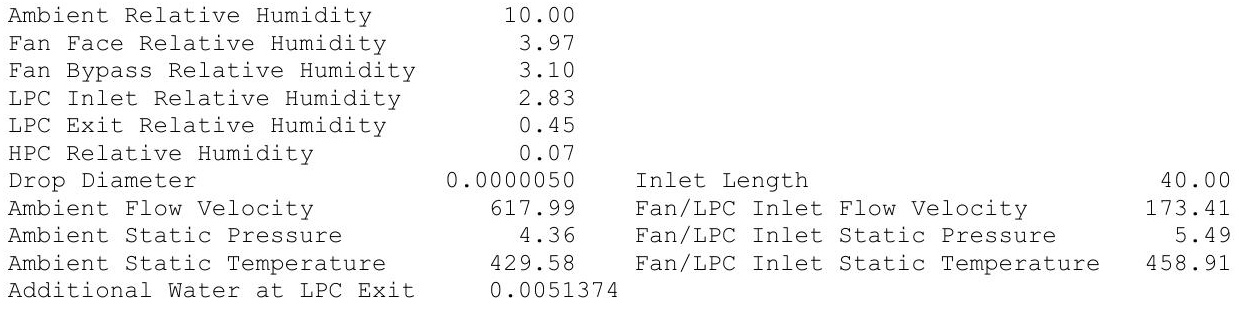

\begin{tabular}{|c|c|c|c|c|c|c|c|c|c|c|c|c|c|c|}
\hline & & dTamb & & JMMARY & TPUT DAT & Wfuel & & VTR & & & & $\pi /$ & T41 & T49 \\
\hline 0.608 & 30029.0 & 18.00 & 305.45 & $\begin{array}{r}\text { m } \\
755.2\end{array}$ & 1.0510 & 793.73 & 8.4645 & 617.99 & 8.214 & 0.828 & $\begin{array}{l}\text { Powerset } \\
10.000\end{array}$ & 1673.3 & 1580.9 & 1107.2 \\
\hline
\end{tabular}

FSO Ambient.Fl O

FS1 Inlet.Fl_o

FS2 Splitter.Fl_02

FS14 Fan.FI_O

S23 LPC.FI_O

FS24 VaporIN.F1_-

FS3 HPC.Fl_O

$\begin{array}{ll}\text { FS3 } & \text { HPC.F1_O } \\ \text { FS36 } & \text { Bleed3.F1 }\end{array}$

FS4 Burner.FI_O

FS45 HPT.FI-

FS49 LPT.FI-O

FS8 Core_Nozz.FI_O

FS17 FanDuctLkg.Fi_o

FS171 Bleed15.FI_O

FS172 FanDuct. Fl-O

TURBOMACHINERY PERFORMANCE DATA

\begin{tabular}{|c|c|c|c|c|c|c|c|c|c|}
\hline & Wc & $P R$ & eff & NC & $\mathrm{TR}$ & efpoly & pwr & SMN & SMW \\
\hline Fan & 677.08 & 1.099 & 0.9028 & 2261.160 & 1.0305 & 0.9041 & -1302.1 &.-- & 52.63 \\
\hline LPC & 79.99 & 1.406 & 0.8519 & 2261.160 & 1.1202 & 0.8589 & -606.9 & 5.94 & 2.66 \\
\hline PC & 51.80 & 5.845 & 0.8144 & 8885.964 & 1.7944 & 0.8532 & -3862.2 & 61.93 & 58.35 \\
\hline HPT & 12.08 & 4.001 & 0.8728 & 218.341 & 1.3535 & 0.8508 & 3862.2 & & \\
\hline LPT & 52.65 & 2.378 & 0.8324 & 63.972 & 1.2116 & 0.8147 & 1909.1 & & \\
\hline JRB & ERY MAP & DATA & & & & & & & \\
\hline & WcMap & PRmap & effmap & NcMap & R/Parm & s WcDes & s PRdes & $s$ effDes & s NcDes \\
\hline an & 786.70 & 1.097 & 0.9046 & 39.600 & 0.0417 & $\overline{0} .8607$ & $\overline{1} .0235$ & 0.9980 & $\overline{0} .9905$ \\
\hline LPC & 60.72 & 1.386 & 0.7658 & 0.597 & 0.0000 & 1.3175 & 1.0522 & 1.1125 & 0.0003 \\
\hline PC & 47.11 & 5.617 & 0.8228 & 8649.070 & 10.9695 & 1.0995 & 1.04 & 0.9 & 0.9733 \\
\hline HPT & 0.96 & 3.917 & 0.8728 & 1.312 & 3.9175 & 12.6299 & 0.9723 & 1.0000 & 0.0003 \\
\hline LPT & 0.8 & 2.037 & 0.8386 & 0.720 & 2.0365 & 65.2590 & 0.7521 & 0.9926 & 0.0005 \\
\hline
\end{tabular}

FLOW STATION DATA

\begin{tabular}{rrrrrr}
\multicolumn{7}{c}{ FLOW } & \multicolumn{1}{c}{ STATION DATA } \\
W & Pt & Tt & ht & FAR & Wc \\
305.45 & 5.595 & 461.42 & -20.30 & 0.0000 & 756.70 \\
305.45 & 5.595 & 461.42 & -20.30 & 0.0000 & 756.70 \\
273.17 & 5.592 & 461.42 & -20.30 & 0.0000 & 677.08 \\
32.27 & 5.592 & 461.42 & -20.30 & 0.0000 & 79.99 \\
273.17 & 6.149 & 475.48 & -16.93 & 0.0000 & 625.13 \\
32.27 & 7.863 & 516.86 & -7.01 & 0.0000 & 60.21 \\
32.44 & 7.863 & 523.98 & -34.80 & 0.0000 & 60.94 \\
27.57 & 7.863 & 523.98 & -34.80 & 0.0000 & 51.80 \\
26.24 & 45.960 & 940.22 & 66.66 & 0.0000 & 11.29 \\
20.33 & 45.960 & 940.22 & 66.66 & 0.0000 & 8.75 \\
20.55 & 44.901 & 1673.26 & 54.66 & 0.0109 & 12.08 \\
27.47 & 11.224 & 1111.42 & -43.56 & 0.0081 & 52.65 \\
27.79 & 4.720 & 915.41 & -91.41 & 0.0080 & 114.97 \\
27.79 & 4.720 & 915.48 & -91.41 & 0.0080 & 114.98 \\
27.79 & 4.720 & 915.55 & -91.41 & 0.0080 & 114.98 \\
273.17 & 6.149 & 475.48 & -16.93 & 0.0000 & 625.13 \\
278.04 & 6.149 & 476.33 & -17.24 & 0.0000 & 636.83 \\
278.04 & 6.149 & 476.33 & -17.24 & 0.0000 & 636.83 \\
278.04 & 6.149 & 476.33 & -17.24 & 0.0000 & 636.83
\end{tabular}

0.720

65.2590

0.0005

Ts
429.58
451.79
450.53
458.91
456.36
510.18
517.01
519.05
907.88
922.01
1649.36
1084.58
905.91
905.98
895.69
456.36
453.50
453.50
431.66

$\begin{array}{rr}\text { Aphy } & \text { MN } \\ 2599.2 & 0.6080\end{array}$ $\begin{array}{lll}2599.2 & 0.6080 & 1.40081\end{array}$ $\begin{array}{lll}4168.2 & 0.3260 & 1.40081 \\ 3531.8 & 0.3472 & 1.40081\end{array}$ $\begin{array}{lll}830.5 & 0.1651 & 1.40081\end{array}$ $\begin{array}{lll}412.6 & 0.2559\end{array}$ $\begin{array}{ll}412.6 & 0.2559 \\ 412.6 & 0.2597\end{array}$ $\begin{array}{llll}49.3 & 0.3206 & 1.38350\end{array}$ $\begin{array}{lll}74.6 & 0.2947 & 1.33294\end{array}$ $\begin{array}{lll}265.4 & 0.3666 & 1.36729\end{array}$ $\begin{array}{lll}860.2 & 0.2349 & 1.37966 \\ 860.2 & 0.2349 & 1.37966\end{array}$ $\begin{array}{llll}613.4 & 0.3415 & 1.37965\end{array}$ $\begin{array}{lll}2606.7 & 0.4572 & 1.40070\end{array}$ $\begin{array}{llll}2481.9 & 0.5012 & 1.40068\end{array}$ $\begin{array}{lll}2481.9 & 0.5012 & 1.40068\end{array}$ $2006.9 \quad 0.7185$

$\begin{array}{rrrr}\text { WAR } & \text { Wair } & \text { WH2O } & \text { H2O frac } \\ 0.0000681 & 305.43 & 0.021 & 0.0001 \\ 0.0000681 & 305.43 & 0.021 & 0.0001 \\ 0.0000681 & 273.16 & 0.019 & 0.0001 \\ 0.0000681 & 32.27 & 0.002 & 0.0001 \\ 0.0000681 & 273.16 & 0.019 & 0.0001 \\ 0.0000681 & 32.27 & 0.002 & 0.0001 \\ 0.0052055 & 32.27 & 0.168 & 0.0052 \\ 0.0052055 & 27.43 & 0.143 & 0.0052 \\ 0.0052555 & 26.10 & 0.136 & 0.0052 \\ 0.0052055 & 20.23 & 0.105 & 0.0052 \\ 0.0052055 & 20.23 & 0.105 & 0.0183 \\ 0.0052055 & 27.11 & 0.141 & 0.0150 \\ 0.0052055 & 27.43 & 0.143 & 0.0149 \\ 0.0052055 & 27.43 & 0.143 & 0.0149 \\ 0.0052055 & 27.43 & 0.143 & 0.0149 \\ 0.0000681 & 273.16 & 0.019 & 0.0001 \\ 0.0001576 & 278.00 & 0.044 & 0.0002 \\ 0.0001576 & 278.00 & 0.044 & 0.0002 \\ 0.0001576 & 278.00 & 0.044 & 0.0002\end{array}$




\begin{tabular}{|c|c|c|c|c|c|c|c|c|}
\hline$===$ INLETS $====$ & eRam & Afs & Fram & \multirow{2}{*}{\multicolumn{2}{|c|}{ BLEEDS - interstg }} & & & \\
\hline Inlet & 1.0000 & 2599.17 & 5867.0 & & & Wb/Win & BldWk & BldP \\
\hline$====$ DUCTS $====$ & dPnorm & MN & Aphy & \multicolumn{2}{|c|}{ HPT_COOLC HPC.C> } & $\begin{array}{l}0.0368 \\
0.0117\end{array}$ & 0.5000 & 0.2200 \\
\hline TEGV & 0.0000 & 0.2349 & 860.21 & WB $2 \bar{x}$ & HPC.B> & 0.0000 & 0.4500 & 0.2200 \\
\hline FanDuct & 0.0000 & 0.5012 & 2481.93 & $\begin{array}{l}\text { WB2Y } \\
\text { WBA2X }\end{array}$ & HPC.B> & 0.0000 & 0.7600 & 0.6200 \\
\hline & & & & & HPC.B> & 0.0000 & 0.4500 & 0.2200 \\
\hline$==$ SPLITTERS $==$ & BPR & $\mathrm{dP} / \mathrm{P} 1$ & $\mathrm{dP} / \mathrm{P} 2$ & $\begin{array}{l}\text { WBA2X } \\
\text { WBLKG }\end{array}$ & HPC. $1>$ & 0.0000 & 1.0000 & 1.0000 \\
\hline Splitter & 8.4645 & 0.0005 & 0.0005 & WBW2X & HPC.B> & 0.0000 & 0.4500 & 0.2200 \\
\hline$===$ SHAFTS $====$ & Nmech & $\operatorname{trg}$ in & pwr in & \multicolumn{2}{|c|}{ BLEEDS - output } & Wb/Win & hscale & Pscale \\
\hline HP Shaft & 8931.3 & 2271.2 & 3862.2 & WB17Y & Bleed> & 0.1500 & 1.0000 & 1.0000 \\
\hline LP_Shaft & 2132.7 & 4701.4 & 1909.1 & HPT COOLA & A Bleed> & 0.1142 & 1.0000 & 1.0000 \\
\hline & & & & $\mathrm{HPT}_{-}^{-} \mathrm{COOLB}$ & B Bleed> & 0.0999 & 1.0000 & 1.0000 \\
\hline & & & & WB3 $3 \bar{X}$ & Bleed> & 0.0000 & 1.0000 & 1.0000 \\
\hline & & & & WBA3X & Bleed> & 0.0000 & 1.0000 & 1.0000 \\
\hline & & & & \multirow{2}{*}{$\begin{array}{l}\text { WBW3X } \\
\text { WBFDLKG }\end{array}$} & Bleed> & 0.0000 & 1.0000 & 1.0000 \\
\hline & & & & & FanDu> & 0.0000 & 1.0000 & 1.0000 \\
\hline & & & & WB15X & Bleed> & 0.0000 & 1.0000 & 1.0000 \\
\hline & & & & $\begin{array}{l}\text { WB15Y } \\
\text { WB17X }\end{array}$ & Bleed> & 0.0000 & 1.0000 & 1.0000 \\
\hline & & & & WB17X & Bleed> & 0.0000 & 1.0000 & 1.0000 \\
\hline$===$ BURNERS $===$ & Ttout & eff & dPnorm & Wfuel & FAR & & & \\
\hline Burner & 1673.20 & 0.9995 & 0.0230 & \multicolumn{2}{|c|}{$0.22048 \quad 0.01090$} & & & \\
\hline$===$ NOZZLES $===$ & PR & $\mathrm{Cfg}$ & CdTh & $\mathrm{Cv}$ & & MNth & Vact & $\mathrm{Fg}$ \\
\hline Core_Nozz & 1.083 & 0.9801 & 1.0000 & 0.9800 & 613.40 & \multirow{2}{*}{$\begin{array}{l}0.341 \\
0.719\end{array}$} & 488.3 & 421.8 \\
\hline Byp_Nozozz & 1.411 & 0.9800 & 1.0000 & \multicolumn{2}{|c|}{2006.86} & & 717.5 & 6200.4 \\
\hline
\end{tabular}


Date:07/05/13 Time:11:15:17 Model: Turbofan Engine - COMDES ON Converge = 1 CASE: 0

Version:NPSS 1.6.5 - Rev: -> Gas Package: Janaf iter/pass/Jacb/Broy= 22/ 50/ 2/19 Run by: Philip C Jorgenson PC: 10

Temperature Stator 1 inlet: 473.27 Stator 1 exit: $477.40 \quad$ Stator 2 inlet: $482.10 \quad$ Stator 2 exit: 483.97 $\begin{array}{lll}\text { Stator } 3 \text { inlet: } 489.18 & \text { Stator } 3 \text { exit: } 490.64 & \text { Stator } 4 \text { inlet: } 495.62 \text { Stator } 4 \text { exit: } 496.68 \\ \text { Stator } 5 \text { inlet: } 500.34 & \text { Stator } 5 \text { exit: } 501.11 & \text { Unblocked Percent Blockage: } 0.00\end{array}$

\begin{tabular}{|c|c|c|c|}
\hline bient Relative & 10.00 & & \\
\hline Fan Face Relative Humidity & 4.83 & & \\
\hline Fan Bypass Relative Humidity & 3.92 & & \\
\hline C Inlet Relative Humidity & 3.81 & & \\
\hline PC Exit Relative Humidity & 1.16 & & \\
\hline C Relative Humidity & 0.08 & & \\
\hline cop Diameter & 0.0000050 & Inlet Length & 40.00 \\
\hline bient Flow Velocity & 571.13 & Fan/LPC Inlet Flow Velocity & 193.27 \\
\hline bient Static Pressure & 5.30 & Fan/LPC Inlet Static Pressure & 6.36 \\
\hline ient static Temperature & 445.14 & Fan/LPC Inlet Static Temperature & 469.21 \\
\hline itional Water at LPC Exi & 0.004632 & & \\
\hline
\end{tabular}

Additional Water at LPC Exit

SUMMARY OUTPUT DATA

\begin{tabular}{|c|c|c|c|c|c|c|c|c|c|c|c|c|c|c|}
\hline $\begin{array}{r}M N \\
0.552\end{array}$ & $\begin{array}{r}\text { alt } \\
25666.0\end{array}$ & $\begin{array}{l}\text { dTamb } \\
18.00\end{array}$ & $\begin{array}{r}W \\
330.91\end{array}$ & $\begin{array}{r}\text { JMMARY } \\
\text { Fn } \\
775.1\end{array}$ & $\begin{array}{r}\text { TPUT DA } \\
\text { TSFC } \\
1.0929\end{array}$ & $\begin{array}{r}\text { Wfuel } \\
847.06\end{array}$ & $\begin{array}{r}\text { BPR } \\
7.1100\end{array}$ & $\begin{array}{r}\text { VTAS } \\
571.13\end{array}$ & $\begin{array}{r}\text { OPR } \\
7.359\end{array}$ & $\begin{array}{c}\text { EPR } \\
0.859\end{array}$ & $\begin{array}{l}\text { Powerset } \\
10.000\end{array}$ & $\begin{array}{c}\text { T4 } \\
1681.9\end{array}$ & $\begin{array}{c}\text { T41 } \\
1587.3\end{array}$ & $\begin{array}{c}\text { T49 } \\
1116.8\end{array}$ \\
\hline
\end{tabular}

\begin{tabular}{|c|c|c|c|c|c|c|c|c|c|c|c|}
\hline & & & & FLOW $s$ & STATION DAT & & & & & & \\
\hline FSO & Ambient.Fl O & & $\begin{array}{r}W \\
330.91\end{array}$ & $\begin{array}{r}\text { Pt } \\
6.516\end{array}$ & $\begin{array}{r}T t \\
472.33\end{array}$ & $\begin{array}{r}h t \\
-18.03\end{array}$ & $\begin{array}{r}\text { FA } \\
0.000\end{array}$ & 712.1 & $\begin{array}{l}\text { Wc } \\
17\end{array}$ & $\begin{array}{l}\text { Ps } \\
97\end{array}$ & \\
\hline FS1 & Inlet.Fl o- & & 330.91 & 6.516 & 472.33 & -18.03 & 0.000 & 712.1 & & & $\begin{array}{l}445 . \\
463 .\end{array}$ \\
\hline FS12 & Splitter.Fl_o & & 290.11 & 6.513 & 472.33 & -18.03 & 0.000 & 624.6 & & & 463. \\
\hline FS2 & Splitter.Fl_o & & 40.80 & 6.513 & 472.33 & -18.03 & 0.000 & $87.8-2-1$ & & & 469. \\
\hline FS14 & Fan.Fl_O & & 290.11 & 7.066 & 484.65 & -15.07 & 0.000 & $583.2-r .2$ & & & 468. \\
\hline FS23 & LPC.FI_O & & 40.80 & 8.252 & 512.38 & -8.42 & 0.000 & 72.2 & & & 502. \\
\hline FS24 & 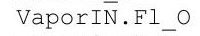 & & 40.99 & 8.252 & 518.87 & -33.48 & 0.000 & 73.0 & & & 508. \\
\hline FS25 & Bleed2.F1_o & & 28.69 & 8.252 & 518.87 & -33.48 & 0.000 & 51.1 & & & 514. \\
\hline FS3 & $\mathrm{HPC} . \mathrm{Fl} O$ & & 27.30 & 47.950 & 930.01 & 66.64 & 0.000 & 11.2 & 42. & & 898. \\
\hline FS36 & Bleed3.Fl_O & & 21.16 & 47.950 & 930.01 & 66.64 & 0.000 & 8.6 & 44. & & 912 \\
\hline FS 4 & Burner.FI_O & & 21.39 & 6.846 & 1681.87 & 54.34 & 0.011 & 12.0 & 44. & & 1657. \\
\hline FS 45 & HPT.FI_O & & 28.59 & 12.061 & 1121.21 & -42.45 & 0.008 & 51.2 & 11. & & 1095. \\
\hline FS49 & LPT.FI_O & & 28.93 & 5.629 & 946.68 & -84.93 & 0.008 & 102.0 & & & 939. \\
\hline FS5 & TEGV.F̄ & & 28.93 & 5.629 & 946.75 & -84.93 & 0.008 & 102.0 & & & 939. \\
\hline FS 8 & Core_Nozz.Fl & & 28.93 & 5.629 & 946.82 & -84.93 & 0.008 & 102.0 & & & 931. \\
\hline FS17 & FanDūctLkg.F̄ & & 290.11 & 7.066 & 484.65 & -15.07 & 0.000 & 583.2 & & & 468. \\
\hline FS171 & Bleed15.FI_O & & 302.40 & 7.066 & 486.05 & -15.82 & 0.000 & 608.8 & & & 465. \\
\hline FS172 & FanDuct.FI_O & & 302.40 & 7.066 & 486.05 & -15.82 & 0.000 & 608.8 & & & 465. \\
\hline FS173 & Byp_Nozz.Fì_C & & 302.40 & 7.066 & 486.05 & -15.82 & 0.000 & 608.8 & & & 447. \\
\hline TURBOI & ER & ORMANCE & DATA & & & & & & & & \\
\hline & WC & $\mathrm{PR}$ & eff & NC & $\mathrm{TR}$ & efPoly & & pwr & SMN & & SMW \\
\hline Fan & 624.67 & 1.085 & 0.9039 & 2054.570 & 1.0261 & 0.9050 & & $212.6 \quad-2$ & -2862.02 & & .81 \\
\hline LPC & 87.86 & 1.267 & 0.8256 & 2054.570 & 1.0848 & 0.8314 & & 554.4 & 6.36 & & .41 \\
\hline HPC & 51.11 & 5.811 & 0.8137 & 8864.644 & 1.7924 & 0.8526 & & 966.1 & 61.03 & & .40 \\
\hline HPT & 12.09 & 3.884 & 0.8741 & 216.197 & 1.3450 & 0.8528 & & 966.2 & & & \\
\hline LPT & 51.23 & 2.142 & 0.8229 & 58.554 & 1.1815 & 0.8067 & & 766.9 & & & \\
\hline TUR & MACH INERY & $\mathrm{DA}$ & & & & & & & & & \\
\hline & WcMap & PRmap & effmap & NcMap & R/Parm & S_W & CDes & S_PRdes & s_effDe & & S_NcDes \\
\hline Fan & 725.81 & 1.083 & 0.9057 & 2034.980 & 0.0426 & $\overline{0}$ & 8607 & $\overline{1} .0235$ & 0.998 & & $\overline{0} .9905$ \\
\hline LPC & 53.80 & 1.315 & 0.7610 & 0.543 & 0.0000 & & 6329 & 0.8487 & 1.085 & & 0.0003 \\
\hline HPC & 46.49 & 5.584 & 0.8221 & 8628.318 & 11.0332 & & 0995 & 1.0494 & 0.989 & & 0.9733 \\
\hline HPT & 0.96 & 3.804 & 0.8741 & 1.299 & 3.8043 & 12. & 6299 & 0.9723 & 1.000 & & 0.0003 \\
\hline LPT & 0.78 & 1.859 & 0.8290 & 0.659 & 1.8593 & $65.2=$ & & 0.7521 & 0.992 & & 0.0005 \\
\hline
\end{tabular}




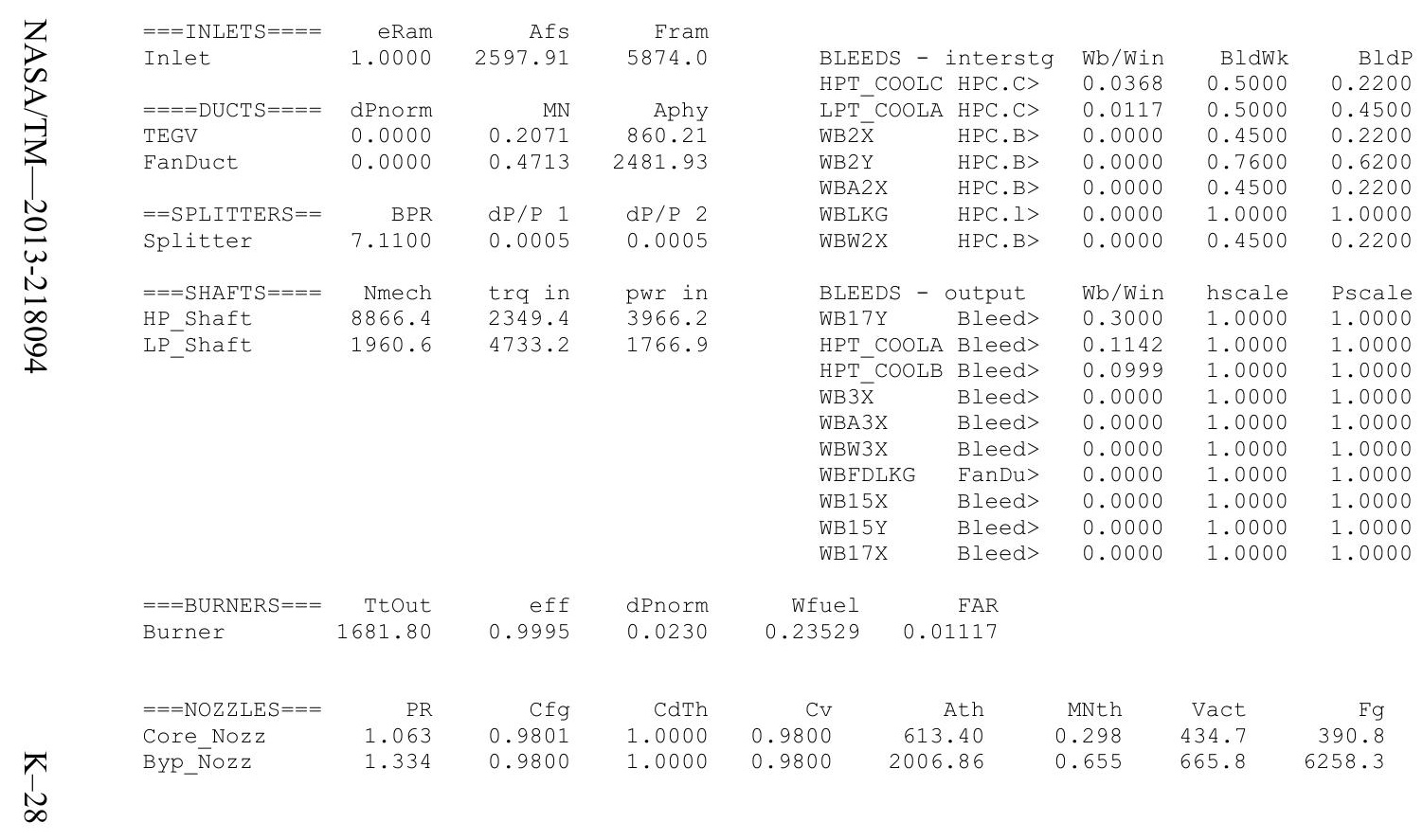


COnverge = 1 CASE: 0

Version:NPSS_1.6.5 - Rev: -> Gas Package: Janaf iter/pass/Jacb/Broy=33/61/2/30 Run by: Philip C Jorgenson PC: 10

$\begin{array}{rllll}\text { Temperature Stator } 1 \text { inlet: } 486.43 & \text { Stator } 1 \text { exit: } 489.44 & \text { Stator } 2 \text { inlet: } 492.29 & \text { Stator } 2 \text { exit: } 493.11 \\ \text { Stator } 3 \text { inlet: } 496.53 & \text { Stator } 3 \text { exit: } 496.92 & \text { Stator } 4 \text { inlet: } 500.25 & \text { Stator } 4 \text { exit: } 500.33\end{array}$ $\begin{array}{lll}\text { Stator } 3 \text { inlet: } 496.53 & \text { Stator } 3 \text { exit: } 496.92 & \text { Stator } 4 \text { inlet: } 500.25 \text { Stator } 4 \text { exit: } 500.33 \\ \text { Stator } 5 \text { inlet: } 502.26 & \text { Stator } 5 \text { exit: } 502.15 & \text { Unblocked Percent Blockage: } 0.00\end{array}$

$\begin{array}{lclll}\text { Ambient Relative Humidity } & 10.00 & & & \\ \text { Fan Face Relative Humidity } & 5.86 & & & \\ \text { Fan Bypass Relative Humidity } & 4.89 & & & \\ \text { LPC Inlet Relative Humidity } & 5.02 & & & \\ \text { LPC Exit Relative Humidity } & 2.54 & & & \\ \text { HPC Relative Humidity } & 0.09 & & & \\ \text { Drop Diameter } & 0.0000050 & \text { Inlet Length } & & \\ \text { Ambient Flow Velocity } & 518.26 & \text { Fan/LPC Inlet Flow Velocity } & 203.16 \\ \text { Ambient Static Pressure } & 6.74 & \text { Fan/LPC Inlet Static Pressure } & 7.75 \\ \text { Ambient Static Temperature } & 465.18 & \text { Fan/LPC Inlet Static Temperature } & 484.12 \\ \text { Additional Water at LPC Exit } & 0.0050450 & & & \\ \end{array}$

\begin{tabular}{|c|c|c|c|c|c|c|c|c|c|c|c|c|c|c|}
\hline MN & alt & dTamb & W & $\begin{array}{l}\text { UMMARY } \\
\text { Fn }\end{array}$ & $\begin{array}{l}\text { DUTPUT DATA } \\
\text { TSFC }\end{array}$ & Wfuel & BPR & VTAS & $O P R$ & EPR & Powerset & T4 & $T 41$ & T49 \\
\hline 0.490 & 20047.0 & 18.00 & 368.61 & 863.8 & 1.0938 & 944.87 & 6.2869 & 518.26 & 6.484 & 0.890 & 10.000 & 1716.0 & 1617.4 & 1148 \\
\hline
\end{tabular}

\begin{tabular}{|c|c|c|c|c|c|c|c|c|c|c|c|}
\hline & & & & FLOW & STATION DAT & & & & & & \\
\hline FSO & Ambient.Fl O & & $\begin{array}{r}W \\
368.61\end{array}$ & $\begin{array}{r}\mathrm{Pt} \\
7.943\end{array}$ & $\begin{array}{r}T t \\
487.56\end{array}$ & $\begin{array}{r}h t \\
-15.16\end{array}$ & $\begin{array}{r}F A \\
0.000\end{array}$ & 661. & $\begin{array}{l}\text { WC } \\
20\end{array}$ & $\begin{array}{l}\text { Ps } \\
40\end{array}$ & \\
\hline FS1 & Inlet.Fl o & & 368.61 & 7.943 & 487.56 & -15.16 & 0.000 & $661.2=1$ & & & 480. \\
\hline FS12 & Splitter.Fl 0 & & 318.03 & 7.939 & 487.56 & -15.16 & 0.000 & 570. & & & 479 \\
\hline FS2 & Splitter.Fl_o & & 50.59 & 7.939 & 487.56 & -15.16 & 0.000 & 90. & & & 484 \\
\hline FS14 & Fan.Fl_O & & 318.03 & 8.522 & 498.58 & -12.52 & 0.000 & 537. & & 719 & 484 \\
\hline FS23 & LPC.Fl_o & & 50.59 & 9.130 & 515.91 & -8.36 & 0.000 & 81.2 & & & 503 \\
\hline FS24 & 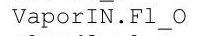 & & 50.84 & 9.130 & 522.91 & -35.64 & 0.000 & 82. & & $\beta 32$ & 509 \\
\hline FS25 & Bleed2.Fl_ō & & 30.50 & 9.130 & 522.91 & -35.64 & 0.000 & 49.3 & & & 518 \\
\hline FS3 & HPC.Fl_O & & 29.02 & 51.507 & 930.22 & 63.61 & 0.000 & 11.0 & 45 & & 899 \\
\hline FS36 & Bleed3.FI_O & & 22.49 & 51.507 & 930.22 & 63.61 & 0.000 & 8.5 & 48 & & 912 \\
\hline FS 4 & Burner.Fl_o & & 22.76 & 50.321 & 1716.02 & 50.74 & 0.011 & 12.0 & 47 & & 1691 \\
\hline FS 45 & HPT.Fl_O & & 30.41 & 13.596 & 1152.90 & -44.99 & 0.008 & 49.0 & 12 & & 1129 \\
\hline FS 49 & LPT.FI_O & & 30.77 & 7.051 & 997.43 & -82.80 & 0.008 & 88.5 & & & \\
\hline FS5 & TEGV.F̄__O & & 30.77 & 7.051 & 997.50 & -82.80 & 0.008 & 88.9 & & & 991 \\
\hline FS 8 & Core Nozz.Fl & & 30.77 & 7.051 & 997.57 & -82.80 & 0.008 & 88.9 & 93 & & 985 \\
\hline FS17 & FanDūctLkg.F̄ & & 318.03 & 8.522 & 498.58 & -12.52 & 0.000 & 537.6 & & & \\
\hline FS171 & Bleed15.FI_O & & 338.36 & 8.522 & 500.04 & -13.91 & 0.000 & 572.9 & & & 481 \\
\hline FS172 & FanDuct.Fl_o & & 338.36 & 8.522 & 500.04 & -13.91 & 0.000 & 572.9 & & 482 & 481 \\
\hline FS173 & Byp_Nozz.Fī_o & & 338.36 & 8.522 & 500.04 & -13.91 & 0.000 & 572.9 & & 740 & \\
\hline TURBOI & MACHINERY PERE & ORMANCE & DATA & & & & & & & & \\
\hline & Wc & $\mathrm{PR}$ & eff & NC & TR & efPoly & & pwr & SMN & & SMW \\
\hline Fan & 570.75 & 1.073 & 0.9059 & 1859.517 & 1.0226 & 0.9069 & & 189.0 & -1898.08 & & .37 \\
\hline LPC & 90.78 & 1.150 & 0.7009 & 1859.517 & 1.0582 & 0.7067 & & 486.8 & 6.92 & & .24 \\
\hline HPC & 49.30 & 5.641 & 0.8098 & 8806.179 & 1.7789 & 0.8490 & & 179.6 & 60.83 & & .00 \\
\hline HPT & 12.09 & 3.701 & 0.8764 & 213.449 & 1.3304 & 0.8563 & & 179.6 & & & \\
\hline LPT & 49.01 & 1.928 & 0.8161 & 53.097 & 1.1526 & 0.8019 & & 675.7 & & & \\
\hline TURB & MACHINERY MAP & DATA & & & & & & & & & \\
\hline & WcMap & PRmap & effMap & NcMap & R/Parm & S_Wc & cDes & S_PRdes & s_effDe & & S_NcDes \\
\hline Fan & 663.16 & 1.072 & 0.9077 & 1841.787 & 0.0439 & $\overline{0} .8$ & 8607 & $\overline{1} .0235$ & -0.998 & & 0.9905 \\
\hline LPC & 47.37 & 1.250 & 0.7561 & 0.491 & 0.0000 & 1. & 9165 & 0.6000 & 0.92 & & 0.0003 \\
\hline HPC & 44.84 & 5.423 & 0.8182 & 8571.412 & 11.0706 & & 0995 & 1.0494 & 0.98 & & 0.9733 \\
\hline HPT & 0.96 & 3.626 & 0.8764 & 1.283 & 3.6262 & 12. & 6299 & 0.9723 & 1.000 & & 0.0003 \\
\hline LPT & 0.75 & 1.698 & 0.8222 & 0.597 & 1.6982 & $65.2 \cdot \ln x$ & 2590 & 0.7521 & 0.992 & & 0.0005 \\
\hline
\end{tabular}




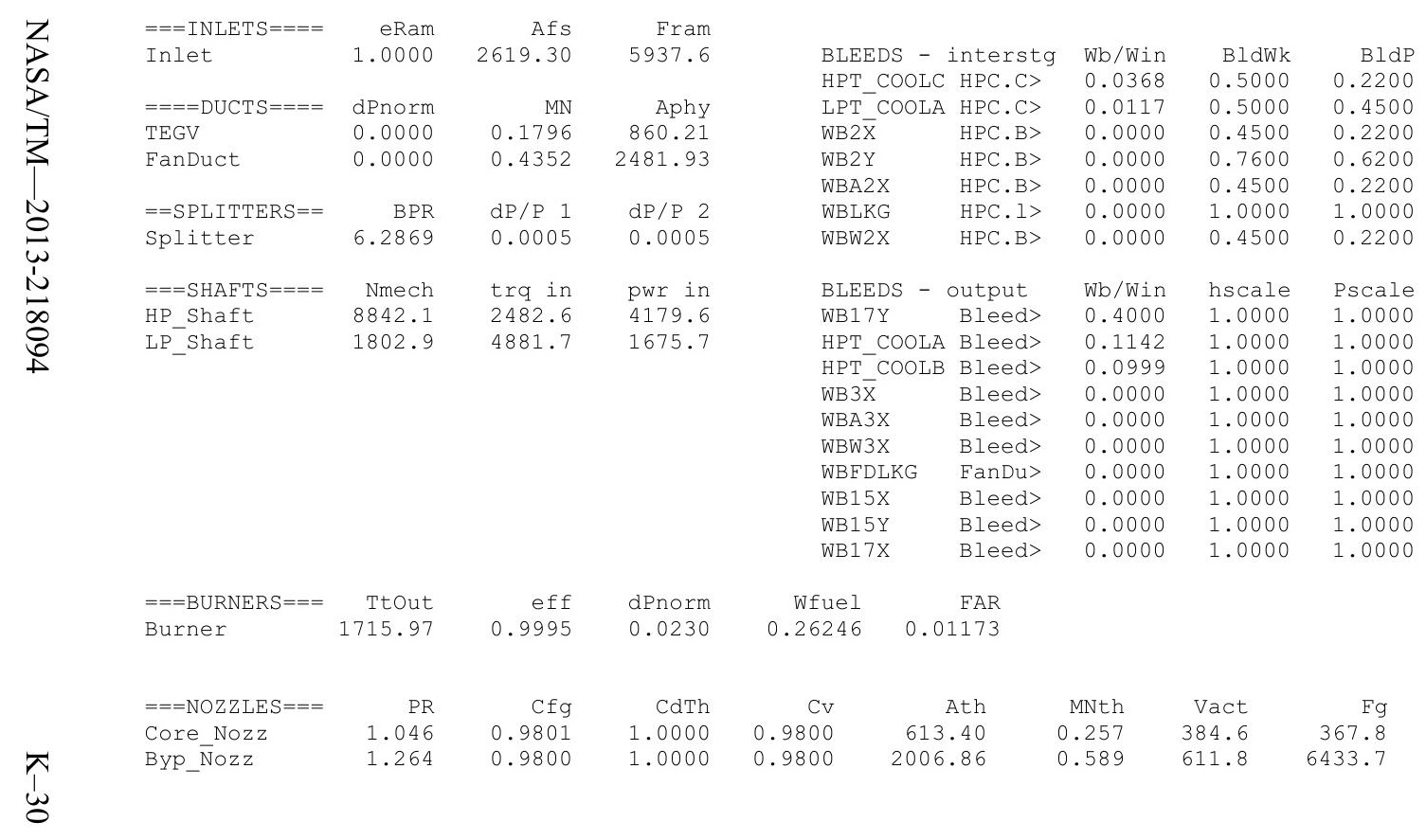


Turbofan Engine - COMDES ON Converge = 1 CASE: 0

Version:NPSS 1.6.5 - Rev: -> Gas Package: Janaf iter/pass/Jacb/Broy= 45/73/2/42 Run by: Philip C Jorgenson PC: 10

Temperature Stator 1 inlet: 500.42 Stator 1 exit: 502.83 Stator 2 inlet: 505.20 Stator 2 exit: 505.94

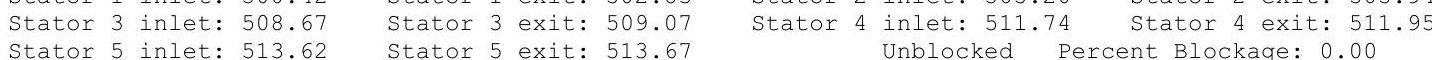

Ambient Relative Humidity $\quad 10.00$

Fan Face Relative Humidity $\quad 6.54$

Fan Bypass Relative Humidity $\quad 5.94$

$\begin{array}{ll}\text { LPC Inlet Relative Humidity } & 5.70 \\ \text { LPC Exit Relative Humidity } & 3.33\end{array}$

Drop Ritive Humidity

0.15

Drop Diameter

0.0000050

479.99
8.15

Inlet Length

Fan/LPC Inlet Flow Velocity $\quad 172.31$

Ambient Flow Velocity

172.31
9.18

mbient Static Temperature

Fan/LPC Inlet Static Temperature 498.34

$\begin{array}{rrrr}\text { MN } & \text { alt } & \text { dTamb } & W \\ 0.446 & 15435.0 & 18.00 & 387.48\end{array}$ SUMMARY OUTPUT DATA

$\begin{array}{rrr} & \text { Fn } & \text { TSFC } \\ 387.48 & 679.7 & 1.3219\end{array}$

$679.7 \quad 1.3219 \quad$ Wfuel 898.46

BPR
6.8471

VTAS 479.99
EPR 0.902
Powerset 10.000 $\begin{array}{ccc}\text { T4 } & \text { T41 } & \text { T49 } \\ 1670.4 & 1573.8 & 1140.1\end{array}$
FSO Ambient.Fl_O

FS1 Inlet.F1_O

$\stackrel{\pi}{\omega}$

FS2 Splitter.

$W$
387.48
387.48
338.10
49.38
338.10
49.38
49.69
29.81
28.37
21.98
22.23
29.71
30.06
30.06
30.06
338.10
357.98
357.98
357.98

FLOW STATION DATA

$\begin{array}{rr}\text { Pt } & \text { Tt } \\ 9.342 & 500.81 \\ 9.342 & 500.81 \\ 9.337 & 500.81 \\ 9.337 & 500.81 \\ 9.826 & 508.99 \\ 10.483 & 523.69 \\ 10.483 & 532.27 \\ 10.483 & 532.27 \\ 49.699 & 902.08 \\ 49.699 & 902.08 \\ 48.554 & 1670.35 \\ 14.322 & 1144.85 \\ 8.400 & 1018.64 \\ 8.400 & 1018.71 \\ 8.400 & 1018.78 \\ 9.826 & 508.99 \\ 9.826 & 510.29 \\ 9.826 & 510.29 \\ 9.826 & 510.29\end{array}$

$\begin{array}{rr}\text { ht } & \text { FAR } \\ -13.05 & 0.0000\end{array}$

FS14 Fan.Fl_O

LPC.FI-O

FS25 Bleed2.F1_-

FS3 HPC.FI_O

FS4 Bleed3.F1_O

FS45 HPT.FI_O

FS49 LPT.FI-O

$\begin{array}{ll}\text { FS5 } & \text { TEGV.FI_O } \\ \text { FS8 } & \text { Core Nozz.FI }\end{array}$

FS17 FanDuctLkg.FI_O

FS171 Bleed15.FI_O

FS172 FanDuct.Fl_O

357.98

TURBOMACHINERY PERFORMANCE DATA

$\begin{array}{lrrrrrrrrr} & \text { WC } & \text { PR } & \text { eff } & \text { NC } & \text { TR } & \text { efPoly } & \text { pwr } & \text { SMN } & \text { SMW } \\ \text { Fan } & 522.90 & 1.052 & 0.9000 & 1622.380 & 1.0163 & 0.9007 & -938.7 & -1530.50 & 44.70 \\ \text { LPC } & 76.37 & 1.123 & 0.7362 & 1622.380 & 1.0457 & 0.7405 & -383.4 & 8.18 & 1.95 \\ \text { HPC } & 42.34 & 4.741 & 0.7956 & 8545.229 & 1.6948 & 0.8340 & -3709.9 & 68.50 & 63.29 \\ \text { HPT } & 12.08 & 3.390 & 0.8682 & 211.807 & 1.3030 & 0.8485 & 3709.9 & & \\ \text { LPT } & 45.30 & 1.705 & 0.8029 & 47.116 & 1.1204 & 0.7907 & 1322.2 & \end{array}$

TURBOMACHINERY MAP DATA

$\begin{array}{lrrrrrrrrr} & \text { WcMap } & \text { PRmap } & \text { effMap } & \text { NcMap } & \text { R/Parm } & \text { S_WcDes } & \text { S_PRdes } & \text { S_effDes } & \text { S_NcDes } \\ \text { Fan } & 607.56 & 1.051 & 0.9018 & 1606.910 & 0.0442 & 0.8607 & 1.0235 & 0.9980 & 0.9905 \\ \text { LPC } & 40.07 & 1.188 & 0.7484 & 0.429 & 0.0000 & 1.9057 & 0.6541 & 0.9837 & 0.0003 \\ \text { HPC } & 38.51 & 4.565 & 0.8038 & 8317.419 & 10.7439 & 1.0995 & 1.0494 & 0.9898 & 0.9733 \\ \text { HPT } & 0.96 & 3.324 & 0.8682 & 1.273 & 3.3240 & 12.6299 & 0.9723 & 1.0000 & 0.0003 \\ \text { LPT } & 0.69 & 1.530 & 0.8089 & 0.530 & 1.5303 & 65.2590 & 0.7521 & 0.9926 & 0.0005\end{array}$

\begin{tabular}{|c|c|}
\hline & \\
\hline 8.14 & 481. \\
\hline 8.93 & 494. \\
\hline 8.90 & 494 \\
\hline 9.17 & 498 \\
\hline 9.03 & \\
\hline 9.84 & 514. \\
\hline 9.82 & 522.5 \\
\hline 10.25 & 528. \\
\hline 44.08 & 872. \\
\hline 46.46 & 885.3 \\
\hline 45.84 & 1646. \\
\hline 13.42 & 1125. \\
\hline 8.27 & 1014. \\
\hline 8.27 & 1014.5 \\
\hline 8.14 & 1010 \\
\hline 9.03 & 496. \\
\hline 8.82 & 494. \\
\hline 8.82 & 494. \\
\hline 8.14 & 483.7 \\
\hline & SMW \\
\hline & 4.70 \\
\hline 8 & 1.95 \\
\hline & 3.29 \\
\hline fDes & S_NCDes \\
\hline 9980 & $\overline{0} .9905$ \\
\hline 9837 & 0.0003 \\
\hline 9898 & 0.9733 \\
\hline 0000 & 0.0003 \\
\hline 9926 & 0.0005 \\
\hline
\end{tabular}

$\begin{array}{llll}0.69 & 1.530 & 0.8089\end{array}$
$0.530 \quad 1.5303$

$\begin{array}{lll}65.2590 & 0.7521 & 0.9926\end{array}$

$\begin{array}{rrr}\text { Aphy } & \text { MN } & \text { gamt } \\ 2546.1 & 0.4460 & 1.40040 \\ 4168.2 & 0.2517 & 1.40040 \\ 3531.8 & 0.2600 & 1.40040 \\ 830.5 & 0.1574 & 1.40040 \\ 2606.7 & 0.3483 & 1.40030 \\ 412.6 & 0.2999 & 1.40010 \\ 412.6 & 0.3056 & 1.39912 \\ 412.6 & 0.1767 & 1.39912 \\ 49.7 & 0.4191 & 1.38547 \\ 49.3 & 0.3131 & 1.38547 \\ 74.6 & 0.2948 & 1.33250 \\ 265.4 & 0.3088 & 1.36464 \\ 860.2 & 0.1481 & 1.37285 \\ 860.2 & 0.1481 & 1.37284 \\ 613.3 & 0.2105 & 1.37284 \\ 2606.7 & 0.3483 & 1.40030 \\ 2481.9 & 0.3959 & 1.40023 \\ 2481.9 & 0.3959 & 1.40023 \\ 2006.9 & 0.5240 & 1.40023\end{array}$

WAR
0.0004484
0.0004484
0.0004484
0.0004484
0.0004484
0.0004484
0.0067224
0.0067224
0.0067224
0.0067224
0.0067224
0.0067224
0.0067224
0.0067224
0.0067224
0.0004484
0.0007946
0.0007946
0.0007946

\section{Wair} 387.31 337.95 49.36 49.36
49.36 49.36
49.36 $\begin{array}{ll}49.36 & 0.332\end{array}$ $\begin{array}{ll}28.18 & 0.189\end{array}$ $\begin{array}{ll}21.84 & 0.147\end{array}$ $\begin{array}{ll}21.84 & 0.147 \\ 29.27 & 0.197\end{array}$ $\begin{array}{ll}29.61 & 0.199\end{array}$ $29.61 \quad 0.199$ $\begin{array}{ll}29.61 & 0.199\end{array}$ $\begin{array}{ll}357.70 & 0.284 \\ 357.70 & 0.284\end{array}$ $\begin{array}{ll}357.70 & 0.284\end{array}$

\begin{tabular}{lr} 
H2O frac \\
4 & 0.0004 \\
4 & 0.0004 \\
2 & 0.0004 \\
2 & 0.0004 \\
2 & 0.0004 \\
2 & 0.0004 \\
2 & 0.0067 \\
9 & 0.0067 \\
9 & 0.0067 \\
7 & 0.0067 \\
7 & 0.0204 \\
7 & 0.0170 \\
9 & 0.0169 \\
9 & 0.0169 \\
9 & 0.0169 \\
52 & 0.0004 \\
\hline 8 & 0.0008 \\
4 & 0.0008 \\
4 & 0.0008
\end{tabular}




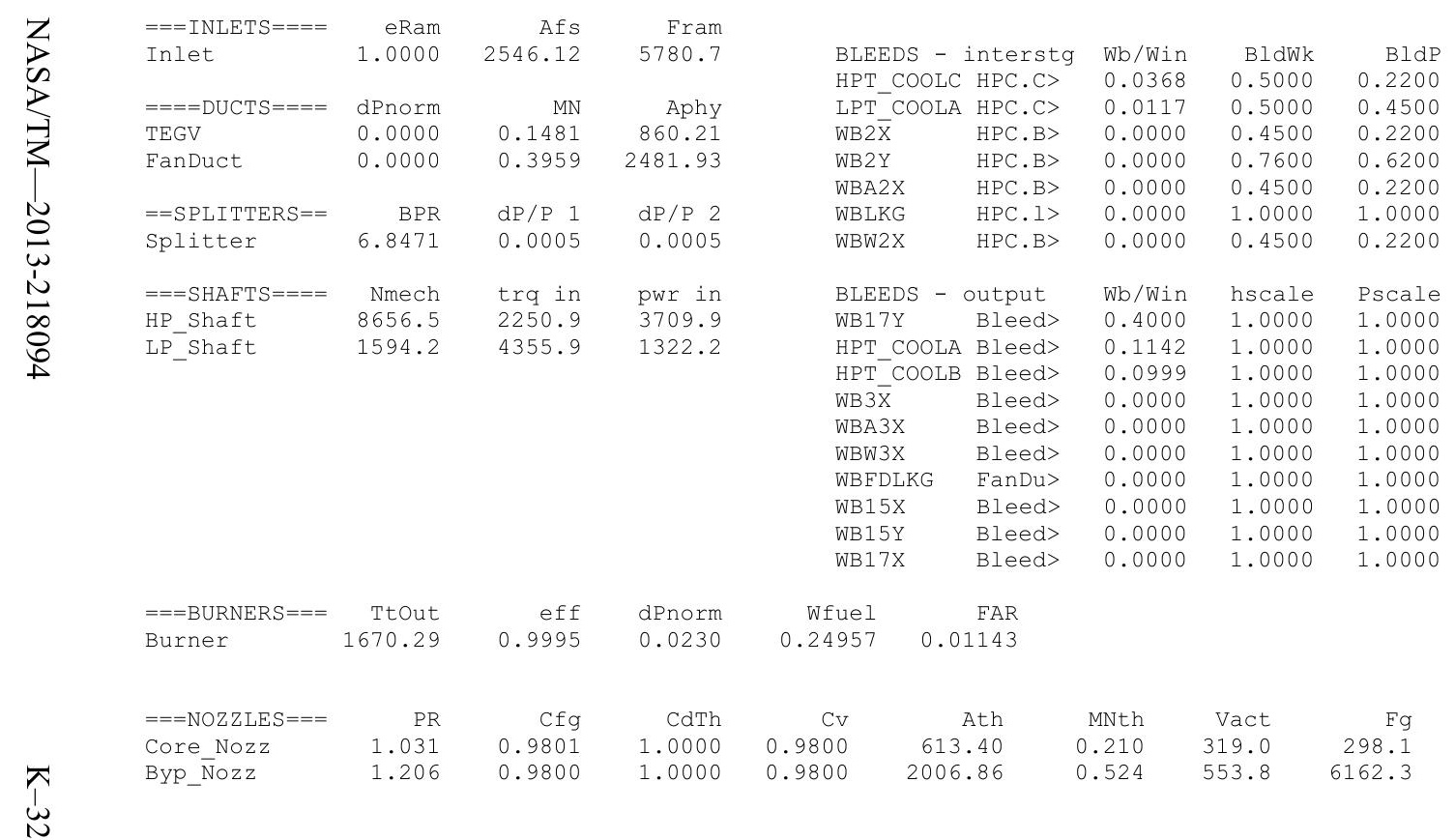


Date: $04 / 04 / 13$ Time: $08: 33: 20$ Model:

Turbofan Engine - COMDES ON Converge $=1$ CASE: 0

Version:NPSS 1.6.5 - Rev: -> Gas Package: Janaf iter/pass/Jacb/Broy=12/ 26/1/10 Run by: Philip C Jorgenson PC: 10

Temperature Stator 1 inlet: 473.74 Stator 1 exit: 481.44 Stator 2 inlet: 491.10 Stator 2 exit: 496.08 $\begin{array}{lll}\text { Stator } 3 \text { inlet: } 506.00 & \text { Stator } 3 \text { exit: } 510.52 & \text { Stator } 4 \text { inlet: } 519.75 \text { Stator } 4 \text { exit: } 523.58 \\ \text { Stator } 5 \text { inlet: } 531.12 & \text { Stator } 5 \text { exit: } 534.43 & \text { Unblocked Percent Blockage: } 0.00\end{array}$

Ambient Relative Humidity $\quad 10.00$

Fan Face Relative Humidity $\quad 1.87$

Fan Bypass Relative Humidity $\quad 1.31$

LPC Inlet Relative Humidity $\quad 1.21$

LPC Exit Relative Humidity $\quad 0.11$

HPC Relative Humidity $\quad 0.04$

0.0000050 Inlet Length

Ambient Flow Velocity

781.11

$\quad 40.00$
188.09

Ambient Static Pressure

Additional Water at LPC Exit

Fan/LPC Inlet Static Temperature 464.88

\section{SUMMARY OUTPUT DATA}

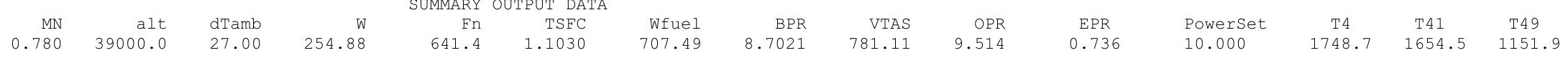

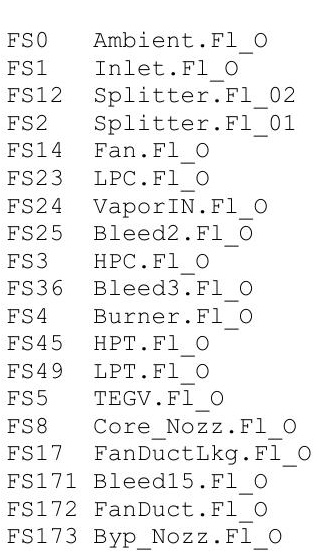

W
254.88
254.88
228.61
26.27
228.61
26.27
26.44
23.80
22.64
17.55
17.74
23.72
23.99
23.99
23.99
228.61
231.25
231.25
231.25

TURBOMACHINERY PERFORMANCE DATA

$\begin{array}{lrrr} & \text { WC } & \text { PR } & \text { eff } \\ \text { Fan } & 748.17 & 1.131 & 0.9059 \\ \text { LPC } & 85.98 & 1.566 & 0.8346 \\ \text { HPC } & 54.08 & 6.078 & 0.8182 \\ \text { HPT } & 12.07 & 4.167 & 0.8768 \\ \text { LPT } & 54.67 & 2.917 & 0.8555\end{array}$

FLOW STATION DATA

\begin{tabular}{rrrr}
\multicolumn{1}{c}{ Pt } & Tt & ht & FAR \\
4.267 & 467.83 & -18.66 & 0.0000 \\
4.267 & 467.83 & -18.66 & 0.0000 \\
4.265 & 467.83 & -18.66 & 0.0000 \\
4.265 & 467.83 & -18.66 & 0.0000 \\
4.825 & 486.40 & -14.21 & 0.0000 \\
6.679 & 544.53 & -0.27 & 0.0000 \\
6.679 & 553.21 & -35.52 & 0.0000 \\
6.679 & 553.21 & -35.52 & 0.0000 \\
40.595 & 1000.91 & 74.13 & 0.0000 \\
40.595 & 1000.91 & 74.13 & 0.0000 \\
39.660 & 1748.67 & 61.66 & 0.0113 \\
9.517 & 1156.08 & -44.59 & 0.0084 \\
3.263 & 905.69 & -106.38 & 0.0083 \\
3.263 & 905.76 & -106.38 & 0.0083 \\
3.263 & 905.84 & -106.38 & 0.0083 \\
4.825 & 486.40 & -14.21 & 0.0000 \\
4.825 & 487.16 & -14.45 & 0.0000 \\
4.825 & 487.16 & -14.45 & 0.0000 \\
4.825 & 487.16 & -14.45 & 0.0000
\end{tabular}

\section{Wc
833.73}

833.73
833.73

833.73
748.17

85.98
674.27

59.23

59.23
60.09

54.08

11.39
8.82

8.82

12.07
54.67

142.79 142.80
142.80 142.80 682.6

Ps
2.854
3.892
3.838
4.171
4.048
6.392
6.382
6.440
35.687
37.780
37.445
8.617
3.071
3.071
2.854
4.048
3.914
3.914
2.854

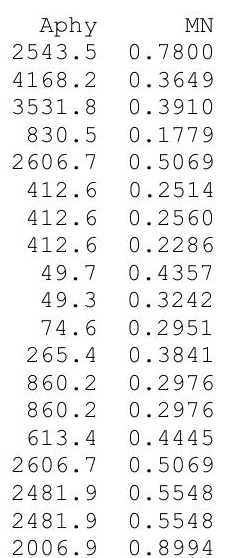

gamt
1.40077
1.40077
1.40077
1.40077
1.40061
1.39985
1.39881
1.39881
1.37964
1.37964
1.32894
1.36401
1.37987
1.37987
1.37986
1.40061
1.40059
1.40059
1.40059

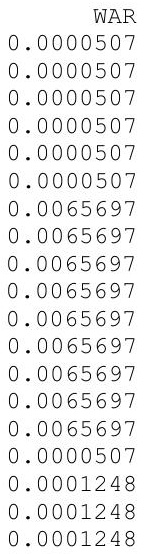

$\begin{array}{rrr}\text { Wair } & \text { WH2O } & \text { H2O frac } \\ 254.86 & 0.013 & 0.0001 \\ 254.86 & 0.013 & 0.0001 \\ 228.59 & 0.012 & 0.0001 \\ 26.27 & 0.001 & 0.0001 \\ 228.59 & 0.012 & 0.0001 \\ 26.27 & 0.001 & 0.0001 \\ 26.27 & 0.173 & 0.0065 \\ 23.64 & 0.155 & 0.0065 \\ 22.50 & 0.148 & 0.0065 \\ 17.43 & 0.115 & 0.0065 \\ 17.43 & 0.115 & 0.0201 \\ 23.37 & 0.154 & 0.0167 \\ 23.64 & 0.155 & 0.0166 \\ 23.64 & 0.155 & 0.0166 \\ 23.64 & 0.155 & 0.0166 \\ 228.59 & 0.012 & 0.0001 \\ 231.22 & 0.029 & 0.0001 \\ 231.22 & 0.029 & 0.0001 \\ 231.22 & 0.029 & 0.0001\end{array}$

TURBOMACHINERY MAP DATA

$\begin{array}{lrrrrrrrrr} & \text { WcMap } & \text { PRmap } & \text { effMap } & \text { NcMap } & \text { R/Parm } & \text { S_WcDes } & \text { S_PRdes } & \text { S_effDes } & \text { S_NcDes } \\ \text { Fan } & 869.30 & 1.128 & 0.9078 & 2553.508 & 0.0415 & 0.8607 & 1.0235 & 0.9980 & 0.9905 \\ \text { LPC } & 74.33 & 1.526 & 0.7804 & 0.681 & 0.0000 & 1.1567 & 1.0754 & 1.0696 & 0.0003 \\ \text { HPC } & 49.18 & 5.839 & 0.8267 & 8721.315 & 10.9601 & 1.0995 & 1.0494 & 0.9898 & 0.9733 \\ \text { HPT } & 0.96 & 4.079 & 0.8767 & 1.330 & 4.0795 & 12.6299 & 0.9723 & 1.0000 & 0.0003 \\ \text { LPT } & 0.84 & 2.441 & 0.8618 & 0.810 & 2.4415 & 65.2590 & 0.7521 & 0.9926 & 0.0005\end{array}$




\begin{tabular}{|c|c|c|c|c|c|c|c|c|}
\hline $\begin{array}{l}===\text { INLETS }==== \\
\text { Inlet }\end{array}$ & $\begin{array}{r}\text { eRam } \\
1.0000\end{array}$ & $\begin{array}{r}\text { Afs } \\
2543.49\end{array}$ & $\begin{array}{r}\text { Fram } \\
6187.8\end{array}$ & & $\mathrm{~Wb} / \mathrm{Win}$ & & B]dP $P$ C \\
\hline Inlet & & & & \multicolumn{2}{|c|}{$\begin{array}{l}\text { BLEEDS - interstg } \\
\text { HPT COOLC HPC.C> }\end{array}$} & 0.0368 & 0.5000 & 0.2200 \\
\hline$====$ DUCTS $====$ & dPnorm & MN & Aphy & \multirow{2}{*}{$\mathrm{LPT}^{-} \mathrm{COOL}$} & A HPC.C> & 0.0117 & 0.5000 & 0.4500 \\
\hline TEGV & 0.0000 & 0.2976 & 860.21 & & HPC.B> & 0.0000 & 0.4500 & 0.2200 \\
\hline FanDuct & 0.0000 & 0.5548 & 2481.93 & WB2Y & HPC. B> & 0.0000 & 0.7600 & 0.6200 \\
\hline & & & & \multirow{2}{*}{ WBA2X } & HPC. B> & 0.0000 & 0.4500 & 0.2200 \\
\hline$==$ SPLITTERS $==$ & BPR & $\mathrm{dP} / \mathrm{P} 1$ & $\mathrm{dP} / \mathrm{P} 2$ & & HPC.I> & 0.0000 & 1.0000 & 1.0000 \\
\hline Splitter & 8.7021 & 0.0005 & 0.0005 & $\begin{array}{l}\text { WBDKG } \\
\text { WBW2X }\end{array}$ & $\mathrm{HPC} . \mathrm{B}>$ & 0.0000 & 0.4500 & 0.2200 \\
\hline$===$ SHAFT $S===$ & Nmech & trq in & pwr in & \multicolumn{2}{|c|}{ BLFEDS - output } & Wb/Win & hscale & Pscale \\
\hline HP_Shaft & 9253.8 & 2044.7 & 3602.5 & WB17Y & Bleed> & 0.1000 & 1.0000 & 1.0000 \\
\hline LP_Shaft & 2448.5 & 4553.5 & 2122.8 & HPT COOLA & A Bleed> & 0.1142 & 1.0000 & 1.0000 \\
\hline & & & & \multirow{2}{*}{$\begin{array}{l}\mathrm{HPT}^{-} \text {COOLB } \\
\text { WB } 3 \overline{\mathrm{X}}\end{array}$} & B Bleed> & 0.0999 & 1.0000 & 1.0000 \\
\hline & & & & & Bleed> & 0.0000 & 1.0000 & 1.0000 \\
\hline & & & & $\begin{array}{l}\text { WB } 3 \bar{X} \\
\text { WBA3X }\end{array}$ & Bleed> & 0.0000 & 1.0000 & 1.0000 \\
\hline & & & & WBW3X & Bleed> & 0.0000 & 1.0000 & 1.0000 \\
\hline & & & & WBFDLKG & FanDu> & 0.0000 & 1.0000 & 1.0000 \\
\hline & & & & WB15X & Bleed> & 0.0000 & 1.0000 & 1.0000 \\
\hline & & & & $\begin{array}{l}\text { WB15Y } \\
\text { WB17X }\end{array}$ & Bleed> & 0.0000 & 1.0000 & 1.0000 \\
\hline & & & & WB17X & Bleed> & 0.0000 & 1.0000 & 1.0000 \\
\hline$===$ BURNERS $===$ & Ttout & eff & dPnorm & & FAR & & & \\
\hline Burner & 1748.62 & 0.9995 & 0.0230 & $\begin{array}{l}\text { Wfuel } \\
.19653\end{array}$ & 01127 & & & \\
\hline$===$ NOZZLES $===$ & PR & $\mathrm{Cfg}$ & CdTh & & & MNth & Vact & $\mathrm{Fg}$ \\
\hline Core_Nozz & 1.143 & 0.9801 & 1.0000 & $\begin{array}{r}\mathrm{Cv} \\
0.9800\end{array}$ & $\begin{array}{r}\text { Ath } \\
613.40\end{array}$ & 0.445 & \multirow{2}{*}{$\begin{array}{l}628.0 \\
885.0\end{array}$} & 468.4 \\
\hline Byp_Nozz & 1.691 & 0.9800 & 1.0000 & \multicolumn{2}{|c|}{2006.86} & 0.899 & & 6360.8 \\
\hline
\end{tabular}


Version:NPSS 1.6.5 - Rev: -> Gas Package: Janaf iter/pass/Jacb/Broy=11/ 39/2/8 Run by: Philip C Jorgenson PC: 10

Temperature Stator 1 inlet: $467.07 \quad$ Stator 1 exit: $474.28 \quad$ Stator 2 inlet: 483.32 Stator 2 exit: 487.97 $\begin{array}{rrrr}\text { Stator } 3 \text { inlet: } 497.25 & \text { Stator } 3 \text { exit: } 501.46 & \text { Stator } 4 \text { inlet: } 510.10 \quad \text { Stator } 4 \text { exit: } 513.67 \\ \text { Stator } 5 \text { inlet: } 520.74 & \text { Stator } 5 \text { exit: } 523.81 & \text { Unblocked Percent Blockage: } 0.00\end{array}$

$\begin{array}{lclll}\text { Ambient Relative Humidity } & 10.00 & & & \\ \text { Fan Face Relative Humidity } & 2.35 & & & \\ \text { Fan Bypass Relative Humidity } & 1.69 & & & \\ \text { LPC Inlet Relative Humidity } & 1.54 & & & \\ \text { LPC Exit Relative Humidity } & 0.15 & & & \\ \text { HPC Relative Humidity } & 0.05 & & & \\ \text { Drop Diameter } & 0.0000050 & \text { Inlet Length } & \\ \text { Ambient Flow Velocity } & 731.04 & \text { Fan/LPC Inlet Flow Velocity } & 181.64 \\ \text { Ambient Static Pressure } & 2.95 & \text { Fan/LPC Inlet Static Pressure } & 4.11 \\ \text { Ambient Static Temperature } & 416.97 & \text { Fan/LPC Inlet Static Temperature } & 458.77 \\ \text { Additional Water at LPC Exit } & 0.0058255 & & & \end{array}$

\begin{tabular}{|c|c|c|c|c|c|c|c|c|c|c|c|c|c|c|}
\hline & & & & JMMARY & TPUT DAT & & & & & & & & & \\
\hline $\begin{array}{r}\mathrm{MN} \\
730\end{array}$ & alt & dTamb & W & $\mathrm{Fn}$ & TSFC & Wfuel & $\begin{array}{r}B P R \\
\quad-7955\end{array}$ & VTAS & OPR & EPR & Powerset & T4 & T41 & $\begin{array}{c}\text { T49 } \\
1134.3\end{array}$ \\
\hline
\end{tabular}

\begin{tabular}{|c|c|c|c|c|c|c|c|c|c|c|c|}
\hline & & & & FLOW & STATION DA? & & & & & & \\
\hline & & & W & Pt & Tt & ht & FAR & & Wc & Ps & \\
\hline FSO & Ambient.Fl_O & & 248.00 & 4.201 & 461.52 & -20.16 & 0.0000 & 818.3 & 2.9 & 47 & 416. \\
\hline FS1 & Inlet.FI_O & & 248.00 & 4.201 & 461.52 & -20.16 & 0.0000 & 818.3 & 3.8 & 47 & \\
\hline FS12 & Splitter.Fl_o & & 222.66 & 4.199 & 461.52 & -20.16 & 0.0000 & 735. & 3.7 & & 448. \\
\hline FS2 & Splitter.Fl-0 & & 25.34 & 4.199 & 461.52 & -20.16 & 0.0000 & 83. & 4.1 & 12 & 458. \\
\hline FS14 & Fan.Fl_O & & 222.66 & 4.718 & 478.81 & -16.02 & 0.0000 & 666.3 & 3.9 & & 456. \\
\hline FS23 & LPC.FI_O & & 25.34 & 6.437 & 533.31 & -2.95 & 0.0000 & 58. & 6.1 & 66 & 526. \\
\hline FS24 & VaporIN.FI_O & & 25.49 & 6.437 & 541.20 & -34.47 & 0.0000 & 59.2. & 6.1 & 57 & 534. \\
\hline FS25 & Bleed2.Fl_o & & 22.94 & 6.437 & 541.20 & -34.47 & 0.0000 & 53.5 & 6.2 & & 535. \\
\hline FS3 & HPC.FI_O & & 21.83 & 38.796 & 977.79 & 72.25 & 0.0000 & 11.3 & 34.1 & & 943. \\
\hline FS36 & Bleed3.Fl_o & & 16.92 & 38.796 & 977.79 & 72.25 & 0.0000 & $8 . \varepsilon$ & 36.1 & & 958. \\
\hline FS4 & Burner.Fl_o & & 17.11 & 37.903 & 1720.20 & 59.95 & 0.0111 & 12. & 35.7 & & 1695 \\
\hline FS45 & HPT.FI_O & & 22.86 & 9.210 & 1138.44 & -43.42 & 0.0083 & 54. & 8.3 & & 1109. \\
\hline FS 49 & LPT.FI_O & & 23.13 & 3.313 & 902.50 & -101.44 & 0.0082 & 135.3 & 3.1 & & 889. \\
\hline FS5 & TEGV.Fİ_O & & 23.13 & 3.313 & 902.57 & -101.44 & 0.0082 & 135.3 & 3.1 & 39 & 889. \\
\hline FS8 & Core_Nozz.FI & & 23.13 & 3.313 & 902.64 & -101.44 & 0.0082 & 135.3 & 2.9 & 47 & 873. \\
\hline FS17 & FanDuctLkg. Fi & & 222.66 & 4.718 & 478.81 & -16.02 & 0.0000 & 666.3 & 3.9 & 81 & 456. \\
\hline FS171 & Bleed15.Fl_o & & 225.21 & 4.718 & 479.52 & -16.23 & 0.0000 & 674.2 & 3.8 & 56 & 452 \\
\hline FS172 & FanDuct.Fl_o & & 225.21 & 4.718 & 479.52 & -16.23 & 0.0000 & 674.2 & 3.8 & 56 & 452. \\
\hline FS173 & Byp_Nozz.Fī_o & & 225.21 & 4.718 & 479.52 & -16.23 & 0.0000 & 674.2 & 2.9 & 47 & 419. \\
\hline TURBO & MACHINERY PERE & ORMANCE & DATA & & & & & & & & \\
\hline & & PR & eff & NC & TR & efPoly & & pwr & SMN & & SMW \\
\hline Fan & 735.05 & 1.124 & 0.9051 & 2513.662 & 1.0375 & 0.9067 & -13 & 305.3 & 2076.94 & & .57 \\
\hline LPC & 83.67 & 1.533 & 0.8353 & 2513.662 & 1.1555 & 0.8449 & & 617.2 & 6.81 & & .37 \\
\hline HPC & 53.50 & 6.027 & 0.8175 & 8941.663 & 1.8067 & 0.8562 & -33 & 380.0 & 61.49 & & .09 \\
\hline HPT & 12.08 & 4.116 & 0.8754 & 220.223 & 1.3611 & 0.8534 & & 380.0 & & & \\
\hline LPT & 54.05 & 2.780 & 0.8497 & 70.275 & 1.2595 & 0.8304 & & 922.5 & & & \\
\hline TURBOM & MACHINERY MAP & DAT & & & & & & & & & \\
\hline & WcMap & PRmap & effMap & NcMap & R/Parm & s WCI & cDes & s PRdes & $s$ effDes & & S NcDes \\
\hline Fan & 854.05 & 1.121 & 0.9070 & 2489.695 & 0.0414 & $\overline{0} .8$ & 8607 & $\overline{1} .0235$ & 0.9980 & & 0.9905 \\
\hline LPC & 71.54 & 1.498 & 0.7773 & 0.664 & 0.0000 & & 1694 & 1.0709 & 1.0745 & & 0.0003 \\
\hline HPC & 48.66 & 5.790 & 0.8260 & 8703.284 & 10.9750 & 1.0 & 0995 & 1.0494 & 0.9898 & & 0.9733 \\
\hline HPT & 0.96 & 4.029 & 0.8754 & 1.324 & 4.0293 & 12.62 & 6299 & 0.9723 & 1.0000 & & 0.0003 \\
\hline LPT & 0.83 & 2.339 & 0.8561 & 0.791 & 2.3387 & 65.25 & 2590 & 0.7521 & 0.9926 & & 0.0005 \\
\hline
\end{tabular}




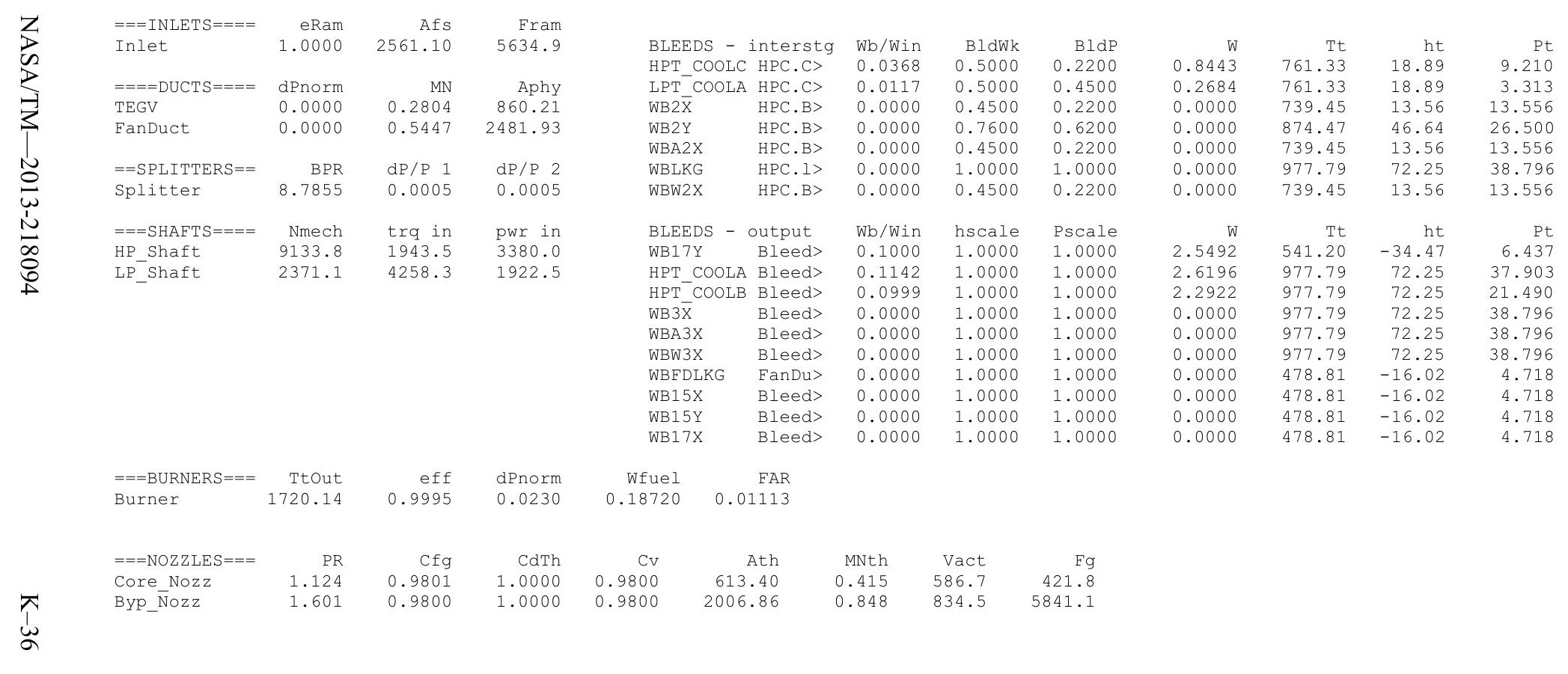


Date:04/04/13 Time:08:34:09 Model: Turbofan Engine - COMDES ON Converge = 1 CASE: 0

Version:NPSS 1.6.5 - Rev: -> Gas Package: Janaf iter/pass/Jacb/Broy= 11/ 39/ 2/ 8 Run by: Philip C Jorgenson PC: 10

Temperature Stator 1 inlet: 465.00 Stator 1 exit: $472.02 \quad$ Stator 2 inlet: 480.81 Stator 2 exit: 485.33

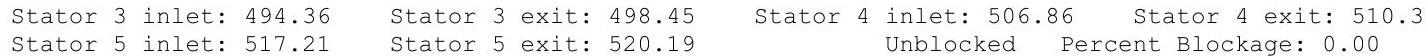

$\begin{array}{lclll}\text { Ambient Relative Humidity } & 10.00 & & & \\ \text { Fan Face Relative Humidity } & 2.52 & & & \\ \text { Fan Bypass Relative Humidity } & 1.84 & & & \\ \text { LPC Inlet Relative Humidity } & 1.66 & & & \\ \text { LPC Exit Relative Humidity } & 0.16 & & & \\ \text { HPC Relative Humidity } & 0.05 & & & \\ \text { Drop Diameter } & 0.0000050 & \text { Inlet Length } & \\ \text { Ambient Flow Velocity } & 715.01 & \text { Fan/LPC Inlet Flow Velocity } & 179.15 \\ \text { Ambient Static Pressure } & 3.09 & \text { Fan/LPC Inlet Static Pressure } & 4.25 \\ \text { Ambient Static Temperature } & 416.97 & \text { Fan/LPC Inlet Static Temperature } & 456.92 \\ \text { Additional Water at LPC Exit } & 0.0052577 & & & \end{array}$

$\begin{array}{rrrrrr} & & & & \text { SUMMARY OUTPUT DATA } \\ \text { MN } & \text { alt } & \text { dTamb } & \text { W } & \text { Fn } & \text { TSFC } \\ 0.714 & 37357.0 & 27.00 & 254.64 & 640.0 & 1.0699\end{array}$

FLOW STATION DATA

FSO Ambient.Fl_O

FS1 Inlet.F1_O

市

FS2 Splitter.

FS14 Fan.FI_O

FS23 LPC.FI_O

$\begin{array}{ll}\text { FS24 } & \text { VaporIN.F1_O } \\ \text { FS25 } & \text { Bleed2.Fl_O }\end{array}$

$\begin{array}{ll}\text { FS25 } & \text { Bleed2.FI_- } \\ \text { FS3 } & \text { HPC.Fl_O }\end{array}$

FS36 Bleed3.F1_O

FS4 Burner.F1_O

FS45 HPT.FI-

FS49 LPT.FI-O

FS8 Core_Nozz.FI_O

FS17 FanDuctLkg.Fi_o

FS171 Bleed15. F1 1 -

FS172 FanDuct.FI-O

FS173 Byp_Nozz.FI_O

\begin{tabular}{rrrrr}
\multicolumn{7}{c}{ FLOW } & STATION DATA & \\
W & Pt & Tt & ht & FAR \\
254.64 & 4.339 & 459.59 & -20.61 & 0.0000 \\
254.64 & 4.339 & 459.59 & -20.61 & 0.0000 \\
228.70 & 4.337 & 459.59 & -20.61 & 0.0000 \\
25.94 & 4.337 & 459.59 & -20.61 & 0.0000 \\
228.70 & 4.859 & 476.38 & -16.59 & 0.0000 \\
25.94 & 6.590 & 529.47 & -3.86 & 0.0000 \\
26.07 & 6.590 & 536.64 & -32.31 & 0.0000 \\
23.46 & 6.590 & 536.64 & -32.31 & 0.0000 \\
22.33 & 39.530 & 968.70 & 73.19 & 0.0000 \\
17.30 & 39.530 & 968.70 & 73.19 & 0.0000 \\
17.49 & 38.620 & 1707.93 & 60.95 & 0.0111 \\
23.38 & 9.425 & 1130.77 & -41.23 & 0.0082 \\
23.66 & 3.453 & 900.41 & -97.78 & 0.0081 \\
23.66 & 3.453 & 900.48 & -97.78 & 0.0081 \\
23.66 & 3.453 & 900.55 & -97.78 & 0.0081 \\
228.70 & 4.859 & 476.38 & -16.59 & 0.0000 \\
231.31 & 4.859 & 477.06 & -16.77 & 0.0000 \\
231.31 & 4.859 & 477.06 & -16.77 & 0.0000 \\
231.31 & 4.859 & 477.06 & -16.77 & 0.0000
\end{tabular}

$\begin{array}{rr}\text { Wfuel } & \text { BPR } \\ 684.75 & 8.8179\end{array}$

VTAS
715.01

OPR
9.110

EPR
0.772

Powerset

10.000

$\begin{array}{ccc}\text { T4 } & \text { T41 } & \text { T49 } \\ 1707.9 & 1614.8 & 1126.6\end{array}$

TURBOMACHINERY PERFORMANCE DATA

\begin{tabular}{|c|c|c|c|c|c|c|c|c|c|}
\hline \\
\hline & $\begin{array}{l}\text { WC } \\
729.46\end{array}$ & PR & eff & $\begin{array}{r}\mathrm{NC} \\
\end{array}$ & $\begin{array}{r}T R \\
T R\end{array}$ & efPoly & $\begin{array}{r}\text { pwr } \\
-13018\end{array}$ & $\begin{array}{r}\text { SMN } \\
2436.79\end{array}$ & SMW \\
\hline Fian & $\begin{array}{r}729.46 \\
82.72\end{array}$ & 1.120 & 0.9047 & 2486.297 & $\begin{array}{l}1.0365 \\
1.1520\end{array}$ & 0.9062 & $\begin{array}{r}-1301.8 \\
-614.8\end{array}$ & $\begin{array}{r}2436.79 \\
6.72\end{array}$ & $\begin{array}{r}54.51 \\
3.30\end{array}$ \\
\hline HPC & $\begin{array}{l}82.12 \\
53.23\end{array}$ & $\begin{array}{l}1.519 \\
5.999\end{array}$ & $\begin{array}{l}0.8351 \\
0.8171\end{array}$ & $\begin{array}{l}2486.291 \\
8932.753\end{array}$ & 1.8051 & 0.8558 & -3417.6 & 61.53 & $\begin{array}{r}3.30 \\
58.10\end{array}$ \\
\hline HPT & 12.08 & 4.097 & 0.8748 & 219.859 & 1.3600 & 0.8526 & 3417.6 & & \\
\hline LPT & 53.83 & 2.730 & 0.8474 & 69.599 & 1.2538 & 0.8282 & 1916.6 & & \\
\hline \multirow{2}{*}{\multicolumn{10}{|c|}{$\begin{array}{c}\text { TURBOMACHINERY MAP DATA } \\
\text { WCMaP PRmap }\end{array}$}} \\
\hline & & PRmap & effmap & & R/Parm & s_WcDes & S_PRdes & S_effDes & S_NcDes \\
\hline Fan & 847.56 & 1.118 & 0.9066 & 2462.591 & 0.0413 & $\overline{0} .8607$ & $\overline{1} .0235$ & 0.9980 & 0.9905 \\
\hline LPC & 70.36 & 1.485 & 0.7761 & 0.657 & 0.0000 & 1.1758 & 1.0699 & 1.0768 & 0.0003 \\
\hline HPC & 48.41 & 5.763 & 0.8255 & 8694.612 & 10.9759 & 1.0995 & 1.0494 & 0.9898 & 0.9733 \\
\hline HPT & 0.96 & 4.012 & 0.8748 & 1.321 & 4.0116 & 12.6299 & 0.9723 & 1.0000 & 0.0003 \\
\hline LPT & 0.82 & 2.301 & 0.8537 & 0.783 & 2.3010 & 65.2590 & 0.7521 & 0.9926 & 0.0005 \\
\hline
\end{tabular}

$\begin{array}{rrrrrrrr}\text { Ts } & \text { Aphy } & \text { MN } & \text { gamt } & \text { WAR } & \text { Wair } & \text { WH2O } & \text { H2O frac } \\ 416.97 & 2565.2 & 0.7140 & 1.40083 & 0.0000469 & 254.62 & 0.012 & 0.0000 \\ 448.35 & 4168.2 & 0.3536 & 1.40083 & 0.0000469 & 254.62 & 0.012 & 0.0000 \\ 446.72 & 3531.8 & 0.3792 & 1.40083 & 0.0000469 & 228.69 & 0.011 & 0.0000 \\ 456.92 & 830.5 & 0.1709 & 1.40083 & 0.0000469 & 25.93 & 0.001 & 0.0000 \\ 454.09 & 2606.7 & 0.4949 & 1.40070 & 0.0000469 & 228.69 & 0.011 & 0.0000 \\ 523.05 & 412.6 & 0.2478 & 1.40008 & 0.0000469 & 25.93 & 0.001 & 0.0000 \\ 529.94 & 412.6 & 0.2515 & 1.39925 & 0.0053046 & 25.93 & 0.138 & 0.0053 \\ 531.28 & 412.6 & 0.2246 & 1.39925 & 0.0053046 & 23.34 & 0.124 & 0.0053 \\ 935.12 & 49.7 & 0.4330 & 1.38180 & 0.0053046 & 22.21 & 0.118 & 0.0053 \\ 949.82 & 49.3 & 0.3224 & 1.38180 & 0.0053046 & 17.21 & 0.091 & 0.0053 \\ 1683.63 & 74.6 & 0.2948 & 1.33117 & 0.0053046 & 17.21 & 0.091 & 0.0186 \\ 1102.10 & 265.4 & 0.3765 & 1.36594 & 0.0053046 & 23.07 & 0.122 & 0.0153 \\ 887.70 & 860.2 & 0.2742 & 1.38046 & 0.0053046 & 23.34 & 0.124 & 0.0152 \\ 887.77 & 860.2 & 0.2742 & 1.38046 & 0.0053046 & 23.34 & 0.124 & 0.0152 \\ 873.26 & 613.4 & 0.4048 & 1.38045 & 0.0053046 & 23.34 & 0.124 & 0.0152 \\ 454.09 & 2606.7 & 0.4949 & 1.40070 & 0.0000469 & 228.69 & 0.011 & 0.0000 \\ 450.68 & 2481.9 & 0.5405 & 1.40068 & 0.0001058 & 231.28 & 0.024 & 0.0001 \\ 450.68 & 2481.9 & 0.5405 & 1.40068 & 0.0001058 & 231.28 & 0.024 & 0.0001 \\ 419.04 & 2006.9 & 0.8311 & 1.40068 & 0.0001058 & 231.28 & 0.024 & 0.0001\end{array}$




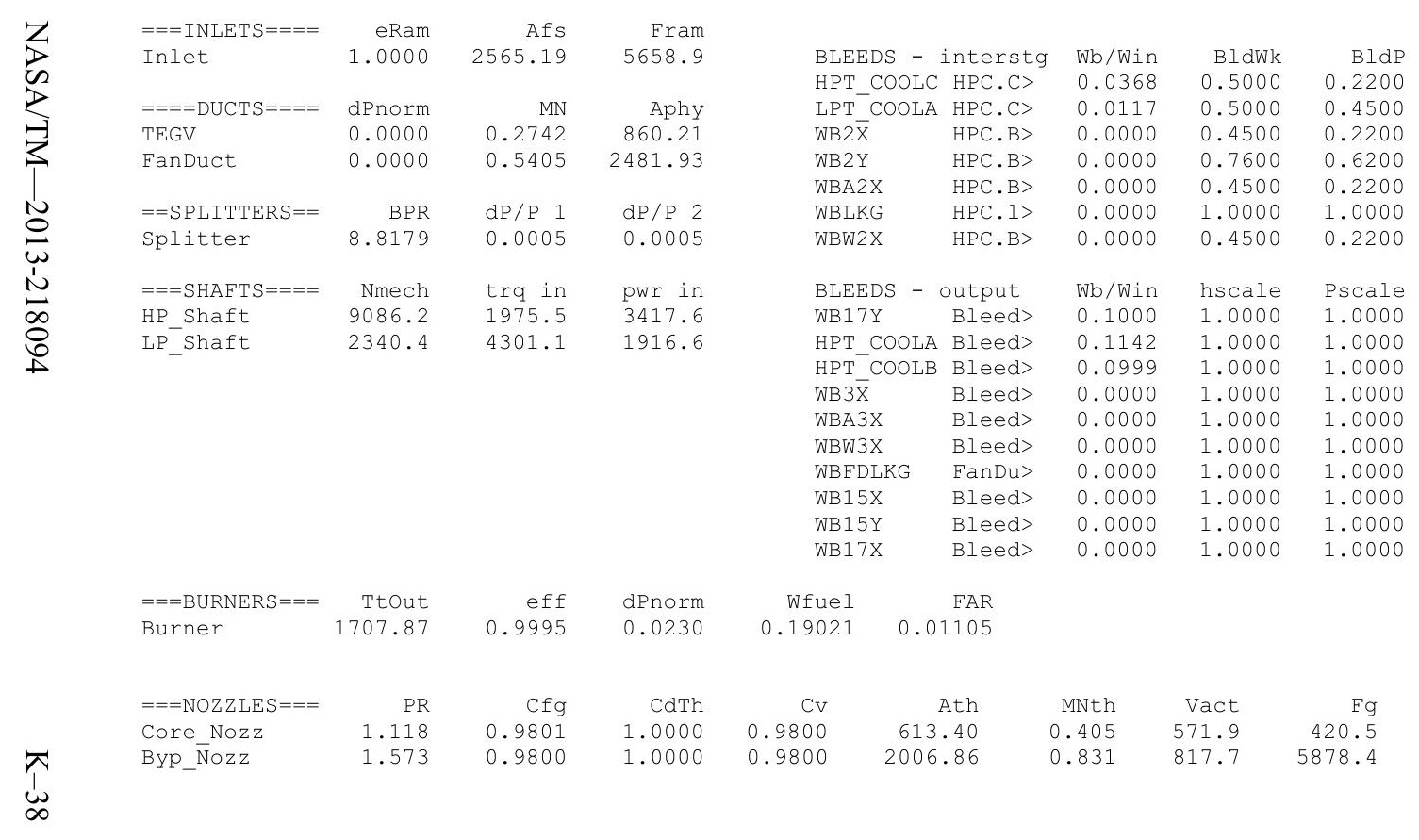


Turbofan Engine - COMDES ON converge $=1$ CASE: 0

Version:NPSS1.6.5 - Rev: -> Gas Package: Janaf iter/pass/Jacb/Broy=12/26/1/10 Run by: Philip C Jorgenson PC: 10

Temperature Stator 1 inlet: $466.50 \quad$ Stator 1 exit: $473.07 \quad$ Stator 2 inlet: 481.29 Stator 2 exit: 485.51 $\begin{array}{llll}\text { Stator } 3 \text { inlet: } 493.94 & \text { Stator } 3 \text { exit: } 497.73 & \text { Stator } 4 \text { inlet: } 505.60 \text { Stator } 4 \text { exit: } 508.79 \\ \text { Stator } 5 \text { inlet: } 515.22 & \text { Stator } 5 \text { exit: } 517.96 & & \text { Unblocked Percent Blockage: } 0.00\end{array}$

Ambient Relative Humidity $\quad 10.00$

Fan Face Relative Humidity $\quad 3.13$

Fan Bypass Relative Humidity $\quad 2.36$

$\begin{array}{ll}\text { LPC Inlet Relative Humidity } & 2.11 \\ \text { LPC Exit Relative Humidity } & 0.24\end{array}$

Drop Diameter

0.05

0.0000050

675.10

Inlet Length

40.00

Drme Flow Velocity

Ambient Static Tressure

3.58

Ean/LPC Inlet Flow Velocity $\quad 173.29$

Additional Water at LPC Exit 0.0047504

an/LPC Inlet Static Temperature 458.91

SUMMARY OUTPUT DATA

$\begin{array}{rrr}\text { MN } & \text { alt } & \text { dTamb } \\ 0.669 & 34281.0 & 27.00\end{array}$

275.81

$\begin{array}{rr}\text { Fn } & \text { TSFC } \\ 689.8 & 1.0611\end{array}$
Wfuel
731.95

8.9045

\section{FLOW STATION DATA}

FSO Ambient.Fl_O

FS1 Inlet.Fl_O

交

FS14 Splitter.Fl_0

$\begin{array}{rrrr}\text { Pt } & \text { Tt } & \text { ht } & \text { FAR } \\ 4.831 & 461.41 & -20.25 & 0.0000\end{array}$

$4.831 \quad 461.41$

$\begin{array}{ll}4.829 & 461.41\end{array}$

$\begin{array}{ll}4.829 & 461.41 \\ 5.368 & 477.12\end{array}$

$\begin{array}{ll}-20.25 & 0.0000\end{array}$

$\begin{array}{ll}-20.25 & 0.0000\end{array}$

FS14 Fan.F1_O

FS24 VaporIN.FI

FS25 Bleed2.F1 O

FS3 HPC.FIO

FS36 Bleed3.F1_O

FS4 Burner.FI_O

$\begin{array}{ll}\text { FS45 } & \text { HPT.F1 } \\ \text { FS49 } & \text { LPT.FI } \\ \text { ES5 } & \text { IEGV. }\end{array}$

FS5 5 LPT.FI-O

FS8 Core_Nozz.FI_O

FS17 FanDuctLkg.F̄

FS171 Bleed15.FI_O

FS173 FanDuct. Fl-O

$W$
275.81
275.81
247.96
27.85
247.96
27.85
27.98
25.18
23.96
18.57
18.77
25.09
25.38
25.38
25.38
247.96
250.76
250.76
250.76

7.146

7.146
7.146

$\begin{array}{rrrr}7.146 & 533.31 & -30.28 & 0.0000 \\ 42.285 & 533.31 & -30.28 & 0.0000 \\ 42.285 & 959.54 & 73.69 & 0.0000\end{array}$

$\begin{array}{lll}477.12 & -16.48 & 0.0000 \\ 526.80 & -4.57 & 0.0000\end{array}$

$\begin{array}{lll}533.31 & -30.28 & 0.0000\end{array}$

$\begin{array}{llll}42.285 & 959.54 & 73.69 & 0.0000\end{array}$

$\begin{array}{llll}41.311 & 1697.41 & 61.50 & 0.0110\end{array}$

$\begin{array}{rrrr}10.194 & 1125.74 & -39.17 & 0.0082 \\ 3.943 & 908.34 & -92.43 & 0.0081\end{array}$

3.943

908.34

5.368

5.368

908.49
477.12

$\begin{array}{ll}-92.43 & 0.0081 \\ -92.43 & 0.0081\end{array}$

$\begin{array}{llll}5.368 & 477.12 & -16.48 & 0.0000 \\ 5.368 & 477.75 & -16.64 & 0.00000\end{array}$

$\begin{array}{lll}477.75 & -16.64 & 0.0000\end{array}$

TURBOMACHINERY PERFORMANCE DATA

\begin{tabular}{lrrrrrrrrr}
\multicolumn{1}{c}{ W } & WR & PR & eff & NC & TR & efPoly & pwr & SMN & SMW \\
Fan & 711.80 & 1.112 & 0.9037 & 2405.476 & 1.0340 & 0.9052 & -1320.5 & 5030.32 & 54.10 \\
LPC & 79.94 & 1.480 & 0.8371 & 2405.476 & 1.1417 & 0.8459 & -617.7 & 6.44 & 3.08 \\
HPC & 52.51 & 5.917 & 0.8157 & 8909.132 & 1.7992 & 0.8544 & -3614.6 & 61.85 & 58.34 \\
HPT & 12.08 & 4.052 & 0.8741 & 219.273 & 1.3569 & 0.8520 & 3614.6 & & \\
LPT & 53.29 & 2.586 & 0.8423 & 67.621 & 1.2371 & 0.8238 & 1938.2 & &
\end{tabular}

TURBOMACHINERY MAP DATA

$\begin{array}{lrrrrrrrrr} & \text { WcMap } & \text { PRmap } & \text { effMap } & \text { NcMap } & \text { R/Parm } & \text { S_WcDes } & \text { S_PRdes } & \text { S_effDes } & \text { S_NcDes } \\ \text { Fan } & 827.04 & 1.109 & 0.9056 & 2382.540 & 0.0413 & 0.8607 & 1.0235 & 0.9980 & 0.9905 \\ \text { LPC } & 66.86 & 1.449 & 0.7723 & 0.635 & 0.0000 & 1.1957 & 1.0683 & 1.0840 & 0.0003 \\ \text { HPC } & 47.76 & 5.685 & 0.8241 & 8671.620 & 10.9653 & 1.0995 & 1.0494 & 0.9898 & 0.9733 \\ \text { HPT } & 0.96 & 3.968 & 0.8741 & 1.318 & 3.9678 & 12.6299 & 0.9723 & 1.0000 & 0.0003 \\ \text { LPT } & 0.82 & 2.193 & 0.8486 & 0.761 & 2.1925 & 65.2590 & 0.7521 & 0.9926 & 0.0005\end{array}$




\begin{tabular}{|c|c|c|c|c|c|c|c|c|}
\hline$===$ INLETS $====$ & eRam & Afs & Fram & \multirow{2}{*}{\multicolumn{2}{|c|}{ BLEEDS - intersta }} & & & \\
\hline Inlet & 1.0000 & 2579.39 & 5787.2 & & & Wb/Win & BldWk & BldP \\
\hline & & & & \multicolumn{2}{|c|}{ 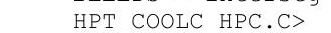 } & 0.0368 & 0.5000 & 0.2200 \\
\hline$====$ DUCTS $====$ & dPnorm & MN & Aphy & LPT_COOLA & A HPC.C> & 0.0117 & 0.5000 & 0.4500 \\
\hline TEGV & 0.0000 & 0.2574 & 860.21 & WB $2 \bar{x}$ & HPC.B> & 0.0000 & 0.4500 & 0.2200 \\
\hline FanDuct & 0.0000 & 0.5262 & 2481.93 & \multirow{2}{*}{$\begin{array}{l}\text { WB2Y } \\
\text { WBA2X }\end{array}$} & HPC.B> & 0.0000 & 0.7600 & 0.6200 \\
\hline & & & & & HPC.B> & 0.0000 & 0.4500 & 0.2200 \\
\hline$==$ SPLITTERS $==$ & BPR & $\mathrm{dP} / \mathrm{P} 1$ & $\mathrm{dP} / \mathrm{P} 2$ & WBLKG & HPC.1> & 0.0000 & 1.0000 & 1.0000 \\
\hline Splitter & 8.9045 & 0.0005 & 0.0005 & WBW2X & HPC.B> & 0.0000 & 0.4500 & 0.2200 \\
\hline$===$ SHAFTS $====$ & Nmech & trg in & pwr in & \multicolumn{2}{|c|}{ BLEEDS - output } & Wb/Win & hscale & Pscale \\
\hline HP_Shaft & 9034.0 & 2101.4 & 3614.6 & WB17Y & Bleed> & 0.1000 & 1.0000 & 1.0000 \\
\hline LP_Shaft & 2268.8 & 4486.7 & 1938.2 & HPT_COOLA & A Bleed> & 0.1142 & 1.0000 & 1.0000 \\
\hline & & & & \multirow{2}{*}{$\begin{array}{l}\mathrm{HPT}^{-} \mathrm{COOLB} \\
\text { WB } 3 \overline{\mathrm{X}}\end{array}$} & B Bleed> & 0.0999 & 1.0000 & 1.0000 \\
\hline & & & & & Bleed> & 0.0000 & 1.0000 & 1.0000 \\
\hline & & & & WBA3X & Bleed> & 0.0000 & 1.0000 & 1.0000 \\
\hline & & & & WBW3X & Bleed> & 0.0000 & 1.0000 & 1.0000 \\
\hline & & & & WBFDLKG & FanDu> & 0.0000 & 1.0000 & 1.0000 \\
\hline & & & & WB15X & Bleed> & 0.0000 & 1.0000 & 1.0000 \\
\hline & & & & \multirow{2}{*}{$\begin{array}{l}\text { WB15Y } \\
\text { WB17X }\end{array}$} & Bleed> & 0.0000 & 1.0000 & 1.0000 \\
\hline & & & & & Bleed> & 0.0000 & 1.0000 & 1.0000 \\
\hline$===$ BURNERS $===$ & Ttout & eff & dPnorm & & & & \\
\hline Burner & 1697.35 & 0.9995 & 0.0230 & \multicolumn{2}{|c|}{$\begin{array}{rr}\text { Wfuel } & \text { FAR } \\
0.20332 & 0.01100\end{array}$} & & & \\
\hline$===$ NOZZLES $===$ & PR & Cfg & CdTh & & & MNth & \multirow{2}{*}{$\begin{array}{r}\text { Vact } \\
536.4\end{array}$} & $\mathrm{Fg}$ \\
\hline Core_Nozz & 1.102 & 0.9801 & 1.0000 & 0.9800 & $\begin{array}{r}\text { Ath } \\
613.40\end{array}$ & \multirow{2}{*}{$\begin{array}{l}0.377 \\
0.784\end{array}$} & & 423.2 \\
\hline Byp_Nozozz & 1.500 & 0.9800 & 1.0000 & \multicolumn{2}{|c|}{2006.86} & & 776.7 & 6053.8 \\
\hline
\end{tabular}


\begin{tabular}{l} 
Model: \\
Time: 08:34:42 Turbofan Engine - COMDES ON COnverge $=1$ CASE: 0 \\
\hline
\end{tabular}

Temperature Stator 1 inlet: 474.95 Stator 1 exit: 480.63 Stator 2 inlet: 487.65 Stator 2 exit: 491.07 Stator 3 inlet: 498.37 Stator 3 exit: 501.38 Stator 4 inlet: 508.23 Stator 4 exit: 510.71 Stator 5 inlet: 516.21 Stator 5 exit: 518.29 Unblocked Percent Blockage: 0.00

Ambient Relative Humidity $\quad 10.00$

Fan Face Relative Humidity $\quad 4.08$

Fan Bypass Relative Humidity $\quad 3.22$

LPC Inlet Relative Humidity $\quad 2.94$

$\begin{array}{ll}\text { LPC Exit Relative Humidity } & 0.50 \\ \text { HPC Relative Humidity } & 0.06\end{array}$

Drop Diative Humidity

Drop Diameter

0.0000050

40.00
Inlet Length

Fan/LPC Inlet Flow Velocity $\quad 174.75$

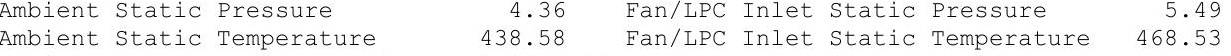

Additional Water at LPC Exit 0.004772

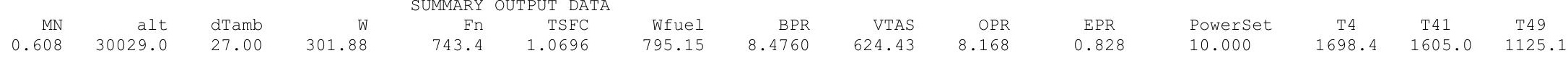

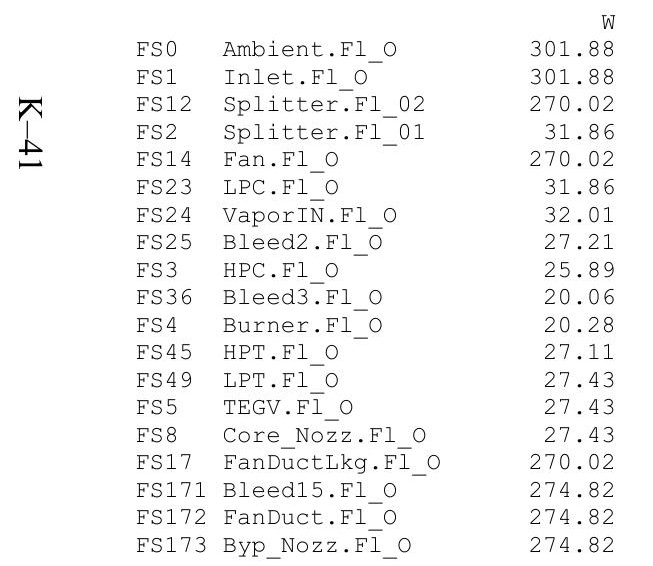

\section{FLOW STATION DATA}

$\begin{array}{rrrr}\text { Pt } & \text { Tt } & \text { ht } & \text { FAR } \\ 5.595 & 471.08 & -18.23 & 0.0000 \\ 5.595 & 471.08 & -18.23 & 0.0000 \\ 5.592 & 471.08 & -18.23 & 0.0000 \\ 5.592 & 471.08 & -18.23 & 0.0000 \\ 6.144 & 485.31 & -14.82 & 0.0000 \\ 7.848 & 527.32 & -4.74 & 0.0000 \\ 7.848 & 533.85 & -30.58 & 0.0000 \\ 7.848 & 533.85 & -30.58 & 0.0000 \\ 45.703 & 956.58 & 72.54 & 0.0000 \\ 45.703 & 956.58 & 72.54 & 0.0000 \\ 44.651 & 1698.45 & 60.30 & 0.0111 \\ 11.175 & 1129.30 & -39.50 & 0.0083 \\ 4.716 & 930.87 & -88.04 & 0.0082 \\ 4.716 & 930.94 & -88.04 & 0.0082 \\ 4.716 & 931.01 & -88.04 & 0.0082 \\ 6.144 & 485.31 & -14.82 & 0.0000 \\ 6.144 & 486.16 & -15.09 & 0.0000 \\ 6.144 & 486.16 & -15.09 & 0.0000 \\ 6.144 & 486.16 & -15.09 & 0.0000\end{array}$

755.65

755.65

755.65

776.24

79.78
624.79

60.15

60.81
51.69
11.30

51.69
11.30
8.76

8.76

12.08
52.61

114.51

114.51
114.52

114.52
624.79

624.79
636.46

636.46
636.46

636.46

Ps
4.358
5.199
5.146
5.487
5.324
7.499
7.490
7.593
40.280
42.588
42.156
10.207
4.542
4.542
4.358
5.324
5.176
5.176
4.358

Ts
438.58
461.28
460.00
468.53
465.82
520.51
526.79
528.84
923.66
938.05
1674.25
1102.15
921.32
921.39
911.04
465.82
462.90
462.90
440.68

$\begin{array}{rr}\text { Aphy } & \text { MN } \\ 2595.7 & 0.6080 \\ 4168.2 & 0.3255 \\ 3531.8 & 0.3467 \\ 830.5 & 0.1646 \\ 2606.7 & 0.4569 \\ 412.6 & 0.2556 \\ 412.6 & 0.2591 \\ 412.6 & 0.2177 \\ 49.7 & 0.4309 \\ 49.3 & 0.3210 \\ 74.6 & 0.2948 \\ 265.4 & 0.3663 \\ 860.2 & 0.2340 \\ 860.2 & 0.2340 \\ 613.5 & 0.3399 \\ 2606.7 & 0.4569 \\ 2481.9 & 0.5008 \\ 2481.9 & 0.5008 \\ 2006.9 & 0.7176\end{array}$

gamt
1.40073
1.40073
1.40073
1.40073
1.40061
1.40010
1.39935
1.39935
1.38259
1.38259
1.33167
1.36609
1.37871
1.37870
1.37870
1.40061
1.40059
1.40059
1.40059

WAR
0.0001104

Wair

WH2O H2O frac $\begin{array}{llll}0.0001104 & 301.84 & 0.033 & 0.0001\end{array}$ $\begin{array}{llll}0.0001104 & 269.99 & 0.033 & 0.0001 \\ 0.0001104 & 31.85 & 0.004 & 0.0001\end{array}$ $\begin{array}{llll}0.0001104 & 31.85 & 0.004 & 0.0001\end{array}$ $\begin{array}{llll}0.0001104 & 31.85 & 0.004 & 0.0001\end{array}$ $\begin{array}{llll}0.0048829 & 31.85 & 0.156 & 0.0049\end{array}$ $\begin{array}{llll}0.0048829 & 31.85 & 0.156 & 0.0049\end{array}$ $\begin{array}{llll}0.0048829 & 25.76 & 0.126 & 0.0049\end{array}$ $\begin{array}{llll}0.0048829 & 19.97 & 0.097 & 0.0049\end{array}$ $\begin{array}{llll}0.0048829 & 19.97 & 0.097 & 0.0182\end{array}$ $\begin{array}{llll}0.0048829 & 26.76 & 0.131 & 0.0149\end{array}$ $\begin{array}{llll}0.0048829 & 27.08 & 0.132 & 0.0148\end{array}$ $\begin{array}{llll}0.0048829 & 27.08 & 0.132 & 0.0148\end{array}$ $\begin{array}{llll}0.0048829 & 27.08 & 0.132 & 0.0148\end{array}$ $\begin{array}{lrrr}0.0001104 & 269.99 & 0.030 & 0.0001\end{array}$ $\begin{array}{llll}0.0001934 & 274.77 & 0.053 & 0.0002\end{array}$ TURBOMACHINERY PERFORMANCE DATA

$\begin{array}{lrrrrrrrrr} & \text { Wc } & \text { PR } & \text { eff } & \text { NC } & \text { TR } & \text { efPoly } & \text { pwr } & \text { SMN } & \text { SMW } \\ \text { Fan } & 676.24 & 1.099 & 0.9025 & 2255.002 & 1.0302 & 0.9038 & -1303.0 & -9896.42 & 52.67 \\ \text { LPC } & 79.78 & 1.403 & 0.8521 & 2255.002 & 1.1194 & 0.8591 & -607.8 & 5.95 & 2.64 \\ \text { HPC } & 51.69 & 5.824 & 0.8140 & 8882.190 & 1.7918 & 0.8528 & -3873.2 & 62.24 & 58.63 \\ \text { HPT } & 12.08 & 3.996 & 0.8740 & 218.654 & 1.3526 & 0.8522 & 3873.2 & & \\ \text { LPT } & 52.61 & 2.370 & 0.8339 & 63.950 & 1.2107 & 0.8164 & 1910.9 & & \end{array}$

TURBOMACHINERY MAP DATA

$\begin{array}{lrrrrrrrrr} & \text { WcMap } & \text { PRmap } & \text { effMap } & \text { NCMap } & \text { R/Parm } & \text { S_WCDes } & \text { S_PRdes } & \text { S_effDes } & \text { S_NcDes } \\ \text { Fan } & 785.73 & 1.096 & 0.9043 & 2233.501 & 0.0417 & 0.8607 & \overline{1} .0235 & 0.9980 & 0.9905 \\ \text { LPC } & 60.51 & 1.384 & 0.7656 & 0.596 & 0.0000 & 1.3184 & 1.0509 & 1.1130 & 0.0003 \\ \text { HPC } & 47.01 & 5.596 & 0.8224 & 8645.397 & 10.9526 & 1.0995 & 1.0494 & 0.9898 & 0.9733 \\ \text { HPT } & 0.96 & 3.913 & 0.8740 & 1.314 & 3.9125 & 12.6299 & 0.9723 & 1.0000 & 0.0003 \\ \text { LPT } & 0.81 & 2.030 & 0.8401 & 0.719 & 2.0302 & 65.2590 & 0.7521 & 0.9926 & 0.0005\end{array}$




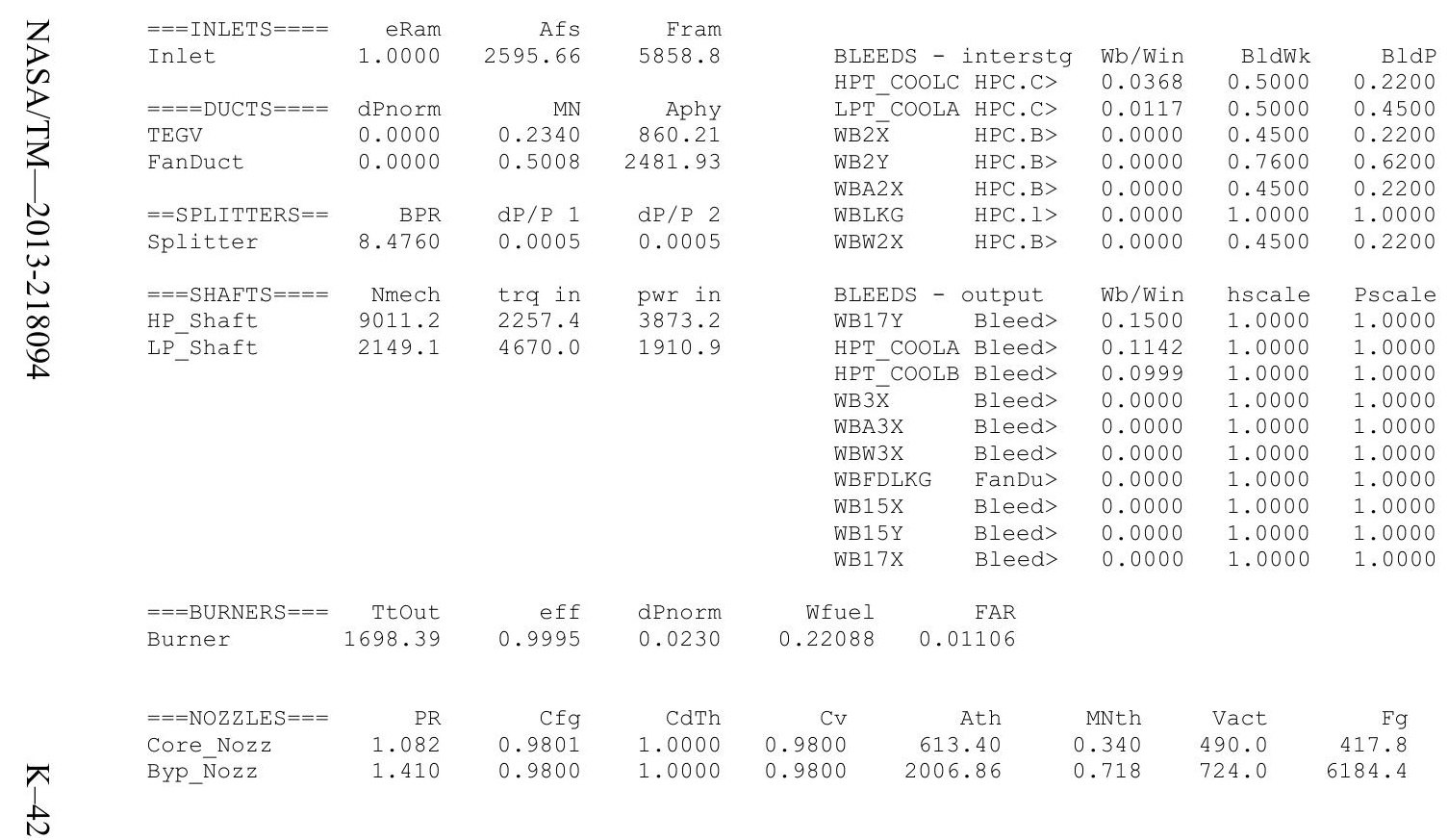


Date:04/04/13 Time:08:35:12 Model: Turbofan Engine - COMDES ON Converge = 1 CASE: 0

Version:NPSS 1.6.5 - Rev: -> Gas Package: Janaf iter/pass/Jacb/Broy= 21/49/2/18 Run by: Philip C Jorgenson PC: 10

Temperature Stator 1 inlet: 482.85 Stator 1 exit: $487.03 \quad$ Stator 2 inlet: 491.79 Stator 2 exit: 493.68 $\begin{array}{lll}\text { Stator } 3 \text { inlet: } 498.94 & \text { Stator } 3 \text { exit: } 500.42 & \text { Stator } 4 \text { inlet: } 505.46 \text { Stator 4 exit: } 506.53 \\ \text { Stator } 5 \text { inlet: } 510.22 & \text { Stator } 5 \text { exit: } 511.00 & \text { Unblocked Percent Blockage: } 0.00\end{array}$

$\begin{array}{lclll}\text { Ambient Relative Humidity } & 10.00 & & & \\ \text { Fan Face Relative Humidity } & 4.93 & & & \\ \text { Fan Bypass Relative Humidity } & 4.06 & & & \\ \text { LPC Inlet Relative Humidity } & 3.92 & & & \\ \text { LPC Exit Relative Humidity } & 1.25 & & & \\ \text { HPC Relative Humidity } & 0.06 & & & \\ \text { Drop Diameter } & 0.0000050 & \text { Inlet Length } & \\ \text { Ambient Flow Velocity } & 576.87 & \text { Fan/LPC Inlet Flow Velocity } & 193.91 \\ \text { Ambient Static Pressure } & 5.30 & \text { Fan/LPC Inlet Static Pressure } & 6.37 \\ \text { Ambient Static Temperature } & 454.14 & \text { Fan/LPC Inlet Static Temperature } & 478.74 \\ \text { Additional Water at LPC Exit } & 0.0044016 & & & \end{array}$

Additional Water at LPC Exit

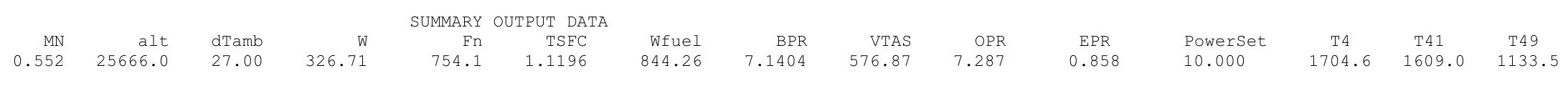

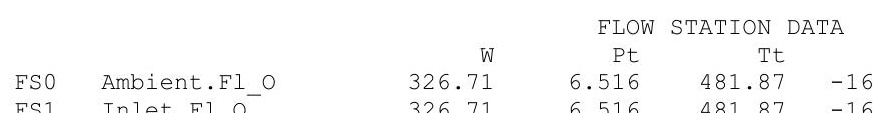

FS1 Inlet. Fi_

FS12 Splitter.F1_02

FS24 VaporIN̄.FI_O

FS25 Bleed2.FI_O

FS3 HPC.FI_O

FS4 Bleed3.F1_O

$\begin{array}{ll}\text { FS4 } & \text { Burner.FI } \\ \text { FS45 } & \text { HPT.FI O }\end{array}$

$\begin{array}{ll}\text { FS } 49 & \text { LPT.FI-FIO } \\ \text { FS5 } & \text { TEGV.FI? }\end{array}$

FS8 Core_Nozz.FI_O

FS17 FanDütLkg.Fì_O

FS171 Bleed15.F1_O

FS172 FanDuct.Fl-O

$\begin{array}{rrrrrr} & \text { Tt } & \text { Tt } & \text { ht } & \text { FAR } & \text { Wc } \\ 326.71 & 6.516 & 481.87 & -16.15 & 0.0000 & 710.20\end{array}$

$\begin{array}{llllll}6.516 & 481.87 & -16.15 & 0.0000 & 710.20\end{array}$

$40.13-6.513 \quad 481.87-16.15-0.0000-623.27$

$\begin{array}{llllll}286.57 & 7.056 & 494.24 & -13.19 & 0.0000 & 582.60\end{array}$

$\begin{array}{llllll}40.13 & 8.235 & 522.34 & -6.45 & 0.0000 & 71.87\end{array}$

$\begin{array}{llrlr}8.235 & 522.34 & -6.45 & 0.0000 & 71.87 \\ 8.235 & 528.40 & -30.27 & 0.0000 & 72.61\end{array}$

$\begin{array}{llllll}28.22 & 8.235 & 528.40 & -30.27 & 0.0000 & 50.82\end{array}$

$\begin{array}{llllll}26.85 & 47.484 & 944.78 & 71.20 & 0.0000 & 11.21\end{array}$

$\begin{array}{llllll}21.04 & 46.390 & 1704.55 & 58.69 & 0.0113 & 12.08\end{array}$

$\begin{array}{llllll}28.12 & 11.977 & 1137.91 & -39.39 & 0.0084 & 51.11\end{array}$

$\begin{array}{llllll}28.45 & 5.624 & 962.12 & -82.25 & 0.0083 & 101.27 \\ 28.45 & 5.624 & 962.19 & -82.25 & 0.0083 & 101.27\end{array}$

$\begin{array}{llllll}28.45 & 5.624 & 962.19 & -82.25 & 0.0083 & 101.2 \\ 28.45 & 5.624 & 962.26 & -82.25 & 0.0083 & 101.27\end{array}$

28.45
286.57

298.66

298.66

$\begin{array}{llll}5.624 & 962.26 & -82.25 & 0.0083 \\ 7.056 & 494.24 & -13.19 & 0.0000\end{array}$

$\begin{array}{llll}7.056 & 495.63 & -13.88 & 0.0000\end{array}$

$\begin{array}{llll}7.056 & 495.63 & -13.88 & 0.0000\end{array}$

$\begin{array}{ll}-13.88 & 0.0000\end{array}$

582.60

608.04

608.04

Ps
5.297
6.113
6.078
6.366
6.258
7.701
7.687
7.977
41.955
44.303
43.797
11.004
5.463
5.463
5.297
6.258
6.063
6.063
5.297

Ts
454.14
473.14
472.43
478.74
477.55
512.41
518.12
523.62
912.87
926.79
1680.29
1112.35
954.52
954.59
946.62
477.55
474.59
474.59
456.59

$\begin{array}{rr}\text { Aphy } & \text { MN } \\ 2590.8 & 0.5520 \\ 4168.2 & 0.3035 \\ 3531.8 & 0.3157 \\ 830.5 & 0.1807 \\ 2606.7 & 0.4177 \\ 412.6 & 0.3111 \\ 412.6 & 0.3153 \\ 412.6 & 0.2138 \\ 49.7 & 0.4264 \\ 49.3 & 0.3180 \\ 74.6 & 0.2949 \\ 265.4 & 0.3541 \\ 860.2 & 0.2055 \\ 860.2 & 0.2055 \\ 613.4 & 0.2960 \\ 2606.7 & 0.4177 \\ 2481.9 & 0.4705 \\ 2481.9 & 0.4705 \\ 2006.9 & 0.6533\end{array}$

gamt
1.40063
1.40063
1.40063
1.40063
1.40050
1.40016
1.39947
1.39947
1.38333
1.38333
1.33125
1.36544
1.37673
1.37673
1.37672
1.40050
1.40046
1.40046
1.40046

WAR
0.0001988
0.0001988
0.0001988
0.0001988
0.0001988
0.0001988
0.0046004
0.0046004
0.0046004
0.0046004
0.0046004
0.0046004
0.0046004
0.0046004
0.0046004
0.0001988
0.0003763
0.0003763
0.0003763

$\begin{array}{rrr}\text { Wair } & \text { WH2O } & \text { H2O frac } \\ 326.64 & 0.065 & 0.0002\end{array}$

TURBOMACHINERY PERFORMANCE DATA

\begin{tabular}{lrrrrrrrrr}
\multicolumn{1}{c}{ Wc } & PR & eff & NC & TR & efPoly & pwr & SMN & SMW \\
Fan & 623.27 & 1.083 & 0.9031 & 2044.393 & 1.0257 & 0.9043 & -1202.5 & -2763.57 & 49.88 \\
LPC & 87.29 & 1.264 & 0.8262 & 2044.393 & 1.0840 & 0.8319 & -551.1 & 6.38 & 2.41 \\
HPC & 50.82 & 5.766 & 0.8128 & 8854.850 & 1.7880 & 0.8517 & -3953.0 & 61.50 & 57.83 \\
HPT & 12.08 & 3.873 & 0.8751 & 216.478 & 1.3437 & 0.8539 & 3952.9 & & \\
LPT & 51.11 & 2.130 & 0.8239 & 58.416 & 1.1798 & 0.8080 & 1753.7 & &
\end{tabular}

TURBOMACHINERY MAP DATA

$\begin{array}{lrrrrrrrrr} & \text { WCMap } & \text { PRmap } & \text { effMap } & \text { NcMap } & \text { R/Parm } & \text { S_WcDes } & \text { S_PRdes } & \text { S_effDes } & \text { S_NCDes } \\ \text { Fan } & 724.18 & 1.082 & 0.9050 & 2024.900 & 0.0425 & 0.8607 & 1.0235 & 0.9980 & 0.9905 \\ \text { LPC } & 53.46 & 1.311 & 0.7607 & 0.540 & 0.0000 & 1.6327 & 0.8502 & 1.0861 & 0.0003 \\ \text { HPC } & 46.22 & 5.541 & 0.8212 & 8618.786 & 11.0083 & 1.0995 & 1.0494 & 0.9898 & 0.9733 \\ \text { HPT } & 0.96 & 3.794 & 0.8750 & 1.301 & 3.7936 & 12.6299 & 0.9723 & 1.0000 & 0.0003 \\ \text { LPT } & 0.78 & 1.850 & 0.8300 & 0.657 & 1.8498 & 65.2590 & 0.7521 & 0.9926 & 0.0005\end{array}$




\begin{tabular}{|c|c|c|c|c|c|c|c|c|}
\hline \multirow{2}{*}{$\begin{array}{ll}Z & ===\text { INLETS }==== \\
& \text { Inlet }\end{array}$} & eRam & Afs & Fram & \multirow{2}{*}{\multicolumn{2}{|c|}{ BLEFDS - intersta }} & \multirow[b]{2}{*}{ Wb/Win } & \multirow[b]{2}{*}{ BldWk } & \multirow{3}{*}{$\begin{array}{r}\text { BldP } \\
0.2200\end{array}$} \\
\hline & 1.0000 & 2590.82 & 5857.7 & & & & & \\
\hline & & & & HPT_COOLC & $C$ HPC.C> & 0.0368 & 0.5000 & \\
\hline$====$ DUCTS $====$ & dPnorm & MN & Aphy & LPT_COOLA & A HPC.C> & 0.0117 & 0.5000 & 0.4500 \\
\hline TEGV & 0.0000 & 0.2055 & 860.21 & WB2 $\bar{x}$ & HPC.B> & 0.0000 & 0.4500 & 0.2200 \\
\hline FanDuct & 0.0000 & 0.4705 & 2481.93 & WB2Y & HPC.B> & 0.0000 & 0.7600 & 0.6200 \\
\hline & & & & WBA2X & HPC.B> & 0.0000 & 0.4500 & 0.2200 \\
\hline$==$ SPLITTERS $==$ & BPR & $\mathrm{dP} / \mathrm{P} 1$ & $\mathrm{dP} / \mathrm{P} 2$ & WBLKG & HPC.1> & 0.0000 & 1.0000 & 1.0000 \\
\hline Splitter & 7.1404 & 0.0005 & 0.0005 & WBW2X & HPC.B> & 0.0000 & 0.4500 & 0.2200 \\
\hline$===$ SHAFTS $====$ & Nmech & trg in & pwr in & BLEEDS - & output & Wb/Win & hscale & Pscale \\
\hline HP Shaft & 8937.6 & 2322.9 & 3952.9 & WB17Y & Bleed> & 0.3000 & 1.0000 & 1.0000 \\
\hline LP_Shaft & 1970.5 & 4674.1 & 1753.7 & HPT_COOLA & A Bleed> & 0.1142 & 1.0000 & 1.0000 \\
\hline & & & & $\mathrm{HPT}_{\text {CCOOLB }}$ & B Bleed> & 0.0999 & 1.0000 & 1.0000 \\
\hline & & & & WB3 $3 \bar{x}$ & Bleed> & 0.0000 & 1.0000 & 1.0000 \\
\hline & & & & WBA3X & Bleed> & 0.0000 & 1.0000 & 1.0000 \\
\hline & & & & WBW3X & Bleed> & 0.0000 & 1.0000 & 1.0000 \\
\hline & & & & WBFDLKG & FanDu> & 0.0000 & 1.0000 & 1.0000 \\
\hline & & & & WB15X & Bleed> & 0.0000 & 1.0000 & 1.0000 \\
\hline & & & & WB15Y & Bleed> & 0.0000 & 1.0000 & 1.0000 \\
\hline & & & & WB17X & Bleed> & 0.0000 & 1.0000 & 1.0000 \\
\hline$===$ BURNERS $===$ & Ttout & eff & dPnorm & Wfuel & FAR & & & \\
\hline Burner & 1704.49 & 0.9995 & 0.0230 & 0.23452 & 01132 & & & \\
\hline$===$ NOZZLES $===$ & PR & $\operatorname{Cfg}$ & CdTh & $\mathrm{Cv}$ & Ath & MNth & Vact & $\mathrm{Fg}$ \\
\hline Core_Nozz & 1.062 & 0.9801 & 1.0000 & 0.9800 & 3.40 & 0.296 & 434.5 & 384.3 \\
\hline Byp_Nozz & 1.332 & 0.9800 & 1.0000 & 0.9800 & 6.86 & 0.653 & 670.9 & 6227.5 \\
\hline
\end{tabular}


Turbofan Engine - COMDES ON Converge = 1 CASE: 0

Version:NPSS_1.6.5 - Rev: -> Gas Package: Janaf iter/pass/Jacb/Broy= 50/78/2/47 Run by: Philip C Jorgenson PC: 10

$\begin{array}{rllll}\text { Temperature Stator } 1 \text { inlet: } 495.90 & \text { Stator } 1 \text { exit: } 498.91 & \text { Stator } 2 \text { inlet: } 501.78 & \text { Stator } 2 \text { exit: } 502.62 \\ \text { Stator } 3 \text { inlet: } 506.05 & \text { Stator } 3 \text { exit: } 506.45 & \text { Stator } 4 \text { inlet: } 509.77 & \text { Stator } 4 \text { exit: } 509.86\end{array}$

Stator 5 inlet: 511.80 Stator 5 exit: 511.68 Unblocked Percent Blockage: 0.00

$\begin{array}{lclll}\text { Ambient Relative Humidity } & 10.00 & & & \\ \text { Fan Face Relative Humidity } & 5.94 & & & \\ \text { Fan Bypass Relative Humidity } & 5.03 & & & \\ \text { LPC Inlet Relative Humidity } & 5.11 & & & \\ \text { LPC Exit Relative Humidity } & 2.66 & & & \\ \text { HPC Relative Humidity } & 0.07 & & & \\ \text { Drop Diameter } & 0.000050 & \text { Inlet Length } & & \\ \text { Ambient Flow Velocity } & 523.25 & \text { Fan/LPC Inlet Flow Velocity } & 202.76 \\ \text { Ambient Static Pressure } & 6.74 & \text { Fan/LPC Inlet Static Pressure } & 7.75 \\ \text { Ambient Static Temperature } & 474.18 & \text { Fan/LPC Inlet Static Temperature } & 493.56 \\ \text { Additional Water at LPC Exit } & 0.0045473 & & & \end{array}$

Additional Water at LPC Exit

SUMMARY OUTPUT DATA

0.490 .20047 .0 .27 .00

FSO Ambient.Fl_O

FS1 Inlet.FI_O

$\pi$

Splitter.Fl_0

FLOW STATION DATA

FS14 Fan.F1_O

FS24 VaporIN.F1

FS25 Bleed2.F1_O

FS3 HPC.FI_O

FS36 Bleed3.F1_O

FS4 Burner.Fl_O

FS49

FS5 5 TEGV FI

FS8 Core Nozz.Fl O

FS17 FanDüctLkg.Fì

FS171 Bleed15.FI_O

FS172 FanDuct.FI_O

$W$
363.33
363.33
313.78
49.54
313.78
49.54
49.77
29.86
28.41
22.02
22.28
29.77
30.12
30.12
30.12
313.78
333.69
333.69
333.69

TURBOMACHINERY PERFORMANCE DATA

$\begin{array}{lrrrrrrrrr} & \text { Wc } & \text { PR } & \text { eff } & \text { NC } & \text { TR } & \text { efPoly } & \text { pwr } & \text { SMN } & \text { SMW } \\ \text { Fan } & 568.56 & 1.071 & 0.9051 & 1842.251 & 1.0220 & 0.9060 & -1163.4 & -1872.26 & 46.44 \\ \text { LPC } & 89.77 & 1.148 & 0.7035 & 1842.251 & 1.0571 & 0.7092 & -477.4 & 7.00 & 2.16 \\ \text { HPC } & 48.76 & 5.564 & 0.8084 & 8787.280 & 1.7718 & 0.8475 & -4123.4 & 61.55 & 57.62 \\ \text { HPT } & 12.09 & 3.680 & 0.8770 & 213.643 & 1.3284 & 0.8571 & 4123.4 & & \\ \text { LPT } & 48.77 & 1.911 & 0.8165 & 52.785 & 1.1502 & 0.8025 & 1640.7 & & \end{array}$

TURBOMACHINERY MAP DATA

$\begin{array}{lrrrrrrrrr} & \text { WcMap } & \text { PRmap } & \text { effMap } & \text { NcMap } & \text { R/Parm } & \text { S_WcDes } & \text { S_PRdes } & \text { S_effDes } & \text { S_NcDes } \\ \text { Fan } & 660.61 & 1.070 & 0.9069 & 1824.685 & 0.0438 & 0.8607 & \overline{1} .0235 & 0.9980 & 0.9905 \\ \text { LPC } & 46.84 & 1.245 & 0.7556 & 0.487 & 0.0000 & 1.9166 & 0.6021 & 0.9310 & 0.0003 \\ \text { HPC } & 44.34 & 5.349 & 0.8167 & 8553.017 & 11.0349 & 1.0995 & 1.0494 & 0.9898 & 0.9733 \\ \text { HPT } & 0.96 & 3.606 & 0.8770 & 1.284 & 3.6060 & 12.6299 & 0.9723 & 1.0000 & 0.0003 \\ \text { LPT } & 0.75 & 1.685 & 0.8226 & 0.594 & 1.6852 & 65.2590 & 0.7521 & 0.9926 & 0.0005\end{array}$

$\begin{array}{clccc}\text { EPR } & \text { PowerSet } & \text { T4 } & \text { T41 } & \text { T49 } \\ 0.889 & 10.000 & 1733.9 & 1634.6 & 1162.4\end{array}$

$\begin{array}{rrrrr}\text { Pt } & \text { Tt } & \text { ht } & \text { FAR } & \text { WC } \\ 7.943 & 496.98 & -13.65 & 0.0000 & 658.00\end{array}$

$\begin{array}{llllll}7.943 & 496.98 & -13.65 & 0.0000 & 658.00\end{array}$

$\begin{array}{lllll}7.939 & 496.98 & -13.65 & 0.0000 & 568.56\end{array}$

$\begin{array}{lllll}7.939 & 496.98 & -13.65 & 0.0000 & 89.77\end{array}$

$\begin{array}{lllll}8.506 & 507.91 & -11.03 & 0.0000 & 536.50\end{array}$

$\begin{array}{lllll}9.112 & 525.37 & -6.84 & 0.0000\end{array}$

$\begin{array}{llll}9.112 & 531.61 & -31.45 & 0.0000\end{array}$

$\begin{array}{rrrr}9.112 & 531.61 & -31.45 & 0.0000\end{array}$

$50.698-941.92 \quad 68.58 \quad 0.0000$

$\begin{array}{llll}49.530 & 1733.93 & 55.53 & 0.0118\end{array}$

$\begin{array}{llll}13.458 & 1167.13 & -40.93 & 0.0088\end{array}$

$\begin{array}{llll}7.042 & 1011.85 & -78.73 & 0.008 \\ 7.042 & 1011.92 & -78.73 & 0.0087\end{array}$

$\begin{array}{llll}7.042 & 1011.85 & -78.73 & 0.0087 \\ 7.042 & 1011.99 & -78.73 & 0.0087\end{array}$

$\begin{array}{llll}8.506 & 507.91 & -11.03 & 0.0000\end{array}$

$\begin{array}{llll}8.506 & 509.33 & -12.25 & 0.0000 \\ 8.506 & 509.33 & -12.25 & 0.0000\end{array}$

8.506

509.33

$\begin{array}{ll}-12.25 & 0.0000\end{array}$

80.42

48.76

11.10
8.60

12.09
48.77
87.79

87.79
87.80

87.80
87.80

536.50

571.33

571.33

PS
6.740
7.525
7.504
7.750
7.708
8.356
8.336
8.850
44.934
47.376
46.760
12.472
6.892
6.892
6.740
7.708
7.474
7.474
6.740

$T S$
474.18
489.36
489.03
493.56
493.81
512.52
518.26
527.20
910.89
924.38
1709.31
1143.66
1005.95
1006.02
999.99
493.81
490.84
490.84
476.55

$\begin{array}{rr}\text { Aphy } & \text { MN } \\ 2606.8 & 0.4900\end{array}$ $\begin{array}{ll}2606.8 & 0.4900 \\ 4168.2 & 0.2788\end{array}$

$\begin{array}{rr}3531.8 & 0.2849 \\ 830.5 & 0.1861\end{array}$

$\begin{array}{rr}830.5 & 0.1861 \\ 2606.7 & 0.3777\end{array}$

$\begin{array}{ll}412.6 & 0.3540\end{array}$

$\begin{array}{ll}412.6 & 0.3591 \\ 412.6 & 0.2047\end{array}$

$\begin{array}{ll}49.7 & 0.4209\end{array}$

$\begin{array}{ll}49.3 & 0.3143 \\ 74.6 & 0.2952\end{array}$

$\begin{array}{rr}74.6 & 0.2952 \\ 265.4 & 0.3357\end{array}$

$\begin{array}{ll}265.4 & 0.3357 \\ 860.2 & 0.1773\end{array}$

$\begin{array}{ll}860.2 & 0.1773\end{array}$

$\begin{array}{ll}613.5 & 0.2534\end{array}$

$\begin{array}{ll}2606.7 & 0.3777 \\ 2481.9 & 0.4338\end{array}$

$\begin{array}{ll}2481.9 & 0.4338 \\ 2481.9 & 0.4338\end{array}$

2006.90 .5861
WAR
0.0003932
0.0003932
0.0003932
0.0003932
0.0003932
0.0003932
0.0049405
0.0049405
0.0049405
0.0049405
0.0049405
0.0049405
0.0049405
0.0049405
0.0049405
0.0003932
0.0006634
0.0006634
0.0006634

Wair
363.18

$\begin{array}{lll}363.18 & 0.143 & \text { H2O frac }\end{array}$ $\begin{array}{lll}363.18 & 0.143 & 0.0004\end{array}$ $\begin{array}{lll}49.52 & 0.019 & 0.0004\end{array}$ $\begin{array}{lll}313.66 & 0.123 & 0.0004\end{array}$ $\begin{array}{lll}49.52 & 0.019 & 0.0004\end{array}$ $\begin{array}{lll}49.52 & 0.245 & 0.0049 \\ 29.71 & 0.147 & 0.0049 \\ 28.27 & 0.140 & 0.0049\end{array}$

$\begin{array}{lll}28.27 & 0.140 & 0.0049\end{array}$

$\begin{array}{lll}21.91 & 0.108 & 0.0049\end{array}$

$\begin{array}{lll}21.91 & 0.108 & 0.0192\end{array}$

$29.37 \quad 0.145 \quad 0.0156$

$\begin{array}{lll}29.71 & 0.147 & 0.0155\end{array}$

$\begin{array}{lll}29.71 & 0.147 & 0.0155\end{array}$

$\begin{array}{lll}29.71 & 0.147 & 0.0155\end{array}$

$\begin{array}{lll}313.66 & 0.123 & 0.0004\end{array}$

$\begin{array}{lll}333.47 & 0.221 & 0.0007\end{array}$

$\begin{array}{ll}333.47 & 0.221 \\ 333.47 & 0.221\end{array}$ 


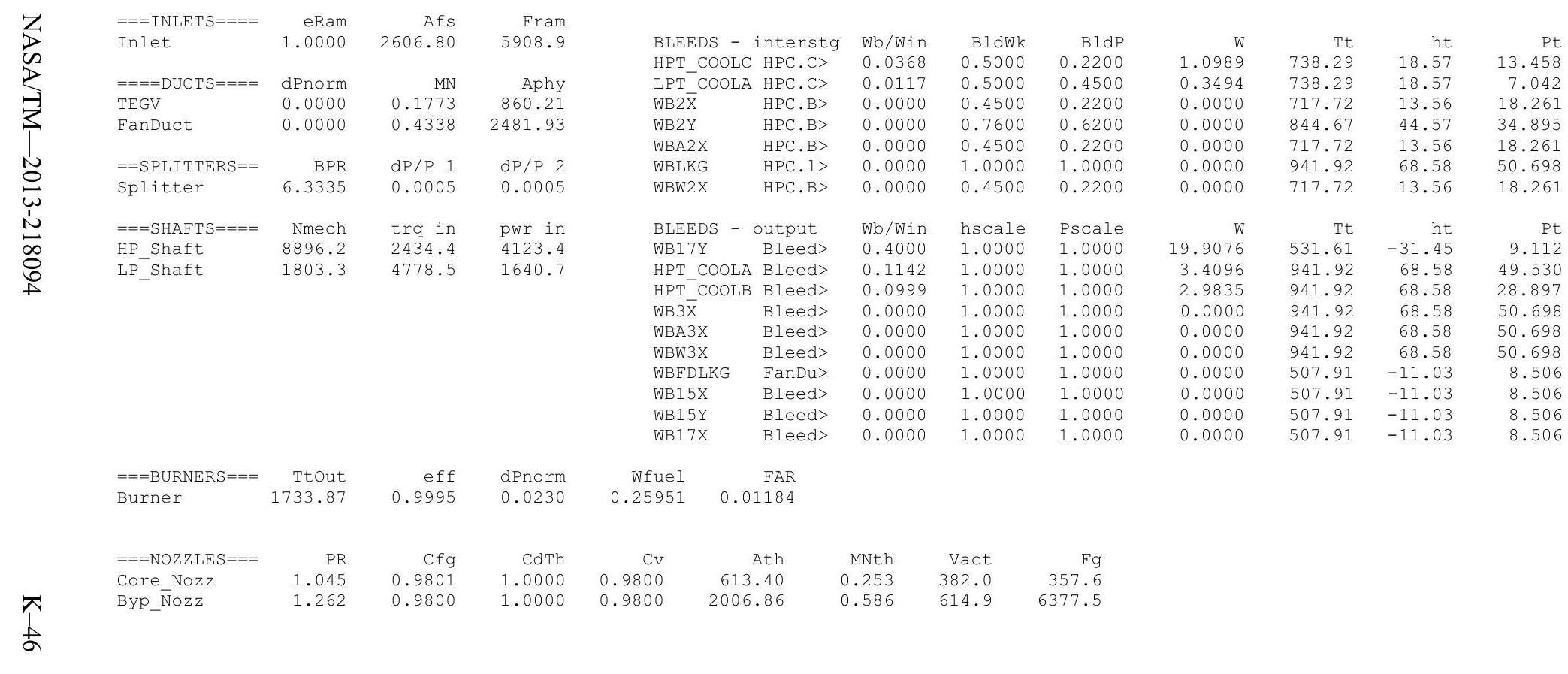


Turbofan Engine - COMDES ON Converge = 1 CASE: 0

Version:NPSS_1.6.5 - Rev: -> Gas Package: Janaf iter/pass/Jacb/Broy= 43/71/2/40 Run by: Philip C Jorgenson PC: 10

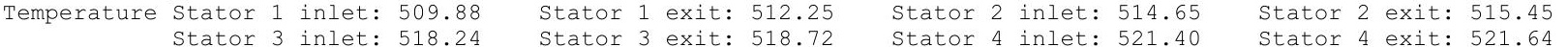
$\begin{array}{lll}\text { Stator } 3 \text { inlet: } 518.24 & \text { Stator } 3 \text { exit: } 518.72 & \text { Stator } 4 \text { inlet: } 521.40 \text { Stator } 4 \text { exit: } 521.64 \\ \text { Stator } 5 \text { inlet: } 523.34 & \text { Stator } 5 \text { exit: } 523.42 & \text { Unblocked Percent Blockage: } 0.00\end{array}$

$\begin{array}{lclll}\text { Ambient Relative Humidity } & 10.00 & & & \\ \text { Fan Face Relative Humidity } & 6.60 & & & \\ \text { Fan Bypass Relative Humidity } & 6.07 & & & \\ \text { LPC Inlet Relative Humidity } & 5.78 & & & \\ \text { LPC Exit Relative Humidity } & 3.42 & & & \\ \text { HPC Relative Humidity } & 0.14 & & & \\ \text { Drop Diameter } & 0.0000050 & \text { Inlet Length } & \\ \text { Ambient Flow Velocity } & 484.46 & \text { Fan/LPC Inlet Flow Velocity } & 170.01 \\ \text { Ambient Static Pressure } & 8.15 & \text { Fan/LPC Inlet Static Pressure } & 9.18 \\ \text { Ambient Static Temperature } & 490.63 & \text { Fan/LPC Inlet Static Temperature } & 507.76 \\ \text { Additional Water at LPC Exit } & 0.0069693 & & & \end{array}$

SUMMARY OUTPUT DATA

\begin{tabular}{|c|c|c|c|c|c|c|c|c|c|c|c|c|c|c|}
\hline $\begin{array}{r}\mathrm{MN} \\
\mathrm{M}\end{array}$ & alt & dTamb & W & $\begin{array}{r}\text { UMMARY } \\
\text { Fn }\end{array}$ & $\begin{array}{r}\text { TPUT DA } \\
\text { TSFC }\end{array}$ & Wfuel & $\begin{array}{r}\text { BPR } \\
5588\end{array}$ & VTAS & $\begin{array}{r}\text { OPR } \\
5 \quad 188\end{array}$ & EPR & Powerset & T4 & T41 & \\
\hline 0.446 & 15435.0 & 27.00 & 380.80 & 629.6 & 1.3999 & 881.42 & 6.9588 & 484.46 & 5.188 & 0.900 & 10.000 & 1688.0 & 1590.7 & 1156.0 \\
\hline
\end{tabular}

\begin{tabular}{|c|c|}
\hline FSO & Ambient.Fl_O \\
\hline FS1 & Inlet.FI_o \\
\hline FS12 & Splitter.Fl_02 \\
\hline FS2 & Splitter.FI_01 \\
\hline FS14 & Fan.Fl_O \\
\hline FS23 & LPC.Fl-O \\
\hline FS24 & VaporIN̄.FI_O \\
\hline FS25 & Bleed2.F1_o \\
\hline FS3 & HPC.Fl_O \\
\hline FS36 & Bleed3.Fl_o \\
\hline FS4 & Burner.Fl-o \\
\hline FS 45 & HPT.FIO \\
\hline FS49 & LPT.FI- \\
\hline FS5 & TEGV.FI $O$ \\
\hline FS8 & Core_Nozz.FI \\
\hline FS17 & FanDuctLkg.Fì \\
\hline FS171 & Bleed15.Fl \\
\hline 'S172 & FanDuct.Fl \\
\hline FS173 & Byp Nozz.Fi \\
\hline
\end{tabular}

\begin{tabular}{|c|c|c|c|c|}
\hline & & STATION & & \\
\hline W & Pt & Tt & ht & FAR \\
\hline .80 & 9.342 & 510.16 & -11.98 & 0.0000 \\
\hline 380.80 & 9.342 & 510.16 & -11.98 & 0.0000 \\
\hline 332.95 & 9.337 & 510.16 & -11.98 & 0.0000 \\
\hline 47.85 & 9.337 & 510.16 & -11.98 & 0.0000 \\
\hline 332.95 & 9.802 & 518.13 & -10.07 & 0.0000 \\
\hline 47.85 & 10.476 & 533.17 & -6.45 & 0.0000 \\
\hline 48.18 & 10.476 & 542.57 & -44.07 & 0.0000 \\
\hline 28.91 & 10.476 & 542.57 & -44.07 & 0.0000 \\
\hline 27.51 & 48.463 & 913.20 & 46.40 & 0.0000 \\
\hline 21.32 & 48.463 & 913.20 & 46.40 & 0.0000 \\
\hline 21.56 & 47.347 & 1688.00 & 33.93 & 0.0116 \\
\hline 28.81 & 14.130 & 1160.80 & -53.16 & 0.0086 \\
\hline 29.15 & 8.389 & 1035.70 & -83.41 & 0.0085 \\
\hline 29.15 & 8.389 & 1035.77 & -83.41 & 0.0085 \\
\hline 29.15 & 8.389 & 1035.85 & -83.41 & 0.0085 \\
\hline 332.95 & 9.802 & 518.13 & -10.07 & 0.0000 \\
\hline 352.23 & 9.802 & 519.47 & -11.93 & 0.0000 \\
\hline 352.23 & 9.802 & 519.47 & -11.93 & 0.0000 \\
\hline 352.23 & 9.802 & 519.47 & -11.93 & 0.0000 \\
\hline
\end{tabular}

WC
594.11
594.11
519.72
74.69
498.93
68.05
69.13
41.48
11.07
8.58
12.07
44.83
72.17
72.17
72.17
498.93
528.49
528.49
528.49

PS
8.149
8.946
8.914
9.184
9.020
9.871
9.847
10.259
42.980
45.302
44.700
13.266
8.269
8.269
8.149
9.020
8.809
8.809
8.149

TS
490.63
503.89
503.44
507.76
505.96
524.19
533.09
539.35
883.20
896.23
1663.96
1141.41
1031.67
1031.74
1027.74
505.96
503.85
503.85
492.76

$\begin{array}{rr}\text { Aphy } & \text { MN } \\ 2525.7 & 0.4460 \\ 4168.2 & 0.2495 \\ 3531.8 & 0.2583 \\ 830.5 & 0.1539 \\ 2606.7 & 0.3467 \\ 412.6 & 0.2927 \\ 412.6 & 0.2987 \\ 412.6 & 0.1730 \\ 49.7 & 0.4197 \\ 49.3 & 0.3135 \\ 74.6 & 0.2949 \\ 265.4 & 0.3054 \\ 860.2 & 0.1451 \\ 860.2 & 0.1451 \\ 613.4 & 0.2060 \\ 2606.7 & 0.3467 \\ 2481.9 & 0.3937 \\ 2481.9 & 0.3937 \\ 2006.9 & 0.5204\end{array}$

gamt
1.40025
1.40025
1.40025
1.40025
1.40015
1.39994
1.39884
1.39884
1.38470
1.38470
1.33142
1.36341
1.37157
1.37156
1.37156
1.40015
1.40008
1.40008
1.40008

WAR
0.0006510
0.0006510
0.0006510
0.0006510
0.0006510
0.0006510
0.0076203
0.0076203
0.0076203
0.0076203
0.0076203
0.0076203
0.0076203
0.0076203
0.0076203
0.0006510
0.0010299
0.0010299
0.0010299

Wair
380.55 $\begin{array}{rrr}380.55 & 0.248 & 0.0007 \\ 380.55 & 0.248 & 0.0007\end{array}$ $\begin{array}{lll}380.55 & 0.248 & 0.0007\end{array}$ $\begin{array}{lll}332.74 & 0.217 & 0.0007 \\ 47.82 & 0.031 & 0.0007\end{array}$ $\begin{array}{rrr}47.82 & 0.031 & 0.0007 \\ 332.74 & 0.217 & 0.0007\end{array}$ $\begin{array}{lll}47.82 & 0.031 & 0.0007\end{array}$ $\begin{array}{lll}47.82 & 0.364 & 0.0076\end{array}$ $\begin{array}{lll}28.69 & 0.219 & 0.0076 \\ 27.30 & 0.208 & 0.0076\end{array}$ $\begin{array}{lll}21.16 & 0.161 & 0.0076\end{array}$ $\begin{array}{lll}21.16 & 0.161 & 0.0215\end{array}$ $\begin{array}{lll}21.16 & 0.161 & 0.0215 \\ 28.35 & 0.216 & 0.0180\end{array}$ $\begin{array}{llll}28.69 & 0.219 & 0.0179\end{array}$ $\begin{array}{lll}28.69 & 0.219 & 0.0179\end{array}$ $\begin{array}{lll}28.69 & 0.219 & 0.0179\end{array}$ $\begin{array}{lll}28.69 & 0.219 & 0.0179 \\ 332.74 & 0.217 & 0.0007\end{array}$ $\begin{array}{lll}351.86 & 0.362 & 0.0010\end{array}$ TURBOMACHINERY PERFORMANCE DATA

\begin{tabular}{|c|c|c|c|c|c|c|c|c|c|}
\hline & WC & $\mathrm{PR}$ & eff & $\mathrm{NC}$ & $\mathrm{TR}$ & efPoly & pwr & SMN & SMW \\
\hline Fan & 519.72 & 1.050 & 0.8960 & 1600.228 & 1.0156 & 0.8967 & -899.9 & -1532.72 & 44.75 \\
\hline LPC & 74.69 & 1.122 & 0.7411 & 1600.228 & 1.0451 & 0.7453 & -373.9 & 8.33 & 1.96 \\
\hline HPC & 41.48 & 4.626 & 0.7926 & 8512.931 & 1.6831 & 0.8310 & -3610.6 & 69.77 & 64.34 \\
\hline HPT & 12.07 & 3.35 & 0.8685 & 211.922 & 1.2991 & 0.8491 & 3610.6 & & \\
\hline LPT & 44.83 & 1.68 & .8032 & 46.582 & 1.1172 & 0.7913 & 1273.6 & & \\
\hline JRB & NERY MAP & DATA & & & & & & & \\
\hline & WcMap & PRmap & effMap & NcMap & R/Parm & S_WCDes & S_PRdes & S_effDes & S_NcDes \\
\hline $\operatorname{Tan}$ & 603.87 & 1.049 & 0.8978 & 1584.970 & 0.0441 & 0.8607 & $\overline{1} .0235$ & 0.9980 & 0.9905 \\
\hline PC & 39.39 & 1.1 & & & & 1.8 & 0.6712 & 0. & 0.0003 \\
\hline $\begin{array}{l}\text { HPC } \\
\text { PPC }\end{array}$ & 37.72 & 4.455 & 0.8008 & 8285.982 & 10.6913 & 1.0995 & 1.0494 & 0.9898 & 0.9733 \\
\hline $\begin{array}{l}\mathrm{HPT} \\
\mathrm{HPT}\end{array}$ & 0.96 & 3.286 & 0.8685 & 1.274 & 3.2856 & 12.6299 & 0.9723 & 1.0000 & 0.0003 \\
\hline LPT & 0.69 & 1.515 & 0.8091 & 0.524 & 1.5147 & 65.2590 & 0.7521 & 0.9926 & 0.0005 \\
\hline
\end{tabular}




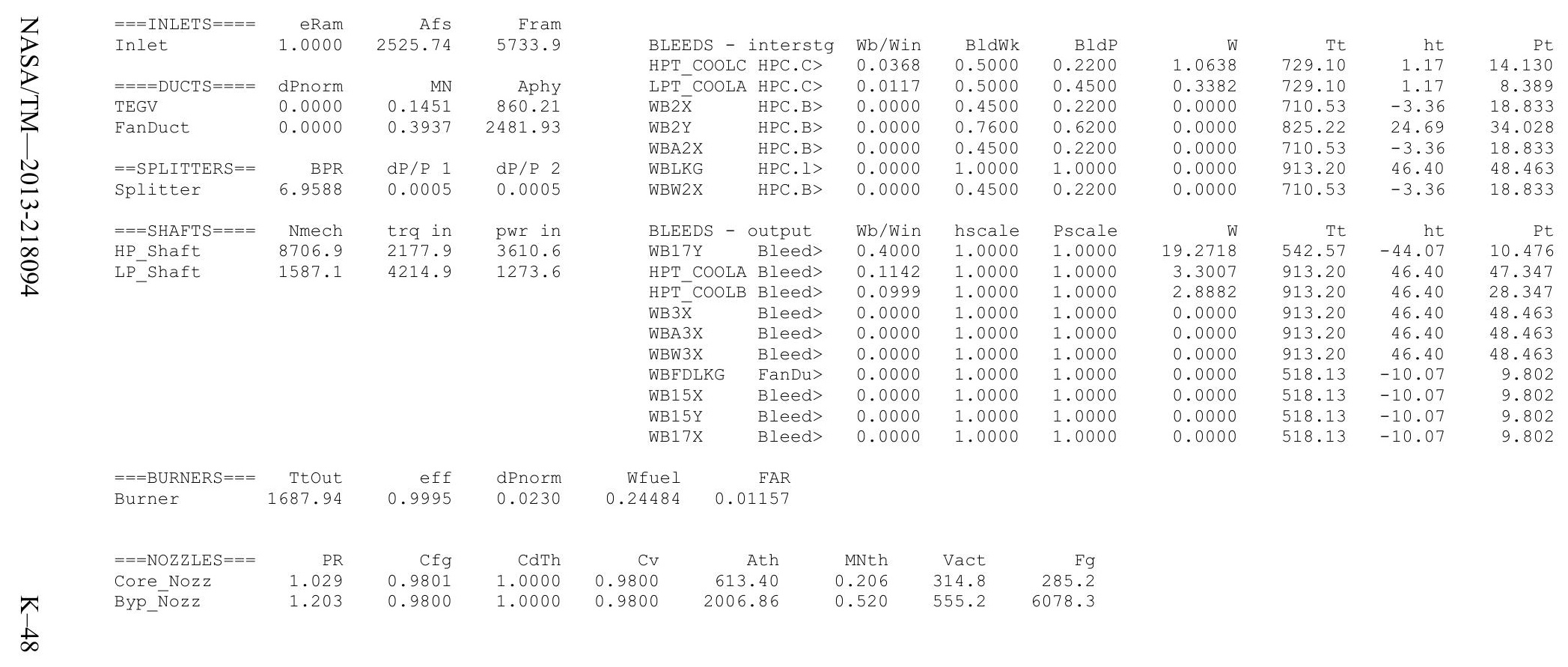


Date: $04 / 04 / 13$ Time: $08: 19: 37$ Model

Turbofan Engine - COMDES ON Converge $=1$
CASE:

Temperature Stator 1 inlet: $473.84 \quad$ Stator 1 exit: $481.57 \quad$ Stator 2 inlet: 491.27 Stator 2 exit: 496.29

Stator 5 inlet: 531.28 Stator 5 exit: 534.66 Unblocked Percent Blockage: 0.00

Ambient Relative Humidity $\quad 10.00$

Fan Face Relative Humidity $\quad 1.87$

Fan Bypass Relative Humidity, 1.30

$\begin{array}{ll}\text { LPC Inlet Relative Humidity } & 1.21 \\ \text { LPC Exit Relative Humidity } & 0.11\end{array}$

Drop Diameter

Drop Diameter

Ambient Static Pressure

781.11
2.85

Inlet Length

Ambient Static Temperature

416.97

an/LPC Inlet Flow Velocity

87.19

Additional Water at LPC Exit

0.0091071

n/LPC Inlet static Temperature

464.91

$\begin{array}{rrrrrr}\text { MN } & \text { alt } & \text { dTamb } & \text { W } & \text { SUMMARY } & \text { OUTPUT DATA } \\ 0.780 & 39000.0 & 27.00 & 254.97 & 652.9 & \text { TSFC }\end{array}$

\section{FLOW STATION DATA}

$\mathrm{Pt} \quad \mathrm{Tt}$

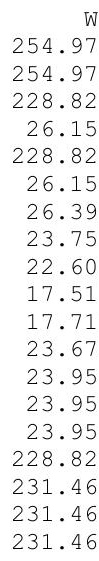

\begin{tabular}{|c|c|}
\hline FS1 & Inlet.FI_O \\
\hline FS12 & Splitter.Fl_02 \\
\hline FS2 & Splitter.Fl_01 \\
\hline FS14 & Fan.Fl_O \\
\hline FS23 & LPC.FI_O \\
\hline FS24 & VaporI⿳亠丷⿵冂丶.FI_O \\
\hline FS25 & Bleed2.FI_o \\
\hline FS 3 & HPC.Fl_O \\
\hline FS36 & Bleed3.FI_O \\
\hline FS 4 & Burner.Fl_o \\
\hline FS 45 & HPT.FI_O \\
\hline FS 49 & LPT.Fl_O \\
\hline FS5 & TEGV.Fİ_O \\
\hline FS8 & Core_Nozzz.FI_O \\
\hline FS17 & FanDüctLkg.Fì \\
\hline FS171 & Bleed15.FI_O \\
\hline FS172 & FanDuct.FI_O \\
\hline FS173 & Byp_Nozz.FI_C \\
\hline
\end{tabular}

TURBOMACHINERY PERFORMANCE DATA

$\begin{array}{lrrr} & \text { WC } & \text { PR } & \text { eff } \\ \text { Fan } & 748.89 & 1.132 & 0.9062 \\ \text { LPC } & 85.58 & 1.569 & 0.8325 \\ \text { HPC } & 54.06 & 6.071 & 0.8181 \\ \text { HPT } & 12.07 & 4.167 & 0.8772 \\ \text { LPT } & 54.68 & 2.917 & 0.8564\end{array}$

$\begin{array}{rrrr}4.267 & 467.83 & -18.66 & 0.0000\end{array}$

$\begin{array}{llll}4.267 & 467.83 & -18.66 & 0.0000\end{array}$

$\begin{array}{llll}4.265 & 467.83 & -18.66 & 0.0000 \\ 4.265 & 467.83 & -18.66 & 0.0000\end{array}$

$\begin{array}{llll}4.265 & 467.83 & -18.66 & 0.0000 \\ 4.830 & 467.83 & -18.66 & 0.0000 \\ 6.690 & 586.54 & -14.18 & 0.0000\end{array}$

$\begin{array}{llll}4.8650 & 486.54 & -14.18 & 0.0000 \\ 6.690 & 545.05 & -0.14 & 0.0000\end{array}$

$\begin{array}{llll}6.690 & 545.05 & -0.14 & 0.0000 \\ 6.690 & 557.10 & -49.28 & 0.0000\end{array}$

$\begin{array}{llll}6.690 & 557.10 & -49.28 & 0.0000 \\ 6.690 & -49.28 & 0.0000\end{array}$

$\begin{array}{llll}40.618 & 1007.03 & 61.22 & 0.0000\end{array}$

$\begin{array}{llll}40.618 & 1007.03 & 61.22 & 0.0000\end{array}$

$\begin{array}{rrrr}39.683 & 1757.48 & 48.80 & 0.0114 \\ 9.523 & 1162.54 & -58.29 & 0.0085\end{array}$

$\begin{array}{llll}9.523 & 1162.54 & -58.29 & 0.0085\end{array}$

$\begin{array}{llll}3.264 & 910.78 & -120.61 & 0.0084\end{array}$

$\begin{array}{llll}3.264 & 910.85 & -120.61 & 0.0084\end{array}$

$\begin{array}{llrl}3.264 & 910.92 & -120.61 & 0.0084 \\ 4.830 & 486.54 & -14.18 & 0.0000\end{array}$

$\begin{array}{llll}4.830 & 486.54 & -14.18 & 0.0000 \\ 4.830 & 487.35 & -14.58 & 0.0000\end{array}$

$\begin{array}{llll}4.830 & 487.35 & -14.58 & 0.0000 \\ 4.830 & 487.35 & -14.58 & 0.0000\end{array}$

4.830

487.35

487.35

$-14.580 .0000$

BPR

VTAS

OPR
9.520

$\mathrm{EPR}$
0.73

PowerSet

T4
1757.5

T41

T49

$\begin{array}{rrrrrr}\text { NC } & \text { TR } & \text { efPoly } & \text { pwr } & \text { SMN } & \text { SMW } \\ 2583.766 & 1.0400 & 0.9078 & -1451.6 & 1508.91 & 54.61 \\ 2583.766 & 1.1650 & 0.8428 & -685.1 & 7.02 & 3.84 \\ 8959.355 & 1.8076 & 0.8567 & -3622.5 & 61.75 & 58.40 \\ 221.489 & 1.3636 & 0.8553 & 3622.4 & & \\ 71.970 & 1.2747 & 0.8369 & 2136.6 & & \end{array}$

TURBOMACHINERY MAP DATA

$\begin{array}{lrrrrrrrrr} & \text { WCMap } & \text { PRmap } & \text { effMap } & \text { NCMap } & \text { R/Parm } & \text { S WCDes } & \text { S.PRdes } & \text { S_effDes } & \text { S NCDes } \\ \text { Fan } & 870.14 & 1.129 & 0.9080 & 2559.130 & 0.0415 & 0.8607 & 1.0235 & 0.9990 & 0.9905 \\ \text { LPC } & 74.58 & 1.529 & 0.7806 & 0.682 & 0.0000 & 1.1475 & 1.0754 & 1.0664 & 0.0003 \\ \text { HPC } & 49.16 & 5.832 & 0.8266 & 8720.505 & 10.9525 & 1.0995 & 1.0494 & 0.9898 & 0.9733 \\ \text { HPT } & 0.96 & 4.079 & 0.8772 & 1.331 & 4.0795 & 12.6299 & 0.9723 & 1.0000 & 0.0003 \\ \text { LPT } & 0.84 & 2.442 & 0.8627 & 0.810 & 2.4419 & 65.2590 & 0.7521 & 0.9926 & 0.0005\end{array}$

$\begin{array}{rrrrrrrr}\text { Ts } & \text { Aphy } & \text { MN } & \text { gamt } & \text { WAR } & \text { Wair } & \text { WH2O } & \text { H2O frac } \\ 416.97 & 2544.5 & 0.7800 & 1.40077 & 0.0000507 & 254.96 & 0.013 & 0.0001 \\ 455.66 & 4168.2 & 0.3651 & 1.40077 & 0.0000507 & 254.96 & 0.013 & 0.0001 \\ 453.89 & 3531.8 & 0.3914 & 1.40077 & 0.0000507 & 228.81 & 0.012 & 0.0001 \\ 464.91 & 830.5 & 0.1770 & 1.40077 & 0.0000507 & 26.15 & 0.001 & 0.0001 \\ 462.71 & 2606.7 & 0.5070 & 1.40061 & 0.0000507 & 228.81 & 0.012 & 0.0001 \\ 538.33 & 412.6 & 0.2498 & 1.39984 & 0.0000507 & 26.15 & 0.001 & 0.0001 \\ 549.91 & 412.6 & 0.2561 & 1.39840 & 0.0091579 & 26.15 & 0.239 & 0.0091 \\ 551.35 & 412.6 & 0.2287 & 1.39840 & 0.0091579 & 23.53 & 0.216 & 0.0091 \\ 971.83 & 49.7 & 0.4365 & 1.37886 & 0.0091579 & 22.39 & 0.205 & 0.0091 \\ 987.27 & 49.3 & 0.3248 & 1.37886 & 0.0091579 & 17.35 & 0.159 & 0.0091 \\ 1732.61 & 74.6 & 0.2954 & 1.32807 & 0.0091579 & 17.35 & 0.159 & 0.0227 \\ 1132.03 & 265.4 & 0.3846 & 1.36318 & 0.0091579 & 23.26 & 0.213 & 0.0193 \\ 895.67 & 860.2 & 0.2981 & 1.37916 & 0.0091579 & 23.53 & 0.216 & 0.0192 \\ 895.74 & 860.2 & 0.2981 & 1.37916 & 0.0091579 & 23.53 & 0.216 & 0.0192 \\ 877.83 & 613.5 & 0.4452 & 1.37915 & 0.0091579 & 23.53 & 0.216 & 0.0192 \\ 462.71 & 2606.7 & 0.5070 & 1.40061 & 0.0000507 & 228.81 & 0.012 & 0.0001 \\ 459.03 & 2481.9 & 0.5549 & 1.40058 & 0.0001536 & 231.43 & 0.036 & 0.0002 \\ 459.03 & 2481.9 & 0.5549 & 1.40058 & 0.0001536 & 231.43 & 0.036 & 0.0002 \\ 419.24 & 2006.8 & 0.9002 & 1.40058 & 0.0001536 & 231.43 & 0.036 & 0.0002\end{array}$




\begin{tabular}{|c|c|c|c|c|c|c|c|c|}
\hline \multirow{2}{*}{$\begin{array}{ll}Z & ===\text { INLETS }==== \\
& \text { Inlet }\end{array}$} & eRam & Afs & Fram & \multirow{2}{*}{\multicolumn{2}{|c|}{ BLEEDS - interstg }} & \multirow{2}{*}{ Wb/Win } & \multirow{2}{*}{$\mathrm{Bl} d W \mathrm{k}$} & \multirow[b]{2}{*}{$\begin{array}{r}\text { BldF } \\
0.2200\end{array}$} \\
\hline & 1.0000 & 2544.46 & 6190.1 & $\begin{array}{l}\text { BLEEDS - } \\
\text { HPT COOL }\end{array}$ & & & & \\
\hline$====$ DUCTS $====$ & dPnorm & MN & Aphy & $\mathrm{LPT}^{-} \mathrm{COOL}$ & A HPC.C> & 0.0117 & 0.5000 & 0.4500 \\
\hline TEGV & 0.0000 & 0.2981 & 860.21 & WB2 $\overline{\mathrm{X}}$ & HPC. B> & 0.0000 & 0.4500 & 0.2200 \\
\hline FanDuct & 0.0000 & 0.5549 & 2481.93 & WB2Y & HPC.B> & 0.0000 & 0.7600 & 0.6200 \\
\hline & & & & WBA2X & HPC.B> & 0.0000 & 0.4500 & 0.2200 \\
\hline$==$ SPLITTERS $==$ & BPR & $\mathrm{dP} / \mathrm{P} 1$ & $\mathrm{dP} / \mathrm{P} 2$ & WBLKG & HPC.I> & 0.0000 & 1.0000 & 1.0000 \\
\hline Splitter & 8.7511 & 0.0005 & 0.0005 & WBW2X & HPC.B> & 0.0000 & 0.4500 & 0.2200 \\
\hline$===$ SHAFTS $====$ & Nmech & trg in & pwr in & BLEEDS - & output & Wb/Win & hscale & Pscale \\
\hline HP Shaft & 9285.3 & 2049.0 & 3622.4 & WB17Y & Bleed> & 0.1000 & 1.0000 & 1.0000 \\
\hline LP_Shaft & 2453.9 & 4573.1 & 2136.6 & HPT_COOL & A Bleed> & 0.1142 & 1.0000 & 1.0000 \\
\hline & & & & $\mathrm{HPT}^{-} \mathrm{COOL}$ & B Bleed> & 0.0999 & 1.0000 & 1.0000 \\
\hline & & & & WB $3 \bar{x}$ & Bleed> & 0.0000 & 1.0000 & 1.0000 \\
\hline & & & & WBA3X & Bleed> & 0.0000 & 1.0000 & 1.0000 \\
\hline & & & & WBW3X & Bleed> & 0.0000 & 1.0000 & 1.0000 \\
\hline & & & & WBFDLKG & FanDu> & 0.0000 & 1.0000 & 1.0000 \\
\hline & & & & WB15X & Bleed> & 0.0000 & 1.0000 & 1.0000 \\
\hline & & & & WB15Y & Bleed> & 0.0000 & 1.0000 & 1.0000 \\
\hline & & & & WB17X & Bleed> & 0.0000 & 1.0000 & 1.0000 \\
\hline$===$ BURNERS $===$ & Ttout & eff & dPnorm & Wfuel & FAR & & & \\
\hline Burner & 1757.42 & 0.9995 & 0.0230 & .19754 & 01138 & & & \\
\hline$===\operatorname{NOZZLES}===$ & $\mathrm{PR}$ & Cfg & CdTh & $\mathrm{Cv}$ & Ath & MNth & Vact & $\mathrm{Fg}$ \\
\hline Core Nozz & 1.144 & 0.9801 & 1.0000 & 0.9800 & 3.40 & 0.445 & 631.1 & 469.7 \\
\hline Byp_Nozz & 1.692 & 0.9800 & 1.0000 & 0.9800 & 6.86 & 0.900 & 885.9 & 6373.4 \\
\hline
\end{tabular}


COMverge = 1 CASE: 0

Version:NPSS_1.6.5 - Rev: -> Gas Package: Janaf iter/pass/Jacb/Broy=13/41/2/10 Run by: Philip C Jorgenson PC: 10

Temperature Stator 1 inlet: $467.15 \quad$ Stator 1 exit: $474.39 \quad$ Stator 2 inlet: $483.46 \quad$ Stator 2 exit: 488.14 $\begin{array}{lll}\text { Stator } 3 \text { inlet: } 497.43 & \text { Stator } 3 \text { exit: } 501.66 & \text { Stator } 4 \text { inlet: 510.30 Stator 4 exit: } 513.8 \\ \text { Stator } 5 \text { inlet: } 520.89 & \text { Stator } 5 \text { exit: } 523.93 & \text { Unblocked Percent Blockage: } 0.00\end{array}$

$\begin{array}{lclll}\text { Ambient Relative Humidity } & 10.00 & & & \\ \text { Fan Face Relative Humidity } & 2.36 & & & \\ \text { Fan Bypass Relative Humidity } & 1.69 & & & \\ \text { LPC Inlet Relative Humidity } & 1.54 & & & \\ \text { LPC Exit Relative Humidity } & 0.15 & & & \\ \text { HPC Relative Humidity } & 0.06 & & & \\ \text { Drop Diameter } & 0.0000050 & \text { Inlet Length } & \\ \text { Ambient Flow Velocity } & 731.04 & \text { Fan/LPC Inlet Flow Velocity } & 180.92 \\ \text { Ambient Static Pressure } & 2.95 & \text { Fan/LPC Inlet Static Pressure } & 4.11 \\ \text { Ambient Static Temperature } & 416.97 & \text { Fan/LPC Inlet Static Temperature } & 458.79 \\ \text { Additional Water at LPC Exit } & 0.0079013 & & & \end{array}$

\begin{tabular}{|c|c|c|c|c|c|c|c|c|c|c|c|c|c|c|}
\hline & alt & dTamb & W & $\begin{array}{l}\text { JMMARY } \\
\text { Fn }\end{array}$ & $\begin{array}{l}\text { DUTPUT DATA } \\
\text { TSFC }\end{array}$ & Wfuel & BPR & VTAS & $O P R$ & EPR & Powerset & T4 & $T 41$ & T49 \\
\hline 0.730 & 38334.0 & 27.00 & 248.09 & 636.9 & 1.0625 & 676.68 & 8.8265 & 731.04 & 9.238 & 0.763 & 10.000 & 1727.2 & 1633.5 & 1139. \\
\hline
\end{tabular}

\begin{tabular}{|c|c|}
\hline FSO & Ambient.Fl O \\
\hline FS1 & Inlet.Fl $0^{-}$ \\
\hline FS12 & Splitter.Fl_02 \\
\hline FS2 & Splitter.Fl_01 \\
\hline FS14 & Fan.Fl O \\
\hline FS23 & LPC.Fl_o \\
\hline FS24 & VaporIN.Flo \\
\hline FS25 & Bleed2.Fl_o \\
\hline FS3 & HPC.Fl O \\
\hline FS36 & Bleed3.F1 O \\
\hline FS4 & Burner.FI_O \\
\hline FS45 & HPT.FI_O \\
\hline FS49 & LPT.FI- \\
\hline FS5 & TEGV.FI_O \\
\hline FS8 & Core_Nozz.F \\
\hline FS17 & FanDūctLkg.Fin_o \\
\hline FS171 & Bleed15.Fl_o \\
\hline FS172 & FanDuct.F \\
\hline & \\
\hline
\end{tabular}

\begin{tabular}{rrrrr}
\multicolumn{7}{c}{ FLOW } & STATION DATA & & \\
$W$ & Pt & Tt & $h t$ & FAR \\
248.09 & 4.201 & 461.52 & -20.16 & 0.0000 \\
248.09 & 4.201 & 461.52 & -20.16 & 0.0000 \\
222.84 & 4.199 & 461.52 & -20.16 & 0.0000 \\
25.25 & 4.199 & 461.52 & -20.16 & 0.0000 \\
222.84 & 4.722 & 478.92 & -15.99 & 0.0000 \\
25.25 & 6.446 & 533.65 & -2.87 & 0.0000 \\
25.45 & 6.446 & 544.30 & -45.53 & 0.0000 \\
22.90 & 6.446 & 544.30 & -45.53 & 0.0000 \\
21.79 & 38.812 & 982.65 & 61.85 & 0.0000 \\
16.89 & 38.812 & 982.65 & 61.85 & 0.0000 \\
17.08 & 37.918 & 1727.24 & 49.59 & 0.0112 \\
22.82 & 9.213 & 1143.60 & -54.44 & 0.0084 \\
23.09 & 3.314 & 906.61 & -112.87 & 0.0083 \\
23.09 & 3.314 & 906.68 & -112.87 & 0.0083 \\
23.09 & 3.314 & 906.75 & -112.87 & 0.0083 \\
222.84 & 4.722 & 478.92 & -15.99 & 0.0000 \\
225.39 & 4.722 & 479.66 & -16.33 & 0.0000 \\
225.39 & 4.722 & 479.66 & -16.33 & 0.0000 \\
225.39 & 4.722 & 479.66 & -16.33 & 0.0000
\end{tabular}

WC
818.60
818.60
735.66
83.35
666.45
58.38
59.43
53.48
11.36
8.80
12.08
54.05
135.38
135.38
135.39
666.45
674.58
674.58
674.58

PS
2.947
3.847
3.795
4.113
3.984
6.177
6.166
6.221
34.145
36.134
35.797
8.363
3.140
3.140
2.947
3.984
3.858
3.858
2.947

Ts
416.97
450.02
448.34
458.79
456.19
527.19
537.44
538.81
948.49
963.45
1702.69
1114.34
893.24
893.31
877.88
456.19
452.73
452.73
419.12

$\begin{array}{rr}\text { Aphy } & \mathrm{MN} \\ 2562.0 & 0.7300 \\ 4168.2 & 0.3571 \\ 3531.8 & 0.3830 \\ 830.5 & 0.1723 \\ 2606.7 & 0.4987 \\ 412.6 & 0.2475 \\ 412.6 & 0.2530 \\ 412.6 & 0.260 \\ 49.7 & 0.4343 \\ 49.3 & 0.3233 \\ 74.6 & 0.2952 \\ 265.4 & 0.3789 \\ 860.2 & 0.2807 \\ 860.2 & 0.2807 \\ 613.4 & 0.4157 \\ 2606.7 & 0.4987 \\ 2481.9 & 0.5449 \\ 2481.9 & 0.5449 \\ 2006.9 & 0.8489\end{array}$

gamt
1.40081
1.40081
1.40081
1.40081
1.40067
1.40002
1.39877
1.39877
1.38055
1.38055
1.32976
1.36465
1.37965
1.37965
1.37964
1.40067
1.40066
1.40066
1.40066

WAR
0.0000491
0.0000491
0.0000491
0.0000491
0.0000491
0.0000491
0.0079504
0.0079504
0.0079504
0.0079504
0.0079504
0.0079504
0.0079504
0.0079504
0.0079504
0.0000491
0.0001376
0.0001376
0.0001376

$\begin{array}{rrr}\text { Wair } & \text { WH2O } & \text { H2O frac } \\ 248.08 & 0.012 & 0.0000 \\ 248.08 & 0.012 & 0.0000 \\ 222.83 & 0.011 & 0.0000 \\ 25.25 & 0.001 & 0.0000 \\ 222.83 & 0.011 & 0.0000 \\ 25.25 & 0.001 & 0.0000 \\ 25.25 & 0.201 & 0.0079 \\ 22.72 & 0.181 & 0.0079 \\ 21.62 & 0.172 & 0.0079 \\ 16.76 & 0.133 & 0.0079 \\ 16.76 & 0.133 & 0.0214 \\ 22.46 & 0.179 & 0.0180 \\ 22.72 & 0.181 & 0.0179 \\ 22.72 & 0.181 & 0.0179 \\ 22.72 & 0.181 & 0.0179 \\ 222.83 & 0.011 & 0.0000 \\ 225.36 & 0.031 & 0.0001 \\ 225.36 & 0.031 & 0.0001 \\ 225.36 & 0.031 & 0.0001\end{array}$

TURBOMACHINERY PERFORMANCE DATA

\begin{tabular}{|c|c|c|c|c|c|c|c|c|c|}
\hline & Wc & PR & eff & $\mathrm{NC}$ & TR & efPoly & pwr & SMN & SMW \\
\hline Fan & 735.66 & 1.124 & 0.9053 & 2518.320 & 1.0377 & 0.9069 & -1314.7 & 2024.64 & 54.51 \\
\hline LPC & 83.35 & 1.535 & 0.8343 & 2518.320 & 1.1563 & 0.8440 & -617.8 & 6.82 & 3.38 \\
\hline $\begin{array}{l}\text { HPC } \\
\text { HP }\end{array}$ & 53.48 & 6.021 & 0.8174 & 8940.949 & 1.8054 & 0.8560 & -3394.8 & 61.61 & 58.21 \\
\hline $\mathrm{HPT}$ & 12.08 & 4.116 & 0.8758 & 220.384 & 1.3607 & 0.8538 & 3394.8 & & \\
\hline LPT & 54.05 & 2.780 & 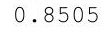 & 70.246 & 1.2594 & 0.8312 & 1932.5 & & \\
\hline \multirow{2}{*}{\multicolumn{10}{|c|}{ TURBOMACHINERY MAP D }} \\
\hline & & & & & & s WcDes & S_PRdes & s_effDes & S NCDes \\
\hline an & 854.77 & 1.122 & 0.9072 & 94.309 & 0.0414 & $\overline{0} .8$ & $\overline{1} .0235$ & 0.9980 & $\overline{0} .9905$ \\
\hline $\mathrm{PC}$ & 71.75 & 1.500 & 0.7776 & 0. & 100 & 1. & 09 & 1.0730 & 0.0003 \\
\hline HPC & 48.64 & 5.784 & 0.8259 & 702.589 & 10.9679 & 1.0995 & 1.0494 & 0.9898 & 0.9733 \\
\hline HРT & 0.96 & 4.029 & 0.8758 & 1.324 & 4.0292 & 12.6299 & 0.9723 & 1.0000 & 0.0003 \\
\hline LPT & 0.83 & 2.339 & 0.8568 & 0.790 & 2.3390 & 65.2590 & 0.7521 & 0.9926 & 0.0005 \\
\hline
\end{tabular}




\begin{tabular}{|c|c|c|c|c|c|c|c|c|c|c|c|c|c|}
\hline$Z$ & $===\operatorname{INLETS} S====$ & eRam & Afs & Fram & \multirow{2}{*}{\multicolumn{2}{|c|}{ BIFFDS - intersto }} & & & & & & & \\
\hline D & Inlet & 1.0000 & 2562.02 & 5637.0 & & & $\begin{array}{l}\text { Wb/Win } \\
0.0368\end{array}$ & $\begin{array}{r}\text { BldWk } \\
0.5000\end{array}$ & $\begin{array}{r}\text { BldP } \\
0.2200\end{array}$ & $\begin{array}{r}W \\
0.8428\end{array}$ & $\begin{array}{r}\mathrm{Tt} \\
765.35\end{array}$ & $\begin{array}{r}h t \\
8.16\end{array}$ & $\begin{array}{r}P t \\
9.213\end{array}$ \\
\hline$=$ & $====$ DUCT $S====$ & dPnorm & MN & Aphy & \multicolumn{2}{|c|}{ LPT COOLA HPC.C> } & 0.0117 & 0.5000 & 0.4500 & 0.2680 & 765.35 & 8.16 & 3.314 \\
\hline ت & TEGV & 0.0000 & 0.2807 & 860.21 & WB $2 \overline{\mathrm{X}}$ & HPC.B> & 0.0000 & 0.4500 & 0.2200 & 0.0000 & 743.38 & 2.79 & 13.567 \\
\hline & FanDuct & 0.0000 & 0.5449 & 2481.93 & WB2Y & HPC.B> & 0.0000 & 0.7600 & 0.6200 & 0.0000 & 878.94 & 36.08 & 26.513 \\
\hline & & & & & \multirow{2}{*}{$\begin{array}{l}\text { WBA2X } \\
\text { WBLKG }\end{array}$} & HPC. B> & 0.0000 & 0.4500 & 0.2200 & 0.0000 & 743.38 & 2.79 & 13.567 \\
\hline 0 & $==$ SPLITTERS $==$ & BPR & $\mathrm{dP} / \mathrm{P} 1$ & $\mathrm{dP} / \mathrm{P} 2$ & & HPC.I> & 0.0000 & 1.0000 & 1.0000 & 0.0000 & 982.65 & 61.85 & 38.812 \\
\hline 0 & Splitter & 8.8265 & 0.0005 & 0.0005 & WBW2X & HPC.B> & 0.0000 & 0.4500 & 0.2200 & 0.0000 & 743.38 & 2.79 & 13.567 \\
\hline$\underline{\simeq}$ & $===$ SHAFTS $====$ & Nmech & trg in & pwr in & BLEEDS - & output & Wb/Win & hscale & Pscale & $\mathrm{W}$ & Tt & ht & Pt \\
\hline$\infty$ & HP_Shaft & 9159.2 & 1946.7 & 3394.8 & WB17Y & Bleed> & 0.1000 & 1.0000 & 1.0000 & 2.5447 & 544.30 & -45.53 & 6.446 \\
\hline$P$ & LP ${ }^{-}$Shaft & 2375.5 & 4272.5 & 1932.5 & \multirow{2}{*}{ HPT_COOLA } & Bleed $>$ & 0.1142 & 1.0000 & 1.0000 & 2.6150 & 982.65 & 61.85 & 37.918 \\
\hline & & & & & & Bleed> & 0.0999 & 1.0000 & 1.0000 & 2.2882 & 982.65 & 61.85 & 21.499 \\
\hline & & & & & WB $3 \bar{X}$ & Bleed> & 0.0000 & 1.0000 & 1.0000 & 0.0000 & 982.65 & 61.85 & 38.812 \\
\hline & & & & & WBA $3 \mathrm{X}$ & Bleed> & 0.0000 & 1.0000 & 1.0000 & 0.0000 & 982.65 & 61.85 & 38.812 \\
\hline & & & & & $\begin{array}{l}\text { WBW3X } \\
\text { WBFDLKG }\end{array}$ & Bleed> & 0.0000 & 1.0000 & 1.0000 & 0.0000 & 982.65 & 61.85 & 38.812 \\
\hline & & & & & WBFDLKG & FanDu> & 0.0000 & 1.0000 & 1.0000 & 0.0000 & 478.92 & -15.99 & 4.722 \\
\hline & & & & & \multirow{2}{*}{ WB15X } & Bleed> & 0.0000 & 1.0000 & 1.0000 & 0.0000 & 478.92 & -15.99 & 4.722 \\
\hline & & & & & & Bleed> & 0.0000 & 1.0000 & 1.0000 & 0.0000 & 478.92 & -15.99 & 4.722 \\
\hline & & & & & $\begin{array}{l}\text { WB15Y } \\
\text { WB17X }\end{array}$ & Bleed> & 0.0000 & 1.0000 & 1.0000 & 0.0000 & 478.92 & -15.99 & 4.722 \\
\hline & $===$ BURNERS $===$ & Ttout & eff & dPnorm & & & & & & & & \\
\hline & Burner & 1727.18 & 0.9995 & 0.0230 & \multicolumn{2}{|c|}{$\begin{array}{rr}\text { Wfuel } & \text { FAR } \\
0.18797 & 0.01122\end{array}$} & & & & & & & \\
\hline & $===\operatorname{NOZZLES}===$ & PR & $\operatorname{Cfg}$ & CdTh & & Ath & MNth & \multirow{3}{*}{$\begin{array}{r}\text { Vact } \\
589.1 \\
835.2\end{array}$} & $\mathrm{Fg}$ & & & & \\
\hline & Core_Nozz & 1.125 & 0.9801 & 1.0000 & \multirow{2}{*}{\multicolumn{2}{|c|}{$\begin{array}{r}\mathrm{CV} \\
0.9800 \\
0.9800\end{array}$}} & 0.416 & & 422.8 & & & & \\
\hline & Byp_Nozoz & 1.602 & 0.9800 & 1.0000 & & & 0.849 & & 5851.1 & & & & \\
\hline
\end{tabular}


COnverge = 1 CASE: 0

Version:NPSS_1.6.5 - Rev: -> Gas Package: Janaf iter/pass/Jacb/Broy=12/40/2/9 Run by: Philip C Jorgenson PC: 10

$\begin{array}{rllll}\text { Temperature Stator } 1 \text { inlet: } 465.08 & \text { Stator } 1 \text { exit: } 472.13 & \text { Stator } 2 \text { inlet: } 480.96 & \text { Stator } 2 \text { exit: } 485.51 \\ \text { Stator } 3 \text { inlet: } 494.56 & \text { Stator } 3 \text { exit: } 498.67 & \text { Stator } 4 \text { inlet: } 507.09 & \text { Stator } 4 \text { exit: 510.56 }\end{array}$

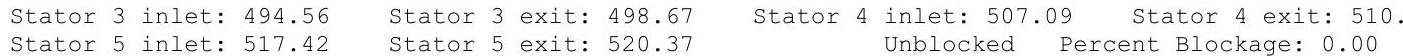

$\begin{array}{lclll}\text { Ambient Relative Humidity } & 10.00 & & & \\ \text { Fan Face Relative Humidity } & 2.52 & & & \\ \text { Fan Bypass Relative Humidity } & 1.83 & & & \\ \text { LPC Inlet Relative Humidity } & 1.66 & & & \\ \text { LPC Exit Relative Humidity } & 0.16 & & & \\ \text { HPC Relative Humidity } & 0.06 & & & \\ \text { Drop Diameter } & 0.000050 & \text { Inlet Length } & \\ \text { Ambient Flow Velocity } & 715.01 & \text { Fan/LPC Inlet Flow Velocity } & 178.44 \\ \text { Ambient Static Pressure } & 3.09 & \text { Fan/LPC Inlet Static Pressure } & 4.25 \\ \text { Ambient Static Temperature } & 416.97 & \text { Fan/LPC Inlet Static Temperature } & 456.94 \\ \text { Additional Water at LPC Exit } & 0.0073898 & & & \end{array}$

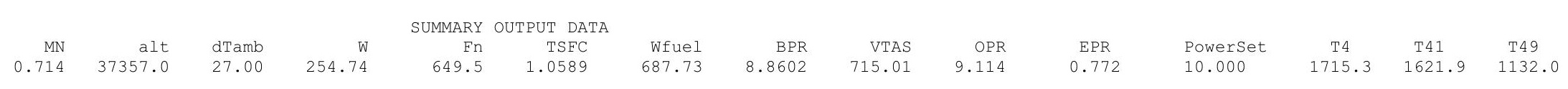

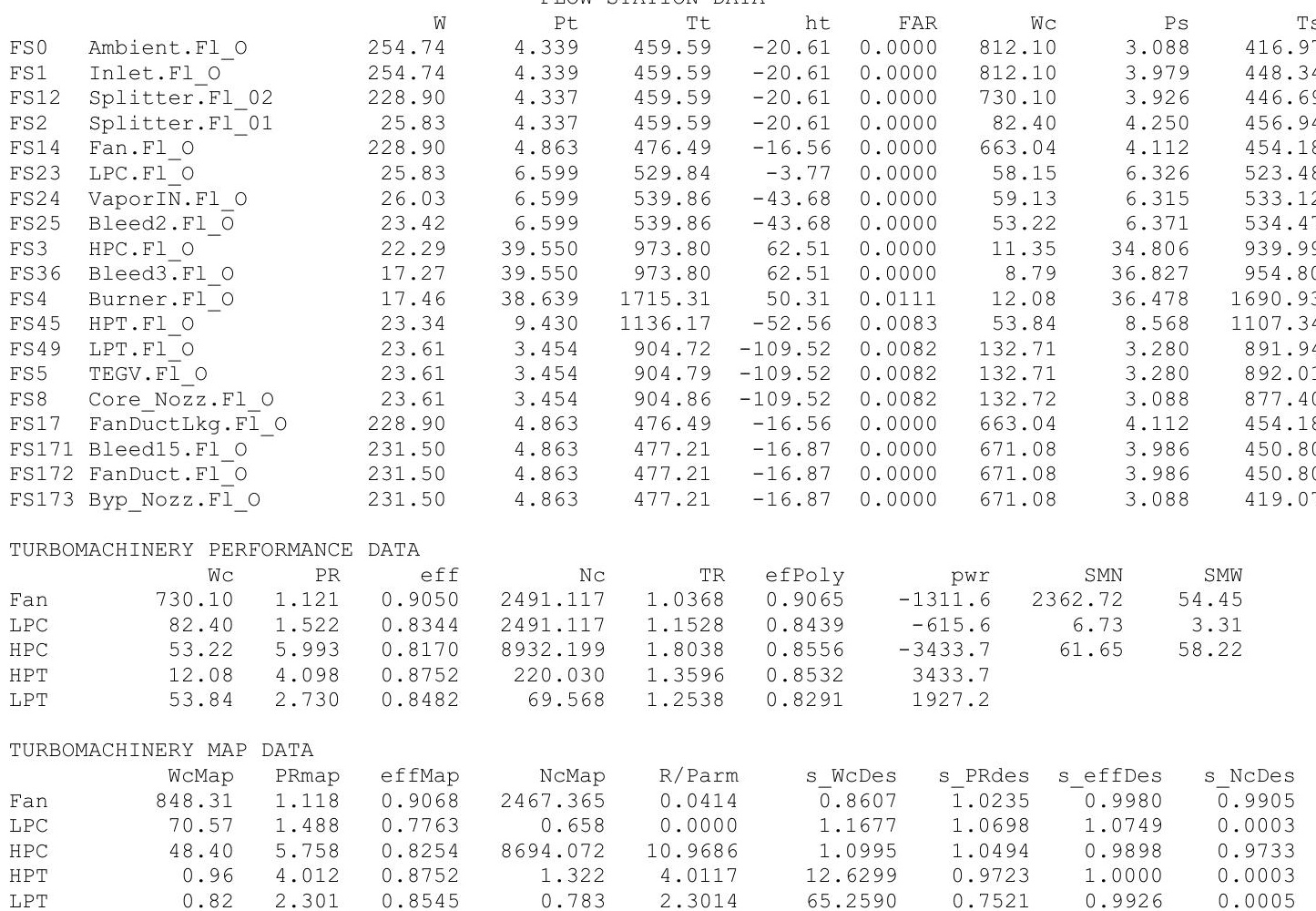




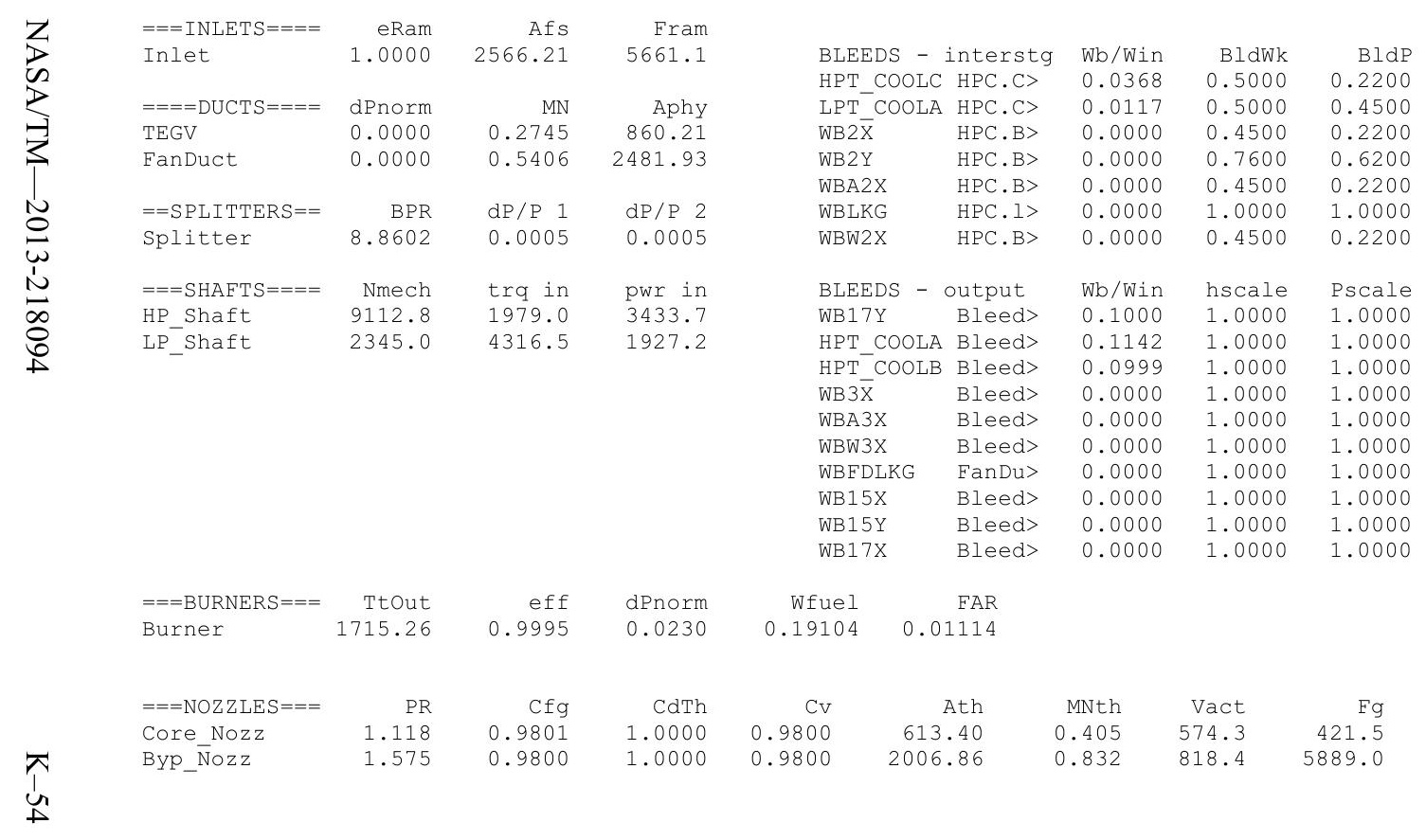


Turbofan Engine - COMDES ON Converge = 1 CASE: 0

Version:NPSS1.6.5 - Rev: -> Gas Package: Janaf iter/pass/Jacb/Broy=14/42/2/11 Run by: Philip C Jorgenson PC: 10

Temperature Stator 1 inlet: 466.56 Stator 1 exit: $473.17 \quad$ Stator 2 inlet: 481.42 $\quad$ Stator 2 exit: 485.66 Stator 5 inlet: 515.41 Stator 5 exit: 518.13 Unblocked Percent Blockage: 0.00

Ambient Relative Humidity $\quad 10.00$

Fan Face Relative Humidity $\quad 3.13$

Fan Bypass Relative Humidity $\quad 2.35$

2.10
LPC Exit Relative Humidity

Dit Relative Humidity

Drop Diameter

0.07

Ambient Flow Velocity

Ambient Static Pressure

Ambient Static Temperature

675.10

Inlet Length

40.00

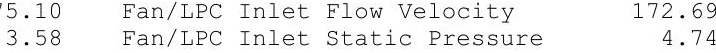

Additional Water at LPC Exit

0.0066923

Fan/LPC Inlet Static Temperature 458.93

MN alt dTamb SUMMARY OUTPUT DATA

$\begin{array}{rrrrrr} & \text { alt } & \text { dTamb } & \text { W } & \text { Fn } & \text { TSFC } \\ 0.669 & 34281.0 & 27.00 & 275.93 & 699.1 & \end{array}$

FLOW STATION DATA

FSO Ambient.FI_O

FS1 Inlet.Fl_o

$\begin{array}{ll}\text { FS12 } & \text { Splitter.FI_02 } \\ \text { FS2 } & \text { Splitter.Fl_01 }\end{array}$

FS14 Fan.F1_O

FS24 VaporIN.F1

FS25 Bleed2.FIO

FS3 HPC.FI O

S36 Bleed3.Fl_O

FS4 Burner.FI_O

FS49

FS5 TEGV.Fīo

FS8 Core_Nozz.FI_O

FS17 FanDúctLkg.Fì

FS171 Bleed15.FI_O

FS172 FanDuct.FI-O

FS173 Byp Nozz.Fì O

$W$
275.93
275.93
248.18
27.75
248.18
27.75
27.94
25.14
23.92
18.54
18.75
25.05
25.35
25.35
25.35
248.18
250.97
250.97
250.97

Pt
4.831

$\begin{array}{rrr}\text { Tt } & \text { ht } & \text { FAR } \\ 461.41 & -20.25 & 0.0000\end{array}$

$\begin{array}{lllll}4.831 & 461.41 & -20.25 & 0.0000 & 791.69\end{array}$

$\begin{array}{llllll}4.829 & 461.41 & -20.25 & 0.0000 & 712.42\end{array}$

$\begin{array}{lllll}4.829 & 461.41 & -20.25 & 0.0000 & 712.42 \\ 5.372 & 477.22 & -20.25 & 0.0000 & 79.67 \\ 7.156 & 527.14 & -16.46 & 0.0000 & 651.27\end{array}$

$\begin{array}{lllll}4.829 & 461.41 & -20.25 & 0.0000 & 79.67 \\ 7.372 & 477.22 & -16.46 & 0.0000 & 651.27\end{array}$

$\begin{array}{lllll}5.372 & 477.22 & -16.46 & 0.0000 & 651.27 \\ 7.156 & 536.26 & -4.49 & 0.0000 & 57.45\end{array}$

$\begin{array}{lllll}7.156 & 527.14 & -4.49 & 0.0000 & 57.45 \\ 7.156 & 536.26 & -40.65 & 0.0000 & 58.34\end{array}$

$7.156-536.26-40.65-0.0000$
$42.313-9640$

$\begin{array}{llll}42.313 & 964.23 & -63.96 & 0.0000\end{array}$

$\begin{array}{lllll}42.313 & 964.23 & 63.96 & 0.0000\end{array}$

$\begin{array}{llll}1 . .338 & 1704.23 & 51.80 & 0.0111\end{array}$

$\begin{array}{rrrr}10.200 & 1130.72 & -49.50 & 0.0083 \\ 3.944 & 912.34 & -103.13 & 0.0082\end{array}$

$\begin{array}{llll}3.944 & 912.34 & -103.13 & 0.0082 \\ 3.944 & 912.41 & -103.13 & 0.0082\end{array}$

$\begin{array}{llll}3.944 & 912.48 & -103.13 & 0.0082\end{array}$

$\begin{array}{lll}477.22 & -16.46 & 0.0000\end{array}$

$\begin{array}{llll}5.372 & 477.22 & -16.46 & 0.0000 \\ 5.372 & 477.88 & -16.73 & 0.0000 \\ 5.372 & 477.88 & -16.73 & 0.0000\end{array}$

$5.372-477.88-16.73-0.0000$

52.50
11.33

11.33
8.78

12.08
53.30

53.30
125.28

125.28

125.29
651.27

659.06

659.06
659.06

659.06

VTAS

675.10

OPR
8.759

EPR
0.795

PowerSet
10.000

$\begin{array}{ccc}\text { T4 } & \text { T41 } & \text { T49 } \\ 1704.2 & 1611.0 & 1126.5\end{array}$

$\begin{array}{rr}\text { Aphy } & \mathrm{MN} \\ 2580.5 & 0.6690 \\ 4168.2 & 0.3435 \\ 3531.8 & 0.3686 \\ 830.5 & 0.1644 \\ 2606.7 & 0.4830 \\ 412.6 & 0.2432 \\ 412.6 & 0.2479 \\ 412.6 & 0.2215 \\ 49.7 & 0.4326 \\ 49.3 & 0.3221 \\ 74.6 & 0.2950 \\ 265.4 & 0.3723 \\ 860.2 & 0.2578 \\ 860.2 & 0.2578 \\ 613.4 & 0.3780 \\ 2606.7 & 0.4830 \\ 2481.9 & 0.5264 \\ 2481.9 & 0.5264 \\ 2006.9 & 0.7843\end{array}$

gamt

1.40081
1.40081

1.40081
1.40081

1.40081

1.40069

1.40011
1.39906

1.39906

1.38184
1.38184

1.38184
1.33111

1.36572
1.37954

1.37954
1.37954

1.37953
1.40069

1.40067

1.40067

TURBOMACHINERY PERFORMANCE DATA

\begin{tabular}{|c|c|c|c|c|c|c|c|c|c|}
\hline & WC & PR & eff & NC & $\mathrm{TR}$ & efPoly & pwr & SMN & SMW \\
\hline Fan & 712.42 & 1.112 & 0.9040 & 2409.995 & 1.0343 & 0.9054 & -1330.0 & 4741.63 & 54.05 \\
\hline LPC & 79.67 & 1.482 & 0.8361 & 2409.995 & 1.1424 & 0.8449 & -618.7 & 6.46 & 3.09 \\
\hline PC & 52.50 & 5.913 & 0.8156 & 8908.784 & 1.7981 & 0.8543 & -3631.2 & 61.96 & 58.44 \\
\hline HPT & 12.08 & 4.053 & 0.8745 & 219.430 & 1.3566 & 0.8525 & 3631.2 & & \\
\hline LPT & 53.30 & 2.586 & 0.8430 & 67.599 & 1.2371 & 0.8245 & 1948.8 & & \\
\hline JRE & NERY MAE & DATA & & & & & & & \\
\hline & WcMap & PRmap & effMap & NcMap & R/Parm & s_WcDes & S_PRdes & S_effDes & S_NCDes \\
\hline Fan & 827.76 & 1.110 & 0.9058 & 2387.016 & 0.0414 & $\overline{0} .8607$ & $\overline{1} .0235$ & 0.9980 & 0.9905 \\
\hline $\mathrm{PC}$ & 67.06 & 1.4 & 0.7725 & 0.637 & 0.0000 & 1.1881 & 1.0682 & 1.0823 & 0.0003 \\
\hline PC & 47.75 & 5.681 & 0.8240 & 8671.282 & 10.9589 & 1.0995 & 1.0494 & 0.9898 & 0.9733 \\
\hline PT & 0.96 & 3.968 & 0.8745 & 1.319 & 3.9681 & 12.6299 & 0.9723 & 1.0000 & 0.0003 \\
\hline LPT & 0.82 & 2.193 & 0.8493 & 0.760 & 2.1932 & 65.2590 & 0.7521 & 0.9926 & 0.0005 \\
\hline
\end{tabular}

\begin{tabular}{|c|c|}
\hline & $s$ \\
\hline 3.5 & 423.4 \\
\hline 4.4 & 450.76 \\
\hline 4.3 & 449.18 \\
\hline 4.7 & 458.9 \\
\hline 4.5 & 455.90 \\
\hline 6.8 & 520.97 \\
\hline 6.8 & 529.7 \\
\hline 6. & 531.06 \\
\hline 37.2 & 930.86 \\
\hline 39.4 & 945.46 \\
\hline 39.0 & 1679.96 \\
\hline 9.2 & 1102.69 \\
\hline 3.7 & 900.97 \\
\hline 3.7 & 901.04 \\
\hline 3.5 & 888.34 \\
\hline 4.5 & 455.90 \\
\hline 4.4 & 452.74 \\
\hline 4.4 & 452.74 \\
\hline 3.5 & 425.42 \\
\hline MII & SMW \\
\hline 63 & 54.05 \\
\hline 46 & 3.09 \\
\hline 96 & 58.44 \\
\hline ffDes & S_NcDes \\
\hline .9980 & $\overline{0} .9905$ \\
\hline .0823 & 0.0003 \\
\hline .9898 & 0.9733 \\
\hline .0000 & 0.0003 \\
\hline .9926 & 0.0005 \\
\hline
\end{tabular}

WAR
0.0000588
0.0000588
0.0000588
0.0000588
0.0000588
0.0000588
0.0067510
0.0067510
0.0067510
0.0067510
0.0067510
0.0067510
0.0067510
0.0067510
0.0067510
0.0000588
0.0001328
0.0001328
0.0001328

Wair 275.91 275.91
248.16 27.75 248.16 27.75
27.75 24.98 $\begin{array}{ll}23.76 & 0.160\end{array}$

$\begin{array}{ll}18.42 & 0.124\end{array}$

$\begin{array}{ll}24.68 & 0.167 \\ 24.98 & 0.169\end{array}$

$\begin{array}{ll}24.98 & 0.169\end{array}$

$\begin{array}{rr}24.98 & 0.169 \\ 248.16 & 0.015\end{array}$

$\begin{array}{ll}248.16 & 0.015 \\ 250.94 & 0.033\end{array}$ $\begin{array}{ll}250.94 & 0.033 \\ 250.94 & 0.033\end{array}$ $250.94 \quad 0.033$

$\begin{array}{rr}20 & \text { H2O frac } \\ 16 & 0.0001 \\ 16 & 0.0001 \\ 15 & 0.0001 \\ 02 & 0.0001 \\ 15 & 0.0001 \\ 02 & 0.0001 \\ 87 & 0.0067 \\ 69 & 0.0067 \\ 60 & 0.0067 \\ 24 & 0.0067 \\ 24 & 0.0201 \\ 67 & 0.0167 \\ 69 & 0.0166 \\ 69 & 0.0166 \\ 69 & 0.0166 \\ 015 & 0.0001 \\ .033 & 0.0001 \\ .033 & 0.0001 \\ 033 & 0.0001\end{array}$

$\begin{array}{lll}.75 & 5.681 & 0.8240 \\ .82 & 2.193 & 0.8745\end{array}$

0.760

$\begin{array}{ll}65.2590 & 0.7521\end{array}$

.0005 


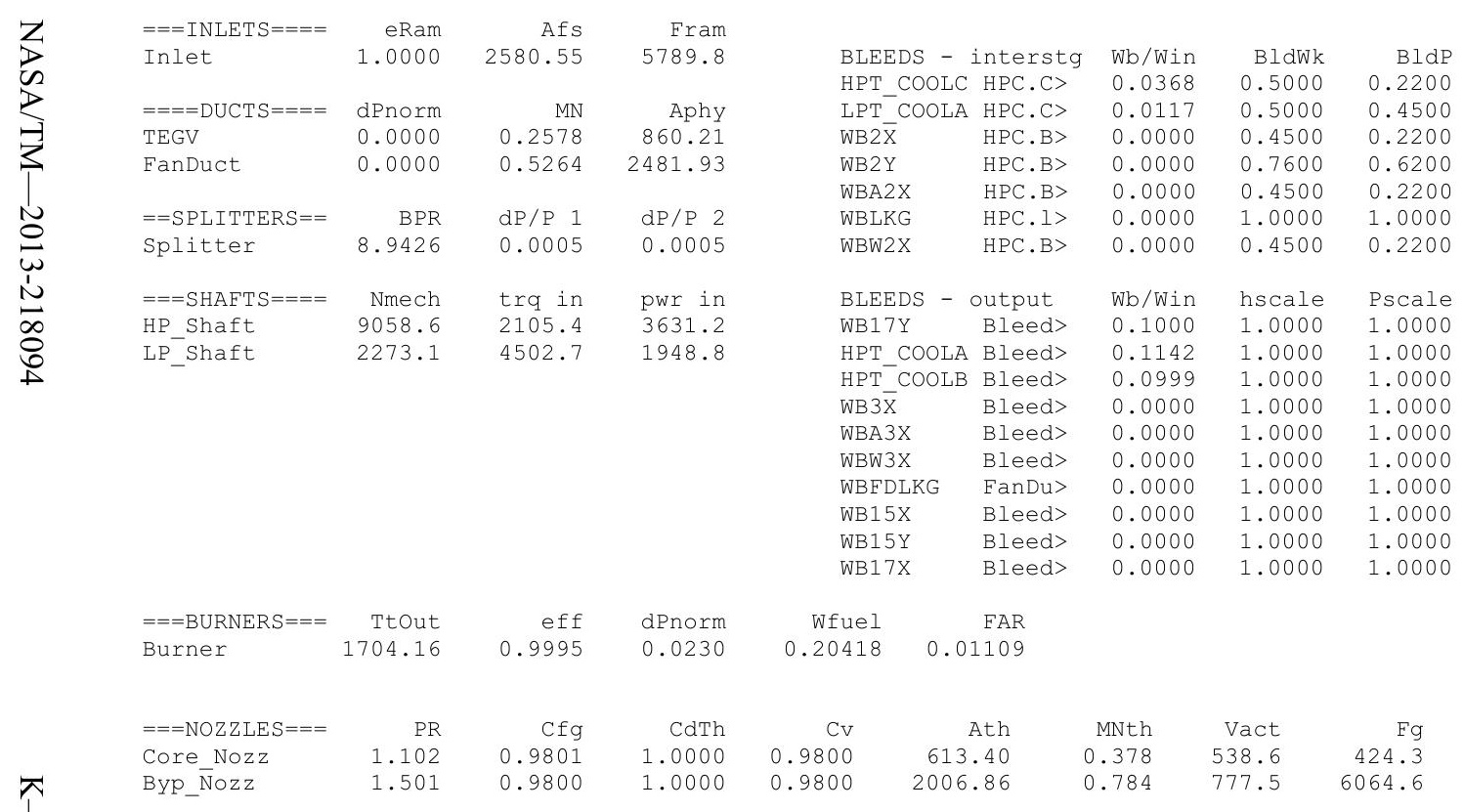


COnverge $=1$ CASE: 0

Version:NPSS_1.6.5 - Rev: -> Gas Package: Janaf iter/pass/Jacb/Broy= 20/48/2/17 Run by: Philip C Jorgenson PC: 10

$\begin{array}{rllll}\text { Temperature Stator } 1 \text { inlet: } 475.00 & \text { Stator } 1 \text { exit: } 480.71 & \text { Stator } 2 \text { inlet: } 487.75 & \text { Stator } 2 \text { exit: } 491.19 \\ \text { Stator } 3 \text { inlet: } 498.51 & \text { Stator } 3 \text { exit: } 501.53 & \text { Stator } 4 \text { inlet: } 508.37 & \text { Stator } 4 \text { exit: } 510.85\end{array}$

$\begin{array}{lll}\text { Stator } 3 \text { inlet: } 498.51 & \text { Stator } 3 \text { exit: } 501.53 & \text { Stator } 4 \text { inlet: 508.37 Stator 4 exit: } 510.85 \\ \text { Stator } 5 \text { inlet: } 516.32 & \text { Stator } 5 \text { exit: } 518.38 & \text { Unblocked Percent Blockage: } 0.00\end{array}$

Ambient Relative Humidity $\quad 10.00$

Fan Face Relative Humidity $\quad 4.08$

$\begin{array}{ll}\text { Fan Bypass Relative Humidity } & 3.22 \\ \text { LPC } & 2.94\end{array}$

LPC Exit Relative Humidity $\quad 0.50$

HPC Relative Humidity

Drop Diameter

0.07

0.0000050
624.43

$\begin{array}{lr} & 40.00\end{array}$

Ambient Static Pressure

4.36 Fan/LPC Inlet Flow Velocity $\quad \begin{aligned} 47.28 \\ 48.58\end{aligned}$

$\begin{array}{llllr}\text { Ambient Static Pressure } & 4.36 & \text { Fan/LPC } & \text { Inlet Static Pressure r } & 5.49 \\ \text { Ambient Static Temperature } & 438.58 & \text { Fan/LPC } & \text { Inlet Static Temperature } & 468.55 \\ \text { Additional Water at LPC Exit } & 0.0064079 & & & \end{array}$

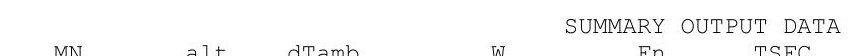

$\begin{array}{rrr}\text { MN } & \text { alt } & \text { dTamb } \\ 0.608 & 30029.0 & 27.00\end{array}$

302.02
Wfuel
797.99 $\begin{array}{rl}752.3 & 1.0607\end{array}$ 797.99

BPR
8.5056

VTAS
624.43

OPR
8.174

$E P R$
0.828

Powerset

10.000

$\begin{array}{ccc}\text { T4 } & \text { T41 } & \text { T49 } \\ 1704.0 & 1610.3 & 1129.1\end{array}$

FSO Ambient.Fl

FS1 Inlet.FI_O

FS12 Splitter.FI_02

FS14 Fan.F1_O

FS24 VaporIN.FI_O

FS3 Bleed2.F1_

FS36 Ble.F3 31

FS4 Burner.FI_O

FS49 LPT.FI_O

FS8 Core Nozz.FI

FS17 FanDüctLkg.Fì

FS171 Bleed15.FI_O

FS172 FanDuct. FI-O

TURBOMACHINERY PERFORMANCE DATA

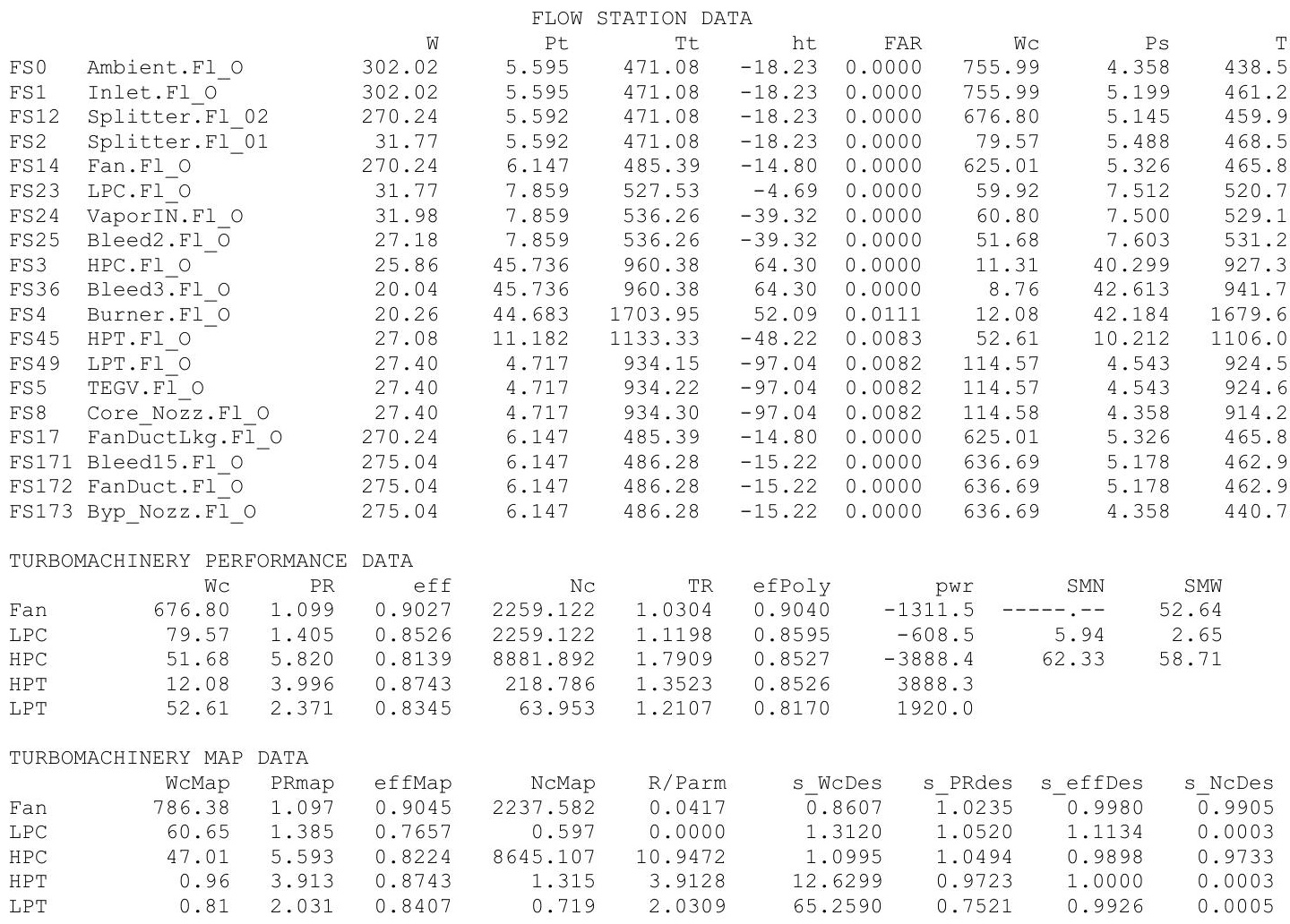

TURBOMACHINERY MAP DATA

\begin{tabular}{|c|c|c|c|c|c|c|c|c|c|c|c|}
\hline & & & $\mathrm{W}$ & $\begin{array}{l}\text { FLOW } S \\
\text { Pt }\end{array}$ & $\begin{array}{c}\text { STATION DA' } \\
\text { Tt }\end{array}$ & ht & FAR & Wo & & Ps & \\
\hline FSO & Ambient.Fl O & & 302.02 & 5.595 & 471.08 & -18.23 & 0.0000 & 755.99 & & & 438.5 \\
\hline FS1 & Inlet.Fl_o- & & 302.02 & 5.595 & 471.08 & -18.23 & 0.0000 & 755.99 & & & 461.2 \\
\hline FS12 & Splitter.Fl 0 & & 270.24 & 5.592 & 471.08 & -18.23 & 0.0000 & 676.80 & & & 459 \\
\hline FS2 & Splitter.Fl_o & & 31.77 & 5.592 & 471.08 & -18.23 & 0.0000 & 79.57 & & & \\
\hline FS14 & Fan.Fl_o & & 270.24 & 6.147 & 485.39 & -14.80 & 0.0000 & 625.01 & & & 465 \\
\hline FS23 & LPC.FI_O & & 31.77 & 7.859 & 527.53 & -4.69 & 0.0000 & 59.92 & & & 520 \\
\hline FS24 & VaporIN.FI_O & & 31.98 & 7.859 & 536.26 & -39.32 & 0.0000 & 60.80 & & & 529 \\
\hline S25 & Bleed2.FI_ō & & 27.18 & 7.859 & 536.26 & -39.32 & 0.0000 & 51.68 & & & 531 \\
\hline FS3 & HPC.FI_O & & 25.86 & 45.736 & 960.38 & 64.30 & 0.0000 & 11.31 & 40. & & \\
\hline S36 & Bleed3.Fl_o & & 20.04 & 45.736 & 960.38 & 64.30 & .0000 & 8.76 & 42. & & 94 \\
\hline S4 & Burner.Fl_-O & & 20.26 & .683 & 1703.95 & 52.09 & 0.0111 & 12.08 & 42. & & 1679 \\
\hline S45 & HPT.FI_O & & 27.08 & 11.182 & 1133.33 & -48.22 & & 52.61 & 10. & & 0 \\
\hline 549 & LPT.FI_O & & 40 & 4.717 & 934.15 & -97.04 & 0 & 114.57 & & & 92 \\
\hline 55 & TEGV.F̄̄_O & & 27.40 & 4.717 & 934.22 & -97.04 & 0. & 114.57 & & & \\
\hline S8 & Core_Nozz.FI & & 27.40 & 4.717 & 934.30 & -97.04 & & 114.58 & & & \\
\hline S17 & FanDūctLkg.Fi & & $27 \mathrm{C}$ & 6.147 & 485.39 & -14.80 & 0 & 625.01 & & & \\
\hline FS171 & Bleed15.Fl_o & & 275 & 6.147 & 486.28 & -15.22 & & 636.69 & & & \\
\hline FS172 & FanDuct.Fl_o & & 275.04 & 6.147 & 486.28 & -15.22 & 0. & 636.69 & & & 46 \\
\hline FS173 & Byp_Nozz.Fin_o & & 275.04 & 6.147 & 486.28 & -15.22 & 0.0000 & 636.69 & & & \\
\hline JRBOI & MACHINERY PERE & ORMANCE & DATA & & & & & & & & \\
\hline & & $\mathrm{PR}$ & eff & $\mathrm{NC}$ & $\mathrm{TR}$ & efPoly & & pwr & SMI & & SMW \\
\hline an & 676.80 & 1.099 & 0.9027 & 2259.122 & 1.0304 & 0.9040 & -13 & 11.5 & & & .64 \\
\hline $\mathrm{PC}$ & 79.57 & 1.40 & 0.8526 & 2259.122 & 1.1198 & 0.8595 & & 08.5 & 5.94 & & .65 \\
\hline PC & 51.68 & 5.820 & 0.8139 & 8881.892 & 1.7909 & 0.8527 & -38 & 88.4 & 62.33 & & 3.71 \\
\hline HPT & 12.08 & 3.996 & 0.8743 & 218.786 & 1.3523 & 0.8526 & & 88.3 & & & \\
\hline $\mathrm{PT}$ & 52.61 & 2.371 & 0.8345 & 63.953 & 1.2107 & 0.8170 & & 20.0 & & & \\
\hline $\mathrm{RBOI}$ & MACH INERY & DA? & & & & & & & & & \\
\hline & WcMap & PRmap & effMap & NcMap & R/Parm & $\mathrm{s}_{-} \mathrm{Wc}$ & cDes & S_PRdes & $s_{-} e f f D e$ & & S_NcDes \\
\hline Fan & 786.38 & 1.097 & 0.9045 & 2237.582 & 0.0417 & & 8607 & $\overline{1} .0235$ & 0.998 & & $\overline{0} .9905$ \\
\hline LPC & 60.65 & 1.385 & 0.7657 & 0.597 & 0.0000 & & 3120 & 1.0520 & 1.113 & & 0.0003 \\
\hline HPC & 47.01 & 5.593 & 0.8224 & 8645.107 & 10.9472 & & 0995 & 1.0494 & 0.989 & & 0.9733 \\
\hline HPT & 0.96 & 3.913 & 0.8743 & 1.315 & 3.9128 & 12. & 6299 & 0.9723 & 1.000 & & 0.0003 \\
\hline LPT & 0.81 & 2.031 & 0.8407 & 0.719 & 2.0309 & $65.2 .2 x \cdot x$ & 2590 & 0.7521 & 0.992 & & 0.0005 \\
\hline
\end{tabular}

$\begin{array}{rrrrrrr}\text { Aphy } & \text { MN } & \text { gamt } & \text { WAR } & \text { Wair } & \text { WH2O } & \text { H2O frac } \\ 2596.9 & 0.6080 & 1.40073 & 0.0001104 & 301.98 & 0.033 & 0.0001 \\ 4168.2 & 0.3257 & 1.40073 & 0.0001104 & 301.98 & 0.033 & 0.0001 \\ 3531.8 & 0.3470 & 1.40073 & 0.0001104 & 270.21 & 0.030 & 0.0001 \\ 830.5 & 0.1642 & 1.40073 & 0.0001104 & 31.77 & 0.004 & 0.0001 \\ 2606.7 & 0.4571 & 1.40061 & 0.0001104 & 270.21 & 0.030 & 0.0001 \\ 412.6 & 0.2545 & 1.40010 & 0.0001104 & 31.77 & 0.004 & 0.0001 \\ 412.6 & 0.2592 & 1.39909 & 0.0065183 & 31.77 & 0.207 & 0.0065 \\ 412.6 & 0.2178 & 1.39909 & 0.0065183 & 27.00 & 0.176 & 0.0065 \\ 49.7 & 0.4314 & 1.38211 & 0.0065183 & 25.69 & 0.167 & 0.0065 \\ 49.3 & 0.3213 & 1.38211 & 0.0065183 & 19.91 & 0.130 & 0.0065 \\ 74.6 & 0.2950 & 1.33112 & 0.0065183 & 19.91 & 0.130 & 0.0199 \\ 265.4 & 0.3666 & 1.36556 & 0.0065183 & 26.69 & 0.174 & 0.0165 \\ 860.2 & 0.2343 & 1.37825 & 0.0065183 & 27.00 & 0.176 & 0.0164 \\ 860.2 & 0.2343 & 1.37825 & 0.0065183 & 27.00 & 0.176 & 0.0164 \\ 613.3 & 0.3405 & 1.37824 & 0.0065183 & 27.00 & 0.176 & 0.0164 \\ 2606.7 & 0.4571 & 1.40061 & 0.0001104 & 270.21 & 0.030 & 0.0001 \\ 2481.9 & 0.5011 & 1.40058 & 0.0002214 & 274.98 & 0.061 & 0.0002 \\ 2481.9 & 0.5011 & 1.40058 & 0.0002214 & 274.98 & 0.061 & 0.0002 \\ 2006.9 & 0.7182 & 1.40058 & 0.0002214 & 274.98 & 0.061 & 0.0002\end{array}$

$\begin{array}{ll}2006.9 & 0.7182\end{array}$ 


\begin{tabular}{|c|c|c|c|c|c|c|c|c|c|c|}
\hline$Z$ & $===$ INLETS $====$ & eRam & Afs & Fram & \multirow{2}{*}{\multicolumn{3}{|c|}{ BLEEDS - interstg }} & & & \\
\hline D & Inlet & 1.0000 & 2596.85 & 5861.5 & & & & $\begin{array}{l}\text { g Wb/Win } \\
0.0368\end{array}$ & $\begin{array}{r}\text { BldWk } \\
0.5000\end{array}$ & $\begin{array}{r}\text { BldP } \\
0.2200\end{array}$ \\
\hline & $====$ DUCTS $====$ & dPnorm & MN & Aphy & \multirow{2}{*}{\multicolumn{3}{|c|}{$\begin{array}{ll}\text { LPT COOLA } & \text { HPC.C } C> \\
\text { WB } 2 \bar{X} & \text { HPC. B> }\end{array}$}} & 0.0117 & 0.5000 & 0.4500 \\
\hline & TEGV & 0.0000 & 0.2343 & 860.21 & & & & 0.0000 & 0.4500 & 0.2200 \\
\hline & FanDuct & 0.0000 & 0.5011 & 2481.93 & \multicolumn{2}{|c|}{$\begin{array}{l}\text { WB2X } \\
\text { WB2Y }\end{array}$} & $\mathrm{HPC} . \mathrm{B}>$ & 0.0000 & 0.7600 & 0.6200 \\
\hline & & & & & \multicolumn{2}{|c|}{ WBA2X } & $\mathrm{HPC} \cdot \mathrm{B}>$ & 0.0000 & 0.4500 & 0.2200 \\
\hline & $==$ SPLITTERS $==$ & BPR & $\mathrm{dP} / \mathrm{P} 1$ & $\mathrm{dP} / \mathrm{P} 2$ & \multicolumn{2}{|c|}{ WBLKG } & HPC.I> & 0.0000 & 1.0000 & 1.0000 \\
\hline & Splitter & 8.5056 & 0.0005 & 0.0005 & \multicolumn{2}{|c|}{ WBW2X } & $\mathrm{HPC} \cdot \mathrm{B}>$ & 0.0000 & 0.4500 & 0.2200 \\
\hline & $===$ SHAFT $S====$ & Nmech & trq in & pwr in & \multicolumn{3}{|c|}{ BLEEDS - output } & Wb/Win & hscale & Pscale \\
\hline & HP_Shaft & 9031.3 & 2261.3 & 3888.3 & \multicolumn{2}{|c|}{ WB17Y } & Bleed> & 0.1500 & 1.0000 & 1.0000 \\
\hline & LP Shaft & 2153.0 & 4683.7 & 1920.0 & \multicolumn{2}{|c|}{ HPT_COOLA } & A Bleed> & 0.1142 & 1.0000 & 1.0000 \\
\hline & & & & & \multirow{2}{*}{\multicolumn{2}{|c|}{$\begin{array}{l}\mathrm{HPT}^{-} \mathrm{COOLB} \\
\mathrm{WB} 3 \overline{\mathrm{X}}\end{array}$}} & B Bleed> & 0.0999 & 1.0000 & 1.0000 \\
\hline & & & & & & & Bleed $>$ & 0.0000 & 1.0000 & 1.0000 \\
\hline & & & & & \multicolumn{2}{|c|}{ WBA3X } & Bleed> & 0.0000 & 1.0000 & 1.0000 \\
\hline & & & & & \multicolumn{2}{|c|}{ WBW3X } & Bleed> & 0.0000 & 1.0000 & 1.0000 \\
\hline & & & & & \multicolumn{2}{|c|}{ WBEDLKG } & FanDu $>$ & 0.0000 & 1.0000 & 1.0000 \\
\hline & & & & & \multicolumn{2}{|c|}{ WB15X } & Bleed> & 0.0000 & 1.0000 & 1.0000 \\
\hline & & & & & \multirow{2}{*}{\multicolumn{2}{|c|}{$\begin{array}{l}\text { WB15Y } \\
\text { WB17X }\end{array}$}} & Bleed> & 0.0000 & 1.0000 & 1.0000 \\
\hline & & & & & & & Bleed> & 0.0000 & 1.0000 & 1.0000 \\
\hline & $===$ BURNERS $===$ & TtOut & eff & dPnorm & & & & \\
\hline & Burner & 1703.89 & 0.9995 & 0.0230 & \multicolumn{3}{|c|}{$\begin{array}{rr}\text { Wfuel } & \text { FAR } \\
0.22166 & 0.01113\end{array}$} & & & \\
\hline & $===\operatorname{NOZZLES}===$ & PR & Cfg & CdTh & $\mathrm{Cv}$ & \multicolumn{2}{|r|}{ Ath } & MNth & Vact & $\mathrm{Fg}$ \\
\hline & Core_Nozz & 1.082 & 0.9801 & 1.0000 & 0.9800 & \multirow{2}{*}{\multicolumn{2}{|c|}{$\begin{array}{r}613.40 \\
2006.86\end{array}$}} & 0.340 & 491.9 & 418.9 \\
\hline & Byp_Nozz & 1.410 & 0.9800 & 1.0000 & 0.9800 & & & 0.718 & 724.7 & 6194.9 \\
\hline
\end{tabular}




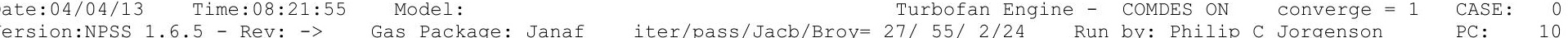

Temperature Stator 1 inlet: 482.91 Stator 1 exit: 487.10 Stator 2 inlet: 491.89 Stator 2 exit: 493.80 Stator 3 inlet: 499.08 Stator 3 exit: 500.58 Stator 4 inlet: 505.62 Stator 4 exit: 506.70 Stator 5 inlet: 510.38 Stator 5 exit: 511.15 Unblocked Percent Blockage: 0.00

Ambient Relative Humidity $\quad 10.00$

Fan Face Relative Humidity $\quad 4.93$

Fan Bypass Relative Humidity $\quad 4.05$

$\begin{array}{ll}\text { LPC Inlet Relative Humidity } & 3.92 \\ \text { LPC Exit Relative Humidity } & 1.24\end{array}$

HPC Relative Humidity $\quad 0.08$

0.0000050

576.87
5.30

Inlet Length

Ambient Flow Velocity

Fan/IPC Inlet $r \begin{array}{lr}193.48 \\ \text { Fan/IPC }\end{array}$

Ambient Static Temperature

454.14

Fan/LPC Inlet Static Temperature $\begin{array}{r}478.75 \\ \hline\end{array}$

Additional Water at LPC Exit

SUMMARY OUTPUT DATA

\begin{tabular}{|c|c|c|c|c|c|c|c|c|c|c|c|c|c|c|}
\hline MN & alt & dTamb & W & $\begin{array}{r}\text { JMMARY } \\
\text { Fn }\end{array}$ & $\begin{array}{l}\text { TPUT DAT } \\
\text { TSFE }\end{array}$ & Wfuel & BPR & VTAS & OPR & EPR & Powerset & T4 & T41 & T49 \\
\hline 0.552 & 25666.0 & 27.00 & 326.86 & 763.7 & 1.1097 & 847.48 & 7.1619 & 576.87 & 7.295 & 0.858 & 10.000 & 1709.9 & 1614.2 & 1137.4 \\
\hline
\end{tabular}

FSO Ambient.Fl O $\quad$ W

FS1 Inlet.FI_O $\quad 326.86$

FS12 Splitter.F1_02 286.81

FS2 Splitter.F1_01

FS14 Fan.FI_O

FS24 VaporIN.F1 O

FS25 Bleed2.FI_o

FS3 HPC.Fl_O

FS36 Bleed3.FI O

FS4 Burner.FI_O

FS45 HPT.FI-

FS49 LPT.FI-O

FS8 Core_Nozz.FI_O

FS17 FanDúctLkg.Fi_o

FS171 Bleed15.FI_O

FS172 FanDuct.FI-O

40.05

286.81
40.05

40.05
40.29

28.20

26.83

20.80

21.03

28.44

28.44

28.44
28.44

28.44
286.81

286.81
298.90 298.90
298.90 298.90

TURBOMACHINERY PERFORMANCE DATA

$\begin{array}{lrrr} & \text { Wc } & \text { PR } & \text { eff } \\ \text { Fan } & 623.79 & 1.084 & 0.9034 \\ \text { LPC } & 87.10 & 1.267 & 0.8287 \\ \text { HPC } & 50.82 & 5.762 & 0.8127 \\ \text { HPT } & 12.08 & 3.874 & 0.8754 \\ \text { LPT } & 51.12 & 2.131 & 0.8245\end{array}$

FLOW STATION DATA

\begin{tabular}{rrrr}
\multicolumn{1}{c}{ F } & Tt & ht & FAR \\
Pt & Tt & -16.15 & 0.0000 \\
6.516 & 481.87 & -16.15 & 0.0000 \\
6.516 & 481.87 & -16.87 \\
6.513 & 481.87 & -16.15 & 0.0000 \\
6.513 & 481.87 & -16.15 & 0.0000 \\
7.060 & 494.31 & -13.17 & 0.0000 \\
8.250 & 522.54 & -6.40 & 0.0000 \\
8.250 & 530.79 & -38.86 & 0.0000 \\
8.250 & 530.79 & -38.86 & 0.0000 \\
47.534 & 948.51 & 63.11 & 0.0000 \\
47.534 & 948.51 & 63.11 & 0.0000 \\
46.440 & 1709.89 & 50.63 & 0.0114 \\
11.988 & 1141.82 & -47.94 & 0.0085 \\
5.625 & 965.31 & -91.07 & 0.0084 \\
5.625 & 965.38 & -91.07 & 0.0084 \\
5.625 & 965.46 & -91.07 & 0.0084 \\
7.060 & 494.31 & -13.17 & 0.0000 \\
7.060 & 495.80 & -14.21 & 0.0000 \\
7.060 & 495.80 & -14.21 & 0.0000 \\
7.060 & 495.80 & -14.21 & 0.0000
\end{tabular}

WC
710.53

710.53
710.53

623.79

87.10
582.84

71.60

72.60

50.82
11.22
8.69

8.69
12.08

12.08
51.12

51.12
101.36

101.36
101.36

101.36
101.37

101.37
582.84

608.31

608.31
608.31

$\begin{array}{rrrr}8854.502 & 1.7870 & 0.8516 & -3969.9 \\ 216.618 & 1.3435 & 0.8543 & 3969.8\end{array}$

$\begin{array}{llll}58.422 & 1.1800 & 0.8086 & 1763.3\end{array}$

Ps
5.297
6.112
6.077
6.367
6.260
7.719
7.701
7.992
41.989
44.345
43.841
11.012
5.464
5.464
5.297
6.260
6.065
6.065
5.297

Ts
454.14
473.13
472.42
478.75
477.60
512.69
520.45
525.98
916.44
930.43
1685.56
1116.16
957.68
957.75
949.73
477.60
474.72
474.72
456.68

$\begin{array}{rr}\text { Aphy } & \text { MN } \\ 2592.0 & 0.5520 \\ 4168.2 & 0.3036 \\ 3531.8 & 0.3160 \\ 830.5 & 0.1803 \\ 2606.7 & 0.4180 \\ 412.6 & 0.3098 \\ 412.6 & 0.3154 \\ 412.6 & 0.2139 \\ 49.7 & 0.4269 \\ 49.3 & 0.3184 \\ 74.6 & 0.2951 \\ 265.4 & 0.3545 \\ 860.2 & 0.2059 \\ 860.2 & 0.2059 \\ 613.4 & 0.2965 \\ 2606.7 & 0.4180 \\ 2481.9 & 0.4708 \\ 2481.9 & 0.4708 \\ 2006.9 & 0.6538\end{array}$

gamt
1.40063
1.40063
1.40063
1.40063
1.40050
1.40016
1.39921
1.39921
1.38286
1.38286
1.33071
1.36493
1.37628
1.37628
1.37627
1.40050
1.40045
1.40045
1.40045

WAR
0.0001988
0.0001988

Wair WH2O H2O frac

$\begin{array}{rlrrr}\text { NC } & \text { TR efPoly } & \text { pwr } & \text { SMN } & \text { SMW }\end{array}$

$\begin{array}{lllrrr}2048.126 & 1.0258 & 0.9045 & -1210.6 & -2798.92 & 49.86\end{array}$

$\begin{array}{llllrr}2048.126 & 1.0844 & 0.8343 & -552.7 & 6.37 & 2.41\end{array}$

TURBOMACHINERY MAP DATA

$\begin{array}{lrrr} & \text { WcMap } & \text { PRmap } & \text { effMap } \\ \text { Fan } & 724.78 & 1.082 & 0.9053 \\ \text { LPC } & 53.59 & 1.312 & 0.7608 \\ \text { HPC } & 46.22 & 5.537 & 0.8212 \\ \text { HPT } & 0.96 & 3.794 & 0.8753 \\ \text { LPT } & 0.78 & 1.851 & 0.8306\end{array}$

$\begin{array}{rr}\text { NcMap } & \text { R/Parm } \\ 2028.597 & 0.0426 \\ 0.541 & 0.0000 \\ 8618.447 & 11.0023 \\ 1.302 & 3.7943 \\ 0.657 & 1.8509\end{array}$

$\begin{array}{rrr}\text { S.WCDes } & \text { S_PRdes } & \text { S_effDes } \\ 0.8607 & \overline{1} .0235 & 0.9980 \\ 1.6253 & 0.8541 & 1.0892 \\ 1.0995 & 1.0494 & 0.9898 \\ 12.6299 & 0.9723 & 1.0000 \\ 65.2590 & 0.7521 & 0.9926\end{array}$

S NCDes

0.9905
0.0003
0.9733

0.0003
0.9733

0.0003

0.0005 


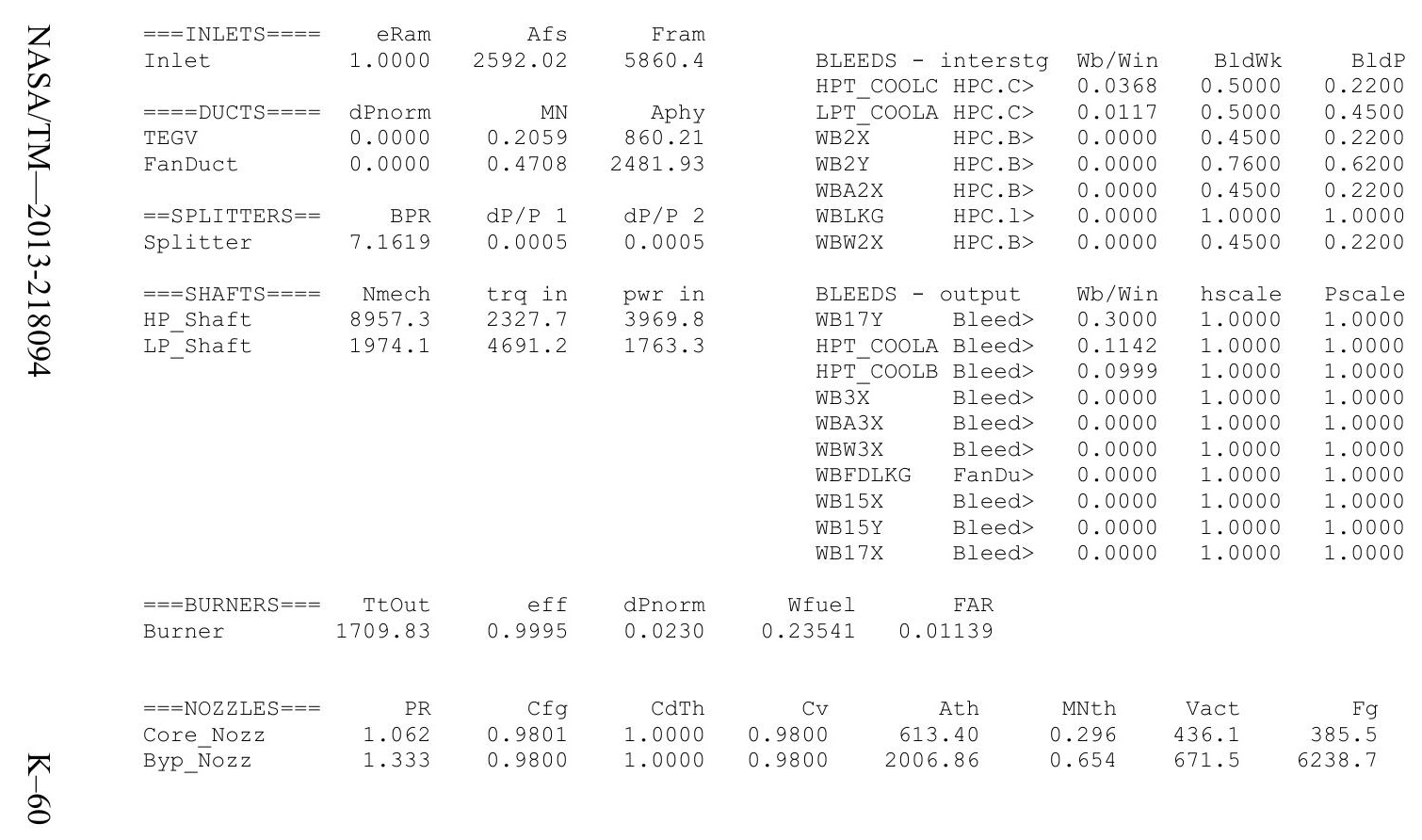


Date:04/04/13 Time:08:22:46 Model: Turbofan Engine - CoMDES ON Converge = 1 CASE: 0

Version:NPSS 1.6.5 - Rev: -> Gas Package: Janaf iter/pass/Jacb/Broy= 56/84/2/53 Run by: Philip C Jorgenson PC: 10

Temperature Stator 1 inlet: $495.95 \quad$ Stator 1 exit: $498.98 \quad$ Stator 2 inlet: 501.87 Stator 2 exit: 502.71 Stator 5 inlet: 511.88 Stator 5 exit: $511.80 \quad$ Unblocked Percent Blockage: 0.00

Ambient Relative Humidity $\quad 10.00$

Fan Face Relative Humidity $\quad 5.94$

Fan Bypass Relative Humidity $\quad 5.02$

LPC Inlet Relative Humidity $\quad 5.10$

HPC Relative Humidity

Drop Diative Humidity

0.09

Ambient Static Pressure

Additional Water at LPC Exit

$$
0.0000050
$$

Inlet Length $\quad 40.00$

523.25 Fan/LPC Inlet Flow Velocity 202.42

6.74 Fan/LPC Inlet Static Pressure $\quad 7.75$

Fan/LPC Inlet Static Temperature 493.57
$\begin{array}{rrr}\text { MN } & \text { alt } & \text { dTamb } \\ 0.490 & 20047.0 & 27.00\end{array}$
W
363.50
$\mathrm{Fn}$
836.0
TSFC
1.1216

Wfuel
937.61

BPR
6.3486

VTAS
523.25

OPR
6.390

EPR
0.889

PowerSet
10.000

$\begin{array}{ccc}\text { T4 } & \text { T41 } & \text { T49 } \\ 1738.6 & 1639.1 & 1165.8\end{array}$
FSO Ambient.Fl_O

FS1 Inlet.FI_O

$\begin{array}{ll}\text { FS12 } & \text { Splitter.FI_02 } \\ \text { FS2 } & \text { Splitter.Fl_01 }\end{array}$

FS14 Fan.Fl_O

FS24 VaporIN.FI

FS25 Bleed2.Fl_

FS3 HPC.F1 O

FS36 Bleed3.FI

ES4 Burner.Fl_

FS49 4 TPT

FS5 TEGV.Fīo

FS8 Core_Nozz.Fl_O

FS17 FanDúctLkg.Fì

FS171 Bleed15.FI

FS172 FanDuct.Fl-O

FS173 Byp Nozz.FİO

TURBOMACHINERY PERFORMANCE DATA

$\begin{array}{lrrr} & \text { WC } & \text { PR } & \text { eff } \\ \text { Fan } & 569.02 & 1.072 & 0.9053 \\ \text { LPC } & 89.63 & 1.149 & 0.7084 \\ \text { HPC } & 48.76 & 5.562 & 0.8084 \\ \text { HPT } & 12.09 & 3.682 & 0.8773 \\ \text { LPT } & 48.79 & 1.912 & 0.8170\end{array}$

FLOW STATION DATA

$\begin{array}{rrrrrr}\text { W } & \text { Pt } & \text { Tt } & \text { ht } & \text { FAR } & \text { Wc } \\ 363.50 & 7.943 & 496.98 & -13.65 & 0.0000 & 658.32 \\ 363.50 & 7.943 & 496.98 & -13.65 & 0.0000 & 658.32 \\ 314.04 & 7.939 & 496.98 & -13.65 & 0.0000 & 569.02 \\ 49.47 & 7.939 & 496.98 & -13.65 & 0.0000 & 89.63 \\ 314.04 & 8.509 & 507.97 & -11.02 & 0.0000 & 536.75 \\ 49.47 & 9.126 & 525.49 & -6.81 & 0.0000 & 80.18 \\ 49.76 & 9.126 & 533.61 & -38.95 & 0.0000 & 81.27 \\ 29.86 & 9.126 & 533.61 & -38.95 & 0.0000 & 48.76 \\ 28.41 & 50.761 & 945.12 & 61.50 & 0.0000 & 11.10 \\ 22.02 & 50.761 & 945.12 & 61.50 & 0.0000 & 8.60 \\ 22.28 & 49.592 & 1738.58 & 48.48 & 0.0119 & 12.09 \\ 29.77 & 13.470 & 1170.54 & -48.41 & 0.0089 & 48.79 \\ 30.12 & 7.043 & 1014.66 & -86.42 & 0.0088 & 87.89 \\ 30.12 & 7.043 & 1014.73 & -86.42 & 0.0088 & 87.89 \\ 30.12 & 7.043 & 1014.80 & -86.42 & 0.0088 & 87.89 \\ 314.04 & 8.509 & 507.97 & -11.02 & 0.0000 & 536.75 \\ 333.94 & 8.509 & 509.51 & -12.68 & 0.0000 & 571.63 \\ 333.94 & 8.509 & 509.51 & -12.68 & 0.0000 & 571.63 \\ 333.94 & 8.509 & 509.51 & -12.68 & 0.0000 & 571.63\end{array}$

PS
6.740

7.503

7.750

7.710
8.373

8.373
8.347

8.863

44.981

47.430

$46.816 \quad 1713.91$

$12.481-1146.97$

$6.893 \quad 1008.80$

$6.740 \quad 1002.73$

$\begin{array}{rr}6.740 & 1002.73 \\ 7.710 & 493.85\end{array}$

$\begin{array}{ll}7.475 & 490.99\end{array}$

$\begin{array}{ll}7.475 & 490.99 \\ 6.740 & 490.99\end{array}$

$6.740 \quad 476.66$ $\begin{array}{lll}474.18 & 2608.1 & 0.4900 \\ 489.02 & 3168.2 & 0.2790\end{array}$

$\begin{array}{rrr}49.57 & 830.5 & 0.1858\end{array}$

$\begin{array}{lll}493.57 & 830.5 & 0.1858 \\ 512.72 & 406.7 & 0.3779\end{array}$

$\begin{array}{ll}412.6 & 0.3528 \\ 412.6 & 0.3594\end{array}$

$\begin{array}{lll}412.6 & 0.3594 \\ 412.6 & 0.2048\end{array}$

$\begin{array}{lll}49.7 & 0.4214\end{array}$

$\begin{array}{lll}49.3 & 0.3146\end{array}$

$\begin{array}{lll}74.6 & 0.2954\end{array}$

860.20 .1776

$860.2 \quad 0.1776$

$\begin{array}{rr}613.4 & 0.2539 \\ 2606.7 & 0.3779\end{array}$

$2481.9 \quad 0.4341$

$2481.9 \quad 0.4341$

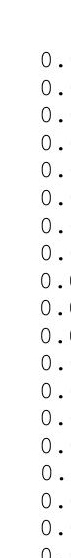

TURBOMACHINERY MAP DATA

$\begin{array}{lrrrrrrrrr} & \text { WcMap } & \text { PRmap } & \text { effMap } & \text { NcMap } & \text { R/Parm } & \text { S_WcDes } & \text { S_PRdes } & \text { S_effDes } & \text { S_NcDes } \\ \text { Fan } & 661.15 & 1.070 & 0.9071 & 1828.227 & 0.0438 & 0.8607 & \overline{1} .0235 & 0.9980 & 0.9905 \\ \text { LPC } & 46.95 & 1.246 & 0.7557 & 0.488 & 0.0000 & 1.9091 & 0.6068 & 0.9375 & 0.0003 \\ \text { HPC } & 44.35 & 5.347 & 0.8167 & 8553.298 & 11.0305 & 1.0995 & 1.0494 & 0.9898 & 0.9733 \\ \text { HPT } & 0.96 & 3.607 & 0.8773 & 1.285 & 3.6072 & 12.6299 & 0.9723 & 1.0000 & 0.0003 \\ \text { LPT } & 0.75 & 1.686 & 0.8231 & 0.594 & 1.6863 & 65.2590 & 0.7521 & 0.9926 & 0.0005\end{array}$




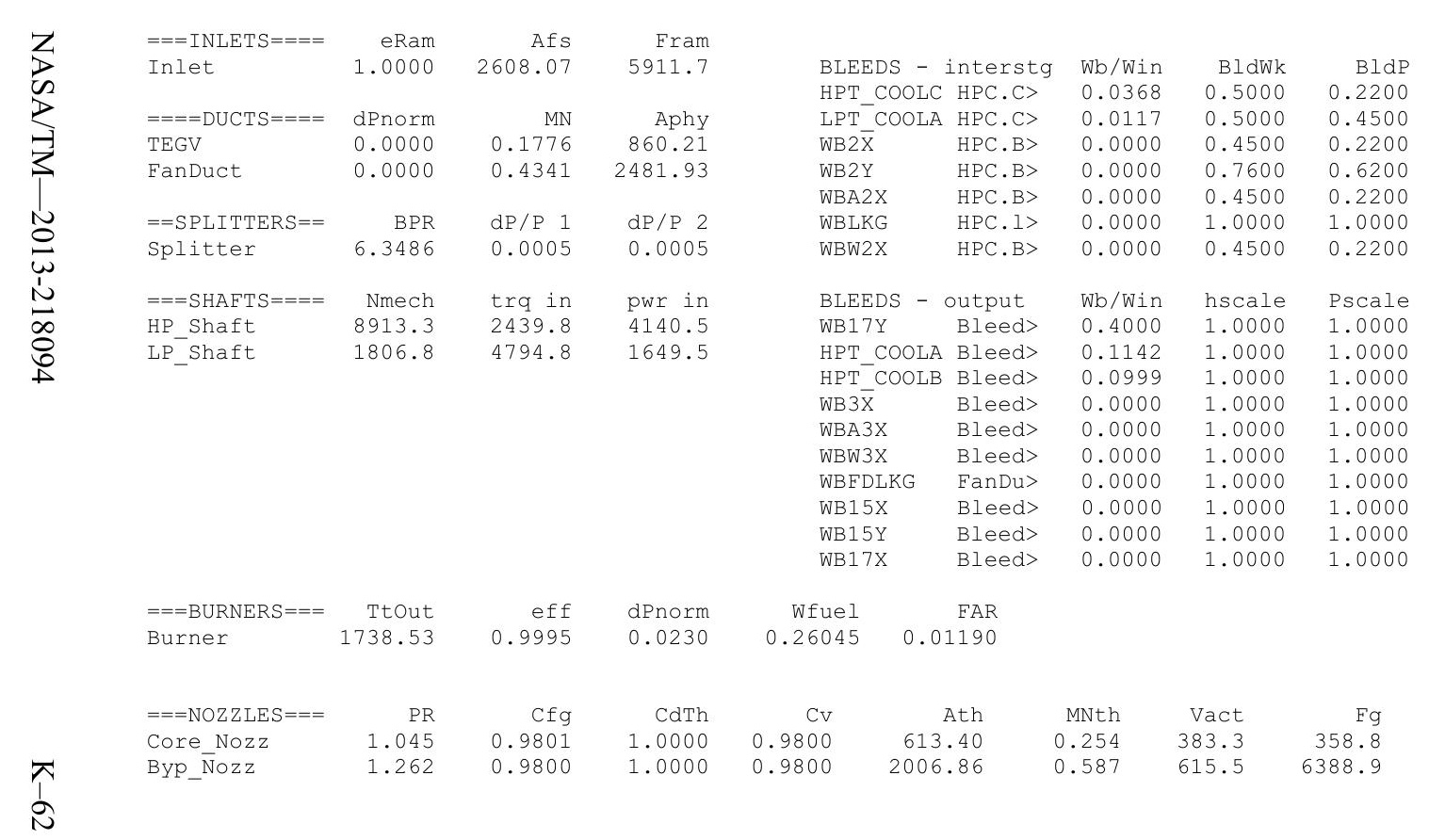


Date:04/04/13 Time:08:23:20 Model: Turbofan Engine - COMDES ON Converge = 1 CASE: 0

Version:NPSS 1.6.5 - Rev: -> Gas Package: Janaf iter/pass/Jacb/Broy= 39/67/2/36 Run by: Philip C Jorgenson PC: 10

Temperature Stator 1 inlet: 509.87 Stator 1 exit: 512.21 Stator 2 inlet: 514.69 Stator 2 exit: 515.51

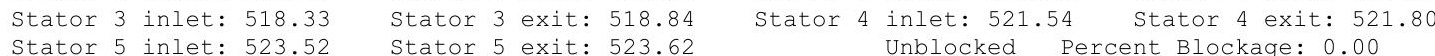

Ambient Relative Humidity $\quad 10.00$

Fan Face Relative Humidity $\quad 6.61$

Fan Bypass Relative Humidity $\quad 6.06$

LPC Inlet Relative Humidity $\quad 5.77$

LPC Exit Relative Humidity

$\begin{array}{lr}\text { HPC Relative Humidity } & 0.18 \\ \text { Drop Diameter } & 0.0000050\end{array}$

0.18

Ambient Flow Velocity

484.46

Inlet Length

Ambient Static Temperatur

Additional Water at LPC Exit

490.63

169.47
Static Pressure $\quad 9.19$ 0.0097470

$\begin{array}{rrrrrc} & & & & \text { SUMMARY OUTPUT DATA } \\ \text { MN } & \text { alt } & \text { dTamb } & \text { W } & \text { Fn } & \text { TSFC } \\ 0.446 & 15435.0 & 27.00 & 381.14 & 646.0 & 1.3738\end{array}$

FS1 Inlet.Fl_o

六

FS14 Fan.F1-

FS23 UPC.FI_

FS25 Bleed2.FI_O

FS3 HPC.Fl_O

FS36 Bleed3.F1_O

FS4 Burner.FI_-

FS45 HPT.FI-

FS49 LPT.FI-O

FS8 Core_Nozz.FI_O

FS17 FanDuctLkg. Fi__o

FS171 Bleed15.FI_O

FS172 FanDuct.Fl_O
FS173 Byp Nozz.FI_O

TURBOMACHINERY PERFORMANCE DATA

\begin{tabular}{|c|c|c|c|c|c|c|c|c|c|c|c|}
\hline & & & & FLOW & STATION D & & & & & & \\
\hline SO & Ambient.Fl 0 & & 381.14 & 9.342 & 510.16 & $\begin{array}{r}\text { ht } \\
-11.98\end{array}$ & 0.0000 & 594.6 & $\begin{array}{l}\text { WC } \\
65\end{array}$ & 49 & 490 \\
\hline FS1 & Inlet.FI_O- & & 381.14 & 9.342 & 510.16 & -11.98 & 0.0000 & 594.6 & & & 503.8 \\
\hline FS12 & Splitter.Fl_o & & 333.45 & 9.337 & 510.16 & -11.98 & 0.0000 & 520.4 & & & 503.4 \\
\hline S2 & Splitter.Fl_o & & 47.70 & 9.337 & 510.16 & -11.98 & 0.0000 & 74.4 & & & 507.7 \\
\hline FS14 & Fan.Fl_o & & 333.45 & 9.808 & 518.21 & -10.05 & 0.0000 & 499.4 & & & 506.0 \\
\hline FS23 & LPC.FI_O & & 47.70 & 10.496 & 533.38 & -6.40 & 0.0000 & 67.7 & & & 524.4 \\
\hline ES24 & 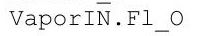 & & 48.16 & 10.496 & 546.45 & -58.87 & 0.0000 & 69.2 & & & 536 \\
\hline S25 & Bleed2.FI $\bar{O}$ & & 28.90 & 10.496 & 546.45 & -58.87 & 0.0000 & 41.5 & 10. & & \\
\hline E3 & HPC.FI_O & & 27.50 & 48.582 & 919.37 & 32.42 & 0.0000 & 11.0 & 43. & & 88 \\
\hline S36 & Bleed3.Fl_O & & 21 & 8.582 & 919.37 & 32.42 & & 8.5 & 45. & & \\
\hline ES 4 & Burner.FI_O & & 21. & 47.463 & 1696.85 & 20.01 & & 12.0 & 44. & & 6 \\
\hline F 45 & HPT.FI_O & & 28.81 & 14.150 & 1167.22 & -67.91 & 0.0087 & 44.8 & 13. & & 1147 \\
\hline FS 49 & LPT.FI_O & & 29.14 & 8.390 & 1041.21 & -98.48 & 0.0 & 72.3 & & & 1037. \\
\hline FS5 & TEGV.F̄̄_O & & 29.14 & 8.390 & 1041.28 & -98.48 & & 72.3 & & & 1037 \\
\hline S8 8 & Core Nozz.Fl & & 29.14 & 8.390 & 1041.35 & -98.48 & 6 & 72.3 & & & 1033 \\
\hline FS17 & FanDuctLkg. Fu & & 333.45 & 9.808 & 518.21 & -10.05 & 0.0000 & 499.4 & & & \\
\hline FS171 & Bleed15.FI_O & & 352.71 & 9.808 & 519.77 & -12.71 & & 529.0 & & & \\
\hline FS172 & FanDuct.Fl-o & & 352.71 & 9.808 & 519.77 & -12.71 & 0.0000 & 529.0 & & & \\
\hline FS173 & Byp_Nozz.Fì_o & & 352.71 & 9.808 & 519.77 & -12.71 & 0.0000 & 529.0 & & & \\
\hline JBBO & ERY PER & ORMANCE & DA & & & & & & & & \\
\hline & WC & $\mathrm{PR}$ & eff & NC & TR & efPoly & & pwr & SMN & & SMW \\
\hline an & 520.49 & .050 & 0.8972 & 1605.437 & 1.0158 & 0.8979 & & 11.0 & 1529.28 & & \\
\hline PC & 74.46 & 1.124 & 0.7470 & 1605.437 & 1.0455 & 0.7512 & & 76.0 & 8.29 & & 96 \\
\hline PC & 41.53 & 4.629 & 0.7926 & 8515.007 & 1.6824 & 0.8310 & -36 & 41.8 & 69.87 & & 45 \\
\hline HPT & 12.07 & 3.35 & 0.8692 & 212.174 & 1.2991 & 0.8499 & & 11.8 & & & \\
\hline LPT & 44.88 & 1.686 & 0.8042 & 46.604 & 1.1175 & 0.7923 & & 37.0 & & & \\
\hline TURBOM & MACHINERY MAP & DA & & & & & & & & & \\
\hline & WcMap & PRmap & effMap & NcMap & R/Parm & S WC & Des & PRdes & $s$ effDe & & NCDes \\
\hline Fan & 604.76 & 1.049 & 0.8990 & 590.130 & 0.0442 & & & $\overline{1} .0235$ & 0.998 & & $\overline{0} .9905$ \\
\hline LPC & 39.55 & 1.183 & 0.7479 & 0.424 & 0.0000 & 1.8 & 825 & 0.6780 & 0.998 & & 0.0003 \\
\hline HPC & 37.77 & 4.458 & 0.8008 & 8288.003 & 10.6846 & & 995 & 1.0494 & 0.989 & & 0.9733 \\
\hline HPT & 0.96 & 3.289 & 0.8692 & 1.275 & 3.2891 & 12. & 5299 & 0.9723 & 1.000 & & 0.0003 \\
\hline LPT & 0.69 & 1.516 & 0.8102 & 0.524 & 1.5163 & 65.2 & 590 & 0.7521 & 0.992 & & 0.0005 \\
\hline
\end{tabular}

$\begin{array}{llccc}\text { EPR } & \text { PowerSet } & \text { T4 } & \text { T41 } & \text { T49 } \\ 0.900 & 10.000 & 1696.9 & 1599.2 & 1162.4\end{array}$

WAR
0.0006510
0.0006510
0.0006510
0.0006510
0.0006510
0.0006510
0.0103980
0.0103980
0.0103980
0.0103980
0.0103980
0.0103980
0.0103980
0.0103980
0.0103980
0.0006510
0.0011786
0.0011786
0.0011786

Wair 380.90
380.90 380.90 47.67 $\begin{array}{ll}333.23 & 0.217\end{array}$ $\begin{array}{ll}47.67 & 0.031 \\ 47.67 & 0.496\end{array}$ $\begin{array}{ll}28.60 & 0.497\end{array}$ $\begin{array}{ll}27.21 & 0.283\end{array}$ $\begin{array}{ll}21.09 & 0.219\end{array}$ $\begin{array}{ll}21.09 & 0.219 \\ 28.27 & 0.294\end{array}$ $\begin{array}{ll}28.27 & 0.294 \\ 28.60 & 0.297\end{array}$ $\begin{array}{ll}28.60 & 0.297 \\ 28.60 & 0.297\end{array}$ $\begin{array}{ll}28.60 & 0.297 \\ 28.60 & 0.297\end{array}$ $\begin{array}{rr}28.60 & 0.297 \\ 333.23 & 0.217\end{array}$ $\begin{array}{lll}352.30 & 0.217 \\ 352.30 & 0.415\end{array}$ $\begin{array}{ll}353.23 & 0.217 \\ 352.30 & 0.415 \\ 352.30 & 0.415\end{array}$ $352.30 \quad 0.415$

$\begin{array}{rr}20 & \text { H2O frac } \\ 248 & 0.0007 \\ 248 & 0.0007 \\ 77 & 0.0007 \\ 31 & 0.0007 \\ 7 & 0.0007 \\ 31 & 0.0007 \\ 96 & 0.0103 \\ 97 & 0.0103 \\ 83 & 0.0103 \\ 9 & 0.0103 \\ 9 & 0.0243 \\ 94 & 0.0208 \\ 97 & 0.0207 \\ 97 & 0.0207 \\ 97 & 0.0207 \\ 7 & 0.0007 \\ 5 & 0.0012 \\ 5 & 0.0012 \\ 5 & 0.0012\end{array}$




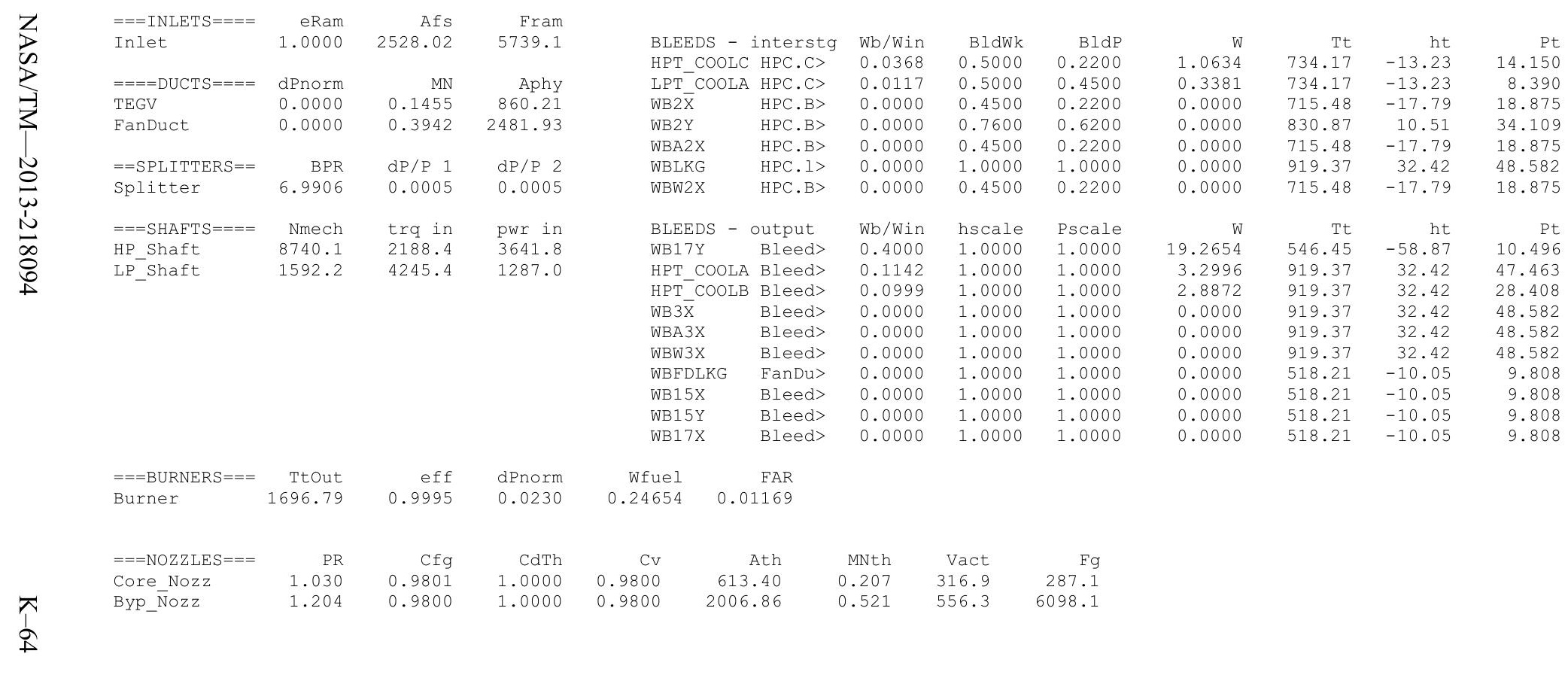


Date: $05 / 10 / 13 \quad$ Time: $11: 40: 17 \quad$ Model

(-)

Version:NPSS 1.6.5 - Rev: -> Gas Package: Janaf iter/pass/Jacb/Broy=12/ $40 / 2 / 9$ Run by: Philip C Jorgenson PC: 10

Temperature Stator 1 inlet: 484.08 Stator 1 exit: 492.01 Stator 2 inlet: 501.96 Stator 2 exit: 507.10

Stator 3 inlet: 517.28 Stator 3 exit: 521.94 Stator 4 inlet: 531.38 stator 4 exit: 535.3

Ambient Relative Humidity $\quad 10.00$

Fan Bypass Relative Humidity 1.98

Fan Bypass Relative Humidity 1.39

LPC Exit Relative Humidity 0.12

HPC Relative Humidity

Diameter

Ambient Flow Velocity

mbient Static Pressure

Ambient Static Temperature

0.0000050

28.45

Inlet Length

40.00

Fan/LPC Inlet Flow Velocity $\quad 189.51$

425.97 Fan/LPC Inlet Static Temperature 474.93

SUMMARY OUTPUT DATA
$\begin{array}{rr}\text { MN alt dTamb } \\ 0.780 \quad 39000.0 & \end{array}$
252.43
Fn $\quad$ TSFC
Wfuel
719.97
BPR
8.7419
VTAS $\quad$ OPR
EPR
Powerset
T4 T41
T 49

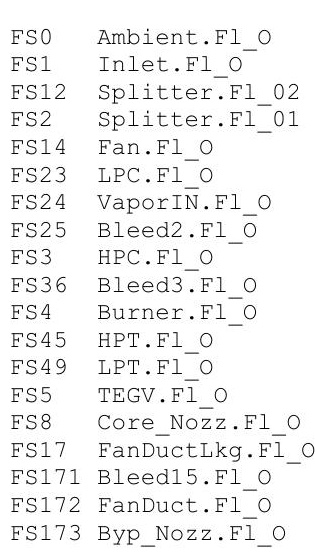

252.43
252.4
226.52
25.9
226.5
25.9
26.1
23.5
22.4
17.3
17.5
23.4
23.7
23.7
23.7
226.5
229.1
229.1
229.1

FLOW STATION DATA

$\begin{array}{rrrrr}\text { Pt } & \text { Tt } & \text { ht } & \text { FAR } & \text { Wc } \\ 4.267 & 477.92 & -16.44 & 0.0000 & 834.59 \\ 4.267 & 477.92 & -16.44 & 0.0000 & 834.59 \\ 4.265 & 477.92 & -16.44 & 0.0000 & 749.30 \\ 4.265 & 477.92 & -16.44 & 0.0000 & 85.71 \\ 4.832 & 497.11 & -11.84 & 0.0000 & 674.40 \\ 6.699 & 557.07 & 2.55 & 0.0000 & 58.91 \\ 6.699 & 569.47 & -48.91 & 0.0000 & 60.13 \\ 6.699 & 569.47 & -48.91 & 0.0000 & 54.11 \\ 40.666 & 1028.50 & 64.03 & 0.0000 & 11.40 \\ 40.666 & 1028.50 & 64.03 & 0.0000 & 8.83 \\ 39.729 & 1791.04 & 51.33 & 0.0116 & 12.07 \\ 9.537 & 1185.91 & -58.12 & 0.0087 & 54.68 \\ 3.266 & 928.81 & -121.97 & 0.0086 & 142.98 \\ 3.266 & 928.88 & -121.97 & 0.0086 & 142.99 \\ 3.266 & 928.95 & -121.97 & 0.0086 & 142.99 \\ 4.832 & 497.11 & -11.84 & 0.0000 & 674.40 \\ 4.832 & 497.95 & -12.26 & 0.0000 & 682.76 \\ 4.832 & 497.95 & -12.26 & 0.0000 & 682.76 \\ 4.832 & 497.95 & -12.26 & 0.0000 & 682.76\end{array}$

Ps
2.85
3.891
3.83
4.172
4.05
6.415
6.400
6.45
35.725
37.83
37.50
8.632
3.07
3.07
2.85
4.05
3.92
3.92
2.85

Ts
425.97
465.47
463.67
474.93
472.76
550.20
562.11
563.58
992.56
1008.33
1765.80
1154.87
913.40
913.47
895.18
472.76
469.01
469.01
428.30

$\begin{array}{rr}\text { Aphy } & \text { MN } \\ 2546.2 & 0.7800 \\ 4168.2 & 0.3654 \\ 3531.8 & 0.3917 \\ 830.5 & 0.1773 \\ 2606.7 & 0.5071 \\ 412.6 & 0.2499 \\ 412.6 & 0.2565 \\ 412.6 & 0.2290 \\ 49.7 & 0.4372 \\ 49.3 & 0.3252 \\ 74.6 & 0.2956 \\ 265.4 & 0.3849 \\ 860.2 & 0.2986 \\ 860.2 & 0.2986 \\ 613.4 & 0.4461 \\ 2606.7 & 0.5071 \\ 2481.9 & 0.5550 \\ 2481.9 & 0.5550 \\ 2006.9 & 0.9008\end{array}$

gamt 1.40068 1.40068 1.40068 1.40049 1.39964
1.39811 1.39811

1.37745

1.37745

1.32633
1.36150

1.37791

1.37790
1.37790

1.40049

1.40046

1.40046 1.40046
WAR 0.0000851 0.0000851 0.0000851 0.0000851 0.0000851 0.0096227 0.0096227 0.0096227 . . . .0096227 0.0096227 0.0000851 0.0001929 0.0001929

TURBOMACHINERY PERFORMANCE DATA

\begin{tabular}{|c|c|c|c|c|c|c|c|c|c|}
\hline & Wc & PR & eff & $\mathrm{NC}$ & $\mathrm{TR}$ & efpoly & pwr & SMN & SMW \\
\hline Fan & 749.30 & 1.133 & 0.9063 & 2587.056 & 1.0402 & 0.9080 & -1474.6 & 1489.03 & 54.55 \\
\hline LPC & 85.71 & 1.571 & 0.8321 & 2587.056 & 1.1656 & 0.8425 & -696.0 & 7.03 & 3.87 \\
\hline HPC & 54.11 & 6.070 & 0.8181 & 8961.021 & 1.8061 & 0.8566 & -3670.8 & 61.93 & 58.58 \\
\hline HPT & 12.07 & 4.166 & 0.8788 & 221.867 & 1.3628 & 0.8572 & 3670.8 & & \\
\hline LPT & 54.68 & 2.920 & 0.8588 & 72.113 & 1.2751 & 0.8396 & 2170.6 & & \\
\hline \multicolumn{10}{|c|}{ TURBOMACHINERY MAP D } \\
\hline & WcMap & PRmap & effMap & NcMap & R/Parm & S_WCDes & S_PRdes & s_effDes & s_NcDes \\
\hline an & 870.61 & 1.130 & 0.9081 & 2562.389 & 0.0415 & $\overline{0} .8607$ & $\overline{1} .0235$ & 0.9980 & $\overline{0} .9905$ \\
\hline LPC & 74.72 & 1.530 & 0.7808 & 0.683 & 0.0000 & 1.1471 & 1.0764 & 1.0658 & 0.0003 \\
\hline HPC & 49.22 & 5.831 & 0.8265 & 8722.126 & 10.9409 & 1.0995 & 1.0494 & 0.9898 & 0.9733 \\
\hline HPT & 0.96 & 4.078 & 0.8788 & 1.333 & 4.0783 & 12.6299 & 0.9723 & 1.0000 & 0.0003 \\
\hline LPT & 0.84 & 2.444 & 0.8652 & 0.811 & 2.4443 & 65.2590 & 0.7521 & 0.9926 & 0.0005 \\
\hline
\end{tabular}


$\begin{array}{lrrr}===\text { INLETS }==== & \text { eRam } & \text { Afs } & \text { Fram } \\ \text { Inlet } & 1.0000 & 2546.18 & 6194.1\end{array}$

$====$ DUCT $S====$
TEGV

FanDuct

0.0000

$0.2986 \quad 860.21$

$0.5550 \quad 2481.93$

$\mathrm{dP} / \mathrm{P} 2$

Splitter

8.7419

0.0005

.0005

LP_Shaft

Nmech

trq in pwr in

$2053.3 \quad 3670.8$

2170.6 1790.99

eff

dPnorm

PR
1.144
1.693

Cfg
0.9801

Byp_Nozz

1.693

0.9800
$===$ BURNERS $===\quad$ TtOut

BLEEDS - interstg Wb/Win BldWk 0.2200 LPT_COOLA HPC.C> $0.0117 \quad 0.5000 \quad 0.4500$ $\begin{array}{lllll}\text { WB2 } \bar{X} & \text { HPC.B> } & 0.0000 & 0.4500 & 0.2200 \\ \text { WB2Y } & \text { HPC. B> } & 0.0000 & 0.7600 & 0.6200\end{array}$ $\begin{array}{lllll}\text { WBA2X HPC.B> } & 0.0000 & 0.4500 & 0.2200\end{array}$ WBLKG HPC.1> $0.0000 \quad 1.0000=1.0000$ $\begin{array}{lllll}\text { WBW2X HPC.B } & 0.0000 & 0.4500 & 0.2200\end{array}$

BLEEDS - output Wb/Win hscale Pscale WB17Y Bleed> $0.1000-1.0000-1.0000$ HPT_COOLA Bleed> $0.1142 \quad 1.0000 \quad 1.0000$ HPT_COOLB Bleed> $0.0999 \quad 1.0000 \quad 1.0000$ WB3X Bleed> $0.0000 \quad 1.0000 \quad 1.0000$ WBA3X Bleed> $0.0000 \quad 1.0000-1.0000$ WBW3X Bleed> $0.0000 \quad 1.0000 \quad 1.0000$ WBPDLKG FanDu> $0.0000-1.0000-1.0000$ WB15Y Bleed> $0.0000-1.0000 \quad 1.00$ $0.0000 \quad 1.0000 \quad 1.0000$

\begin{tabular}{|c|c|c|c|}
\hline $\mathrm{W}$ & Tt & ht & Pt \\
\hline 0.8664 & 801.23 & 7.56 & 9.537 \\
\hline 0.2755 & 801.23 & 7.56 & 3.266 \\
\hline 0.0000 & 778.22 & 1.91 & 14.172 \\
\hline 0.0000 & 920.10 & 36.92 & 27.758 \\
\hline 0.0000 & 778.22 & 1.91 & 14.172 \\
\hline 0.0000 & 1028.50 & 64.03 & 40.666 \\
\hline 0.0000 & 778.22 & 1.91 & 14.172 \\
\hline W & $\mathrm{Tt}$ & ht & Pt \\
\hline 2.6159 & 569.47 & -48.91 & 6.699 \\
\hline 2.6881 & 1028.50 & 64.03 & 39.729 \\
\hline 2.3522 & 1028.50 & 64.03 & 22.459 \\
\hline 0.0000 & 1028.50 & 64.03 & 40.666 \\
\hline 0.0000 & 1028.50 & 64.03 & 40.666 \\
\hline 0.0000 & 1028.50 & 64.03 & 40.666 \\
\hline 0.0000 & 497.11 & -11.84 & 4.832 \\
\hline 0.0000 & 497.11 & -11.84 & 4.832 \\
\hline 0.0000 & 497.11 & -11.84 & 4.832 \\
\hline 0.0000 & 497.11 & -11.84 & 4.832 \\
\hline
\end{tabular}

Wfue

FAR
Ath
613.40
2006.86

MNth

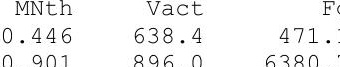

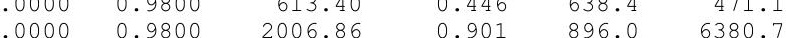


Date:05/10/13 Time:11:40:42 Model: COMDES ON Converge = 1 CASE: 0

Version:NPSS 1.6.5 - Rev: -> Gas Package: Janaf iter/pass/Jacb/Broy=11/39/2/8 Run by: Philip C Jorgenson PC: 10

Temperature Stator 1 inlet: 477.21 Stator 1 exit: 484.61 Stator 2 inlet: 493.88 Stator 2 exit: 498.66 $\begin{array}{ll}\text { Stator } 3 \text { inlet: } 508.16 & \text { Stator } 3 \text { exit: } 512.48 \text { Stator } 4 \text { inlet: } 521.31 \text { Stator } 4 \text { exit: } 524.94 \\ \text { Stator } 5 \text { inlet: } 532.15 & \text { Stator } 5 \text { exit: } 535.32\end{array}$

Ambient Relative Humidity $\quad 10.00$

Fan Face Relative Humidity 2.47

Fan Bypass Relative Humidity $\quad 1.78$

LPC Inlet Relative Humidity $\quad 1.63$

LPC Exit Relative Humidity

HPC Relative Humidity

Drop Diameter
Ambient Flow Velocity

0.05
0.0000050

738.88
2.95

Inlet Length

40.00

Ambient static Pressure

Additional Water at LPC Exit

Fan/LPC Inlet Flow Velocity $\quad 183.43$

$\begin{array}{llr}425.97 & \text { Fan/LPC Inlet Static Temperature } & 468.67 \\ 0.0074386 & & \end{array}$

$\begin{array}{rrrrrr} & & & & \text { SUMMARY } & \text { OUTPUT DATA } \\ \text { MN } & \text { alt } & \text { dTamb } & \text { W } & \text { Fn } & \text { TSFC } \\ 0.730 & 38334.0 & 36.00 & 245.58 & 637.7 & 1.0723\end{array}$

$\begin{array}{rr}\text { Fn } & \text { TSFC } \\ 637.7 & 1.0723\end{array}$

Wfuel
683.80

BPR
8.8021

VTAS

738.88

$O P R$
9.246

EPR
0.764

PowerSet
10.000

$\begin{array}{cc}T 4 & \text { T41 } \\ 1757.5 & 1662 .\end{array}$

T49

FLOW STATION DATA

FSO Ambient.FI_O

FS1 Inlet.FI_O

衣

FS12 Splitter.Fl_O2

FS14 Fan.Fl_O

S23 LPC.FI_O

$\begin{array}{ll}\text { FS24 } & \text { VaporIN.Fl_O } \\ \text { FS25 } & \text { Bleed2.FI O }\end{array}$

FS3 HPC.Fl O

FS36 Bleed3.F1_O

FS4 Burner.FI_O

FS45 HPT.FI_

ES49 LPT.FI-O

FS8 Core_Nozz.Fl_O

FS17 FanDüctLkg.Fì_O

FS171 Bleed15.F1_O

ES172 FanDuct.FI-O

$\begin{array}{rrrr}\text { Pt } & \text { Tt } & \text { ht } & \text { FAR } \\ 4.201 & 471.48 & -17.97 & 0.0000 \\ 4.201 & 471.48 & -17.97 & 0.0000 \\ 4.199 & 471.48 & -17.97 & 0.0000 \\ 4.199 & 471.48 & -17.97 & 0.0000 \\ 4.723 & 489.28 & -13.70 & 0.0000 \\ 6.449 & 545.22 & -0.28 & 0.0000 \\ 6.449 & 555.10 & -40.48 & 0.0000 \\ 6.449 & 555.10 & -40.48 & 0.0000 \\ 38.845 & 1001.70 & 69.01 & 0.0000 \\ 38.845 & 1001.70 & 69.01 & 0.0000 \\ 37.950 & 1757.46 & 56.45 & 0.0114 \\ 9.224 & 1164.53 & -49.60 & 0.0085 \\ 3.315 & 922.92 & -109.30 & 0.0084 \\ 3.315 & 922.99 & -109.30 & 0.0084 \\ 3.315 & 923.06 & -109.30 & 0.0084 \\ 4.723 & 489.28 & -13.70 & 0.0000 \\ 4.723 & 490.03 & -14.00 & 0.0000 \\ 4.723 & 490.03 & -14.00 & 0.0000 \\ 4.723 & 490.03 & -14.00 & 0.0000\end{array}$

WC
819.02

819.02
819.02

735.83

83.60
666.47

58.54

59.50
53.55

11.36

8.81
12.08
5.05

54.05
135.48
135.48

135.48

135.48

135.48
666.47

674.61

674.61
674.61

674.61
674.61

Ps
2.947
3.846
3.794
4.113
3.985
6.178
6.168
6.223
34.168
36.162
35.828
8.373
3.140
3.140
2.947
3.985
3.859
3.859
2.947

Ts
425.97
459.71
458.00
468.67
466.06
538.59
548.08
549.49
966.90
982.15
1732.59
1134.82
909.31
909.38
893.68
466.06
462.51
462.51
428.16

$\begin{array}{rr}\text { Aphy } & \text { MN } \\ 2563.4 & 0.7300 \\ 4168.2 & 0.3574 \\ 3531.8 & 0.3832 \\ 830.5 & 0.1728 \\ 2606.7 & 0.4987 \\ 412.6 & 0.2482 \\ 412.6 & 0.2534 \\ 412.6 & 0.2263 \\ 49.7 & 0.4348 \\ 49.3 & 0.3236 \\ 74.6 & 0.2953 \\ 265.4 & 0.3790 \\ 860.2 & 0.2810 \\ 860.2 & 0.2810 \\ 613.4 & 0.4161 \\ 2606.7 & 0.4987 \\ 2481.9 & 0.5450 \\ 2481.9 & 0.5450 \\ 2006.9 & 0.8491\end{array}$

gamt
1.40073
1.40073
1.40073
1.40073
1.40057
1.39983
1.39865
1.39865
1.37944
1.37944
1.32829
1.36326
1.37865
1.37864
1.37864
1.40057
1.40055
1.40055
1.40055

WAR
0.0000824

$0.0000824 \quad$ Wair $0.0000824 \quad 245.56$

0.0000824

0.020

0.020

0.018

$0.0000824-25.051-0.002$

$\begin{array}{lrl}0.0000824 & 25.05 & 0.002\end{array}$

$\begin{array}{lll}0.00075210 & 25.05 & 0.002 \\ 0.0075210 & 22.55 & 0.180\end{array}$

$0.0075210-21.45 \quad 0.170$

$\begin{array}{lll}0.0075210 & 21.45 & 0.161 \\ 0.0075210 & 16.63 & 0.125\end{array}$

$\begin{array}{ll}16.63 & 0.125\end{array}$

$\begin{array}{lll}0.0075210 & 16.63 & 0.125 \\ 0.0075210 & 16.63 & 0.125 \\ 0.0075210 & 22.28 & 0.168 \\ 0.0075210 & 22.55 & 0.170\end{array}$

$\begin{array}{lll}0.0075210 & 22.28 & 0.168 \\ 0.0075210 & 22.55 & 0.170 \\ 0.0075210 & 22.55 & 0.170\end{array}$

$\begin{array}{lrr}0.0075210 & 22.55 & 0.170\end{array}$

$\begin{array}{lll}0.000824 & 220.51 & 0.170 \\ 0.0001660 & 223.02 & 0.018\end{array}$

$\begin{array}{lll}0.0000824 & 220.51 & 0.018 \\ 0.0001660 & 223.02 & 0.03\end{array}$

$\begin{array}{llllllll}2.947 & 428.16 & 2006.9 & 0.8491 & 1.40055 & 0.0001660 & 223.02 & 0.037\end{array}$

TURBOMACHINERY PERFORMANCE DATA

$\begin{array}{lrrrrrrrrr} & \text { WC } & \text { PR } & \text { eff } & \text { NC } & \text { TR } & \text { efPoly } & \text { pwr } & \text { SMN } & \text { SMW } \\ \text { Fan } & 735.83 & 1.125 & 0.9054 & 2519.750 & 1.0378 & 0.9070 & -1331.8 & 2009.04 & 54.49 \\ \text { LPC } & 83.60 & 1.536 & 0.8342 & 2519.750 & 1.1564 & 0.8440 & -627.0 & 6.83 & 3.39 \\ \text { HPC } & 53.55 & 6.023 & 0.8174 & 8943.039 & 1.8045 & 0.8560 & -3433.6 & 61.71 & 58.31 \\ \text { HPT } & 12.08 & 4.114 & 0.8773 & 220.690 & 1.3601 & 0.8556 & 3433.6 & & \\ \text { LPT } & 54.05 & 2.783 & 0.8526 & 70.399 & 1.2598 & 0.8336 & 1958.7 & \end{array}$

TURBOMACHINERY MAP DATA

$\begin{array}{lrrrrrrrrr} & \text { WcMap } & \text { PRmap } & \text { effMap } & \text { NcMap } & \text { R/Parm } & \text { S_WcDes } & \text { S_PRdes } & \text { S_effDes } & \text { S NcDes } \\ \text { Fan } & 854.97 & 1.122 & 0.9073 & 2495.725 & 0.0414 & 0.8607 & 1.0235 & 0.9980 & 0.9905 \\ \text { LPC } & 71.81 & 1.500 & 0.7776 & 0.666 & 0.0000 & 1.1642 & 1.0708 & 1.0728 & 0.0003 \\ \text { HPC } & 48.71 & 5.787 & 0.8259 & 8704.623 & 10.9607 & 1.0995 & 1.0494 & 0.9898 & 0.9733 \\ \text { HPT } & 0.96 & 4.028 & 0.8773 & 1.326 & 4.0280 & 12.6299 & 0.9723 & 1.0000 & 0.0003 \\ \text { LPT } & 0.83 & 2.341 & 0.8589 & 0.792 & 2.3410 & 65.2590 & 0.7521 & 0.9926 & 0.0005\end{array}$




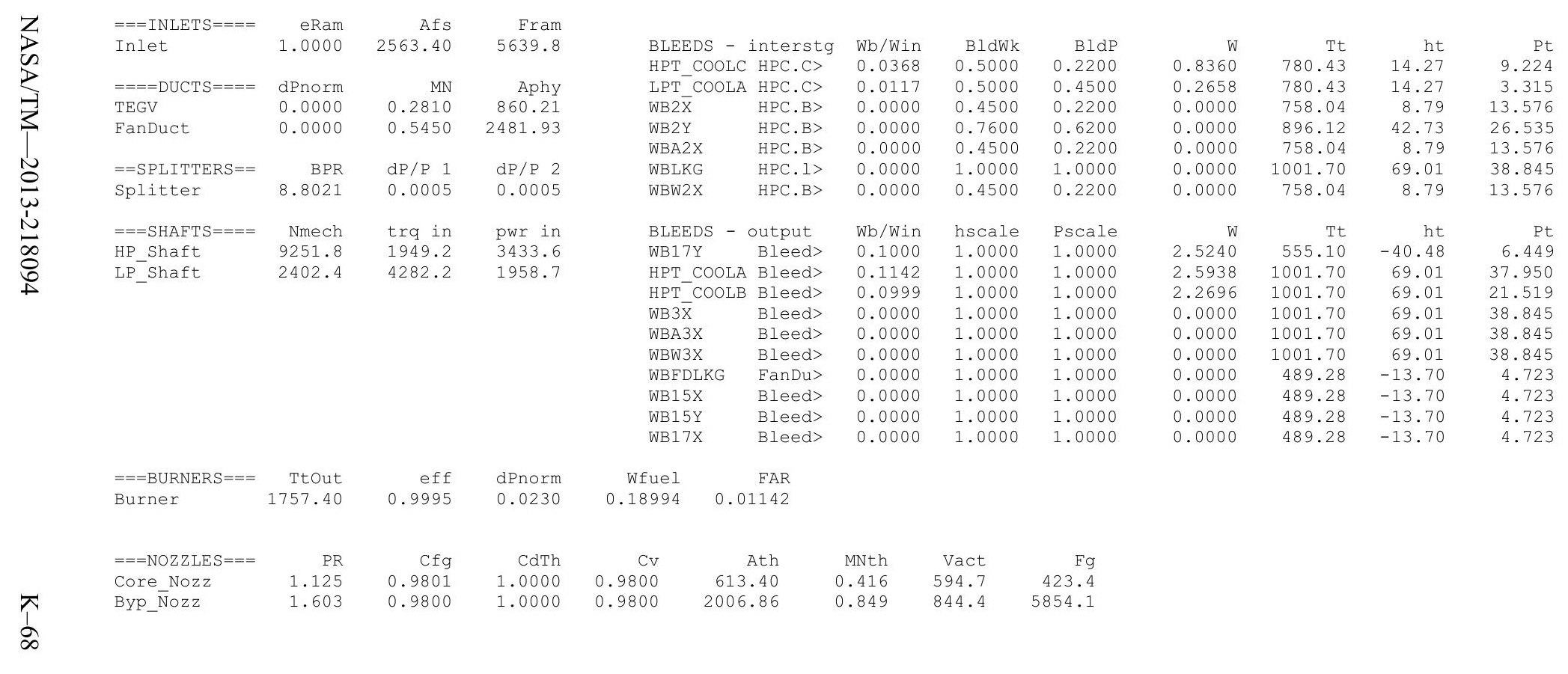


Date:05/10/13 Time:11:41:06 Model: Turbofan Engine - CoMDES ON Converge = 1 CASE: 0

Version:NPSS 1.6.5 - Rev: -> Gas Package: Janaf iter/pass/Jacb/Broy=11/39/2/8 Run by: Philip C Jorgenson PC: 10

Temperature Stator 1 inlet: 475.09 Stator 1 exit: $482.29 \quad$ Stator 2 inlet: $491.30 \quad$ Stator 2 exit: 495.94 $\begin{array}{lll}\text { Stator } 3 \text { inlet: } 505.19 & \text { Stator } 3 \text { exit: } 509.38 \text { Stator } 4 \text { inlet: } 517.98 \text { Stator } 4 \text { exit: } 521.5 \\ \text { Stator } 5 \text { inlet: } 528.52 & \text { Stator } 5 \text { exit: } 531.56 & \end{array}$

Ambient Relative Humidity $\quad 10.00$

Fan Face Relative Humidity $\quad 2.64$

Fan Bypass Relative Humidity $\quad 1.93$

LPC Inlet Relative Humidity 1.76

Cxit Relative Humidity

Drop Diameter

0.0000050

Inlet Length

40.00

Ambient Flow Velocity

Ambient Static Pressure

7.08
3.09

$\mathrm{Fan/LPC}$ Inl

180.89
4.25
466.78

Additional Water at LPC Exit 0.0066979

an/LPC Inlet Static Temperature $\quad 466.78$

$\begin{array}{ll}\text { MN alt } & \text { SUMMARY OUTPUT DATA }\end{array}$
$\begin{array}{rrr}\text { MN } & \text { alt } & \text { dTamb } \\ 0.714 & 37357.0 & 36.00\end{array}$

252.12

$\mathrm{Fn}$
648.4

TSFC
1.0703

Wfuel
694.02

$\mathrm{BPR}$
8.8351

VTAS
722.68

$\mathrm{OPR}$
9.116

$E P R$
0.772

PowerSet
10.000

$\begin{array}{ccc}\text { T4 } & \text { T41 } & \text { T49 } \\ 1744.1 & 1649.4 & 1151.9\end{array}$
FSO Ambient.FI_O

FS1 Inlet.Fl_o

$\begin{array}{ll}\text { FS12 } & \text { Splitter.FI_02 } \\ \text { FS2 } & \text { Splitter.Fl_01 }\end{array}$

FS14 Fan.Fl_

FS23 LPC.FI_O

FS25 Bleed2.F1_O

FS3 HPC.FI_O

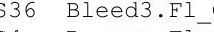

ES4

FS49 4 TPT

FS4 5 TEGV.FI

FS8 Core_Nozz.Fl_O

FS17 FanDūctLkg.F̄_

FS171 Bleed15.FI_O

FS172 FanDuct.Fl-O

FS173 Byp Nozz.F̄̄o

TURBOMACHINERY PERFORMANCE DATA

$\begin{array}{lrrr} & \text { WC } & \text { PR } & \text { eff } \\ \text { Fan } & 730.14 & 1.121 & 0.9050 \\ \text { LPC } & 82.64 & 1.522 & 0.8352 \\ \text { HPC } & 53.26 & 5.993 & 0.8169 \\ \text { HPT } & 12.08 & 4.095 & 0.8766 \\ \text { LPT } & 53.82 & 2.732 & 0.8501\end{array}$

FLOW STATION DATA

\begin{tabular}{rrrrrr} 
W & \multicolumn{1}{c}{ Pt } & Tt & ht & FAR & WC \\
252.12 & 4.339 & 469.50 & -18.42 & 0.0000 & 812.38 \\
252.12 & 4.339 & 469.50 & -18.42 & 0.0000 & 812.38 \\
226.48 & 4.337 & 469.50 & -18.42 & 0.0000 & 730.14 \\
25.63 & 4.337 & 469.50 & -18.42 & 0.0000 & 82.64 \\
226.48 & 4.863 & 486.78 & -14.28 & 0.0000 & 663.03 \\
25.63 & 6.600 & 541.20 & -1.23 & 0.0000 & 58.31 \\
25.81 & 6.600 & 550.16 & -37.44 & 0.0000 & 59.18 \\
23.23 & 6.600 & 550.16 & -37.44 & 0.0000 & 53.26 \\
22.10 & 39.555 & 991.90 & 70.72 & 0.0000 & 11.35 \\
17.13 & 39.555 & 991.90 & 70.72 & 0.0000 & 8.80 \\
17.32 & 38.644 & 1744.08 & 58.23 & 0.0113 & 12.08 \\
23.15 & 9.436 & 1156.11 & -46.52 & 0.0085 & 53.82 \\
23.42 & 3.454 & 920.40 & -104.64 & 0.0084 & 132.74 \\
23.42 & 3.454 & 920.47 & -104.64 & 0.0084 & 132.74 \\
23.42 & 3.454 & 920.54 & -104.64 & 0.0084 & 132.75 \\
226.48 & 4.863 & 486.78 & -14.28 & 0.0000 & 663.03 \\
229.06 & 4.863 & 487.49 & -14.54 & 0.0000 & 671.08 \\
229.06 & 4.863 & 487.49 & -14.54 & 0.0000 & 671.08 \\
229.06 & 4.863 & 487.49 & -14.54 & 0.0000 & 671.08
\end{tabular}

PS
3.088

$\begin{array}{lrrr}3.088 & \text { T25. } & \text { Aphy } & \text { MN }\end{array}$

$\begin{array}{llll}3.979 & 458.00 & 4168.2 & 0.3539 \\ 3.926 & 456.33 & 3531.8 & 0.3796\end{array}$

4.250

4.113

6.325

6.315
6.372

34.805

36.82

$8.574 \quad 1126.8$

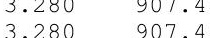

$3.088 \quad 892.64$

$3.986-463.99$

$3.986-460.52$

$3.088 \quad 428.11$

$\begin{array}{ll}2481.9 & 0.5407 \\ 2481.9 & 0.5407\end{array}$ $\begin{array}{lll}2567.1 & 0.7140 & 1.40075 \\ 3531.2 & 0.3539 & 1.40075 \\ 830.8 & 0.3796 & 1.40075\end{array}$

$\begin{array}{rrrr}458.00 & 4168.2 & 0.3539 & 1.40075 \\ 466.78 & 830.5 & 0.1707 & 1.40075 \\ 463.99 & 260.7 & 0.4951 & 1.40060\end{array}$

$\begin{array}{rr}2606.7 & 0.4951\end{array}$

$\begin{array}{ll}412.6 & 0.2471 \\ 412.6 & 0.2518\end{array}$

$\begin{array}{ll}412.6 & 0.2249\end{array}$

$\begin{array}{ll}49.7 & 0.4341 \\ 49.3 & 0.3231\end{array}$

$\begin{array}{ll}74.6 & 0.2952 \\ 265.4 & 0.3769\end{array}$

$\begin{array}{ll}265.4 & 0.3769\end{array}$

$\begin{array}{ll}860.2 & 0.2746 \\ 860.2 & 0.2746\end{array}$

$\begin{array}{ll}613.4 & 0.4056\end{array}$

$2606.7 \quad 0.4951$

$\begin{array}{ll}2481.9 & 0.5407 \\ 2006.9 & 0.831\end{array}$
1.40075

1.40060
1.39990

1.39883
1.39883

1.39883
1.38017

1.38017

1.32908
1.36395

1.37894
1.37894

1.37893
1.40060

1.40060
1.40058
1.40058

1.40058
1.40058

$\begin{array}{rrrr}\text { WAR } & \text { Wair } & \text { WH2O } & \text { H2O frac } \\ 0.0000786 & 252.10 & 0.020 & 0.0001 \\ 0.0000786 & 252.10 & 0.020 & 0.0001 \\ 0.0000786 & 226.46 & 0.018 & 0.0001 \\ 0.0000786 & 25.63 & 0.002 & 0.0001 \\ 0.0000786 & 226.46 & 0.018 & 0.0001 \\ 0.0000786 & 25.63 & 0.002 & 0.0001 \\ 0.0067765 & 25.63 & 0.174 & 0.0067 \\ 0.0067765 & 23.07 & 0.156 & 0.0067 \\ 0.0067765 & 21.95 & 0.149 & 0.0067 \\ 0.0067765 & 17.01 & 0.115 & 0.0067 \\ 0.0067765 & 17.01 & 0.115 & 0.0204 \\ 0.0067765 & 22.80 & 0.154 & 0.0170 \\ 0.0067765 & 23.07 & 0.156 & 0.0168 \\ 0.0067765 & 23.07 & 0.156 & 0.0168 \\ 0.0067765 & 23.07 & 0.156 & 0.0168 \\ 0.0000786 & 226.46 & 0.018 & 0.0001 \\ 0.0001536 & 229.03 & 0.035 & 0.0002 \\ 0.0001536 & 229.03 & 0.035 & 0.0002 \\ 0.0001536 & 229.03 & 0.035 & 0.0002\end{array}$

TURBOMACHINERY MAP DATA

$\begin{array}{lrrrrrrrrr} & \text { WcMap } & \text { PRmap } & \text { effMap } & \text { NcMap } & \text { R/Parm } & \text { S_WcDes } & \text { S_PRdes } & \text { S_effDes } & \text { S_NcDes } \\ \text { Fan } & 848.35 & 1.119 & 0.9068 & 2467.742 & 0.0414 & 0.8607 & \overline{1} .0235 & 0.9980 & 0.9905 \\ \text { LPC } & 70.59 & 1.488 & 0.7763 & 0.658 & 0.0000 & 1.1708 & 1.0698 & 1.0758 & 0.0003 \\ \text { HPC } & 48.44 & 5.758 & 0.8254 & 8695.377 & 10.9612 & 1.0995 & 1.0494 & 0.9898 & 0.9733 \\ \text { HPT } & 0.96 & 4.010 & 0.8766 & 1.324 & 4.0097 & 12.6299 & 0.9723 & 1.0000 & 0.0003 \\ \text { LPT } & 0.82 & 2.303 & 0.8565 & 0.784 & 2.3027 & 65.2590 & 0.7521 & 0.9926 & 0.0005\end{array}$


$\begin{array}{lrrr}===\text { INLETS }==== & \text { eRam } & \text { Afs } & \text { Fram } \\ \text { Inlet } & 1.0000 & 2567.14 & 5662.9\end{array}$

$====$ DUCTS $====$
TEGV

Aphy

FanDuct

0.0000

0.0000

$==$ SPLITTERS $==$

Splitter

8.8351

0.5407

860.21
2481.93

$===\mathrm{SHAFTS}===$

8.8351

$\mathrm{dP} / \mathrm{P}$

$\mathrm{dP} / \mathrm{P} 2$

HP_Shaft

Nmech

0.0005

0.0005

LP_Shaft

2370.5

$\begin{array}{ll}\text { trq in } & \text { pwr in } \\ 1979.6 & 3467.9\end{array}$

$4320.8 \quad 1950.1$

$===$ BURNERS $===\quad$ TtOut

Burner 1744.03

eff

dPnorm

$===$ NOZZLES $===$

Core $\overline{N O Z Z}^{2}$

PR
1.118
1.575

$\mathrm{Cfg}$
0.9801

0.9800

ป̃
BLEEDS - interstg Wb/Win BldWk BldP HPT_COOLC HPC.C> $0.0368 \quad 0.5000=0.2200$ $\begin{array}{lllll}\text { LPT COOLA HPC.C }> & 0.0117 & 0.5000 & 0.4500 \\ \text { WB } 2 \bar{X} & \text { HPC. B> } & 0.0000 & 0.4500 & 0.2200\end{array}$ $\begin{array}{lllll}\text { WB2X } & \text { HPC.B } & 0.0000 & 0.4500 & 0.2200 \\ \text { WB2Y } & \text { HPC.B } & 0.0000 & 0.7600 & 0.6200\end{array}$ $\begin{array}{lllll}\text { WBA2X HPC.B } & 0.0000 & 0.4500 & 0.2200\end{array}$ WBLKG HPC.1> $0.0000 \quad 1.0000-1.0000$ $\begin{array}{lllll}\text { WBW2X HPC.B } & 0.0000 & 0.4500 & 0.2200\end{array}$

BLEEDS - output Wb/Win hscale Pscale WB17Y Bleed> $0.10001 .0000 \quad 1.0000$ HPT_COOLA Bleed> $0.1142 \quad 1.0000 \quad 1.0000$ HPT_COOLB Bleed> $0.0999 \quad 1.0000 \quad 1.0000$ $\begin{array}{lllll}\text { WB3X } & \text { Bleed> } & 0.0000 & 1.0000 & 1.0000 \\ \text { WBA3X } & \text { Bleed> } & 0.0000 & 1.0000 & 1.0000\end{array}$

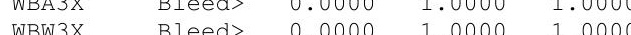
$\begin{array}{lllll}\text { WBW3X } & \text { Bleed } & 0.00000 & 1.0000 & 1.0000\end{array}$ $\begin{array}{lllll}\text { WBFDLKG } & \text { FanDu> } & 0.0000 & 1.0000 & 1.0000 \\ \text { WB15X } & \text { Bleed }> & 0.0000 & 1.0000 & 1.0000\end{array}$ WB15X Bleed> $0.0000 \quad 1.0000 \quad 1.0000$ $\begin{array}{lllll}\text { WB15Y } & \text { Bleed> } & 0.0000 & 1.0000 & 1.0000 \\ \text { WB17X } & \text { Bleed }> & 0.0000 & 1.0000 & 1.0000\end{array}$

$\begin{array}{rrrr}\text { W } & \text { Tt } & \text { ht } & \text { Pt } \\ 0.8547 & 772.97 & 16.64 & 9.436 \\ 0.2717 & 772.97 & 16.64 & 3.454 \\ 0.0000 & 750.83 & 11.23 & 13.850 \\ 0.0000 & 887.42 & 44.76 & 27.032 \\ 0.0000 & 750.83 & 11.23 & 13.850 \\ 0.0000 & 991.90 & 70.72 & 39.555 \\ 0.0000 & 750.83 & 11.23 & 13.850 \\ \text { W } & \text { Tt } & \text { ht } & \text { Pt } \\ 2.5806 & 550.16 & -37.44 & 6.600 \\ 2.6519 & 991.90 & 70.72 & 38.644 \\ 2.3204 & 991.90 & 70.72 & 21.937 \\ 0.0000 & 991.90 & 70.72 & 39.555 \\ 0.0000 & 991.90 & 70.72 & 39.555 \\ 0.0000 & 991.90 & 70.72 & 39.555 \\ 0.0000 & 486.78 & -14.28 & 4.863 \\ 0.0000 & 486.78 & -14.28 & 4.863 \\ 0.0000 & 486.78 & -14.28 & 4.863 \\ 0.0000 & 486.78 & -14.28 & 4.863\end{array}$

FAR $0.19278 \quad 0.01133$$$
0.19278-0.01133
$$

$\begin{array}{rrrrrr}\text { CdTh } & \text { Cv } & \text { Ath } & \text { MNth } & \text { Vact } & \text { Fg } \\ 1.0000 & 0.9800 & 613.40 & 0.406 & 579.2 & 421.6 \\ 1.0000 & 0.9800 & 2006.86 & 0.832 & 827.3 & 5889.8\end{array}$


Date:05/10/13 Time:11:41:22 Model: Turbofan Engine - ComDES ON Converge = 1 CASE: 0

Version:NPSS 1.6.5 - Rev: -> Gas Package: Janaf iter/pass/Jacb/Broy=12/26/1/10 Run by: Philip C Jorgenson PC: 10

Temperature Stator 1 inlet: $476.45 \quad$ Stator 1 exit: $483.18 \quad$ Stator 2 inlet: 491.59 Stator 2 exit: 495.91 Stator 5 inlet: 526.21 Stator 5 exit: $528.99 \quad$ Unblocked Percent Blockage: 0.00

Ambient Relative Humidity $\quad 10.00$

Fan Face Relative Humidity $\quad 3.25$

Fan Bypass Relative Humidity $\quad 2.46$

LPC Inlet Relative Humidity

LPC Exit Relative Humidity

Drop Ditive Humidity

Ambient Flow Velocity

Ambient Static Pressure

0.0000050

682.24

Inlet Length

40.00

Ambient Static Temperature

174.69

Additional Water at LPC Exit

32.42

4.74

0.0060647

$\begin{array}{rrrrrr}\text { MN } & \text { alt } & \text { dTamb } & \text { W } & \text { Fn } & \text { TSFC } \\ 0.669 & 34281.0 & 36.00 & 272.97 & 694.5 & 1.0652\end{array}$

172.97

694.5

1.0652

Wfuel
739.78

BPR
8.9302

VTAS
682.24

$O P R$
8.742

EPR
0.795

Powerset

10.000

$\begin{array}{cc}\text { T4 } & \text { T41 } \\ 1731.5 & 1637 .\end{array}$

T49

FLOW STATION DATA

FS0 Ambient.F1_O

FS1 Inlet.Fl_O

$\begin{array}{ll}\text { FS12 } & \text { Splitter.F1_02 } \\ \text { FS2 } & \text { Splitter.Fl_01 }\end{array}$

FS14 Fan.Fl_

FS24 VaporIN.FI

FS25 Bleed2.F1_O

FS3 HPC.FIO

ES36 Bleed3.FI_-

FS4 Burner.Fl_

FS49

FS5

FS8 Core_Nozz.Fl_

FS17 FanDuctLkg.Fi_

FS171 Bleed15.FI

FS172 FanDuct.FI-O

$\begin{array}{rrr}\text { Pt } & \text { Tt } \\ 4.831 & 471.21 & -18 \\ 4.831 & 471.21 & -18\end{array}$

$W$
272.97
272.97
245.48
27.49
245.48
27.49
27.66
24.89
23.68
18.35
18.56
24.80
25.10
25.10
25.10
245.48
248.25
248.25
248.25

471.21
471.21

ht FAR

$\begin{array}{rr}\text { Wc } & \text { Ps } \\ 791.49 & 3.578\end{array}$

$\begin{array}{lllll}4.829-4929 & 471.21 & -18.12 & 0.0000 & 791.49\end{array}$

$\begin{array}{llll}5.370-487.31 & -14.26 & 0.00000\end{array}$

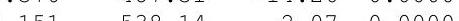

$\begin{array}{llll}7.151 & 546.29 & -34.87 & 0.0000\end{array}$

$\begin{array}{llll}7.151 & 546.29 & -34.87 & 0.0000 \\ 42.231 & 981.50 & -34.87 & 0.0000\end{array}$

$\begin{array}{llll}42.231 & 981.50 & 71.57 & 0.0000\end{array}$

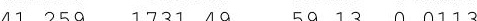

$\begin{array}{llll}10.189 & 1749.77 & -43.92 & 0.0113\end{array}$

$\begin{array}{lllll}10.189 & 1149.77 & -43.92 & 0.0084\end{array}$

$\begin{array}{llll}3.942 & 927.91 & -98.52 & 0.0083\end{array}$

$\begin{array}{llll}3.942 & 927.98 & -98.52 & 0.0083\end{array}$

$\begin{array}{lllll}5.370 & 487.97 & -14.49 & 0.0000\end{array}$

$\begin{array}{lllll}5.370 & 487.97 & -14.49 & 0.0000\end{array}$

$\begin{array}{lllll}5.370 & 487.97 & -14.49 & 0.0000 & 658.95 \\ 5.370 & 487.97 & -14.49 & 0.0000 & 658.95\end{array}$

TURBOMACHINERY PERFORMANCE DATA

$\begin{array}{lrrrrrrrrr} & \text { WC } & \text { PR } & \text { eff } & \text { NC } & \text { TR } & \text { efPoly } & \text { pwr } & \text { SMN } & \text { SMW } \\ \text { Fan } & 712.14 & 1.112 & 0.9039 & 2408.079 & 1.0342 & 0.9053 & -1340.1 & 4859.75 & 54.07 \\ \text { LPC } & 79.75 & 1.481 & 0.8367 & 2408.079 & 1.1420 & 0.8455 & -624.2 & 6.45 & 3.08 \\ \text { HPC } & 52.49 & 5.905 & 0.8155 & 8908.345 & 1.7967 & 0.8542 & -3657.4 & 62.13 & 58.61 \\ \text { HPT } & 12.08 & 4.049 & 0.8758 & 219.712 & 1.3559 & 0.8540 & 3657.4 & & \\ \text { LPT } & 53.27 & 2.584 & 0.8447 & 67.690 & 1.2370 & 0.8264 & 1964.3 & \end{array}$

TURBOMACHINERY MAP DATA

$\begin{array}{lrrrrrrrrr} & \text { WcMap } & \text { PRmap } & \text { effMap } & \text { NcMap } & \text { R/Parm } & \text { S_WcDes } & \text { S_PRdes } & \text { S_effDes } & \text { S_NcDes } \\ \text { Fan } & 827.44 & 1.110 & 0.9057 & 2385.118 & 0.0414 & 0.8607 & \overline{1} .0235 & 0.9980 & 0.9905 \\ \text { LPC } & 66.97 & 1.450 & 0.7724 & 0.636 & 0.0000 & 1.1907 & 1.0679 & 1.0833 & 0.0003 \\ \text { HPC } & 47.74 & 5.674 & 0.8239 & 8670.855 & 10.9485 & 1.0995 & 1.0494 & 0.9898 & 0.9733 \\ \text { HPT } & 0.96 & 3.965 & 0.8758 & 1.320 & 3.9648 & 12.6299 & 0.9723 & 1.0000 & 0.0003 \\ \text { LPT } & 0.82 & 2.192 & 0.8510 & 0.761 & 2.1917 & 65.2590 & 0.7521 & 0.9926 & 0.0005\end{array}$

$\begin{array}{lll}0.82 & 2.192 & 0.8510\end{array}$

0.761

$65.2590 \quad 0.7521$

0.0005

$\begin{array}{rrrrrrrr}\text { Ts } & \text { Aphy } & \text { MN } & \text { gamt } & \text { WAR } & \text { Wair } & \text { WH2O } & \text { H2O frac } \\ 432.42 & 2580.0 & 0.6690 & 1.40074 & 0.0000968 & 272.95 & 0.026 & 0.0001 \\ 460.34 & 4168.2 & 0.3434 & 1.40074 & 0.0000968 & 272.95 & 0.026 & 0.0001 \\ 458.73 & 3531.8 & 0.3684 & 1.40074 & 0.0000968 & 245.46 & 0.024 & 0.0001 \\ 468.67 & 830.5 & 0.1646 & 1.40074 & 0.0000968 & 27.49 & 0.003 & 0.0001 \\ 465.55 & 2606.7 & 0.4830 & 1.40059 & 0.0000968 & 245.46 & 0.024 & 0.0001 \\ 531.82 & 412.6 & 0.2437 & 1.39994 & 0.0000968 & 27.49 & 0.003 & 0.0001 \\ 539.68 & 412.6 & 0.2478 & 1.39898 & 0.0061615 & 27.49 & 0.169 & 0.0061 \\ 541.00 & 412.6 & 0.2214 & 1.39898 & 0.0061615 & 24.74 & 0.152 & 0.0061 \\ 947.55 & 49.7 & 0.4330 & 1.38090 & 0.0061615 & 23.54 & 0.145 & 0.0061 \\ 962.41 & 49.3 & 0.3224 & 1.38090 & 0.0061615 & 18.24 & 0.112 & 0.0061 \\ 1706.91 & 74.6 & 0.2951 & 1.32979 & 0.0061615 & 18.24 & 0.112 & 0.0197 \\ 1121.38 & 265.4 & 0.3721 & 1.36448 & 0.0061615 & 24.45 & 0.151 & 0.0163 \\ 916.33 & 860.2 & 0.2575 & 1.37862 & 0.0061615 & 24.74 & 0.152 & 0.0162 \\ 916.40 & 860.2 & 0.2575 & 1.37861 & 0.0061615 & 24.74 & 0.152 & 0.0162 \\ 903.56 & 613.4 & 0.3774 & 1.37861 & 0.0061615 & 24.74 & 0.152 & 0.0162 \\ 465.55 & 2606.7 & 0.4830 & 1.40059 & 0.0000968 & 245.46 & 0.024 & 0.0001 \\ 462.31 & 2481.9 & 0.5263 & 1.40057 & 0.0001640 & 248.21 & 0.041 & 0.0002 \\ 462.31 & 2481.9 & 0.5263 & 1.40057 & 0.0001640 & 248.21 & 0.041 & 0.0002 \\ 434.45 & 2006.9 & 0.7840 & 1.40057 & 0.0001640 & 248.21 & 0.041 & 0.0002\end{array}$

0.0002 


\begin{tabular}{|c|c|c|c|c|c|c|c|c|c|c|}
\hline Z & $===$ INLET $S====$ & eRam & Afs & Fram & & & & \\
\hline 胥 & Inlet & 1.0000 & 2579.98 & 5788.3 & & & & $9 \quad \begin{array}{l}\text { Wb/Win } \\
0.0368\end{array}$ & $\begin{array}{r}\text { BldWk } \\
0.5000\end{array}$ & $\begin{array}{r}\text { BldP } \\
0.2200\end{array}$ \\
\hline 2 & $====$ DUCTS $====$ & dPnorm & MN & Aphy & \multicolumn{3}{|c|}{ LPT_COOLA HPC.C> } & 0.0117 & 0.5000 & 0.4500 \\
\hline$\exists$ & TEGV & 0.0000 & 0.2575 & 860.21 & \multicolumn{2}{|c|}{ WB2 $\bar{x}$} & HPC.B> & 0.0000 & 0.4500 & 0.2200 \\
\hline 3 & FanDuct & 0.0000 & 0.5263 & 2481.93 & \multirow{2}{*}{\multicolumn{2}{|c|}{$\begin{array}{l}\text { WB2Y } \\
\text { WBA2X }\end{array}$}} & HPC.B> & 0.0000 & 0.7600 & 0.6200 \\
\hline & & & & & & & HPC.B> & 0.0000 & 0.4500 & 0.2200 \\
\hline N & $==$ SPLITTERS $==$ & BPR & $\mathrm{dP} / \mathrm{P} \quad 1$ & $\mathrm{dP} / \mathrm{P} 2$ & \multicolumn{2}{|c|}{ WBLKG } & HPC.I> & 0.0000 & 1.0000 & 1.0000 \\
\hline 0 & Splitter & 8.9302 & 0.0005 & 0.0005 & \multicolumn{2}{|c|}{ WBW2X } & HPC.B> & 0.0000 & 0.4500 & 0.2200 \\
\hline 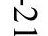 & $===$ SHAFTS $====$ & Nmech & trg in & pwr in & \multicolumn{3}{|c|}{ BLEEDS - output } & Wb/Win & hscale & Pscale \\
\hline$\infty$ & HP_Shaft & 9142.5 & 2101.1 & 3657.4 & \multicolumn{2}{|c|}{ WB17Y } & Bleed> & 0.1000 & 1.0000 & 1.0000 \\
\hline 8 & LP_Shaft & 2295.3 & 4494.7 & 1964.3 & \multicolumn{2}{|c|}{ HPT COOLA } & A Bleed> & 0.1142 & 1.0000 & 1.0000 \\
\hline & & & & & \multicolumn{2}{|c|}{ HPT_COOLB } & 3 Bleed> & 0.0999 & 1.0000 & 1.0000 \\
\hline & & & & & \multicolumn{2}{|l|}{ WB $3 \bar{x}$} & Bleed> & 0.0000 & 1.0000 & 1.0000 \\
\hline & & & & & \multirow{2}{*}{\multicolumn{2}{|c|}{$\begin{array}{l}\text { WBA3X } \\
\text { WBW3X }\end{array}$}} & Bleed> & 0.0000 & 1.0000 & 1.0000 \\
\hline & & & & & & & Bleed> & 0.0000 & 1.0000 & 1.0000 \\
\hline & & & & & & FanDu> & 0.0000 & 1.0000 & 1.0000 \\
\hline & & & & & \multicolumn{2}{|c|}{$\begin{array}{l}\text { WBFDLKG } \\
\text { WB15X }\end{array}$} & Bleed> & 0.0000 & 1.0000 & 1.0000 \\
\hline & & & & & \multicolumn{2}{|c|}{ WB15Y } & Bleed> & 0.0000 & 1.0000 & 1.0000 \\
\hline & & & & & \multicolumn{2}{|c|}{ WB17X } & Bleed> & 0.0000 & 1.0000 & 1.0000 \\
\hline & $===$ BURNERS $===$ & Ttout & eff & dPnorm & & & & & \\
\hline & Burner & 1731.44 & 0.9995 & 0.0230 & \multicolumn{3}{|c|}{$0.20549 \quad 0.01126$} & & & \\
\hline & $===$ NOZZLES $===$ & PR & $\mathrm{Cfg}$ & CdTh & & \multicolumn{2}{|r|}{ Ath } & MNth & Vact & $\mathrm{Fg}$ \\
\hline & Core_Nozz & 1.102 & 0.9801 & 1.0000 & 0.9800 & \multicolumn{2}{|c|}{613.40} & \multirow{2}{*}{$\begin{array}{l}0.377 \\
0.784\end{array}$} & 542.1 & 422.9 \\
\hline & 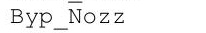 & 1.501 & 0.9800 & 1.0000 & \multicolumn{3}{|c|}{2006.86} & & 785.4 & 6059.9 \\
\hline
\end{tabular}


Turbofan Engine - COMDES ON converge $=1$ CASE: 0

Version:NPSS 1.6.5 - Rev: -> Gas Package: Janaf iter/pass/Jacb/Broy=13/27/1/11 Run by: Philip C Jorgenson PC: 10

Temperature Stator 1 inlet: 484.73 Stator 1 exit: 490.51 Stator 2 inlet: 497.66 Stator 2 exit: 501.14 $\begin{array}{ll}\text { Stator } 3 \text { inlet: } 508.56 & \text { Stator } 3 \text { exit: } 511.63 \text { Stator 4 lnbloked Percent Blockage: } 0.00 \\ \text { Stator } 5 \text { inlet: } 526.62 & \text { Stator } 5 \text { exit: } 528.77\end{array}$

Ambient Relative Humidity $\quad 10.00$

Fan Face Relative Humidity $\quad 4.19$

Fan Bypass Relative Humidity $\quad 3.34$

$\begin{array}{ll}\text { LPC Inlet Relative Humidity } & 3.05 \\ \text { LC Exit Relative Humidity } & 0.55\end{array}$

HPC Relative Humidity

0.06

0.0000050

Inlet Length

40.00

Amblent Flow Velocity

Ambient Static Pressure

Additional Water at LPC Exi

630.80 Fan/LPC Inlet Flow Velocity $\quad 175.45$

4.36 Fan/LPC Inlet Static Pressure

447.58 Fan/LPC Inlet Static Temperature 478.17

\section{MN alt dTamb SUMMARY OUTPUT DAT}

$\begin{array}{rrrrrr}\text { MN } & \text { alt } & \text { dTamb } & \text { W } & \text { Fn } & \text { TSFC } \\ 0.608 & 30029.0 & 36.00 & 298.46 & 738.9 & 1.0811\end{array}$
FSO Ambient.FI_O

FS1 Inlet.F1_O

FS12 Splitter.F1_02

FS2 Splitter.F1_01

FS14 Fan.F1_O

FS24 VaporIN̄.El_O

FS25 Bleed2.F1_o

ES3 HPC.FI O

S36 Bleed3.F1_O

FS4 Burner.Fl_O

FS45 HPT.FI

FS49 LPT.FI-O

FS8 Core_Nozz.FI_O

FS17 FanDüctLkg.Fì O

FS171 Bleed15.F1_O

FS172 FanDuct.Fl-O

FS173 Byp Nozz.Fì O

TURBOMACHINERY PERFORMANCE DATA

$\begin{array}{lrrr} & \text { WC } & \text { PR } & \text { eff } \\ \text { Fan } & 675.80 & 1.098 & 0.9023 \\ \text { LPC } & 79.30 & 1.402 & 0.8515 \\ \text { HPC } & 51.56 & 5.796 & 0.8135 \\ \text { HPT } & 12.08 & 3.990 & 0.8755 \\ \text { LPT } & 52.56 & 2.361 & 0.8358\end{array}$

FLOW STATION DATA

\begin{tabular}{crrrr}
\multicolumn{4}{c}{ FLOW } & STATION DATA \\
Pt & Tt & ht & FAR & WC \\
5.595 & 480.74 & -16.28 & 0.0000 & 754.73 \\
5.595 & 480.74 & -16.28 & 0.0000 & 754.73 \\
5.592 & 480.74 & -16.28 & 0.0000 & 675.80 \\
5.592 & 480.74 & -16.28 & 0.0000 & 79.30 \\
6.141 & 495.19 & -12.82 & 0.0000 & 624.61 \\
7.841 & 538.01 & -2.55 & 0.0000 & 59.83 \\
7.841 & 546.24 & -35.66 & 0.0000 & 60.66 \\
7.841 & 546.24 & -35.66 & 0.0000 & 51.56 \\
45.449 & 976.73 & 69.61 & 0.0000 & 11.32 \\
45.449 & 976.73 & 69.61 & 0.0000 & 8.77 \\
44.402 & 1729.03 & 57.17 & 0.0113 & 12.08 \\
1.128 & 1151.23 & -44.72 & 0.0084 & 52.56 \\
4.713 & 949.82 & -94.18 & 0.0083 & 114.04 \\
4.713 & 949.89 & -94.18 & 0.0083 & 114.05 \\
4.713 & 949.96 & -94.18 & 0.0083 & 114.05 \\
6.141 & 495.19 & -12.82 & 0.0000 & 624.61 \\
6.141 & 496.09 & -13.22 & 0.0000 & 636.24 \\
6.141 & 496.09 & -13.22 & 0.0000 & 636.24 \\
6.141 & 496.09 & -13.22 & 0.0000 & 636.24
\end{tabular}

VTAS

Wfuel

798.85

8.5220

630.80

8.123

EPR
0.827

Powerset
10.000

$\begin{array}{ccc}\text { T4 } & \text { T41 } & \text { T49 } \\ 1729.0 & 1634.3 & 1146.9\end{array}$

$\begin{array}{rrr}\text { Aphy } & \text { MN } & \text { gamt } \\ 2592.6 & 0.6080 & 1.40064 \\ 4168.2 & 0.3251 & 1.40064 \\ 3531.8 & 0.3465 & 1.40064 \\ 830.5 & 0.1636 & 1.40064 \\ 2606.7 & 0.4568 & 1.40049 \\ 412.6 & 0.2542 & 1.39993 \\ 412.6 & 0.2586 & 1.39896 \\ 412.6 & 0.2172 & 1.39896 \\ 49.7 & 0.4320 & 1.38116 \\ 49.3 & 0.3218 & 1.38116 \\ 74.6 & 0.2951 & 1.32986 \\ 265.4 & 0.3664 & 1.36435 \\ 860.2 & 0.2332 & 1.37726 \\ 860.2 & 0.2332 & 1.37725 \\ 613.3 & 0.3388 & 1.37725 \\ 2606.7 & 0.4568 & 1.40049 \\ 2481.9 & 0.5006 & 1.40047 \\ 2481.9 & 0.5006 & 1.40047 \\ 2006.9 & 0.7172 & 1.40047\end{array}$

WAR
0.0001750
0.0001750
0.0001750
0.0001750
0.0001750
0.0001750
0.0062976
0.0062976
0.0062976
0.0062976
0.0062976
0.0062976
0.0062976
0.0062976
0.0062976
0.0001750
0.0002809
0.0002809
0.0002809

TURBOMACHINERY MAP DATA

\begin{tabular}{|c|c|c|c|c|c|c|c|c|}
\hline WC & PR & eff & Nc & $\mathrm{TR}$ & efPoly & pwr & SMN & SMW \\
\hline 675.80 & 1.098 & 0.9023 & 2251.777 & 1.0301 & 0.9036 & $-1309.6-$ & -9547.00 & 52.70 \\
\hline 79.30 & 1.402 & 0.8515 & 2251.777 & 1.1191 & 0.8584 & -609.2 & 5.95 & 2.63 \\
\hline 51.56 & 5.796 & 0.8135 & 8877.522 & 1.7881 & 0.8523 & -3895.8 & 62.65 & 59.01 \\
\hline 12.08 & 3.990 & 0.8755 & 219.098 & 1.3514 & 0.8539 & 3895.7 & & \\
\hline 52.56 & 2.361 & 0.8358 & 63.893 & 1.2096 & 0.8186 & 1918.8 & & \\
\hline NERY MAP & DATA & & & & & & & \\
\hline WcMap & PRmap & effmap & NcMap & R/Parm & S WcDes & s PRdes & $s$ s effDes & s NcDes \\
\hline 785.22 & 1.096 & 0.9041 & 2230.307 & 0.0416 & $\overline{0} .8607$ & $\overline{1} .0235$ & 0.9980 & $\overline{0} .9905$ \\
\hline 60.40 & 1.383 & 0.7655 & 0.595 & 0.0000 & 1.3129 & 1.0509 & 1.1123 & 0.0003 \\
\hline 46.89 & 5.570 & 0.8219 & 8640.853 & 10.9296 & 1.0995 & 1.0494 & 0.9898 & 0.9733 \\
\hline 0.96 & 3.907 & 0.8754 & 1.317 & 3.9074 & 12.6299 & 0.9723 & 1.0000 & 0.0003 \\
\hline 0.81 & 2.024 & 0.8420 & 0.719 & 2.0237 & 65.2590 & 0.7521 & 0.9926 & 0.0005 \\
\hline
\end{tabular}

\begin{tabular}{|c|c|}
\hline & \\
\hline 5.2 & \\
\hline 5.1 & 469.4 \\
\hline 5.4 & 478.1 \\
\hline 5.3 & 475.3 \\
\hline 7.4 & 531.1 \\
\hline 7.4 & 539.0 \\
\hline 7.5 & \\
\hline 40.0 & 943.07 \\
\hline 42.3 & 957. \\
\hline 41.92 & 1704.4 \\
\hline 10.1 & 1123.6 \\
\hline 4.5 & 940.1 \\
\hline 4.5 & 940.24 \\
\hline 4.3 & 929. \\
\hline 5.3. & 475. \\
\hline 5.17 & 472.37 \\
\hline 5.17 & 472.3 \\
\hline 4.35 & 449. \\
\hline $\mathbb{N}$ & SMW \\
\hline & 52.70 \\
\hline 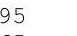 & 2.63 \\
\hline 65 & 59.01 \\
\hline ffDes & $S_{-} N_{C}$ \\
\hline . 9980 & $\overline{0} .9905$ \\
\hline .1123 & 0.0003 \\
\hline 9898 & 0.9733 \\
\hline .0000 & 0.0003 \\
\hline .9926 & 0.0005 \\
\hline
\end{tabular}

$\begin{array}{rrr}\text { Wair } & \text { WH2O } & \text { H2O frac } \\ 298.41 & 0.052 & 0.0002 \\ 298.41 & 0.052 & 0.0002 \\ 267.07 & 0.047 & 0.0002 \\ 31.34 & 0.005 & 0.0002 \\ 267.07 & 0.047 & 0.0002 \\ 31.34 & 0.005 & 0.0002 \\ 31.34 & 0.197 & 0.0063 \\ 26.64 & 0.168 & 0.0063 \\ 25.35 & 0.160 & 0.0063 \\ 19.64 & 0.124 & 0.0063 \\ 19.64 & 0.124 & 0.0199 \\ 26.33 & 0.166 & 0.0165 \\ 26.64 & 0.168 & 0.0163 \\ 26.64 & 0.168 & 0.0163 \\ 26.64 & 0.168 & 0.0163 \\ 267.07 & 0.047 & 0.0002 \\ 271.77 & 0.076 & 0.0003 \\ 271.77 & 0.076 & 0.0003 \\ 271.77 & 0.076 & 0.0003\end{array}$




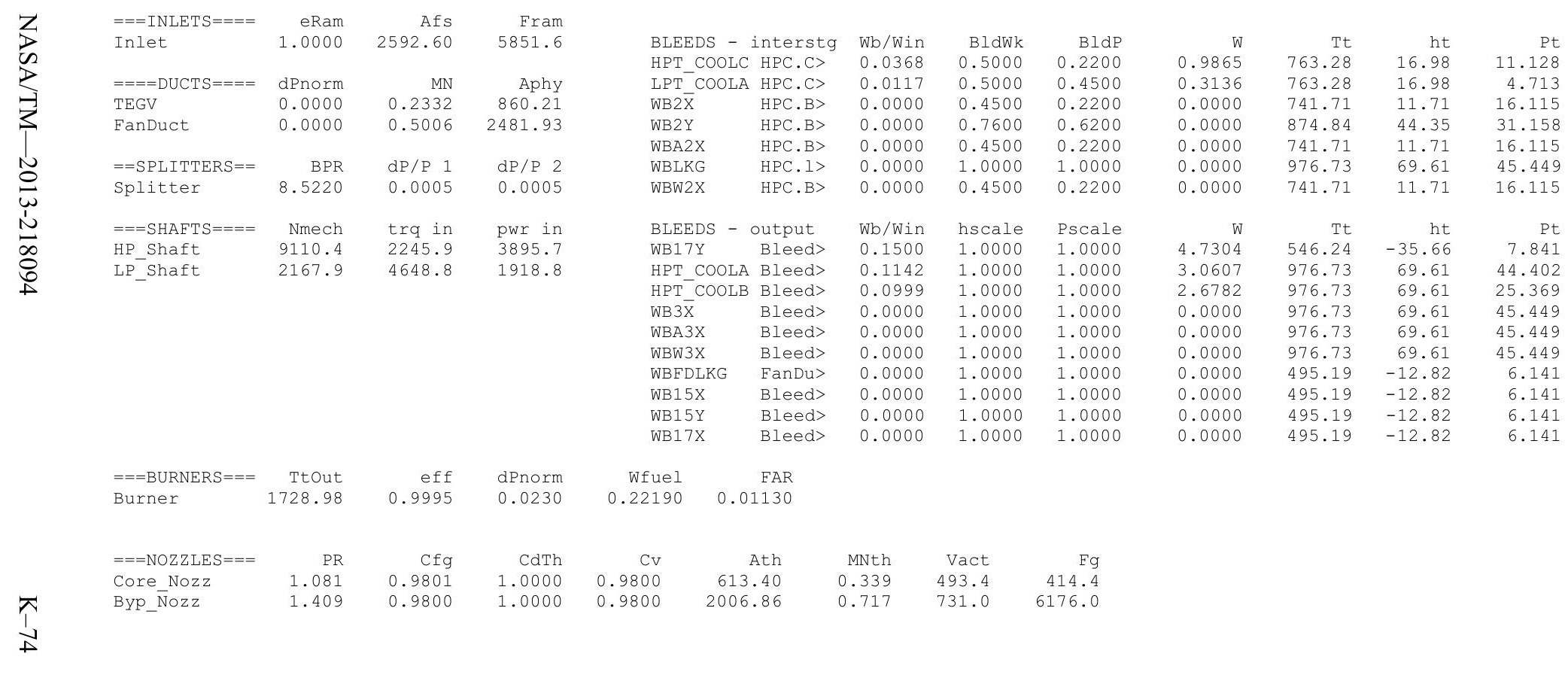


Turbofan Engine - COMDES ON Converge = 1 CASE: 0

Version:NPSS_1.6.5 - Rev: -> Gas Package: Janaf iter/pass/Jacb/Broy= 27/55/2/24 Run by: Philip C Jorgenson PC: 10

$\begin{array}{rllll}\text { Temperature Stator } 1 \text { inlet: } 492.48 & \text { Stator } 1 \text { exit: } 496.71 & \text { Stator } 2 \text { inlet: } 501.55 & \text { Stator } 2 \text { exit: } 503.48 \\ \text { Stator } 3 \text { inlet: } 508.81 & \text { Stator } 3 \text { exit: } 510.32 & \text { Stator } 4 \text { inlet: 515.40 } & \text { Stator 4 exit: 516.48 }\end{array}$ Stator 5 inlet: 520.20 Stator 5 exit: $521.01 \quad$ Unblocked Percent Blockage: 0.00

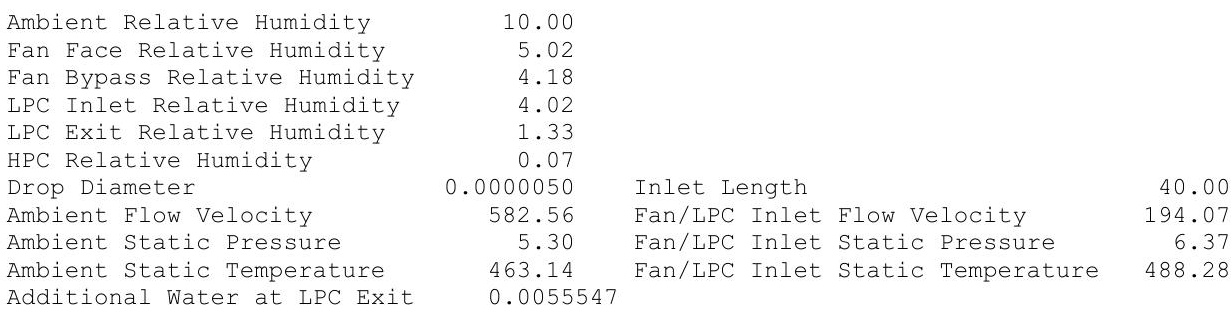

Wfuel BPR
7.1911 VTAS
582.56 OPR
7.220 $\begin{array}{cl}\text { EPR } & \text { Powerset } \\ 0.857 & 10.000\end{array}$ $\begin{array}{ccc}\text { T4 } & \text { T41 } & \text { T49 } \\ 1731.5 & 1634.9 & 1153.3\end{array}$

FSO Ambient.FI_O

FS1 Inlet.FI_O

FS12 Splitter.Fl_02

FS14 Fan.FI_O

VaporIN.FI_O

FS3 3 (

FS36 Bleed3.F1_O

FS4 Burner.Fl_O

FS45 HPT.FI-O

FS49 LPT.FI-O

FS8 Core_Nozz.FI_O

FS17 FanDuctLkg.F̄_o

FS171 Bleed15.FI_O

FS173 FanDuct.Fl-O

TURBOMACHINERY PERFORMANCE DATA

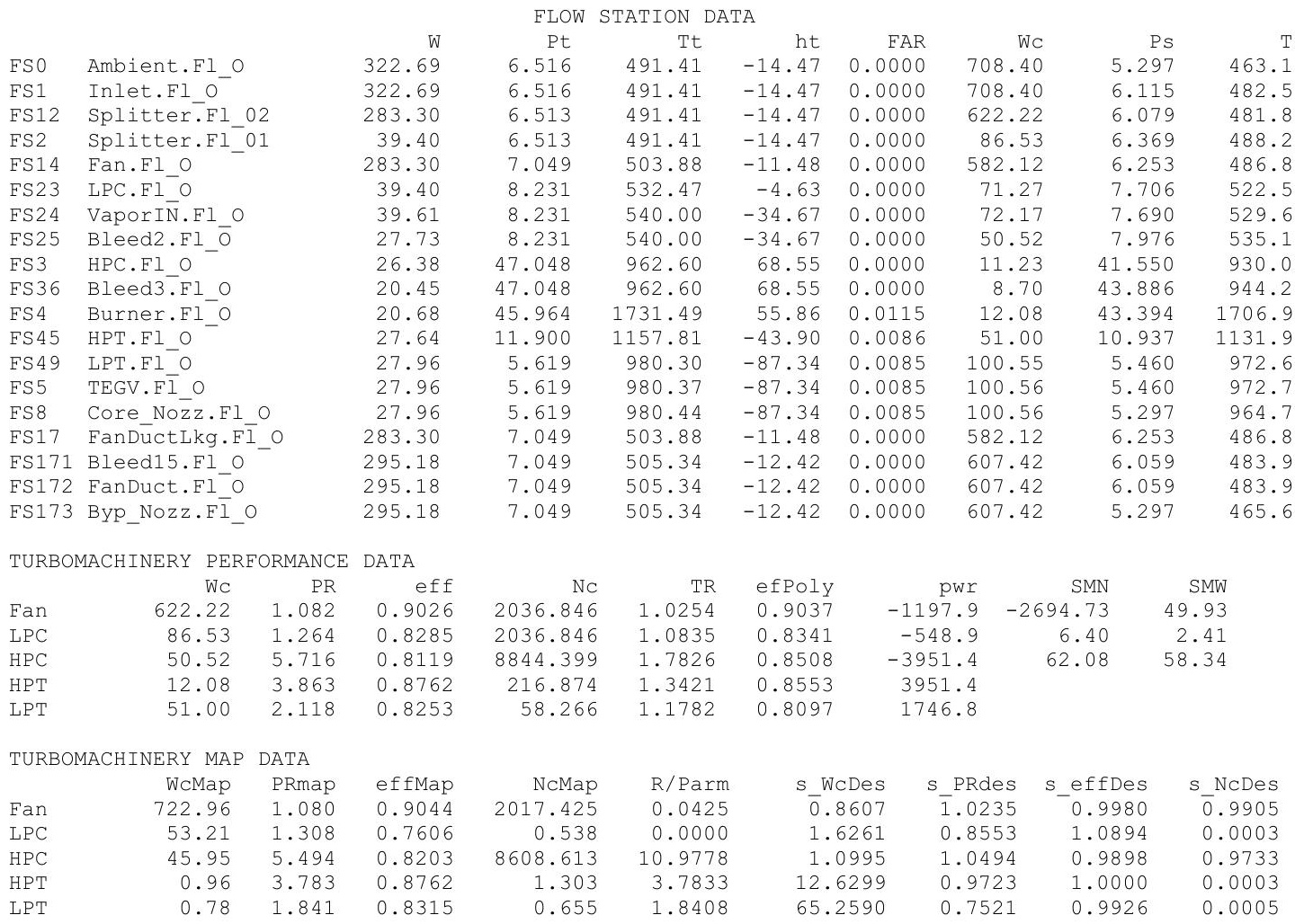

TURBOMACHINERY MAP DATA

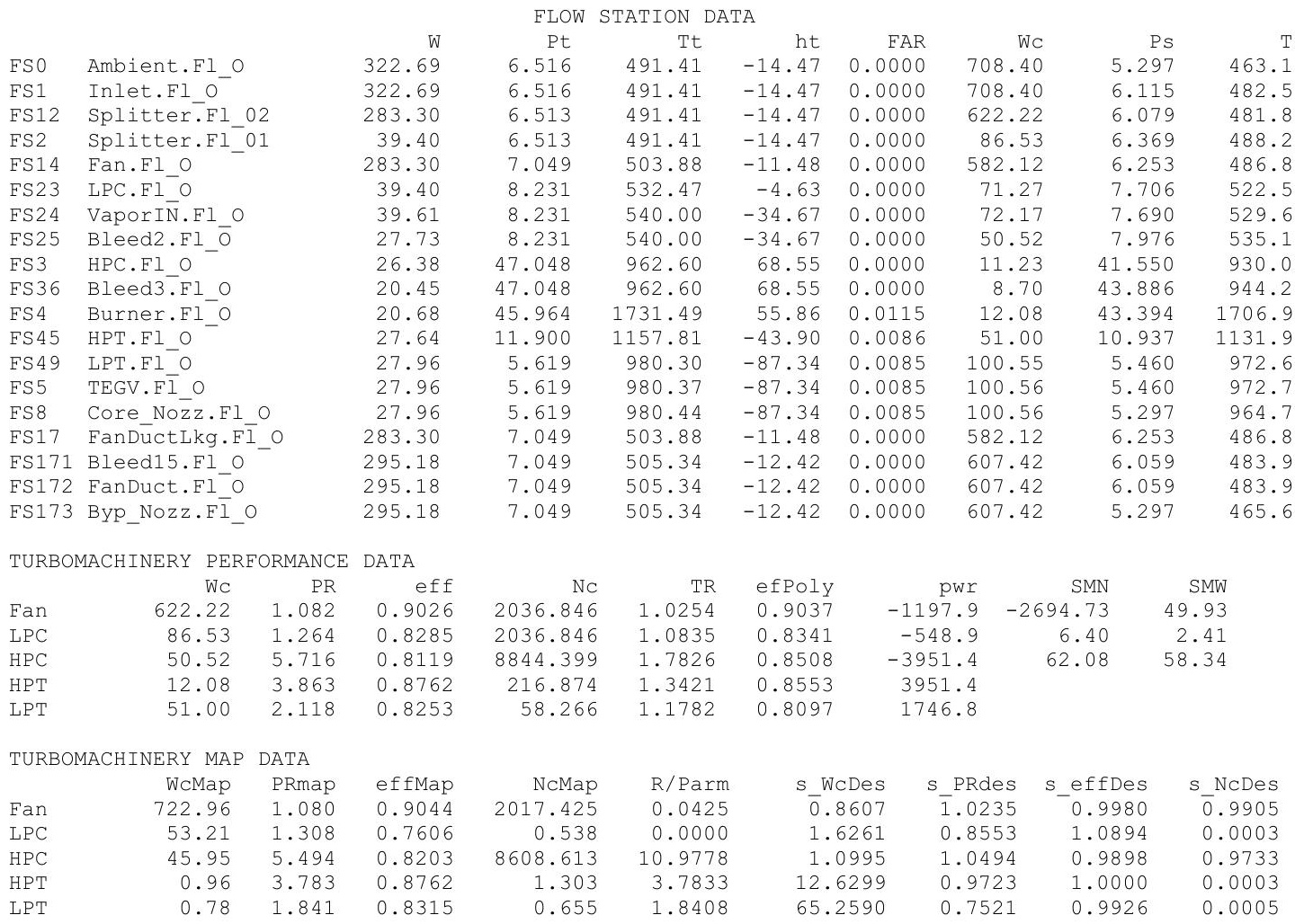

$\begin{array}{rr}\text { Aphy } & \text { MN } \\ 2584.4 & 0.5520 \\ 4168.2 & 0.3026 \\ 3531.8 & 0.3152 \\ 830.5 & 0.1791 \\ 2606.7 & 0.4173 \\ 412.6 & 0.3082 \\ 412.6 & 0.3133 \\ 412.6 & 0.2126 \\ 49.7 & 0.4275 \\ 49.3 & 0.3187 \\ 74.6 & 0.2951 \\ 265.4 & 0.3536 \\ 860.2 & 0.2042 \\ 860.2 & 0.2042 \\ 613.4 & 0.2939 \\ 2606.7 & 0.4173 \\ 2481.9 & 0.4699 \\ 2481.9 & 0.4699 \\ 2006.9 & 0.6521\end{array}$

gamt
1.40052
1.40052
1.40052
1.40052
1.40038
1.40000
1.39912
1.39912
1.38208
1.38208
1.32966
1.36388
1.37534
1.37534
1.37533
1.40038
1.40033
1.40033
1.40033
$0.0003043 \quad 322.59$ $\begin{array}{llll}0.098 & 0.0003 \\ 0.0003043 & 322.59 & 0.098\end{array}$ $\begin{array}{llll}0.0003043 & 322.59 & 0.098 & 0.0003\end{array}$ $\begin{array}{lrll}0.0003043 & 39.38 & 0.012 & 0.0003\end{array}$ $\begin{array}{llll}0.0003043 & 39.38 & 0.012 & 0.0003 \\ 0.0003043 & 283.21 & 0.086 & 0.0003\end{array}$ $\begin{array}{llll}0.00038591 & 39.38 & 0.012 & 0.0003\end{array}$ $\begin{array}{llll}0.0058591 & 27.57 & 0.162 & 0.0058\end{array}$ $\begin{array}{llll}0.0058591 & 26.23 & 0.162 & 0.0058\end{array}$ $\begin{array}{llll}0.0058591 & 26.23 & 0.154 & 0.0058\end{array}$ $\begin{array}{llll}0.0058591 & 20.33 & 0.119 & 0.0197\end{array}$ $\begin{array}{llll}0.0058591 & 27.25 & 0.160 & 0.0162\end{array}$ $\begin{array}{llll}0.0058591 & 27.57 & 0.162 & 0.0161\end{array}$ $\begin{array}{llll}0.0058591 & 27.57 & 0.162 & 0.0161\end{array}$ $\begin{array}{llll}0.0058591 & 27.57 & 0.162 & 0.0161\end{array}$ $\begin{array}{llll}0.0003043 & 283.21 & 0.086 & 0.0003\end{array}$ $\begin{array}{llll}0.0005268 & 295.03 & 0.155 & 0.0005\end{array}$ $\begin{array}{llll}0.0005268 & 295.03 & 0.155 & 0.0005\end{array}$ 0.0005

$$
\left(\frac{10}{20}\right.
$$




\begin{tabular}{|c|c|c|c|c|c|c|c|c|c|c|c|c|}
\hline$===$ INLETS $====$ & eRam & Afs & Fram & \multirow{2}{*}{\multicolumn{2}{|c|}{ BLEEDS - interstg }} & & & & & & & \\
\hline Inlet & 1.0000 & 2584.38 & 5842.8 & & & Wb/Win & BldWk & $\mathrm{BldP}$ & W & $\mathrm{Tt}$ & ht & $\mathrm{Pt}$ \\
\hline & & & & \multirow{2}{*}{\multicolumn{2}{|c|}{$\begin{array}{l}\text { HPT_COOLC HPC.C> } \\
\text { LPT COOLA HPC.C> }>\end{array}$}} & 0.0368 & 0.5000 & 0.2200 & 1.0205 & 752.98 & 16.94 & 11.900 \\
\hline$====$ DUCTS $====$ & dPnorm & MN & Aphy & & & 0.0117 & 0.5000 & 0.4500 & 0.3244 & 752.98 & 16.94 & 5.619 \\
\hline TEGV & 0.0000 & 0.2042 & 860.21 & \multicolumn{2}{|c|}{ WB $2 \overline{\mathrm{X}} \quad$ HPC. $\mathrm{B}>$} & 0.0000 & 0.4500 & 0.2200 & 0.0000 & 731.80 & 11.78 & 16.771 \\
\hline FanDuct & 0.0000 & 0.4699 & 2481.93 & WB2Y & HPC.B> & 0.0000 & 0.7600 & 0.6200 & 0.0000 & 862.52 & 43.78 & 32.297 \\
\hline & & & & \multirow{2}{*}{$\begin{array}{l}\text { WBA2X } \\
\text { WBLKG }\end{array}$} & HPC.B> & 0.0000 & 0.4500 & 0.2200 & 0.0000 & 731.80 & 11.78 & 16.771 \\
\hline$==$ SPLITTERS $==$ & BPR & $\mathrm{dP} / \mathrm{P} 1$ & $\mathrm{dP} / \mathrm{P} 2$ & & HPC. $1>$ & 0.0000 & 1.0000 & 1.0000 & 0.0000 & 962.60 & 68.55 & 47.048 \\
\hline Splitter & 7.1911 & 0.0005 & 0.0005 & WBW2X & $\mathrm{HPC} . \mathrm{B}>$ & 0.0000 & 0.4500 & 0.2200 & 0.0000 & 731.80 & 11.78 & 16.771 \\
\hline$===$ SHAFT $\mathrm{S}====$ & Nmech & trq in & pwr in & \multicolumn{2}{|c|}{ BLEEDS - output } & Wb/Win & hscale & Pscale & W & $\mathrm{Tt}$ & ht & Pt \\
\hline HP_Shaft & 9024.4 & 2299.7 & 3951.4 & WB17Y & Bleed> & 0.3000 & 1.0000 & 1.0000 & 11.8842 & 540.00 & -34.67 & 8.231 \\
\hline LP_Shaft & 1982.6 & 4627.6 & 1746.8 & HPT_COOLA & A Bleed> & 0.1142 & 1.0000 & 1.0000 & 3.1662 & 962.60 & 68.55 & 45.964 \\
\hline & & & & \multirow{2}{*}{$\begin{array}{l}\text { HPT COOLB } \\
\text { WB3 } 3 \overline{\mathrm{X}}\end{array}$} & B Bleed> & 0.0999 & 1.0000 & 1.0000 & 2.7705 & 962.60 & 68.55 & 26.479 \\
\hline & & & & & Bleed $>$ & 0.0000 & 1.0000 & 1.0000 & 0.0000 & 962.60 & 68.55 & 47.048 \\
\hline & & & & WBA3X & Bleed> & 0.0000 & 1.0000 & 1.0000 & 0.0000 & 962.60 & 68.55 & 47.048 \\
\hline & & & & WBW3X & Bleed> & 0.0000 & 1.0000 & 1.0000 & 0.0000 & 962.60 & 68.55 & 47.048 \\
\hline & & & & WBFDLKG & FanDu> & 0.0000 & 1.0000 & 1.0000 & 0.0000 & 503.88 & -11.48 & 7.049 \\
\hline & & & & \multirow{2}{*}{$\begin{array}{l}\text { WB15X } \\
\text { WB15Y }\end{array}$} & Bleed> & 0.0000 & 1.0000 & 1.0000 & 0.0000 & 503.88 & -11.48 & 7.049 \\
\hline & & & & & Bleed> & 0.0000 & 1.0000 & 1.0000 & 0.0000 & 503.88 & -11.48 & 7.049 \\
\hline & & & & WB17X & Bleed> & 0.0000 & 1.0000 & 1.0000 & 0.0000 & 503.88 & -11.48 & 7.049 \\
\hline$===$ BURNERS $===$ & TtOut & eff & dPnorm & \multirow{2}{*}{\multicolumn{2}{|c|}{$\begin{array}{rr}\text { Wfuel } & \text { FAR } \\
.23438 & 0.01153\end{array}$}} & & & & & & & \\
\hline Burner & 1731.44 & 0.9995 & 0.0230 & & & & & & & & & \\
\hline$===$ NOZZLES $===$ & $\mathrm{PR}$ & $\mathrm{C} f \mathrm{~g}$ & CdTh & $\begin{array}{r}\mathrm{Cv} \\
0.9800\end{array}$ & \multirow{2}{*}{$\begin{array}{r}\text { Ath } \\
613.40\end{array}$} & \multirow{3}{*}{$\begin{array}{r}\text { MNth } \\
0.294 \\
0.652\end{array}$} & \multirow{2}{*}{$\begin{array}{r}\text { Vact } \\
435.6\end{array}$} & $\mathrm{Fg}$ & & & & \\
\hline Core_Nozz & 1.061 & 0.9801 & 1.0000 & \multirow{2}{*}{\multicolumn{2}{|c|}{2006.86}} & & & 378.6 & & & & \\
\hline Byp_Nozzz & 1.331 & 0.9800 & 1.0000 & & & & 676.3 & 6204.7 & & & & \\
\hline
\end{tabular}




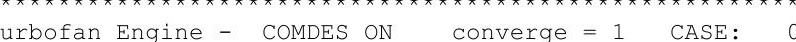

Version:NPSS_1.6.5 - Rev: -> Gas Package: Janaf iter/pass/Jacb/Broy= 43/71/2/40 Run by: Philip C Jorgenson PC: 10

Temperature Stator 1 inlet: 505.44 Stator 1 exit: 508.48 Stator 2 inlet: 511.40 Stator 2 exit: 512.25 $\begin{array}{llll}\text { Stator } 3 \text { inlet: } 515.70 & \text { Stator } 3 \text { exit: } 516.12 & \text { Stator } 4 \text { inlet: } 519.49 \text { Stator } 4 \text { exit: } 519.63 \\ \text { Stator } 5 \text { inlet: } 521.64 & \text { Stator } 5 \text { exit: } 521.58 & & \text { Unblocked Percent Blockage: } 0.00\end{array}$

$\begin{array}{lclll}\text { Ambient Relative Humidity } & 10.00 & & & \\ \text { Fan Face Relative Humidity } & 6.02 & & & \\ \text { Fan Bypass Relative Humidity } & 5.16 & & & \\ \text { LPC Inlet Relative Humidity } & 5.19 & & & \\ \text { LPC Exit Relative Humidity } & 2.75 & & & \\ \text { HPC Relative Humidity } & 0.09 & & & \\ \text { Drop Diameter } & 0.0000050 & \text { Inlet Length } & \\ \text { Ambient Flow Velocity } & 528.20 & \text { Fan/LPC Inlet Flow Velocity } & 201.72 \\ \text { Ambient Static Pressure } & 6.74 & \text { Fan/LPC Inlet Static Pressure } & 7.75 \\ \text { Ambient Static Temperature } & 483.18 & \text { Fan/LPC Inlet Static Temperature } & 503.02 \\ \text { Additional Water at LPC Exit } & 0.0064451 & & & \end{array}$

MN alt dTamb

W
358.30
SUMMARY OUTPUT DATA

$\begin{array}{rr}\text { Fn } & \text { TSFC } \\ 801.9 & 1.1573\end{array}$
Wfuel
928.02
BPR
6.4043 VTAS
528.20
OPR $\begin{array}{ll}\text { EPR } & \text { PowerSet } \\ 0.888 & 10.000\end{array}$ $\begin{array}{ccc}\text { T4 } & \text { T41 } & \text { T49 } \\ 1758.3 & 1658.1 & 1181.6\end{array}$
FSO Ambient.FI_O

FS1 Inlet.F1_O

FS12 Splitter.Fl_02

FS14 Fan.F1_O

FS23 LPC.FI-O

FS24 VaporIN.FI_O

FS3 3 Bleed2.F1-

FS36 Bleed3.F1_O

FS4 Burner.FI_O

FS49 HPT.FI-

FS4 LPT.FI-O

FS8 Core_Nozz.Fl_O

FS17 FanDüctLkg.F̄__o

FS171 Bleed15.FI_O

FS172 FanDuct. F1-O

TURBOMACHINERY PERFORMANCE DATA

\begin{tabular}{|c|c|c|c|c|c|c|c|c|c|}
\hline & WC & $\mathrm{PR}$ & eff & NG & $T R$ & efPoly & pwr & SMN & SMWT \\
\hline Fan & 566.84 & 1.070 & 0.9044 & 1828.989 & 1.0215 & 0.9053 & -1146.2 & -1852.73 & 46.50 \\
\hline LPC & 88.51 & 1.148 & 0.7107 & 1828.989 & 1.0567 & 0.7163 & -471.9 & 7.06 & 2.10 \\
\hline HPC & 48.23 & 5.482 & 0.8076 & 8767.052 & 1.7628 & 0.8465 & -4091.0 & 62.46 & 58.43 \\
\hline HPT & 12.08 & 3.661 & 0.8778 & 214.084 & 1.3260 & 0.8581 & 4090.9 & & \\
\hline LPT & 48.56 & 1.896 & 0.8176 & 52.469 & 1.1479 & 0.8039 & 1618.2 & & \\
\hline RD & NERY & DATA & & & & & & & \\
\hline & WcMap & PRmap & effMap & NcMap & R/Parm & S_WcDes & s_PRdes & S_effDes & s_NCDes \\
\hline Fan & 658.62 & 1.068 & 0.9062 & 1811.550 & 0.0438 & $\overline{0} .8607$ & $\overline{1} .0235$ & 0.9980 & $\overline{0.9905}$ \\
\hline LPC & 46.43 & 1.242 & 0.7552 & 0.483 & 0.0000 & 1.9 & 0.6129 & 0.9411 & 0.0003 \\
\hline HPC & 43.87 & 5.271 & .8159 & 533.328 & 10.9880 & 1.0995 & 1.0494 & 0.9898 & 0.9733 \\
\hline HPT & 0.96 & 3.587 & 0.8778 & 1.287 & 3.5875 & 12.6299 & 0.9723 & 1.0000 & 0.0003 \\
\hline LPT & 0.74 & 1.674 & 0.8237 & 0.590 & 1.6736 & 65.2590 & 0.7521 & 0.9926 & 0.0005 \\
\hline
\end{tabular}

FLOW STATION DATA

\begin{tabular}{rrrrrrrrrr} 
W & \multicolumn{1}{c}{ Pt } & Tt & ht & FAR & Wc & Ps & Ts & Aphy & MN \\
358.30 & 7.943 & 506.41 & -12.46 & 0.0000 & 655.03 & 6.740 & 483.18 & 2595.3 & 0.4900 \\
358.30 & 7.943 & 506.41 & -12.46 & 0.0000 & 655.03 & 7.529 & 498.72 & 4168.2 & 0.2775 \\
309.91 & 7.939 & 506.41 & -12.46 & 0.0000 & 566.84 & 7.507 & 498.36 & 3531.8 & 0.2840 \\
48.39 & 7.939 & 506.41 & -12.46 & 0.0000 & 88.51 & 7.755 & 503.02 & 830.5 & 0.1834 \\
309.91 & 8.493 & 517.30 & -9.85 & 0.0000 & 535.56 & 7.699 & 503.00 & 2606.7 & 0.3769 \\
48.39 & 9.116 & 535.13 & -5.57 & 0.0000 & 79.24 & 8.384 & 522.47 & 412.6 & 0.3480 \\
48.70 & 9.116 & 543.81 & -40.38 & 0.0000 & 80.39 & 8.357 & 530.48 & 412.6 & 0.3549 \\
29.22 & 9.116 & 543.81 & -40.38 & 0.0000 & 48.23 & 8.860 & 539.40 & 412.6 & 0.2025 \\
27.80 & 49.978 & 958.61 & 61.03 & 0.0000 & 11.11 & 44.269 & 926.94 & 49.7 & 0.4222 \\
21.55 & 49.978 & 958.61 & 61.03 & 0.0000 & 8.61 & 46.690 & 940.72 & 49.3 & 0.3152 \\
21.81 & 48.827 & 1758.30 & 47.88 & 0.0120 & 12.08 & 46.093 & 1733.41 & 74.6 & 0.2955 \\
29.14 & 13.336 & 1186.38 & -49.92 & 0.0090 & 48.56 & 12.366 & 1162.79 & 265.4 & 0.3345 \\
29.48 & 7.035 & 1030.55 & -88.02 & 0.0089 & 86.80 & 6.889 & 1024.69 & 860.2 & 0.1754 \\
29.48 & 7.035 & 1030.62 & -88.02 & 0.0089 & 86.80 & 6.889 & 1024.76 & 860.2 & 0.1754 \\
29.48 & 7.035 & 1030.69 & -88.02 & 0.0089 & 86.81 & 6.740 & 1018.78 & 613.5 & 0.2506 \\
309.91 & 8.493 & 517.30 & -9.85 & 0.0000 & 535.56 & 7.699 & 503.00 & 2606.7 & 0.3769 \\
329.39 & 8.493 & 518.88 & -11.65 & 0.0000 & 570.09 & 7.467 & 500.14 & 2481.9 & 0.4326 \\
329.39 & 8.493 & 518.88 & -11.65 & 0.0000 & 570.09 & 7.467 & 500.14 & 2481.9 & 0.4326 \\
329.39 & 8.493 & 518.88 & -11.65 & 0.0000 & 570.09 & 6.740 & 485.70 & 2006.8 & 0.5841
\end{tabular}

gamt
1.40031
1.40031
1.40031
1.40031
1.40017
1.39992
1.39890
1.39890
1.38214
1.38214
1.32791
1.36163
1.37175
1.37175
1.37174
1.40017
1.40010
1.40010
1.40010

WAR
0.0005787
0.0005787
0.0005787
0.0005787
0.0005787
0.0005787
0.0070238
0.0070238
0.0070238
0.0070238
0.0070238
0.0070238
0.0070238
0.0070238
0.0070238
0.0005787
0.0009575
0.0009575
0.0009575

Wair $\begin{array}{lll}58.09 & 0.207 \quad \mathrm{H} 20 \mathrm{frac}\end{array}$ $\begin{array}{lll}358.09 & 0.207 & 0.0006\end{array}$ $\begin{array}{lll}48.36 & 0.028 & 0.0006\end{array}$ $\begin{array}{lll}309.73 & 0.179 & 0.0006\end{array}$ $\begin{array}{lll}48.36 & 0.028 & 0.0006\end{array}$ $\begin{array}{ll}48.36 & 0.340=0.0070\end{array}$ $\begin{array}{lll}29.02 & 0.204 & 0.0070\end{array}$ $\begin{array}{lll}27.61 & 0.194 & 0.0070\end{array}$ $\begin{array}{lll}21.40 & 0.150 & 0.0070\end{array}$ $\begin{array}{lll}21.40 & 0.150 & 0.0215\end{array}$ $\begin{array}{lll}28.68 & 0.201 & 0.0178\end{array}$ $\begin{array}{lll}29.02 & 0.204 & 0.0177\end{array}$ $\begin{array}{lll}29.02 & 0.204 & 0.0177\end{array}$ $\begin{array}{lll}29.02 & 0.204 & 0.0177\end{array}$ $\begin{array}{lll}309.73 & 0.179 & 0.0006\end{array}$ $\begin{array}{lll}329.07 & 0.315 & 0.0010 \\ 329.07 & 0.315 & 0.0010\end{array}$ $329.07 \quad 0.315$ 


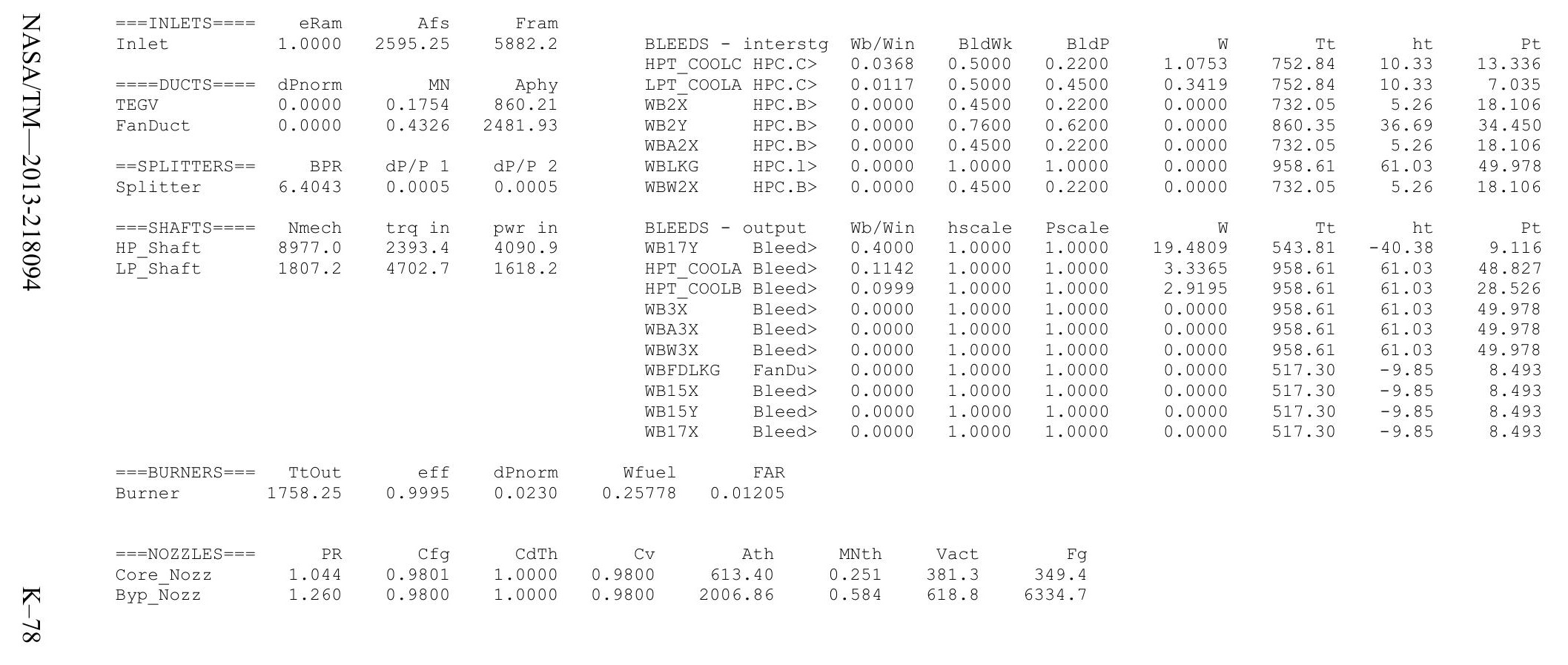


Date: 07/05/13 Time:11:40:21 Model

Turbofan Engine - COMDES ON Converge $=1$ CASE: 0

1.6.5 - Rev: -> Gas Package: Janaf iter/pass/Jacb/Broy=13/27/1/11 Run by: Philip C Jorgenson PC: 10

$\begin{array}{lllll}\text { Temperature Stator } 1 \text { inlet: } 484.24 & \text { Stator } 1 \text { exit: } 492.23 & \text { Stator } 2 \text { inlet: } 502.23 & \text { Stator } 2 \text { exit: 507.43 } \\ \text { Stator } 3 \text { inlet: 517.61 } & \text { Stator } 3 \text { exit: 522.26 } & \text { Stator } 4 \text { inlet: 531.67 } & \text { Stator } 4 \text { exit: 535.70 }\end{array}$

Stator 5 inlet: 543.48 Stator 5 exit: 546.96 Unblocked Percent Blockage: 0.00

Ambient Relative Humidity $\quad 10.00$

Fan Face Relative Humidity $\quad 1.98$

Fan Bypass Relative Humidity $\quad 1.38$

LPC Inlet Relative Humidity $\quad 1.30$

LPC Exit Relative Humidity $\quad 0.12$

HPC Relative Humidity 0.07

Drop Dianterer

0.0000050

Ambient Flow Velocity

Inlet Length 40.00

$\begin{array}{lrlr} & 789.49 & \text { Fan/LPC Inlet Flow Velocity } & 188.03 \\ \text { Ambient Static Pressure } & 2.85 & \text { Fan/LPC Inlet Static Pressure } & 4.17\end{array}$

$\begin{array}{llllr}\text { Ambient Static Pressure } & 2.85 & \text { Fan/LPC } & \text { Inlet Static Pressure } & 4.17 \\ \text { Ambient Static Temperature } & 425.97 & \text { Fan/LPC } & \text { Inlet Static Temperature } & 474.97 \\ \text { Additional Water at LPC Exit } & 0.0136991 & & & \end{array}$ 0.0136991

SUMMARY OUTPUT DATA

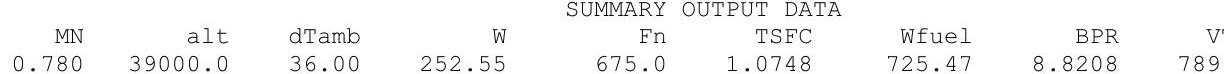

\section{FLOW STATION DATA}

WC
834.98

834.98
834.98

$W$
252.55
252.55
226.83
25.72
226.83
25.72
26.07
23.46
22.32
17.30
17.50
23.39
23.66
23.66
23.66
226.83
229.44
229.44
229.44

\begin{tabular}{|c|c|c|c|}
\hline Pt & Tt & ht & FAR \\
\hline .267 & 477.92 & -16.44 & 0.0000 \\
\hline 4.267 & 477.92 & -16.44 & 0.0000 \\
\hline 4.265 & 477.92 & -16.44 & 0.0000 \\
\hline 4.265 & 477.92 & -16.44 & 0.0000 \\
\hline 4.839 & 497.33 & -11.79 & 0.0000 \\
\hline 6.717 & 557.91 & 2.75 & 0.0000 \\
\hline 6.717 & 575.55 & -70.86 & 0.0000 \\
\hline 6.717 & 575.55 & -70.86 & 0.0000 \\
\hline 0.686 & 1037.91 & 43.38 & 0.0000 \\
\hline 0.686 & 1037.91 & 43.38 & 0.0000 \\
\hline 9.749 & 1804.55 & 30.77 & 0.0118 \\
\hline 9.542 & 1195.90 & -79.98 & 0.0088 \\
\hline 3.268 & 936.79 & -144.66 & 0.0087 \\
\hline 3.268 & 936.86 & -144.66 & 0.0087 \\
\hline 3.268 & 936.93 & -144.66 & 0.0087 \\
\hline 4.839 & 497.33 & -11.79 & 0.0000 \\
\hline 4.839 & 498.23 & -12.46 & 0.0000 \\
\hline 4.839 & 498.23 & -12.46 & 0.0000 \\
\hline 4.839 & 498.23 & -12.46 & 0.0000 \\
\hline
\end{tabular}
834.98
750.33 85.06 674.54 58.35 60.08 11.41

8.84
12.07

12.07
54.69

54.69
143.03 143.03
143.03 143.03 143.04
674.54 674.54 682.90 682.90
682.90
VTAS $\begin{array}{ll}\text { EPR } & \text { PowerSet } \\ 0.736 & 10.000\end{array}$ $\begin{array}{ccc}\text { T4 } & \text { T41 } & \text { T49 } \\ 1804.6 & 1708.2 & 1191.6\end{array}$

TURBOMACHINERY PERFORMANCE DATA

\begin{tabular}{|c|c|c|c|c|c|c|c|c|c|}
\hline & Wc & $\mathrm{PR}$ & eff & NC & TR & efpoly & pwr & SMN & SMW \\
\hline Fan & 750.33 & 1.135 & 0.9066 & 2595.218 & 1.0406 & 0.9083 & -1492.9 & 1442.03 & 54.43 \\
\hline LPC & 85.06 & 1.575 & 0.8285 & 2595.218 & 1.1674 & 0.8391 & -698.1 & 7.05 & 3.93 \\
\hline HPC & 54.07 & 6.057 & 0.8178 & 8959.420 & 1.8033 & 0.8563 & -3700.2 & 62.16 & 58.80 \\
\hline HPT & 12.07 & 4.166 & 0.8795 & 222.173 & 1.3620 & 0.8580 & 3700.2 & & \\
\hline LPT & 54.69 & 2.920 & 0.8601 & 72.037 & 1.2749 & 0.8411 & 2191.0 & & \\
\hline JRB & NERY & DATA & & & & & & & \\
\hline & WcMap & PRmap & effMap & NcMap & R/Parm & S_WCDes & S_PRdes & $S_{\text {S_effDes }}$ & S_NcDes \\
\hline Fan & 871.82 & 1.132 & 0.9085 & 2570.473 & 0.0416 & $\overline{0} .8607$ & $\overline{1} .0235$ & 0.9980 & $\overline{0} .9905$ \\
\hline $\mathrm{PC}$ & 75.08 & 1.534 & 0.7812 & 0.685 & 0.0000 & 1.1330 & 1.0768 & 1.0606 & 0.0003 \\
\hline PC & 49.18 & 5.819 & .8263 & 20.568 & 10.9274 & 1.0 & 1.0494 & 0.9898 & 0.9733 \\
\hline PT & 0. & 4.078 & 0.8795 & 1.335 & 779 & 12.6 & 0.9723 & 1.0000 & 0.0003 \\
\hline LPT & 0.84 & 2.444 & 0.8665 & 0.810 & 2.4443 & 65.2590 & 0.7521 & 0.9926 & 0.0005 \\
\hline
\end{tabular}

\begin{tabular}{|c|c|}
\hline & Ts \\
\hline 2.85 & 425.97 \\
\hline 3.89 & 465.4 \\
\hline 3.83 & 463. \\
\hline 4.17 & 474.9 \\
\hline 4.05 & 472.95 \\
\hline 6.43 & 551.16 \\
\hline 6.41 & 568.11 \\
\hline 6.47 & 569.61 \\
\hline 35.72 & 1001.55 \\
\hline 37.84 & 1017.51 \\
\hline 37.51 & 1779.1 \\
\hline 8.63 & 1164.58 \\
\hline 3.07 & 921.23 \\
\hline 3.07 & 921.3 \\
\hline 2.85 & 902.8 \\
\hline 4.05 & 472.9 \\
\hline 3.92 & 469.2 \\
\hline 3.92 & 469.2 \\
\hline 2.85 & 428. \\
\hline & SMW \\
\hline & 4.43 \\
\hline 5 & 3.93 \\
\hline & 8.80 \\
\hline fDes & S_NCDes \\
\hline 9980 & $\overline{0} .9905$ \\
\hline 0606 & 0.0003 \\
\hline 9898 & 0.9733 \\
\hline 0000 & 0.0003 \\
\hline 9926 & 0.0005 \\
\hline
\end{tabular}

$\begin{array}{rrr}\text { Aphy } & \text { MN } & \text { gamt } \\ 2547.4 & 0.7800 & 1.40068 \\ 4168.2 & 0.3656 & 1.40068 \\ 3531.8 & 0.3923 & 1.40068 \\ 830.5 & 0.1759 & 1.40068 \\ 2606.7 & 0.5072 & 1.40048 \\ 412.6 & 0.2474 & 1.39963 \\ 412.6 & 0.2567 & 1.39743 \\ 412.6 & 0.2292 & 1.39743 \\ 49.7 & 0.4384 & 1.37623 \\ 49.3 & 0.3261 & 1.37623 \\ 74.6 & 0.2961 & 1.32499 \\ 265.4 & 0.3858 & 1.36020 \\ 860.2 & 0.2992 & 1.37678 \\ 860.2 & 0.2992 & 1.37677 \\ 613.4 & 0.4472 & 1.37677 \\ 2606.7 & 0.5072 & 1.40048 \\ 2481.9 & 0.5552 & 1.40045 \\ 2481.9 & 0.5552 & 1.40045 \\ 2006.9 & 0.9021 & 1.40045\end{array}$

WAR
0.0000851
0.0000851
0.0000851
0.0000851
0.0000851
0.0000851
0.0137842
0.0137842
0.0137842
0.0137842
0.0137842
0.0137842
0.0137842
0.0137842
0.0137842
0.0000851
0.0002386
0.0002386
0.0002386

Wair
252.52

$\begin{array}{rr}\text { WH2O } & \text { H2O frac } \\ 0.021 & 0.0001 \\ 0.021 & 0.0001\end{array}$ $\begin{array}{lll}252.52 & 0.021 & 0.0001\end{array}$ $\begin{array}{lll}226.81 & 0.019 & 0.0001\end{array}$ $\begin{array}{rrr}25.71 & 0.002 & 0.0001 \\ 226.81 & 0.019 & 0.0001\end{array}$ $\begin{array}{lll}25.71 & 0.002 & 0.0001\end{array}$ $\begin{array}{lll}25.71 & 0.354 & 0.0136 \\ 23.14 & 0.319 & 0.0136\end{array}$ $\begin{array}{lll}23.14 & 0.319 & 0.0136\end{array}$ $\begin{array}{lll}22.02 & 0.304 & 0.0136\end{array}$ $\begin{array}{lll}17.07 & 0.235 & 0.0276\end{array}$ $\begin{array}{lll}22.87 & 0.315 & 0.0241\end{array}$ $\begin{array}{lll}23.14 & 0.319 & 0.0240\end{array}$ $\begin{array}{lll}23.14 & 0.319 & 0.0240\end{array}$ $\begin{array}{lll}23.14 & 0.319 & 0.0240\end{array}$ $\begin{array}{lll}226.81 & 0.019 & 0.0001\end{array}$ $\begin{array}{lll}229.38 & 0.055 & 0.0002\end{array}$ $\begin{array}{llllll}2006.9 & 0.9021 & 1.40045 & 0.0002386 & 229.38 & 0.055\end{array}$ 


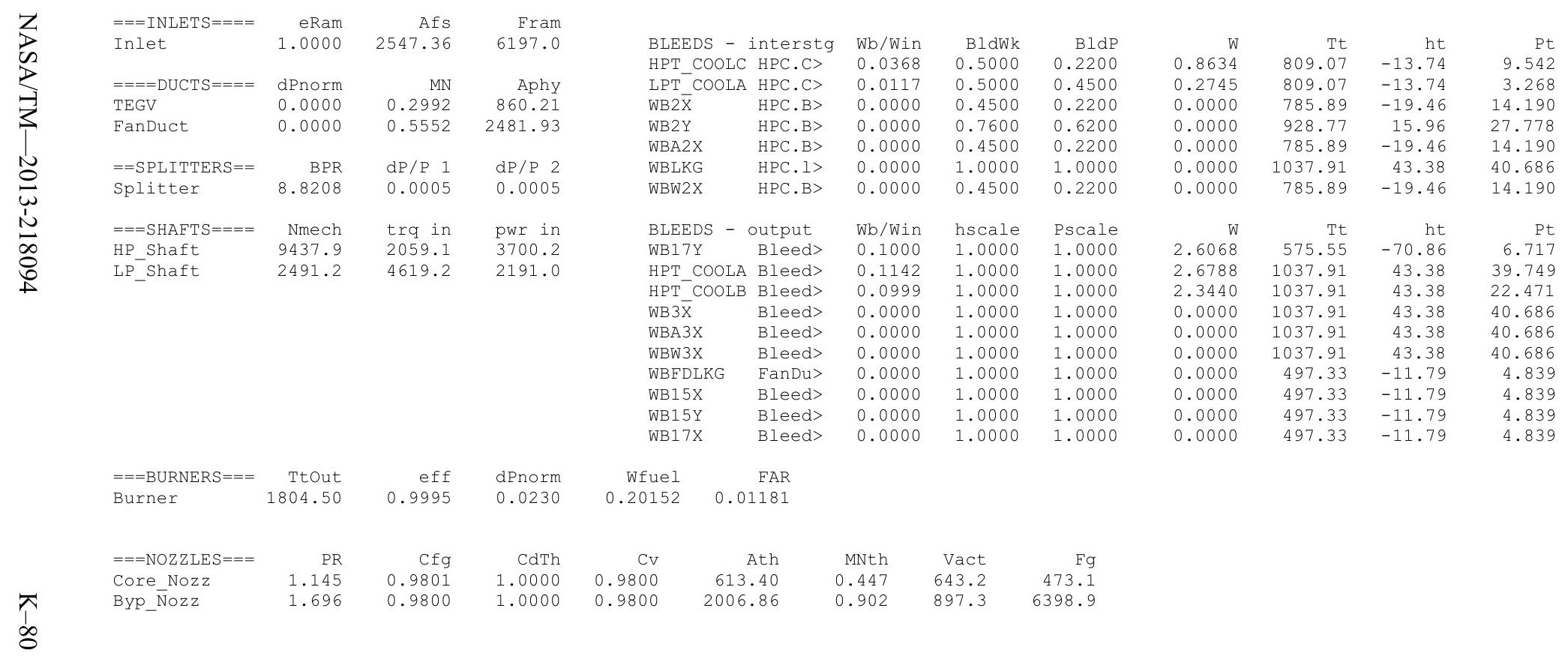


Turbofan Engine - COMDES ON converge $=1$ CASE: 0

Version:NPSS_1.6.5 - Rev: -> Gas Package: Janaf iter/pass/Jacb/Broy=13/27/1/11 Run by: Philip C Jorgenson PC: 10

Temperature Stator 1 inlet: $477.31 \quad$ Stator 1 exit: $484.74 \quad$ Stator 2 inlet: 494.04 Stator 2 exit: 498.85 $\begin{array}{lll}\text { Stator } 3 \text { inlet: } 508.36 & \text { Stator } 3 \text { exit: } 512.69 & \text { Stator } 4 \text { inlet: } 521.47 \\ \text { Stator } 5 \text { inlet: } 532.29 & \text { Stator } 5 \text { exit: } 535.49 & \text { Unblocked Percent Blockage: } 0.00\end{array}$

Ambient Relative Humidity $\quad 10.00$

Fan Face Relative Humidity $\quad 2.47$

Fan Bypass Relative Humidity $\quad 1.77$

$\begin{array}{ll}\text { LPC Inlet Relative Humidity } & 1.63 \\ \text { LPC Exit Relative Humidity } & 0.16\end{array}$

LPC Exit Relative Humidity

Drop Diameter

0.06

0.0000050

Inlet Length $\quad 40.00$

Ambent Flow Velocity

Fan/LPC Inlet Flow velocity 182.53

Ambient Static Pressure

0.010

Fan/LPC Inlet Static Temperature 468.70

Additional Water at LPC Exit 0.0100863

SUMMARY OUTPUT DATA

$\begin{array}{rrr}\text { MN } & \text { alt } & \text { dTamb } \\ 0.730 & 38334.0 & 36.00\end{array}$ 245.68

$\begin{array}{rr}\text { Fn } & \text { TSFC } \\ 648.7 & 1.0595\end{array}$

Wfuel
687.25 BPR
8.8534
VTAS
738.88
OPR
9.250

FLOW STATION DATA

$\begin{array}{lrrr}\text { FLOW } & \text { STATION } & \text { DATA } & \\ \text { Pt } & \text { Tt } & \text { ht } & \text { FAR } \\ 4.201 & 471.48 & -17.97 & 0.0000 \\ 4.201 & 471.48 & -17.97 & 0.0000 \\ 4.199 & 471.48 & -17.97 & 0.0000 \\ 4.199 & 471.48 & -17.97 & 0.0000 \\ 4.727 & 489.42 & -13.67 & 0.0000 \\ 6.461 & 545.68 & -0.17 & 0.0000 \\ 6.461 & 558.99 & -54.53 & 0.0000 \\ 6.461 & 558.99 & -54.53 & 0.0000 \\ 38.862 & 1007.76 & 55.78 & 0.0000 \\ 38.862 & 1007.76 & 55.78 & 0.0000 \\ 37.967 & 1766.18 & 43.27 & 0.0115 \\ 9.229 & 1170.97 & -63.61 & 0.0086 \\ 3.316 & 928.08 & -123.81 & 0.0085 \\ 3.316 & 928.15 & -123.81 & 0.0085 \\ 3.316 & 928.22 & -123.81 & 0.0085 \\ 4.727 & 489.42 & -13.67 & 0.0000 \\ 4.727 & 490.21 & -14.13 & 0.0000 \\ 4.727 & 490.21 & -14.13 & 0.0000 \\ 4.727 & 490.21 & -14.13 & 0.0000\end{array}$

WC
819.36 819.36
819.36
736.57

83.20

666.62

58.17

59.47

53.53
11.37

8.81

12.08
54.06

54.06
135.52

135.52
135.53

135.53

666.62

674.77

674.77
674.77

674.77

PS
2.947
3.846
3.794
4.113
3.988
6.193
6.179
6.234
34.170
36.171
35.841
8.375
3.141
3.141
2.947
3.988
3.862
3.862
2.947

Ts
425.97
459.70
457.97
468.70
466.17
539.13
551.93
553.35
972.69
988.06
1741.19
1141.07
914.38
914.45
898.63
466.17
462.66
462.66
428.20

$\begin{array}{rr}\text { Aphy } & \text { MN } \\ 2564.5 & 0.7300 \\ 4168.2 & 0.3575 \\ 3531.8 & 0.3836 \\ 830.5 & 0.1719 \\ 2606.7 & 0.4989 \\ 412.6 & 0.2466 \\ 412.6 & 0.2535 \\ 412.6 & 0.2264 \\ 49.7 & 0.4356 \\ 49.3 & 0.3241 \\ 74.6 & 0.2956 \\ 265.4 & 0.3796 \\ 860.2 & 0.2814 \\ 860.2 & 0.2814 \\ 613.4 & 0.4168 \\ 2606.7 & 0.4989 \\ 2481.9 & 0.5452 \\ 2481.9 & 0.5452 \\ 2006.9 & 0.8500\end{array}$

Powerset 10.000 $\begin{array}{ccc}\text { T4 } & \text { T41 } & \text { T49 } \\ 1766.2 & 1670.8 & 1166.7\end{array}$

TURBOMACHINERY PERFORMANCE DATA

\begin{tabular}{|c|c|c|c|c|c|c|c|c|c|}
\hline & WC & $P R$ & eff & Ne & $T R$ & $e f \mathrm{PO}_{\mathrm{y}}$ & pwr & SMN & SMWT \\
\hline Fan & 736.57 & 1.126 & 0.9057 & 2525.346 & 1.0381 & 0.9073 & -1343.2 & 1950.43 & 54.42 \\
\hline LPC & 83.20 & 1.539 & 0.8328 & 2525.346 & 1.1574 & 0.8426 & -627.9 & 6.84 & 3.41 \\
\hline HPC & 53.53 & 6.015 & 0.8173 & 8942.041 & 1.8028 & 0.8558 & -3452.0 & 61.87 & 58.46 \\
\hline HPT & 12.08 & 4.114 & 0.8778 & 220.891 & 1.3596 & 0.8561 & 3452.0 & & \\
\hline LPT & 54.06 & 2.783 & 0.8534 & 70.361 & 1.2598 & 0.8346 & 1971.1 & & \\
\hline$J H_{-}$ & ERY M & DATA & & & & & & & \\
\hline & WcMap & PRmap & effMap & NcMap & R/Parm & S_WcDes & S_PRdes & s_effDes & S_NCDes \\
\hline Fan & 855.83 & 1.123 & 0.9075 & 2501.267 & 0.0415 & $\overline{0} .8607$ & $\overline{1} .0235$ & 0.9980 & 0.9905 \\
\hline ec & 72.05 & 1.503 & 0.7779 & 0.667 & 0.0000 & 1.1 & 1.0710 & 1.0 & 0.0003 \\
\hline ec & 48.68 & 5.779 & 0.8257 & 8703.652 & 10.9516 & 1.0995 & 1.0494 & 0.9898 & 0.9733 \\
\hline T & 0.96 & 4.028 & 0.8778 & 1.328 & 4.0278 & 12.6299 & 0.9723 & 1.0000 & 0.00 \\
\hline $\mathrm{PT}$ & 0.83 & 2.341 & 0.8598 & 0.791 & 2.3413 & 65.2590 & 0.7521 & 0.9926 & 0.0005 \\
\hline
\end{tabular}




\begin{tabular}{|c|c|c|c|c|c|c|c|c|c|}
\hline Z & $===$ INLETS $====$ & eRam & Afs & Fram & \multirow{2}{*}{\multicolumn{2}{|c|}{ BLEEDS - interstg }} & & & \\
\hline$\stackrel{s}{\infty}$ & Inlet & 1.0000 & 2564.47 & 5642.2 & & & $\begin{array}{l}\text { Wb/Win } \\
0.0368\end{array}$ & $\begin{array}{r}\text { BldWk } \\
0.5000\end{array}$ & $\begin{array}{r}\text { BldP } \\
0.2200\end{array}$ \\
\hline 7 & $====$ DUCTS $====$ & dPnorm & MN & Aphy & LPT_COOLA & HPC.C> & 0.0117 & 0.5000 & 0.4500 \\
\hline$\exists$ & TEGV & 0.0000 & 0.2814 & 860.21 & WB $2 \overline{\mathrm{X}}$ & HPC. B> & 0.0000 & 0.4500 & 0.2200 \\
\hline & FanDuct & 0.0000 & 0.5452 & 2481.93 & WB2Y & HPC.B> & 0.0000 & 0.7600 & 0.6200 \\
\hline & & & & & \multirow{2}{*}{$\begin{array}{l}\text { WBA2X } \\
\text { WBLKG }\end{array}$} & HPC.B> & 0.0000 & 0.4500 & 0.2200 \\
\hline N & $==$ SPLITTERS $==$ & $\mathrm{BPR}$ & $\mathrm{dP} / \mathrm{P} 1$ & $\mathrm{dP} / \mathrm{P} 2$ & & HPC.I> & 0.0000 & 1.0000 & 1.0000 \\
\hline & Splitter & 8.8534 & 0.0005 & 0.0005 & WBW2X & HPC. B> & 0.0000 & 0.4500 & 0.2200 \\
\hline & $===$ SHAFT $\mathrm{S}====$ & Nmech & trq in & pwr in & \multicolumn{2}{|c|}{ BLEEDS - output } & Wb/Win & hscale & Pscale \\
\hline & HP Shaft & 9283.1 & 1953.0 & 3452.0 & \multicolumn{2}{|c|}{ WB17Y Bleed> } & 0.1000 & 1.0000 & 1.0000 \\
\hline & LP_Shaft & 2407.7 & 4299.6 & 1971.1 & \multirow{2}{*}{\multicolumn{2}{|c|}{$\begin{array}{l}\text { HPT_COOLA Bleed> } \\
\text { HPT COOLB Bleed> }\end{array}$}} & 0.1142 & 1.0000 & 1.0000 \\
\hline & & & & & & & 0.0999 & 1.0000 & 1.0000 \\
\hline & & & & & WB $3 \bar{x}$ & Bleed> & 0.0000 & 1.0000 & 1.0000 \\
\hline & & & & & WBA3X & Bleed> & 0.0000 & 1.0000 & 1.0000 \\
\hline & & & & & WBW3X & Bleed> & 0.0000 & 1.0000 & 1.0000 \\
\hline & & & & & \multirow{2}{*}{$\begin{array}{l}\text { WBFDLKG } \\
\text { WB1 } 15\end{array}$} & FanDu> & 0.0000 & 1.0000 & 1.0000 \\
\hline & & & & & & Bleed $>$ & 0.0000 & 1.0000 & 1.0000 \\
\hline & & & & & $\begin{array}{l}\text { WB15X } \\
\text { WB15Y }\end{array}$ & Bleed> & 0.0000 & 1.0000 & 1.0000 \\
\hline & & & & & WB17X & Bleed> & 0.0000 & 1.0000 & 1.0000 \\
\hline & $===$ BURNERS $===$ & Ttout & eff & dPnorm & \multirow{2}{*}{\multicolumn{2}{|c|}{ FAR }} & & & \\
\hline & Burner & 1766.13 & 0.9995 & 0.0230 & & 1154 & & & \\
\hline & $===$ NOZZLES $===$ & PR & $C f g$ & CdTh & $\mathrm{Cv}$ & & MNth & Vact & $\mathrm{Fg}$ \\
\hline & Core_Nozz & 1.125 & 0.9801 & 1.0000 & \multirow{2}{*}{0.9800} & \multirow{2}{*}{$\begin{array}{r}613.40 \\
2006.86\end{array}$} & 0.417 & 597.7 & 424.6 \\
\hline & Byp Nozz & 1.604 & 0.9800 & 1.0000 & & & 0.850 & 845.3 & 5866.2 \\
\hline
\end{tabular}


Turbofan Engine - COMDES ON Converge $=1$ CASE: 0

Version:NPSS 1.6.5 - Rev: -> Gas Package: Janaf iter/pass/Jacb/Broy=13/27/1/11 Run by: Philip C Jorgenson PC: 10

Temperature Stator 1 inlet: 475.18 Stator 1 exit: 482.41 Stator 2 inlet: 491.46 Stator 2 exit: 496.13 Stator 5 inlet: 528.65 Stator 5 exit: $531.75 \quad$ Stator 4 Unblocked Percent Blockage: 0.00

Ambient Relative Humidity $\quad 10.00$

Fan Face Relative Humidity $\quad 2.64$

Fan Bypass Relative Humidity $\quad 1.92$

$\begin{array}{ll}\text { LPC Inlet Relative Humidity } & 1.75 \\ \text { LC Exit Relative Humidity } & 0.18\end{array}$

HPC Relative Humidity

0.06

0.0000050

Inlet Length

40.00

Ambient Flow Velocity

Ambient Static Pressure

Additional Water at LPC Exit

722.68 Fan/LPC Inlet Flow Velocity $\quad 180.06$

3.09 Fan/LPC Inlet Static Pressure

425.97 Fan/LPC Inlet Static Temperature 466.80

$\begin{array}{rrrrrr} & & & & \text { SUMMARY } & \text { OUTPUT DAT } \\ \text { MN } & \text { alt } & \text { dTamb } & \text { W } & \text { Fn } & \text { TSFC } \\ 0.714 & 37357.0 & 36.00 & 252.23 & 659.2 & 1.0582\end{array}$

252.23

$\begin{array}{rr}\text { Fn } & \text { TSFC } \\ 659.2 & 1.0582\end{array}$

Wfuel
697.55

BPR
8.8837

VTAS
722.68

OPR
9.121

EPR
0.772

PowerSet
10.000

$\begin{array}{ccc}\text { T4 } & \text { T41 } & \text { T49 } \\ 1752.6 & 1657.6 & 1158.1\end{array}$

FSO Ambient.FI O

FS1 Inlet.F1_O

FS12 Splitter.F1_02

FS2 Splitter.Fl_01

FS14 Fan.F1_O

FS24 VaporIN.FI_O

FS25 Bleed2.F1 $\bar{O}$

FS3 HPC.FIO

FS36 Bleed3.F1_O

FS4 Burner.FI_O

HPT. FI

LPT.FI-O

FS8 Core_Nozz.Fl_O

FS17 FanDuctlkg.FI_O

FS171 Bleed15.F1_O

FS172 FanDuct.Fl-O

FS173 Byp Nozz.Fì O

W
252.23
252.23
226.71
25.52
226.71
25.52
25.75
23.18
22.05
17.09
17.29
23.10
23.37
23.37
23.37
226.71
229.28
229.28
229.28

FLOW STATION DATA

\begin{tabular}{lrrrr}
\multicolumn{1}{c}{ FLOW } & STATION DATA \\
Pt & Tt & ht & FAR & WC \\
4.339 & 469.50 & -18.42 & 0.0000 & 812.74 \\
4.339 & 469.50 & -18.42 & 0.0000 & 812.74 \\
4.337 & 469.50 & -18.42 & 0.0000 & 730.87 \\
4.337 & 469.50 & -18.42 & 0.0000 & 82.27 \\
4.867 & 486.90 & -14.25 & 0.0000 & 663.20 \\
6.610 & 541.65 & -1.12 & 0.0000 & 57.98 \\
6.610 & 553.85 & -50.63 & 0.0000 & 59.17 \\
6.610 & 553.85 & -50.63 & 0.0000 & 53.25 \\
39.578 & 997.72 & 58.33 & 0.0000 & 11.36 \\
39.578 & 997.72 & 58.33 & 0.0000 & 8.80 \\
38.666 & 1752.62 & 45.89 & 0.0114 & 12.08 \\
9.441 & 1162.36 & -59.66 & 0.0085 & 53.83 \\
3.455 & 925.40 & -118.27 & 0.0084 & 132.79 \\
3.455 & 925.47 & -118.27 & 0.0084 & 132.80 \\
3.455 & 925.54 & -118.27 & 0.0084 & 132.80 \\
4.867 & 486.90 & -14.25 & 0.0000 & 663.20 \\
4.867 & 487.66 & -14.66 & 0.0000 & 671.26 \\
4.867 & 487.66 & -14.66 & 0.0000 & 671.26 \\
4.867 & 487.66 & -14.66 & 0.0000 & 671.26
\end{tabular}

Ps
3.088
3.979
3.925
4.250
4.116
6.338
6.325
6.381
34.812
36.844
36.501
8.577
3.281
3.281
3.088
4.11
3.989
3.98
3.088

Ts
425.97
457.99
456.30
466.80
464.09
535.19
546.93
548.31
963.06
978.25
1727.80
1132.96
912.33
912.40
897.46
464.09
460.66
460.66
428.15

$\begin{array}{rr}\text { Aphy } & M N \\ 2568.3 & 0.7140 \\ 4168.2 & 0.3541 \\ 3531.8 & 0.3801 \\ 830.5 & 0.1700 \\ 2606.7 & 0.4953 \\ 412.6 & 0.2457 \\ 412.6 & 0.2520 \\ 412.6 & 0.2251 \\ 49.7 & 0.4348 \\ 49.3 & 0.3236 \\ 74.6 & 0.2955 \\ 265.4 & 0.3775 \\ 860.2 & 0.2751 \\ 860.2 & 0.2751 \\ 613.4 & 0.4062 \\ 2606.7 & 0.4953 \\ 2481.9 & 0.5409 \\ 2481.9 & 0.5409 \\ 2006.9 & 0.8328\end{array}$

gamt
1.40075
1.40075
1.40075
1.40075
1.40060
1.39989
1.39844
1.39844
1.37943
1.37943
1.32824
1.36315
1.37825
1.37825
1.37824
1.40060
1.40058
1.40058
1.40058

WAR
0.0000786 $\begin{array}{ll}0.0000786 & \text { Wair } \\ 0.0252 .21\end{array}$ $0.0000786 \quad 252.21$ 0.0000786 $0.0092574-25.52$ 0.0092574 0.0092574 25.52
22.97 22.97
21.85 21.85
16.94 $\begin{array}{lll}0.0092574 & 16.94 & 0.157\end{array}$ $\begin{array}{lll}0.0092574 & 16.94 & 0.157 \\ 0.0092574 & 22.707 & 0.210\end{array}$ $\begin{array}{lll}0.0092574 & 22.97 & 0.213 \\ 0.0092574 & 22.97 & 0.213\end{array}$ $\begin{array}{llll}0.0092574 & 22.97 & 0.213\end{array}$ $\begin{array}{lll}0.00000786 & 226.69 & 0.018\end{array}$ $0.0001808 \quad 229.24 \quad 0.041$ $\begin{array}{lll}0.0000786 & 226.69 & 0.018 \\ 0.0001808 & 229.24 & 0.041\end{array}$ $0.0001808 \quad 229.24 \quad 0.041$
2O H2O frac $0.020 \quad 0.0001$ 0.018 0.018 0.002 0.002 0.00092
0.0092 0.0092 0.0092 0.0092
0.0229
0.0194 0.0193 0.0193 0.0193 0.0001 0.0002 0.0002 0.0002

\begin{tabular}{|c|c|c|c|c|c|c|c|c|c|}
\hline \multirow{2}{*}{\multicolumn{2}{|c|}{ TURBOMACH INERY }} & PERFORMANCE & DATA $_{\text {eff }}$ & \multirow[b]{2}{*}{ NC } & \multirow[b]{2}{*}{ TR } & \multirow[b]{2}{*}{ efPoly } & \multirow[b]{2}{*}{ pwr } & \multirow[b]{2}{*}{ SMN } & \multirow[b]{2}{*}{ SMW } \\
\hline & & PR & eff & & & & & & \\
\hline Fan & 730.87 & 1.122 & 0.9052 & 2496.952 & 1.0371 & 0.9068 & -1337.7 & 2278.73 & 54.38 \\
\hline LPC & 82.27 & 1.524 & 0.8333 & 2496.952 & 1.1537 & 0.8429 & -624.7 & 6.75 & 3.33 \\
\hline HPC & 53.25 & 5.987 & 0.8168 & 8933.068 & 1.8014 & 0.8554 & -3486.5 & 61.89 & 58.46 \\
\hline HPT & 12.08 & 4.095 & 0.8771 & 220.499 & 1.3585 & 0.8554 & 3486.5 & & \\
\hline LPT & 53.83 & 2.733 & 0.8510 & 69.681 & 1.2540 & 0.8322 & 1962.4 & & \\
\hline \multicolumn{10}{|c|}{ INERY MAP DATA } \\
\hline & WcMap & PRmap & effMap & NcMap & R/Parm & S_WcDes & S_PRdes & S_effDes & S_NCDes \\
\hline Fan & 849.20 & 1.119 & 0.9071 & 2473.144 & 0.0414 & $\overline{0} .8607$ & 1.0235 & 0.9980 & 0.9905 \\
\hline LPC & 70.82 & 1.490 & 0.7766 & 0.660 & 0.0000 & 1.1617 & 1.0693 & 1.0730 & 0.0003 \\
\hline HPC & 48.43 & 5.752 & 0.8253 & 8694.918 & 10.9534 & 1.0995 & 1.0494 & 0.9898 & 0.9733 \\
\hline HPT & 0.96 & 4.010 & 0.8771 & 1.325 & 4.0097 & 12.6299 & 0.9723 & 1.0000 & 0.0003 \\
\hline LPT & 0.82 & 2.303 & 0.8573 & 0.784 & 2.3032 & 65.2590 & 0.7521 & 0.9926 & 0.0005 \\
\hline
\end{tabular}




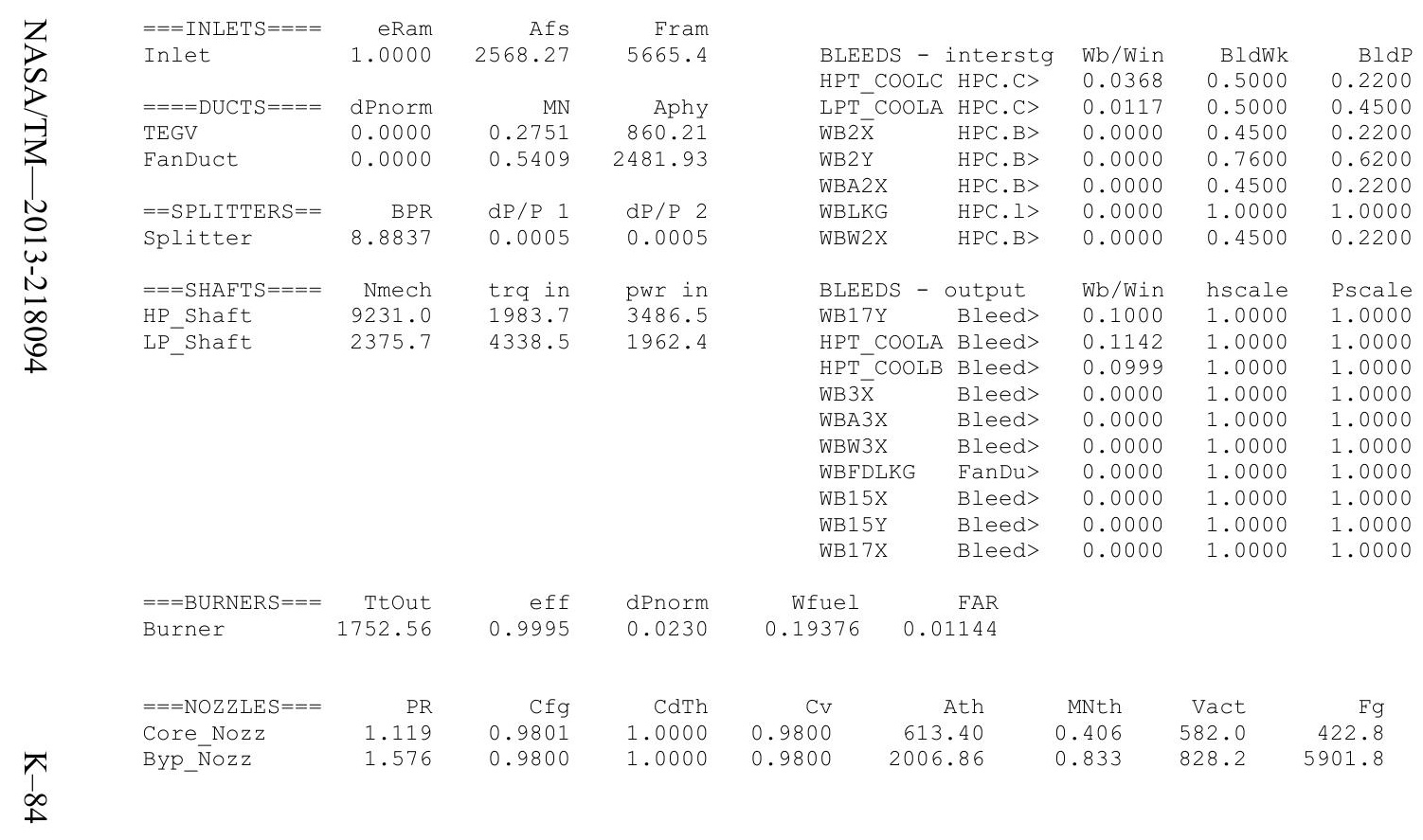


Date:07/05/13 Time:11:41:11 Model: Turbofan Engine - COMDES ON Converge = 1 CASE: 0

Version:NPSS 1.6.5 - Rev: -> Gas Package: Janaf iter/pass/Jacb/Broy= 12/ 26/1/10 Run by: Philip C Jorgenson PC: 10

Temperature Stator 1 inlet: $476.52 \quad$ Stator 1 exit: 483.28 Stator 2 inlet: 491.72 Stator 2 exit: 496.06 $\begin{array}{llll}\text { Stator } 3 \text { inlet: } 504.69 & \text { Stator } 3 \text { exit: } 508.58 & \text { Stator } 4 \text { inlet: } 516.57 \text { Stator } 4 \text { exit: } 519.79 \\ \text { Stator } 5 \text { inlet: } 526.29 & \text { Stator } 5 \text { exit: } 529.14 & & \end{array}$

Ambient Relative Humidity $\quad 10.00$

Fan Face Relative Humidity $\quad 3.25$

Fan Bypass Relative Humidity $\quad 2.45$

LPC Exit Relative Humidity 0.27

HPC Relative Humidity

Drop Diameter

0.07
.000050

Ambient Flow Velocity

Inlet Length

Ambient Static Pressure 0.0082762

\begin{tabular}{rr} 
& 40.00 \\
\hline
\end{tabular}$\quad 4.74$

Additional Water at LPC Exit

\section{$\begin{array}{rllr}\text { MN alt dTamb } & \text { W } & \text { SUMMARY OUTPUT DATA } \\ 0.669 & \text { TSFC }\end{array}$}

$\begin{array}{llll}0.669 & 34281.0 & 36.00 & 273.09\end{array}$
$704.6 \quad 1.0548$

\section{FLOW STATION DATA}

FSO Ambient.Fl_O

FS1 Inlet.Fl_O

$\pi$

Splitter.Fl_01

FS14 Fan.F1_

FS24 LPC.FI-O

FS25 Bleed2.FI_O

$\begin{array}{ll}\text { FS25 } & \text { Bleed2.FI } \\ \text { FS3 HPC.FI_O }\end{array}$

FS36 Bleed3.Fl_O

FS4 Burner.FI_O

FS45 HPT.FI-

FS49 LPT.FI-O

FS8 Core_Nozz.FI_O

FS17 FanDuctLkg.Fi_o

FS171 Bleed15.FI_O

FS172 FanDuct.Fl_O

W
273.09
273.09
245.71
27.38
245.71
27.38
27.61
24.85
23.64
18.32
18.53
24.76
25.05
25.05
25.05
245.71
248.47
248.47
248.47

$\begin{array}{rrrr}\text { Pt } & \text { Tt } & \text { ht } & \text { FAR } \\ 4.831 & 471.21 & -18.12 & 0.0000 \\ 4.831 & 471.21 & -18.12 & 0.0000 \\ 4.829 & 471.21 & -18.12 & 0.0000 \\ 4.829 & 471.21 & -18.12 & 0.0000 \\ 5.374 & 487.42 & -14.23 & 0.0000 \\ 7.161 & 538.52 & -1.98 & 0.0000 \\ 7.161 & 549.59 & -46.65 & 0.0000 \\ 7.161 & 549.59 & -46.65 & 0.0000 \\ 42.255 & 986.70 & 60.50 & 0.0000 \\ 42.255 & 986.70 & 60.50 & 0.0000 \\ 41.281 & 1739.08 & 48.11 & 0.0114 \\ 0.194 & 1155.34 & -55.65 & 0.0085 \\ 3.943 & 932.36 & -110.66 & 0.0084 \\ 3.943 & 932.43 & -110.66 & 0.0084 \\ 3.943 & 932.50 & -110.66 & 0.0084 \\ 5.374 & 487.42 & -14.23 & 0.0000 \\ 5.374 & 488.11 & -14.59 & 0.0000 \\ 5.374 & 488.11 & -14.59 & 0.0000 \\ 5.374 & 488.11 & -14.59 & 0.0000\end{array}$

Wfuel

BPR
8.9740

VTAS
682.24

$\mathrm{OPR}$
8.747

EPR
0.795

PowerSet
10.000

$\begin{array}{ccc}\text { T4 } & \text { T41 } & \text { T49 } \\ 1739.1 & 1644.4 & 1151.1\end{array}$

TURBOMACHINERY PERFORMANCE DATA

\begin{tabular}{|c|c|c|c|c|c|c|c|c|c|}
\hline & WC & PR & eff & NC & $T R$ & efpoly & pwr & SMN & SMW \\
\hline Fan & 712.80 & 1.113 & 0.9041 & 2412.821 & 1.0344 & 0.9056 & -1350.2 & 4577.10 & 54.02 \\
\hline LPC & 79.43 & 1.483 & 0.8351 & 2412.821 & 1.1428 & 0.8440 & -625.3 & 6.47 & 3.10 \\
\hline${ }^{2} \mathrm{C}$ & 52.48 & 5.900 & .8154 & 8907.943 & 1.7953 & 0.8541 & -3675.2 & 62.25 & 58.72 \\
\hline HPT & 12.08 & 4.049 & 0.8762 & 219.883 & 1.3555 & 0.8546 & 3675.2 & & \\
\hline LPT & 53.28 & 2.585 & 0.8455 & 67.660 & 1.2370 & 0.8273 & 1975.5 & & \\
\hline RI & IERY MAP & DATA & & & & & & & \\
\hline & WcMap & PRmap & effMap & NcMap & R/Parm & s_WCDes & S_PRdes & s_effDes & S_NCDes \\
\hline Fan & 828.20 & 1.110 & 0.9060 & 2389.815 & 0.0414 & 0.8607 & 1.0235 & 0.9980 & 0.9905 \\
\hline PC & 67.18 & 1.4 & & 0.637 & 00 & 1.1 & 1.0675 & 1.0 & 0.0003 \\
\hline PC & 47.73 & 5.669 & 0.8238 & 8670.463 & 10.9417 & 1.0995 & 1.0494 & 0.9898 & 0.9733 \\
\hline IPT & 0.96 & 3.965 & 0.8762 & 1.321 & 3.9650 & 12.6299 & 0.9723 & 1.0000 & 0.0003 \\
\hline $\mathrm{PT}$ & 0.82 & 2.192 & 0.8517 & 0.761 & 2.1922 & 65.2590 & 0.7521 & 0.9926 & 0.0005 \\
\hline
\end{tabular}


$\begin{array}{lrrr}===\text { INLETS }==== & \text { eRam } & \text { Afs } & \text { Fram } \\ \text { Inlet } & 1.0000 & 2581.09 & 5790.8\end{array}$

$====$ DUCTS $====$ dPnorm
TEGV

FanDuct

0.0000

0.0000

$==$ SPLITTERS $==$

Splitter

BPR

8.9740

$===$ SHAFTS $===$

HP_Shaft

Nmech
9169.6

2299.8

LP_Shaft

$=$ BURNERS $==$

TtOut

Burner

eff

dPnorm

0.0230

$===$ NOZZLES $===$

Core $\mathrm{NOZZ}^{-}$

PR
1.102
1.502

Cfg
0.9801

0.9800

CdTh
1.0000

$1.0000 \quad 0.9800$

BLEEDS - interstg Wb/Win BldWk

0.2200

$\begin{array}{lllll}\text { LPT_COOLA HPC.C }> & 0.0117 & 0.5000 & 0.4500\end{array}$

$\begin{array}{lllll}\text { WB2X } & \text { HPC.B } & 0.0000 & 0.4500 & 0.2200 \\ \text { WB2Y } & \text { HPC.B } & 0.0000 & 0.7600 & 0.6200\end{array}$

$\begin{array}{lllll}\text { WBA2X HPC.B } & 0.0000 & 0.4500 & 0.2200\end{array}$

$\begin{array}{lllll}\text { WBLKG HPC.I> } & 0.0000 & 1.0000 & 1.0000\end{array}$

$\begin{array}{lllll}\text { WBW2X HPC.B } & 0.0000 & 0.4500 & 0.2200\end{array}$

BLEEDS - output Wb/Win hscale Pscale

$\begin{array}{lllll}\text { WB17Y Bleed } & 0.1000 & 1.0000 & 1.0000\end{array}$

HPT_COOLA Bleed> $0.1142 \quad 1.0000 \quad 1.0000$

HPT COOLB Bleed> $0.0999 \quad 1.0000 \quad 1.0000$

$\begin{array}{lllll}\text { WB3X } & \text { Bleed> } & 0.0000 & 1.0000 & 1.0000 \\ \text { WBA3X } & \text { Bleed> } & 0.0000 & 1.0000 & 1.0000\end{array}$

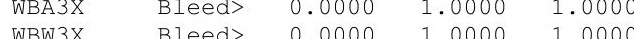

$\begin{array}{lllll}\text { WBW3X } & \text { Bleed> } & 0.0000 & 1.0000 & 1.0000 \\ \text { WBFDLKG } & \text { FanDu> } & 0.0000 & 1.0000 & 1.0000\end{array}$

$\begin{array}{lllll}\text { WBFDLKG } & \text { FanDu> } & 0.0000 & 1.0000 & 1.0000 \\ \text { WB15X } & \text { Bleed }> & 0.0000 & 1.0000 & 1.0000\end{array}$

WB15X Bleed> $0.0000 \quad 1.0000 \quad 1.0000$

$\begin{array}{lllll}\text { WB15Y } & \text { Bleed> } & 0.0000 & 1.0000 & 1.0000 \\ \text { WB17X } & \text { Bleed> } & 0.0000 & 1.0000 & 1.0000\end{array}$

$\begin{array}{rrrr}\text { W } & \text { Tt } & \text { ht } & \text { Pt } \\ 0.9143 & 770.04 & 6.93 & 10.194 \\ 0.2907 & 770.04 & 6.93 & 3.943 \\ 0.0000 & 748.13 & 1.57 & 14.882 \\ 0.0000 & 883.30 & 34.78 & 28.919 \\ 0.0000 & 748.13 & 1.57 & 14.882 \\ 0.0000 & 986.70 & 60.50 & 42.255 \\ 0.0000 & 748.13 & 1.57 & 14.882\end{array}$

त
$\infty$
$\infty$

FAR

$\begin{array}{rrrr}\text { W } & \text { Tt } & \text { ht } & \text { Pt } \\ 2.7607 & 549.59 & -46.65 & 7.161 \\ 2.8369 & 986.70 & 60.50 & 4.281\end{array}$

$\begin{array}{llll}2.8369 & 986.70 & 60.50 & 41.281\end{array}$

$\begin{array}{llll}2.4824 & 986.70 & 60.50 & 23.500\end{array}$

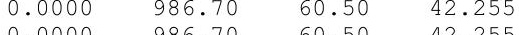

$0.0000-986.70-60.50-42.255$

$\begin{array}{llll}0.0000 & 986.70 & 60.50 & 42.255 \\ 0.0000 & 487.42 & -14.23 & 5.374\end{array}$

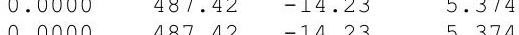

$0.0000-487.42-14.23-5.374$

$\begin{array}{llll}0.0000 & 487.42 & -14.23 & 5.374\end{array}$

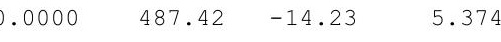


Turbofan Engine - COMDES ON Converge $=1$ CASE: 0

Version:NPSS 1.6.5 - Rev: -> Gas Package: Janaf iter/pass/Jacb/Broy=15/43/2/12 Run by: Philip C Jorgenson PC: 10

Temperature Stator 1 inlet: 484.81 Stator 1 exit: 490.63 Stator 2 inlet: 497.82 Stator 2 exit: 501.33 Stator 3 inlet: $508.76 \quad$ Stator 3 exit: 511.83 Stator 4 inlet: 518.73 Stator 4 exit: 521.22

Ambient Relative Humidity $\quad 10.00$

Fan Face Relative Humidity $\quad 4.20$

Fan Bypass Relative Humidity $\quad 3.33$

$\begin{array}{ll}\text { LPC Inlet Relative Humidity } & 3.05 \\ \text { LPC Exit Relative Humidity } & 0.54\end{array}$

作

Drop Diameter

0.08

Ambient Flow Velocity

Ambient Static Pressure

Ambient Static Temperature
Additional Water at LPC Exit

$\begin{array}{lr} & 40.00 \\ \text { Fanlet Length } & 174.79\end{array}$

$\begin{array}{lrr}4.35 & \text { Fan/LPC Inlet Static Pressure r } & 5.49 \\ 447.58 & \text { Fan/LPC Inlet Static Temperature } & 478.19\end{array}$

0.0086245

MN alt dTamb SUMMARY OUTPUT DATA

$\begin{array}{rrr}0.608 & 30029.0 & 36.00\end{array}$

298.69 $\begin{array}{rr}\text { Fn } & \text { TSFC } \\ 752.6 & 1.0677\end{array}$

Wfuel
803.55

BPR
8.5644

VTAS
630.80

OPR
8.134

EPR
0.827

PowerSet
10.000

$\begin{array}{ccc}\text { T4 } & \text { T41 } & \text { T49 } \\ 1737.5 & 1642.5 & 1153.2\end{array}$
FSO Ambient.FI_O

FS1 Inlet.FI_O

ז

FS2 Splitter.F1_01

FS14 Fan.Fl_

FS24 VaporIN . FI

ES25 Bleed2.FI_O

FS HPC.Fl_O

BSt

ES4

ES49 IPT. FI

FS5 TEGV.Fīo

FS8 Core_Nozz.Fl_O

FS17 FanDüctLkg.Fì_o

FS171 Bleed15.FI

FS172 FanDuct.Fl-O

FS173 Byp Nozz.Fì O

W
298.69
298.69
267.46
31.23
267.46
31.23
31.50
26.77
25.48
19.74
19.97
26.68
27.00
27.00
27.00
267.46
272.19
272.19
272.19

FLOW STATION DATA

$\begin{array}{rrrrr}\text { Pt } & \mathrm{Tt} & \mathrm{ht} & \mathrm{FAR} & \text { Wc } \\ 5.595 & 480.74 & -16.28 & 0.0000 & 755.31 \\ 5.595 & 480.74 & -16.28 & 0.0000 & 755.31 \\ 5.592 & 480.74 & -16.28 & 0.0000 & 676.68 \\ 5.592 & 480.74 & -16.28 & 0.0000 & 79.01 \\ 6.146 & 495.32 & -12.79 & 0.0000 & 624.95 \\ 7.860 & 538.42 & -2.45 & 0.0000 & 59.49 \\ 7.860 & 549.94 & -48.98 & 0.0000 & 60.64 \\ 7.860 & 549.94 & -48.98 & 0.0000 & 51.55 \\ 45.513 & 982.53 & 57.08 & 0.0000 & 11.32 \\ 45.513 & 982.53 & 57.08 & 0.0000 & 8.77 \\ 4.465 & 1737.49 & 44.68 & 0.0114 & 12.08 \\ 1.143 & 1157.45 & -57.99 & 0.0085 & 52.57 \\ 4.715 & 954.80 & -107.91 & 0.0084 & 114.18 \\ 4.715 & 954.87 & -107.91 & 0.0084 & 114.18 \\ 4.715 & 954.94 & -107.91 & 0.0084 & 114.19 \\ 6.146 & 495.32 & -12.79 & 0.0000 & 624.95 \\ 6.146 & 496.28 & -13.42 & 0.0000 & 636.61 \\ 6.146 & 496.28 & -13.42 & 0.0000 & 636.61 \\ 6.146 & 496.28 & -13.42 & 0.0000 & 636.61\end{array}$

PS
4.358

$\begin{array}{lll}44.58 & 2594.6 & 0.6080\end{array}$

$\begin{array}{llll}4.358 & 447.58 & 2594.6 & 0.6080 \\ 5.199 & 470.75 & 4168.2 & 0.3254 \\ 5.489 & 469.42 & 3531.8 & 0.3470\end{array}$

5.489

5.325

7.325
7.519
7.503

7.503

7.606
40.077

40.077

$10.176 \quad 1129.73$

$4.541 \quad 945.07$

.541
4.358
5.325

4.541
5.325
5.177

$\begin{array}{ll}5.177 & 472.52\end{array}$

5.177

4.358

449.80 $3531.8 \quad 0.3470$ $\begin{array}{rr}830.5 & 0.1630 \\ 2606.7 & 0.4571\end{array}$ $\begin{array}{ll}412.6 & 0.2526\end{array}$ $\begin{array}{ll}412.6 & 0.2587 \\ 412.6 & 0.2174\end{array}$ $\begin{array}{ccc}49.7 & 0.4328\end{array}$ $\begin{array}{ll}49.3 & 0.3223\end{array}$ $\begin{array}{lll}74.6 & 0.2954 \\ 265.4 & 0.3669\end{array}$ $\begin{array}{lll}265.4 & 0.3669\end{array}$ $\begin{array}{ll}860.2 & 0.2337\end{array}$

$\begin{array}{ll}613.5 & 0.3395\end{array}$ $\begin{array}{ll}2606.7 & 0.4571 \\ 2481.9 & 0.5010\end{array}$

$\begin{array}{ll}2481.9 & 0.501 \\ 2481.9 & 0.5010\end{array}$

$2006.9 \quad 0.718$

gamt
1.40064
1.40064
1.40064
1.40064
1.40049
1.39993
1.39856
1.39856
1.38043
1.38043
1.32903
1.36355
1.37656
1.37655
1.37655
1.40049
1.40046
1.40046
1.40046

WAR
0.0001750
0.0001750
0.0001750
0.0001750
0.0001750
0.0001750
0.0087995
0.0087995
0.0087995
0.0087995
0.0087995
0.0087995
0.0087995
0.0087995
0.0087995
0.0001750
0.0003234
0.0003234
0.0003234

Wair 298.64
298.64 298.64
267.42 31.22 267.42 31.22 31.22
26.54 26.54
25.25 $\begin{array}{ll}9.57 & 0.172\end{array}$ $\begin{array}{ll}19.57 & 0.172\end{array}$ $\begin{array}{ll}26.54 & 0.234\end{array}$ $\begin{array}{ll}26.54 & 0.234 \\ 26.54 & 0.234\end{array}$ $\begin{array}{rr}26.54 & 0.234 \\ 267.42 & 0.047\end{array}$ $272.10 \quad 0.088$ $\begin{array}{ll}272.10 & 0.088 \\ 272.10 & 0.088\end{array}$ $272.10 \quad 0.088$

\begin{tabular}{|c|c|c|c|c|c|c|c|c|c|}
\hline & Wc & PR & eff & $\mathrm{NC}$ & TR & efPoly & pwr & SMN & SMW \\
\hline Fan & 676.68 & 1.099 & 0.9026 & 2258.248 & 1.0303 & 0.9040 & -1323.1 &.--- & 52.65 \\
\hline LPC & 79.01 & 1.406 & 0.8518 & 2258.248 & 1.1200 & 0.8588 & -611.3 & 5.94 & 2.65 \\
\hline HPC & 51.55 & 5.790 & 0.8134 & 8877.027 & 1.7866 & 0.8522 & -3920.1 & 62.78 & 59.14 \\
\hline HPT & 12.08 & 3.990 & 0.8759 & 219.291 & 1.3510 & 0.8545 & 3920.1 & & \\
\hline LPT & 52.57 & 2.364 & 0.83 & 63.904 & 1.2098 & 0.8195 & 1934.4 & & \\
\hline 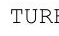 & NERY MAP & DATA & & & & & & & \\
\hline & WcMap & PRmap & effMap & NcMap & R/Parm & S_WcDes & S_PRdes & S_effDes & S_NCDes \\
\hline an & 786.23 & 1.097 & 0.9045 & 2236.716 & 0.0417 & $\overline{0} .8607$ & $\overline{1} .0235$ & 0.9980 & $\overline{0} .9905$ \\
\hline PC & 60.62 & 1.385 & .7657 & 0.596 & 0.0000 & 1.3034 & 1.0536 & 1.1125 & 0.0003 \\
\hline${ }^{\mathrm{C}} \mathrm{C}$ & 46.88 & 5.565 & .8218 & 8640.372 & 10.9217 & 1.0995 & 1.0494 & 0.9898 & 0.9733 \\
\hline 1 & 0.96 & 3.908 & .8759 & 1.318 & 3.9076 & 12.6299 & 0.9723 & 1.0000 & 0.0003 \\
\hline LPT & 0.81 & 2.026 & 0.8429 & 0.719 & 2.0255 & 65.2590 & 0.7521 & 0.9926 & 0.00 \\
\hline
\end{tabular}


$\begin{array}{lrrr}===\text { INLETS }==== & \text { eRam } & \text { Afs } & \text { Fram } \\ \text { Inlet } & 1.0000 & 2594.60 & 5856.1\end{array}$

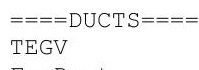

FanDuct

dPnorm
0.0000
0.0000

0.0000

$==$ SPLITTERS $==$

Splitter

8.5644

$===$ SHAFTS $===$

HP_Shaft

Nmech
9140.7
2174.1

LP_Shaft

2174.1

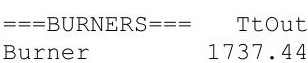

eff
0.9995

dPnorm

0.0230

BLEEDS - interstg Wb/Win BldWk

$\begin{array}{llllll}\text { HPT COOLC HPC.C }> & 0.0368 & 0.5000 & 0.2200\end{array}$

$\begin{array}{lllll}\text { LPT_COOLA } & \text { HPC.C> } & 0.0117 & 0.5000 & 0.4500 \\ \text { WB } 2 \bar{X} & \text { HPC. B> } & 0.0000 & 0.4500 & 0.2200\end{array}$

$\begin{array}{lllll}\text { WB2Y HPC.B> } & 0.0000 & 0.7600 & 0.6200\end{array}$

$\begin{array}{lllll}\text { WBA2X HPC.B } & 0.0000 & 0.4500 & 0.2200\end{array}$

WBLKG HPC.1> $0.0000 \quad 1.0000 \quad 1.0000$

$\begin{array}{lllll}\text { WBW2X HPC.B } & 0.0000 & 0.4500 & 0.2200\end{array}$

BLEEDS - output

Wb/win hscale Pscale

HPT_COOLA Bleed> $0.1142 \quad 1.0000 \quad 1.0000$

${ }_{\text {HPT COOLB Bleed> }} 0.0999 \quad 1.0000 \quad 1.0000$

$\begin{array}{lllll}\text { WB3X } & \text { Bleed> } & 0.0000 & 1.0000 & 1.0000 \\ \text { WBA3X } & \text { Bleed }> & 0.0000 & 1.0000 & 1.0000\end{array}$

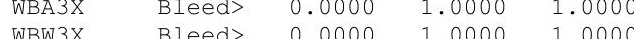

$\begin{array}{lllll}\text { WBW3X } & \text { Bleed> } & 0.0000 & 1.0000 & 1.0000 \\ \text { WBFDLKG } & \text { FanDu> } & 0.0000 & 1.0000 & 1.0000\end{array}$

$\begin{array}{lllll}\text { WBFDLKG } & \text { FanDu> } & 0.0000 & 1.0000 & 1.0000 \\ \text { WB15X } & \text { Bleed> } & 0.0000 & 1.0000 & 1.0000\end{array}$

WB15X Bleed> $0.0000 \quad 1.0000 \quad 1.0000$

$\begin{array}{lllll}\text { WB15Y } & \text { Bleed> } & 0.0000 & 1.0000 & 1.0000 \\ \text { WB17X } & \text { Bleed }> & 0.0000 & 1.0000 & 1.0000\end{array}$

Core Nozz

$\mathrm{PR}$
1.082

$\mathrm{Cfg}$

$\pi$

$\begin{array}{rrrrrr}\text { CdTh } & \text { Cv } & \text { Ath } & \text { MNth } & \text { Vact } & \text { Fg } \\ 1.0000 & 0.9800 & 613.40 & 0.340 & 496.0 & 416.2 \\ 1.0000 & 0.9800 & 2006.86 & 0.718 & 732.0 & 6192.5\end{array}$

$\begin{array}{rrrr}\text { W } & \text { Tt } & \text { ht } & \text { Pt } \\ 0.9853 & 768.08 & 4.05 & 11.143 \\ 0.0133 & 768.08 & 4.05 & 4.715 \\ 0.0000 & 746.40 & -1.25 & 16.144 \\ 0.0000 & 880.18 & 31.62 & 31.205 \\ 0.0000 & 746.40 & -1.25 & 16.144 \\ 0.0000 & 982.53 & 57.08 & 45.513 \\ 0.0000 & 746.40 & -1.25 & 16.144 \\ \text { W } & \text { Tt } & \text { ht } & \mathrm{Pt} \\ 4.7248 & 549.94 & -48.98 & 7.860 \\ 3.0571 & 982.53 & 57.08 & 44.465 \\ 2.6750 & 982.53 & 57.08 & 25.405 \\ 0.0000 & 982.53 & 57.08 & 45.513 \\ 0.0000 & 982.53 & 57.08 & 45.513 \\ 0.0000 & 982.53 & 57.08 & 45.513 \\ 0.0000 & 495.32 & -12.79 & 6.146 \\ 0.0000 & 495.32 & -12.79 & 6.146 \\ 0.0000 & 495.32 & -12.79 & 6.146 \\ 0.0000 & 495.32 & -12.79 & 6.146\end{array}$


Date:07/05/13 Time:11:42:08 Model: Turbofan Engine - COMDES ON Converge = 1 CASE: 0

Version:NPSS 1.6.5 - Rev: -> Gas Package: Janaf iter/pass/Jacb/Broy= 22/ 50/ 2/19 Run by: Philip C Jorgenson PC: 10

Temperature Stator 1 inlet: 492.56 Stator 1 exit: 496.81 Stator 2 inlet: 501.69 Stator 2 exit: 503.65 Stator 3 inlet: 508.98 Stator 3 exit: 510.49 Stator 4 inlet: 515.55 Stator 4 exit: 516.62

$\begin{array}{lclll}\text { Ambient Relative Humidity } & 10.00 & & & \\ \text { Fan Face Relative Humidity } & 5.03 & & & \\ \text { Fan Bypass Relative Humidity } & 4.17 & & & \\ \text { LPC Inlet Relative Humidity } & 4.02 & & & \\ \text { LPC Exit Relative Humidity } & 1.31 & & & \\ \text { HPC Relative Humidity } & 0.09 & & & \\ \text { Drop Diameter } & 0.0000050 & \text { Inlet Length } & & \\ \text { Ambient Flow Velocity } & 582.56 & \text { Fan/LPC Inlet Flow Velocity } & 193.47 \\ \text { Ambient Static Pressure } & 5.30 & \text { Fan/LPC Inlet Static Pressure } & 6.37 \\ \text { Ambient Static Temperature } & 463.14 & \text { Fan/LPC Inlet Static Temperature } & 488.30 \\ \text { Additional Water at LPC Exit } & 0.0078492 & & & \\ \end{array}$

Additional Water at LPC Exit

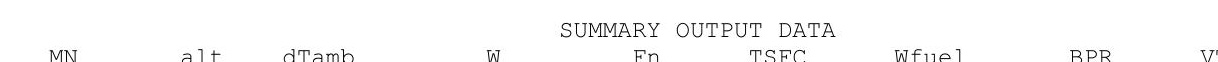

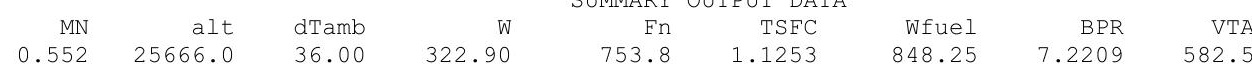

FSO Ambient.Fl_O

FS1 Inlet.F1_O

$\pi$
1
$\infty$

(1)

FLOW STATION DATA

FS14 Fan.Fl_O

$\begin{array}{ll}\text { FS23 } & \text { LPC.FI-O } \\ \text { FS24 } & \text { VaporIN.FI_O }\end{array}$

FS25 Bleed2.F1_O

FS3 HPC.FI_O

FS36 Bleed3.F1_O

FS45 HPT.Fl_o

$\begin{array}{ll}\text { FS45 } & \text { HPT.FI_O } \\ \text { FS49 } & \text { LPT.FI-O } \\ \text { FS5 } & \text { TEGV.FI? }\end{array}$

FS8 Core_Nozz.Fl_o

FS17 FanDuctLkg.Fi_o

FS171 Bleed15.FI_O

FS172 FanDuct.FI_O

$W$
322.90
322.90
283.62
39.28
283.62
39.28
39.59
27.71
26.37
20.43
20.67
27.62
27.95
27.95
27.95
283.62
295.50
295.50
295.50

$\begin{array}{rrrrr}\text { FLOW } & \text { STATION DATA } \\ \text { Pt } & \text { Tt } & \text { ht } & \text { FAR } & \text { Wc } \\ 6.516 & 491.41 & -14.47 & 0.0000 & 708.85 \\ 6.516 & 491.41 & -14.47 & 0.0000 & 708.85 \\ 6.513 & 491.41 & -14.47 & 0.0000 & 622.94 \\ 6.513 & 491.41 & -14.47 & 0.0000 & 86.27 \\ 7.054 & 503.98 & -11.46 & 0.0000 & 582.45 \\ 8.252 & 532.75 & -4.56 & 0.0000 & 70.89 \\ 8.252 & 543.34 & -46.91 & 0.0000 & 72.15 \\ 8.252 & 543.34 & -46.91 & 0.0000 & 50.51 \\ 47.121 & 967.79 & 57.00 & 0.0000 & 11.23 \\ 47.121 & 967.79 & 57.00 & 0.0000 & 8.71 \\ 46.036 & 1738.85 & 44.36 & 0.0116 & 12.08 \\ 11.915 & 1163.24 & -56.09 & 0.0087 & 51.02 \\ 5.620 & 984.73 & -99.91 & 0.0086 & 100.69 \\ 5.620 & 984.80 & -99.90 & 0.0086 & 100.69 \\ 5.620 & 984.87 & -99.90 & 0.0086 & 100.70 \\ 7.054 & 503.98 & -11.46 & 0.0000 & 582.45 \\ 7.054 & 505.57 & -12.88 & 0.0000 & 607.80 \\ 7.054 & 505.57 & -12.88 & 0.0000 & 607.80 \\ 7.054 & 505.57 & -12.88 & 0.0000 & 607.80\end{array}$

TURBOMACHINERY PERFORMANCE DATA

\begin{tabular}{|c|c|c|c|c|c|c|c|c|c|}
\hline & WC & PR & eff & $\mathrm{NC}$ & TR & efpoly & pwr & SMN & SMW \\
\hline Fan & 622.94 & 1.083 & 0.9030 & 2042.002 & 1.0256 & 0.9041 & -1209.2 & -2741.38 & 49.90 \\
\hline LPC & 86.27 & 1.267 & 0.8322 & 2042.002 & 1.0841 & 0.8377 & -551.1 & 6.39 & 2.41 \\
\hline HPC & 50.51 & 5.710 & 0.8118 & 8843.919 & 1.7812 & 0.8506 & -3975.2 & 62.22 & 58.48 \\
\hline $\mathrm{HPT}$ & 1 & 3.8 & & 217.072 & 1.3 & 0.8558 & 3975.1 & & \\
\hline LPT & 51.02 & 2.120 & 0.8262 & 58.277 & 1.1784 & 0.8106 & 1760.4 & & \\
\hline
\end{tabular}

TURBOMACHINERY MAP DATA

$\begin{array}{lrrrrrrrrr} & \text { WcMap } & \text { PRmap } & \text { effMap } & \text { NcMap } & \text { R/Parm } & \text { S_WcDes } & \text { S_PRdes } & \text { s_effDes } & \text { S_NcDes } \\ \text { Fan } & 723.79 & 1.081 & 0.9048 & 2022.532 & 0.0425 & 0.8607 & 1.0235 & 0.9980 & 0.9905 \\ \text { LPC } & 53.38 & 1.310 & 0.7607 & 0.539 & 0.0000 & 1.6160 & 0.8609 & 1.0940 & 0.0003 \\ \text { HPC } & 45.94 & 5.488 & 0.8202 & 8608.145 & 10.9695 & 1.0995 & 1.0494 & 0.9898 & 0.9733 \\ \text { HPT } & 0.96 & 3.784 & 0.8766 & 1.305 & 3.7843 & 12.6299 & 0.9723 & 1.0000 & 0.0003 \\ \text { LPT } & 0.78 & 1.842 & 0.8323 & 0.656 & 1.8424 & 65.2590 & 0.7521 & 0.9926 & 0.0005\end{array}$

$\begin{array}{clccc}\text { EPR } & \text { PowerSet } & \text { T4 } & \text { T41 } & \text { T49 } \\ 0.857 & 10.000 & 1738.8 & 1642.0 & 1158.8\end{array}$

WAR
0.0003043
0.0003043
0.0003043
0.0003043
0.0003043
0.0003043
0.0081535
0.0081535
0.0081535
0.0081535
0.0081535
0.0081535
0.0081535
0.0081535
0.0081535
0.0003043
0.0006174
0.0006174
0.0006174

Wair 322.80
322.80 0.0003 (283.54 $0.098-0.0003$ $\begin{array}{lll}39.27 & 0.012 & 0.0003\end{array}$ $\begin{array}{lll}38.54 & 0.086 & 0.0003 \\ 39.27 & 0.012 & 0.0003\end{array}$ $\begin{array}{lll}39.27 & 0.012 & 0.0003 \\ 27.49 & 0.320 & 0.0081\end{array}$ $\begin{array}{lll}39.27 & 0.012 & 0.0003 \\ 27.49 & 0.320 & 0.0081\end{array}$ $\begin{array}{lll}27.45 & 0.224 & 0.0081 \\ 26.15 & 0.213 & 0.0081\end{array}$ $\begin{array}{lll}26.15 & 0.213 & 0.0081 \\ 20.27 & 0.165 & 0.0081\end{array}$ $\begin{array}{lll}20.27 & 0.165 & 0.0221\end{array}$ $\begin{array}{lll}27.16 & 0.221 & 0.0185\end{array}$ $\begin{array}{lll}27.49 & 0.224 & 0.0184\end{array}$ $\begin{array}{lll}27.49 & 0.224 & 0.0184\end{array}$ $\begin{array}{lll}27.49 & 0.224 & 0.0184\end{array}$ $\begin{array}{lll}283.54 & 0.086 & 0.0003\end{array}$ $\begin{array}{lll}295.32 & 0.182 & 0.0006 \\ 295.32 & 0.182 & 0.0006\end{array}$ $\begin{array}{lll}295.32 & 0.182 & 0.0006\end{array}$ 


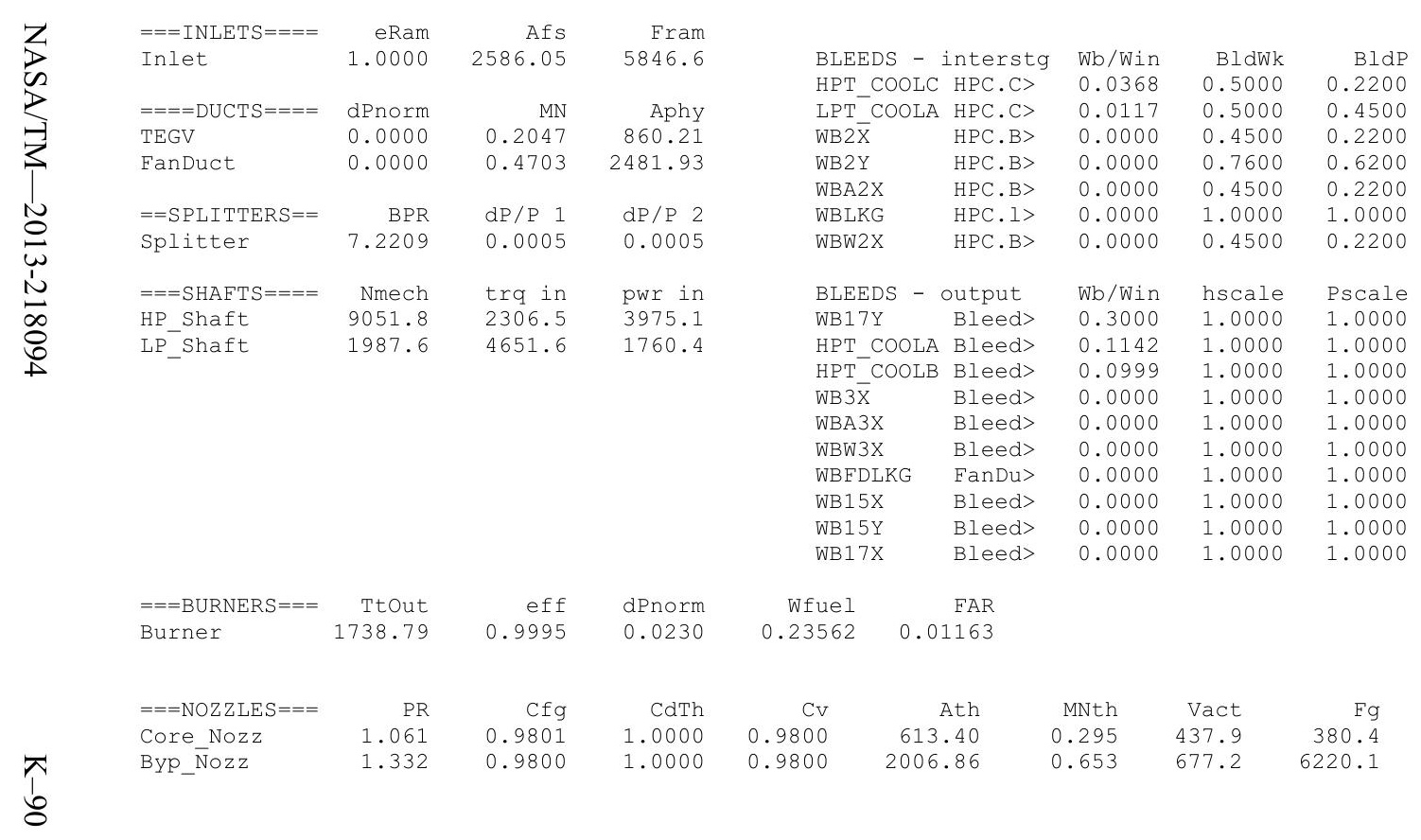


Date:07/05/13 Time:11:42:59 Model: Turbofan Engine - COMDES ON Converge = 1 CASE: 0

Version:NPSS 1.6.5 - Rev: -> Gas Package: Janaf iter/pass/Jacb/Broy= 67/ 95/2/64 Run by: Philip C Jorgenson PC: 10

Temperature Stator 1 inlet: $505.52 \quad$ Stator 1 exit: $508.54 \quad$ Stator 2 inlet: $511.46 \quad$ Stator 2 exit: 512.30 $\begin{array}{lll}\text { Stator } 3 \text { inlet: } 515.80 & \text { Stator } 3 \text { exit: } 516.28 & \text { Stator } 4 \text { inlet: } 519.69 \text { Stator } 4 \text { exit: } 519.86 \\ \text { Stator } 5 \text { inlet: } 521.92 & \text { Stator } 5 \text { exit: } 521.88 & \text { Unblocked Percent Blockage: } 0.00\end{array}$

Ambient Relative Humidity $\quad 10.00$

Fan Face Relative Humidity $\quad 6.02$

Fan Bypass Relative Humidity $\quad 5.15$

$\begin{array}{ll}\text { LPC Inlet Relative Humidity } & 5.19 \\ \text { LPC Exit Relative Humidity } & 2.72\end{array}$

HPC Relative Humidi

0.1

Inlet Length

Fan/LPC Inlet Flow Velocity $\quad 201.18$

Ambient Flow Velocity

Ambient Static Pressure

Additional Water at LPC Exit

$\begin{array}{lrr}483.18 & \text { Fan/LPC Inlet Static Pressure r } & 7.76 \\ & \text { Fan/LPC Inlet Static Temperature } & 503.04\end{array}$

\section{MN alt dTamb W SUMMARY OUTPUT DATA}

$\begin{array}{rrrrrr}\text { MN } & \text { alt } & \text { dTamb } & \text { W } & \text { Fn } & \text { TSFC } \\ 0.490 & 20047.0 & 36.00 & 358.54 & 816.5 & 1.1432\end{array}$

933.41

BPR
6.4284

VTAS
528.20

OPR
6.305

EPR
0.888

PowerSet
10.000

T4
1765.8

T41

T49

FLOW STATION DATA

FSO Ambient.Fl_O

FS1 Inlet.F1_O

FS12 Splitter.F1_02

FS2 Splitter.F1_01

FS14 Fan.F1_

FS24 VaporIN.FI_O

FS25 Bleed2.F1_O

FS3 HPC.Fl O

FS36 Bleed3.F1_O

FS4 Burner.Fl_O

$\begin{array}{ll}\text { FS4 } & \text { Burner.FI } \\ \text { FS45 HPT.FIO }\end{array}$

FS49 LPT.FI-O

FS5 TEGV.FI_O

FS8 Core_Nozz.FI_O

FS17 FanDüctLkg.Fì O

FS171 Bleed15.F1_O

FS172 FanDuct. Fl_O

$\begin{array}{crrr}\text { FLOW } & \text { STATION DATA } & & \\ \text { Pt } & \text { Tt } & \text { ht } & \text { FAR } \\ 7.943 & 506.41 & -12.46 & 0.0000 \\ 7.943 & 506.41 & -12.46 & 0.0000\end{array}$

358.54

$\begin{array}{lllll}7.943 & 506.41 & -12.46 & 0.0000 & 655.46\end{array}$

$\begin{array}{lllll}7.939 & 506.41 & -12.46 & 0.0000 & 567.51 \\ 7.939 & 506.41 & -12.46 & 0.0000 & 88.28\end{array}$

$\begin{array}{rrrrr}7.939 & 506.41 & -12.46 & 0.0000 & 58.51 \\ 8.498 & 517.40 & -9.82 & 0.0000 & 535.92\end{array}$

$\begin{array}{lllll}8.498 & 517.40 & -9.82 & 0.0000 & 535.92 \\ 9.140 & 535.38 & -5.51 & 0.0000 & 78.85\end{array}$

$\begin{array}{llll}9.140 & 535.38 & -5.51 & 0.0000\end{array}$

$\begin{array}{llll}9.140 & 547.12 & -52.71 & 0.0000\end{array}$

78.85
80.40

80.40
48.24

$\begin{array}{rrrrrr}29.21 & 9.140 & 547.12 & -52.71 & 0.0000 & 48.24 \\ 27.80 & 50.079 & 963.81 & 49.39 & 0.0000 & 11.12\end{array}$

$\begin{array}{llll}50.079 & 963.81 & 49.39 & 0.0000\end{array}$

$\begin{array}{llllll}21.80 & 48.925 & 1765.81 & 36.30 & 0.0121\end{array}$

$\begin{array}{llllll}29.47 & 7.037 & 1035.14 & -100.64 & 0.0090\end{array}$

12.08
48.59
86.95

$\begin{array}{lllll}7.037 & 1035.14 & -100.64 & 0.0090 & 86.95 \\ 7.037 & 1035.21 & -100.64 & 0.0090 & 86.96\end{array}$

$\begin{array}{rrrrrr}29.47 & 7.037 & 1035.28 & -100.64 & 0.0090 & 86.96 \\ 310.27 & 8.498 & 517.40 & -9.82 & 0.0000 & 535.92\end{array}$

310.2

329.75
329.75
329.75

$\begin{array}{llrrr}8.498 & 517.40 & -9.82 & 0.0000 & 535.92 \\ 8.498 & 519.16 & -12.36 & 0.0000 & 570.53\end{array}$

$\begin{array}{lllll}8.498 & 519.16 & -12.36 & 0.0000 & 570.53 \\ 8.498 & 519.16 & -12.36 & 0.0000 & 570.53\end{array}$

$\begin{array}{lllll}8.498 & 519.16 & -12.36 & 0.0000 & 570.53\end{array}$

Ps
6.740
7.529
7.505
7.756
7.703
8.414
8.377
8.882
44.343
46.776
46.183
12.381
6.890
6.890
6.740
7.703
7.470
7.470
6.740

TS
483.18
498.71
498.34
503.04
503.07
522.86
533.69
542.68
931.92
945.80
1740.82
1168.15
1029.23
1029.30
1023.27
503.07
500.38
500.38
485.89

$\begin{array}{rrr}\text { Aphy } & \text { MN } & \text { gamt } \\ 2597.0 & 0.4900 & 1.40031 \\ 4168.2 & 0.2777 & 1.40031 \\ 3531.8 & 0.2844 & 1.40031 \\ 830.5 & 0.1829 & 1.40031 \\ 2606.7 & 0.3772 & 1.40017 \\ 412.6 & 0.3460 & 1.39992 \\ 412.6 & 0.3553 & 1.39854 \\ 412.6 & 0.2027 & 1.39854 \\ 49.7 & 0.4229 & 1.38147 \\ 49.3 & 0.3157 & 1.38147 \\ 74.6 & 0.2958 & 1.32715 \\ 265.4 & 0.3351 & 1.36090 \\ 860.2 & 0.1759 & 1.37109 \\ 860.2 & 0.1759 & 1.37108 \\ 613.4 & 0.2514 & 1.37108 \\ 2606.7 & 0.3772 & 1.40017 \\ 2481.9 & 0.4331 & 1.40008 \\ 2481.9 & 0.4331 & 1.40008 \\ 2006.9 & 0.5849 & 1.40008\end{array}$

$$
\begin{aligned}
& 0 . \\
& 0 . \\
& 0 . \\
& 0 . \\
& 0 . \\
& 0 . \\
& 0 . \\
& 0 . \\
& 0 . \\
& 0 . \\
& 0 . \\
& 0 . \\
& 0 . \\
& 0 . \\
& 0 . \\
& 0 . \\
& 0 . \\
& 0.00 \\
& 0.00
\end{aligned}
$$

$\begin{array}{rrrr}\text { WAR } & \text { Wair } & \text { WH2O } & \text { H2O frac } \\ 0.0005787 & 358.33 & 0.207 & 0.0006\end{array}$ $\begin{array}{llll}0.0005787 & 310.09 & 0.179 & 0.0006\end{array}$ $\begin{array}{rrrr}0.0005787 & 48.24 & 0.028 & 0.0006\end{array}$ $\begin{array}{rrrr}0.0005787 & 310.09 & 0.179 & 0.0006\end{array}$ $\begin{array}{llll}0.0005787 & 48.24 & 0.028 & 0.0006 \\ 0.0093388 & 48.24 & 0.450 & 0.0093\end{array}$ $\begin{array}{llll}0.0093388 & 28.94 & 0.270 & 0.0093\end{array}$ $\begin{array}{llll}0.0093388 & 27.54 & 0.257 & 0.0093\end{array}$ $\begin{array}{llll}0.0093388 & 21.34 & 0.2199 & 0.0093\end{array}$ $\begin{array}{llll}0.0093388 & 21.34 & 0.199 & 0.0238\end{array}$ $\begin{array}{llll}0.0093388 & 21.34 & 0.199 & 0.0238 \\ 0.0093388 & 28.60 & 0.267 & 0.0202 \\ 0.009388 & 28.94 & 0.270 & 0.0200\end{array}$ $\begin{array}{llll}0.0093388 & 28.94 & 0.270 & 0.0200\end{array}$ $\begin{array}{llll}0.0093388 & 28.94 & 0.270 & 0.0200\end{array}$ $\begin{array}{llll}0.0093388 & 28.94 & 0.270 & 0.0200 \\ 0.0005787 & 310.09 & 0.179 & 0.0006\end{array}$ $\begin{array}{llll}0.0010918 & 329.39 & 0.360 & 0.0011\end{array}$ $\begin{array}{lllll}0.0010918 & 329.39 & 0.360 & 0.0011\end{array}$ TURBOMACHINERY PERFORMANCE DATA

\begin{tabular}{|c|c|c|c|c|c|c|c|c|c|}
\hline & Wc & $\mathrm{PR}$ & eff & $\mathrm{NC}$ & $\mathrm{TR}$ & efPoly & pwr & SMN & SMW \\
\hline Fan & 567.51 & 1.070 & 0.9047 & 1834.139 & 1.0217 & 0.9056 & $-1157.2-$ & -1860.24 & 46.48 \\
\hline LPC & 88.28 & 1.151 & 0.7179 & 1834.139 & 1.0572 & 0.7235 & -474.8 & 7.04 & 2.12 \\
\hline HPC & 48.24 & 5.479 & 0.8075 & 8767.345 & 1.7616 & 0.8464 & -4118.0 & 62.59 & 58.56 \\
\hline HPT & 12.08 & 3.663 & 0.8782 & 214.285 & 1.3257 & 0.8586 & 4118.1 & & \\
\hline LPT & 48.59 & 1.898 & 0.8184 & 52.495 & 1.1481 & 0.8047 & 1632.0 & & \\
\hline TURE & NERY MAP & DATA & & & & & & & \\
\hline & WcMap & PRmap & effMap & NcMap & R/Parm & S_WcDes & S_PRdes & S_effDes & S_NcDes \\
\hline Fan & 659.38 & 1.069 & 0.9065 & 1816.650 & 0.0438 & $\overline{0} .8607$ & 1.0235 & 0.9980 & 0.9905 \\
\hline LPC & 46.59 & 1.243 & 0.7553 & 0.484 & 0.0000 & 1.8950 & 0.6217 & 0.9505 & 0.0003 \\
\hline $\mathrm{HPC}$ & 43.88 & 5.268 & 0.8159 & 8533.614 & 10.9802 & 1.0995 & 1.0494 & 0.9898 & 0.9733 \\
\hline HPT & 0.96 & 3.589 & 0.8782 & 1.288 & 3.5895 & 12.6299 & 0.9723 & 1.0000 & 0.0003 \\
\hline LPT & 0.74 & 1.675 & 0.8244 & 0.591 & 1.6754 & 65.2590 & 0.7521 & 0.9926 & 0.0005 \\
\hline
\end{tabular}




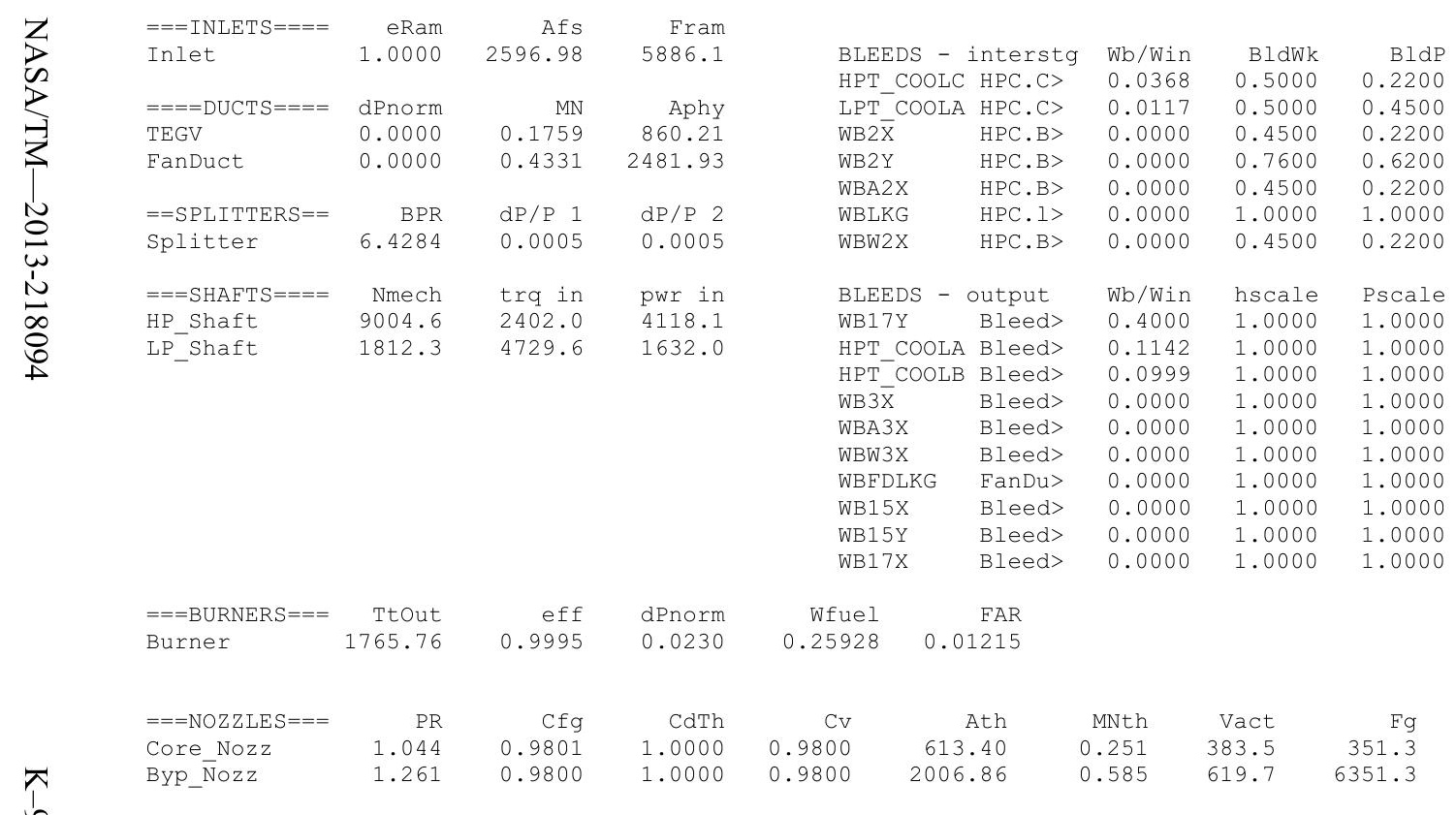




\section{Appendix L: Compressor code analysis for the descent conditions}

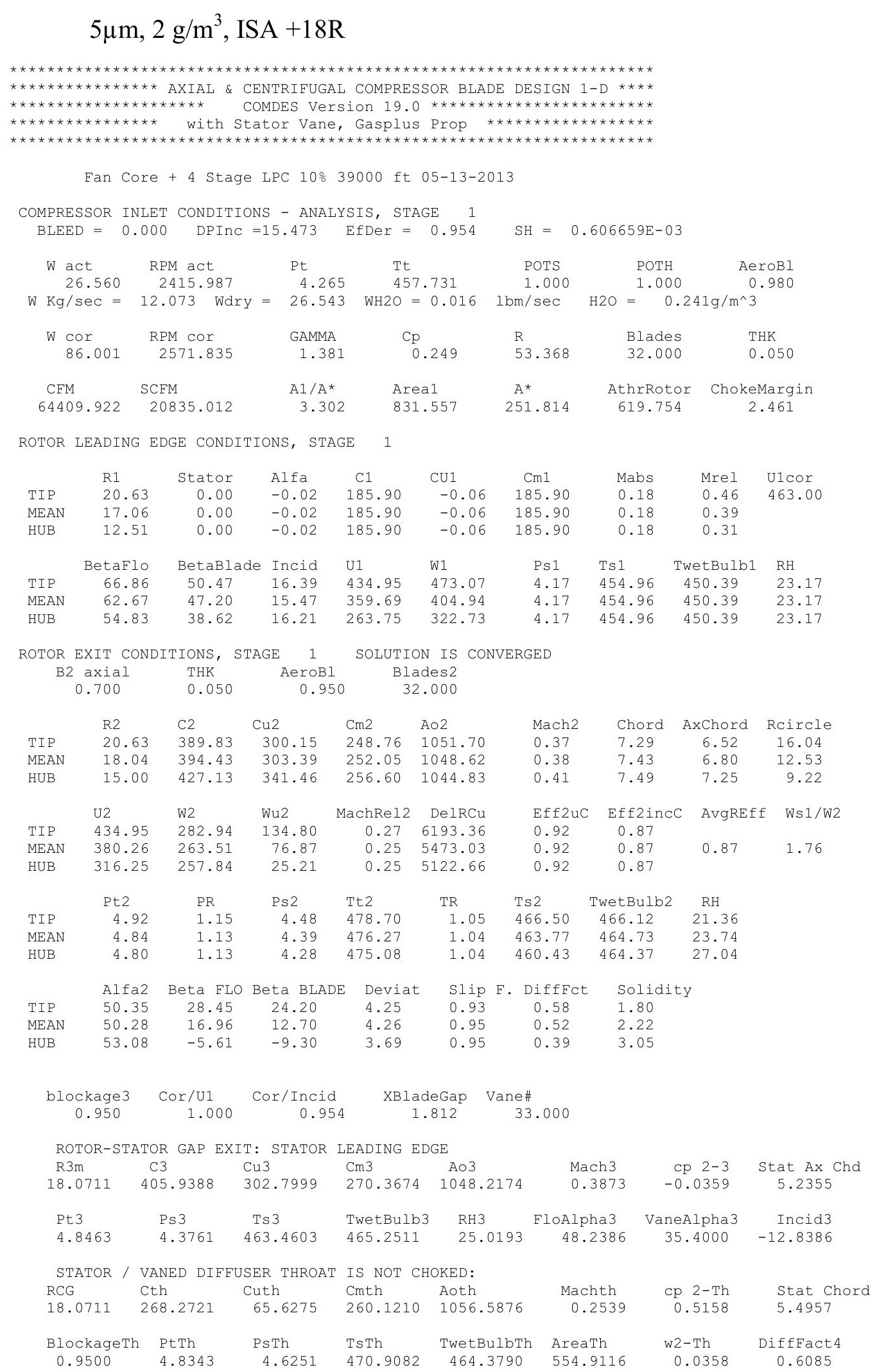




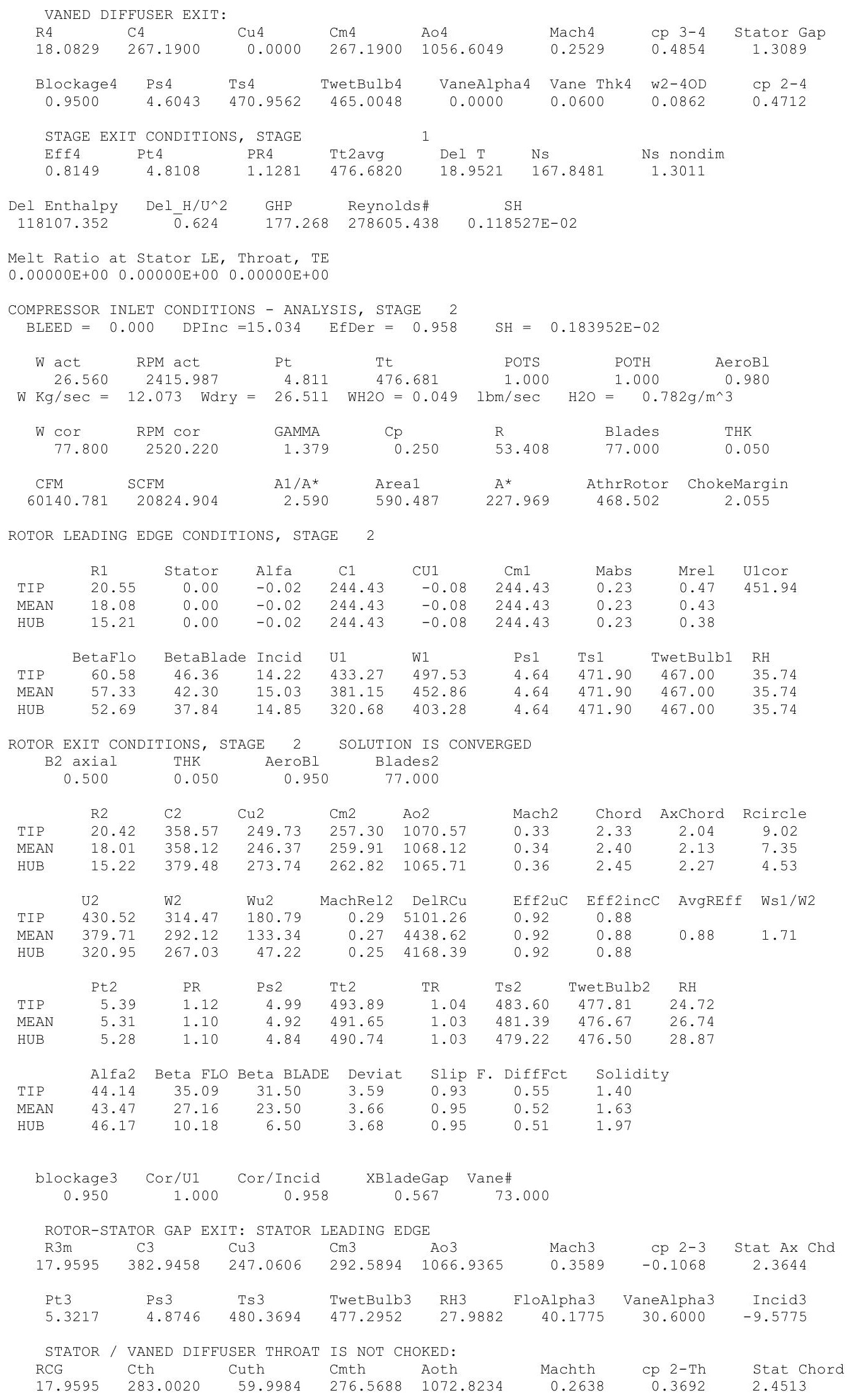




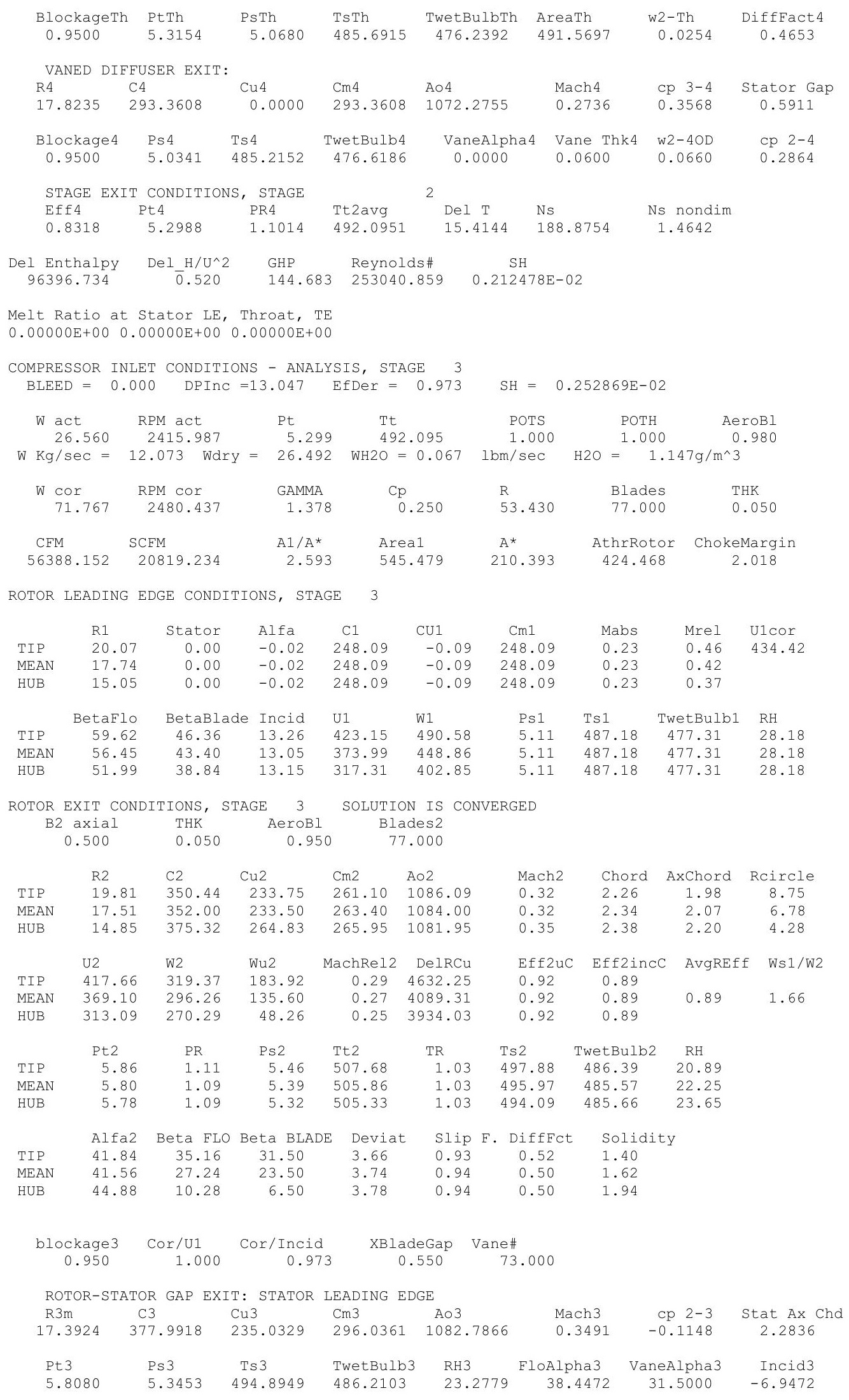




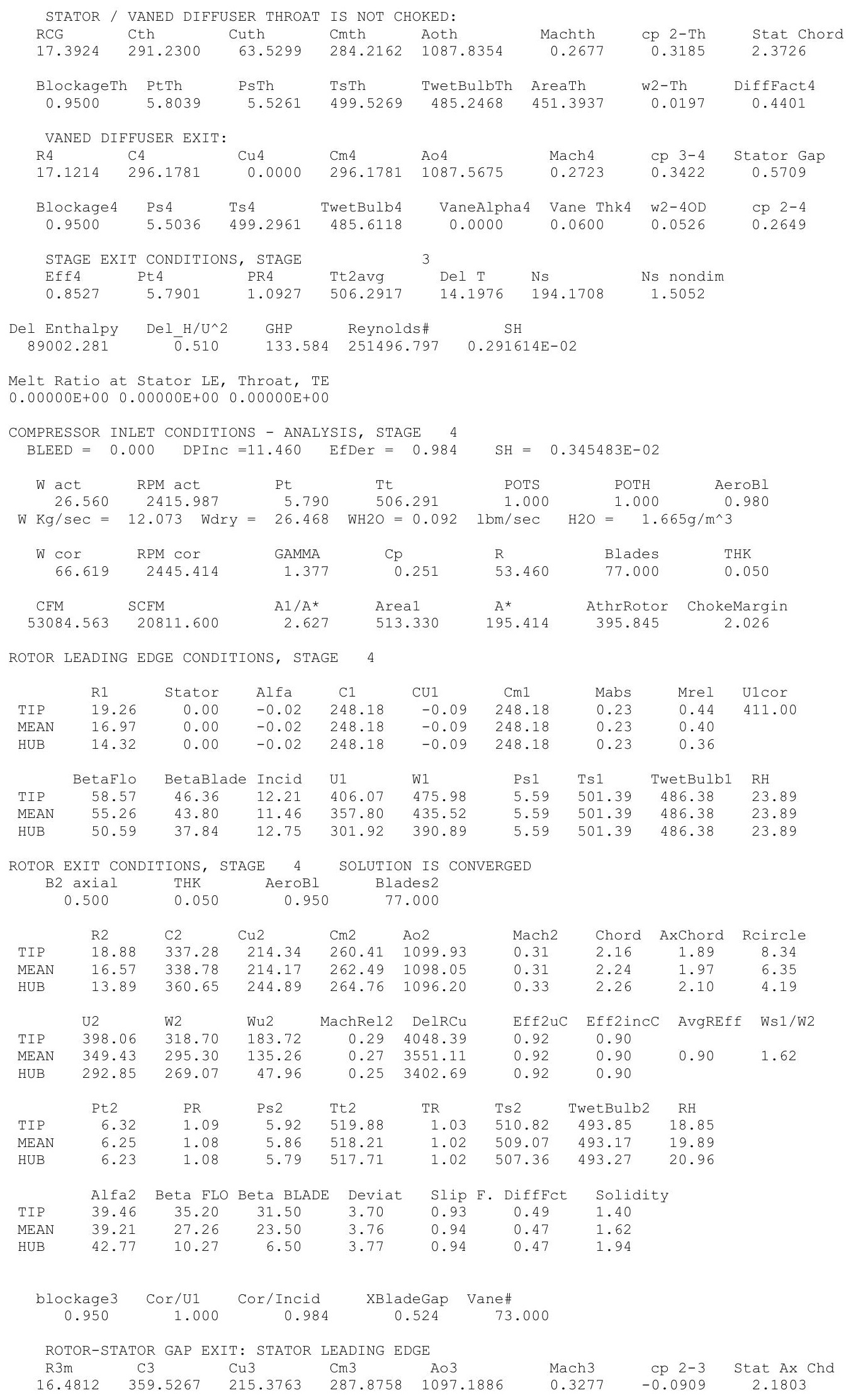




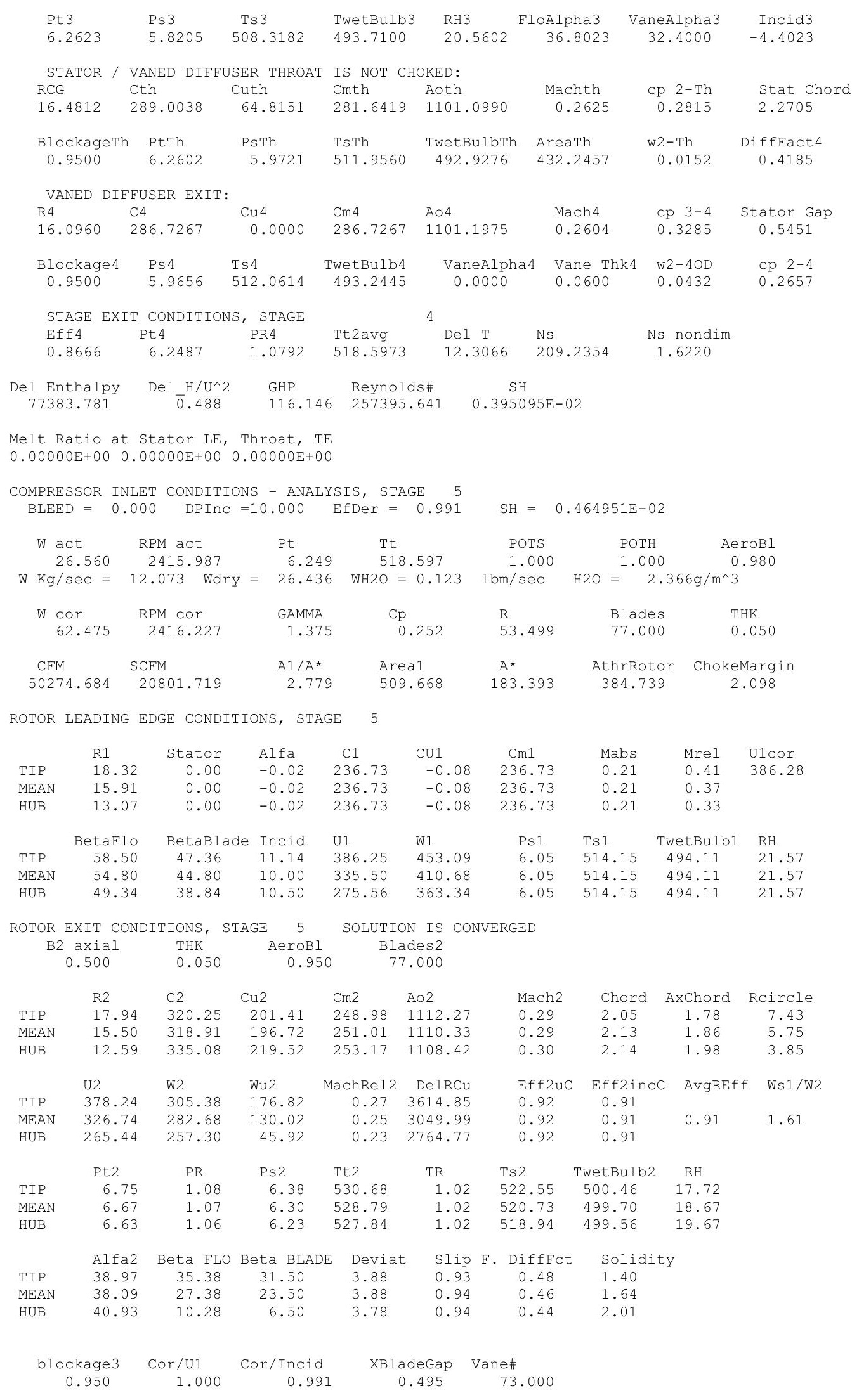




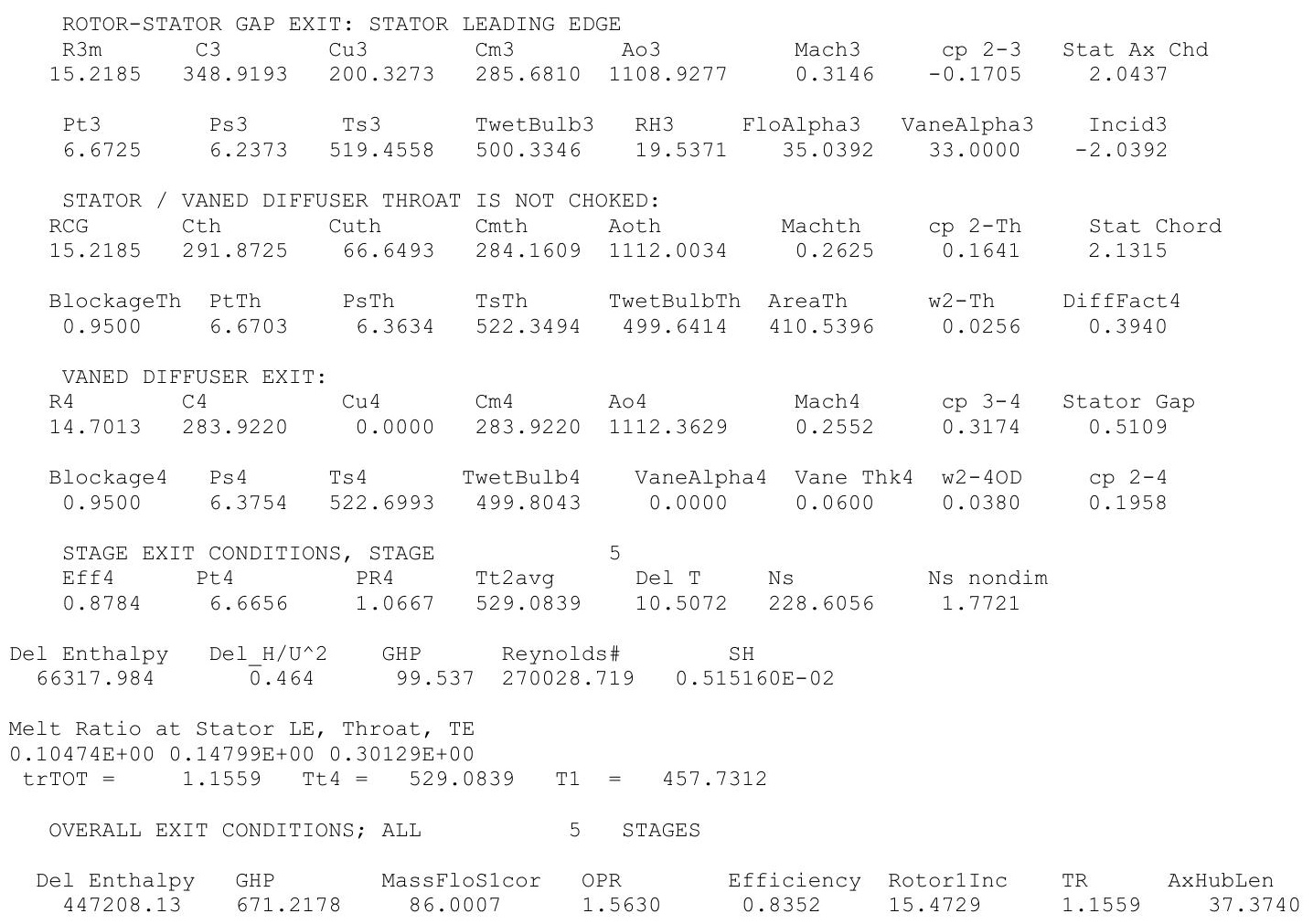




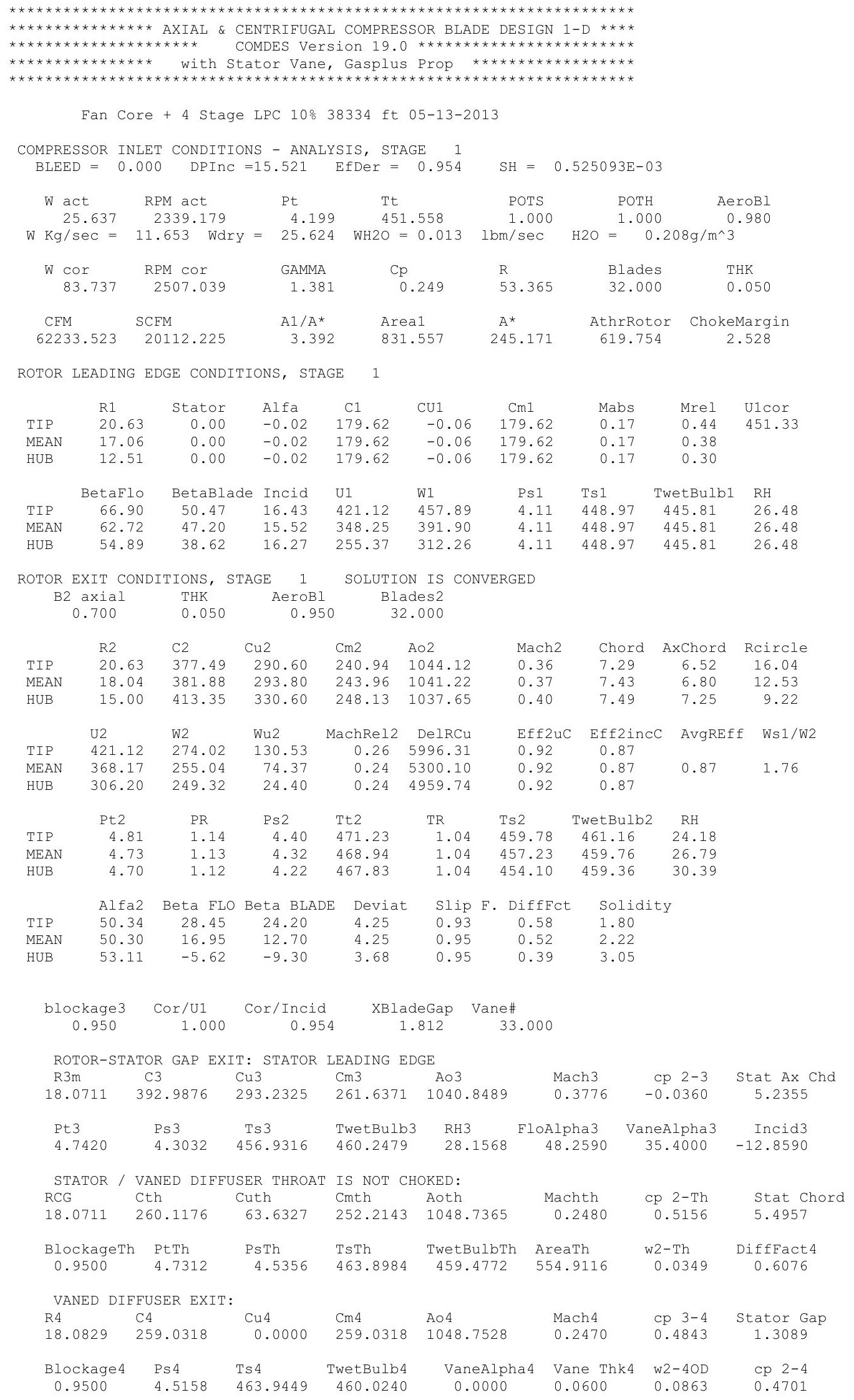




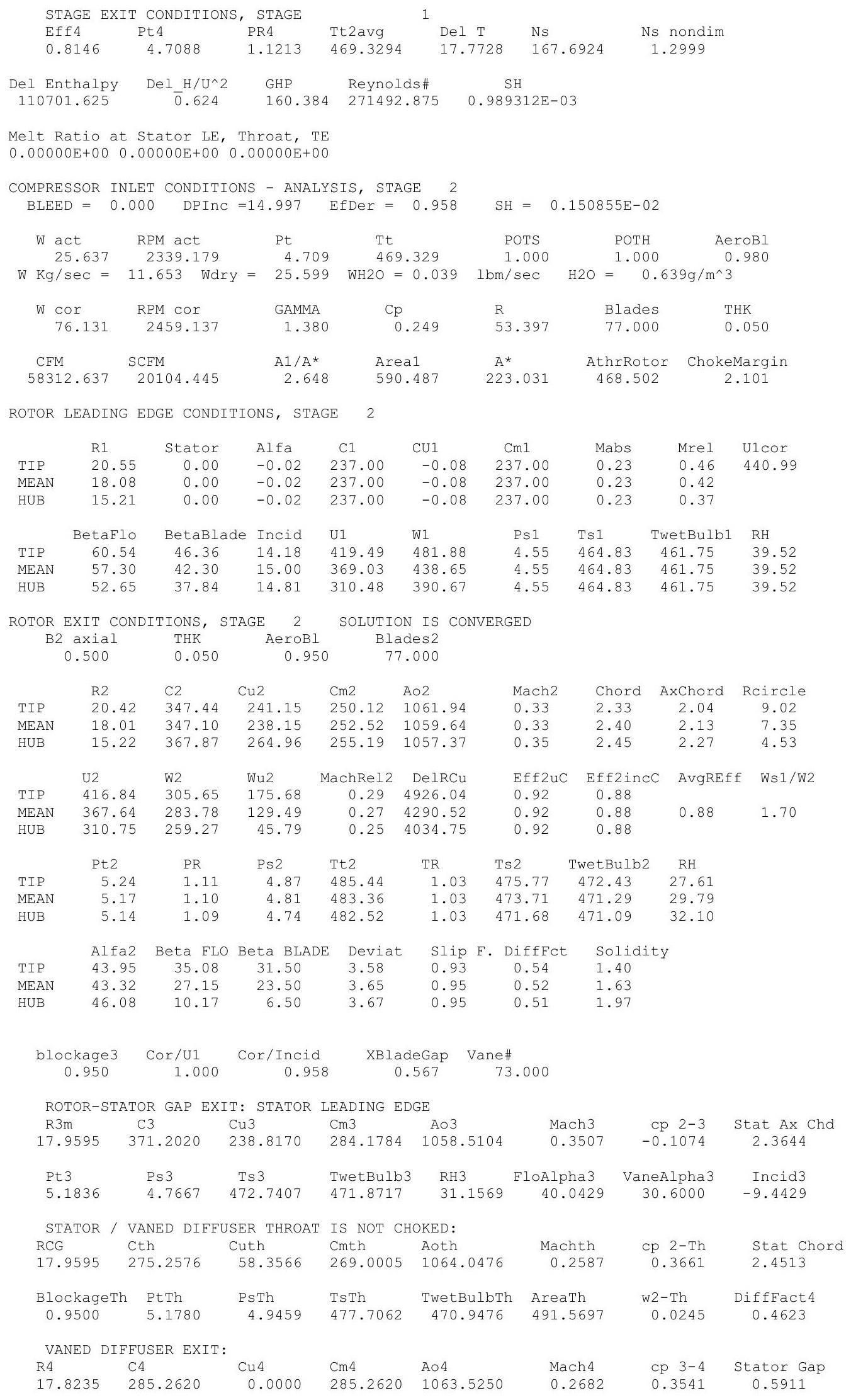




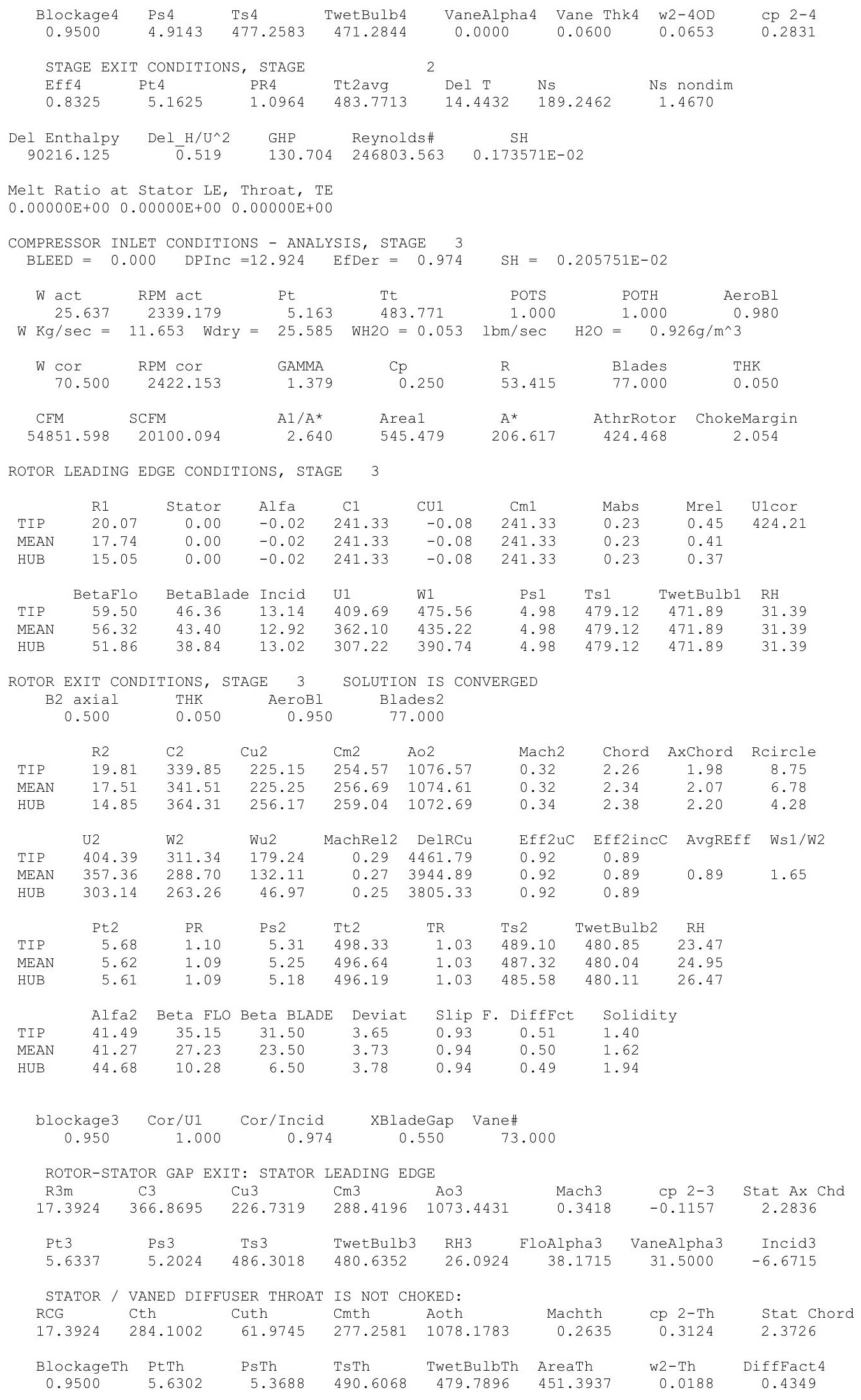




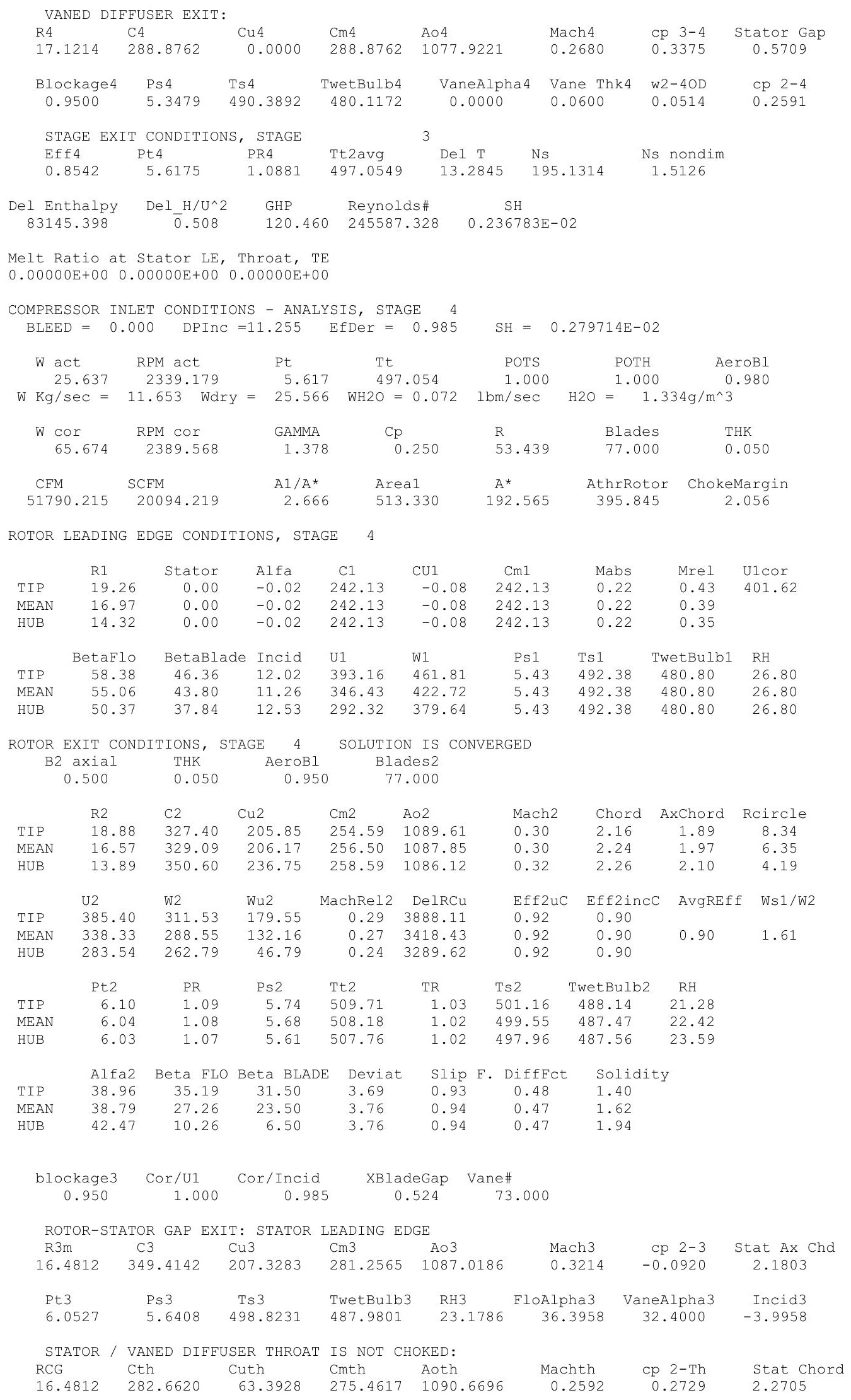




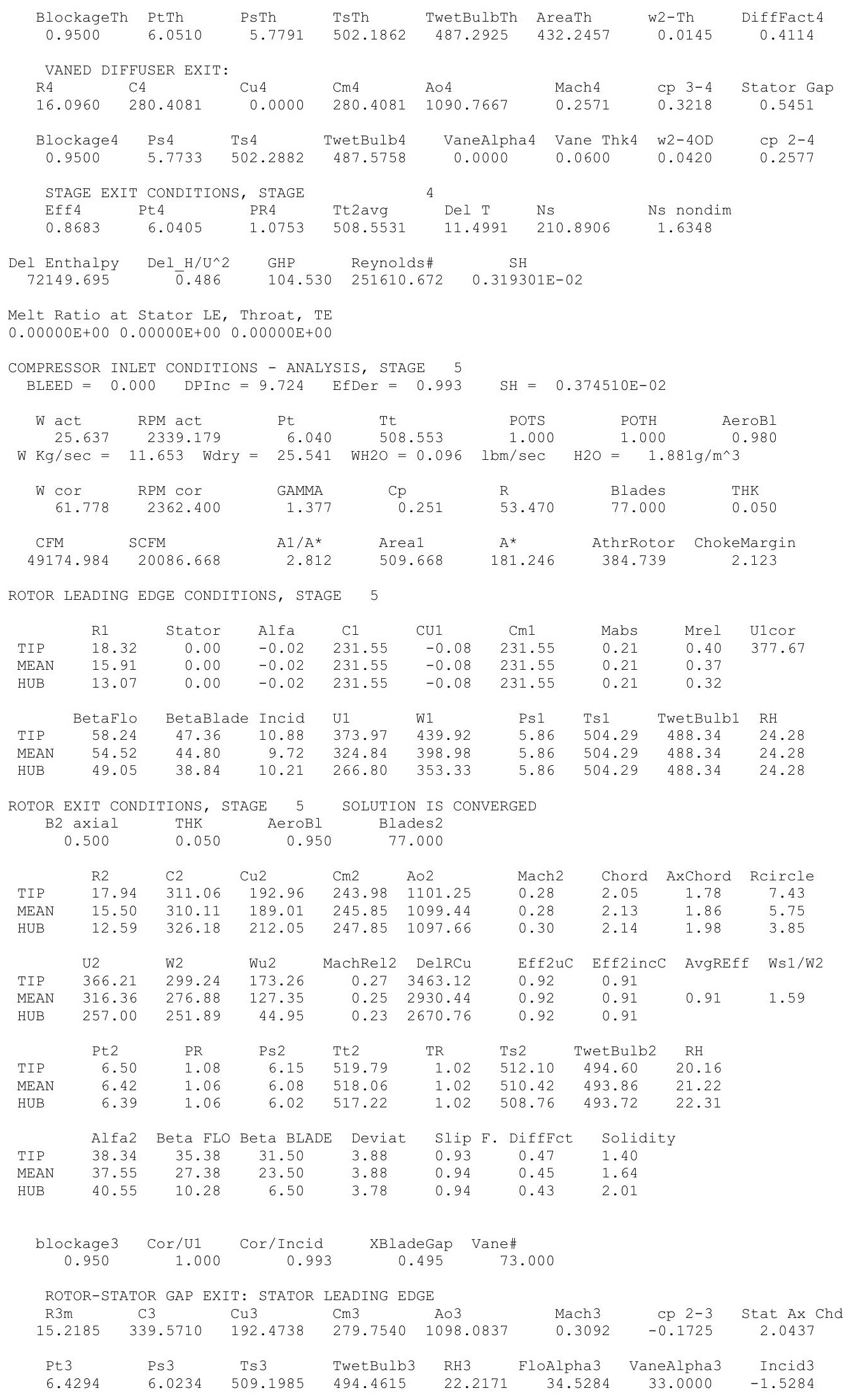




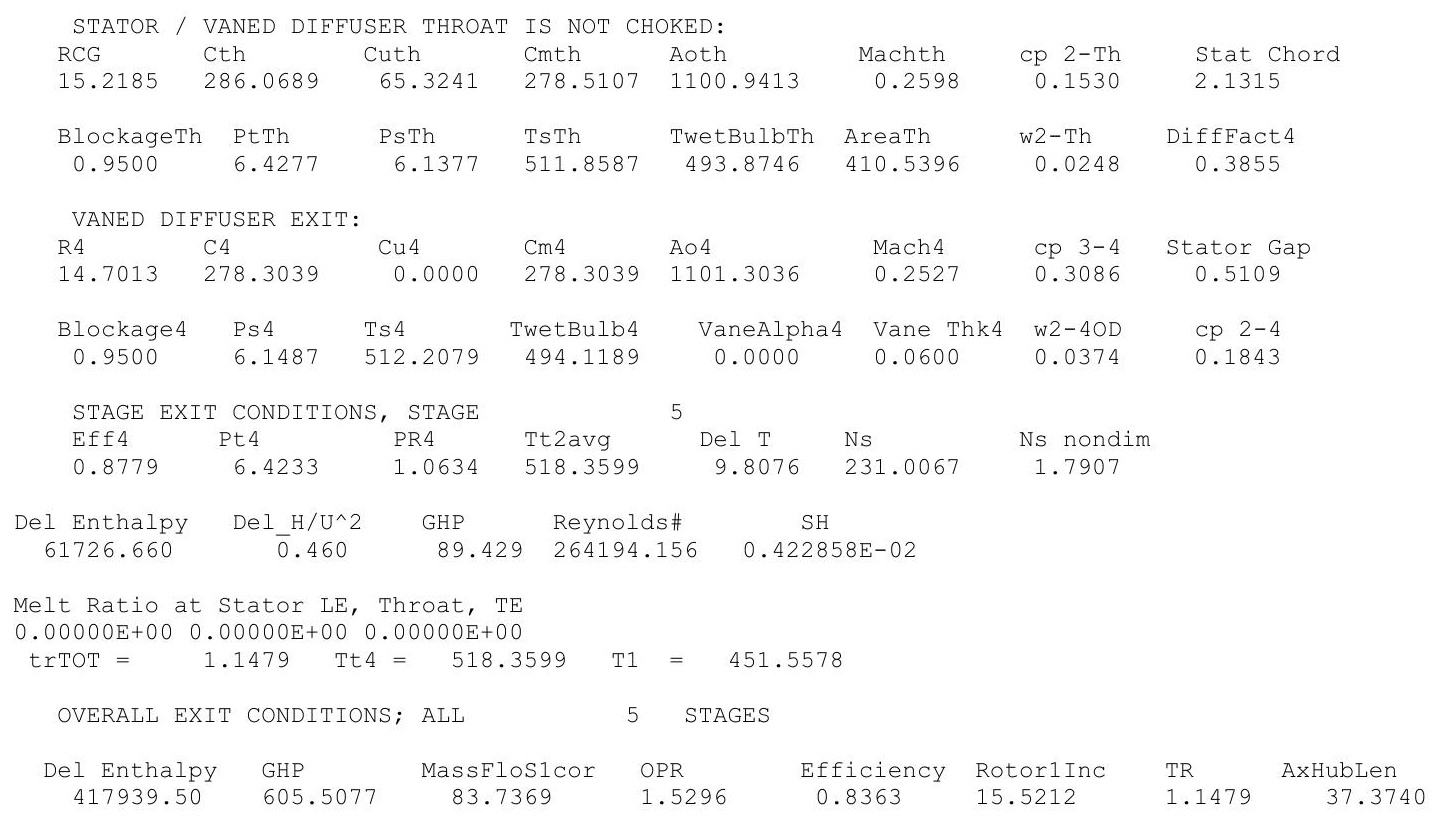




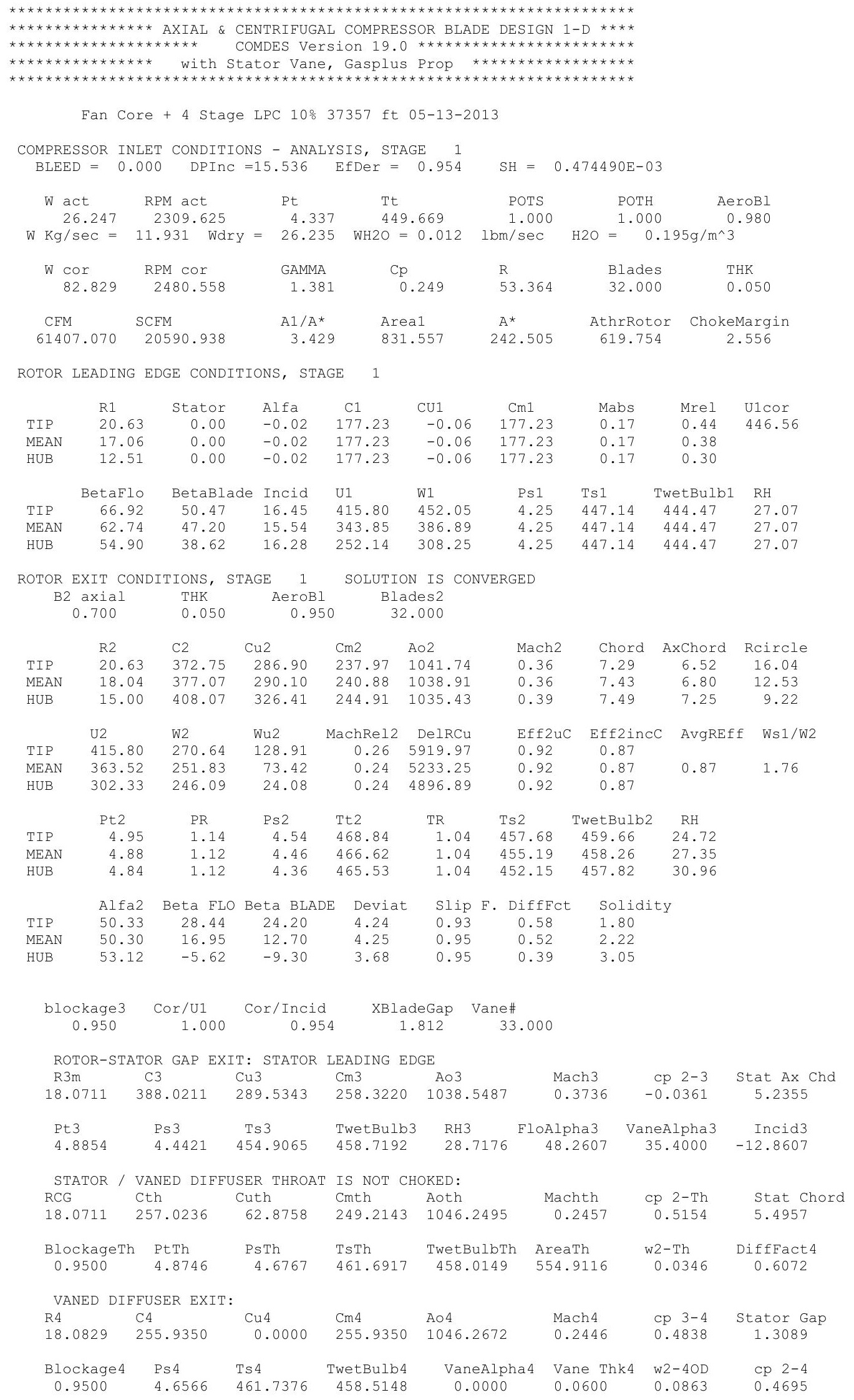




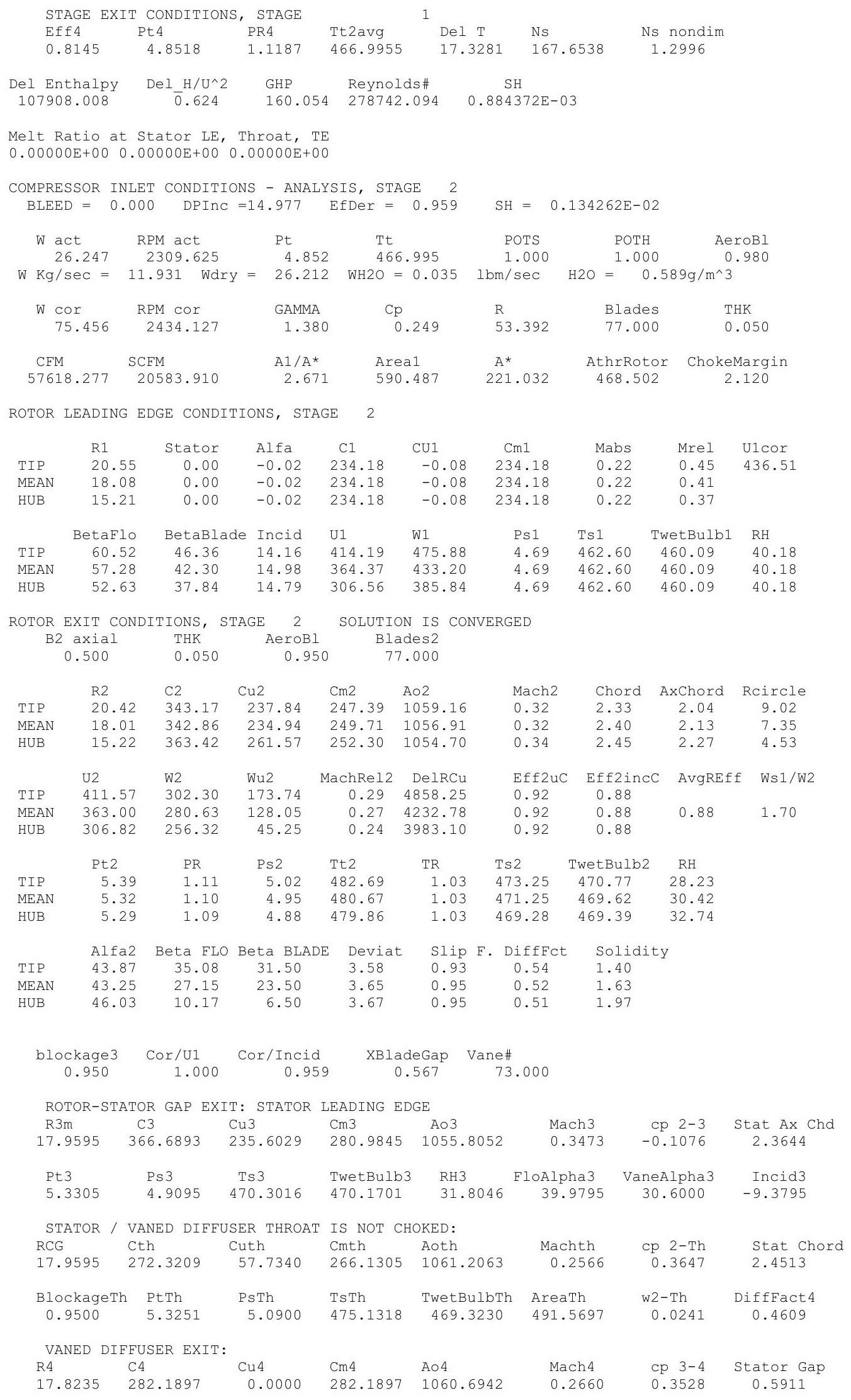




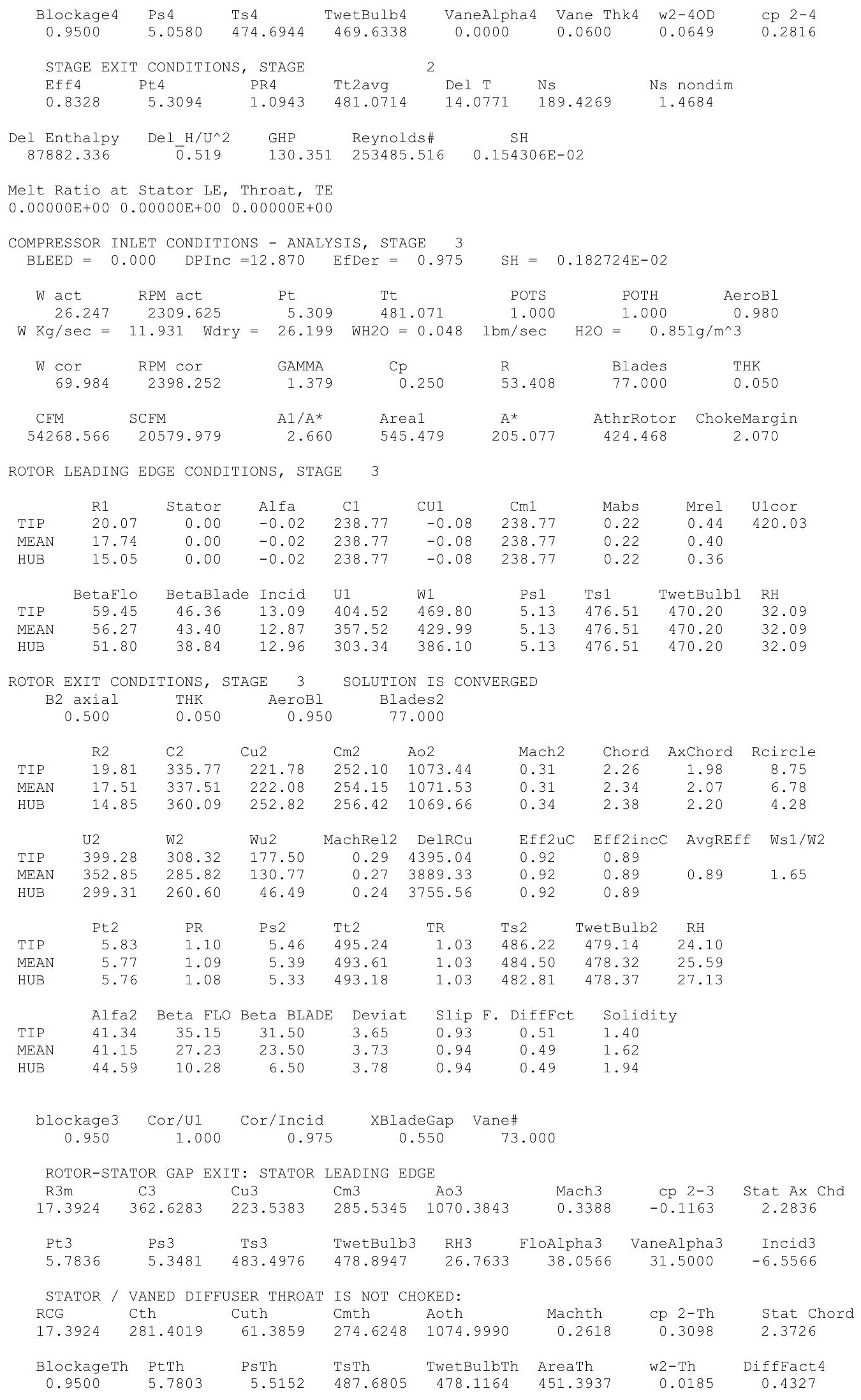




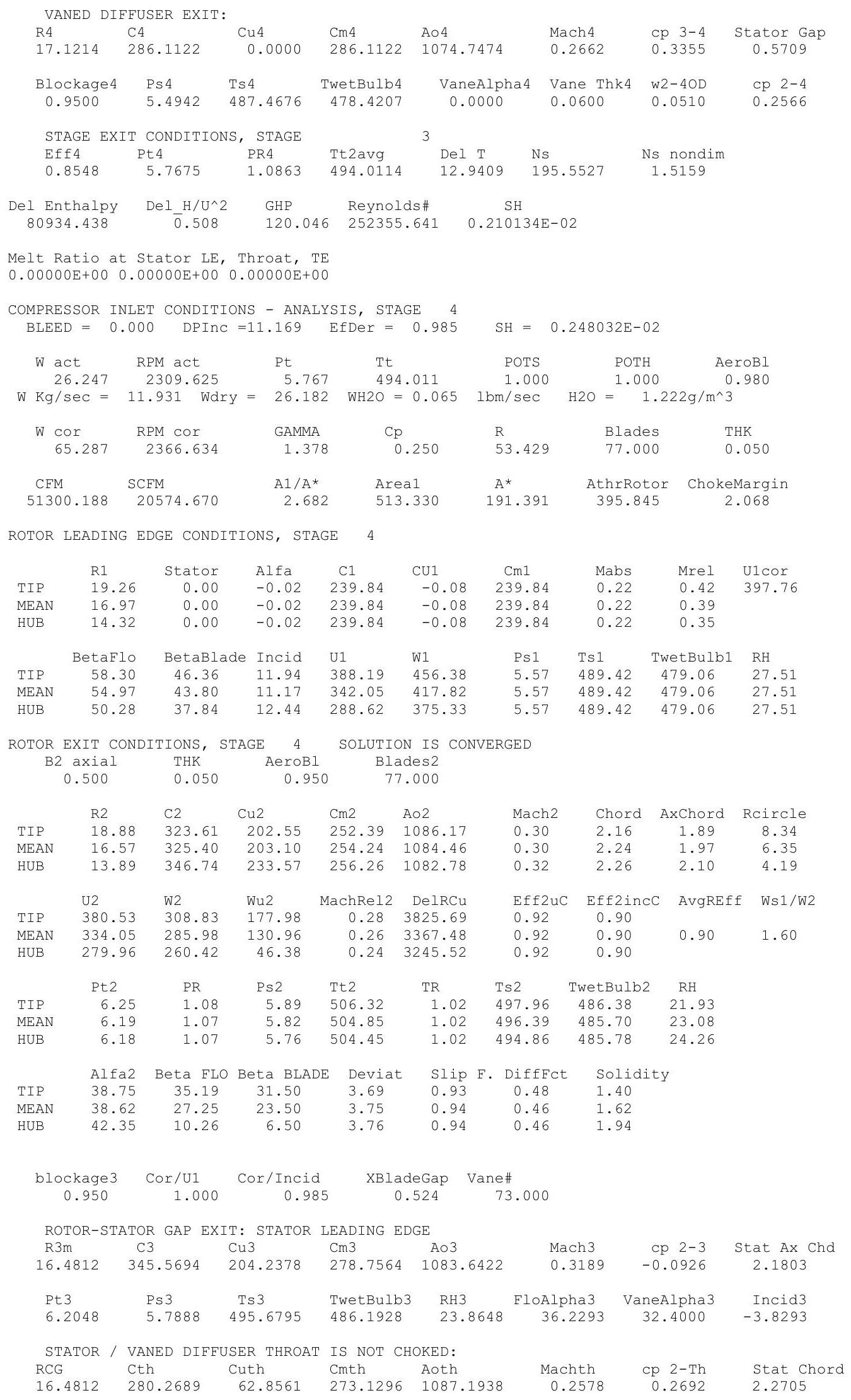




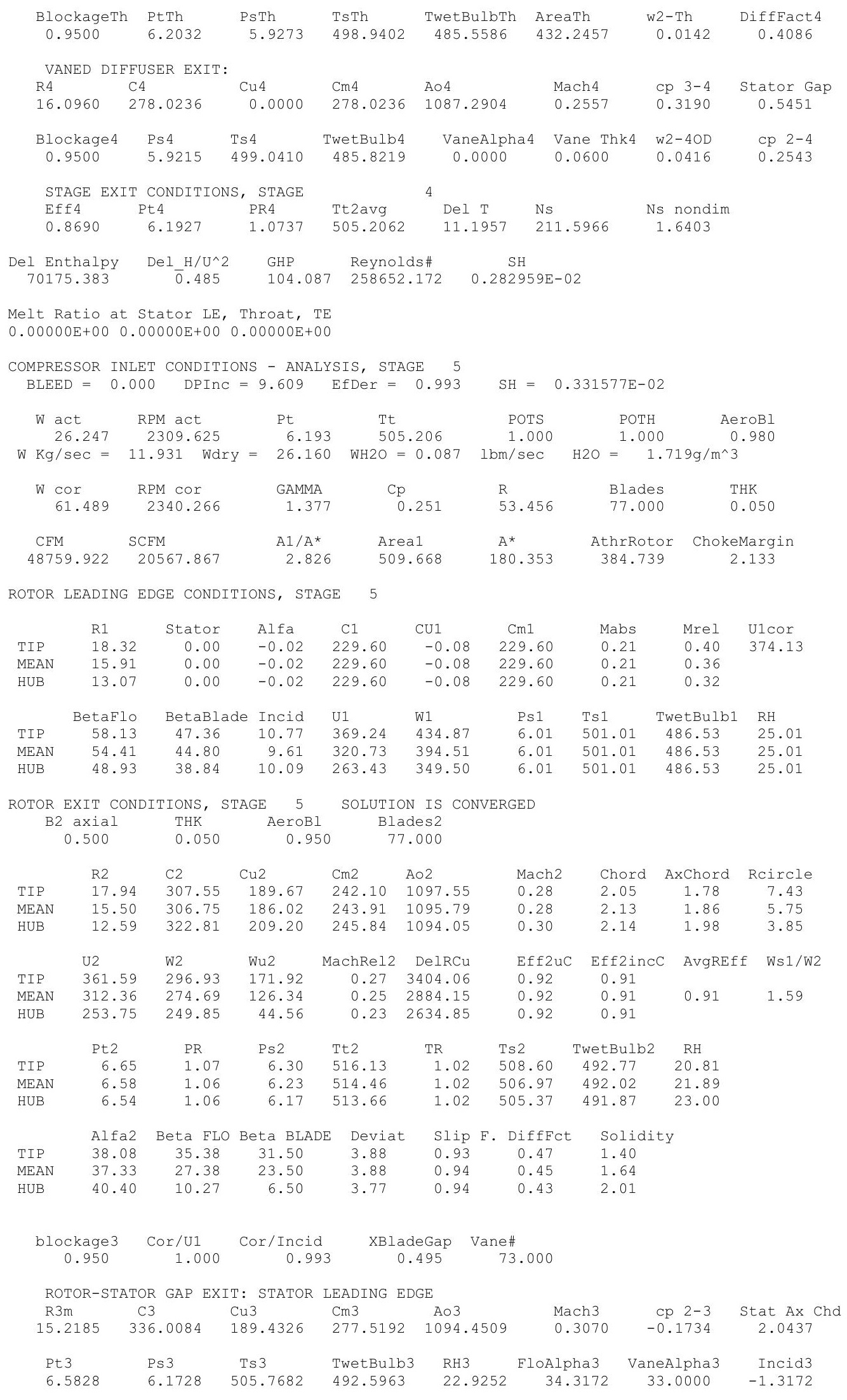




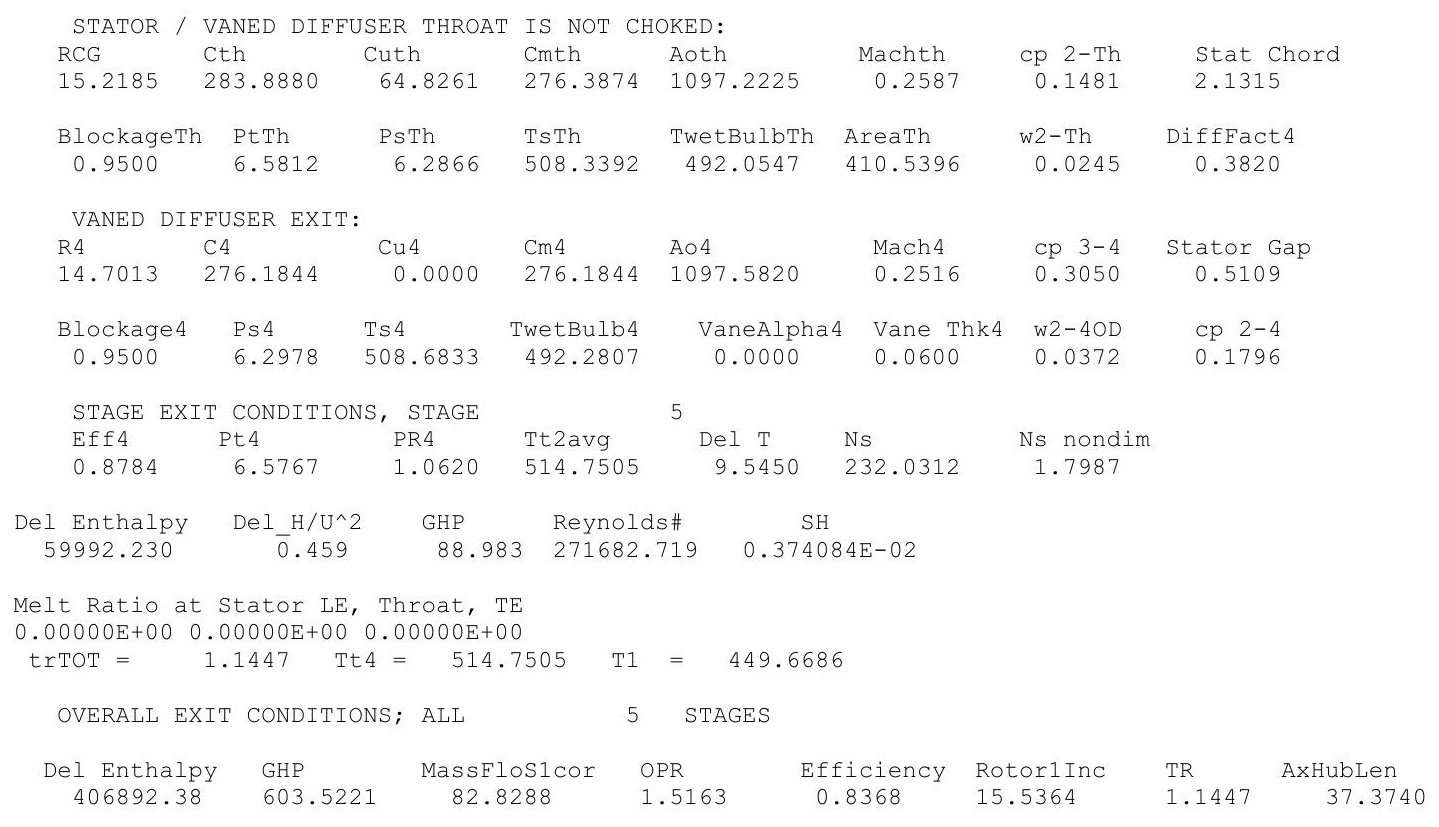




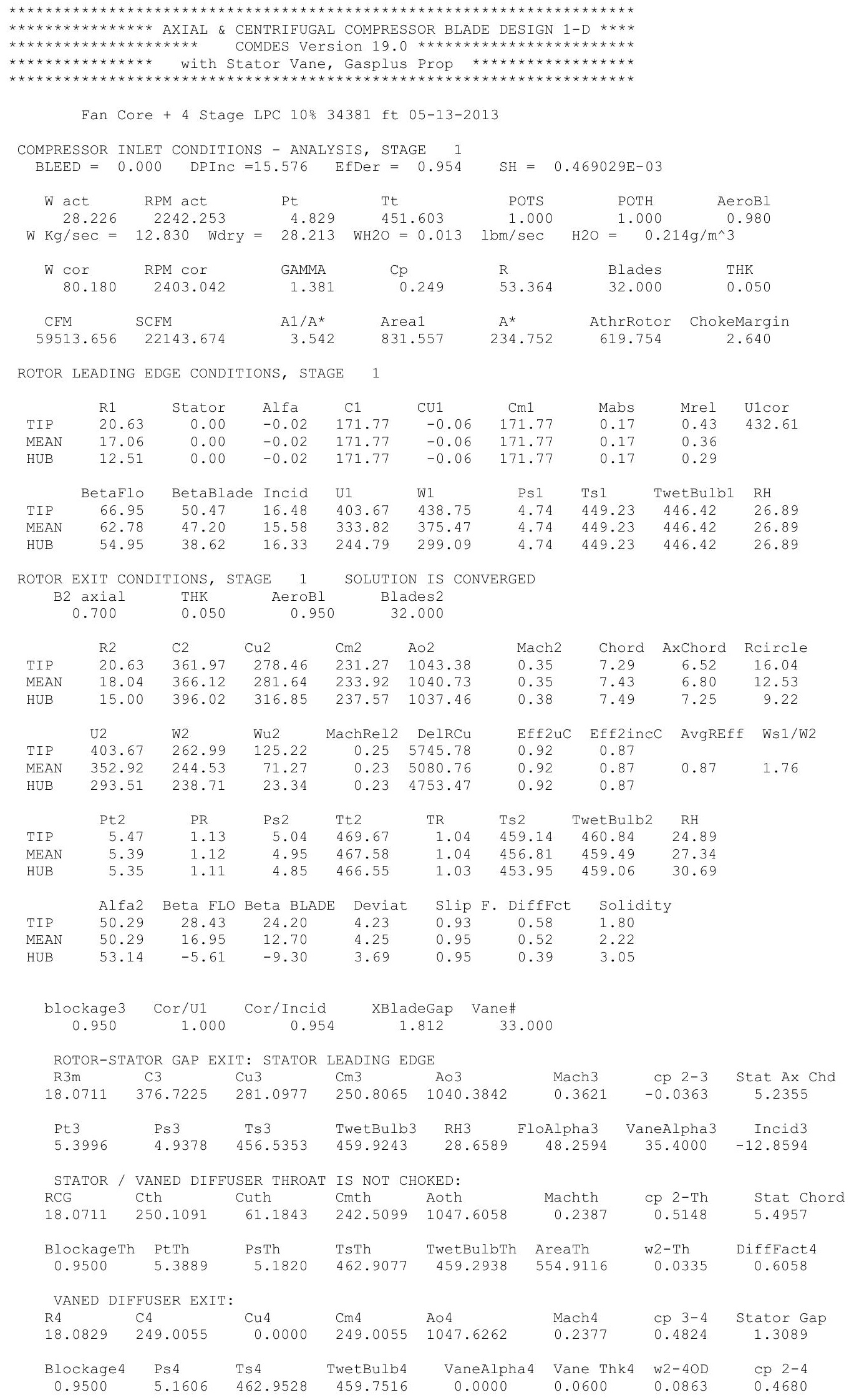




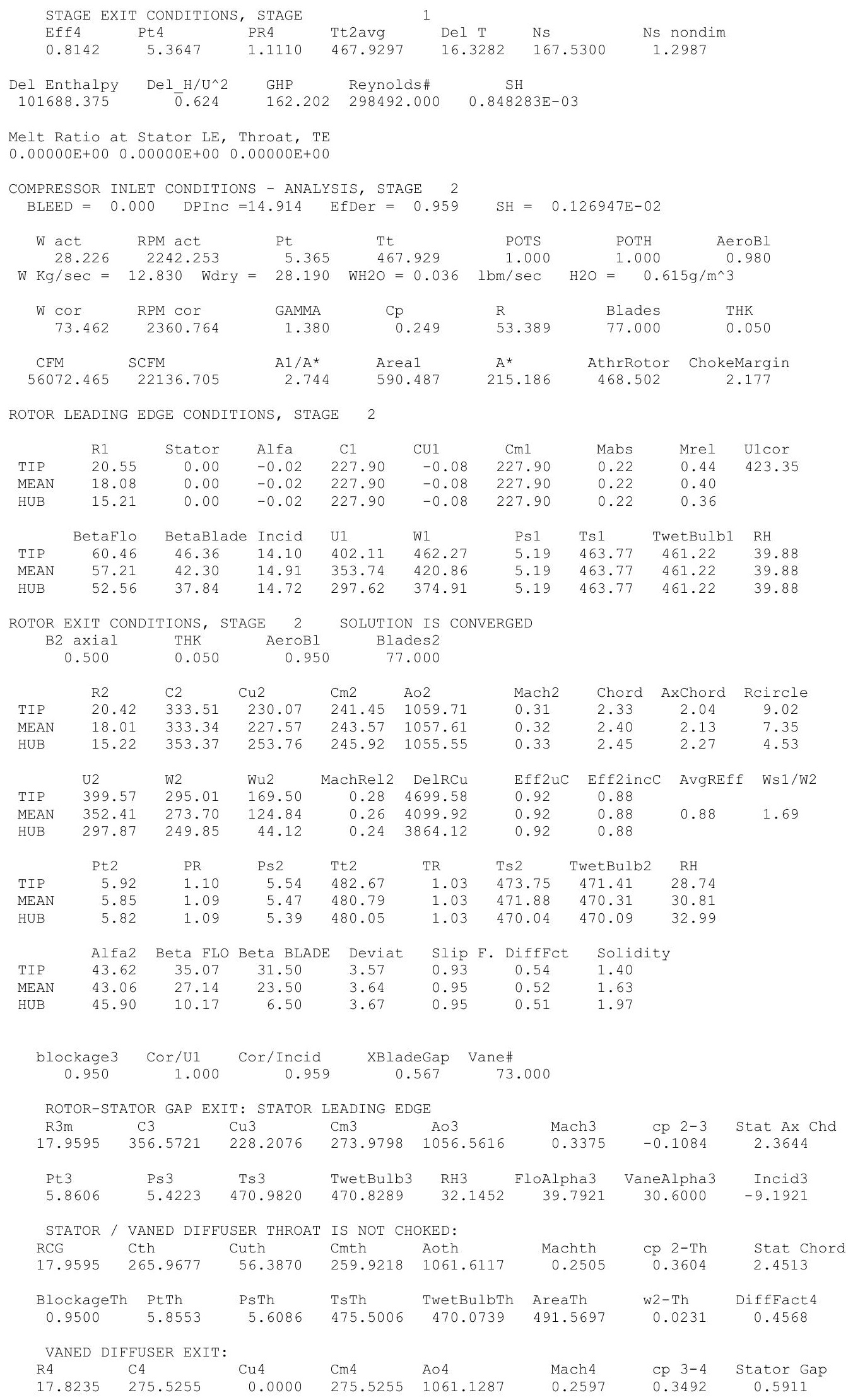




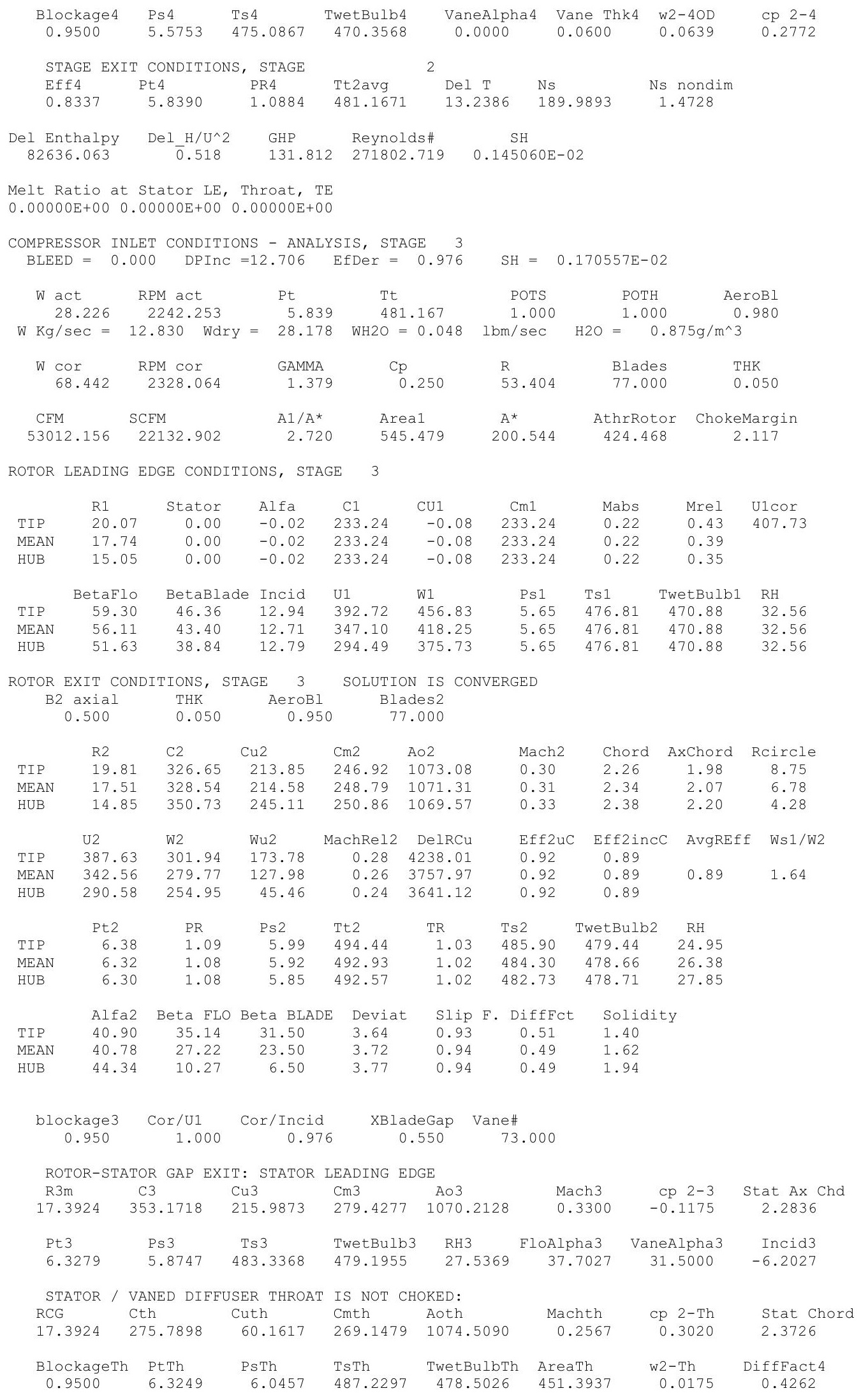




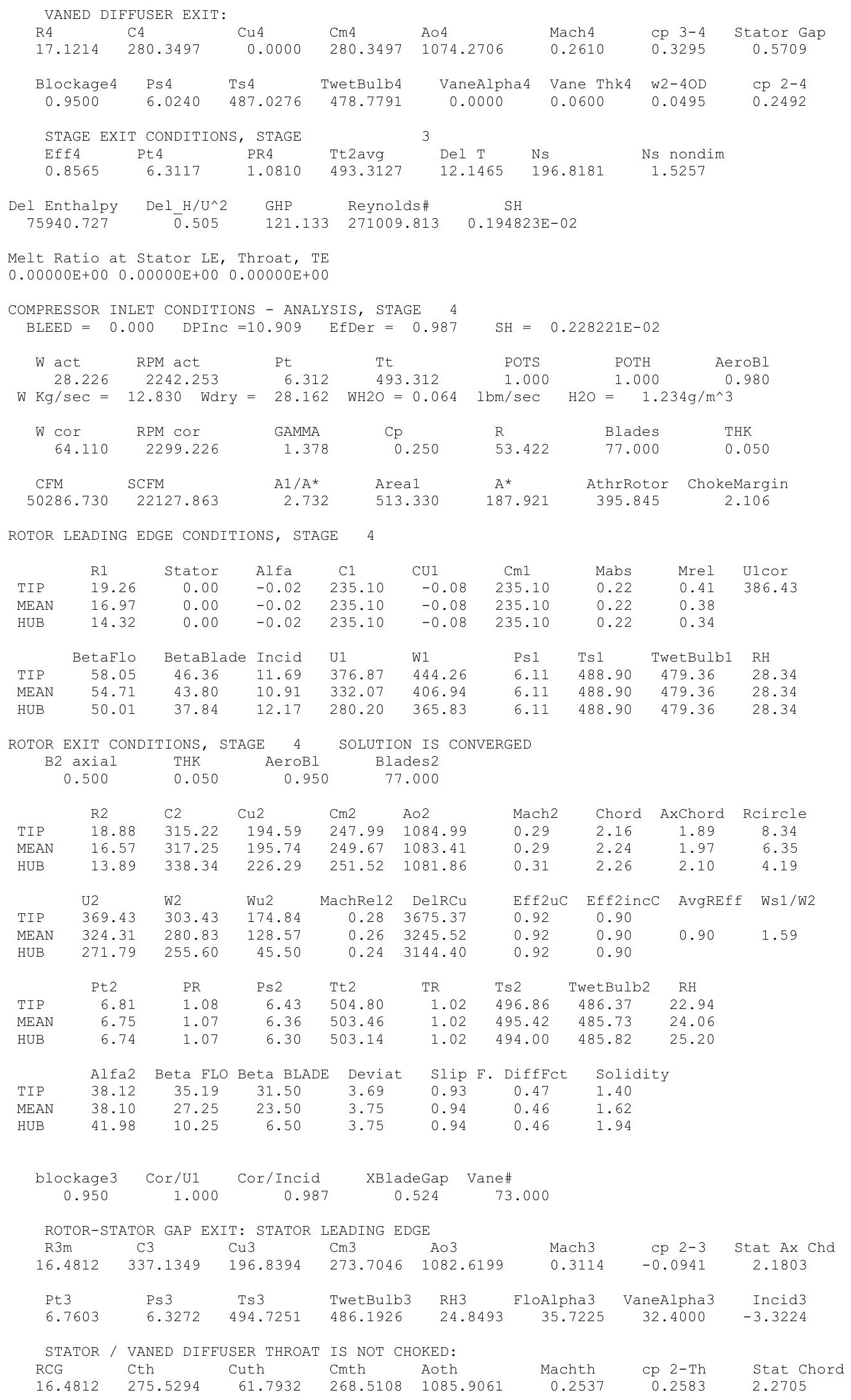




\begin{tabular}{|c|c|c|c|c|c|c|c|}
\hline $\begin{array}{l}\text { BlockageTh } \\
0.9500\end{array}$ & $\begin{array}{l}\text { PtTh } \\
6.7590\end{array}$ & $\begin{array}{l}\text { PsTh } \\
\quad 6.4674\end{array}$ & $\begin{array}{l}\text { TsTh } \\
497.7382\end{array}$ & $\begin{array}{c}\text { TwetBulbTh } \\
485.6314\end{array}$ & $\begin{array}{l}\text { AreaTh } \\
432.2457\end{array}$ & $\begin{array}{l}\text { w2-Th } \\
0.0135\end{array}$ & $\begin{array}{c}\text { DiffFact } 4 \\
0.3999\end{array}$ \\
\hline $\begin{array}{lr}\text { VANED } & \text { DIFF } \\
\text { R4 } \\
16.0960\end{array}$ & $\begin{array}{l}\text { EUSER EXIT: } \\
\text { C4 } \\
273.2949\end{array}$ & $\begin{array}{l}\mathrm{Cu} 4 \\
0.0000\end{array}$ & $\begin{array}{l}\mathrm{Cm} 4 \\
273.2949\end{array}$ & $\begin{array}{l}\text { Ao4 } \\
1086.0015\end{array}$ & $\begin{array}{l}\text { Mach4 } \\
0.2517\end{array}$ & $\begin{array}{l}\mathrm{cp} 3-4 \\
0.3106\end{array}$ & $\begin{array}{c}\text { Stator Gap } \\
0.5451\end{array}$ \\
\hline $\begin{array}{c}\text { Blockage } 4 \\
0.9500\end{array}$ & $\begin{array}{l}\text { Ps } 4 \\
6.4617\end{array}$ & $\begin{array}{l}\text { Ts } 4 \\
497.8368\end{array}$ & $\begin{array}{r}\text { TwetBulb4 } \\
485.8696\end{array}$ & $\begin{array}{c}\text { VaneAlpha } 4 \\
0.0000\end{array}$ & $\begin{array}{l}\text { Vane Thk } 4 \\
0.0600\end{array}$ & $\begin{array}{l}\text { w2-4OD } \\
0.0404\end{array}$ & $\begin{array}{l}\text { cp } 2-4 \\
0.2442\end{array}$ \\
\hline $\begin{array}{l}\text { STAGE EXIT } \\
\text { Eff } 4 \\
0.8709\end{array}$ & $\begin{array}{l}\text { T CONDITIONS } \\
\text { Pt } 4 \\
\quad 6.7482\end{array}$ & $\begin{array}{l}\text { IS, STAGE } \\
\text { PR4 } \\
\quad 1.0691\end{array}$ & $\begin{array}{l}\text { Tt2avg } \\
503.7977\end{array}$ & $\begin{array}{l}\text { Del T } \\
10.4859\end{array}$ & $\begin{array}{l}\text { Ns } \\
213.7209\end{array}$ & $\begin{array}{l}\text { Ns nondim } \\
1.6568\end{array}$ & \\
\hline $\begin{array}{c}\text { Del Enthalpy } \\
65687.422\end{array}$ & $\begin{array}{c}\mathrm{De}{ }_{\overline{0}} \mathrm{H} / \mathrm{U}^{\wedge} 2 \\
0.481\end{array}$ & $\begin{array}{l}\text { GHP } \\
104.778\end{array}$ & $\begin{array}{l}\text { Reynold } \\
278157 \text {. }\end{array}$ & $\begin{array}{c}\mathrm{SH} \\
0.25872\end{array}$ & $20 \mathrm{E}-02$ & & \\
\hline
\end{tabular}

Melt Ratio at Stator LE, Throat, TE

$0.00000 \mathrm{E}+00 \quad 0.00000 \mathrm{E}+00 \quad 0.00000 \mathrm{E}+00$

COMPRESSOR INLET CONDITIONS - ANALYSIS, STAGE 5 $\mathrm{BLEED}=0.000 \quad$ DPInC $=9.263 \quad$ EfDer $=0.995$

$\begin{array}{llrllrr}W \text { act } & \text { RPM act } & \text { Pt } & \text { Tt } & \text { POTS } & \text { POTH } & \text { AeroBI } \\ 28.226 & 2242.253 & 6.748 & 503.797 & 1.000 & 1.000 & 0.980\end{array}$

$\mathrm{SH}=0.301009 \mathrm{E}-02$ $\mathrm{W} \mathrm{Kg} / \mathrm{sec}=12.830$ Wary $=28.141 \quad \mathrm{WH} 2 \mathrm{O}=0.085 \quad \mathrm{lbm} / \mathrm{sec} \quad \mathrm{H} 2 \mathrm{O}=1.707 \mathrm{~g} / \mathrm{m}^{\wedge} 3$

$\begin{array}{ccccccc}\text { W cor } & \text { RPM cor } & \text { GAMMA } & \text { Cp } & \text { R } & \text { Blades } & \text { THK } \\ 60.597 & 2275.175 & 1.377 & 0.251 & 53.446 & 77.000 & 0.050 \\ & & & & & & \\ \text { CFM } & \text { SCFM } & \text { A1/A* } & \text { Areal } & A^{*} & \text { AthrRotor } & \text { ChokeMargin } \\ 47944.465 & 22121.494 & 2.868 & 509.668 & 177.707 & 384.739 & 2.165\end{array}$

ROTOR LEADING EDGE CONDITIONS, STAGE 5

$\begin{array}{lccccccccc} & \text { R1 } & \text { Stator } & \text { Alfa } & \text { C1 } & \text { CU1 } & \text { Cm1 } & \text { Mabs } & \text { Mrel } & \text { U1cor } \\ \text { TIP } & 18.32 & 0.00 & -0.02 & 225.76 & -0.08 & 225.76 & 0.21 & 0.39 & 363.73 \\ \text { MEAN } & 15.91 & 0.00 & -0.02 & 225.76 & -0.08 & 225.76 & 0.21 & 0.35 & \\ \text { HUB } & 13.07 & 0.00 & -0.02 & 225.76 & -0.08 & 225.76 & 0.21 & 0.31 & \\ & & & & & & & & & \\ & & & & & & & & \\ \text { TIP } & 57.80 & 47.36 & 10.44 & 358.47 & 423.71 & 6.55 & 499.74 & 486.51 & 26.02 \\ \text { MEAN } & 54.06 & 44.80 & 9.26 & 311.38 & 384.67 & 6.55 & 499.74 & 486.51 & 26.02 \\ \text { HUB } & 48.57 & 38.84 & 9.73 & 255.75 & 341.19 & 6.55 & 499.74 & 486.51 & 26.02\end{array}$

ROTOR EXIT CONDITIONS, STAGE 5 SOLUTION IS CONVERGED

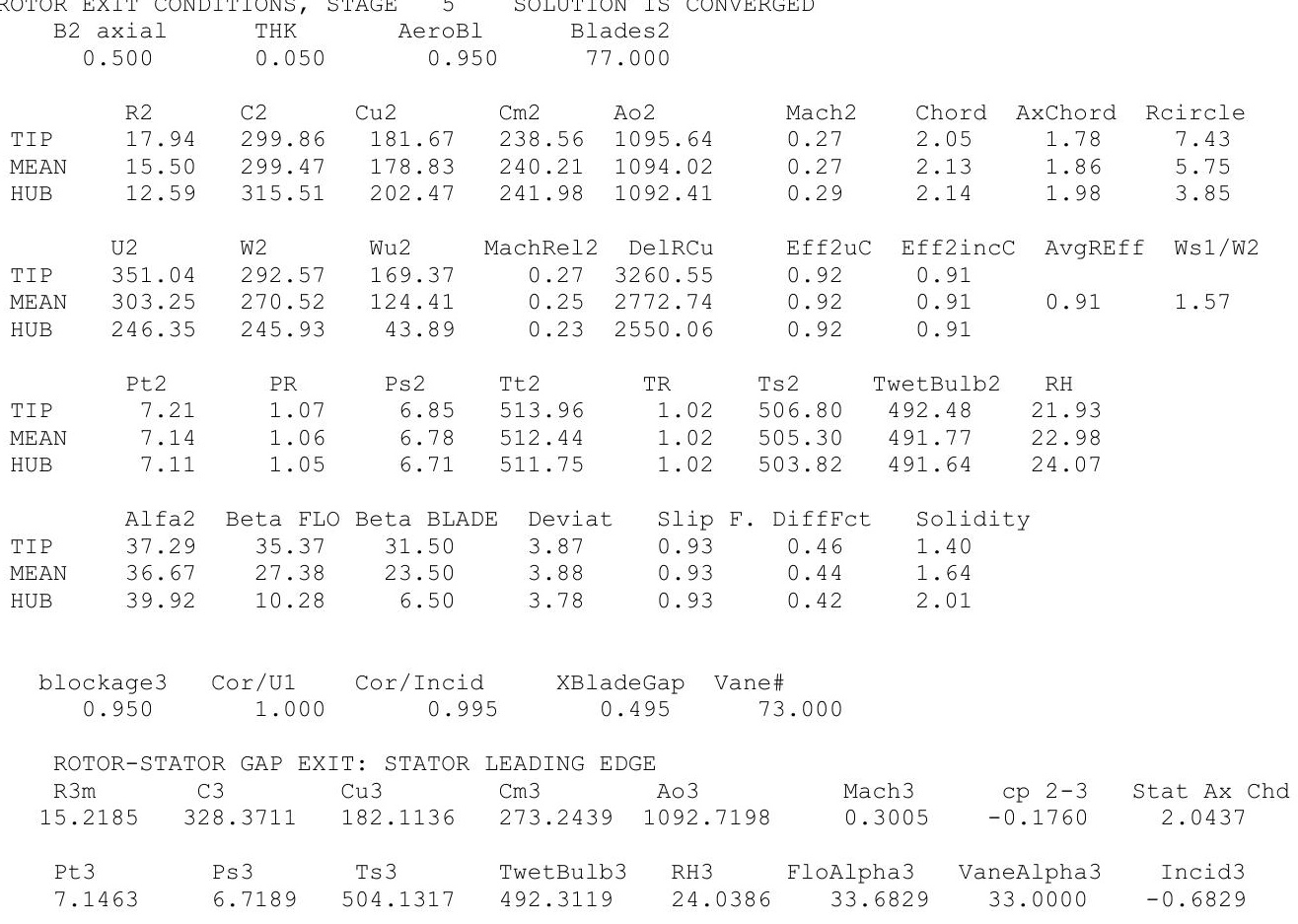




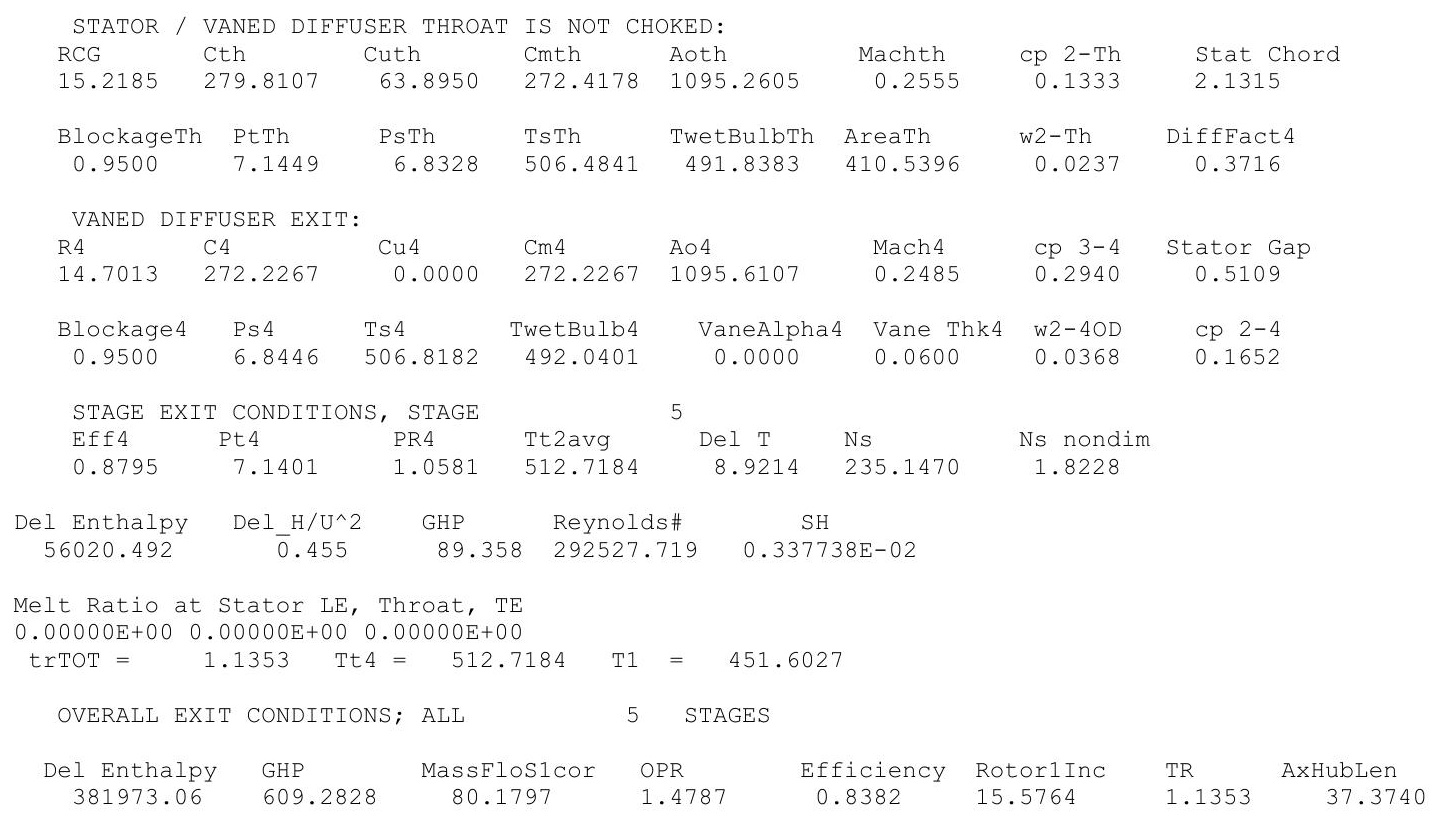




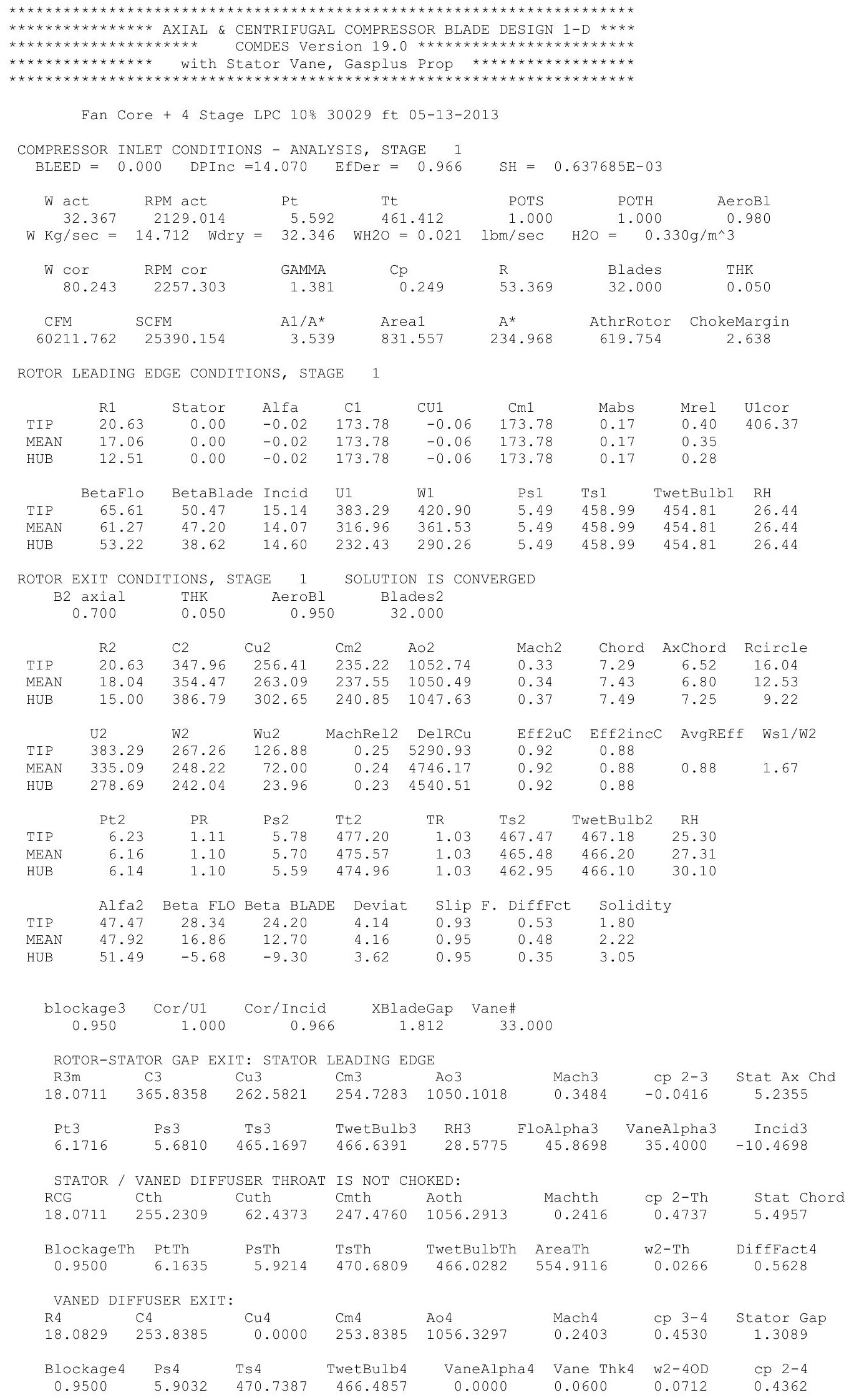




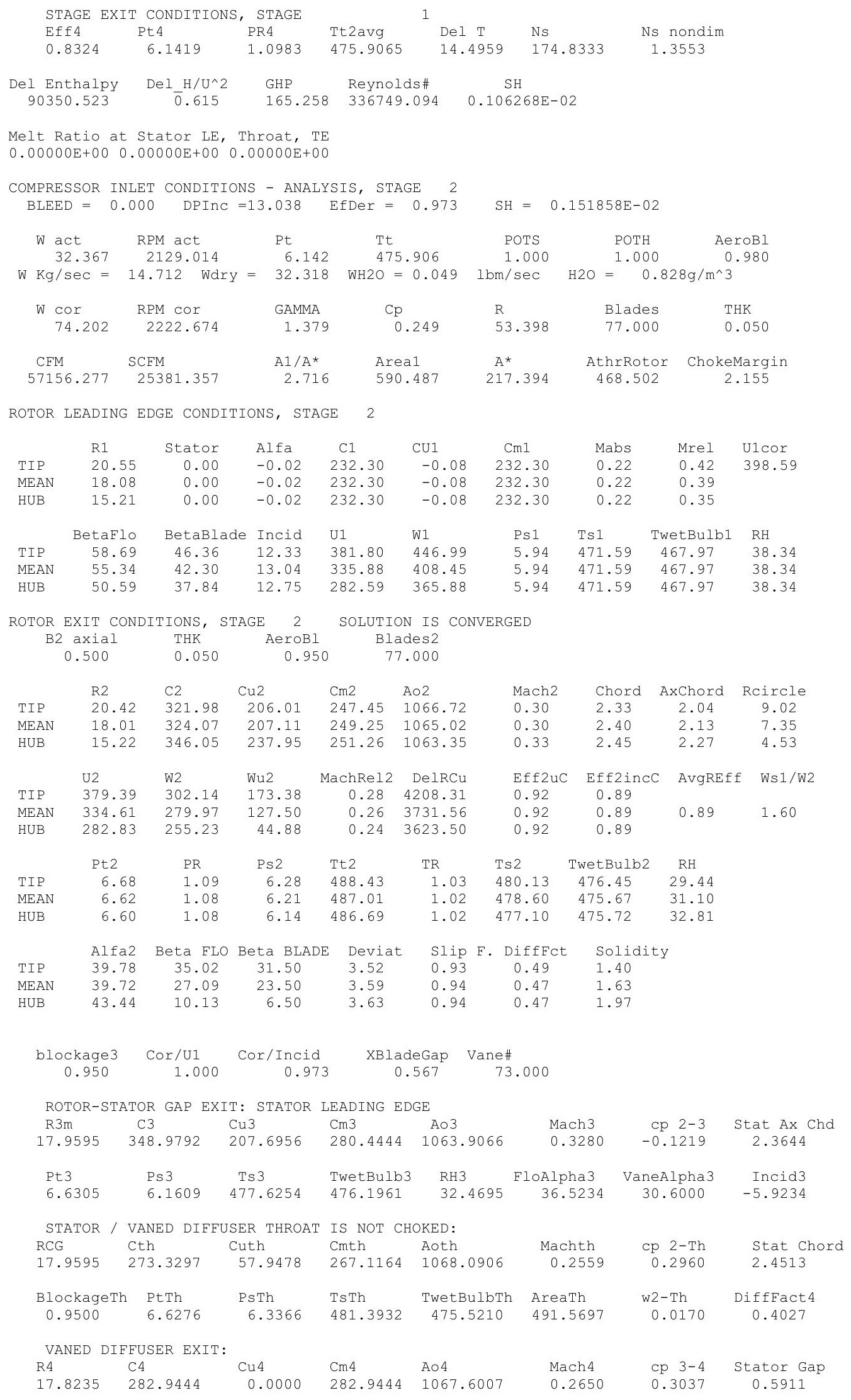




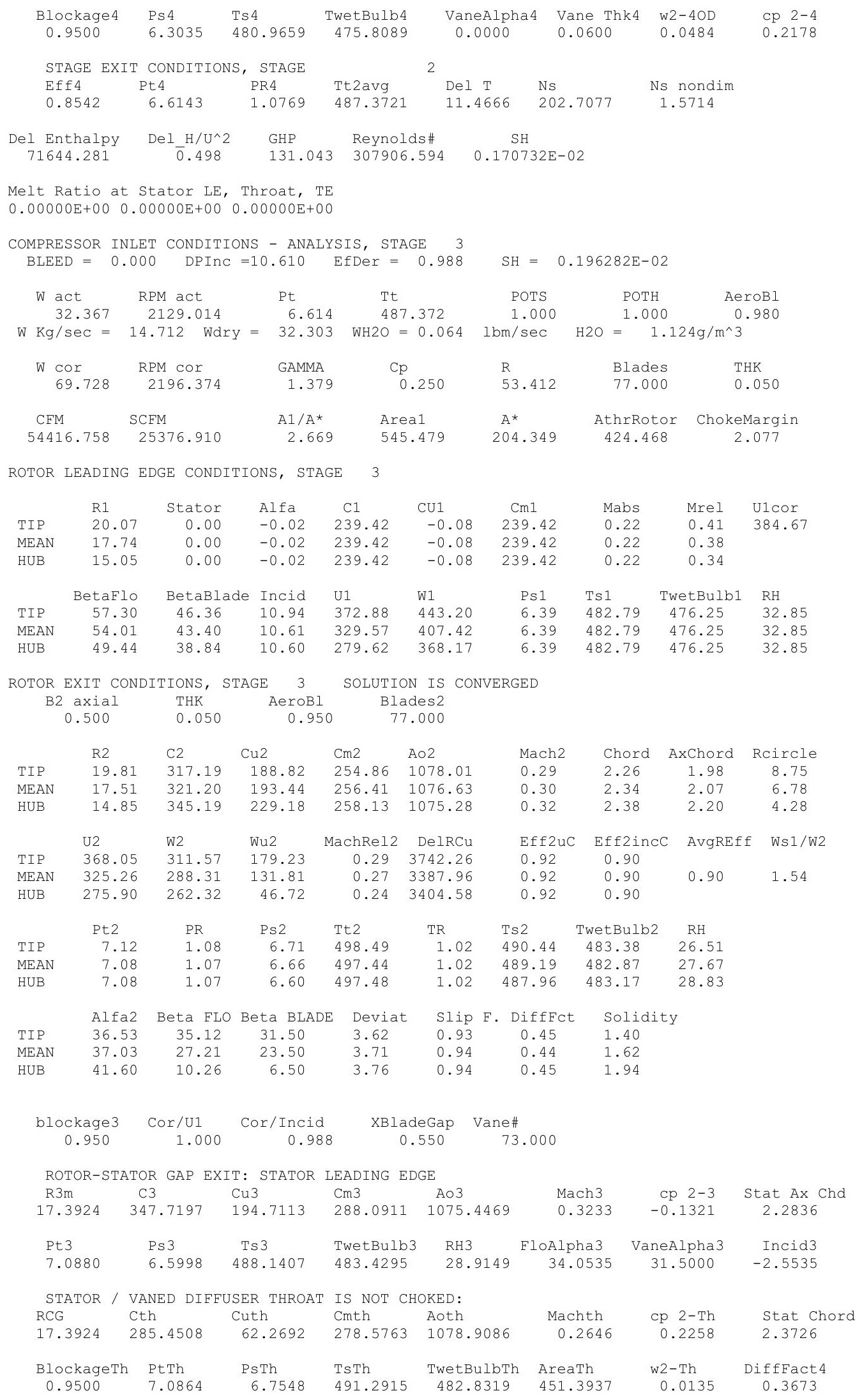




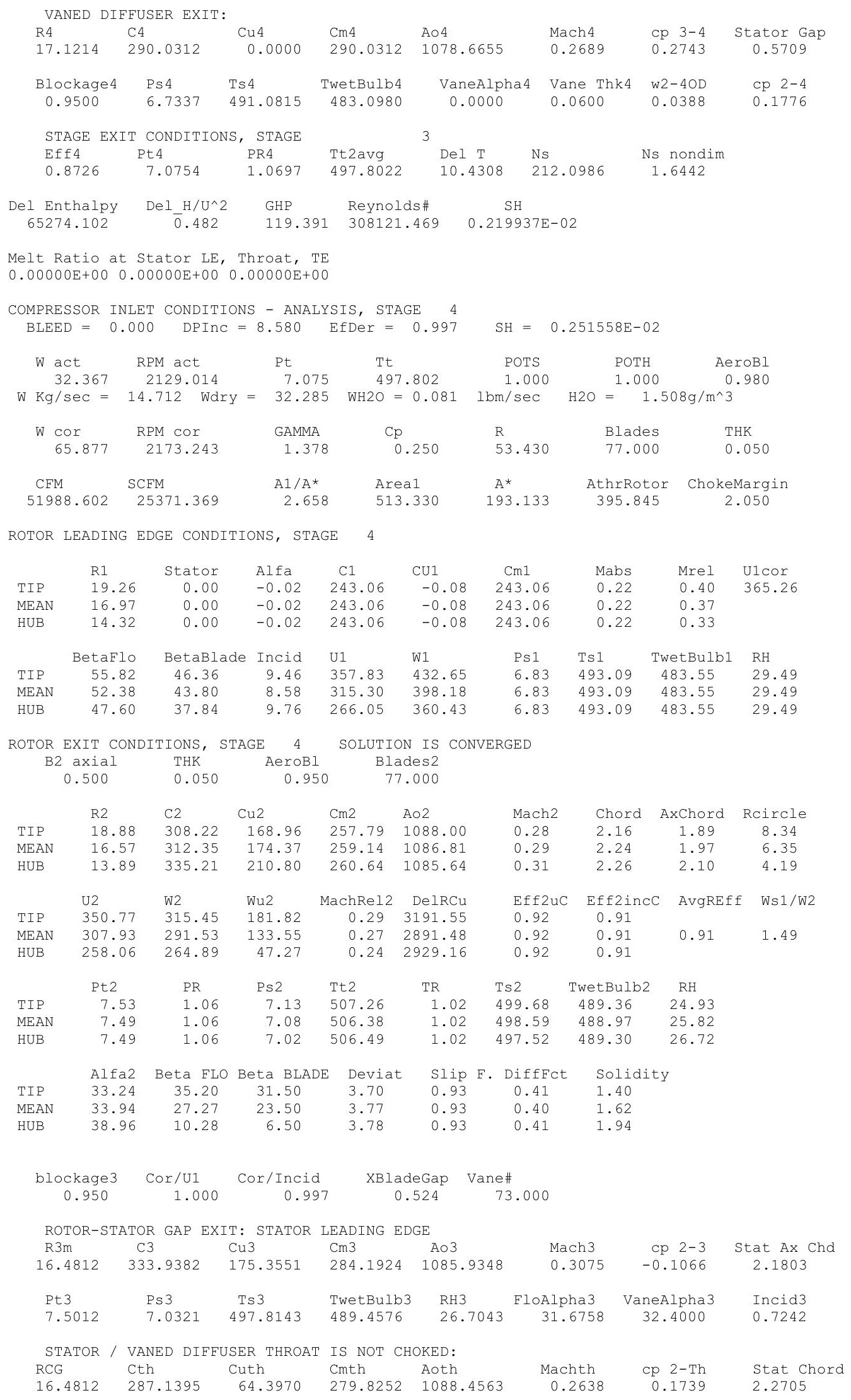




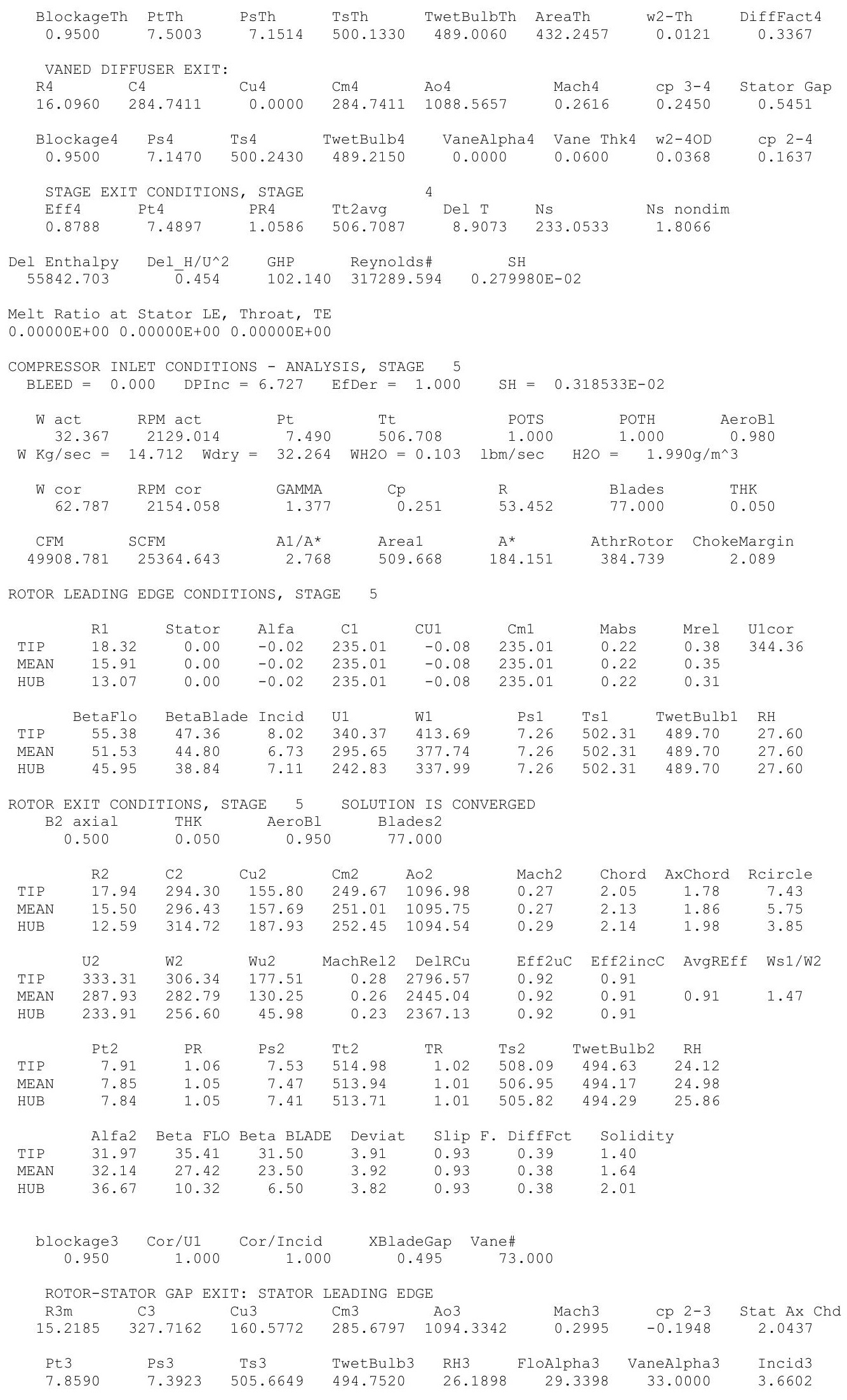




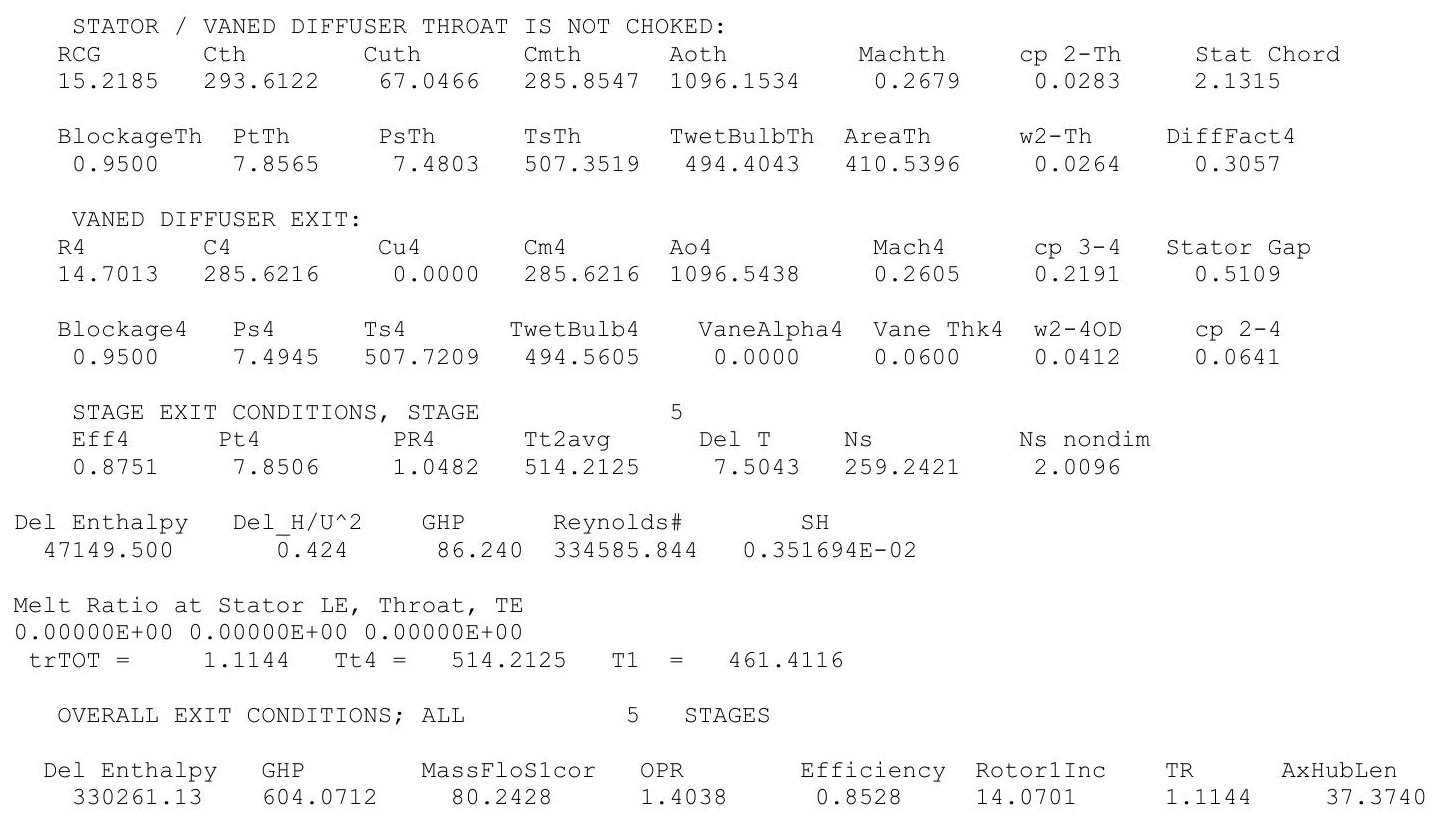




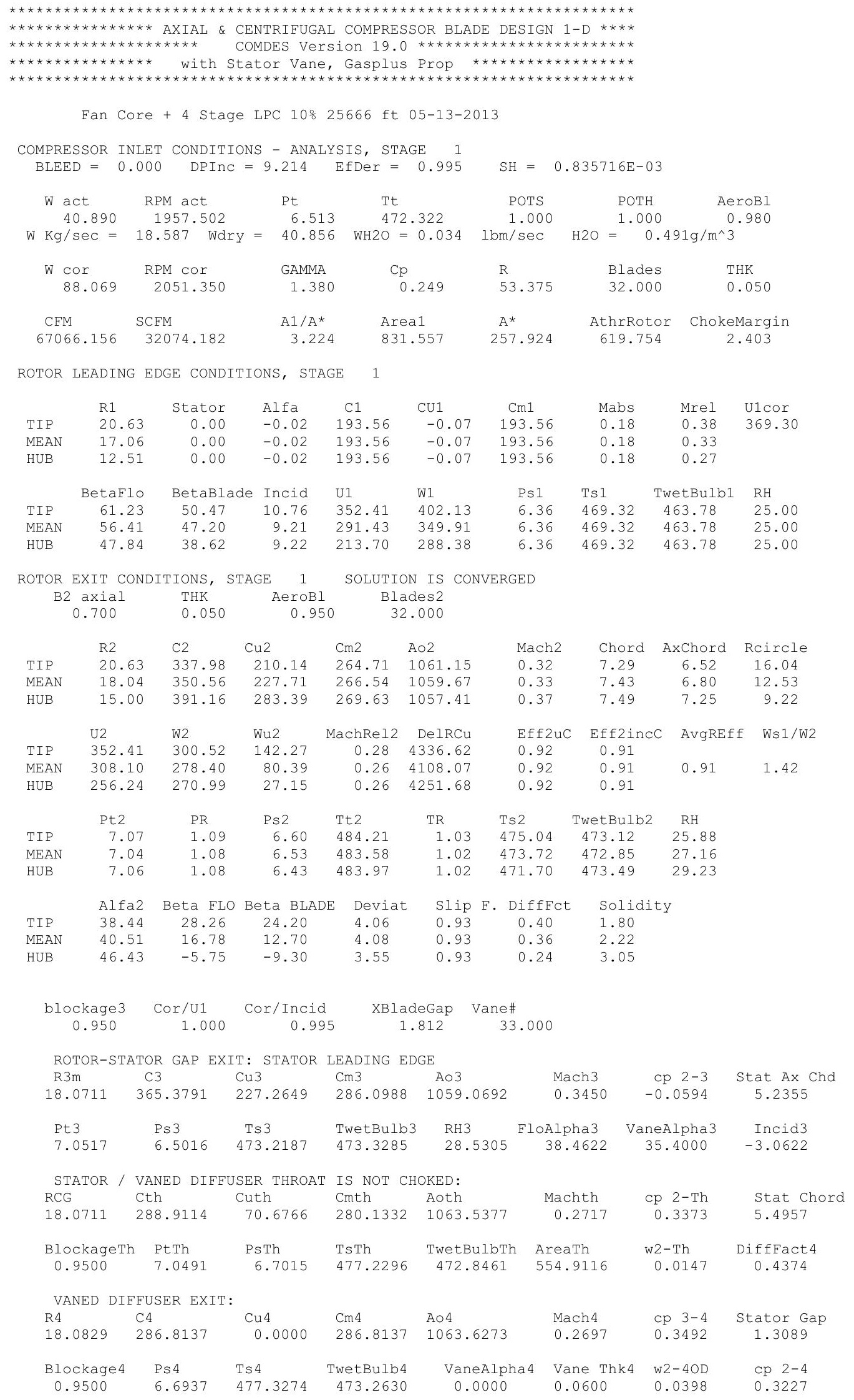




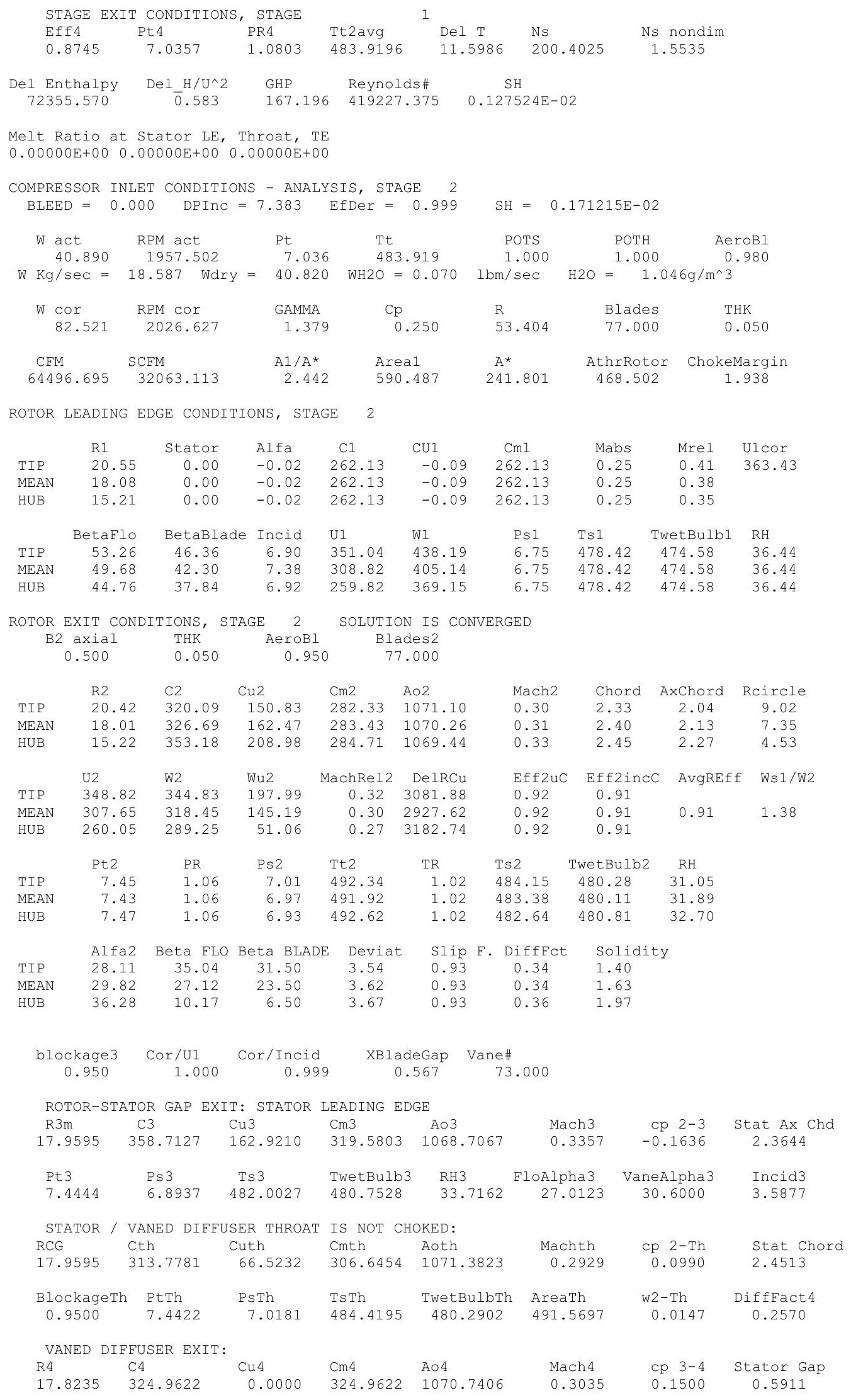




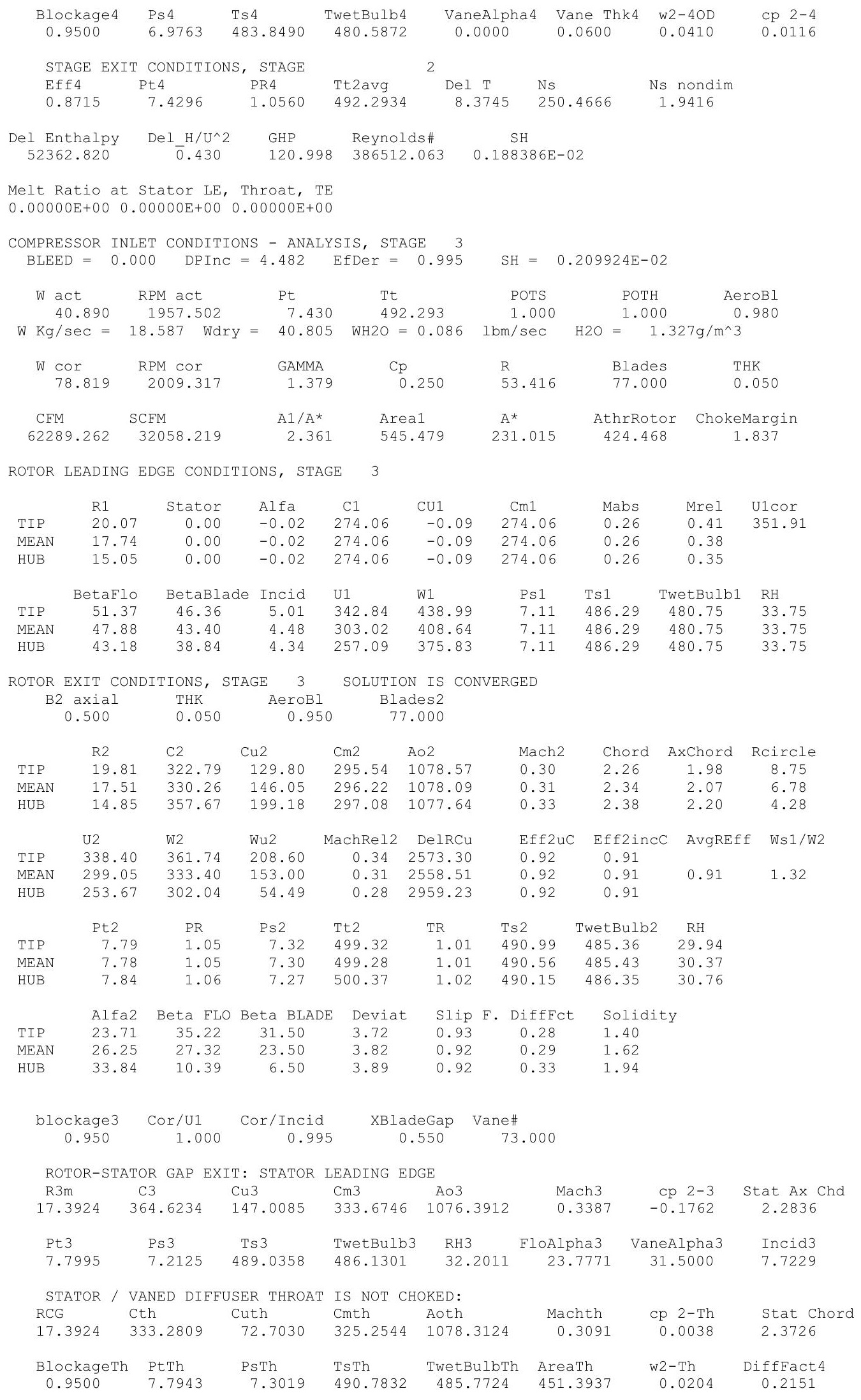




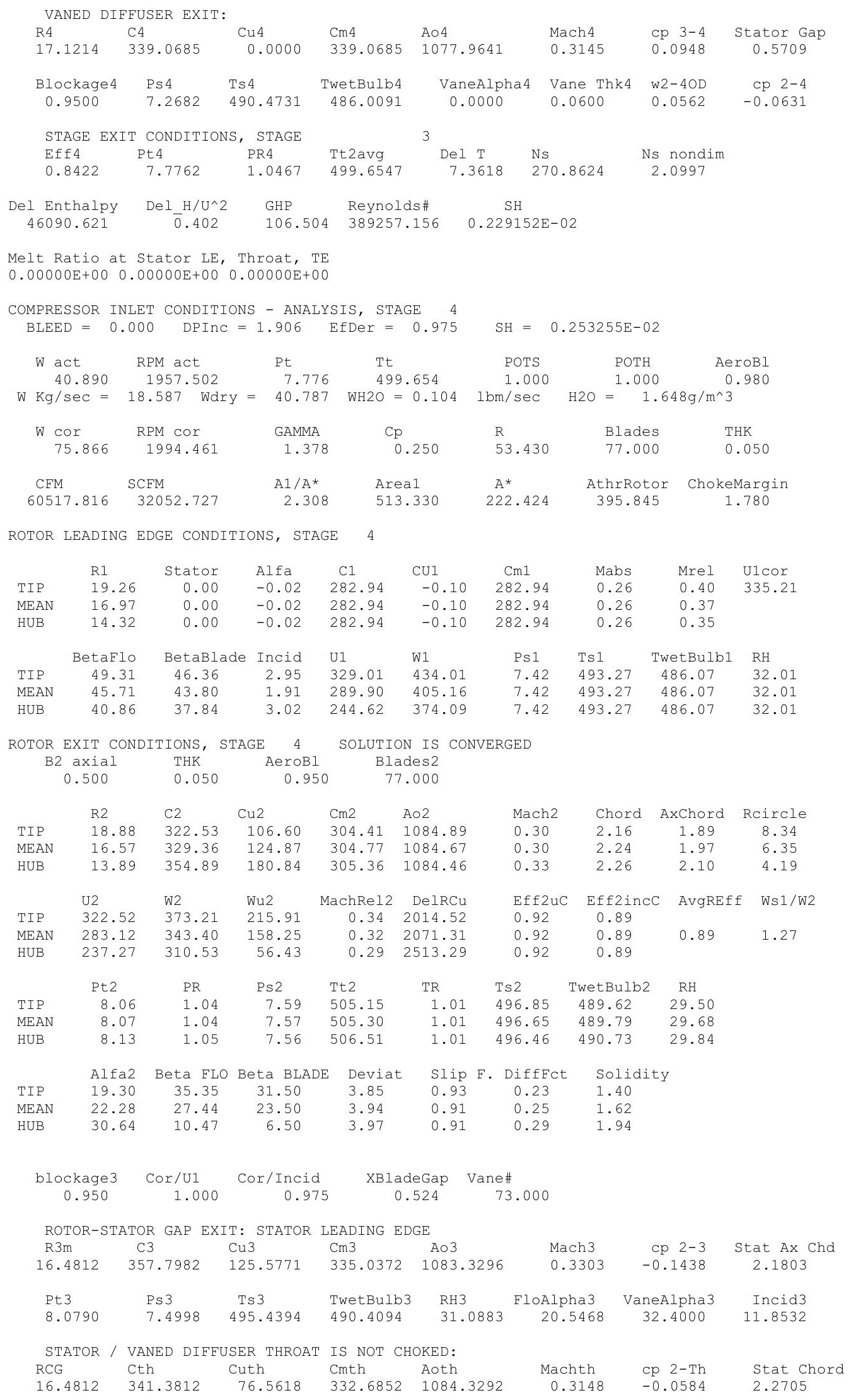




\begin{tabular}{|c|c|c|c|c|c|c|c|}
\hline $\begin{array}{l}\text { BlockageTh } \\
0.9500\end{array}$ & $\begin{array}{l}\text { PtTh } \\
8.0696\end{array}$ & $\begin{array}{l}\text { PsTh } \\
\quad 7.5417\end{array}$ & $\begin{array}{l}\text { TsTh } \\
496.3554\end{array}$ & $\begin{array}{c}\text { TwetBulbTh } \\
490.2257\end{array}$ & $\begin{array}{l}\text { Areath } \\
432.2457\end{array}$ & $\begin{array}{l}\text { w2-Th } \\
0.0282\end{array}$ & $\begin{array}{c}\text { DiffFact } 4 \\
0.1787\end{array}$ \\
\hline $\begin{array}{l}\text { VANED DIFF } \\
\text { R4 } \\
16.0960\end{array}$ & $\begin{array}{l}\text { FUSER EXIT: } \\
\text { C4 } \\
339.1375\end{array}$ & $\begin{array}{l}\mathrm{Cu} 4 \\
0.0000\end{array}$ & $\begin{array}{l}\mathrm{Cm} 4 \\
339.1375\end{array}$ & $\begin{array}{l}\text { A०4 } \\
1084.4576\end{array}$ & $\begin{array}{l}\text { Mach } 4 \\
0.3127\end{array}$ & $\begin{array}{l}\text { cp } 3-4 \\
0.0414\end{array}$ & $\begin{array}{c}\text { Stator Gap } \\
0.5451\end{array}$ \\
\hline $\begin{array}{c}\text { Blockage } 4 \\
0.9500\end{array}$ & $\begin{array}{l}\text { Ps } 4 \\
7.5238\end{array}$ & $\begin{array}{l}\text { Ts4 } \\
496.4778\end{array}$ & $\begin{array}{r}\text { TwetBulb4 } \\
490.3412\end{array}$ & $\begin{array}{c}\text { VaneAlpha } 4 \\
0.0000\end{array}$ & $\begin{array}{l}\text { Vane Thk4 } \\
0.0600\end{array}$ & $\begin{array}{l}\mathrm{w} 2-4 \mathrm{OD} \\
0.0798\end{array}$ & $\begin{array}{r}\mathrm{cp} 2-4 \\
-0.0935\end{array}$ \\
\hline $\begin{array}{l}\text { STAGE EXIT } \\
\text { Eff } 4 \\
0.7749\end{array}$ & $\begin{array}{l}\text { T CONDITIONS, } \\
\text { Pt } 4 \\
8.0432\end{array}$ & $\begin{array}{l}\text { S, STAGE } \\
\text { PR4 } \\
1.0343\end{array}$ & $\begin{array}{l}\text { Tt2avg } \\
505.6503\end{array}$ & $\begin{array}{l}\text { Del T } \\
\quad 5.9961\end{array}$ & $\begin{array}{l}\text { Ns } \\
311.0760\end{array}$ & $\begin{array}{l}\text { Ns nondim } \\
2.4114\end{array}$ & \\
\hline $\begin{array}{c}\text { Del Enthalpy } \\
37592.535\end{array}$ & $\begin{array}{c}\mathrm{De}{ }_{\overline{0}} \mathrm{H} / \mathrm{U}^{\wedge} 2 \\
0.361\end{array}$ & $\begin{array}{l}\text { GHP } \\
86.867\end{array}$ & $\begin{array}{ll} & \text { Reynold } \\
7 & 403297 .\end{array}$ & $\begin{array}{r}\mathrm{SH} \\
0.27431\end{array}$ & $.7 \mathrm{E}-02$ & & \\
\hline
\end{tabular}

Melt Ratio at Stator LE, Throat, TE

$0.00000 \mathrm{E}+00 \quad 0.00000 \mathrm{E}+00 \quad 0.00000 \mathrm{E}+00$

COMPRESSOR INLET CONDITIONS - ANALYSIS, STAGE 5 BLEED $=0.000 \quad$ DPInC $=-0.483 \quad$ EfDer $=0.942$

$\begin{array}{llllll}W & \text { Ret } & \text { RPM act } & \text { Pt } & \text { POTS } & \text { POTH }\end{array}$ $\mathrm{W} \mathrm{Kg} / \mathrm{sec}=18.587$ Wdry $=40.767 \quad \mathrm{WH} 2 \mathrm{O}=0.123 \quad \mathrm{lbm} / \mathrm{sec} \quad \mathrm{H} 2 \mathrm{O}=2.008 \mathrm{~g} / \mathrm{m}^{\wedge} 3$

$\begin{array}{llllll}W & \text { Cor } & \text { GPM cor } & \text { B } & \text { Blades } & \text { THK }\end{array}$

$\begin{array}{lccclll}\text { Ror } & \text { RPM Cor } & \text { GAMMA } & \text { Cp } & \text { R } & \text { Blades } & \text { THK } \\ 73.787 & 1982.601 & 1.377 & 0.251 & 53.446 & 77.000 & 0.050\end{array}$

$\begin{array}{cccccc}\text { CFM } & \text { SCFM } & \text { A1/A* } & \text { Area1 } & A^{*} & \text { AthrRotor ChokeMargin }\end{array}$

$\begin{array}{lllllll}59142.027 & 32046.602 & 2.355 & 509.668 & 216.392 & 384.739 & 1.778\end{array}$

ROTOR LEADING EDGE CONDITIONS, STAGE 5

$\begin{array}{lccccccccc} & \text { R1 } & \text { Stator } & \text { Alfa } & \text { C1 } & \text { CU1 } & \text { Cm1 } & \text { Mabs } & \text { Mrel } & \text { U1cor } \\ \text { TIP } & 18.32 & 0.00 & -0.02 & 278.49 & -0.10 & 278.49 & 0.26 & 0.39 & 316.95 \\ \text { MEAN } & 15.91 & 0.00 & -0.02 & 278.49 & -0.10 & 278.49 & 0.26 & 0.36 & \\ \text { HUB } & 13.07 & 0.00 & -0.02 & 278.49 & -0.10 & 278.49 & 0.26 & 0.33 & \\ & & & & & & & & & \\ & \text { BetaFlo } & \text { BetaBlade } & \text { Incid } & \text { U1 } & \text { W } 1 & \text { Ts1 } & \text { TwetBulb1 } & \text { RH } \\ \text { TIP } & 48.34 & 47.36 & 0.98 & 312.95 & 418.99 & 7.69 & 499.47 & 490.33 & 30.91 \\ \text { MEAN } & 44.32 & 44.80 & -0.48 & 271.83 & 389.23 & 7.69 & 499.47 & 490.33 & 30.91 \\ \text { HUB } & 38.73 & 38.84 & -0.11 & 223.27 & 357.00 & 7.69 & 499.47 & 490.33 & 30.91\end{array}$

ROTOR EXIT CONDITIONS, STAGE 5 SOLUTION IS CONVERGED

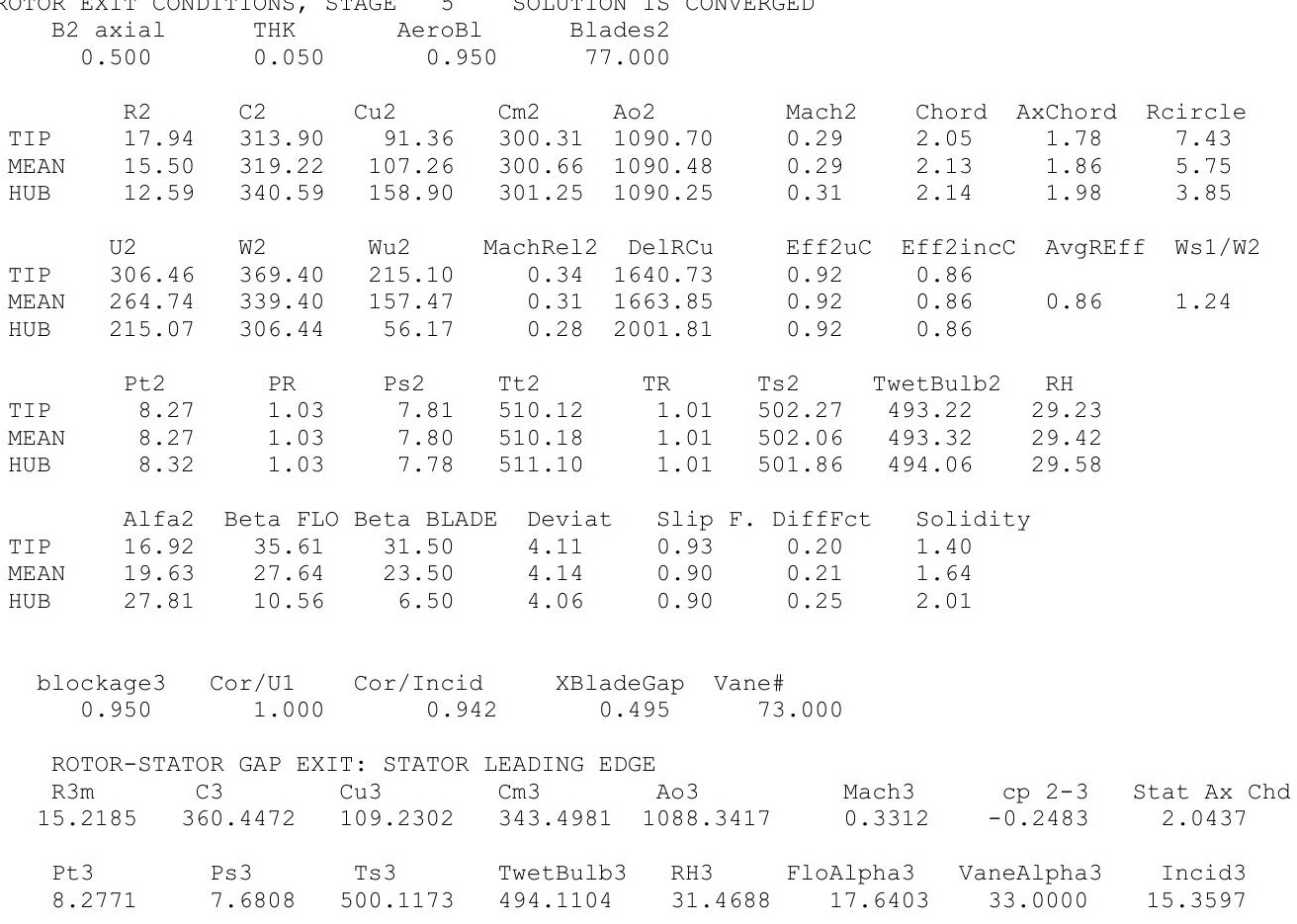




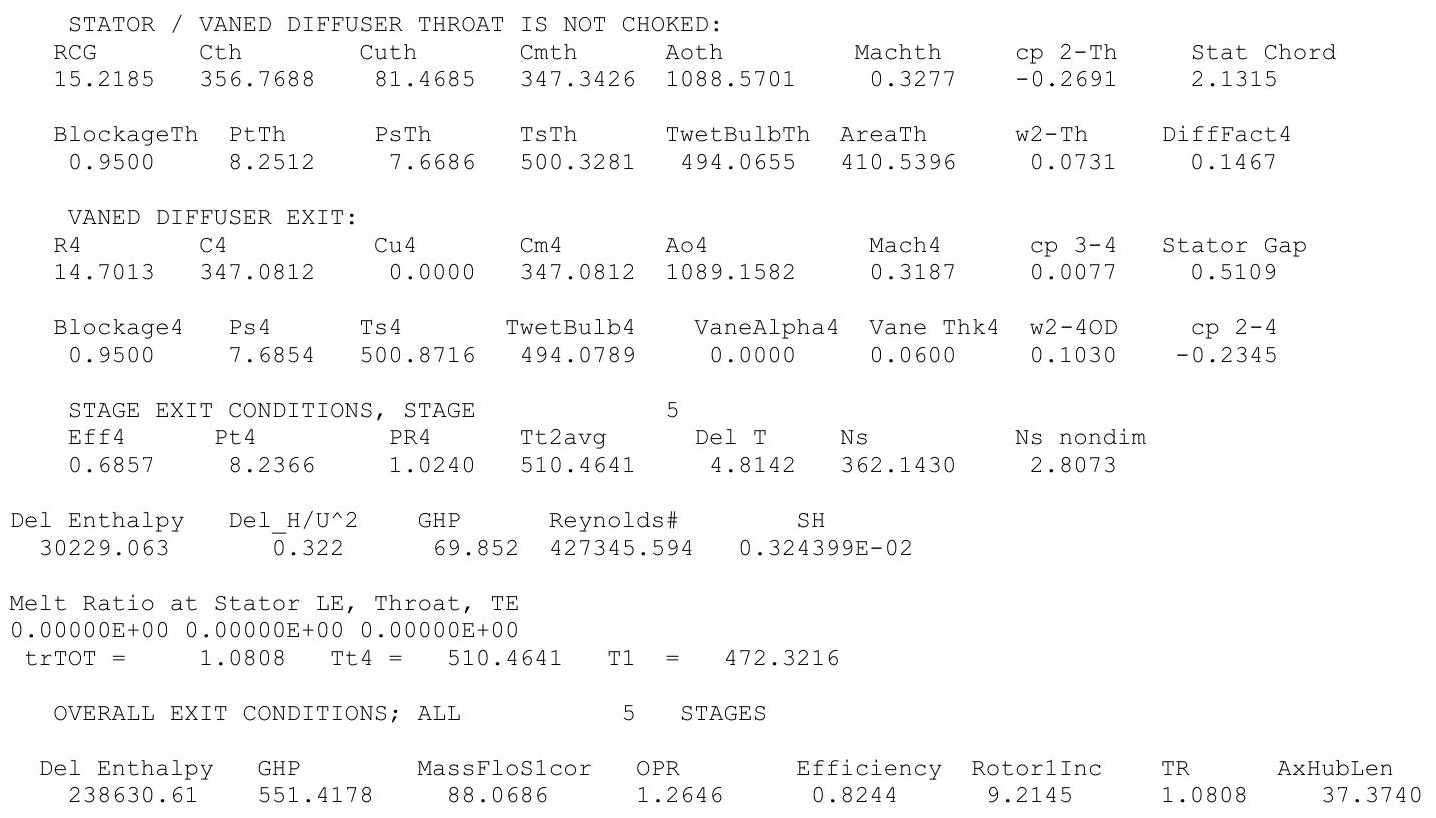




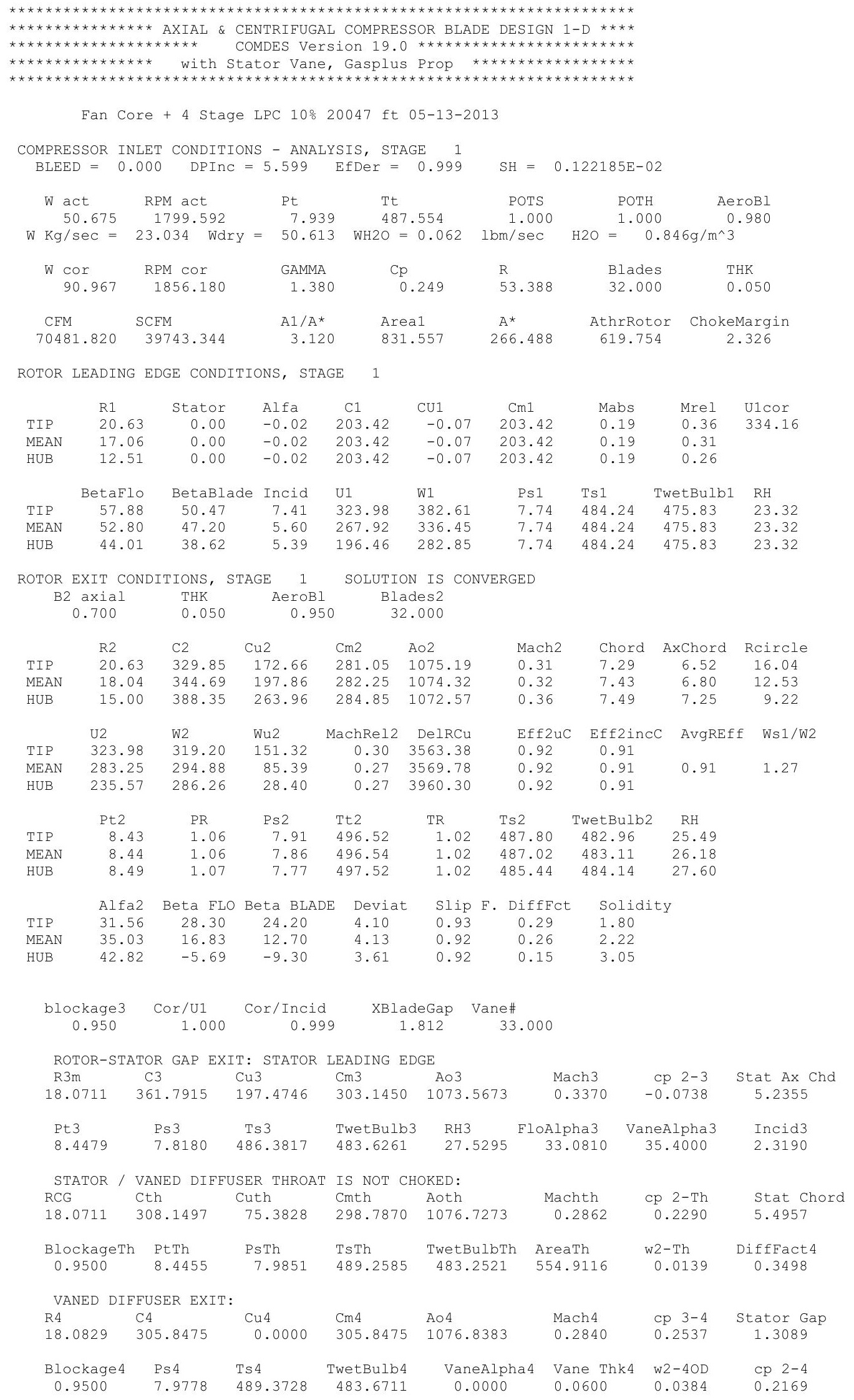




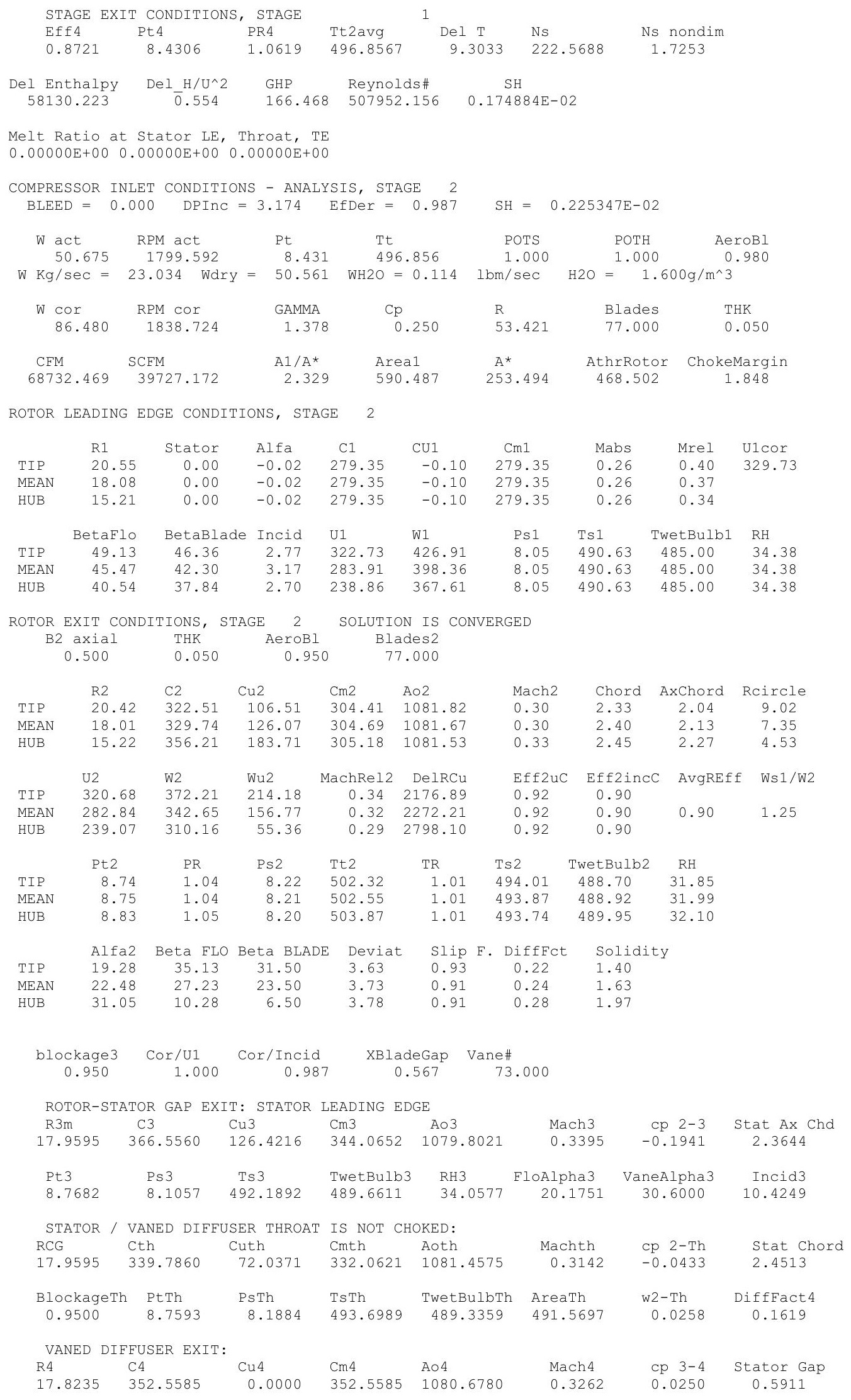




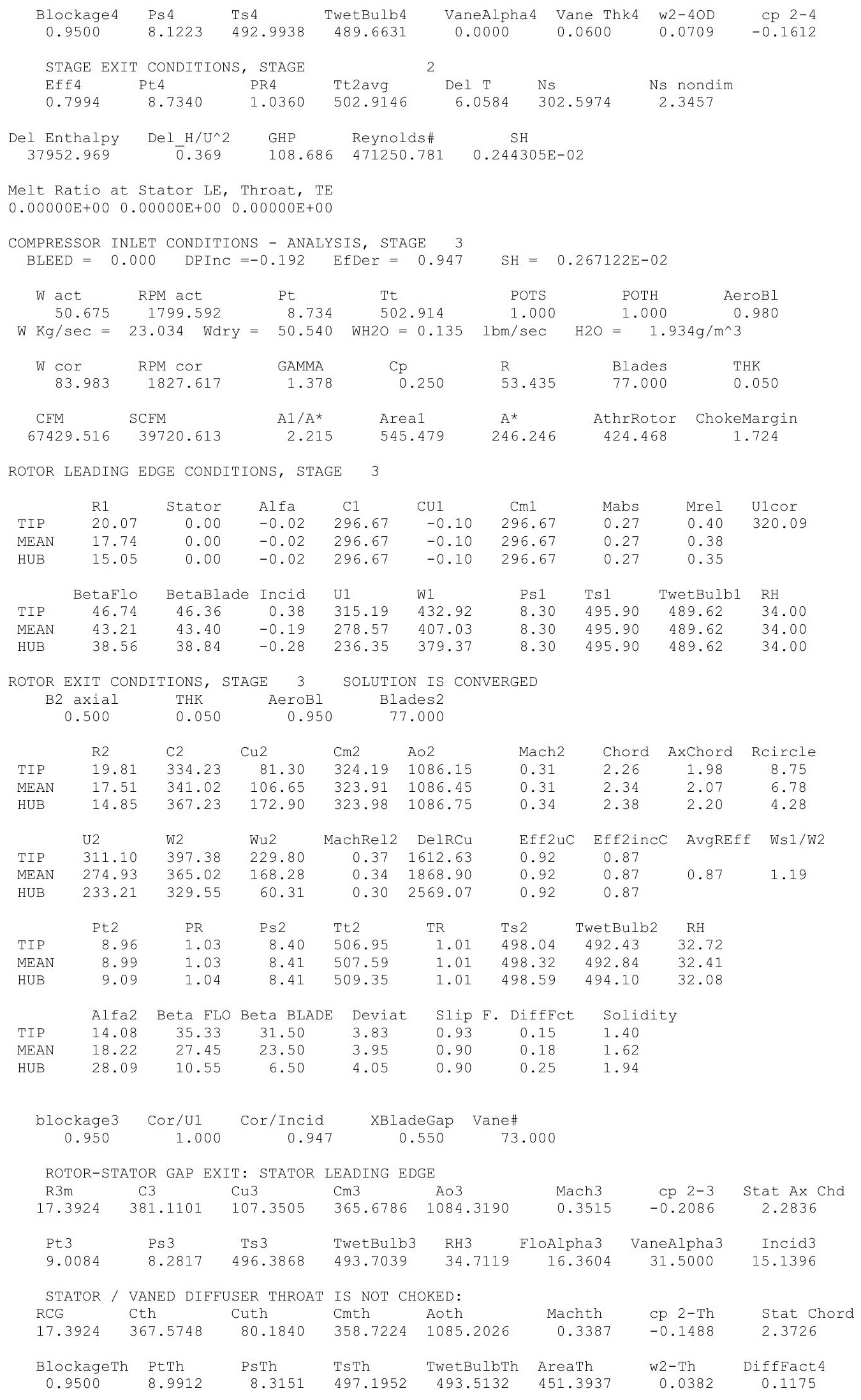




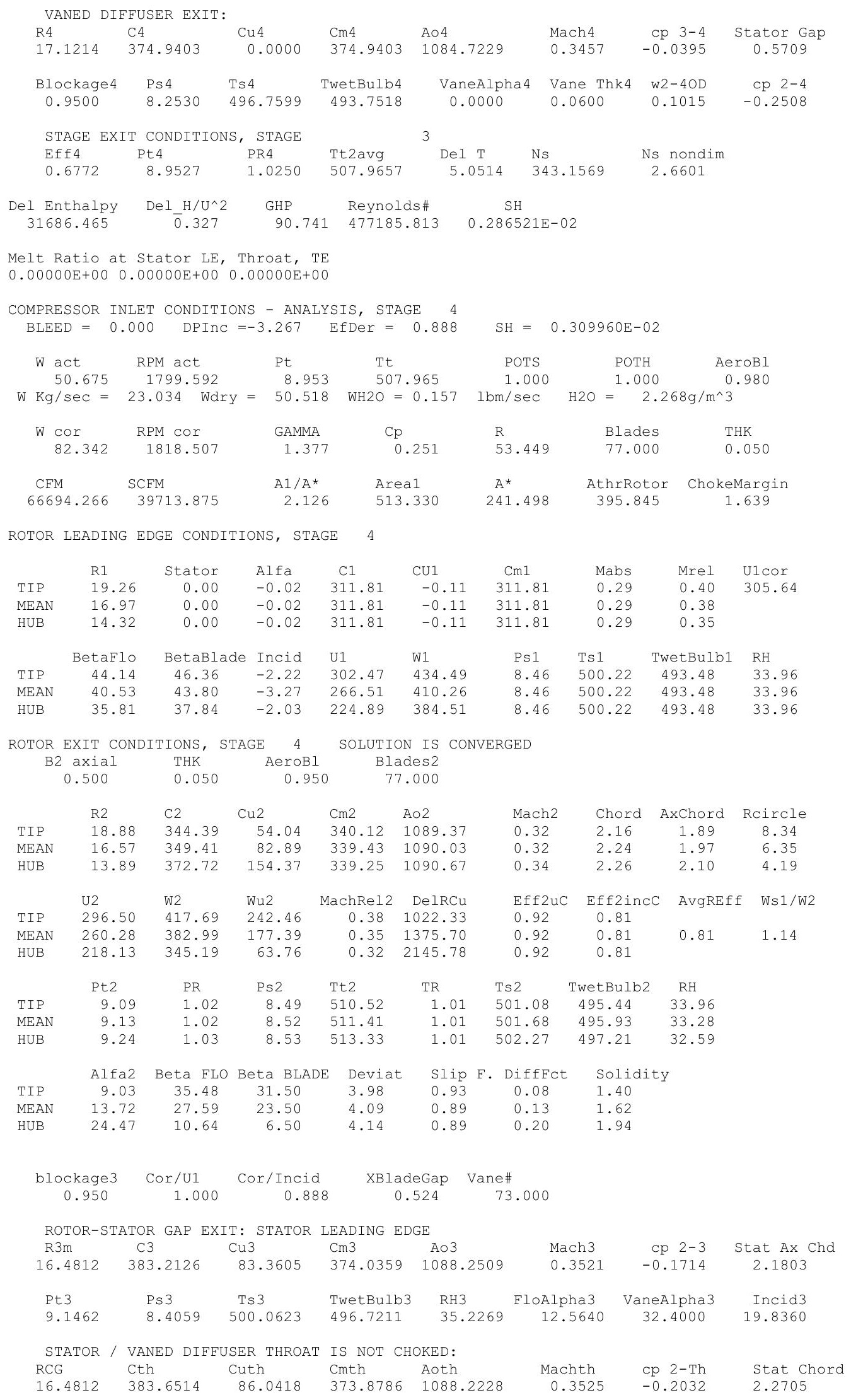




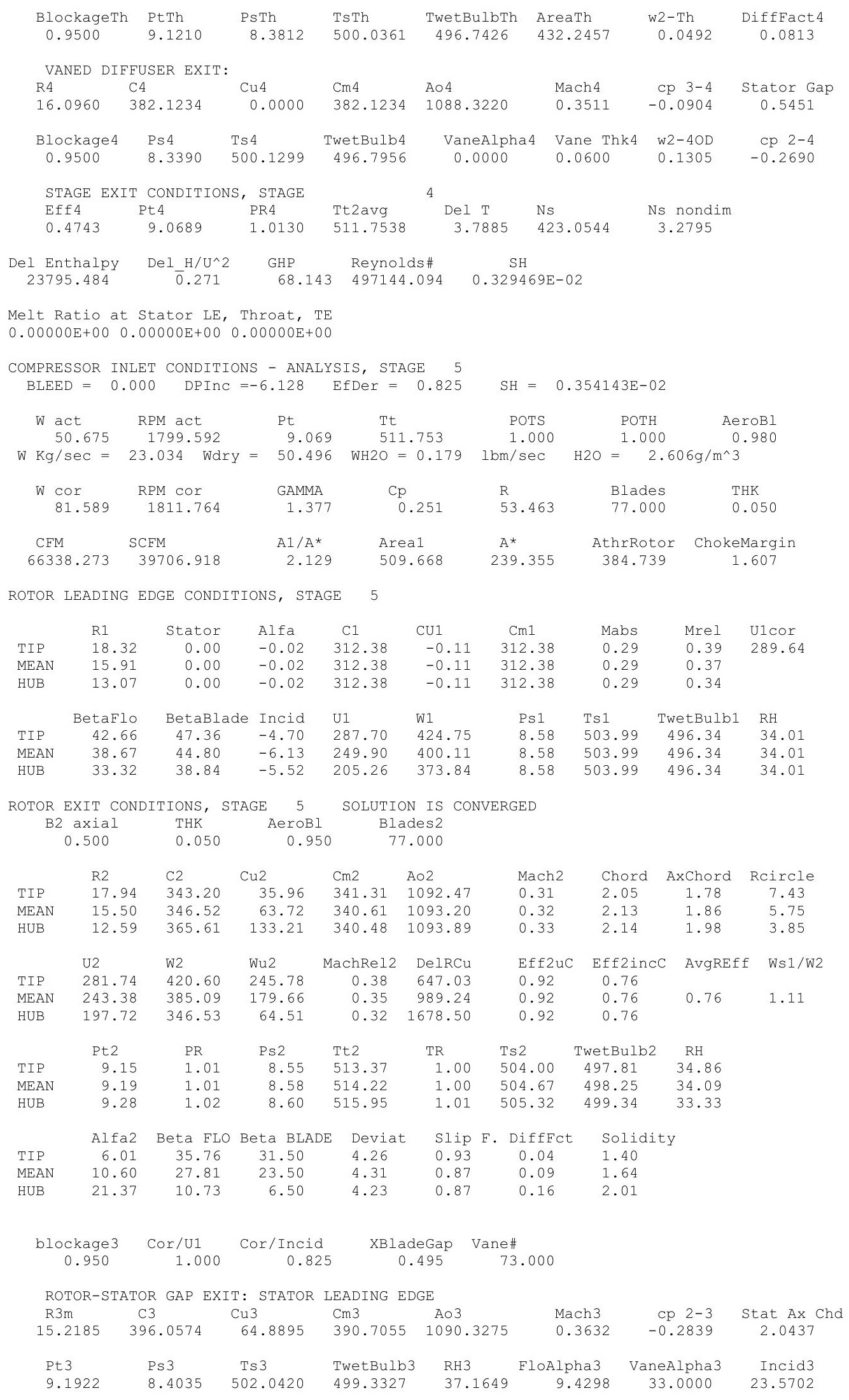




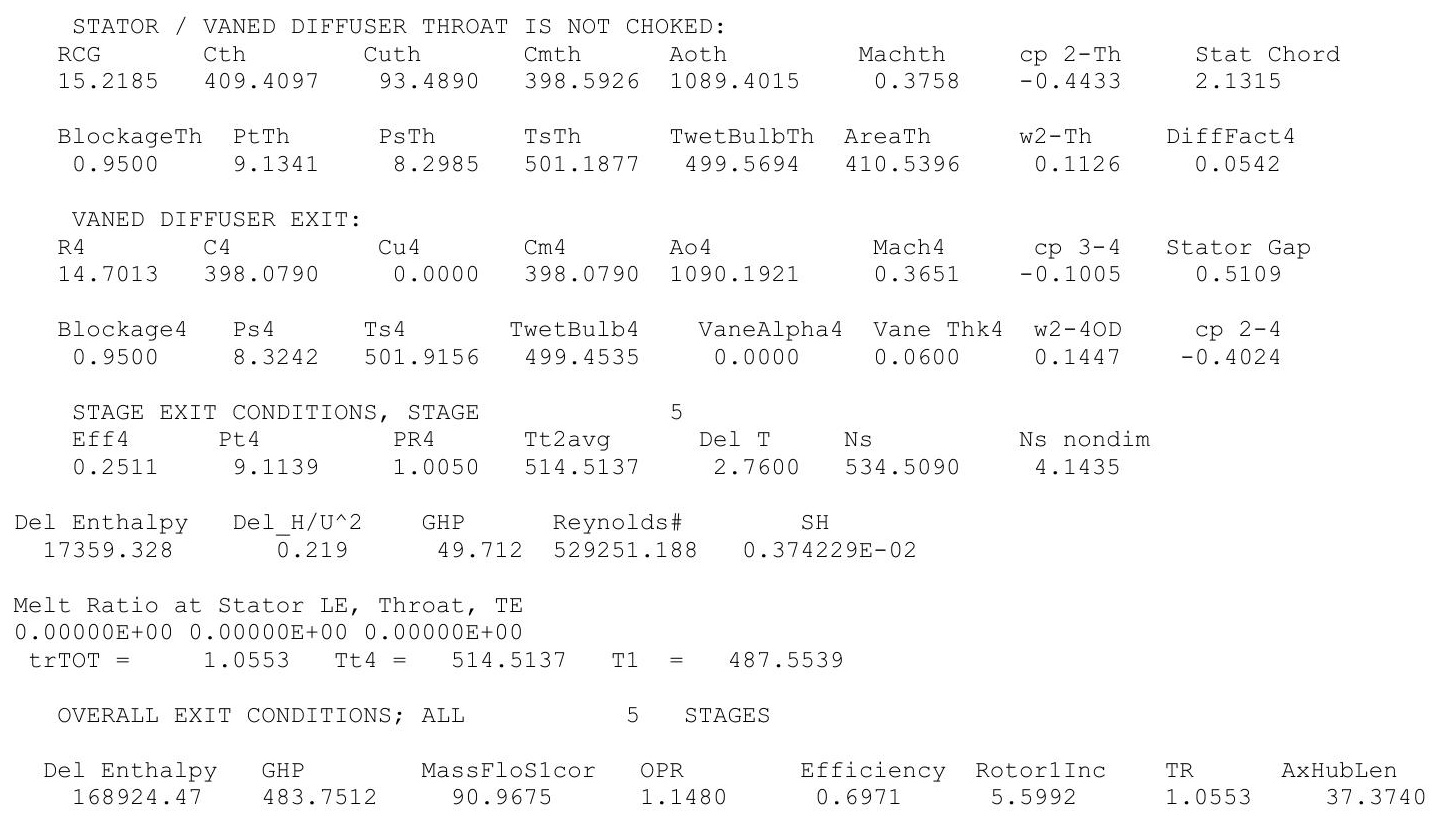




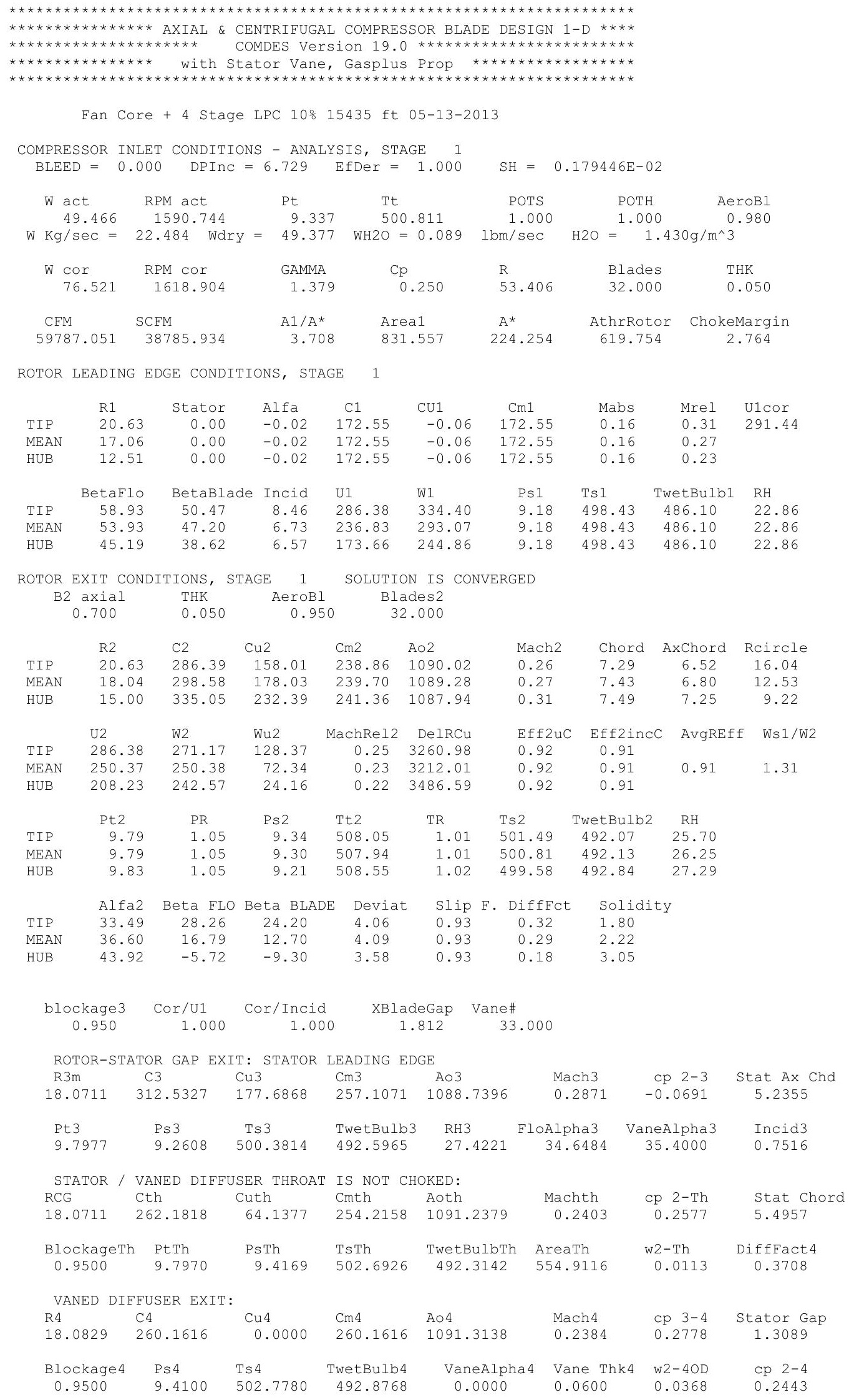




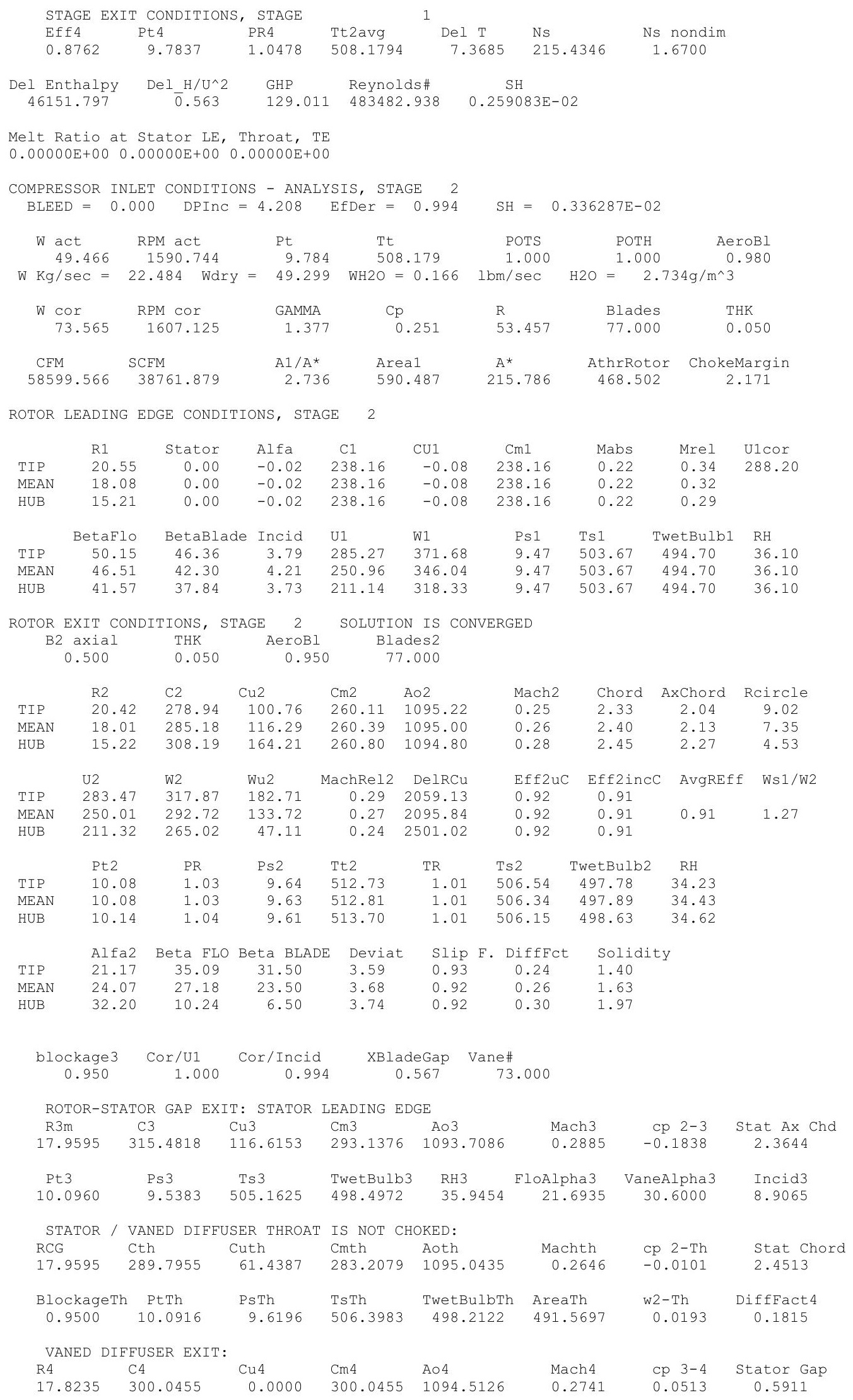




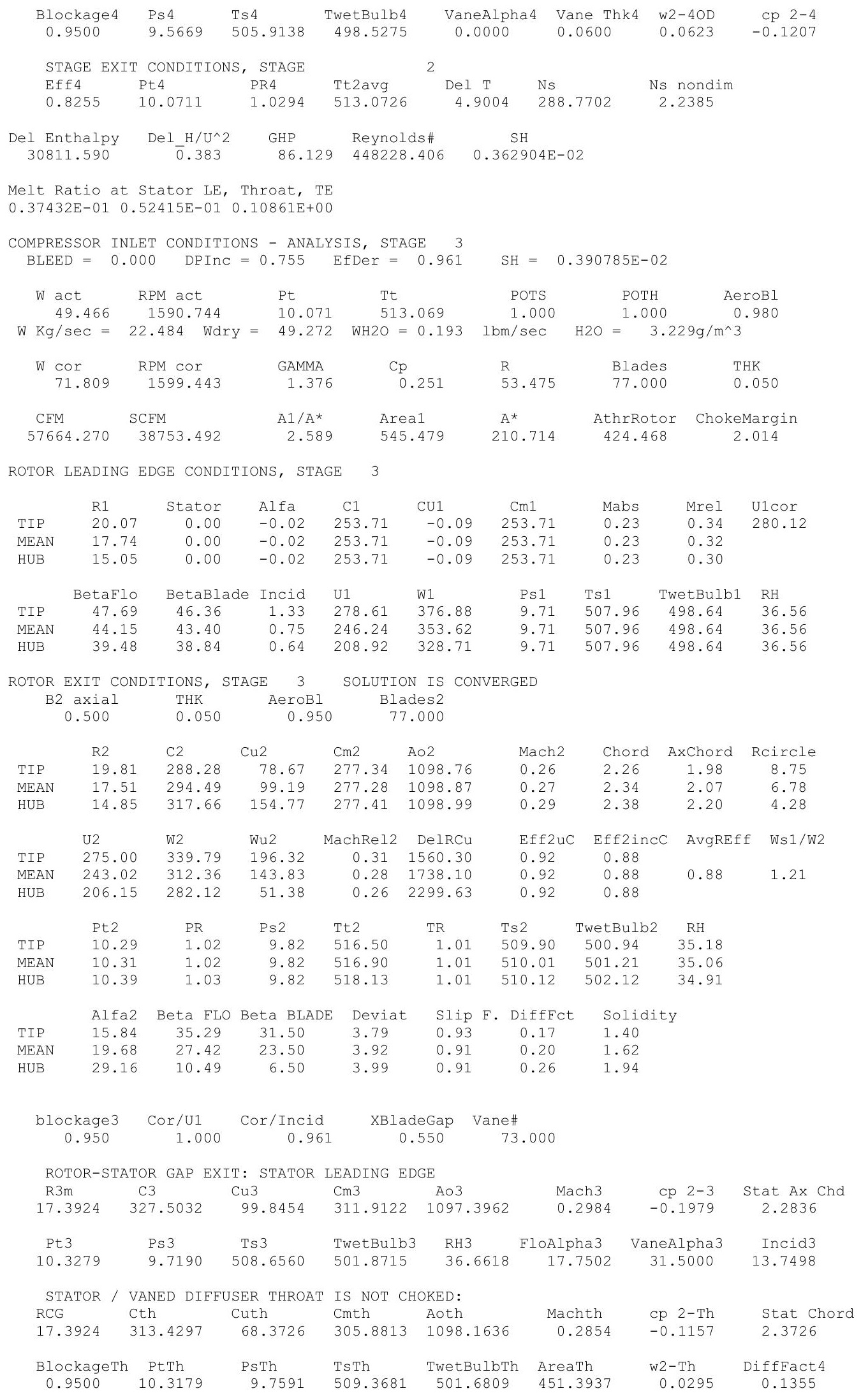




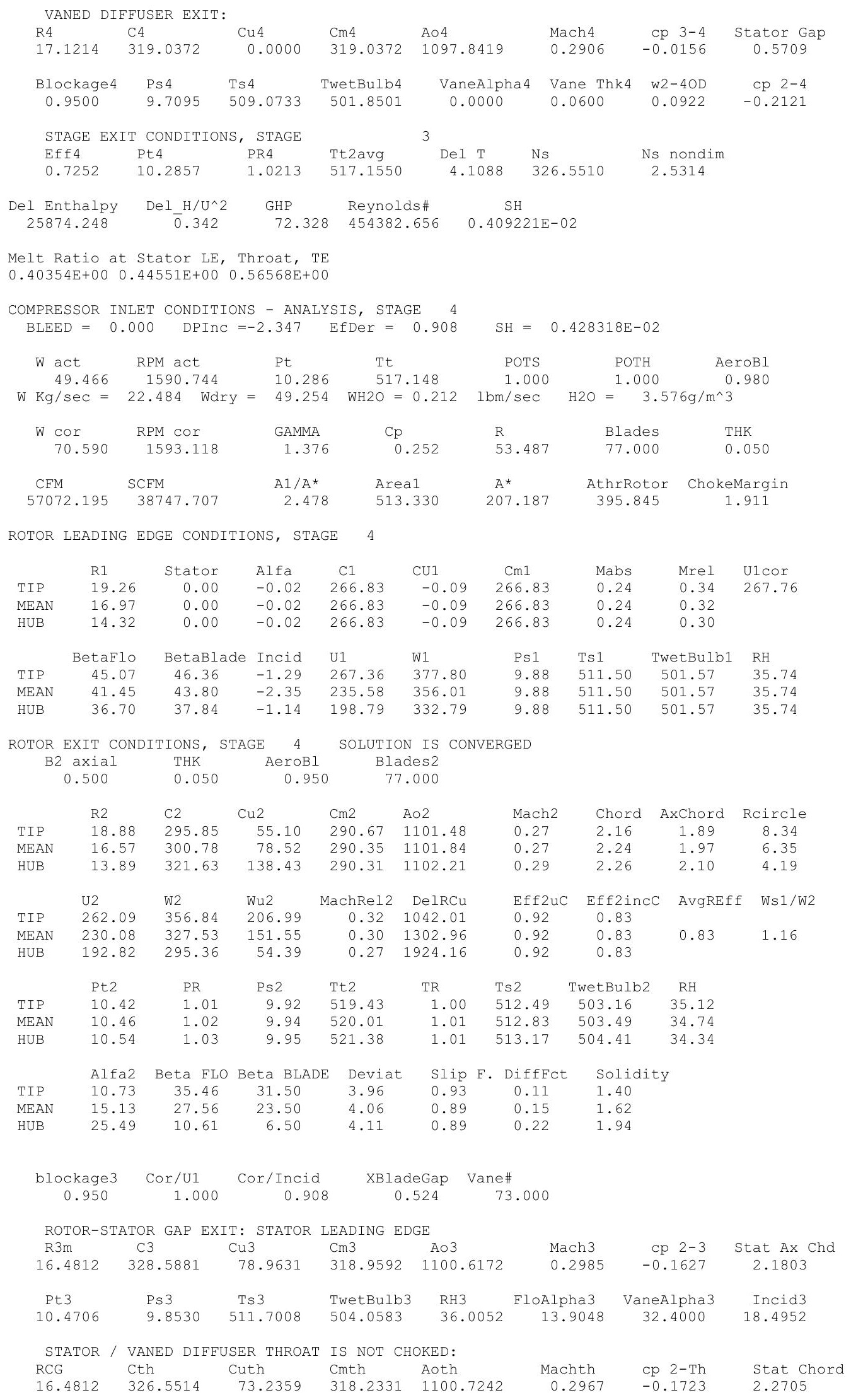




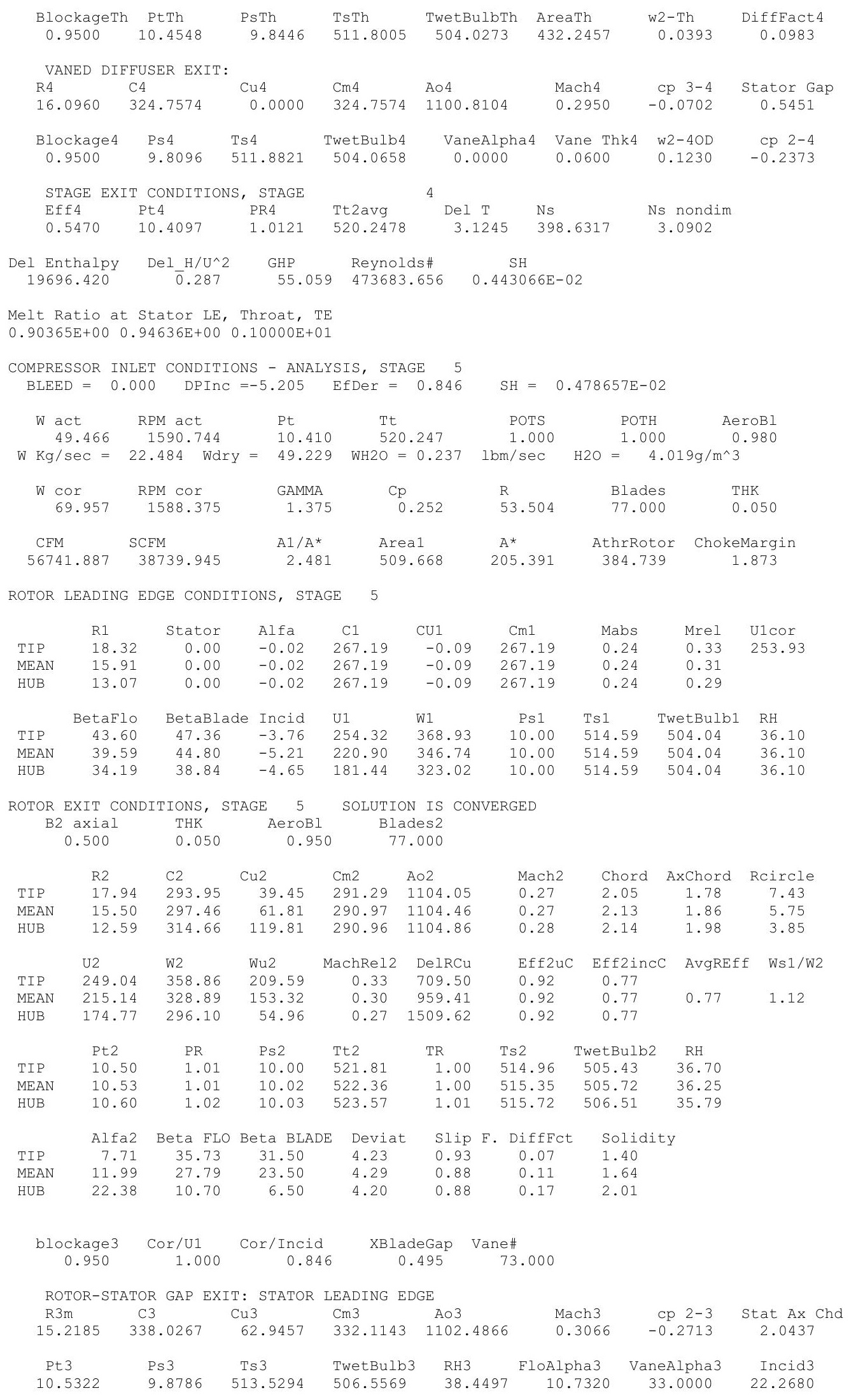




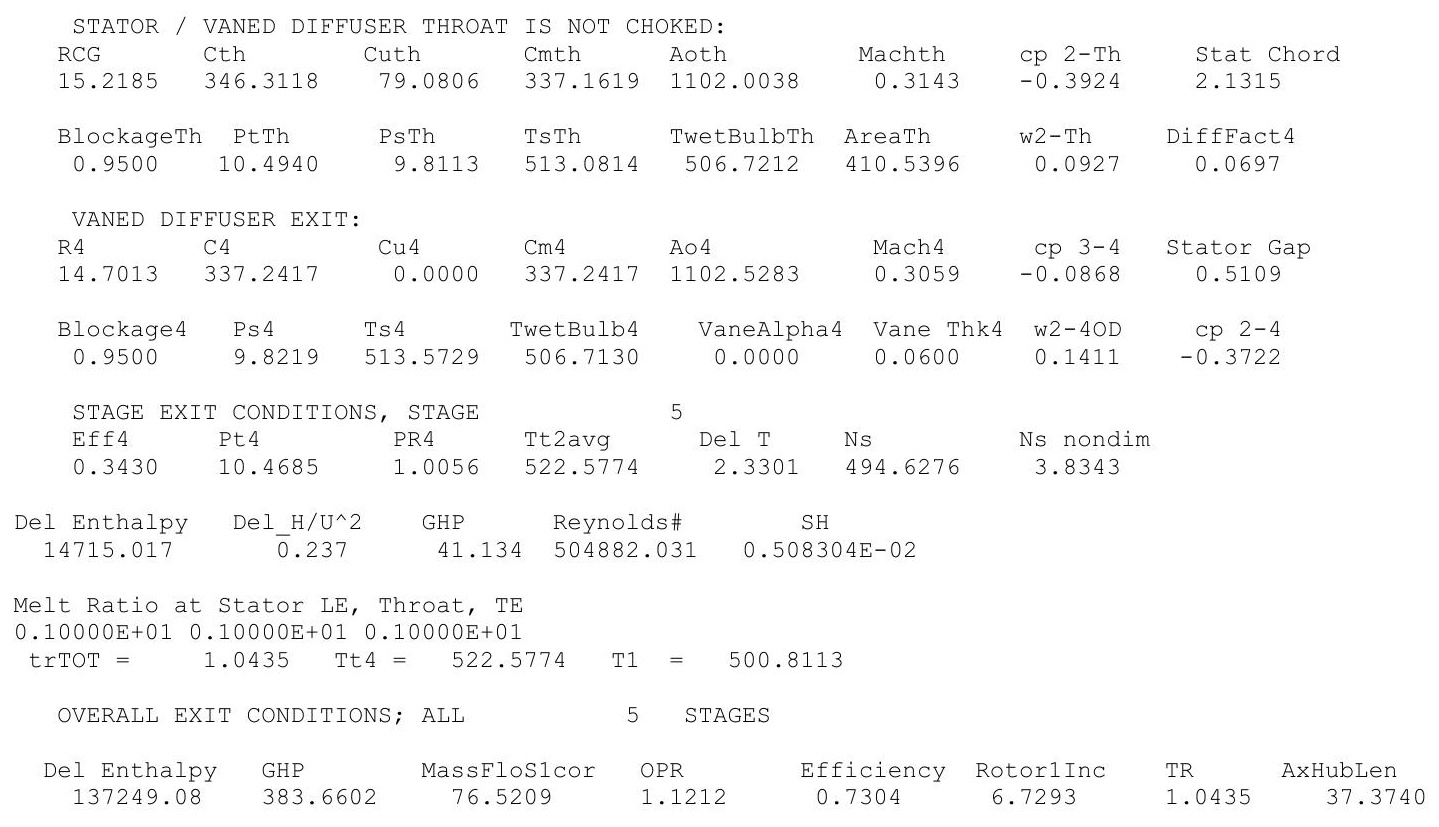


$5 \mu \mathrm{m}, 4 \mathrm{~g} / \mathrm{m}^{3}, \mathrm{ISA}+18 \mathrm{R}$

\begin{abstract}
A AXIAL \& CENTRIFUGAL COMPRESSOR BLADE DESIGN 1-D $* * * *$

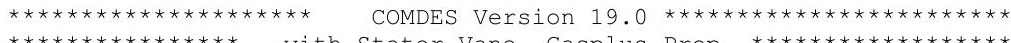

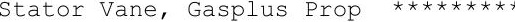

Fan Core + 4 Stage LPC 10\%39000 ft 05-13-2013

COMPRESSOR INLET CONDITIONS - ANALYSIS, STAGE 1

$\mathrm{BLEED}=0.000 \quad$ DPInC $=15.608 \quad$ EfDer $=0.953$

$\mathrm{SH}=0.992095 \mathrm{E}-03$

W act RPM act $\quad$ Pt $\quad$ POTS POTH

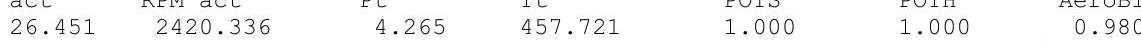

$\mathrm{W} \mathrm{Kg} / \mathrm{sec}=12.023$ Wdry $=26.425 \mathrm{WH} 2 \mathrm{O}=0.026 \mathrm{lbm} / \mathrm{sec} \quad \mathrm{H} 2 \mathrm{O}=0.394 \mathrm{~g} / \mathrm{m}^{\wedge} 3$

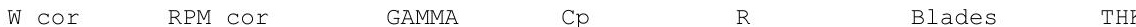

$\begin{array}{lrl}1.380 & \mathrm{CP} & \mathrm{R} \\ & 0.249 & 53.380\end{array}$

CFM SCFM A1/A* Area1 A* AthrRotor ChokeMargin

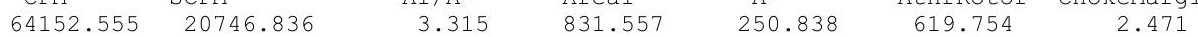

ROTOR LEADING EDGE CONDITIONS, STAGE 1

$\begin{array}{lccccccccc} & \text { R1 } & \text { Stator } & \text { Alfa } & \text { C1 } & \text { CU1 } & \text { Cm1 } & \text { Mabs } & \text { Mrel } & \text { U1cor } \\ \text { TIP } & 20.63 & 0.00 & -0.02 & 185.15 & -0.06 & 185.15 & 0.18 & 0.46 & 463.83 \\ \text { MEAN } & 17.06 & 0.00 & -0.02 & 185.15 & -0.06 & 185.15 & 0.18 & 0.39 & \\ \text { HUB } & 12.51 & 0.00 & -0.02 & 185.15 & -0.06 & 185.15 & 0.18 & 0.31 & \\ & & & & & & & & & \\ & \text { BetaFlo } & \text { BetaBlade } & \text { Incid } & \text { U1 } & \text { W1 } & \text { Ps1 } & \text { Ts1 } & \text { TwetBulb1 } & \text { RH } \\ \text { TIP } & 66.98 & 50.47 & 16.51 & 435.73 & 473.50 & 4.17 & 454.97 & 451.85 & 37.87 \\ \text { MEAN } & 62.81 & 47.20 & 15.61 & 360.33 & 405.18 & 4.17 & 454.97 & 451.85 & 37.87 \\ \text { HUB } & 54.99 & 38.62 & 16.37 & 264.23 & 322.70 & 4.17 & 454.97 & 451.85 & 37.87\end{array}$

ROTOR EXIT CONDITIONS, STAGE 1 SOLUTION IS CONVERGED

\begin{tabular}{|c|c|c|c|c|c|c|c|c|c|}
\hline & $\begin{array}{l}\text { axial } \\
700\end{array}$ & $\begin{array}{l}\text { THK } \\
0.050\end{array}$ & $\begin{array}{l}\text { AeroBl } \\
0.950\end{array}$ & $\begin{array}{r}\mathrm{Bla} \\
32\end{array}$ & $\begin{array}{l}\text { des } 2 \\
.000\end{array}$ & & & & \\
\hline & $\mathrm{R} 2$ & $\mathrm{C} 2$ & $\mathrm{Cu} 2$ & $\mathrm{Cm} 2$ & $\mathrm{~A} \circ 2$ & Mach2 & Chord & AxChord & Rcircle \\
\hline TIP & 20.63 & 390.17 & 301.44 & 247.72 & 1051.77 & 0.37 & 7.29 & 6.52 & 16.04 \\
\hline MEAN & 18.04 & 394.52 & 304.36 & 251.01 & 1048.67 & 0.38 & 7.43 & 6.80 & 12.53 \\
\hline HUB & 15.00 & 426.85 & 341.90 & 255.55 & 1044.87 & 0.41 & 7.49 & 7.25 & 9.22 \\
\hline & $\mathrm{U} 2$ & W2 & Wu2 & MachRel2 & DelRCu & Eff $2 u C$ & Eff2incC & AvgREff & Ws $1 /$ W2 \\
\hline TIP & 435.73 & 281.78 & 134.29 & 0.27 & 6220.07 & 0.92 & 0.87 & & \\
\hline MEAN & 380.95 & 262.43 & 76.58 & 0.25 & 5490.56 & 0.92 & 0.87 & 0.87 & 1.77 \\
\hline $\mathrm{HUB}$ & 316.82 & 256.78 & 25.08 & 0.25 & 5129.36 & 0.92 & 0.87 & & \\
\hline & Pt2 & $\mathrm{PR}$ & Ps2 & Tt2 & TR & Ts2 & TwetBulb2 & RH & \\
\hline$\Gamma \perp P$ & 4.92 & 1.15 & 4.48 & 478.80 & 1.05 & 466.59 & 468.49 & 34.41 & \\
\hline SAN & 4.84 & 1.13 & 4.39 & 476.33 & 1.04 & 463.84 & 467.17 & 38.27 & \\
\hline JB & 4.80 & 1.13 & 4.29 & 475.11 & 1.04 & 460.49 & 467.01 & 43.60 & \\
\hline & Alfa2 & Beta FLO & Beta BLADE & Deviat & Slip & F. DiffFct & Solidit & & \\
\hline P & 50.59 & 28.46 & 24.20 & 4.26 & 0.93 & 0.58 & 1.80 & & \\
\hline AN & 50.49 & 16.97 & 12.70 & 4.27 & 0.95 & 0.53 & 2.22 & & \\
\hline & 53.22 & -5.61 & -9.30 & 3.69 & 0.95 & 0.39 & 3.05 & & \\
\hline
\end{tabular}

$\begin{array}{ccccc}\text { blockage } 3 & \text { Cor/U1 } & \text { Cor/Incid } & \text { XBladeGap } & \text { Vane\# } \\ 0.950 & 1.000 & 0.953 & 1.812 & 33.000\end{array}$

ROTOR-STATOR GAP EXIT: STATOR LEADING EDGE

\begin{tabular}{|c|c|c|c|c|c|c|c|}
\hline $\begin{array}{l}\text { R3m } \\
18.0711\end{array}$ & $\begin{array}{l}\text { C3 } \\
405.9364\end{array}$ & $\begin{array}{l}\text { Cu3 } \\
303.7706\end{array}$ & $\begin{array}{l}\mathrm{Cm} 3 \\
269.2726\end{array}$ & $\begin{array}{l}\text { A०3 } \\
1048.2499\end{array}$ & $\begin{array}{l}\text { Mach3 } \\
0.3873\end{array}$ & $\begin{array}{r}\mathrm{cp} 2-3 \\
-0.0354\end{array}$ & $\begin{array}{c}\text { Stat Ax Chd } \\
5.2355\end{array}$ \\
\hline Pt 3 & Ps 3 & Ts 3 & TwetBulb3 & RH3 & FloAlpha 3 & VaneAlpha3 & Incid3 \\
\hline 4.8484 & 4.3782 & 463.5425 & 467.8000 & 40.1117 & 48.4451 & 35.4000 & -13.0451 \\
\hline STATOR / & VANED DIF & USER THROAT & IS NOT CHC & DKED : & & & \\
\hline $\begin{array}{l}\text { RCG } \\
18.0711\end{array}$ & $\begin{array}{l}\text { Cth } \\
267.1325\end{array}$ & $\begin{array}{l}\text { Cuth } \\
65.3488\end{array}$ & $\begin{array}{l}\text { Cmth } \\
259.0161\end{array}$ & $\begin{array}{l}\text { Aoth } \\
1056.6517\end{array}$ & $\begin{array}{l}\text { Machth } \\
0.2528\end{array}$ & $\begin{array}{r}\mathrm{cp} 2-\mathrm{Th} \\
0.5190\end{array}$ & $\begin{array}{l}\text { Stat Chord } \\
5.4957\end{array}$ \\
\hline $\begin{array}{c}\text { BlockageTh } \\
0.9500\end{array}$ & $\begin{array}{l}\text { PtTh } \\
4.8361\end{array}$ & $\begin{array}{l}\text { PsTh } \\
4.6287\end{array}$ & $\begin{array}{l}\text { TsTh } \\
471.0272\end{array}$ & $\begin{array}{c}\text { TwetBulbTh } \\
466.4515\end{array}$ & $\begin{array}{l}\text { AreaTh } \\
554.9116\end{array}$ & $\begin{array}{l}\text { w2-Th } \\
0.0363\end{array}$ & DiffFact 4 \\
\hline VANED DII & FEUSER EX & & & & & & \\
\hline R4 & $\mathrm{C} 4$ & $\mathrm{Cu} 4$ & $\mathrm{Cm} 4$ & A०4 & Mach 4 & cp 3-4 & tator Gap \\
\hline 18.0829 & 266.0807 & 0.0000 & 266.0807 & 1056.6504 & 0.2518 & 0.4877 & 1.3089 \\
\hline
\end{tabular}




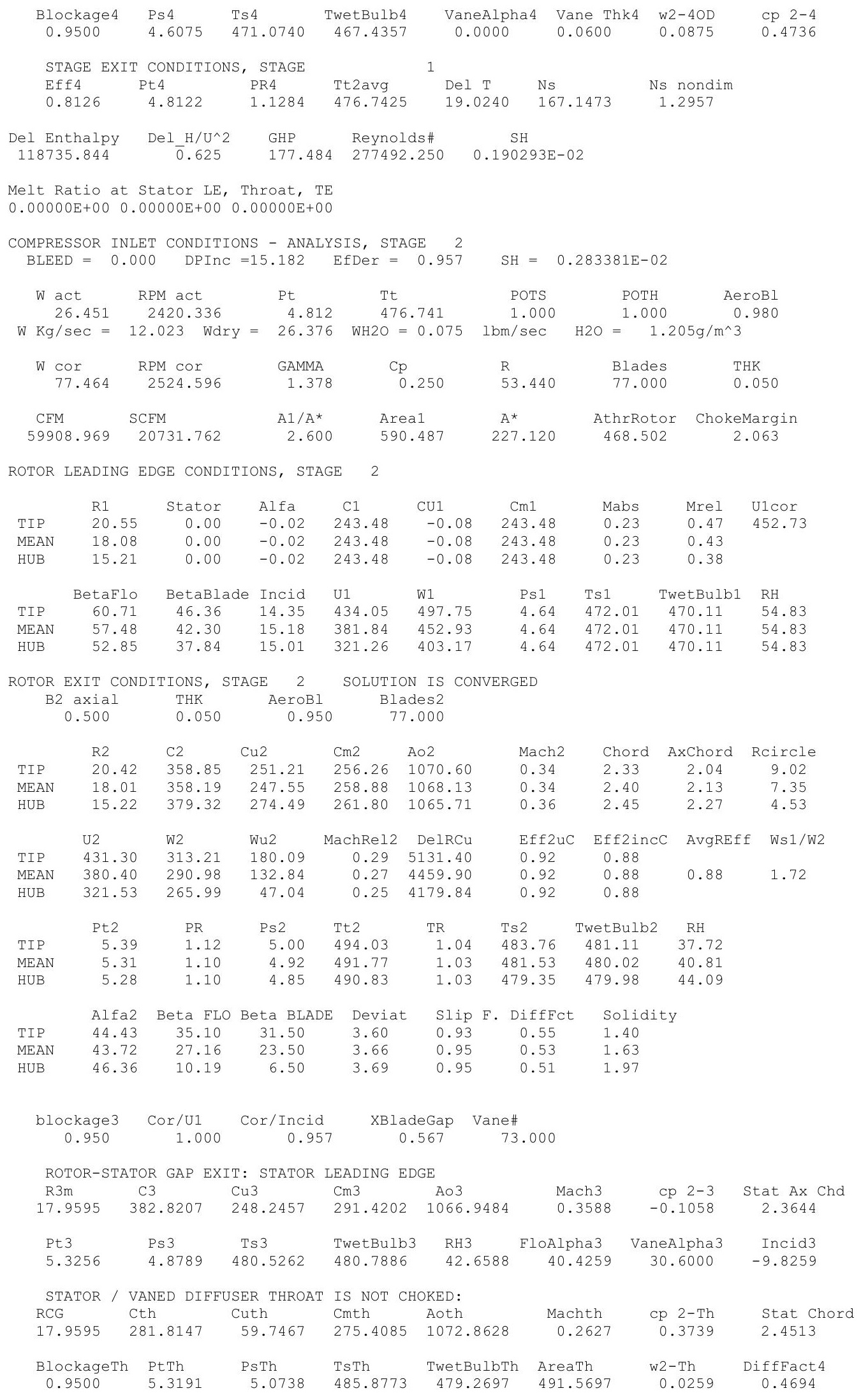




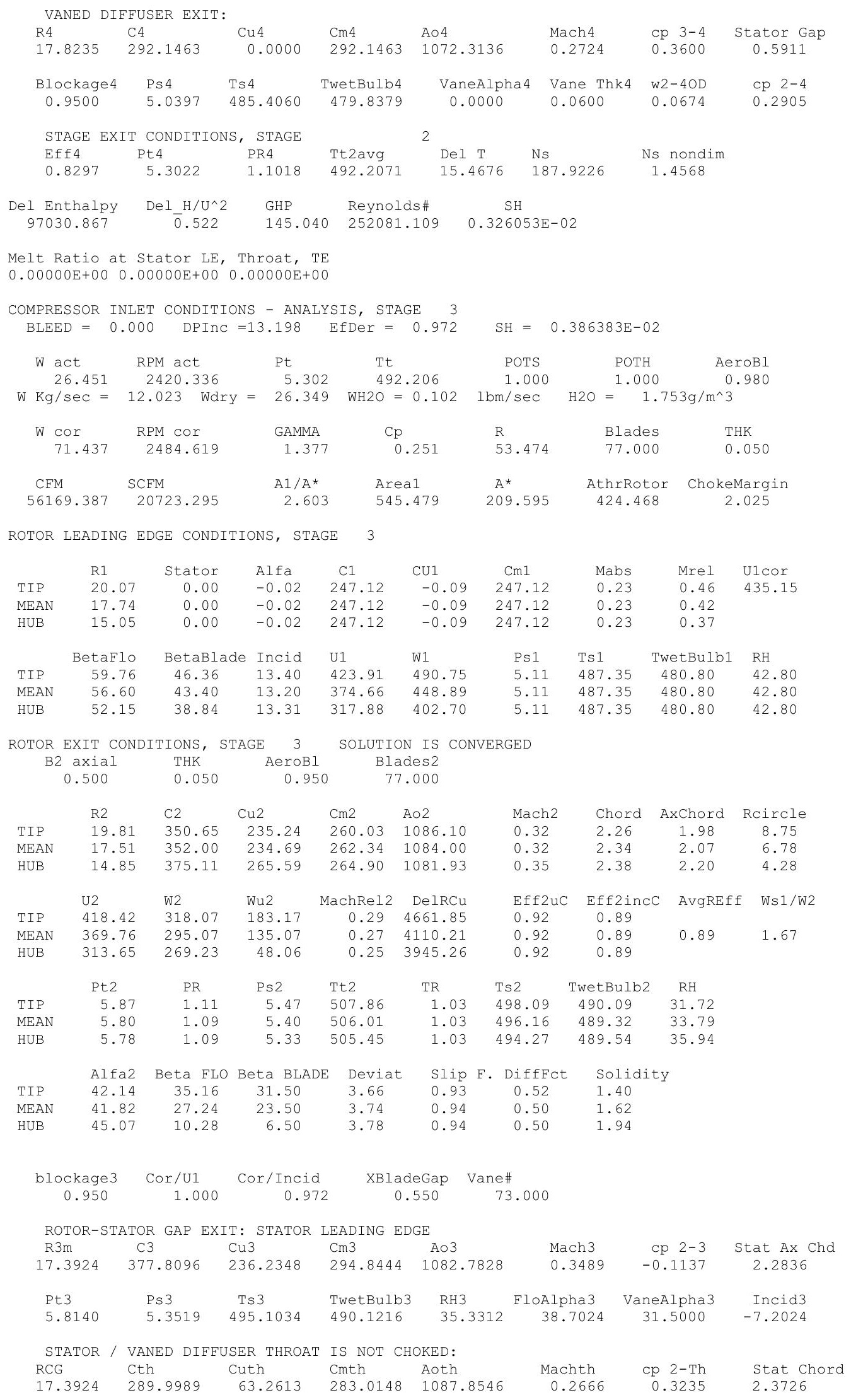




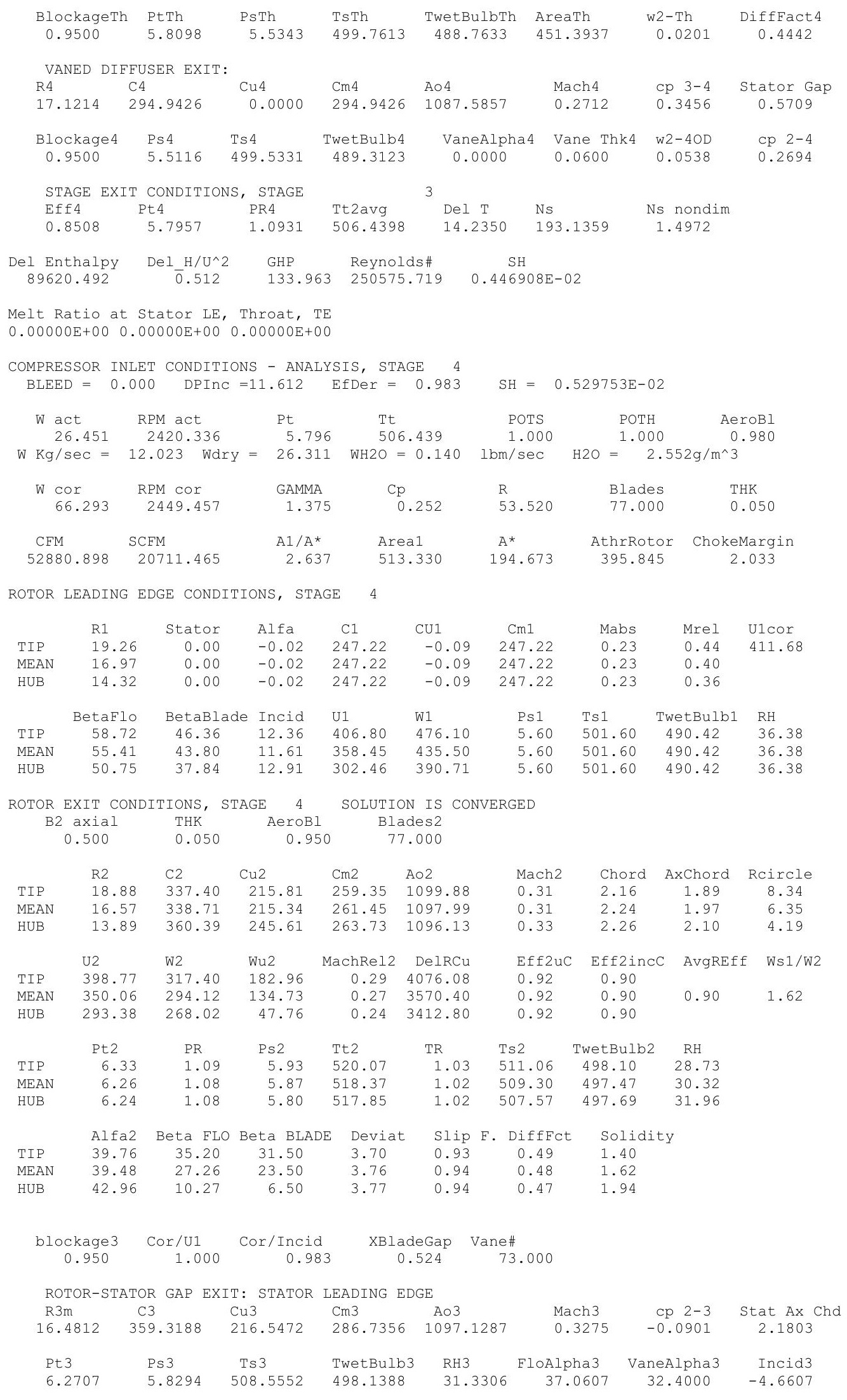




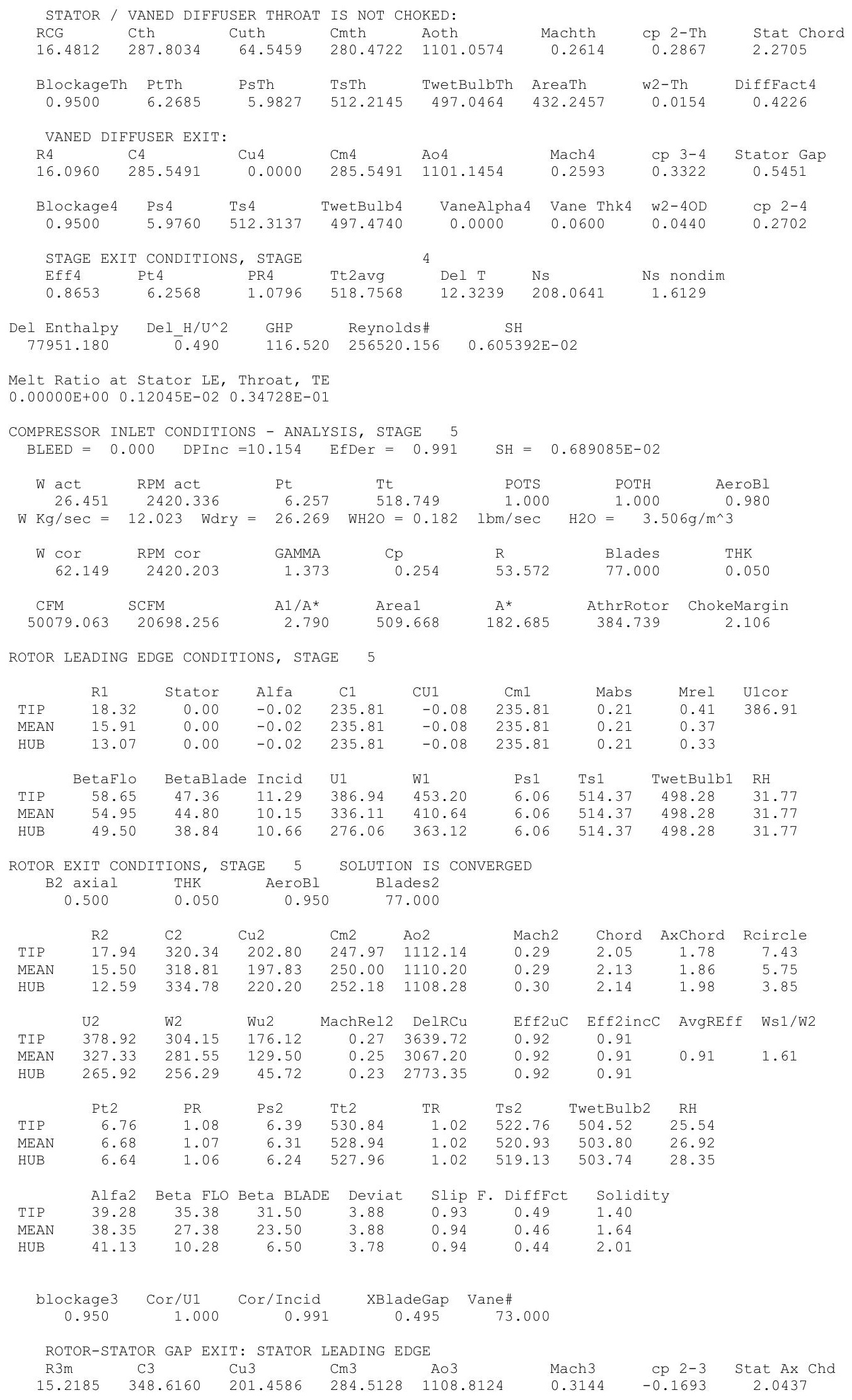




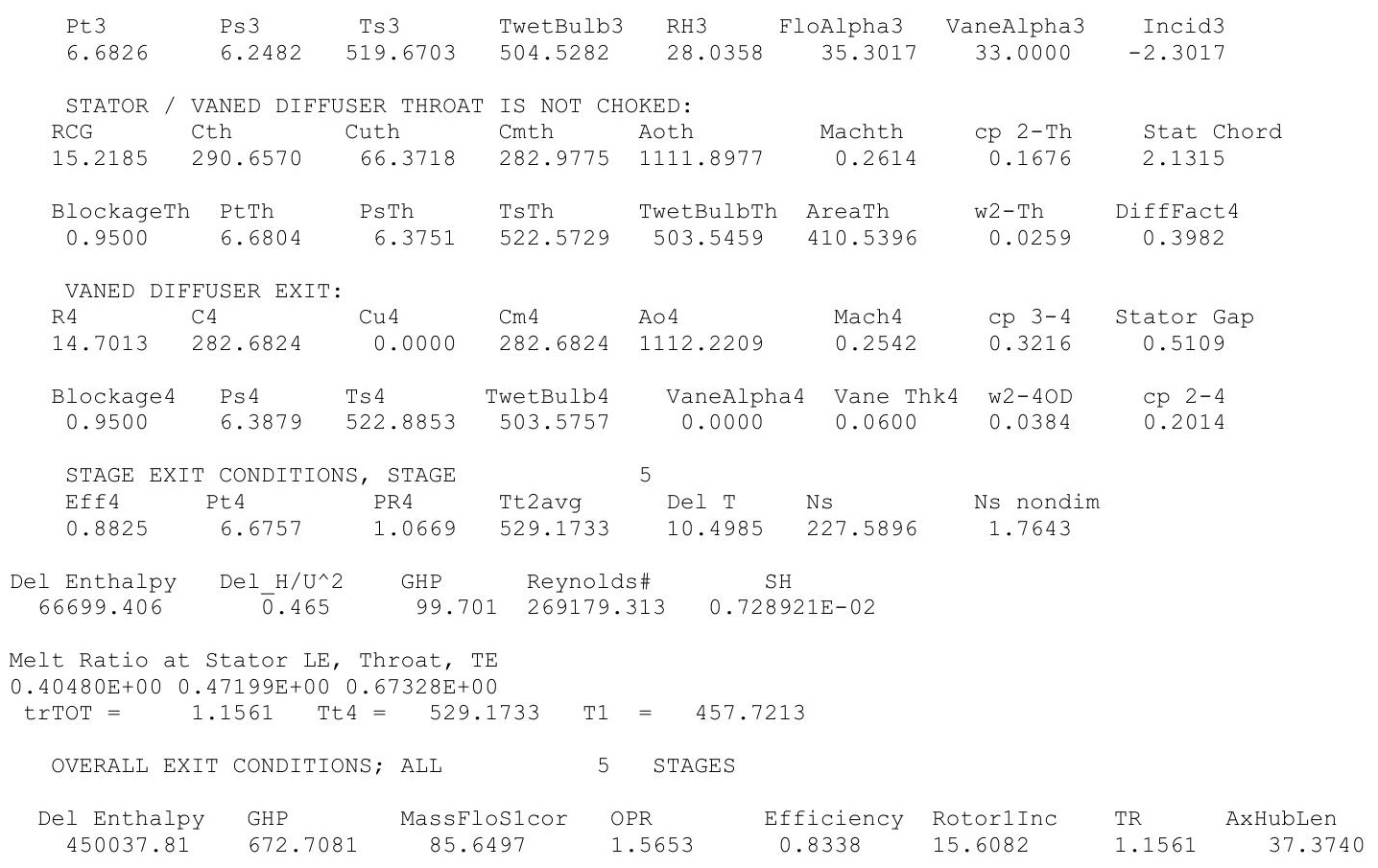




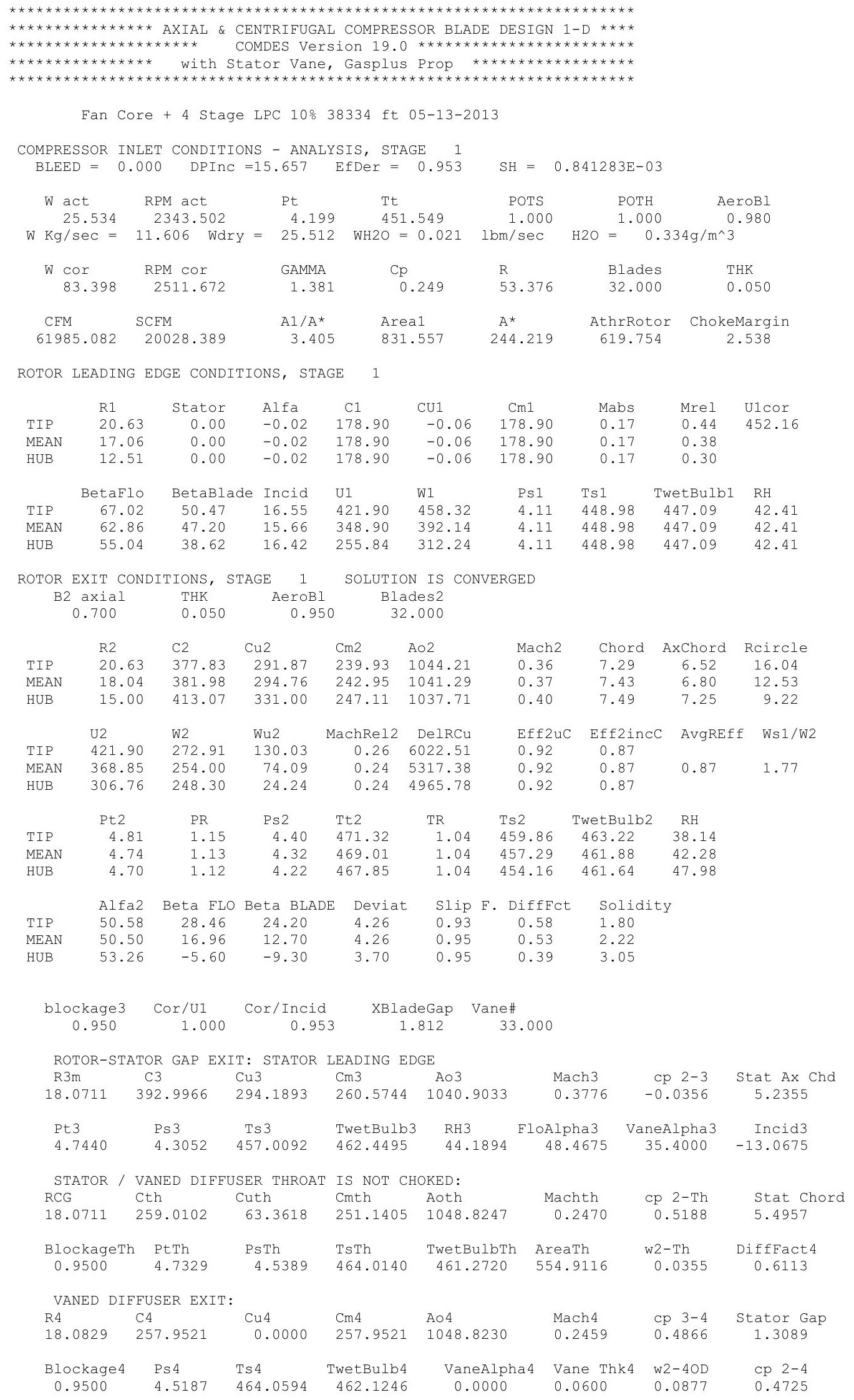




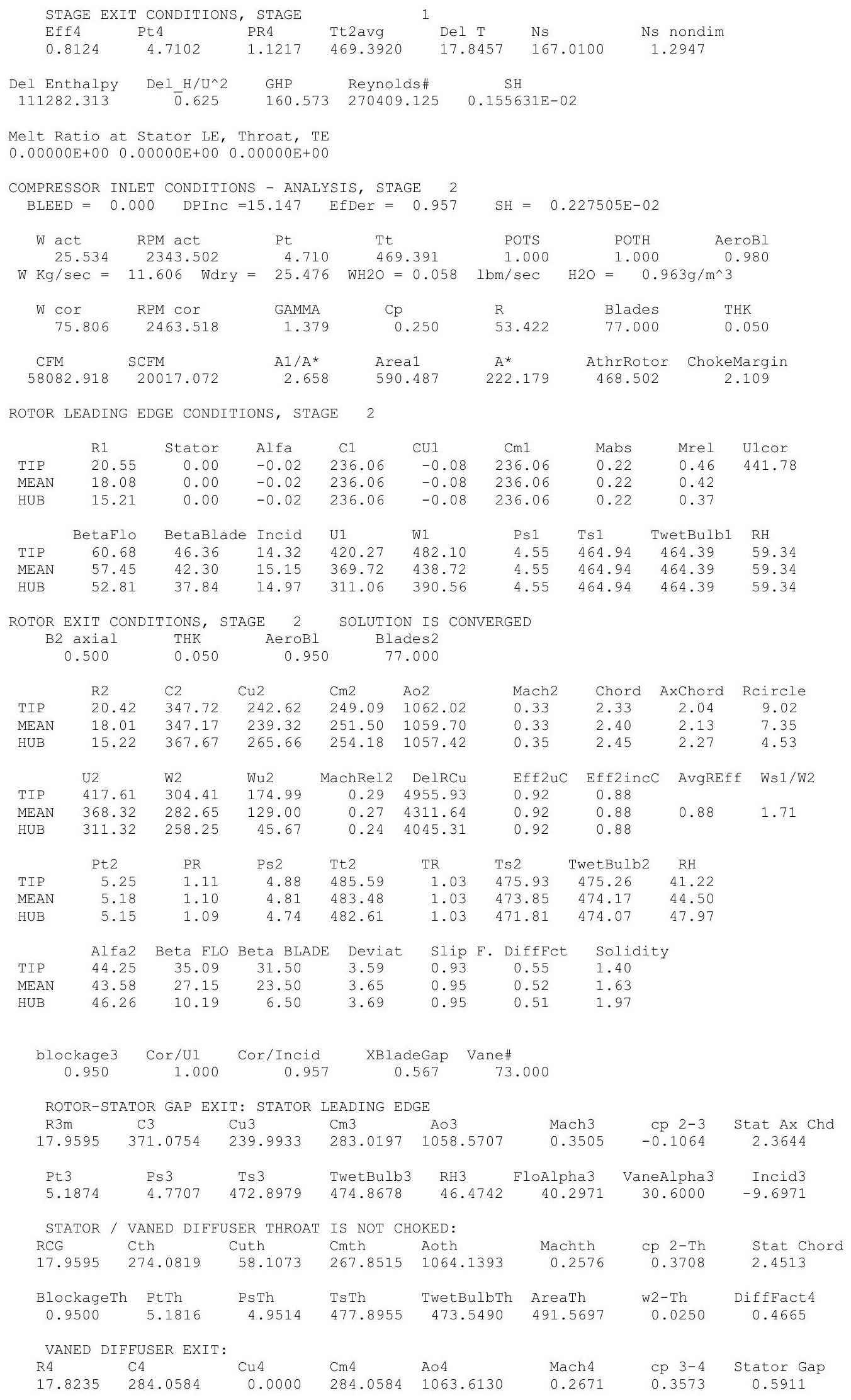




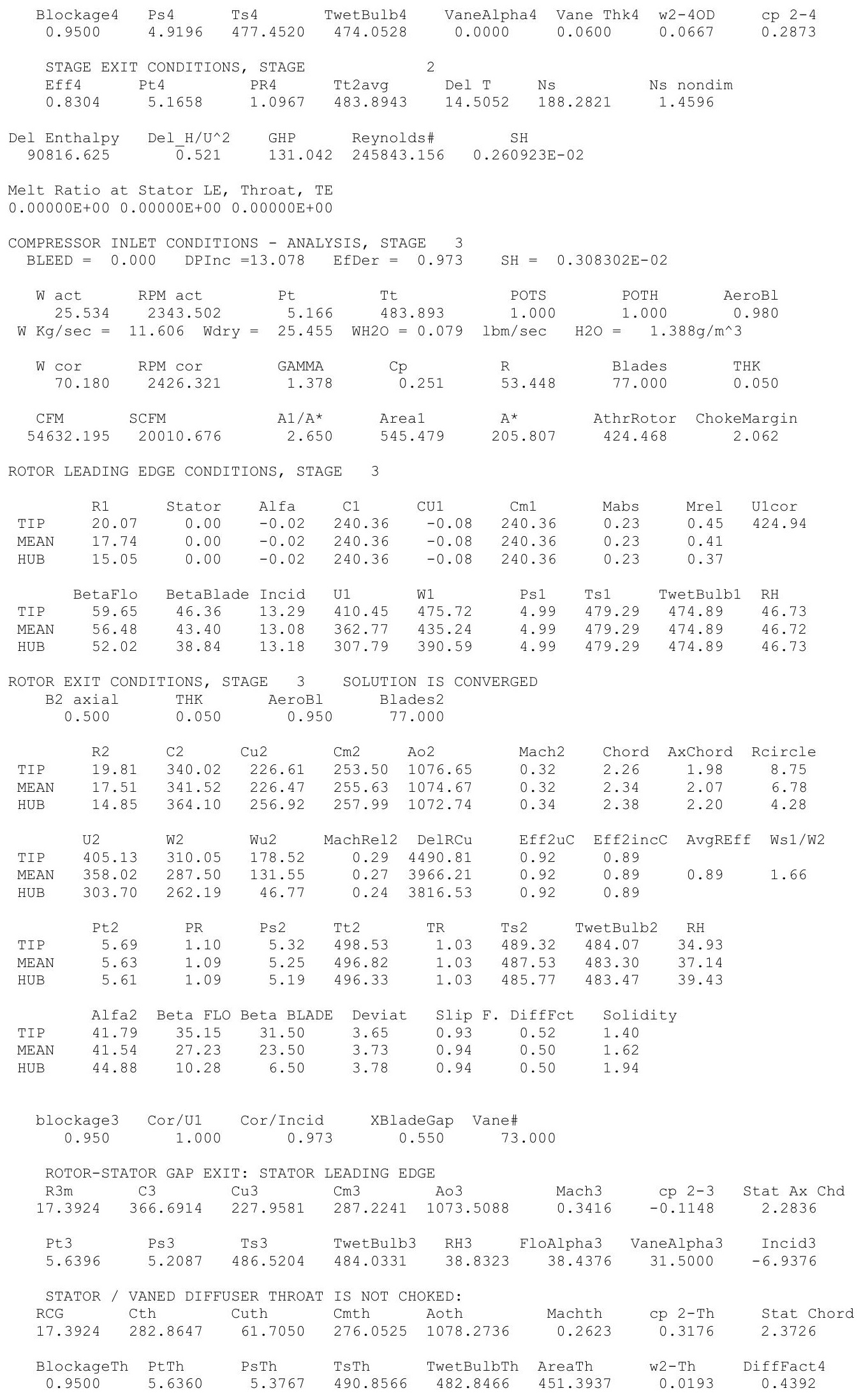




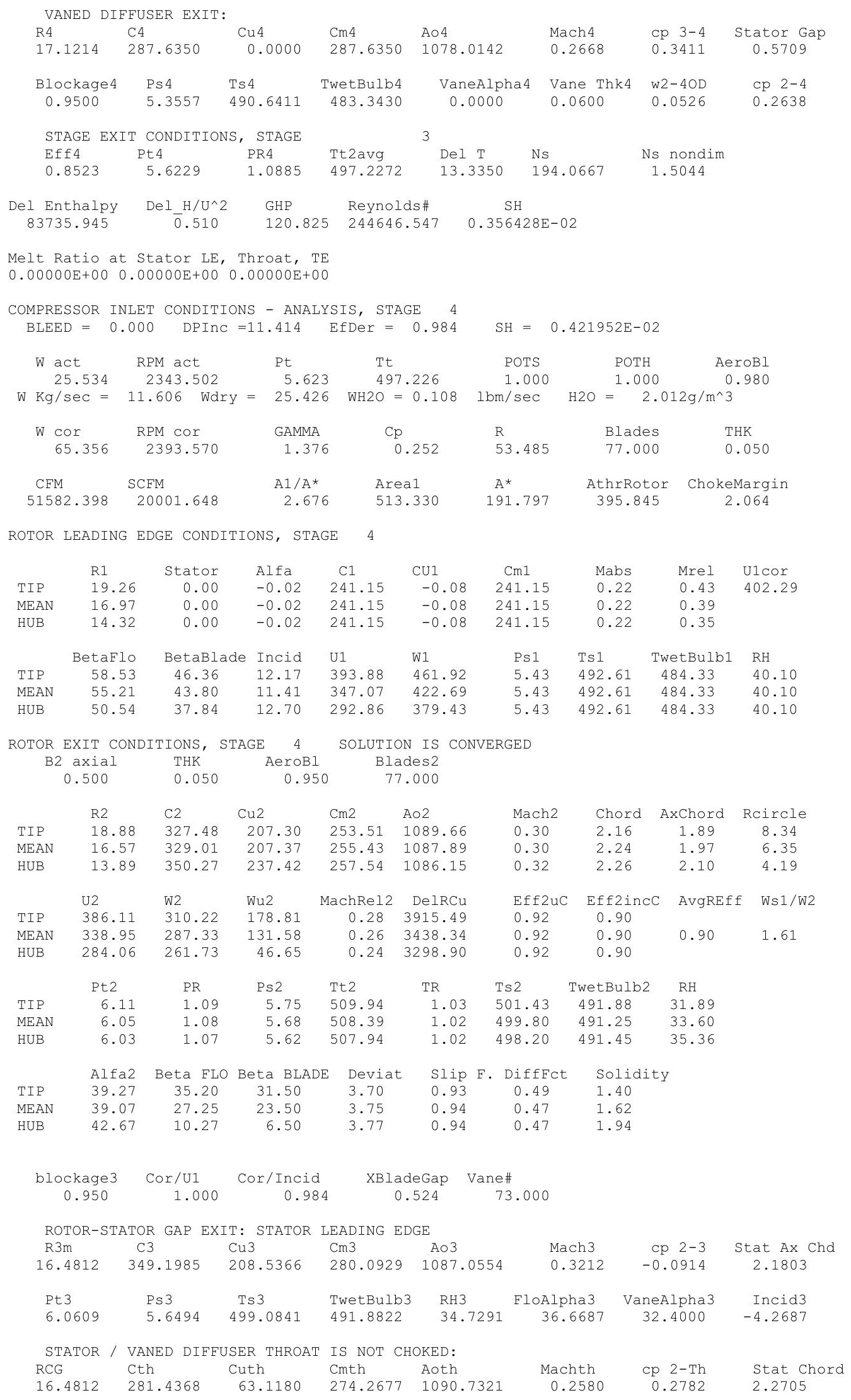




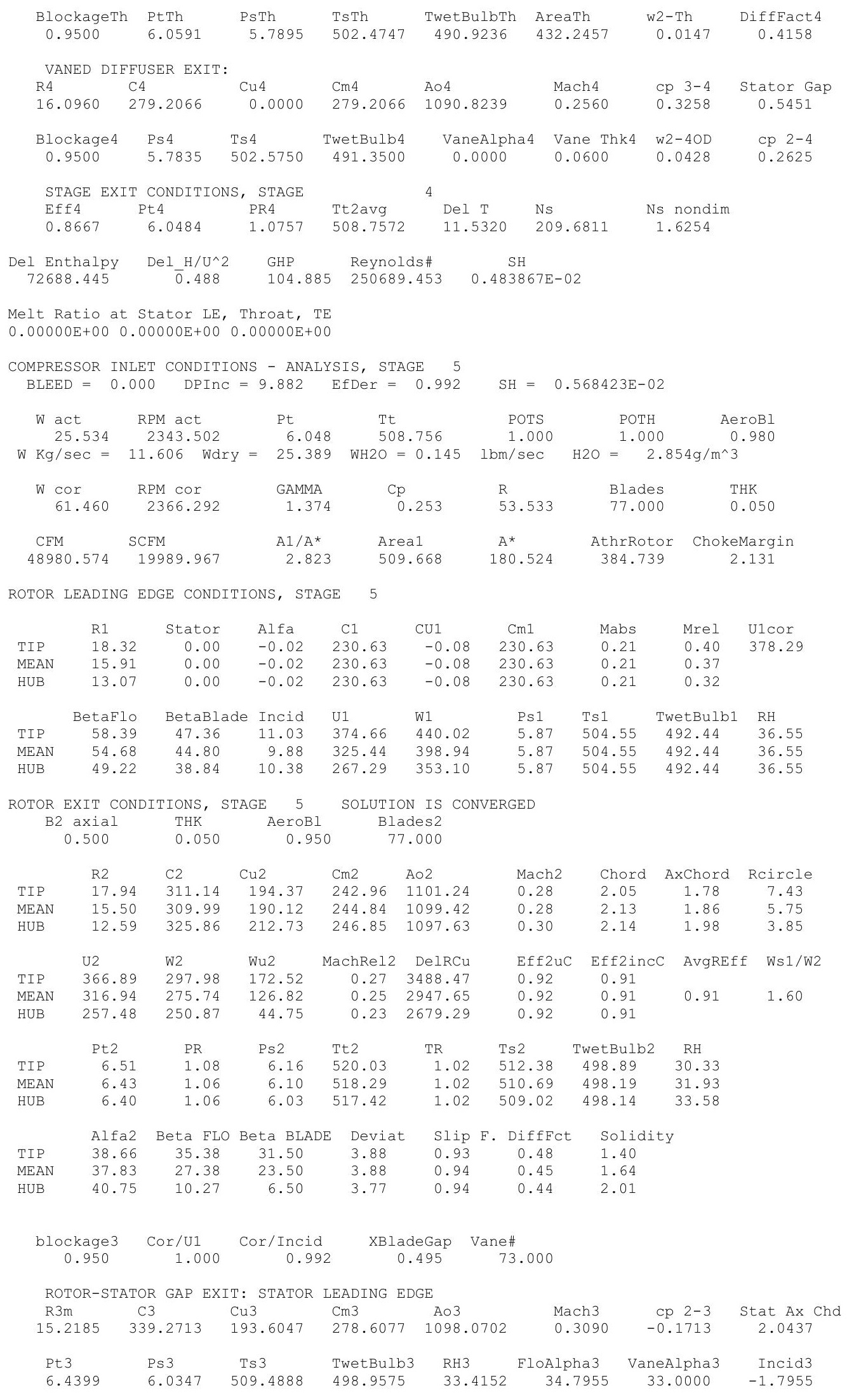




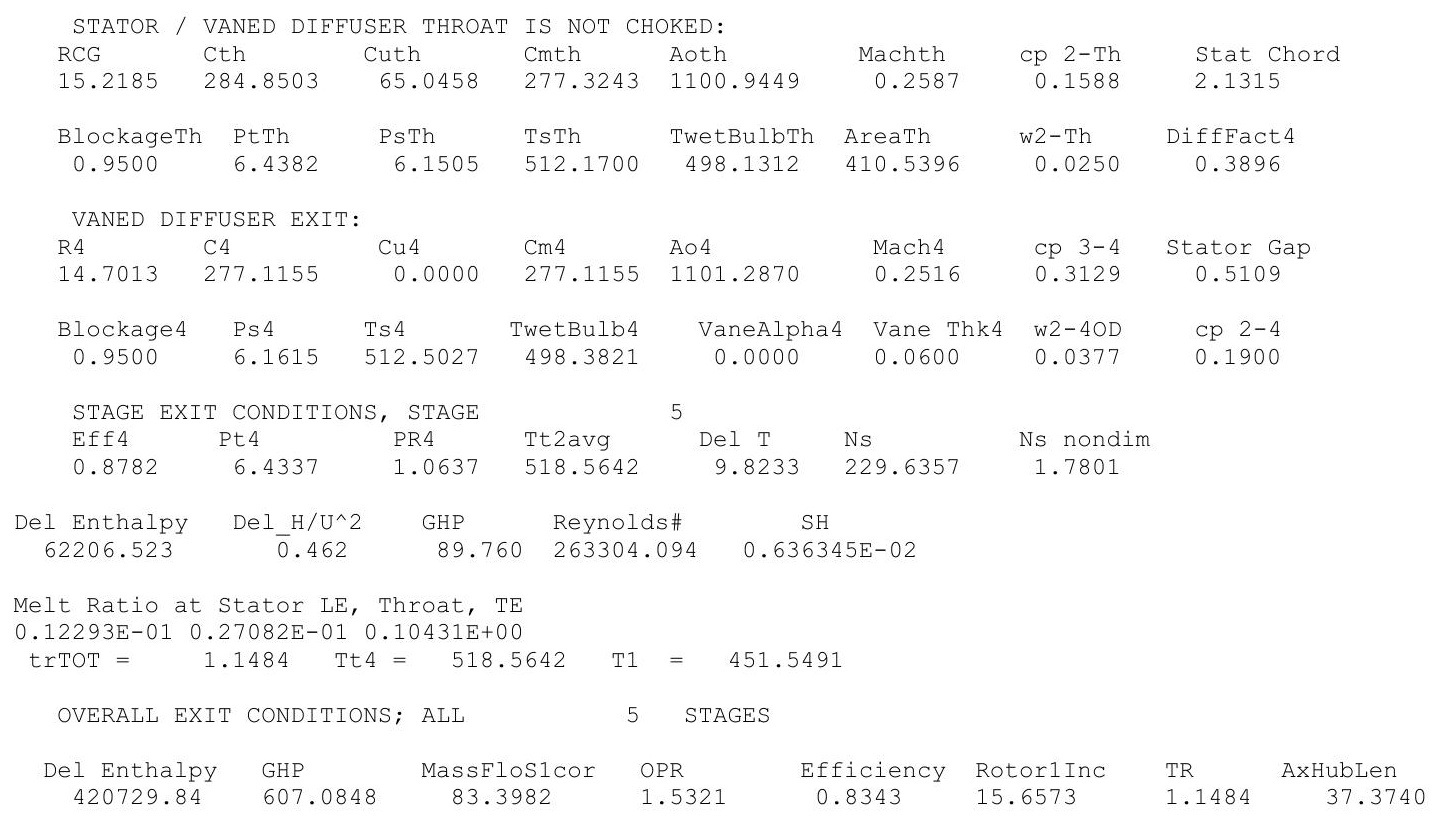




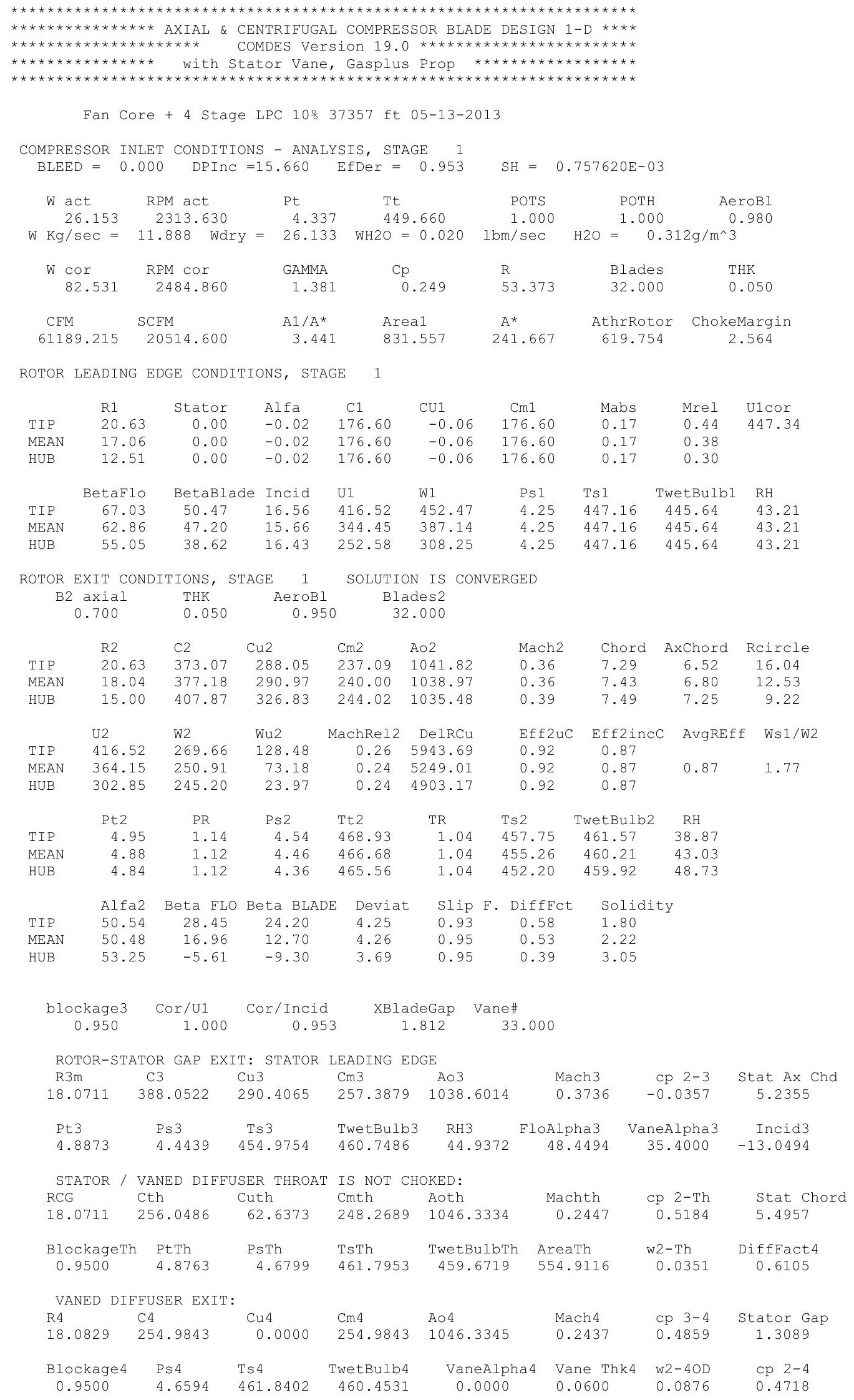




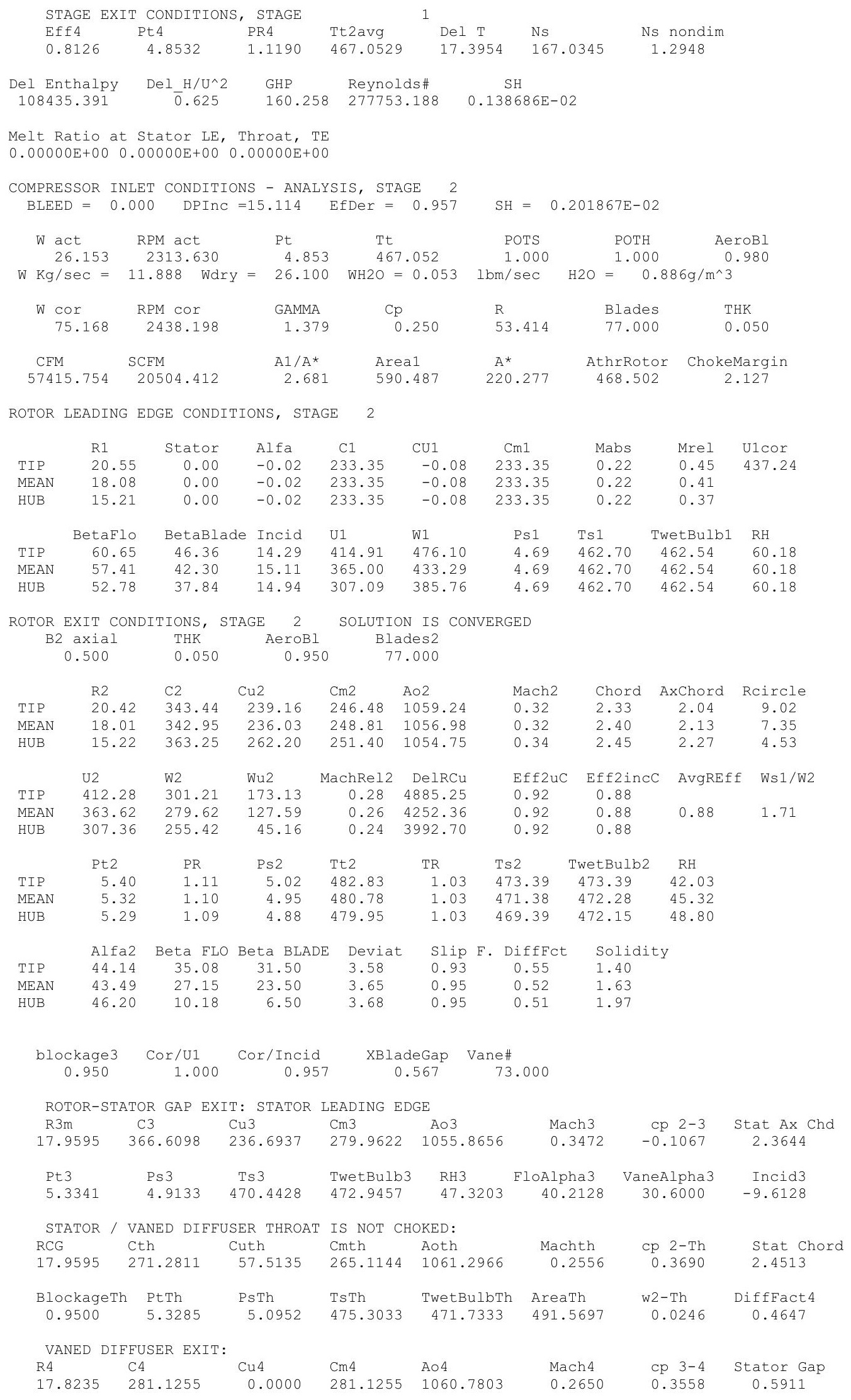




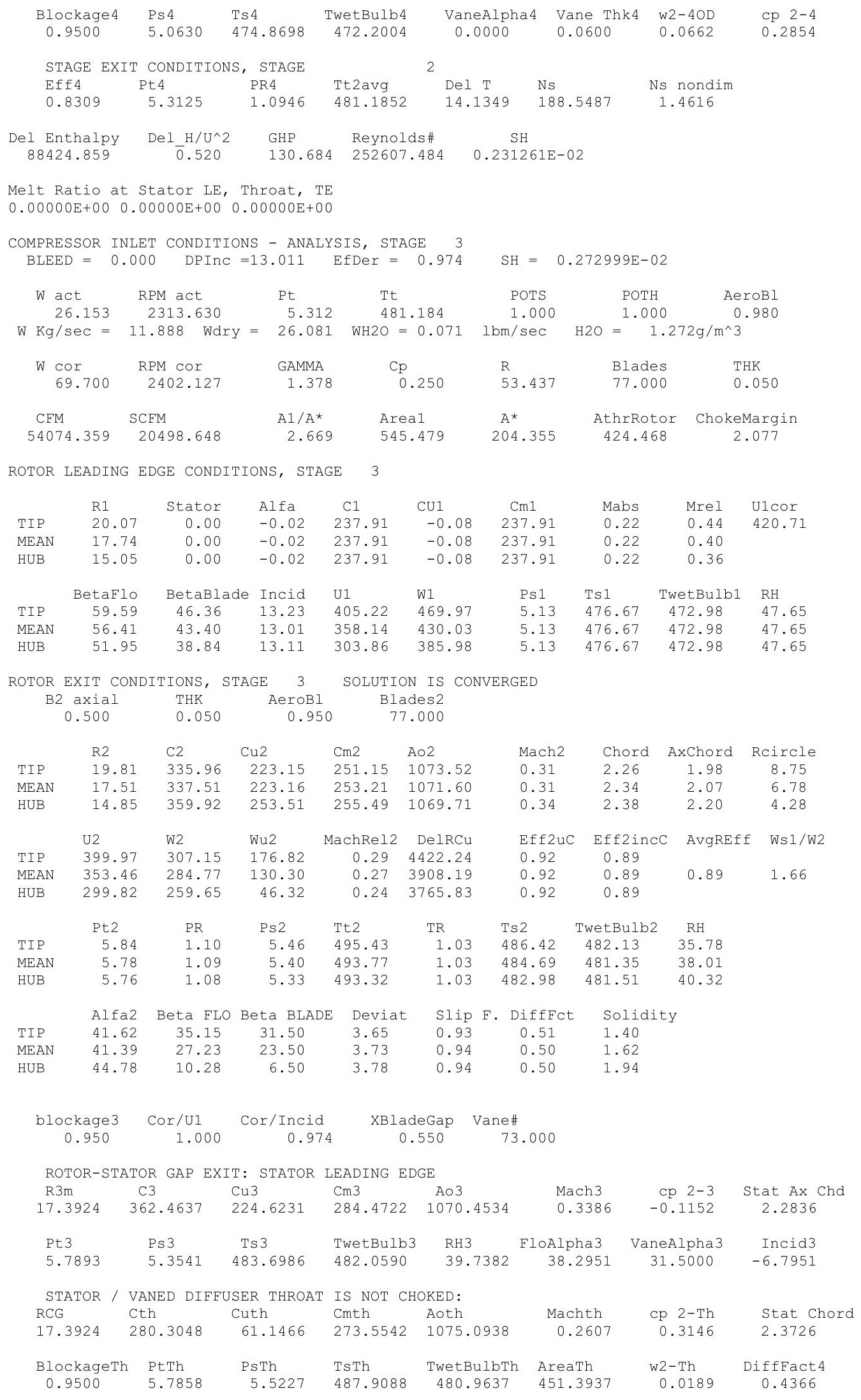




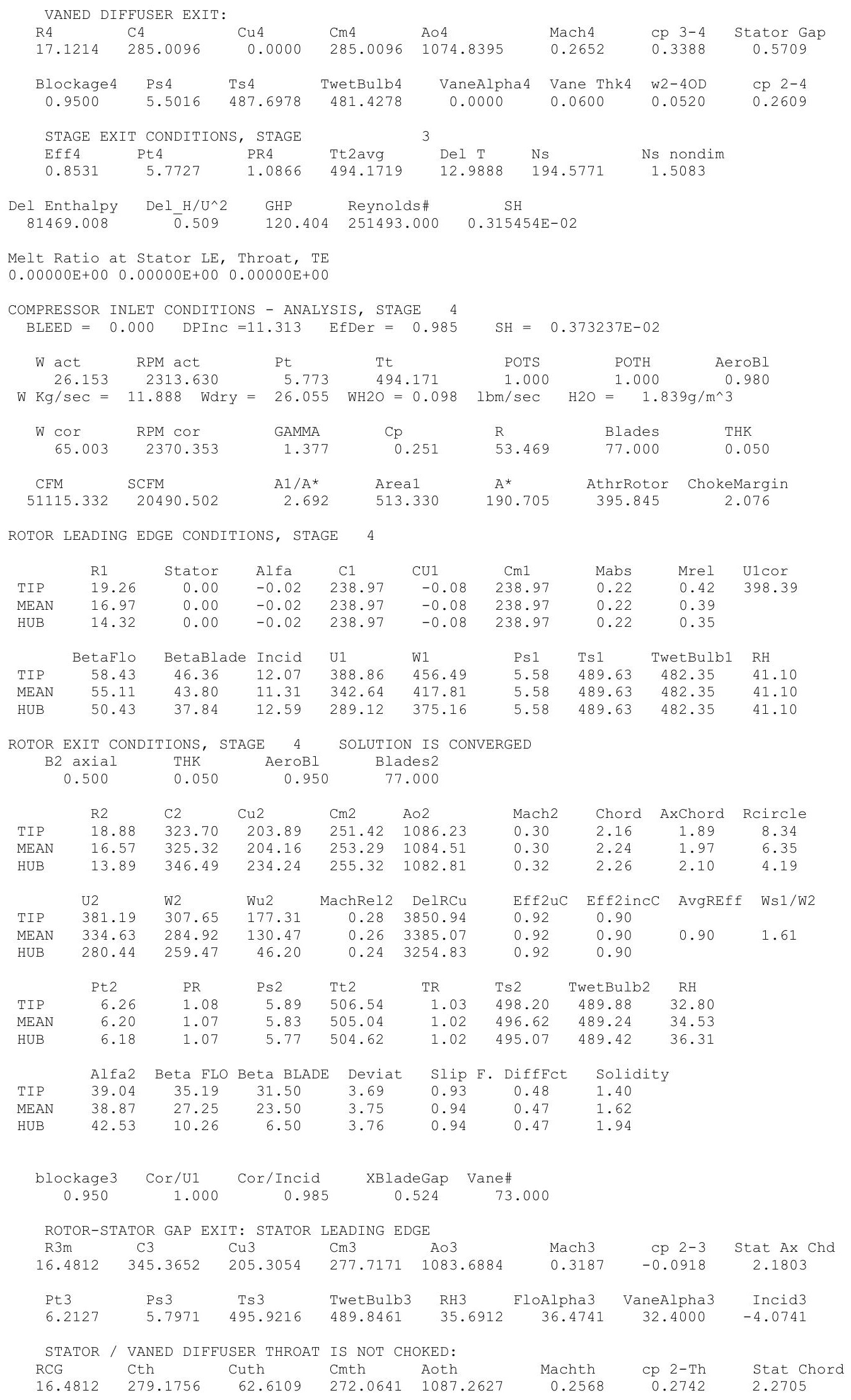




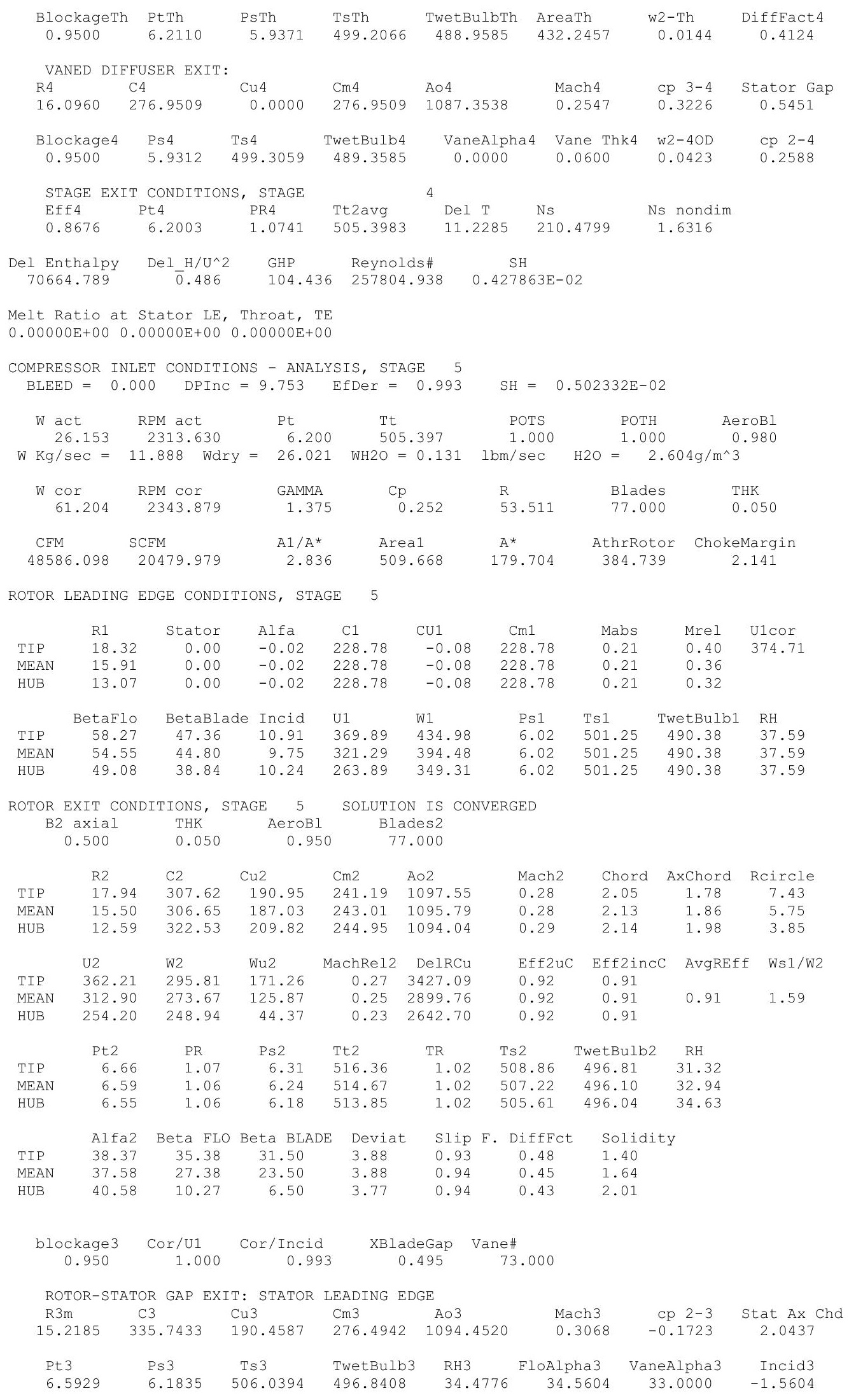




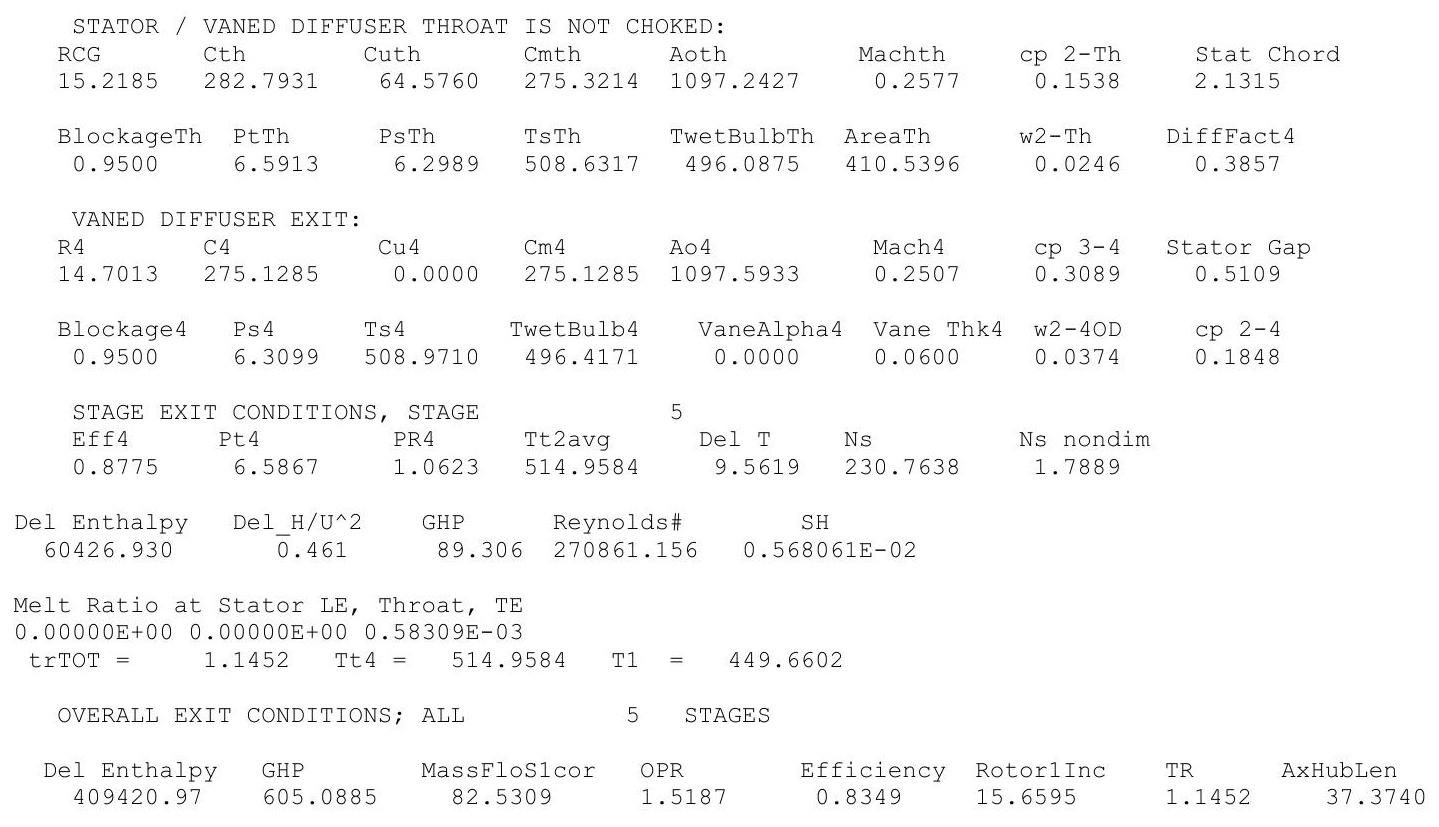




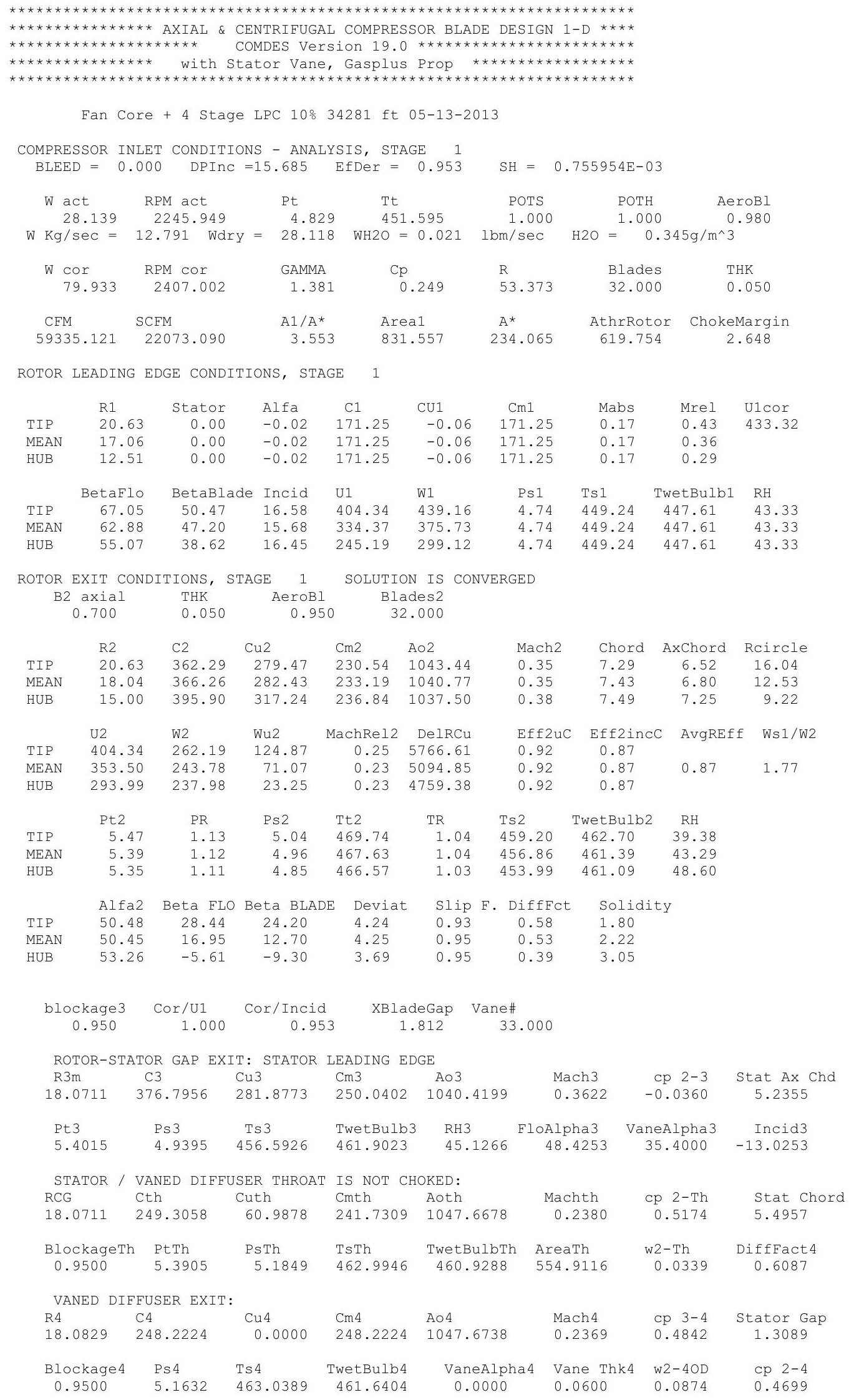




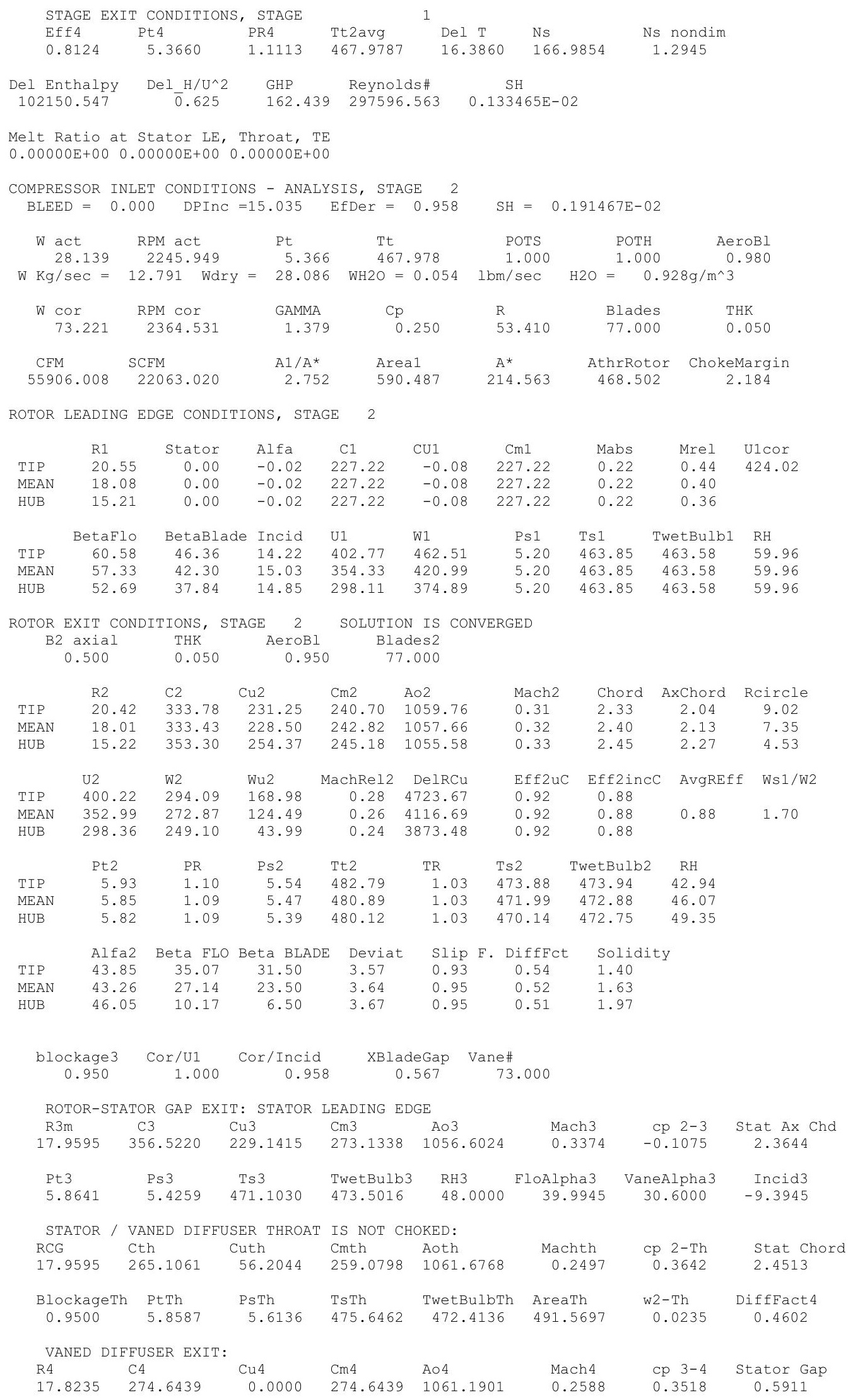




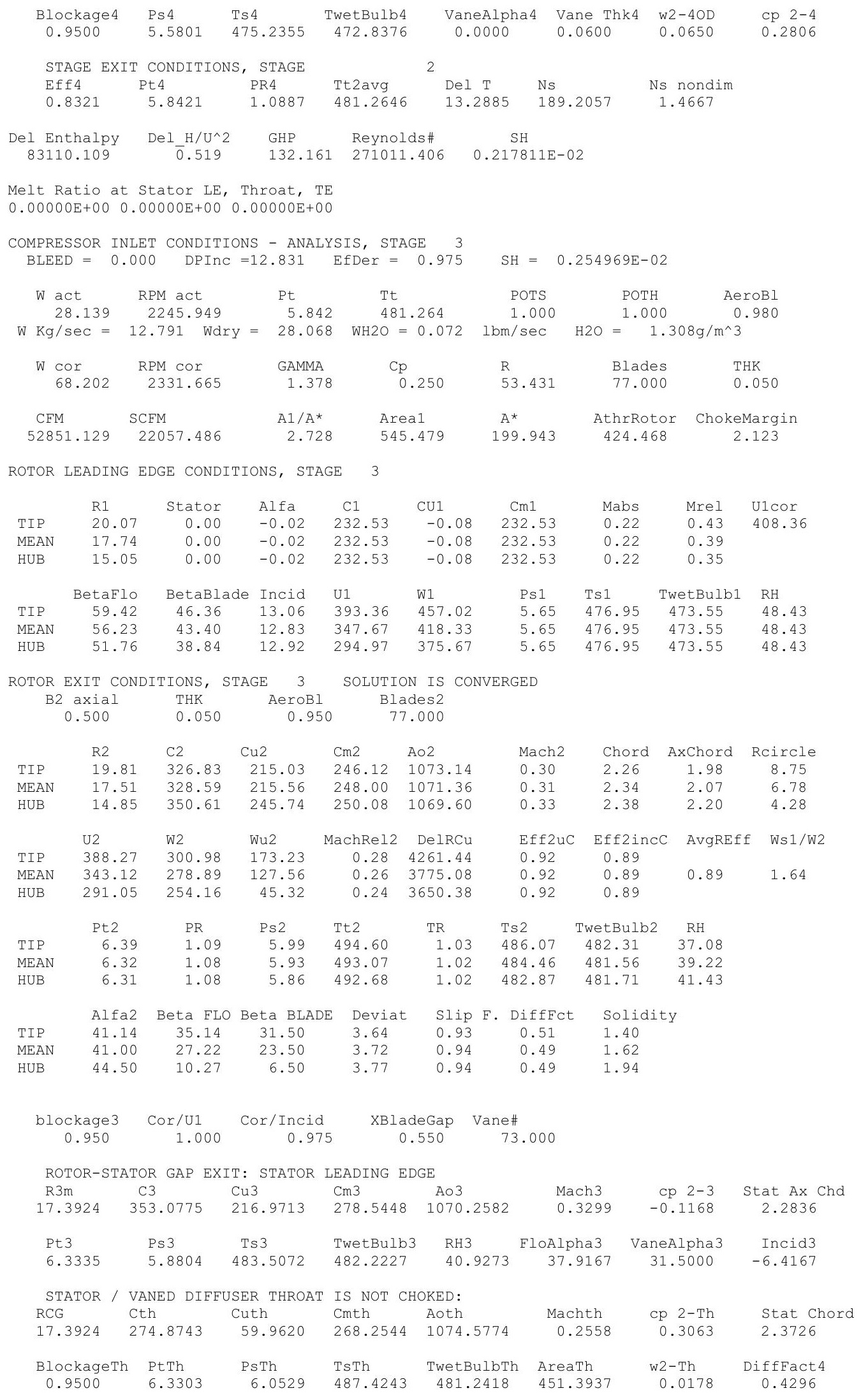




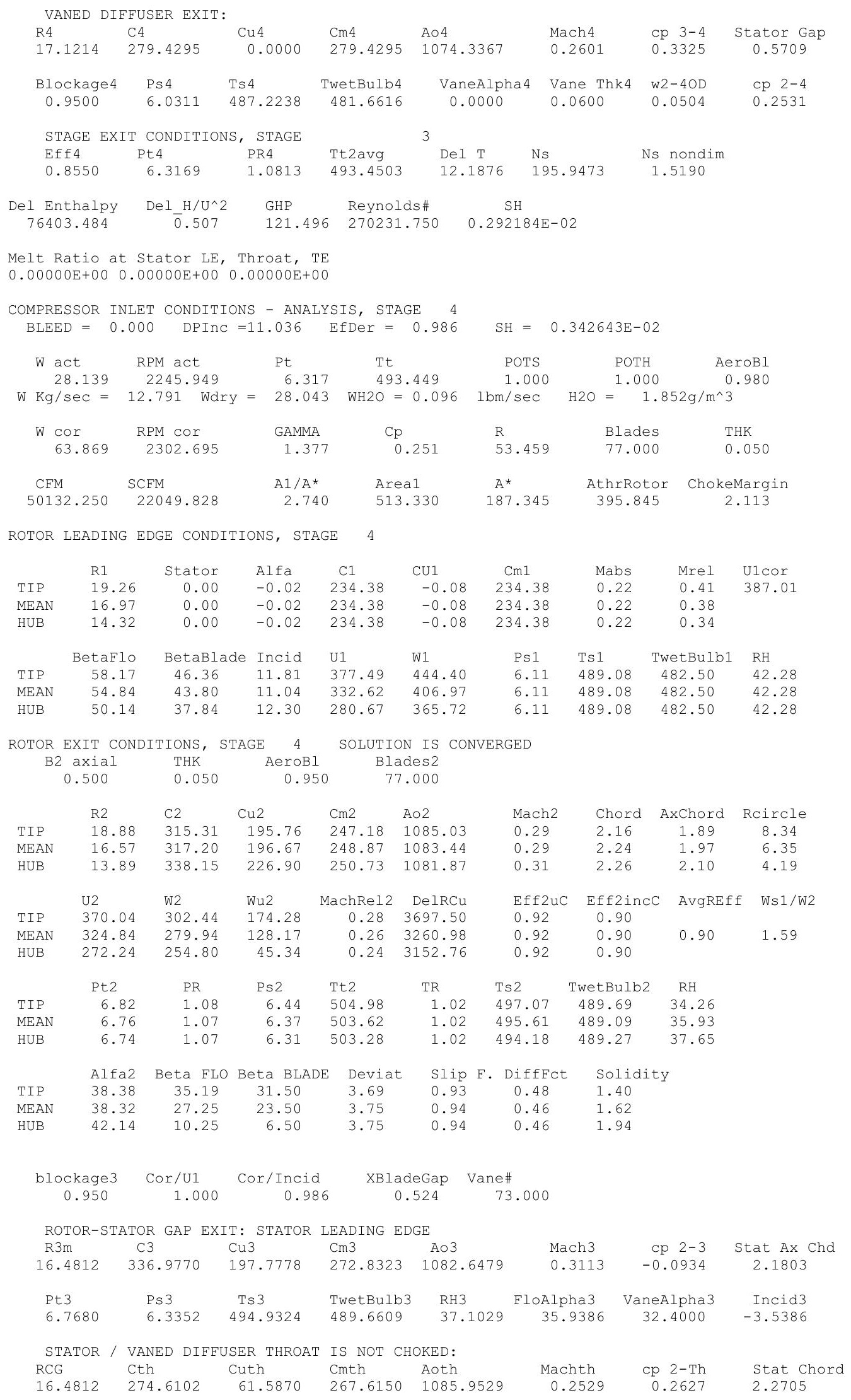




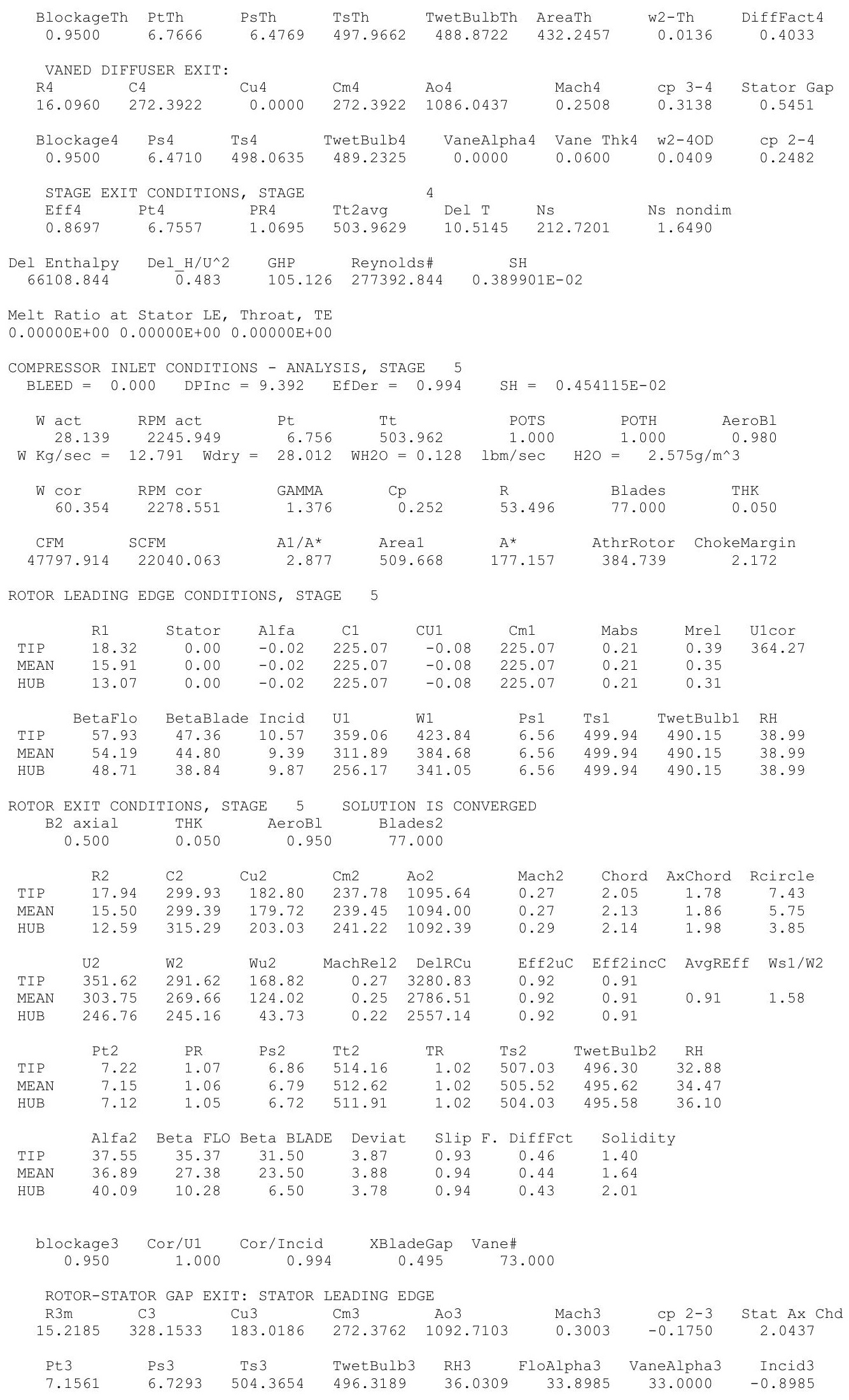




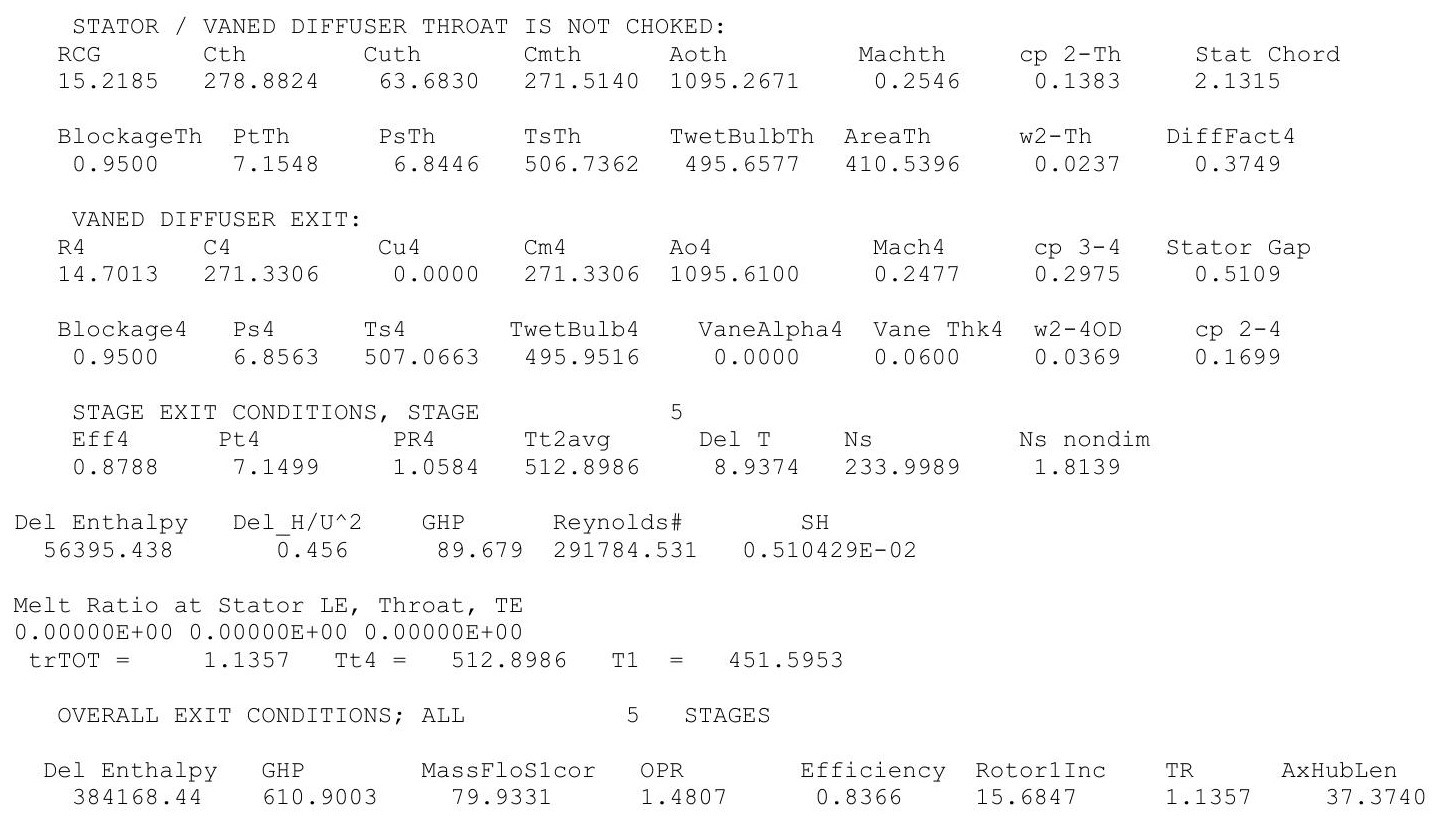




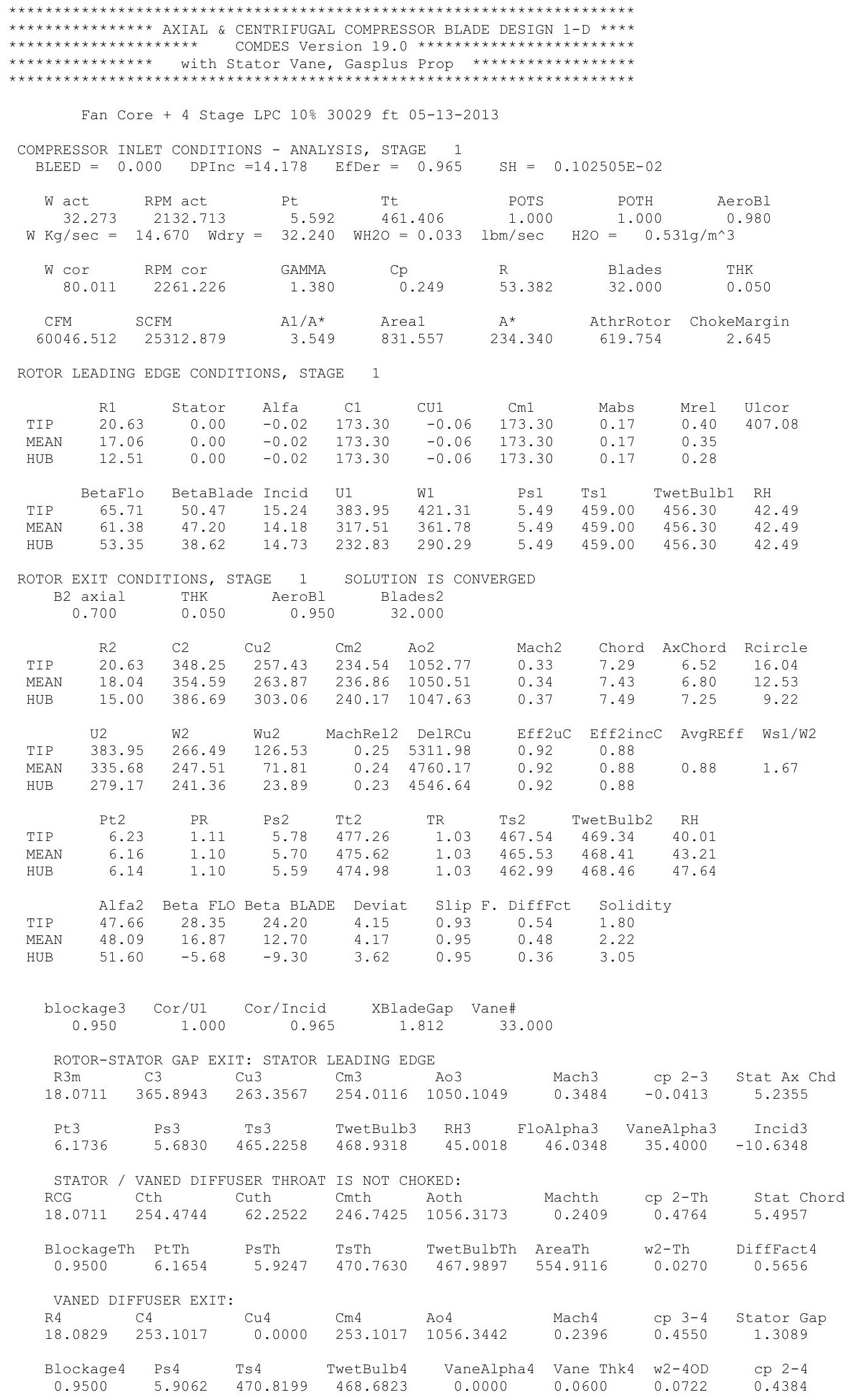




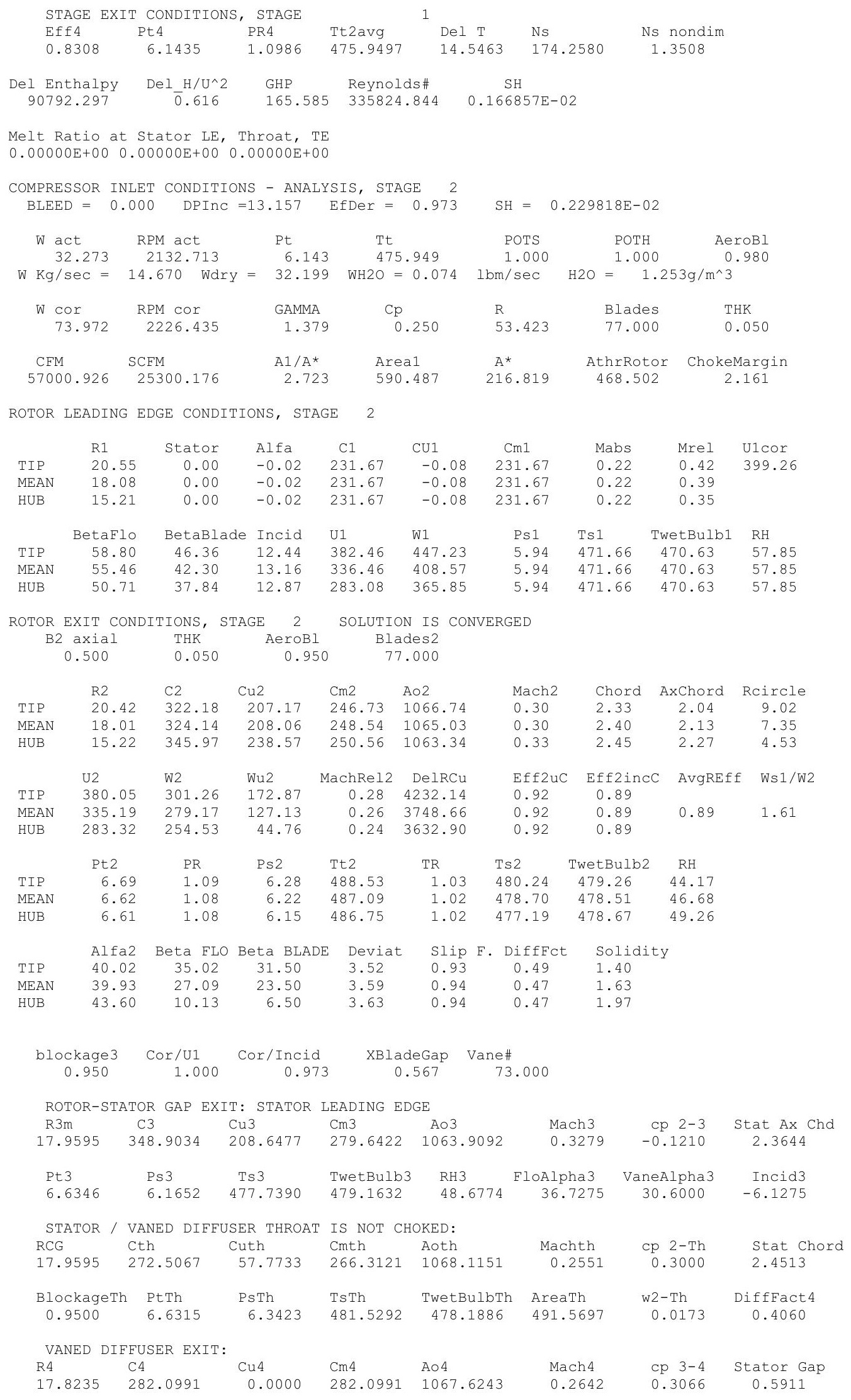




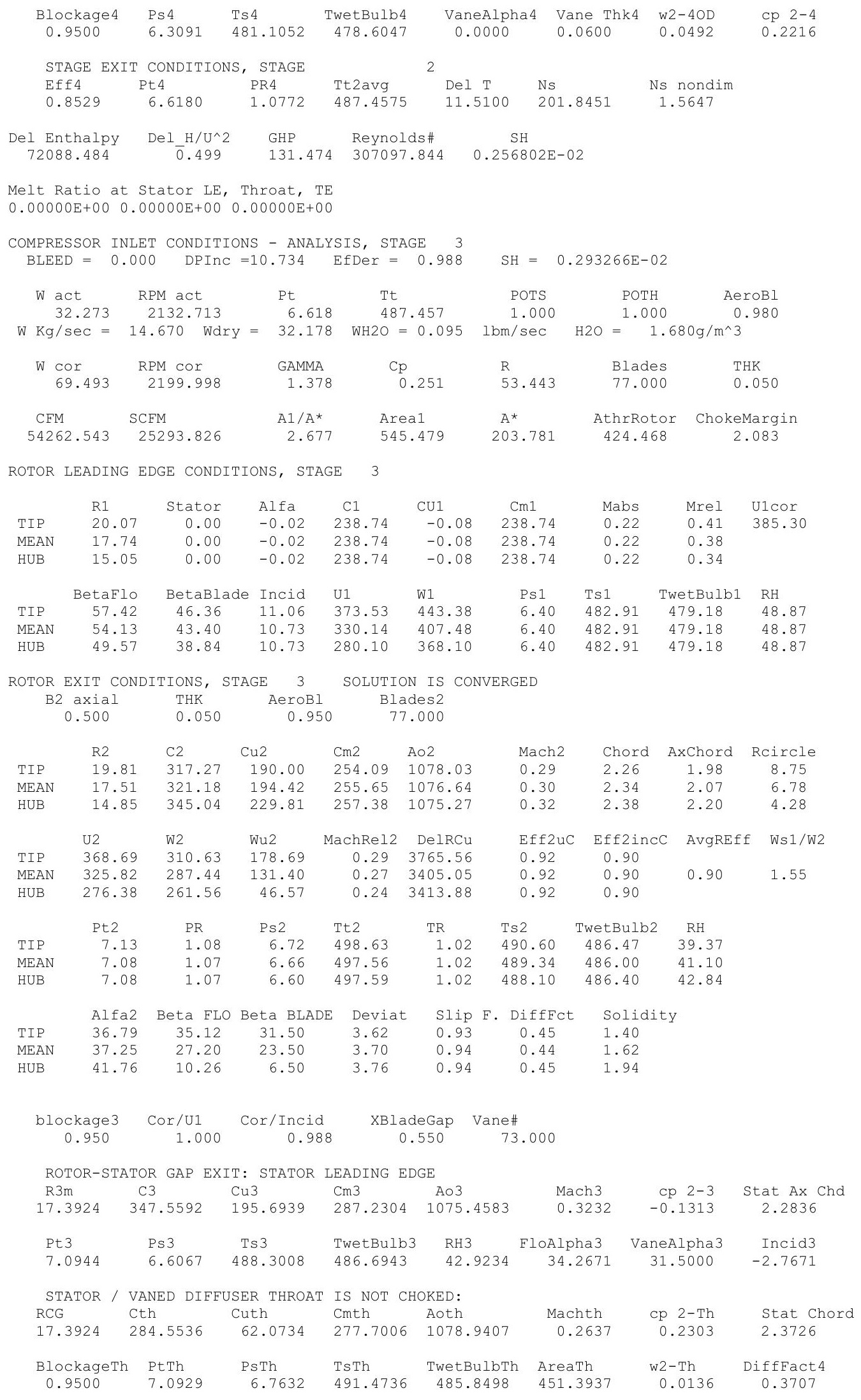




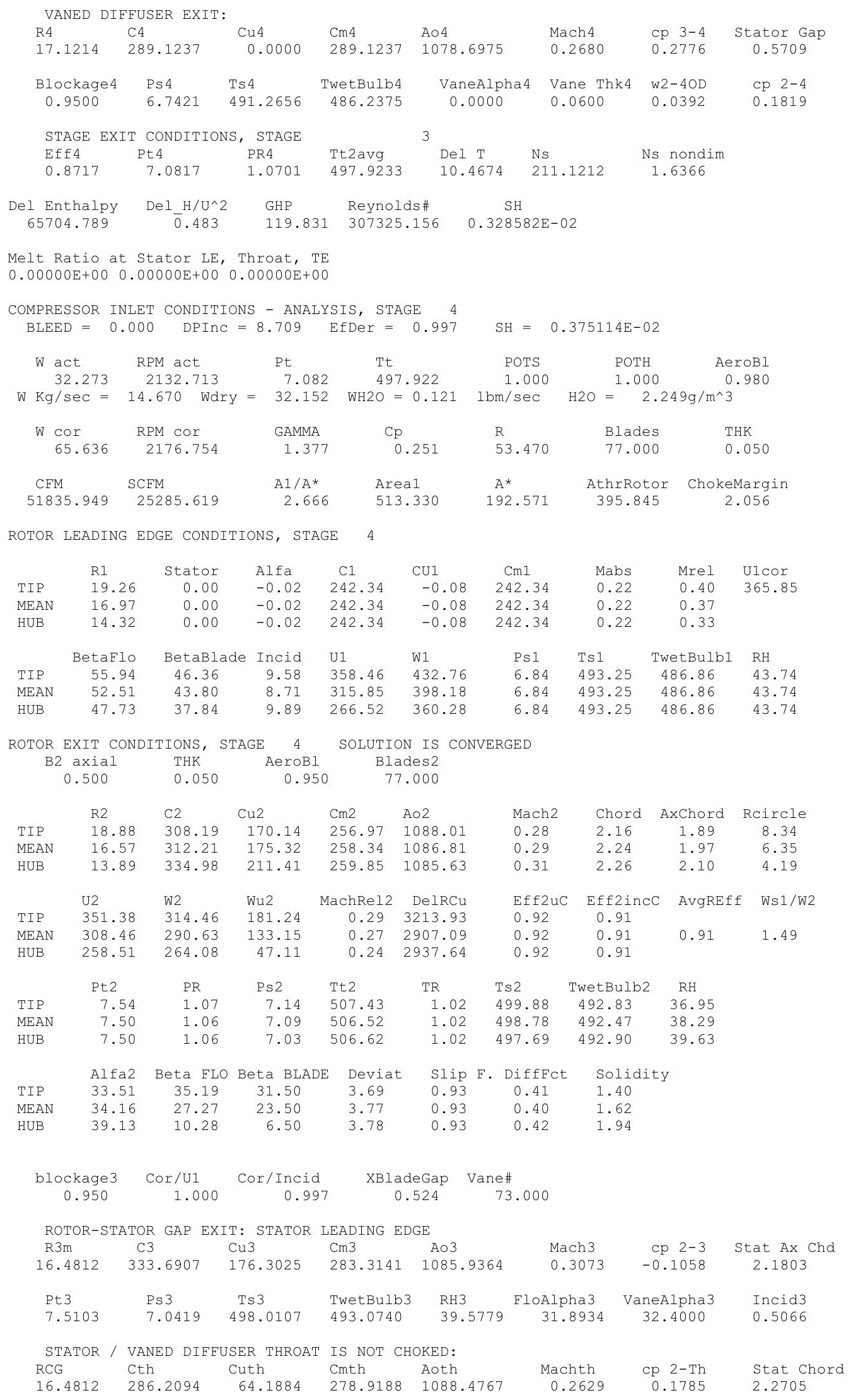




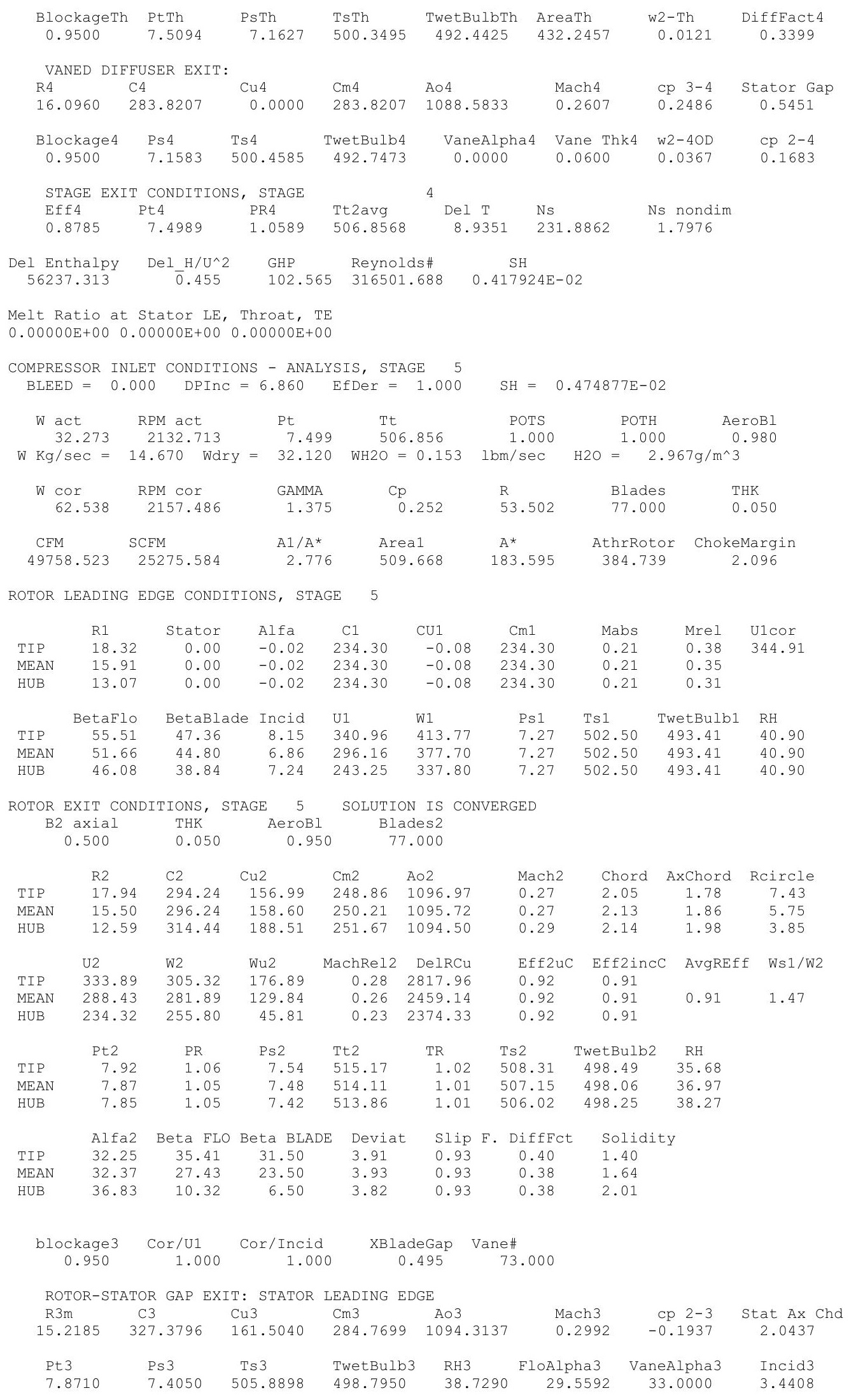




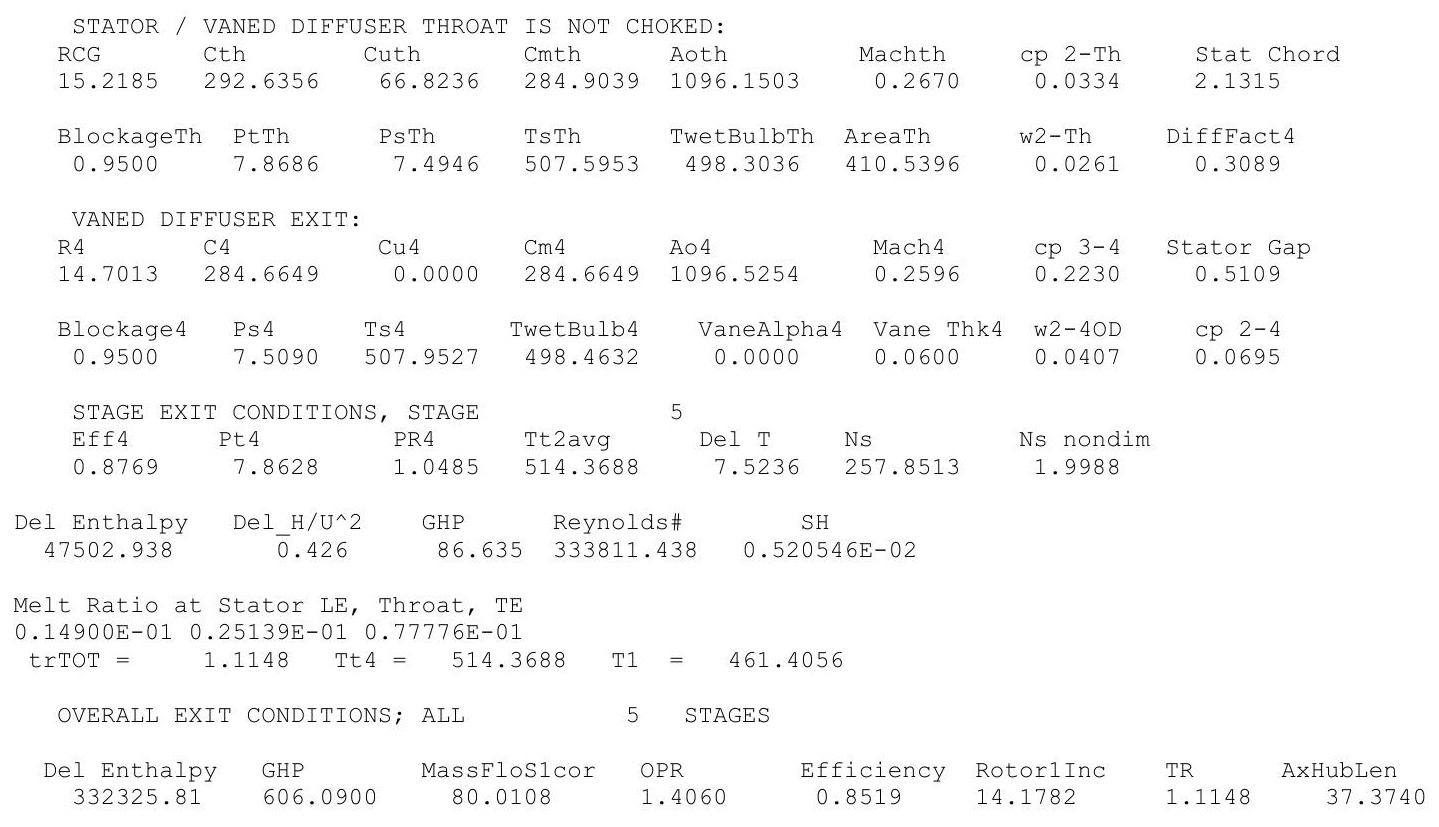




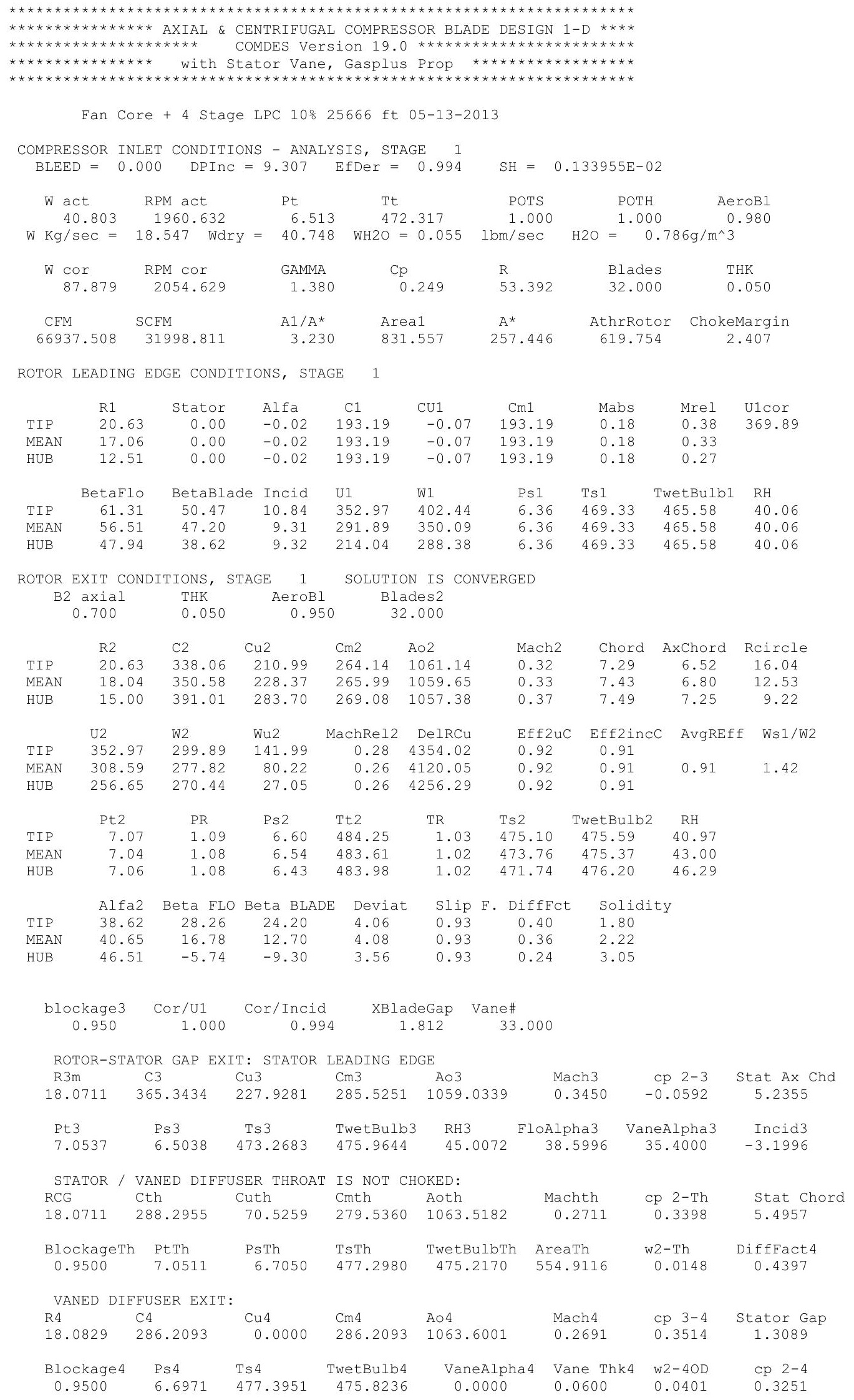




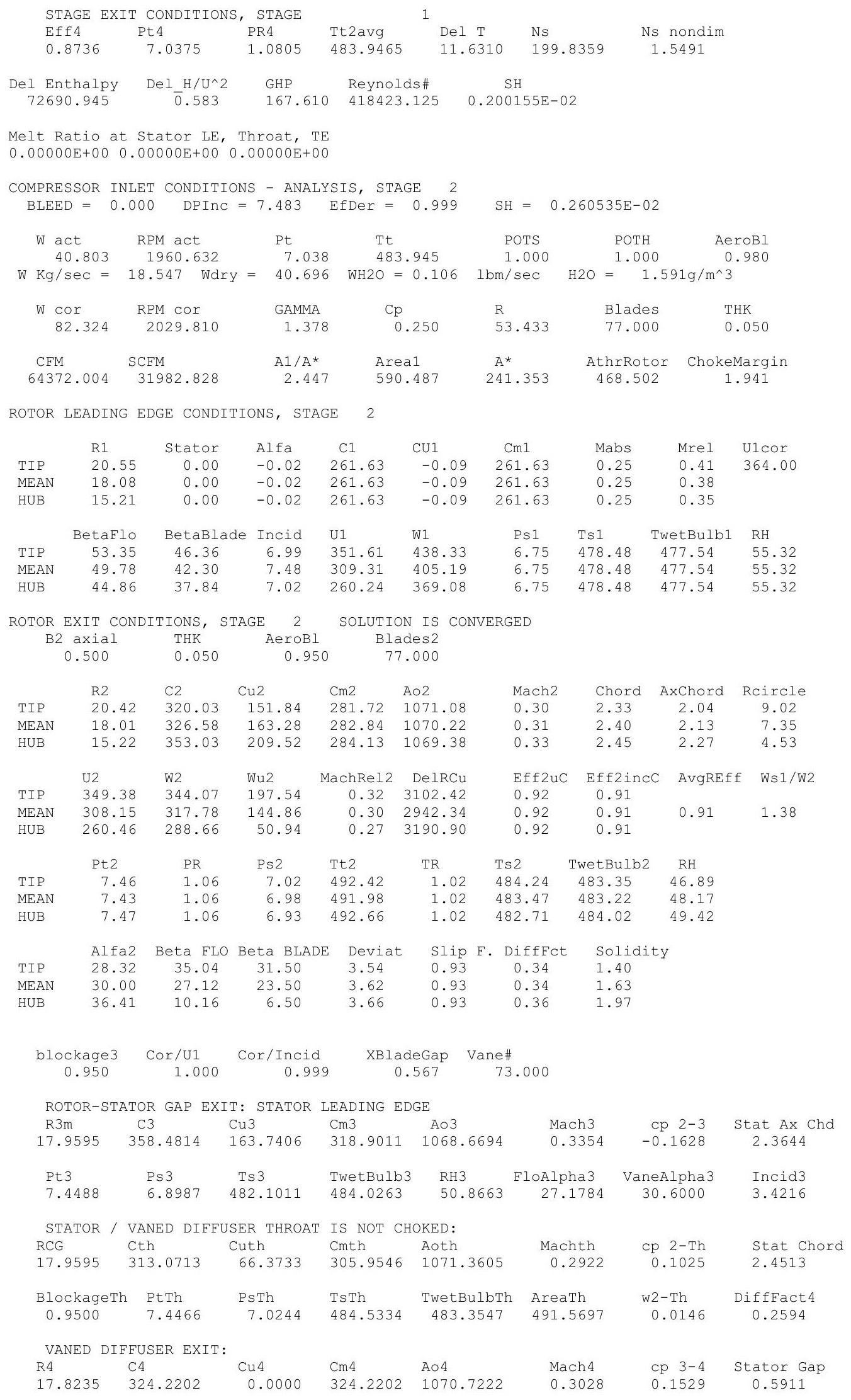




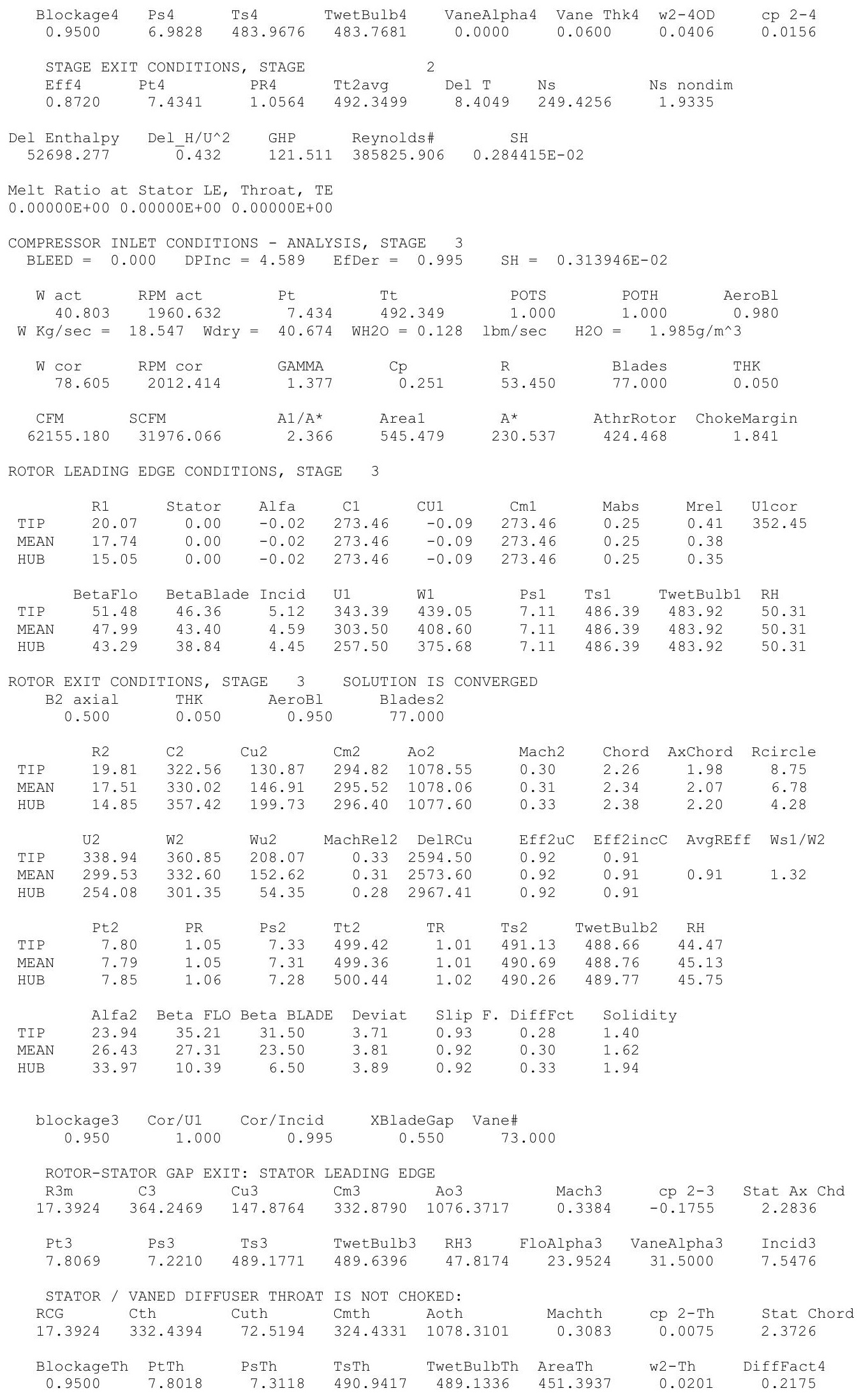




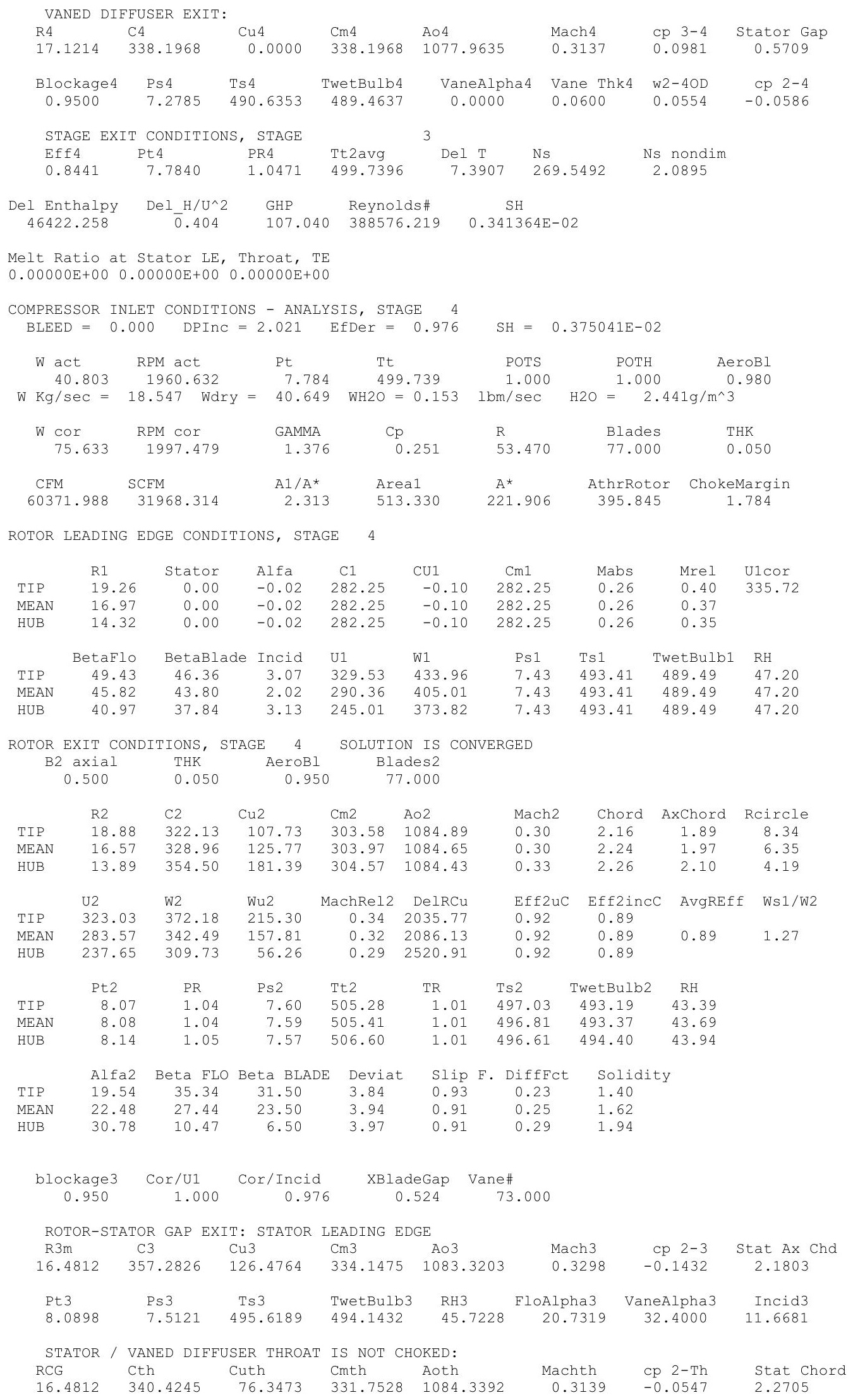




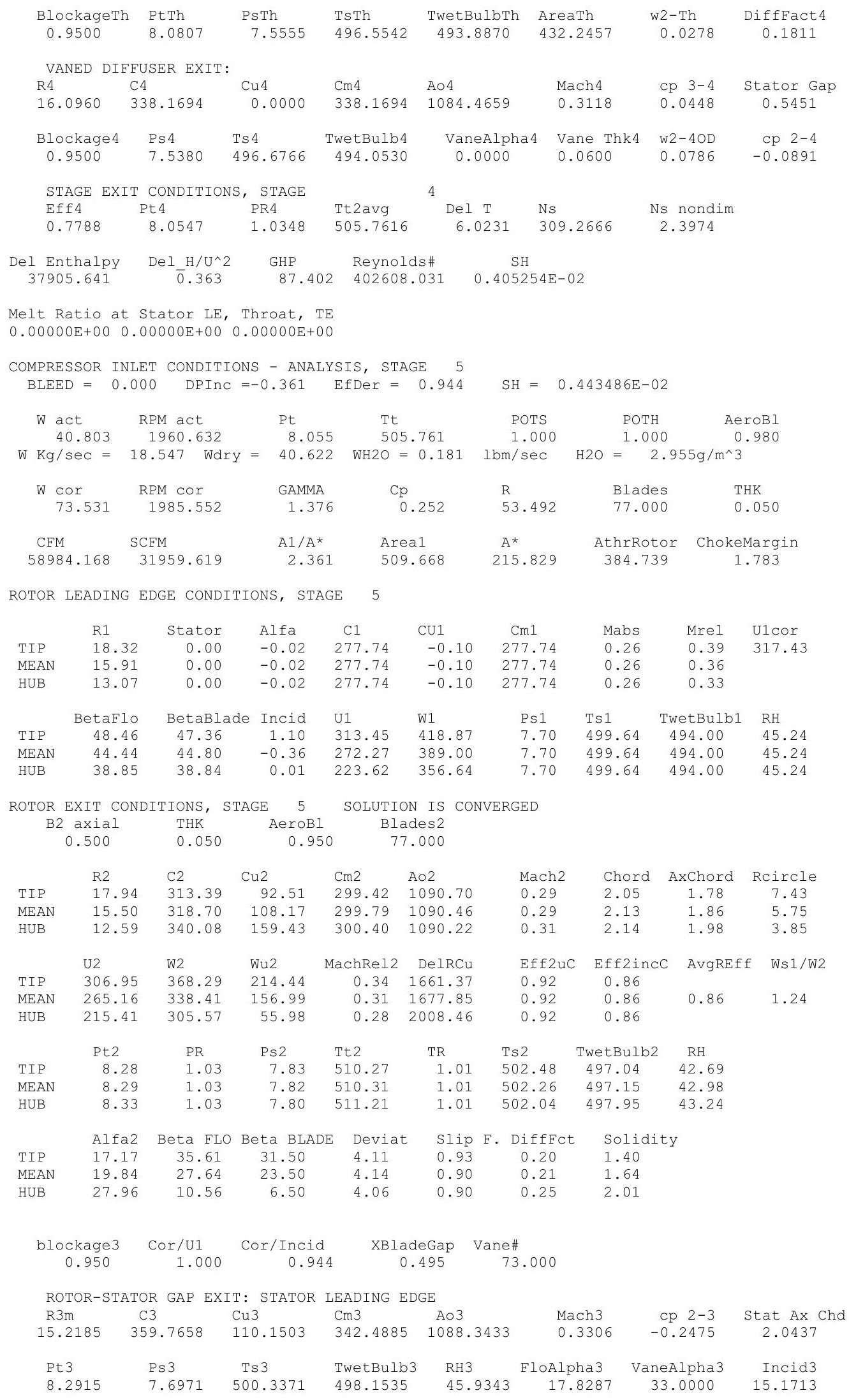




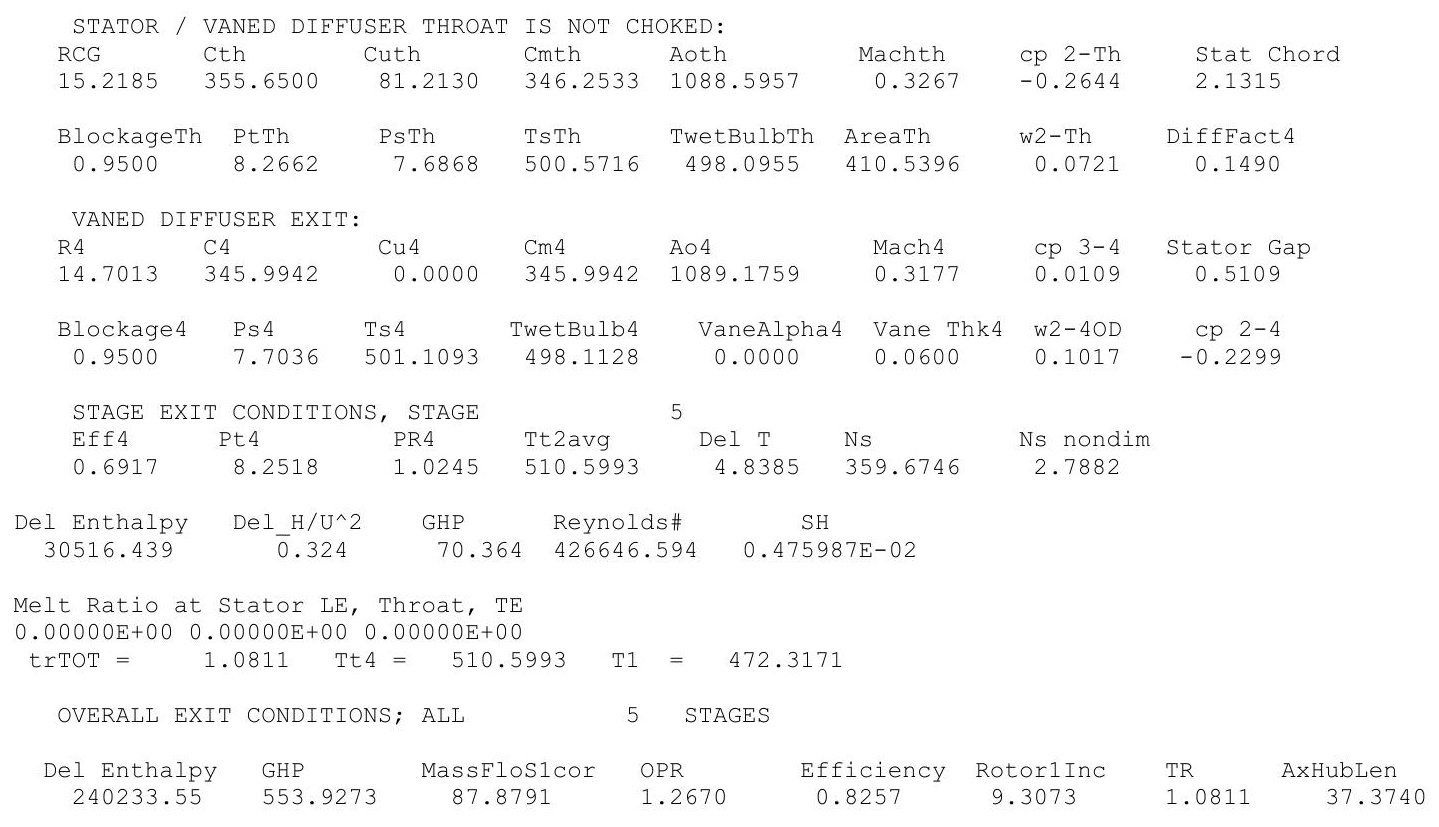




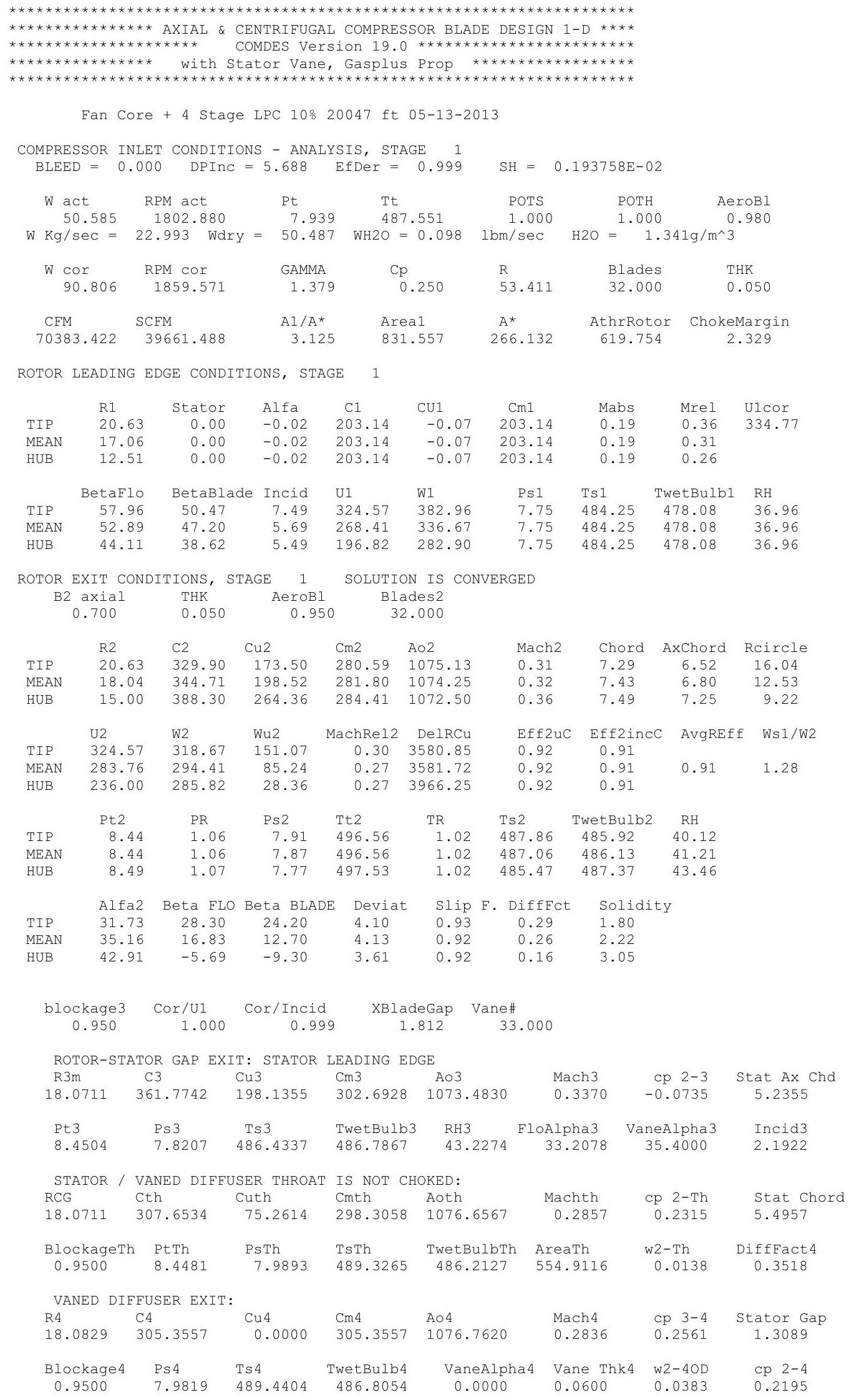




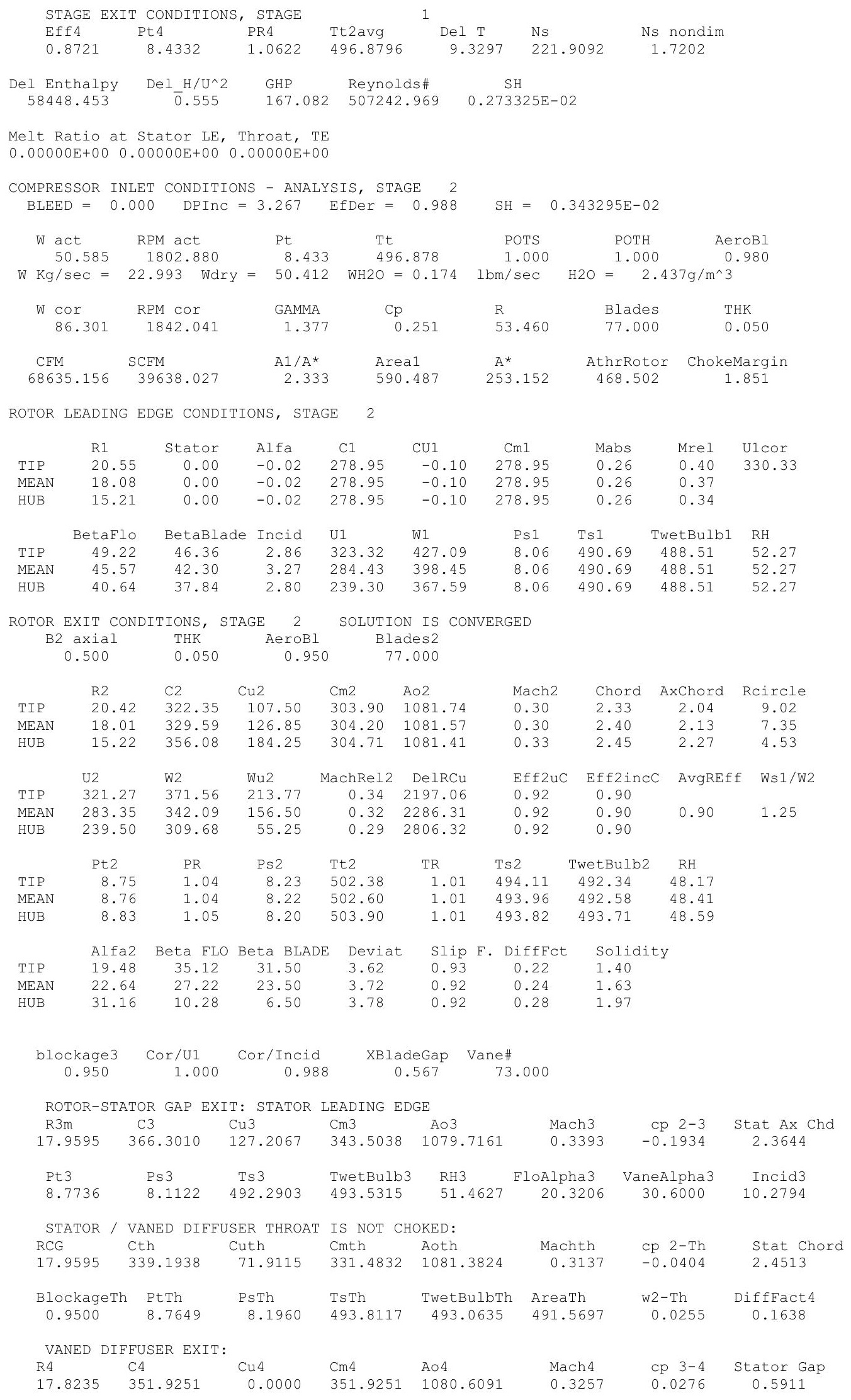




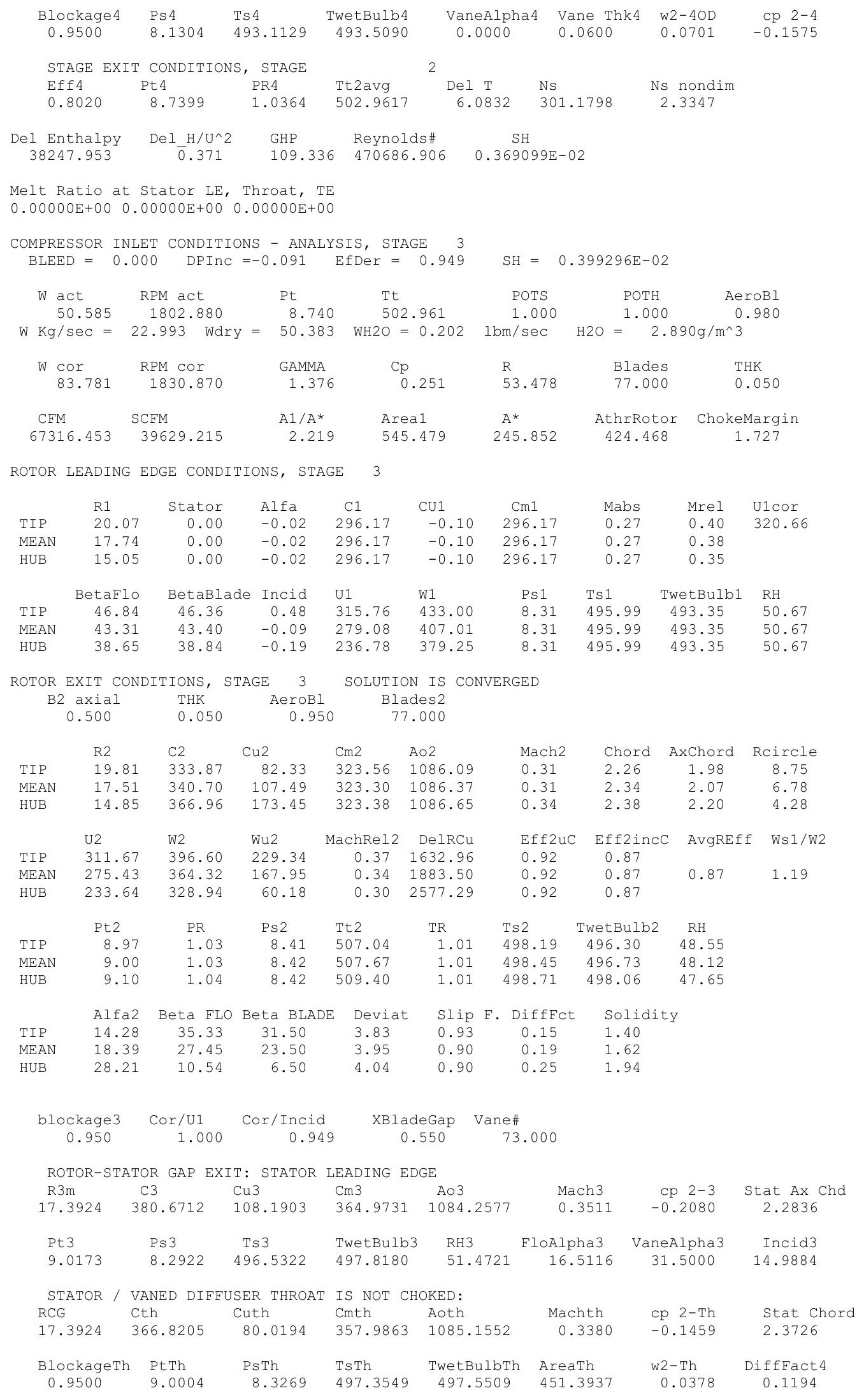




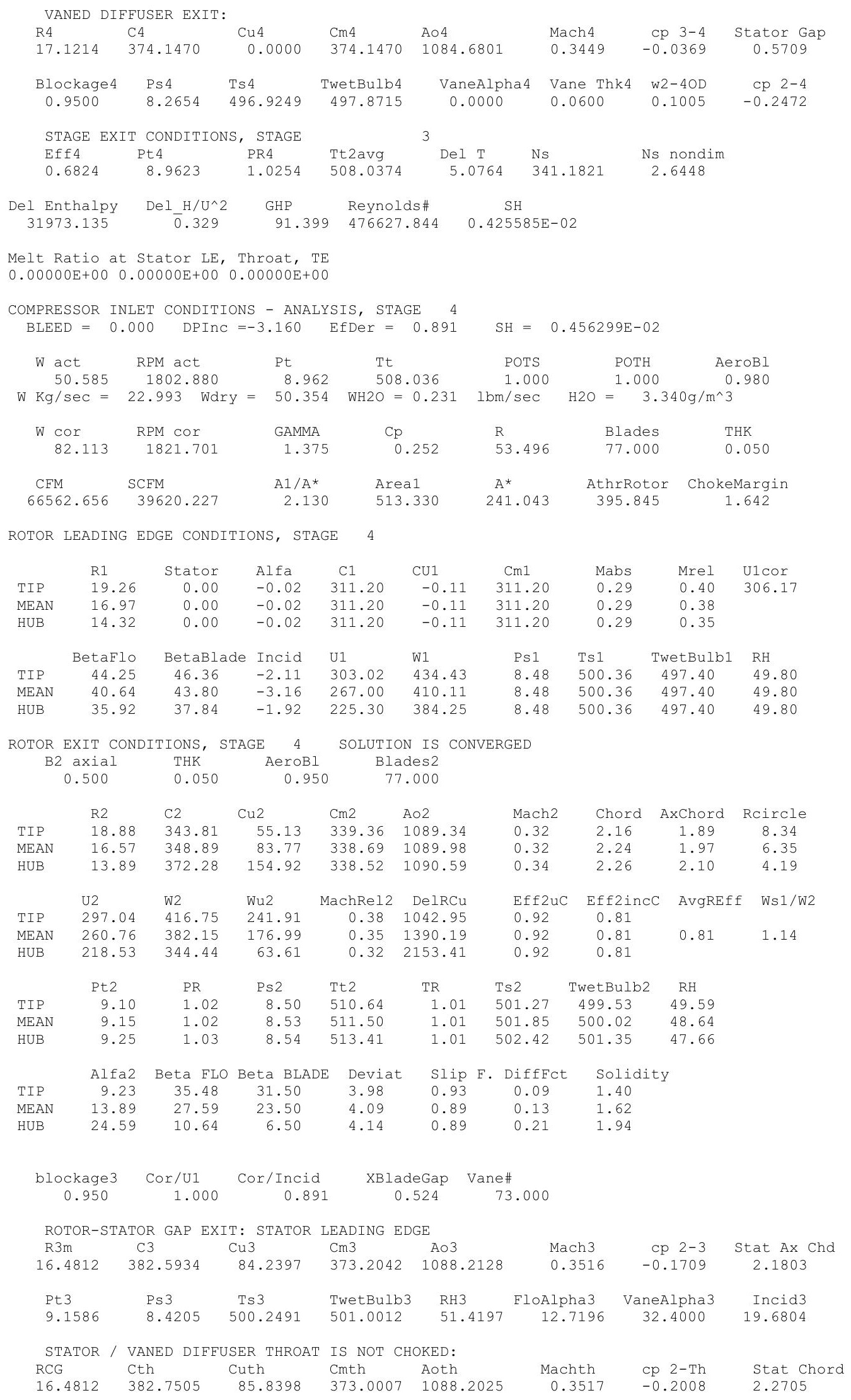




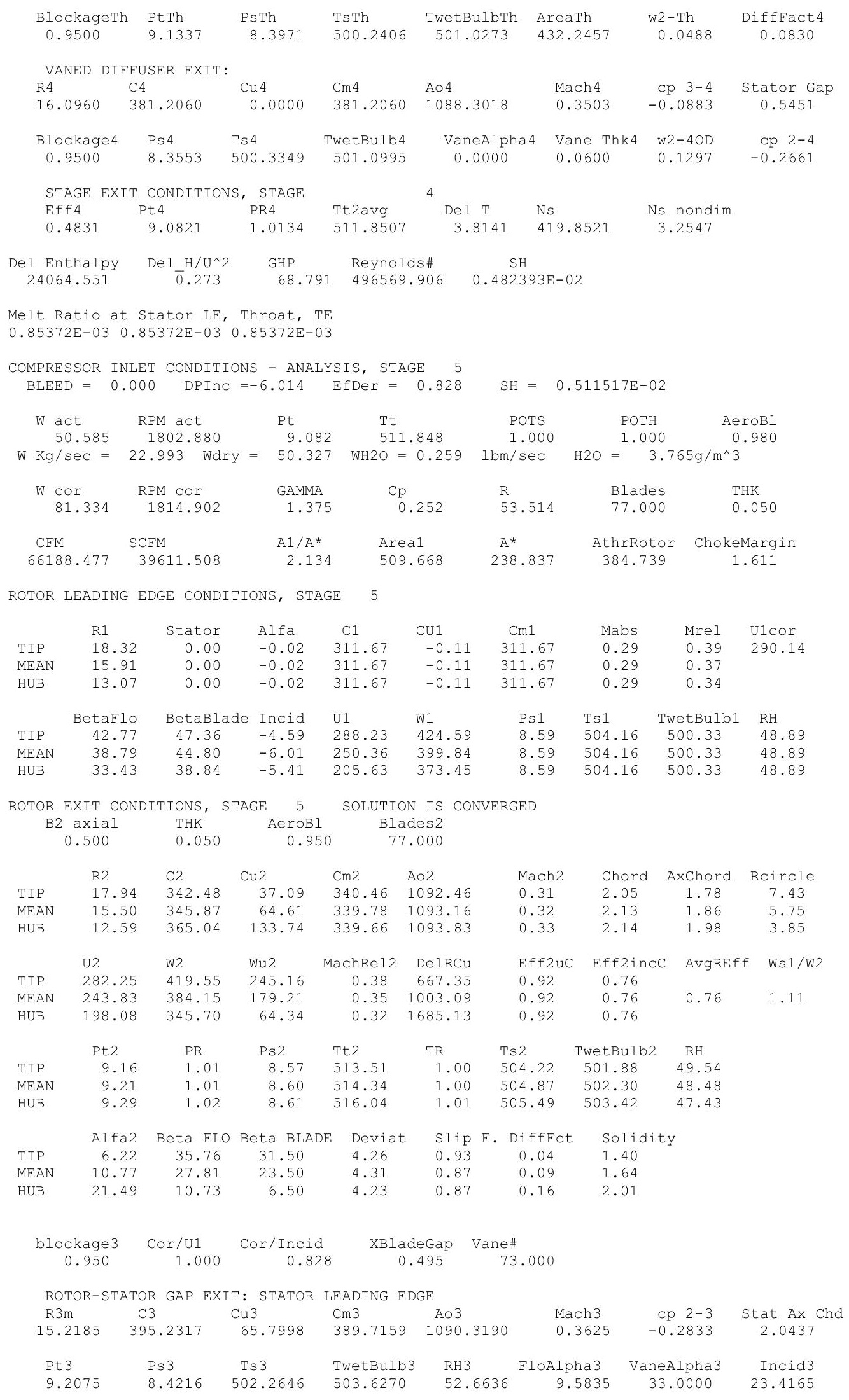




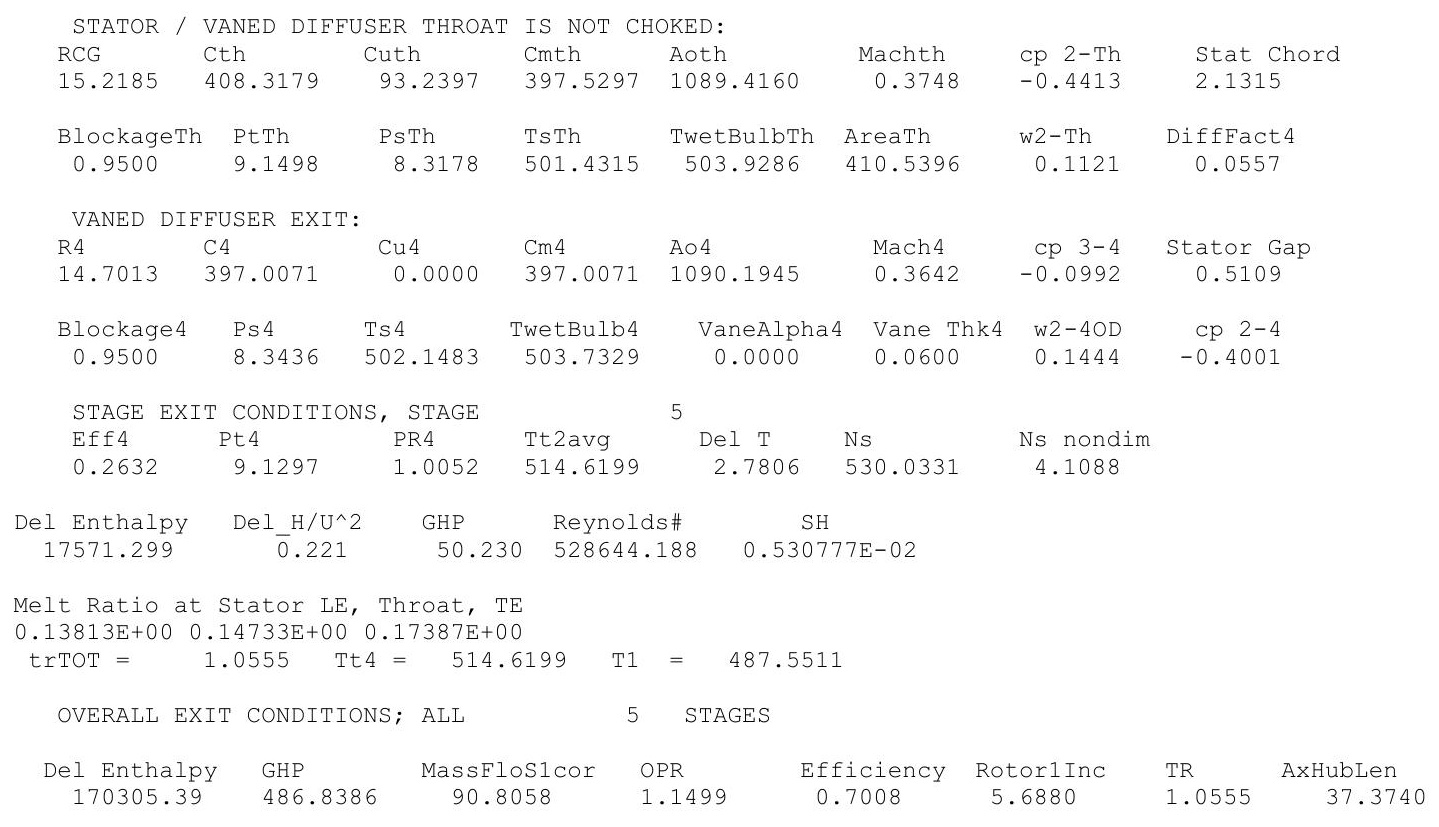




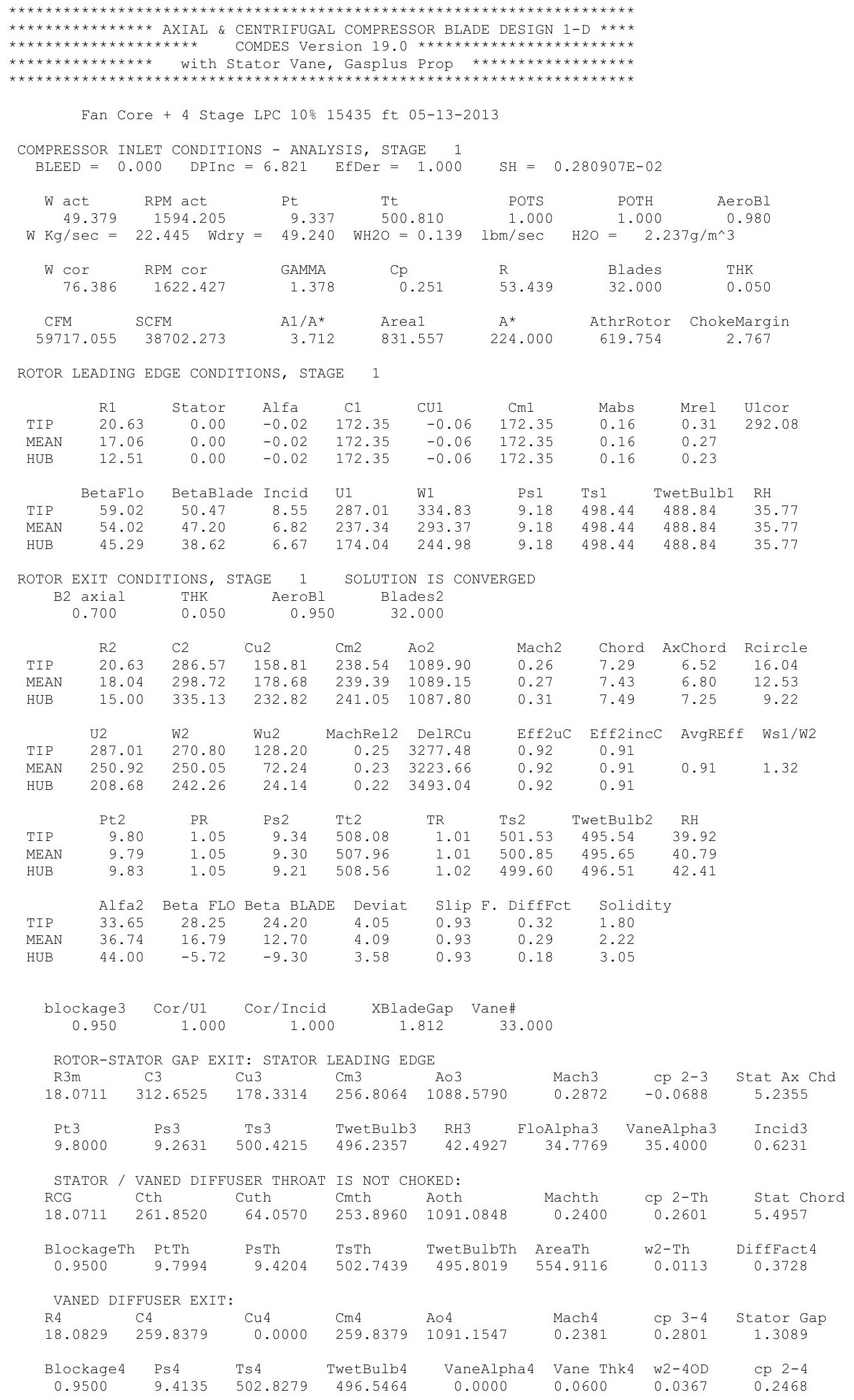




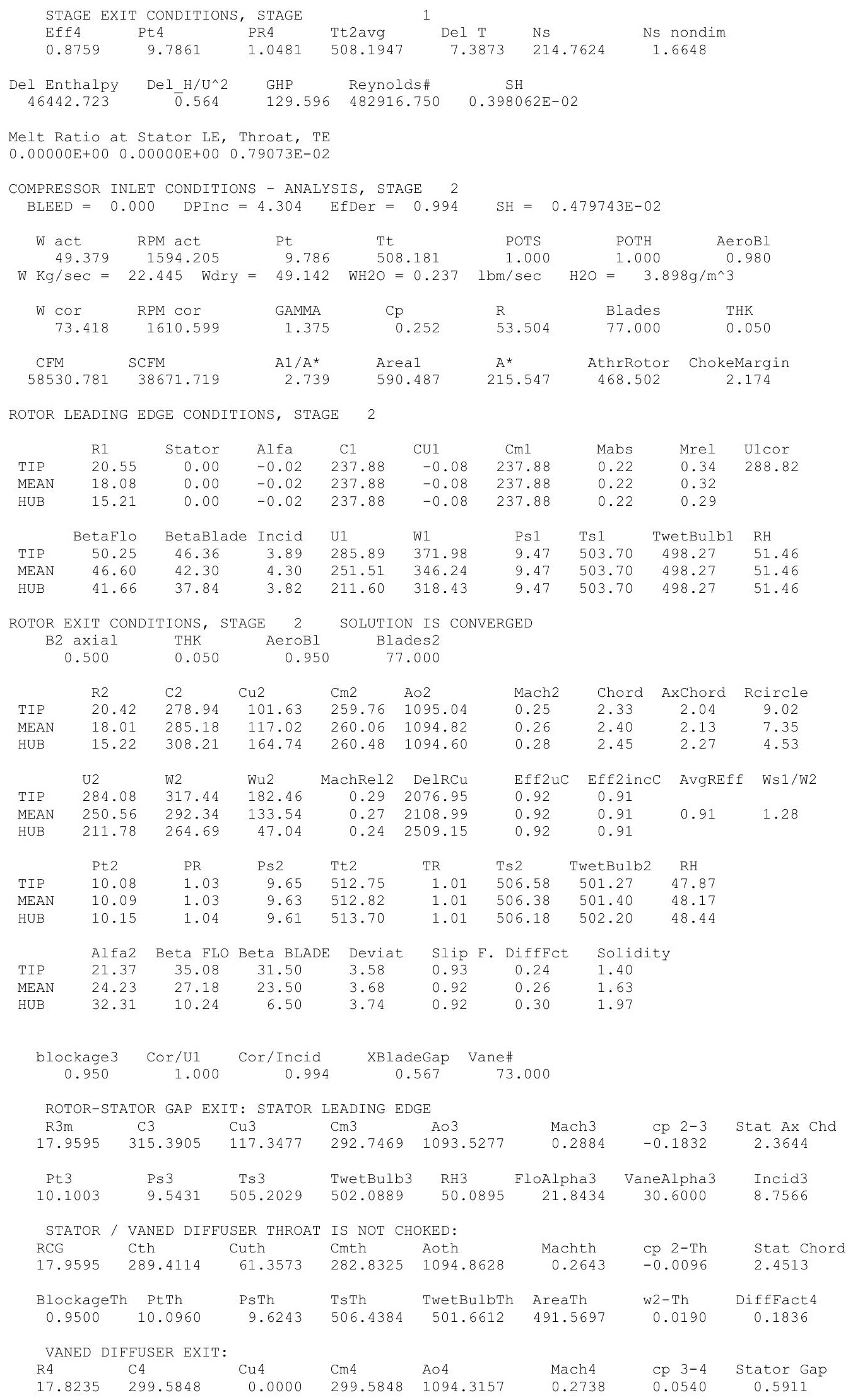




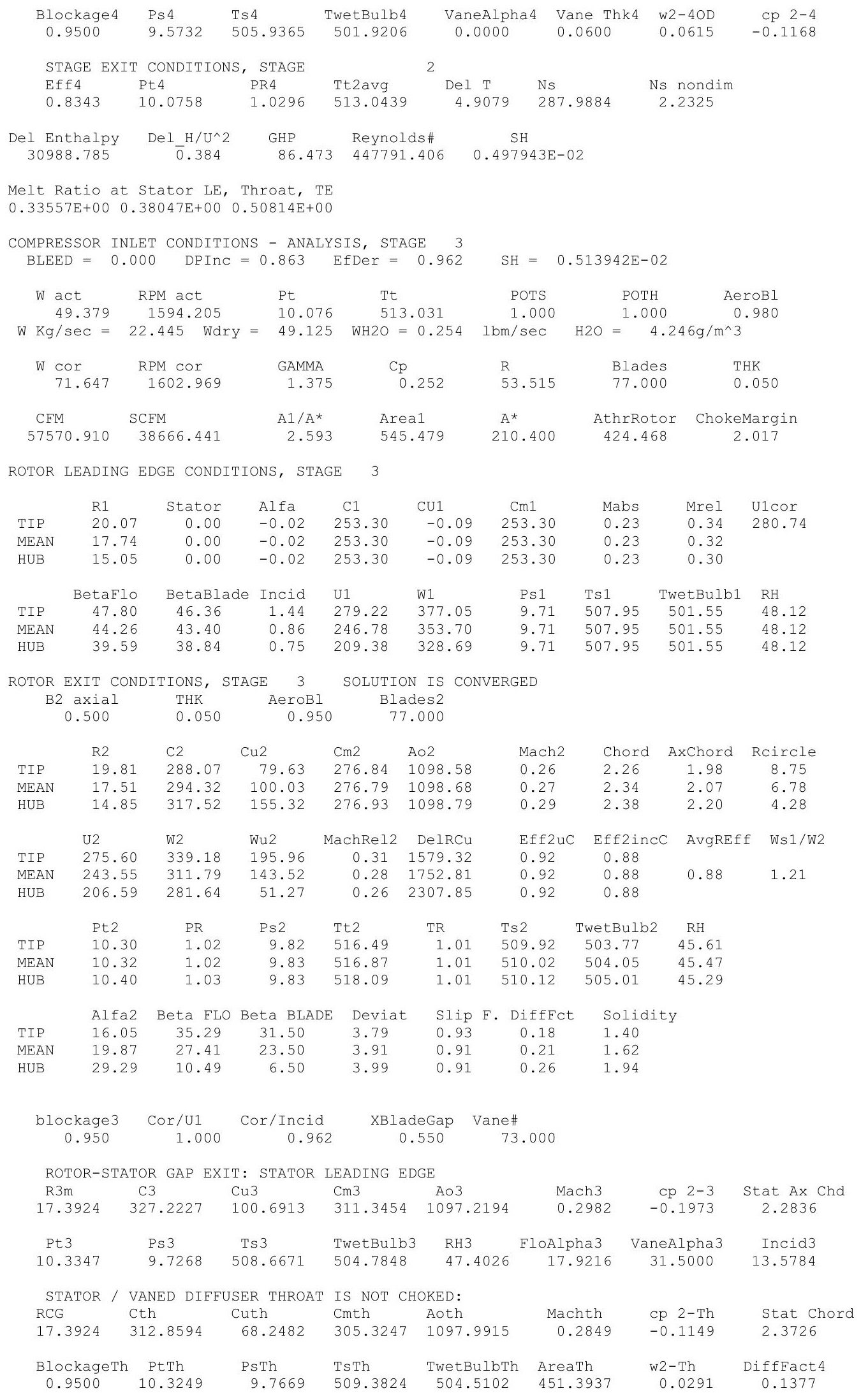




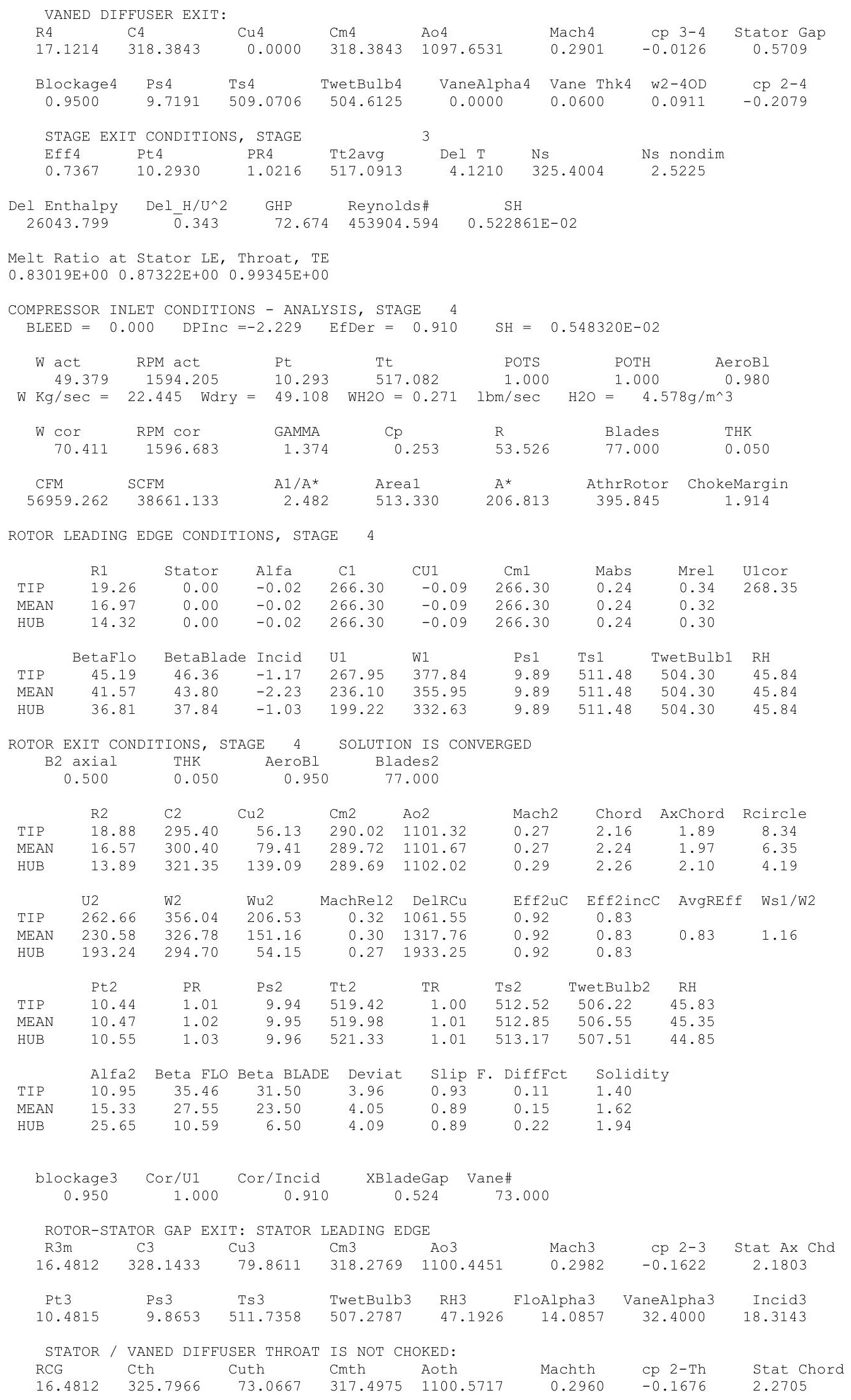




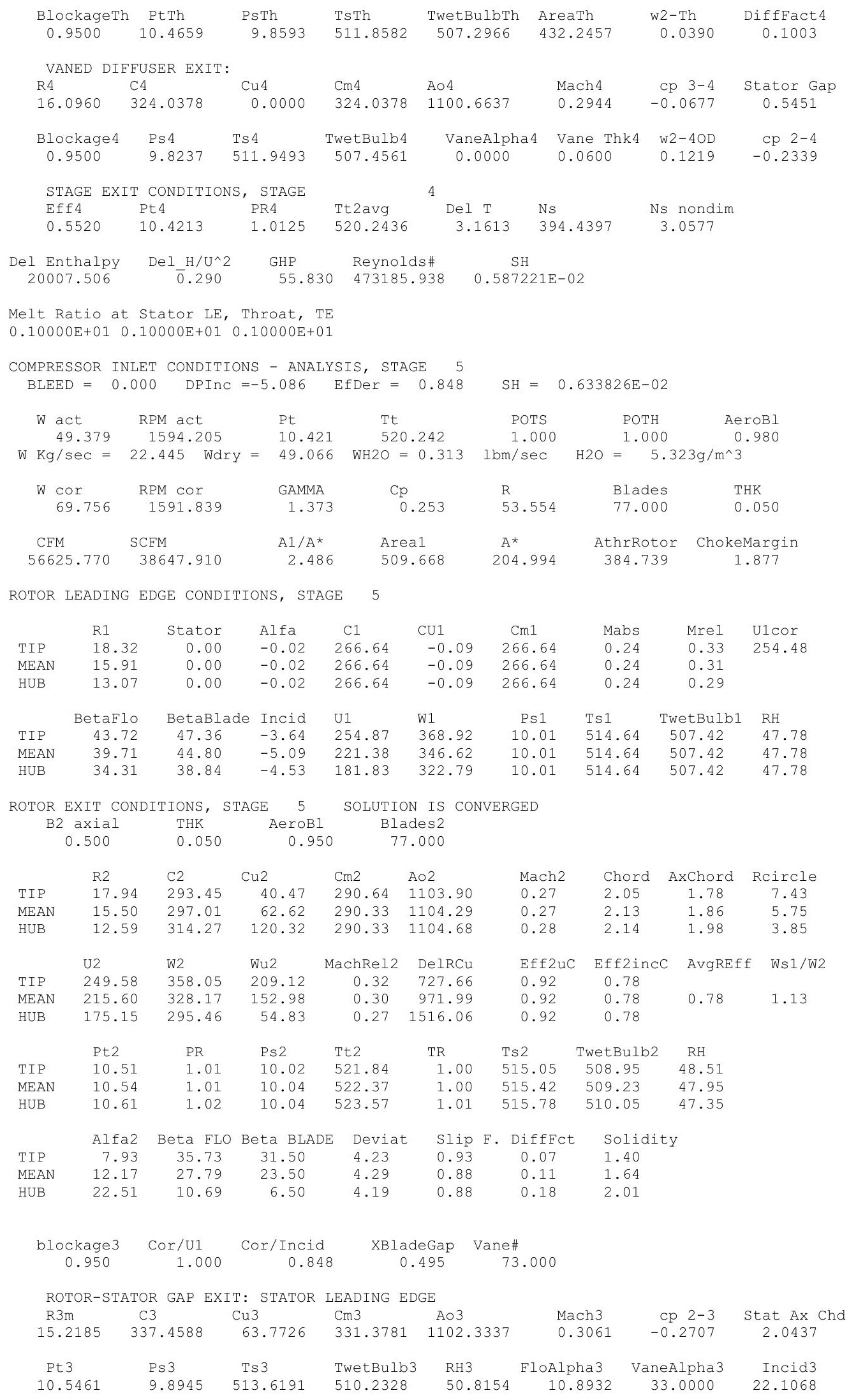




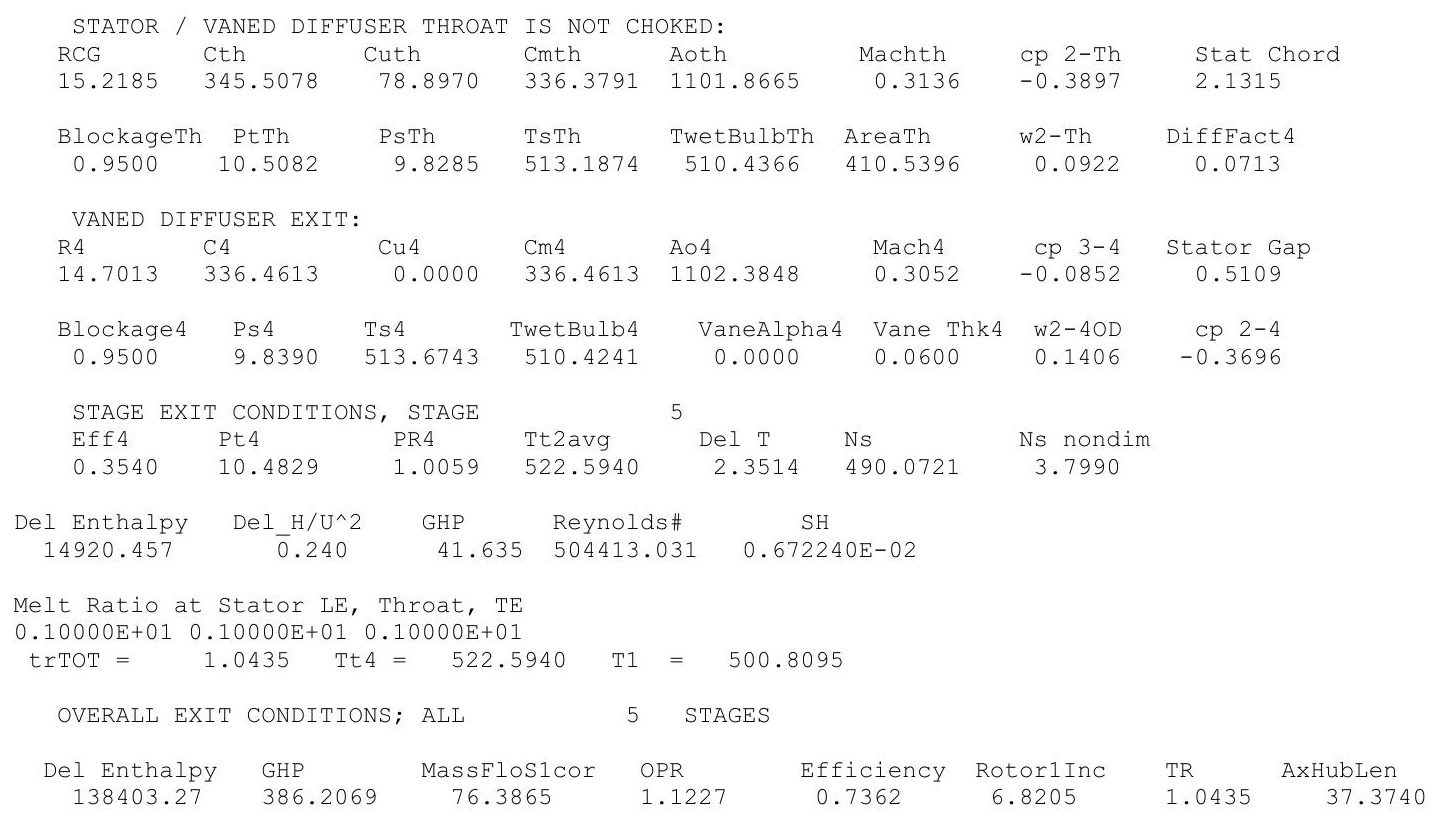


$5 \mu \mathrm{m}, 2 \mathrm{~g} / \mathrm{m}^{3}, \mathrm{ISA}+27 \mathrm{R}$

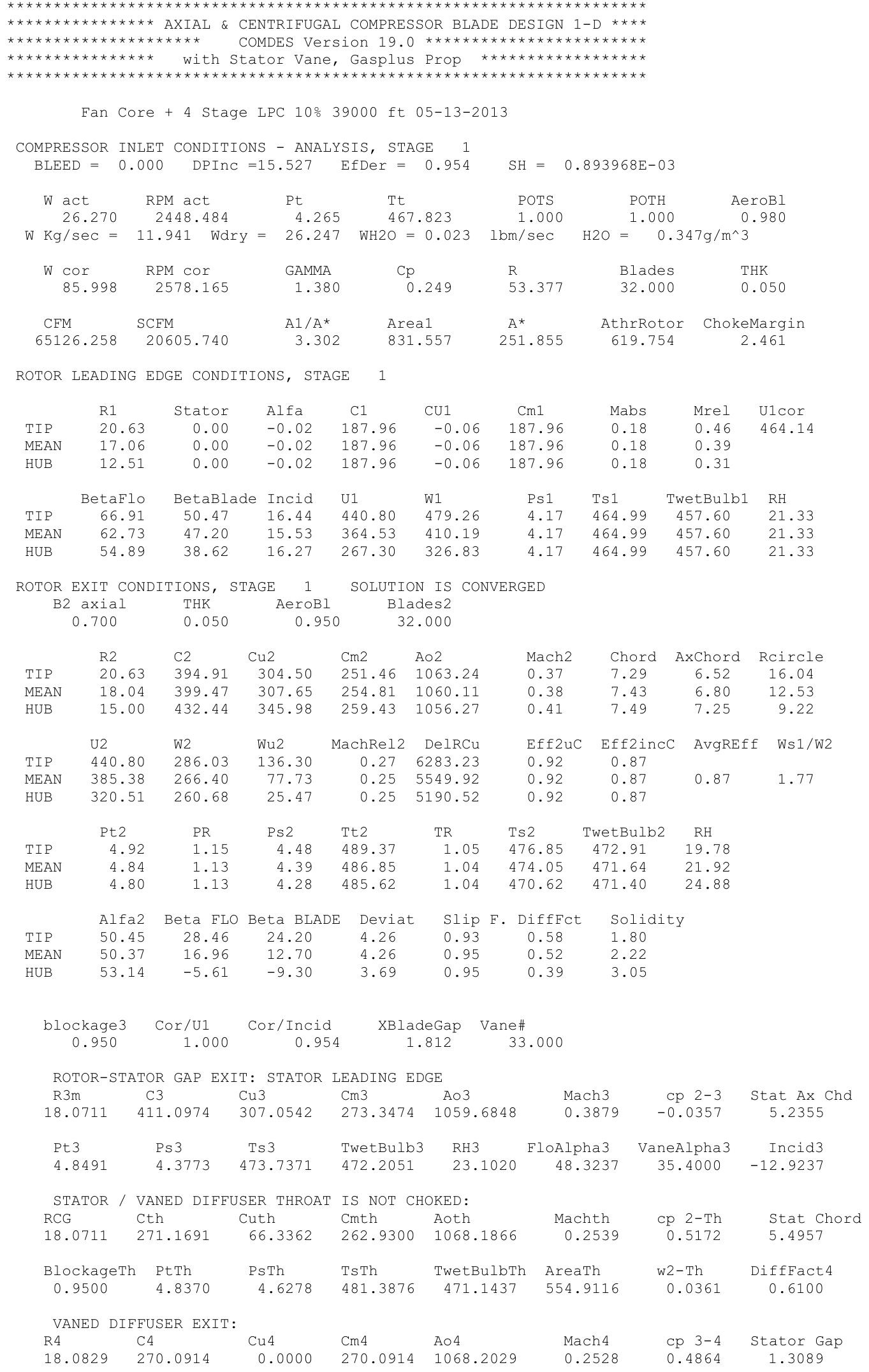




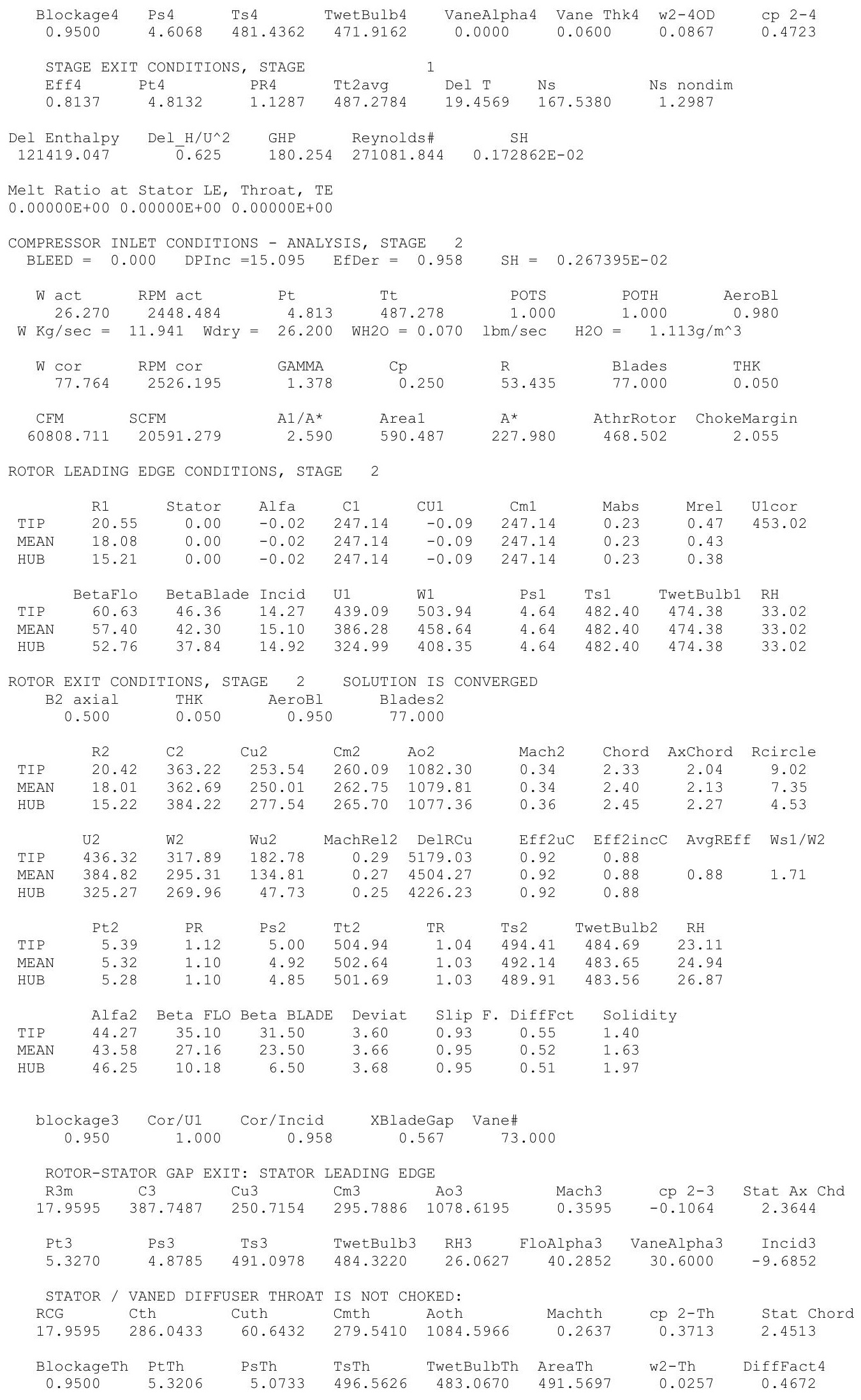




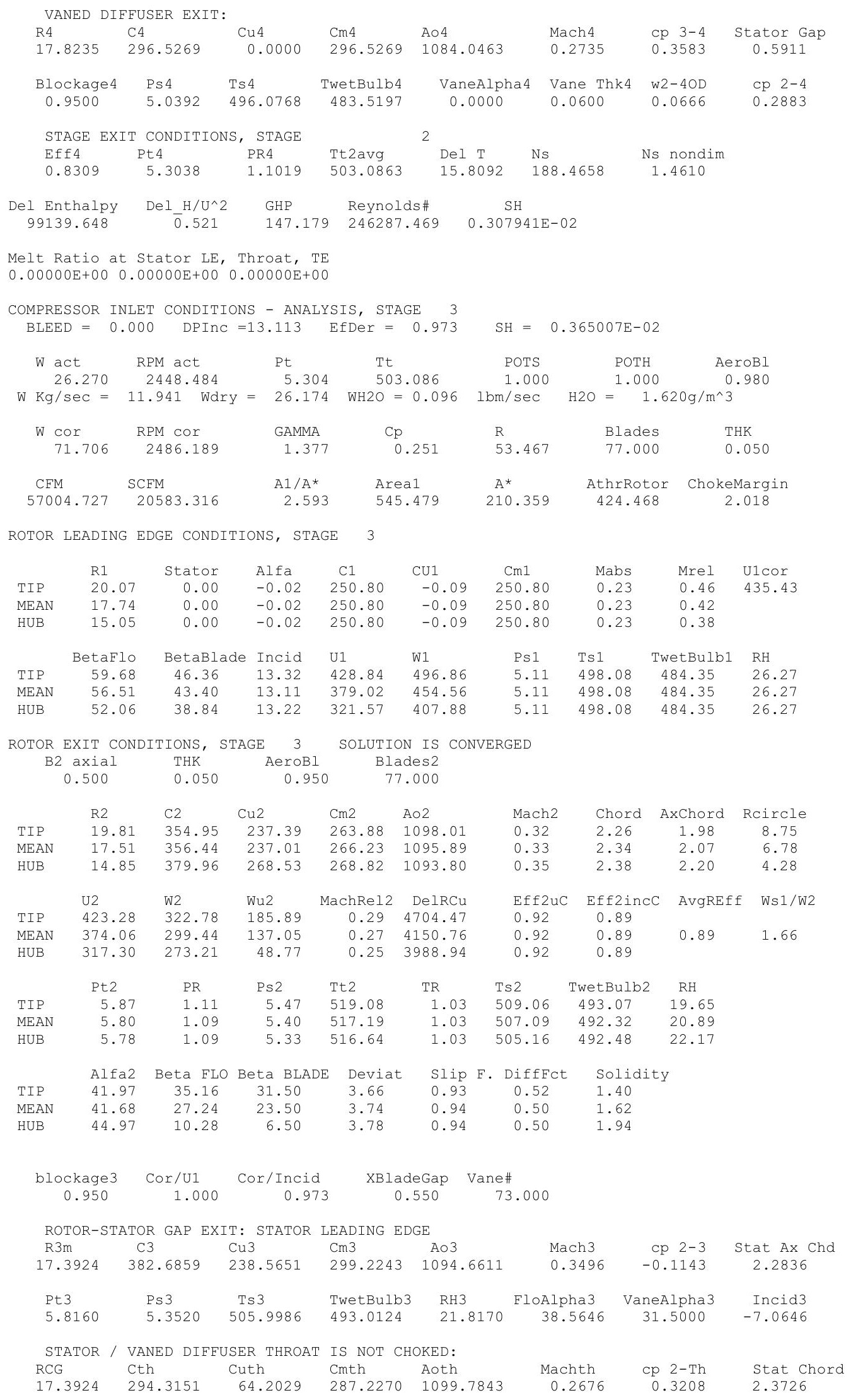




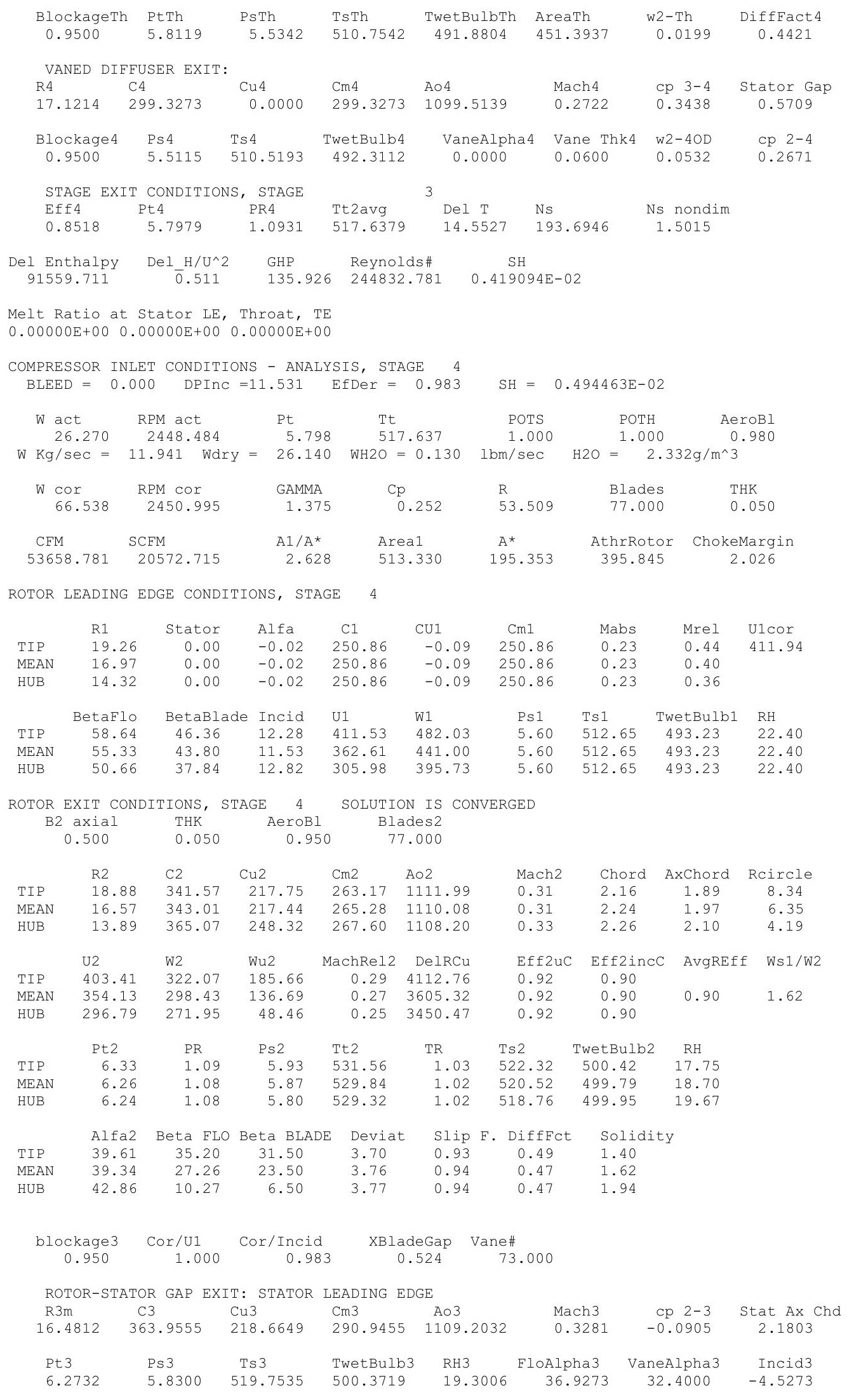




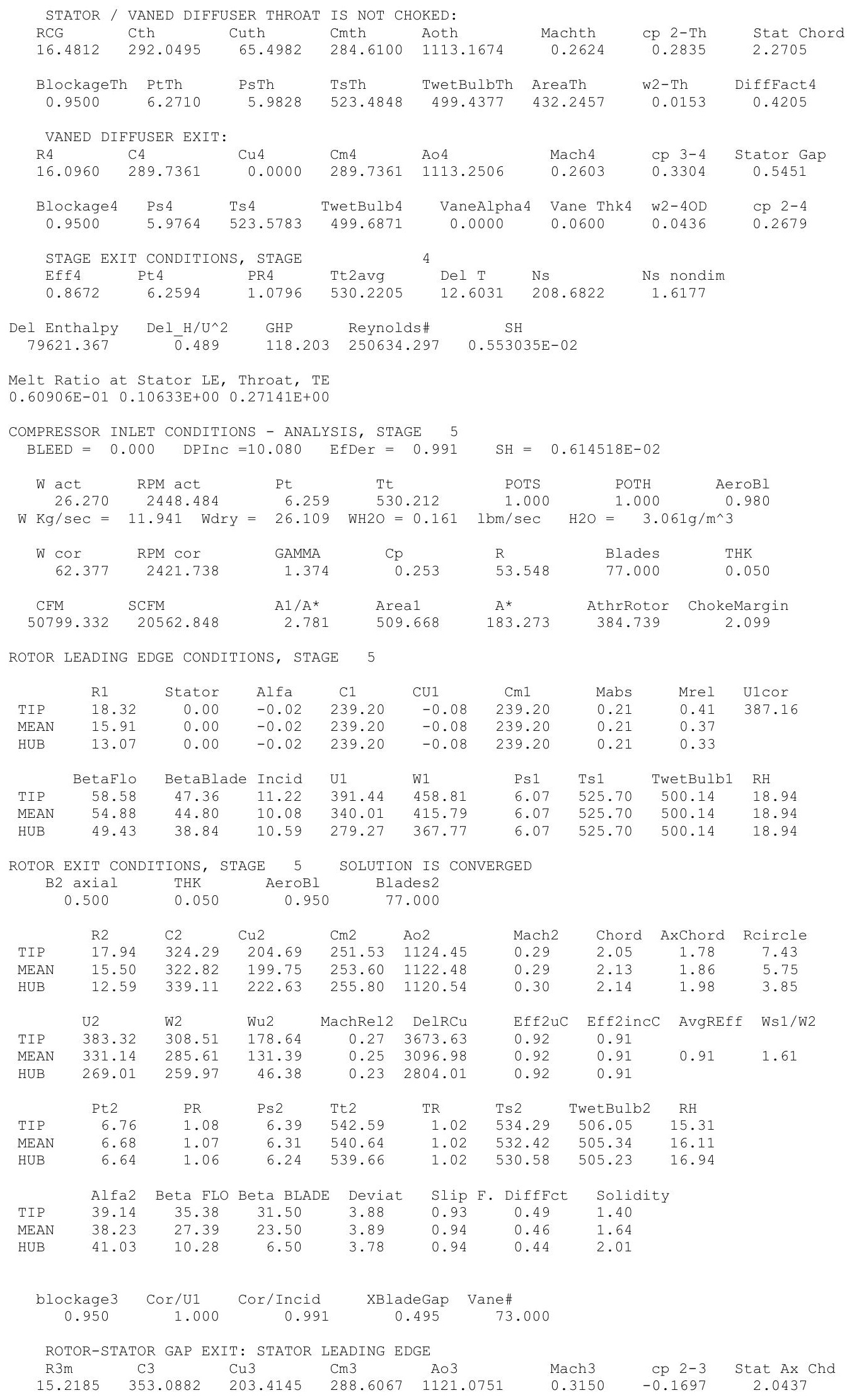




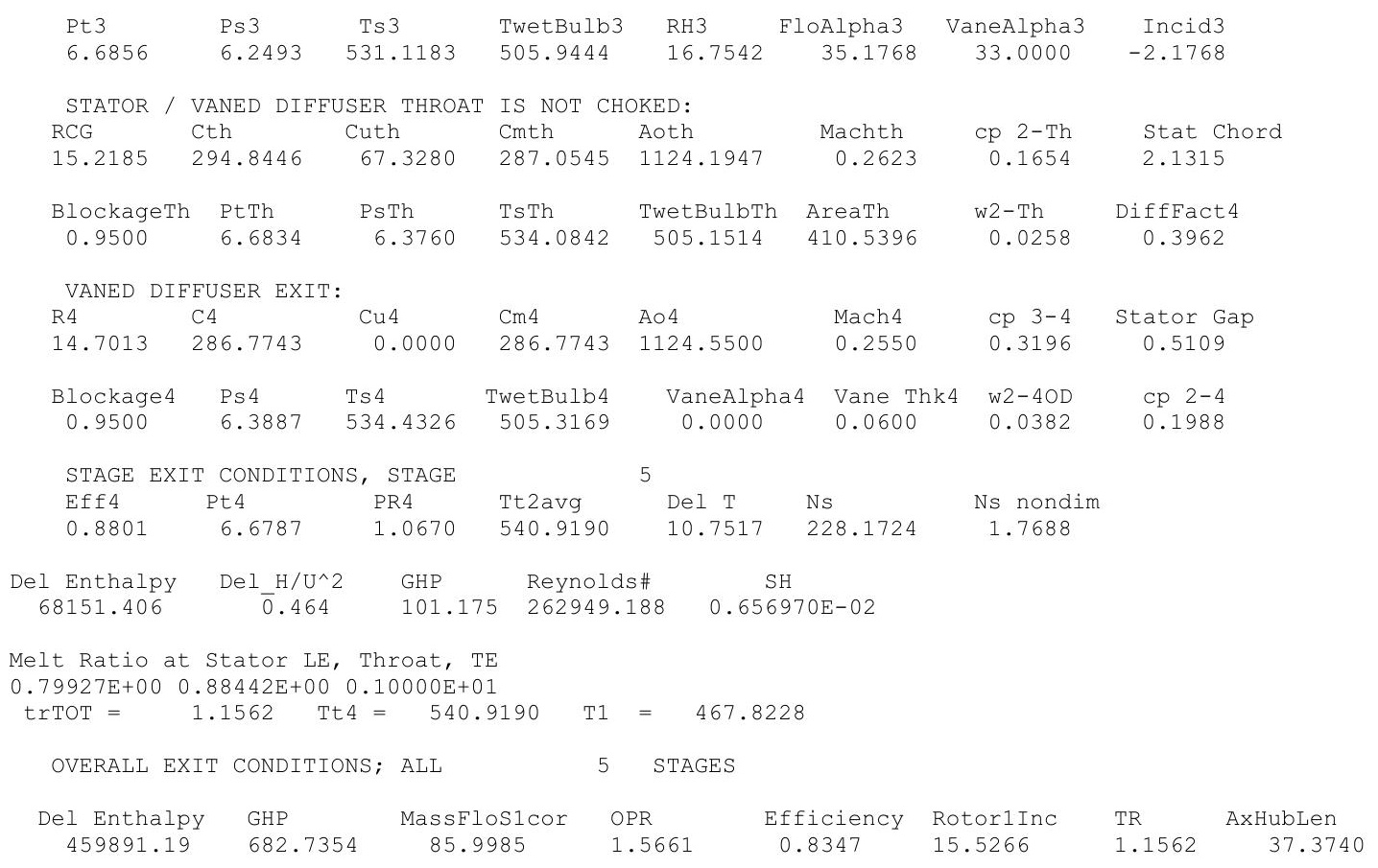




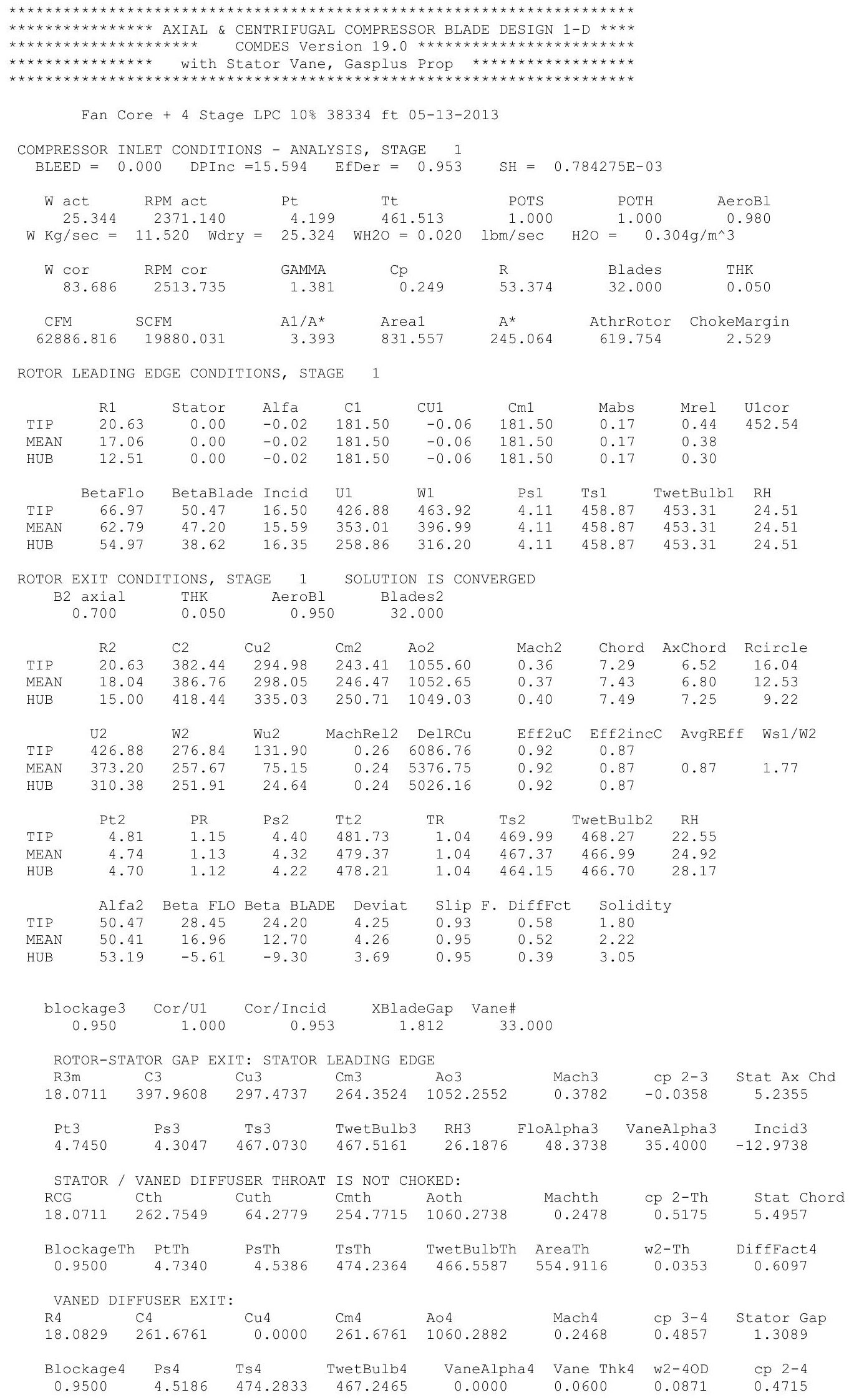




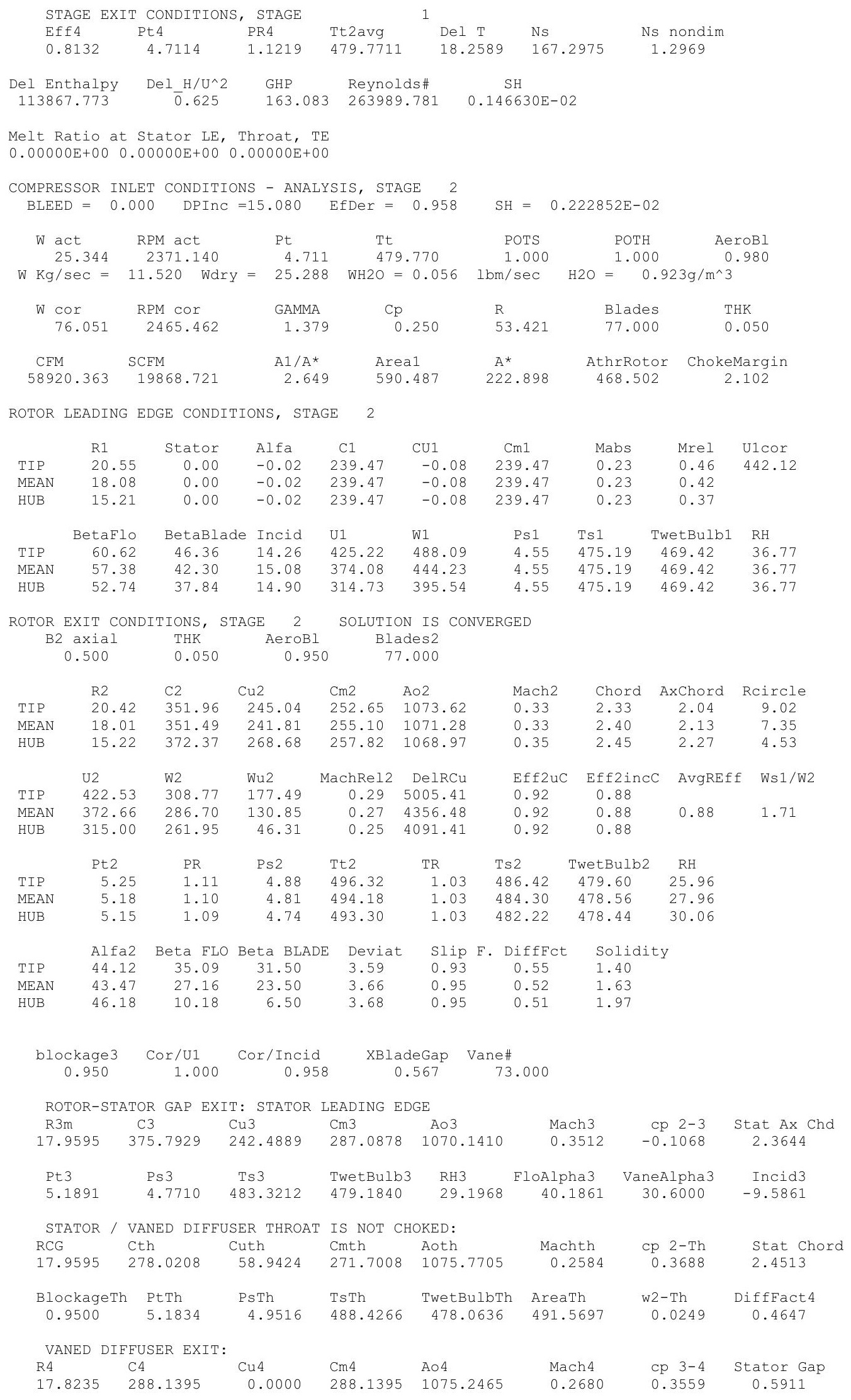




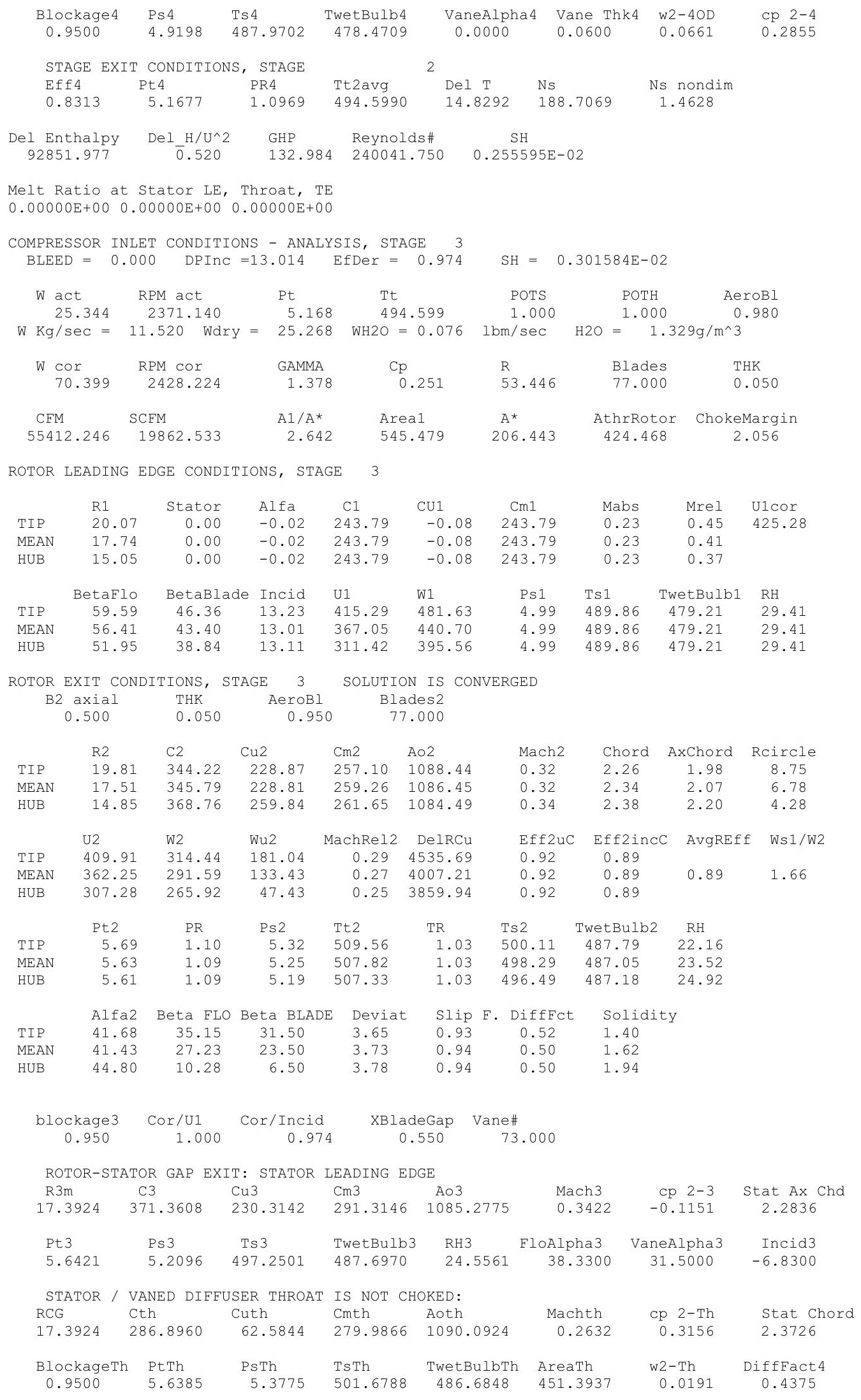




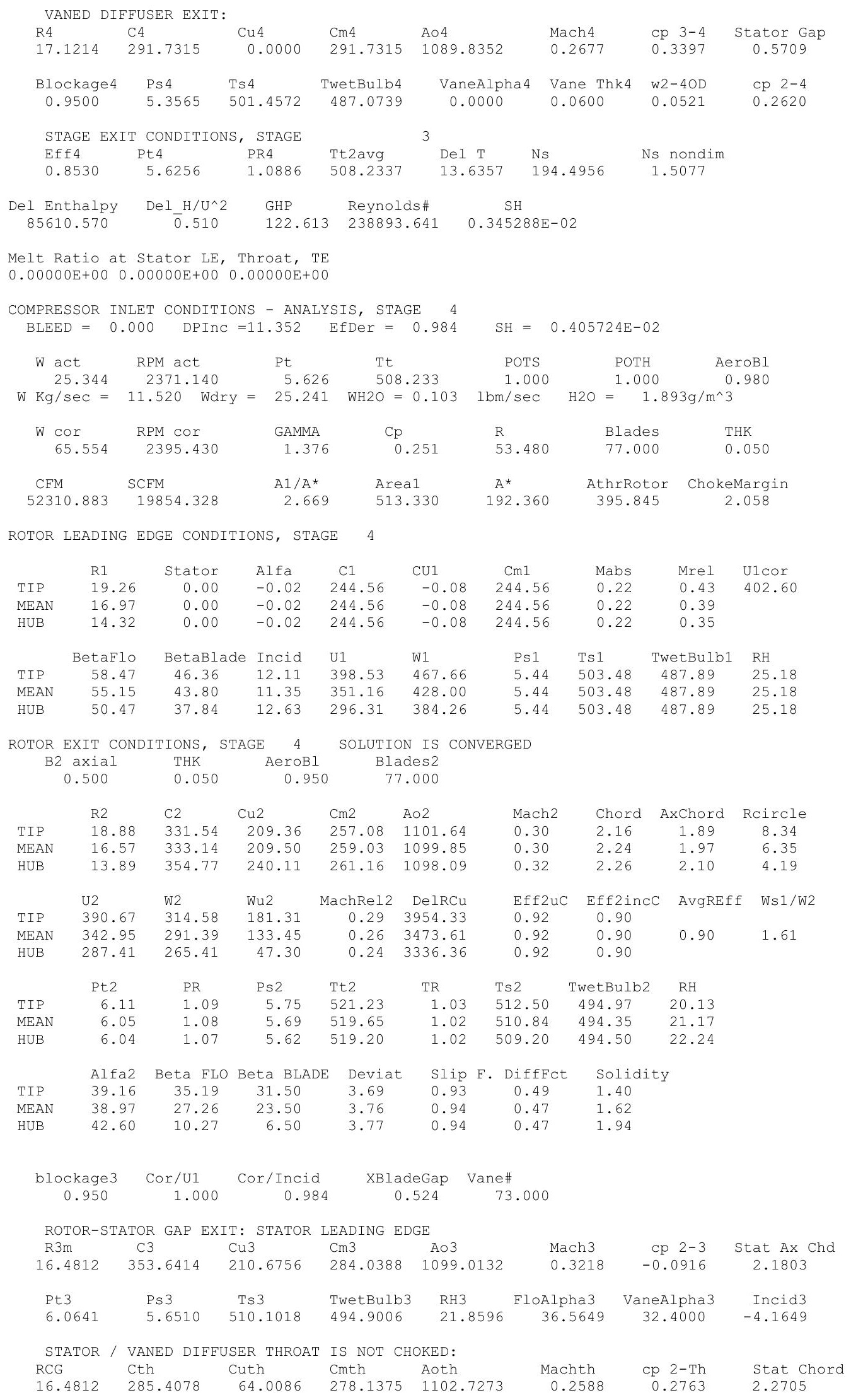




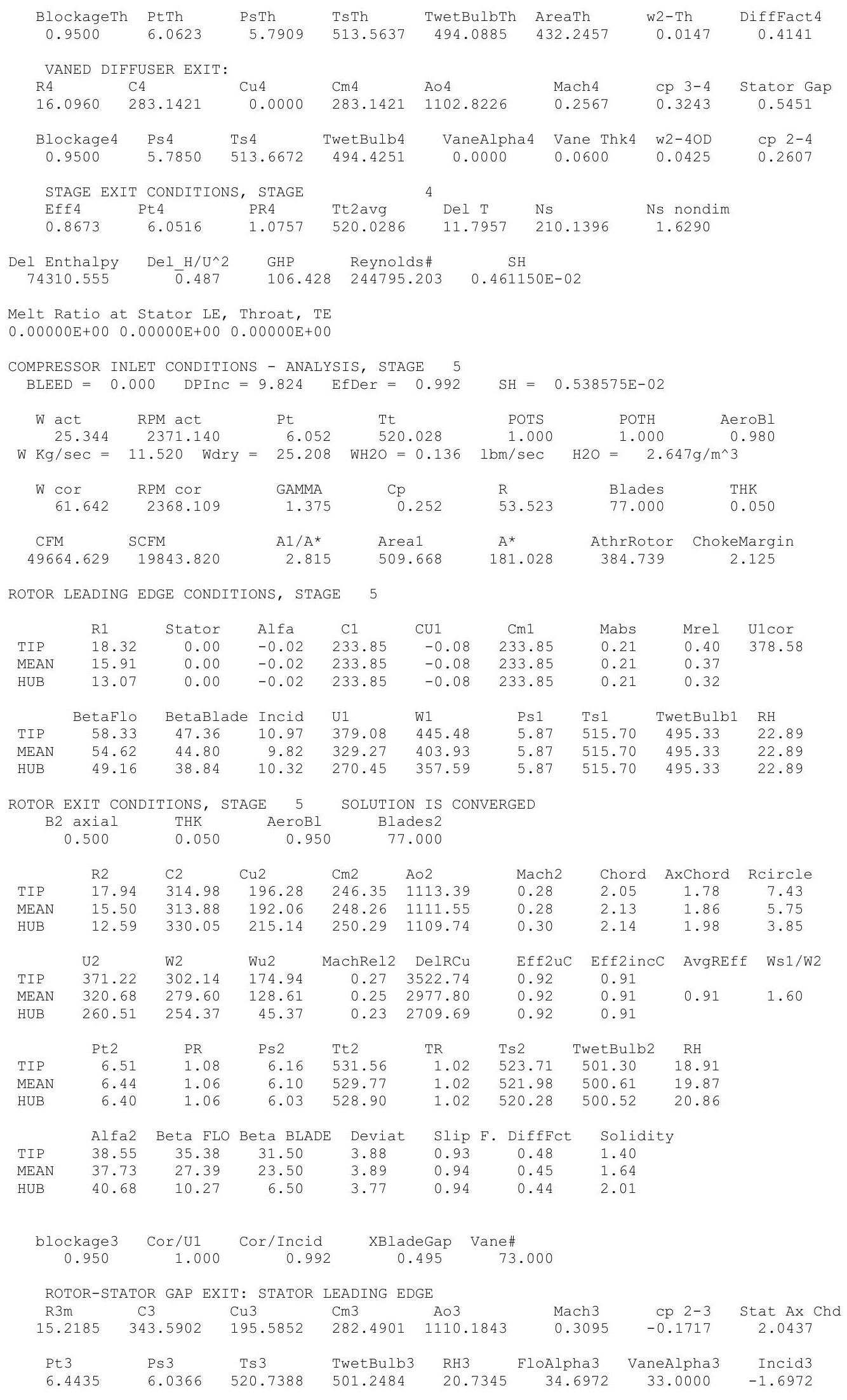




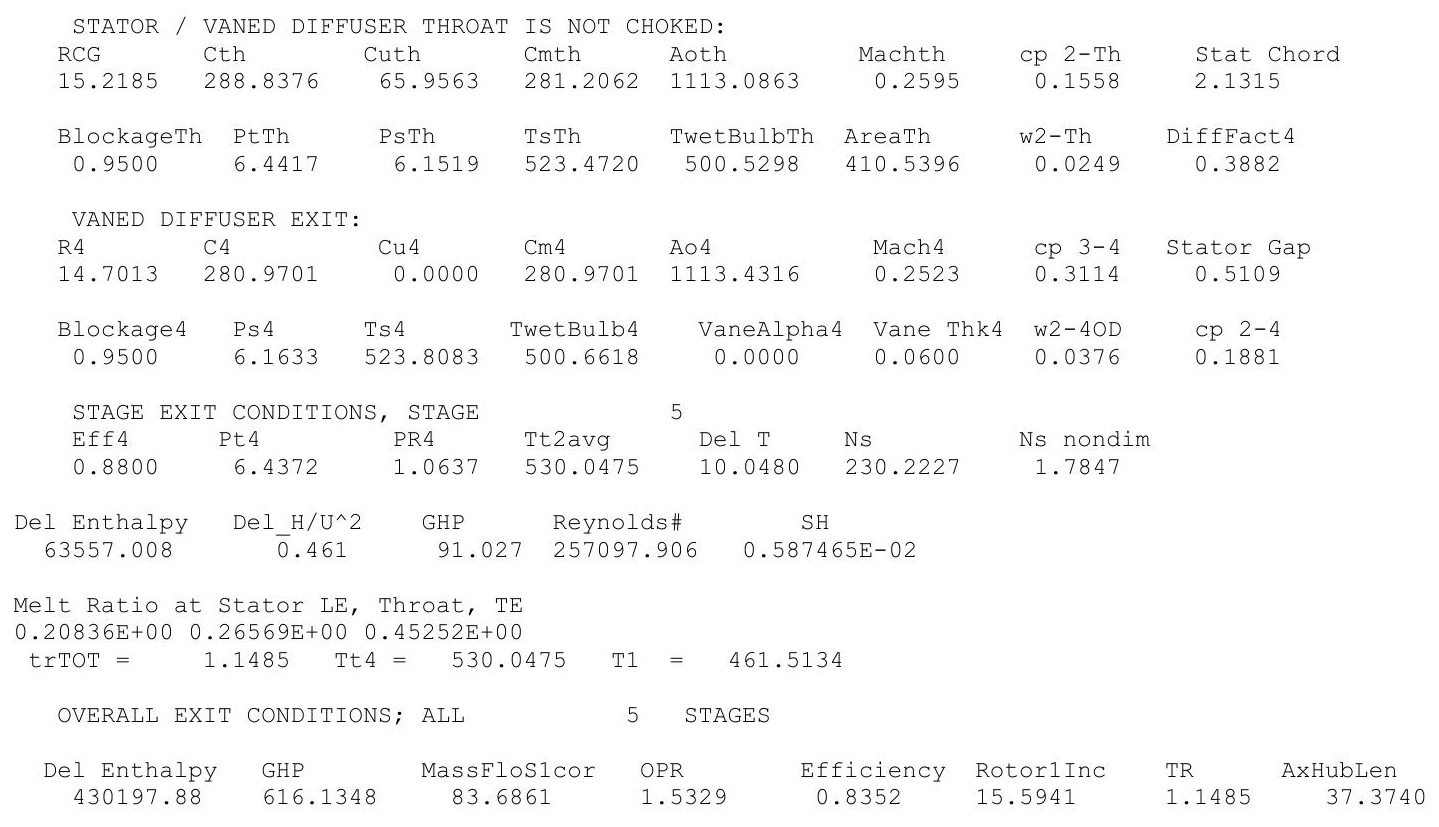




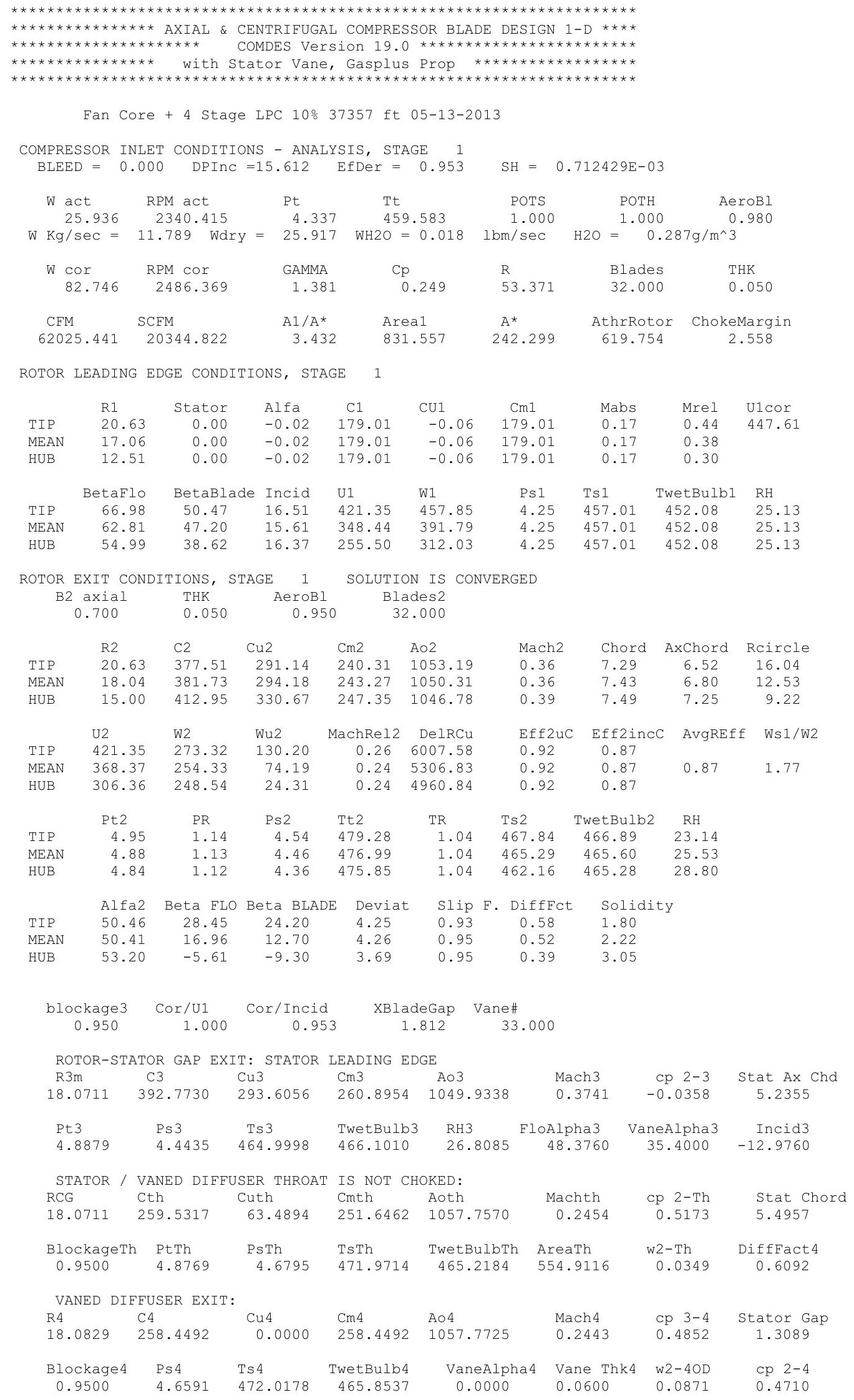




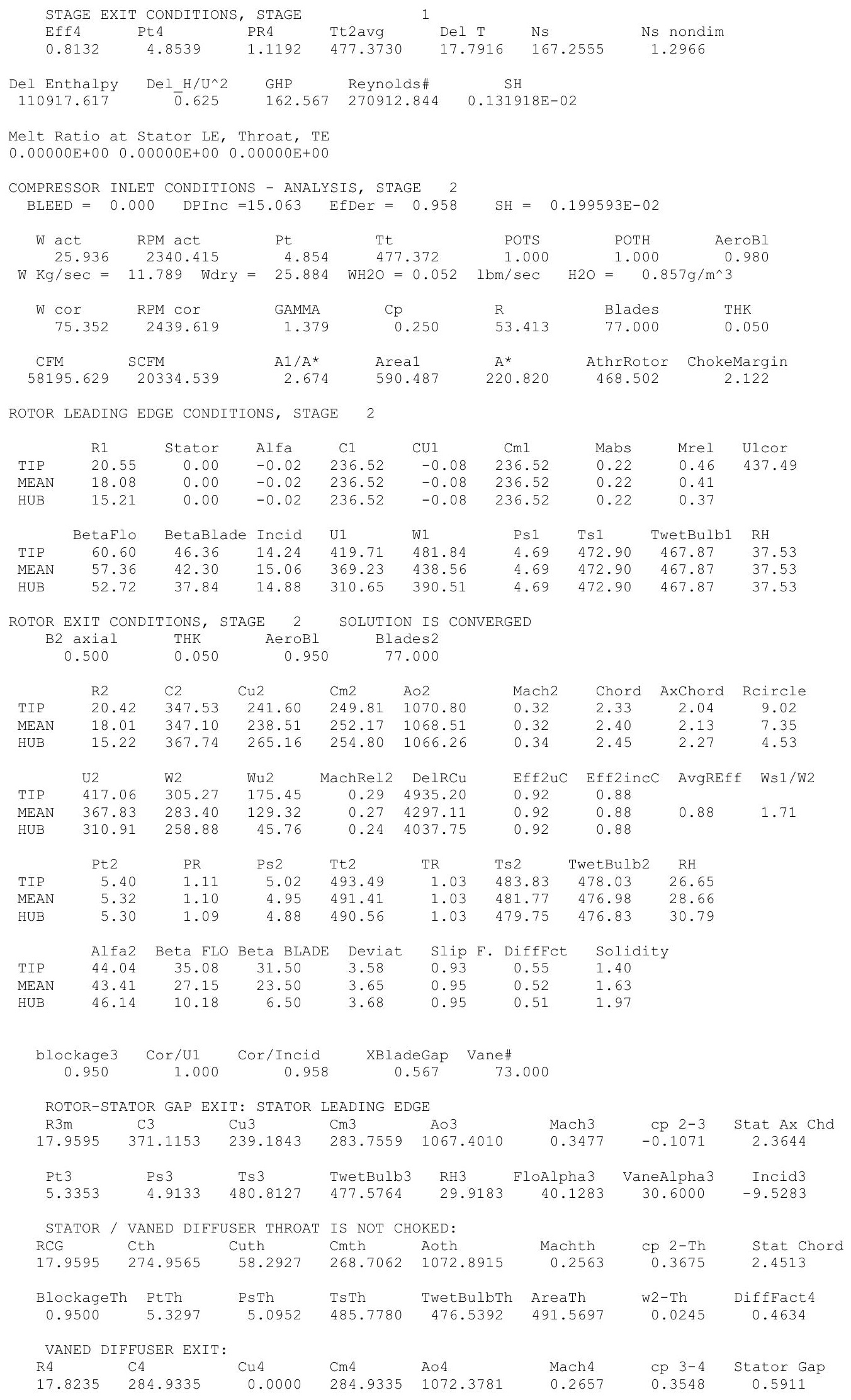




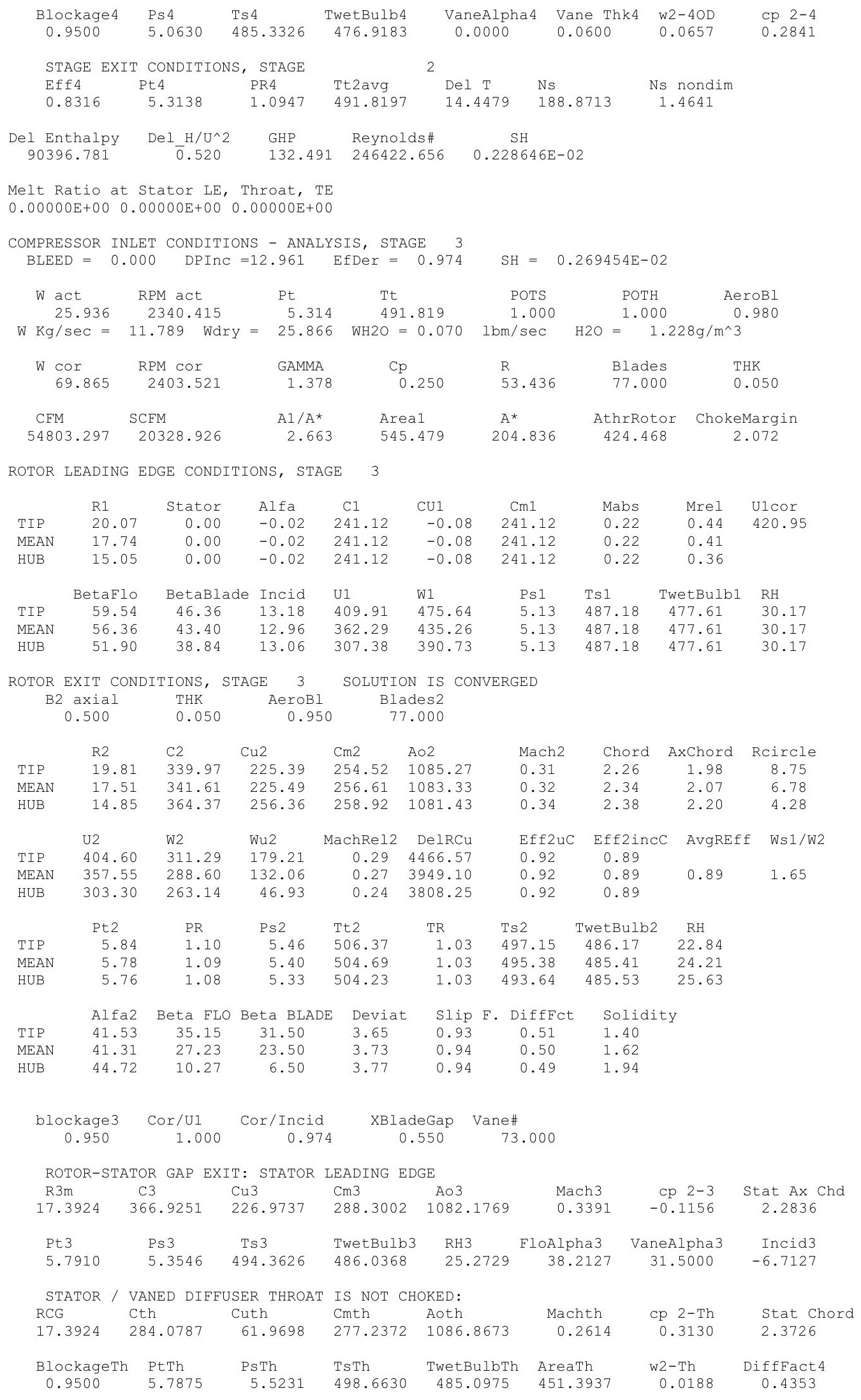




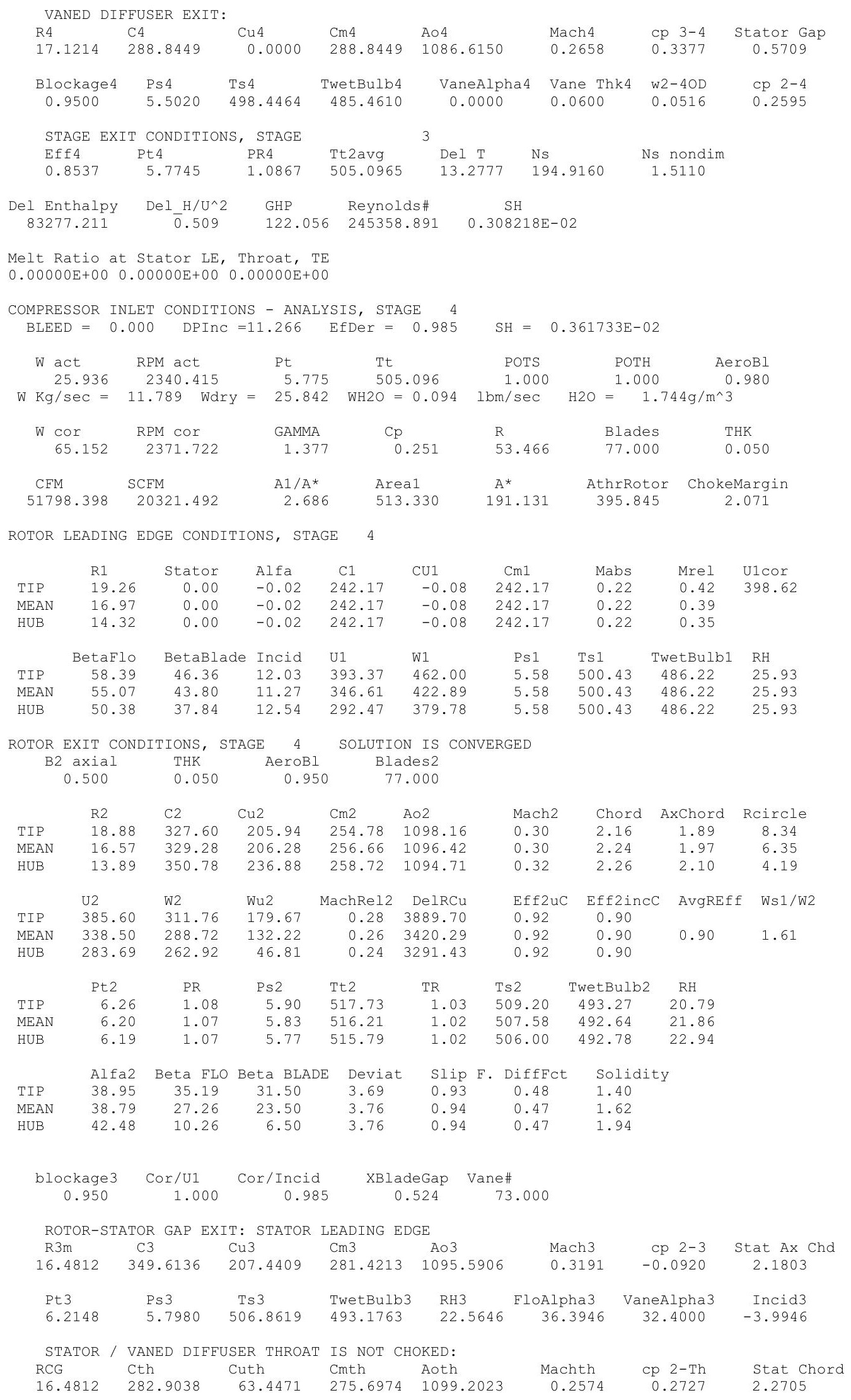




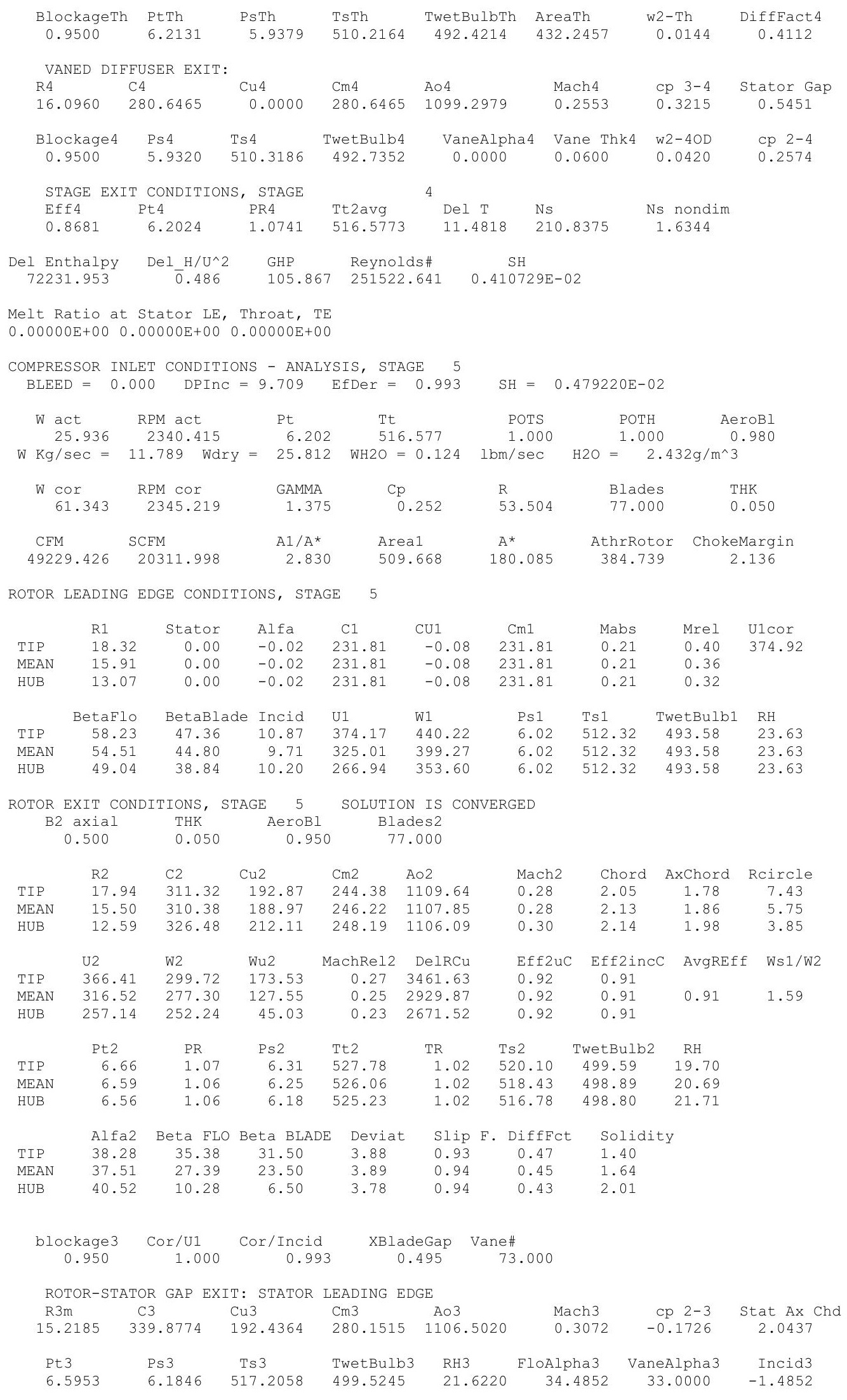




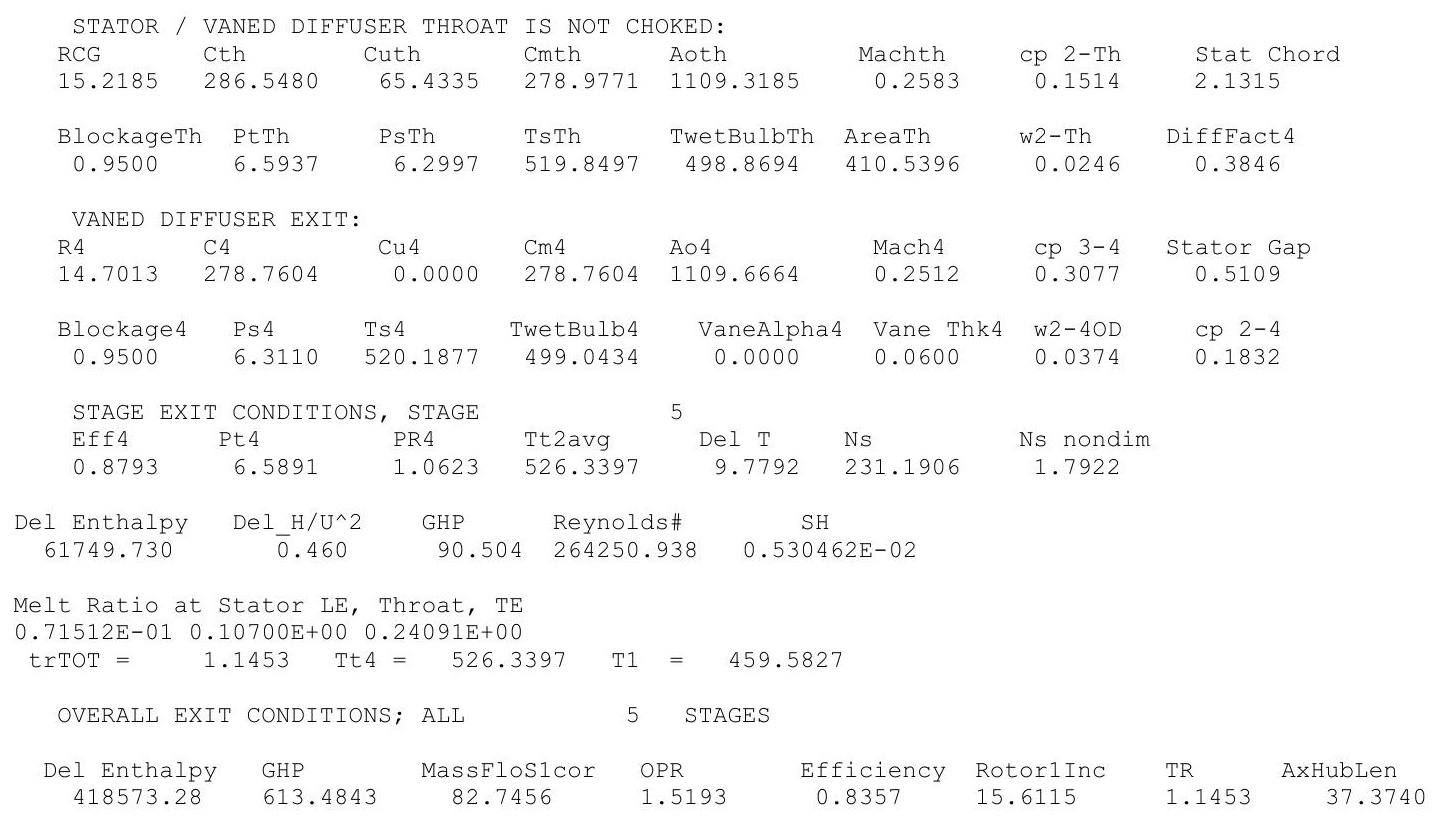




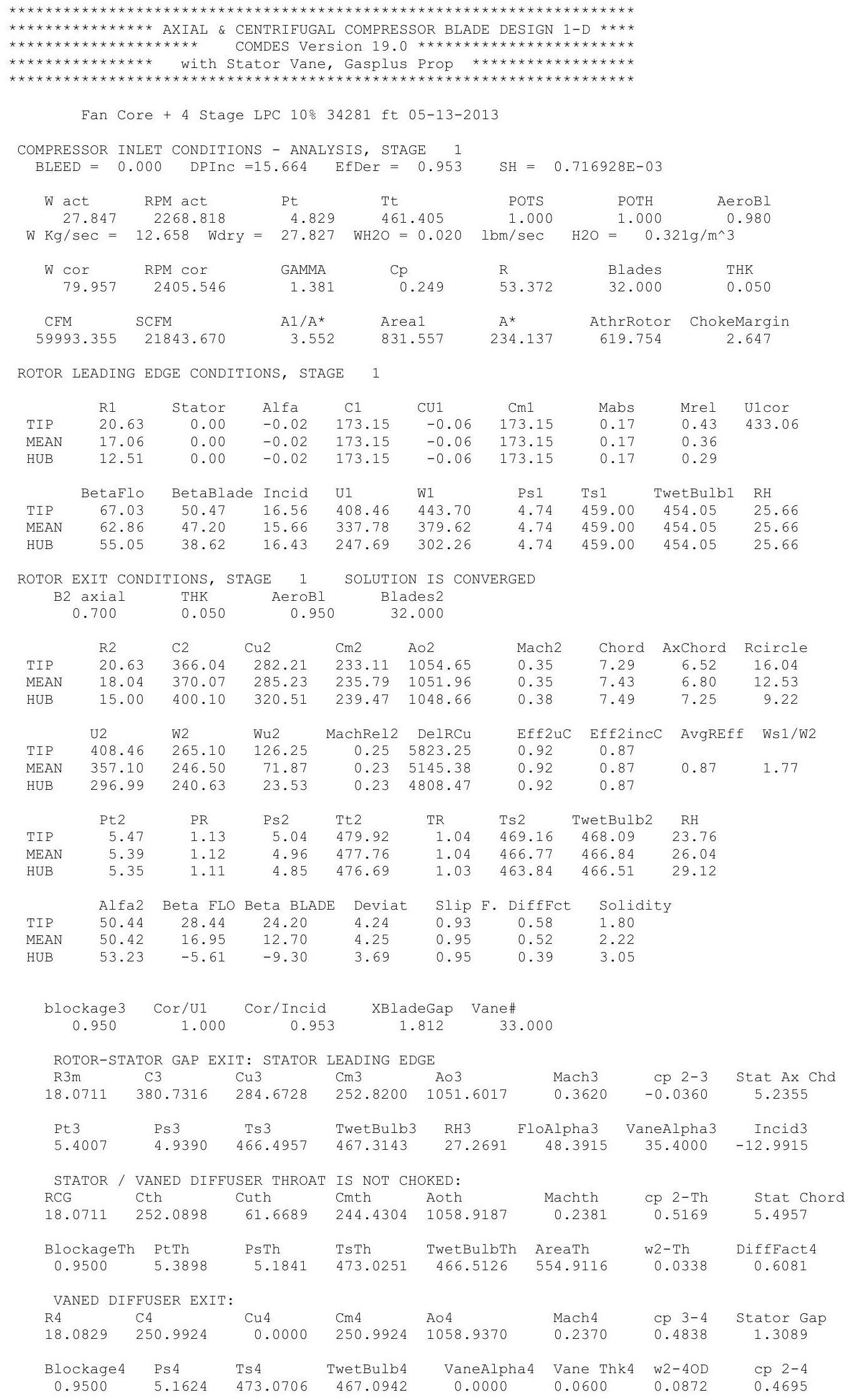




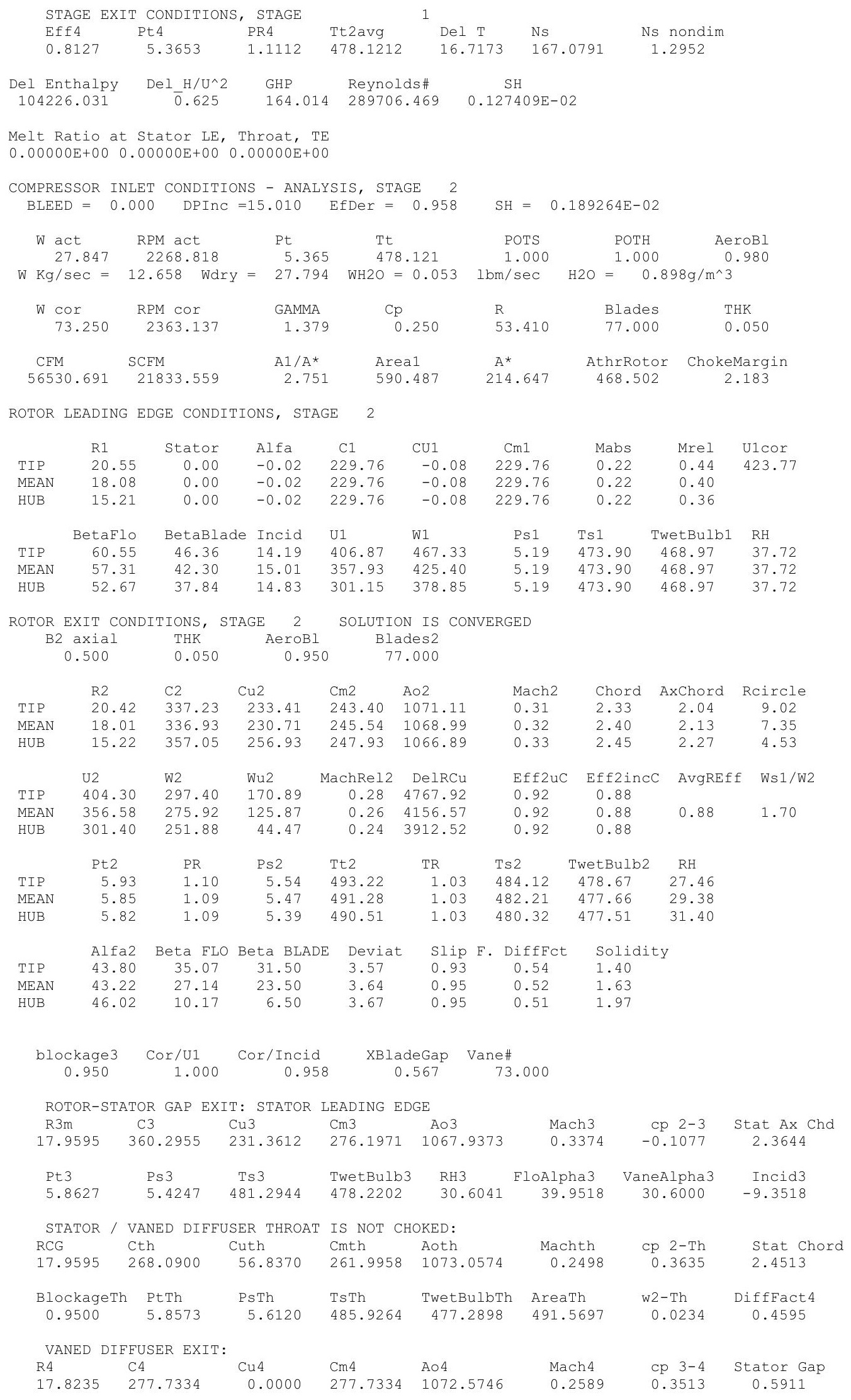




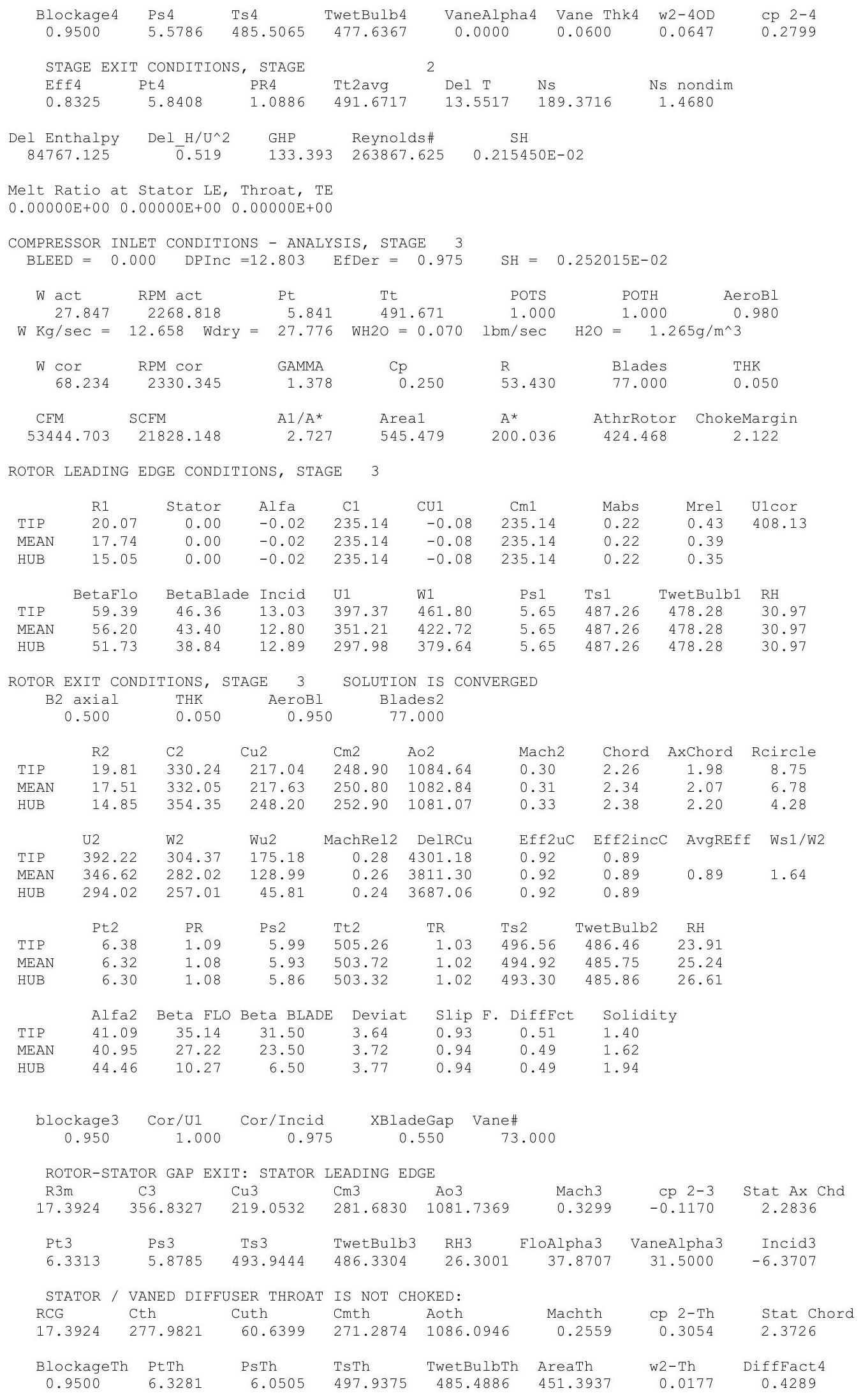




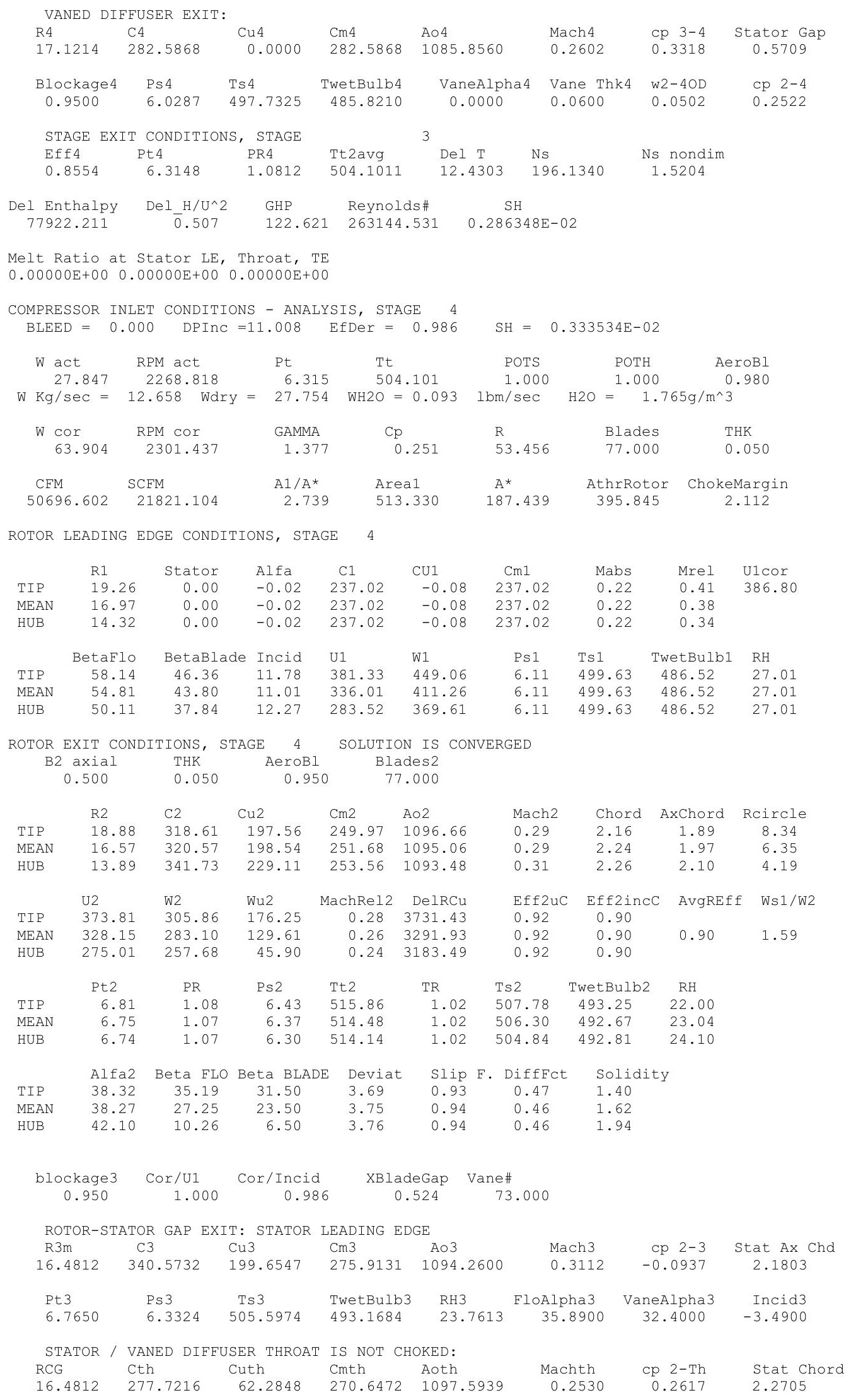




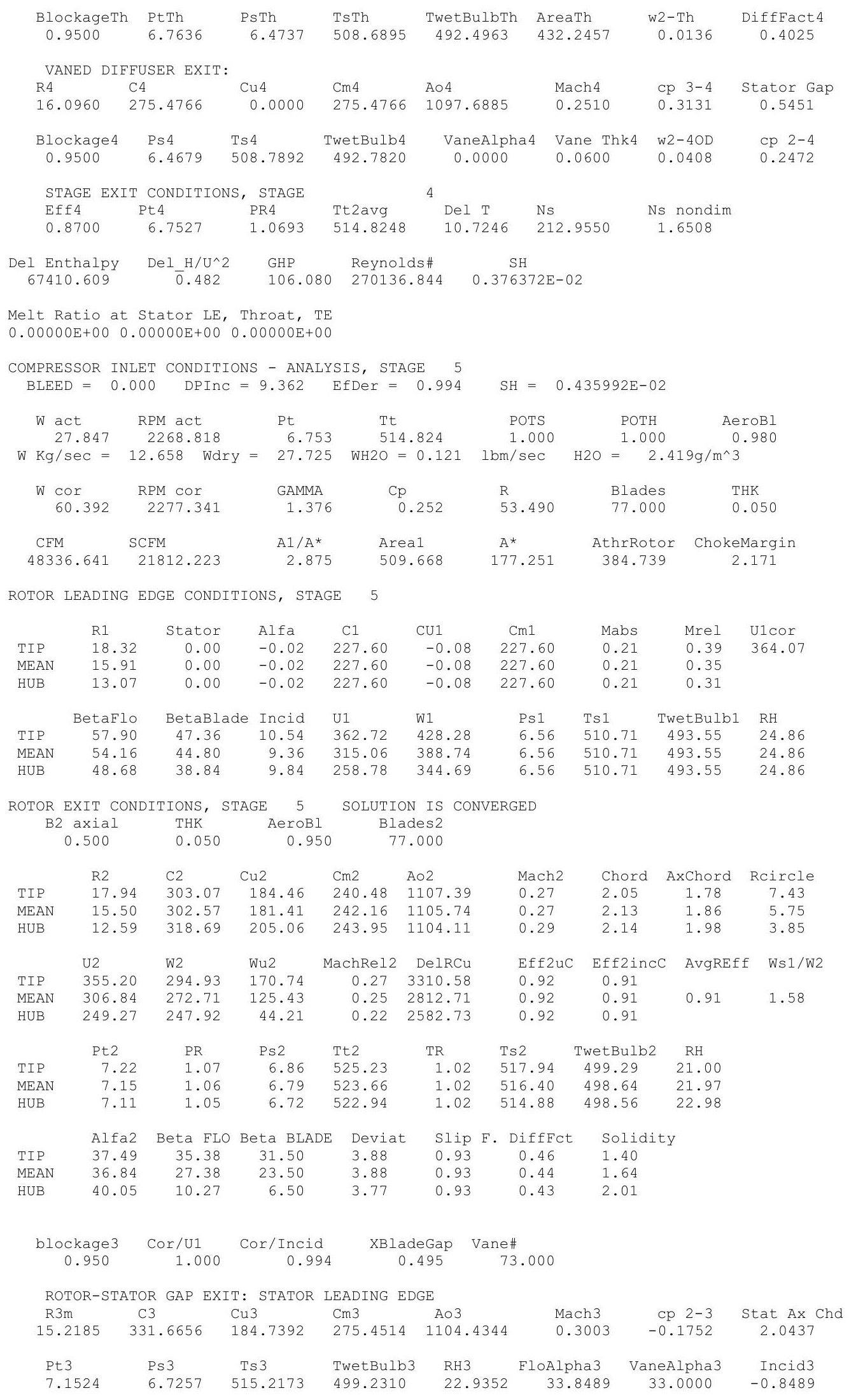




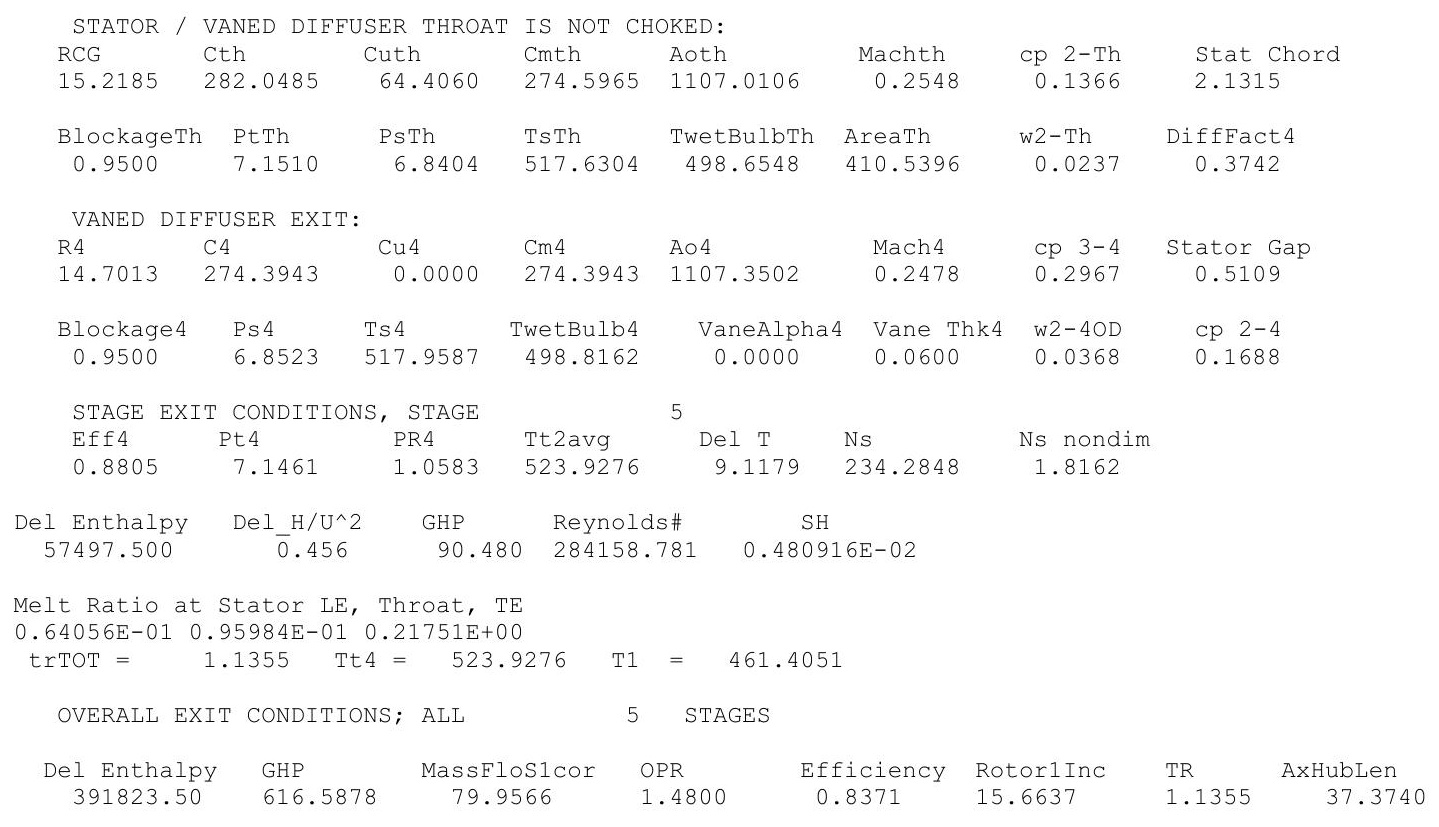




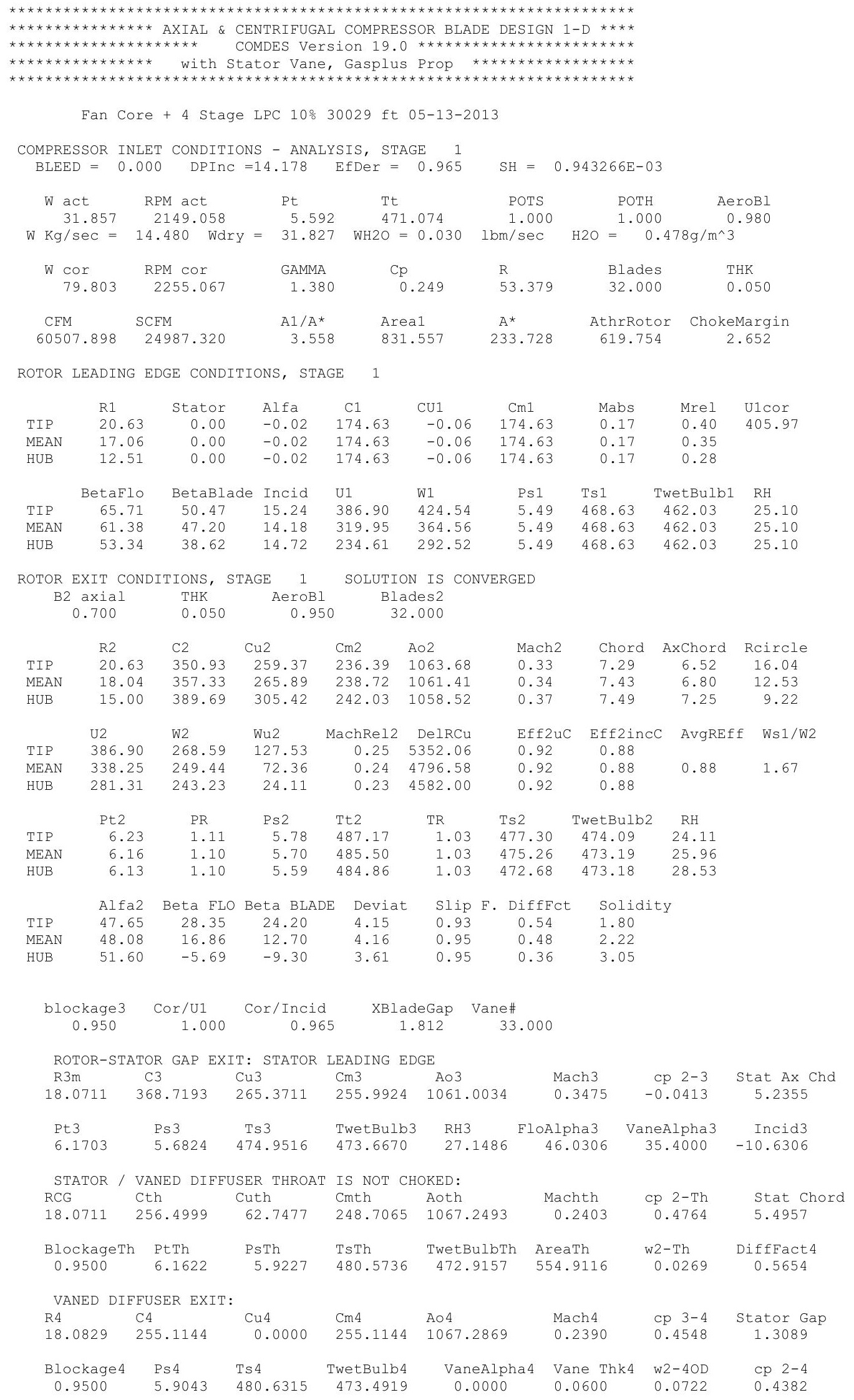




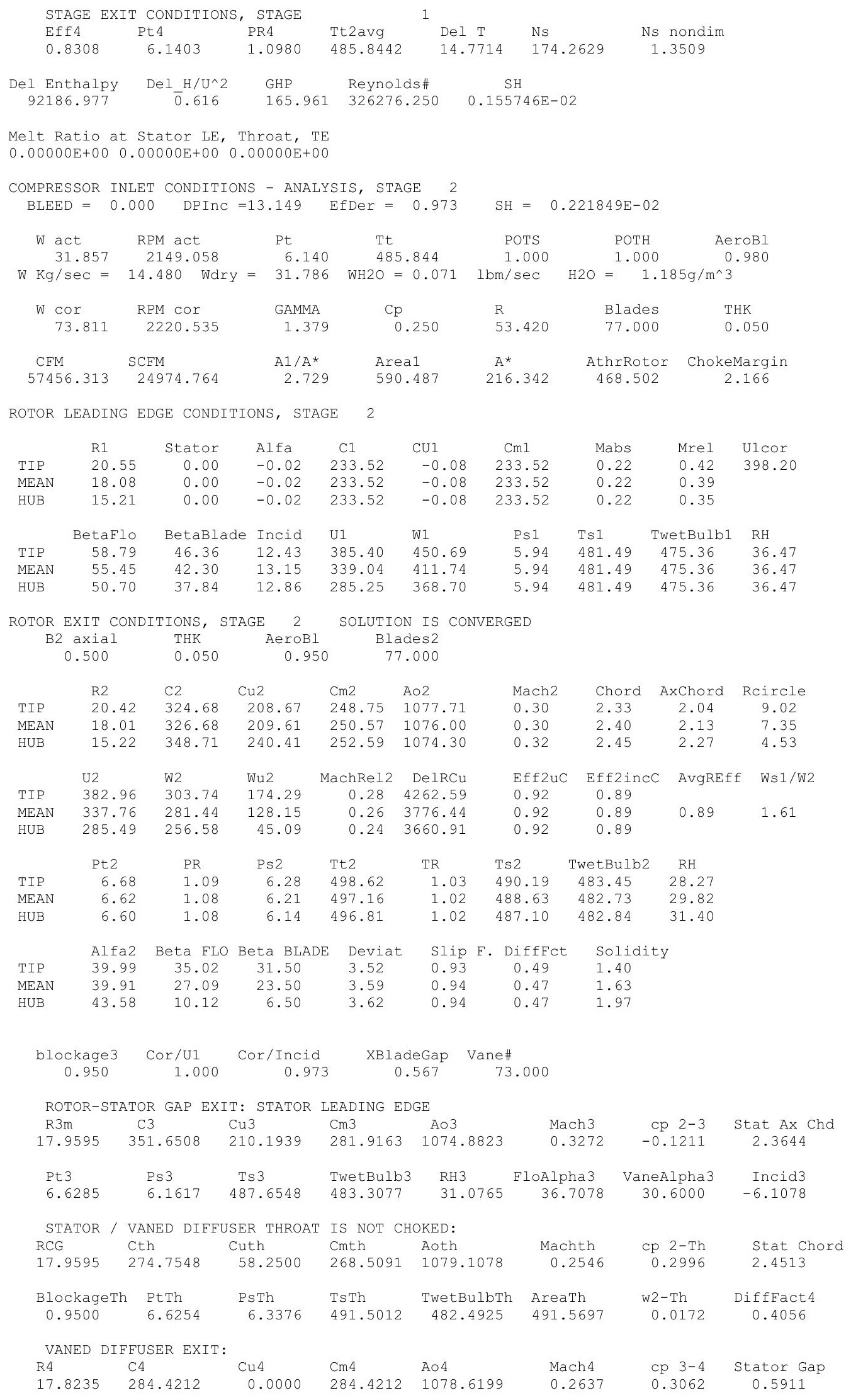




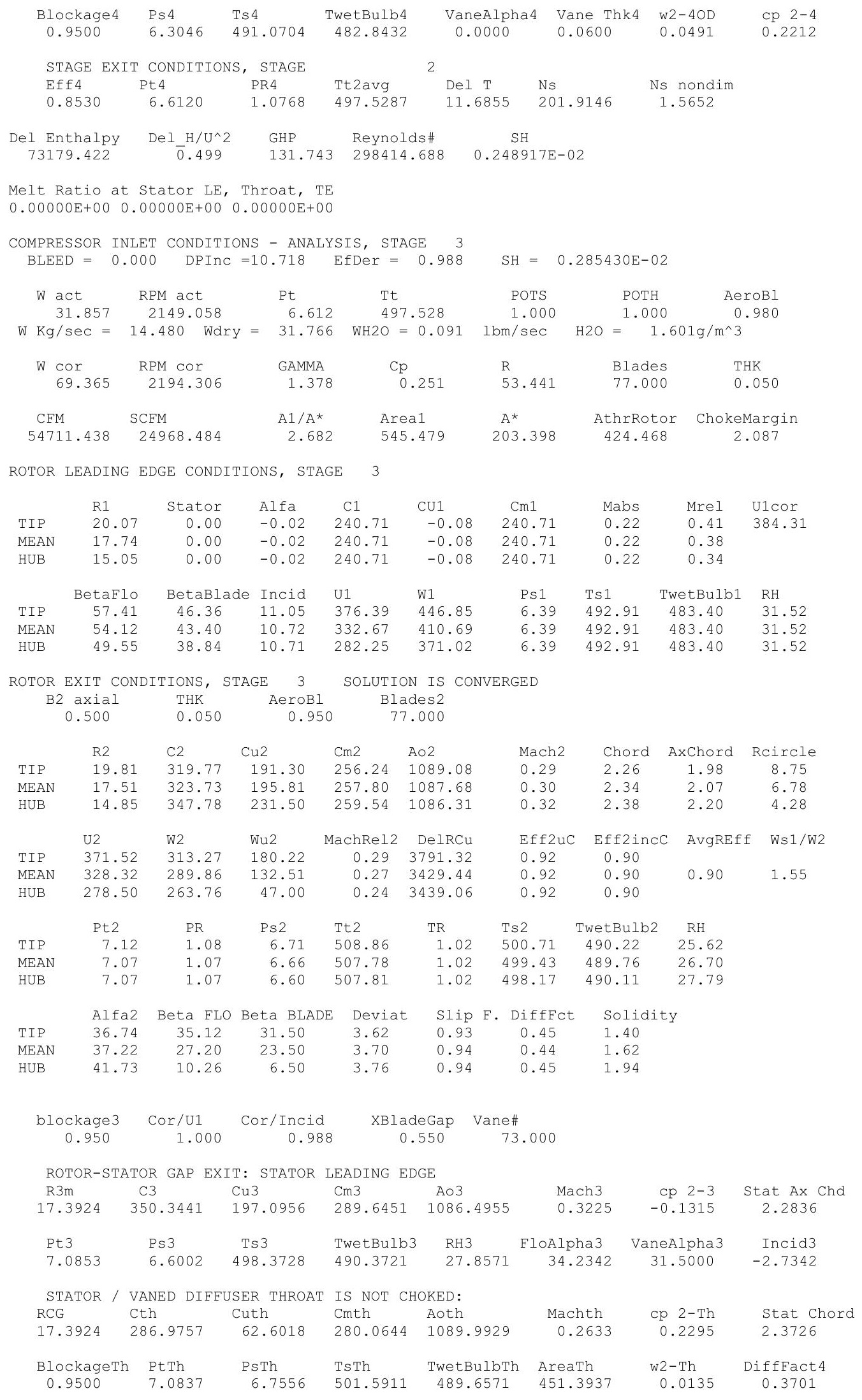




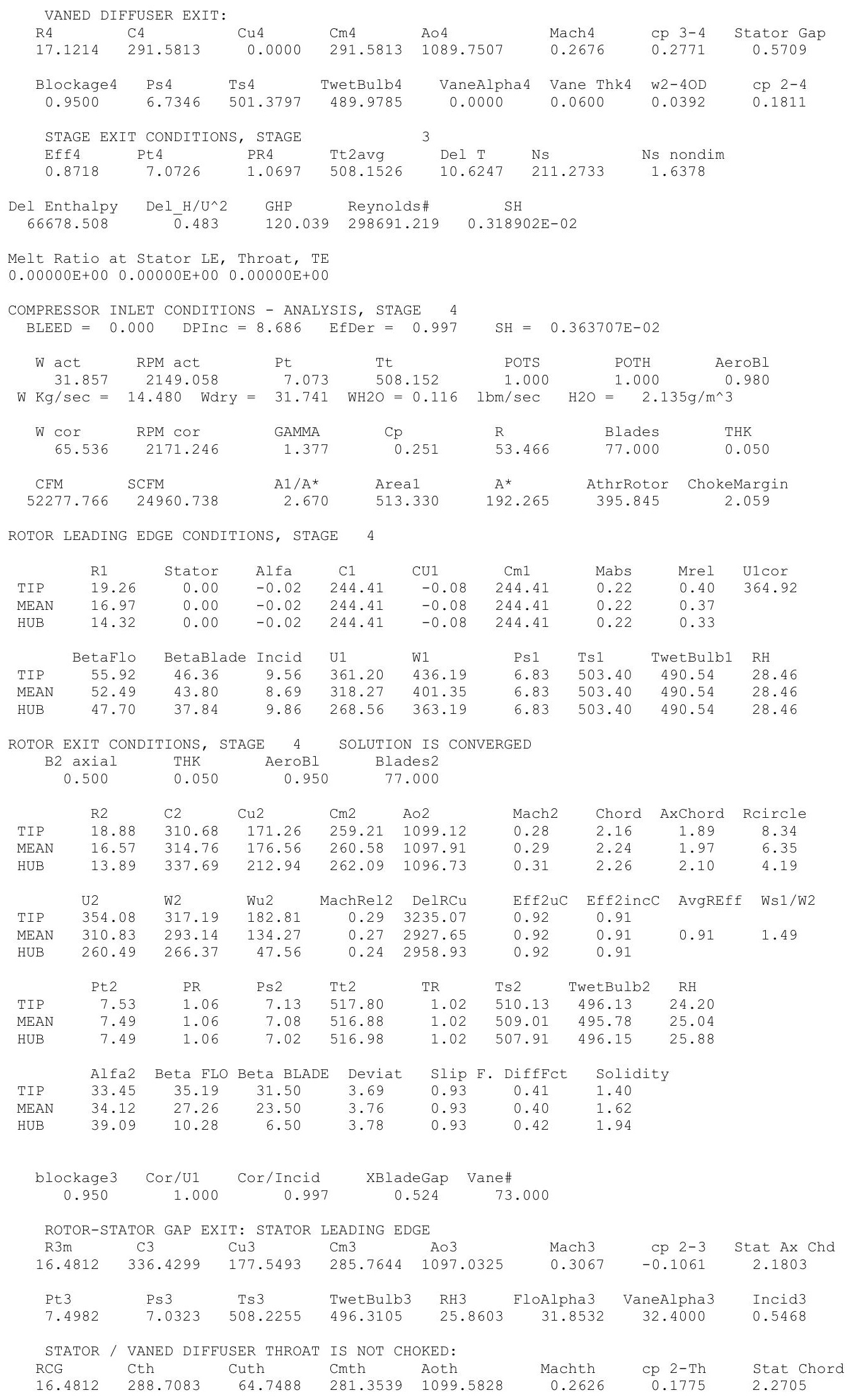




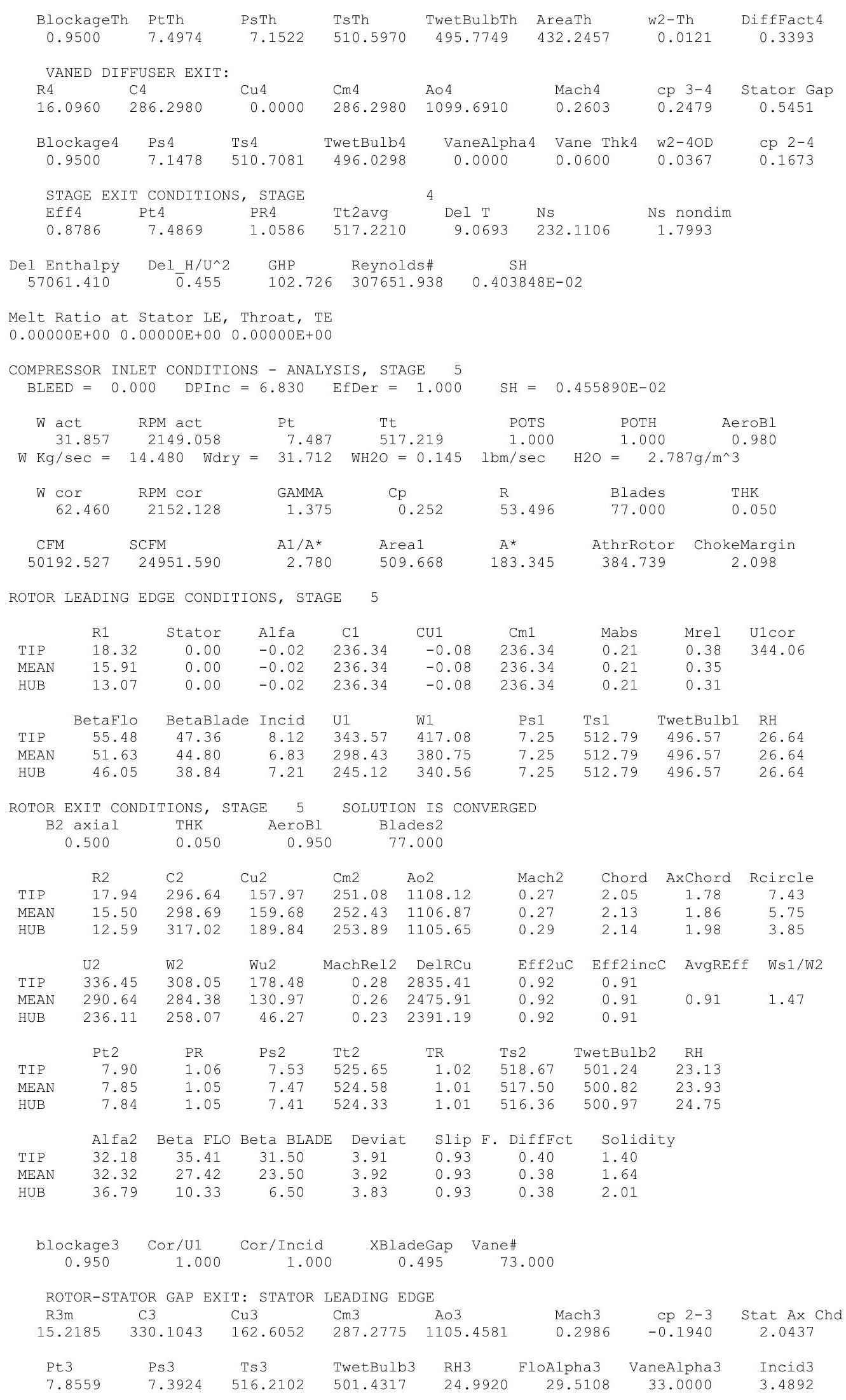




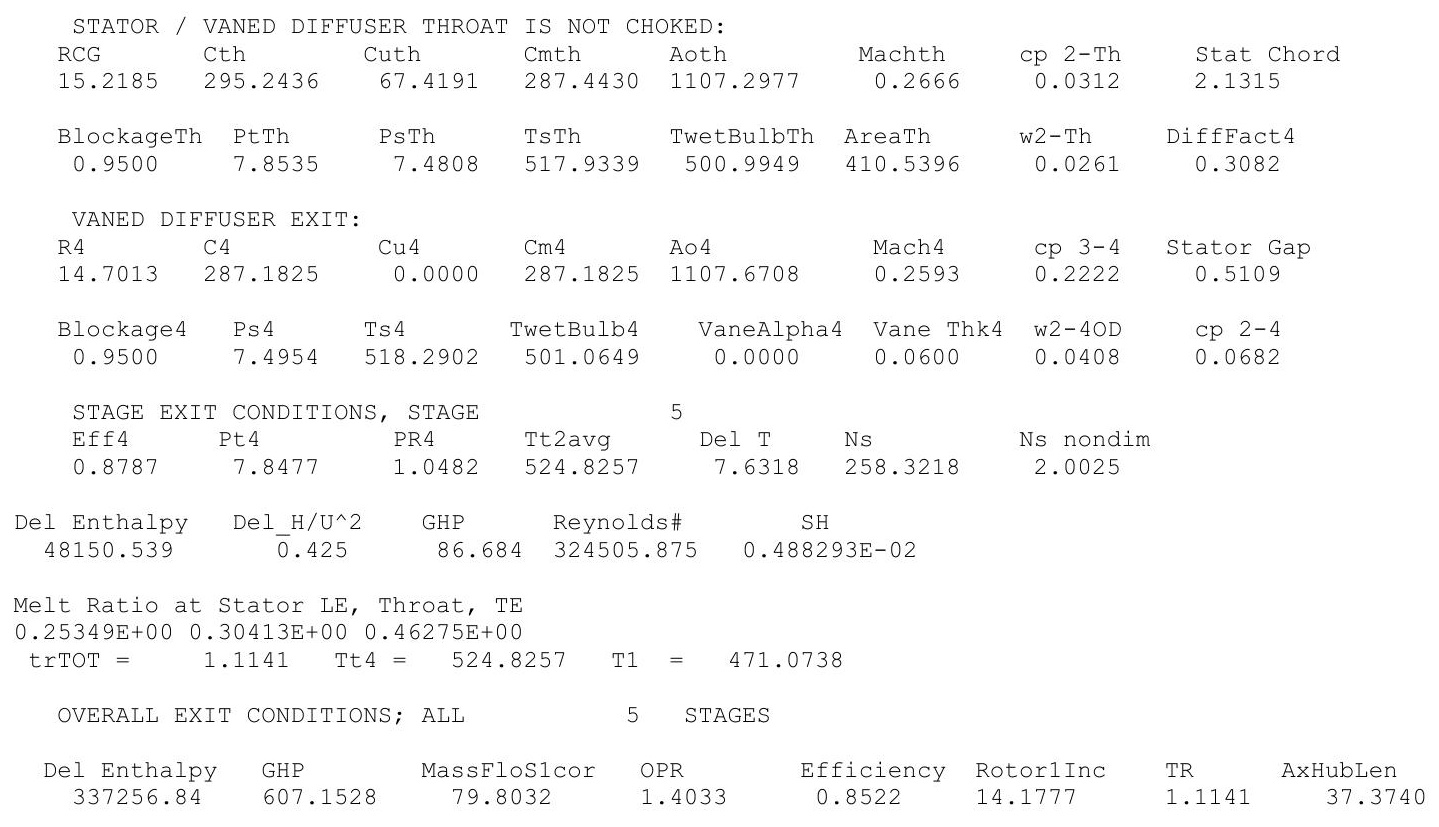




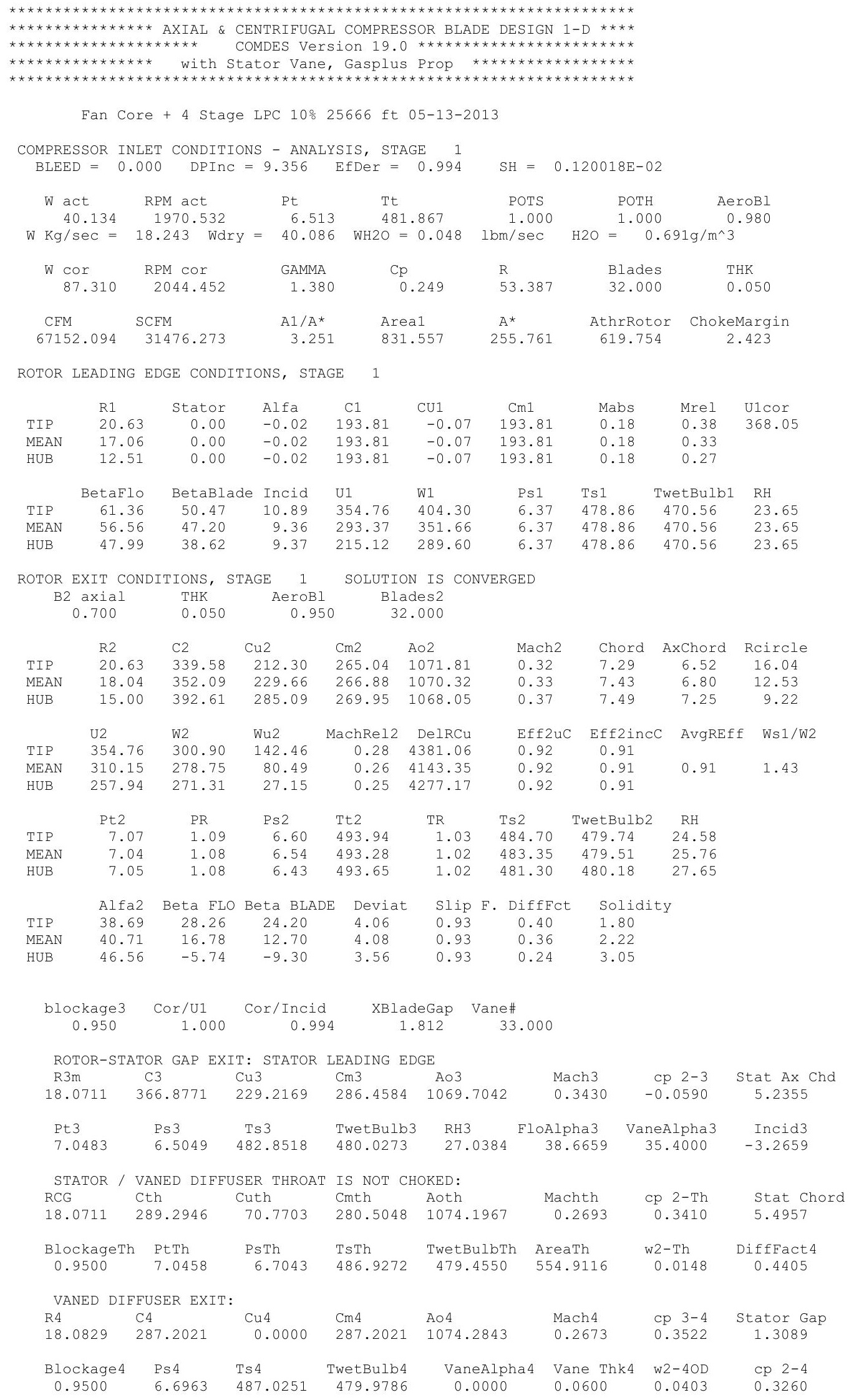




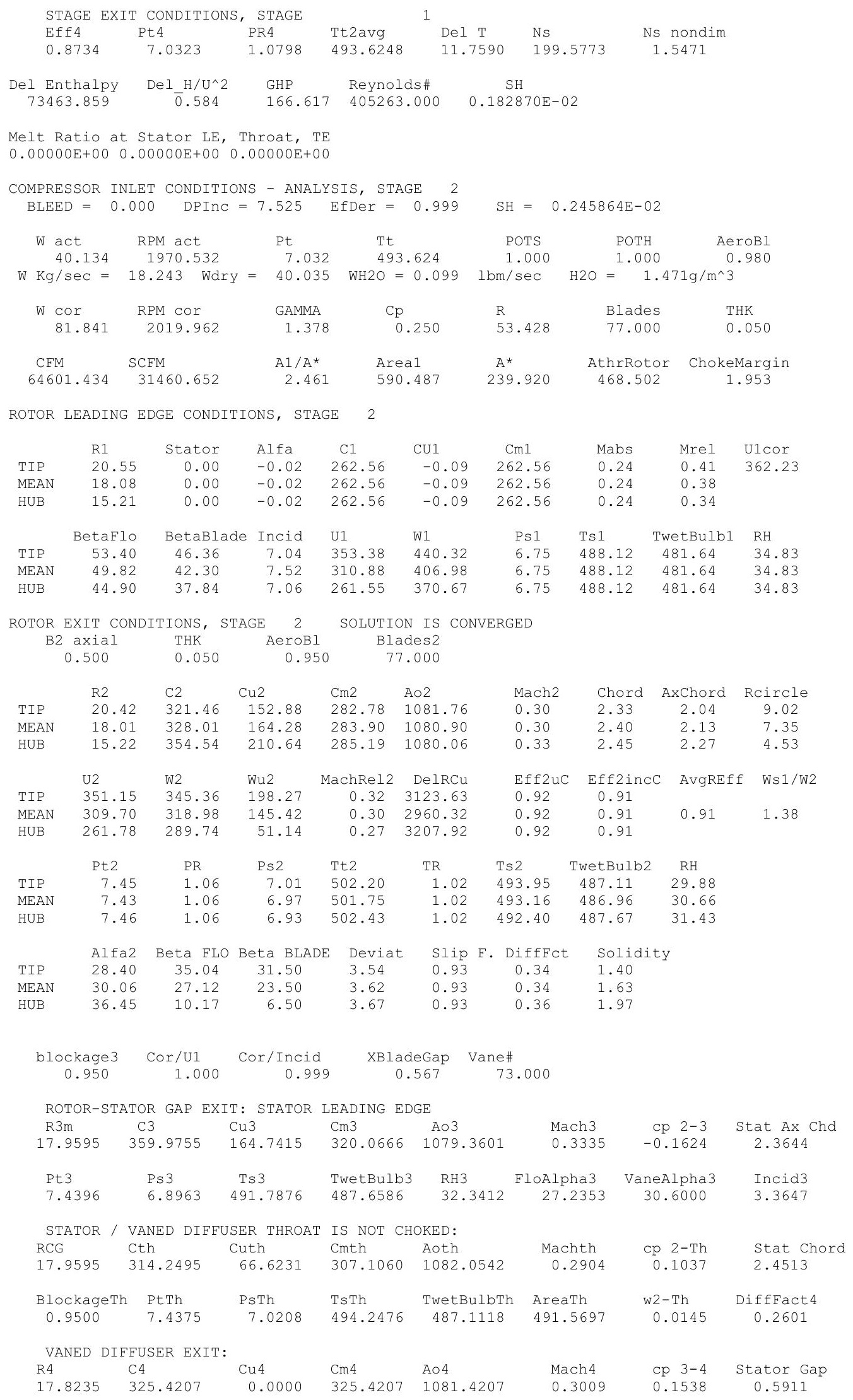




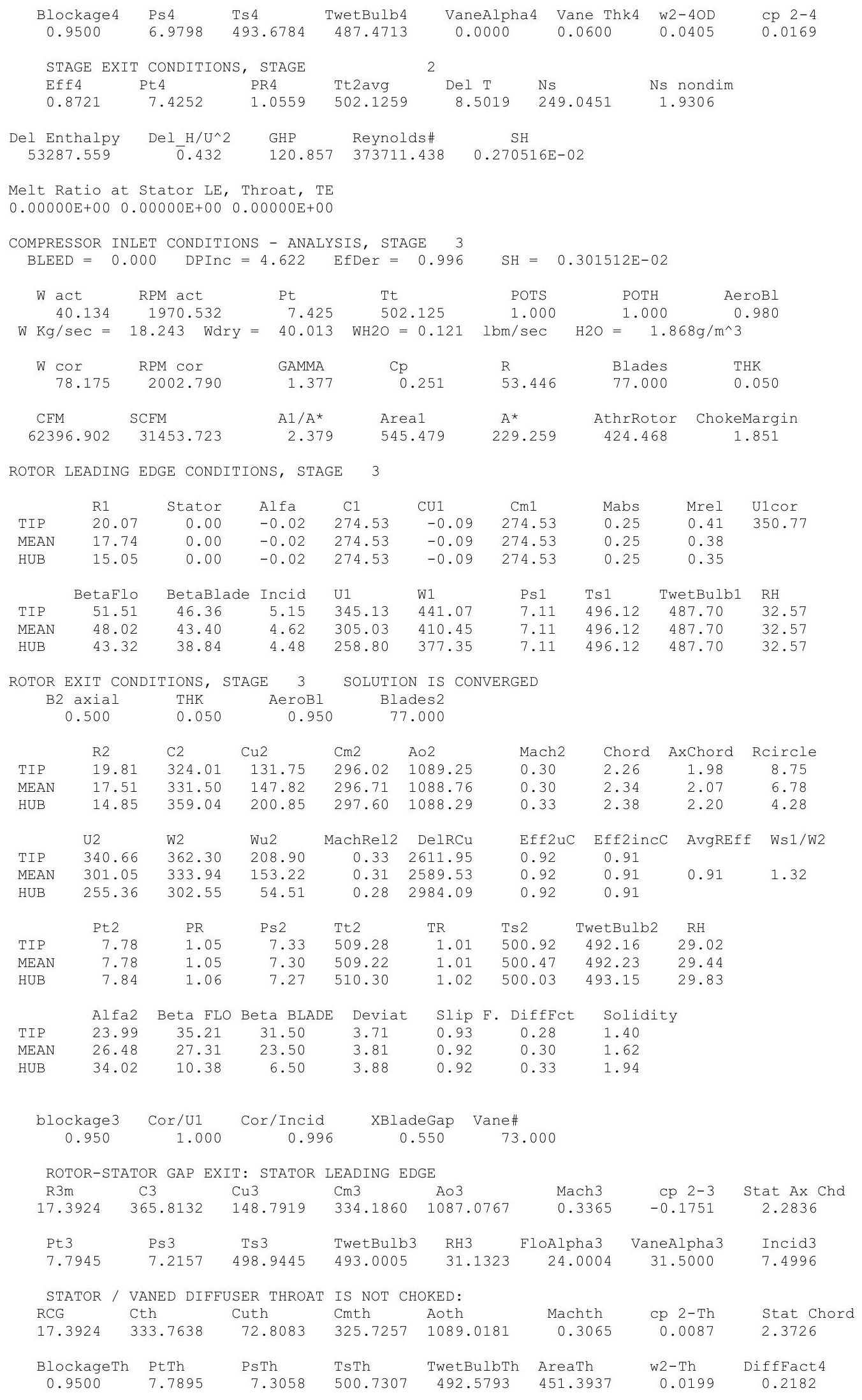




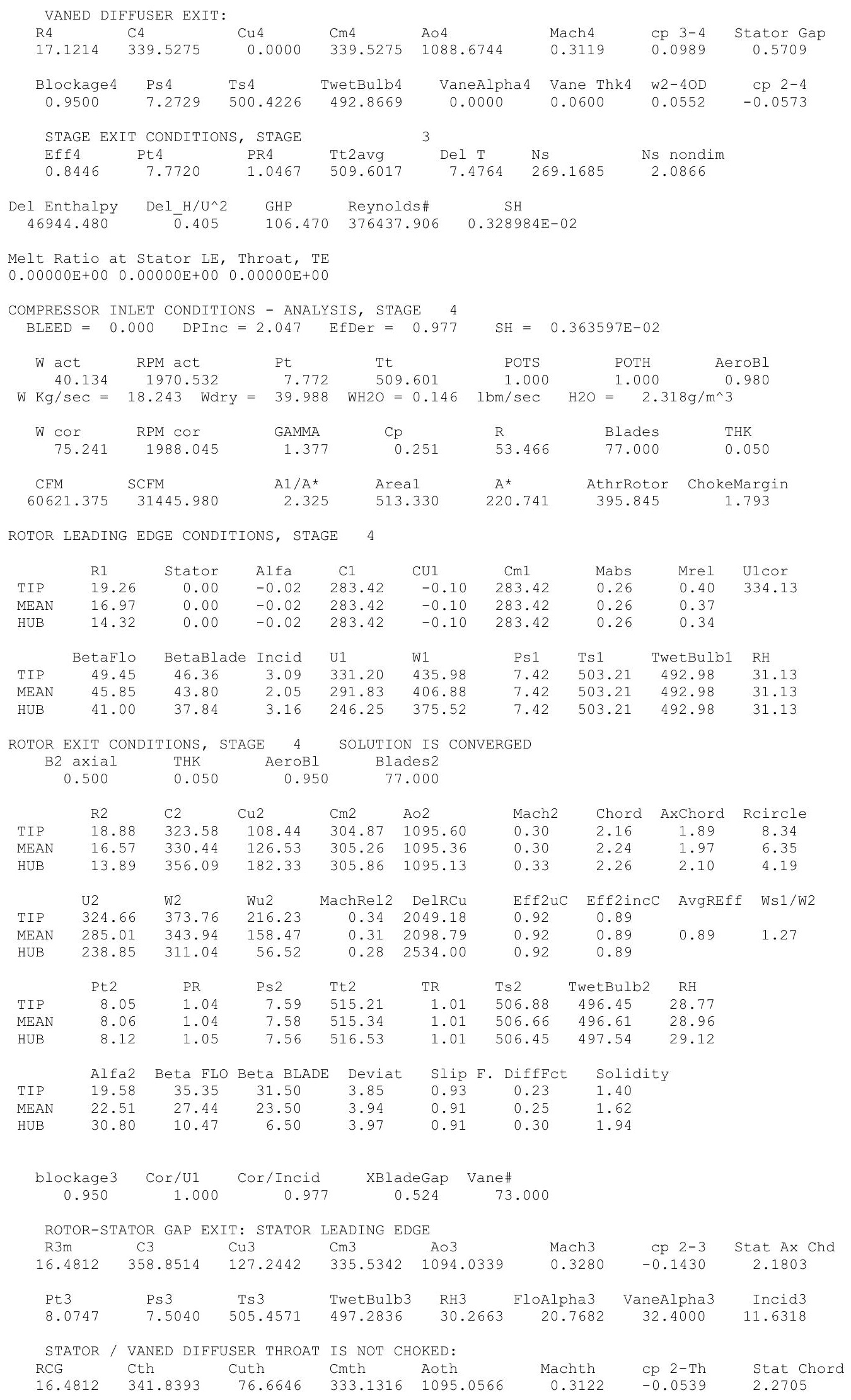




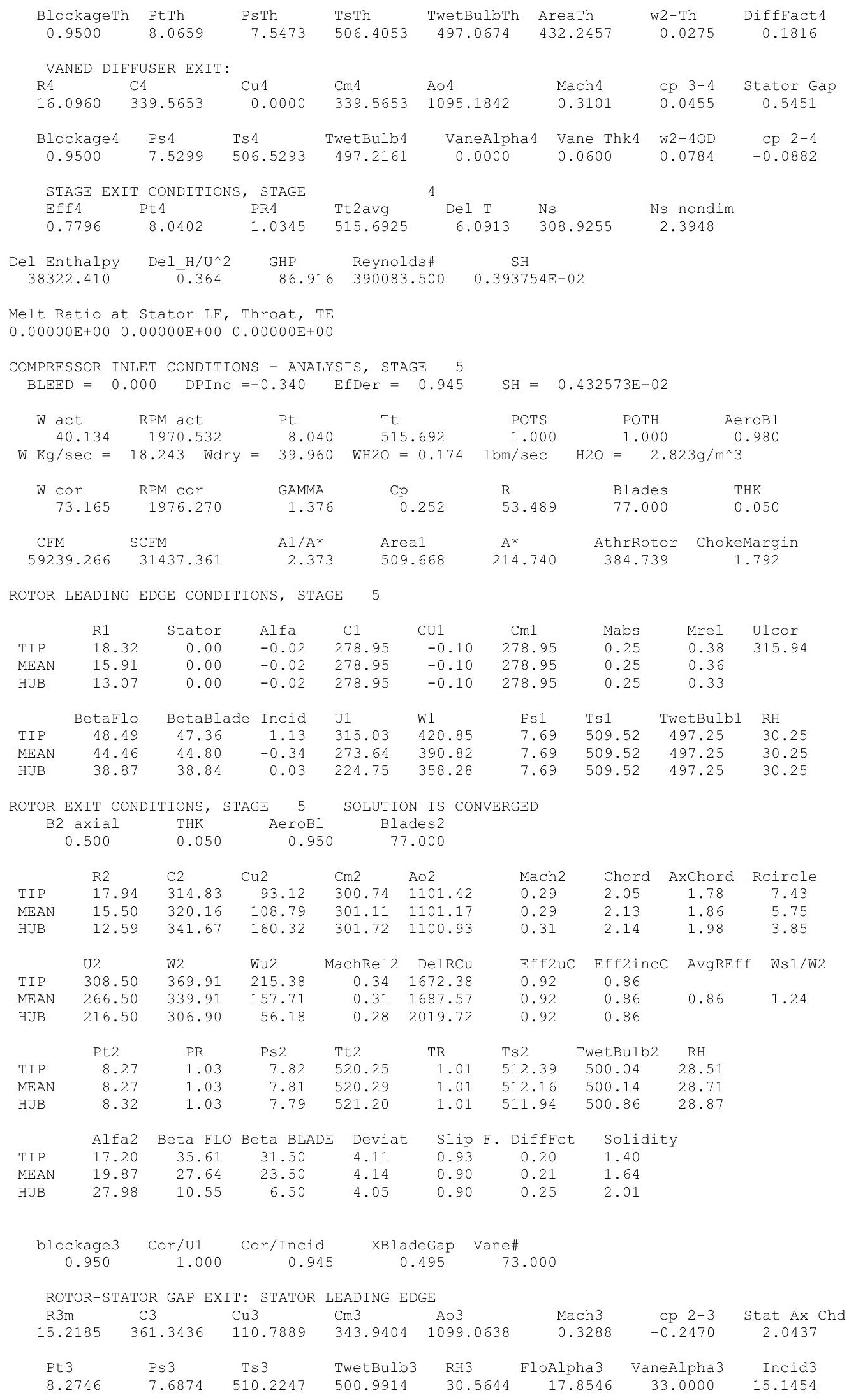




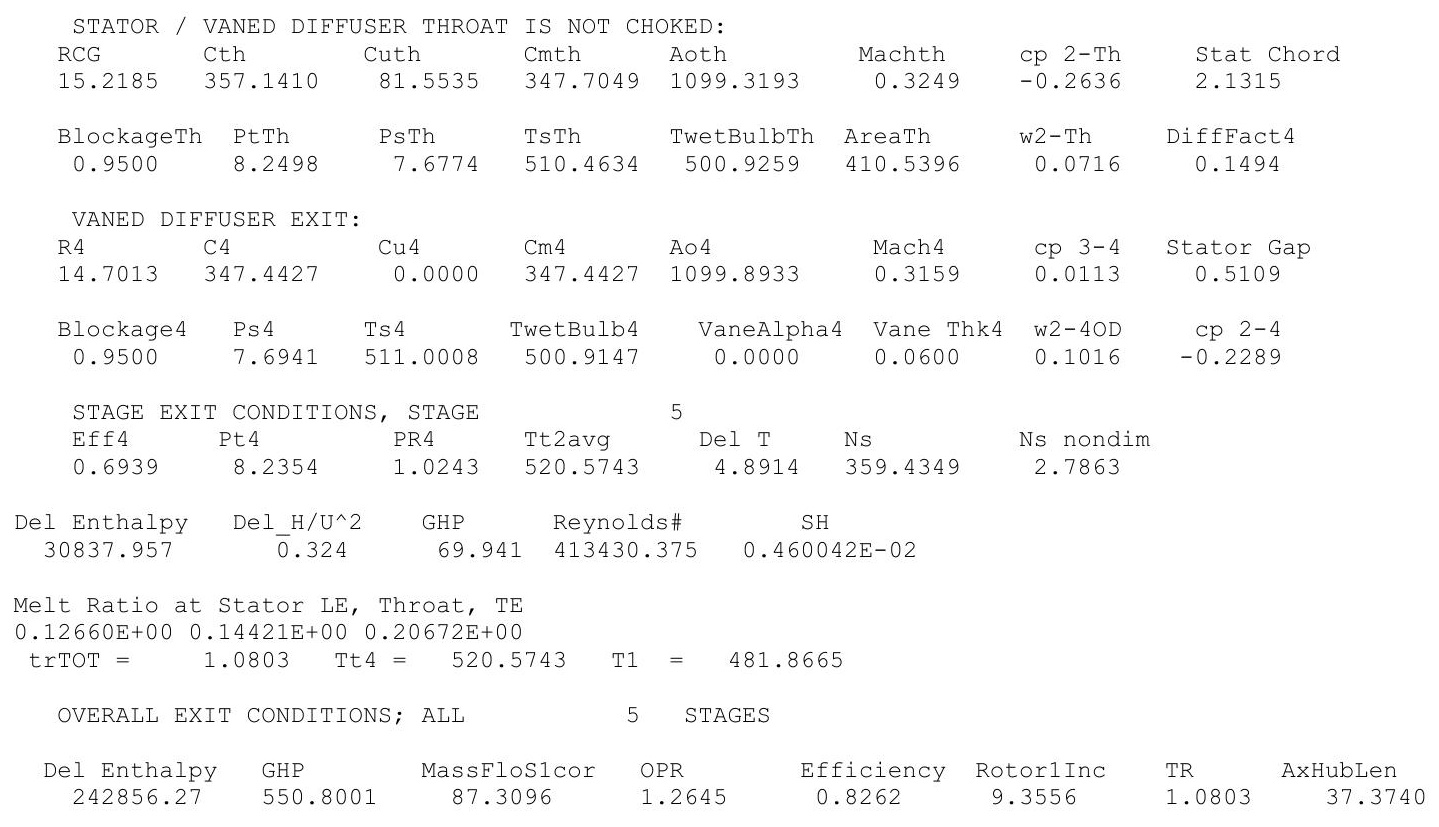




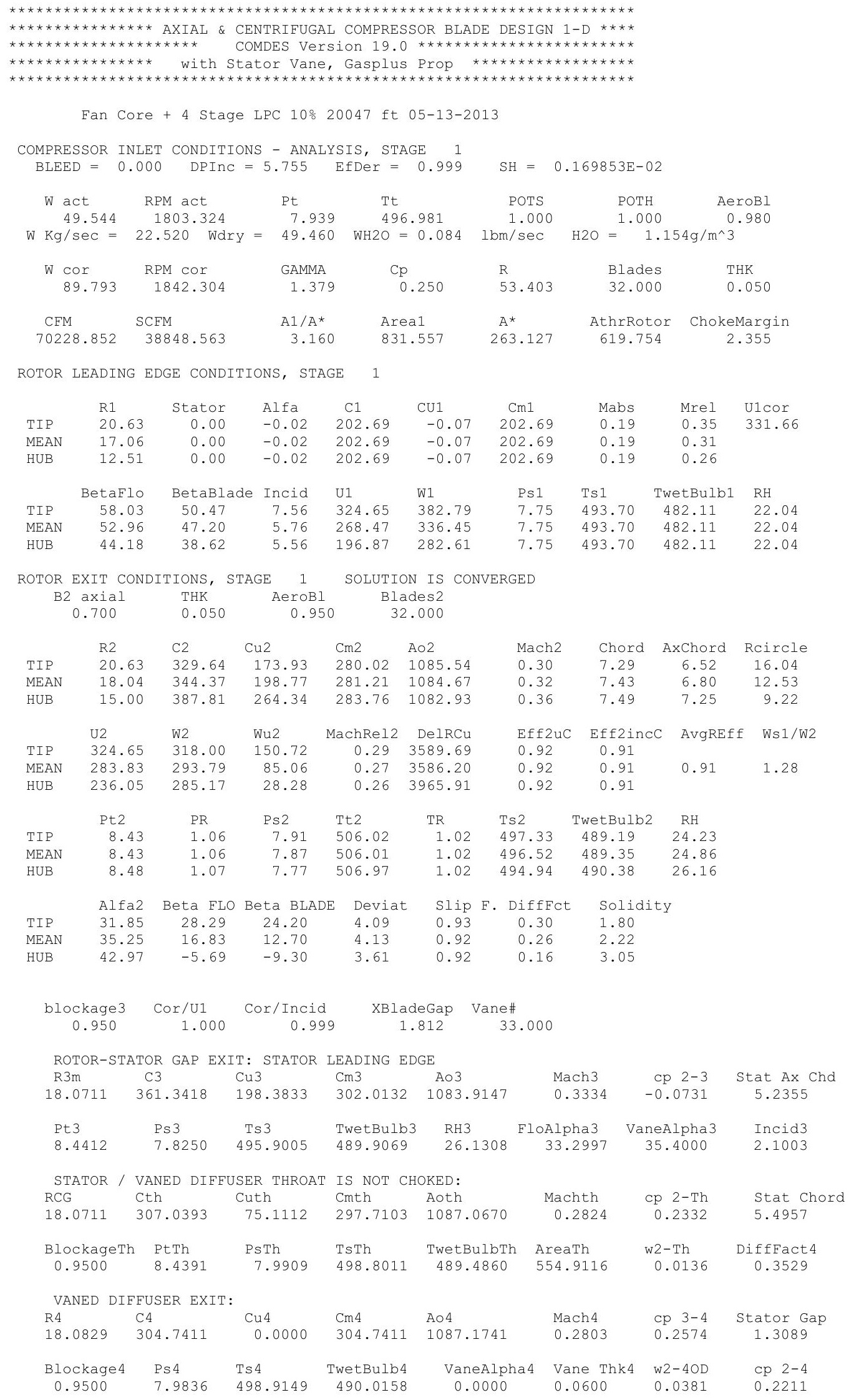




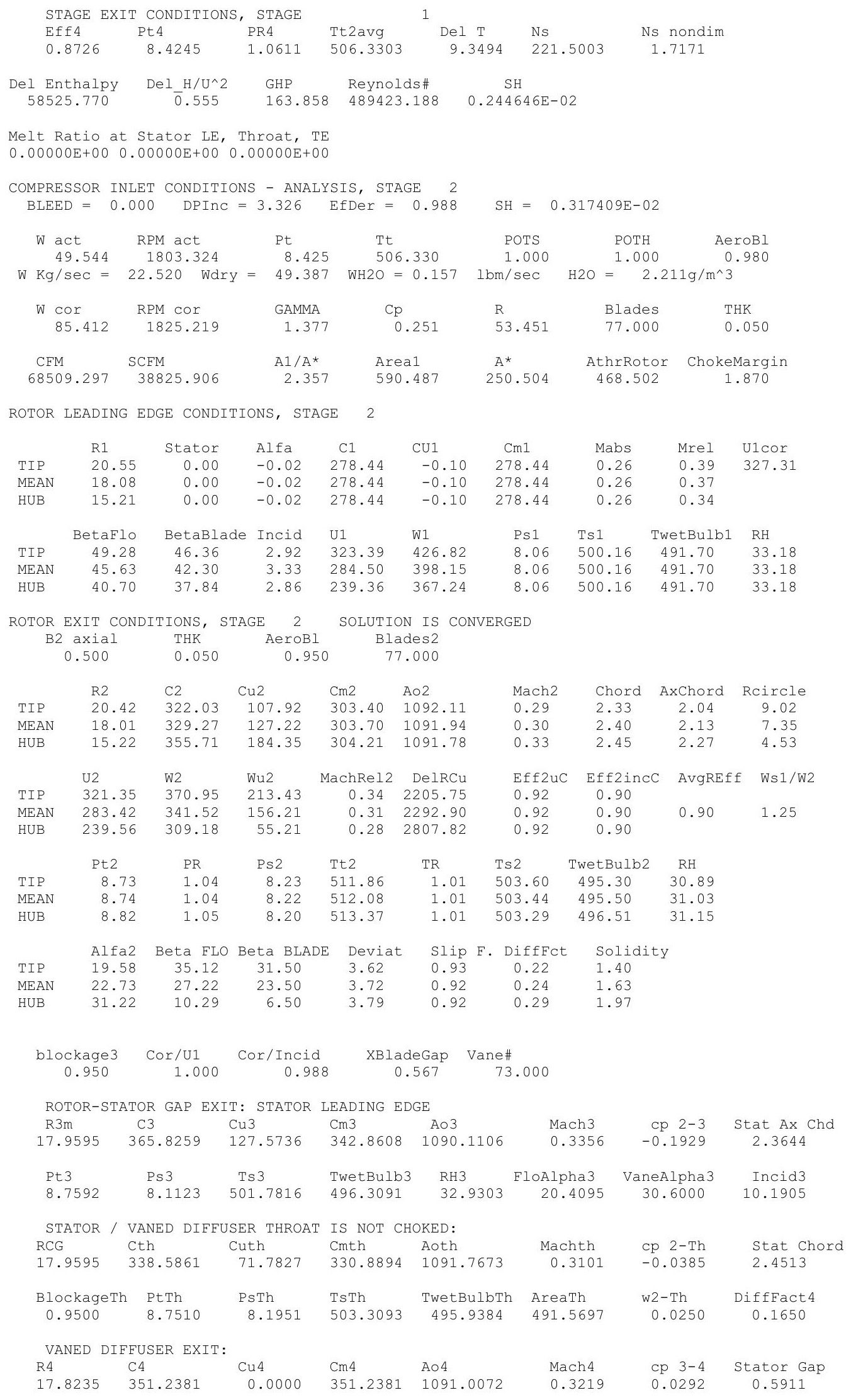




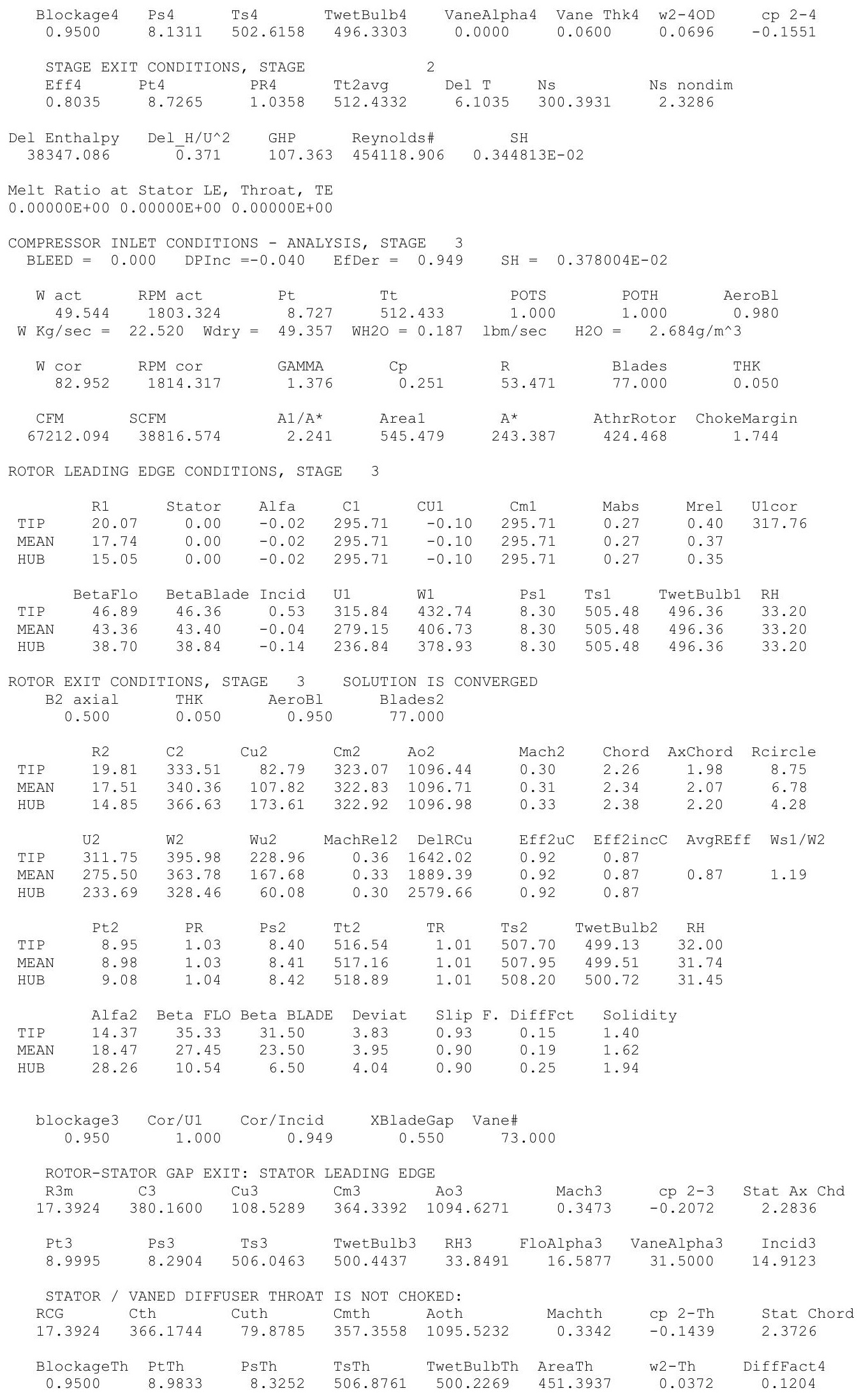




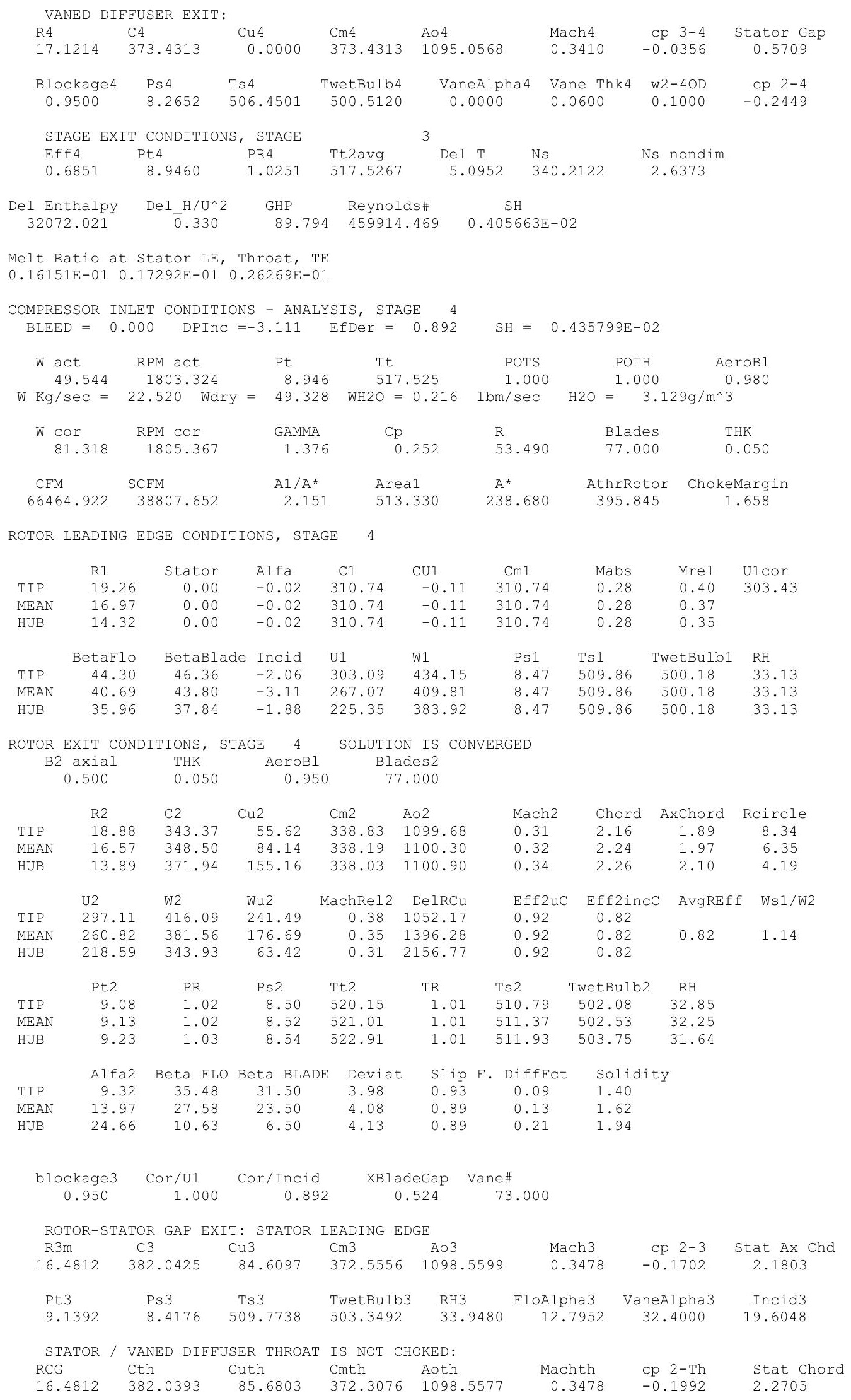




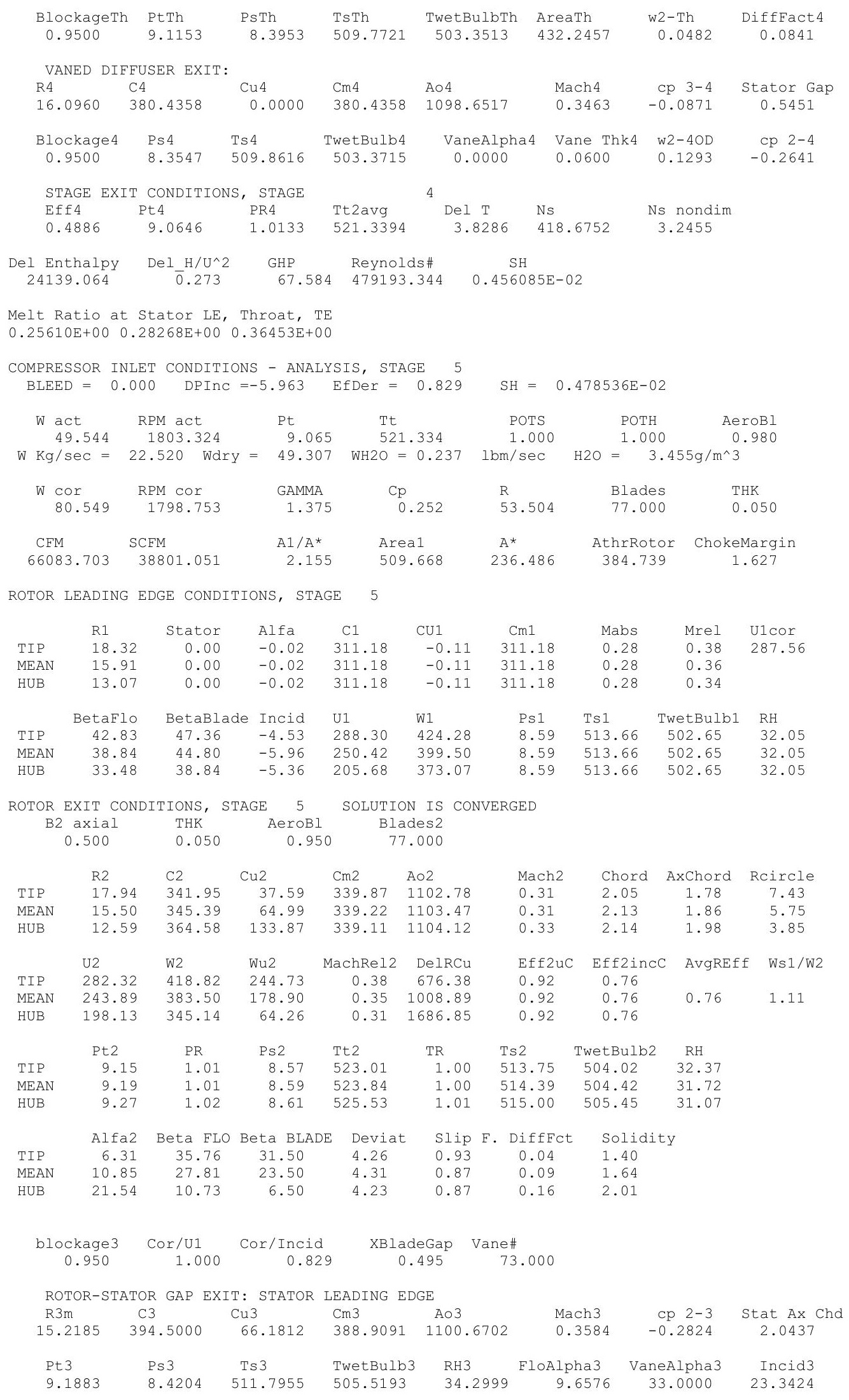




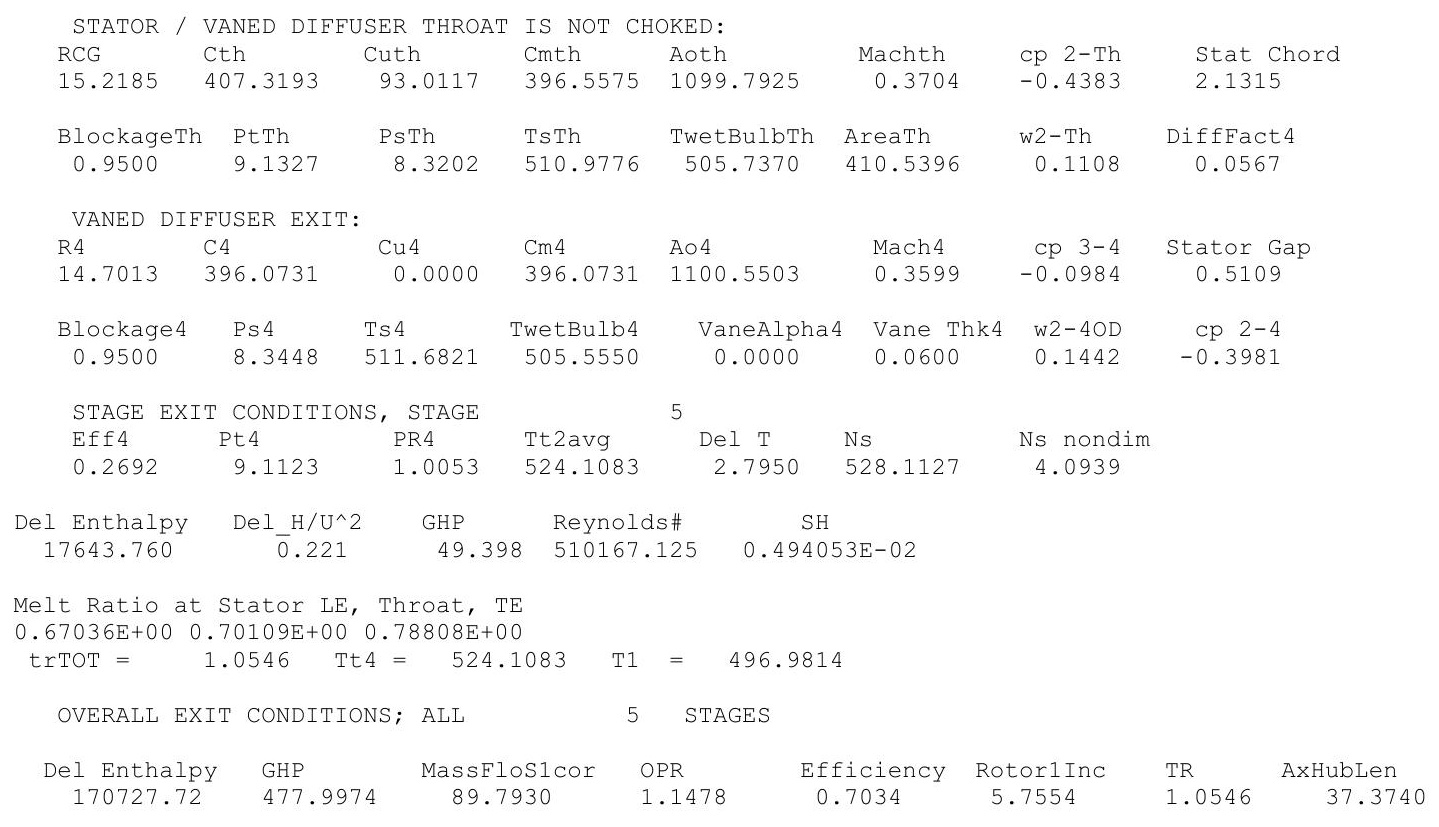




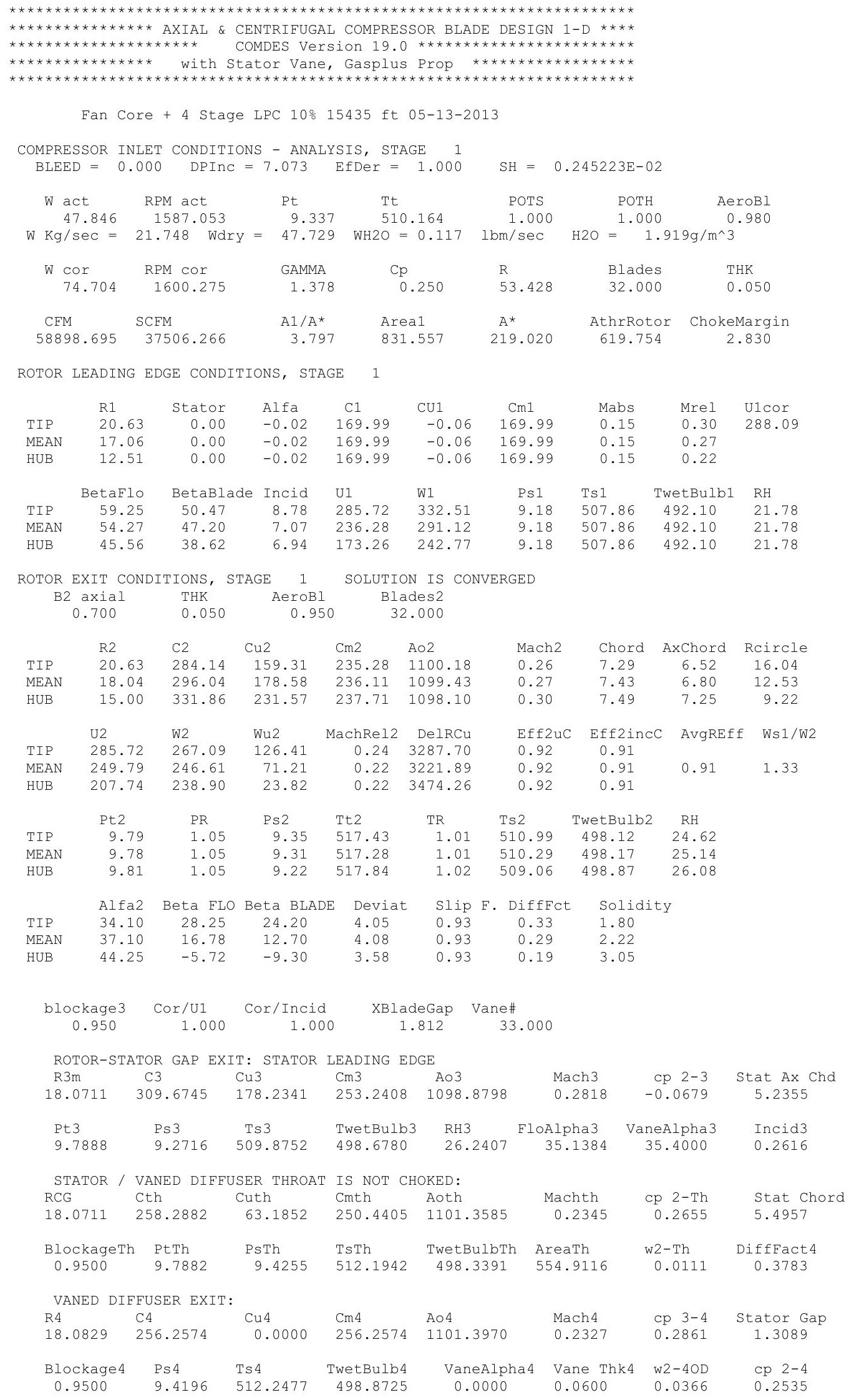




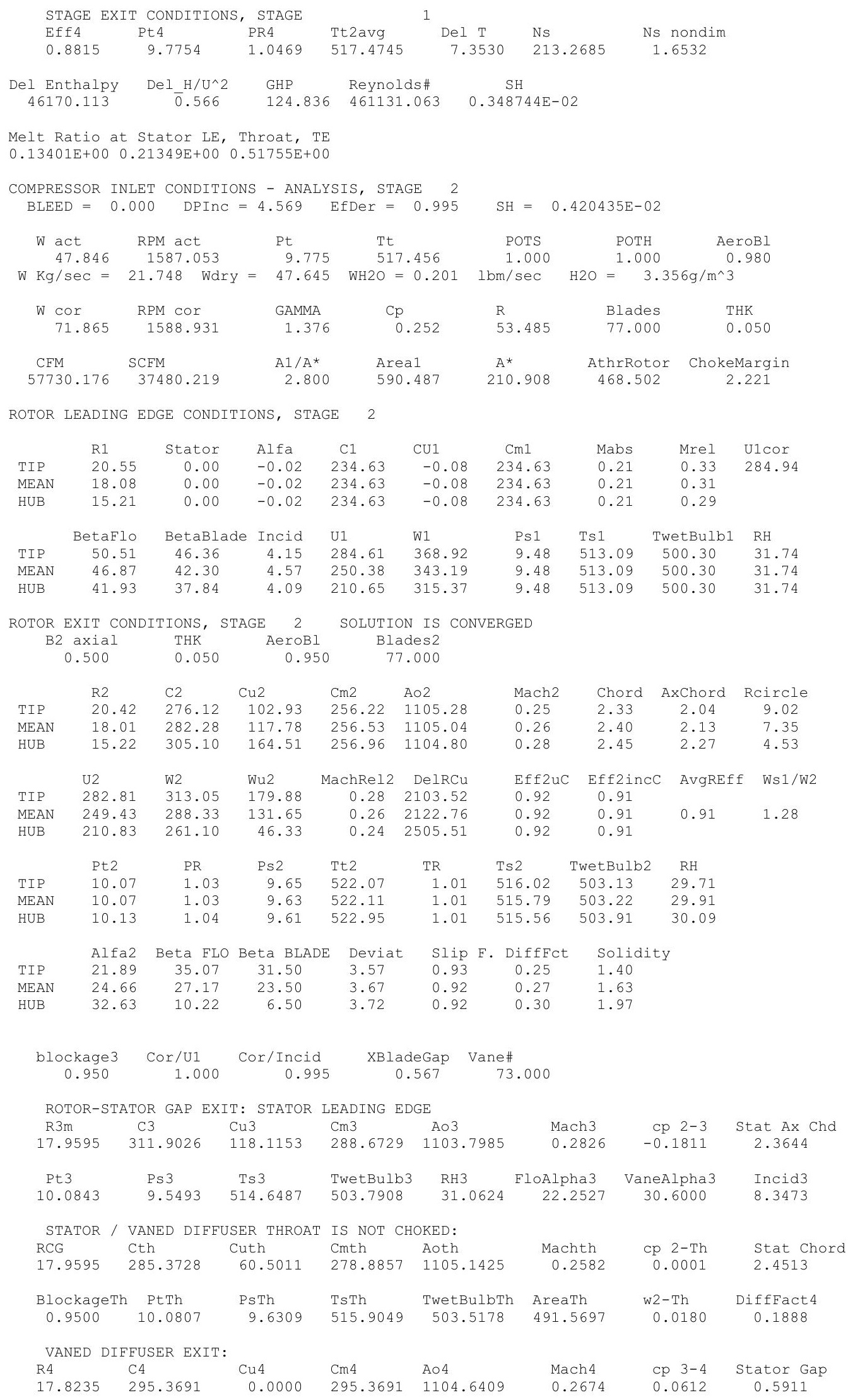




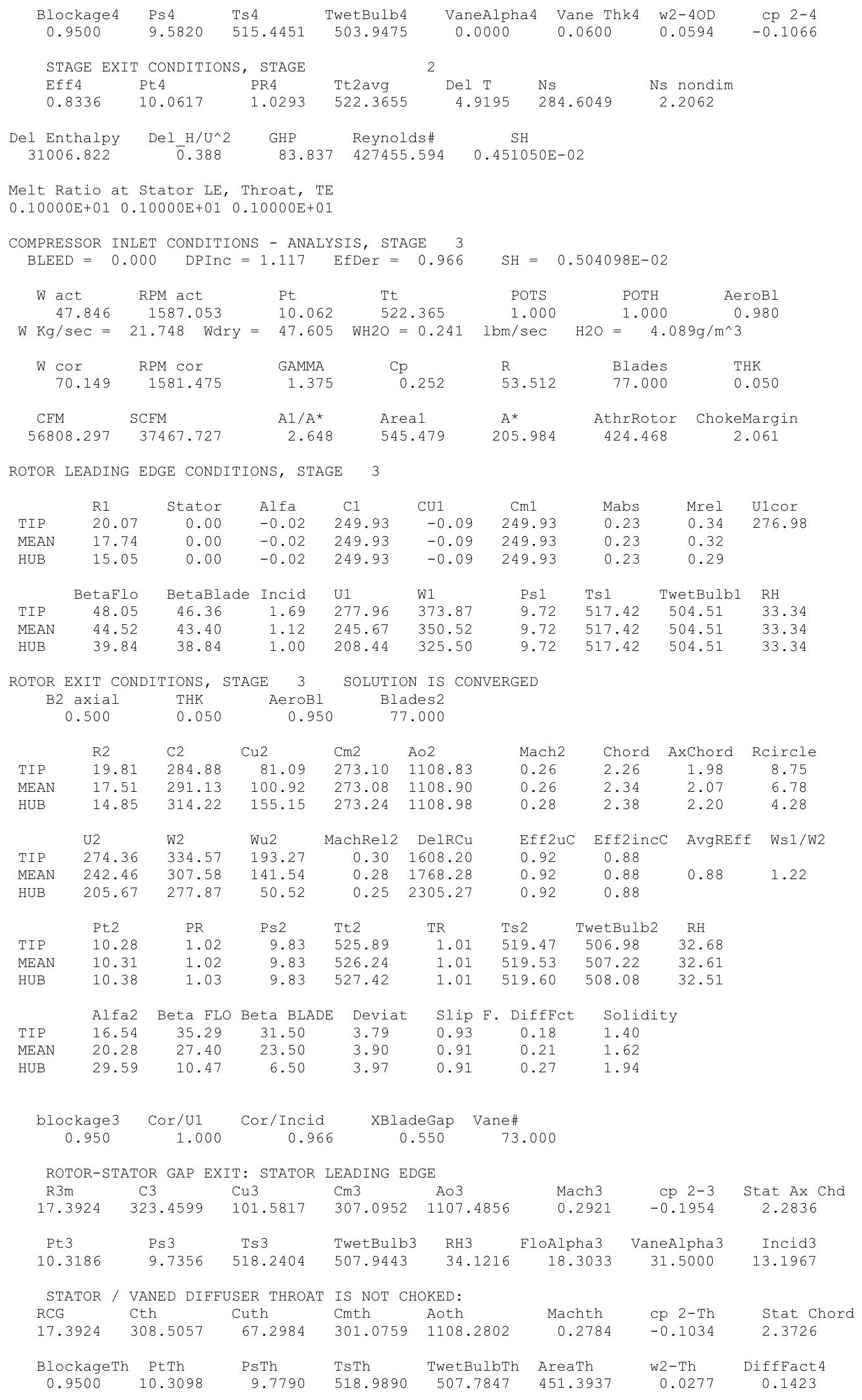




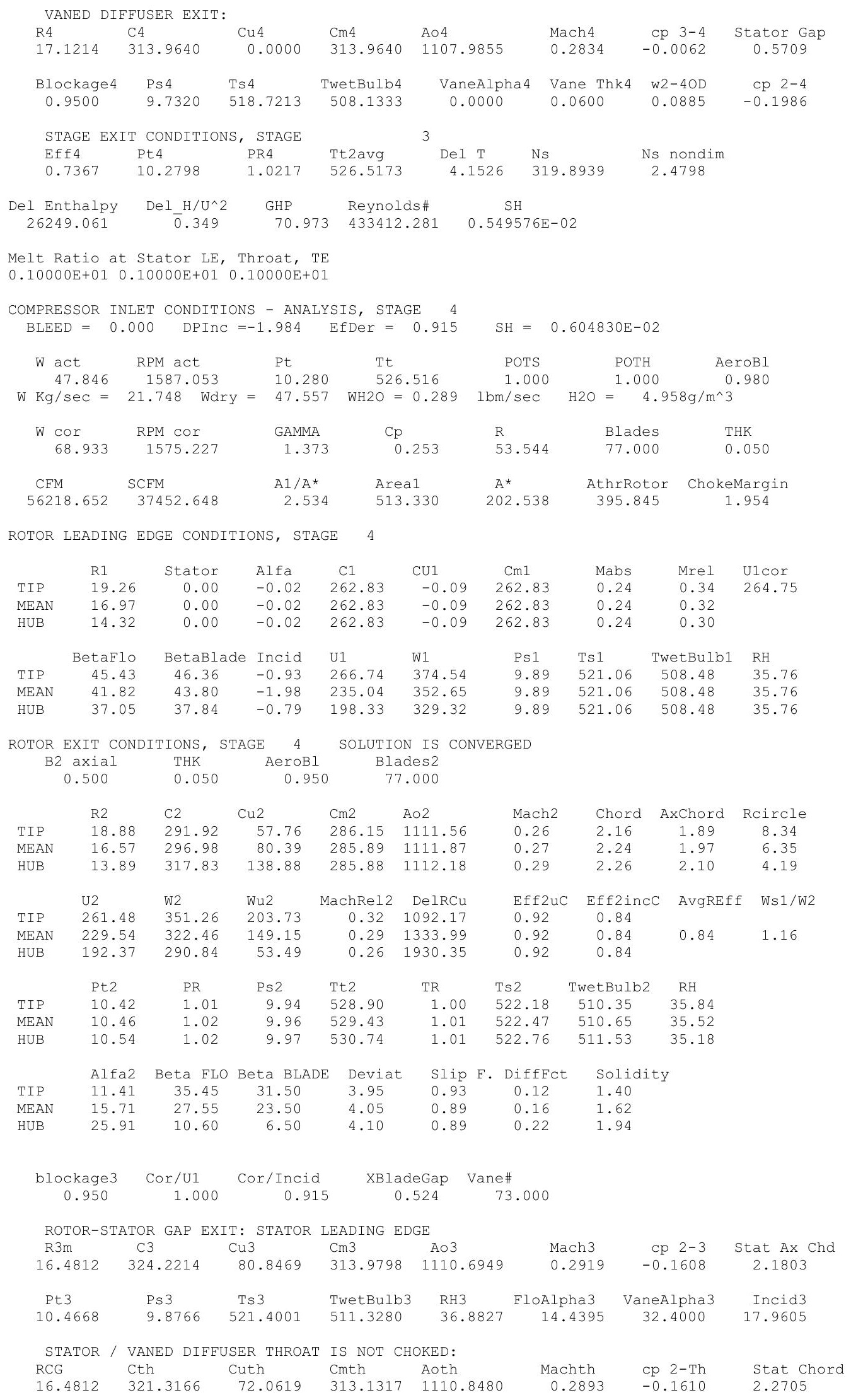




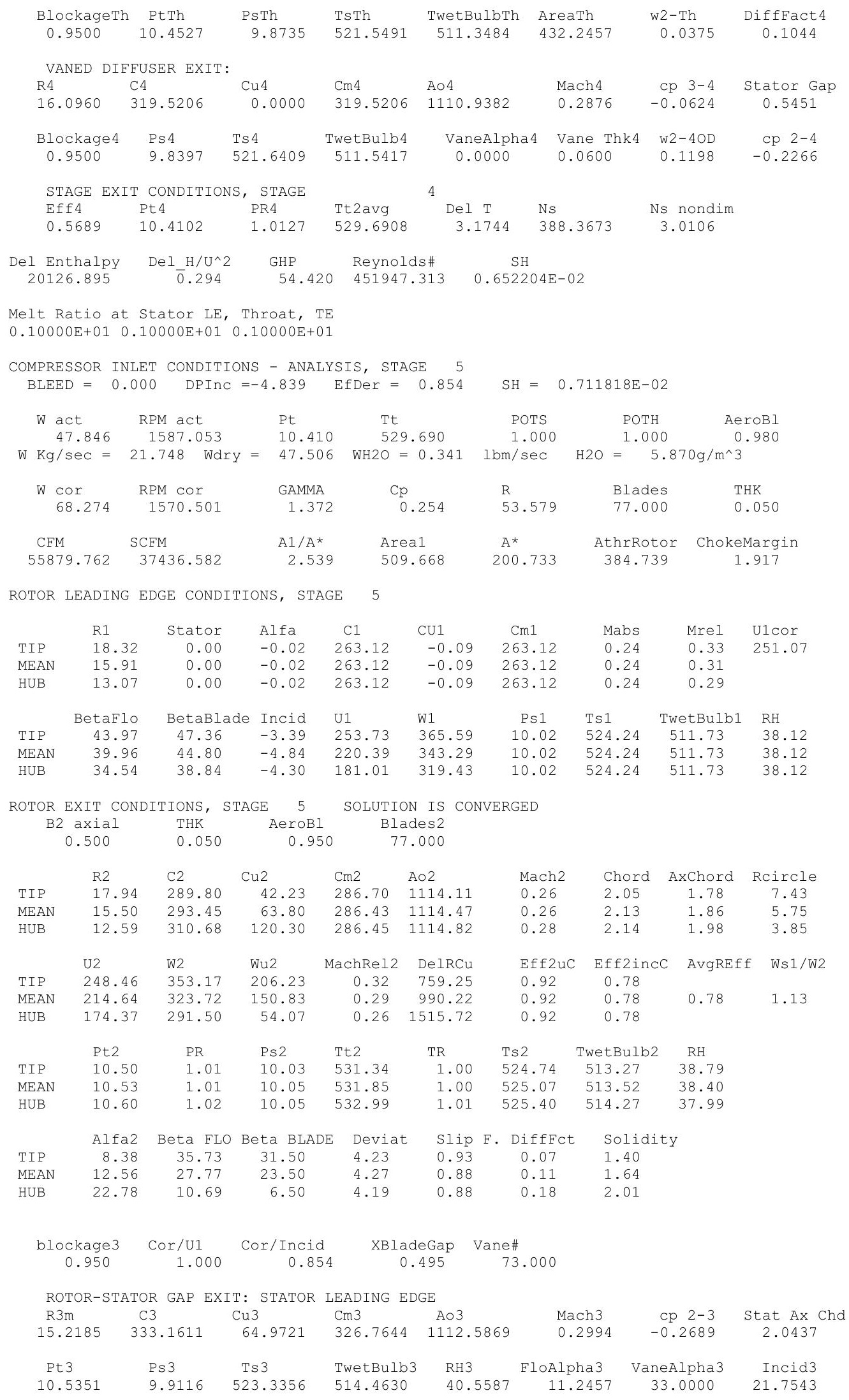




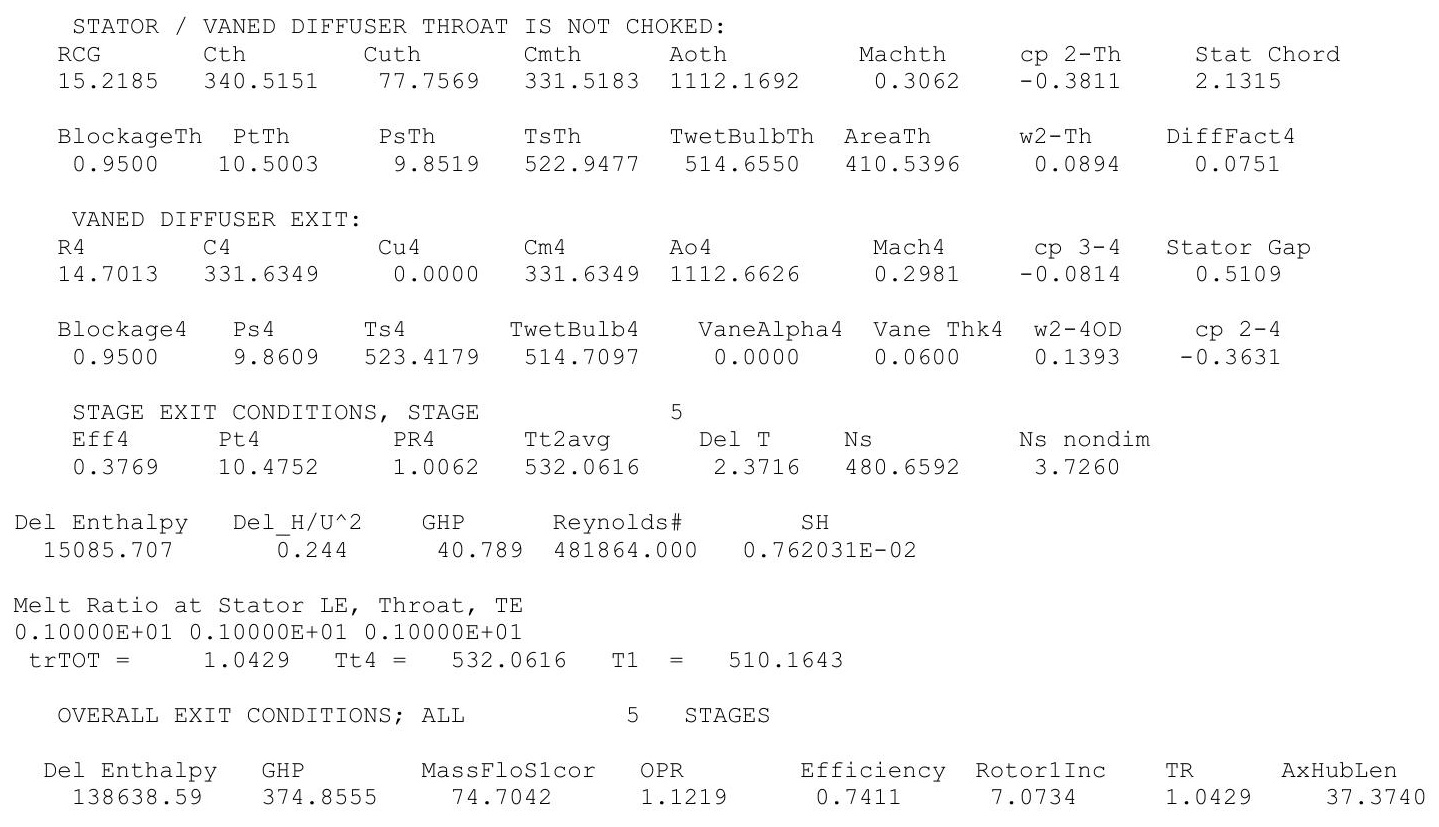


$5 \mu \mathrm{m}, 4 \mathrm{~g} / \mathrm{m}^{3}, \mathrm{ISA}+27 \mathrm{R}$

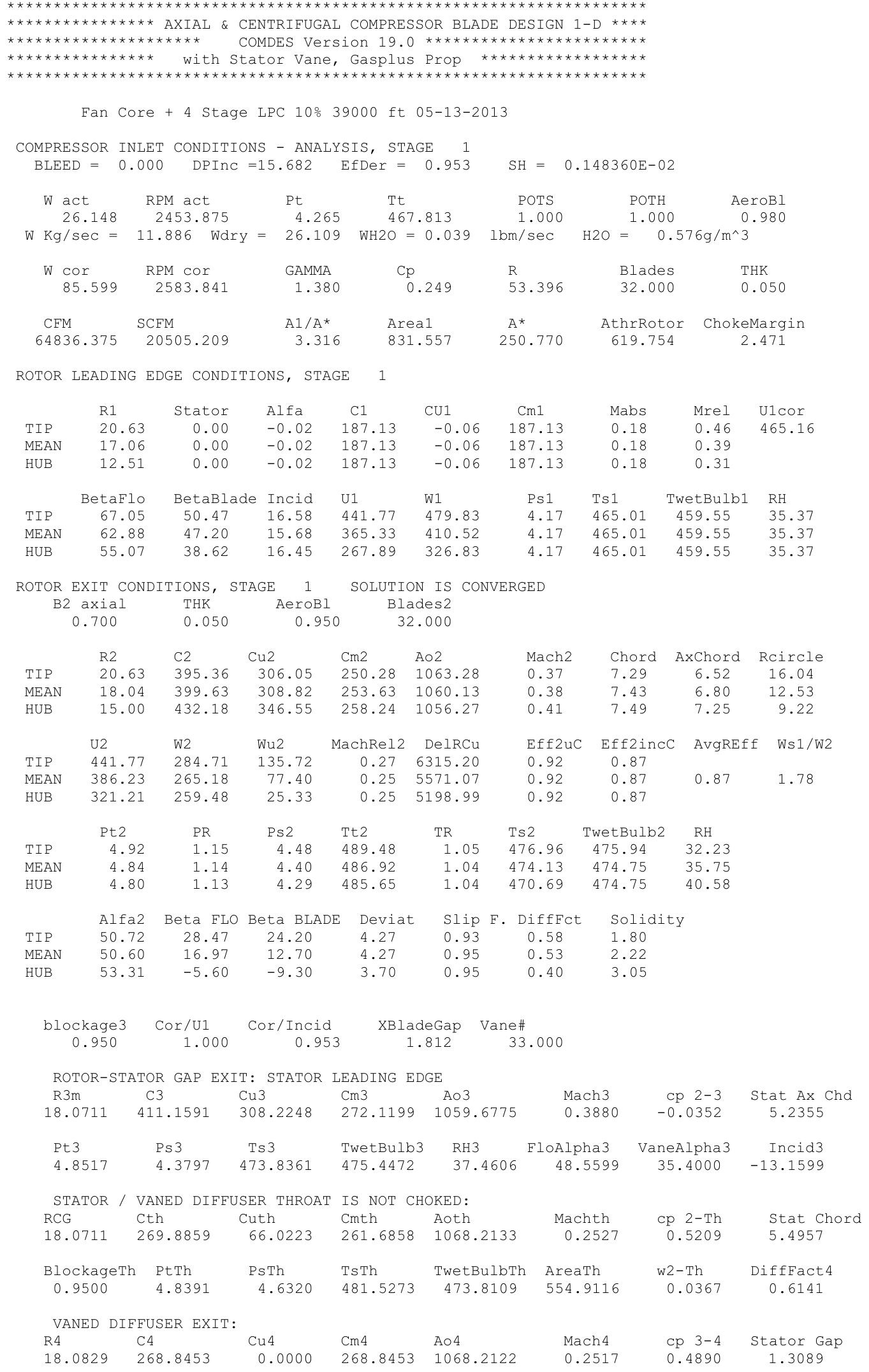




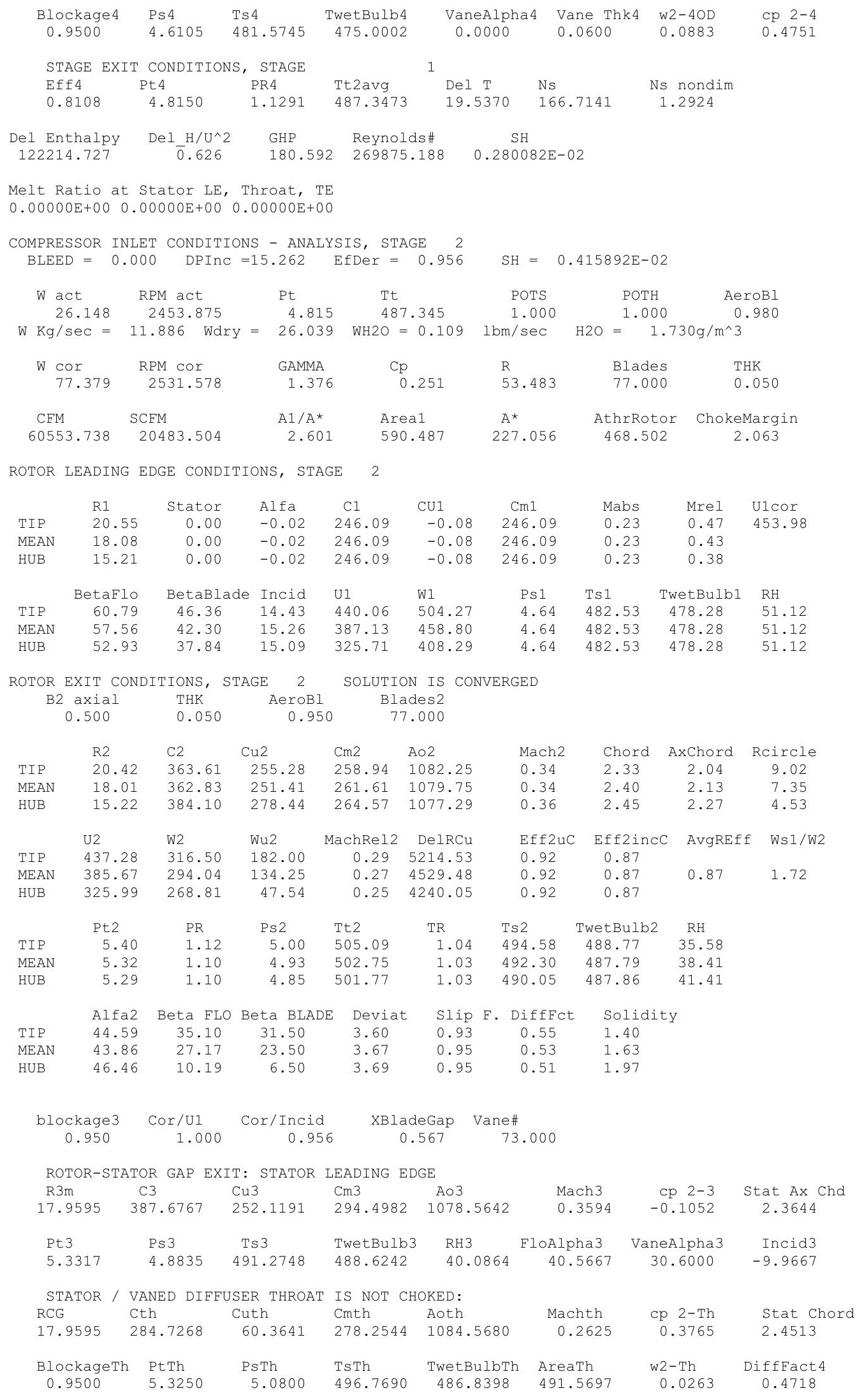




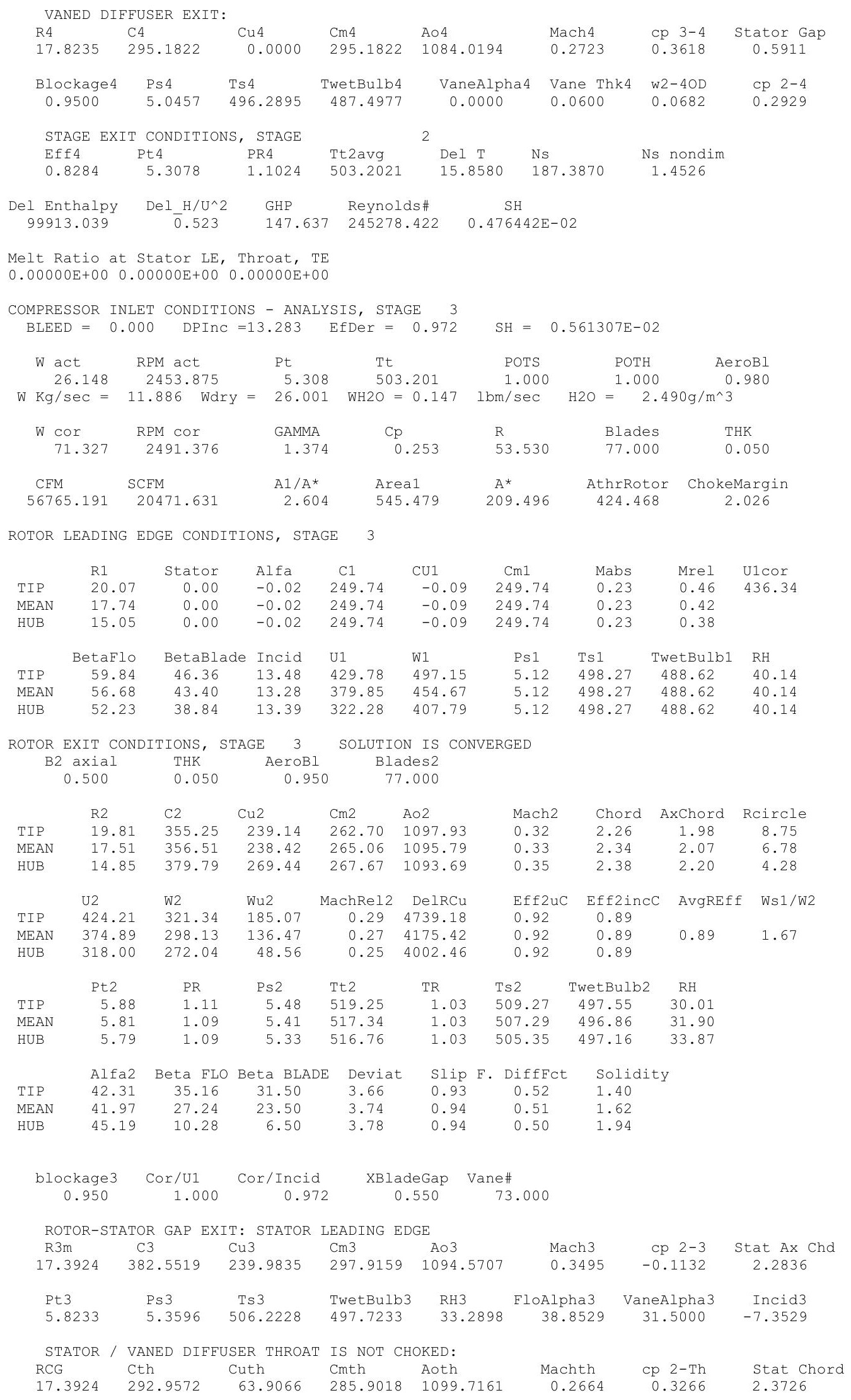




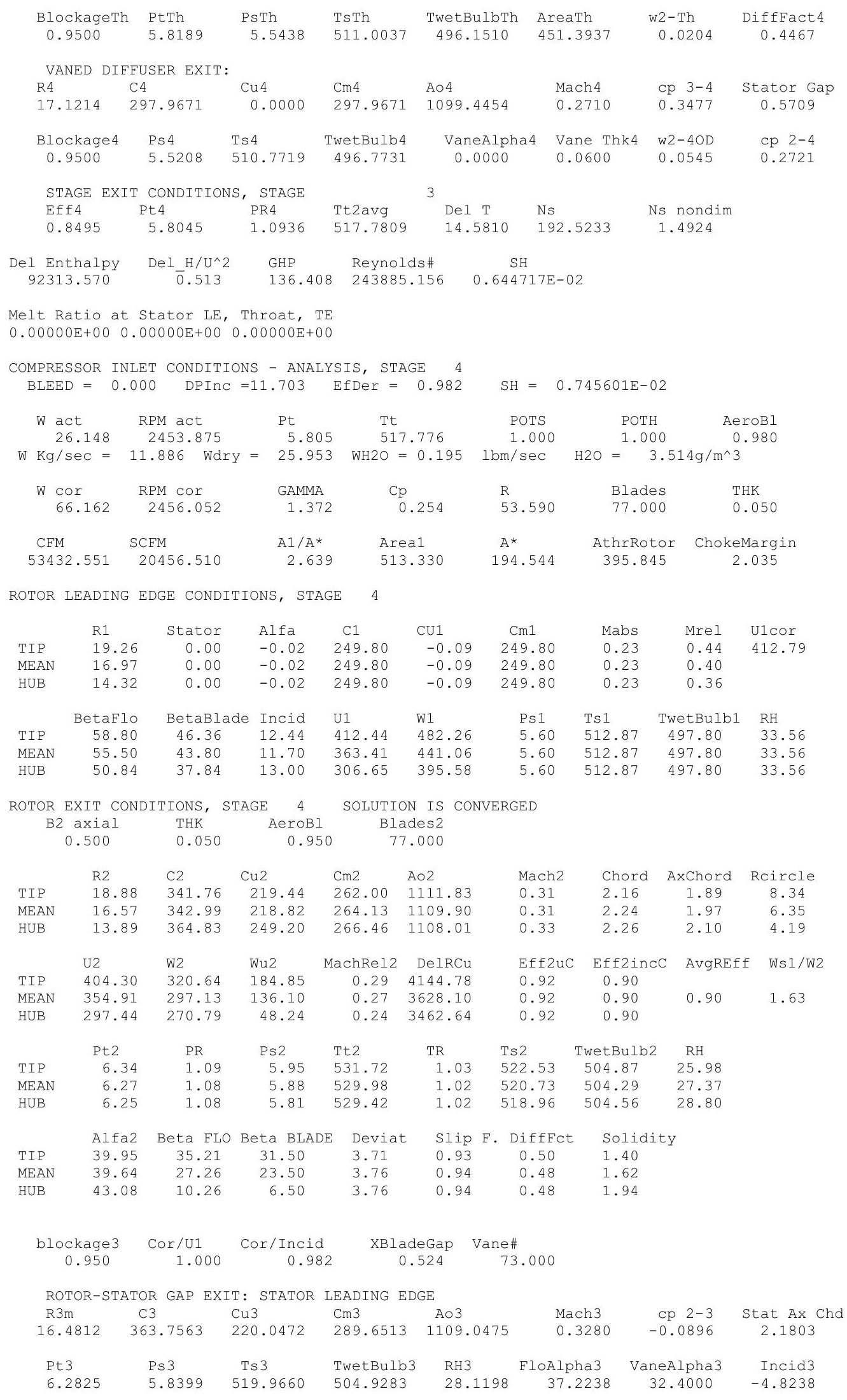




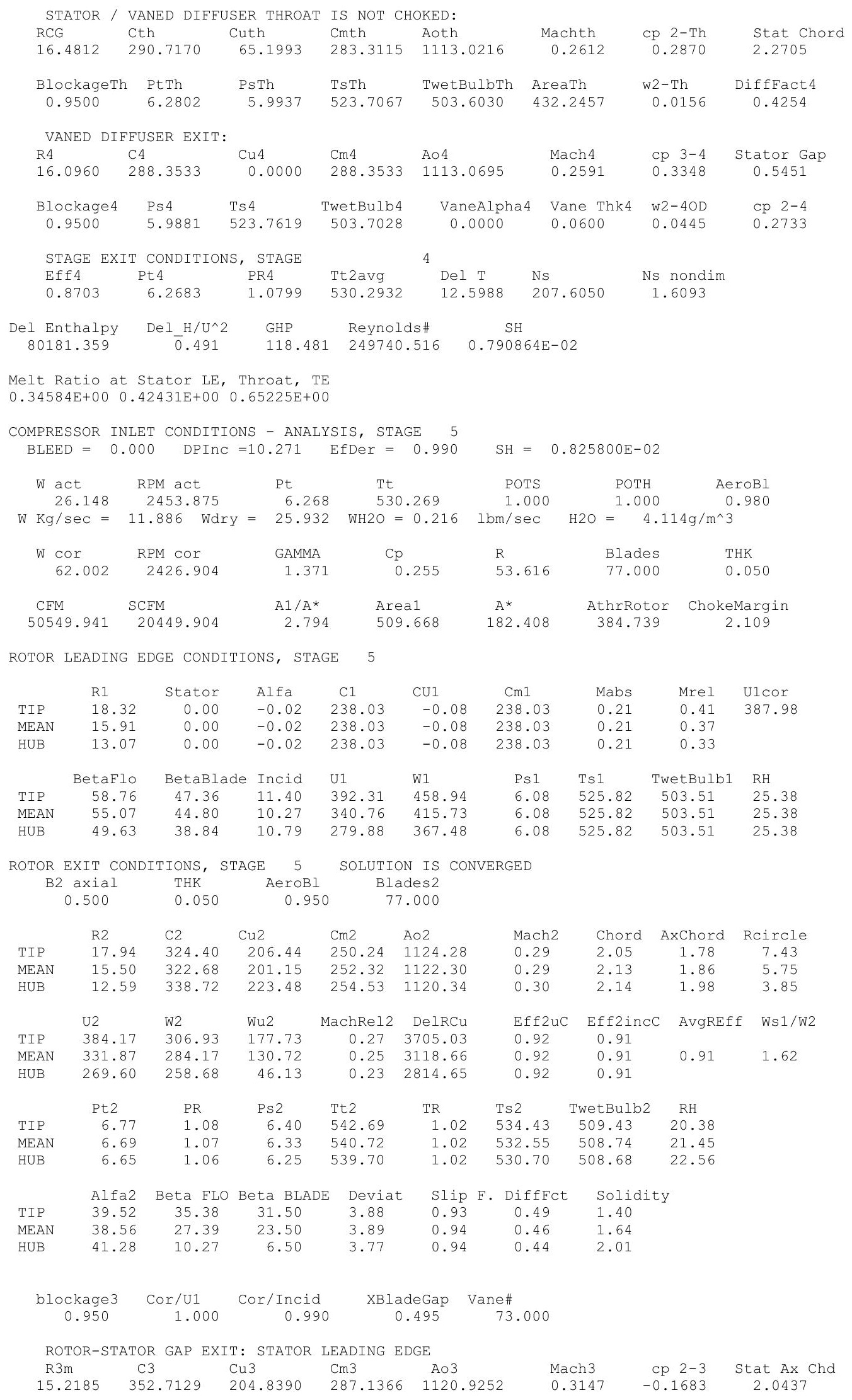




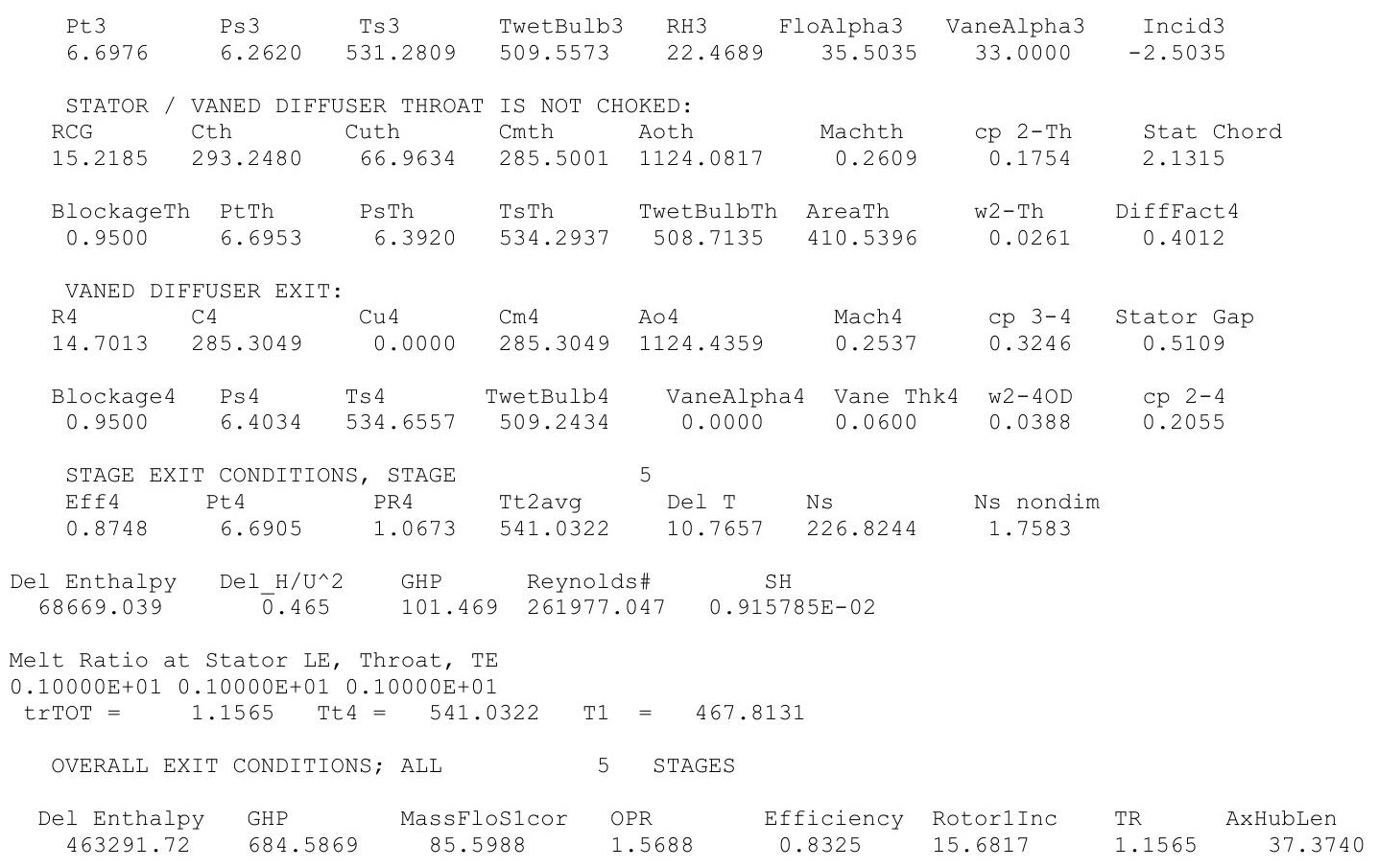




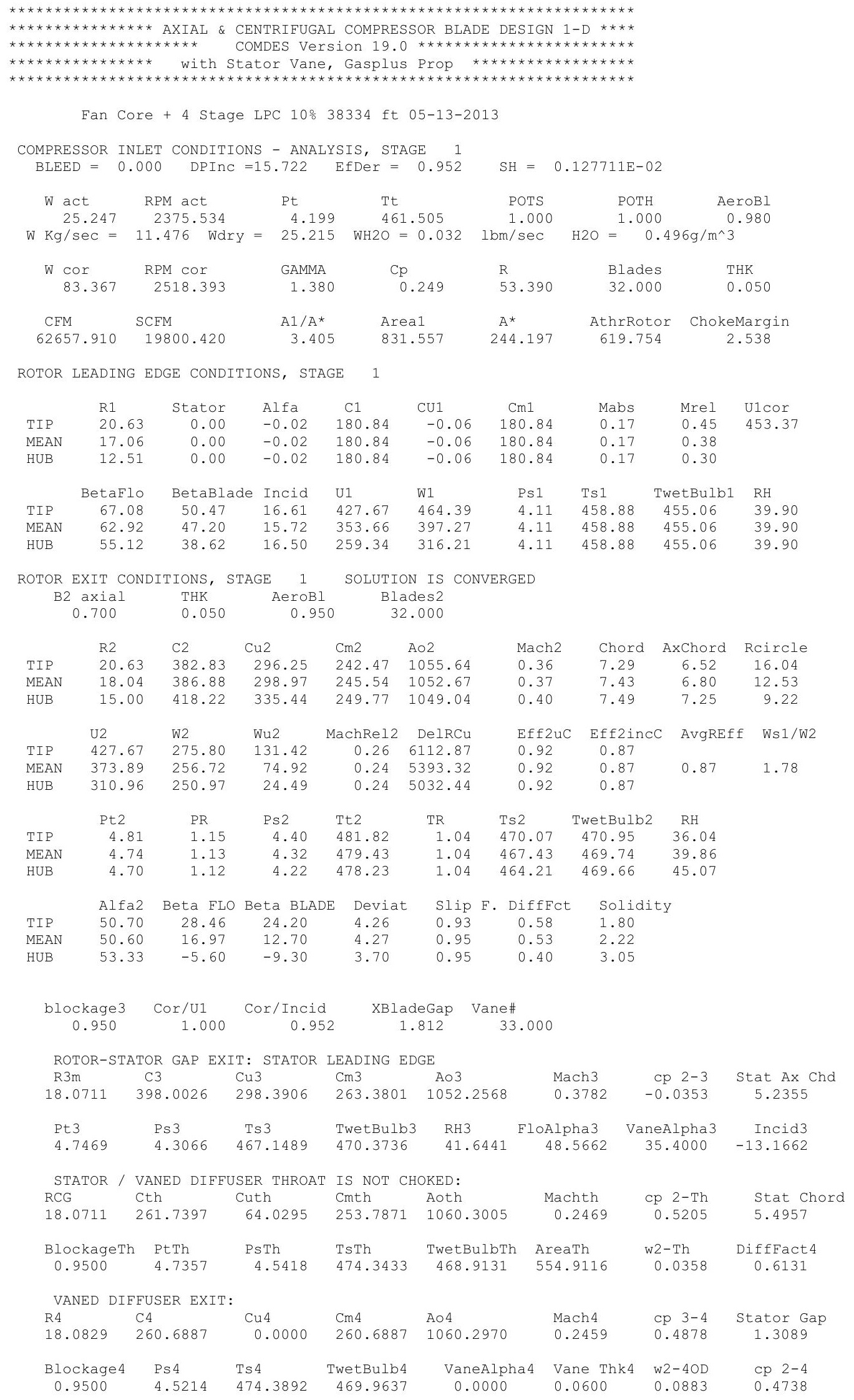




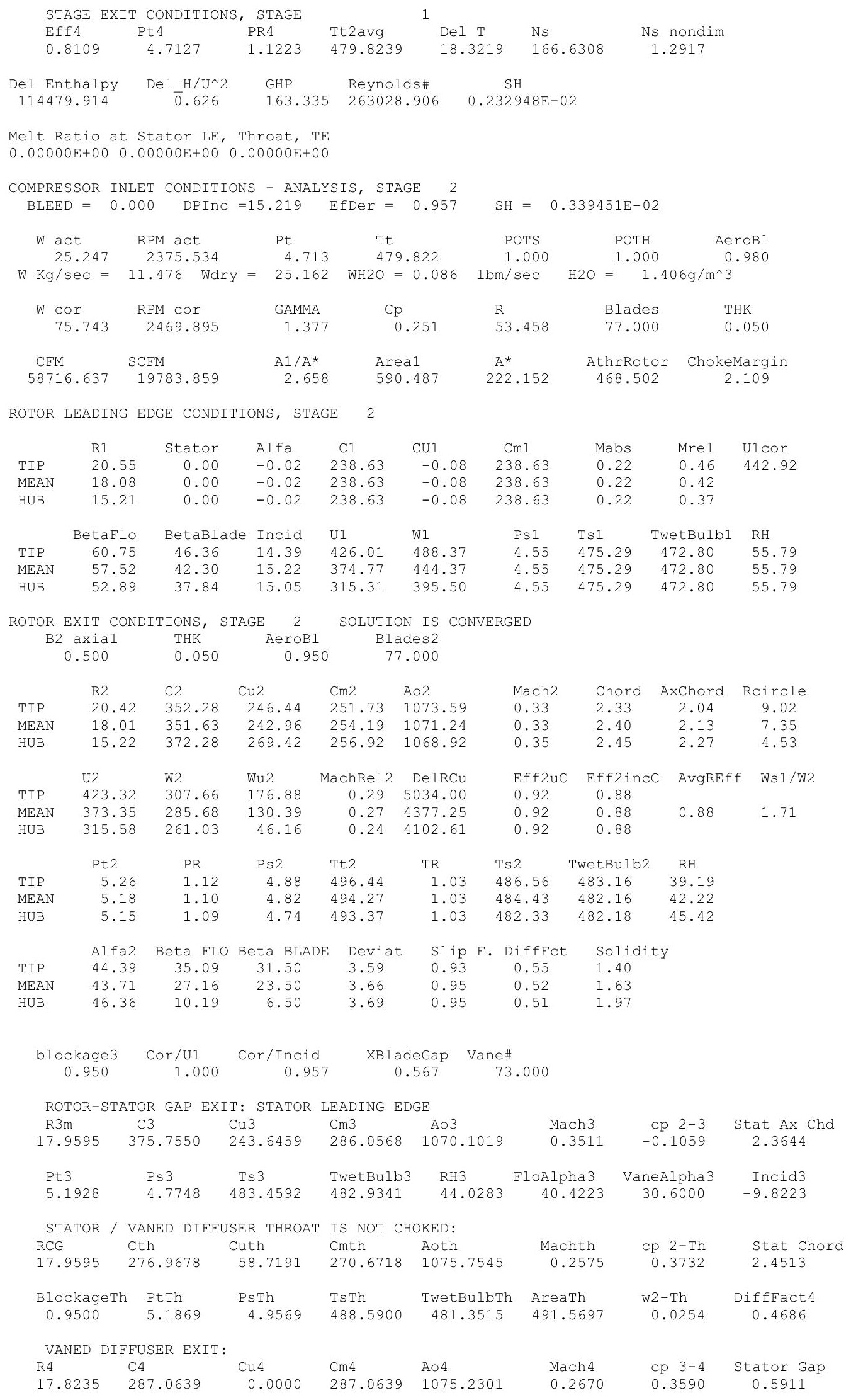




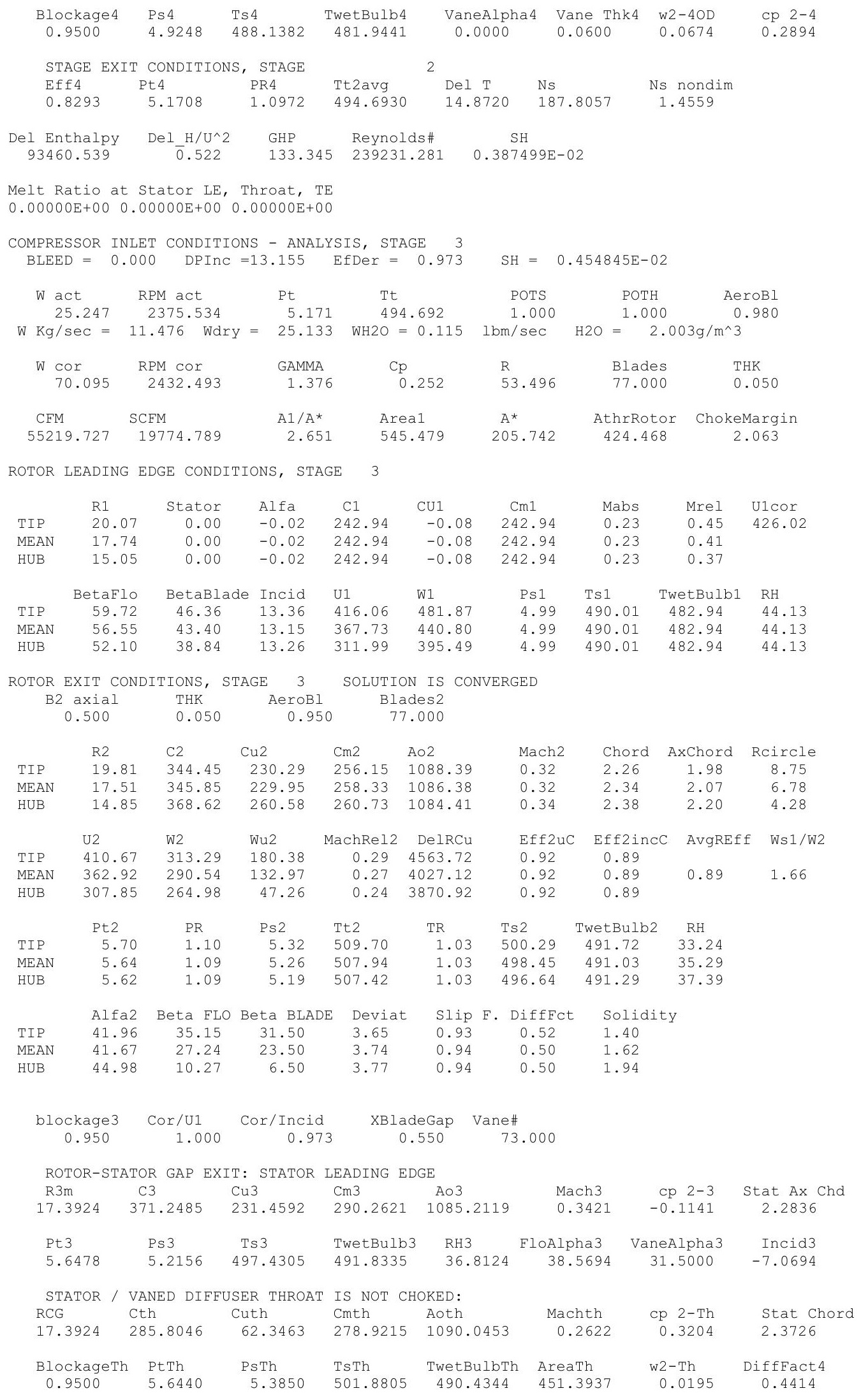




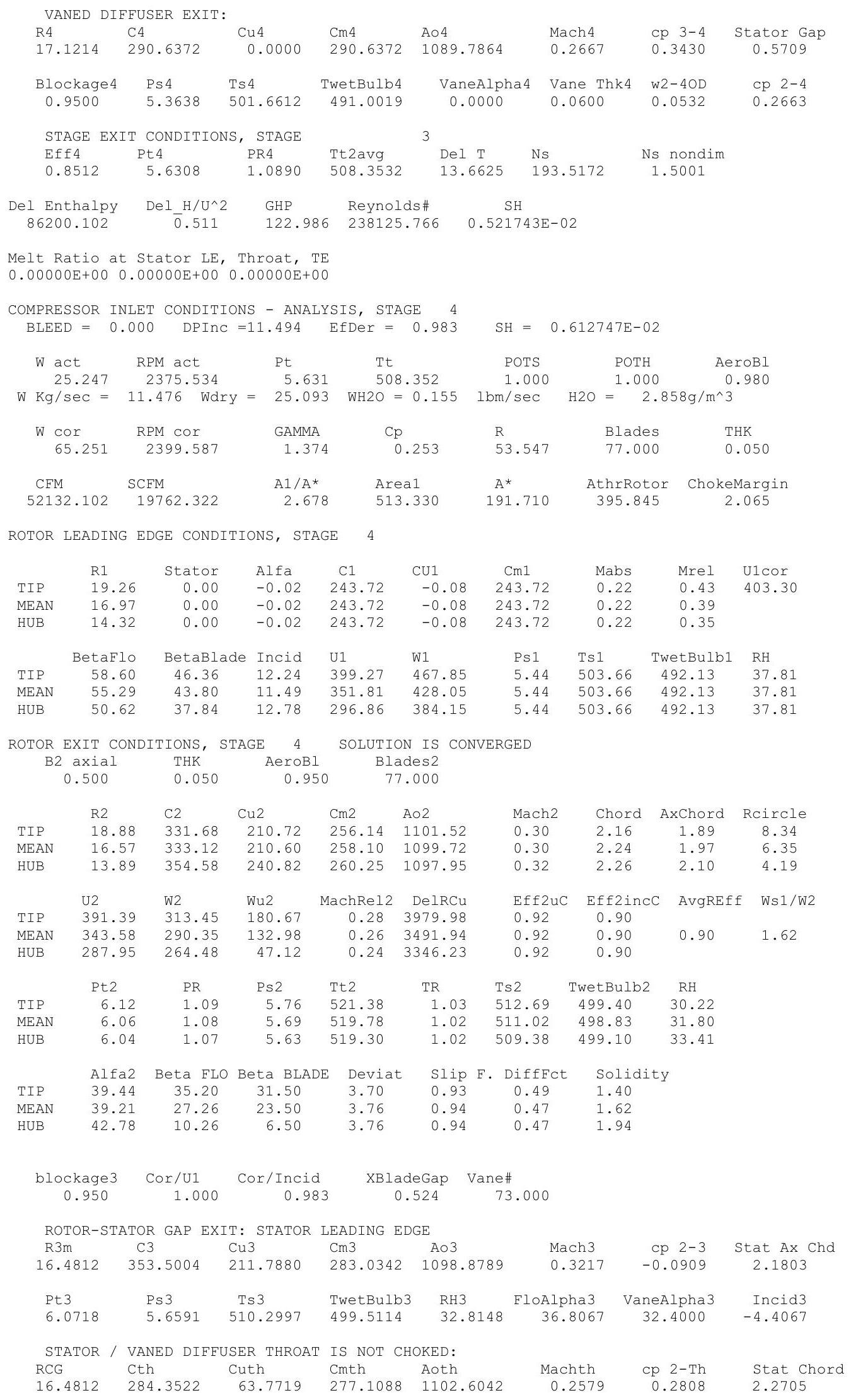




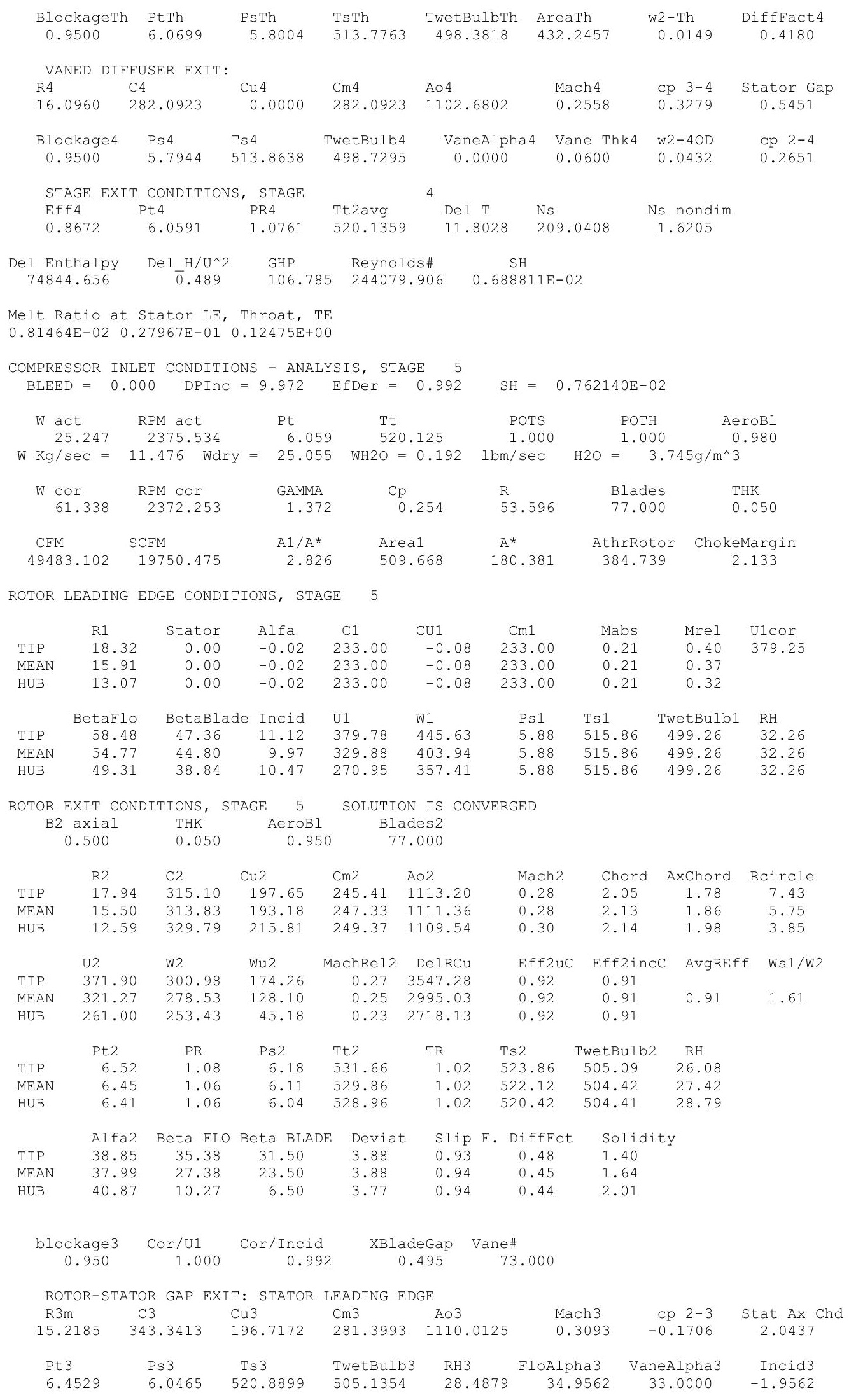




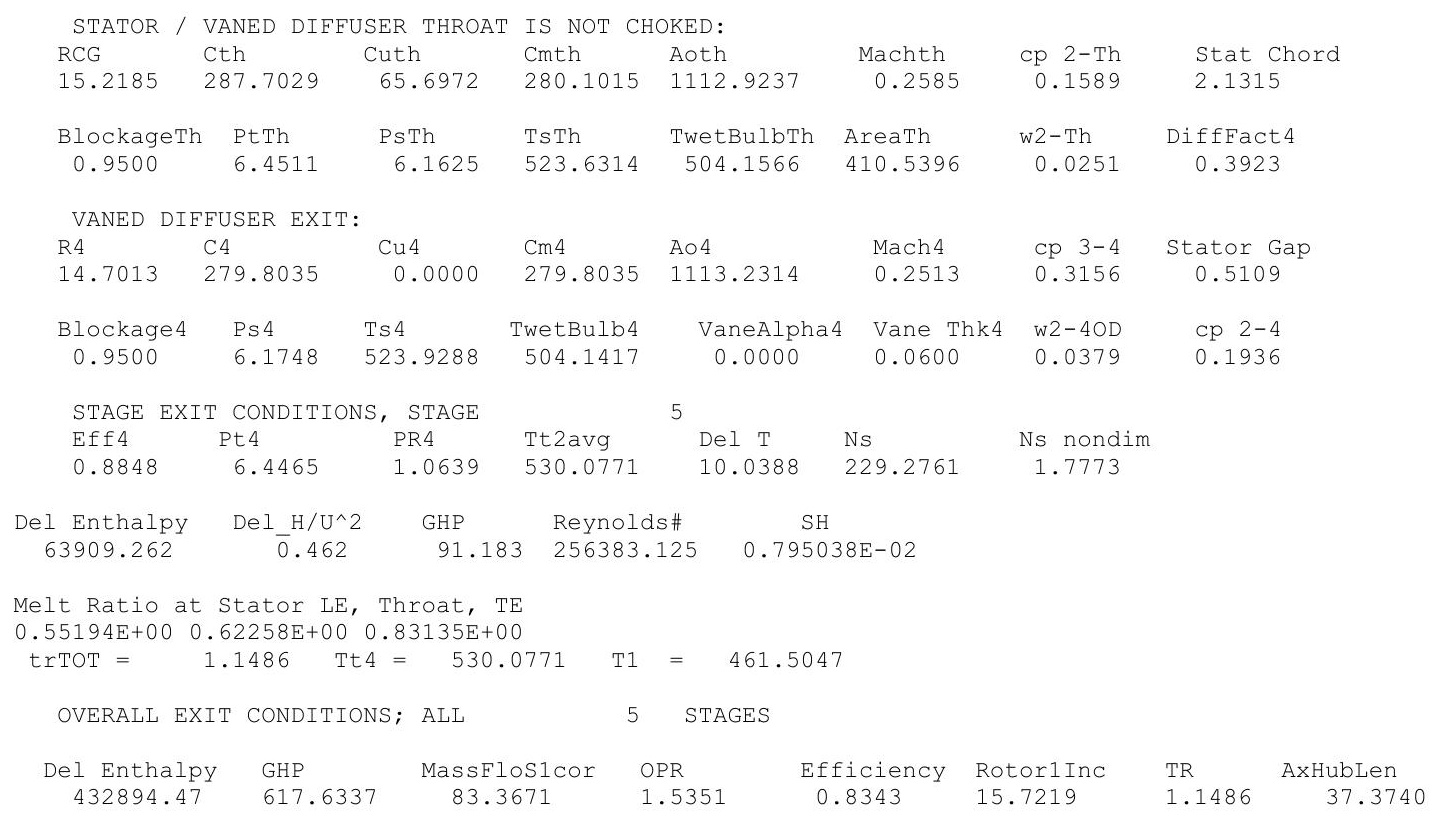




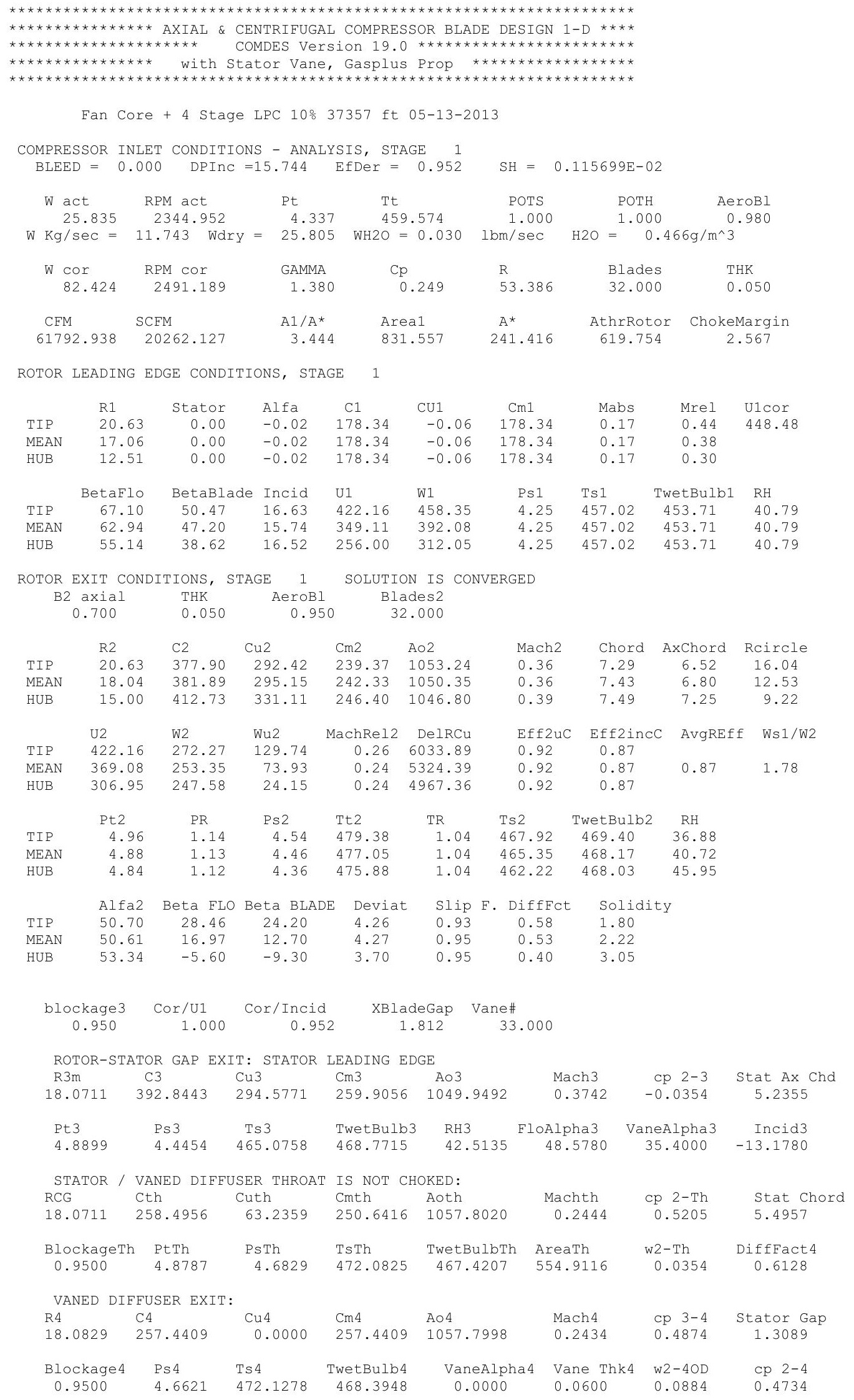




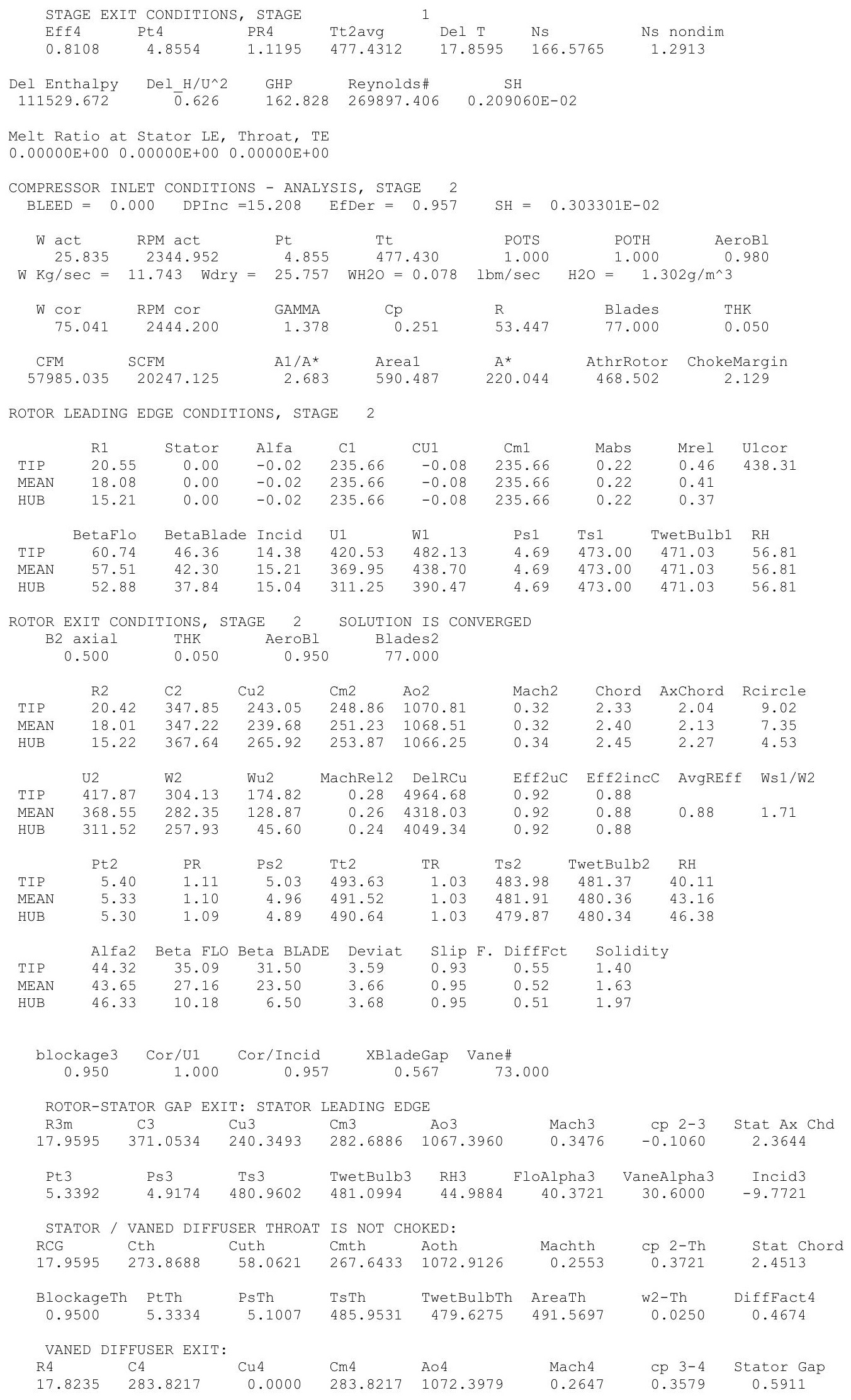




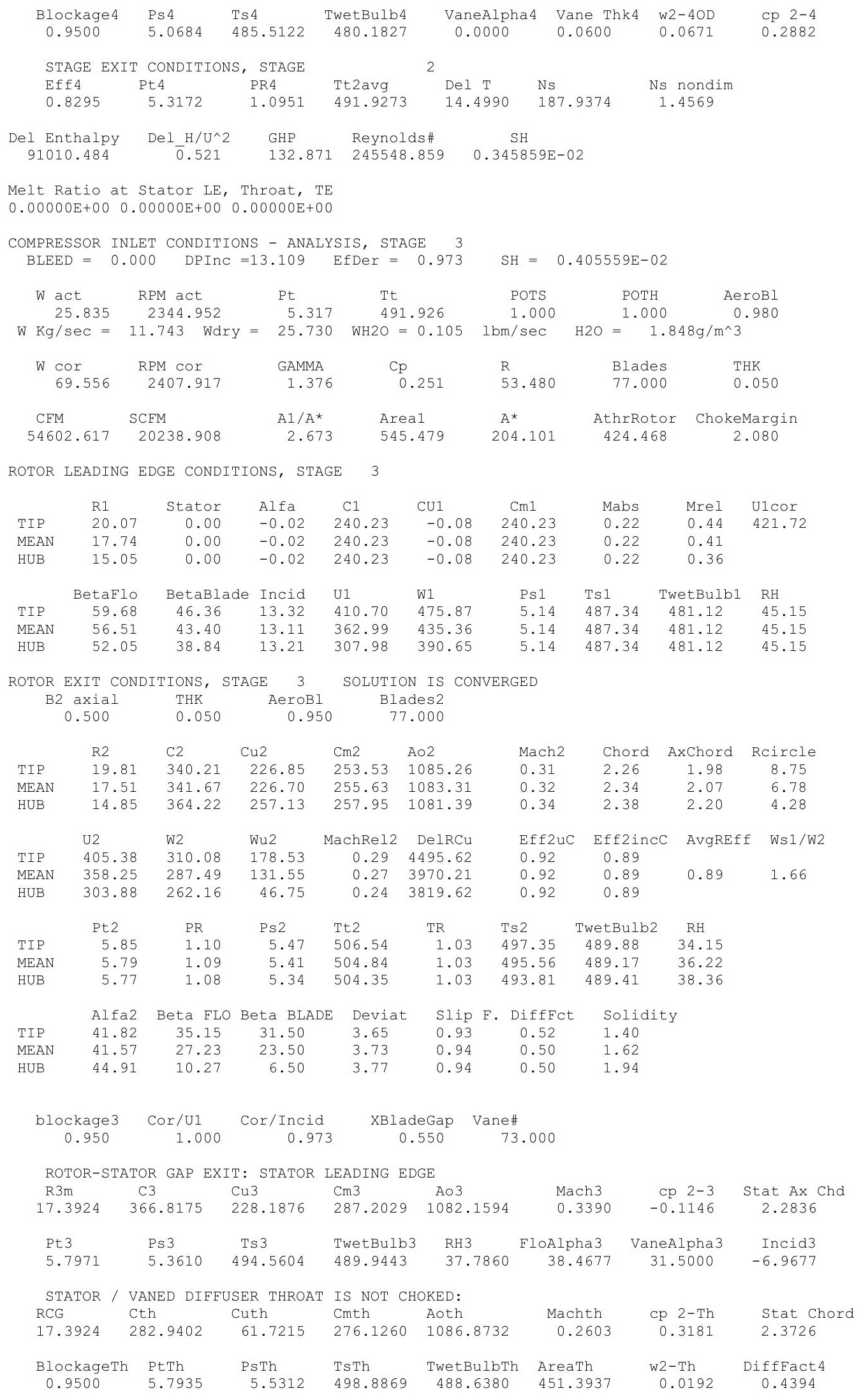




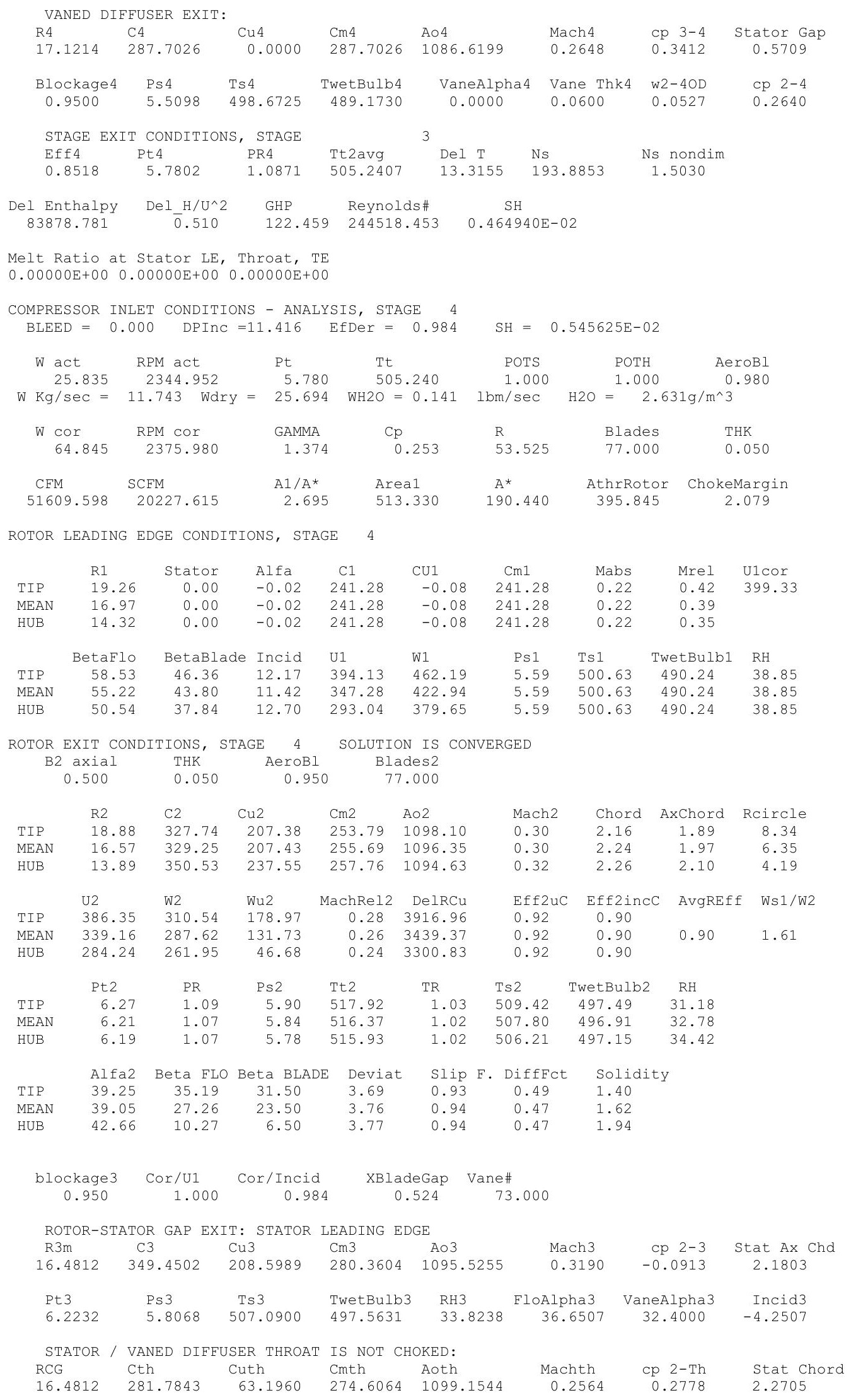




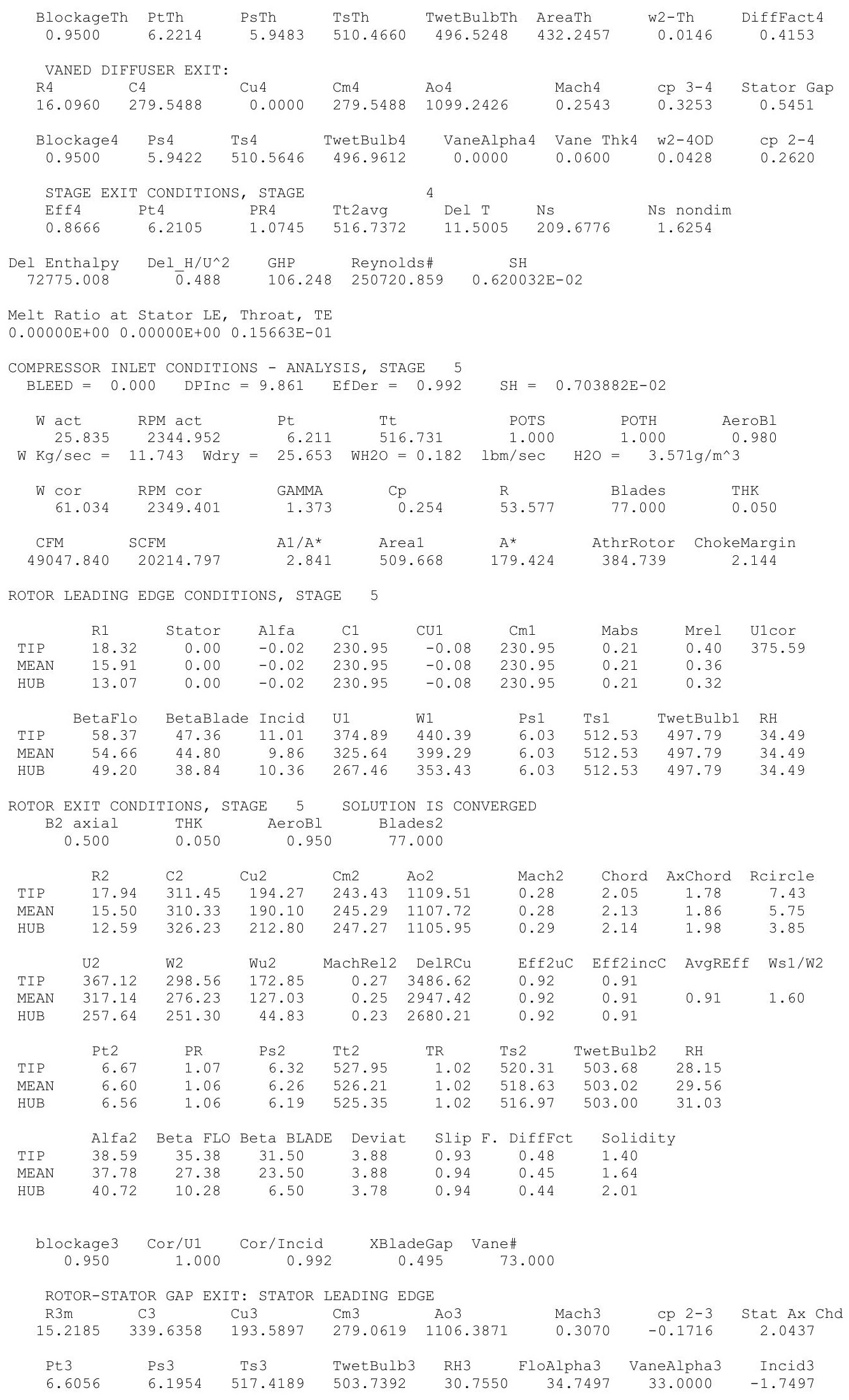




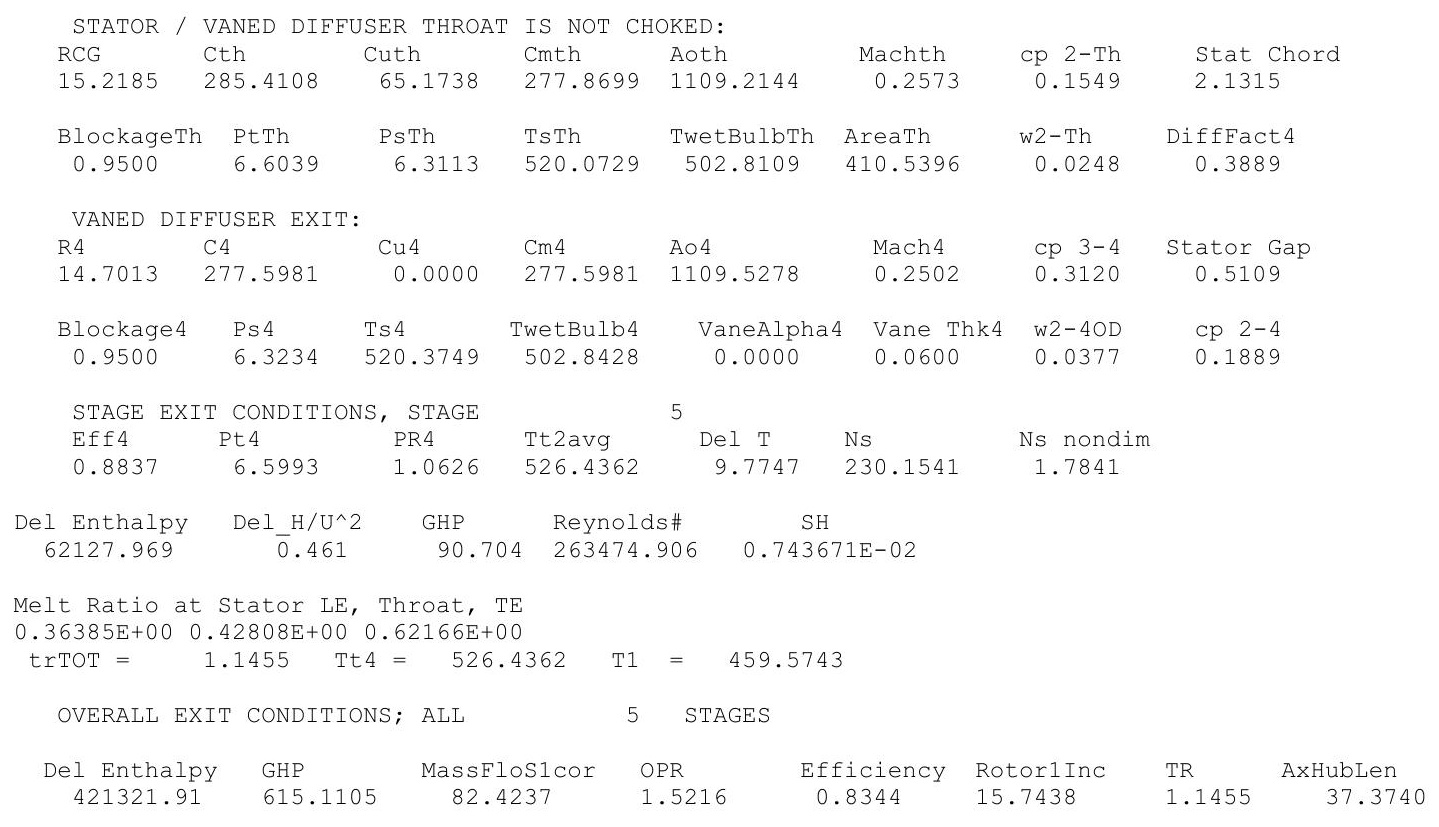




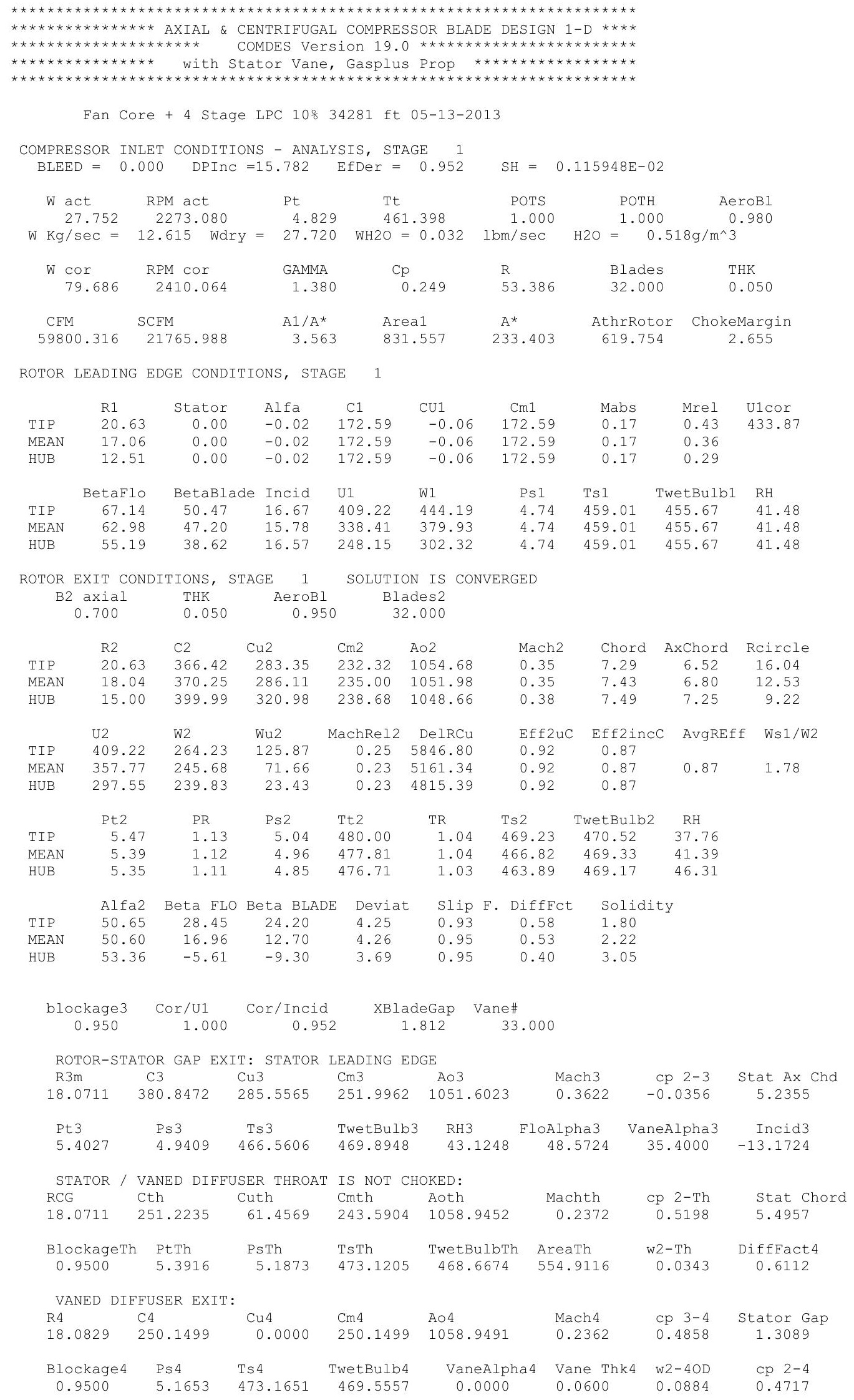




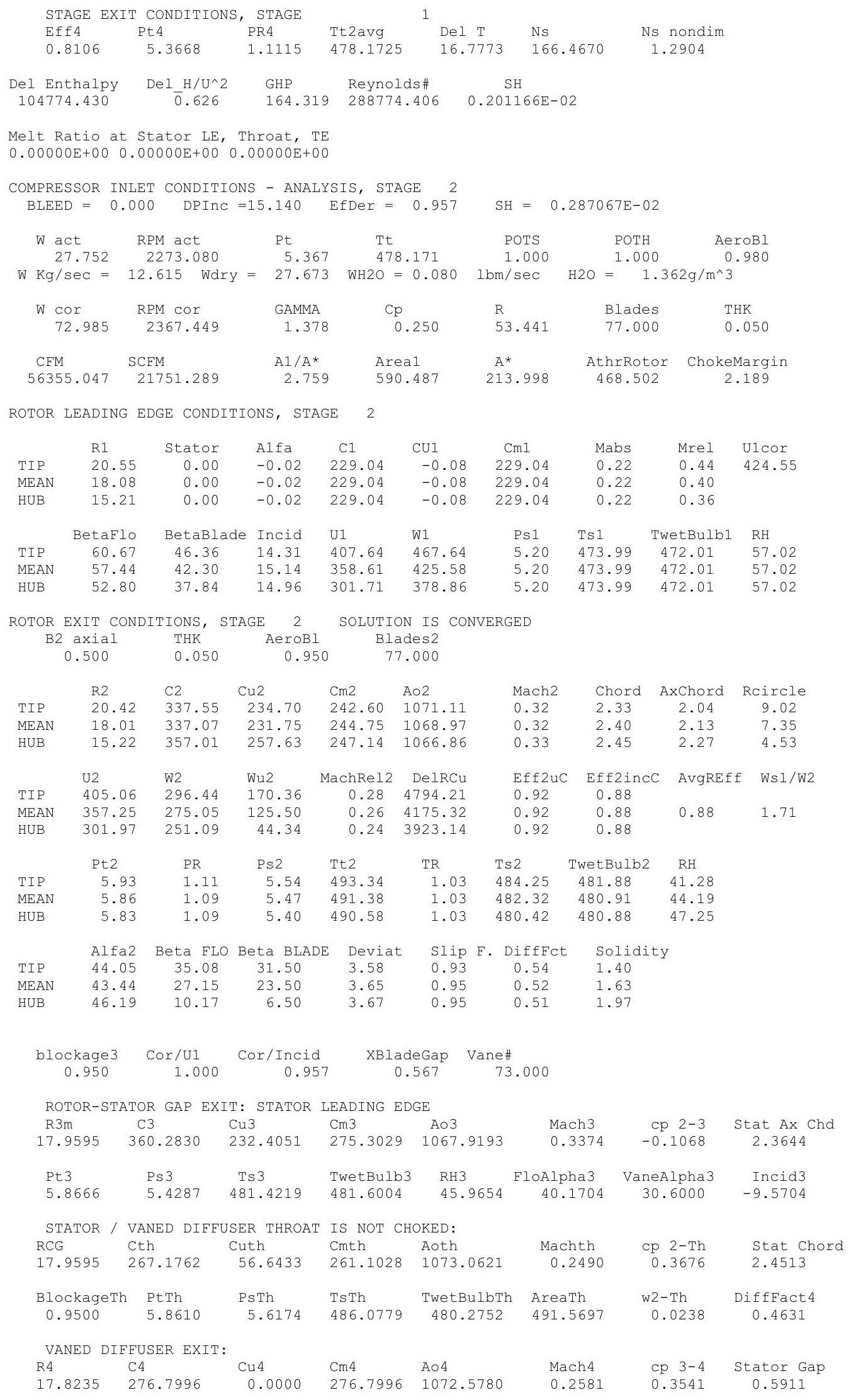




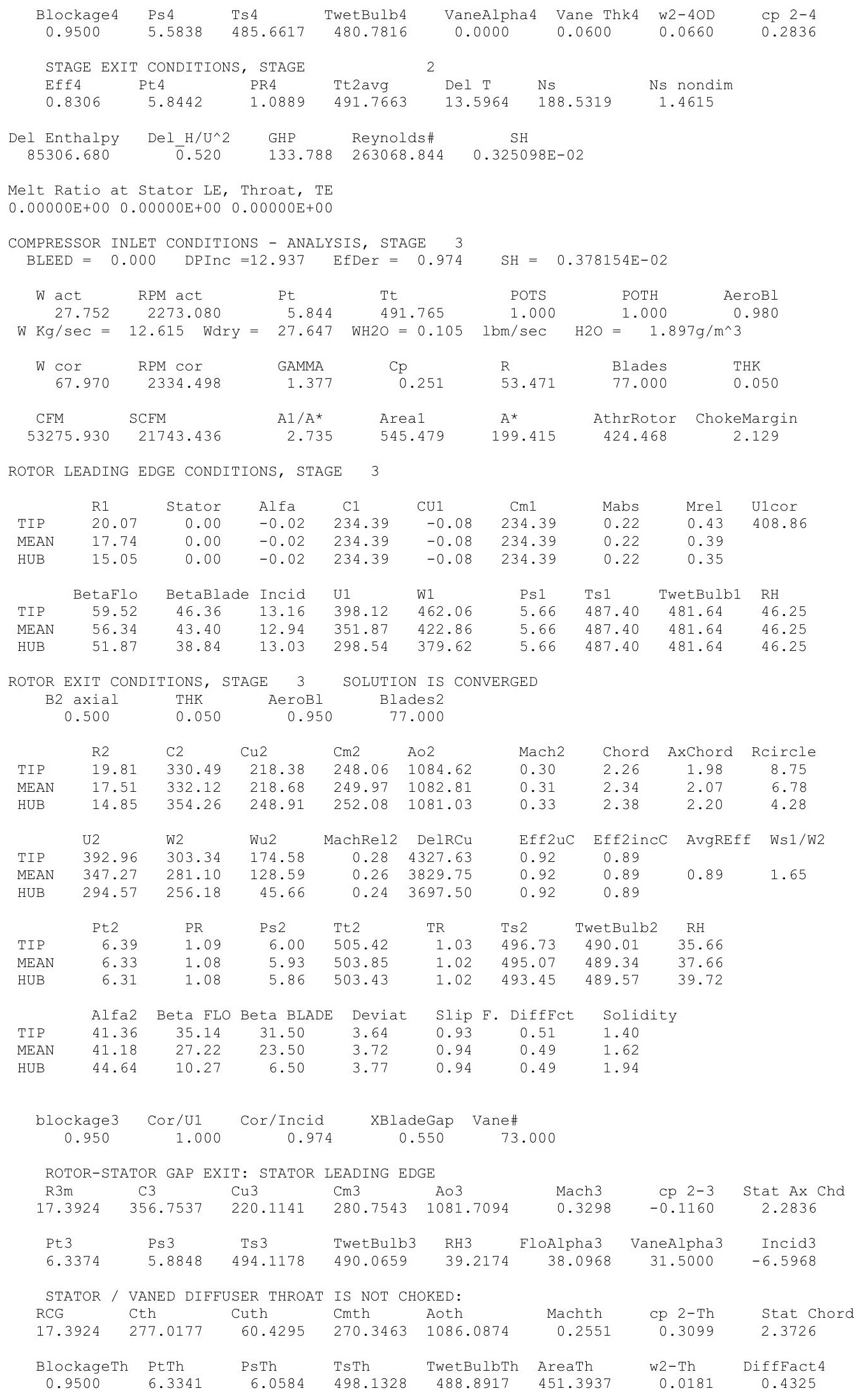




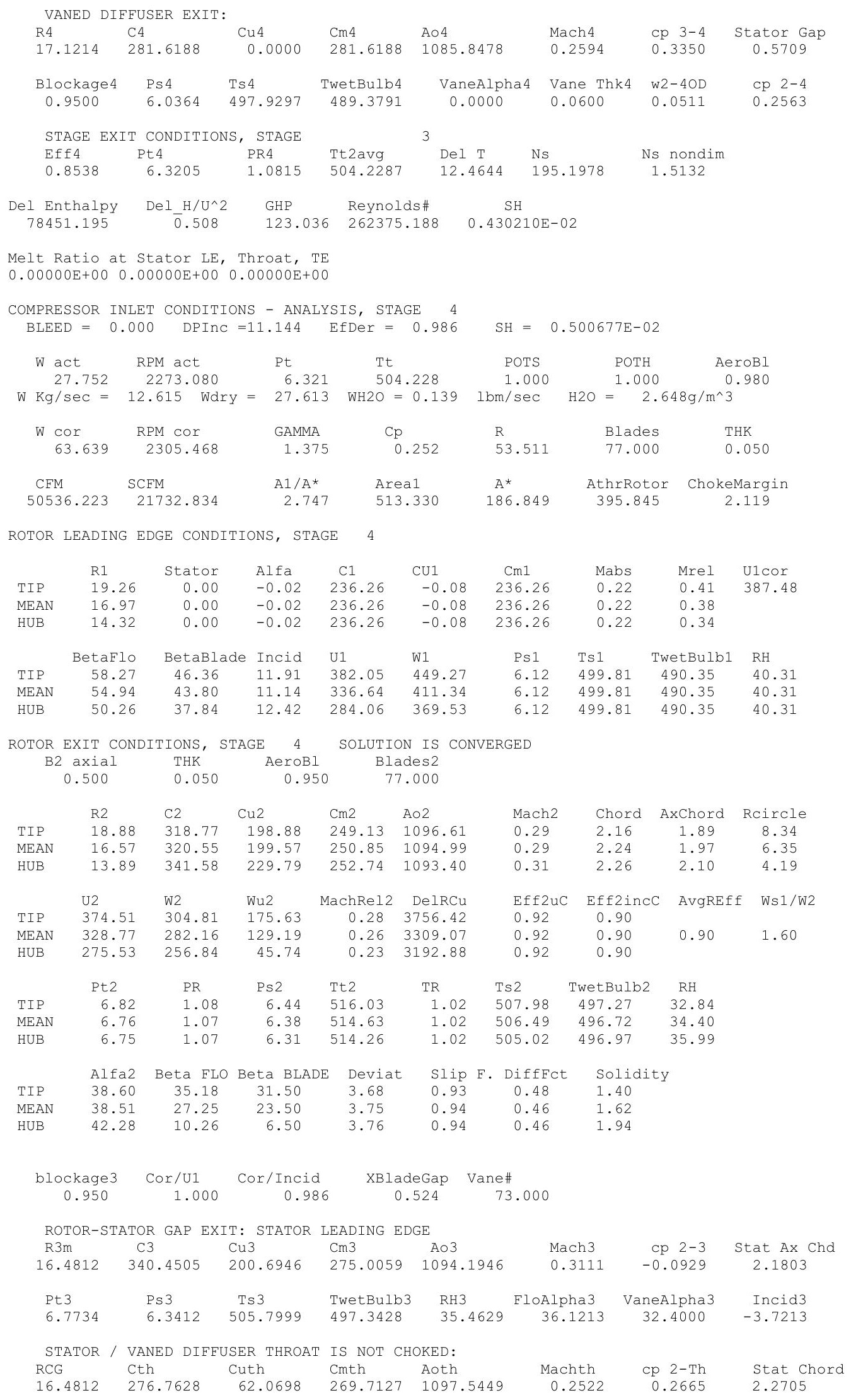




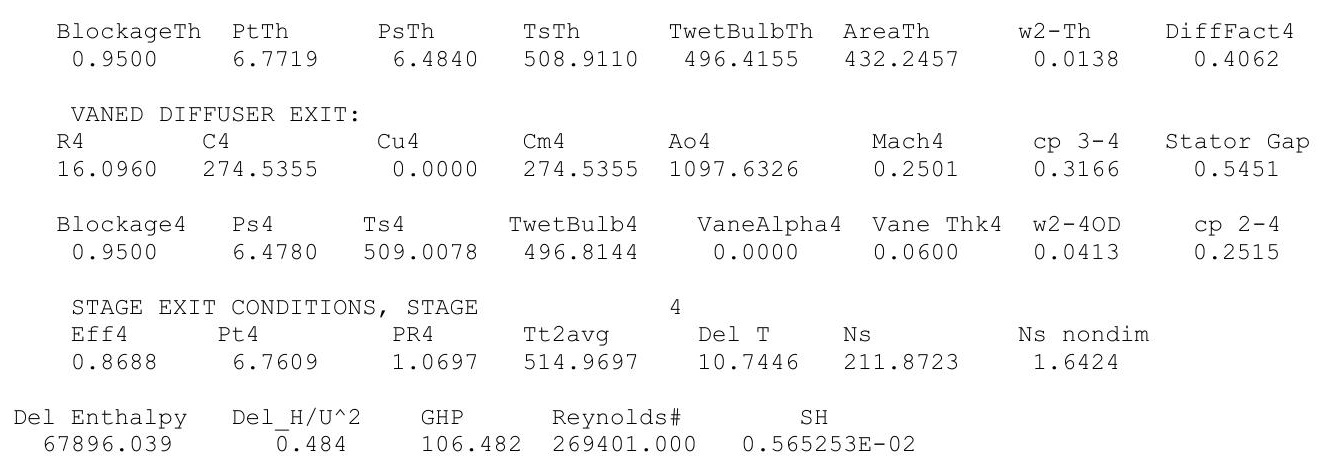

Melt Ratio at Stator LE, Throat, TE

$0.00000 \mathrm{E}+000.00000 \mathrm{E}+000.12626 \mathrm{E}-01$

COMPRESSOR INLET CONDITIONS - ANALYSIS, STAGE 5 BLEED $=0.000 \quad$ DPInC $=9.501 \quad$ EfDer $=0.994$

$\begin{array}{llllll}W & \text { Ret } & \text { PPM act } & \text { POTS } & \text { POTH } & \text { AeroBI }\end{array}$

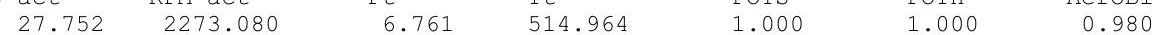

$\mathrm{W} \mathrm{Kg} / \mathrm{sec}=12.615$ WdrY $=27.575 \quad \mathrm{WH} 2 \mathrm{O}=0.177 \quad \mathrm{lbm} / \mathrm{sec} \quad \mathrm{H} 2 \mathrm{O}=3.544 \mathrm{~g} / \mathrm{m}^{\wedge} 3$

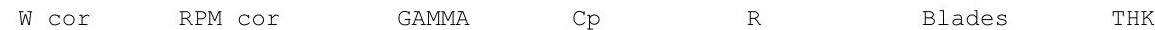

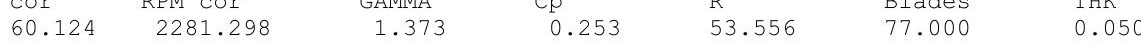

CFM SCFM A1/A* Area1 A* AthrRotor ChokeMargin

$\begin{array}{lllllll}48180.926 & 21720.826 & 2.885 & 509.668 & 176.681 & 384.739 & 2.178\end{array}$

ROTOR LEADING EDGE CONDITIONS, STAGE 5

$\begin{array}{lccccccccc} & \text { R1 } & \text { Stator } & \text { Alfa } & \text { C1 } & \text { CU1 } & \text { Cm1 } & \text { Mabs } & \text { Mrel } & \text { U1cor } \\ \text { TIP } & 18.32 & 0.00 & -0.02 & 226.87 & -0.08 & 226.87 & 0.21 & 0.39 & 364.71 \\ \text { MEAN } & 15.91 & 0.00 & -0.02 & 226.87 & -0.08 & 226.87 & 0.21 & 0.35 & \\ \text { HUB } & 13.07 & 0.00 & -0.02 & 226.87 & -0.08 & 226.87 & 0.21 & 0.31 & \\ & & & & & & & & & \\ & & & & & & & & & \\ \text { TIP } & 58.03 & 47.36 & 10.67 & 363.40 & 428.47 & 6.57 & 510.90 & 497.58 & 36.23 \\ \text { MEAN } & 54.30 & 44.80 & 9.50 & 315.66 & 388.79 & 6.57 & 510.90 & 497.58 & 36.23 \\ \text { HUB } & 48.82 & 38.84 & 9.98 & 259.26 & 344.57 & 6.57 & 510.90 & 497.58 & 36.23\end{array}$

ROTOR EXIT CONDITIONS, STAGE 5 SOLUTION IS CONVERGED

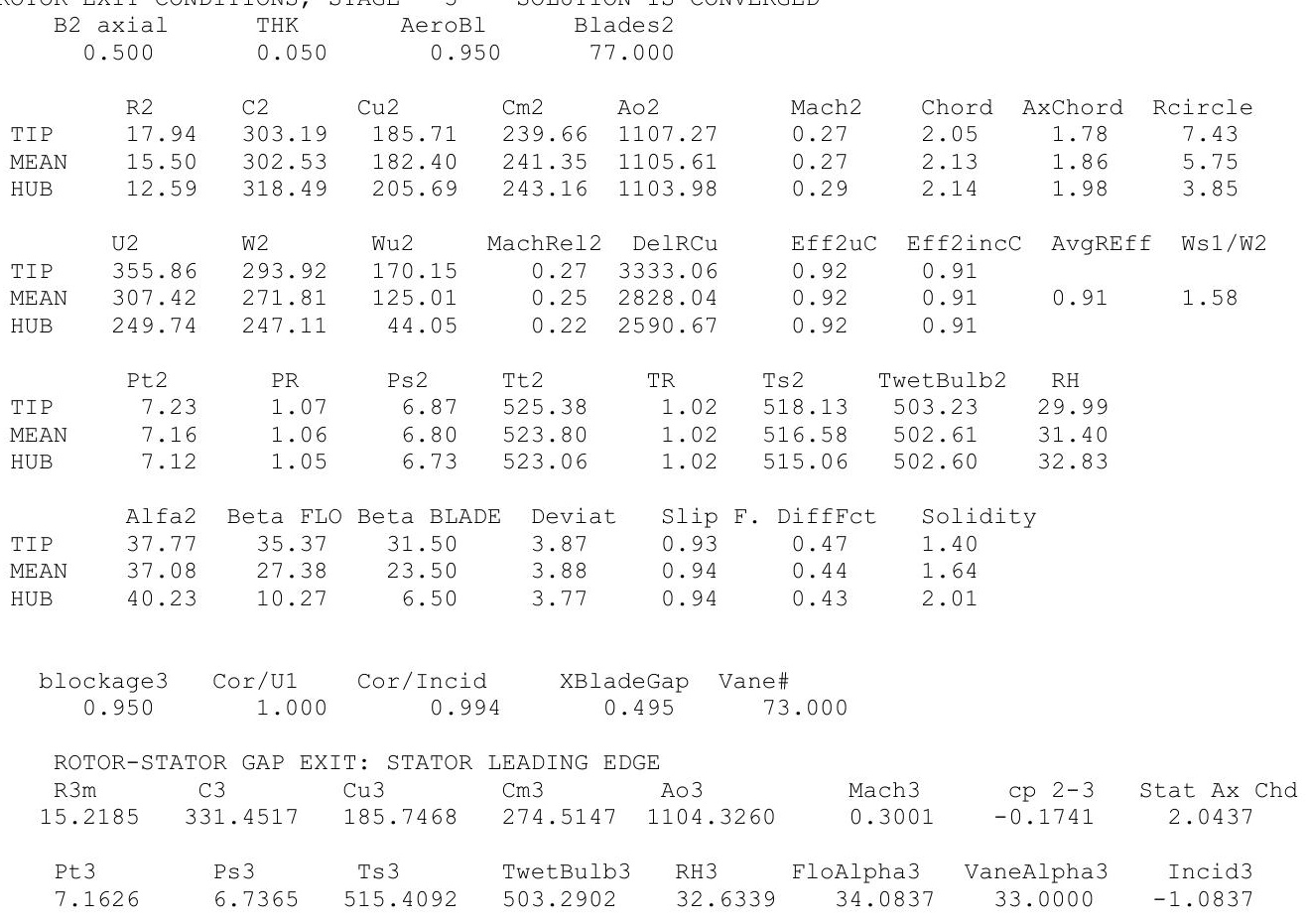




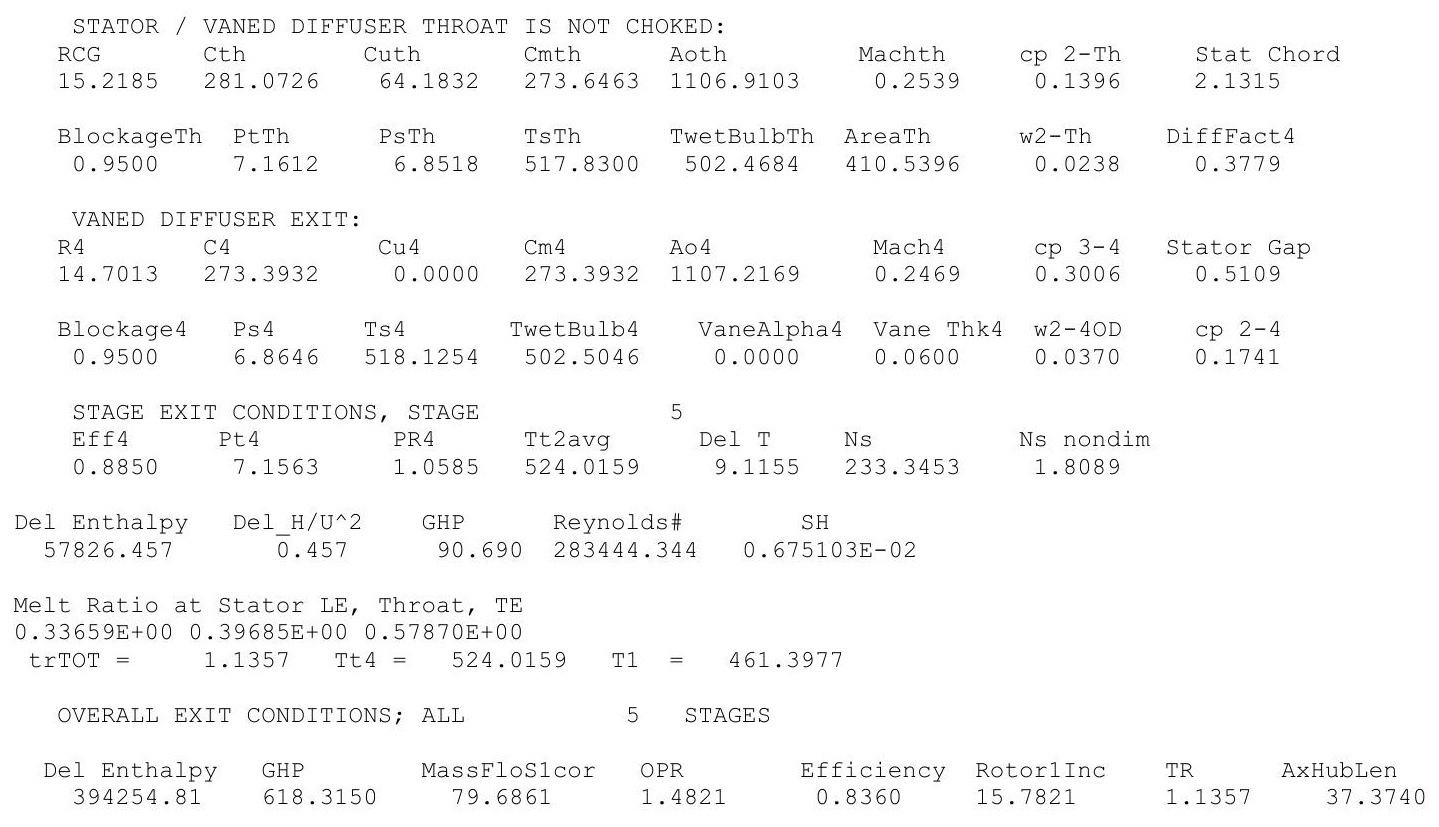




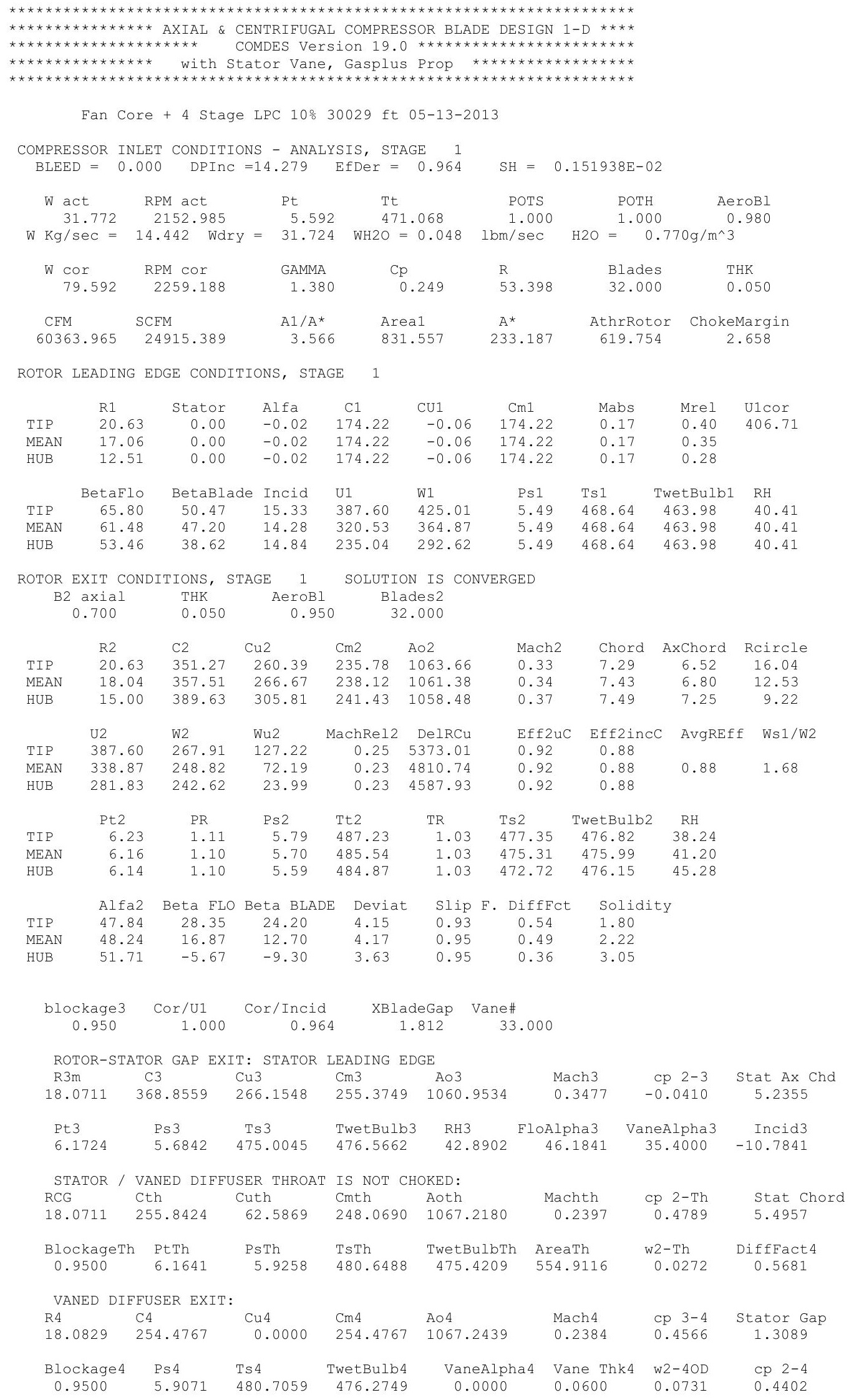




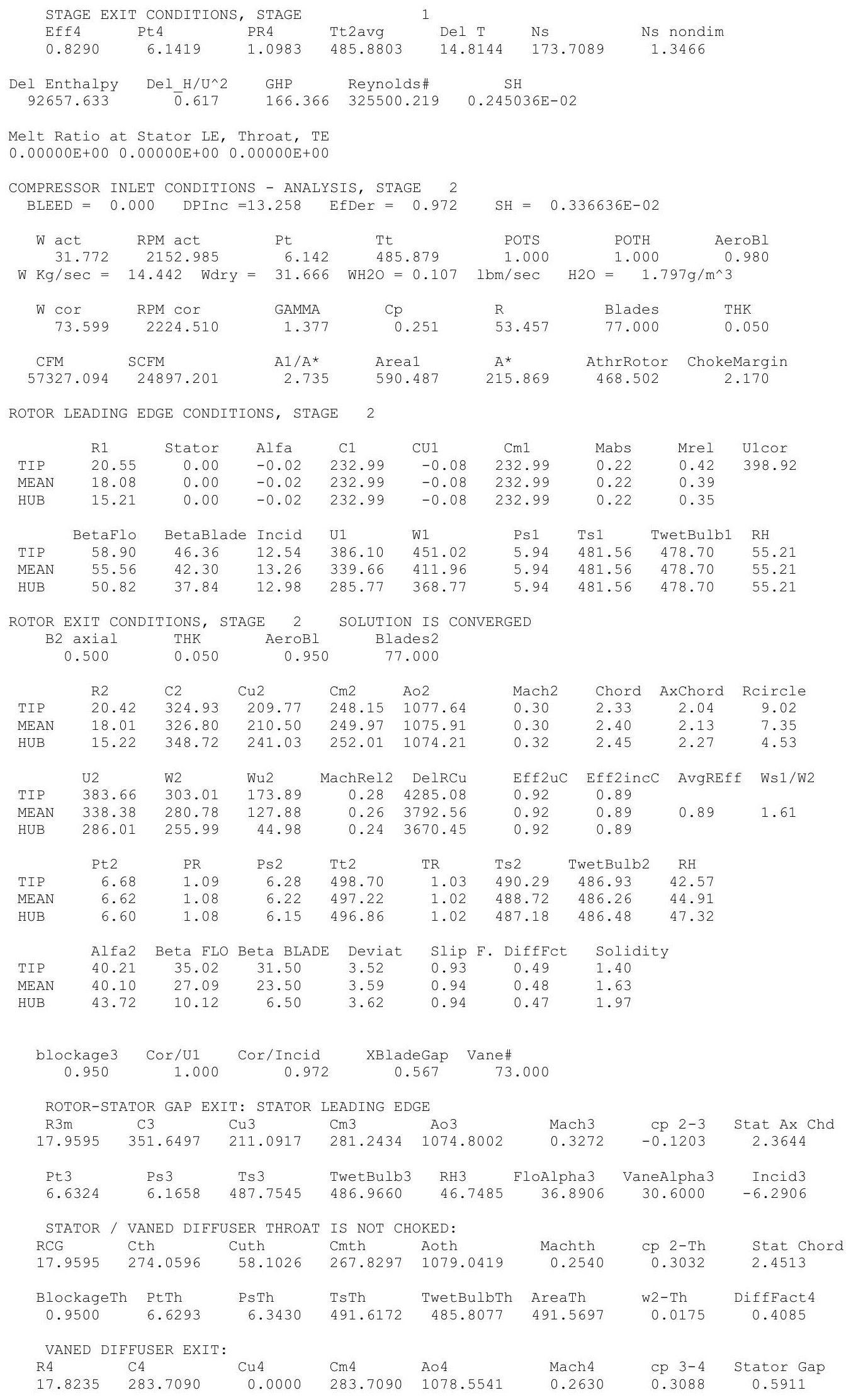




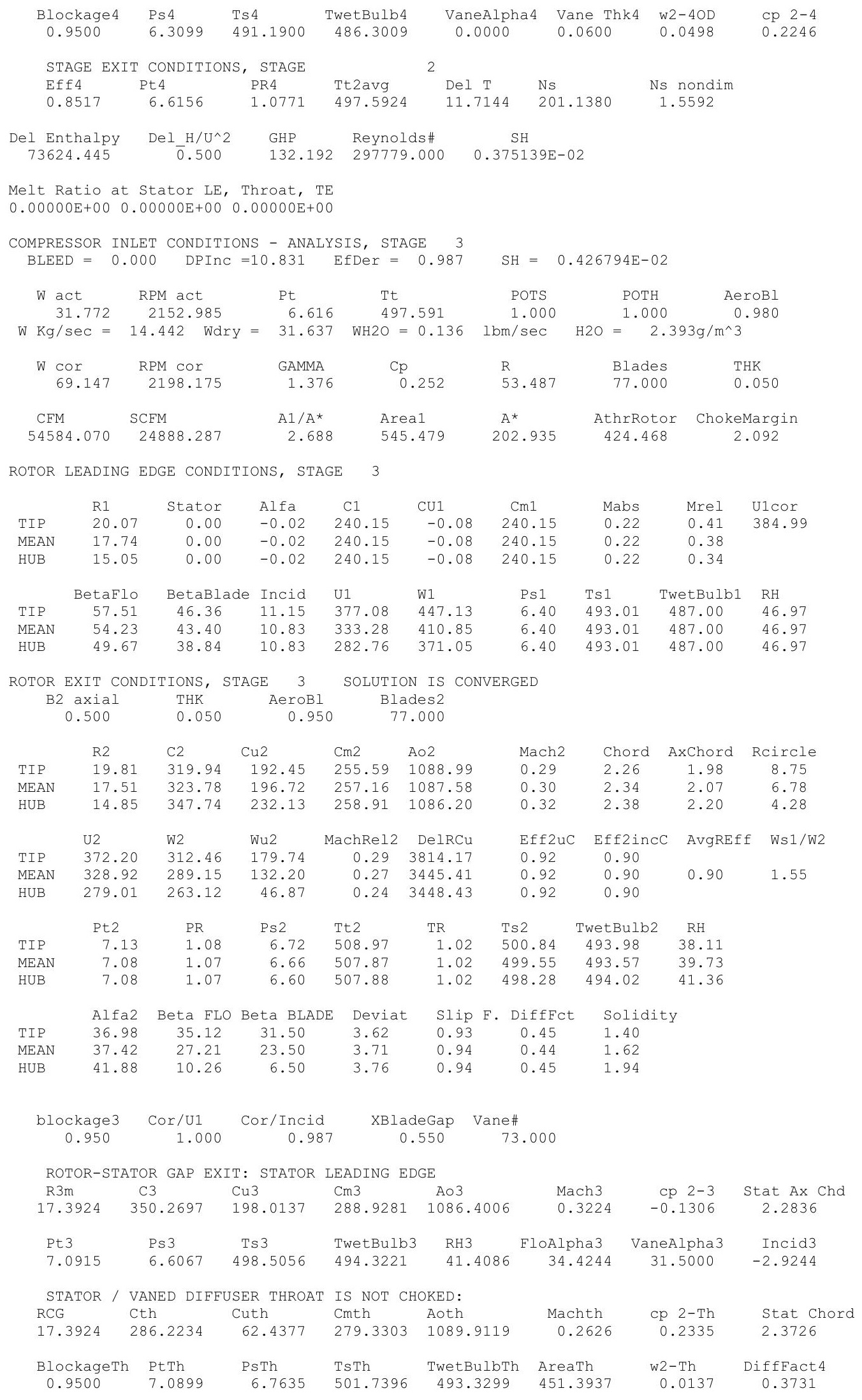




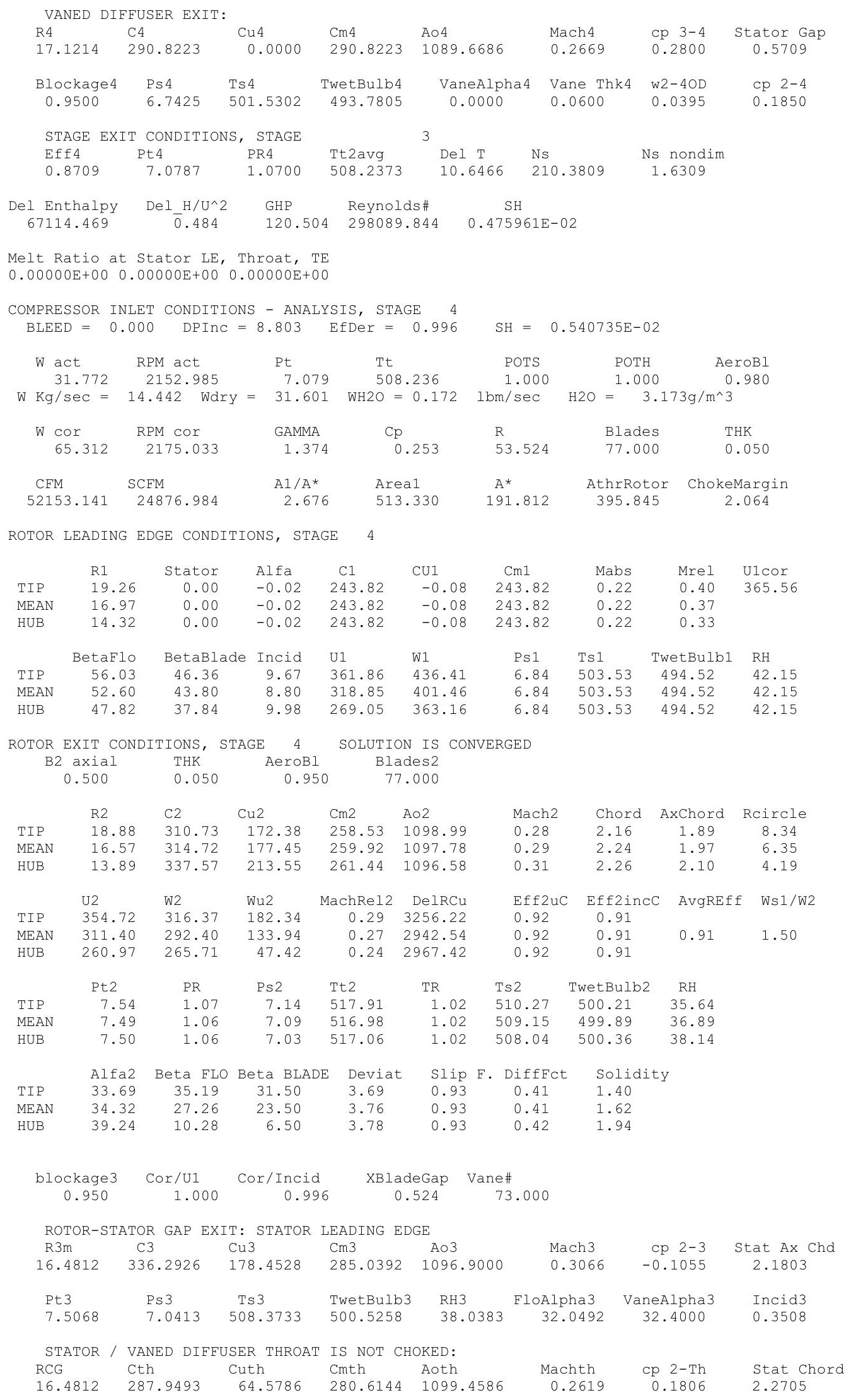




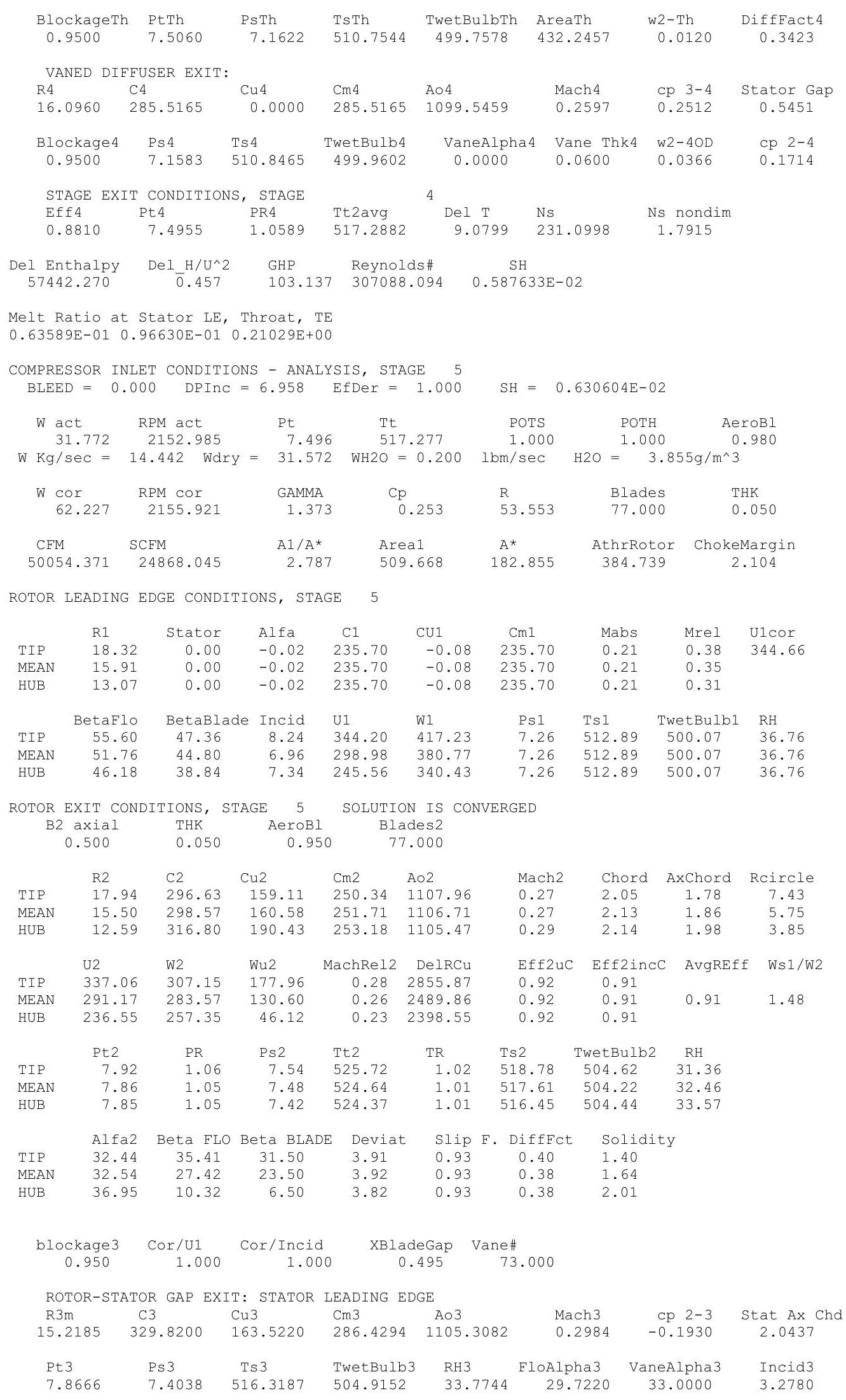




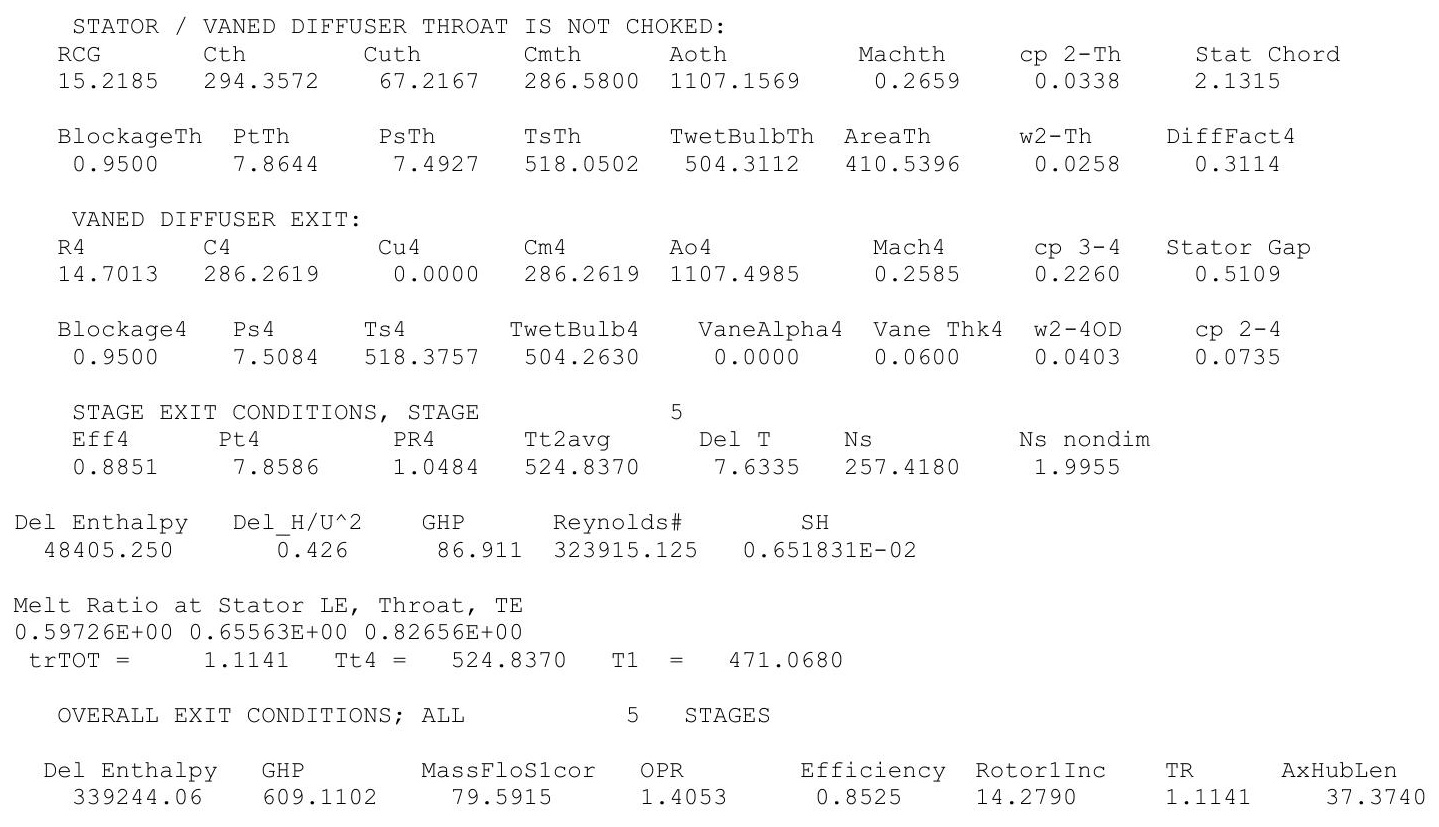




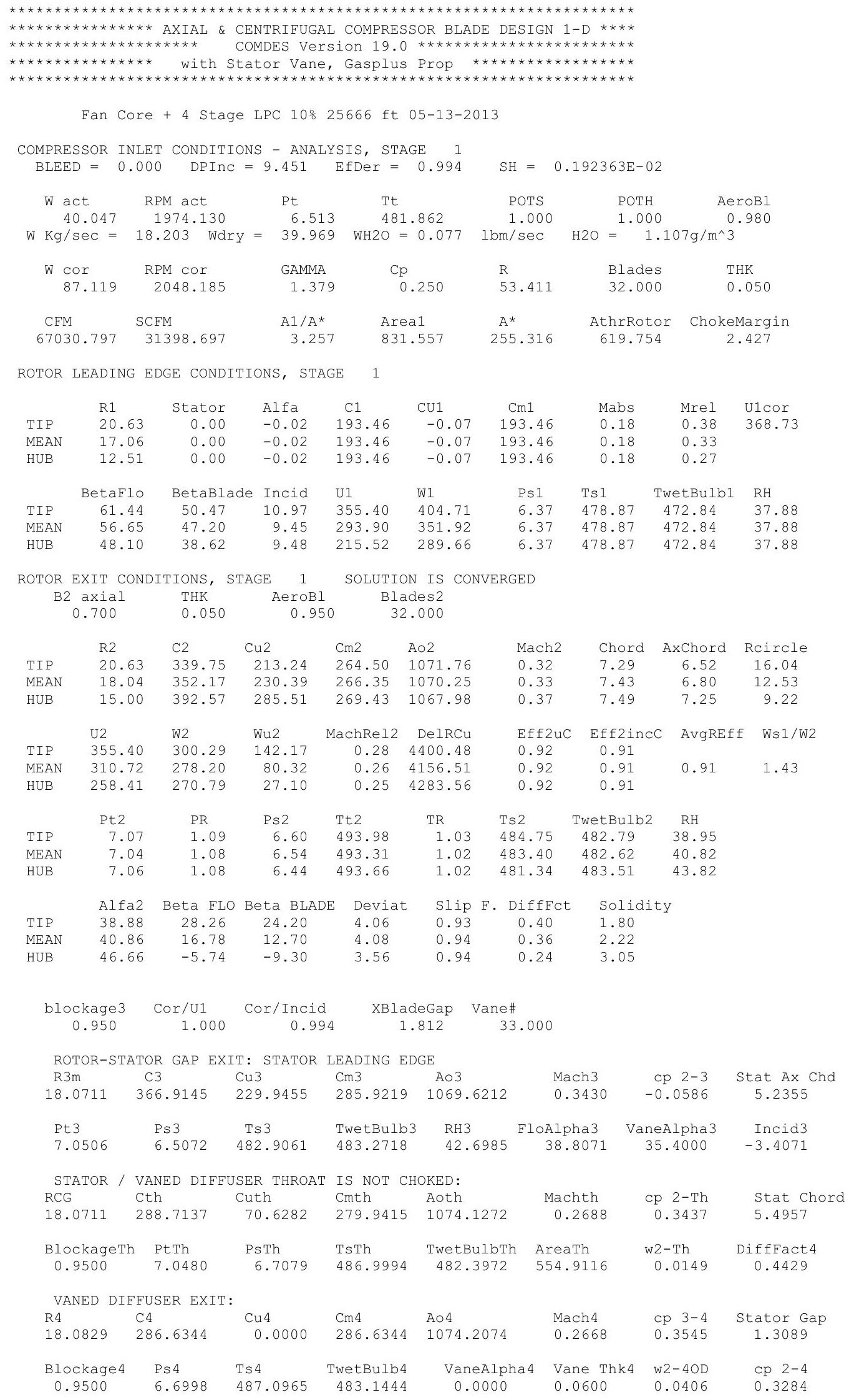




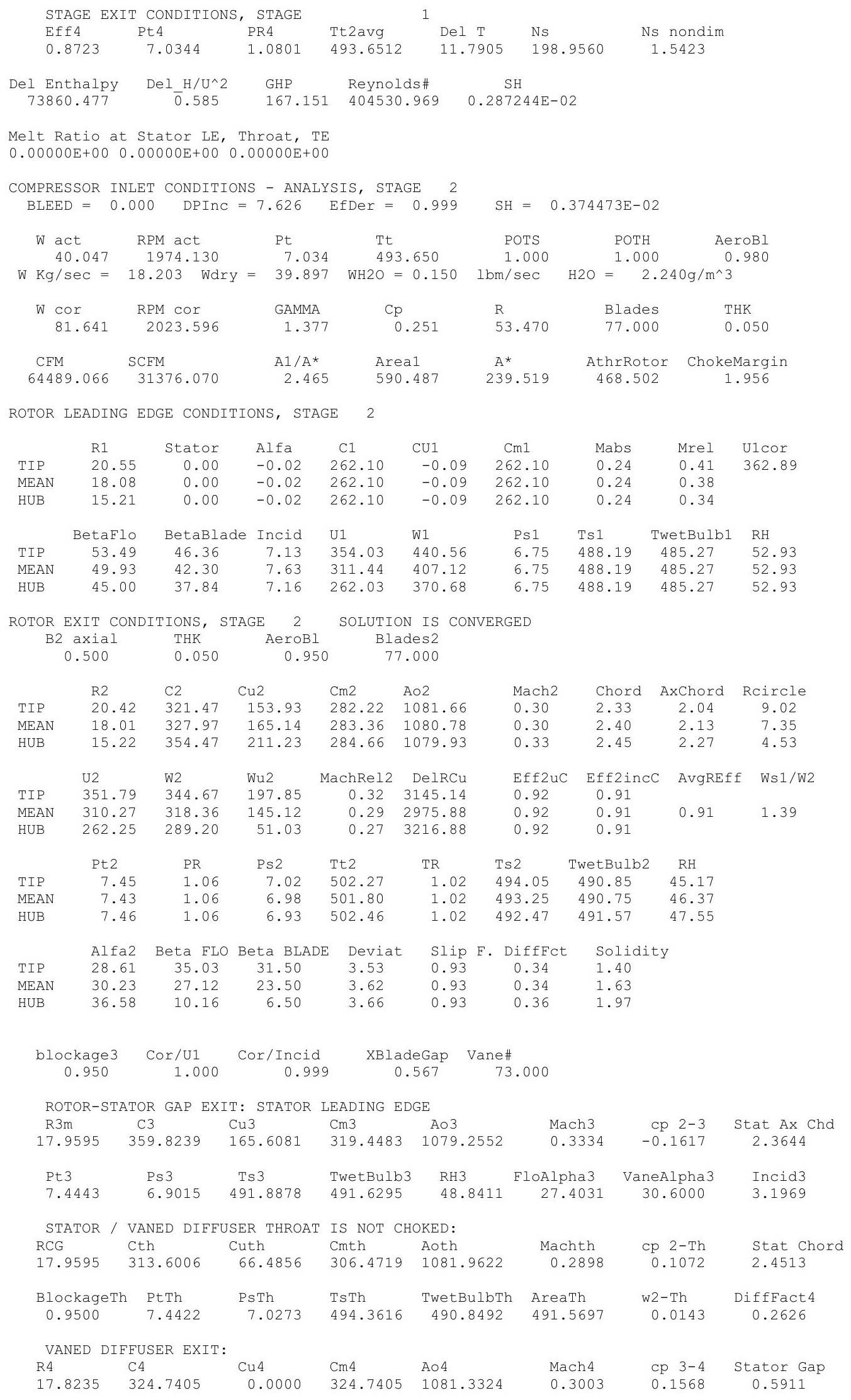




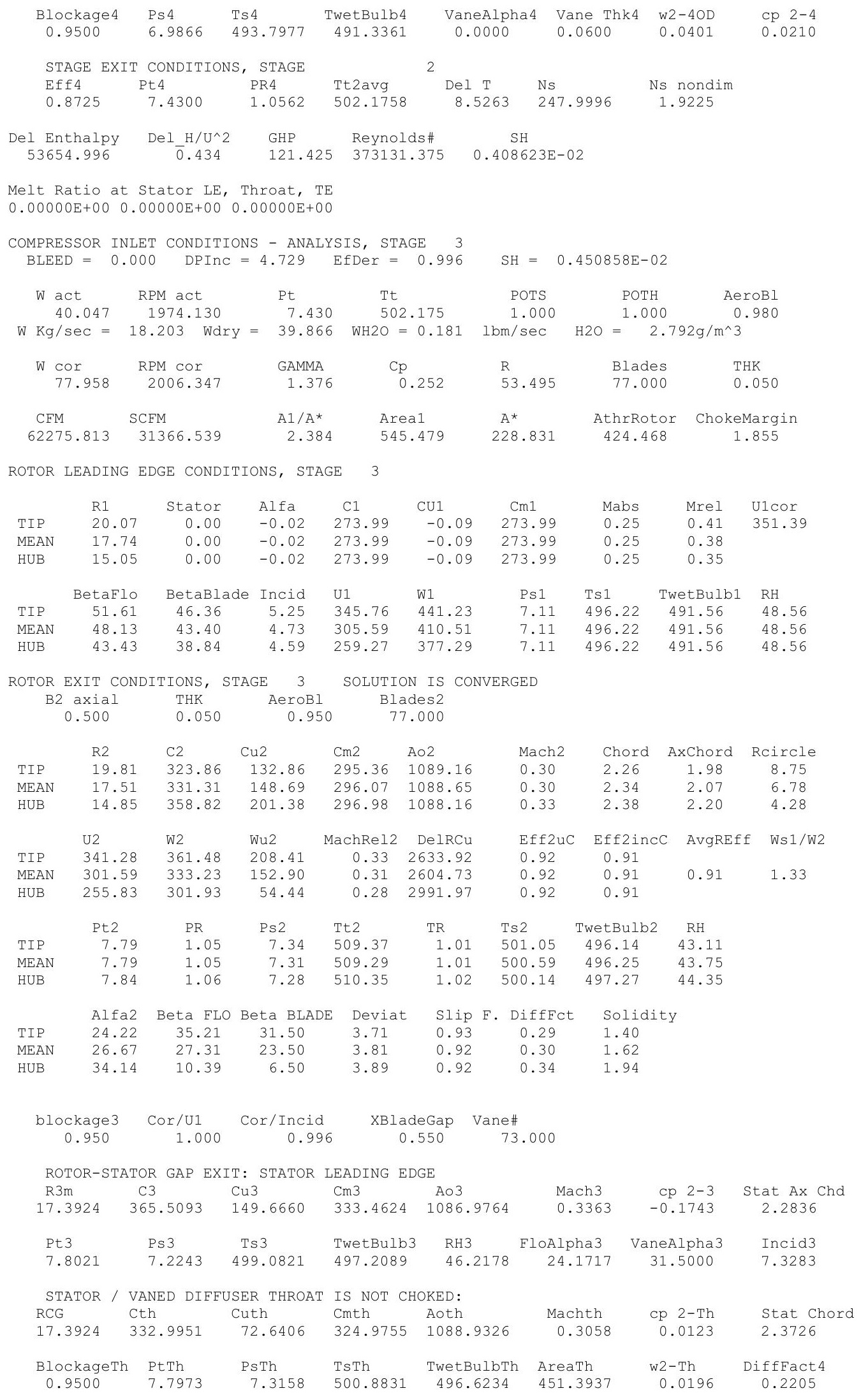




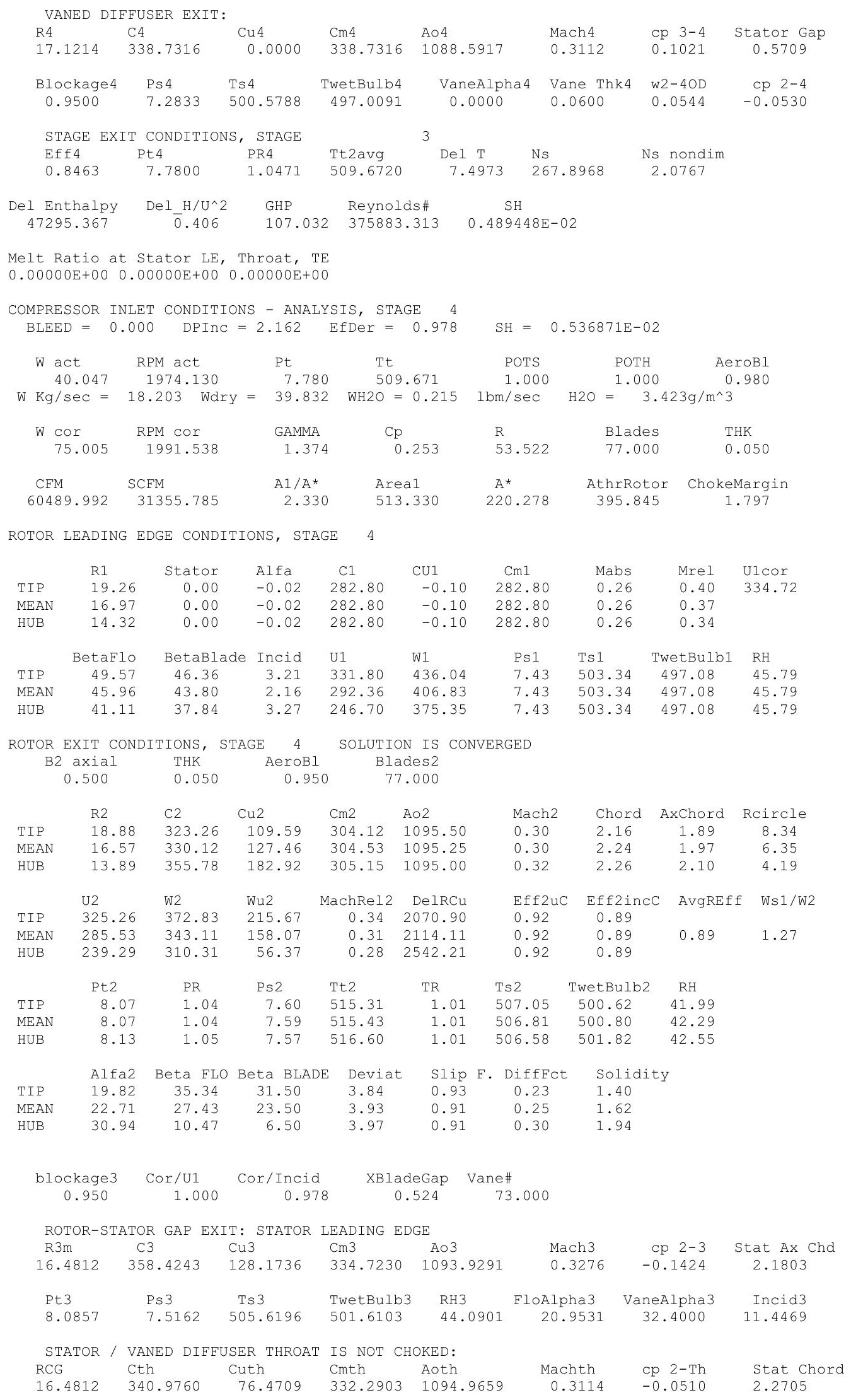




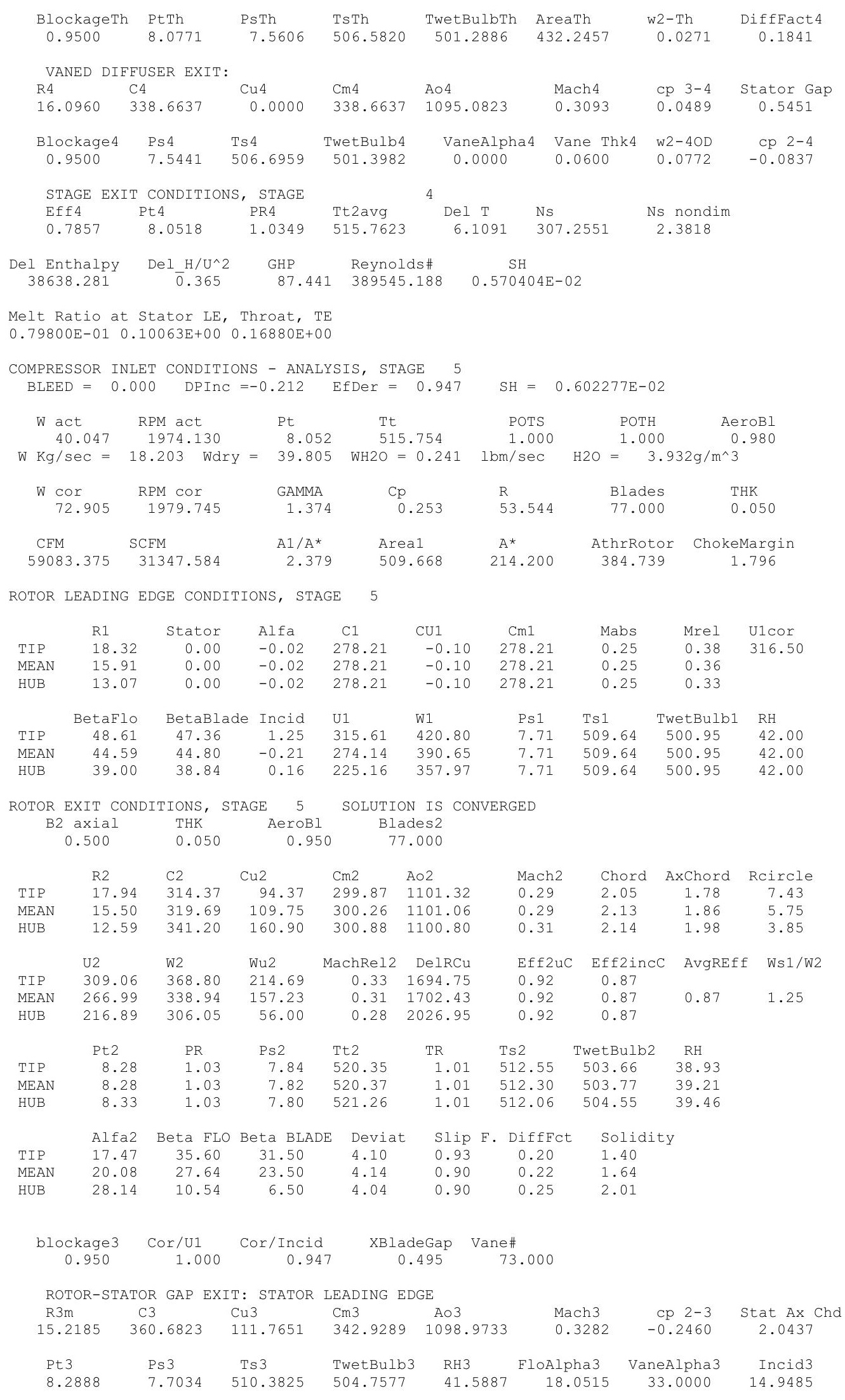




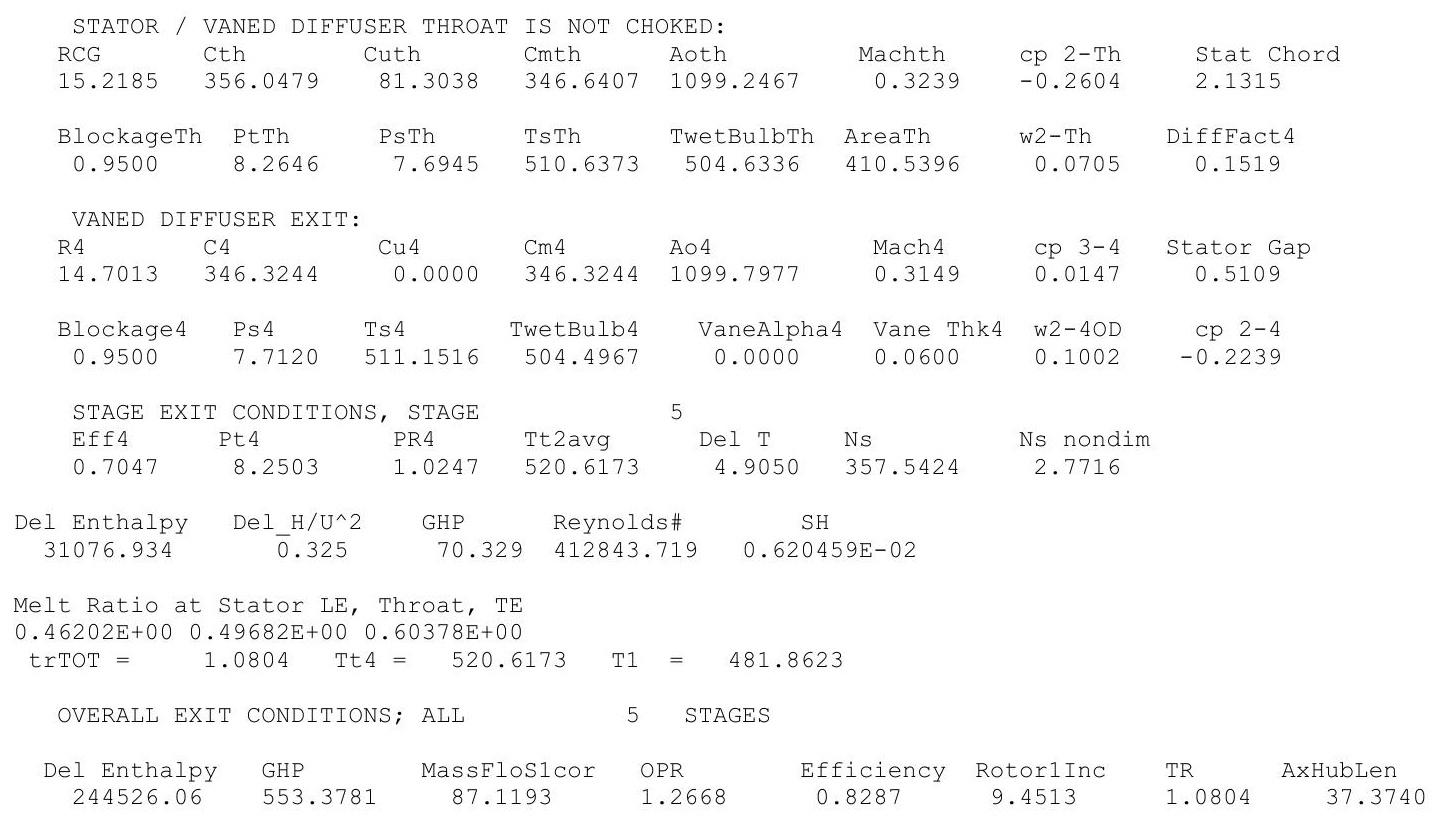




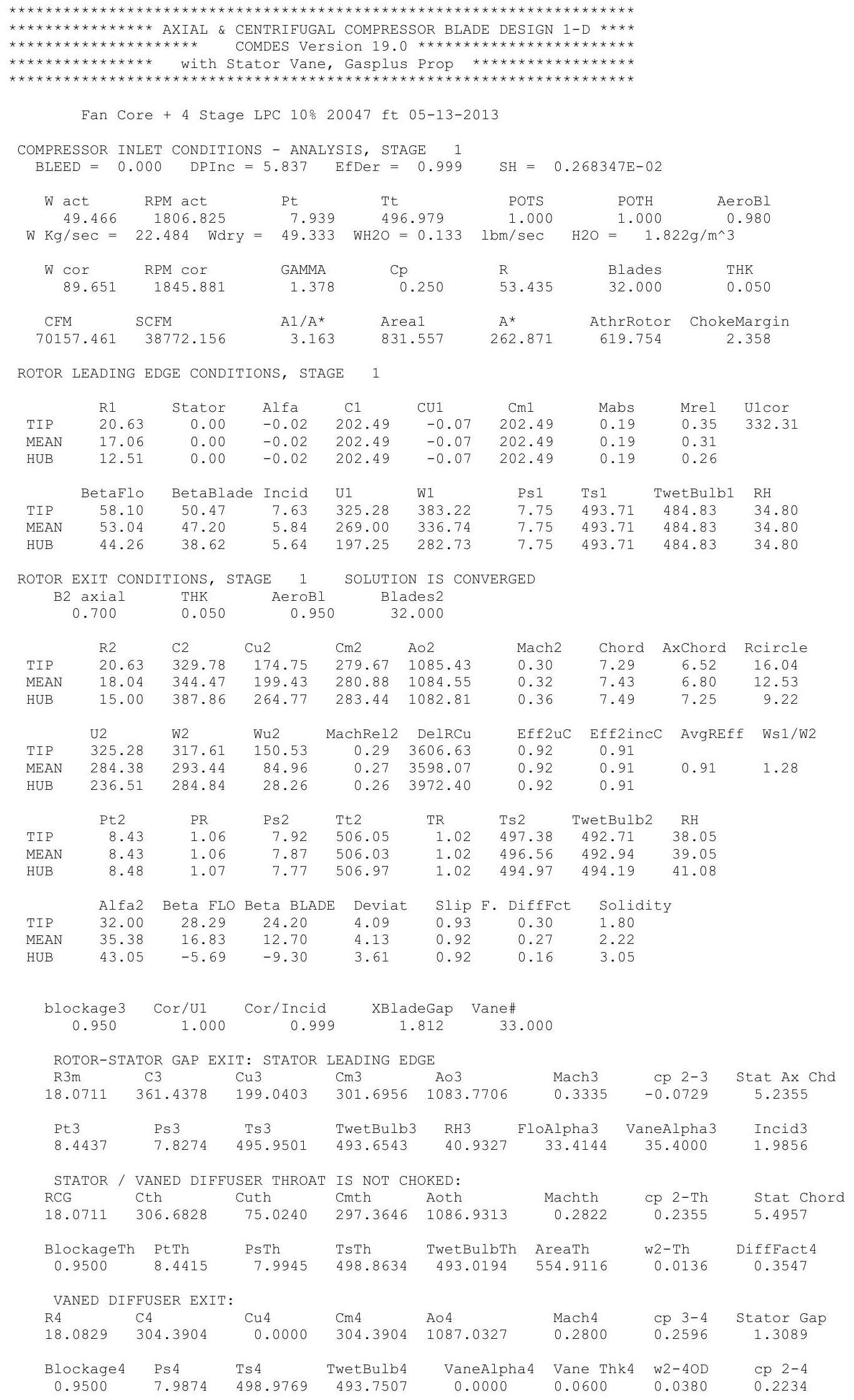




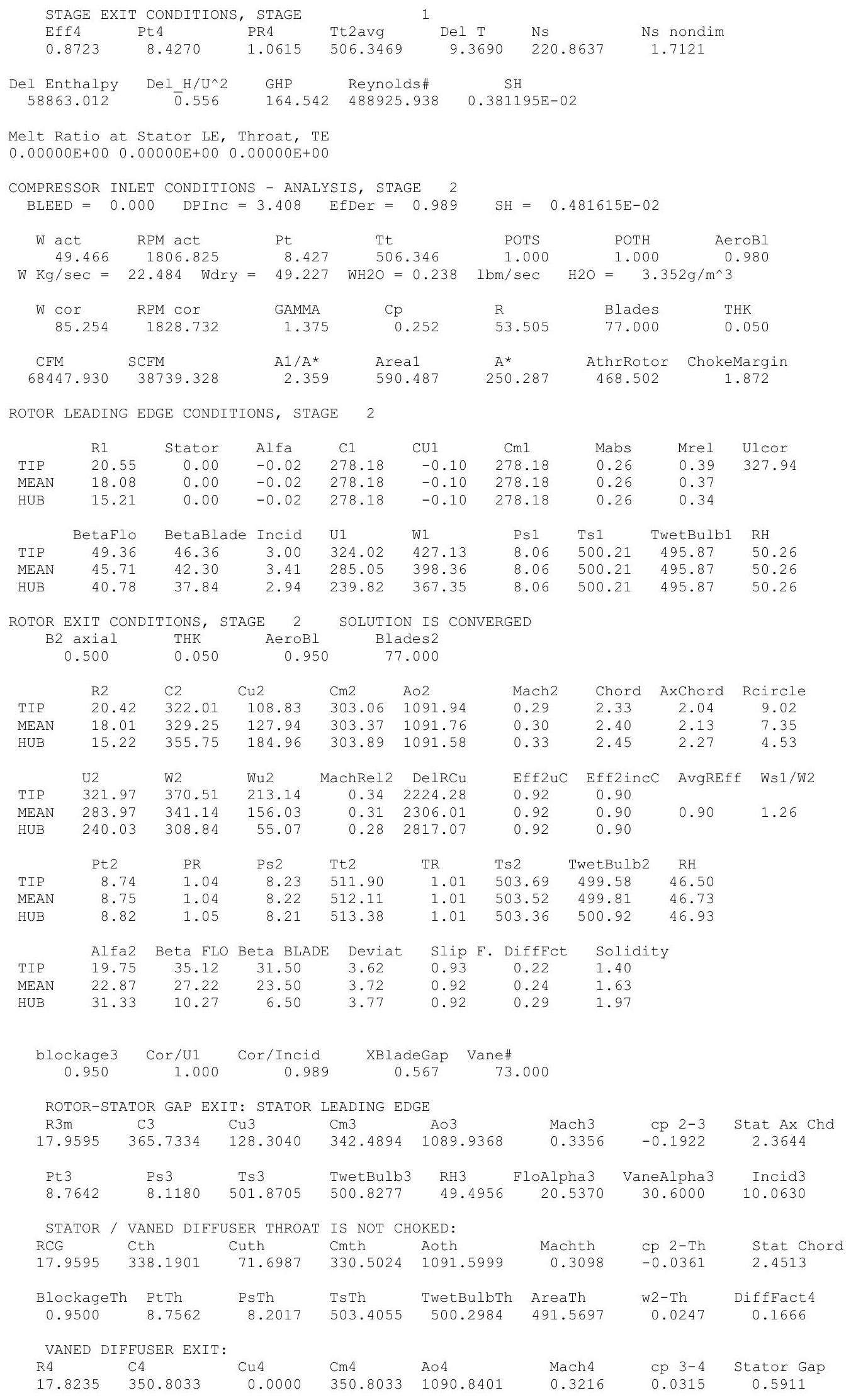




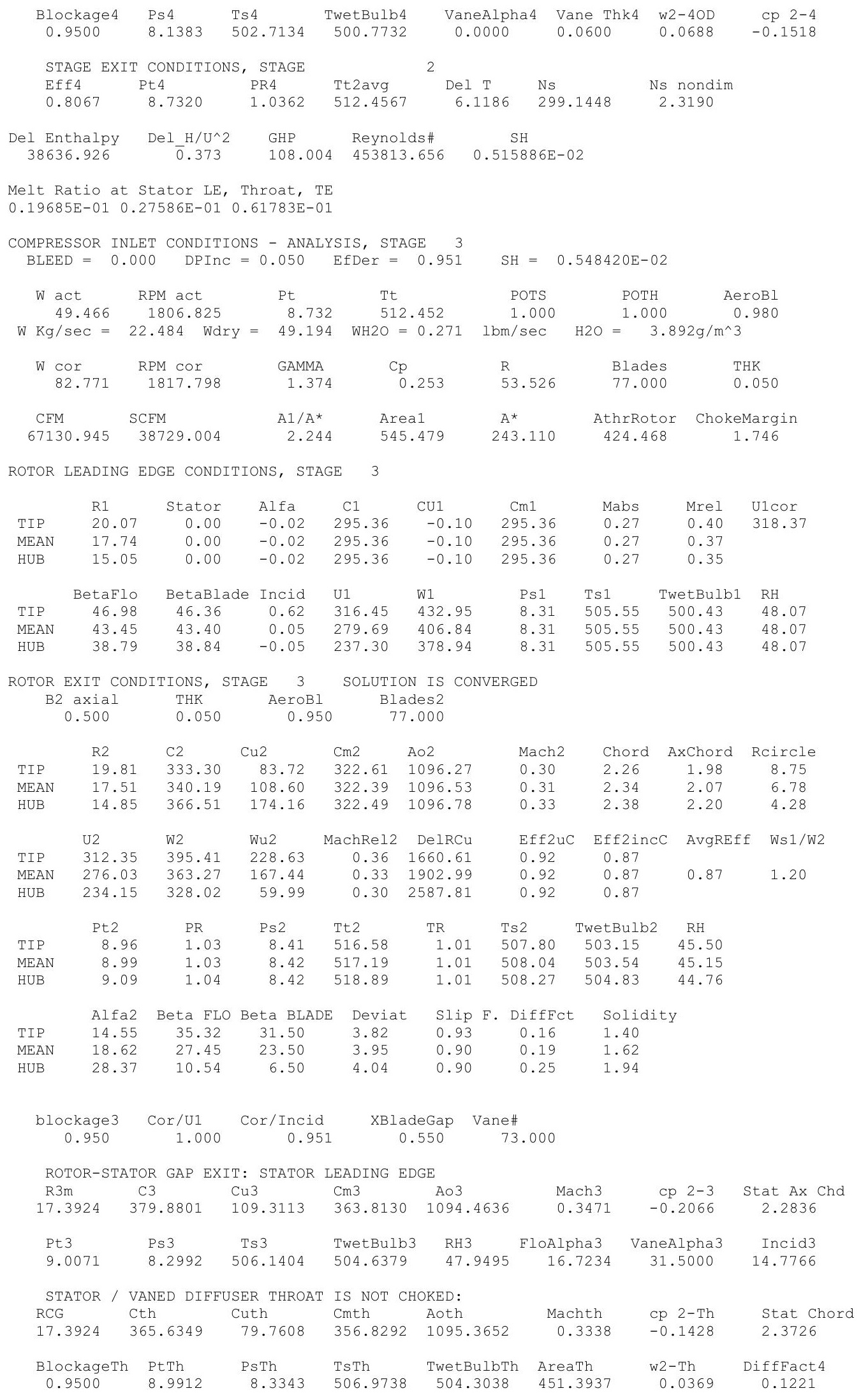




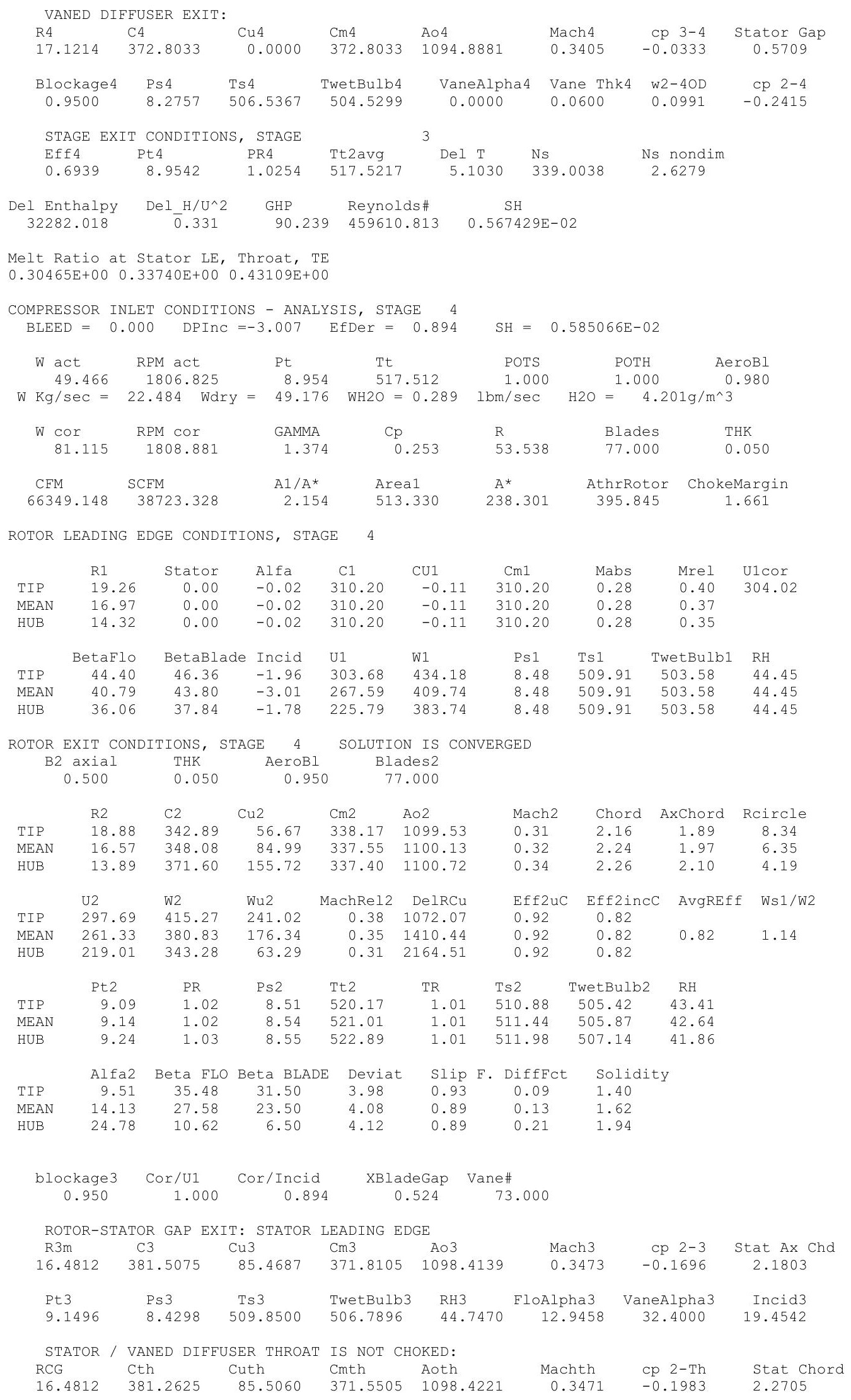




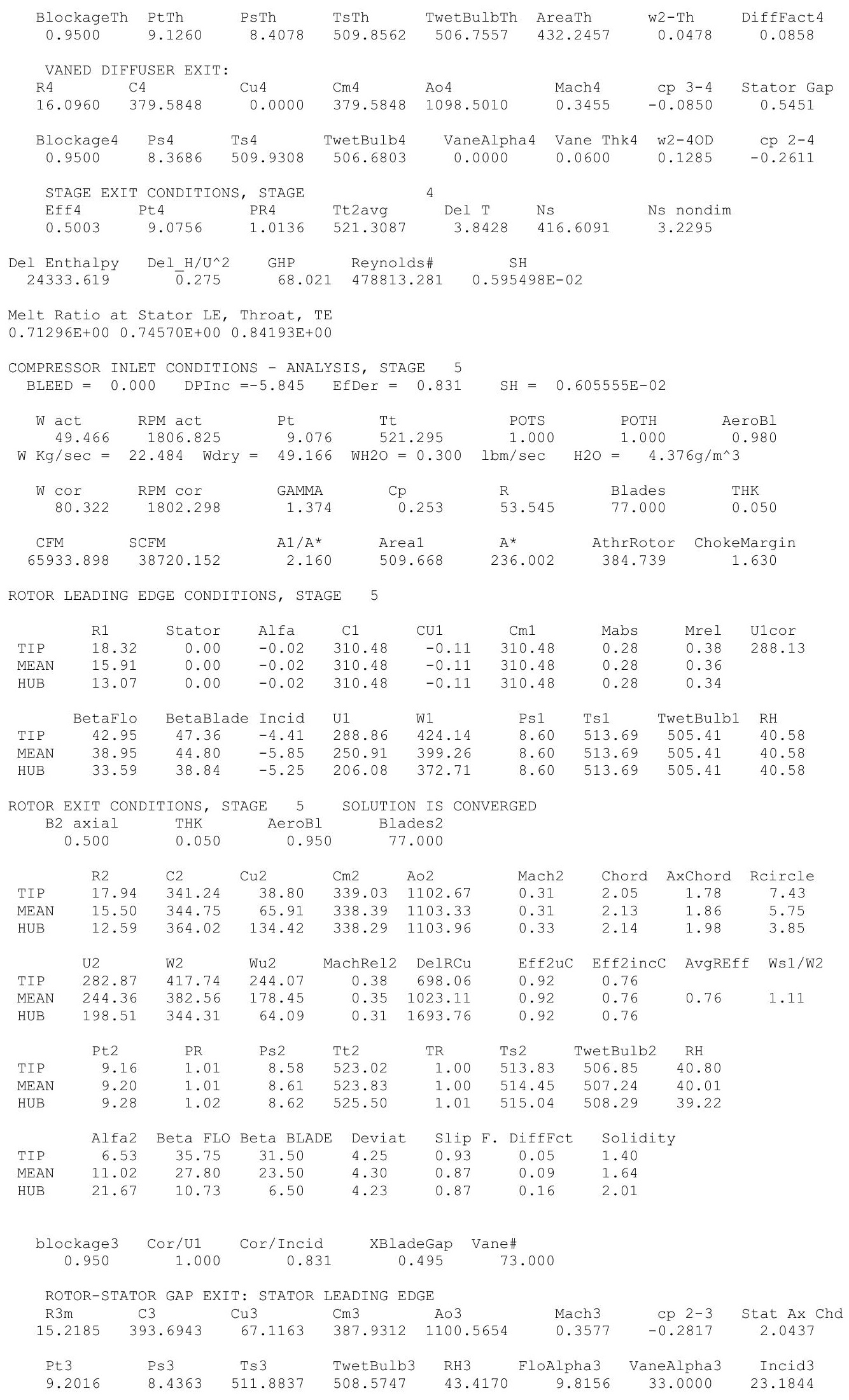




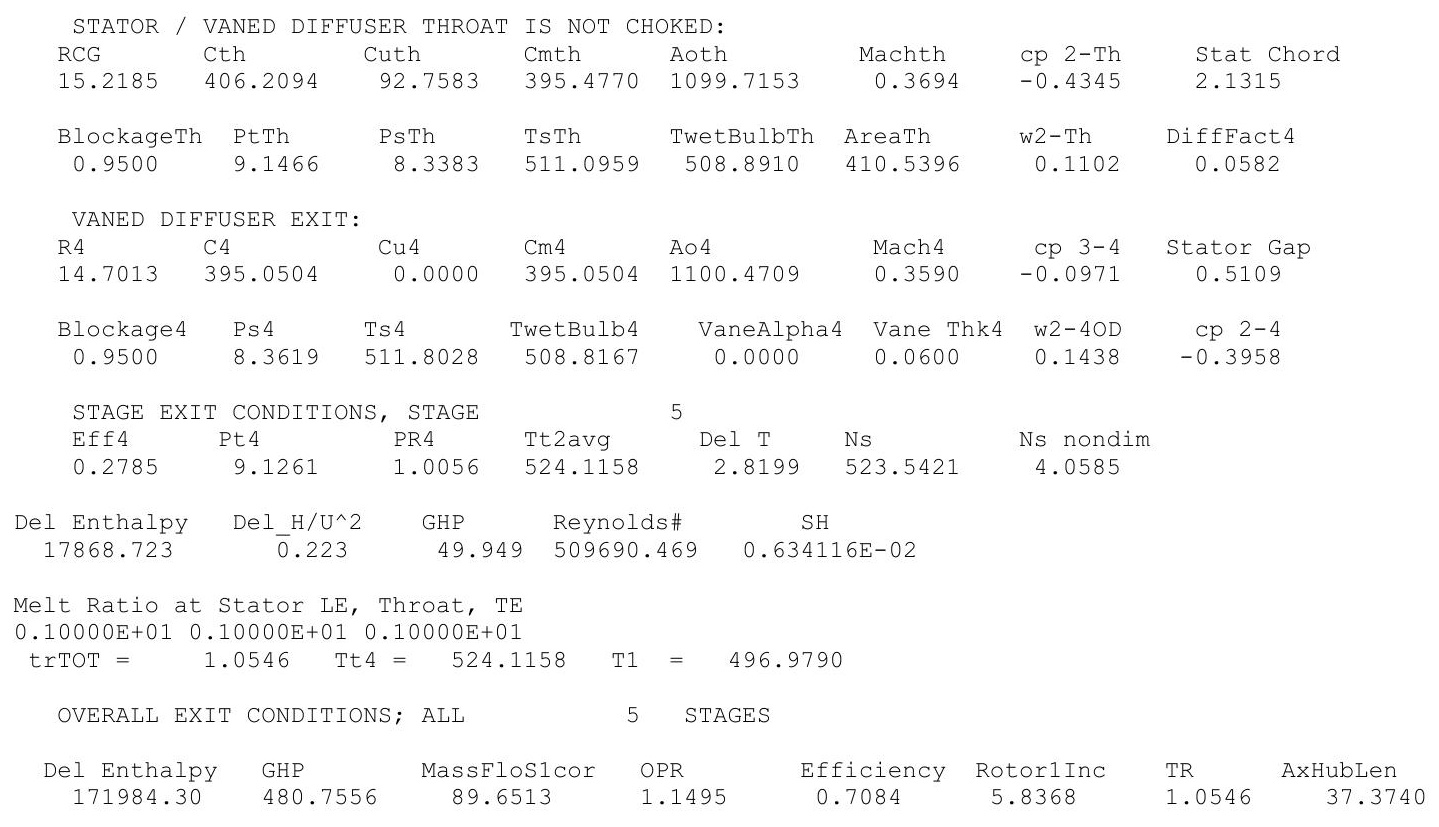




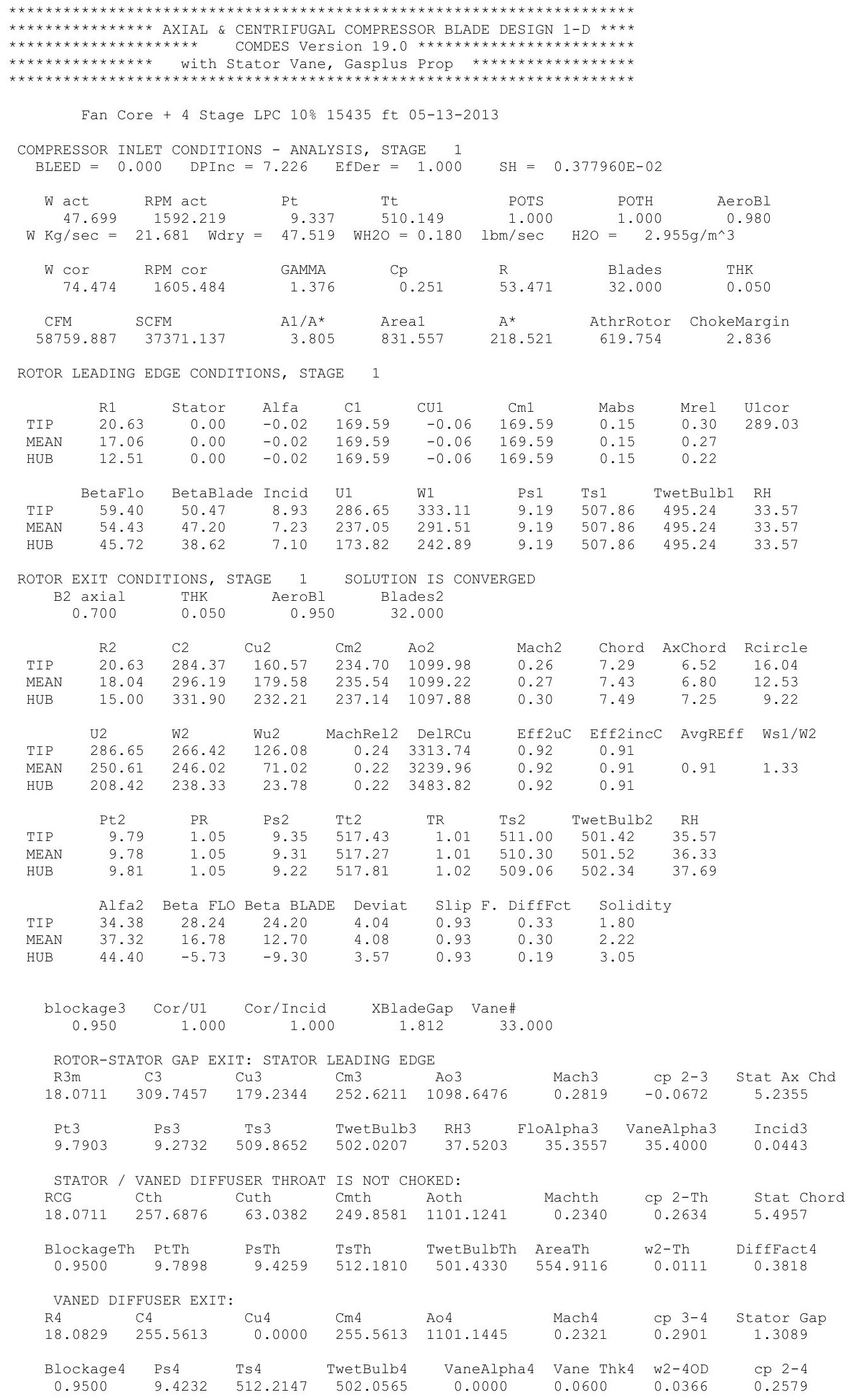




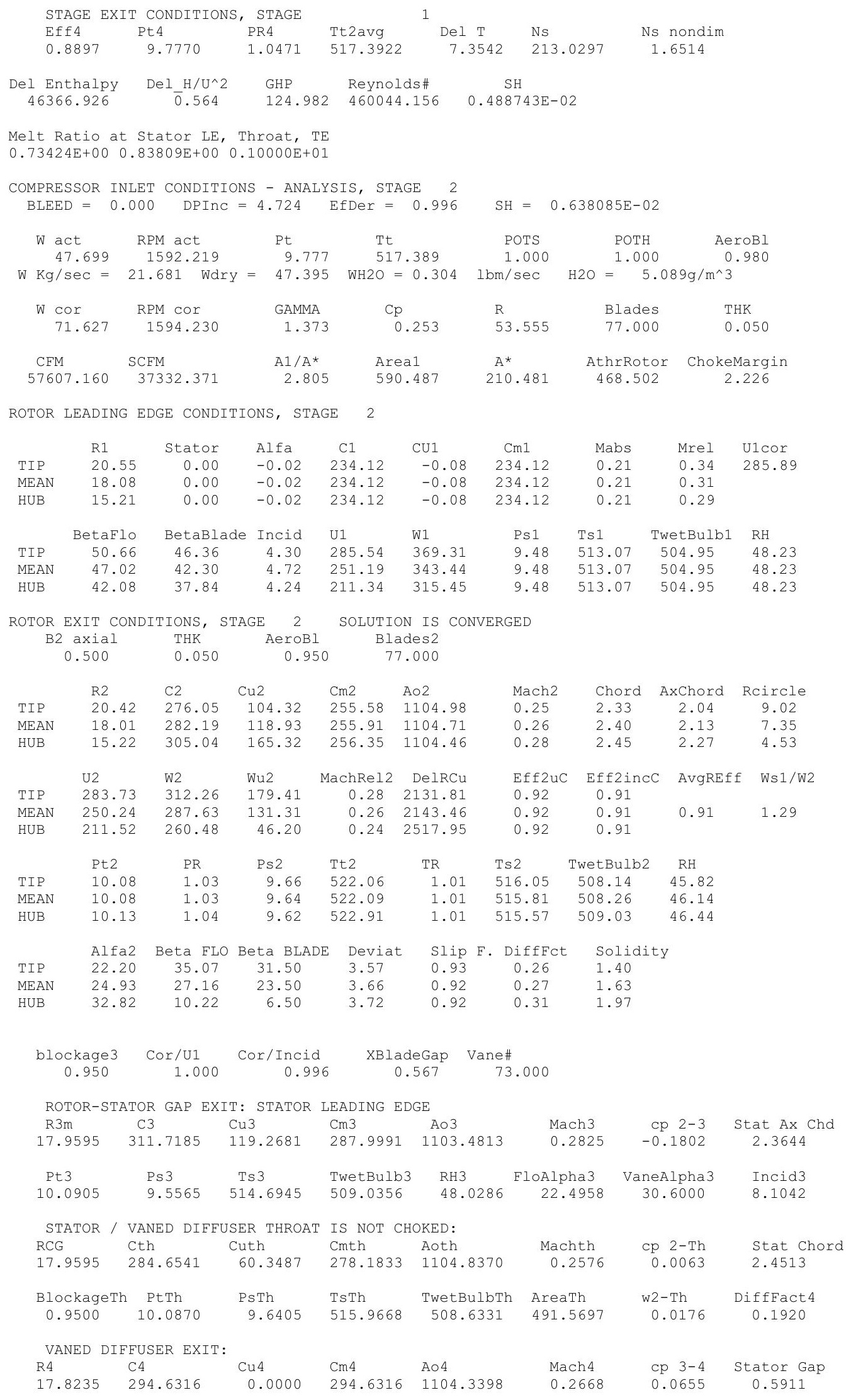




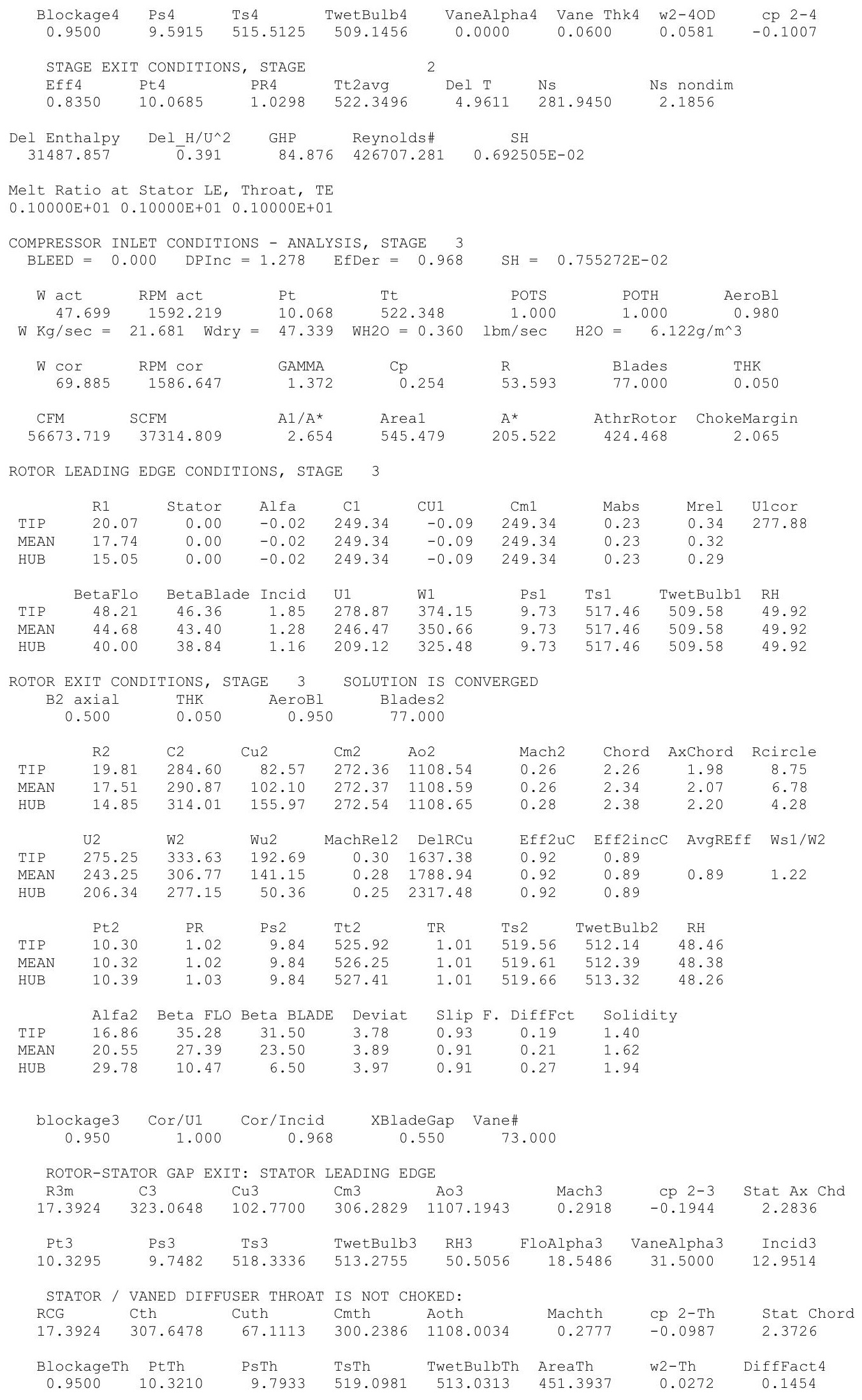




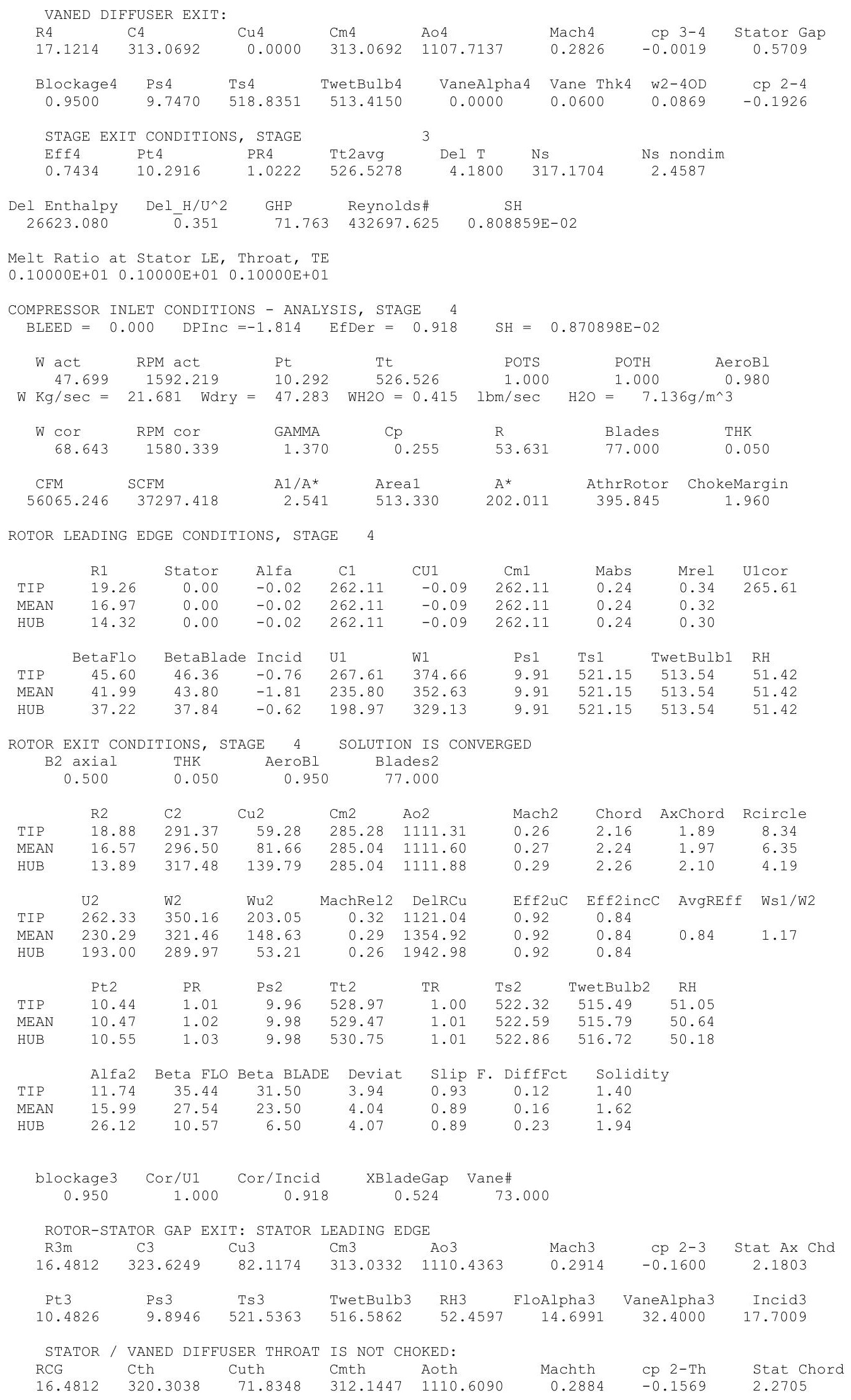




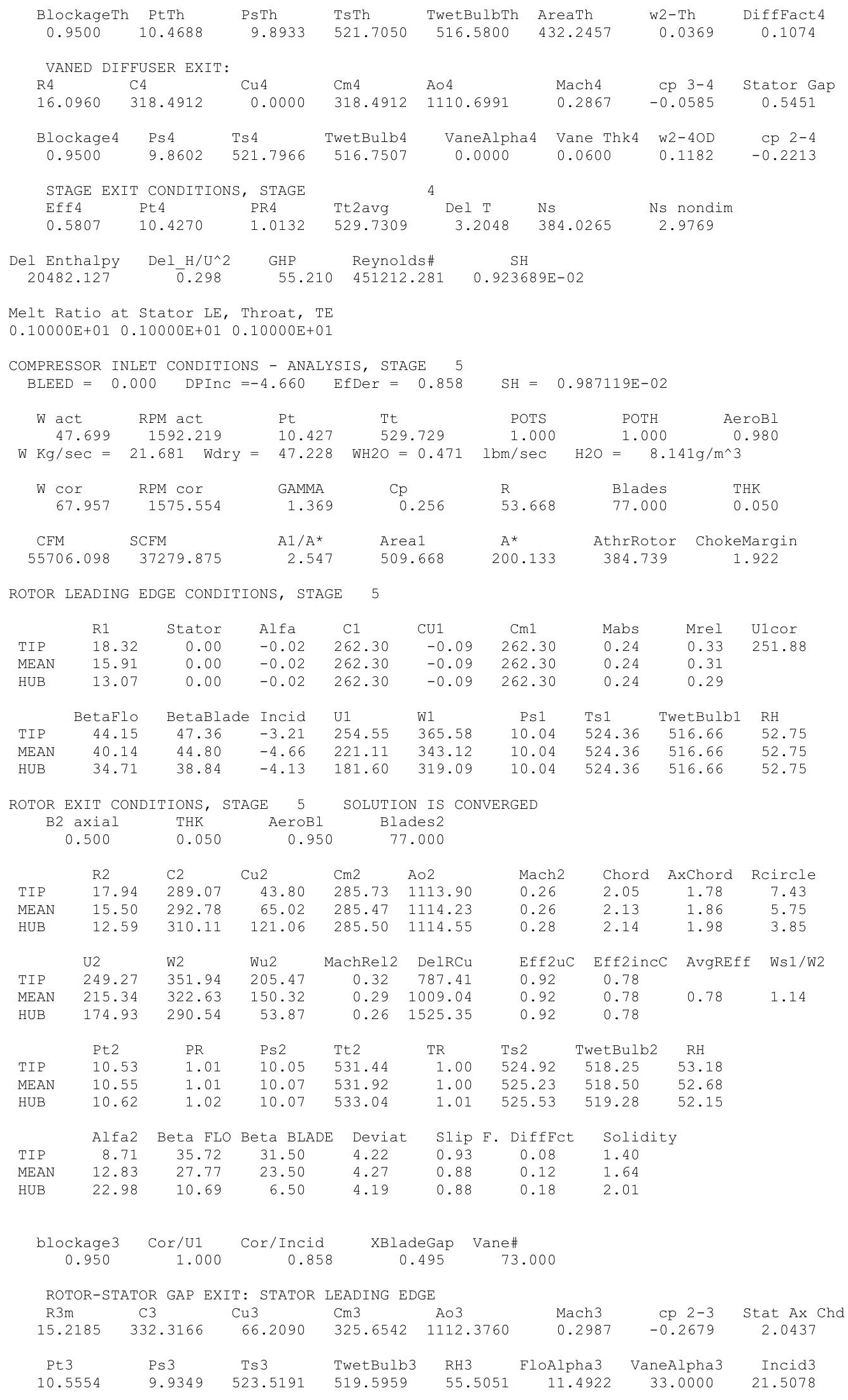




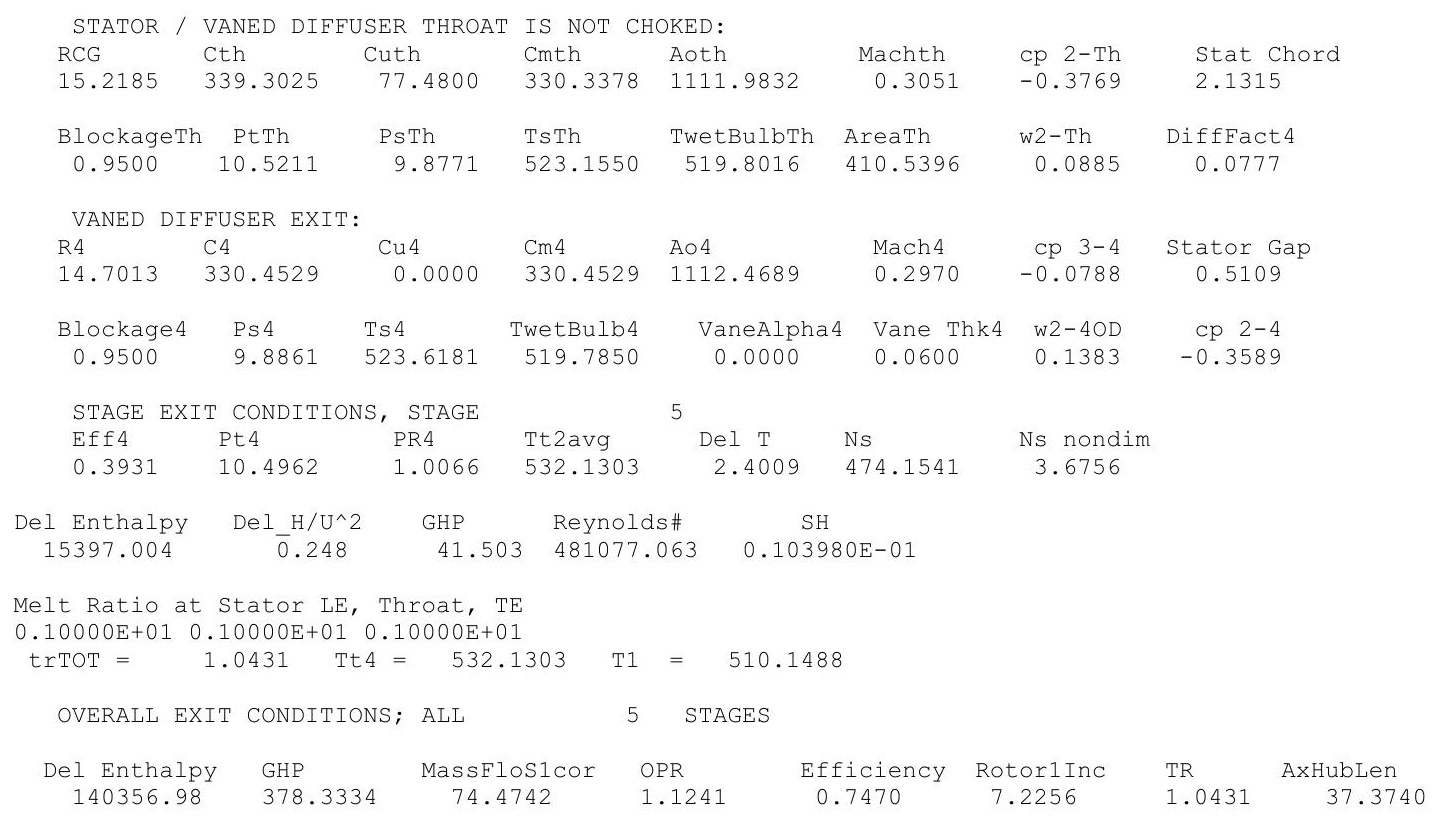


$5 \mu \mathrm{m}, 2 \mathrm{~g} / \mathrm{m}^{3}, \mathrm{ISA}+36 \mathrm{R}$

\begin{abstract}
AXIAL \& CENTRIFUGAL COMPRESSOR BLADE DESIGN $1-\mathrm{D} * \star * *$

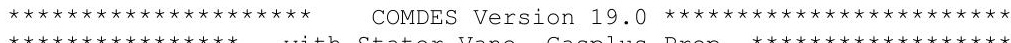

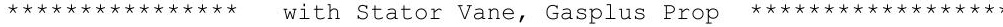

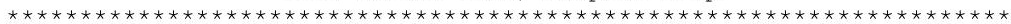

Fan Core + 4 Stage LPC 10\% 39000 Ft 05-13-2013

COMPRESSOR INLET CONDITIONS - ANALYSIS, STAGE 1

$\mathrm{BLEED}=0.000 \quad$ DPInC $=15.675 \quad$ EfDer $=0.953 \quad \mathrm{SH}=0.130125 \mathrm{E}-02$

$\begin{array}{lrrlllr}W \text { act } & \text { RPM act } & \text { Pt } & \text { Tt } & \text { POTS } & \text { POTH } & \text { AeroBl } \\ 25.912 & 2483.351 & 4.265 & 477.912 & 1.000 & 1.000 & 0.980\end{array}$

$\mathrm{W} \mathrm{Kg} / \mathrm{sec}=11.778$ Wdry $=25.878$ WH2O $=0.034 \quad \mathrm{lbm} / \mathrm{sec} \quad \mathrm{H} 2 \mathrm{O}=0.495 \mathrm{~g} / \mathrm{m}^{\wedge} 3$

$\begin{array}{llllll}W & \text { RPM cor } & \text { GAMMA } & \text { Cp } & \text { Blades } & \text { THK }\end{array}$

$\begin{array}{lll}1.380 & \mathrm{Cp} & \mathrm{R} \\ & 0.249 & 53.390\end{array}$

CFM SCFM A1/A* Area1 A* AthrRotor ChokeMargin

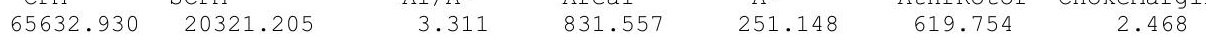

ROTOR LEADING EDGE CONDITIONS, STAGE 1

\begin{tabular}{|c|c|c|c|c|c|c|c|c|c|}
\hline & R1 & Stator & Alfa & $\mathrm{C} 1$ & CU1 & $\mathrm{Cm} 1$ & Mabs & Mrel & U1cor \\
\hline TIP & 20.63 & 0.00 & -0.02 & 189.43 & -0.07 & 189.43 & 0.18 & 0.46 & 465.75 \\
\hline MEAN & 17.06 & 0.00 & -0.02 & 189.43 & -0.07 & 189.43 & 0.18 & 0.39 & \\
\hline HUB & 12.51 & 0.00 & -0.02 & 189.43 & -0.07 & 189.43 & 0.18 & 0.31 & \\
\hline & BetaFlo & BetaBlade & Incid & U1 & W1 & Ps 1 & Ts 1 & TwetBulb1 & $\mathrm{RH}$ \\
\hline TIP & 67.04 & 50.47 & 16.57 & 447.08 & 485.61 & 4.17 & 475.04 & 464.31 & 19.8 \\
\hline MEAN & 62.88 & 47.20 & 15.68 & 369.72 & 415.48 & 4.17 & 475.04 & 464.31 & 19.8 \\
\hline HUB & 55.06 & 38.62 & 16.44 & 271.11 & 330.78 & 4.17 & 475.04 & 464.31 & 19.8 \\
\hline
\end{tabular}

ROTOR EXIT CONDITIONS, STAGE 1 SOLUTION IS CONVERGED

\begin{tabular}{|c|c|c|c|c|c|c|c|c|c|}
\hline & $\begin{array}{l}\text { axial } \\
700\end{array}$ & $\begin{array}{l}\text { THK } \\
0.050\end{array}$ & $\begin{array}{l}\text { AeroBl } \\
0.950\end{array}$ & $\begin{array}{r}\mathrm{Bla} \\
32\end{array}$ & $\begin{array}{l}\text { des } 2 \\
.000\end{array}$ & & & & \\
\hline & $\mathrm{R} 2$ & $\mathrm{C} 2$ & $\mathrm{Cu} 2$ & $\mathrm{Cm} 2$ & $\mathrm{~A} \circ 2$ & Mach2 & Chord & AxChord & Rcircle \\
\hline TIP & 20.63 & 400.12 & 309.72 & 253.32 & 1074.72 & 0.37 & 7.29 & 6.52 & 16.04 \\
\hline MEAN & 18.04 & 404.43 & 312.50 & 256.72 & 1071.52 & 0.38 & 7.43 & 6.80 & 12.53 \\
\hline HUB & 15.00 & 437.42 & 350.72 & 261.41 & 1067.61 & 0.41 & 7.49 & 7.25 & 9.22 \\
\hline & $\mathrm{U} 2$ & W2 & Wu2 & MachRel2 & DelRCu & Eff $2 u C$ & Eff2incC & AvgREff & Ws $1 /$ W2 \\
\hline TIP & 447.08 & 288.17 & 137.36 & 0.27 & 6390.87 & 0.92 & 0.87 & & \\
\hline MEAN & 390.86 & 268.42 & 78.37 & 0.25 & 5637.31 & 0.92 & 0.87 & 0.87 & 1.78 \\
\hline HUB & 325.07 & 262.66 & 25.65 & 0.25 & 5261.58 & 0.92 & 0.87 & & \\
\hline & Pt2 & $\mathrm{PR}$ & Ps2 & Tt2 & $\mathrm{TR}$ & Ts2 & TwetBulb2 & RH & \\
\hline TIP & 4.93 & 1.16 & 4.48 & 500.11 & 1.05 & 487.28 & 479.32 & 18.34 & \\
\hline SAN & 4.84 & 1.14 & 4.40 & 497.49 & 1.04 & 484.38 & 478.15 & 20.29 & \\
\hline JB & 4.80 & 1.13 & 4.29 & 496.19 & 1.04 & 480.85 & 478.02 & 22.95 & \\
\hline & Alfa2 & Beta FLO & Beta BLADE & Deviat & Slip & F. DiffFct & Solidit & & \\
\hline & 50.72 & 28.47 & 24.20 & 4.27 & 0.93 & 0.58 & 1.80 & & \\
\hline AN & 50.60 & 16.98 & 12.70 & 4.28 & 0.95 & 0.53 & 2.22 & & \\
\hline & 53.30 & -5.60 & -9.30 & 3.70 & 0.95 & 0.40 & 3.05 & & \\
\hline
\end{tabular}

$\begin{array}{ccccc}\text { blockage } & \text { Cor/U1 } & \text { Cor/Incid } & \text { XBladeGap } & \text { Vane\# } \\ 0.950 & 1.000 & 0.953 & 1.812 & 33.000\end{array}$

ROTOR-STATOR GAP EXIT: STATOR LEADING EDGE

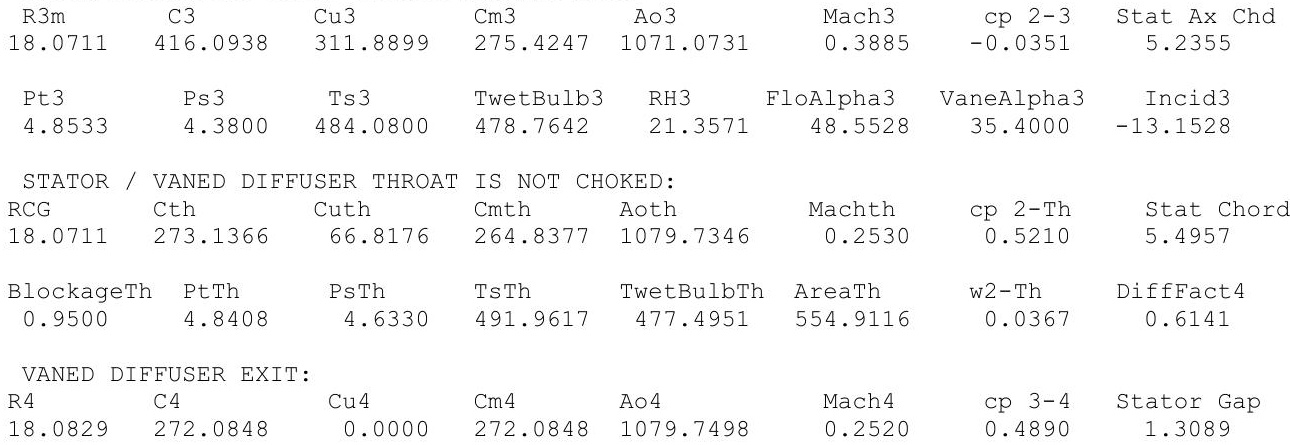




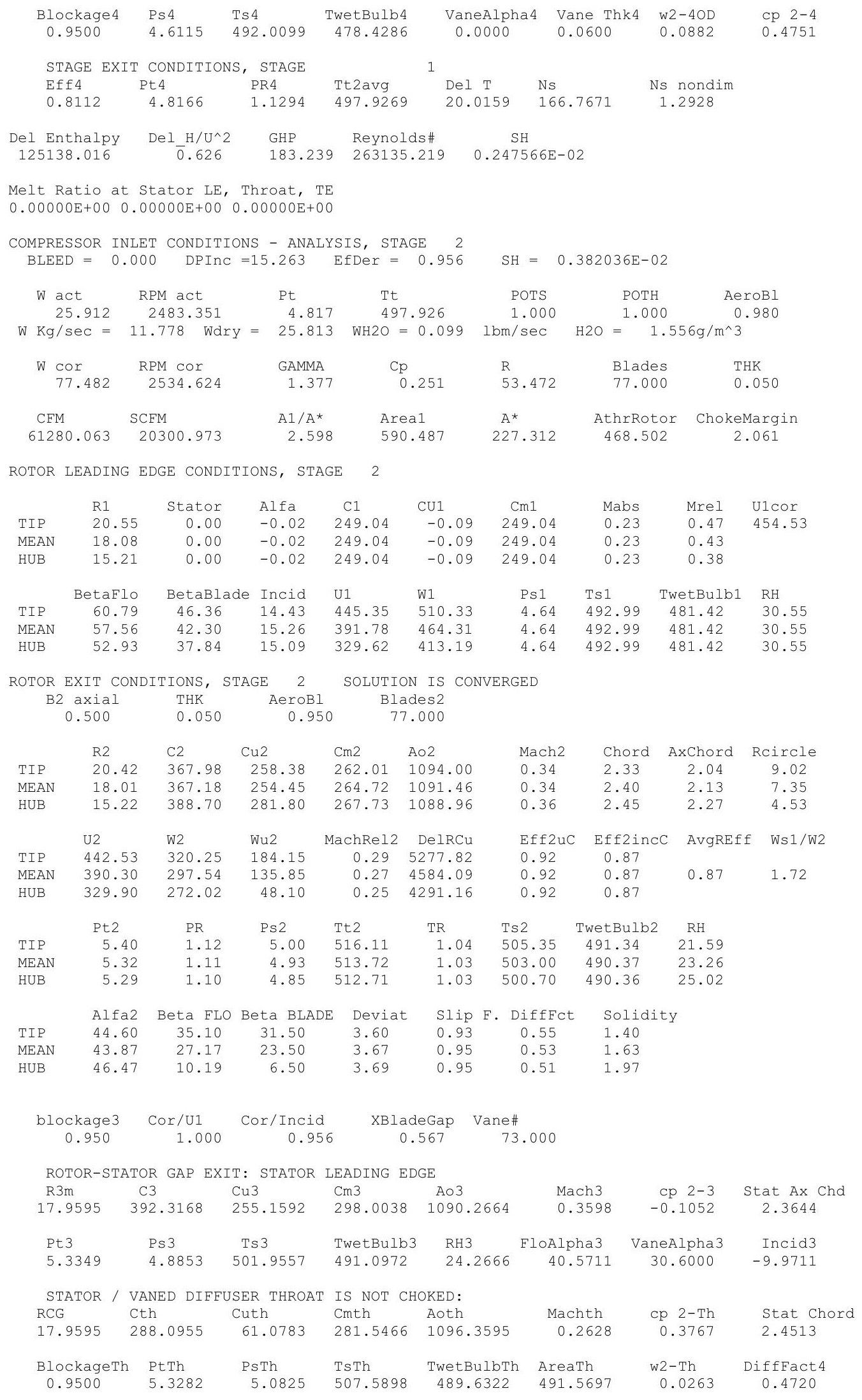




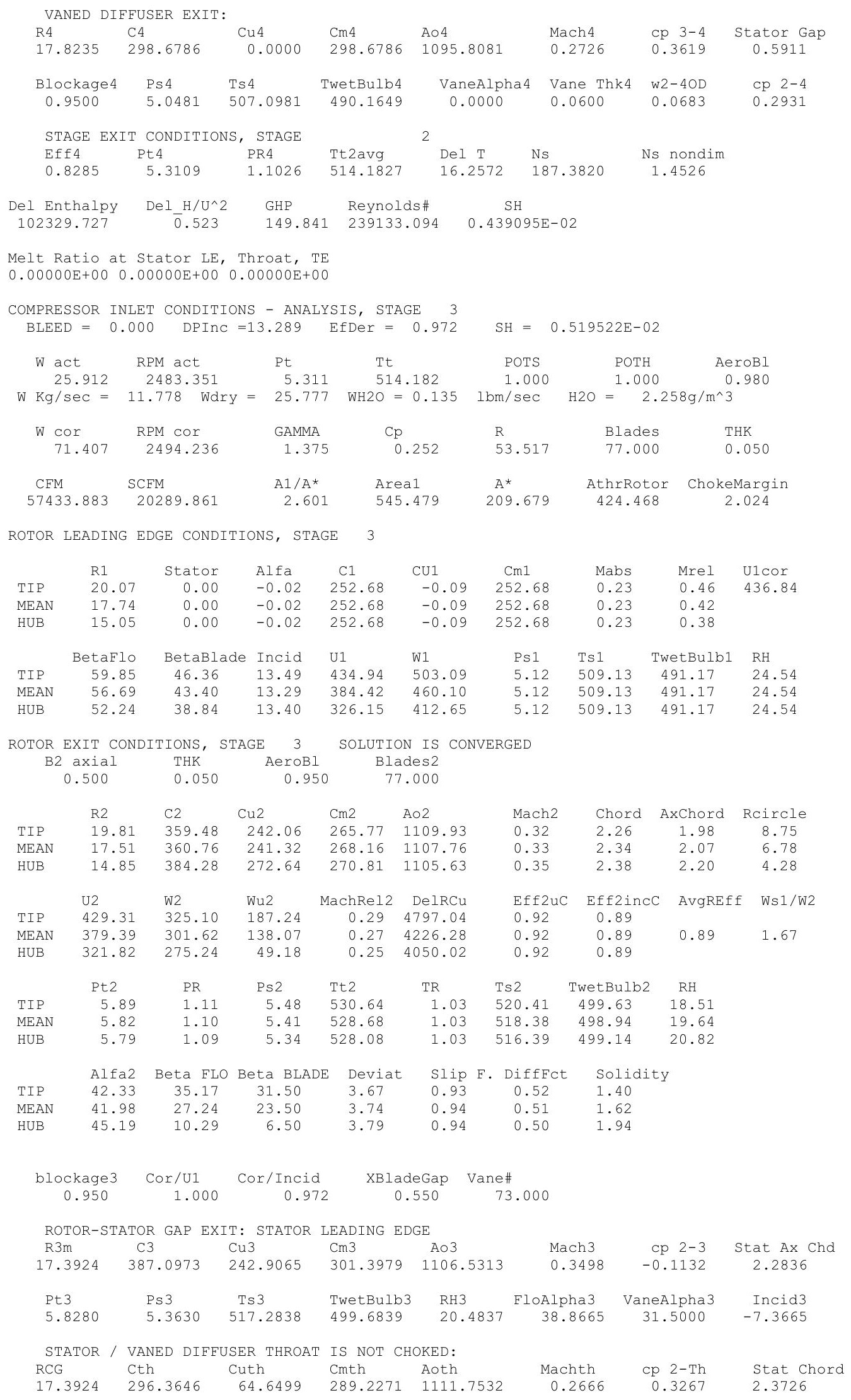




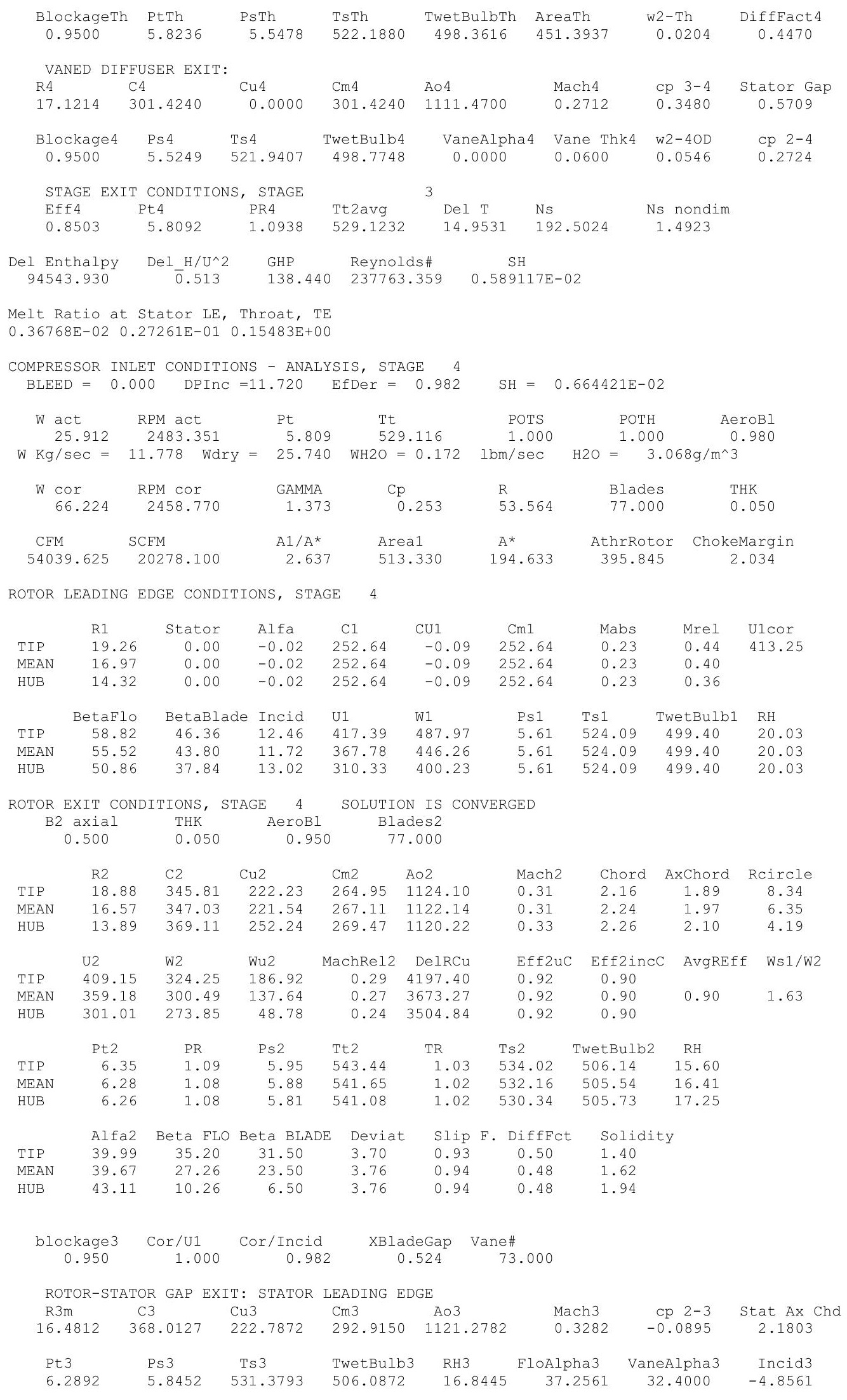




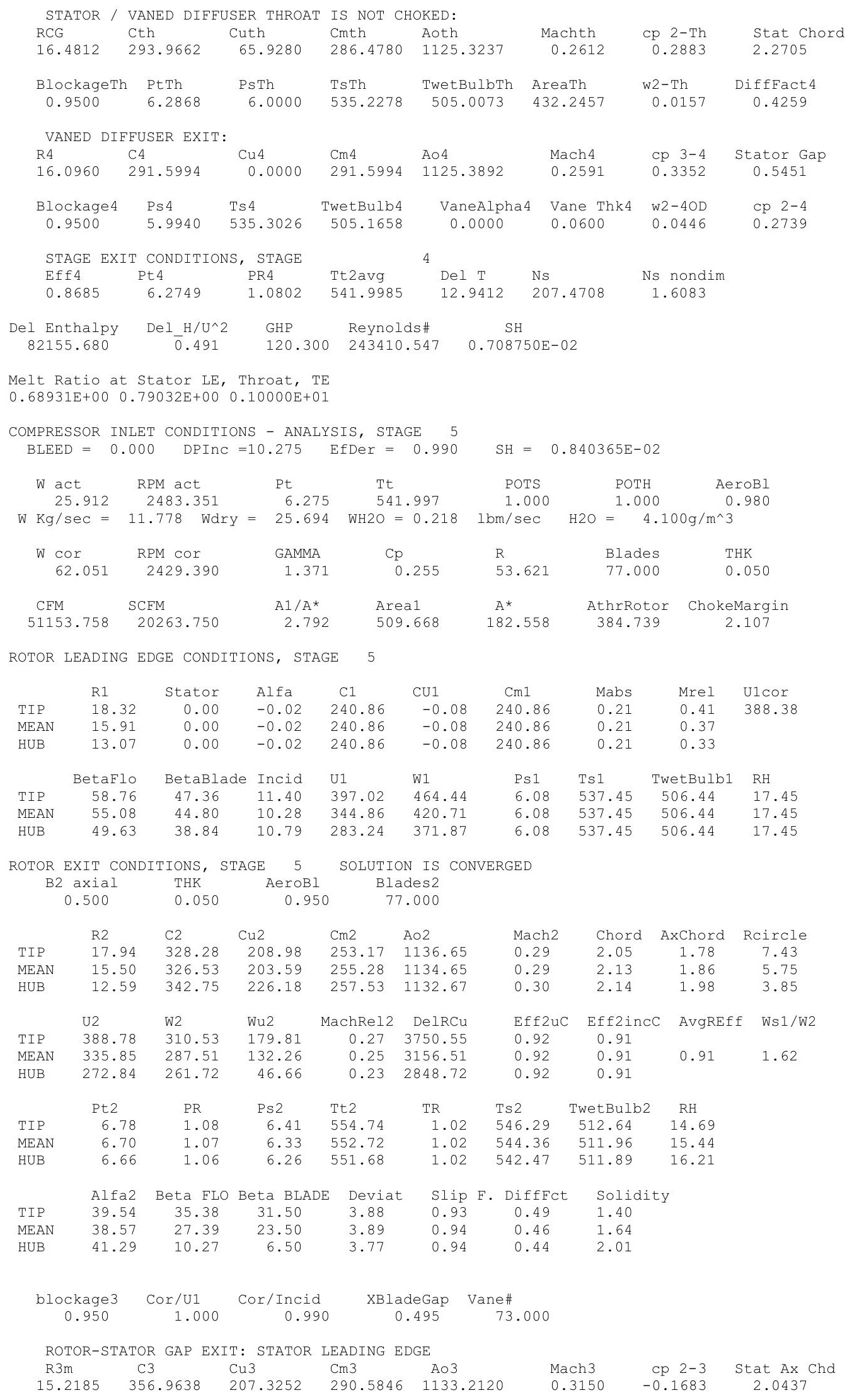




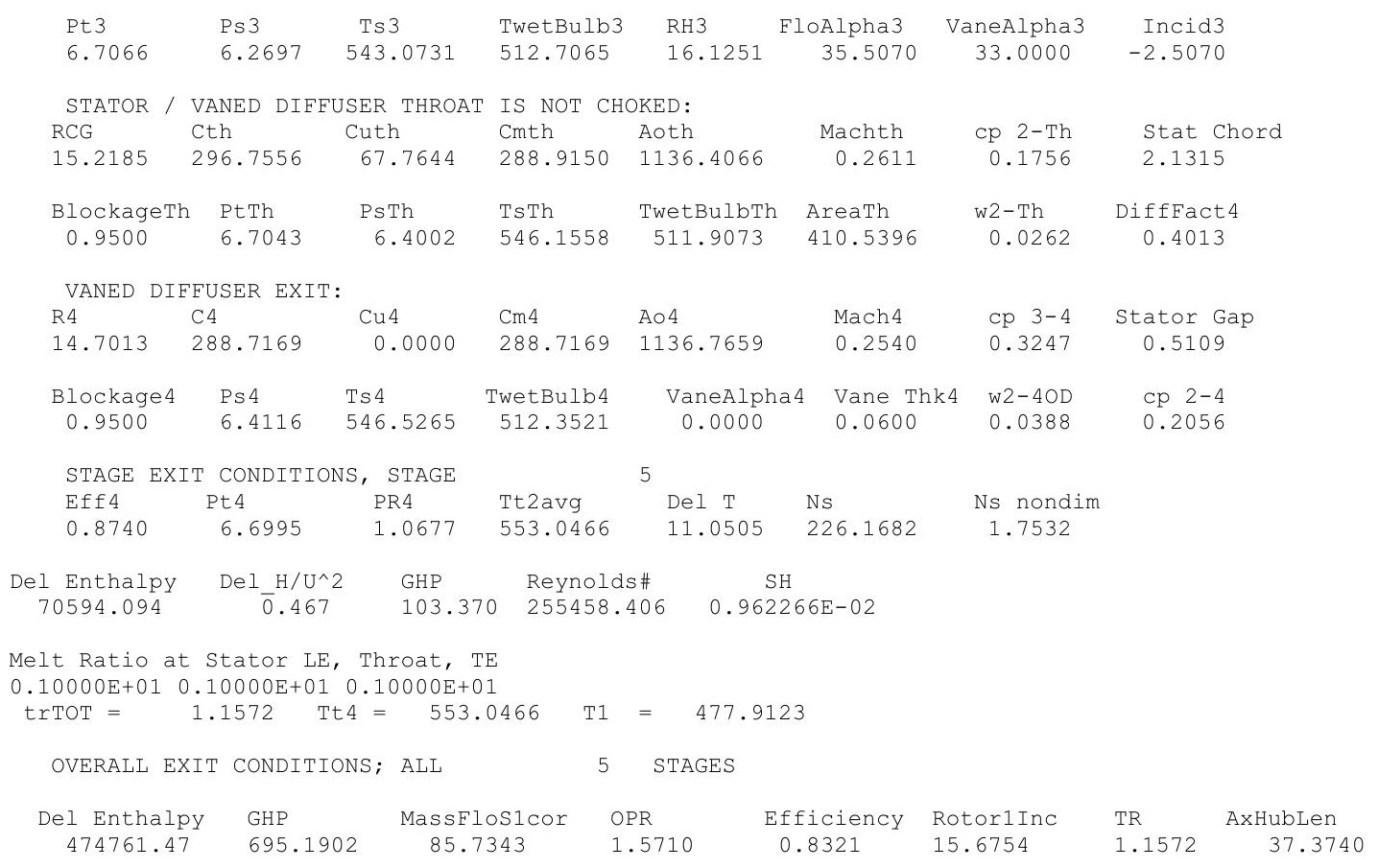




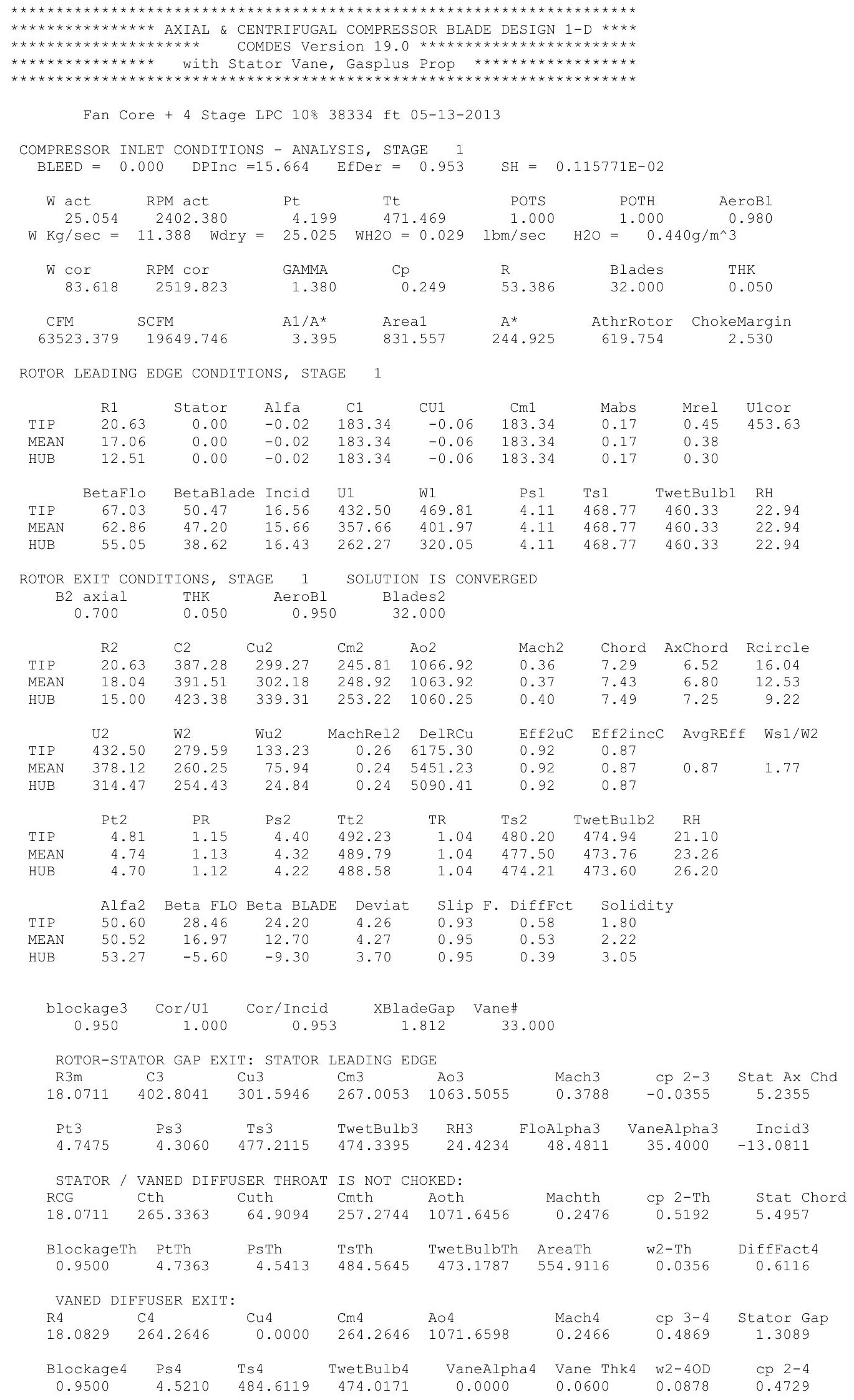




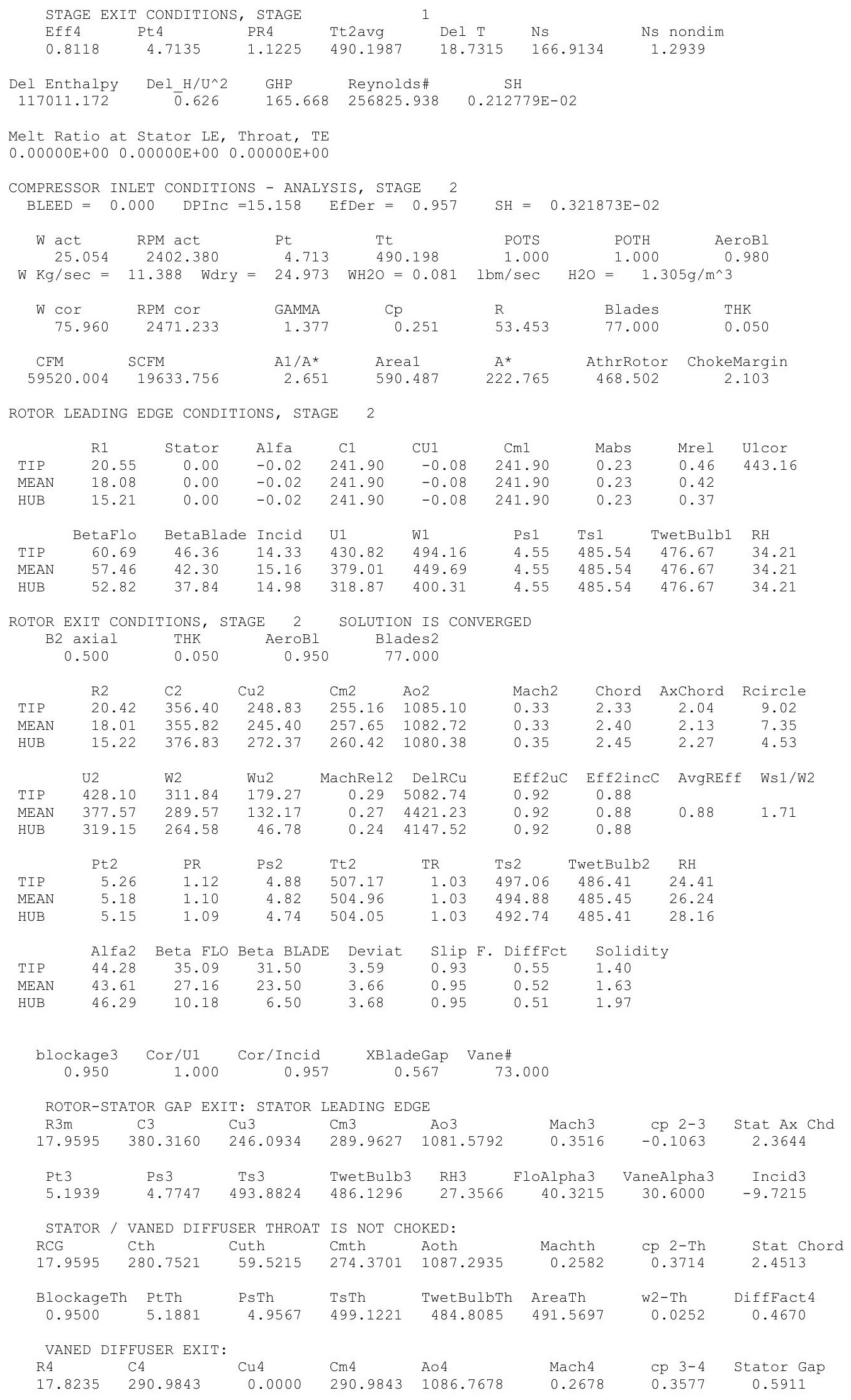




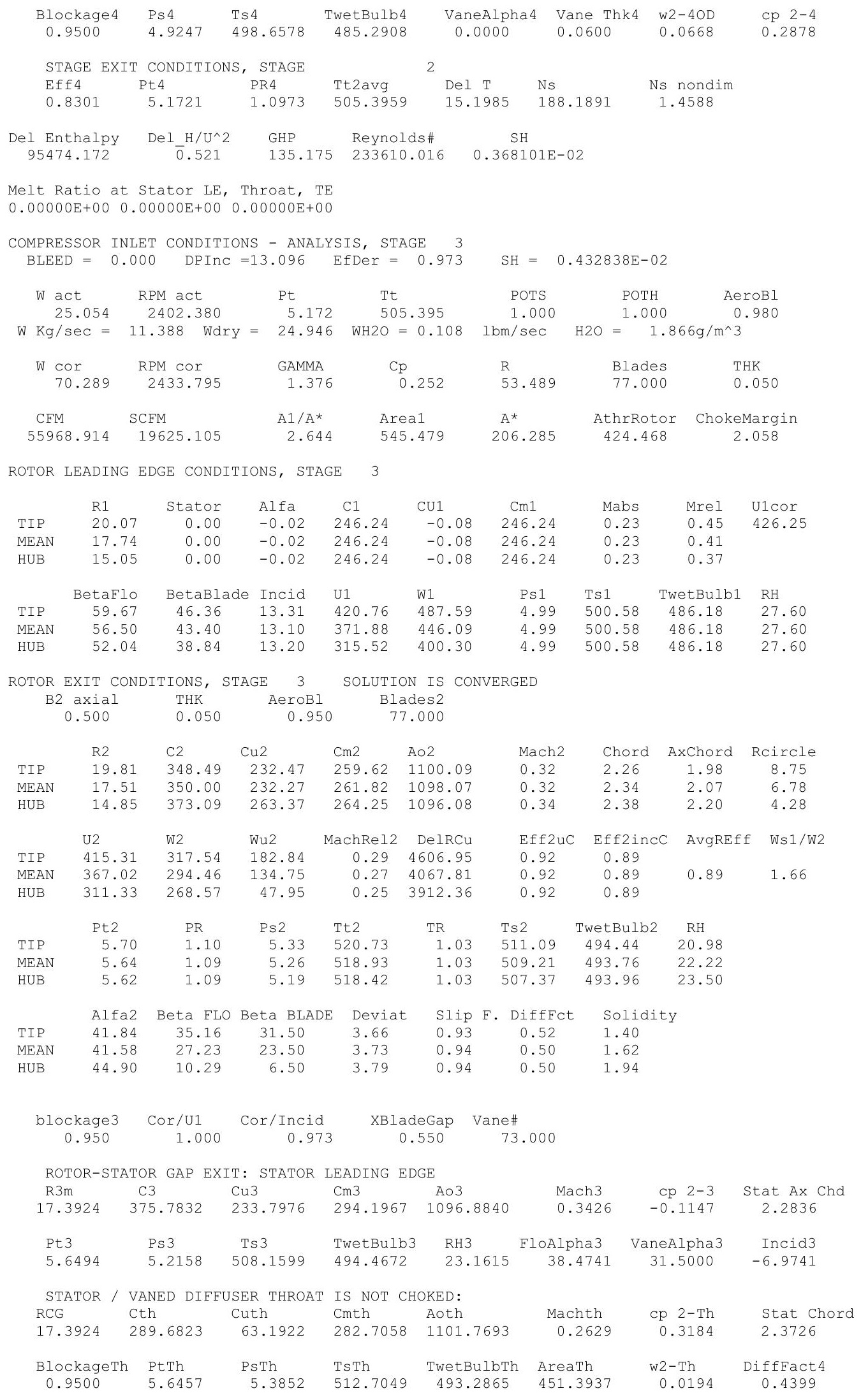




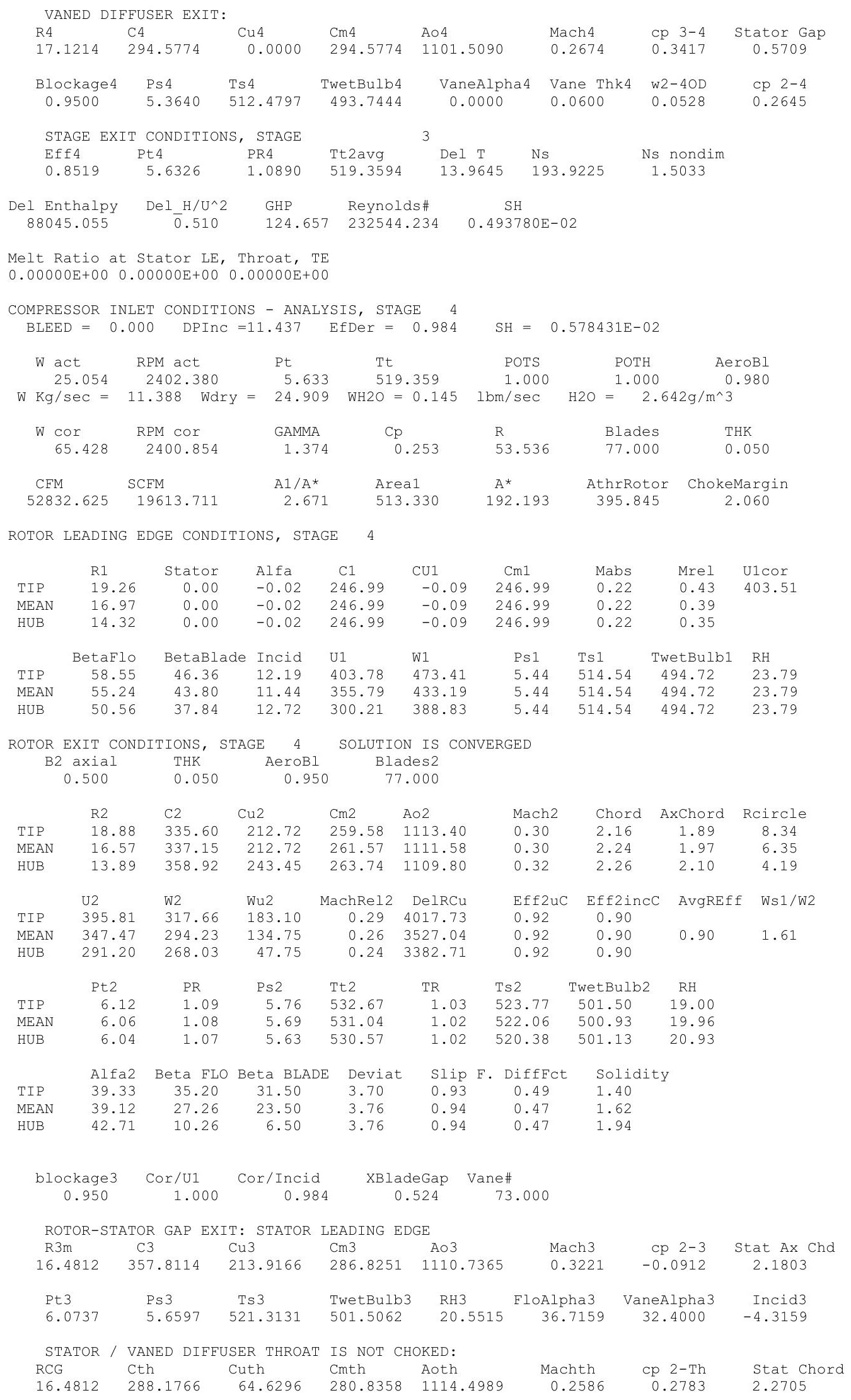




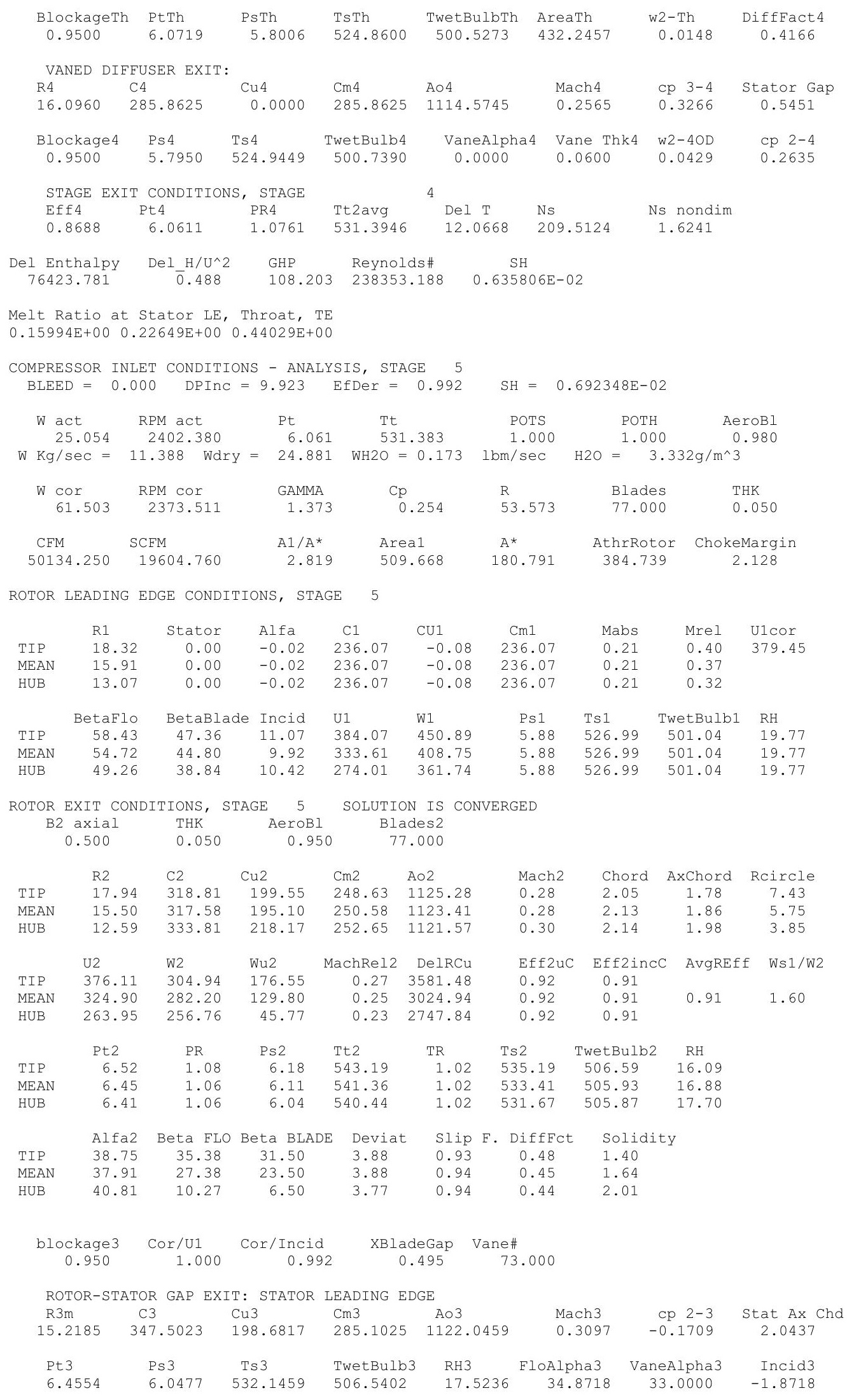




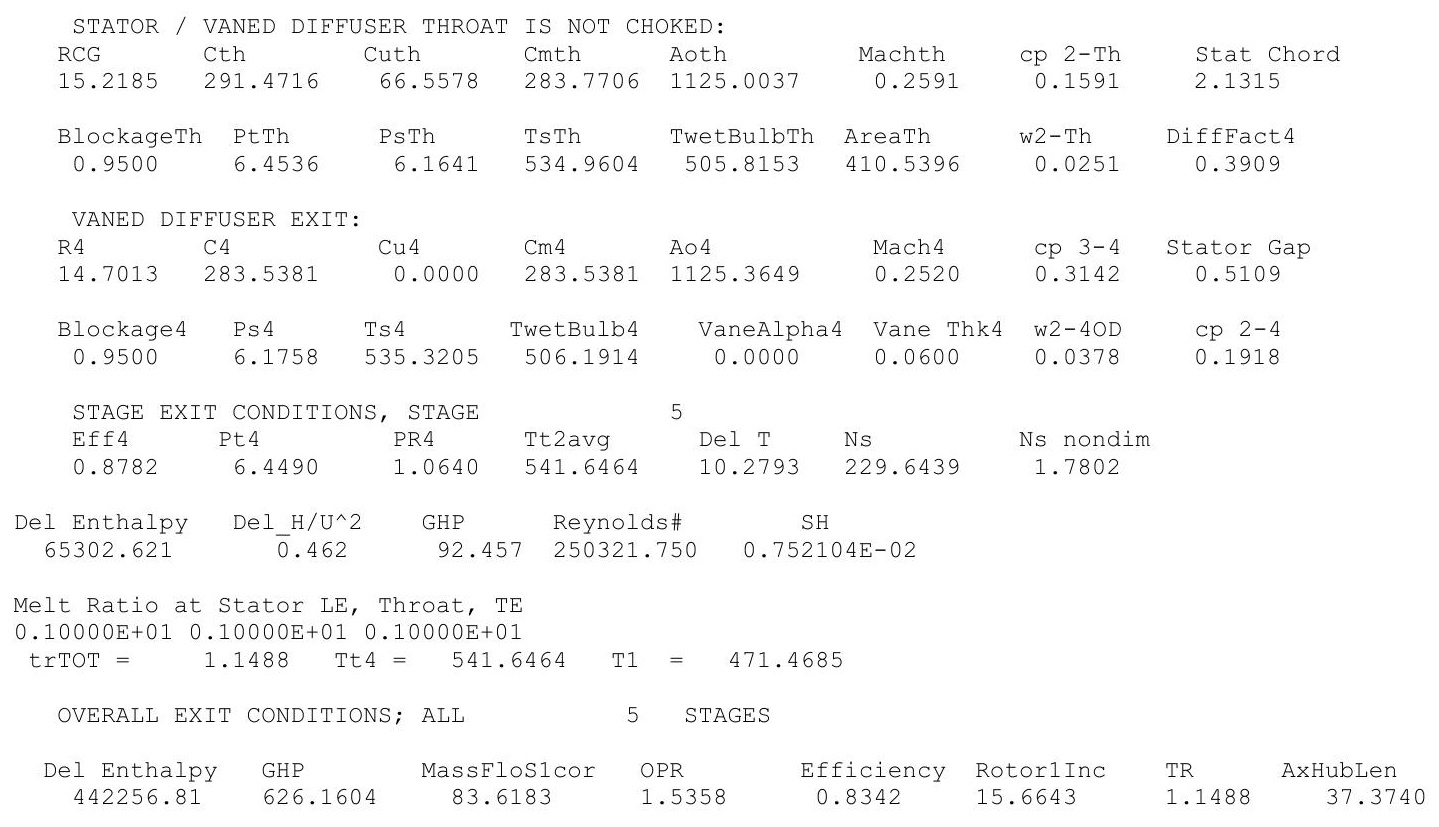




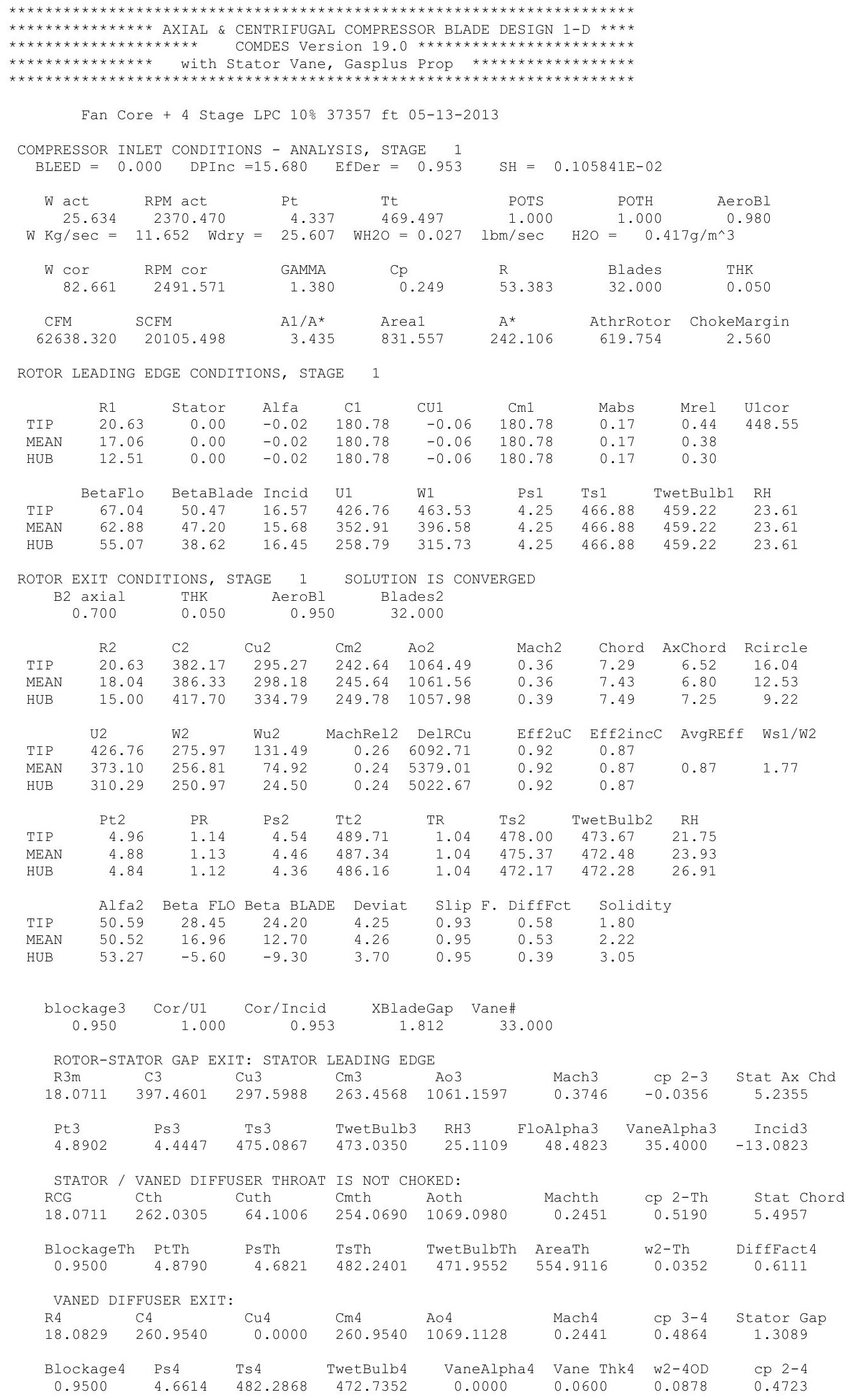




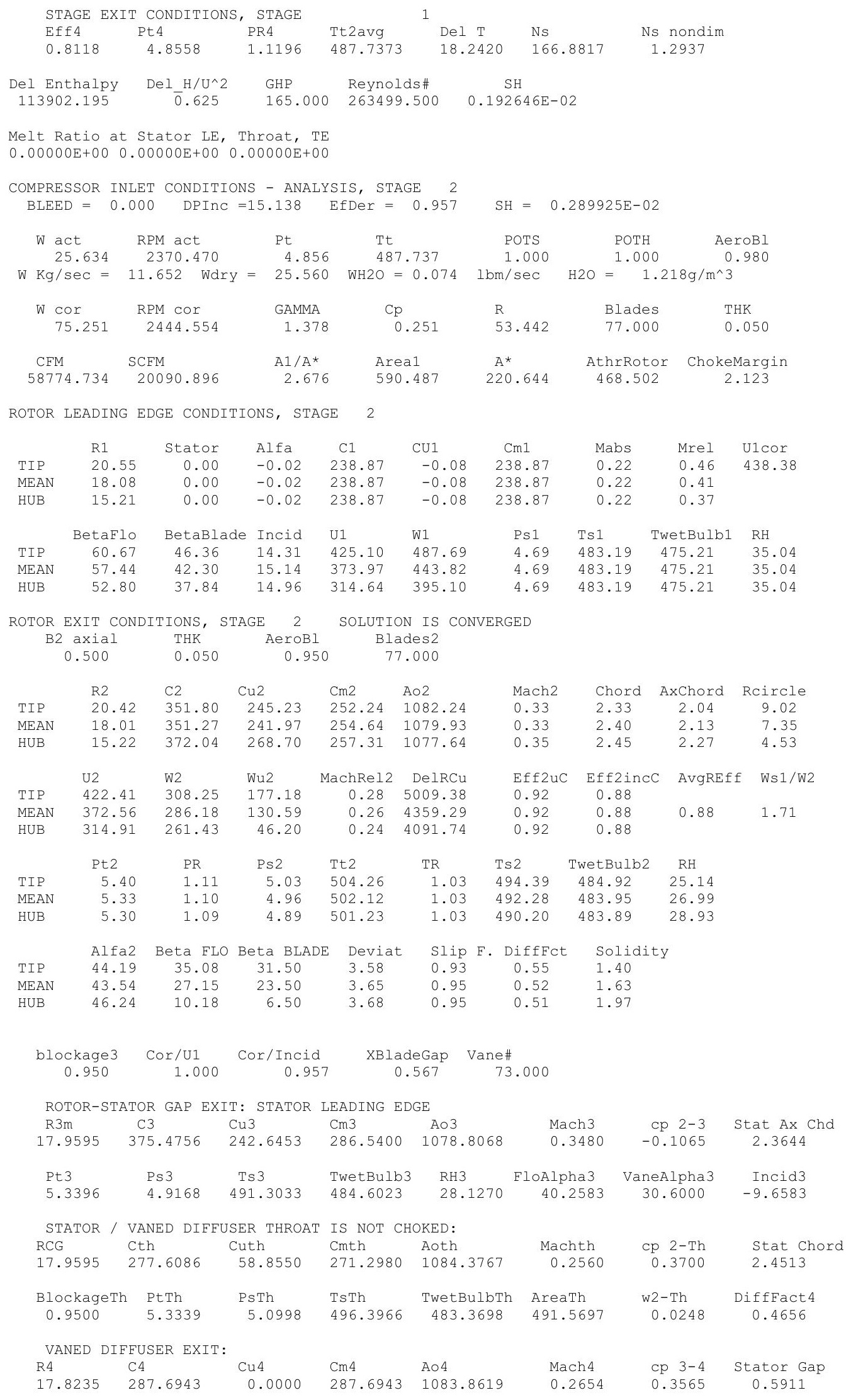




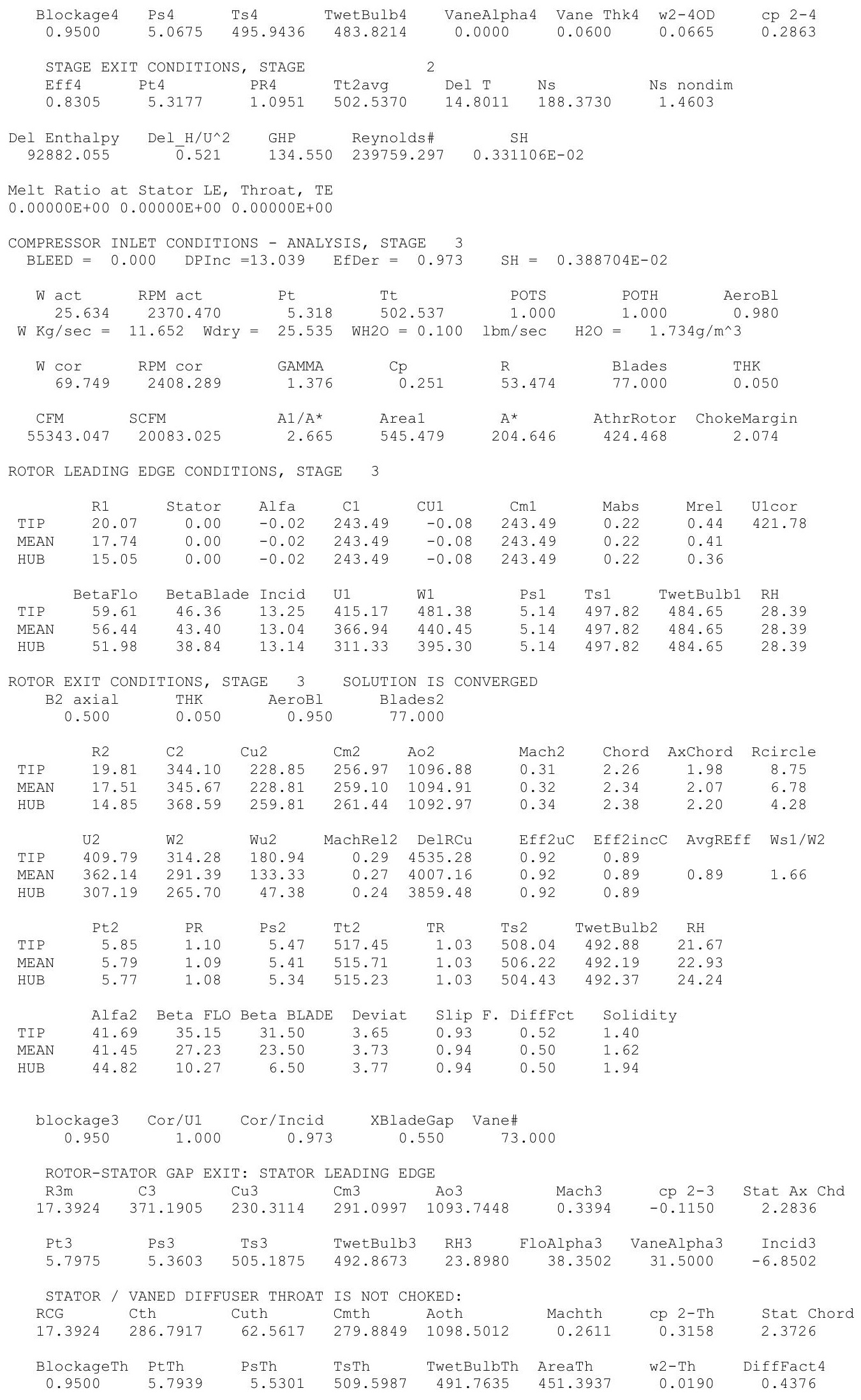




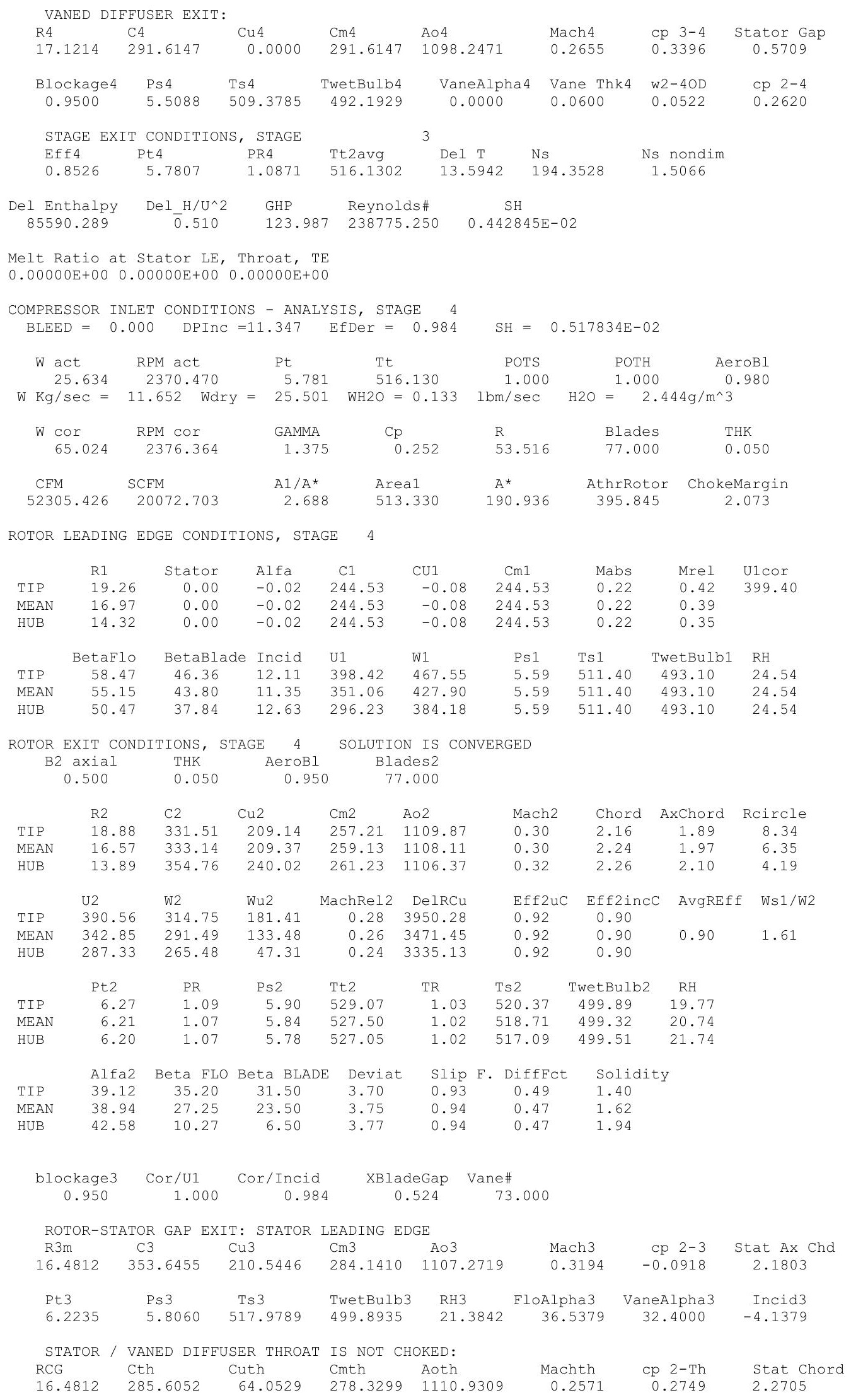




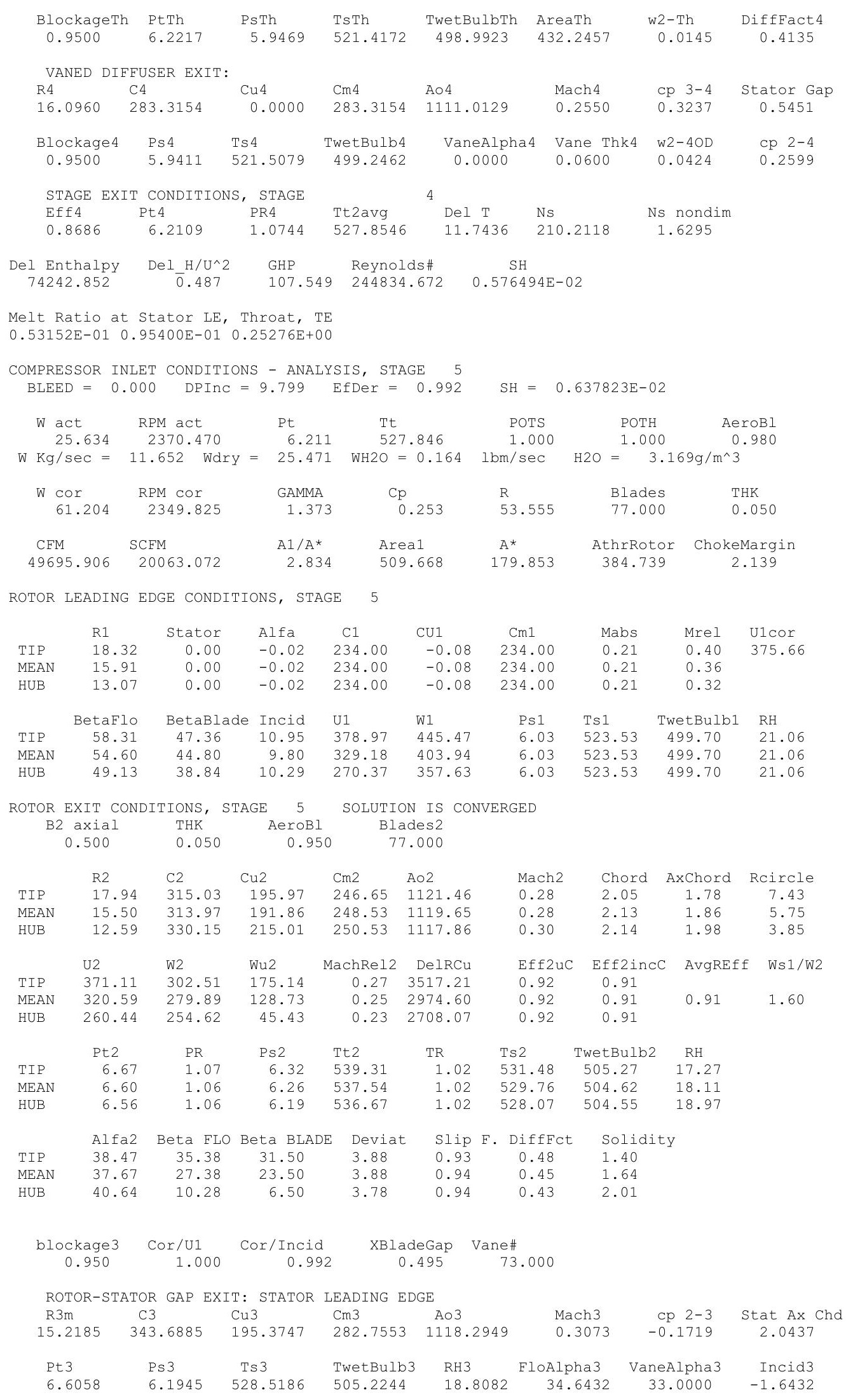




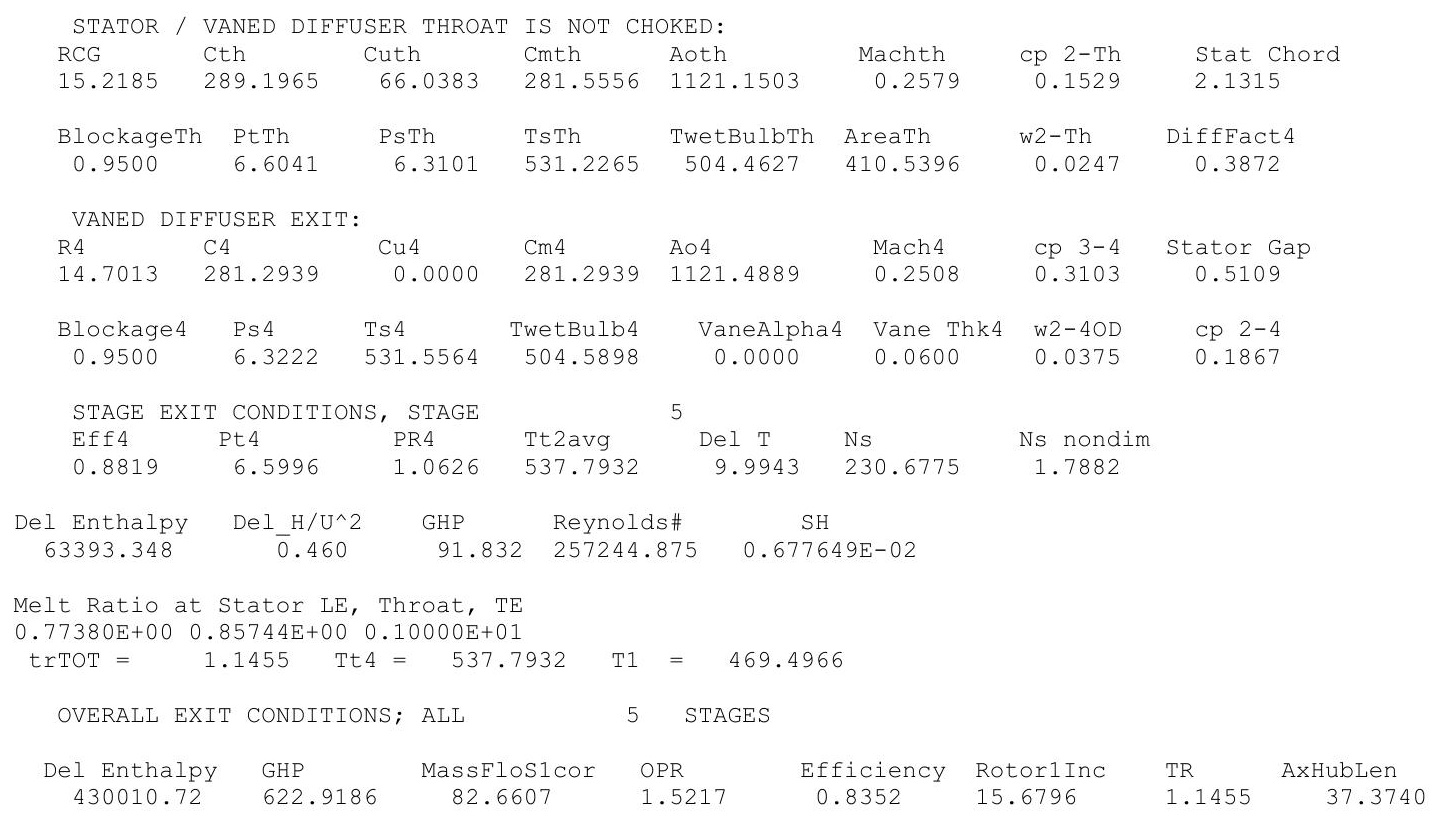




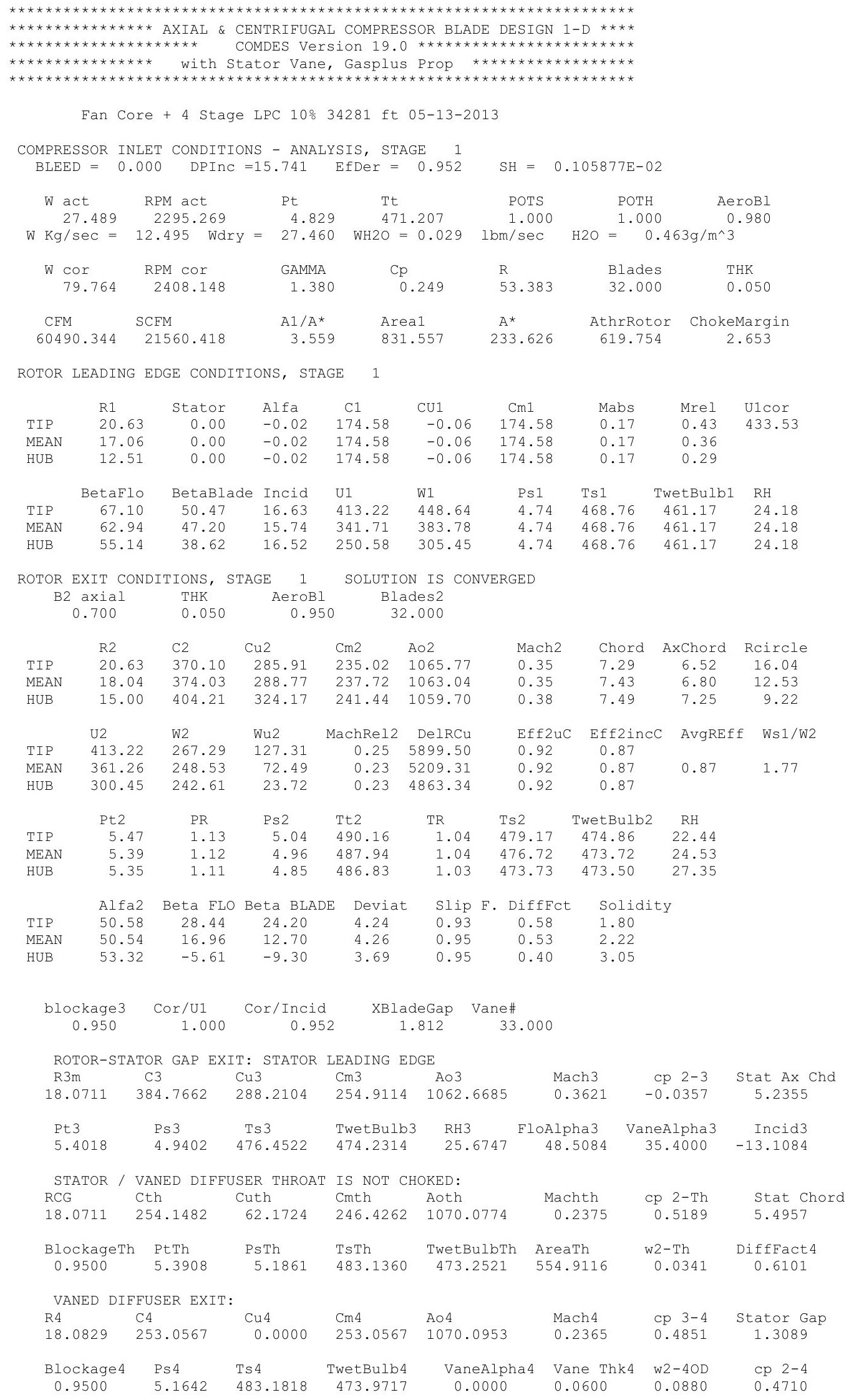




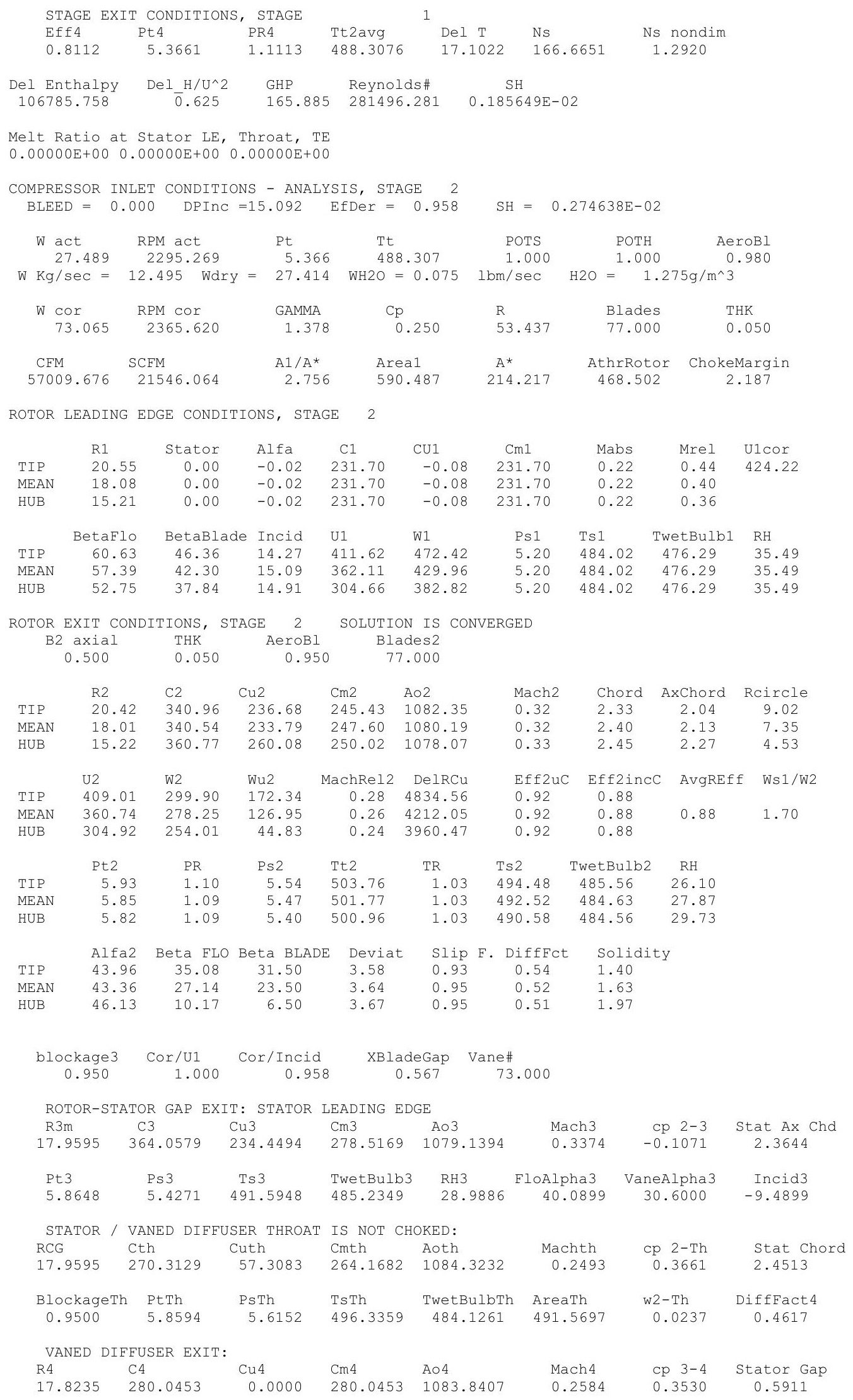




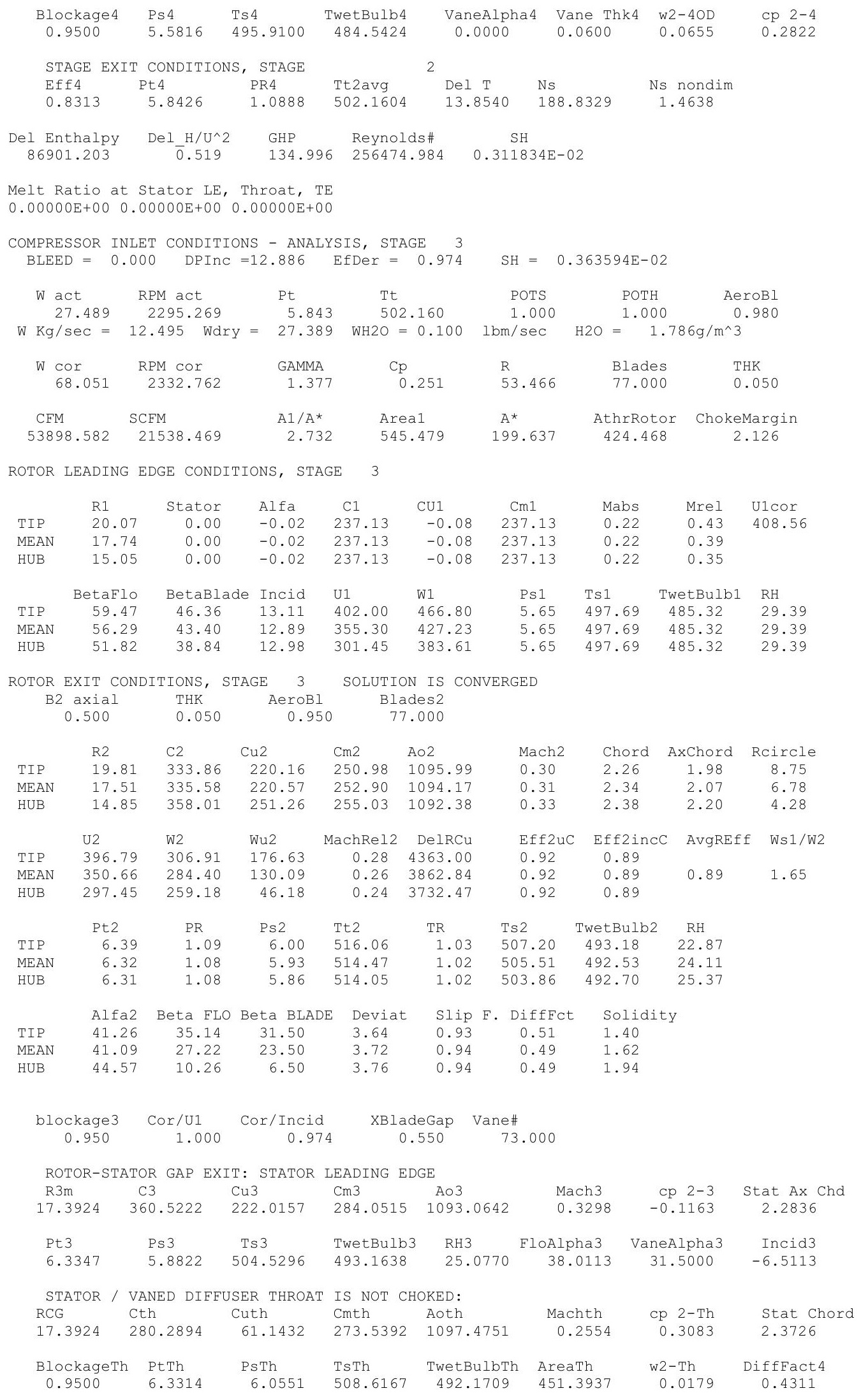




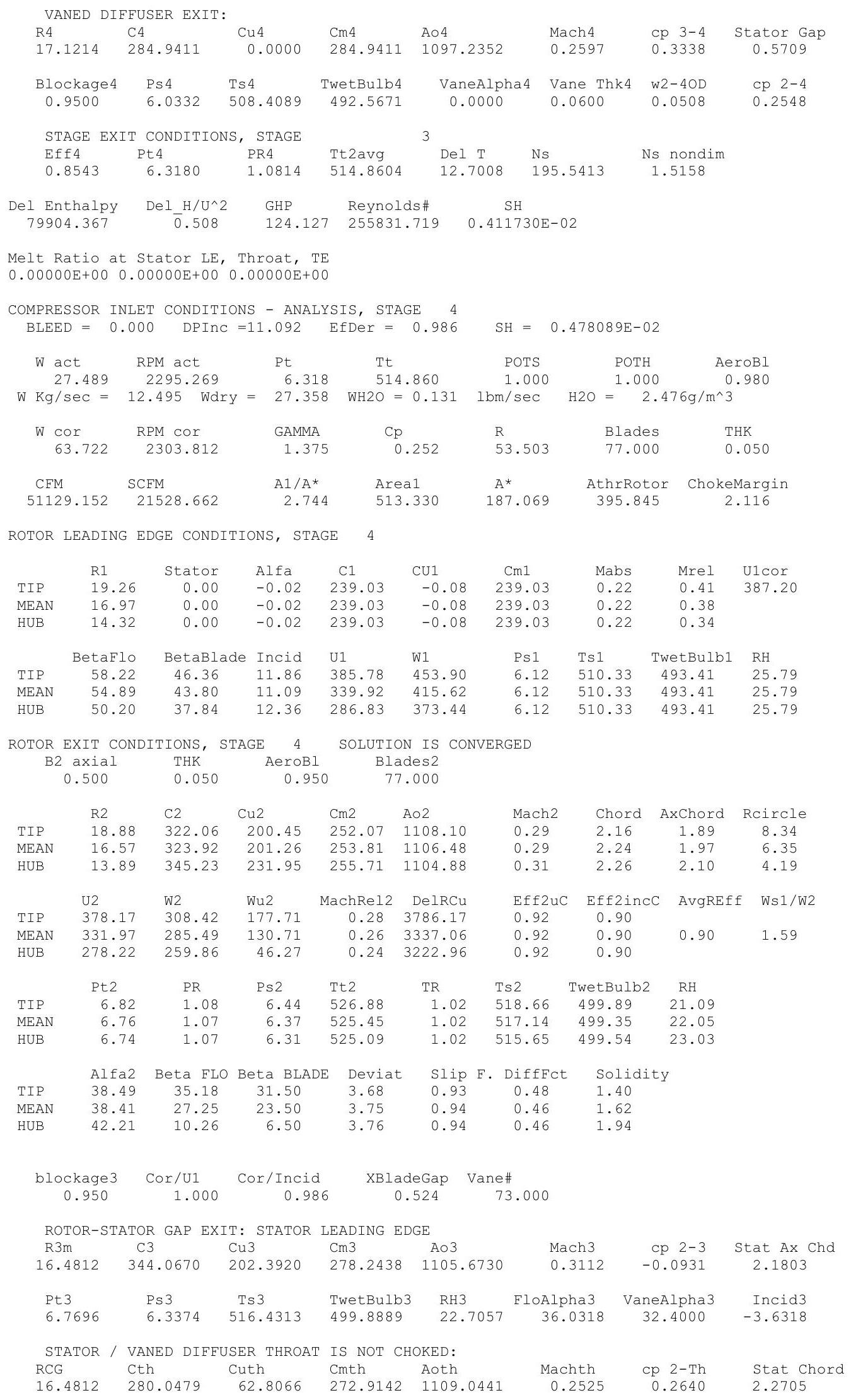




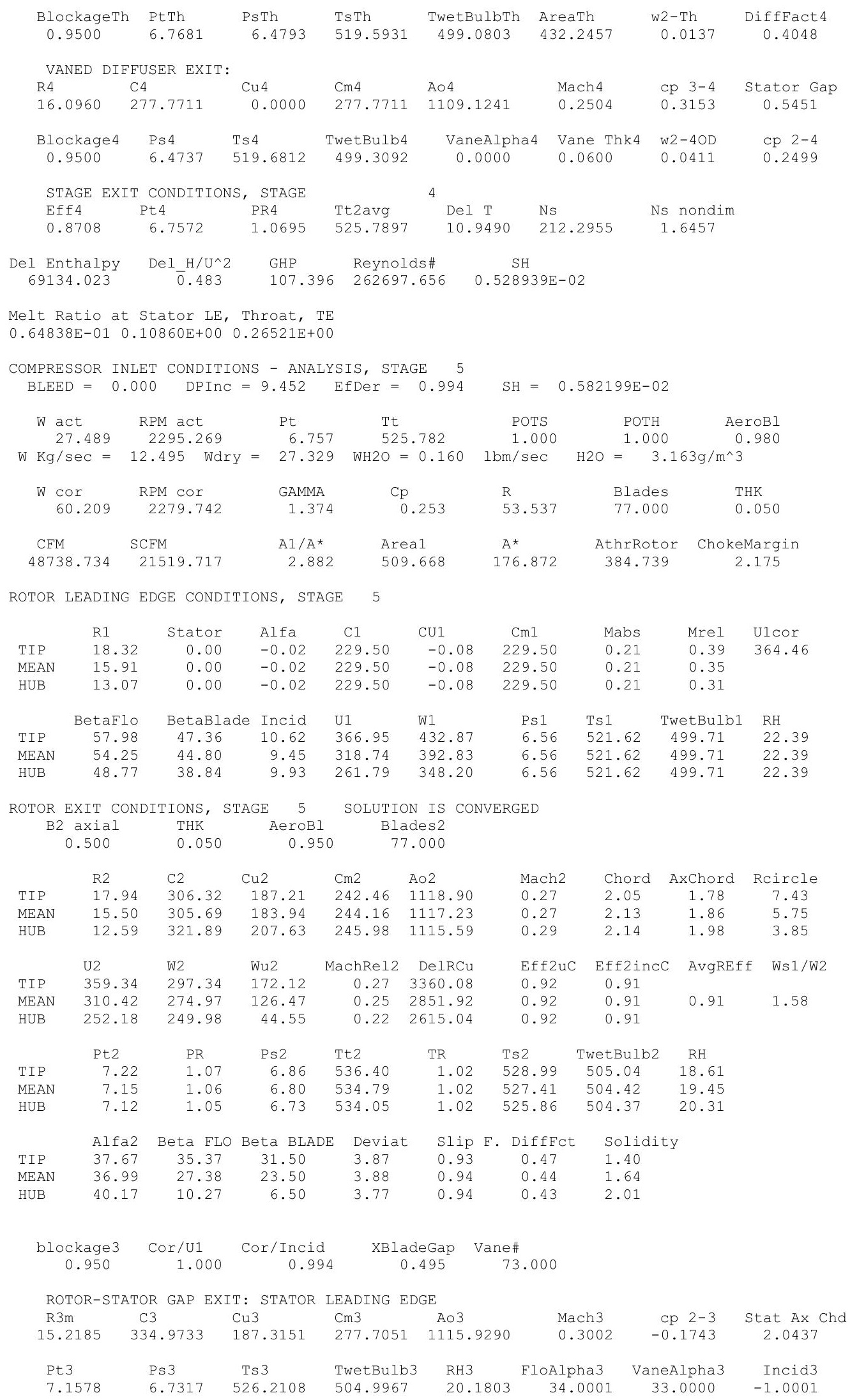




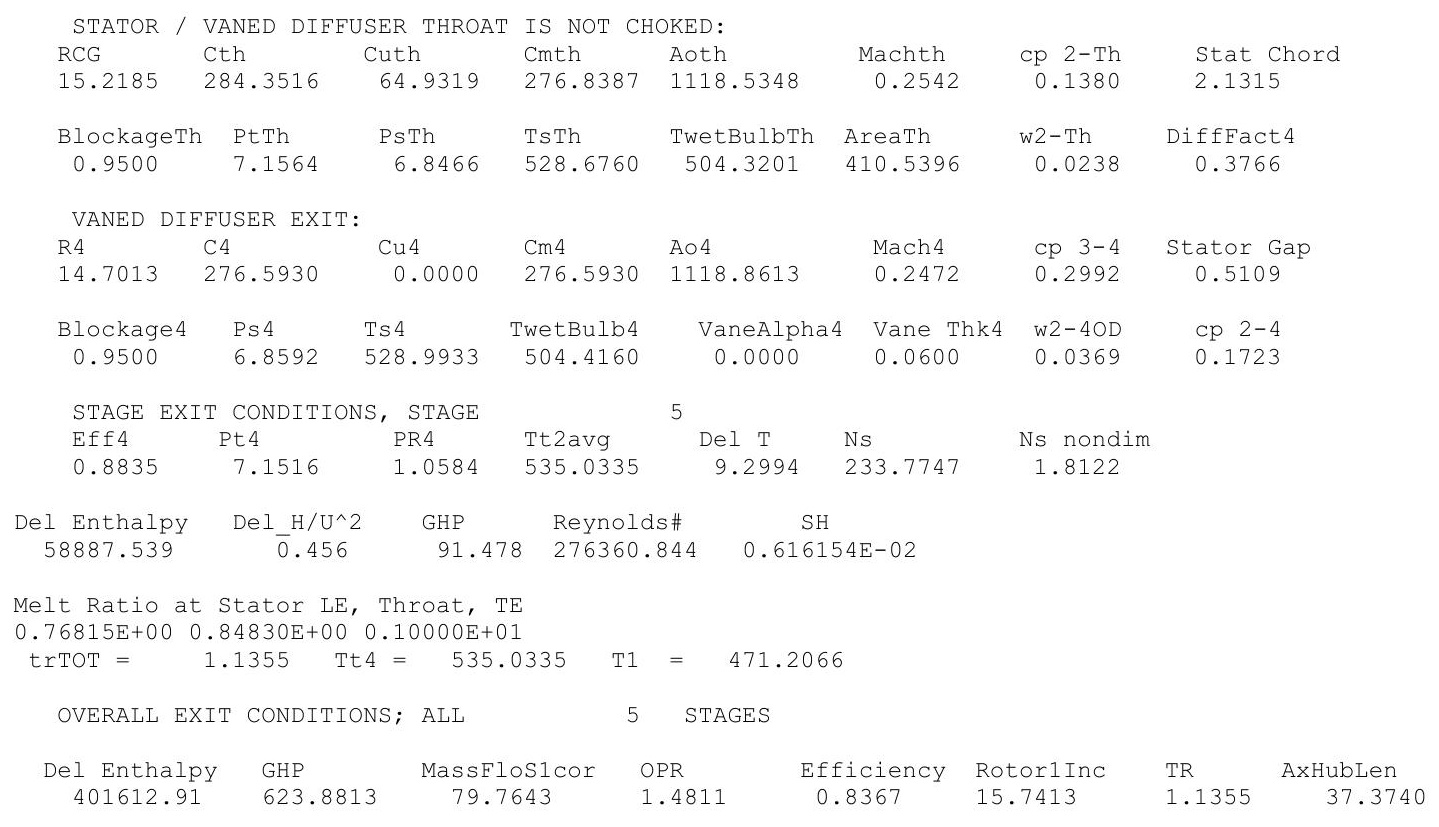




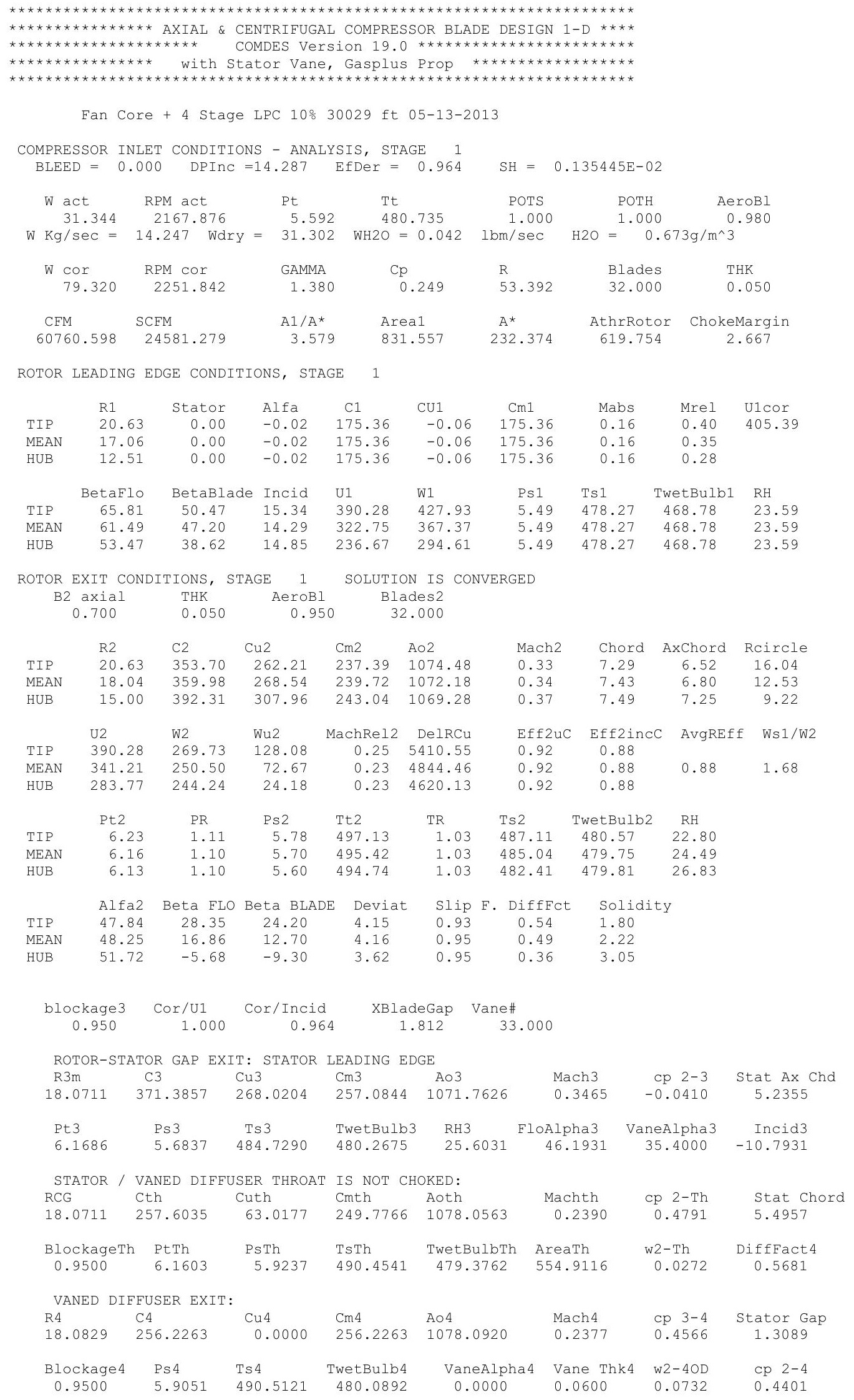




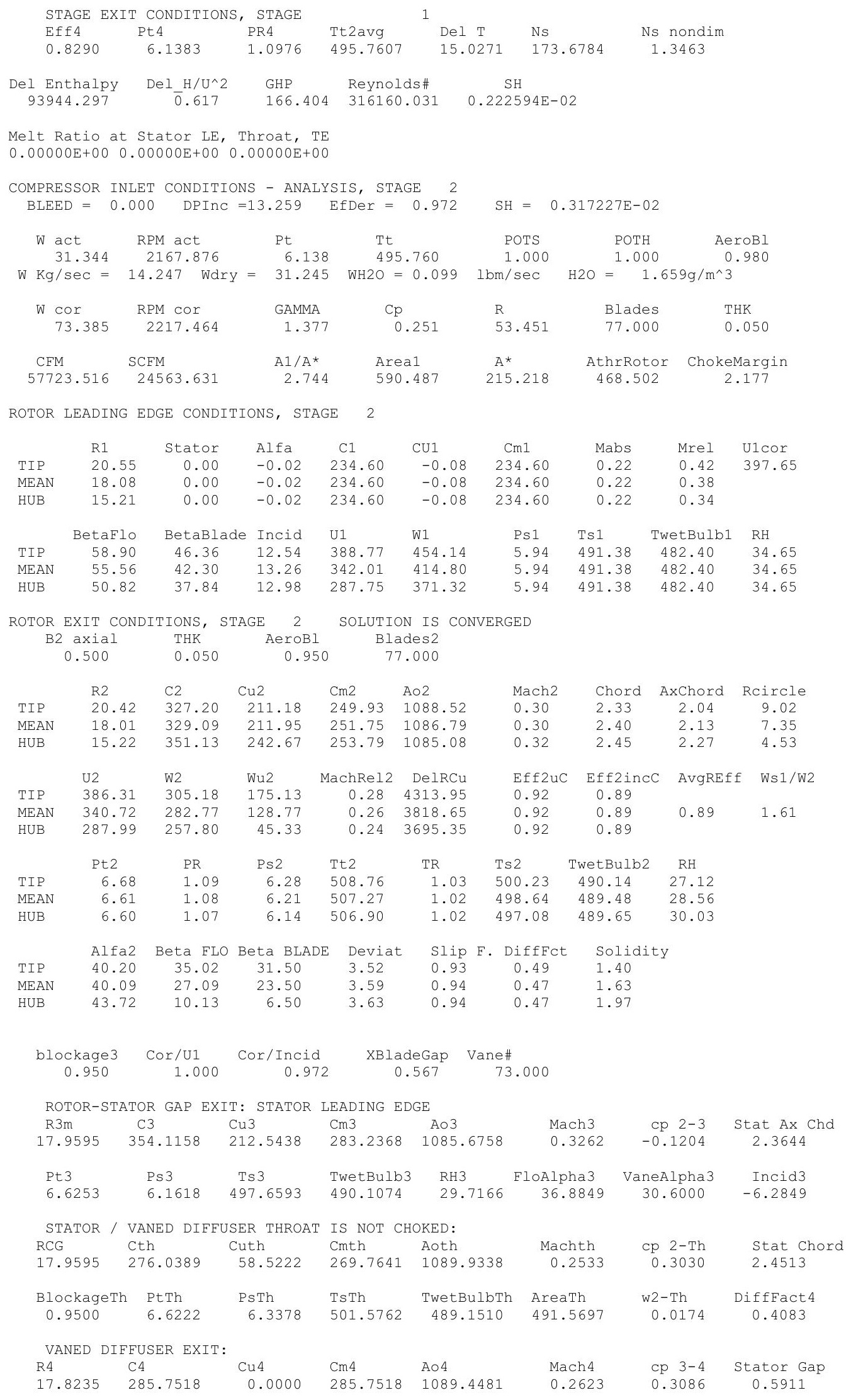




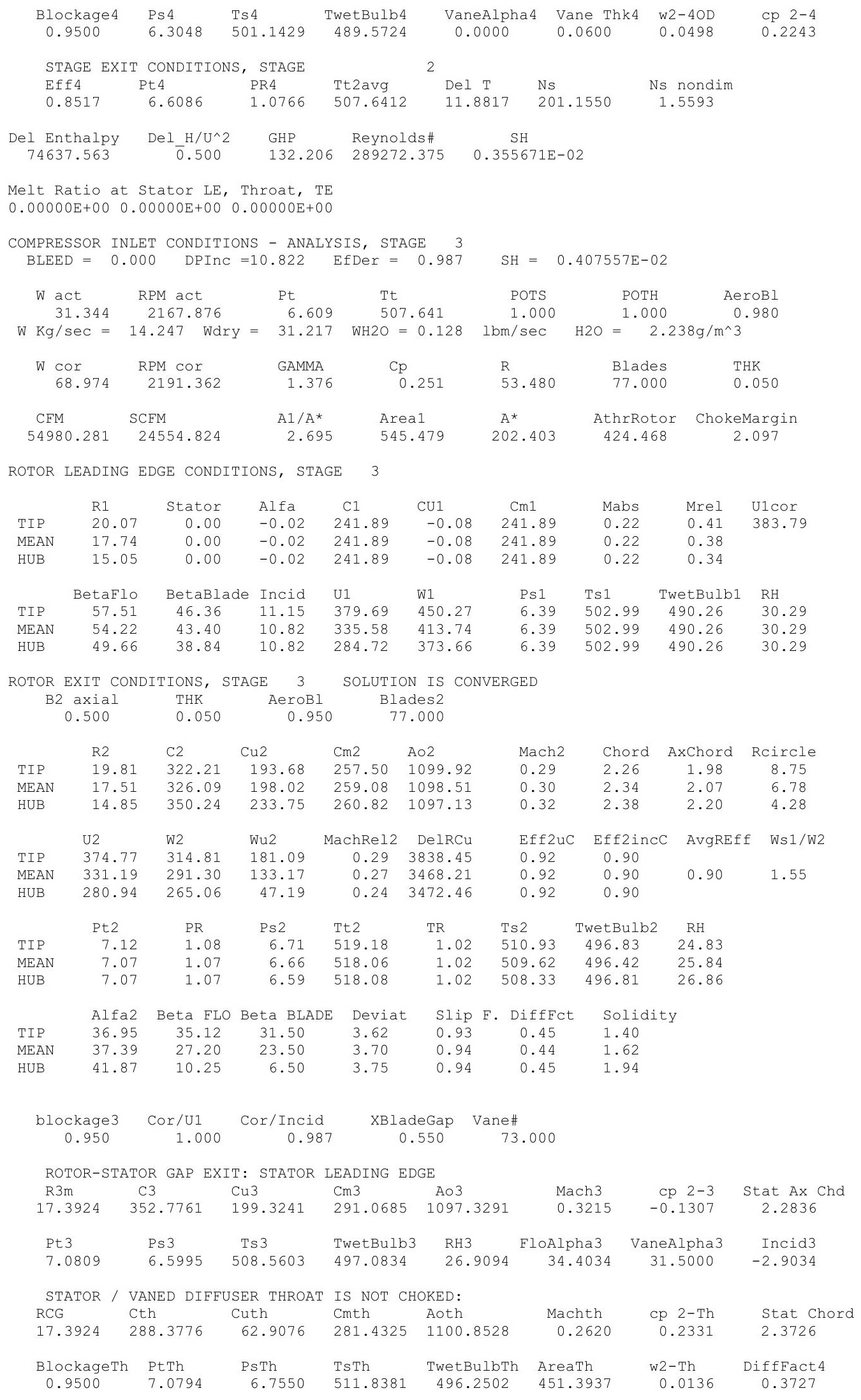




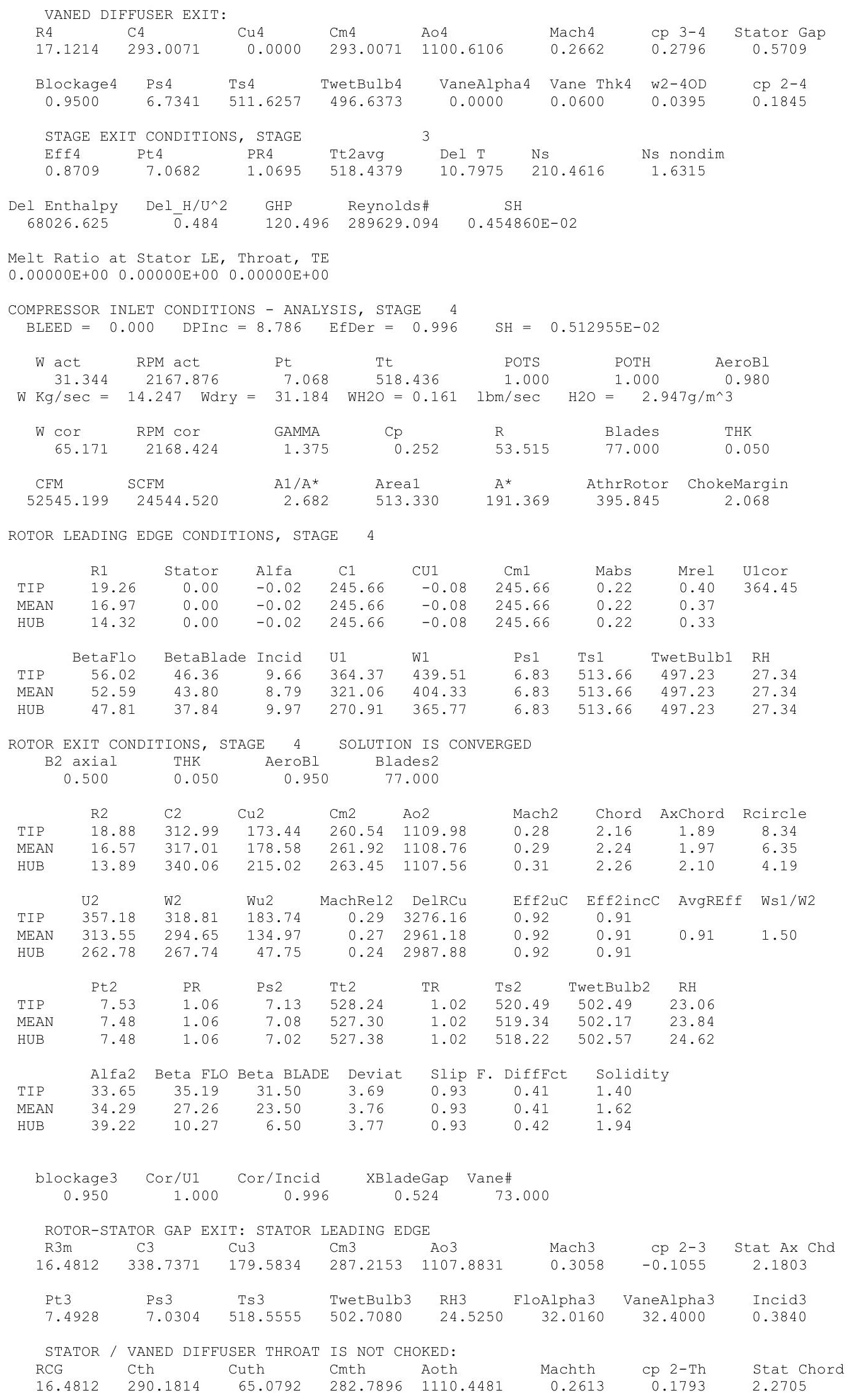




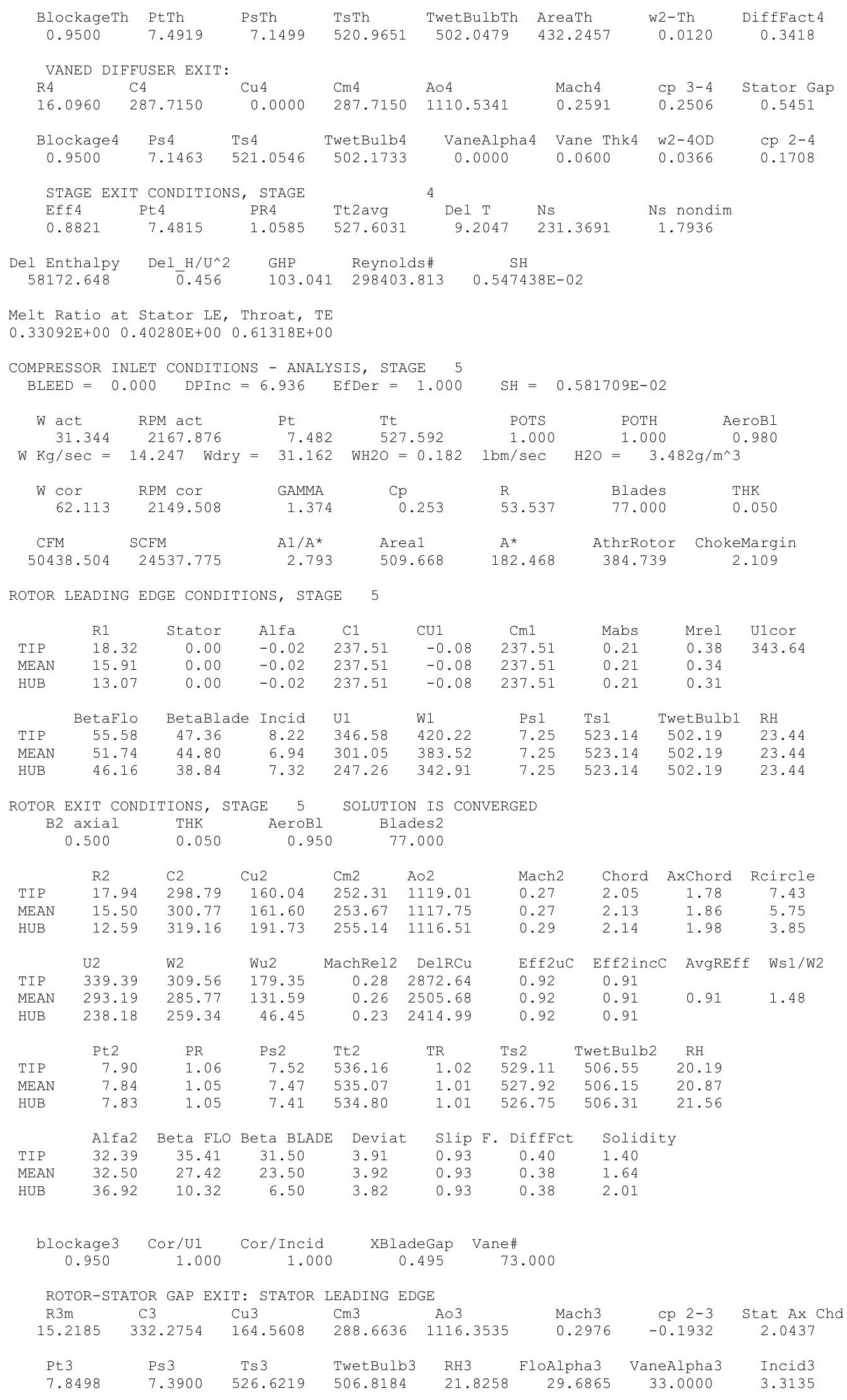




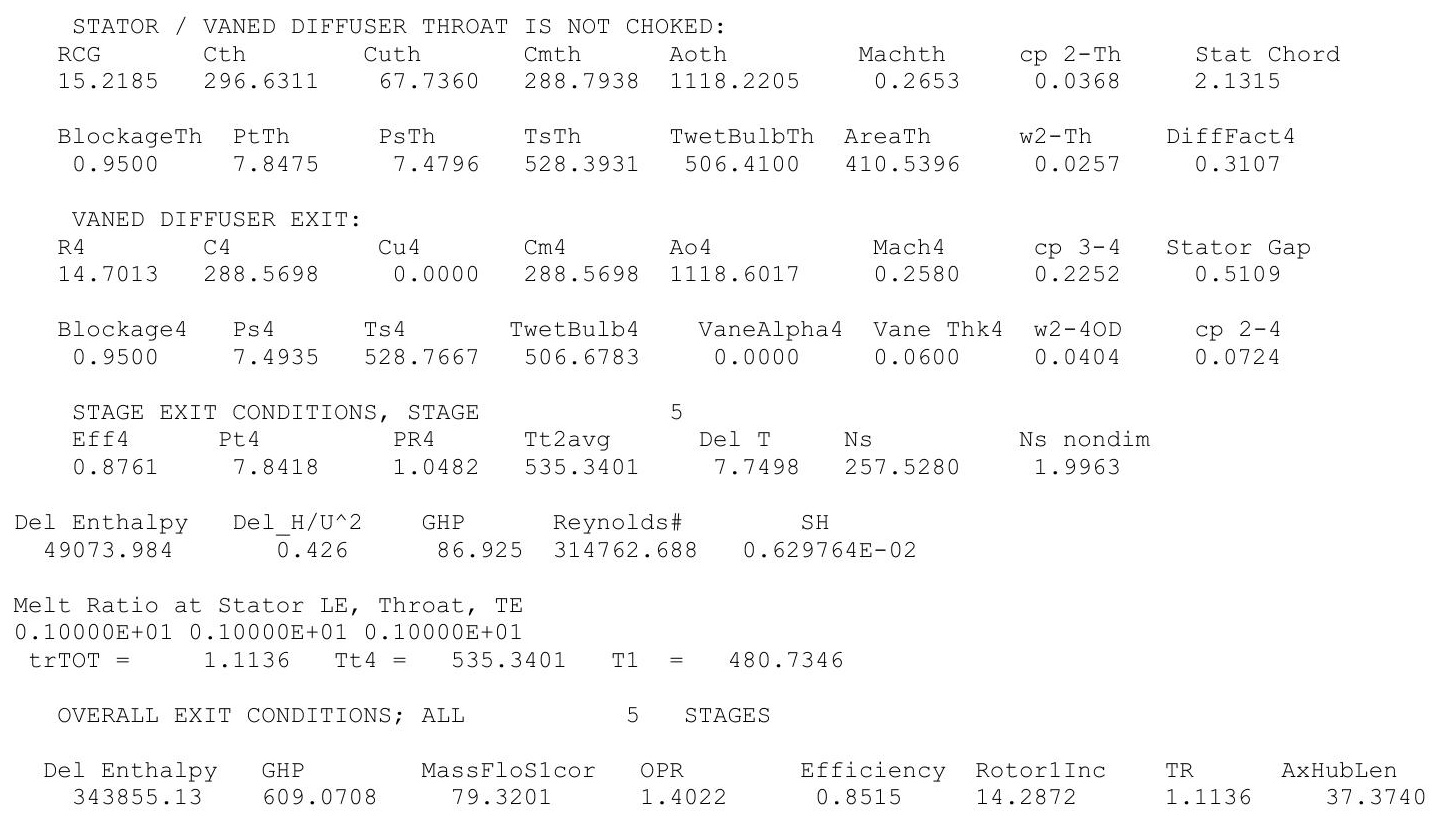




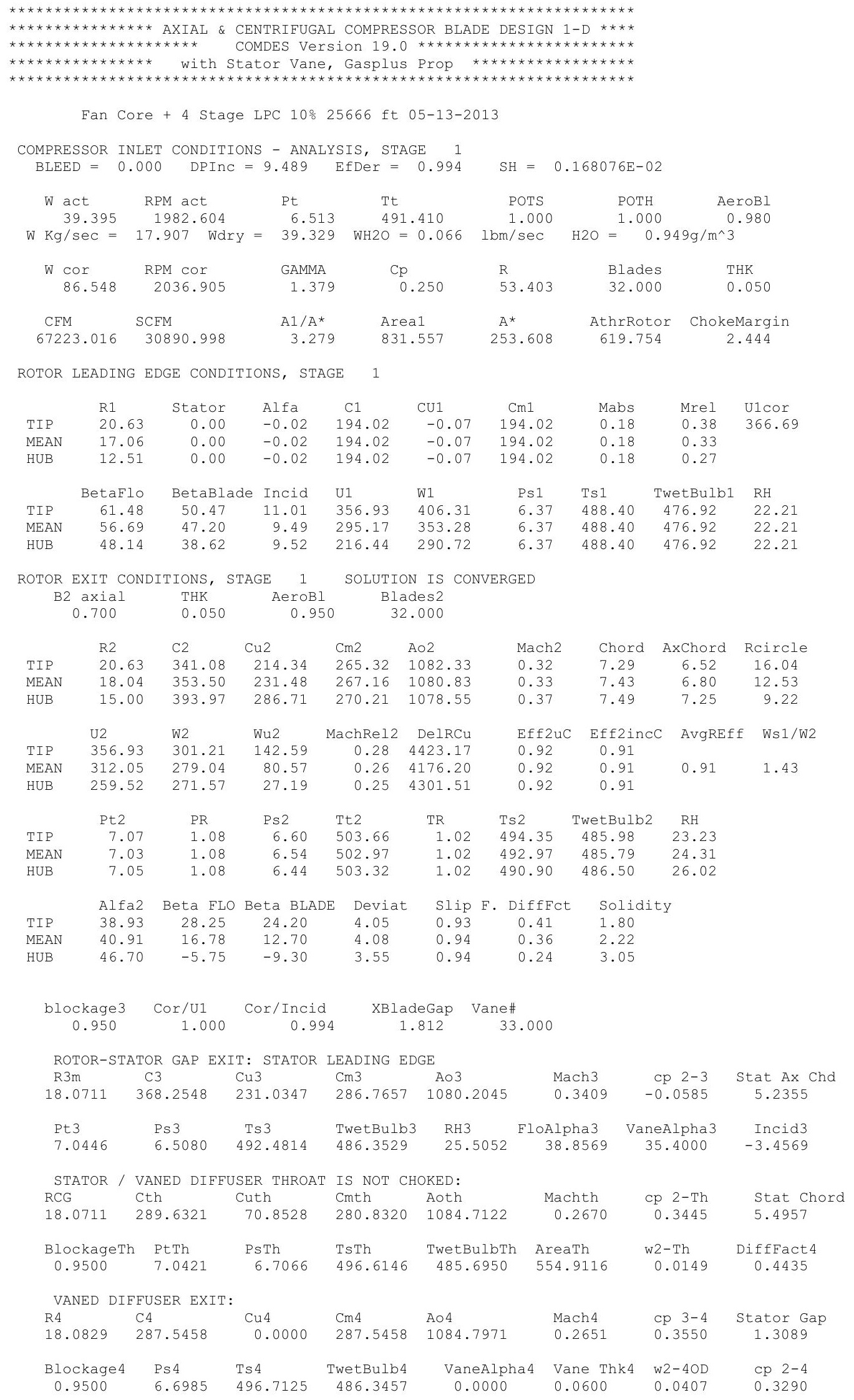




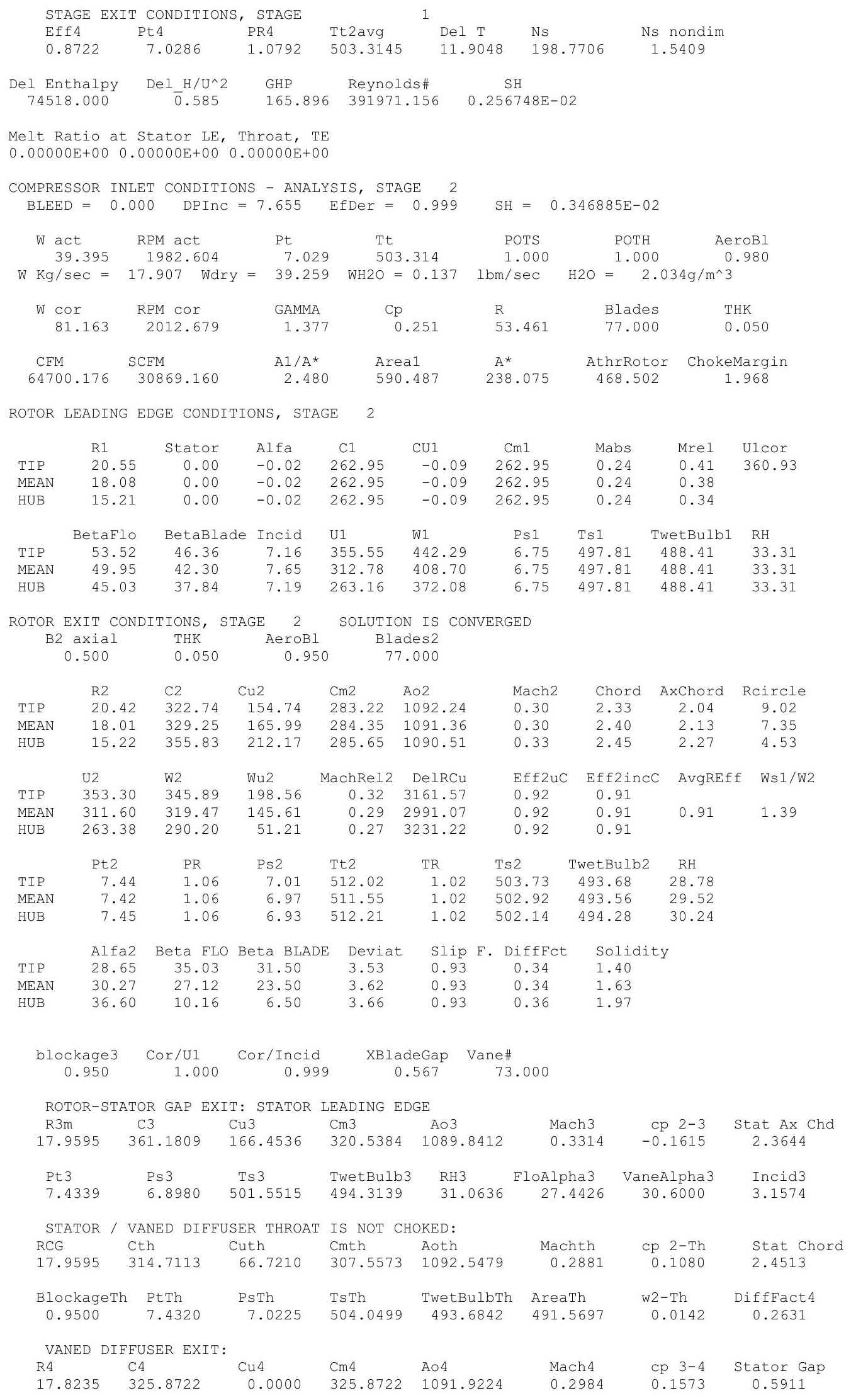




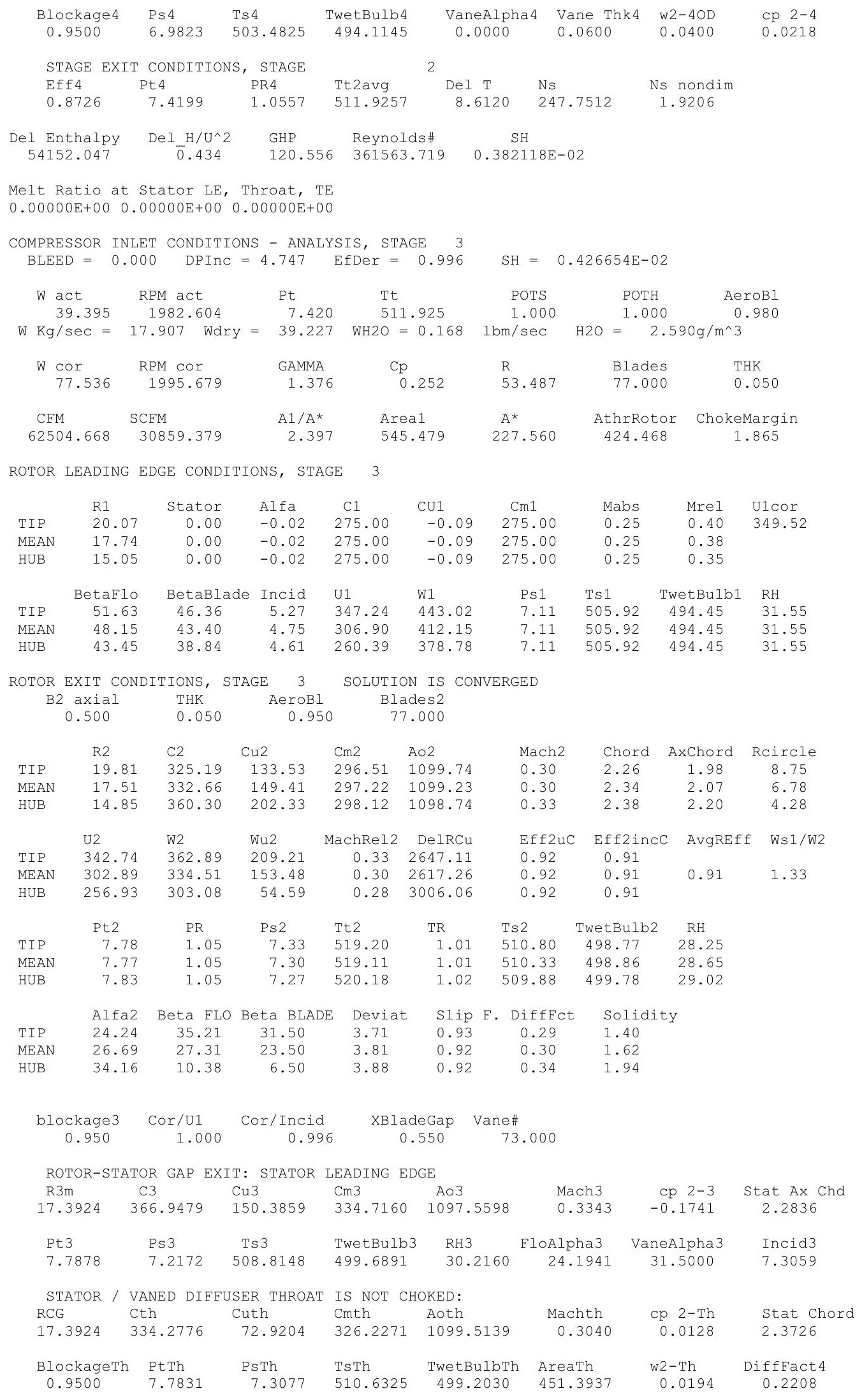




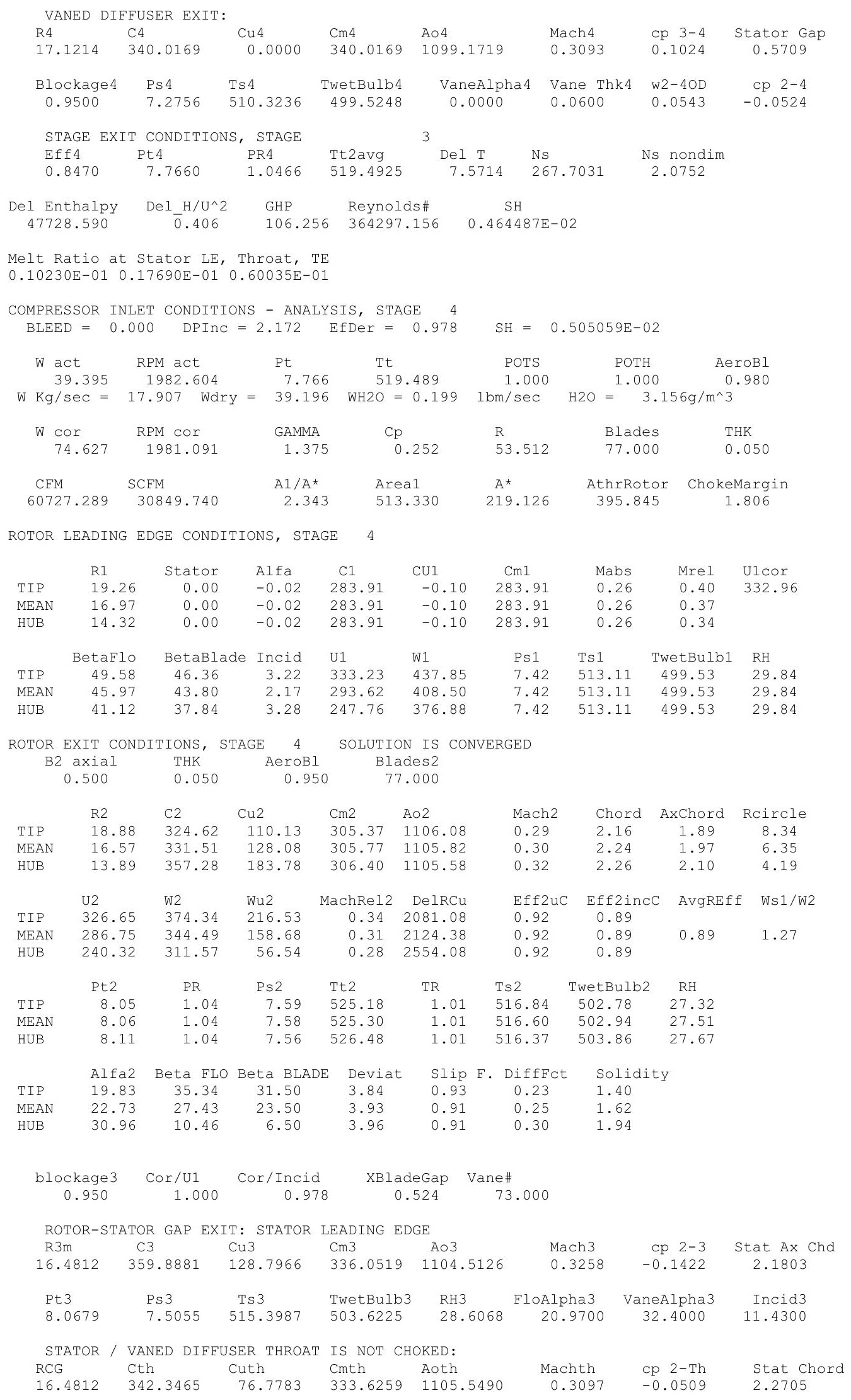




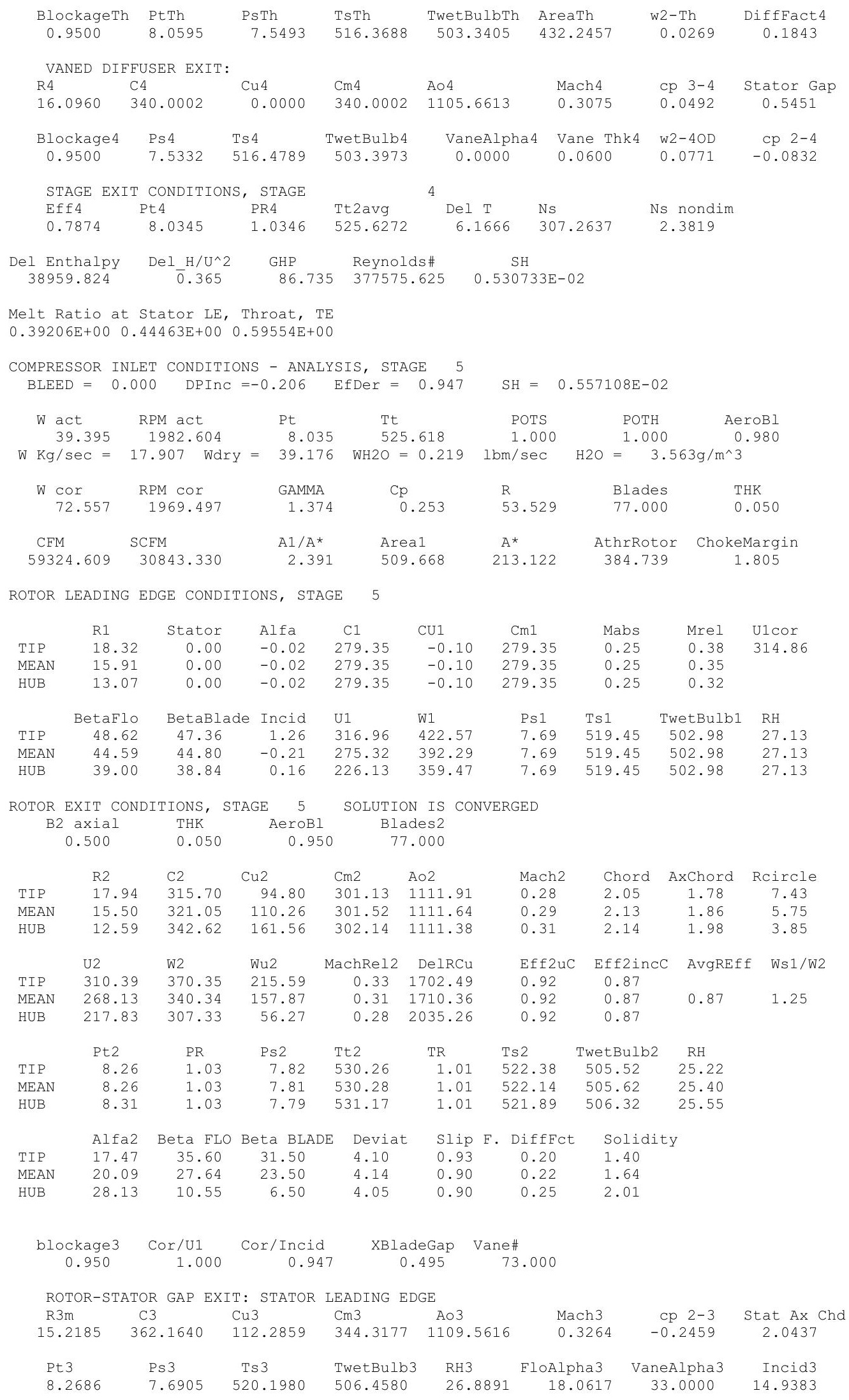




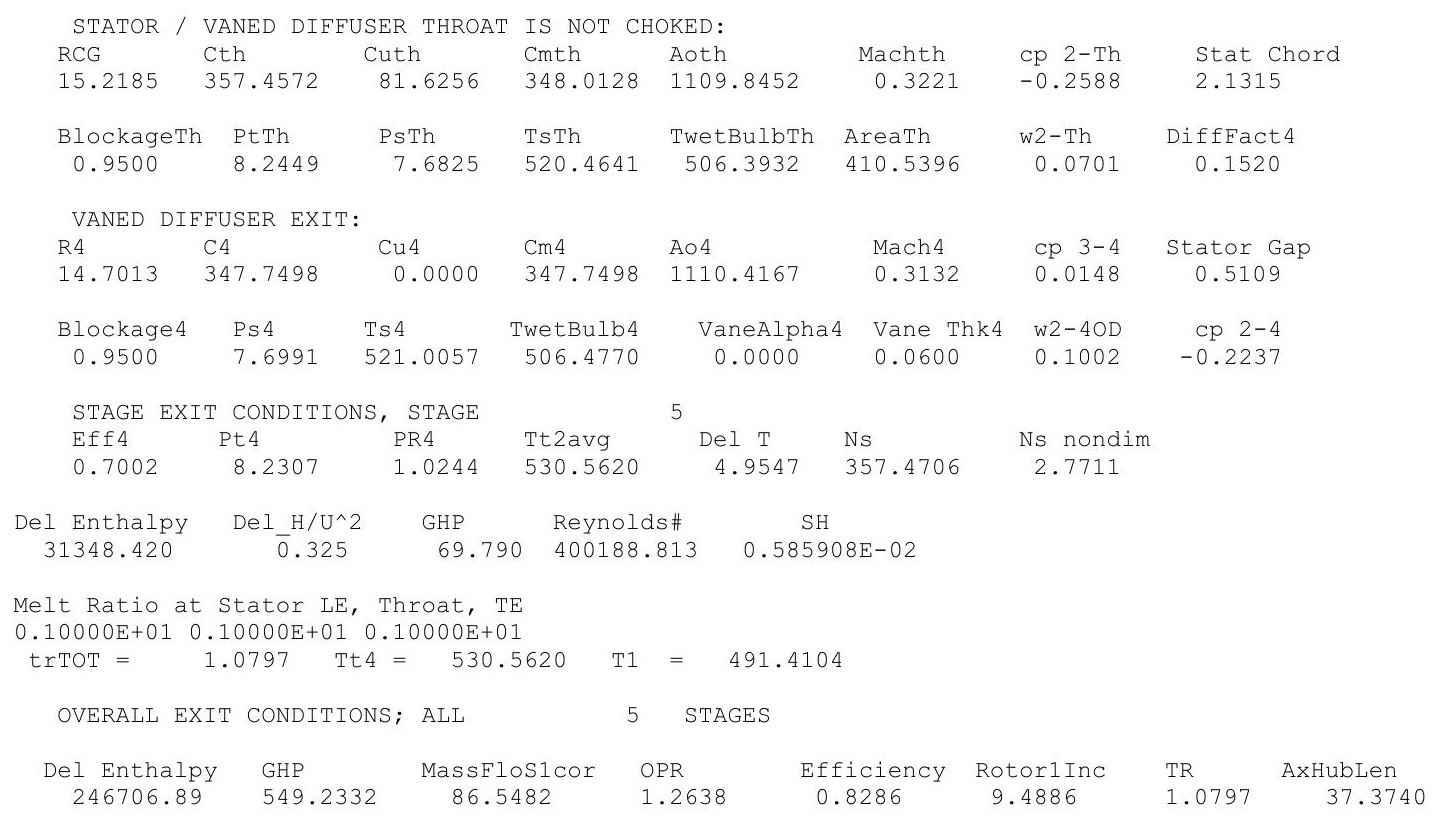




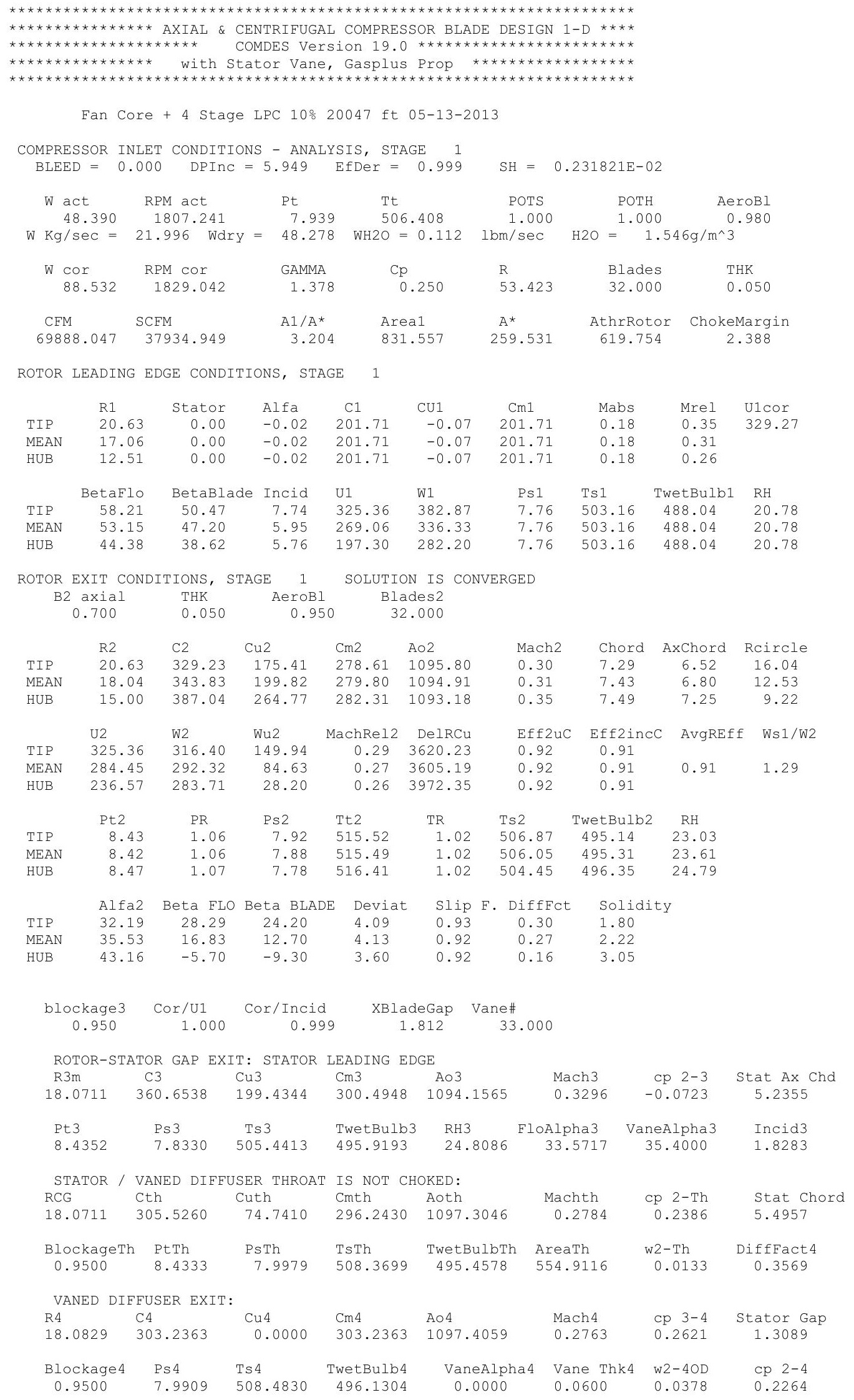




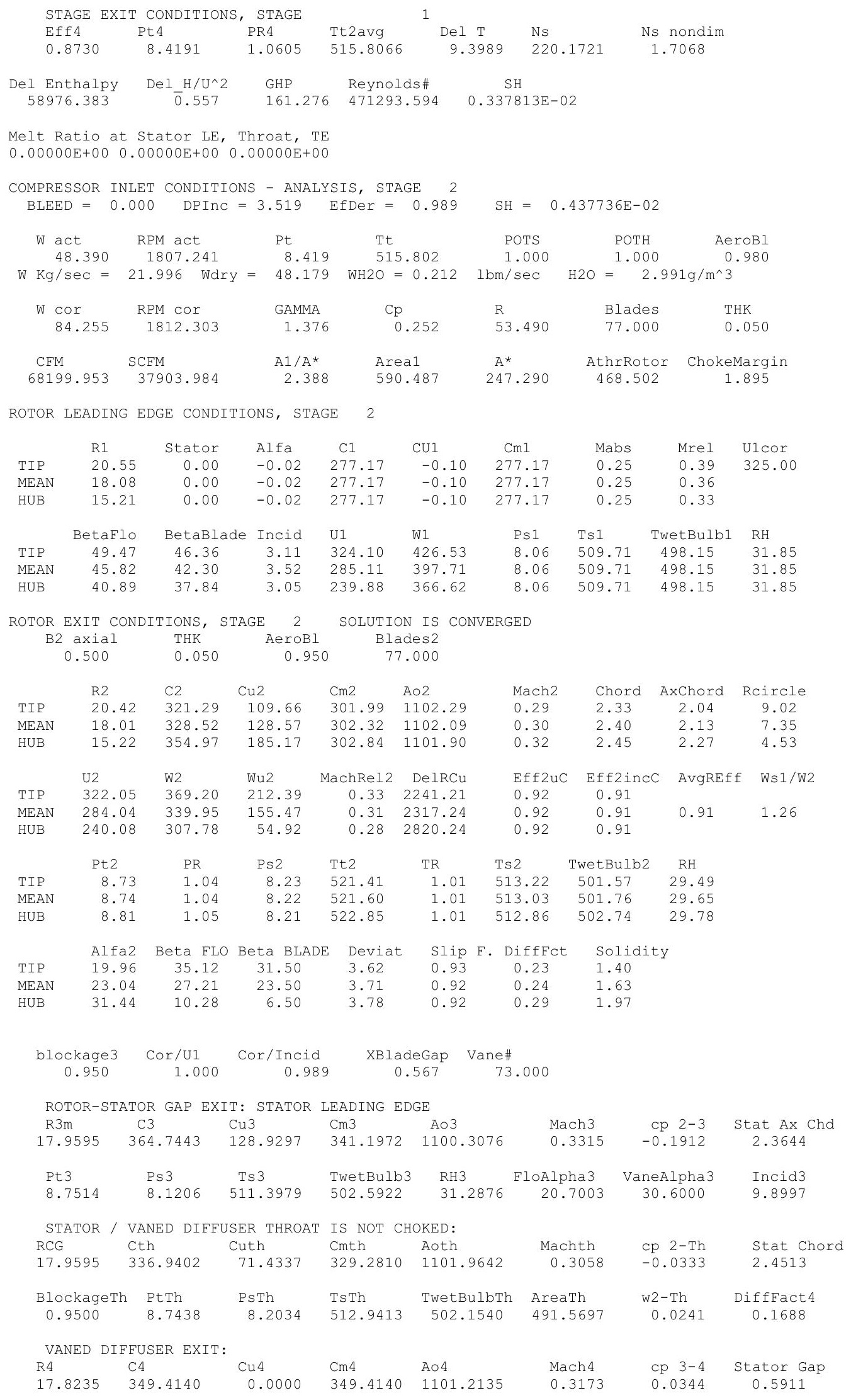




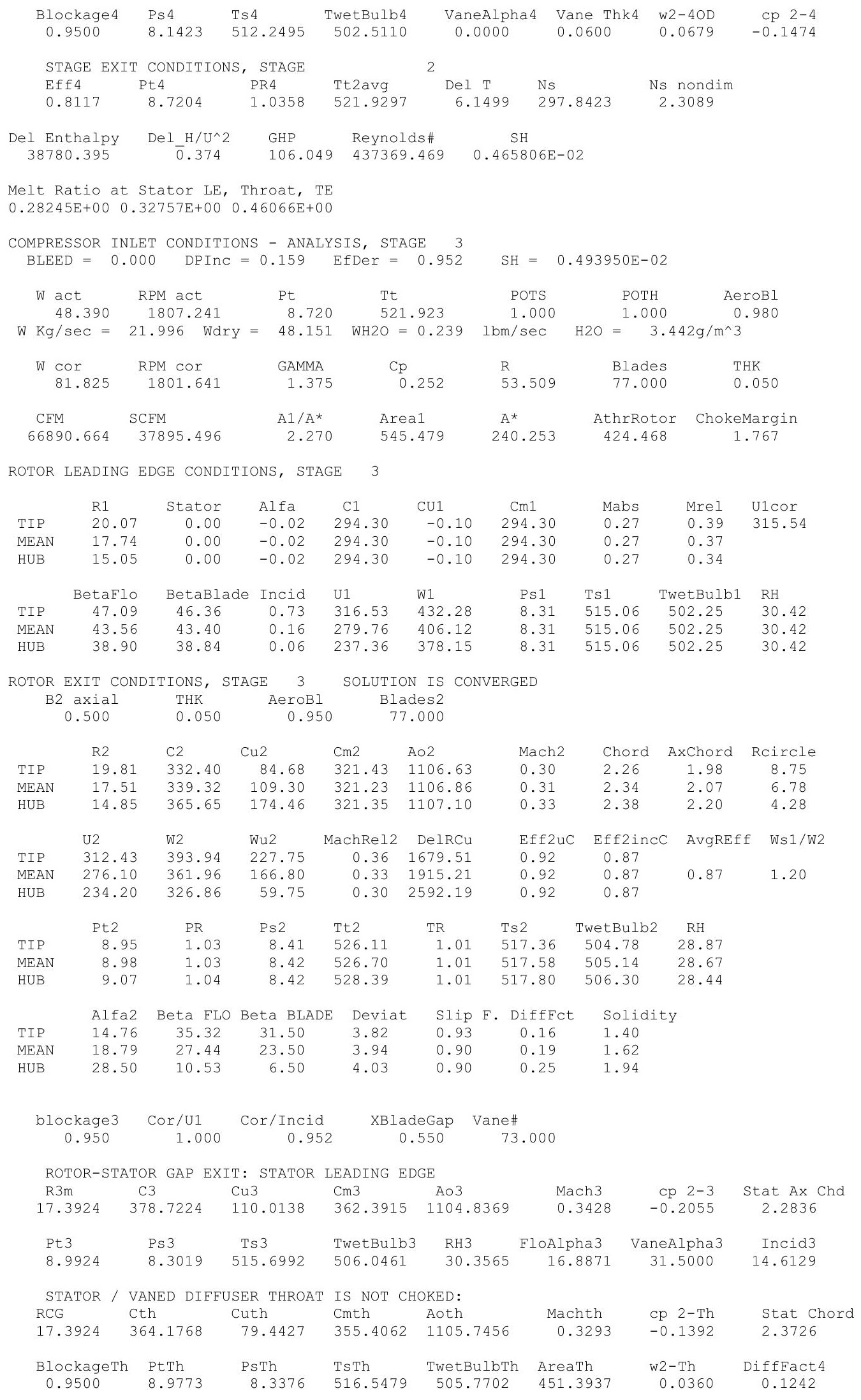




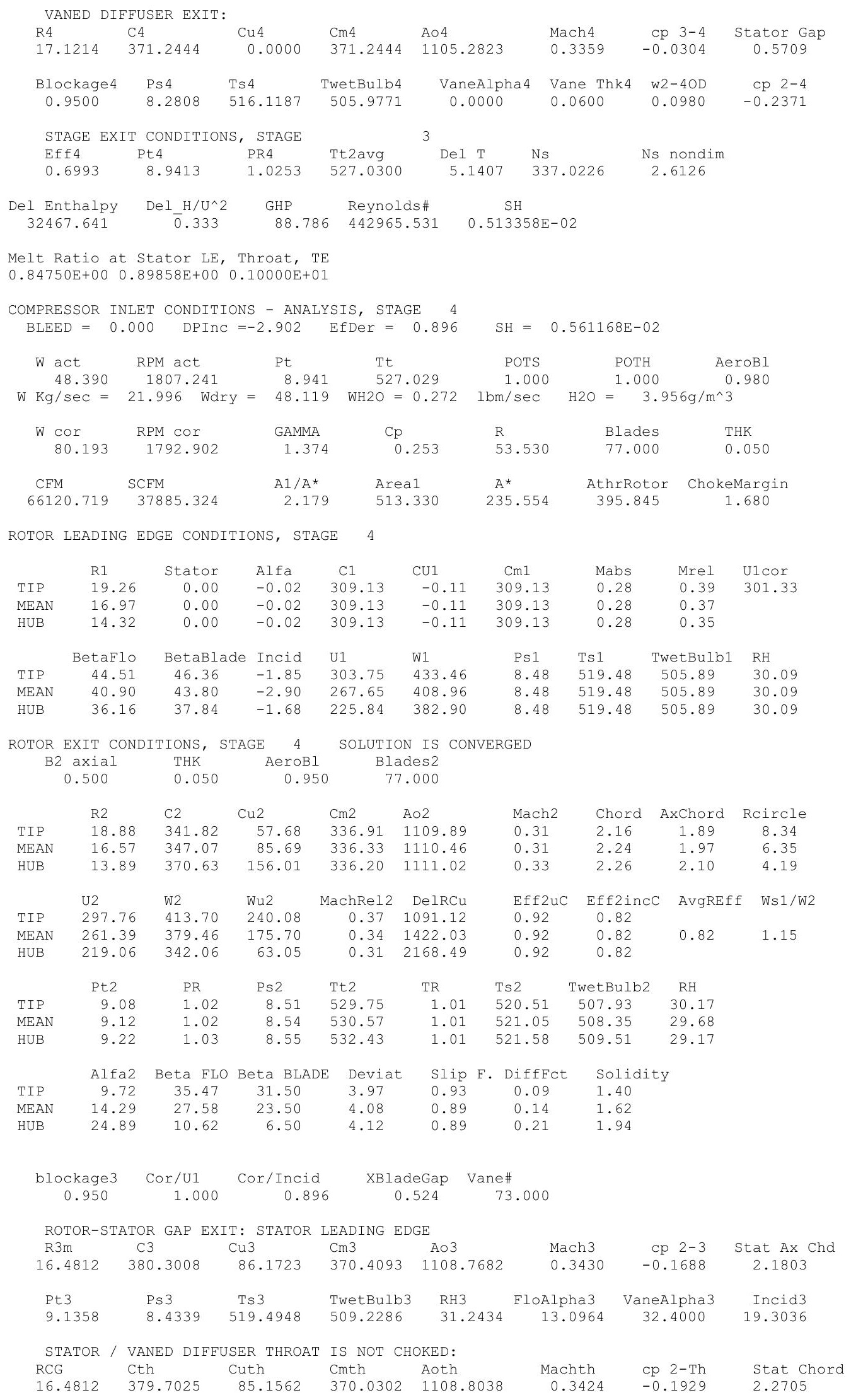




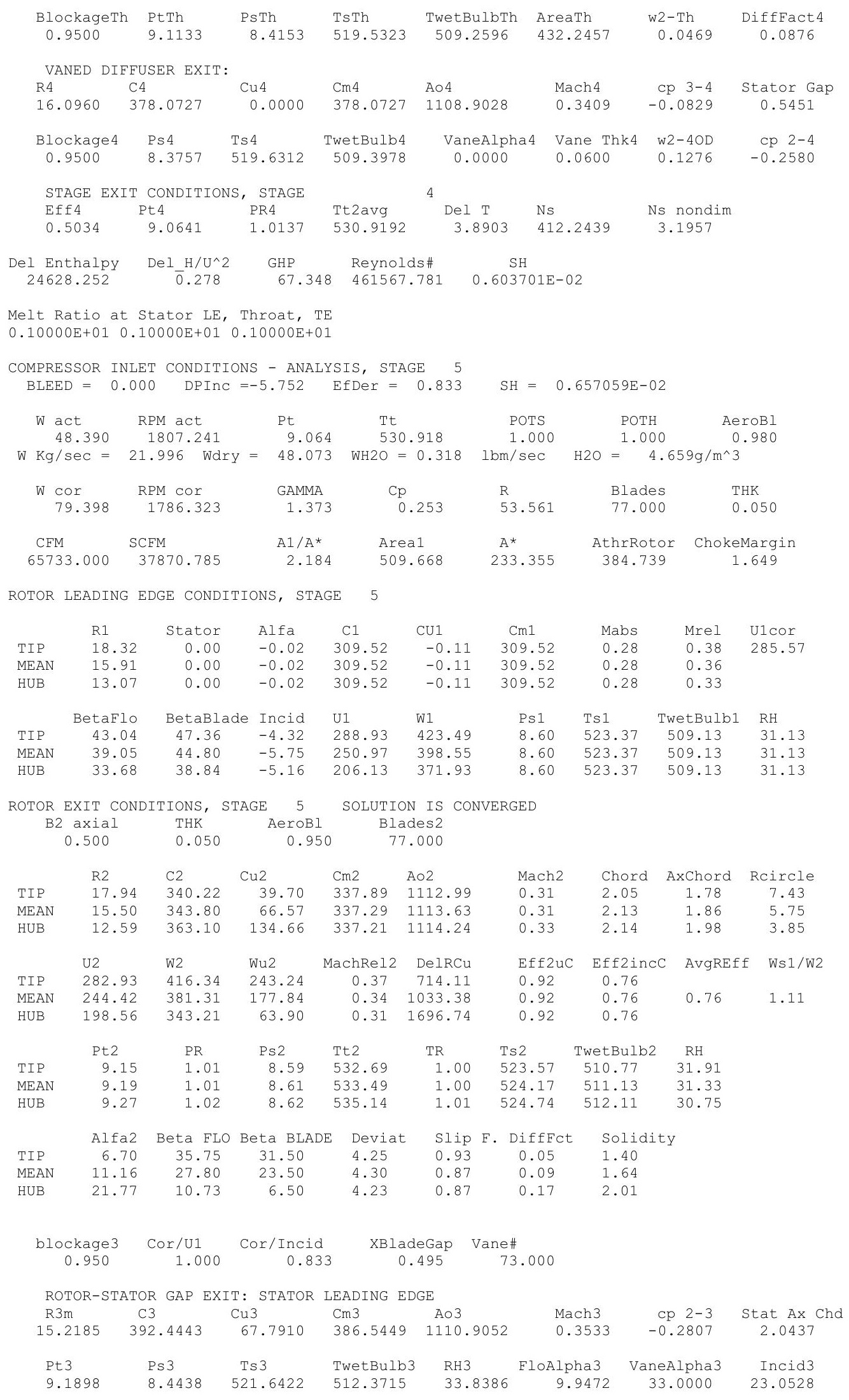




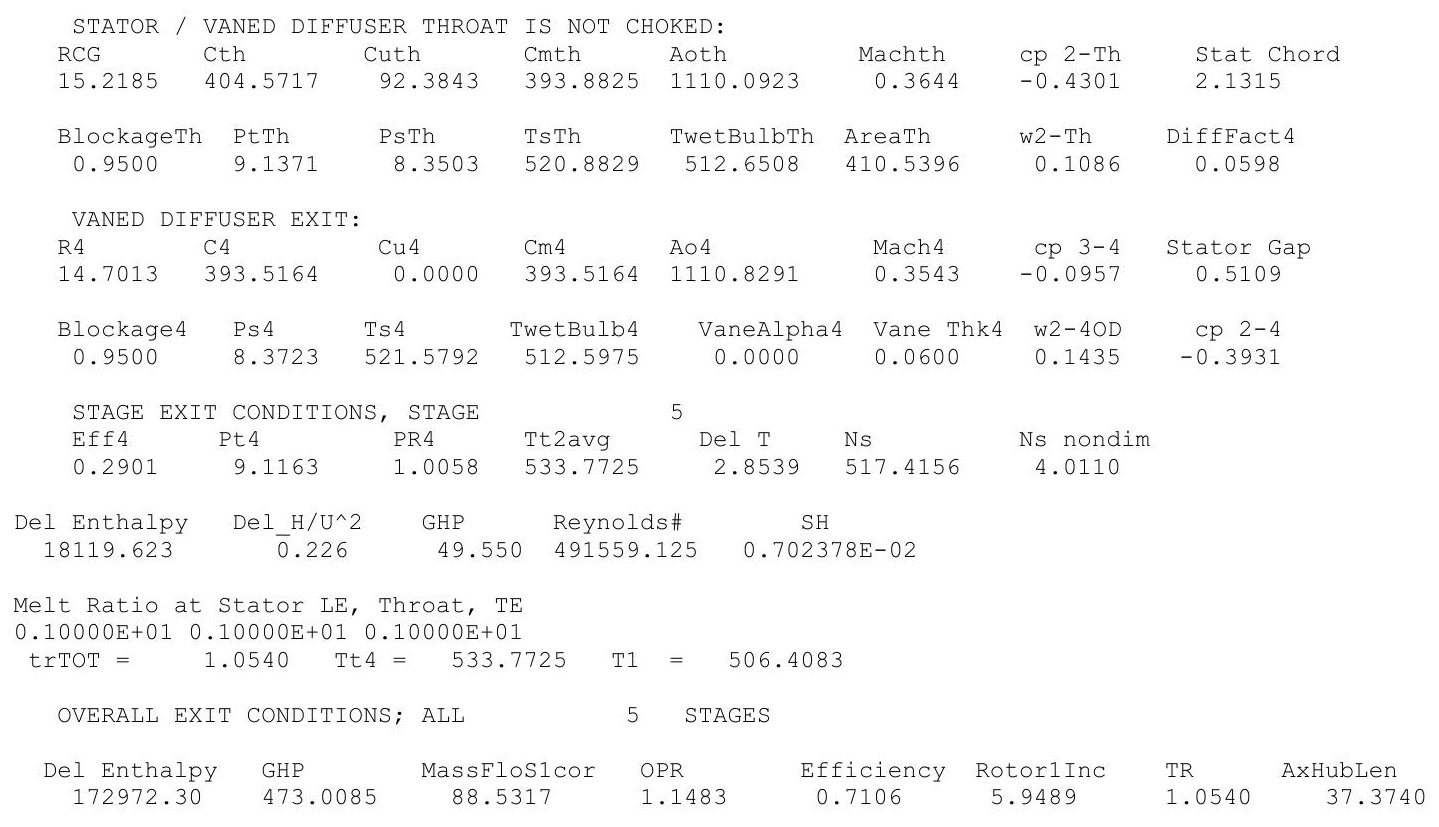


$5 \mu \mathrm{m}, 4 \mathrm{~g} / \mathrm{m}^{3}, \mathrm{ISA}+36 \mathrm{R}$

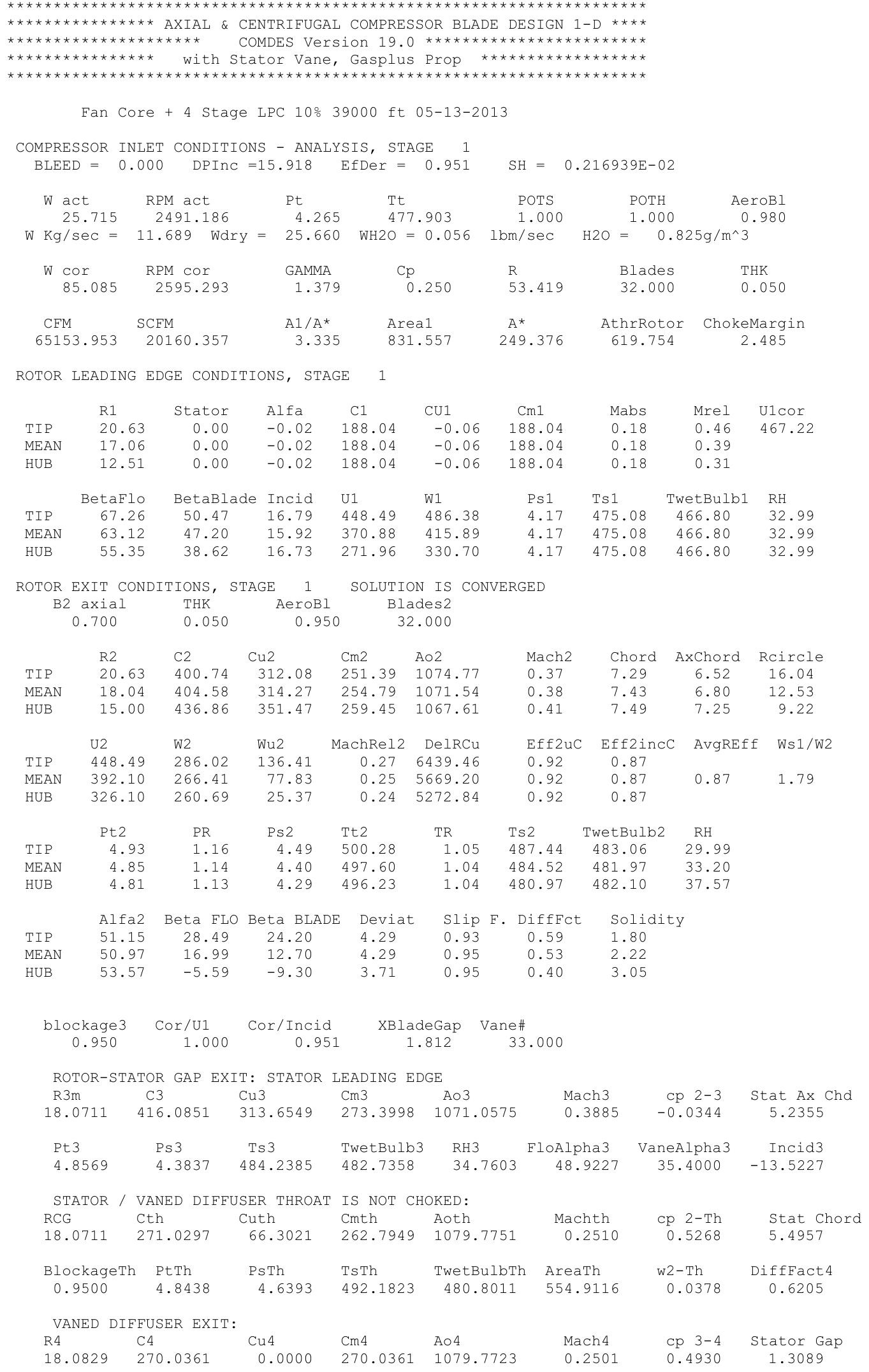




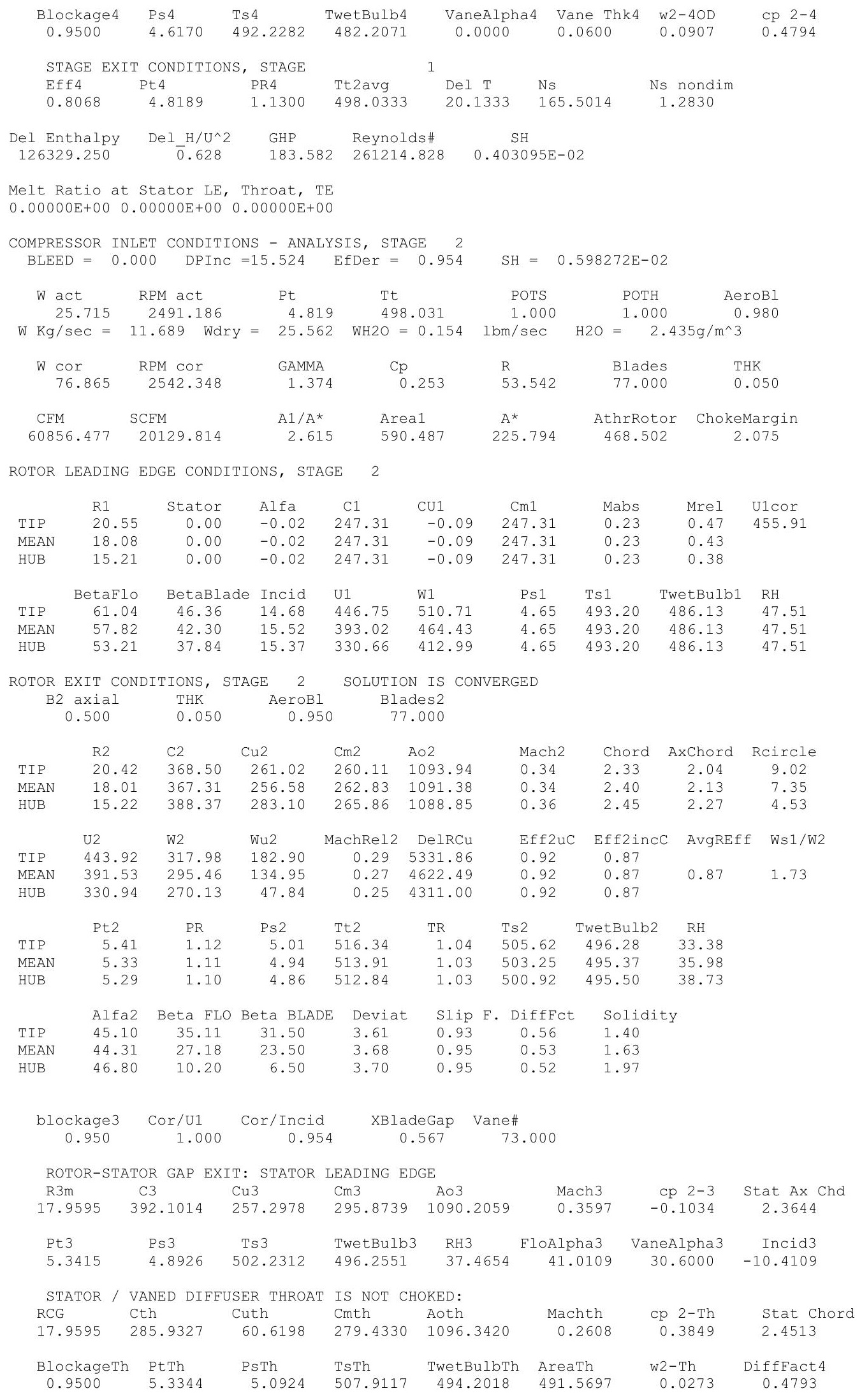




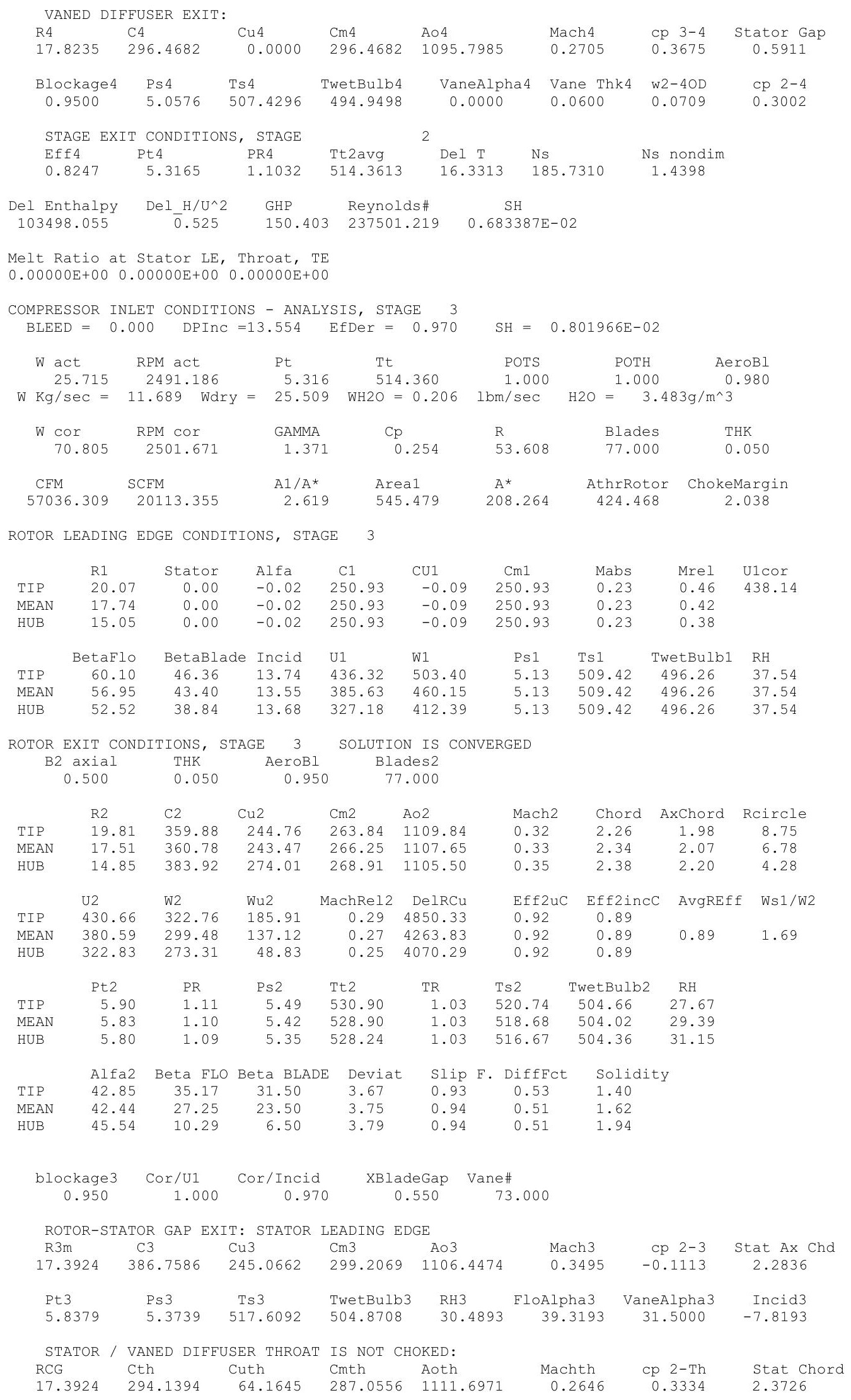




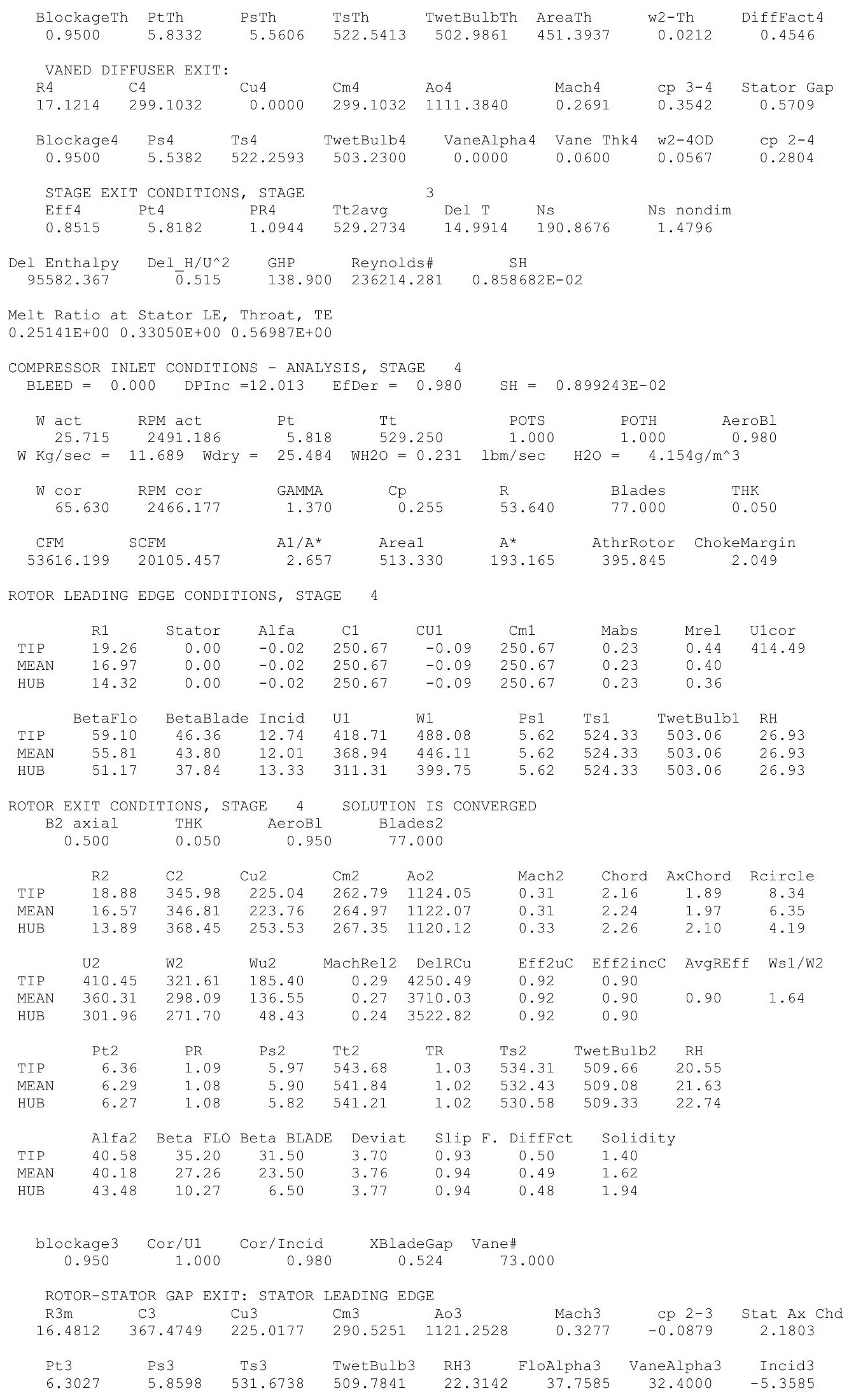




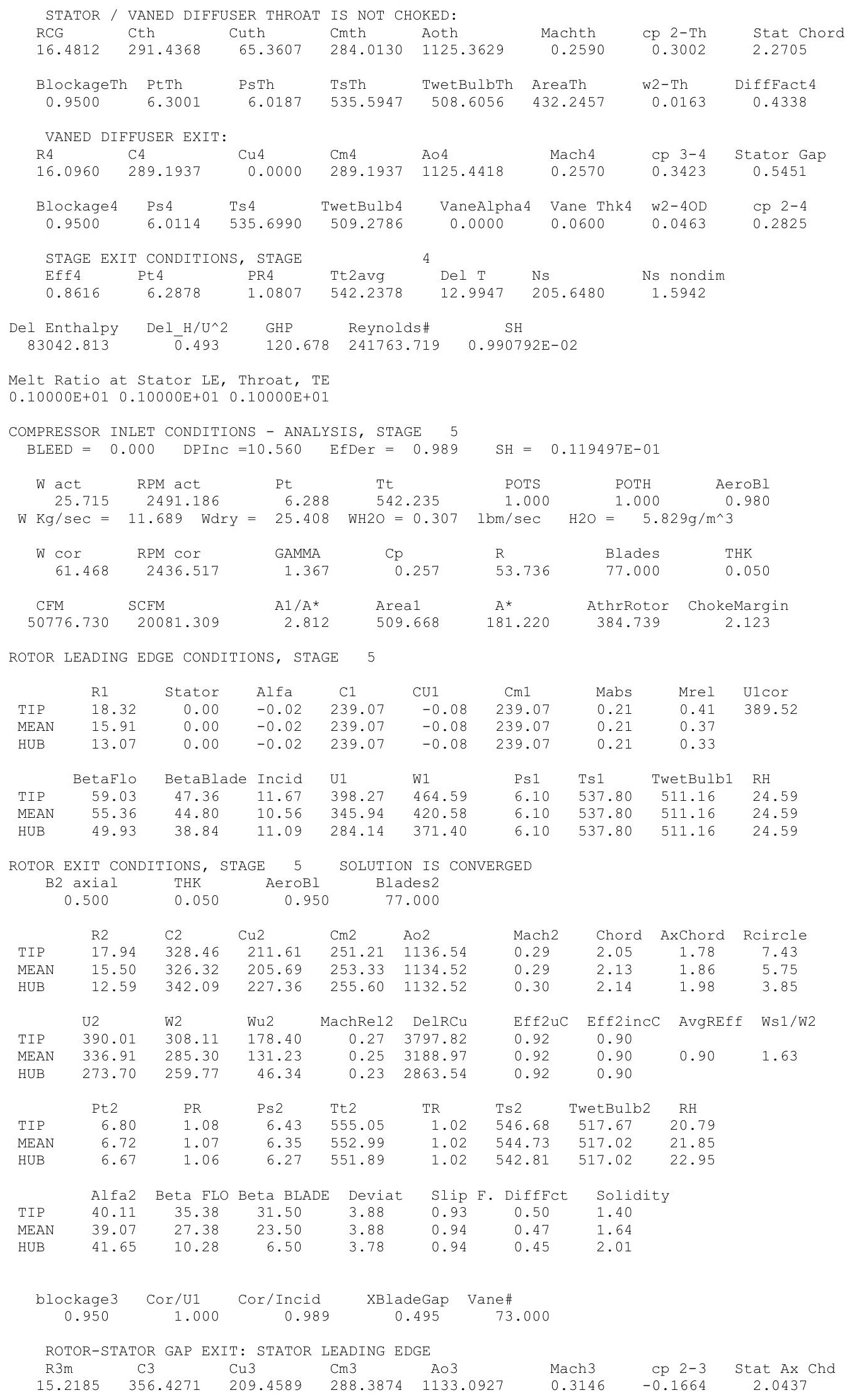




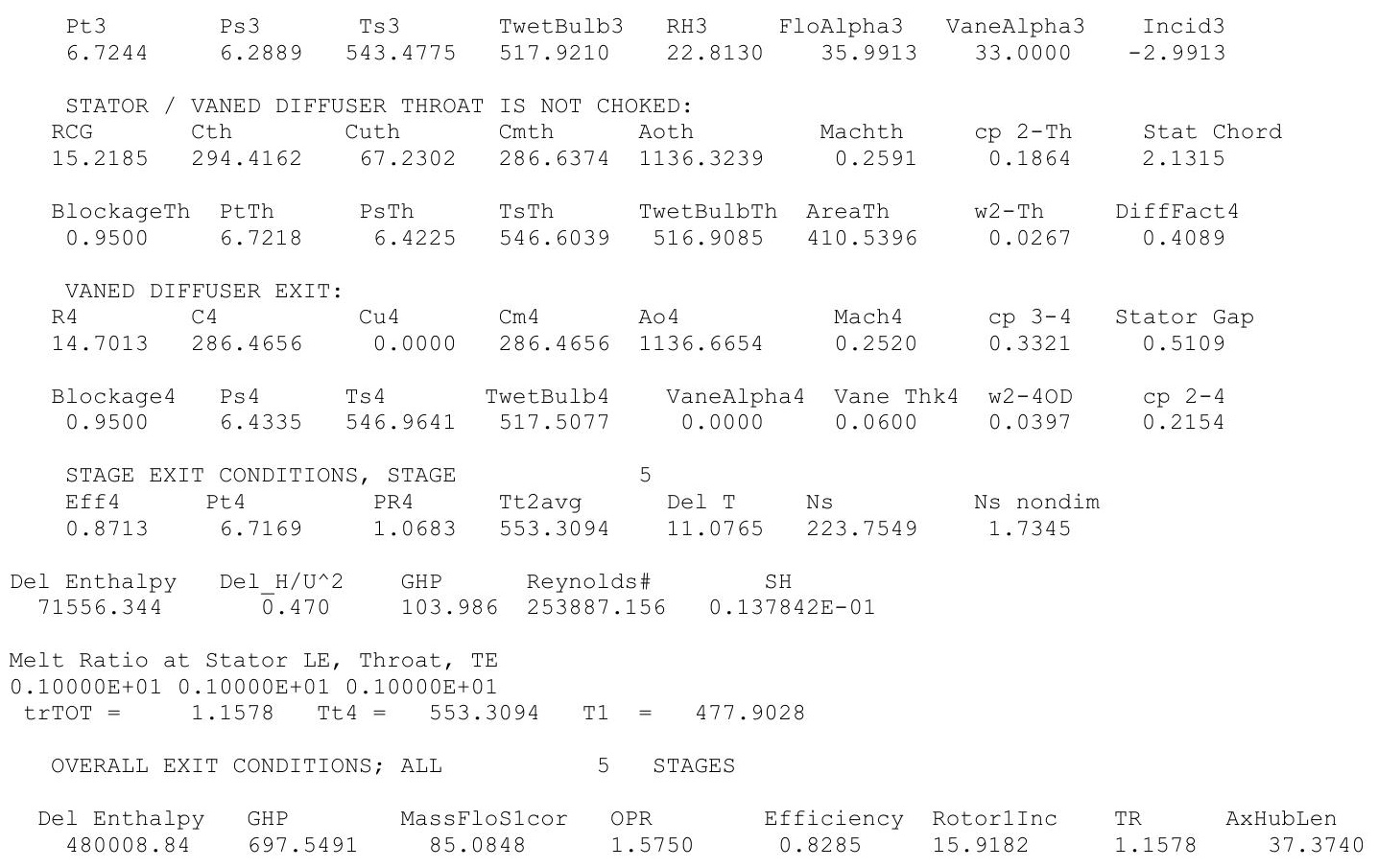




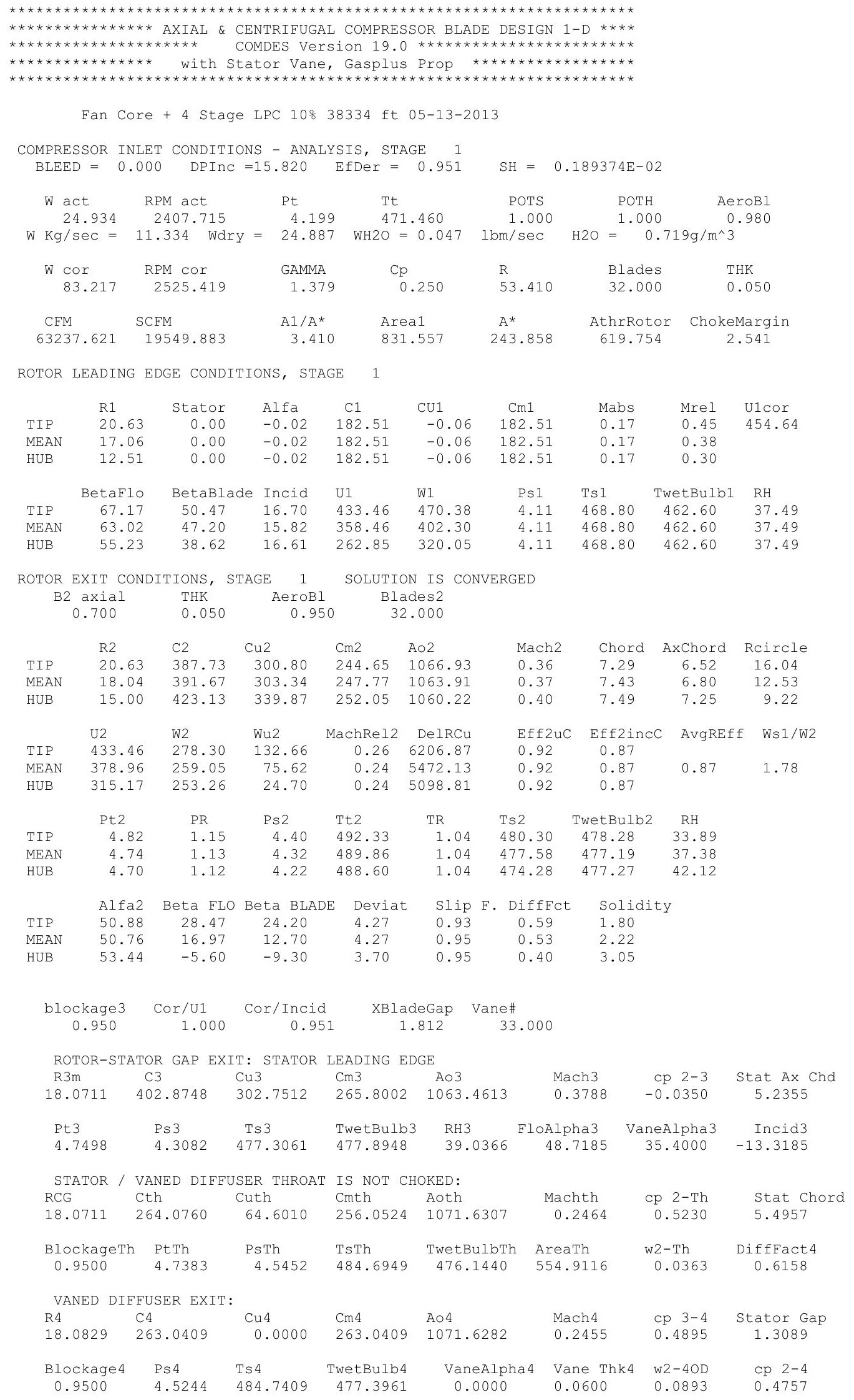




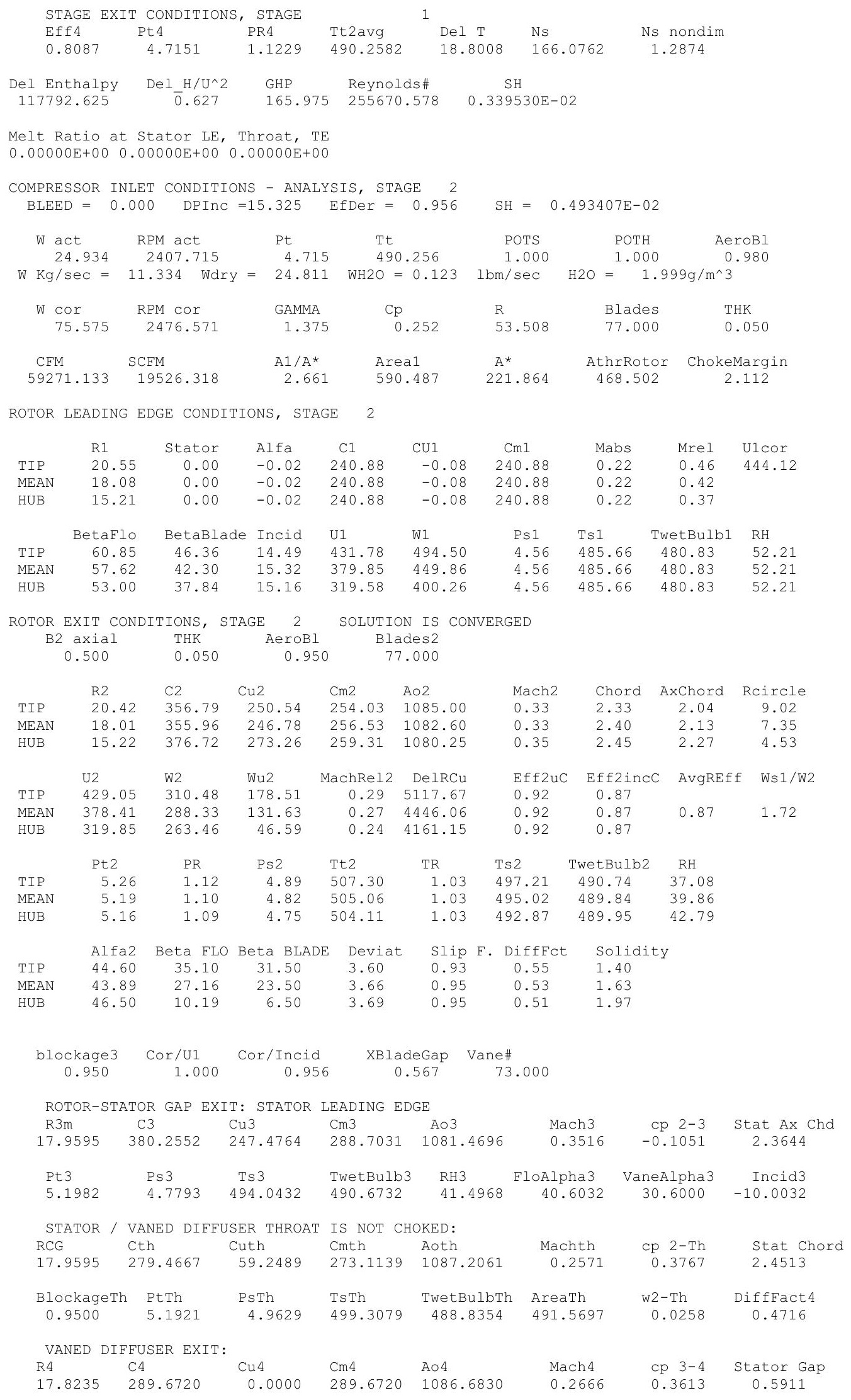




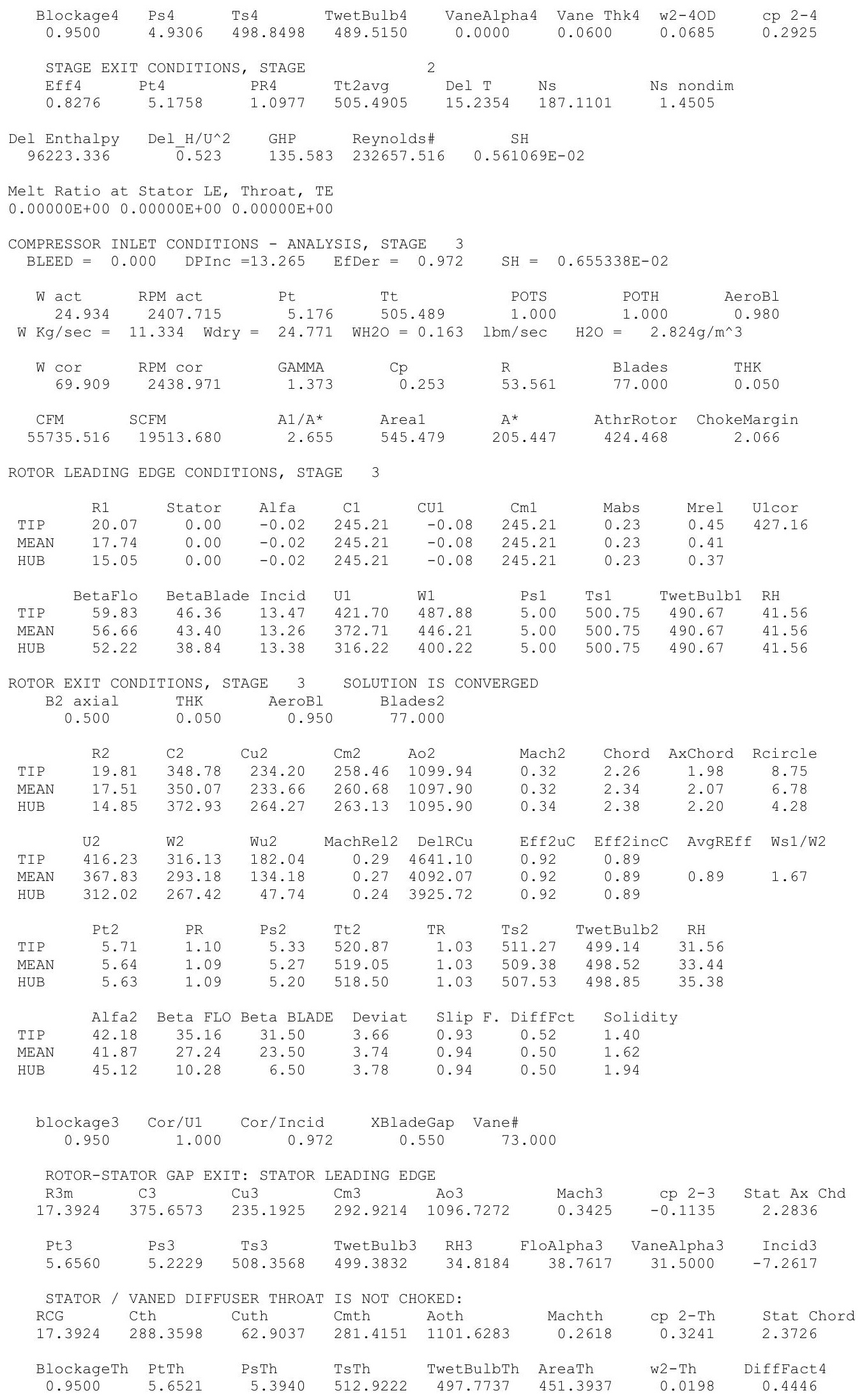




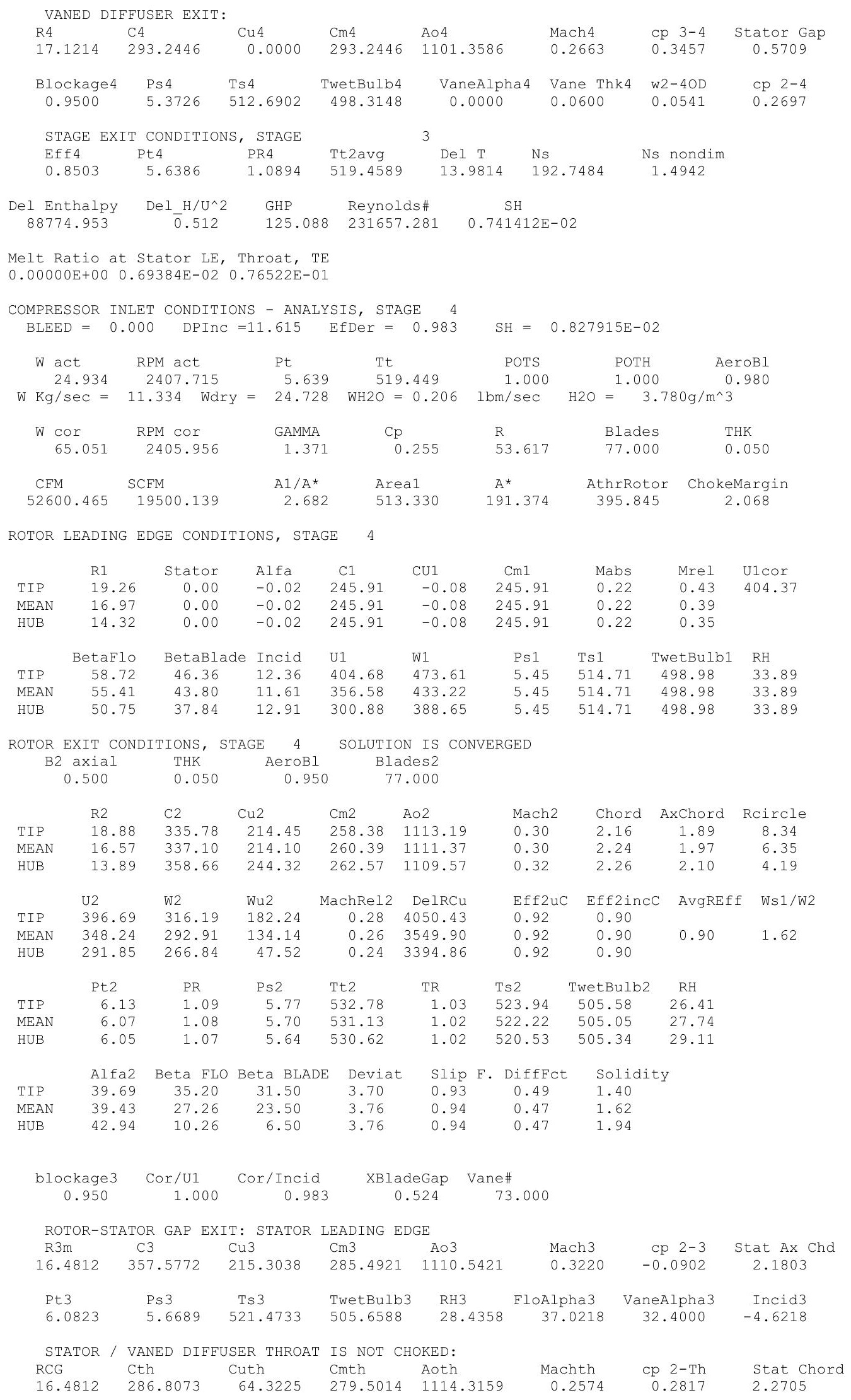




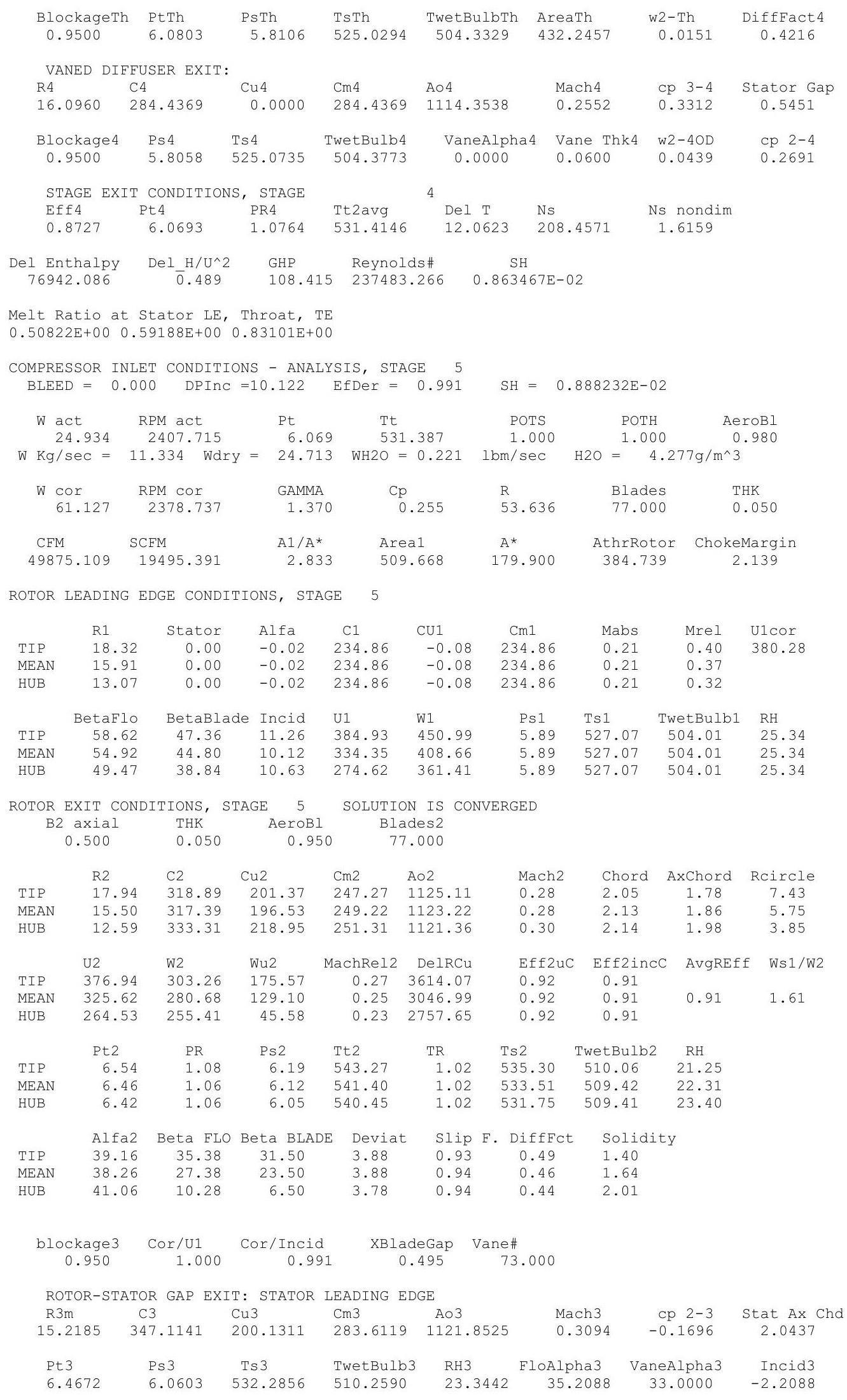




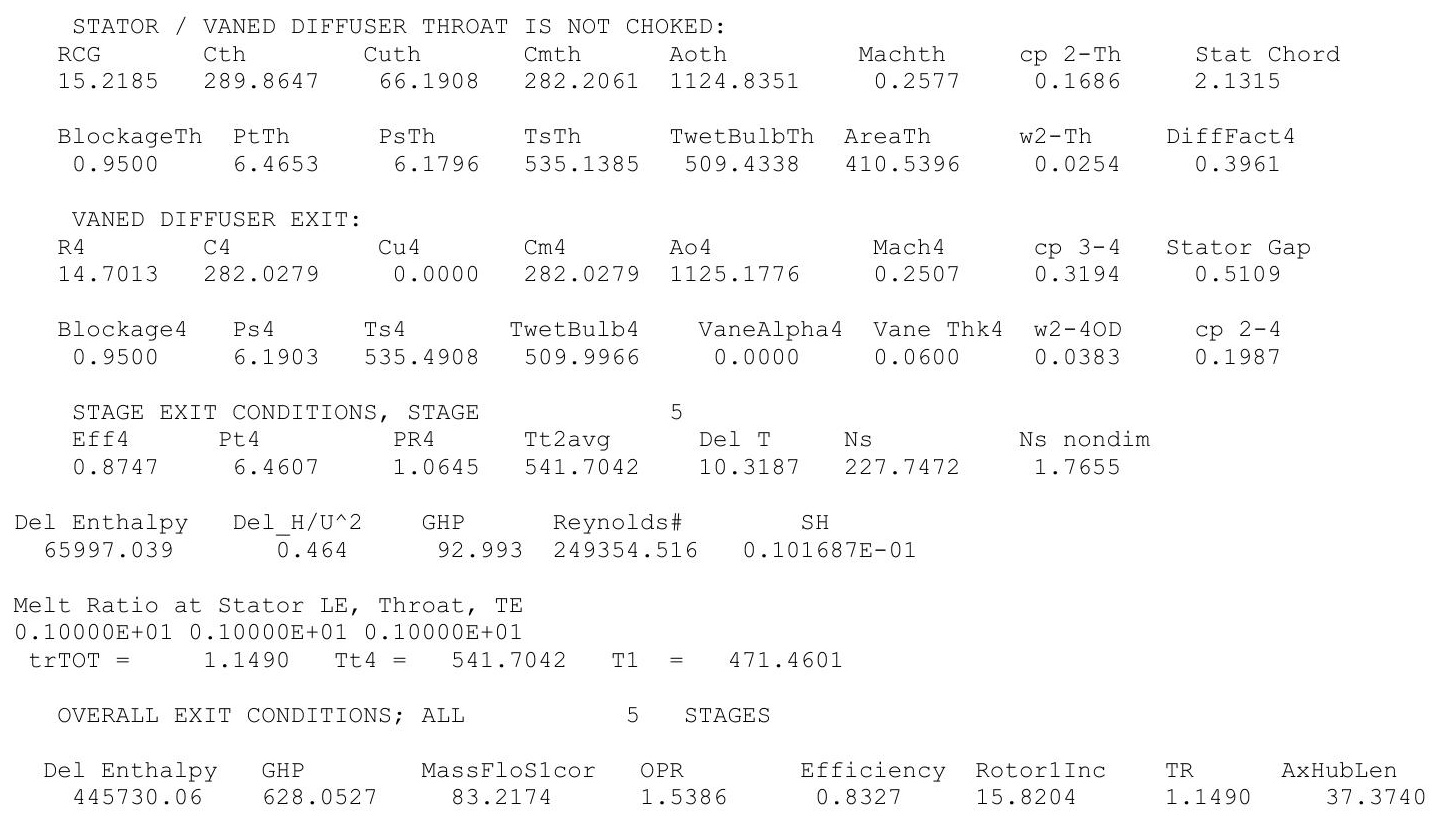




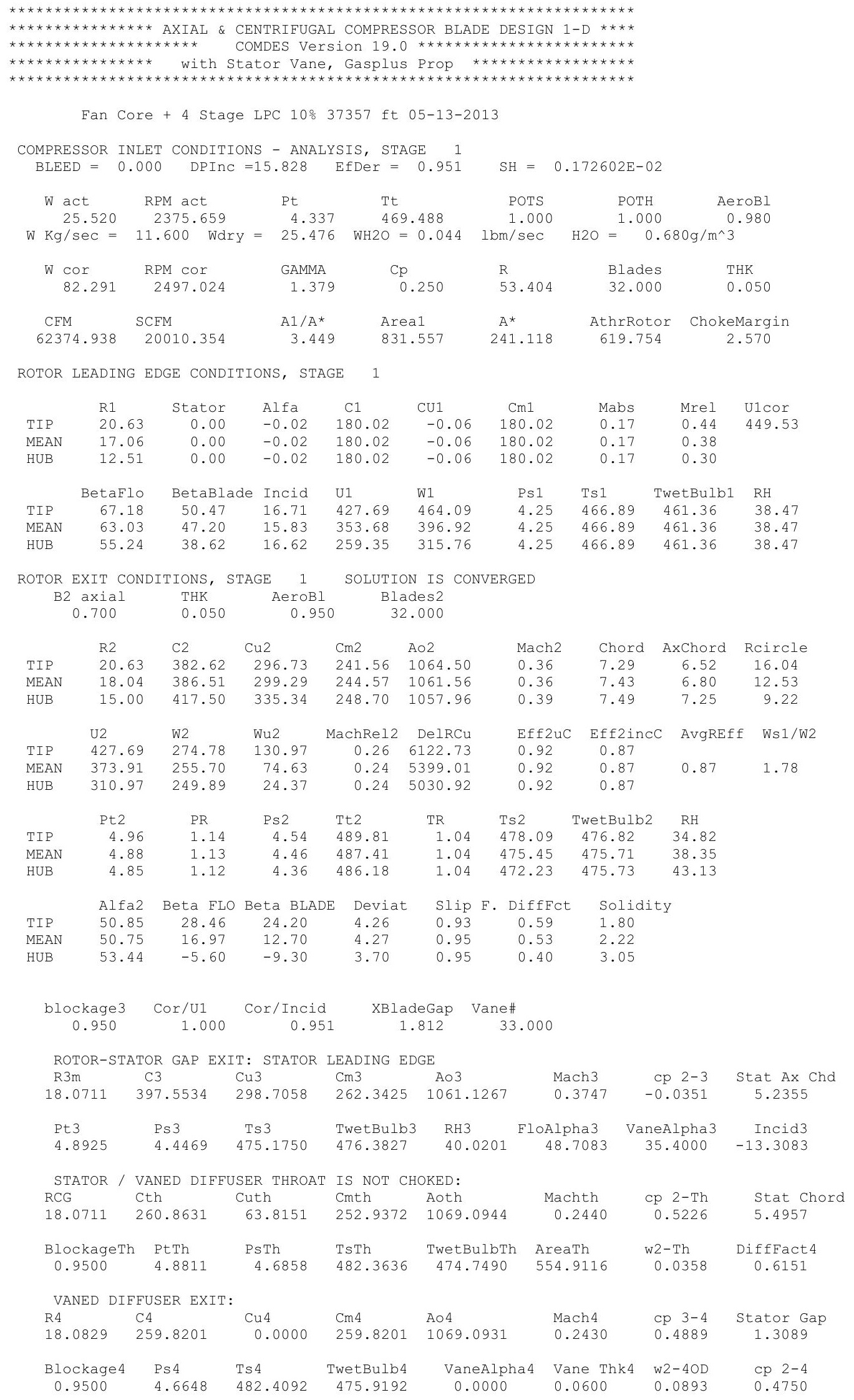




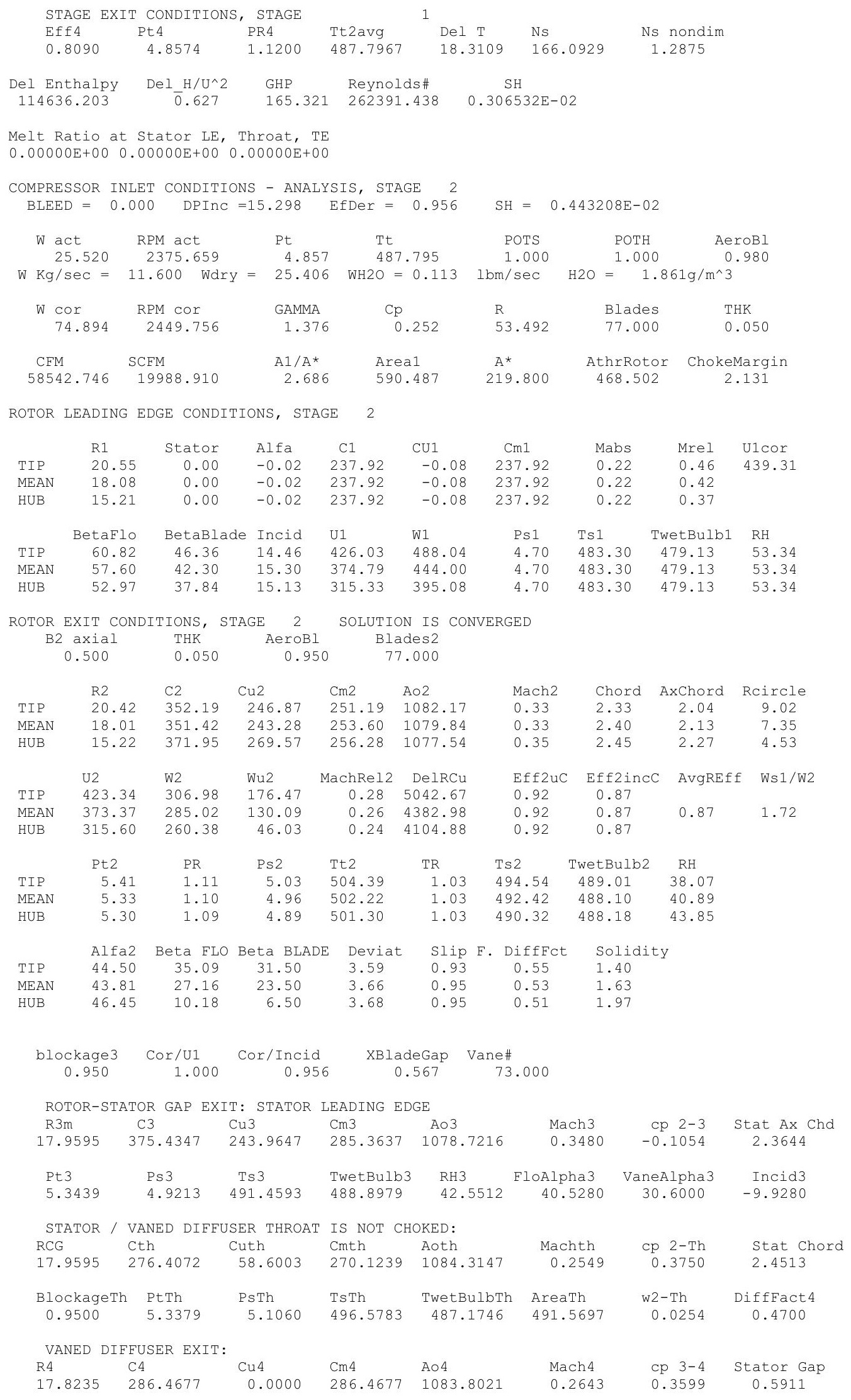




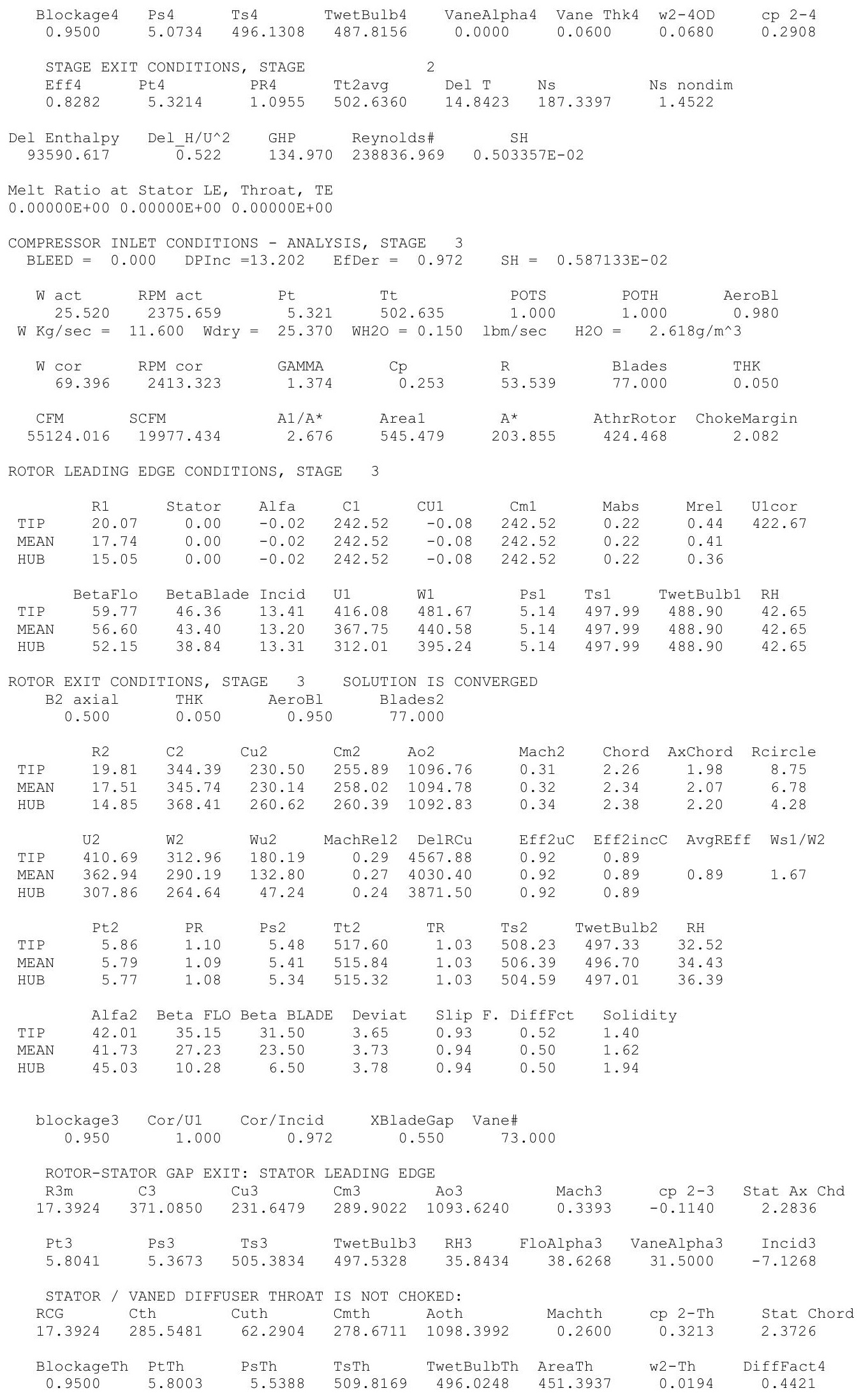




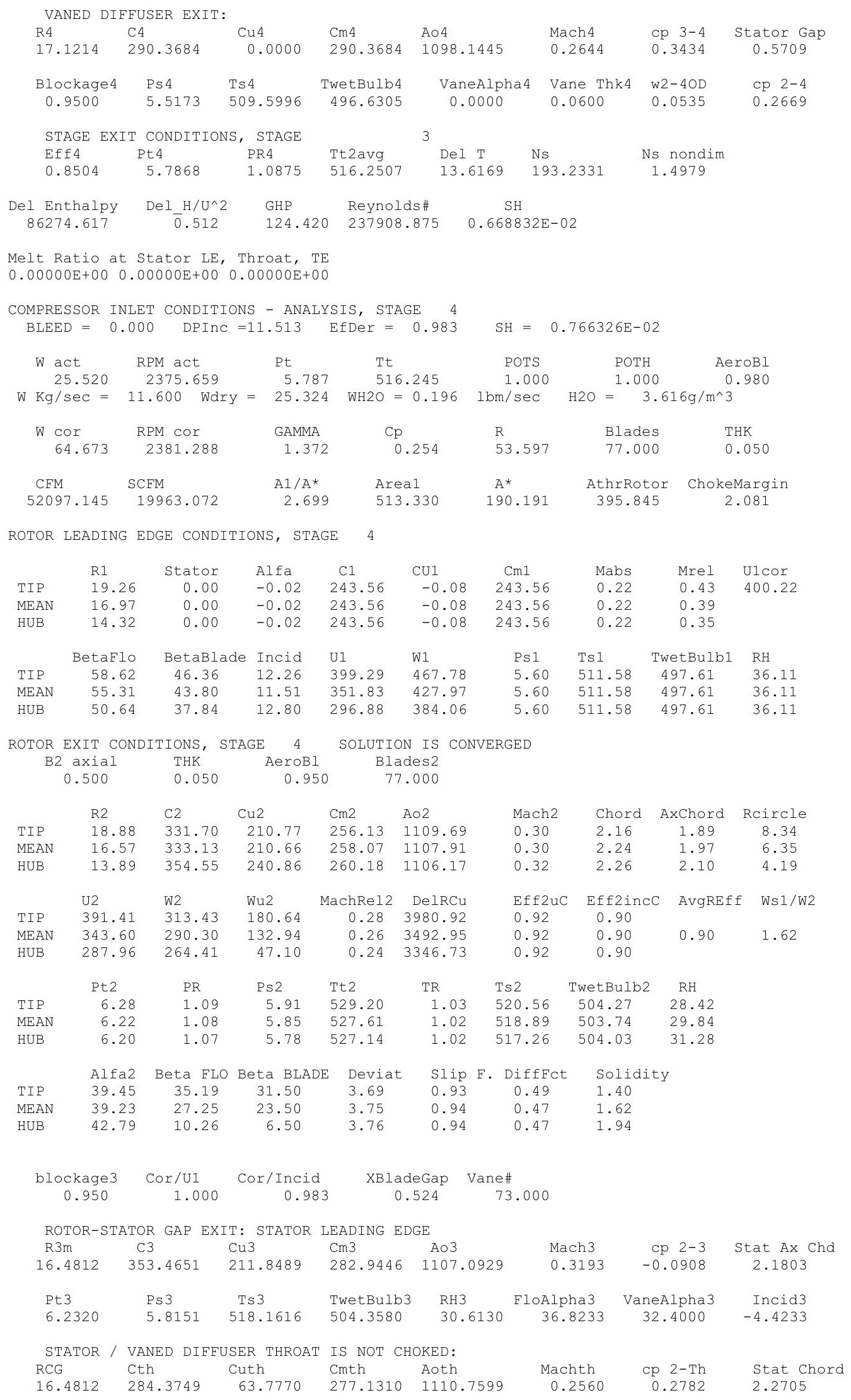




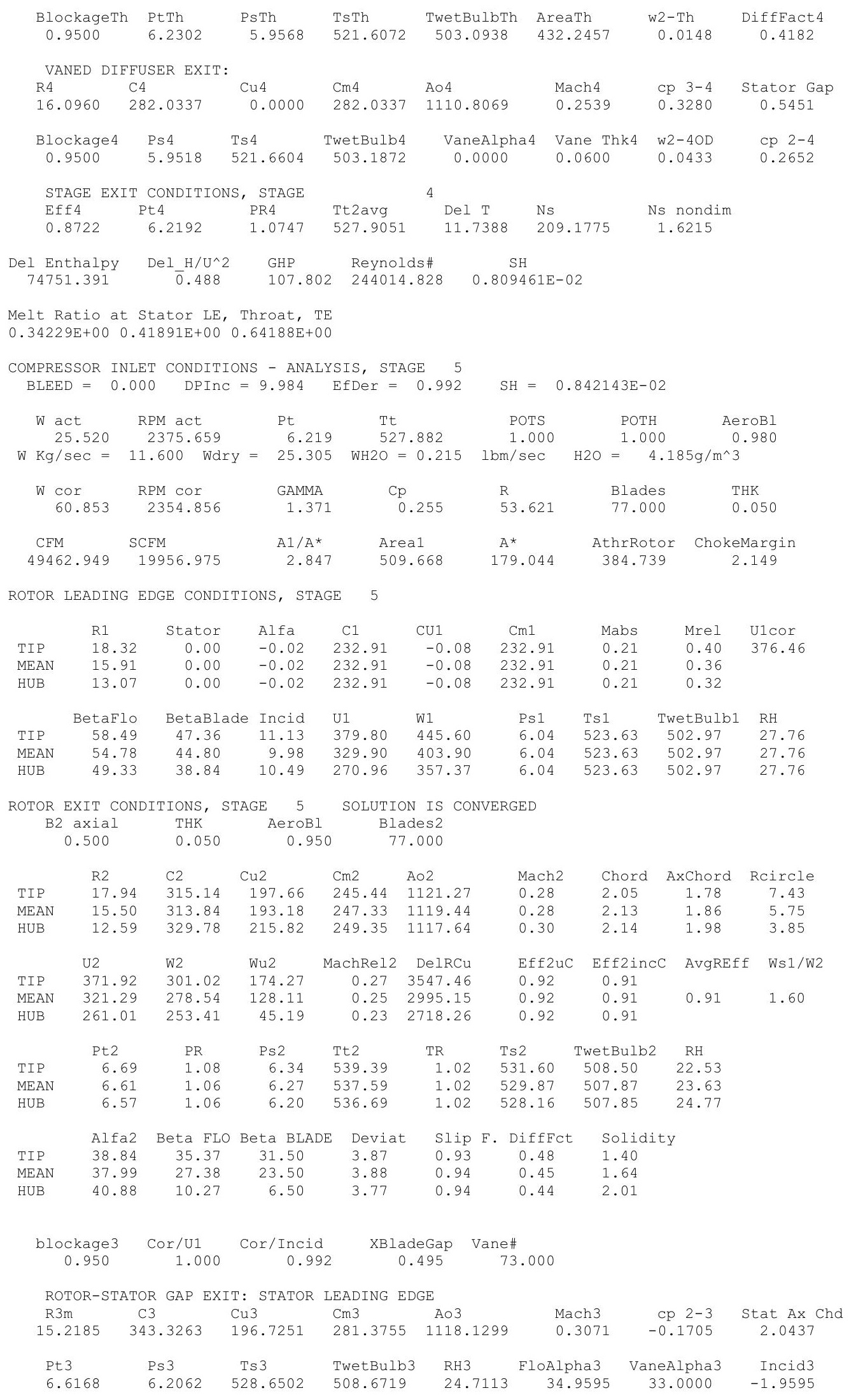




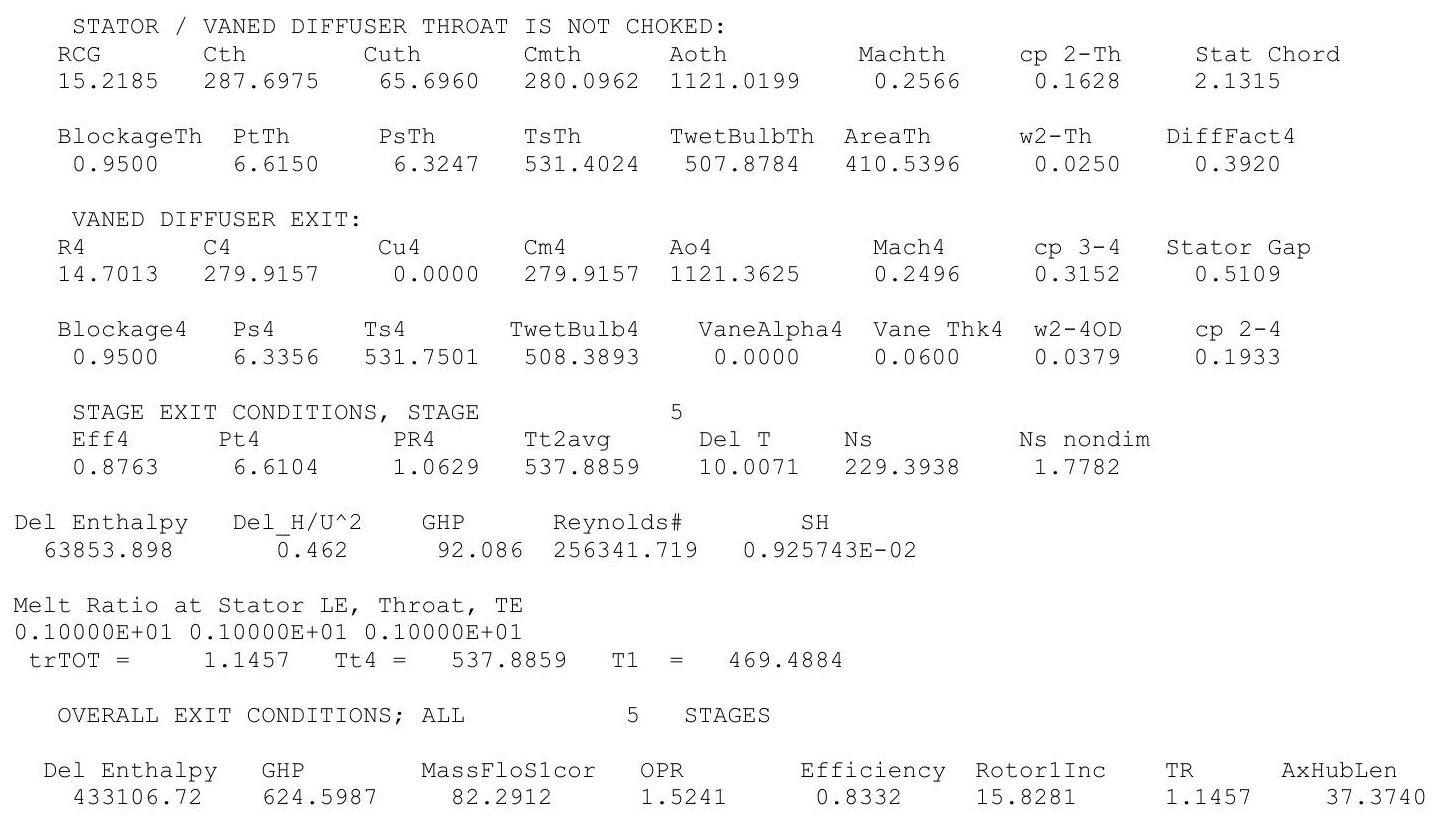




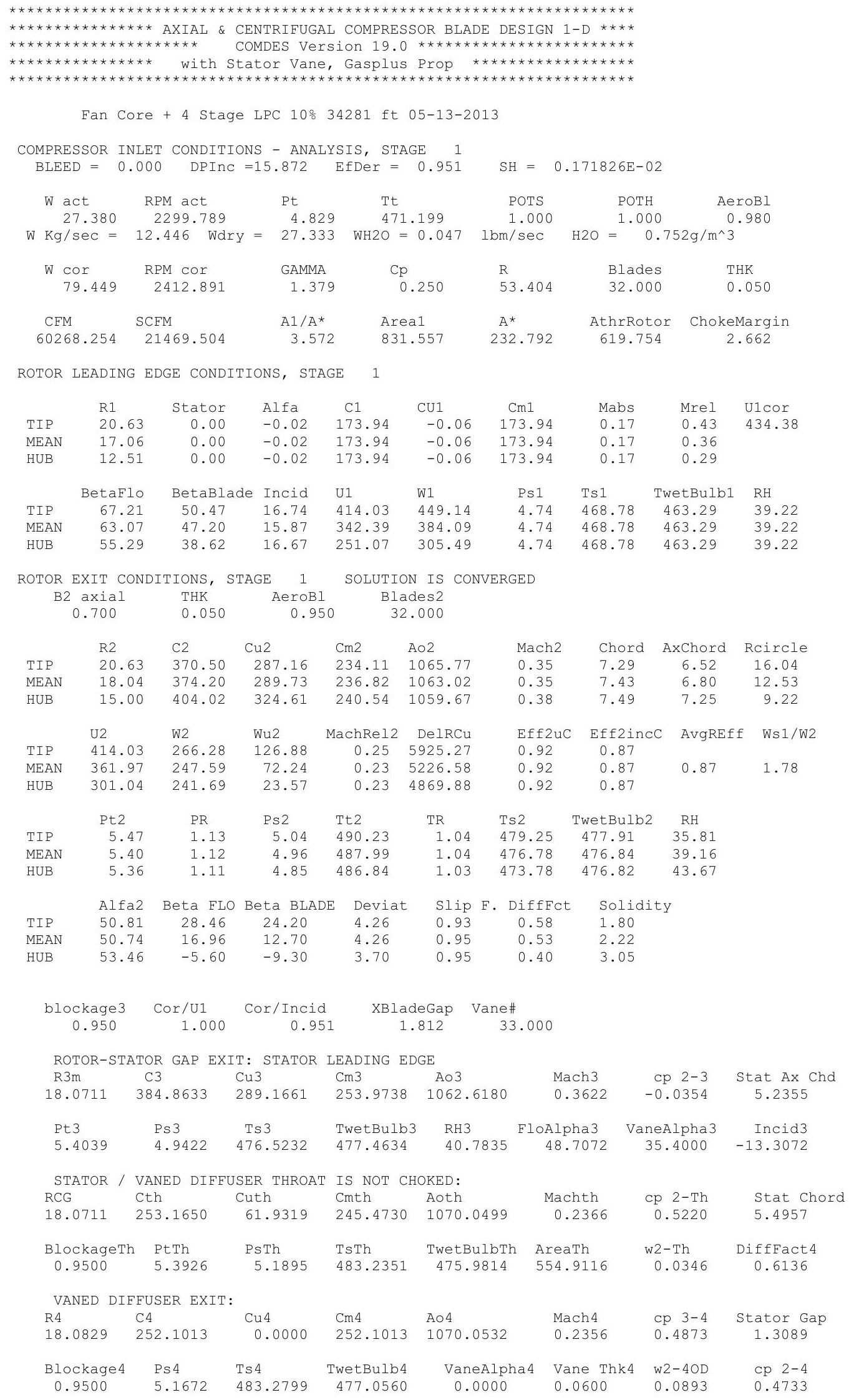




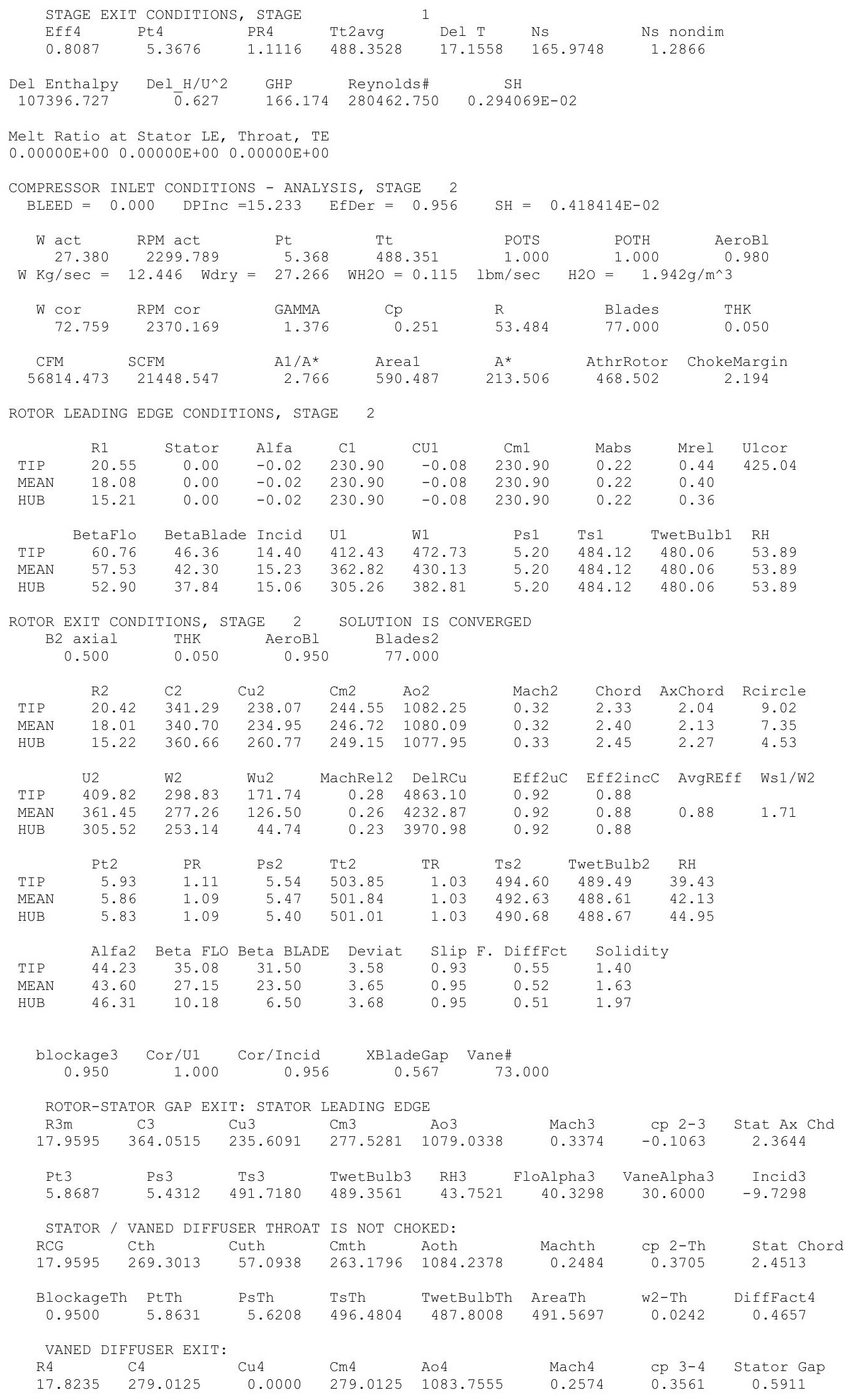




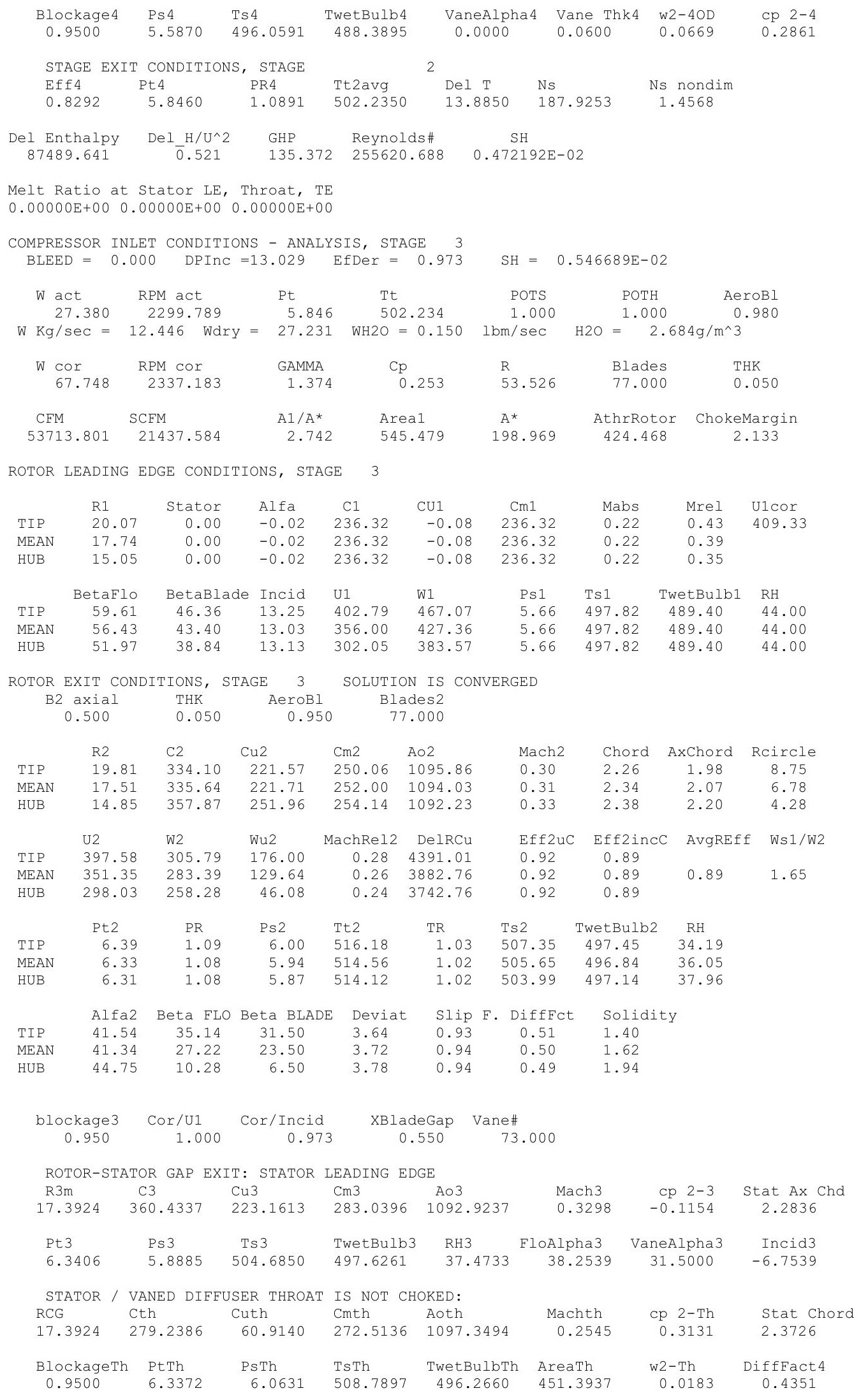




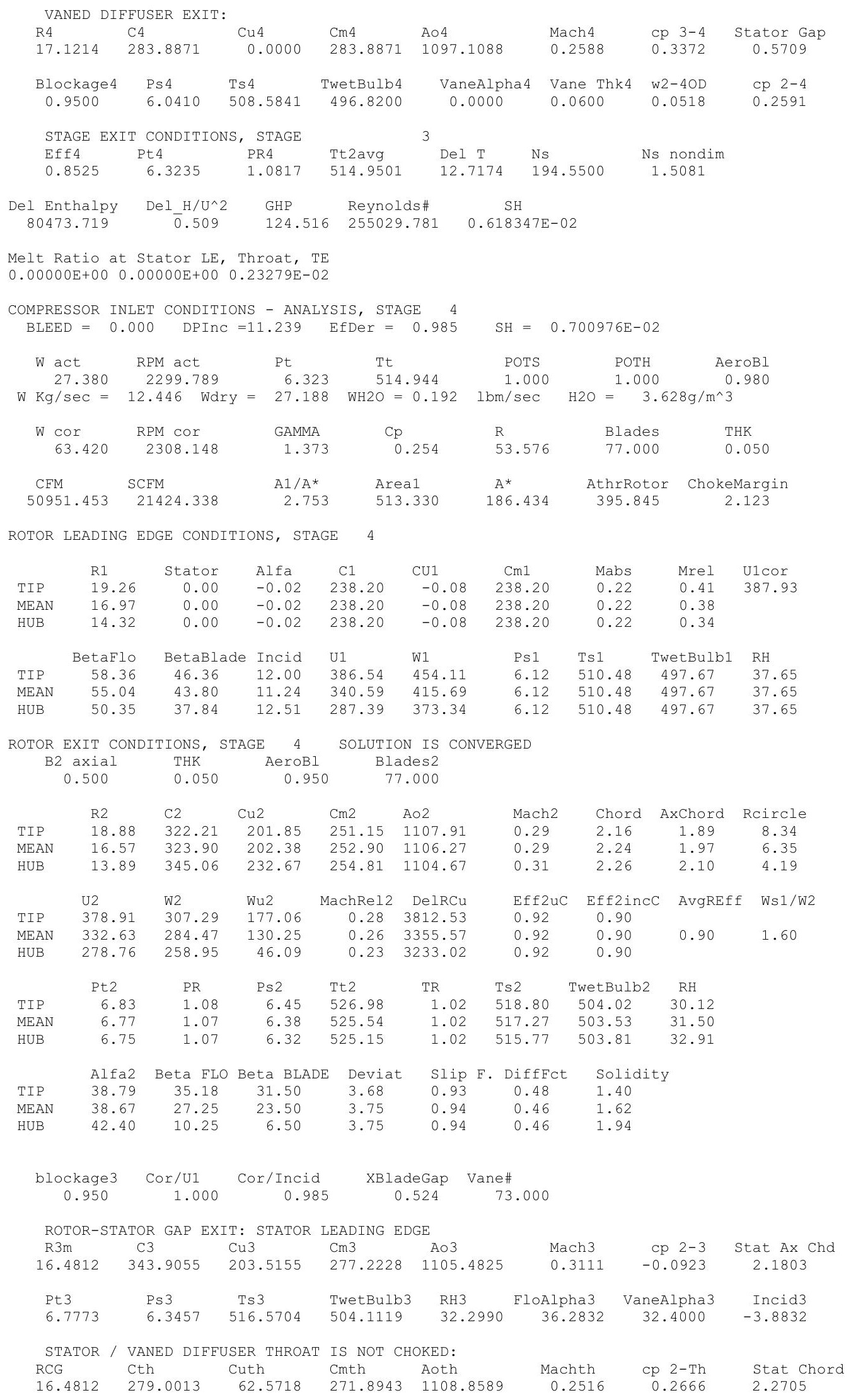




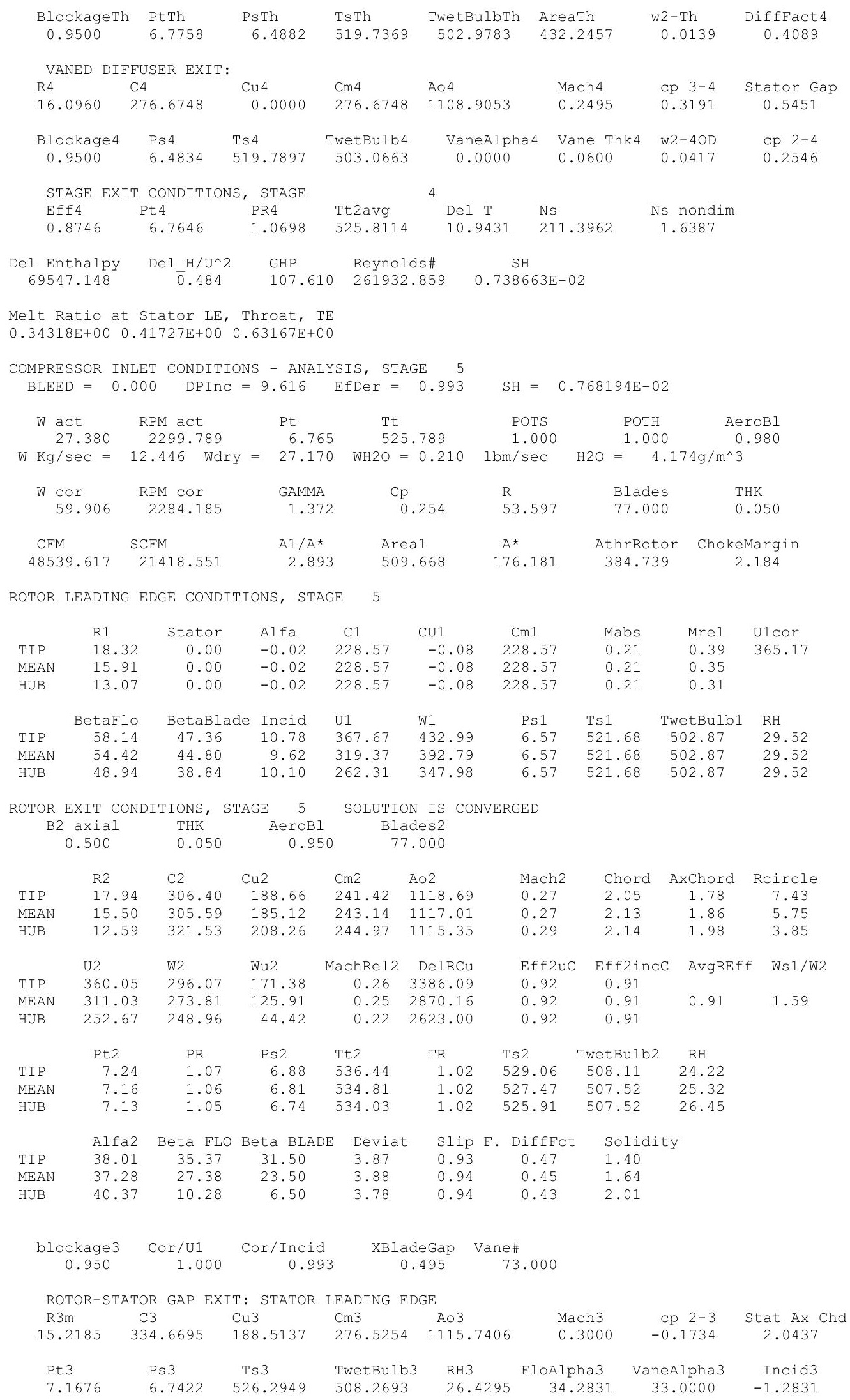




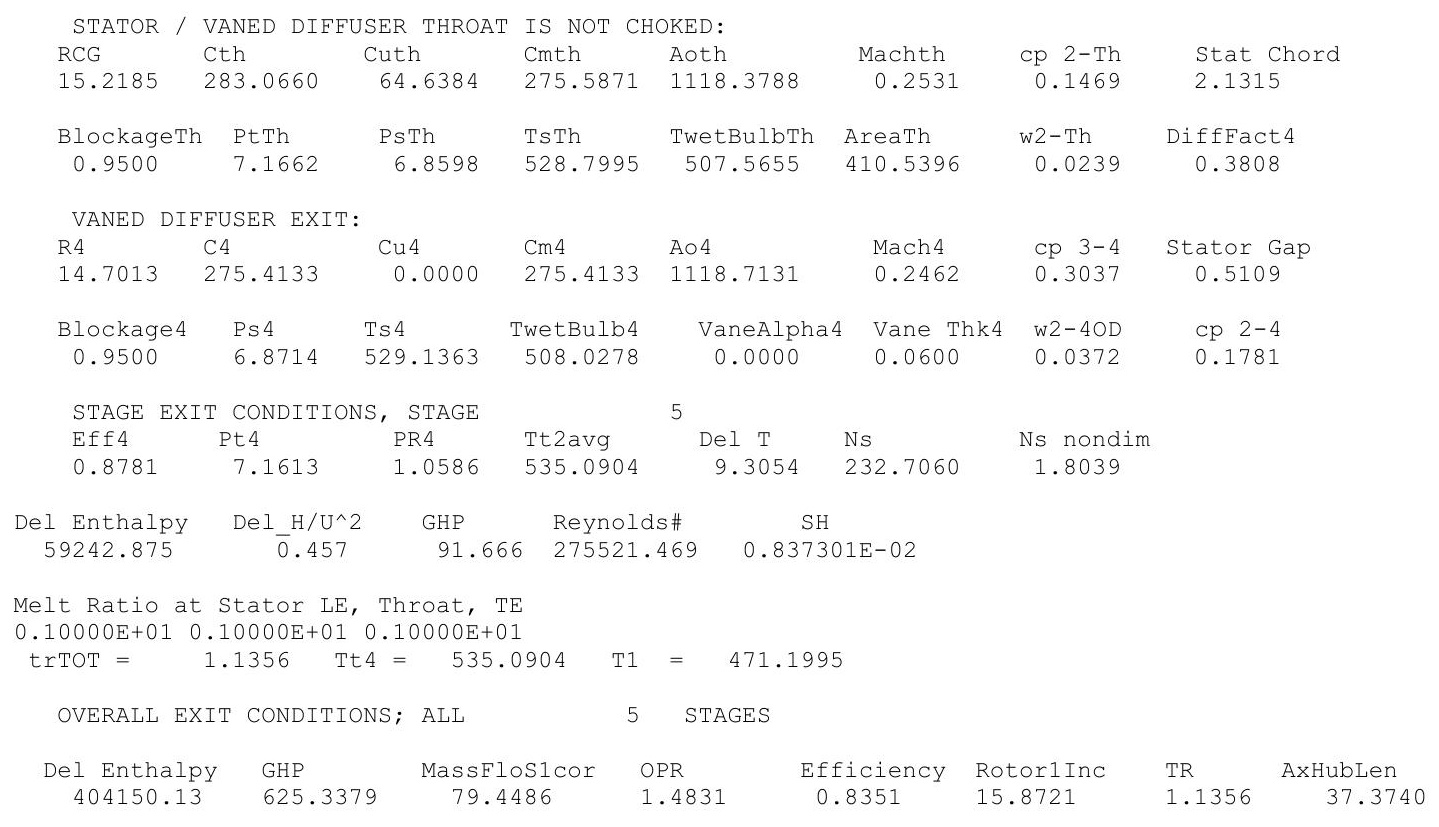




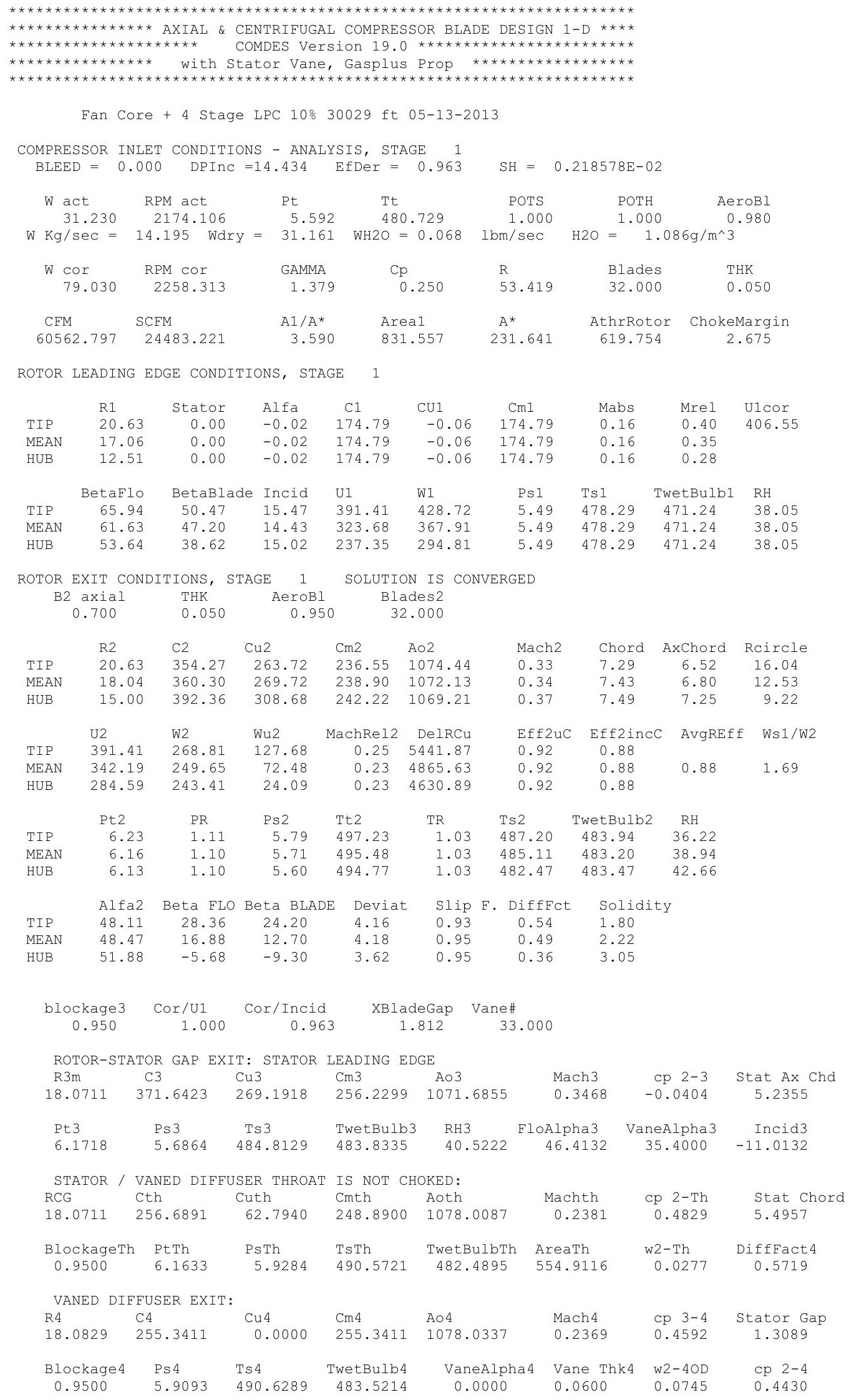




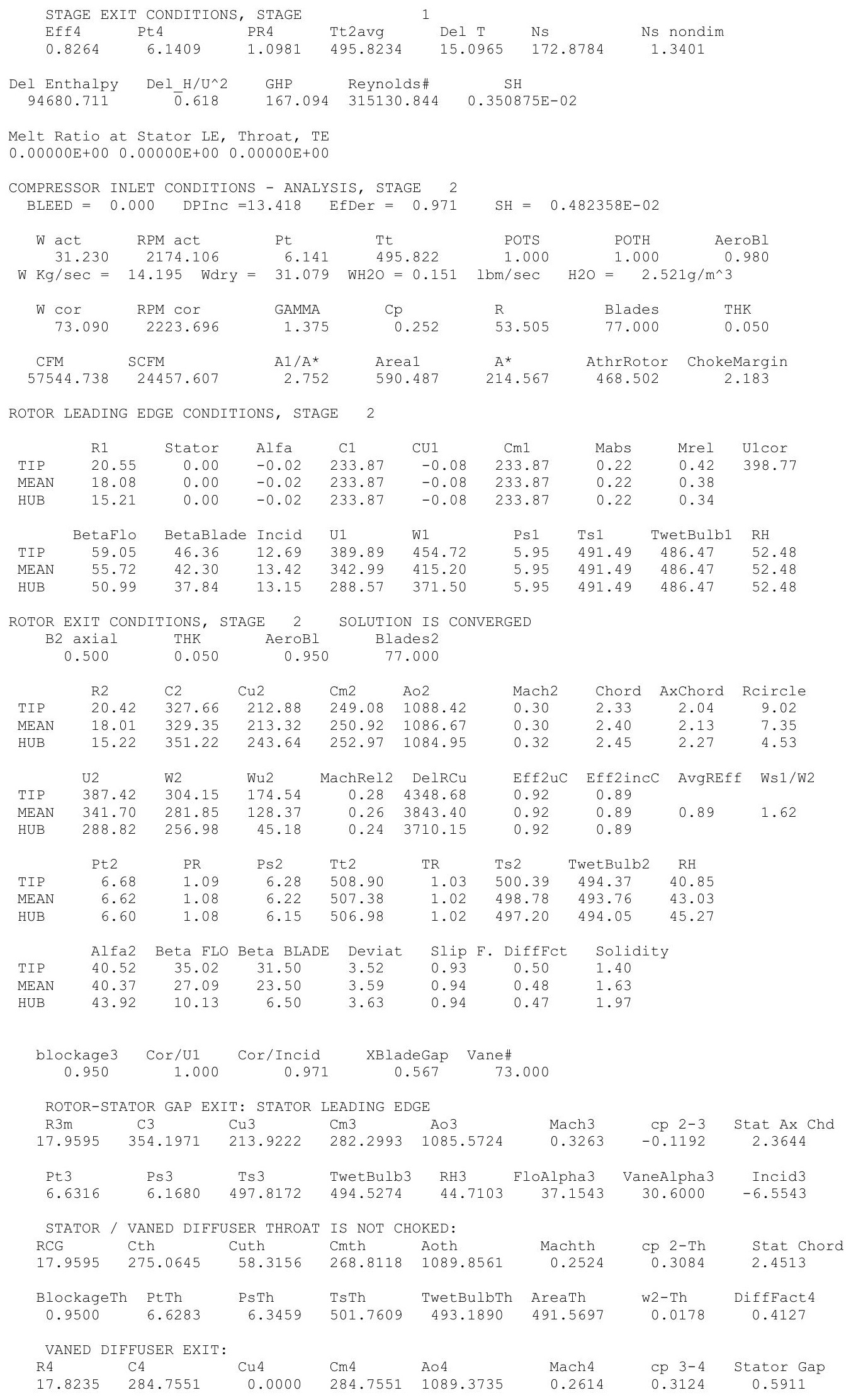




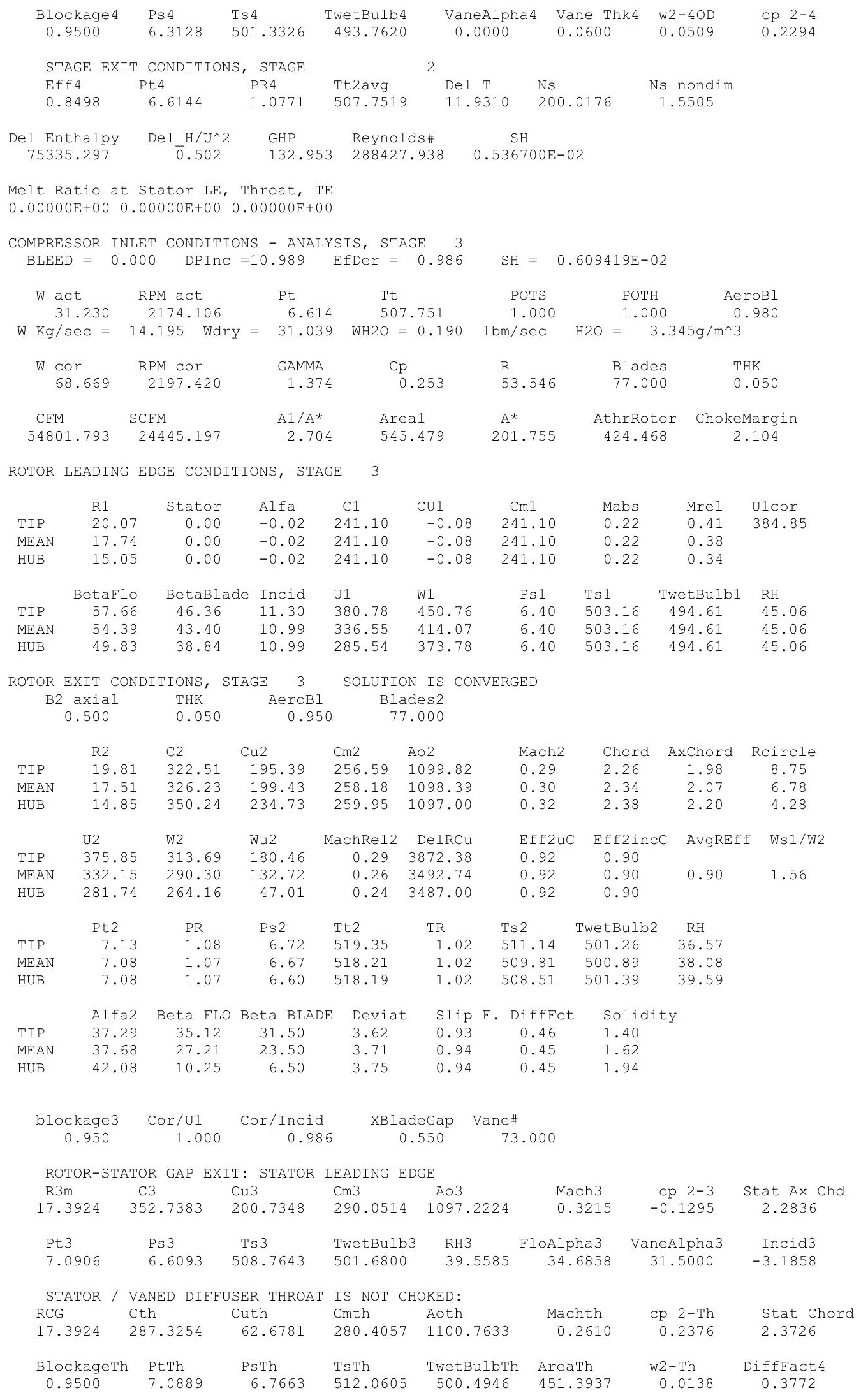




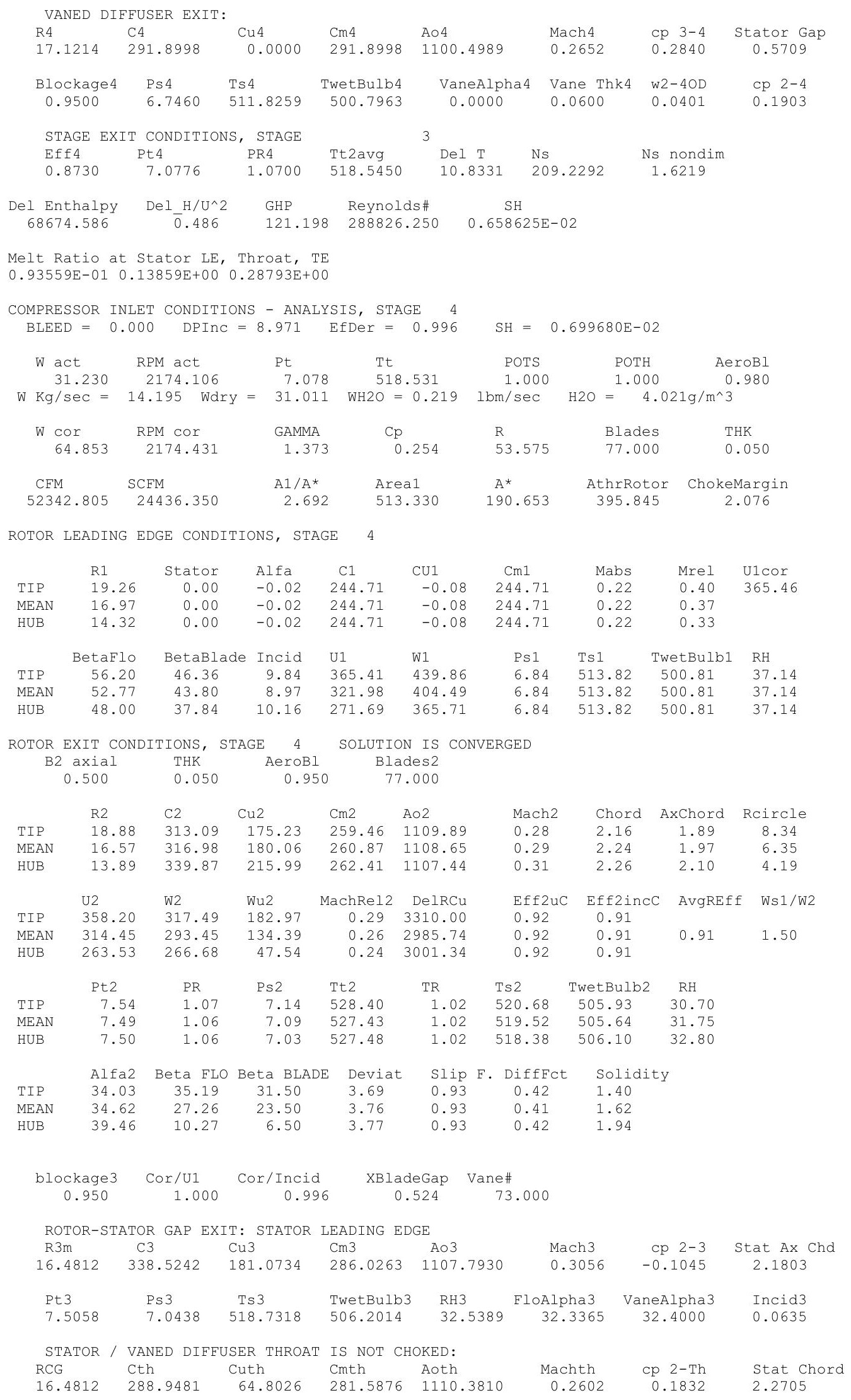




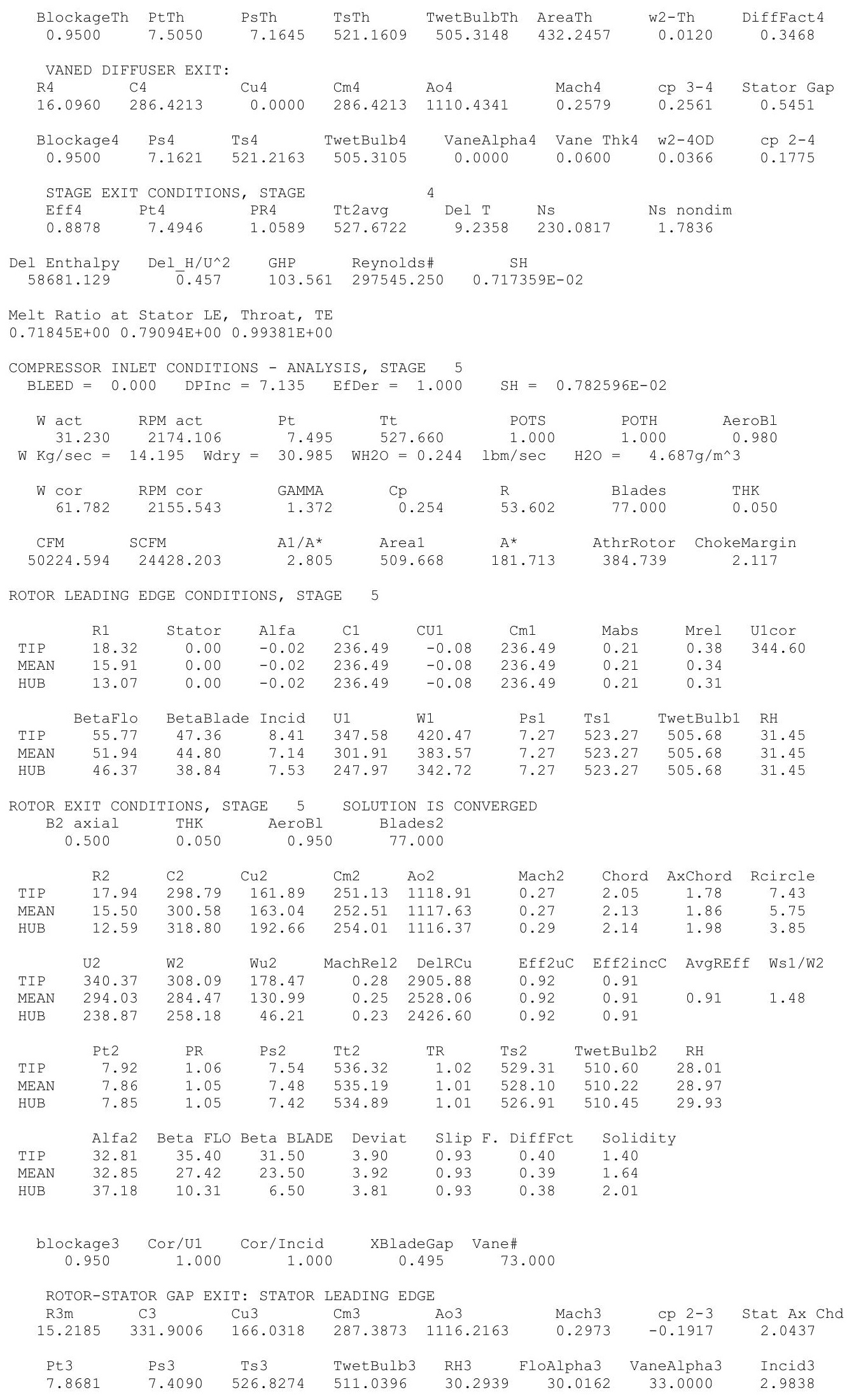




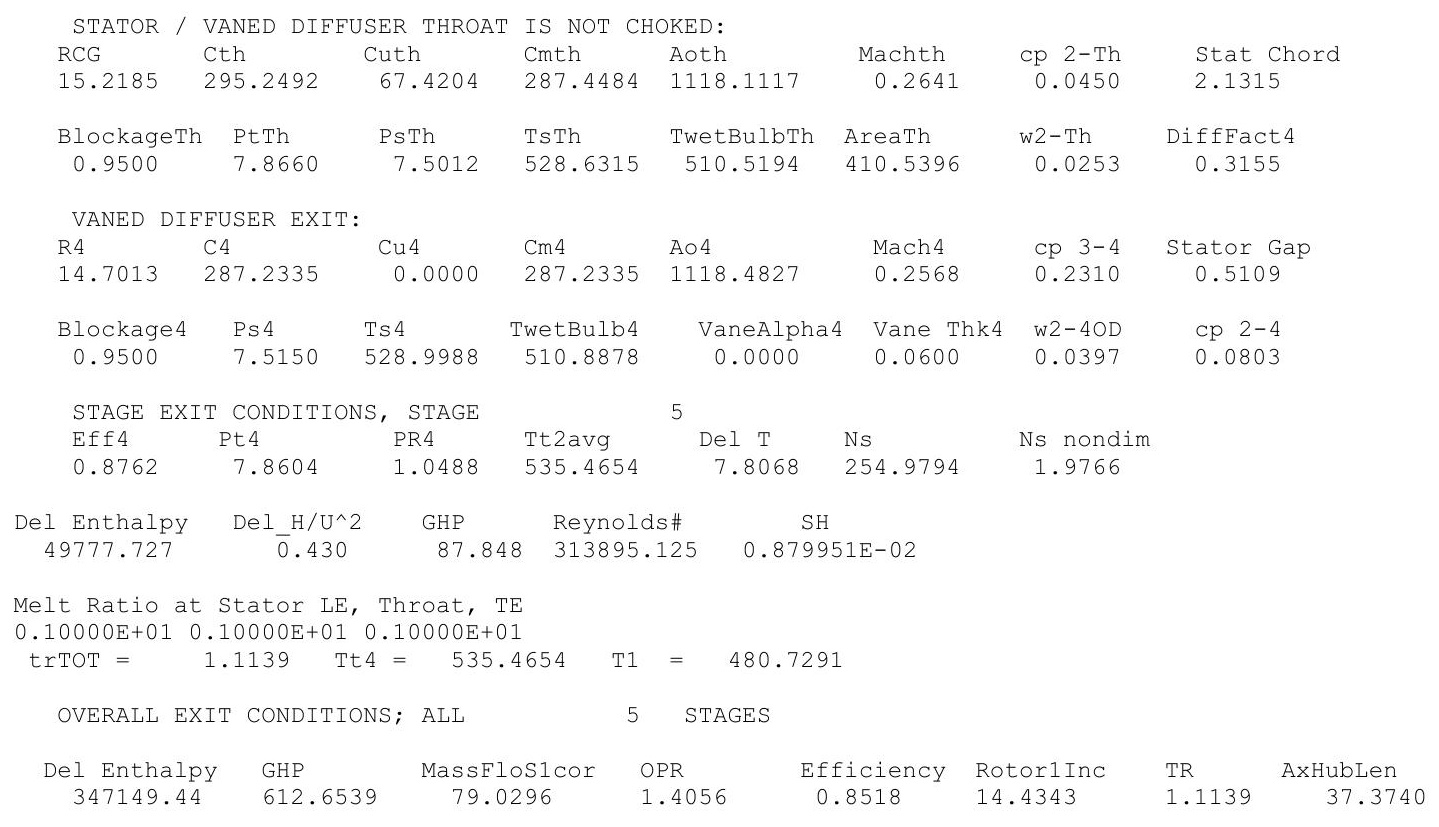




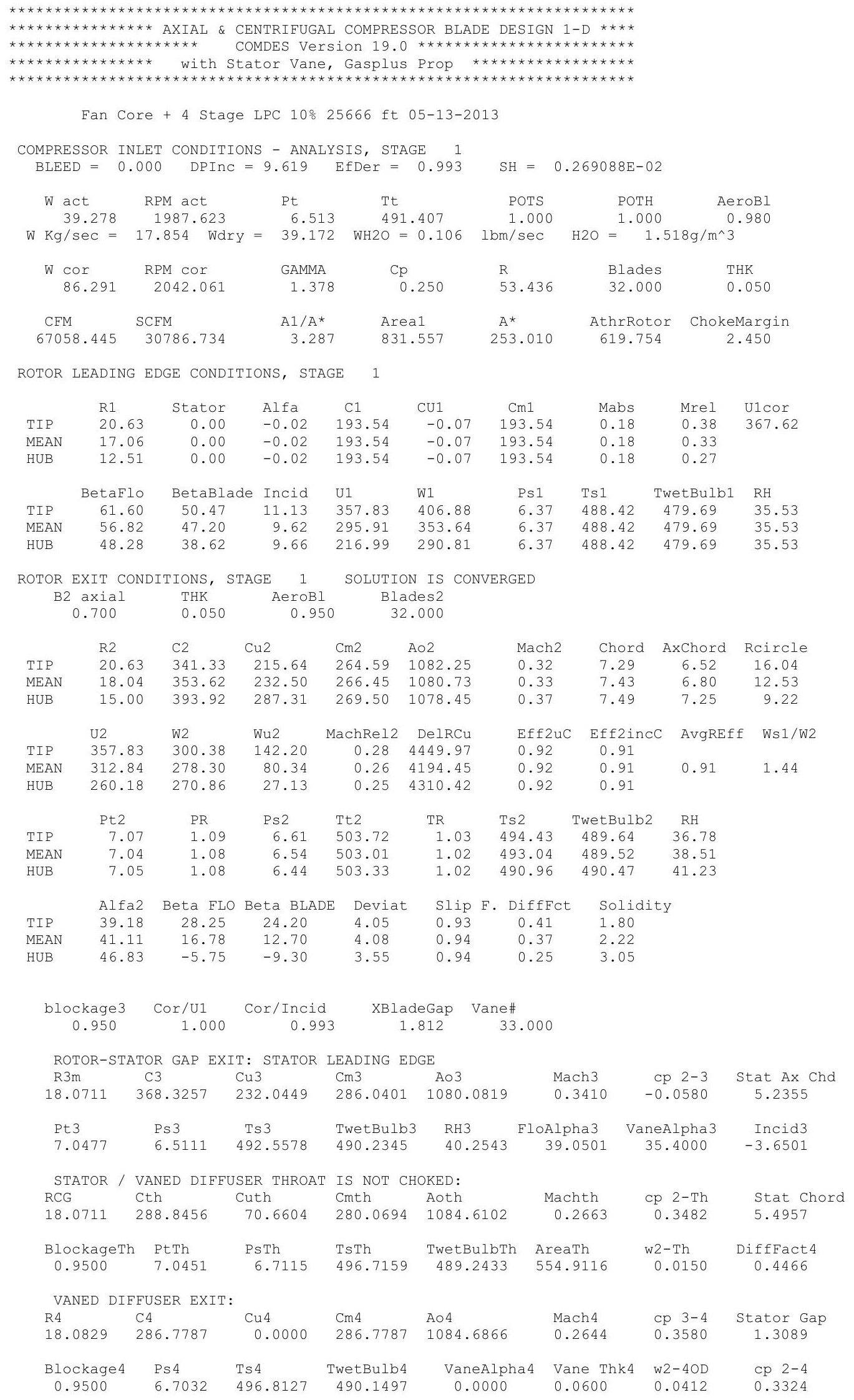




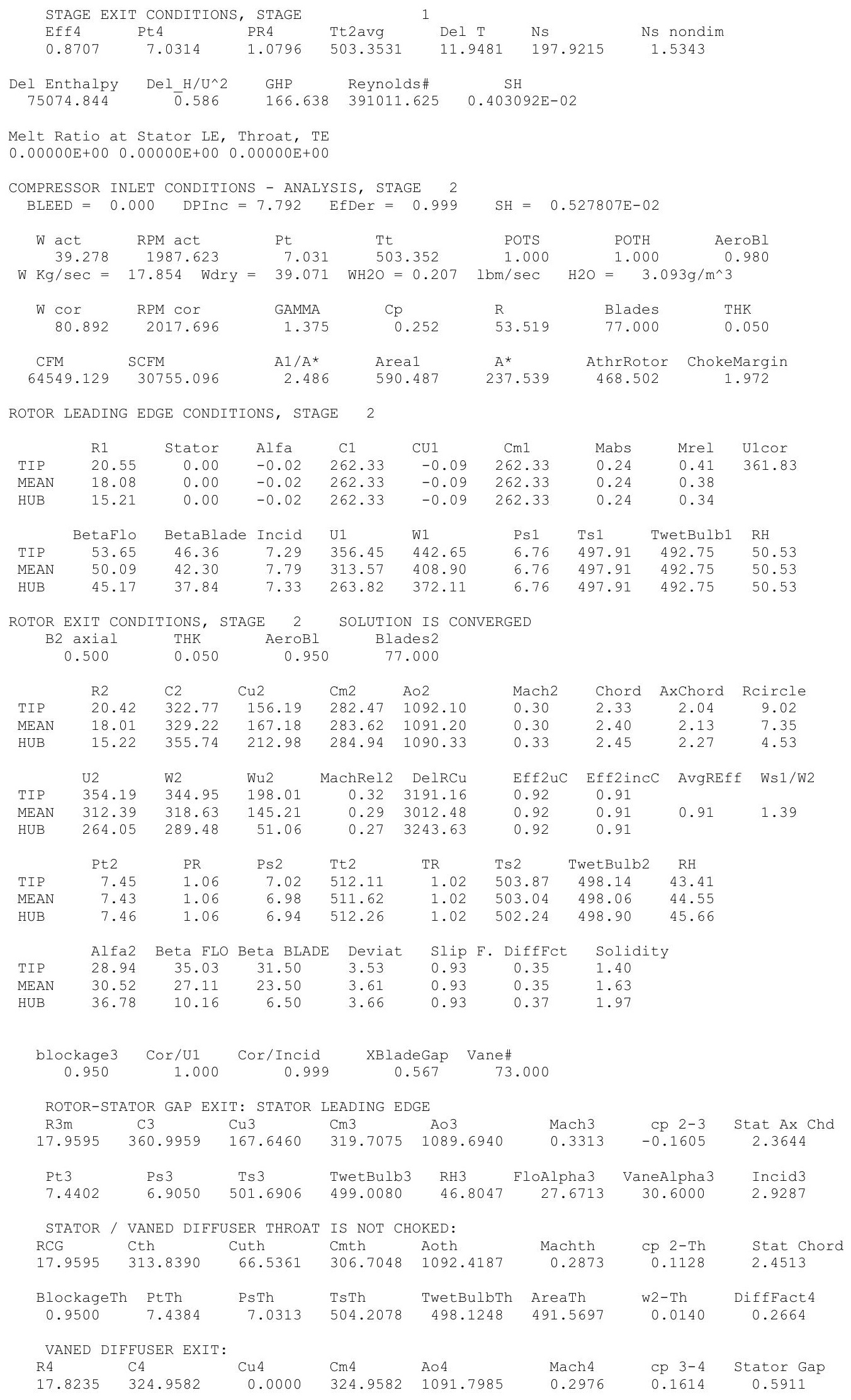




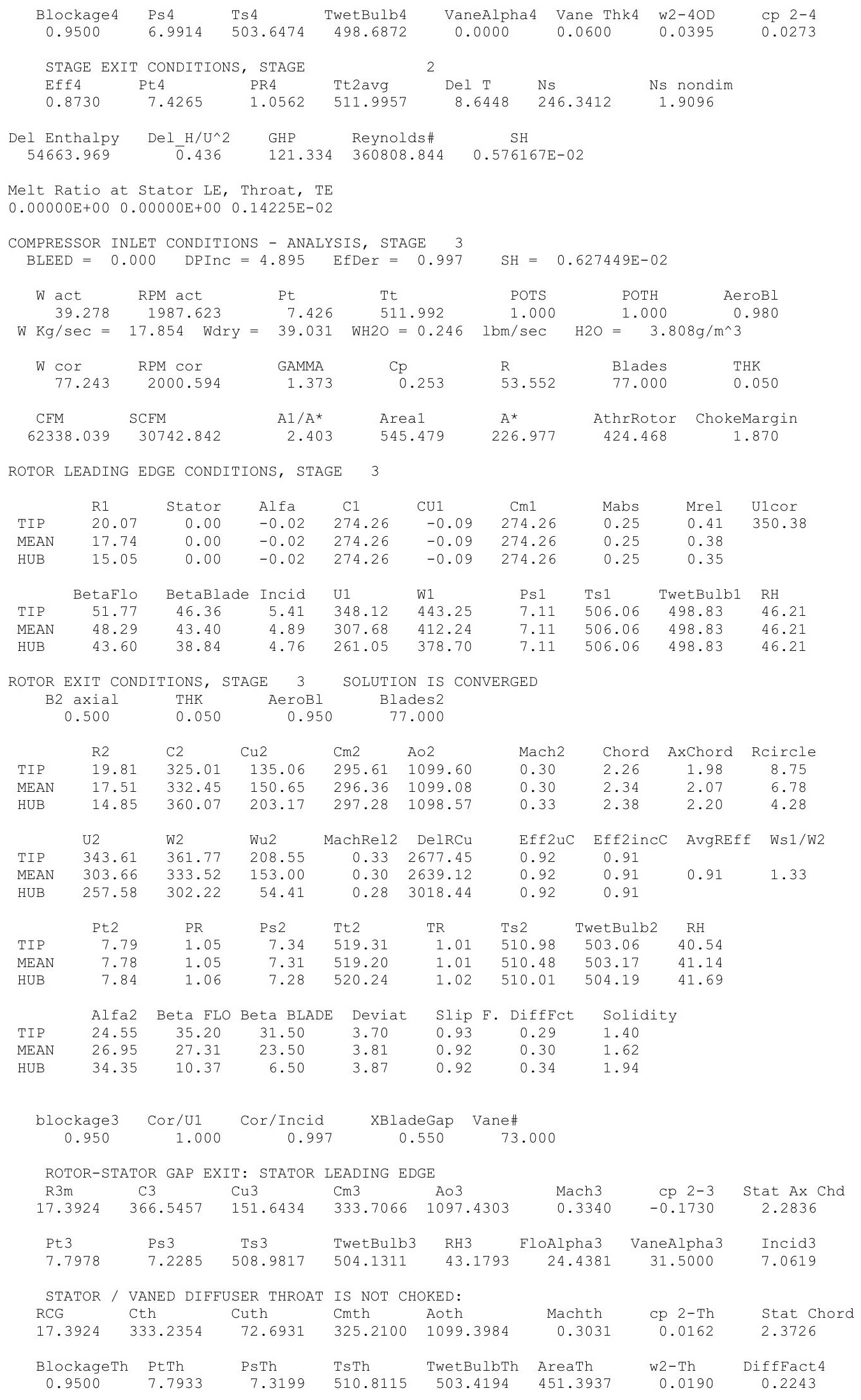




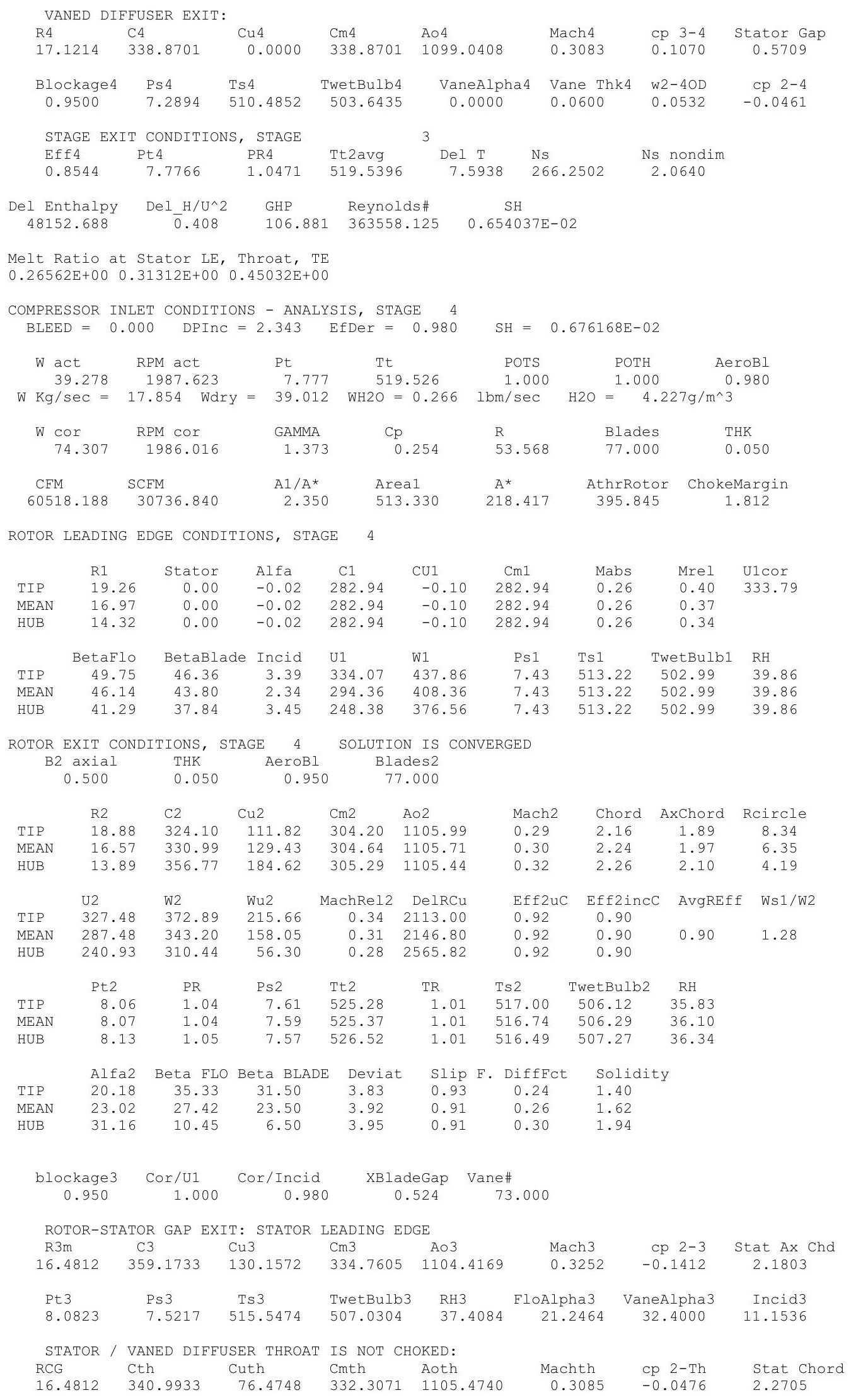




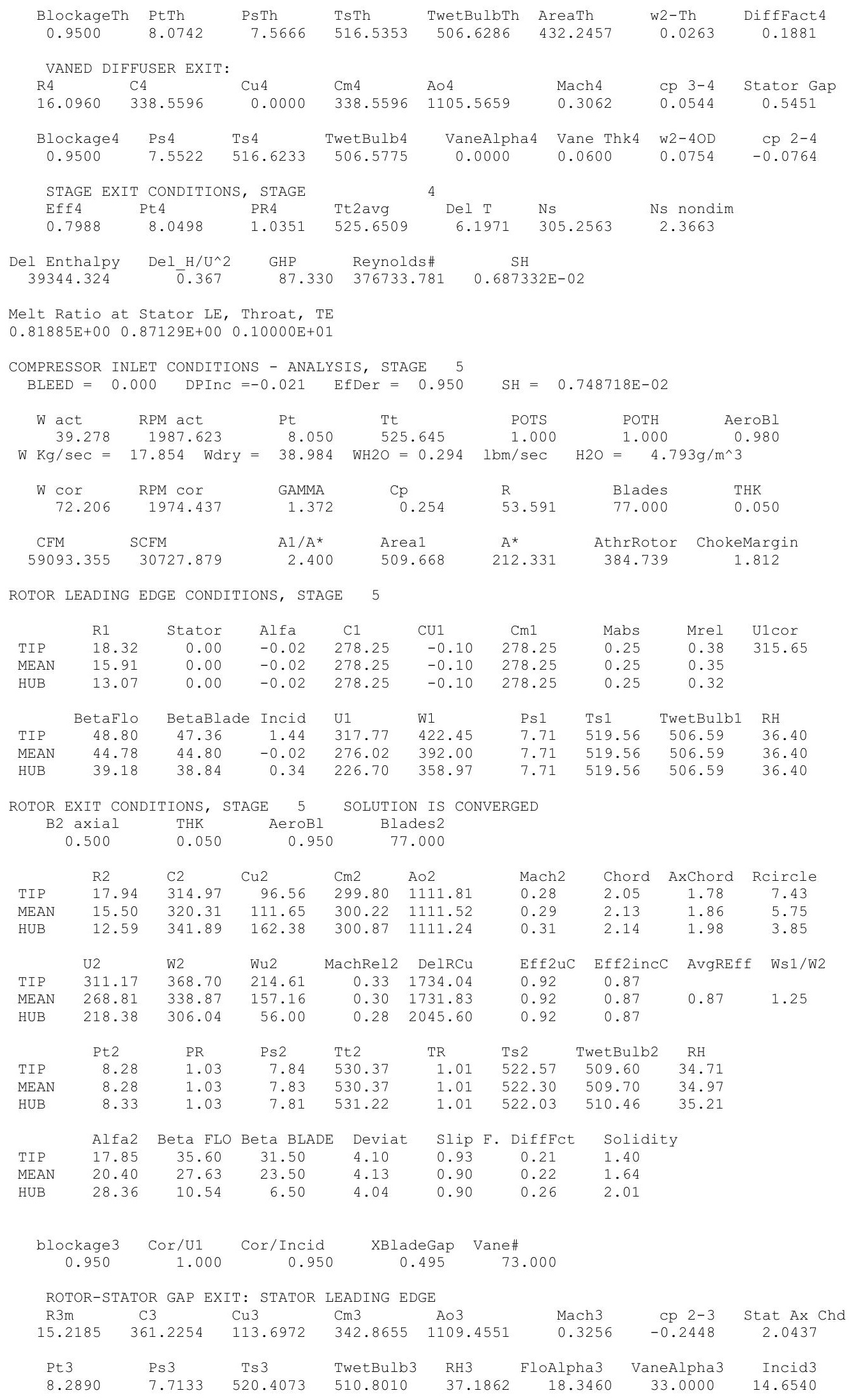




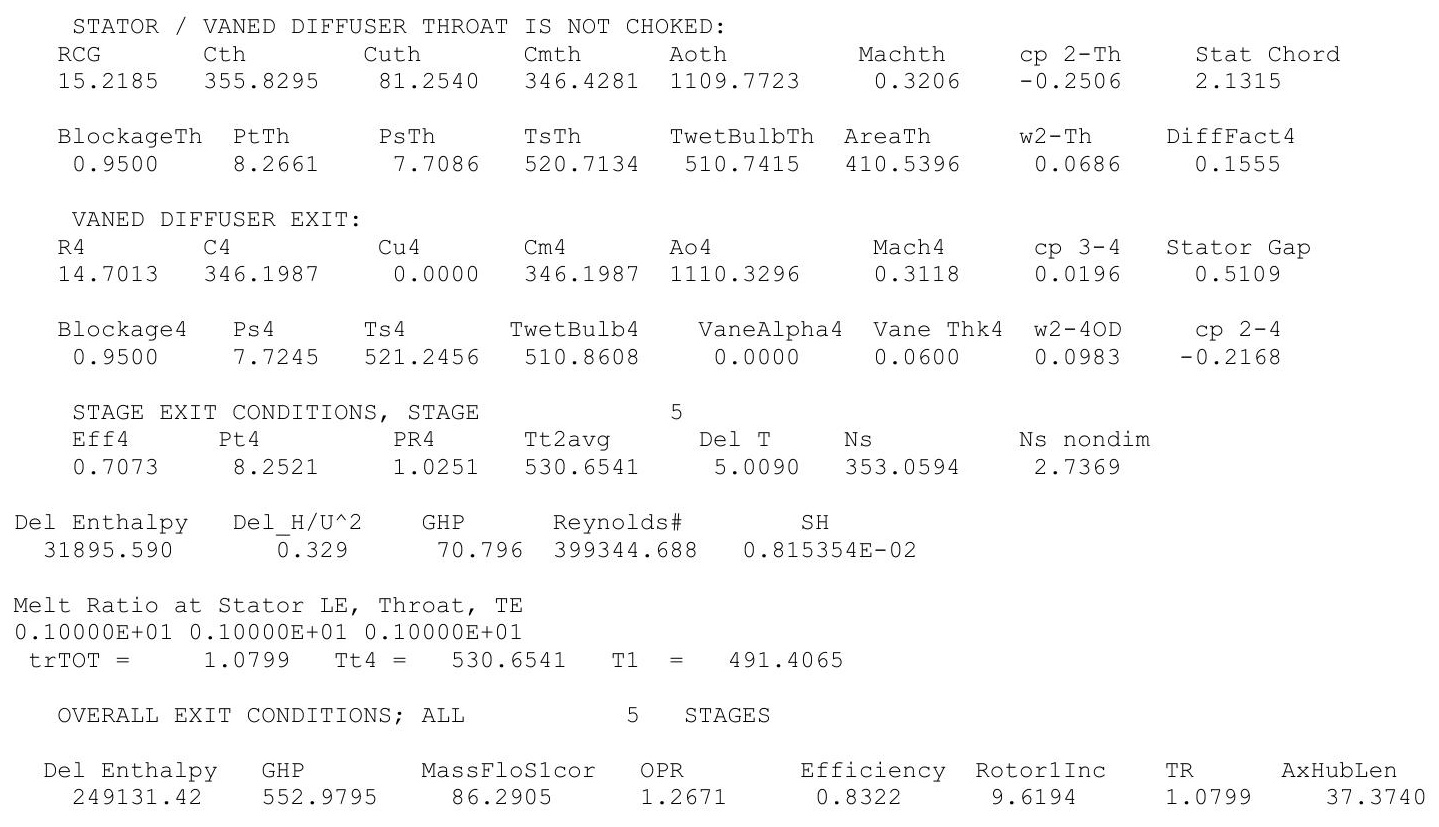




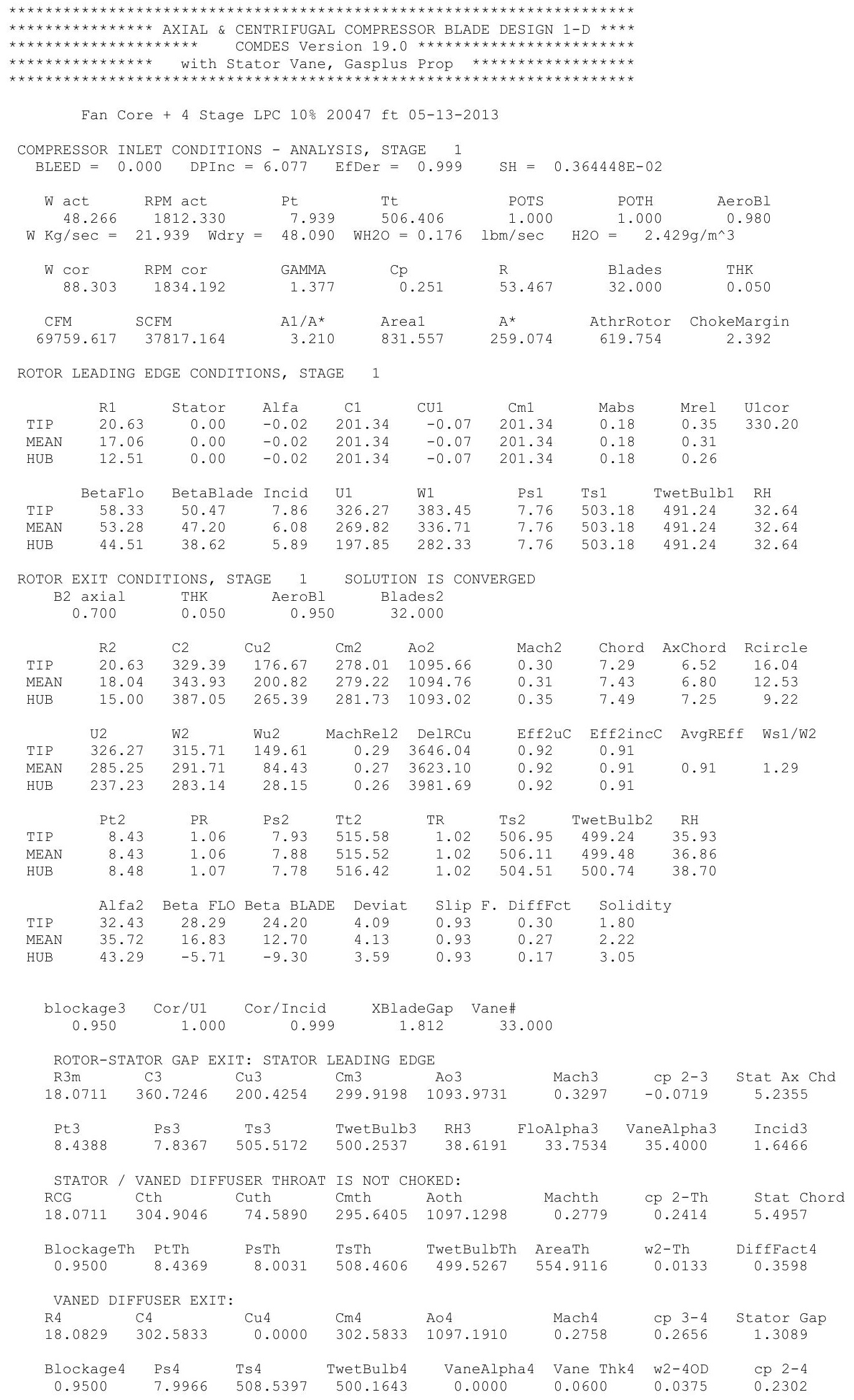




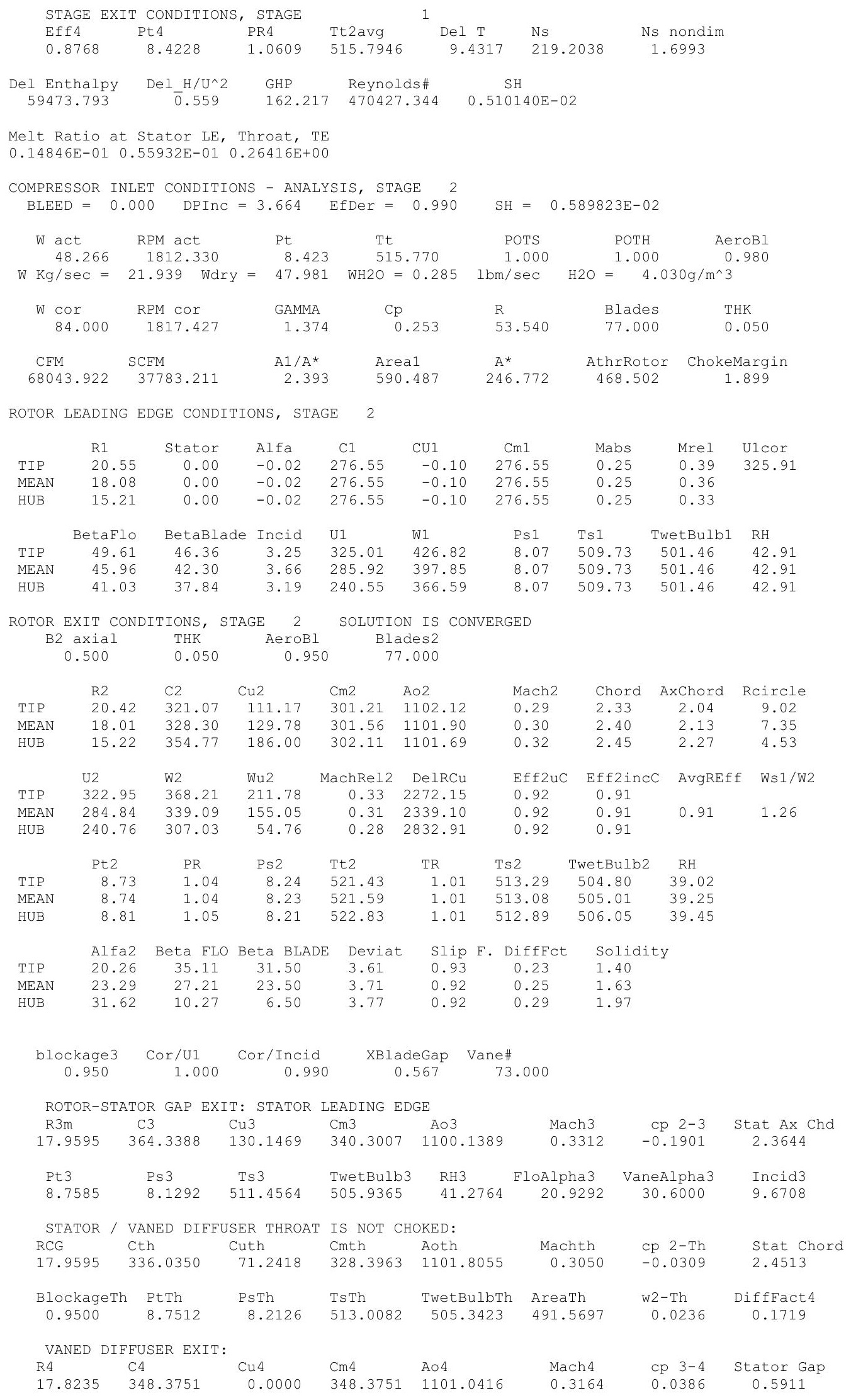




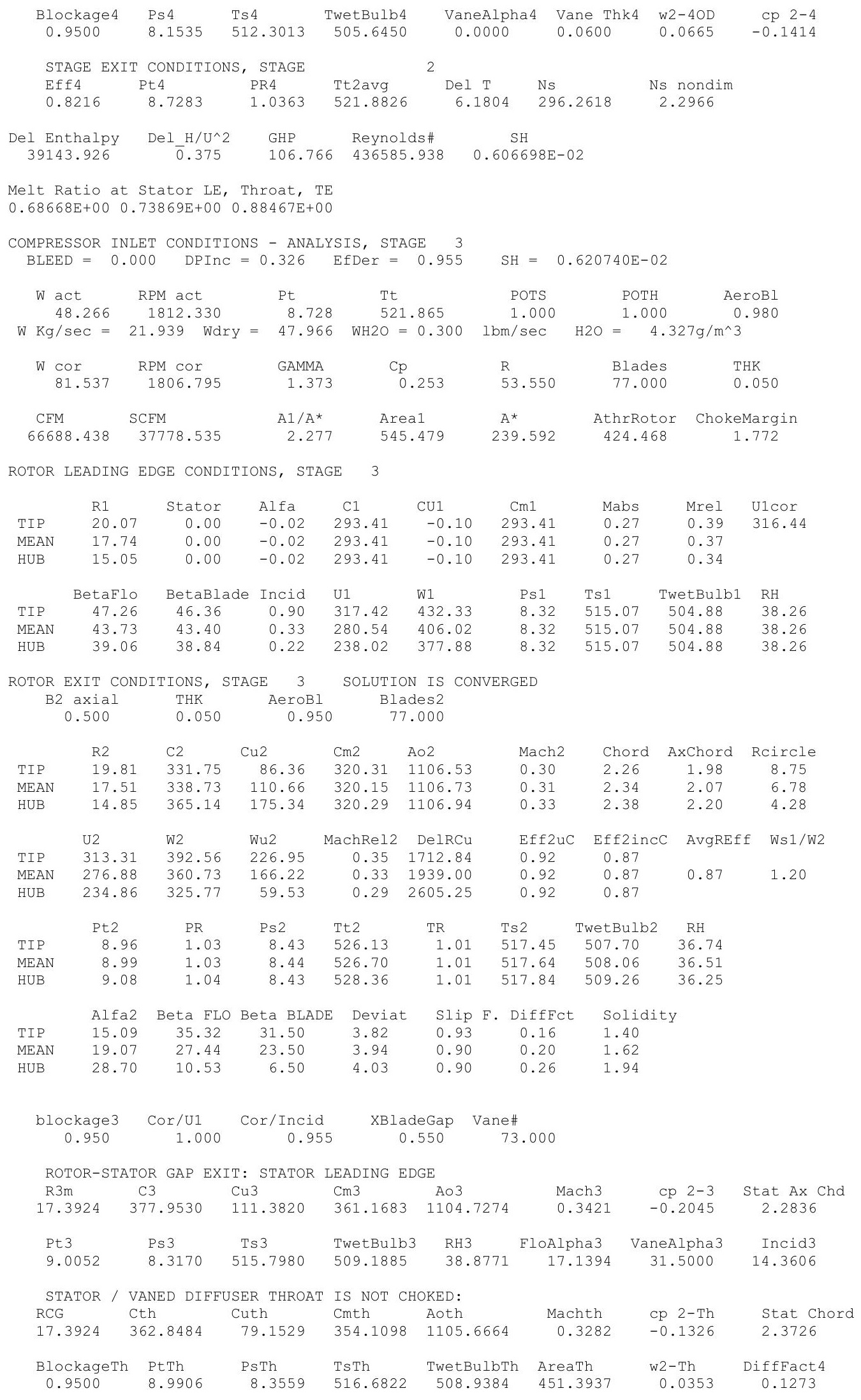




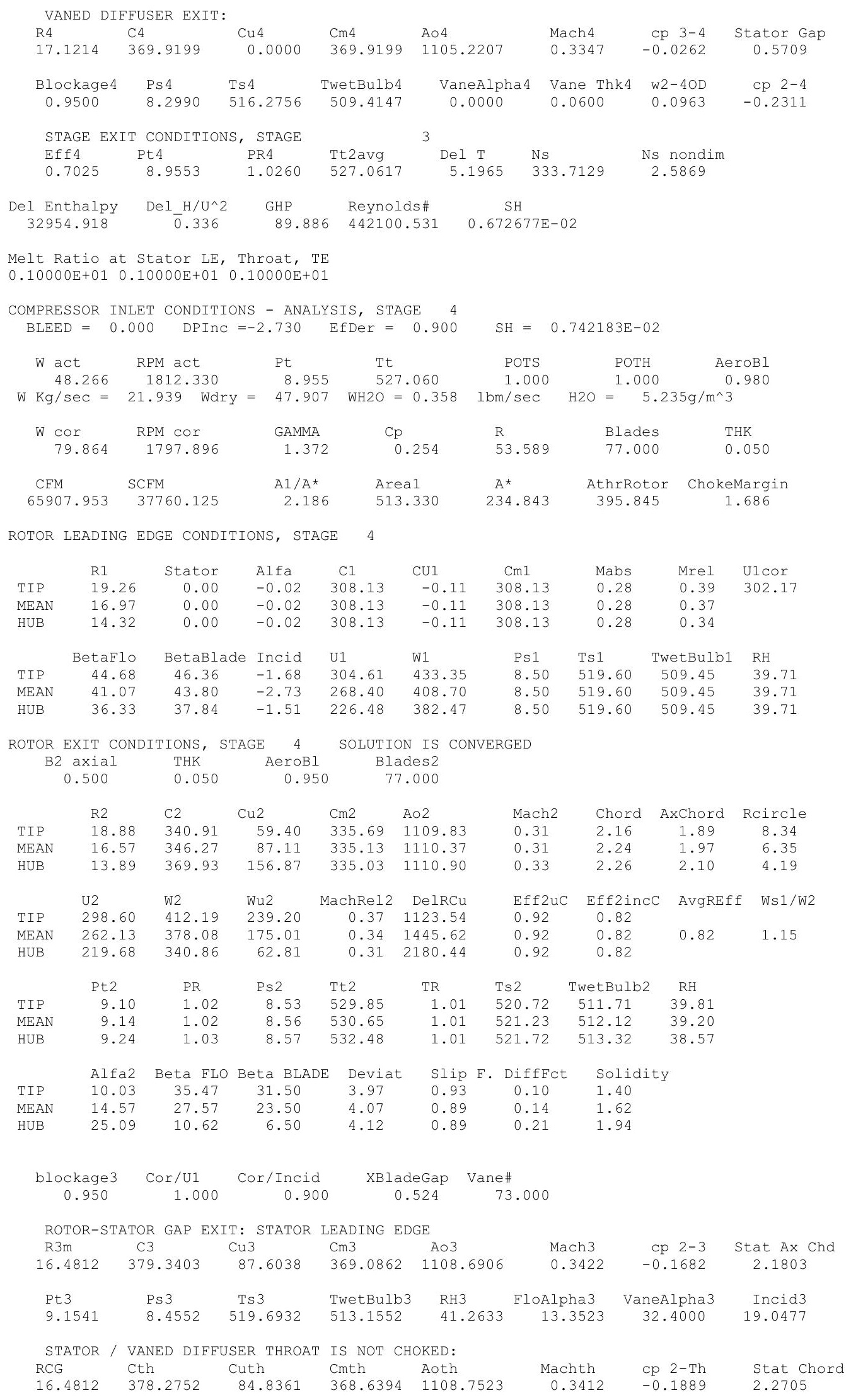




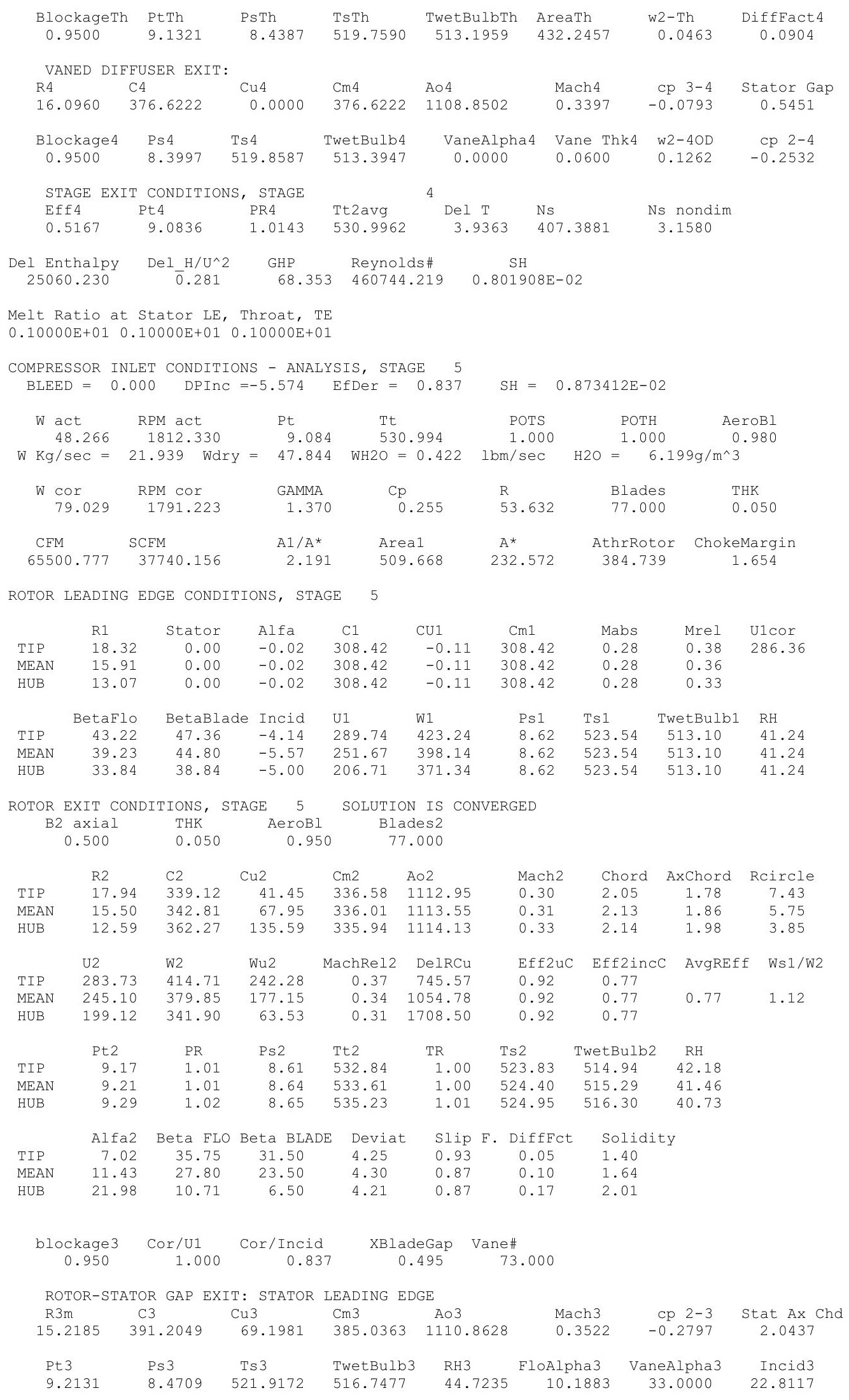




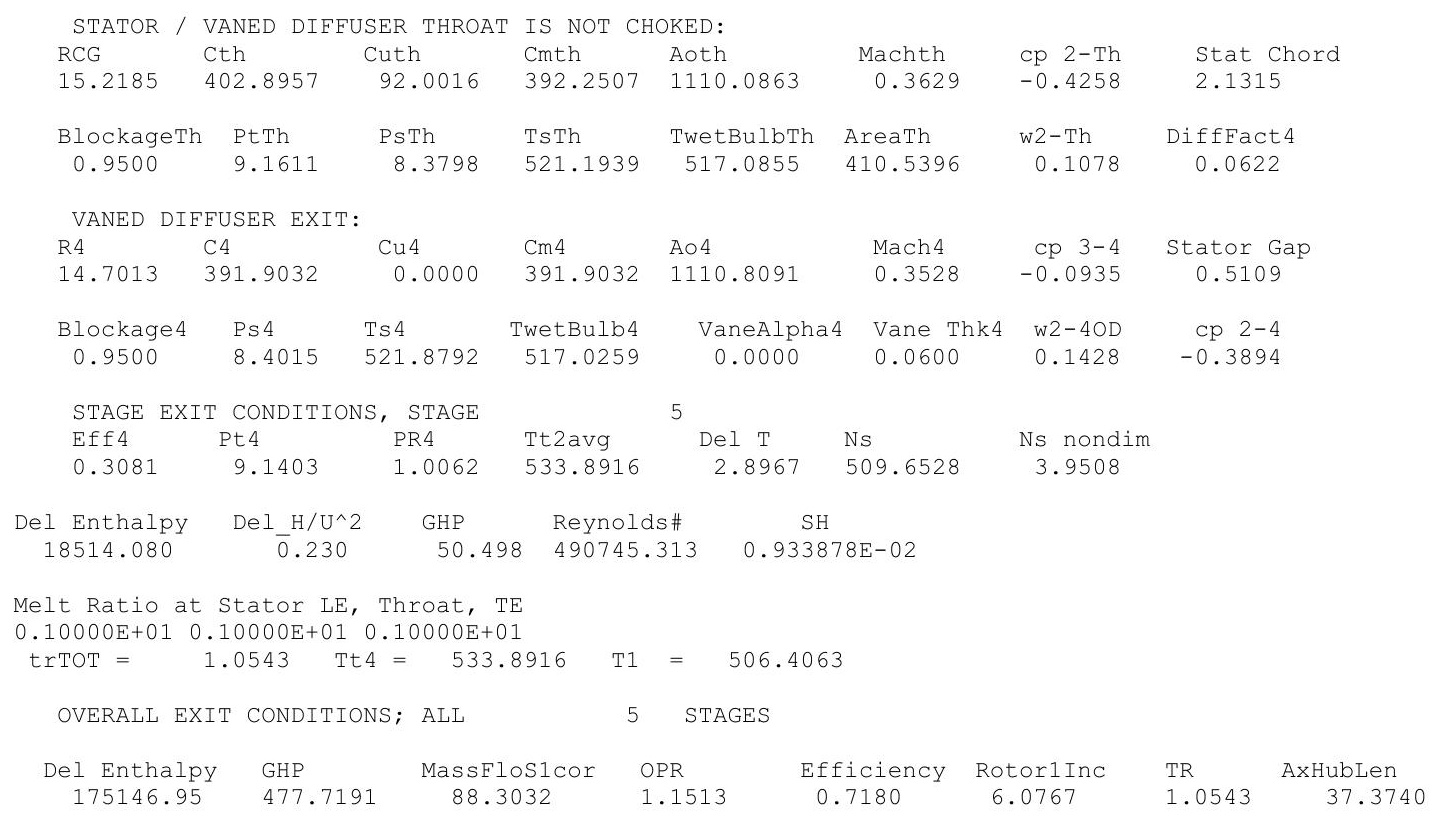


Appendix M: NPSS cycle analysis and compressor code analysis for the descent conditions

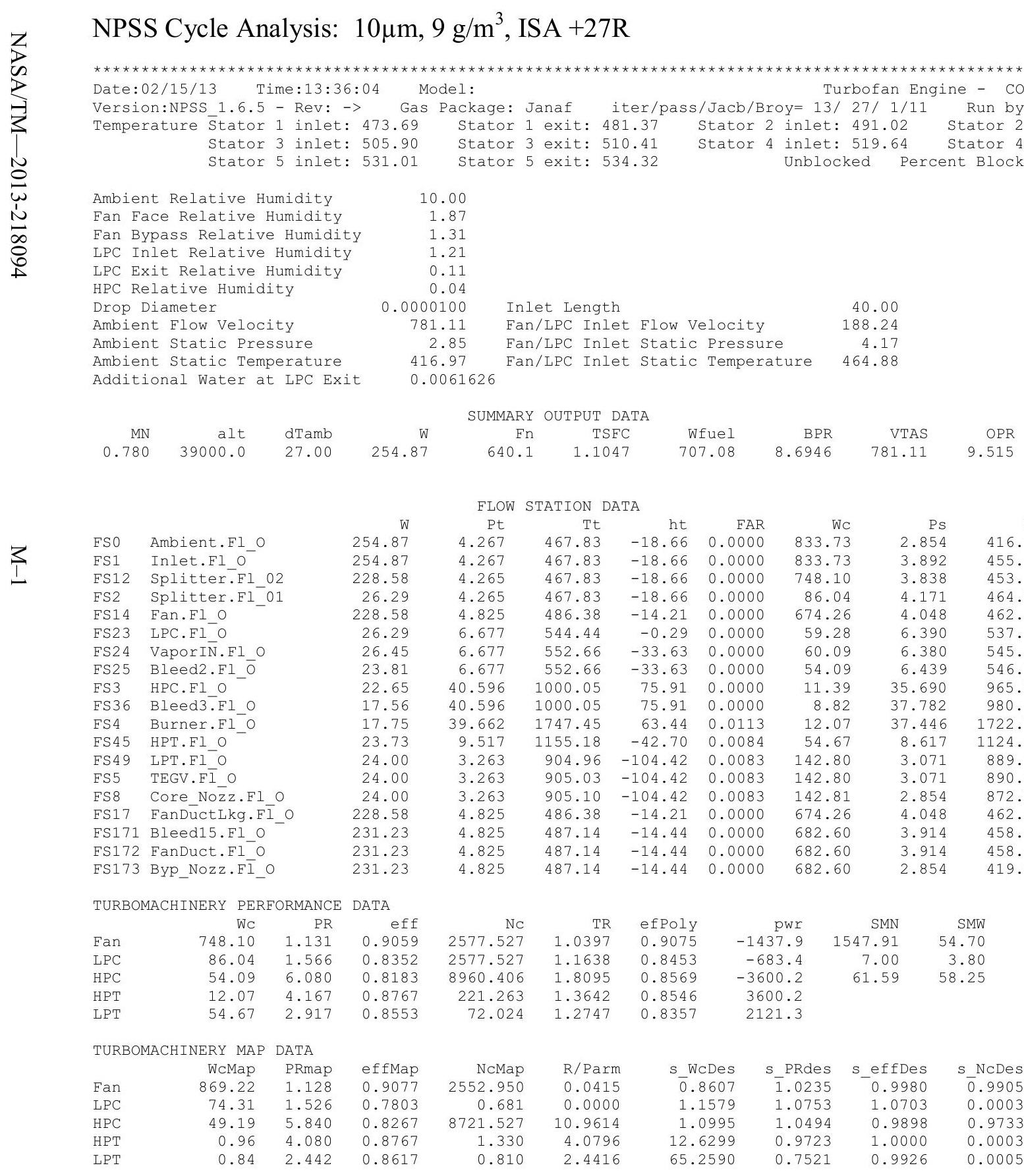




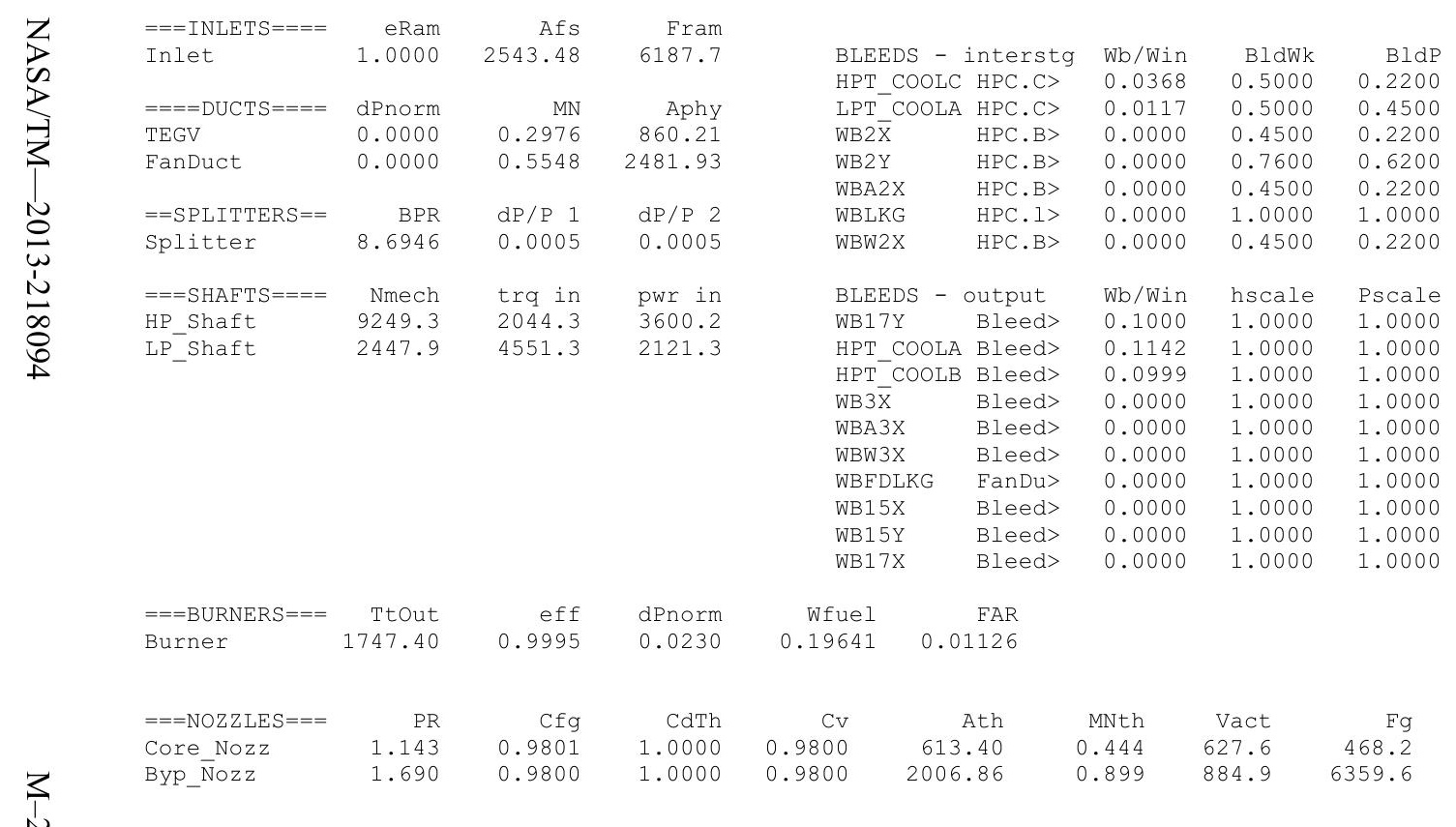


Date:02/15/13 Time:13:36:29 Model: Turbofan Engine - COMDES ON Converge = 1 CASE: 0

Version:NPSS 1.6.5 - Rev: -> Gas Package: Janaf iter/pass/Jacb/Broy=11/ 39/ 2/ 8 Run by: Philip C Jorgenson PC: 10

Temperature Stator 1 inlet: 467.03 Stator 1 exit: 474.23 Stator 2 inlet: $483.26 \quad$ Stator 2 exit: 487.89 $\begin{array}{llll}\text { Stator } 3 \text { inlet: } 497.16 & \text { Stator } 3 \text { exit: } 501.36 & \text { Stator } 4 \text { inlet: } 510.00 \text { Stator } 4 \text { exit: } 513.56 \\ \text { Stator } 5 \text { inlet: } 520.63 & \text { Stator } 5 \text { exit: } 523.71 & \text { Unblocked Percent Blockage: } 0.00\end{array}$

Ambient Relative Humidity $\quad 10.00$

Fan Face Relative Humidity $\quad 2.35$

Fan Bypass Relative Humidity $\quad 1.69$

LPC Inlet Relative Humidity $\quad 1.5$

LPC Exit Relative Humidity $\quad 0.15$

HPC Relative Humidity

Drop Diameter

0.05
000100

Ambient Flow Velocity

0.0000100

731.04
2.95

Inlet Length

40.00

Ambient Static Pressure

Additional Water at LPC Exit

0.005568

$\begin{array}{lr}\text { Fan/LPC Inlet } & 181.73 \\ & \end{array}$

$\begin{array}{lr} & 4.11 \\ \text { Fan/LPC Inlet Static Temperature } & 458.77\end{array}$
MN SUMMARY OUTPUT DATA

$\begin{array}{rrrr}\text { MN } & \text { alt } & \text { dTamb } & \text { W } \\ 0.730 & 38334.0 & 27.00 & 247.99\end{array}$

$\begin{array}{rr}\text { Fn } & \text { TSFC } \\ 626.9 & 1.0745\end{array}$

Wfuel
673.55

BPR
8.7804

VTAS

OPR

EPR

Powerset

$\begin{array}{ccc}\text { T4 } & \text { T41 } & \text { T49 } \\ 1719.3 & 1625.8 & 1133.6\end{array}$

FSO Ambient.Fl_O

FS1 Inlet.Fl_o

$W$
247.99
247.99
222.64
25.36
222.64
25.36
25.50
22.95
21.83
16.92
17.11
22.87
23.13
23.13
23.13
222.64
225.19
225.19
225.19

FLOW STATION DATA

FS12 Splitter.F1_02

FS14 Fan.F1_O

IS23 LPC.FI-O

FS25 Bleed2.FI_O

FS3 HPC.Fl_O

FS36 Bleed3.F1_O

FS4 Burner.Fl_O

FS45 HPT.FI_-

FS49 LPT.FI-O

FS8 Core_Nozz.FI_O

FS17 FanDuctlkg.Fi_O

FS171 Bleed15.F1_O

FS172 FanDuct.FI_O

$\begin{array}{rrrr}\text { Pt } & \text { Tt } & \text { ht } & \text { FAR } \\ 4.201 & 461.52 & -20.16 & 0.0000 \\ 4.201 & 461.52 & -20.16 & 0.0000 \\ 4.199 & 461.52 & -20.16 & 0.0000 \\ 4.199 & 461.52 & -20.16 & 0.0000 \\ 4.718 & 478.80 & -16.02 & 0.0000 \\ 6.436 & 533.24 & -2.97 & 0.0000 \\ 6.436 & 540.79 & -33.10 & 0.0000 \\ 6.436 & 540.79 & -33.10 & 0.0000 \\ 3.794 & 977.14 & 73.53 & 0.0000 \\ 38.794 & 977.14 & 73.53 & 0.0000 \\ 7.901 & 1719.25 & 61.22 & 0.0111 \\ 9.209 & 1137.76 & -42.06 & 0.0083 \\ 3.313 & 901.95 & -100.03 & 0.0082 \\ 3.313 & 902.02 & -100.03 & 0.0082 \\ 3.313 & 902.09 & -100.03 & 0.0082 \\ 4.718 & 478.80 & -16.02 & 0.0000 \\ 4.718 & 479.50 & -16.22 & 0.0000 \\ 4.718 & 479.50 & -16.22 & 0.0000 \\ 4.718 & 479.50 & -16.22 & 0.0000\end{array}$

WC
818.27

PS
2.947
3.847

Ts
416.97
450.03
448.37
458.77
456.09
526.71
533.97
535.34
943.22
958.07
1694.83
1108.66
888.66
888.73
873.41
456.09
452.59
452.59
419.08

$\begin{array}{rr}\text { Aphy } & \text { MN } \\ 2561.0 & 0.7300 \\ 4168.2 & 0.3570 \\ 3531.8 & 0.3826 \\ 830.5 & 0.1730 \\ 2606.7 & 0.4985 \\ 412.6 & 0.2490 \\ 412.6 & 0.2529 \\ 412.6 & 0.2259 \\ 49.7 & 0.4336 \\ 49.3 & 0.3228 \\ 74.6 & 0.2949 \\ 265.4 & 0.3784 \\ 860.2 & 0.2803 \\ 860.2 & 0.2803 \\ 613.4 & 0.4150 \\ 2606.7 & 0.4985 \\ 2481.9 & 0.5447 \\ 2481.9 & 0.5447 \\ 2006.9 & 0.8481\end{array}$

gamt
1.40081
1.40081
1.40081
1.40081
1.40067
1.40002
1.39914
1.39914
1.38124
1.38124
1.33055
1.36541
1.38030
1.38029
1.38029
1.40067
1.40066
1.40066
1.40066

WAR
0.0000491
0.0000491
0.0000491
0.0000491
0.0000491
0.0000491
0.0056179
0.0056179
0.0056179
0.0056179
0.0056179
0.0056179
0.0056179
0.0056179
0.0056179
0.0000491
0.0001118
0.0001118
0.0001118

$\begin{array}{rrr}\text { Wair } & \text { WH2O } & \text { H2O frac } \\ 247.98 & 0.012 & 0.0000 \\ 247.98 & 0.012 & 0.0000 \\ 222.63 & 0.011 & 0.0000 \\ 25.35 & 0.001 & 0.0000 \\ 222.63 & 0.011 & 0.0000 \\ 25.35 & 0.001 & 0.0000 \\ 25.35 & 0.142 & 0.0056 \\ 22.82 & 0.128 & 0.0056 \\ 21.71 & 0.122 & 0.0056 \\ 16.83 & 0.095 & 0.0056 \\ 16.83 & 0.095 & 0.0190 \\ 22.55 & 0.127 & 0.0156 \\ 22.82 & 0.128 & 0.0155 \\ 22.82 & 0.128 & 0.0155 \\ 22.82 & 0.128 & 0.0155 \\ 222.63 & 0.011 & 0.0000 \\ 225.16 & 0.025 & 0.0001 \\ 225.16 & 0.025 & 0.0001 \\ 225.16 & 0.025 & 0.0001\end{array}$

TURBOMACHINERY PERFORMANCE DATA

\begin{tabular}{|c|c|c|c|c|c|c|c|c|c|}
\hline & WC & PR & eff & $\mathrm{NC}$ & $\mathrm{TR}$ & efpoly & pwr & SMN & SMW \\
\hline Fan & 734.98 & 1.124 & 0.9051 & 2513.129 & 1.0374 & 0.9067 & -1304.2 & 2083.10 & 54.58 \\
\hline LPC & 83.71 & 1.533 & 0.8357 & 2513.129 & 1.1554 & 0.8453 & -616.9 & 6.80 & 3.37 \\
\hline HPC & 53.51 & 6.028 & 0.8175 & 8941.716 & 1.8069 & 0.8562 & -3378.0 & 61.47 & 58.08 \\
\hline HPT & 12.08 & 4.116 & 0.8754 & 220.202 & 1.3611 & 0.8533 & 3378.0 & & \\
\hline LPT & 54.05 & 2.780 & 0.8497 & 70.281 & 1.2595 & 0.8303 & 1921.2 & & \\
\hline TURB & JERY MAP & DATA & & & & & & & \\
\hline & WcMap & PRmap & effMap & NcMap & R/Parm & s WcDes & S PRdes & $s$ effDes & s NcDes \\
\hline Fan & 853.97 & 1.121 & 0.9069 & 2489.166 & 0.0414 & $\overline{0} .8607$ & $\overline{1} .0235$ & 0.9980 & $\overline{0} .9905$ \\
\hline LPC & 71.52 & 1.497 & 0.7773 & 0.664 & 0.0000 & 1.1704 & 1.0709 & 1.0751 & 0.0003 \\
\hline HPC & 48.66 & 5.791 & 0.8260 & 8703.336 & 10.9759 & 1.0995 & 1.0494 & 0.9898 & 0.9733 \\
\hline HPT & 0.96 & 4.029 & 0.8754 & 1.323 & 4.0293 & 12.6299 & 0.9723 & 1.0000 & 0.0003 \\
\hline LPT & 0.83 & 2.339 & 0.8560 & 0.791 & 2.3386 & 65.2590 & 0.7521 & 0.9926 & 0.0005 \\
\hline
\end{tabular}




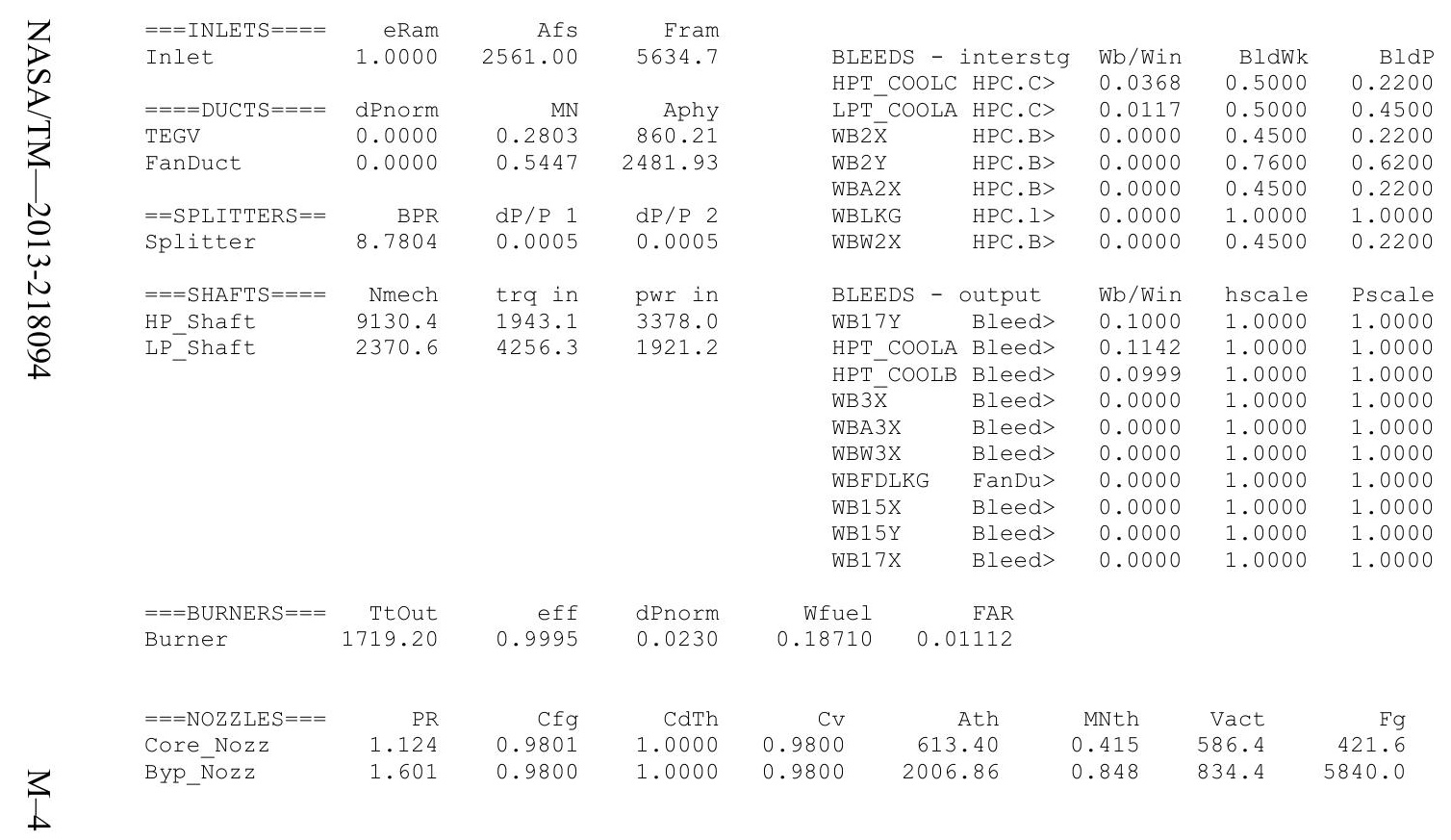


Date:02/15/13 Time:13:36:53 Model: Turbofan Engine - COMDES ON CONverge $=1$ CASE: 0

Version:NPSS 1.6.5 - Rev: -> Gas Package: Janaf iter/pass/Jacb/Broy=11/39/2/8 Run by: Philip C Jorgenson PC: 10

Temperature Stator 1 inlet: 464.96 Stator 1 exit: 471.97 Stator 2 inlet: 480.76 Stator 2 exit: 485.27

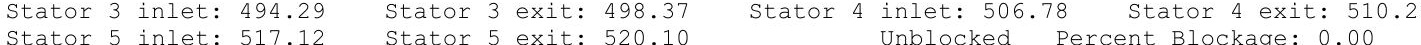

$\begin{array}{lclll}\text { Ambient Relative Humidity } & 10.00 & & & \\ \text { Fan Face Relative Humidity } & 2.52 & & & \\ \text { Fan Bypass Relative Humidity } & 1.84 & & & \\ \text { LPC Inlet Relative Humidity } & 1.66 & & & \\ \text { LPC Exit Relative Humidity } & 0.16 & & & \\ \text { HPC Relative Humidity } & 0.05 & & & \\ \text { Drop Diameter } & 0.0000100 & \text { Inlet Length } & & \\ \text { Ambient Flow Velocity } & 715.01 & \text { Fan/LPC Inlet Flow Velocity } & 179.23 \\ \text { Ambient Static Pressure } & 3.09 & \text { Fan/LPC Inlet Static Pressure } & 4.25 \\ \text { Ambient Static Temperature } & 416.97 & \text { Fan/LPC Inlet Static Temperature } & 456.91 \\ \text { Additional Water at LPC Exit } & 0.0050613 & & & \\ \end{array}$

SUMMARY OUTPUT DATA

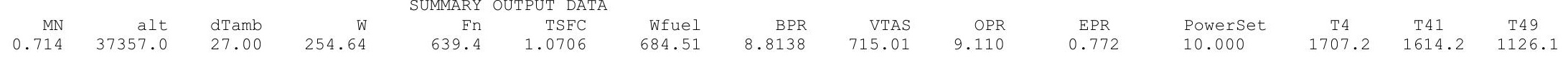

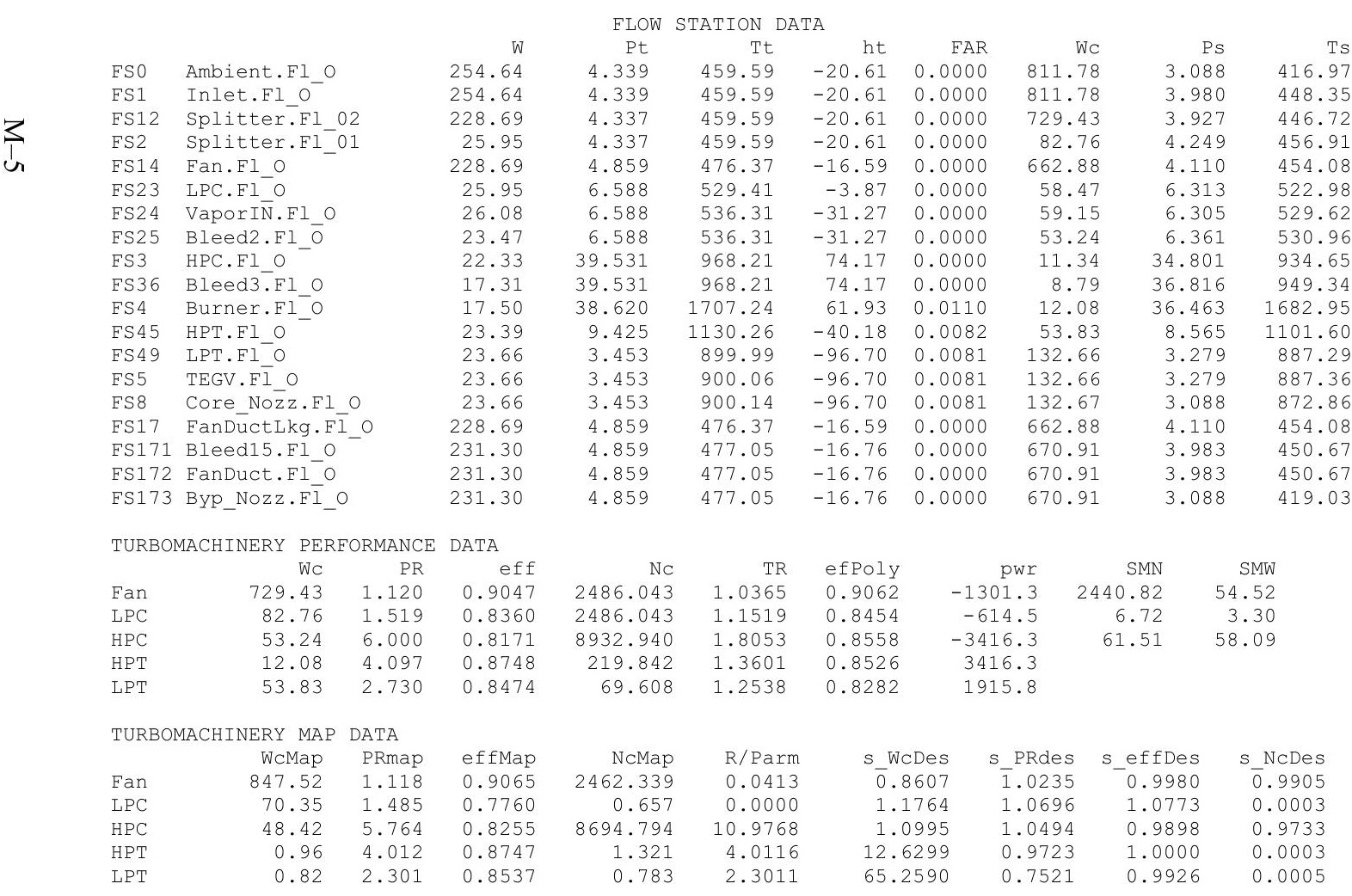

$\begin{array}{rrrrrrr}\text { Aphy } & \text { MN } & \text { gamt } & \text { WAR } & \text { Wair } & \text { WH2O } & \text { H2O frac } \\ 2565.2 & 0.7140 & 1.40083 & 0.0000469 & 254.62 & 0.012 & 0.0000 \\ 4168.2 & 0.3536 & 1.40083 & 0.0000469 & 254.62 & 0.012 & 0.0000 \\ 3531.8 & 0.3791 & 1.40083 & 0.0000469 & 228.68 & 0.011 & 0.0000 \\ 830.5 & 0.1710 & 1.40083 & 0.0000469 & 25.95 & 0.001 & 0.0000 \\ 2606.7 & 0.4949 & 1.40070 & 0.0000469 & 228.68 & 0.011 & 0.0000 \\ 412.6 & 0.2479 & 1.40008 & 0.0000469 & 25.95 & 0.001 & 0.0000 \\ 412.6 & 0.2515 & 1.39928 & 0.0051081 & 25.95 & 0.133 & 0.0051 \\ 412.6 & 0.2246 & 1.39928 & 0.0051081 & 23.35 & 0.119 & 0.0051 \\ 49.7 & 0.4329 & 1.38186 & 0.0051081 & 22.22 & 0.113 & 0.0051 \\ 49.3 & 0.3224 & 1.38186 & 0.0051081 & 17.22 & 0.088 & 0.0051 \\ 74.6 & 0.2948 & 1.33124 & 0.0051081 & 17.22 & 0.088 & 0.0184 \\ 265.4 & 0.3764 & 1.36601 & 0.0051081 & 23.08 & 0.118 & 0.0151 \\ 860.2 & 0.2742 & 1.38052 & 0.0051081 & 23.35 & 0.119 & 0.0150 \\ 860.2 & 0.2742 & 1.38052 & 0.0051081 & 23.35 & 0.119 & 0.0150 \\ 613.4 & 0.4048 & 1.38051 & 0.0051081 & 23.35 & 0.119 & 0.0150 \\ 2606.7 & 0.4949 & 1.40070 & 0.0000469 & 228.68 & 0.011 & 0.0000 \\ 2481.9 & 0.5404 & 1.40068 & 0.0001037 & 231.27 & 0.024 & 0.0001 \\ 2481.9 & 0.5404 & 1.40068 & 0.0001037 & 231.27 & 0.024 & 0.0001 \\ 2006.9 & 0.8311 & 1.40068 & 0.0001037 & 231.27 & 0.024 & 0.0001\end{array}$




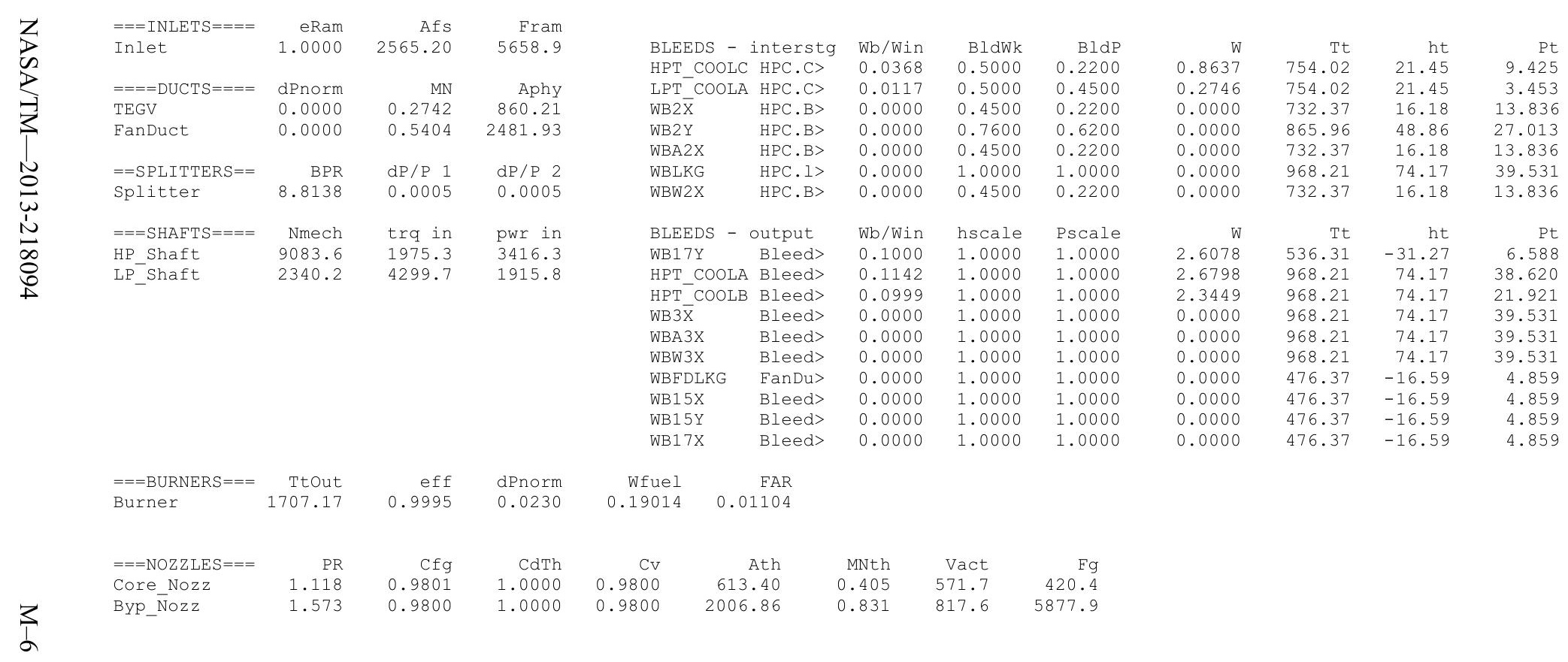


Date:02/15/13 Time:13:37:09 Model: Turbofan Engine - COMDES ON Converge = 1 CASE: 0

Version:NPSS_1.6.5 - Rev: -> Gas Package: Janaf iter/pass/Jacb/Broy=12/ 26/1/10 Run by: Philip C Jorgenson PC: 10

Temperature Stator 1 inlet: 466.46 Stator 1 exit: $473.03 \quad$ Stator 2 inlet: 481.24 $\quad$ Stator 2 exit: 485.45 $\begin{array}{llll}\text { Stator } 3 \text { inlet: } 493.88 & \text { Stator } 3 \text { exit: } 497.66 & \text { Stator } 4 \text { inlet: } 505.52 \text { Stator } 4 \text { exit: } 508.7 \\ \text { Stator } 5 \text { inlet: } 515.14 & \text { Stator } 5 \text { exit: } 517.88 & & \text { Unblocked Percent Blockage: } 0.00\end{array}$

Ambient Relative Humidity $\quad 10.00$

Fan Face Relative Humidity $\quad 3.13$

Fan Bypass Relative Humidity $\quad 2.36$

LPC Inlet Relative Humidity

LPC Exit Relative Humidity

De Relative Humidity

Drop Diameter

2.11
0.24

Ambient Flow Velocity

Ambient static Pressure

Additional Water at LPC Exit

0.05

0.0000100

675.10

Inlet Length

Fan/LPC Inlet Flow Velocity $\quad 173.34$

3.58 Fan/LPC Inlet Static Pressure $\quad 4.74$

0.004589

Fan/LPC Inlet Static Temperature 458.91

SUMMARY OUTPUT DATA

$\begin{array}{rrrr}\text { MN } & \text { alt } & \text { dTamb } & \text { W } \\ 0.669 & 34281.0 & 27.00 & 275.80\end{array}$

FSO Ambient.Fl_O

FS1 Inlet.Fl_O

$\sqrt[3]{4}$

FS2 Splitter.F1_02
FS14 Fan.F1_O

FS23 LPC.F1-O

FS25 Bleod2.FI-

FS25 Bleed2.F1

FS36 BPC.F1-O

FS36 Bleed3.F1_O

$\begin{array}{ll}\text { FS4 } & \text { Burner.FI } \\ \text { FS45 } & \text { HPT.FI O }\end{array}$

FS49 LPT.FI_O

FS5 TEGV.Fì_O

FS8 Core_Nozz.Fl_O

FS171 FanDuctLkg.F1

FS172 FanDuct. F1-

FS173 Byp Nozz. Fi

TURBOMACHINERY PERFORMANCE DATA

\begin{tabular}{|c|c|c|c|c|c|c|c|c|c|}
\hline & WC & PR & eff & $\mathrm{NC}$ & $T R$ & efPoly & pwr & SMN & SMW \\
\hline Fan & 711.76 & 1.112 & 0.9037 & 2405.184 & 1.0340 & 0.9051 & -1319.9 & 5050.19 & 54.11 \\
\hline LPC & 79.96 & 1.480 & 0.8375 & 2405.184 & 1.1416 & 0.8462 & -617.4 & 6.44 & 3.07 \\
\hline HPC & 52.51 & 5.917 & 0.8157 & 8909.144 & 1.7993 & 0.8545 & -3613.2 & 61.84 & 58.33 \\
\hline HPT & 12.08 & 4.052 & 0.8741 & 219.259 & 1.3570 & 0.8520 & 3613.2 & & \\
\hline LPT & 53.28 & 2.585 & 0.8423 & 67.626 & 1.2371 & 0.8237 & 1937.3 & & \\
\hline TURB & JERY MAP & DATA & & & & & & & \\
\hline & WcMap & PRmap & effMap & NcMap & R/Parm & s WcDes & s PRdes & $s$ effDes & s NCDes \\
\hline Fan & 826.99 & 1.109 & 0.9055 & 2382.251 & 0.0413 & $\overline{0} .8607$ & $\overline{1} .0235$ & 0.9980 & $\overline{0.9905}$ \\
\hline LPC & 66.85 & 1.449 & 0.7723 & 0.635 & 0.0000 & 1.1962 & 1.0683 & 1.0844 & 0.0003 \\
\hline HPC & 47.76 & 5.686 & 0.8241 & 8671.632 & 10.9658 & 1.0995 & 1.0494 & 0.9898 & 0.9733 \\
\hline $\mathrm{HPT}$ & 0.96 & 3.968 & 0.8740 & 1.318 & 3.9678 & 12.6299 & 0.9723 & 1.0000 & 0.0003 \\
\hline LPT & 0.82 & 2.192 & 0.8485 & 0.761 & 2.1925 & 65.2590 & 0.7521 & 0.9926 & 0.0005 \\
\hline
\end{tabular}

$\begin{array}{llccc}\text { EPR } & \text { PowerSet } & \text { T4 } & \text { T41 } & \text { T49 } \\ 0.795 & 10.000 & 1696.8 & 1603.9 & 1121.1\end{array}$

\begin{tabular}{|c|c|c|c|c|c|c|c|c|c|}
\hline 275.80 & 4.831 & 461.41 & -20.25 & 0.0000 & 791.32 & 3.578 & 423.42 & 2579.3 & 0.6690 \\
\hline 275.80 & 4.831 & 461.41 & -20.25 & 0.0000 & 791.32 & 4.453 & 450.77 & 4168.2 & 0.3433 \\
\hline 247.95 & 4.829 & 461.41 & -20.25 & 0.0000 & 711.76 & 4.397 & 449.21 & 3531.8 & 0.3682 \\
\hline 27.86 & 4.829 & 461.41 & -20.25 & 0.0000 & 79.96 & 4.738 & 458.91 & 830.5 & 0.1650 \\
\hline 247.95 & 5.368 & 477.11 & -16.48 & 0.0000 & 651.06 & 4.576 & 455.82 & 2606.7 & 0.4828 \\
\hline 27.86 & 7.146 & 526.76 & -4.58 & 0.0000 & 57.73 & 6.854 & 520.53 & 412.6 & 0.2445 \\
\hline 27.98 & 7.146 & 533.05 & -29.43 & 0.0 & 58.34 & 6.847 & 526.59 & 412.6 & 0.2477 \\
\hline 25.18 & 7.146 & 533.05 & -29.43 & 0.0000 & 52.51 & 6.906 & 527.88 & 412.6 & 0.2213 \\
\hline 23.96 & 42.284 & 959.12 & 74.50 & 0.0 & 11.33 & 37.245 & 925.98 & 49.7 & 0.4319 \\
\hline 18.57 & 42.284 & 959.12 & 74.50 & 0.0 & 8.78 & 39.390 & 940.48 & 49.3 & 0.3217 \\
\hline 18.77 & 41.310 & 1696.80 & 62.30 & 0.0110 & 12.08 & 39.002 & 1672.62 & 74.6 & 0.2947 \\
\hline 25.09 & 10.194 & 1125.30 & -38.32 & 0.0 & 53.28 & 9.285 & 1097.42 & 265.4 & 0.3718 \\
\hline 25.39 & 3.943 & 907.98 & -91.54 & 0.0081 & 125.20 & 3.768 & 896.68 & 860.2 & 0.2574 \\
\hline 25.39 & 3.943 & 908.05 & -91.54 & 0.0081 & 125.21 & 3.768 & 896.75 & 860.2 & 0.2574 \\
\hline 25.39 & 3.943 & 908.13 & -91.54 & 0.0 & 125.21 & 3.578 & 884.14 & 613.3 & 0.3774 \\
\hline 247.95 & 5.368 & 477.11 & -16.48 & 0.0 & 651.06 & 4.576 & 455.82 & 2606.7 & 0.4828 \\
\hline 0.74 & 5.368 & 477.74 & -16.63 & 0.0 & 658.84 & 4.445 & 452.63 & 2481.9 & 0.5261 \\
\hline 250.74 & 5.368 & 477.74 & -16.63 & 0.0000 & 658.84 & 4.445 & 452.63 & 2481.9 & 0.5261 \\
\hline 250.74 & 5.368 & 477.74 & -16.63 & 0.0000 & 658.84 & 3.578 & 425.39 & 2006.9 & 0.7836 \\
\hline
\end{tabular}

gamt
1.40081
1.40081
1.40081
1.40081
1.40069
1.40011
1.39939
1.39939
1.38247
1.38247
1.33183
1.36642
1.38013
1.38013
1.38013
1.40069
1.40068
1.40068
1.40068

WAR
0.0000588
0.0000588
0.0000588
0.0000588
0.0000588
0.0000588
0.0046478
0.0046478
0.0046478
0.0046478
0.0046478
0.0046478
0.00466478
0.0046678
0.0046478
0.0000588
0.0001097
0.0001097
0.0001097

Wair
275.79 $\begin{array}{lll}275.79 & 0.016 & 0.0001 \\ 275.79 & 0.016 & 0.0001\end{array}$ $247.93 \quad 0.015 \quad 0.0001$ $\begin{array}{rrr}27.85 & 0.002 & 0.0001\end{array}$ $\begin{array}{rrr}247.93 & 0.015 & 0.0001 \\ 27.85 & 0.002 & 0.0001\end{array}$ $\begin{array}{lll}27.85 & 0.002 & 0.0001 \\ 27.85 & 0.129 & 0.0046\end{array}$ $\begin{array}{lll}27.85 & 0.002 & 0.0001 \\ 25.07 & 0.117 & 0.0046\end{array}$ $\begin{array}{lll}25.07 & 0.117 & 0.0046 \\ 23.85 & 0.111 & 0.0046\end{array}$ $\begin{array}{lll}23.85 & 0.111 & 0.0046\end{array}$ $\begin{array}{lll}18.49 & 0.086 & 0.0046 \\ 18.49 & 0.086 & 0.0179\end{array}$ $\begin{array}{lll}18.49 & 0.086 & 0.0179 \\ 25.07 & 0.115 & 0.0146\end{array}$ $\begin{array}{lll}24.78 & 0.115 & 0.0146 \\ 25.07 & 0.117 & 0.0145\end{array}$ $\begin{array}{lll}25.07 & 0.117 & 0.0145\end{array}$ $\begin{array}{lll}25.07 & 0.117 & 0.0145\end{array}$ $\begin{array}{lll}247.93 & 0.015 & 0.0001\end{array}$ $\begin{array}{lll}250.72 & 0.028 & 0.0001\end{array}$ $\begin{array}{lll}250.72 & 0.028 & 0.0001\end{array}$

0.0005




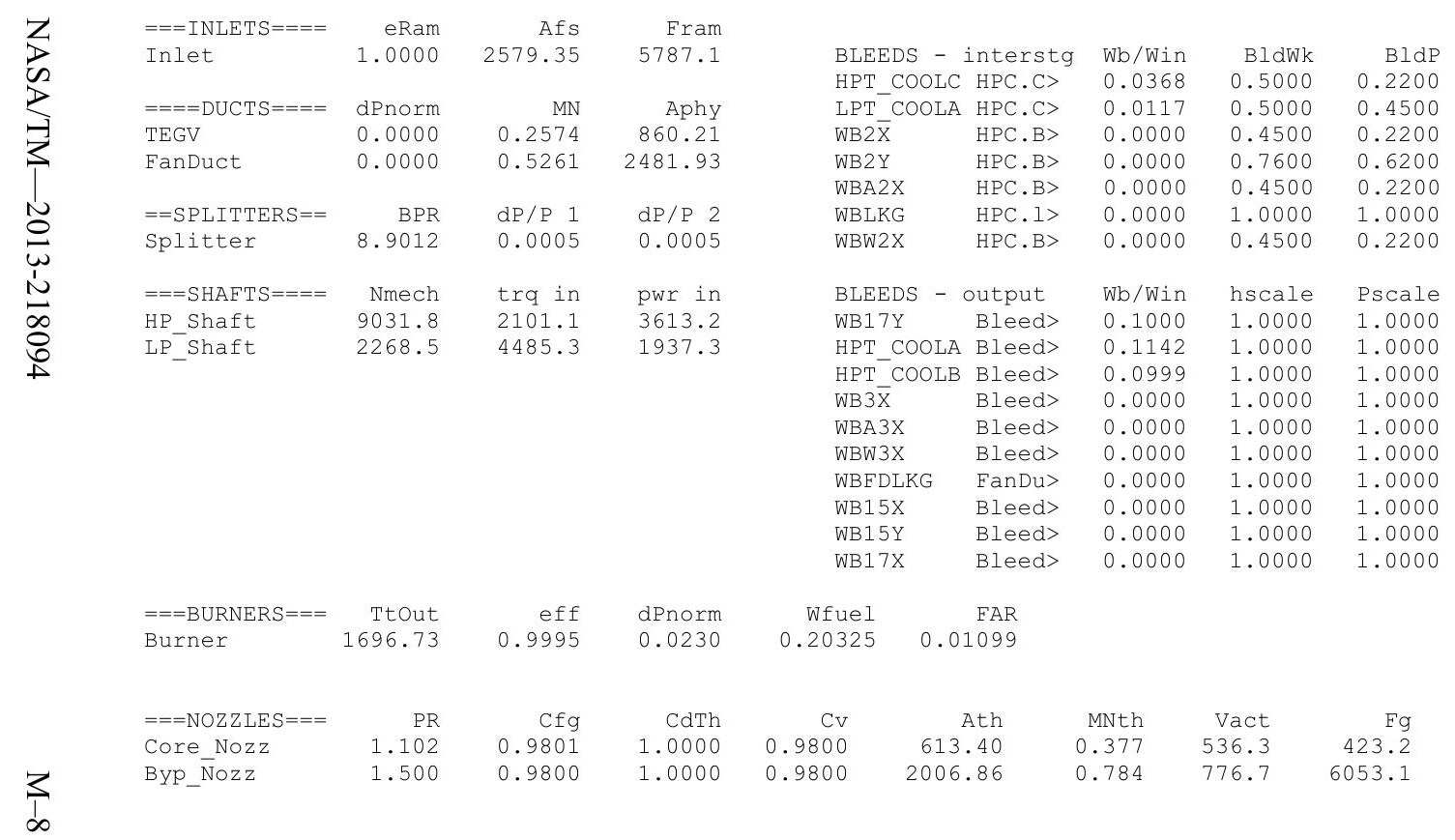


Date:02/15/13 Time:13:37:27 Model: Turbofan Engine - COMDES ON Converge = 1 CASE: 0

Version:NPSS1.6.5 - Rev: -> Gas Package: Janaf iter/pass/Jacb/Broy=16/30/1/14 Run by: Philip C Jorgenson PC: 10

Temperature Stator 1 inlet: 474.92 Stator 1 exit: $480.59 \quad$ Stator 2 inlet: 487.60 Stator 2 exit: 491.01 $\begin{array}{ll}\text { Stator } 3 \text { inlet: } 498.31 & \text { Stator } 3 \text { exit: } 501.31 \quad \text { Stator } 4 \text { inlet: } 508.15 \text { Stator } 4 \text { exit: } 510.62 \\ \text { Stator } 5 \text { inlet: } 516.13 & \text { Stator } 5 \text { exit: } 518.21\end{array}$

Ambient Relative Humidity $\quad 10.00$

Fan Face Relative Humidity

Fan Bypass Relative Humidity

LPC Exit Relative Humidity

HPC Relative Humidity

Drop Diameter

Ambient Flow Velocity

Ambient Static Pressure

Ambient Static Temperature
Additional Water at LPC Exit

3.23

2.94
0.50

0.05

0.0000100

624.43

4.36

Inlet Length

Fan/LPC Inlet Flow Velocity $\quad \begin{aligned} 40.00 \\ \text { Fan/LPC }\end{aligned}$ 0.004573

$\begin{array}{lr} & 5.49 \\ \text { Fan/LPC Inlet Static Temperature } & 468.53\end{array}$

$\begin{array}{rrr}\text { MN } & \text { alt } & \text { dTamb } \\ 0.608 & 30029.0 & 27.00\end{array}$

$\begin{array}{crc} & \text { SUMMARY OUTPUT DATA } \\ \text { W } & \text { Fn } & \text { TSFC }\end{array}$

Wfuel

BPR
8.4735

VTAS
624.43

$\mathrm{OPR}$
8.166

EPR
0.828

Powerset

$\begin{array}{ccc}\text { T4 } & \text { T41 } & \text { T49 } \\ 1697.6 & 1604.2 & 1124.5\end{array}$

FSO Ambient.Fl_O

FS1 Inlet.FI_O

FS14 Fan.Fl_o

FS23 LPC.FI $O$

FS25 Bleed2.FI-

$\begin{array}{ll}\text { FS25 } & \text { Bleed2.Fl- } \\ \text { FS3 } & \text { HPC.Fl O }\end{array}$

FS36 Bleed3.F1_O

FS4 Burner.FI_O

FS45 HPT.FI_-

FS5 4 LPT.FI-O

FS8 Core_Nozz.FI_O

FS17 FanDuctLkg.Fi_O

FS171 Bleed15.F1_O

FS172 Fanduct.Fl_o

$W$
301.85
301.85
269.99
31.86
269.99
31.86
32.01
27.21
25.89
20.06
20.28
27.11
27.43
27.43
27.43
269.99
274.79
274.79
274.79

FLOW STATION DATA

\begin{tabular}{|c|c|c|c|}
\hline Pt & Tt & ht & FAR \\
\hline 595 & 471.08 & -18.23 & 0.0000 \\
\hline 59 & 471.08 & -18.23 & 0.0000 \\
\hline 592 & 471.08 & -18.23 & 0.0000 \\
\hline 592 & 471.08 & -18.23 & 0.0000 \\
\hline 143 & 485.30 & -14.82 & 0.0000 \\
\hline 846 & 527.25 & -4.76 & 0.0000 \\
\hline 846 & 533.51 & -29.52 & 0.0000 \\
\hline 846 & 533.51 & -29.52 & 0.0000 \\
\hline 691 & 956.02 & 73.52 & 0.0000 \\
\hline & 956.02 & 73.52 & 0.0000 \\
\hline 639 & 1697.62 & 61.28 & 0.0111 \\
\hline & 1128.71 & -38.44 & 0.0082 \\
\hline 16 & 930.43 & -86.93 & 0.0082 \\
\hline 716 & 930.50 & -86.93 & 0.0082 \\
\hline 716 & 930.57 & -86.93 & 0.0082 \\
\hline & 485.30 & -14.82 & 0.0000 \\
\hline 143 & 486.14 & -15.08 & 0.0000 \\
\hline 143 & 486.14 & -15.08 & 0.0000 \\
\hline & 486.14 & -15.08 & 0.0000 \\
\hline
\end{tabular}

WC
755.58
755.58

PS
4.358

Ts
438.58
461.29
460.00
468.53
465.81
520.44
526.46
528.51
923.12
937.51
1673.43
1101.58
920.88
920.95
910.61
465.81
462.88
462.88
440.67

$\begin{array}{rr}\text { Aphy } & \text { MN } \\ 2595.4 & 0.6080 \\ 4168.2 & 0.3255 \\ 3531.8 & 0.3467 \\ 830.5 & 0.1647 \\ 2606.7 & 0.4569 \\ 412.6 & 0.2557 \\ 412.6 & 0.2591 \\ 412.6 & 0.2177 \\ 49.7 & 0.4309 \\ 49.3 & 0.3210 \\ 74.6 & 0.2948 \\ 265.4 & 0.3662 \\ 860.2 & 0.2339 \\ 860.2 & 0.2339 \\ 613.4 & 0.3398 \\ 2606.7 & 0.4569 \\ 2481.9 & 0.5008 \\ 2481.9 & 0.5008 \\ 2006.9 & 0.7175\end{array}$

gamt
1.40073
1.40073
1.40073
1.40073
1.40061
1.40010
1.39938
1.39938
1.38265
1.38265
1.33175
1.36616
1.37877
1.37876
1.37876
1.40061
1.40059
1.40059
1.40059

WAR
0.0001104

Wair

0.0001104 269.96
31.86

0.0001104

269.96
31.86

$\begin{array}{ll}0.004 \\ 0.011045-31.86 & 0.004\end{array}$

60.17

51.69

11.30

12.08

52.60
114.48

114.48

114.49
624.76

636.42

636.42
636.42

TURBOMACHINERY PERFORMANCE DATA

$\begin{array}{lrrrrrrrrr} & \text { WC } & \text { PR } & \text { eff } & \text { NC } & \text { TR } & \text { efPoly } & \text { pwr } & \text { SMN } & \text { SMW } \\ \text { Fan } & 676.16 & 1.098 & 0.9024 & 2254.369 & 1.0302 & 0.9037 & -1301.7 & -9825.89 & 52.68 \\ \text { LPC } & 79.80 & 1.403 & 0.8525 & 2254.369 & 1.1192 & 0.8594 & -607.2 & 5.95 & 2.64 \\ \text { HPC } & 51.69 & 5.824 & 0.8140 & 8882.085 & 1.7919 & 0.8528 & -3870.2 & 62.23 & 58.62 \\ \text { HPT } & 12.08 & 3.995 & 0.8740 & 218.636 & 1.3526 & 0.8521 & 3870.2 & & \\ \text { LPT } & 52.60 & 2.369 & 0.8338 & 63.949 & 1.2106 & 0.8163 & 1908.9 & & \end{array}$

TURBOMACHINERY MAP DATA

$\begin{array}{lrrrrrrrrr} & \text { WcMap } & \text { PRmap } & \text { effMap } & \text { NCMap } & \text { R/Parm } & \text { S_WcDes } & \text { S_PRdes } & \text { s_effDes } & \text { S_NcDes } \\ \text { Fan } & 785.63 & 1.096 & 0.9042 & 2232.874 & 0.0417 & 0.8607 & 1.0235 & 0.9980 & 0.9905 \\ \text { LPC } & 60.49 & 1.384 & 0.7656 & 0.595 & 0.0000 & 1.3192 & 1.0505 & 1.1135 & 0.0003 \\ \text { HPC } & 47.01 & 5.596 & 0.8224 & 8645.295 & 10.9531 & 1.0995 & 1.0494 & 0.9898 & 0.9733 \\ \text { HPT } & 0.96 & 3.912 & 0.8740 & 1.314 & 3.9123 & 12.6299 & 0.9723 & 1.0000 & 0.0003 \\ \text { LPT } & 0.81 & 2.030 & 0.8400 & 0.719 & 2.0298 & 65.2590 & 0.7521 & 0.9926 & 0.0005\end{array}$




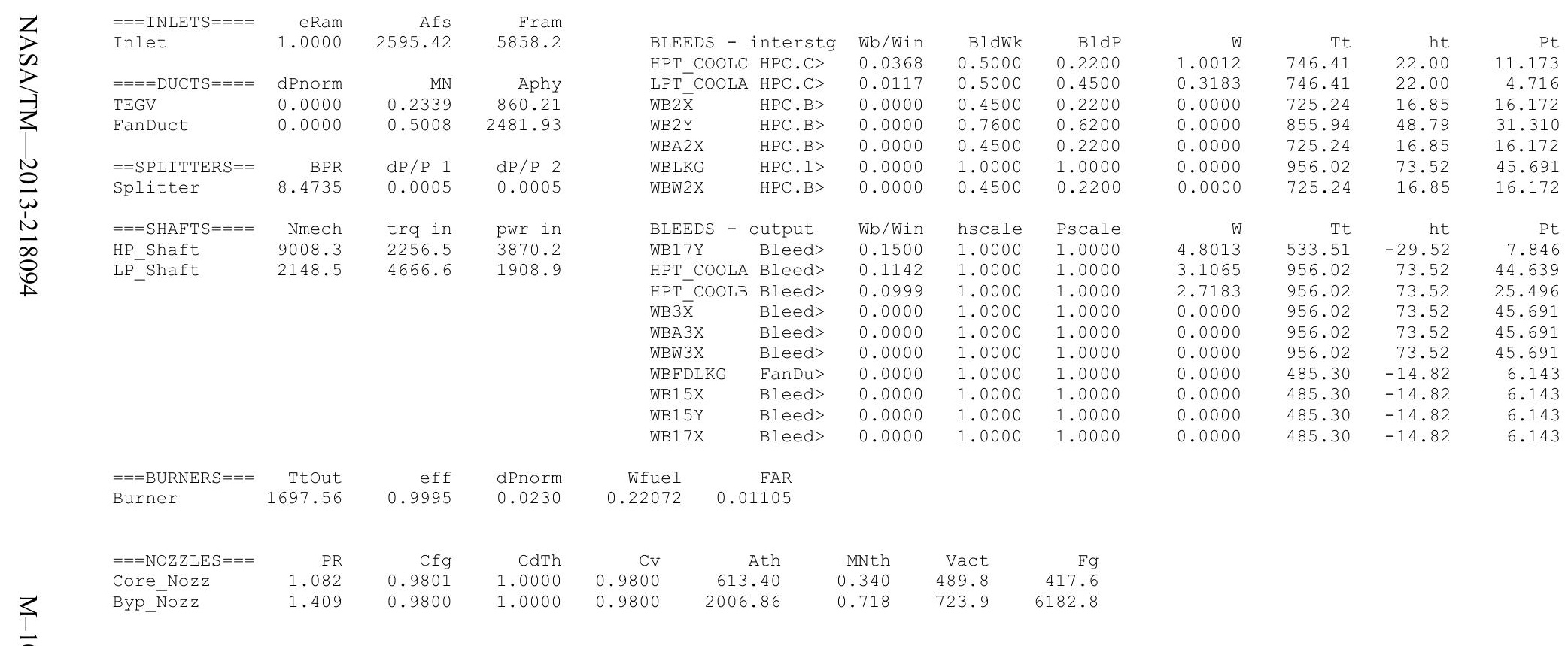


Date:02/15/13 Time:13:37:53 Model: Turbofan Engine - COMDES ON COnverge = 1 CASE: 0

Version:NPSS 1.6.5 - Rev: -> Gas Package: Janaf iter/pass/Jacb/Broy=28/42/1/26 Run by: Philip C Jorgenson PC: 10

Temperature Stator 1 inlet: $482.83 \quad$ Stator 1 exit: $487.00 \quad$ Stator 2 inlet: 491.75 Stator 2 exit: 493.63

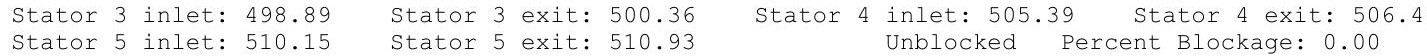

$\begin{array}{lclll}\text { Ambient Relative Humidity } & 10.00 & & & \\ \text { Fan Face Relative Humidity } & 4.92 & & & \\ \text { Fan Bypass Relative Humidity } & 4.06 & & & \\ \text { LPC Inlet Relative Humidity } & 3.92 & & & \\ \text { LPC Exit Relative Humidity } & 1.25 & & & \\ \text { HPC Relative Humidity } & 0.06 & & & \\ \text { Drop Diameter } & 0.0000100 & \text { Inlet Length } & \\ \text { Ambient Flow Velocity } & 576.87 & \text { Fan/LPC Inlet Flow Velocity } & 193.94 \\ \text { Ambient Static Pressure } & 5.30 & \text { Fan/LPC Inlet Static Pressure } & 6.37 \\ \text { Ambient Static Temperature } & 454.14 & \text { Fan/LPC Inlet Static Temperature } & 478.74 \\ \text { Additional Water at LPC Exit } & 0.0041775 & & & \end{array}$

SUMMARY OUTPUT DATA

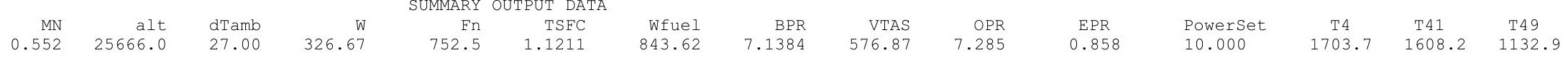

\begin{tabular}{|c|c|c|}
\hline & FSO & Ambient.Fl_o \\
\hline & FS1 & Inlet.Fl_O- \\
\hline & FS12 & Splitter.Fl_02 \\
\hline & FS2 & Splitter.FI_01 \\
\hline & FS14 & Fan.Fl_O \\
\hline & FS23 & LPC.FI_O \\
\hline & FS24 & VaporIN.FI_O \\
\hline & FS25 & Bleed2.FI_ō \\
\hline & FS3 & HPC.Fl_O \\
\hline & FS36 & Bleed3.Fl_O \\
\hline & FS4 & Burner.Fl_o \\
\hline & FS 45 & HPT.FI_O ${ }^{-}$ \\
\hline & FS 49 & LPT.FI_O \\
\hline & FS5 & TEGV.F̄ \\
\hline & FS 8 & Core_Nozz.Fl_o \\
\hline & FS17 & FanDüctLkg.F̄_o \\
\hline & FS171 & Bleed15.FI_O \\
\hline & FS172 & FanDuct.Fl_o \\
\hline & FS173 & Byp_Nozz.Fi \\
\hline
\end{tabular}

\begin{tabular}{rrrrr}
\multicolumn{7}{c}{ FLOW } & STATION DATA \\
W & Pt & Tt & ht & FAR \\
326.67 & 6.516 & 481.87 & -16.15 & 0.0000 \\
326.67 & 6.516 & 481.87 & -16.15 & 0.0000 \\
286.53 & 6.513 & 481.87 & -16.15 & 0.0000 \\
40.14 & 6.513 & 481.87 & -16.15 & 0.0000 \\
286.53 & 7.056 & 494.23 & -13.19 & 0.0000 \\
40.14 & 8.233 & 522.29 & -6.46 & 0.0000 \\
40.31 & 8.233 & 528.05 & -29.08 & 0.0000 \\
28.22 & 8.233 & 528.05 & -29.08 & 0.0000 \\
26.85 & 47.467 & 944.18 & 72.31 & 0.0000 \\
20.81 & 47.467 & 944.18 & 72.31 & 0.0000 \\
21.04 & 46.374 & 1703.69 & 59.79 & 0.0113 \\
28.12 & 11.974 & 1137.29 & -38.19 & 0.0084 \\
28.45 & 5.623 & 961.64 & -81.02 & 0.0083 \\
28.45 & 5.623 & 961.71 & -81.02 & 0.0083 \\
28.45 & 5.623 & 961.78 & -81.02 & 0.0083 \\
286.53 & 7.056 & 494.23 & -13.19 & 0.0000 \\
298.62 & 7.056 & 495.60 & -13.83 & 0.0000 \\
298.62 & 7.056 & 495.60 & -13.83 & 0.0000 \\
298.62 & 7.056 & 495.60 & -13.83 & 0.0000
\end{tabular}

Wc
710.13
710.13
623.18
87.30
582.56
71.90
72.60
50.82
11.21
8.69
12.08
51.10
101.24
101.24
101.24
582.56
607.99
607.99
607.99

Ps
5.297
6.113
6.078
6.366
6.257
7.698
7.685
7.975
41.942
44.288
43.782
11.002
5.463
5.463
5.297
6.257
6.063
6.063
5.297

Ts
454.14
473.14
472.44
478.74
477.54
512.35
517.77
523.27
912.29
926.20
1679.43
1111.75
954.06
954.13
946.16
477.54
474.57
474.57
456.58

$\begin{array}{rr}\text { Aphy } & \text { MN } \\ 2590.6 & 0.5520 \\ 4168.2 & 0.3034 \\ 3531.8 & 0.3157 \\ 830.5 & 0.1808 \\ 2606.7 & 0.4177 \\ 412.6 & 0.3113 \\ 412.6 & 0.3152 \\ 412.6 & 0.2138 \\ 49.7 & 0.4264 \\ 49.3 & 0.3180 \\ 74.6 & 0.2949 \\ 265.4 & 0.3540 \\ 860.2 & 0.2054 \\ 860.2 & 0.2054 \\ 613.4 & 0.2959 \\ 2606.7 & 0.4177 \\ 2481.9 & 0.4704 \\ 2481.9 & 0.4704 \\ 2006.9 & 0.6532\end{array}$

gamt
1.40063
1.40063
1.40063
1.40063
1.40050
1.40016
1.39950
1.39950
1.38340
1.38340
1.33133
1.36552
1.37680
1.37680
1.37679
1.40050
1.40046
1.40046
1.40046

WAR
0.0001988
0.0001988
0.0001988
0.0001988
0.0001988
0.0001988
0.0043763
0.0043763
0.0043763
0.0043763
0.0043763
0.0043763
0.0043763
0.0043763
0.0043763
0.0001988
0.0003673
0.0003673
0.0003673

Wair
326.61
326.61
286.47
40.13
286.47
40.13
40.13
28.09
26.73
20.72
20.72
27.76
28.09
28.09
28.09
286.47
298.51
298.51
298.51

WH2O H2O frac $0.057 \quad 0.0002$ $0.008-0.0002$ 0.0002 0.0002 0.00002
0.0044 0.0044 0.0044
0.0044 0.0044 0.0180 0.0146 0.0145 0.0145 0.0145 0.0002 0.0004 0.0004 0.0004

\begin{tabular}{|c|c|c|c|c|c|c|c|c|c|}
\hline & $\begin{array}{l}\text { PER } \\
\text { WC }\end{array}$ & PR & DATA eff & Nc & $\mathrm{TR}$ & efpoly & pwr & SMN & SMW \\
\hline Fan & 623.18 & 1.083 & 0.9031 & 2043.757 & 1.0256 & 0.9042 & -1201.1 & -2757.63 & 49.89 \\
\hline LPC & 87.30 & 1.264 & 0.8262 & 2043.757 & 1.0839 & 0.8319 & -550.5 & 6.38 & 2.41 \\
\hline HPC & 50.82 & 5.766 & 0.8128 & 8854.683 & 1.7881 & 0.8517 & -3949.4 & 61.50 & 57.82 \\
\hline HPT & 12.08 & 3.873 & 0.8750 & 216.456 & 1.3437 & 0.8539 & 3949.4 & & \\
\hline LPT & 51.10 & 2.129 & 0.8238 & 58.413 & 1.1798 & 0.8079 & 1751.6 & & \\
\hline \multicolumn{10}{|c|}{ TURBOMACHINERY MAP DATA } \\
\hline & WcMap & PRmap & effmap & NcMap & R/Parm & S_WcDes & S_PRdes & S_effDes & S_NCDes \\
\hline Fan & 724.08 & 1.081 & 0.9049 & 2024.270 & 0.0425 & $\overline{0} .8607$ & $\overline{1} .0235$ & 0.9980 & 0.9905 \\
\hline $\begin{array}{ll}\text { LPC } \\
\text { LP }\end{array}$ & 53.44 & 1.311 & 0.7607 & 0.540 & 0.0000 & 1.6336 & 0.8496 & 1.0861 & 0.0003 \\
\hline HPC & 46.22 & 5.541 & 0.8212 & 8618.623 & 11.0087 & 1.0995 & 1.0494 & 0.9898 & 0.9733 \\
\hline HPT & 0.96 & 3.793 & 0.8750 & 1.301 & 3.7932 & 12.6299 & 0.9723 & 1.0000 & 0.0003 \\
\hline LPT & 0.78 & 1.849 & 0.8299 & 0.657 & 1.8494 & 65.2590 & 0.7521 & 0.9926 & 0.0005 \\
\hline
\end{tabular}




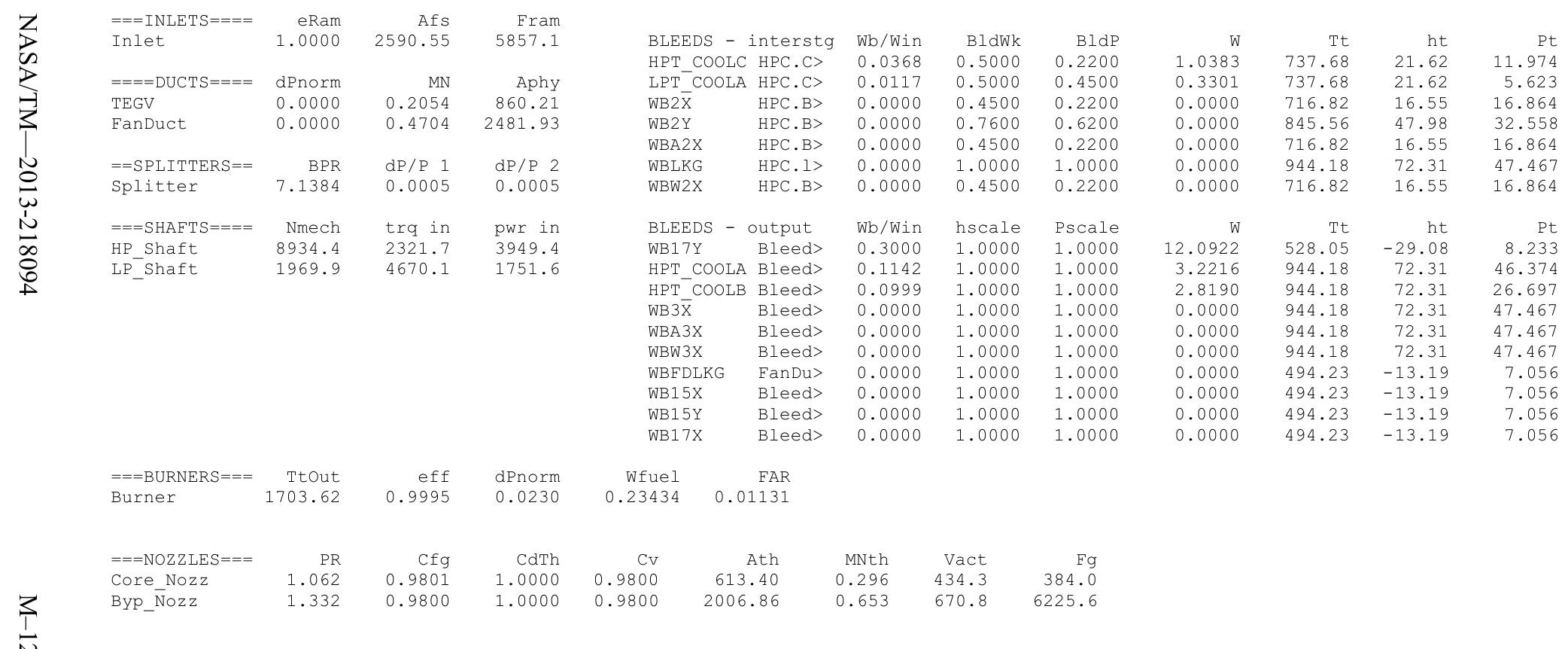


Date:02/15/13 Time:13:38:36 Model: Turbofan Engine - COMDES ON Converge = 1 CASE: 0

Version:NPSS 1.6.5 - Rev: -> Gas Package: Janaf iter/pass/Jacb/Broy= 44/72/ 2/41 Run by: Philip C Jorgenson PC: 10

Temperature Stator 1 inlet: 495.88 Stator 1 exit: 498.89 Stator 2 inlet: 501.74 $\quad$ Stator 2 exit: 502.57 $\begin{array}{llll}\text { Stator } 3 \text { inlet: } 506.00 & \text { Stator } 3 \text { exit: } 506.40 & \text { Stator } 4 \text { inlet: } 509.72 \text { Stator } 4 \text { exit: } 509.81 \\ \text { Stator } 5 \text { inlet: } 511.74 & \text { Stator } 5 \text { exit: } 511.63 & & \text { Unblocked Percent Blockage: } 0.00\end{array}$

Ambient Relative Humidity $\quad 10.00$

Fan Face Relative Humidity $\quad 5.94$

Fan Bypass Relative Humidity $\quad 5.03$

LPC Inlet Relative Humidity $\quad 5.11$

LPC Exit Relative Humidity

Drop Diameter

0.07

Ambient Flow Velocity

0.0000100

Ambient static Pressure

Additional Water at LPC Exit

523.25

Inlet Iength

Fan/LPC Inlet Flow Velocity

40.00

0.74

Fan/LPC Inlet Static Pressure

7.75
493.56

0.004256
493.56

$\begin{array}{rrr}\text { MN } & \text { alt } & \text { dTamb } \\ 0.490 & 20047.0 & 27.00\end{array}$

W
363.30

$\begin{array}{rr}\text { Fn } & \text { TSFC } \\ 824.5 & 1.1323\end{array}$

Wfuel
933.61

BPR
6.3306

VTAS
523.25

$\mathrm{OPR}$
6.381

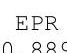

Powerset

$\begin{array}{ccc}\text { T4 } & \text { T41 } & \text { T49 } \\ 1733.0 & 1633.7 & 1161.7\end{array}$

FSO Ambient.Fl_O

FS1 Inlet.Fl_O

FS12 Splitter.F1_0

FS14 Fan.F1_o

FS23 LPC.FI-O

FS24 VaporIñ.FI_

FS25 Bleed2.F1_ $\overline{0}$

HPC.FI_O

FS36 Bleed3.Fl_O

FS45 HPT.FI_O

FS49 LPT.FI_O

FS5 5 TPT.FI-

FS8 Core_Nozz.Fl_O

FS17 FanDuctLkg.FI_O

FS172 FanDuct.FI_O

FLOW STATION DATA

$W$
363.30
363.30
313.74
49.56
313.74
49.56
49.77
29.86
28.41
22.02
22.28
29.77
30.12
30.12
30.12
313.74
333.65
333.65
333.65

\begin{tabular}{lrrr}
\multicolumn{1}{c}{ FLOW } & STATION DATA \\
Pt & Tt & ht & FAR \\
7.943 & 496.98 & -13.65 & 0.0000 \\
7.943 & 496.98 & -13.65 & 0.0000 \\
7.939 & 496.98 & -13.65 & 0.0000 \\
7.939 & 496.98 & -13.65 & 0.0000 \\
8.505 & 507.90 & -11.03 & 0.0000 \\
9.109 & 525.34 & -6.85 & 0.0000 \\
9.109 & 531.18 & -29.89 & 0.0000 \\
9.109 & 531.18 & -29.89 & 0.0000 \\
0.687 & 941.26 & 70.05 & 0.0000 \\
0.687 & 941.26 & 70.05 & 0.0000 \\
9.519 & 1733.03 & 56.99 & 0.0118 \\
3.456 & 1166.48 & -39.38 & 0.0088 \\
7.042 & 1011.30 & -77.14 & 0.0087 \\
7.042 & 1011.37 & -77.14 & 0.0087 \\
7.042 & 1011.44 & -77.14 & 0.0087 \\
8.505 & 507.90 & -11.03 & 0.0000 \\
8.505 & 509.29 & -12.16 & 0.0000 \\
8.505 & 509.29 & -12.16 & 0.0000 \\
8.505 & 509.29 & -12.16 & 0.0000
\end{tabular}

WC
657.95

657.95
657.95

568.48

89.80
536.46

536.46
80.47

80.47
81.26

48.75

11.10
8.60

8.60
12.09

12.09
48.76

87.77
87.78
87.78

87.77
87.78
536.46

536.46

FS173 Byp Nozz.F̄̄ O

TURBOMACHINERY PERFORMANCE DATA

\begin{tabular}{|c|c|c|c|c|c|c|c|c|c|}
\hline & Wc & $\mathrm{PR}$ & eff & $\mathrm{NC}$ & $\mathrm{TR}$ & efpoly & pwr & SMN & SMW \\
\hline Fan & 568.48 & 1.071 & 0.9050 & 1841.626 & 1.0220 & 0.9060 & -1162.0 & -1871.34 & 46.45 \\
\hline LPC & 89.80 & 1.147 & 0.7025 & 1841.626 & 1.0571 & 0.7082 & -476.9 & 7.00 & 2.16 \\
\hline HPC & 48.75 & 5.564 & 0.8084 & 8787.337 & 1.7720 & 0.8475 & -4120.0 & 61.53 & 57.60 \\
\hline HРT & 12.09 & 3.680 & 0.8770 & 213.613 & 1.3284 & 0.8570 & 4120.0 & & \\
\hline LPT & 48.76 & 1.911 & 0.8164 & 52.782 & 1.1501 & 0.8024 & 1639.0 & & \\
\hline TURB & NERY MAP & DATA & & & & & & & \\
\hline & WcMap & PRmap & effMap & NcMap & R/Parm & s WcDes & S PRdes & $s$ effDes & s NcDes \\
\hline Fan & 660.52 & 1.070 & 0.9069 & 1824.067 & 0.0438 & $\overline{0} .8607$ & 1.0235 & 0.9980 & 0.9905 \\
\hline LPC & 46.82 & 1.245 & 0.7556 & 0.486 & 0.0000 & 1.9180 & 0.6009 & 0.9297 & 0.0003 \\
\hline $\begin{array}{l}\text { HPC } \\
\text { He }\end{array}$ & 44.34 & 5.349 & 0.8168 & 8553.073 & 11.0361 & $\begin{array}{l}1.0995 \\
1.09\end{array}$ & 1.0494 & 0.9898 & 0.9733 \\
\hline HPT & 0.96 & 3.606 & 0.8770 & 1.284 & 3.6058 & 12.6299 & 0.9723 & 1.0000 & 0.0003 \\
\hline LPT & 0.75 & 1.685 & 0.8225 & 0.594 & 1.6850 & 65.2590 & 0.7521 & 0.9926 & 0.0005 \\
\hline
\end{tabular}




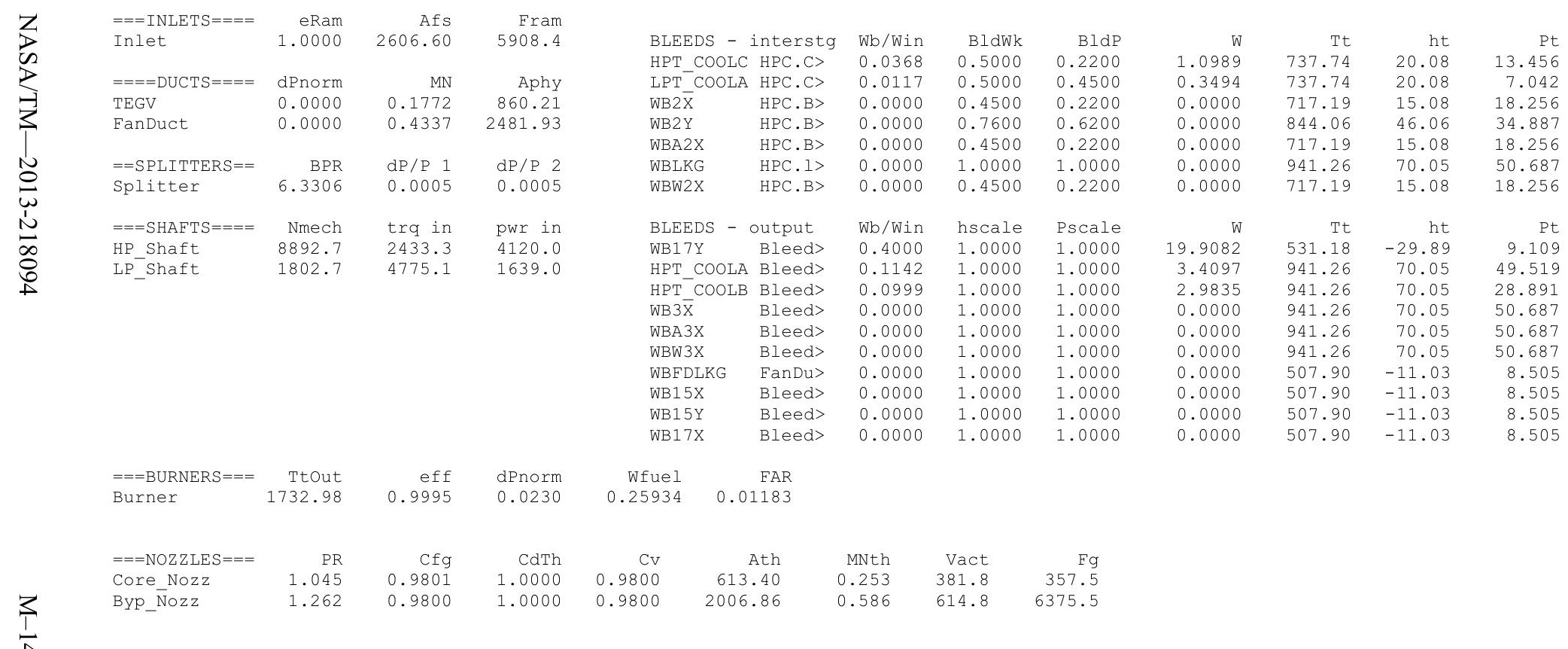


Date:02/15/13 Time:13:39:21 Model: Turbofan Engine - COMDES ON COnverge = 1 CASE: 0

Version:NPSS_1.6.5 - Rev: -> Gas Package: Janaf iter/pass/Jacb/Broy=41/83/3/37 Run by: Philip C Jorgenson PC: 10

Temperature Stator 1 inlet: 509.81 Stator 1 exit: 512.17 Stator 2 inlet: 514.54 Stator 2 exit: 515.29 $\begin{array}{llll}\text { Stator } 3 \text { inlet: } 518.02 & \text { Stator } 3 \text { exit: } 518.45 & \text { Stator } 4 \text { inlet: } 521.06 \text { Stator } 4 \text { exit: } 521.2 \\ \text { Stator } 5 \text { inlet: } 522.88 & \text { Stator } 5 \text { exit: } 522.92 & \text { Unblocked Percent Blockage: } 0.00\end{array}$

Ambient Relative Humidity $\quad 10.00$

Fan Face Relative Humidity $\quad 6.60$

$\begin{array}{ll}\text { Fan Bypass Relative Humidity } & 6.08 \\ \text { LPC Inlet Relative Humidity } & 5.78\end{array}$

LPC Inlet Relative Humidity

LPC Exit Relative Humidity

DrC Relative Humidity

Drop Diameter

0.10

Ambient Flow Velocity

Ambient Static Pressure

Additional Water at LPC Exit

Inlet Length

Fan/LPC Inlet Flow Velocity $\quad \begin{aligned} 40.00 \\ \text { Fan/LPC Inlet }\end{aligned}$

4.15

Fan/LPC Inlet Static Pressure $\quad 9.18$

0.004123

9.18
07.74

\section{MN alt dTamb SUMMARY OUTPUT DATA}

$0.446 \quad 15435.0-27.00$

W
380.62

$\begin{array}{cc}\text { Fn } & \text { TSFC } \\ 615.9 & 1.4205\end{array}$

Wfuel

BPR
6.9316

VTAS

$\mathrm{OPR}$
5.173

EPR
0.900

Powerset

10.000

$\begin{array}{ccc}\text { T4 } & \text { T41 } & \text { T49 } \\ 1678.4 & 1581.4 & 1149.1\end{array}$

FSO Ambient.Fl_O

FS1 Inlet.Fl_O

$W$
380.62
380.62
332.63
47.99
332.63
47.99
48.19
28.91
27.51
21.32
21.56
28.82
29.15
29.15
29.15
332.63
351.90
351.90
351.90

FLOW STATION DATA

FS2 Splitter. 1 - 02

FS14 Fan.FI_O

FS24 VaporIN.FI_O

FS25 Bleed2.FI_O

HPC.Fl_O

FS4 Bleed3.Fl_O

FS45 HPT.FI_O

FS49 LPT.EI_O

FS5 TEGV.FI_O

FS17 FanDuctlkg.F̄_O

FS171 Bleed15.Fl_O

FS172 FanDuct. Fl-O

\begin{tabular}{rrrr}
\multicolumn{1}{c}{ Pt } & Tt & ht & FAR \\
Pt & 510.16 & -11.98 & 0.0000 \\
9.342 & 510.16 & -11.98 & 0.0000 \\
9.342 & 510.16 & -11.98 & 0.0000 \\
9.337 & 510.16 & -11.98 & 0.0000 \\
9.337 & 510.06 & 5 \\
9.798 & 518.06 & -10.08 & 0.0000 \\
10.453 & 532.69 & -6.57 & 0.0000 \\
10.453 & 538.30 & -28.89 & 0.0000 \\
10.453 & 538.30 & -28.89 & 0.0000 \\
48.322 & 906.32 & 60.70 & 0.0000 \\
48.322 & 906.32 & 60.70 & 0.0000 \\
47.209 & 1678.36 & 48.15 & 0.0115 \\
14.109 & 1153.86 & -38.04 & 0.0085 \\
8.387 & 1029.77 & -67.94 & 0.0084 \\
8.387 & 1029.84 & -67.94 & 0.0084 \\
8.387 & 1029.91 & -67.94 & 0.0084 \\
9.798 & 518.06 & -10.08 & 0.0000 \\
9.798 & 519.17 & -11.11 & 0.0000 \\
9.798 & 519.17 & -11.11 & 0.0000 \\
9.798 & 519.17 & -11.11 & 0.0000
\end{tabular}

Wc
593.82

Ps
8.149

Ts
490.63
503.89
503.46
507.74
505.91
523.62
528.93
535.11
876.62
889.52
1654.46
1134.66
1025.78
1025.85
1021.89
505.91
503.59
503.59
492.54

$\begin{array}{rr}\text { Aphy } & \text { MN } \\ 2524.5 & 0.4460\end{array}$

$\begin{array}{llr}2524.5 & 0.4460 & \text { gamt } \\ 4168.2 & 0.2494 & 1.40025\end{array}$

$\begin{array}{lll}4168.2 & 0.2494 & 1.40025 \\ 3531.8 & 0.2580 & 1.40025\end{array}$

$830.5 \quad 0.1543$

1.40025
1.40025

WAR

$\begin{array}{rrrr}9.183 & 507.74 & 830.5 & 0.1543 \\ 9.017 & 505.91 & 2606.7 & 0.3464\end{array}$

1.40015

$\begin{array}{lr}0.0006510 & 380.37 \\ 0.0006510 & 380.37\end{array}$

$\begin{array}{ll}0.0006510 & 332.41\end{array}$

$\begin{array}{lllllllll}9.843 & 523.62 & 412.6 & 0.2943 & 1.39995 & 0.0006510 & 47.96 & 0.031 & 0.0007 \\ 9.829 & 528.93 & 412.6 & 0.2978 & 1.39930 & 0.0047747 & 47.96 & 0.229 & 0.0048\end{array}$

$\begin{array}{llll}0.0006510 & 332.41 & 0.031 & 0.0007 \\ 0.0006510 & 47.96 & 0.031 & 0.0007\end{array}$

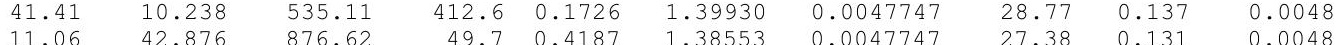

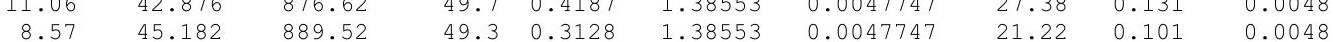

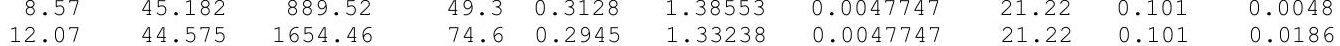

$\begin{array}{llllllllll}44.77 & 13.250 & 1134.66 & 265.4 & 0.3045 & 1.36432 & 0.0047747 & 28.44 & 0.136 & 0.0151 \\ 71.98 & 8.268 & 1025.78 & 860.2 & 0.1445 & 1.37240 & 0.0047747 & 28.77 & 0.137 & 0.0150\end{array}$

$\begin{array}{llllllllll}71.98 & 8.268 & 1025.78 & 860.2 & 0.1445 & 1.37240 & 0.0047747 & 28.77 & 0.137 & 0.0150 \\ 71.98 & 8.268 & 1025.85 & 860.2 & 0.1445 & 1.37240 & 0.0047747 & 28.77 & 0.137 & 0.0150\end{array}$

$\begin{array}{llllllllll}71.98 & 8.268 & 1025.85 & 860.2 & 0.1445 & 1.37240 & 0.0047747 & 28.77 & 0.137 & 0.0150 \\ 71.99 & 8.149 & 1021.89 & 613.4 & 0.2052 & 1.37239 & 0.0047747 & 28.77 & 0.137 & 0.0150\end{array}$

498.62

$\begin{array}{rr}9.017 & 505.91 \\ 8.807 & 503.59\end{array}$

$2606.7 \quad 0.3464$

1.37239
1.40015

0.0006510

1.40011

528.08

$8.149 \quad 492.54$

201.90 .3933

1.40011

0.0008760

0.00008760
0.0008760

$\begin{array}{ll}351.59 & 0.308 \\ 351.59 & 0.308\end{array}$

0.0007

0.0007
0.0009
0.0009

0.0009

TURBOMACHINERY PERFORMANCE DATA

\begin{tabular}{|c|c|c|c|c|c|c|c|c|c|}
\hline & Wc & PR & eff & NC & TR & efPoly & pwr & SMN & SMW \\
\hline Fan & 519.22 & 1.049 & 0.8951 & 1596.527 & 1.0155 & 0.8958 & -891.9 & -1535.28 & 44.76 \\
\hline LPC & 74.91 & 1.119 & 0.7424 & 1596.527 & 1.0442 & 0.7465 & -367.1 & 8.35 & 1.96 \\
\hline HPC & 41.41 & 4.623 & 0.7925 & 8510.501 & 1.6837 & 0.8310 & -3575.5 & 69.67 & 64.23 \\
\hline HPT & 12.07 & 3.346 & 0.8678 & 211.630 & 1.2990 & 0.8482 & 3575.5 & & \\
\hline LPT & 44.77 & 1.682 & 0.8019 & 46.613 & 1.1169 & 0.7900 & 1259.0 & & \\
\hline TURB & NERY MAP & DATA & & & & & & & \\
\hline & WcMap & PRmap & effmap & NcMap & R/Parm & s WcDes & s PRdes & $s \quad s$ effDes & $s$ NCDes \\
\hline Fan & 603.28 & 1.048 & 0.8969 & 1581.304 & 0.0441 & $\overline{0} .8607$ & $\overline{1} .0235$ & 0.9980 & $\overline{0.9905}$ \\
\hline LPC & 39.28 & 1.181 & 0.7476 & 0.422 & 0.0000 & 1.9071 & 0.6613 & 0.9931 & 0.0003 \\
\hline HPC & 37.66 & 4.452 & 0.8007 & 8283.616 & 10.6983 & 1.0995 & 1.0494 & 0.9898 & 0.9733 \\
\hline $\mathrm{HPT}$ & 0.96 & 3.281 & 0.8677 & 1.272 & 3.2811 & 12.6299 & 0.9723 & 1.0000 & 0.0003 \\
\hline LPT & 0.69 & 1.513 & 0.8079 & 0.524 & 1.5131 & 65.2590 & 0.7521 & 0.9926 & 0.0005 \\
\hline
\end{tabular}




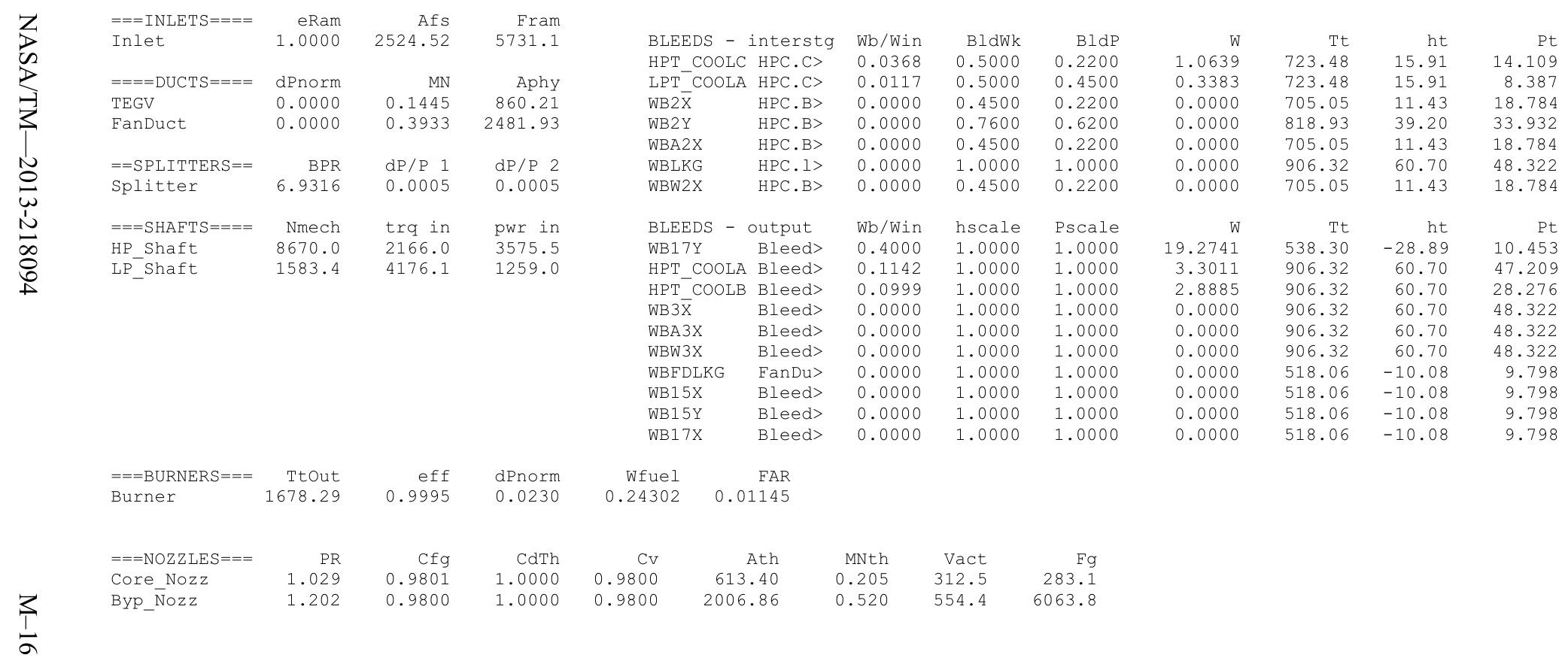




\section{Compressor Code Analysis: $10 \mu \mathrm{m}, 9 \mathrm{~g} / \mathrm{m}^{3}$, ISA $+27 \mathrm{R}$}

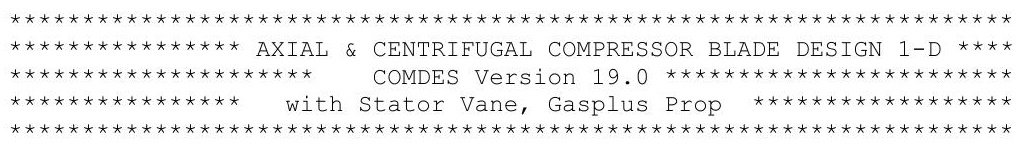

Fan Core + 4 Stage LPC 10\%39000 ft 02-15-2013

COMPRESSOR INLET CONDITIONS - ANALYSIS, STAGE 1 $\mathrm{BLEED}=0.000 \quad$ DPInC $=15.503 \quad$ EfDer $=0.954$ $\mathrm{SH}=0.100590 \mathrm{E}-02$

\begin{tabular}{|c|c|c|c|c|c|c|c|}
\hline $\begin{array}{l}\text { W act } \\
26.291\end{array}$ & $\begin{array}{l}\text { RPM act } \\
2447.949\end{array}$ & $\begin{array}{l}\text { Pt } \\
4.265\end{array}$ & $\begin{array}{l}\text { Tt } \\
467.792\end{array}$ & $\begin{array}{l}\text { POTS } \\
1.000\end{array}$ & & $\begin{array}{l}\text { POTH } \\
1.000\end{array}$ & $\begin{array}{r}\text { AeroBl } \\
0.980\end{array}$ \\
\hline $\mathrm{Kg} / \mathrm{sec}=$ & 11.950 & 26.264 & $\mathrm{WH} 2 \mathrm{O}=0.026$ & $1 \mathrm{bm} / \mathrm{sec}$ & $\mathrm{H} 2 \mathrm{O}$ & 0.3 & $\mathrm{lg} / \mathrm{m}^{\wedge} 3$ \\
\hline $\begin{array}{l}\text { W } \operatorname{cor} \\
\quad 86.065\end{array}$ & $\begin{array}{l}\text { RPM cor } \\
2577.601\end{array}$ & $\begin{array}{l}\text { GAMMA } \\
1.380\end{array}$ & $\begin{array}{l}\text { Cp } \\
0.249\end{array}$ & $\begin{array}{l}R \\
53.381\end{array}$ & & $\begin{array}{l}\text { Blades } \\
32.000\end{array}$ & $\begin{array}{l}\text { THK } \\
0.050\end{array}$ \\
\hline $\begin{array}{l}\text { CFM } \\
65178.254\end{array}$ & $\begin{array}{l}\text { SCFM } \\
20620.678\end{array}$ & $\begin{array}{l}\mathrm{A} 1 / \mathrm{A}^{*} \\
3.299\end{array}$ & $\begin{array}{l}\text { Areal } \\
831.557\end{array}$ & $\begin{array}{c}A^{\star} \\
252.057\end{array}$ & & $\begin{array}{l}\text { thrRotor } \\
619.754\end{array}$ & $\begin{array}{c}\text { ChokeMargin } \\
2.459\end{array}$ \\
\hline
\end{tabular}

ROTOR LEADING EDGE CONDITIONS, STAGE 1

\begin{tabular}{|c|c|c|c|c|c|c|c|c|c|}
\hline & R1 & Stator & Alfa & $\mathrm{C} 1$ & CU1 & $\mathrm{Cm} 1$ & Mabs & Mrel & U1cor \\
\hline TIP & 20.63 & 0.00 & -0.02 & 188.11 & -0.06 & 188.11 & 0.18 & 0.46 & 464.03 \\
\hline MEAN & 17.06 & 0.00 & -0.02 & 188.11 & -0.06 & 188.11 & 0.18 & 0.39 & \\
\hline HUB & 12.51 & 0.00 & -0.02 & 188.11 & -0.06 & 188.11 & 0.18 & 0.31 & \\
\hline & Betaflo & BetaBlade & Incid & U1 & W1 & Ps 1 & Ts1 & TwetBulb1 & $\mathrm{RH}$ \\
\hline TIP & 66.89 & 50.47 & 16.42 & 440.71 & 479.23 & 4.17 & 464.95 & 457.96 & 24.04 \\
\hline MEAN & 62.70 & 47.20 & 15.50 & 364.45 & 410.19 & 4.17 & 464.95 & 457.96 & 24.04 \\
\hline HUB & 54.86 & 38.62 & 16.24 & 267.24 & 326.86 & 4.17 & 464.95 & 457.96 & 24.04 \\
\hline
\end{tabular}

ROTOR EXIT CONDITIONS, STAGE 1 SOLUTION IS CONVERGED

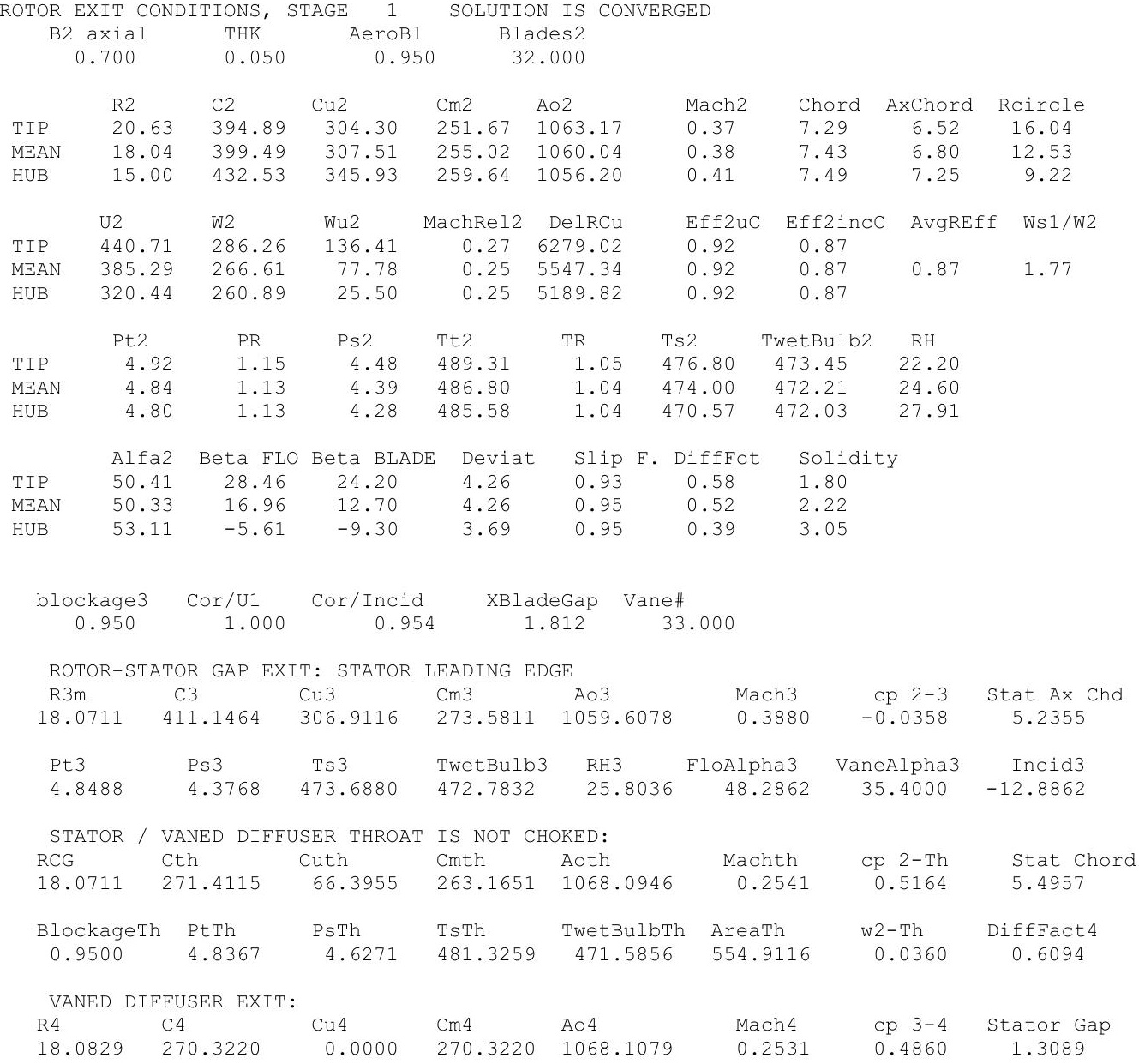




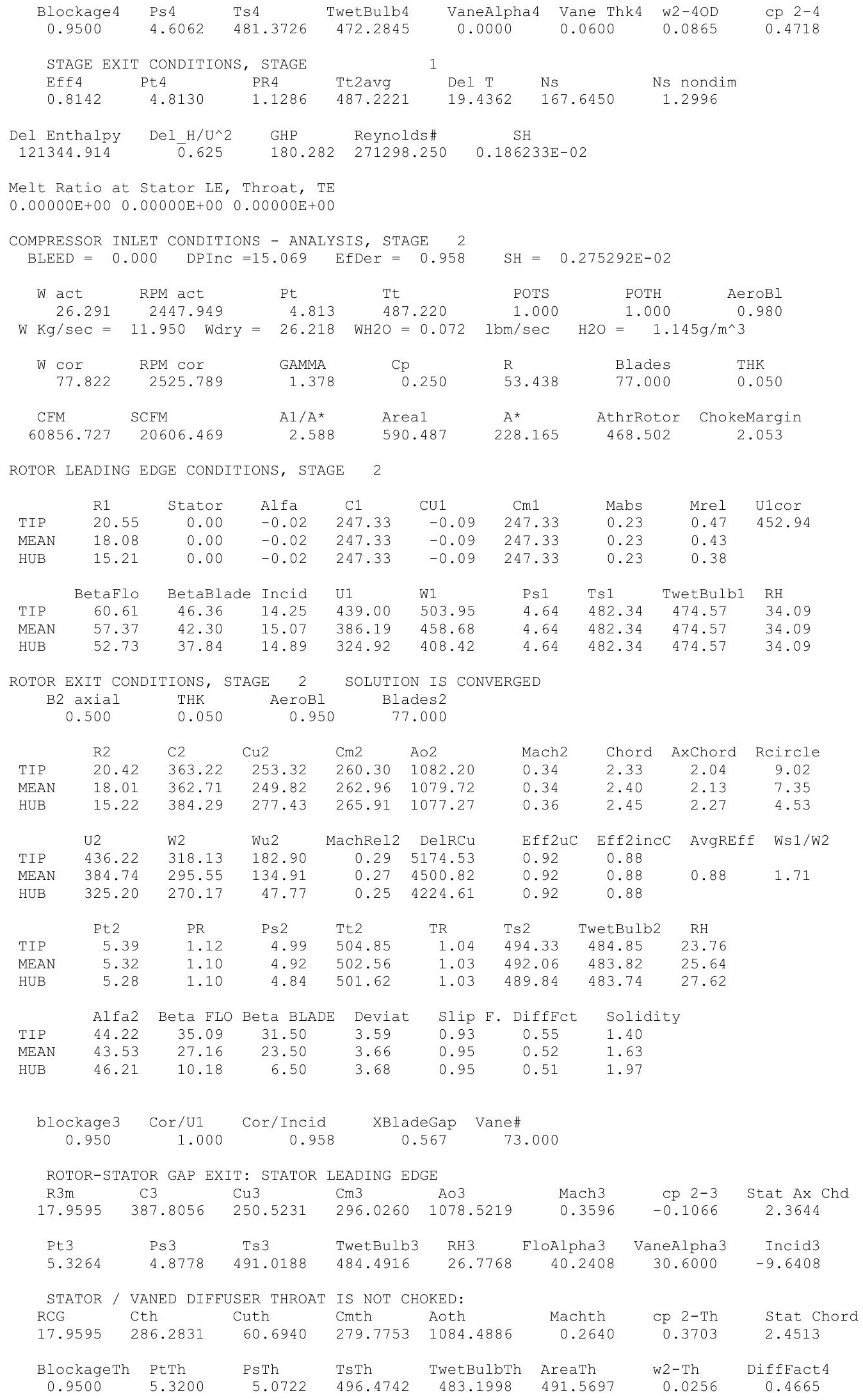

$\begin{array}{lcclcccccc} & \text { R1 } & \text { Stator } & \text { Alfa } & \text { C1 } & \text { CU1 } & \text { Cm1 } & \text { Mabs } & \text { Mrel } & \text { U1cor } \\ \text { TIP } & 20.55 & 0.00 & -0.02 & 247.33 & -0.09 & 247.33 & 0.23 & 0.47 & 452.94 \\ \text { MEAN } & 18.08 & 0.00 & -0.02 & 247.33 & -0.09 & 247.33 & 0.23 & 0.43 & \\ \text { HUB } & 15.21 & 0.00 & -0.02 & 247.33 & -0.09 & 247.33 & 0.23 & 0.38 & \\ & & & & & & & & & \\ & \text { BetaFlo } & \text { BetaBlade } & \text { Incid } & \text { U1 } & \text { W1 } & \text { Ps1 } & \text { Ts1 } & \text { TwetBulb1 } & \text { RH } \\ \text { TIP } & 60.61 & 46.36 & 14.25 & 439.00 & 503.95 & 4.64 & 482.34 & 474.57 & 34.09 \\ \text { MEAN } & 57.37 & 42.30 & 15.07 & 386.19 & 458.68 & 4.64 & 482.34 & 474.57 & 34.09 \\ \text { HUB } & 52.73 & 37.84 & 14.89 & 324.92 & 408.42 & 4.64 & 482.34 & 474.57 & 34.09\end{array}$

ROTOR EXIT CONDITIONS, STAGE 2 SOLUTION IS CONVERGED

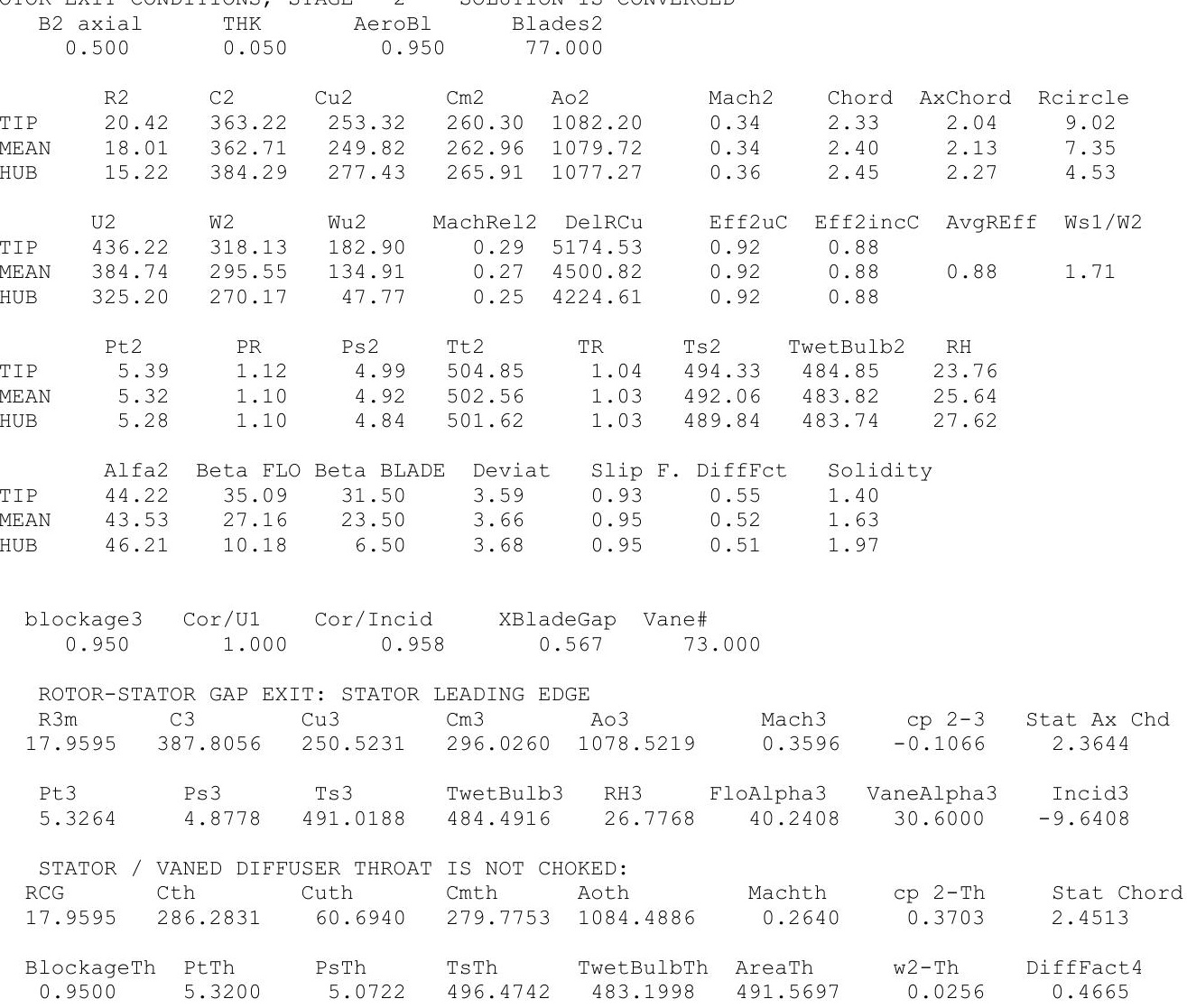




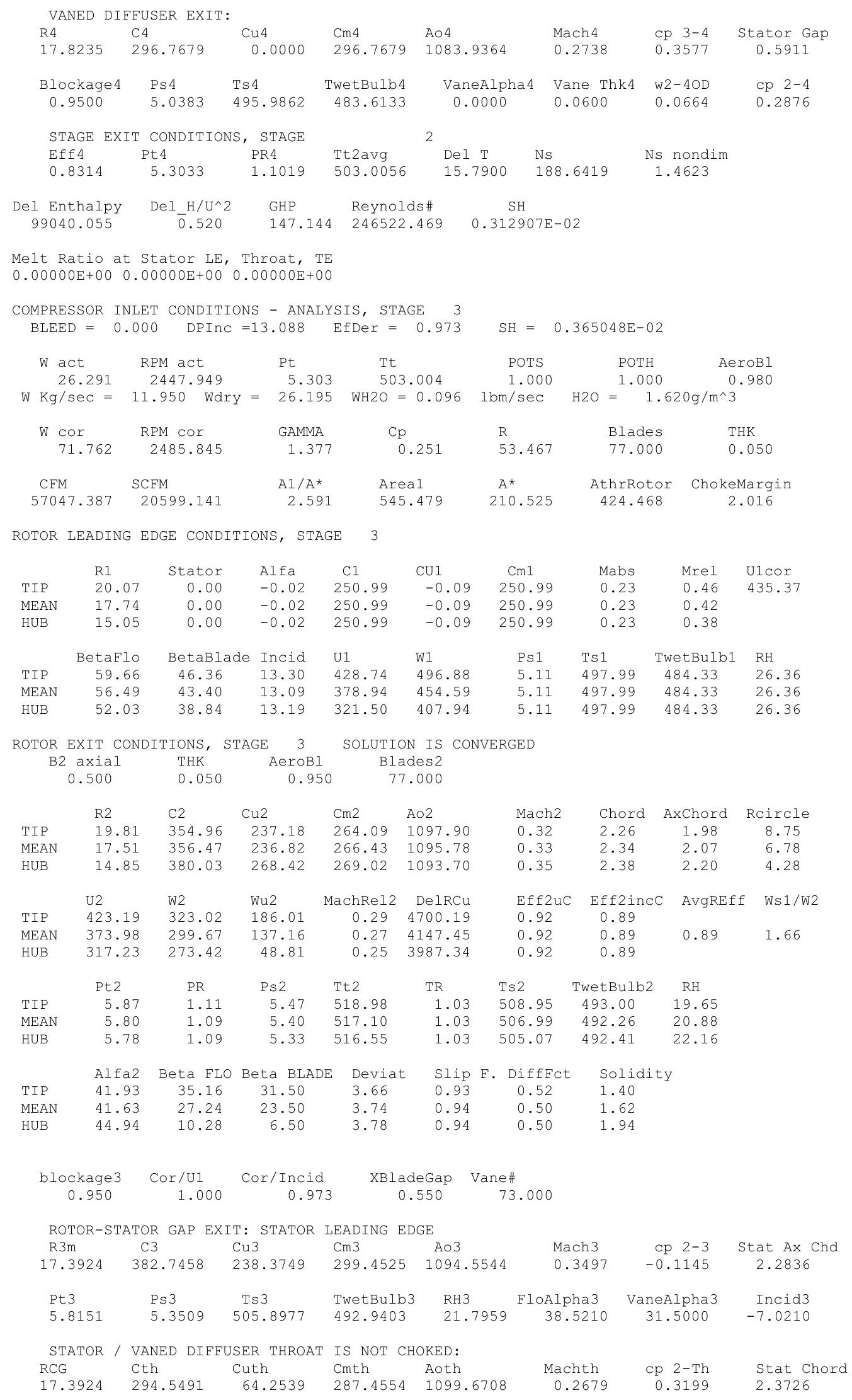




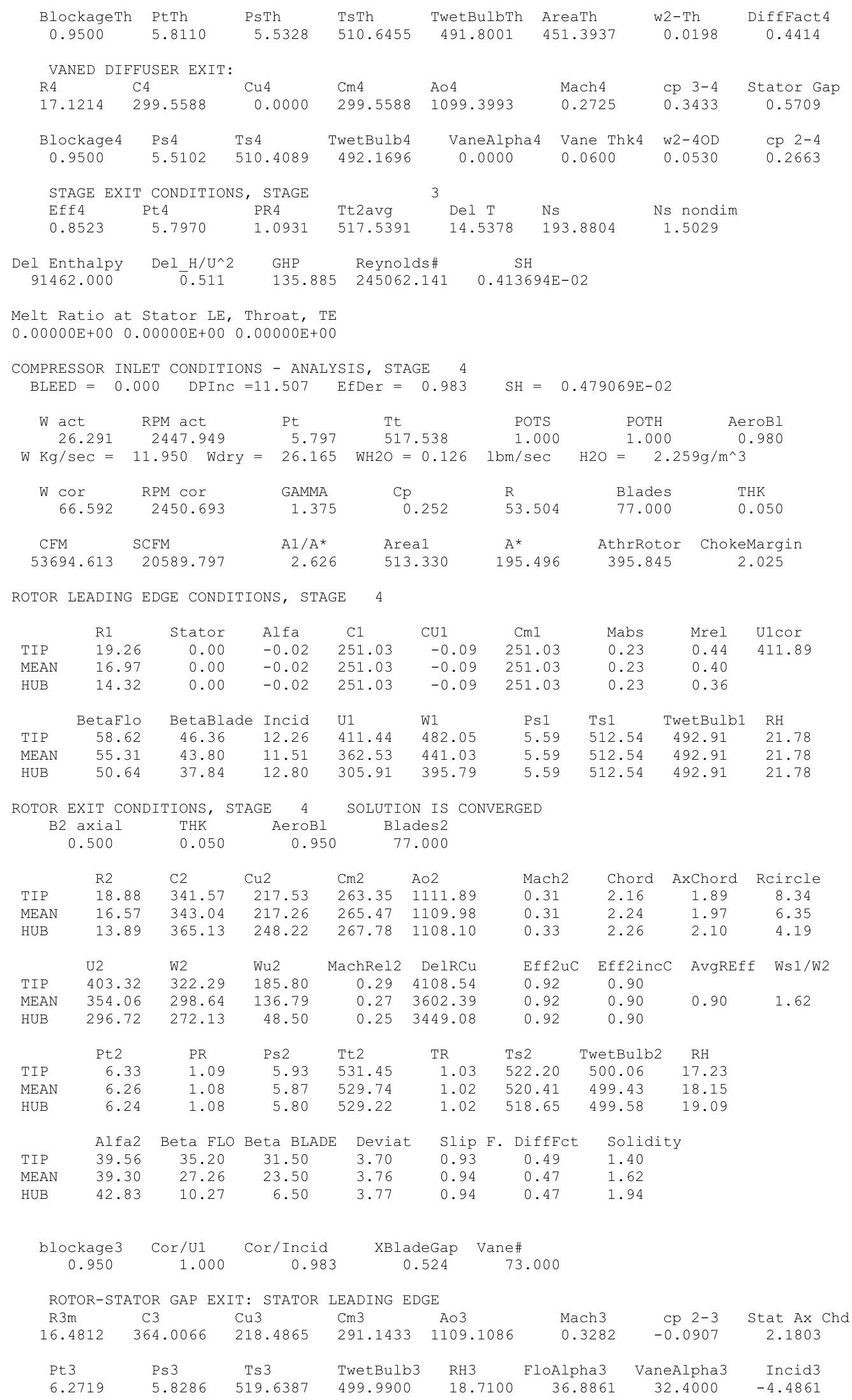




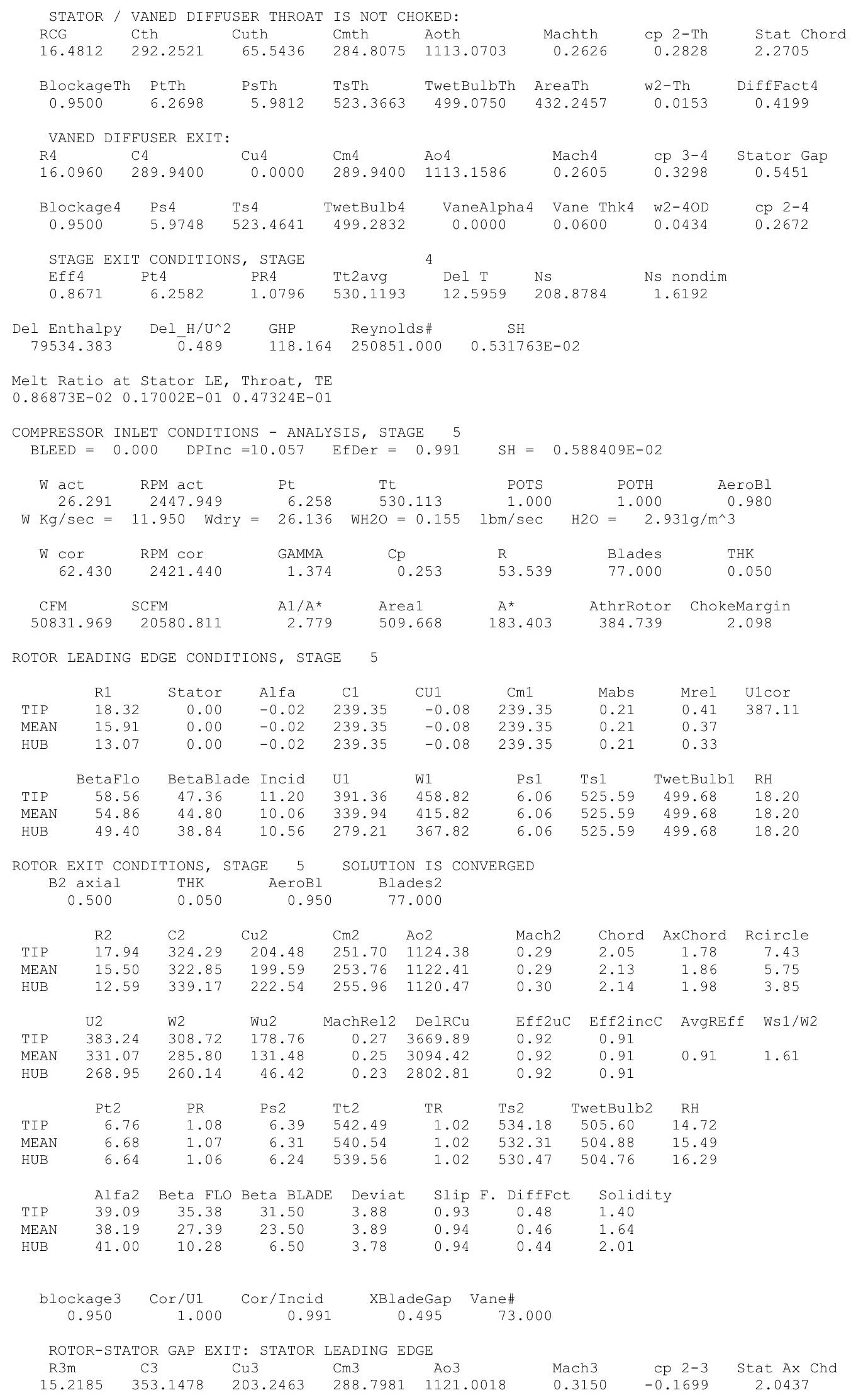




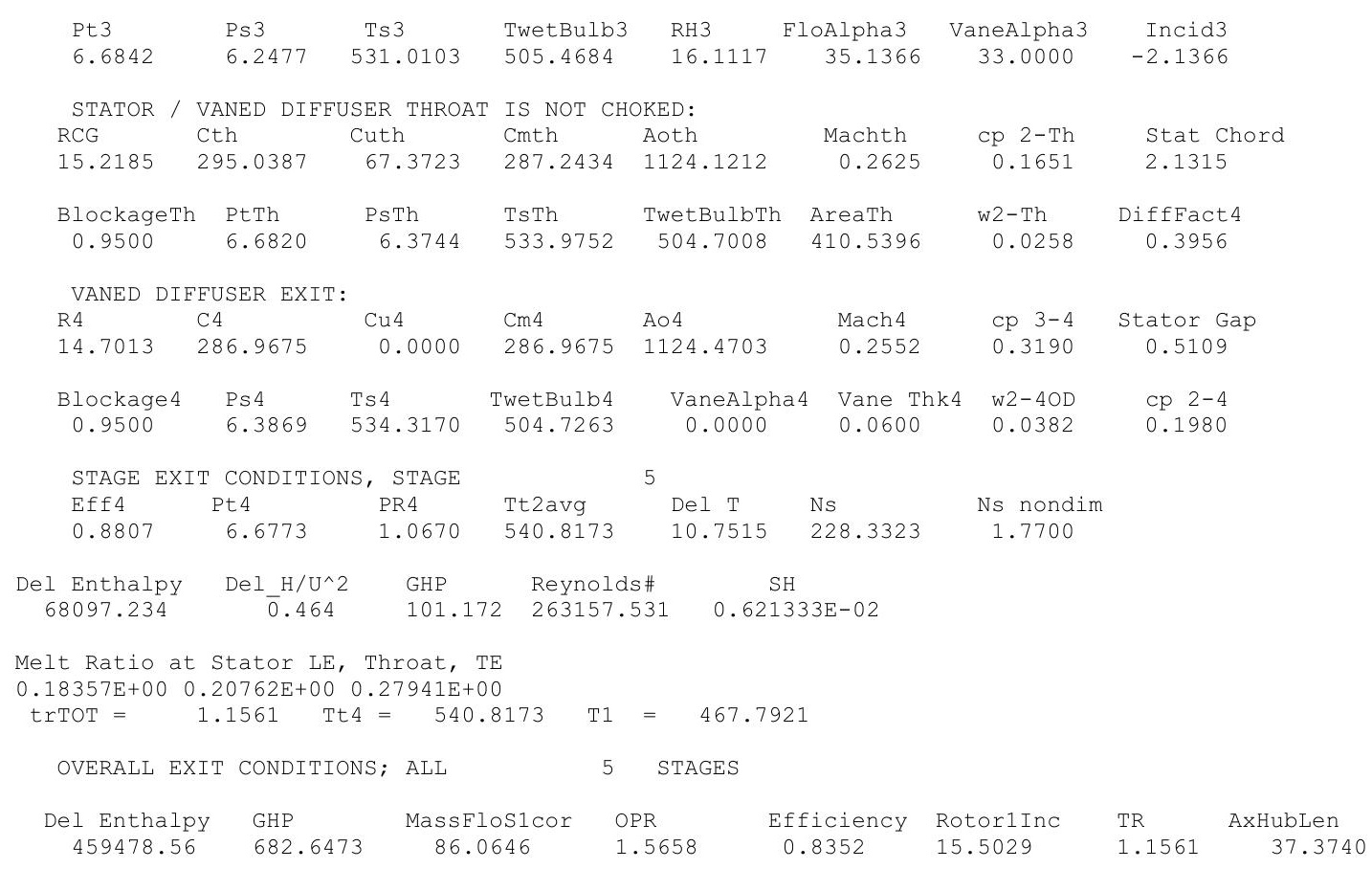




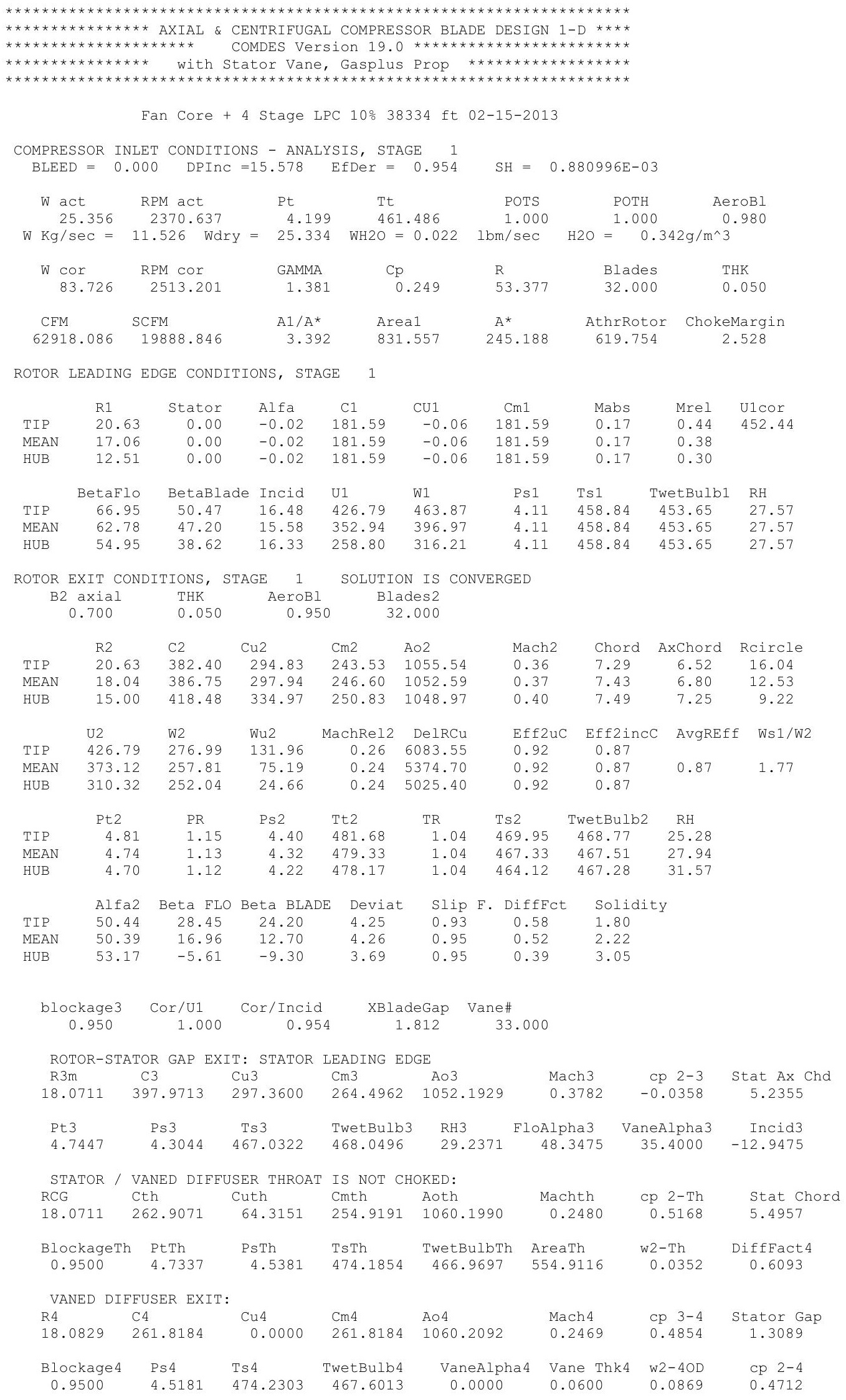




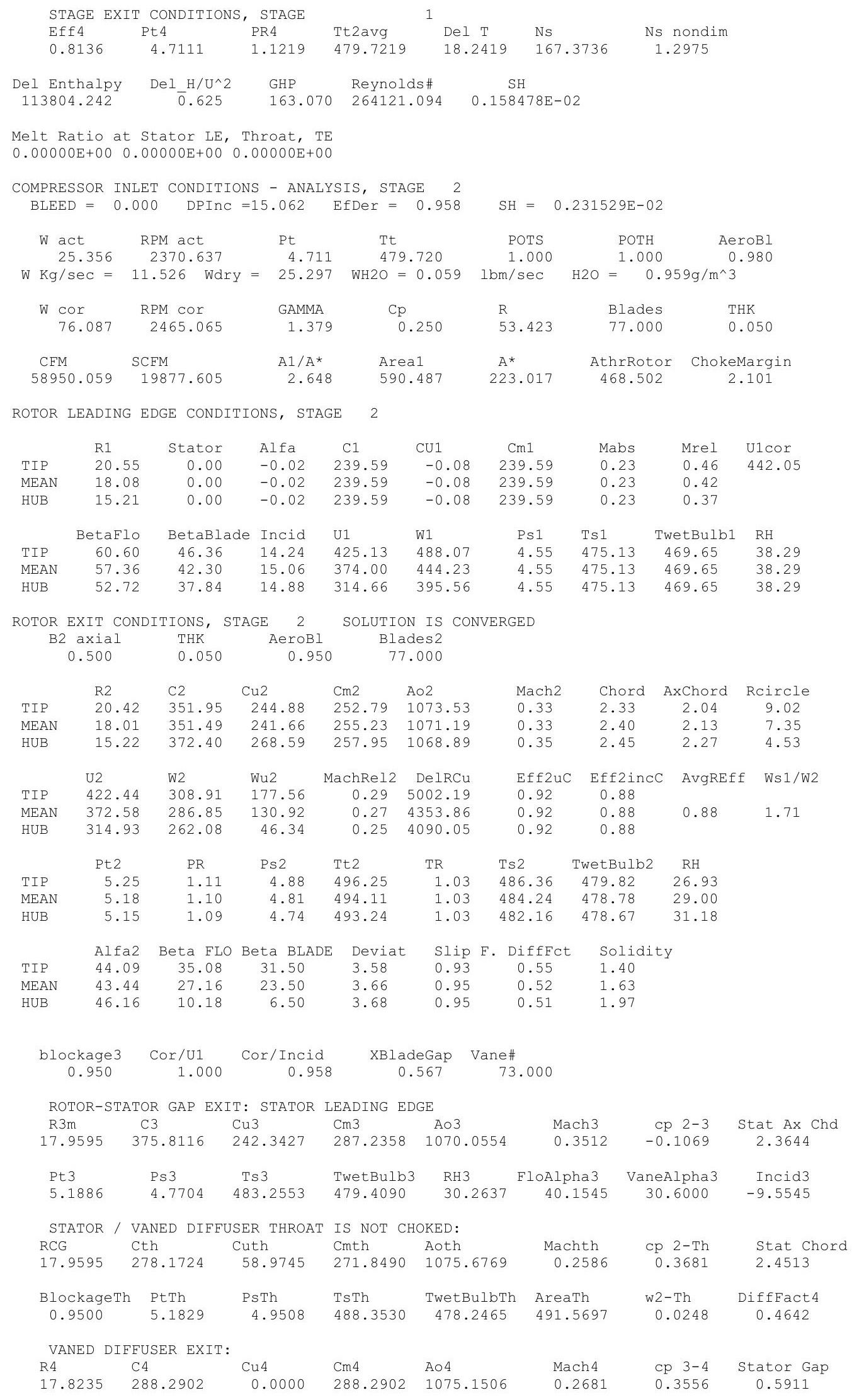




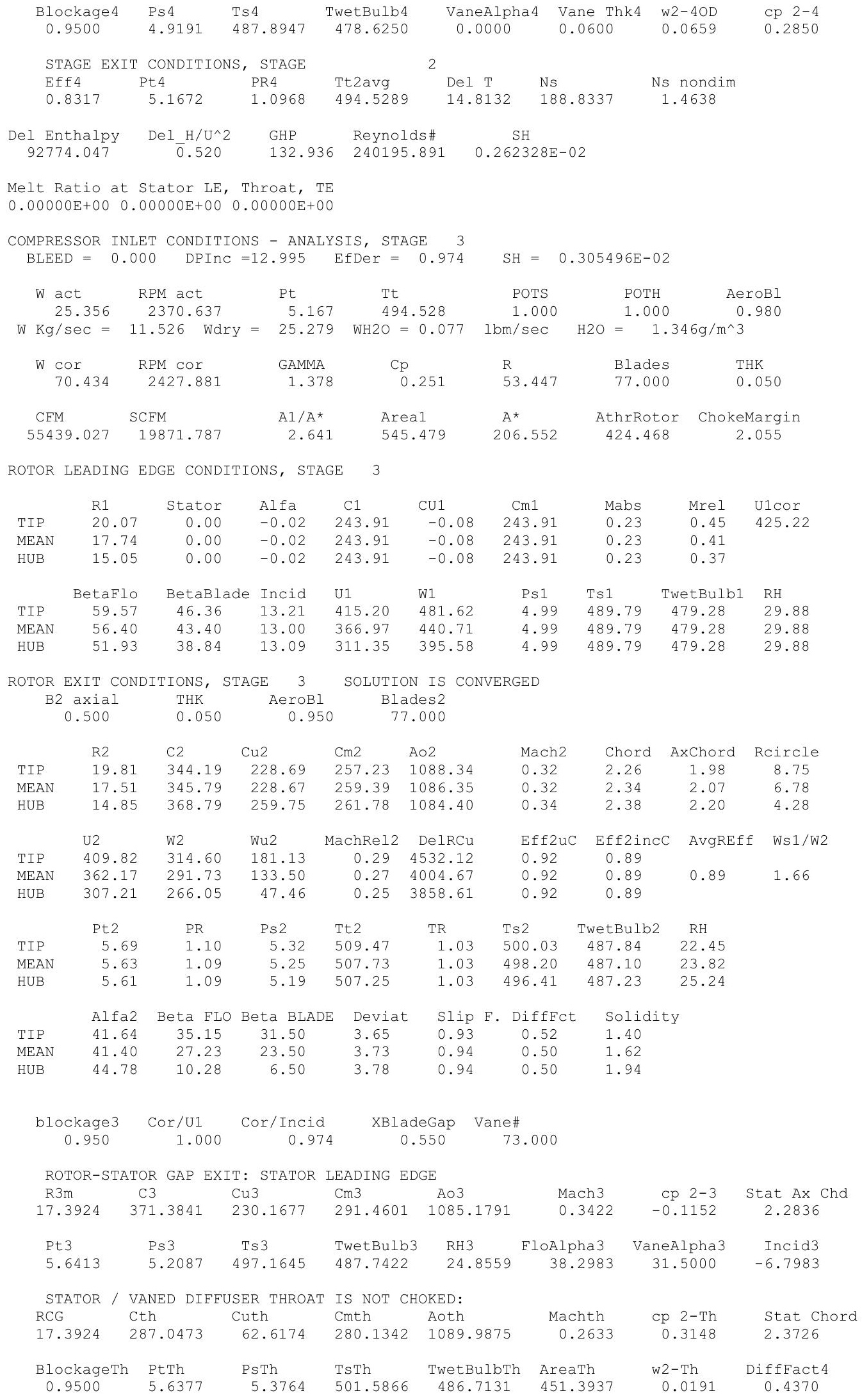

$\begin{array}{lccccccccc} & \text { R1 } & \text { Stator } & \text { Alfa } & \text { C1 } & \text { CU1 } & \text { Cm1 } & \text { Mabs } & \text { Mrel } & \text { U1Cor } \\ \text { TIP } & 20.07 & 0.00 & -0.02 & 243.91 & -0.08 & 243.91 & 0.23 & 0.45 & 425.22 \\ \text { MEAN } & 17.74 & 0.00 & -0.02 & 243.91 & -0.08 & 243.91 & 0.23 & 0.41 & \\ \text { HUB } & 15.05 & 0.00 & -0.02 & 243.91 & -0.08 & 243.91 & 0.23 & 0.37 & \\ & & & & & & & & & \\ & \text { BetaFlo } & \text { BetaBlade } & \text { Incid } & \text { U1 } & \text { W1 } & \text { Ts } 1 & \text { TwetBulb1 } & \text { RH } \\ \text { TIP } & 59.57 & 46.36 & 13.21 & 415.20 & 481.62 & 4.99 & 489.79 & 479.28 & 29.88 \\ \text { MEAN } & 56.40 & 43.40 & 13.00 & 366.97 & 440.71 & 4.99 & 489.79 & 479.28 & 29.88 \\ \text { HUB } & 51.93 & 38.84 & 13.09 & 311.35 & 395.58 & 4.99 & 489.79 & 479.28 & 29.88\end{array}$

ROTOR EXIT CONDITIONS, STAGE 3 SOLUTION IS CONVERGED

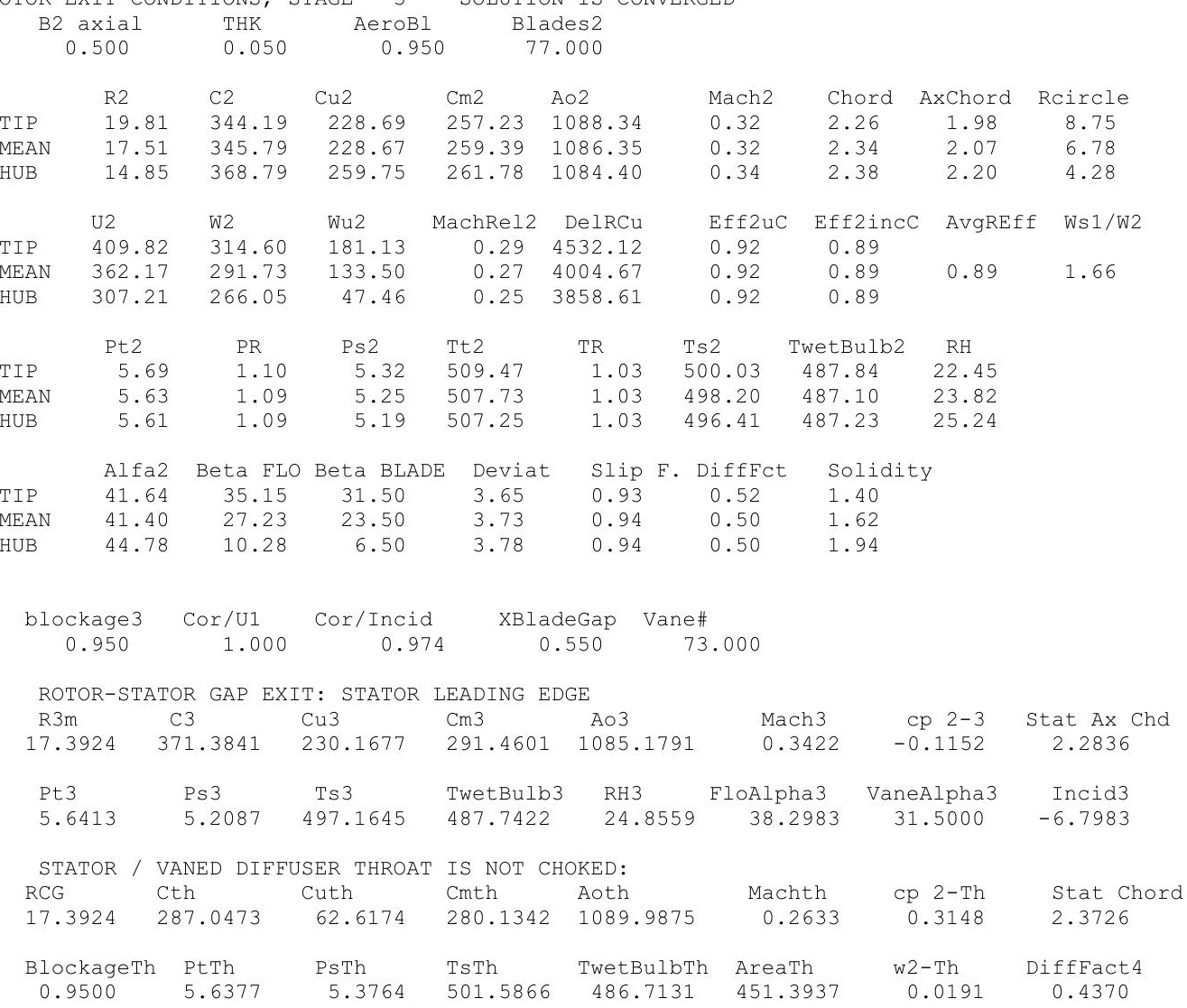




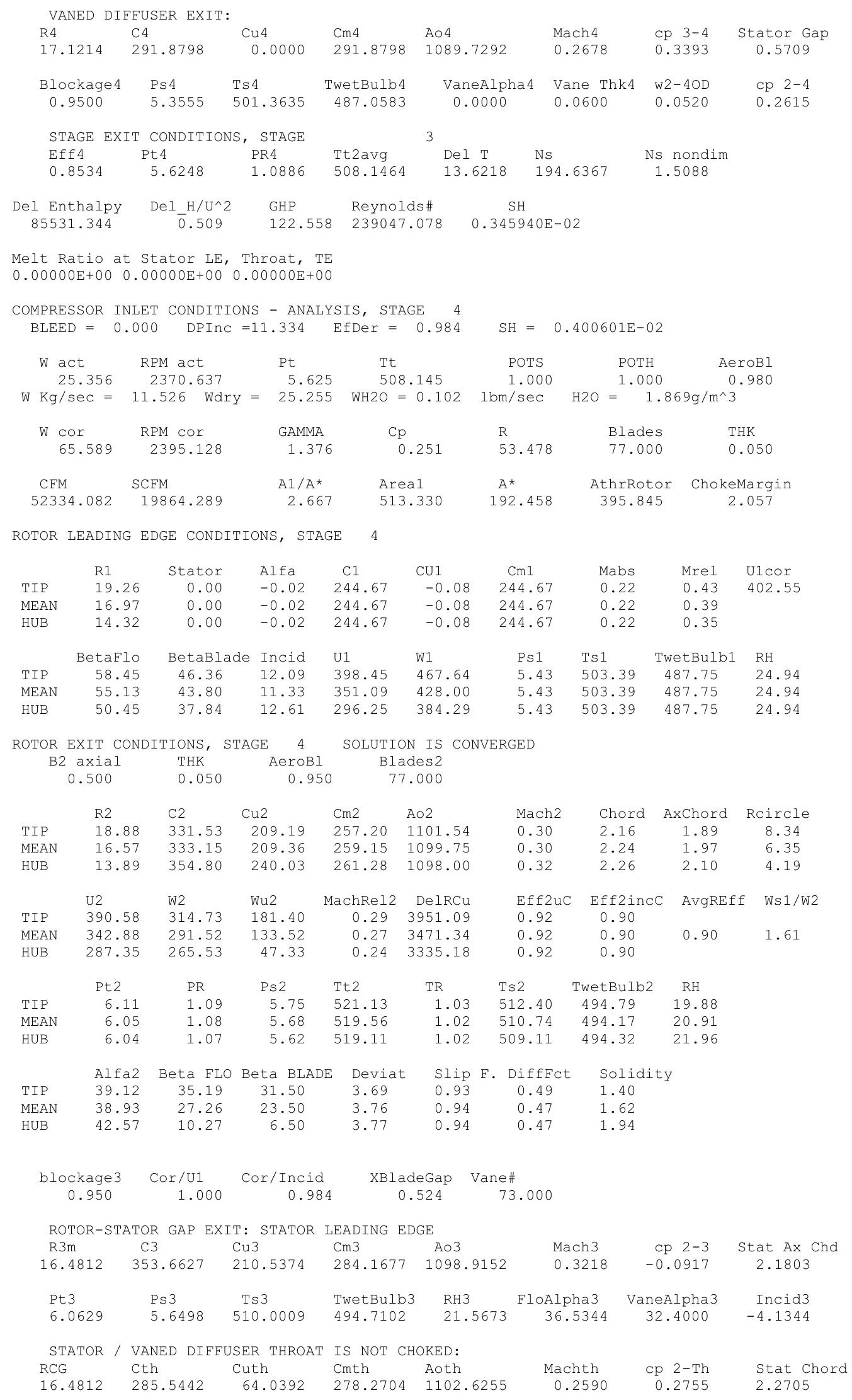




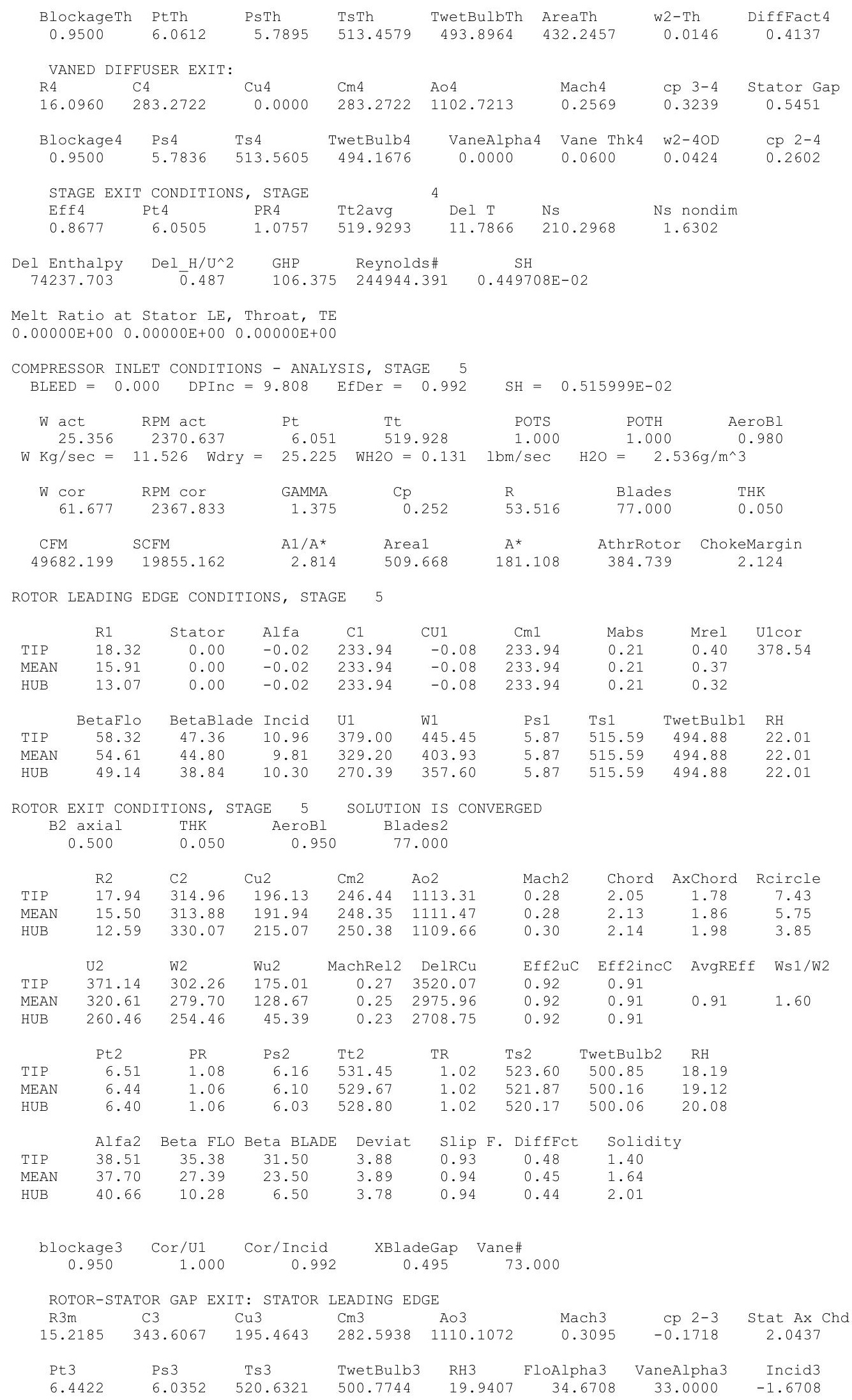




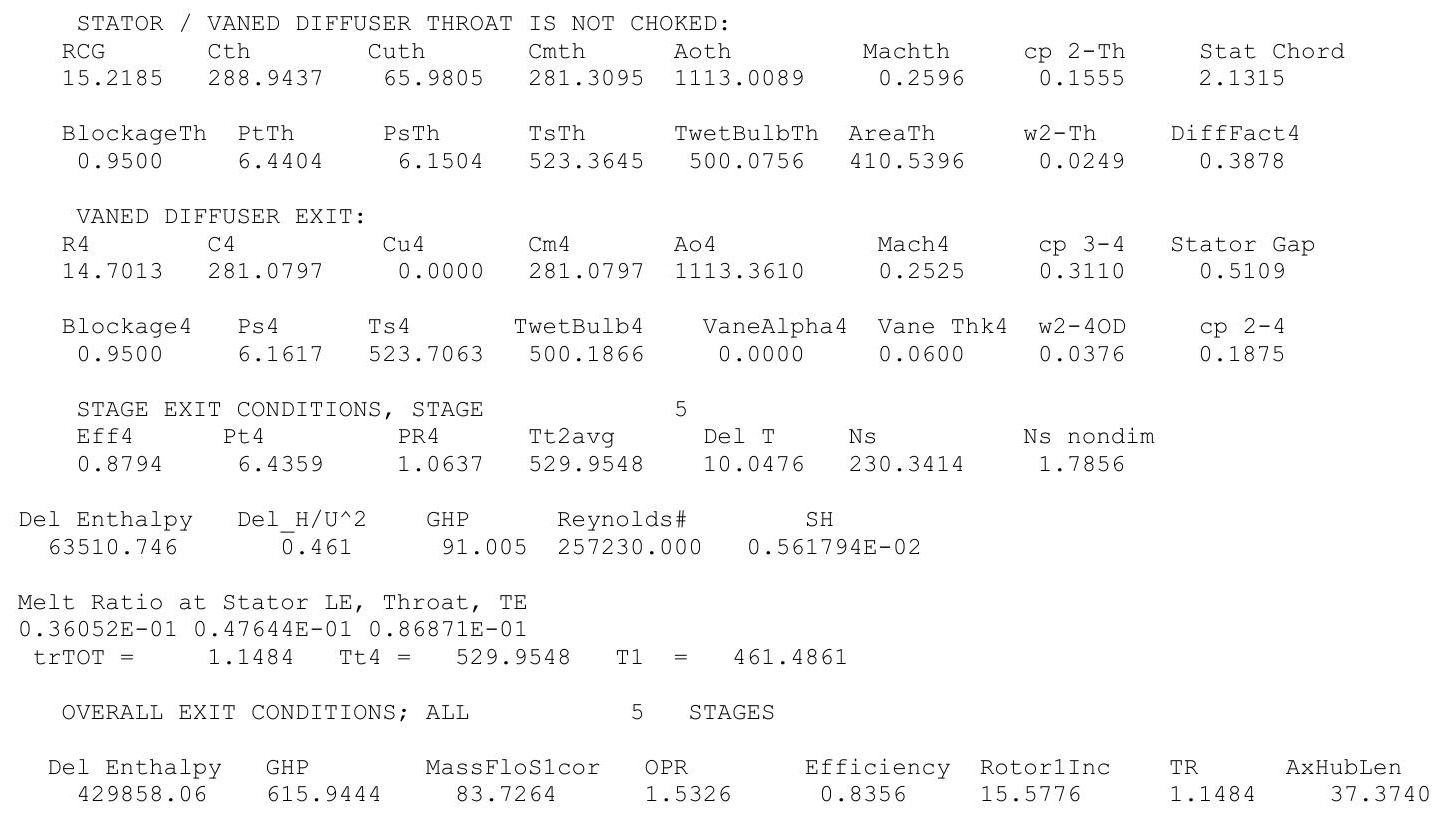




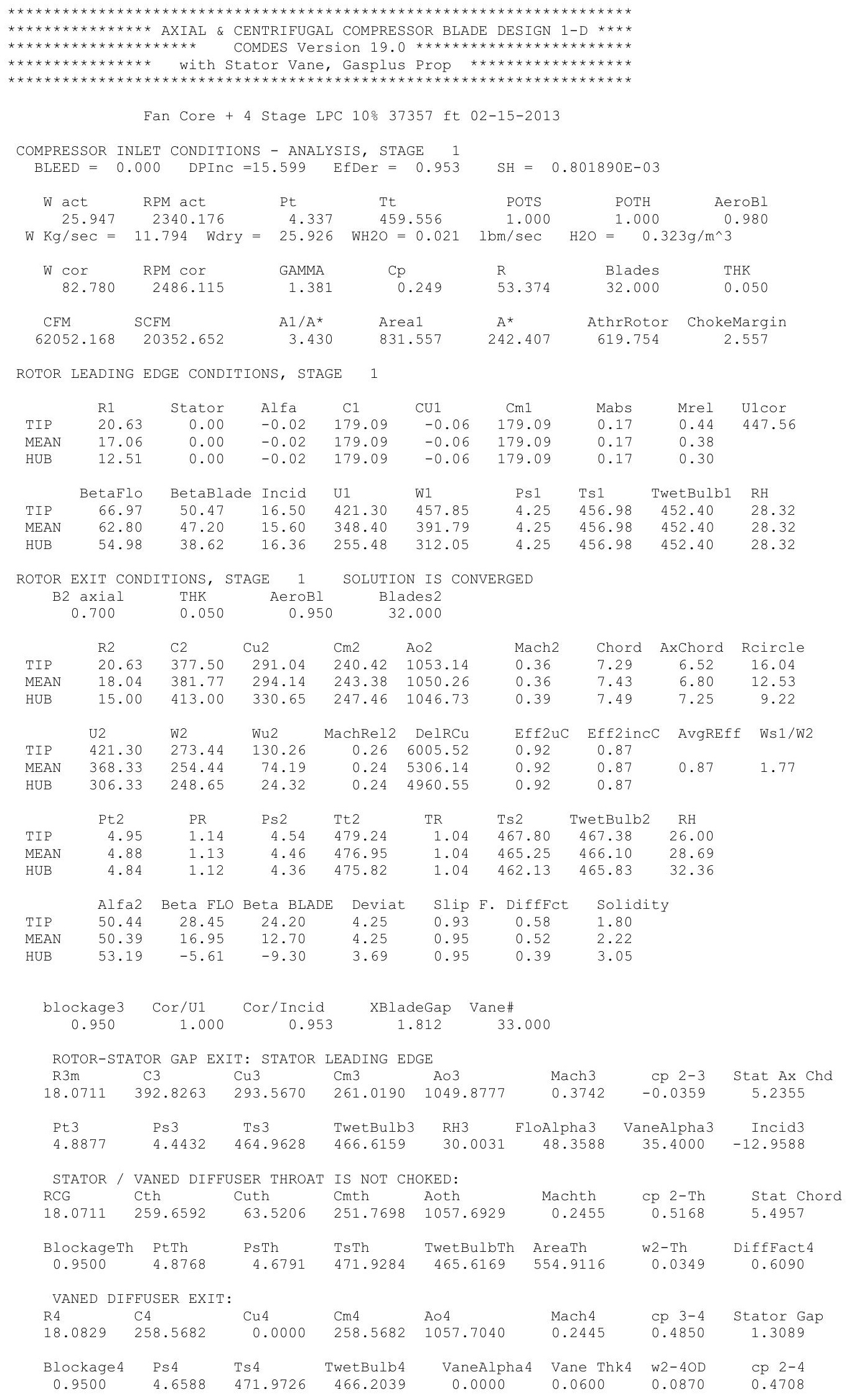




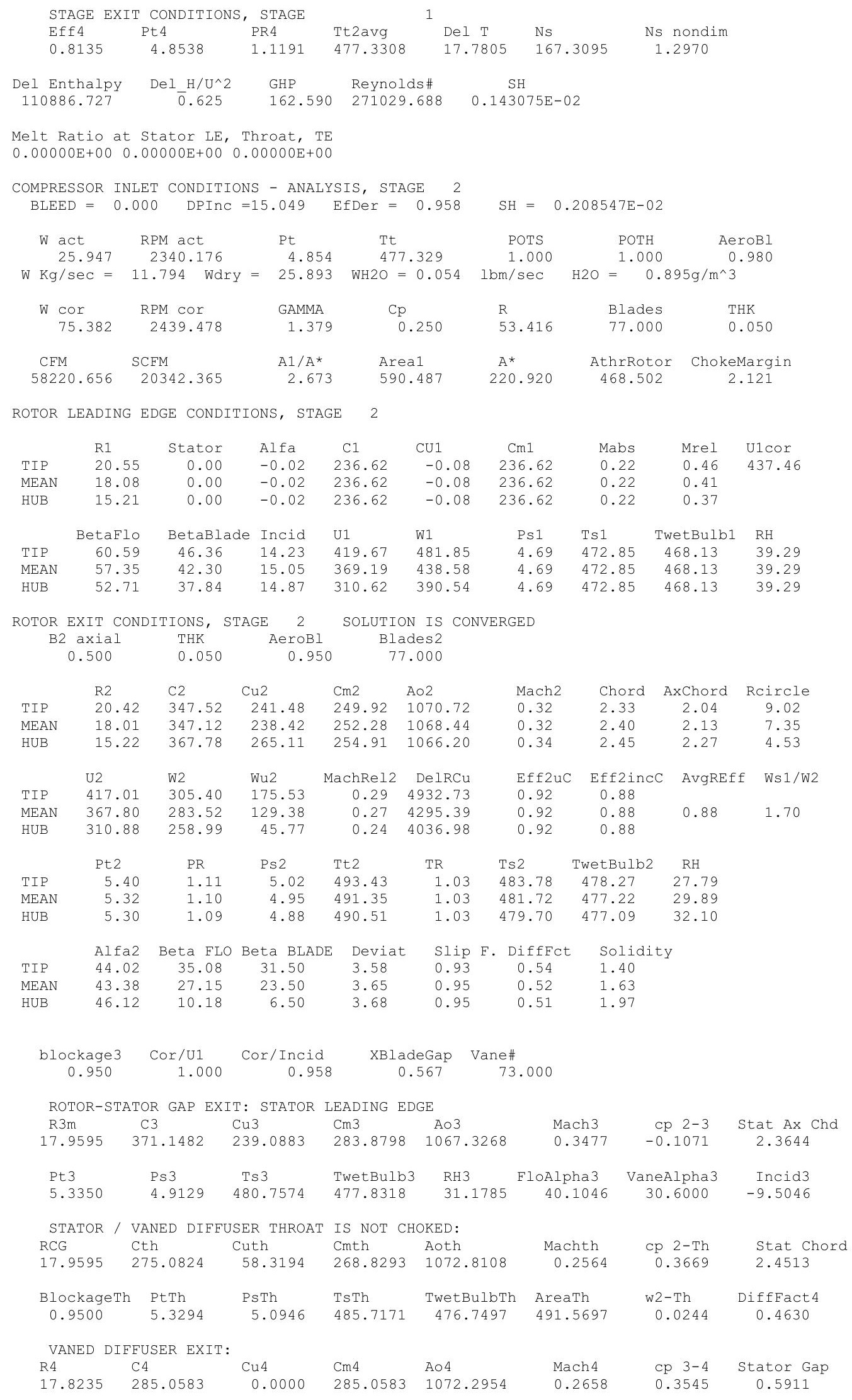




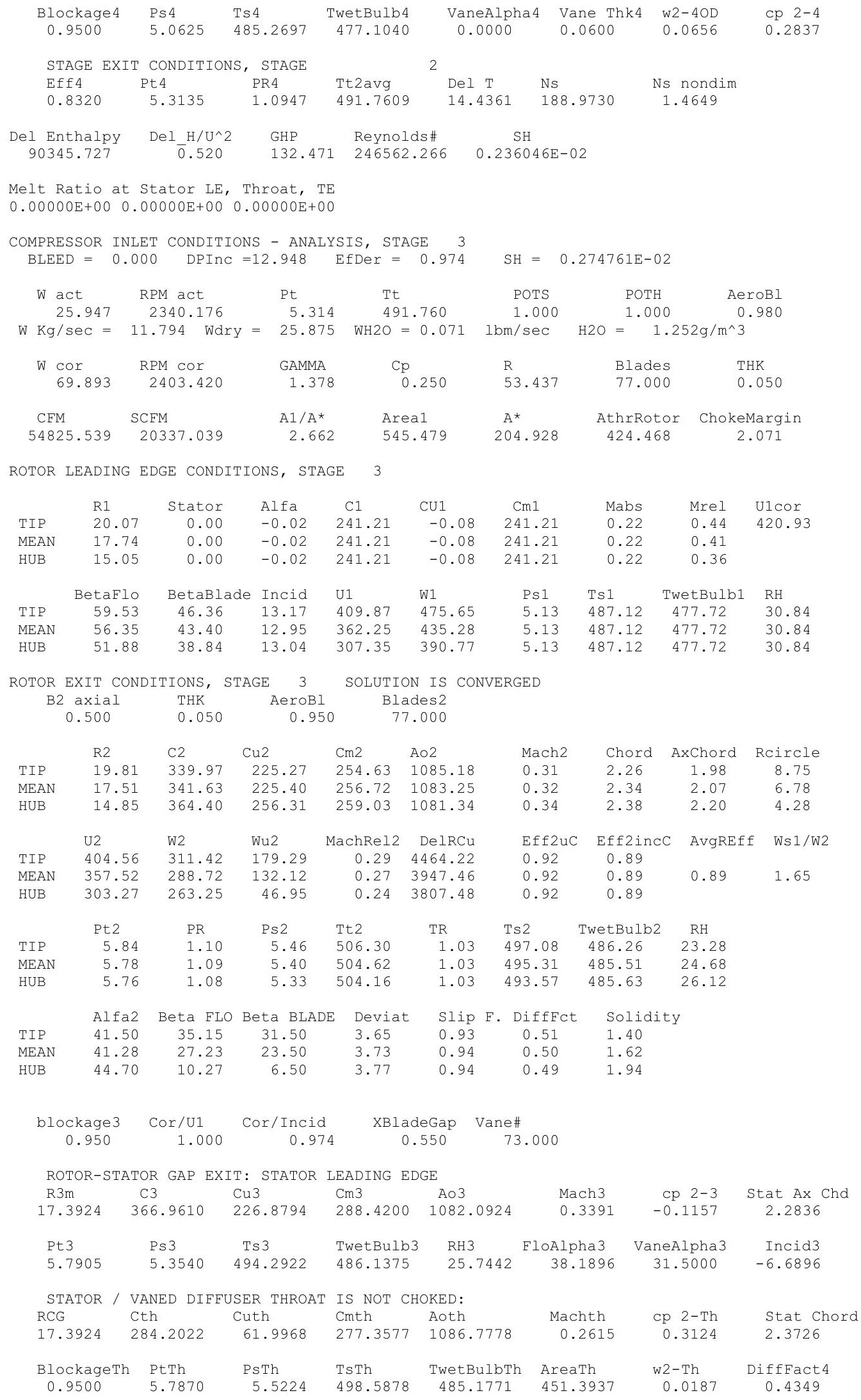

$\begin{array}{lccccccccc} & \text { R1 } & \text { Stator } & \text { Alfa } & \text { C1 } & \text { CU1 } & \text { Cm1 } & \text { Mabs } & \text { Mrel } & \text { U1cor } \\ \text { TIP } & 20.07 & 0.00 & -0.02 & 241.21 & -0.08 & 241.21 & 0.22 & 0.44 & 420.93 \\ \text { MEAN } & 17.74 & 0.00 & -0.02 & 241.21 & -0.08 & 241.21 & 0.22 & 0.41 & \\ \text { HUB } & 15.05 & 0.00 & -0.02 & 241.21 & -0.08 & 241.21 & 0.22 & 0.36 & \\ & & & & & & & & & \\ & \text { BetaFlo } & \text { BetaBlade } & \text { Incid } & \text { U1 } & \text { W1 } & \text { Ps1 } & \text { Ts1 } & \text { TwetBulb1 } & \text { RH } \\ \text { TIP } & 59.53 & 46.36 & 13.17 & 409.87 & 475.65 & 5.13 & 487.12 & 477.72 & 30.84 \\ \text { MEAN } & 56.35 & 43.40 & 12.95 & 362.25 & 435.28 & 5.13 & 487.12 & 477.72 & 30.84 \\ \text { HUB } & 51.88 & 38.84 & 13.04 & 307.35 & 390.77 & 5.13 & 487.12 & 477.72 & 30.84\end{array}$

ROTOR EXIT CONDITIONS, STAGE 3 SOLUTION IS CONVERGED

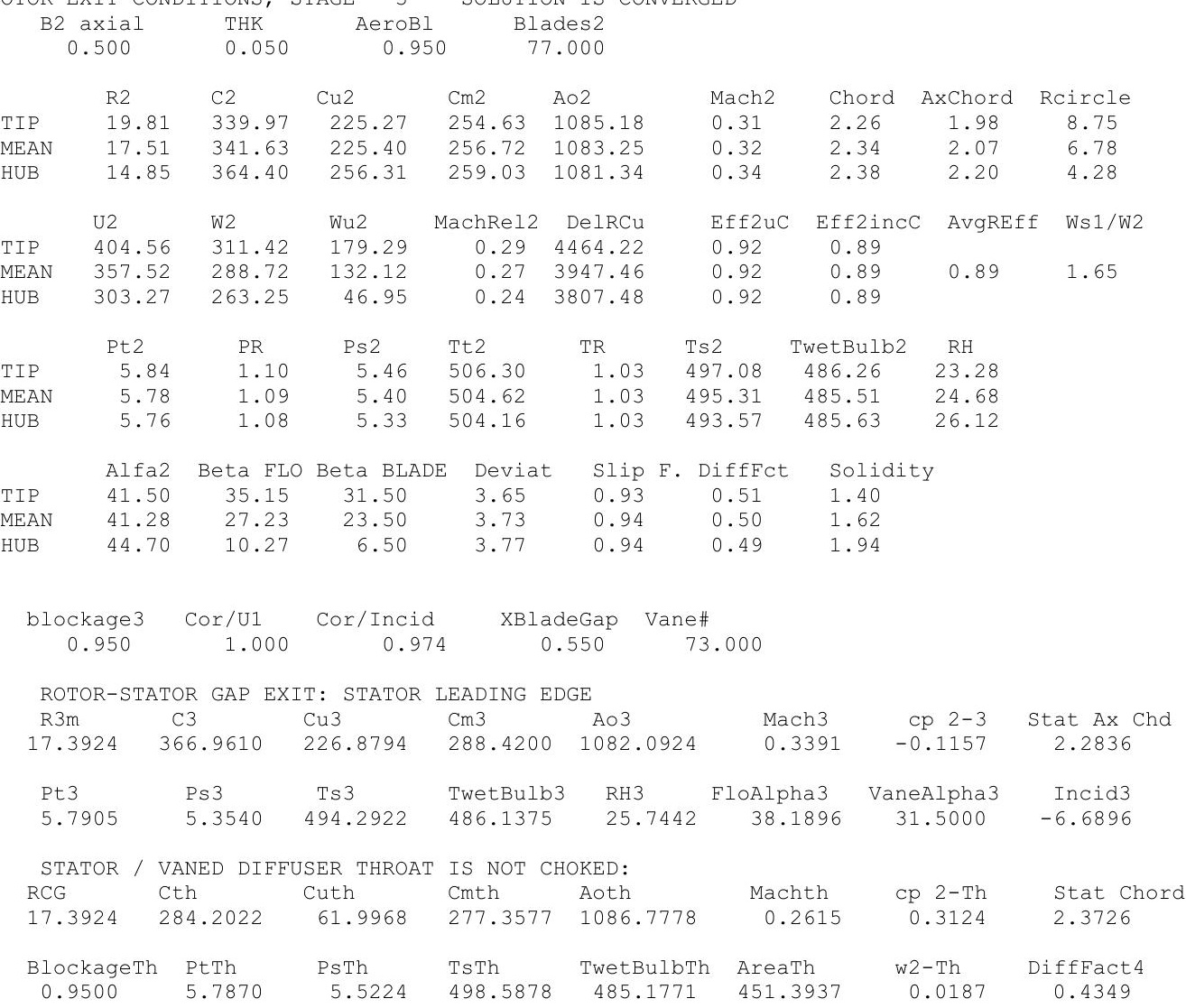




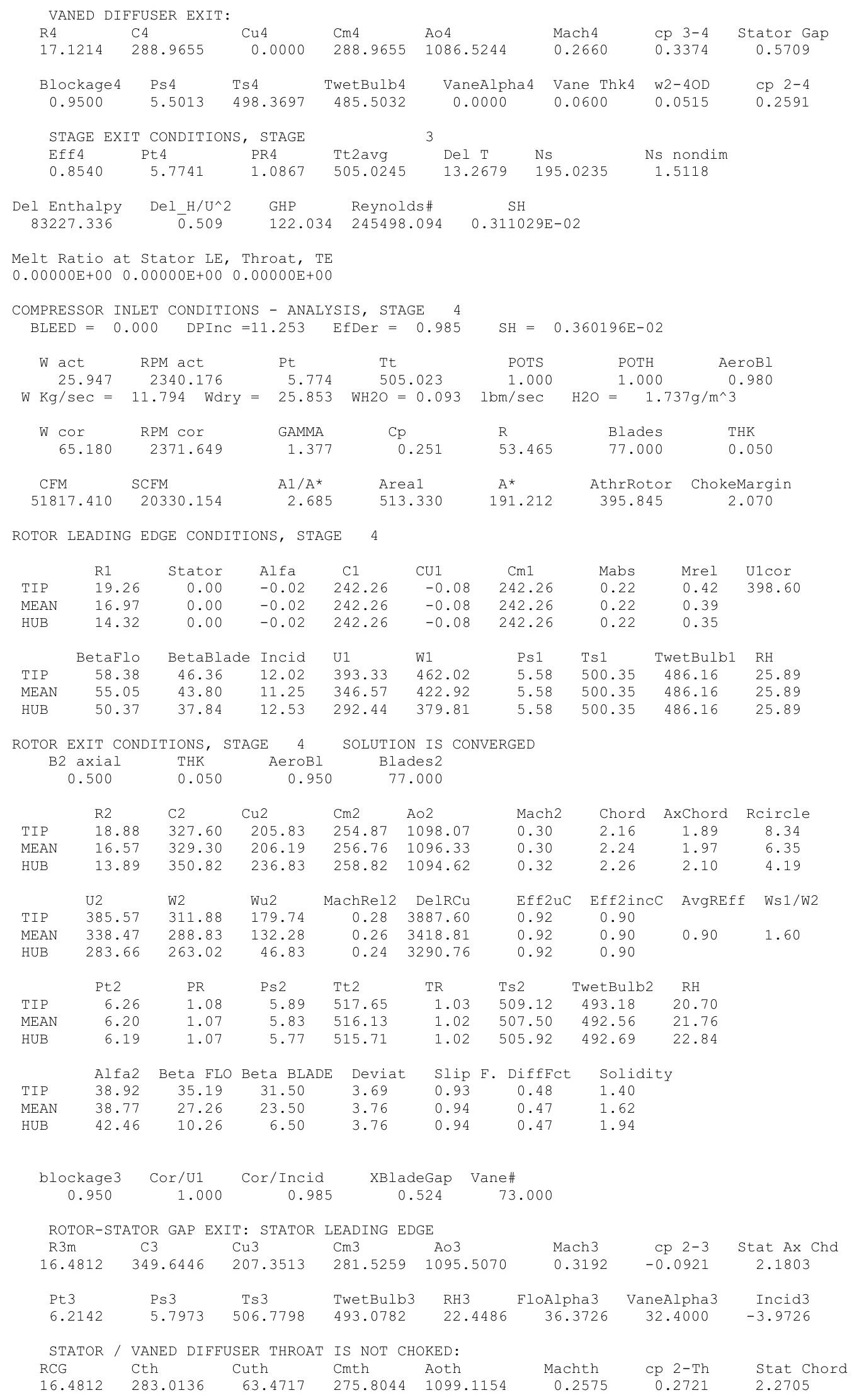




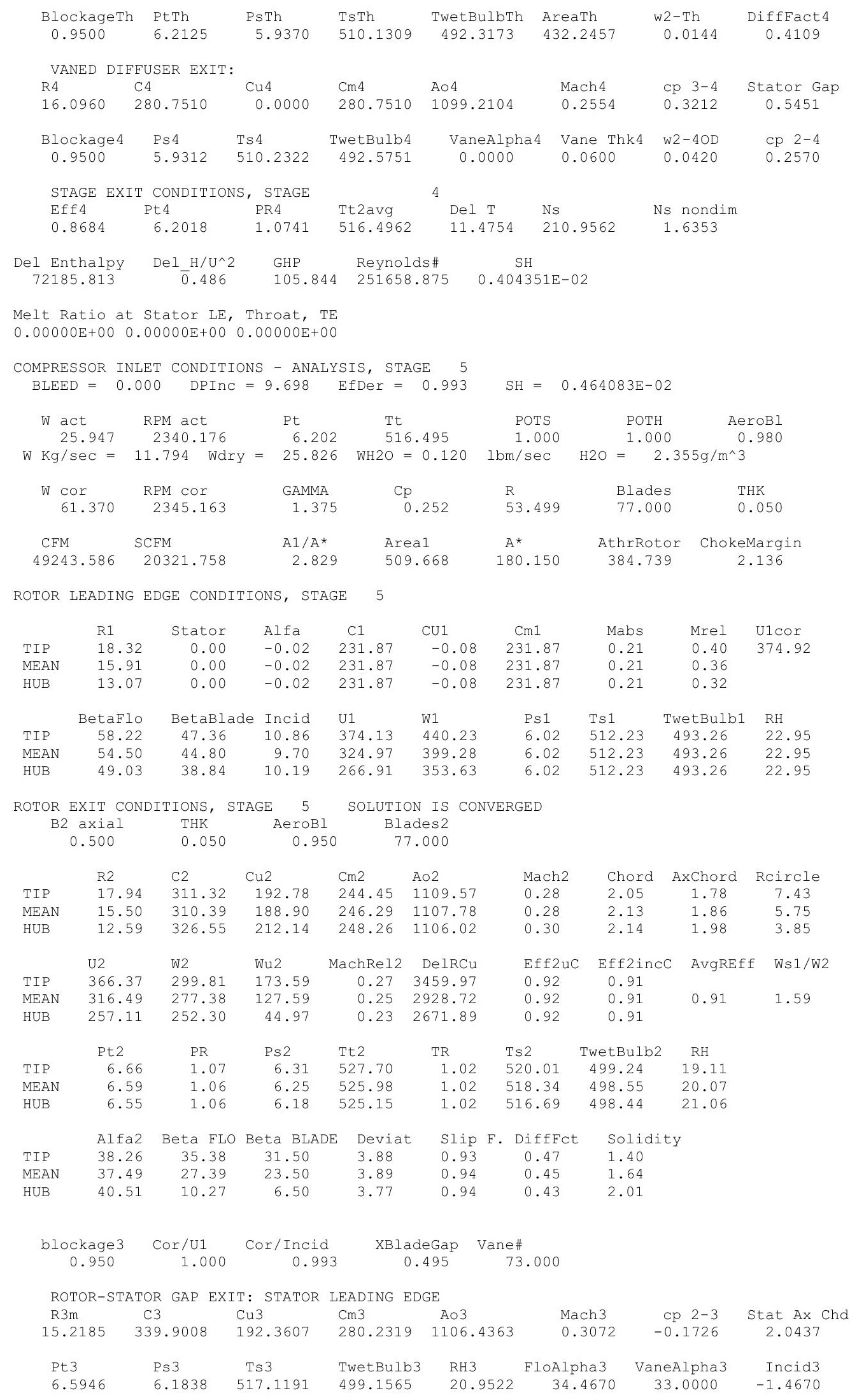




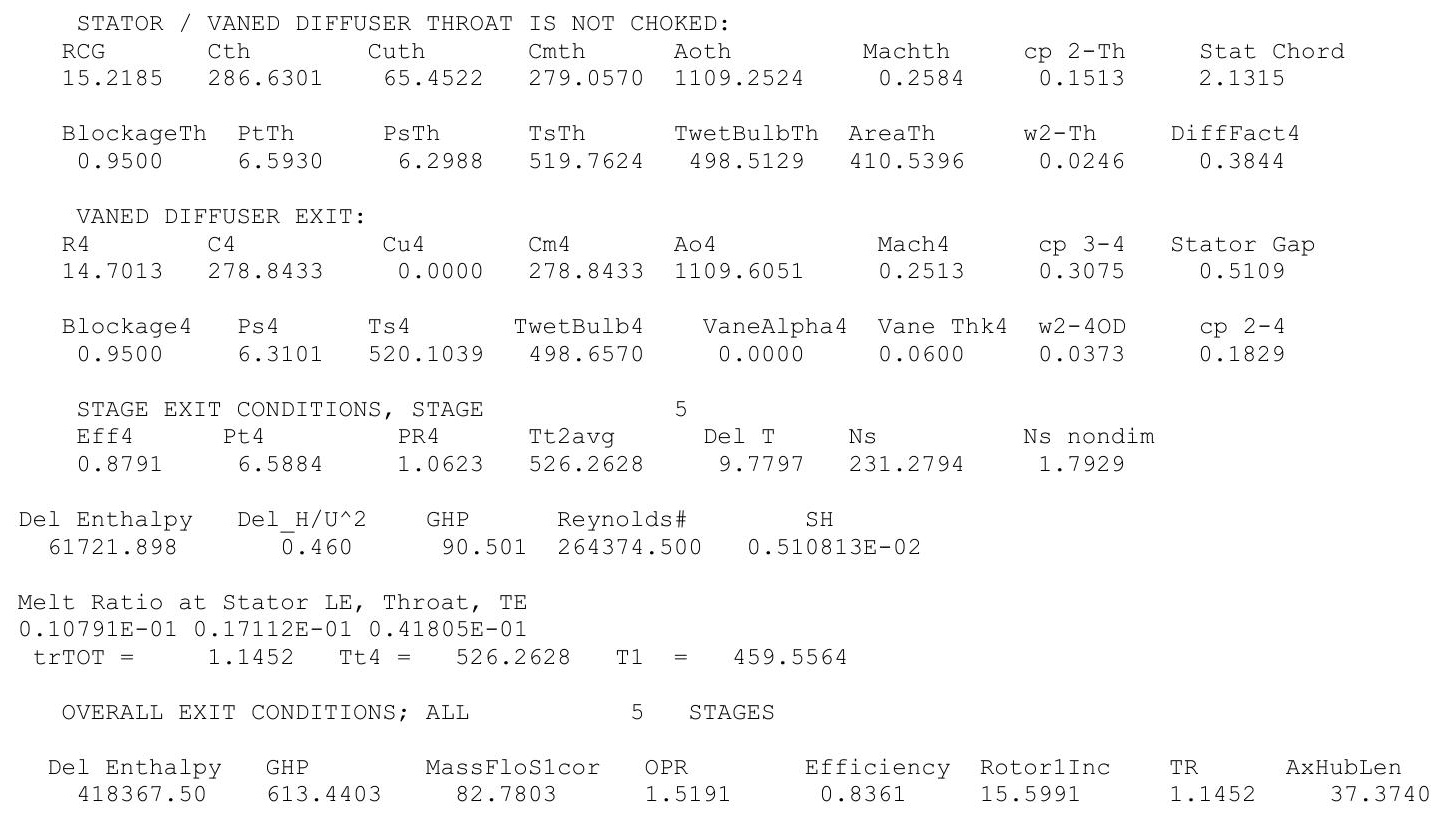




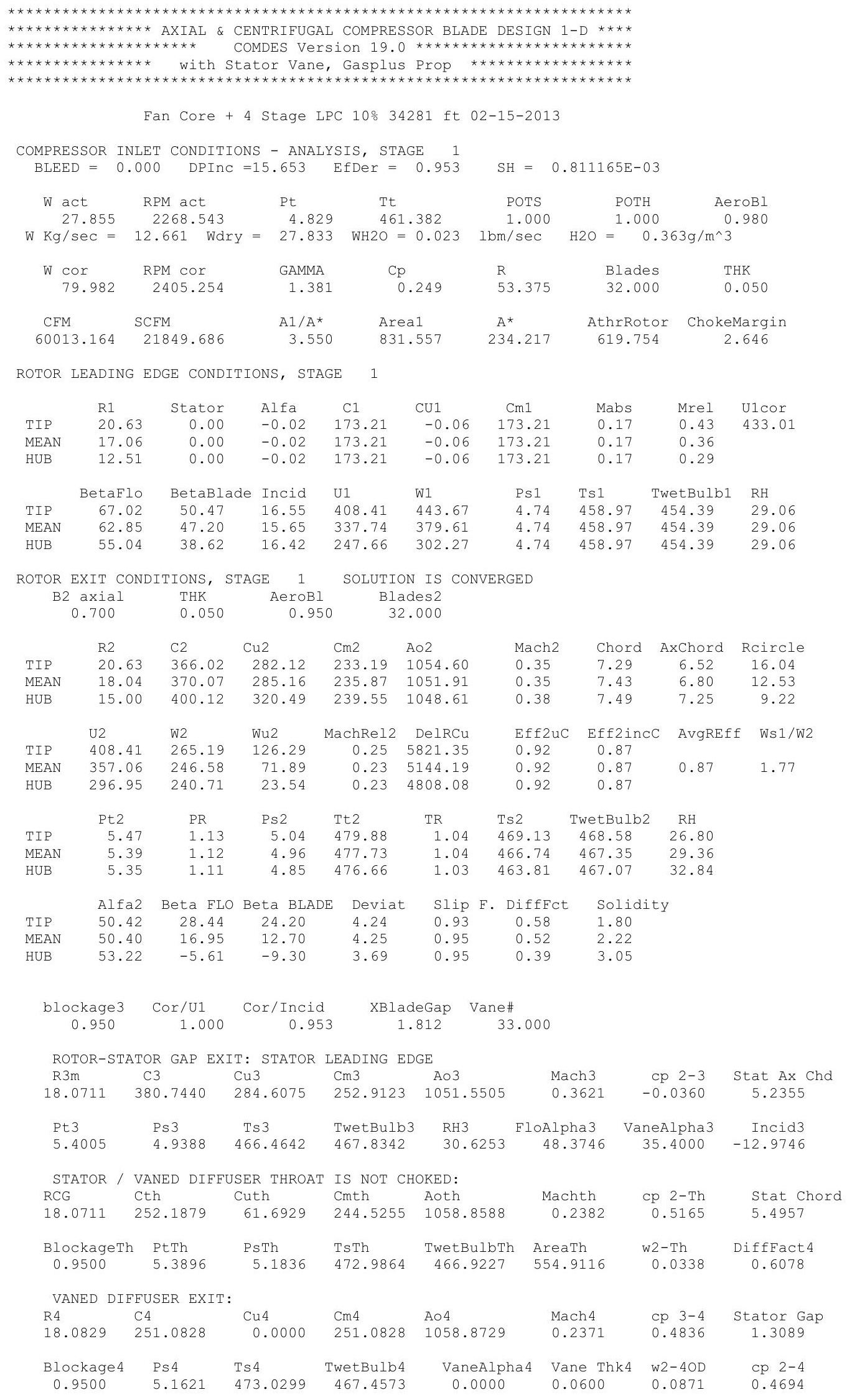




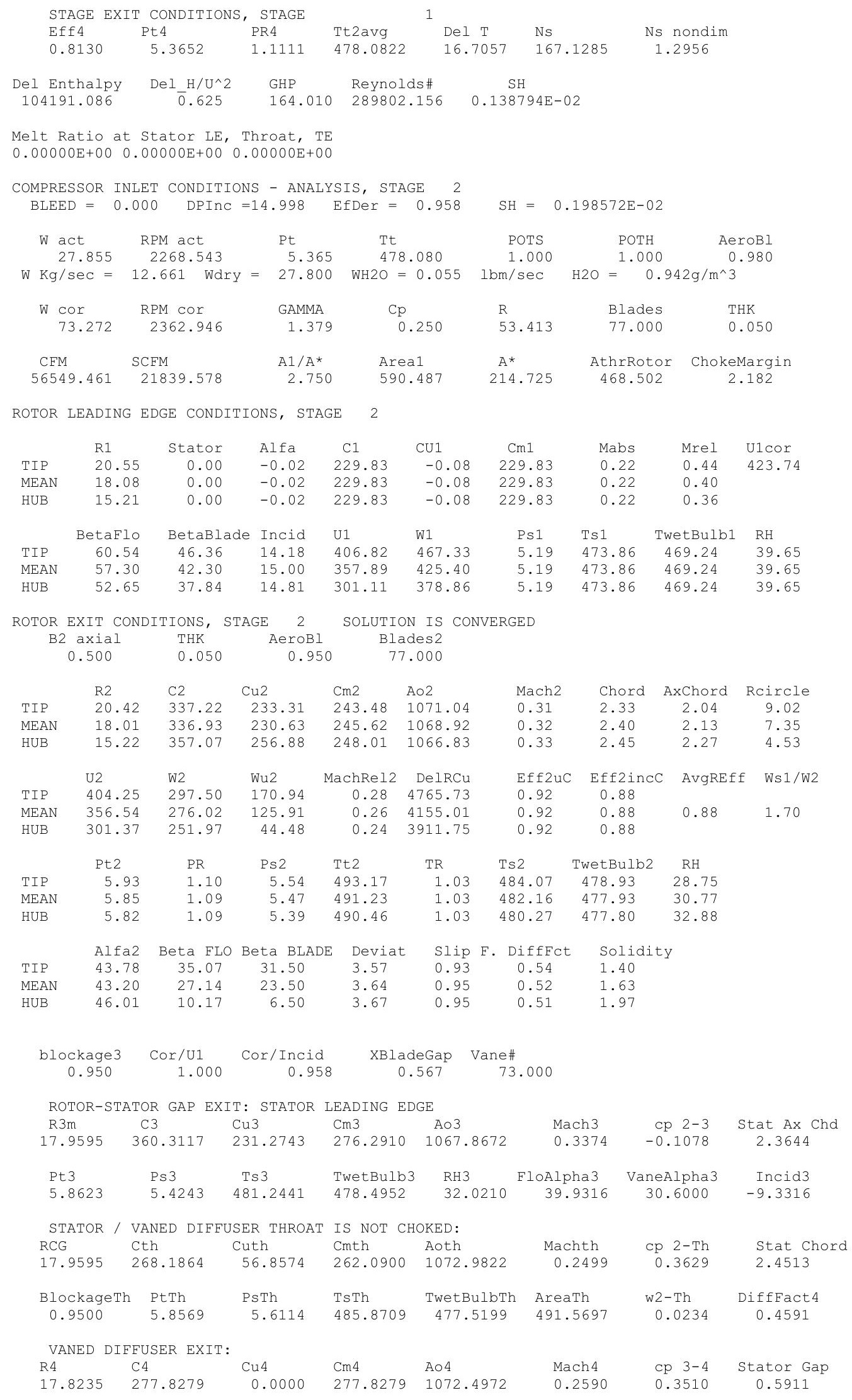




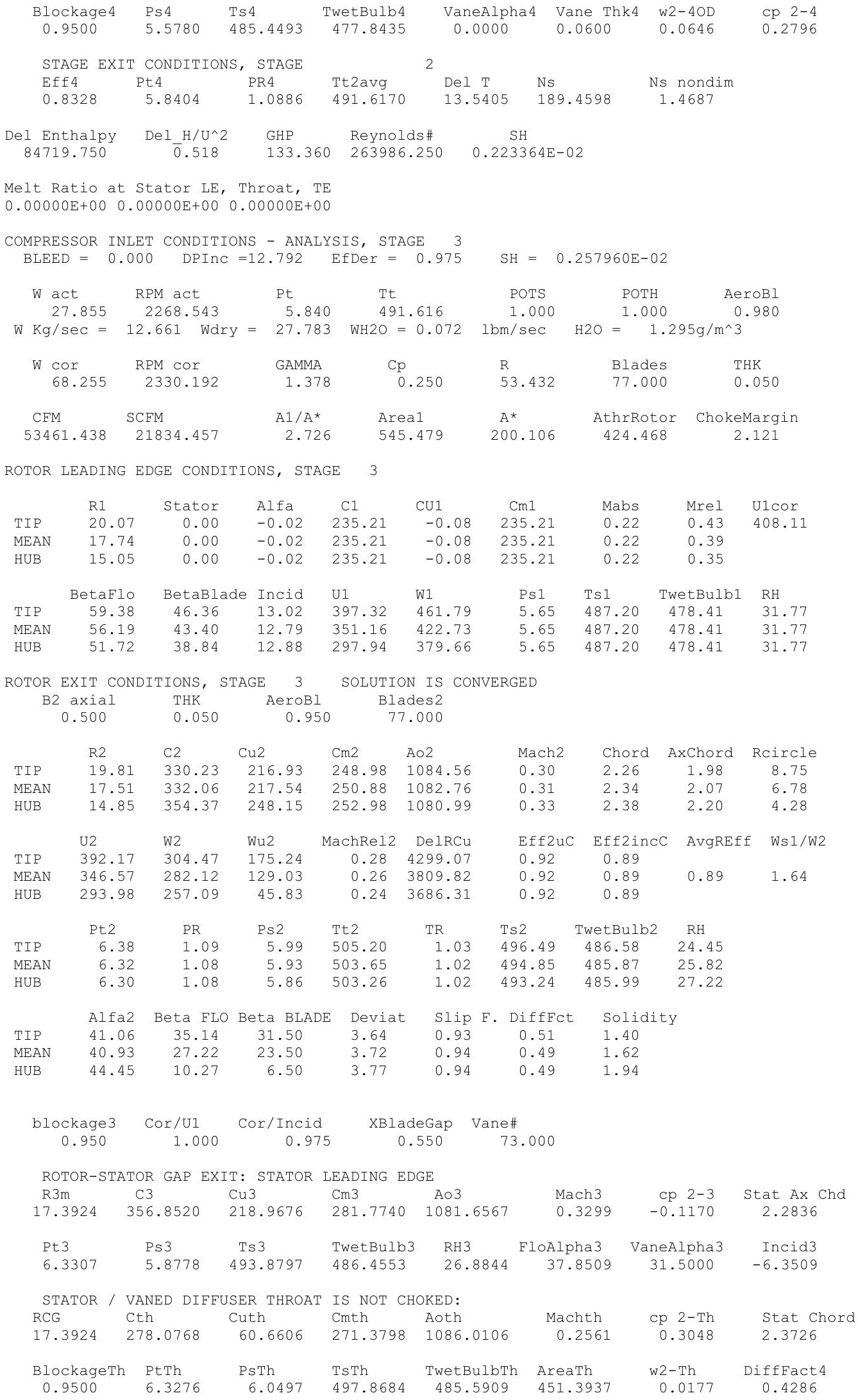




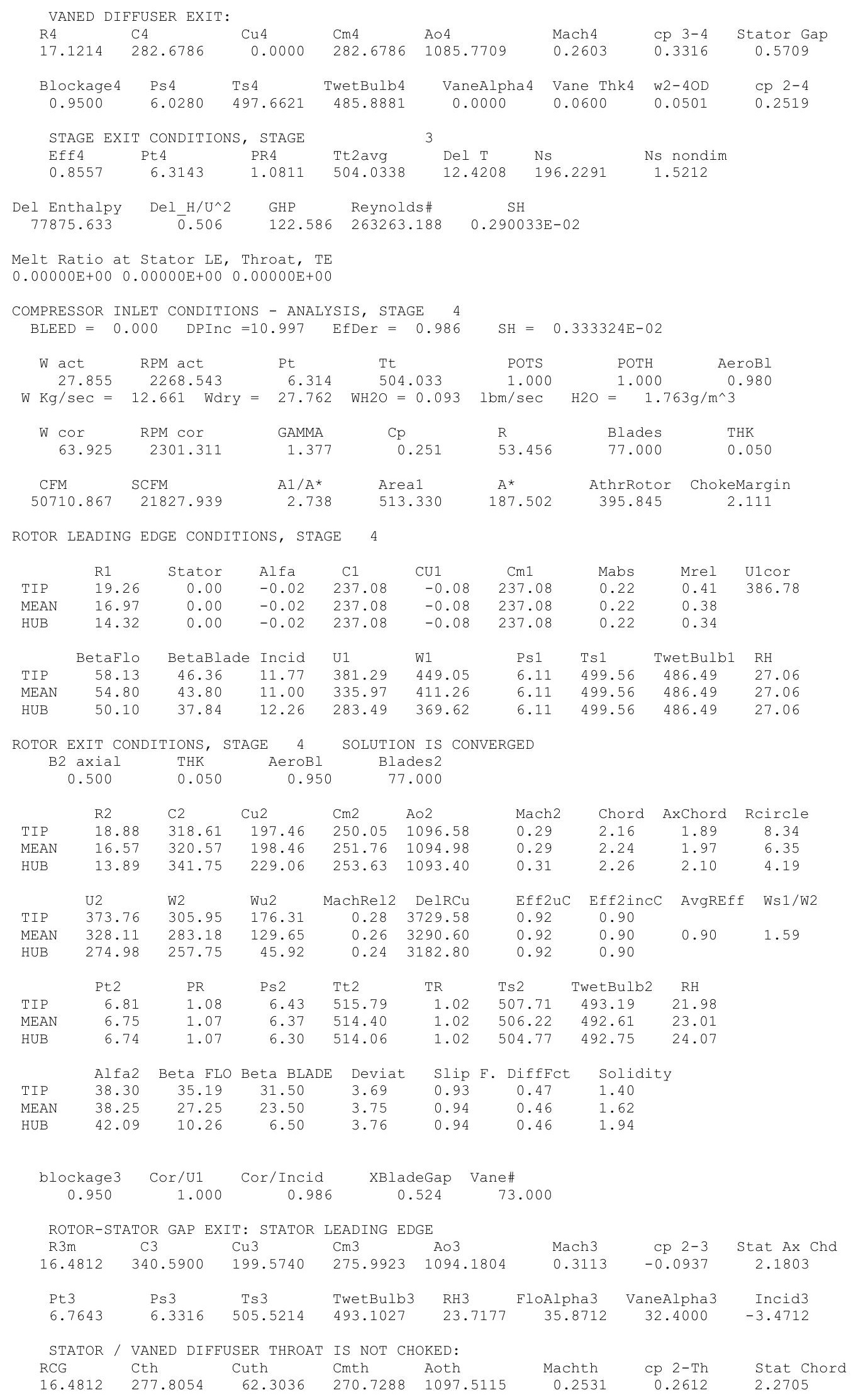




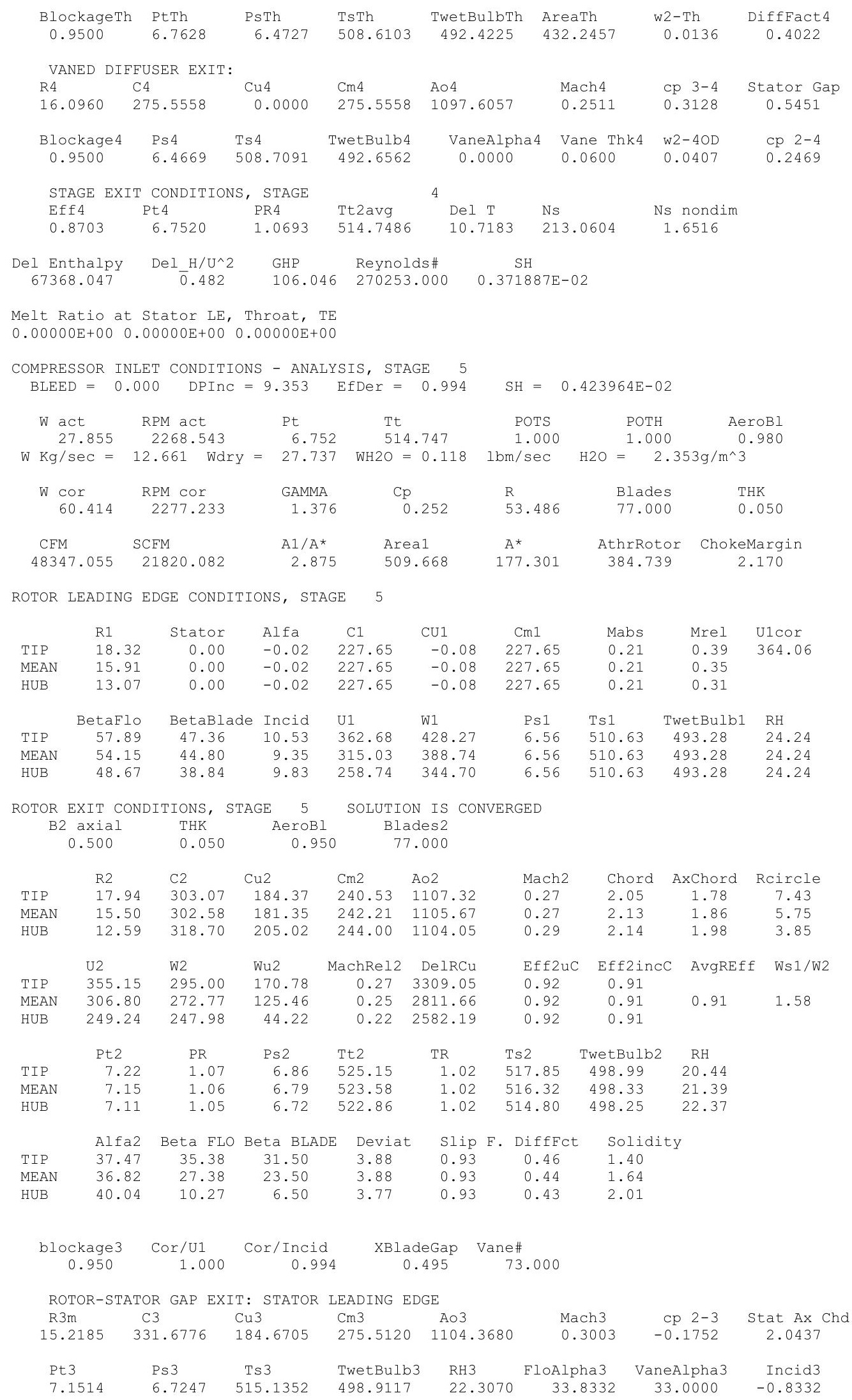




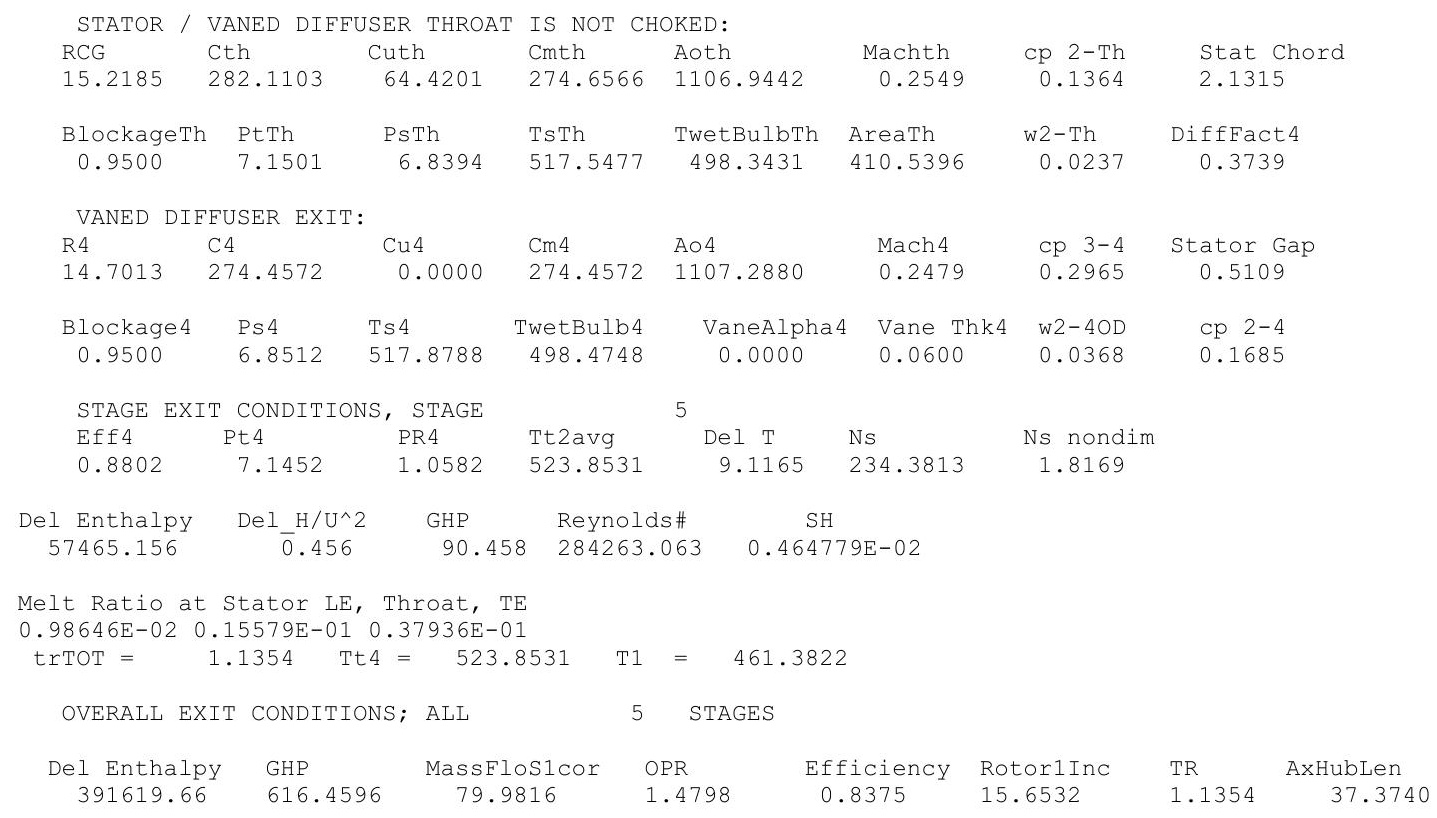




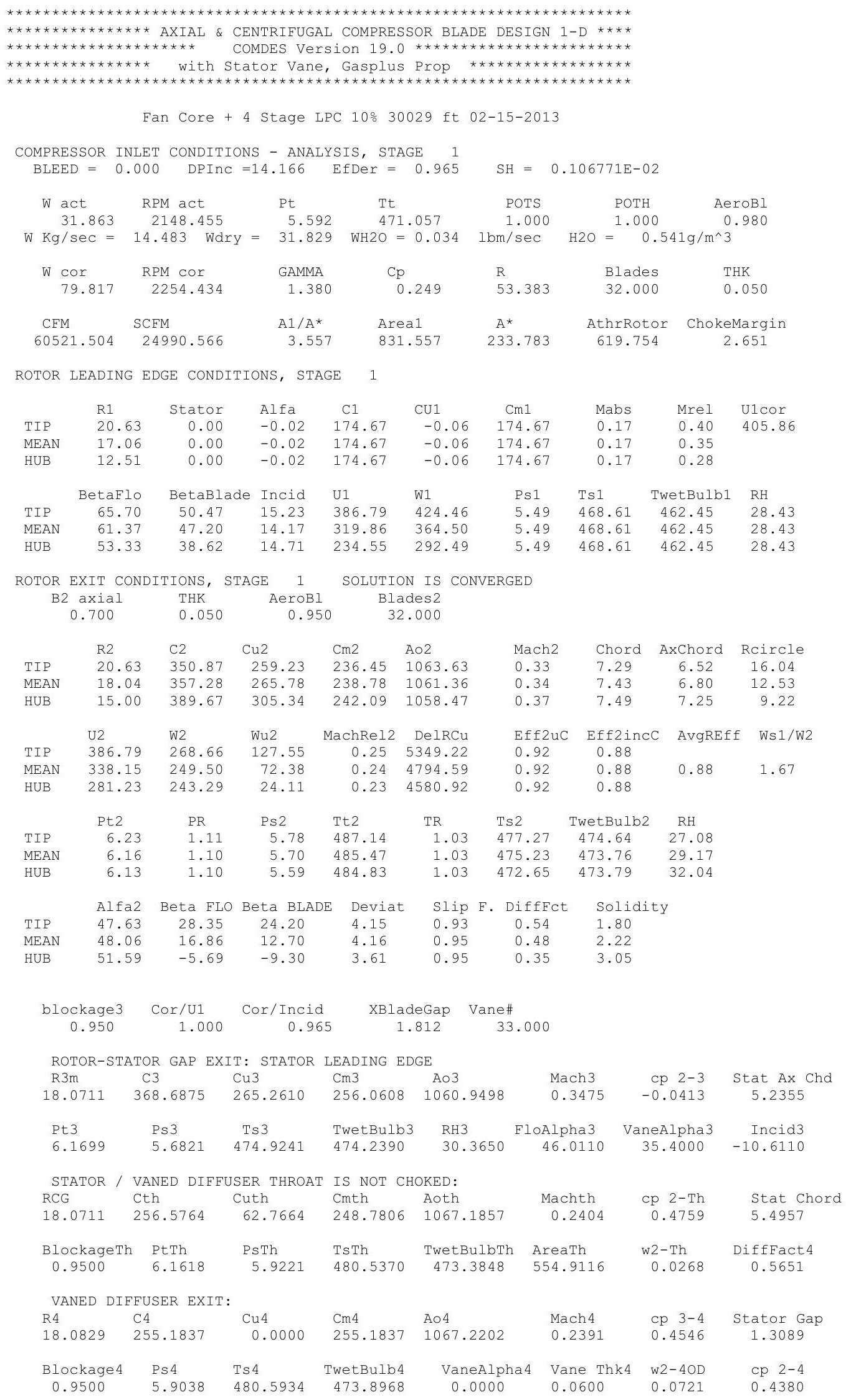




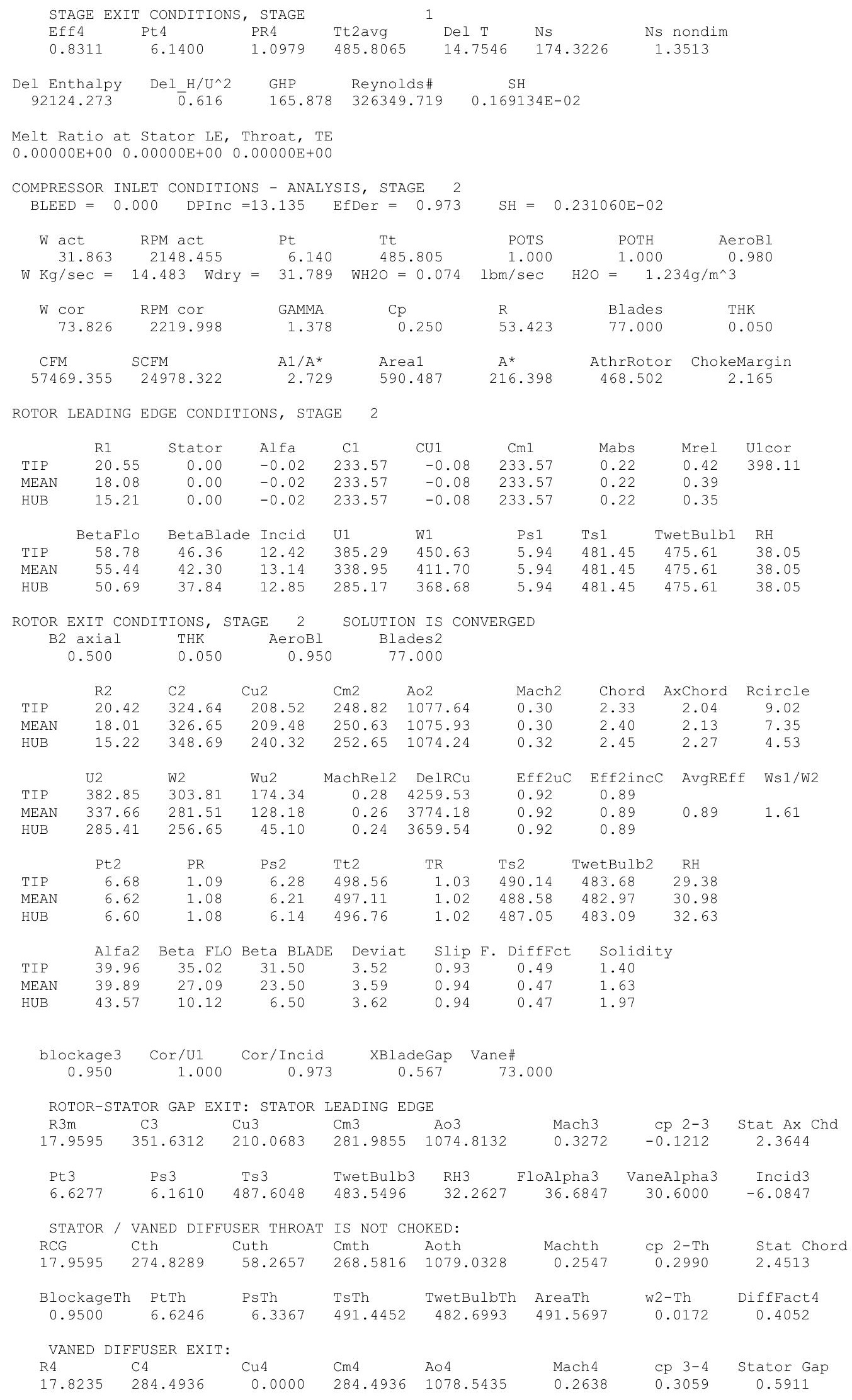




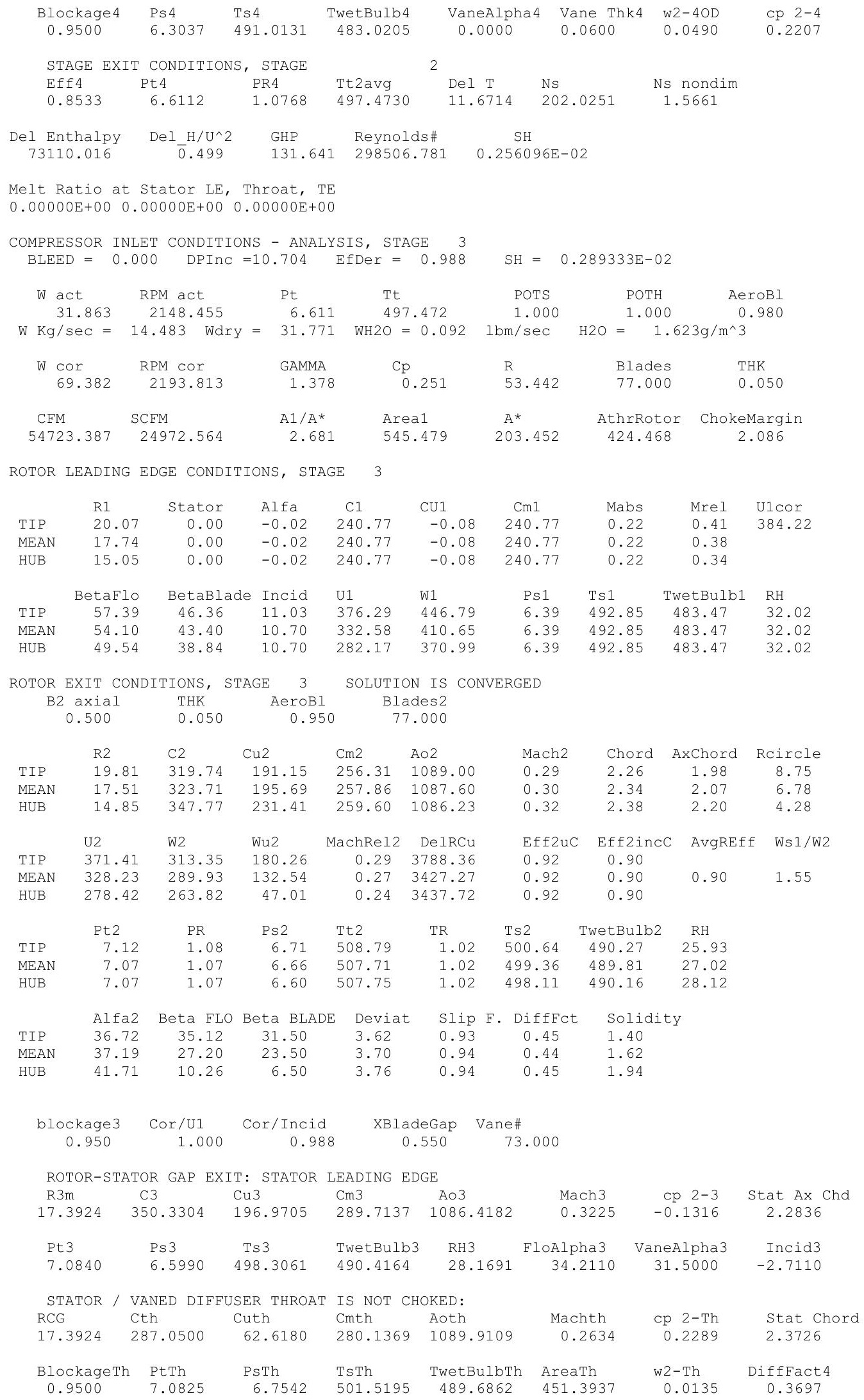

$\begin{array}{lcclcccccc} & \text { R1 } & \text { Stator } & \text { Alfa } & \text { C1 } & \text { CU1 } & \text { Cm1 } & \text { Mabs } & \text { Mrel } & \text { U1cor } \\ \text { TIP } & 20.07 & 0.00 & -0.02 & 240.77 & -0.08 & 240.77 & 0.22 & 0.41 & 384.22 \\ \text { MEAN } & 17.74 & 0.00 & -0.02 & 240.77 & -0.08 & 240.77 & 0.22 & 0.38 & \\ \text { HUB } & 15.05 & 0.00 & -0.02 & 240.77 & -0.08 & 240.77 & 0.22 & 0.34 & \\ & & & & & & & & & \\ & \text { BetaFlo } & \text { BetaBlade } & \text { Incid } & \text { U1 } & \text { W1 } & \text { Ps1 } & \text { Ts1 } & \text { TwetBulb1 } & \text { RH } \\ \text { TIP } & 57.39 & 46.36 & 11.03 & 376.29 & 446.79 & 6.39 & 492.85 & 483.47 & 32.02 \\ \text { MEAN } & 54.10 & 43.40 & 10.70 & 332.58 & 410.65 & 6.39 & 492.85 & 483.47 & 32.02 \\ \text { HUB } & 49.54 & 38.84 & 10.70 & 282.17 & 370.99 & 6.39 & 492.85 & 483.47 & 32.02\end{array}$

ROTOR EXIT CONDITIONS, STAGE 3 SOLUTION IS CONVERGED

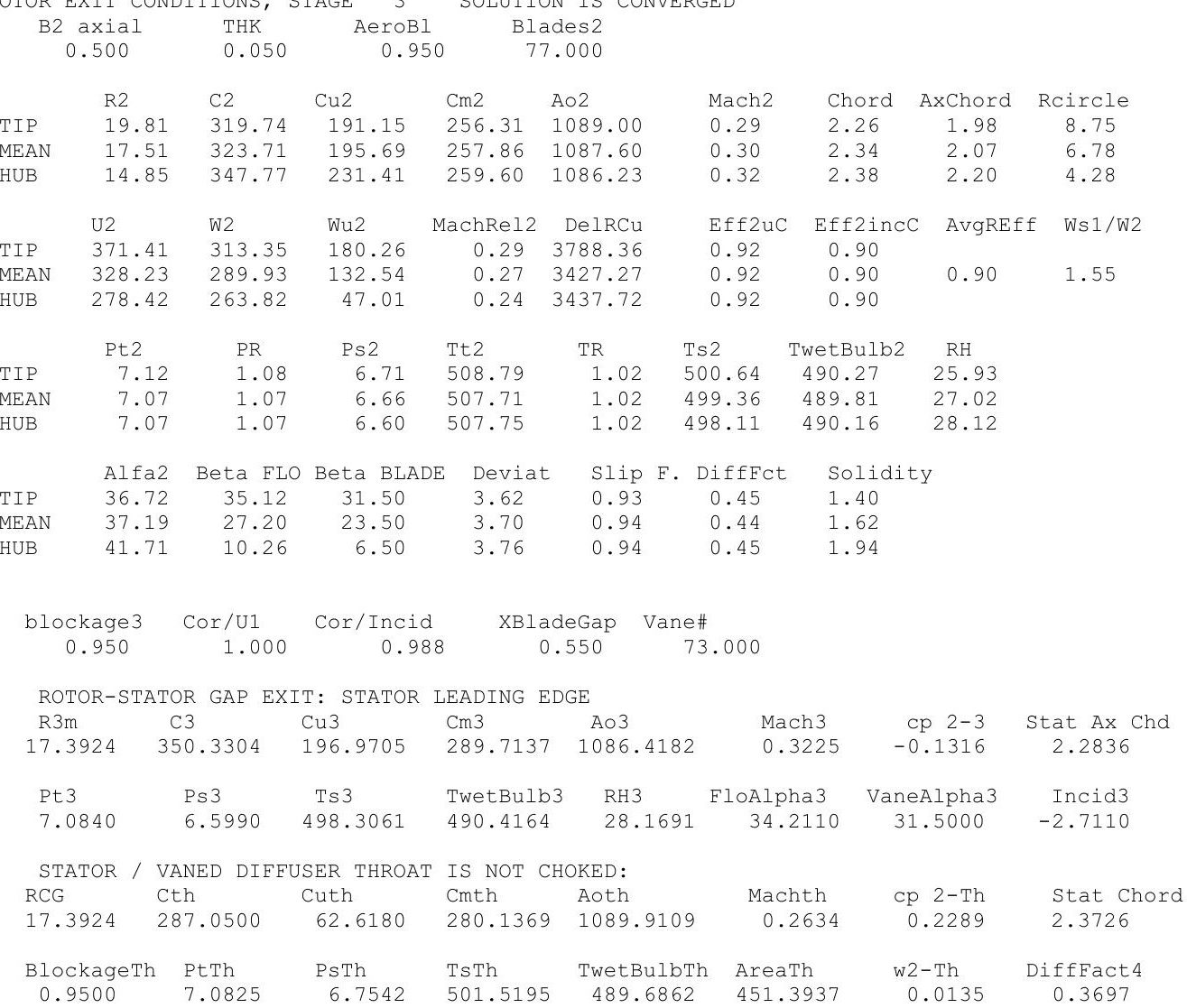




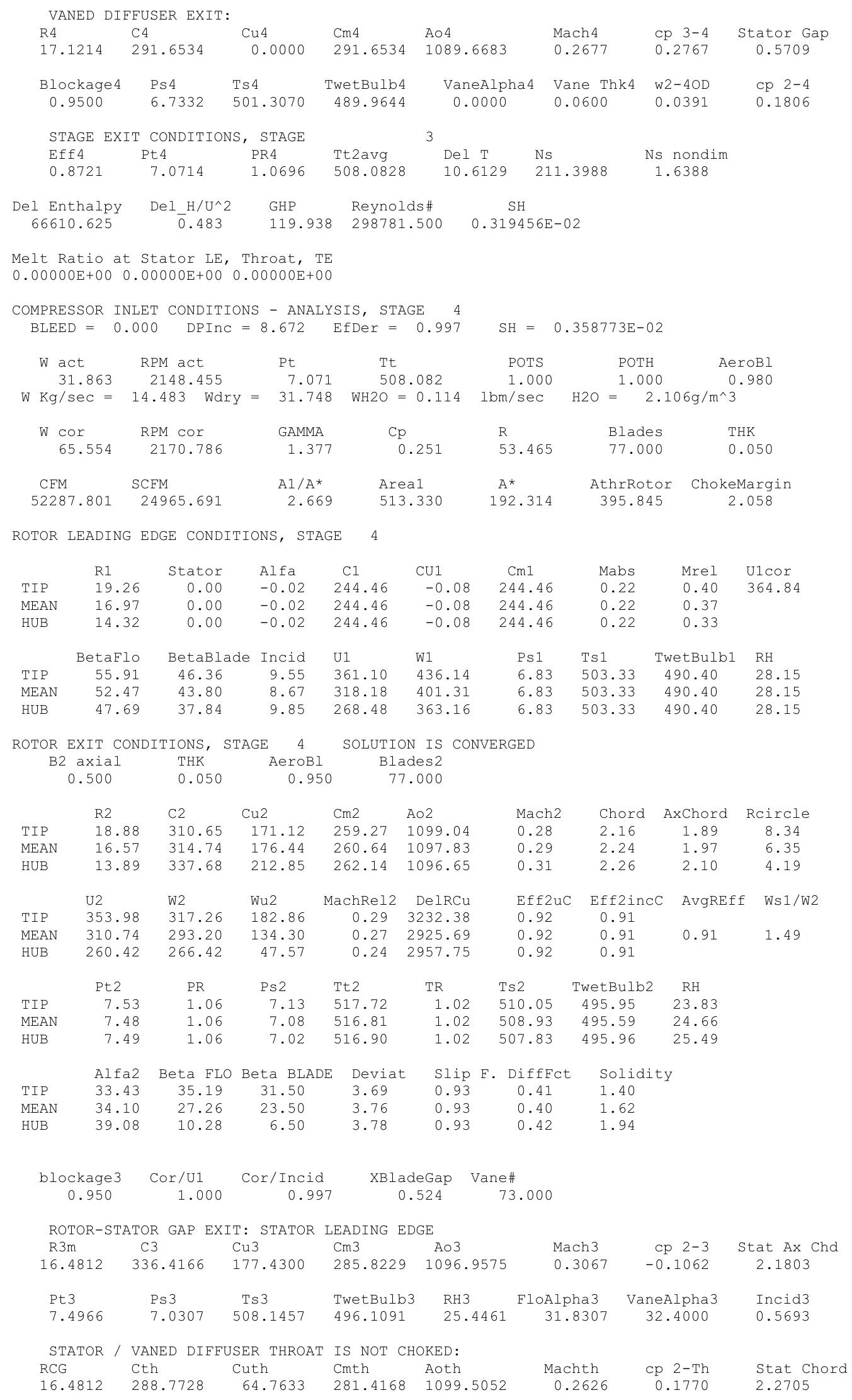




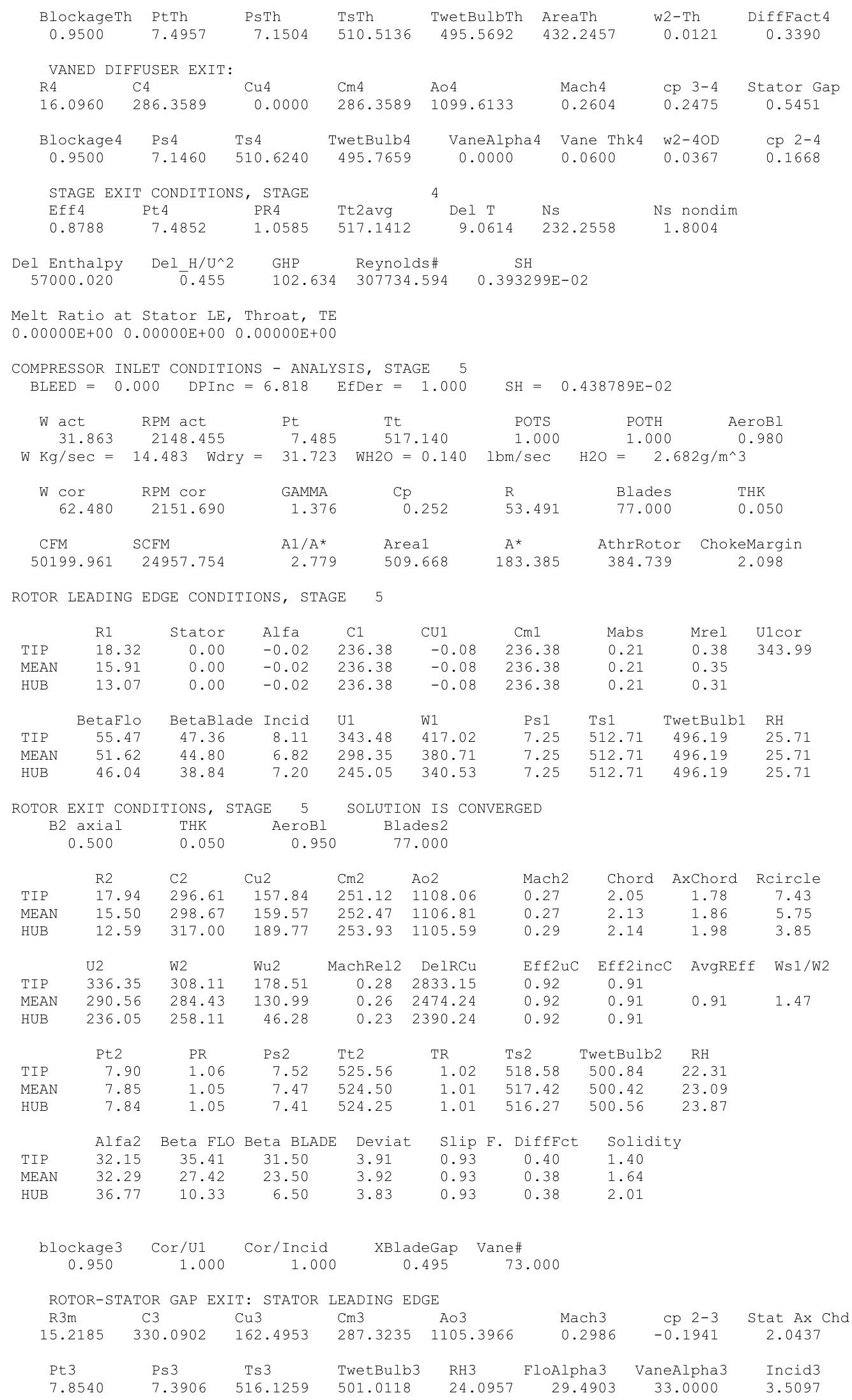




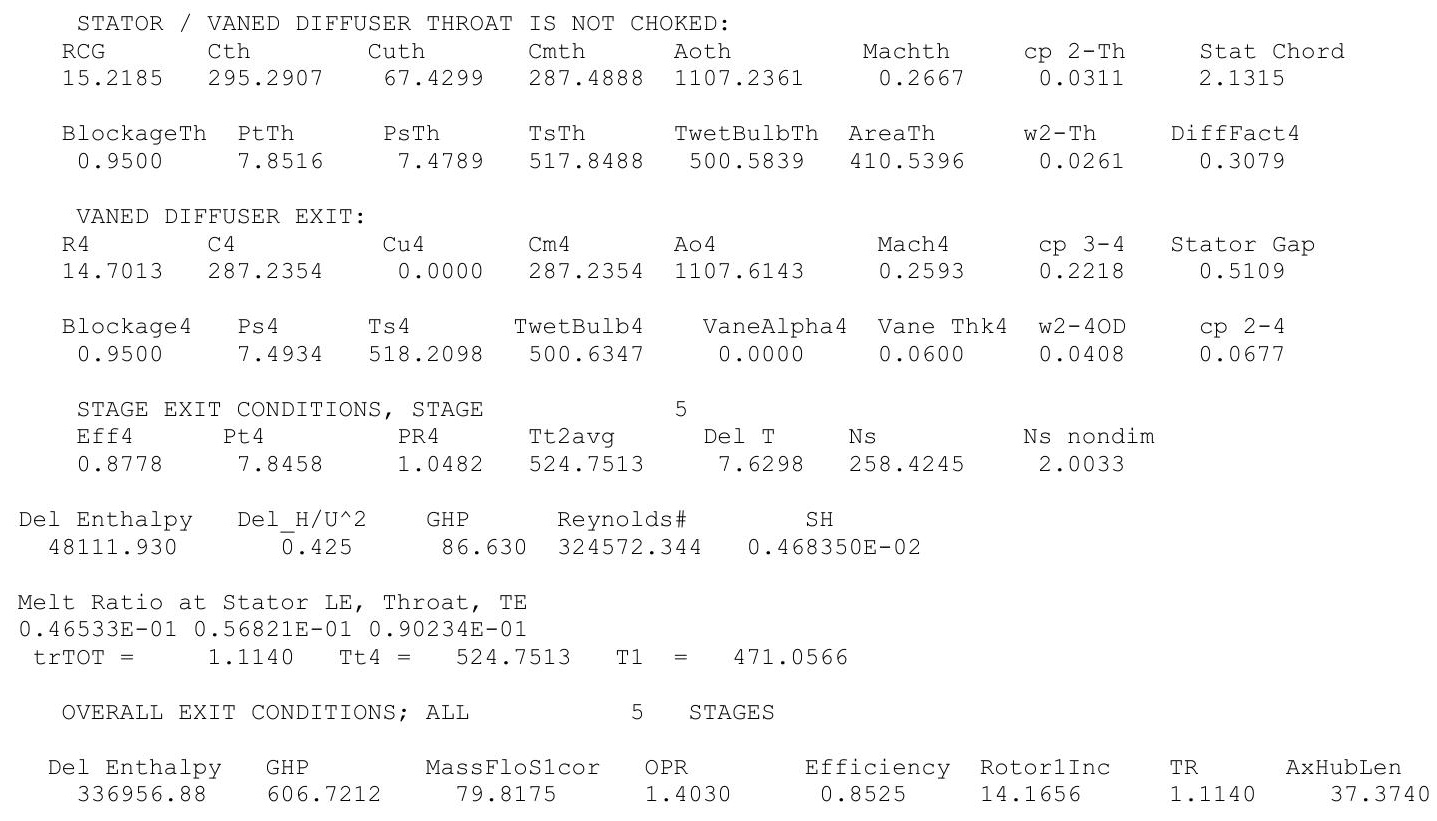




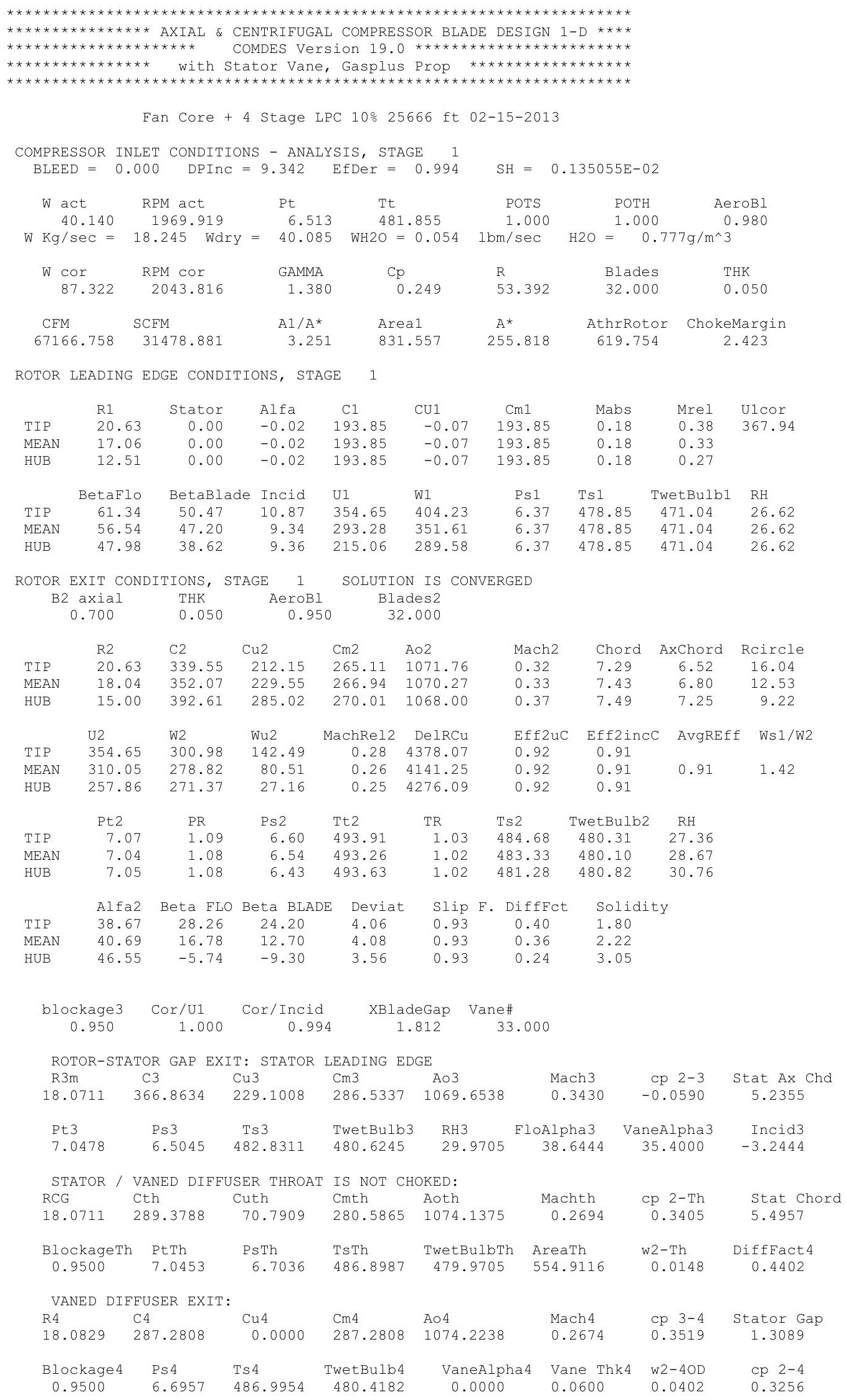




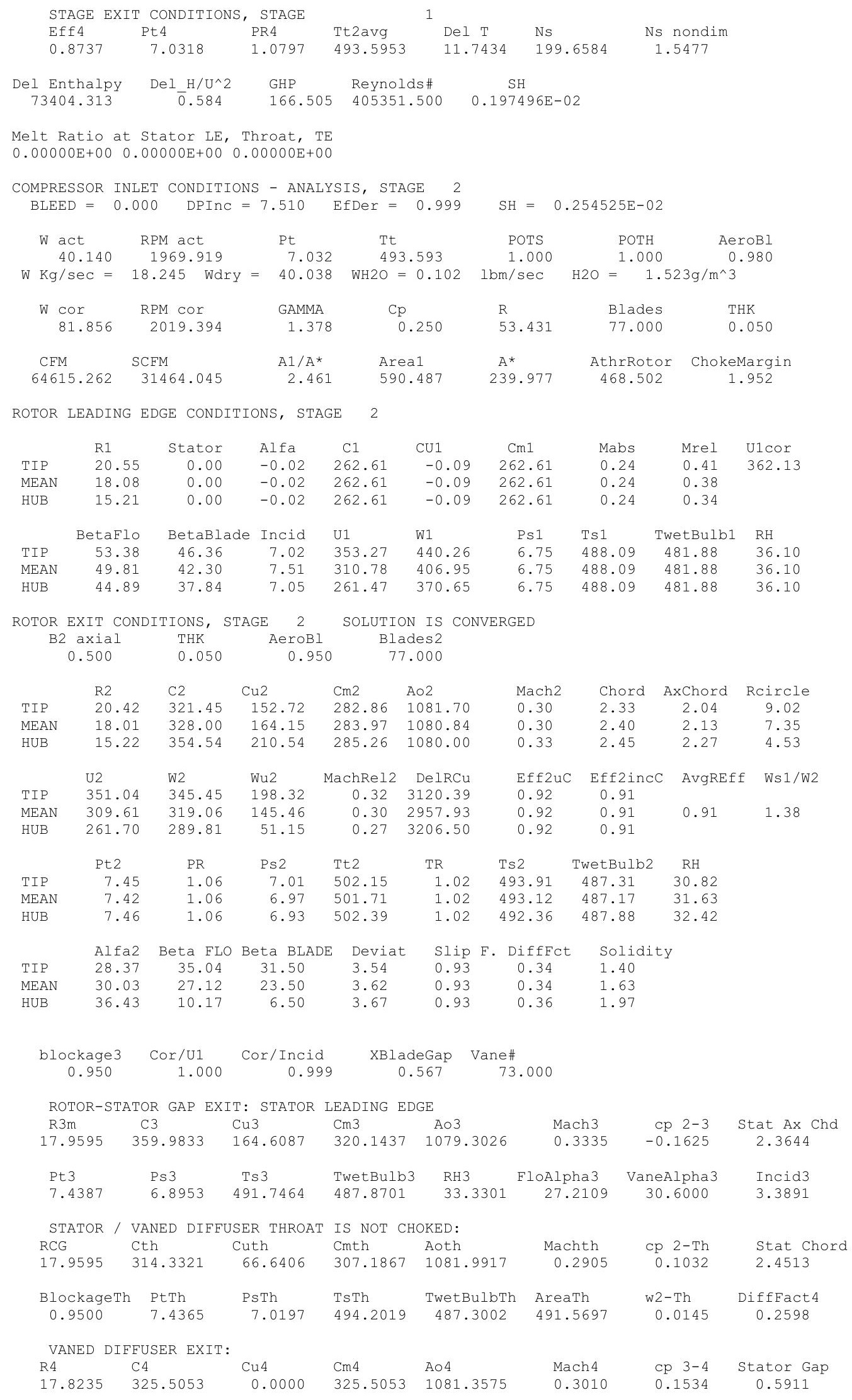




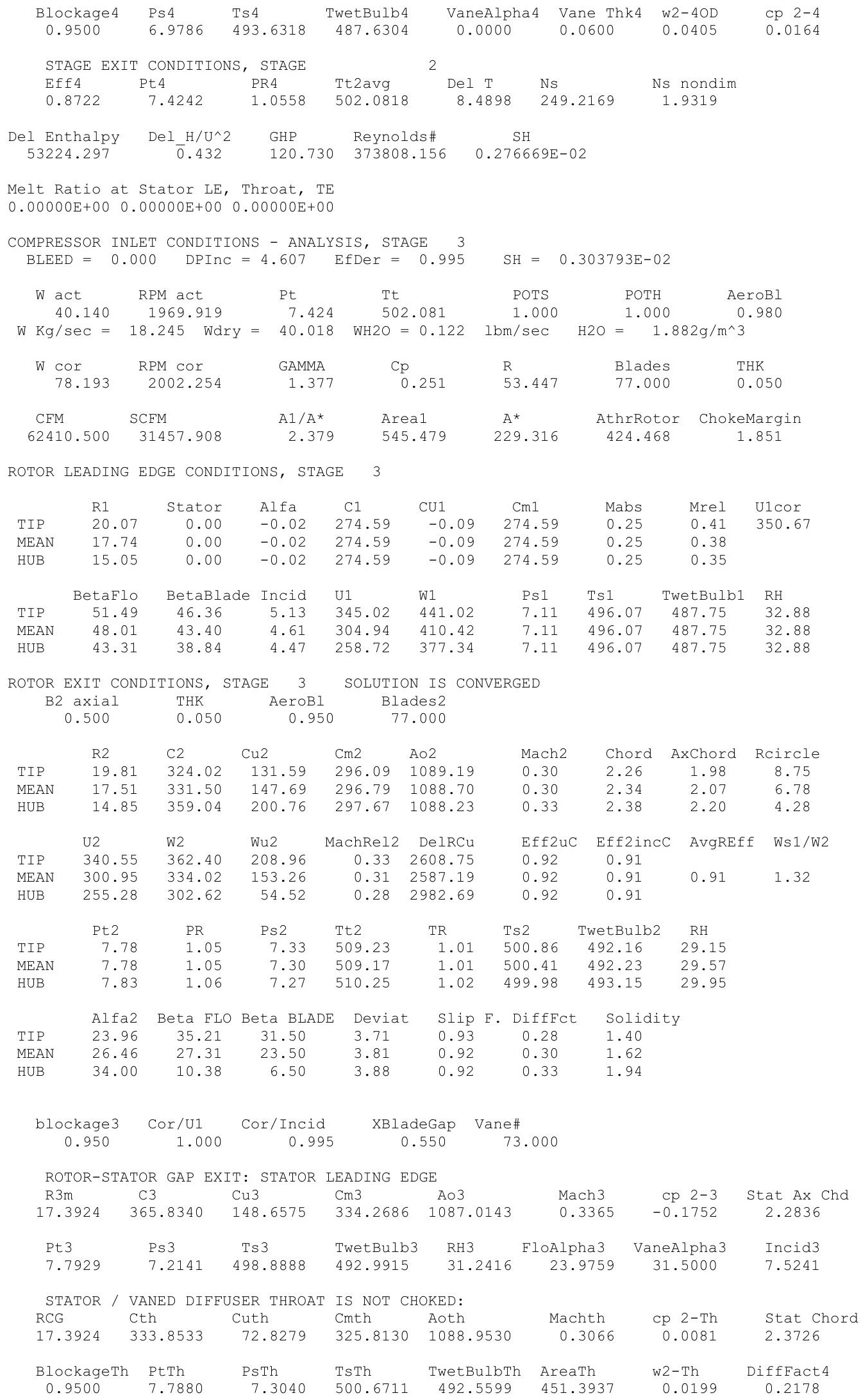

$\begin{array}{lccccccccc} & \text { R1 } & \text { Stator } & \text { Alfa } & \text { C1 } & \text { CU1 } & \text { Cm1 } & \text { Mabs } & \text { Mrel } & \text { U1cor } \\ \text { TIP } & 20.07 & 0.00 & -0.02 & 274.59 & -0.09 & 274.59 & 0.25 & 0.41 & 350.67 \\ \text { MEAN } & 17.74 & 0.00 & -0.02 & 274.59 & -0.09 & 274.59 & 0.25 & 0.38 & \\ \text { HUB } & 15.05 & 0.00 & -0.02 & 274.59 & -0.09 & 274.59 & 0.25 & 0.35 & \\ & & & & & & & & & \\ & \text { BetaFlo } & \text { BetaBlade } & \text { Incid } & \text { U1 } & \text { W1 } & \text { Ps1 } & \text { Ts1 } & \text { TwetBulb1 } & \text { RH } \\ \text { TIP } & 51.49 & 46.36 & 5.13 & 345.02 & 441.02 & 7.11 & 496.07 & 487.75 & 32.88 \\ \text { MEAN } & 48.01 & 43.40 & 4.61 & 304.94 & 410.42 & 7.11 & 496.07 & 487.75 & 32.88 \\ \text { HUB } & 43.31 & 38.84 & 4.47 & 258.72 & 377.34 & 7.11 & 496.07 & 487.75 & 32.88\end{array}$

ROTOR EXIT CONDITIONS, STAGE 3 SOLUTION IS CONVERGED

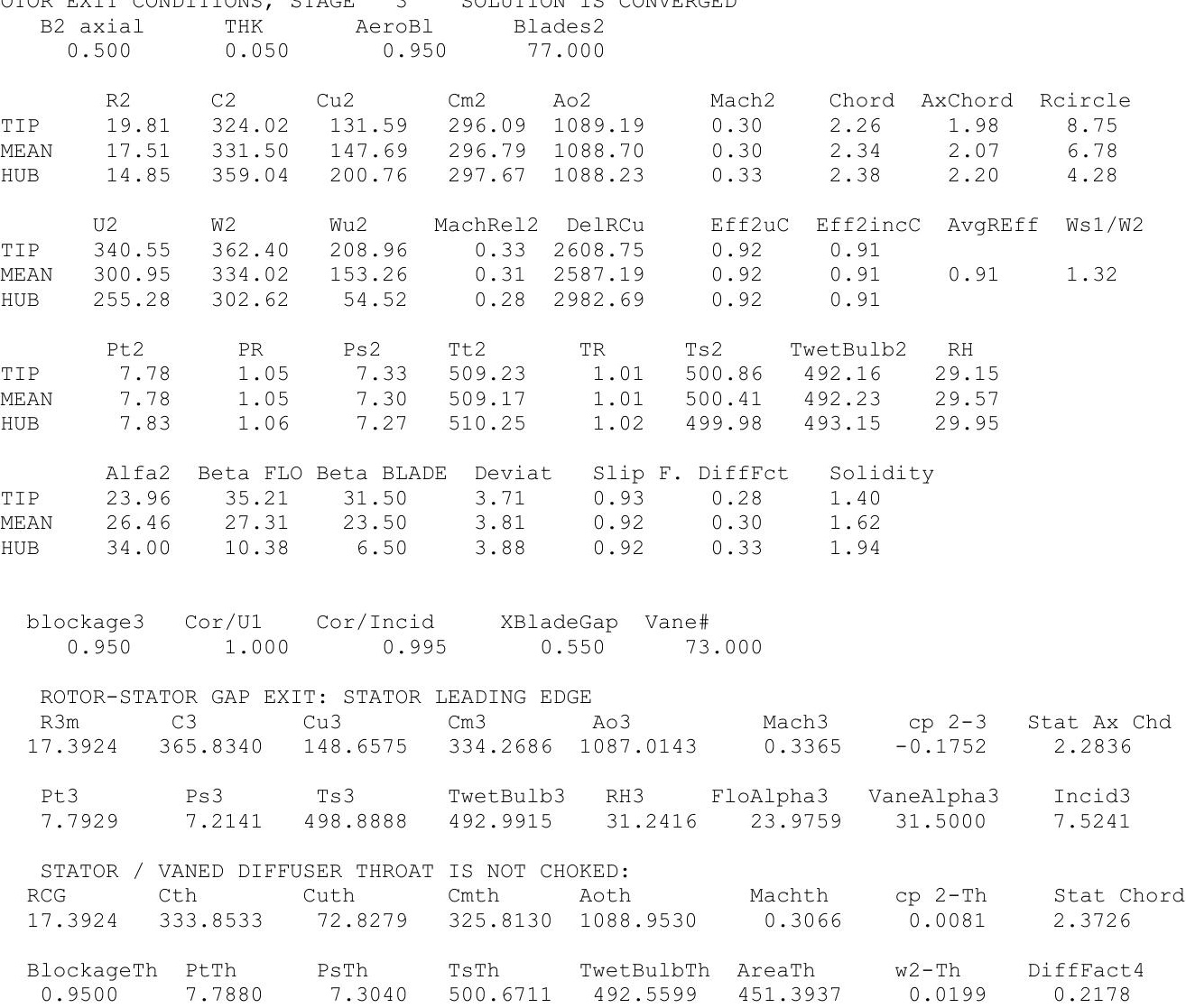




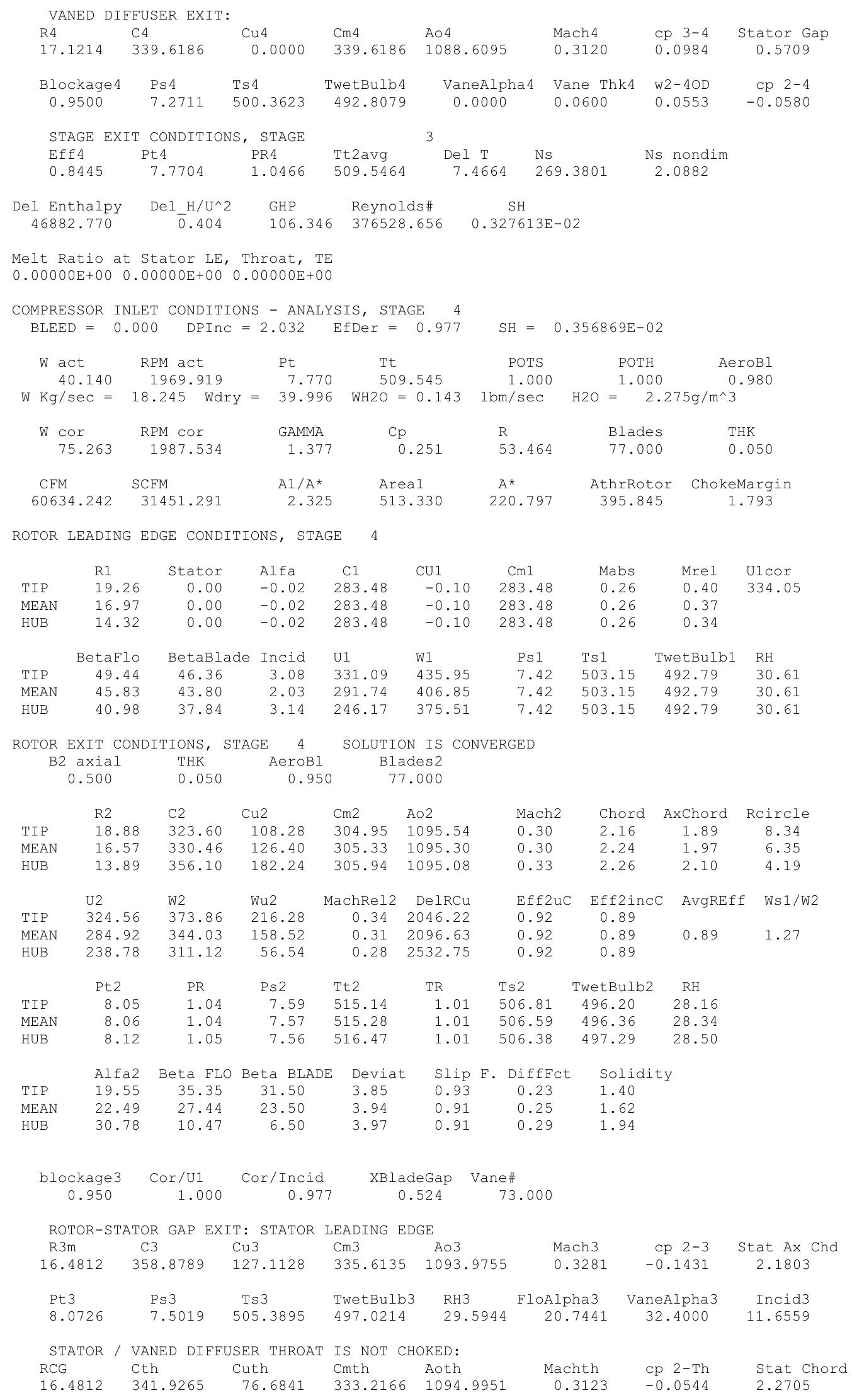




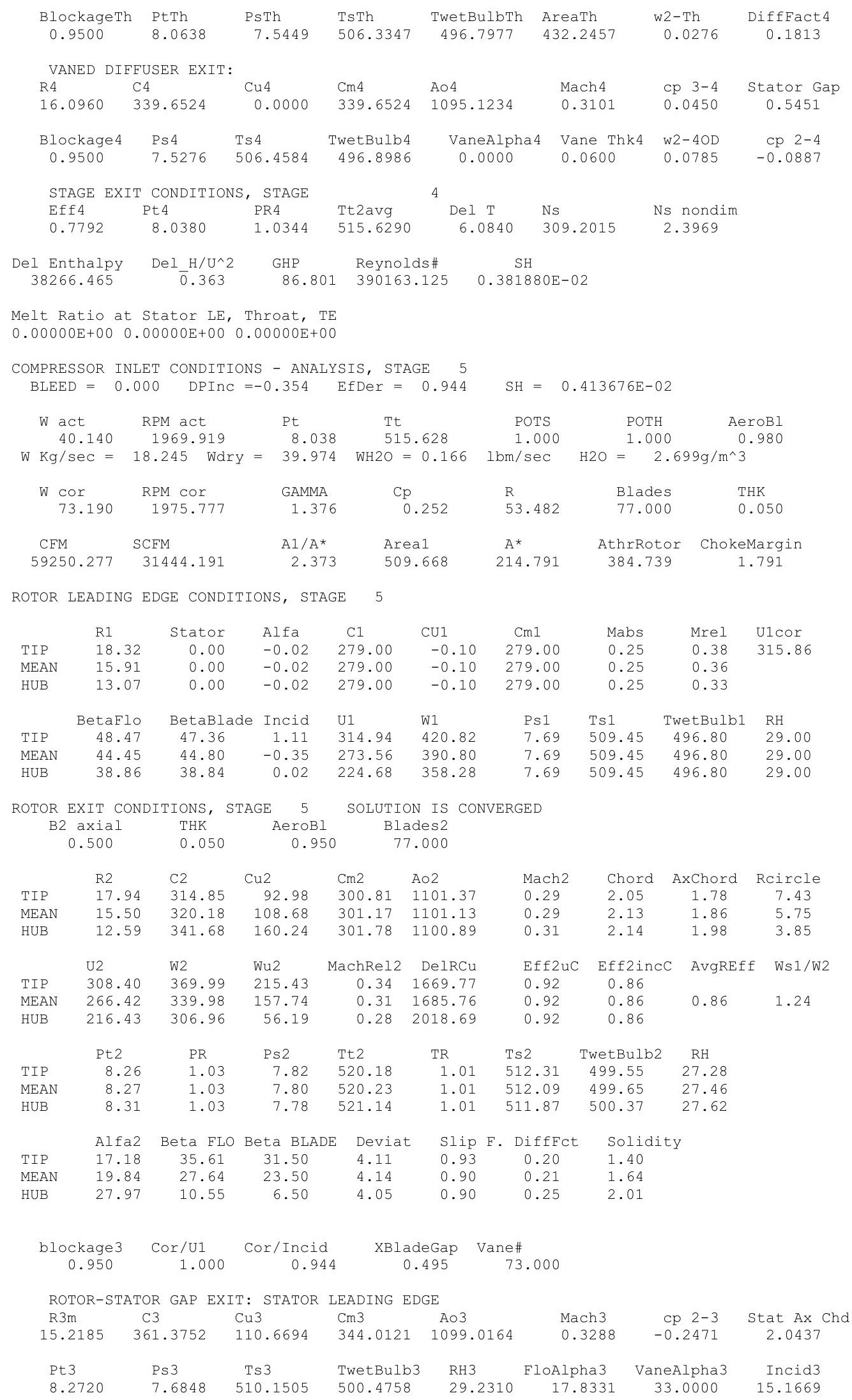




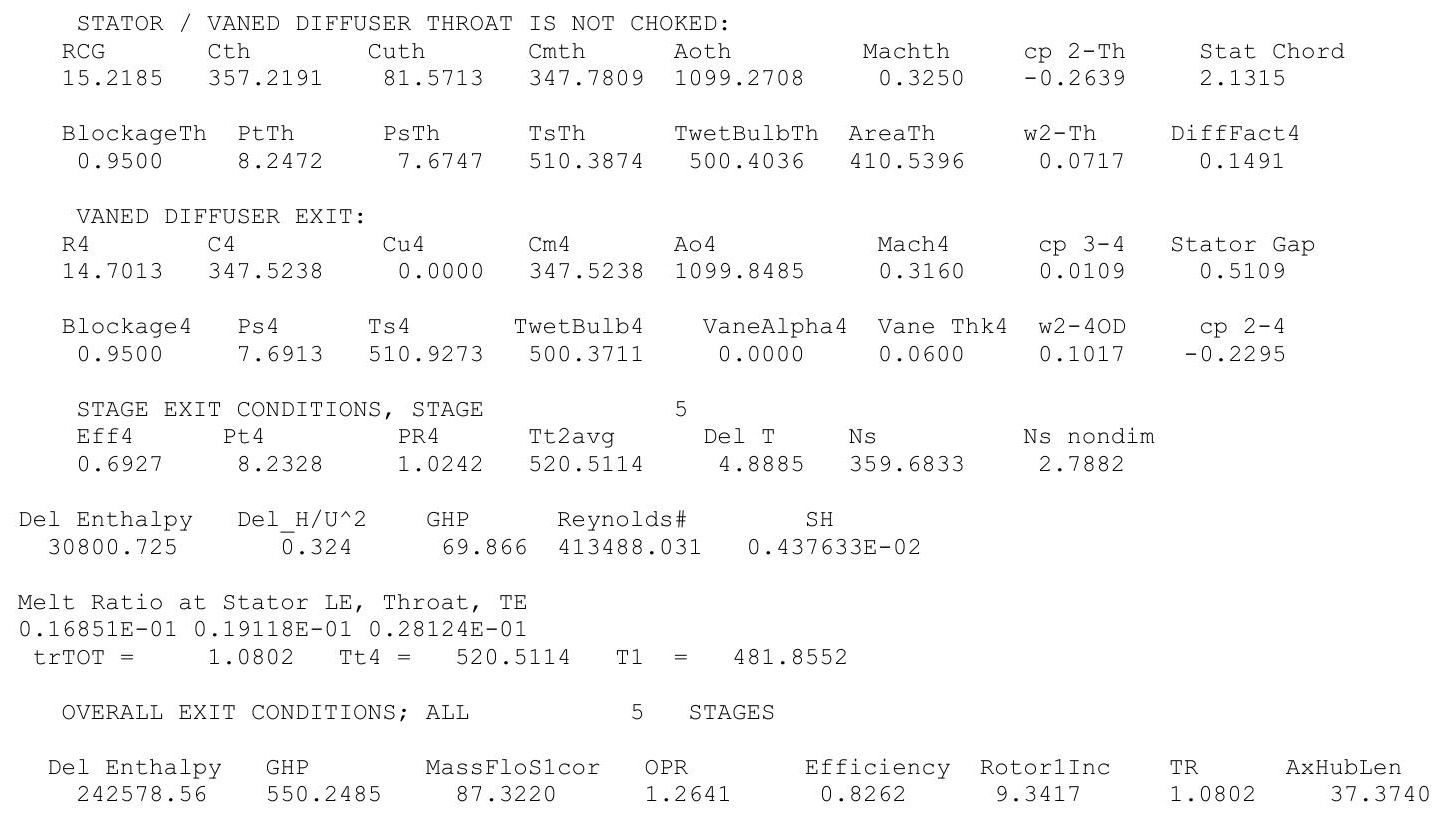




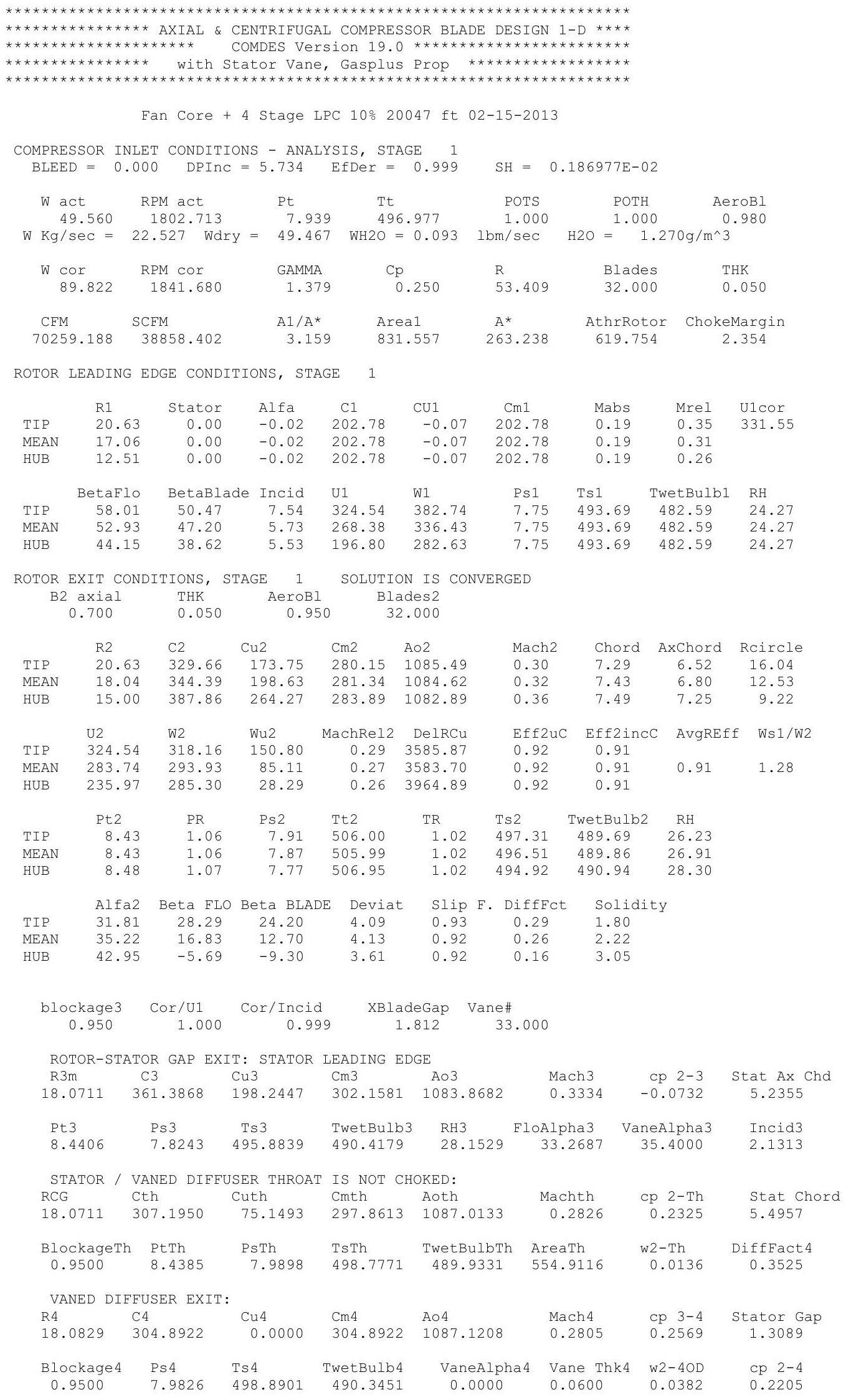




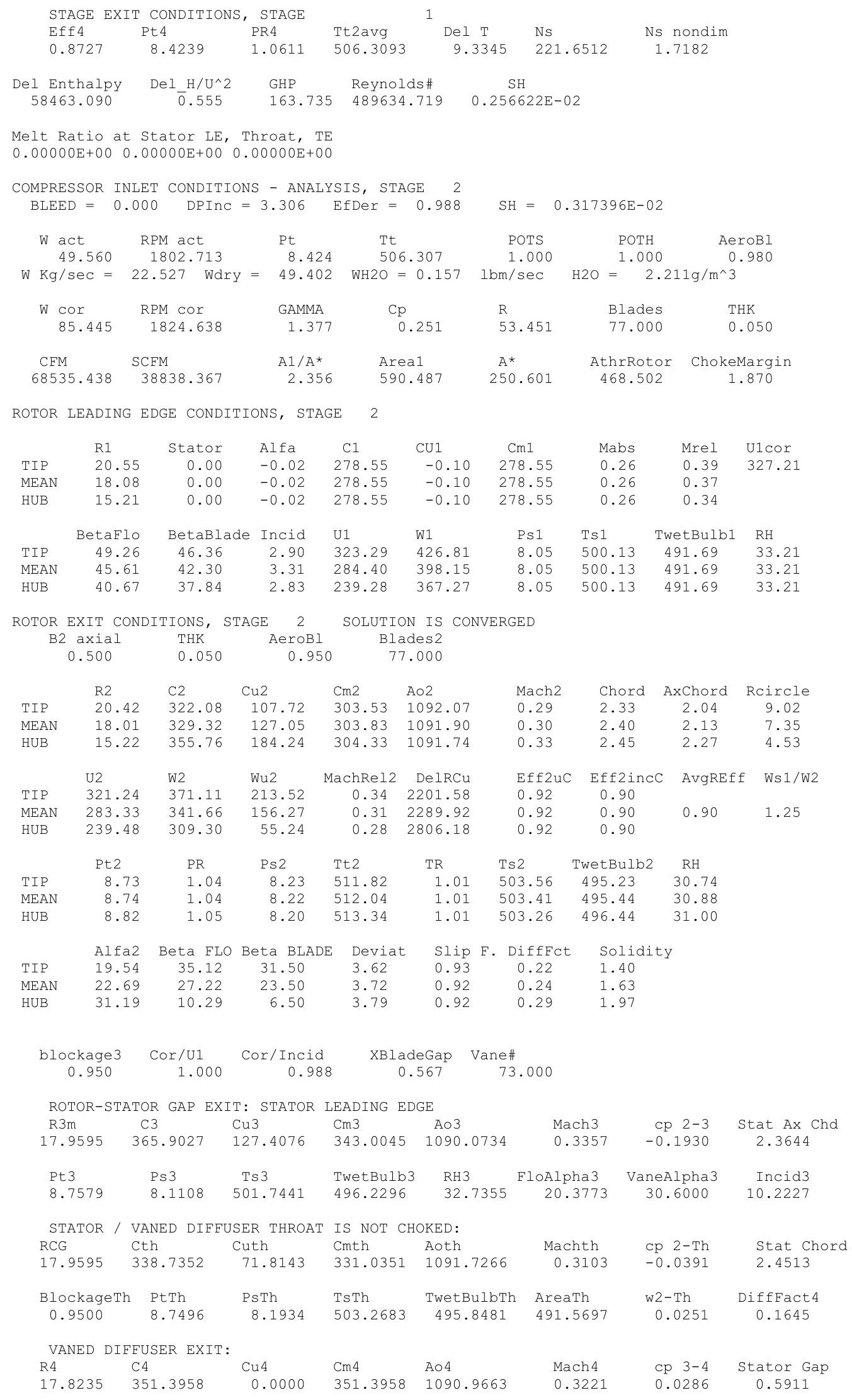




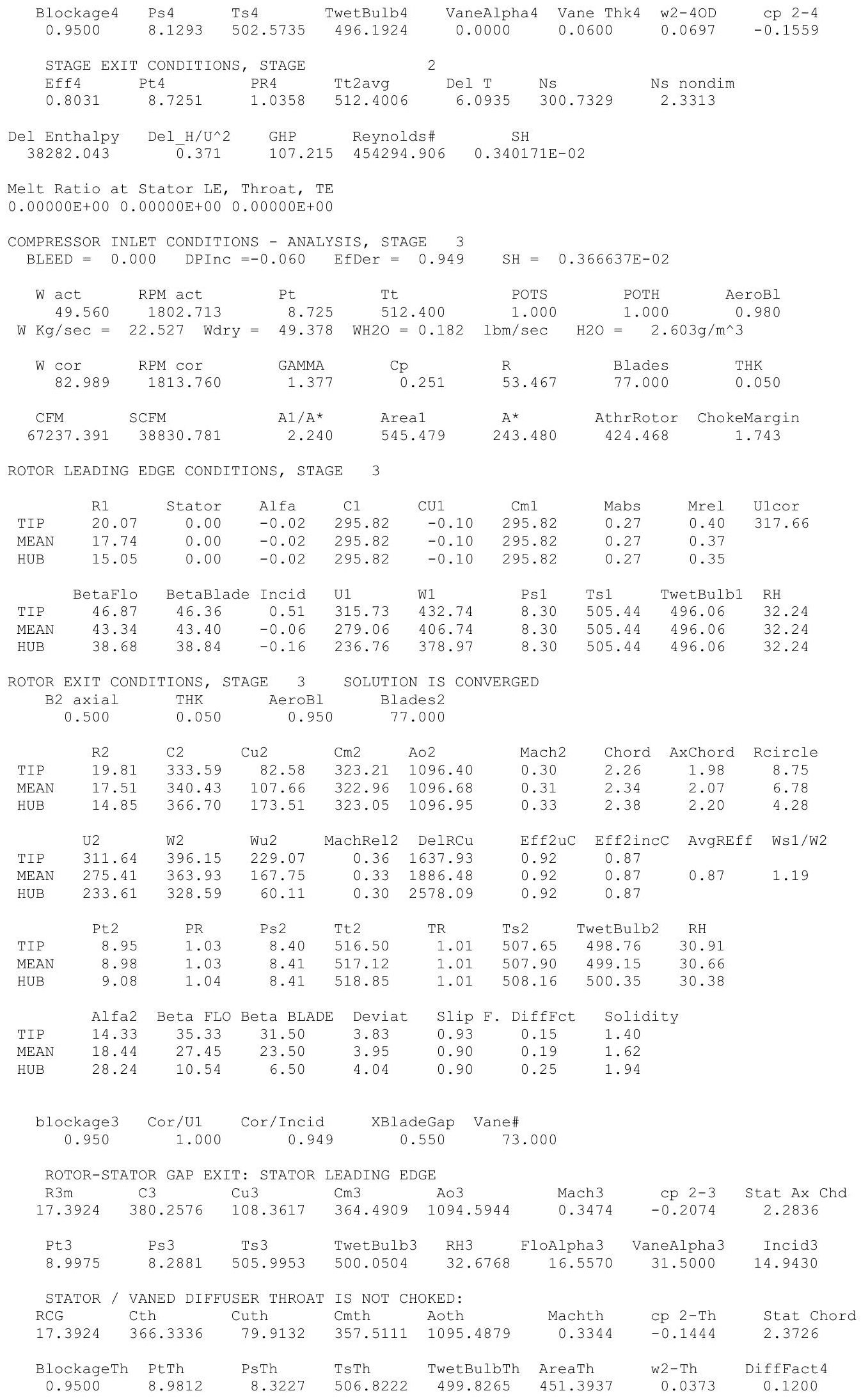

\begin{tabular}{|c|c|c|c|c|c|c|c|c|c|}
\hline & R1 & stator & Alfa & $\mathrm{C} 1$ & CU1 & $\mathrm{Cm} 1$ & Mabs & Mrel & U1cor \\
\hline TIP & 20.07 & 0.00 & -0.02 & 295.82 & -0.10 & 295.82 & 0.27 & 0.40 & 317.66 \\
\hline MEAN & 17.74 & 0.00 & -0.02 & 295.82 & -0.10 & 295.82 & 0.27 & 0.37 & \\
\hline HUB & 15.05 & 0.00 & -0.02 & 295.82 & -0.10 & 295.82 & 0.27 & 0.35 & \\
\hline & BetaFlo & BetaBlade & Incid & U1 & W1 & Ps 1 & Ts1 & TwetBulb1 & $\mathrm{RH}$ \\
\hline TIP & 46.87 & 46.36 & 0.51 & 315.73 & 432.74 & 8.30 & 505.44 & 496.06 & 32.24 \\
\hline MEAN & 43.34 & 43.40 & -0.06 & 279.06 & 406.74 & 8.30 & 505.44 & 496.06 & 32.24 \\
\hline HUB & 38.68 & 38.84 & -0.16 & 236.76 & 378.97 & 8.30 & 505.44 & 496.06 & 32.24 \\
\hline
\end{tabular}

ROTOR EXIT CONDITIONS, STAGE 3 SOLUTION IS CONVERGED

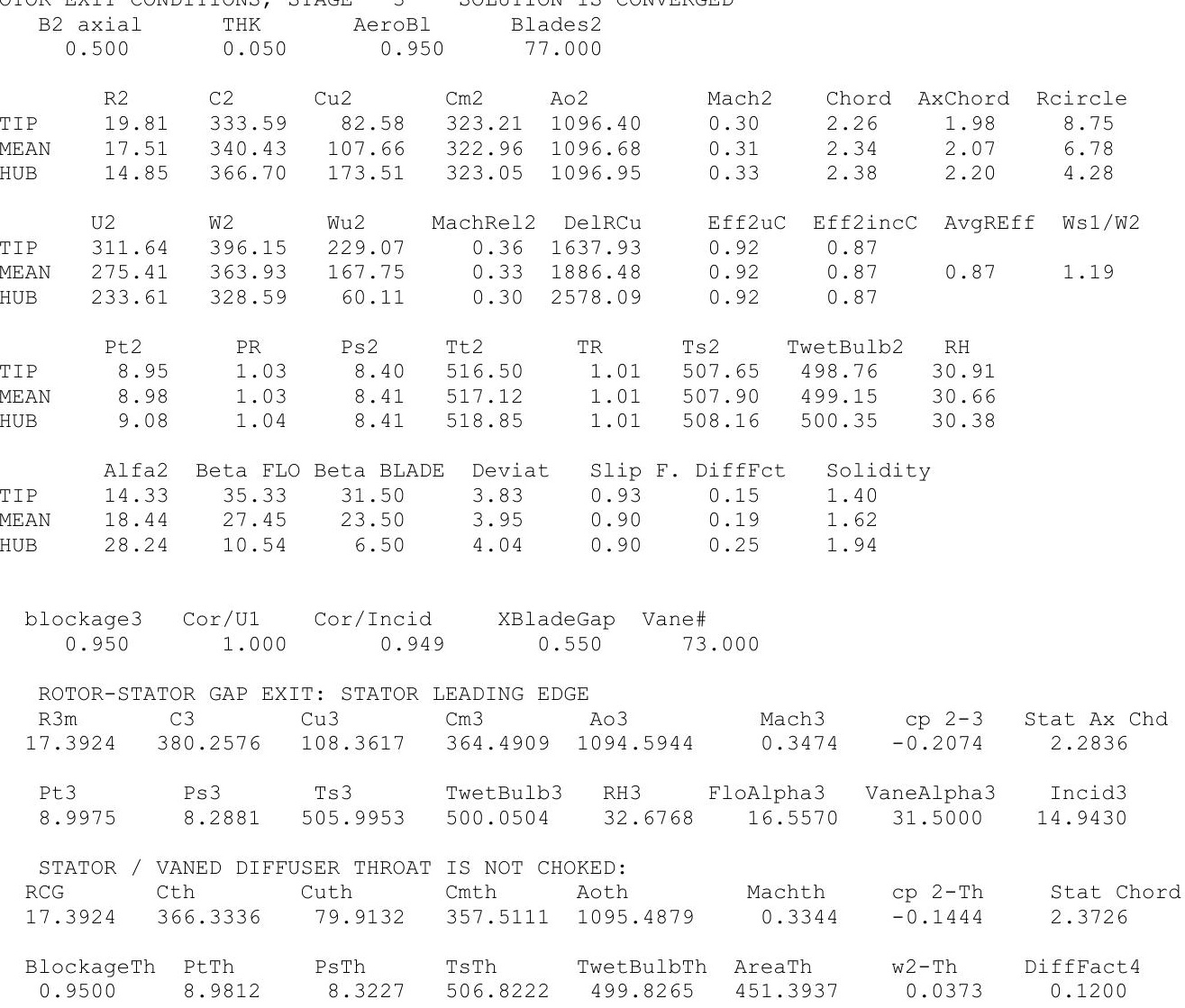




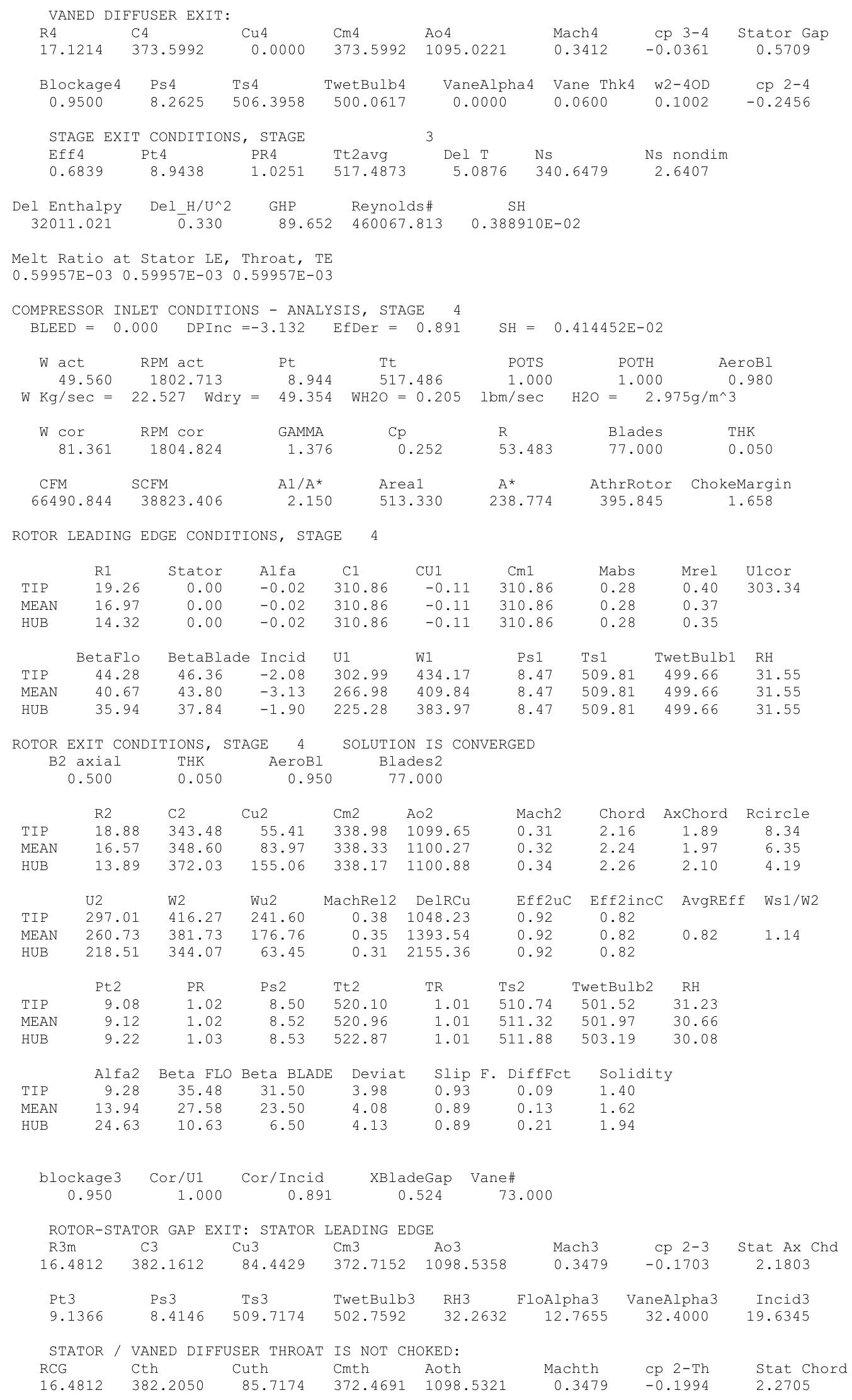




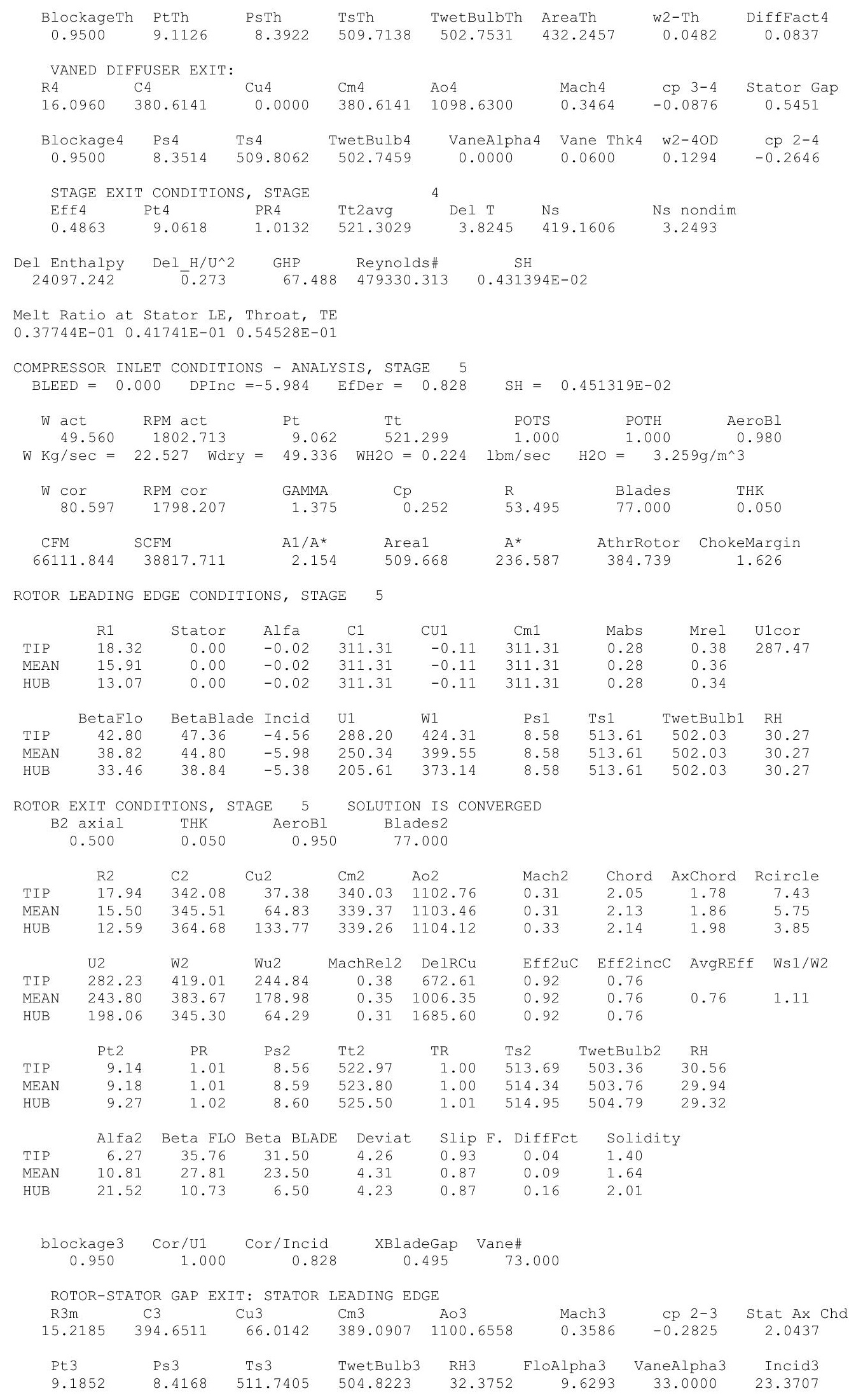




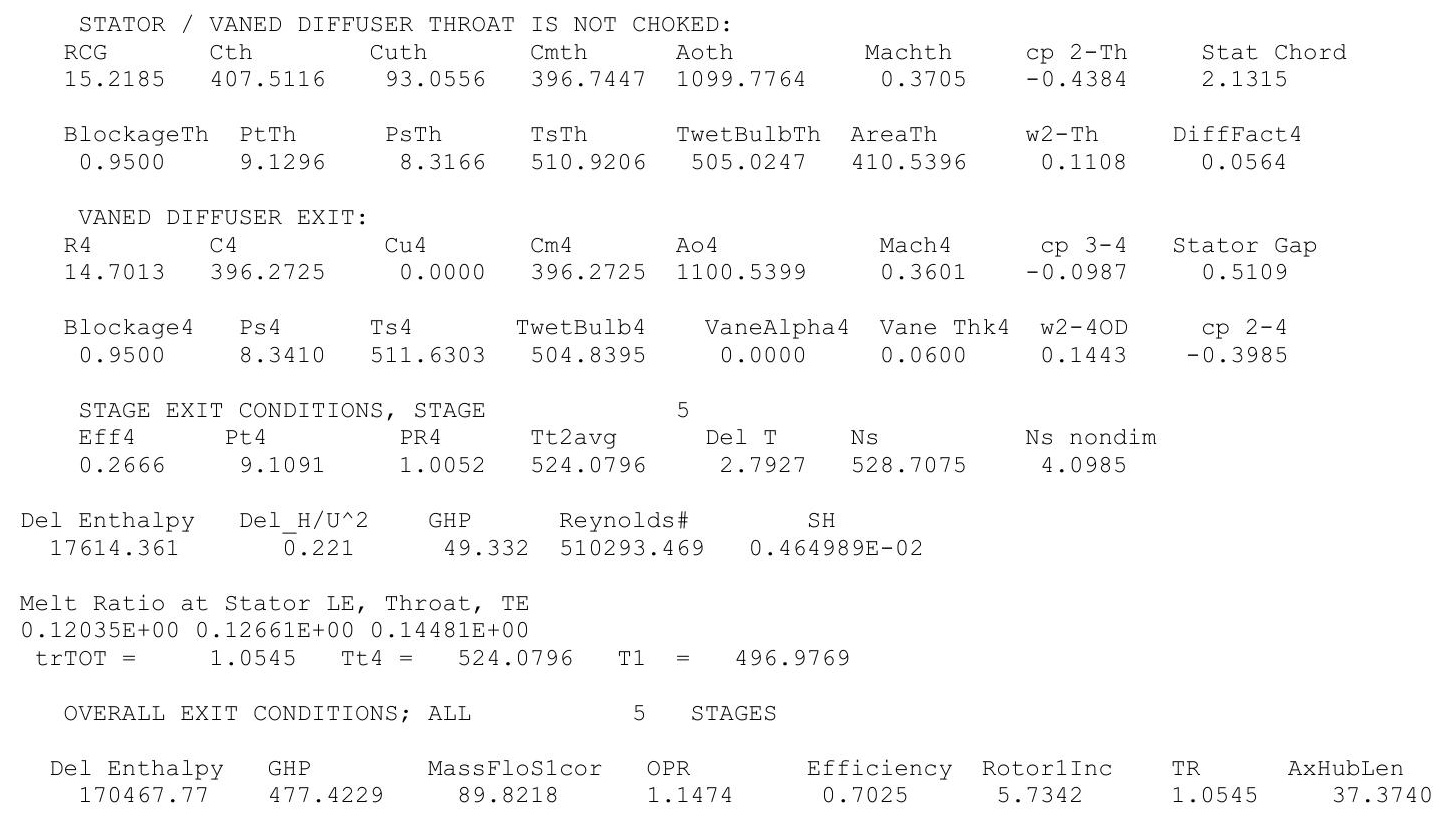




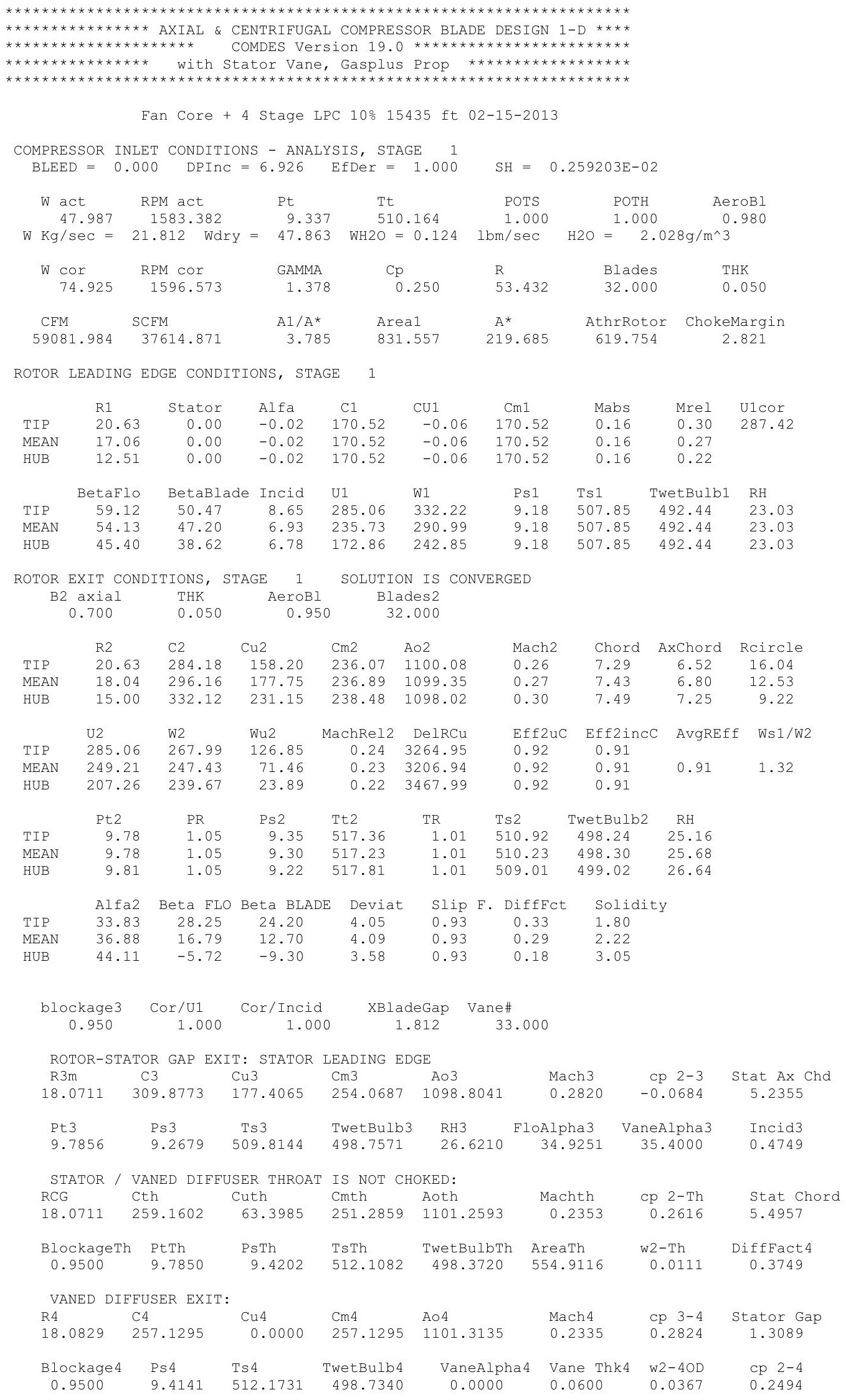




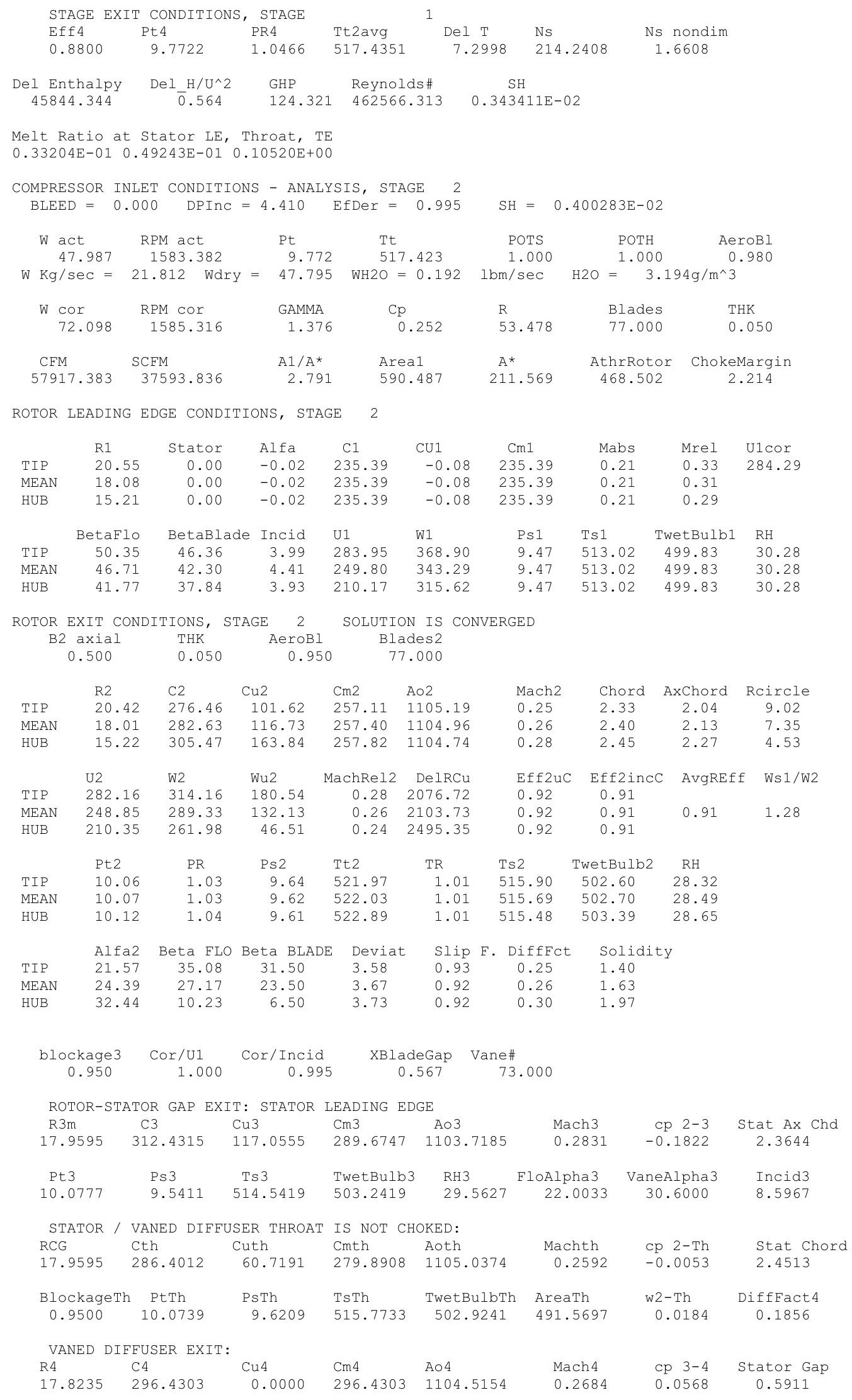




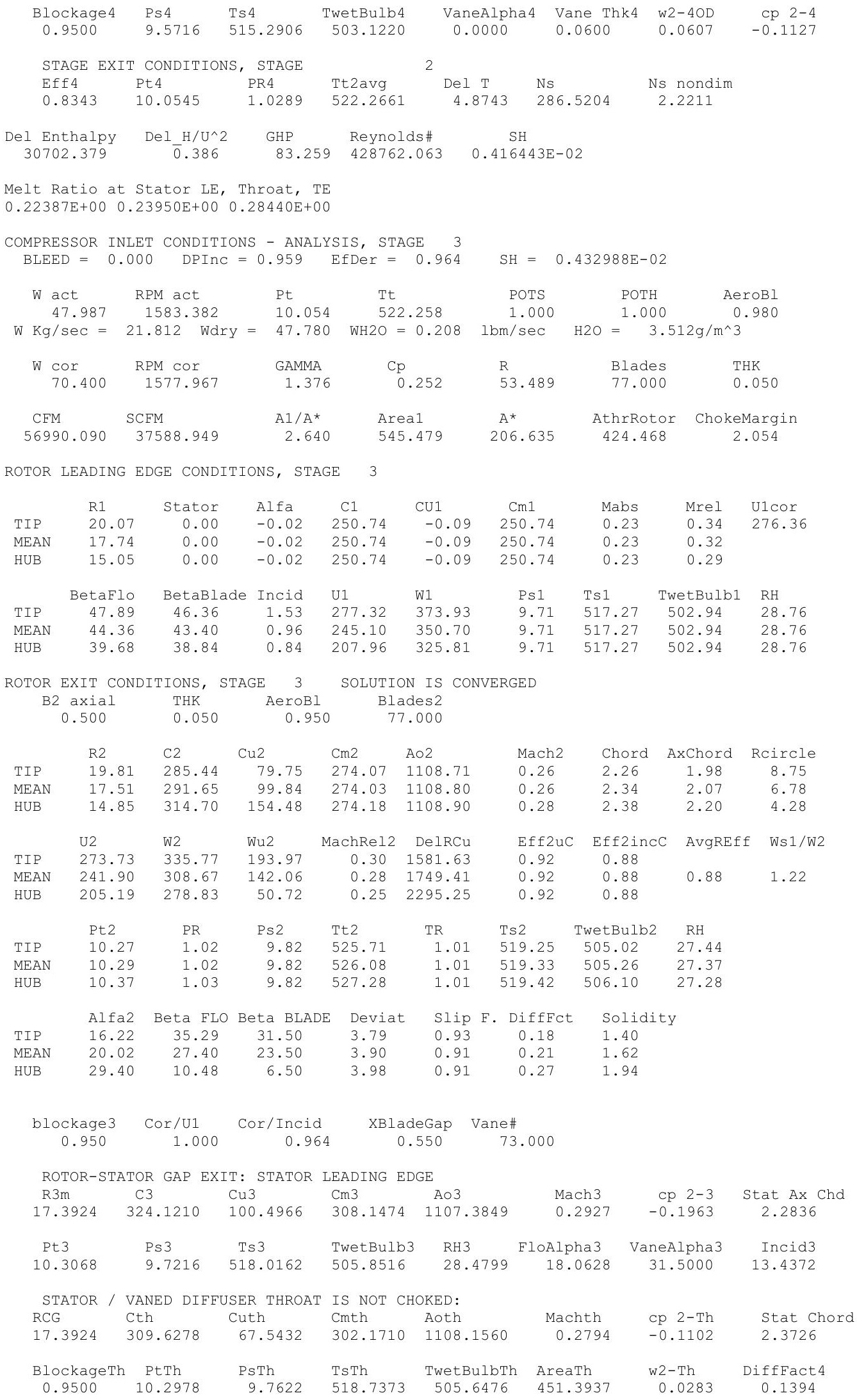




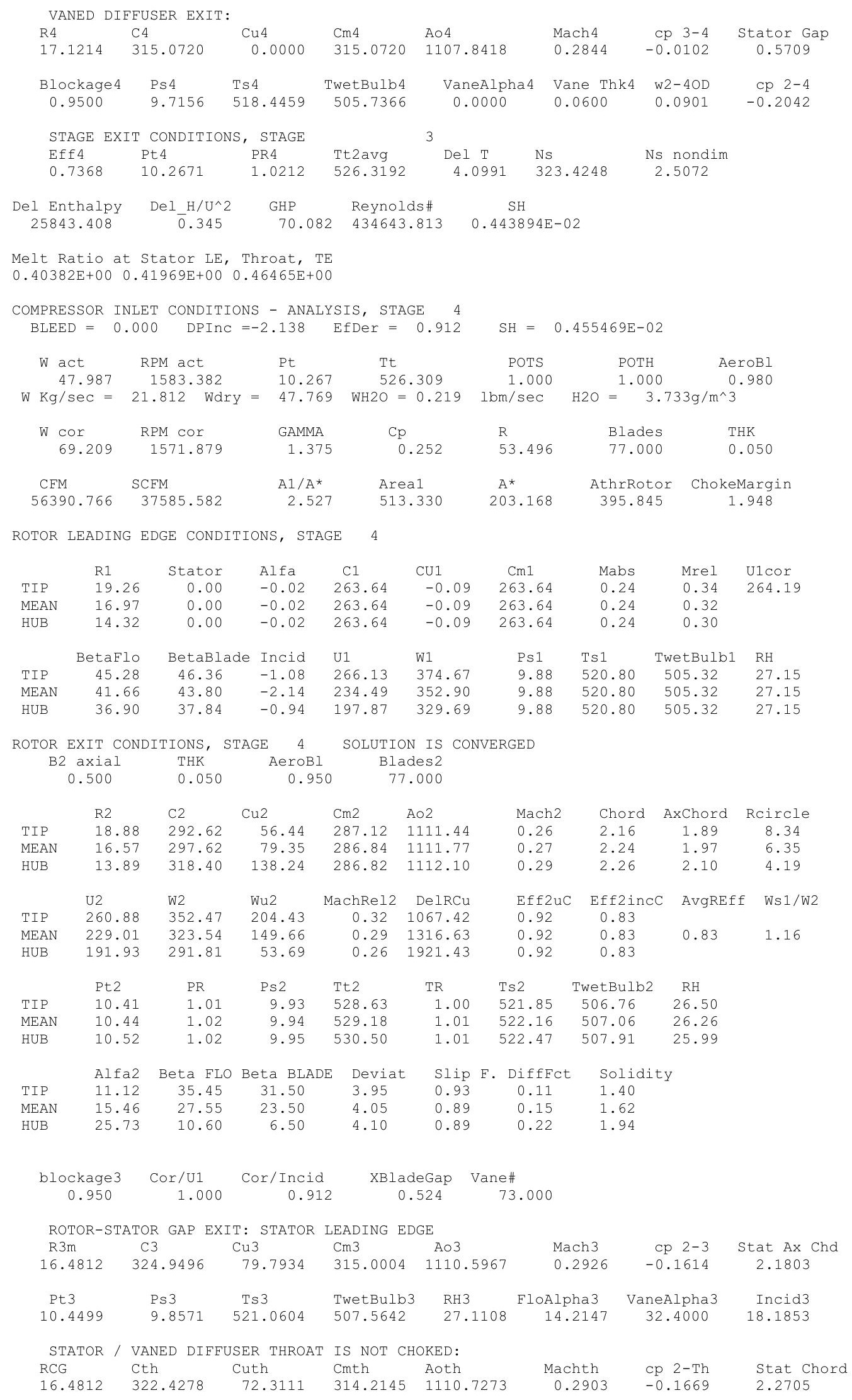




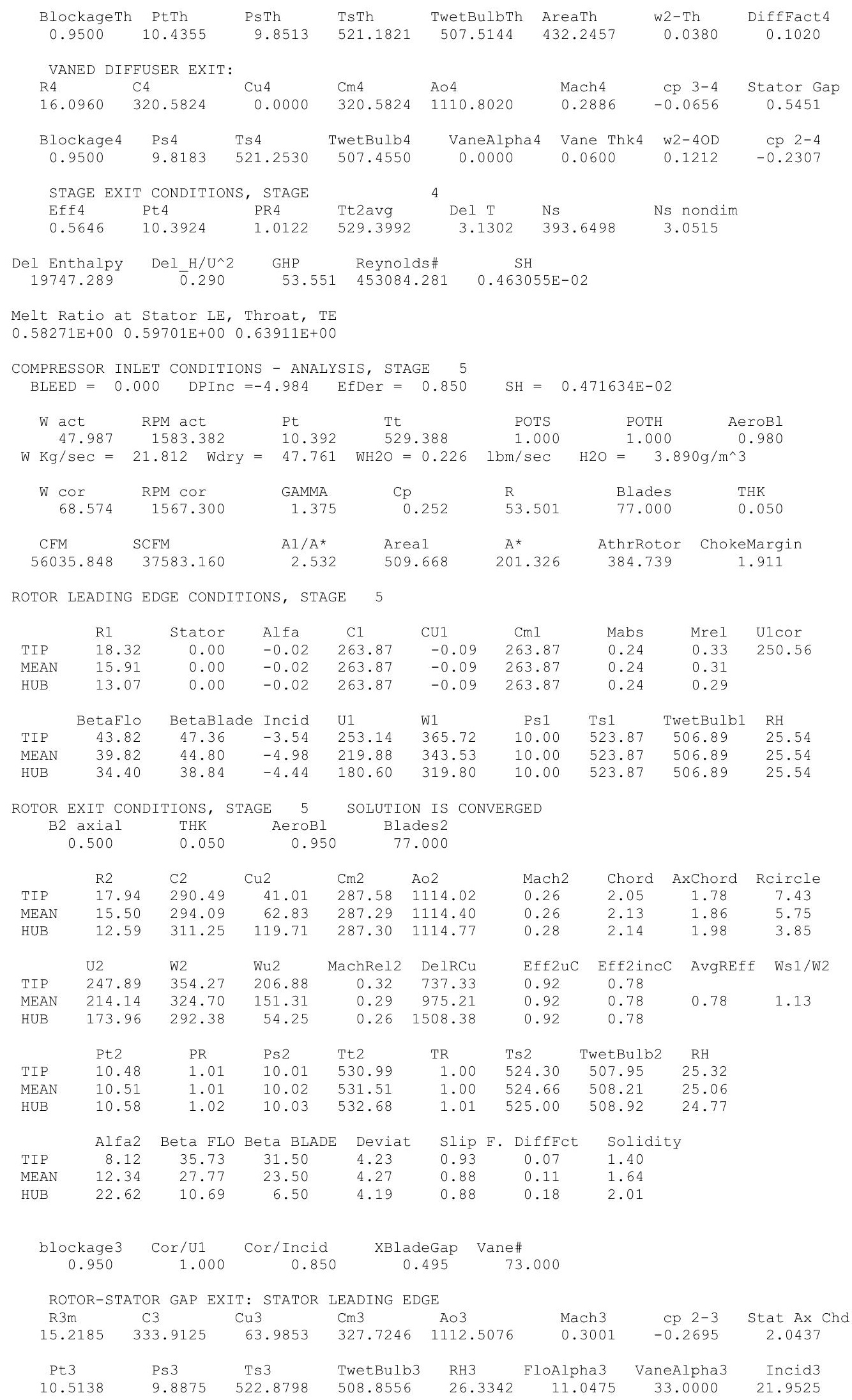




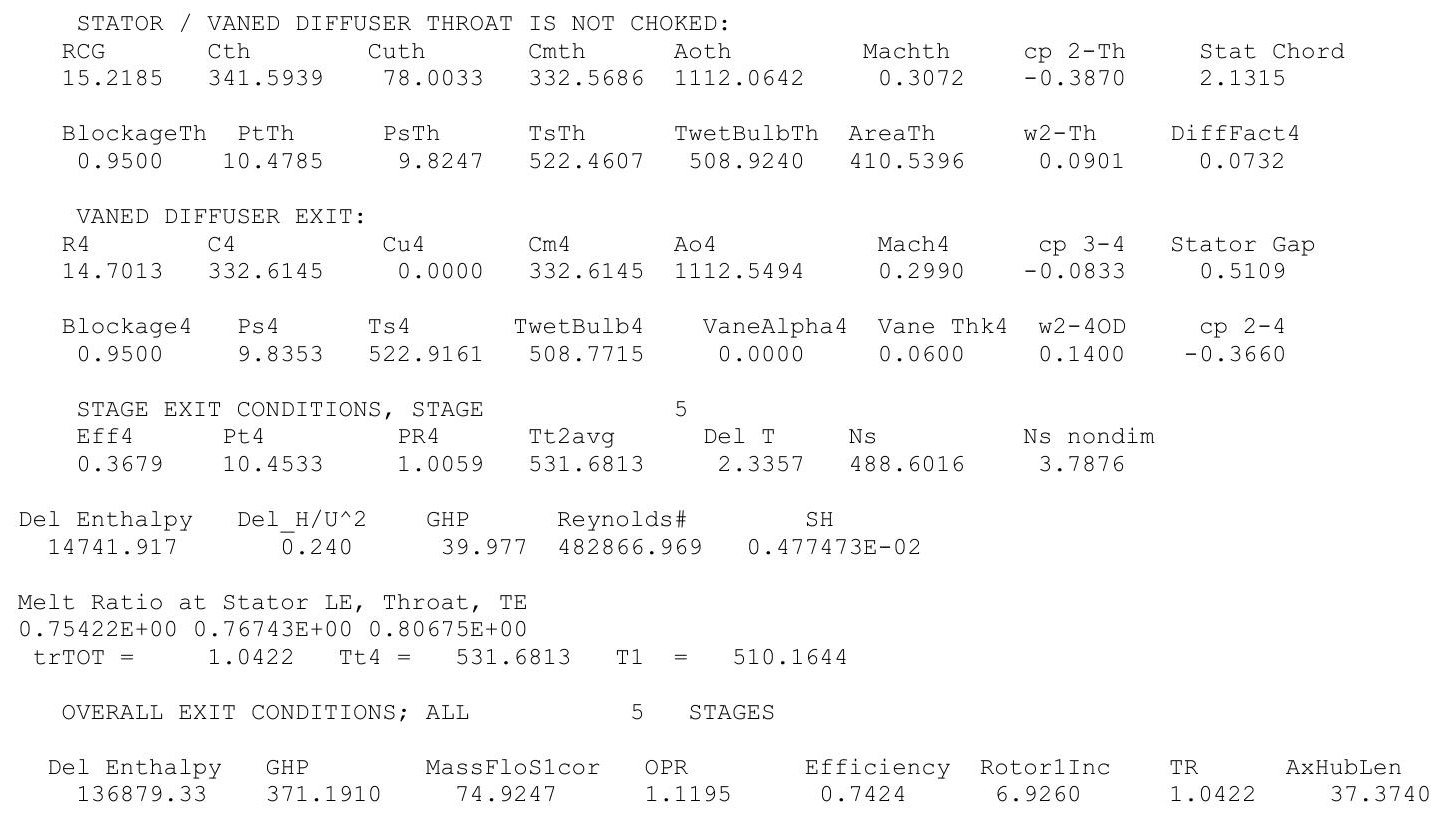




\section{Appendix N: NPSS cycle analysis for the descent conditions}

\section{$10 \mu \mathrm{m}, \mathrm{ISA}+18 \mathrm{R}$}

Date:05/13/13 Time:07:06:30 Model: Turbofan Engine - COMDES ON CONverge = 1 CASE: 0

Dev: -> Gas Package: Janaf iter/pass/Jacb/Broy $=12 /$

Temperature Stator 1 inlet: 463.30 Stator 1 exit: 470.73 Stator 2 inlet: 480.06 Stator 2 exit: 484.84

Stator 3 inlet: $494.48 \quad$ Stator 3 exit: 498.82 Stator 4 inlet: 507.82 Stator 4 exit: 511.52
Stator 5 inlet: $518.91 \quad$ Stator 5 exit: $522.14 \quad$ Unblocked Percent Blockage: 0.00

Ambient Relative Humidity

Fan Face Relative Humidity $\quad 1.76$

Fan Bypass Relative Humidity $\quad 1.24$

LPC Inlet Relative Humidity $\quad 1.13$

LPC Exit Relative Humidity

Drop Diameter

0.01

Drop Diameter

0.0000100

772.64 Inlet Length $\quad 40.00$

2.85 Fan/LPC Inlet Static Pressure 187.38

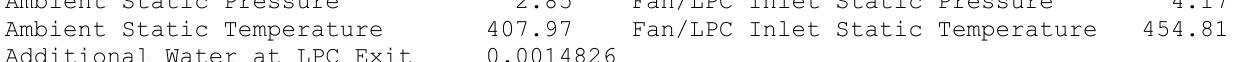

0.0014826

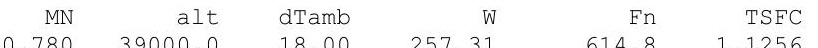

$614.8 \quad 1.1256$

Wfuel
692.01

BPR
8213

VTAS

9.491

EPR
0.73

PowerSet

T4 T41

T49

$\begin{array}{cc}\text { FLOW } & \text { STATION DATA } \\ \text { Pt } & \text { Tt }\end{array}$

\begin{tabular}{|c|c|}
\hline FSO & Ambient.Fl_O \\
\hline FS1 & Inlet.Fl_o \\
\hline FS12 & Splitter.Fl_02 \\
\hline FS2 & Splitter.Fl_01 \\
\hline FS14 & Fan.Fl O \\
\hline FS23 & LPC.FI- \\
\hline FS24 & VaporIN .FI_O \\
\hline FS25 & Bleed2.Fl_o \\
\hline FS3 & HPC.FI O \\
\hline FS36 & Bleed3.F1_O \\
\hline FS4 & Burner.Fl_o \\
\hline FS45 & HPT.FI O \\
\hline FS49 & LPT.FI- \\
\hline FS5 & TEGV.Fİ_O \\
\hline FS8 & Core_Nozz.FI \\
\hline FS17 & FanDúctLkg.Fi o \\
\hline FS171 & Bleed15.Fl O \\
\hline FS172 & FanDuct.Fl ${ }^{-} \mathrm{O}$ \\
\hline FS173 & Byp Nozz.F \\
\hline
\end{tabular}

W
257.31

$\begin{array}{llrrr}4.267 & 457.74 & -20.96 & 0.0000 & 832.56\end{array}$

$\begin{array}{llllll}257.31 & 4.267 & 457.74 & -20.96 & 0.0000 & 832.56 \\ 230.57 & 4.265 & 457.74 & -20.96 & 0.0000 & 746.40\end{array}$

$\begin{array}{rrrrrr}230.57 & 4.265 & 457.74 & -20.96 & 0.0000 & 746.40 \\ 26.74 & 4.265 & 457.74 & -20.96 & 0.0000 & 86.58\end{array}$

$\begin{array}{llllll}230.57 & 4.813 & 475.56 & -16.68 & 0.0000 & 674.05\end{array}$

$\begin{array}{llllll}26.74 & 6.647 & 531.58 & -3.25 & 0.0000 & 59.86\end{array}$

$\begin{array}{llllll}26.78 & 6.647 & 533.63 & -11.30 & 0.0000 & 60.07 \\ 24.11 & 6.647 & 533.63 & -11.30 & 0.0000 & 54.06\end{array}$

$\begin{array}{llll}6.647 & 533.63 & -11.30 & 0.0000\end{array}$

$\begin{array}{lllll}22.94 & 40.498 & 967.89 & 94.37 & 0.0000 \\ 17.78 & 40.498 & 967.89 & 94.37 & 0.0000\end{array}$

17.98

24.02

24.30
24.30
24.30

24.30
24.30

24.30
230.57

233.2

233.2

$39.565 \quad 1698.47$

$9.492 \quad 1120.65$

$\begin{array}{ll}3.259 & 878.24 \\ 3.259 & 878.32 \\ 3.259 & 878.39\end{array}$

$\begin{array}{ll}3.259 & 878.32 \\ 3.259 & 878.39\end{array}$

$4.813 \quad 475.56$

$4.813 \quad 476.23$

$\begin{array}{ll}4.813 & 476.23 \\ 4.813 & 476.23\end{array}$

$\begin{array}{rr}82.11 & 0.0108 \\ -20.25 & 0.0081\end{array}$

$\begin{array}{rr}-79.54 & 0.0080 \\ -79.53 & 0.0080\end{array}$

$\begin{array}{ll}-79.53 & 0.0080\end{array}$

$\begin{array}{ll}-79.53 & 0.0080 \\ -16.68 & 0.0000\end{array}$

$\begin{array}{ll}-16.68 & 0.0000 \\ -16.62 & 0.0000 \\ -16.62 & 0.0000\end{array}$

$\begin{array}{ll}-16.62 & 0.0000\end{array}$

54.06
11.37

1.87
8.81
12.08

12.08
54.66

142.56
142.56

142.56
142.57

674.05

682.36

682.36

Ps
2.854
3.893
3.840
4.170
4.039
6.354
6.352
6.410
35.639
37.709
37.361
8.598
3.069
3.069
2.854
4.039
3.906
3.906
2.854

Ts
407.97
445.88
444.21
454.81
452.29
524.79
526.77
528.14
934.20
948.95
1674.31
1091.21
863.73
863.80
846.65
452.29
448.59
448.59
410.06

$\begin{array}{rr}\text { Aphy } & \text { MN } \\ 2539.9 & 0.7800 \\ 4168.2 & 0.3643 \\ 3531.8 & 0.3898 \\ 830.5 & 0.1792 \\ 2606.7 & 0.5067 \\ 412.6 & 0.2543 \\ 412.6 & 0.2554 \\ 412.6 & 0.2280 \\ 49.7 & 0.4335 \\ 49.3 & 0.3228 \\ 74.6 & 0.2943 \\ 265.4 & 0.3827 \\ 860.2 & 0.2963 \\ 860.2 & 0.2963 \\ 613.4 & 0.4422 \\ 2606.7 & 0.5067 \\ 2481.9 & 0.5544 \\ 2481.9 & 0.5544 \\ 2006.9 & 0.8971\end{array}$

gamt

1.40084
1.40084

1.40084

1.40084
1.40071

1.40005

1.39981

1.39981
1.38244

1.38244
1.38244

1.33234
1.36726

1.38236

1.38236
1.38235

1.38235
1.40071

1.40070

1.40070

TURBOMACHINERY PERFORMANCE DATA

\begin{tabular}{|c|c|c|c|c|c|c|c|c|c|}
\hline & Wr & & & & & & & CMNT & SMTT \\
\hline Fan & 746.40 & 1.129 & $\begin{array}{r}\text { ett } \\
0.9053\end{array}$ & 2563.961 & $\begin{array}{r}1.0389 \\
1.03\end{array}$ & 0.9069 & $\begin{array}{r}\text { pWr } \\
-1393.0\end{array}$ & 1639.98 & 54.90 \\
\hline LPC & 86.58 & 1.559 & 0.8387 & 2563.961 & 1.1613 & 0.8485 & -669.9 & 6.96 & 3.70 \\
\hline $\mathrm{APC}$ & 54.06 & 6.093 & .81 & 8959.996 & 1.8138 & 0.8573 & -3516.5 & 61.17 & 57.84 \\
\hline $\mathrm{HPT}$ & 12.08 & 4.168 & 0.8742 & 220.524 & 1.3658 & 0.8516 & 3516.5 & & \\
\hline LPT & 54.66 & 2.912 & 0.8512 & 71.952 & 1.2742 & 0.8310 & 2062.9 & & \\
\hline URB & NERY MAP & DATA & & & & & & & \\
\hline & WcMap & PRmap & effMap & NcMap & R/Parm & s_WCDes & S_PRdes & s_effDes & S_NCDes \\
\hline an & 867.24 & 1.126 & 0.9071 & 2539.514 & 0.0413 & $\overline{0} .8607$ & $\overline{1} .0235$ & 0.9980 & $\overline{0} .9905$ \\
\hline $\mathrm{PC}$ & 73.72 & 1.520 & 0.7797 & 0.677 & 0.0000 & 1.1742 & 1.0739 & 1.0757 & 0.0003 \\
\hline HPC & 49.17 & 5.853 & 0.8270 & 8721.128 & 10.9874 & 1.0995 & 1.0494 & 0.9898 & 0.9733 \\
\hline $\mathrm{HPT}$ & 0.96 & 4.081 & 0.8742 & 1.325 & 4.0806 & 12.6299 & 0.9723 & 1.0000 & 0.0003 \\
\hline LPT & 0.84 & 2.438 & 0.8575 & 0.809 & 2.4382 & 65.2590 & 0.7521 & 0.9926 & 0.0005 \\
\hline
\end{tabular}

WAR Wair WH2O H2O frac $\begin{array}{llll}0.0000295 & 257.30 & 0.008 & 0.0000 \\ 0.0000295 & 257.30 & 0.008 & 0.0000\end{array}$ $\begin{array}{llll}0.0000295 & 257.30 & 0.008 & 0.0000 \\ 0.000295 & 257.30 & 0.008 & 0.0000 \\ 0.0000295 & 230.56 & 0.007 & 0.0000\end{array}$ $\begin{array}{llll}0.0000295 & 26.74 & 0.001 & 0.0000\end{array}$ $\begin{array}{llll}0.0000295 & 230.56 & 0.007 & 0.0000\end{array}$ $\begin{array}{llll}0.0000295 & 26.74 & 0.001 & 0.0000\end{array}$ $\begin{array}{llll}0.0015120 & 26.74 & 0.040 & 0.0015\end{array}$ $\begin{array}{llll}0.0015120 & 26.74 & 0.040 & 0.0015 \\ 0.0015120 & 24.07 & 0.036 & 0.0015 \\ 0.0015120 & 22.90 & 0.035 & 0.0015\end{array}$ $\begin{array}{llll}0.0015120 & 17.75 & 0.027 & 0.0015\end{array}$ $\begin{array}{llll}0.0015120 & 17.75 & 0.027 & 0.0147\end{array}$ $\begin{array}{llll}0.0015120 & 17.75 & 0.027 & 0.0147 \\ 0.0015120 & 23.79 & 0.036 & 0.0114 \\ 0.0015120 & 24.07 & 0.036 & 0.0113\end{array}$ $\begin{array}{llll}0.0015120 & 24.07 & 0.036 & 0.0113\end{array}$ $\begin{array}{llll}0.0015120 & 24.07 & 0.036 & 0.0113\end{array}$ $\begin{array}{llll}0.0000295 & 230.56 & 0.007 & 0.0000\end{array}$ $\begin{array}{llll}0.0000465 & 233.23 & 0.011 & 0.0000\end{array}$ $\begin{array}{llll}0.0000465 & 233.23 & 0.011 & 0.0000\end{array}$ 0.0000 


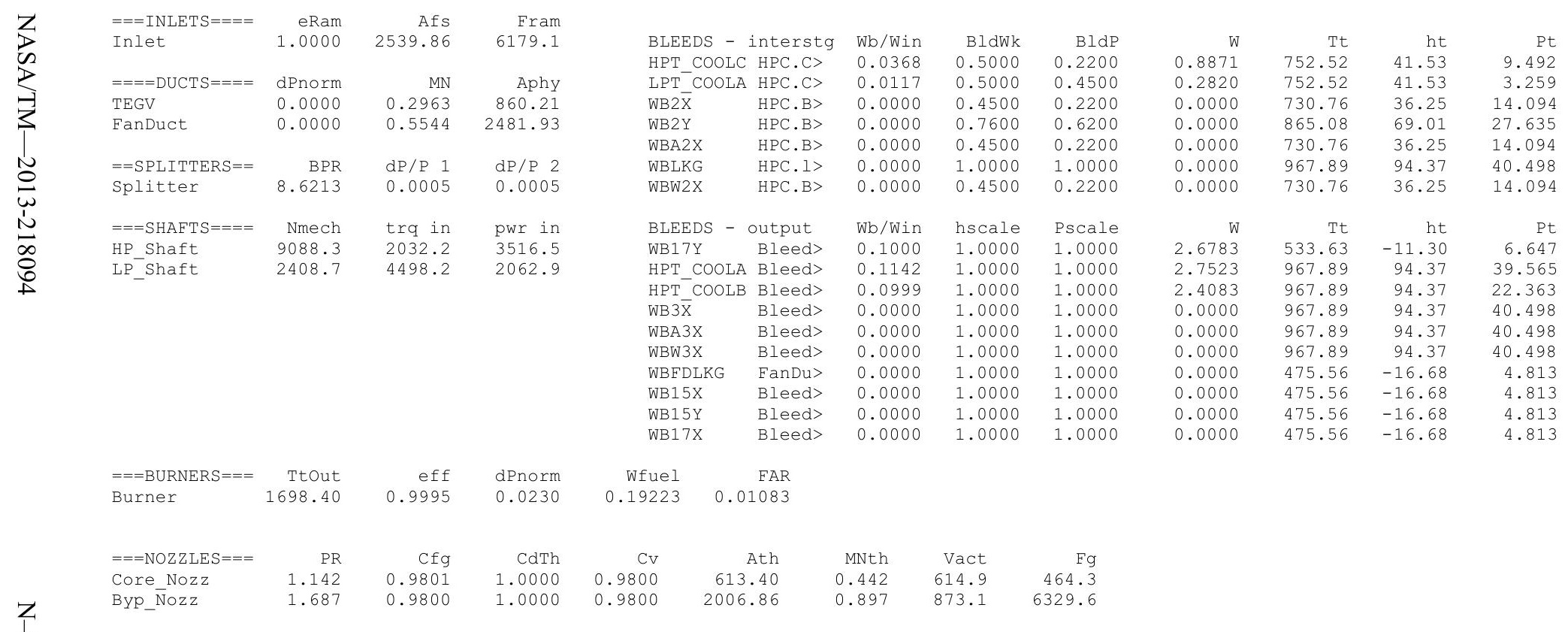


Turbofan Engine - COMDES ON Converge $=1$ CASE: 0

Version:NPSS 1.6.5 - Rev: -> Gas Package: Janaf iter/pass/Jacb/Broy=12/26/1/10 Run by: Philip C Jorgenson PC: 10

Temperature Stator 1 inlet: 456.81 Stator 1 exit: 463.77 Stator 2 inlet: 472.50 Stator 2 exit: 476.96 $\begin{array}{ll}\text { Stator } 3 \text { inlet: } 485.96 & \text { Stator } 3 \text { exit: } 490.01 \\ \text { Stator } 5 \text { inlet: } 508.75 & \text { Stator } 5 \text { exit: } 511.73\end{array}$

Ambient Relative Humidity $\quad 10.00$

Fan Face Relative Humidity $\quad 2.23$

Fan Bypass Relative Humidity 1.61

LPC Inlet Relative Humidity $\quad 1.44$

LPC Exit Relative Humidity

Drelative Humidity

Drop Diameter

0.01

Ambient Flow Velocity

0.0000100

723.11

Inlet Length

40.00

Ambient Static Pressure

407.97

Inlet Length $\quad 180.82$

Additional Water at LPC Exit 0.0012504

SUMMARY OUTPUT DATA

$\begin{array}{rrr}\text { MN } & \text { alt } & \text { dTamb } \\ 0.730 & 38334.0 & 18.00\end{array}$

250.36

604.5

1.0913

Wfuel
659.66

BPR
8.7106

VTAS
723.11

OPR
9.212

EPR
0.763

Powerset

10.000 $\begin{array}{ccc}\text { T4 } & \text { T41 } & \text { T49 } \\ 1671.6 & 1580.1 & 1100.2\end{array}$
FSO Ambient.FI O

FS1 Inlet.F1_O

FS12 Splitter.F1_02

FS14 Fan.F1_O

FS24 VaporIN.FI_O

FS25 Bleed2.F1-

FS36 Bleed3.F1_O

FS4 Burner.Fl_o

$\begin{array}{ll}\text { FS45 } & \text { HPT.F1 } \\ \text { FS49 } & \text { LPT.FI }\end{array}$

FS5 5 LPT.FI-O

FS8 Core_Nozz.FI_O

FS17 FanDüctLkg.Fì

FS171 Bleed15.FI_

FS173 Byp Nozz. Fì

TURBOMACHINERY PERFORMANCE DATA

$\begin{array}{lrrrrrrrrr} & \text { Wc } & \text { PR } & \text { eff } & \text { NC } & \text { TR } & \text { efPoly } & \text { pwr } & \text { SMN } & \text { SMW } \\ \text { Fan } & 733.33 & 1.121 & 0.9045 & 2500.478 & 1.0368 & 0.9060 & -1265.0 & 2240.58 & 54.74 \\ \text { LPC } & 84.19 & 1.526 & 0.8394 & 2500.478 & 1.1531 & 0.8487 & -604.5 & 6.76 & 3.34 \\ \text { HPC } & 53.47 & 6.040 & 0.8178 & 8941.099 & 1.8108 & 0.8565 & -3301.0 & 61.07 & 57.68 \\ \text { HPT } & 12.08 & 4.116 & 0.8729 & 219.483 & 1.3626 & 0.8503 & 3301.0 & & \\ \text { LPT } & 54.03 & 2.775 & 0.8456 & 70.211 & 1.2589 & 0.8258 & 1869.5 & & \end{array}$

TURBOMACHINERY MAP DATA

\begin{tabular}{|c|c|c|c|c|c|c|c|c|c|c|c|}
\hline so & & Ambient.FI_O & 250.36 & $\begin{array}{r}P t \\
4.201\end{array}$ & $\begin{array}{r}\mathrm{Tt} \\
451.57\end{array}$ & $\begin{array}{r}h t \\
-22.43\end{array}$ & $\begin{array}{r}\text { FA } \\
0.000\end{array}$ & 817. & $\begin{array}{l}\text { Wc } \\
10\end{array}$ & 2.947 & 407 \\
\hline 1 & \multicolumn{2}{|l|}{ Inlet.Fl_o- } & 250.36 & 4.201 & 451.57 & -22.43 & 0.000 & 817.10 & & 440. \\
\hline 12 & \multicolumn{2}{|c|}{ Splitter.F1 02} & 224.57 & 4.199 & 451.57 & -22.43 & 0.000 & 733.33 & & & 438 \\
\hline 2 & \multicolumn{2}{|l|}{ Splitter.Fl_01 } & 25.78 & 4.199 & 451.57 & -22.43 & 0.000 & 84.19 & .19 & 3.798 & 448 \\
\hline 14 & \multicolumn{2}{|l|}{ Fan.Fl_O } & 224.57 & 4.708 & 468.18 & -18.45 & 0.00 & 665.97 & \multicolumn{2}{|c|}{3.974} & 446 \\
\hline 23 & \multicolumn{2}{|l|}{ LPC.FI $\mathrm{O}$} & 25.78 & 6.408 & 520.70 & -5.86 & 0.000 & 59.24 & & 6.133 & 14 \\
\hline 24 & \multicolumn{2}{|l|}{ VaporIN. FI_O } & 81 & 88 & 522.46 & -12.64 & 0.0 & 59.42 & \multicolumn{2}{|c|}{6.131} & \\
\hline 25 & \multicolumn{2}{|l|}{ Bleed2.FI_O } & 23.23 & 6.408 & 522.46 & -12.64 & 0.000 & 53.47 & .47 & & 517 \\
\hline 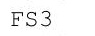 & HPC.FI_O & & 1 & 38.704 & 946.04 & 90.28 & 0.0 & 11. & 34. & & 913. \\
\hline 36 & Bleed3.Fl_O & & 1 & 8.704 & 946.04 & 90.28 & & 8. & 36. & & \\
\hline 4 & Burner.FI-O & & 2 & 37.813 & 1671.63 & 78.19 & 0.0 & 12. & 35. & & 1647 \\
\hline 45 & HPT.FI_O & & & 9.186 & 1104.24 & -21.47 & 0. & 54. & & & 1075 \\
\hline 49 & LPT.FI_O & & & 3.310 & & -77.20 & 0.0 & 135. & & & \\
\hline 5 & Fì & & & 3.310 & & -77.20 & 0. & 13 & & & 862 \\
\hline 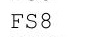 & Nozz.FI & & 2 & 3.310 & 86 & -77.20 & & 135. & & & 848.1 \\
\hline 17 & Fan & & & 4. & & -18 & & 665. & & & 44 \\
\hline 171 & Blee & & & 4. & & -18 & & 674 & & & \\
\hline 172 & FanDuct.FI_O & & 2 & 4.708 & & -18.38 & 0. & 674. & & & 442 \\
\hline 173 & Byp_Nozz.FI'_ & & 227.16 & 4.708 & 468.80 & -18.38 & 0.000 & 674.0 & & & 409 \\
\hline 1DOrt & NERY PER & $\mathrm{CE}$ & $\mathrm{DA}$ & & & & & & & & \\
\hline & & $\mathrm{PR}$ & eff & $\mathrm{NC}$ & TR & efPoly & & pwr & SM & & SMW \\
\hline & 733.33 & 1.12 & 9045 & 2500.478 & 1.0368 & 0.9060 & & 65.0 & 2240.5 & & \\
\hline & 84.19 & 1.5 & 0.8394 & 2500.478 & 1.1531 & 0.8487 & & 04.5 & 6.76 & & sat \\
\hline & 53.47 & 6. & & 8941.099 & 1.8108 & 0.8565 & & 01.0 & 61.07 & & .68 \\
\hline T & 12.08 & 4. & & 21 & & 0.8 & & 01.0 & & & \\
\hline & 54.03 & 2.775 & 0.8456 & 70.211 & 1.2589 & 0.8258 & & 69.5 & & & \\
\hline & CHINERY & DA & & & & & & & & & \\
\hline & WcMap & PRmap & ef & $\mathrm{NCM} a$ & R/Parm & $s_{-}+$ & cDes & S_PRdes & S_effD & & S_NcDes \\
\hline & 852.05 & 1 & & 2476.637 & & & 8607 & $\overline{1} .0235$ & & & 905 \\
\hline & 70.97 & 1.492 & & 0.660 & 0.0000 & & 1861 & 1.0697 & 1.0 & & 0.0003 \\
\hline & 48.63 & 5.802 & 0.8262 & 8702.736 & 11.0009 & & 0995 & 1.0494 & 0.989 & & 0.9733 \\
\hline & 0.96 & 4.030 & 0.8729 & 1.319 & 4.0301 & & 6299 & 0.9723 & 1.000 & & 0.0003 \\
\hline & 0.83 & 2.335 & 0.8519 & 0.7 & & & & 7521 & 0.992 & & .0005 \\
\hline
\end{tabular}

$\begin{array}{rrrrrrr}\text { Aphy } & \text { MN } & \text { gamt } & \text { WAR } & \text { Wair } & \text { WH2O } & \text { H2O frac } \\ 2557.3 & 0.7300 & 1.40089 & 0.0000285 & 250.35 & 0.007 & 0.0000 \\ 4168.2 & 0.3563 & 1.40089 & 0.0000285 & 250.35 & 0.007 & 0.0000 \\ 3531.8 & 0.3816 & 1.40089 & 0.0000285 & 224.57 & 0.006 & 0.0000 \\ 830.5 & 0.1740 & 1.40089 & 0.0000285 & 25.78 & 0.001 & 0.0000 \\ 2606.7 & 0.4981 & 1.40077 & 0.0000285 & 224.57 & 0.006 & 0.0000 \\ 412.6 & 0.2514 & 1.40020 & 0.0000285 & 25.78 & 0.001 & 0.0000 \\ 412.6 & 0.2523 & 1.40001 & 0.0012789 & 25.78 & 0.033 & 0.0013 \\ 412.6 & 0.2254 & 1.40001 & 0.0012789 & 23.20 & 0.030 & 0.0013 \\ 49.7 & 0.4316 & 1.38377 & 0.0012789 & 22.08 & 0.028 & 0.0013 \\ 49.3 & 0.3215 & 1.38377 & 0.0012789 & 17.11 & 0.022 & 0.0013 \\ 74.6 & 0.2942 & 1.33374 & 0.0012789 & 17.11 & 0.022 & 0.0143 \\ 265.4 & 0.3772 & 1.36842 & 0.0012789 & 22.93 & 0.029 & 0.0110 \\ 860.2 & 0.2791 & 1.38259 & 0.0012789 & 23.20 & 0.030 & 0.0109 \\ 860.2 & 0.2791 & 1.38259 & 0.0012789 & 23.20 & 0.030 & 0.0109 \\ 613.4 & 0.4131 & 1.38258 & 0.0012789 & 23.20 & 0.030 & 0.0109 \\ 2606.7 & 0.4981 & 1.40077 & 0.0000285 & 224.57 & 0.006 & 0.0000 \\ 2481.9 & 0.5442 & 1.40076 & 0.0000427 & 227.15 & 0.010 & 0.0000 \\ 2481.9 & 0.5442 & 1.40076 & 0.0000427 & 227.15 & 0.010 & 0.0000 \\ 2006.9 & 0.8461 & 1.40076 & 0.0000427 & 227.15 & 0.010 & 0.0000\end{array}$

$2006.9 \quad 0.8461$

1.40076 


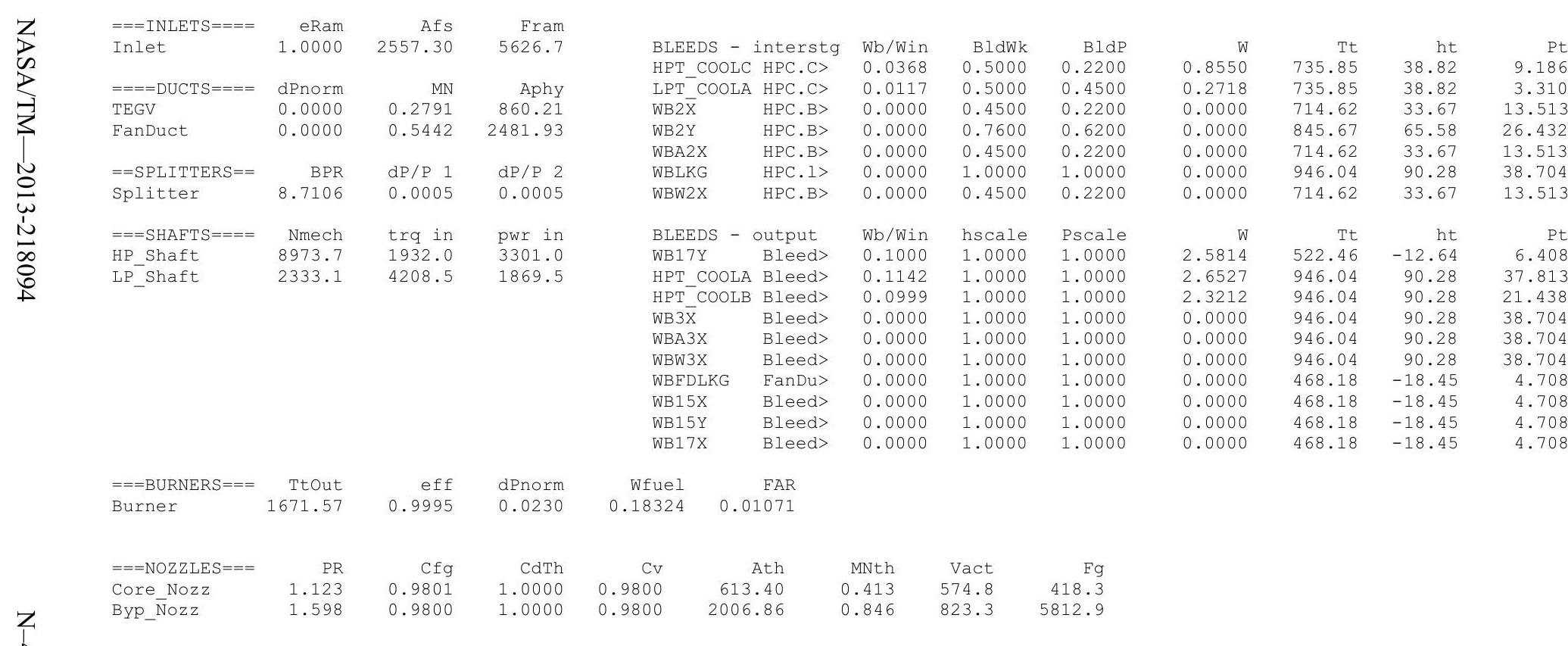


Turbofan Engine - COMDES ON Converge $=1$ CASE: 0

Version:NPSS 1.6.5 - Rev: -> Gas Package: Janaf iter/pass/Jacb/Broy=11/25/1/9 Run by: Philip C Jorgenson PC: 10

Temperature Stator 1 inlet: $454.80 \quad$ Stator 1 exit: 461.58 $\quad$ Stator 2 inlet: $470.09 \quad$ Stator 2 exit: 474.44 Stator 3 inlet: $483.21 \quad$ Stator 3 exit: 487.14 Stator 4 inlet: 495.33 Stator 4 exit: 498.66

Ambient Relative Humidity $\quad 10.00$

Fan Face Relative Humidity $\quad 2.40$

Fan Bypass Relative Humidity $\quad 1.75$

$\begin{array}{ll}\text { LPC Init Relative Humidity } & 1.56 \\ \text { LPC Exit Relative Humidity } & 0.15\end{array}$

Drop Diameter

0.01

0.0000100

$\begin{array}{ll}\text { Inlet Length } & 40.00\end{array}$

Ambient Flow Velocity

Fan/LPC Inlet Flow velocity 178.30

Ambient static Pressure

$\begin{array}{rrr}3.09 & \text { Fan/LPC Inlet Static Pressure } & 4.25 \\ 407.97 & \text { Fan/LPC Inlet Static Temperature } & 447.03\end{array}$

Additional Water at LPC Exit

0.0011211

$\begin{array}{rrr}\text { MN } & \text { alt } & \text { dTamb } \\ 0.714 & 37357.0 & 18.00\end{array}$

257.11

SUMMARY OUTPUT DATA

Fn TSFC

Wfuel

BPR
8.7472

VTAS
707.26

OPR
9.095

$E P R$
0.772

Powerset

10.000

$\begin{array}{ccc}\text { T4 } & \text { T41 } & \text { T49 } \\ 1661.3 & 1570.1 & 1093.8\end{array}$
FSO Ambient.Fl O

FS1 Inlet.F1_O

FS12 Splitter.F1_02

FS14 Fan.F1_O

FS23 LPC.FI_O

FS24 VaporIN.FI_O

FS25 Bleed2.F1-

FS36 Bleed3.F1_O

FS4 Burner.Fl_o

$\begin{array}{ll}\text { FS45 } & \text { HPT.FI_O } \\ \text { FS49 } & \text { LPT.FI-O }\end{array}$

FS49 LPT.FIO

FS8 Core_Nozz.FI_O

FS17 FanDüctLkg.F̄

FS171 Bleed15.FI_O

FS172 FanDuct.Fl_O

TURBOMACHINERY PERFORMANCE DATA

$\begin{array}{lrrrrrrrrr} & \text { Wc } & \text { PR } & \text { eff } & \text { NC } & \text { TR } & \text { efPoly } & \text { pwr } & \text { SMN } & \text { SMW } \\ \text { Fan } & 727.95 & 1.118 & 0.9041 & 2474.843 & 1.0360 & 0.9056 & -1264.8 & 2632.78 & 54.65 \\ \text { LPC } & 83.22 & 1.513 & 0.8396 & 2474.843 & 1.1498 & 0.8487 & -602.7 & 6.68 & 3.27 \\ \text { HPC } & 53.21 & 6.013 & 0.8173 & 8932.634 & 1.8090 & 0.8561 & -3343.3 & 61.11 & 57.70 \\ \text { HPT } & 12.08 & 4.099 & 0.8723 & 219.152 & 1.3615 & 0.8497 & 3343.3 & & \\ \text { LPT } & 53.81 & 2.726 & 0.8435 & 69.546 & 1.2534 & 0.8239 & 1867.6 & & \end{array}$

TURBOMACHINERY MAP DATA

$\begin{array}{lrrrrrrrrr} & \text { WcMap } & \text { PRmap } & \text { effMap } & \text { NcMap } & \text { R/Parm } & \text { S_WCDes } & \text { S_PRdes } & \text { S_effDes } & \text { S_NcDes } \\ \text { Fan } & 845.80 & 1.116 & 0.9060 & 2451.245 & 0.0412 & 0.8607 & 1.0235 & 0.9980 & 0.9905 \\ \text { LPC } & 69.86 & 1.480 & 0.7755 & 0.654 & 0.0000 & 1.1912 & 1.0688 & 1.0827 & 0.0003 \\ \text { HPC } & 48.40 & 5.777 & 0.8258 & 8694.496 & 11.0014 & 1.0995 & 1.0494 & 0.9898 & 0.9733 \\ \text { HPT } & 0.96 & 4.013 & 0.8723 & 1.317 & 4.0130 & 12.6299 & 0.9723 & 1.0000 & 0.0003 \\ \text { LPT } & 0.82 & 2.298 & 0.8498 & 0.782 & 2.2984 & 65.2590 & 0.7521 & 0.9926 & 0.0005\end{array}$

FLOW STATION DATA

$\begin{array}{rrrrrrrrrr}\text { W } & \text { Pt } & \text { Tt } & \text { ht } & \text { FAR } & \text { WC } & \text { PS } & \text { Ts } & \text { Aphy } & \text { MN } \\ 257.11 & 4.339 & 449.68 & -22.87 & 0.0000 & 810.76 & 3.088 & 407.97 & 2561.9 & 0.7140 \\ 257.11 & 4.339 & 449.68 & -22.87 & 0.0000 & 810.76 & 3.981 & 438.71 & 4168.2 & 0.3531 \\ 230.73 & 4.337 & 449.68 & -22.87 & 0.0000 & 727.95 & 3.929 & 437.14 & 3531.8 & 0.3782 \\ 26.38 & 4.337 & 449.68 & -22.87 & 0.0000 & 83.22 & 4.249 & 447.03 & 830.5 & 0.1720 \\ 230.73 & 4.850 & 465.85 & -19.00 & 0.0000 & 662.53 & 4.103 & 444.08 & 2606.7 & 0.4946 \\ 26.38 & 6.563 & 517.05 & -6.72 & 0.0000 & 58.97 & 6.284 & 510.66 & 412.6 & 0.2502 \\ 26.41 & 6.563 & 518.64 & -12.81 & 0.0000 & 59.13 & 6.282 & 512.19 & 412.6 & 0.2510 \\ 23.77 & 6.563 & 518.64 & -12.81 & 0.0000 & 53.21 & 6.338 & 513.48 & 412.6 & 0.2242 \\ 22.61 & 39.464 & 938.25 & 89.09 & 0.0000 & 11.33 & 34.774 & 905.81 & 49.7 & 0.4311 \\ 17.53 & 39.464 & 938.25 & 89.09 & 0.0000 & 8.78 & 36.770 & 919.99 & 49.3 & 0.3211 \\ 17.71 & 38.555 & 1661.28 & 77.08 & 0.0107 & 12.08 & 36.406 & 1637.53 & 74.6 & 0.2942 \\ 23.67 & 9.406 & 1097.90 & -21.59 & 0.0079 & 53.81 & 8.551 & 1070.00 & 265.4 & 0.3754 \\ 23.95 & 3.450 & 874.50 & -76.00 & 0.0079 & 132.48 & 3.278 & 862.18 & 860.2 & 0.2732 \\ 23.95 & 3.450 & 874.58 & -76.00 & 0.0079 & 132.48 & 3.278 & 862.25 & 860.2 & 0.2732 \\ 23.95 & 3.450 & 874.65 & -76.00 & 0.0079 & 132.49 & 3.088 & 848.21 & 613.4 & 0.4032 \\ 230.73 & 4.850 & 465.85 & -19.00 & 0.0000 & 662.53 & 4.103 & 444.08 & 2606.7 & 0.4946 \\ 233.37 & 4.850 & 466.45 & -18.93 & 0.0000 & 670.55 & 3.977 & 440.69 & 2481.9 & 0.5400 \\ 233.37 & 4.850 & 466.45 & -18.93 & 0.0000 & 670.55 & 3.977 & 440.69 & 2481.9 & 0.5400 \\ 233.37 & 4.850 & 466.45 & -18.93 & 0.0000 & 670.55 & 3.088 & 409.92 & 2006.9 & 0.8293\end{array}$

gamt
1.40090
1.40090
1.40090
1.40090
1.40079
1.40025
1.40008
1.40008
1.38425
1.38425
1.33430
1.36889
1.38271
1.38270
1.38270
1.40079
1.40078
1.40078
1.40078

WAR
0.0000272
0.0000272
0.0000272
0.0000272
0.0000272
0.0000272
0.0011483
0.0011483
0.0011483
0.0011483
0.0011483
0.0011483
0.0011483
0.0011483
0.0011483
0.0000272
0.0000399
0.0000399
0.0000399

Wair
257.10

WH2O H2O frac $\begin{array}{lll}257.10 & 0.007 & 0.0000\end{array}$ $\begin{array}{lll}230.72 & 0.006 & 0.0000\end{array}$ $\begin{array}{rrr}26.38 & 0.001 & 0.0000\end{array}$ $\begin{array}{lll}230.72 & 0.001 & 0.0000 \\ 26.38 & 0.001 & 0.0000\end{array}$ $\begin{array}{lll}26.38 & 0.030-0.0011\end{array}$ $\begin{array}{lll}23.74 & 0.027 & 0.0011\end{array}$ $\begin{array}{lll}22.59 & 0.026 & 0.0011\end{array}$ $\begin{array}{lll}17.51 & 0.020 & 0.0011\end{array}$ $\begin{array}{lll}17.51 & 0.020 & 0.0141\end{array}$ $\begin{array}{lll}23.46 & 0.027 & 0.0109\end{array}$ $\begin{array}{lll}23.74 & 0.027 & 0.0108\end{array}$ $\begin{array}{lll}23.74 & 0.027 & 0.0108\end{array}$ $\begin{array}{lll}23.74 & 0.027 & 0.0108\end{array}$ $\begin{array}{lll}230.72 & 0.006 & 0.0000\end{array}$ $\begin{array}{lll}233.36 & 0.009 & 0.0000\end{array}$ $0.009 \quad 0.0000$ $0.009 \quad 0.0000$ 


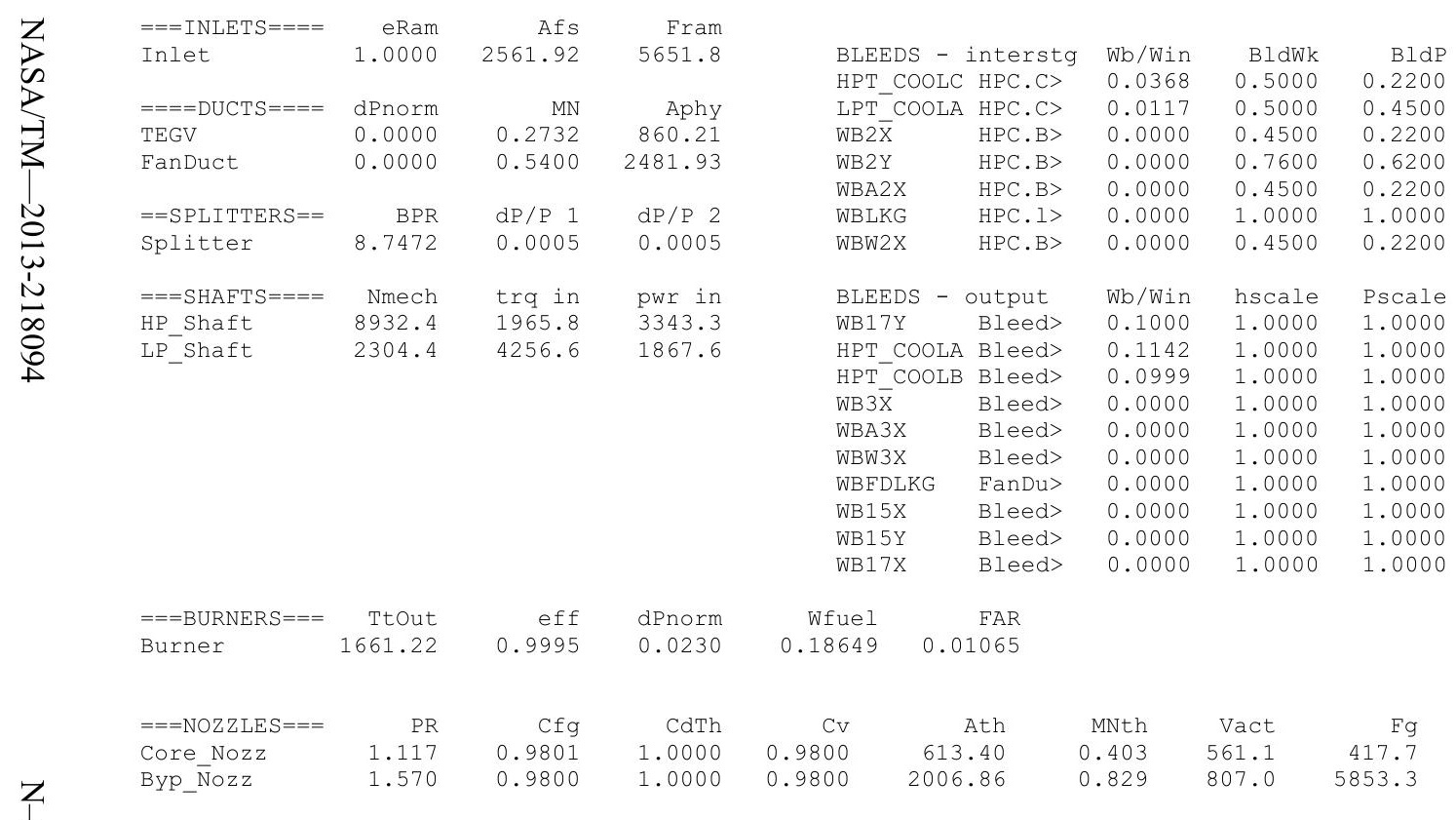


Date:05/13/13 Time:07:07:18 Model: Turbofan Engine - COMDES ON Converge = 1 CASE: 0

Version:NPSS 1.6.5 - Rev: -> Gas Package: Janaf iter/pass/Jacb/Broy=10/24/1/8 Run by: Philip C Jorgenson PC: 10

Temperature Stator 1 inlet: $456.45 \quad$ Stator 1 exit: $462.82 \quad$ Stator 2 inlet: $470.81 \quad$ Stator 2 exit: 474.87

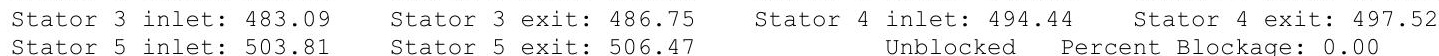

$\begin{array}{lclll}\text { Ambient Relative Humidity } & 10.00 & & & \\ \text { Fan Face Relative Humidity } & 3.00 & & & \\ \text { Fan Bypass Relative Humidity } & 2.26 & & & \\ \text { LPC Inlet Relative Humidity } & 2.00 & & & \\ \text { LPC Exit Relative Humidity } & 0.22 & & & \\ \text { HPC Relative Humidity } & 0.01 & & & \\ \text { Drop Diameter } & 0.0000100 & \text { Inlet Length } & \\ \text { Ambient Flow Velocity } & 667.90 & \text { Fan/LPC Inlet Flow Velocity } & 172.72 \\ \text { Ambient Static Pressure } & 3.58 & \text { Fan/LPC Inlet Static Pressure } & 4.74 \\ \text { Ambient Static Temperature } & 414.42 & \text { Fan/LPC Inlet Static Temperature } & 449.12 \\ \text { Additional Water at LPC Exit } & 0.0010079 & & & \end{array}$

\section{$\begin{array}{llll}\text { MN alt dTamb } & \text { W } & \text { SUMMAR OUTPUT DATA } \\ \text { TSFC }\end{array}$}

$\begin{array}{rrrr}0.669 & 34281.0 & \text { dTamb } & \text { W } \\ & & 18.00 & 278.63\end{array}$ $\begin{array}{rr}\text { Fn } & \text { TSFC } \\ 674.3 & 1.0687\end{array}$

Wfuel
720.58

$\mathrm{BPR}$
8.8272

FLOW STATION DATA

\begin{tabular}{|c|c|}
\hline FSO & Ambient.Fl_o \\
\hline FS1 & Inlet.Fl_o \\
\hline FS12 & Splitter.Fl_02 \\
\hline FS2 & Splitter.FI_01 \\
\hline FS14 & Fan.Fl_O \\
\hline FS23 & LPC.FI_O \\
\hline FS24 & 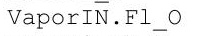 \\
\hline FS25 & Bleed2.FI_O \\
\hline FS3 & HPC.Fl_O ${ }^{-}$ \\
\hline FS36 & Bleed3.Fl O \\
\hline FS4 & Burner.Fl_o \\
\hline FS45 & HPT.FI_O \\
\hline FS49 & LPT.FI_O \\
\hline FS5 & TEGV.F̄'_O \\
\hline FS8 & Core_Nozzz. \\
\hline FS17 & FanDüctLkg.Fi \\
\hline FS171 & Bleed15.Fl \\
\hline FS172 & FanDuct.Fl-o \\
\hline
\end{tabular}

$W$
278.63
278.63
250.28
28.35
250.28
28.35
28.38
25.54
24.30
18.84
19.04
25.44
25.74
25.74
25.74
250.28
253.12
253.12
253.12

TURBOMACHINERY PERFORMANCE DATA

\begin{tabular}{|c|c|c|c|c|c|c|c|c|c|}
\hline & Wc & PR & eff & $\mathrm{NC}$ & $T R$ & efpoly & pwr & SMN & SMW \\
\hline Fan & 710.78 & 1.110 & 0.9033 & 2398.017 & 1.0337 & 0.9047 & -1290.9 & 5592.06 & 54.18 \\
\hline LPC & 80.52 & 1.476 & 0.8406 & 2398.017 & 1.1402 & 0.8491 & -608.6 & 6.41 & 3.05 \\
\hline HPC & 52.55 & 5.938 & 0.8161 & 8910.844 & 1.8034 & 0.8549 & -3550.9 & 61.40 & 57.91 \\
\hline HPT & 12.08 & 4.055 & 0.8718 & 218.602 & 1.3584 & 0.8493 & 3550.9 & & \\
\hline LPT & 53.29 & 2.586 & 0.8389 & 67.618 & 1.2372 & 0.8199 & 1899.5 & & \\
\hline TURI & NERY MAP & DATA & & & & & & & \\
\hline & WcMap & PRmap & effMap & NcMap & R/Parm & s WCDes & s PRdes & s effDes & s NCDes \\
\hline Fan & 825.85 & 1.108 & 0.9051 & 2375.152 & 0.0413 & $\overline{0} .8607$ & 1.0235 & 0.9980 & 0.9905 \\
\hline LPC & 66.54 & 1.446 & 0.7719 & 0.633 & 0.0000 & 1.2101 & 1.0675 & 1.0890 & 0.0003 \\
\hline HPC & 47.79 & 5.705 & 0.8245 & 8673.287 & 10.9923 & 1.0995 & 1.0494 & 0.9898 & 0.9733 \\
\hline HPT & 0.96 & 3.971 & 0.8718 & 1.314 & 3.9705 & 12.6299 & 0.9723 & 1.0000 & 0.0003 \\
\hline LPT & 0.82 & 2.193 & 0.8452 & 0.761 & 2.1932 & 65.2590 & 0.7521 & 0.9926 & 0.0005 \\
\hline
\end{tabular}

$\begin{array}{llccc}\text { EPR } & \text { PowerSet } & \text { T4 } & \text { T41 } & \text { T49 } \\ 0.795 & 10.000 & 1654.1 & 1562.9 & 1091.0\end{array}$

\begin{tabular}{rrrrrrrrrr}
\multicolumn{1}{c}{ FLOW } & STATION DATA \\
Pt & Tt & ht & FAR & Wc & Ps & Ts & Aphy & MN & gamt \\
4.831 & 451.61 & -22.45 & 0.0000 & 790.90 & 3.578 & 414.42 & 2577.9 & 0.6690 & 1.40088 \\
4.831 & 451.61 & -22.45 & 0.0000 & 790.90 & 4.453 & 441.20 & 4168.2 & 0.3430 & 1.40088 \\
4.829 & 451.61 & -22.45 & 0.0000 & 710.78 & 4.398 & 439.70 & 3531.8 & 0.3676 & 1.40088 \\
4.829 & 451.61 & -22.45 & 0.0000 & 80.52 & 4.736 & 449.12 & 830.5 & 0.1662 & 1.40088 \\
5.362 & 466.82 & -18.81 & 0.0000 & 650.77 & 4.572 & 446.01 & 2606.7 & 0.4825 & 1.40078 \\
7.127 & 514.90 & -7.28 & 0.0000 & 58.25 & 6.832 & 508.70 & 412.6 & 0.2469 & 1.40028 \\
7.127 & 516.34 & -12.75 & 0.0000 & 58.39 & 6.830 & 510.08 & 412.6 & 0.2476 & 1.40012 \\
7.127 & 516.34 & -12.75 & 0.0000 & 52.55 & 6.889 & 511.33 & 412.6 & 0.2212 & 1.40012 \\
42.320 & 931.18 & 87.94 & 0.0000 & 11.31 & 37.308 & 899.09 & 49.7 & 0.4301 & 1.38467 \\
42.320 & 931.18 & 87.94 & 0.0000 & 8.76 & 39.440 & 913.11 & 49.3 & 0.3205 & 1.38467 \\
41.345 & 1654.14 & 75.96 & 0.0106 & 12.08 & 39.040 & 1630.46 & 74.6 & 0.2941 & 1.33467 \\
10.196 & 1095.08 & -21.51 & 0.0079 & 53.29 & 9.289 & 1067.87 & 265.4 & 0.3710 & 1.36909 \\
3.942 & 883.50 & -72.98 & 0.0078 & 125.26 & 3.767 & 872.47 & 860.2 & 0.2570 & 1.38222 \\
3.942 & 883.57 & -72.98 & 0.0078 & 125.27 & 3.767 & 872.54 & 860.2 & 0.2570 & 1.38222 \\
3.942 & 883.64 & -72.98 & 0.0078 & 125.27 & 3.578 & 860.25 & 613.4 & 0.3768 & 1.38222 \\
5.362 & 466.82 & -18.81 & 0.0000 & 650.77 & 4.572 & 446.01 & 2606.7 & 0.4825 & 1.40078 \\
5.362 & 467.38 & -18.74 & 0.0000 & 658.54 & 4.441 & 442.84 & 2481.9 & 0.5258 & 1.40077 \\
5.362 & 467.38 & -18.74 & 0.0000 & 658.54 & 4.441 & 442.84 & 2481.9 & 0.5258 & 1.40077 \\
5.362 & 467.38 & -18.74 & 0.0000 & 658.54 & 3.578 & 416.28 & 2006.9 & 0.7824 & 1.40077
\end{tabular}

WAR
0.0000348
0.0000348
0.0000348
0.0000348
0.0000348
0.0000348
0.0010427
0.0010427
0.0010427
0.0010427
0.0010427
0.0010427
0.0010427
0.0010427
0.0010427
0.0000348
0.0000461
0.0000461
0.0000461

Wair

WH2O H2O frac $278.62 \quad 0.010 \quad 0.0000$ 25.27 $0.009=0.0000$ $\begin{array}{lll}28.35 & 0.001 & 0.0000\end{array}$ $28.35-0.001 \quad 0.0000$ $\begin{array}{lll}28.35 & 0.001 & 0.0000\end{array}$ $\begin{array}{lll}28.35 & 0.030 & 0.0010 \\ 25.52 & 0.027 & 0.0010\end{array}$ $24.28 \quad 0.025-0.0010$ $\begin{array}{lll}24.28 & 0.025 & 0.0010 \\ 18.82 & 0.020 & 0.0010\end{array}$ $\begin{array}{lll}18.82 & 0.020 & 0.0140\end{array}$ $\begin{array}{lll}18.82 & 0.020 & 0.0140\end{array}$ $\begin{array}{lll}25.52 & 0.027 & 0.0106\end{array}$ $\begin{array}{lll}25.52 & 0.027 & 0.0106\end{array}$ $\begin{array}{lll}25.52 & 0.027 & 0.0106\end{array}$ $\begin{array}{rrr}250.27 & 0.009 & 0.0000\end{array}$ $\begin{array}{lll}253.11 & 0.012 & 0.0000\end{array}$ $\begin{array}{lll}253.11 & 0.012 & 0.0000\end{array}$ 0.0000 
$\begin{array}{lrr}===\text { INLETS }==== & \text { eRam } & \text { Afs } \\ \text { Inlet } & \text { Fram }\end{array}$

$\begin{array}{ll}====\text { DUCTS }==== & \text { dPnorm } \\ \text { TEGV } & 0.0000\end{array}$

FanDuct $\quad 0.0000$

$==$ SPLITTERS $==$

Splitter

BPR

8.8272

$===$ SHAFTS $===$

HP_Shaft

Nmech
8890.8

LP_Shaft

2237.6

4458.5

1899.5

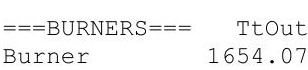

eff
0.9995

dPnorm

$\mathrm{PR}$
1.102
1.499

$\mathrm{Cfg}$
0.9801

0.9800
BLEEDS - interstg Wb/Win BldWk 0.2200 LPT_COOLA HPC.C> $0.0117 \quad 0.5000 \quad 0.4500$ $\begin{array}{lllll}\text { WB2 } \bar{X} & \text { HPC.B> } & 0.0000 & 0.4500 & 0.2200 \\ \text { WB2Y } & \text { HPC. B> } & 0.0000 & 0.7600 & 0.6200\end{array}$ $\begin{array}{lllll}\text { WB2Y } & \text { HPC.B> } & 0.0000 & 0.7500 & 0.6200 \\ \text { WBA2X } & \text { HPC.B> } & 0.0000 & 0.4500 & 0.2200\end{array}$ $\begin{array}{lllll}\text { WBA2X } & \text { HPC.B } & 0.0000 & 0.4500 & 0.2200 \\ \text { WBLKG } & \text { HPC. }> & 0.0000 & 1.0000 & 1.0000\end{array}$ $\begin{array}{lllll}\text { WBW2X HPC.B> } & 0.0000 & 0.4500 & 0.2200\end{array}$

BLEEDS - output Wb/Win hscale Pscale WB17Y Bleed> $0.1000 \quad 1.0000 \quad 1.0000$ HPT_COOLA Bleed> $0.1142 \quad 1.0000 \quad 1.0000$ HPT_COOLB Bleed> $0.0999 \quad 1.0000 \quad 1.0000$ WB3X Bleed> $0.0000 \quad 1.0000 \quad 1.0000$ WBA3X Bleed> $0.00001 .0000-1.0000$ WBW3X Bleed> $0.0000 \quad 1.0000 \quad 1.0000$ WB15Y Bleed> $0.0000 \quad 1.0000 \quad 1.0000$ $0.0000 \quad 1.0000 \quad 1.0000$

$\begin{array}{rrrr}\text { W } & \text { Tt } & \text { ht } & \text { Pt } \\ 0.9400 & 725.24 & 37.60 & 10.196 \\ 0.2989 & 725.24 & 37.60 & 3.942 \\ 0.0000 & 704.45 & 32.56 & 14.870 \\ 0.0000 & 832.81 & 63.78 & 28.947 \\ 0.0000 & 704.45 & 32.56 & 14.870 \\ 0.0000 & 931.18 & 87.94 & 42.320 \\ 0.0000 & 704.45 & 32.56 & 14.870\end{array}$

$\begin{array}{rrrr}\text { W } & \text { Tt } & \text { ht } & \text { Pt } \\ 2.8382 & 516.34 & -12.75 & 7.127 \\ 2.9166 & 931.18 & 87.94 & 4.345\end{array}$ $\begin{array}{llll}2.9166 & 931.18 & 87.94 & 41.345\end{array}$ $\begin{array}{llll}2.5521 & 931.18 & 87.94 & 23.528\end{array}$ $\begin{array}{llll}0.0000 & 931.18 & 87.94 & 42.320\end{array}$ $\begin{array}{llll}0.0000 & 931.18 & 87.94 & 42.320\end{array}$

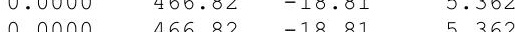
$\begin{array}{llll}0.0000 & 466.82 & -18.81 & 5.362 \\ 0.0000 & 466.82 & -18.81 & 5.362\end{array}$ $\begin{array}{llll}0.0000 & 466.82 & -18.81 & 5.362\end{array}$

$t$
96
92
70
47
70
20
70
$7 t$
pt
27
345
58
20
20
20
62
362
62
62
WBFDLKG FanDu> $0.0000-1.0000-1.0000$

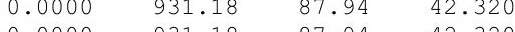

FAR

$\begin{array}{rrrrr}\text { CV } & \text { Ath } & \text { MNth } & \text { Vact } & \text { Fg } \\ 0.9800 & 613.40 & 0.377 & 527.9 & 422.4 \\ 0.9800 & 2006.86 & 0.782 & 767.2 & 6036.0\end{array}$


Date:05/13/13 Time:07:07:44 Model: Turbofan Engine - COMDES ON Converge = 1 CASE: 0

Version:NPSS 1.6.5 - Rev: -> Gas Package: Janaf iter/pass/Jacb/Broy= 15/43/2/12 Run by: Philip C Jorgenson PC: 10

Temperature Stator 1 inlet: $465.08 \quad$ Stator 1 exit: $470.61 \quad$ Stator 2 inlet: $477.45 \quad$ Stator 2 exit: 480.75 Stator 5 inlet: 505.31 Stator 5 exit: 507.35 Unblocked Percent Blockage: 0.00

Ambient Relative Humidity $\quad 10.00$

Fan Face Relative Humidity 3.96

Fan Bypass Relative Humidity $\quad 3.11$

LPC Inlet Relative Humidity

LPC Exit Relative Humidity

Drop Diatve Humidity

Drop Diameter

Ambient Static Pressure

Additional Water at LPC Exit

Inlet Length 40.00

$\begin{array}{rrr}617.99 & \text { Fan/LPC Inlet Flow Velocity } & 174.69 \\ 4.36 & \text { Fan/LPC Inlet Static Pressure } & 5.49\end{array}$

$\begin{array}{rrr}4.36 & \text { Fan/LPC Inlet Static Pressure r } & 5.49 \\ 429.58 & \text { Fan/LPC Inlet Static Temperature } & 458.87\end{array}$

0.0010284

SUMMARY OUTPUT DATA

$\begin{array}{rrr}\text { MN } & \text { alt } & \text { dTamb } \\ 0.608 & 30029.0 & 18.0\end{array}$

305.17

1.0714

Wfuel
786.52

BPR
8.3887

VTAS
617.99

OPR
8.201

EPR
0.828

PowerSet
10.000

$\begin{array}{ccc}\text { T4 } & \text { T41 } & \text { T49 } \\ 1658.5 & 1566.7 & 1096.5\end{array}$
FSO Ambient.Fl O

FS1 Inlet.FI_O

FS12 Splitter.F1_02

FS14 Fan.Fl_O

S23 LPC.F1-

SS24 Vaporin. FI-

(ES25

FS36 Bleed3 Fi

FS4 Burner.Fl_O

FS5 5 TEGV.FI

FS8 Core Nozz.FI

SS17 FanDüctikg. $\overline{1}$

FS171 Bleed15.FIO

FS172 FanDuct.Fl-O

FS173 Byp Nozz.F̄̄o

TURBOMACHINERY PERFORMANCE DATA

$\begin{array}{lrrr} & \text { WC } & \text { PR } & \text { eff } \\ \text { Fan } & 675.82 & 1.098 & 0.9022 \\ \text { LPC } & 80.56 & 1.401 & 0.8545 \\ \text { HPC } & 51.83 & 5.858 & 0.8146 \\ \text { HPT } & 12.08 & 4.000 & 0.8720 \\ \text { LPT } & 52.63 & 2.376 & 0.8307\end{array}$

FLOW STATION DATA

\begin{tabular}{rrrrrr} 
W & \multicolumn{1}{c}{ Pt } & Tt & ht & FAR & Wc \\
305.17 & 5.595 & 461.42 & -20.30 & 0.0000 & 756.00 \\
305.17 & 5.595 & 461.42 & -20.30 & 0.0000 & 756.00 \\
272.66 & 5.592 & 461.42 & -20.30 & 0.0000 & 675.82 \\
32.50 & 5.592 & 461.42 & -20.30 & 0.0000 & 80.56 \\
272.66 & 6.141 & 475.29 & -16.97 & 0.0000 & 624.63 \\
32.50 & 7.833 & 516.04 & -7.20 & 0.0000 & 60.83 \\
32.54 & 7.833 & 517.50 & -12.78 & 0.0000 & 60.98 \\
27.66 & 7.833 & 517.50 & -12.78 & 0.0000 & 51.83 \\
26.32 & 45.884 & 929.98 & 87.34 & 0.0000 & 11.29 \\
20.39 & 45.884 & 929.98 & 87.34 & 0.0000 & 8.75 \\
20.61 & 44.827 & 1658.51 & 75.27 & 0.0107 & 12.08 \\
27.55 & 11.207 & 1100.65 & -21.61 & 0.0080 & 52.63 \\
27.87 & 4.717 & 906.68 & -68.72 & 0.0079 & 114.81 \\
27.87 & 4.717 & 906.75 & -68.72 & 0.0079 & 114.82 \\
27.87 & 4.717 & 906.82 & -68.72 & 0.0079 & 114.82 \\
272.66 & 6.141 & 475.29 & -16.97 & 0.0000 & 624.63 \\
277.54 & 6.141 & 476.04 & -16.90 & 0.0000 & 636.31 \\
277.54 & 6.141 & 476.04 & -16.90 & 0.0000 & 636.31 \\
277.54 & 6.141 & 476.04 & -16.90 & 0.0000 & 636.31
\end{tabular}

Ps
4.358
5.199
5.147
5.485
5.322
7.477
7.475
7.578
40.476
42.775
42.328
10.23
4.543
4.543
4.35
5.32
5.17
5.17

Ts
429.58
451.81
450.58
458.87
456.22
509.22
510.62
512.63
898.09
912.02
1634.78
1074.10
897.30
897.37
887.22
456.22
453.27
453.27
431.55

$\begin{array}{rr}\text { Aphy } & \text { MN } \\ 2596.8 & 0.6080\end{array}$ $\begin{array}{ll}2596.8 & 0.6080 \quad 1.40081\end{array}$ $\begin{array}{lll}4531.8 & 0.3257-1.40081\end{array}$ $\begin{array}{rr}3531.8 & 0.3464 \\ 830.5 & 0.1663\end{array}$ $\begin{array}{llll}412.6 & 0.2587\end{array}$ $412.6 \quad 0.2595$ $412.6 \quad 0.25180$ $\begin{array}{rrr}49.7 & 0.4290\end{array}$ $\begin{array}{ll}49.3 & 0.3197\end{array}$ $\begin{array}{ll}74.6 & 0.2942\end{array}$ $\begin{array}{lll}265.4 & 0.3656 \\ 860.2 & 0.2342\end{array}$ $\begin{array}{lll}860.2 & 0.2342\end{array}$ $\begin{array}{lll}660.2 & 0.2342\end{array}$ $\begin{array}{ll}2606.7 & 0.4567\end{array}$ 2481.90 .5006 $2481.9 \quad 0.5006$ $2006.9 \quad 0.7172$

$\begin{array}{rrrr}\text { WAR } & \text { Wair } & \text { WH2O } & \text { H2O frac } \\ 0.0000681 & 305.15 & 0.021 & 0.0001 \\ 0.0000681 & 305.15 & 0.021 & 0.0001 \\ 0.0000681 & 272.64 & 0.019 & 0.0001 \\ 0.0000681 & 32.50 & 0.002 & 0.0001 \\ 0.0000681 & 272.64 & 0.019 & 0.0001 \\ 0.0000681 & 32.50 & 0.002 & 0.0001 \\ 0.0010965 & 32.50 & 0.036 & 0.0011 \\ 0.0010965 & 27.63 & 0.030 & 0.0011 \\ 0.0010965 & 26.29 & 0.029 & 0.0011 \\ 0.0010965 & 20.37 & 0.022 & 0.0011 \\ 0.0010965 & 20.37 & 0.022 & 0.0142 \\ 0.0010965 & 27.30 & 0.030 & 0.0109 \\ 0.0010965 & 27.63 & 0.030 & 0.0108 \\ 0.0010965 & 27.63 & 0.030 & 0.0108 \\ 0.0010965 & 27.63 & 0.030 & 0.0108 \\ 0.0000681 & 272.64 & 0.019 & 0.0001 \\ 0.0000862 & 277.52 & 0.024 & 0.0001 \\ 0.0000862 & 277.52 & 0.024 & 0.0001 \\ 0.0000862 & 277.52 & 0.024 & 0.0001\end{array}$

TURBOMACHINERY MAP DATA

$\begin{array}{lrrrrrrrrr} & \text { WcMap } & \text { PRmap } & \text { effMap } & \text { NcMap } & \text { R/Parm } & \text { S_WcDes } & \text { S_PRdes } & \text { S_effDes } & \text { S_NcDes } \\ \text { Fan } & 785.23 & 1.096 & 0.9041 & 2230.293 & 0.0416 & 0.8607 & \overline{1} .0235 & 0.9980 & 0.9905 \\ \text { LPC } & 60.40 & 1.383 & 0.7655 & 0.595 & 0.0000 & 1.3338 & 1.0471 & 1.1162 & 0.0003 \\ \text { HPC } & 47.14 & 5.629 & 0.8231 & 8650.338 & 10.9851 & 1.0995 & 1.0494 & 0.9898 & 0.9733 \\ \text { HPT } & 0.96 & 3.917 & 0.8720 & 1.310 & 3.9168 & 12.6299 & 0.9723 & 1.0000 & 0.0003 \\ \text { LPT } & 0.81 & 2.035 & 0.8369 & 0.720 & 2.0347 & 65.2590 & 0.7521 & 0.9926 & 0.0005\end{array}$




\begin{tabular}{|c|c|c|c|c|c|c|c|c|c|c|c|c|}
\hline \multirow{2}{*}{$\begin{array}{ll}Z & ===\text { INLETS }==== \\
& \text { Inlet }\end{array}$} & eRam & Afs & Fram & & \multirow[b]{2}{*}{$\begin{array}{l}\text { Wb/Win } \\
0.0368\end{array}$} & \multirow[b]{2}{*}{$\begin{array}{r}\text { BldWk } \\
0.5000\end{array}$} & \multirow[b]{2}{*}{ BldP } & \multirow[b]{2}{*}{$\begin{array}{r}W \\
1.0178\end{array}$} & & & \multirow[b]{2}{*}{$\begin{array}{r}P t \\
11.207\end{array}$} \\
\hline & 1.0000 & 2596.78 & 5861.6 & $\begin{array}{l}\text { BLEEDS - } \\
\text { HPT COOLC }\end{array}$ & $\begin{array}{l}\text { interstg } \\
\text { HPC.C> }\end{array}$ & & & & & & & \\
\hline$====$ DUCTS $====$ & dPnorm & MN & Aphy & LPT_COOLA & A HPC.C> & 0.0117 & 0.5000 & 0.4500 & 0.3236 & 725.21 & 37.28 & 4.717 \\
\hline TEGV & 0.0000 & 0.2342 & 860.21 & WB2 $\bar{x}$ & HPC.B> & 0.0000 & 0.4500 & 0.2200 & 0.0000 & 704.54 & 32.27 & 16.204 \\
\hline FanDuct & 0.0000 & 0.5006 & 2481.93 & WB2Y & HPC. B> & 0.0000 & 0.7600 & 0.6200 & 0.0000 & 832.17 & 63.31 & 31.424 \\
\hline & & & & WBA2X & HPC.B> & 0.0000 & 0.4500 & 0.2200 & 0.0000 & 704.54 & 32.27 & 16.204 \\
\hline$==$ SPLITTERS $==$ & BPR & $\mathrm{dP} / \mathrm{P} 1$ & $\mathrm{dP} / \mathrm{P} 2$ & WBLKG & HPC.1> & 0.0000 & 1.0000 & 1.0000 & 0.0000 & 929.98 & 87.34 & 45.884 \\
\hline Splitter & 8.3887 & 0.0005 & 0.0005 & WBW2X & HPC. B> & 0.0000 & 0.4500 & 0.2200 & 0.0000 & 704.54 & 32.27 & 16.204 \\
\hline$===$ SHAFT $S====$ & Nmech & trq in & pwr in & BLEEDS - & output & Wb/Win & hscale & Pscale & W & Tt & ht & Pt \\
\hline HP_Shaft & 8877.3 & 2261.7 & 3822.9 & WB17Y & Bleed> & 0.1500 & 1.0000 & 1.0000 & 4.8805 & 517.50 & -12.78 & 7.833 \\
\hline LP_Shaft & 2123.9 & 4661.9 & 1885.2 & HPT_COOLA & A Bleed> & 0.1142 & 1.0000 & 1.0000 & 3.1578 & 929.98 & 87.34 & 44.827 \\
\hline & & & & $\mathrm{HPT}_{-}^{-} \mathrm{COOLB}$ & 3 Bleed> & 0.0999 & 1.0000 & 1.0000 & 2.7632 & 929.98 & 87.34 & 25.596 \\
\hline & & & & WB3 $\bar{x}$ & Bleed> & 0.0000 & 1.0000 & 1.0000 & 0.0000 & 929.98 & 87.34 & 45.884 \\
\hline & & & & WBA3X & Bleed> & 0.0000 & 1.0000 & 1.0000 & 0.0000 & 929.98 & 87.34 & 45.884 \\
\hline & & & & WBW3X & Bleed> & 0.0000 & 1.0000 & 1.0000 & 0.0000 & 929.98 & 87.34 & 45.884 \\
\hline & & & & WBFDLKG & FanDu> & 0.0000 & 1.0000 & 1.0000 & 0.0000 & 475.29 & -16.97 & 6.141 \\
\hline & & & & WB15X & Bleed> & 0.0000 & 1.0000 & 1.0000 & 0.0000 & 475.29 & -16.97 & 6.141 \\
\hline & & & & WB15Y & Bleed> & 0.0000 & 1.0000 & 1.0000 & 0.0000 & 475.29 & -16.97 & 6.141 \\
\hline & & & & WB17X & Bleed> & 0.0000 & 1.0000 & 1.0000 & 0.0000 & 475.29 & -16.97 & 6.141 \\
\hline$===$ BURNERS $===$ & Ttout & eff & dPnorm & Wfuel & FAR & & & & & & & \\
\hline Burner & 1658.44 & 0.9995 & 0.0230 & .21848 & 01072 & & & & & & & \\
\hline$===$ NOZZLES $===$ & PR & $\mathrm{Cfg}$ & CdTh & $\mathrm{Cv}$ & Ath & MNth & Vact & $\mathrm{Fg}$ & & & & \\
\hline Core_Nozz & 1.082 & 0.9801 & 1.0000 & 0.9800 & 3.40 & 0.340 & 484.0 & 419.3 & & & & \\
\hline Byp_Nozz & 1.409 & 0.9800 & 1.0000 & 0.9800 & 6.86 & 0.717 & 716.0 & 6176.4 & & & & \\
\hline
\end{tabular}


Date:05/13/13 Time:07:08:15 Model: Turbofan Engine - COMDES ON COnverge = 1 CASE: 0

Version:NPSS 1.6.5 - Rev: -> Gas Package: Janaf iter/pass/Jacb/Broy= 23/51/2/20 Run by: Philip C Jorgenson PC: 10

Temperature Stator 1 inlet: $473.14 \quad$ Stator 1 exit: $477.22 \quad$ Stator 2 inlet: $481.84 \quad$ Stator 2 exit: 483.66 Stator 5 inlet: $499.77 \quad$ Stator 5 exit: $500.50 \quad$ Stator 4 inlet: 495.16 Stator 4 exit: 496.17

Ambient Relative Humidity $\quad 10.00$

Fan Face Relative Humidity $\quad 4.82$

Fan Bypass Relative Humidity $\quad 3.94$

LPC Inlet Relative Humidity $\quad 3.82$

LPC Exit Relative Humidity

Dre Relative Humidity

0.02

0.0000100

Inlet Length

40.00

Ambient Flow Velocity

Ambient Static Pressure

Additional Water at LPC Exit

5.30
5.11

$\begin{array}{rr}\text { Fan/LPC Inlet Static Pressure } & 6.36 \\ \text { Fan/LPC Inlet Static Temperature } & 469.18\end{array}$ 0.0009281

$\begin{array}{rrrrrr} & & & & \text { SUMMARY } & \text { OUTPUT DATA } \\ \text { MN } & \text { alt } & \text { dTamb } & \text { W } & \text { Fn } & \text { TSFC } \\ 0.552 & 25666.0 & 18.00 & 330.61 & 753.5 & 1.1140\end{array}$

330.61

TSFC
1.1140
Wfuel
839.45
BPR
7.0610
VTAS
571.13
OPR
7.339

7.339 $\begin{array}{cl}\text { EPR } & \text { PowerSet } \\ 0.859 & 10.000\end{array}$
FSO Ambient.Fl_O

FS1 Inlet.F1_O

FS12 Splitter.F1_02

FS2 Splitter.F1_01

FS24 VaporIN.F1_O

FS25 Bleed2.F1_o

HPC.Fl_

FS4 Bleed3.Fl_O

Burner.FI

FS45 HPT.FI-

FS49 LPT.FI-

FS8 Core Nozz. El

FS17 Candütr.

FS171 Bleed15. F1

FS171 Bleed15.FI_-

FS173 Byp Nozz. Fi

TURBOMACHINERY PERFORMANCE DATA

$\begin{array}{lrrr} & \text { WC } & \text { PR } & \text { eff } \\ \text { Fan } & 623.58 & 1.084 & 0.9033 \\ \text { LPC } & 88.31 & 1.261 & 0.8225 \\ \text { HPC } & 51.13 & 5.822 & 0.8139 \\ \text { HPT } & 12.09 & 3.882 & 0.8734 \\ \text { LPT } & 51.18 & 2.139 & 0.8214\end{array}$

FLOW STATION DATA

\begin{tabular}{rrrrrr} 
W & \multicolumn{1}{c}{ Pt } & Tt & ht & FAR & Wc \\
330.61 & 6.516 & 472.33 & -18.03 & 0.0000 & 711.54 \\
330.61 & 6.516 & 472.33 & -18.03 & 0.0000 & 711.54 \\
289.60 & 6.513 & 472.33 & -18.03 & 0.0000 & 623.58 \\
41.01 & 6.513 & 472.33 & -18.03 & 0.0000 & 88.31 \\
289.60 & 7.058 & 484.50 & -15.11 & 0.0000 & 582.75 \\
41.01 & 8.214 & 511.73 & -8.58 & 0.0000 & 72.88 \\
41.05 & 8.214 & 513.06 & -13.61 & 0.0000 & 73.05 \\
28.74 & 8.214 & 513.06 & -13.61 & 0.0000 & 51.13 \\
27.34 & 47.823 & 920.81 & 85.31 & 0.0000 & 11.20 \\
21.19 & 47.823 & 920.81 & 85.31 & 0.0000 & 8.68 \\
21.42 & 46.722 & 1668.79 & 72.94 & 0.0110 & 12.09 \\
28.63 & 12.036 & 1111.68 & -22.65 & 0.0082 & 51.18 \\
28.97 & 5.627 & 938.91 & -64.51 & 0.0081 & 101.80 \\
28.97 & 5.627 & 938.98 & -64.51 & 0.0081 & 101.80 \\
28.97 & 5.627 & 939.05 & -64.51 & 0.0081 & 101.81 \\
289.60 & 7.058 & 484.50 & -15.11 & 0.0000 & 582.75 \\
301.92 & 7.058 & 485.66 & -15.05 & 0.0000 & 608.26 \\
301.92 & 7.058 & 485.66 & -15.05 & 0.0000 & 608.26 \\
301.92 & 7.058 & 485.66 & -15.05 & 0.0000 & 608.26
\end{tabular}

Ps
5.297
6.111
6.077
6.363
6.259
7.665
7.662
7.954
42.292
44.640
44.115
11.057
5.465
5.465
5.297
6.259
6.064
6.064
5.297

Ts
445.14
463.73
463.07
469.18
468.12
501.70
502.95
508.36
889.82
903.32
1644.95
1086.57
931.39
931.47
923.58
468.12
465.02
465.02
447.36

$\begin{array}{rr}\text { Aphy } & \text { MN } \\ 2595.6 & 0.5520 \\ 4168.2 & 0.3041 \\ 3531.8 & 0.3159 \\ 830.5 & 0.1829 \\ 2606.7 & 0.4178 \\ 412.6 & 0.3161 \\ 412.6 & 0.3170 \\ 412.6 & 0.2149 \\ 49.7 & 0.4246 \\ 49.3 & 0.3168 \\ 74.6 & 0.2943 \\ 265.4 & 0.3540 \\ 860.2 & 0.2063 \\ 860.2 & 0.2063 \\ 613.4 & 0.2971 \\ 2606.7 & 0.4178 \\ 2481.9 & 0.4707 \\ 2481.9 & 0.4707 \\ 2006.9 & 0.6536\end{array}$

gamt
1.40072
1.40072
1.40072
1.40072
1.40061
1.40031
1.40016
1.40016
1.38527
1.38527
1.33370
1.36781
1.37879
1.37879
1.37878
1.40061
1.40060
1.40060
1.40060$$
0.0
$$

$\begin{array}{rrrr}\text { WAR } & \text { Wair } & \text { WH2O } & \text { H2O frac } \\ 0.0001274 & 330.57 & 0.042 & 0.0001 \\ 0.0001274 & 330.57 & 0.042 & 0.0001 \\ 0.0001274 & 289.56 & 0.037 & 0.0001 \\ 0.0001274 & 41.01 & 0.005 & 0.0001 \\ 0.0001274 & 289.56 & 0.037 & 0.0001 \\ 0.0001274 & 41.01 & 0.005 & 0.0001 \\ 0.0010555 & 41.01 & 0.043 & 0.0011 \\ 0.0010555 & 28.71 & 0.030 & 0.0011 \\ 0.0010555 & 27.31 & 0.029 & 0.0011 \\ 0.0010555 & 21.17 & 0.022 & 0.011 \\ 0.0010555 & 21.17 & 0.022 & 0.0145 \\ 0.0010555 & 28.37 & 0.030 & 0.0111 \\ 0.0010555 & 28.71 & 0.030 & 0.0110 \\ 0.0010555 & 28.71 & 0.030 & 0.0110 \\ 0.0010555 & 28.71 & 0.030 & 0.0110 \\ 0.0001274 & 289.56 & 0.037 & 0.0001 \\ 0.0001652 & 301.87 & 0.050 & 0.0002 \\ 0.0001652 & 301.87 & 0.050 & 0.0002 \\ 0.0001652 & 301.87 & 0.050 & 0.0002\end{array}$

TURBOMACHINERY MAP DATA

\begin{tabular}{lrrrrrrrrr}
\multicolumn{1}{l}{ TURBOMACHINERY MAP } & DATA & & & & & & \\
WCMap & PRmap & effMap & NcMap & R/Parm & S_WcDes & S_PRdes & S_effDes & S_NcDes \\
Fan & 724.54 & 1.082 & 0.9051 & 2027.101 & 0.0425 & 0.8607 & 1.0235 & 0.9980 & 0.9905 \\
LPC & 53.54 & 1.312 & 0.7608 & 0.541 & 0.0000 & 1.6496 & 0.8378 & 1.0812 & 0.0003 \\
HPC & 46.50 & 5.595 & 0.8223 & 8629.182 & 11.0483 & 1.0995 & 1.0494 & 0.9898 & 0.9733 \\
HPT & 0.96 & 3.802 & 0.8734 & 1.297 & 3.8020 & 12.6299 & 0.9723 & 1.0000 & 0.0003 \\
LPT & 0.78 & 1.857 & 0.8275 & 0.659 & 1.8567 & 65.2590 & 0.7521 & 0.9926 & 0.0005
\end{tabular}




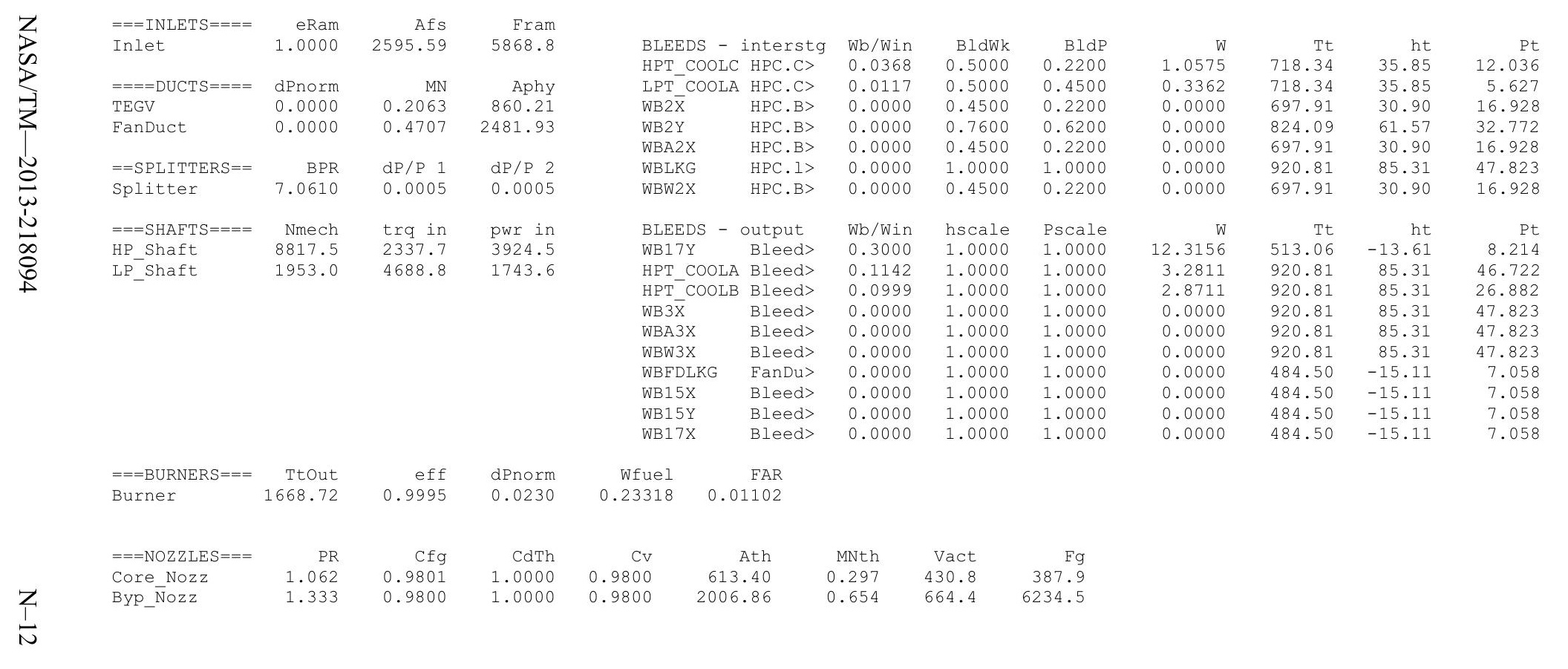


Date:05/13/13 Time:07:08:46 Model: Turbofan Engine - COMDES ON Converge = 1 CASE: 0

Version:NPSS 1.6.5 - Rev: -> Gas Package: Janaf iter/pass/Jacb/Broy= 26/54/2/23 Run by: Philip C Jorgenson PC: 10

Temperature Stator 1 inlet: $486.29 \quad$ Stator 1 exit: $489.25 \quad$ Stator 2 inlet: $492.01 \quad$ Stator 2 exit: 492.78

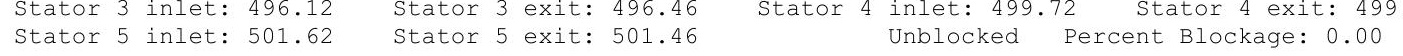

Ambient Relative Humidity $\quad 10.00$

Fan Face Relative Humidity $\quad 5.85$

Fan Bypass Relative Humidity $\quad 4.91$

LPC Inlet Relative Humidity $\quad 5.02$

PC Relative Humidity

Drop Diameter

0.02

mbient Flow Velocity

Ambient Static Pressure

Ambient Static Temperature
Additional Water at LPC Exit

0.0000100

518.26
6.74

Inlet Length

Fan/LPC Inlet Flow Velocity $\quad 204.13$

465.18

7.74

0.0009833

an/LPC Inlet Static Temperature 484.08

$\begin{array}{rrrrrr}\text { MN } & \text { alt } & \text { dTamb } & \text { W } & \text { SUMMARY } & \text { OUTPUT DATA } \\ 0.490 & 20047.0 & 18.00 & 368.24 & 837.7 & 1.1161\end{array}$

$\begin{array}{lll}0.490 .720047 .0 & 837.7 & 1.1161\end{array}$

FSO Ambient.Fl_O

FS1 Inlet.FI_O

FS12 Splitter.Fl_02

FS2 Splitter.Fl_01

FS14 Fan.FI_-

FS24 VaporIN.EI_O

FS25 Bleed2.F1_o

FS3 HPC.Fl 0

FS36 Bleed3.F1_O

FS4 Burner.FI_O

FS45 HPT.FI O

FS49 LPT.FI-O

FS8 COR.FIO

FS17 FanDuctIrgi_.

FS171 B1

FS171 Bleed15.FI_-

FS173 Byp Nozz.Fİ

W
368.24
368.24
317.42
50.82
317.42
50.82
50.87
30.52
29.04
22.51
22.77
30.42
30.78
30.78
30.78
317.42
337.77
337.77
337.77

$\begin{array}{cc}\text { FLOW } & \text { STATION DATA } \\ \text { Pt } & \text { Tt }\end{array}$

\begin{tabular}{rrrrr}
\multicolumn{1}{c}{ Pt } & Tt & ht & FAR & Wc \\
7.943 & 487.56 & -15.16 & 0.0000 & 660.53 \\
7.943 & 487.56 & -15.16 & 0.0000 & 660.53 \\
7.939 & 487.56 & -15.16 & 0.0000 & 569.66 \\
7.939 & 487.56 & -15.16 & 0.0000 & 91.21 \\
8.514 & 498.42 & -12.55 & 0.0000 & 537.10 \\
9.087 & 515.32 & -8.50 & 0.0000 & 81.92 \\
9.087 & 516.72 & -13.83 & 0.0000 & 82.11 \\
9.087 & 516.72 & -13.83 & 0.0000 & 49.27 \\
51.310 & 920.26 & 84.09 & 0.0000 & 11.08 \\
51.310 & 920.26 & 84.09 & 0.0000 & 8.59 \\
50.129 & 1701.92 & 71.14 & 0.0116 & 12.09 \\
13.561 & 1142.72 & -23.26 & 0.0086 & 48.94 \\
7.048 & 989.02 & -60.45 & 0.0085 & 88.63 \\
7.048 & 989.09 & -60.45 & 0.0085 & 88.63 \\
7.048 & 989.16 & -60.45 & 0.0085 & 88.64 \\
8.514 & 498.42 & -12.55 & 0.0000 & 537.10 \\
8.514 & 499.53 & -12.63 & 0.0000 & 572.16 \\
8.514 & 499.53 & -12.63 & 0.0000 & 572.16 \\
8.514 & 499.53 & -12.63 & 0.0000 & 572.16
\end{tabular}

Wfuel
934.98

BPR
6.2459

VTAS
518.26

OPR
6.460

EPR
0.890

Powerset
10.000

$\begin{array}{cc}T 4 & T 41 \\ 1701.9 & 1603 .\end{array}$

T49

TS
465.18
480.02
479.72
484.08
484.55
502.16
503.45
512.34
890.05
903.18
1677.69
1119.52
983.12
983.19
977.17
484.55
481.33
481.33
467.24

$\begin{array}{rr}\text { Aphy } & \mathrm{MN} \\ 2616.6 & 0.4900 \\ 4168.2 & 0.2800 \\ 3531.8 & 0.2855 \\ 830.5 & 0.1892 \\ 2606.7 & 0.3781 \\ 412.6 & 0.3618 \\ 412.6 & 0.3629 \\ 412.6 & 0.2066 \\ 49.7 & 0.4191 \\ 49.3 & 0.3131 \\ 74.6 & 0.2947 \\ 265.4 & 0.3364 \\ 860.2 & 0.1787 \\ 860.2 & 0.1787 \\ 613.5 & 0.2555 \\ 2606.7 & 0.3781 \\ 2481.9 & 0.4345 \\ 2481.9 & 0.4345 \\ 2006.9 & 0.5873\end{array}$

gamt
1.40057
1.40057
1.40057
1.40057
1.40045
1.40024
1.40009
1.40009
1.38528
1.38528
1.33172
1.3655
1.3754
1.3754
1.3754
1.4004
1.4004
1.40043
1.40043

$$
\begin{aligned}
& 0.000 \\
& 0.000 \\
& 0.000 \\
& 0.000 \\
& 0.000 \\
& 0.000 \\
& 0.001 \\
& 0.001 \\
& 0.001 \\
& 0.001 \\
& 0.001 \\
& 0.001 \\
& 0.001 \\
& 0.001 \\
& 0.001 \\
& 0.000 \\
& 0.000 \\
& 0.000
\end{aligned}
$$

$\begin{array}{rrrr}\text { WAR } & \text { Wair } & \text { WH2O } & \text { H2O frac } \\ 0.0002627 & 368.14 & 0.097 & 0.0003\end{array}$ $\begin{array}{llll}0.0002627 & 368.14 & 0.097 & 0.0003\end{array}$ $\begin{array}{llll}0.0002627 & 368.14 & 0.097 & 0.0003 \\ 0.002627 & 517.33 & 0.083 & 0.0003\end{array}$ $\begin{array}{lrll}0.0002627 & 50.81 & 0.013 & 0.0003\end{array}$ $\begin{array}{lrrr}0.0002627 & 317.33 & 0.083 & 0.0003 \\ 0.0002627 & 50.81 & 0.013 & 0.0003\end{array}$ $\begin{array}{llll}0.0002627 & 50.81 & 0.013 & 0.0003 \\ 0.0012460 & 50.81 & 0.063 & 0.0012\end{array}$ $\begin{array}{llll}0.0012466 & 0.81 & 0.063 & 0.0012 \\ 0.0012460 & 30.48 & 0.038 & 0.0012\end{array}$ $\begin{array}{llll}0.0012460 & 29.01 & 0.036 & 0.0012\end{array}$ $\begin{array}{llll}0.0012460 & 22.48 & 0.036 & 0.0012\end{array}$ $\begin{array}{llll}.0012460 & 22.48 & 0.028 & 0.0153\end{array}$ $\begin{array}{llll}0.0012460 & 22.48 & 0.028 & 0.0153 \\ 0.0012460 & 30.13 & 0.038 & 0.0118 \\ 0.0012460 & 30.48 & 0.038 & 0.0117\end{array}$ $\begin{array}{llll}0.0012460 & 30.48 & 0.038 & 0.0117\end{array}$ $\begin{array}{llll}0.0012460 & 30.48 & 0.038 & 0.0117\end{array}$ $\begin{array}{llll}0.0002627 & 317.33 & 0.083 & 0.0003\end{array}$ $\begin{array}{llll}0.0003219 & 337.66 & 0.109 & 0.0003\end{array}$ $0.0003219 \quad 337.66 \quad 0.109 \quad 0.0003$ 0.0003

\begin{tabular}{|c|c|c|c|c|c|c|c|c|c|}
\hline \multicolumn{2}{|c|}{ TURBOMACH INERY } & PERFORMANCE & \multicolumn{2}{|l|}{ DATA } & \multirow[b]{2}{*}{ TR } & \multirow[b]{2}{*}{ efPoly } & \multirow[b]{2}{*}{ pwr } & \multirow[b]{2}{*}{ SMN } & \multirow[b]{2}{*}{ SMW } \\
\hline & Wc & PR & eff & $\mathrm{NC}$ & & & & & \\
\hline Fan & 569.66 & 1.072 & 0.9055 & 1850.759 & 1.0223 & 0.9064 & $-1170.2-1$ & -1884.98 & 46.41 \\
\hline LPC & 91.21 & 1.145 & 0.6913 & 1850.759 & 1.0569 & 0.6972 & -478.8 & 6.96 & 2.20 \\
\hline HPC & 49.27 & 5.646 & 0.8099 & 8805.262 & 1.7810 & 0.8491 & -4126.3 & 60.59 & 56.76 \\
\hline HPT & 12.09 & 3.697 & 0.8755 & 213.036 & 1.3307 & 0.8552 & 4126.2 & & \\
\hline LPT & 48.94 & 1.924 & 0.8145 & 53.082 & 1.1521 & 0.8002 & 1649.1 & & \\
\hline TURB & JERY MAP & DATA & & & & & & & \\
\hline & WcMap & PRmap & effMap & NcMap & R/Parm & s WcDes & S PRdes & $s$ effDes & S NCDes \\
\hline Fan & 661.89 & 1.071 & 0.9073 & 1833.113 & 0.0439 & $\overline{0} .8607$ & $\overline{1} .0235$ & 0.9980 & $\overline{0} .9905$ \\
\hline LPC & 47.10 & 1.248 & 0.7559 & 0.489 & 0.0000 & 1.9364 & 0.5839 & 0.9146 & 0.0003 \\
\hline HPC & 44.81 & 5.427 & 0.8183 & 8570.520 & 11.0857 & 1.0995 & 1.0494 & 0.9898 & 0.9733 \\
\hline HPT & 0.96 & 3.622 & 0.8755 & 1.280 & 3.6218 & 12.6299 & 0.9723 & 1.0000 & 0.0003 \\
\hline LPT & 0.75 & 1.695 & 0.8205 & 0.597 & 1.6951 & 65.2590 & 0.7521 & 0.9926 & 0.0005 \\
\hline
\end{tabular}




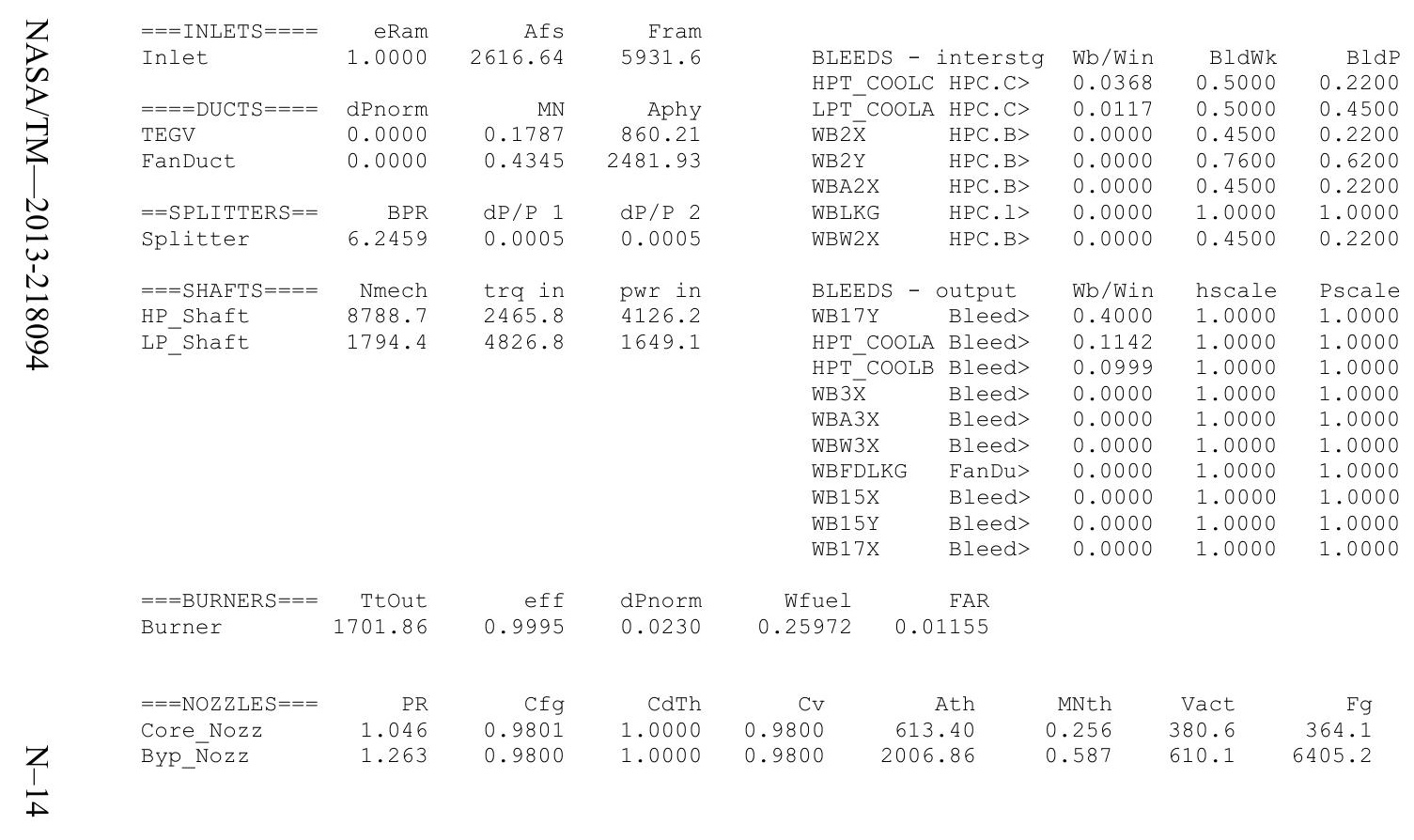


Turbofan Engine - COMDES ON Converge $=1$ CASE: 0

Version:NPSS_1.6.5 - Rev: -> Gas Package: Janaf iter/pass/Jacb/Broy=30/58/2/27 Run by: Philip C Jorgenson PC: 10

Temperature Stator 1 inlet: $500.30 \quad$ Stator 1 exit: $502.66 \quad$ Stator 2 inlet: $504.99 \quad$ Stator 2 exit: 505.72 $\begin{array}{llll}\text { Stator } 3 \text { inlet: } 508.44 & \text { Stator } 3 \text { exit: } 508.85 & \text { Stator } 4 \text { inlet: } 511.46 \text { Stator } 4 \text { exit: } 511.64 \\ \text { Stator } 5 \text { inlet: } 513.24 & \text { Stator } 5 \text { exit: } 513.26 & \text { Unblocked Percent Blockage: } 0.00\end{array}$

$\begin{array}{lclll}\text { Ambient Relative Humidity } & 10.00 & & & \\ \text { Fan Face Relative Humidity } & 6.53 & & & \\ \text { Fan Bypass Relative Humidity } & 5.96 & & & \\ \text { LPC Inlet Relative Humidity } & 5.71 & & & \\ \text { LPC Exit Relative Humidity } & 3.38 & & & \\ \text { HPC Relative Humidity } & 0.04 & & & \\ \text { Drop Diameter } & 0.0000100 & \text { Inlet Length } & \\ \text { Ambient Flow Velocity } & 479.99 & \text { Fan/LPC Inlet Flow Velocity } & 173.25 \\ \text { Ambient Static Pressure } & 8.15 & \text { Fan/LPC Inlet Static Pressure } & 9.18 \\ \text { Ambient Static Temperature } & 481.63 & \text { Fan/LPC Inlet Static Temperature } & 498.31 \\ \text { Additional Water at LPC Exit } & 0.0012964 & & & \end{array}$

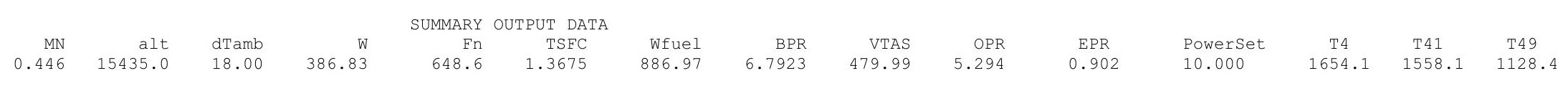

\begin{tabular}{|c|c|}
\hline FSO & Ambient.Fl_O \\
\hline FS1 & Inlet.Fl_O \\
\hline FS12 & Splitter.Fl_02 \\
\hline FS2 & Splitter.Fl-01 \\
\hline & Fan.Fl_O \\
\hline FS23 & LPC.FI_O \\
\hline ES24 & 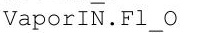 \\
\hline S25 & Bleed2.Fl $\bar{O}$ \\
\hline ES 3 & HPC.El_O \\
\hline 536 & Bleed3.FI_O \\
\hline FS 4 & Burner.FI-O \\
\hline FS 45 & HPT.FI_O \\
\hline ES 49 & LPT.FI_O \\
\hline & TEGV.FI $O$ \\
\hline FS 8 & Core_Nozz.Fl_c \\
\hline S17 & FanDuctLkg.Fí \\
\hline 171 & Bleed15.Fl_o \\
\hline & FanDuct.Fl-o \\
\hline .73 & Byp Nozz.FI \\
\hline
\end{tabular}

\begin{tabular}{rrrrrr}
\multicolumn{7}{c}{ FLOW } & STATION DATA \\
W & Pt & Tt & ht & FAR & Wc \\
386.83 & 9.342 & 500.81 & -13.05 & 0.0000 & 597.95 \\
386.83 & 9.342 & 500.81 & -13.05 & 0.0000 & 597.95 \\
337.18 & 9.337 & 500.81 & -13.05 & 0.0000 & 521.48 \\
49.64 & 9.337 & 500.81 & -13.05 & 0.0000 & 76.77 \\
337.18 & 9.816 & 508.83 & -11.13 & 0.0000 & 500.02 \\
49.64 & 10.441 & 523.31 & -7.65 & 0.0000 & 70.18 \\
49.71 & 10.441 & 525.12 & -14.69 & 0.0000 & 70.39 \\
29.82 & 10.441 & 525.12 & -14.69 & 0.0000 & 42.24 \\
28.38 & 49.459 & 890.59 & 73.96 & 0.0000 & 11.05 \\
21.99 & 49.459 & 890.59 & 73.96 & 0.0000 & 8.56 \\
22.24 & 48.320 & 1654.07 & 61.48 & 0.0112 & 12.08 \\
29.72 & 14.283 & 1133.11 & -23.80 & 0.0084 & 45.20 \\
30.07 & 8.397 & 1008.63 & -53.65 & 0.0083 & 73.39 \\
30.07 & 8.397 & 1008.70 & -53.65 & 0.0083 & 73.39 \\
30.07 & 8.397 & 1008.77 & -53.65 & 0.0083 & 73.40 \\
337.18 & 9.816 & 508.83 & -11.13 & 0.0000 & 500.02 \\
357.07 & 9.816 & 509.73 & -11.33 & 0.0000 & 529.98 \\
357.07 & 9.816 & 509.73 & -11.33 & 0.0000 & 529.98 \\
357.07 & 9.816 & 509.73 & -11.33 & 0.0000 & 529.98
\end{tabular}

$\begin{array}{rrrr}\text { PS } & \text { Ts } & \text { Aphy } & \text { MN } \\ 8.149 & 481.63 & 2541.8 & 0.4460 \\ 8.941 & 494.56 & 4168.2 & 0.2512 \\ 8.911 & 494.16 & 3531.8 & 0.2592 \\ 9.175 & 498.31 & 830.5 & 0.1583 \\ 9.029 & 496.81 & 2606.7 & 0.3475 \\ 9.797 & 513.87 & 412.6 & 0.3030 \\ 9.792 & 515.58 & 412.6 & 0.3041 \\ 10.218 & 521.89 & 412.6 & 0.1760 \\ 43.910 & 861.48 & 49.7 & 0.4175 \\ 46.258 & 874.11 & 49.3 & 0.3120 \\ 45.628 & 1630.44 & 74.6 & 0.2941 \\ 13.397 & 1113.81 & 265.4 & 0.3073 \\ 8.273 & 1004.55 & 860.2 & 0.1472 \\ 8.273 & 1004.63 & 860.2 & 0.1472 \\ 8.149 & 1000.57 & 613.4 & 0.2091 \\ 9.029 & 496.81 & 2606.7 & 0.3475 \\ 8.815 & 494.30 & 2481.9 & 0.3949 \\ 8.815 & 494.30 & 2481.9 & 0.3949 \\ 8.149 & 483.33 & 2006.9 & 0.5224\end{array}$

gamt
1.40040
1.40040
1.40040
1.40040
1.40030
1.40011
1.39991
1.39991
1.38687
1.38687
1.33416
1.36622
1.37429
1.37429
1.37428
1.40030
1.40028
1.40028
1.40028

WAR
0.0004484
0.0004484
0.0004484
0.0004484
0.0004484
0.0004484
0.0017448
0.0017448
0.0017448
0.0017448
0.0017448
0.0017448
0.0017448
0.0017448
0.0017448
0.0004484
0.0005205
0.0005205
0.0005205

Wair $386.65 \quad 0.173 \quad \mathrm{H} 2 \mathrm{O}$ frac $\begin{array}{lll}49.62 & 0.022 & 0.0004 \\ 337.03 & 0.151 & 0.0004\end{array}$ $\begin{array}{lll}337.03 & 0.151 & 0.0004\end{array}$ $\begin{array}{lll}49.62 & 0.022 & 0.0004 \\ 49.62 & 0.087 & 0.0017\end{array}$ $\begin{array}{lll}49.62 & 0.087 & 0.0017 \\ 29.77 & 0.052 & 0.0017\end{array}$ $\begin{array}{lll}28.33 & 0.049 & 0.0017\end{array}$ $\begin{array}{lll}28.33 & 0.049 & 0.0017 \\ 21.95 & 0.038 & 0.0017\end{array}$ $\begin{array}{lll}21.95 & 0.038 & 0.0154\end{array}$ $\begin{array}{lll}29.42 & 0.051 & 0.0120\end{array}$ $\begin{array}{lll}29.77 & 0.052 & 0.0118\end{array}$ $\begin{array}{lll}29.77 & 0.052 & 0.0118\end{array}$ $\begin{array}{lll}29.77 & 0.052 & 0.0119\end{array}$ $\begin{array}{rrr}337.03 & 0.151 & 0.0004\end{array}$ $\begin{array}{lll}356.88 & 0.186 & 0.0005\end{array}$ FS173 Byp Nozz.Fl_O 357.07

TURBOMACHINERY PERFORMANCE DATA

\begin{tabular}{|c|c|c|c|c|c|c|c|c|c|}
\hline & WC & PR & eff & $\mathrm{NC}$ & $\mathrm{TR}$ & efPoly & pwr & SMN & SMW \\
\hline Fan & 521.48 & 1.051 & 0.8987 & 1612.378 & 1.0160 & 0.8994 & -917.2 & -1524.62 & 44.72 \\
\hline LPC & 76.77 & 1.118 & 0.7226 & 1612.378 & 1.0449 & 0.7270 & -379.1 & 8.25 & 1.96 \\
\hline HPC & 42.24 & 4.737 & 0.7956 & 8541.451 & 1.6960 & 0.8340 & -3649.6 & 68.30 & 63.08 \\
\hline HPT & 12.08 & 3.383 & 0.8669 & 211.319 & 1.3031 & 0.8470 & 3649.6 & & \\
\hline LPT & 45.20 & 1.70 & 0.8009 & 47.068 & 1.1199 & 0.7886 & 1296.2 & & \\
\hline$J R$ & NERY MAP & DATA & & & & & & & \\
\hline & WcMap & PRmap & effMap & NcMap & R/Parm & s_WCDes & S_PRdes & S_effDes & S_NCDe \\
\hline Fan & 605.90 & 1.050 & 0.9005 & 1597.004 & 0.0442 & $\overline{0} .8607$ & $\overline{1} .0235$ & 0.9980 & 0.9905 \\
\hline PC & 39. & 1.1 & 0 & 0.426 & & 1. & 0.6395 & 0.9659 & 0.0003 \\
\hline HPC & 38.41 & 4.561 & 0.8038 & 8313.741 & 10.7568 & 1.0995 & 1.0494 & 0.9898 & 0.9733 \\
\hline $\begin{array}{l}\text { HPT } \\
\text { HPT }\end{array}$ & 0.96 & 3.317 & 0.8669 & 1.270 & 3.3171 & 12.6299 & 0.9723 & 1.0000 & 0.0003 \\
\hline LPT & 0.69 & 1.527 & 0.8069 & 0.529 & 1.5272 & 65.2590 & 0.7521 & 0.9926 & 0.0005 \\
\hline
\end{tabular}




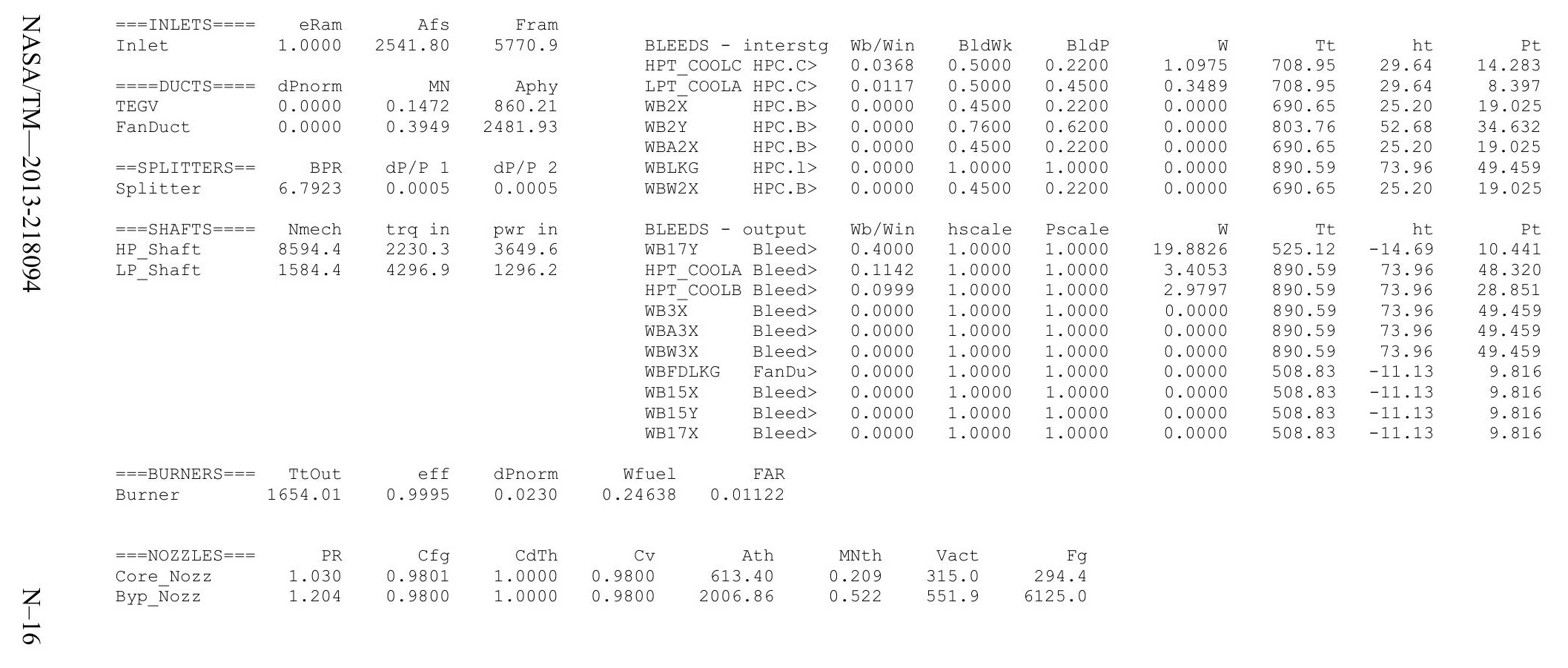


Date:05/13/13 Time:07:26:11 Model:

Version:NPSS 1.6.5 - Rev: -> Gas Package: Janaf iter/pass/Jacb/Broy=11/25/1/9 Run by: Philip C Jorgenson PC: 10

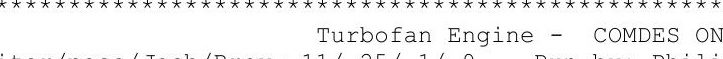

converge $=1$ CAS:

Temperature Stator 1 inlet: 483.77 Stator 1 exit: 491.58 Stator 2 inlet: 501.39 Stator 2 exit: 506.41

Stator 5 inlet: $542.19 \quad$ Stator 5 exit: $545.58 \quad$ Stator 4 inlet: 530.55 Stator 4 exit: 534.44

Ambient Relative Humidity

Fan Face Relative Humidity $\quad 1.97$

Fan Bypass Relative Humidity 1.40

LPC Inlet Relative Humidity $\quad 1.30$

LPC Exit Relative Humidity

Drop Diameter

0.13

$\begin{array}{lr}\text { Drop Diameter } & 0.0000100 \\ \text { Ambient Flow Velocity } & 789.49\end{array}$

Ambient Static Pressure

8.45
2.85

$\begin{array}{lr}\text { Inlet Length } & 40.00 \\ \text { Fan/LPC Inlet Flow Velocity } & 192.08\end{array}$

Ambient Static Temperature

Additional Water at LPC Exit

0.0024853

\begin{tabular}{lr}
4.17 \\
\hline
\end{tabular}

SUMMARY OUTPUT DATA

MN alt dTamb W

\section{FLOW STATION DATA}

\begin{tabular}{|c|c|}
\hline FSO & Ambient.FI_O \\
\hline FS1 & Inlet.Fl o \\
\hline FS12 & Splitter.Fl_02 \\
\hline FS2 & Splitter.Fl_o1 \\
\hline FS14 & Fan.Fl O \\
\hline FS23 & LPC.Fl_O \\
\hline FS24 & VaporIN̄.FIO \\
\hline FS25 & Bleed2.Fl $\bar{O}$ \\
\hline FS3 & HPC.FI_O \\
\hline FS36 & Bleed3.Fl_o \\
\hline FS4 & Burner.Fl_o \\
\hline FS45 & HPT.FI_O \\
\hline FS49 & LPT.FI_O \\
\hline FS5 & TEGV.F̄_O \\
\hline FS8 & Core_Noz̄z.Fl_ \\
\hline FS17 & FanDuctLkg.Fín \\
\hline FS171 & Bleed15.FI_o \\
\hline FS172 & FanDuct.Fl_ \\
\hline FS173 & Byp_Nozz.F $\overline{1}$ \\
\hline
\end{tabular}

\begin{tabular}{rrrrr}
\multicolumn{7}{c}{ W } & Pt & \multicolumn{1}{l}{ STATION } & hAt & FAR \\
252.23 & 4.267 & 477.92 & -16.44 & 0.0000 \\
252.23 & 4.267 & 477.92 & -16.44 & 0.0000 \\
225.98 & 4.265 & 477.92 & -16.44 & 0.0000 \\
26.25 & 4.265 & 477.92 & -16.44 & 0.0000 \\
225.98 & 4.821 & 496.75 & -11.93 & 0.0000 \\
26.25 & 6.667 & 555.57 & 2.19 & 0.0000 \\
26.32 & 6.667 & 558.88 & -11.31 & 0.0000 \\
23.69 & 6.667 & 558.88 & -11.31 & 0.0000 \\
22.54 & 40.628 & 1012.12 & 99.39 & 0.0000 \\
17.47 & 40.628 & 1012.12 & 99.39 & 0.0000 \\
17.66 & 39.693 & 1767.67 & 86.54 & 0.0113 \\
23.61 & 9.526 & 1168.62 & -20.65 & 0.0085 \\
23.88 & 3.263 & 915.06 & -83.10 & 0.0084 \\
23.88 & 3.263 & 915.13 & -83.10 & 0.0084 \\
23.88 & 3.263 & 915.20 & -83.10 & 0.0084 \\
225.98 & 4.821 & 496.75 & -11.93 & 0.0000 \\
228.61 & 4.821 & 497.47 & -11.92 & 0.0000 \\
228.61 & 4.821 & 497.47 & -11.92 & 0.0000 \\
228.61 & 4.821 & 497.47 & -11.92 & 0.0000
\end{tabular}

Wc
833.95
833.95
747.52
86.84
674.16
59.90
60.22
54.20
11.39
8.83
12.07
54.66
142.89
142.90
142.90
674.16
682.50
682.50
682.50

Ps
2.854
3.891
3.839
4.169
4.045
6.373
6.369
6.428
35.730
37.818
37.481
8.628
3.071
3.071
2.854
4.045
3.911
3.911
2.854

Ts
425.97
465.49
463.75
474.85
472.44
548.48
551.65
553.10
976.89
992.33
1742.72
1138.08
899.92
899.99
882.05
472.44
468.59
468.59
428.18

$\begin{array}{rr}\text { Aphy } & \text { MN } \\ 2544.2 & 0.7800 \\ 4168.2 & 0.3651 \\ 3531.8 & 0.3906 \\ 830.5 & 0.1798 \\ 2606.7 & 0.5068 \\ 412.6 & 0.2545 \\ 412.6 & 0.2562 \\ 412.6 & 0.2288 \\ 49.7 & 0.4351 \\ 49.3 & 0.3238 \\ 74.6 & 0.2947 \\ 265.4 & 0.3835 \\ 860.2 & 0.2975 \\ 860.2 & 0.2975 \\ 613.4 & 0.4442 \\ 2606.7 & 0.5068 \\ 2481.9 & 0.5547 \\ 2481.9 & 0.5547 \\ 2006.9 & 0.8986\end{array}$

gamt
1.40068
1.40068
1.40068
1.40068
1.40049
1.39967
1.39927
1.39927
1.37957
1.37957
1.32864
1.36376
1.37985
1.37985
1.37984
1.40049
1.40048
1.40048
1.40048

WAR
0.0000851
0.0000851
0.0000851
0.0000851
0.0000851
0.0000851
0.0025704
0.0025704
0.0025704
0.0025704
0.0025704
0.0025704
0.0025704
0.0025704
0.0025704
0.0000851
0.0001136
0.0001136
0.0001136

$\begin{array}{rrr}\text { Wair } & \text { WH2O } & \text { H2O frac } \\ 252.21 & 0.021 & 0.0001 \\ 252.21 & 0.021 & 0.0001 \\ 225.96 & 0.019 & 0.0001 \\ 26.25 & 0.002 & 0.0001 \\ 225.96 & 0.019 & 0.0001 \\ 26.25 & 0.002 & 0.0001 \\ 26.25 & 0.067 & 0.0026 \\ 23.63 & 0.061 & 0.0026 \\ 22.48 & 0.058 & 0.0026 \\ 17.42 & 0.045 & 0.0026 \\ 17.42 & 0.045 & 0.0163 \\ 23.35 & 0.060 & 0.0129 \\ 23.63 & 0.061 & 0.0127 \\ 23.63 & 0.061 & 0.0128 \\ 23.63 & 0.061 & 0.0128 \\ 225.96 & 0.019 & 0.0001 \\ 228.59 & 0.026 & 0.0001 \\ 228.59 & 0.026 & 0.0001 \\ 228.59 & 0.026 & 0.0001\end{array}$

TURBOMACHINERY PERFORMANCE DATA

\begin{tabular}{|c|c|c|c|c|c|c|c|c|c|}
\hline & & & & & & & & & \\
\hline & Wc & $\mathrm{PR}$ & eff & NC & TR & efPoly & pwr & SMN & SMW \\
\hline $\operatorname{Ean}$ & 747.52 & 1.130 & 0.9057 & 2573.075 & 1.0394 & 0.9073 & -1443.6 & 1576.92 & 54.76 \\
\hline LPC & 86.84 & 1.563 & 0.8383 & 2573.075 & 1.1625 & 0.8482 & -691.9 & 6.99 & 3.76 \\
\hline HPC & 54.20 & 6.094 & 0.8185 & 8964.236 & 1.8110 & 0.8571 & -3620.0 & 61.50 & 58.17 \\
\hline $\mathrm{HPT}$ & 12.07 & 4.167 & 0.87 & 221.323 & 1.3643 & 0.8557 & 3620.0 & & \\
\hline LPT & 54.66 & 2.920 & 0.8565 & 72.252 & 1.2754 & 0.8369 & 2135.5 & & \\
\hline JRE & $\mathrm{NEH}$ & DA & & & & & & & \\
\hline & WcMap & PRmap & effMap & NcMap & R/Parm & s_WcDes & S_PRdes & S_effDes & $\mathrm{S}_{-} \mathrm{N}$ CDes \\
\hline$a n$ & 868.55 & 1.127 & 0.9075 & 2548.541 & 0.0414 & $\overline{0} .8607$ & $\overline{1} .0235$ & 0.9980 & $\overline{0} .9905$ \\
\hline PC & 74.12 & 1.524 & 0.7801 & 0.680 & 0.0000 & 1.1716 & 1.0745 & 1.0746 & 0.0003 \\
\hline PC & 49.29 & 5.8 & 0.82 & 725.256 & 10.9657 & 1.0 & 1.0494 & 0.9898 & 0.9733 \\
\hline PT & 0.96 & 4.079 & 0.877 & 1.330 & 4.0789 & 12.6299 & 0.9723 & 1.0000 & 0.0003 \\
\hline LPT & 0.84 & 2.444 & 0.8628 & 0.813 & 2.4440 & 65.2590 & 0.7521 & 0.9926 & 0.0005 \\
\hline
\end{tabular}




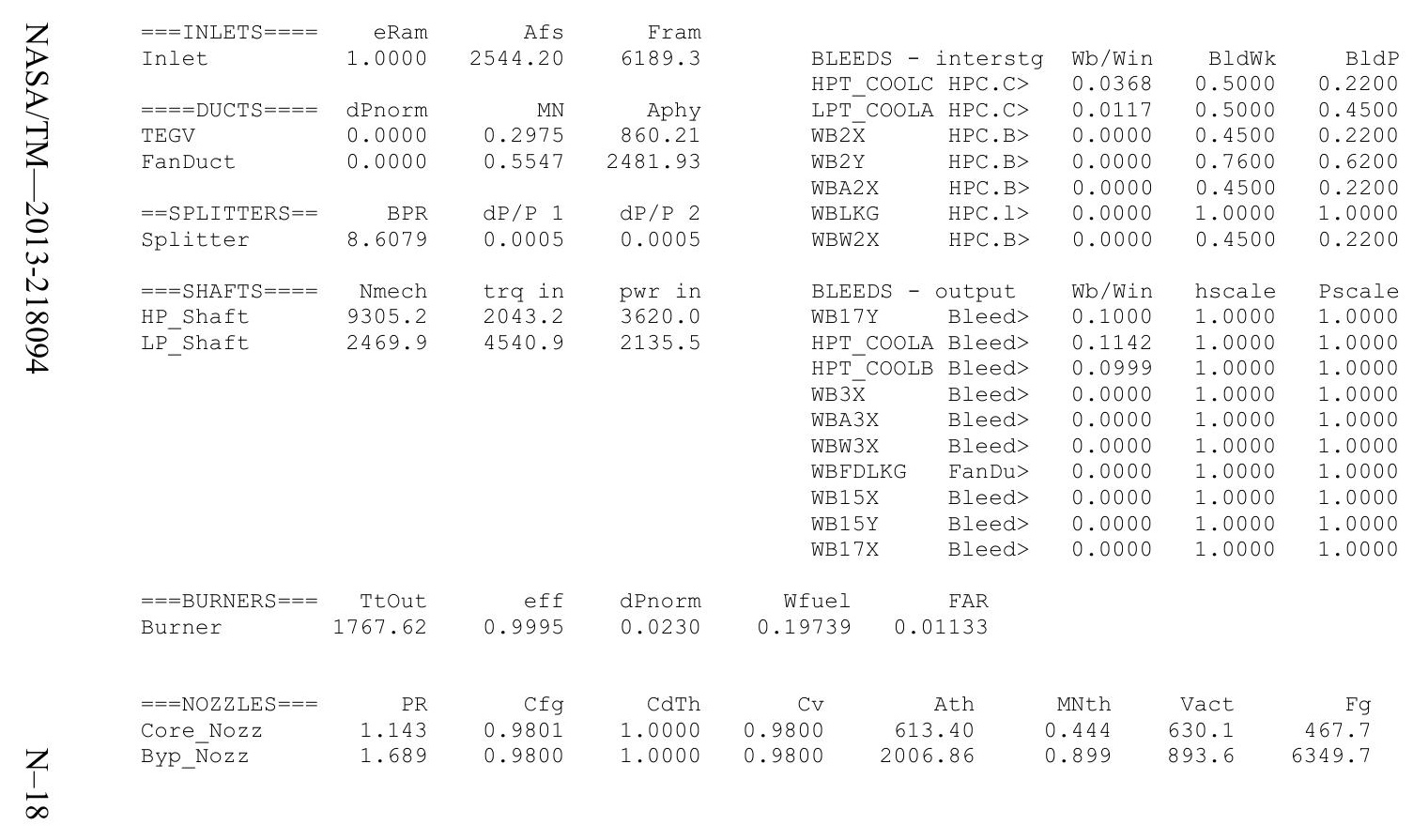


Date:05/13/13 Time:07:26:26 Model: Turbofan Engine - COMDES ON Converge = 1 CASE: 0

Version:NPSS 1.6.5 - Rev: -> Gas Package: Janaf iter/pass/Jacb/Broy=11/25/1/9 Run by: Philip C Jorgenson PC: 10

Temperature Stator 1 inlet: $476.99 \quad$ Stator 1 exit: $484.31 \quad$ Stator 2 inlet: 493.48 Stator 2 exit: 498.17 Stator 5 inlet: 531.55 Stator 5 exit: $534.68 \quad$ Unblocked Percent Blockage: 0.00

Ambient Relative Humidity $\quad 10.00$

Fan Face Relative Humidity 2.46

Fan Bypass Relative Humidity $\quad 1.80$

IPC Inlet Relative Humidity $\quad 1.64$

rop Diative Humidity

0.02

Ambient static Pressure

Additional Water at LPC Exit

Inlet Length

40.00

95 Fan/LPC Inlet Flow Velocity $\quad 185.26$

2.95 Fan/LPC Inlet Static Pressure

0.0022286

an/LPC Inlet Static Temperature 468.62

$\begin{array}{rrrrrr} & & & & \text { SUMMARY } & \text { OUTPUT DATA } \\ \text { MN } & \text { alt } & \text { dTamb } & \text { W } & \text { Fn } & \text { TSFC } \\ 0.730 & 38334.0 & 36.00 & 245.39 & 616.1 & 1.0985\end{array}$

245.39

Fn

TSFC
1.0985

Wfuel

676.77

BPR
8.7010

VTAS
738.88

OPR
9.236

EPR
0.764

Powerset
10.000

T4
1739.5

T4 T41

$\begin{array}{cc}1645.1 & \text { T49 } \\ 1147.1\end{array}$
FSO Ambient.FI_O

FS1 Inlet.Fl_o

$\begin{array}{ll}\text { FS12 } & \text { Splitter.FI_02 } \\ \text { FS2 } & \text { Splitter.Fl_01 }\end{array}$

FS14 Fan.Fl_-

FS23 LPC.FI_O

FS25 Bleed2.F1_O

FS3 HPC.FI_O

Bleed3.FI_O

Burner.F1

FS49

FS5 5 TEGV.FI

FS8 Core_Nozz.Fl_

FS17 FanDüctLkg.Fì

FS171 Bleed15.FI

FS172 FanDuct.Fl-O

FS173 Byp Nozz.Fì O

TURBOMACHINERY PERFORMANCE DATA

$\begin{array}{lrrr} & \text { WC } & \text { PR } & \text { eff } \\ \text { Fan } & 734.39 & 1.123 & 0.9049 \\ \text { LPC } & 84.40 & 1.530 & 0.8388 \\ \text { HPC } & 53.60 & 6.039 & 0.8177 \\ \text { HPT } & 12.08 & 4.114 & 0.8763 \\ \text { LPT } & 54.03 & 2.782 & 0.8508\end{array}$

\begin{tabular}{rrrrrr} 
W & \multicolumn{9}{c}{ FLOW } & STATION & DATA & Tt & $\mathrm{ht}$ & FAR & WC \\
245.39 & 4.201 & 471.48 & -17.97 & 0.0000 & 818.38 \\
245.39 & 4.201 & 471.48 & -17.97 & 0.0000 & 818.38 \\
220.10 & 4.199 & 471.48 & -17.97 & 0.0000 & 734.39 \\
25.30 & 4.199 & 471.48 & -17.97 & 0.0000 & 84.40 \\
220.10 & 4.715 & 489.02 & -13.76 & 0.0000 & 666.16 \\
25.30 & 6.425 & 544.15 & -0.54 & 0.0000 & 59.26 \\
25.35 & 6.425 & 547.17 & -12.64 & 0.0000 & 59.56 \\
22.82 & 6.425 & 547.17 & -12.64 & 0.0000 & 53.60 \\
21.71 & 38.805 & 989.28 & 95.17 & 0.0000 & 11.36 \\
16.83 & 38.805 & 989.28 & 95.17 & 0.0000 & 8.80 \\
17.01 & 37.911 & 1739.52 & 82.50 & 0.0112 & 12.08 \\
22.74 & 9.214 & 1151.35 & -21.86 & 0.0084 & 54.03 \\
23.00 & 3.312 & 912.39 & -80.52 & 0.0083 & 135.37 \\
23.00 & 3.312 & 912.46 & -80.52 & 0.0083 & 135.38 \\
23.00 & 3.312 & 912.53 & -80.52 & 0.0083 & 135.38 \\
220.10 & 4.715 & 489.02 & -13.76 & 0.0000 & 666.16 \\
222.63 & 4.715 & 489.68 & -13.75 & 0.0000 & 674.29 \\
222.63 & 4.715 & 489.68 & -13.75 & 0.0000 & 674.29 \\
222.63 & 4.715 & 89.68 & -13.75 & 0000 & 674.29
\end{tabular}

PS
2.947
3.847
3.79
4.111
3.978
6.14
6.14
6.201
34.159
36.138
35.79
8.36
3.13
3.13
2.94
3.97
3.85
3.85
2.947

Ts
425.97
459.73
458.06
468.62
465.84
537.36
540.26
541.64
955.02
970.02
1714.88
1122.01
898.97
899.04
883.58
465.84
462.22
462.22
428.07

$\begin{array}{rr}\text { Aphy } & \text { MN } \\ 2561.4 & 0.7300\end{array}$

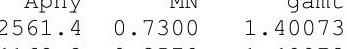

$\begin{array}{lll}2561.4 & 0.7300 & 1.40073 \\ 3531.8 & 0.35703 & 1.40073\end{array}$

$\begin{array}{ll}2606.7 & 0.4984\end{array}$

$\begin{array}{ll}412.6 & 0.2515 \\ 412.6 & 0.2531\end{array}$

$\begin{array}{ll}412.6 & 0.2531 \\ 412.6 & 0.2261 \\ 49.7 & 0.4332\end{array}$

$\begin{array}{ll}49.7 & 0.4332\end{array}$

$\begin{array}{lll}49.3 & 0.33226 \\ 74.6 & 0.2946\end{array}$

$\begin{array}{ll}265.4 & 0.3779\end{array}$

$860.2 \quad 0.2801$

$860.2 \quad 0.2801$

$\begin{array}{rr}613.4 & 0.4147 \\ 2606.7 & 0.4984\end{array}$

$\begin{array}{ll}2481.9 & 0.5446\end{array}$

$2481.9 \quad 0.5446$

$2006.9 \quad 0.8474$

$\begin{array}{rrrr}\text { WAR } & \text { Wair } & \text { WH2O } & \text { H2O frac } \\ 0.0000824 & 245.37 & 0.020 & 0.0001 \\ 0.0000824 & 245.37 & 0.020 & 0.0001 \\ 0.0000824 & 220.08 & 0.018 & 0.0001 \\ 0.0000824 & 25.29 & 0.002 & 0.0001 \\ 0.0000824 & 220.08 & 0.018 & 0.0001 \\ 0.0000824 & 25.29 & 0.002 & 0.0001 \\ 0.0023110 & 25.29 & 0.058 & 0.0023 \\ 0.0023110 & 22.76 & 0.053 & 0.0023 \\ 0.0023110 & 21.66 & 0.050 & 0.0023 \\ 0.0023110 & 16.79 & 0.039 & 0.0023 \\ 0.0023110 & 16.79 & 0.039 & 0.0159 \\ 0.0023110 & 22.50 & 0.052 & 0.0125 \\ 0.0023110 & 22.76 & 0.053 & 0.0124 \\ 0.0023110 & 22.76 & 0.053 & 0.0124 \\ 0.0023110 & 22.76 & 0.053 & 0.0124 \\ 0.0000824 & 220.08 & 0.018 & 0.0001 \\ 0.0001077 & 222.61 & 0.024 & 0.0001 \\ 0.0001077 & 222.61 & 0.024 & 0.0001 \\ 0.0001077 & 222.61 & 0.024 & 0.0001\end{array}$

TURBOMACHINERY MAP DATA

$\begin{array}{lrrrrrrrrr} & \text { WcMap } & \text { PRmap } & \text { effMap } & \text { NcMap } & \text { R/Parm } & \text { S_WcDes } & \text { S_PRdes } & \text { S_effDes } & \text { S_NcDes } \\ \text { Fan } & 853.29 & 1.120 & 0.9067 & 2484.881 & 0.0413 & 0.8607 & \overline{1} .0235 & 0.9980 & 0.9905 \\ \text { LPC } & 71.33 & 1.495 & 0.7771 & 0.663 & 0.0000 & 1.1832 & 1.0700 & 1.0794 & 0.0003 \\ \text { HPC } & 48.75 & 5.802 & 0.8262 & 8706.431 & 10.9784 & 1.0995 & 1.0494 & 0.9898 & 0.9733 \\ \text { HPT } & 0.96 & 4.028 & 0.8763 & 1.324 & 4.0281 & 12.6299 & 0.9723 & 1.0000 & 0.0003 \\ \text { LPT } & 0.83 & 2.340 & 0.8571 & 0.793 & 2.3402 & 65.2590 & 0.7521 & 0.9926 & 0.0005\end{array}$




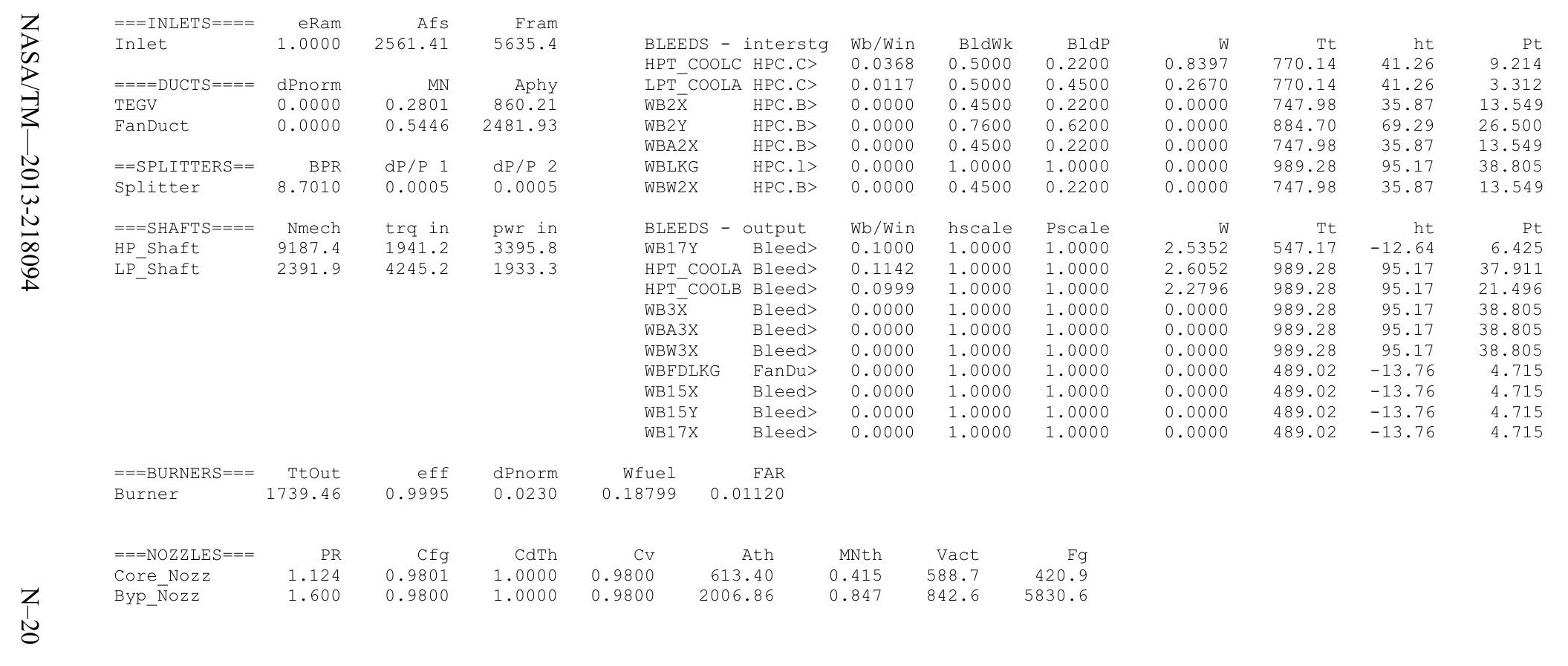


Date:05/13/13 Time:07:26:42 Model: Turbofan Engine - COMDES ON Converge = 1 CASE: 0

Version:NPSS 1.6.5 - Rev: -> Gas Package: Janaf iter/pass/Jacb/Broy=12/26/1/10 Run by: Philip C Jorgenson PC: 10

Temperature Stator 1 inlet: $474.90 \quad$ Stator 1 exit: $482.02 \quad$ Stator 2 inlet: 490.95 Stator 2 exit: 495.51 $\begin{array}{lll}\text { Stator } 3 \text { inlet: } 504.71 & \text { Stator } 3 \text { exit: } 508.84 & \text { Stator } 4 \text { inlet: } 517.43 \\ \text { Stator } 5 \text { inlet: } 527.97 & \text { Stator } 5 \text { exit: } 531.01 & \text { Unblocked Percent Blockage: } 0.00\end{array}$

Ambient Relative Humidity $\quad 10.00$

Fan Face Relative Humidity $\quad 2.63$

Fan Bypass Relative Humidity $\quad 1.94$

$\begin{array}{ll}\text { LPC Inlet Relative Humidity } & 1.76 \\ \text { LPC Exit Relative Humidity } & 0.19\end{array}$

HPC Relative Humidity

Drop Delative Humidity

Ambient Flow Velocity

Ambient Static Pressure

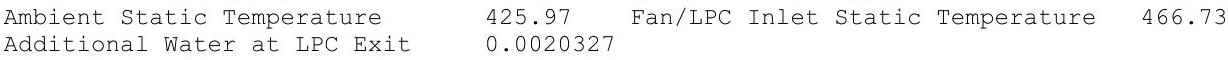

0.0000100

722.68 Fan/LPC Inlet Flow Velocity $\quad \begin{aligned} 40.00 \\ 3.09\end{aligned}$

3.09 Fan/LPC Inlet Static Pressure $\quad 4.25$

$\begin{array}{rrrrrr} & & & & \text { SUMMARY } & \text { OUTPUT DATA } \\ \text { MN } & \text { alt } & \text { dTamb } & \text { W } & \text { Fn } & \text { TSFC } \\ 0.714 & 37357.0 & 36.00 & 251.93 & 628.7 & 1.0937\end{array}$

$628.7 \quad 1.0937 \quad 687.60$

687.60

BPR
8.7439

VTAS
722.68

OPR
9.107

EPR
0.772

Powerset
10.000

$\begin{array}{cc}\text { T4 } & \text { T41 } \\ 1728.0 & 1633.9\end{array}$

T4 9

FLOW STATION DATA

FSO Ambient.FI_O

FS1 Inlet.Fl_o

$\begin{array}{ll}\text { FS12 } & \text { Splitter.F1_02 } \\ \text { FS2 } & \text { Splitter.Fl_01 }\end{array}$

FS14 Fan.F1_O

FS24 VaporIN.FI

FS25 Bleed2.FI_

FS3 HPC.FIO

FS36 Bleed3.FI_O

FS4 Burner.FI_O

FS49 IPT FI-

FS4 5 TEGT.FI

FS8 Core_Nozz.Fl_O

FS17 FanDuctLkg.Fi_

FS171 Bleed15.FI

FS173 FanDuct.FI-O

$\begin{array}{rrr}\text { Tt } & \text { ht } & \text { FAR } \\ 469.50 & -18.42 & 0.0000 \\ 469.50 & -18.42 & 0.0000 \\ 469.50 & -18.42 & 0.0000 \\ 469.50 & -18.42 & 0.0000 \\ 486.54 & -14.34 & 0.0000 \\ 540.27 & -1.45 & 0.0000 \\ 543.04 & -12.48 & 0.0000 \\ 543.04 & -12.48 & 0.0000 \\ 980.74 & 94.17 & 0.0000 \\ 980.74 & 94.17 & 0.0000 \\ 1728.02 & 81.59 & 0.0111 \\ 1144.30 & -21.65 & 0.0083 \\ 910.93 & -78.87 & 0.0082 \\ 911.00 & -78.87 & 0.0082 \\ 911.07 & -78.87 & 0.0082 \\ 486.54 & -14.34 & 0.0000 \\ 487.19 & -14.32 & 0.0000 \\ 487.19 & -14.32 & 0.0000 \\ 487.19 & -14.32 & 0.0000\end{array}$

WC
811.77
811.77

811.77

728.83
83.35

83.35
662.72
58.96

662.72
58.96

58.96
59.23

59.23

53.31
11.35
-8.79

11.35
8.79

12.08

53.80
132.64

132.64
132.64
132.65

132.65
662.72

662.72
670.75

670.75
670.75

670.75

PS
3.088
3.980
3.928
4.248
4.107
6.298
6.294
6.350
34.796
36.806
36.454
8.569
3.279
3.279
3.088
4.107
3.981
3.981
3.088

Ts
425.97
458.02
456.38
466.73
463.79
533.60
536.26
537.62
946.82
961.67
1703.50
1115.40
898.11
898.18
883.55
463.79
460.27
460.27
428.03

$\begin{array}{rr}\text { Aphy } & \text { MN } \\ 2565.2 & 0.7140\end{array}$

$\begin{array}{rrr}\text { Aphy } & \text { MN } & \text { gamt } \\ 2565.2 & 0.7140 & 1.40075\end{array}$ $\begin{array}{ll}3531.8 & 0.3536 \\ 830.5 & 0.1723\end{array}$ $\begin{array}{ll}830.5 & 0.1723\end{array}$ 2606.70 .4948 $\begin{array}{ll}412.6 & 0.2501 \\ 412.6 & 0.2516\end{array}$ $\begin{array}{ll}412.6 & 0.2516 \\ 412.6 & 0.2247\end{array}$ $\begin{array}{ll}49.7 & 0.4326\end{array}$ $\begin{array}{lll}74.6 & 0.3222\end{array}$ $\begin{array}{lll}74.6 & 0.2946\end{array}$ $\begin{array}{ll}265.4 & 0.3759 \\ 860.2 & 0.2739\end{array}$ $\begin{array}{ll}860.2 & 0.2739\end{array}$ $\begin{array}{rr}613.4 & 0.4043\end{array}$ $2606.7-0.4948$ $\begin{array}{ll}2481.9 & 0.5403 \\ 2481.9 & 0.5403\end{array}$

$2006.9 \quad 0.830$ 1.40075
1.40075
1.40075 1.40075
1.40075 1.40060 1.39991 1.39958
1.39958

1.38157 1.38157 1.36548 1.38025
1.38025

1.38025
1.30060

1.40060
1.40059
1.40059

1.40059

$\begin{array}{rrrr}\text { WAR } & \text { Wair } & \text { WH2O } & \text { H2O frac } \\ 0.0000786 & 251.91 & 0.020 & 0.0001 \\ 0.0000786 & 251.91 & 0.020 & 0.0001 \\ 0.0000786 & 226.06 & 0.018 & 0.0001 \\ 0.0000786 & 25.85 & 0.002 & 0.0001 \\ 0.0000786 & 226.06 & 0.018 & 0.0001 \\ 0.0000786 & 25.85 & 0.002 & 0.0001 \\ 0.0021113 & 25.85 & 0.055 & 0.0021 \\ 0.0021113 & 23.27 & 0.049 & 0.0021 \\ 0.0021113 & 22.14 & 0.047 & 0.0021 \\ 0.0021113 & 17.16 & 0.036 & 0.0021 \\ 0.0021113 & 17.16 & 0.036 & 0.0156 \\ 0.0021113 & 23.00 & 0.049 & 0.0122 \\ 0.0021113 & 23.27 & 0.049 & 0.0121 \\ 0.0021113 & 23.27 & 0.049 & 0.0121 \\ 0.0021113 & 23.27 & 0.049 & 0.0121 \\ 0.0000786 & 226.06 & 0.018 & 0.0001 \\ 0.0001016 & 228.64 & 0.023 & 0.0001 \\ 0.0001016 & 228.64 & 0.023 & 0.0001 \\ 0.0001016 & 228.64 & 0.023 & 0.0001\end{array}$

\begin{tabular}{|c|c|c|c|c|c|c|c|c|c|}
\hline IURI & NERY PER & ORMANCE & \multicolumn{2}{|l|}{ DATA } & & \multirow[b]{2}{*}{ efpoly } & \multirow[b]{2}{*}{ pwr } & \multirow[b]{2}{*}{ SMN } & \multirow[b]{2}{*}{ SMW } \\
\hline & Wc & $\mathrm{PR}$ & eff & $\mathrm{NC}$ & TR & & & & \\
\hline Fan & 728.83 & 1.120 & 0.9045 & 2481.637 & 1.0363 & 0.9060 & -1306.4 & 2512.84 & 54.57 \\
\hline LPC & 83.35 & 1.517 & 0.8389 & 2481.637 & 1.1507 & 0.8481 & -620.8 & 6.70 & 3.29 \\
\hline HPC & 53.31 & 6.008 & 0.8172 & 8935.248 & 1.8060 & 0.8559 & -3433.3 & 61.49 & 58.07 \\
\hline PT & 12.08 & 4.095 & .8757 & 219.940 & 1.3 & 0.8 & 3433.3 & & \\
\hline LPT & 53.80 & 2.731 & .8485 & 69.798 & 1.2541 & 0.8295 & 1927.2 & & \\
\hline & $\mathrm{NES}$ & DATA & & & & & & & \\
\hline & WcMap & PRmap & effmap & NcMap & R/Parm & S_WcDes & S_PRdes & S_effDes & s_NcDes \\
\hline an & 846.83 & 1.117 & 0.9063 & 2457.975 & 0.0413 & $\overline{0} .8607$ & $\overline{1} .0235$ & 0.9980 & 0.9905 \\
\hline PC & 70.16 & 1.483 & 0.7758 & 0.655 & 0.0000 & 1.1881 & 1.0689 & 1.0813 & 0.0003 \\
\hline${ }^{\mathrm{C}}$ & 48.48 & 5.772 & 3257 & 8697.040 & $10.9^{\prime}$ & 1.0 & 1.0494 & 0.9898 & 0.9733 \\
\hline TT & 0.96 & 4.010 & 0.8757 & 1.322 & 4.0097 & 12.6299 & 0.9723 & 1.0000 & 0.0003 \\
\hline & 0.82 & 2.302 & .8548 & 0.785 & 2.3019 & 65.2590 & 0.7521 & 0.9926 & 0.00 \\
\hline
\end{tabular}




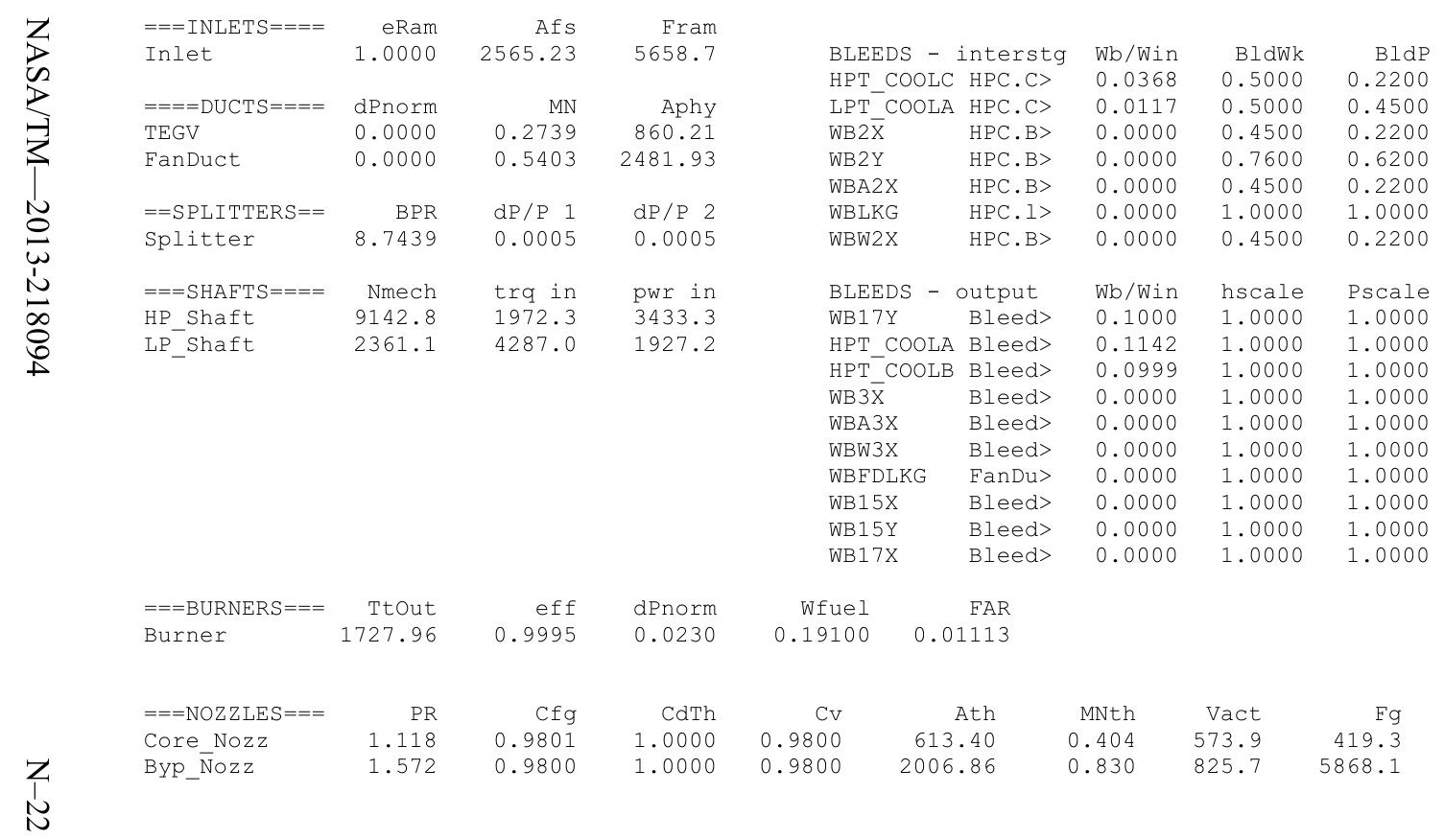


Date:05/13/13 Time:07:26:57 Model: Turbofan Engine - COMDES ON Converge = 1 CASE: 0

Version:NPSS 1.6.5 - Rev: -> Gas Package: Janaf iter/pass/Jacb/Broy=10/24/1/8 Run by: Philip C Jorgenson PC: 10

Temperature Stator 1 inlet: $476.29 \quad$ Stator 1 exit: $482.95 \quad$ Stator 2 inlet: 491.29 Stator 2 exit: 495.54 $\begin{array}{lll}\text { Stator } 3 \text { inlet: } 504.12 & \text { Stator } 3 \text { exit: } 507.94 & \text { Stator } 4 \text { inlet: } 515.96 \\ \text { Stator } 5 \text { inlet: } 525.74 & \text { Stator } 5 \text { exit: } 528.52 & \text { Unblocked Percent Blockage: } 0.00\end{array}$

Ambient Relative Humidity $\quad 10.00$

Fan Face Relative Humidity $\quad 3.24$

Fan Bypass Relative Humidity 2.48

LPC Inlet Relative Humidity $\quad 0.22$

HPC Relative Humidity

Drop Diameter

0.02

0.0000100

Inlet Length

40.00

Ambient Flow Velocity

682.24

Fan/LPC Inlet

176.05
4.74

Ambient Static Pressure

32.42

Fan/LPC Inlet Static Temperature 468.63

Additional Water at LPC Exit 0.0018496

MN alt dTamb SUMMARY OUTPUT DATA

$\begin{array}{rrrrrr}\text { MN } & \text { alt } & \text { dTamb } & \text { W } & \text { Fn } & \text { TSFC } \\ 0.669 & 34281.0 & 36.00 & 272.75 & 675.2 & \end{array}$

FLOW STATION DATA

FSO Ambient. 1 (

FS1 Inlet.FI_O

FS12 Splitter.F1_02

FS2 Splitter.Fl_01

FS14 Fan.F1_

FS24 VaporIN.FI

FS25 Bleed2.Fl_

FS3 HPC.FI_O

ES46. Bled3.

FS4 Burner.F1-

(5)

FS5 TEGV.FĪO

FS8 Core_Nozz.Fl_O

FS17 FanDuctLkg.Fì_o

FS171 Bleed15.FI_O

FS172 FanDuct.FI-O

TURBOMACHINERY PERFORMANCE DATA

$\begin{array}{lrrr} & \text { WC } & \text { PR } & \text { eff } \\ \text { Fan } & 710.89 & 1.111 & 0.9034 \\ \text { LPC } & 80.35 & 1.477 & 0.8398 \\ \text { HPC } & 52.52 & 5.916 & 0.8157 \\ \text { HPT } & 12.08 & 4.049 & 0.8750 \\ \text { LPT } & 53.25 & 2.583 & 0.8433\end{array}$

$\begin{array}{crrr}\text { FLOW } & \text { STATION DATA } & \\ \text { Pt } & \text { Tt } & \text { ht } & \text { FAR } \\ 4.831 & 471.21 & -18.12 & 0.0000 \\ 4.831 & 471.21 & -18.12 & 0.0000 \\ 4.829 & 471.21 & -18.12 & 0.0000 \\ 4.829 & 471.21 & -18.12 & 0.0000 \\ 5.363 & 487.11 & -14.31 & 0.0000 \\ 7.130 & 537.35 & -2.26 & 0.0000 \\ 7.130 & 539.88 & -12.30 & 0.0000 \\ 7.130 & 539.88 & -12.30 & 0.0000 \\ 42.182 & 971.40 & 92.78 & 0.0000 \\ 42.182 & 971.40 & 92.78 & 0.0000 \\ 41.210 & 1716.81 & 80.27 & 0.0111 \\ 10.178 & 1139.03 & -21.42 & 0.0083 \\ 3.940 & 919.16 & -75.23 & 0.0082 \\ 3.940 & 919.23 & -75.23 & 0.0082 \\ 3.940 & 919.30 & -75.23 & 0.0082 \\ 5.363 & 487.11 & -14.31 & 0.0000 \\ 5.363 & 487.70 & -14.28 & 0.0000 \\ 5.363 & 487.70 & -14.28 & 0.0000 \\ 5.363 & 487.70 & -14.28 & 0.0000\end{array}$

Wfuel

BPR
8.8476

VTAS
682.24

$\mathrm{OPR}$
8.731

EPR
0.795

PowerSet
10.000

$\begin{array}{cc}\text { T4 } & \text { T41 } \\ 1716.8 & 1623 .\end{array}$

T49

TURBOMACHINERY MAP DATA

$\begin{array}{lrrrrrrrrr} & \text { WcMap } & \text { PRmap } & \text { effMap } & \text { NcMap } & \text { R/Parm } & \text { S_WcDes } & \text { S_PRdes } & \text { S_effDes } & \text { S_NcDes } \\ \text { Fan } & 825.99 & 1.108 & 0.9052 & 2376.137 & 0.0413 & 0.8607 & \overline{1} .0235 & 0.9980 & 0.9905 \\ \text { LPC } & 66.58 & 1.446 & 0.7720 & 0.634 & 0.0000 & 1.2067 & 1.0675 & 1.0878 & 0.0003 \\ \text { HPC } & 47.77 & 5.685 & 0.8241 & 8671.855 & 10.9625 & 1.0995 & 1.0494 & 0.9898 & 0.9733 \\ \text { HPT } & 0.96 & 3.964 & 0.8750 & 1.318 & 3.9644 & 12.6299 & 0.9723 & 1.0000 & 0.0003 \\ \text { LPT } & 0.82 & 2.191 & 0.8496 & 0.762 & 2.1907 & 65.2590 & 0.7521 & 0.9926 & 0.0005\end{array}$


$\begin{array}{lr}===\text { INLETS }==== & \text { eRam } \\ \text { Inlet } & 1.0000\end{array}$

$\begin{array}{ll}====\text { DUCTS }==== & \text { dPnorm } \\ \text { TEGV } & 0.0000\end{array}$

FanDuct

0.0000

$==$ SPLITTERS $==$

Splitter

BPR

8.8476

$===\operatorname{SHAFT} S===$

HP_Shaft
LP_Shaft

Nmech
9089.7

2286.6

$-$

$=\begin{array}{r}\text { BURNERS }===\begin{array}{r}\text { TtOut } \\ 1716.76\end{array} \\ \text { rner }\end{array}$

eff
0.9995

dPnorm$$
\text { Burner }
$$

$$
1716.76
$$

0.9995

$===$ NOZZLES $===$

Core $\mathrm{NOZZ}$

PR
1.101
1.499

Cfg
0.9801

0.9800

Fram
5783.5

Aphy
860.21 2481.93

0.0005

$\mathrm{dP} / \mathrm{P} 2$

0.0005

trq in pwr in

3622.8
1942.5

$\stackrel{1}{\perp}$
BLEEDS - interstg Wb/Win BldWk BldP HPT COOLC HPC.C> $>0.0368 \quad 0.5000=0.2200$ $\begin{array}{lllll}\text { LPT_COOLA HPC.C> } & 0.0117 & 0.5000 & 0.4500 \\ \text { WB } 2 \bar{X} & \text { HPC. B> } & 0.0000 & 0.4500 & 0.2200\end{array}$ $\begin{array}{lllll}\text { WB2X } & \text { HPC.B }> & 0.0000 & 0.4500 & 0.2200 \\ \text { WB2Y } & \text { HPC.B } & 0.0000 & 0.7600 & 0.6200\end{array}$ $\begin{array}{lllll}\text { WBA2X HPC.B } & 0.0000 & 0.4500 & 0.2200\end{array}$ WBLKG HPC.1> $0.0000 \quad 1.0000 \quad 1.0000$ $\begin{array}{lllll}\text { WBW2X HPC.B } & 0.0000 & 0.4500 & 0.2200\end{array}$

BLEEDS - output Wb/Win hscale Pscale $\begin{array}{llll}\text { WB17Y Bleed } & 0.1000 & 1.0000 & 1.0000\end{array}$ HPT_COOLA Bleed> $0.1142 \quad 1.0000 \quad 1.0000$ HPT_COOLB Bleed> $0.0999 \quad 1.0000 \quad 1.0000$

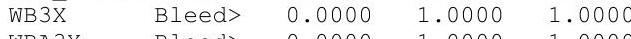
WBA3X Bleed> $0.0000 \quad 1.0000=1.0000$ WBW3X Bleed> $0.0000 \quad 1.0000 \quad 1.0000$ WBFDLKG EanDu> $0.0000 \quad 1.0000 \quad 1.0000$ WB15X Bleed> $0.0000-1.0000 \quad 1.0000$ WB15Y Bleed $0.0000 \quad 1.0000 \quad 1.0000$ $0.0000 \quad 1.0000 \quad 1.0000$

\begin{tabular}{|c|c|c|c|}
\hline $\mathrm{W}$ & Tt & $h t$ & Pt \\
\hline 0.9190 & 757.41 & 40.24 & 10.178 \\
\hline 0.2922 & 757.41 & 40.24 & 3.940 \\
\hline 0.0000 & 735.78 & 34.99 & 14.841 \\
\hline 0.0000 & 869.24 & 67.56 & 28.862 \\
\hline 0.0000 & 735.78 & 34.99 & 14.841 \\
\hline 0.0000 & 971.40 & 92.78 & 42.182 \\
\hline 0.0000 & 735.78 & 34.99 & 14.841 \\
\hline W & $\mathrm{Tt}$ & ht & Pt \\
\hline 2.7748 & 539.88 & -12.30 & 7.130 \\
\hline 2.8515 & 971.40 & 92.78 & 41.210 \\
\hline 2.4951 & 971.40 & 92.78 & 23.460 \\
\hline 0.0000 & 971.40 & 92.78 & 42.182 \\
\hline 0.0000 & 971.40 & 92.78 & 42.182 \\
\hline 0.0000 & 971.40 & 92.78 & 42.182 \\
\hline 0.0000 & 487.11 & -14.31 & 5.363 \\
\hline 0.0000 & 487.11 & -14.31 & 5.363 \\
\hline 0.0000 & 487.11 & -14.31 & 5.363 \\
\hline 0.0000 & 487.11 & -14.31 & 5.363 \\
\hline
\end{tabular}

$\begin{array}{rr}\text { Wfuel } & \text { FAR } \\ 0.20370 & 0.01108\end{array}$

$$
0.0230-0.20370-0.01108
$$

$\begin{array}{rrrrrr}\text { CdTh } & \text { Cv } & \text { Ath } & \text { MNth } & \text { Vact } & \text { Fg } \\ 1.0000 & 0.9800 & 613.40 & 0.376 & 537.4 & 420.6 \\ 1.0000 & 0.9800 & 2006.86 & 0.783 & 783.9 & 6038.1\end{array}$


Date:05/13/13 Time:07:27:15 Model: Turbofan Engine - CoMDES ON Converge = 1 CASE: 0

Version:NPSS 1.6.5 - Rev: -> Gas Package: Janaf iter/pass/Jacb/Broy= 15/29/1/13 Run by: Philip C Jorgenson PC: 10

Temperature Stator 1 inlet: 484.57 Stator 1 exit: 490.29 Stator 2 inlet: 497.36 Stator 2 exit: 500.79 $\begin{array}{llrl}\text { Stator } 3 \text { inlet: } 508.16 & \text { Stator } 3 \text { exit: } 511.17 & \text { Stator } 4 \text { inlet: } 518.10 & \text { Stator } 4 \text { exit: } 520.5 \\ \text { Stator } 5 \text { inlet: } 526.17 & \text { Stator } 5 \text { exit: } 528.27 & \text { Unblocked Percent Blockage: } 0.00\end{array}$

Ambient Relative Humidity $\quad 10.00$

Fan Face Relative Humidity $\quad 4.19$

Fan Bypass Relative Humidity $\quad 3.36$

3.36
LPC Inlet Relative Humidity

(

Drop Diameter

0.02

0.0000100

630.80

Inlet Length

40.00

Ambient Flow Velocity

Ambient Static Pressure

Additional Water at LPC Exit

447.58 Fan/LPC Inlet Static Temperature 478.13

$\begin{array}{rrrrrr} & & & & \text { SUMMARY OUTPUT DATA } \\ \text { MN } & \text { alt } & \text { dTamb } & \text { W } & \text { Fn } & \text { TSFC } \\ 0.608 & 30029.0 & 36.00 & 298.22 & 718.6 & 1.1019\end{array}$

718.6

1.1019

Wfuel
791.89

BPR
8.4428

VTAS
630.80

$\mathrm{OPR}$
8.114

EPR
0.827

Powerset
10.000 $\begin{array}{ccr}\text { T4 } & \text { T41 } & \text { T49 } \\ 1714.5 & 1620.4 & 1136.3\end{array}$
FSO Ambient.FI_O

FS1 Inlet.FI_O

FS12 Splitter.F1_02

FS2 Splitter.Fl_01

FS14 Fan.F1_-

FS24 VaporIN. FI

S25 Bleed2.FI

FS3 HPC.FI_O

ESt_-

ES45 Burner.FI-

FS49 IPT.FI-O

FS5 5 TEGV.

FS8 Core_Nozz.Fl_O

FS17 FanDūctLkg.F̄̄

FS171 Bleed15.FI_O

FS172 FanDuct.Fl-O

FS173 Byp Nozz.FİO

W
298.22
298.22
266.64
31.58
266.64
31.58
31.64
26.89
25.59
19.83
20.05
26.80
27.11
27.11
27.11
266.64
271.39
271.39
271.39

FLOW STATION DATA

$\begin{array}{rrrrr}\text { Pt } & \text { Tt } & \text { ht } & \text { FAR } & \text { Wc } \\ 5.595 & 480.74 & -16.28 & 0.0000 & 754.13 \\ 5.595 & 480.74 & -16.28 & 0.0000 & 754.13 \\ 5.592 & 480.74 & -16.28 & 0.0000 & 674.60 \\ 5.592 & 480.74 & -16.28 & 0.0000 & 79.90 \\ 6.134 & 495.01 & -12.86 & 0.0000 & 624.13 \\ 7.815 & 537.27 & -2.73 & 0.0000 & 60.45 \\ 7.815 & 539.78 & -12.67 & 0.0000 & 60.70 \\ 7.815 & 539.78 & -12.67 & 0.0000 & 51.59 \\ 45.396 & 966.66 & 91.26 & 0.0000 & 11.31 \\ 45.396 & 966.66 & 91.26 & 0.0000 & 8.76 \\ 44.351 & 1714.49 & 78.73 & 0.0111 & 12.08 \\ 11.115 & 1140.52 & -21.80 & 0.0083 & 52.54 \\ 4.711 & 941.00 & -70.55 & 0.0082 & 113.93 \\ 4.711 & 941.07 & -70.55 & 0.0082 & 113.93 \\ 4.711 & 941.14 & -70.55 & 0.0082 & 113.94 \\ 6.134 & 495.01 & -12.86 & 0.0000 & 624.13 \\ 6.134 & 495.80 & -12.86 & 0.0000 & 635.74 \\ 6.134 & 495.80 & -12.86 & 0.0000 & 635.74 \\ 6.134 & 495.80 & -12.86 & 0.0000 & 635.74\end{array}$

PS
4.358
5.201
5.148
5.48
5.31
7.46
7.46
7.563
40.01
42.30
41.87
10.15
4.54
4.54
4.35
5.31
5.17
5.17
4.35

Ts
447.58
470.79
469.49
478.13
475.19
530.27
532.67
534.74
933.44
947.96
1690.16
1113.25
931.46
931.53
921.23
475.19
472.15
472.15
449.63

$\begin{array}{rr}\text { Aphy } & \text { MN } \\ 2590.5 & 0.6080\end{array}$ $\begin{array}{ll}4168.2 & 0.3248 \\ 3531.8 & 0.3457\end{array}$ $\begin{array}{rr}3531.8 & 0.3457 \\ 830.5 & 0.1649\end{array}$ $\begin{array}{ll}2606.7 & 0.4563\end{array}$ $\begin{array}{ll}412.6 & 0.2570 \\ 412.6 & 0.2583\end{array}$ $\begin{array}{ll}412.6 & 0.2583 \\ 412.6 & 0.2170\end{array}$ $\begin{array}{lll}49.7 & 0.4307 \\ 49.3 & 0.3209\end{array}$ $\begin{array}{lll}49.7 & 0.4307\end{array}$ $\begin{array}{ll}74.6 & 0.2945\end{array}$ $\begin{array}{ll}265.4 & 0.3654 \\ 86.2 & 0.2325\end{array}$ $\begin{array}{ll}860.2 & 0.2325\end{array}$ 860.20 .2325 $\begin{array}{ll}613.4 & 0.3376\end{array}$ $2606.7 \quad 0.4563$ $2481.9 \quad 0.5000$

$2006.9 \quad 0.7159$

WAR
0.0001750
0.0001750
0.0001750
0.0001750
0.0001750
0.0001750
0.0020069
0.0020069
0.0020069
0.0020069
0.0020069
0.0020069
0.0020069
0.0020069
0.0020069
0.0001750
0.0002070
0.0002070
0.0002070

TURBOMACHINERY PERFORMANCE DATA

\begin{tabular}{lrrrrrrrrr}
\multicolumn{1}{c}{ Nan } & WC & PR & eff & NC & TR & efPoly & pwr & SMN & SMW \\
LPC & 674.60 & 1.097 & 0.9017 & 2242.889 & 1.0297 & 0.9030 & -1291.2 & -8700.18 & 52.77 \\
HPC & 79.90 & 1.397 & 0.8538 & 2242.889 & 1.1176 & 0.8605 & -605.9 & 5.97 & 2.61 \\
HPT & 51.59 & 5.809 & 0.8137 & 8878.896 & 1.7908 & 0.8526 & -3858.9 & 62.39 & 58.77 \\
LPT & 12.08 & 3.990 & 0.8747 & 218.753 & 1.3522 & 0.8529 & 3858.9 & &
\end{tabular}

TURBOMACHINERY MAP DATA

$\begin{array}{lrrrrrrrrr} & \text { WcMap } & \text { PRmap } & \text { effMap } & \text { NcMap } & \text { R/Parm } & \text { S_WcDes } & \text { S_PRdes } & \text { S_effDes } & \text { S_NcDes } \\ \text { Fan } & 783.82 & 1.095 & 0.9035 & 2221.503 & 0.0416 & 0.8607 & \overline{1} .0235 & 0.9980 & 0.9905 \\ \text { LPC } & 60.10 & 1.380 & 0.7653 & 0.592 & 0.0000 & 1.3295 & 1.0471 & 1.1156 & 0.0003 \\ \text { HPC } & 46.92 & 5.582 & 0.8222 & 8642.190 & 10.9446 & 1.0995 & 1.0494 & 0.9898 & 0.9733 \\ \text { HPT } & 0.96 & 3.907 & 0.8746 & 1.315 & 3.9072 & 12.6299 & 0.9723 & 1.0000 & 0.0003 \\ \text { LPT } & 0.81 & 2.022 & 0.8405 & 0.719 & 2.0225 & 65.2590 & 0.7521 & 0.9926 & 0.0005\end{array}$




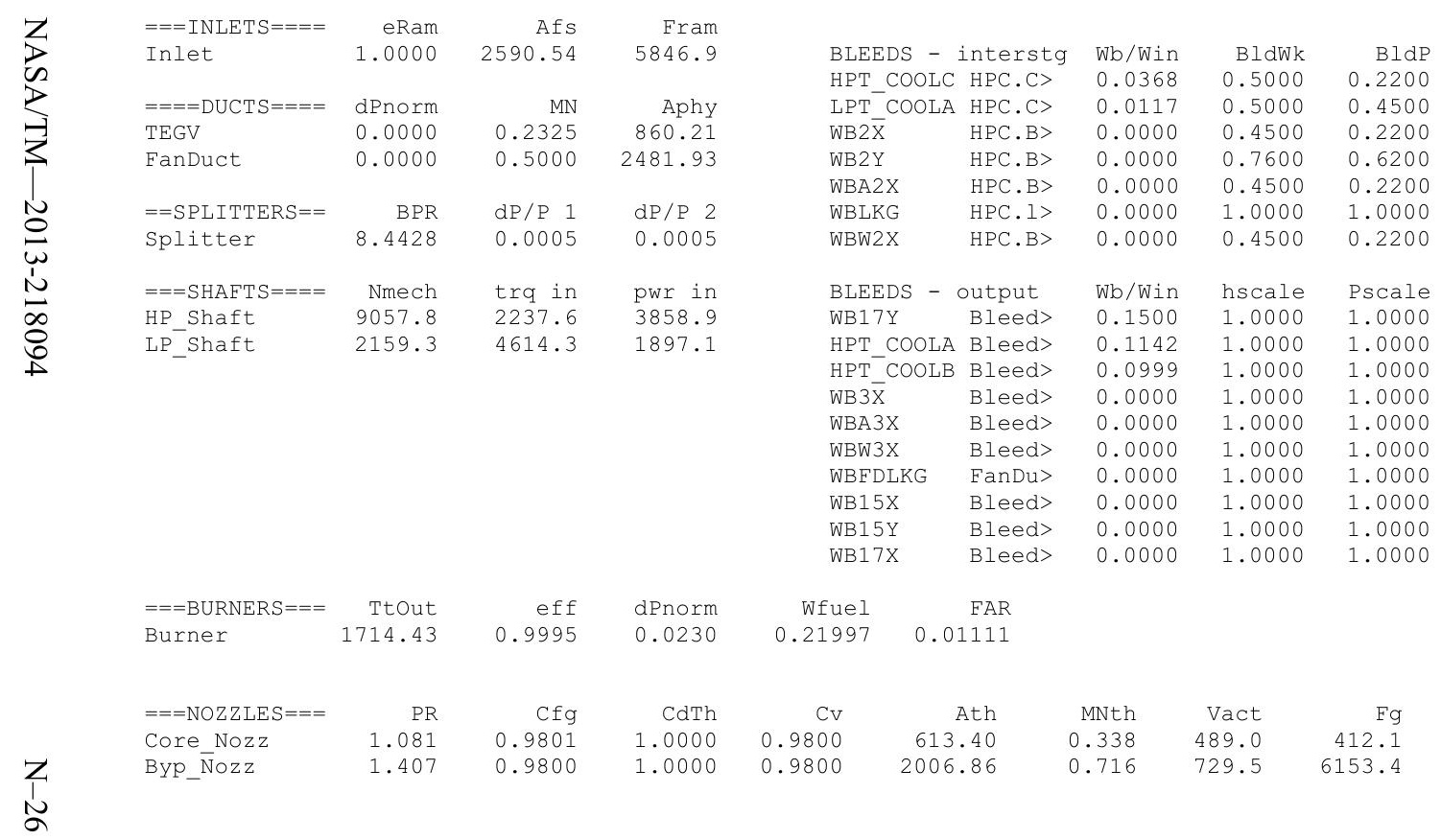


COnverge = 1 CASE: 0

Version:NPSS_1.6.5 - Rev: -> Gas Package: Janaf iter/pass/Jacb/Broy= 22/ 50/2/19 Run by: Philip C Jorgenson PC: 10

Temperature Stator 1 inlet: 492.34 Stator 1 exit: $496.52 \quad$ Stator 2 inlet: 501.28 Stator 2 exit: 503.16 $\begin{array}{lll}\text { Stator } 3 \text { inlet: } 508.43 & \text { Stator } 3 \text { exit: } 509.89 & \text { Stator } 4 \text { inlet: 514.95 Stator } 4 \text { exit: } 516.00 \\ \text { Stator } 5 \text { inlet: } 519.70 & \text { Stator } 5 \text { exit: } 520.47 & \text { Unblocked Percent Blockage: } 0.00\end{array}$

$\begin{array}{lclll}\text { Ambient Relative Humidity } & 10.00 & & & \\ \text { Fan Face Relative Humidity } & 5.02 & & & \\ \text { Fan Bypass Relative Humidity } & 4.20 & & & \\ \text { LPC Inlet Relative Humidity } & 4.03 & & & \\ \text { LPC Exit Relative Humidity } & 1.36 & & & \\ \text { HPC Relative Humidity } & 0.02 & & & \\ \text { Drop Diameter } & 0.000100 & \text { Inlet Length } & \\ \text { Ambient Flow Velocity } & 582.56 & \text { Fan/LPC Inlet Flow Velocity } & 195.14 \\ \text { Ambient Static Pressure } & 5.30 & \text { Fan/LPC Inlet Static Pressure } & 6.37 \\ \text { Ambient Static Temperature } & 463.14 & \text { Fan/LPC Inlet Static Temperature } & 488.24 \\ \text { Additional Water at LPC Exit } & 0.0016385 & & & \end{array}$

Additional Water at LPC Exit

SUMMARY OUTPUT DATA

\begin{tabular}{|c|c|c|c|c|c|c|c|c|c|c|c|c|c|c|}
\hline $\begin{array}{r}\mathrm{MN} \\
552\end{array}$ & alt & dTamb & $\begin{array}{r}W \\
322 \quad 38\end{array}$ & $\begin{array}{r}\text { UMMARY } \\
\text { Fn }\end{array}$ & $\begin{array}{r}\text { TPUT DA } \\
\text { TSFC }\end{array}$ & Wfuel & BPR & $\begin{array}{r}\text { VTAS } \\
582\end{array}$ & OPR & EPR & Powerset & T4 & $\begin{array}{r}\text { T41 } \\
\end{array}$ & $\begin{array}{r}\text { T49 } \\
\end{array}$ \\
\hline 0.552 & 25666.0 & 36.00 & 322.38 & 718.6 & 1.1634 & 836.02 & 7.1400 & 582.56 & 7.201 & 0.857 & 10.000 & 1718.4 & 1622.2 & 1143.8 \\
\hline
\end{tabular}

FSO Ambient.Fl_o

FS1 Inlet.F1_O

FS12 Splitter.Fl_02

FS14 Fan.Fl_O

FS24 VaporIN.F1_O

$\begin{array}{ll}\text { FS25 } & \text { Bleed2.FI__ } \\ \text { FS3 } & \text { HPC.Fl_O }\end{array}$

FS36 Bleed3.F1_O

FS4 Burner.FI_O

FS45 HPT.F1_O

FS49 LPT.FI-O

FS8 Core_Nozz.FI_O

FS17 Fanductikg.Fì

FS171 Bleed15.FI_O

FS171 Bleed15.F1_O
FS172 FanDuct.FI-O
FS173 Byp Nozz.FI O

TURBOMACHINERY PERFORMANCE DATA

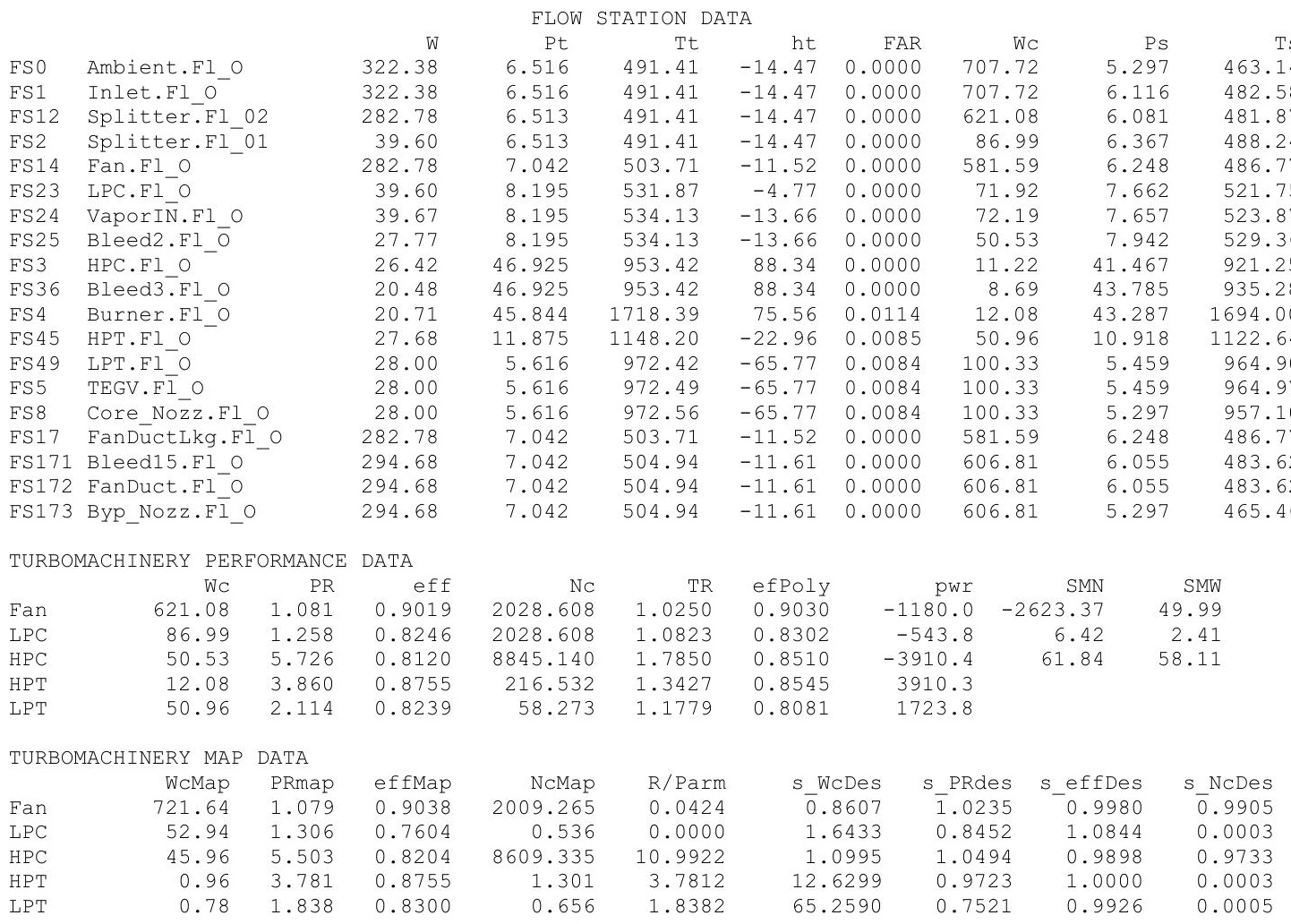

TURBOMACHINERY MAP DATA

\begin{tabular}{|c|c|c|c|c|c|c|c|c|c|c|c|}
\hline & & & W & $\begin{array}{l}\text { FLOW } S \\
\text { Pt }\end{array}$ & $\begin{array}{c}\text { STATION DA } \\
\text { Tt }\end{array}$ & ht & $\mathrm{FE}$ & & Wc & Ps & \\
\hline FSO & Ambient.Fl O & & 322.38 & 6.516 & 491.41 & -14.47 & 0.000 & 707. & .72 & & 463 \\
\hline FS1 & Inlet.Fl_O & & 322.38 & 6.516 & 491.41 & -14.47 & 0.000 & 707. & & & \\
\hline FS12 & Splitter.Fl 0 & & 282.78 & 6.513 & 491.41 & -14.47 & 0.000 & 621. & .08 & & 481 \\
\hline FS2 & Splitter.Fl_o & & 39.60 & 6.513 & 491.41 & -14.47 & 0.000 & 86. & .99 & & \\
\hline FS14 & Fan.Fl_O & & 282.78 & 7.042 & 503.71 & -11.52 & 0.000 & 581. & .59 & & 486 \\
\hline FS23 & LPC.FI_O & & 39.60 & 8.195 & 531.87 & -4.77 & 0.000 & 71. & .92 & & 521 \\
\hline FS24 & VaporI⿳亠丷⿵冂丶.FI_O & & 39.67 & 8.195 & 534.13 & -13.66 & 0.000 & 72 . & .19 & & \\
\hline FS25 & Bleed2.FI_ō & & 27.77 & 8.195 & 534.13 & -13.66 & 0.000 & 50.5 & .53 & & 529 \\
\hline 53 & HPC.FI_O & & 26.42 & 46.925 & 953.42 & 88.34 & 0.000 & 11.2 & .22 & & \\
\hline$\$ 36$ & Bleed3.Fl_O & & 20.48 & 46.925 & 953.42 & 88.34 & 0.000 & & .69 & & 93 \\
\hline$\$ 4$ & Burner.Fl_-O & & 20.71 & 45.844 & 1718.39 & 75.56 & 0.011 & 12.0 & .08 & & 169 \\
\hline 54 & HPT.FI_O & & 27.68 & 11.875 & 1148.20 & -22.96 & 0.008 & 50. & .96 & & \\
\hline 549 & LPT.FI_O & & 28.00 & 5.616 & 972.42 & -65.77 & 0.008 & 100.3 & .33 & & \\
\hline 5 & TEGV.F̄̄_O & & 00 & 5.616 & 972.49 & -65.77 & 0.0 & 100.3 & .33 & & \\
\hline 8 & Core_Nozz.FI & & 28.00 & 5.616 & 972.56 & -65.77 & 0.0 & 100.3 & .33 & & \\
\hline 517 & FanDúctLkg.Fi & & 282.78 & 7.042 & 503.71 & -11.52 & 0.0 & 581.5 & .59 & 48 & \\
\hline 171 & Bleed15.Fl_o & & 29 & 7.042 & 504.94 & -11.61 & 0.000 & $606.8-\gamma=1.8$ & .81 & 55 & \\
\hline 5172 & FanDuct.Fl_o & & 294.68 & 7.042 & 504.94 & -11.61 & 0.00 & 606.8 & .81 & 55 & 483 \\
\hline 173 & Byp_Nozz.Fin_o & & 294.68 & 7.042 & 504.94 & -11.61 & 0.000 & $606 . \varepsilon=1-2$ & .81 & 297 & \\
\hline TURBOM & MACHINERY PERE & ORMANCE & DATA & & & & & & & & \\
\hline & & PR & eff & $\mathrm{NC}$ & $T R$ & efPoly & & pwr & SMI & & SMW \\
\hline Fan & 621.08 & 1.081 & 0.9019 & 2028.608 & 1.0250 & 0.9030 & & 180.0 & -2623.37 & & 9.99 \\
\hline & 86.99 & 1.258 & 0.8246 & 2028.608 & 1.0823 & 0.8302 & & 543.8 & 6.42 & & 2.41 \\
\hline ?c & 50.53 & 5.726 & 0.8120 & 8845.140 & 1.7850 & 0.8510 & & 910.4 & 61.84 & & 3.11 \\
\hline HPT & 12.08 & 3.860 & 0.8755 & 216.532 & 1.3427 & 0.8545 & & 910.3 & & & \\
\hline LPT & 50.96 & 2.114 & 0.8239 & 58.273 & 1.1779 & 0.8081 & & 723.8 & & & \\
\hline JRBOM & MACHINERY MAP & DAT & & & & & & & & & \\
\hline & WcMap & PRmap & effMap & NcMap & R/Parm & $s_{n} n$ & Ees & S_PRdes & s_effL & & $\mathrm{S}_{-} \mathrm{NCDe}$ \\
\hline 111 & 721.64 & 1.079 & 0.9038 & 2009.265 & 0.0424 & $\overline{0} .8$ & 3607 & $\overline{1} .0235$ & 0.99 & & $\overline{0} .9905$ \\
\hline${ }^{2} \mathrm{C}$ & 52.94 & 1.306 & 0.7604 & 0.536 & 0.0000 & & 5433 & 0.8452 & 1.08 & & 0.0003 \\
\hline PC & 45.96 & 5.503 & 0.8204 & 8609.335 & 10.9922 & & 999 & 1.0494 & 0.98 & & 0.9733 \\
\hline PT & 0.96 & 3.781 & 0.8755 & 1.301 & 3.7812 & 12.6 & 5299 & 0.9723 & 1.00 & & 0.0003 \\
\hline $\mathrm{PT}$ & 0.78 & 1.838 & 0.8300 & 0.656 & 1.8382 & 65.2 & 2590 & 0.7521 & 0.99 & & 0.0005 \\
\hline
\end{tabular}




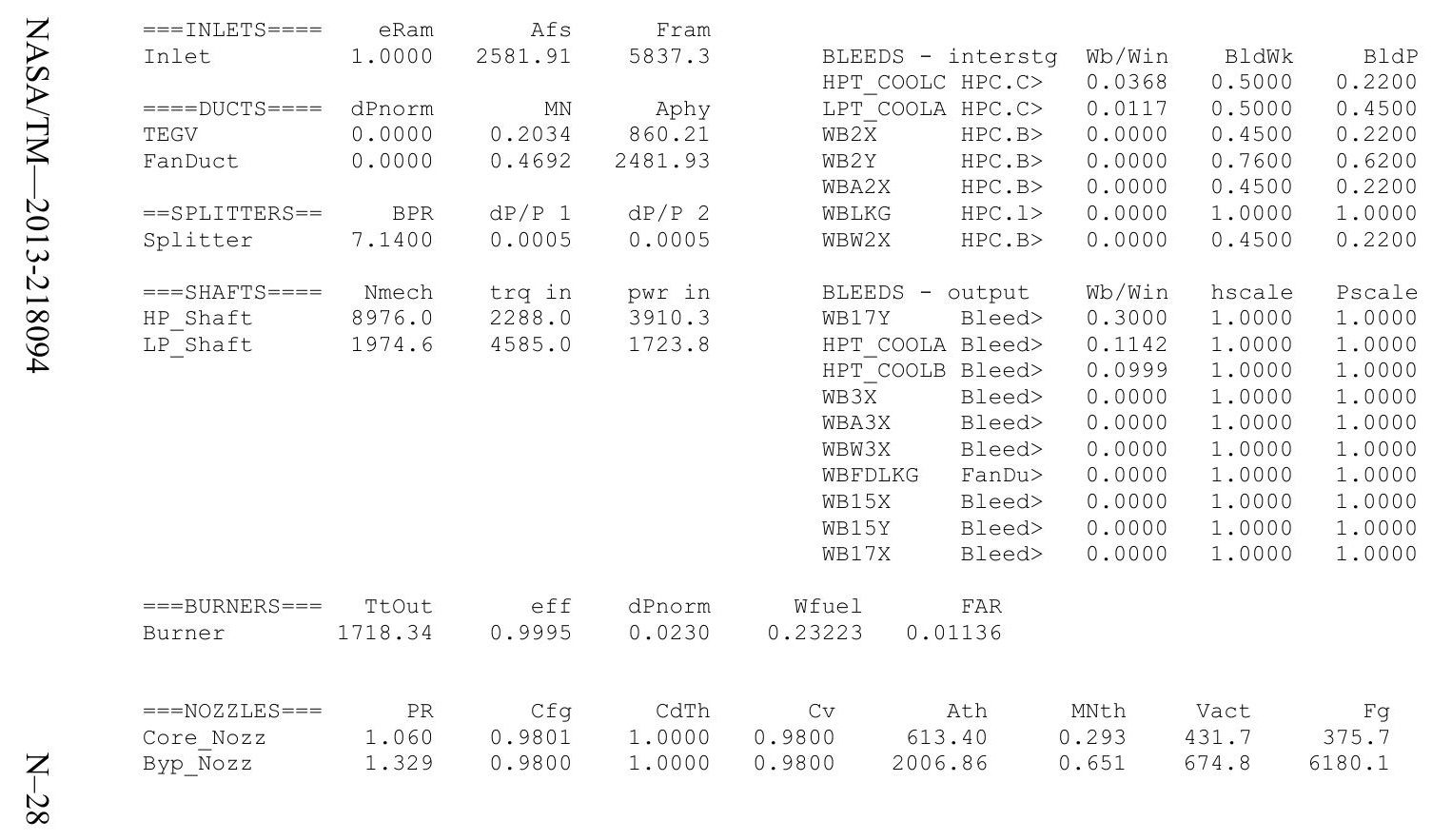


Tonverge = 1 CASE: 0

Version:NPSS_1.6.5 - Rev: -> Gas Package: Janaf iter/pass/Jacb/Broy= 29/57/2/26 Run by: Philip C Jorgenson PC: 10

Temperature Stator 1 inlet: 505.27 $\quad$ Stator 1 exit: $508.26 \quad$ Stator 2 inlet: 511.07 $\quad$ Stator 2 exit: 511.88 $\begin{array}{llll}\text { Stator } 3 \text { inlet: } 515.27 & \text { Stator } 3 \text { exit: } 515.65 & \text { Stator } 4 \text { inlet: 518.94 Stator 4 exit: } 519.02 \\ \text { Stator } 5 \text { inlet: } 520.94 & \text { Stator } 5 \text { exit: } 520.82 & \text { Unblocked Percent Blockage: } 0.00\end{array}$

$\begin{array}{lclll}\text { Ambient Relative Humidity } & 10.00 & & & \\ \text { Fan Face Relative Humidity } & 6.01 & & & \\ \text { Fan Bypass Relative Humidity } & 5.18 & & & \\ \text { LPC Inlet Relative Humidity } & 5.20 & & & \\ \text { LPC Exit Relative Humidity } & 2.82 & & & \\ \text { HPC Relative Humidity } & 0.03 & & & \\ \text { Drop Diameter } & 0.000100 & \text { Inlet Length } & & \\ \text { Ambient Flow Velocity } & 528.20 & \text { Fan/LPC Inlet Flow Velocity } & 202.80 \\ \text { Ambient Static Pressure } & 6.74 & \text { Fan/LPC Inlet Static Pressure } & 7.75 \\ \text { Ambient Static Temperature } & 483.18 & \text { Fan/LPC Inlet Static Temperature } & 502.99 \\ \text { Additional Water at LPC Exit } & 0.0016391 & & & \end{array}$

Additional Water at LPC Exit

MN alt dTamb SUMMARY OUTPUT DATA

$\begin{array}{rrr}\text { MN } & \text { alt } & \text { dTamb } \\ 0.490 & 20047.0 & 36.00\end{array}$

357.84

772.0

1.1870

Wfuel
916.37

BPR
6.3566

VTAS
528.20

OPR
6.264

EPR
0.888

Powerset

10.000
FSO Ambient.Fl_O

FS1 Inlet.FI_O

FS12 Splitter.F1_02

FS14 Fan.FI_O

FS24 VaporIN.FI_O

FS25 Bleed2.F1-

FS3 HPC.Fl_O

FS4 Burner.FI_O

FS49 LPT.FI_O

FS8 Core Nozz.Flo

FS17 FanDuctLkg.F̄_o

FS171 Bleed15.FI_O

FS172 FanDuct. F1-0

FS173 Byp Nozz.Fí

$W$
357.84

FLOW STATION DATA

$\begin{array}{rrrr}\text { Pt } & \text { Tt } & \text { ht } & \text { FAR } \\ 7.943 & 506.41 & -12.46 & 0.0000 \\ 7.943 & 506.41 & -12.46 & 0.0000 \\ 7.939 & 506.41 & -12.46 & 0.0000 \\ 7.939 & 506.41 & -12.46 & 0.0000 \\ 8.483 & 517.12 & -9.89 & 0.0000 \\ 9.069 & 534.49 & -5.72 & 0.0000 \\ 9.069 & 536.74 & -14.61 & 0.0000 \\ 9.069 & 536.74 & -14.61 & 0.0000 \\ 49.753 & 947.33 & 85.28 & 0.0000 \\ 49.753 & 947.33 & 85.28 & 0.0000 \\ 48.608 & 1742.04 & 72.01 & 0.0118 \\ 3.295 & 1174.54 & -24.25 & 0.0088 \\ 7.032 & 1020.75 & -61.62 & 0.0087 \\ 7.032 & 1020.82 & -61.62 & 0.0087 \\ 7.032 & 1020.89 & -61.62 & 0.0087 \\ 8.483 & 517.12 & -9.89 & 0.0000 \\ 8.483 & 518.28 & -10.17 & 0.0000 \\ 8.483 & 518.28 & -10.17 & 0.0000 \\ 8.483 & 518.28 & -10.17 & 0.0000\end{array}$

WC
654.18

654.18

654.18
565.54

88.97

534.8

80.02

80.32
48.19

48.19

8.61

12.09

48.48

86.45

86.46
86.46

86.46
534.86

534.86
569.22

569.22
569.22

569.22

Ps
6.740
7.530
7.509
7.753
7.693
8.324
8.317
8.814
44.103
46.497
45.894
12.335
6.887
6.887
6.740
7.693
7.463
7.463
6.740

Ts
483.18
498.74
498.40
502.99
502.86
521.56
523.64
532.40
916.15
929.71
1717.37
1151.28
1014.99
1015.06
1009.20
502.86
499.64
499.64
485.31

2591.9 MN

$\begin{array}{ll}3531.8 & 0.2833\end{array}$

$\begin{array}{rr}830.5 & 0.1844 \\ 2606.7 & 0.3763\end{array}$

$\begin{array}{ll}412.6 & 0.3520\end{array}$

$\begin{array}{ll}412.6 & 0.3538 \\ 412.6 & 0.2020\end{array}$

$\begin{array}{rrr}412.6 & 0.2020 \\ 49.7 & 0.4207\end{array}$

$\begin{array}{lll}49.3 & 0.3142\end{array}$

$\begin{array}{ll}74.6 & 0.2949 \\ 265.4 & 0.3332\end{array}$

$\begin{array}{ll}74.6 & 0.2949 \\ 860.2 & 0.3332\end{array}$

$\begin{array}{ll}860.2 & 0.1743\end{array}$

$\begin{array}{ll}613.4 & 0.2491 \\ 2481.9 & 0.3763\end{array}$

$\begin{array}{ll}2606.7 & 0.3763\end{array}$

$\begin{array}{ll}2481.9 & 0.4317 \\ 2481.9 & 0.4317\end{array}$

$2006.9 \quad 0.5826$

gamt
1.40031
1.40031
1.40031
1.40031
1.40017
1.39993
1.39967
1.39967
1.38355
1.38355
1.32952
1.36317
1.37316
1.37315
1.37315
1.40017
1.40015
1.40015
1.40015

1.40015

WAR
0.0005787
0.0005787
0.0005787
0.0005787
0.0005787
0.0005787
0.0022178
0.0022178
0.0022178
0.0022178
0.0022178
0.0022178
0.0022178
0.0022178
0.0022178
0.0005787
0.0006757
0.0006757
0.0006757

Wair $\begin{array}{lll}357.63 & 0.207 & 0.0006\end{array}$ $\begin{array}{lll}48.61 & 0.028 & 0.0006\end{array}$ $\begin{array}{lll}38.62 & 0.028 & 0.0006\end{array}$ $\begin{array}{lll}48.61 & 0.028 & 0.0006\end{array}$ $\begin{array}{lll}48.61 & 0.108 & 0.0002\end{array}$ $\begin{array}{lll}29.17 & 0.065 & 0.0022\end{array}$ $\begin{array}{lll}27.75 & 0.062 & 0.0022\end{array}$ $\begin{array}{lll}21.51 & 0.048 & 0.0022\end{array}$ $\begin{array}{lll}21.51 & 0.048 & 0.0166\end{array}$ $\begin{array}{lll}28.83 & 0.064 & 0.0130\end{array}$ $\begin{array}{lll}29.17 & 0.065 & 0.0129\end{array}$ $\begin{array}{lll}29.17 & 0.065 & 0.0129\end{array}$ $\begin{array}{lll}29.17 & 0.065 & 0.0129\end{array}$ $\begin{array}{lll}309.02 & 0.179 & 0.0006\end{array}$ $\begin{array}{lll}328.46 & 0.222 & 0.0007\end{array}$ $\begin{array}{lll}328.46 & 0.222 & 0.0007\end{array}$ 0.0007

\begin{tabular}{|c|c|c|c|c|c|c|c|c|c|}
\hline & W & & & & & & & & \\
\hline Fan & 565.54 & 1.068 & $\begin{array}{r}\text { etf } \\
0.9037\end{array}$ & 1818.644 & 1.0211 & $\begin{array}{l}\text { efPoly } \\
0.9046\end{array}$ & $\begin{array}{r}\text { pwr } \\
-1124.1\end{array}$ & -1837.84 & $\begin{array}{r}\text { SMW } \\
46.55\end{array}$ \\
\hline LPC & 88.97 & 1.142 & 0.6988 & 1818.644 & 1.0554 & 0.7044 & -463.8 & 7.11 & 2.05 \\
\hline HPC & 48.19 & 5.486 & 0.8076 & 8765.766 & 1.7650 & 0.8467 & -4031.4 & 62.22 & 58.20 \\
\hline HPT & 12.09 & 3.656 & 87 & 213.647 & 1.3264 & 0.8570 & 4031.4 & & \\
\hline LPT & 48.48 & 1.891 & .81 & 52.435 & 1.1473 & 0.8020 & 1587.9 & & \\
\hline & NERY M & DA & & & & & & & \\
\hline & WcMap & PRmap & effmap & NcMap & R/Parm & S_WcDes & S_PRdes & S_effDes & S_NCDes \\
\hline an & 657.10 & 1.067 & 0.9056 & 1801.304 & 0.0437 & $\overline{0} .8607$ & $\overline{1} .0235$ & 0.9980 & $\overline{0} .9905$ \\
\hline${ }^{\mathrm{C}} \mathrm{C}$ & 46.11 & 1.2 & .73 & 0. & 0.0000 & 1.9 & 0.5948 & 0.9257 & 0.0003 \\
\hline${ }^{\mathrm{C}} \mathrm{C}$ & 43.83 & 5.275 & .816 & 8532.076 & 11.0030 & 1.0995 & 1.0494 & 0.9898 & 0.9733 \\
\hline $\mathrm{T}$ & 0.96 & 3.582 & 证 & 1.284 & 3.5824 & 12.6299 & 0.9723 & 1.0000 & 0.00 \\
\hline $\mathrm{PT}$ & 0.74 & 1.670 & 0.8218 & 0.590 & 1.6699 & 65.2590 & 0.7521 & 0.9926 & 0.0005 \\
\hline
\end{tabular}




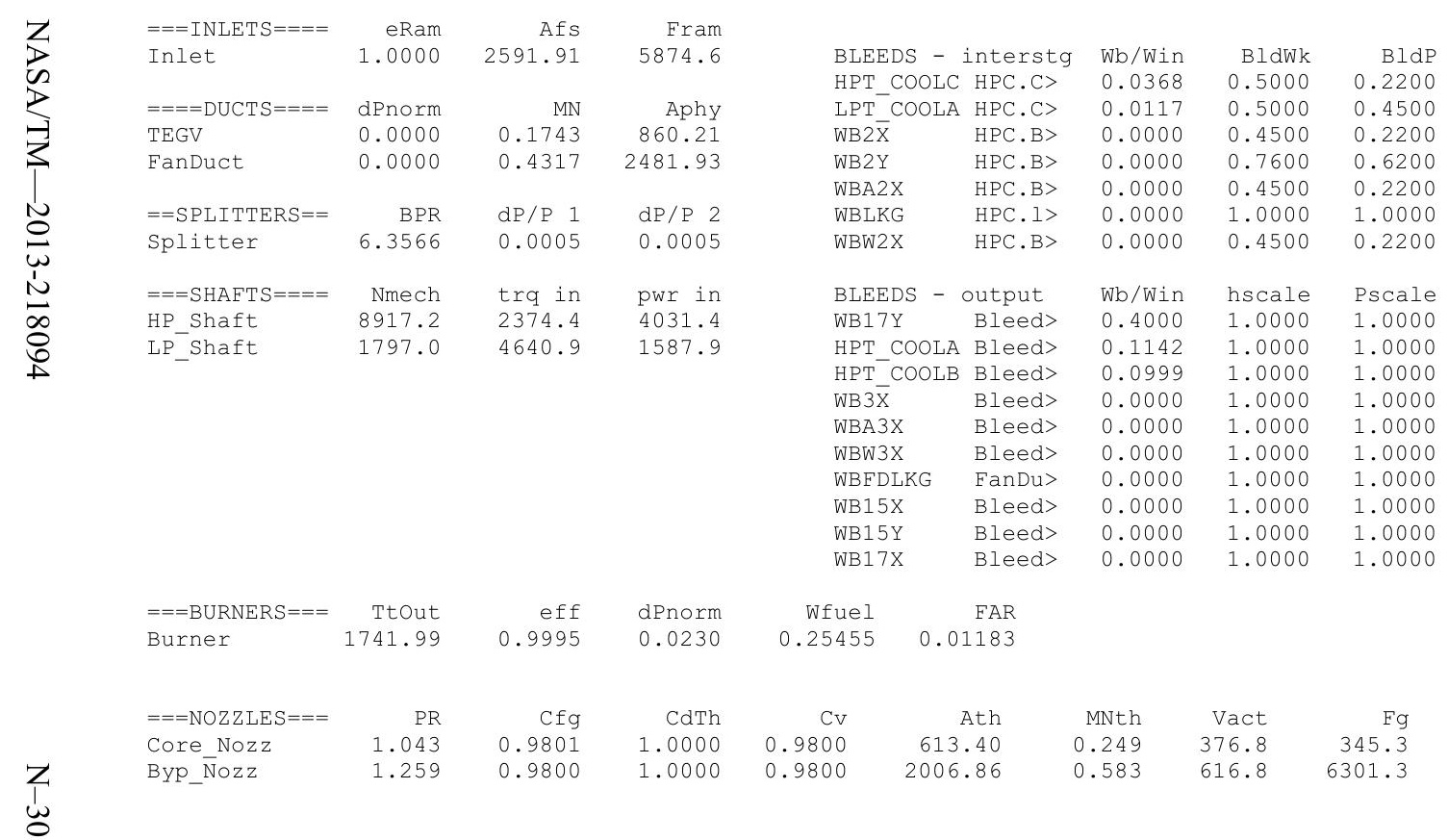




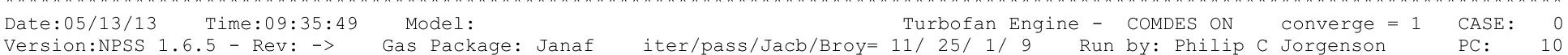

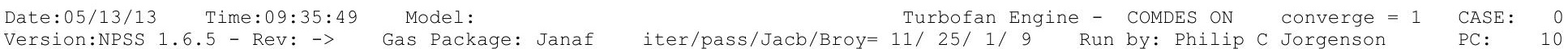

$\begin{array}{lllll}\text { Temperature Stator } 1 \text { inlet: } 463.25 & \text { Stator } 1 \text { exit: } 470.65 & \text { Stator } 2 \text { inlet: } 479.96 & \text { Stator } 2 \text { exit: } 484.71\end{array}$

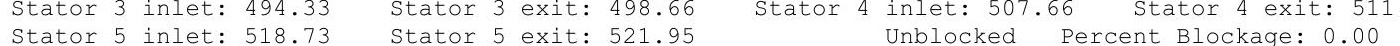

Ambient Relative Humidity $\quad 10.00$

Humidity $\quad 1.76$

Fan Bypass Relative Humidity 1.24

$\begin{array}{ll}\text { LPC Exit Relative Humidity } & 1.13 \\ \text { HPC Relative Humidity } & 0.00\end{array}$

Drop Diameter

Ambient Flow Velocity

0.0000250

Ambient Static Pressure

Ambient Static Temperature

772.64
2.85

Inlet Length $\quad 40.00$

Fan/IPC Inlet Static Pressure $\quad 187.85$

407.97 Fan/LPC Inlet Static Temperature 454.80

SUMMARY OUTPUT DATA

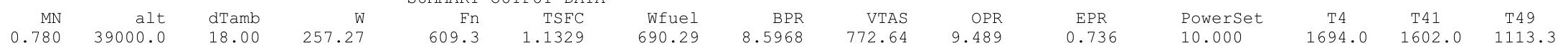

\begin{tabular}{|c|c|}
\hline FSO & Ambient.Fl_o \\
\hline FS1 & Inlet.Fl_o \\
\hline FS12 & Splitter.Fl_02 \\
\hline FS2 & Splitter.Fl-01 \\
\hline FS14 & Fan.Fl_O \\
\hline FS23 & LPC.FI_O \\
\hline FS24 & VaporIN. \\
\hline FS25 & Bleed2.Fl o \\
\hline FS3 & HPC.FI_O ${ }^{-}$ \\
\hline FS36 & Bleed3.FI_O \\
\hline FS 4 & Burner.Fl_o \\
\hline FS45 & HPT.FI_O \\
\hline FS49 & LPT.FI_O \\
\hline FS5 & TEGV.F̄__O \\
\hline FS 8 & Core_Nozzz.FI_O \\
\hline FS17 & FanDuctLkg.FI_o \\
\hline FS171 & Bleed15.F1 O \\
\hline FS172 & FanDuct.Fl_o \\
\hline FS173 & Byp Nozz.Fi 0 \\
\hline
\end{tabular}

\begin{tabular}{rrrr} 
& \multicolumn{1}{c}{ FLOW } & STATION DATA \\
W & Pt & Tt & \\
257.27 & 4.267 & 457.74 & -20.
\end{tabular}

FLOW STATION DATE

$\begin{array}{llllll} & 4.267 & 457.74 & -20.96 & 0.0000 & 832.43 \\ 257.27 & 4.267 & 457.74 & -20.96 & 0.0000 & 832.43\end{array}$

$\begin{array}{llllll}4.265 & 457.74 & -20.96 & 0.0000 & 832.43 \\ 230.46 & 4.26000 & 746.07\end{array}$

$\begin{array}{llllll}26.81 & 4.265 & 457.74 & -20.96 & 0.0000 & 86.78\end{array}$

$\begin{array}{llllll}230.46 & 4.811 & 475.50 & -16.70 & 0.0000 & 674.00\end{array}$

$\begin{array}{llllll}26.81 & 6.640 & 531.29 & -3.32 & 0.0000 & 60.05\end{array}$

$\begin{array}{llllll}26.81 & 6.640 & 531.65 & -4.61 & 0.0000 & 60.08\end{array}$

$\begin{array}{rrrrrr}24.13 & 6.640 & 531.65 & -4.61 & 0.0000 & 54.08 \\ 22.96 & 40.489 & 964.75 & 100.64 & 0.0000 & 11.37\end{array}$

$\begin{array}{llllll}17.80 & 40.489 & 964.75 & 100.64 & 0.0000 & 8.8\end{array}$

$\begin{array}{rrrrrr}17.99 & 39.556 & 1694.00 & 88.37 & 0.0108 & 12.08 \\ 24.04 & 9.490 & 1117.39 & -13.58 & 0.0080 & 54.65\end{array}$

$\begin{array}{rrrrrr}24.04 & 9.490 & 1117.39 & -13.58 & 0.0080 & 54.65 \\ 24.32 & 3.259 & 875.68 & -72.60 & 0.0079 & 142.53\end{array}$

$\begin{array}{llllll}24.32 & 3.259 & 875.68 & -72.60 & 0.0079 & 142.53 \\ 24.32 & 3.259 & 875.75 & -72.60 & 0.0079 & 142.5\end{array}$

$\begin{array}{llllll}24.32 & 3.259 & 875.82 & -72.60 & 0.0079 & 142.54\end{array}$

230.46

233.14

233.14

4.811

4.811

4.811

475.50

$\begin{array}{llll}476.14 & -16.56 & 0.0000 & 682.31\end{array}$

$\begin{array}{lll}-16.56 & 0.0000 & 682.31\end{array}$

$\begin{array}{llll}476.14 & -16.56 & 0.0000 & 682.31\end{array}$

TURBOMACHINERY PERFORMANCE DATA

\begin{tabular}{|c|c|c|c|c|c|c|c|c|c|}
\hline & WC & $\mathrm{PR}$ & eff & Ne & $T R$ & efPoly & nwr & SMN & SMW \\
\hline Fan & 746.07 & 1.128 & 0.9051 & 2561.318 & 1.0388 & 0.9068 & $\begin{array}{r}\text { PWI } \\
-1387.4\end{array}$ & 1659.17 & 54.93 \\
\hline LPC & 86.78 & 1.557 & 0.8401 & 2561.318 & 1.1607 & 0.8498 & -668.8 & 6.95 & 3.68 \\
\hline HPC & 54.08 & 6.098 & 0.8186 & 8960.575 & 1.8147 & 0.8574 & -3506.7 & 61.09 & 57.76 \\
\hline HPT & 12.08 & 4.168 & 0.8739 & 220.416 & 1.3661 & 0.8513 & 3506.7 & & \\
\hline LPT & 54.65 & 2.912 & 0.8507 & 71.982 & 1.2742 & 0.8305 & 2056.2 & & \\
\hline URB & NERY M & DATA & & & & & & & \\
\hline & WcMap & PRmap & effMap & NcMap & R/Parm & s_WCDes & S_PRdes & S_effDes & S_NCDes \\
\hline an & 866.85 & 1.125 & 0.9070 & 2536.896 & 0.0413 & $\overline{0} .8607$ & $\overline{1} .0235$ & 0.9980 & $\overline{0} .9905$ \\
\hline $\mathrm{PC}$ & 73.61 & 1.519 & 0.7796 & 0.677 & 0.0000 & 1.1790 & 1.0734 & 1.0776 & 0.0003 \\
\hline HPC & 49.18 & 5.857 & 0.8271 & 8721.692 & 10.9923 & 1.0995 & 1.0494 & 0.9898 & 0.9733 \\
\hline $\mathrm{HPT}$ & 0.96 & 4.081 & 0.87 & 1.325 & 4.0806 & 12.6299 & 0.9723 & 1.0000 & 0.0003 \\
\hline LPT & 0.84 & 2.438 & 0.8570 & 0.810 & 2.4380 & 65.2590 & 0.7521 & 0.9926 & 0.0005 \\
\hline
\end{tabular}

\begin{tabular}{|c|c|}
\hline \\
\hline 2.85 & 407 \\
\hline 3.89 & 445 \\
\hline 3.84 & 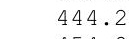 \\
\hline 4.17 & 454 \\
\hline 4.03 & 452 \\
\hline 6.34 & 524 \\
\hline 6.34 & 524 \\
\hline 6.40 & 526 \\
\hline 35.63 & 931 \\
\hline 37.70 & 945 \\
\hline 37.35 & 1669 \\
\hline 8.59 & 1088 \\
\hline 3.06 & 861 \\
\hline 3.06 & 861. \\
\hline 2.85 & 844 \\
\hline 4.03 & 452 \\
\hline 3.90 & 448 \\
\hline 3.90 & 448 \\
\hline 2.85 & 410 \\
\hline & SMW \\
\hline & 4.93 \\
\hline 5 & 3.68 \\
\hline 9 & 7.76 \\
\hline fDes & S_NcDes \\
\hline 9980 & $\overline{0} .9905$ \\
\hline 0776 & 0.0003 \\
\hline 9898 & 0.9733 \\
\hline 0000 & 0.0003 \\
\hline 9926 & 0.0005 \\
\hline
\end{tabular}

$\operatorname{LP}$

$\begin{array}{rrr}\text { Aphy } & \text { MN } & \text { gamt } \\ 2539.5 & 0.7800 & 1.40084 \\ 4168.2 & 0.3642 & 1.40084 \\ 3531.8 & 0.3896 & 1.40084 \\ 830.5 & 0.1796 & 1.40084 \\ 2606.7 & 0.5066 & 1.40071 \\ 412.6 & 0.2551 & 1.40005 \\ 412.6 & 0.2553 & 1.40001 \\ 412.6 & 0.2280 & 1.40001 \\ 49.7 & 0.4331 & 1.38282 \\ 49.3 & 0.3225 & 1.38282 \\ 74.6 & 0.2941 & 1.33277 \\ 265.4 & 0.3824 & 1.36768 \\ 860.2 & 0.2961 & 1.38271 \\ 860.2 & 0.2961 & 1.38270 \\ 613.4 & 0.4418 & 1.38270 \\ 2606.7 & 0.5066 & 1.40071 \\ 2481.9 & 0.5544 & 1.40070 \\ 2481.9 & 0.5544 & 1.40070 \\ 2006.9 & 0.8967 & 1.40070\end{array}$

$\begin{array}{rrrr}\text { WAR } & \text { Wair } & \text { WH2O } & \text { H2O frac } \\ 0.0000295 & 257.26 & 0.008 & 0.0000 \\ 0.0000295 & 257.26 & 0.008 & 0.0000 \\ 0.0000295 & 230.46 & 0.007 & 0.0000 \\ 0.0000295 & 26.81 & 0.001 & 0.0000 \\ 0.0000295 & 230.46 & 0.007 & 0.0000 \\ 0.0000295 & 26.81 & 0.001 & 0.0000 \\ 0.0002677 & 26.81 & 0.007 & 0.0003 \\ 0.0002677 & 24.13 & 0.006 & 0.0003 \\ 0.0002677 & 22.96 & 0.006 & 0.0003 \\ 0.0002677 & 17.79 & 0.005 & 0.0003 \\ 0.0002677 & 17.79 & 0.005 & 0.0134 \\ 0.0002677 & 23.84 & 0.006 & 0.0101 \\ 0.0002677 & 24.13 & 0.006 & 0.0100 \\ 0.0002677 & 24.13 & 0.006 & 0.0100 \\ 0.0002677 & 24.13 & 0.006 & 0.0100 \\ 0.0000295 & 230.46 & 0.007 & 0.0000 \\ 0.0000322 & 233.14 & 0.008 & 0.0000 \\ 0.0000322 & 233.14 & 0.008 & 0.0000 \\ 0.0000322 & 233.14 & 0.008 & 0.0000\end{array}$




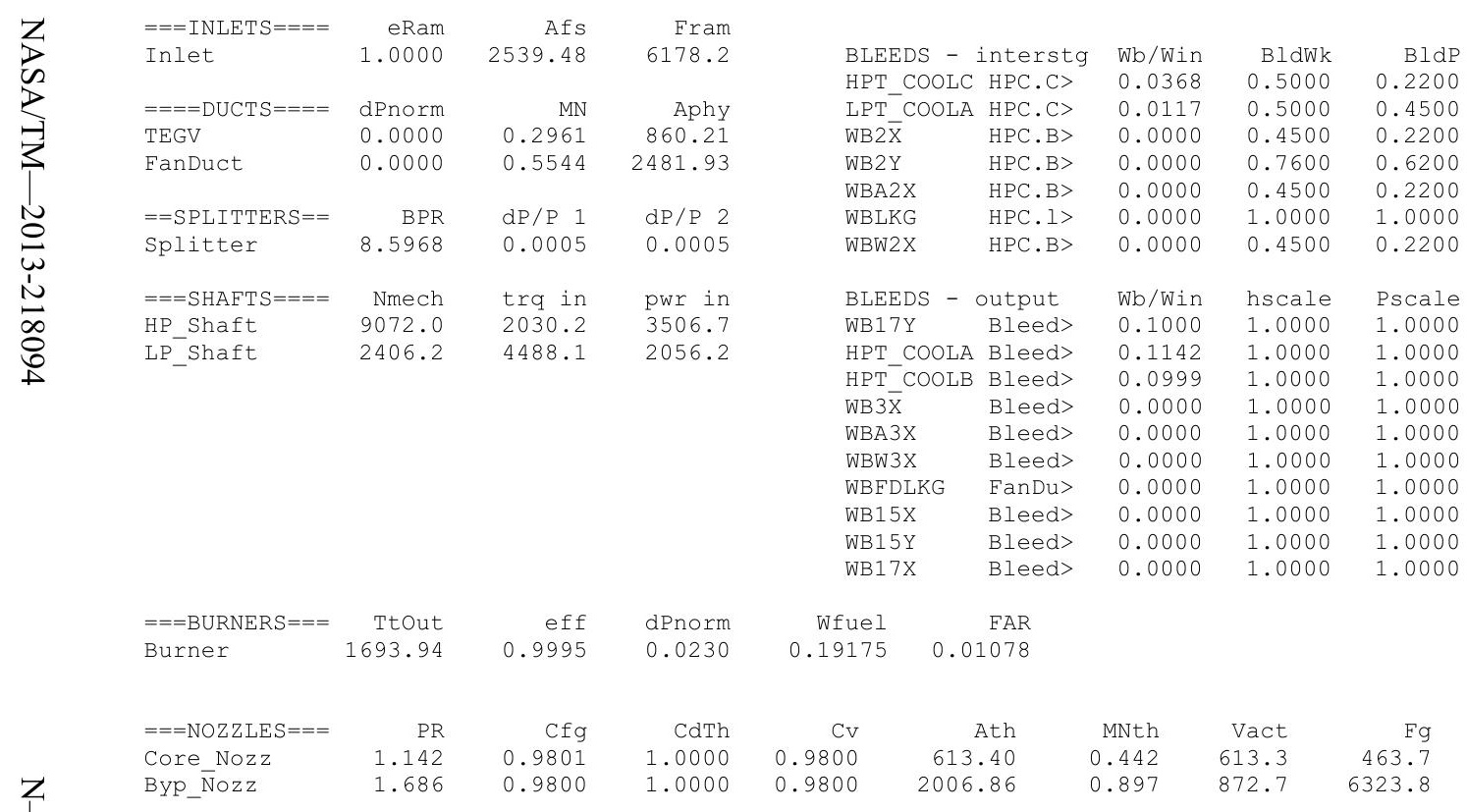


Date:05/13/13 Time:09:36:06 Model: Turbofan Engine - COMDES ON Converge = 1 CASE: 0

Version:NPSS 1.6.5 - Rev: -> Gas Package: Janaf iter/pass/Jacb/Broy=11/25/1/9 Run by: Philip C Jorgenson PC: 10

Temperature Stator 1 inlet: 456.76 Stator 1 exit: 463.70 Stator 2 inlet: 472.41 Stator 2 exit: 476.85

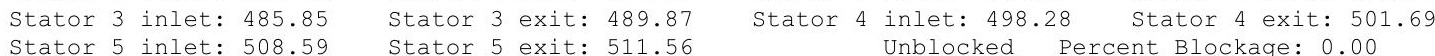

Ambient Relative Humidity $\quad 10.00$

Fan Face Relative Humidity $\quad 2.23$

Fan Bypass Relative Humidity $\quad 1.61$

LPC Exit Relative Humidity $\quad 0.13$

HPC Relative Humidity

Drop Diameter 0.00
0.0000250
723.11

723.11
2.95

Inlet Length

40.00

Ambient Static Pressure

Ambient Static Temperature

407.97

Fan/LPC Inlet Flow Velocity $\quad 181.19$ 0.0002037

Fan/LPC Inlet Static Temperature 448.83

MUMMARY OUTPUT DATA

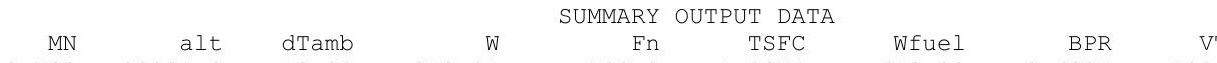

\section{FLOW STATION DATA}

FSO Ambient.Fl_O

FS1 Inlet.F1_O

FS12 Splitter.F1_02

FS14 Fan.F1

FS23 Fan.F1-

FS24 VaporIN.FI_O

FS25 Bleed2.F1_0

FS3 HPC.FI_O

FS4 Bleed3.F1_O

$\begin{array}{ll}\text { FS4 } & \text { Burner.FI } \\ \text { FS45 } & \text { HPT.FI O }\end{array}$

$\begin{array}{ll}\text { FS49 } & \text { LPT.FI-O } \\ \text { FS5 } & \text { TEGV.FI' }\end{array}$

FS8 Core_Nozz.FI_O

FS17 FanDuctLkg.Fi_o

FS171 Bleed15.F1_O

FS172 FanDuct.Fl_O

$W$
250.31
250.31
224.48
25.83
224.48
25.83
25.84
23.25
22.13
17.15
17.33
23.17
23.44
23.44
23.44
224.48
227.06
227.06
227.06

$\begin{array}{rrrr}\text { Pt } & \text { Tt } & \text { ht } & \text { FAR } \\ 4.201 & 451.57 & -22.43 & 0.0000\end{array}$

$\begin{array}{llll}4.201 & 451.57 & -22.43 & 0.0000\end{array}$

$\begin{array}{llll}4.199 & 451.57 & -22.43 & 0.0000\end{array}$

$\begin{array}{llll}4.707 & 468.13 & -18.46 & 0.0000\end{array}$

$\begin{array}{llll}6.403 & 520.46 & -5.92 & 0.0000\end{array}$

$\begin{array}{ll}6.403 & 520.46 \\ 6.403 & 520.77\end{array}$

$\begin{array}{ll}6.403 & 520.46 \\ 6.403 & 520.77\end{array}$

$\begin{array}{rr}6.403 & 520.77 \\ 38.693 & 943.36\end{array}$

$38.693 \quad 943.36$

$37.802 \quad 1667.78$

$9.183 \quad 1101.44$

$3.309 \quad 873.50$

$3.309 \quad 873.57$

3.309

4.707

4.707
4.707

4.707

873.65

468.13
468.73

468.73

468.73

TURBOMACHINERY PERFORMANCE DATA

$\begin{array}{lrrrrrrrrr} & \text { Wc } & \text { PR } & \text { eff } & \text { NC } & \text { TR } & \text { efPoly } & \text { pwr } & \text { SMN } & \text { SMW } \\ \text { Fan } & 733.02 & 1.121 & 0.9043 & 2498.137 & 1.0367 & 0.9059 & -1260.4 & 2272.33 & 54.77 \\ \text { LPC } & 84.35 & 1.525 & 0.8404 & 2498.137 & 1.1526 & 0.8496 & -603.6 & 6.76 & 3.33 \\ \text { HPC } & 53.48 & 6.043 & 0.8178 & 8941.494 & 1.8115 & 0.8566 & -3292.8 & 61.00 & 57.62 \\ \text { HPT } & 12.08 & 4.116 & 0.8727 & 219.391 & 1.3628 & 0.8500 & 3292.8 & & \\ \text { LPT } & 54.02 & 2.775 & 0.8452 & 70.235 & 1.2589 & 0.8254 & 1864.0 & & \end{array}$

TURBOMACHINERY MAP DATA

$\begin{array}{lrrrrrrrrr} & \text { WcMap } & \text { PRmap } & \text { effMap } & \text { NCMap } & \text { R/Parm } & \text { S_WcDes } & \text { S_PRdes } & \text { S_effDes } & \text { S_NcDes } \\ \text { Fan } & 851.70 & 1.118 & 0.9062 & 2474.317 & 0.0412 & 0.8607 & \overline{1} .0235 & 0.9980 & 0.9905 \\ \text { LPC } & 70.87 & 1.491 & 0.7766 & 0.660 & 0.0000 & 1.1901 & 1.0693 & 1.0822 & 0.0003 \\ \text { HPC } & 48.64 & 5.806 & 0.8263 & 8703.120 & 11.0050 & 1.0995 & 1.0494 & 0.9898 & 0.9733 \\ \text { HPT } & 0.96 & 4.030 & 0.8726 & 1.319 & 4.0300 & 12.6299 & 0.9723 & 1.0000 & 0.0003 \\ \text { LPT } & 0.83 & 2.335 & 0.8515 & 0.790 & 2.3350 & 65.2590 & 0.7521 & 0.9926 & 0.0005\end{array}$

$\begin{array}{lll}0.83 & 2.335 & 0.8515\end{array}$
0.790

65.2590

0.0005

$\begin{array}{llccc}\text { EPR } & \text { PowerSet } & \text { T4 } & \text { T41 } & \text { T49 } \\ 0.763 & 10.000 & 1667.8 & 1576.4 & 1097.4\end{array}$
0.0000285 0.0000285 0.0000285 0000285 0.0002323 0.0002323

0.0002323

0.0002323

0.0002323

0.0002323

0.0002323

0.0002323

0.0000285

0.0000308

0.0000308

$\begin{array}{rrr}\text { Wair } & \text { WH2O } & \text { H2O frac } \\ 250.31 & 0.007 & 0.0000 \\ 250.31 & 0.007 & 0.0000 \\ 224.47 & 0.006 & 0.0000 \\ 25.83 & 0.001 & 0.0000 \\ 224.47 & 0.006 & 0.0000 \\ 25.83 & 0.001 & 0.0000 \\ 25.83 & 0.006 & 0.0002 \\ 23.25 & 0.005 & 0.0002 \\ 22.12 & 0.005 & 0.0002 \\ 17.14 & 0.004 & 0.0002 \\ 17.14 & 0.004 & 0.0132 \\ 22.98 & 0.005 & 0.0100 \\ 23.25 & 0.005 & 0.0099 \\ 23.25 & 0.005 & 0.0099 \\ 23.25 & 0.005 & 0.0099 \\ 224.47 & 0.006 & 0.0000 \\ 227.06 & 0.007 & 0.0000 \\ 227.06 & 0.007 & 0.0000 \\ 227.06 & 0.007 & 0.0000\end{array}$




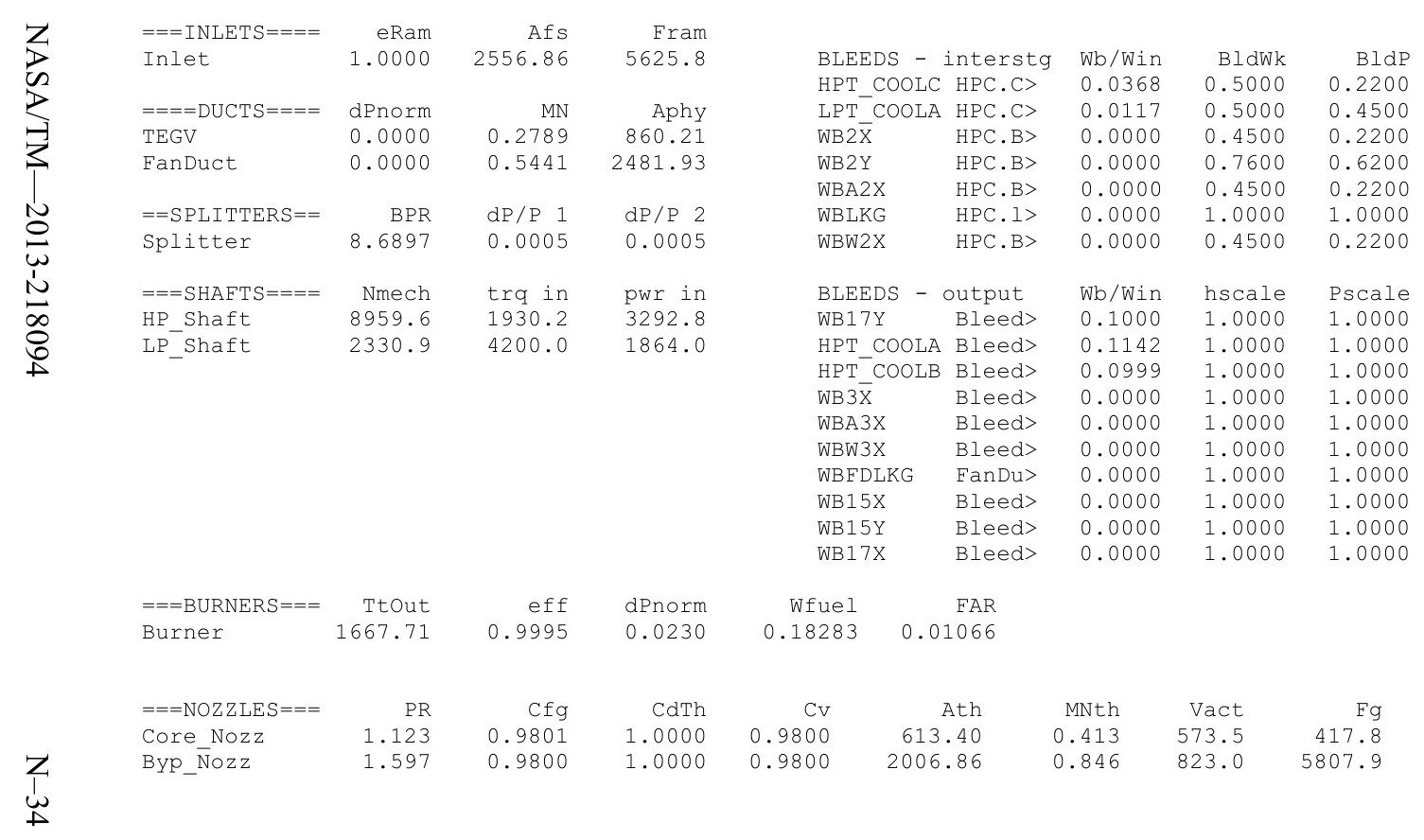


Date:05/13/13 Time:09:36:22 Model: Turbofan Engine - ComDES ON Converge = 1 CASE: 0

Version:NPSS 1.6.5 - Rev: -> Gas Package: Janaf iter/pass/Jacb/Broy=10/24/1/8 Run by: Philip C Jorgenson PC: 10

Temperature Stator 1 inlet: $454.76 \quad$ Stator 1 exit: $461.53 \quad$ Stator 2 inlet: $470.02 \quad$ Stator 2 exit: 474.35 $\begin{array}{llll}\text { Stator } 3 \text { inlet: } 483.10 & \text { Stator } 3 \text { exit: } 487.02 & \text { Stator } 4 \text { inlet: } 495.21 & \text { Stator } 4 \text { exit: } 498.5 \\ \text { Stator } 5 \text { inlet: } 505.24 & \text { Stator } 5 \text { exit: } 508.12 & \text { Unblocked Percent Blockage: } 0.00\end{array}$

Ambient Relative Humidity $\quad 10.00$

Fan Face Relative Humidity $\quad 2.40$

Fan Bypass Relative Humidity $\quad 1.75$

LPC Inlet Relative Humidity $\quad 1.56$

rop Diative Humidity

Drop Diameter

Ambient static Pressure

Additional Water at LPC Exit

Inlet Length

40.00

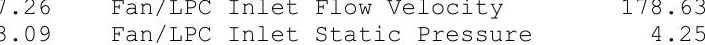
0.0001836 an/LPC Inlet static Temperature

$\begin{array}{rrrrrr} & & & & \text { SUMMARY } & \text { OUTPUT DATA } \\ \text { MN } & \text { alt } & \text { dTamb } & \text { W } & \text { Fn } & \text { TSFC } \\ 0.714 & 37357.0 & 18.00 & 257.07 & 615.0 & 1.0895\end{array}$

W
257.07

$\mathrm{Fn}$
615.0

TSFC
1.0895

Wfuel
670.02

BPR
8.7284

VTAS
707.26

OPR
9.092

EPR
0.772

PowerSet
10.000

$\begin{array}{ccc}\text { T4 } & \text { T41 } & \text { T49 } \\ 1657.8 & 1566.8 & 1091.3\end{array}$
FSO Ambient.Fl O

FS1 Inlet.Fl_o

FS12 Splitter.F1_02

FS2 Splitter.Fl_01

FS14 Fan.F1-

FS24 VaporIN. FI

S25 Bleed2.F1

FS3 HPC.FI_O

ES46 Bleed3.FI_O

ES4

FS49

FS4 5 TEGV.FI

FS8 Core_Nozz.Fl_O

FS17 FanDūctLkg.F̄

FS171 Bleed15.FI

FS172 FanDuct.Fl-O

FS173 Byp Nozz.FİO

TURBOMACHINERY PERFORMANCE DATA

$\begin{array}{lrrr} & \text { WC } & \text { PR } & \text { eff } \\ \text { Fan } & 727.67 & 1.118 & 0.9040 \\ \text { LPC } & 83.37 & 1.512 & 0.8405 \\ \text { HPC } & 53.22 & 6.016 & 0.8174 \\ \text { HPT } & 12.08 & 4.099 & 0.8721 \\ \text { LPT } & 53.81 & 2.726 & 0.8431\end{array}$

FLOW STATION DATA

$W$
257.07
257.07
230.64
26.42
230.64
26.42
26.43
23.79
22.63
17.54
17.73
23.69
23.97
23.97
23.97
230.64
233.28
233.28
233.28

Pt
4.339

Tt
449.68

$\begin{array}{rr}\text { ht } & \text { FAR } \\ -22.87 & 0.0000\end{array}$

4.339
4.337

4.337

4.849

6.558

6.558

39.454

38.545

38.545
9.404
3.450

3.450

3.450

3.450

4.849

4.849

4.849

4.849

$\begin{array}{lllll}44.68 & -22.87 & 0.0000 & 810.63 \\ 4.68 & -22.87 & 0.0000 & 810.63\end{array}$

$\begin{array}{lllll}449.68 & -22.87 & 0.0000 & 810.63 \\ 449.68 & -22.87 & 0.0000 & 727.67\end{array}$

$\begin{array}{lllll}449.68 & -22.87 & 0.0000 & 83.37\end{array}$

$\begin{array}{rrrr}516.85 & -6.77 & 0.0000 & 59.11\end{array}$

$\begin{array}{llll}517.13 & -7.76 & 0.0000 & 59.14 \\ 517.13 & -7.76 & 0.0000 & 53.22\end{array}$

$\begin{array}{llll}517.13 & -7.76 & 0.0000 & 59.14 \\ 535.85 & -7.76 & 0.0000 & 53.22 \\ 935.85 & 93.82 & 0.0000 & 11.32\end{array}$

$\begin{array}{rrrr}935.85 & 93.82 & 0.0000 & 8.78 \\ 935.85 & 81.79 & 0.0106 & 12.08\end{array}$

$\begin{array}{rr}93.82 & 0.0000 \\ -16.56 & 0.0106\end{array}$

872.52

$\begin{array}{lll}872.52 & -70.78 & 0.0078 \\ 872.59 & -70.78 & 0.0078\end{array}$

$\begin{array}{lll}872.66 & -70.78 & 0.0078\end{array}$

465.80

$-19.010 .0000$

466.38

$\begin{array}{ll}-18.88 & 0.0000\end{array}$

$\begin{array}{lll}6.38 & -18.88 & 0.0000\end{array}$

$\begin{array}{ll}-18.88 & 0.0000\end{array}$

12.08

132.45

132.46

132.46
662.47

670.48

670.48

670.48

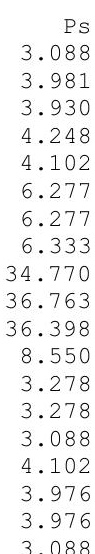

Ts
407.97
438.71
437.15
447.02
444.04
510.42
510.70
511.98
903.51
917.65
1634.13
1067.57
860.23
860.30
846.30
444.04
440.64
440.64
409.90

$\begin{array}{rr}\text { Aphy } & \text { MN } \\ 2561.5 & 0.7140\end{array}$

$\begin{array}{rrr}2561.5 & 0.7140 & 1.40090\end{array}$

$\begin{array}{ll}4168.2 & 0.3530 \\ 3531.8 & 0.3780\end{array}$

$830.5 \quad 0.1723$

$2606.7 \quad 0.4945$

$\begin{array}{ll}412.6 & 0.2509\end{array}$

$\begin{array}{cc}412.6 & 0.2241 \\ 49.7 & 0.4308\end{array}$

$\begin{array}{ll}49.7 & 0.4308 \\ 49.3 & 0.3209\end{array}$

$\begin{array}{lll}74.6 & 0.2940\end{array}$

$\begin{array}{ll}265.4 & 0.3752\end{array}$

860.20 .2730

$\begin{array}{ll}860.2 & 0.2730 \\ 613.4 & 0.4029\end{array}$

$\begin{array}{ll}2606.7 & 0.4945 \\ 2481.9 & 0.5399\end{array}$

$\begin{array}{ll}2481.9 & 0.5399 \\ 2481.9 & 0.5399\end{array}$

$2006.9 \quad 0.8290$ gamt
1.40090
1.40090
1.40090
1.40090
1.40079
1.40025
1.40023
1.40023
1.38453
1.38453
1.33463
1.36921
1.38297
1.38296
1.38296
1.40079
1.40078
1.40078
1.40078

WAR
0.0000272
0.0000272
0.0000272
0.0000272
0.0000272
0.0000272
0.0002108
0.0002108
0.0002108
0.0002108
0.0002108
0.0002108
0.0002108
0.0002108
0.0002108
0.0000272
0.0000293
0.0000293
0.0000293
Wair WH2O H2O frac 257.06 257.06 $26.42 \quad 0.001$ $\begin{array}{rr}230.64 & 0.006\end{array}$ $26.42 \quad 0.006$ $23.78 \quad 0.005$ $22.63 \quad 0.005$

$17.54 \quad 0.004$

$23.50-0.004$
23.78

$\begin{array}{ll}23.78 & 0.005\end{array}$

$\begin{array}{ll}23.78 & 0.005\end{array}$

$\begin{array}{rr}23.78 & 0.005 \\ 230.64 & 0.006\end{array}$

$\begin{array}{ll}230.64 & 0.006 \\ 233.28 & 0.007\end{array}$

$\begin{array}{ll}233.28 & 0.007 \\ 233.28 & 0.007\end{array}$
0.0000 0.0000 0.0000 0.0000 0.0002 0.0002 0.0002 .0132 0.0098 0.0098 0.0098 0.0000 0.0000 0.0000 0.0000

TURBOMACHINERY MAP DATA

$\begin{array}{lrrrrrrrrr} & \text { WcMap } & \text { PRmap } & \text { effMap } & \text { NcMap } & \text { R/Parm } & \text { S_WcDes } & \text { S_PRdes } & \text { S_effDes } & \text { S_NcDes } \\ \text { Fan } & 845.48 & 1.115 & 0.9058 & 2449.180 & 0.0412 & 0.8607 & \overline{1} .0235 & 0.9980 & 0.9905 \\ \text { LPC } & 69.77 & 1.479 & 0.7754 & 0.653 & 0.0000 & 1.1947 & 1.0683 & 1.0839 & 0.0003 \\ \text { HPC } & 48.41 & 5.780 & 0.8259 & 8694.837 & 11.0051 & 1.0995 & 1.0494 & 0.9898 & 0.9733 \\ \text { HPT } & 0.96 & 4.013 & 0.8721 & 1.317 & 4.0129 & 12.6299 & 0.9723 & 1.0000 & 0.0003 \\ \text { LPT } & 0.82 & 2.298 & 0.8494 & 0.783 & 2.2982 & 65.2590 & 0.7521 & 0.9926 & 0.0005\end{array}$




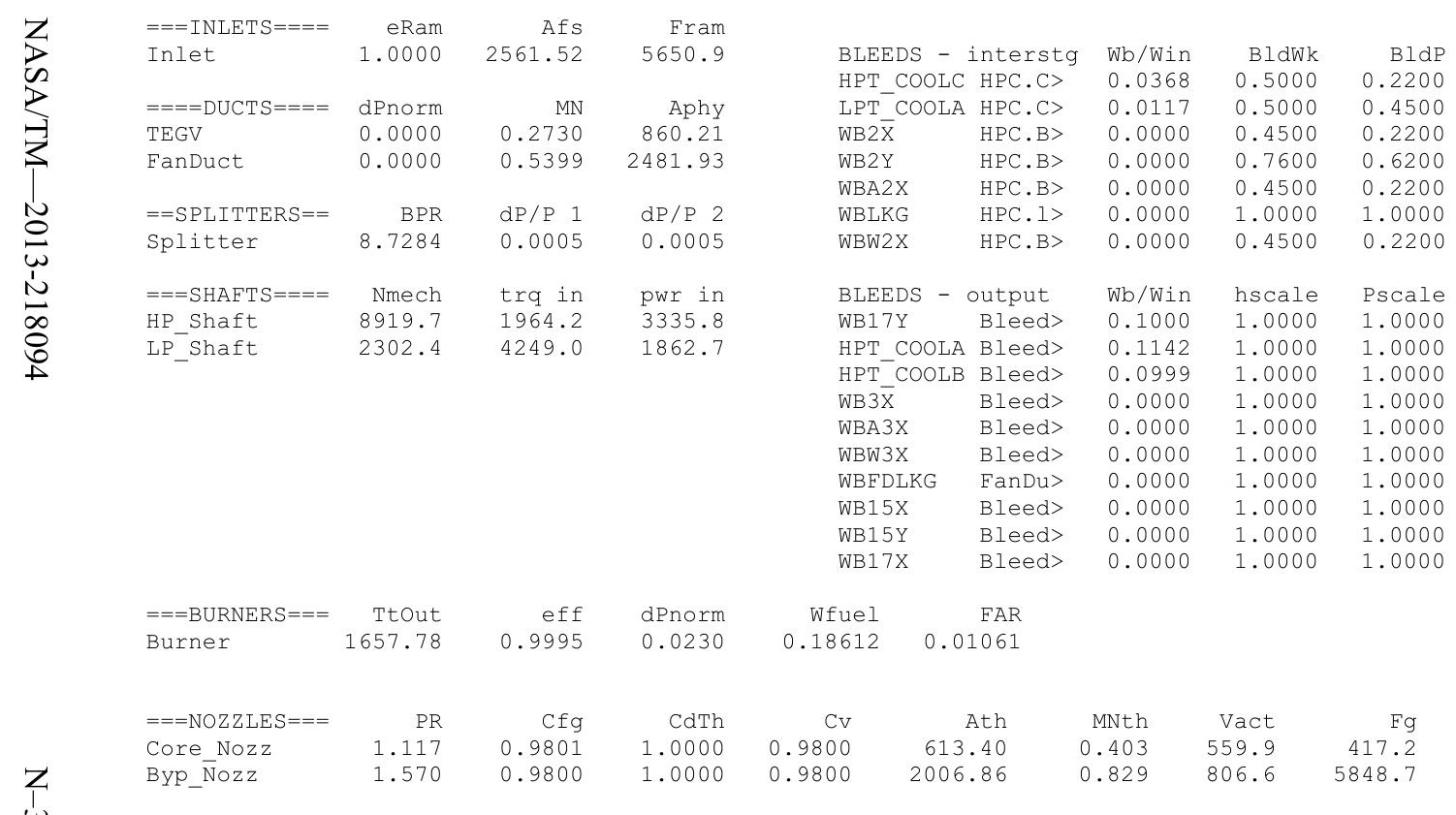




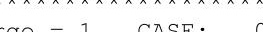

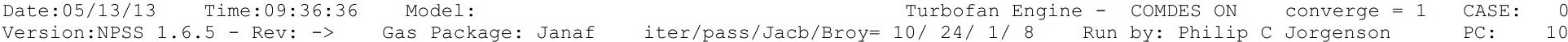

Temperature Stator 1 inlet: $456.41 \quad$ Stator 1 exit: $462.77 \quad$ Stator 2 inlet: 470.74 Stator 2 exit: 474.79 Stator 3 inlet: 483.00 Stator 3 exit: 486.65 Stator 4 inlet: 494.33 Stator 4 exit: 497.40 Stator 5 inlet: 503.68 Stator 5 exit: $506.34 \quad$ Unblocked Percent Blockage: 0.00

Ambient Relative Humidity $\quad 10.00$

Fan Face Relative Humidity $\quad 3.00$

2.00

LPC Exit Relative Humidity

HPC Relative Humidity

Drop Diameter

0.00

low velocity

Ambient Static Pressure

Additional Water at LPC Exi

$\begin{array}{clr}0.0000250 & \text { Inlet Length } & 40.00 \\ 667.90 & \text { Fan/LPC Inlet Flow Velocity } & 173.00 \\ 3.58 & \text { Fan/LPC Inlet Static Pressure } & 4.74 \\ 414.42 & \text { Fan/LPC Inlet Static Temperature } & 449.11 \\ 0.0001599 & & \end{array}$

\section{SUMMARY OUTPUT DATA}

$0.669 \quad 34281.0 \quad$ alt $\quad$ dTamb

W
58

$18.00 \quad 278.58$

$\begin{array}{rr}\text { Fn } & \text { TSFC } \\ 670.2 & 1.0732\end{array}$

VTAS
667.90

$\mathrm{OPR}$
8.757

EPR
0.795

Powerset

$\mathrm{T} 4$

T41

T49

FLOW STATION DATA

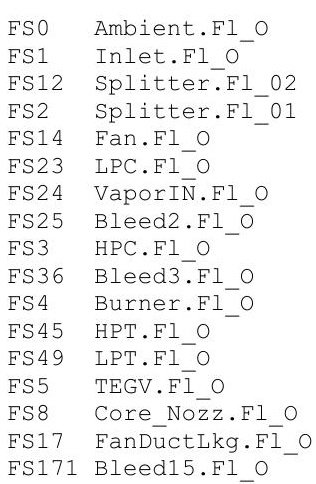

$\begin{array}{lll}\mathrm{Pt} & \mathrm{Tt} \\ 4.831 & 451.61 & -22 .\end{array}$

$W$
278.58
278.58
250.18
28.40
250.18
28.40
28.40
25.56
24.32
18.85
19.05
25.46
25.76
25.76
25.76
250.18
253.02
253.02
253.02

4.831

4.831
4.829

4.829

5.360

7.123
7.123

7.123

2.307

451.61
451.61

ht FAR

$\begin{array}{lll}451.61 & -22.45 & 0.0000\end{array}$

$\begin{array}{lll}451.61 & -22.45 & 0.0000\end{array}$

$\begin{array}{lll}466.78 & -18.82 & 0.0000\end{array}$

$\begin{array}{lll}514.73 & -7.33 & 0.0000 \\ 514.98 & -8.19 & 0.0000\end{array}$

$\begin{array}{lll}514.98 & -8.19 & 0.0000\end{array}$

$\begin{array}{lrrr}.307 & 928.99 & 92.22 & 0.0000\end{array}$

$\begin{array}{rrrr}41.333 & 1650.94 & 80.22 & 0.0106 \\ 10.193 & 1092.77 & -16.96 & 0.0079 \\ 3.942 & 881.65 & -68.26 & 0.0078\end{array}$

$\begin{array}{rrrr}0.193 & 1092.77 & -16.96 & 0.0079 \\ 3.942 & 881.65 & -68.26 & 0.0078\end{array}$

$\begin{array}{llll}3.942 & 881.65 & -68.26 & 0.0078 \\ 3.942 & 881.72 & -68.26 & 0.0078\end{array}$

$\begin{array}{llll}3.942 & 881.79 & -68.26 & 0.0078 \\ 5.360 & 466.78 & -18.82 & 0.0000\end{array}$

$\begin{array}{llll}5.360 & 467.32 & -18.82 & 0.0000\end{array}$

$\begin{array}{llll}5.360 & 467.32 & -18.70 & 0.0000\end{array}$

$\begin{array}{llll}5.360 & 467.32 & -18.70 & 0.0000\end{array}$

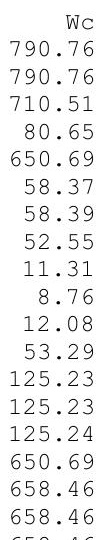

PS
3.578

$\begin{array}{ll}3.578 & 414.42 \\ 4.453 & 44.21\end{array}$

4.453

4.736

4.571

6.826

6.826
6.884

37.302

$\begin{array}{rr}39.030 & 910.97\end{array}$

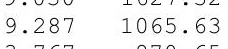

$\begin{array}{ll}9.287 & 1065.63 \\ 3.767 & 870.65 \\ 3.578 & 870.72\end{array}$

870.72
858.47

$3.578 \quad 858.47$

4.440

445.98
442.80

$\begin{array}{ll}4.440 & 442.80 \\ 3.578 & 416.27\end{array}$

$\begin{array}{rr}\text { Aphy } & \mathrm{MN} \\ 2577.4 & 0.6690 \\ 4168.2 & 0.3430 \\ 3531.8 & 0.3674 \\ 830.5 & 0.1665 \\ 2606.7 & 0.4824 \\ 412.6 & 0.2474 \\ 412.6 & 0.2475 \\ 412.6 & 0.2211 \\ 49.7 & 0.4299 \\ 49.3 & 0.3203 \\ 74.6 & 0.2940 \\ 265.4 & 0.3708 \\ 860.2 & 0.2569 \\ 860.2 & 0.2569 \\ 613.3 & 0.3765 \\ 2606.7 & 0.4824 \\ 2481.9 & 0.5257 \\ 2481.9 & 0.5257 \\ 2006.9 & 0.7821\end{array}$

gamt
1.40088
1.40088
1.40088
1.40088
1.40078
1.40028
1.40026
1.40026
1.38493
1.38493
1.33498
1.36939
1.38247
1.38246
1.38246
1.40078
1.40078
1.40078
1.40078

WAR

0.00000348
0.0000348

0.0000348

0.0000348

0.0000348

0.0001947

0.0001947
0.0001947

0.0001947
0.00001947

0.0001947
0.0001947

0.0001947

0.0001947

0.0001947

0.0001947

0.0000366

0.0000366
0.0000366

TURBOMACHINERY PERFORMANCE DATA

\begin{tabular}{|c|c|c|c|c|c|c|c|c|c|}
\hline & WC & PR & eff & NC & $T R$ & efPoly & pwr & SMN & SMW \\
\hline Fan & 710.51 & 1.110 & 0.9032 & 2396.066 & 1.0336 & 0.9046 & -1286.9 & 5760.24 & 54.20 \\
\hline LPC & 80.65 & 1.475 & 0.8415 & 2396.066 & 1.1398 & 0.8500 & -607.9 & 6.41 & 3.05 \\
\hline HPC & 52.55 & 5.940 & 0.8161 & 8910.957 & 1.8039 & 0.8550 & -3543.2 & 61.35 & 57.86 \\
\hline HPT & 12.08 & 4.055 & 0.8716 & 218.528 & 1.3585 & 0.8490 & 3543.3 & & \\
\hline LPT & 53.29 & 2.586 & 0.8386 & 67.635 & 1.2372 & 0.8196 & 1894.7 & & \\
\hline \multicolumn{10}{|c|}{ TURBOMACHINERY MAP DATA } \\
\hline & WcMap & PRmap & effmap & NcMap & R/Parm & s_WcDes & S_PRdes & s_effDes & S_NCDes \\
\hline Fan & 825.54 & 1.108 & 0.9050 & 2373.220 & 0.0413 & $\overline{0} .8607$ & $\overline{1} .0235$ & 0.9980 & $\overline{0} .9905$ \\
\hline PC & 66.45 & 1.445 & 18 & 0.633 & 0.0000 & 1.2 & 1.0675 & 1.0 & 0.0003 \\
\hline HPC & 47.79 & 5.707 & 0.8245 & 8673.396 & 10.9953 & 1. & 1.0494 & 0.9898 & 0. \\
\hline HPT & 0.96 & 3.9 & 0. & 1.313 & 3.9704 & 12. & 0.9723 & 1.0000 & 0.000 \\
\hline PT & 0.82 & 2.193 & 0.8448 & 0.761 & 2.1929 & 65.2590 & 0.7521 & 0.9926 & 0.000 \\
\hline
\end{tabular}




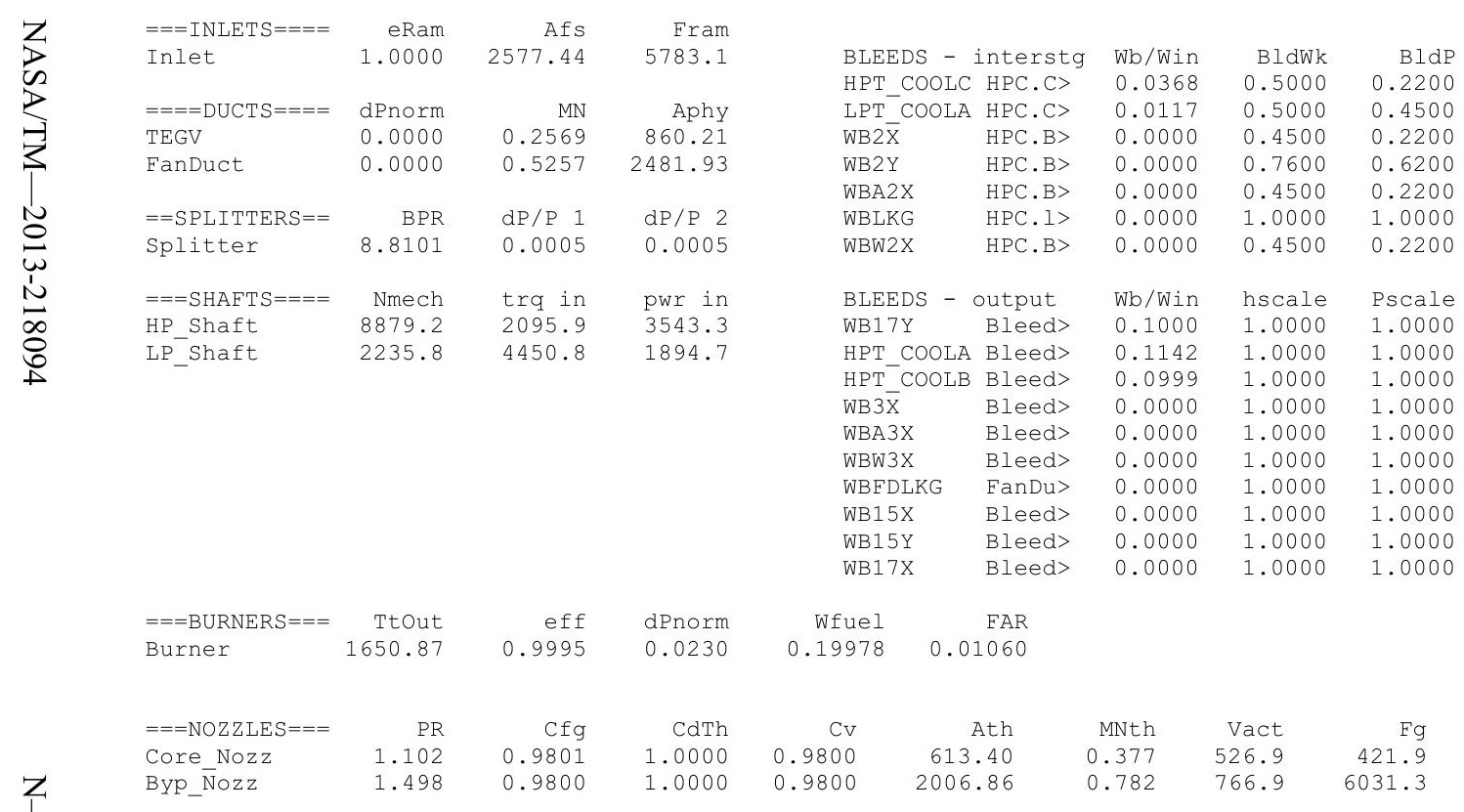


Turbofan Engine - COMDES ON Converge = 1 CASE: 0

Version:NPSS_1.6.5 - Rev: -> Gas Package: Janaf iter/pass/Jacb/Broy=13/27/1/11 Run by: Philip C Jorgenson PC: 10

Temperature Stator 1 inlet: $465.05 \quad$ Stator 1 exit: $470.56 \quad$ Stator 2 inlet: 477.38 Stator 2 exit: 480.67

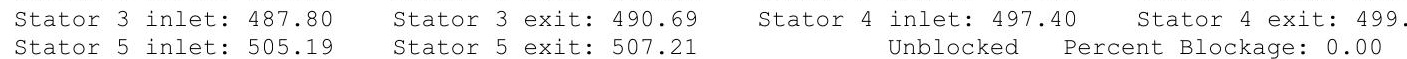

$\begin{array}{lclll}\text { Ambient Relative Humidity } & 10.00 & & & \\ \text { Fan Face Relative Humidity } & 3.96 & & & \\ \text { Fan Bypass Relative Humidity } & 3.12 & & & \\ \text { LPC Inlet Relative Humidity } & 2.83 & & & \\ \text { LPC Exit Relative Humidity } & 0.47 & & & \\ \text { HPC Relative Humidity } & 0.00 & & & \\ \text { Drop Diameter } & 0.0000250 & \text { Inlet Length } & \\ \text { Ambient Flow Velocity } & 617.99 & \text { Fan/LPC Inlet Flow Velocity } & 174.95 \\ \text { Ambient Static Pressure } & 4.36 & \text { Fan/LPC Inlet Static Pressure } & 5.49 \\ \text { Ambient Static Temperature } & 429.58 & \text { Fan/LPC Inlet Static Temperature } & 458.87 \\ \text { Additional Water at LPC Exit } & 0.0001731 & & & \\ \end{array}$
W
305.10
SUMMARY OUTPUT DATA

$\begin{array}{rr}\text { Fn } & \text { TSFC } \\ 729.6 & 1.0759\end{array}$

Wfuel
784.96 784.96
BPR
8.3732

VTAS
617.99
$\mathrm{OPR}$
8.197
Powerset 10.000 $\begin{array}{ccc}\text { T4 } & \text { T41 } & \text { T49 } \\ 1655.4 & 1563.6 & 1094.2\end{array}$
FSO Ambient. F1_O

FS1 Inlet.F1_O

FS12 Splitter.Fl_02

FS14 Fan.F1_O

F24 VaporIN.F1_O

Fileed2.F1-O

FS36 Bleed3.F1_O

FS4 Burner.FI_-

FS45 HPT.Fl_

FS49 LPT.FI-O

FS8 Core_Nozz.FI_O

FS17 FanDuctLkg.Fì

FS171 Bleed15.FI_O

FS172 FanDuct.FI-O

TURBOMACHINERY PERFORMANCE DATA

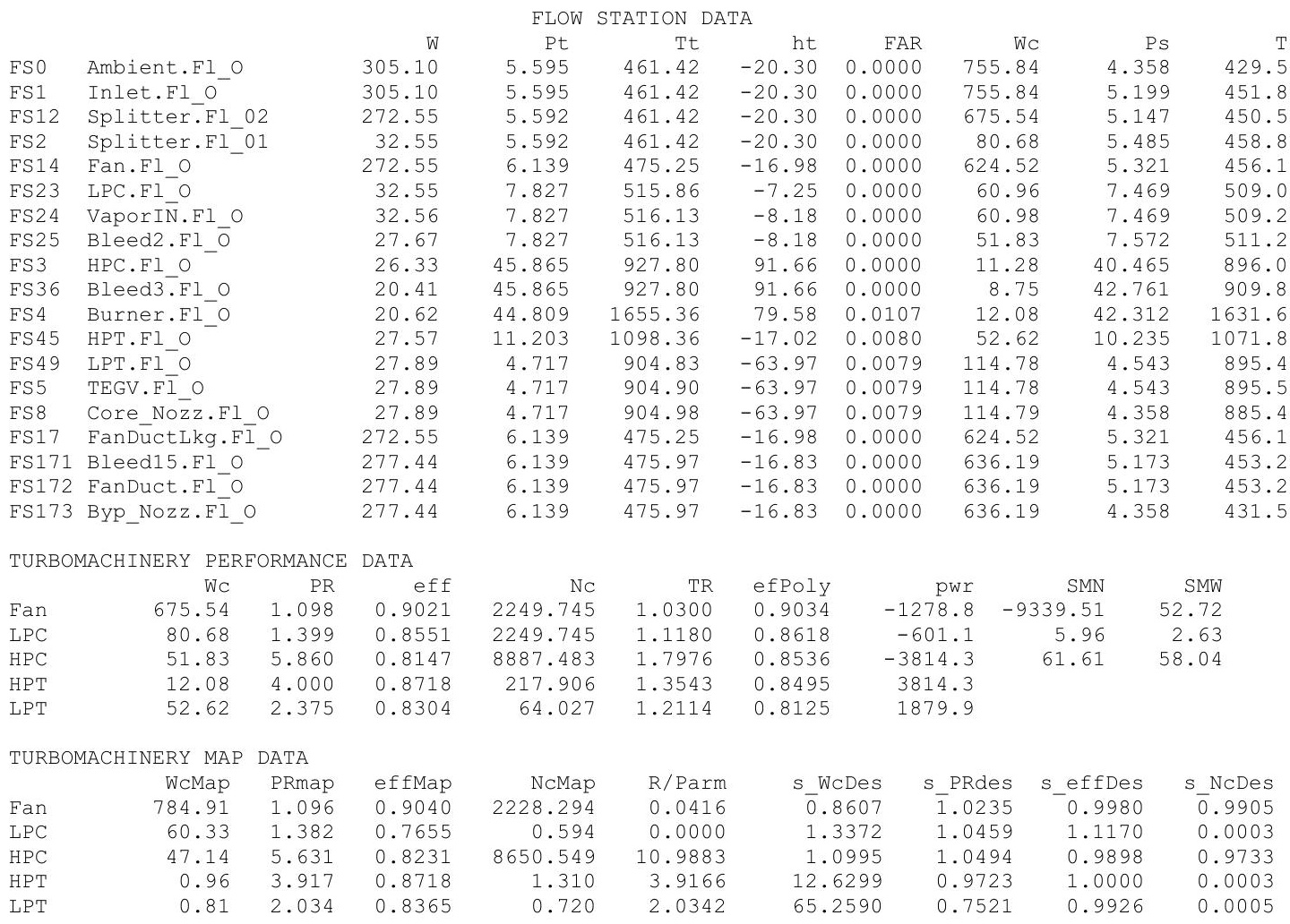

TURBOMACHINERY MAP DATA

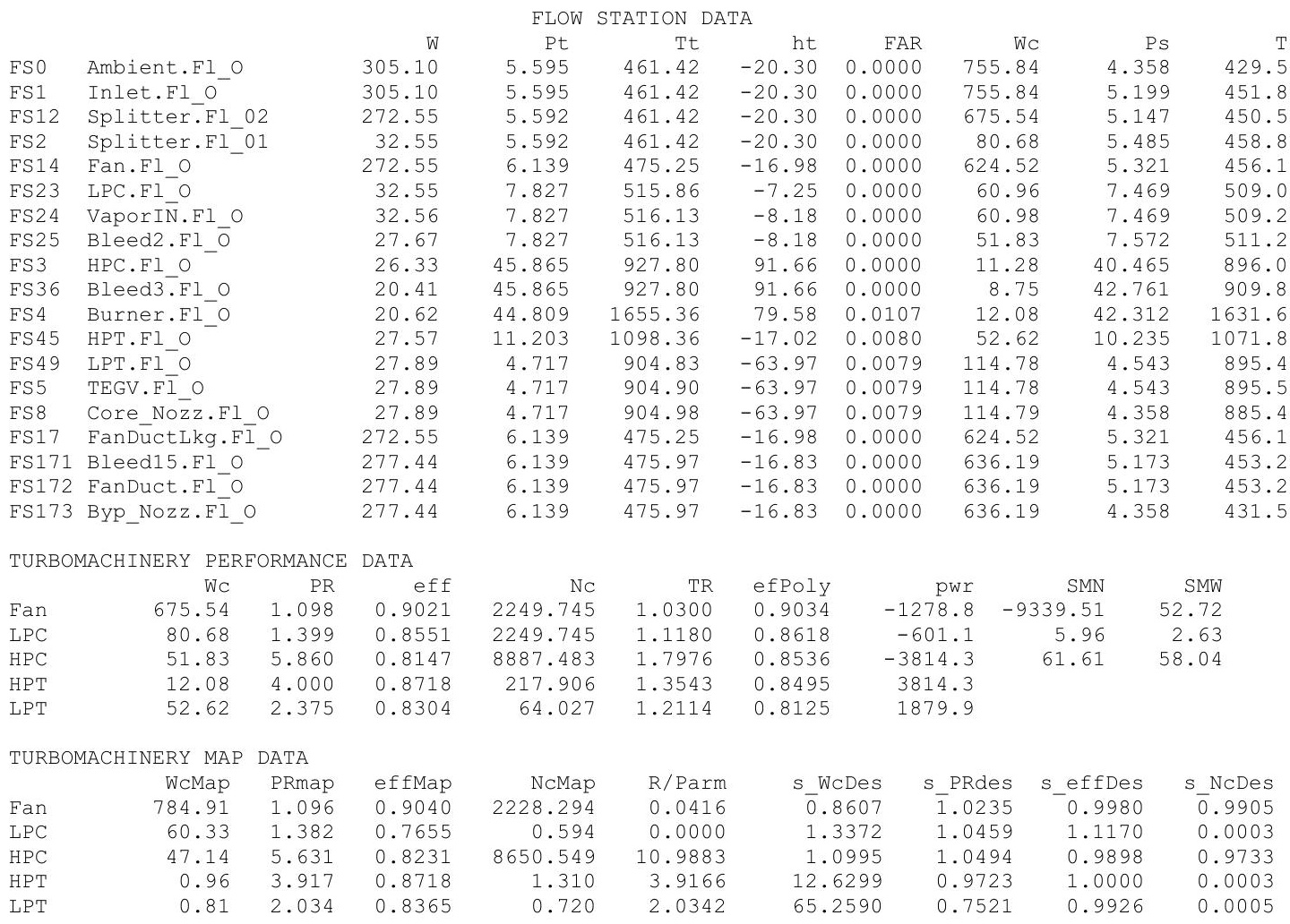

$\begin{array}{rrrrrrr}\text { Aphy } & \text { MN } & \text { gamt } & \text { WAR } & \text { Wair } & \text { WH2O } & \text { H2O frac } \\ 2596.2 & 0.6080 & 1.40081 & 0.0000681 & 305.08 & 0.021 & 0.0001 \\ 4168.2 & 0.3256 & 1.40081 & 0.0000681 & 305.08 & 0.021 & 0.0001 \\ 3531.8 & 0.3463 & 1.40081 & 0.0000681 & 272.54 & 0.019 & 0.0001 \\ 830.5 & 0.1665 & 1.40081 & 0.0000681 & 32.55 & 0.002 & 0.0001 \\ 2606.7 & 0.4566 & 1.40070 & 0.0000681 & 272.54 & 0.019 & 0.0001 \\ 412.6 & 0.2593 & 1.40026 & 0.0000681 & 32.55 & 0.002 & 0.0001 \\ 412.6 & 0.2594 & 1.40023 & 0.0002412 & 32.55 & 0.008 & 0.0002 \\ 412.6 & 0.2179 & 1.40023 & 0.0002412 & 27.67 & 0.007 & 0.0002 \\ 49.7 & 0.4288 & 1.38500 & 0.0002412 & 26.32 & 0.006 & 0.0002 \\ 49.3 & 0.3196 & 1.38500 & 0.0002412 & 20.40 & 0.005 & 0.0002 \\ 74.6 & 0.2941 & 1.33469 & 0.0002412 & 20.40 & 0.005 & 0.0133 \\ 265.4 & 0.3654 & 1.36897 & 0.0002412 & 27.34 & 0.007 & 0.0100 \\ 860.2 & 0.2340 & 1.38109 & 0.0002412 & 27.67 & 0.007 & 0.0099 \\ 860.2 & 0.2340 & 1.38108 & 0.0002412 & 27.67 & 0.007 & 0.0099 \\ 613.4 & 0.3400 & 1.38108 & 0.0002412 & 27.67 & 0.007 & 0.0099 \\ 2606.7 & 0.4566 & 1.40070 & 0.0000681 & 272.54 & 0.019 & 0.0001 \\ 2481.9 & 0.5005 & 1.40070 & 0.0000711 & 277.42 & 0.020 & 0.0001 \\ 2481.9 & 0.5005 & 1.40070 & 0.0000711 & 277.42 & 0.020 & 0.0001 \\ 2006.9 & 0.7169 & 1.40070 & 0.0000711 & 277.42 & 0.020 & 0.0001\end{array}$




\begin{tabular}{|c|c|c|c|c|c|c|c|c|c|}
\hline Z & $===$ INLET $S===$ & eRam & Afs & Fram & \multirow{2}{*}{\multicolumn{2}{|c|}{ BLEEDS - interstg }} & & & \\
\hline D & Inlet & 1.0000 & 2596.24 & 5860.4 & & & $\begin{array}{l}\text { Wb/Win } \\
0.0368\end{array}$ & $\begin{array}{r}\text { BldWk } \\
0.5000\end{array}$ & $\begin{array}{r}\text { BldP } \\
0.2200\end{array}$ \\
\hline 2 & $====$ DUCT $S====$ & dPnorm & MN & Aphy & \multicolumn{2}{|c|}{$\begin{array}{l}\text { HPT_COOLC HPC.C> } \\
\text { LPT COOLA HPC.C> }\end{array}$} & 0.0117 & 0.5000 & 0.4500 \\
\hline F & TEGV & 0.0000 & 0.2340 & 860.21 & WB $2 \overline{\mathrm{X}}$ & HPC.B> & 0.0000 & 0.4500 & 0.2200 \\
\hline & FanDuct & 0.0000 & 0.5005 & 2481.93 & WB2Y & HPC.B> & 0.0000 & 0.7600 & 0.6200 \\
\hline & & & & & & HPC.B> & 0.0000 & 0.4500 & 0.2200 \\
\hline$\hat{\jmath}$ & $==$ SPLITTERS $==$ & BPR & $\mathrm{dP} / \mathrm{P} 1$ & $\mathrm{dP} / \mathrm{P} 2$ & WBLKG & HPC.1> & 0.0000 & 1.0000 & 1.0000 \\
\hline & splitter & 8.3732 & 0.0005 & 0.0005 & WBW2X & HPC.B> & 0.0000 & 0.4500 & 0.2200 \\
\hline & $===$ SHAFT $\mathrm{S}====$ & Nmech & trq in & pwr in & \multicolumn{2}{|c|}{ BLEEDS - output } & Wb/Win & hscale & Pscale \\
\hline & HP_Shaft & 8865.7 & 2259.6 & 3814.3 & WB17Y & Bleed> & 0.1500 & 1.0000 & 1.0000 \\
\hline & LP_Shaft & 2121.9 & 4653.1 & 1879.9 & HPT_COOLA & A Bleed> & 0.1142 & 1.0000 & 1.0000 \\
\hline & & & & & $\mathrm{HPT}_{-}^{-} \mathrm{COOLB}$ & B Bleed> & 0.0999 & 1.0000 & 1.0000 \\
\hline & & & & & \multirow{2}{*}{$\begin{array}{l}\text { WB3X } \\
\text { WBA3X }\end{array}$} & Bleed> & 0.0000 & 1.0000 & 1.0000 \\
\hline & & & & & & Bleed> & 0.0000 & 1.0000 & 1.0000 \\
\hline & & & & & WBW3X & Bleed> & 0.0000 & 1.0000 & 1.0000 \\
\hline & & & & & WBEDLKG & FanDu> & 0.0000 & 1.0000 & 1.0000 \\
\hline & & & & & $\begin{array}{l}\text { WB15X } \\
\text { WB15Y }\end{array}$ & Bleed> & 0.0000 & 1.0000 & 1.0000 \\
\hline & & & & & \multirow{2}{*}{$\begin{array}{l}\text { WB15Y } \\
\text { WB17X }\end{array}$} & Bleed> & 0.0000 & 1.0000 & 1.0000 \\
\hline & & & & & & Bleed> & 0.0000 & 1.0000 & 1.0000 \\
\hline & $===$ BURNERS $===$ & Ttout & eff & dPnorm & \multirow{2}{*}{\multicolumn{2}{|c|}{$\begin{array}{rr}\text { Wfuel } & \text { FAR } \\
21804 & 0.01069\end{array}$}} & & & \\
\hline & Burner & 1655.29 & 0.9995 & 0.0230 & & & & & \\
\hline & $===$ NOZZLES $===$ & PR & $\mathrm{Cfg}$ & CdTh & & Ath & MNth & Vact & $\mathrm{Fg}$ \\
\hline & Core Nozz & 1.082 & 0.9801 & 1.0000 & \multirow{2}{*}{$\begin{array}{l}0.9800 \\
0.9800\end{array}$} & 613.40 & 0.340 & 483.0 & 418.7 \\
\hline & Byp Nozz & 1.409 & 0.9800 & 1.0000 & & 006.86 & 0.717 & 715.7 & 6171.3 \\
\hline
\end{tabular}


Date:05/13/13 Time:09:37:15 Model: Turbofan Engine - COMDES ON Converge = 1 CASE: 0

Version:NPSS 1.6.5 - Rev: -> Gas Package: Janaf iter/pass/Jacb/Broy= 22/36/1/20 Run by: Philip C Jorgenson PC: 10

Temperature Stator 1 inlet: $473.11 \quad$ Stator 1 exit: $477.18 \quad$ Stator 2 inlet: 481.79 Stator 2 exit: 483.59 $\begin{array}{lll}\text { Stator } 3 \text { inlet: } 488.73 & \text { Stator } 3 \text { exit: } 490.13 & \text { Stator } 4 \text { inlet: } 495.05 \text { Stator } 4 \text { exit: } 496.05 \\ \text { Stator } 5 \text { inlet: } 499.65 & \text { Stator } 5 \text { exit: } 500.37 & \text { Unblocked Percent Blockage: } 0.00\end{array}$

\begin{tabular}{|c|c|c|c|}
\hline bient Relative & 10.00 & & \\
\hline Fan Face Relative Humidity & 4.82 & & \\
\hline Fan Bypass Relative Humidity & 3.94 & & \\
\hline Inlet Relative Humidity & 3.82 & & \\
\hline PC Exit Relative Humidity & 1.19 & & \\
\hline Relative Humidity & 0.01 & & \\
\hline cop Diameter & 0.0000250 & Inlet Length & 40.00 \\
\hline bient Flow Velocity & 571.13 & Fan/LPC Inlet Flow Velocity & 194.52 \\
\hline bient Static Pressure & 5.30 & Fan/LPC Inlet Static Pressure & 6.36 \\
\hline ient Static Temperature & 445.14 & Fan/LPC Inlet Static Temperature & 469.17 \\
\hline itional Water at LPC Exi & 0.00016 & & \\
\hline
\end{tabular}

Additional Water at LPC Exit

SUMMARY OUTPUT DATA

$\begin{array}{rrr}\text { MN } & \text { alt } & \text { dTamb } \\ 0.552 & 25666.0 & 18.00\end{array}$

330.55 $\begin{array}{rr}\text { Fn } & \text { TSFC } \\ 749.0 & 1.1186\end{array}$

Wfuel $\begin{array}{rr}\text { BPR } \\ 837.85 & 7.0509\end{array}$
VTAS
571.13
$\mathrm{OPR}$
7.335 7.335 $\begin{array}{llccc}\text { EPR } & \text { Powerset } & \text { T4 } & \text { T41 } & \text { T49 } \\ 0.859 & 10.000 & 1666.1 & 1572.0 & 1105.4\end{array}$
FSO Ambient.Fl O

FS1 Inlet.Fi_o

FS12 Splitter.Fl 02

FS2 Splitter.F1_01

FS14 Fan.F1_-

FS24 VaporIN.FIO

FS25 Bleed2.Fl_o

FS3 HPC.FI_O

FS36 Bleed3.F1_O

FS4 Burner.Fl_o

FS45 HPT.Fl_

FS49 LPT. FI-O

FS8 Core_Nozz.Fl_O

FS17 FanDuctLkg.Fi_o

FS171 Bleed15.FI_O

FS172 FanDuct.FI-O

$W$
330.55
330.55
289.49
41.06
289.49
41.06
41.06
28.75
27.35
21.20
21.43
28.64
28.98
28.98
28.98
289.49
301.81
301.81
301.81

FLOW STATION DATA

$\begin{array}{rrrr}\text { Pt } & \text { Tt } & \text { ht } & \text { FAR } \\ 6.516 & 472.33 & -18.03 & 0.0000 \\ 6.516 & 472.33 & -18.03 & 0.0000 \\ 6.513 & 472.33 & -18.03 & 0.0000 \\ 6.513 & 472.33 & -18.03 & 0.0000 \\ 7.057 & 484.46 & -15.12 & 0.0000 \\ 8.207 & 511.60 & -8.61 & 0.0000 \\ 8.207 & 511.87 & -9.51 & 0.0000 \\ 8.207 & 511.87 & -9.51 & 0.0000 \\ 47.796 & 918.91 & 89.17 & 0.0000 \\ 47.796 & 918.91 & 89.17 & 0.0000 \\ 46.696 & 1666.06 & 76.78 & 0.0110 \\ 12.031 & 1109.70 & -18.56 & 0.0082 \\ 5.626 & 937.29 & -60.29 & 0.0081 \\ 5.626 & 937.36 & -60.29 & 0.0081 \\ 5.626 & 937.44 & -60.29 & 0.0081 \\ 7.057 & 484.46 & -15.12 & 0.0000 \\ 7.057 & 485.58 & -14.89 & 0.0000 \\ 7.057 & 485.58 & -14.89 & 0.0000 \\ 7.057 & 485.58 & -14.89 & 0.0000\end{array}$

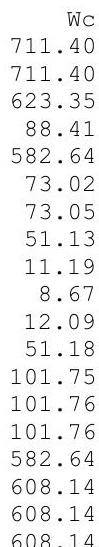

Ps
5.297
6.111
6.078
6.362
6.258
7.656
7.655
7.947
42.274
44.618
44.092
11.053
5.464
5.464
5.297
6.258
6.064
6.064
5.297

$\begin{array}{rr}\text { Aphy } & \text { MN } \\ 2595.1 & 0.5520 \\ 4168.2 & 0.3040 \\ 3531.8 & 0.3158 \\ 830.5 & 0.1831 \\ 2606.7 & 0.4178 \\ 412.6 & 0.3167 \\ 412.6 & 0.3169 \\ 412.6 & 0.2148 \\ 49.7 & 0.4244 \\ 49.3 & 0.3166 \\ 74.6 & 0.2942 \\ 265.4 & 0.3538 \\ 860.2 & 0.2061 \\ 860.2 & 0.2061 \\ 613.4 & 0.2969 \\ 2606.7 & 0.4178 \\ 2481.9 & 0.4705 \\ 2481.9 & 0.4705 \\ 2006.9 & 0.6533\end{array}$

gamt
1.40072
1.40072
1.40072
1.40072
1.40061
1.40031
1.40028
1.40028
1.38550
1.38550
1.33397
1.36807
1.37901
1.37901
1.37900
1.40061
1.40060
1.40060
1.40060

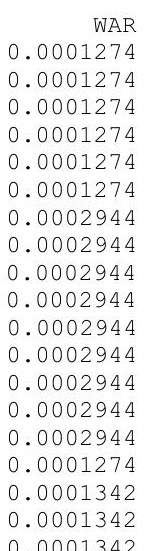

Wair 330.51 330.51 41.05 41.05
289.45 \begin{tabular}{l}
$41.05 \quad 0.037$ \\
\hline
\end{tabular} $\begin{array}{ll}41.05 & 0.005\end{array}$ $\begin{array}{lll}28.74 & 0.012 \\ 27.34 & 0.008\end{array}$ $27.34 \quad 0.008$ $\begin{array}{ll}21.19 & 0.006\end{array}$ $21.19 \quad 0.006$ $\begin{array}{ll}28.40 & 0.008\end{array}$ $28.74 \quad 0.008$ $28.74 \quad 0.008$ $28.74 \quad 0.008$ $289.45 \quad 0.037$ $301.77 \quad 0.040$ $301.77 \quad 0.040$ $301.77 \quad 0.040$

$\begin{array}{rr} & \text { H2O frac } \\ .042 & 0.0001 \\ 042 & 0.0001 \\ 037 & 0.0001 \\ 005 & 0.0001 \\ 037 & 0.0001 \\ 005 & 0.0001 \\ 2 & 0.0003 \\ 00 & 0.0003 \\ 08 & 0.0003 \\ 006 & 0.0003 \\ 0.0137 \\ 08 & 0.0103 \\ 08 & 0.0102 \\ 08 & 0.0102 \\ 037 & 0.0102 \\ 0.0001 \\ 000 & 0.0001 \\ 0.0001 \\ 00 & 0.0001\end{array}$

\begin{tabular}{|c|c|c|c|c|c|c|c|c|c|}
\hline \\
\hline & Wc & PR & eff & $\mathrm{NC}$ & TR & efPoly & pwr & SMN & SMW \\
\hline Tan & 623.35 & 1.084 & 0.9032 & 2044.928 & 1.0257 & 0.9043 & $-1191.7-$ & -2768.59 & 49.88 \\
\hline LPC & 88.41 & 1.260 & 0.8219 & 2044.928 & 1.0832 & 0.8277 & -547.1 & 6.38 & 2.41 \\
\hline HPC & 51.13 & 5.824 & 0.8139 & 8865.652 & 1.7952 & 0.8529 & -3915.9 & 60.73 & 57.11 \\
\hline $\mathrm{HPT}$ & 12.09 & 3.881 & 0.8732 & 215.773 & 1.3456 & 0.8517 & 3915.9 & & \\
\hline LPT & 51.18 & 2.138 & 0.8211 & 58.580 & 1.1810 & 0.8048 & 1738.7 & & \\
\hline URB & $\mathrm{NEF}$ & DATA & & & & & & & \\
\hline & WcMap & PRmap & effMap & NcMap & R/Parm & s_WCDes & S_PRdes & $S$ S_effDes & S_NCDes \\
\hline an & 724.27 & 1.082 & 0.9050 & 2025.429 & 425 & $\overline{0} .8607$ & $\overline{1} .0235$ & 0.9980 & $\overline{0} .9905$ \\
\hline LPC & 53.48 & 1.311 & 0.7608 & 0.540 & 0.0000 & 1.6530 & 0.8357 & 1.0804 & 0.0003 \\
\hline HPC & 46.51 & 5.597 & 0.8223 & 8629.300 & 11.0513 & 1.0995 & 1.0494 & 0.9898 & 0.9733 \\
\hline $\mathrm{HPT}$ & 0 & 3.802 & 0.8732 & 1.297 & 3.8015 & 12.6299 & 0.9723 & 1.0000 & 0.0003 \\
\hline $\mathrm{CPT}$ & 0.78 & 1.856 & 0.8272 & 0.659 & 1.8562 & 65.2590 & 0.7521 & 0.9926 & 0.0005 \\
\hline
\end{tabular}




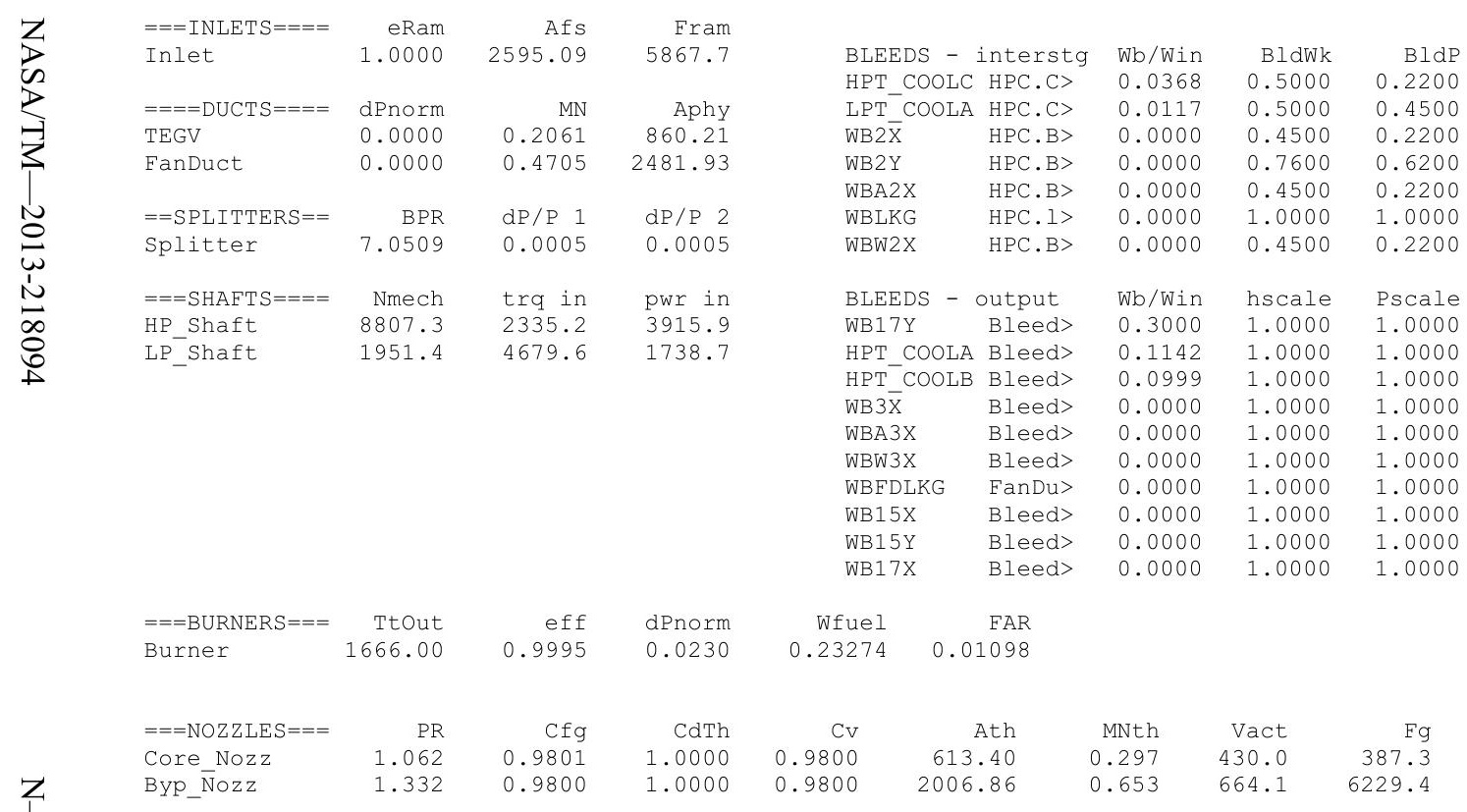


Tol COMDES ON Converge = 1 CASE: 0

Version:NPSS_1.6.5 - Rev: -> Gas Package: Janaf iter/pass/Jacb/Broy= 28/56/2/25 Run by: Philip C Jorgenson PC: 10

Temperature Stator 1 inlet: 486.26 Stator 1 exit: 489.21 $\quad$ Stator 2 inlet: 491.95 $\quad$ Stator 2 exit: 492.71 $\begin{array}{llll}\text { Stator } 3 \text { inlet: } 496.04 & \text { Stator } 3 \text { exit: } 496.37 & \text { Stator } 4 \text { inlet: } 499.61 \text { Stator } 4 \text { exit: } 499.64 \\ \text { Stator } 5 \text { inlet: } 501.49 & \text { Stator } 5 \text { exit: } 501.32 & \text { Unblocked Percent Blockage: } 0.00\end{array}$

$\begin{array}{lclll}\text { Ambient Relative Humidity } & 10.00 & & & \\ \text { Fan Face Relative Humidity } & 5.85 & & & \\ \text { Fan Bypass Relative Humidity } & 4.91 & & & \\ \text { LPC Inlet Relative Humidity } & 5.02 & & & \\ \text { LPC Exit Relative Humidity } & 2.62 & & & \\ \text { HPC Relative Humidity } & 0.01 & & & \\ \text { Drop Diameter } & 0.0000250 & \text { Inlet Length } & \\ \text { Ambient Flow Velocity } & 518.26 & \text { Fan/LPC Inlet Flow Velocity } & 204.30 \\ \text { Ambient Static Pressure } & 6.74 & \text { Fan/LPC Inlet Static Pressure } & 7.74 \\ \text { Ambient Static Temperature } & 465.18 & \text { Fan/LPC Inlet Static Temperature } & 484.08 \\ \text { Additional Water at LPC Exit } & 0.0001863 & & & \end{array}$

SUMMARY OUTPUT DATA

\begin{tabular}{|c|c|c|c|c|c|c|c|c|c|c|c|c|c|c|}
\hline MN & alt & dTamb & W & $\begin{array}{r}\text { UMMARY } \\
\text { Fn }\end{array}$ & $\begin{array}{r}\text { TPUT DA? } \\
\text { TSFC }\end{array}$ & Wfuel & BPR & VTAS & OPR & EPR & Powerset & T4 & $\mathrm{T} 41$ & T49 \\
\hline 0.490 & 20047.0 & 18.00 & 368.15 & 832.5 & 1.1207 & 932.96 & 6.2384 & 518.26 & 6.454 & 0.890 & 10.000 & 1699.1 & 1601.1 & 1136.0 \\
\hline
\end{tabular}

\begin{tabular}{|c|c|}
\hline FSO & Ambient.Fl_O \\
\hline FS1 & Inlet.Fl_o \\
\hline FS12 & Splitter.Fl_02 \\
\hline FS2 & Splitter.Fl-01 \\
\hline FS14 & Fan.Fl_o \\
\hline FS23 & LPC.FI_O \\
\hline FS24 & 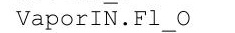 \\
\hline FS25 & Bleed2.FI_o \\
\hline FS3 & HPC.Fl_O \\
\hline FS36 & Bleed3.Fl_O \\
\hline FS4 & Burner.Fl_o \\
\hline FS45 & HPT.Fl_O \\
\hline FS 49 & LPT.FI_O \\
\hline FS5 & TEGV.FI__o \\
\hline FS8 & Core Nozz.Fl O \\
\hline FS17 & FanDüctLkg.Fì o \\
\hline FS171 & Bleed15.Fl o \\
\hline FS172 & FanDuct.Fl \\
\hline FS173 & Byp_Nozz.Fì_o \\
\hline
\end{tabular}

\begin{tabular}{rrrrr}
\multicolumn{7}{c}{ FLOW } & STATION DATA \\
W & Pt & Tt & $h t$ & FAR \\
368.15 & 7.943 & 487.56 & -15.16 & 0.0000 \\
368.15 & 7.943 & 487.56 & -15.16 & 0.0000 \\
317.29 & 7.939 & 487.56 & -15.16 & 0.0000 \\
50.86 & 7.939 & 487.56 & -15.16 & 0.0000 \\
317.29 & 8.512 & 498.39 & -12.56 & 0.0000 \\
50.86 & 9.079 & 515.20 & -8.53 & 0.0000 \\
50.87 & 9.079 & 515.49 & -9.53 & 0.0000 \\
30.52 & 9.079 & 515.49 & -9.53 & 0.0000 \\
29.04 & 51.266 & 918.25 & 88.12 & 0.0000 \\
22.51 & 51.266 & 918.25 & 88.12 & 0.0000 \\
22.77 & 50.085 & 1699.12 & 75.15 & 0.0115 \\
30.42 & 13.553 & 1140.71 & -18.98 & 0.0086 \\
30.78 & 7.047 & 987.36 & -56.04 & 0.0085 \\
30.78 & 7.047 & 987.44 & -56.04 & 0.0085 \\
30.78 & 7.047 & 987.51 & -56.04 & 0.0085 \\
317.29 & 8.512 & 498.39 & -12.56 & 0.0000 \\
337.64 & 8.512 & 499.42 & -12.38 & 0.0000 \\
337.64 & 8.512 & 499.42 & -12.38 & 0.0000 \\
337.64 & 8.512 & 499.42 & -12.38 & 0.0000
\end{tabular}

WC
660.38
660.38
569.43
91.28
536.98
82.05
82.09
49.25
11.08
8.58
12.09
48.92
88.56
88.57
88.57
536.98
572.01
572.01
572.01

Ps
6.740
7.522
7.503
7.743
7.712
8.291
8.290
8.813
45.484
77.932
77.290
12.556
6.895
6.895
6.740
7.712
7.477
7.477
6.740

Ts
465.18
480.02
479.73
484.08
484.52
501.99
502.26
511.12
888.13
901.21
1674.93
1117.56
981.49
981.56
975.55
484.52
481.24
481.24
467.17

$\begin{array}{rr}\text { Aphy } & \text { MN } \\ 2616.0 & 0.4900 \\ 4168.2 & 0.2799 \\ 3531.8 & 0.2854 \\ 830.5 & 0.1894 \\ 2606.7 & 0.3780 \\ 412.6 & 0.3625 \\ 412.6 & 0.3627 \\ 412.6 & 0.2065 \\ 49.7 & 0.4188 \\ 49.3 & 0.3129 \\ 74.6 & 0.2946 \\ 265.4 & 0.3361 \\ 860.2 & 0.1785 \\ 860.2 & 0.1785 \\ 613.4 & 0.2553 \\ 2606.7 & 0.3780 \\ 2481.9 & 0.4343 \\ 2481.9 & 0.4343 \\ 2006.9 & 0.5871\end{array}$

gamt
1.40057
1.40057
1.40057
1.40057
1.40045
1.40024
1.40021
1.40021
1.38552
1.38552
1.33200
1.36576
1.37568
1.37567
1.37567
1.40045
1.40044
1.40044
1.40044

WAR
0.0002627
0.0002627
0.0002627
0.0002627
0.0002627
0.0002627
0.0004491
0.0004491
0.0004491
0.0004491
0.0004491
0.0004491
0.0004491
0.0004491
0.0004491
0.0002627
0.0002740
0.0002740
0.0002740

$\begin{array}{rrr}\text { Wair } & \text { WH2O } & \text { H2O frac } \\ 368.06 & 0.097 & 0.0003 \\ 368.06 & 0.097 & 0.0003 \\ 317.21 & 0.083 & 0.0003 \\ 50.85 & 0.013 & 0.0003 \\ 317.21 & 0.083 & 0.0003 \\ 50.85 & 0.013 & 0.0003 \\ 50.85 & 0.023 & 0.0004 \\ 30.51 & 0.014 & 0.0004 \\ 29.03 & 0.013 & 0.0004 \\ 22.50 & 0.010 & 0.0004 \\ 22.50 & 0.010 & 0.0145 \\ 30.15 & 0.014 & 0.0110 \\ 30.51 & 0.014 & 0.0108 \\ 30.51 & 0.014 & 0.0108 \\ 30.51 & 0.014 & 0.0108 \\ 317.21 & 0.083 & 0.0003 \\ 337.55 & 0.092 & 0.0003 \\ 337.55 & 0.092 & 0.0003 \\ 337.55 & 0.092 & 0.0003\end{array}$

TURBOMACHINERY PERFORMANCE DATA

\begin{tabular}{|c|c|c|c|c|c|c|c|c|c|}
\hline & Wc & $\mathrm{PR}$ & eff & NC & TR & efPoly & pwr & SMN & SMW \\
\hline Fan & 569.43 & 1.072 & 0.9054 & 1848.947 & 1.0222 & 0.9063 & $-1166.4 \quad-$ & -1882.29 & 46.42 \\
\hline LPC & 91.28 & 1.144 & 0.6896 & 1848.947 & 1.0567 & 0.6954 & -477.0 & 6.97 & 2.19 \\
\hline HPC & 49.25 & 5.647 & 0.8099 & 8804.937 & 1.7813 & 0.8491 & -4115.0 & 60.55 & 56.72 \\
\hline HPT & 12.09 & 3.695 & 0.8753 & 212.950 & 1.3307 & 0.8549 & 4115.0 & & \\
\hline LPT & 48.92 & 1.923 & 0.8141 & 53.077 & 1.1520 & 0.7998 & 1643.5 & & \\
\hline \multirow{2}{*}{\multicolumn{10}{|c|}{ TURBOMACHINERY MAP DATA }} \\
\hline & & PRmap & effmap & NcMap & R/Parm & S_WcDes & S_PRdes & S S_effDes & S_NCDes \\
\hline Fan & 661.62 & 1.070 & 0.9072 & 1831.317 & 0.0438 & $\overline{0} .8607$ & $\overline{1} .0235$ & 0.9980 & $\overline{0} .9905$ \\
\hline LPC & 47.04 & 1.247 & 0.7558 & 0.488 & 0.0000 & 1.9402 & 0.5807 & 0.9124 & 0.0003 \\
\hline HPC & 44.80 & 5.428 & 0.8182 & 8570.203 & 11.0884 & 1.0995 & 1.0494 & 0.9898 & 0.9733 \\
\hline HPT & 0.96 & 3.621 & 0.8753 & 1.280 & 3.6208 & 12.6299 & 0.9723 & 1.0000 & 0.0003 \\
\hline LPT & 0.75 & 1.694 & 0.8202 & 0.597 & 1.6944 & 65.2590 & 0.7521 & 0.9926 & 0.0005 \\
\hline
\end{tabular}




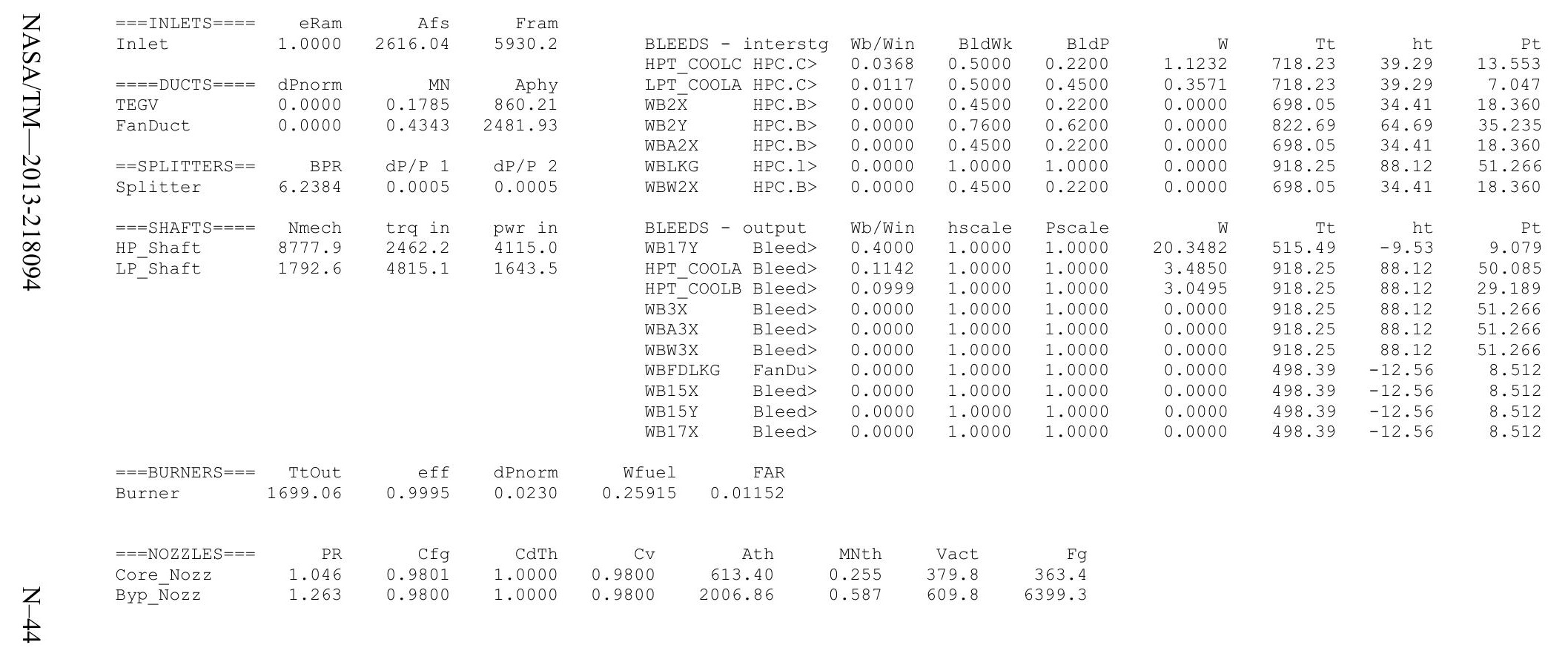


Date:05/13/13 Time:09:38:20 Model: Turbofan Engine - COMDES ON Converge = 1 CASE: 0

Version:NPSS 1.6.5 - Rev: -> Gas Package: Janaf iter/pass/Jacb/Broy=30/58/2/27 Run by: Philip C Jorgenson PC: 10

Temperature Stator 1 inlet: 500.27 Stator 1 exit: 502.63 Stator 2 inlet: 504.94 Stator 2 exit: 505.66 Stator 3 inlet: $508.36 \quad$ Stator 3 exit: $508.76 \quad$ Stator 4 inlet: 511.37 Stator 4 exit: 511.54
Stator 5 inlet: $513.13 \quad$ Stator 5 exit: 513.14

Ambient Relative Humidity $\quad 10.00$

Fan Face Relative Humidity $\quad 6.53$

Fan Bypass Relative Humidity $\quad 5.96$

LPC Inlet Relative Humidity

HPC Relativerive Humidity

Drop Diameter

0.02

0.0000250

Ambient Flow Velocity

Ambient Static Pressure

Additional Water at LPC Exit

479.99

Inlet Length

40.00

$\begin{array}{lrr}\text { 8.15 Fan/LPC Inlet Flow Velocity } & 173.45 \\ & \text { Fan/LPC Inlet Static Pressure } & 9.18\end{array}$

481.63 Fan/LPC Inlet Static Temperature 498.31

$\begin{array}{rrrrrr} & & & & \text { SUMMARY OUTPUT DATA } \\ \text { MN } & \text { alt } & \text { dTamb } & \text { W } & \text { Fn } & \text { TSFC } \\ 0.446 & 15435.0 & 18.00 & 386.71 & 642.4 & 1.3769\end{array}$

W
386.71

Fn
642.4

TSFC
1.3769

Wfuel
884.50

BPR
6.7810

VTAS
479.99

OPR
5.289

EPR
0.902

PowerSet
10.000

$\begin{array}{ccc}\text { T4 } & \text { T41 } & \text { T49 } \\ 1650.5 & 1554.6 & 1125.9\end{array}$
FSO Ambient.Fl O

FS1 Inlet.Fl_o

FS12 Splitter.Fl_02

FS2 Splitter.FI_0

FS14 Fan.F1-

FS24 VaporIN..$F 1$

ES25 Bleed2.Fl_

F3 HPC.Fl_O

Bleed3.FI_O

Burner.F1-O

FS49 4 TP .

FS5 TEGV.Fīo

FS8 Core_Nozz.Fl_O

FS17 FanDüctLkg.Fì

FS171 Bleed15.FI

FS172 FanDuct.Fl-O

FS173 Byp Nozz.Fì O

TURBOMACHINERY PERFORMANCE DATA

$\begin{array}{lrrr} & \text { WC } & \text { PR } & \text { eff } \\ \text { Fan } & 521.20 & 1.051 & 0.8983 \\ \text { LPC } & 76.86 & 1.117 & 0.7208 \\ \text { HPC } & 42.21 & 4.736 & 0.7955 \\ \text { HPT } & 12.08 & 3.381 & 0.8666 \\ \text { LPT } & 45.18 & 1.700 & 0.8005\end{array}$

FLOW STATION DATA

$W$
386.71
386.71
337.01
49.70
337.01
49.70
49.71
29.83
28.38
21.99
22.24
29.72
30.07
30.07
30.07
337.01
356.89
356.89

Pt
9.342
9.342

Tt
500.81

ht FAR $\begin{array}{lll}-13.05 & 0.0000 & 597.77\end{array}$ $\begin{array}{lllll}9.342 & 500.81 & -13.05 & 0.0000 & 597.77 \\ 9.337 & 500.81 & -13.05 & 0.0000 & 597.77\end{array}$ $\begin{array}{lllll}9.337 & 500.81 & -13.05 & 0.0000 & 597.77\end{array}$

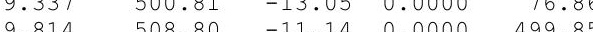

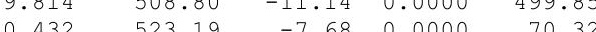

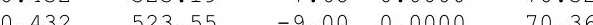

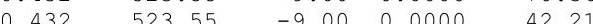
$\begin{array}{lll}49.407-888.07 & 79.31 & 0.0000\end{array}$ $\begin{array}{lrrr}49.407 & 888.07 & 79.31 & 0.0000\end{array}$ $\begin{array}{llll}48.269 & 1650.48 & 66.82 & 0.0112\end{array}$ $\begin{array}{llll}14.275 & 1130.53 & -18.14 & 0.0083\end{array}$ $\begin{array}{llll}8.396 & 1006.43 & -47.86 & 0.0082\end{array}$ $\begin{array}{llll}8.396 & 1006.50 & -47.85 & 0.0082\end{array}$ $\begin{array}{llll}8.396 & 1006.57 & -47.85 & 0.0082\end{array}$ $\begin{array}{llll}9.814 & 508.80 & -11.14 & 0.0000\end{array}$ $\begin{array}{llll}9.814 & 509.62 & -11.02 & 0.0000 \\ 9.814 & 509.62 & -11.02 & 0.0000\end{array}$

$\begin{array}{llll}9.814 & 509.62 & -11.02 & 0.0000\end{array}$

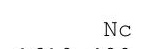

$\begin{array}{rr}\text { TR } & \text { efPoly } \\ 1.0159 & 0.8990 \\ 1.0447 & 0.7252 \\ 1.6962 & 0.8340 \\ 1.3031 & 0.8467 \\ 1.1197 & 0.7882\end{array}$

$\begin{array}{rrr}\text { pwr } & \text { SMN } & \text { SMW } \\ -913.2 & -1525.87 & 44.72 \\ -377.4 & 8.26 & 1.96 \\ -3636.5 & 68.26 & 63.04 \\ 3636.5 & & \end{array}$
211.212 1.1197

.7882

1290.6

TURBOMACHINERY MAP DATA

\begin{tabular}{|c|c|c|c|c|c|c|c|c|c|}
\hline & WcMap & PRmap & effMap & NcMap & R/Parm & s WCDes & S PRdes & s effDes & S_NcDes \\
\hline Fan & 605.59 & 1.050 & 0.9001 & 1595.132 & 0.0442 & 0.8607 & $\overline{1} .0235$ & 0.9980 & 0.9905 \\
\hline LPC & 39.71 & 1.184 & 0.7480 & 0.425 & 0.0000 & 1.9357 & 0.6360 & 0.9636 & 0.0003 \\
\hline HPC & 38.39 & 4.560 & 0.8038 & 8312.935 & 10.7596 & 1.0995 & 1.0494 & 0.9898 & 0.9733 \\
\hline HPT & 0.96 & 3.315 & 0.8666 & 1.269 & 3.3155 & 12.6299 & 0.9723 & 1.0000 & 0.0003 \\
\hline LPT & 0.69 & 1.527 & 0.8064 & 0.529 & 1.5266 & 65.2590 & 0.7521 & 0.9926 & 0.0005 \\
\hline
\end{tabular}

\begin{tabular}{|c|c|}
\hline & \\
\hline 8.1 & 9 \\
\hline 8.9 & 494 \\
\hline 8.9 & 494 \\
\hline 9.1 & 498 \\
\hline 9.0 & 49 \\
\hline 9.78 & 513 \\
\hline 9.78 & 51 \\
\hline 10.2 & 520 \\
\hline 43.8 & 859 \\
\hline 46.2 & 871 \\
\hline 45.58 & 1626 \\
\hline 13.3 & 1111 \\
\hline 8.2 & 1002 \\
\hline 8.2 & 1002 \\
\hline 8.1 & 998 \\
\hline 9.0 & 496 \\
\hline 8.8 & 494 \\
\hline 8.8 & \\
\hline 8.1 & 483 \\
\hline & SMW \\
\hline & 44.72 \\
\hline & 1.96 \\
\hline & 63.04 \\
\hline & S_NCDe \\
\hline & $\overline{0} .990$ \\
\hline 9636 & 0.000 \\
\hline & 0.9 \\
\hline 00 & 0.0 \\
\hline 992 & 0 \\
\hline
\end{tabular}

$.529 \quad 1.3155$
-5266

$\begin{array}{rrrrrrr}\text { Aphy } & \text { MN } & \text { gamt } & \text { WAR } & \text { Wair } & \text { WH2O } & \text { H2O frac } \\ 2541.0 & 0.4460 & 1.40040 & 0.0004484 & 386.53 & 0.173 & 0.0004 \\ 4168.2 & 0.2511 & 1.40040 & 0.0004484 & 386.53 & 0.173 & 0.0004 \\ 3531.8 & 0.2590 & 1.40040 & 0.0004484 & 366.86 & 0.151 & 0.0004 \\ 830.5 & 0.1585 & 1.40040 & 0.0004484 & 49.68 & 0.022 & 0.0004 \\ 2606.7 & 0.3474 & 1.40030 & 0.0004484 & 336.86 & 0.151 & 0.0004 \\ 412.6 & 0.3036 & 1.40011 & 0.0004484 & 49.68 & 0.022 & 0.0004 \\ 412.6 & 0.3038 & 1.40007 & 0.0006925 & 49.68 & 0.034 & 0.0007 \\ 412.6 & 0.1758 & 1.40007 & 0.0006925 & 29.81 & 0.021 & 0.0007 \\ 49.7 & 0.4171 & 1.38717 & 0.0006925 & 28.36 & 0.020 & 0.0007 \\ 49.3 & 0.3117 & 1.38717 & 0.0006925 & 21.98 & 0.015 & 0.0007 \\ 74.6 & 0.2940 & 1.33453 & 0.0006925 & 21.98 & 0.015 & 0.0143 \\ 265.4 & 0.3070 & 1.36656 & 0.0006925 & 29.46 & 0.020 & 0.0109 \\ 860.2 & 0.1470 & 1.37460 & 0.0006925 & 29.81 & 0.021 & 0.0108 \\ 860.2 & 0.1470 & 1.37460 & 0.0006925 & 29.81 & 0.021 & 0.0108 \\ 613.4 & 0.2088 & 1.37459 & 0.0006925 & 29.81 & 0.021 & 0.0108 \\ 2606.7 & 0.3474 & 1.40030 & 0.0004484 & 336.86 & 0.151 & 0.0004 \\ 2481.9 & 0.3947 & 1.40029 & 0.0004619 & 356.73 & 0.165 & 0.0005 \\ 2481.9 & 0.3947 & 1.40029 & 0.0004619 & 356.73 & 0.165 & 0.0005 \\ 2006.9 & 0.5221 & 1.40029 & 0.0004619 & 356.73 & 0.165 & 0.0005\end{array}$

$2006.9 \quad 0.5221$ 


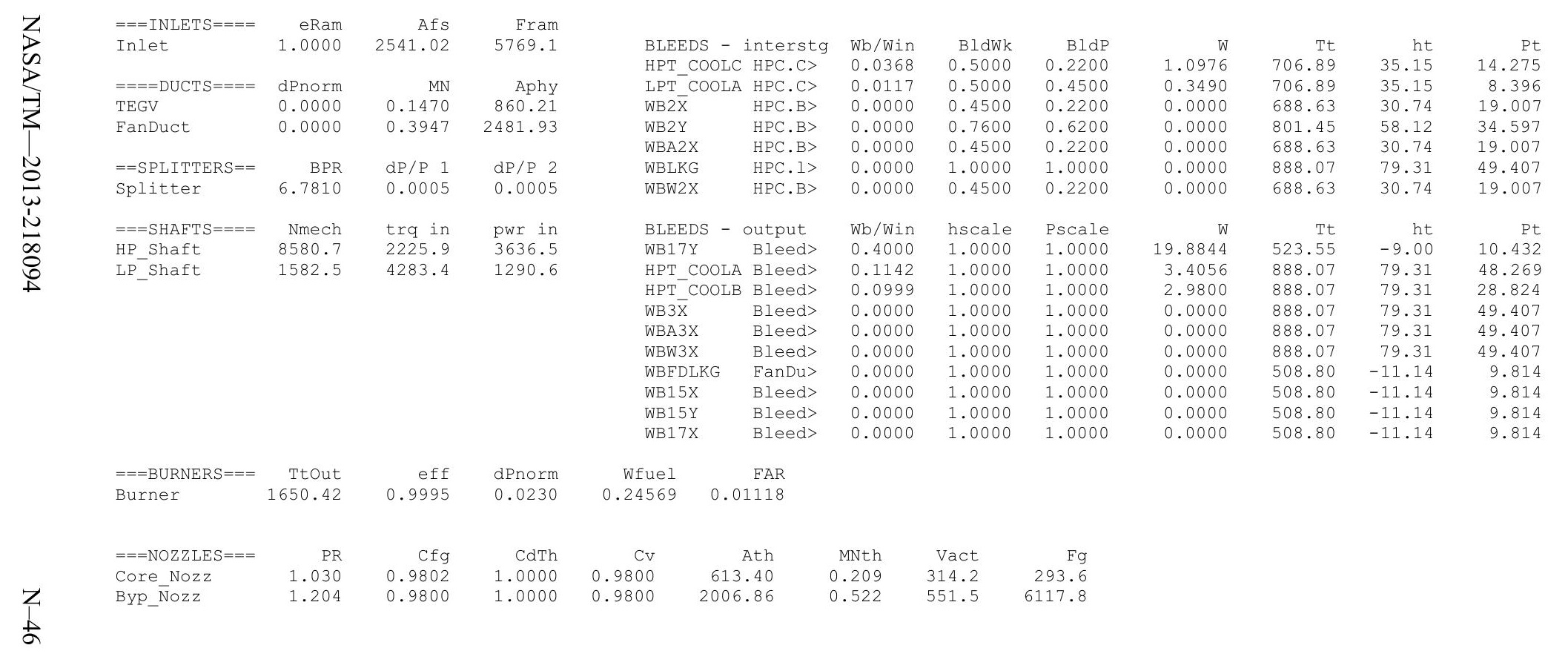




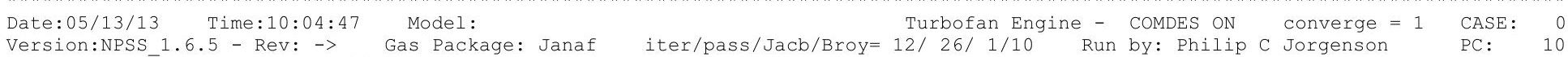

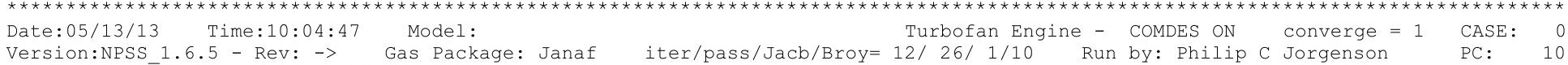

Temperature Stator 1 inlet: 483.68 Stator 1 exit: $491.45 \quad$ Stator 2 inlet: 501.22 Stator 2 exit: 506.21

Stator 3 inlet: 516.31 Stator 3 exit: 520.84 Stator 4 inlet: 530.29 stator 4 exit: 534.15

Ambient Relative Humidity $\quad 10.00$

Fan Face Relative Humidity $\quad 1.97$

$\begin{array}{ll}\text { Fan Bypass Relative Humidity } & 1.41 \\ \text { LPC Inlet Relative Humidity } & 1.30\end{array}$

LPC Exit Relative Humidity 0.13

HPC Relative Humidity

Drop Diameter

Ambient Flow Velocity

0.0000250

789.49

Ambient Static Pressure

Ambient Static Temperature

789.49
2.85

Inlet Length

Additi static Temperature

Inlet 192.84

425.97 Fan/LPC Inlet Static Temperature 474.82

SUMMARY OUTPUT DATA

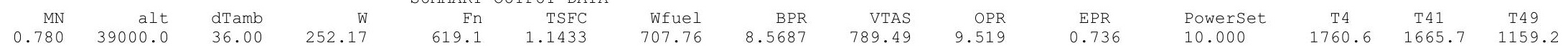

$\begin{array}{ll}\text { FS0 } & \text { Ambient.F1_O } \\ \text { FS1 } & \text { Inlet.F1_O } \\ \text { FS12 } & \text { Splitter.F1_02 } \\ \text { FS2 } & \text { Splitter.F1_01 } \\ \text { FS14 } & \text { Fan.F1_O } \\ \text { FS23 } & \text { LPC.Fl_O } \\ \text { FS24 } & \text { VaporIN.F1_O } \\ \text { FS25 } & \text { Bleed2.F1_O } \\ \text { FS3 } & \text { HPC.F1_O } \\ \text { FS36 } & \text { Bleed3.F1_O } \\ \text { FS4 } & \text { Burner.F1_O } \\ \text { FS45 } & \text { HPT.F1_O } \\ \text { FS49 } & \text { LPT.F1_O } \\ \text { FS5 } & \text { TEGV.FI_O } \\ \text { FS8 } & \text { Core_Nozz.F1_O } \\ \text { FS17 } & \text { FanDuctLkg.F1_O } \\ \text { FS171 } & \text { Bleed15.F1_O } \\ \text { FS172 } & \text { FanDuct.Fl_O } \\ \text { FS173 } & \text { Byp_Nozz.F1_O } \\ & \end{array}$

252.17
252.17
225.82
26.35
225.82
26.35
26.36
23.73
22.58
17.50
17.69
23.65
23.92
23.92
23.92
225.82
228.45
228.45
228.45

228.45

TURBOMACHINERY PERFORMANCE DATA

\begin{tabular}{|c|c|c|c|c|c|c|c|c|c|}
\hline & & & & & & & & & \\
\hline & Wc & PR & eff & $\mathrm{Nc}$ & TR & efPoly & pwr & SMN & SMW \\
\hline Fan & 746.98 & 1.130 & 0.9055 & 2568.797 & 1.0392 & 0.9071 & -1434.2 & 1605.87 & 54.82 \\
\hline LPC & 87.17 & 1.561 & 0.8402 & 2568.797 & 1.1615 & 0.8499 & -690.4 & 6.97 & 3.73 \\
\hline HPC & 54.23 & 6.102 & 0.8186 & 8965.163 & 1.8124 & 0.8573 & -3604.3 & 61.37 & 58.05 \\
\hline HPT & 12.07 & 4.167 & 0.8772 & 221.157 & 1.3647 & 0.8552 & 3604.3 & & \\
\hline LPT & 54.66 & 2.920 & 0.8557 & 72.294 & 1.2754 & 0.8361 & 2124.5 & & \\
\hline & NERY MAP & DA & & & & & & & \\
\hline & WcMap & PRmap & effMap & NcMap & R/Parm & S_WCDes & S_PRdes & s_effDes & S_NcDes \\
\hline an & 867.92 & 1.127 & 0.9073 & 2544.304 & 0.0414 & $\overline{0} .8607$ & $\overline{1} .0235$ & 0.9980 & 0.9905 \\
\hline $\mathrm{PC}$ & 73.93 & 1.522 & 0.7799 & 0.678 & 0.0000 & 1.1791 & 1.0738 & 1.0773 & 0.0003 \\
\hline PC & 49.32 & 5.8 & 71 & 8726.157 & 10.9732 & 1.0995 & 1.0494 & 0.9898 & 0.9733 \\
\hline PT & 0.96 & 4.079 & 3772 & 1.329 & 4.0789 & 12.6299 & 0.9723 & 1.0000 & 0.0003 \\
\hline LPT & 0.84 & 2.444 & .8621 & 0.813 & 2.4438 & 65.2590 & 0.7521 & 0.9926 & 0.0005 \\
\hline
\end{tabular}

FLOW STATION DATA

$\begin{array}{rrrrrrrrr}\text { Pt } & \text { Tt } & \text { ht } & \text { FAR } & \text { Wc } & \text { Ps } & \text { Ts } & \text { Aphy } & \text { MN } \\ 4.267 & 477.92 & -16.44 & 0.0000 & 833.74 & 2.854 & 425.97 & 2543.6 & 0.7800 \\ 4.267 & 477.92 & -16.44 & 0.0000 & 833.74 & 3.891 & 465.50 & 4168.2 & 0.3649 \\ 4.265 & 477.92 & -16.44 & 0.0000 & 746.98 & 3.839 & 463.77 & 3531.8 & 0.3902 \\ 4.265 & 477.92 & -16.44 & 0.0000 & 87.17 & 4.169 & 474.82 & 830.5 & 0.1805 \\ 4.817 & 496.65 & -11.95 & 0.0000 & 674.09 & 4.043 & 472.35 & 2606.7 & 0.5067 \\ 6.656 & 555.11 & 2.08 & 0.0000 & 60.19 & 6.360 & 547.94 & 412.6 & 0.2558 \\ 6.656 & 555.68 & -0.13 & 0.0000 & 60.25 & 6.359 & 548.49 & 412.6 & 0.2561 \\ 6.656 & 555.68 & -0.13 & 0.0000 & 54.23 & 6.418 & 549.94 & 412.6 & 0.2287 \\ 40.613 & 1007.14 & 109.89 & 0.0000 & 11.38 & 35.728 & 972.14 & 49.7 & 0.4344 \\ 40.613 & 1007.14 & 109.89 & 0.0000 & 8.82 & 37.810 & 987.47 & 49.3 & 0.3234 \\ 39.678 & 1760.56 & 97.00 & 0.0112 & 12.07 & 37.470 & 1735.71 & 74.6 & 0.2944 \\ 9.523 & 1163.38 & -9.52 & 0.0084 & 54.66 & 8.626 & 1133.00 & 265.4 & 0.3830 \\ 3.262 & 910.91 & -71.53 & 0.0083 & 142.86 & 3.070 & 895.85 & 860.2 & 0.2971 \\ 3.262 & 910.98 & -71.53 & 0.0083 & 142.86 & 3.070 & 895.92 & 860.2 & 0.2971 \\ 3.262 & 911.06 & -71.53 & 0.0083 & 142.87 & 2.854 & 878.09 & 613.4 & 0.4436 \\ 4.817 & 496.65 & -11.95 & 0.0000 & 674.09 & 4.043 & 472.35 & 2606.7 & 0.5067 \\ 4.817 & 497.33 & -11.82 & 0.0000 & 682.42 & 3.909 & 468.47 & 2481.9 & 0.5546 \\ 4.817 & 497.33 & -11.82 & 0.0000 & 682.42 & 3.909 & 468.47 & 2481.9 & 0.5546 \\ 4.817 & 497.33 & -11.82 & 0.0000 & 682.42 & 2.854 & 428.14 & 2006.9 & 0.8979\end{array}$

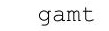

1.40068 1.40068 1.40068 1.40068 1.40049 1.39961 1.39961 1.38021

1.38021

1.32934

$1.38043-0.0004925$

$1.38043-0.0004925$

$\begin{array}{ll}1.38043 & 0.0004925 \\ 1.40049 & 0.0004925 \\ 1.40048 & 0.0000851\end{array}$

$\begin{array}{ll}1.40048 & 0.0000898\end{array}$

$1.40048 \quad 0.0000898$

$\begin{array}{ll}1.40048 & 0.0000898\end{array}$
WAR Wair $\begin{array}{r}252.15 \\ 252.15 \\ \hline\end{array}$ 26.35
225.80 $26.35 \quad 0.002$ $\begin{array}{ll}23.72 & 0.012\end{array}$ $\begin{array}{ll}22.57 & 0.011\end{array}$

$\begin{array}{ll}17.49 & 0.009 \\ 17.49 & 0.009\end{array}$

$23.44 \quad 0.012$

$\begin{array}{ll}23.72 & 0.012\end{array}$

$\begin{array}{ll}23.72 & 0.012 \\ 23.72 & 0.012\end{array}$

$225.80 \quad 0.01$

$228.43 \quad 0.021$ $\begin{array}{ll}228.43 & 0.021 \\ 228.43 & 0.021\end{array}$
$\mathrm{H} 2 \mathrm{O} \mathrm{H} 2 \mathrm{O}$ frac $\begin{array}{ll}0.0001 \\ 021 & 0.0001\end{array}$ 0.0001 0.0001 0.0001 0.0005 0.0005 0.0005 0.0005 0.0142 0.0107 0.0106 0.0106 0.0106 0.0001 0.0001 0.0001 0.0001 


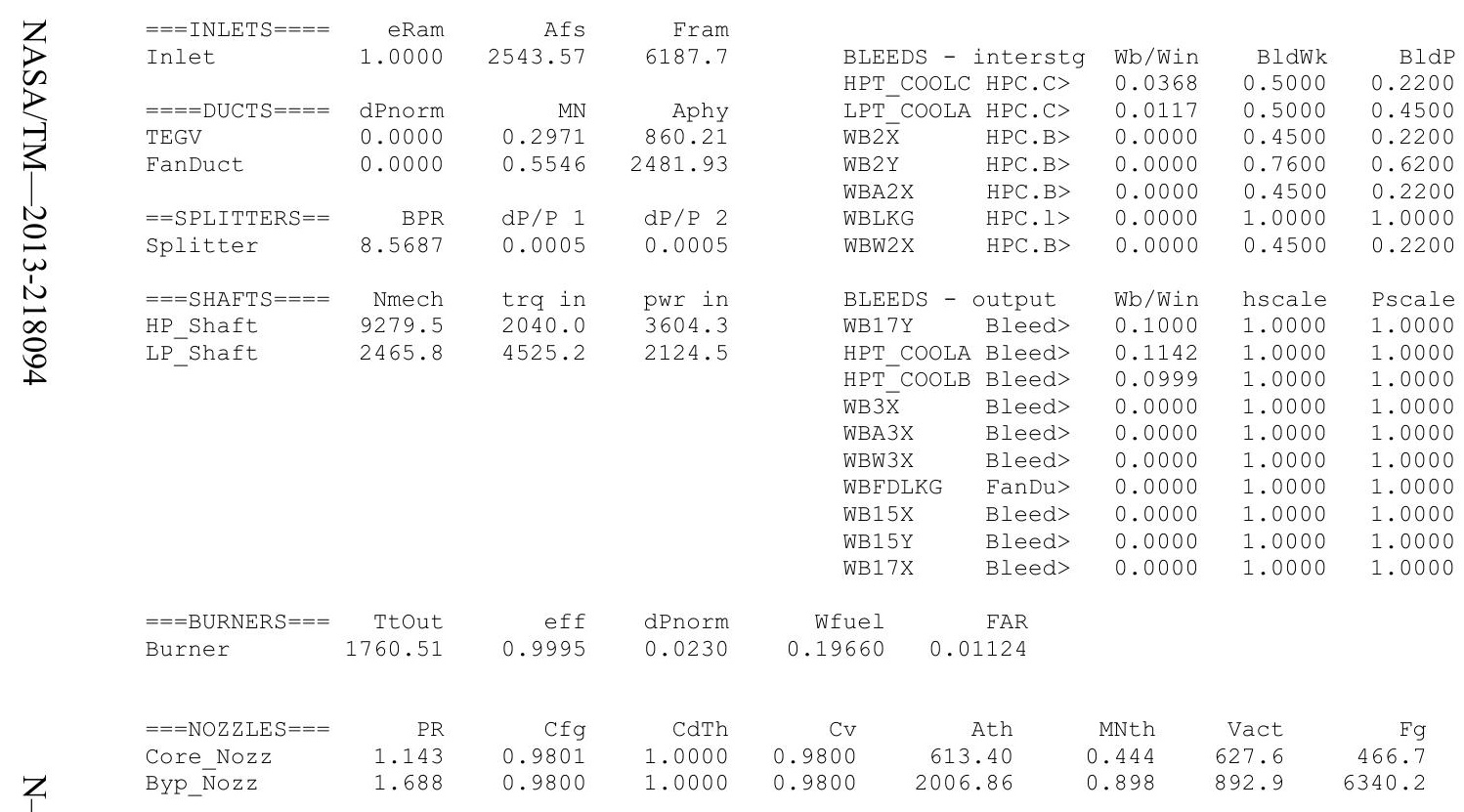


Date:05/13/13 Time:10:05:02 Model: Turbofan Engine - COMDES ON Converge = 1 CASE: 0

Version:NPSS 1.6.5 - Rev: -> Gas Package: Janaf iter/pass/Jacb/Broy=11/25/1/9 Run by: Philip C Jorgenson PC: 10

Temperature Stator 1 inlet: 476.92 Stator 1 exit: 484.20 Stator 2 inlet: 493.33 Stator 2 exit: 497.99

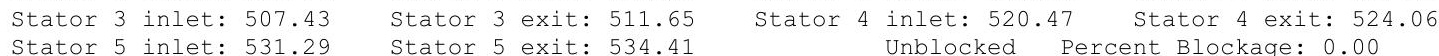

$\begin{array}{lrlll}\text { Ambient Relative Humidity } & 10.00 & & & \\ \text { Fan Face Relative Humidity } & 2.46 & & & \\ \text { Fan Bypass Relative Humidity } & 1.80 & & & \\ \text { LPC Inlet Relative Humidity } & 1.64 & & & \\ \text { LPC Exit Relative Humidity } & 0.18 & & & \\ \text { HPC Relative Humidity } & 0.00 & & & \\ \text { Drop Diameter } & 0.0000250 & \text { Inlet Length } & \\ \text { Ambient Flow Velocity } & 738.88 & \text { Fan/LPC Inlet Flow Velocity } & 185.92 \\ \text { Ambient Static Pressure } & 2.95 & \text { Fan/LPC Inlet Static Pressure } & 4.11 \\ \text { Ambient Static Temperature } & 425.97 & \text { Fan/LPC Inlet Static Temperature } & 468.60 \\ \text { Additional Water at LPC Exit } & 0.0003590 & & & \\ \end{array}$

\begin{tabular}{|c|c|c|c|c|c|c|c|c|c|c|c|c|c|c|}
\hline & & & & JMMARY & TPUT DAT & & & & & & & & & \\
\hline MN & alt & dTamb & W & Fn & TSFC & Wfuel & BPR & VTAS & OPR & EPR & Powerset & T4 & T41 & T49 \\
\hline
\end{tabular}

\begin{tabular}{|c|c|c|c|c|c|c|c|c|c|c|c|}
\hline & & & & FLOW & TAATION DA? & & & & & & \\
\hline & & & W & Pt & Tt & ht & FAR & & Wc & Ps & \\
\hline FSO & Ambient.Fl_O & & 245.33 & 4.201 & 471.48 & -17.97 & 0.0000 & 818. & 2.9 & 47 & 425 \\
\hline FS1 & Inlet.FI_O & & 245.33 & 4.201 & 471.48 & -17.97 & 0.0000 & 818.1 & 3.8 & 47 & 459 \\
\hline FS12 & Splitter.Fl_o & & 219.94 & 4.199 & 471.48 & -17.97 & 0.0000 & 733.8 & 3.7 & 97 & 458. \\
\hline FS2 & Splitter.Fl_o & & 25.38 & 4.199 & 471.48 & -17.97 & 0.0000 & 84. & 4.1 & 10 & 468 \\
\hline FS14 & Fan.Fl_o & & 219.94 & 4.712 & 488.93 & -13.79 & 0.0000 & 666. & 3.9 & 76 & 465 \\
\hline FS23 & LPC.Fl-O & & 25.38 & 6.416 & 543.74 & -0.64 & 0.0000 & 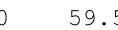 & 6.1 & & 536. \\
\hline FS24 & VaporIN.FI_O & & 25.39 & 6.416 & 544.25 & -2.59 & 0.0000 & 59.5 & 6.1 & 37 & 537. \\
\hline FS25 & Bleed2.Fl_o & & 22.85 & 6.416 & 544.25 & -2.59 & 0.0000 & 53. & 6.1 & & 538. \\
\hline FS3 & HPC.FI O & & 21.74 & 38.789 & 984.71 & 104.61 & 0.0000 & 11.3 & 34.1 & & 950. \\
\hline FS36 & Bleed3.Fl O & & 16.85 & 38.789 & 984.71 & 104.61 & 0.0000 & 8.8 & 36.1 & & 965. \\
\hline FS 4 & Burner.Fl_o & & 17.04 & 37.896 & 1732.95 & 91.91 & 0.0111 & 12. & 35.7 & & 1708 \\
\hline FS45 & HPT.FI O & & 22.77 & 9.211 & 1146.52 & -11.84 & 0.0083 & 54. & 8.3 & & 1117. \\
\hline FS49 & LPT.FI_O & & 23.04 & 3.311 & 908.54 & -70.13 & 0.0082 & 135. & 3.1 & 38 & 895. \\
\hline FS5 & TEGV.FI__O & & 23.04 & 3.311 & 908.62 & -70.13 & 0.0082 & 135. & 3.1 & 38 & 895 \\
\hline FS8 & Core_Nozz.FI & & 23.04 & 3.311 & 908.69 & -70.13 & 0.0082 & 135.3 & 2.9 & 47 & 879. \\
\hline FS17 & FanDüctLkg.Fi & & 219.94 & 4.712 & 488.93 & -13.79 & 0.0000 & 666. & 3.9 & 76 & 465. \\
\hline FS171 & Bleed15.Fl_o & & 222.48 & 4.712 & 489.56 & -13.66 & 0.0000 & 674.7 & 3.8 & & $462-1$ \\
\hline FS172 & FanDuct.Fl_o & & 222.48 & 4.712 & 489.56 & -13.66 & 0.0000 & 674. & 3.8 & 51 & 462 \\
\hline FS173 & Byp_Nozz.FI__o & & 222.48 & 4.712 & 489.56 & -13.66 & 0.0000 & 674.7 & 2.9 & 47 & 428 \\
\hline BOI & PERE & ORMANCE & DATA & & & & & & & & \\
\hline & & PR & eff & NC & TR & efpoly & & pwr & SMN & & SMW \\
\hline Fan & 733.88 & 1.122 & 0.9047 & 2504.901 & 1.0370 & 0.9063 & -13 & 301.6 & 2182.81 & & .68 \\
\hline LPC & 84.70 & 1.528 & 0.8406 & 2504.901 & 1.1533 & 0.8499 & & 622.4 & 6.78 & & .35 \\
\hline HPC & 53.62 & 6.046 & 0.8178 & 8945.664 & 1.8093 & 0.8565 & & 381.9 & 61.29 & & .92 \\
\hline HPT & 12.08 & 4.114 & 0.8760 & 220.128 & 1.3615 & 0.8539 & & 381.9 & & & \\
\hline LPT & 54.02 & 2.781 & 0.8501 & 70.532 & 1.2600 & 0.8308 & & 924.0 & & & \\
\hline TURBOI & MACHINERY MAP & DATA & & & & & & & & & \\
\hline & WcMap & PRmap & effmap & NcMap & R/Parm & s WCI & cDes & s PRdes & $s$ effDes & & S NcDes \\
\hline Fan & 852.70 & 1.119 & 0.9065 & 2481.017 & 0.0413 & $\overline{0} .8$ & 8607 & $\overline{1} .0235$ & 0.9980 & & 0.9905 \\
\hline LPC & 71.17 & 1.494 & 0.7769 & 0.662 & 0.0000 & 1.1 & 1901 & 1.0693 & 1.0820 & & 0.0003 \\
\hline HPC & 48.77 & 5.808 & 0.8263 & 8707.178 & 10.9852 & 1.0 & 0995 & 1.0494 & 0.9898 & & 0.9733 \\
\hline HPT & 0.96 & 4.028 & 0.8760 & 1.323 & 4.0281 & 12.6 & 6299 & 0.9723 & 1.0000 & & 0.0003 \\
\hline LPT & 0.83 & 2.340 & 0.8564 & 0.793 & 2.3399 & 65.2 & 2590 & 0.7521 & 0.9926 & & 0.0005 \\
\hline
\end{tabular}




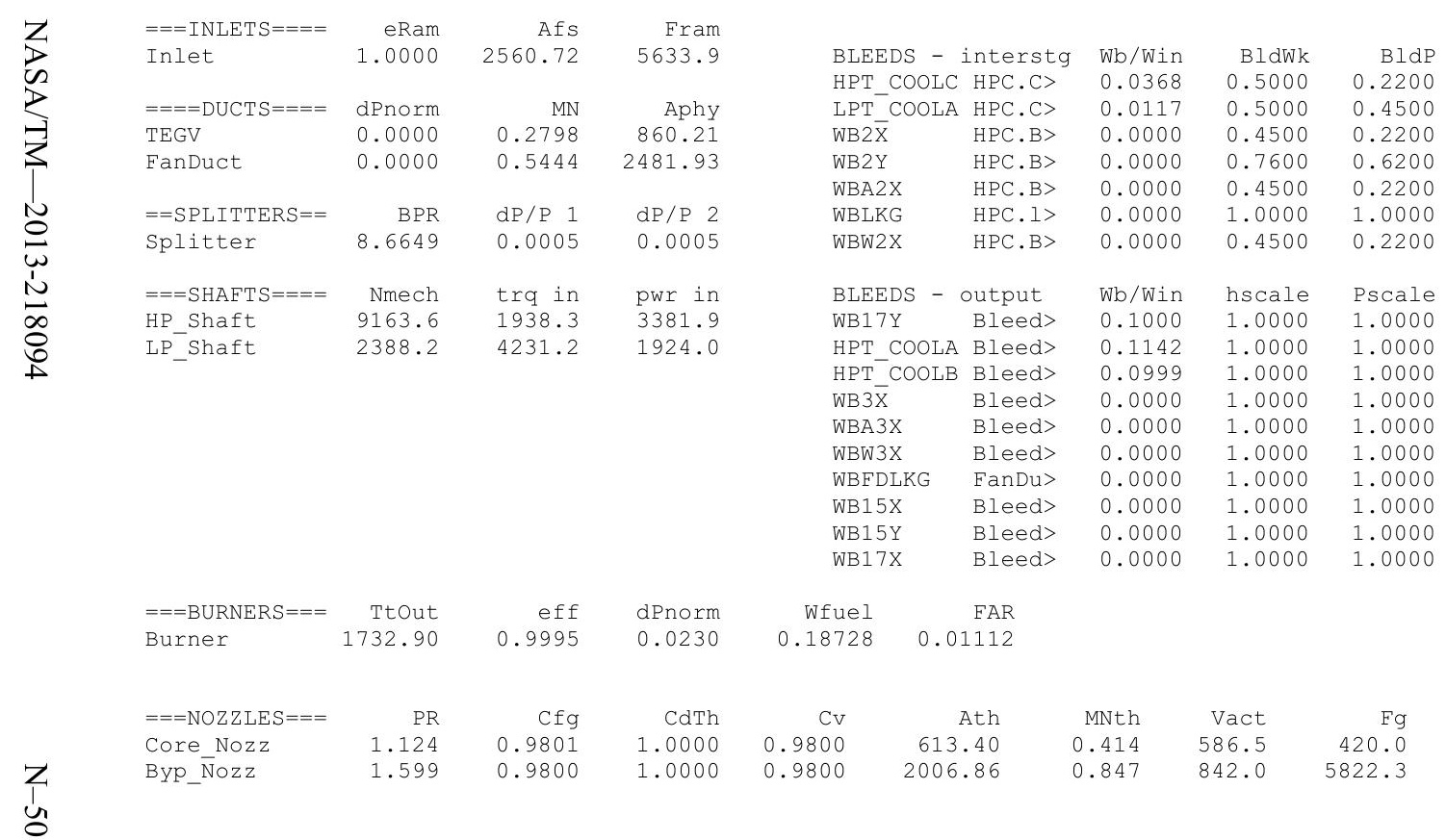


Turbofan Engine - COMDES ON Converge $=1$ CASE: 0

Version:NPSS_1.6.5 - Rev: -> Gas Package: Janaf iter/pass/Jacb/Broy=11/25/1/9 Run by: Philip C Jorgenson PC: 10

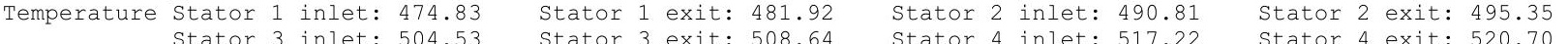
$\begin{array}{llll}\text { Stator } 3 \text { inlet: } 504.53 & \text { Stator } 3 \text { exit: } 508.64 & \text { Stator } 4 \text { inlet: } 517.22 & \text { Stator 4 exit: } 520.70 \\ \text { Stator } 5 \text { inlet: } 527.74 & \text { Stator } 5 \text { exit: } 530.76 & \text { Unblocked Percent Blockage: } 0.00\end{array}$

\begin{tabular}{|c|c|c|c|c|}
\hline bient Relative Humidi & 10.00 & & & \\
\hline Fan Face Relative Humidity & 2.63 & & & \\
\hline Fan Bypass Relative Humidity & 1.95 & & & \\
\hline LPC Inlet Relative Humidity & 1.76 & & & \\
\hline LPC Exit Relative Humidity & 0.19 & & & \\
\hline HPC Relative Humidity & 0.00 & & & \\
\hline Drop Diameter & 0.0000250 & Inlet Length & & 40.00 \\
\hline Ambient Flow Velocity & 722.68 & Fan/LPC Inlet & Flow Velocity & 183.09 \\
\hline bient Static Pressure & 3.09 & Fan/LPC Inlet & Static Pressure & 4.25 \\
\hline bient Static Temperature & 425.97 & Fan/LPC Inlet & Static Temperature & 466.71 \\
\hline ditional Water at LPC Exi & 0.000327 & & & \\
\hline
\end{tabular}

\begin{tabular}{|c|c|c|c|c|c|c|c|c|c|c|c|c|c|c|}
\hline MN & alt & dTamb & W & $\begin{array}{r}\text { UMMARY } \\
\text { Fn }\end{array}$ & $\begin{array}{c}\text { JTPUT DAT } \\
\text { TSFC }\end{array}$ & Wfuel & BPR & VTAS & OPR & EPR & Powerset & $\mathrm{T} 4$ & T41 & T49 \\
\hline 0.714 & 37357.0 & 36.00 & 251.86 & 621.4 & 1.1026 & 685.21 & 8.7107 & 722.68 & 9.103 & 0.772 & 10.000 & 1722.0 & 1628.1 & 1135.7 \\
\hline
\end{tabular}

FSO Ambient.FI_O

FS1 Inlet.Fl_O

$\begin{array}{ll}\text { FS12 } & \text { Splitter.Fl_02 } \\ \text { FS2 } & \text { Splitter.Fl_01 }\end{array}$

FS14 Fan.F1_O

$\begin{array}{ll}\text { FS23 } & \text { LPC.FI } \\ \text { FS24 } & \text { VaporIN.FI }\end{array}$

FS24 VaporIN.FI-

FS3 HPC.FI O

FS36 Bleed3.F1_O

FS4 Burner.F1_

FS49

FS5 5 TEGV F1

FS8 Core_Nozz.FI_O

FS17 FanDuctLkg.Fí

FS171 Bleed15.FI_O

FS172 FanDuct.FI-O

TURBOMACHINERY PERFORMANCE DATA

\begin{tabular}{|c|c|c|c|c|c|c|c|c|c|}
\hline & & & & & & & & & \\
\hline Fan & $\begin{array}{r}\text { Wc } \\
728.35\end{array}$ & $\begin{array}{r}\text { PR } \\
119\end{array}$ & $\begin{array}{r}\text { eff } \\
0.9043\end{array}$ & $\begin{array}{r}\mathrm{NC} \\
2478.068\end{array}$ & $\begin{array}{r}\text { TR } \\
1.0361\end{array}$ & $\begin{array}{l}\text { efPoly } \\
0.9058\end{array}$ & $\begin{array}{r}\text { pwr } \\
-1299\end{array}$ & $\begin{array}{r}\text { SMN } \\
2574.39\end{array}$ & $\begin{array}{r}\text { SMW } \\
54.61\end{array}$ \\
\hline LPC & $\begin{array}{r}128.35 \\
83.62\end{array}$ & $\begin{array}{l}1.119 \\
1.515\end{array}$ & $\begin{array}{l}0.9043 \\
0.8406\end{array}$ & 2478.068 & $\begin{array}{l}1.0361 \\
1.1499\end{array}$ & $\begin{array}{l}.0 .8498 \\
0.8497\end{array}$ & -619.4 & $\begin{array}{r}6.69 \\
6.69\end{array}$ & $\begin{array}{r}54.61 \\
3.28\end{array}$ \\
\hline HPC & 53.32 & $\begin{array}{l}1.515 \\
6.013\end{array}$ & $\begin{array}{l}0.8406 \\
0.8173\end{array}$ & 935.880 & 1.8072 & .8560 & -3420.2 & 61.38 & 57.97 \\
\hline HPT & 12.0 & 4.095 & 0.8754 & 219.799 & 1.3603 & 0.8533 & 3420.2 & & \\
\hline LPT & 53.80 & 2.731 & 0.847 & 69.833 & 1.2542 & 0.8288 & 1918.6 & & \\
\hline JBB & NE & $D F$ & & & & & & & \\
\hline & WcMap & PRmap & effMap & NcMap & R/Parm & S_WcDes & S_PRdes & s_effDes & S_NCDes \\
\hline an & 846.2 & 1.116 & 0.9061 & 454.440 & 0.0413 & 0.8 & $\overline{1} .0235$ & 0.9980 & $\overline{0} .9905$ \\
\hline LPC & 70.00 & 1.482 & 0.77 & 0.655 & 0.0000 & 1.1 & 1.0683 & 1.0837 & 0.0003 \\
\hline $\mathrm{APC}$ & $4 \xi$ & 5. & & 597.655 & & & & & 0.9733 \\
\hline $\mathrm{APT}$ & 0.9 & .010 & 0.8754 & 1.321 & 4.0096 & 12.6299 & 0.9723 & 1.0000 & 0.0003 \\
\hline LPT & 0.82 & .302 & .8542 & 0.786 & 2.3016 & 65.2590 & 0.7521 & 0.9926 & 0.0005 \\
\hline
\end{tabular}

$\begin{array}{lll}0.82 & 2.302 & 0.8542\end{array}$
0.786

65.2590

0.0005
FLOW STATION DATA

\begin{tabular}{rrrrrrrrrr} 
W & \multicolumn{1}{c}{ Ft } & Tt & ht & FAR & Wc & Ps & Ts & Aphy & MN \\
251.86 & 4.339 & 469.50 & -18.42 & 0.0000 & 811.56 & 3.088 & 425.97 & 2564.6 & 0.7140 \\
251.86 & 4.339 & 469.50 & -18.42 & 0.0000 & 811.56 & 3.980 & 458.03 & 4168.2 & 0.3535 \\
225.93 & 4.337 & 469.50 & -18.42 & 0.0000 & 728.35 & 3.929 & 456.40 & 3531.8 & 0.3785 \\
25.94 & 4.337 & 469.50 & -18.42 & 0.0000 & 83.62 & 4.248 & 466.71 & 830.5 & 0.1728 \\
225.93 & 4.853 & 486.46 & -14.36 & 0.0000 & 662.60 & 4.105 & 463.72 & 2606.7 & 0.4947 \\
25.94 & 6.569 & 539.89 & -1.54 & 0.0000 & 59.20 & 6.287 & 533.16 & 412.6 & 0.2512 \\
25.95 & 6.569 & 540.37 & -3.32 & 0.0000 & 59.25 & 6.286 & 533.62 & 412.6 & 0.2515 \\
23.35 & 6.569 & 540.37 & -3.32 & 0.0000 & 53.32 & 6.342 & 534.97 & 412.6 & 0.2246 \\
22.22 & 39.501 & 976.54 & 102.78 & 0.0000 & 11.34 & 34.792 & 942.80 & 49.7 & 0.4321 \\
17.22 & 39.501 & 976.54 & 102.78 & 0.0000 & 8.79 & 36.796 & 957.56 & 49.3 & 0.3218 \\
17.41 & 38.591 & 1721.96 & 90.16 & 0.0111 & 12.08 & 36.442 & 1697.53 & 74.6 & 0.2944 \\
23.27 & 9.423 & 1139.87 & -12.51 & 0.0083 & 53.80 & 8.567 & 1111.09 & 265.4 & 0.3755 \\
23.54 & 3.451 & 907.39 & -69.39 & 0.0082 & 132.60 & 3.278 & 894.63 & 860.2 & 0.2736 \\
23.54 & 3.451 & 907.46 & -69.39 & 0.0082 & 132.60 & 3.278 & 894.70 & 860.2 & 0.2736 \\
23.54 & 3.451 & 907.53 & -69.39 & 0.0082 & 132.61 & 3.088 & 880.14 & 613.4 & 0.4038 \\
225.93 & 4.853 & 486.46 & -14.36 & 0.0000 & 662.60 & 4.105 & 463.72 & 2606.7 & 0.4947 \\
228.52 & 4.853 & 487.07 & -14.23 & 0.0000 & 670.64 & 3.979 & 460.17 & 2481.9 & 0.5401 \\
228.52 & 4.853 & 487.07 & -14.23 & 0.0000 & 670.64 & 3.979 & 460.17 & 2481.9 & 0.5401 \\
228.52 & 4.853 & 487.07 & -14.23 & 0.0000 & 670.64 & 3.088 & 428.00 & 2006.9 & 0.8298
\end{tabular}

gamt
1.40075
1.40075
1.40075
1.40075
1.40060
1.39992
1.39986
1.39986
1.38210
1.38210
1.33125
1.36606
1.38074
1.38073
1.38073
1.40060
1.40060
1.40060
1.40060

WAR
0.0000786
0.0000786
0.0000786
0.0000786
0.0000786
0.0000786
0.0004064
0.0004064
0.0004064
0.0004064
0.0004064
0.0004064
0.0004064
0.0004064
0.0004064
0.0000786
0.0000823
0.0000823
0.0000823
$\begin{array}{rrr}\text { Wair } & \text { WH2O } & \text { H2O frac } \\ 251.84 & 0.020 & 0.0001\end{array}$ $\begin{array}{lll}251.84 & 0.020 & 0.0001\end{array}$ $25.93-0.002-0.0001$ $\begin{array}{lll}225.91 & 0.018 & 0.0001\end{array}$ $\begin{array}{lll}25.93 & 0.002 & 0.0001 \\ 25.93 & 0.011 & 0.0004\end{array}$ $\begin{array}{lll}25.93 & 0.011 & 0.0004 \\ 23.34 & 0.009 & 0.0004\end{array}$ $\begin{array}{lll}22.21 & 0.009 & 0.0004\end{array}$ $\begin{array}{lll}17.21 & 0.007 & 0.0004\end{array}$ $\begin{array}{lll}17.21 & 0.007 & 0.0139\end{array}$ $\begin{array}{lll}23.07 & 0.009 & 0.0105\end{array}$ $\begin{array}{lll}23.34 & 0.009 & 0.0104\end{array}$ $\begin{array}{lll}23.34 & 0.009 & 0.0104\end{array}$ $\begin{array}{lll}23.34 & 0.009 & 0.0104\end{array}$ $\begin{array}{lll}225.91 & 0.018 & 0.0001\end{array}$ $\begin{array}{lll}228.50 & 0.019 & 0.0001\end{array}$ $228.50 \quad 0.019 \quad 0.0001$ 
$\begin{array}{lrrr}===\text { INLETS }==== & \text { eRam } & \text { Afs } & \text { Fram } \\ \text { Inlet } & 1.0000 & 2564.57 & 5657.3\end{array}$

$====$ DUCTS $====$
TEGV

Aphy

FanDuct

0.0000

0.2736

860.21
2481.93

$==$ SPLITTERS $==$

0.0000

$\mathrm{dP} / \mathrm{P} 2$

Splitter

BPR
8.7107

0.0005

0.0005

$===\mathrm{SHAFTS}===$

LP_Shaft

Nmech

$\begin{array}{ll}\text { trq in } & \text { pwr in } \\ 1969.5 & 3420.2\end{array}$

1918.6

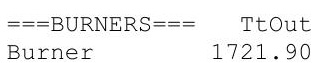

eff
0.9995

dPnorm

$\mathrm{PR}$
1.117
1.571

Core_Nozz

1.117
1.571

Cfg
0.9801

0.9801
BLEEDS - interstg Wb/Win BldWk BldP
0.2200 LPT COOLA HPC.C> $0.0117 \quad 0.5000 \quad 0.4500$ $\begin{array}{lllll}\text { WB2 } \bar{X} & \text { HPC.B> } & 0.0000 & 0.4500 & 0.2200 \\ \text { WB2Y } & \text { HPC.B> } & 0.0000 & 0.7600 & 0.6200\end{array}$ WBA2X HPC.B $>0.0000 \quad 0.4500-0.2200$ WBLKG HPC.1> $0.0000-1.0000-1.0000$ $\begin{array}{lllll}\text { WBW2X HPC.B } & 0.0000 & 0.4500 & 0.2200\end{array}$

BLEEDS - output Wb/Win hscale Pscale WB17Y Bleed> $0.1000-1.0000 \quad 1.0000$ HPT_COOLA Bleed> $0.1142 \quad 1.0000 \quad 1.0000$ HPT_COOLB Bleed> $0.0999 \quad 1.0000 \quad 1.0000$ $\begin{array}{lllll}\text { WB3X } & \text { Bleed> } & 0.0000 & 1.0000 & 1.0000 \\ \text { WBA3X } & \text { Bleed> } & 0.0000 & 1.0000 & 1.0000\end{array}$

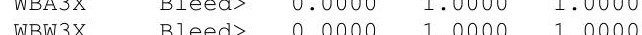
$\begin{array}{lllll}\text { WBW3X } & \text { Bleed> } & 0.0000 & 1.0000 & 1.0000 \\ \text { WBFDLKG } & \text { FanDu> } & 0.0000 & 1.0000 & 1.0000\end{array}$ $\begin{array}{lllll}\text { WBFDLKG } & \text { FanDu> } & 0.0000 & 1.0000 & 1.0000 \\ \text { WB15X } & \text { Bleed }> & 0.0000 & 1.0000 & 1.0000\end{array}$ WB15X Bleed> $0.00001 .0000-1.0000$ WB15Y Bleed> $0.0000 \quad 1.0000=1.0000$ $0.0000 \quad 1.0000 \quad 1.0000$

\begin{tabular}{|c|c|c|c|}
\hline $\mathrm{W}$ & $\mathrm{Tt}$ & ht & Pt \\
\hline 0.8593 & 760.27 & 49.73 & 9.423 \\
\hline 0.2732 & 760.27 & 49.73 & 3.451 \\
\hline 0.0000 & 738.41 & 44.43 & 13.814 \\
\hline 0.0000 & 873.31 & 77.32 & 26.987 \\
\hline 0.0000 & 738.41 & 44.43 & 13.814 \\
\hline 0.0000 & 976.54 & 102.78 & 39.501 \\
\hline 0.0000 & 738.41 & 44.43 & 13.814 \\
\hline W & Tt & ht & Pt \\
\hline 2.5945 & 540.37 & -3.32 & 6.569 \\
\hline 2.6662 & 976.54 & 102.78 & 38.591 \\
\hline 2.3330 & 976.54 & 102.78 & 21.907 \\
\hline 0.0000 & 976.54 & 102.78 & 39.501 \\
\hline 0.0000 & 976.54 & 102.78 & 39.501 \\
\hline 0.0000 & 976.54 & 102.78 & 39.501 \\
\hline 0.0000 & 486.46 & -14.36 & 4.853 \\
\hline 0.0000 & 486.46 & -14.36 & 4.853 \\
\hline 0.0000 & 486.46 & -14.36 & 4.853 \\
\hline 0.0000 & 486.46 & -14.36 & 4.853 \\
\hline
\end{tabular}

$\begin{array}{rrrrrr}\text { CdTh } & \text { Cv } & \text { Ath } & \text { MNth } & \text { Vact } & \text { Fg } \\ 1.0000 & 0.9800 & 613.40 & 0.404 & 571.9 & 418.5 \\ 1.0000 & 0.9800 & 2006.86 & 0.830 & 825.1 & 5860.2\end{array}$


Date:05/13/13 Time:10:05:32 Model: Turbofan Engine - COMDES ON Converge = 1 CASE: 0

Version:NPSS 1.6.5 - Rev: -> Gas Package: Janaf iter/pass/Jacb/Broy=10/ 24/1/8 Run by: Philip C Jorgenson PC: 10

Temperature Stator 1 inlet: $476.23 \quad$ Stator 1 exit: 482.87 Stator 2 inlet: 491.17 Stator 2 exit: 495.40

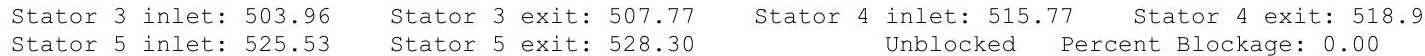

$\begin{array}{lclll}\text { Ambient Relative Humidity } & 10.00 & & & \\ \text { Fan Face Relative Humidity } & 3.24 & & & \\ \text { Fan Bypass Relative Humidity } & 2.48 & & & \\ \text { LPC Inlet Relative Humidity } & 2.22 & & & \\ \text { LPC Exit Relative Humidity } & 0.28 & & & \\ \text { HPC Relative Humidity } & 0.00 & & & \\ \text { Drop Diameter } & 0.0000250 & \text { Inlet Length } & \\ \text { Ambient Flow Velocity } & 682.24 & \text { Fan/LPC Inlet Flow Velocity } & 176.55 \\ \text { Ambient Static Pressure } & 3.58 & \text { Fan/LPC Inlet Static Pressure } & 4.74 \\ \text { Ambient Static Temperature } & 432.42 & \text { Fan/LPC Inlet Static Temperature } & 468.62 \\ \text { Additional Water at LPC Exit } & 0.0003050 & & & \end{array}$

\begin{tabular}{|c|c|c|c|c|c|c|c|c|c|c|c|c|c|c|}
\hline MN & a]t & dTamb & WT & JMMARY & TPUT DA & Wfuel & BPR & VTAS & OPR & FPR & Powerset & T4 3 & T41 & T49 \\
\hline 0.669 & 34281.0 & 36.00 & 272.67 & 668.1 & 1.0939 & 730.83 & 8.8177 & 682.24 & 8.727 & 0.795 & 10.000 & 1711.3 & 1617.6 & 1130.8 \\
\hline
\end{tabular}

\begin{tabular}{|c|c|c|c|c|c|c|c|c|c|c|c|}
\hline & & & W & $\begin{array}{l}\text { FLOW } \\
\text { Pt }\end{array}$ & $\begin{array}{c}\text { STATION DAT } \\
\text { Tt }\end{array}$ & TA & $F A$ & & Wc & Ps & \\
\hline FSO & Ambient.Fl_o & & 272.67 & 4.831 & 471.21 & -18.12 & 0.000 & 790.6 & & & 432.4 \\
\hline FS1 & Inlet.Fl $0^{-}$ & & 272.67 & 4.831 & 471.21 & -18.12 & 0.000 & 790.6 & & 453 & 460. \\
\hline FS12 & Splitter.Fl_o & & 244.89 & 4.829 & 471.21 & -18.12 & 0.000 & 710.4 & & 399 & 458 \\
\hline FS2 & Splitter.Fl_0 & & 27.77 & 4.829 & 471.21 & $-18 \cdot 12$ & 0.000 & 80.5 & & & 468 \\
\hline FS14 & Fan.Fl_o & & 244.89 & 5.360 & 487.03 & -14.32 & 0.000 & 650.6 & & 571 & 465. \\
\hline FS23 & LPC.Fl_O & & 27.77 & 7.122 & 537.04 & -2.33 & 0.000 & 58.3 & & & 530. \\
\hline FS24 & VaporIN̄.FI_O & & 27.78 & 7.122 & 537.49 & -3.98 & 0.000 & 58.3 & & & 530. \\
\hline FS25 & Bleed2.FI__o & & 25.00 & 7.122 & 537.49 & -3.98 & 0.000 & 52.5 & & 384 & 532. \\
\hline FS3 & HPC.Fl_O & & 23.79 & 42.162 & 967.59 & 100.59 & 0.000 & 11.3 & 37. & & 934 \\
\hline FS36 & Bleed3.FI_O & & 18.44 & 42.162 & 967.59 & 100.59 & 0.000 & 8.7 & 39. & & 948 \\
\hline FS 4 & Burner.Fl_o & & 18.64 & 41.191 & 1711.28 & 88.04 & 0.011 & 12.0 & 38. & & 1686 \\
\hline FS45 & HPT.FI_O & & 24.91 & 10.174 & 1135.00 & -13.13 & 0.008 & 53.2 & & & 1107 \\
\hline FS 49 & LPT.FI-O & & 25.21 & 3.940 & 915.91 & -66.65 & 0.008 & 124.9 & & 766 & 904 \\
\hline FS5 & TEGV.F̄_ $\mathrm{O}$ & & 25.21 & 3.940 & 915.99 & -66.65 & 0.008 & 124.9 & & 766 & \\
\hline FS 8 & Core_Nozz.FI_ & & 25.21 & 3.940 & 916.06 & -66.65 & 0.008 & 124.9 & & & $892-2 x \cdot h$ \\
\hline FS17 & FanDuctLkg.Fi & & 244.89 & 5.360 & 487.03 & -14.32 & 0.000 & 650.6 & & 571 & 46 \\
\hline FS171 & Bleed15.FI_O & & 247.67 & 5.360 & 487.60 & -14.21 & 0.000 & 658.4 & & 440 & 462 \\
\hline FS172 & FanDuct.Fl_o & & 247.67 & 5.360 & 487.60 & -14.21 & 0.000 & 658.4 & & 440 & 462 \\
\hline FS173 & Byp_Nozz.FI__O & & 247.67 & 5.360 & 487.60 & -14.21 & 0.000 & 658.4 & & 578 & 434 \\
\hline TURBON & MACHINERY PERE & ORMANCE & DATA & & & & & & & & \\
\hline & & $\mathrm{PR}$ & eff & $\mathrm{NC}$ & TR & efpoly & & pwr & SMN & & SMW \\
\hline Fan & 710.43 & 1.110 & 0.9032 & 2395.649 & 1.0336 & 0.9046 & & 313.8 & 5797.22 & & .20 \\
\hline LPC & 80.57 & 1.475 & 0.8412 & 2395.649 & 1.1397 & 0.8497 & & 620.3 & 6.41 & & .05 \\
\hline HPC & 52.52 & 5.920 & 0.8157 & 8909.582 & 1.8002 & 0.8545 & & 609.5 & 61.81 & & .30 \\
\hline HPT & 12.08 & 4.049 & 0.8747 & 219.248 & 1.3569 & 0.8527 & & 609.5 & & & \\
\hline LPT & 53.24 & 2.582 & 0.8428 & 67.778 & 1.2370 & 0.8242 & & 934.1 & & & \\
\hline TURBON & MACHINERY MAP & DATA & & & & & & & & & \\
\hline & WcMap & PRmap & effMap & NcMap & R/Parm & S_WC & Ees & S_PRdes & s_effDe & & S_NCDes \\
\hline Fan & 825.45 & 1.108 & 0.9050 & 372.807 & 0.0413 & & 3607 & $\overline{1} .0235$ & 0.998 & & $\overline{0} .9905$ \\
\hline LPC & 66.44 & 1.445 & 0.7718 & 0.633 & 0.0000 & 1.2 & 2128 & 1.0676 & 1.090 & & 0.0003 \\
\hline HPC & 47.77 & 5.688 & 0.8242 & 8672.058 & 10.9678 & 1.0 & 9995 & 1.0494 & 0.989 & & 0.9733 \\
\hline HPT & 0.96 & 3.964 & 0.8746 & 1.318 & 3.9642 & 12.6 & 5299 & 0.9723 & 1.000 & & 0.0003 \\
\hline LPT & 0.82 & 2.190 & 0.8490 & 0.762 & 2.1902 & 65.2 & 2590 & 0.7521 & 0.992 & & 0.0005 \\
\hline
\end{tabular}




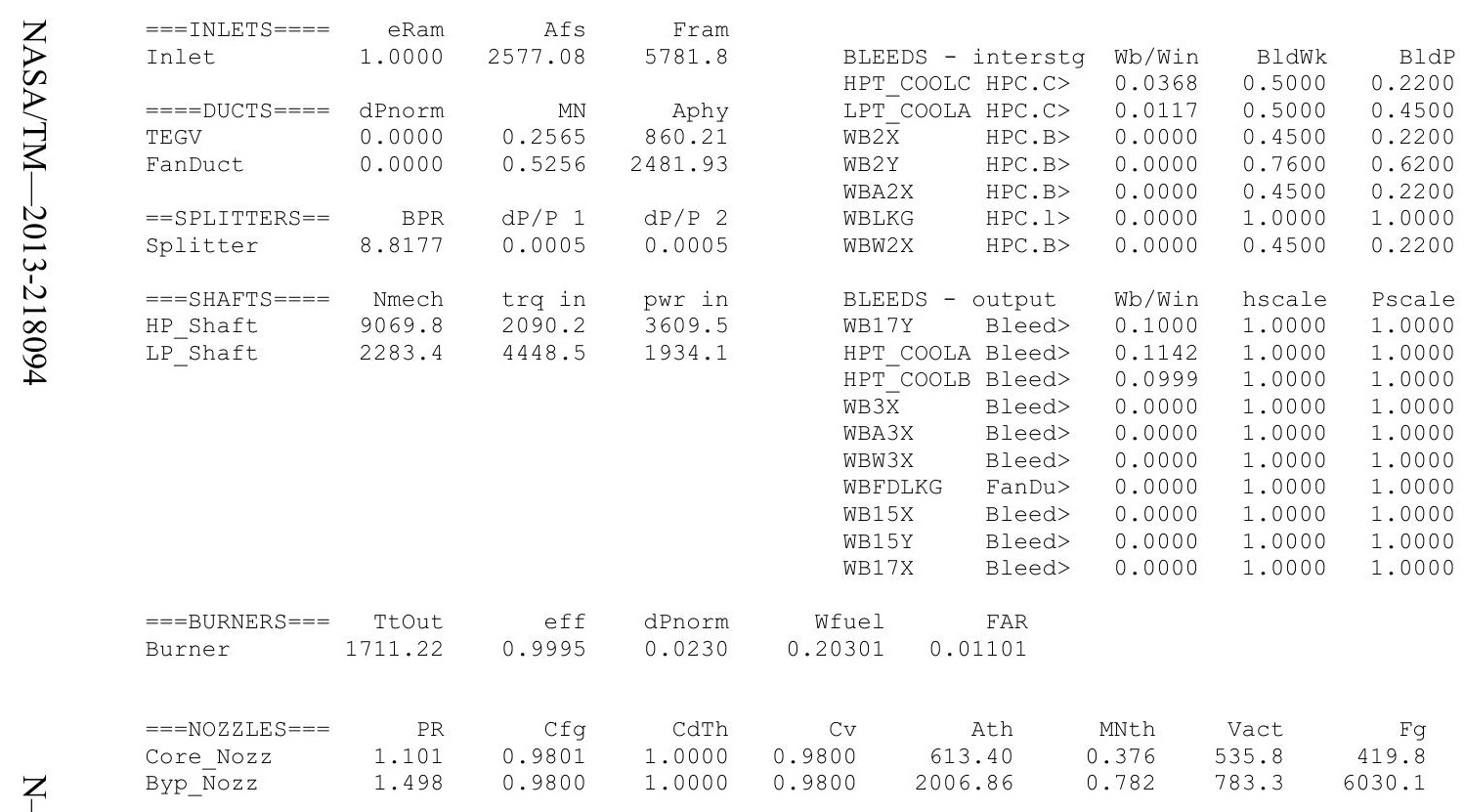


Date:05/13/13 Time:10:05:49 Model: Turbofan Engine - ComDES ON Converge = 1 CASE: 0

Version:NPSS 1.6.5 - Rev: -> Gas Package: Janaf iter/pass/Jacb/Broy=14/28/1/12 Run by: Philip C Jorgenson PC: 10

Temperature Stator 1 inlet: $484.51 \quad$ Stator 1 exit: $490.21 \quad$ Stator 2 inlet: 497.25 Stator 2 exit: 500.66 Stator 5 inlet: 525.96 Stator 5 exit: $528.05 \quad$ Unblocked Percent Blockage: 0.00

Ambient Relative Humidity $\quad 10.00$

Fan Face Relative Humidity $\quad 4.18$

Fan Bypass Relative Humidity $\quad 3.37$

LPC Inlet Relative Humidity

rop Diane Humidity

0.01

Ambient Flow Velocity

Ambient Static Pressure

Additional Water at LPC Exit

$$
0.0000250
$$

Inlet Length $\quad 40.00$

$\begin{array}{rrr}630.80 & \text { Fan/LPC Inlet Flow Velocity } & 177.28 \\ 4.36 & \text { Fan/LPC Inlet Static Pressure } & 5.49\end{array}$

447.58 Fan/LPC Inlet Static Temperature 478.12

\section{SUMMARY OUTPUT DATA}

$\begin{array}{rrr}\text { MN } & \text { alt } & \text { dTamb } \\ 0.608 & 30029.0 & 36.00\end{array}$

W 298.12

Fn
711.1

TSFC
1.1099

Wfuel
789.23

BPR
8.4157

VTAS
630.80

OPR
8.109

EPR
0.827

PowerSet
10.000

$\begin{array}{ccc}\text { T4 } & \text { T41 } & \text { T49 } \\ 1709.2 & 1615.2 & 1132.4\end{array}$

FSO Ambient.Fl 0

FS1 Inlet.FI_O

FS12 Splitter.F1_02

FS14 Fan.Fl_O

S23 LPC.F1-

S24 Vaporin. HIO

(ES25

FS36 Bleed3rito

FS4 Burner.Fl_-

FS5 TEGV.Fīo

FS8 Core_Nozz.Fl_O

FS17 FanDüctLkg.Fì_o

FS171 Bleed15.FI_O

FS172 FanDuct.FI-O

FS173 Byp Nozz.F̄̄o

TURBOMACHINERY PERFORMANCE DATA

$\begin{array}{lrrr} & \text { WC } & \text { PR } & \text { eff } \\ \text { Fan } & 674.15 & 1.096 & 0.9014 \\ \text { LPC } & 80.11 & 1.396 & 0.8547 \\ \text { HPC } & 51.60 & 5.813 & 0.8138 \\ \text { HPT } & 12.08 & 3.990 & 0.8744 \\ \text { LPT } & 52.53 & 2.359 & 0.8338\end{array}$

FLOW STATION DATA

\begin{tabular}{rrrrrr} 
W & \multicolumn{1}{c}{ Pt } & Tt & ht & FAR & WC \\
298.12 & 5.595 & 480.74 & -16.28 & 0.0000 & 753.87 \\
298.12 & 5.595 & 480.74 & -16.28 & 0.0000 & 753.87 \\
266.46 & 5.592 & 480.74 & -16.28 & 0.0000 & 674.15 \\
31.66 & 5.592 & 480.74 & -16.28 & 0.0000 & 80.11 \\
266.46 & 6.131 & 494.95 & -12.88 & 0.0000 & 623.95 \\
31.66 & 7.805 & 536.97 & -2.80 & 0.0000 & 60.66 \\
31.67 & 7.805 & 537.43 & -4.51 & 0.0000 & 60.71 \\
26.92 & 7.805 & 537.43 & -4.51 & 0.0000 & 51.60 \\
25.62 & 45.368 & 962.98 & 98.94 & 0.0000 & 11.31 \\
19.85 & 45.368 & 962.98 & 98.94 & 0.0000 & 8.76 \\
20.07 & 44.323 & 1709.17 & 86.38 & 0.0110 & 12.08 \\
26.83 & 11.109 & 1136.62 & -13.67 & 0.0082 & 52.53 \\
27.14 & 4.710 & 937.83 & -62.14 & 0.0081 & 113.87 \\
27.14 & 4.710 & 937.90 & -62.14 & 0.0081 & 113.87 \\
27.14 & 4.710 & 937.98 & -62.14 & 0.0081 & 113.88 \\
266.46 & 6.131 & 494.95 & -12.88 & 0.0000 & 623.95 \\
271.21 & 6.131 & 495.69 & -12.73 & 0.0000 & 635.55 \\
271.21 & 6.131 & 495.69 & -12.73 & 0.0000 & 635.55 \\
271.21 & 6.131 & 495.69 & -12.73 & 0.0000 & 635.55
\end{tabular}

PS
4.358

$\begin{array}{lrrr}4.358 & 447.58 & \text { Aphy } & \text { MN }\end{array}$

5.201
5.149

5.487

5.315

7.452

7.451

7.553
40.000

42.284

$41.854 \quad 1684.90$

$10.152 \quad 1109.4$

$\begin{array}{ll}4.539 & 928.34 \\ 4.539 & 928.41\end{array}$

$4.358 \quad 918.16$

5.315

5.169

5.169

4.358

$\begin{array}{lll}928.41 & 860.2 & 0.2322 \\ 975.14 & 613.4 & 0.3372\end{array}$

$\begin{array}{ll}263.7 & 0.3372 \\ 2481.9 & 0.4561\end{array}$

$\begin{array}{llll}495.69 & -12.73 & 0.0000 & 635.55\end{array}$

449.59

$2006.9 \quad 0.7154$

$\begin{array}{rrrrrr}\text { NC } & \text { TR } & \text { efPoly } & \text { pwr } & \text { SMN } & \text { SMW } \\ 2239.512 & 1.0296 & 0.9027 & -1284.2 & -8416.53 & 52.79 \\ 2239.512 & 1.1170 & 0.8614 & -604.2 & 5.98 & 2.60 \\ 8879.291 & 1.7918 & 0.8527 & -3844.7 & 62.30 & 58.68 \\ 218.626 & 1.3525 & 0.8526 & 3844.7 & & \\ 63.952 & 1.2095 & 0.8163 & 1888.4 & & \end{array}$

gamt
1.40064
1.40064
1.40064
1.40064
1.40050
1.39995
1.39990
1.39990
1.38289
1.38289
1.33184
1.36626
1.37892
1.37892
1.37891
1.40050
1.40049
1.40049
1.40049

WAR
0.0001750
0.0001750
0.0001750
0.0001750
0.0001750
0.0001750
0.0004905
0.0004905
0.0004905
0.0004905
0.0004905
0.0004905
0.0004905
0.0004905
0.0004905
0.0001750
0.0001805
0.0001805
0.0001805

Wair 298.07
298.07 298.07
266.42 $31.66 \quad 0.006$ $\begin{array}{rr}266.42 & 0.047 \\ 31.66 & 0.006\end{array}$ $31.66 \quad 0.016$ $26.91 \quad 0.013$ $25.60 \quad 0.013$ $\begin{array}{ll}19.84 & 0.010 \\ 19.84 & 0.010\end{array}$ $26.59 \quad 0.013$ $\begin{array}{ll}26.91 & 0.013 \\ 26.91 & 0.013\end{array}$ $26.91 \quad 0.013$ $\begin{array}{ll}266.42 & 0.047 \\ 271.16 & 0.049\end{array}$ $\begin{array}{ll}271.16 & 0.049 \\ 271.16 & 0.049\end{array}$

TURBOMACHINERY MAP DATA

$\begin{array}{lrrrrrrrrr} & \text { WcMap } & \text { PRmap } & \text { effMap } & \text { NcMap } & \text { R/Parm } & \text { S_WcDes } & \text { S_PRdes } & \text { S_effDes } & \text { S_NcDes } \\ \text { Fan } & 783.29 & 1.094 & 0.9033 & 2218.159 & 0.0416 & 0.8607 & \overline{1} .0235 & 0.9980 & 0.9905 \\ \text { LPC } & 59.99 & 1.378 & 0.7653 & 0.592 & 0.0000 & 1.3353 & 1.0454 & 1.1169 & 0.0003 \\ \text { HPC } & 46.93 & 5.586 & 0.8222 & 8642.575 & 10.9499 & 1.0995 & 1.0494 & 0.9898 & 0.9733 \\ \text { HPT } & 0.96 & 3.907 & 0.8743 & 1.314 & 3.9070 & 12.6299 & 0.9723 & 1.0000 & 0.0003 \\ \text { LPT } & 0.81 & 2.022 & 0.8399 & 0.719 & 2.0218 & 65.2590 & 0.7521 & 0.9926 & 0.0005\end{array}$




\begin{tabular}{|c|c|c|c|c|c|c|c|c|c|c|c|c|c|}
\hline Z & $===$ INLETS $S===$ & eRam & Afs & Fram & & & & & & & & \\
\hline D & Inlet & 1.0000 & 2589.67 & 5845.0 & BLEEDS - interstg & & $\begin{array}{l}\text { Wb/Win } \\
0.0368\end{array}$ & $\begin{array}{r}\text { BldWk } \\
0.5000\end{array}$ & $\begin{array}{r}\text { BldP } \\
0.2200\end{array}$ & $\begin{array}{r}W \\
0.9907\end{array}$ & $\begin{array}{r}\text { Tt } \\
89\end{array}$ & $\begin{array}{r}\text { ht } \\
47.22\end{array}$ & $\begin{array}{r}P t \\
11.109\end{array}$ \\
\hline R & $====$ DUCT $S====$ & dPnorm & MN & Aphy & \multicolumn{2}{|c|}{ LPT COOLA HPC.C> } & 0.0117 & 0.5000 & 0.4500 & 0.3150 & 751.89 & 47.22 & 4.710 \\
\hline$\exists$ & TEGV & 0.0000 & 0.2322 & 860.21 & WB2 $\bar{x}$ & HPC.B> & 0.0000 & 0.4500 & 0.2200 & 0.0000 & 730.57 & 42.04 & 16.069 \\
\hline & FanDuct & 0.0000 & 0.4998 & 2481.93 & WB2Y & HPC.B> & 0.0000 & 0.7600 & 0.6200 & 0.0000 & 862.20 & 74.11 & 31.094 \\
\hline & & & & & \multirow{2}{*}{$\begin{array}{l}\text { WBA2X } \\
\text { WBLKG }\end{array}$} & HPC.B> & 0.0000 & 0.4500 & 0.2200 & 0.0000 & 730.57 & 42.04 & 16.069 \\
\hline 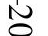 & $==$ SPLITTERS $==$ & BPR & $\mathrm{dP} / \mathrm{P} 1$ & $\mathrm{dP} / \mathrm{P} 2$ & & HPC.I> & 0.0000 & 1.0000 & 1.0000 & 0.0000 & 962.98 & 98.94 & 45.368 \\
\hline & Splitter & 8.4157 & 0.0005 & 0.0005 & WBW2X & HPC.B> & 0.0000 & 0.4500 & 0.2200 & 0.0000 & 730.57 & 42.04 & 16.069 \\
\hline$\underline{二}$ & $===$ SHAFTS $====$ & Nmech & trg in & pwr in & \multicolumn{2}{|c|}{ BLEEDS - output } & Wb/Win & hscale & Pscale & W & Tt & ht & Pt \\
\hline$\infty$ & HP_Shaft & 9038.5 & 2234.1 & 3844.7 & WB17Y & Bleed> & 0.1500 & 1.0000 & 1.0000 & 4.7509 & 537.43 & -4.51 & 7.805 \\
\hline & LP ${ }^{-}$Shaft & 2156.1 & 4600.2 & 1888.4 & HPT COOLA & Bleed> & 0.1142 & 1.0000 & 1.0000 & 3.0739 & 962.98 & 98.94 & 44.323 \\
\hline & & & & & \multirow{2}{*}{$\begin{array}{l}\text { HPT_COOLB } \\
\text { WB } 3 \bar{X}\end{array}$} & Bleed> & 0.0999 & 1.0000 & 1.0000 & 2.6897 & 962.98 & 98.94 & 25.325 \\
\hline & & & & & & Bleed> & 0.0000 & 1.0000 & 1.0000 & 0.0000 & 962.98 & 98.94 & 45.368 \\
\hline & & & & & WBA $3 \mathrm{X}$ & Bleed> & 0.0000 & 1.0000 & 1.0000 & 0.0000 & 962.98 & 98.94 & 45.368 \\
\hline & & & & & $\begin{array}{l}\text { WBW3X } \\
\text { WBFDLKG }\end{array}$ & Bleed> & 0.0000 & 1.0000 & 1.0000 & 0.0000 & 962.98 & 98.94 & 45.368 \\
\hline & & & & & \multirow{2}{*}{$\begin{array}{l}\text { WBFDLKG } \\
\text { WB1 } 5 \mathrm{x}\end{array}$} & FanDu> & 0.0000 & 1.0000 & 1.0000 & 0.0000 & 494.95 & -12.88 & 6.131 \\
\hline & & & & & & Bleed> & 0.0000 & 1.0000 & 1.0000 & 0.0000 & 494.95 & -12.88 & 6.131 \\
\hline & & & & & $\begin{array}{l}\text { WB15X } \\
\text { WB15Y }\end{array}$ & Bleed> & 0.0000 & 1.0000 & 1.0000 & 0.0000 & 494.95 & -12.88 & 6.131 \\
\hline & & & & & WB17X & Bleed> & 0.0000 & 1.0000 & 1.0000 & 0.0000 & 494.95 & -12.88 & 6.131 \\
\hline & $===$ BURNERS $===$ & TtOut & eff & dPnorm & & & & & & & & \\
\hline & Burner & 1709.11 & 0.9995 & 0.0230 & \multicolumn{2}{|c|}{$\begin{array}{rr}\text { Wfuel } & \text { FAR } \\
0.21923 & 0.01105\end{array}$} & & & & & & & \\
\hline & $===$ NOZZLES $===$ & $\mathrm{PR}$ & $\mathrm{Cfg}$ & CdTh & & Ath & MNth & \multirow{3}{*}{$\begin{array}{r}\text { Vact } \\
487.5 \\
729.0\end{array}$} & \multirow{2}{*}{$\mathrm{Fg}$} & & & & \\
\hline & Core Nozz & 1.081 & 0.9801 & 1.0000 & \multirow{2}{*}{\multicolumn{2}{|c|}{$\begin{array}{r}\mathrm{CV} \\
0.9800 \\
0.9800\end{array}$}} & 0.337 & & & & & & \\
\hline & Byp_Nozoz & 1.407 & 0.9800 & 1.0000 & & & 0.715 & & 6144.8 & & & & \\
\hline
\end{tabular}


Date:05/13/13 Time:10:06:18 Model: Turbofan Engine - ComDES ON Converge = 1 CASE: 0

Version:NPSS 1.6.5 - Rev: -> Gas Package: Janaf iter/pass/Jacb/Broy=19/47/2/16 Run by: Philip C Jorgenson PC: 10

Temperature Stator 1 inlet: $492.29 \quad$ Stator 1 exit: $496.45 \quad$ Stator 2 inlet: 501.18 Stator 2 exit: 503.04 Stator 5 inlet: 519.50 Stator 5 exit: $520.25 \quad$ Unblocked Percent Blockage: 0.00

Ambient Relative Humidity $\quad 10.00$

Fan Face Relative Humidity $\quad 5.02$

Fan Bypass Relative Humidity 4.21

LPC Inlet Relative Humidity $\quad 4.03$

Drop Diameter

0.01

0.0000250

40.00

Ambient Flow Velocity

Ambient Static Pressure

Additional Water at LPC Exit

582.56 Fan/LPC Inlet Flow Velocity 195.51

$\begin{array}{lrr}463.14 & \text { Fan/LPC Inlet Static Pressure } & 6.37 \\ & \text { Fan/LPC Inlet Static Temperature } & 488.23\end{array}$

MN SUMMARY OUTPUT DATA

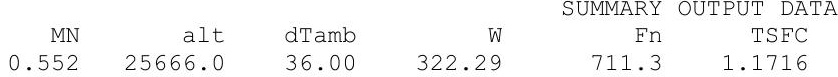

\section{(20)}

FSO Ambient.FI_O

FS1 Inlet.Fl_o

FS12 Splitter.F1_02

FS2 Splitter.FI_01

FS14 Fan.F1_O

FS24 VaporIN . FI

FS25 Bleed2.F1_O

S3 HPC.FI_O

FS4 Bleed3. F1-

FS4 Burner.F1-

BS4

FS5 5 TEGV.FI

FS8 Core_Nozz.Fl_O

FS17 FanDūctLkg.F̄̄

FS171 Bleed15.F1

FS172 FanDuct.FI-O

FS173 Byp Nozz.FİO

W
322.29
322.29
282.61
39.68
282.61
39.68
39.69
27.78
26.44
20.49
20.72
27.69
28.01
28.01
28.01
282.61
294.52
294.52
294.52

$\begin{array}{cc}\text { FLOW } & \text { STATION DATA } \\ \text { Pt } & \text { Tt }\end{array}$

\begin{tabular}{|c|c|c|c|c|}
\hline $\mathrm{Pt}$ & $\mathrm{Tt}$ & ht & FAR & Wc \\
\hline 516 & 491.41 & -14.47 & 0.0000 & 707.51 \\
\hline 5.516 & 491.41 & -14.47 & 0.0000 & 707.51 \\
\hline 6.513 & 491.41 & -14.47 & 0.0000 & 620.72 \\
\hline 6.513 & 491.41 & -14.47 & 0.0000 & 87.15 \\
\hline .039 & 503.66 & -11.54 & 0.0000 & 581.42 \\
\hline 8.183 & 531.64 & -4.82 & 0.0000 & 72.15 \\
\hline 8.183 & 532.07 & -6.42 & 0.0000 & 72.20 \\
\hline 8.183 & 532.07 & -6.42 & 0.0000 & 50.54 \\
\hline 46.883 & 950.19 & 95.16 & 0.0000 & 11.22 \\
\hline 46.883 & 950.19 & 95.16 & 0.0000 & 8.69 \\
\hline 45.803 & 1713.83 & 82.35 & 0.0113 & 12.08 \\
\hline 1.867 & 1144.85 & -15.75 & 0.0084 & 50.94 \\
\hline 5.615 & 969.67 & -58.34 & 0.0083 & 100.25 \\
\hline 5.615 & 969.74 & -58.34 & 0.0083 & 100.25 \\
\hline 5.615 & 969.81 & -58.34 & 0.0083 & 100.26 \\
\hline 7.039 & 503.66 & -11.54 & 0.0000 & 581.42 \\
\hline & 504.81 & -11.33 & 0. & 606.61 \\
\hline 039 & 04.81 & 11.33 & 0.0000 & 606.61 \\
\hline
\end{tabular}

VTAS
582.56

Wfuel BPR

OPR
7.195

EPR
0.857

Powerset
10.000

$\begin{array}{cc}\text { T4 } & \text { T41 } \\ 1713.8 & 1617.8\end{array}$

T49

TURBOMACHINERY PERFORMANCE DATA

$\begin{array}{lrrrrrrrrr} & \text { WC } & \text { PR } & \text { eff } & \text { NC } & \text { TR } & \text { efPoly } & \text { pwr } & \text { SMN } & \text { SMW } \\ \text { Fan } & 620.72 & 1.081 & 0.9017 & 2025.930 & 1.0249 & 0.9028 & -1174.2 & -2600.98 & 50.00 \\ \text { LPC } & 87.15 & 1.256 & 0.8236 & 2025.930 & 1.0819 & 0.8292 & -541.7 & 6.43 & 2.41 \\ \text { HPC } & 50.54 & 5.730 & 0.8121 & 8845.414 & 1.7858 & 0.8511 & -3896.1 & 61.75 & 58.03 \\ \text { HPT } & 12.08 & 3.860 & 0.8752 & 216.408 & 1.3429 & 0.8542 & 3896.1 & & \\ \text { LPT } & 50.94 & 2.113 & 0.8234 & 58.281 & 1.1778 & 0.8076 & 1715.9 & \end{array}$

TURBOMACHINERY MAP DATA

$\begin{array}{lrrrrrrrrr} & \text { WcMap } & \text { PRmap } & \text { effMap } & \text { NcMap } & \text { R/Parm } & \text { S_WcDes } & \text { S_PRdes } & \text { S_effDes } & \text { S_NcDes } \\ \text { Fan } & 721.21 & 1.079 & 0.9035 & 2006.613 & 0.0424 & 0.8607 & \overline{1} .0235 & 0.9980 & 0.9905 \\ \text { LPC } & 52.85 & 1.305 & 0.7603 & 0.535 & 0.0000 & 1.6491 & 0.8415 & 1.0832 & 0.0003 \\ \text { HPC } & 45.97 & 5.507 & 0.8205 & 8609.601 & 10.9973 & 1.0995 & 1.0494 & 0.9898 & 0.9733 \\ \text { HPT } & 0.96 & 3.780 & 0.8752 & 1.301 & 3.7804 & 12.6299 & 0.9723 & 1.0000 & 0.0003 \\ \text { LPT } & 0.78 & 1.837 & 0.8295 & 0.656 & 1.8374 & 65.2590 & 0.7521 & 0.9926 & 0.0005\end{array}$

\begin{tabular}{|c|c|}
\hline \multicolumn{2}{|c|}{ Ps } \\
\hline 5.2 & 7 \\
\hline 6.1 & 482 \\
\hline 6.0 & 481 \\
\hline 6.3 & 488 \\
\hline 6.2 & 48 \\
\hline 7.6 & 521 \\
\hline 7.6 & 521 \\
\hline 7.9 & 527 \\
\hline 11.4 & 918 \\
\hline 43.7 & 932 \\
\hline 43.2 & 1689 \\
\hline 10.9 & 1119 \\
\hline 5.4 & 962 \\
\hline 5.4 & 962 \\
\hline 5.2 & 954 \\
\hline 6.2 & 486 \\
\hline 6.0 & 48 \\
\hline 6.0 & \\
\hline 5.2 & 465 \\
\hline & SMW \\
\hline & 50.00 \\
\hline & 2.41 \\
\hline & 58.03 \\
\hline & ${ }_{S}{ }_{N}$ Deses \\
\hline & $\overline{0} .990$ \\
\hline 0832 & 0.000 \\
\hline & $0.9^{\circ}$ \\
\hline & 0.00 \\
\hline 2 & 0 \\
\hline
\end{tabular}

$\begin{array}{rr}\text { Aphy } & \mathrm{MN} \\ 2581.2 & 0.5520 \\ 4168.2 & 0.3022 \\ 3531.8 & 0.3143 \\ 830.5 & 0.1805 \\ 2606.7 & 0.4167 \\ 412.6 & 0.3125 \\ 412.6 & 0.3128 \\ 412.6 & 0.2122 \\ 49.7 & 0.4258 \\ 49.3 & 0.3176 \\ 74.6 & 0.2945 \\ 265.4 & 0.3523 \\ 860.2 & 0.2031 \\ 860.2 & 0.2031 \\ 613.4 & 0.2923 \\ 2606.7 & 0.4167 \\ 2481.9 & 0.4690 \\ 2481.9 & 0.4690 \\ 2006.9 & 0.6504\end{array}$

gamt
1.40052
1.40052
1.40052
1.40052
1.40039
1.40001
1.39996
1.39996
1.38363
1.38363
1.33143
1.36558
1.37685
1.37684
1.37684
1.40039
1.40037
1.40037
1.40037

$\begin{array}{rrrr}\text { WAR } & \text { Wair } & \text { WH2O } & \text { H2O frac } \\ 0.0003043 & 322.19 & 0.098 & 0.0003 \\ 0.0003043 & 322.19 & 0.098 & 0.0003 \\ 0.0003043 & 282.52 & 0.086 & 0.0003 \\ 0.0003043 & 39.67 & 0.012 & 0.0003 \\ 0.0003043 & 282.52 & 0.086 & 0.0003 \\ 0.0003043 & 39.67 & 0.012 & 0.0003 \\ 0.0005989 & 39.67 & 0.024 & 0.0006 \\ 0.0005989 & 27.77 & 0.017 & 0.0006 \\ 0.0005989 & 26.42 & 0.016 & 0.0006 \\ 0.0005989 & 20.48 & 0.012 & 0.0006 \\ 0.0005989 & 20.48 & 0.012 & 0.0144 \\ 0.0005989 & 27.44 & 0.016 & 0.0109 \\ 0.0005989 & 27.77 & 0.017 & 0.0108 \\ 0.0005989 & 27.77 & 0.017 & 0.0108 \\ 0.0005989 & 27.77 & 0.017 & 0.0108 \\ 0.0003043 & 282.52 & 0.086 & 0.0003 \\ 0.0003162 & 294.42 & 0.093 & 0.0003 \\ 0.0003162 & 294.42 & 0.093 & 0.0003 \\ 0.0003162 & 294.42 & 0.093 & 0.0003\end{array}$




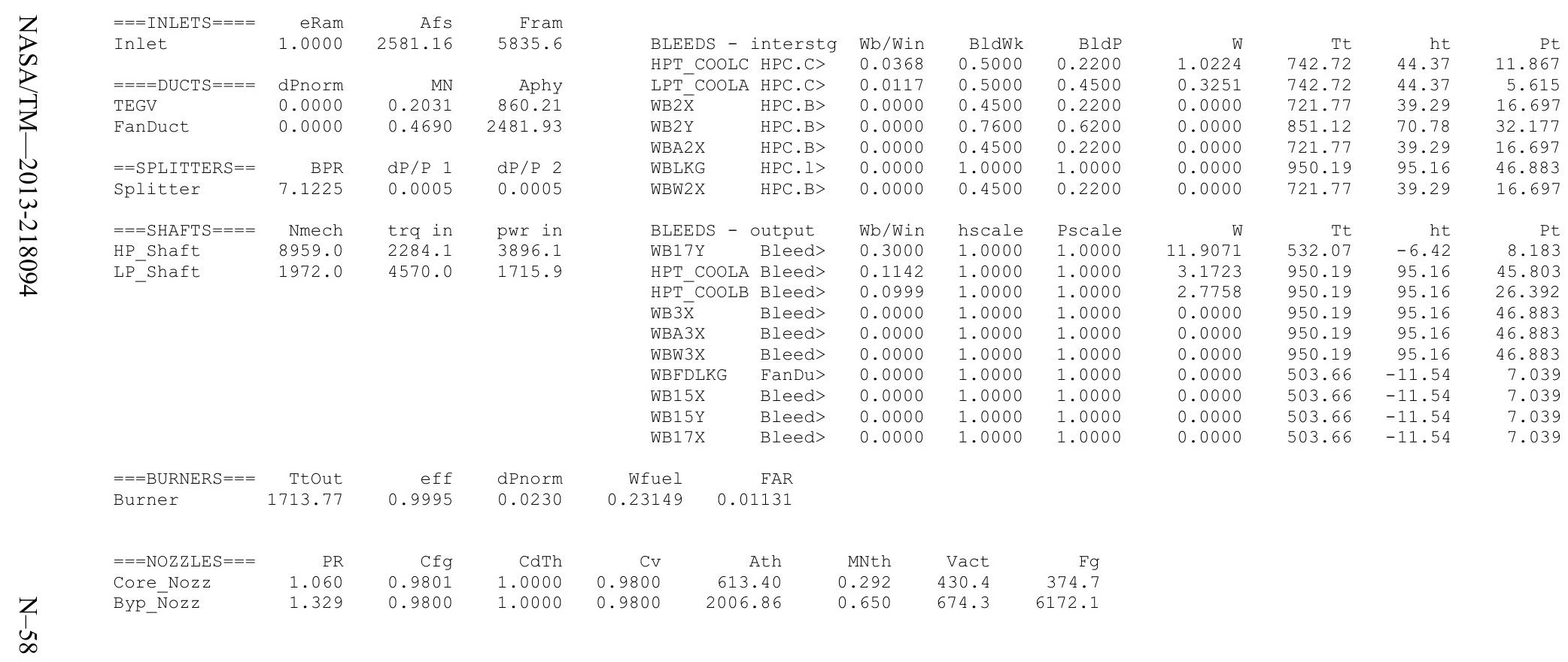


Date:05/13/13 Time:10:06:48 Model: Turbofan Engine - COMDES ON Converge = 1 CASE: 0

Version:NPSS 1.6.5 - Rev: -> Gas Package: Janaf iter/pass/Jacb/Broy= 24/52/2/21 Run by: Philip C Jorgenson PC: 10

Temperature Stator 1 inlet: $505.23 \quad$ Stator 1 exit: $508.19 \quad$ Stator 2 inlet: 510.98 Stator 2 exit: 511.77 Stator 5 inlet: 520.73 Stator 5 exit: $520.60 \quad$ Unblocked Percent Blockage: 0.00

Ambient Relative Humidity $\quad 10.00$

Fan Face Relative Humidity $\quad 6.01$

Fan Bypass Relative Humidity $\quad 5.19$

$\begin{array}{ll}\text { LPC Inlet Relative Humidity } & 5.20 \\ \text { LPC Exit Relative Humidity } & 2.84\end{array}$

HPC

Drop Diative Humidity

Ambient Flow Velocity

Ambient Static Pressure

Ambient Static Temperature$$
0.01
$$

0.0000250

Inlet Length $\quad 40.00$

$\begin{array}{rrr} & & \\ 6.74 & \text { Fan/LPC Inlet Flow Velocity } & 203.11 \\ 63.18 & \text { Fanlet Static Pressure } & 7.75\end{array}$

Additional Water at LPC Exit 0.0003085

an/LPC Inlet Static Temperature 502.97

$\begin{array}{rrrrrr} & & & & \text { SUMMARY OUTPUT DATA } \\ \text { MN } & \text { alt } & \text { dTamb } & \text { W } & \text { Fn } & \text { TSFC } \\ 0.490 & 20047.0 & 36.00 & 357.72 & 763.9 & 1.1954\end{array}$

\section{FLOW STATION DATA}

FSO Ambient.Fl

FS1 Inlet.Fl_O

FS12 Splitter.F1_02

FS2 Splitter.FI_01

FS14 Fan.F1_O

FS24 VaporIN.FI

FS25 Bleed2.Fl $\bar{O}$

FS3 HPC.FIO

S36 Bleed3.FI_-

FS4 Burner.Fl_

FS49

FS5 TEGV.Fīo

FS8 Core_Nozz.Fl_

FS17 FanDúctLkg.Fì

FS171 Bleed15.FI_O

FS172 FanDuct.FI- 0

FS173 Byp Nozz.Fì O

$W$
357.72
357.72
309.01
48.71
309.01
48.71
48.73
29.24
27.82
21.56
21.81
29.15
29.49
29.49
29.49
309.01
328.50
328.50
328.50

$\begin{array}{rrrr}\text { Pt } & \text { Tt } & \text { ht } & \text { FAR } \\ 7.943 & 506.41 & -12.46 & 0.0000\end{array}$

$\begin{array}{llll}7.943 & 506.41 & -12.46 & 0.0000 \\ 7.939 & 506.41 & -12.46 & 0.0000\end{array}$

$\begin{array}{llll}7.949 & 506.41 & -12.46 & 0.0000\end{array}$

$\begin{array}{llll}7.939 & 506.41 & -12.46 & 0.0000 \\ 8.480 & 517.07 & -12.46 & 0.0000\end{array}$

$\begin{array}{llll}8.480 & 517.07 & -9.96 & 0.00000\end{array}$

$9.056-534.30$

$9.056 \quad 534.75$

$\begin{array}{rr}-5.77 & 0.0000\end{array}$

$\begin{array}{ll}-5.77 & 0.0000 \\ -7.44 & 0.0000 \\ 92.03 & 0.0000\end{array}$

$\begin{array}{ll}92.03 & 0.0000\end{array}$

$\begin{array}{ll}92.03 & 0.0000\end{array}$

$49.690 \quad 944.13$

$48.546 \quad 1737.49$

$\begin{array}{ll}78.73 & 0.0118\end{array}$

$\begin{array}{llll}13.284 & 1171.24 & -17.10 & 0.0088\end{array}$

$\begin{array}{ll}7.031 & 1018.01\end{array}$

$\begin{array}{rr}-54.27 & 0.0087 \\ -54.27 & 0.0087\end{array}$

$\begin{array}{llll}7.031 & 1018.01 & -54.27 & 0.0087 \\ 7.031 & 1018.08 & -54.27 & 0.0087\end{array}$

$\begin{array}{llll}8.480 & 517.07 & -9.90 & 0.0000\end{array}$

$8.480 \quad 518.12$

$8.480 \quad 518.12$

$8.480 \quad 518.12$

TURBOMACHINERY PERFORMANCE DATA

$\begin{array}{lrrrrrrrrr} & \text { WC } & \text { PR } & \text { eff } & \text { NC } & \text { TR } & \text { efPoly } & \text { pwr } & \text { SMN } & \text { SMW } \\ \text { Fan } & 565.19 & 1.068 & 0.9035 & 1815.905 & 1.0211 & 0.9044 & -1118.3 & -1833.94 & 46.56 \\ \text { LPC } & 89.10 & 1.141 & 0.6960 & 1815.905 & 1.0551 & 0.7016 & -461.3 & 7.12 & 2.04 \\ \text { HPC } & 48.18 & 5.487 & 0.8076 & 8765.283 & 1.7656 & 0.8467 & -4014.5 & 62.15 & 58.13 \\ \text { HPT } & 12.09 & 3.655 & 0.8766 & 213.518 & 1.3265 & 0.8567 & 4014.5 & & \\ \text { LPT } & 48.46 & 1.889 & 0.8152 & 52.429 & 1.1472 & 0.8014 & 1579.6 & & \end{array}$

TURBOMACHINERY MAP DATA

$\begin{array}{lrrrrrrrrr} & \text { WcMap } & \text { PRmap } & \text { effMap } & \text { NcMap } & \text { R/Parm } & \text { S_WcDes } & \text { S_PRdes } & \text { S_effDes } & \text { S_NcDes } \\ \text { Fan } & 656.70 & 1.067 & 0.9054 & 1798.590 & 0.0437 & 0.8607 & \overline{1} .0235 & 0.9980 & 0.9905 \\ \text { LPC } & 46.03 & 1.238 & 0.7547 & 0.480 & 0.0000 & 1.9357 & 0.5898 & 0.9221 & 0.0003 \\ \text { HPC } & 43.82 & 5.276 & 0.8160 & 8531.607 & 11.0073 & 1.0995 & 1.0494 & 0.9898 & 0.9733 \\ \text { HPT } & 0.96 & 3.581 & 0.8766 & 1.283 & 3.5810 & 12.6299 & 0.9723 & 1.0000 & 0.0003 \\ \text { LPT } & 0.74 & 1.669 & 0.8213 & 0.590 & 1.6689 & 65.2590 & 0.7521 & 0.9926 & 0.0005\end{array}$

$\begin{array}{clccc}\text { EPR } & \text { PowerSet } & \text { T4 } & \text { T41 } & \text { T49 } \\ 0.888 & 10.000 & 1737.5 & 1638.0 & 1166.5\end{array}$ 


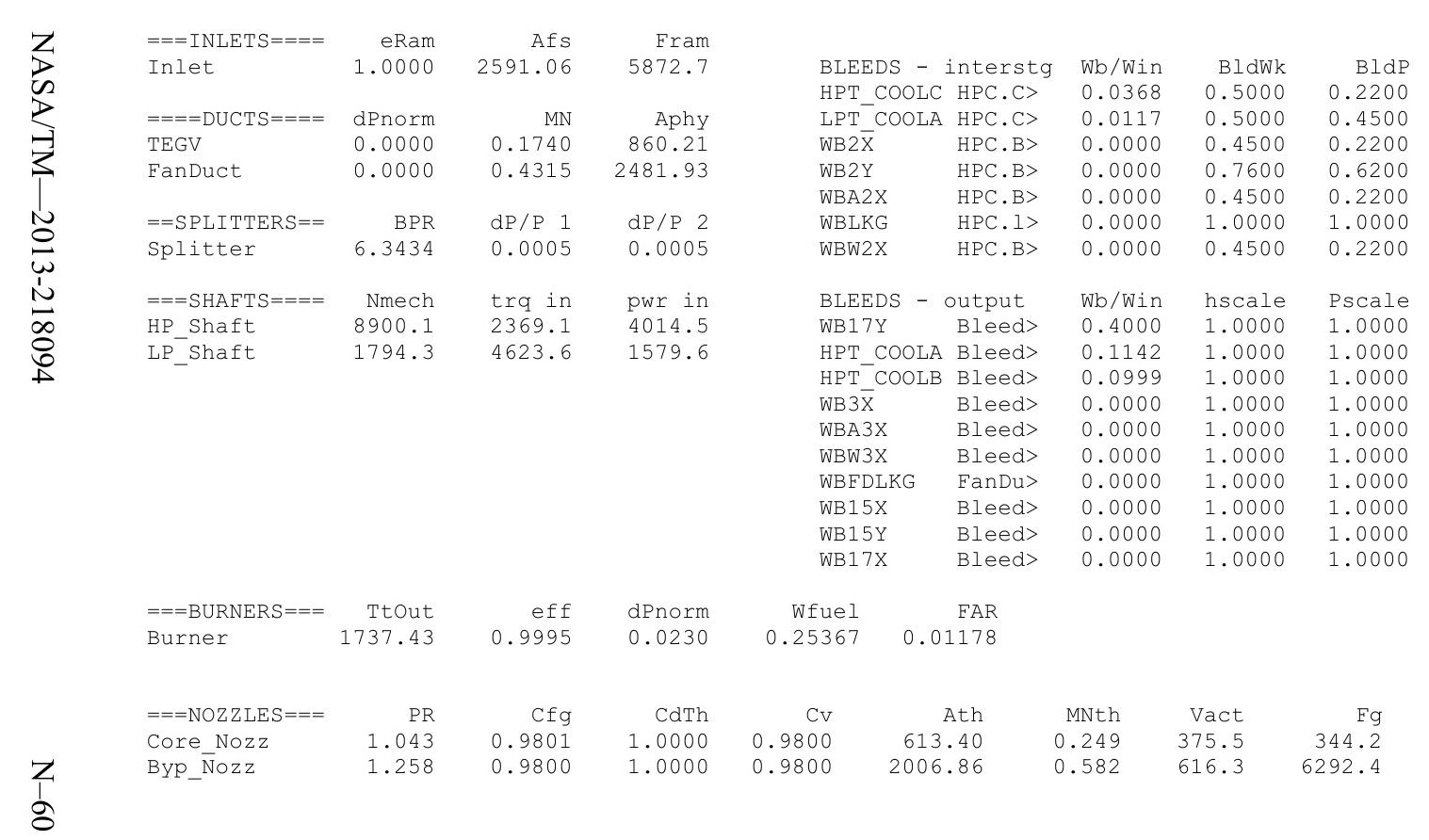




\section{$50 \mu \mathrm{m}, \mathrm{ISA}+18 \mathrm{R}$}

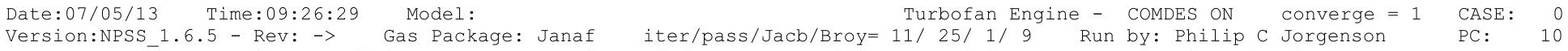

$\begin{array}{lllll}\text { Version:NPSS 1.6.5 - Rev: -> Gas Package: Janat } & \\ \text { Temperature Stator } 1 \text { inlet: } 463.24 & \text { Stator } 1 \text { exit: } 470.64 & \text { Stator } 2 \text { inlet: } 479.94 & \text { Stator } 2 \text { exit: } 484.70\end{array}$

Stator 3 inlet: 494.32 Stator 3 exit: 498.64 stator

$$
\text { Stator } 5 \text { inlet: } 518.70 \text { Stator } 5 \text { exit: } 521.92 \text { Unblocked Percent Blockage: } 0.00
$$

$\quad 1.76$

Fan Bypass Relative Humidity 1.24

IPC Exit Relative Humidity

HPC Relative Humidity

Drop Diameter

0.10

Ambient

0.0000500

Ambient Static Pressure

Ambient Static Temperature

Additional water at IPC Exit

772.64

40.00

Fan/LPC Inlet Flow Velocity $\quad 187.91$

407.97 Fan/LPC Inlet Static Temperature 454.80

SUMMARY OUTPUT DATA

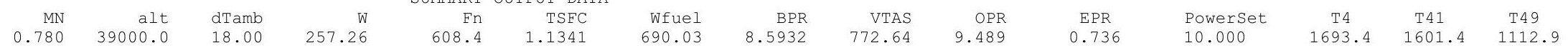

\begin{tabular}{|c|c|}
\hline FSO & Ambient.Fl O \\
\hline FS1 & Inlet.FI_O \\
\hline FS12 & Splitter.Fl 02 \\
\hline FS2 & Splitter.FI- 01 \\
\hline FS14 & Fan.Fl_O \\
\hline FS23 & LPC.FI_O \\
\hline FS24 & 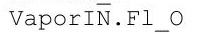 \\
\hline FS25 & Bleed2.Fl_o \\
\hline FS3 & HPC.Fl_O \\
\hline FS36 & Bleed3.Fl O \\
\hline FS4 & Burner.Fl_o \\
\hline FS45 & HPT.FI_O \\
\hline FS49 & LPT.Fl-O \\
\hline FS5 & TEGV.FI_O \\
\hline FS 8 & Core Nozz.Fl O \\
\hline FS17 & FanDuctLkg.FI_C \\
\hline FS171 & Bleed15.FI_o \\
\hline FS172 & FanDuct.FI_O \\
\hline FS173 & Byp_Nozz.FI__O \\
\hline
\end{tabular}

\begin{tabular}{rrrrr}
\multicolumn{6}{c}{ FLOW } & STATION DATA & \\
W & Pt & Tt & $h t$ & FAR \\
257.26 & 4.267 & 457.74 & -20.96 & 0.0000 \\
257.26 & 4.267 & 457.74 & -20.96 & 0.0000 \\
230.45 & 4.265 & 457.74 & -20.96 & 0.0000 \\
26.82 & 4.265 & 457.74 & -20.96 & 0.0000 \\
230.45 & 4.811 & 475.49 & -16.70 & 0.0000 \\
26.82 & 6.639 & 531.26 & -3.33 & 0.0000 \\
26.82 & 6.639 & 531.36 & -3.62 & 0.0000 \\
24.14 & 6.639 & 531.36 & -3.62 & 0.0000 \\
22.97 & 40.488 & 964.30 & 101.58 & 0.0000 \\
17.80 & 40.488 & 964.30 & 101.58 & 0.0000 \\
17.99 & 39.555 & 1693.36 & 89.30 & 0.0108 \\
24.05 & 9.489 & 1116.92 & -12.58 & 0.0080 \\
24.33 & 3.259 & 875.30 & -71.58 & 0.0079 \\
24.33 & 3.259 & 875.38 & -71.58 & 0.0079 \\
24.33 & 3.259 & 875.45 & -71.58 & 0.0079 \\
230.45 & 4.811 & 475.49 & -16.70 & 0.0000 \\
233.13 & 4.811 & 476.13 & -16.55 & 0.0000 \\
233.13 & 4.811 & 476.13 & -16.55 & 0.0000 \\
233.13 & 4.811 & 476.13 & -16.55 & 0.0000
\end{tabular}

Wc
832.41
832.41
746.01
86.81
673.99
60.08
60.09
54.08
11.37
8.81
12.08
54.65
142.53
142.53
142.54
673.99
682.30
682.30
682.30

PS
2.854
3.893
3.841
4.170
4.037
6.345
6.345
6.403
35.638
37.704
37.354
8.596
3.069
3.069
2.854
4.037
3.904
3.904
2.854

Ts
407.97
445.88
444.23
454.80
452.23
524.42
524.52
525.89
930.77
945.45
1669.26
1087.58
860.85
860.92
843.84
452.23
448.50
448.50
410.03

$\begin{array}{rr}\text { Aphy } & \text { MN } \\ 2539.4 & 0.7800 \\ 4168.2 & 0.3642 \\ 3531.8 & 0.3896 \\ 830.5 & 0.1797 \\ 2606.7 & 0.5066 \\ 412.6 & 0.2553 \\ 412.6 & 0.2553 \\ 412.6 & 0.2280 \\ 49.7 & 0.4331 \\ 49.3 & 0.3224 \\ 74.6 & 0.2941 \\ 265.4 & 0.3824 \\ 860.2 & 0.2960 \\ 860.2 & 0.2960 \\ 613.4 & 0.4418 \\ 2606.7 & 0.5066 \\ 2481.9 & 0.5544 \\ 2481.9 & 0.5544 \\ 2006.9 & 0.8967\end{array}$

gamt
1.40084
1.40084
1.40084
1.40084
1.40071
1.40005
1.40004
1.40004
1.38288
1.38288
1.33284
1.36774
1.38276
1.38275
1.38275
1.40071
1.40070
1.40070
1.40070

WAR
0.0000295
0.0000295
0.0000295
0.0000295
0.0000295
0.0000295
0.0000837
0.0000837
0.0000837
0.0000837
0.0000837
0.0000837
0.0000837
0.0000837
0.0000837
0.0000295
0.0000301
0.0000301
0.0000301

WAir

TURBOMACHINERY PERFORMANCE DATA

\begin{tabular}{|c|c|c|c|c|c|c|c|c|c|}
\hline & & & & & & & & & \\
\hline & Wc & PR & eff & $\mathrm{NC}$ & TR & efpoly & pwr & SMN & SMW \\
\hline Fan & 746.01 & 1.128 & 0.9051 & 2560.883 & 1.0388 & 0.9067 & -1386.4 & 1662.37 & 54.94 \\
\hline LPC & 86.81 & 1.557 & 0.8402 & 2560.883 & 1.1606 & 0.8499 & -668.8 & 6.95 & 3.67 \\
\hline HPC & 54.08 & 6.098 & 0.8187 & 8960.639 & 1.8148 & 0.8574 & -3505.3 & 61.08 & 57.75 \\
\hline HPT & 12.08 & 4.168 & 0.8739 & 220.402 & 1.3661 & 0.8512 & 3505.3 & & \\
\hline$\angle P T$ & 54.65 & 2.912 & .8506 & 71.985 & 1.2742 & 0.8304 & 2055.2 & & \\
\hline JRE & NE & DF & & & & & & & \\
\hline & WcMap & PRmap & effmap & NcMap & R/Parm & S_WcDes & S_PRdes & s_effDes & S_NCDes \\
\hline an & 866.79 & 1.125 & 0.9070 & 2536.465 & 0.0413 & $\overline{0} .8607$ & 1.0235 & 0.9980 & 0.9905 \\
\hline PC & 73.59 & 1.519 & 0.7796 & 0.676 & 0.0000 & 1.1797 & 1.0734 & 1.0778 & 0.0003 \\
\hline PC & 49.18 & 5.858 & 0. & 8721.754 & 10.9930 & 1.0 & 1.0494 & 0.9898 & 0.9733 \\
\hline $\mathrm{PT}$ & 0.96 & 4.081 & 0.8739 & 1.325 & 4.0806 & 12.6299 & 0.9723 & 1.0000 & 0.0003 \\
\hline$\angle P T$ & 0.84 & 2.438 & .8569 & 0.810 & 2.4380 & 65.2590 & 0.7521 & 0.9926 & 0.0005 \\
\hline
\end{tabular}




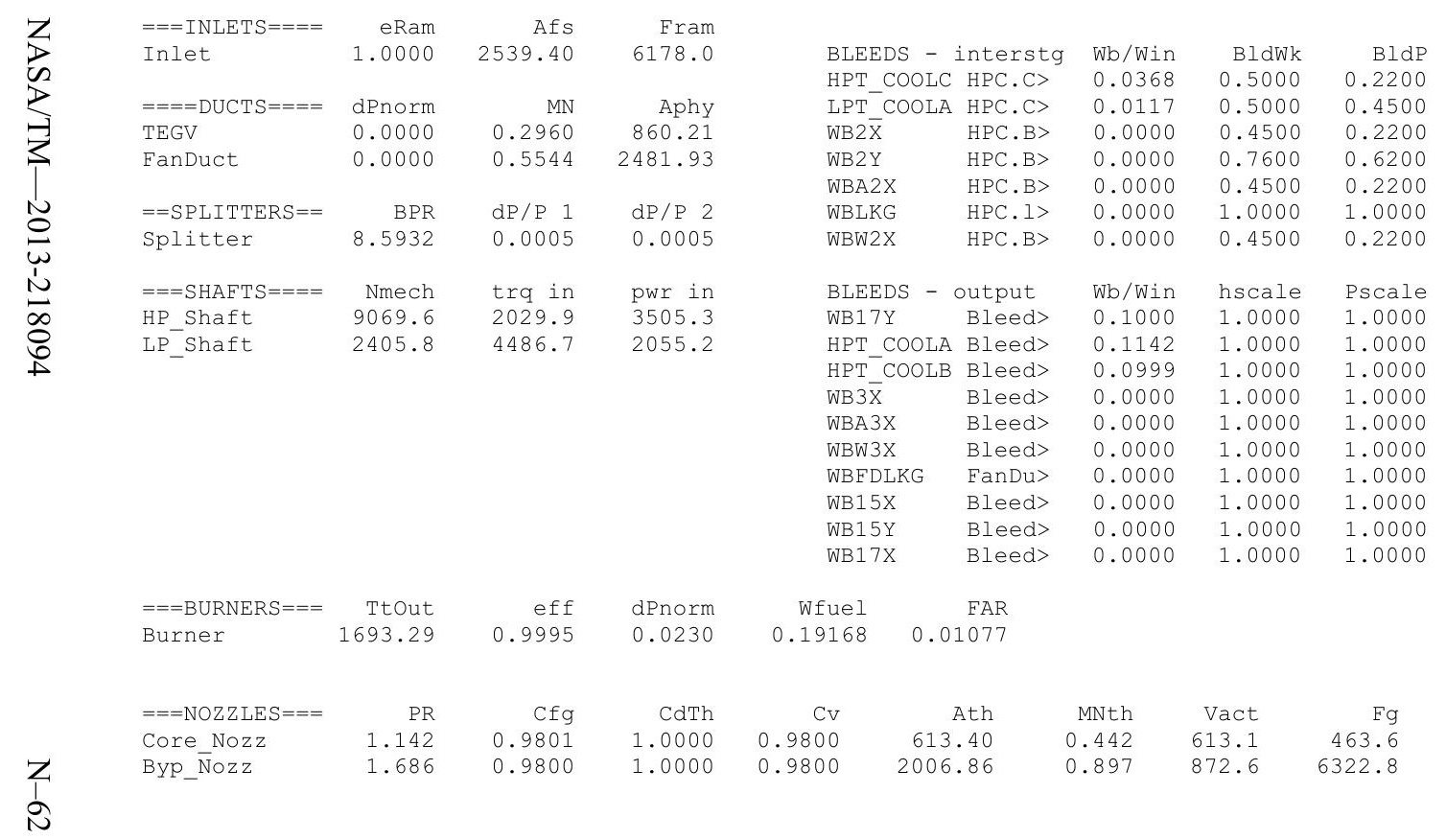


Date:07/05/13 Time:09:26:46 Model: Turbofan Engine - COMDES ON Converge = 1 CASE: 0

Version:NPSS 1.6.5 - Rev: -> Gas Package: Janaf iter/pass/Jacb/Broy=11/25/1/9 Run by: Philip C Jorgenson PC: 10

Temperature Stator 1 inlet: $456.76 \quad$ Stator 1 exit: $463.69 \quad$ Stator 2 inlet: $472.40 \quad$ Stator 2 exit: 476.84 Stator 5 inlet: 508.57 Stator 5 exit: 511.54 Unblocked Percent Blockage: 0.00

Ambient Relative Humidity $\quad 10.00$

Fan Face Relative Humidity $\quad 2.23$

Fan Bypass Relative Humidity $\quad 1.61$

LPC Inlet Relative Humidity

LPC Exit Relative Humidity

Hrop Riative Humidity

Ambient Flow Velocity

Ambient Static Pressure

Additional Water at LPC Exit

$$
0.0000500
$$

Inlet Length

40.00

2.95 Fan/LPC Inlet Static Pressure

0.0000485

an/LPC Inlet Static Temperature 448.83
$\begin{array}{rrr}0.730 & 38334.0 & 18.00\end{array}$
250.31
$\begin{array}{rr}\text { Fn } & \text { TSFC } \\ 59.3 & 1.0980\end{array}$

Wfuel
657.99
$B P R$
8.6866
VTAS
723.11
OPR
9.209
Powerset 10.000 $\begin{array}{ccc}\text { T4 } & \text { T41 } & \text { T49 } \\ 1667.2 & 1575.9 & 1097.0\end{array}$
FSO Ambient.FI O

FS1 Inlet.FI_O

FS12 Splitter.F1_02

FS2 Splitter.FI_01

FS14 Fan.F1-

FS24 VaporIN. FI

S25 Bleed2.FI

FS HPC.Fl_

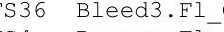

FS4 Burner.FI-

FS49 4 TP .

FS5 TEGV.Fīo

FS8 Core_Nozz.Fl_O

FS17 FanDüctLkg.Fì

FS171 Bleed15.FI

FS172 FanDuct.Fl-O

FS173 Byp Nozz.Fì O

TURBOMACHINERY PERFORMANCE DATA

$\begin{array}{lrrr} & \text { WC } & \text { PR } & \text { eff } \\ \text { Fan } & 732.97 & 1.121 & 0.9043 \\ \text { LPC } & 84.38 & 1.524 & 0.8406 \\ \text { HPC } & 53.49 & 6.044 & 0.8179 \\ \text { HPT } & 12.08 & 4.116 & 0.8726 \\ \text { LPT } & 54.02 & 2.775 & 0.8451\end{array}$

FLOW STATION DATA

$\begin{array}{rrrrr}\text { W } & \text { Pt } & \text { Tt } & \text { ht } & \text { FAR } \\ 250.31 & 4.201 & 451.57 & -22.43 & 0.0000 \\ 250.31 & 4.201 & 451.57 & -22.43 & 0.0000 \\ 224.47 & 4.199 & 451.57 & -22.43 & 0.0000 \\ 25.84 & 4.199 & 451.57 & -22.43 & 0.0000 \\ 224.47 & 4.706 & 468.12 & -18.46 & 0.0000 \\ 25.84 & 6.402 & 520.42 & -5.92 & 0.0000 \\ 25.84 & 6.402 & 520.52 & -6.18 & 0.0000 \\ 23.26 & 6.402 & 520.52 & -6.18 & 0.0000 \\ 22.13 & 38.692 & 942.96 & 96.34 & 0.0000 \\ 17.15 & 38.692 & 942.96 & 96.34 & 0.0000 \\ 17.33 & 37.801 & 1667.21 & 84.23 & 0.0107 \\ 23.17 & 9.183 & 1101.02 & -15.03 & 0.0080 \\ 23.44 & 3.309 & 873.17 & -70.51 & 0.0079 \\ 23.44 & 3.309 & 873.25 & -70.51 & 0.0079 \\ 23.44 & 3.309 & 873.32 & -70.51 & 0.0079 \\ 224.47 & 4.706 & 468.12 & -18.46 & 0.0000 \\ 227.05 & 4.706 & 468.72 & -18.32 & 0.0000 \\ 227.05 & 4.706 & 468.72 & -18.32 & 0.0000 \\ 227.05 & 4.706 & 468.72 & -18.32 & 0.0000\end{array}$

$\begin{array}{rr}\text { WC } & \text { Ps } \\ 816.95 & 2.947\end{array}$ 816.95 732.97
84.38 84.38
665.89 665.89 59.42 59.43 11.33 11.33
8.78

12.08

12.08
54.02
135.06

135.06

135.08

135.08

673.99

673.99
673.99

673.99 $\begin{array}{rrrrr} & \text { Ts } & \text { Aphy } & \text { MN }\end{array}$ $\begin{array}{llll}2.947 & 407.97 & 2556.8 & 0.7300\end{array}$ $\begin{array}{llll}2.947 & 407.97 & 2556.8 & 0.7300 \\ 3.798 & 438.77 & 358.2 & 0.3563\end{array}$ $\begin{array}{llrl}4.111 & 448.83 & 830.5 & 0.1745\end{array}$ $\begin{array}{llll}4.111 & 448.83 & 830.5 & 0.1745 \\ 3.972 & 445.95 & 2606.7 & 0.4981\end{array}$ $\begin{array}{llll}6.125 & 513.88 & 412.6 & 0.2522\end{array}$ $\begin{array}{llll}6.125 & 513.98 & 412.6 & 0.2523\end{array}$ 34.090 924.60 1643.41 $\begin{array}{ll}.341 & 1072.82 \\ 3.137 & 860.35\end{array}$ $3.137 \quad 860.42$ $2.947 \quad 845.68$ $\begin{array}{ll}2.947 & 845.68 \\ 3.972 & 445.95\end{array}$ $3.848 \quad 442.46$ $\begin{array}{lllll}.99 & 2.947 & 409.94 & 2006.9 & 0.8457\end{array}$ $\begin{array}{lllll}.99 & 2.947 & 409.94 & 2006.9 & 0.8457\end{array}$ $\begin{array}{ll}42.6 & 0.2253 \\ 49.7 & 0.2313\end{array}$ $\begin{array}{ll}49.7 & 0.4313 \\ 49.3 & 0.3212\end{array}$ $\begin{array}{ll}74.6 & 0.2940\end{array}$ $\begin{array}{ll}265.4 & 0.3770 \\ 860.2 & 0.2789\end{array}$ $\begin{array}{lll}860.2 & 0.2789 \\ 860.2 & 0.2789\end{array}$ $\begin{array}{lll}613.4 & 0.4127\end{array}$ $2606.7 \quad 0.4981$ $\begin{array}{ll}2481.9 & 0.544\end{array}$ 2481.90 .5441
1.40089 $\begin{array}{lr}0.0000285 & 250.30 \\ 0.0000285 & 250.30\end{array}$ 1.40089 1.40089
1.40077 1.40021 1.40020 1.38414 1.38414 1.36883 1.38293
1.38293 1.38292 1.40077
1.40077 1.40077

$\begin{array}{rrrr}\text { WAR } & \text { Wair } & \text { WH2O } & \text { H2O frac } \\ 0.0000285 & 250.30 & 0.007 & 0.0000 \\ 0.0000285 & 250.30 & 0.007 & 0.0000 \\ 0.0000285 & 224.46 & 0.006 & 0.0000 \\ 0.0000285 & 25.84 & 0.001 & 0.0000 \\ 0.0000285 & 224.46 & 0.006 & 0.0000 \\ 0.0000285 & 25.84 & 0.001 & 0.0000 \\ 0.0000770 & 25.84 & 0.002 & 0.0001 \\ 0.0000770 & 23.26 & 0.002 & 0.0001 \\ 0.0000770 & 22.13 & 0.002 & 0.0001 \\ 0.0000770 & 17.15 & 0.001 & 0.0001 \\ 0.0000770 & 17.15 & 0.001 & 0.0131 \\ 0.0000770 & 22.98 & 0.002 & 0.0098 \\ 0.0000770 & 23.26 & 0.002 & 0.0097 \\ 0.0000770 & 23.26 & 0.002 & 0.0097 \\ 0.0000770 & 23.26 & 0.002 & 0.0097 \\ 0.0000285 & 224.46 & 0.006 & 0.0000 \\ 0.0000291 & 227.04 & 0.007 & 0.0000 \\ 0.0000291 & 227.04 & 0.007 & 0.0000 \\ 0.0000291 & 227.04 & 0.007 & 0.0000\end{array}$

TURBOMACHINERY MAP DATA

$\begin{array}{lrrrrrrrrr} & \text { WcMap } & \text { PRmap } & \text { effMap } & \text { NcMap } & \text { R/Parm } & \text { S_WcDes } & \text { S_PRdes } & \text { S_effDes } & \text { S_NcDes } \\ \text { Fan } & 851.64 & 1.118 & 0.9062 & 2473.988 & 0.0412 & 0.8607 & \overline{1} .0235 & 0.9980 & 0.9905 \\ \text { LPC } & 70.86 & 1.491 & 0.7766 & 0.660 & 0.0000 & 1.1908 & 1.0692 & 1.0824 & 0.0003 \\ \text { HPC } & 48.64 & 5.806 & 0.8263 & 8703.194 & 11.0056 & 1.0995 & 1.0494 & 0.9898 & 0.9733 \\ \text { HPT } & 0.96 & 4.030 & 0.8726 & 1.318 & 4.0300 & 12.6299 & 0.9723 & 1.0000 & 0.0003 \\ \text { LPT } & 0.83 & 2.335 & 0.8514 & 0.790 & 2.3350 & 65.2590 & 0.7521 & 0.9926 & 0.0005\end{array}$




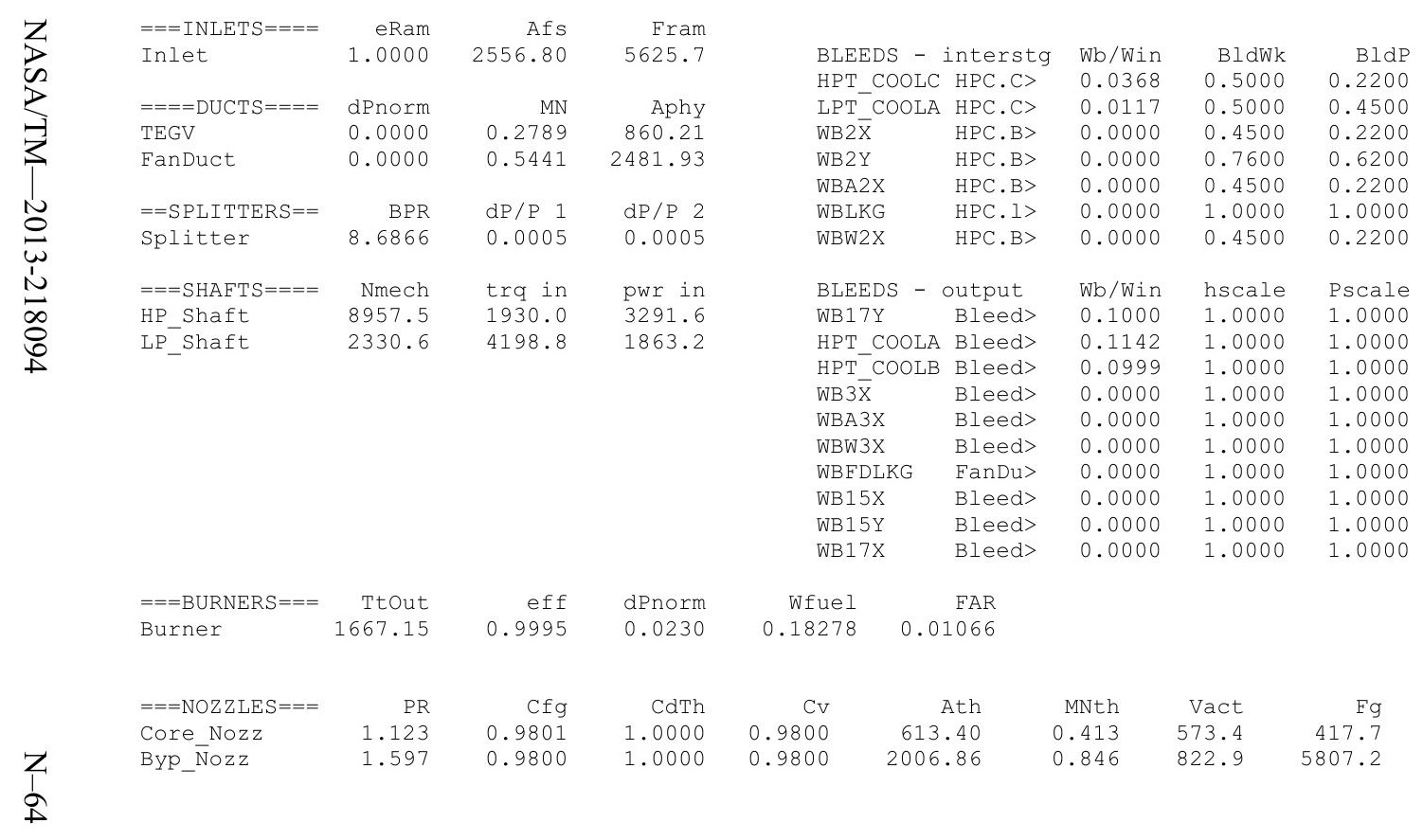


Turbofan Engine - COMDES ON Converge $=1$ CASE: 0

Version:NPSS 1.6.5 - Rev: -> Gas Package: Janaf iter/pass/Jacb/Broy=11/25/1/9 Run by: Philip C Jorgenson PC: 10

Temperature Stator 1 inlet: 454.76 Stator 1 exit: $461.52 \quad$ Stator 2 inlet: 470.01 Stator 2 exit: 474.34 Stator 3 inlet: 483.09 Stator 3 exit: 487.01 Stator 4 inlet: 495.20 Stator 4 exit: 498.51

Ambient Relative Humidity $\quad 10.00$

Fan Face Relative Humidity $\quad 2.40$

Fan Bypass Relative Humidity $\quad 1.75$

LPC Inlet Relative Humidity $\quad 1.56$

LPC Exit Relative Humidity

HPC Relative Humidity

Drop Diameter

0.00

0.0000500

Ambient Flow Velocity

707.26

Inlet Length

Ambient static Pressure

3.09

Ean/LPC Inlet Flow Velocity $\quad 178.68$

Additional Water at LPC Exit 0.0000445

SUMMARY OUTPUT DATA

$\begin{array}{rrr}\text { MN } & \text { alt } & \text { dTamb } \\ 0.714 & 37357.0 & 18.00\end{array}$

257.06

614

1.0902

Wfuel
669.83

BPR
8.7256

VTAS
707.26

OPR
9.092

$E P R$
0.772

PowerSet
10.000 $\begin{array}{ccc}\text { T4 } & \text { T41 } & \text { T49 } \\ 1657.3 & 1566.3 & 1091.0\end{array}$

\begin{tabular}{|c|c|}
\hline so & Ambient.Fl O \\
\hline FS1 & Inlet.Fl $0^{-}$ \\
\hline FS12 & Splitter.Fl 02 \\
\hline FS2 & Splitter.FI-01 \\
\hline FS14 & Fan.Fl O \\
\hline FS23 & LPC.FI \\
\hline FS24 & VaporIN.FIO \\
\hline FS25 & Bleed2.Fl $\overline{0}$ \\
\hline FS3 & HPC.FI O \\
\hline FS36 & Bleed3.F1 O \\
\hline FS4 & Burner.Fl-o \\
\hline FS45 & HPT.FI O \\
\hline FS49 & LPT.FI \\
\hline FS5 & TEGV.FI_O \\
\hline FS 8 & Core Nozz.Fl \\
\hline FS17 & FanDuctLkg.Fi \\
\hline FS171 & Bleed15.FI \\
\hline FS172 & FanDuct.FI- \\
\hline
\end{tabular}

257.06
257.06
230.63
26.43
230.63
26.43
26.43
23.79
22.64
17.54
17.73
23.70
23.98
23.98
23.98
230.63
233.27
233.27
233.27

FS173 Byp Nozz. Fì

TURBOMACHINERY PERFORMANCE DATA

\begin{tabular}{|c|c|c|c|c|c|c|c|c|c|}
\hline & Wc & $\mathrm{PR}$ & eff & $\mathrm{NC}$ & TR & efPoly & pwr & SMN & SMW \\
\hline Fan & 727.63 & 1.118 & 0.9040 & 2472.475 & 1.0358 & 0.9055 & -1260.2 & 2677.24 & 54.68 \\
\hline LPC & 83.39 & 1.512 & 0.8407 & 2472.475 & 1.1493 & 0.8497 & -601.8 & 6.67 & 3.26 \\
\hline PC & 53.22 & 6.016 & 0.8174 & 8933.056 & 1.8098 & 0.8562 & -3334.7 & 61.04 & 57.63 \\
\hline HPT & 12.08 & 4.099 & .8721 & 219.055 & 1.3617 & 0.8494 & 3334.7 & & \\
\hline LPT & 53.81 & 2.726 & .8431 & 69.571 & 1.2534 & 0.8234 & 1861.9 & & \\
\hline & NERY M & DAT: & & & & & & & \\
\hline & WcMap & PRmap & effMap & NcMap & R/Parm & S_WcDes & S_PRdes & s_effDes & S_NCDe \\
\hline an & 845.44 & 1.115 & 0.9058 & 2448.900 & 0.0412 & $\overline{0} .8607$ & $\overline{1} .0235$ & 0.9980 & 0.9905 \\
\hline 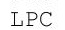 & 69.76 & 1.479 & & 0.653 & 00 & 1.1 & 1.0682 & 1.0842 & 0.0003 \\
\hline${ }^{\mathrm{C}} \mathrm{C}$ & 48.41 & 5.780 & 0.8259 & 8694.906 & 11.0056 & 1.0995 & 1.0494 & 0.9898 & 0.9733 \\
\hline $\mathrm{T}$ & 0.96 & 4.013 & 0.8721 & 1.316 & 4.0128 & 12.6299 & 0.9723 & 1.0000 & 0.0003 \\
\hline PT & 0.82 & 2.298 & 0.8493 & 0.783 & 2.2982 & 65.2590 & 0.7521 & 0.9926 & 0.000 \\
\hline
\end{tabular}

FLOW STATION DATA

\begin{tabular}{rrrrrrrrr}
\multicolumn{1}{c}{ FLOW STATION DATA } & Tt & ht & FAR & Wc & Ps & Ts & Aphy & MN \\
4.339 & 449.68 & -22.87 & 0.0000 & 810.62 & 3.088 & 407.97 & 2561.5 & 0.7140 \\
4.339 & 449.68 & -22.87 & 0.0000 & 810.62 & 3.981 & 438.72 & 4168.2 & 0.3530 \\
4.337 & 449.68 & -22.87 & 0.0000 & 727.63 & 3.930 & 437.15 & 3531.8 & 0.3780 \\
4.337 & 449.68 & -22.87 & 0.0000 & 83.39 & 4.248 & 447.01 & 830.5 & 0.1723 \\
4.848 & 465.79 & -19.01 & 0.0000 & 662.46 & 4.102 & 444.03 & 2606.7 & 0.4945 \\
6.557 & 516.81 & -6.78 & 0.0000 & 59.13 & 6.276 & 510.38 & 412.6 & 0.2509 \\
6.557 & 516.90 & -7.02 & 0.0000 & 59.14 & 6.276 & 510.47 & 412.6 & 0.2509 \\
6.557 & 516.90 & -7.02 & 0.0000 & 53.22 & 6.332 & 511.75 & 412.6 & 0.2241 \\
39.452 & 935.48 & 94.52 & 0.0000 & 11.32 & 34.769 & 903.16 & 49.7 & 0.4307 \\
39.452 & 935.48 & 94.52 & 0.0000 & 8.78 & 36.762 & 917.29 & 49.3 & 0.3209 \\
38.544 & 1657.32 & 82.49 & 0.0106 & 12.08 & 36.397 & 1633.62 & 74.6 & 0.2940 \\
9.404 & 1095.02 & -15.81 & 0.0079 & 53.81 & 8.550 & 1067.20 & 265.4 & 0.3751 \\
3.450 & 872.21 & -70.01 & 0.0078 & 132.45 & 3.278 & 859.93 & 860.2 & 0.2730 \\
3.450 & 872.29 & -70.01 & 0.0078 & 132.45 & 3.278 & 860.00 & 860.2 & 0.2730 \\
3.450 & 872.36 & -70.01 & 0.0078 & 132.46 & 3.088 & 846.01 & 613.4 & 0.4028 \\
4.848 & 465.79 & -19.01 & 0.0000 & 662.46 & 4.102 & 444.03 & 2606.7 & 0.4945 \\
4.848 & 466.37 & -18.88 & 0.0000 & 670.47 & 3.976 & 440.63 & 2481.9 & 0.5399 \\
4.848 & 466.37 & -18.88 & 0.0000 & 670.47 & 3.976 & 440.63 & 2481.9 & 0.5399 \\
4.848 & 466.37 & -18.88 & 0.0000 & 670.47 & 3.088 & 409.90 & 2006.9 & 0.8289
\end{tabular}

gamt
1.40090
1.40090
1.40090
1.40090
1.40079
1.40025
1.40025
1.40025
1.38458
1.38458
1.33468
1.36925
1.38301
1.38300
1.38300
1.40079
1.40078
1.40078
1.40078

WAR
0.0000272
0.0000272
0.0000272
0.0000272
0.0000272
0.0000272
0.0000717
0.0000717
0.0000717
0.0000717
0.0000717
0.0000717
0.0000717
0.0000717
0.0000717
0.0000272
0.0000277
0.0000277
0.0000277

$\begin{array}{rr} & \text { Wair } \\ & \end{array}$ $\begin{array}{ll} & \\ & \end{array} 2 \mathrm{frac}$ $\begin{array}{lll}257.05 & 0.007 & 0.0000\end{array}$ $\begin{array}{lll}230.62 & 0.006 & 0.0000\end{array}$ $230.62-0.006-0.0000$ $\begin{array}{lll}26.43 & 0.001 & 0.0000\end{array}$ $\begin{array}{lll}26.43 & 0.002 & 0.0001\end{array}$ $\begin{array}{lll}22.63 & 0.002 & 0.0001\end{array}$ $\begin{array}{lll}17.54 & 0.001 & 0.0001\end{array}$ $\begin{array}{lll}17.54 & 0.001 & 0.0130\end{array}$ $\begin{array}{lll}23.51 & 0.002 & 0.0098\end{array}$ $\begin{array}{lll}23.79 & 0.002 & 0.0097\end{array}$ $\begin{array}{lll}23.79 & 0.002 & 0.0097\end{array}$ $\begin{array}{ll}23.79 & 0.002\end{array}$ $230.62 \quad 0.006$ $\begin{array}{ll}233.27 & 0.006 \\ 233.27 & 0.006\end{array}$ $233.27 \quad 0.006$ 


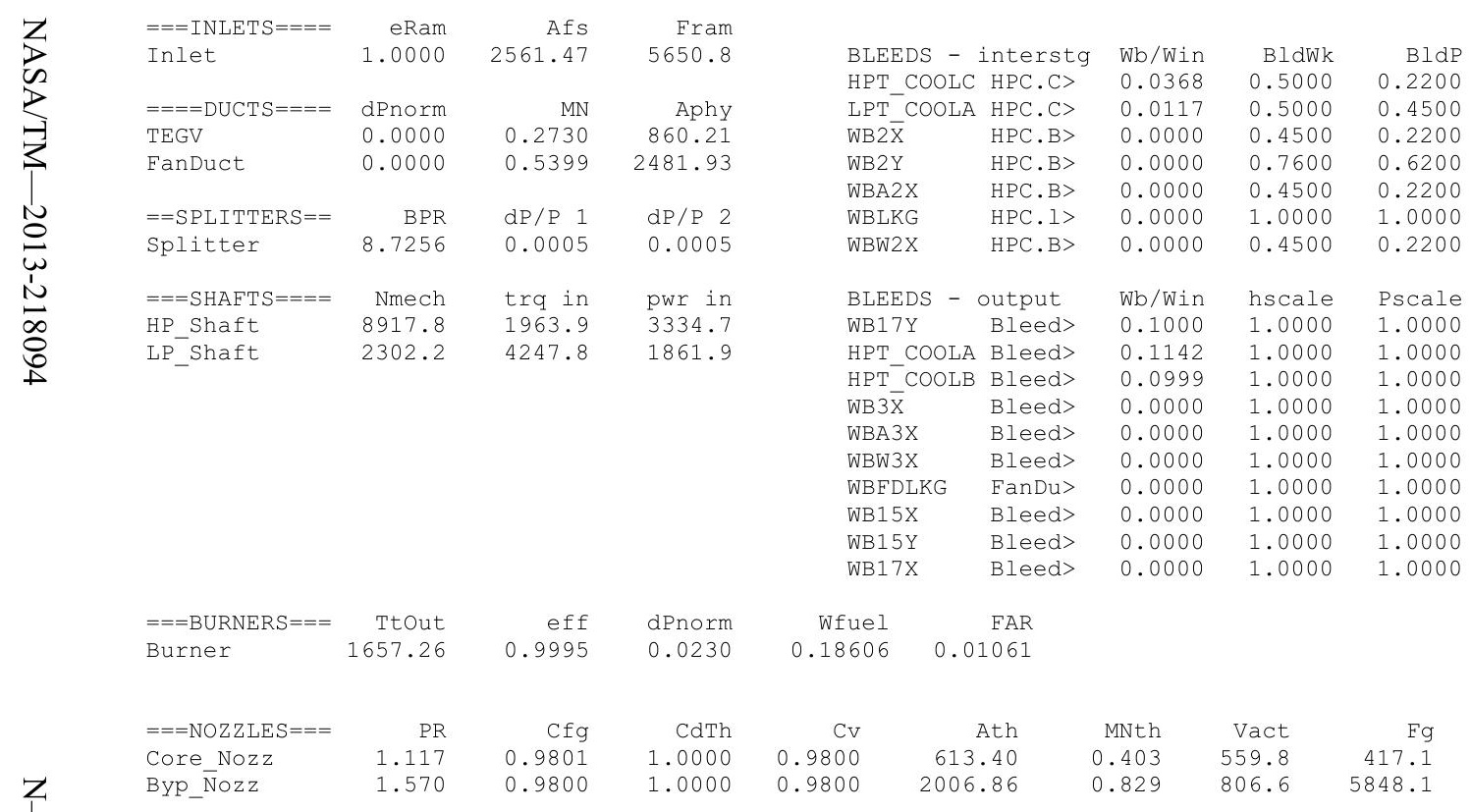


Date:07/05/13 Time:09:27:18 Model: Turbofan Engine - CoMDES ON Converge = 1 CASE: 0

Version:NPSS 1.6.5 - Rev: -> Gas Package: Janaf iter/pass/Jacb/Broy=11/25/1/9 Run by: Philip C Jorgenson PC: 10

Temperature Stator 1 inlet: $456.41 \quad$ Stator 1 exit: $462.77 \quad$ Stator 2 inlet: $470.73 \quad$ Stator 2 exit: 474.78

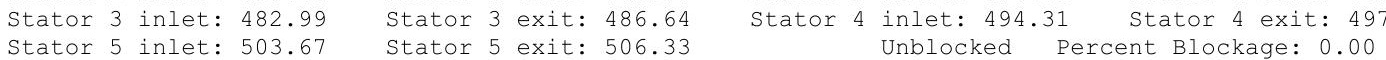

Ambient Relative Humidity $\quad 10.00$

Fan Face Relative Humidity $\quad 3.00$

Re

LPC Exit Relative Humidity 0.22

HPC Relative Humidity

Drop Diameter

0.00

Ambient Flow Velocity

0.0000500

Inlet Length

40.00

67.90 Fan/LPC Inlet Flow Velocity 173.04

Ambient Static Pressure

Additional Water at LPC Exit

3.58

tic Pressure

4.74
49.11

MN SUMMARY OUTPUT DATA

$\begin{array}{rrr}\text { MN } & \text { alt } & \text { dTamb } \\ 0.669 & 34281.0 & 18.00\end{array}$

278.58

Fn
669.5

TSFC
1.0739

Wfuel
719.01

BPR
8.8076

VTAS
667.90

OPR
8.757

EPR
0.795

Powerset

T4 T41

T49

FLOW STATION DATA

FSO Ambient.FI_O

FS1 Inlet.Fl_o

$\begin{array}{ll}\text { FS12 } & \text { Splitter.Fl_02 } \\ \text { FS2 } & \text { Splitter.Fl_01 }\end{array}$

FS14 Fan.Fl_-

$\begin{array}{ll}\text { FS23 } & \text { LPC.FI-O } \\ \text { FS24 } & \text { VaporIN.FI }\end{array}$

FS25 Bleed2.FI_O

FS3 HPC.Fl_O

S36 Bleed3.FI_-

FS4 Burner.FI

FS49 4 PT.

FS5 TEGV.FI_O

FS8 Core_Nozz.FI_O

FS17 FanDuctLkg.Fì_o

FS171 Bleed15.F1

FS173 Byp

$W$
278.58
278.58
250.17
28.40
250.17
28.40
28.40
25.56
24.32
18.85
19.05
25.47
25.76
25.76
25.76
250.17
253.01
253.01
253.01

$\begin{array}{rrrr}\text { Pt } & \text { Tt } & \text { ht } & \text { FAR } \\ 4.831 & 451.61 & -22.45 & 0.0000 \\ 4.831 & 451.61 & -22.45 & 0.0000\end{array}$

$\begin{array}{lllll}4.831 & 451.61 & -22.45 & 0.0000 & 790.74 \\ 4.831 & 451.61 & -22.45 & 0.0000 & 790.74\end{array}$

$\begin{array}{llll}451.61 & -22.45 & 0.0000 & 710.47\end{array}$

$\begin{array}{lllll}4.829 & 451.61 & -22.45 & 0.0000 & 710.47 \\ 5.360 & 466.78 & -18.82 & 0.0000 & 80.67\end{array}$

$\begin{array}{lllll}7.122 & 514.69 & -7.33 & 0.0000 & 58.39\end{array}$

$\begin{array}{lllll}7.122 & 514.69 & -7.33 & 0.0000 & 58.39 \\ 7.122 & 514.77 & -7.50 & 0.0000 & 58.39 \\ 7.122 & 514.77 & -7.50 & 0.0000 & 52.55\end{array}$

$\begin{array}{llll}7.122 & 514.77 & -7.50 & 0.0000\end{array}$

$\begin{array}{llll}42.305 & 928.66 & 92.86 & 0.0000\end{array}$

$\begin{array}{lllll}42.305 & 928.66 & 92.86 & 0.0000 & 8.76\end{array}$

Ps
3.578

$\begin{array}{rrr}\text { Aphy } & \text { MN } \\ .14 .42 & 2577.4 & 0.6690\end{array}$

$\begin{array}{lllll} & & & & \\ 4.578 & 441.21 & 4168.2 & 0.3430 & 1.40088\end{array}$

$\begin{array}{rrr}439.71 & 3531.8 & 0.3674\end{array}$

1.40088
1.40088

$\begin{array}{rrrr}4.736 & 445.97 & 2606.7 & 0.4824 \\ 6.571 & 508.46 & 412.6 & 0.2475\end{array}$

$\begin{array}{llll}508.46 & 412.6 & 0.2475 & 1.40028 \\ 508.53 & 412.6 & 0.2475 & 1.40028\end{array}$

1.40028
1.40028

$509.78 \quad 412.6 \quad 0.2211$

$\begin{array}{rrr}412.6 & 0.2211 \\ 49.7 & 0.4298\end{array}$

$\begin{array}{llll}37.301 & 896.67 & 49.7 & 0.4298 \\ 39.429 & 910.65 & 49.3 & 0.3203\end{array}$

1.40028
1.38497

910.65

$\begin{array}{ll}49.7 & 0.4298 \\ 74.6 & 0.3203 \\ 265.4 & 0.2940\end{array}$

1.38497
1.33503

$\begin{array}{rr}39.027 & 1626.86 \\ 9.287 & 1065.30 \\ 3.767 & 870.38\end{array}$

$\begin{array}{lllll}10.192 & 1092.43 & -16.28 & 0.0079 & 53.29\end{array}$

$\begin{array}{lllll}3.941 & 881.38 & -67.55 & 0.0078 & 125.23 \\ 3.941 & 881.45 & -67.55 & 0.0078 & 125.23 \\ 3.941 & 881.52 & -67.55 & 0.0078 & 125.24\end{array}$

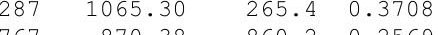

870.38
870.45

$\begin{array}{ll}860.2 & 0.2569 \\ 860.2 & 0.2569\end{array}$

$1.36943 \quad 0.0000670$

$\begin{array}{ll}3.767 & 870.45 \\ 3.578 & 858.21\end{array}$

$\begin{array}{llll}881.45 & -67.55 & 0.0078 & 125.23 \\ 881.52 & -67.55 & 0.0078 & 125.24\end{array}$

$\begin{array}{lllll}3.941 & 881.52 & -67.55 & 0.0078 & 125.24 \\ 5.360 & 466.78 & -18.82 & 0.0000 & 650.68\end{array}$

$\begin{array}{llllll}5.360 & 467.32 & -18.69 & 0.0000 & 658.44\end{array}$

$\begin{array}{lllll}5.360 & 467.32 & -18.69 & 0.0000 & 658.44\end{array}$

4.571

$\begin{array}{rrr}858.21 & 613.4 & 0.3764 \\ 445.97 & 2606.7 & 0.4824\end{array}$

$1.38250-0.0000670$

$\begin{array}{ll}1.38249 & 0.0000670 \\ 1.40078 & 0.0000348\end{array}$

$\begin{array}{llll}442.79 & 2481.9 & 0.5256\end{array}$

1.30078
1.40078

4.440
4.440

442.79
442.79

$2481.9 \quad 0.5256$

$\begin{array}{lllll}467.32 & -18.69 & 0.0000 & 658.44 & 3.578\end{array}$

$\begin{array}{lll}416.27 & 2006.9 & 0.7821\end{array}$

$1.40078-0.0000351$

$1.40078 \quad 0.0000351$

TURBOMACHINERY PERFORMANCE DATA

$\begin{array}{lrrrrrrrrr} & \text { WC } & \text { PR } & \text { eff } & \text { NC } & \text { TR } & \text { efPoly } & \text { pwr } & \text { SMN } & \text { SMW } \\ \text { Fan } & 710.47 & 1.110 & 0.9032 & 2395.792 & 1.0336 & 0.9046 & -1286.3 & 5784.67 & 54.21 \\ \text { LPC } & 80.67 & 1.475 & 0.8416 & 2395.792 & 1.1397 & 0.8501 & -607.7 & 6.41 & 3.05 \\ \text { HPC } & 52.55 & 5.940 & 0.8161 & 8911.057 & 1.8040 & 0.8550 & -3542.1 & 61.34 & 57.85 \\ \text { HPT } & 12.08 & 4.055 & 0.8716 & 218.517 & 1.3586 & 0.8490 & 3542.1 & & \\ \text { LPT } & 53.29 & 2.586 & 0.8385 & 67.638 & 1.2372 & 0.8195 & 1894.0 & \end{array}$

TURBOMACHINERY MAP DATA

$\begin{array}{lrrrrrrrrr} & \text { WcMap } & \text { PRmap } & \text { effMap } & \text { NcMap } & \text { R/Parm } & \text { S_WcDes } & \text { S_PRdes } & \text { S_effDes } & \text { S_NcDes } \\ \text { Fan } & 825.49 & 1.108 & 0.9050 & 2372.948 & 0.0413 & 0.8607 & \overline{1} .0235 & 0.9980 & 0.9905 \\ \text { LPC } & 66.44 & 1.445 & 0.7718 & 0.633 & 0.0000 & 1.2141 & 1.0673 & 1.0904 & 0.0003 \\ \text { HPC } & 47.80 & 5.707 & 0.8246 & 8673.494 & 10.9958 & 1.0995 & 1.0494 & 0.9898 & 0.9733 \\ \text { HPT } & 0.96 & 3.970 & 0.8716 & 1.313 & 3.9703 & 12.6299 & 0.9723 & 1.0000 & 0.0003 \\ \text { LPT } & 0.82 & 2.193 & 0.8448 & 0.761 & 2.1929 & 65.2590 & 0.7521 & 0.9926 & 0.0005\end{array}$




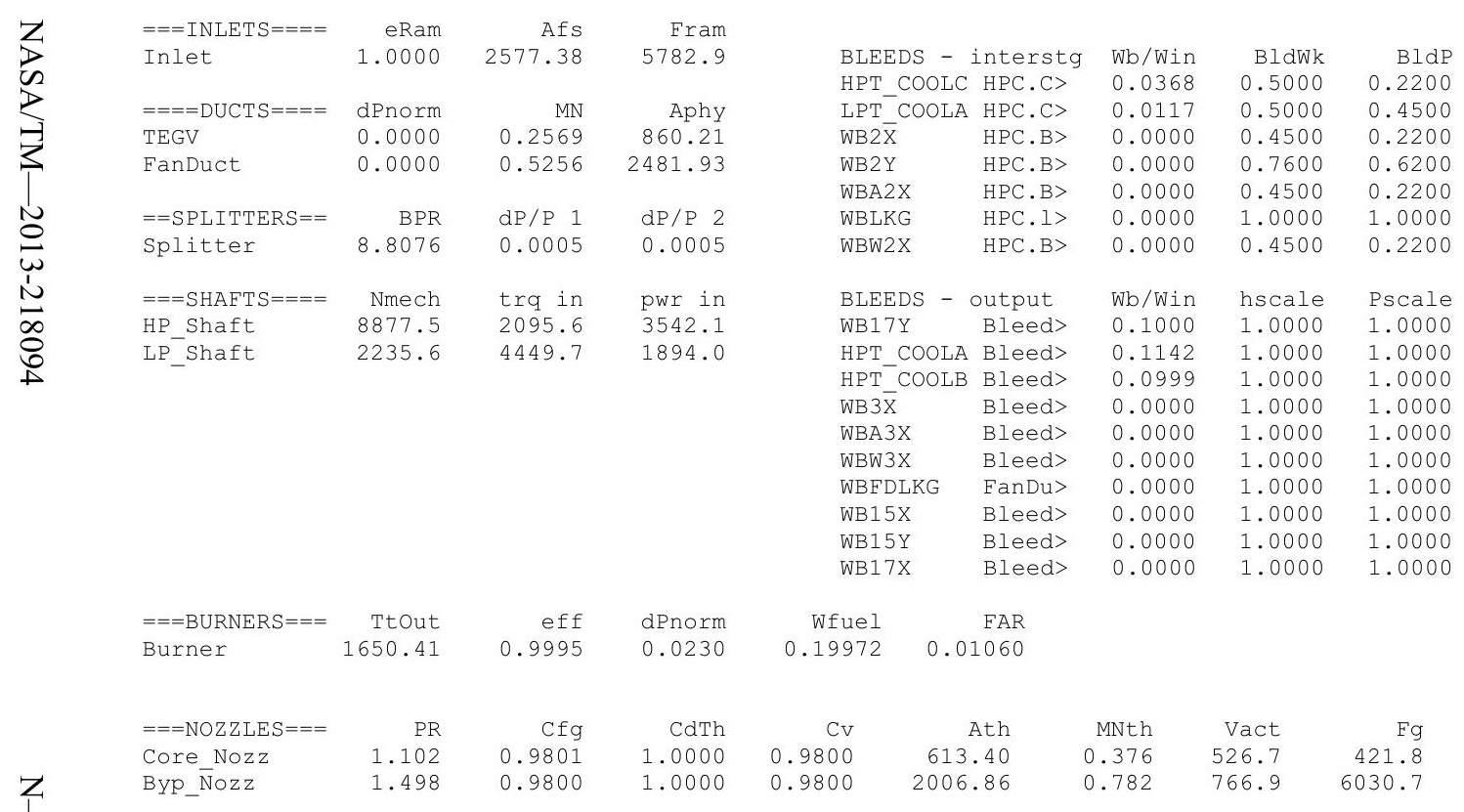


Date:07/05/13 Time:09:27:35 Model: Turbofan Engine - COMDES ON Converge = 1 CASE: 0

Version:NPSS1.6.5 - Rev: -> Gas Package: Janaf iter/pass/Jacb/Broy= 14/28/1/12 Run by: Philip C Jorgenson PC: 10

Temperature Stator 1 inlet: 465.04 Stator 1 exit: $470.56 \quad$ Stator 2 inlet: 477.37 Stator 2 exit: 480.66 $\begin{array}{lll}\text { Stator } 3 \text { inlet: } 487.79 & \text { Stator } 3 \text { exit: } 490.68 & \text { Stator } 4 \text { inlet: } 497.38 \text { Stator 4 exit: } 499.77 \\ \text { stator } 5 \text { inlet: } 505.17 & \text { Stator } 5 \text { exit: } 507.19 & \text { Unblocked Percent Blockage: } 0.00\end{array}$

Ambient Relative Humidity $\quad 10.00$

Fan Face Relative Humidity $\quad 3.96$

Fan Bypass Relative Humidity $\quad 3.12$

LPC Inlet Relative Humidity $\quad 2.83$

HPC Relative Humidity

Drop Diameter

0.00

Ambient Flow Velocity

Ambient Static Pressure

Ambient Static Temperature
Additional Water at LPC Exit

0.0000500

617.99 Fan/LPC Inlet Flow Velocity $\quad \begin{aligned} 40.00 \\ 4.99\end{aligned}$

4.36 Fan/LPC Inlet Static Pressure

429.58
0.0000397

Fan/LPC Inlet Static Temperature 458.86

$\begin{array}{rrrrrr}\text { MN } & \text { alt } & \text { dTamb } & \text { W } & \text { SUMMARY } & \text { FUTPUT DATA } \\ 0.608 & 30029.0 & 18.00 & 305.09 & 728.9 & 1.0766\end{array}$ $\begin{array}{rrr}728.9 & 1.0766 & \text { Wfuel } \\ & & 784.71\end{array}$

FLOW STATION DATA

FSO Ambient.FI_O

FS1 Inlet.F1_O

FS12 Splitter.F1_02

FS2 Splitter.Fl_01

FS14 Fan.F1_

FS24 VaporIN.EI_O

FS25 Bleed2.F1_-

FS3 HPC.Fl 0

FS36 Bleed3.F1 O

FS4 Burner.FI_O

FS45 HPT.FI O

FS49 LPT.FI_O

FSB COI.

FS17 FanDuctI.F1_O

FS171 Bled15. E1.

FS171 Bleed15.FI_-

FS173 Byp Nozz.FI-

$W$
305.09
305.09
272.54
32.56
272.54
32.56
32.56
27.68
26.33
20.41
20.63
27.57
27.89
27.89
27.89
272.54
277.42
277.42
277.42

Pt
5.595

Tt
461.42

$\begin{array}{rr}\text { ht } & \text { FAR } \\ -20.30 & 0.0000\end{array}$

$\begin{array}{llllll}5.595 & 461.42 & -20.30 & 0.0000 & 755.82\end{array}$

$\begin{array}{lllll}5.592 & 461.42 & -20.30 & 0.0000 & 675.50\end{array}$

$\begin{array}{lllll}5.592 & 461.42 & -20.30 & 0.0000 & 80.70 \\ 6.139 & 475.25 & -16.98 & 0.0000 & 624.50\end{array}$

$\begin{array}{lllll}5.592 & 461.42 & -20.30 & 0.0000 & 80.70 \\ 7.139 & 475.25 & -16.98 & 0.0000 & 624.50 \\ 7.826 & 515.84 & -7.25 & 0.0000 & 60.97\end{array}$

$\begin{array}{lllll}7.826 & 515.84 & -7.25 & 0.0000 & 60.97 \\ 7.826 & 515.92 & -7.46 & 0.0000 & 60.98\end{array}$

$\begin{array}{lllll}7.826 & 515.84 & -7.25 & 0.0000 & 60.97 \\ 7.826 & 515.92 & -7.46 & 0.0000 & 60.98\end{array}$

$\begin{array}{lllll}7.826 & 515.92 & -7.46 & 0.0000 & 60.98 \\ 45.862 & 915.92 & -7.46 & 0.0000 & 51.83\end{array}$

$\begin{array}{rrrrr}75.862 & 927.46 & 92.34 & 0.0000 & 11.28 \\ 45.862 & 927.46 & 92.34 & 0.0000 & 8.74\end{array}$

$\begin{array}{llrlr}44.806 & 1654.85 & 80.25 & 0.0107 & 12.08\end{array}$

$\begin{array}{rrrrr}11.202 & 1098.00 & -16.30 & 0.0080 & 52.62 \\ 4.717 & 904.54 & -63.23 & 0.0079 & 114.77\end{array}$

$\begin{array}{lllll}1.202 & 1098.00 & -16.30 & 0.0080 & 52.62 \\ 4.717 & 904.54 & -63.23 & 0.0079 & 114.77 \\ 4.717 & 904.61 & -63.23 & 0.0079 & 114.77\end{array}$

$\begin{array}{lllll}4.717 & 904.61 & -63.23 & 0.0079 & 114.77 \\ 4.717 & 904.68 & -63.23 & 0.0079 & 114.78\end{array}$

$\begin{array}{lllll}4.717 & 904.68 & -63.23 & 0.0079 & 114.77 \\ 6.139 & 475.25 & -16.98 & 0.0000 & 624.50\end{array}$

$\begin{array}{lllll}6.139 & 475.96 & -16.82 & 0.0000 & 636.17\end{array}$

$\begin{array}{lllll}6.139 & 475.96 & -16.82 & 0.0000 & 636.1\end{array}$

$\begin{array}{llll}475.96 & -16.82 & 0.0000 & 636.17 \\ 475.96 & -16.82 & 0.0000 & 636.17\end{array}$

TURBOMACHINERY PERFORMANCE DATA

$\begin{array}{lrrr} & \text { WC } & \text { PR } & \text { eff } \\ \text { Fan } & 675.50 & 1.098 & 0.9021 \\ \text { LPC } & 80.70 & 1.399 & 0.8552 \\ \text { HPC } & 51.83 & 5.860 & 0.8147 \\ \text { HPT } & 12.08 & 4.000 & 0.8718 \\ \text { LPT } & 52.62 & 2.375 & 0.8303\end{array}$

$N C$
2249.411
2249.411
8887.489
217.895
64.028

TR
1.0300
1.1179
1.7977
1.3544
1.2114

efPoly
0.9034
0.8619
0.8536
0.8495
0.8124

pwr
-1278.1
-601.0

$-3813.0$

3812.9

61.61

0.8124

1879.1

TURBOMACHINERY MAP DATA

$\begin{array}{lrrrrrrrrr} & \text { WcMap } & \text { PRmap } & \text { effMap } & \text { NcMap } & \text { R/Parm } & \text { S_WcDes } & \text { S_PRdes } & \text { S_effDes } & \text { S_NcDes } \\ \text { Fan } & 784.86 & 1.096 & 0.9039 & 227.963 & 0.0416 & 0.8607 & \overline{1} .0235 & 0.9980 & 0.9905 \\ \text { LPC } & 60.32 & 1.382 & 0.7655 & 0.594 & 0.0000 & 1.3379 & 1.0459 & 1.1172 & 0.0003 \\ \text { HPC } & 47.14 & 5.631 & 0.8231 & 8650.554 & 10.9887 & 1.0995 & 1.0494 & 0.9898 & 0.9733 \\ \text { HPT } & 0.96 & 3.917 & 0.8718 & 1.310 & 3.9166 & 12.6299 & 0.9723 & 1.0000 & 0.0003 \\ \text { LPT } & 0.81 & 2.034 & 0.8365 & 0.720 & 2.0342 & 65.2590 & 0.7521 & 0.9926 & 0.0005\end{array}$

\begin{tabular}{|c|c|}
\hline & \\
\hline 4.3 & 429 \\
\hline 5.1 & 451 \\
\hline 5.1 & 450 \\
\hline 5.4 & 458 \\
\hline 5.3 & 456 \\
\hline 7.4 & 508 \\
\hline 7.4 & 509 \\
\hline 7.5 & 511 \\
\hline 40.4 & 895 \\
\hline 42.7 & 909 \\
\hline 42.3 & 1631 \\
\hline 10.2 & 1071 \\
\hline 4.5 & 895 \\
\hline 4.5 & 895 \\
\hline 4.3 & 885 \\
\hline 5.3 & 456 \\
\hline 5.1 & 453 \\
\hline 5.1 & \\
\hline 4.3 & 431 \\
\hline & SMW \\
\hline & 52.72 \\
\hline & 2.63 \\
\hline & 58.03 \\
\hline EfDes & S_NCDes \\
\hline .9980 & $\overline{0} .9905$ \\
\hline . 1172 & 0.00 \\
\hline 8 & 0.9733 \\
\hline 0000 & 0.000 \\
\hline 9926 & 0 \\
\hline
\end{tabular}

$\begin{array}{lll}0.81 & 2.034 & 0.8365\end{array}$
$0.720 \quad 2.0342$

$\begin{array}{lll}65.2590 & 0.7521 & 0.9926\end{array}$

.0005

$\begin{array}{llccc}\text { EPR } & \text { PowerSet } & \text { T4 } & \text { T41 } & \text { T49 } \\ 0.828 & 10.000 & 1654.9 & 1563.1 & 1093.8\end{array}$

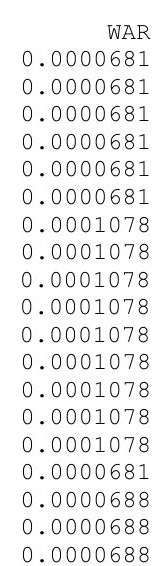

Wair 305.07
305.07 305.07
272.52 272.52 32.56
32.56 0.004 $27.67 \quad 0.003$ $\begin{array}{lll}20.41 & 0.002\end{array}$ $\begin{array}{ll}20.41 & 0.002\end{array}$ $\begin{array}{ll}20.45 & 0.002 \\ 27.35 & 0.003\end{array}$ $\begin{array}{ll}27.67 & 0.003 \\ 27.67 & 0.003\end{array}$ $\begin{array}{ll}27.67 & 0.003\end{array}$ $\begin{array}{rr}27.67 & 0.003\end{array}$ $\begin{array}{ll}272.52 & 0.019\end{array}$ $\begin{array}{ll}277.40 & 0.019 \\ 277.40 & 0.019\end{array}$ $\begin{array}{ll}277.40 & 0.019\end{array}$

$\begin{array}{rr}20 & \text { H2O frac } \\ 21 & 0.0001 \\ 21 & 0.0001 \\ 19 & 0.0001 \\ 02 & 0.0001 \\ 19 & 0.0001 \\ 02 & 0.0001 \\ 04 & 0.0001 \\ 03 & 0.0001 \\ 03 & 0.0001 \\ 02 & 0.0001 \\ 02 & 0.0131 \\ 03 & 0.0099 \\ 03 & 0.0098 \\ 03 & 0.0098 \\ 03 & 0.0098 \\ 19 & 0.0001 \\ 19 & 0.0001 \\ 19 & 0.0001 \\ 19 & 0.0001\end{array}$




\begin{tabular}{|c|c|c|c|c|c|c|c|c|}
\hline \multirow{2}{*}{$\begin{array}{ll}Z & ===\text { INLETS }==== \\
& \text { Inlet }\end{array}$} & eRam & Afs & Fram & & \multirow{2}{*}{ Wb/Win } & \multirow{2}{*}{$B l d W k$} & \multirow[b]{2}{*}{$\begin{array}{r}\text { BldF } \\
0.2200\end{array}$} \\
\hline & 1.0000 & 2596.15 & 5860.2 & $\begin{array}{l}\text { BLEEDS - } \\
\text { HPT COOL }\end{array}$ & $\begin{array}{l}\text { intersto } \\
\text { C HPC.C> }\end{array}$ & & & \\
\hline$====$ DUCTS $====$ & dPnorm & MN & Aphy & $\mathrm{LPT}^{-} \mathrm{COOL}$ & A HPC.C> & 0.0117 & 0.5000 & 0.4500 \\
\hline TEGV & 0.0000 & 0.2340 & 860.21 & WB2 $\overline{\mathrm{X}}$ & HPC. B> & 0.0000 & 0.4500 & 0.2200 \\
\hline FanDuct & 0.0000 & 0.5005 & 2481.93 & WB2Y & HPC.B> & 0.0000 & 0.7600 & 0.6200 \\
\hline & & & & WBA2X & HPC.B> & 0.0000 & 0.4500 & 0.2200 \\
\hline$==$ SPLITTERS $==$ & BPR & $\mathrm{dP} / \mathrm{P} 1$ & $\mathrm{dP} / \mathrm{P} 2$ & WBLKG & HPC.I> & 0.0000 & 1.0000 & 1.0000 \\
\hline Splitter & 8.3707 & 0.0005 & 0.0005 & WBW2X & HPC.B> & 0.0000 & 0.4500 & 0.2200 \\
\hline$===$ SHAFTS $====$ & Nmech & trg in & pwr in & BLEEDS - & output & Wb/Win & hscale & Pscale \\
\hline HP Shaft & 8863.9 & 2259.3 & 3812.9 & WB17Y & Bleed> & 0.1500 & 1.0000 & 1.0000 \\
\hline LP_Shaft & 2121.6 & 4651.8 & 1879.1 & HPT_COOL & A Bleed> & 0.1142 & 1.0000 & 1.0000 \\
\hline & & & & $\mathrm{HPT}^{-} \mathrm{COOL}$ & B Bleed> & 0.0999 & 1.0000 & 1.0000 \\
\hline & & & & WB $3 \bar{x}$ & Bleed> & 0.0000 & 1.0000 & 1.0000 \\
\hline & & & & WBA3X & Bleed> & 0.0000 & 1.0000 & 1.0000 \\
\hline & & & & WBW3X & Bleed> & 0.0000 & 1.0000 & 1.0000 \\
\hline & & & & WBFDLKG & FanDu> & 0.0000 & 1.0000 & 1.0000 \\
\hline & & & & WB15X & Bleed> & 0.0000 & 1.0000 & 1.0000 \\
\hline & & & & WB15Y & Bleed> & 0.0000 & 1.0000 & 1.0000 \\
\hline & & & & WB17X & Bleed> & 0.0000 & 1.0000 & 1.0000 \\
\hline$===$ BURNERS $===$ & Ttout & eff & dPnorm & Wfuel & FAR & & & \\
\hline Burner & 1654.79 & 0.9995 & 0.0230 & 21797 & 01068 & & & \\
\hline$===\operatorname{NOZZLES} S===$ & $\mathrm{PR}$ & Cfg & CdTh & $\mathrm{Cv}$ & Ath & MNth & Vact & $\mathrm{Fg}$ \\
\hline Core Nozz & 1.082 & 0.9801 & 1.0000 & 0.9800 & 3.40 & 0.340 & 482.9 & 418.6 \\
\hline Byp_Nozz & 1.409 & 0.9800 & 1.0000 & 0.9800 & 6.86 & 0.717 & 715.6 & 6170.4 \\
\hline
\end{tabular}


Date:07/05/13 Time:09:27:57 Model: Turbofan Engine - COMDES ON Converge = 1 CASE: 0

Version:NPSS 1.6.5 - Rev: -> Gas Package: Janaf iter/pass/Jacb/Broy= 22/36/1/20 Run by: Philip C Jorgenson PC: 10

Temperature Stator 1 inlet: $473.11 \quad$ Stator 1 exit: $477.17 \quad$ Stator 2 inlet: 481.78 Stator 2 exit: 483.58 Stator 5 inlet: 499.63 Stator 5 exit: $500.35 \quad$ Unblocked Percent Blockage: 0.00

Ambient Relative Humidity $\quad 10.00$

Fan Face Relative Humidity $\quad 4.82$

Fan Bypass Relative Humidity $\quad 3.94$

LPC Inlet Relative Humidity $\quad 3.82$

(

Drop Diameter

0.00

0.0000500

Inlet Length

40.00

Ambient Flow Velocity

Ambient Static Pressure

Additional Water at LPC Exit

571.13

Inlet Iength

$\begin{array}{rrr}5.30 & \text { Fan/LPC Inlet Static Pressure } & 6.36 \\ 45.14 & \text { Fan/LPC Inlet Static Temperature } & 469.17\end{array}$

MN SUMMARY OUTPUT DATA

\begin{tabular}{|c|c|c|c|c|c|c|c|c|c|c|c|c|c|c|}
\hline MN & alt & dTamb & W & $\begin{array}{r}\text { UMMARY } \\
\text { Fn }\end{array}$ & $\begin{array}{r}\text { TPUT DA? } \\
\text { TSFC }\end{array}$ & Wfuel & BPR & VTAS & OPR & EPR & Powerset & T4 & T41 & T49 \\
\hline 0.552 & 25666.0 & 18.00 & 330.54 & 748.3 & 1.1193 & 837.60 & 7.0492 & 571.13 & 7.334 & 0.859 & 10.000 & 1665.6 & 1571.6 & 1105.0 \\
\hline
\end{tabular}

FSO Ambient.Fl 0

FS1 Inlet.FI_O

FS12 Splitter.F1_02

FS14 4 Sanctiter.

FS23 LPC.Fl-O

FS24 VaporIN . FI_O

S25 Bleed2.Fl_

.

FS4 Burner. Fl-

FS4 Burner.FI

FS49 IPT. F1-

FS5 5 TEGV.FI

FS8 Core_Nozz.Fl_O

FS17 FanDúctLkg.Fì O

FS171 Bleed15.F1_O

FS172 FanDuct.FI-O

FS173 Byp Nozz.FİO

TURBOMACHINERY PERFORMANCE DATA

$\begin{array}{lrrr} & \text { WC } & \text { PR } & \text { eff } \\ \text { Fan } & 623.31 & 1.083 & 0.9032 \\ \text { LPC } & 88.42 & 1.260 & 0.8218 \\ \text { HPC } & 51.14 & 5.824 & 0.8139 \\ \text { HPT } & 12.09 & 3.881 & 0.8732 \\ \text { LPT } & 51.17 & 2.138 & 0.8210\end{array}$

FLOW STATION DATA

$\begin{array}{lrrrr}\text { FLOW } & \text { STATION DATA } \\ \text { Pt } & \text { Tt } & \text { ht } & \text { FAR } & \text { Wc } \\ 6.516 & 472.33 & -18.03 & 0.0000 & 711.38 \\ 6.516 & 472.33 & -18.03 & 0.0000 & 711.38 \\ 6.513 & 472.33 & -18.03 & 0.0000 & 623.31 \\ 6.513 & 472.33 & -18.03 & 0.0000 & 88.42 \\ 7.057 & 484.46 & -15.12 & 0.0000 & 582.63 \\ 8.206 & 511.58 & -8.61 & 0.0000 & 73.04 \\ 8.206 & 511.67 & -8.85 & 0.0000 & 73.05 \\ 8.206 & 511.67 & -8.85 & 0.0000 & 51.14 \\ 47.792 & 918.60 & 89.79 & 0.0000 & 11.19 \\ 47.792 & 918.60 & 89.79 & 0.0000 & 8.67 \\ 46.691 & 1665.62 & 77.40 & 0.0110 & 12.09 \\ 12.030 & 1109.37 & -17.89 & 0.0082 & 51.17 \\ 5.626 & 937.03 & -59.60 & 0.0081 & 101.74 \\ 5.626 & 937.10 & -59.60 & 0.0081 & 101.75 \\ 5.626 & 937.18 & -59.60 & 0.0081 & 101.75 \\ 7.057 & 484.46 & -15.12 & 0.0000 & 582.63 \\ 7.057 & 485.57 & -14.86 & 0.0000 & 608.12 \\ 7.057 & 485.57 & -14.86 & 0.0000 & 608.12 \\ 7.057 & 485.57 & -14.86 & 0.0000 & 608.12\end{array}$

$\begin{array}{rrrr}\text { Ps } & \text { Ts } & \text { Aphy } & \text { MN } \\ 5.297 & 445.14 & 2595.0 & 0.5520\end{array}$

6.078

6.362

6.362
7.654

7.654
7.654

7.654

7.946
42.271

$44.614 \quad 901.16$

$11.053 \quad 1084.34$

$\begin{array}{ll}5.464 & 929.54 \\ 5.464 & 929.61\end{array}$

$5.297 \quad 921.76$

$\begin{array}{ll}5.258 & 468.09\end{array}$

$6.063 \quad 464.95$

$6.063 \quad 464.95$

$5.297 \quad 447.31$

TR
1.0257
1.0831
1.7953
1.3457
1.1810

efPoly
0.9043
0.8276
0.8529
0.8517
0.8047

pwr
-1191.1
-546.8
-3914.5
3914.5
1737.9
SMN SMW

$\begin{array}{rr}6.38 & 2.41 \\ 60.72 & 57.10\end{array}$

$60.72-57.10$

TURBOMACHINERY MAP DATA

$\begin{array}{lrrrrrrrrr} & \text { WcMap } & \text { PRmap } & \text { effMap } & \text { NcMap } & \text { R/Parm } & \text { S_WcDes } & \text { S_PRdes } & \text { S_effDes } & \text { S_NcDes } \\ \text { Fan } & 724.23 & 1.082 & 0.9050 & 2025.172 & 0.0425 & 0.8607 & \overline{1} .0235 & 0.9980 & 0.9905 \\ \text { LPC } & 53.47 & 1.311 & 0.7607 & 0.540 & 0.0000 & 1.6537 & 0.8353 & 1.0803 & 0.0003 \\ \text { HPC } & 46.51 & 5.597 & 0.8223 & 8629.340 & 11.0519 & 1.0995 & 1.0494 & 0.9898 & 0.9733 \\ \text { HPT } & 0.96 & 3.801 & 0.8732 & 1.297 & 3.8014 & 12.6299 & 0.9723 & 1.0000 & 0.0003 \\ \text { LPT } & 0.78 & 1.856 & 0.8271 & 0.659 & 1.8561 & 65.2590 & 0.7521 & 0.9926 & 0.0005\end{array}$




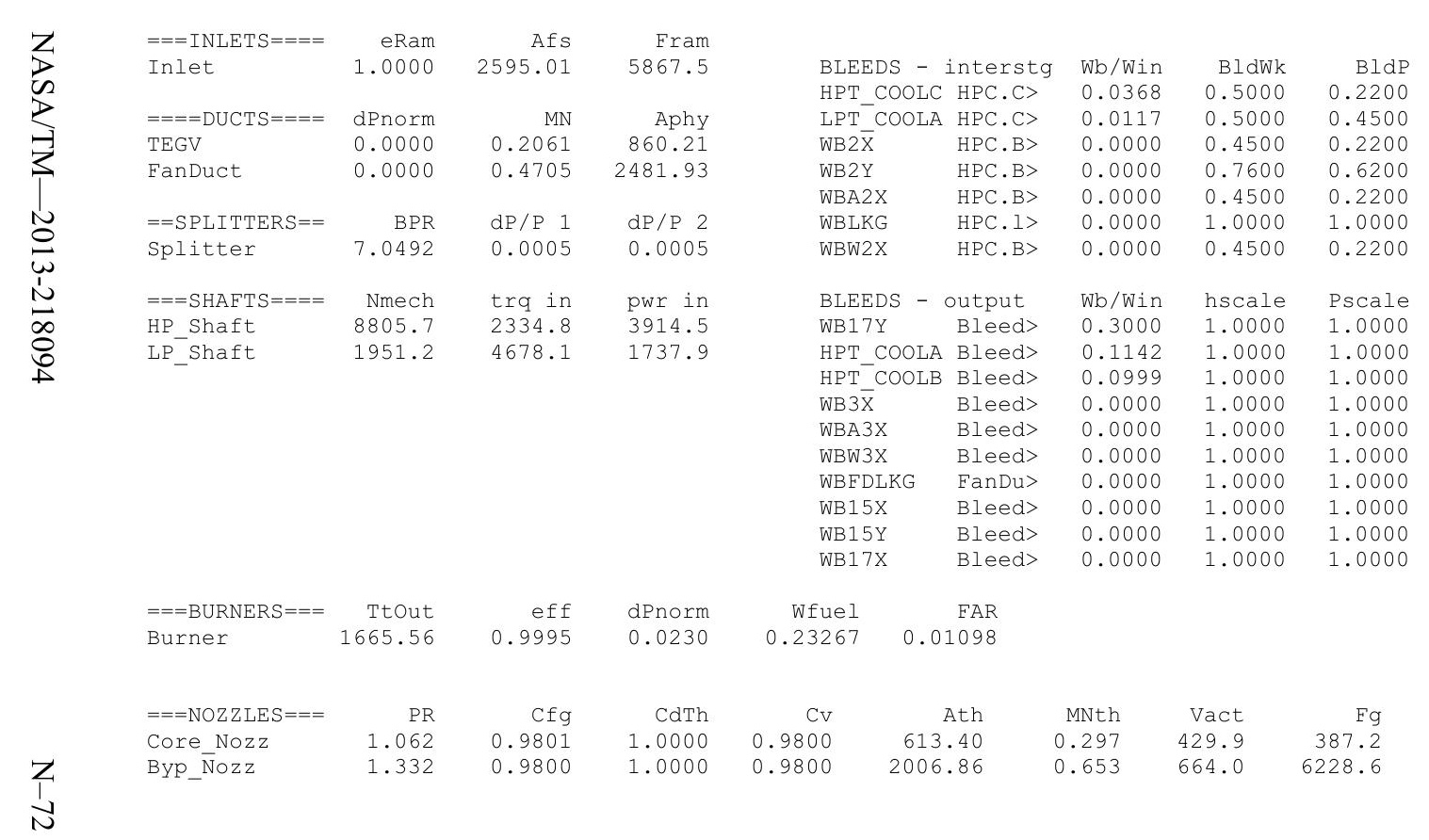


Turbofan Engine - COMDES ON Converge = 1 CASE: 0

Version:NPSS_1.6.5 - Rev: -> Gas Package: Janaf iter/pass/Jacb/Broy= 26/40/1/24 Run by: Philip C Jorgenson PC: 10

Temperature Stator 1 inlet: 486.26 Stator 1 exit: $489.21 \quad$ Stator 2 inlet: $491.94 \quad$ Stator 2 exit: 492.70 $\begin{array}{lll}\text { Stator } 3 \text { inlet: } 496.03 & \text { Stator } 3 \text { exit: } 496.35 & \text { Stator } 4 \text { inlet: 499.60 Stator 4 exit: } 499.62 \\ \text { Stator } 5 \text { inlet: } 501.46 & \text { Stator } 5 \text { exit: } 501.30 & \text { Unblocked Percent Blockage: } 0.00\end{array}$

$\begin{array}{lclll}\text { Ambient Relative Humidity } & 10.00 & & & \\ \text { Fan Face Relative Humidity } & 5.85 & & & \\ \text { Fan Bypass Relative Humidity } & 4.91 & & & \\ \text { LPC Inlet Relative Humidity } & 5.02 & & & \\ \text { LPC Exit Relative Humidity } & 2.62 & & & \\ \text { HPC Relative Humidity } & 0.01 & & & \\ \text { Drop Diameter } & 0.0000500 & \text { Inlet Length } & \\ \text { Ambient Flow Velocity } & 518.26 & \text { Fan/LPC Inlet Flow Velocity } & 204.34 \\ \text { Ambient Static Pressure } & 6.74 & \text { Fan/LPC Inlet Static Pressure } & 7.74 \\ \text { Ambient Static Temperature } & 465.18 & \text { Fan/LPC Inlet Static Temperature } & 484.08 \\ \text { Additional Water at LPC Exit } & 0.0000541 & & & \end{array}$ $\begin{array}{rrr} & \text { SUMMARY } & \text { OUTPUT DATA } \\ \text { W } & \text { Fn } & \text { TSFC } \\ 368.15 & 831.7 & 1.1214\end{array}$

\begin{abstract}
Fn
831.7
\end{abstract}
Wfuel $\begin{array}{rr}\text { BPR } \\ 932.67 & 6.2370\end{array}$
VTAS
518.26
$\mathrm{OPR}$
6.453 6.453 $\begin{array}{ll}\text { EPR } & \text { PowerSet } \\ 0.890 & 10.000\end{array}$ $\begin{array}{ccc}\text { T4 } & \text { T41 } & \text { T49 } \\ 1698.7 & 1600.6 & 1135.7\end{array}$
FSO Ambient.FI O

FS1 Inlet.F1_O

FS12 Splitter.FI_02

FS14 Fan.FI_O

FS24 VaporIN.F1_O

FS3 HPC.F1_O

FS36 Bleed3.F1_O

FS4 Burner.Fl_o

FS45 HPT.FI_O

FS49 LPT.FI-O

FS8 Core_Nozz.FI_O

FS17 FanDuctLkg.Fí

FS171 Bleed15.FI_O

FS172 FanDuct.FI-O

TURBOMACHINERY PERFORMANCE DATA

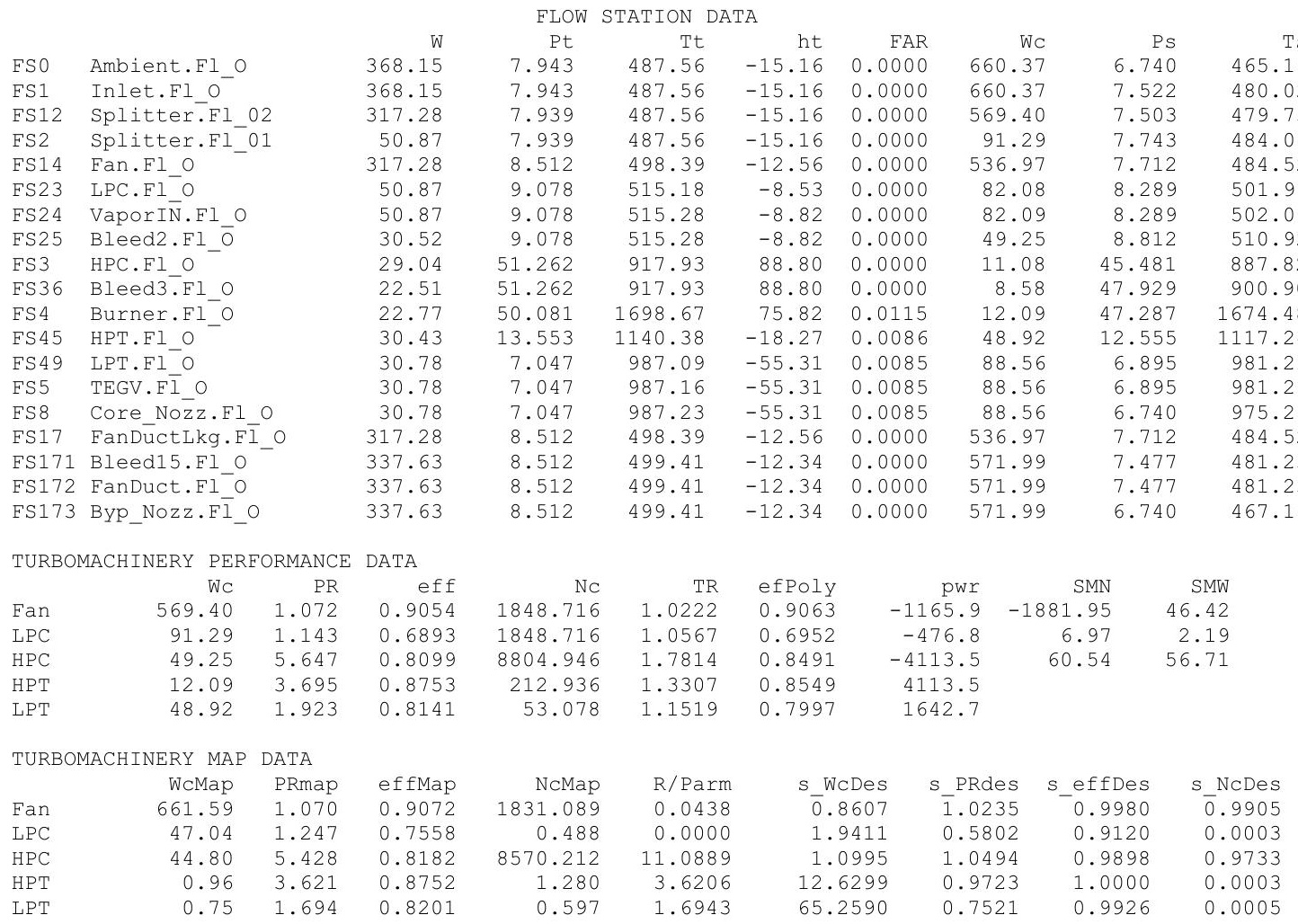

TURBOMACHINERY MAP DATA

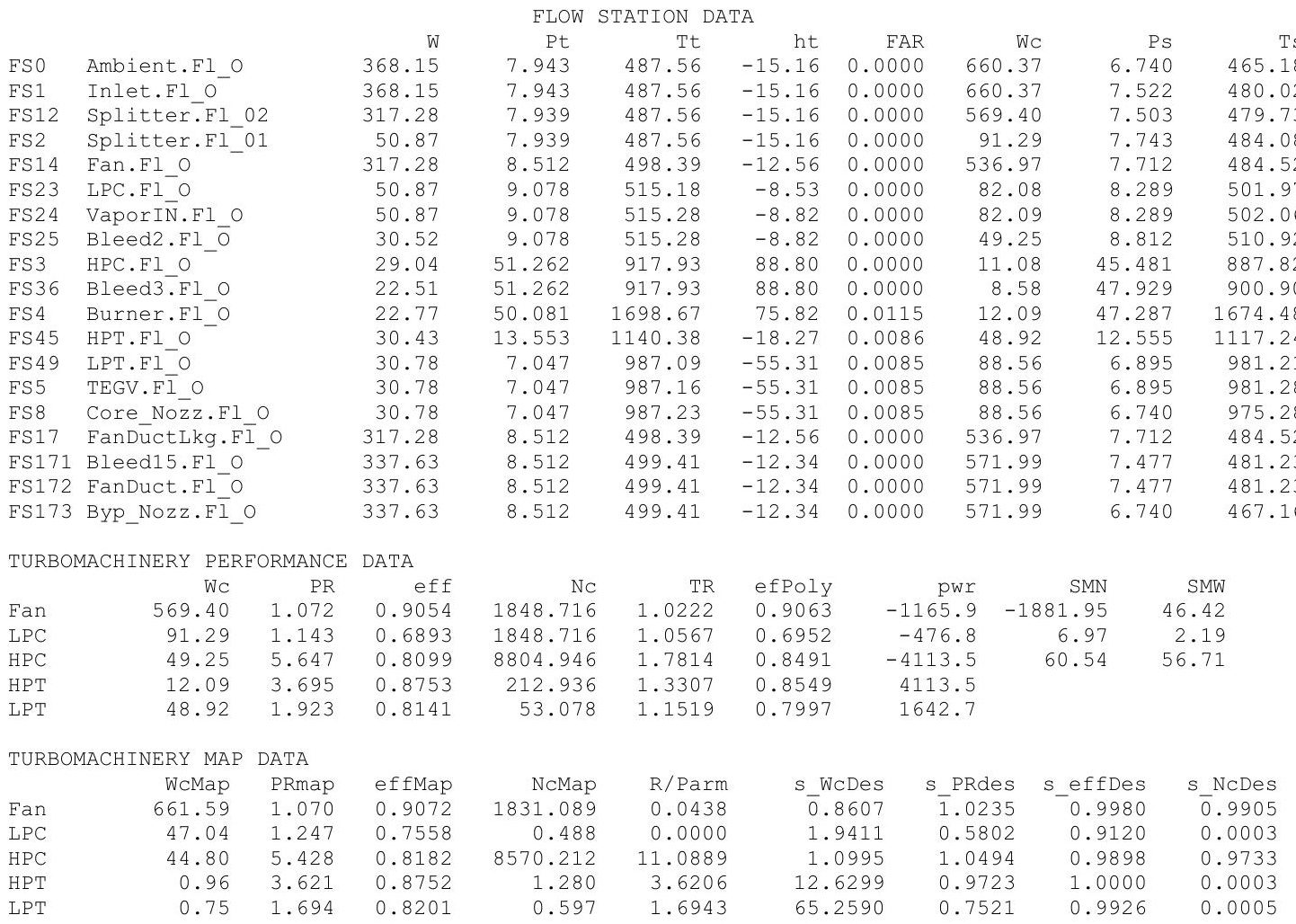

$\begin{array}{rrrrrrr}\text { Aphy } & \text { MN } & \text { gamt } & \text { WAR } & \text { Wair } & \text { WH2O } & \text { H2O frac } \\ 2616.0 & 0.4900 & 1.40057 & 0.0002627 & 368.05 & 0.097 & 0.0003 \\ 4168.2 & 0.2799 & 1.40057 & 0.0002627 & 368.05 & 0.097 & 0.0003 \\ 3531.8 & 0.2854 & 1.40057 & 0.0002627 & 317.19 & 0.083 & 0.0003 \\ 830.5 & 0.1894 & 1.40057 & 0.0002627 & 50.86 & 0.013 & 0.0003 \\ 2606.7 & 0.3780 & 1.40045 & 0.0002627 & 317.19 & 0.083 & 0.0003 \\ 412.6 & 0.3626 & 1.40024 & 0.0002627 & 50.86 & 0.013 & 0.0003 \\ 412.6 & 0.3627 & 1.40023 & 0.0003168 & 50.86 & 0.016 & 0.0003 \\ 412.6 & 0.2065 & 1.40023 & 0.0003168 & 30.51 & 0.010 & 0.0003 \\ 49.7 & 0.4188 & 1.38556 & 0.0003168 & 29.03 & 0.009 & 0.0003 \\ 49.3 & 0.3129 & 1.38556 & 0.0003168 & 22.50 & 0.007 & 0.0003 \\ 74.6 & 0.2946 & 1.33204 & 0.0003168 & 22.50 & 0.007 & 0.0143 \\ 265.4 & 0.3361 & 1.36580 & 0.0003168 & 30.16 & 0.010 & 0.0108 \\ 860.2 & 0.1785 & 1.37572 & 0.0003168 & 30.51 & 0.010 & 0.0107 \\ 860.2 & 0.1785 & 1.37571 & 0.0003168 & 30.51 & 0.010 & 0.0107 \\ 613.4 & 0.2552 & 1.37571 & 0.0003168 & 30.51 & 0.010 & 0.0107 \\ 2606.7 & 0.3780 & 1.40045 & 0.0002627 & 317.19 & 0.083 & 0.0003 \\ 2481.9 & 0.4343 & 1.40044 & 0.0002660 & 337.54 & 0.090 & 0.0003 \\ 2481.9 & 0.4343 & 1.40044 & 0.0002660 & 337.54 & 0.090 & 0.0003 \\ 2006.9 & 0.5870 & 1.40044 & 0.0002660 & 337.54 & 0.090 & 0.0003\end{array}$




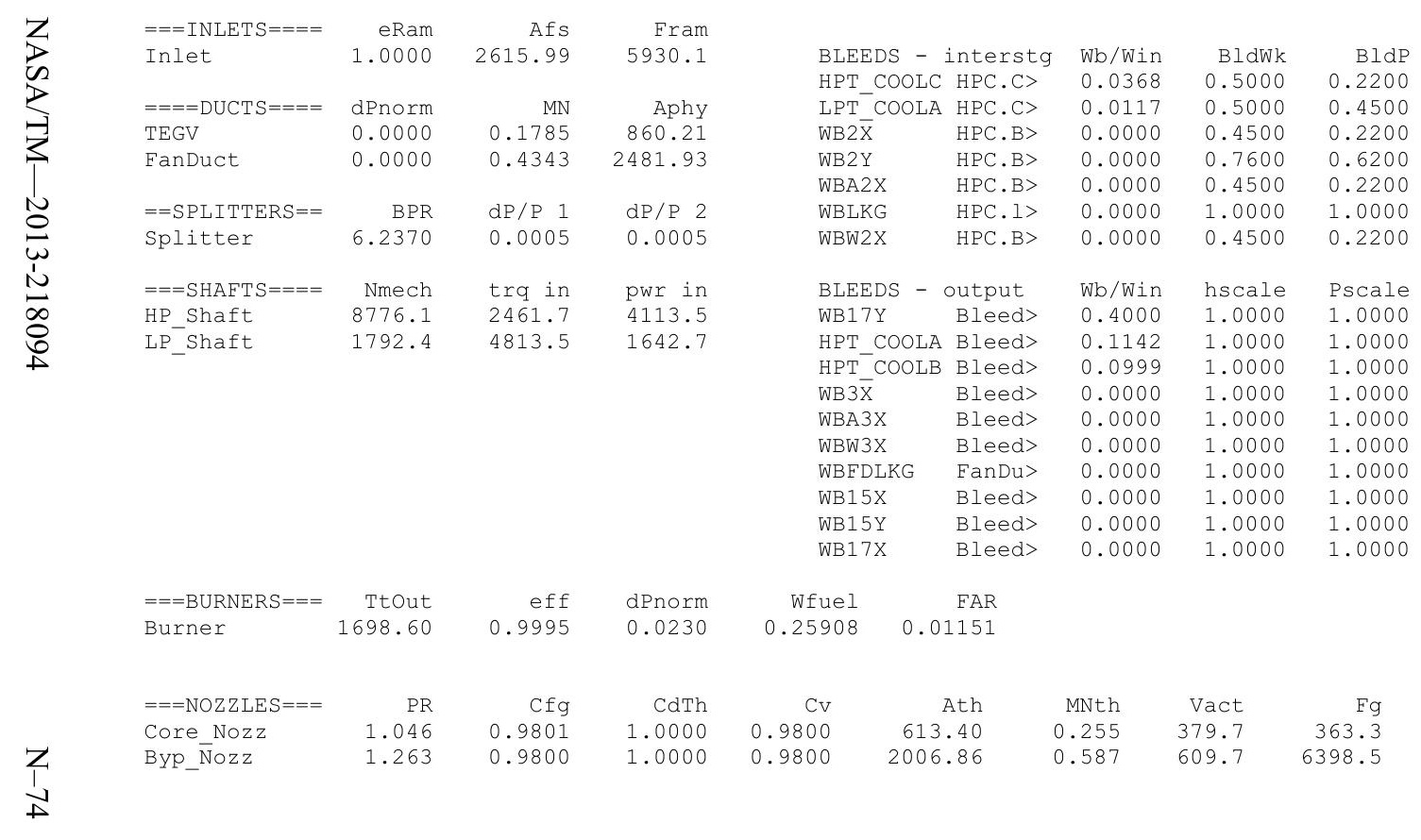


Date:07/05/13 Time:09:28:48 Model: Turbofan Engine - COMDES ON Converge = 1 CASE: 0

Version:NPSS 1.6.5 - Rev: -> Gas Package: Janaf iter/pass/Jacb/Broy= 23/51/2/20 Run by: Philip C Jorgenson PC: 10

Temperature Stator 1 inlet: 500.26 Stator 1 exit: $502.62 \quad$ Stator 2 inlet: 504.93 Stator 2 exit: 505.65 $\begin{array}{llll}\text { Stator } 3 \text { inlet: } 508.35 & \text { Stator } 3 \text { exit: } 508.75 & \text { Stator } 4 \text { inlet: } 511.35 & \text { Stator } 4 \text { exit: } 511.5 \\ \text { Stator } 5 \text { inlet: } 513.11 & \text { Stator } 5 \text { exit: } 513.12 & & \text { Unblocked Percent Blockage: } 0.00\end{array}$

Ambient Relative Humidity $\quad 10.00$

Fan Face Relative Humidity $\quad 6.53$

Fan Bypass Relative Humidity $\quad 5.96$

$\begin{array}{ll}\text { LPC Inlet Relative Humidity } & 5.71 \\ \text { LPC Exit Relative Humidity } & 3.40\end{array}$

HPC Relative Humidity

Drop Diameter

0.0

0.0000500

479.99
8.15

Inlet Length

40.00

Ambient Flow Velocity

Ambient Static Pressure

Additional Water at LPC Exit

/LPC Inlet Static Pressure $\quad 9.17$

481.63 Fan/LPC Inlet Static Temperature 498.31

$\begin{array}{rrrrrr}\text { MN } & \text { alt } & \text { dTamb } & \text { W } & \text { Fn } & \text { TSFC } \\ 0.446 & 15435.0 & 18.00 & 386.68 & 641.2 & 1.3788\end{array}$

$\begin{array}{rr}\text { Wfuel } \\ 64.2-1.3788 & 884.08\end{array}$

FLOW STATION DATA

FSO Ambient.FI_O

FS1 Inlet.Fl_O

FS12 Splitter.F1_02

FS2 Splitter.Fl_0

FS14 Fan.Fl_o

FS24 VaporIN .FI_O

FS25 Bleed2.F1_O

ES3 36 Bleed3.FI-O

FS4 Burner. Fl-

$\begin{array}{ll}\text { FS4 } & \text { Burner.F1 } \\ \text { FS45 } & \text { HPT.FI O }\end{array}$

FS49 LPT.FIO

FS5 TEGV.FI O

FS8 Core_Nozz.FI_O

FS17 FanDúctLkg.Fì

FS171 Bleed15.FI_O

FS172 FanDuct.FI-O

FS173 Byp Nozz.Fì O

$W$
386.68
386.68
336.97
49.71
336.97
49.71
49.71
29.83
28.38
22.00
22.24
29.72
30.07
30.07
30.07
336.97
356.86
356.86
356.86

$\begin{array}{cc}\mathrm{Pt} & \mathrm{Tt} \\ 9.342 & 500.81\end{array}$

$\begin{array}{llll}9.342 & 500.81 & -13.05 & \text { FAR }\end{array}$ $\begin{array}{llll}9.342 & 500.81 & -13.05 & 0.0000 \\ 9.337 & 500.81 & -13.05 & 0.0000\end{array}$ $\begin{array}{llll}9.337 & 500.81 & -13.05 & 0.0000 \\ 9.337 & 500.81 & -13.05 & 0.0000\end{array}$ $\begin{array}{llll}9.337 & 500.81 & -13.05 & 0.0000 \\ 9.813 & 508.79 & -11.14 & 0.0000\end{array}$ $\begin{array}{llll}9.337 & 500.81 & -13.05 & 0.0000 \\ 10.431 & 523.17 & -11.14 & 0.0000\end{array}$ $\begin{array}{llll}10.431 & 523.17 & -7.69 & 0.0000 \\ 10.431 & 523.30 & -8.08 & 0.0000\end{array}$ $\begin{array}{llll}10.431 & 523.30 & -8.08 & 0.0000 \\ 10.431 & 523.30 & -8.08 & 0.0000\end{array}$ $\begin{array}{llll}10.499 & 887.67 & 80.18 & 0.0000\end{array}$ $\begin{array}{llll}49.399 & 887.67 & 80.18 & 0.0000\end{array}$ $\begin{array}{lrrr}48.261 & 1649.87 & 67.68 & 0.0112\end{array}$ $\begin{array}{llll}14.273 & 1130.09 & -17.22 & 0.0083\end{array}$ $\begin{array}{rrrr}14.273 & 1130.09 & -17.22 & 0.0083 \\ 8.396 & 1006.05 & -46.91 & 0.0082\end{array}$ $\begin{array}{llll}8.396 & 1006.13 & -46.91 & 0.0082\end{array}$ $\begin{array}{llll}8.396 & 1006.20 & -46.91 & 0.0082\end{array}$ $\begin{array}{llll}9.813 & 508.79 & -11.14 & 0.0000\end{array}$ $\begin{array}{llll}9.813 & 509.60 & -10.97 & 0.0000\end{array}$ $\begin{array}{llll}9.813 & 509.60 & -10.97 & 0.0000\end{array}$ 9.813

509.60

$-10.97 \quad 0.0000$
BPR
6.7788

VTAS
479.99
OPR
5.288 0.902 PowerSet
10.000 $\begin{array}{ccc}\text { T4 } & \text { T41 } & \text { T49 } \\ 1649.9 & 1554.0 & 1125.4\end{array}$
TURBOMACHINERY PERFORMANCE DATA

\begin{tabular}{|c|c|c|c|c|c|c|c|c|c|}
\hline & WC & PR & eff & NC & $\mathrm{TR}$ & efpoly & pwr & SMN & SMW \\
\hline Fan & 521.15 & 1.051 & 0.8982 & 1610.128 & 1.0159 & 0.8989 & -912.4 & -1526.11 & 44.72 \\
\hline LPC & 76.88 & 1.117 & 0.7204 & 1610.128 & 1.0447 & 0.7248 & -377.3 & 8.26 & 1.96 \\
\hline PC & 42.21 & 4.736 & 0.7955 & 8540.487 & 1.6963 & 0.8340 & -3634.6 & 68.26 & 63.03 \\
\hline HPT & 12.08 & 3.381 & 0.8666 & 211.198 & 1.3031 & 0.8466 & 3634.5 & & \\
\hline LPT & 45.18 & 1.700 & 0.8004 & 47.065 & 1.1197 & 0.7881 & 1289.8 & & \\
\hline JRE & NERY MAE & DATA & & & & & & & \\
\hline & WcMap & PRmap & effMap & NcMap & R/Parm & s_WcDes & S_PRdes & S_effDes & S_NcDes \\
\hline $\operatorname{Tan}$ & 605.53 & 1.050 & 0.9000 & 1594.776 & 0.0442 & $\overline{0} .8607$ & $\overline{1} .0235$ & 0.9980 & 0.9905 \\
\hline PC & 39.70 & 1.184 & 0.7480 & 0.425 & 0.0000 & 1.9 & 0.6356 & 0.9631 & 0.0003 \\
\hline PC & 38.39 & 4.560 & 0.8038 & 8312.803 & 10.7600 & 1.0995 & 1.0494 & 0.9898 & 0.9733 \\
\hline PT & 0.96 & 3.315 & & 1.269 & 3.3153 & 12.6299 & 0.9723 & 1.0000 & 0.0003 \\
\hline LPT & 0.69 & 1.527 & 0.8063 & 0.529 & 1.5265 & 65.2590 & 0.7521 & 0.9926 & 0.0005 \\
\hline
\end{tabular}




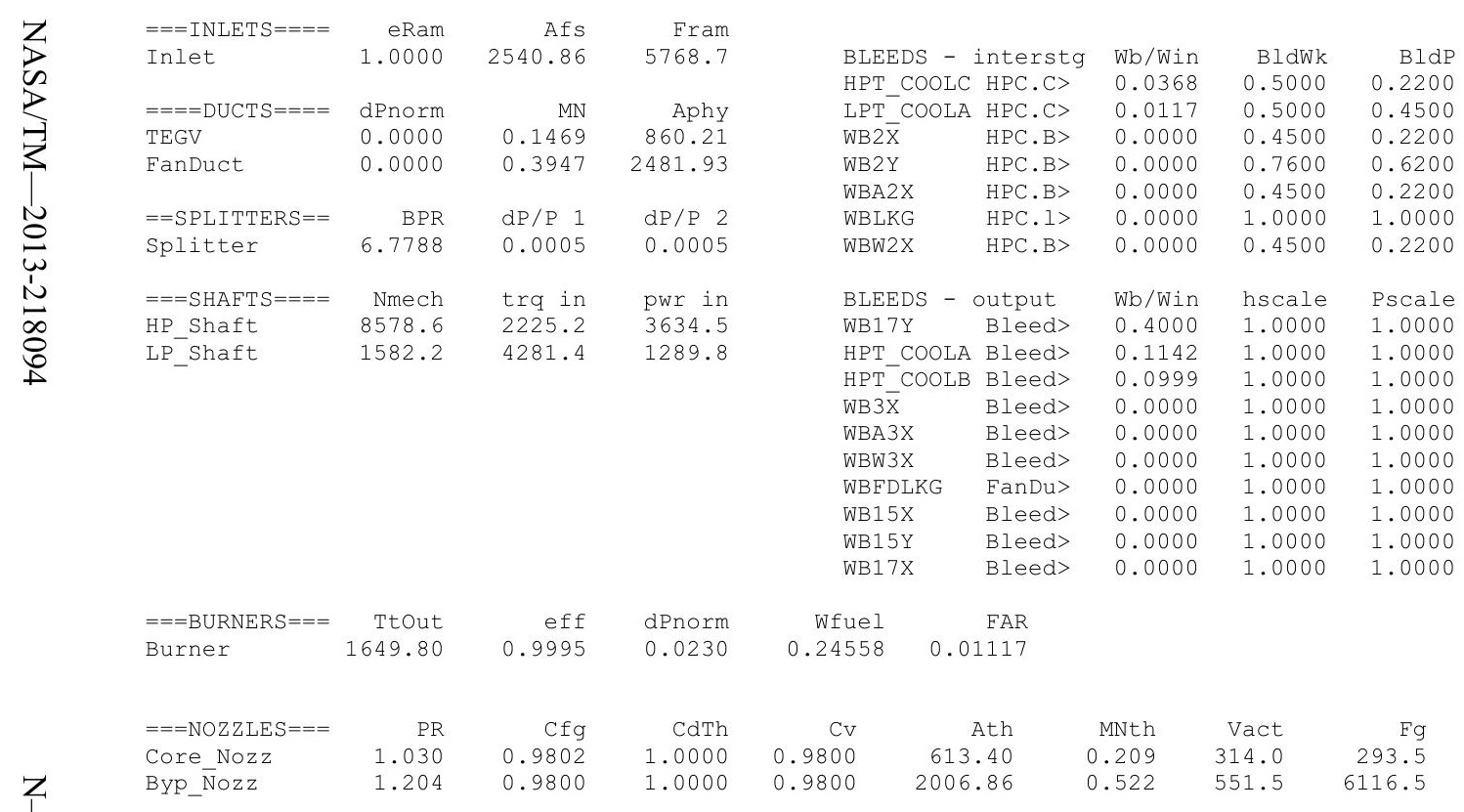




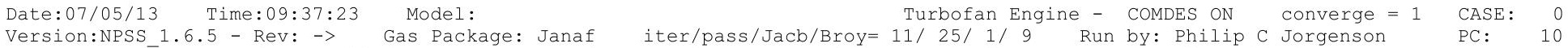

Version:NPSS 1.6.5 - Rev: -> Gas Package: Janaf iter/pass/Jacb/Broy=11/ $25 / 1 / 9$ Run by: Philip C Jorge
Temperature Stator 1 inlet: $483.67 \quad$ Stator 1 exit: 491.43 Stator 2 inlet: 501.20 Stator 2 exit: 506.18

Stator 3 inlet: 516.28 Stator 3 exit: 520.81 Stator 4 inlet: 530.25 stator 4 exit: 534.12

Ambient Relative Humidity $\quad 10.00$

Fan Bypass Relative Humidity 1.97

Fan Bypass Relative Humidity 1.41

LPC Exit Relative Humidity $\quad 0.13$

0.0000500

7.000500

Ambient Flow Velocity

Ambient Static Pressure

Ambient Static Temperature $\quad 425.97 \quad$ Fan/LPC Inlet Static Temperature 474.82

789.49

40.00

SUMMARY OUTPUT DATA

\begin{tabular}{|c|c|c|c|c|c|c|c|c|c|c|c|c|}
\hline $\begin{array}{r}\mathrm{MN} \\
0.780\end{array}$ & $\begin{array}{r}\text { alt } \\
39000.0\end{array}$ & $\begin{array}{l}\text { dTamb } \\
36.00\end{array}$ & $\begin{array}{r}\text { W } \\
252.16\end{array}$ & $\begin{array}{r}\mathrm{Fn} \\
617.7\end{array}$ & $\begin{array}{r}\text { TSFC } \\
1.1451\end{array}$ & $\begin{array}{r}\text { Wfuel } \\
707.33\end{array}$ & $\begin{array}{r}\text { BPR } \\
8.5627\end{array}$ & $\begin{array}{r}\text { VTAS } \\
789.49\end{array}$ & $\begin{array}{r}\text { OPR } \\
9.518\end{array}$ & $\begin{array}{c}\text { EPR } \\
0.736\end{array}$ & $\begin{array}{l}\text { Powerset } \\
10.000\end{array}$ & $\begin{array}{c}T 4 \\
1759.5\end{array}$ \\
\hline
\end{tabular}

$\begin{array}{ll}\text { FS0 } & \text { Ambient.F1_O } \\ \text { FS1 } & \text { Inlet.F1_O } \\ \text { FS12 } & \text { Splitter.F1_02 } \\ \text { FS2 } & \text { Splitter.F1_01 } \\ \text { FS14 } & \text { Fan.F1_O } \\ \text { FS23 } & \text { LPC.Fl_O } \\ \text { FS24 } & \text { VaporIN.F1_O } \\ \text { FS25 } & \text { Bleed2.F1_O } \\ \text { FS3 } & \text { HPC.F1_O } \\ \text { FS36 } & \text { Bleed3.F1_O } \\ \text { FS4 } & \text { Burner.F1_O } \\ \text { FS45 } & \text { HPT.F1_O } \\ \text { FS49 } & \text { LPT.F1_O } \\ \text { FS5 } & \text { TEGV.FI_O } \\ \text { FS8 } & \text { Core_Nozz.F1_O } \\ \text { FS17 } & \text { FanDuctLkg.FI_O } \\ \text { FS171 } & \text { Bleed15.F1_O } \\ \text { FS172 } & \text { FanDuct.Fl_O } \\ \text { FS173 } & \text { Byp_Nozz.F1_O }\end{array}$

$W$
252.16
252.16
225.79
26.37
225.79
26.37
26.37
23.73
22.58
17.50
17.70
23.65
23.93
23.93
23.93
225.79
228.43
228.43
228.43

DATA

FLOW STATION DATA

$\begin{array}{rrrrrr}\text { Pt } & \text { Tt } & \text { ht } & \text { FAR } & \text { Wc } & \text { Ps } \\ 4.267 & 477.92 & -16.44 & 0.0000 & 833.71 & 2.854 \\ 4.267 & 477.92 & -16.44 & 0.0000 & 833.71 & 3.892 \\ 4.265 & 477.92 & -16.44 & 0.0000 & 746.90 & 3.839 \\ 4.265 & 477.92 & -16.44 & 0.0000 & 87.23 & 4.169 \\ 4.817 & 496.63 & -11.96 & 0.0000 & 674.07 & 4.042 \\ 6.654 & 555.04 & 2.06 & 0.0000 & 60.24 & 6.358 \\ 6.654 & 555.19 & 1.60 & 0.0000 & 60.26 & 6.358 \\ 6.654 & 555.19 & 1.60 & 0.0000 & 54.23 & 6.416 \\ 40.611 & 1006.37 & 111.52 & 0.0000 & 11.38 & 35.727 \\ 40.611 & 1006.37 & 111.52 & 0.0000 & 8.82 & 37.809 \\ 39.676 & 1759.47 & 98.62 & 0.0112 & 12.07 & 37.468 \\ 9.522 & 1162.57 & -7.79 & 0.0084 & 54.65 & 8.626 \\ 3.261 & 910.27 & -69.74 & 0.0083 & 142.85 & 3.070 \\ 3.261 & 910.34 & -69.74 & 0.0083 & 142.86 & 3.070 \\ 3.261 & 910.42 & -69.74 & 0.0083 & 142.86 & 2.854 \\ 4.817 & 496.63 & -11.96 & 0.0000 & 674.07 & 4.042 \\ 4.817 & 497.31 & -11.80 & 0.0000 & 682.41 & 3.908 \\ 4.817 & 497.31 & -11.80 & 0.0000 & 682.41 & 3.908 \\ 4.817 & 497.31 & -11.80 & 0.0000 & 682.41 & 2.854\end{array}$

Ts
425.97
465.50
463.77
474.82
472.33
547.86
548.00
549.44
971.40
986.71
1734.63
1132.22
895.22
895.30
877.48
472.33
468.45
468.45
428.14

$\begin{array}{rr}\text { Aphy } & \text { MN } \\ 2543.5 & 0.7800 \\ 4168.2 & 0.3649 \\ 3531.8 & 0.3902 \\ 830.5 & 0.1806 \\ 2606.7 & 0.5067 \\ 412.6 & 0.2561 \\ 412.6 & 0.2561 \\ 412.6 & 0.2287 \\ 49.7 & 0.4343 \\ 49.3 & 0.3233 \\ 74.6 & 0.2944 \\ 265.4 & 0.3829 \\ 860.2 & 0.2970 \\ 860.2 & 0.2971 \\ 613.4 & 0.4435 \\ 2606.7 & 0.5067 \\ 2481.9 & 0.5546 \\ 2481.9 & 0.5546 \\ 2006.9 & 0.8978\end{array}$

gamt
1.40068
1.40068
1.40068
1.40068
1.40049
1.39968
1.39966
1.39966
1.38031
1.38031
1.32945
1.36454
1.38052
1.38052
1.38051
1.40049
1.40048
1.40048
1.40048

WAR 0.0000851 0.0000851 0.0000851 0.0000851 0.0000851 0.0001710 0.0001710 0.0001710 0.0001710 0.0001710 0.0001710 0.0001710 0.0001710 0.0001710 0.0000851 0.0000861 0.0000861 0.0000861

TURBOMACHINERY PERFORMANCE DATA

\begin{tabular}{|c|c|c|c|c|c|c|c|c|c|}
\hline & WC & PR & eff & $\mathrm{NC}$ & $T R$ & efPoly & pwr & SMN & SMW \\
\hline Fan & 746.90 & 1.130 & 0.9055 & 2568.150 & 1.0391 & 0.9071 & -1432.7 & 1610.34 & 54.83 \\
\hline LPC & 87.23 & 1.560 & 0.8405 & 2568.150 & 1.1614 & 0.8502 & -690.1 & 6.97 & 3.73 \\
\hline HPC & 54.23 & 6.103 & 0.8187 & 8965.332 & 1.8127 & 0.8573 & -3601.9 & 61.35 & 58.03 \\
\hline HPT & 12.07 & 4.167 & 0.8772 & 221.131 & 1.3648 & 0.8551 & 3601.9 & & \\
\hline LPT & 54.65 & 2.920 & 0.8556 & 72.301 & 1.2754 & 0.8360 & 2122.9 & & \\
\hline JRE & NERY M & DATA & & & & & & & \\
\hline & WcMap & PRmap & effMap & NcMap & R/Parm & S_WcDes & S_PRdes & $S_{\text {S_effDes }}$ & S_NCDes \\
\hline an & 867.82 & 1.127 & 0.9073 & 2543.663 & 0.0414 & $\overline{0} .8607$ & $\overline{1} .0235$ & 0.9980 & $\overline{0} .9905$ \\
\hline PC & 73.90 & 1.522 & 0.7799 & 0.678 & 0.0000 & 1.1803 & 1.0736 & 1.0777 & 0.0003 \\
\hline HPC & 49.32 & 5.862 & 0.8271 & 8726.322 & 10.9744 & 1.0995 & 1.0494 & 0.9898 & 0.9733 \\
\hline $\mathrm{PT}$ & 0.96 & 4.079 & 0.8772 & 1.329 & 4.0790 & 12.6299 & 0.9723 & 1.0000 & 0.0003 \\
\hline $\mathrm{PT}$ & 0.84 & 2.444 & 0.8620 & 0.813 & 2.4438 & 65.2590 & 0.7521 & 0.9926 & 0.0005 \\
\hline
\end{tabular}




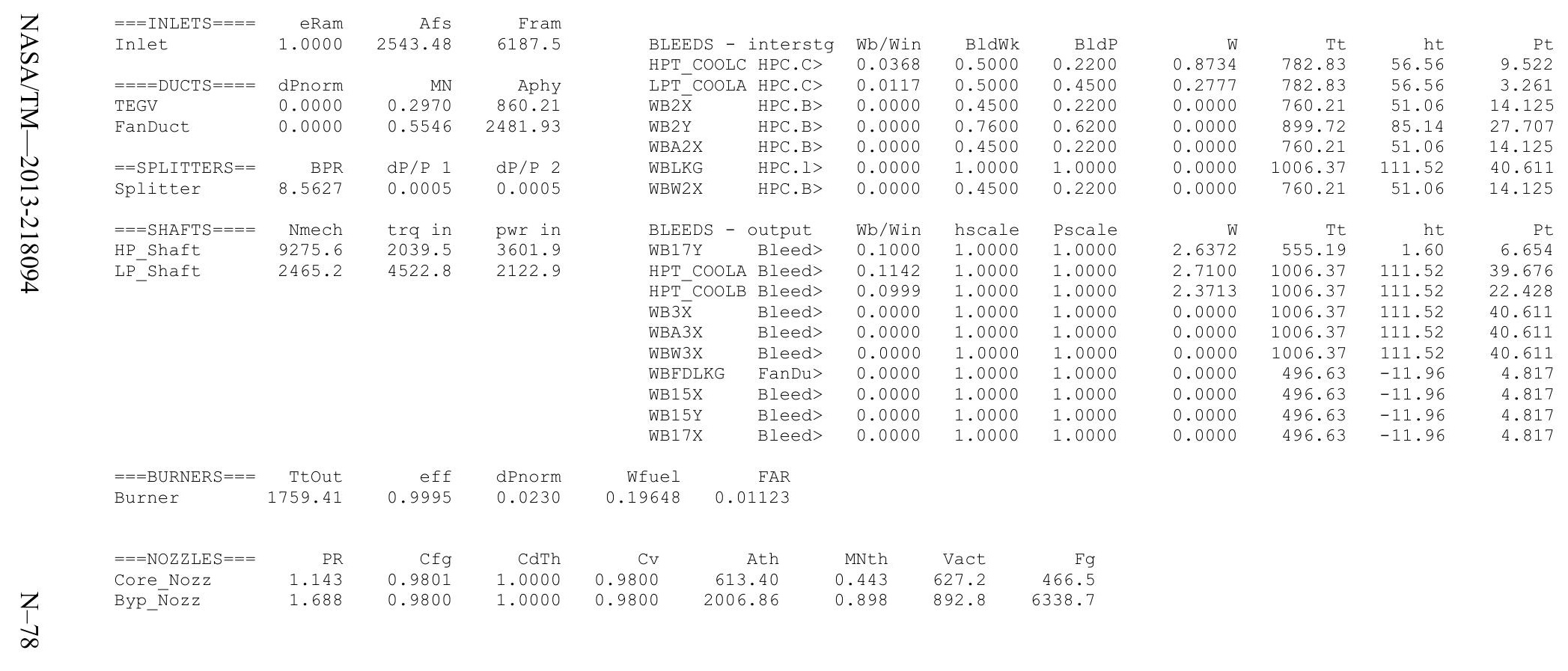


列

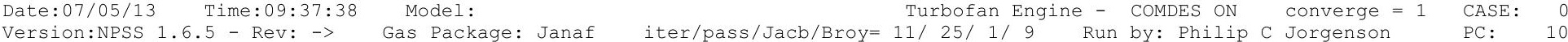

Temperature Stator 1 inlet: $476.90 \quad$ Stator 1 exit: $484.18 \quad$ Stator 2 inlet: 493.31 Stator 2 exit: 497.97 Stator 3 inlet: 507.40 Stator 3 exit: 511.62 Stator 4 inlet: 520.44 Stator 4 exit: 524.02 Stator 5 inlet: 531.26 Stator 5 exit: $534.37 \quad$ Unblocked Percent Blockage: 0.00

Ambient Relative Humidity $\quad 10.00$

Fan Face Relative Humidity $\quad 2.46$

LPC Bypass Relative Humid

LPC Exit Relative Humidity

HPC Relative Humidity

Drop Diameter

0.00

Ambient Flow Velocity

Ambient Static Pressure

Additional Water at LPC Exit

$\begin{array}{clr}0.0000500 & \text { Inlet Length } & 40.00 \\ 738.88 & \text { Fan/LPC Inlet Flow Velocity } & 186.02 \\ 2.95 & \text { Fan/LPC Inlet Static Pressure } & 4.11 \\ 425.97 & \text { Fan/LPC Inlet Static Temperature } & 468.59 \\ 0.0000765 & & \end{array}$

$\begin{array}{rrrrrr}\text { MN } & \text { alt } & \text { dTamb } & & \text { SUMMARY } & \text { OUTPUT DATA } \\ 0.730 & 38334.0 & 36.00 & 245.32 & 607.1 & 1.1099\end{array}$

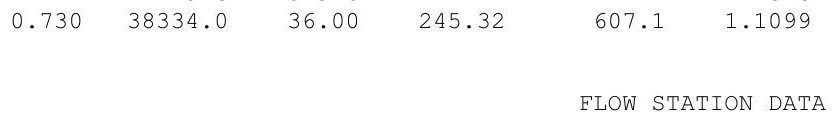

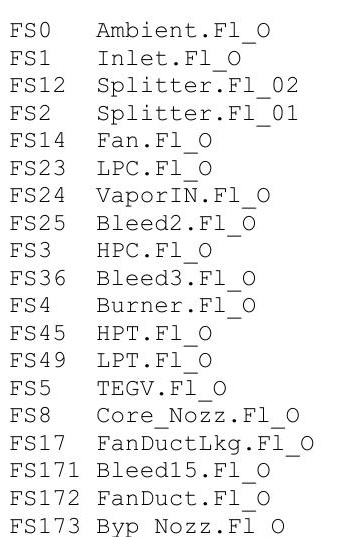

$W$
245.32
245.32
219.92
25.40
219.92
25.40
25.40
22.86
21.75
16.86
17.04
22.78
23.05
23.05
23.05
219.92
222.46
222.46
222.46

$\begin{array}{ccc}\mathrm{Pt} & \mathrm{Tt} \\ 4.201 & 471.48 & -17 \\ 4.201 & 471.48 & -17\end{array}$

VTAS

$738.88 \quad 9.232$ $\begin{array}{clccc}\text { EPR } & \text { PowerSet } & \text { T4 } & \text { T41 } & \text { T49 } \\ 0.764 & 10.000 & 1731.9 & 1637.7 & 1141.6\end{array}$

TURBOMACHINERY PERFORMANCE DATA

\begin{tabular}{|c|c|c|c|c|c|c|c|c|c|}
\hline & WC & PR & eff & $\mathrm{NC}$ & TR & efPoly & pwr & SMN & SMW \\
\hline Fan & 733.80 & 1.122 & 0.9047 & 2504.280 & 1.0370 & 0.9062 & -1300.4 & 2190.73 & 54.69 \\
\hline LPC & 84.74 & 1.528 & 0.8409 & 2504.280 & 1.1531 & 0.8501 & -622.2 & 6.78 & 3.35 \\
\hline HPC & 53.62 & 6.046 & 0.8178 & 8945.742 & 1.8095 & 0.8565 & -3379.7 & 61.28 & 57.90 \\
\hline HPT & 12.08 & 4.114 & 0.8759 & 220.105 & 1.3615 & 0.8539 & 3379.7 & & \\
\hline LPT & 54.02 & 2.781 & 0.8500 & 70.537 & 1.2600 & 0.8307 & 1922.6 & & \\
\hline \multicolumn{10}{|c|}{ TURBOMACHINERY MAP DATA } \\
\hline & WcMap & PRmap & effMap & NcMap & R/Parm & s WcDes & s PRdes & $s$ effDes & S NCDes \\
\hline Fan & 852.60 & 1.119 & 0.9065 & 2480.402 & 0.0413 & $\overline{0} .8607$ & $\overline{1} .0235$ & 0.9980 & 0.9905 \\
\hline PC & 71.14 & 1.493 & 0 . & & 0.0000 & 1. & 1.0692 & 1.0824 & 0.0 \\
\hline HPC & 48.77 & 5.809 & 0.8263 & 8707.255 & 10.9862 & 1.0 & 1.0494 & 0.9898 & 0.9 \\
\hline HPT & 0.96 & 4.0 & 0.8 & 1.323 & 4.0281 & 12.6299 & 0.9723 & 1.0000 & 0.0003 \\
\hline $\mathrm{PT}$ & 0.83 & 2.340 & 0.8563 & 0.793 & 2.3398 & 65.2590 & 0.7521 & 0.9926 & 0.0005 \\
\hline
\end{tabular}




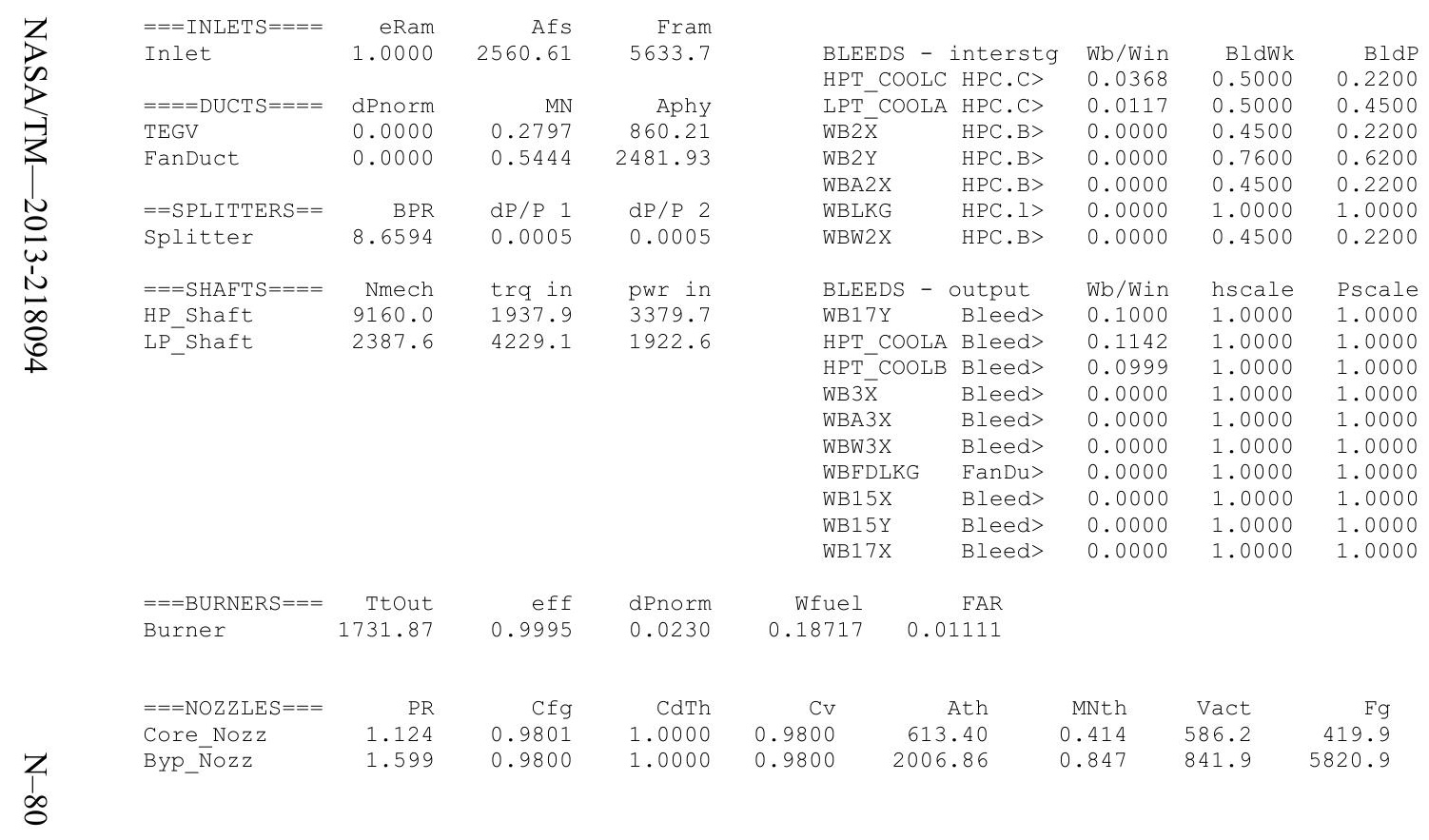


Date:07/05/13 Time:09:37:54 Model: Turbofan Engine - COMDES ON COnverge $=1$ CASE: O

Temperature Stator 1 inlet: 474.82 Stator 1 exit: $481.90 \quad$ Stator 2 inlet: 490.80 Sun by: Philip C Jorg Stator 3 inlet: 504.51 Stator 3 exit: 508.61 Stator 4 inlet: 517.19 stator 4 exit: 520.67 Stator 5 inlet: 527.71 Stator 5 exit: 530.72 Unblocked Percent Blockage: 0.00

Ambient Relative Humidity $\quad 10.00$

Fan Face Relative Humidity 2.63

Fan Bypass Relative Humidity $\quad 1.95$

LPC Inlet Relative Humidity $\quad 1.76$

LPC Exit Relative Humidity

HPC Relative Humidity

Drop Diameter

0.00

0.0000500

Ambient Flow Velocity

722.68

Inlet Length

Ambient static Pressure

Additional Water at LPC Exit

SUMMARY OUTPUT DATA

$$
0.714 .37357 .02 .00
$$

\section{FLOW STATION DATA}

FSO Ambient.FI_O

FS1 Inlet.Fl_O

Splitter.FI_01

25.95

225.90

FS14 Fan.F1_O

FS24 VaporIN.FI

FS25 Bleed2.F1_O

FS3 HPC.FI O

FS36 Bleed3.F1_O

FS4 Burner.Fl_O

FS49 HPT.FI-

FS5 TEGV.FI

FS8 Core Nozz.Fl O

FS17 FanDüctLkg.F̄_o

FS171 Bleed15.FI_O

FS172 FanDuct.Fl_O
FS173 Byp_Nozz.FI_O

$\begin{array}{rrrrr}\text { Pt } & \text { Tt } & \text { ht } & \text { FAR } & \text { WC } \\ 4.339 & 469.50 & -18.42 & 0.0000 & 811.53\end{array}$

$\begin{array}{llllll}225.90 & 4.337 & 469.50 & -18.42 & 0.0000 & 811.53\end{array}$

25.95

25.95

22.22

17.22

17.41
23.27

23.55

23.55
23.55

23.55
225.90

228.50

TURBOMACHINERY PERFORMANCE DATA

$\begin{array}{lrrrrrrrrr} & \text { Wc } & \text { PR } & \text { eff } & \text { NC } & \text { TR } & \text { efPoly } & \text { pwr } & \text { SMN } & \text { SMW } \\ \text { Fan } & 728.28 & 1.119 & 0.9043 & 2477.503 & 1.0361 & 0.9058 & -1298.0 & 2584.42 & 54.62 \\ \text { LPC } & 83.66 & 1.514 & 0.8408 & 2477.503 & 1.1498 & 0.8498 & -619.3 & 6.69 & 3.27 \\ \text { HPC } & 53.32 & 6.014 & 0.8173 & 8935.972 & 1.8073 & 0.8561 & -3418.2 & 61.37 & 57.96 \\ \text { HPT } & 12.08 & 4.095 & 0.8753 & 219.778 & 1.3603 & 0.8533 & 3418.2 & & \\ \text { LPT } & 53.79 & 2.730 & 0.8478 & 69.837 & 1.2542 & 0.8287 & 1917.3 & & \end{array}$

TURBOMACHINERY MAP DATA

$\begin{array}{lrrrrrrrrr} & \text { WcMap } & \text { PRmap } & \text { effMap } & \text { NCMap } & \text { R/Parm } & \text { S_WCDes } & \text { S_PRdes } & \text { S_effDes } & \text { S_NcDes } \\ \text { Fan } & 846.19 & 1.116 & 0.9061 & 2453.880 & 0.0413 & 0.8607 & 1.0235 & 0.9980 & 0.9905 \\ \text { LPC } & 69.98 & 1.481 & 0.7756 & 0.654 & 0.0000 & 1.1954 & 1.0682 & 1.0840 & 0.0003 \\ \text { HPC } & 48.50 & 5.778 & 0.8258 & 8697.745 & 10.9845 & 1.0995 & 1.0494 & 0.9898 & 0.9733 \\ \text { HPT } & 0.96 & 4.010 & 0.8753 & 1.321 & 4.0096 & 12.6299 & 0.9723 & 1.0000 & 0.0003 \\ \text { LPT } & 0.82 & 2.302 & 0.8541 & 0.786 & 2.3015 & 65.2590 & 0.7521 & 0.9926 & 0.0005\end{array}$


$\begin{array}{lrrr}===\text { INLETS }==== & \text { eRam } & \text { Afs } & \text { Fram } \\ \text { Inlet } & 1.0000 & 2564.46 & 5657.0\end{array}$

$====$ DUCT $S====$
TEGV

FanDuct

0.0000

0.0000

$==$ SPLITTERS $==$

$\mathrm{BPR}$

Splitter

8.7056

$===$ SHAFTS $==$

LP_Shaft

Nmech
9117.6

2357.2

4272 .

272.0

418.2

1917.3

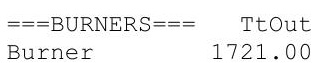

$e f f$
0.9995

dPnorm

PR
1.117
1.571

$===$ NOZZLES $===$

Core Nozz

Cfg
0.9801

0.9801

CdTh

1.0000

$C V$
0.9800

800

(n)

$\begin{array}{rrr}\text { Ath } & \text { MNth } & \text { Vact } \\ 613.40 & 0.404 & 571.6 \\ 2006.86 & 0.830 & 825.0\end{array}$

$\mathrm{Fg}$
8.3

\begin{tabular}{|c|c|c|c|}
\hline W & Tt & ht & Pt \\
\hline 8595 & 759.75 & 51.08 & 9.422 \\
\hline 0.2733 & 759.75 & 51.08 & 3.451 \\
\hline 0.0000 & 737.90 & 45.78 & 13.812 \\
\hline 0.0000 & 872.73 & 78.64 & 26.985 \\
\hline 0.0000 & 737.90 & 45.78 & 13.812 \\
\hline 0.0000 & 975.91 & 104.09 & 39.498 \\
\hline 0.0000 & 737.90 & 45.78 & 13.812 \\
\hline & $\mathrm{Tt}$ & ht & \\
\hline 2.5951 & 539.97 & -1.93 & 6.568 \\
\hline 2.6668 & 975.91 & 104.09 & 38.589 \\
\hline 2.3335 & 975.91 & 104.09 & 21.906 \\
\hline 0.0000 & 975.91 & 104.09 & 39.498 \\
\hline 0.0000 & 975.91 & 104.09 & 39.498 \\
\hline 0.0000 & 975.91 & 104.09 & 39.498 \\
\hline 0.0000 & 486.45 & -14.36 & 4.852 \\
\hline 0.0000 & 486.45 & -14.36 & 4.852 \\
\hline 0.0000 & 486.45 & -14.36 & 4.852 \\
\hline 0.0000 & 486.45 & -14.36 & 4.852 \\
\hline
\end{tabular}

4.852 
Date:07/05/13 Time:09:38:09 Model: Turbofan Engine - COMDES ON Converge $=1$ CASE: 0

Temperature Stator 1 inlet: 476.22 Stator 1 exit: 482.86 Stator 2 inlet: 491.16 Stator 2 exit: 495.38 $\begin{array}{llll}\text { Stator } 3 \text { inlet: } 503.94 & \text { Stator } 3 \text { exit: } 507.74 & \text { Stator } 2 \text { inlet: } 491.16 & \text { Stator } 2 \text { exit: } 495.38 \\ \text { Stator } 5 \text { inlet: } 525.50 & \text { Stator } 5 \text { exit: } 528.27 & \text { inlet: } 515.75 & \text { Stator 4 exit: } 518.95 \\ \text { Stablocked Percent Blockage: } 0.00\end{array}$

Ambient Relative Humidity $\quad 10.00$

an Face Relative Humidity $\quad 3.24$

Tive Humidity 2.22

LPC Exit Relative Humidity

HPC Relative Humidity

0.28

Drop Diameter

0.0000500

mbient Flow velocity

ambient Static Pressure

Additional Water at LPC Exit

$\begin{array}{rr} & 40.00 \\ & \end{array}$

3.58 Fan/LPC Inlet Static Pressure $\quad 4.74$

432.42 Fan/LPC Inlet Static Temperature 468.61

\section{SUMMARY OUTPUT DATA}

$\begin{array}{rrr}\text { MN } & \text { alt } & \text { dTamb } \\ 0.669 & 34281.0 & 36.00\end{array}$

W
272.66

$\begin{array}{rr}\text { Fn } & \text { TSFC } \\ 667.0 & 1.0952\end{array}$

Wfuel

730.45
BPR

8.8130
VTAS

682.24
OPR
8.726
PowerSet 10.000
T4
1710.
49

FLOW STATION DATA

$\begin{array}{lrrrr}\text { FLOW } & \text { STATION DATA } & & \\ \text { Pt } & \text { Tt } & \text { ht } & \text { FAR } & \text { Wc } \\ 4.831 & 471.21 & -18.12 & 0.0000 & 790.57 \\ 4.831 & 471.21 & -18.12 & 0.000 & 790.57 \\ 4.829 & 471.21 & -18.12 & 0.0000 & 710.36 \\ 4.829 & 471.21 & -18.12 & 0.0000 & 80.60 \\ 5.360 & 487.02 & -14.33 & 0.0000 & 650.62 \\ 7.120 & 536.98 & -2.35 & 0.0000 & 58.35 \\ 7.120 & 537.10 & -2.71 & 0.0000 & 58.36 \\ 7.120 & 537.10 & -2.71 & 0.0000 & 52.53 \\ 42.157 & 966.99 & 101.78 & 0.0000 & 11.33 \\ 42.157 & 966.99 & 101.78 & 0.0000 & 8.78 \\ 41.186 & 1710.41 & 89.23 & 0.0110 & 12.08 \\ 10.173 & 1134.37 & -11.86 & 0.0082 & 53.24 \\ 3.939 & 915.40 & -65.33 & 0.0081 & 124.94 \\ 3.939 & 915.48 & -65.33 & 0.0081 & 124.95 \\ 3.939 & 915.55 & -65.33 & 0.0081 & 124.95 \\ 5.360 & 487.02 & -14.33 & 0.0000 & 650.62 \\ 5.360 & 487.58 & -14.20 & 0.0000 & 658.38 \\ 5.360 & 487.58 & -14.20 & 0.0000 & 658.38 \\ 5.360 & 487.58 & -14.20 & 0.0000 & 658.38\end{array}$

$\begin{array}{rr}\text { PS } & \text { Ts } \\ 3.578 & 432.42 \\ 4.453 & 460.37 \\ 4.399 & 458.81 \\ 4.736 & 468.61 \\ 4.570 & 465.32 \\ 6.823 & 530.49 \\ 6.823 & 530.61 \\ 6.882 & 531.91 \\ 37.149 & 933.67 \\ 39.280 & 948.24 \\ 38.892 & 1686.13 \\ 9.270 & 1106.40 \\ 3.766 & 904.09 \\ 3.766 & 904.16 \\ 3.578 & 891.56 \\ 4.570 & 465.32 \\ 4.440 & 462.01 \\ 4.440 & 462.01 \\ 3.578 & 434.35\end{array}$

3.578

$\begin{array}{rr}\text { Aphy } & \text { MN } \\ 2577.0 & 0.6690 \\ 4168.2 & 0.3429 \\ 3531.8 & 0.3674 \\ 830.5 & 0.1664 \\ 2606.7 & 0.4824 \\ 412.6 & 0.2474 \\ 412.6 & 0.2474 \\ 412.6 & 0.2210 \\ 49.7 & 0.4312 \\ 49.3 & 0.3212 \\ 74.6 & 0.2943 \\ 265.4 & 0.3708 \\ 860.2 & 0.2564 \\ 860.2 & 0.2564 \\ 613.4 & 0.3757 \\ 2606.7 & 0.4824 \\ 2481.9 & 0.5256 \\ 2481.9 & 0.5256 \\ 2006.9 & 0.7820\end{array}$

gamt
1.40074
1.40074
1.40074
1.40074
1.40059
1.39996
1.39995
1.39995
1.38270
1.38270
1.33186
1.36647
1.38033
1.38032
1.38032
1.40059
1.40059
1.40059
1.40059

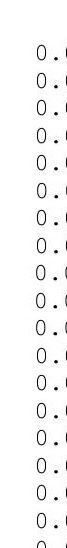

TURBOMACHINERY PERFORMANCE DATA

\begin{tabular}{|c|c|c|c|c|c|c|c|c|c|}
\hline & WC & $\mathrm{PR}$ & eff & $\mathrm{NC}$ & TR & efPoly & pwr & SMN & SMW \\
\hline Fan & 710.36 & 1.110 & 0.9031 & 2395.161 & 1.0335 & 0.9046 & -1312.8 & 5841.49 & 54.21 \\
\hline LPC & 80.60 & 1.475 & 0.8415 & 2395.161 & 1.1396 & 0.8499 & -620.0 & 6.40 & 3.05 \\
\hline HPC & 52.53 & 5.921 & 0.8158 & 8909.698 & 1.8004 & 0.8545 & -3607.5 & 61.79 & 58.29 \\
\hline HPT & 12.08 & 4.049 & 0.8746 & 219.228 & 1.3570 & 0.8526 & 3607.5 & & \\
\hline LPT & 53.24 & 2.582 & 0.8427 & 67.783 & 1.2370 & 0.8241 & 1932.8 & & \\
\hline$K E$ & NERY & DATA & & & & & & & \\
\hline & WcMap & PRmap & effmap & NcMap & R/Parm & S_WcDes & S_PRdes & S_effDes & S_NCDes \\
\hline Pan & 825.37 & 1.107 & 0.9050 & 2372.323 & 0.0413 & $\overline{0} .8607$ & $\overline{1} .0235$ & 0.9980 & $\overline{0} .9905$ \\
\hline PC & 66 & 1.4 & 0.7718 & & 0 & 1.2 & 1.0672 & 1.0903 & 0.0003 \\
\hline HPC & 47.7 & 5.689 & 0.8242 & 672.172 & 10. & 1.0 & 1.0494 & 0.9 & 0.97 \\
\hline $\mathrm{APT}$ & & & 0.8 & 1.3 & & 12.6 & 0.9723 & 1.0000 & 0.0 \\
\hline LPT & 0.82 & 2.190 & 0.8489 & 0.762 & 2.1901 & 65.2590 & 0.7521 & 0.9926 & 0.0005 \\
\hline
\end{tabular}




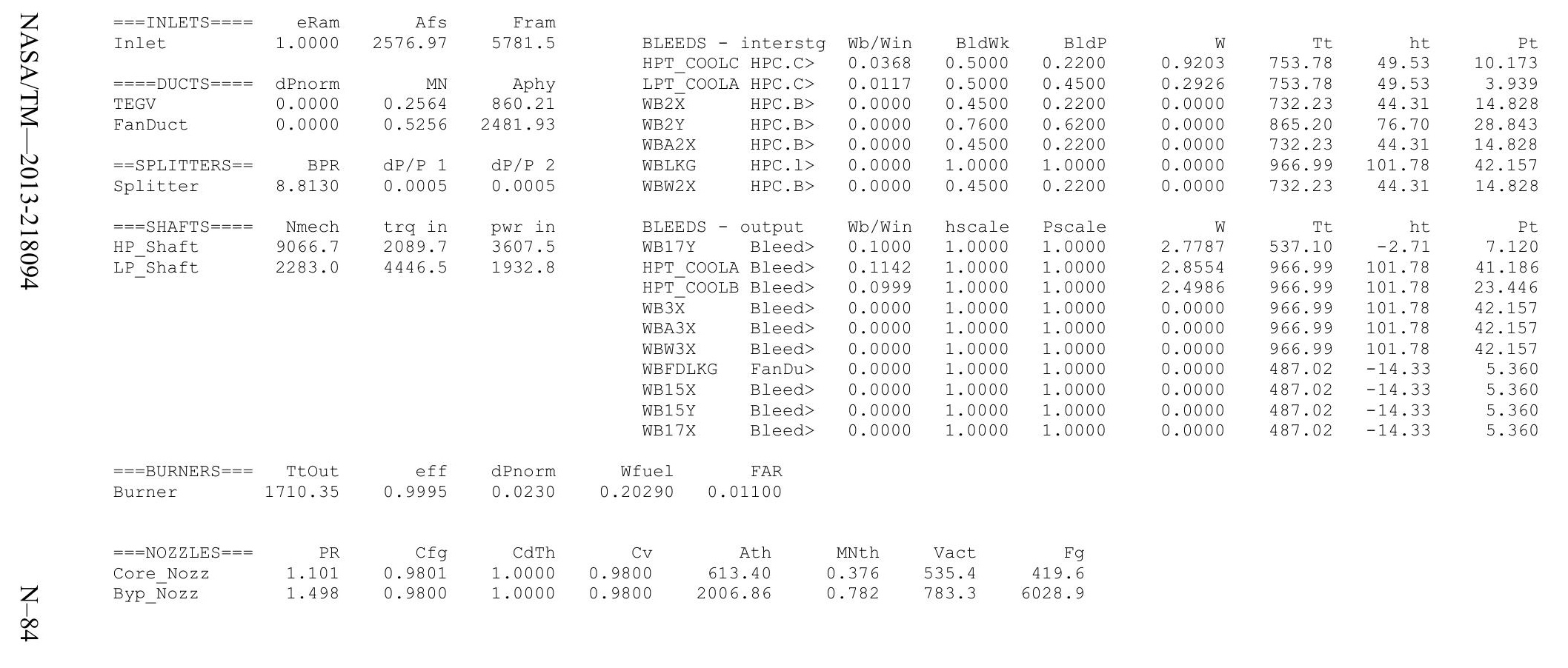


Turbofan Engine - COMDES ON Converge $=1$ CASE: 0

Version:NPSS_1.6.5 - Rev: -> Gas Package: Janaf iter/pass/Jacb/Broy=14/28/1/12 Run by: Philip C Jorgenson PC: 10

Temperature Stator 1 inlet: 484.51 Stator 1 exit: $490.20 \quad$ Stator 2 inlet: 497.24 $\quad$ Stator 2 exit: 500.64 $\begin{array}{lll}\text { Stator } 3 \text { inlet: } 507.99 & \text { Stator } 3 \text { exit: } 510.98 \text { Stator } 4 \text { inlet: } 517.90 \text { Stator } 4 \text { exit: } 520.36 \\ \text { Stator } 5 \text { inlet: } 525.93 & \text { Stator } 5 \text { exit: } 528.02 & \text { Unblocked Percent Blockage: } 0.00\end{array}$

Ambient Relative Humidity $\quad 10.00$

Fan Face Relative Humidity $\quad 4.18$

Fan Bypass Relative Humidity $\quad 3.37$

$\begin{array}{ll}\text { LPC Inlet Relative Humidity } & 3.05 \\ \text { LPC Exit Relative Humidity } & 0.57\end{array}$

LPC Exit Relative Humidity

Drop Diameter

0.0000500

Inlet Length $\quad 40.00$

Fan/IPC Inlet Static Pressure $\quad 5.35$

mbient Static Pressure

4.36

Fan/LPC Inlet Static Temperature 478.12

Additional Water at LPC Exit 0.0000791

MN SUMMARY OUTPUT DATA

$\begin{array}{rrr}\text { MN } & \text { alt } & \text { dTamb } \\ 0.608 & 30029.0 & 36.00\end{array}$

298.11

710.0

1.1111

Wfuel
788.84 B. 4114

VTAS
630.80

FLOW STATION DATA

FSO Ambient.Fl_O

FS1 Inlet.Fl_O

FS2 Spliter. 1 -02

FS14 Fan.FI_O

FS25 VIPOINT.F1_O

FS3 3 BPC. E1 1 -

FS36 Bleed 3 . F1

FS4 Burner.FI_O

FS49 LPT.FI-O

FS8 Core Nozz.Flo

FS17 FanDuctLkg.F̄_ 0

FS171 Bleed15.FI_O

FS172 FanDuct Fl-0

FS173 Byp Nozz.Fì

$W$
298.11
298.11
266.44
31.68
266.44
31.68
31.68
26.93
25.62
19.86
20.08
26.83
27.15
27.15
27.15
266.44
271.19
271.19
271.19

Pt
5.595
5.595

$\begin{array}{rrr}\text { Tt } & \text { ht } & \text { FAR } \\ 80.74 & -16.28 & 0.0000\end{array}$

$\begin{array}{llllll}5.595 & 480.74 & -16.28 & 0.0000 & 753.84\end{array}$

$\begin{array}{lllll}5.592 & 480.74 & -16.28 & 0.0000 & 674.08\end{array}$

$\begin{array}{rrrrr}.592 & 480.74 & -16.28 & 0.0000 & 80.14\end{array}$

$\begin{array}{lllll}6.130 & 494.94 & -12.88 & 0.0000 & 623.92\end{array}$

$\begin{array}{lllll}7.803 & 536.93 & -2.81 & 0.0000 & 60.70 \\ 7.803 & 537.07 & -3.23 & 0.0000 & 60.71\end{array}$

$\begin{array}{lllll}7.803 & 536.93 & -2.81 & 0.0000 & 60.70 \\ 7.803 & 537.07 & -3.23 & 0.0000 & 60.7\end{array}$

$\begin{array}{lllll}45.365 & 962.40 & 100.14 & 0.0000 & 11.31\end{array}$

$\begin{array}{rrrrr}45.365 & 962.40 & 100.14 & 0.0000 & 8.76\end{array}$

$\begin{array}{lllll}11.108 & 1136.01 & -12.40 & 0.0082\end{array}$

$\begin{array}{lllll}4.710 & 937.33 & -60.83 & 0.0081\end{array}$

$\begin{array}{llll}4.710 & 937.40 & -60.83 & 0.0081\end{array}$

$\begin{array}{lllll}4.710 & 937.48 & -60.83 & 0.0081\end{array}$

$\begin{array}{llll}6.130 & 494.94 & -12.88 & 0.0000\end{array}$

$\begin{array}{lllll}6.130 & 495.68 & -12.71 & 0.0000\end{array}$

$\begin{array}{llll}6.130 & 495.68 & -12.71 & 0.0000\end{array}$

6.130

495.68

$\begin{array}{ll}-12.71 & 0.0000\end{array}$

TURBOMACHINERY PERFORMANCE DATA

\begin{tabular}{lrrrrrrrrr}
\multicolumn{1}{c}{ W } & WC & PR & eff & NC & TR & efPoly & pwr & SMN & SMW \\
Fan & 674.08 & 1.096 & 0.9014 & 2239.031 & 1.0295 & 0.9027 & -1283.3 & -8377.61 & 52.80 \\
LPC & 80.14 & 1.395 & 0.8549 & 2239.031 & 1.1169 & 0.8616 & -604.0 & 5.98 & 2.60 \\
HPC & 51.60 & 5.814 & 0.8138 & 8879.365 & 1.7920 & 0.8527 & -3842.6 & 62.28 & 58.66 \\
HPT & 12.08 & 3.990 & 0.8743 & 218.606 & 1.3525 & 0.8525 & 3842.6 & & \\
LPT & 52.53 & 2.358 & 0.8337 & 63.955 & 1.2095 & 0.8162 & 1887.2 & &
\end{tabular}

TURBOMACHINERY MAP DATA

$\begin{array}{lrlrrrrrrr} & \text { WcMap } & \text { PRmap } & \text { effMap } & \text { NcMap } & \text { R/Parm } & \text { S_WcDes } & \text { S_PRdes } & \text { S_effDes } & \text { S_NcDes } \\ \text { Fan } & 783.22 & 1.094 & 0.9032 & 2217.682 & 0.0416 & 0.8607 & 1.0235 & 0.9980 & 0.9905 \\ \text { LPC } & 59.98 & 1.378 & 0.7652 & 0.591 & 0.0000 & 1.3363 & 1.0451 & 1.1172 & 0.0003 \\ \text { HPC } & 46.93 & 5.587 & 0.8222 & 8642.647 & 10.9508 & 1.0995 & 1.0494 & 0.9898 & 0.9733 \\ \text { HPT } & 0.96 & 3.907 & 0.8743 & 1.314 & 3.9070 & 12.6299 & 0.9723 & 1.0000 & 0.0003 \\ \text { LPT } & 0.80 & 2.022 & 0.8399 & 0.719 & 2.0217 & 65.2590 & 0.7521 & 0.9926 & 0.0005\end{array}$

\begin{tabular}{|c|c|}
\hline & \\
\hline 4.3 & 447 \\
\hline 5.2 & 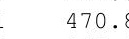 \\
\hline 5.1 & 469 \\
\hline 5.4 & 478 \\
\hline 5.3 & 47 \\
\hline 7.4 & 529 \\
\hline 7.4 & 530 \\
\hline 7.5 & 532 \\
\hline 39.9 & 925 \\
\hline 42.2 & 943 \\
\hline 41.8 & 1684 \\
\hline 10.1 & 1108 \\
\hline 4.5 & 927 \\
\hline 4.5 & 927 \\
\hline 4.3 & 917 \\
\hline 5.3 & \\
\hline 5.1 & 47 \\
\hline 5.1 & 47 \\
\hline 4.3 & 449 \\
\hline & SMW \\
\hline & 52.80 \\
\hline & 2.60 \\
\hline & 58.66 \\
\hline EfDes & NCDes \\
\hline & \\
\hline 172 & 0.0003 \\
\hline 98 & 0.9733 \\
\hline 00 & 0.0003 \\
\hline 26 & 0.0 \\
\hline
\end{tabular}

$\begin{array}{llccc}\text { EPR } & \text { PowerSet } & \text { T4 } & \text { T41 } & \text { T49 } \\ 0.827 & 10.000 & 1708.3 & 1614.4 & 1131.8\end{array}$

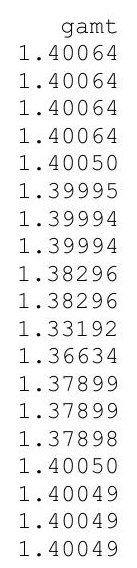

WAR
0.0001750
0.0001750
0.0001750
0.0001750
0.0001750
0.0001750
0.0002541
0.0002541
0.0002541
0.0002541
0.0002541
0.0002541
0.0002541
0.0002541
0.0002541
0.0001750
0.0001764
0.0001764
0.0001764

Wair $\begin{array}{lll} & & \\ 298.06 & 0.052 & \text { H2O frac }\end{array}$ $\begin{array}{lll}298.06 & 0.052 & 0.0002\end{array}$ $\begin{array}{lll}31.67 & 0.047 & 0.0002\end{array}$ $\begin{array}{lll}266.39 & 0.047 & 0.0002\end{array}$ $\begin{array}{lll}31.67 & 0.006 & 0.0002\end{array}$ $\begin{array}{lll}31.67 & 0.008 & 0.0003\end{array}$ $\begin{array}{lll}26.92 & 0.007 & 0.0003\end{array}$ $\begin{array}{lll}25.61 & 0.007 & 0.0003\end{array}$ $\begin{array}{lll}19.85 & 0.005 & 0.0003\end{array}$ $\begin{array}{lll}19.85 & 0.005 & 0.0137\end{array}$ $\begin{array}{lll}26.60 & 0.007 & 0.0103\end{array}$ $\begin{array}{lll}26.92 & 0.007 & 0.0102\end{array}$ $\begin{array}{lll}26.92 & 0.007 & 0.0102\end{array}$ $\begin{array}{lll}26.92 & 0.007 & 0.0102\end{array}$ $\begin{array}{lll}266.39 & 0.047 & 0.0002\end{array}$ $271.14 \quad 0.048 \quad 0.0002$ $271.14 \quad 0.048 \quad 0.0002$ 


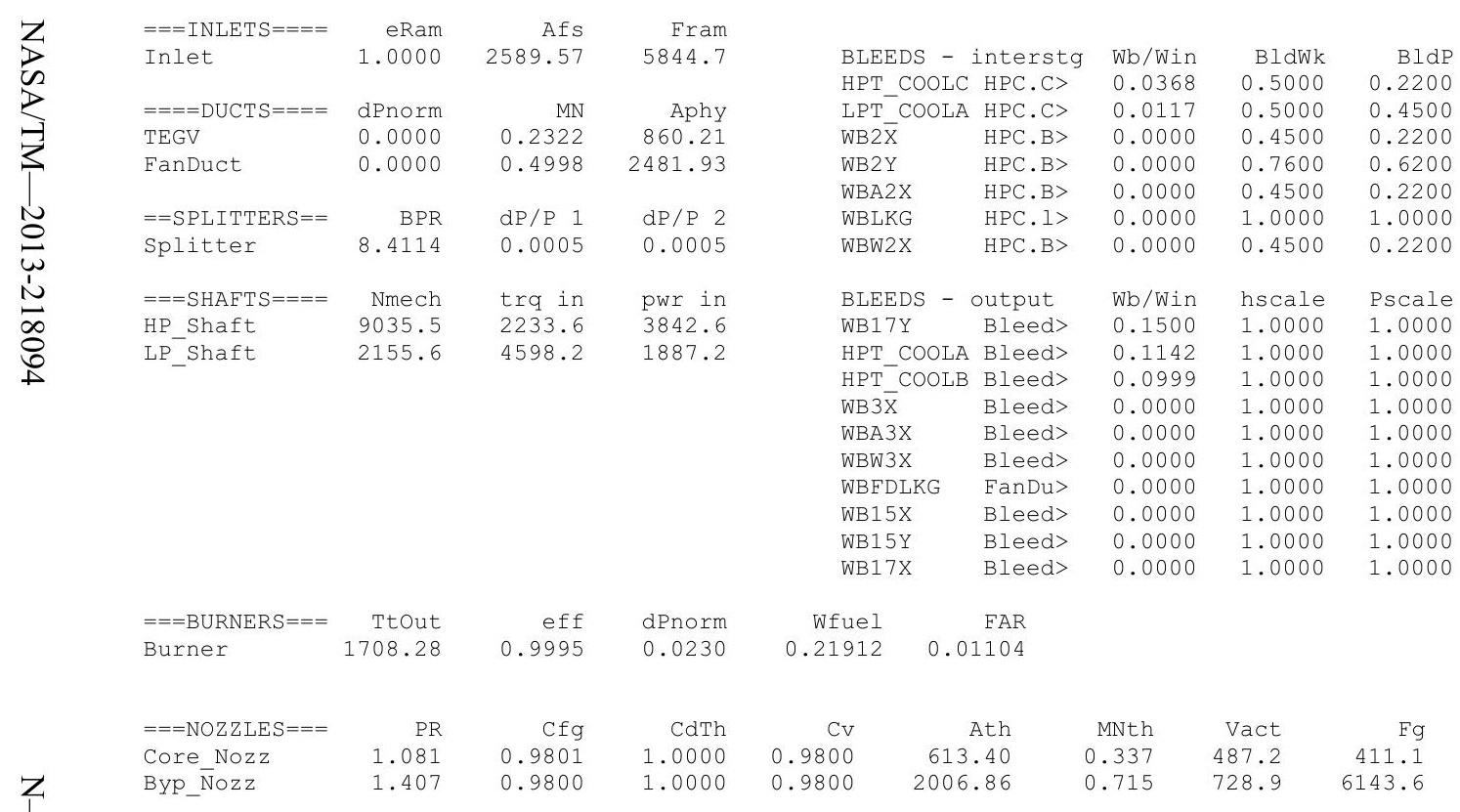


Date:07/05/13 Time:09:38:56 Model: Turbofan Engine - CoMDES ON Converge = 1 CASE: 0

Version:NPSS 1.6.5 - Rev: -> Gas Package: Janaf iter/pass/Jacb/Broy= 22/50/2/19 Run by: Philip C Jorgenson PC: 10

Temperature Stator 1 inlet: $492.28 \quad$ Stator 1 exit: $496.44 \quad$ Stator 2 inlet: 501.17 Stator 2 exit: 503.03 Stator 5 inlet: 519.47 Stator 5 exit: $520.22 \quad$ Unblocked Percent Blockage: 0.00

Ambient Relative Humidity $\quad 10.00$

Fan Face Relative Humidity $\quad 5.02$

Fan Bypass Relative Humidity -4.21

LPC Exit Relative Humidity

(

Drop Diameter

0.00

0.0000500

Inlet Length

40.00

Ambient Flow Velocity

582.56

Fan/LPC Inl

6.37

Ambient Static Pressure

Additional Water at LPC Exit

463.14

Fan/LPC Inlet Static Temperature 488.23

MN alt dTamb SUMMARY OUTPUT DATA

$\begin{array}{rrrrrr}\text { MN } & \text { alt } & \text { dTamb } & \text { W } & \text { Fn } & \text { TSFC } \\ 0.552 & 25666.0 & 36.00 & 322.28 & 710.2 & \end{array}$

FLOW STATION DATA

FSO Ambient.FI_O

FS1 Inlet.Fl_O

$\begin{array}{ll}\text { FS12 } & \text { Splitter.FI_02 } \\ \text { FS2 } & \text { Splitter.Fl_01 }\end{array}$

FS14 Fan.FI_O

FS23 LPC.FI-O

FS25 Bleed2.FIO

FS3 HPC.FIO

S36 Bleed3.Fl_O

FS4 Burner.FI_O

FS49

FS5 TEGV.Fīo

FS8 Core_Nozz.FI_O

FS17 FanDúctLkg.Fì_O

FS171 Bleed15.F1

FS173 Fanduct.FI_O

TURBOMACHINERY PERFORMANCE DATA

$\begin{array}{lrrr} & \text { WC } & \text { PR } & \text { eff } \\ \text { Fan } & 620.66 & 1.081 & 0.9017 \\ \text { LPC } & 87.18 & 1.256 & 0.8234 \\ \text { HPC } & 50.54 & 5.730 & 0.8121 \\ \text { HPT } & 12.08 & 3.860 & 0.8752 \\ \text { LPT } & 50.94 & 2.113 & 0.8233\end{array}$

$\begin{array}{crrr}\text { FLOW } & \text { STATION DATA } & \\ \text { Pt } & \text { Tt } & \text { ht } & \text { FAR } \\ 6.516 & 491.41 & -14.47 & 0.0000 \\ 6.516 & 491.41 & -14.47 & 0.0000 \\ 6.513 & 491.41 & -14.47 & 0.0000 \\ 6.513 & 491.41 & -14.47 & 0.0000 \\ 7.039 & 503.65 & -11.54 & 0.0000 \\ 8.181 & 531.61 & -4.83 & 0.0000 \\ 8.181 & 531.75 & -5.27 & 0.0000 \\ 8.181 & 531.75 & -5.27 & 0.0000 \\ 46.878 & 949.69 & 96.25 & 0.0000 \\ 46.878 & 949.69 & 96.25 & 0.0000 \\ 45.799 & 1713.12 & 83.43 & 0.0113 \\ 11.866 & 1144.33 & -14.60 & 0.0084 \\ 5.615 & 969.24 & -57.16 & 0.0083 \\ 5.615 & 969.31 & -57.16 & 0.0083 \\ 5.615 & 969.38 & -57.16 & 0.0083 \\ 7.039 & 503.65 & -11.54 & 0.0000 \\ 7.039 & 504.79 & -11.28 & 0.0000 \\ 7.039 & 504.79 & -11.28 & 0.0000 \\ 7.039 & 504.79 & -11.28 & 0.0000\end{array}$

Wfuel
833.00

BPR
7.1195

VTAS
582.56

OPR
7.194

EPR
0.857

PowerSet
10.000

$\begin{array}{ccc}\text { T4 } & \text { T41 } & \text { T49 } \\ 1713.1 & 1617.1 & 1139.9\end{array}$

TURBOMACHINERY MAP DATA

\begin{tabular}{|c|c|c|c|c|c|c|c|c|c|}
\hline & Wc & $\mathrm{PR}$ & eff & NC & $\mathrm{TR}$ & efpoly & pwr & SMN & SMW \\
\hline Fan & 620.66 & 1.081 & 0.9017 & 2025.533 & 1.0249 & 0.9028 & -1173.3 & -2597.69 & 50.01 \\
\hline LPC & 87.18 & 1.256 & 0.8234 & 2025.533 & 1.0818 & 0.8290 & -541.4 & 6.43 & 2.41 \\
\hline HPC & 50.54 & 5.730 & 0.8121 & 8845.497 & 1.7860 & 0.8511 & -3894.1 & 61.74 & 58.02 \\
\hline HPT & 12.08 & 3.860 & 0.8752 & 216.389 & 1.3429 & 0.8541 & 3894.1 & & \\
\hline $\mathrm{LPT}$ & 50.94 & 2.113 & 0.8233 & 58.283 & 1.1777 & 0.8075 & 1714.7 & & \\
\hline & NERY MAP & DATA & & & & & & & \\
\hline & WcMap & PRmap & effMap & NcMap & R/Parm & s WcDes & s PRdes & $s \quad s$ effDes & S NcDes \\
\hline in & 721.15 & 1.079 & 0.9035 & 2006.219 & 0.0424 & $\overline{0} .8607$ & $\overline{1} .0235$ & 0.9980 & $\overline{0} .9905$ \\
\hline${ }^{\mathrm{C}} \mathrm{C}$ & 52.83 & 1.305 & 0.7603 & 0.535 & 0.0000 & 1.6500 & 0.8409 & 1.0830 & 0.0003 \\
\hline & 45.97 & 5.507 & 0.8205 & 8609.682 & 10.9982 & 1.0995 & 1.0494 & 0.9898 & 0.9733 \\
\hline PT & 0.96 & 3.780 & 0.8752 & 1.300 & 3.7804 & 12.6299 & 0.9723 & 1.0000 & 0.0003 \\
\hline & 0.78 & 1.837 & 0.8294 & 0.656 & 1.8373 & 65.2590 & 0.7521 & 0.9926 & 0.0005 \\
\hline
\end{tabular}




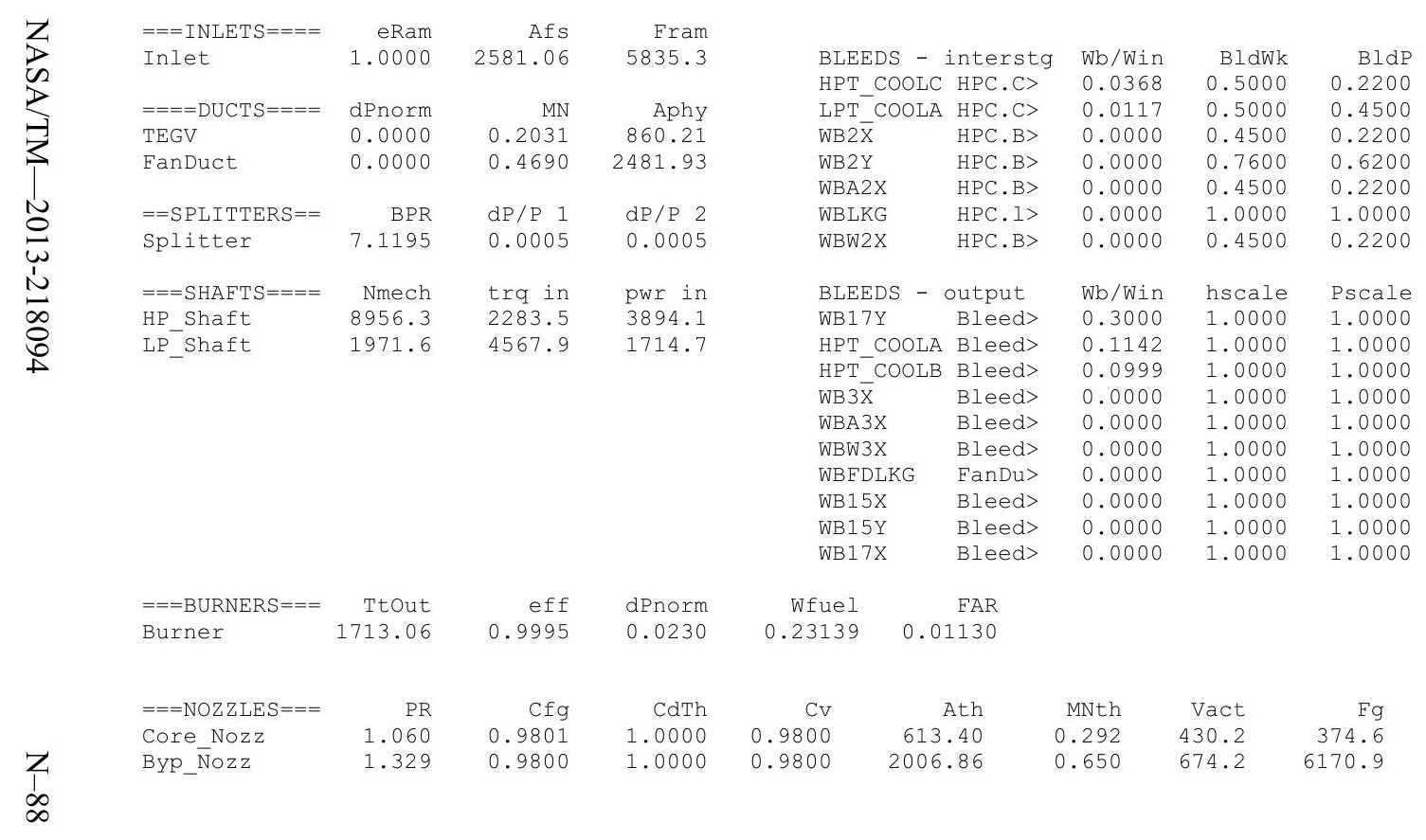


Date: 07/05/13 Time:09:39:25 Model: Turbofan Engine - CoMDES ON Converge $=1$ CASE: 0

Version:NPSS 1.6.5 - Rev: -> Gas Package: Janaf iter/pass/Jacb/Broy=22/50/2/19 Run by: Philip C Jorgenson PC: 10

Temperature Stator 1 inlet: 505.22 Stator 1 exit: 508.18 Stator 2 inlet: 510.96 Stator 2 exit: 511.76 Stator 5 inlet: 520.69 Stator 5 exit: 520.56 Unblocked Percent Blockage: 0.00

Ambient Relative Humidity $\quad 10.00$

Fan Face Relative Humidity $\quad 6.01$

$\begin{array}{ll} & 5.19 \\ & 5.20\end{array}$

LPC Exit Relative Humidity $\quad 2.84$

HPC Relative Humidity

0.0000500

Drop Diameter

Inlet Length $\quad 40.00$

Ambient Flow Velocity

Ambient Static Temperature

Additional Water at LPC Exit

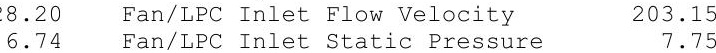

483.18 Fan/LPC Inlet Static Temperature 502.97

0.0000933

$\begin{array}{rrr}\text { MN } & \text { alt } & \text { dTamb } \\ 0.490 & 20047.0 & 36.00\end{array}$

W
357.70

SUMMARY OUTPUT DATA

$\begin{array}{rrr}762.5 & 1.1969 \\ & & \\ \end{array}$

FS0 Ambient.Fl_O

FS1 Inlet.Fl_o

$\begin{array}{ll}\text { FS12 } & \text { Splitter.F1_02 } \\ \text { FS2 } & \text { Splitter.FI_01 }\end{array}$

FS14 Fan.Fl_O

$\begin{array}{ll}\text { FS23 } & \text { LPC.FI-O } \\ \text { FS24 } & \text { VaporIN.F1 }\end{array}$

FS25 Bleed2.FI_

FS3 HPC.FI_O

FS36 Bleed3.F1_O

FS45 HPT.FI O

FS49 LPT.FI-

FS5 TEGV.FI'

FS8 Core_Nozz.FI_O

S17 FanDuct $\mathrm{kg}$. Fl_

.S171 Bleedis. F1

FS173 Fanduct. $1-0$

$W$
357.70
357.70
308.97
48.72
308.97
48.72
48.73
29.24
27.82
21.56
21.81
29.15
29.49
29.49
29.49
308.97
328.46
328.46
328.46

$\begin{array}{cc}\text { FLOW } & \text { STATION DATA } \\ \text { Pt } & \text { Tt }\end{array}$

$\begin{array}{rrrr}\text { Pt } & \text { Tt } & \text { ht } & \text { FAR } \\ 7.943 & 506.41 & -12.46 & 0.0000\end{array}$

7.943
7.939

$\begin{array}{llll}7.943 & 506.41 & -12.46 & 0.0000 \\ 7.939 & 506.41 & -12.46 & 0.0000\end{array}$

7.939

8.480

9.054

$\begin{array}{lll}506.41 & -12.46 & 0.0000\end{array}$

$\begin{array}{lll}517.06 & -9.90 & 0.0000 \\ 534.27 & -5.77 & 0.0000\end{array}$

$\begin{array}{llll}534.27 & -5.77 & 0.0000 & 80.27\end{array}$

$\begin{array}{llll}9.054 & 534.43 & -6.27 & 0.0000\end{array}$

$\begin{array}{llll}49.678 & 943.60 & 93.12 & 0.0000\end{array}$

$\begin{array}{rrrr}49.678 & 943.60 & 93.12 & 0.0000 \\ 48.534 & 1736.73 & 79.81 & 0.0118\end{array}$

$\begin{array}{llll}48.534 & 1736.73 & 79.81 & 0.0118\end{array}$

$\begin{array}{llll}7.282 & 1170.70 & -15.95 & 0.0088\end{array}$

$7.031-1017.56$

$\begin{array}{ll}-53.08 & 0.0087 \\ -53.08 & 0.0087\end{array}$

$\begin{array}{llll}7.031 & 1017.56 & -53.08 & 0.0087 \\ 7.031 & 1017.70 & -53.08 & 0.0087\end{array}$

$\begin{array}{llll}8.480 & 517.06 & -9.90 & 0.0000\end{array}$

$\begin{array}{llll}8.480 & 517.06 & -9.90 & 0.0000\end{array}$

518.09

$\begin{array}{rr}-9.69 & 0.0000 \\ -9.69 & 0.0000\end{array}$

\section{BPR}

14

VTAS

0.888

PowerSet

10.000

T4
1736.7

T41

T49

\section{Ps
6.740}

7.531
7.509

7.752

7.690

8.305

8.800

44.047

46.433

45.827

12.324
6.886

6.886

6.740

7.690

7.461

6.740

TS
483.18
498.75
498.41
502.97
502.82
521.26
521.40
530.11
912.57
926.06
1712.12
1147.54
1011.84
1011.91
1006.09
502.82
499.48
499.48
485.18

$\begin{array}{rrr}\text { Aphy } & \text { MN gamt } \\ 2590.9 & 0.4900 & 1.40031\end{array}$

$\begin{array}{ll}2590.9 & 0.4900 \\ 4168.2 & 0.2770\end{array}$

$\begin{array}{ll}4168.2 & 0.2770 \\ 3531.8 & 0.2831\end{array}$

$\begin{array}{rr}3531.8 & 0.2831 \\ 830.5 & 0.1847\end{array}$

$\begin{array}{rr}2606.7 & 0.3761 \\ 412.6 & 0.3533\end{array}$

$\begin{array}{ll}412.6 & 0.3533 \\ 412.6 & 0.3534\end{array}$

$\begin{array}{rr}412.6 & 0.2018 \\ 49.7 & 0.4202\end{array}$

$\begin{array}{ll}49.7 & 0.4202 \\ 49.3 & 0.3138\end{array}$

$\begin{array}{lll}49.3 & 0.3138\end{array}$

$\begin{array}{ll}265.4 & 0.3327\end{array}$

$\begin{array}{ll}860.2 & 0.1740 \\ 860.2 & 0.1740\end{array}$

$\begin{array}{ll}860.2 & 0.1740 \\ 613.5 & 0.2485\end{array}$

$\begin{array}{ll}2606.7 & 0.3761\end{array}$

$\begin{array}{ll}2481.9 & 0.4314\end{array}$

$2006.9 \quad 0.5821$ gamt
1.40031
1.40031
1.40031
1.40031
1.40018
1.39993
1.39992
1.39992
1.38401
1.38401
1.33004
1.36368
1.37361
1.37361
1.37360
1.40018
1.40016
1.40016
1.40016

WAR
0.0005787

0.000578787
0.0005787
0.0005787

0.0005787

0.0005787

0.0005787

0.0006720

0.0006720

0.0006720

0.0006720

0.0006720

0.0006720

0.0006720

0.0006720

0.0006720

0.0005842

0.0005842

0.0005842

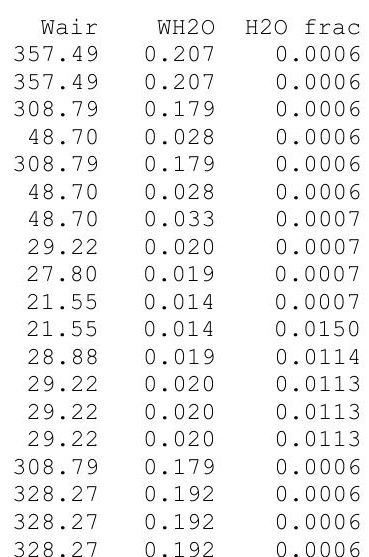

TURBOMACHTNERY PERFORMANCE DATA

\begin{tabular}{|c|c|c|c|c|c|c|c|c|c|}
\hline & WC & $P R$ & eff & $\mathrm{NC}$ & $\mathrm{TR}$ & efPoly & pwr & SMN & SMW \\
\hline Fan & 565.13 & 1.068 & 0.9035 & 1815.414 & 1.0210 & 0.9044 & -1117.3 & -1833.24 & 46.56 \\
\hline LPC & 89.12 & 1.140 & 0.6954 & 1815.414 & 1.0550 & 0.7011 & -460.9 & 7.12 & 2.04 \\
\hline HPC & 48.17 & 5.487 & 0.8076 & 8765.136 & 1.7656 & 0.8467 & -4011.6 & 62.14 & 58.12 \\
\hline HPT & 12.09 & 3.654 & 0.8765 & 213.497 & 1.3265 & 0.8566 & 4011.6 & & \\
\hline $\mathrm{LPT}$ & 48.45 & 1.889 & 0.8151 & 52.427 & 1.1472 & 0.8013 & 1578.1 & & \\
\hline JRE & NERY & DATA & & & & & & & \\
\hline & WcMap & PRmap & effMap & NcMap & R/Parm & s_WcDes & S_PRdes & S_effDes & s_NCDes \\
\hline Tan & 656.63 & 1.067 & 0.9053 & 1798.104 & 0.0437 & 0.8607 & 1.0235 & 0.9980 & $\overline{0} .9905$ \\
\hline $\mathrm{LPC}$ & 46 & & 0. & 0.479 & 00 & 1.9 & 0.5891 & 0.9214 & 0.0003 \\
\hline HPC & 43.81 & 5.276 & 0.8160 & 8531.463 & 11. & 1.0 & 1.0494 & 0.9898 & 0.9733 \\
\hline HPT & & & 0.8 & 1.283 & & 12.6 & 0.9723 & 1.0000 & 0.0 \\
\hline LPT & 0.74 & 1.669 & 0.8212 & 0.590 & 1.6687 & 65.2590 & 0.7521 & 0.9926 & 0.0005 \\
\hline
\end{tabular}




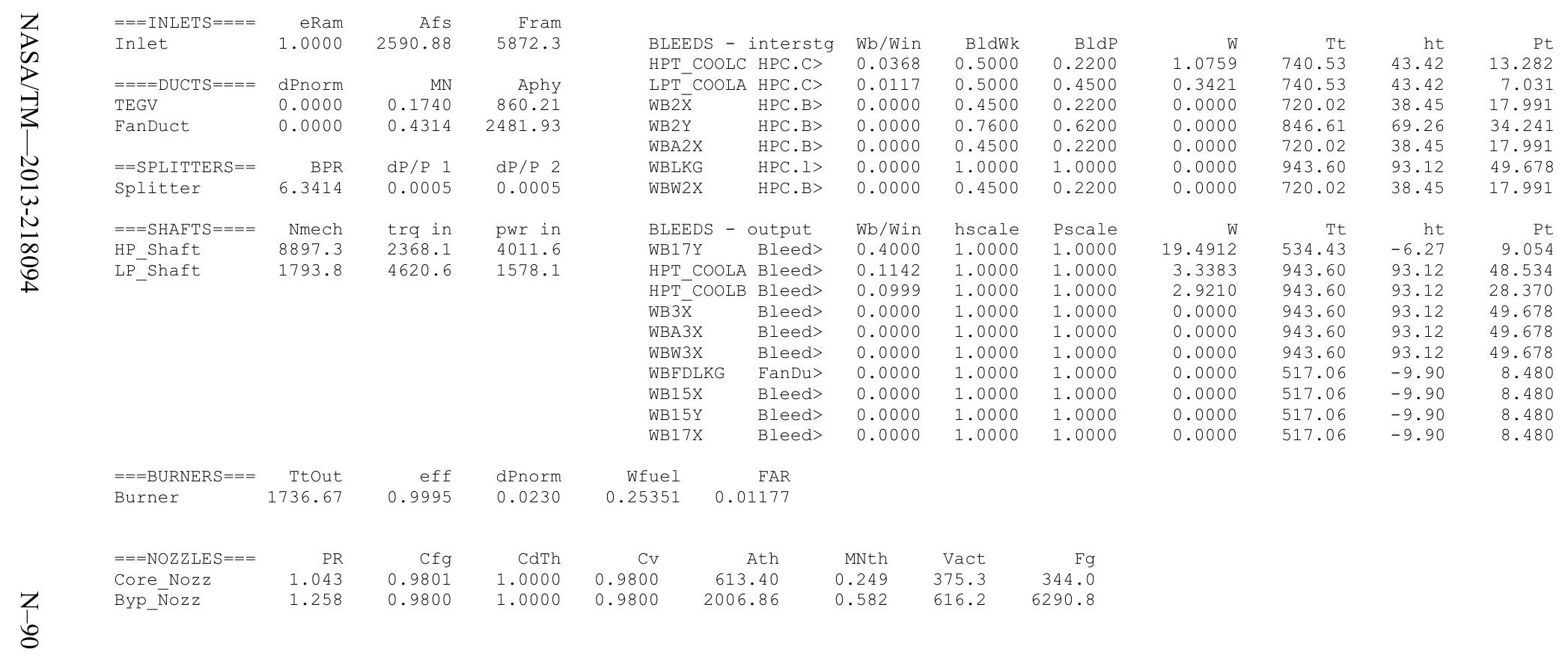


Date: 07/05/13 Time:09:49:59 Model:

Gas Package: Janaf iter/pass/Jacb/Broy=11/25/1/9 Run by: Philip C Jorgenson PC: 10

converge = 1 (CAS:

Temperature Stator 1 inlet: 463.24 Stator 1 exit: 470.64 Stator 2 inlet: 479.94 Stator 2 exit: 484.70

$\begin{array}{ccc}\text { Stator } 3 \text { inlet: } 494.31 & \text { Stator } 3 \text { exit: } 498.63 & \text { Stator } 4 \text { inlet: } 507.63 \text { Stator } 4 \text { exit: } 511.31 \\ \text { Stator } 5 \text { inlet: } 518.70 & \text { Stator } 5 \text { exit: } 521.92 & \text { Unblocked Percent Blockage: } 0.00\end{array}$

Ambient Relative Humidity 10.00

Fan Face Relative Humidity $\quad 1.76$

Fan Bypass Relative Humidity $\quad 1.24$

LPC Inlet Relative Humidity $\quad 1.13$

LPC Exit Relative Humidity

Drop Diameter

0.10
0.00
0001000

Ambient Flew Velocity

772.64
2.85

Inlet Length

40.00

Ambient Static Pressure

Ambient Static Temperature

Fan/LPC Inlet Static Pressure $\quad 187.93$

Fan/LPC Inlet Static Temperature 454.80

MN alt dTamb WU SUMMARY OUTPUT DATA

\section{FLOW STATION DATA}

\begin{tabular}{rrrrr}
\multicolumn{7}{c}{ W } & Pt & \multicolumn{1}{l}{ Tt } & ht & FAR \\
257.26 & 4.267 & 457.74 & -20.96 & 0.0000 \\
257.26 & 4.267 & 457.74 & -20.96 & 0.0000 \\
230.44 & 4.265 & 457.74 & -20.96 & 0.0000 \\
26.82 & 4.265 & 457.74 & -20.96 & 0.0000 \\
230.44 & 4.811 & 475.48 & -16.70 & 0.0000 \\
26.82 & 6.639 & 531.25 & -3.33 & 0.0000 \\
26.82 & 6.639 & 531.30 & -3.42 & 0.0000 \\
24.14 & 6.639 & 531.30 & -3.42 & 0.0000 \\
22.97 & 40.487 & 964.20 & 101.76 & 0.0000 \\
17.80 & 40.487 & 964.20 & 101.76 & 0.0000 \\
17.99 & 39.555 & 1693.20 & 89.48 & 0.0108 \\
24.05 & 9.489 & 1116.81 & -12.39 & 0.0080 \\
24.33 & 3.259 & 875.22 & -71.37 & 0.0079 \\
24.33 & 3.259 & 875.29 & -71.37 & 0.0079 \\
24.33 & 3.259 & 875.36 & -71.37 & 0.0079 \\
230.44 & 4.811 & 475.48 & -16.70 & 0.0000 \\
233.12 & 4.811 & 476.13 & -16.55 & 0.0000 \\
233.12 & 4.811 & 476.13 & -16.55 & 0.0000 \\
233.12 & 4.811 & 476.13 & -16.55 & 0.0000
\end{tabular}

Wc
832.40
832.40
746.00
86.82
673.99
60.08
60.08
54.08
11.37
8.81
12.08
54.65
142.53
142.53
142.54
673.99
682.30
682.30
682.30

VTAS
$O P R$
.489

0.736

Powerset

T4

1601.3

T49

$\begin{array}{rrrrrrrrr}\text { PS } & \text { TS } & \text { Aphy } & \text { MN } & \text { gamt } & \text { WAR } & \text { Wair } & \text { WH2O } & \text { H2O frac } \\ 2.854 & 407.97 & 2539.4 & 0.7800 & 1.40084 & 0.0000295 & 257.25 & 0.008 & 0.0000 \\ 3.893 & 445.88 & 4168.2 & 0.3642 & 1.40084 & 0.0000295 & 257.25 & 0.008 & 0.0000 \\ 3.841 & 444.23 & 3531.8 & 0.3896 & 1.40084 & 0.0000295 & 230.44 & 0.007 & 0.0000 \\ 4.170 & 454.80 & 830.5 & 0.1797 & 1.40084 & 0.0000295 & 26.82 & 0.001 & 0.0000 \\ 4.037 & 452.22 & 2606.7 & 0.5066 & 1.40071 & 0.0000295 & 230.44 & 0.007 & 0.0000 \\ 6.345 & 524.41 & 412.6 & 0.2553 & 1.40005 & 0.0000295 & 26.82 & 0.001 & 0.0000 \\ 6.345 & 524.47 & 412.6 & 0.2553 & 1.40005 & 0.0000475 & 26.82 & 0.001 & 0.0000 \\ 6.403 & 525.84 & 412.6 & 0.2280 & 1.40005 & 0.0000475 & 24.14 & 0.001 & 0.0000 \\ 35.638 & 930.68 & 49.7 & 0.4331 & 1.38289 & 0.0000475 & 22.97 & 0.001 & 0.0000 \\ 37.704 & 945.35 & 49.3 & 0.3224 & 1.38289 & 0.0000475 & 17.80 & 0.001 & 0.0000 \\ 37.353 & 1669.10 & 74.6 & 0.2941 & 1.33285 & 0.0000475 & 17.80 & 0.001 & 0.0132 \\ 8.596 & 1087.47 & 265.4 & 0.3824 & 1.36775 & 0.0000475 & 23.85 & 0.001 & 0.0099 \\ 3.069 & 860.76 & 860.2 & 0.2960 & 1.38277 & 0.0000475 & 24.14 & 0.001 & 0.0098 \\ 3.069 & 860.84 & 860.2 & 0.2960 & 1.38276 & 0.0000475 & 24.14 & 0.001 & 0.0098 \\ 2.854 & 843.76 & 613.4 & 0.4418 & 1.38276 & 0.0000475 & 24.14 & 0.001 & 0.0098 \\ 4.037 & 452.22 & 2606.7 & 0.5066 & 1.40071 & 0.0000295 & 230.44 & 0.007 & 0.0000 \\ 3.904 & 448.50 & 2481.9 & 0.5544 & 1.40070 & 0.0000297 & 233.12 & 0.007 & 0.0000 \\ 3.904 & 448.50 & 2481.9 & 0.5544 & 1.40070 & 0.0000297 & 233.12 & 0.007 & 0.0000 \\ 2.854 & 410.03 & 2006.9 & 0.8966 & 1.40070 & 0.0000297 & 233.12 & 0.007 & 0.0000\end{array}$

TURBOMACHINERY PERFORMANCE DATA

\begin{tabular}{|c|c|c|c|c|c|c|c|c|c|}
\hline & WC & PR & ff & & & $f=1$ & nwr & SMNT & SMVT \\
\hline Fan & 746.00 & $\begin{array}{r}1.128 \\
1.128\end{array}$ & $\begin{array}{r}\text { eft } \\
0.9051\end{array}$ & $\begin{array}{r}\text { NC } \\
2560.797\end{array}$ & $\begin{array}{r}1.0388 \\
1.038\end{array}$ & $\begin{array}{l}\text { eIPOIY } \\
0.9067\end{array}$ & $\begin{array}{r}\text { pWr } \\
-1386.3\end{array}$ & 1663.00 & 54.94 \\
\hline 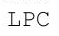 & 86.82 & 1.557 & 0.8403 & 2560.797 & 1.1606 & 0.8500 & -668.7 & 6.95 & 3.67 \\
\hline HPC & 54.08 & 6.098 & 0.8187 & 8960.611 & 1.8148 & 0.8574 & -3505.0 & 61.08 & 57.75 \\
\hline $\mathrm{HPT}$ & 12.08 & 4.168 & 0.8739 & 220.399 & 1.3661 & 0.8512 & 3505.0 & & \\
\hline PT & 54.65 & 2.912 & 0.8506 & 71.986 & 1.2742 & 0.8304 & 2055.0 & & \\
\hline & NEF & DATA & & & & & & & \\
\hline & WcMap & PRmap & effMap & NCMap & R/Parm & S_WcDes & s_PRdes & 5 s_effDes & S_NcDes \\
\hline & 866.78 & 1.125 & 0.9070 & 2536.380 & 0.0413 & $\overline{0} .8607$ & $\overline{1} .0235$ & 0.9980 & $\overline{0} .9905$ \\
\hline ec & 73.59 & 1.519 & 0.7795 & 0.676 & 0.0000 & 1.1798 & 1.0735 & 1.0779 & 0.0003 \\
\hline PC & 49.18 & 5.858 & 0.8271 & 8721.727 & 10.9930 & 1.0995 & 1.0494 & 0.9898 & 0.9733 \\
\hline & 0.96 & 4.081 & 0.8739 & 1.325 & 4.0806 & 12.6299 & 0.9723 & 1.0000 & 0.0003 \\
\hline $\mathrm{PT}$ & 0.84 & 2.438 & 0.8569 & 0.810 & 2.4380 & 65.2590 & 0.7521 & 0.9926 & 0.0005 \\
\hline
\end{tabular}




\begin{tabular}{|c|c|c|c|c|c|c|c|c|c|c|c|c|}
\hline Z $\quad===$ INLETS $S===$ & eRam & Afs & Fram & \multirow{2}{*}{\multicolumn{2}{|c|}{ BLFEDS - intersta }} & \multirow[b]{2}{*}{ Wb/Win } & \multirow[b]{2}{*}{ BldWk } & \multirow[b]{2}{*}{ BldP } & \multirow[b]{2}{*}{$\begin{array}{r}W \\
08883\end{array}$} & & \multirow[b]{2}{*}{ ht } & \multirow[b]{2}{*}{$\begin{array}{r}P t \\
9.489\end{array}$} \\
\hline $\mathbb{S}_{\infty} \quad$ Inlet & 1.0000 & 2539.39 & 6178.0 & & & & & & & & & \\
\hline$====$ DUCTS $====$ & dPnorm & MN & Aphy & LPT COOLA & A HPC.C> & 0.0117 & 0.5000 & 0.4500 & 0.2824 & 749.49 & 49.17 & 3.259 \\
\hline TEGV & 0.0000 & 0.2960 & 860.21 & WB2 $\bar{x}$ & $\mathrm{HPC} \cdot \mathrm{B}>$ & 0.0000 & 0.4500 & 0.2200 & 0.0000 & 727.79 & 43.91 & 14.086 \\
\hline FanDuct & 0.0000 & 0.5544 & 2481.93 & WB2Y & HPC.B> & 0.0000 & 0.7600 & 0.6200 & 0.0000 & 861.70 & 76.51 & 27.625 \\
\hline & & & & WBA2X & HPC. B> & 0.0000 & 0.4500 & 0.2200 & 0.0000 & 727.79 & 43.91 & 14.086 \\
\hline$==$ SPLITTERS $==$ & BPR & $\mathrm{dP} / \mathrm{P} 1$ & $\mathrm{dP} / \mathrm{P} 2$ & WBLKG & HPC.I> & 0.0000 & 1.0000 & 1.0000 & 0.0000 & 964.20 & 101.76 & 40.487 \\
\hline Splitter & 8.5924 & 0.0005 & 0.0005 & WBW2X & HPC.B> & 0.0000 & 0.4500 & 0.2200 & 0.0000 & 727.79 & 43.91 & 14.086 \\
\hline$===$ SHAFTS $====$ & Nmech & trg in & pwr in & BLEEDS - & - output & Wb/Win & hscale & Pscale & W & Tt & ht & Pt \\
\hline HP_Shaft & 9069.1 & 2029.8 & 3505.0 & WB17Y & Bleed> & 0.1000 & 1.0000 & 1.0000 & 2.6820 & 531.30 & -3.42 & 6.639 \\
\hline LP Shaft & 2405.7 & 4486.4 & 2055.0 & HPT COOLA & A B Bleed> & 0.1142 & 1.0000 & 1.0000 & 2.7561 & 964.20 & 101.76 & 39.555 \\
\hline & & & & HPT COOLB & B Bleed> & 0.0999 & 1.0000 & 1.0000 & 2.4116 & 964.20 & 101.76 & 22.357 \\
\hline & & & & WB $3 \bar{X}$ & Bleed> & 0.0000 & 1.0000 & 1.0000 & 0.0000 & 964.20 & 101.76 & 40.487 \\
\hline & & & & WBA3X & Bleed> & 0.0000 & 1.0000 & 1.0000 & 0.0000 & 964.20 & 101.76 & 40.487 \\
\hline & & & & WBW3X & Bleed> & 0.0000 & 1.0000 & 1.0000 & 0.0000 & 964.20 & 101.76 & 40.487 \\
\hline & & & & WBFDLKG & FanDu> & 0.0000 & 1.0000 & 1.0000 & 0.0000 & 475.48 & -16.70 & 4.811 \\
\hline & & & & WB15X & Bleed> & 0.0000 & 1.0000 & 1.0000 & 0.0000 & 475.48 & -16.70 & 4.811 \\
\hline & & & & WB15Y & Bleed> & 0.0000 & 1.0000 & 1.0000 & 0.0000 & 475.48 & -16.70 & 4.811 \\
\hline & & & & WB17X & Bleed> & 0.0000 & 1.0000 & 1.0000 & 0.0000 & 475.48 & -16.70 & 4.811 \\
\hline$===$ BURNERS $===$ & Ttout & eff & dPnorm & Wfuel & FAR & & & & & & & \\
\hline Burner & 1693.14 & 0.9995 & 0.0230 & .19166 & 01077 & & & & & & & \\
\hline$===$ NOZZLES $===$ & $\mathrm{PR}$ & Cfg & CdTh & $\mathrm{Cv}$ & Ath & MNth & Vact & $\mathrm{Fg}$ & & & & \\
\hline Core_Nozz & 1.142 & 0.9801 & 1.0000 & 0.9800 & 3.40 & 0.442 & 613.1 & 463.6 & & & & \\
\hline Byp_Nozoz & 1.686 & 0.9800 & 1.0000 & 0.9800 & 6.86 & 0.897 & 872.6 & 6322.7 & & & & \\
\hline
\end{tabular}


Date:07/05/13 Time:09:50:15 Model:_ Turbofan Engine - COMDES ON Converge = 1 CASE: 0 Version:NPSS 1.6.5 - Rev: -> Gas Package: Janaf iter/pass/Jacb/Broy=10/24/1/8 Run by: Philip C Jorgenson PC: 10 Temperature Stator 1 inlet: 456.76 Stator 1 exit: 463.69 Stator 2 inlet: 472.40 Stator 2 exit: 476.84 $\begin{array}{ccc}\text { Stator } 3 \text { inlet: } 485.83 & \text { Stator } 3 \text { exit: } 489.85 & \text { Stator } 4 \text { inlet: } 498.26 \text { Stator } 4 \text { exit: } 501.67 \\ \text { Stator } 5 \text { inlet: } 508.57 & \text { Stator } 5 \text { exit: } 511.54 & \text { Unblocked Percent Blockage: } 0.00\end{array}$

Ambient Relative Humidity $\quad 10.00$

Fan Face Relative Humidity $\quad 2.23$

an Bypass Relative Humidity

LPC Exit Relative Humidity 0.13

Drop Diameter

0.00

Ambient Flow Velocity

Ambient Static Pressure

$\begin{array}{lll}\text { Ambient Static Temperature } & 407.97 \\ \text { Additional Water at LPC Exit } & 0.0000171\end{array}$ Fan/LPC Inlet Static Temperature 448.83

Inlet Length $\quad 40.00$

$\begin{array}{rrr}23.11 & \text { Fan/LPC Inlet Flow Velocity } & 181.26 \\ 2.95 & \text { Fan/LPC Inlet Static Pressure } & 4.11\end{array}$

$\begin{array}{rrrrrr}\text { MN } & \text { alt } & \text { dTamb } & \text { W } & \text { SUMMARY } & \text { OUTPUT DATA } \\ 0.730 & 38334.0 & 18.00 & 250.31 & 599.2 & 1.0981\end{array}$

\section{FLOW STATION DATA}

FSO Ambient.

FS1 Inlet.Fl_o

FS12 Splitter.F1_02

FS2 Splitter.FI_01

FS14 Fan.F1_O

FS24 VaporIñ.FI_O

FS25 Bleed2.F1_O

FS3 HPC.F1_O

$\begin{array}{ll}\text { FS36 } & \text { Bleed3.F1_O } \\ \text { FS4 } & \text { Burner.FI_O }\end{array}$

FS45 HPT.FI_O

EST

FS8 Core_Nozz.Fl_

FS17 FanDuctLkg.Fì_O

ES171 Bleed15.F1_O

ES1712 Banduct. Fi -0

W
250.31
250.31
224.46
25.84
224.46
25.84
25.84
23.26
22.13
17.15
17.33
23.17
23.44
23.44
23.44
224.46
227.05
227.05
227.05

$\begin{array}{ccc}\mathrm{Pt} & \mathrm{Tt} \\ 4.201 & 451.57 & -22 .\end{array}$ $\begin{array}{rrrr}4.201 & 451.57 & -22.43 & 0.0000\end{array}$ 4.201

4.199

4.199

4.706

6.402
6.402

6.402

38.693

38.693

37.801
9.183

3.309

3.309
3.309

4.706

4.706

4.706

4.706

451.57
451.57

$\begin{array}{lll}451.57 & -22.43 & 0.0000\end{array}$

$\begin{array}{lll}451.57 & -22.43 & 0.0000\end{array}$

$\begin{array}{lll}458.12 & -18.43 & 0.0000\end{array}$

$\begin{array}{lll}520.48 & -6.01 & 0.0000\end{array}$

$\begin{array}{lll}520.48 & -6.01 & 0.0000 \\ 942.89 & 96.50 & 0.0000\end{array}$ $\begin{array}{lll}942.89 & 96.50 & 0.0000\end{array}$ $\begin{array}{lll}942.89 & 96.50 & 0.0000\end{array}$ 1667.10 $\begin{array}{ll}84.39 & 0.0107\end{array}$ $\begin{array}{lll}87.11 & -70.33 & 0.0079\end{array}$ $\begin{array}{lll}873.11 & -70.33 & 0.0079 \\ 873.26 & -70.33 & 0.0079\end{array}$

$\begin{array}{llll}878.12 & -18.46 & 0.0079\end{array}$

$\begin{array}{lll}873.26 & -70.33 & 0.0079 \\ 468.71 & -18.42 & 0.0000\end{array}$

$\begin{array}{lll}468.71 & -18.32 & 0.0000\end{array}$

$\begin{array}{lll}468.71 & -18.32 & 0.0000\end{array}$
BPR
VTAS
723.11
EPR
0.763

PowerSet
10.000 $\begin{array}{cr}\text { T4 } & \text { T41 } \\ 1667.1 & 1575\end{array}$

T49

TURBOMACHINERY PERFORMANCE DATA

$\begin{array}{lrrrrrrrrr} & \text { Wc } & \text { PR } & \text { eff } & \text { NC } & \text { TR } & \text { efPoly } & \text { pwr } & \text { SMN } & \text { SMW } \\ \text { Fan } & 732.96 & 1.121 & 0.9043 & 2497.715 & 1.0367 & 0.9059 & -1259.6 & 2278.15 & 54.78 \\ \text { LPC } & 84.39 & 1.525 & 0.8406 & 2497.715 & 1.1525 & 0.8498 & -603.5 & 6.75 & 3.33 \\ \text { HPC } & 53.48 & 6.044 & 0.8179 & 8941.539 & 1.8116 & 0.8566 & -3291.4 & 60.99 & 57.61 \\ \text { HPT } & 12.08 & 4.116 & 0.8726 & 219.375 & 1.3628 & 0.8499 & 3291.4 & & \\ \text { LPT } & 54.02 & 2.775 & 0.8451 & 70.239 & 1.2589 & 0.8253 & 1863.1 & & \end{array}$

TURBOMACHINERY MAP DATA

$\begin{array}{lrrrrrrrrr} & \text { WcMap } & \text { PRmap } & \text { effMap } & \text { NcMap } & \text { R/Parm } & \text { S_WcDes } & \text { S_PRdes } & \text { S_effDes } & \text { S_NcDes } \\ \text { Fan } & 851.63 & 1.118 & 0.9062 & 2473.899 & 0.0412 & 0.8607 & 1.0235 & 0.9980 & 0.9905 \\ \text { LPC } & 70.85 & 1.490 & 0.7766 & 0.660 & 0.0000 & 1.1909 & 1.0694 & 1.0825 & 0.0003 \\ \text { HPC } & 48.64 & 5.806 & 0.8263 & 8703.164 & 11.0057 & 1.0995 & 1.0494 & 0.9898 & 0.9733 \\ \text { HPT } & 0.96 & 4.030 & 0.8726 & 1.318 & 4.0300 & 12.6299 & 0.9723 & 1.0000 & 0.0003 \\ \text { LPT } & 0.83 & 2.335 & 0.8514 & 0.790 & 2.3350 & 65.2590 & 0.7521 & 0.9926 & 0.0005\end{array}$

0.8514

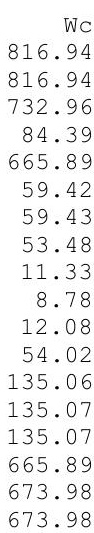

PS
2.947
3.848

3.848
3.798

4.111
3.972

6.125

6.180

34.091
36.049

35.696

8. 342

3.137

2.947

3.84

3.847

409.94
SMW
.78
33

1

$\begin{array}{rrrrrrrr}\text { Ts } & \text { Aphy } & \text { MN } & \text { gamt } & \text { WAR } & \text { Wair } & \text { WH2O } & \text { H2O frac } \\ 407.97 & 2556.8 & 0.7300 & 1.40089 & 0.0000285 & 250.30 & 0.007 & 0.0000 \\ 440.36 & 4168.2 & 0.3563 & 1.40089 & 0.0000285 & 250.30 & 0.007 & 0.0000 \\ 438.77 & 3531.8 & 0.3813 & 1.40089 & 0.0000285 & 224.46 & 0.006 & 0.0000 \\ 448.83 & 830.5 & 0.1745 & 1.40089 & 0.0000285 & 25.84 & 0.001 & 0.0000 \\ 445.95 & 2606.7 & 0.4981 & 1.40077 & 0.0000285 & 224.46 & 0.006 & 0.0000 \\ 513.88 & 412.6 & 0.2522 & 1.40021 & 0.0000285 & 25.84 & 0.001 & 0.0000 \\ 513.93 & 412.6 & 0.2523 & 1.40020 & 0.0000457 & 25.84 & 0.001 & 0.0000 \\ 515.24 & 412.6 & 0.2253 & 1.40020 & 0.0000457 & 23.26 & 0.001 & 0.0000 \\ 910.27 & 49.7 & 0.4312 & 1.38415 & 0.0000457 & 22.13 & 0.001 & 0.0000 \\ 924.53 & 49.3 & 0.3212 & 1.38415 & 0.0000457 & 17.15 & 0.001 & 0.0000 \\ 1643.30 & 74.6 & 0.2940 & 1.33418 & 0.0000457 & 17.15 & 0.001 & 0.0130 \\ 1072.74 & 265.4 & 0.3770 & 1.36884 & 0.0000457 & 22.99 & 0.001 & 0.0098 \\ 860.29 & 860.2 & 0.2789 & 1.38294 & 0.0000457 & 23.26 & 0.001 & 0.0097 \\ 860.36 & 860.2 & 0.2789 & 1.38293 & 0.0000457 & 23.26 & 0.001 & 0.0097 \\ 845.61 & 613.3 & 0.4127 & 1.38293 & 0.0000457 & 23.26 & 0.001 & 0.0097 \\ 445.95 & 2606.7 & 0.4981 & 1.40077 & 0.0000285 & 224.46 & 0.006 & 0.0000 \\ 442.46 & 2481.9 & 0.5441 & 1.40077 & 0.0000287 & 227.04 & 0.007 & 0.0000 \\ 442.46 & 2481.9 & 0.5441 & 1.40077 & 0.0000287 & 227.04 & 0.007 & 0.0000 \\ 409.94 & 2006.9 & 0.8457 & 1.40077 & 0.0000287 & 227.04 & 0.007 & 0.0000\end{array}$




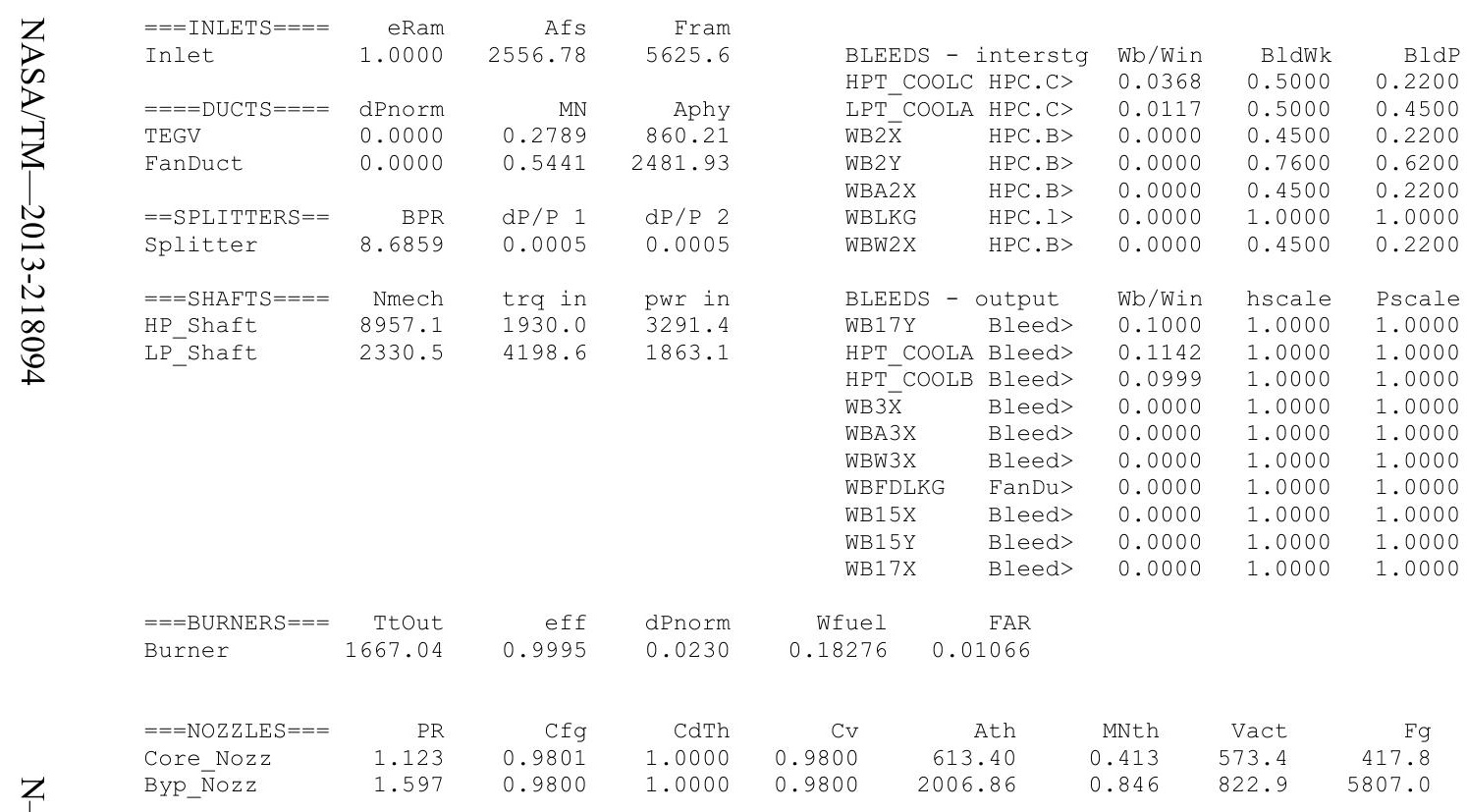


Version:NPSS Time:09:50:32 Model: Turbofan Engine - COMDES ON CONverge = 1 CASE: 0 $\begin{array}{llll}\text { Stator } 1 \text { inlet: } 454.76 & \text { Stator } 1 \text { exit: } 461.52 & \text { Stator } 2 \text { inlet: } 470.01 & \text { Stator } 2 \text { exit: } 474.34 \\ \text { Stator } 3 \text { inlet: } 483.09 & \text { Stator } 3 \text { exit: } 487.00 & \text { Stator } 4 \text { inlet: } 495.19 & \text { Stator } 4 \text { exit: } 498.51\end{array}$ Stator 5 inlet: 505.23 Stator 5 exit: $508.11 \quad$ Unblocked Percent Blockage: 0.00

Ambient Relative Humidity $\quad 10.00$

Fan Face Relative Humidity $\quad 2.40$

Fan Bypass Relative Humidity $\quad 1.75$

LPC Inlet Relative Humidity $\quad 1.56$

Deit Relative Humidity

Drop Diameter

0.00

0.0001000
707.26
3.09

Inlet Length

40.00

Ambient Flow Velocity

Ambient Static Pressure

Additional Water at LPC Exit

$\begin{array}{rrr}3.09 & \text { Fan/LPC Inlet Static Pressure } & 4.25 \\ 407.97 & \text { Fan/LPC Inlet Static Temperature } & 447.01\end{array}$

an/LPC lniet Flow velocity

$\begin{array}{rrr}707.26 & \text { Fan/LPC Inlet Flow Velocity } & 178.69 \\ 3.09 & \text { Fan/LPC Inlet Static Pressure } & 4.25 \\ 407.97 & \text { Fan/LPC Inlet Static Temperature } & 447.01\end{array}$

MN $\quad$ SUMMARY OUTPUT DATA

$\begin{array}{rrr}\text { MN } & \text { alt } & \text { dTamb } \\ 0.714 & 37357.0 & 18.00\end{array}$

257.06

Fn

Fn TSFC

1.0904

Wfuel
669.79

BPR
8.7249

VTAS
707.26

OPR
9.092

EPR
0.772

PowerSet
10.000

$\begin{array}{ccc}\text { T4 } & \text { T41 } & \text { T49 } \\ 1657.2 & 1566.2 & 1090.9\end{array}$
FSO Ambient.Fl O

FS1 Inlet.FI_O-

FS12 Splitter.F1_02

FS2 Splitter.Fl_0

FS14 Fan.F1-

FS24 VaporIN..$F 1$

ES25 Bleed2.FI_O

F3 HPC.Fl_O

ES46 Bleed3.F1_O

ES4

FS49 4 TP .

FS5 5 TEGV.FI

FS8 Core_Nozz.Fl_

FS17 FanDūctLkg.F̄̄

FS171 Bleed15.FI_O

FS172 FanDuct.FI-O

FS173 Byp Nozz.FİO

TURBOMACHINERY PERFORMANCE DATA

$\begin{array}{lrrrr} & \text { WC } & \text { PR } & \text { eff } \\ \text { Fan } & 727.62 & 1.118 & 0.9040 & 247 \\ \text { LPC } & 83.40 & 1.512 & 0.8407 & 247 \\ \text { HPC } & 53.22 & 6.017 & 0.8174 & 893 \\ \text { HPT } & 12.08 & 4.099 & 0.8721 \\ \text { LPT } & 53.81 & 2.726 & 0.8431\end{array}$

FLOW STATION DATA

$\begin{array}{rrrrr}\text { W } & \text { Pt } & \text { Tt } & \text { ht } & \text { FAR } \\ 257.06 & 4.339 & 449.68 & -22.87 & 0.0000 \\ 257.06 & 4.339 & 449.68 & -22.87 & 0.0000 \\ 230.63 & 4.337 & 449.68 & -22.87 & 0.0000 \\ 26.43 & 4.337 & 449.68 & -22.87 & 0.0000 \\ 230.63 & 4.848 & 465.79 & -19.01 & 0.0000 \\ 26.43 & 6.557 & 516.81 & -6.78 & 0.0000 \\ 26.43 & 6.557 & 516.86 & -6.86 & 0.0000 \\ 23.79 & 6.557 & 516.86 & -6.86 & 0.0000 \\ 22.64 & 39.453 & 935.42 & 94.66 & 0.0000 \\ 17.54 & 39.453 & 935.42 & 94.66 & 0.0000 \\ 17.73 & 38.544 & 1657.22 & 82.64 & 0.0106 \\ 23.70 & 9.404 & 1094.94 & -15.66 & 0.0079 \\ 23.98 & 3.450 & 872.15 & -69.85 & 0.0078 \\ 23.98 & 3.450 & 872.23 & -69.85 & 0.0078 \\ 23.98 & 3.450 & 872.30 & -69.85 & 0.0078 \\ 230.63 & 4.848 & 465.79 & -19.01 & 0.0000 \\ 233.27 & 4.848 & 466.37 & -18.88 & 0.0000 \\ 233.27 & 4.848 & 466.37 & -18.88 & 0.0000 \\ 233.27 & 4.848 & 466.37 & -18.88 & 0.0000\end{array}$

WC
810.61

810.61 810.61 727.62 83.40 662.46
59.13 59.13 59.14 53.22 11.32
8.78 12.78 12.08 132.45 132.45
132.45 132.46 662.46 670.47
670.47 $\begin{array}{llll}466.37 & -18.88 & 0.0000 & 670.47\end{array}$

$\begin{array}{rrr}\text { TS } & \text { Aphy } & \text { MN } \\ 407.97 & 2561.5 & 0.7140 \\ 438.72 & 4168.2 & 0.3530 \\ 437.15 & 3531.8 & 0.3780 \\ 447.01 & 830.5 & 0.1723 \\ 444.03 & 2606.7 & 0.4945 \\ 510.38 & 412.6 & 0.2509 \\ 510.43 & 412.6 & 0.2509 \\ 511.71 & 412.6 & 0.2241 \\ 903.10 & 49.7 & 0.4307 \\ 917.23 & 49.3 & 0.3209 \\ 1633.52 & 74.6 & 0.2940 \\ 1067.13 & 265.4 & 0.3751 \\ 859.87 & 860.2 & 0.2730 \\ 859.94 & 860.2 & 0.2730 \\ 845.95 & 613.4 & 0.4028 \\ 444.03 & 2606.7 & 0.4945 \\ 440.63 & 2481.9 & 0.5399 \\ 440.63 & 2481.9 & 0.5399 \\ 409.90 & 2006.9 & 0.8289\end{array}$

gamt
1.40090
1.40090
1.40090
1.40090
1.40079
1.40025
1.40025
1.40025
1.38459
1.38459
1.33469
1.36926
1.38302
1.38301
1.38301
1.40079
1.40078
1.40078
1.40078

WAR
0.0000272
0.0000272
0.0000272
0.0000272
0.0000272
0.0000272
0.0000433
0.0000433
0.0000433
0.0000433
0.0000433
0.0000433
0.0000433
0.0000433
0.0000433
0.0000272
0.0000274
0.0000274
0.0000274

WAR
00272 257.05 257.05
257.05 257.05
230.62 26.43 230.62 26.43
26.43 26.43
23.79 22.64
17.54 17.54
17.54 17.54
23.51 23.79
23.79

23.79
23.79
230.62

23.79
230.62 233.26
233.26 233.26
WH2O H2O frac $0.007 \quad 0.0000$ $0.001 \quad 0.0000$ $0.001 \quad 0.0000$ $0.001 \quad 0.0000$ $0.001 \quad 0.0000$ $0.001-0.0130$ $0.001 \quad 0.0096$ $0.006 \quad 0.0000$ $0.006 \quad 0.0000$ 0.0000

TURBOMACHINERY MAP DATA

$\begin{array}{lrrrrrrrrr} & \text { WcMap } & \text { PRmap } & \text { effMap } & \text { NcMap } & \text { R/Parm } & \text { S_WcDes } & \text { S_PRdes } & \text { S_effDes } & \text { S_NcDes } \\ \text { Fan } & 845.43 & 1.115 & 0.9058 & 2448.831 & 0.0412 & 0.8607 & \overline{1} .0235 & 0.9980 & 0.9905 \\ \text { LPC } & 69.76 & 1.479 & 0.7754 & 0.653 & 0.0000 & 1.1955 & 1.0683 & 1.0842 & 0.0003 \\ \text { HPC } & 48.41 & 5.780 & 0.8259 & 8694.906 & 11.0057 & 1.0995 & 1.0494 & 0.9898 & 0.9733 \\ \text { HPT } & 0.96 & 4.013 & 0.8721 & 1.316 & 4.0128 & 12.6299 & 0.9723 & 1.0000 & 0.0003 \\ \text { LPT } & 0.82 & 2.298 & 0.8493 & 0.783 & 2.2982 & 65.2590 & 0.7521 & 0.9926 & 0.0005\end{array}$




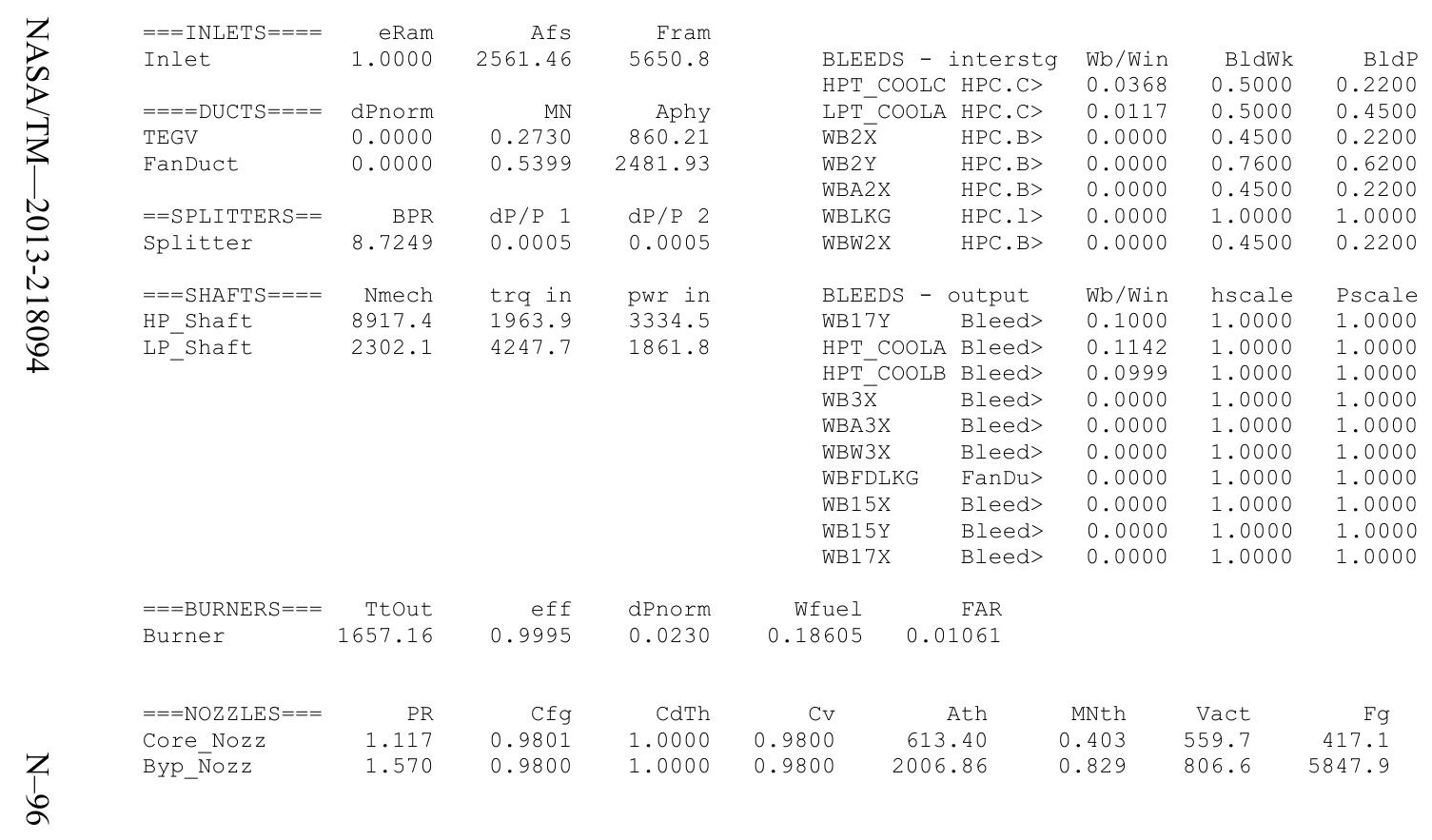


Date:07/05/13 Time:09:50:46 Model: Turbofan Engine - COMDES ON Converge = 1 CASE: 0

Version:NPSS 1.6.5 - Rev: -> Gas Package: Janaf iter/pass/Jacb/Broy=10/24/1/8 Run by: Philip C Jorgenson PC: 10

Temperature Stator 1 inlet: $456.41 \quad$ Stator 1 exit: $462.77 \quad$ Stator 2 inlet: $470.73 \quad$ Stator 2 exit: 474.78 $\begin{array}{llll}\text { Stator } 3 \text { inlet: } 482.99 & \text { Stator } 3 \text { exit: } 486.63 & \text { Stator } 4 \text { inlet: } 494.31 & \text { Stator } 4 \text { exit: } 497.3 \\ \text { Stator } 5 \text { inlet: } 503.67 & \text { Stator } 5 \text { exit: } 506.32 & \text { Unblocked Percent Blockage: } 0.00\end{array}$

Ambient Relative Humidity $\quad 10.00$

Fan Face Relative Humidity $\quad 3.00$

Fan Bypass Relative Humidity 2.26

LPC Inlet Relative Humidity $\quad 2.00$

Relt Relative Humidity

Drop Diameter

0.00

0.0001000

667.90

Inlet Length

40.00

Ambient Flow Velocity

Ambient Static Pressure

Additional Water at LPC Exit

414.42

Fan/LPC Inlet Static Pressure $\quad 173.05$

0.0000045

Fan/LPC Inlet Static Temperature 449.11

$\begin{array}{rrrrrr} & & & & \text { SUMMARY OUTPUT DATA } \\ \text { MN } & \text { alt } & \text { dTamb } & \text { W } & \text { Fn } & \text { TSFC } \\ 0.669 & 34281.0 & 18.00 & 278.57 & 669.4 & 1.0741\end{array}$

W
278.57

669.4

1.0741

Wfuel
718.95

8.8070

VTAS
667.90

FLOW STATION DATA

FSO Ambient.Fl_O

FS1 Inlet.FI_O

$\begin{array}{ll}\text { FS12 } & \text { Splitter.F1_02 } \\ \text { FS2 } & \text { Splitter.Fl_01 }\end{array}$

FS14 Fan.FI_-

FS24 VaporIN.FI

FS25 Bleed2.F1_O

FS3 HPC.FIO

IS36 Bleed3.FI_O

IS4 Burner.Fl_O

FS49 4 TPT

FS5 5 TEGV.

FS8 Core_Nozz.Fl_O

FS17 FanDūctLkg.F̄̄

FS171 Bleed15.FI

ES172 FanDuct.Fl-O

FS173 Byp Nozz.FİO

$W$
278.57
278.57
250.17
28.41
250.17
28.41
28.41
25.57
24.33
18.85
19.05
25.47
25.76
25.76
25.76
250.17
253.01
253.01
253.01

$\begin{array}{rrrr}\text { Pt } & \mathrm{Tt} & \mathrm{ht} & \mathrm{FAR} \\ 4.831 & 451.61 & -22.45 & 0.0000 \\ 4.831 & 451.61 & -22.45 & 0.0000\end{array}$

$\begin{array}{lllll}4.831 & 451.61 & -22.45 & 0.0000 & 790.73 \\ 4.831 & 451.61 & -22.45 & 0.0000 & 790.73\end{array}$

$\begin{array}{llll}451.61 & -22.45 & 0.00000 & 710.46\end{array}$

$\begin{array}{lllll}4.360 & 456.77 & -18.45 & 0.0000 & 80.67\end{array}$

$\begin{array}{lllll}7.122 & 514.69 & -7.82 & 0.0000 & 650.67\end{array}$

$\begin{array}{lllll}7.122 & 514.73 & -7.35 & 0.0000 & 58.39\end{array}$

$\begin{array}{lllll}7.122 & 514.73 & -7.35 & 0.0000 & 58.39 \\ 42.305 & 928.59 & -7.35 & 0.0000 & 52.5\end{array}$

$\begin{array}{llll}72.305 & 928.59 & -7.35 & 0.0000\end{array}$

$\begin{array}{llll}41.330 & 1650.37 & 81.01 & 0.0106\end{array}$

$\begin{array}{lllll}1.302 & 1650.37 & 81.01 & 0.0106\end{array}$

$3.941 \quad 881.32$

$3.941-881.32$

$\begin{array}{ll}-16.13 & 0.0079 \\ -67.40 & 0.0078\end{array}$

$\begin{array}{lll}881.39 & -67.40 & 0.0078\end{array}$

$\begin{array}{llll}3.941 & 881.46 & -67.39 & 0.0078 \\ 5.360 & 466.77 & -18.82 & 0.0000\end{array}$

$\begin{array}{lllll}5.360 & 467.31 & -18.69 & 0.0000\end{array}$

$\begin{array}{lllll}5.360 & 467.31 & -18.69 & 0.0000\end{array}$

$\begin{array}{lllll}5.360 & 467.31 & -18.69 & 0.0000 & 658.44 \\ 5.360 & 467.31 & -18.69 & 0.0000 & 658.44\end{array}$

11.31

8.76
12.08
53.29

53.29

125.22
125.23

125.23

658.44

658.44

TURBOMACHINERY PERFORMANCE DATA

$\begin{array}{lrrr} & \text { WC } & \text { PR } & \text { eff } \\ \text { Fan } & 710.46 & 1.110 & 0.9032 \\ \text { LPC } & 80.67 & 1.475 & 0.8416 \\ \text { HPC } & 52.55 & 5.940 & 0.8161 \\ \text { HPT } & 12.08 & 4.055 & 0.8716 \\ \text { LPT } & 53.29 & 2.586 & 0.8385\end{array}$

\section{NC
2395.702
2395.702}

TR
1.0336
1.1397

efPoly

2395.702
8911.013

218.515

$\begin{array}{ll}1.03367 & 0.80401 \\ 1.8040 & 0.8550 \\ 1.3586 & 0.8490\end{array}$

1.3586

0.8550

0.8195

pwr
-1286.1
-607.7
-3541.9
3541.9
1893.8

$\begin{array}{rrr}\text { pwr } & \text { SMN } & \text { SMW } \\ 286.1 & 5792.75 & 54.21\end{array}$

TURBOMACHINERY MAP DATA

$\begin{array}{lrrrrrrrrr} & \text { WcMap } & \text { PRmap } & \text { effMap } & \text { NcMap } & \text { R/Parm } & \text { S_WcDes } & \text { S_PRdes } & \text { S_effDes } & \text { S_NcDes } \\ \text { Fan } & 825.48 & 1.108 & 0.9050 & 2372.859 & 0.0413 & 0.8607 & \overline{1} .0235 & 0.9980 & 0.9905 \\ \text { LPC } & 66.44 & 1.445 & 0.7718 & 0.633 & 0.0000 & 1.2142 & 1.0674 & 1.0904 & 0.0003 \\ \text { HPC } & 47.80 & 5.707 & 0.8246 & 8673.452 & 10.9959 & 1.0995 & 1.0494 & 0.9898 & 0.9733 \\ \text { HPT } & 0.96 & 3.970 & 0.8716 & 1.313 & 3.9703 & 12.6299 & 0.9723 & 1.0000 & 0.0003 \\ \text { LPT } & 0.82 & 2.193 & 0.8448 & 0.761 & 2.1928 & 65.2590 & 0.7521 & 0.9926 & 0.0005\end{array}$

\begin{tabular}{|c|c|}
\hline \multicolumn{2}{|c|}{ Ps } \\
\hline 3.5 & 414 \\
\hline 4.4 & 441 \\
\hline 4.3 & 439 \\
\hline 4.7 & 449 \\
\hline 4.5 & 445 \\
\hline 6.8 & 50 \\
\hline 6.8 & 508 \\
\hline 6.8 & 50 \\
\hline 37.3 & \\
\hline 39.4 & 910 \\
\hline 39.0 & 162 \\
\hline 9.2 & 1065 \\
\hline 3.7 & 870 \\
\hline 3.7 & 870 \\
\hline 3.5 & 858 \\
\hline 4.5 & \\
\hline 4.4 & 442 \\
\hline 4.4 & 442 \\
\hline 3.5 & 416 \\
\hline & SMW \\
\hline & 54.21 \\
\hline & 3.05 \\
\hline & 57.85 \\
\hline $\begin{array}{l}\text { fDes } \\
9980\end{array}$ & $\begin{array}{l}s^{S} \text { NCDes } \\
0.9905\end{array}$ \\
\hline .09980 & 0.000 \\
\hline .9898 & 0.9733 \\
\hline 0000 & 0.000 \\
\hline & \\
\hline
\end{tabular}

$\begin{array}{lll}0.193 & 0.8716 \\ 0.8448\end{array}$

2.1928

$65.2590 \quad 0.7521$ $\begin{array}{llccc}\text { EPR } & \text { PowerSet } & \text { T4 } & \text { T41 } & \text { T49 } \\ 0.795 & 10.000 & 1650.4 & 1559.3 & 1088.3\end{array}$

gamt
1.40088
1.40088
1.40088
1.40088
1.40078
1.40028
1.40028
1.40028
1.38498
1.38498
1.33504
1.36944
1.38251
1.38251
1.38250
1.40078
1.40078
1.40078
1.40078

WAR
0.0000348
0.0000348
0.0000348
0.0000348
0.0000348
0.0000348
0.0000393
0.0000393
0.0000393
0.0000393
0.0000393
0.0000393
0.0000393
0.0000393
0.0000393
0.0000348
0.0000348
0.0000348
0.0000348

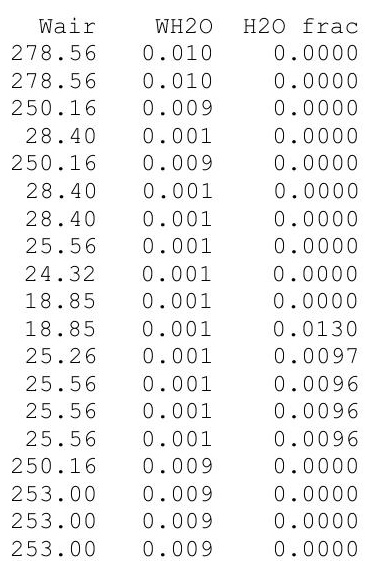




\begin{tabular}{|c|c|c|c|c|c|c|c|c|}
\hline \multirow{2}{*}{$\begin{array}{ll}Z & ===\text { INLETS }==== \\
& \text { Inlet }\end{array}$} & eRam & Afs & Fram & & \multirow[b]{2}{*}{ Wb/Win } & \multirow[b]{2}{*}{$\begin{array}{r}\text { BldWk } \\
0.5000\end{array}$} & \multirow[b]{2}{*}{$\begin{aligned} & B l d F \\
& 0.2200\end{aligned}$} \\
\hline & 1.0000 & 2577.36 & 5782.9 & $\begin{array}{l}\text { BLEEDS - } \\
\text { HPT COOLC }\end{array}$ & $\begin{array}{l}\text { intersto } \\
\text { C HPC.C }>\end{array}$ & & & \\
\hline$====$ DUCT $S===$ & dPnorm & MN & Aphy & $\mathrm{LPT}^{-} \mathrm{COOLA}$ & A $\mathrm{HPC} . \mathrm{C}>$ & 0.0117 & 0.5000 & 0.4500 \\
\hline TEGV & 0.0000 & 0.2568 & 860.21 & WB $2 \overline{\mathrm{X}}$ & HPC. B> & 0.0000 & 0.4500 & 0.2200 \\
\hline FanDuct & 0.0000 & 0.5256 & 2481.93 & WB2Y & HPC.B> & 0.0000 & 0.7600 & 0.6200 \\
\hline & & & & WBA2X & HPC.B> & 0.0000 & 0.4500 & 0.2200 \\
\hline$==$ SPLITTERS $==$ & BPR & $\mathrm{dP} / \mathrm{P} 1$ & $\mathrm{dP} / \mathrm{P} 2$ & WBLKG & HPC.I> & 0.0000 & 1.0000 & 1.0000 \\
\hline Splitter & 8.8070 & 0.0005 & 0.0005 & WBW2X & HPC.B> & 0.0000 & 0.4500 & 0.2200 \\
\hline$===$ SHAFTS $====$ & Nmech & trq in & pwr in & BLEEDS - & - output & Wb/Win & hscale & Pscale \\
\hline HP Shaft & 8877.1 & 2095.5 & 3541.9 & WB17Y & Bleed> & 0.1000 & 1.0000 & 1.0000 \\
\hline LP_Shaft & 2235.5 & 4449.5 & 1893.8 & HPT COOLA & A B Bleed> & 0.1142 & 1.0000 & 1.0000 \\
\hline & & & & HPT_COOLB $^{-}$ & B Bleed> & 0.0999 & 1.0000 & 1.0000 \\
\hline & & & & WB $3 \bar{X}$ & Bleed> & 0.0000 & 1.0000 & 1.0000 \\
\hline & & & & WBA3X & Bleed> & 0.0000 & 1.0000 & 1.0000 \\
\hline & & & & WBW3X & Bleed> & 0.0000 & 1.0000 & 1.0000 \\
\hline & & & & WBFDLKG & FanDu> & 0.0000 & 1.0000 & 1.0000 \\
\hline & & & & WB15X & Bleed> & 0.0000 & 1.0000 & 1.0000 \\
\hline & & & & WB15Y & Bleed> & 0.0000 & 1.0000 & 1.0000 \\
\hline & & & & WB17X & Bleed> & 0.0000 & 1.0000 & 1.0000 \\
\hline$===$ BURNERS $===$ & Ttout & eff & dPnorm & Wfuel & FAR & & & \\
\hline Burner & 1650.30 & 0.9995 & 0.0230 & 0.19971 & 01059 & & & \\
\hline$===$ NOZZLES $===$ & PR & $\mathrm{Cfg}$ & CdTh & $\mathrm{Cv}$ & Ath & MNth & Vact & $\mathrm{Fg}$ \\
\hline Core_Nozz & 1.102 & 0.9801 & 1.0000 & 0.9800 & 3.40 & 0.376 & 526.7 & 421.8 \\
\hline Byp Nozz & 1.498 & 0.9800 & 1.0000 & 0.9800 & 6.86 & 0.782 & 766.9 & 6030.4 \\
\hline
\end{tabular}


Date:07/05/13 Time:09:51:03 Model: Turbofan Engine - COMDES ON Converge $=1$ CASE: 0

Temperature Stator 1 inlet: $465.04 \quad$ Stator 1 exit: $470.56 \quad$ Stator 2 inlet: 477.37 Stator 2 exit: 480.66 Stator 3 inlet: $487.79 \quad$ Stator 3 exit: 490.68 Stator 4 inlet: 497.38 Stator 4 exit: 499.7

$$
\text { Stator } 5 \text { inlet: 505.17 Stator } 5 \text { exit: } 507.19 \text { Unblocked Percent Blockage: } 0.00
$$

Ambient Relative Humidity $\quad 10.00$

Fan Face Relative Humidity $\quad 3.96$

PC Bypass Relative Humid

LPC Exit Relative Humidity

HPC Relative Humidity

0.47
0.00

Drop Diameter

0.0001000

mbient Flow Velocity

ambient Static Pressure

Additional Water at LPC Exit

4.36 Fan/LPC Inlet Static Pressure

429.58 Fan/LPC Inlet Static Temperature 458.86

\section{SUMMARY OUTPUT DATA}

$\begin{array}{rrr}\text { MN } & \text { alt } & \text { dTamb } \\ 0.608 & 30029.0 & 18.00\end{array}$

W

$\begin{array}{rr}\text { Fn } & \text { TSFC } \\ 728.7 & 1.0768\end{array}$

728.7

.0768
Wfuel

784.65
BPR
8.3701

$\begin{array}{r}617.99 \\ \hline\end{array}$
OPR
8.197
EPR
0.828
PowerSet
10.000 $\begin{array}{cc}\text { T4 } & \text { T41 } \\ 1654.7 & 1563.0\end{array}$
FSO Ambient.Fl_O

FS1 Inlet.FI_O

FS12 Splitter.F1_02

FS2 Splitter.Fl_01

FS14 Fan.Fl_

$\begin{array}{ll}\text { FS23 } & \text { LPC.FI_O } \\ \text { FS24 } & \text { VaporIN. F1 }\end{array}$

FS25 Bleed2.FI_O

FS3 HPC.FIO

FS36 Bleed3.F1_O

FS4 Burner.FI_O

FS45 HPT.FI_O

TS5

FS8 Core_Nozz.Fl

FS17 FanDüctLkg.Fì_O

FS171 Bleed15.FI

FS172 FanDuct.Fl-0

FS173 Byp Nozz.Fì O

W
305.09
305.09
272.53
32.56
272.53
32.56
32.56
27.68
26.33
20.41
20.63
27.57
27.89
27.89
27.89
272.53
277.42
277.42
277.42

FLOW STATION DATA

$\begin{array}{rrrrr}\text { Pt } & \text { Tt } & \text { ht } & \text { FAR } & \text { Wc } \\ 5.595 & 461.42 & -20.30 & 0.0000 & 755.81 \\ 5.595 & 461.42 & -20.30 & 0.0000 & 755.81 \\ 5.592 & 461.42 & -20.30 & 0.0000 & 675.49 \\ 5.592 & 461.42 & -20.30 & 0.0000 & 80.70 \\ 6.139 & 475.25 & -16.98 & 0.0000 & 624.50 \\ 7.825 & 515.83 & -7.25 & 0.0000 & 60.98 \\ 7.825 & 515.87 & -7.29 & 0.0000 & 60.98 \\ 7.825 & 515.87 & -7.29 & 0.0000 & 51.84 \\ 45.861 & 927.38 & 92.50 & 0.0000 & 11.28 \\ 45.861 & 927.38 & 92.50 & 0.0000 & 8.74 \\ 44.805 & 1654.74 & 80.41 & 0.0107 & 12.08 \\ 1.202 & 1097.92 & -16.13 & 0.0080 & 52.62 \\ 4.717 & 904.47 & -63.05 & 0.0079 & 114.77 \\ 4.717 & 904.54 & -63.05 & 0.0079 & 114.77 \\ 4.717 & 904.62 & -63.05 & 0.0079 & 114.78 \\ 6.139 & 475.25 & -16.98 & 0.0000 & 624.50 \\ 6.139 & 475.96 & -16.81 & 0.0000 & 636.17 \\ 6.139 & 475.96 & -16.81 & 0.0000 & 636.17 \\ 6.139 & 475.96 & -16.81 & 0.0000 & 636.17\end{array}$

P
4.35
5.19
5.14
5.48
5.32
7.468
7.468
7.57
0.463
2.75
2.309
0.23
4.543
4.543
4.35
5.321
5.173
5.173

Ts
429.58
451.82
450.59
458.86
456.19
508.98
509.02
511.01
895.60
909.48
1631.07
1071.44
895.13
895.20
885.09
456.19
453.22
453.2

$\begin{array}{rr}\text { Aphy } & \text { MN } \\ 2596.1 & 0.6080 \\ 4168.2 & 0.3256 \\ 3531.8 & 0.3462 \\ 830.5 & 0.1666 \\ 2606.7 & 0.4566 \\ 412.6 & 0.2594 \\ 412.6 & 0.2594 \\ 412.6 & 0.2179 \\ 49.7 & 0.4287 \\ 49.3 & 0.3195 \\ 74.6 & 0.2940 \\ 265.4 & 0.3654 \\ 860.2 & 0.2340 \\ 860.2 & 0.2340 \\ 613.4 & 0.3400 \\ 2606.7 & 0.4566 \\ 2481.9 & 0.5004 \\ 2481.9 & 0.5004 \\ 2006.9 & 0.7168\end{array}$

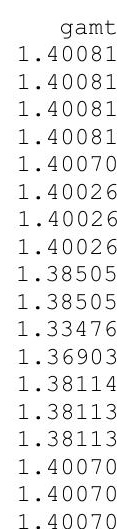

1.40081

1.40081

1.40081

1.40026

1.40026

1.38505

1.36903

1.38113

1.40070

1.40070

$\begin{array}{rrrr}\text { WAR } & \text { Wair } & \text { WH2O } & \text { H2O frac } \\ 0.0000681 & 305.07 & 0.021 & 0.0001 \\ 0.0000681 & 305.07 & 0.021 & 0.0001 \\ 0.0000681 & 272.51 & 0.019 & 0.0001 \\ 0.0000681 & 32.56 & 0.002 & 0.0001 \\ 0.0000681 & 272.51 & 0.019 & 0.0001 \\ 0.0000681 & 32.56 & 0.002 & 0.0001 \\ 0.0000756 & 32.56 & 0.002 & 0.0001 \\ 0.0000756 & 27.67 & 0.002 & 0.0001 \\ 0.0000756 & 26.33 & 0.002 & 0.0001 \\ 0.0000756 & 20.41 & 0.002 & 0.001 \\ 0.0000756 & 20.41 & 0.002 & 0.0131 \\ 0.0000756 & 27.35 & 0.002 & 0.0098 \\ 0.0000756 & 27.67 & 0.002 & 0.0097 \\ 0.0000756 & 27.67 & 0.002 & 0.0097 \\ 0.0000756 & 27.67 & 0.002 & 0.0097 \\ 0.0000681 & 272.51 & 0.019 & 0.0001 \\ 0.0000682 & 277.40 & 0.019 & 0.0001 \\ 0.0000682 & 277.40 & 0.019 & 0.0001 \\ 0.0000682 & 277.40 & 0.019 & 0.0001\end{array}$

TURBOMACHINERY PERFORMANCE DATA

$\begin{array}{rrrrrr}\text { NC } & \text { TR } & \text { efPoly } & \text { pwr } & \text { SMN } & \text { SMW } \\ 2249.340 & 1.0300 & 0.9034 & -1278.0 & -9299.10 & 52.72 \\ 2249.340 & 1.1179 & 0.8619 & -601.0 & 5.96 & 2.63 \\ 8887.506 & 1.7977 & 0.8536 & -3812.6 & 61.60 & 58.03 \\ 217.891 & 1.3544 & 0.8495 & 3812.6 & & \\ 64.028 & 1.2114 & 0.8124 & 1879.0 & & \end{array}$

TURBOMACHINERY MAP DATA

\begin{tabular}{|c|c|c|c|c|c|c|c|c|c|}
\hline & WcMap & PRmap & effMap & NcMap & R/Parm & s WcDes & s PRdes & $s$ effDes & s NcDes \\
\hline Fan & 784.85 & 1.095 & 0.9039 & 2227.892 & 0.0416 & 0.8607 & 1.0235 & 0.9980 & 0.9905 \\
\hline LPC & 60.32 & 1.382 & 0.7655 & 0.594 & 0.0000 & 1.3379 & 1.0458 & 1.1172 & 0.0003 \\
\hline HPC & 47.14 & 5.632 & 0.8231 & 8650.571 & 10.9889 & 1.0995 & 1.0494 & 0.9898 & 0.9733 \\
\hline HPT & 0.96 & 3.917 & 0.8718 & 1.310 & 3.9166 & 12.6299 & 0.9723 & 1.0000 & 0.0003 \\
\hline LPT & 0.81 & 2.034 & 0.8365 & 0.720 & 2.0342 & 65.2590 & 0.7521 & 0.9926 & 0.0005 \\
\hline
\end{tabular}




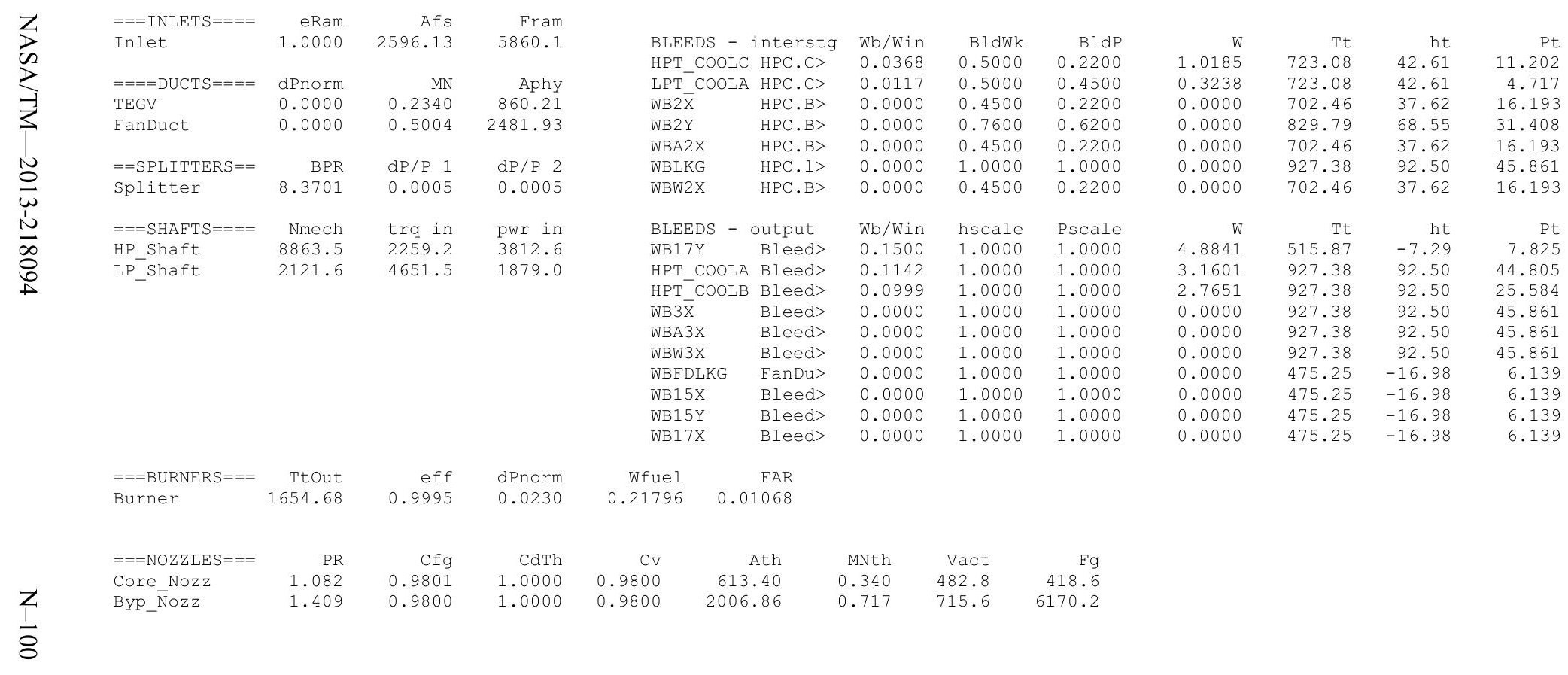


Date:07/05/13 Time:09:51:32 Model: Turbofan Engine - COMDES ON Converge = 1 CASE: 0

Version:NPSS 1.6.5 - Rev: -> Gas Package: Janaf iter/pass/Jacb/Broy= 20/48/2/17 Run by: Philip C Jorgenson PC: 10

Temperature Stator 1 inlet: $473.10 \quad$ Stator 1 exit: $477.17 \quad$ Stator 2 inlet: 481.78 Stator 2 exit: 483.58 $\begin{array}{lll}\text { Stator } 3 \text { inlet: } 488.71 & \text { Stator } 3 \text { exit: } 490.11 & \text { Stator } 4 \text { inlet: } 495.03 \text { Stator } 4 \text { exit: } 496.03 \\ \text { Stator } 5 \text { inlet: } 499.63 & \text { Stator } 5 \text { exit: } 500.34 & \text { Unblocked Percent Blockage: } 0.00\end{array}$

Ambient Relative Humidity $\quad 10.00$

Fan Face Relative Humidity $\quad 4.82$

$\begin{array}{ll}\text { Fan Bypass Relative Humidity } & 3.94 \\ & 3.82\end{array}$

LPC Exit Relative Humidity 1.20

HPC Relative Humidity

Drop Diameter

0.00
0.0001000
571.13

Ambient Flow Velocity

571.13
5.30

Inlet Length

Ambient Static Pressure

Additional Water at LPC Exit

445.14 0.0000104

$\begin{array}{rrrrrr}\text { MN } & \text { alt } & \text { dTamb } & \text { W } & \text { SUMMAR OUTPUT DATA } \\ 0.552 & 25666.0 & \text { Fn } & \text { TSFC }\end{array}$

FSO Ambient.FI O

FS12 Split. 1 - 0

FS2 Splitter.

$\begin{array}{ccc}\text { FLOW } & \text { STATION DATA } \\ \text { Pt } & \text { Tt }\end{array}$

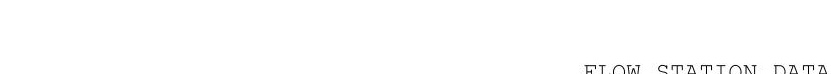

FS14 Fan.FI_O

FS23 LPC.FI_O

$\begin{array}{ll}\text { FS24 } & \text { VaporIN.F1_O } \\ \text { FS25 } & \text { Bleed2.Fl_O }\end{array}$

$\begin{array}{ll}\text { FS25 } & \text { Bleed2.FI- } \\ \text { FS3 } & \text { HPC.Fl_O }\end{array}$

FS36 Bleed3.F1_O

FS4 Burner.F1_O

FS45 HPT.FI-

FS49 LPT.FI-O

FS8 Core_Nozz.FI_O

FS17 FanDuctikg. Fi__o

FS171 Bleed15.FI_O

FS172 Fanduct.Fl_O
FS173 Byp Nozz.FI_O

W
330.53
330.53
289.47
41.07
289.47
41.07
41.07
28.75
27.35
21.20
21.43
28.64
28.98
28.98
28.98
289.47
301.79
301.79
301.79

$\begin{array}{rrrr}\text { Pt } & \text { Tt } & \text { ht } & \text { FAR } \\ 6.516 & 472.33 & -18.03 & 0.0000 \\ 6.516 & 472.33 & -18.03 & 0.0000 \\ 6.513 & 472.33 & -18.03 & 0.0000 \\ 6.513 & 472.33 & -18.03 & 0.0000 \\ 7.057 & 484.46 & -15.12 & 0.0000 \\ 8.205 & 511.58 & -8.62 & 0.0000 \\ 8.205 & 511.62 & -8.66 & 0.0000 \\ 8.205 & 511.62 & -8.66 & 0.0000 \\ 47.789 & 918.52 & 89.96 & 0.0000 \\ 47.789 & 918.52 & 89.96 & 0.0000 \\ 46.689 & 1665.49 & 77.57 & 0.0110 \\ 12.029 & 1109.28 & -17.71 & 0.0082 \\ 5.626 & 936.96 & -59.42 & 0.0081 \\ 5.626 & 937.04 & -59.42 & 0.0081 \\ 5.626 & 937.11 & -59.42 & 0.0081 \\ 7.057 & 484.46 & -15.12 & 0.0000 \\ 7.057 & 485.57 & -14.86 & 0.0000 \\ 7.057 & 485.57 & -14.86 & 0.0000 \\ 7.057 & 485.57 & -14.86 & 0.0000\end{array}$

Wfuel

BPR
7.0489

VTAS
571.13

OPR
7.334

EPR
0.859

PowerSet
10.000

$\begin{array}{ccc}\text { T4 } & \text { T41 } & \text { T49 } \\ 1665.5 & 1571.5 & 1104.9\end{array}$

TURBOMACHINERY PERFORMANCE DATA

\begin{tabular}{|c|c|c|c|c|c|c|c|c|c|}
\hline & Wc & PR & eff & $\mathrm{NC}$ & $T R$ & efpoly & pwr & SMN & SMW \\
\hline Fan & 623.29 & 1.083 & 0.9032 & 2044.521 & 1.0257 & 0.9043 & -1190.8 & -2764.78 & 49.88 \\
\hline LPC & 88.42 & 1.260 & 0.8217 & 2044.521 & 1.0831 & 0.8274 & -546.8 & 6.38 & 2.41 \\
\hline PC & 51.13 & 5.824 & 0.8139 & 8865.647 & 1.7953 & 0.8529 & -3913.9 & 60.72 & 57.10 \\
\hline HPT & 12.09 & 3.881 & 0.8732 & 215.758 & 1.3457 & 0.8517 & 3913.9 & & \\
\hline LPT & 51.17 & 2.138 & 0.8210 & 58.579 & 1.1810 & 0.8047 & 1737.6 & & \\
\hline$J R B$ & IERY MAP & DATA & & & & & & & \\
\hline & WcMap & PRmap & effMap & NcMap & R/Parm & s_WCDes & S_PRdes & $s_{\text {S_effDes }}$ & S_NCDes \\
\hline Fan & 724.20 & 1.082 & 0.9050 & 2025.027 & 0.0425 & 0.8607 & 1.0235 & 0.9980 & 0.9905 \\
\hline PC & 53.47 & 1.3 & & 0.540 & 0 & 1.6 & 0.8353 & 1.0 & 0.0003 \\
\hline PC & 46.51 & 5.597 & 0.8223 & 8629.294 & 11.0519 & 1.0995 & 1.0494 & 0.9898 & 0.9733 \\
\hline IPT & 0.96 & 3.801 & 0.8732 & 1.297 & 3.8014 & 12.6299 & 0.9723 & 1.0000 & 0.0003 \\
\hline $\mathrm{PT}$ & 0.78 & 1.856 & 0.8271 & 0.659 & 1.8560 & 65.2590 & 0.7521 & 0.9926 & 0.0005 \\
\hline
\end{tabular}




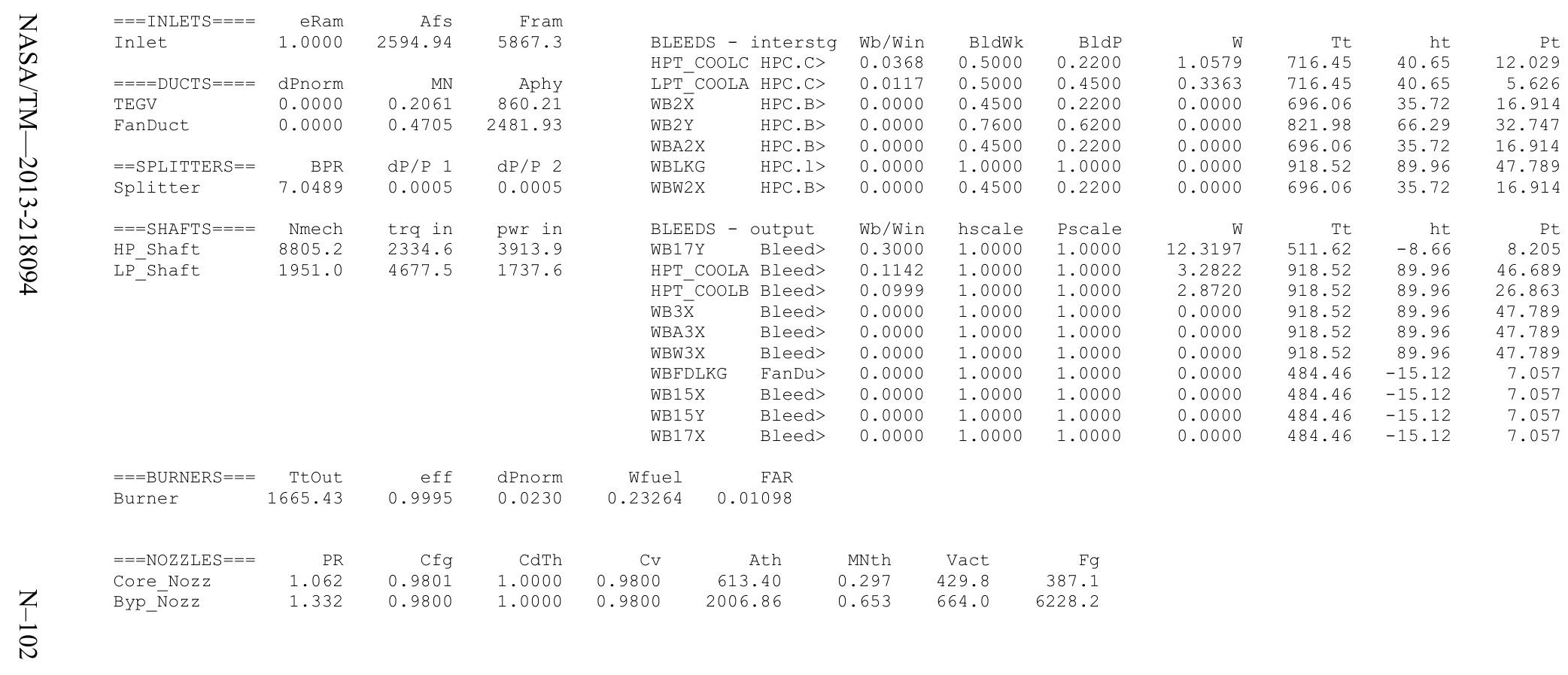


Date:07/05/13 Time:09:52:03 Model: Turbofan Engine - COMDES ON Converge = 1 CASE: 0

Version:NPSS 1.6.5 - Rev: -> Gas Package: Janaf iter/pass/Jacb/Broy= 26/54/2/23 Run by: Philip C Jorgenson PC: 10

Temperature Stator 1 inlet: $486.26 \quad$ Stator 1 exit: $489.21 \quad$ Stator 2 inlet: $491.94 \quad$ Stator 2 exit: 492.70 Stator 5 inlet: 501.46 Stator 5 exit: $501.30 \quad$ Unblocked Percent Blockage: 0.00

Ambient Relative Humidity $\quad 10.00$

Fan Face Relative Humidity $\quad 5.85$

Fan Bypass Relative Humidity $\quad 4.91$

LPC Inlet Relative Humidity $\quad 5.02$

HPC Relatiative Humidity

Drop Diameter

0.01

0.0001000

518.26

Inlet Length

40.00

Ambient Flow Velocity

Ambient Static Pressure

Additional Water at LPC Exit

$\begin{array}{rrr}6.74 & \text { Fan/LPC Inlet Static Pressure } & 7.74 \\ 465.18 & \text { Fan/LPC Inlet Static Temperature } & 484.08\end{array}$

flow velocity

204.34
7.74

MN alt dTamb SUMMARY OUTPUT DATA

$\begin{array}{rrr}\text { MN } & \text { alt } & \text { dTamb } \\ 0.490 & 20047.0 & 18.00\end{array}$

368.14

Fn

TSFC
1.1216

Wfuel
932.52

BPR
6.2369

VTAS
518.26

OPR
6.453

EPR

PowerSet

$\begin{array}{cc}\text { T4 } & \text { T41 } \\ 1698.5 & 1600.5\end{array}$

T49

FLOW STATION DATA

FSO Ambient.FI_O

FS1 Inlet.FI_O

$\begin{array}{ll}\text { FS12 } & \text { Splitter.FI_02 } \\ \text { FS2 } & \text { Splitter.Fl_01 }\end{array}$

FS14 Fan.F1_O

FS24 VaporIN.FI

FS25 Bleed2.FI_

FS3 HPC.Fl_O

ES36 Bleed3.FI_-

Burner.FI-O

FS49

FS5 TEGV.FİO

FS8 Core_Nozz.FI_O

FS17 FanDuctLkg.FI

FS171 Bleed15.F1

FS173 Byp Nozz.

$W$
368.14
368.14
317.27
50.87
317.27
50.87
50.87
30.52
29.04
22.51
22.77
30.42
30.78
30.78
30.78
317.27
337.62
337.62
337.62

$\begin{array}{cc}\text { Pt } & \text { Tt } \\ 7.943 & 487.56\end{array}$

ht FAR

$\begin{array}{llllll}7.943 & 487.56 & -15.16 & 0.0000 & 660.35\end{array}$

$\begin{array}{lllll}7.939 & 487.56 & -15.16 & 0.0000 & 569.39\end{array}$

$\begin{array}{rrrrr}7.939 & 487.56 & -15.16 & 0.0000 & 91.29 \\ 8.512 & 498.38 & -12.56 & 0.0000 & 536.96\end{array}$

$\begin{array}{rrrrr}8.512 & 498.38 & -12.56 & 0.0000 & 536.96 \\ 9.077 & 515.17 & -8.54 & 0.0000 & 82.08\end{array}$

$\begin{array}{lllll}9.077 & 515.17 & -8.54 & 0.0000 & 82.08 \\ 9.077 & 515.22 & -8.62 & 0.0000 & 82.09\end{array}$

$\begin{array}{lllll}9.077 & 515.22 & -8.62 & 0.0000 & 49.25\end{array}$

$\begin{array}{lllll}51.257 & 917.82 & 88.98 & 0.0000 & 11.08 \\ 51.257 & 917.82 & 88.98 & 0.0000 & 8.58\end{array}$

$\begin{array}{llll}51.257 & 917.82 & 88.98 & 0.0000 \\ 50.076 & 1698.52 & 76.01 & 0.0115\end{array}$

$\begin{array}{llrr}50.076 & 1698.52 & 76.01 & 0.0115 \\ 13.552 & 1140.27 & -18.07 & 0.0086\end{array}$

$13.552-1140.27$

$\begin{array}{lll}-18.07 & 0.0086 & 48.92\end{array}$

Ps
6.740
7.522

$\begin{array}{rrrr}\text { Ts } & \text { Aphy } & \text { MN } & \text { gamt } \\ 465.18 & 2615.9 & 0.4900 & 1.40057\end{array}$

$\begin{array}{llll}7.522 & 485.18 & 2615.9 & 0.4900 \\ 7.503 & 480.73 & 4168.2 & 0.2799\end{array}$

$\begin{array}{llll}7.522 & 479.73 & 3531.8 & 0.2854\end{array}$

$\begin{array}{rrrr}480.02 & 4168.2 & 0.2799 & 1.40057 \\ 479.73 & 3531.8 & 0.2854 & 1.40057 \\ 484.08 & 830.5 & 0.1894 & 1.40057\end{array}$

$\begin{array}{lrr}484.52 & 2606.7 & 0.3780 \\ 501.96 & 412.6 & 0.3626\end{array}$

$\begin{array}{lll}501.96 & 412.6 & 0.3626 \\ 502.01 & 412.6 & 0.3627\end{array}$

$\begin{array}{rr}412.6 & 0.3627 \\ 412.6 & 0.2065 \\ 49.7 & 0.4188\end{array}$

510.86
887.72

$\begin{array}{llll}47.924 & 900.79 & 49.3 & 0.3129\end{array}$

$\begin{array}{ll}74.6 & 0.2946 \\ 265.4 & 0.3361\end{array}$

12.5551117 .14

$\begin{array}{ll}265.4 & 0.3361 \\ 860.2 & 0.1785\end{array}$

$\begin{array}{lll}88.55 & 6.895 & 981.1 \\ 88.55 & 6.895 & 981.21\end{array}$

$\begin{array}{lllll}7.047 & 987.08 & -55.10 & 0.0085 & 88.55\end{array}$

$\begin{array}{llllr}7.047 & 987.08 & -55.10 & 0.0085 & 88.55 \\ 7.047 & 987.15 & -55.10 & 0.0085 & 88.56 \\ 8.512 & 498.38 & -12.56 & 0.0000 & 536.96\end{array}$

$\begin{array}{lllll}8.512 & 498.38 & -12.56 & 0.0085 & 88.56 \\ 8.512 & 499.40 & -12.32 & 0.0000 & 571.98\end{array}$

$\begin{array}{lllll}8.512 & 499.40 & -12.32 & 0.0000 & 536.96 \\ 8.512 & 499.40 & -12.32 & 0.0000 & 571.98\end{array}$

$\begin{array}{ll}6.8955 & 981.21 \\ 6.740 & 975.21 \\ 7.712 & 484.52\end{array}$

$\begin{array}{ll}860.2 & 0.1785\end{array}$

$\begin{array}{ll}6.895 & 981.21 \\ 7.740 & 975.21 \\ 7.477 & 484.52\end{array}$

$\begin{array}{rr}613.4 & 0.2552 \\ 2606.7 & 0.3780 \\ 2481.9 & 0.4343\end{array}$

$2481.9 \quad 0.4343$

$\begin{array}{ll}7.712 & 484.52 \\ 7.477 & 481.22 \\ 6.740 & 467.15\end{array}$

$6.740 \quad 467.15$

$\begin{array}{ll}2481.9 & 0.4343 \\ 2006.9 & 0.5870\end{array}$

$\begin{array}{lllll}499.40 & -12.32 & 0.0000 & 571.98 & 6.740\end{array}$

1.40057
1.40045

1.40024
1.40024

1.40024
1.40024
1.38557

1.38557
1.38557

1.33205

0.0002790

$.37572-0.0002790$

1.37572
1.40045

0.0002790

$\begin{array}{ll}1.40045 & 0.0002627 \\ 1.40044 & 0.0002637 \\ 1.40044 & 0.0002637\end{array}$

0.0002637

WAR
02627 .0002627
.0002627 .0002627 .0002627 0.0002790

TURBOMACHINERY PERFORMANCE DATA

$\begin{array}{lrrr} & \text { WC } & \text { PR } & \text { eff } \\ \text { Fan } & 569.39 & 1.072 & 0.9054 \\ \text { LPC } & 91.29 & 1.143 & 0.6892 \\ \text { HPC } & 49.25 & 5.647 & 0.8099 \\ \text { HPT } & 12.09 & 3.695 & 0.8752 \\ \text { LPT } & 48.92 & 1.923 & 0.8141\end{array}$

$\mathrm{NC}$
1848.588
1848.588
8804.864
212.931
53.076

$\mathrm{TR}$
1.0222
1.0566
1.7814
1.3307
1.1519

efPoly
0.9063
0.6951
0.8491
0.8549
0.7997

$\begin{array}{rrr}\text { PWr } & \text { SMN } & \text { SMW } \\ -1165.6 & -1881.76 & 46.42 \\ -476.7 & 6.97 & 2.19 \\ -4112.6 & 60.54 & 56.71 \\ 4112.6 & & \\ 1642.3 & & \end{array}$

TURBOMACHINERY MAP DATA

$\begin{array}{lrrr} & \text { WcMap } & \text { PRmap } & \text { effMap } \\ \text { Fan } & 661.57 & 1.070 & 0.9072 \\ \text { LPC } & 47.03 & 1.247 & 0.7558 \\ \text { HPC } & 44.79 & 5.428 & 0.8182 \\ \text { HPT } & 0.96 & 3.621 & 0.8752 \\ \text { LPT } & 0.75 & 1.694 & 0.8201\end{array}$

$\begin{array}{rr}\text { NcMap } & \text { R/Parm } \\ 1830.961 & 0.0438 \\ 0.488 & 0.0000 \\ 8570.132 & 11.0891 \\ 1.280 & 3.6205 \\ 0.597 & 1.6942\end{array}$

\begin{tabular}{rrrr} 
S_WCDes & S_PRdes & S_effDes & S_NCDes \\
\hline 0.8607 & $\overline{1} .0235$ & 0.9980 & 0.9905 \\
1.9411 & 0.5800 & 0.9119 & 0.0003 \\
1.0995 & 1.0494 & 0.9898 & 0.9733 \\
12.6299 & 0.9723 & 1.0000 & 0.0003 \\
65.2590 & 0.7521 & 0.9926 & 0.0005
\end{tabular}

$\begin{array}{lll}0.75 & 1.694 & 0.8201\end{array}$
0.597

1.6942

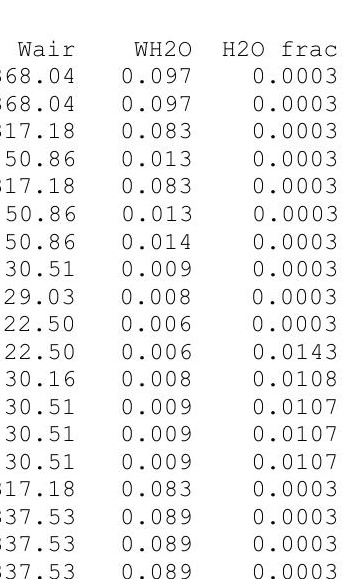




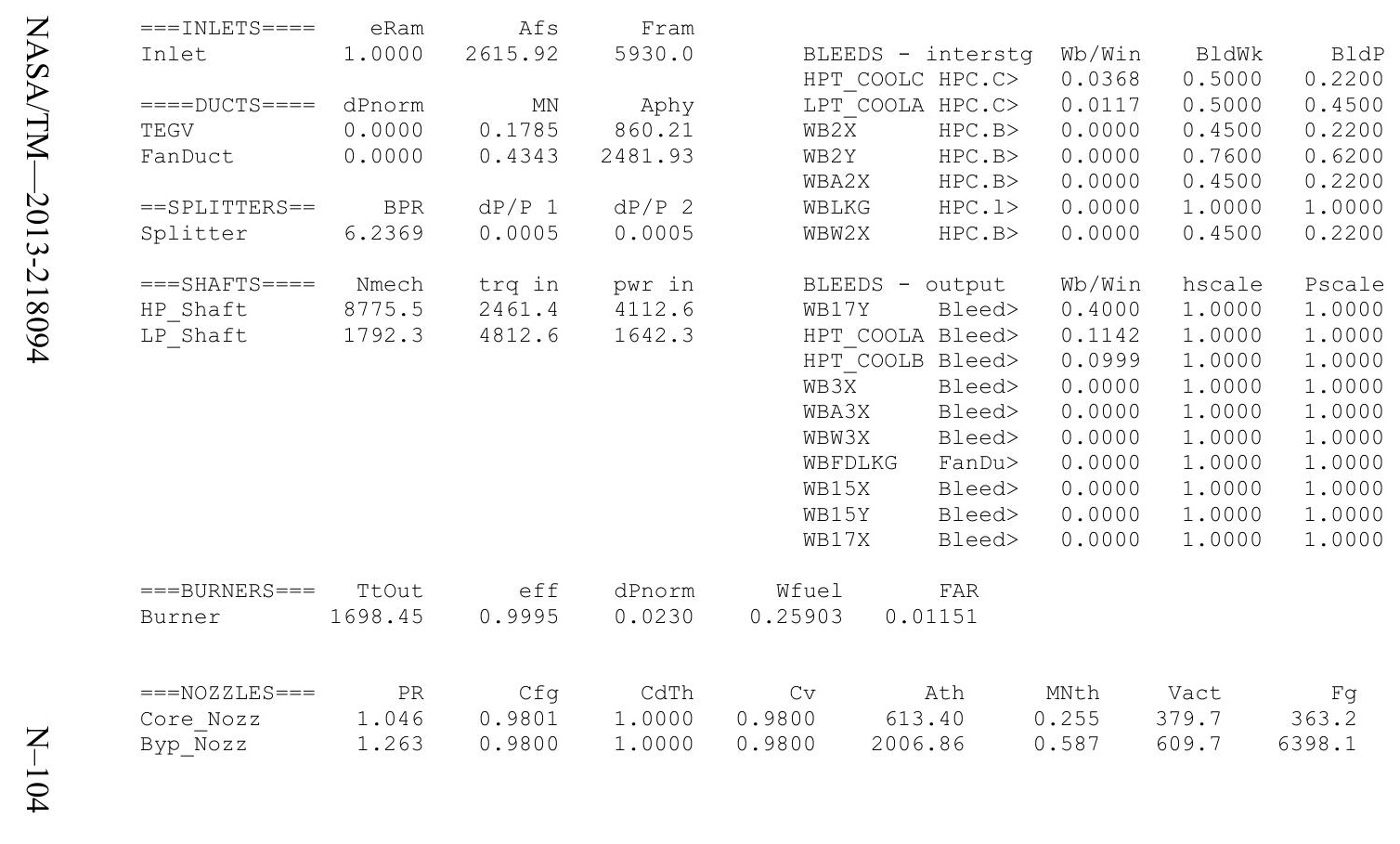


Date: 07/05/13 Time:09:52:30 Model: Turbofan Engine - COMDES ON Converge = 1 CASE: 0

Version:NPSS 1.6.5 - Rev: -> Gas Package: Janaf iter/pass/Jacb/Broy= 22/50/2/19 Run by: Philip C Jorgenson PC: 10

Temperature Stator 1 inlet: 500.26 Stator 1 exit: 502.62 Stator 2 inlet: 504.93 Stator 2 exit: 505.65 $\begin{array}{llll}\text { Stator } 3 \text { inlet: } 508.35 & \text { Stator } 3 \text { exit: } 508.74 & \text { Stator } 4 \text { inlet: } 511.35 & \text { Stator } 4 \text { exit: } 511.5 \\ \text { Stator } 5 \text { inlet: } 513.10 & \text { Stator } 5 \text { exit: } 513.12 & \end{array}$

Ambient Relative Humidity $\quad 10.00$

Fan Face Relative Humidity $\quad 6.53$

Fan Bypass Relative Humidity $\quad 5.96$

LPC Inlet Relative Humidity $\quad 5.71$

HPC Relative Humidity

Drop Diameter

0.01

0.0001000

Ambient Flow Velocity

Ambient Static Pressure

Additional Water at LPC Exit

479.99

Inlet Length

40.00

$\begin{array}{llr}8.15 & \text { Fan/LPC Inlet Flow Velocity } & 173.50 \\ & \text { Fan/LPC Inlet Static Pressure } & 9.17\end{array}$

481.63 Fan/LPC Inlet Static Temperature 498.31
$\begin{array}{rrr}\text { MN } & \text { alt } & \text { dTamb } \\ 0.446 & 15435.0 & 18.00\end{array}$
W
386.68
$\begin{array}{rr}\text { Fn } & \text { TSFC } \\ 641.0 & 1.3790\end{array}$
Wfuel
884.01
BPR
6.7784
VTAS
479.99
OPR
5.288
EPR
Powerset
$\begin{array}{ccc}\text { T4 } & \text { T41 } & \text { T49 } \\ 1649.7 & 1553.9 & 1125.3\end{array}$

\section{FLOW STATION DATA}

FSO Ambient.Fl_O

FS1 Inlet.FI_O

$\begin{array}{ll}\text { FS12 } & \text { Splitter.F1_02 } \\ \text { FS2 } & \text { Splitter.FI_01 }\end{array}$

FS14 Fan.Fl_O

FS24 VaporIN.FI

FS25 Bleed2.F1-

FS3 HPC.FIO

FS36 Bleed3.FI_O

FS4 Burner.FI_O

FS49

FS5 TEGV.Fīo

FS8 Core_Nozz.FI_O

SS17 FanDuctLkg.Fl_o

FS171 Bleed15.F1

FS173

$W$
386.68
386.68
336.97
49.71
336.97
49.71
49.71
29.83
28.38
22.00
22.24
29.72
30.07
30.07
30.07
336.97
356.86
356.86
356.86

$\begin{array}{rrrr}\text { Pt } & \text { Tt } & \text { ht } & \text { FAR } \\ 9.342 & 500.81 & -13.05 & 0.0000 \\ 9.342 & 500.81 & -13.05 & 0.0000\end{array}$

$\begin{array}{llllll}9.342 & 500.81 & -13.05 & 0.0000 & 597.73\end{array}$

$\begin{array}{lllll}9.337 & 500.81 & -13.05 & 0.0000 & 521.14 \\ 9.337 & 500.81 & -13.05 & 0.0000 & 76.88\end{array}$

$\begin{array}{lllll}9.337 & 500.81 & -13.05 & 0.0000 & 521.84 \\ 9.813 & 508.81 & -13.05 & 0.0000 & 76.88\end{array}$

$\begin{array}{lllll}9.813 & 500.81 & -13.05 & 0.0000 & 76.88 \\ 10.430 & 523.17 & -7.69 & 0.0000 & 499.81\end{array}$

$\begin{array}{lllll}10.430 & 523.17 & -7.69 & 0.0000 & 70.35 \\ 10.430 & 523.23 & -7.81 & 0.0000 & 70.35\end{array}$

$\begin{array}{lllll}10.430 & 523.23 & -7.81 & 0.0000 & 70.35 \\ 10.430 & 523.23 & -7.81 & 0.0000 & 42.21\end{array}$

$49.398-887.55-80.44=0.0000$
$49.398-887.55$

$\begin{array}{llll}49.398 & 887.55 & 80.44 & 0.0000\end{array}$

$\begin{array}{llll}48.260 & 1649.74 & 67.94 & 0.0112\end{array}$

$\begin{array}{rrrr}14.273 & 1129.99 & -16.95 & 0.0112 \\ 8.396 & 1005.97 & -46.64 & 0.0082\end{array}$

$\begin{array}{llll}8.396 & 1006.04 & -46.64 & 0.0082\end{array}$

$\begin{array}{llll}8.396 & 1006.11 & -46.64 & 0.0082\end{array}$

$\begin{array}{llll}9.813 & 508.79 & -11.14 & 0.0000\end{array}$

$\begin{array}{llll}9.813 & 509.59 & -10.95 & 0.0000\end{array}$

$\begin{array}{llll}9.813 & 509.59 & -10.95 & 0.0000\end{array}$

9.813

509.59

$\begin{array}{ll}-10.95 & 0.0000\end{array}$

TURBOMACHINERY PERFORMANCE DATA

$\begin{array}{lrrr} & \text { WC } & \text { PR } & \text { eff } \\ \text { Fan } & 521.14 & 1.051 & 0.8982 \\ \text { LPC } & 76.88 & 1.117 & 0.7202 \\ \text { HPC } & 42.21 & 4.736 & 0.7955 \\ \text { HPT } & 12.08 & 3.381 & 0.8666 \\ \text { LPT } & 45.17 & 1.700 & 0.8004\end{array}$

$$
\begin{array}{r}
\text { NC } \\
1610.080 \\
1610.080 \\
8540.497
\end{array}
$$

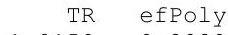

$\begin{array}{ll}1.6963 & 0.8340 \\ 1.3031 & 0.8466\end{array}$

47.065

1.3031
1.1197

0.7881

$\begin{array}{rrr}\text { pwr } & \text { SMN } & \text { SMW } \\ -912.3 & -1526.14 & 44.72 \\ -377.2 & 8.26 & 1.96 \\ -3634.0 & 68.25 & 63.03 \\ 3634.0 & & \end{array}$

TURBOMACHINERY MAP DATA

$\begin{array}{lrrrrrrrrr} & \text { WcMap } & \text { PRmap } & \text { effMap } & \text { NcMap } & \text { R/Parm } & \text { S_WcDes } & \text { S_PRdes } & \text { S_effDes } & \text { S_NcDes } \\ \text { Fan } & 605.52 & 1.050 & 0.9000 & 1594.728 & 0.0442 & 0.8607 & \overline{1} .0235 & 0.9980 & 0.9905 \\ \text { LPC } & 39.69 & 1.184 & 0.7480 & 0.425 & 0.0000 & 1.9368 & 0.6353 & 0.9629 & 0.0003 \\ \text { HPC } & 38.39 & 4.560 & 0.8038 & 8312.813 & 10.7602 & 1.0995 & 1.0494 & 0.9898 & 0.9733 \\ \text { HPT } & 0.96 & 3.315 & 0.8666 & 1.269 & 3.3152 & 12.6299 & 0.9723 & 1.0000 & 0.0003 \\ \text { LPT } & 0.69 & 1.526 & 0.8063 & 0.529 & 1.5265 & 65.2590 & 0.7521 & 0.9926 & 0.0005\end{array}$




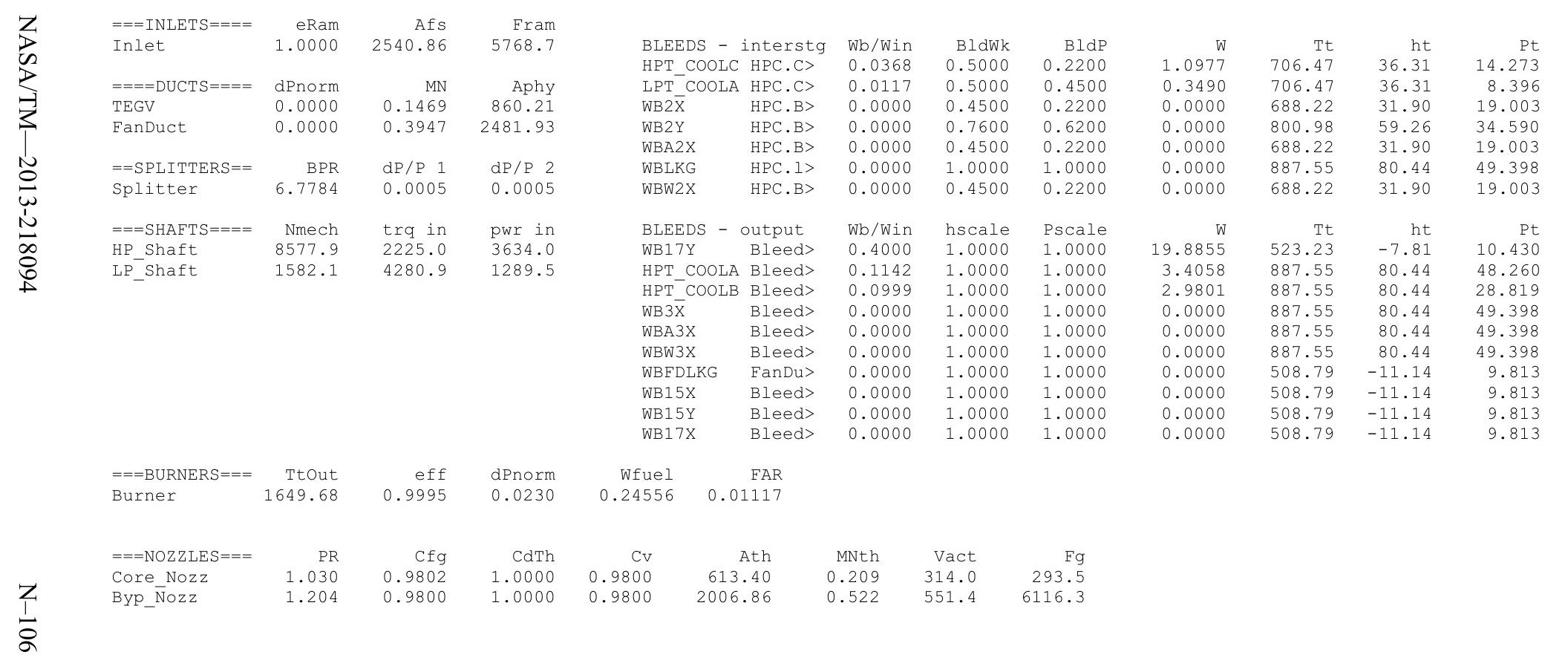


Date: 07/05/13 Time:10:02:31 Model:

$\rightarrow$ Gas Package: Janaf iter/pass/Jacb/Broy=11/25/1/9 Run by: Philip C Jorgenson PC: 10

COMDES ON C Converge = I CASE:

Temperature Stator 1 inlet: 483.67 Stator 1 exit: 491.43 Stator 2 inlet: 501.19 Stator 2 exit: 506.18

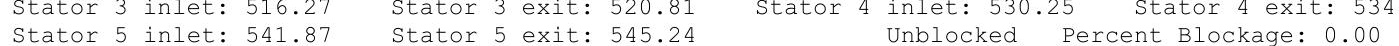

Ambient Relative Humidity 10.00

Fan Face Relative Humidity $\quad 1.97$

Fan Bypass Relative Humidity $\quad 1.41$

LPC Inlet Relative Humidity $\quad 1.30$

LPC Exit Relative Humidity

Drop Diameter

0.00

Ambient Flow Velocity

789.49
2.85

Inlet Length

40.00

Ambient Static Pressure

Additional Water at LPC Exit

425.97

Fan/LPC Inlet Flow Velocity $\quad 192.99$

Fan/LPC Inlet Static Temperature 474.82

MN alt dTamb W

\begin{tabular}{|c|c|}
\hline FSO & Ambient.Fl_o \\
\hline FS1 & Inlet.Fl_o \\
\hline FS12 & Splitter.Fl_02 \\
\hline FS2 & Splitter.Fl_01 \\
\hline FS14 & Fan.Fl_O \\
\hline FS23 & LPC.FI-O \\
\hline FS24 & VaporIN..$E I \_O$ \\
\hline FS25 & Bleed2.Fl $\overline{0}$ \\
\hline FS3 & HPC.FIO \\
\hline FS36 & Bleed3.FI_O \\
\hline FS4 & Burner.FI_O \\
\hline FS 45 & HPT.FI O \\
\hline FS49 & LPT.FI-O \\
\hline FS5 & TEGV.FI_O \\
\hline FS8 & Core Nozz.E \\
\hline FS17 & FanDuctLkg.Fi \\
\hline FS171 & Bleed15.Fl o \\
\hline FS172 & FanDuct.Fl \\
\hline FS173 & Byp Nozz.Fī o \\
\hline
\end{tabular}

TURBOMACHINERY PERFORMANCE DATA

\begin{tabular}{|c|c|c|c|}
\hline 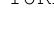 & $\mathrm{w}$ & PR & eff \\
\hline Fan & 746.88 & 1.129 & 0.9055 \\
\hline LPC & 87.24 & 1.560 & 0.8406 \\
\hline HPC & 54.23 & 6.103 & 0.8187 \\
\hline HPT & 12.07 & 4.167 & 0.8772 \\
\hline LPT & 54.65 & 2.920 & 0.8556 \\
\hline TURI & NERY MAP & DATA & \\
\hline & WcMap & PRmap & effMap \\
\hline Fan & 867.80 & 1.127 & 0.9073 \\
\hline LPC & 73.90 & 1.522 & 0.7799 \\
\hline $\mathrm{HPC}$ & 49.32 & 5.863 & 0.8271 \\
\hline HPT & 0.96 & 4.079 & 0.8771 \\
\hline LPT & 0.84 & 2.444 & 0.8620 \\
\hline
\end{tabular}

FLOW STATION DATA

\begin{tabular}{rrrrrr} 
W & \multicolumn{1}{c}{ Pt } & Tt & ht & FAR & WC \\
252.16 & 4.267 & 477.92 & -16.44 & 0.0000 & 833.70 \\
252.16 & 4.267 & 477.92 & -16.44 & 0.0000 & 833.70 \\
225.79 & 4.265 & 477.92 & -16.44 & 0.0000 & 746.88 \\
26.37 & 4.265 & 477.92 & -16.44 & 0.0000 & 87.24 \\
225.79 & 4.817 & 496.63 & -11.96 & 0.0000 & 674.07 \\
26.37 & 6.654 & 555.02 & 2.05 & 0.0000 & 60.25 \\
26.37 & 6.654 & 555.07 & 1.98 & 0.0000 & 60.26 \\
23.74 & 6.654 & 555.07 & 1.98 & 0.0000 & 54.23 \\
22.58 & 40.610 & 1006.19 & 111.88 & 0.0000 & 11.38 \\
17.50 & 40.610 & 1006.19 & 111.88 & 0.0000 & 8.82 \\
17.70 & 39.675 & 1759.21 & 98.98 & 0.0112 & 12.07 \\
23.65 & 9.522 & 1162.39 & -7.41 & 0.0084 & 54.65 \\
23.93 & 3.261 & 910.13 & -69.34 & 0.0083 & 142.85 \\
23.93 & 3.261 & 910.20 & -69.34 & 0.0083 & 142.86 \\
23.93 & 3.261 & 910.27 & -69.34 & 0.0083 & 142.86 \\
225.79 & 4.817 & 496.63 & -11.96 & 0.0000 & 674.07 \\
228.42 & 4.817 & 497.30 & -11.80 & 0.0000 & 682.41 \\
228.42 & 4.817 & 497.30 & -11.80 & 0.0000 & 682.41 \\
228.42 & 4.817 & 497.30 & -11.80 & 0.0000 & 682.41 \\
& & & & &
\end{tabular}

VTA
OPR

EPR
0.736

PowerSet
10.000

T4
1759.2

664

T 49

$\begin{array}{rrrrrrrrr}\text { PS } & \text { Ts } & \text { Aphy } & \text { MN } & \text { gamt } & \text { WAR } & \text { Wair } & \text { WH2O } & \text { H2O frac } \\ 2.854 & 425.97 & 2543.5 & 0.7800 & 1.40068 & 0.0000851 & 252.14 & 0.021 & 0.0001 \\ 3.892 & 465.50 & 4168.2 & 0.3649 & 1.40068 & 0.0000851 & 252.14 & 0.021 & 0.0001 \\ 3.839 & 463.78 & 3531.8 & 0.3902 & 1.40068 & 0.0000851 & 225.77 & 0.019 & 0.0001 \\ 4.169 & 474.82 & 830.5 & 0.1806 & 1.40068 & 0.0000851 & 26.37 & 0.002 & 0.0001 \\ 4.042 & 472.33 & 2606.7 & 0.5067 & 1.40049 & 0.0000851 & 225.77 & 0.019 & 0.0001 \\ 6.357 & 547.83 & 412.6 & 0.2561 & 1.39968 & 0.0000851 & 26.37 & 0.002 & 0.0001 \\ 6.357 & 547.89 & 412.6 & 0.2561 & 1.39967 & 0.0000996 & 26.37 & 0.003 & 0.0001 \\ 6.416 & 549.33 & 412.6 & 0.2287 & 1.39967 & 0.000996 & 23.73 & 0.002 & 0.0001 \\ 35.727 & 971.22 & 49.7 & 0.4343 & 1.38033 & 0.0000996 & 22.58 & 0.002 & 0.0001 \\ 37.808 & 986.54 & 49.3 & 0.3233 & 1.38033 & 0.0000996 & 17.50 & 0.002 & 0.0001 \\ 37.468 & 1734.38 & 74.6 & 0.2944 & 1.32947 & 0.0000996 & 17.50 & 0.002 & 0.0138 \\ 8.626 & 1132.04 & 265.4 & 0.3829 & 1.36457 & 0.0000996 & 23.46 & 0.002 & 0.0103 \\ 3.070 & 895.08 & 860.2 & 0.2970 & 1.38054 & 0.0000996 & 23.73 & 0.002 & 0.0102 \\ 3.070 & 895.15 & 860.2 & 0.2970 & 1.38054 & 0.0000996 & 23.73 & 0.002 & 0.0102 \\ 2.854 & 877.34 & 613.4 & 0.4435 & 1.38053 & 0.0000996 & 23.73 & 0.002 & 0.0102 \\ 4.042 & 472.33 & 2606.7 & 0.5067 & 1.40049 & 0.0000851 & 225.77 & 0.019 & 0.0001 \\ 3.908 & 468.44 & 2481.9 & 0.5546 & 1.40048 & 0.0000852 & 228.40 & 0.019 & 0.0001 \\ 3.908 & 468.44 & 2481.9 & 0.5546 & 1.40048 & 0.0000852 & 228.40 & 0.019 & 0.0001 \\ 2.854 & 428.14 & 2006.9 & 0.8978 & 1.40048 & 0.0000852 & 228.40 & 0.019 & 0.0001\end{array}$

$\begin{array}{rrrrrr}\text { NC } & \text { TR } & \text { efPoly } & \text { pwr } & \text { SMN } & \text { SMW } \\ 2568.019 & 1.0391 & 0.9071 & -1432.4 & 1611.24 & 54.83 \\ 2568.019 & 1.1613 & 0.8503 & -690.1 & 6.97 & 3.73 \\ 8965.376 & 1.8127 & 0.8573 & -3601.3 & 61.35 & 58.03 \\ 221.125 & 1.3648 & 0.8551 & 3601.3 & & \end{array}$

$\begin{array}{llll}221.125 & 1.3648 & 0.8551 & 3601.3\end{array}$

$\begin{array}{rr}\text { NCMap } & \text { R/Parm } \\ 2543.533 & 0.0414 \\ 0.678 & 0.0000 \\ 8726.365 & 10.9747 \\ 1.329 & 4.0790 \\ 0.813 & 2.4438\end{array}$

$\begin{array}{rrrr}\text { S_WCDes } & \text { S_PRdes } & \text { S_effDes } & \text { S_NcDes } \\ 0.8607 & 1.0235 & 0.9980 & 0.9905 \\ 1.1805 & 1.0736 & 1.0778 & 0.0003 \\ 1.0995 & 1.0494 & 0.9898 & 0.9733 \\ 12.6299 & 0.9723 & 1.0000 & 0.0003 \\ 65.2590 & 0.7521 & 0.9926 & 0.0005\end{array}$




\begin{tabular}{|c|c|c|c|c|c|c|c|c|c|c|c|c|c|}
\hline$Z$ & $\begin{array}{l}===\text { INLETS }==== \\
\text { Inlet }\end{array}$ & $\begin{array}{r}\text { eRam } \\
1.0000\end{array}$ & $\begin{array}{r}\text { Afs } \\
2543.46\end{array}$ & $\begin{array}{r}\text { Fram } \\
6187.5\end{array}$ & & Wb/Win & BldWk & BldP & W & $\mathrm{Tt}$ & ht & $\mathrm{Pt}$ \\
\hline 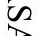 & & & & & \multicolumn{2}{|c|}{ HPT_COOLC HPC.C> } & 0.0368 & 0.5000 & 0.2200 & 0.8735 & 782.68 & 56.93 & 9.522 \\
\hline z & $====$ DUCTS $====$ & dPnorm & MN & Aphy & \multirow{2}{*}{$\begin{array}{l}\text { LPT }{ }^{-} \text {COOLA } \\
\text { WB } 2 \overline{\mathrm{X}}\end{array}$} & HPC.C> & 0.0117 & 0.5000 & 0.4500 & 0.2777 & 782.68 & 56.93 & 3.261 \\
\hline E & TEGV & 0.0000 & 0.2970 & 860.21 & & $\mathrm{HPC} . \mathrm{B}>$ & 0.0000 & 0.4500 & 0.2200 & 0.0000 & 760.07 & 51.44 & 14.124 \\
\hline & FanDuct & 0.0000 & 0.5546 & 2481.93 & WB2Y & HPC.B> & 0.0000 & 0.7600 & 0.6200 & 0.0000 & 899.55 & 85.51 & 27.707 \\
\hline & & & & & \multirow{2}{*}{$\begin{array}{l}\text { WBA2X } \\
\text { WBLKG }\end{array}$} & HPC.B> & 0.0000 & 0.4500 & 0.2200 & 0.0000 & 760.07 & 51.44 & 14.124 \\
\hline š & $==$ SPLITTERS $==$ & BPR & $\mathrm{dP} / \mathrm{P} 1$ & $\mathrm{dP} / \mathrm{P} 2$ & & HPC.1> & 0.0000 & 1.0000 & 1.0000 & 0.0000 & 1006.19 & 111.88 & 40.610 \\
\hline & Splitter & 8.5613 & 0.0005 & 0.0005 & WBW2X & HPC. B> & 0.0000 & 0.4500 & 0.2200 & 0.0000 & 760.07 & 51.44 & 14.124 \\
\hline & $===$ SHAFT $S===$ & Nmech & trq in & pwr in & \multicolumn{2}{|c|}{ BLEEDS - output } & $\mathrm{Wb} / \mathrm{Win}$ & hscale & Pscale & W & Tt & ht & Pt \\
\hline & HP_Shaft & 9274.6 & 2039.4 & 3601.3 & WB17Y & Bleed> & 0.1000 & 1.0000 & 1.0000 & 2.6373 & 555.07 & 1.98 & 6.654 \\
\hline & LP_Shaft & 2465.1 & 4522.2 & 2122.5 & \multirow{2}{*}{$\begin{array}{l}\text { HPT_COOLA } \\
\text { HPT COOLB }\end{array}$} & Bleed> & 0.1142 & 1.0000 & 1.0000 & 2.7102 & 1006.19 & 111.88 & 39.675 \\
\hline & & & & & & 3 Bleed> & 0.0999 & 1.0000 & 1.0000 & 2.3715 & 1006.19 & 111.88 & 22.428 \\
\hline & & & & & WB $3 \bar{X}$ & Bleed> & 0.0000 & 1.0000 & 1.0000 & 0.0000 & 1006.19 & 111.88 & 40.610 \\
\hline & & & & & WBA3X & Bleed> & 0.0000 & 1.0000 & 1.0000 & 0.0000 & 1006.19 & 111.88 & 40.610 \\
\hline & & & & & \multirow{2}{*}{$\begin{array}{l}\text { WBW3X } \\
\text { WBFDLKG }\end{array}$} & Bleed> & 0.0000 & 1.0000 & 1.0000 & 0.0000 & 1006.19 & 111.88 & 40.610 \\
\hline & & & & & & FanDu> & 0.0000 & 1.0000 & 1.0000 & 0.0000 & 496.63 & -11.96 & 4.817 \\
\hline & & & & & WB15X & Bleed> & 0.0000 & 1.0000 & 1.0000 & 0.0000 & 496.63 & -11.96 & 4.817 \\
\hline & & & & & \multirow{2}{*}{$\begin{array}{l}\text { WB15Y } \\
\text { WB17X }\end{array}$} & Bleed> & 0.0000 & 1.0000 & 1.0000 & 0.0000 & 496.63 & -11.96 & 4.817 \\
\hline & & & & & & Bleed> & 0.0000 & 1.0000 & 1.0000 & 0.0000 & 496.63 & -11.96 & 4.817 \\
\hline & $===$ BURNERS $===$ & Ttout & eff & dPnorm & \multicolumn{2}{|c|}{ Wfuel FAR } & & & & & & & \\
\hline & Burner & 1759.16 & 0.9995 & 0.0230 & \multicolumn{2}{|c|}{$0.19645 \quad 0.01122$} & & & & & & & \\
\hline \multirow{3}{*}{\multicolumn{2}{|c|}{$\begin{array}{l}===\text { NOZZLE } \\
\text { Core_Nozz } \\
\text { Byp_NNozz }\end{array}$}} & PR & $\mathrm{Cfg}$ & CdTh & $\mathrm{Cv}$ & Ath & MNth & Vact & $\mathrm{Fg}$ & & & & \\
\hline & & 1.143 & 0.9801 & 1.0000 & \multirow{2}{*}{$\begin{array}{l}0.9800 \\
0.9800\end{array}$} & \multirow{2}{*}{$\begin{array}{r}613.40 \\
2006.86\end{array}$} & 0.443 & 627.1 & 466.5 & & & & \\
\hline & & 1.688 & 0.9800 & 1.0000 & & & 0.898 & 892.8 & 6338.4 & & & & \\
\hline
\end{tabular}


Date:07/05/13 Time:10:02:47 Model: Turbofan Engine - COMDES ON Converge = 1 CASE: 0

Version:NPSS 1.6.5 - Rev: -> Gas Package: Janaf iter/pass/Jacb/Broy=11/25/1/9 Run by: Philip C Jorgenson PC: 10

Temperature Stator 1 inlet: 476.90 Stator 1 exit: 484.18 Stator 2 inlet: 493.31 Stator 2 exit: 497.96

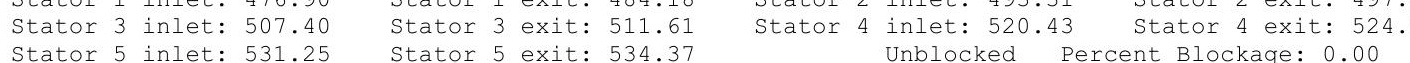

Ambient Relative Humidity $\quad 10.00$

Fan Face Relative Humidity 2.46

Fan Bypass Relative Humidity $\quad 1.80$

LPC Inlet Relative Humidity $\quad 1.64$

LPC Exit Relative Humidity

0.00

Drop Diameter Velocity

Ambient Static Pressure

Ambient Static Temperature
Additional Water at LPC Exit

0.0001000

738.88

Inlet Length

$\begin{array}{rr} & 40.00 \\ \text { Ean/LPC Inlet Flow Velocity } & 186.04\end{array}$

0.0000133

Fan/LPC Inlet Static Temperature 468.59

MN alt dTamb SUMMARY OUTPUT DATA

$\begin{array}{rrrrrr}\text { MN } & \text { alt } & \text { dTamb } & \text { W } & \text { Fn } & \text { TSFC } \\ 0.730 & 38334.0 & 36.00 & 245.31 & 606.9 & 1.1102\end{array}$

$\begin{array}{rr}.1102 & \text { Wfuel } \\ & 673.74\end{array}$

8.6582

VTAS
738.88

OPR
9.232

EPR
0.764

PowerSet
10.000

$\begin{array}{cc}T 4 & T 41 \\ 1731.7 & 1637.5\end{array}$

T49

FLOW STATION DATA

FSO Ambient.Fl_O

FS1 Inlet.F1_O

$\begin{array}{ll}\text { FS12 } & \text { Splitter.Fl_02 } \\ \text { FS2 } & \text { Splitter.Fl_01 }\end{array}$

FS14 Fan.F1_-

$\begin{array}{ll}\text { FS23 } & \text { LPC.FI_O } \\ \text { FS24 } & \text { VaporIN.FI_O }\end{array}$

FS25 Bleed2.F1_-

FS3 HPC.FIO

FS36 Bleed3.F1_O

FS4 Burner.FI_O

FS45 HPT.FI_

FS4 LPT.FI-

FS8 Core_Nozz.Fl_O

FS17 FanDuctLkg.FI_O

FS171 Bleed15.F1_O

FS172 FanDuct.Fl-O

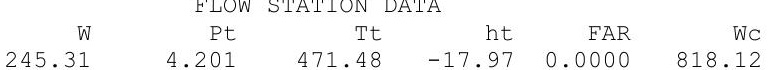

$\begin{array}{llllll}245.31 & 4.201 & 471.48 & -17.97 & 0.0000 & 818.12\end{array}$

$\begin{array}{llllll}219.91 & 4.199 & 471.48 & -17.97 & 0.0000 & 733.78\end{array}$

$\begin{array}{rrrrrr}25.40 & 4.199 & 471.48 & -17.97 & 0.0000 & 84.75 \\ 219.91 & 4.711 & 488.91 & -13.79 & 0.0000 & 666.03\end{array}$

$\begin{array}{rrrrrr}219.91 & 4.711 & 488.91 & -13.79 & 0.0000 & 666.03 \\ 25.40 & 6.414 & 543.66 & -0.66 & 0.0000 & 59.58\end{array}$

$\begin{array}{llllll}25.40 & 6.414 & 543.66 & -0.66 & 0.0000 & 59.58 \\ 25.40 & 6.414 & 543.71 & -0.72 & 0.0000 & 59.58\end{array}$

$\begin{array}{lllrll}22.86 & 6.414 & 543.71 & -0.72 & 0.0000 & 53.62 \\ 21.75 & 38.786 & 983.85 & 106.36 & 0.000 & 11.35\end{array}$

$\begin{array}{rrrrrr}21.75 & 38.786 & 983.85 & 106.36 & 0.0000 & 11.35 \\ 16.86 & 38.786 & 983.85 & 106.36 & 0.0000 & 8.80\end{array}$

$\begin{array}{rrrrrr}17.04 & 37.892 & 1731.72 & 93.65 & 0.0111 & 12.08 \\ 22.78 & 9.210 & 1145.62 & -9.98 & 0.083 & 54.02\end{array}$

$\begin{array}{rrrrrr}22.78 & 9.210 & 1145.62 & -9.98 & 0.0083 & 54.02 \\ 23.05 & 3.311 & 907.82 & -68.20 & 0.0082 & 135.33 \\ 23.05 & 3.311 & 907.90 & -68.20 & 0.0082 & 135.33\end{array}$

$\begin{array}{llllll}23.05 & 3.311 & 907.82 & -68.20 & 0.0082 & 135.33 \\ 23.05 & 3.311 & 907.90 & -68.20 & 0.0082 & 135.33\end{array}$

219.91

219.91
222.45

$\begin{array}{llll}.311 & 907.90 & -68.20 & 0.0082\end{array}$

$\begin{array}{lllll}.311 & 907.97 & -68.20 & 0.0082 & 135.34 \\ 4.711 & 488.91 & -13.79 & 0.0000 & 666.03\end{array}$

$\begin{array}{lllll}4.711 & 489.53 & -13.64 & 0.0000 & 674.15\end{array}$

$\begin{array}{lllll}4.711 & 488.91 & -13.79 & 0.0000 & 666.03 \\ 4.711 & 489.53 & -13.64 & 0.0000 & 674.15 \\ 4.711 & 489.53 & -13.64 & 0.0000 & 674.15\end{array}$

$\begin{array}{lllll}4.711 & 489.53 & -13.64 & 0.0000 & 674.15\end{array}$

Ps
2.947
3.847
3.797
4.110
3.97
6.135
6.135
6.190
34.154
36.126
35.783
8.365
3.138
3.138
2.947
3.97
3.851
3.851
2.947

Ts
425.97
459.74
458.09
468.59
465.74
536.79
536.84
538.22
949.83
964.72
1707.18
1116.44
894.49
894.56
879.20
465.74
462.10
462.10
428.03

$\begin{array}{rr}\text { Aphy } & \text { MN } \\ 2560.6 & 0.7300\end{array}$

$\begin{array}{rrr}2560.6 & 0.7300 & 1.40073\end{array}$

$\begin{array}{lll}4168.2 & 0.3569 & 1.40073 \\ 3531.8 & 0.3819 & 1.40073\end{array}$

$\begin{array}{lll}830.5 & 0.1753 & 1.40073\end{array}$

$\begin{array}{lll}2606.7 & 0.4982 & 1.40058 \\ 412.6 & 0.2530 & 1.39986\end{array}$

$\begin{array}{lll}412.6 & 0.2530 & 1.39986 \\ 412.6 & 0.2530 & 1.39985\end{array}$

$\begin{array}{lll}412.6 & 0.2530 & 1.39985 \\ 412.6 & 0.2260 & 1.39985\end{array}$

$412.6 \quad 0.2260-1.39985$

$\begin{array}{llll}49.3 & 0.3221 & 1.38170\end{array}$

$\begin{array}{lll}74.6 & 0.3221\end{array}$

$\begin{array}{lll}74.6 & 0.2944 \\ 265.4 & 0.3774\end{array}$

1.38170

1.33081
1.36571

$\begin{array}{lll}860.2 & 0.2797 & 1.38074 \\ 860.2 & 0.2797 & 1.38073\end{array}$

$86.2-0.2797-1.38073$

$\begin{array}{lll}613.4 & 0.4141 & 1.38073 \\ 2606.7 & 0.4982 & 1.40058\end{array}$

$\begin{array}{lll}2481.9 & 0.5444 & 1.40057\end{array}$

$\begin{array}{lll}2481.9 & 0.5444 & 1.40057\end{array}$

$2006.9 \quad 0.8467$

$\begin{array}{rrrr}\text { WAR } & \text { Wair } & \text { WH2O } & \text { H2O frac } \\ 0.0000824 & 245.29 & 0.020 & 0.0001 \\ 0.0000824 & 245.29 & 0.020 & 0.0001 \\ 0.0000824 & 219.89 & 0.018 & 0.0001 \\ 0.0000824 & 25.40 & 0.002 & 0.0001 \\ 0.0000824 & 219.89 & 0.018 & 0.0001 \\ 0.0000824 & 25.40 & 0.002 & 0.0001 \\ 0.0000957 & 25.40 & 0.002 & 0.0001 \\ 0.0000957 & 22.86 & 0.002 & 0.0001 \\ 0.0000957 & 21.75 & 0.002 & 0.0001 \\ 0.0000957 & 16.86 & 0.002 & 0.0001 \\ 0.0000957 & 16.86 & 0.002 & 0.0136 \\ 0.0000957 & 22.59 & 0.002 & 0.0102 \\ 0.0000957 & 22.86 & 0.002 & 0.0101 \\ 0.0000957 & 22.86 & 0.002 & 0.0101 \\ 0.0000957 & 22.86 & 0.002 & 0.0101 \\ 0.0000824 & 219.89 & 0.018 & 0.0001 \\ 0.0000825 & 222.43 & 0.018 & 0.0001 \\ 0.0000825 & 222.43 & 0.018 & 0.0001 \\ 0.0000825 & 222.43 & 0.018 & 0.0001\end{array}$

TURBOMACHINERY PERFORMANCE DATA

\begin{tabular}{|c|c|c|c|c|c|c|c|c|c|}
\hline & WC & PR & eff & $\mathrm{NC}$ & TR & efPoly & pwr & SMN & SMW \\
\hline Fan & 733.78 & 1.122 & 0.9047 & 2504.141 & 1.0370 & 0.9062 & -1300.1 & 2192.51 & 54.69 \\
\hline LPC & 84.75 & 1.527 & 0.8409 & 2504.141 & 1.1531 & 0.8501 & -622.2 & 6.78 & 3.35 \\
\hline HPC & 53.62 & 6.047 & 0.8179 & 8945.792 & 1.8095 & 0.8566 & -3379.3 & 61.27 & 57.90 \\
\hline HPT & 12.08 & 4.114 & 0.8759 & 220.099 & 1.3615 & 0.8539 & 3379.3 & & \\
\hline LPT & 54.02 & 2.781 & .850 & 70.538 & 1.2600 & 0.8307 & 1922.3 & & \\
\hline $\mathrm{RE}$ & NERY MAP & DATA & & & & & & & \\
\hline & WcMap & PRmap & effMap & NcMap & R/Parm & s_WcDes & S_PRdes & S_effDes & S_NcDes \\
\hline an & 852.58 & 1.119 & 0.9065 & 2480.264 & 0.0413 & $\overline{0} .8607$ & $\overline{1} .0235$ & 0.9980 & $\overline{0} .9905$ \\
\hline PC & 71.13 & 1.493 & 0.7769 & 0.661 & 0.0000 & 1.1914 & 1.0692 & 1.0824 & 0.0003 \\
\hline HPC & 48.77 & 5.809 & 0.8263 & 8707.303 & 10.9865 & 1.0995 & 1.0494 & 0.9898 & 0.9733 \\
\hline $\mathrm{APT}$ & 0.96 & 4.028 & 0.8759 & 1.323 & 4.0281 & 12.6299 & 0.9723 & 1.0000 & 0.0003 \\
\hline PT & 0.83 & 2.340 & 0.8563 & 0.793 & 2.3398 & 65.2590 & 0.7521 & 0.9926 & 0.0005 \\
\hline
\end{tabular}




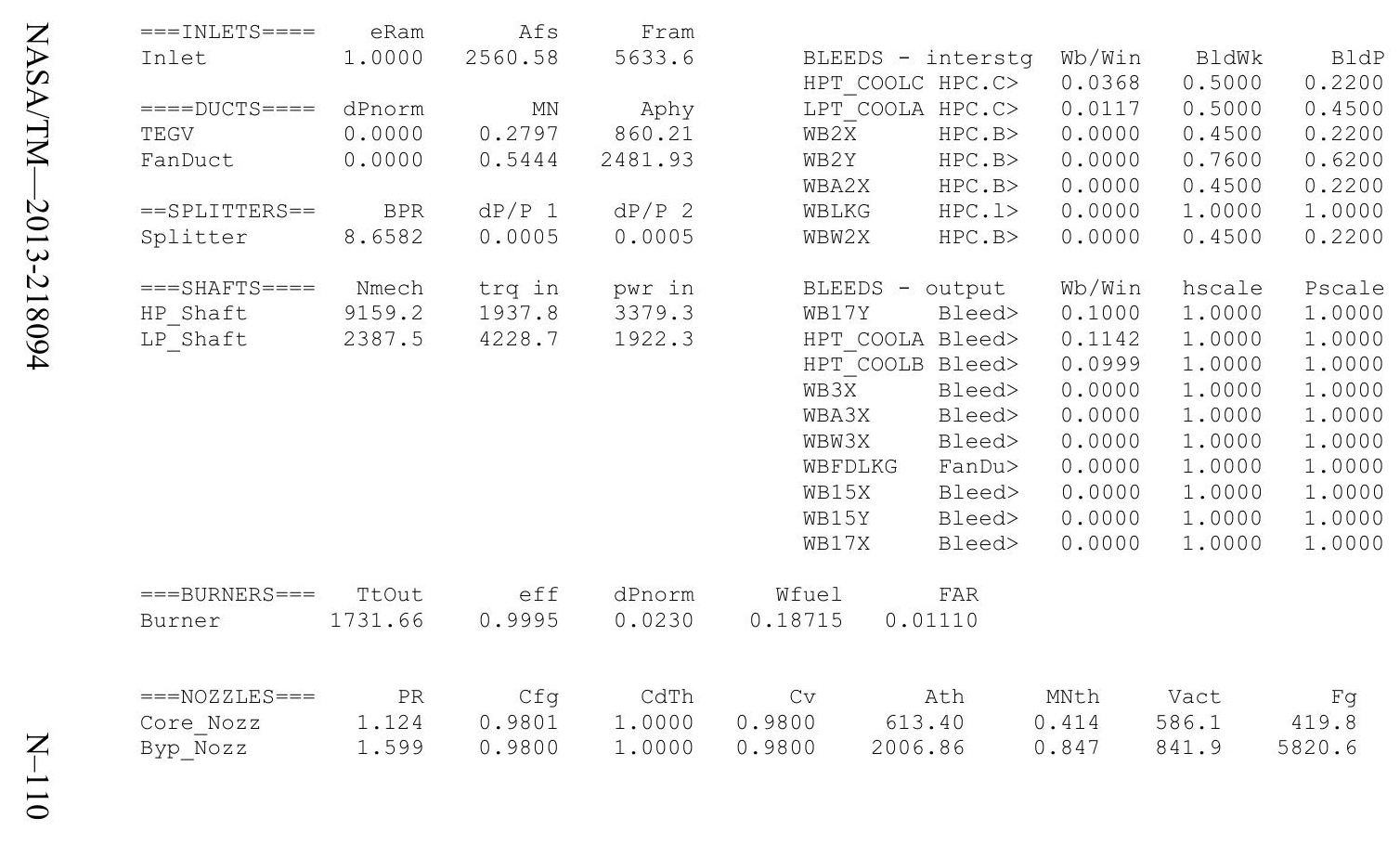


Date:07/05/13 Time:10:03:02 Model: Turbofan Engine - COMDES ON Converge = 1 CASE: 0

Version:NPSS 1.6.5 - Rev: -> Gas Package: Janaf iter/pass/Jacb/Broy=11/25/1/9 Run by: Philip C Jorgenson PC: 10

Temperature Stator 1 inlet: $474.82 \quad$ Stator 1 exit: $481.90 \quad$ Stator 2 inlet: $490.79 \quad$ Stator 2 exit: 495.33 Stator 5 inlet: 527.70 Stator 5 exit: 530.72 Unblocked Percent Blockage: 0.00

Ambient Relative Humidity $\quad 10.00$

Fan Face Relative Humidity $\quad 2.63$

Fan Bypass Relative Humidity $\quad 1.95$

IPC Inlet Relative Humidity $\quad 1.76$

LPC Exit Relative Humidity

Dre Relative Humidity

0.00

Delocity

Ambient Static Pressure

Ambient Static Temperature
Additional Water at LPC Exit

0.0001000

722.68

Inlet Length

Inlet Inlet Flow Velocity $\quad 183.20$

$\begin{array}{rrr}425.97 \quad \text { Fan/LPC Inlet Static Temperature } & 466.71\end{array}$

$\begin{array}{crrrr}\text { MN alt dTamb } & \text { SUMMARY OUTPUT DATA } \\ 0.714 & \text { Fn } & \text { TSFC }\end{array}$

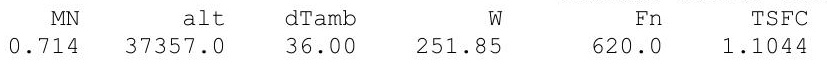

684.74

8.7044

VTAS
722.68

OPR
9.102

0.772

PowerSet
10.000

$\begin{array}{cc}T 4 & T 41 \\ 1720.8 & 1627 .\end{array}$

T49

FLOW STATION DATA

FSO Ambient.Fl_O

FS1 Inlet.F1_O

FS12 Splitter.F1_02

FS2 Splitter.FI_01

FS23 LPC.FI-O

FS24 VaporIN.EI_O

FS25 Bleed2.F1 $\bar{O}$

FS3 HPC.FIO

FS36 Bleed3.F1_O

FS4 Burner.FI_O

FS45 HPT.FI_O

FS5 LPT.FI-

FS8 Core_Nozz.Fl_O

FS17 FanDuctLkg.FI_O

FS171 Bleed15.F1_O

FS172 FanDuct.FI-O

\begin{tabular}{rrrrrr} 
W & \multicolumn{1}{c}{ Pt } & Tt & ht & FAR & WC \\
251.85 & 4.339 & 469.50 & -18.42 & 0.0000 & 811.52
\end{tabular}

$\begin{array}{llllll}251.85 & 4.339 & 469.50 & -18.42 & 0.0000 & 811.52\end{array}$

$\begin{array}{rrrrrr}225.90 & 4.337 & 469.50 & -18.42 & 0.0000 & 728.26 \\ 25.95 & 4.337 & 469.50 & -18.42 & 0.0000 & 83.67\end{array}$

$\begin{array}{rrrrrr}25.95 & 4.337 & 469.50 & -18.42 & 0.0000 & 83.67 \\ 225.90 & 4.852 & 486.44 & -14.36 & 0.0000 & 662.58 \\ 25.95 & 6.568 & 539.83 & -1.56 & 0.0000 & 59.24\end{array}$

$\begin{array}{rrrrrr}25.95 & 6.568 & 539.83 & -1.56 & 0.0000 & 59.24\end{array}$

$\begin{array}{llllll}25.95 & 6.568 & 539.88 & -1.62 & 0.0000 & 59.25 \\ 23.36 & 6.568 & 539.88 & -1.62 & 0.0000 & 53.32\end{array}$

$\begin{array}{llllll}23.36 & 39.468 & 539.88 & -1.62 & 0.0000 & 59.25 \\ 22.22 & 39.498 & 975.76 & 104.37 & 0.0000 & 11.34\end{array}$

$\begin{array}{rrrrrr}17.22 & 39.498 & 975.76 & 104.37 & 0.0000 & 8.79\end{array}$

$\begin{array}{rrrrrr}17.41 & 38.588 & 1720.81 & 91.75 & 0.0110 & 12.08\end{array}$

$\begin{array}{llllll}23.27 & 9.422 & 1139.03 & -10.81 & 0.0082 & 53.79 \\ 23.55 & 3.451 & 906.72 & -67.63 & 0.0081 & 132.59\end{array}$

$\begin{array}{llllll}23.55 & 3.451 & 906.72 & -67.63 & 0.0081 & 132.59 \\ 23.55 & 3.451 & 906.79 & -67.63 & 0.0081 & 132.59 \\ 23.55 & 3.451 & 906.87 & -67.63 & 0.0081 & 132.60\end{array}$

$\begin{array}{llllll}23.55 & 3.451 & 906.87 & -67.63 & 0.0081 & 132.60\end{array}$

$\begin{array}{llllll}225.90 & 4.852 & 486.44 & -14.36 & 0.0000 & 662.58\end{array}$

$\begin{array}{llllll}228.49 & 4.852 & 487.05 & -14.22 & 0.0000 & 670.61\end{array}$

$\begin{array}{llllll}228.49 & 4.852 & 487.05 & -14.22 & 0.0000 & 670.61 \\ 228.49 & 4.852 & 487.05 & -14.22 & 0.0000 & 670.61 \\ 228.49 & 4.852 & 487.05 & -14.22 & 0.0000 & 670.61\end{array}$

FS173 Byp Nozz.Fì O

228.49

TURBOMACHINERY PERFORMANCE DATA

$\begin{array}{lrrr} & \text { WC } & \text { PR } & \text { eff } \\ \text { Fan } & 728.26 & 1.119 & 0.9043 \\ \text { LPC } & 83.67 & 1.514 & 0.8409 \\ \text { HPC } & 53.32 & 6.014 & 0.8173 \\ \text { HPT } & 12.08 & 4.095 & 0.8753 \\ \text { LPT } & 53.79 & 2.730 & 0.8478\end{array}$

$\begin{array}{rr}\text { NC } & \text { TR } \\ 2477.354 & 1.0361 \\ 2477.354 & 1.1498 \\ 8935.941 & 1.8074 \\ 219.774 & 1.3603 \\ 69.838 & 1.2542\end{array}$

$-14.22 \quad 0.0000$

670.61

$P s$
3.088
3.980
3.929
4.247
4.105
6.285
6.285
6.341
34.790
36.794
36.439
8.567
3.278
3.278
3.088
4.105
3.978
3.978
3.088

TS
425.97
458.03
456.41
466.71
463.71
533.09
533.13
534.48
942.05
956.80
1696.40
1110.28
893.97
894.04
879.50
463.71
460.16
460.16
427.99

$$
\begin{aligned}
& 256 \\
& 416 \\
& 353 \\
& 260 \\
& 261 \\
& 412 \\
& 4 \\
& 4 \\
& 24 \\
& 264 \\
& 248 \\
& 200 \\
& 26
\end{aligned}
$$

$\begin{array}{rrr}\text { Aphy MN } & \text { gamt }\end{array}$ $\begin{array}{lll}4168.2 & 0.7140 & 1.40075 \\ 3531.8 & 0.3784 & 1.40075\end{array}$ $\begin{array}{lll}3531.8 & 0.3784 & 1.40075\end{array}$ $\begin{array}{rrr}830.5 & 0.1729 & 1.40075\end{array}$ $\begin{array}{lll}830.5 & 0.1729 & 1.40075 \\ 412.6 & 0.4946 & 1.40060\end{array}$ $\begin{array}{lll}412.6 & 0.2514 & 1.39992 \\ 412.6 & 0.2515 & 1.39991\end{array}$ $\begin{array}{lll}412.6 & 0.2515 & 1.39991 \\ 412.6 & 0.2246 & 1.39991\end{array}$ $\begin{array}{lll}49.7 & 0.4320 & 1.38219\end{array}$ $\begin{array}{lll}49.3 & 0.3217 & 1.38219 \\ 74.6 & 0.2943 & 1.33136\end{array}$ $\begin{array}{lll}765.4 & 0.3755 & 1.36616\end{array}$ $\begin{array}{lll}265.4 & 0.3755 & 1.36616 \\ 860.2 & 0.2735 & 1.38083\end{array}$ $\begin{array}{lll}860.2 & 0.2735 & 1.38083 \\ 860.2 & 0.2735 & 1.38082\end{array}$ $\begin{array}{rrr}613.4 & 0.4037 & 1.38082\end{array}$ $2481.9 \quad 0.49461-1.40060$ $2481.90 .5401-1.40060$ $2006.9 \quad 0.8297 \quad 1.40060$

TURBOMACHINERY MAP DATA

\begin{tabular}{lrrrrrrrrr}
\multicolumn{1}{l}{ TURBOMACHINERY MAP } & DATA & & & & & \\
WCMap & PRmap & effMap & NcMap & R/Parm & S_WcDes & S_PRdes & S_effDes & S_NcDes \\
Fan & 846.16 & 1.116 & 0.9061 & 2453.732 & 0.0413 & 0.8607 & 1.0235 & 0.9980 & 0.9905 \\
LPC & 69.97 & 1.481 & 0.7756 & 0.654 & 0.0000 & 1.1956 & 1.0683 & 1.0841 & 0.0003 \\
HPC & 48.50 & 5.778 & 0.8258 & 8697.714 & 10.9846 & 1.0995 & 1.0494 & 0.9898 & 0.9733 \\
HPT & 0.96 & 4.010 & 0.8753 & 1.321 & 4.0096 & 12.6299 & 0.9723 & 1.0000 & 0.0003 \\
LPT & 0.82 & 2.302 & 0.8541 & 0.786 & 2.3015 & 65.2590 & 0.7521 & 0.9926 & 0.0005
\end{tabular}




\begin{tabular}{|c|c|c|c|c|c|c|c|c|c|c|c|c|c|}
\hline \multirow{2}{*}{\multicolumn{2}{|c|}{$\begin{array}{ll}Z & ===\text { INLETS }==== \\
& \text { Inlet }\end{array}$}} & $\begin{array}{r}\text { eRam } \\
1.0000\end{array}$ & $\begin{array}{r}\text { Afs } \\
2564.42\end{array}$ & $\begin{array}{r}\text { Fram } \\
5656.9\end{array}$ & \multicolumn{2}{|c|}{ BTFEDS - intersto } & \multirow{2}{*}{$\begin{array}{l}\text { Wb/Win } \\
0.0368\end{array}$} & \multirow{2}{*}{$\begin{array}{r}\text { BldWk } \\
0.50000\end{array}$} & \multirow{2}{*}{$\begin{array}{r}\text { BldP } \\
0.2200\end{array}$} & \multirow{2}{*}{$\begin{array}{r}W \\
0.8595\end{array}$} & \multirow{2}{*}{ Tt } & \multirow{2}{*}{$\begin{array}{r}h t \\
51.38\end{array}$} & \multirow{2}{*}{$\begin{array}{r}P t \\
9.422\end{array}$} \\
\hline & & & & & HPT_COOLC & C HPC.C> & & & & & & & \\
\hline E & $====$ DUCTS $====$ & dPnorm & MN & Aphy & LPT COOLA & A HPC.C> & 0.0117 & 0.5000 & 0.4500 & 0.2733 & 759.62 & 51.38 & 3.451 \\
\hline E & TEGV & 0.0000 & 0.2735 & 860.21 & WB $2 \overline{\mathrm{X}}$ & HPC.B> & 0.0000 & 0.4500 & 0.2200 & 0.0000 & 737.78 & 46.08 & 13.812 \\
\hline$\leq 2$ & FanDuct & 0.0000 & 0.5401 & 2481.93 & WB2Y & HPC. B> & 0.0000 & 0.7600 & 0.6200 & 0.0000 & 872.59 & 78.94 & 26.984 \\
\hline & & & & & WBA2X & HPC. B> & 0.0000 & 0.4500 & 0.2200 & 0.0000 & 737.78 & 46.08 & 13.812 \\
\hline & $==$ SPLITTERS $==$ & BPR & $\mathrm{dP} / \mathrm{P} 1$ & $\mathrm{dP} / \mathrm{P} 2$ & WBLKG & HPC. $1>$ & 0.0000 & 1.0000 & 1.0000 & 0.0000 & 975.76 & 104.37 & 39.498 \\
\hline & Splitter & 8.7044 & 0.0005 & 0.0005 & WBW2X & $\mathrm{HPC} . \mathrm{B}>$ & 0.0000 & 0.4500 & 0.2200 & 0.0000 & 737.78 & 46.08 & 13.812 \\
\hline & $===$ SHAFTS $====$ & Nmech & trq in & pwr in & BLEEDS - & output & Wb/Win & hscale & Pscale & W & $\mathrm{Tt}$ & ht & Pt \\
\hline 0 & HP_Shaft & 9116.8 & 1968.9 & 3417.7 & WB17Y & Bleed> & 0.1000 & 1.0000 & 1.0000 & 2.5952 & 539.88 & -1.62 & 6.568 \\
\hline & LP_Shaft & 2357.0 & 4271.5 & 1916.9 & HPT_COOLA & A Bleed> & 0.1142 & 1.0000 & 1.0000 & 2.6669 & 975.76 & 104.37 & 38.588 \\
\hline & & & & & $\mathrm{HPT}_{\text {COCOLB }}^{-}$ & 3 Bleed> & 0.0999 & 1.0000 & 1.0000 & 2.3336 & 975.76 & 104.37 & 21.905 \\
\hline & & & & & WB $3 \bar{X}$ & Bleed> & 0.0000 & 1.0000 & 1.0000 & 0.0000 & 975.76 & 104.37 & 39.498 \\
\hline & & & & & WBA3X & Bleed $>$ & 0.0000 & 1.0000 & 1.0000 & 0.0000 & 975.76 & 104.37 & 39.498 \\
\hline & & & & & WBW3X & Bleed> & 0.0000 & 1.0000 & 1.0000 & 0.0000 & 975.76 & 104.37 & 39.498 \\
\hline & & & & & WBFDLKG & FanDu> & 0.0000 & 1.0000 & 1.0000 & 0.0000 & 486.44 & -14.36 & 4.852 \\
\hline & & & & & WB15X & Bleed> & 0.0000 & 1.0000 & 1.0000 & 0.0000 & 486.44 & -14.36 & 4.852 \\
\hline & & & & & WB15Y & Bleed> & 0.0000 & 1.0000 & 1.0000 & 0.0000 & 486.44 & -14.36 & 4.852 \\
\hline & & & & & WB17X & Bleed> & 0.0000 & 1.0000 & 1.0000 & 0.0000 & 486.44 & -14.36 & 4.852 \\
\hline & $===$ BURNERS $===$ & Ttout & eff & dPnorm & Wfuel & FAR & & & & & & & \\
\hline & Burner & 1720.76 & 0.9995 & 0.0230 & 0.19021 & 01104 & & & & & & & \\
\hline & $===$ NOZZLES $===$ & PR & Cfg & CdTh & $\mathrm{Cv}$ & Ath & MNth & Vact & $\mathrm{Fg}$ & & & & \\
\hline & Core_Nozz & 1.117 & 0.9801 & 1.0000 & 0.9800 & 3.40 & 0.404 & 571.6 & 418.3 & & & & \\
\hline & Byp_̄Nozz & 1.571 & 0.9800 & 1.0000 & 0.9800 & 6.86 & 0.830 & 825.0 & 5858.7 & & & & \\
\hline
\end{tabular}


Date:07/05/13 Time:10:03:18 Model: Turbofan Engine - COMDES ON Converge = 1 CASE: 0

Version:NPSS1.6.5 - Rev: -> Gas Package: Janaf iter/pass/Jacb/Broy=11/25/1/9 Run by: Philip C Jorgenson PC: 10

Temperature Stator 1 inlet: 476.22 $\quad$ Stator 1 exit: 482.85 $\quad$ Stator 2 inlet: $491.16 \quad$ Stator 2 exit: 495.38

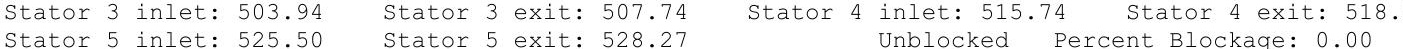

Ambient Relative Humidity $\quad 10.00$

Fan Face Relative Humidity $\quad 3.24$

Fan Bypass Relative Humidity $\quad 2.48$

LPC Inlet Relative Humidity $\quad 2.22$

LPC Exit Relative Humidity

HPC Relative Humidity

0.00

Drop Diameter Velocity

Ambient Static Pressure

Ambient Static Temperature
Additional Water at LPC Exit

0.0001000

$\begin{array}{rrr}682.24 & \text { Fan/LPC Inlet Flow Velocity } & 176.64 \\ 3.58 & \text { Fan/LPC Inlet Static Pressure } & 4.74\end{array}$

Inlet Length

40.00

0.0000139

Fan/LPC Inlet Static Temperature 468.61

$\begin{array}{rrrrrr}\text { MN } & \text { alt } & \text { dTamb } & \text { W } & \text { SUMMARY } & \text { FUTPUT DATA } \\ 0.669 & 34281.0 & 36.00 & 272.65 & 666.7 & 1.0955\end{array}$

666.
FLOW STATION DATA

FSO Ambient.FI_O

FS1 Inlet.F1_O

FS12 Splitter.F1_02

FS2 Splitter.F1_01

FS14 Fan.F1_O

FS24 VaporIN.F1_O

FS25 Bleed2.F1_o

ES3 HPC.FI_O

Bleed3.FI_O

Burner.FI

FS45 HPT.Fl

FS49 LPT.FI-

FS8 Core_Nozz.Fl_O

FS17 FanDuctLkg.FI_O

FS171 Bleed15.F1_O

FS172 FanDuct.Fl-O

FS173 Byp Nozz.Fì O

W
272.65
272.65
244.86
27.79
244.86
27.79
27.79
25.01
23.80
18.44
18.64
24.92
25.21
25.21
25.21
244.86
247.64
247.64
247.64

$\begin{array}{ccc}\text { Pt } & \text { Tt } \\ 4.831 & 471.21 & -18 . \\ 4.831 & 471.21 & -18 .\end{array}$

$\begin{array}{llrrr}4.831 & 471.21 & -18.12 & 0.0000 & \text { Wh }\end{array}$

$\begin{array}{lllll}4.831 & 471.21 & -18.12 & 0.0000 & 790.56\end{array}$

$\begin{array}{lllll}4.829 & 471.21 & -18.12 & 0.0000 & 710.34 \\ 4.829 & 471.21 & -18.12 & 0.0000 & 80.61\end{array}$

$\begin{array}{lllll}4.829 & 471.21 & -18.12 & 0.0000 & 710.34 \\ 5.360 & 487.02 & -18.12 & 0.0000 & 80.61\end{array}$

$\begin{array}{lllll}5.360 & 487.02 & -14.33 & 0.0000 & 650.61\end{array}$

$\begin{array}{lllll}5.120 & 536.97 & -2.35 & 0.00000 & 58.36 \\ 7.120 & 537.03 & -2.41 & 0.0000 & 58.36\end{array}$

$\begin{array}{lllll}7.120 & 537.03 & -2.41 & 0.0000 & 58.36 \\ 7.120 & 537.03 & -2.41 & 0.0000 & 52.53\end{array}$

$7.1207-537.03-2.410 .0000$
$966.86-102.06 \quad 0.0000$

$\begin{array}{llll}42.157 & 966.86 & 102.06 & 0.0000\end{array}$

$\begin{array}{llll}4.157 & 966.86 & 102.06 & 0.0000\end{array}$

$\begin{array}{llll}41.186 & 1710.22 & -89.51 & 0.0110\end{array}$

10.173
3.939
3.939

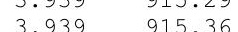

-65.03
-6.0081

$\begin{array}{llll}3.939 & 915.36 & -65.03 & 0.0081\end{array}$

$5.360 \quad 487.02$

$\begin{array}{ll}5.360-487.58 \\ 5.360 & 487.58\end{array}$

$5.360 \quad 487.58$

$\begin{array}{ll}-14.33 & 0.0000\end{array}$

$\begin{array}{ll}-14.19 & 0.0000\end{array}$

52.53

11.33
8.78

12.08

124.94

124.95

124.95

658.37

658.37
658.37

658.37
VTAS

682.24
OPR
8.726

EPR
0.795
Powerset

10.000
T4
1710. $\begin{array}{cc}\text { T41 } & \text { T49 } \\ 1616.6 & 1130.0\end{array}$

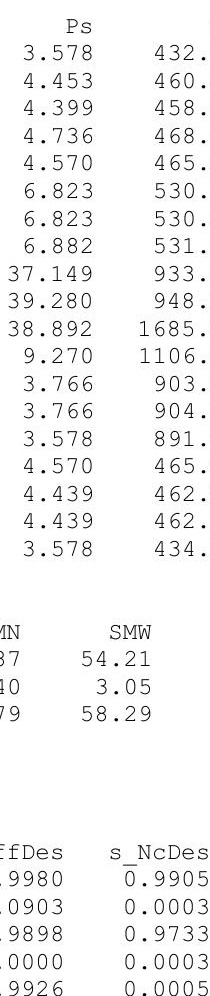

$\begin{array}{rrr}\text { Aphy } & \text { MN } & \text { gamt } \\ 2576.9 & 0.6690 & 1.40074 \\ 4168.2 & 0.3429 & 1.40074 \\ 3531.8 & 0.3673 & 1.40074 \\ 830.5 & 0.1664 & 1.40074 \\ 2606.7 & 0.4824 & 1.40059 \\ 412.6 & 0.2474 & 1.39996 \\ 412.6 & 0.2474 & 1.39996 \\ 412.6 & 0.2210 & 1.39996 \\ 49.7 & 0.4311 & 1.38272 \\ 49.3 & 0.3212 & 1.38272 \\ 74.6 & 0.2943 & 1.33188 \\ 265.4 & 0.3708 & 1.36649 \\ 860.2 & 0.2564 & 1.38034 \\ 860.2 & 0.2564 & 1.38034 \\ 613.4 & 0.3757 & 1.38033 \\ 2606.7 & 0.4824 & 1.40059 \\ 2481.9 & 0.5256 & 1.40059 \\ 2481.9 & 0.5256 & 1.40059 \\ 2006.9 & 0.7820 & 1.40059\end{array}$
$\begin{array}{ll}0.0000968 & 272.62 \\ 0.0000968 & 272.62\end{array}$ 0.0000968 0.0000968 0.0000968 0.0001107 0.0001107 0.0001107 0.0001107

0.0001107

0.0001107

0.0001107

0.0001107

0.00000968
0.0000970
0.0000970

0.0000970
0.0000970

0.0000970
WAR
0.0000968

$\begin{array}{rrr}\text { Wair } & \text { WH2O } & \text { H2O frac } \\ 272.62 & 0.026 & 0.0001 \\ 272.62 & 0.026 & 0.0001 \\ 44.84 & 0.024 & 0.0001 \\ 27.79 & 0.003 & 0.0001 \\ 44.84 & 0.024 & 0.0001 \\ 27.79 & 0.003 & 0.0001 \\ 27.79 & 0.003 & 0.0001 \\ 25.01 & 0.003 & 0.0001 \\ 23.79 & 0.003 & 0.0001 \\ 18.44 & 0.002 & 0.0001 \\ 18.44 & 0.002 & 0.0135 \\ 24.71 & 0.003 & 0.0102 \\ 25.01 & 0.003 & 0.0100 \\ 25.01 & 0.003 & 0.0100 \\ 25.01 & 0.003 & 0.0101 \\ 44.84 & 0.024 & 0.0001 \\ 47.62 & 0.024 & 0.0001 \\ 47.62 & 0.024 & 0.0001 \\ 24.62 & 0.024 & 0.0001\end{array}$




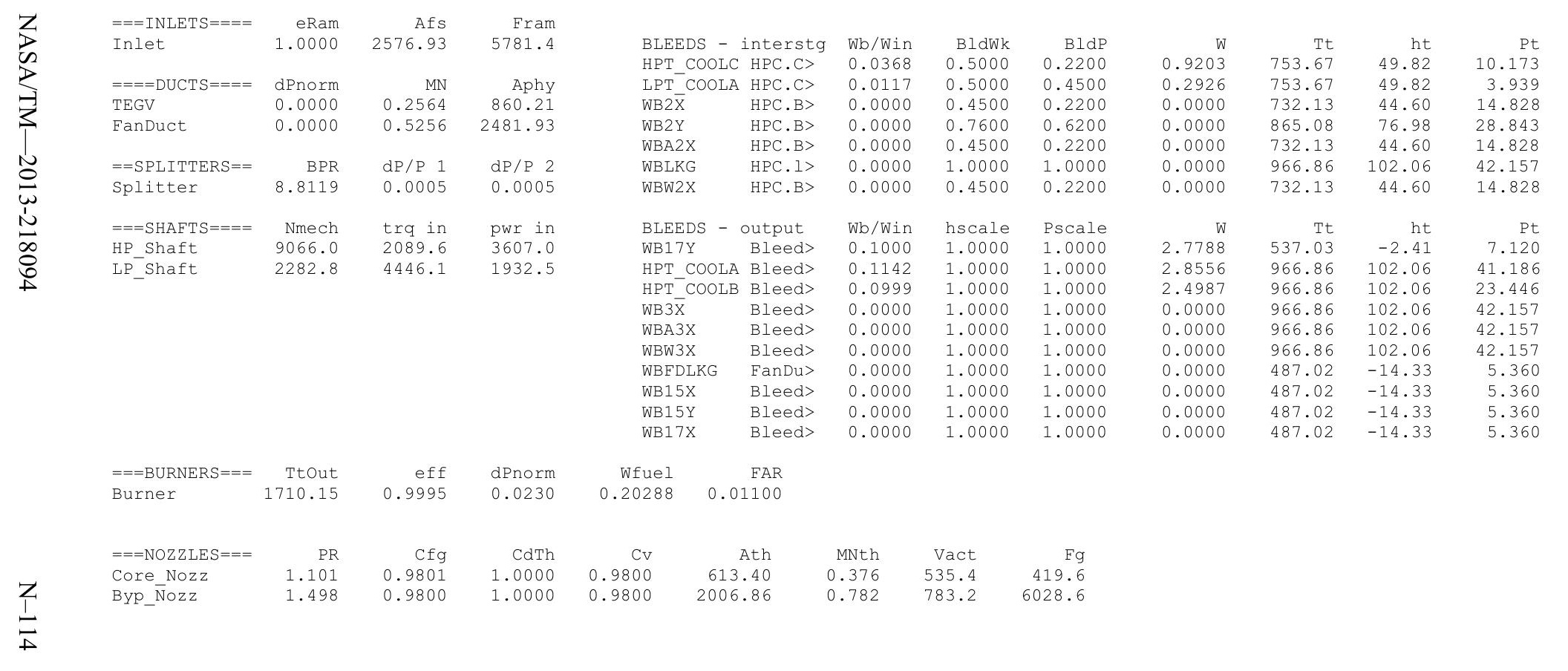


Date:07/05/13 Time:10:03:34 Model: Turbofan Engine - COMDES ON Converge = 1 CASE: 0

Version:NPSS 1.6.5 - Rev: -> Gas Package: Janaf iter/pass/Jacb/Broy= 13/27/1/11 Run by: Philip C Jorgenson PC: 10

Temperature Stator 1 inlet: $484.50 \quad$ Stator 1 exit: $490.20 \quad$ Stator 2 inlet: $497.24 \quad$ Stator 2 exit: 500.63 Stator 5 inlet: 525.92 Stator 5 exit: $528.01 \quad$ Unblocked Percent Blockage: 0.00

Ambient Relative Humidity $\quad 10.00$

Fan Face Relative Humidity $\quad 4.18$

Fan Bypass Relative Humidity $\quad 3.37$

LPC Inlet Relative Humidity $\quad 3.05$

LPC Exit Relative Humidity

HPC Relative Humidity

0.00

0.0001000

630.80
4.36

Inlet Length

40.00

Ambient Static Pressure

Ambient Static Temperature

Additional Water at LPC Exit

447.58 Fan/LPC Inlet Static Temperature 478.12

$\begin{array}{lllr}\text { MN alt dTamb } & \text { W } & \text { SUMMAR OUTPUT DATA } \\ \text { Mn } & \text { TSFC }\end{array}$

$0.608 \quad 30029.0 \quad 36.00$ $36.00 \quad 298.11$

$\begin{array}{rr}\text { Fn } & \text { TSFC } \\ 709.6 & 1.1115\end{array}$
Wfuel
788.72
BPR
8.4102
OPR
8.108 PowerSet
10.000 $\begin{array}{ccc}\text { T4 } & \text { T41 } & \text { T49 } \\ \text { 1708.1 } & 1614.2 & 1131.6\end{array}$

FSO Ambient.Fl_O

FS1 Inlet.F1_O

FS12 Splitter.F1_02

FS2 Splitter.F1_01

FS14 Fan.FI_-

FS24 VaporIN.FI_O

FS25 Bleed2.F1_ $\bar{O}$

FS3 HPC.FI_O

S36 Bleed3.F1_O

FS4 Burner.FI_O

FS45 HPT.F1_-

ES49 LPT.FI-

FS8 Core_Nozz.Fl_o

FS17 FanDuctLkg.FI_O

FS171 Bleed15.F1_O

FS172 FanDuct.Fl-O

FS173 Byp Nozz.Fì O

$W$
298.11
298.11
266.43
31.68
266.43
31.68
31.68
26.93
25.62
19.86
20.08
26.83
27.15
27.15
27.15
266.43
271.18
271.18
271.18

FLOW STATION DATA

$\begin{array}{lrrrr}\text { Pt } & \text { Tt } & \text { ht } & \text { FAR } & \text { Wc } \\ 5.595 & 480.74 & -16.28 & 0.0000 & 753.83 \\ 5.595 & 480.74 & -16.28 & 0.0000 & 753.83 \\ 5.592 & 480.74 & -16.28 & 0.0000 & 674.06 \\ 5.592 & 480.74 & -16.28 & 0.0000 & 80.15 \\ 6.130 & 494.93 & -12.88 & 0.0000 & 623.91 \\ 7.803 & 536.92 & -2.81 & 0.0000 & 60.70 \\ 7.803 & 536.98 & -2.91 & 0.0000 & 60.71 \\ 7.803 & 536.98 & -2.91 & 0.0000 & 51.60 \\ 45.364 & 962.26 & 100.44 & 0.0000 & 11.31 \\ 45.364 & 962.26 & 100.44 & 0.0000 & 8.76 \\ 44.319 & 1708.12 & 87.87 & 0.0110 & 12.08 \\ 1.108 & 1135.85 & -12.08 & 0.0082 & 52.53 \\ 4.710 & 937.20 & -60.49 & 0.0081 & 113.86 \\ 4.710 & 937.28 & -60.49 & 0.0081 & 113.86 \\ 4.710 & 937.35 & -60.49 & 0.0081 & 113.87 \\ 6.130 & 494.93 & -12.88 & 0.0000 & 623.91 \\ 6.130 & 495.67 & -12.71 & 0.0000 & 635.51 \\ 6.130 & 495.67 & -12.71 & 0.0000 & 635.51 \\ 6.130 & 495.67 & -12.71 & 0.0000 & 635.51\end{array}$

Ps
4.358
5.201
5.149
5.486
5.315
7.449
7.449
7.551
39.998
42.281
41.850
10.151
4.539
4.539
4.358
5.315
5.168
5.168
4.358

TS
447.58
470.80
469.52
478.12
475.13
529.86
529.91
531.97
929.23
943.67
1683.86
1108.71
927.72
927.79
917.55
475.13
472.05
472.05
449.58

$\begin{array}{rr}\text { Aphy } & \mathrm{MN} \\ 2589.5 & 0.6080 \\ 4168.2 & 0.3247 \\ 3531.8 & 0.3454 \\ 830.5 & 0.1654 \\ 2606.7 & 0.4561 \\ 412.6 & 0.2582 \\ 412.6 & 0.2582 \\ 412.6 & 0.2169 \\ 49.7 & 0.4301 \\ 49.3 & 0.3205 \\ 74.6 & 0.2943 \\ 265.4 & 0.3650 \\ 860.2 & 0.2322 \\ 860.2 & 0.2322 \\ 613.4 & 0.3371 \\ 2606.7 & 0.4561 \\ 2481.9 & 0.4998 \\ 2481.9 & 0.4998 \\ 2006.9 & 0.7153\end{array}$

gamt
1.40064
1.40064
1.40064
1.40064
1.40050
1.39995
1.39995
1.39995
1.38298
1.38298
1.33194
1.3663
1.3790
1.3790
1.37900
1.4005
1.40049
1.40049
1.40049

$$
\begin{aligned}
& 0.000 .000 \\
& 0.000 \\
& 0.000 \\
& 0.000 \\
& 0.000 \\
& 0.000 \\
& 0.000 \\
& 0.000 \\
& 0.000 \\
& 0.000 \\
& 0.000 \\
& 0.000 \\
& 0.000 \\
& 0.000 \\
& 0.000 \\
& 0.000 \\
& 0.000 \\
& 0.000
\end{aligned}
$$

$\begin{array}{rrrr}\text { WAR } & \text { Wair } & \text { WH2O } & \text { H2O frac } \\ .0001750 & 298.05 & 0.052 & 0.0002 \\ .0001750 & 298.05 & 0.052 & 0.0002 \\ .0001750 & 266.38 & 0.047 & 0.0002 \\ .0001750 & 31.67 & 0.006 & 0.0002 \\ .0001750 & 266.38 & 0.047 & 0.0002 \\ .0001750 & 31.67 & 0.006 & 0.0002 \\ .0001947 & 31.67 & 0.006 & 0.0002 \\ .0001947 & 26.92 & 0.005 & 0.0002 \\ .0001947 & 25.62 & 0.005 & 0.0002 \\ .0001947 & 19.85 & 0.004 & 0.0002 \\ .0001947 & 19.85 & 0.004 & 0.0136 \\ .0001947 & 26.61 & 0.005 & 0.0103 \\ .0001947 & 26.92 & 0.005 & 0.0102 \\ .0001947 & 26.92 & 0.005 & 0.0102 \\ .0001947 & 26.92 & 0.005 & 0.0102 \\ .0001750 & 266.38 & 0.047 & 0.0002 \\ .0001753 & 271.13 & 0.048 & 0.0002 \\ .0001753 & 271.13 & 0.048 & 0.0002 \\ .0001753 & 271.13 & 0.048 & 0.0002\end{array}$

TURBOMACHINERY PERFORMANCE DATA

\begin{tabular}{|c|c|c|c|c|c|c|c|c|c|}
\hline & WC & PR & eff & $\mathrm{NC}$ & $\mathrm{TR}$ & efPoly & pwr & SMN & SMW \\
\hline Fan & 674.06 & 1.096 & 0.9014 & 2238.852 & 1.0295 & 0.9027 & -1282.9 & -8363.19 & 52.80 \\
\hline LPC & 80.15 & 1.395 & 0.8549 & 2238.852 & 1.1169 & 0.8616 & -604.0 & 5.98 & 2.60 \\
\hline HPC & 51.60 & 5.814 & 0.8138 & 8879.345 & 1.7920 & 0.8527 & -3842.0 & 62.28 & 58.66 \\
\hline НPT & 12.08 & 3.990 & 0.8743 & 218.602 & 1.3526 & 0.8525 & 3842.0 & & \\
\hline LPT & 52.53 & 2.358 & 0.8336 & 63.955 & 1.2095 & 0.8162 & 1886.8 & & \\
\hline
\end{tabular}

TURBOMACHINERY MAP DATA

$\begin{array}{lrrrrrrrrr} & \text { WcMap } & \text { PRmap } & \text { effMap } & \text { NcMap } & \text { R/Parm } & \text { S_WcDes } & \text { S_PRdes } & \text { S_effDes } & \text { S_NCDes } \\ \text { Fan } & 783.19 & 1.094 & 0.9032 & 2217.505 & 0.0416 & 0.8607 & \overline{1} .0235 & 0.9980 & 0.9905 \\ \text { LPC } & 59.97 & 1.378 & 0.7652 & 0.591 & 0.0000 & 1.3365 & 1.0452 & 1.1172 & 0.0003 \\ \text { HPC } & 46.93 & 5.587 & 0.8222 & 8642.628 & 10.9509 & 1.0995 & 1.0494 & 0.9898 & 0.9733 \\ \text { HPT } & 0.96 & 3.907 & 0.8743 & 1.314 & 3.9070 & 12.6299 & 0.9723 & 1.0000 & 0.0003 \\ \text { LPT } & 0.80 & 2.022 & 0.8398 & 0.719 & 2.0217 & 65.2590 & 0.7521 & 0.9926 & 0.0005\end{array}$




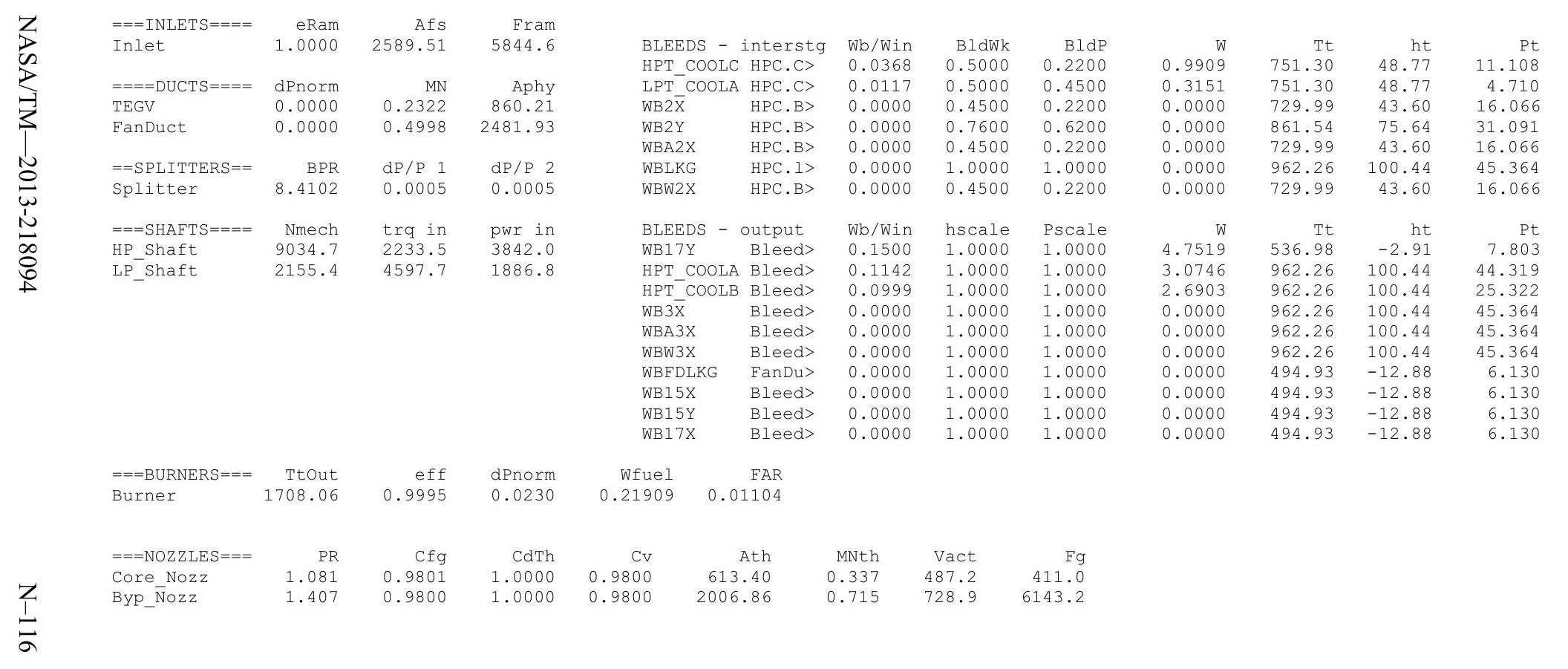


Date:07/05/13 Time:10:04:05 Model: Turbofan Engine - COMDES ON COnverge = 1 CASE: 0

Version:NPSS 1.6.5 - Rev: -> Gas Package: Janaf iter/pass/Jacb/Broy= 23/51/2/20 Run by: Philip C Jorgenson PC: 10

Temperature Stator 1 inlet: 492.28 Stator 1 exit: 496.44 $\quad$ Stator 2 inlet: $501.16 \quad$ Stator 2 exit: 503.02 stator 5 inlet: 519.46 Stator 5 exit: $520.21 \quad$ Unblocked Percent Blockage: 0.00

Ambient Relative Humidity $\quad 10.00$

Fan Face Relative Humidity $\quad 5.01$

Fan Bypass Relative Humidity $\quad 4.21$

LPC Inlet Relative Humidity $\quad 4.03$

LPC Exit Relative Humidity

HPC Relative Humidity

0.00

0.0001000

582.56

Inlet Length

40.00

Ambient Static Pressure

Ambient Static Temperature

Additional Water at LPC Exit

\begin{tabular}{rr}
195.59 \\
\hline an/LPC Inlet Static Pressure & 6.37
\end{tabular}

$463.14 \quad$ Fan/LPC Inlet Static Temperature 488.23

$\begin{array}{rrrrrr} & & & \text { SUMMARY OUTPUT DATA } \\ \text { MN } & \text { alt } & \text { dTamb } & \text { W } & \text { Fn } & \text { TSFC }\end{array}$

$\begin{array}{llll}0.552 & 25666.0 & 36.00 & 322.27\end{array}$
Wfuel 832.84
BPR
7.1190
VTAS 582.56
$\mathrm{OPR}$
7.194 7.194 $\begin{array}{llccc}\text { EPR } & \text { PowerSet } & \text { T4 } & \text { T41 } & \text { T49 } \\ 0.857 & 10.000 & 1712.9 & 1616.9 & 1139.7\end{array}$
FSO Ambient.Fl O

FS1 Inlet.F1_O

FS12 Splitter.F1_02

FS2 Splitter.F1_01

FS14 Fan.FI_-

FS24 VaporIN.F1_O

FS25 Bleed2.F1_o

HPC.FI_O

FS4 Bleed3.FI_O

Burner.FI

FS49

FS49 LPT.FI-

FS8 Core Nozz.F1

FS17 CanDüctivg.

FS171 Bleed15. F1.

FS171 Bleed15.FI_-

FS173 Byp NozZ. FI

TURBOMACHINERY PERFORMANCE DATA

$\begin{array}{lrrr} & \text { WC } & \text { PR } & \text { eff } \\ \text { Fan } & 620.64 & 1.081 & 0.9017 \\ \text { LPC } & 87.18 & 1.256 & 0.8233 \\ \text { HPC } & 50.54 & 5.730 & 0.8121 \\ \text { HPT } & 12.08 & 3.860 & 0.8752 \\ \text { LPT } & 50.94 & 2.113 & 0.8233\end{array}$

FLOW STATION DATA

\begin{tabular}{rrrrrr} 
W & \multicolumn{1}{c}{ Pt } & Tt & ht & FAR & WC \\
322.27 & 6.516 & 491.41 & -14.47 & 0.0000 & 707.46 \\
322.27 & 6.516 & 491.41 & -14.47 & 0.0000 & 707.46 \\
282.57 & 6.513 & 491.41 & -14.47 & 0.0000 & 620.64 \\
39.69 & 6.513 & 491.41 & -14.47 & 0.0000 & 87.18 \\
282.57 & 7.039 & 503.65 & -11.54 & 0.0000 & 581.38 \\
39.69 & 8.180 & 531.59 & -4.83 & 0.0000 & 72.19 \\
39.69 & 8.180 & 531.66 & -4.95 & 0.0000 & 72.20 \\
27.79 & 8.180 & 531.66 & -4.95 & 0.0000 & 50.54 \\
26.44 & 46.874 & 949.54 & 96.54 & 0.0000 & 11.22 \\
20.49 & 46.874 & 949.54 & 96.54 & 0.0000 & 8.69 \\
20.72 & 45.795 & 1712.91 & 83.72 & 0.0113 & 12.08 \\
27.69 & 11.865 & 1144.17 & -14.29 & 0.0084 & 50.94 \\
28.02 & 5.615 & 969.12 & -56.84 & 0.0083 & 100.23 \\
28.02 & 5.615 & 969.19 & -56.84 & 0.0083 & 100.24 \\
28.02 & 5.615 & 969.26 & -56.84 & 0.0083 & 100.24 \\
282.57 & 7.039 & 503.65 & -11.54 & 0.0000 & 581.38 \\
294.48 & 7.039 & 504.78 & -11.27 & 0.0000 & 606.56 \\
294.48 & 7.039 & 504.78 & -11.27 & 0.0000 & 606.56 \\
294.48 & 7.039 & 504.78 & -11.27 & 0.0000 & 606.56
\end{tabular}

Ps
5.297
6.116
6.082
6.366
6.246
7.644
7.644
7.927
41.433
43.743
43.242
10.911
5.459
5.459
5.297
6.246
6.054
6.054
5.297

TS
463.14
482.59
481.88
488.23
486.72
521.39
521.45
526.91
917.55
931.50
1688.59
1118.73
961.64
961.71
953.89
486.72
483.49
483.49
465.36

$\begin{array}{rr}\text { Aphy } & \text { MN } \\ 2581.0 & 0.5520 \\ 4168.2 & 0.3022 \\ 3531.8 & 0.3143 \\ 830.5 & 0.1805 \\ 2606.7 & 0.4167 \\ 412.6 & 0.3127 \\ 412.6 & 0.3128 \\ 412.6 & 0.2122 \\ 49.7 & 0.4257 \\ 49.3 & 0.3175 \\ 74.6 & 0.2944 \\ 265.4 & 0.3522 \\ 860.2 & 0.2030 \\ 860.2 & 0.2030 \\ 613.4 & 0.2922 \\ 2606.7 & 0.4167 \\ 2481.9 & 0.4690 \\ 2481.9 & 0.4690 \\ 2006.9 & 0.6503\end{array}$

gamt
1.40052
1.40052
1.40052
1.40052
1.40039
1.40001
1.40001
1.40001
1.38371
1.38371
1.33152
1.36567
1.37693
1.37692
1.37692
1.40039
1.40037
1.40037
1.40037

WAR
0.0003043
0.0003043
0.0003043
0.0003043
0.0003043
0.0003043
0.0003276
0.0003276
0.0003276
0.0003276
0.0003276
0.0003276
0.0003276
0.0003276
0.0003276
0.0003043
0.0003053
0.0003053
0.0003053

Wair 322.17
322.17 322.17
282.49 39.68 282.49
39.68 39.68 39.68
27.78 .013 $\begin{array}{ll}26.43 & 0.009\end{array}$ $20.48 \quad 0.007$ $\begin{array}{ll}20.48 & 0.007 \\ 27.45 & 0.009\end{array}$ $\begin{array}{ll}27.45 & 0.009 \\ 27.78 & 0.009\end{array}$ $\begin{array}{ll}27.78 & 0.009 \\ 27.78 & 0.009\end{array}$ $\begin{array}{ll}27.78 & 0.009 \\ 27.78 & 0.009\end{array}$ $282.49 \quad 0.086$ $294.39 \quad 0.090$ $294.39 \quad 0.090$ $294.39 \quad 0.090$

$\begin{array}{rrrrrr}\text { NC } & \text { TR } & \text { efPoly } & \text { pwr } & \text { SMN } & \text { SMW } \\ 2025.350 & 1.0249 & 0.9027 & -1172.9 & -2596.18 & 50.01 \\ 2025.350 & 1.0818 & 0.8289 & -541.3 & 6.43 & 2.41 \\ 8845.465 & 1.7860 & 0.8511 & -3893.2 & 61.73 & 58.01 \\ 216.383 & 1.3429 & 0.8541 & 3893.2 & & \\ 58.282 & 1.1777 & 0.8074 & 1714.2 & & \end{array}$

TURBOMACHINERY MAP DATA

$\begin{array}{lrrrrrrrrr} & \text { WcMap } & \text { PRmap } & \text { effMap } & \text { NcMap } & \text { R/Parm } & \text { S_WcDes } & \text { S_PRdes } & \text { S_effDes } & \text { S_NcDes } \\ \text { Fan } & 721.12 & 1.079 & 0.9035 & 2006.039 & 0.0424 & 0.8607 & 1.0235 & 0.9980 & 0.9905 \\ \text { LPC } & 52.83 & 1.304 & 0.7603 & 0.535 & 0.0000 & 1.6504 & 0.8407 & 1.0829 & 0.0003 \\ \text { HPC } & 45.97 & 5.507 & 0.8205 & 8609.651 & 10.9984 & 1.0995 & 1.0494 & 0.9898 & 0.9733 \\ \text { HPT } & 0.96 & 3.780 & 0.8752 & 1.300 & 3.7803 & 12.6299 & 0.9723 & 1.0000 & 0.0003 \\ \text { LPT } & 0.78 & 1.837 & 0.8294 & 0.656 & 1.8372 & 65.2590 & 0.7521 & 0.9926 & 0.0005\end{array}$




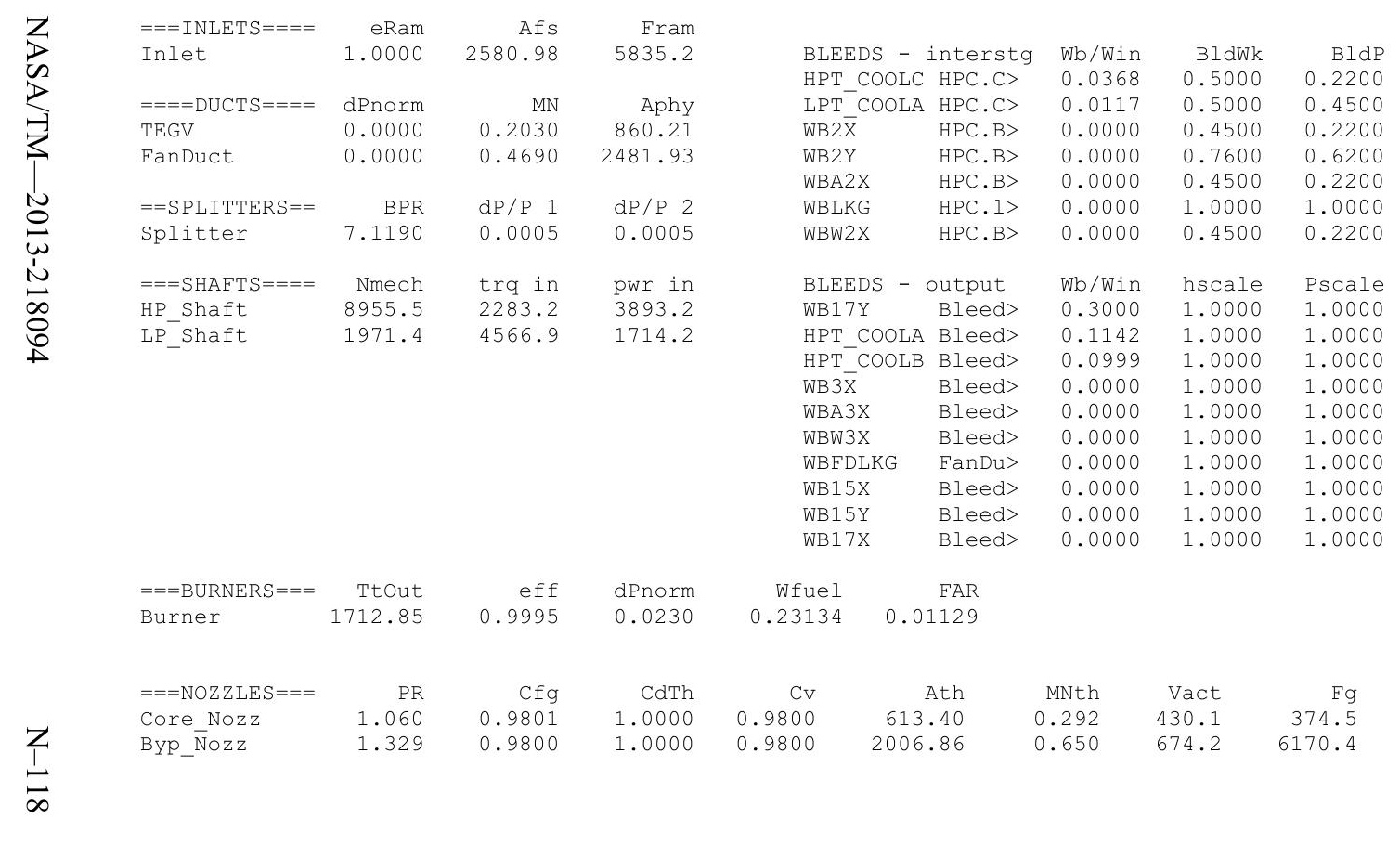


Date:07/05/13 Time:10:04:28 Model: Turbofan Engine - COMDES ON Converge = 1 CASE: 0

Version:NPSS 1.6.5 - Rev: -> Gas Package: Janaf iter/pass/Jacb/Broy= 27/41/1/25 Run by: Philip C Jorgenson PC: 10

Temperature Stator 1 inlet: $505.22 \quad$ Stator 1 exit: $508.18 \quad$ Stator 2 inlet: $510.96 \quad$ Stator 2 exit: 511.75 $\begin{array}{llll}\text { Stator } 3 \text { inlet: } 515.11 & \text { Stator } 3 \text { exit: } 515.47 & \text { Stator } 4 \text { inlet: } 518.74 \text { Stator } 4 \text { exit: } 518.7 \\ \text { Stator } 5 \text { inlet: } 520.69 & \text { Stator } 5 \text { exit: } 520.56 & \end{array}$

Ambient Relative Humidity $\quad 10.00$

Fan Face Relative Humidity $\quad 6.01$

Bypass Relative Humid

LPC Exit Relative Humidity

HPC Relative Humidity

Drop Diameter

0.01

Ambient Flow Velocity

Ambient Static Pressure

Ambient Static Temperature
Additional Water at LPC Exit

528.20

Inlet Length

40.00

$\begin{array}{lrr}6.74 & \text { Fan/LPC Inlet Flow Velocity } & 203.17 \\ 6 \text { 6an Inlet Static Pressure } & 7.75\end{array}$

0.0000304

an/LPC Inlet Static Temperature 502.97

$\begin{array}{rrrrrr}\text { MN } & \text { alt } & \text { dTamb } & \text { W } & \text { Fn } & \text { TSFC } \\ 0.490 & 20047.0 & 36.00 & 357.70 & 762.3 & 1.1971\end{array}$

762.3

1.1971

912.54

BPR
6.3407

VTAS
528.20

$\mathrm{OPR}$
6.254

EPR
0.888

PowerSet
10.000

$\begin{array}{ccc}\text { T4 } & \text { T41 } & \text { T49 } \\ 1736.5 & 1637.1 & 1165.8\end{array}$
FSO Ambient.Fl O

FS1 Inlet.FI_-

FS12 Splitter.F1_02

FS14 Fan.FI_O

S23 LPC.FI-

S24 Vaporin. HI-O

(ES25

ES36 Bleed3rito

ES4 Burner. Fl-

FS45 HPT.FI O

FS49 LPT.FI-O

FS8 Core Nozzz.F1

S17 FanDüctikg.

FS171 Bleed15.FI

FS171 Bleed15.F1-

FS173 Byp Nozz. $\overline{1}$

TURBOMACHINERY PERFORMANCE DATA

$\begin{array}{lrrr} & \text { WC } & \text { PR } & \text { eff } \\ \text { Fan } & 565.13 & 1.068 & 0.9035 \\ \text { LPC } & 89.13 & 1.140 & 0.6953 \\ \text { HPC } & 48.17 & 5.487 & 0.8076 \\ \text { HPT } & 12.09 & 3.654 & 0.8765 \\ \text { LPT } & 48.45 & 1.889 & 0.8151\end{array}$

FLOW STATION DATA

$\begin{array}{rrrrr}\text { W } & \text { Pt } & \text { Tt } & \text { ht } & \text { FAR } \\ 357.70 & 7.943 & 506.41 & -12.46 & 0.0000 \\ 357.70 & 7.943 & 506.41 & -12.46 & 0.0000 \\ 308.97 & 7.939 & 506.41 & -12.46 & 0.0000 \\ 48.73 & 7.939 & 506.41 & -12.46 & 0.0000 \\ 308.97 & 8.480 & 517.06 & -9.90 & 0.0000 \\ 48.73 & 9.053 & 534.25 & -5.78 & 0.0000 \\ 48.73 & 9.053 & 534.33 & -5.94 & 0.0000 \\ 29.24 & 9.053 & 534.33 & -5.94 & 0.0000 \\ 27.82 & 49.677 & 943.46 & 93.44 & 0.0000 \\ 21.56 & 49.677 & 943.46 & 93.44 & 0.0000 \\ 21.81 & 48.533 & 1736.55 & 80.13 & 0.0118 \\ 29.15 & 13.281 & 1170.56 & -15.61 & 0.0088 \\ 29.49 & 7.031 & 1017.45 & -52.74 & 0.0087 \\ 29.49 & 7.031 & 1017.52 & -52.73 & 0.0087 \\ 29.49 & 7.031 & 1017.59 & -52.73 & 0.0087 \\ 308.97 & 8.480 & 517.06 & -9.90 & 0.0000 \\ 328.46 & 8.480 & 518.08 & -9.67 & 0.0000 \\ 328.46 & 8.480 & 518.08 & -9.67 & 0.0000 \\ 328.46 & 8.480 & 518.08 & -9.67 & 0.0000\end{array}$

$\begin{array}{rr}\text { Wc } & \text { Ps } \\ 653.92 & 6.740\end{array}$

565.13

89.13

534.64

80.28

80.29
48.17

48.17

11.10
8.60

8.60
12.09

48.45
86.34

86.34

86.34
86.34

86.34
534.64

534.64

568.93

568.93

$\begin{array}{rrrrrr}\text { NC } & \text { TR } & \text { efPoly } & \text { pwr } & \text { SMN } & \text { SMW } \\ 1815.354 & 1.0210 & 0.9044 & -1117.1 & -1833.16 & 46.56 \\ 1815.354 & 1.0550 & 0.7009 & -460.7 & 7.12 & 2.04 \\ 8765.201 & 1.7657 & 0.8467 & -4011.0 & 62.14 & 58.11 \\ 213.490 & 1.3265 & 0.8566 & 4011.0 & & \\ 52.429 & 1.1472 & 0.8013 & 1577.8 & & \end{array}$
7.531 7.509
7.752 7.690 8.304 8.304

44.047

45.432

45.826
12.324

6.886

$\begin{array}{rr}6.740 & 1005.97\end{array}$

7.461

7.461

6.740

Ts
483.18
498.75
498.41
502.97
502.82
521.24
521.30
530.01
912.44
925.92
1711.94
1147.41
1011.73
1011.80
1005.97
502.82
499.47
499.47
485.17

485.17

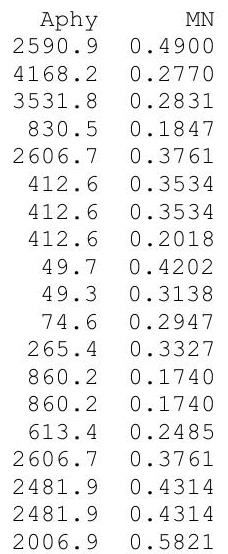

1.40031 1.40031
1.40031 1.40031
1.40031 1.40031
1.40018

1.39993

1.39993
1.39993

1.38403

1.38403

1.33006
1.36370

1.37363
1.37362

1.37362
1.40018

1.40016

1.40016

1.40016
1.40016
WAR
0.0005787
0.0005787
0.0005787
0.0005787
0.0005787
0.0005787
0.0006090
0.0006090
0.0006090
0.0006090
0.0006090
0.0006090
0.0006090
0.0006090
0.0006090
0.0005787
0.0005805
0.0005805
0.0005805

Wair
357.49
357.49 357.49
357.49 $357.49 \quad 0.28$ $\begin{array}{ll}308.79 & 0.179 \\ 308.70 & 0.028\end{array}$

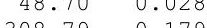

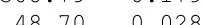
$48.70 \quad 0.030$ $29.22 \quad 0.018$ $27.80 \quad 0.017$ $\begin{array}{ll}21.55 & 0.013\end{array}$ $\begin{array}{ll}21.55 & 0.013 \\ 28.88 & 0.018\end{array}$ $\begin{array}{ll}29.22 & 0.018\end{array}$ 29.22 29.22
308.79 328.27 328.27
328.27 328.27

$\begin{array}{lr}20 & \text { H2O frac } \\ .07 & 0.0006 \\ 207 & 0.0006 \\ 179 & 0.0006 \\ 028 & 0.0006 \\ 179 & 0.0006 \\ 08 & 0.0006 \\ 30 & 0.0006 \\ 18 & 0.0006 \\ 17 & 0.0006 \\ 13 & 0.0006 \\ 13 & 0.0149 \\ 18 & 0.0113 \\ 018 & 0.0112 \\ 018 & 0.0112 \\ 018 & 0.0112 \\ 179 & 0.0006 \\ 191 & 0.0006 \\ 191 & 0.0006 \\ 191 & 0.0006\end{array}$

TURBOMACHINERY MAP DATA

$\begin{array}{lrrrrrrrrr} & \text { WcMap } & \text { PRmap } & \text { effMap } & \text { NcMap } & \text { R/Parm } & \text { S_WcDes } & \text { S_PRdes } & \text { S_effDes } & \text { S_NcDes } \\ \text { Fan } & 656.62 & 1.067 & 0.9053 & 1798.045 & 0.0437 & 0.8607 & \overline{1} .0235 & 0.9980 & 0.9905 \\ \text { LPC } & 46.01 & 1.238 & 0.7547 & 0.479 & 0.0000 & 1.9370 & 0.5887 & 0.9213 & 0.0003 \\ \text { HPC } & 43.81 & 5.276 & 0.8160 & 8531.527 & 11.0083 & 1.0995 & 1.0494 & 0.9898 & 0.9733 \\ \text { HPT } & 0.96 & 3.581 & 0.8765 & 1.283 & 3.5806 & 12.6299 & 0.9723 & 1.0000 & 0.0003 \\ \text { LPT } & 0.74 & 1.669 & 0.8212 & 0.590 & 1.6687 & 65.2590 & 0.7521 & 0.9926 & 0.0005\end{array}$




\begin{tabular}{|c|c|c|c|c|c|c|c|c|c|c|c|c|}
\hline $\begin{array}{l}===\text { INLETS }==== \\
\text { Inlet }\end{array}$ & $\begin{array}{r}\text { eRam } \\
1.0000\end{array}$ & $\begin{array}{r}\text { Afs } \\
2590.89\end{array}$ & $\begin{array}{r}\text { Fram } \\
5872.3\end{array}$ & \multirow{2}{*}{\multicolumn{2}{|c|}{ BLEEDS - interstg }} & & & & W & & & \\
\hline Inlet & & & & & & $9 \begin{array}{l}\text { Wb/Win } \\
0.0368\end{array}$ & $\begin{array}{l}\text { B1aWk } \\
0.5000\end{array}$ & $\begin{array}{l}\mathrm{B} \perp \mathrm{dP} \\
0.2200\end{array}$ & $\begin{array}{r}\mathrm{W} \\
1.0759\end{array}$ & $\begin{array}{r}\text { Tt } \\
740.40\end{array}$ & $\begin{array}{r}h t \\
43.75\end{array}$ & $\begin{array}{r}P t \\
13.281\end{array}$ \\
\hline$====$ DUCTS $====$ & dPnorm & MN & Aphy & \multicolumn{2}{|c|}{$\begin{array}{l}\text { HPT_COOLC HPC.C> } \\
\text { LPT COOLA HPC.C> }\end{array}$} & 0.0117 & 0.5000 & 0.4500 & 0.3421 & 740.40 & 43.75 & 7.031 \\
\hline TEGV & 0.0000 & 0.1740 & 860.21 & WB $2 \overline{\mathrm{X}}$ & HPC.B> & 0.0000 & 0.4500 & 0.2200 & 0.0000 & 719.90 & 38.78 & 17.990 \\
\hline FanDuct & 0.0000 & 0.4314 & 2481.93 & \multirow{2}{*}{$\begin{array}{l}\text { WB2Y } \\
\text { WBA2X }\end{array}$} & HPC.B> & 0.0000 & 0.7600 & 0.6200 & 0.0000 & 846.48 & 69.59 & 34.240 \\
\hline & & & & & HPC.B> & 0.0000 & 0.4500 & 0.2200 & 0.0000 & 719.90 & 38.78 & 17.990 \\
\hline$==$ SPLITTERS $==$ & BPR & $\mathrm{dP} / \mathrm{P} 1$ & $\mathrm{dP} / \mathrm{P} 2$ & WBLKG & HPC.I> & 0.0000 & 1.0000 & 1.0000 & 0.0000 & 943.46 & 93.44 & 49.677 \\
\hline Splitter & 6.3407 & 0.0005 & 0.0005 & WBW2X & HPC.B> & 0.0000 & 0.4500 & 0.2200 & 0.0000 & 719.90 & 38.78 & 17.990 \\
\hline$===$ SHAFTS $====$ & Nmech & trq in & pwr in & BLEEDS - & output & Wb/Win & hscale & Pscale & W & $\mathrm{Tt}$ & ht & Pt \\
\hline HP_Shaft & 8896.5 & 2367.9 & 4011.0 & WB17Y & Bleed> & 0.4000 & 1.0000 & 1.0000 & 19.4917 & 534.33 & -5.94 & 9.053 \\
\hline LP_Shaft & 1793.8 & 4619.9 & 1577.8 & \multirow{2}{*}{ HPT_COOLA } & A Bleed> & 0.1142 & 1.0000 & 1.0000 & 3.3383 & 943.46 & 93.44 & 48.533 \\
\hline & & & & & B Bleed> & 0.0999 & 1.0000 & 1.0000 & 2.9211 & 943.46 & 93.44 & 28.369 \\
\hline & & & & $\begin{array}{l}\text { HPT_COOLB } \\
\text { WB } 3 \bar{X}\end{array}$ & Bleed> & 0.0000 & 1.0000 & 1.0000 & 0.0000 & 943.46 & 93.44 & 49.677 \\
\hline & & & & WBA3X & Bleed> & 0.0000 & 1.0000 & 1.0000 & 0.0000 & 943.46 & 93.44 & 49.677 \\
\hline & & & & WBW3X & Bleed> & 0.0000 & 1.0000 & 1.0000 & 0.0000 & 943.46 & 93.44 & 49.677 \\
\hline & & & & WBFDLKG & FanDu> & 0.0000 & 1.0000 & 1.0000 & 0.0000 & 517.06 & -9.90 & 8.480 \\
\hline & & & & $\begin{array}{l}\text { WB15X } \\
\text { WB15Y }\end{array}$ & Bleed> & 0.0000 & 1.0000 & 1.0000 & 0.0000 & 517.06 & -9.90 & 8.480 \\
\hline & & & & \multirow{2}{*}{$\begin{array}{l}\text { WB15Y } \\
\text { WB17X }\end{array}$} & Bleed> & 0.0000 & 1.0000 & 1.0000 & 0.0000 & 517.06 & -9.90 & 8.480 \\
\hline & & & & & Bleed> & 0.0000 & 1.0000 & 1.0000 & 0.0000 & 517.06 & -9.90 & 8.480 \\
\hline$===$ BURNERS $===$ & Ttout & eff & dPnorm & \multirow{2}{*}{\multicolumn{2}{|c|}{$\begin{array}{rr}\text { Wfuel } & \text { FAR } \\
0.25348 & 0.01176\end{array}$}} & & & & & & & \\
\hline Burner & 1736.49 & 0.9995 & 0.0230 & & & & & & & & & \\
\hline$===$ NOZZLES $===$ & PR & $\mathrm{C} f \mathrm{~g}$ & CdTh & \multirow{2}{*}{$\begin{array}{r}\mathrm{Cv} \\
0.9800\end{array}$} & \multirow{2}{*}{$\begin{array}{r}\text { Ath } \\
613.40\end{array}$} & MNth & Vact & $\mathrm{Fg}$ & & & & \\
\hline Core_Nozz & 1.043 & 0.9801 & 1.0000 & & & 0.249 & 375.3 & 344.0 & & & & \\
\hline Byp__Nozz & 1.258 & 0.9800 & 1.0000 & 0.9800 & 6.86 & 0.582 & 616.2 & 6290.6 & & & & \\
\hline
\end{tabular}




\title{
Appendix O: Compressor code analysis for the descent conditions
}

\section{$10 \mu \mathrm{m}, \mathrm{ISA}+18 \mathrm{R}$}

\begin{abstract}
$\star * \star * * * * * * * * * * * *$ AXIAL \& CENTRIFUGAL COMPRESSOR BLADE DESIGN 1-D $* * * *$

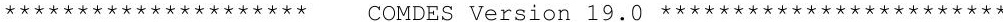

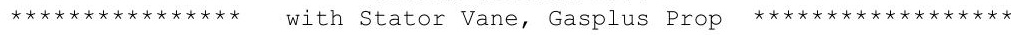

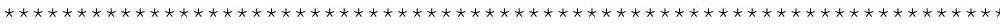

Fan Core + 4 Stage LPC 10\%39000 ft 05-13-2013

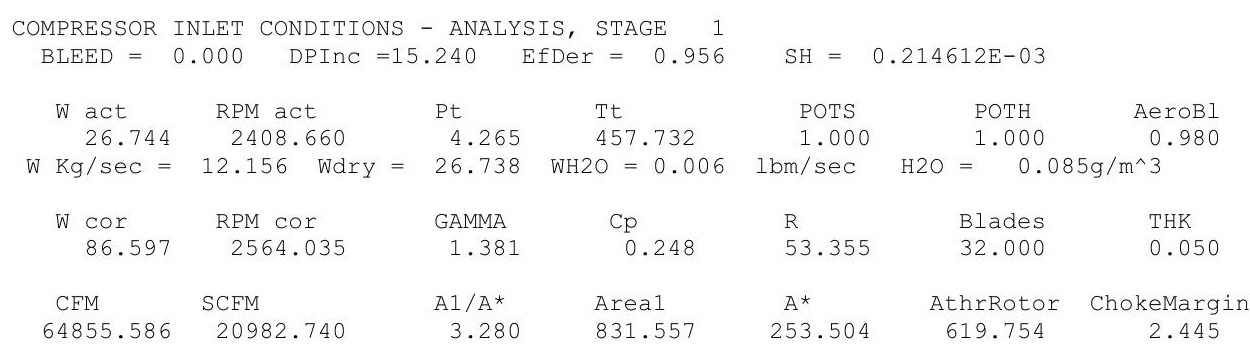

ROTOR LEADING EDGE CONDITIONS, STAGE 1

\begin{tabular}{|c|c|c|c|c|c|c|c|c|c|}
\hline & R1 & Stator & Alfa & $\mathrm{C} 1$ & CU1 & $\mathrm{Cm} 1$ & Mabs & Mrel & U1cor \\
\hline TIP & 20.63 & 0.00 & -0.02 & 187.18 & -0.06 & 187.18 & 0.18 & 0.45 & 461.59 \\
\hline MEAN & 17.06 & 0.00 & -0.02 & 187.18 & -0.06 & 187.18 & 0.18 & 0.39 & \\
\hline HUB & 12.51 & 0.00 & -0.02 & 187.18 & -0.06 & 187.18 & 0.18 & 0.31 & \\
\hline & Betaflo & BetaBlade & Incid & $\mathrm{U} 1$ & W1 & Ps 1 & Ts 1 & TwetBulb1 & $\mathrm{RH}$ \\
\hline TIP & 66.66 & 50.47 & 16.19 & 433.63 & 472.37 & 4.17 & 454.91 & 448.87 & 8 . \\
\hline MEAN & 62.44 & 47.20 & 15.24 & 358.60 & 404.57 & 4.17 & 454.91 & 448.87 & 8 . \\
\hline HUB & 54.56 & 38.62 & 15.94 & 262.95 & 322.83 & 4.17 & 454.91 & 448.87 & \\
\hline
\end{tabular}

ROTOR EXIT CONDITIONS, STAGE 1 SOLUTION IS CONVERGED

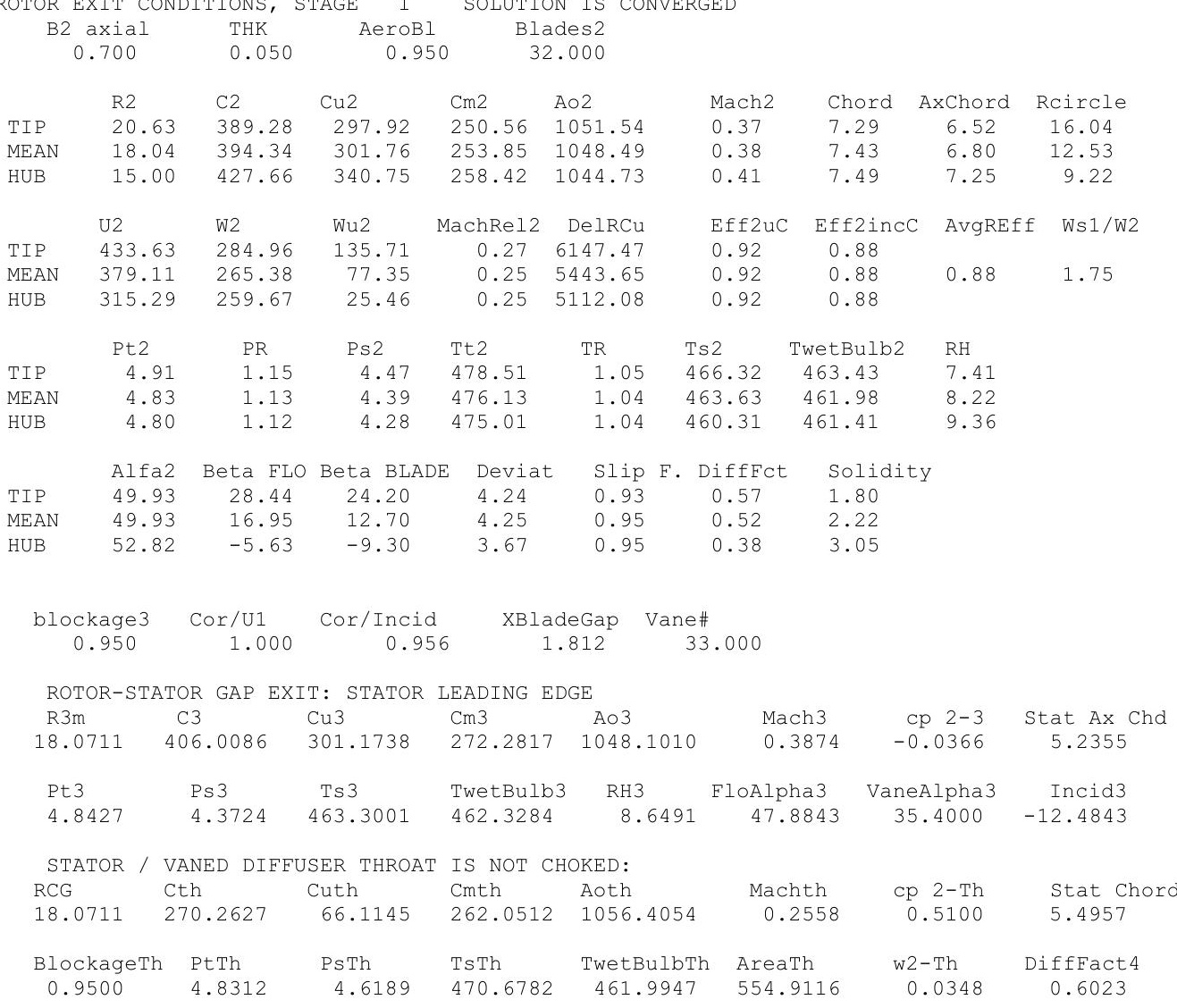




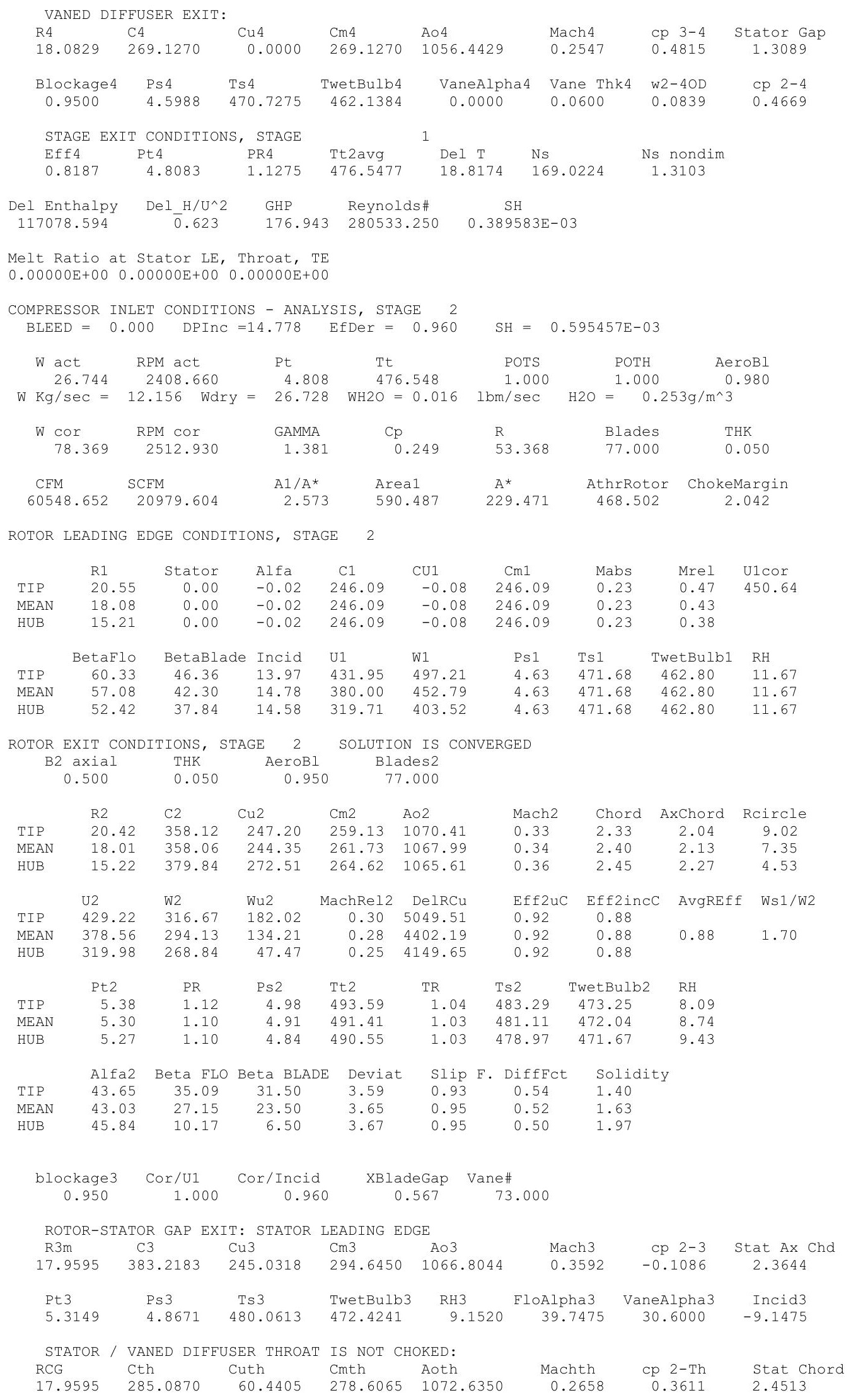




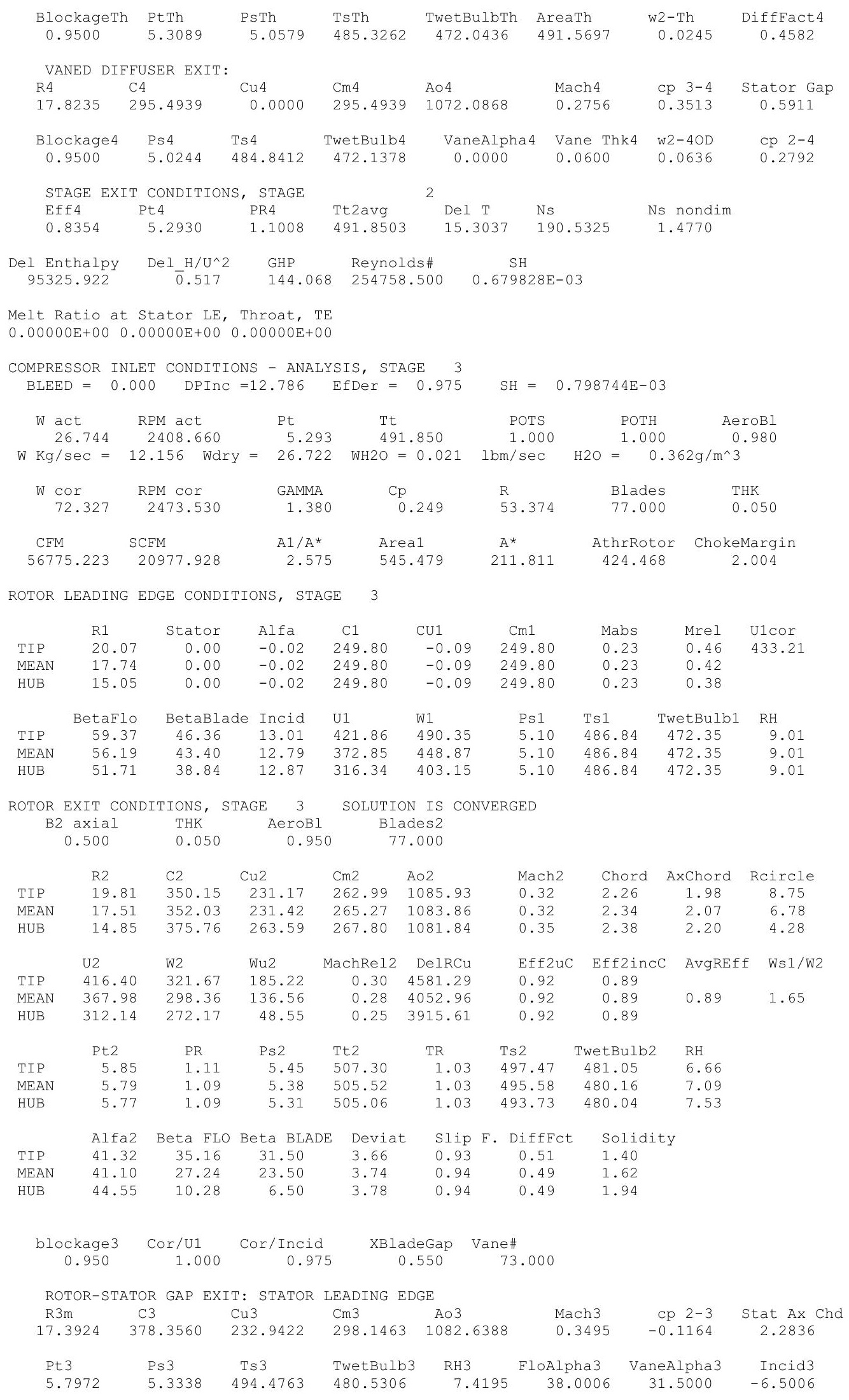




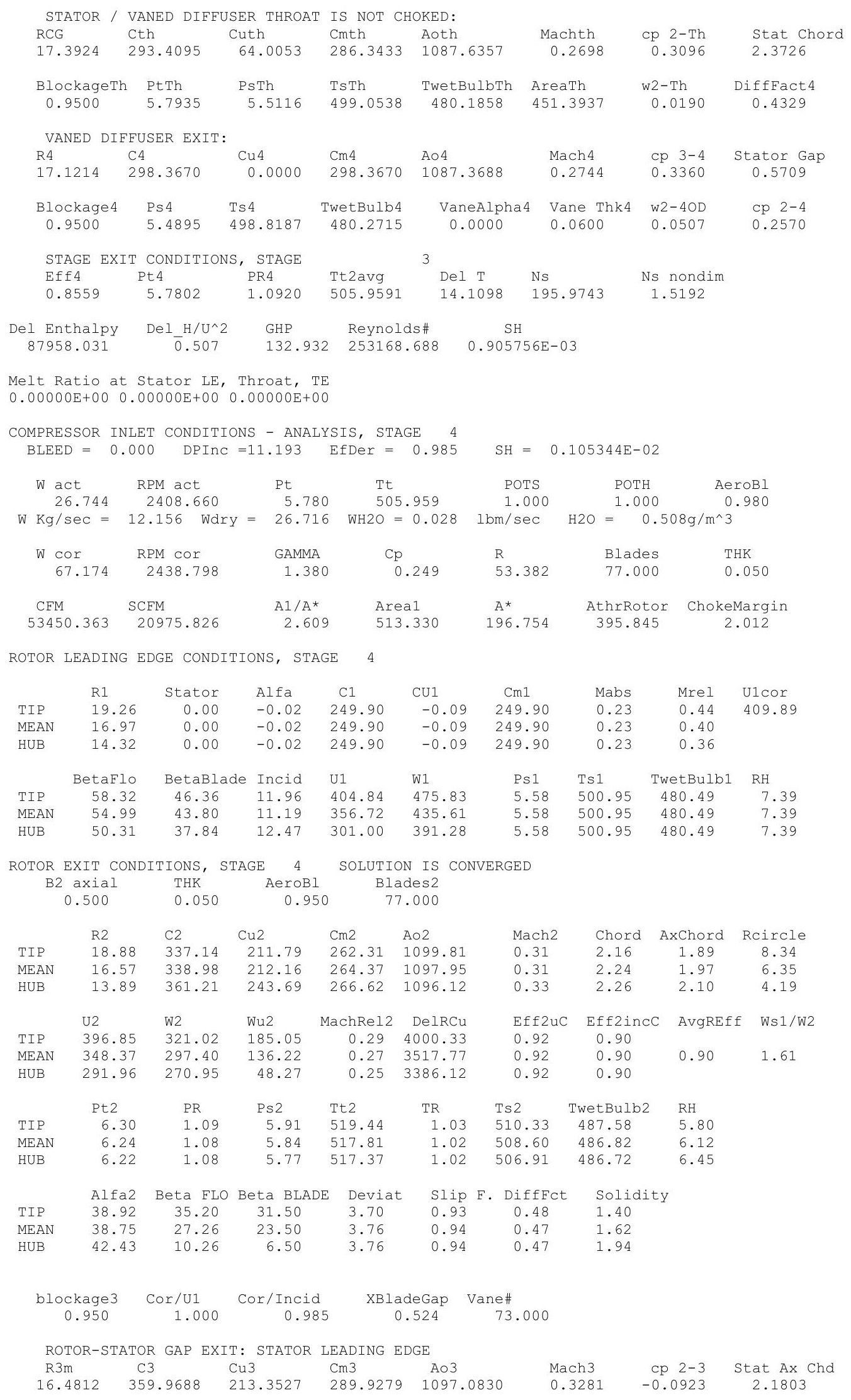




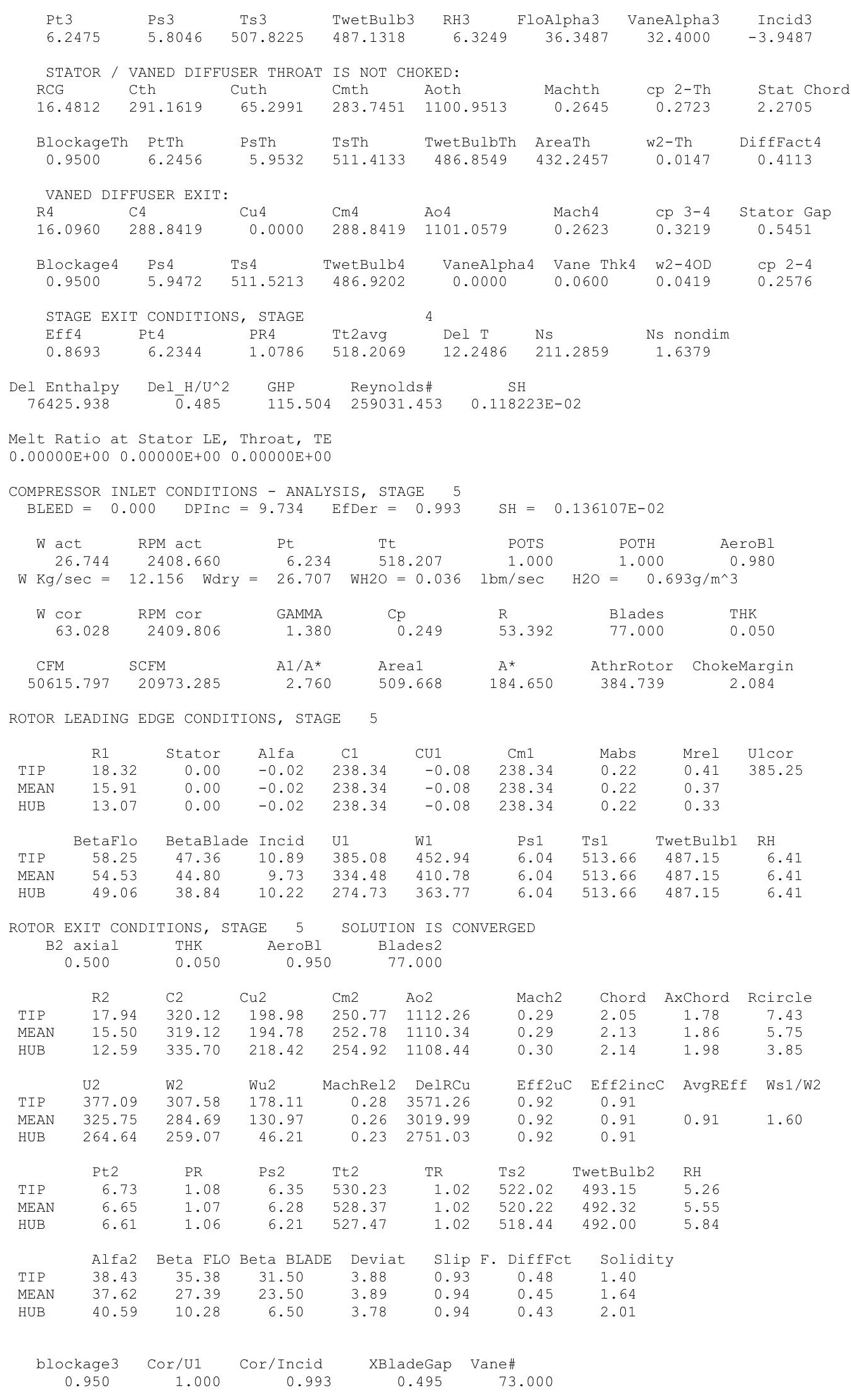

ROTOR-STATOR GAP EXIT: STATOR LEADING EDGE 


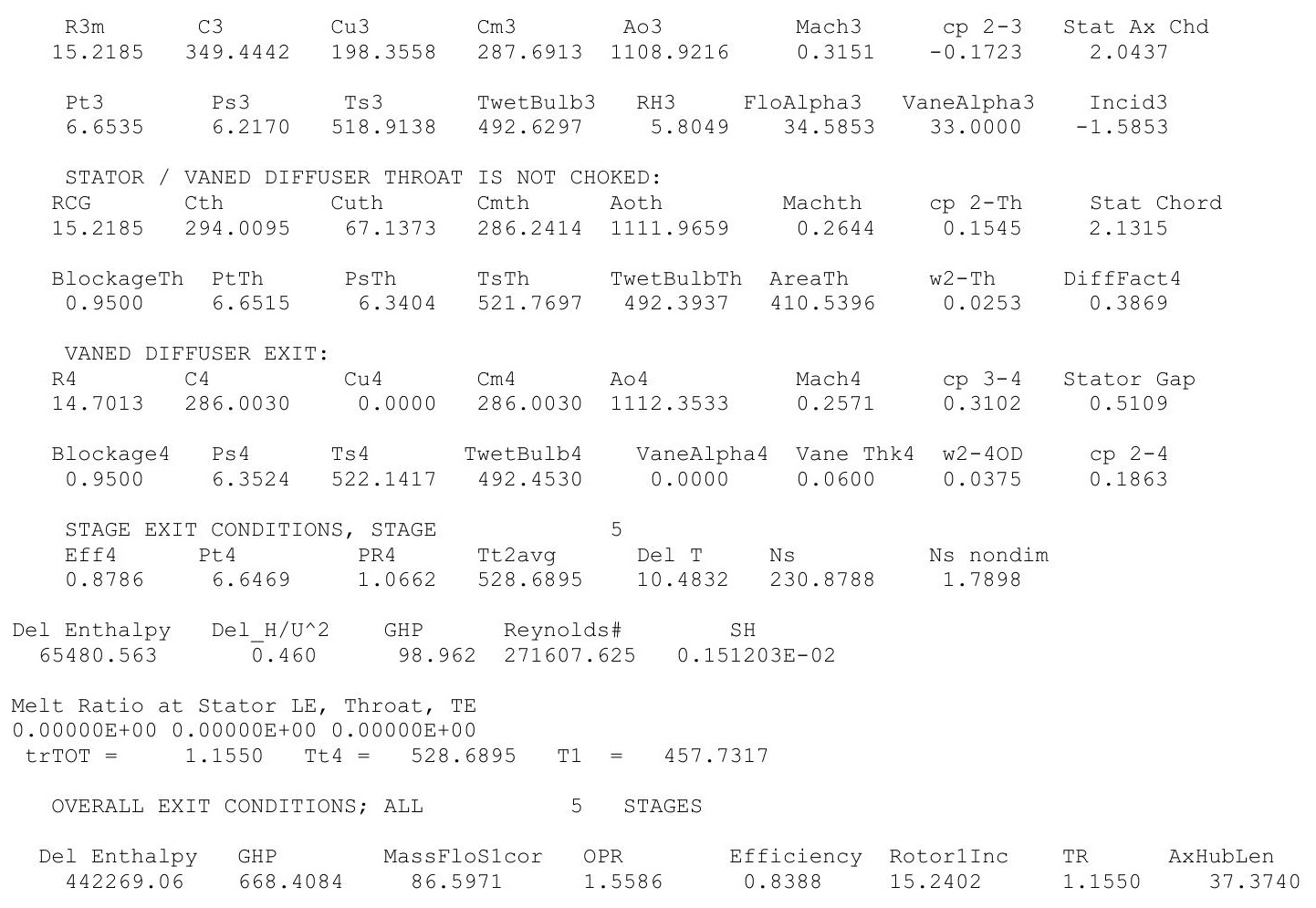




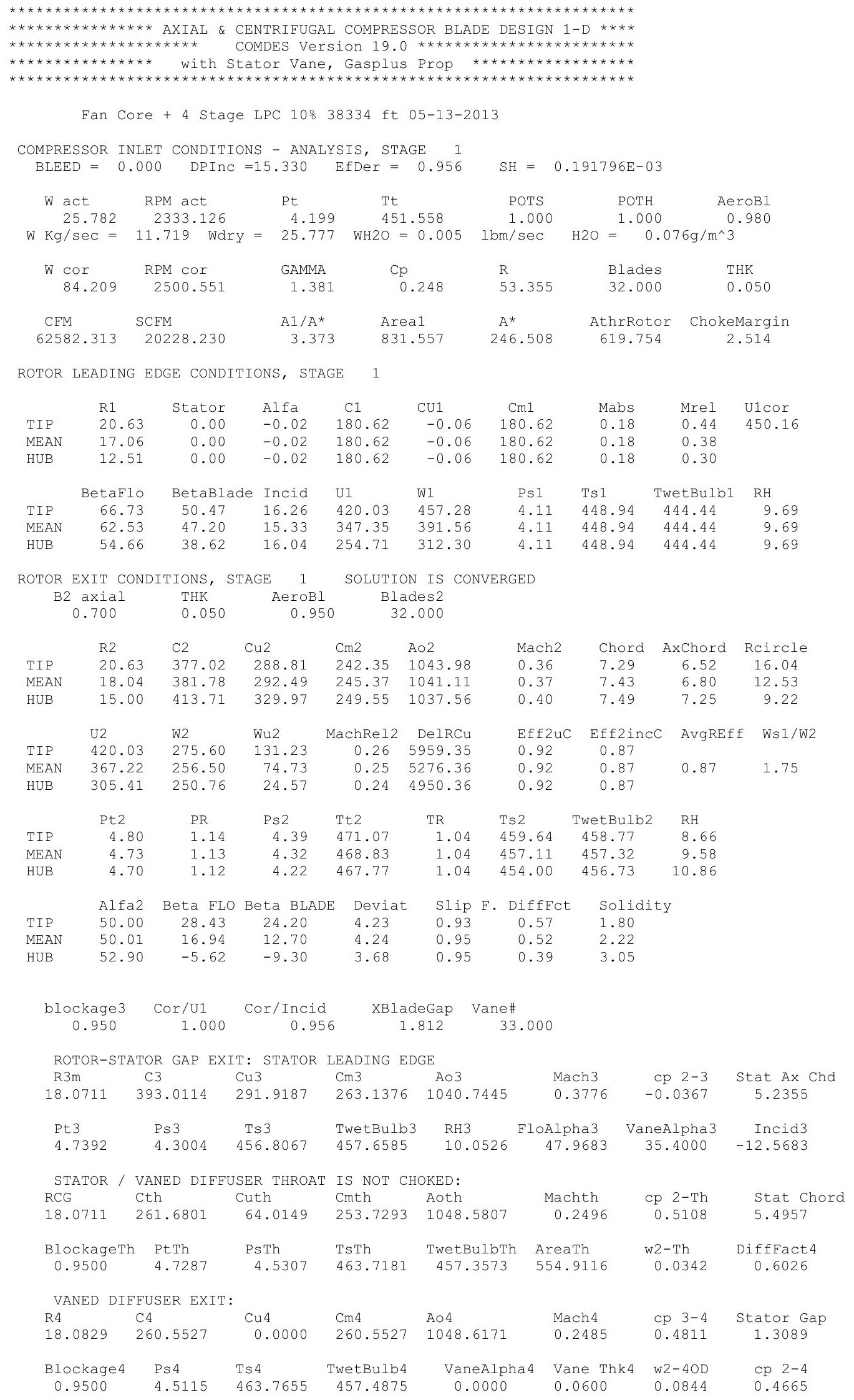




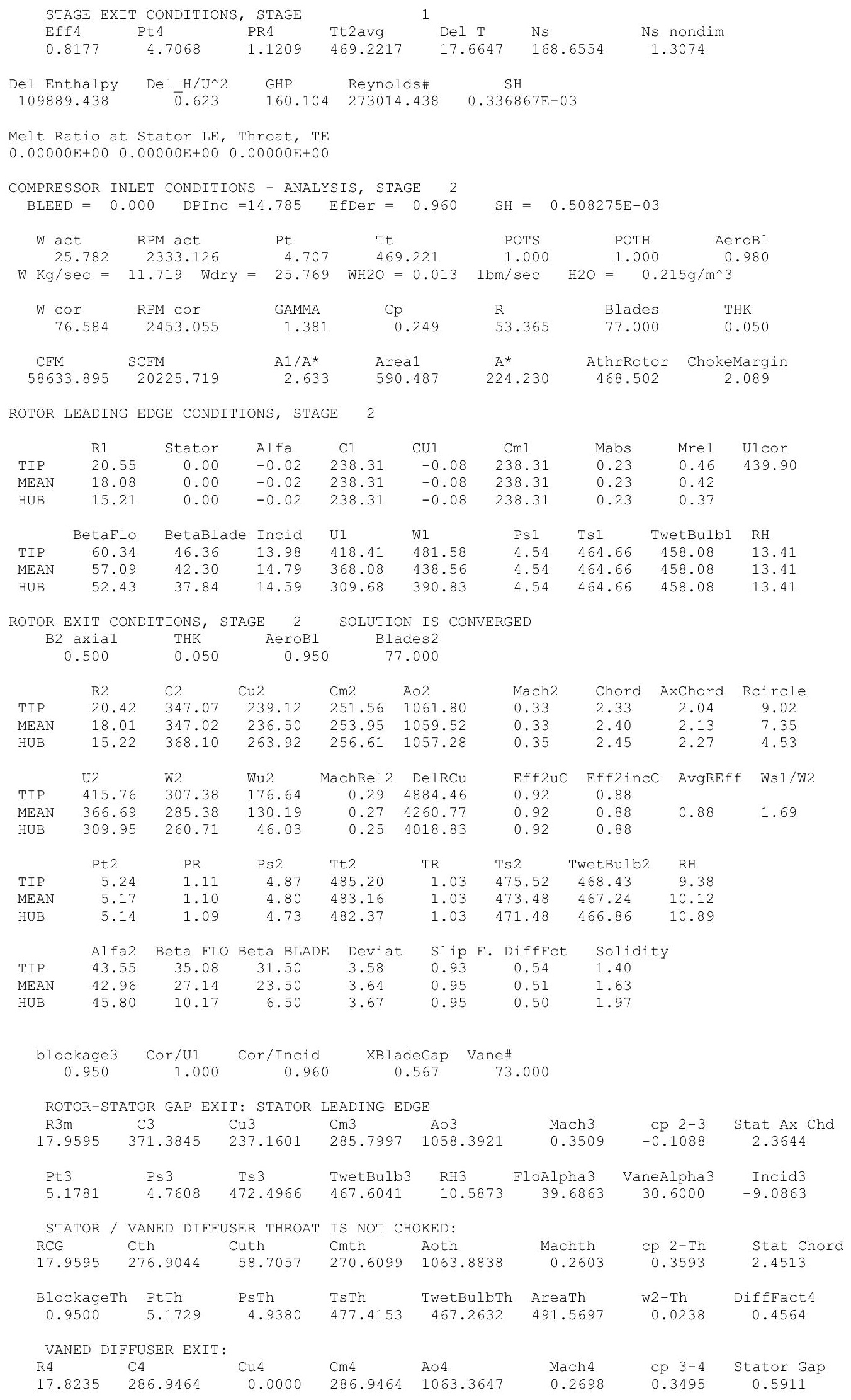




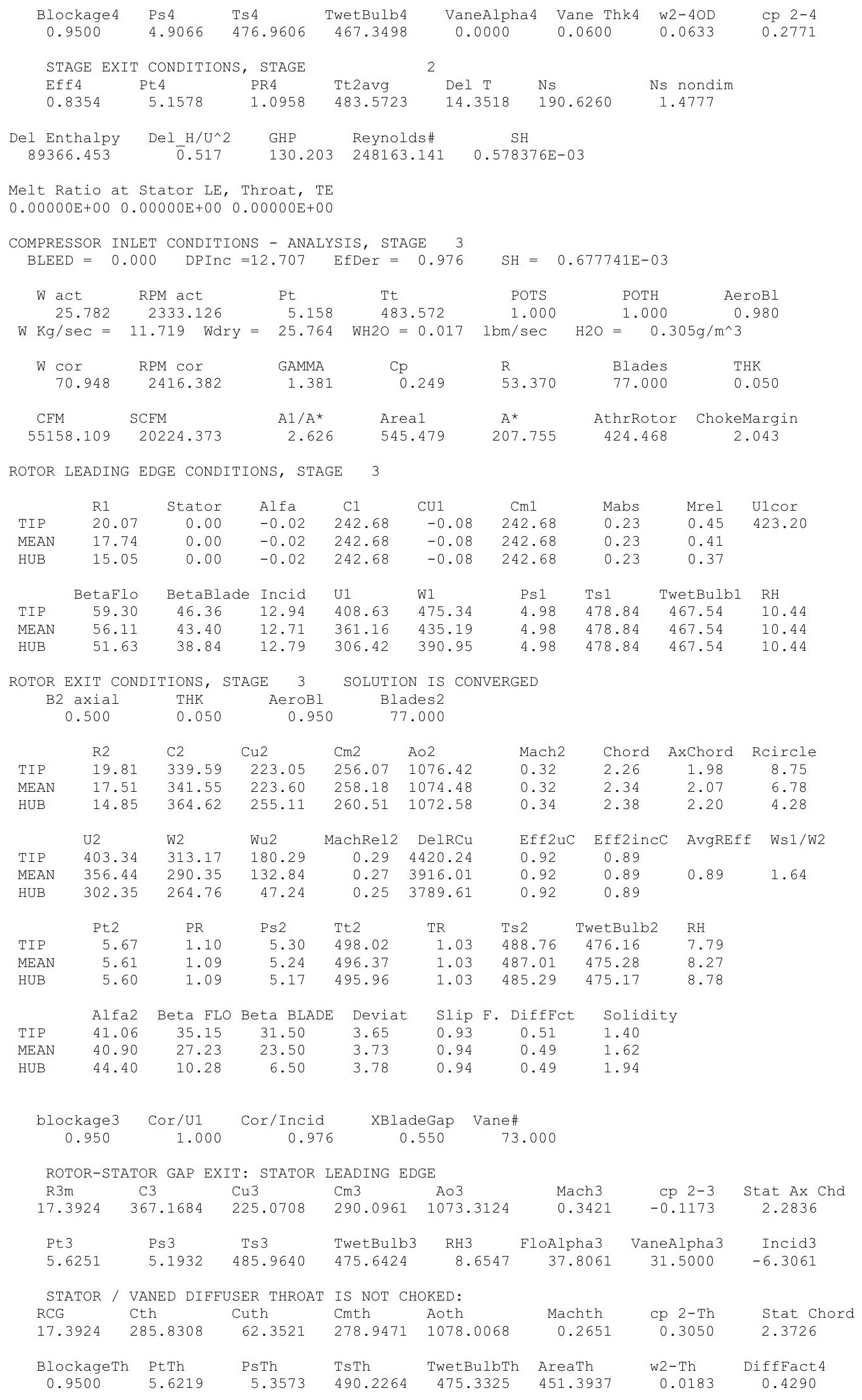




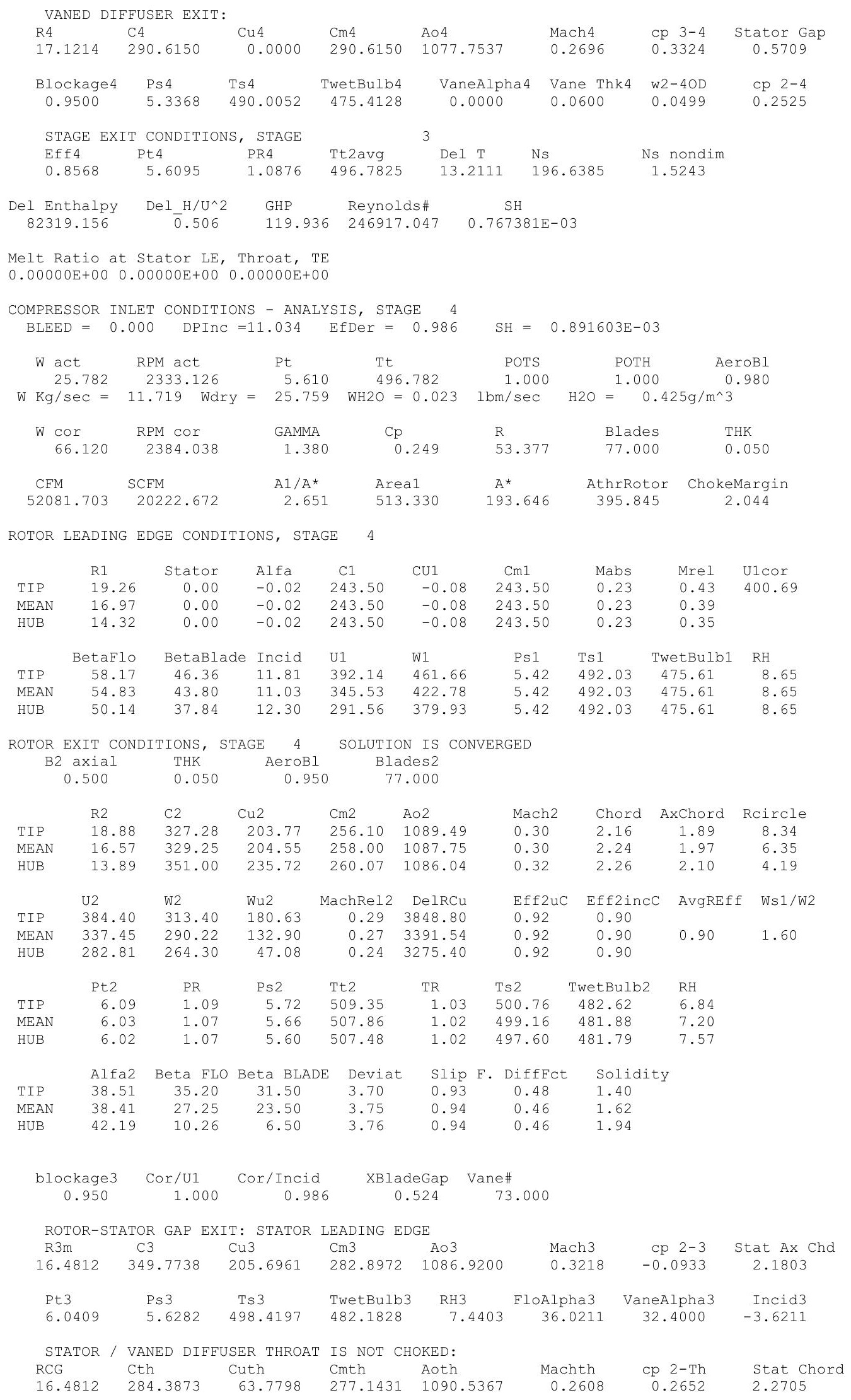




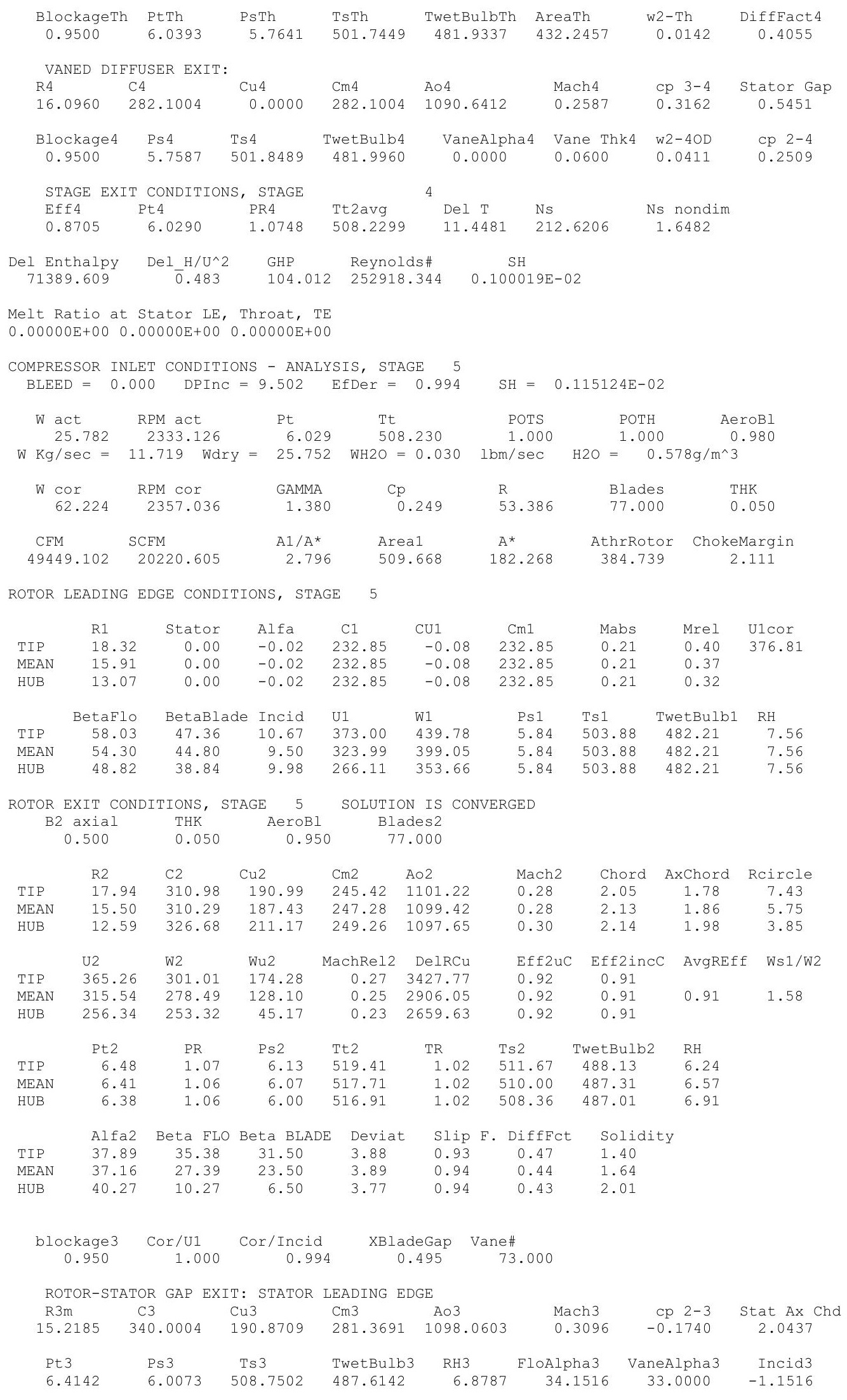




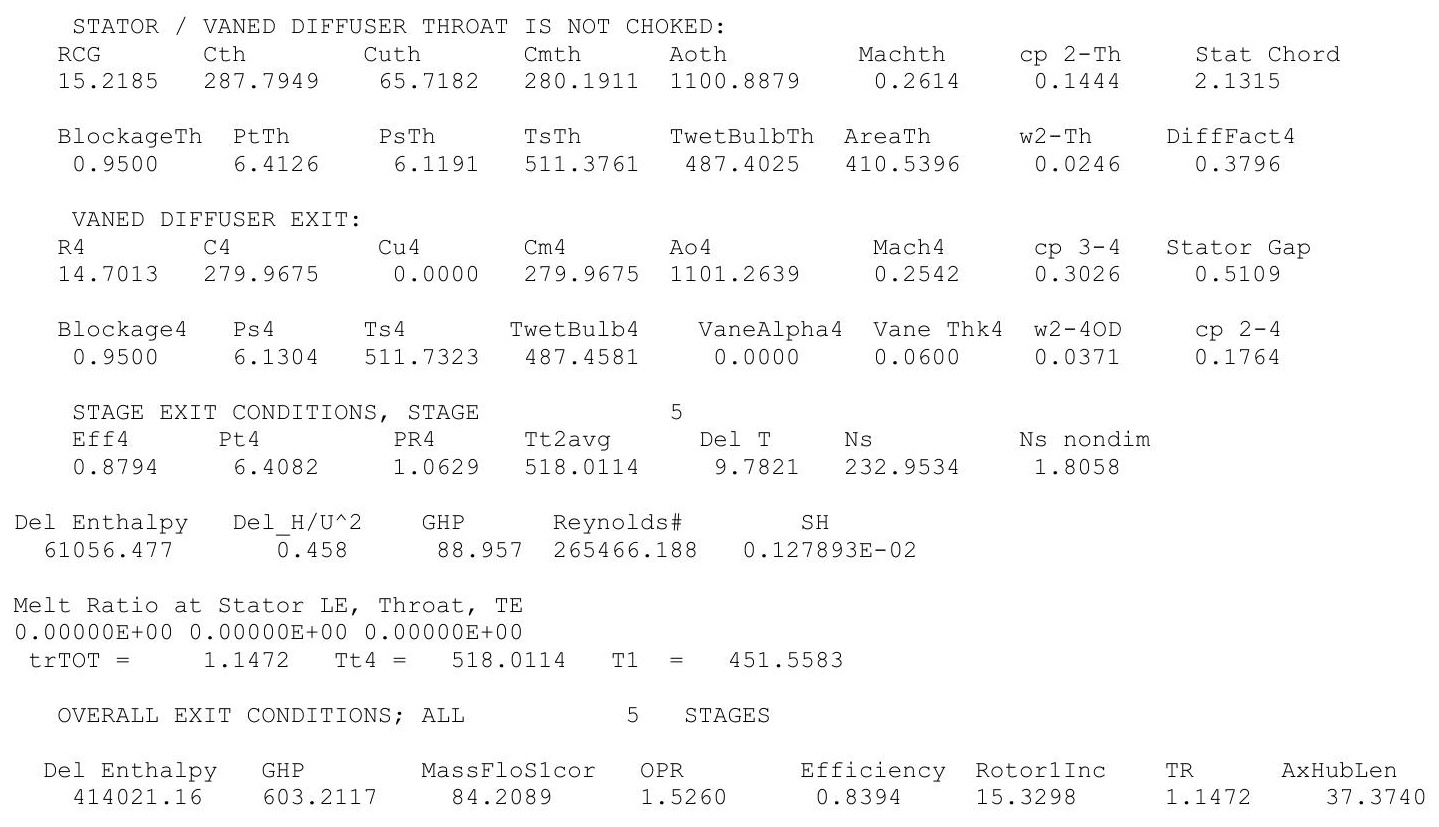




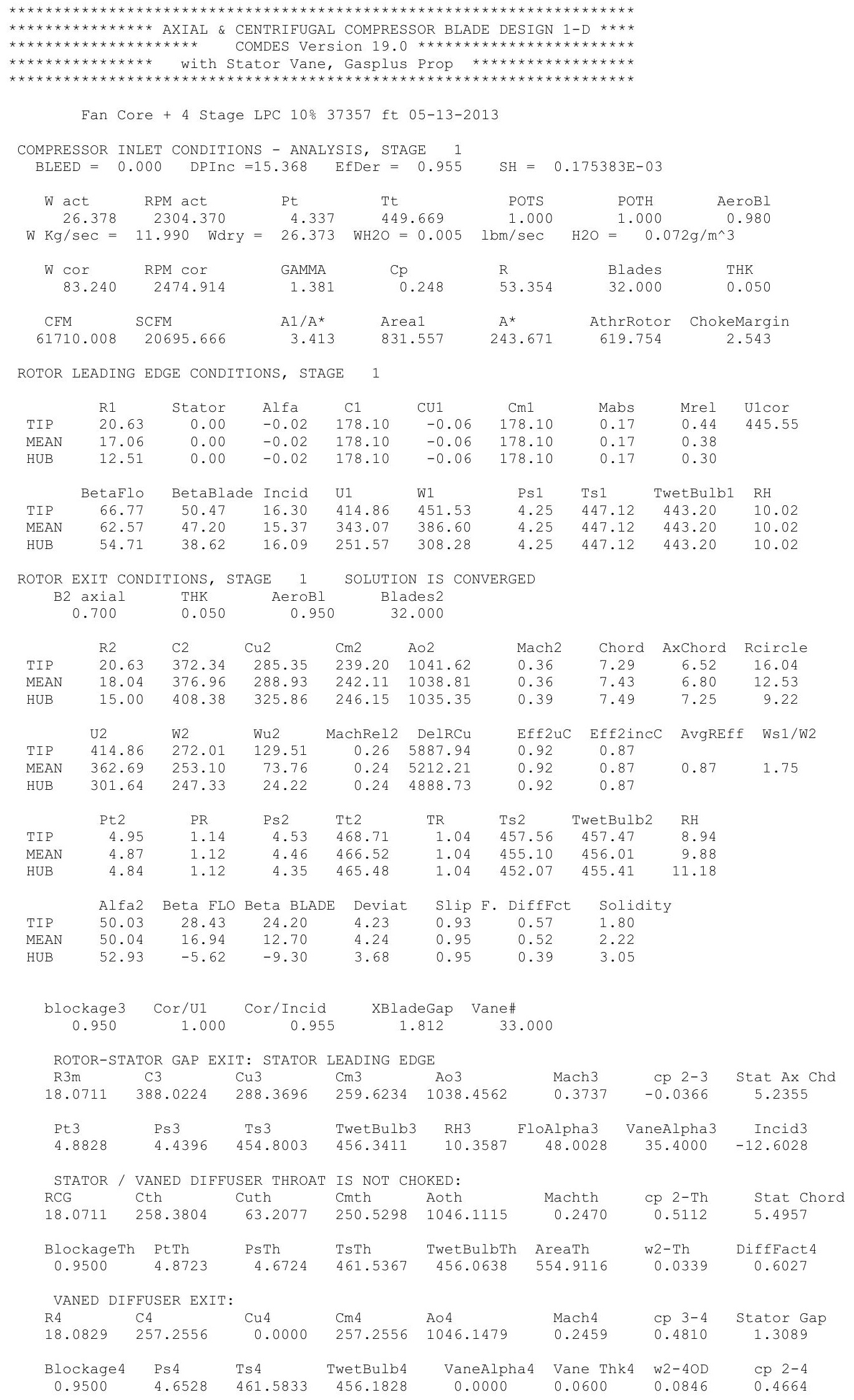




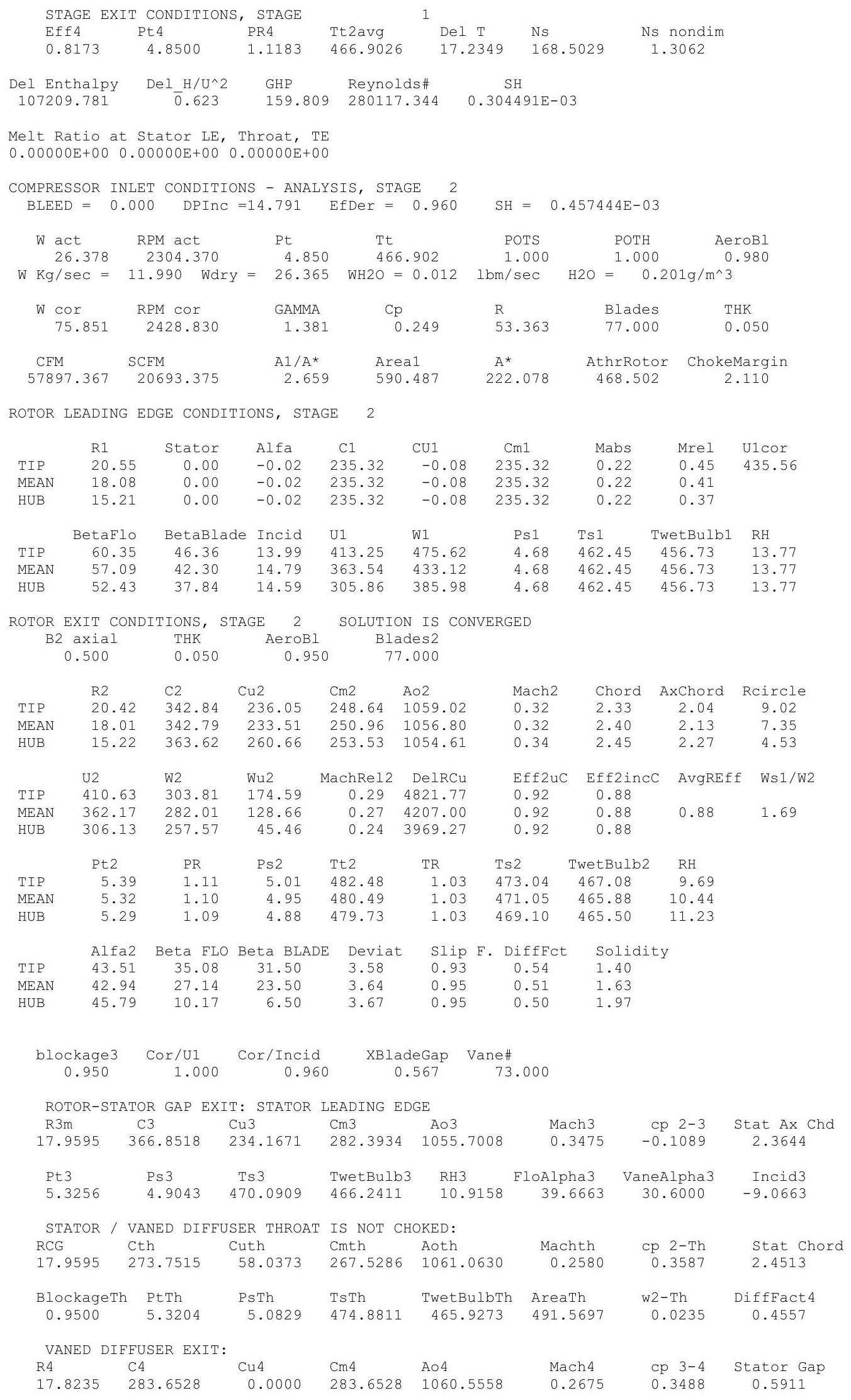




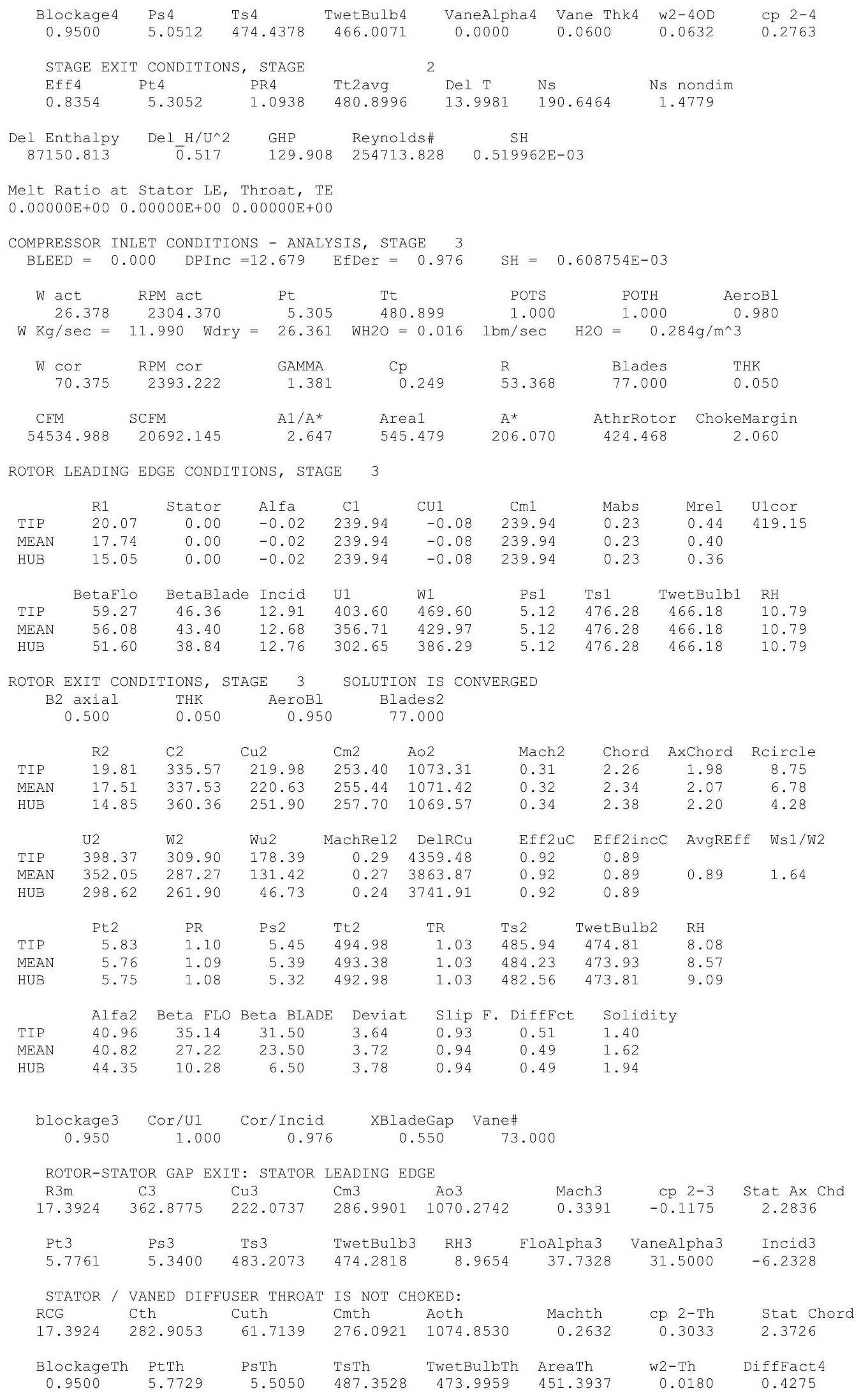




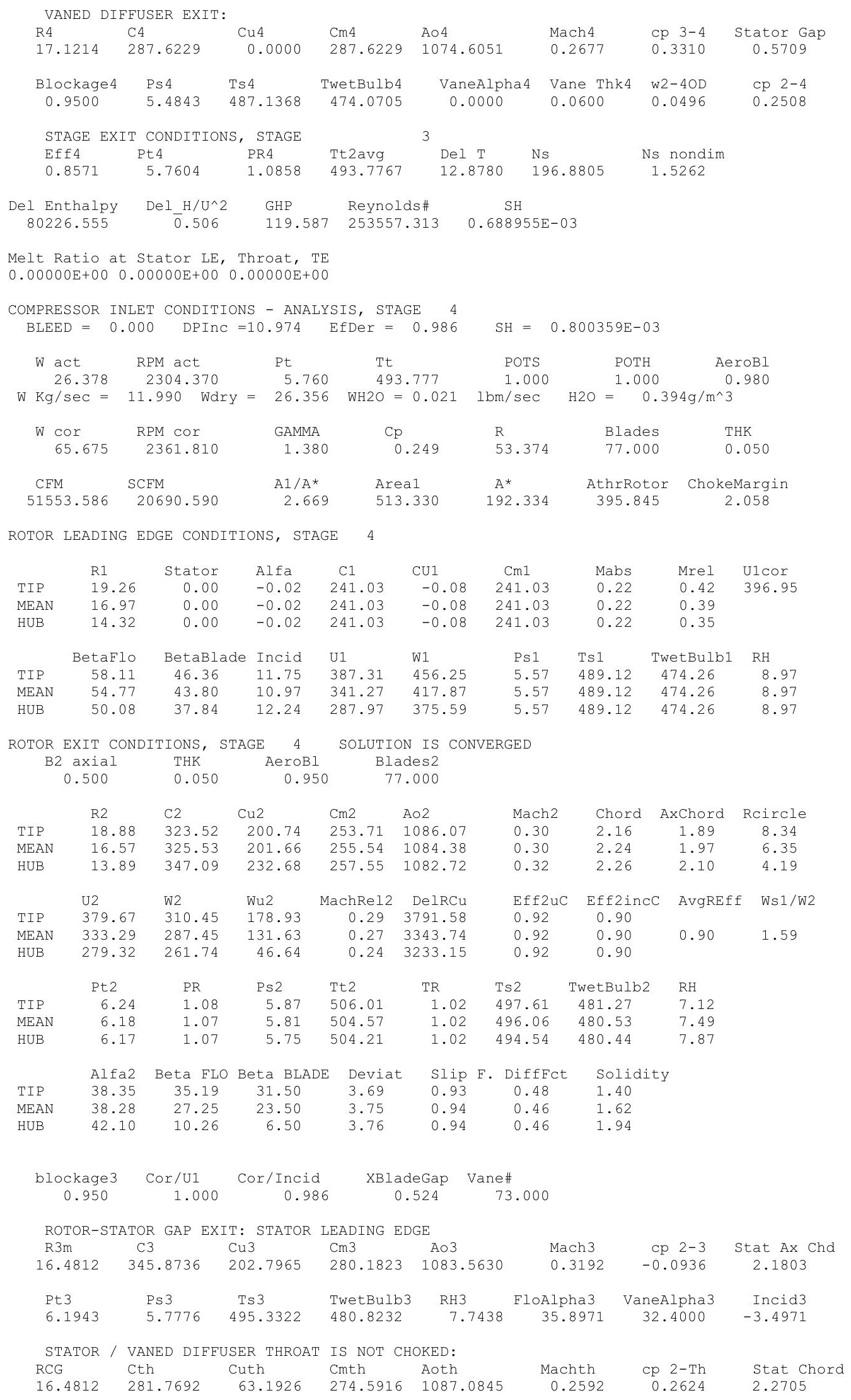




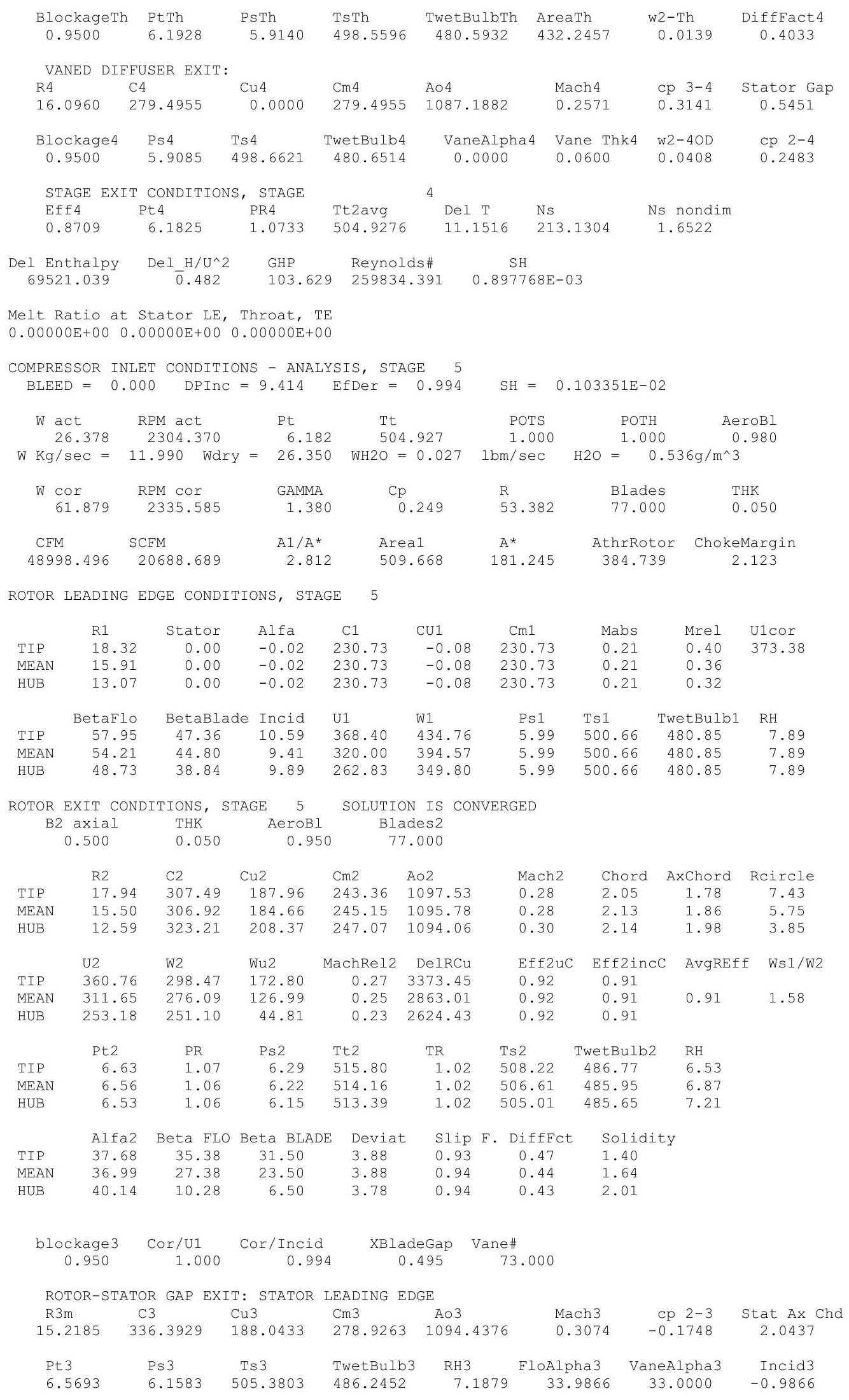




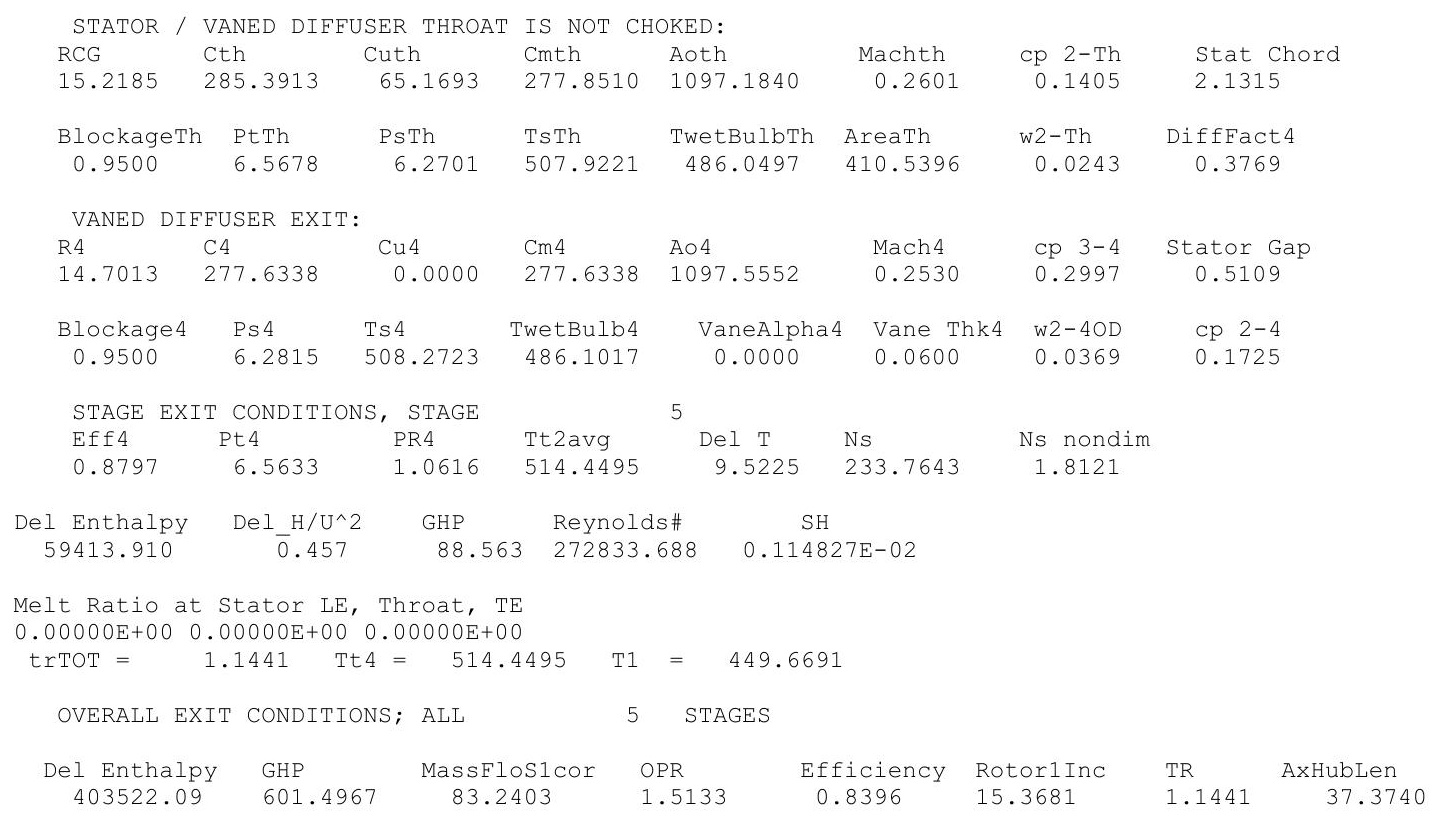




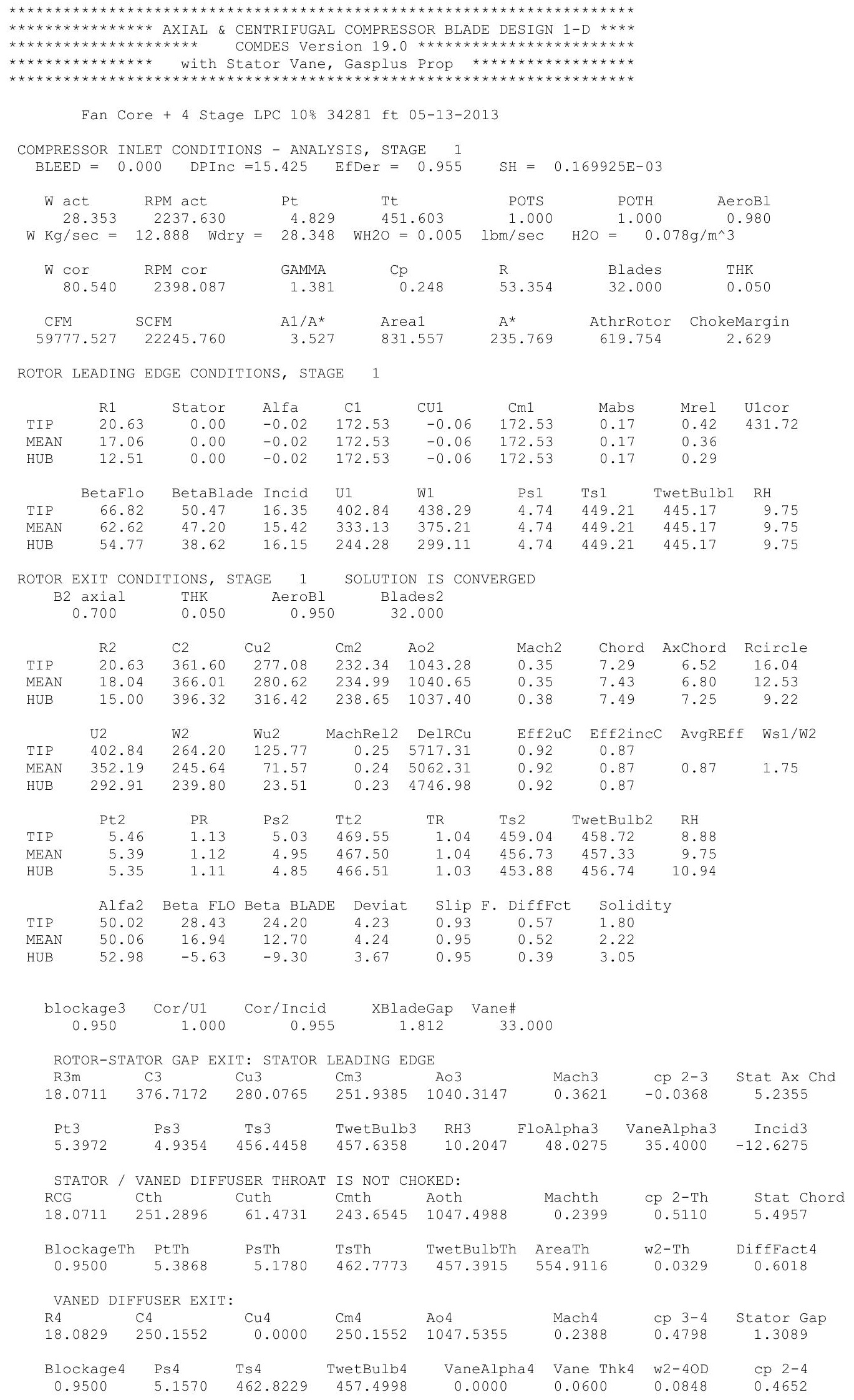




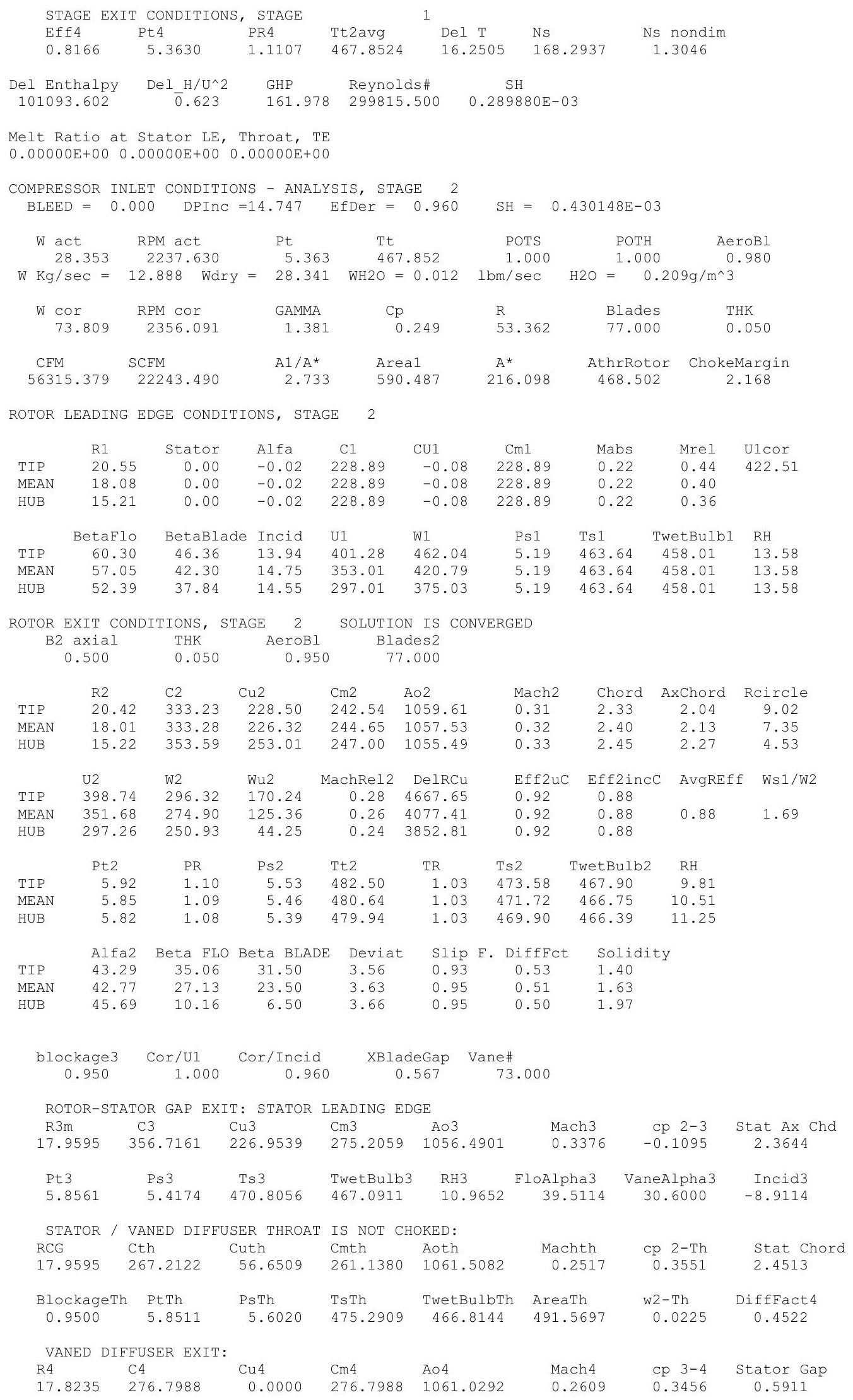




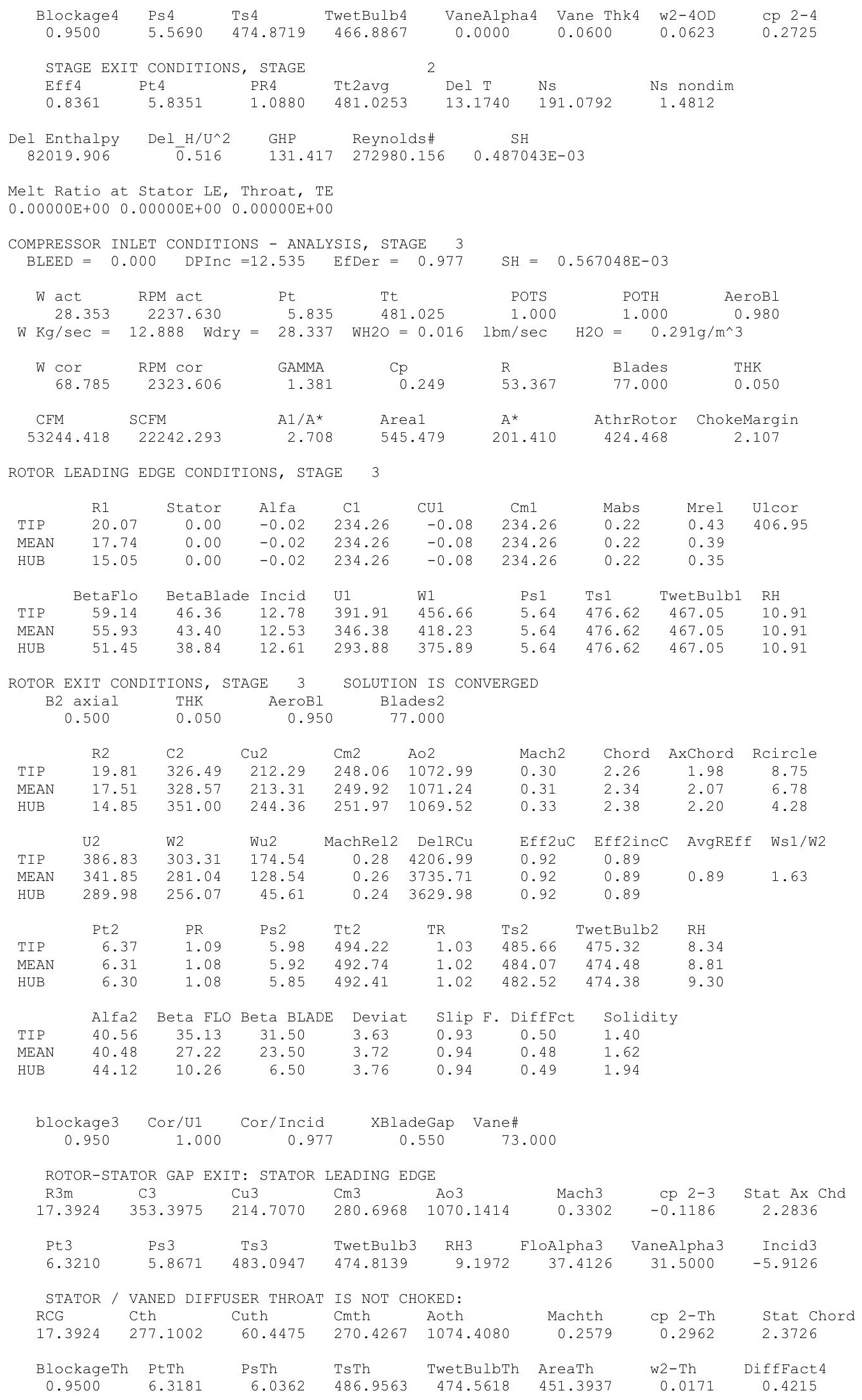




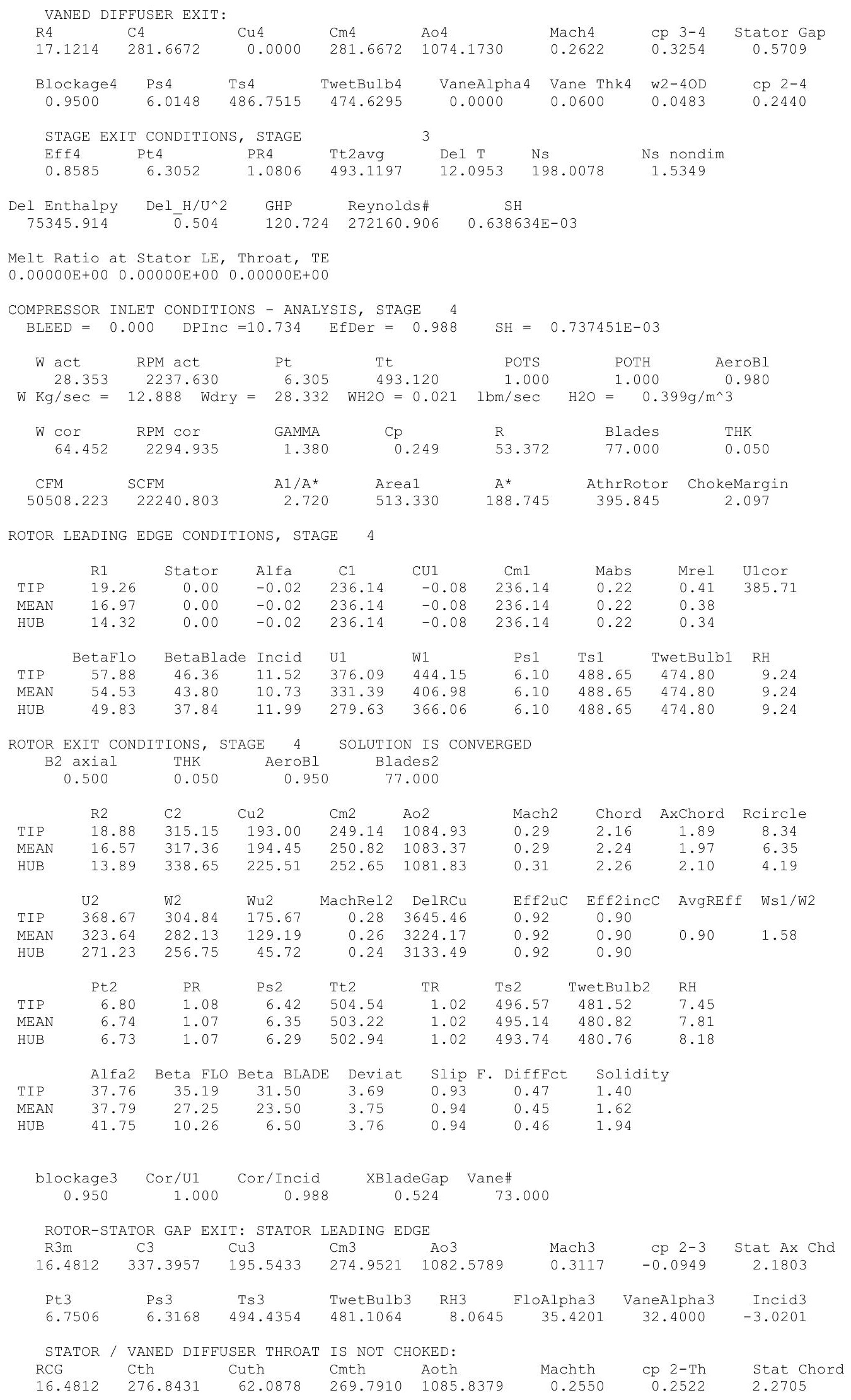




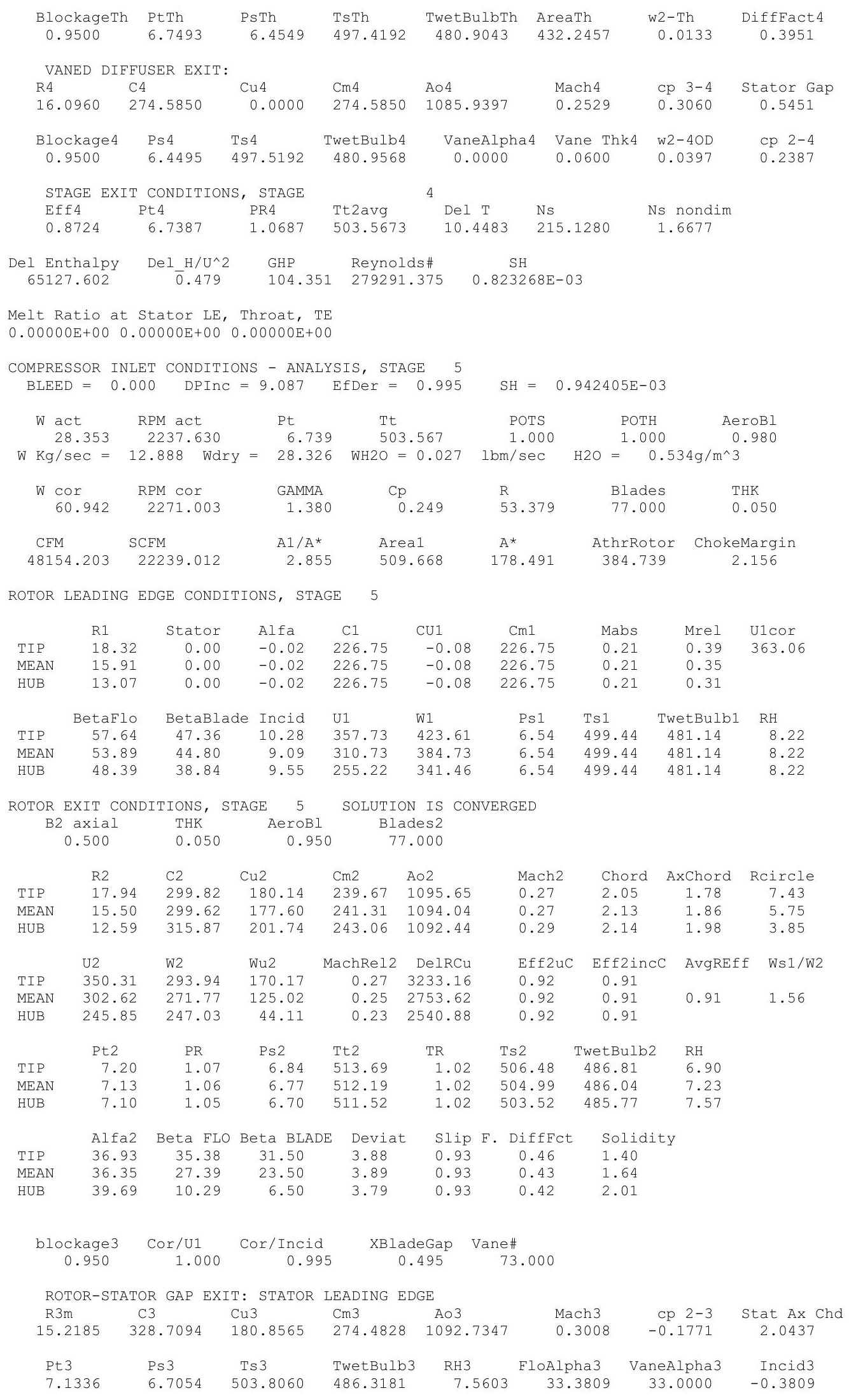




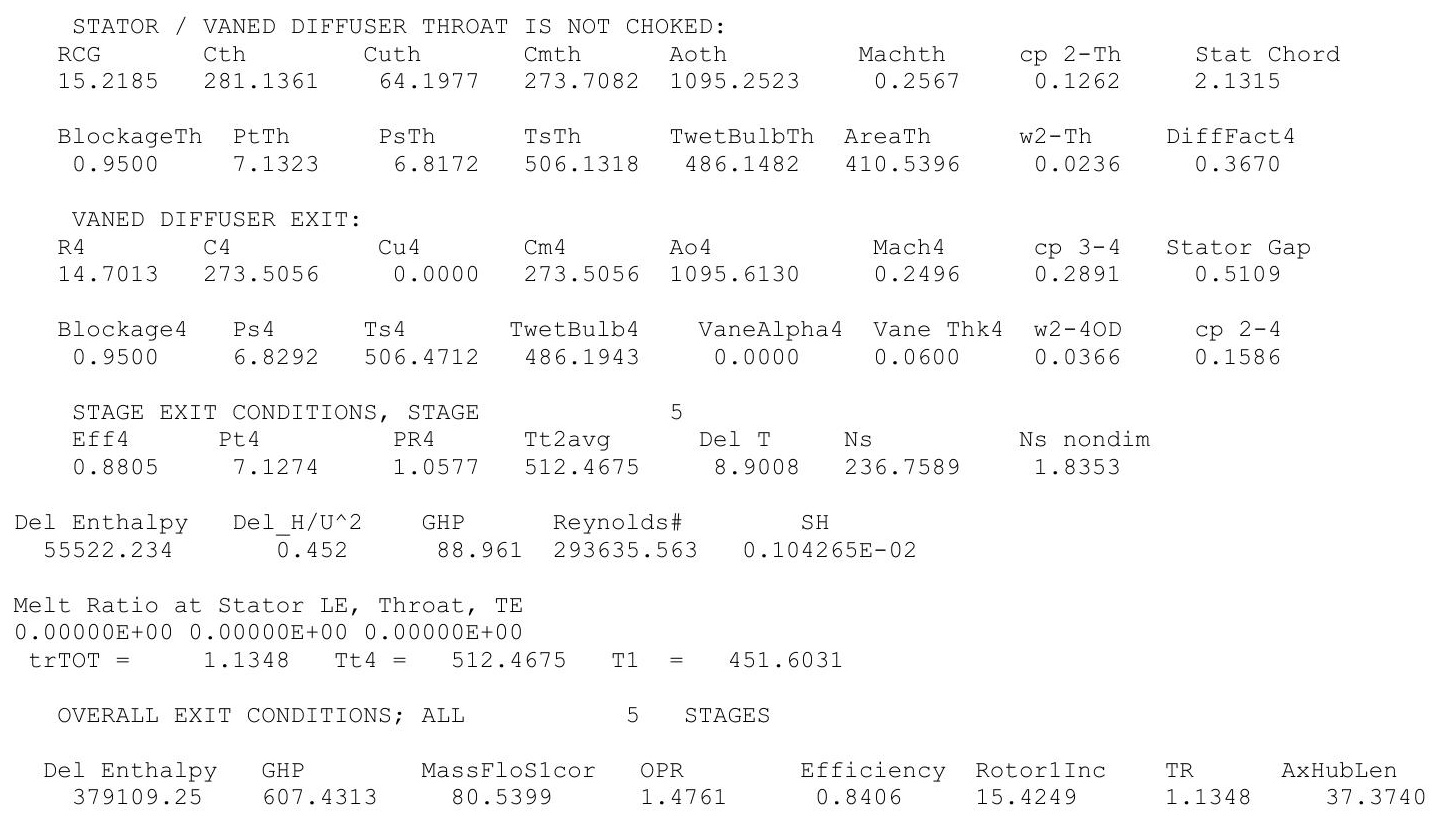




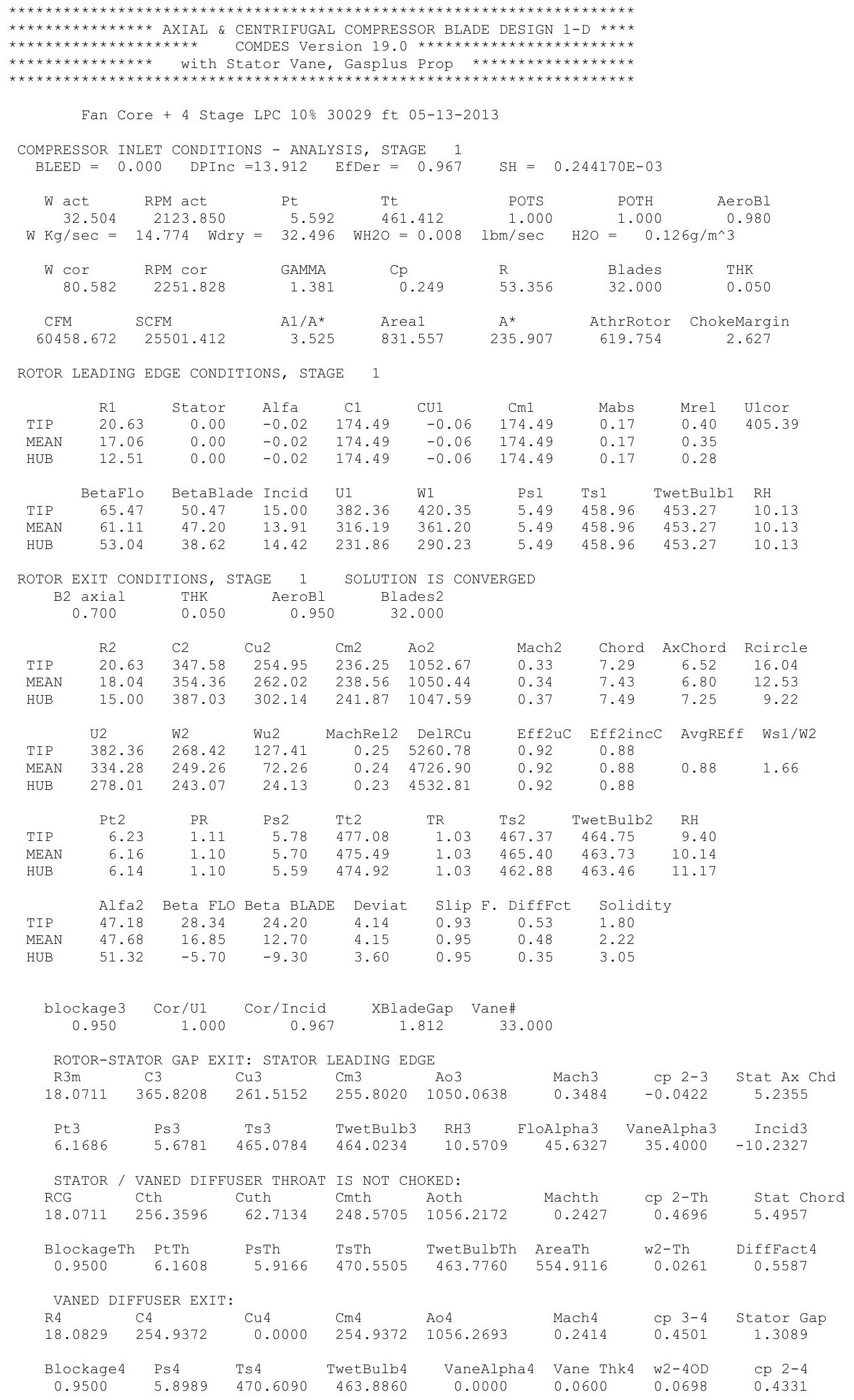




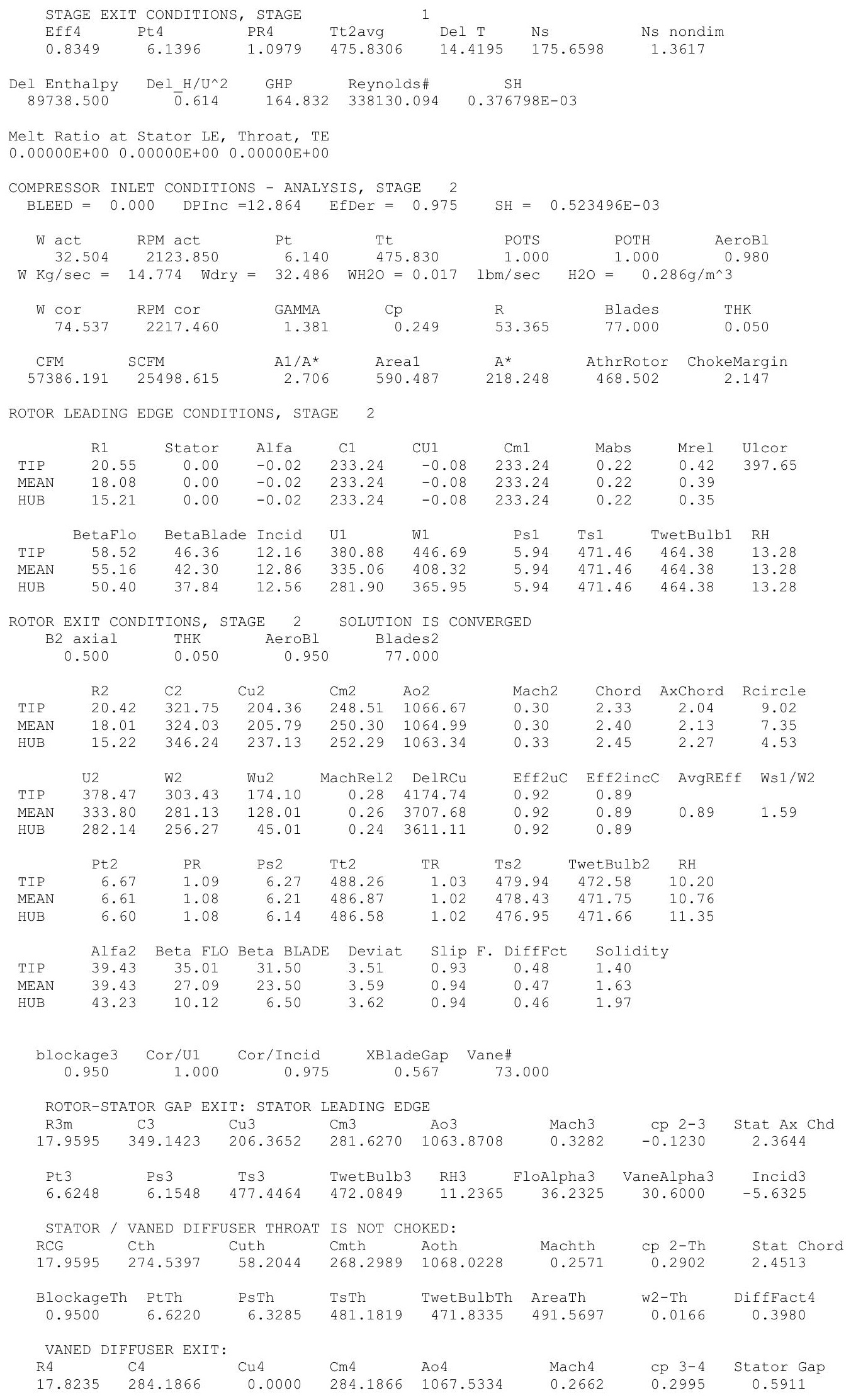




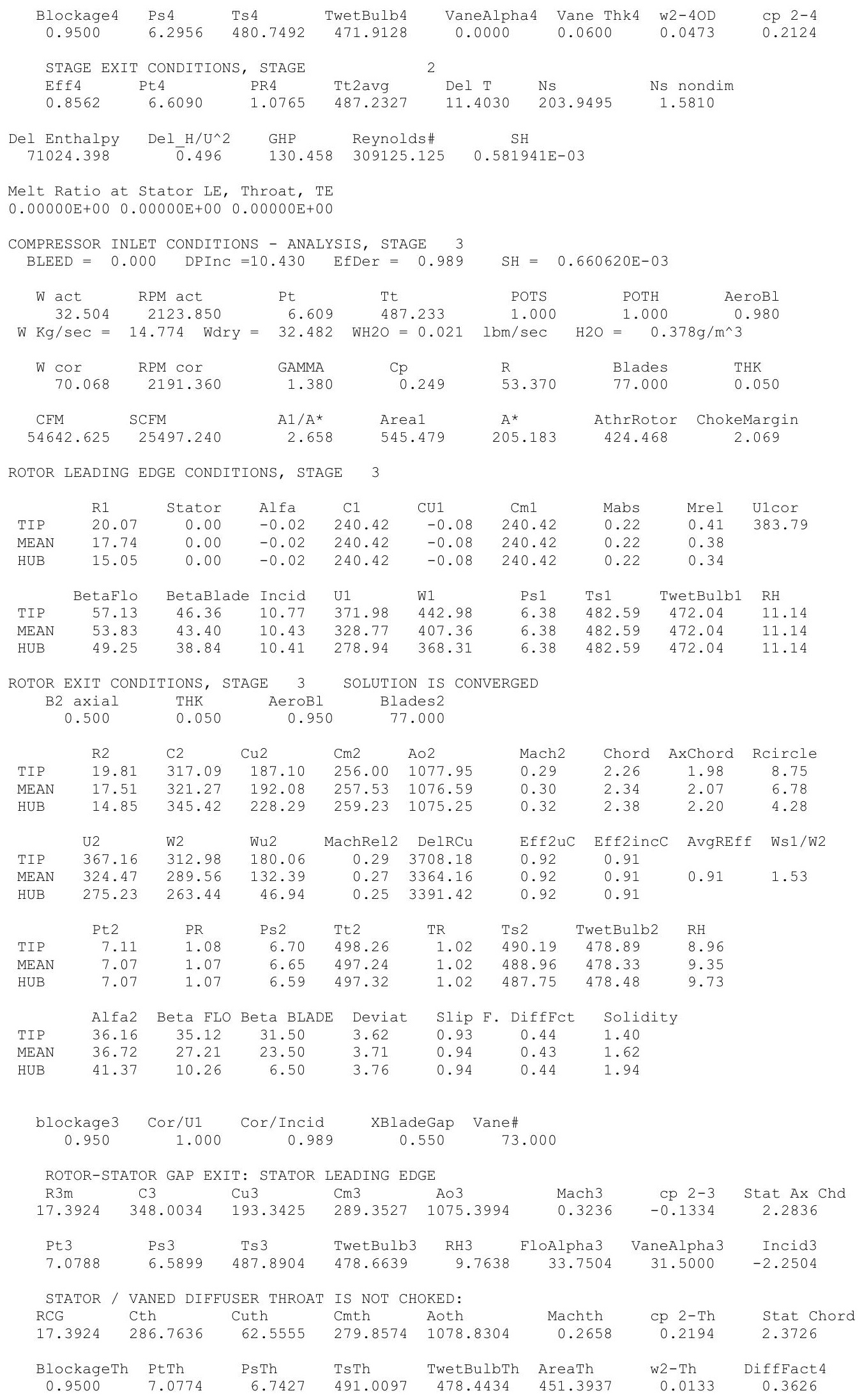




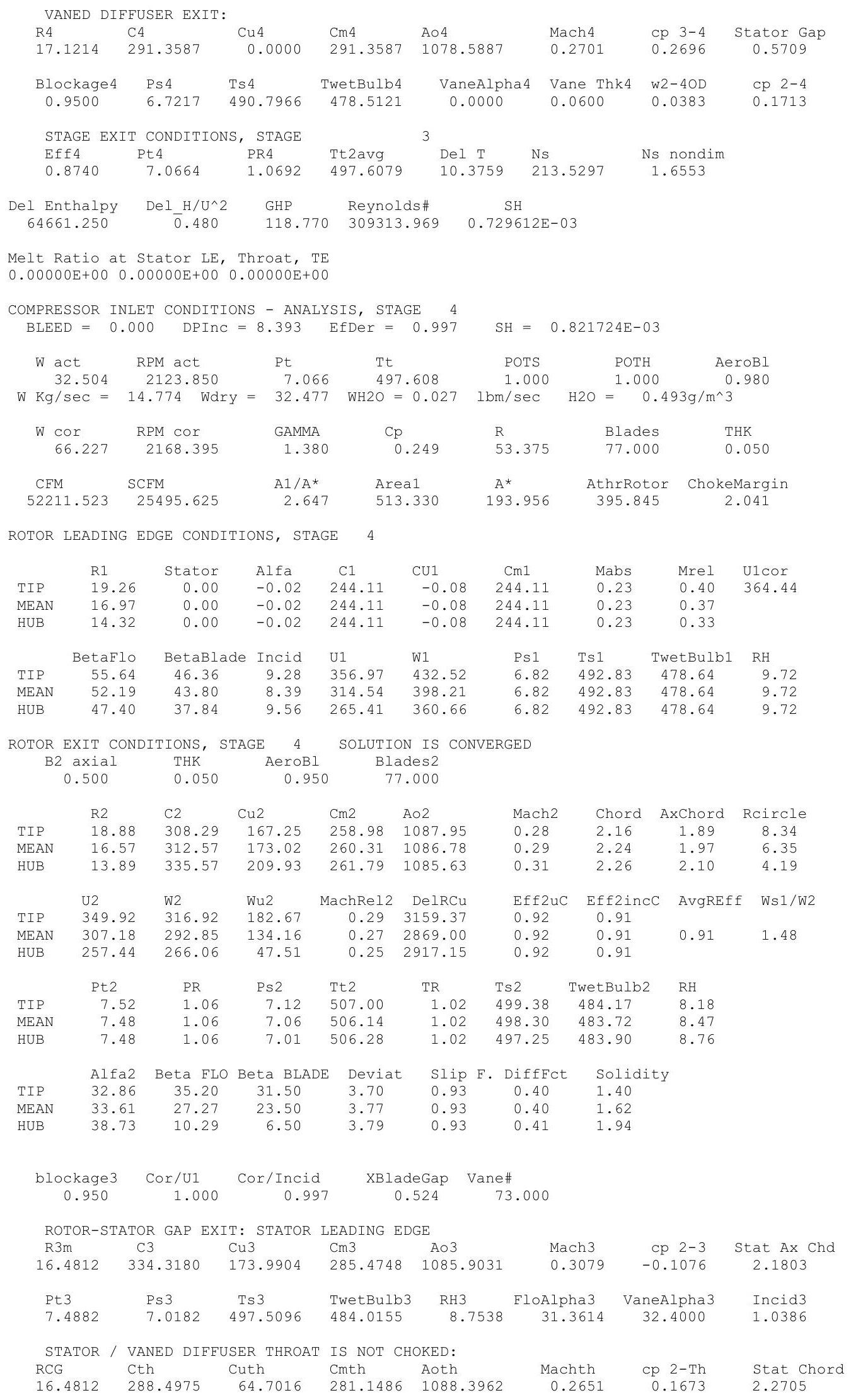




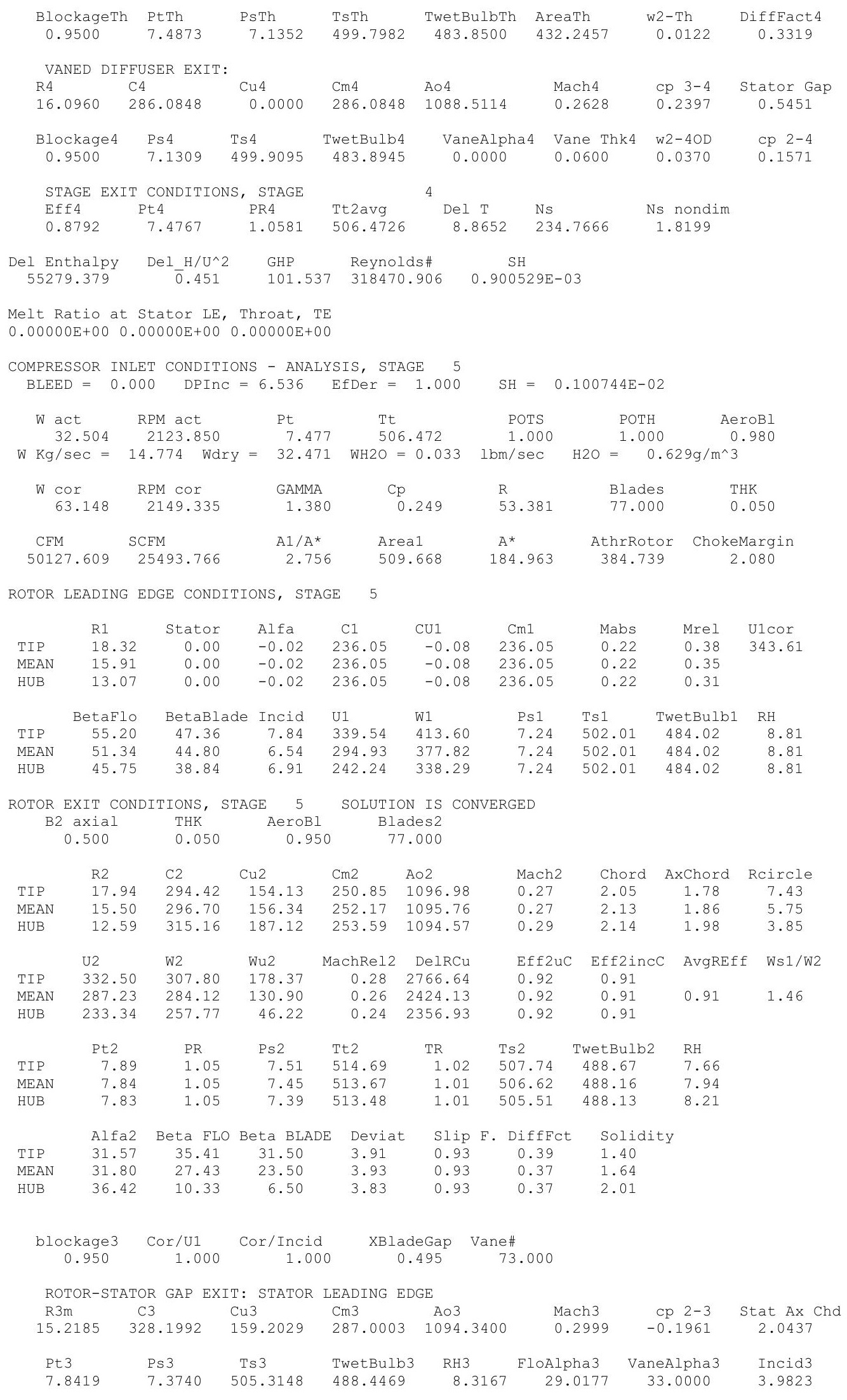




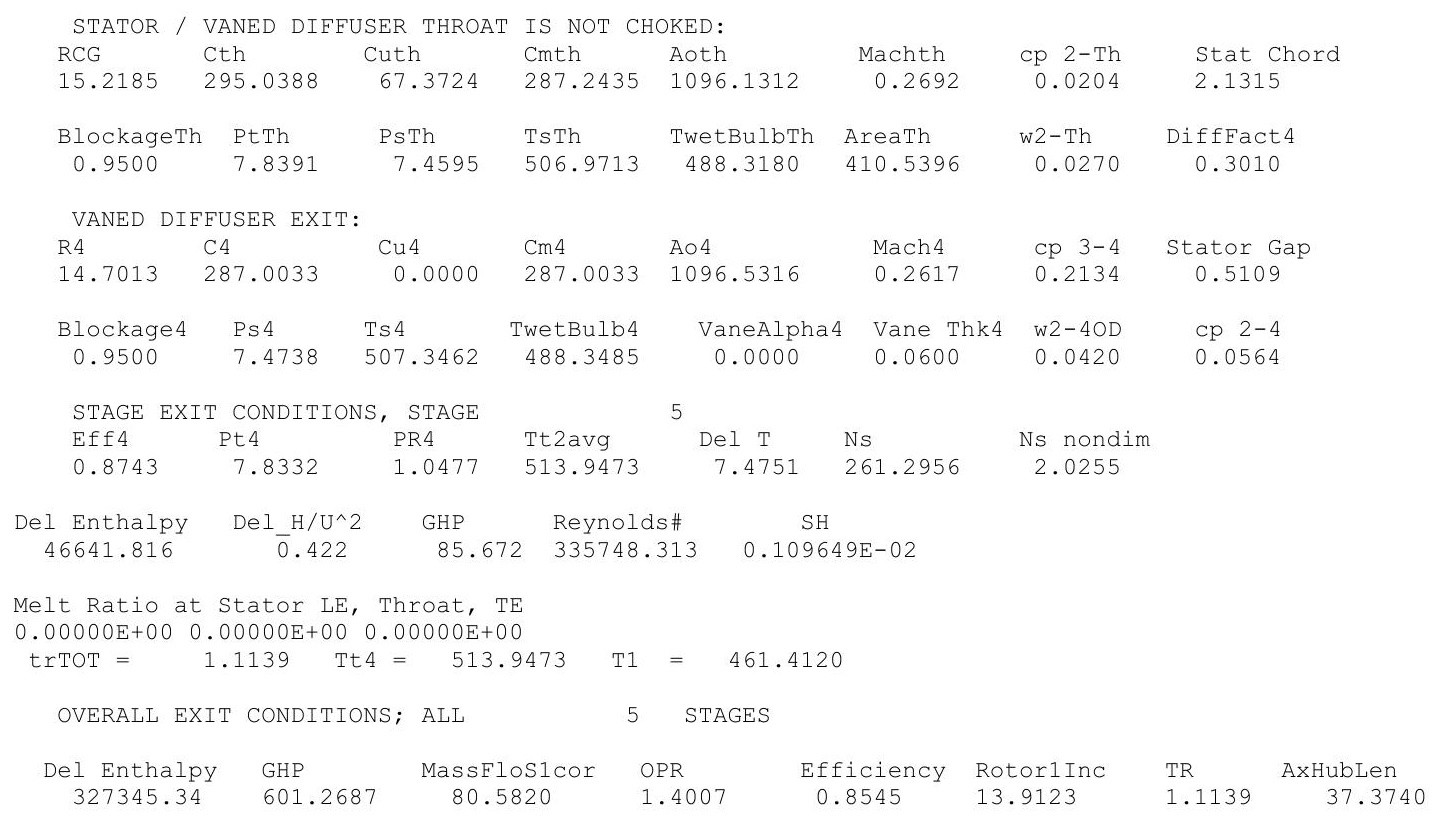




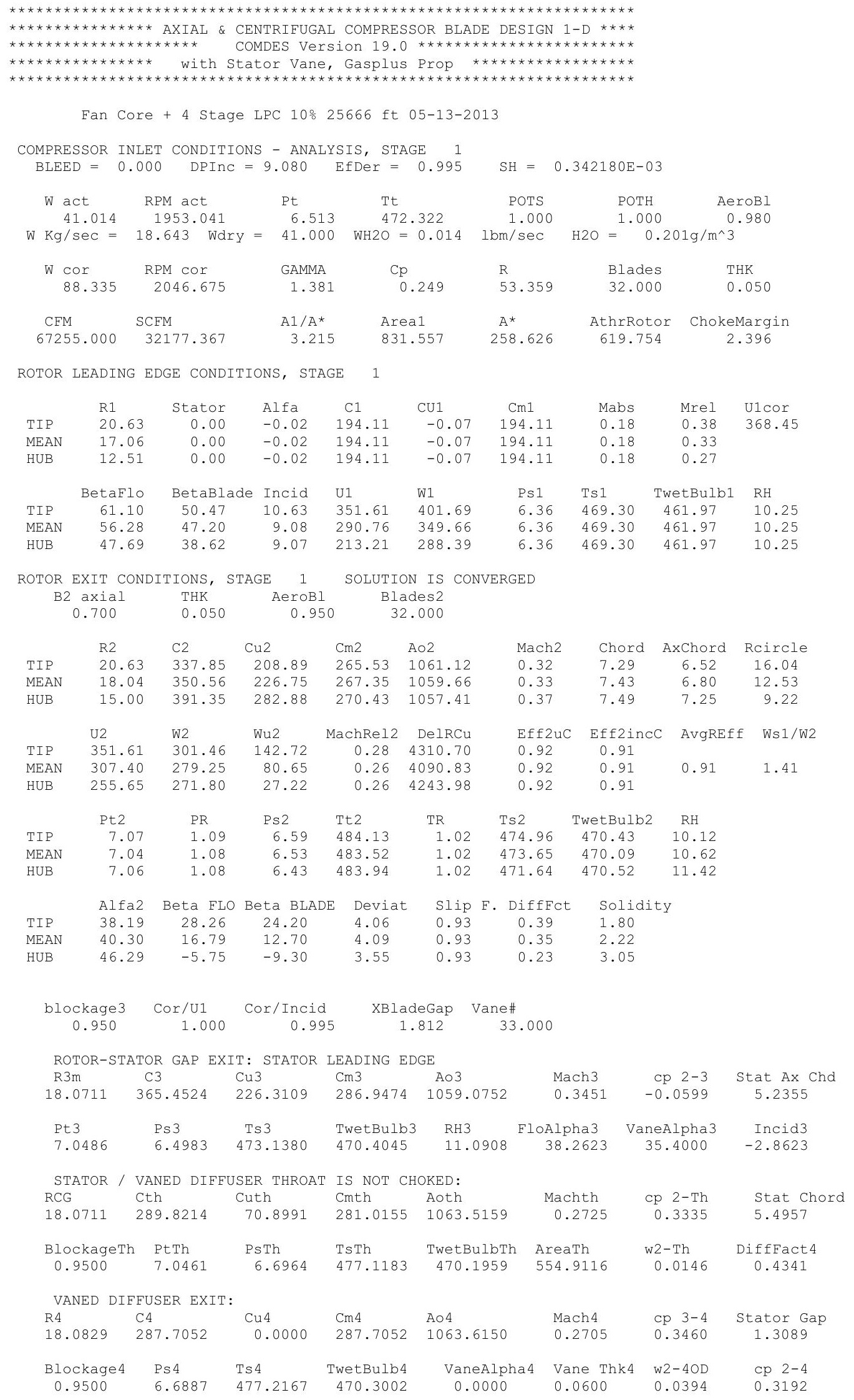




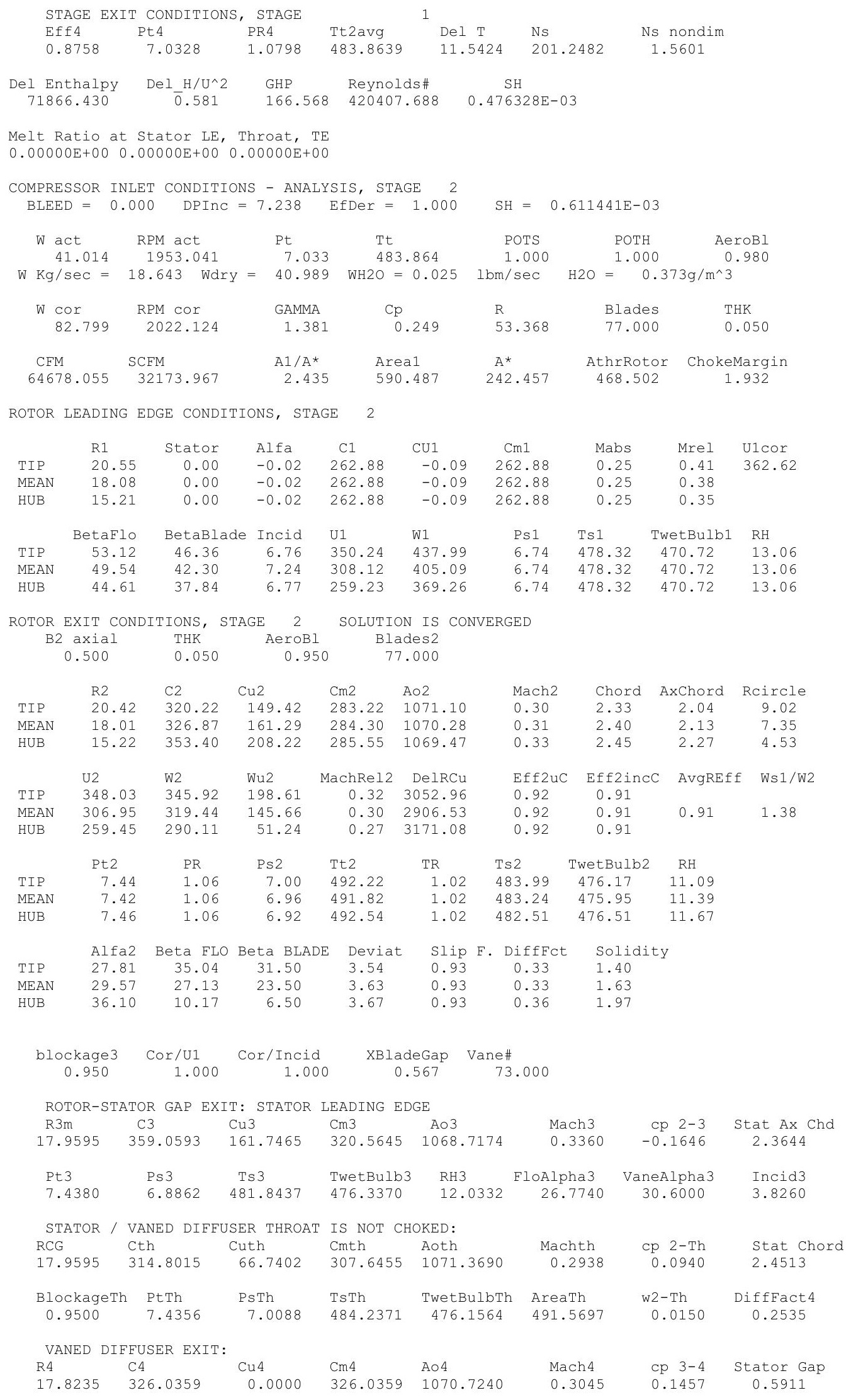




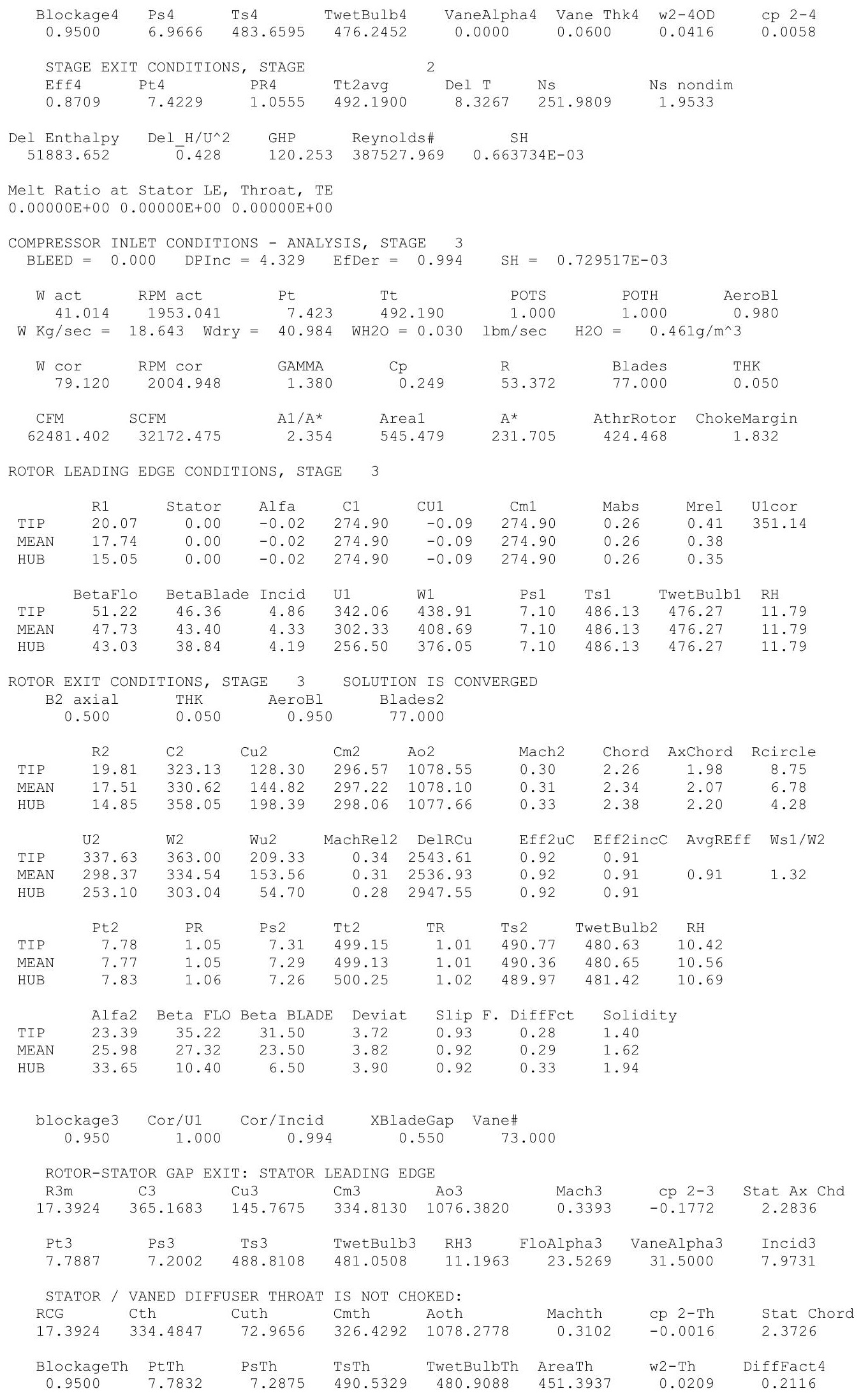




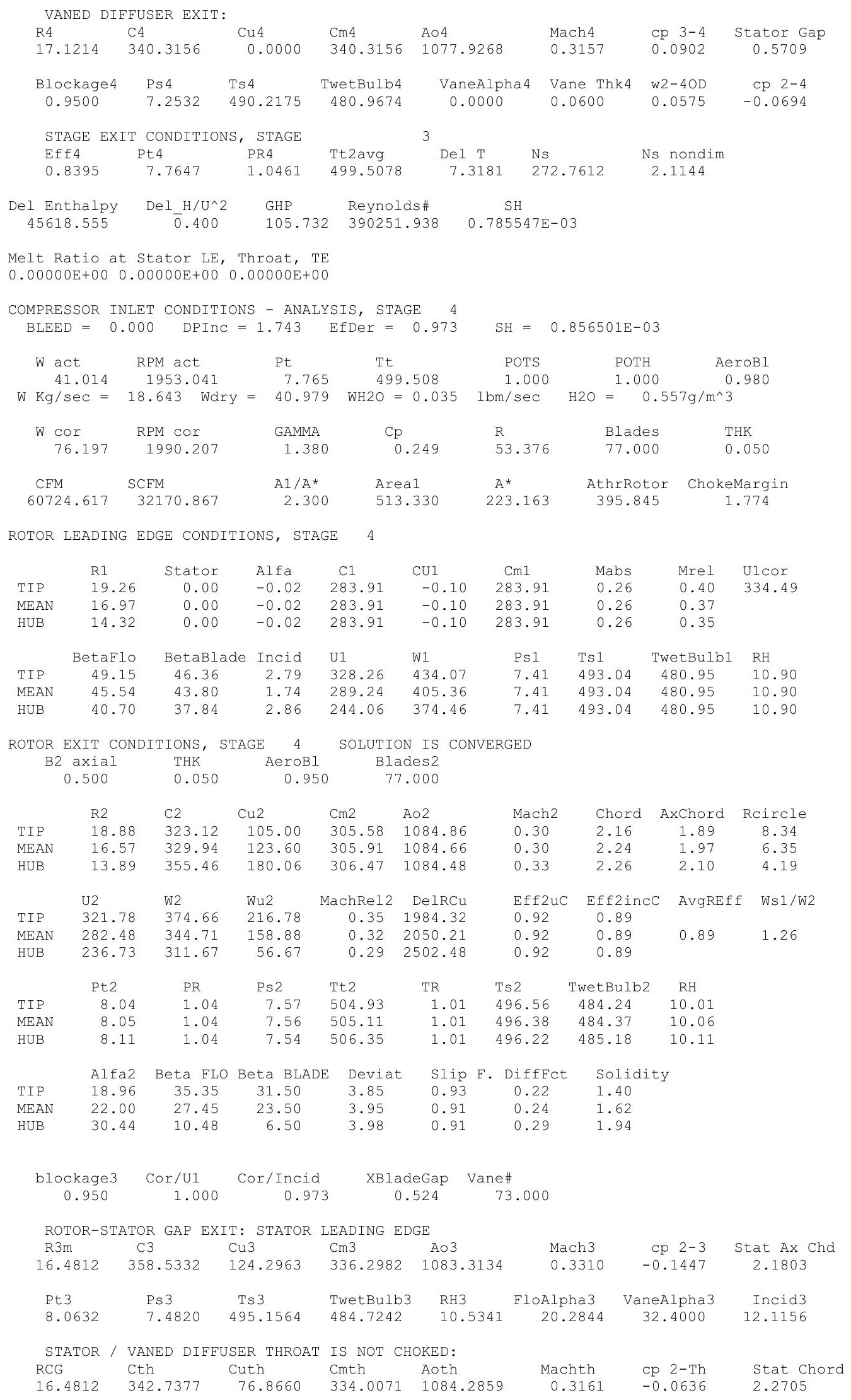




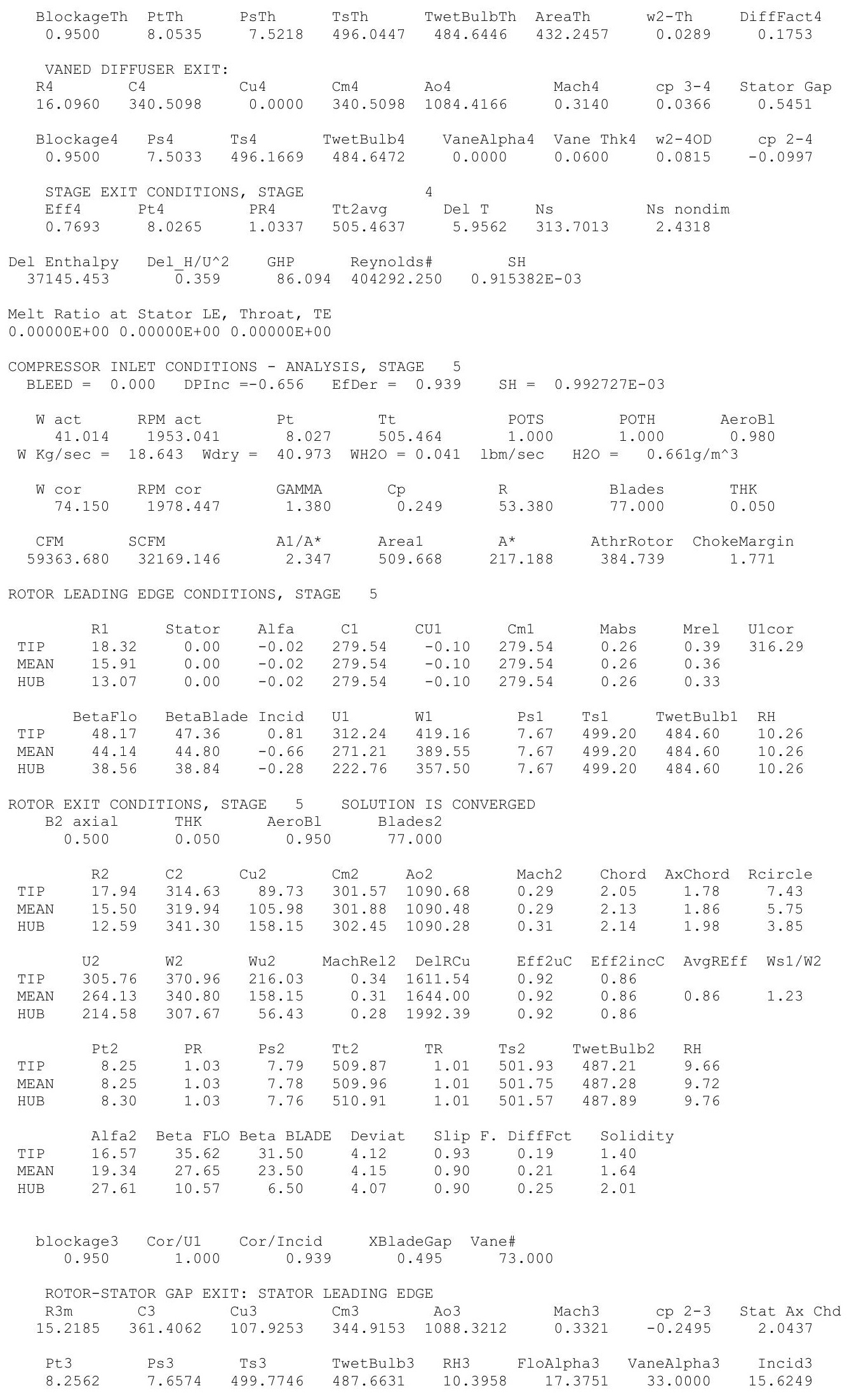




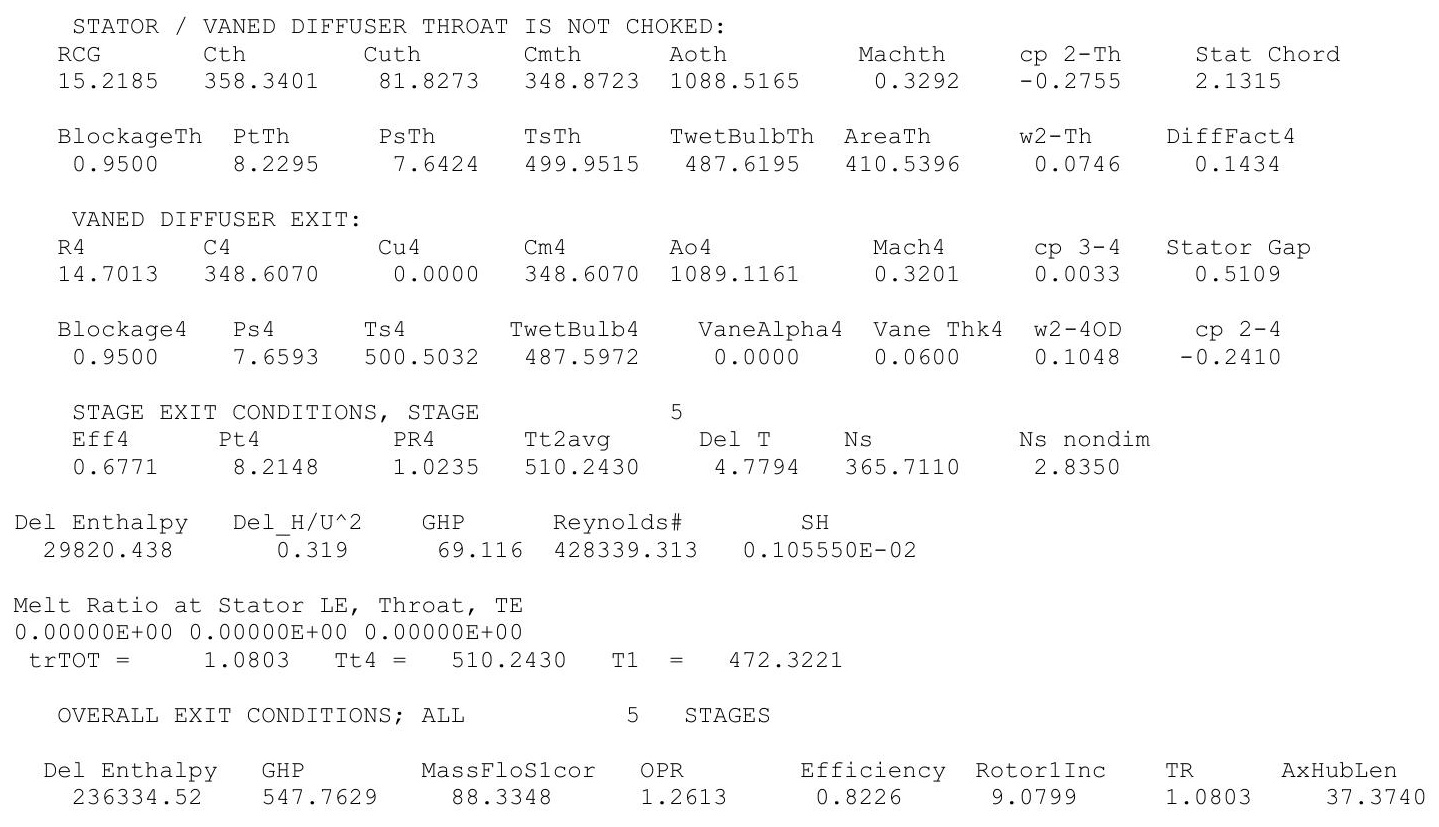




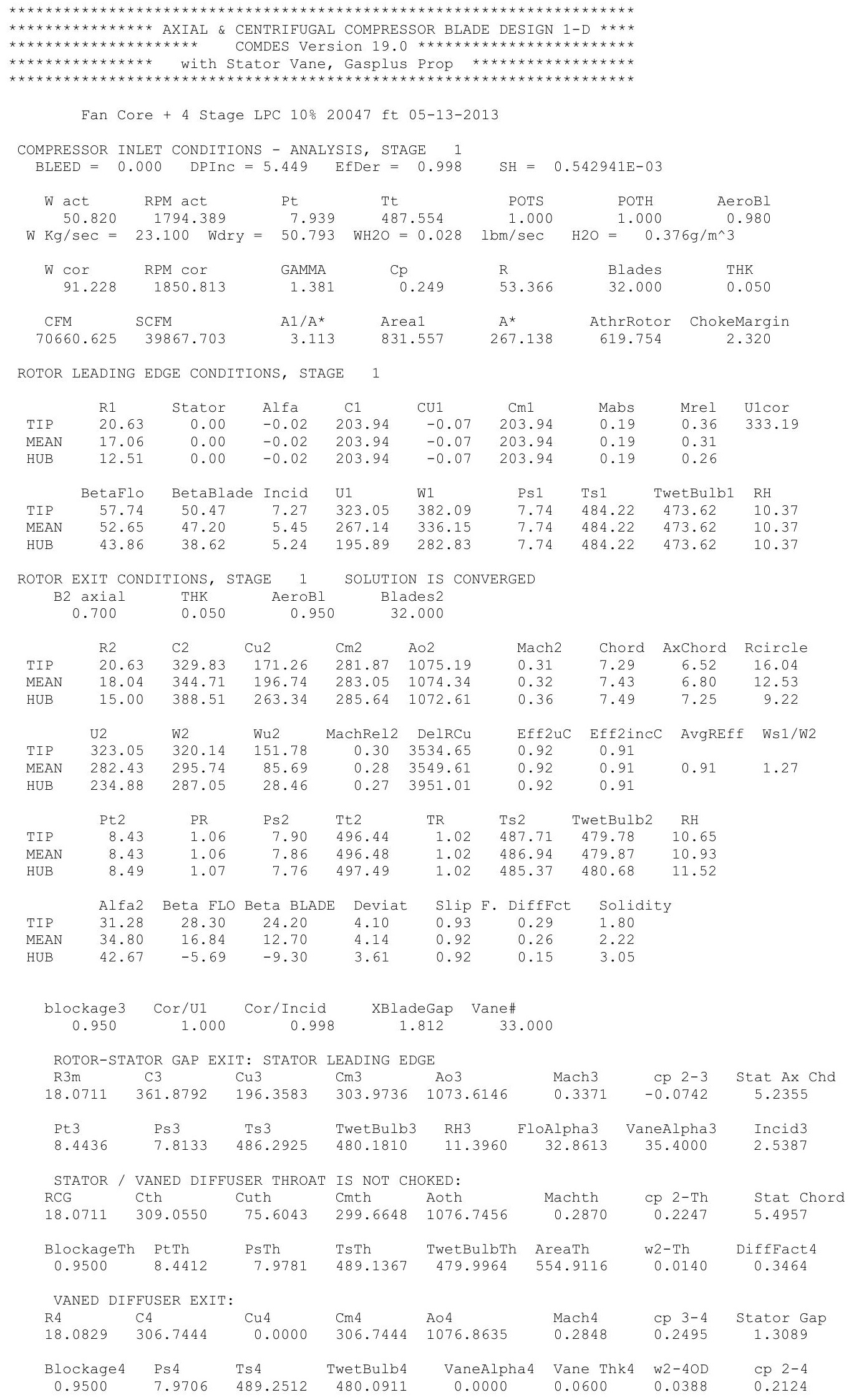




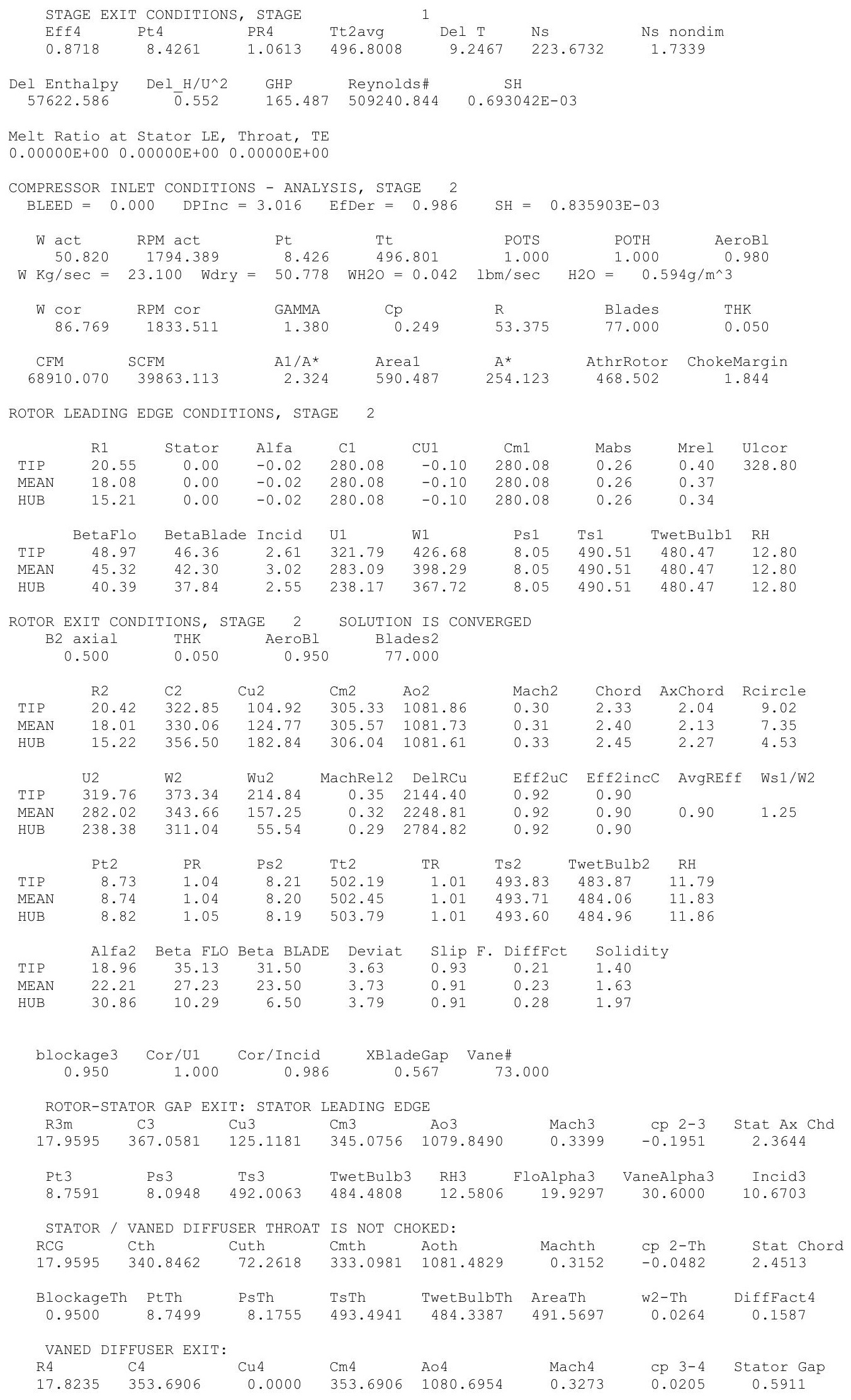




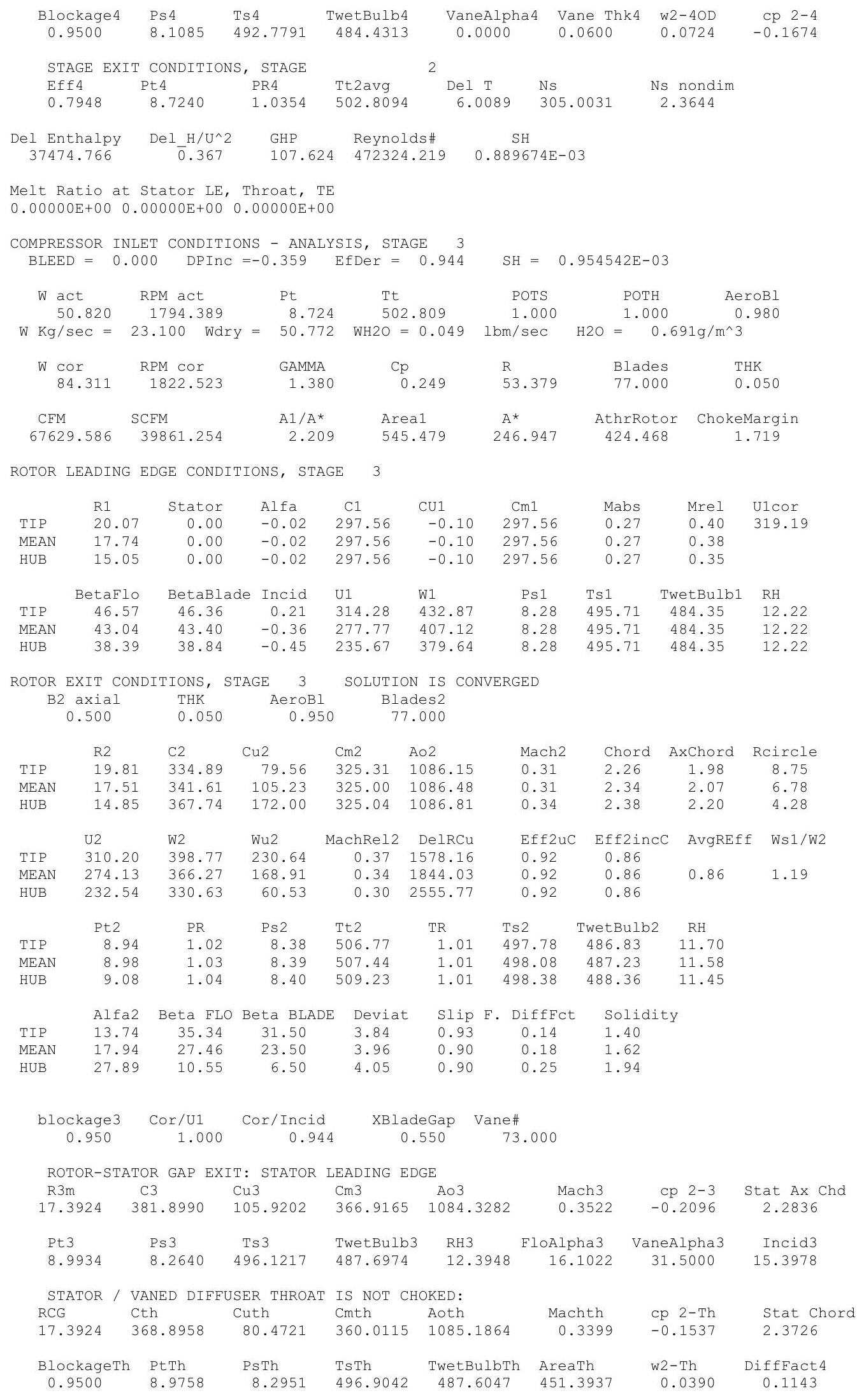




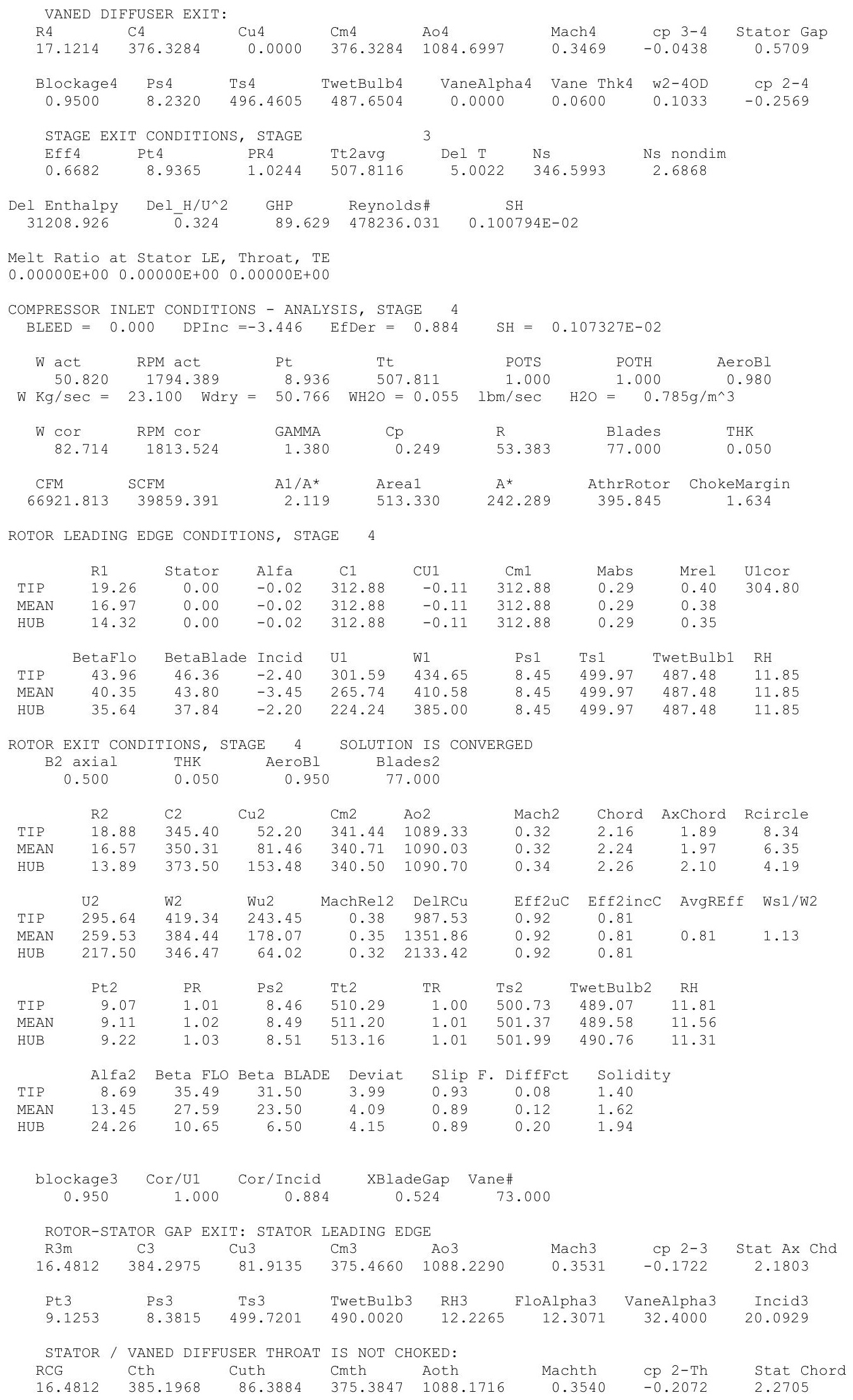




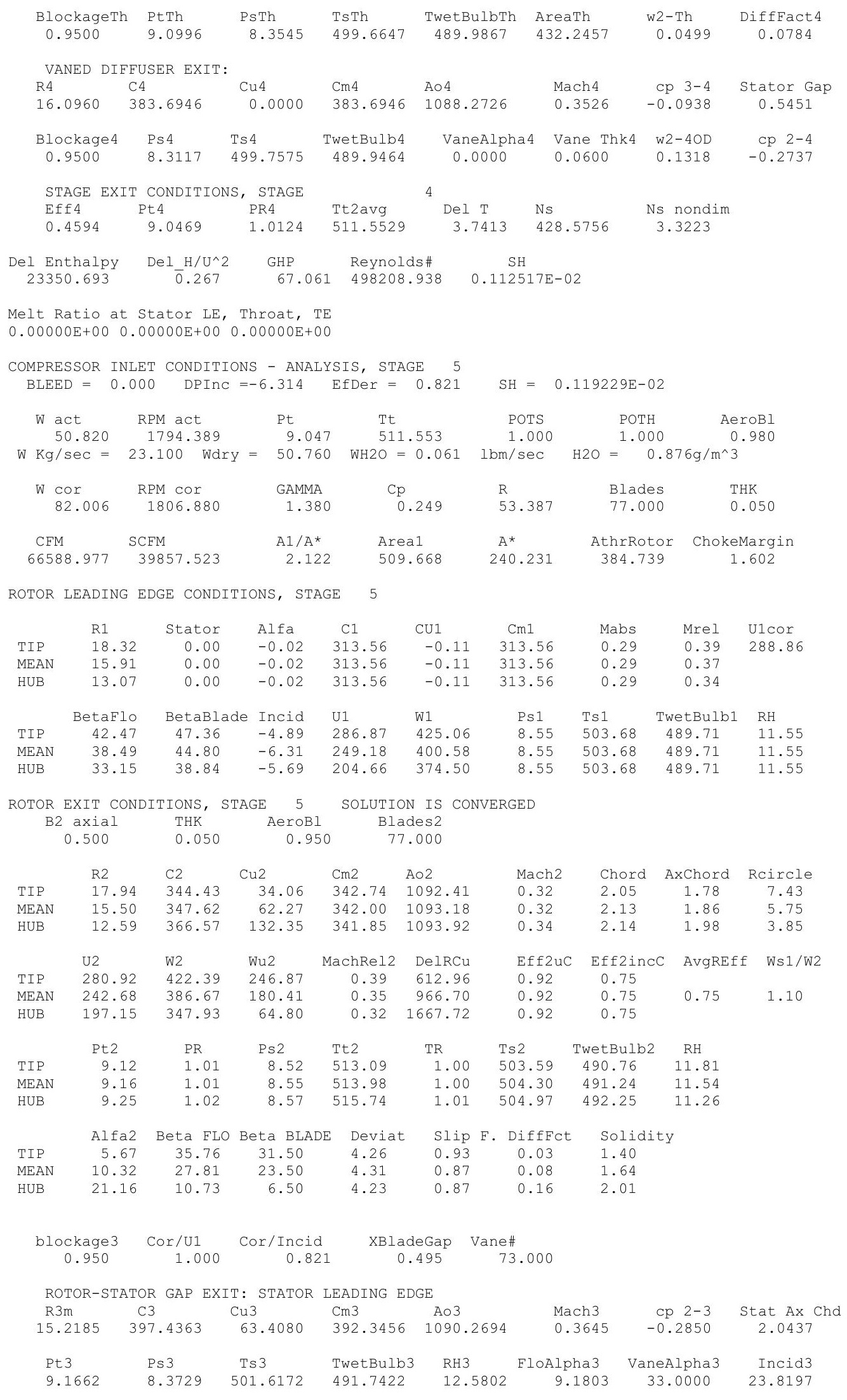




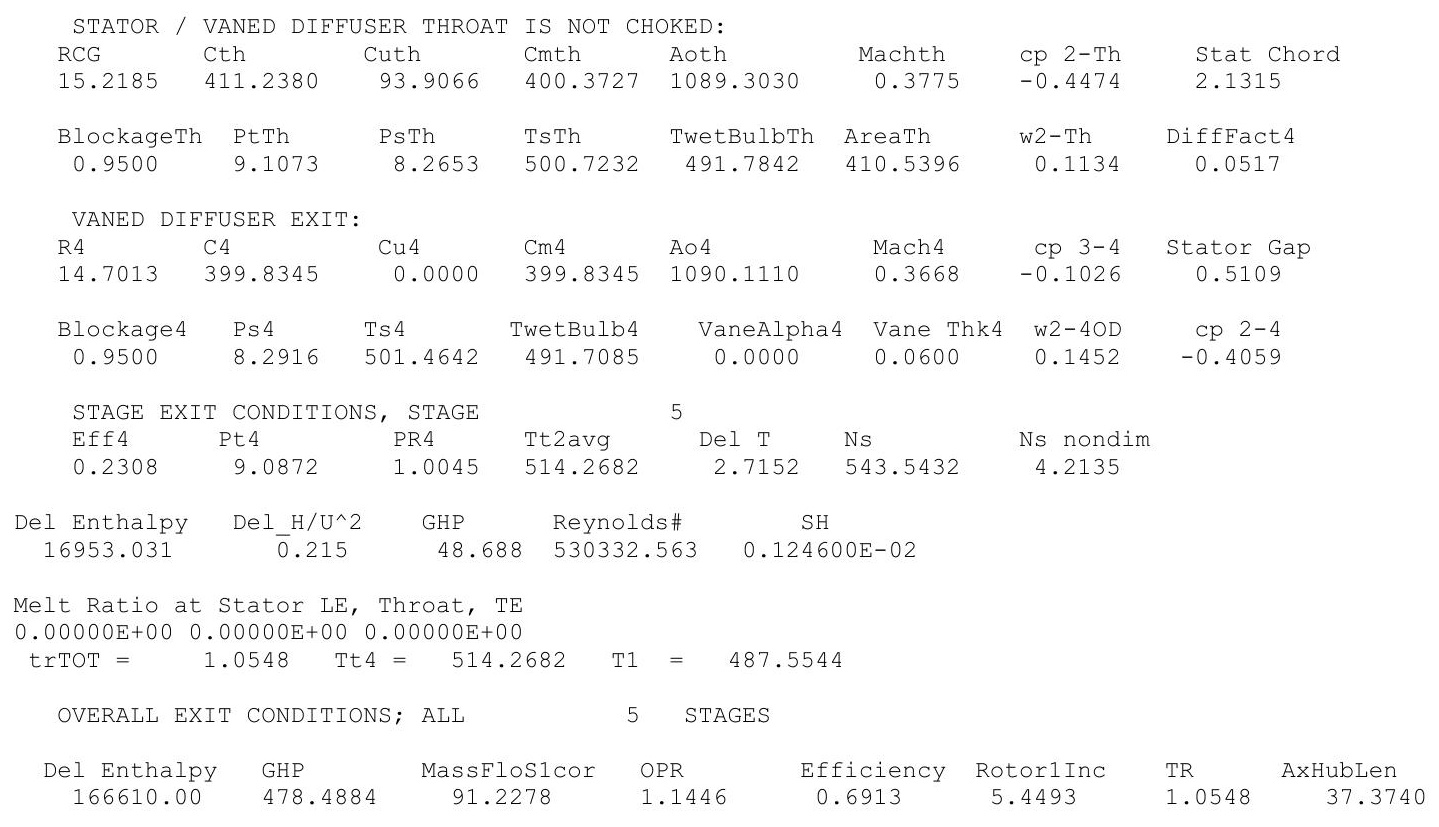




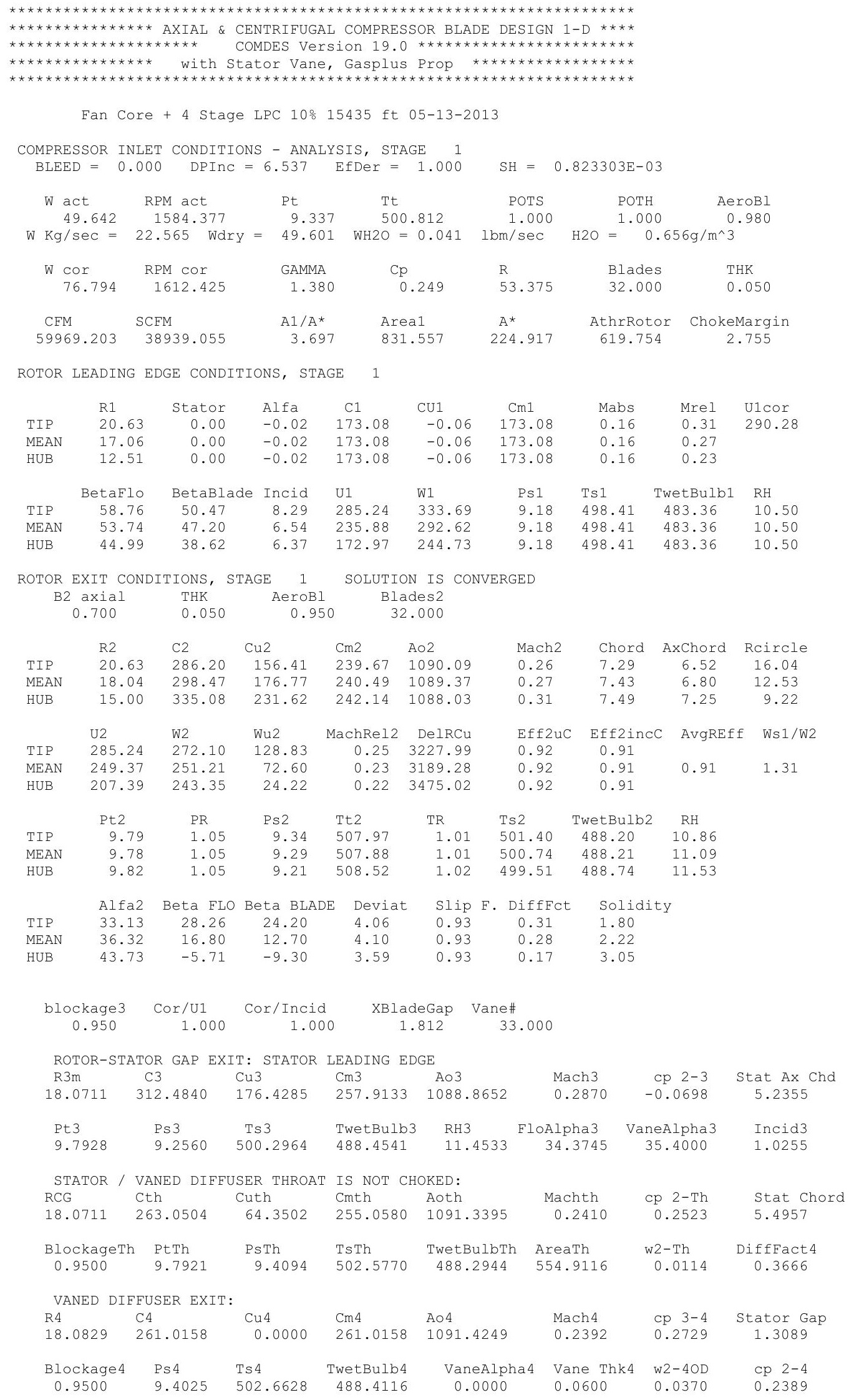




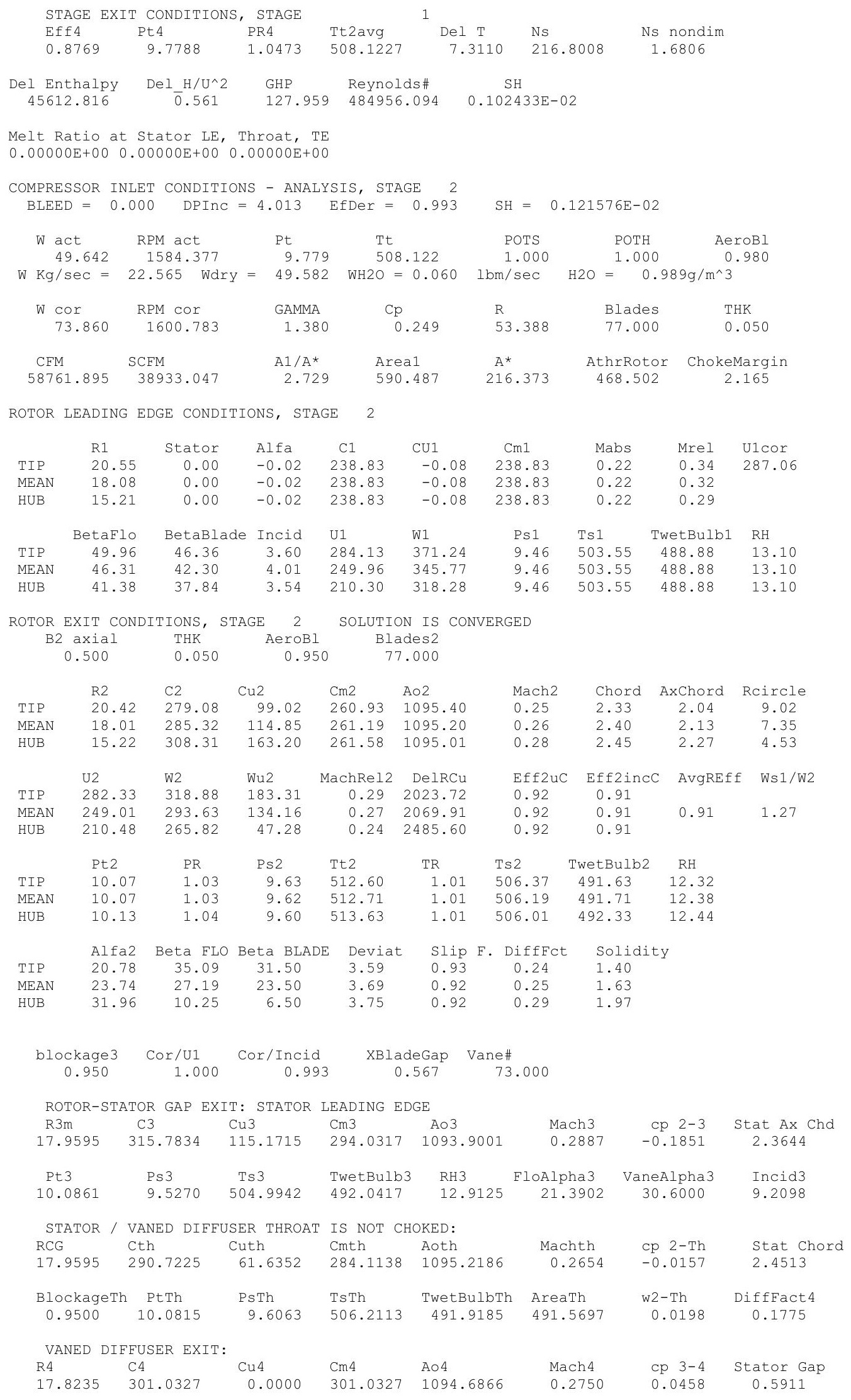




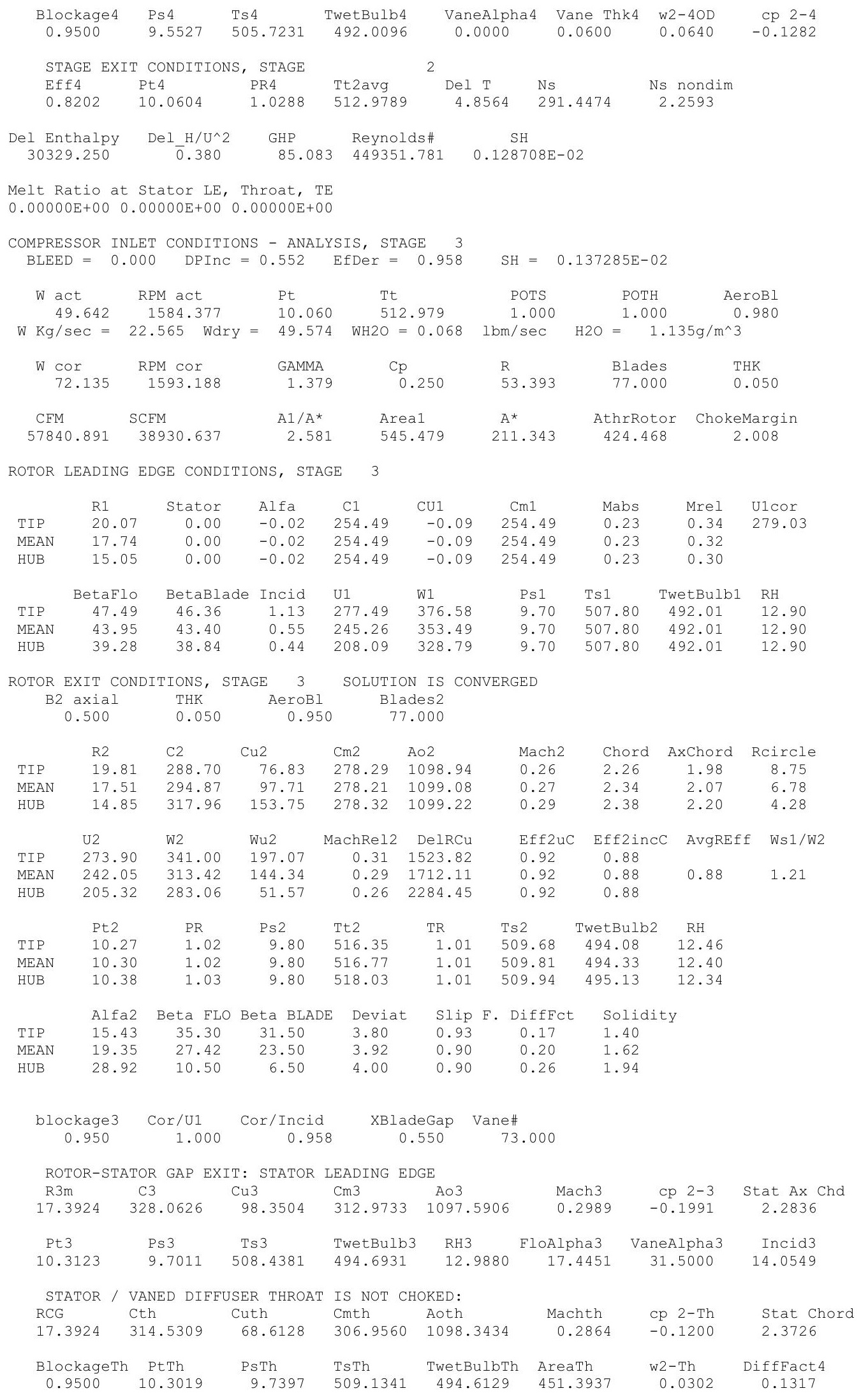




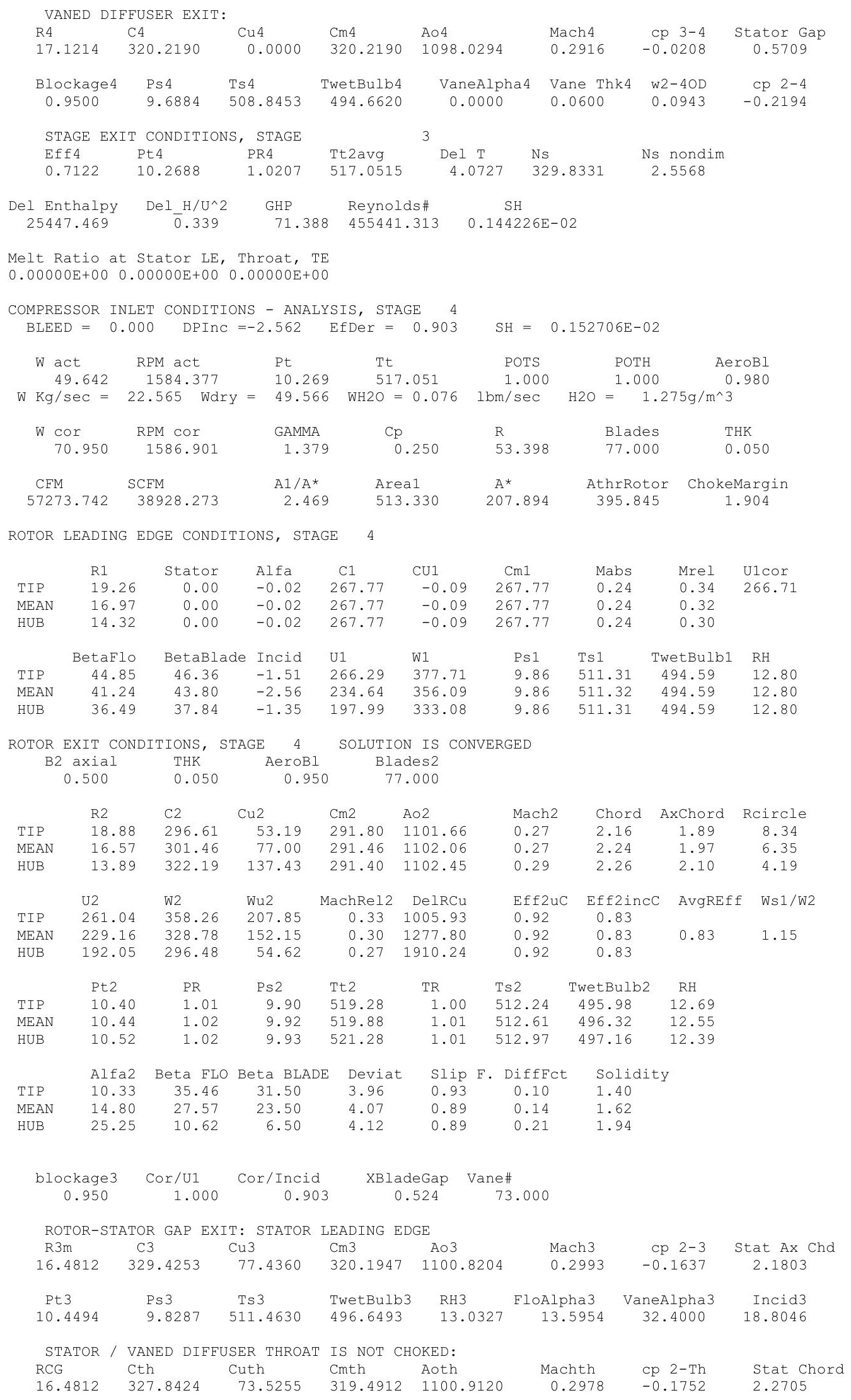




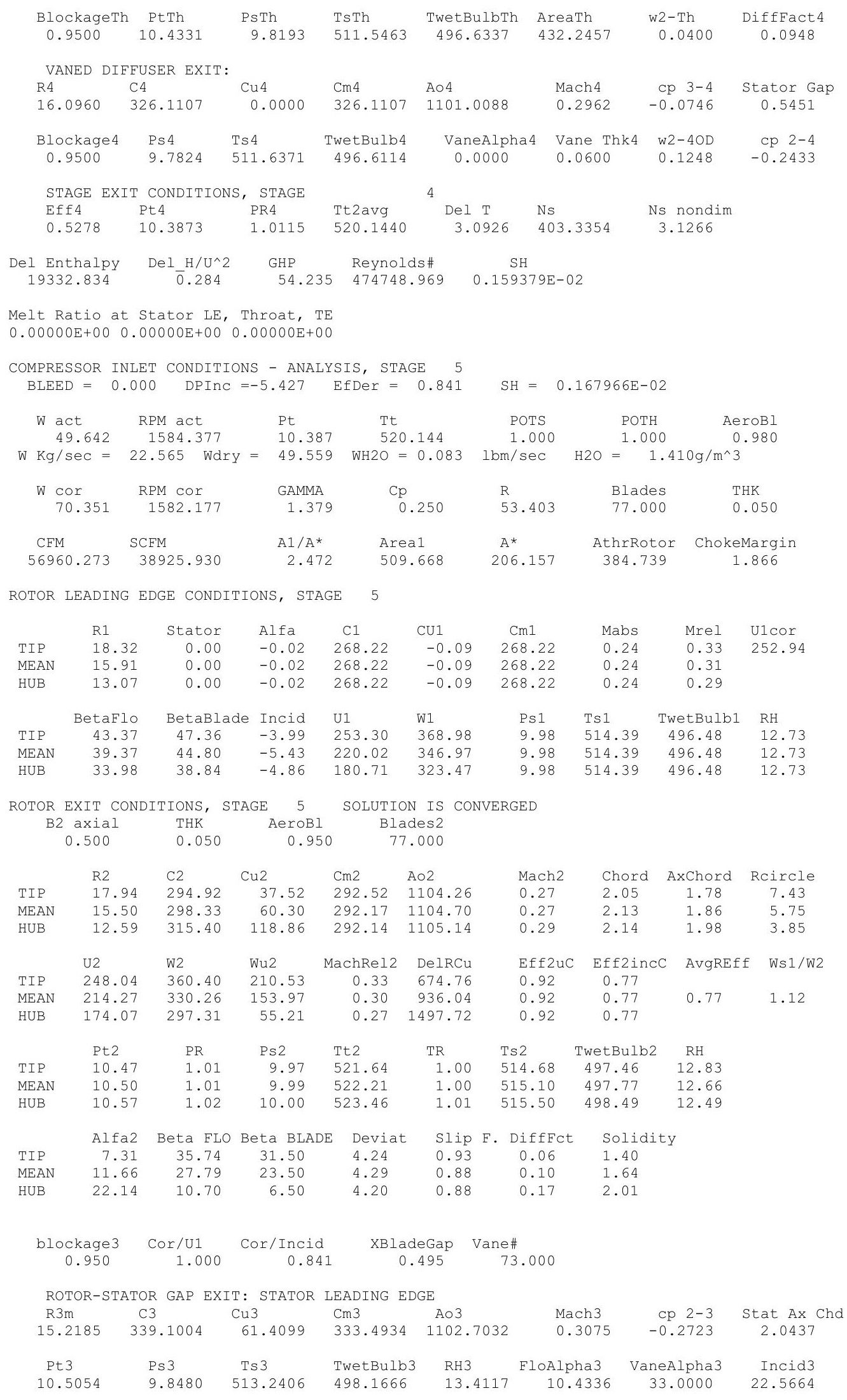




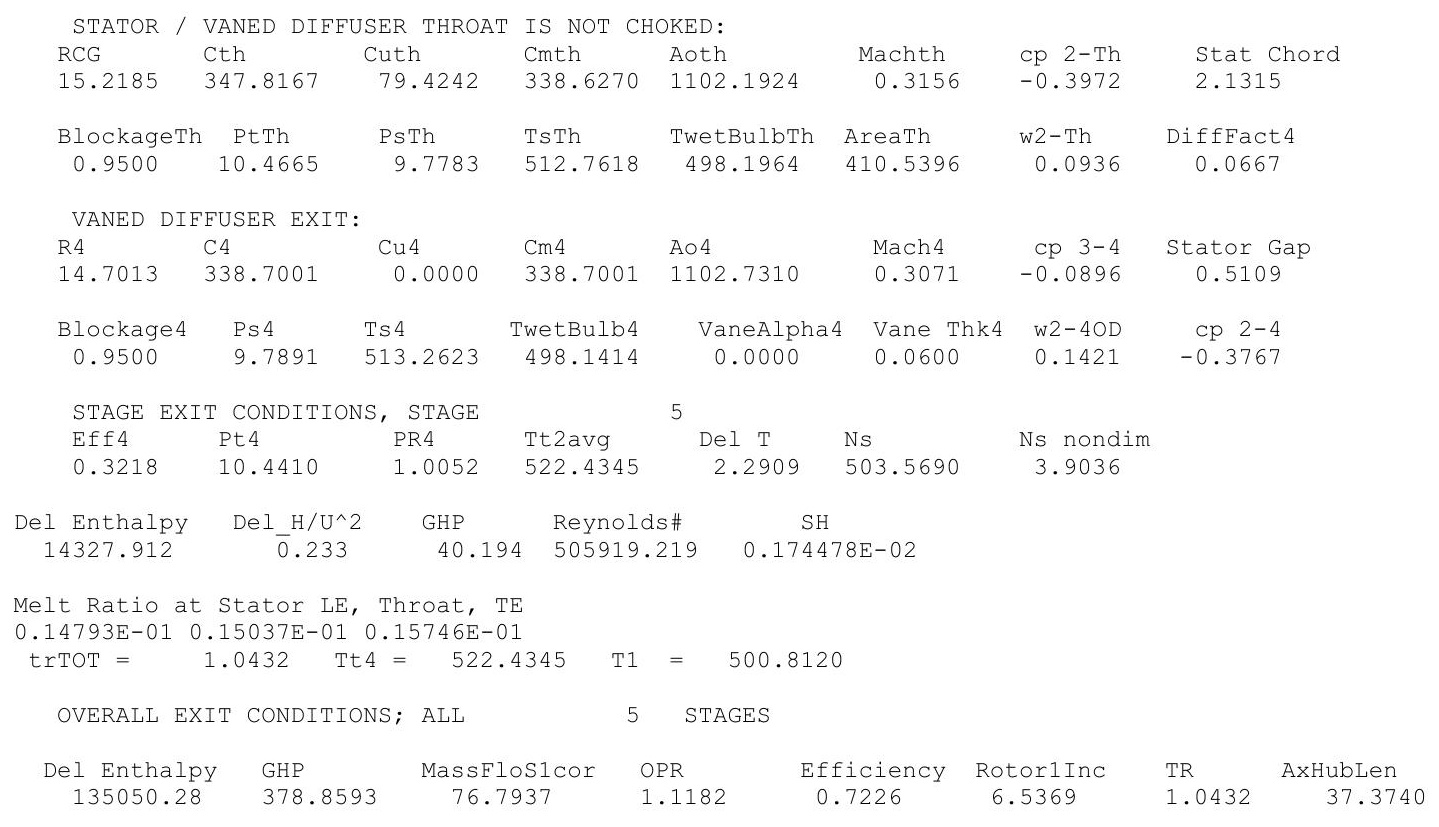


$10 \mu \mathrm{m}, \mathrm{ISA}+36 \mathrm{R}$

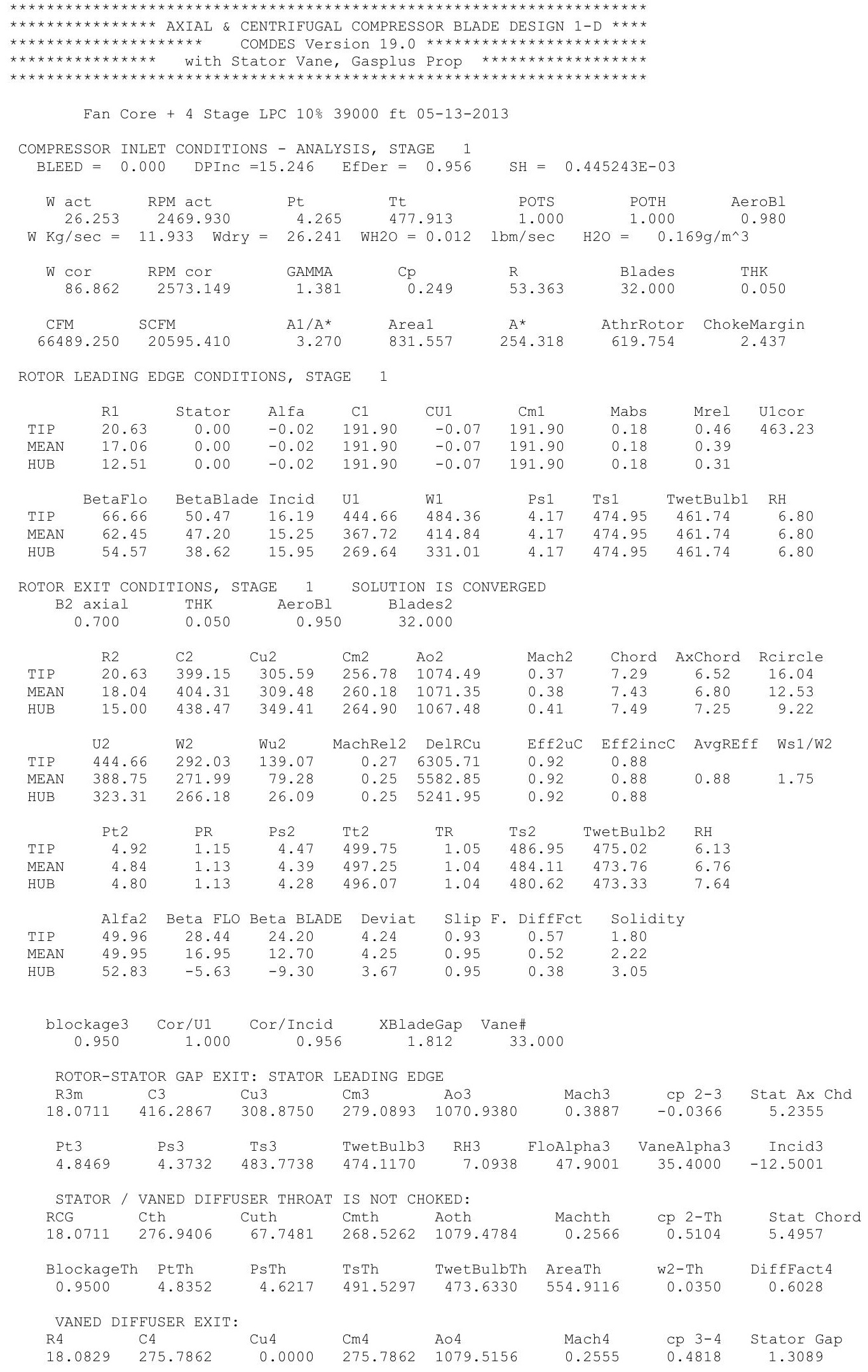




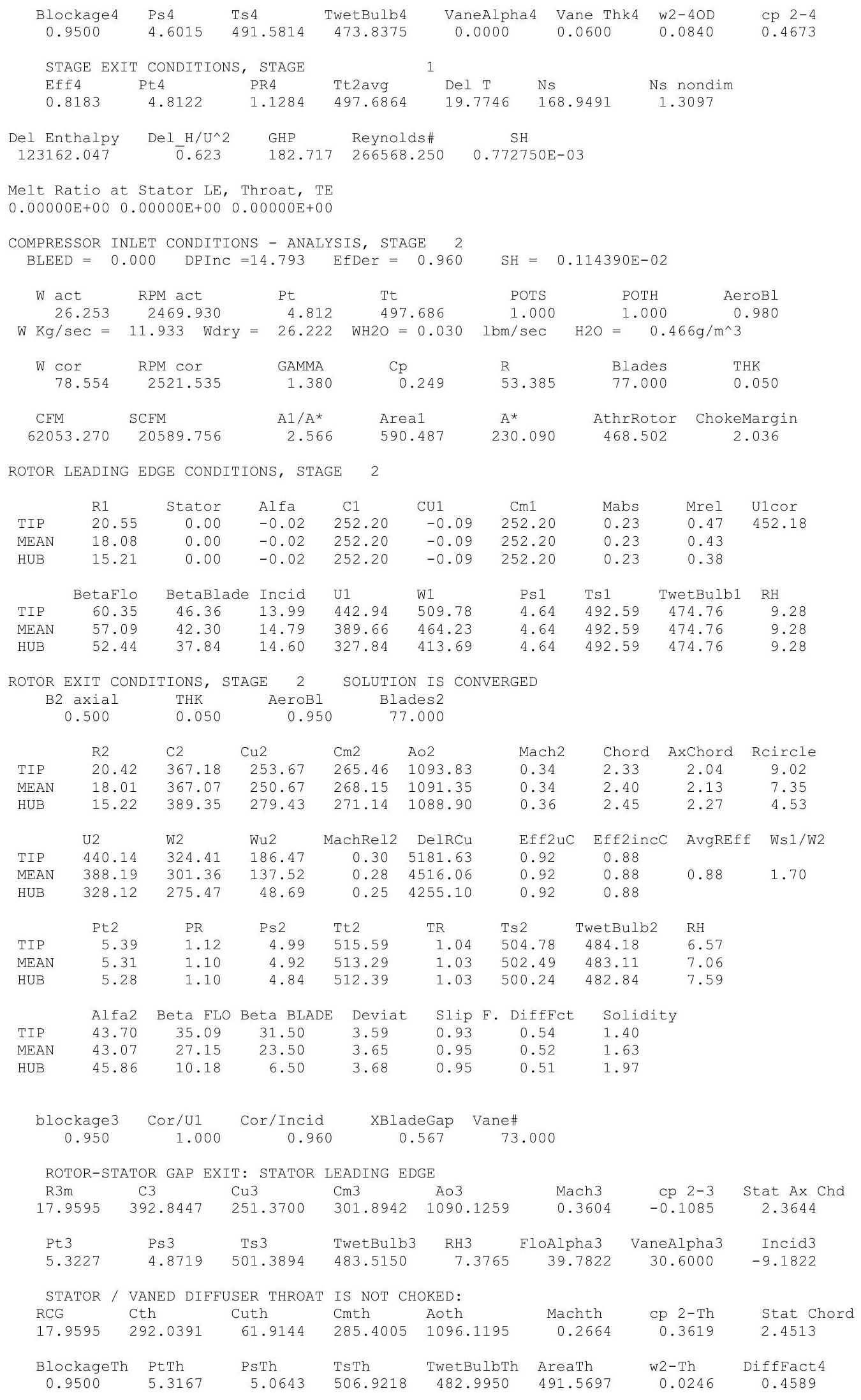




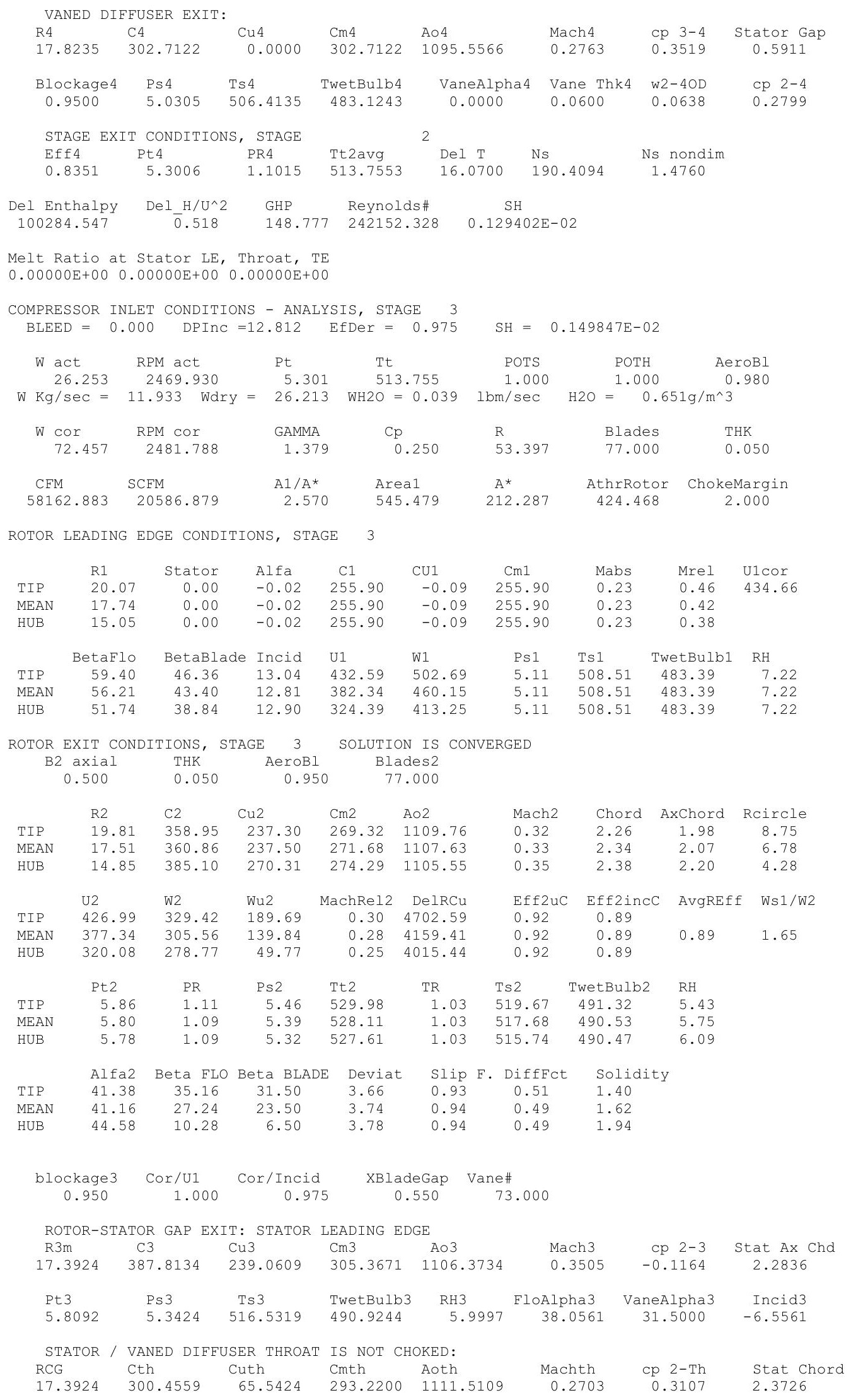




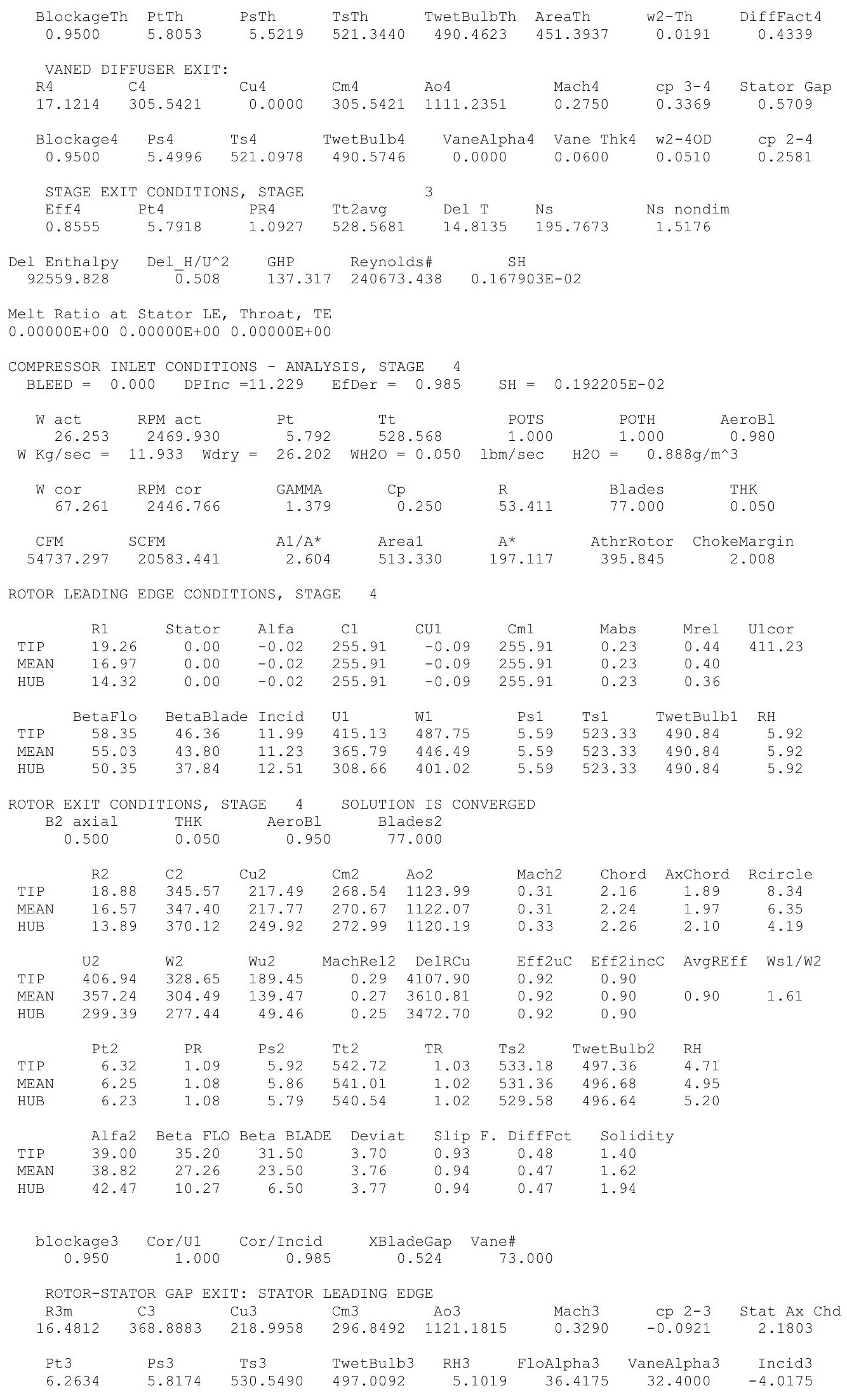




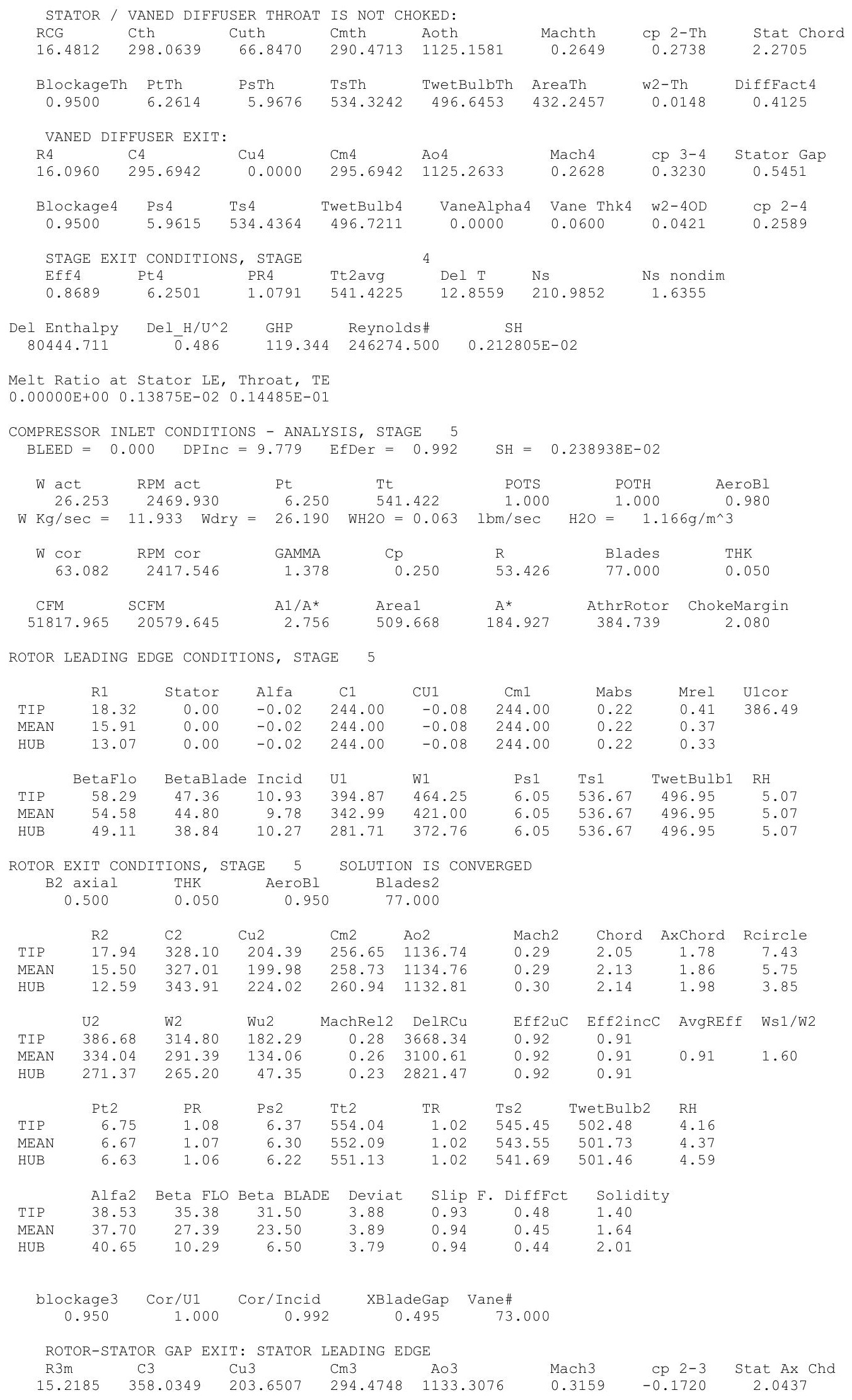




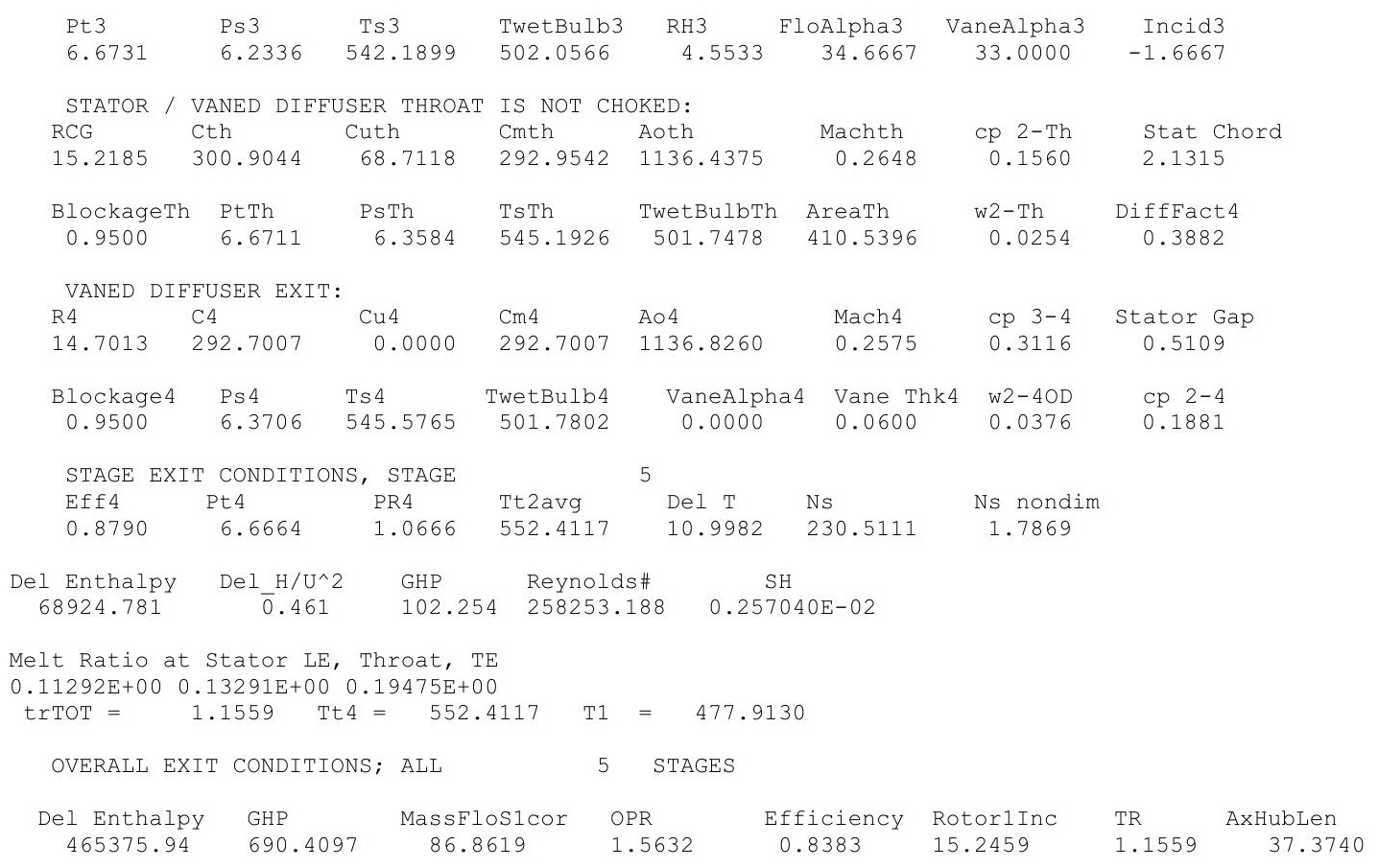




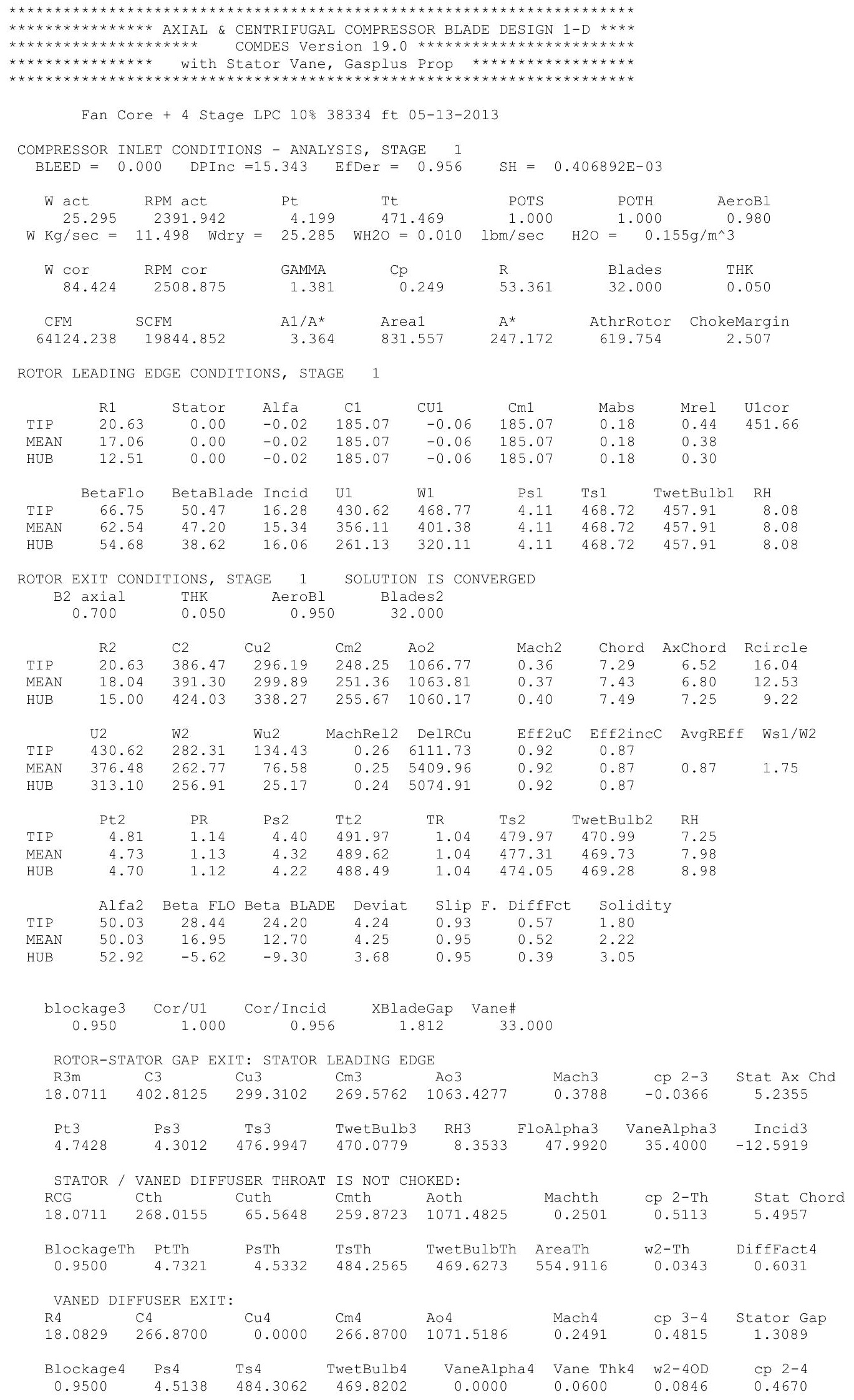




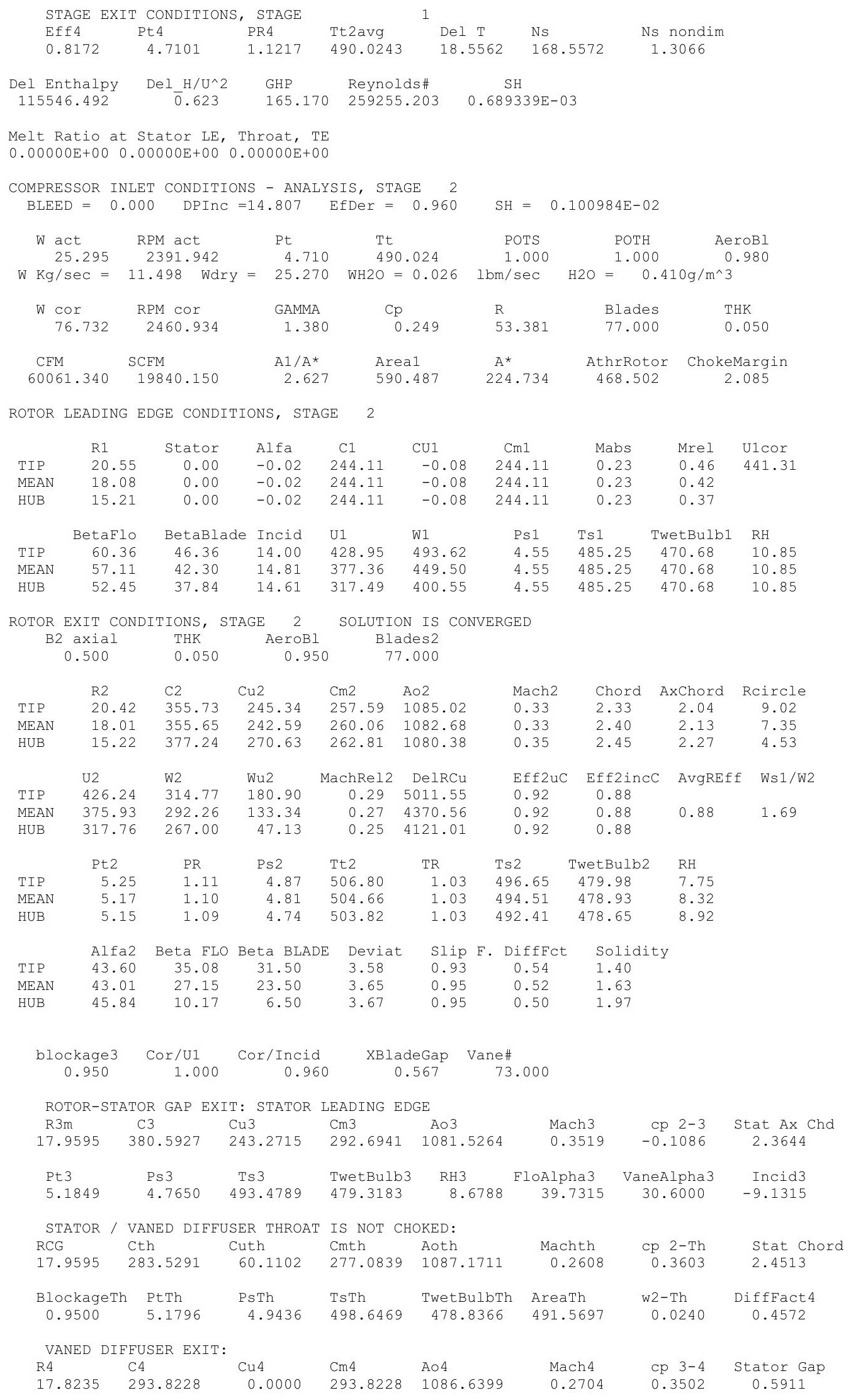




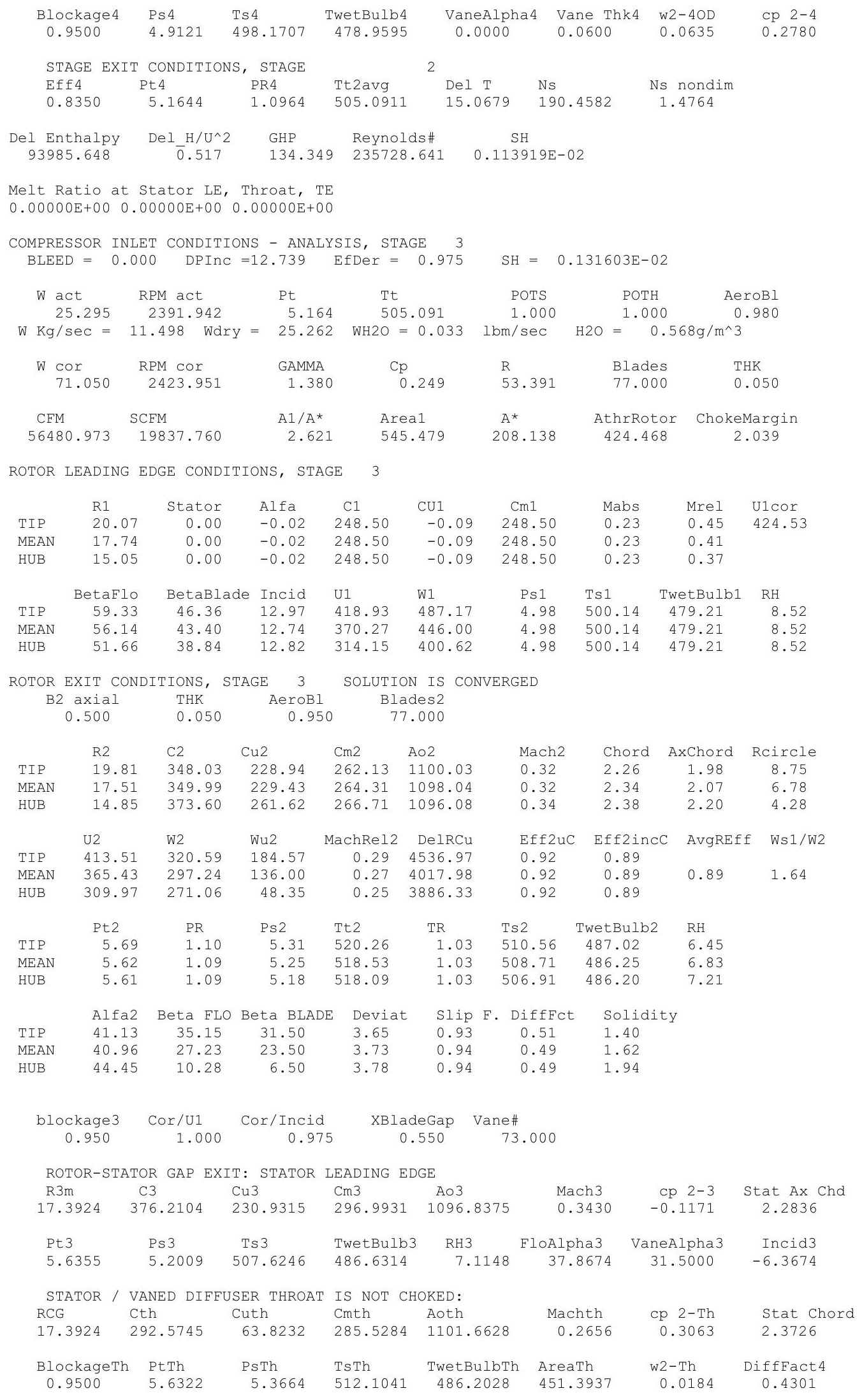




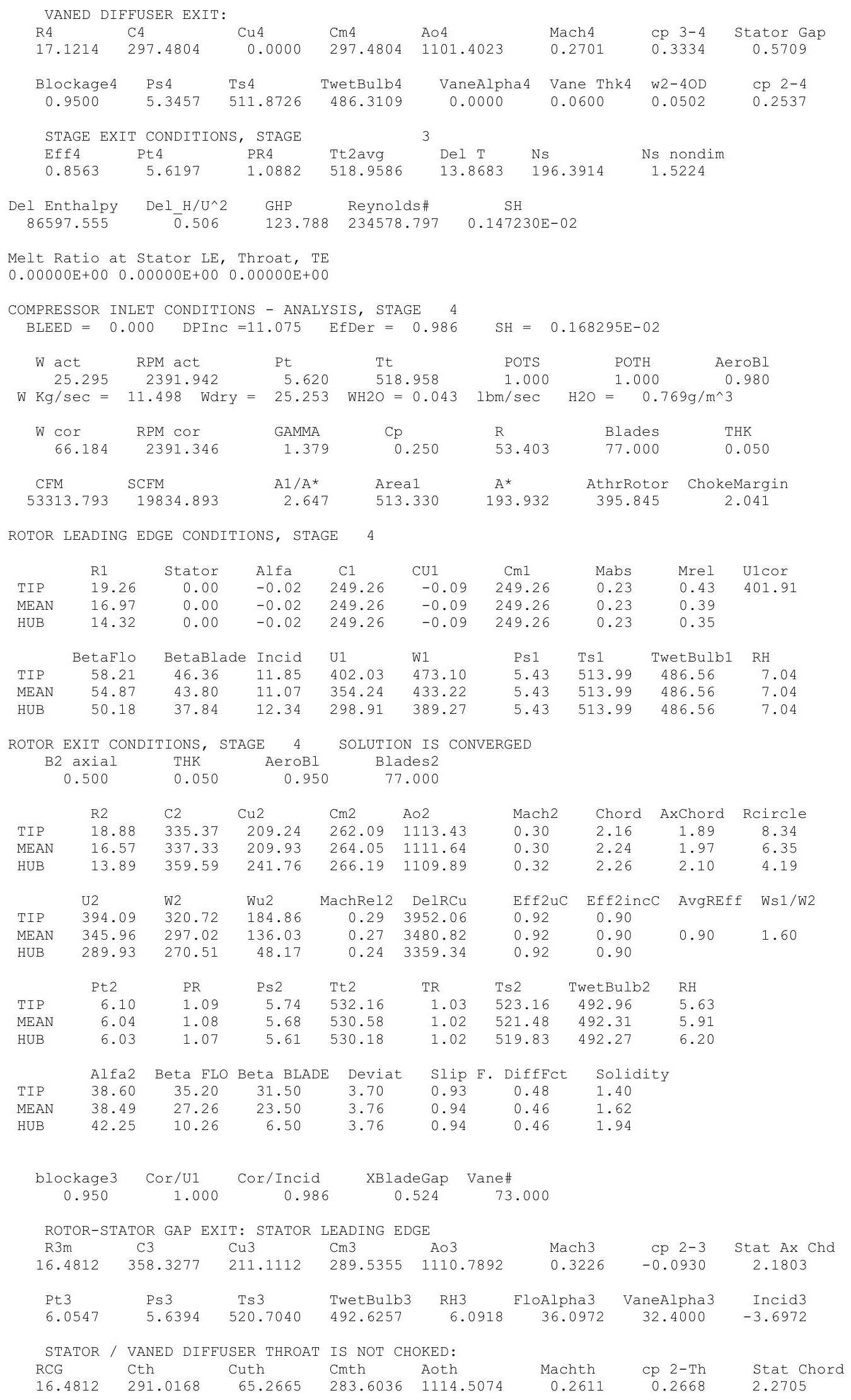




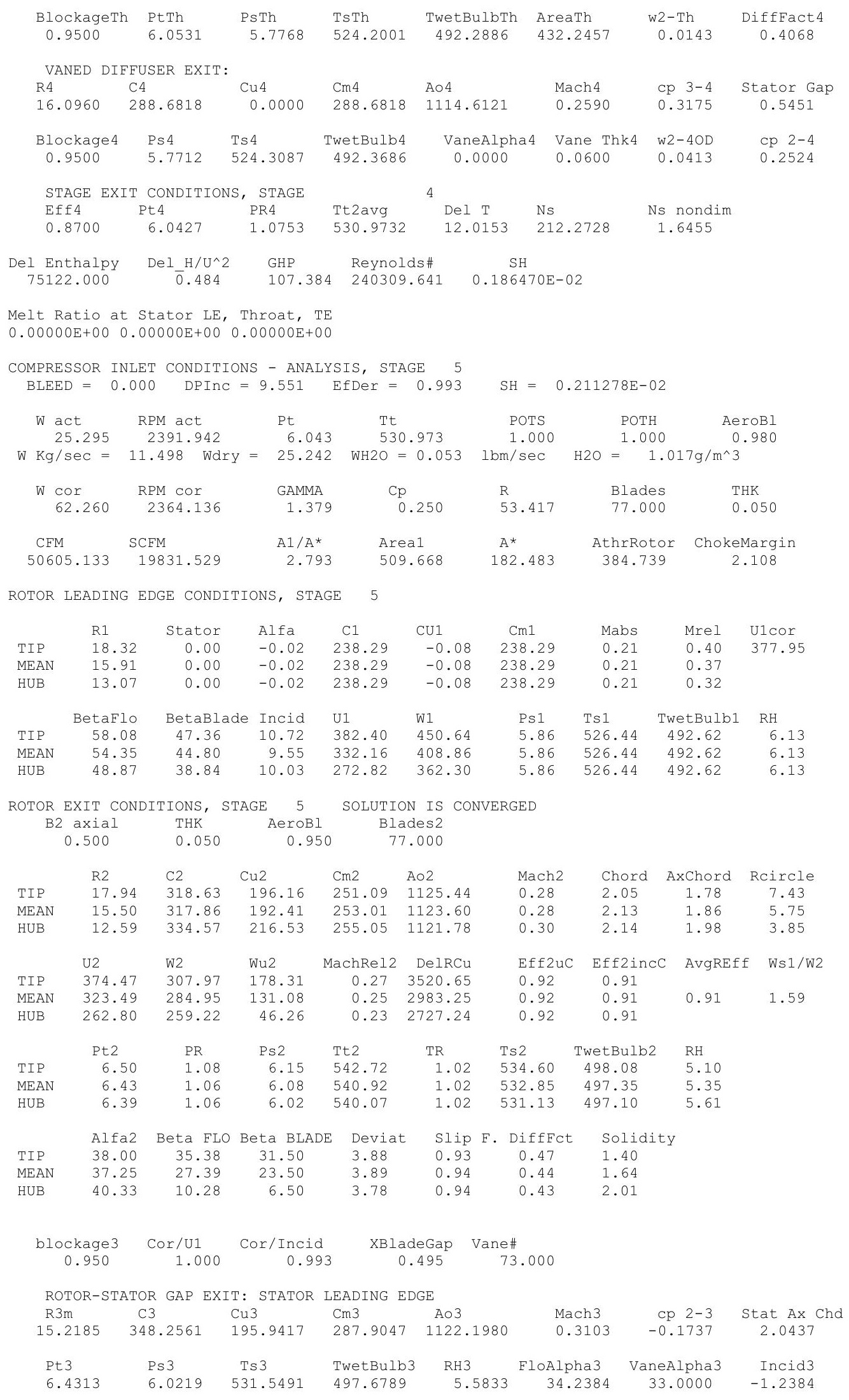




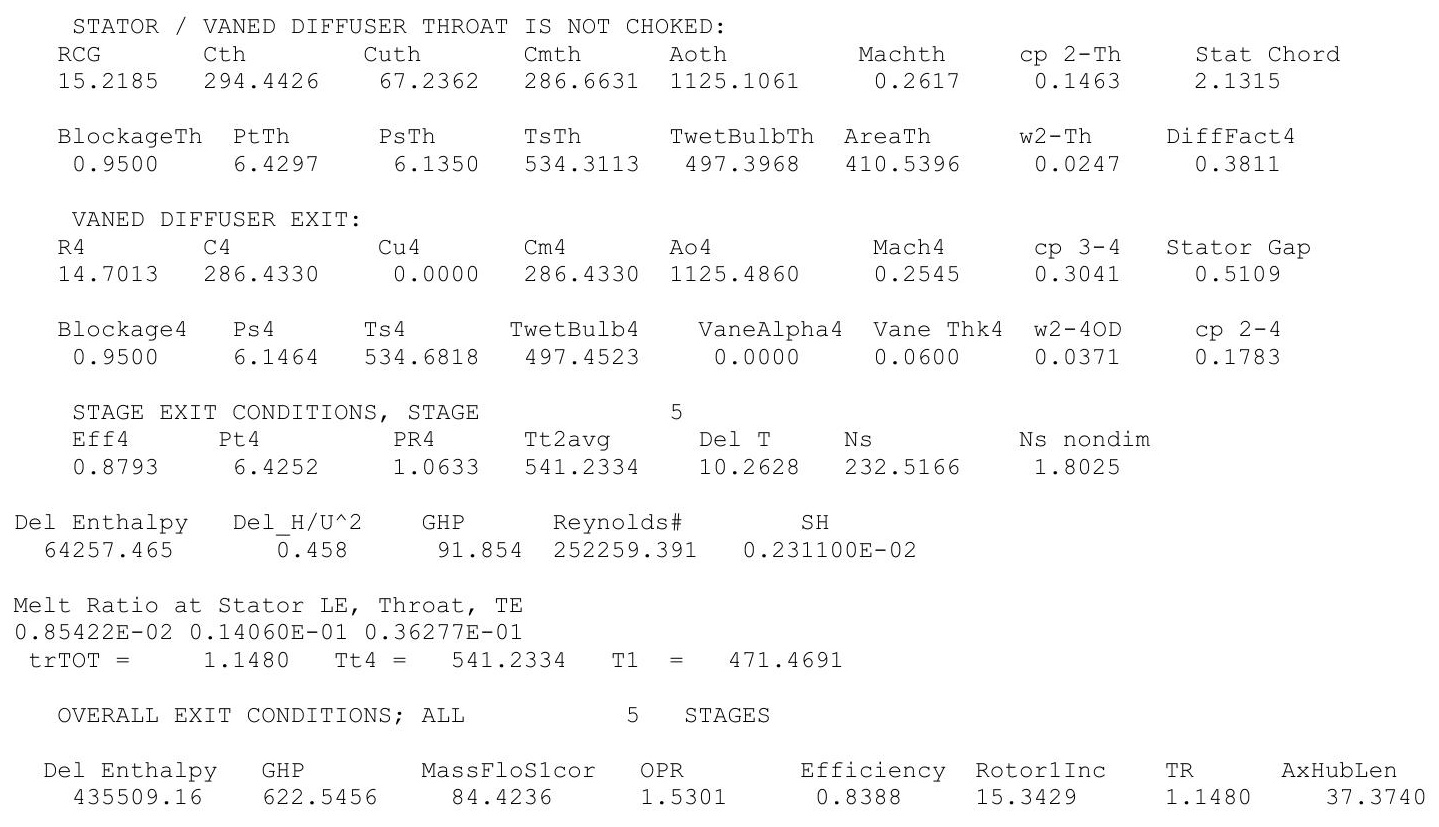




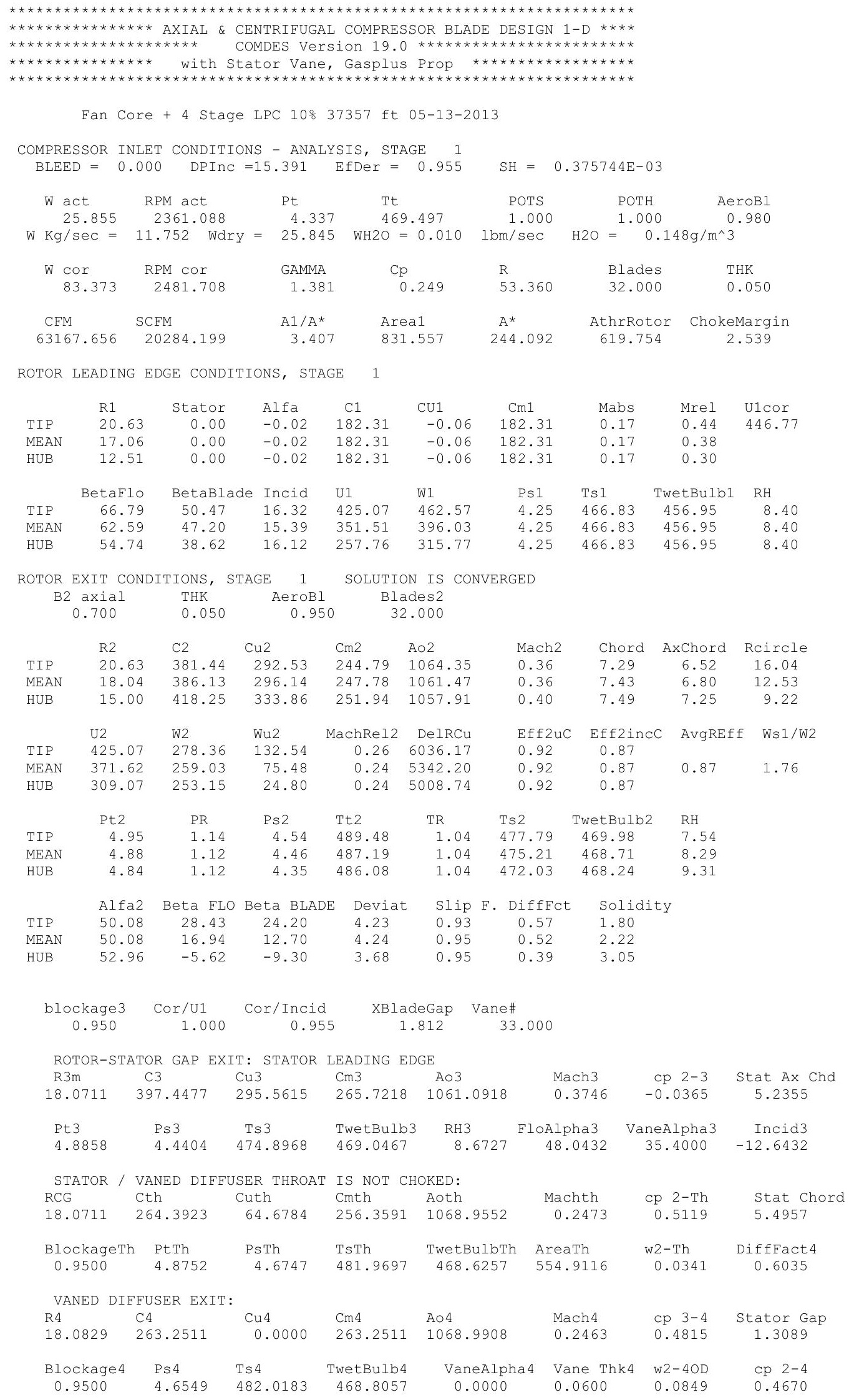




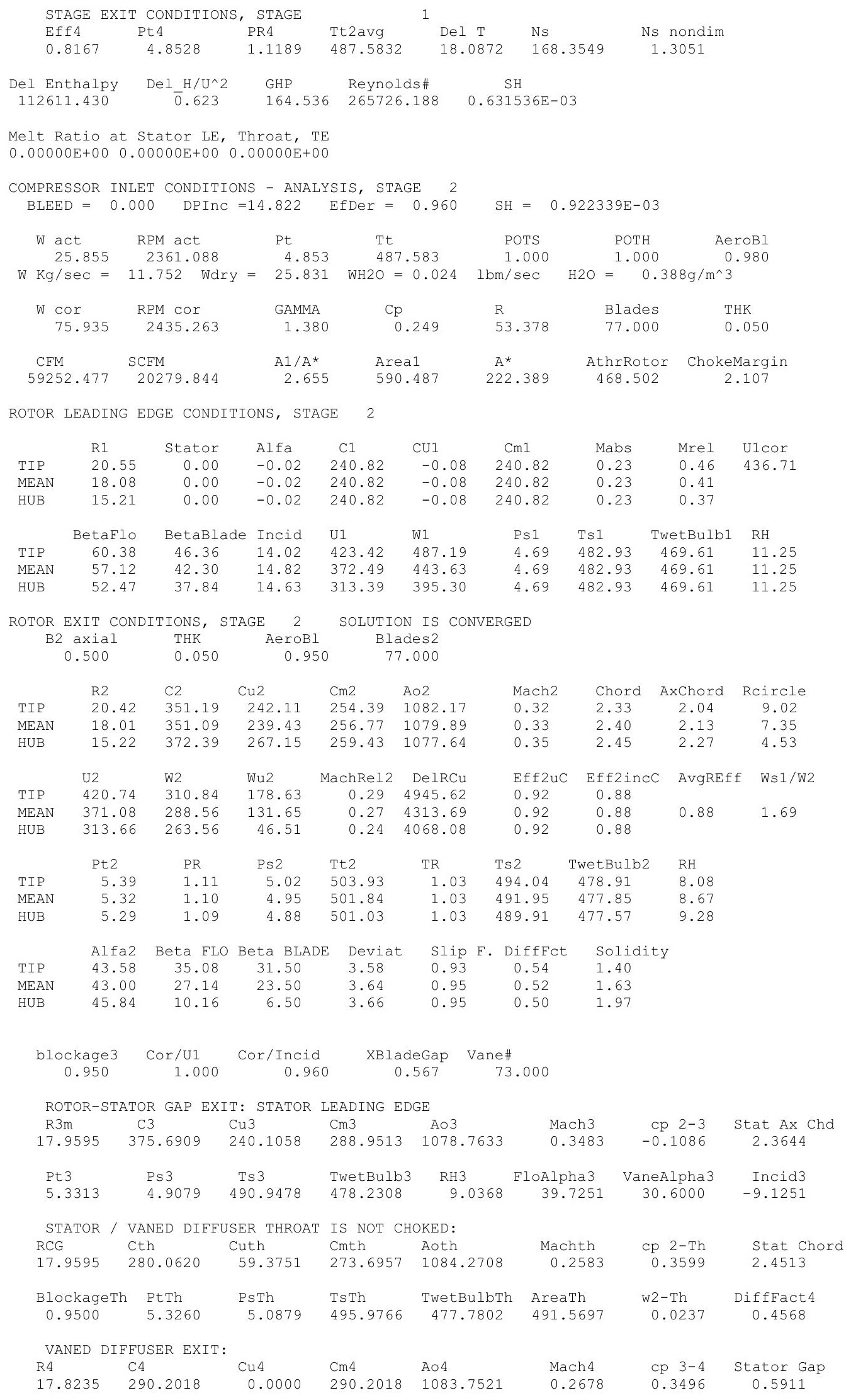




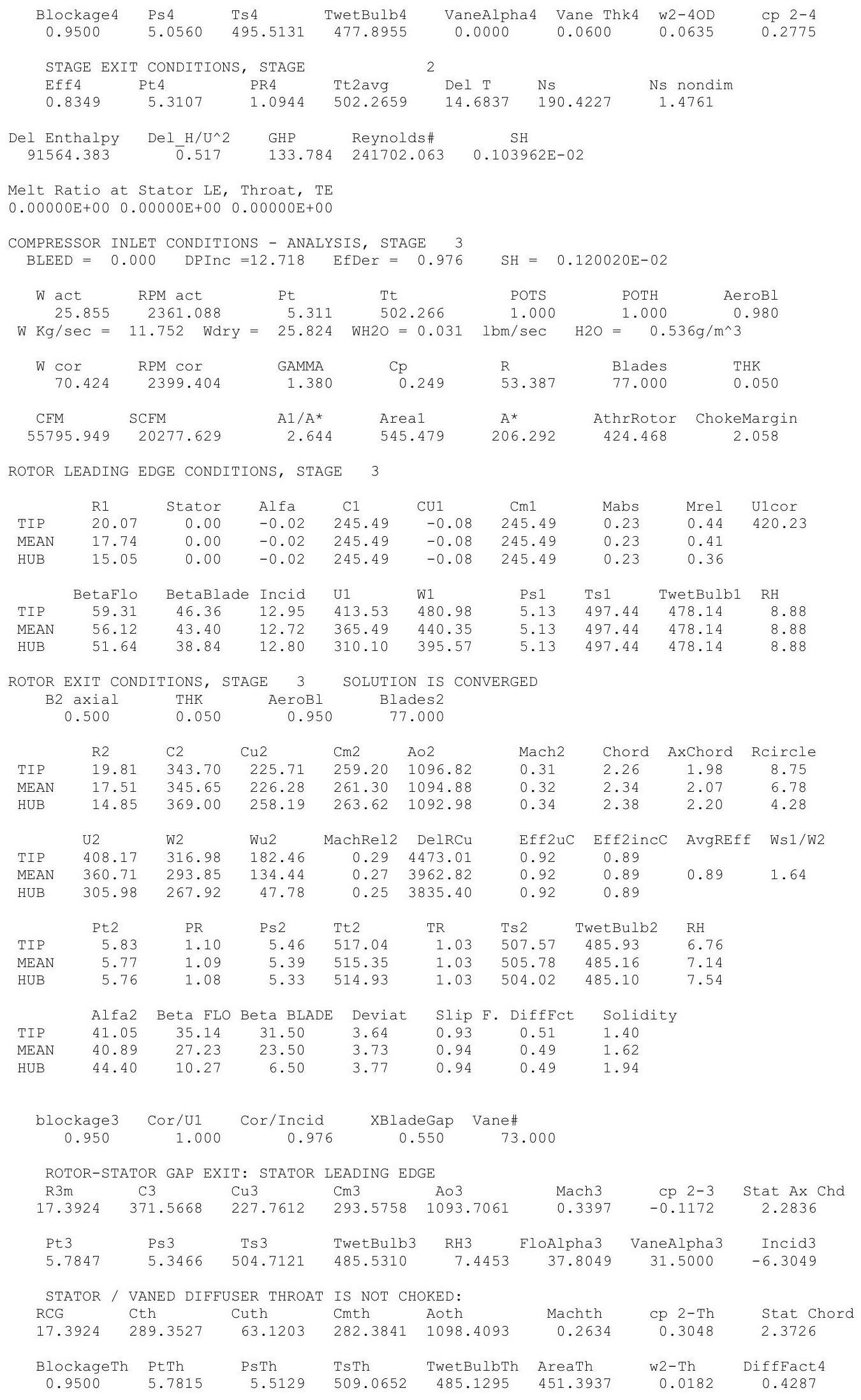




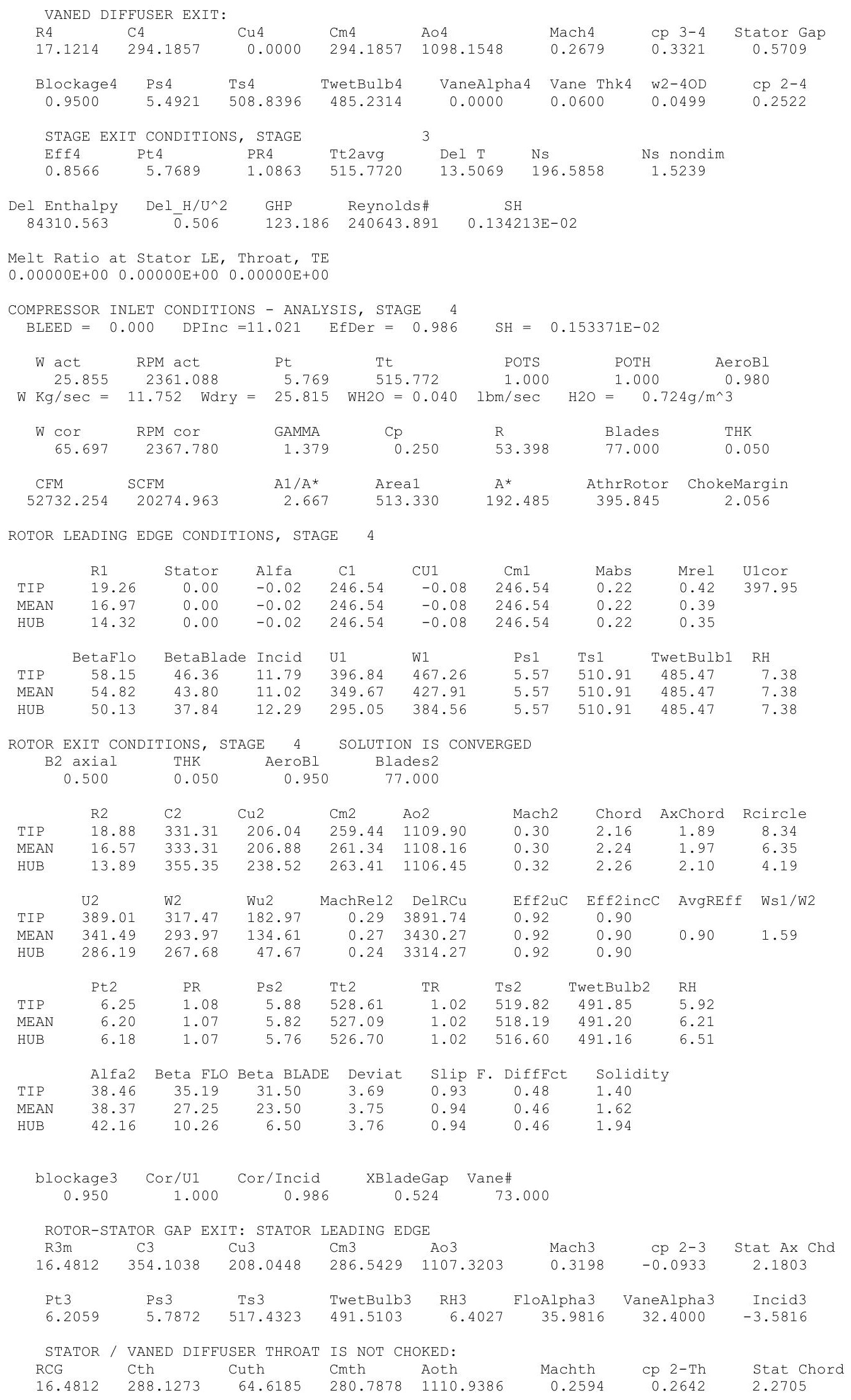




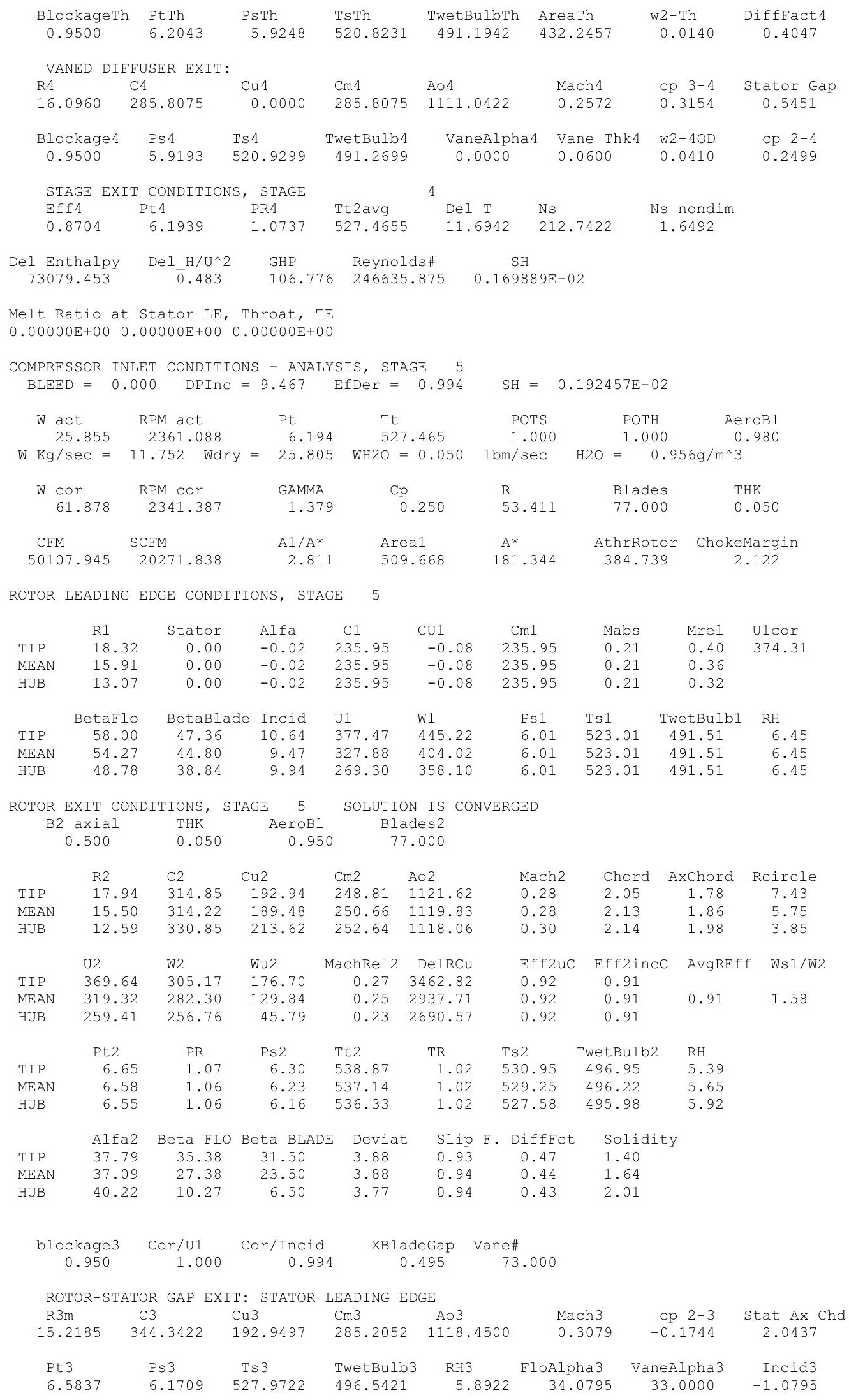




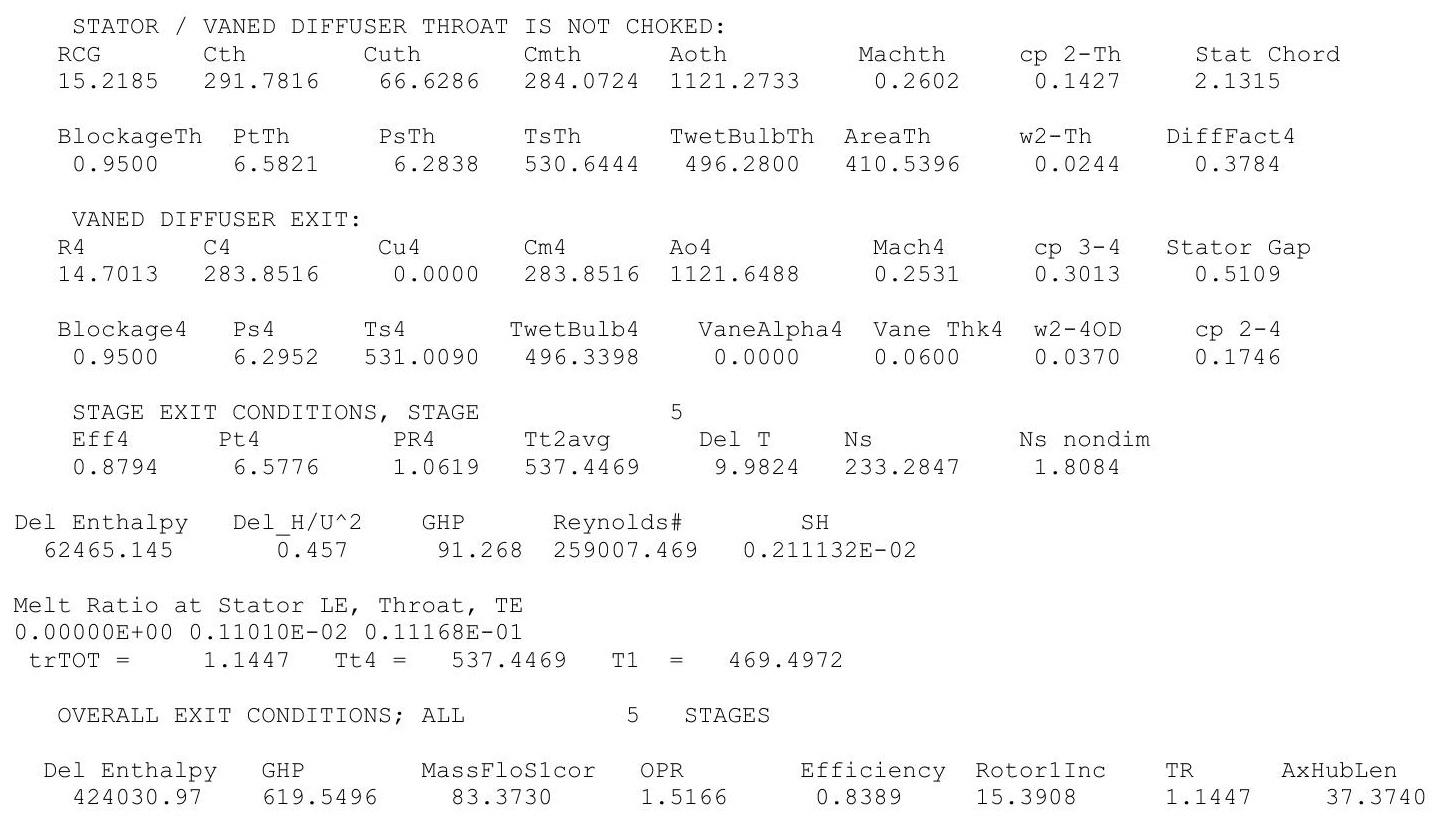




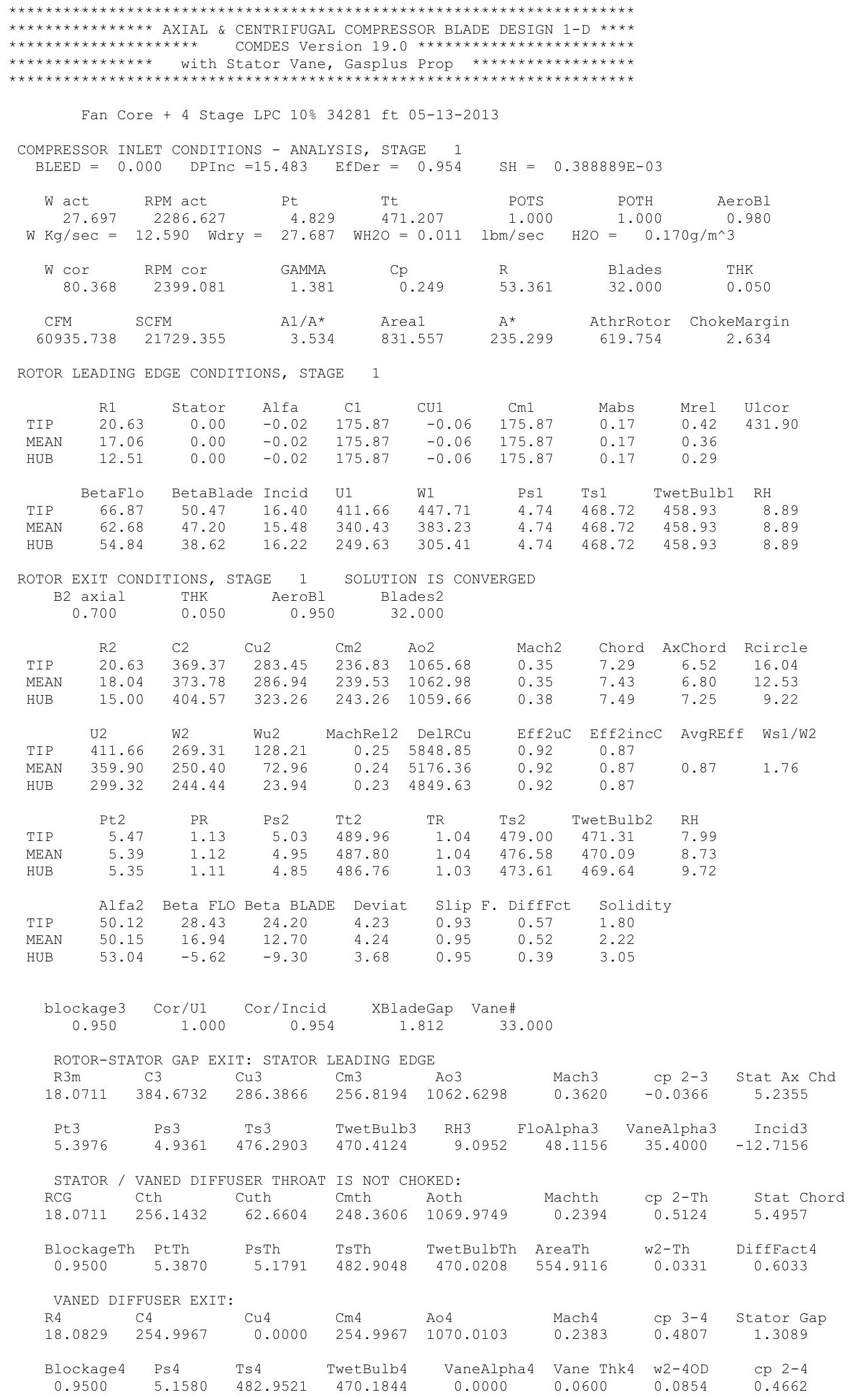




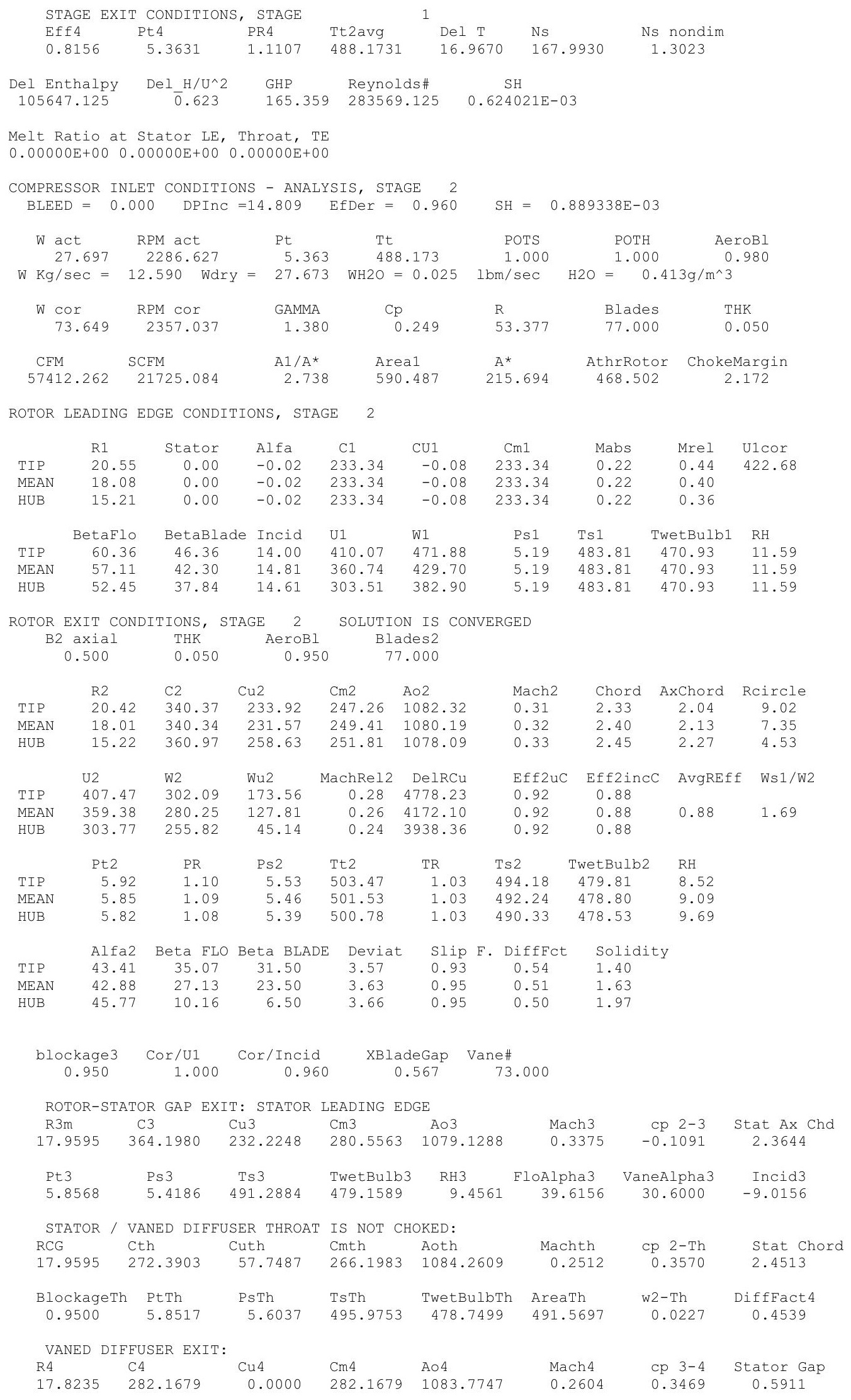




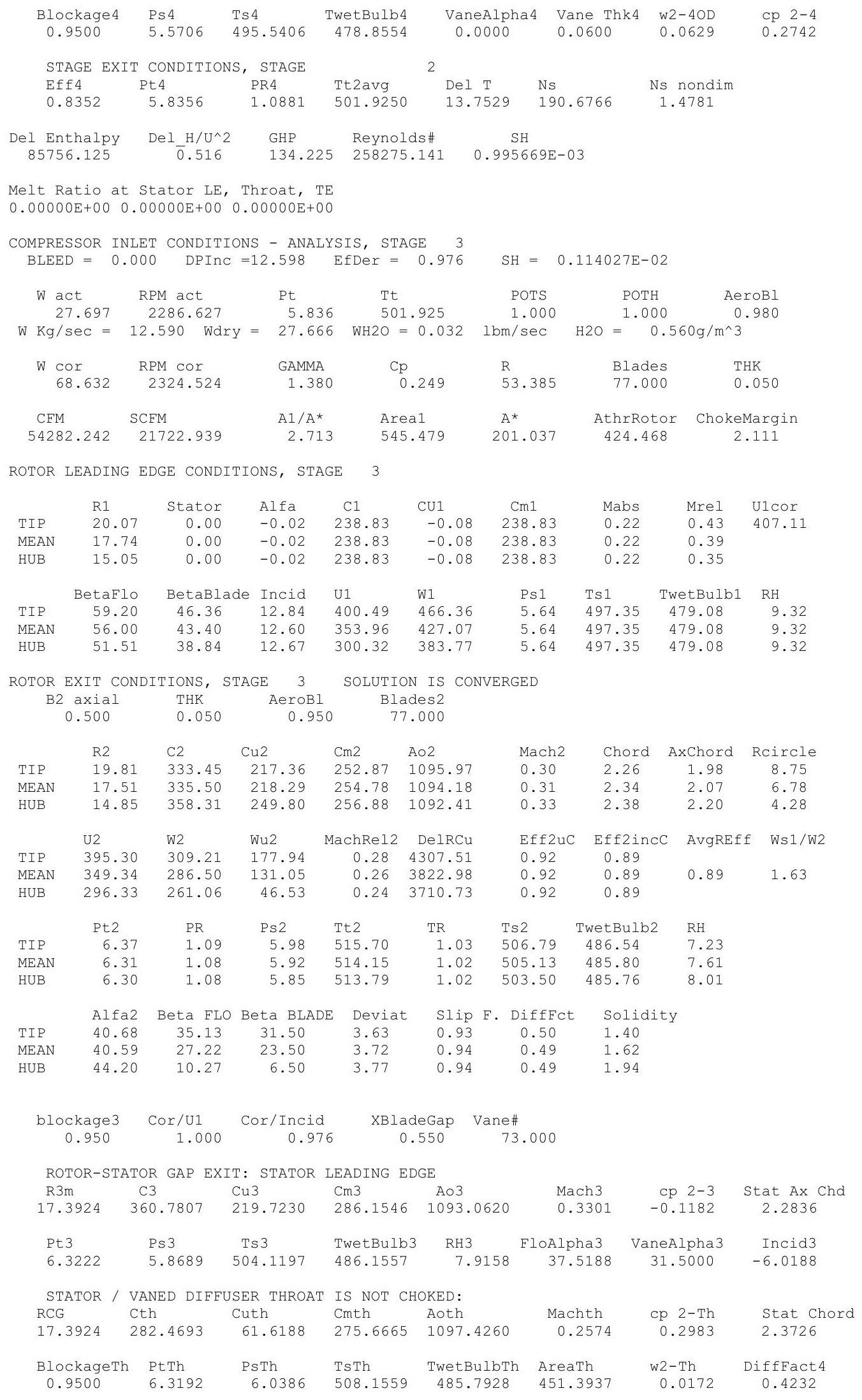




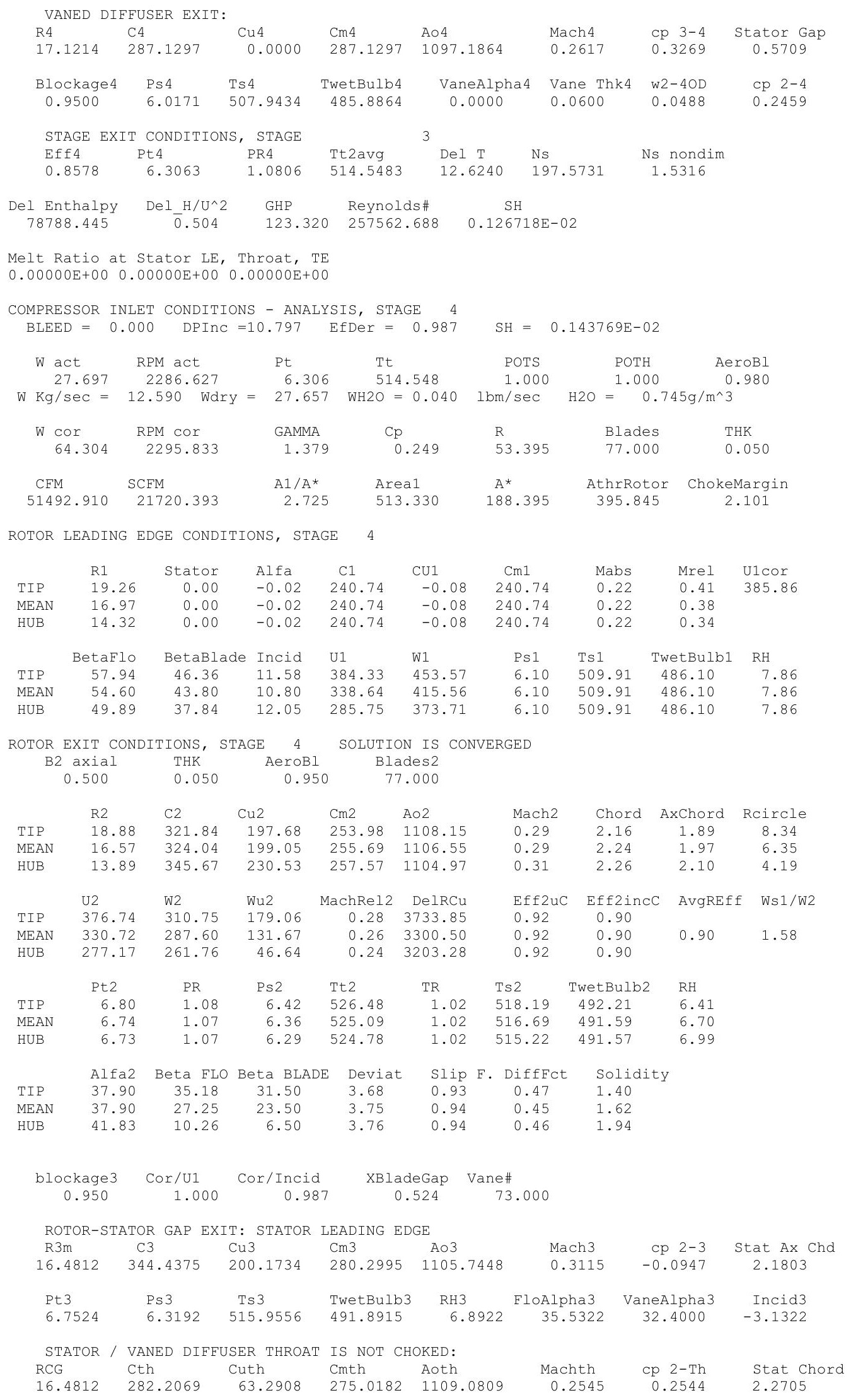




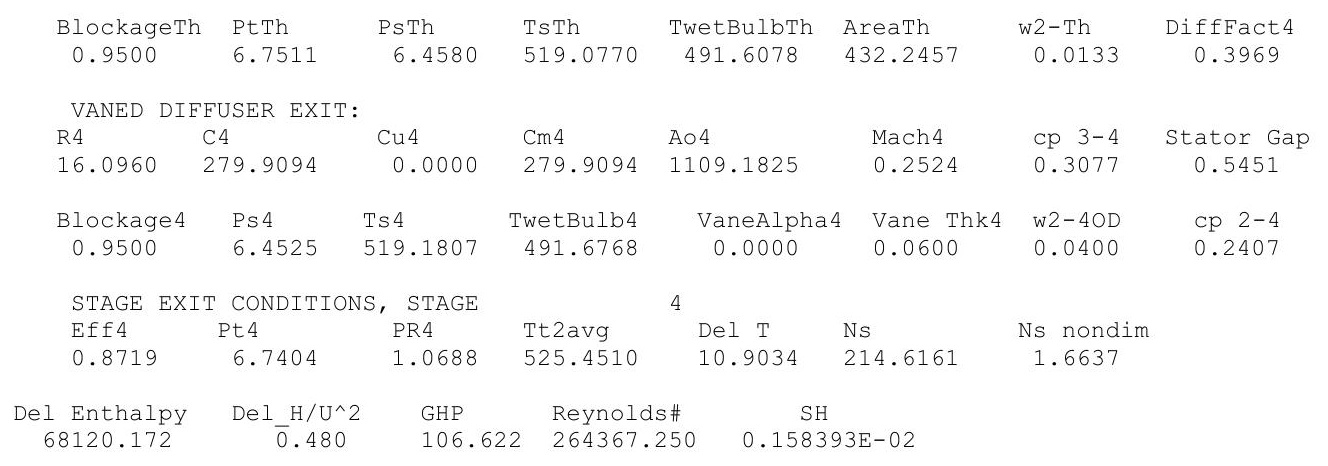

Melt Ratio at Stator LE, Throat, TE

$0.00000 \mathrm{E}+00 \quad 0.00000 \mathrm{E}+00 \quad 0.00000 \mathrm{E}+00$

COMPRESSOR INLET CONDITIONS - ANALYSIS, STAGE 5 BLEED $=0.000 \quad$ DPInC $=9.152 \quad$ EfDer $=0.995$

$\begin{array}{llllll}\text { W act } & \text { RPM act } & \text { Pt } & \text { POTS } & \text { POTH } & \text { AeroBl }\end{array}$

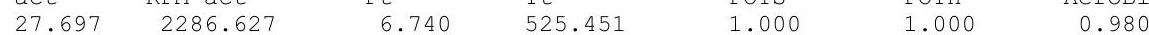

$\mathrm{W} \mathrm{Kg} / \mathrm{sec}=12.590$ Wdry $=27.648 \quad \mathrm{WH} 2 \mathrm{O}=0.049 \mathrm{lbm} / \mathrm{sec} \quad \mathrm{H} 2 \mathrm{O}=0.969 \mathrm{~g} / \mathrm{m}^{\wedge} 3$

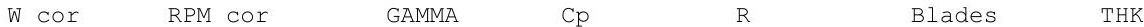

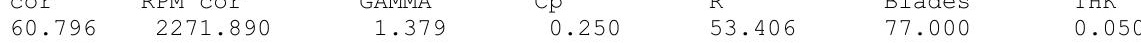

CFM SCFM A1/A* Area1 A* AthrRotor ChokeMargin

$\begin{array}{lllllll}49093.113 & 21717.436 & 2.861 & 509.668 & 178.158 & 384.739 & 2.160\end{array}$

ROTOR LEADING EDGE CONDITIONS, STAGE 5

$\begin{array}{lccccccccc} & \text { R1 } & \text { Stator } & \text { Alfa } & \text { C1 } & \text { CU1 } & \text { Cm1 } & \text { Mabs } & \text { Mrel } & \text { U1cor } \\ \text { TIP } & 18.32 & 0.00 & -0.02 & 231.17 & -0.08 & 231.17 & 0.21 & 0.39 & 363.20 \\ \text { MEAN } & 15.91 & 0.00 & -0.02 & 231.17 & -0.08 & 231.17 & 0.21 & 0.35 & \\ \text { HUB } & 13.07 & 0.00 & -0.02 & 231.17 & -0.08 & 231.17 & 0.21 & 0.31 & \\ & & & & & & & & & \\ & \text { BetaFlo } & \text { BetaBlade } & \text { Incid } & \text { U1 } & \text { W1 } & \text { Ps1 } & \text { Ts1 } & \text { TwetBulb1 } & \text { RH } \\ \text { TIP } & 57.70 & 47.36 & 10.34 & 365.57 & 432.60 & 6.54 & 521.18 & 491.90 & 6.95 \\ \text { MEAN } & 53.95 & 44.80 & 9.15 & 317.54 & 392.84 & 6.54 & 521.18 & 491.90 & 6.95 \\ \text { HUB } & 48.46 & 38.84 & 9.62 & 260.81 & 348.57 & 6.54 & 521.18 & 491.90 & 6.95\end{array}$

ROTOR EXIT CONDITIONS, STAGE 5 SOLUTION IS CONVERGED

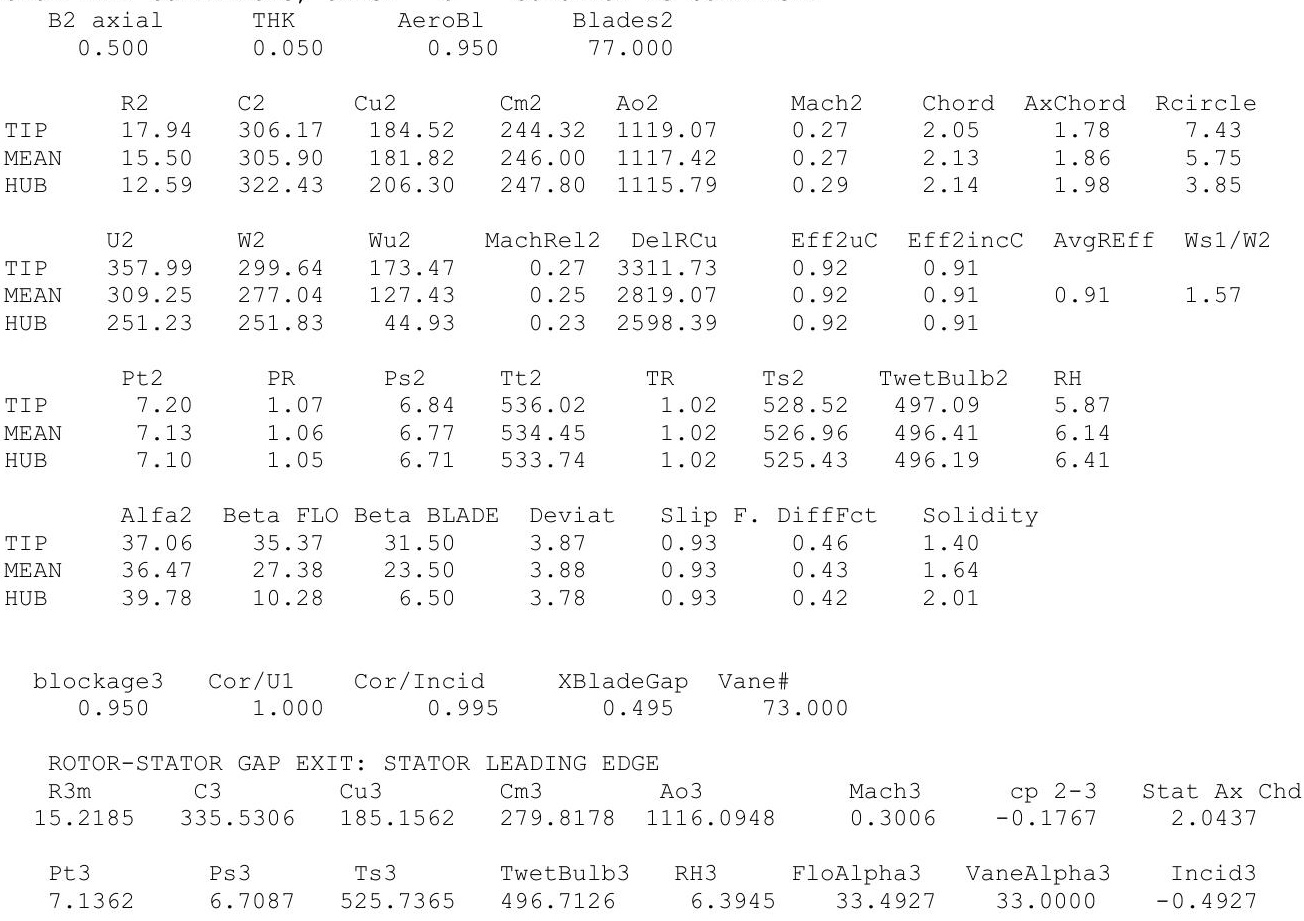




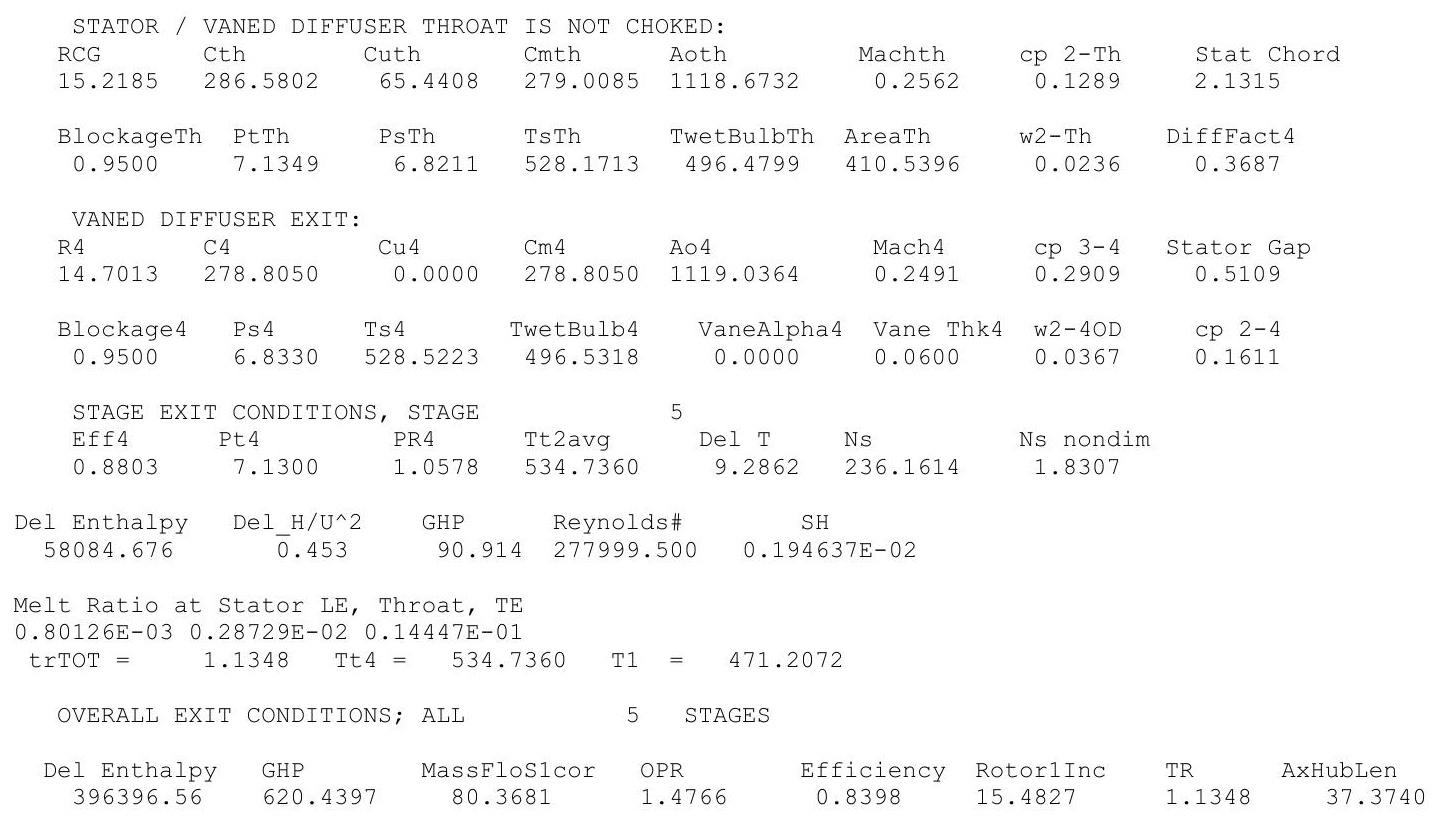




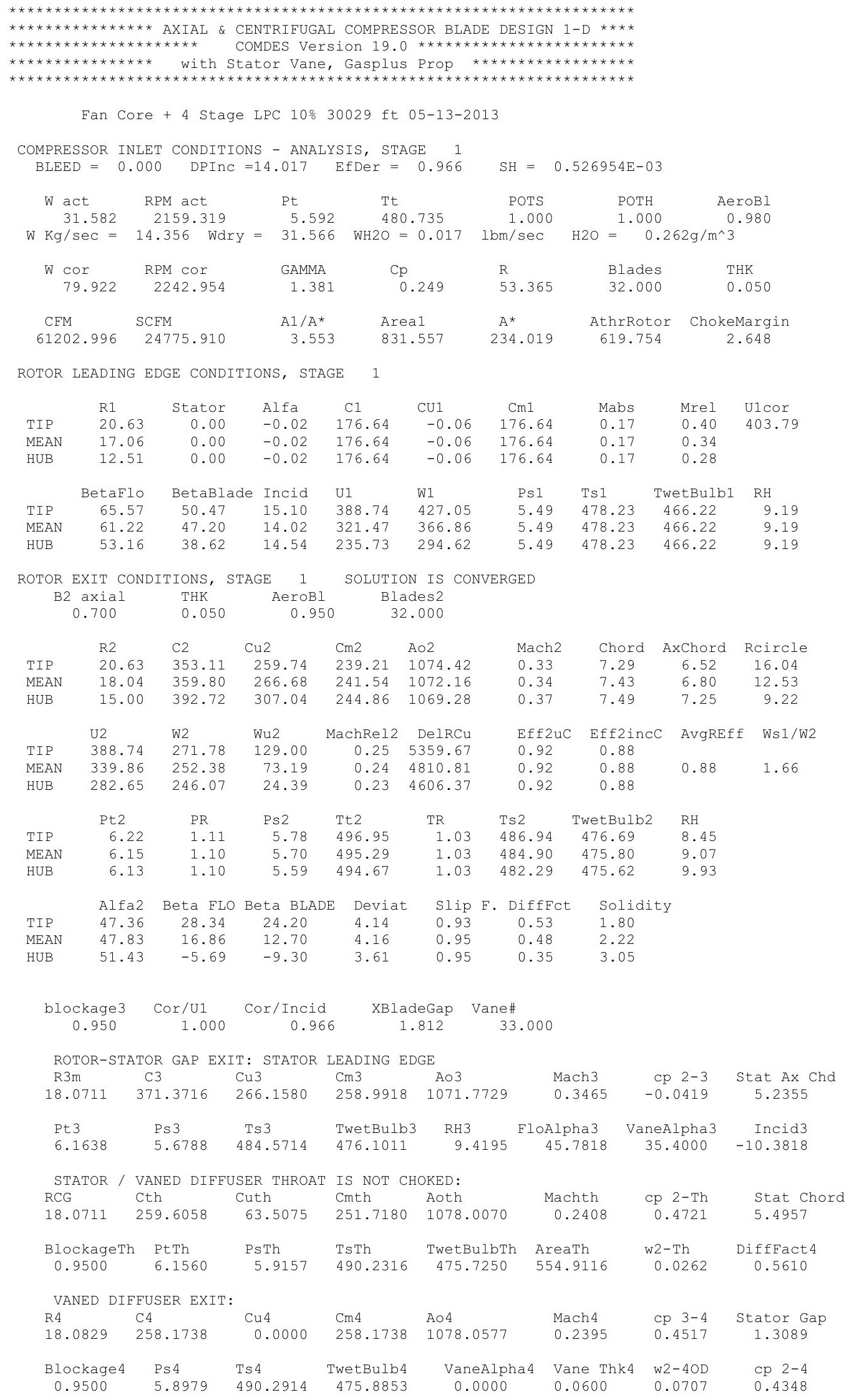




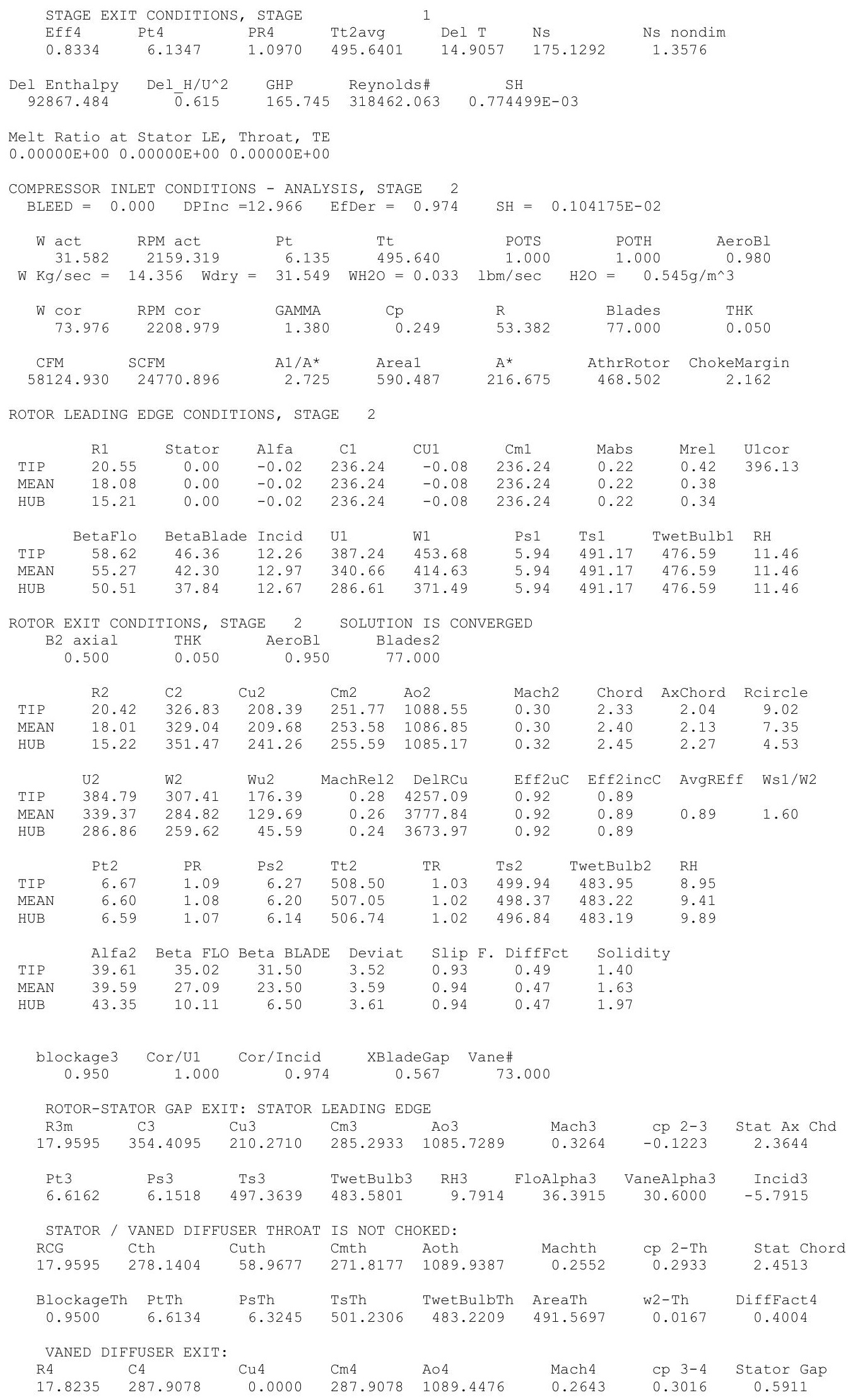




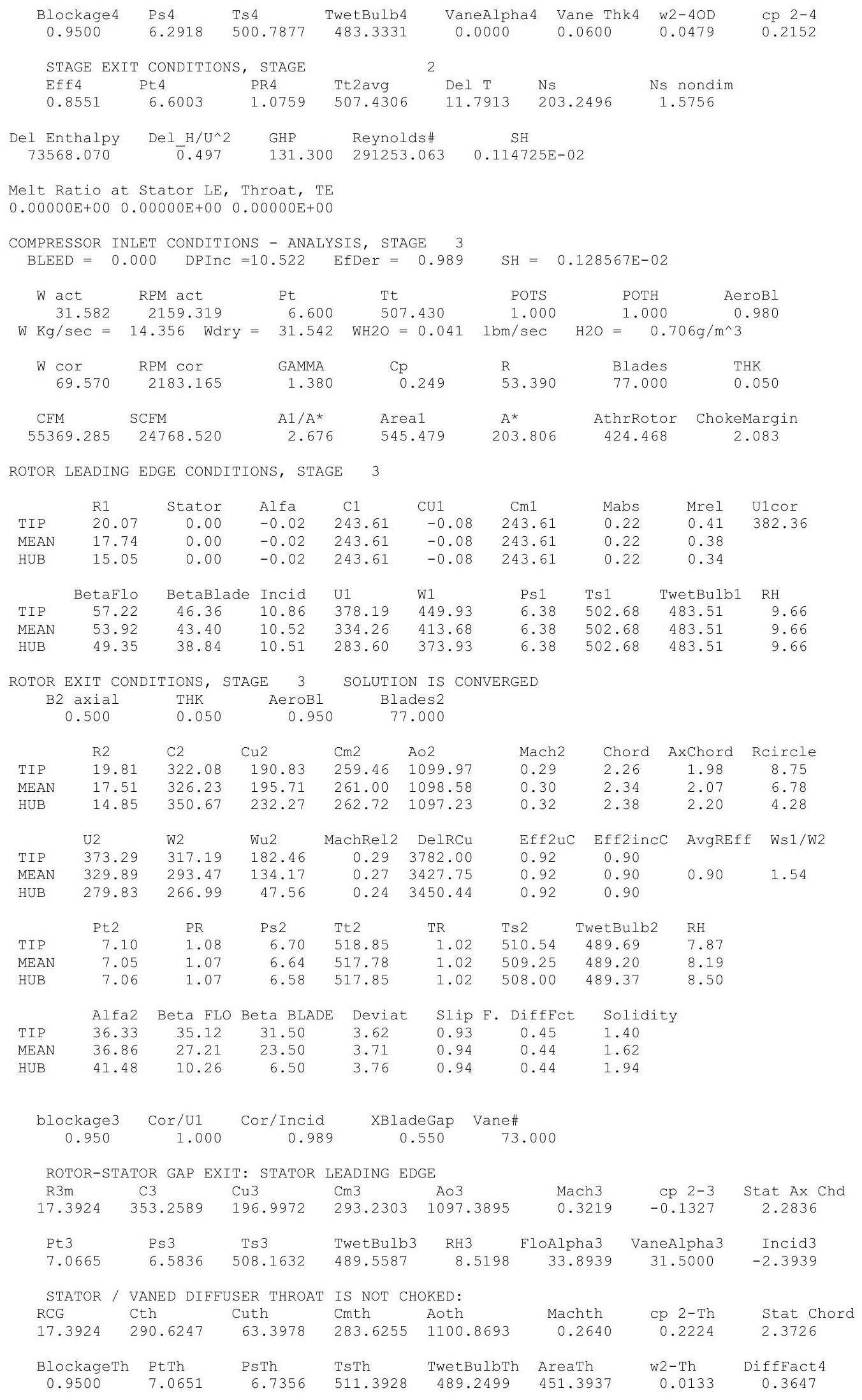




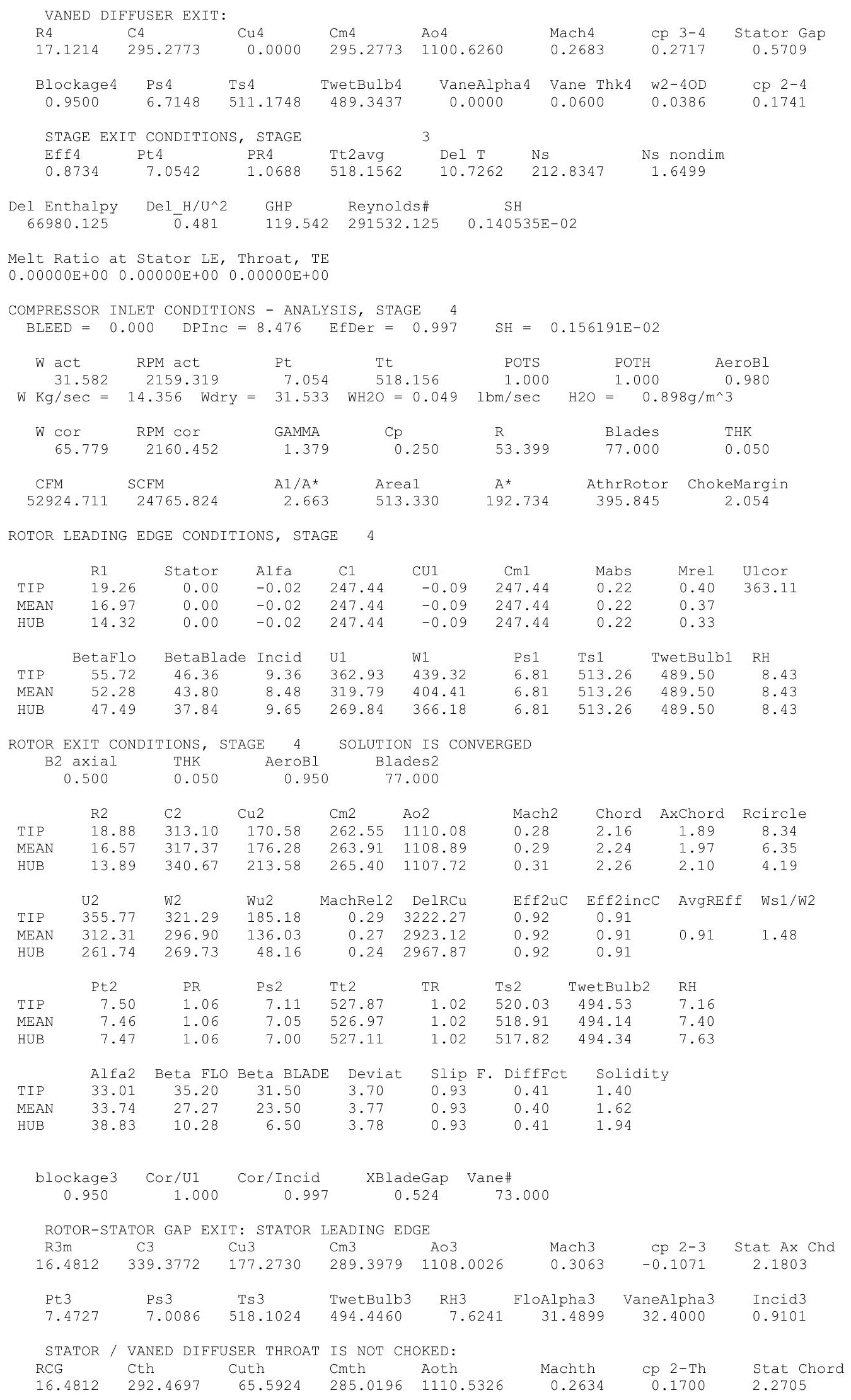




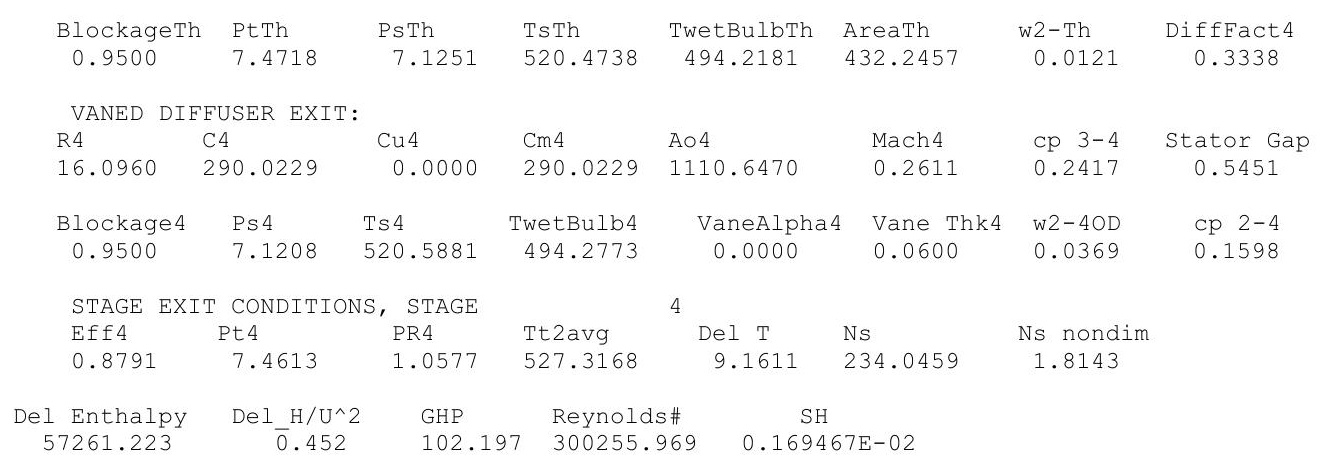

Melt Ratio at Stator LE, Throat, TE

$0.00000 \mathrm{E}+000.00000 \mathrm{E}+000.00000 \mathrm{E}+00$

COMPRESSOR INLET CONDITIONS - ANALYSIS, STAGE 5 BLEED $=0.000 \quad$ DPInC $=6.611 \quad$ EfDer $=1.000$

$\begin{array}{llllll}W & \text { Ret } & \text { RPM act } & \text { POTS } & \text { POTH } & \text { AeroBI }\end{array}$

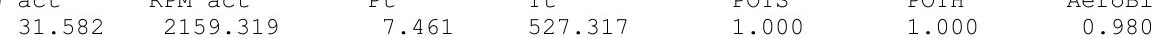

$\mathrm{W} \mathrm{Kg} / \mathrm{sec}=14.356$ Wdry $=31.523 \mathrm{WH} 2 \mathrm{O}=0.059 \mathrm{lbm} / \mathrm{sec} \quad \mathrm{H} 2 \mathrm{O}=1.120 \mathrm{~g} / \mathrm{m}^{\wedge} 3$

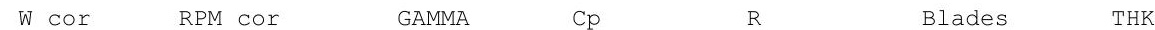

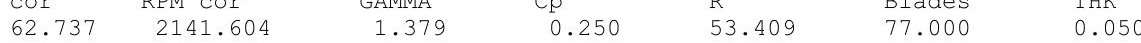

$\begin{array}{ccccc}\text { CFM } & \text { SCFM } & \text { A1/A* } & \text { Area1 } & \text { A* AthrRotor ChokeMargin }\end{array}$

$\begin{array}{lllll}2.772 & 509.668 & 183.859 & 384.739 & 2.093\end{array}$

ROTOR LEADING EDGE CONDITIONS, STAGE 5

$\begin{array}{lccccccccc} & \text { R1 } & \text { Stator } & \text { Alfa } & \text { C1 } & \text { CU1 } & \text { Cm1 } & \text { Mabs } & \text { Mrel } & \text { U1cor } \\ \text { TIP } & 18.32 & 0.00 & -0.02 & 239.34 & -0.08 & 239.34 & 0.22 & 0.38 & 342.37 \\ \text { MEAN } & 15.91 & 0.00 & -0.02 & 239.34 & -0.08 & 239.34 & 0.22 & 0.34 & \\ \text { HUB } & 13.07 & 0.00 & -0.02 & 239.34 & -0.08 & 239.34 & 0.22 & 0.31 & \\ & & & & & & & & & \\ & & & & & & & & & \\ \text { TIP } & 55.27 & 47.36 & 7.91 & 345.21 & 420.14 & 7.23 & 522.74 & 494.43 & 7.62 \\ \text { MEAN } & 51.41 & 44.80 & 6.61 & 299.86 & 383.73 & 7.23 & 522.74 & 494.43 & 7.62 \\ \text { HUB } & 45.83 & 38.84 & 6.99 & 246.29 & 343.49 & 7.23 & 522.74 & 494.43 & 7.62\end{array}$

ROTOR EXIT CONDITIONS, STAGE 5 SOLUTION IS CONVERGED

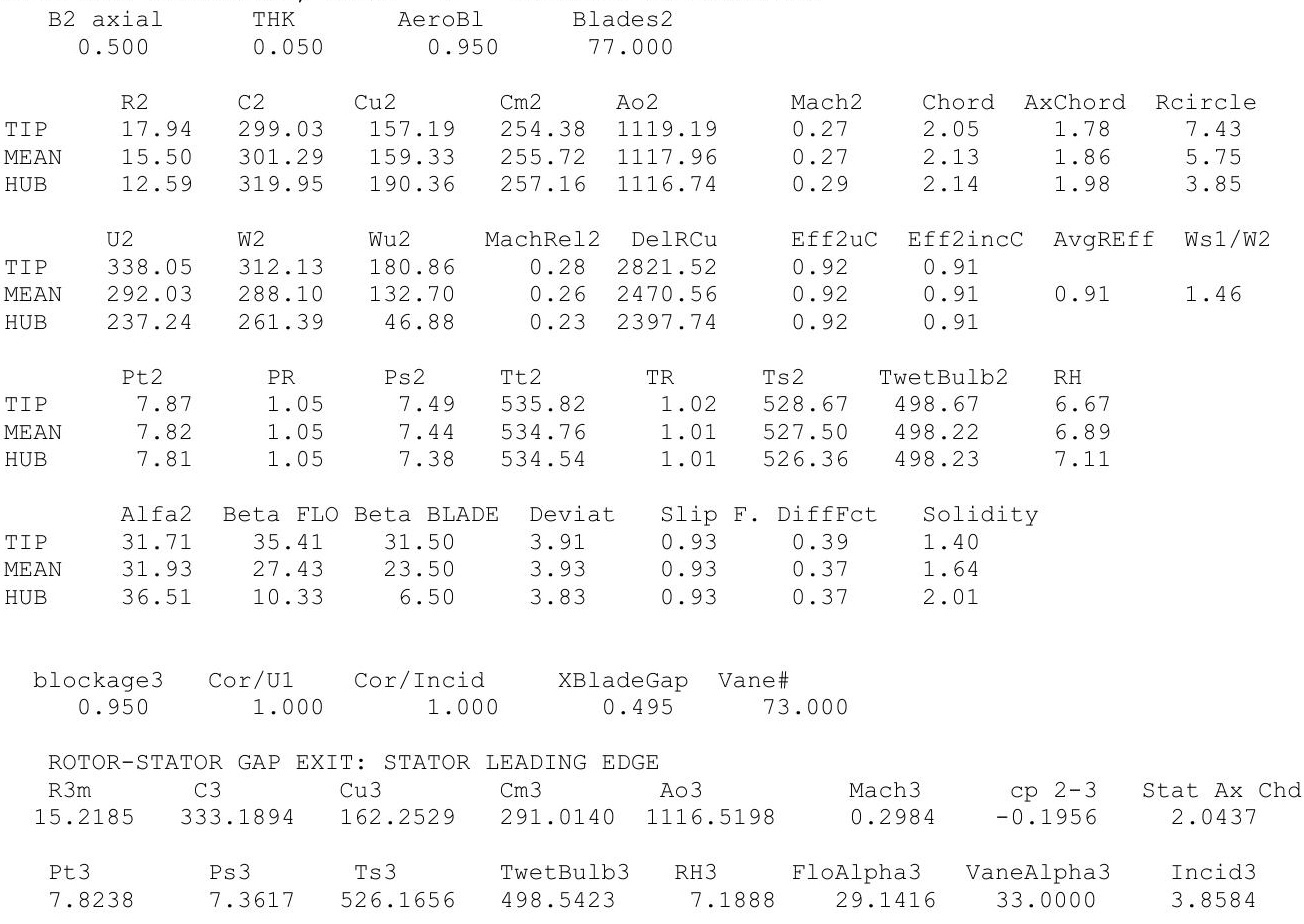




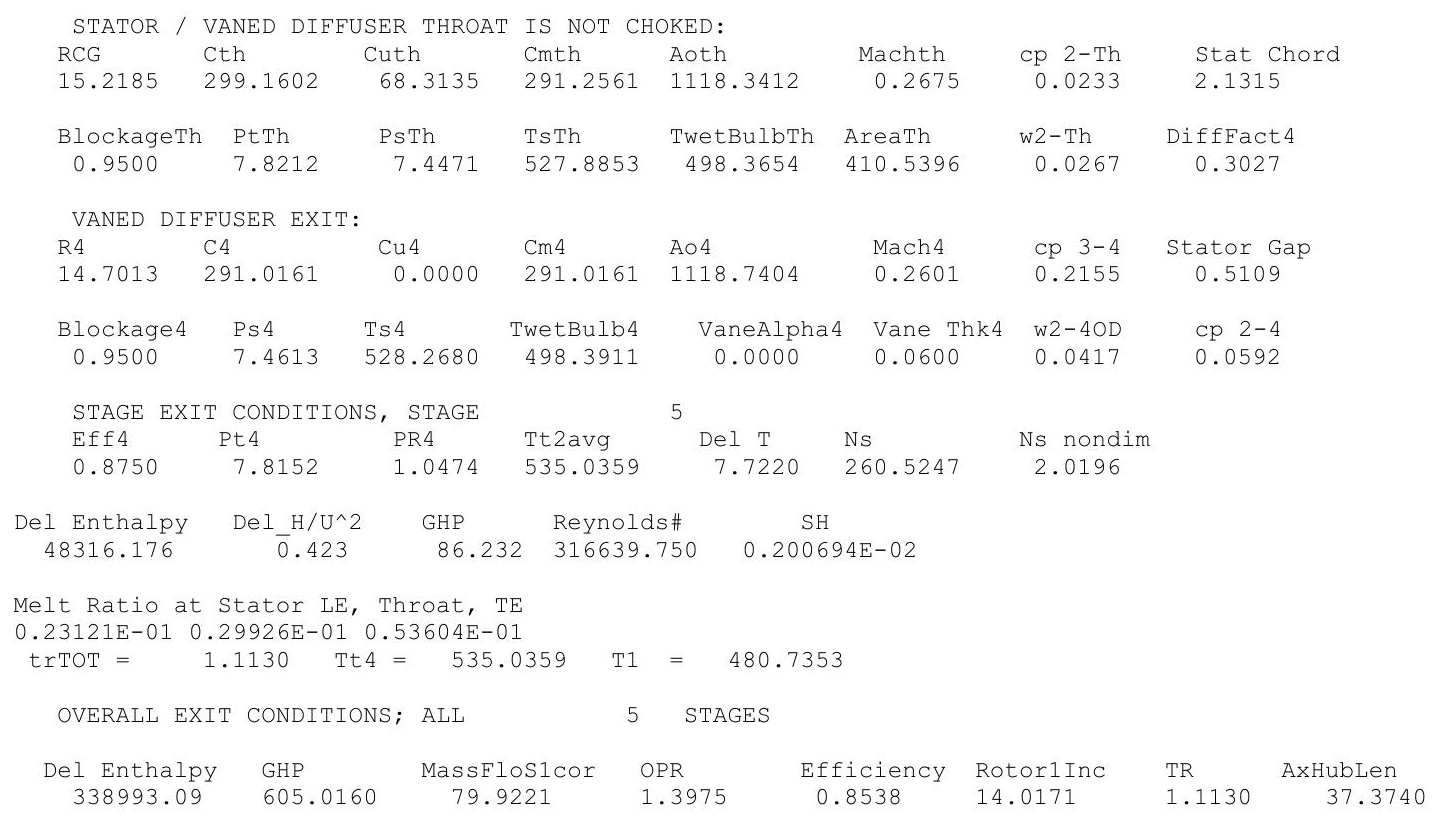




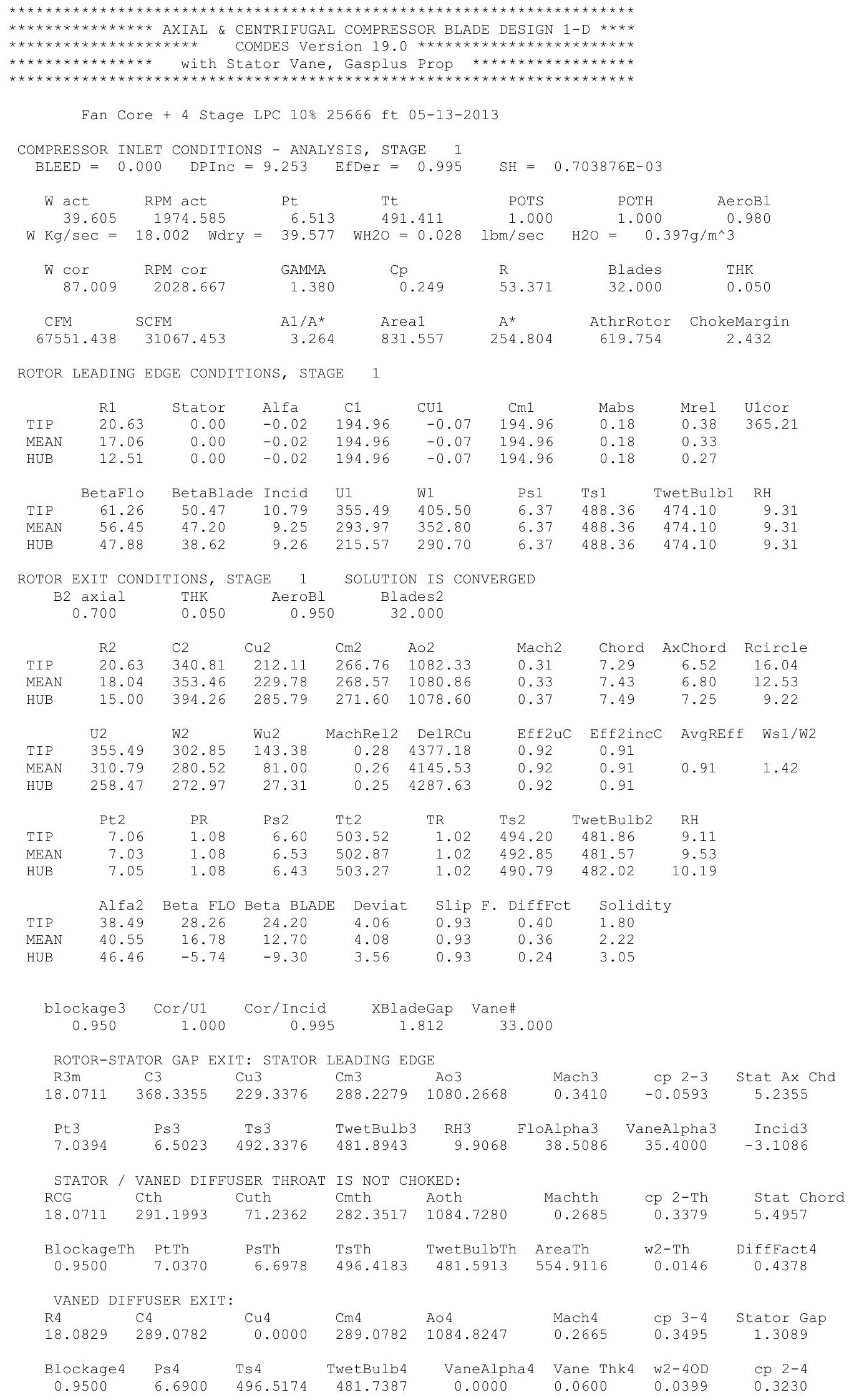




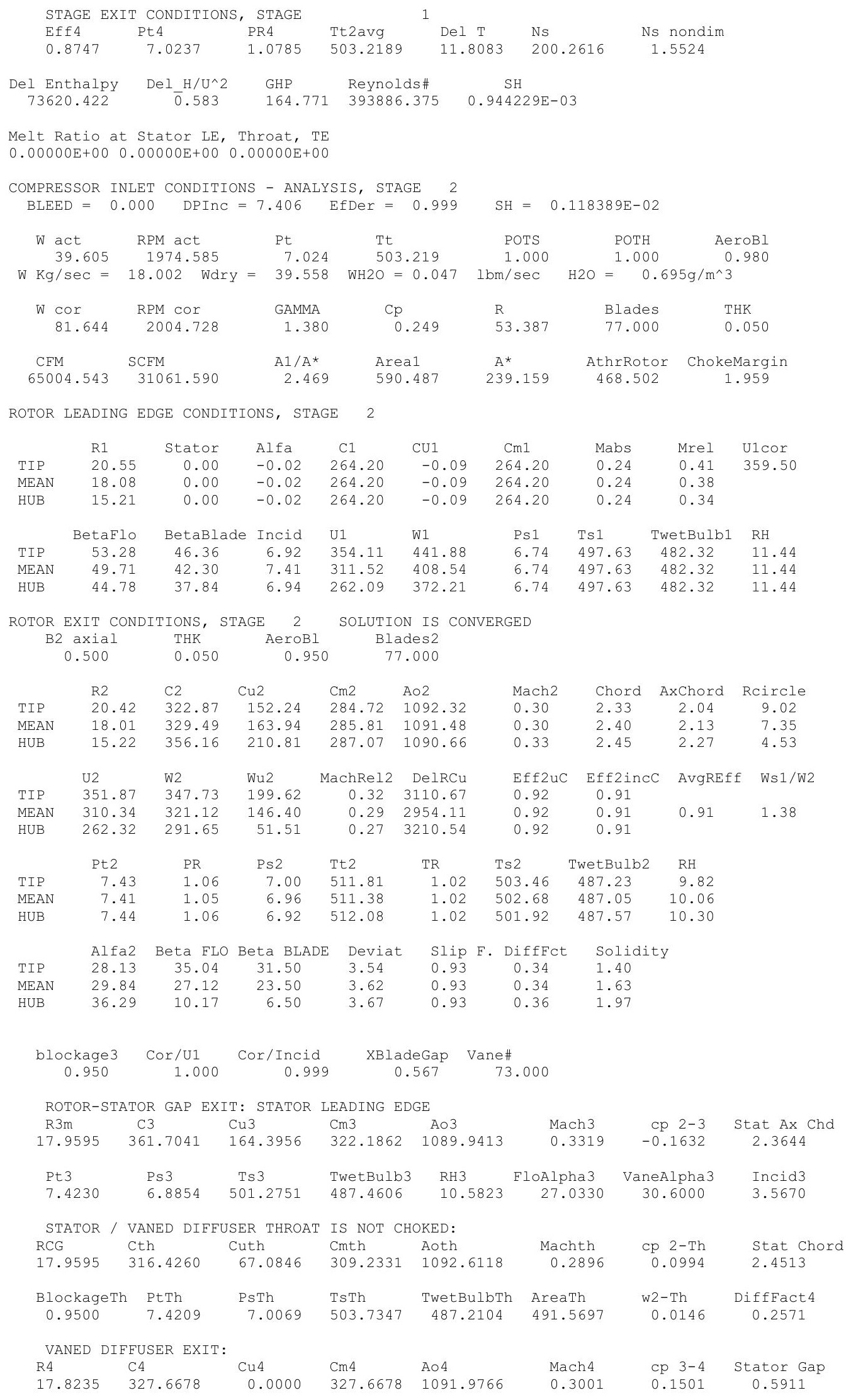




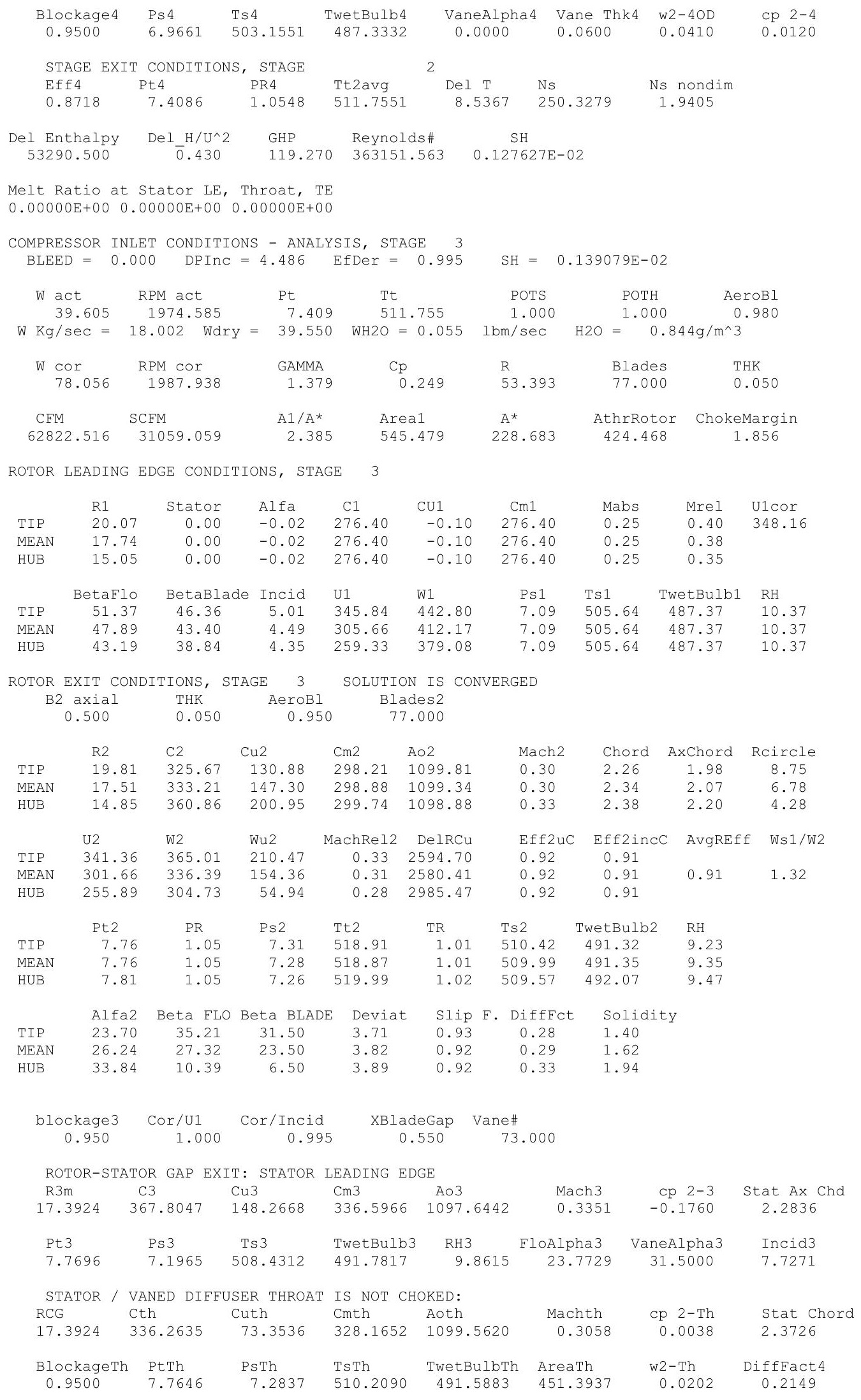




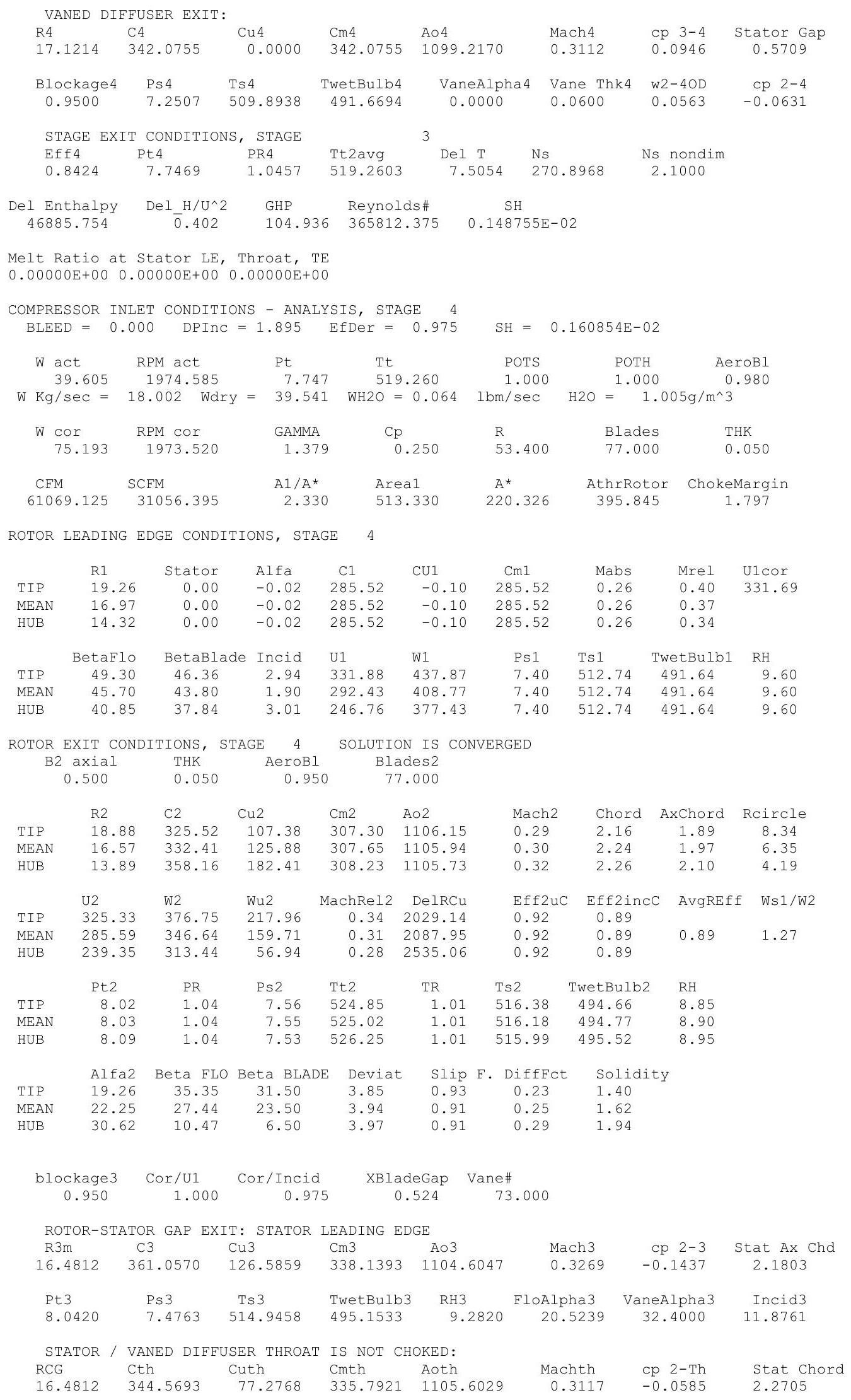




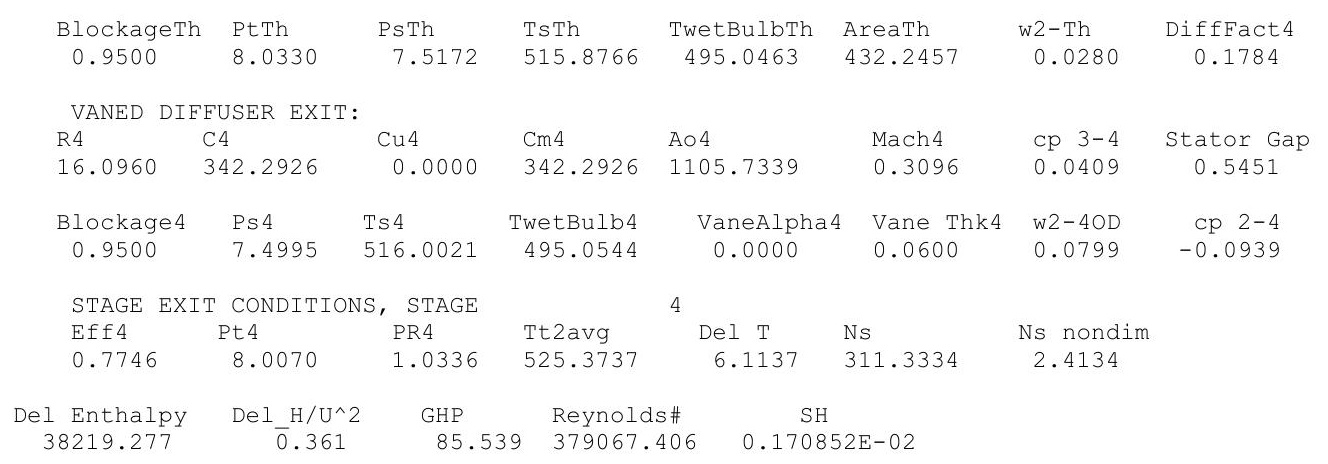

Melt Ratio at Stator LE, Throat, TE

$0.00000 \mathrm{E}+00 \quad 0.00000 \mathrm{E}+00 \quad 0.00000 \mathrm{E}+00$

COMPRESSOR INLET CONDITIONS - ANALYSIS, STAGE 5 BLEED $=0.000 \quad$ DPInC $=-0.506 \quad$ EfDer $=0.942$

$\begin{array}{llllll}W & \text { Ret } & \text { RPM act } & \text { POTS } & \text { POTH } & \text { AeroBI }\end{array}$

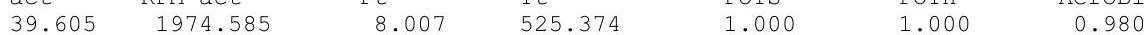

$\mathrm{W} \mathrm{Kg} / \mathrm{sec}=18.002$ Wdry $=39.532 \mathrm{WH} 2 \mathrm{O}=0.073 \mathrm{lbm} / \mathrm{sec} \quad \mathrm{H} 2 \mathrm{O}=1.175 \mathrm{~g} / \mathrm{m}^{\wedge} 3$

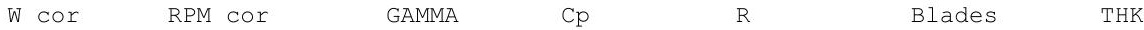

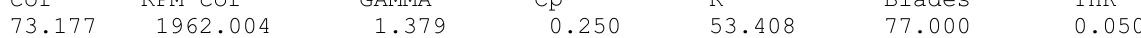

CFM SCFM A1/A* Area1 A* AthrRotor ChokeMargin

$\begin{array}{lllllll}59705.633 & 31053.580 & 2.377 & 509.668 & 214.451 & 384.739 & 1.794\end{array}$

ROTOR LEADING EDGE CONDITIONS, STAGE 5

$\begin{array}{lrrrrrrrrr} & \text { R1 } & \text { Stator } & \text { Alfa } & \text { C1 } & \text { CU1 } & \text { Cm1 } & \text { Mabs } & \text { Mrel } & \text { U1cor } \\ \text { TIP } & 18.32 & 0.00 & -0.02 & 281.15 & -0.10 & 281.15 & 0.25 & 0.38 & 313.66 \\ \text { MEAN } & 15.91 & 0.00 & -0.02 & 281.15 & -0.10 & 281.15 & 0.25 & 0.35 & \\ \text { HUB } & 13.07 & 0.00 & -0.02 & 281.15 & -0.10 & 281.15 & 0.25 & 0.32 & \\ & & & & & & & & & \\ & & & & & & & & & \\ \text { TIP } & 48.32 & 47.36 & 0.96 & 315.68 & 422.80 & 7.66 & 519.05 & 494.99 & 9.04 \\ \text { MEAN } & 44.29 & 44.80 & -0.51 & 274.20 & 392.79 & 7.66 & 519.05 & 494.99 & 9.04 \\ \text { HUB } & 38.71 & 38.84 & -0.13 & 225.22 & 360.29 & 7.66 & 519.05 & 494.99 & 9.04\end{array}$

ROTOR EXIT CONDITIONS, STAGE 5 SOLUTION IS CONVERGED

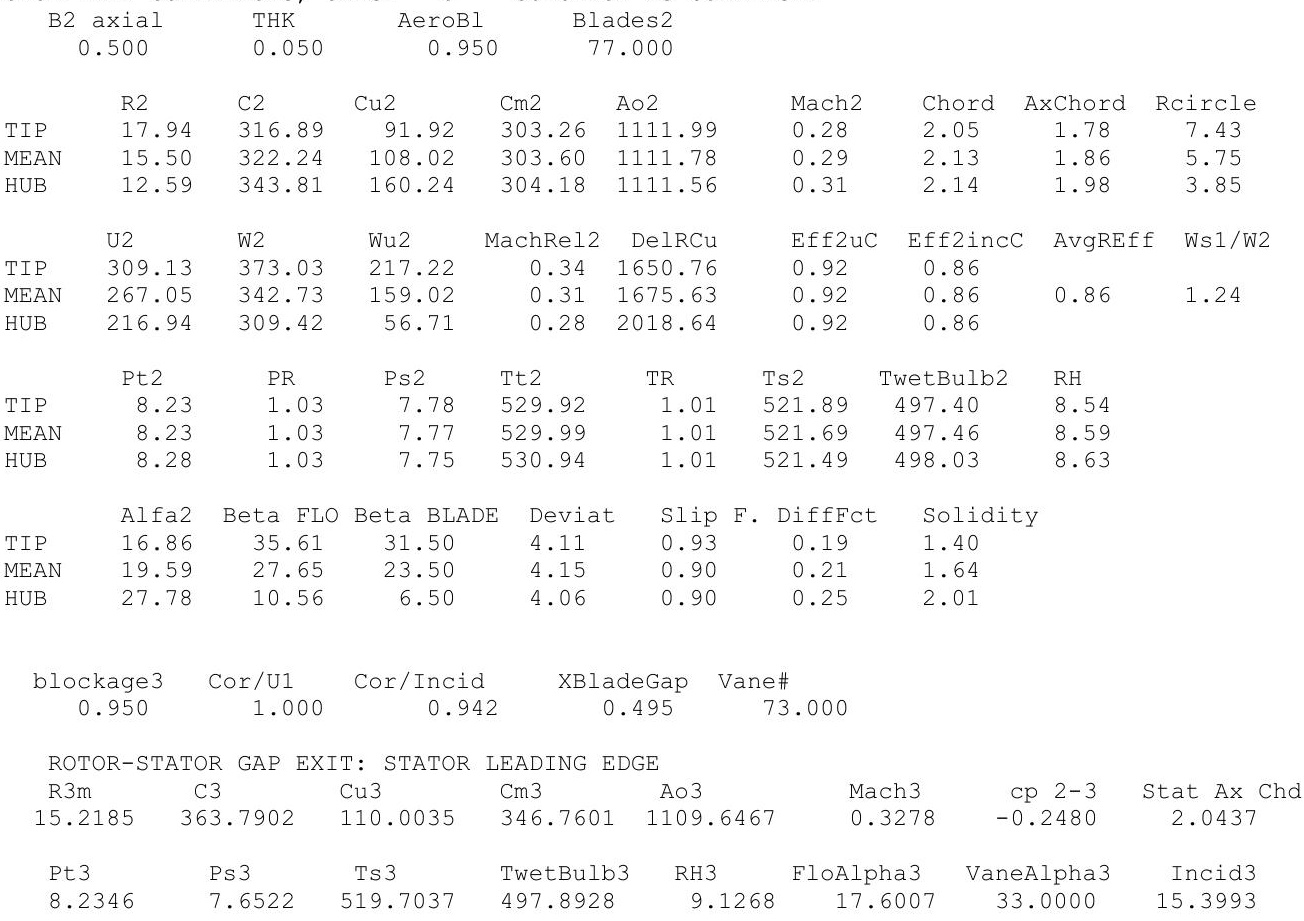




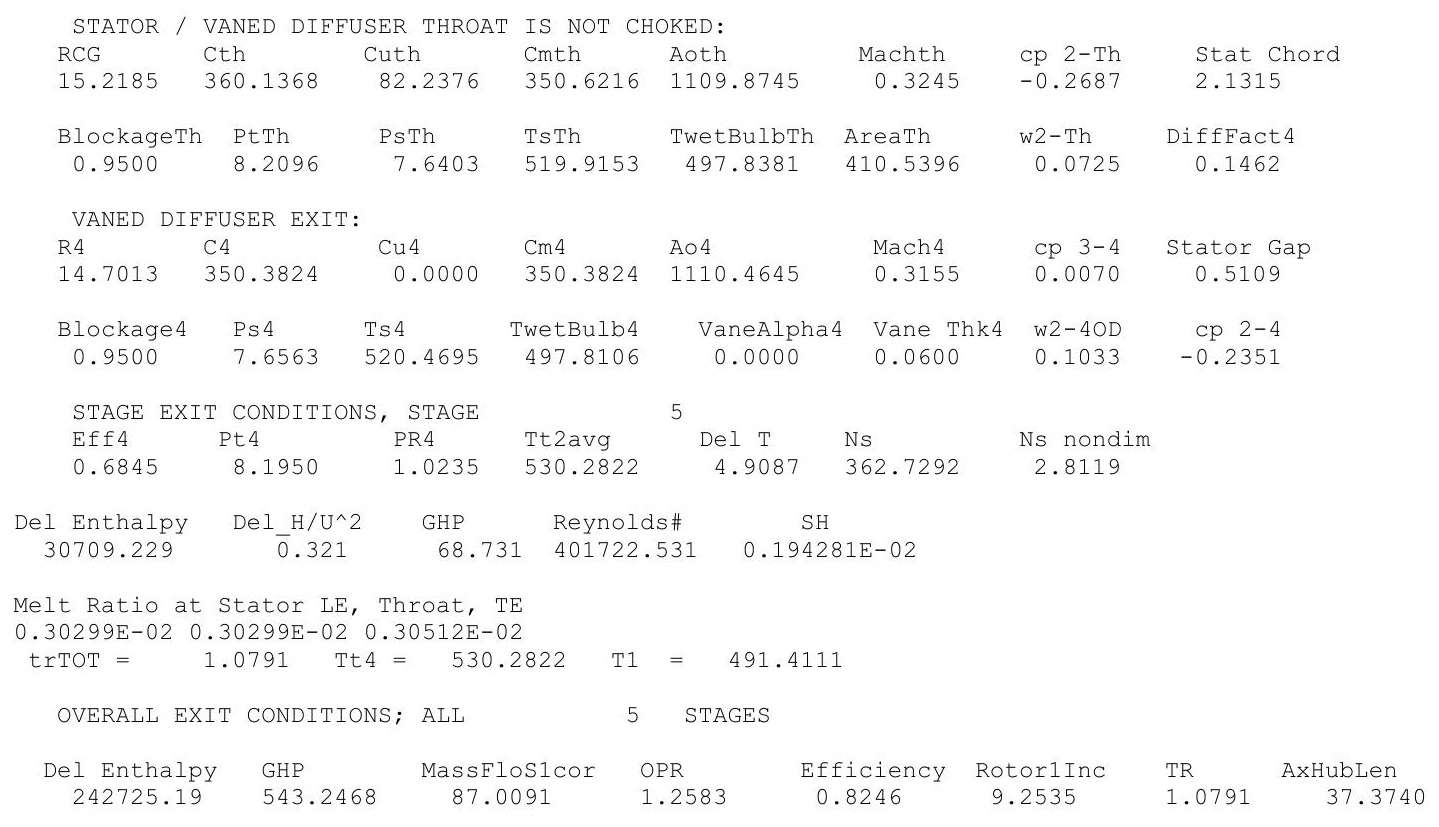




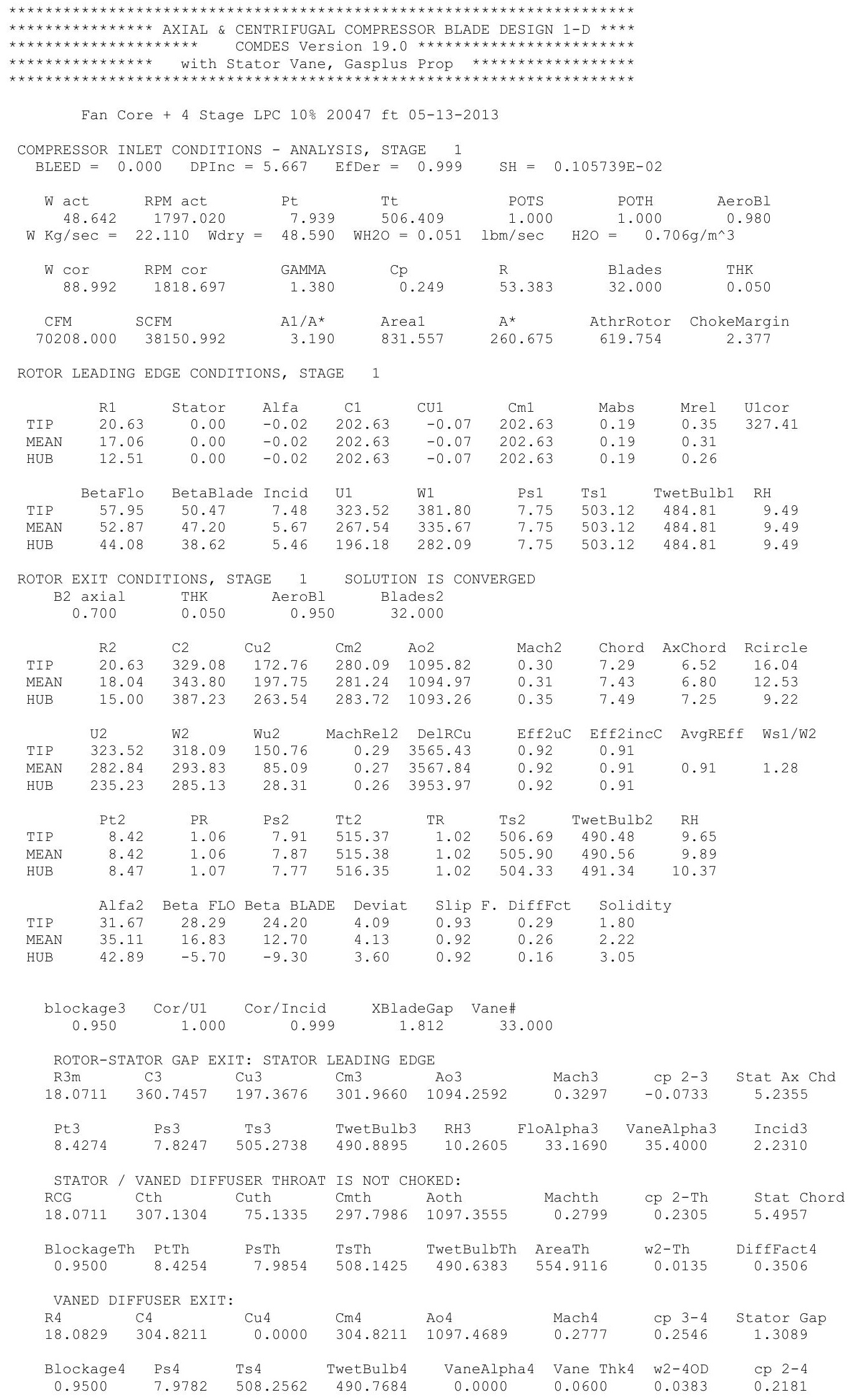




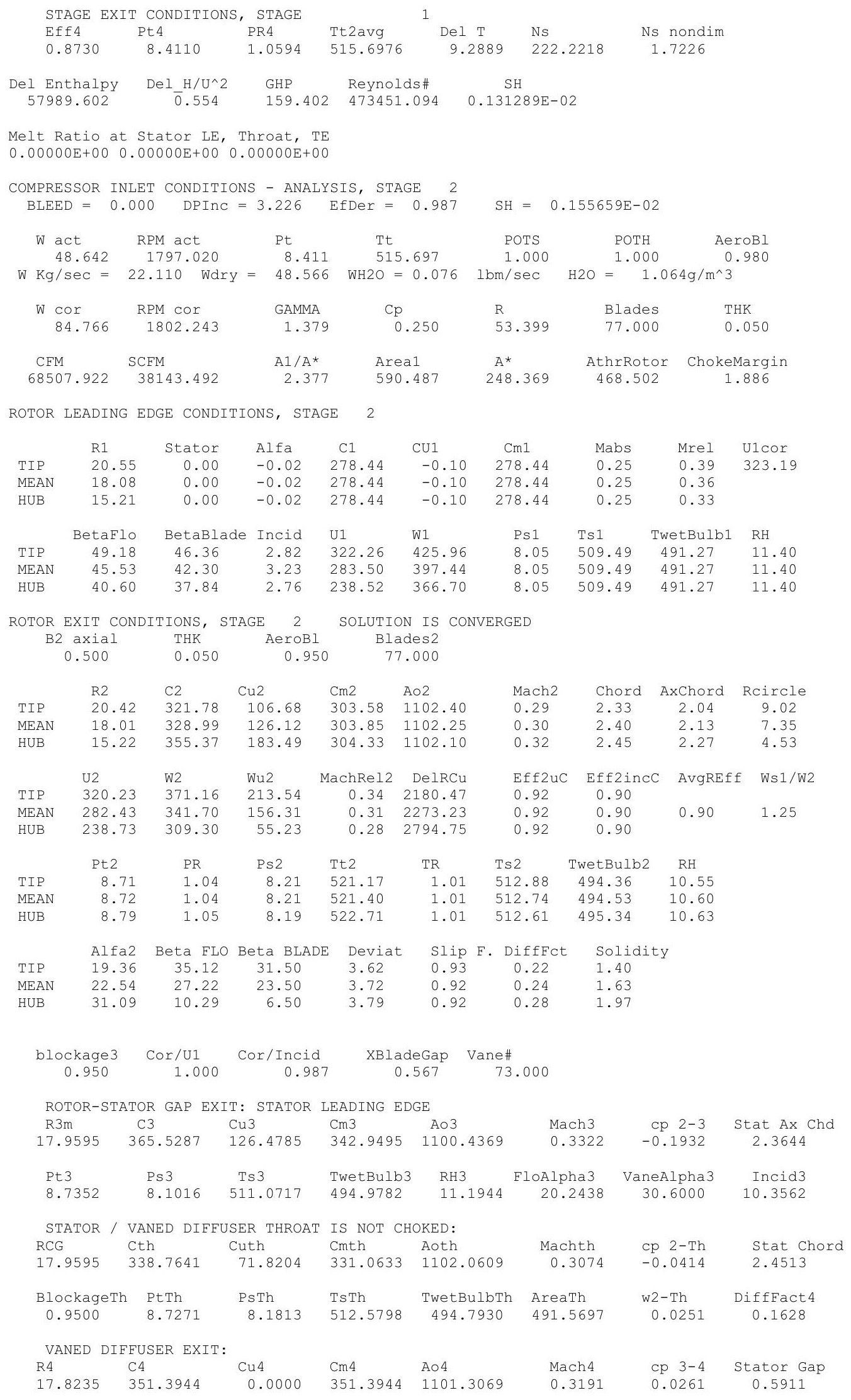




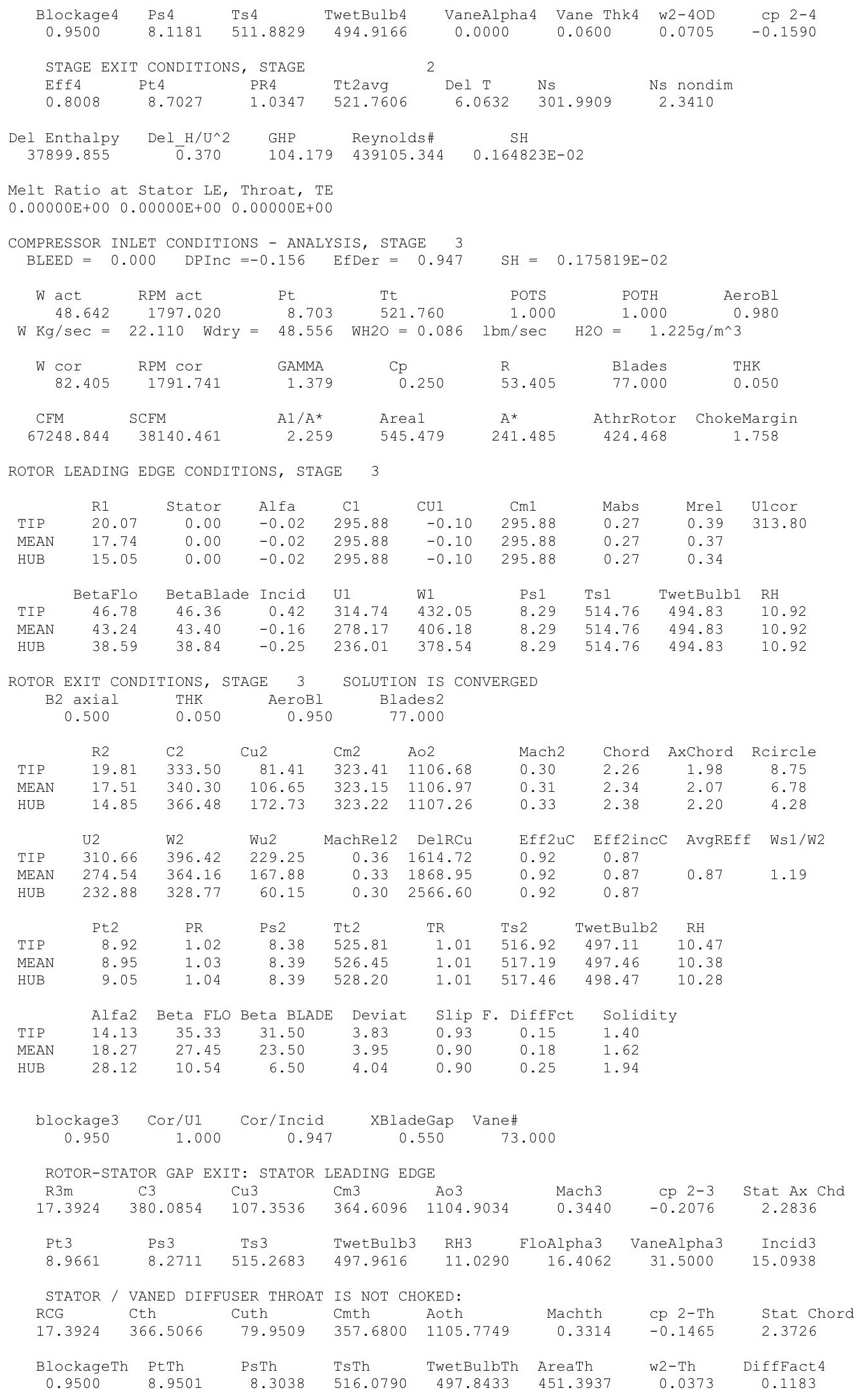




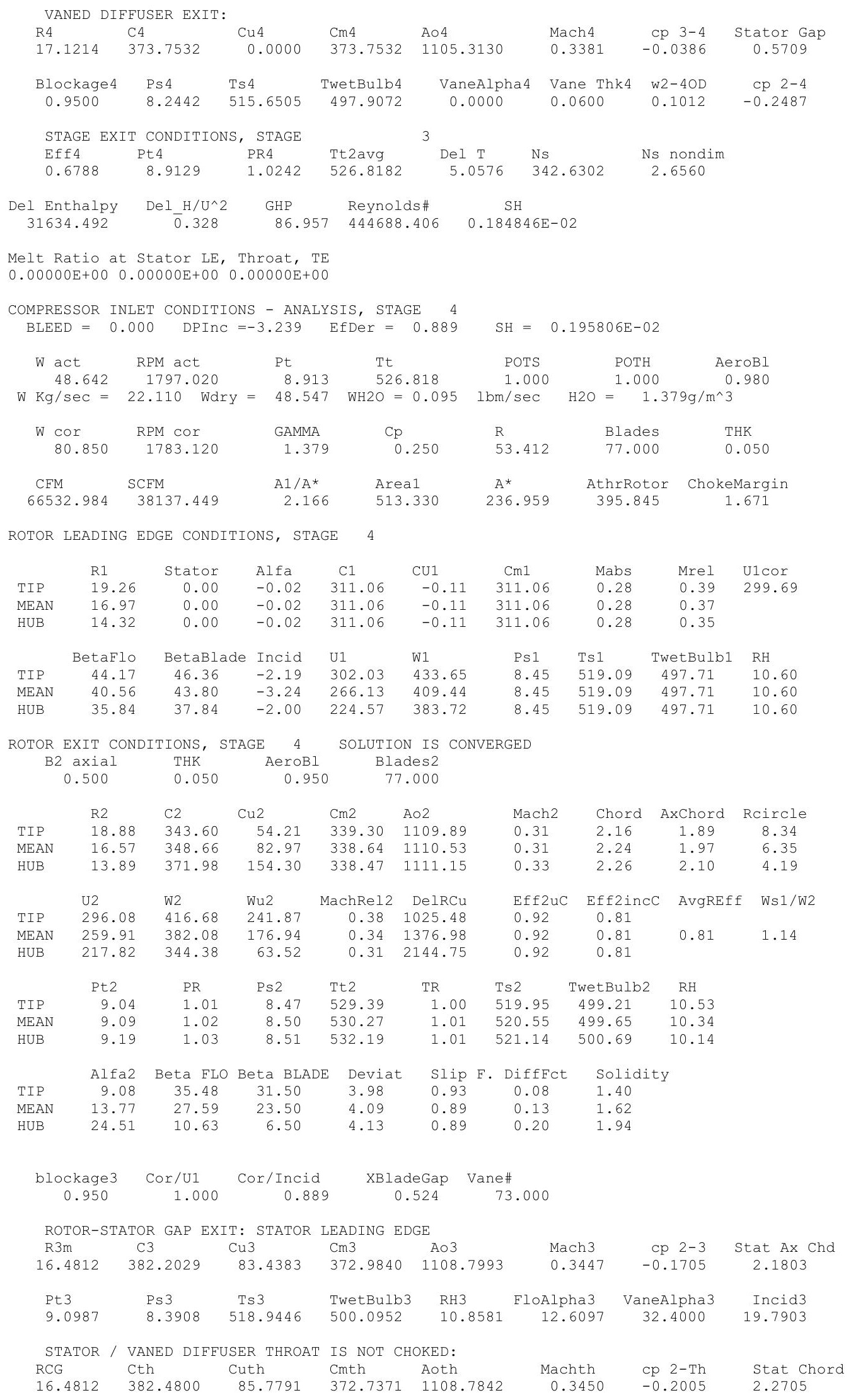




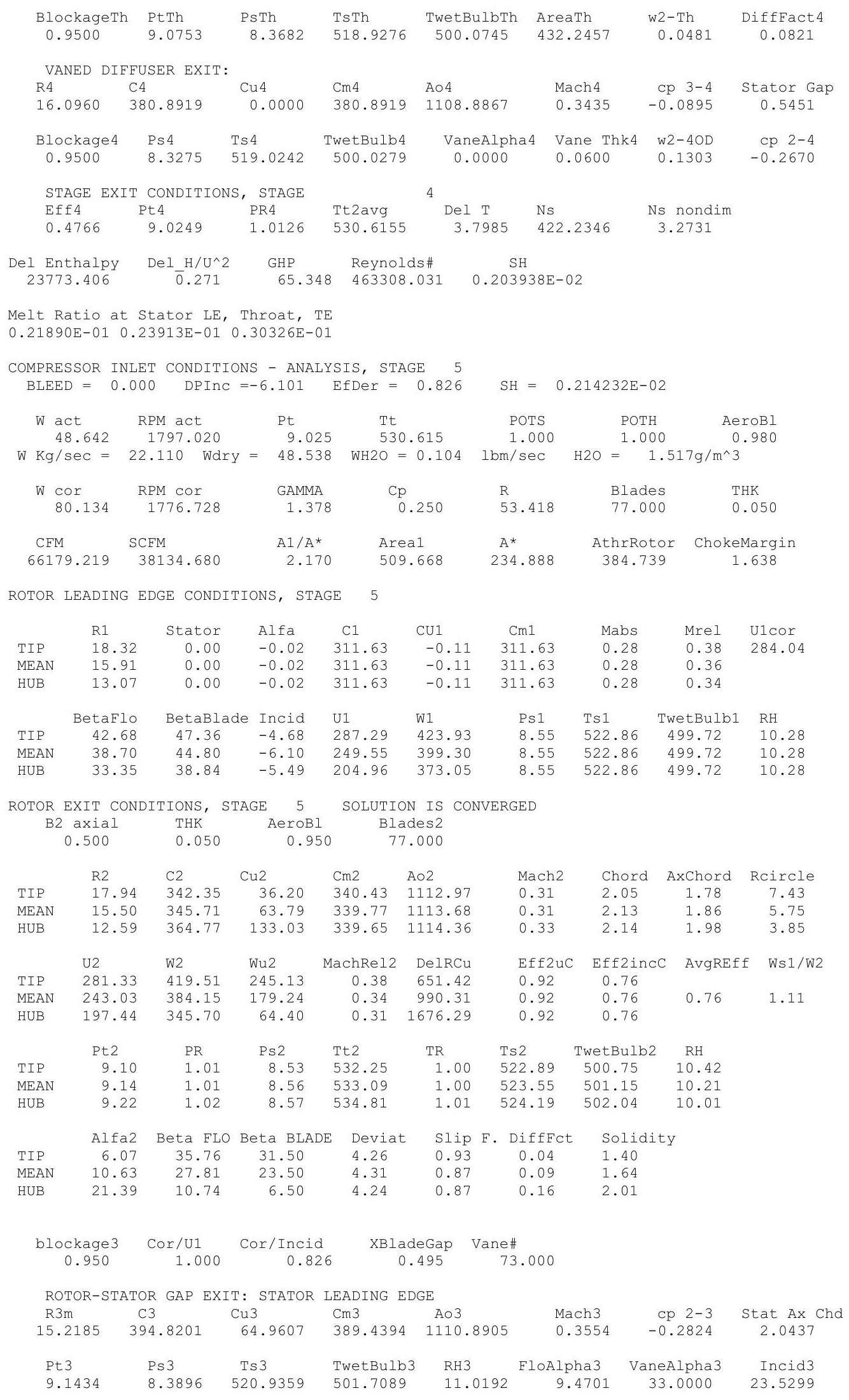




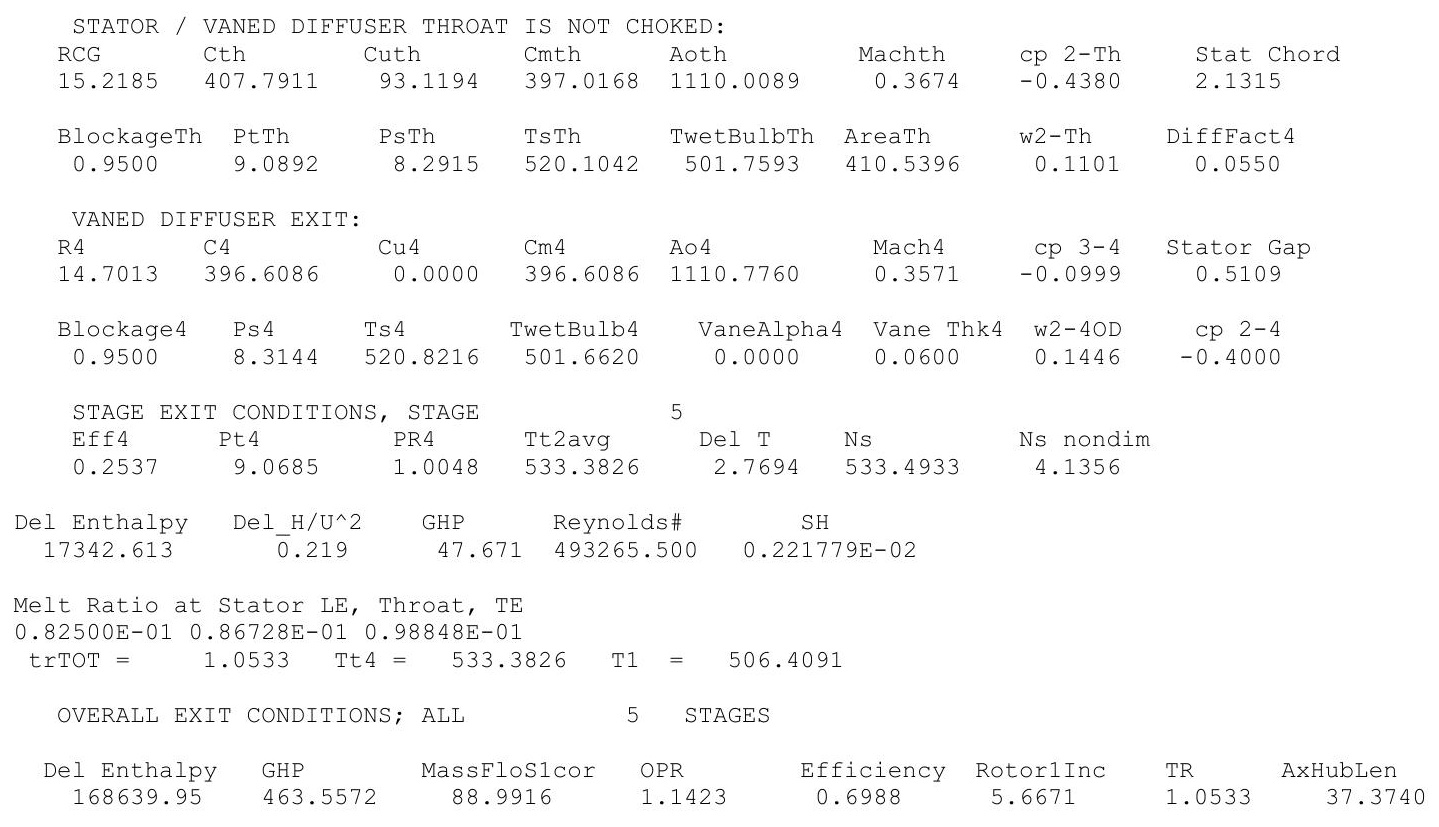




\title{
$25 \mu \mathrm{m}, \mathrm{ISA}+18 \mathrm{R}$
}

\begin{abstract}
$1-D * \star \star x \star * x$

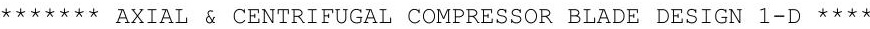

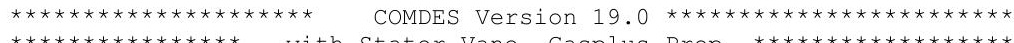

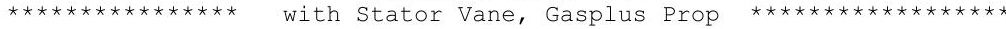

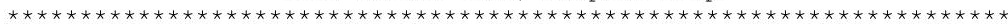

Fan Core + 4 Stage LPC 10\%39000 ft 05-13-2013

COMPRESSOR INLET CONDITIONS - ANALYSIS, STAGE 1

$\mathrm{BLEED}=0.000 \quad$ DPInC $=15.160 \quad$ EfDer $=0.957$

$\mathrm{SH}=0.757291 \mathrm{E}-04$

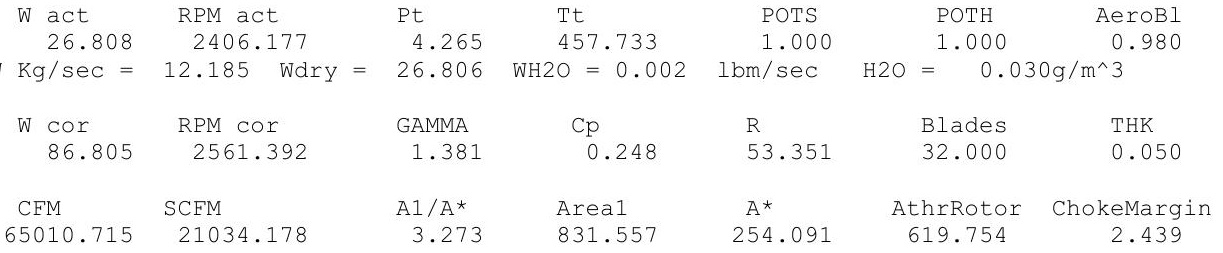

ROTOR LEADING EDGE CONDITIONS, STAGE 1

\begin{tabular}{|c|c|c|c|c|c|c|c|c|c|}
\hline & R1 & Stator & Alfa & $\mathrm{C} 1$ & CU1 & $\mathrm{Cm} 1$ & Mabs & Mrel & U1cor \\
\hline TIP & 20.63 & 0.00 & -0.02 & 187.63 & -0.06 & 187.63 & 0.18 & 0.45 & 461.12 \\
\hline MEAN & 17.06 & 0.00 & -0.02 & 187.63 & -0.06 & 187.63 & 0.18 & 0.39 & \\
\hline HUB & 12.51 & 0.00 & -0.02 & 187.63 & -0.06 & 187.63 & 0.18 & 0.31 & \\
\hline & BetaFlo & BetaBlade & Incid & $\mathrm{U} 1$ & W1 & Ps 1 & Ts 1 & TwetBulb1 & $\mathrm{RH}$ \\
\hline TIP & 66.58 & 50.47 & 16.11 & 433.19 & 472.13 & 4.17 & 454.90 & 448.33 & 2. \\
\hline MEAN & 62.36 & 47.20 & 15.16 & 358.23 & 404.45 & 4.17 & 454.90 & 448.33 & 2.9 \\
\hline HUB & 54.47 & 38.62 & 15.85 & 262.68 & 322.86 & 4.17 & 454.90 & 448.33 & 2.9 \\
\hline
\end{tabular}

ROTOR EXIT CONDITIONS, STAGE 1 SOLUTION IS CONVERGED

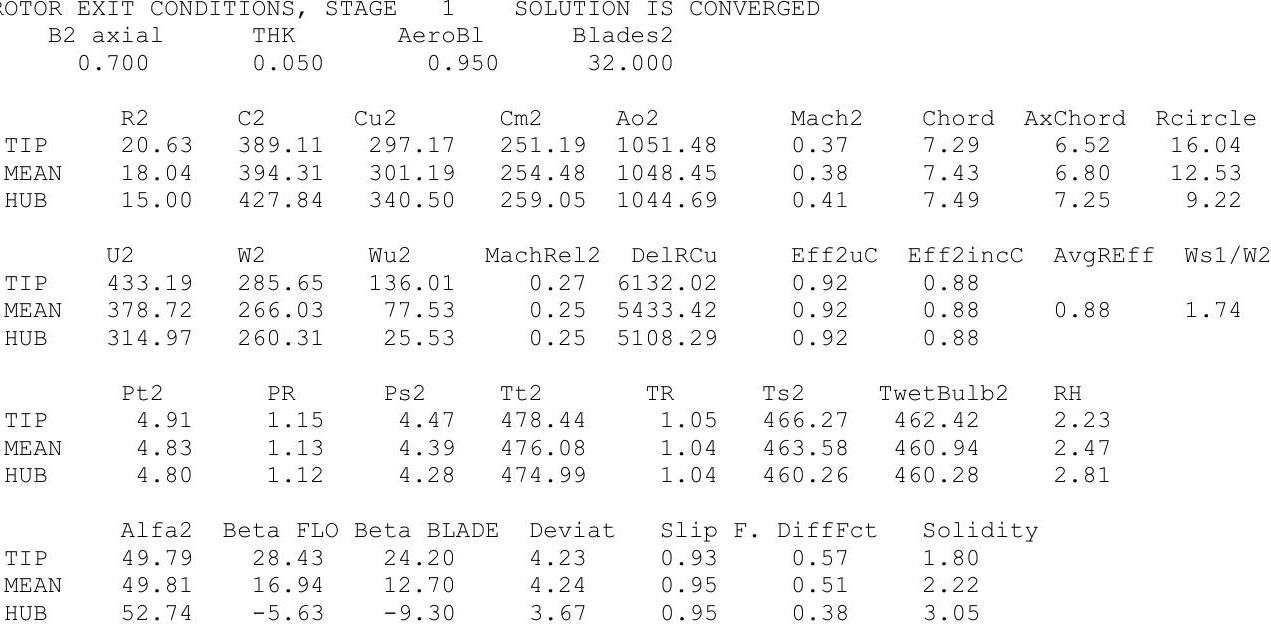

\footnotetext{
blockage 3 Cor/U1 Cor/Incid XBladeGap Vane\#

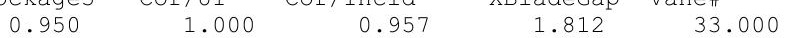

ROTOR-STATOR GAP EXIT: STATOR LEADING EDGE

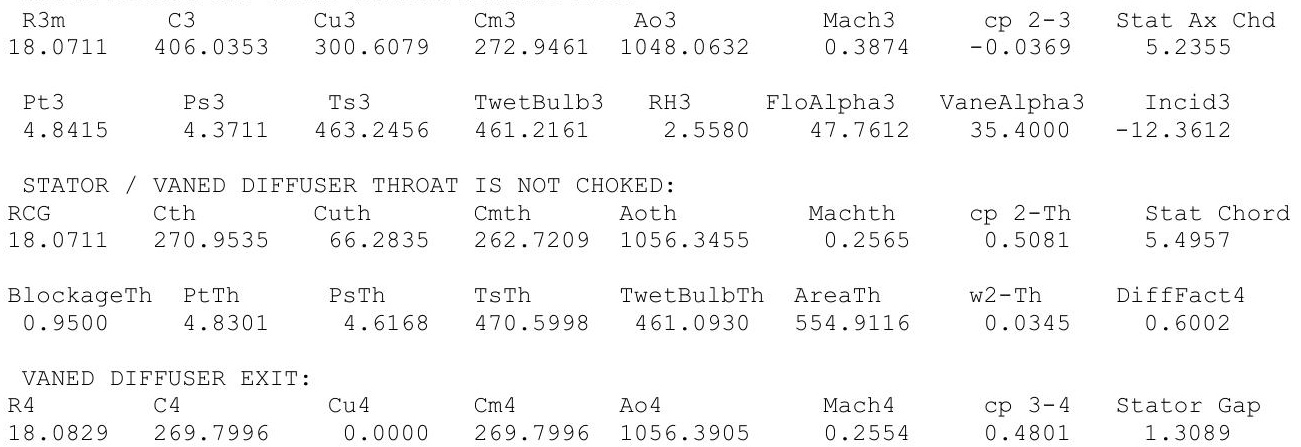




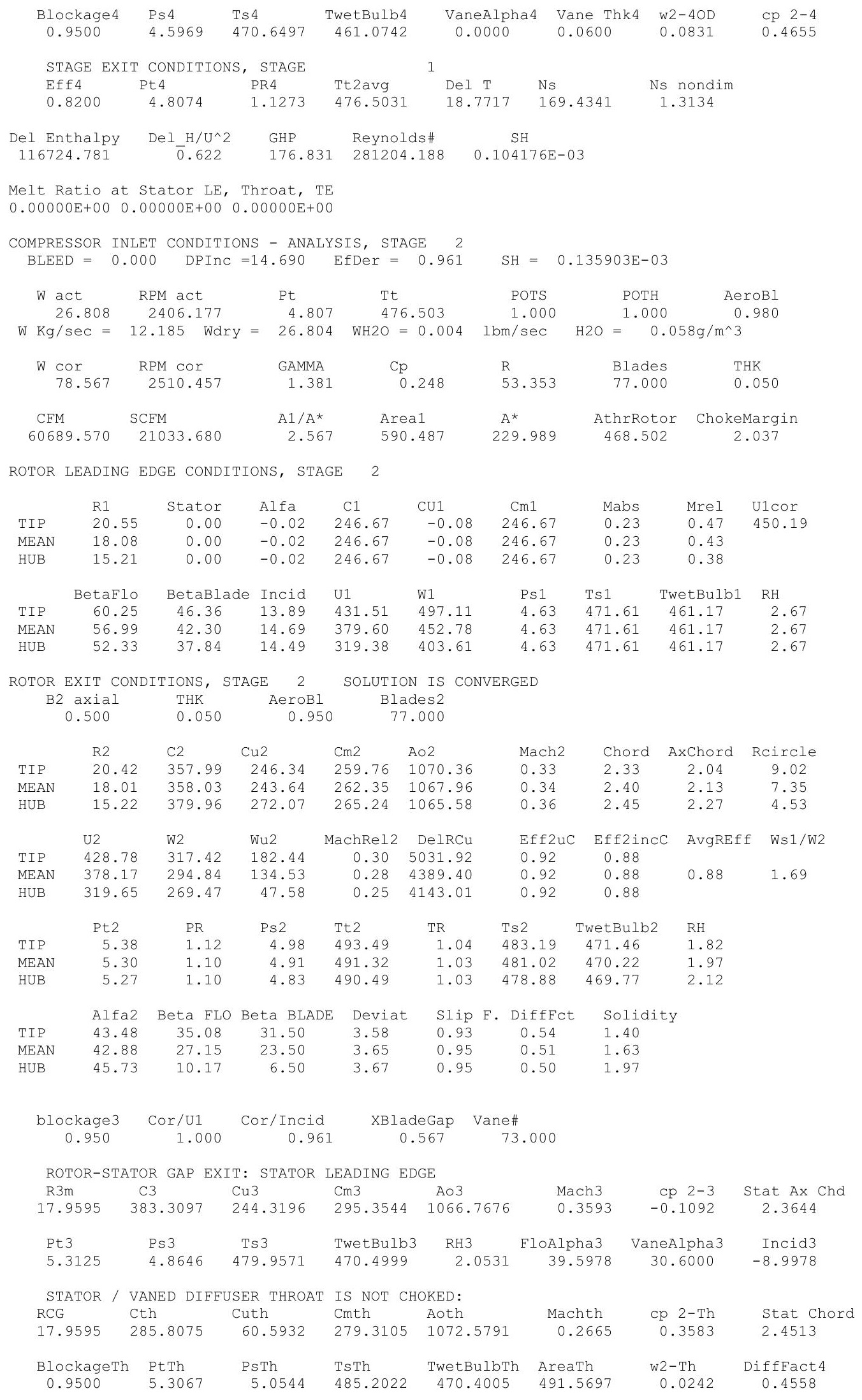




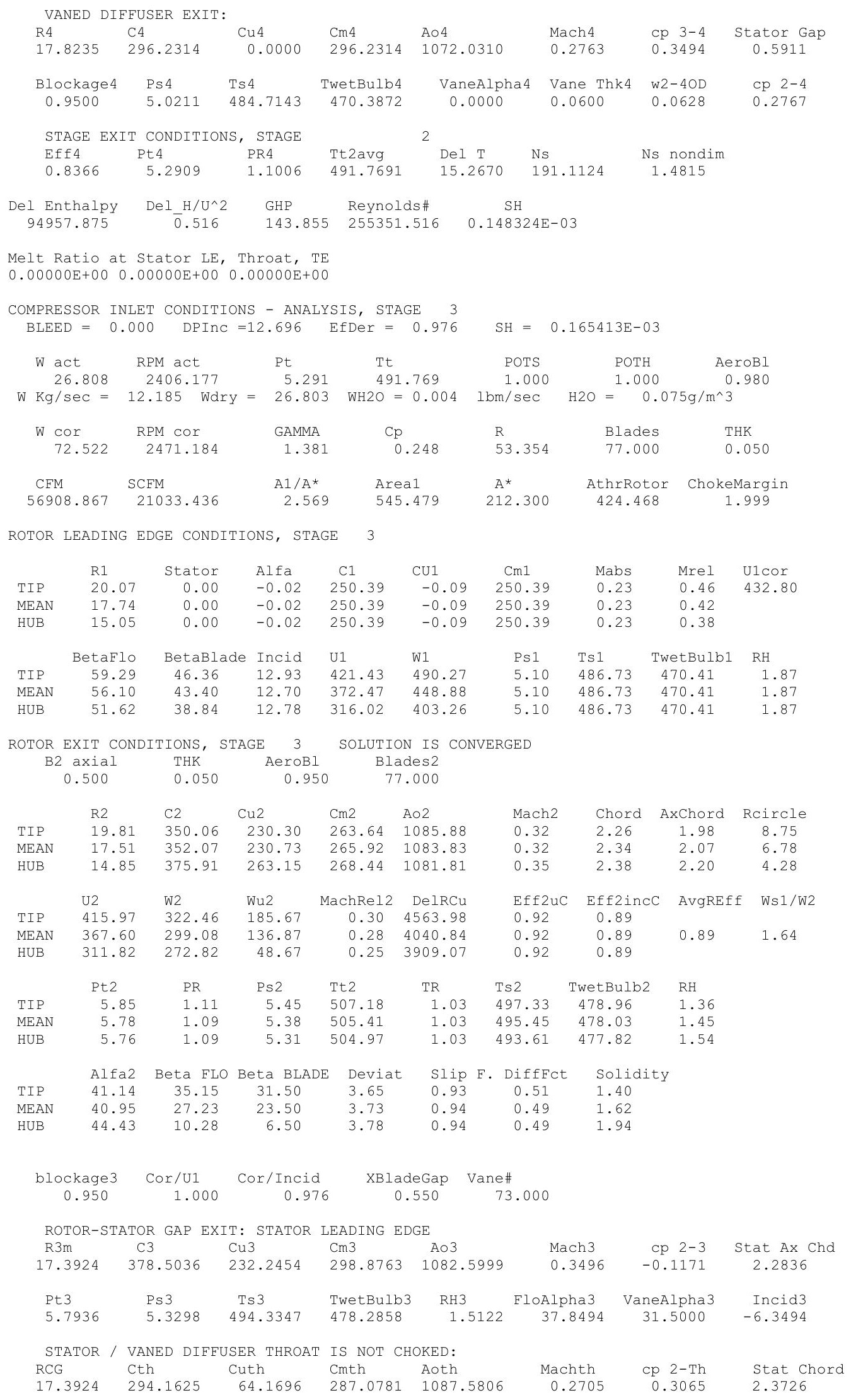




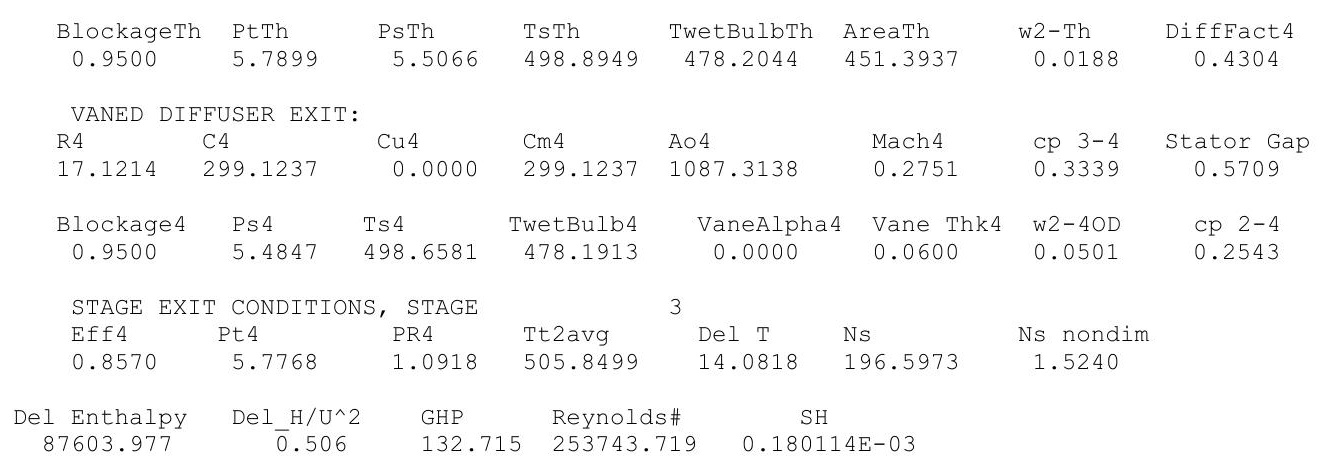

Melt Ratio at Stator LE, Throat, TE

$0.00000 \mathrm{E}+000.00000 \mathrm{E}+000.00000 \mathrm{E}+00$

COMPRESSOR INLET CONDITIONS - ANALYSIS, STAGE 4 $\mathrm{BLEED}=0.000 \quad$ DPInC $=11.102 \quad$ EfDer $=0.986$

ROTOR LEADING EDGE CONDITIONS, STAGE 4

$\begin{array}{lccccccccc} & \text { R1 } & \text { Stator } & \text { Alfa } & \text { C1 } & \text { CU1 } & \text { Cm1 } & \text { Mabs } & \text { Mrel } & \text { U1cor } \\ \text { TIP } & 19.26 & 0.00 & -0.02 & 250.49 & -0.09 & 250.49 & 0.23 & 0.44 & 409.51 \\ \text { MEAN } & 16.97 & 0.00 & -0.02 & 250.49 & -0.09 & 250.49 & 0.23 & 0.40 & \\ \text { HUB } & 14.32 & 0.00 & -0.02 & 250.49 & -0.09 & 250.49 & 0.23 & 0.36 & \\ & & & & & & & & & \\ \text { TIP } & \text { BetaFlo } & \text { BetaBlade } & \text { Incid } & \text { U1 } & \text { W1 } & \text { Ts1 } & \text { TwetBulb1 } & \text { RH } \\ \text { MEAN } & 58.23 & 46.36 & 11.87 & 404.42 & 475.78 & 5.57 & 500.81 & 478.21 & 1.42 \\ \text { HUB } & 50.21 & 37.84 & 12.37 & 300.69 & 391.42 & 5.57 & 500.81 & 478.21 & 1.42\end{array}$

ROTOR EXIT CONDITIONS, STAGE 4 SOLUTION IS CONVERGED

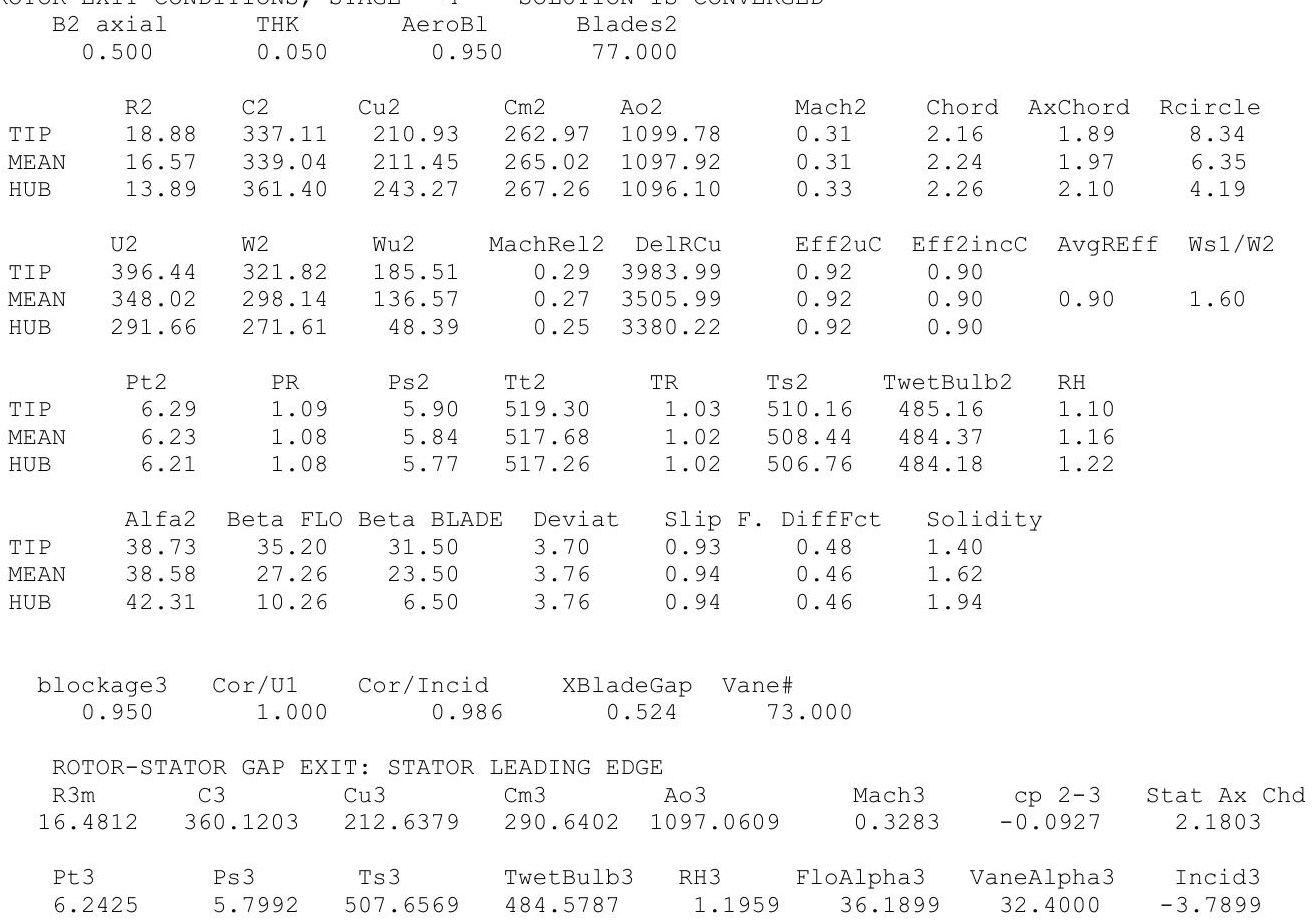




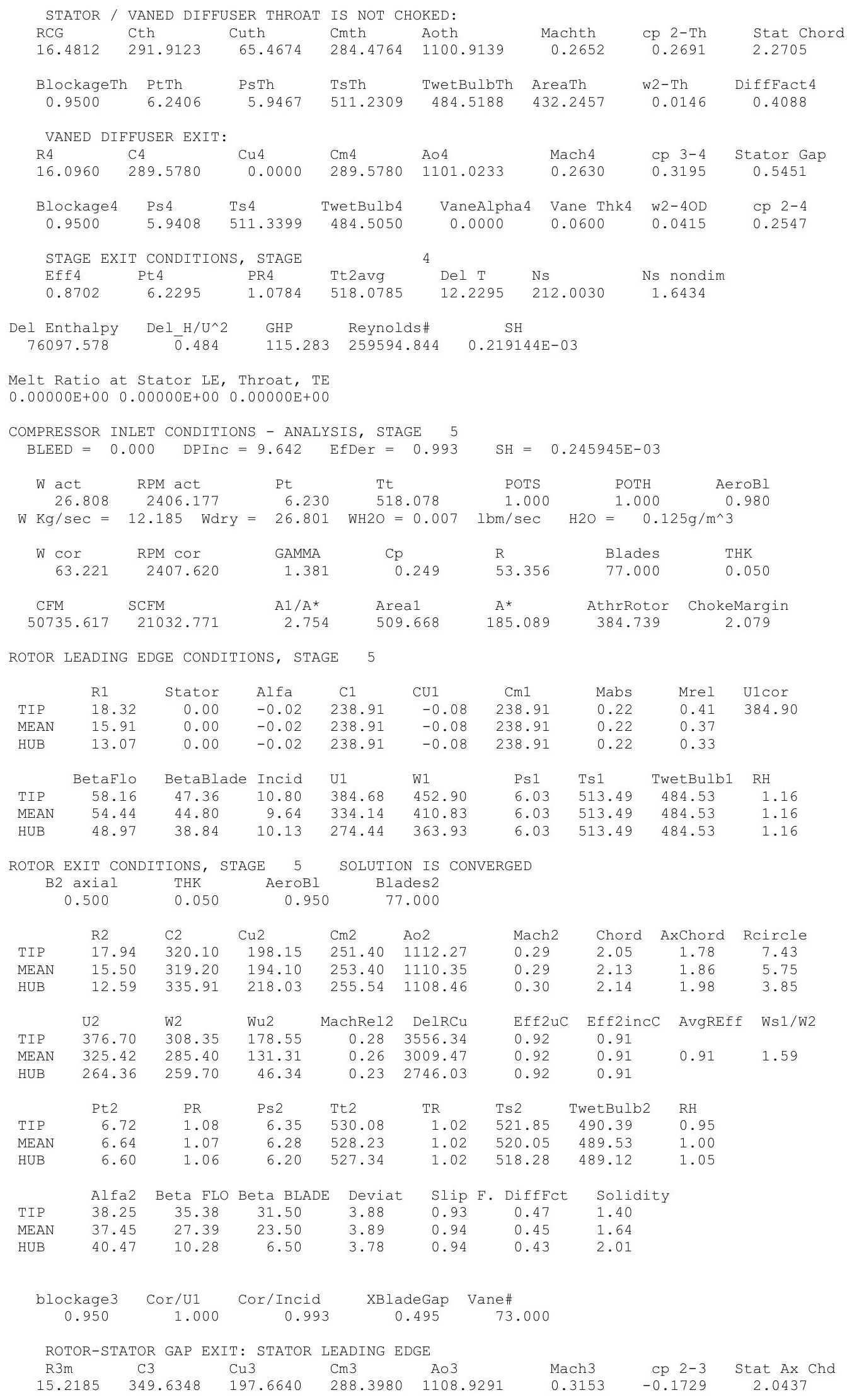




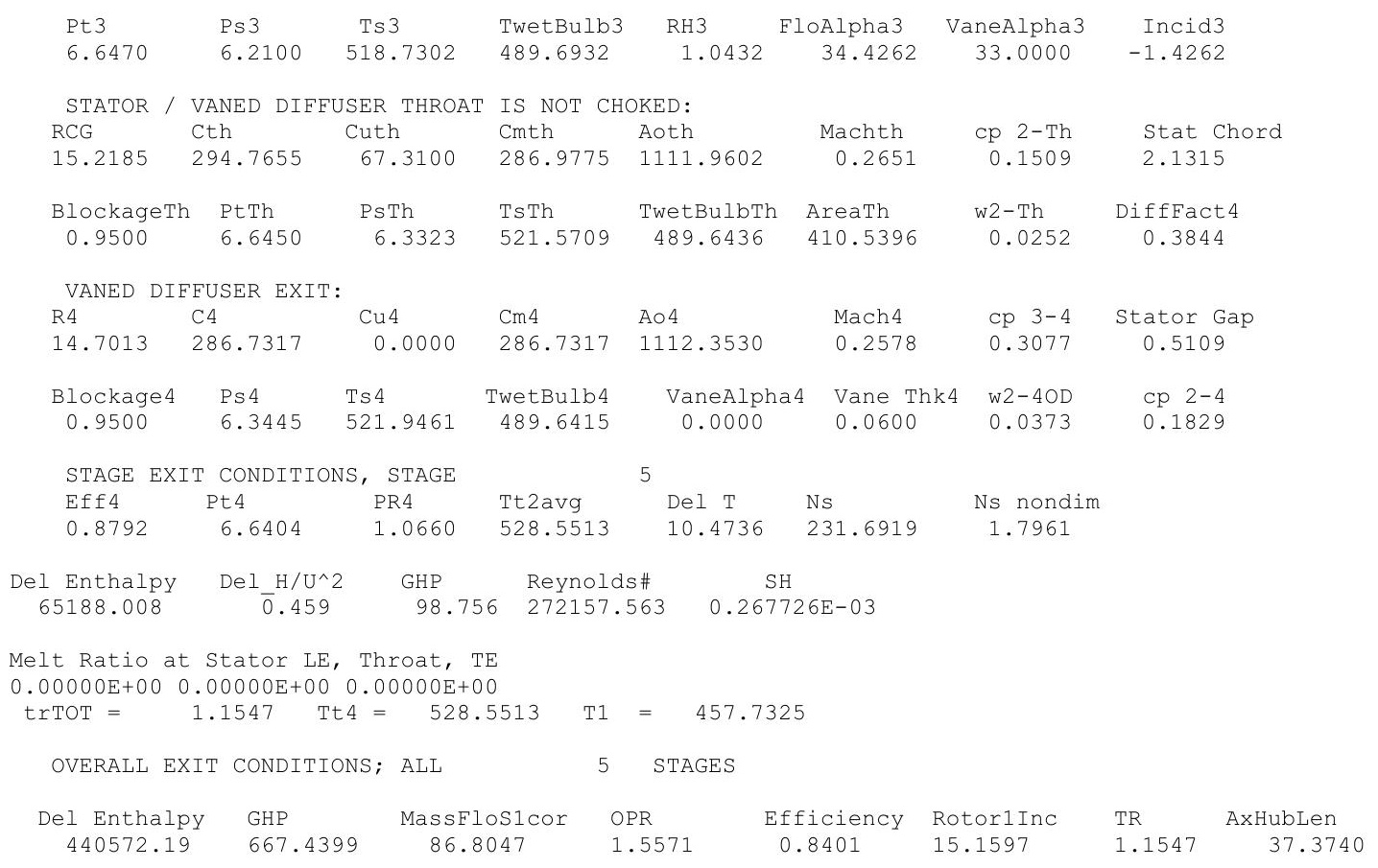




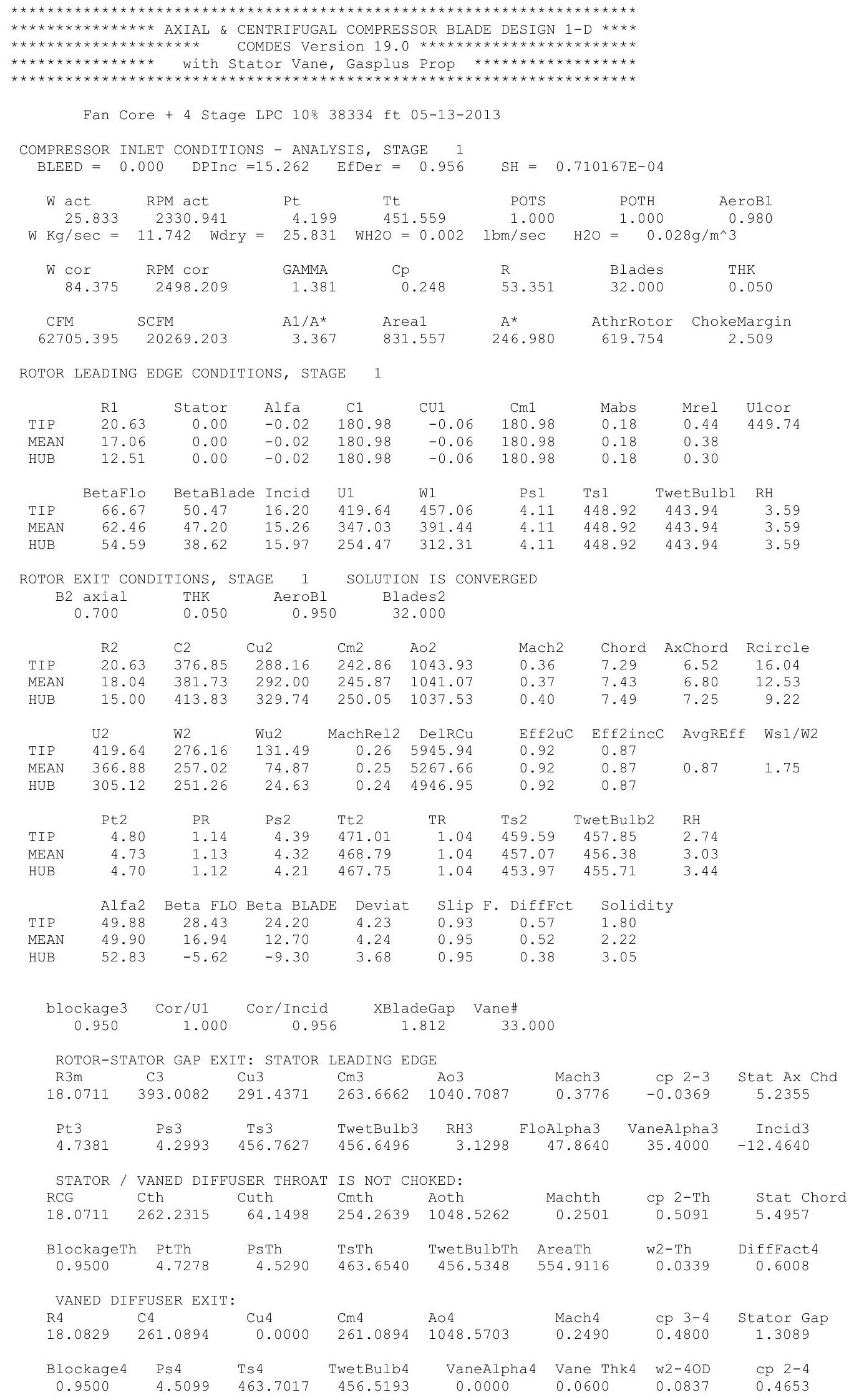




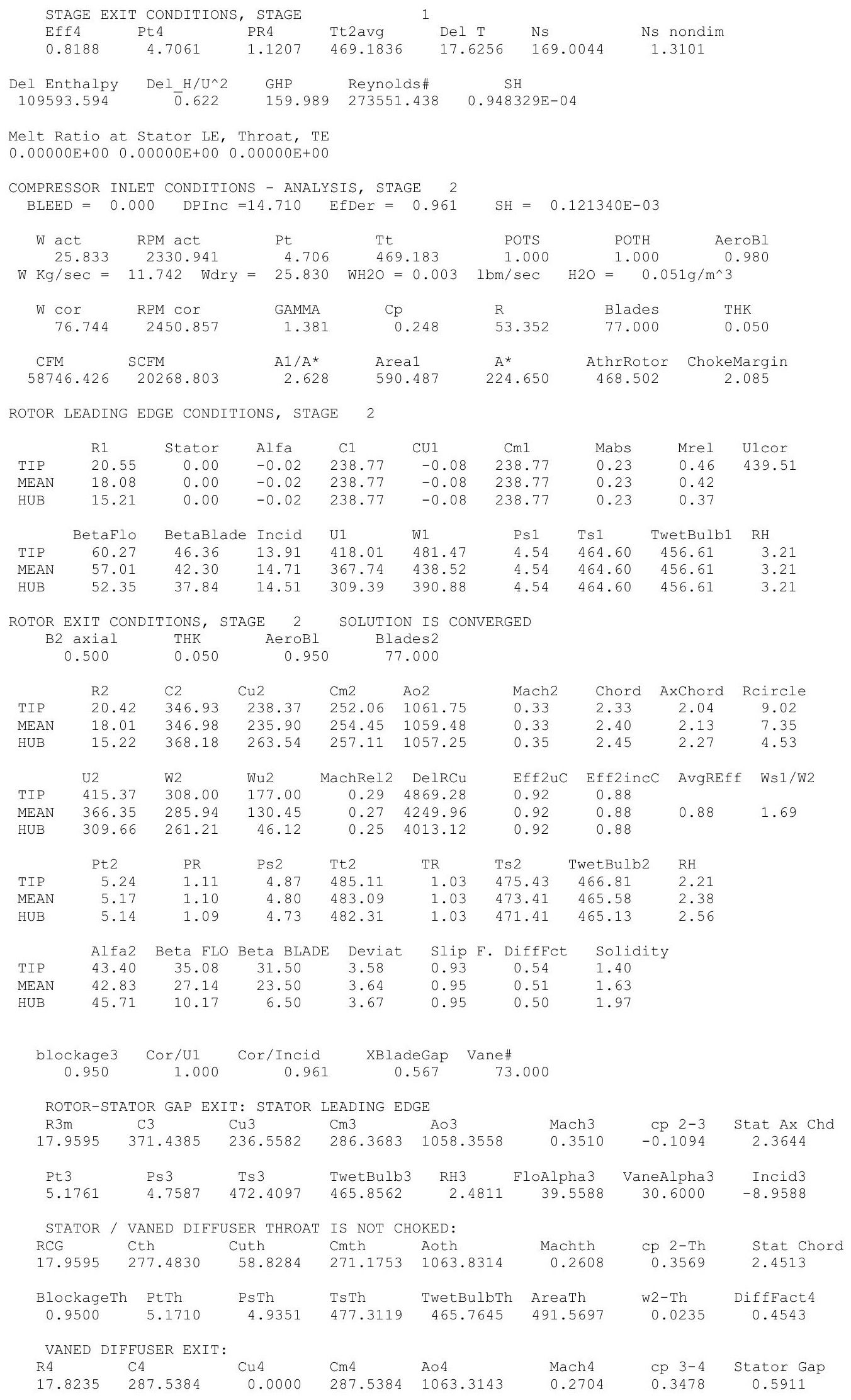




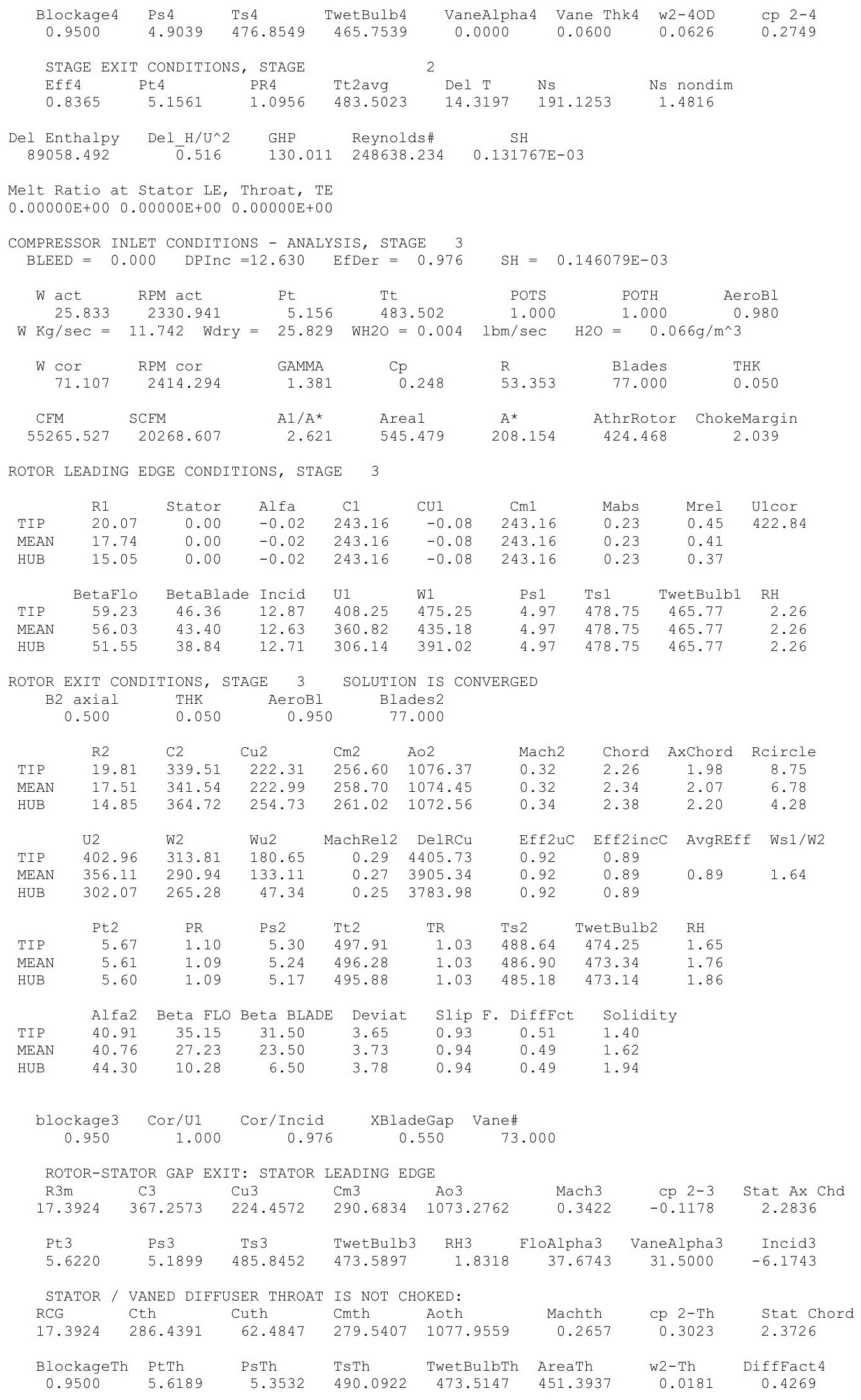




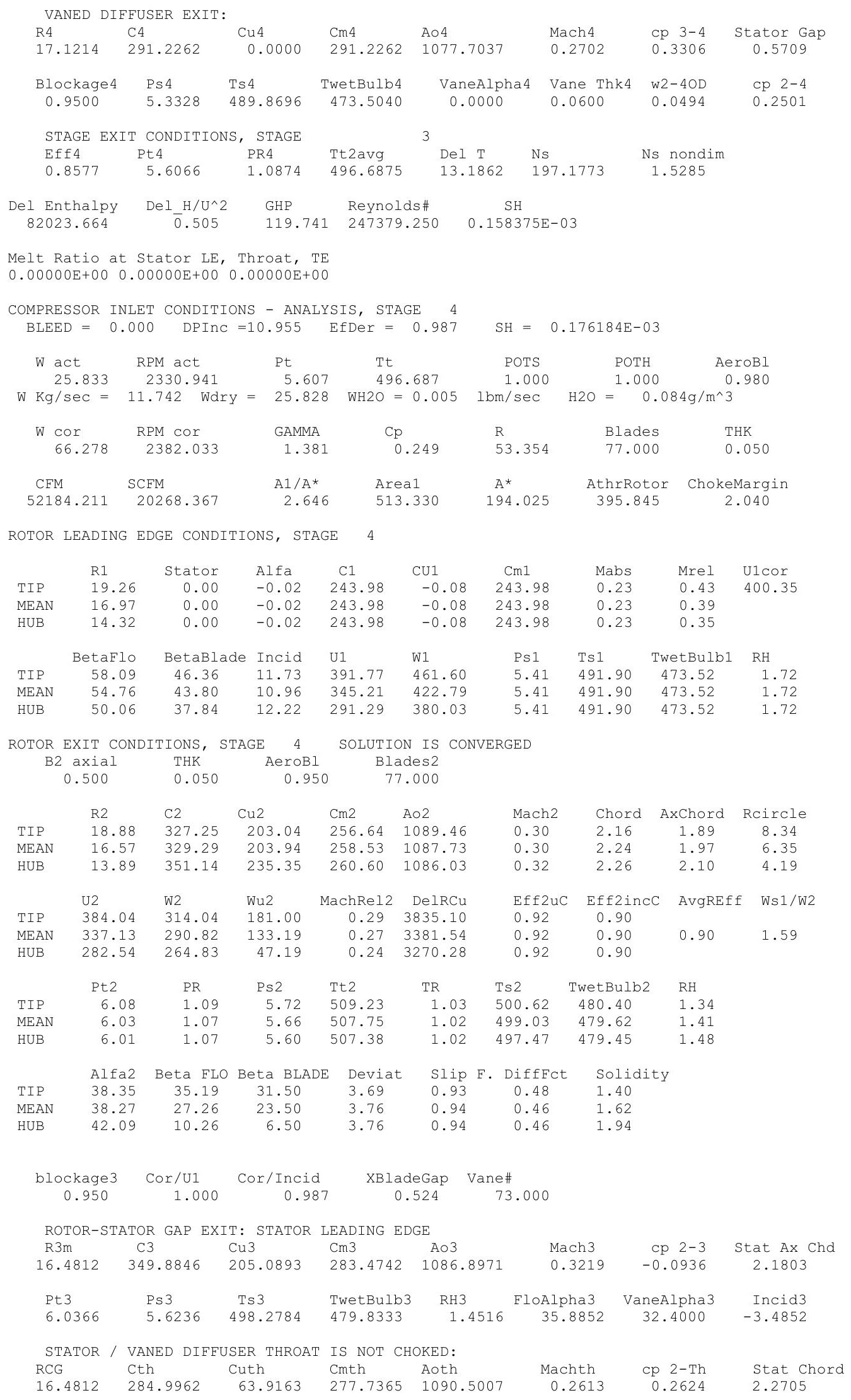




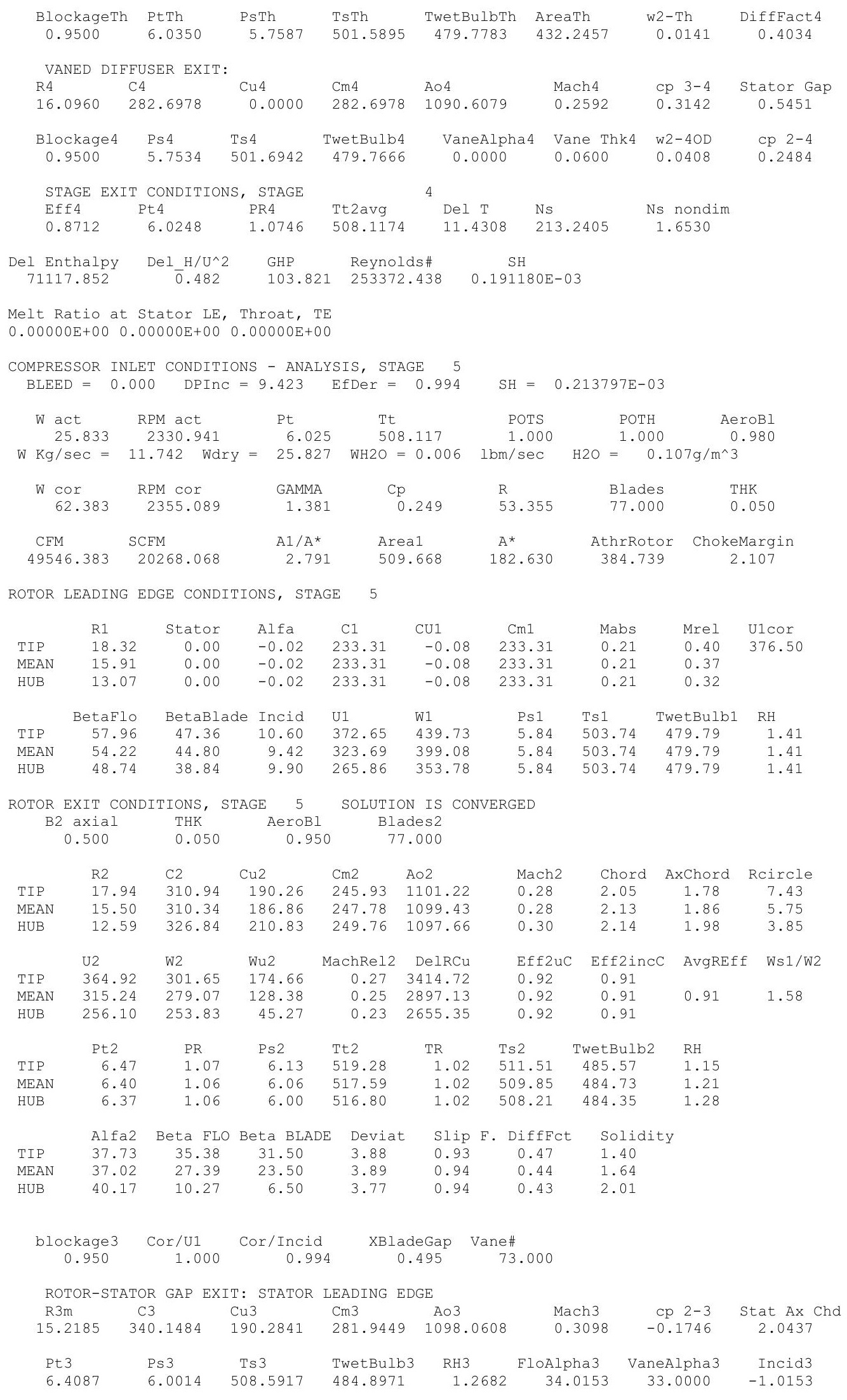




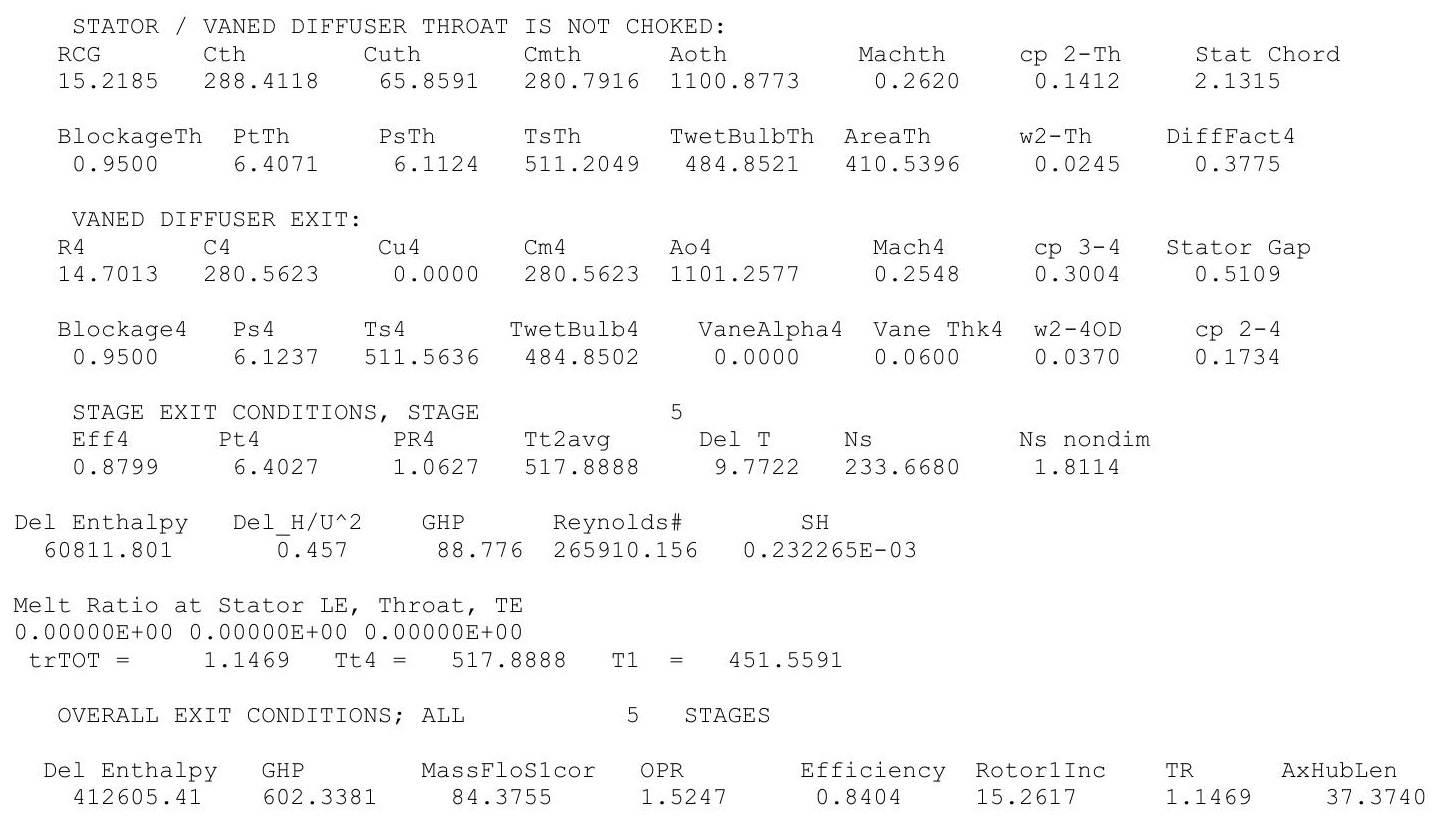




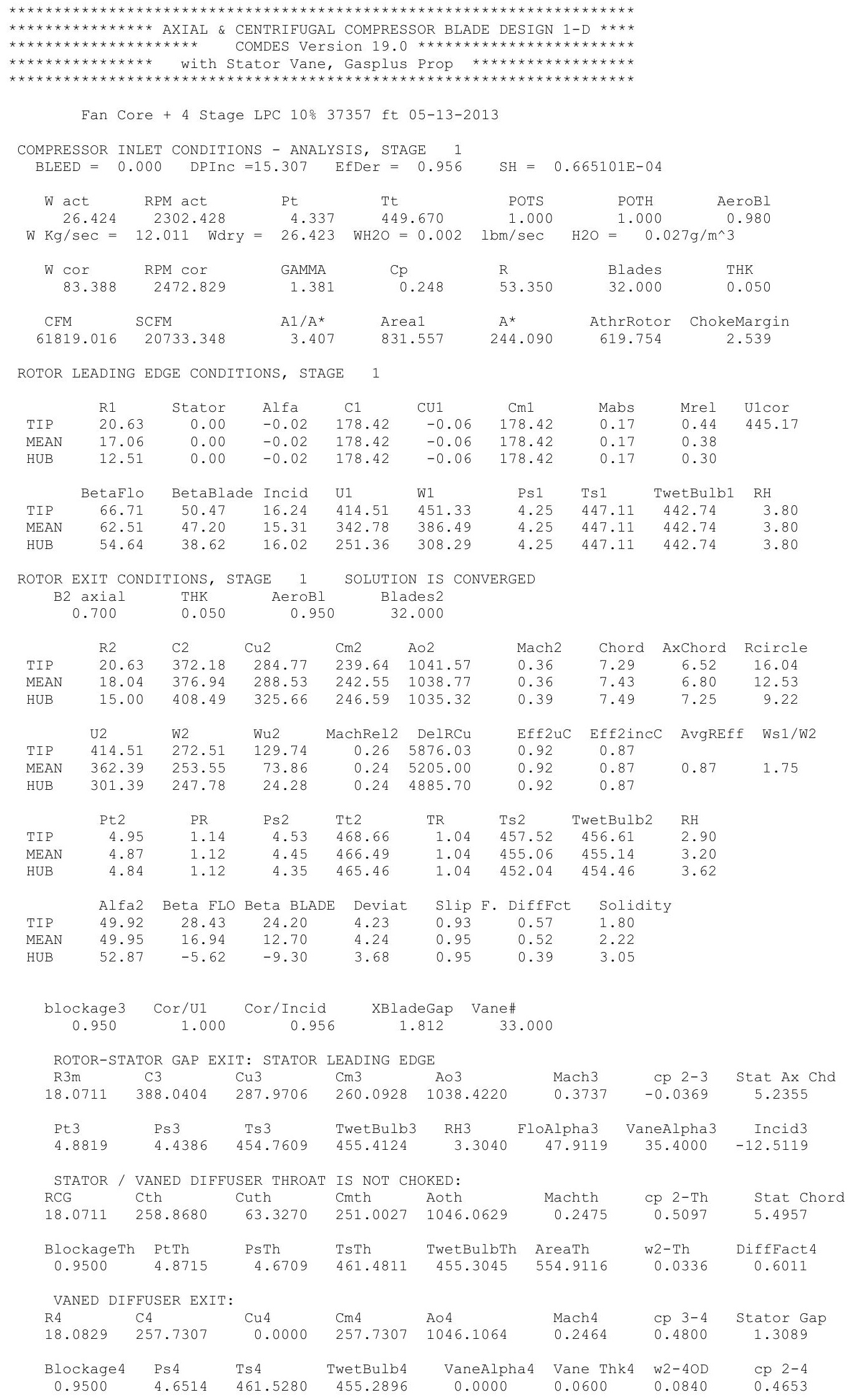




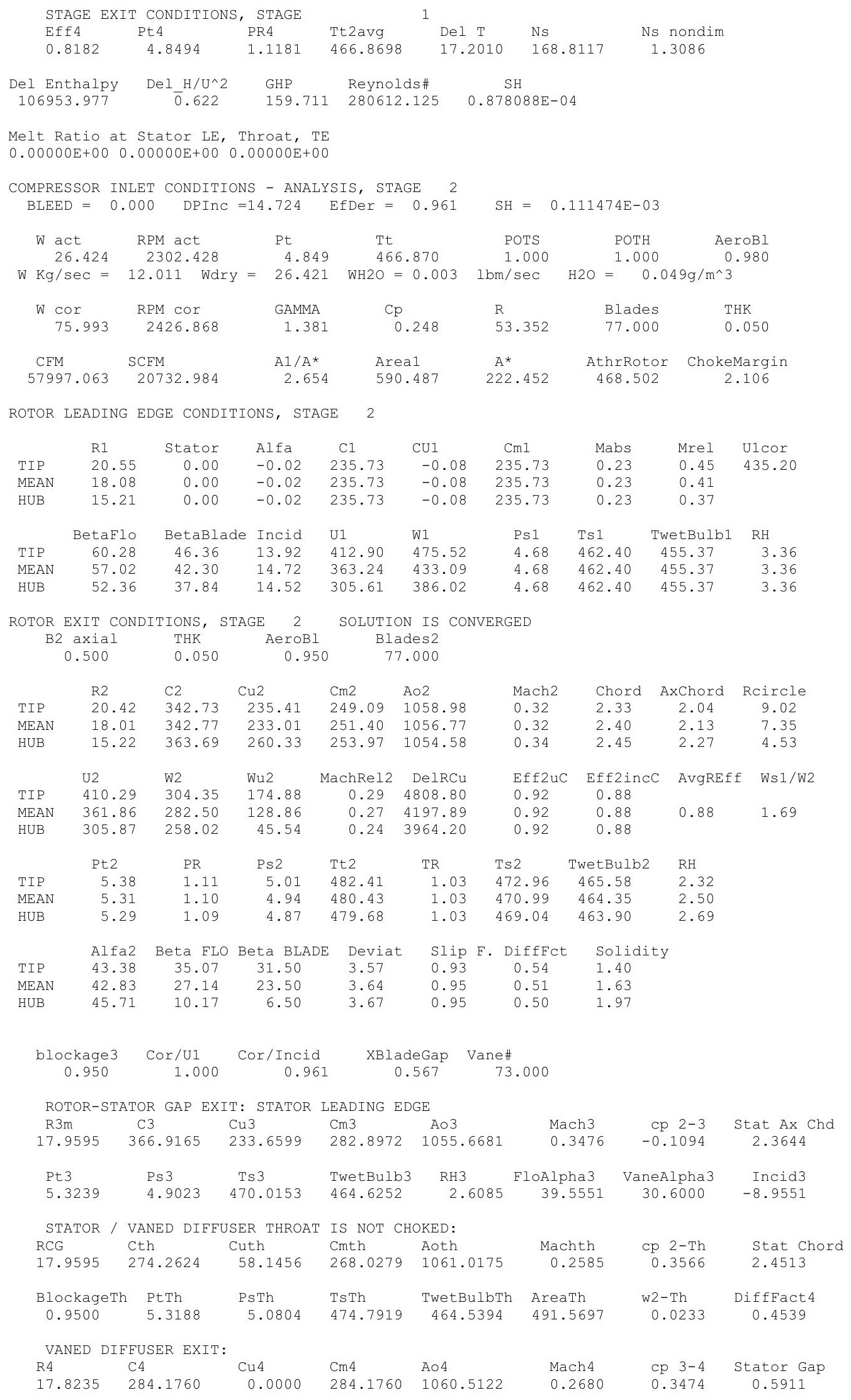




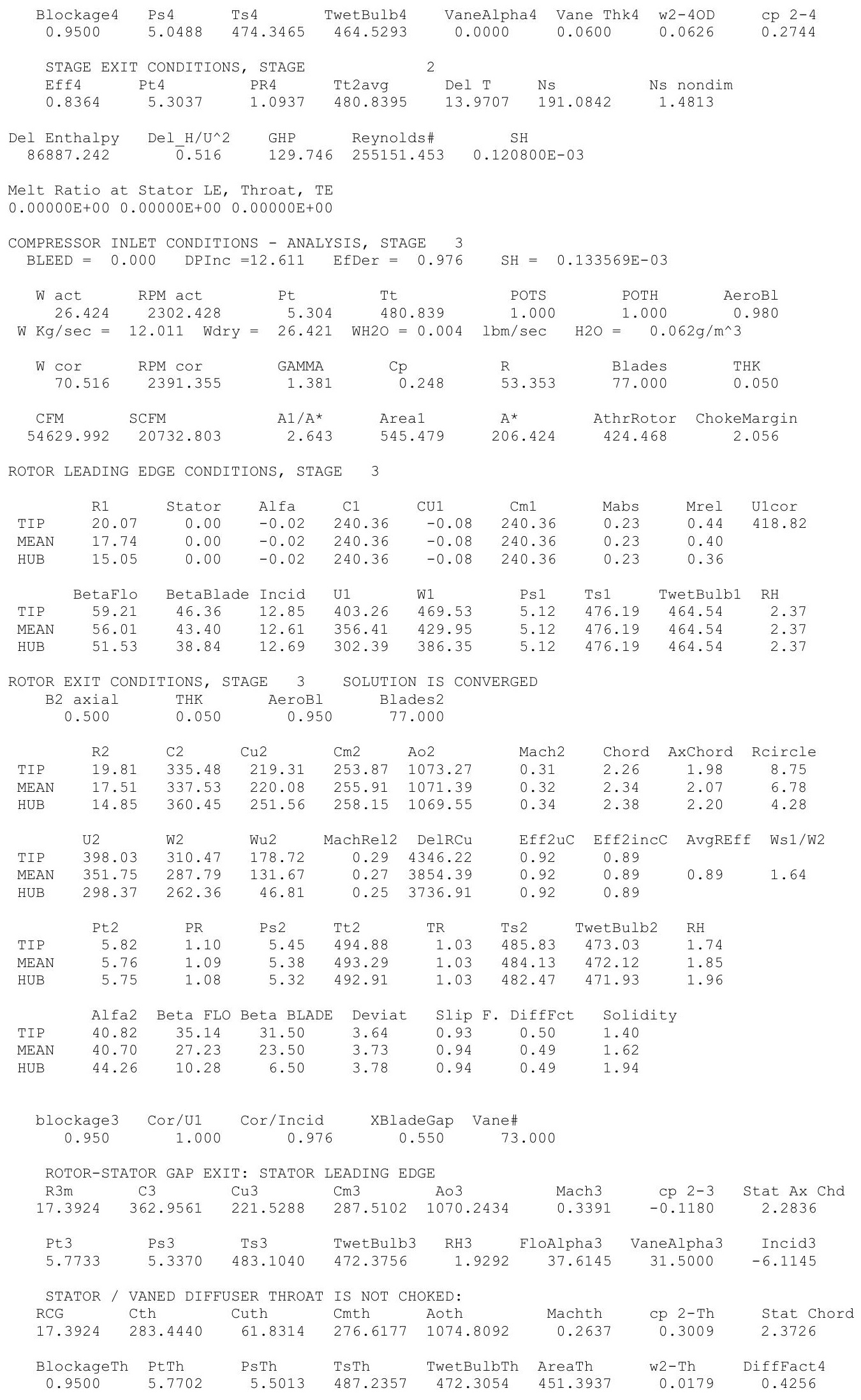




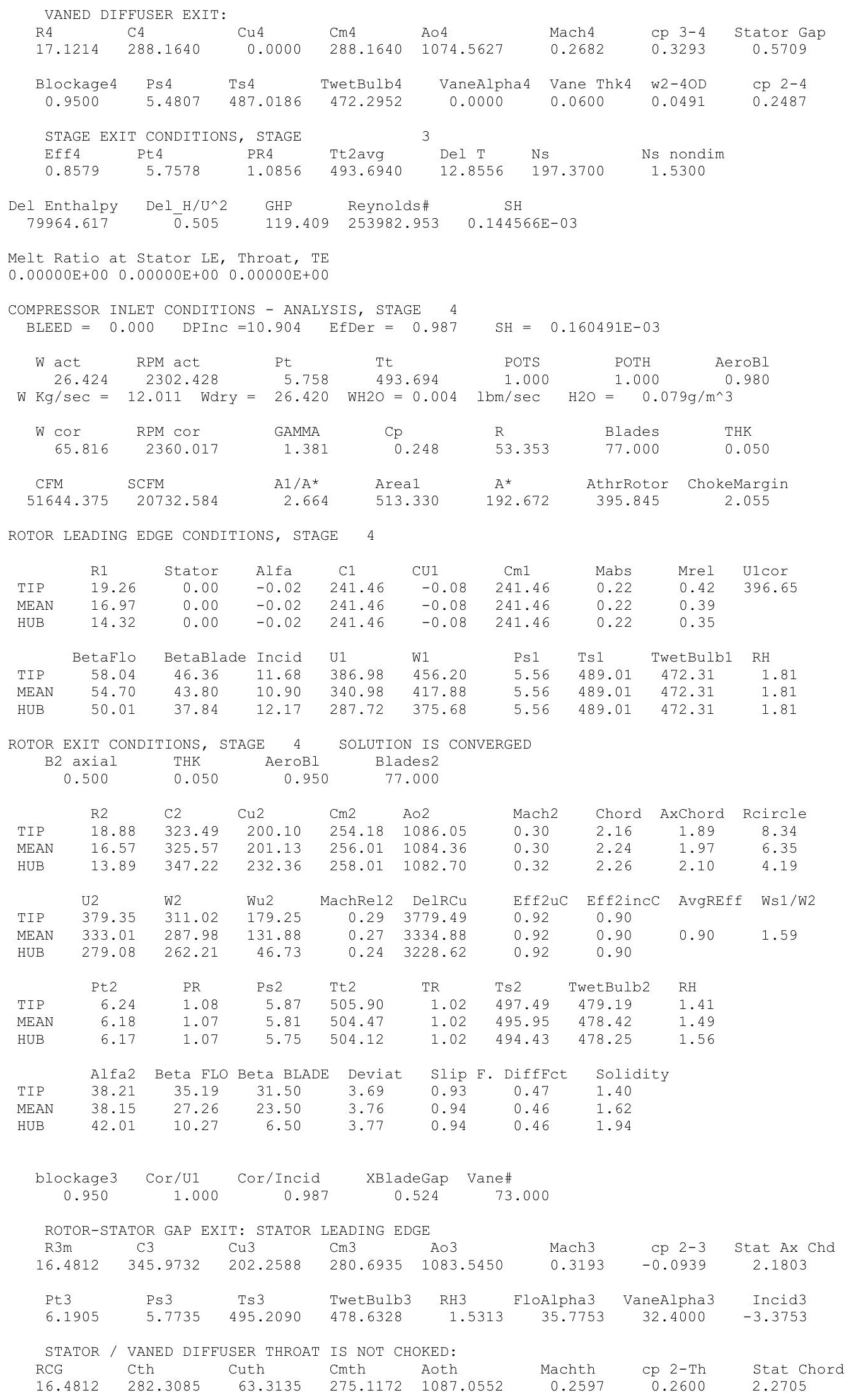




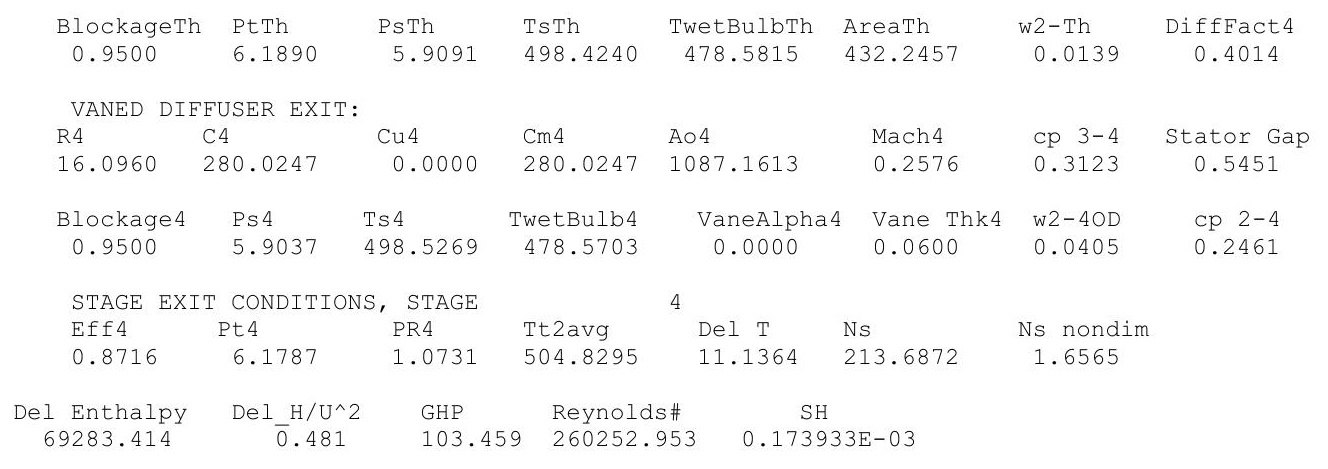

Melt Ratio at Stator LE, Throat, TE

$0.00000 \mathrm{E}+00 \quad 0.00000 \mathrm{E}+00 \quad 0.00000 \mathrm{E}+00$

COMPRESSOR INLET CONDITIONS - ANALYSIS, STAGE 5 $\mathrm{BLEED}=0.000 \quad$ DPInC $=9.343 \quad$ EfDer $=0.994$

$\begin{array}{lrrl}\text { act } & \text { RPM act } & \text { Pt } & \text { Tt } \\ 26.424 & 2302.428 & 6.179 & 504.829\end{array}$

$\mathrm{W} \mathrm{Kg} / \mathrm{sec}=12.011$ Wdry $=$

$\mathrm{SH}=0.194232 \mathrm{E}-03$

$\begin{array}{ccccccc}\text { W cor } & \text { RPM cor } & \text { GAMMA } & \text { Cp } & \text { R } & \text { Blades } & \text { THK } \\ \text { 62.021 } & 2333.844 & 1.381 & 0.249 & 53.355 & 77.000 & 0.050 \\ \text { CFM } & \text { SCFM } & \text { A1/A* } & \text { Area1 } & \text { A* } & \text { AthrRotor } & \text { ChokeMargin } \\ 49084.703 & 20732.309 & 2.807 & 509.668 & 181.567 & 384.739 & 2.119\end{array}$

ROTOR LEADING EDGE CONDITIONS, STAGE 5

$\begin{array}{lccccccccc} & \text { R1 } & \text { Stator } & \text { Alfa } & \text { C1 } & \text { CU1 } & \text { Cm1 } & \text { Mabs } & \text { Mrel } & \text { U1cor } \\ \text { TIP } & 18.32 & 0.00 & -0.02 & 231.14 & -0.08 & 231.14 & 0.21 & 0.40 & 373.11 \\ \text { MEAN } & 15.91 & 0.00 & -0.02 & 231.14 & -0.08 & 231.14 & 0.21 & 0.36 & \\ \text { HUB } & 13.07 & 0.00 & -0.02 & 231.14 & -0.08 & 231.14 & 0.21 & 0.32 & \\ & & & & & & & & & \\ & & & & & & & & & \\ \text { TIP } & 57.88 & 47.36 & 10.52 & 368.09 & 434.71 & 5.99 & 500.54 & 478.59 & 1.49 \\ \text { MEAN } & 54.14 & 44.80 & 9.34 & 319.73 & 394.59 & 5.99 & 500.54 & 478.59 & 1.49 \\ \text { HUB } & 48.66 & 38.84 & 9.82 & 262.61 & 349.90 & 5.99 & 500.54 & 478.59 & 1.49\end{array}$

ROTOR EXIT CONDITIONS, STAGE 5 SOLUTION IS CONVERGED

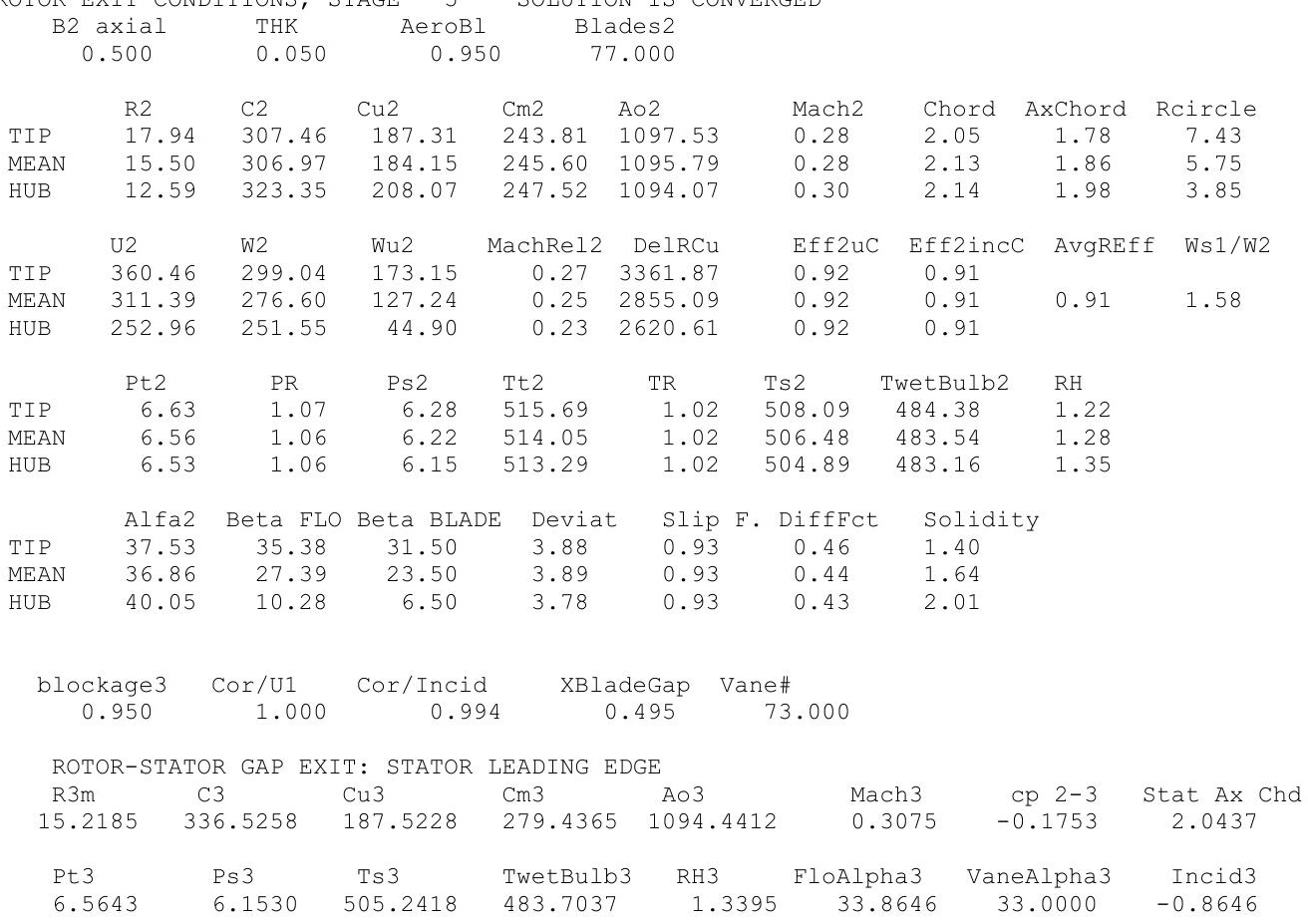




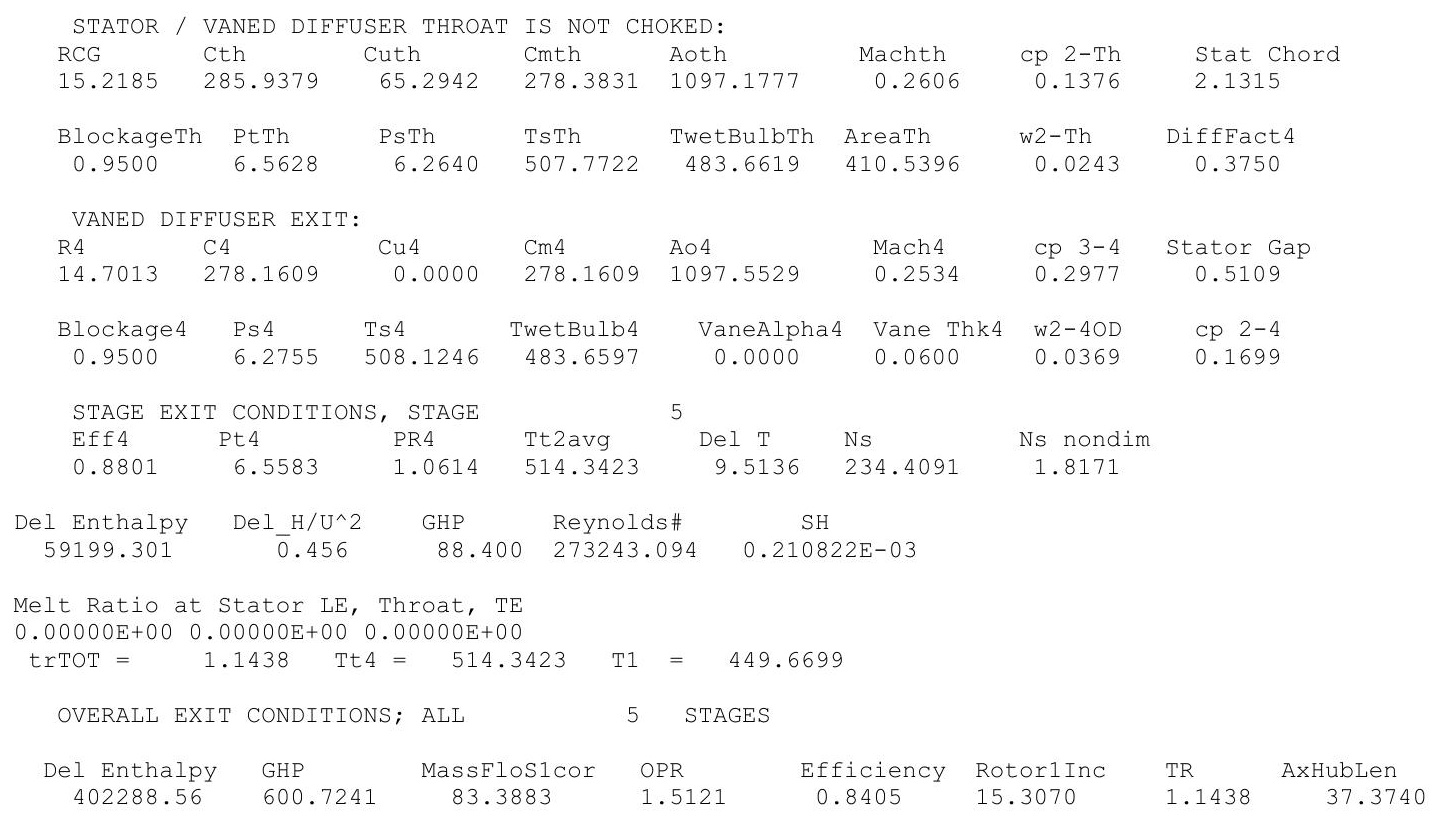




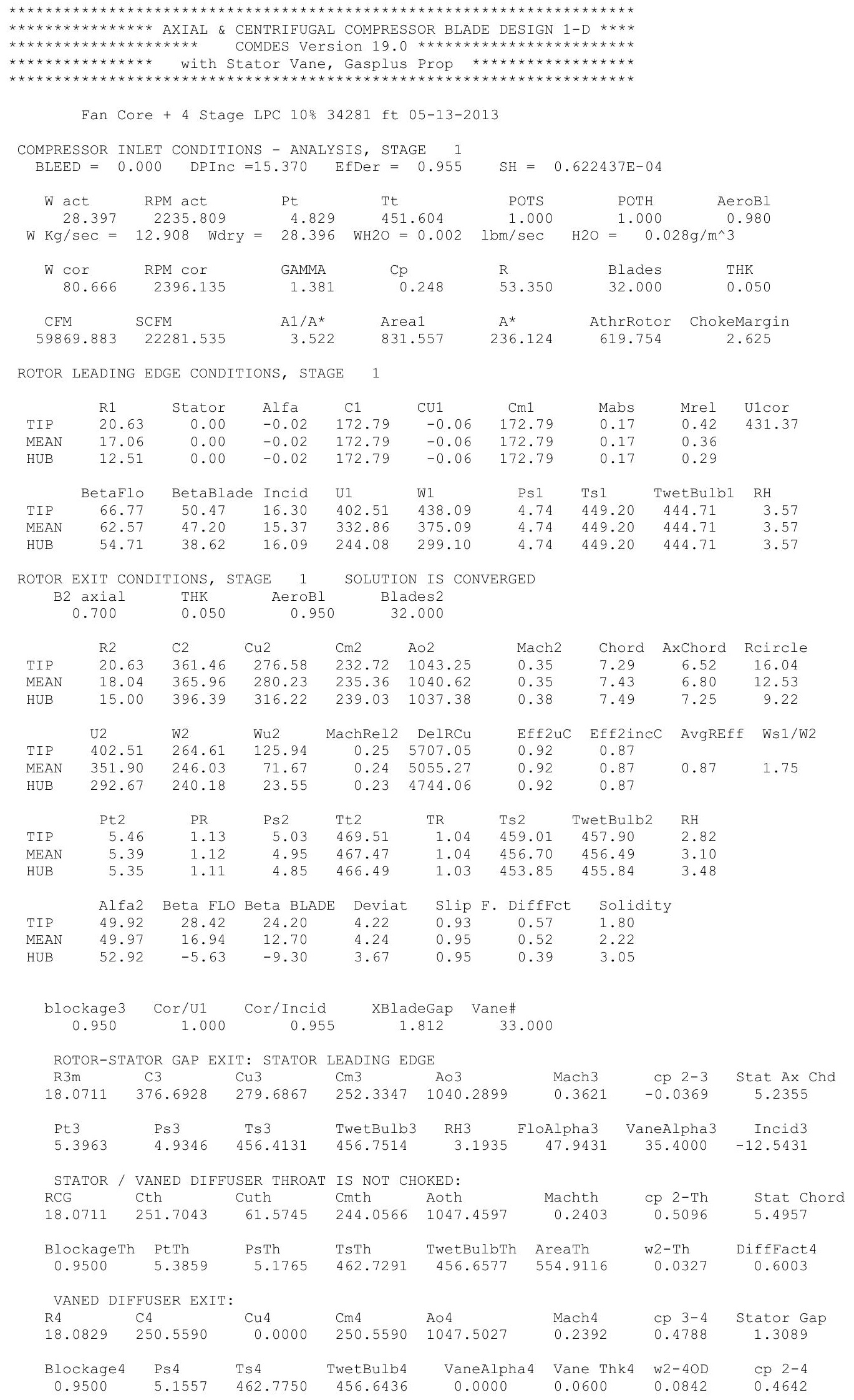




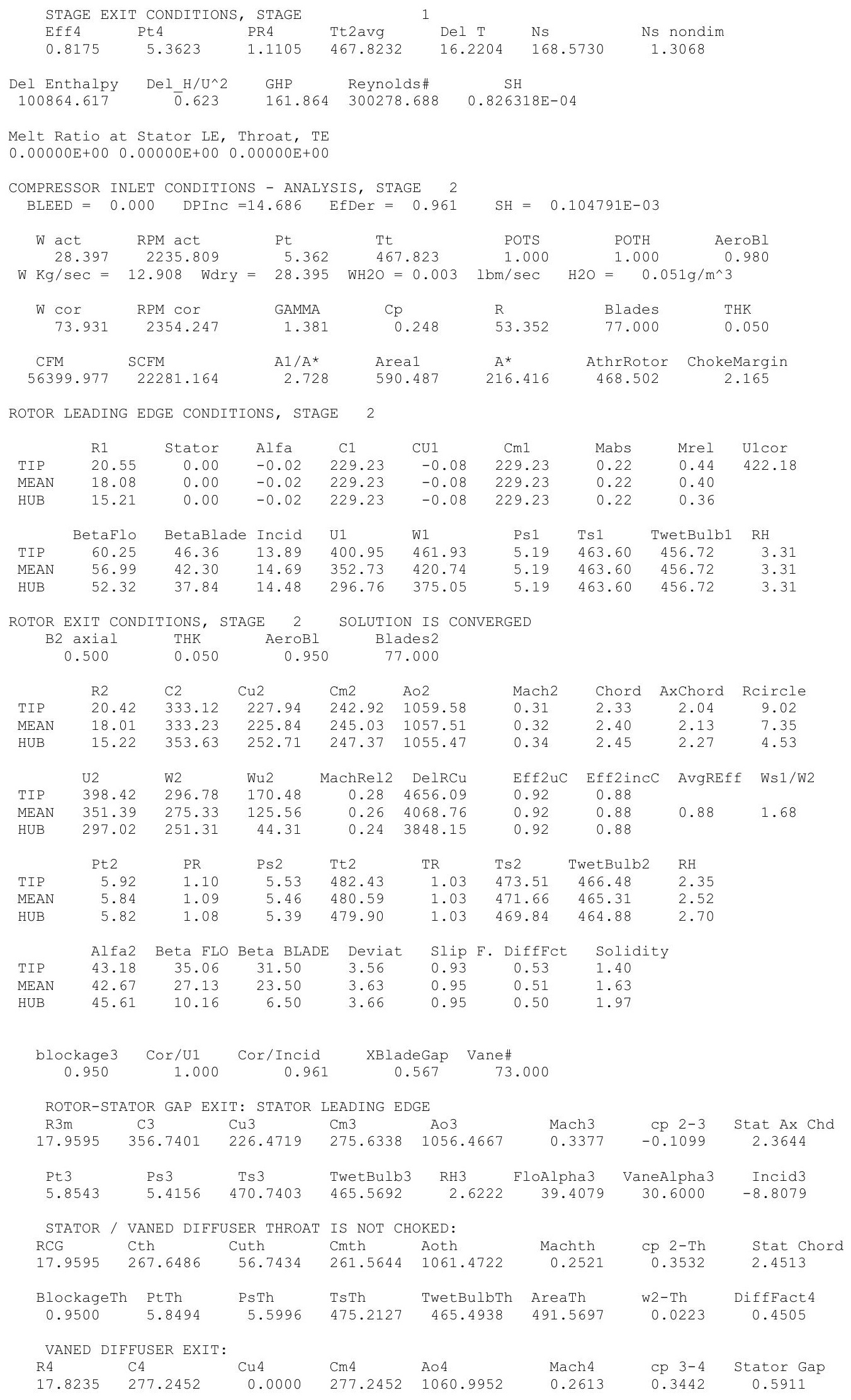




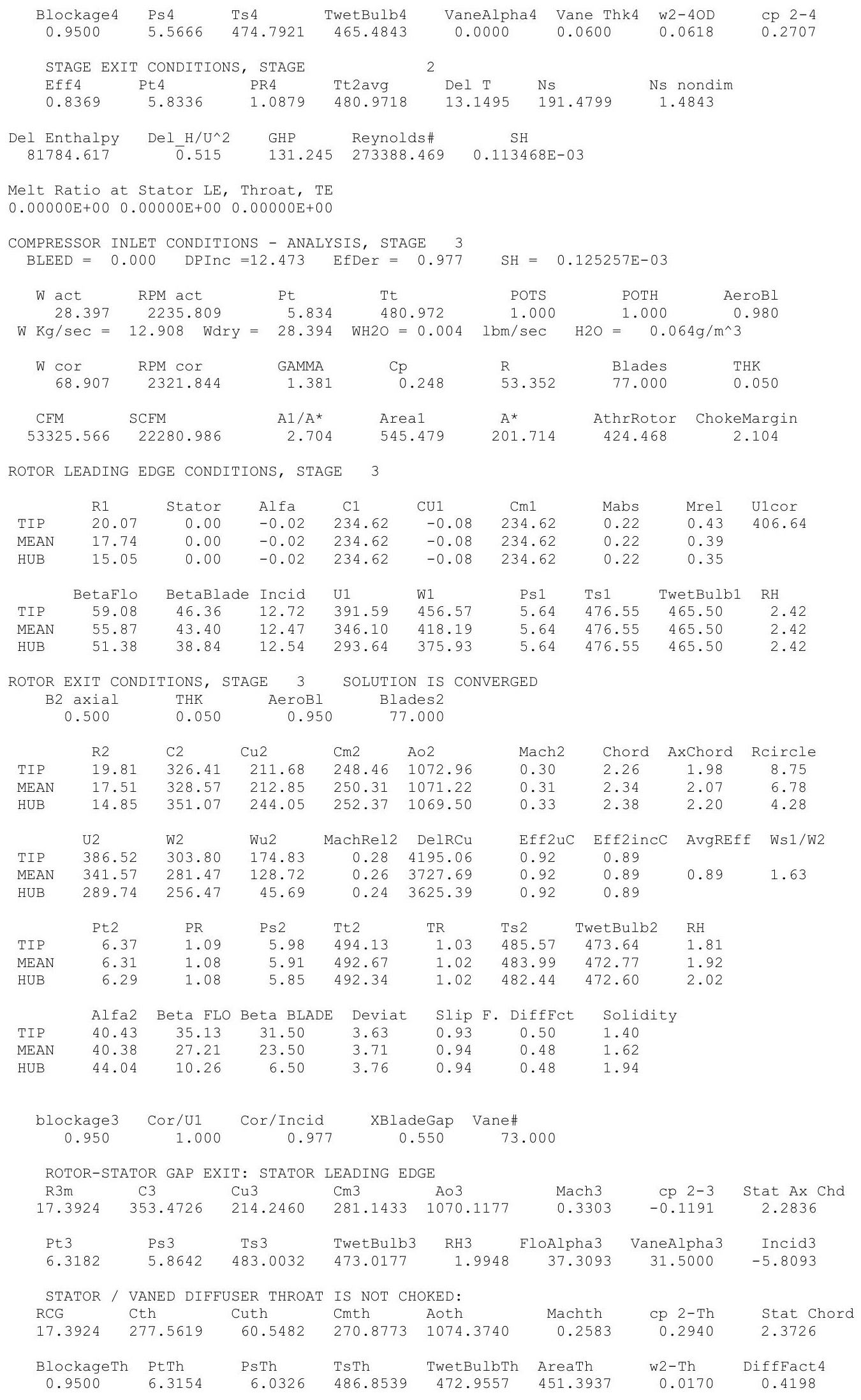




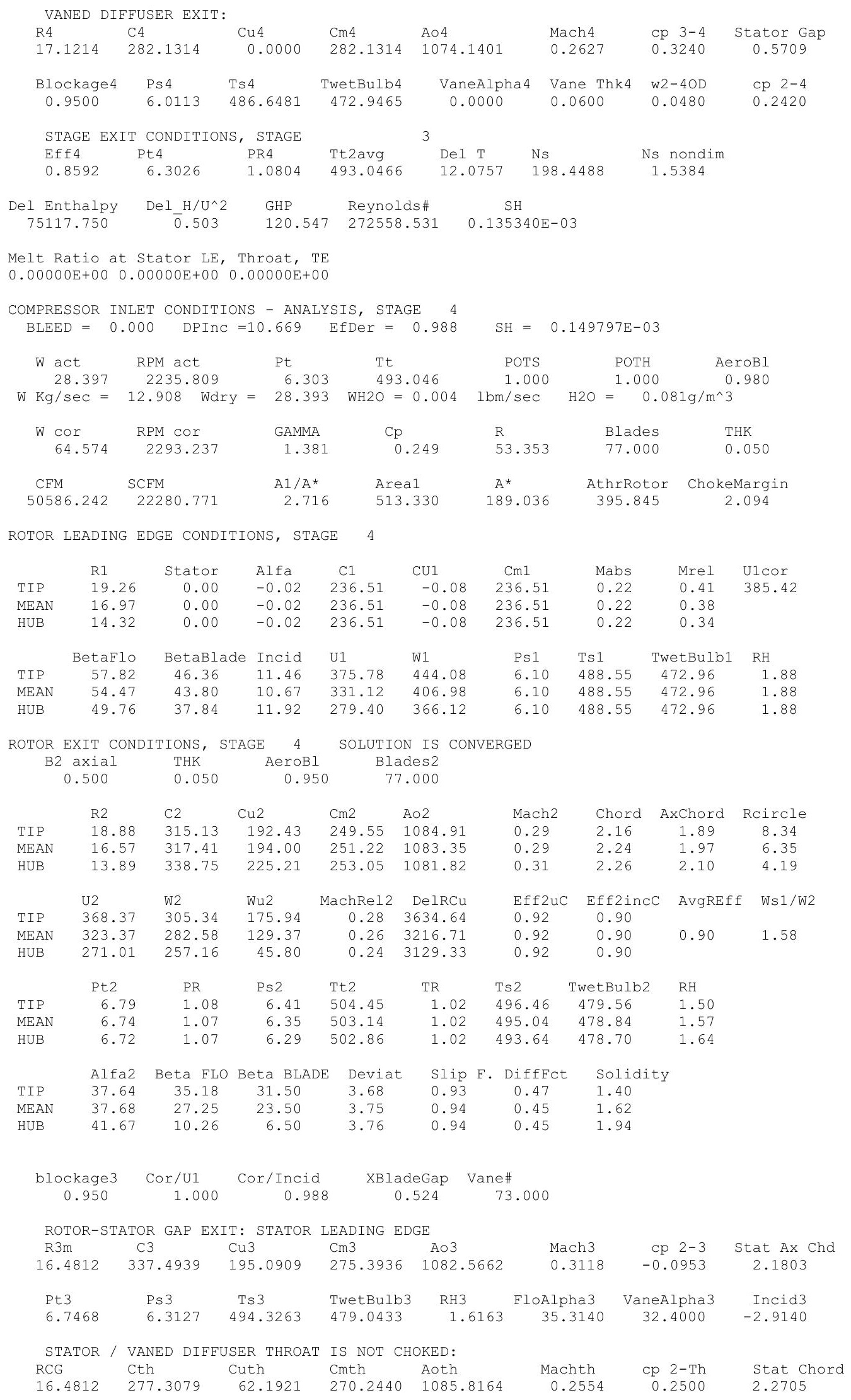




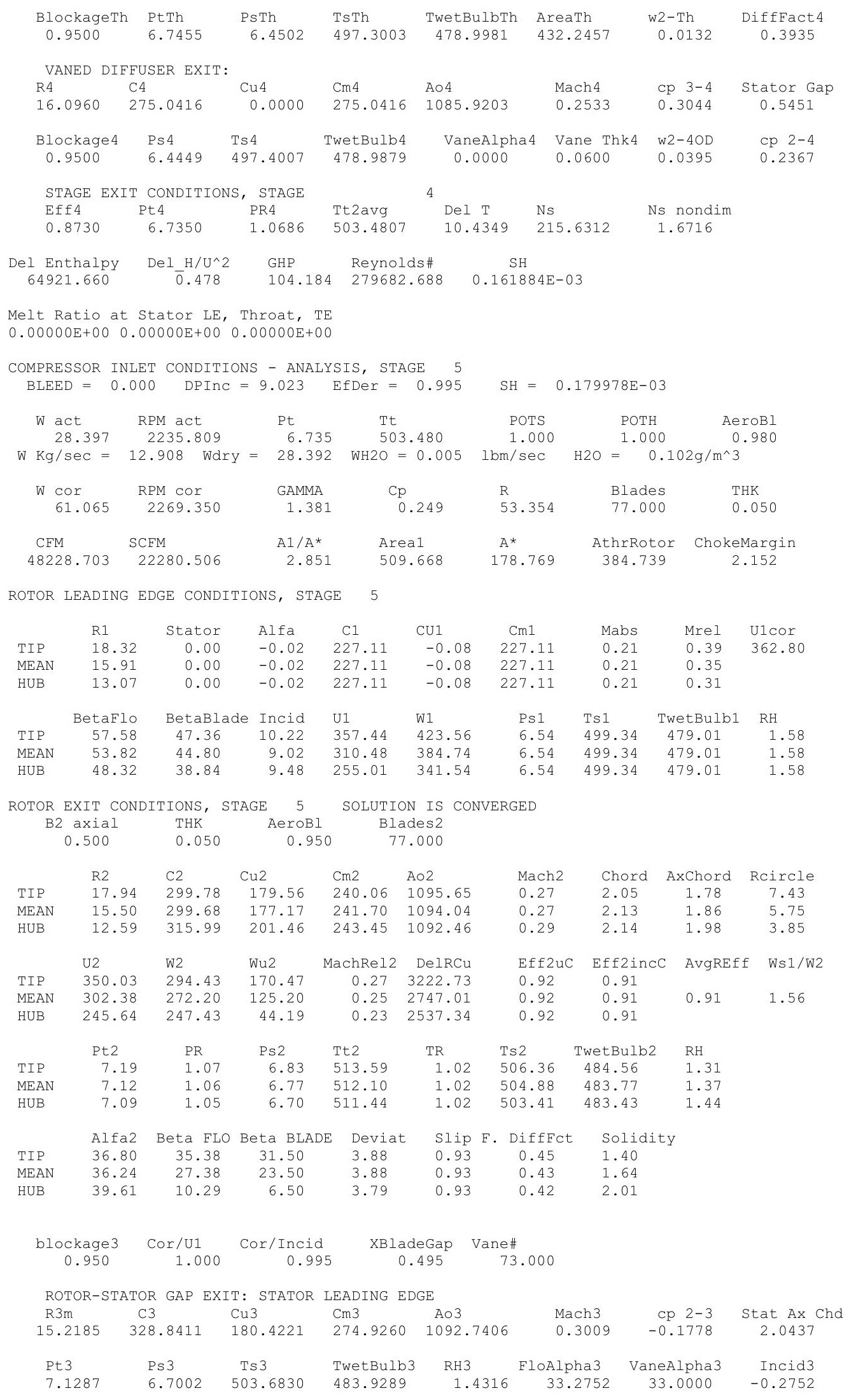




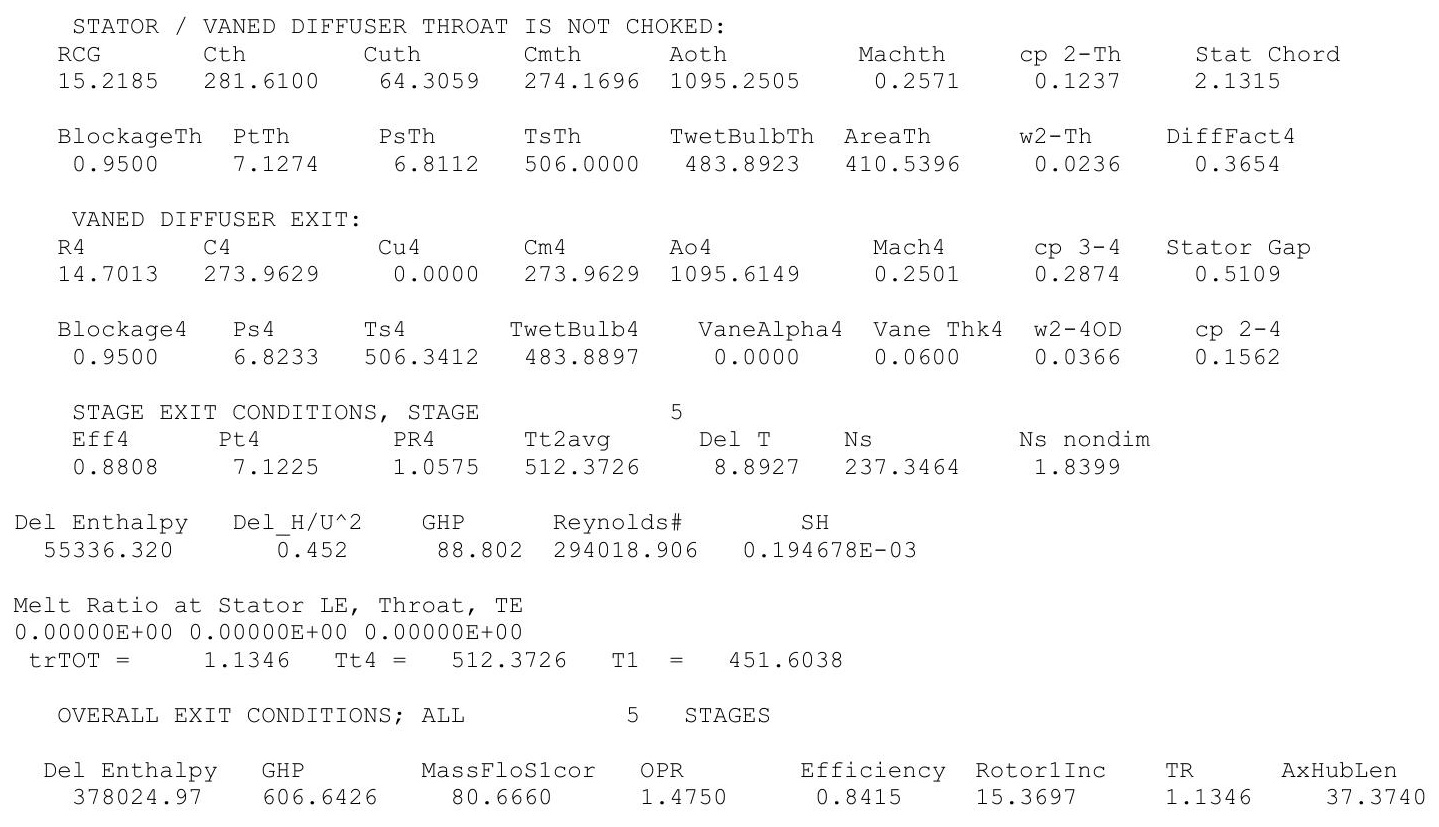




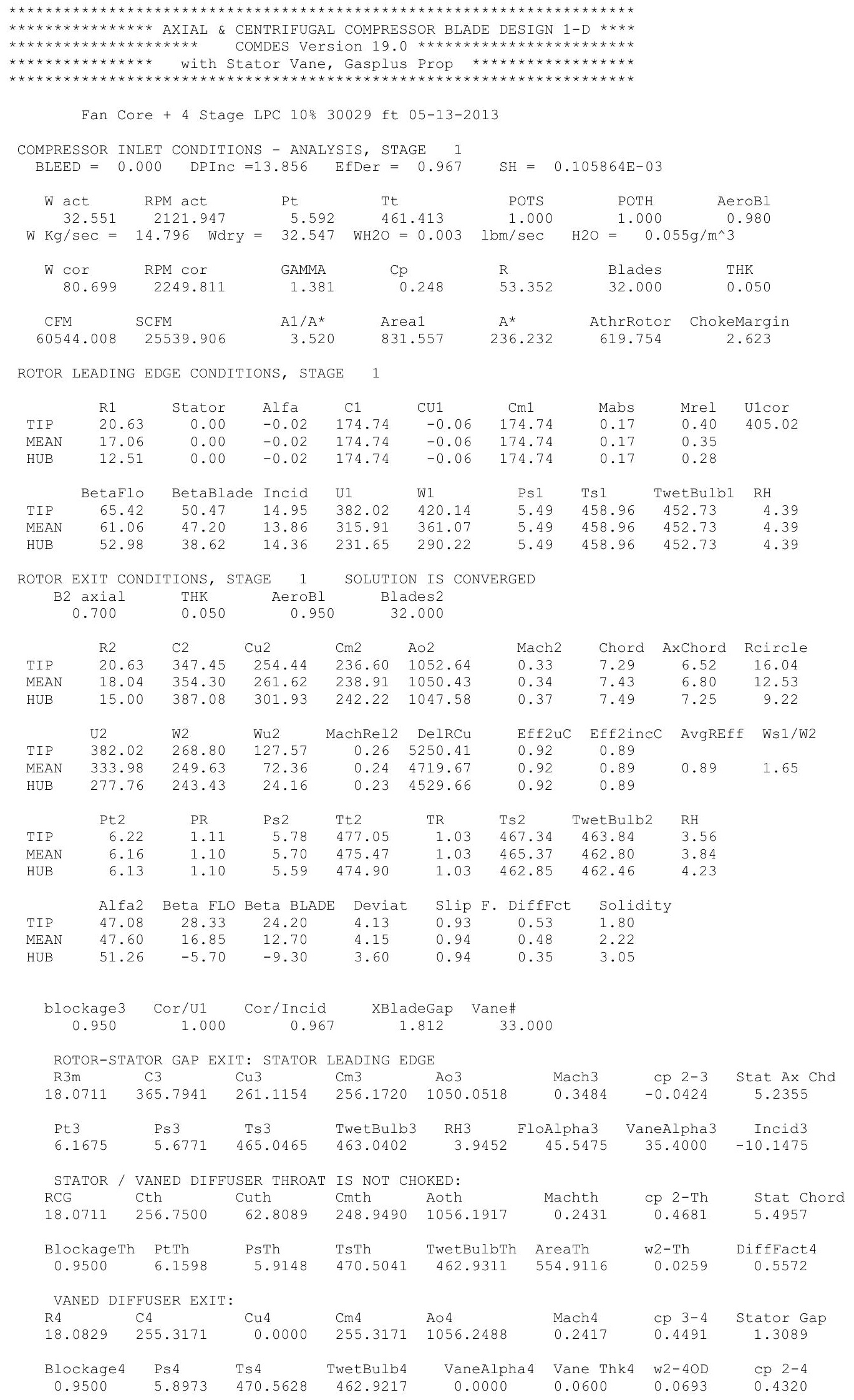




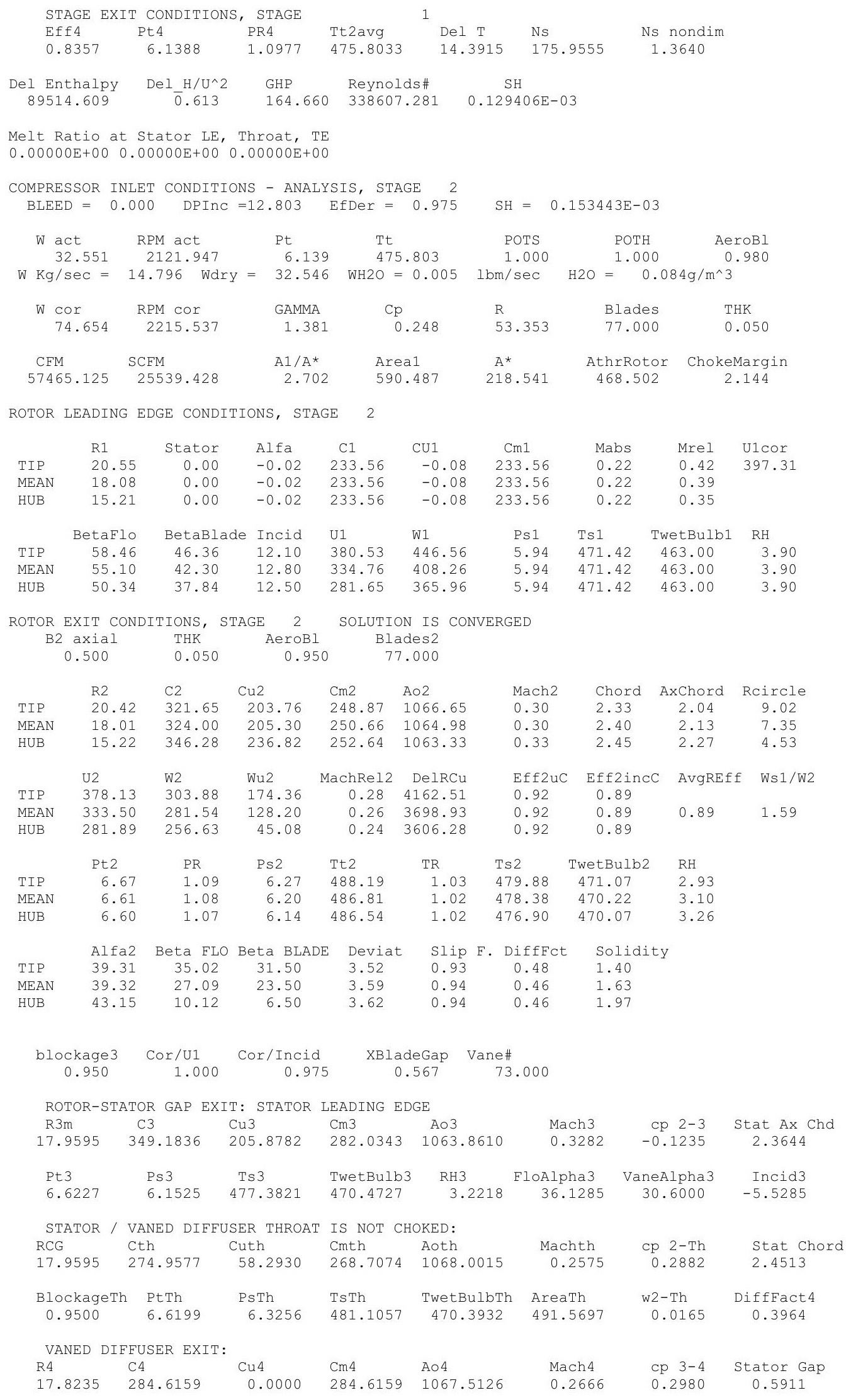




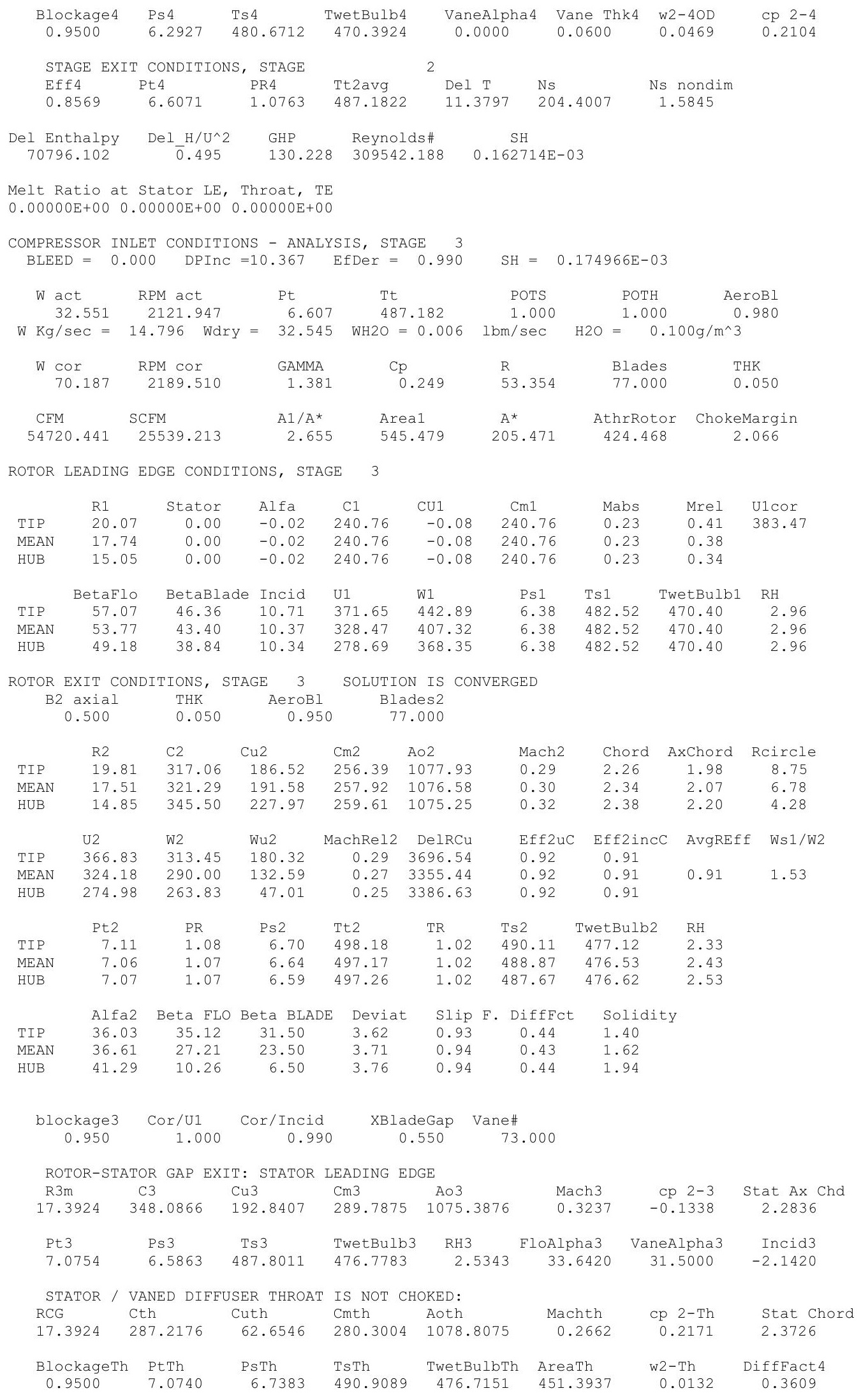




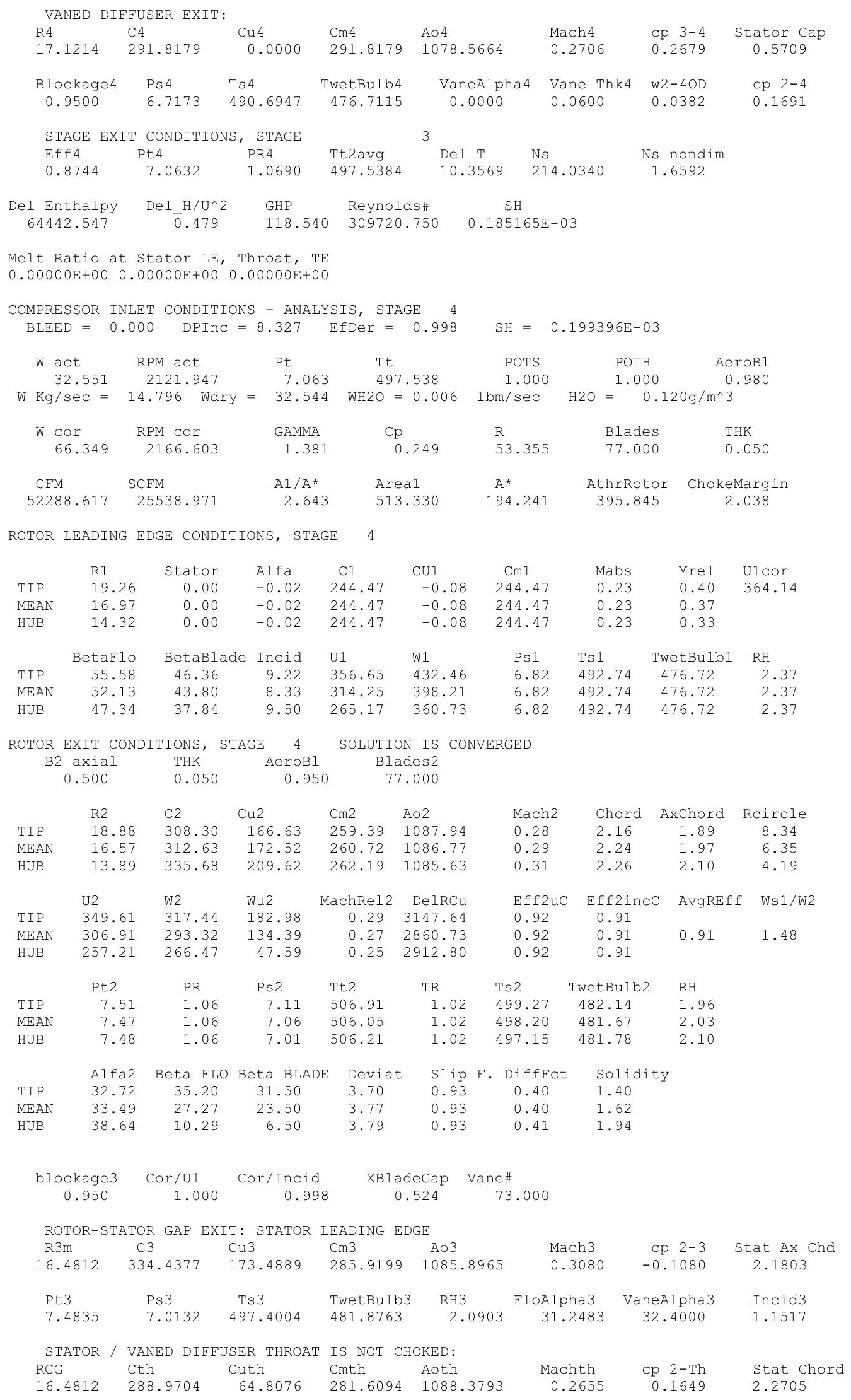




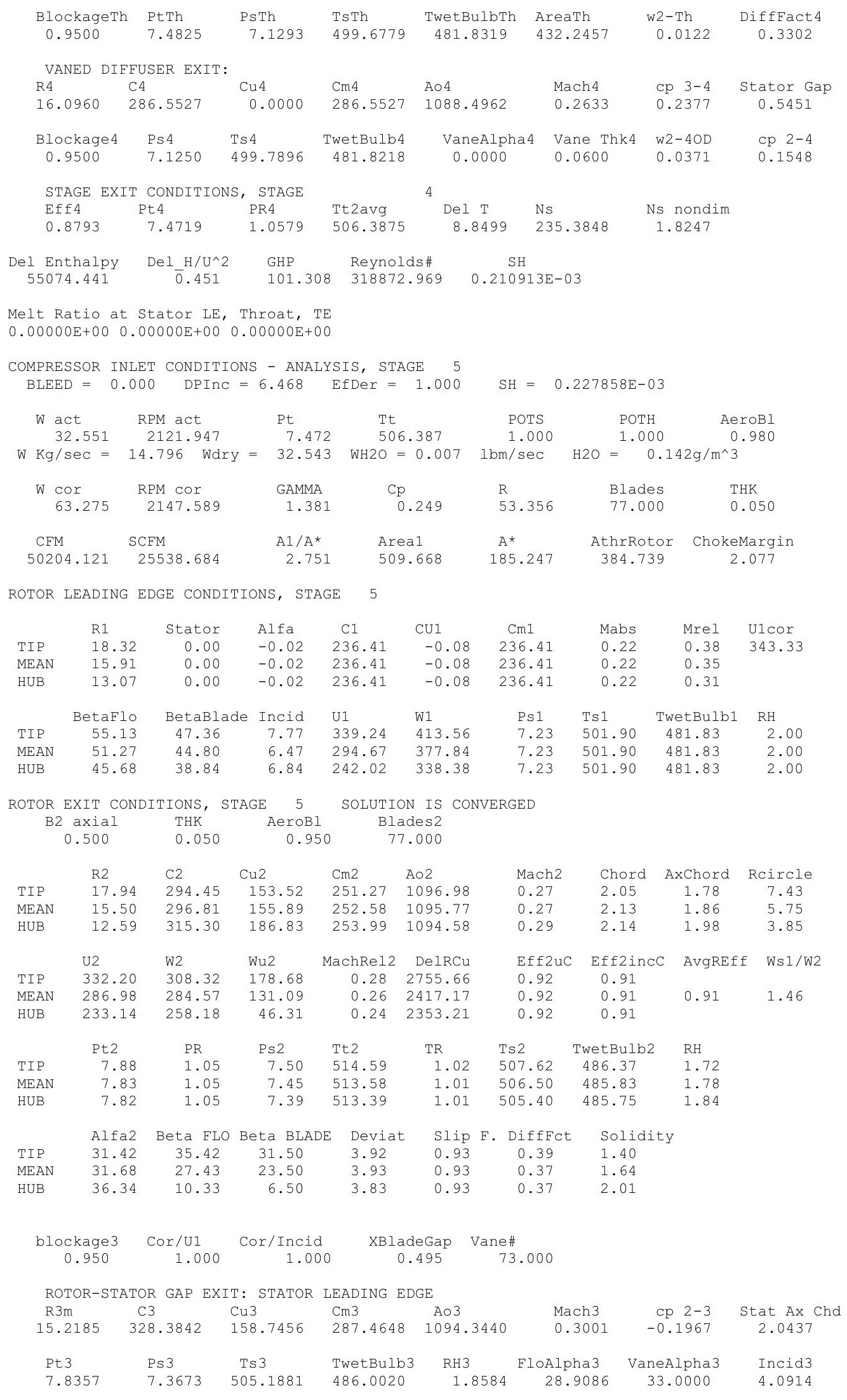




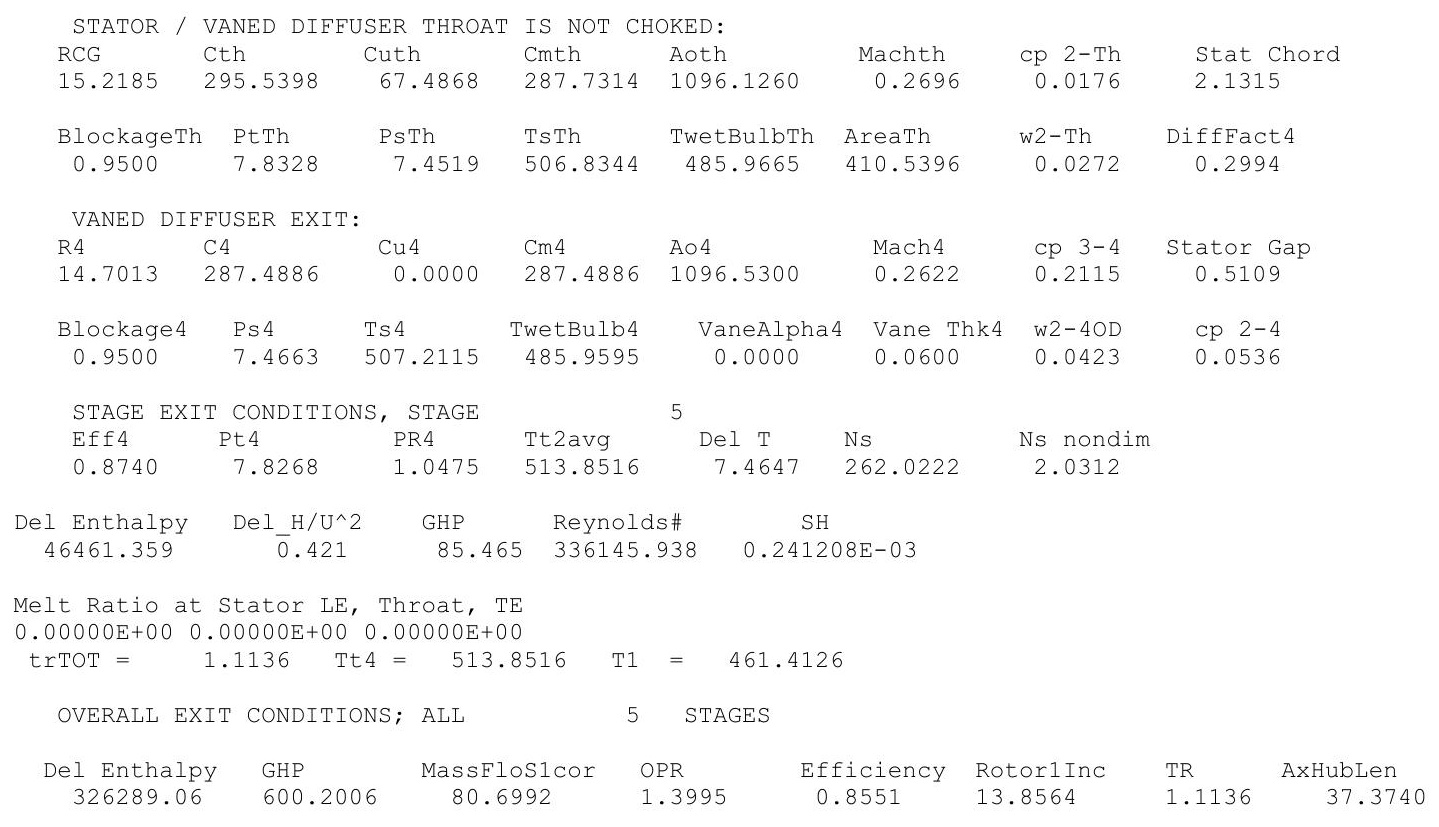




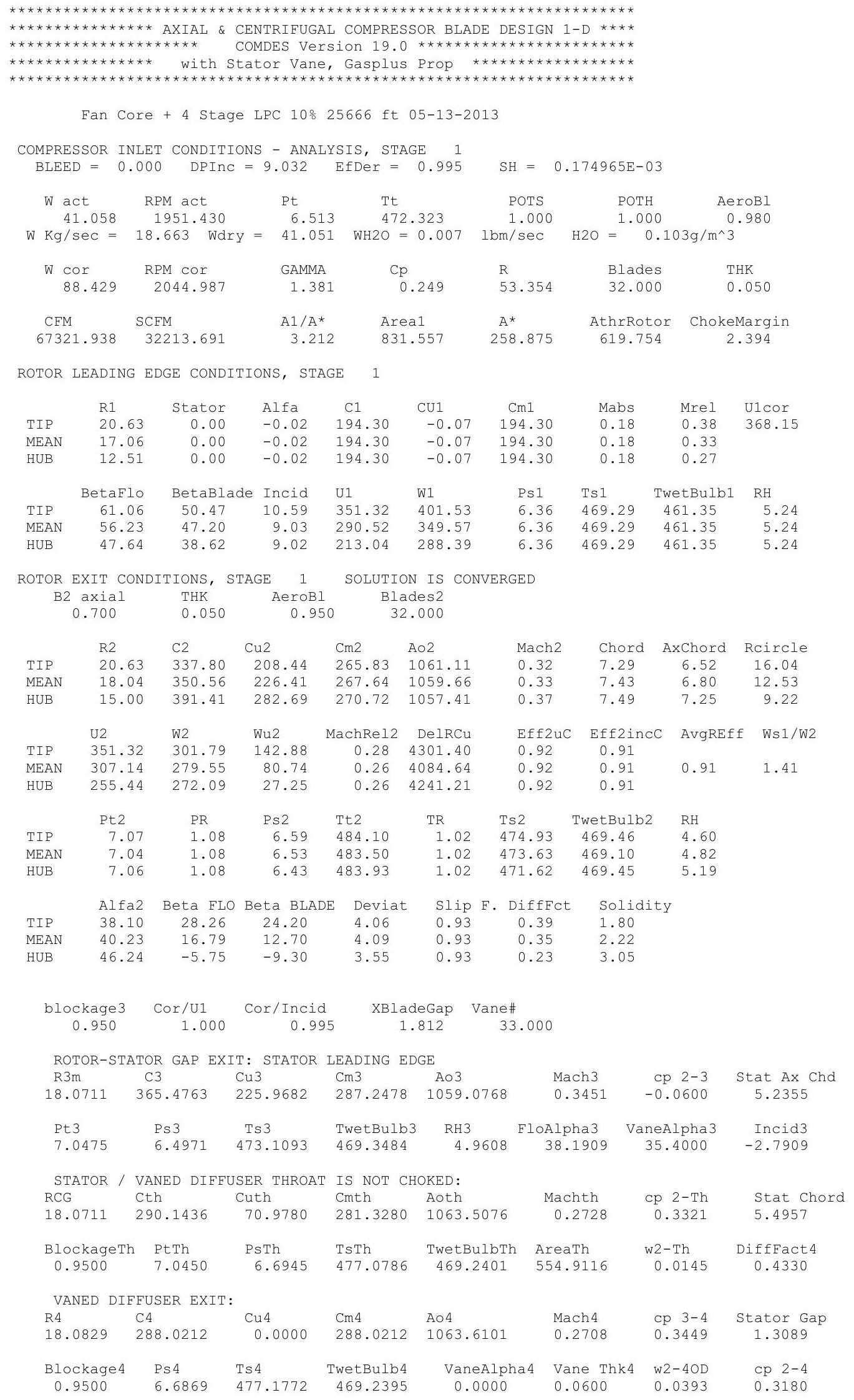




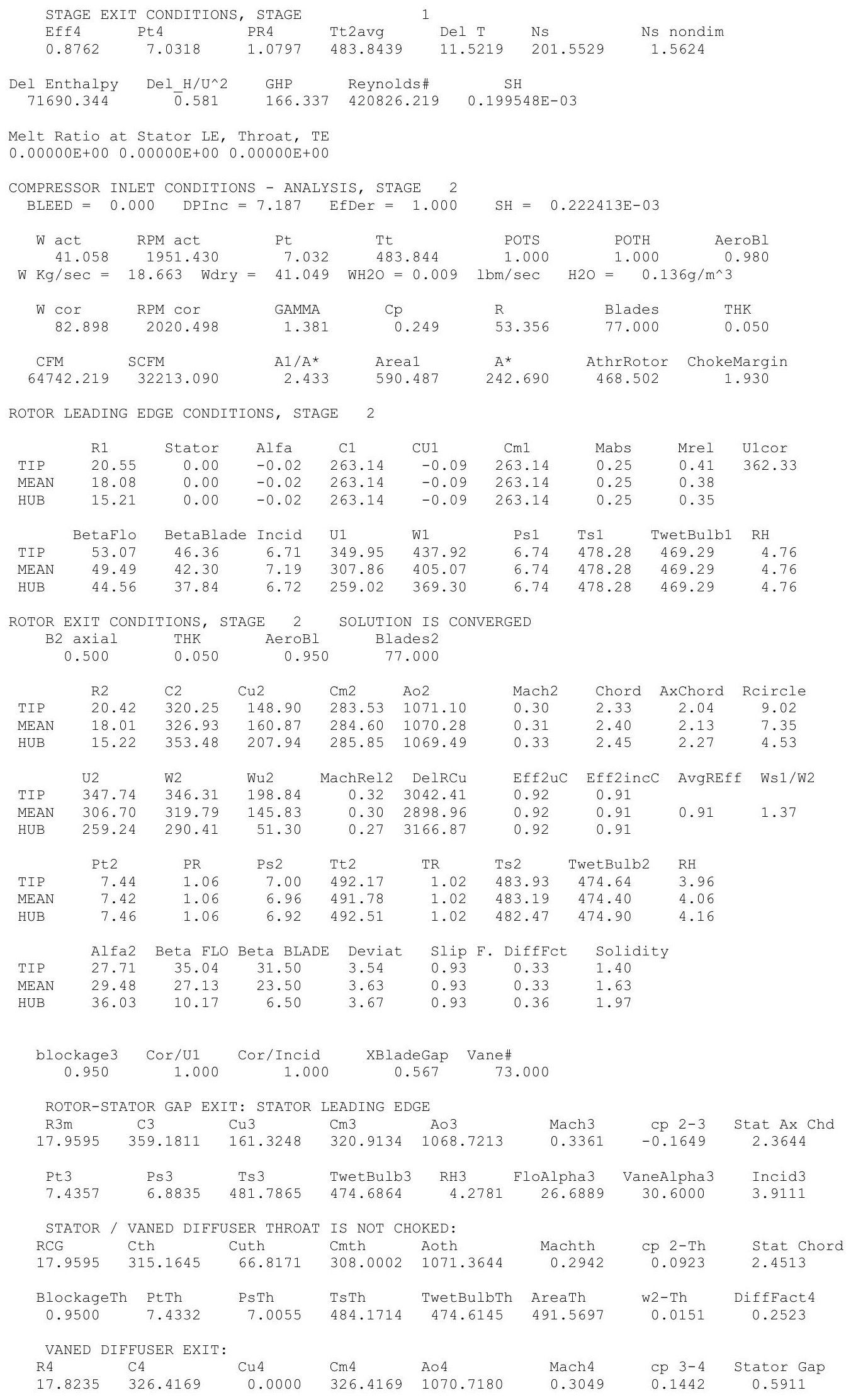




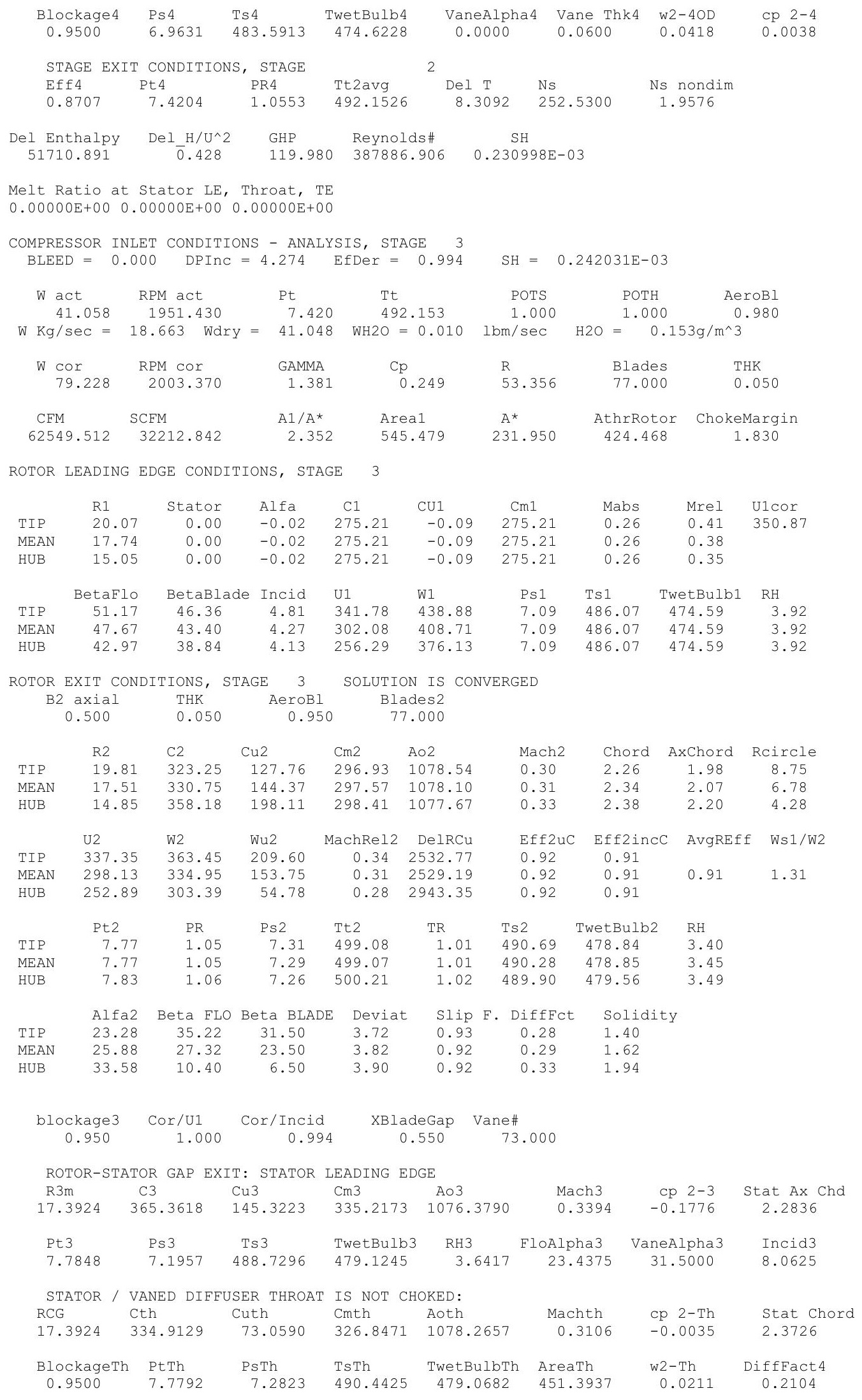




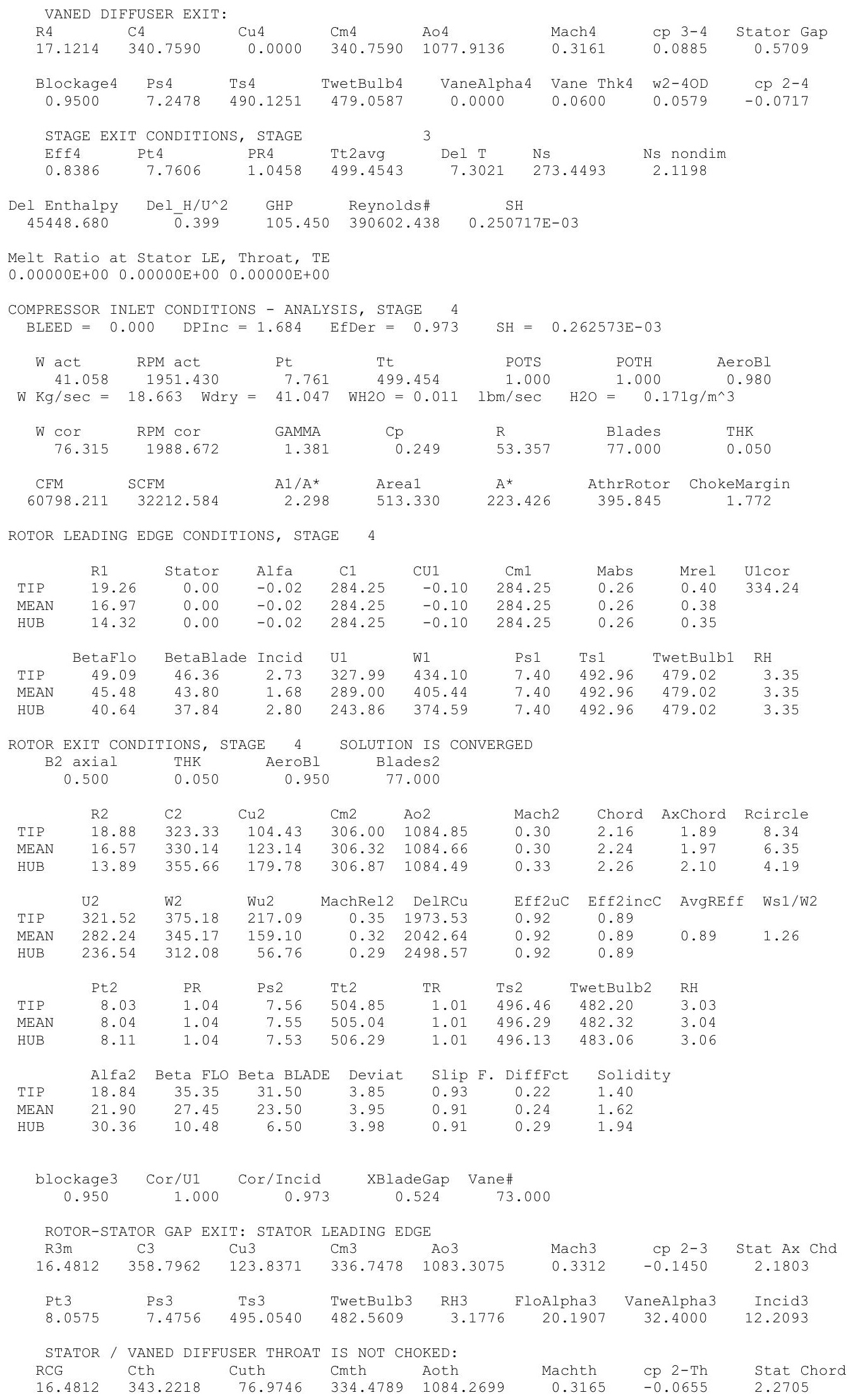




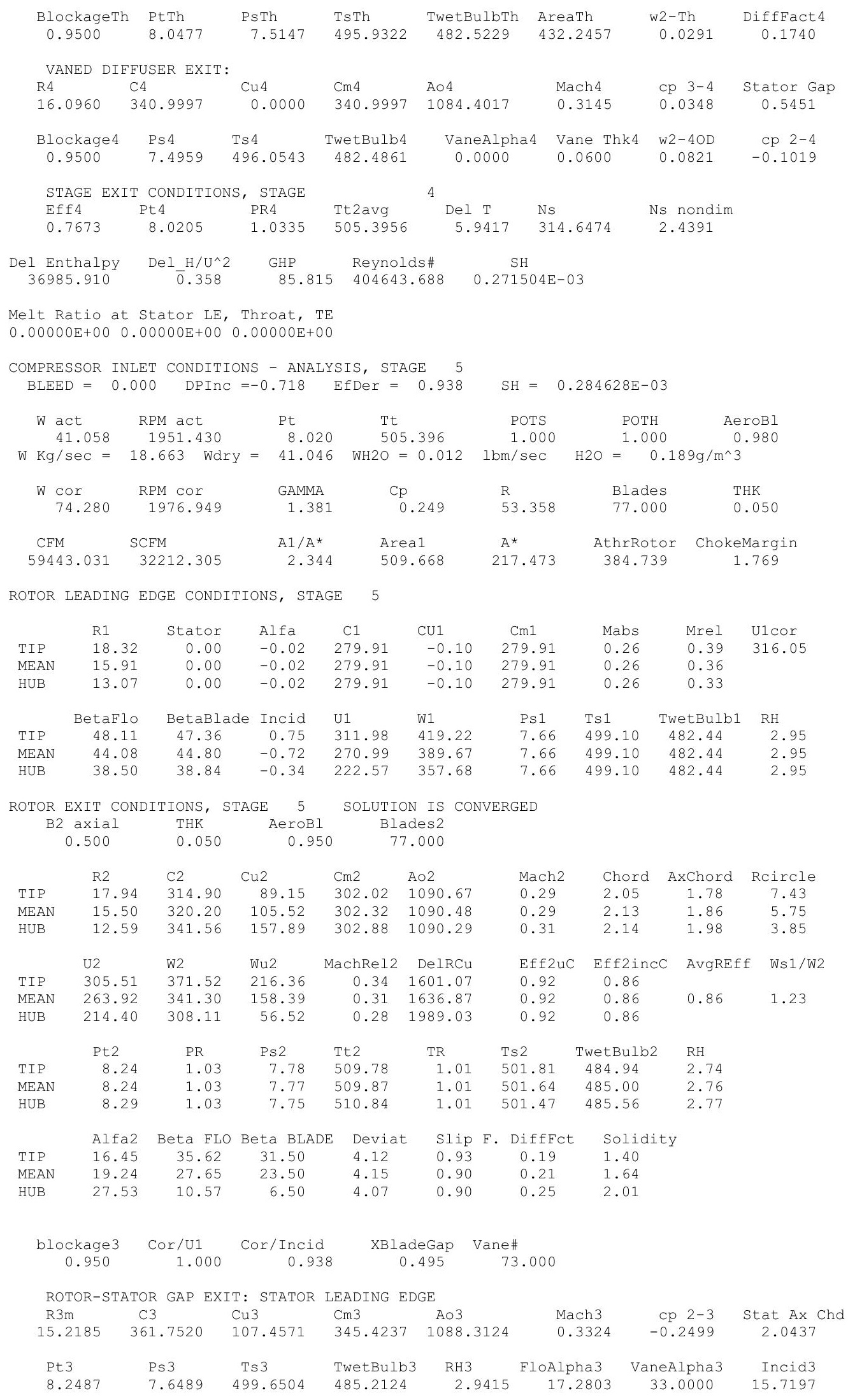




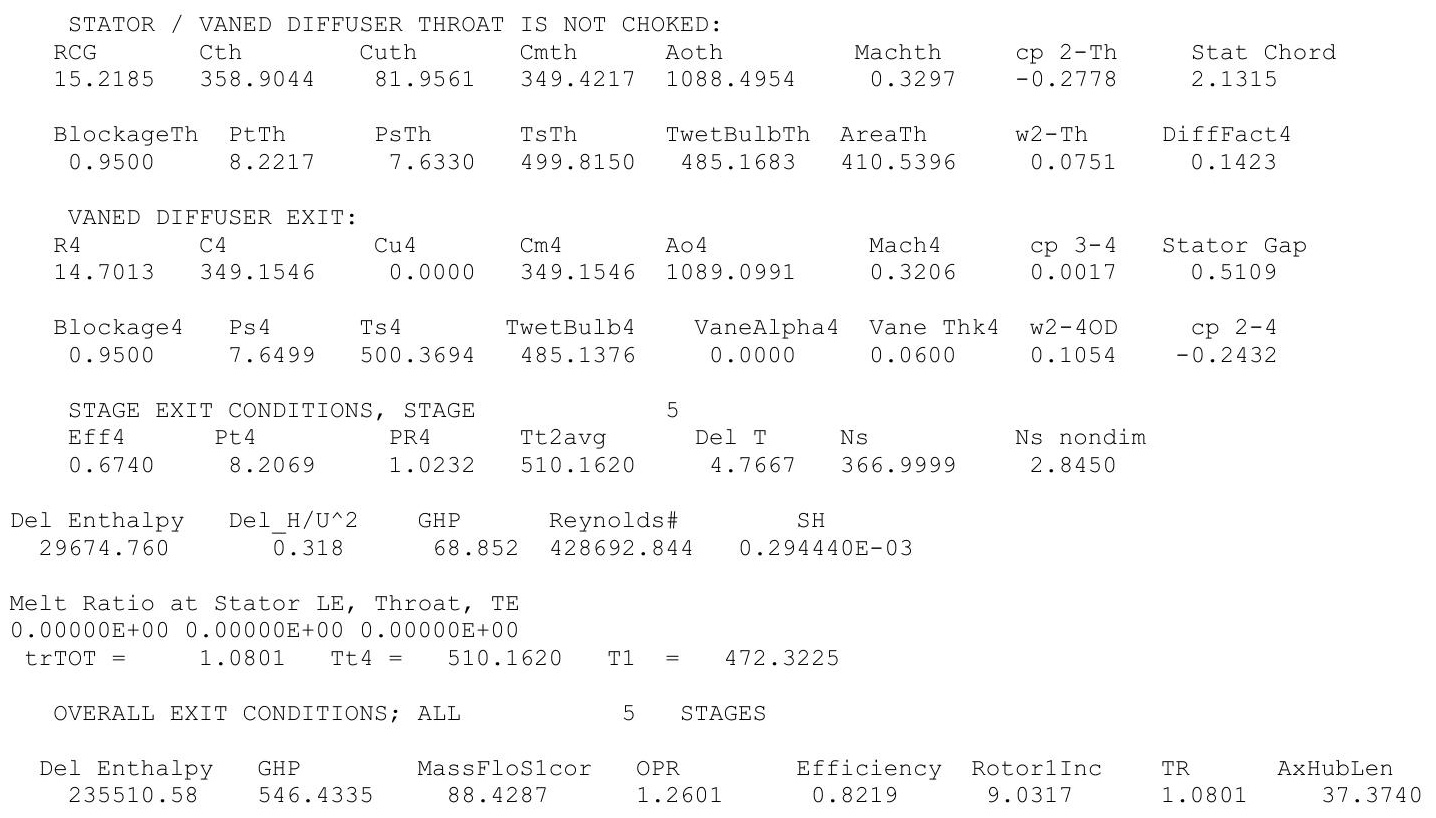




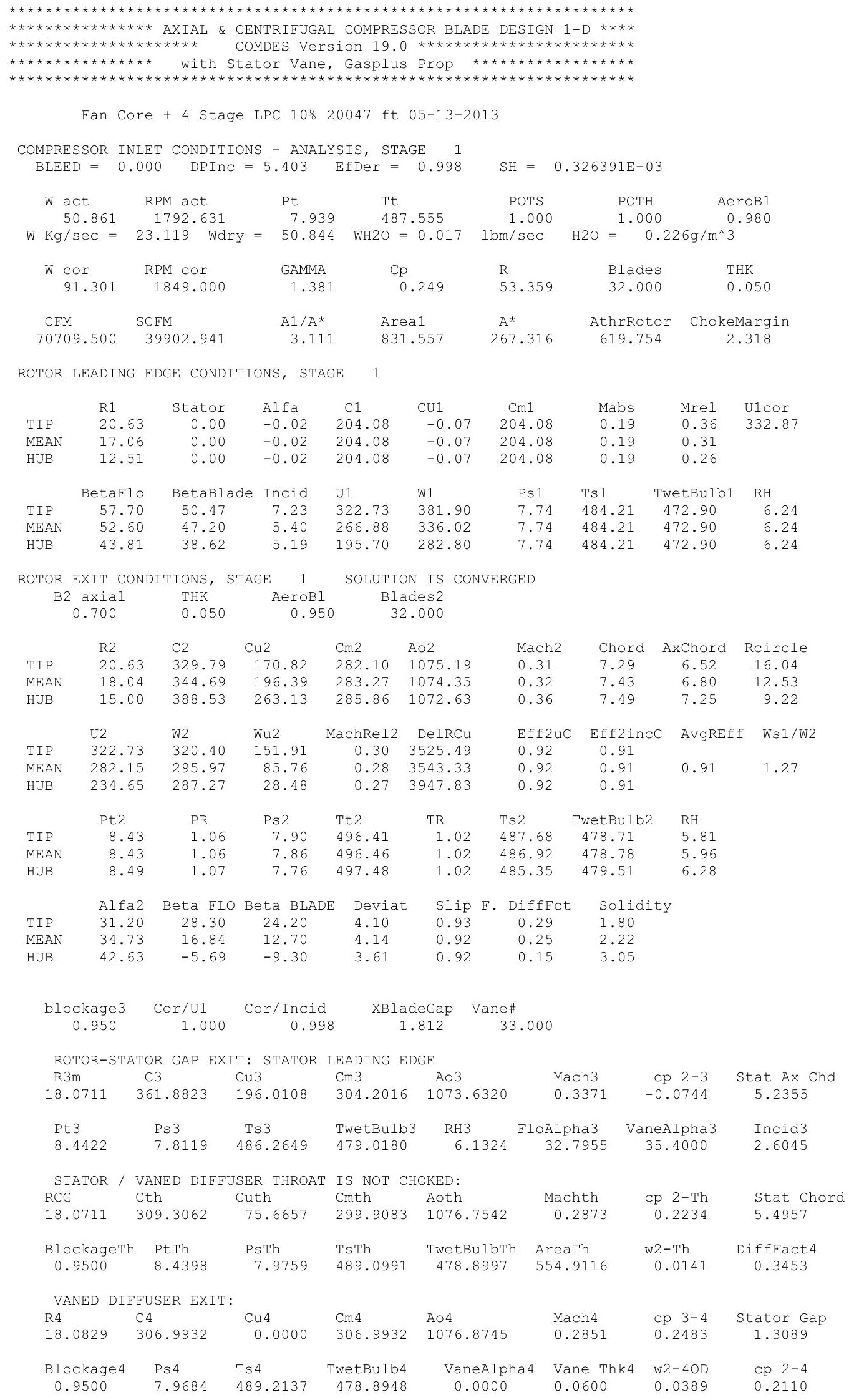




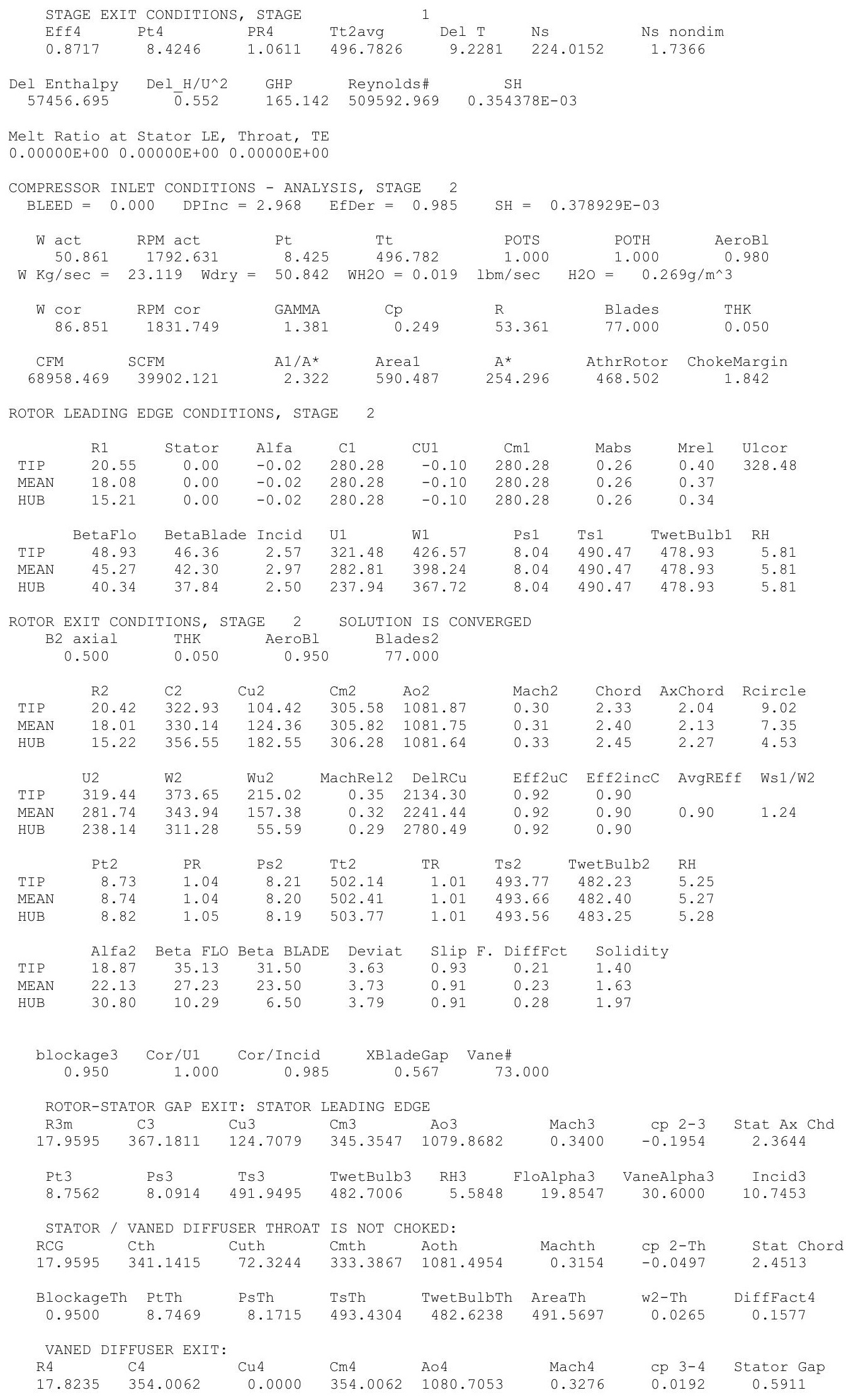




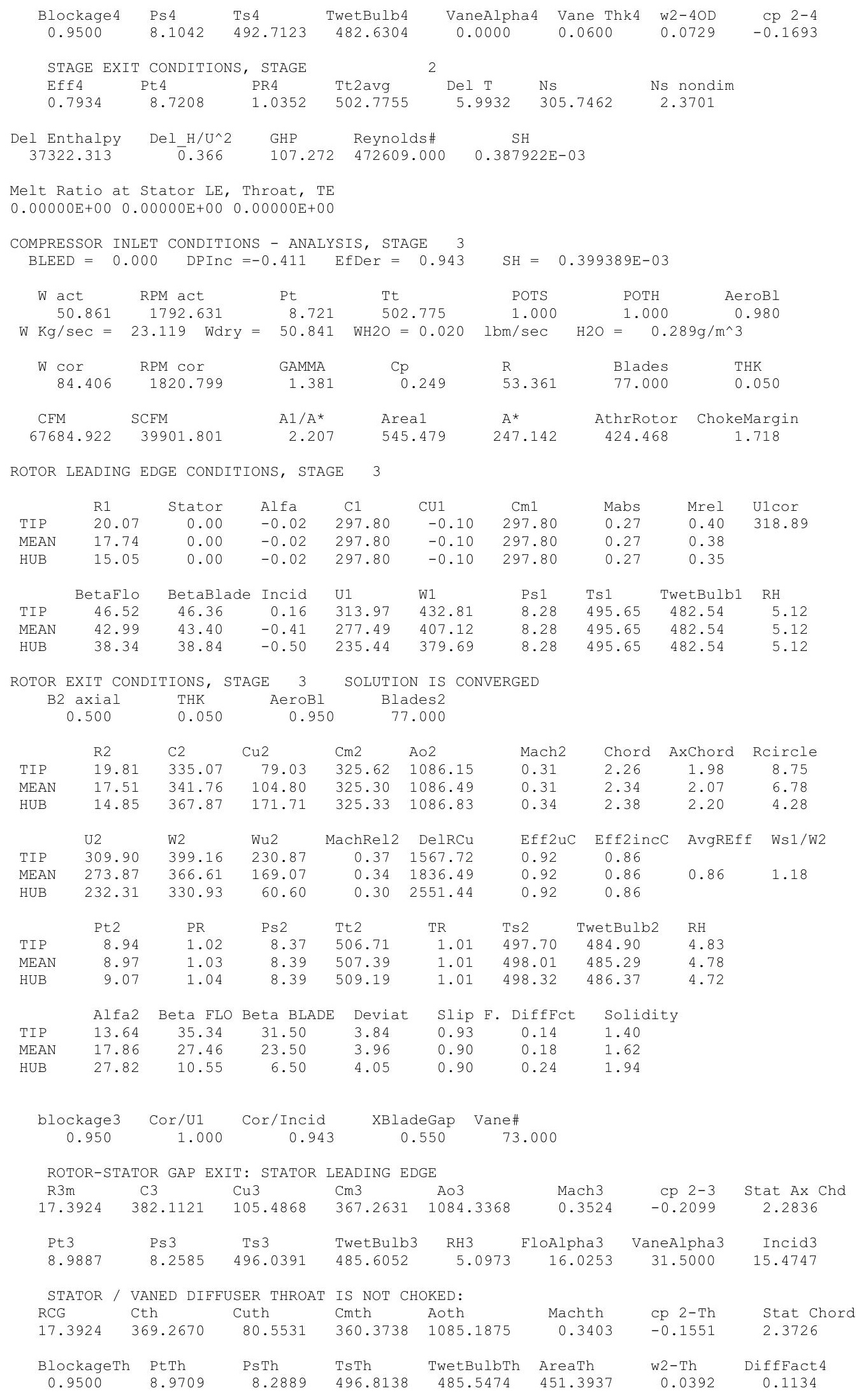




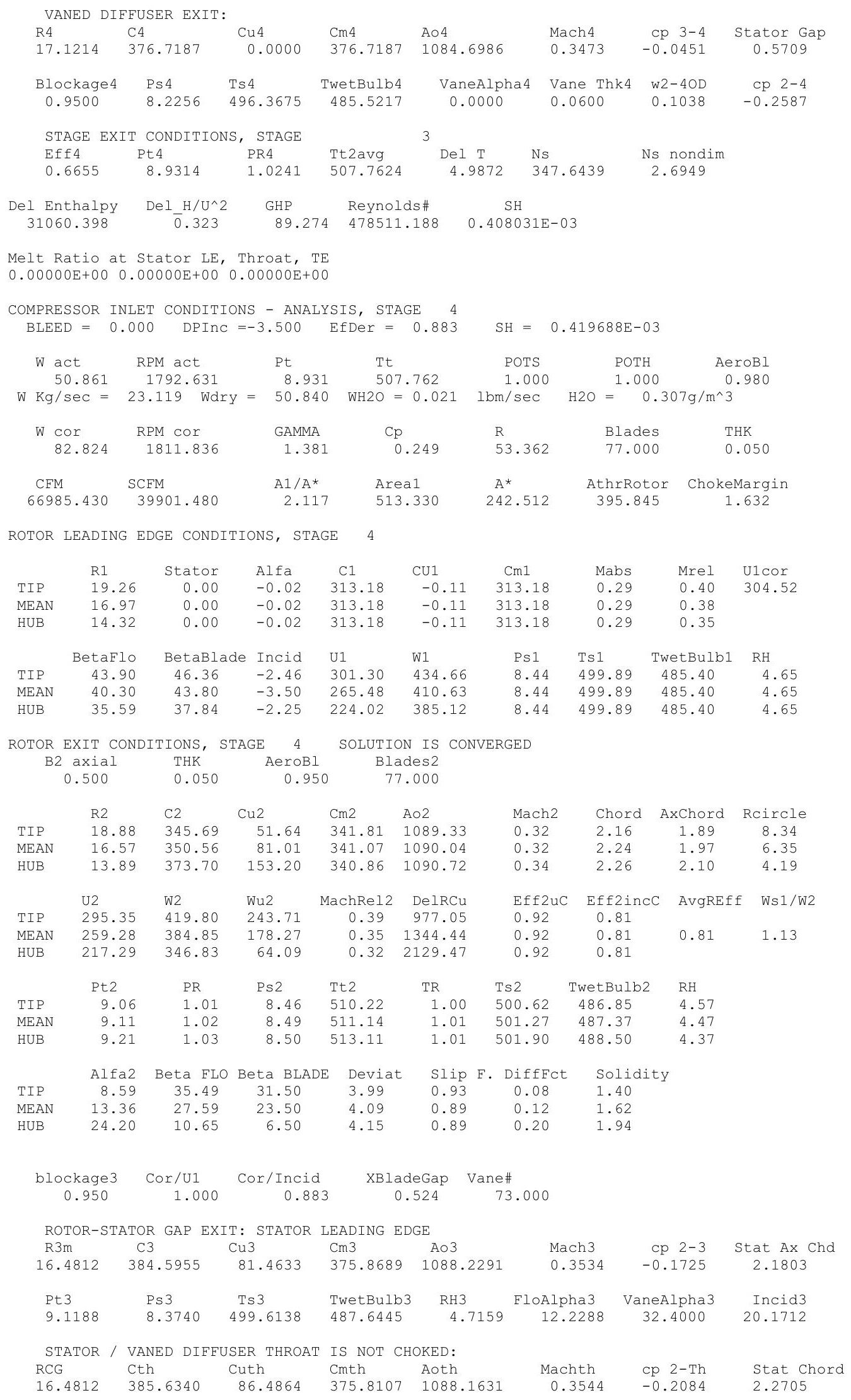




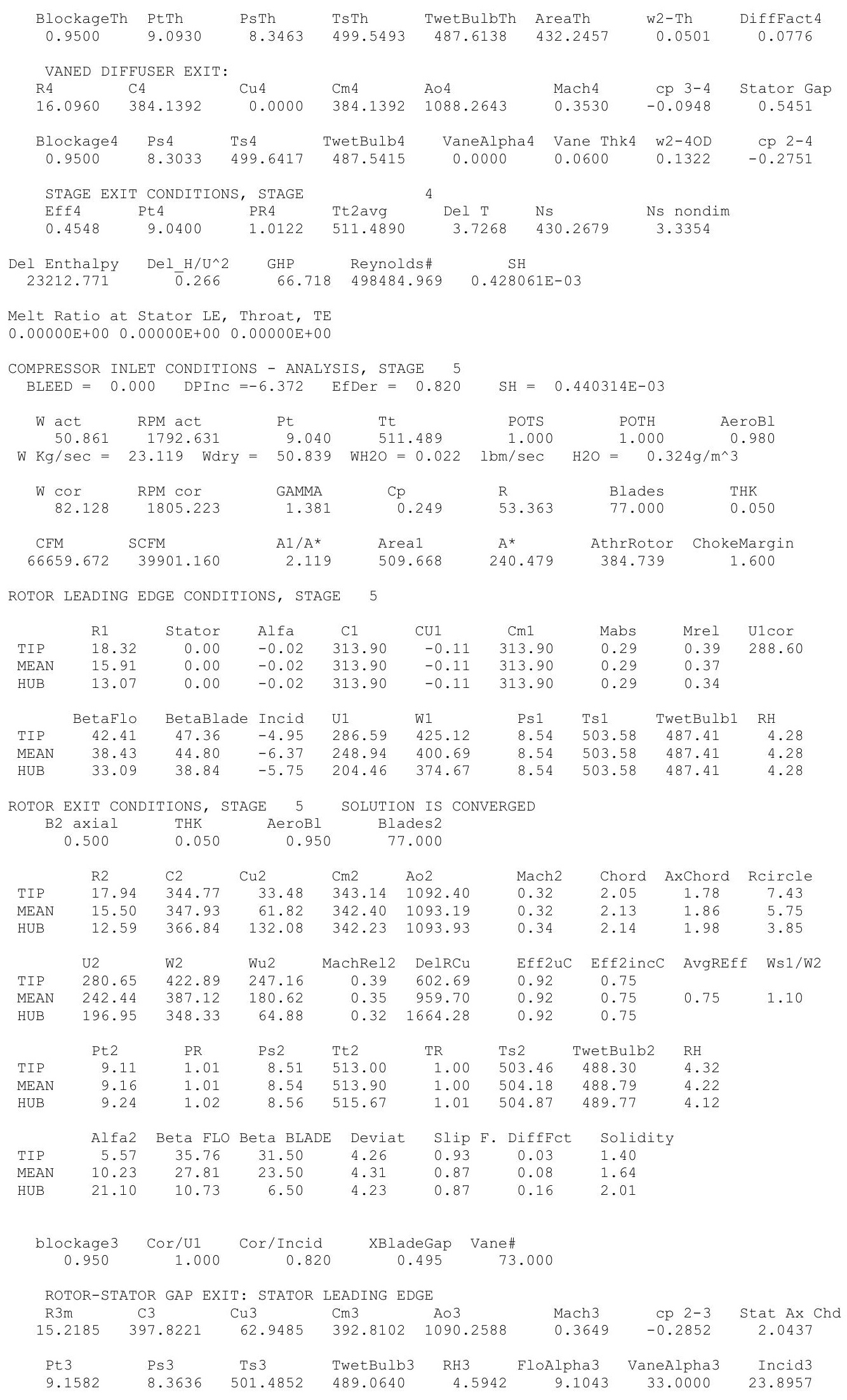




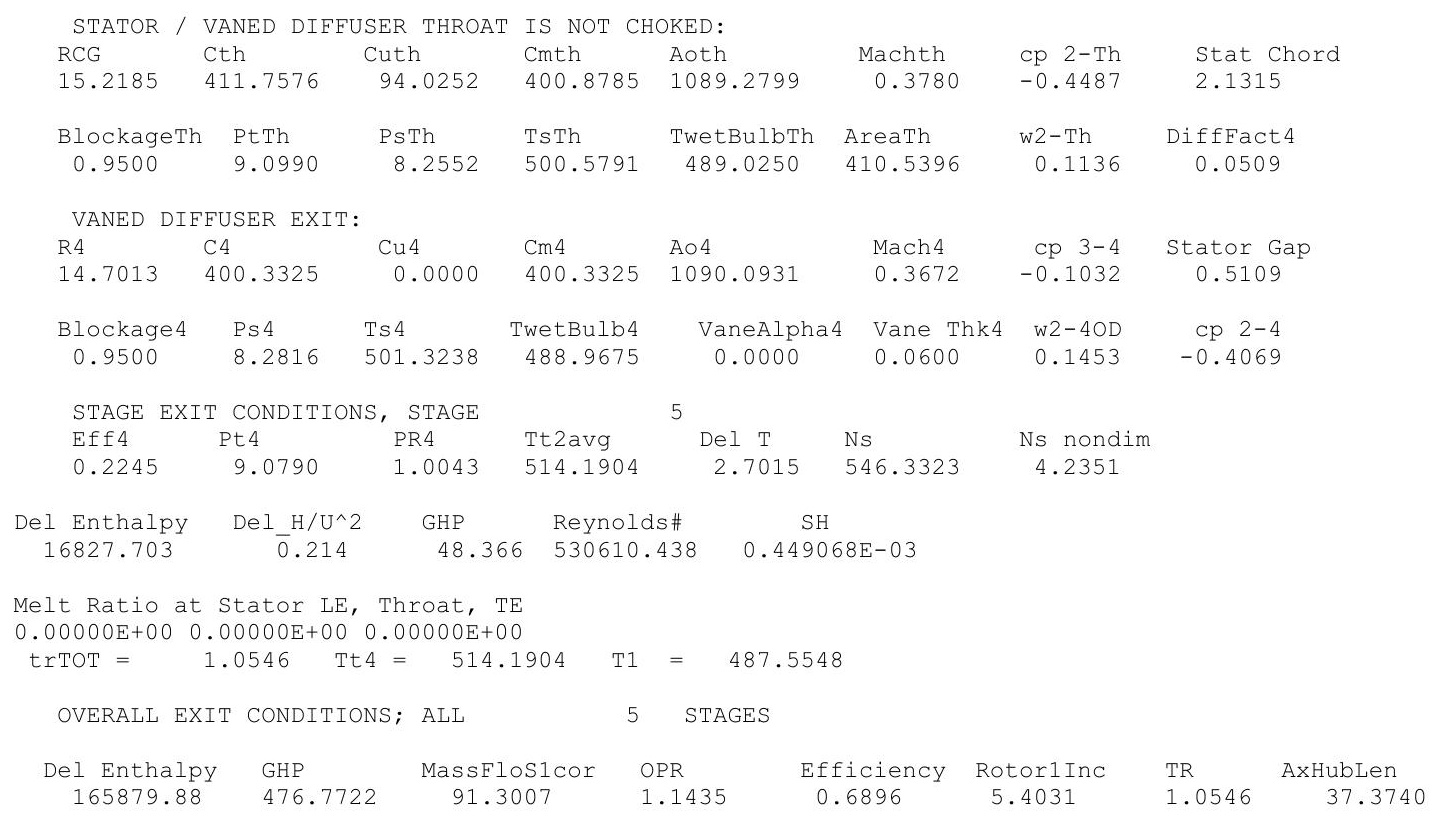




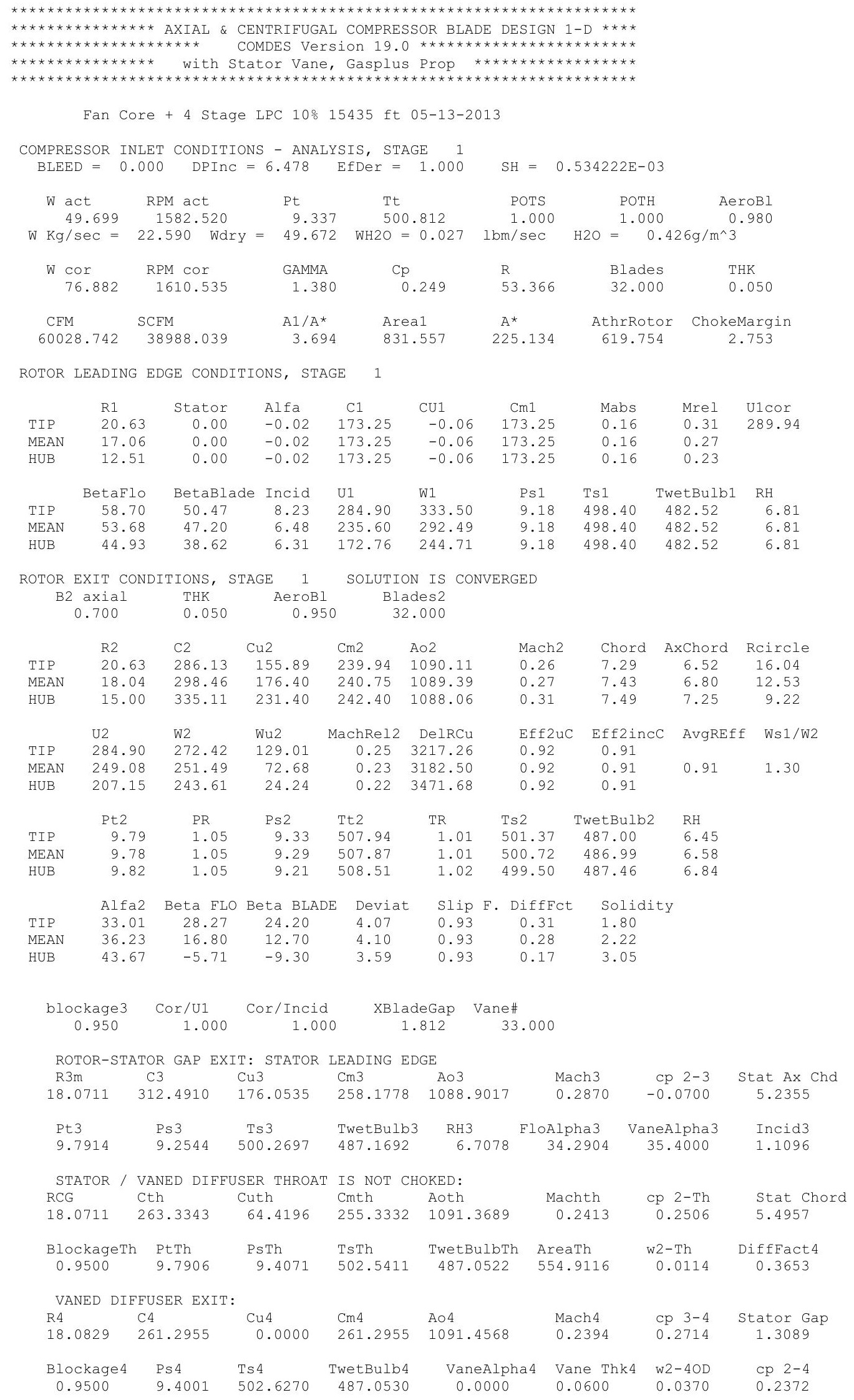




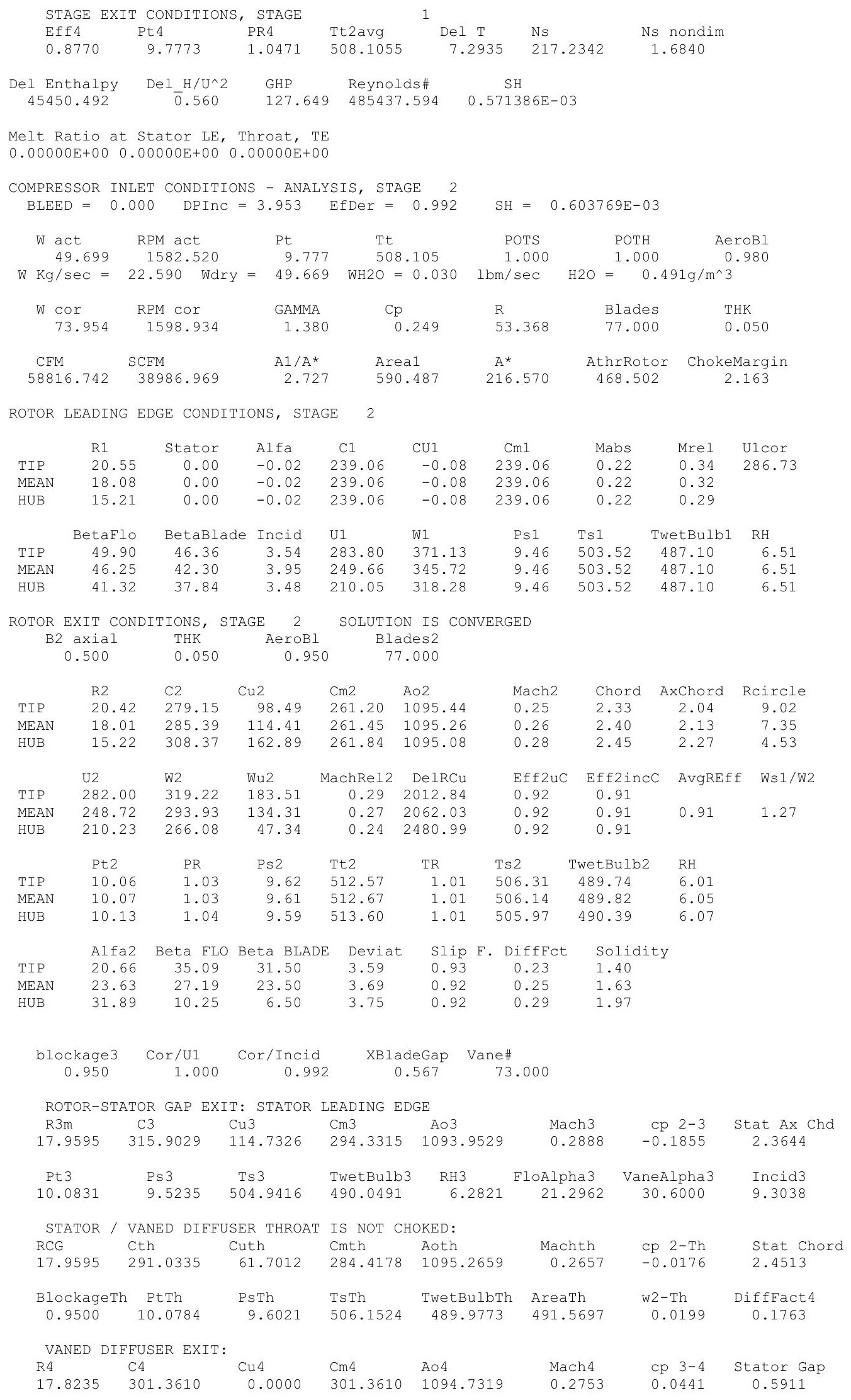




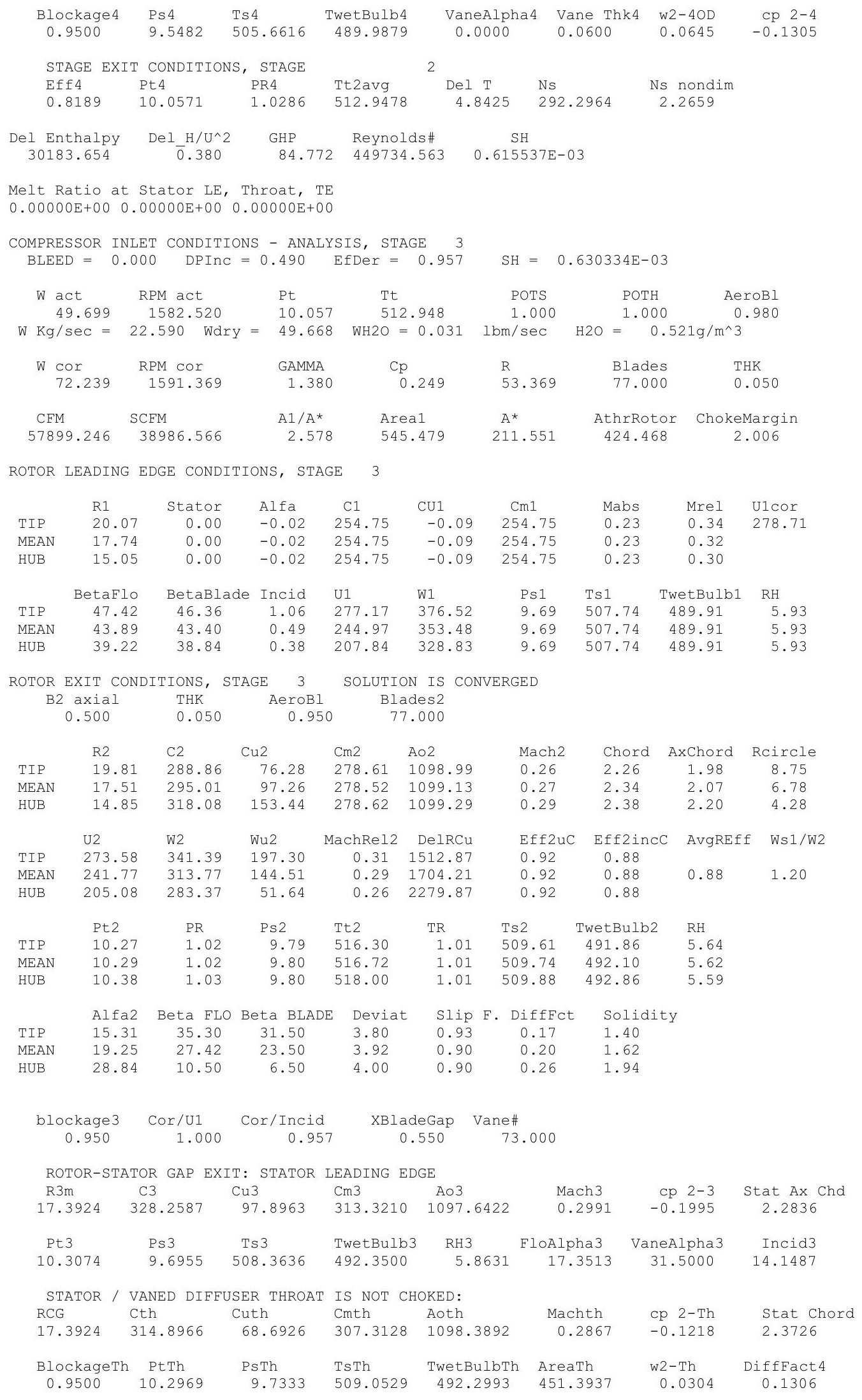




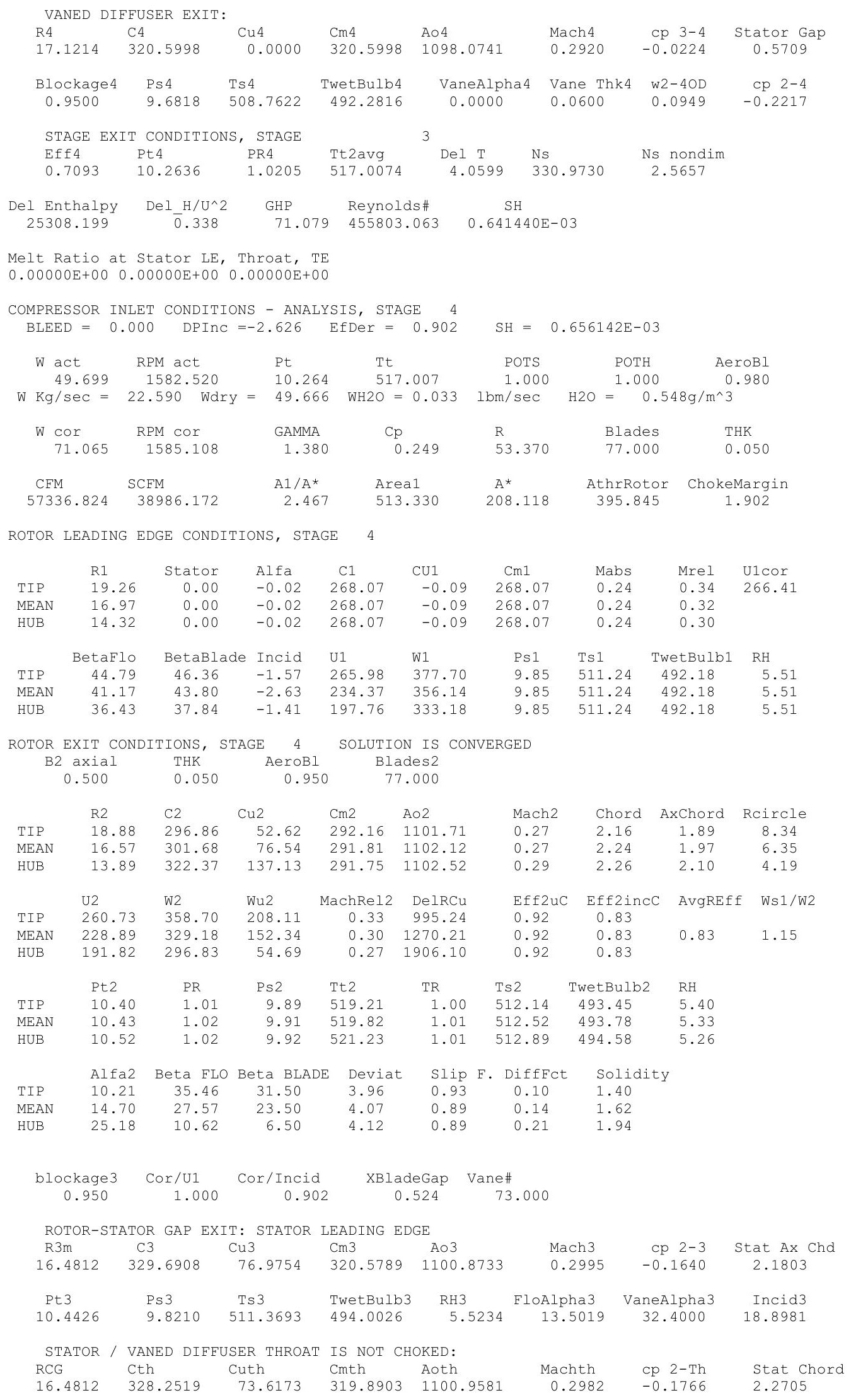




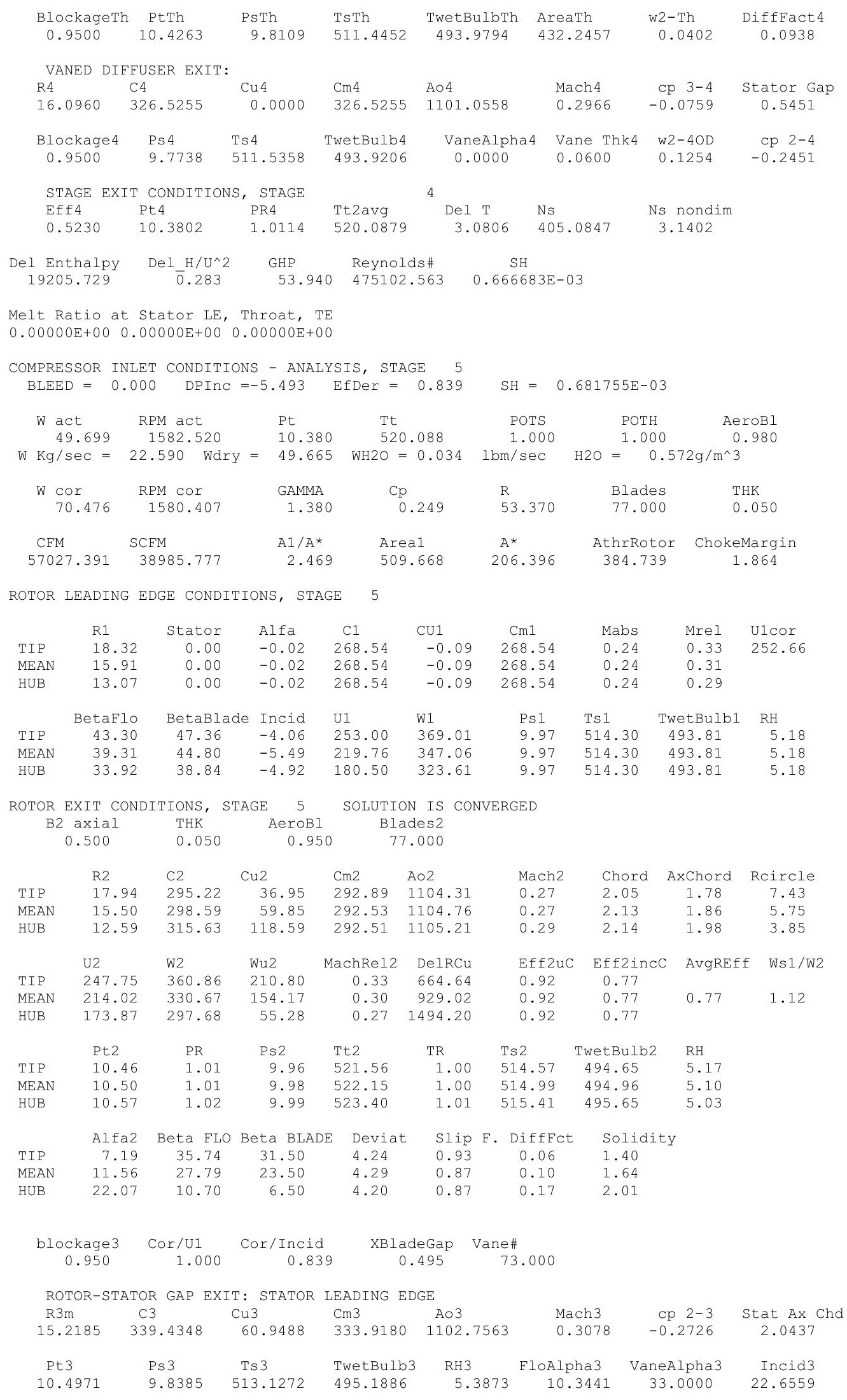




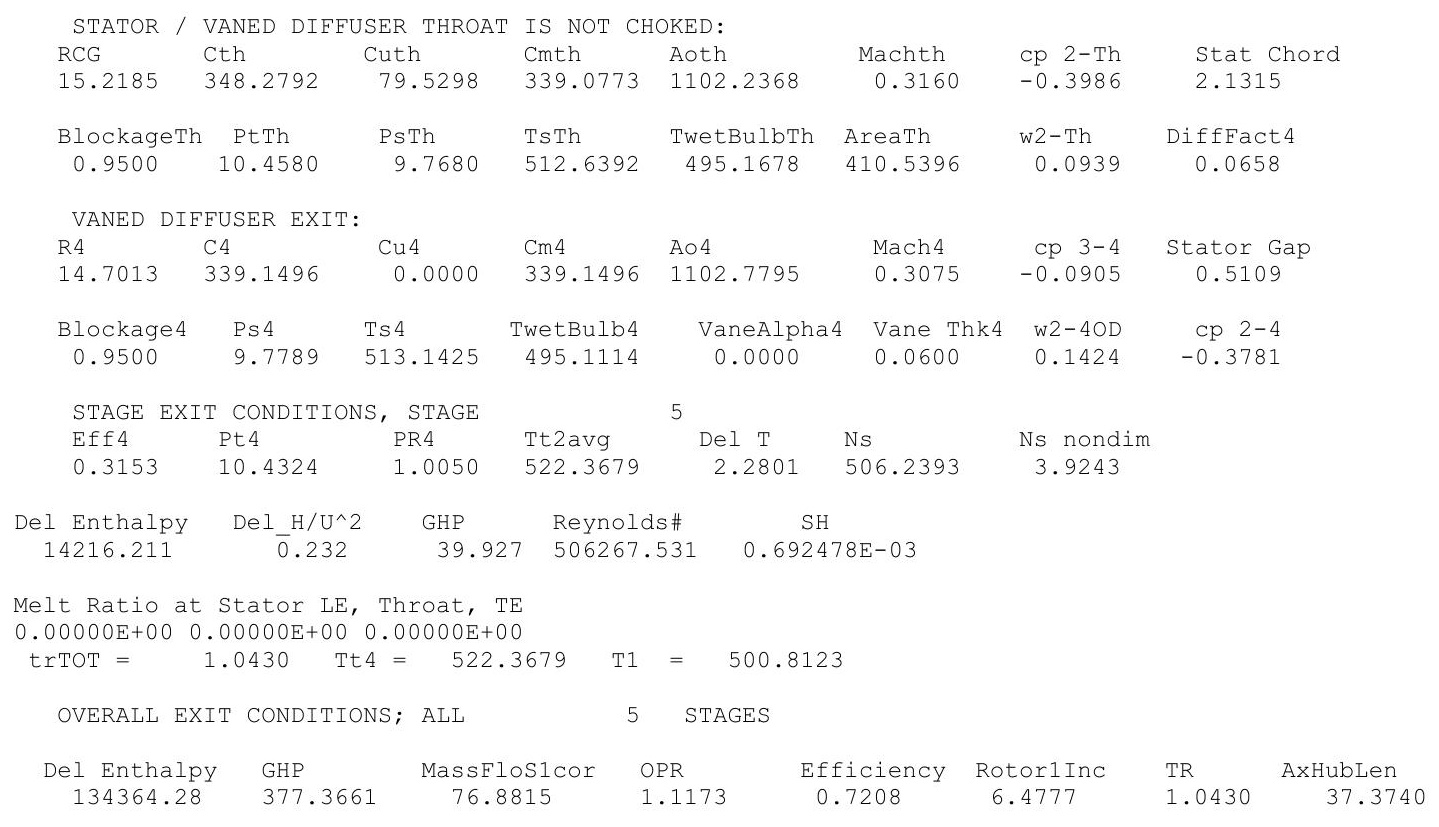




\title{
$25 \mu \mathrm{m}, \mathrm{ISA}+36 \mathrm{R}$
}

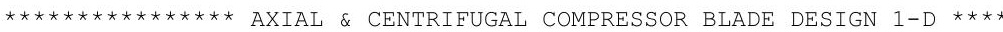

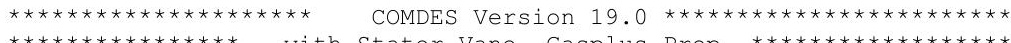

Sane, Gasplus Prop $x * x+x * t+$
\end{abstract}

Fan Core + 4 Stage LPC 10\%39000 ft 05-13-2013

COMPRESSOR INLET CONDITIONS - ANALYSIS, STAGE 1

$\mathrm{BLEED}=0.000 \quad$ DPInC $=15.118 \quad$ EfDer $=0.957 \quad \mathrm{SH}=0.157989 \mathrm{E}-03$

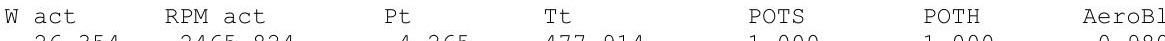

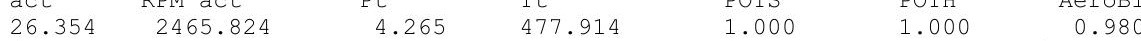

$\mathrm{W} \mathrm{Kg} / \mathrm{sec}=11.979$ Wdry $=26.349 \mathrm{WH} 2 \mathrm{O}=0.004 \mathrm{lbm} / \mathrm{sec} \quad \mathrm{H} 2 \mathrm{O}=0.060 \mathrm{~g} / \mathrm{m}^{\wedge} 3$

$\begin{array}{lccclcl}\text { W cor } & \text { RPM cor } & \text { GAMMA } & \text { Cp } & \text { R } & \text { Blades } & \text { THK } \\ 87.196 & 2568.871 & 1.381 & 0.248 & 53.353 & 32.000 & 0.050\end{array}$

CFM SCFM A1/A* Area1 A* AthrRotor ChokeMargin

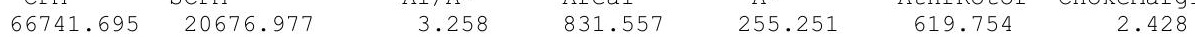

ROTOR LEADING EDGE CONDITIONS, STAGE 1

$\begin{array}{lccccccccc} & \text { R1 } & \text { Stator } & \text { Alfa } & \text { C1 } & \text { CU1 } & \text { Cm1 } & \text { Mabs } & \text { Mrel } & \text { U1cor } \\ \text { TIP } & 20.63 & 0.00 & -0.02 & 192.63 & -0.07 & 192.63 & 0.18 & 0.46 & 462.46 \\ \text { MEAN } & 17.06 & 0.00 & -0.02 & 192.63 & -0.07 & 192.63 & 0.18 & 0.39 & \\ \text { HUB } & 12.51 & 0.00 & -0.02 & 192.63 & -0.07 & 192.63 & 0.18 & 0.31 & \\ & & & & & & & & & \\ & \text { BetaFlo } & \text { BetaBlade } & \text { Incid } & \text { U1 } & \text { W1 } & \text { Ps1 } & \text { Ts1 } & \text { TwetBulb1 } & \text { RH } \\ \text { TIP } & 66.55 & 50.47 & 16.08 & 443.92 & 483.98 & 4.17 & 474.93 & 460.84 & 2.42 \\ \text { MEAN } & 62.32 & 47.20 & 15.12 & 367.11 & 414.63 & 4.17 & 474.93 & 460.84 & 2.42 \\ \text { HUB } & 54.42 & 38.62 & 15.80 & 269.19 & 331.07 & 4.17 & 474.93 & 460.84 & 2.42\end{array}$

ROTOR EXIT CONDITIONS, STAGE 1 SOLUTION IS CONVERGED

\begin{tabular}{|c|c|c|c|c|c|c|c|c|c|}
\hline & $\begin{array}{l}\text { axial } \\
700\end{array}$ & $\begin{array}{l}\text { THK } \\
0.050\end{array}$ & $\begin{array}{l}\text { AeroBl } \\
0.950\end{array}$ & $\begin{array}{r}\mathrm{Bla} \\
32\end{array}$ & $\begin{array}{l}\text { des } 2 \\
.000\end{array}$ & & & & \\
\hline & $\mathrm{R} 2$ & $\mathrm{C} 2$ & $\mathrm{Cu} 2$ & $\mathrm{Cm} 2$ & $\mathrm{~A} \circ 2$ & Mach2 & Chord & AxChord & Rcircle \\
\hline TIP & 20.63 & 398.85 & 304.32 & 257.81 & 1074.42 & 0.37 & 7.29 & 6.52 & 16.04 \\
\hline MEAN & 18.04 & 404.26 & 308.54 & 261.20 & 1071.31 & 0.38 & 7.43 & 6.80 & 12.53 \\
\hline HUB & 15.00 & 438.76 & 348.99 & 265.93 & 1067.45 & 0.41 & 7.49 & 7.25 & 9.22 \\
\hline & $\mathrm{U} 2$ & W2 & Wu2 & MachRel2 & DelRCu & Eff $2 u C$ & Eff2incC & AvgREff & Ws $1 /$ W2 \\
\hline TIP & 443.92 & 293.18 & 139.60 & 0.27 & 6279.55 & 0.92 & 0.88 & & \\
\hline MEAN & 388.11 & 273.05 & 79.56 & 0.25 & 5566.04 & 0.92 & 0.88 & 0.88 & 1.74 \\
\hline HUB & 322.78 & 267.22 & 26.21 & 0.25 & 5235.66 & 0.92 & 0.88 & & \\
\hline & Pt2 & PR & Ps2 & Tt2 & $\mathrm{TR}$ & Ts2 & TwetBulb2 & $\mathrm{RH}$ & \\
\hline TIP & 4.91 & 1.15 & 4.47 & 499.64 & 1.05 & 486.85 & 473.47 & 1.88 & \\
\hline SAN & 4.84 & 1.13 & 4.39 & 497.17 & 1.04 & 484.03 & 472.17 & 2.08 & \\
\hline JB & 4.80 & 1.13 & 4.28 & 496.03 & 1.04 & 480.55 & 471.61 & 2.35 & \\
\hline & Alfa2 & Beta FLO & Beta BLADE & Deviat & Slip & F. DiffFct & Solidity & & \\
\hline & 49.73 & 28.44 & 24.20 & 4.24 & 0.93 & 0.57 & 1.80 & & \\
\hline & 49.75 & 16.94 & 12.70 & 4.24 & 0.95 & 0.51 & 2.22 & & \\
\hline & 52.69 & -5.63 & -9.30 & 3.67 & 0.95 & 0.38 & 3.05 & & \\
\hline
\end{tabular}

$\begin{array}{ccccc}\text { blockage } & \text { Cor/U1 } & \text { Cor/Incid } & \text { XBladeGap } & \text { Vane\# } \\ 0.950 & 1.000 & 0.957 & 1.812 & 33.000\end{array}$

ROTOR-STATOR GAP EXIT: STATOR LEADING EDGE

\begin{tabular}{|c|c|c|c|c|c|c|c|}
\hline $\begin{array}{l}\text { R3m } \\
18.0711\end{array}$ & $\begin{array}{l}\text { C3 } \\
416.3228\end{array}$ & $\begin{array}{l}\text { Cu3 } \\
307.9446\end{array}$ & $\begin{array}{l}\mathrm{Cm} 3 \\
280.1692\end{array}$ & $\begin{array}{l}\text { Ao3 } \\
1070.9060\end{array}$ & $\begin{array}{l}\text { Mach3 } \\
0.3888\end{array}$ & $\begin{array}{r}\mathrm{cp} 2-3 \\
-0.0370\end{array}$ & $\begin{array}{c}\text { Stat Ax Chd } \\
5.2355\end{array}$ \\
\hline Pt 3 & Ps 3 & Ts 3 & TwetBulb3 & RH3 & FloAlpha 3 & VaneAlpha3 & Incid3 \\
\hline 4.8449 & 4.3712 & 483.6812 & 472.4266 & 2.1461 & 47.7040 & 35.4000 & -12.3040 \\
\hline STATOR / & VANED DIF & USER THROAT & IS NOT CHC & KED : & & & \\
\hline $\begin{array}{l}\text { RCG } \\
18.0711\end{array}$ & $\begin{array}{l}\text { Cth } \\
278.0645\end{array}$ & $\begin{array}{l}\text { Cuth } \\
68.0231\end{array}$ & $\begin{array}{l}\text { Cmth } \\
269.6159\end{array}$ & $\begin{array}{l}\text { Aoth } \\
1079.4109\end{array}$ & $\begin{array}{l}\text { Machth } \\
0.2576\end{array}$ & $\begin{array}{c}\text { cp } 2-T h \\
0.5072\end{array}$ & $\begin{array}{l}\text { Stat Chord } \\
5.4957\end{array}$ \\
\hline BlockageTh & PtTh & PsTh & TsTh & TwetBulbTh & AreaTh & w2-Th & DiffFact 4 \\
\hline 0.9500 & 4.8334 & 4.6182 & 491.3993 & 472.2449 & 554.9116 & 0.0344 & 0.5994 \\
\hline VANED DI & PFUSER & & & & & & \\
\hline R4 & $\mathrm{C} 4$ & $\mathrm{Cu} 4$ & $\mathrm{Cm} 4$ & A०4 & $\operatorname{Mach} 4$ & cp 3-4 & Stator Gap \\
\hline 18.0829 & 276.8794 & 0.0000 & 276.8794 & 1079.4552 & 0.2565 & 0.4796 & 1.3089 \\
\hline
\end{tabular}




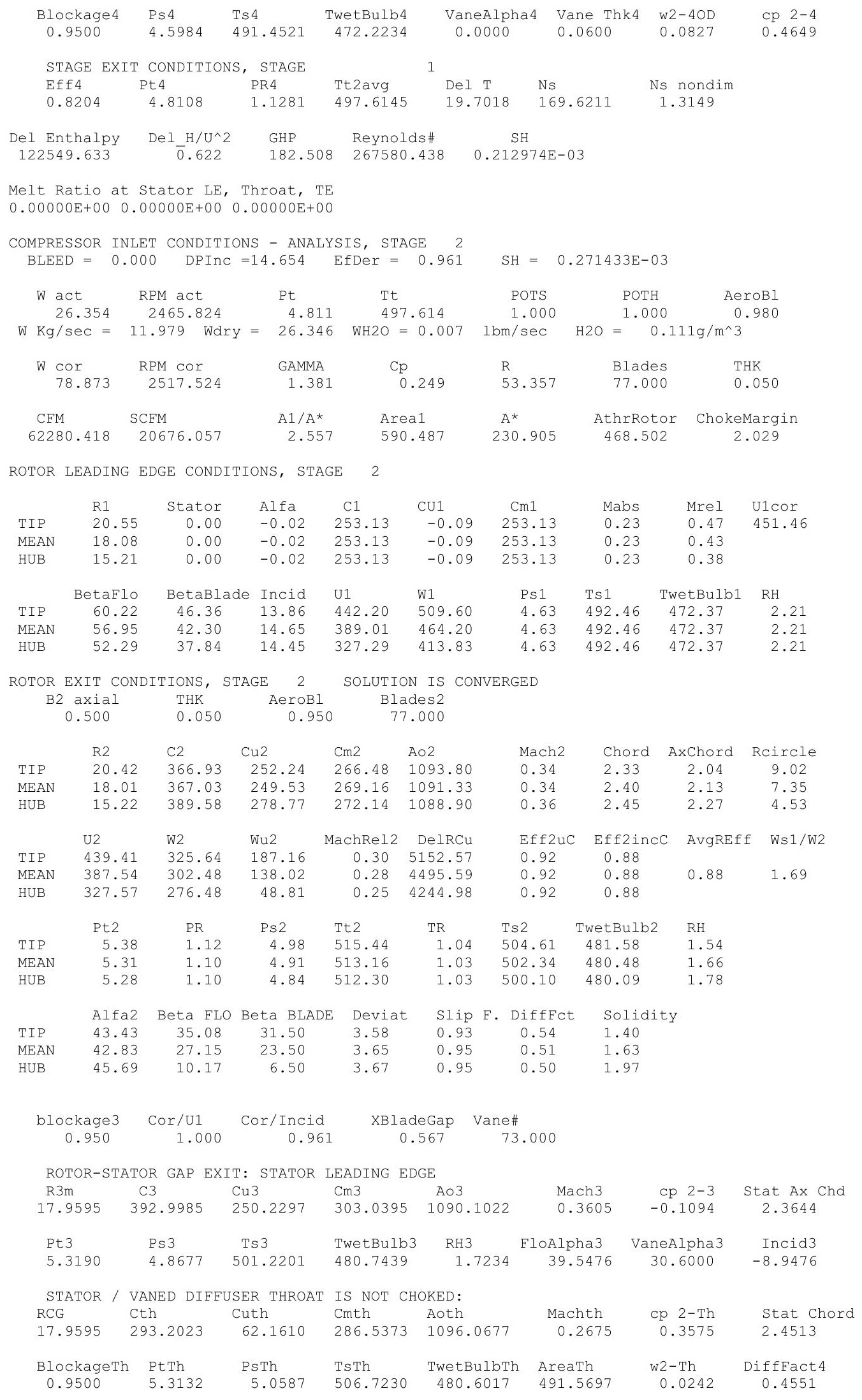




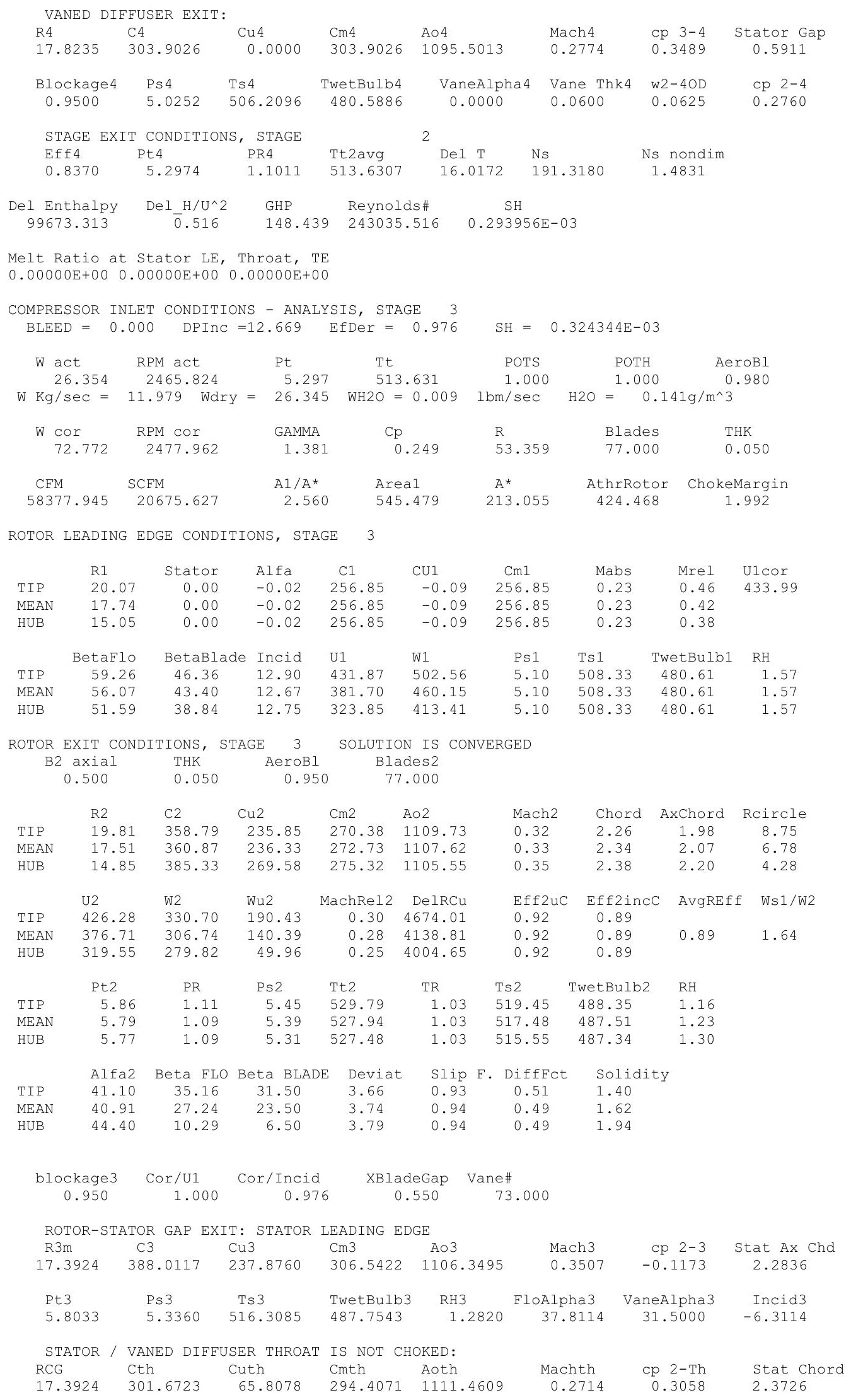




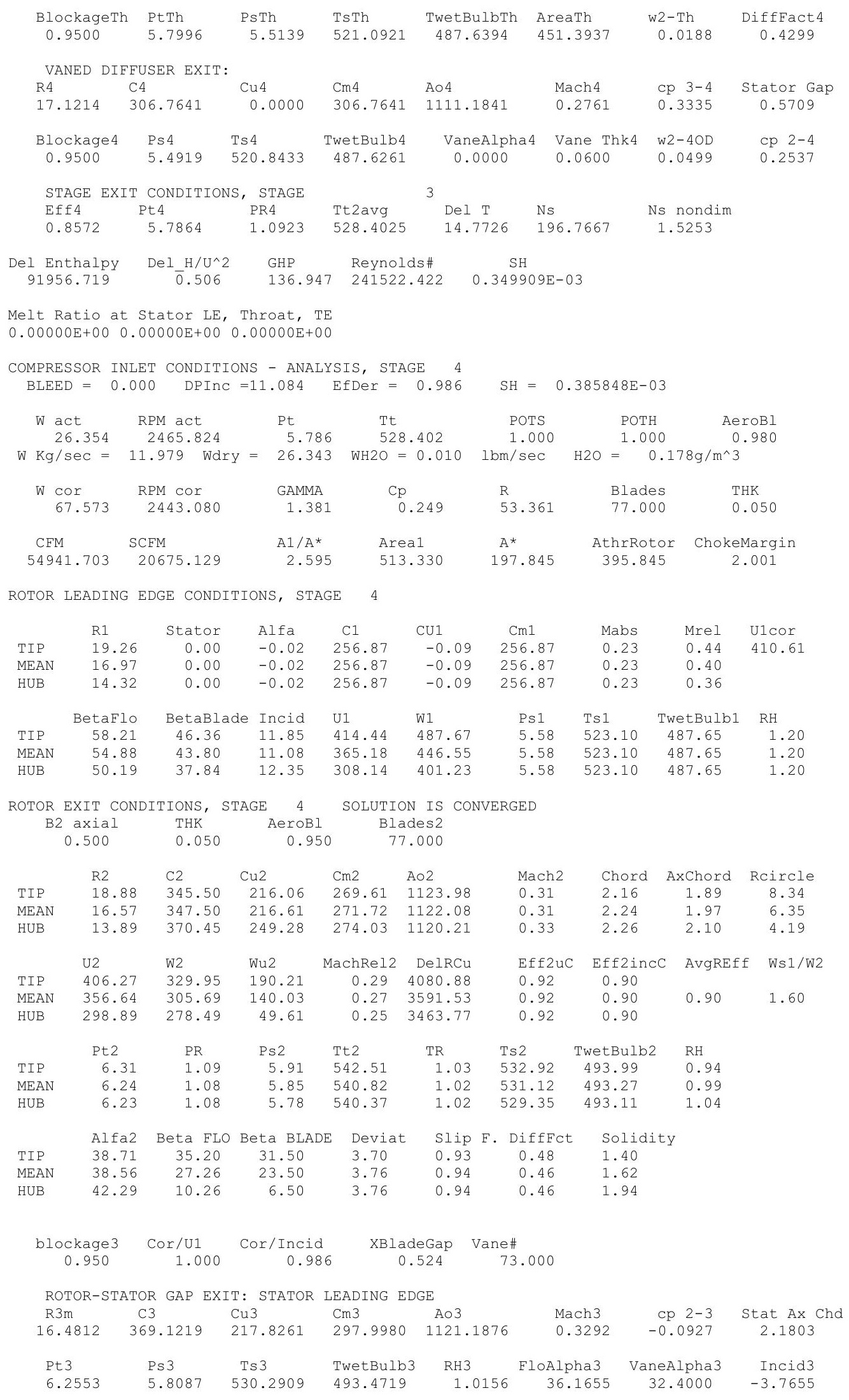




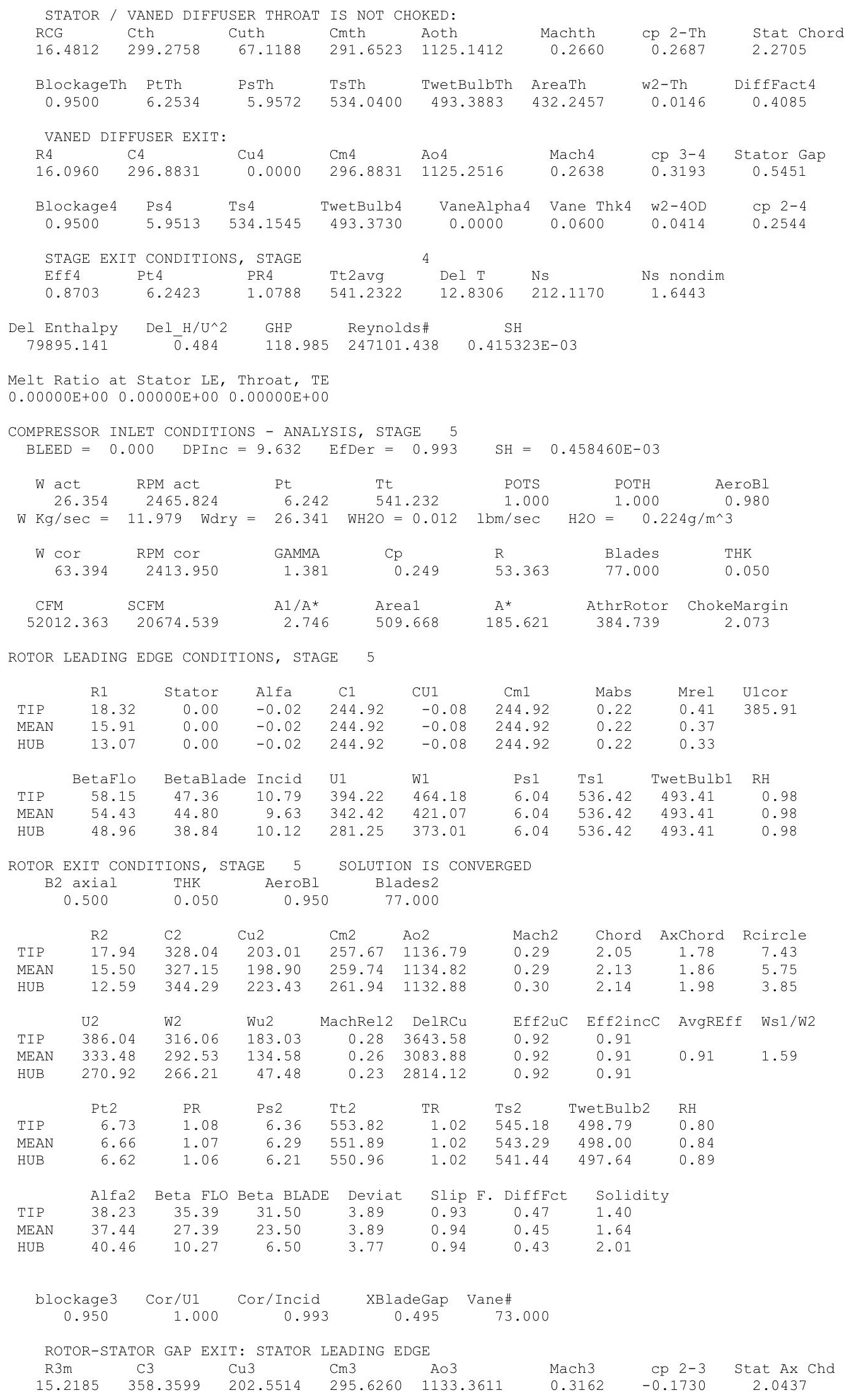




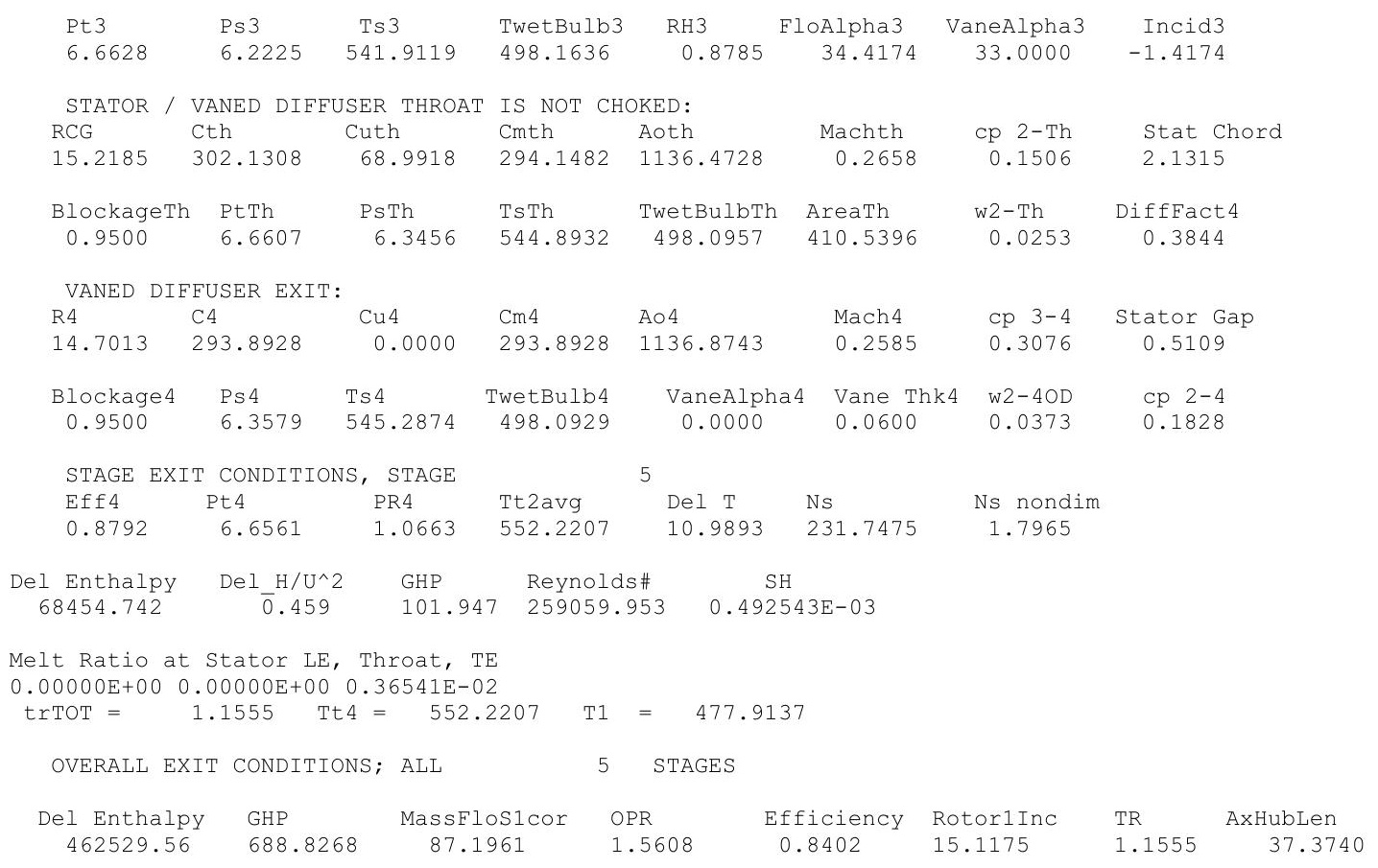




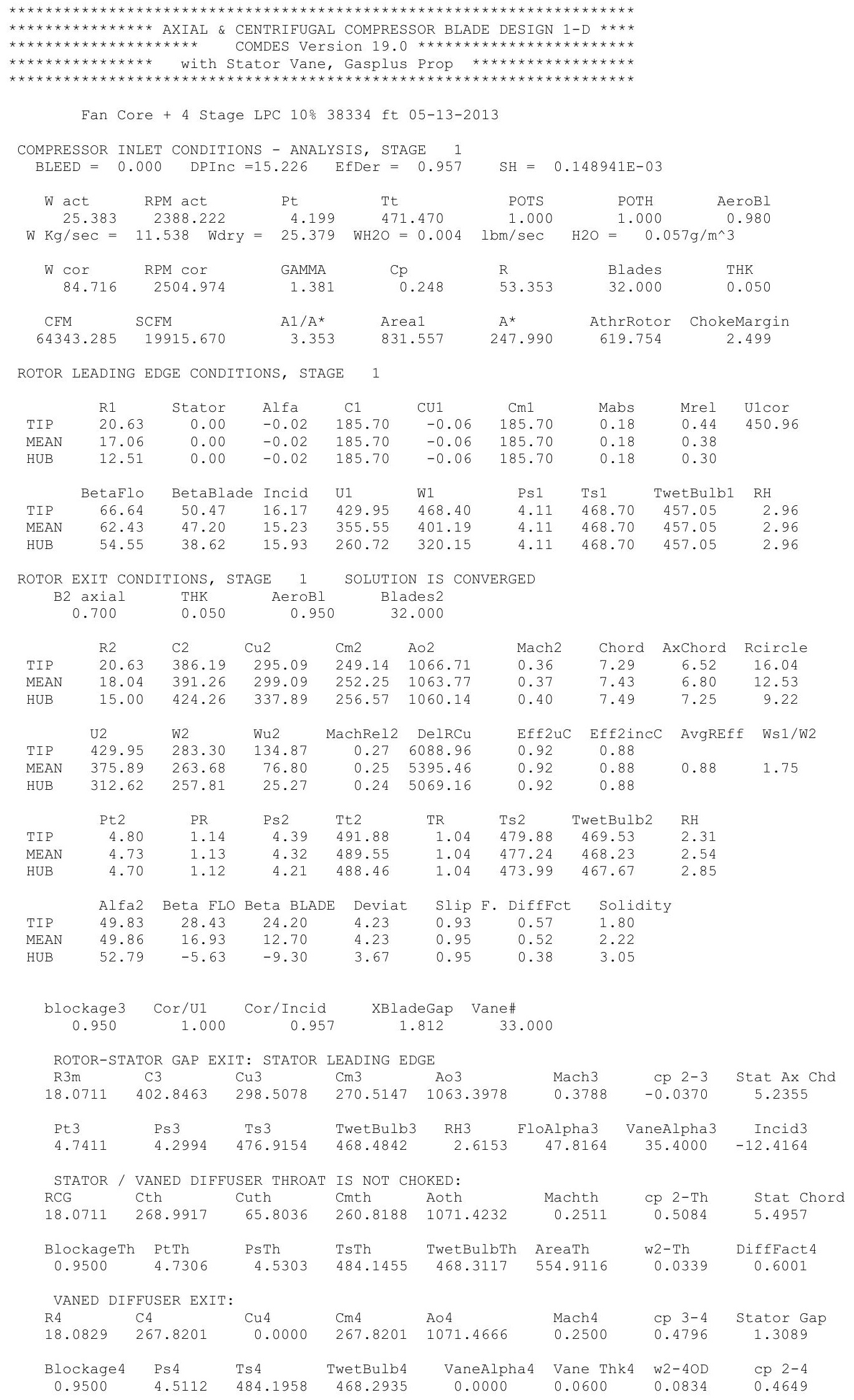




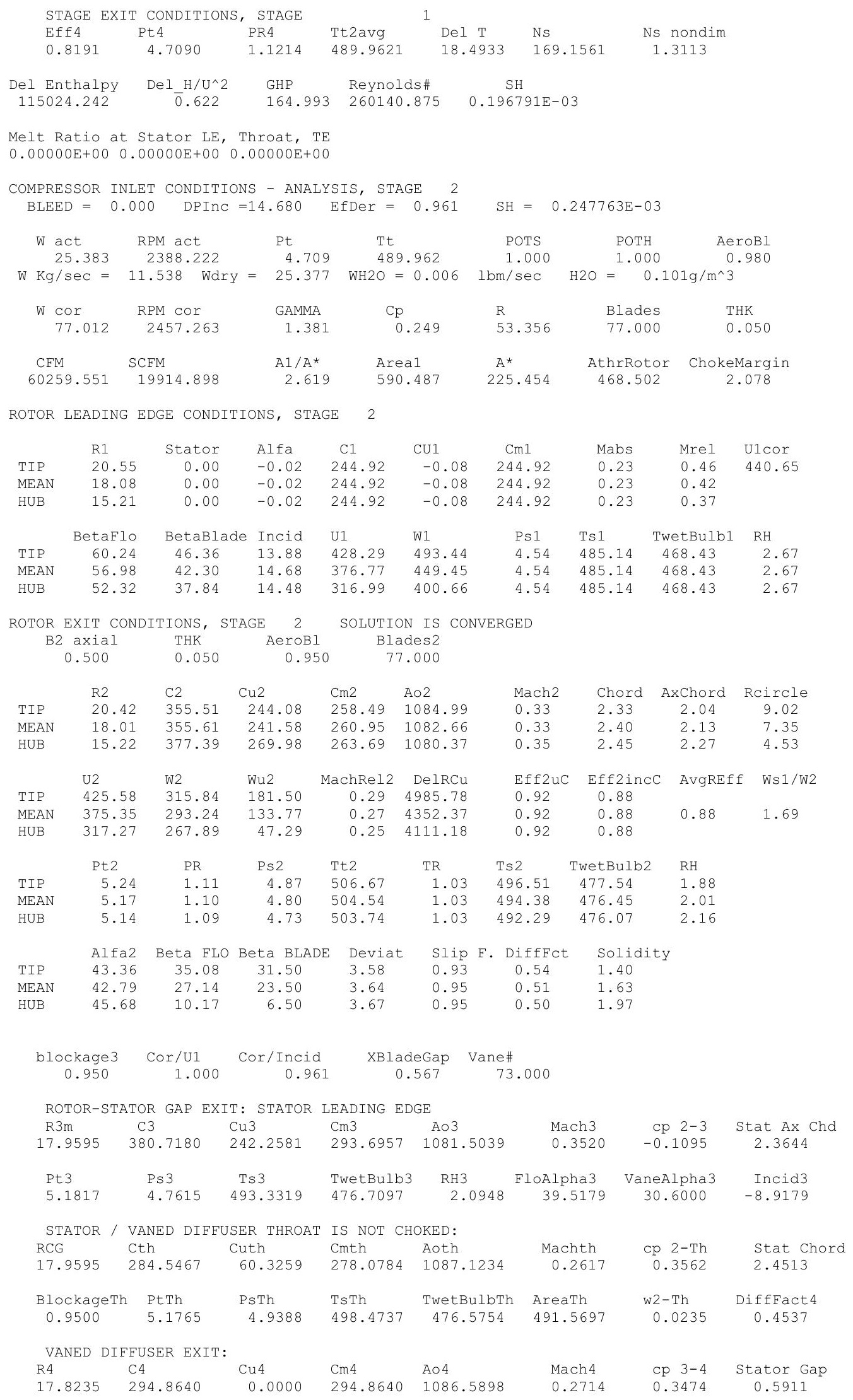




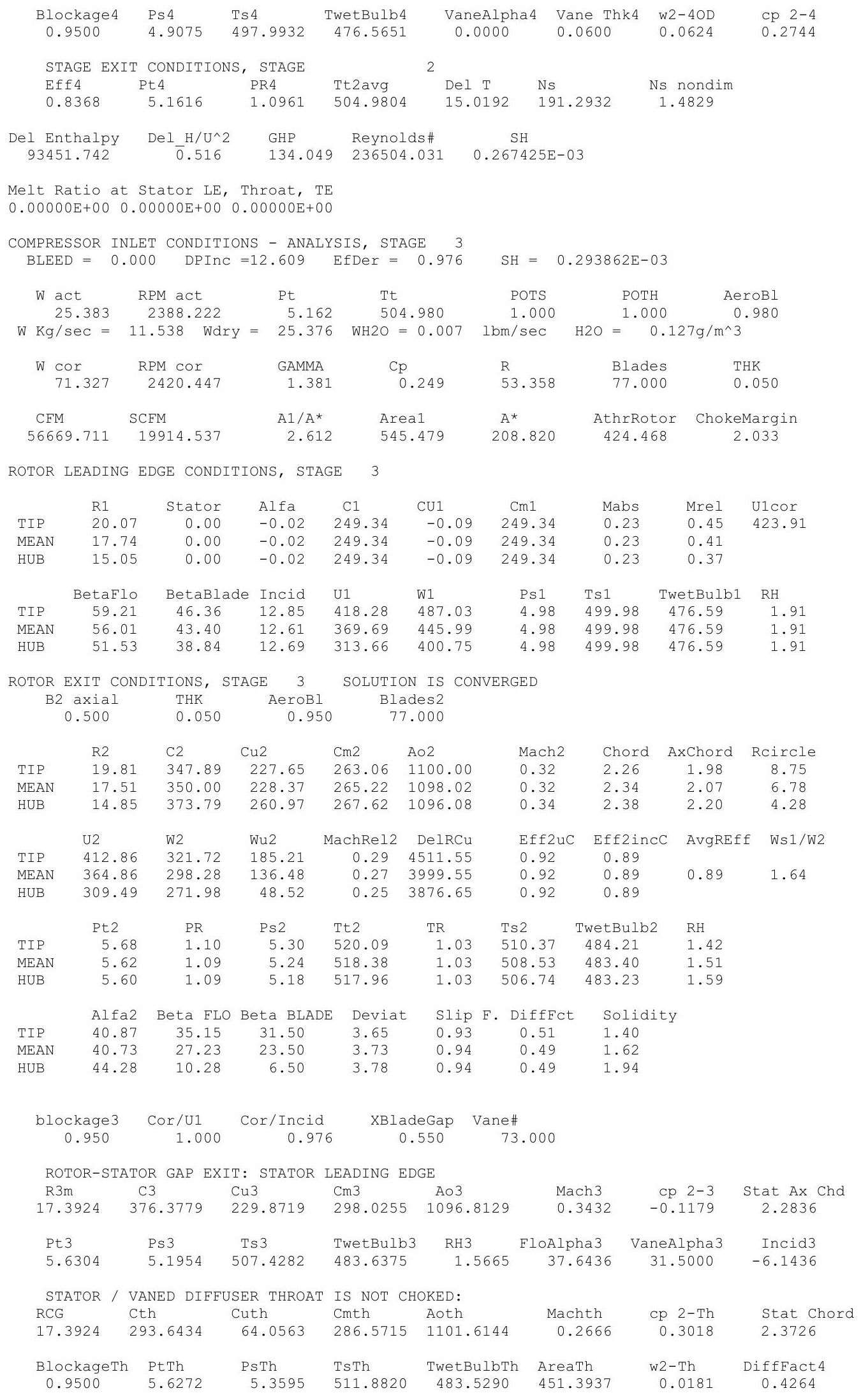




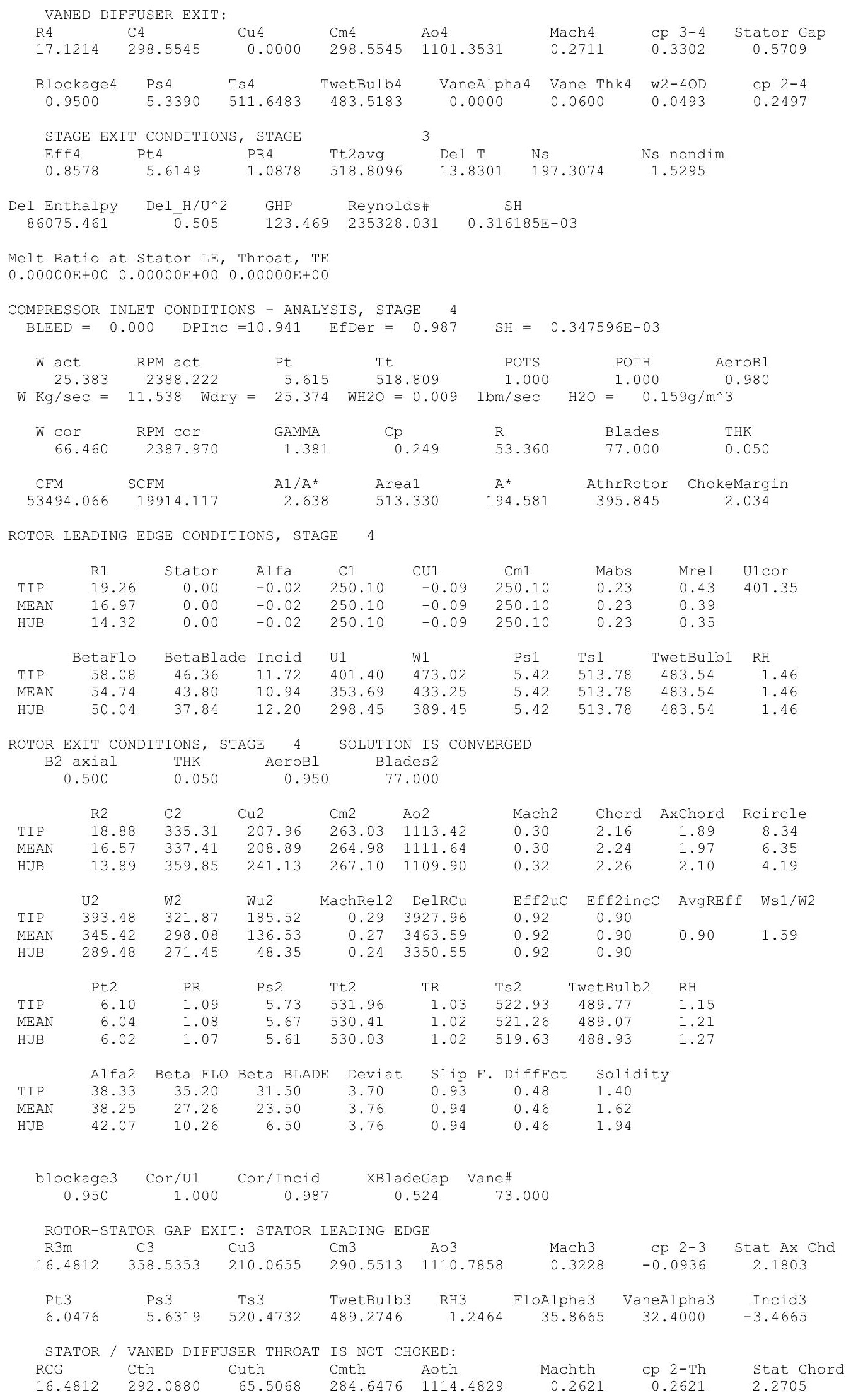




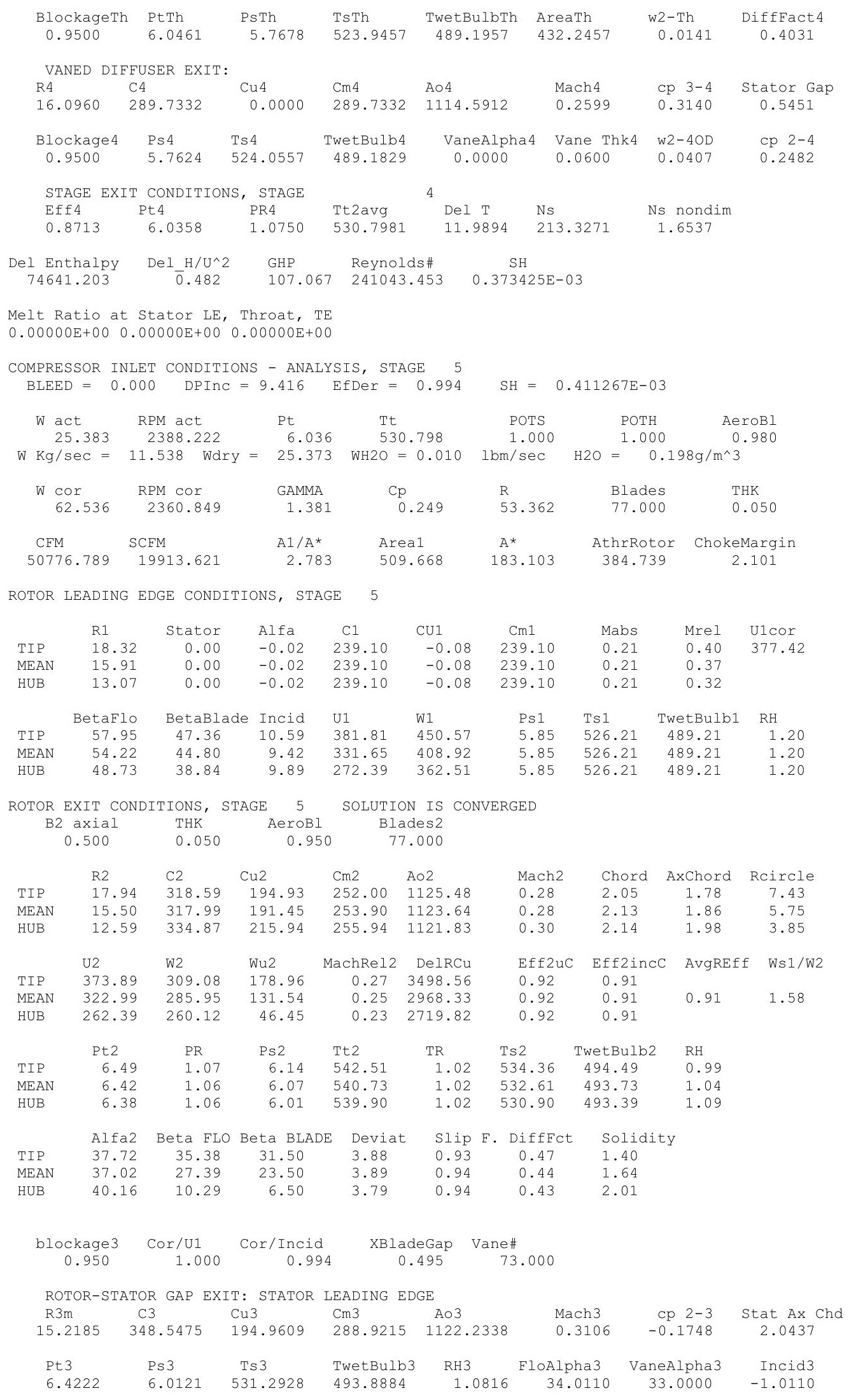




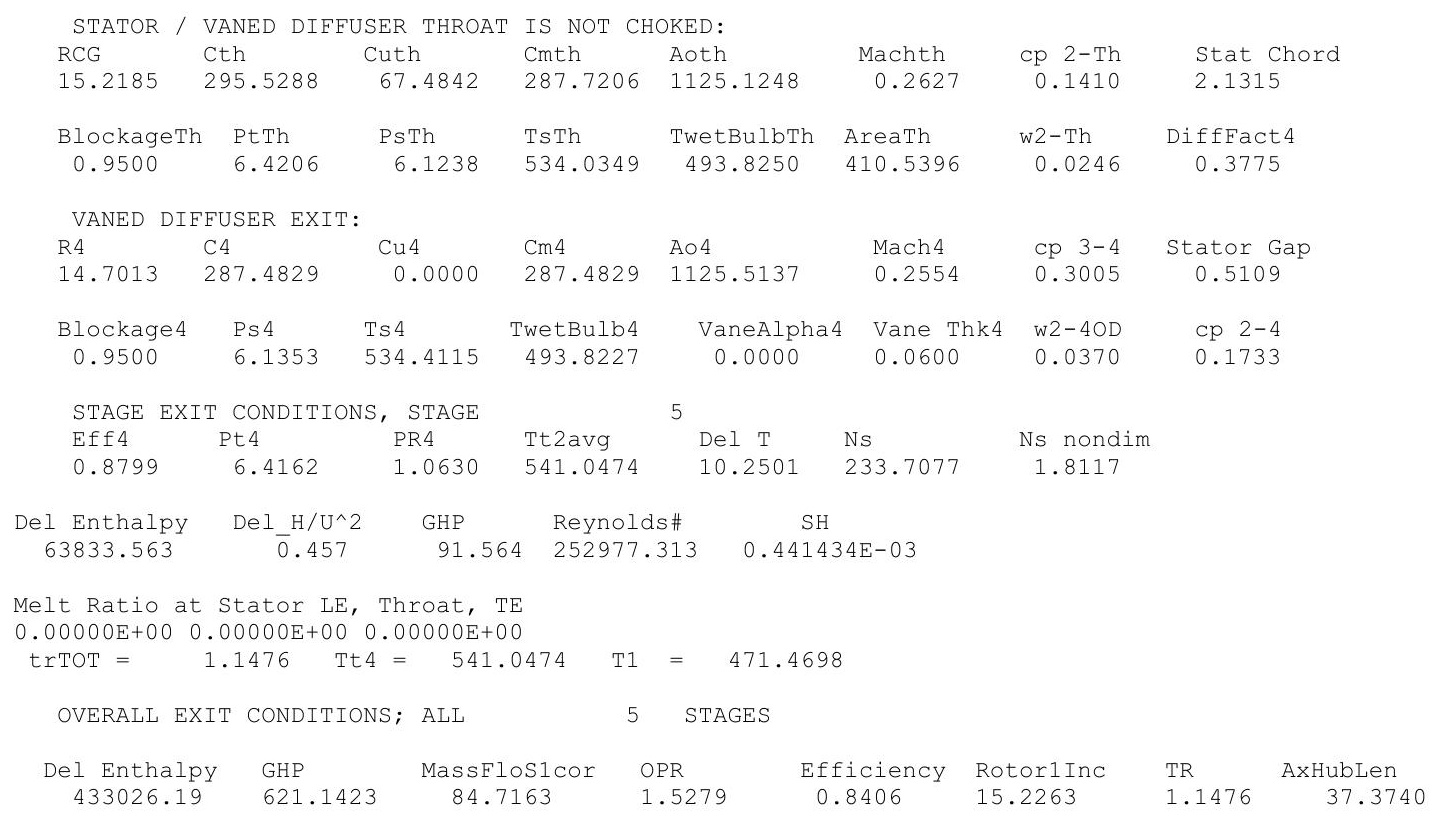




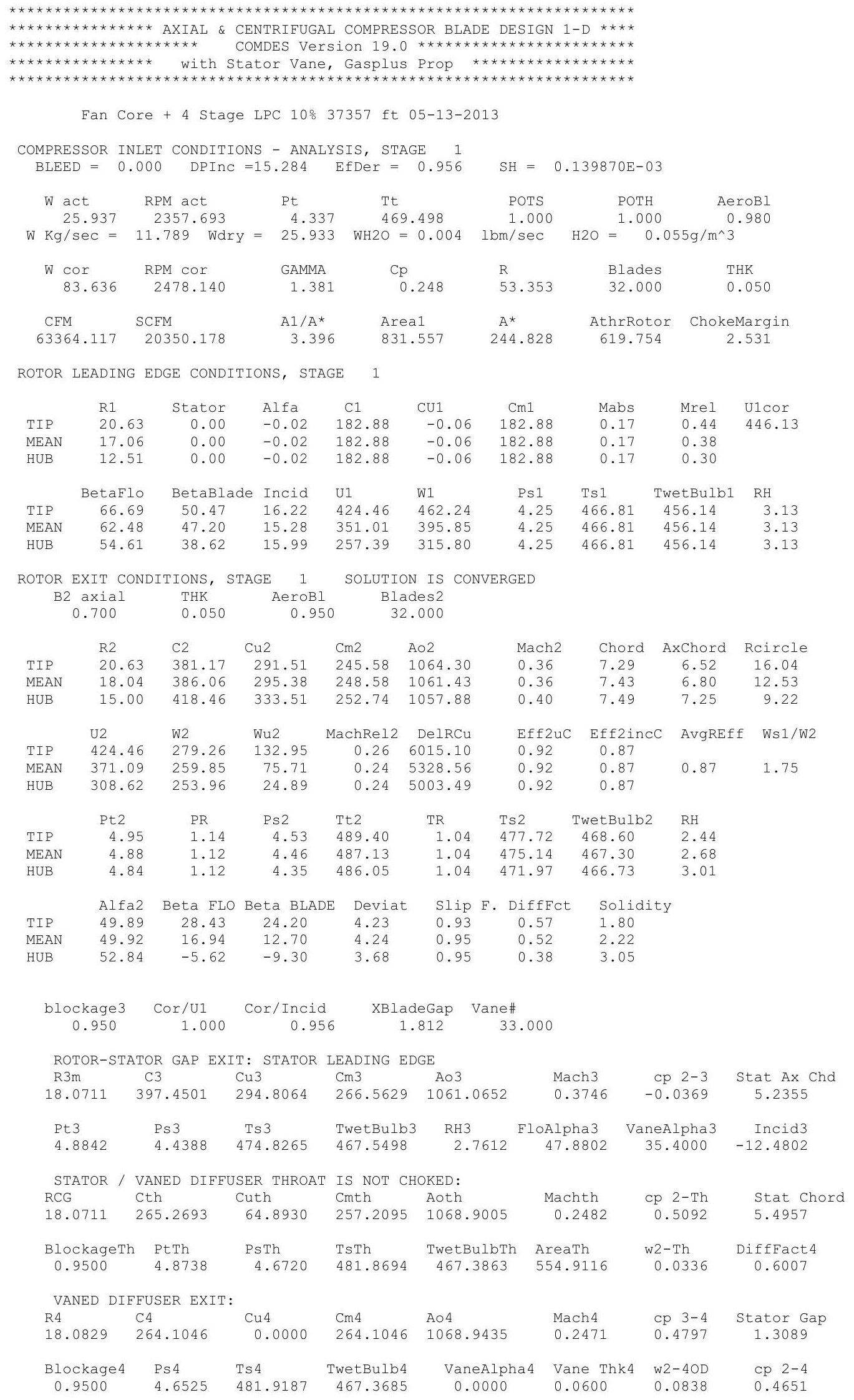




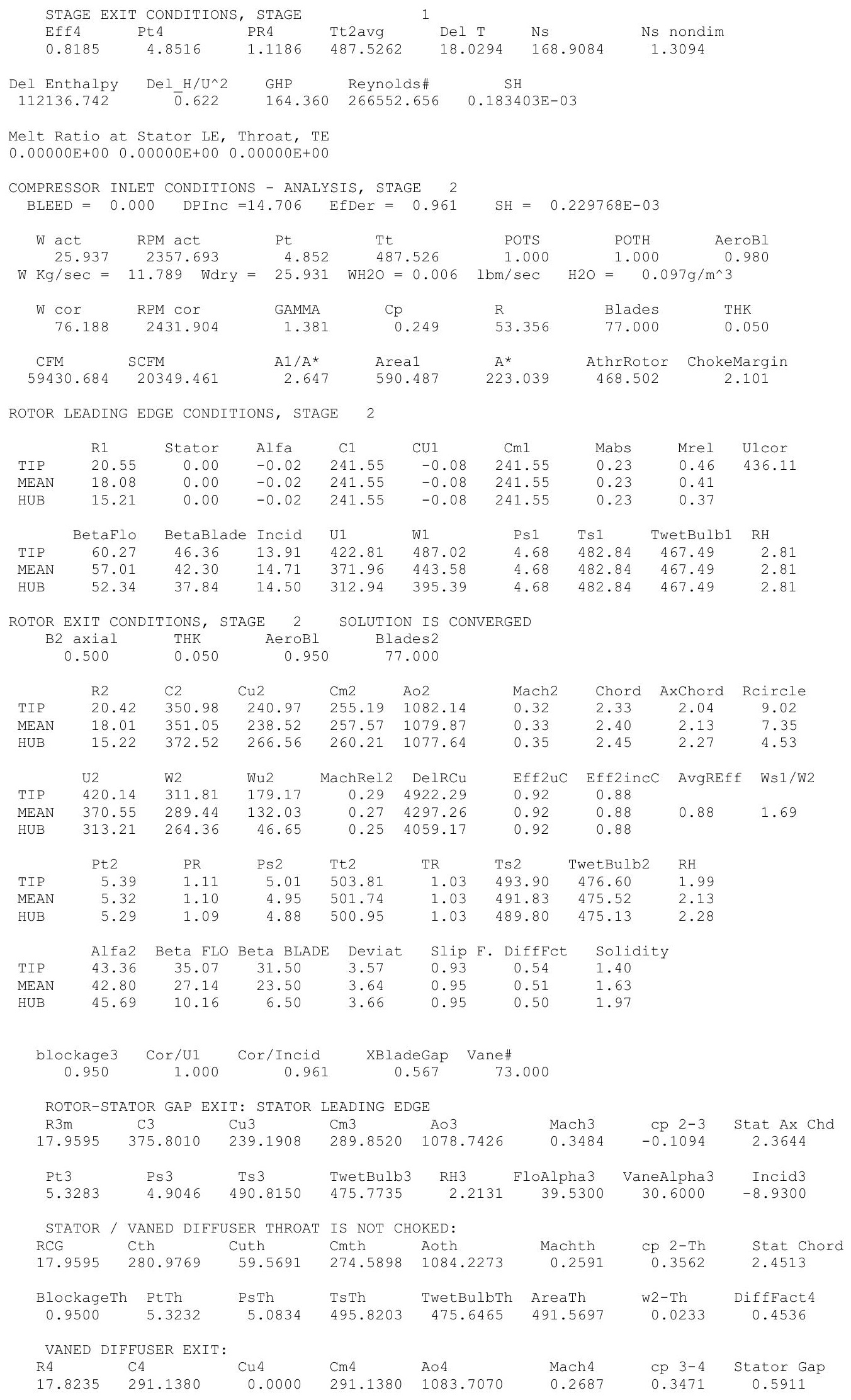




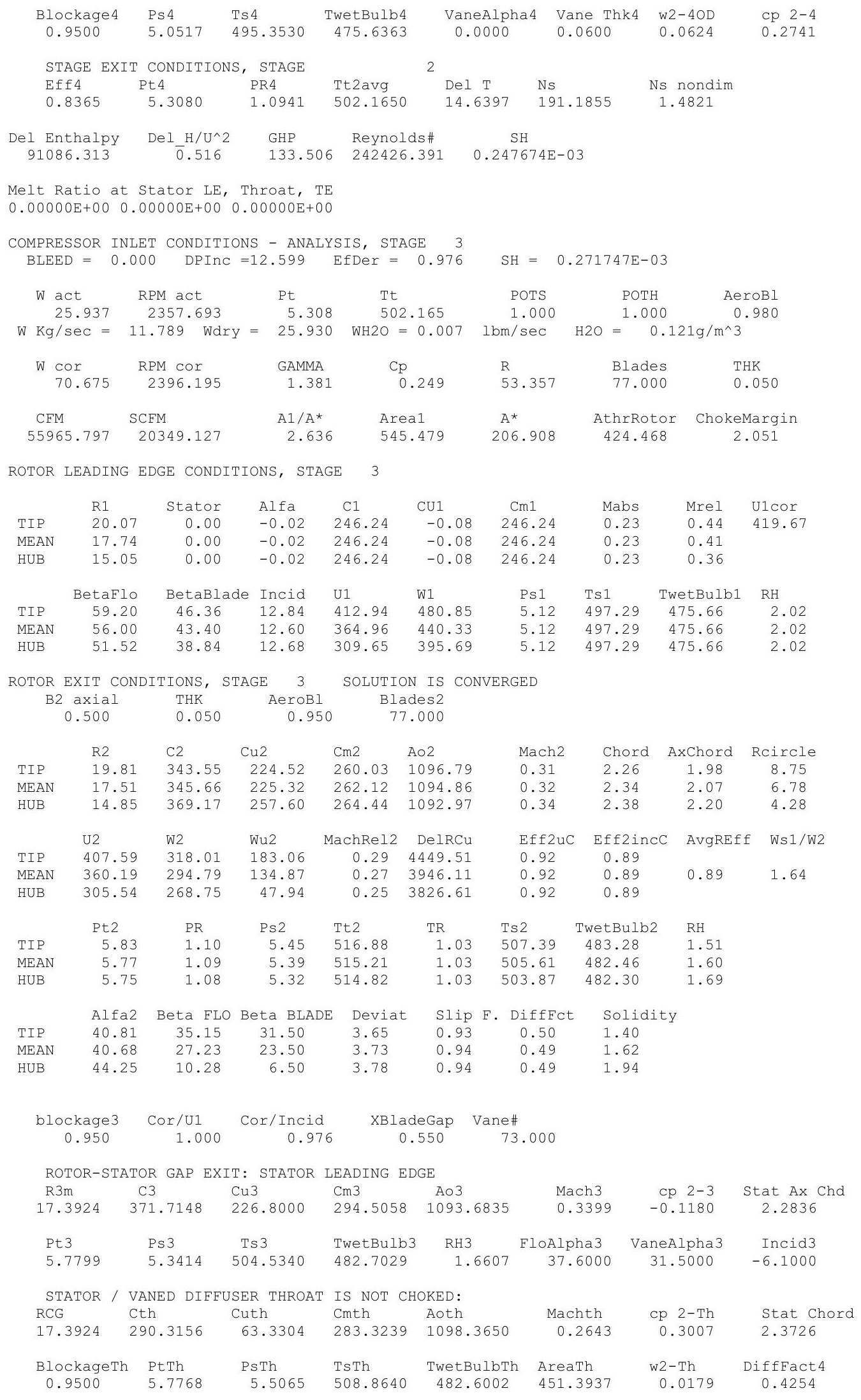




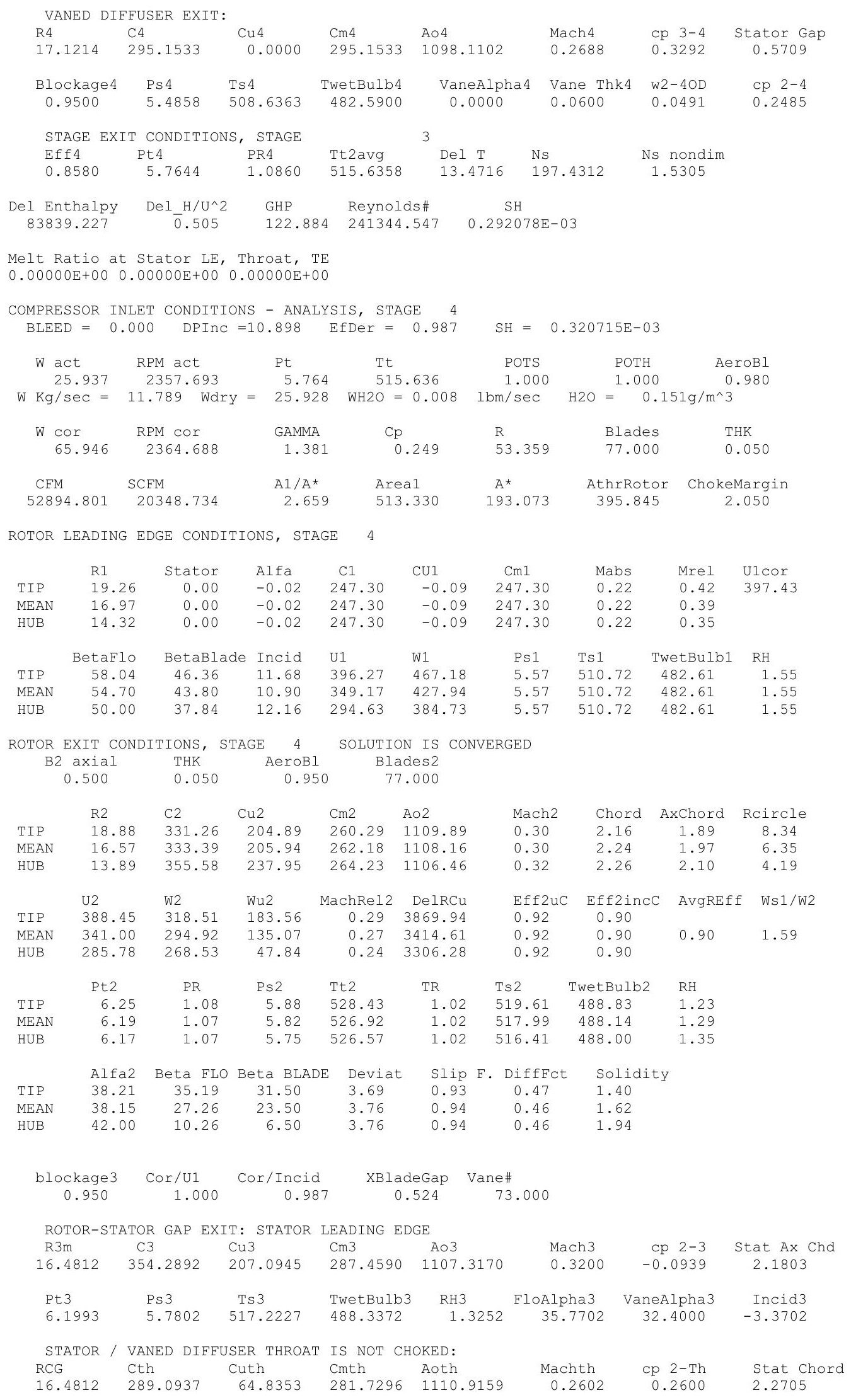




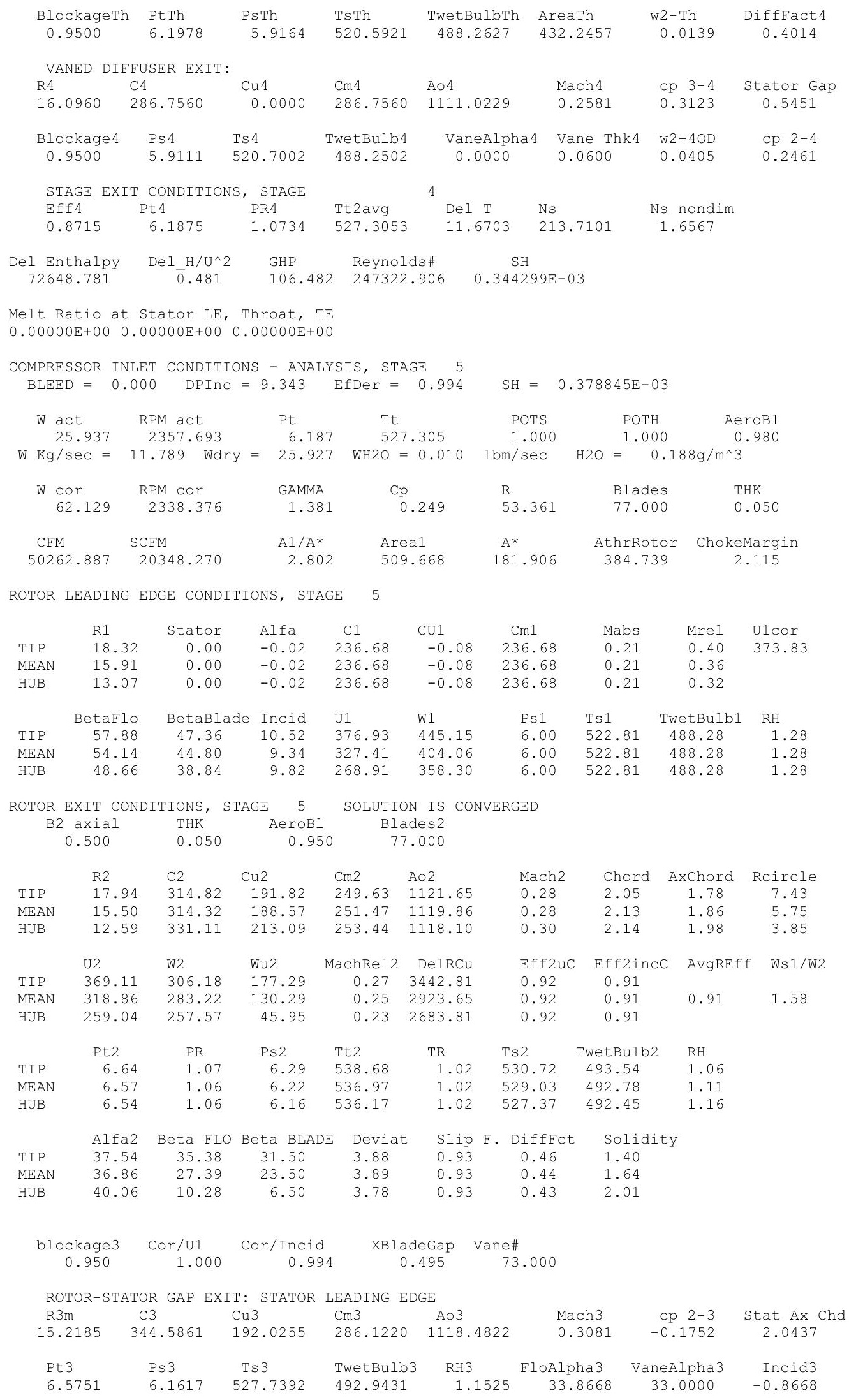




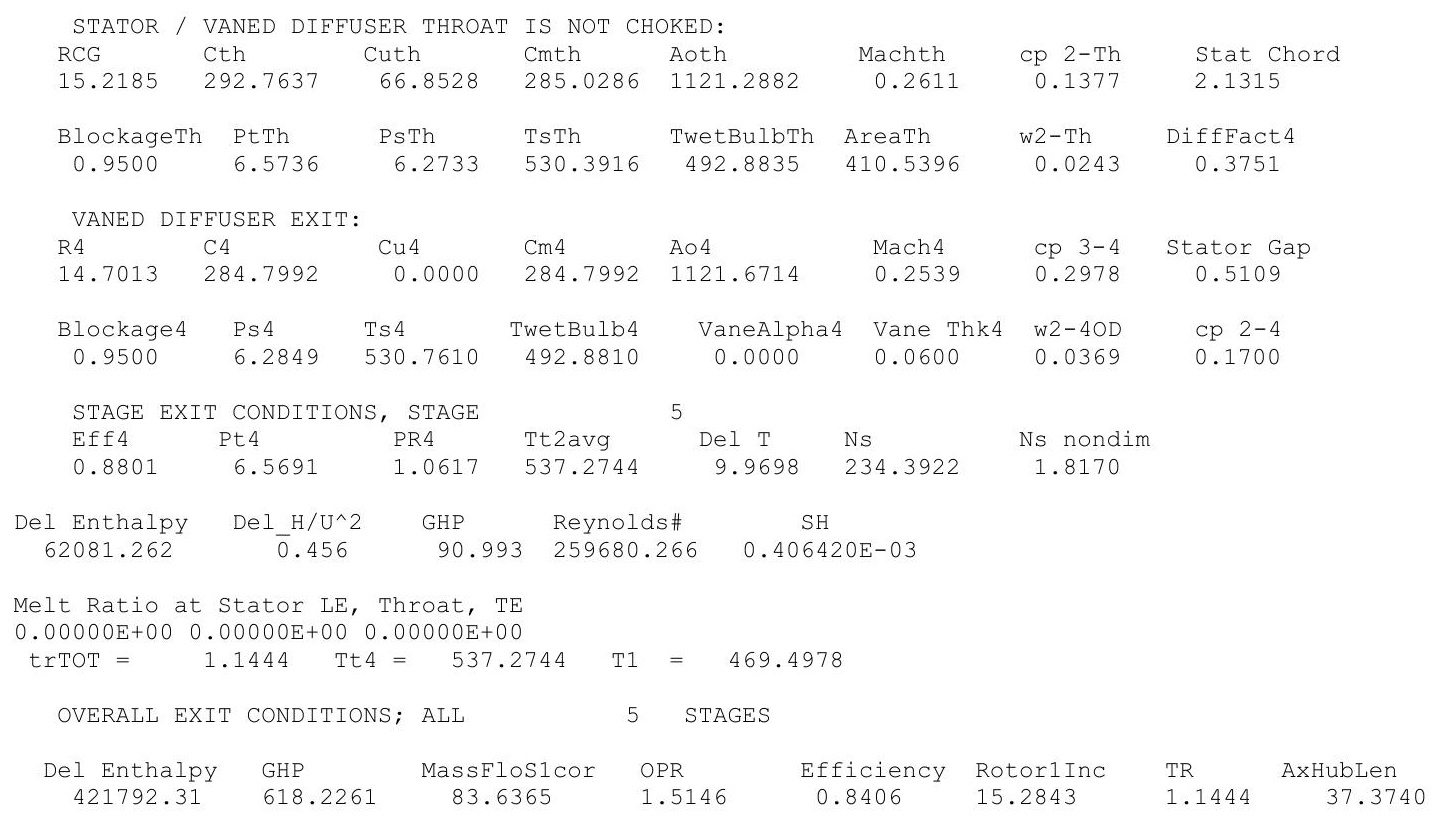




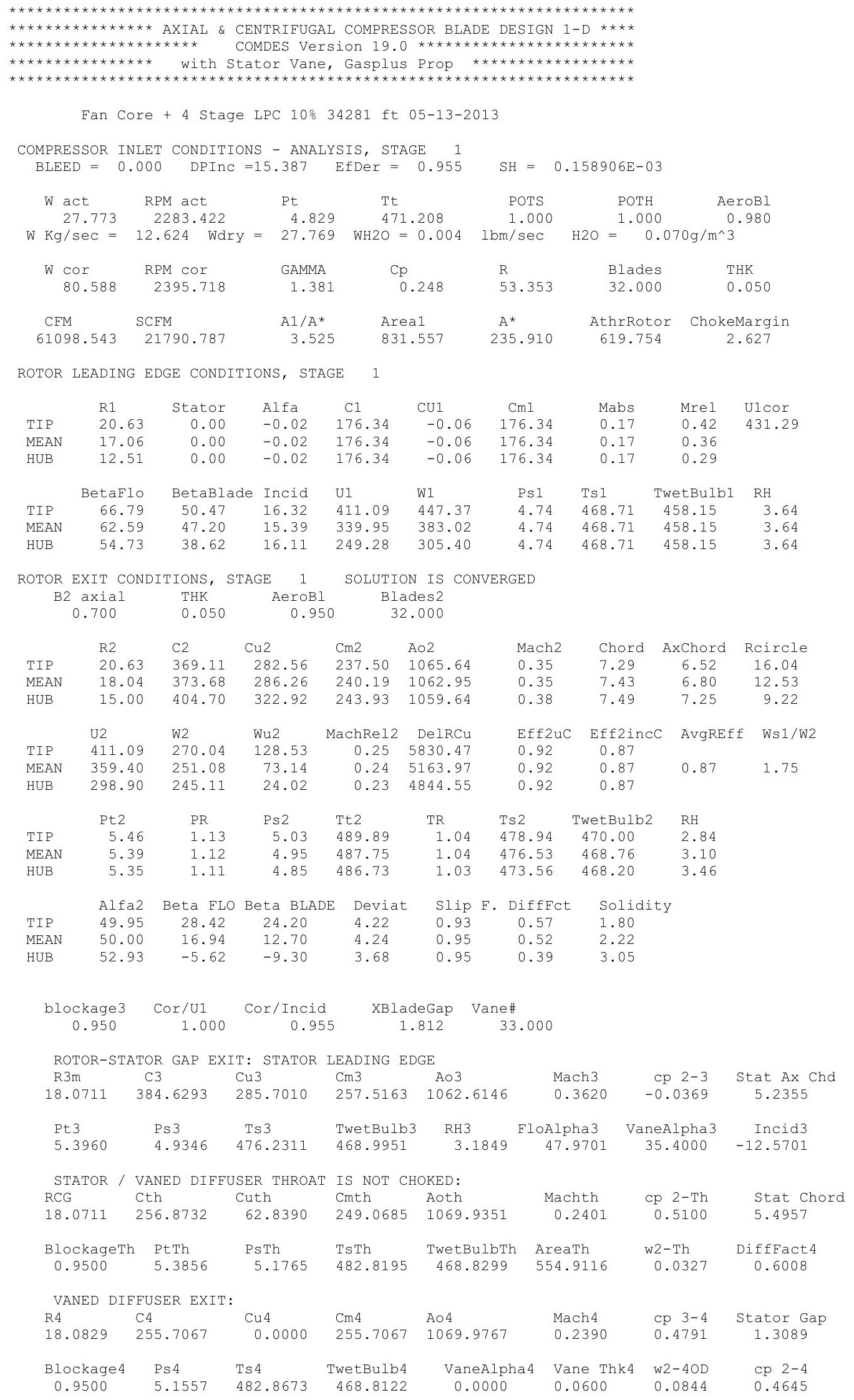




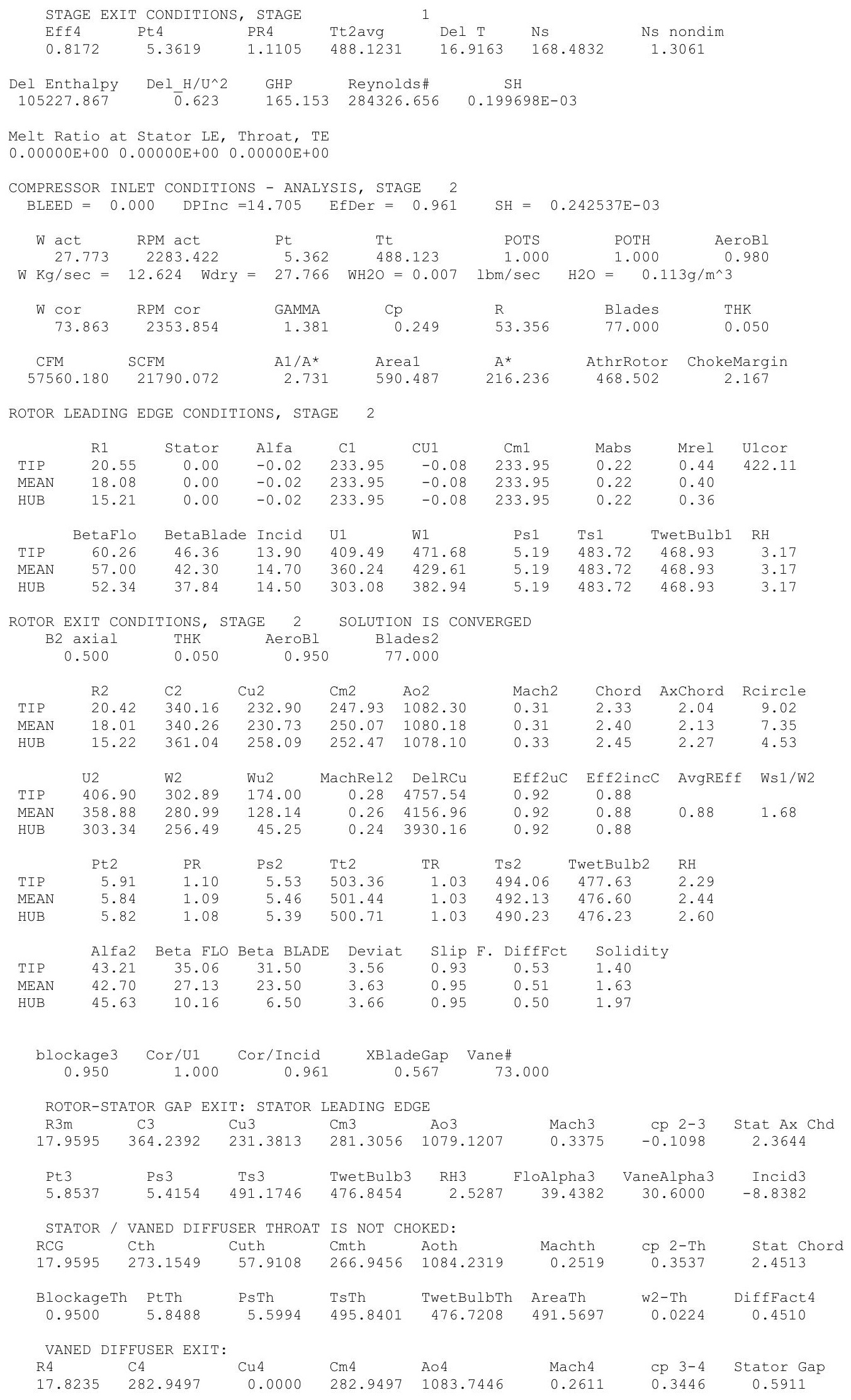




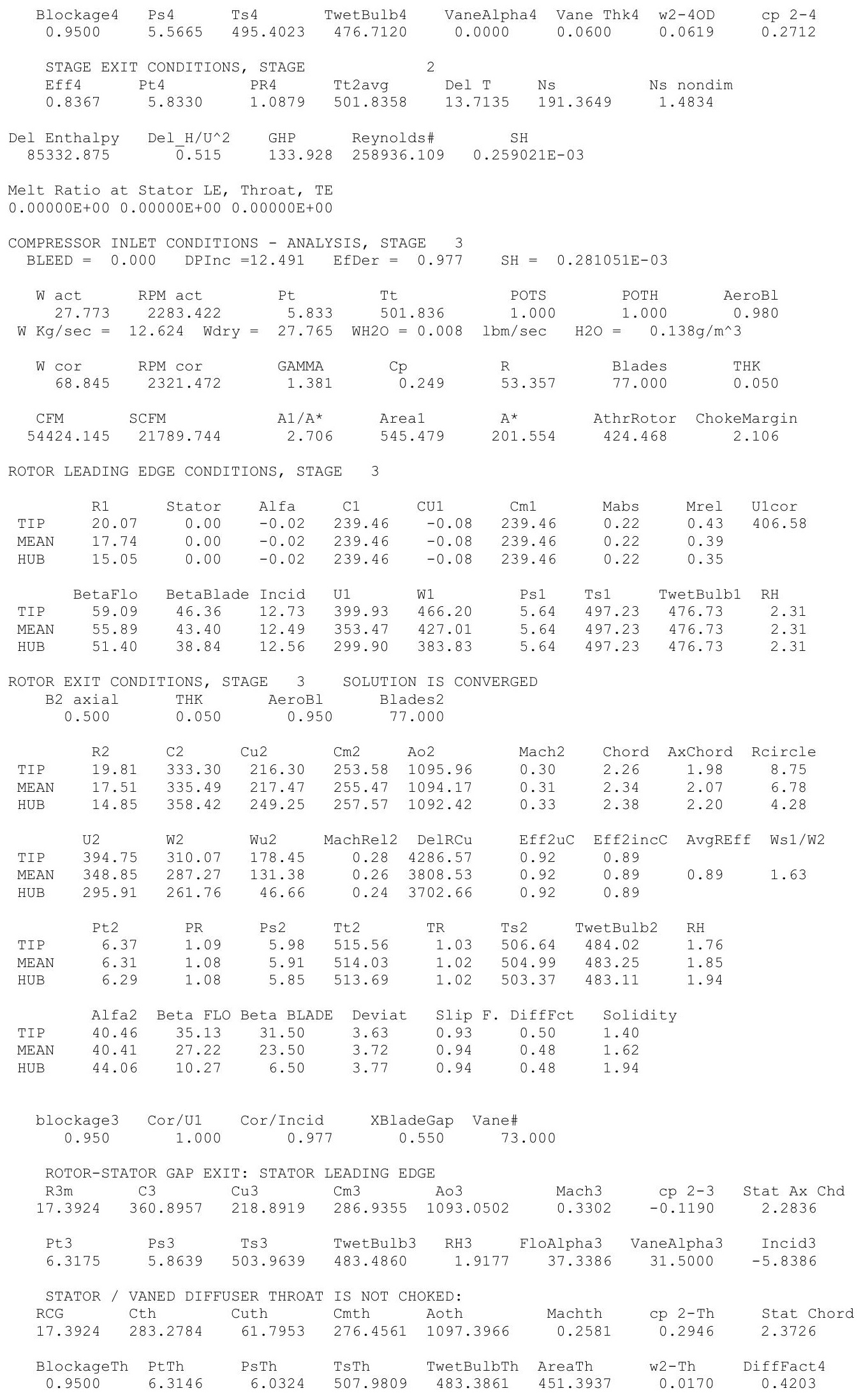




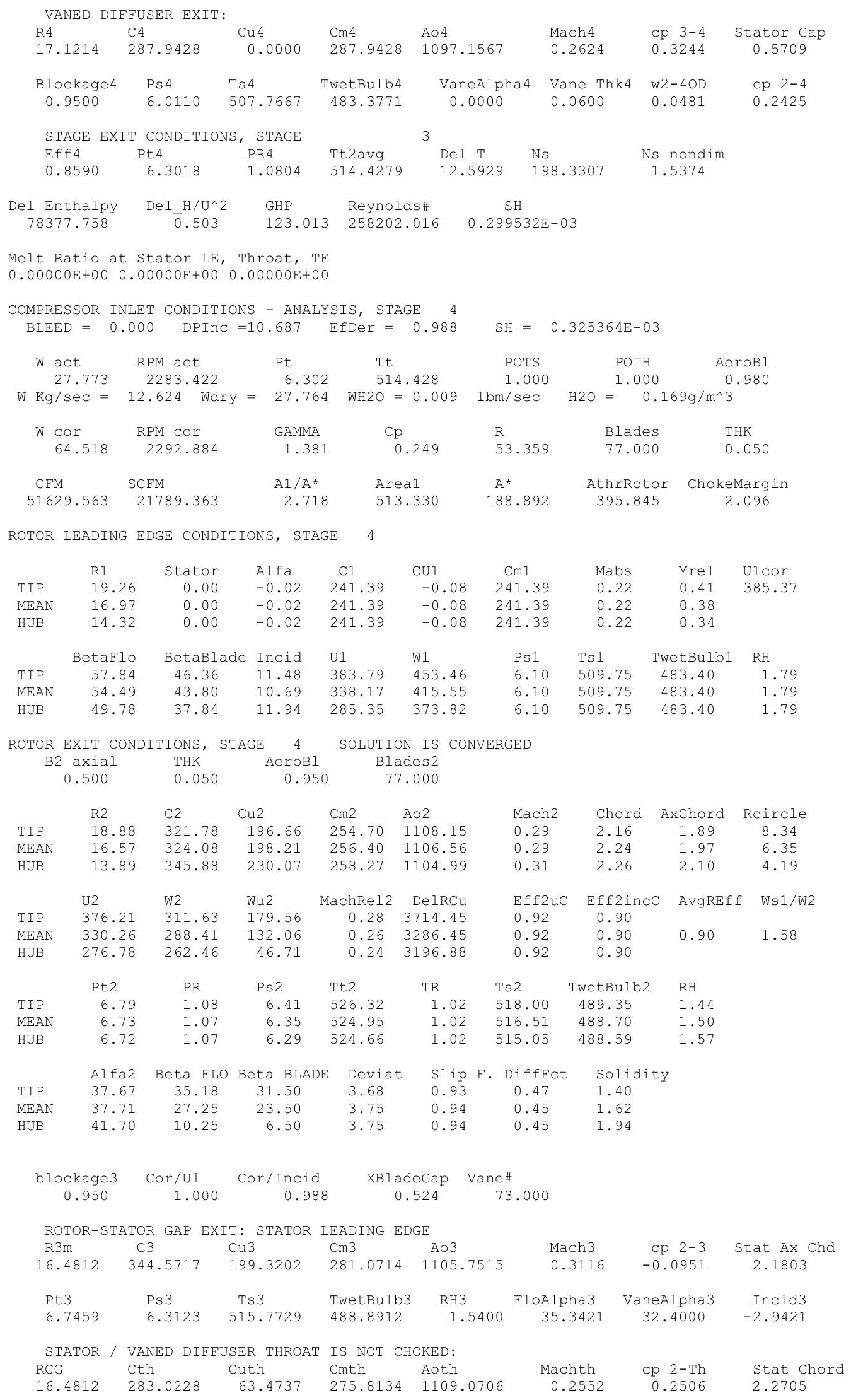




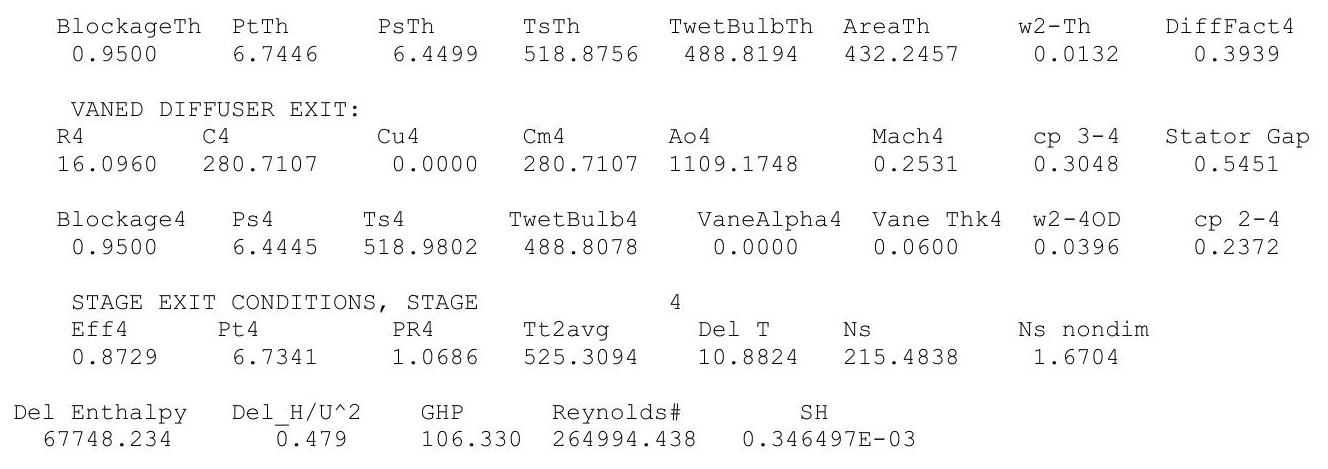

Melt Ratio at Stator LE, Throat, TE

$0.00000 \mathrm{E}+00 \quad 0.00000 \mathrm{E}+00 \quad 0.00000 \mathrm{E}+00$

COMPRESSOR INLET CONDITIONS - ANALYSIS, STAGE 5 BLEED $=0.000 \quad$ DPInC $=9.040 \quad$ EfDer $=0.995$

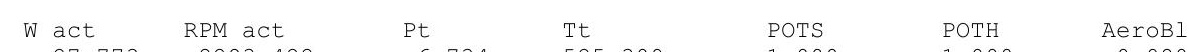

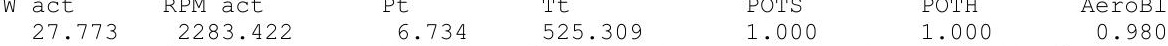

$\mathrm{W} \mathrm{Kg} / \mathrm{sec}=12.624$ Wdry $=27.763 \mathrm{WH} 2 \mathrm{O}=0.010 \mathrm{lbm} / \mathrm{sec} \quad \mathrm{H} 2 \mathrm{O}=0.205 \mathrm{~g} / \mathrm{m}^{\wedge} 3$

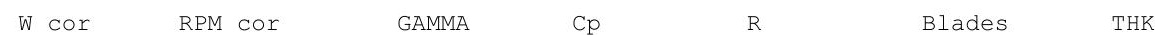

$\begin{array}{lcccccc}61.012 & 2269.011 & 1.381 & 0.249 & 53.361 & 77.000 & 0.050\end{array}$

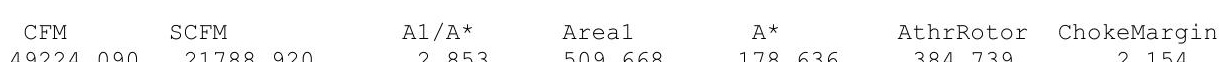

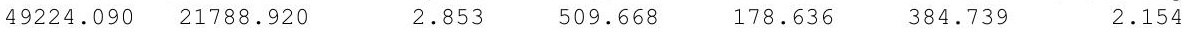

ROTOR LEADING EDGE CONDITIONS, STAGE 5

$\begin{array}{lccccccccc} & \text { R1 } & \text { Stator } & \text { Alfa } & \text { C1 } & \text { CU1 } & \text { Cm1 } & \text { Mabs } & \text { Mrel } & \text { U1cor } \\ \text { TIP } & 18.32 & 0.00 & -0.02 & 231.79 & -0.08 & 231.79 & 0.21 & 0.39 & 362.74 \\ \text { MEAN } & 15.91 & 0.00 & -0.02 & 231.79 & -0.08 & 231.79 & 0.21 & 0.35 & \\ \text { HUB } & 13.07 & 0.00 & -0.02 & 231.79 & -0.08 & 231.79 & 0.21 & 0.31 & \\ & & & & & & & & & \\ & \text { BetaFlo } & \text { BetaBlade } & \text { Incid } & \text { U1 } & \text { W1 } & \text { Ts1 } 1 & \text { TwetBulb1 } & \text { RH } \\ \text { TIP } & 57.59 & 47.36 & 10.23 & 365.06 & 432.49 & 6.54 & 520.99 & 488.83 & 1.48 \\ \text { MEAN } & 53.84 & 44.80 & 9.04 & 317.09 & 392.84 & 6.54 & 520.99 & 488.83 & 1.48 \\ \text { HUB } & 48.34 & 38.84 & 9.50 & 260.44 & 348.71 & 6.54 & 520.99 & 488.83 & 1.48\end{array}$

ROTOR EXIT CONDITIONS, STAGE 5 SOLUTION IS CONVERGED

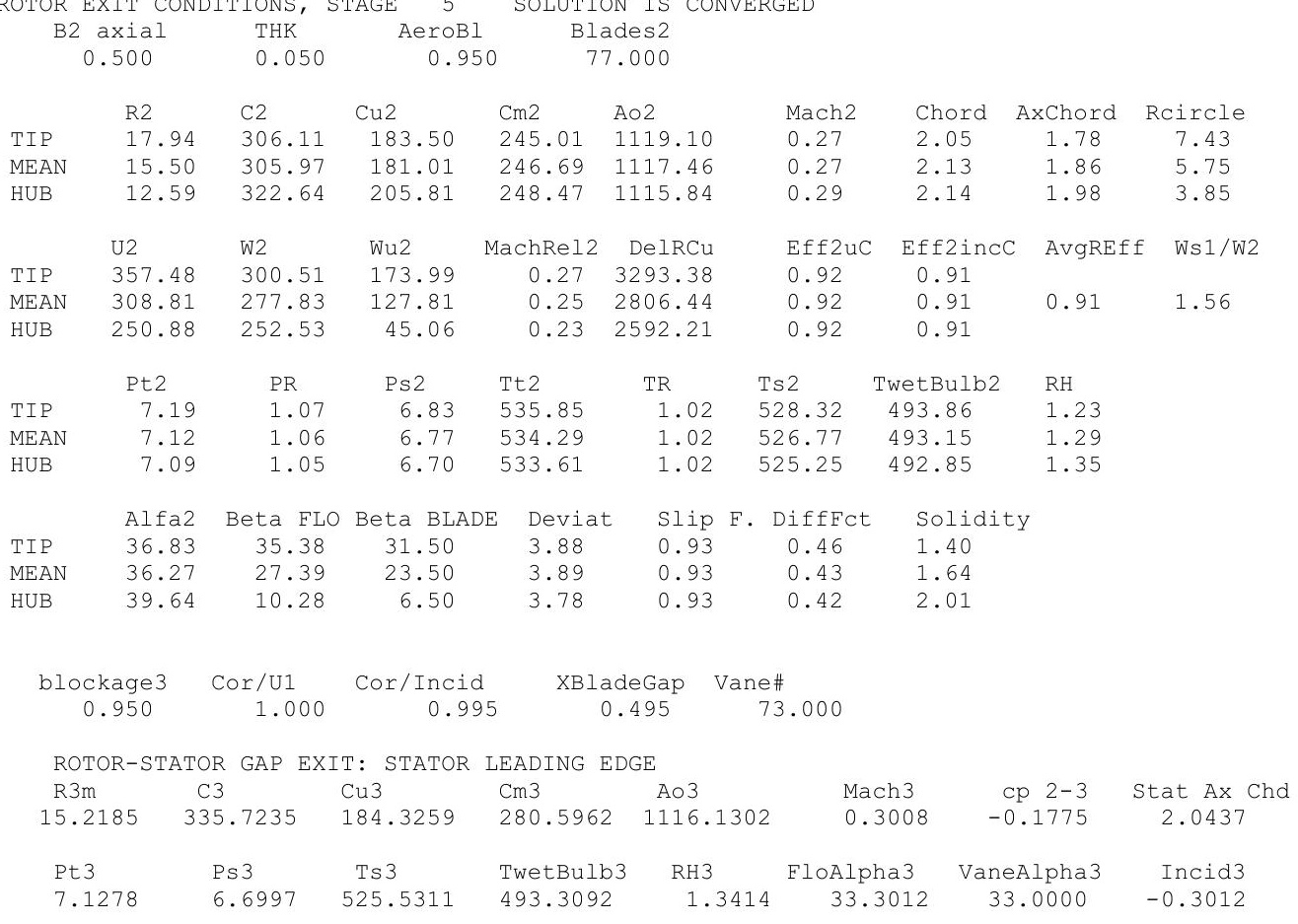




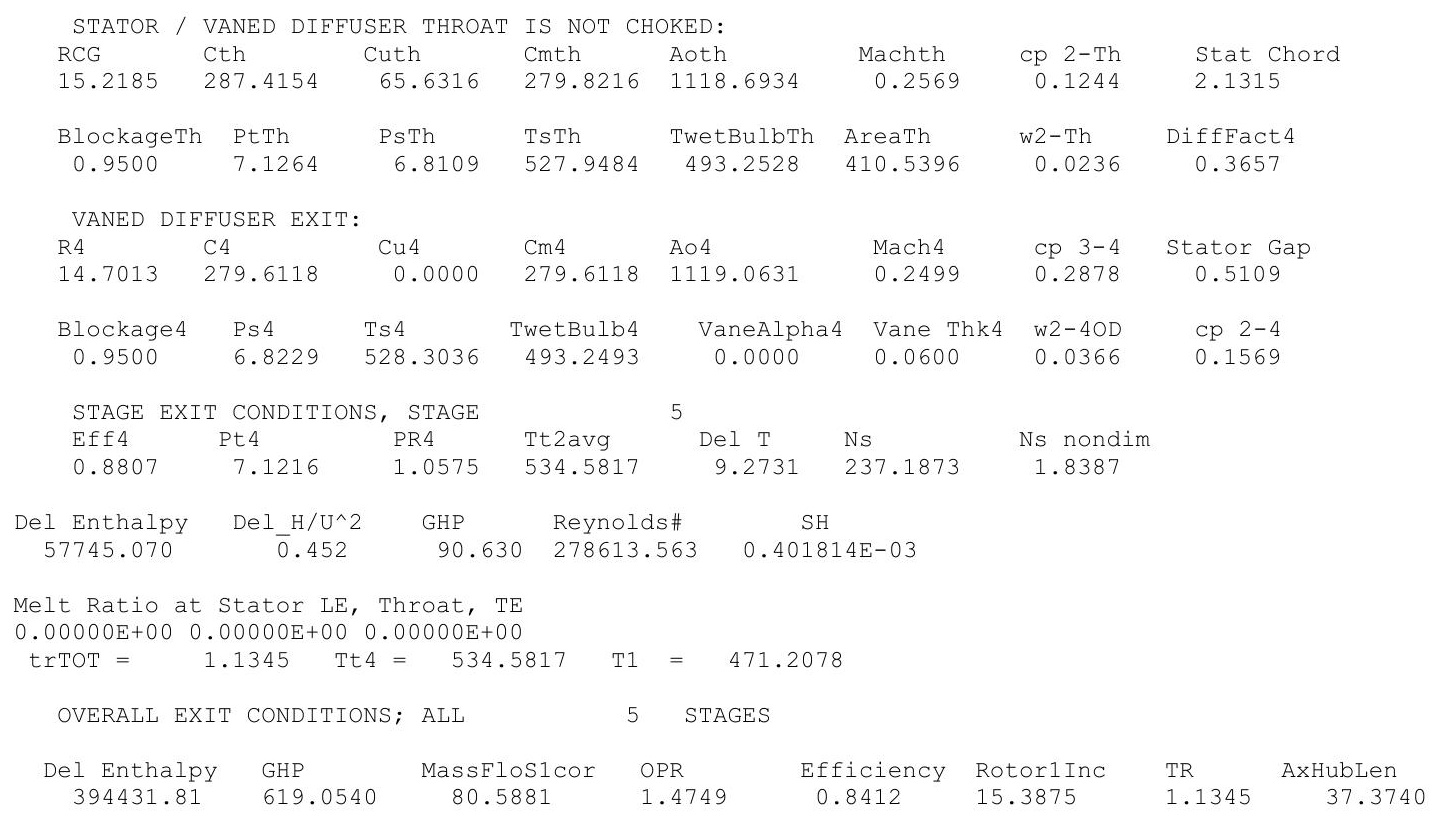




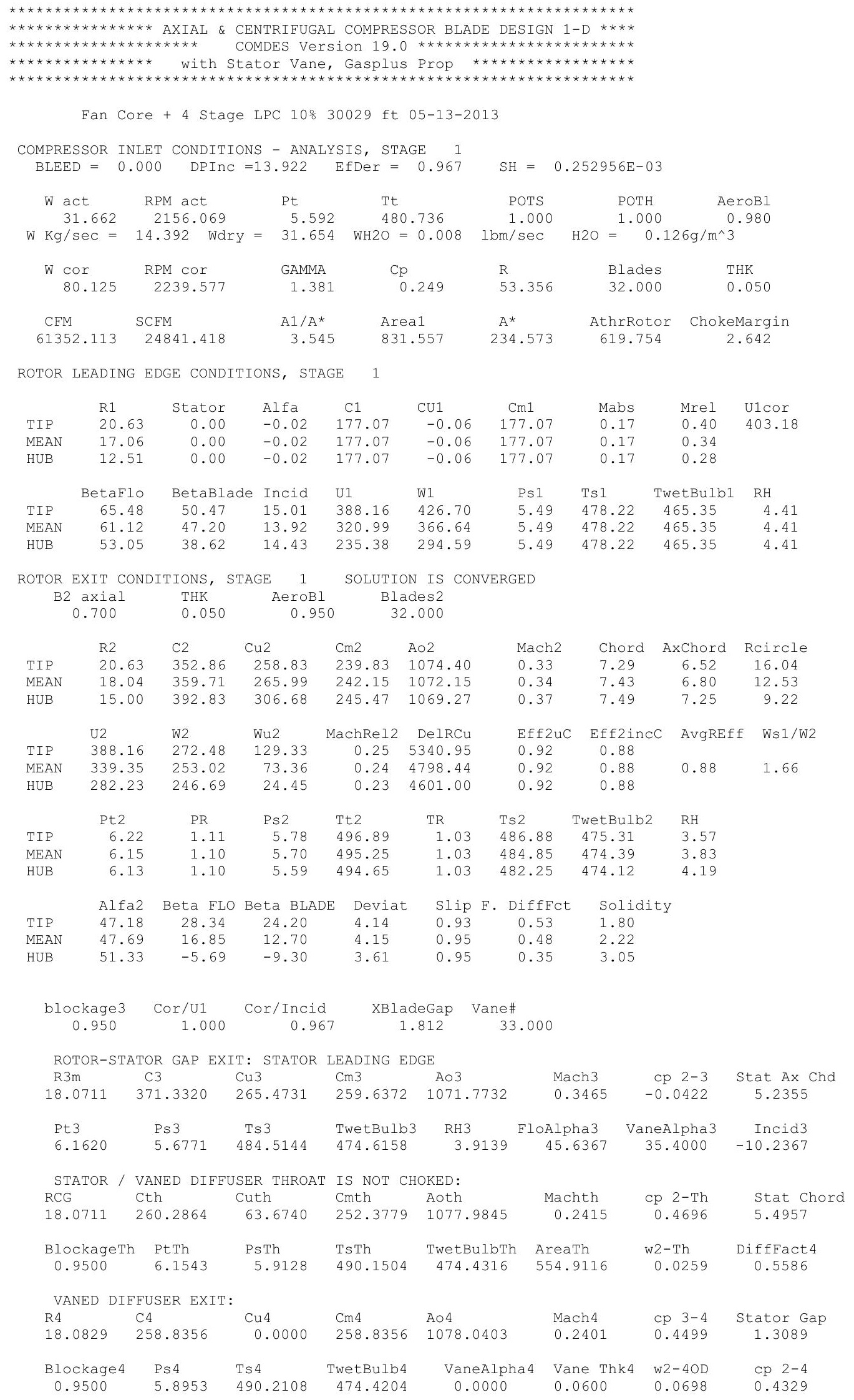




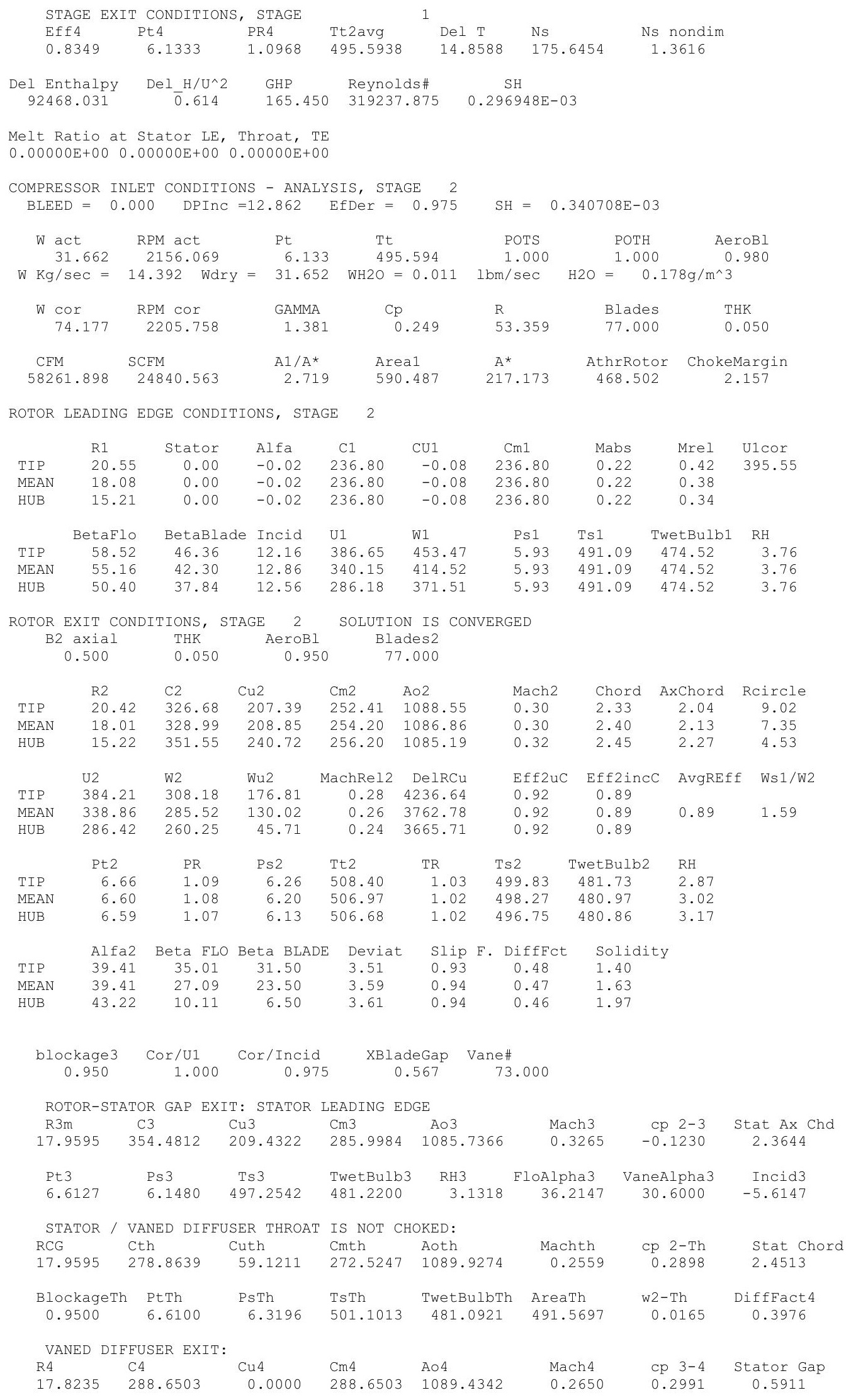




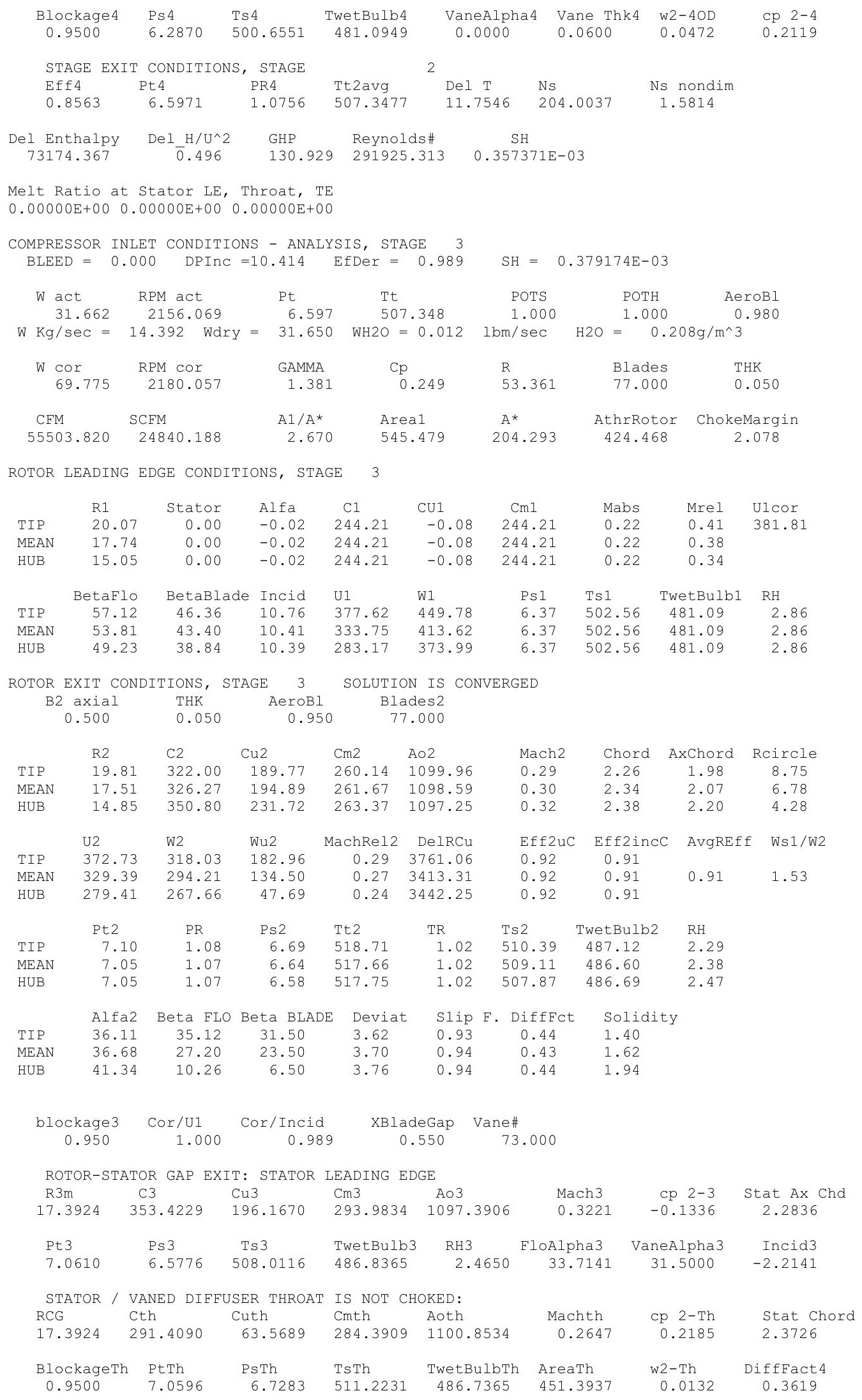




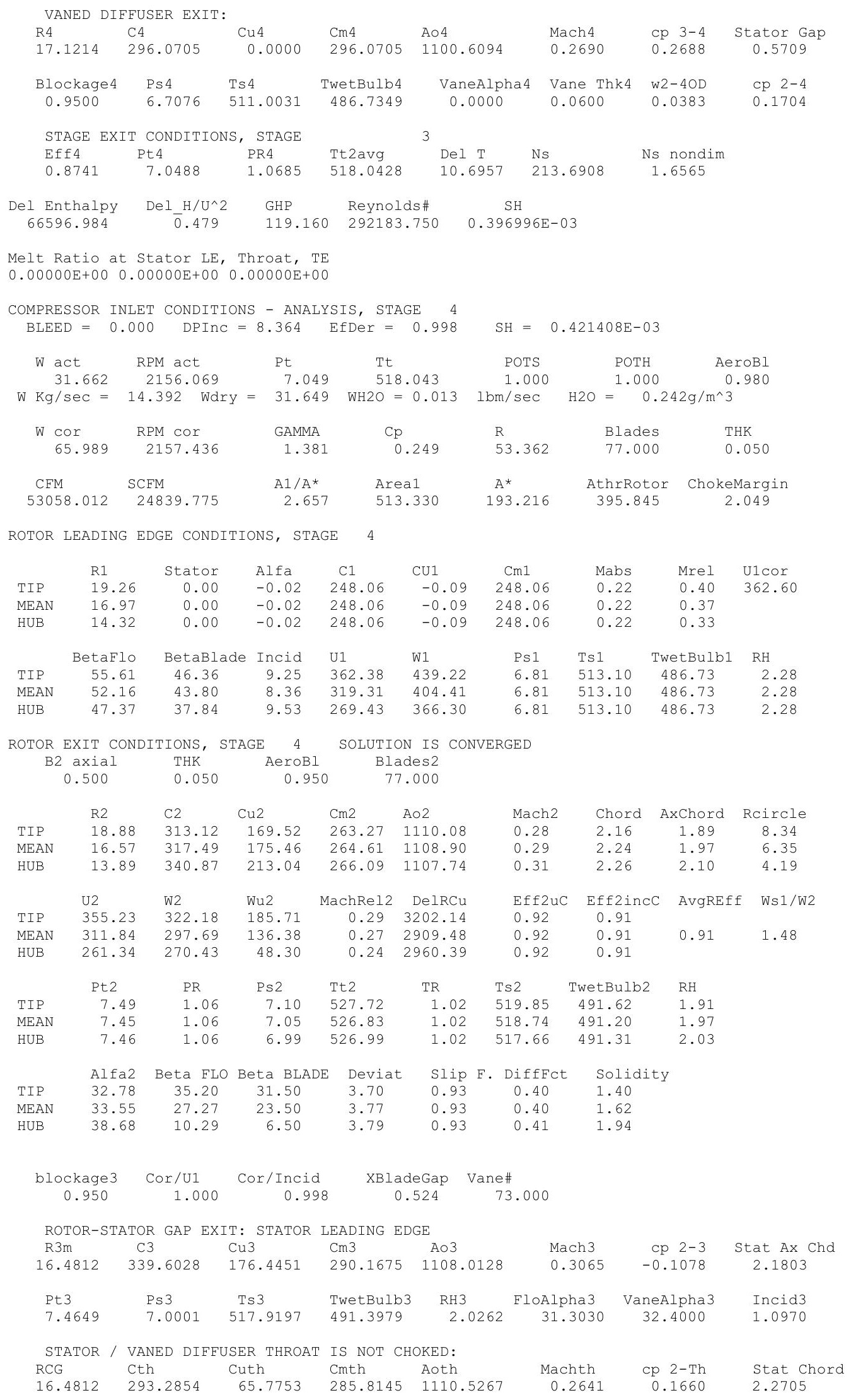




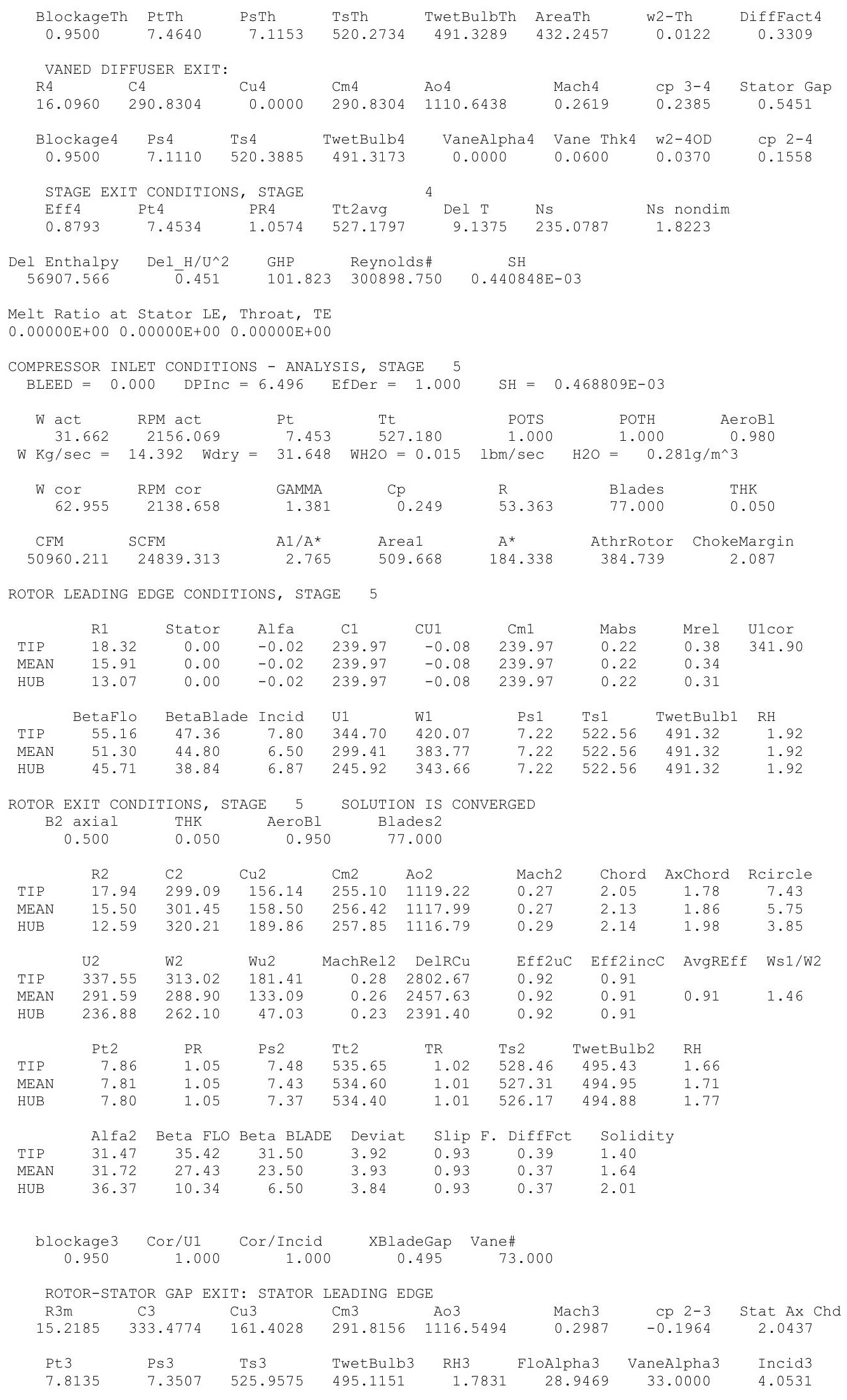




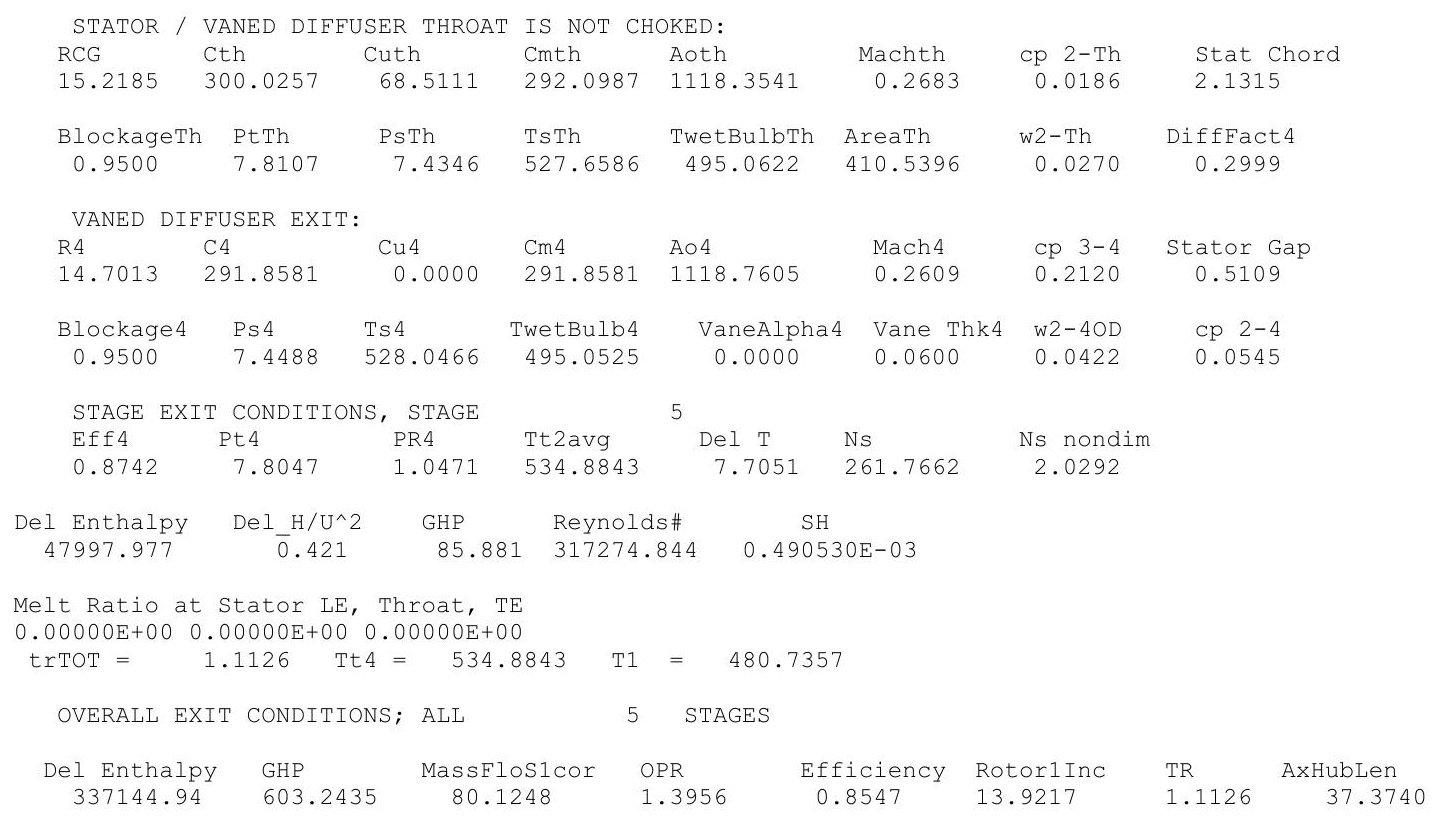




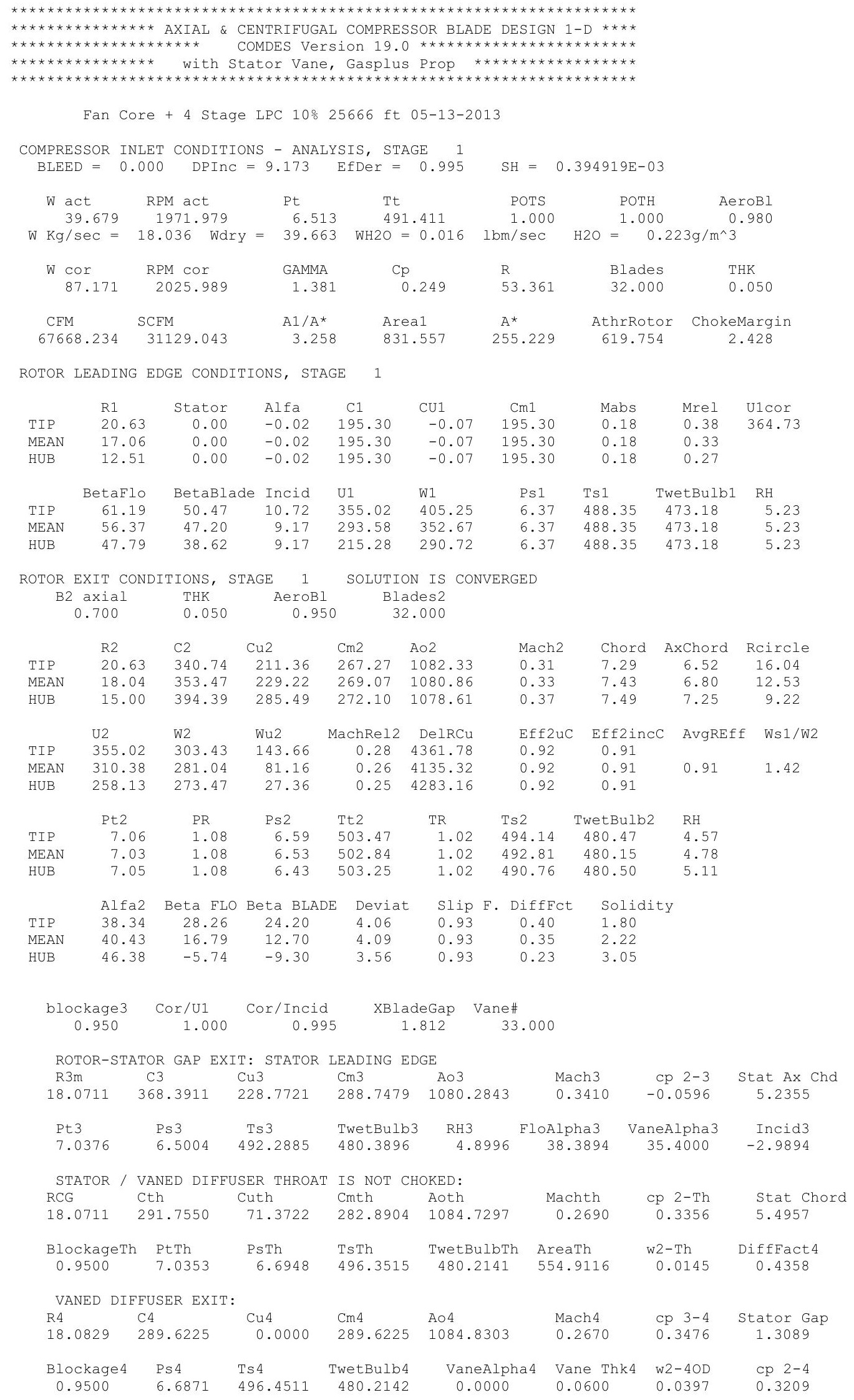




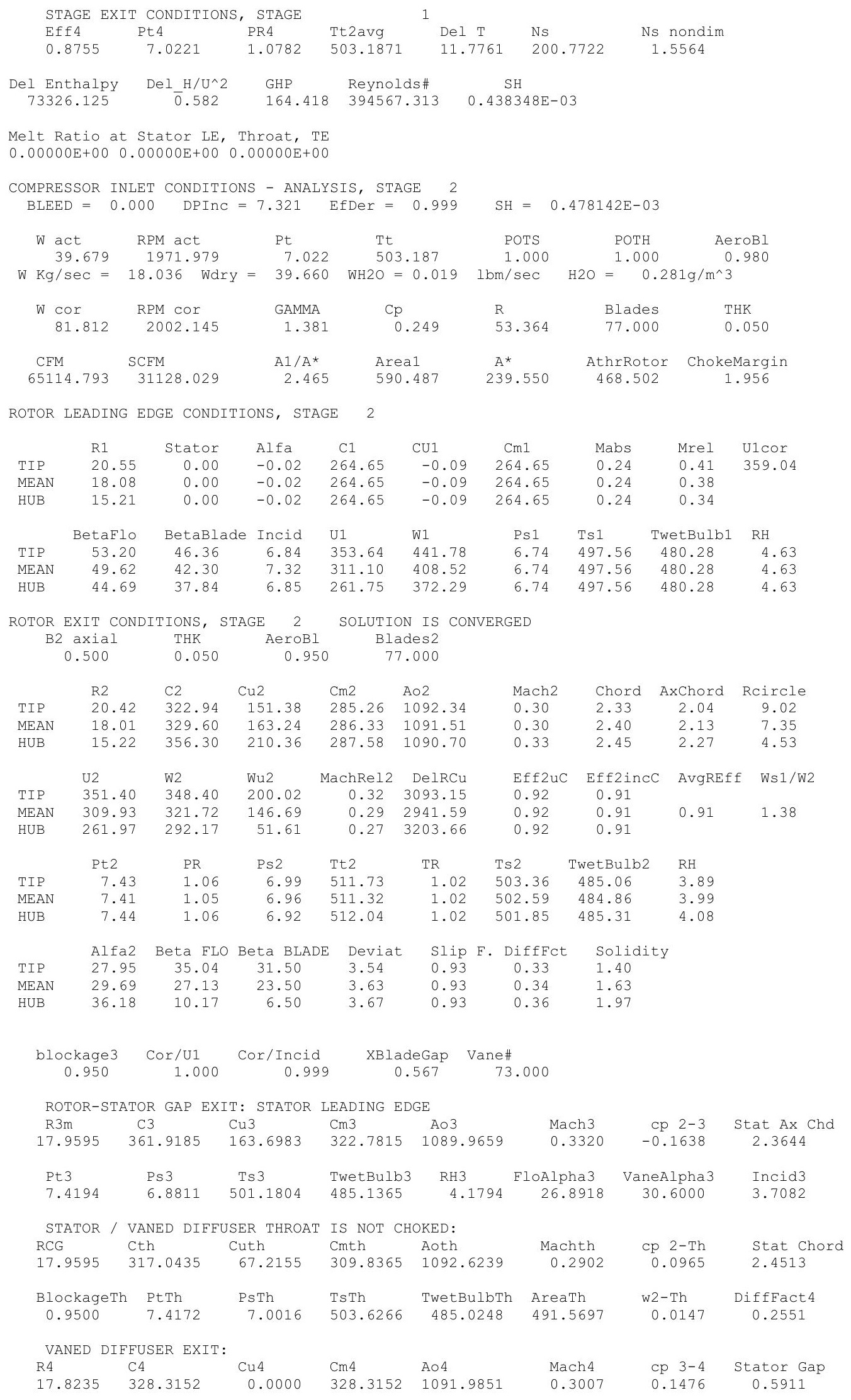




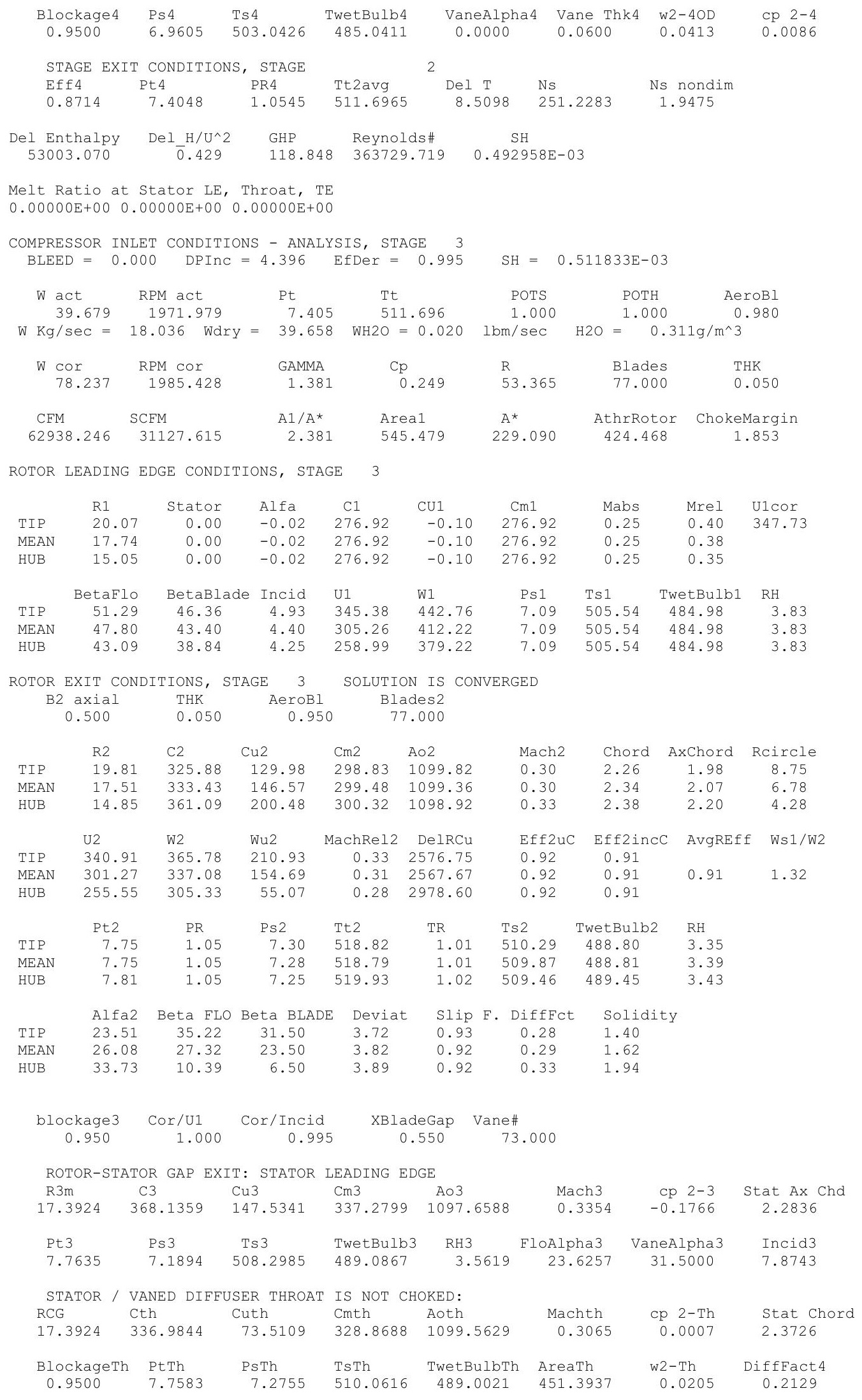




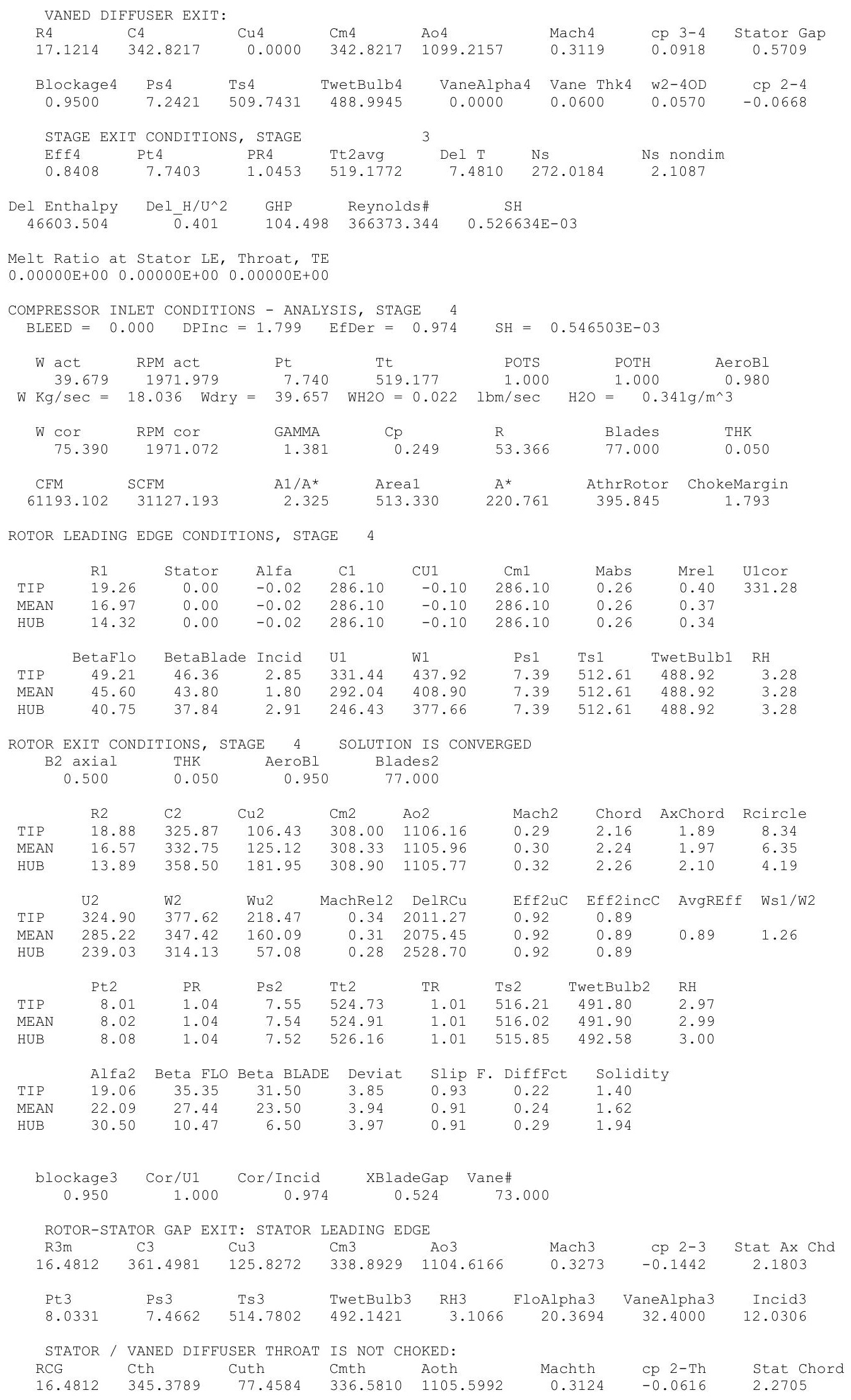




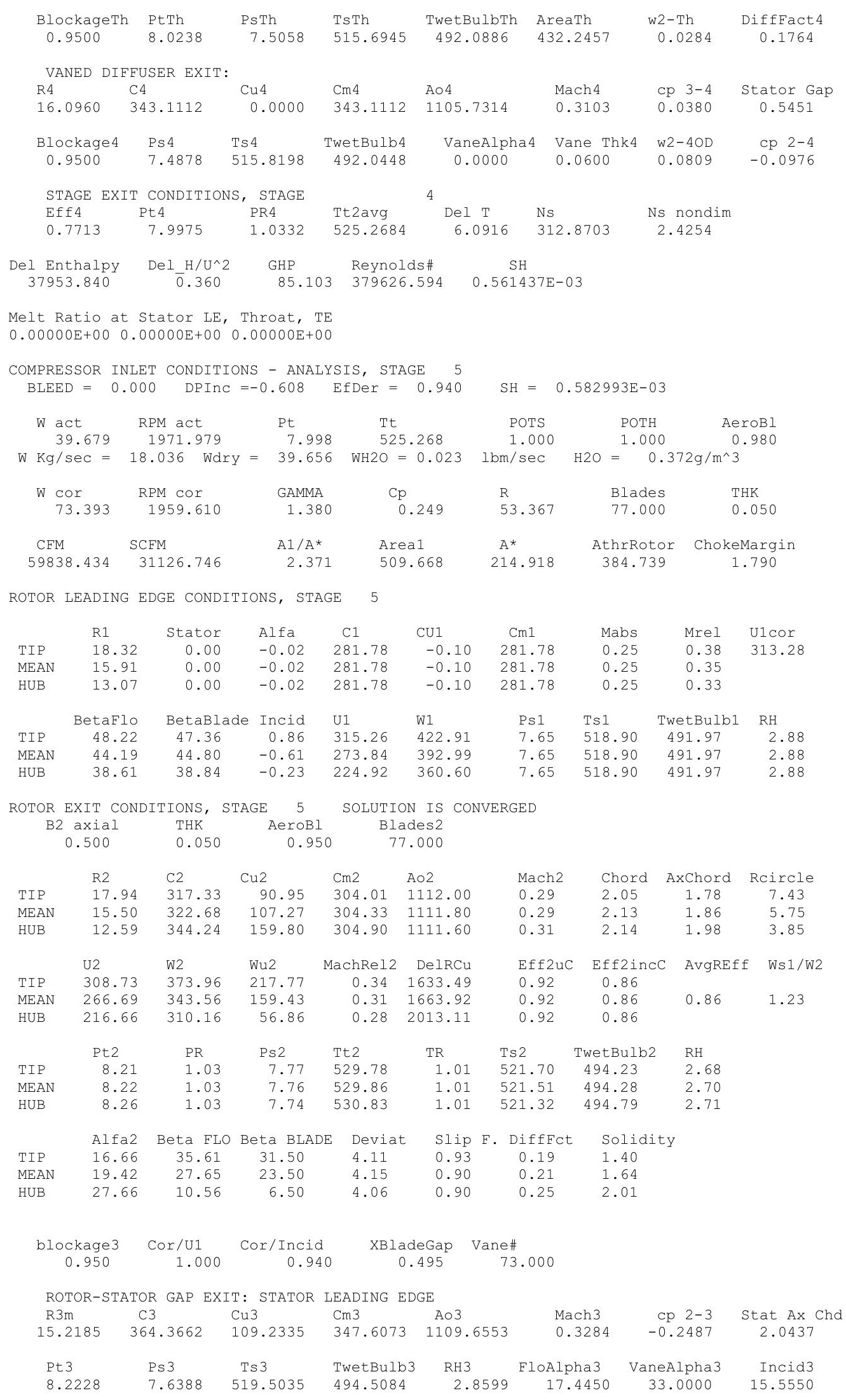




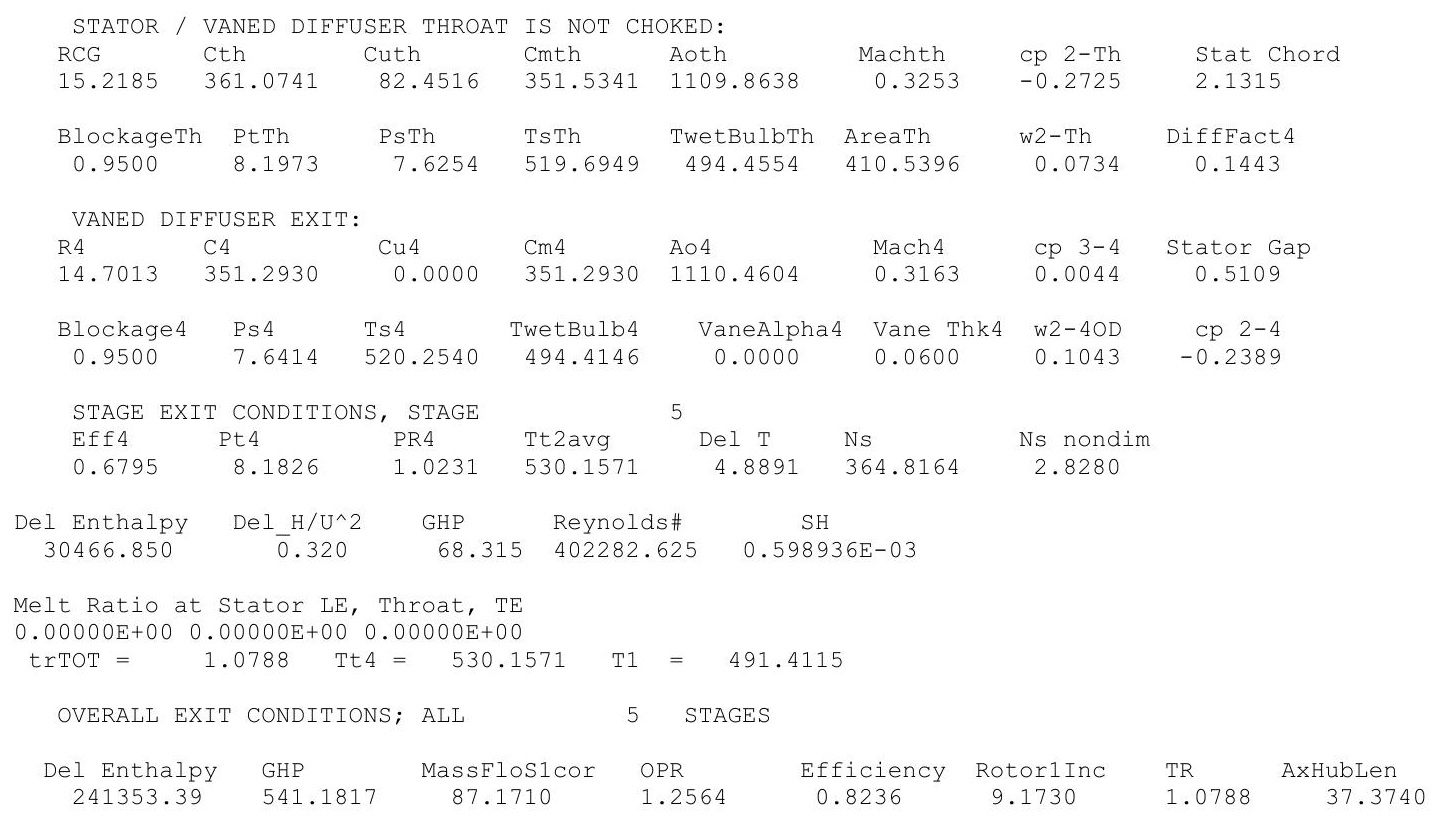




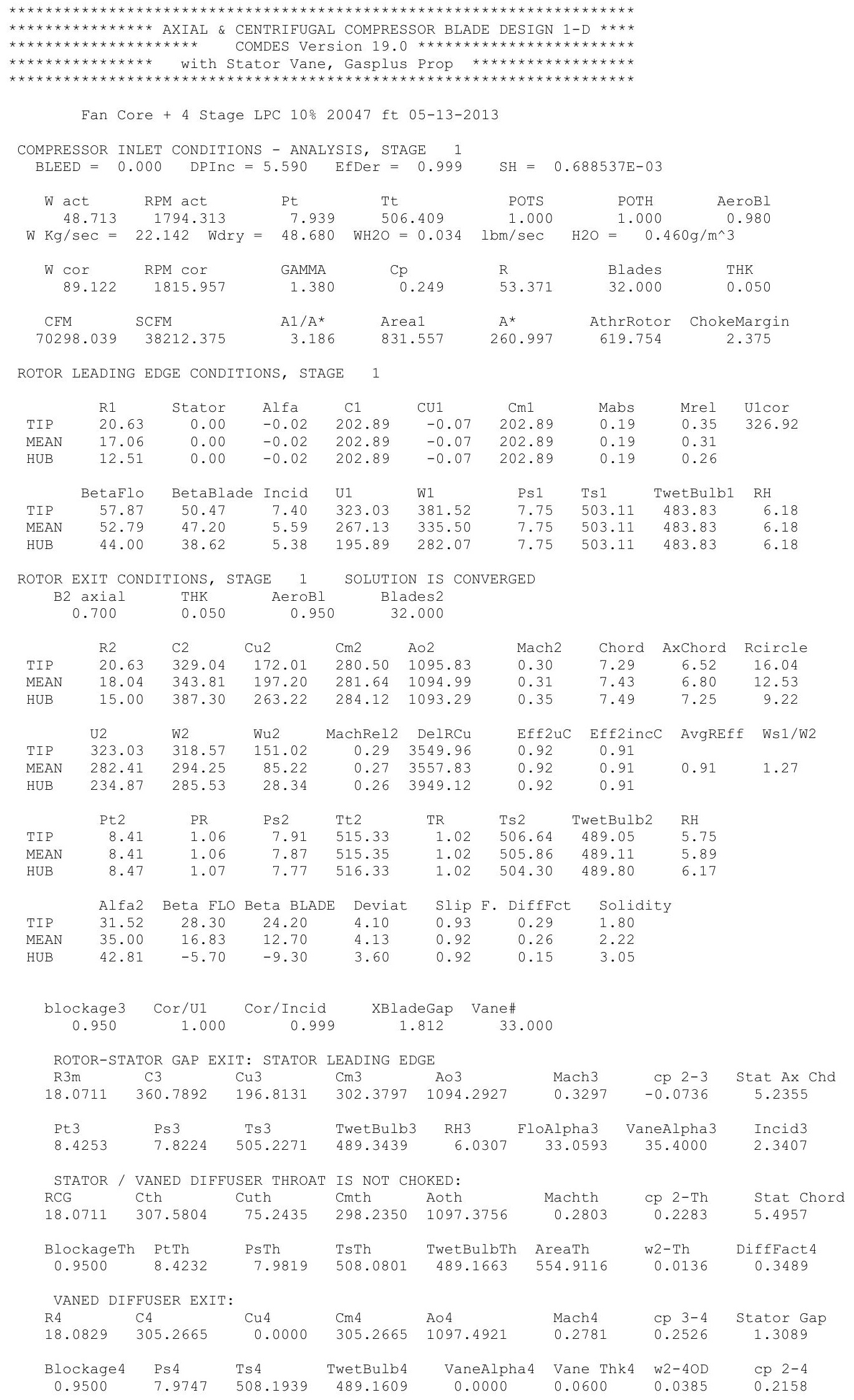




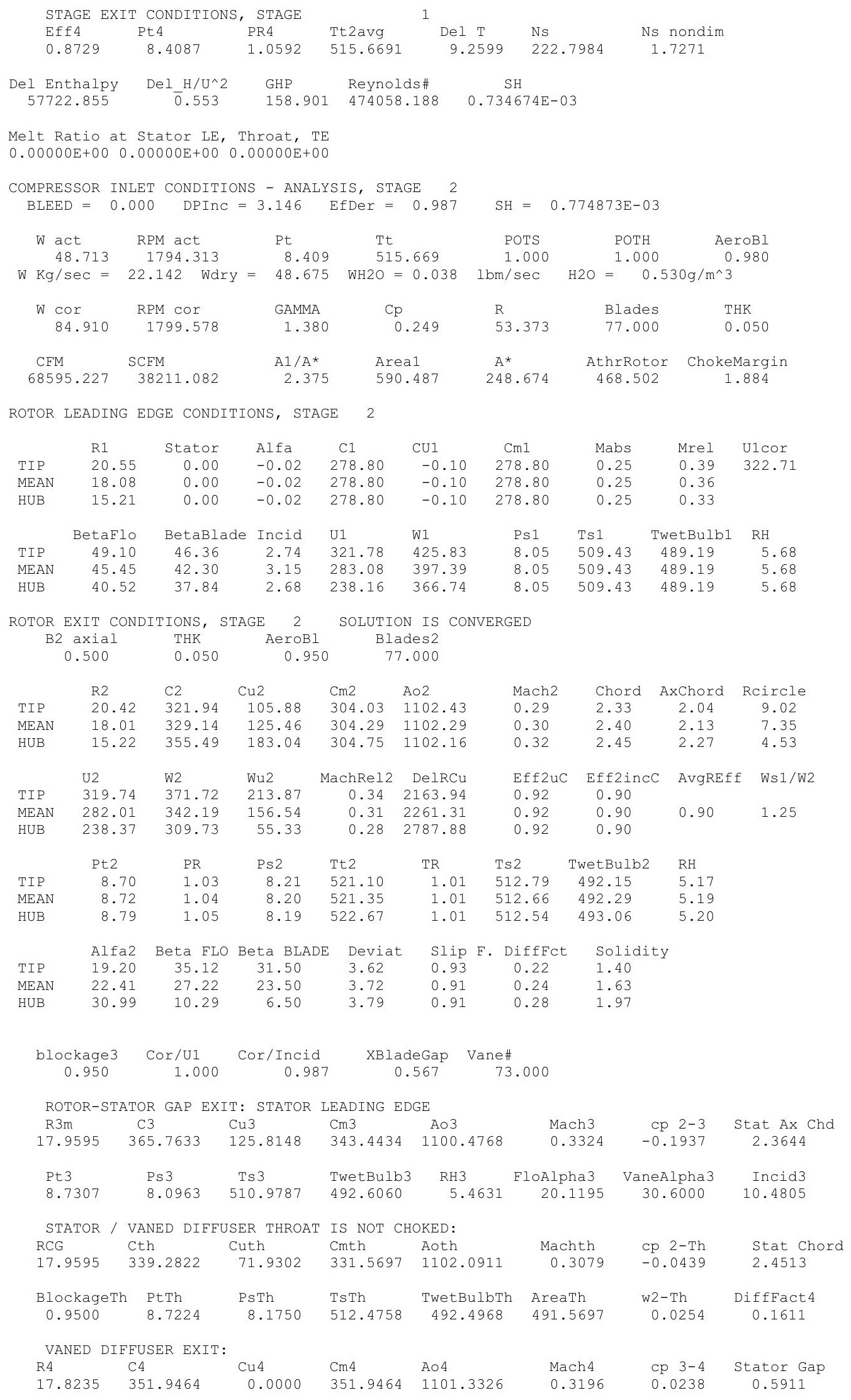




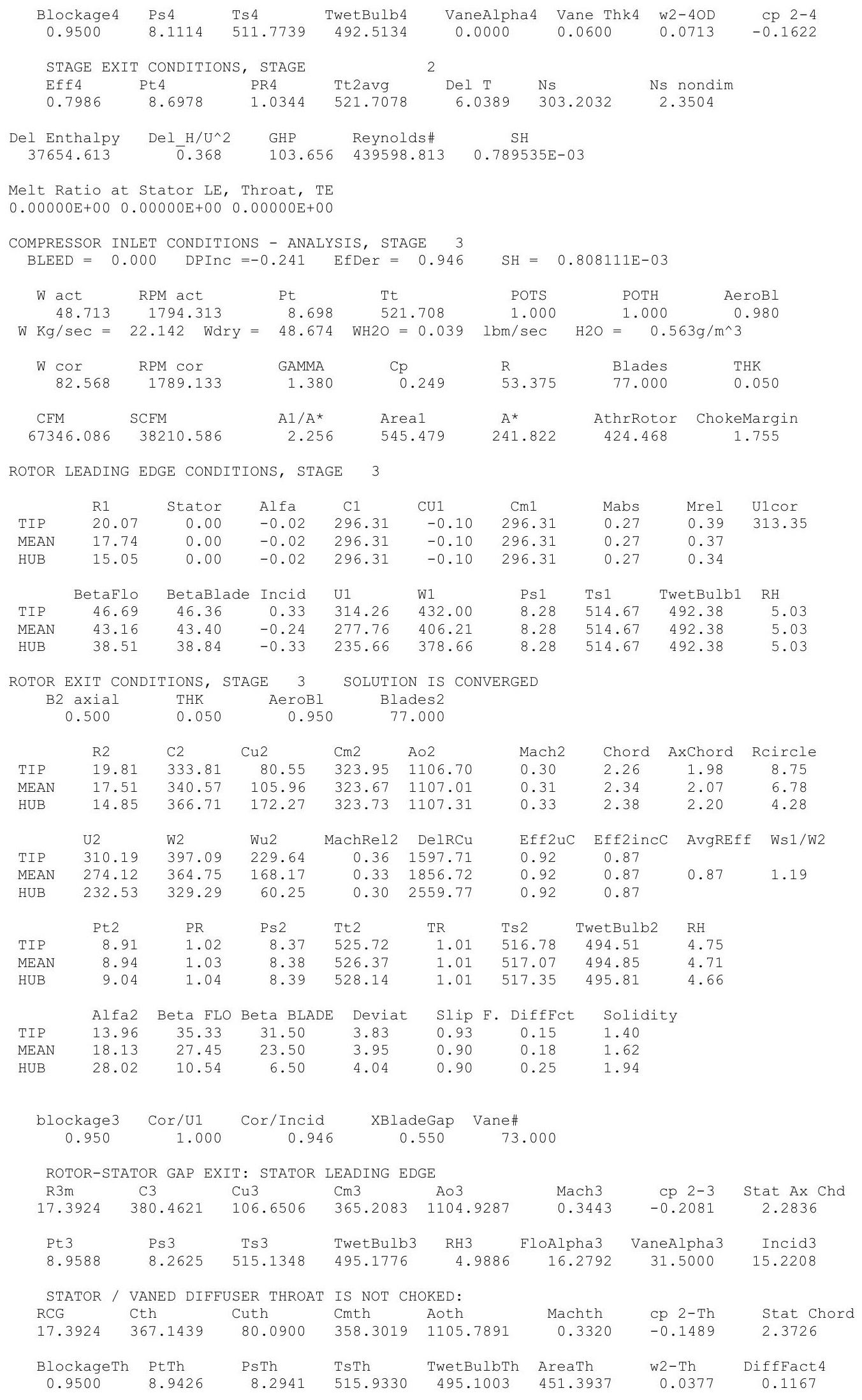




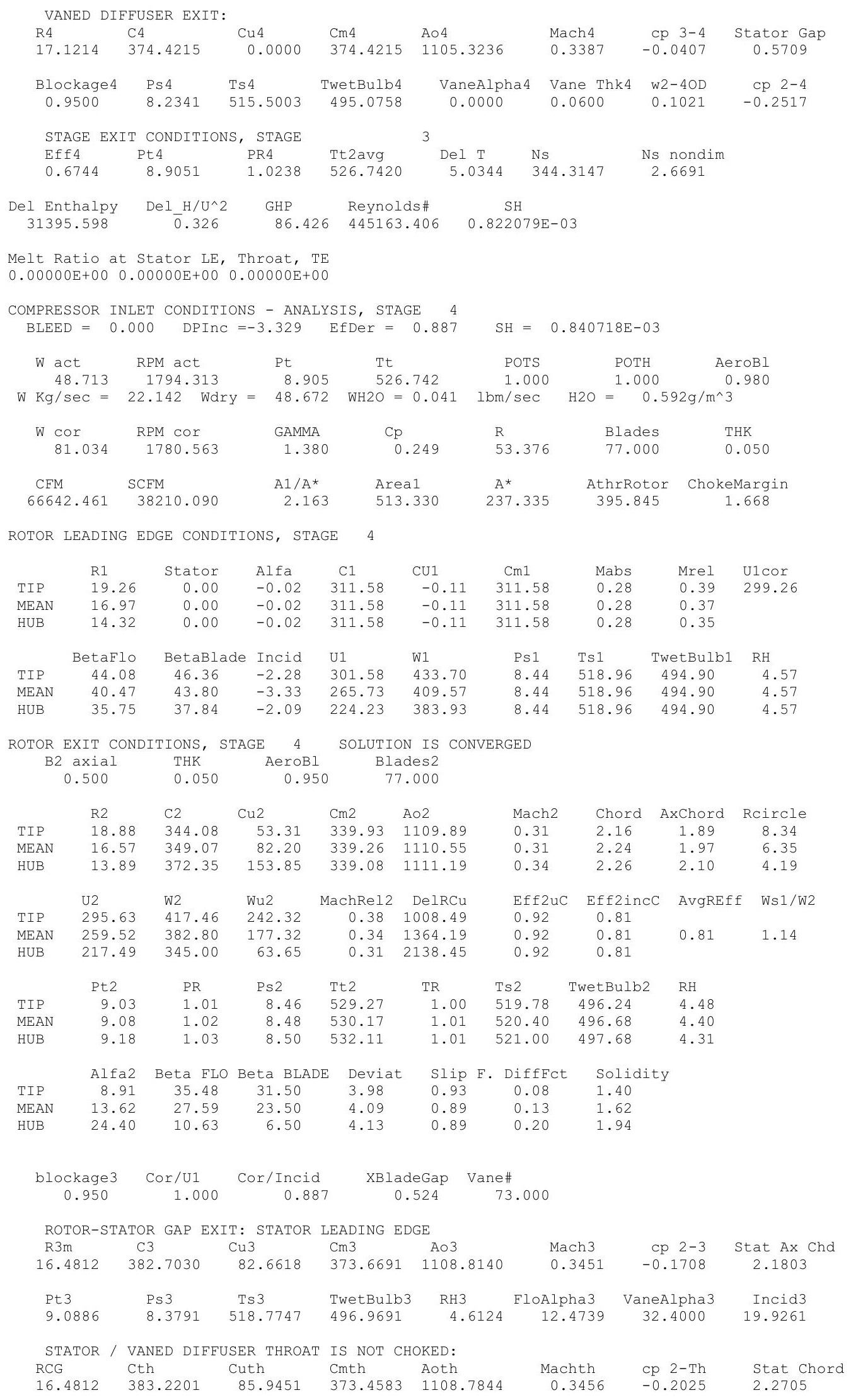




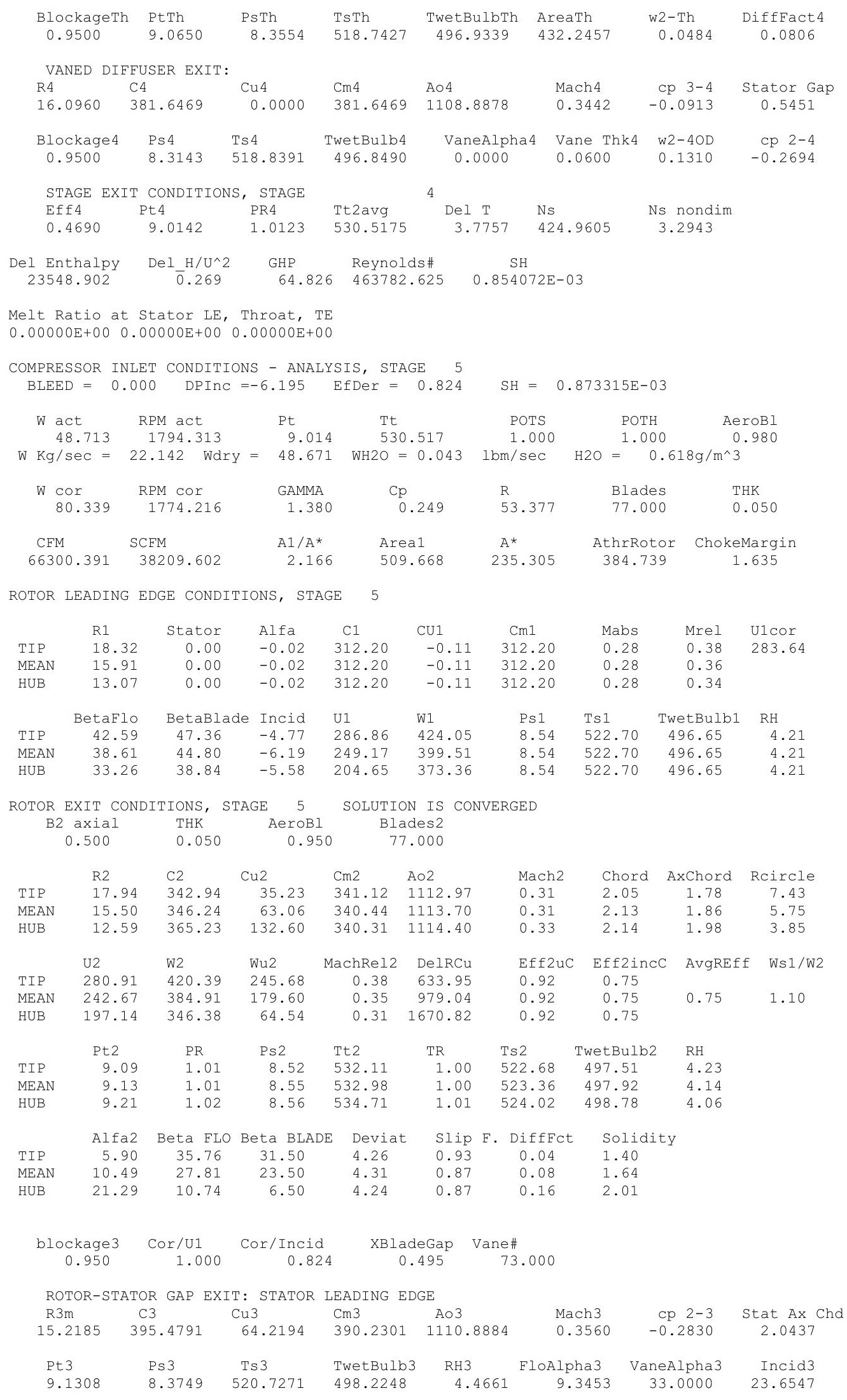




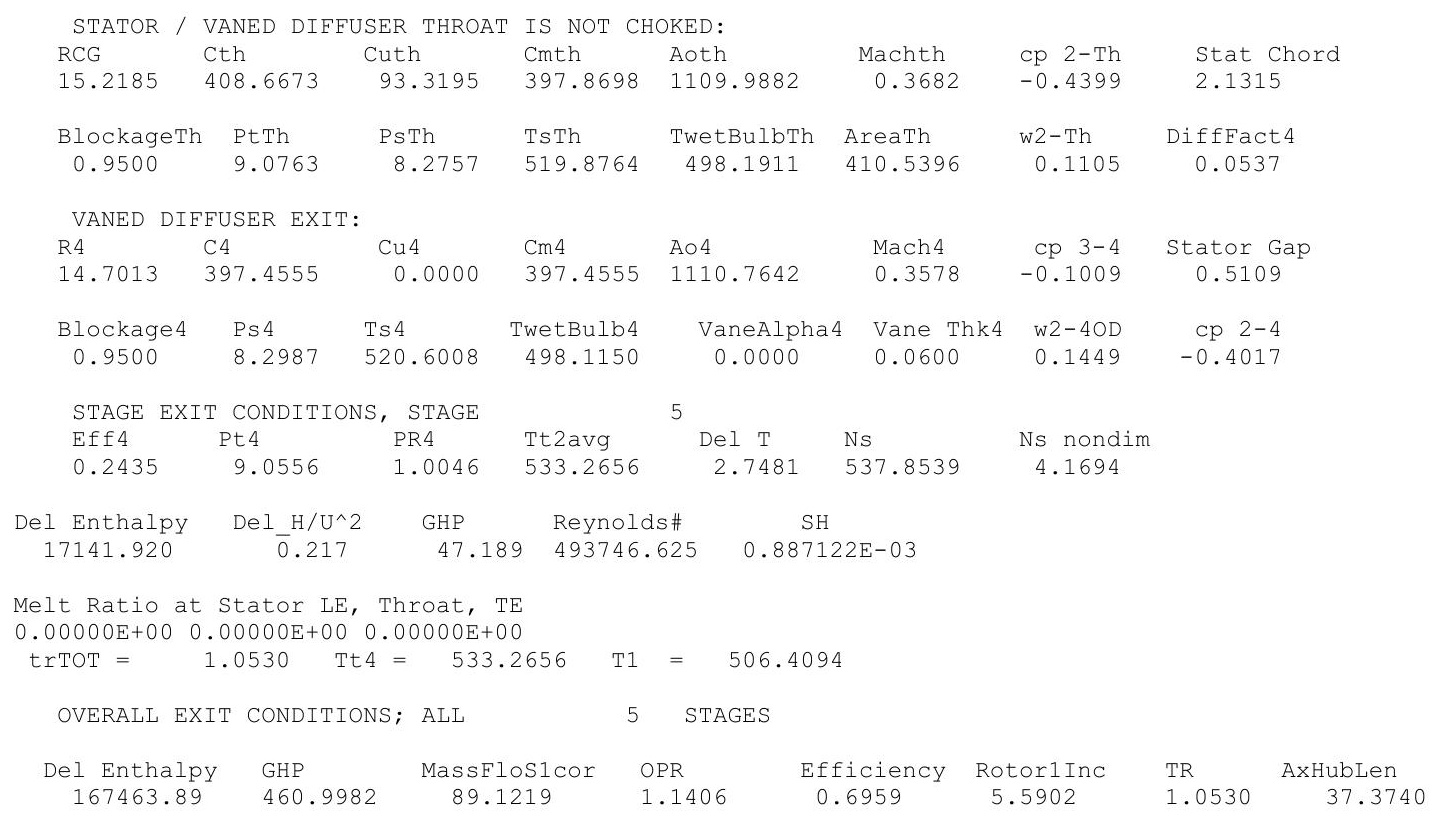




\title{
$50 \mu \mathrm{m}, \mathrm{ISA}+18 \mathrm{R}$
}

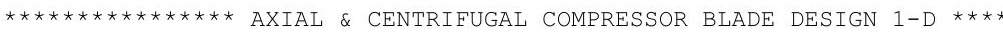

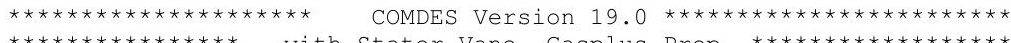

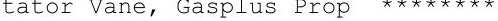

Fan Core + 4 Stage LPC 10\%39000 ft 05-13-2013

COMPRESSOR INLET CONDITIONS - ANALYSIS, STAGE 1

$\mathrm{BLEED}=0.000 \quad$ DPInC $=15.147 \quad$ EfDer $=0.957$

$\mathrm{SH}=0.515696 \mathrm{E}-04$

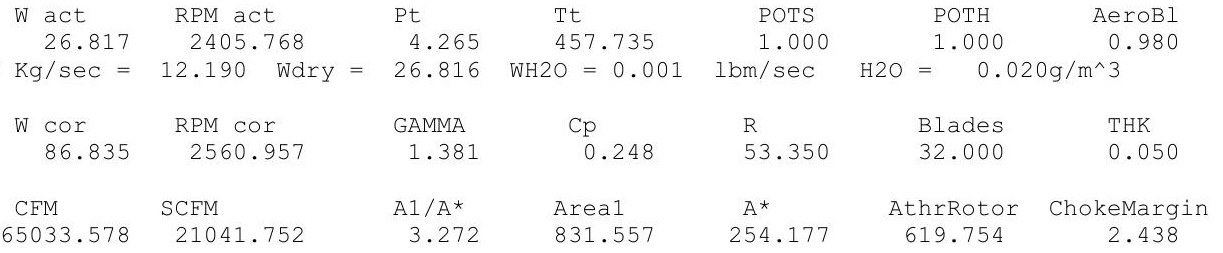

ROTOR LEADING EDGE CONDITIONS, STAGE 1

$\begin{array}{lccccccccc} & \text { R1 } & \text { Stator } & \text { Alfa } & \text { C1 } & \text { CU1 } & \text { Cm1 } & \text { Mabs } & \text { Mrel } & \text { U1cor } \\ \text { TIP } & 20.63 & 0.00 & -0.02 & 187.70 & -0.06 & 187.70 & 0.18 & 0.45 & 461.04 \\ \text { MEAN } & 17.06 & 0.00 & -0.02 & 187.70 & -0.06 & 187.70 & 0.18 & 0.39 & \\ \text { HUB } & 12.51 & 0.00 & -0.02 & 187.70 & -0.06 & 187.70 & 0.18 & 0.31 & \\ & & & & & & & & & \\ & \text { BetaFlo } & \text { BetaBlade } & \text { Incid } & \text { U1 } & \text { W1 } & \text { Ts1 } & \text { TwetBulb1 } & \text { RH } \\ \text { TIP } & 66.57 & 50.47 & 16.10 & 433.11 & 472.09 & 4.17 & 454.90 & 448.23 & 1.97 \\ \text { MEAN } & 62.35 & 47.20 & 15.15 & 358.17 & 404.42 & 4.17 & 454.90 & 448.23 & 1.97 \\ \text { HUB } & 54.45 & 38.62 & 15.83 & 262.64 & 322.87 & 4.17 & 454.90 & 448.23 & 1.97\end{array}$

ROTOR EXIT CONDITIONS, STAGE 1 SOLUTION IS CONVERGED

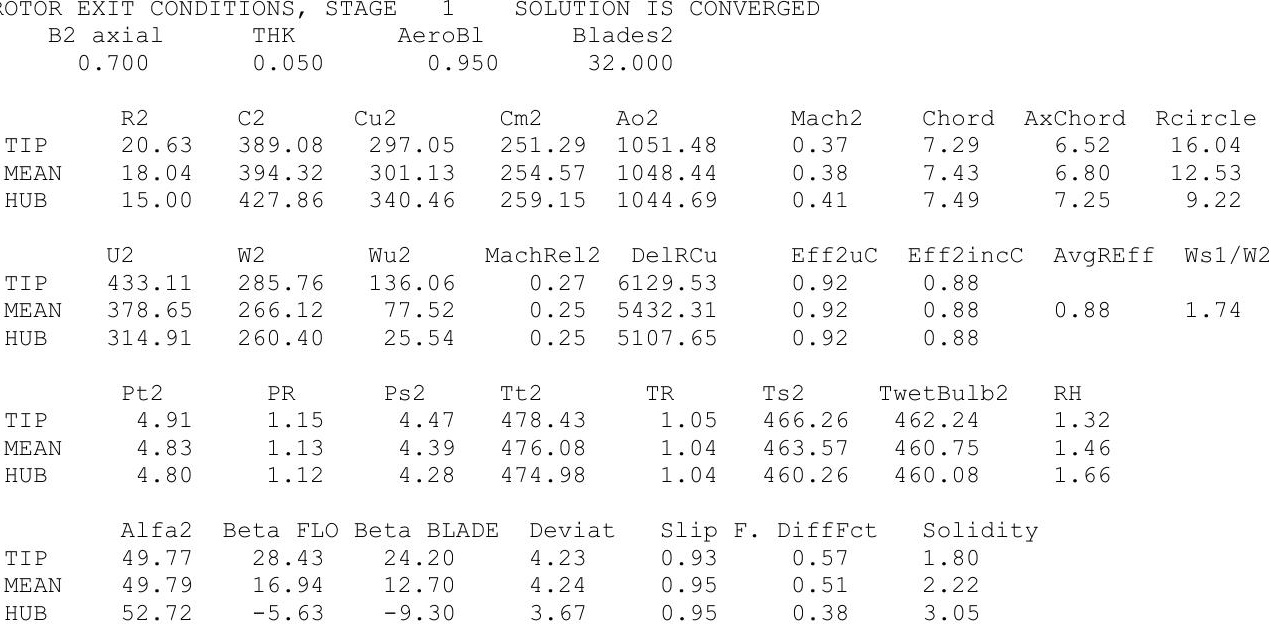

$$
\begin{array}{ccccc}
\text { blockage3 } & \text { Cor/U1 } & \text { Cor/Incid } & \text { XBladeGap } & \text { Vane\# } \\
0.950 & 1.000 & 0.957 & 1.812 & 33.000
\end{array}
$$

ROTOR-STATOR GAP EXIT: STATOR LEADING EDGE

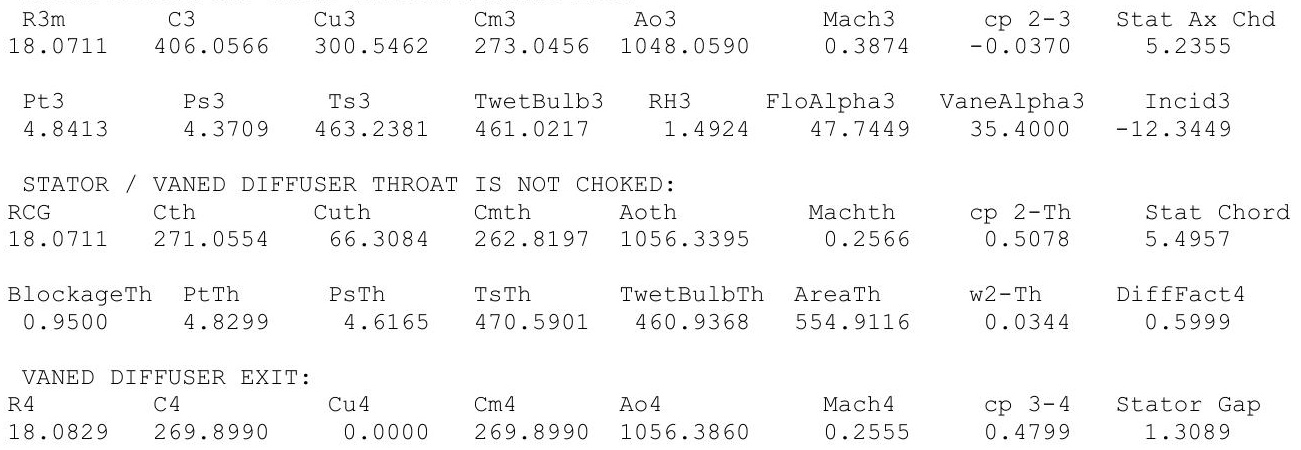




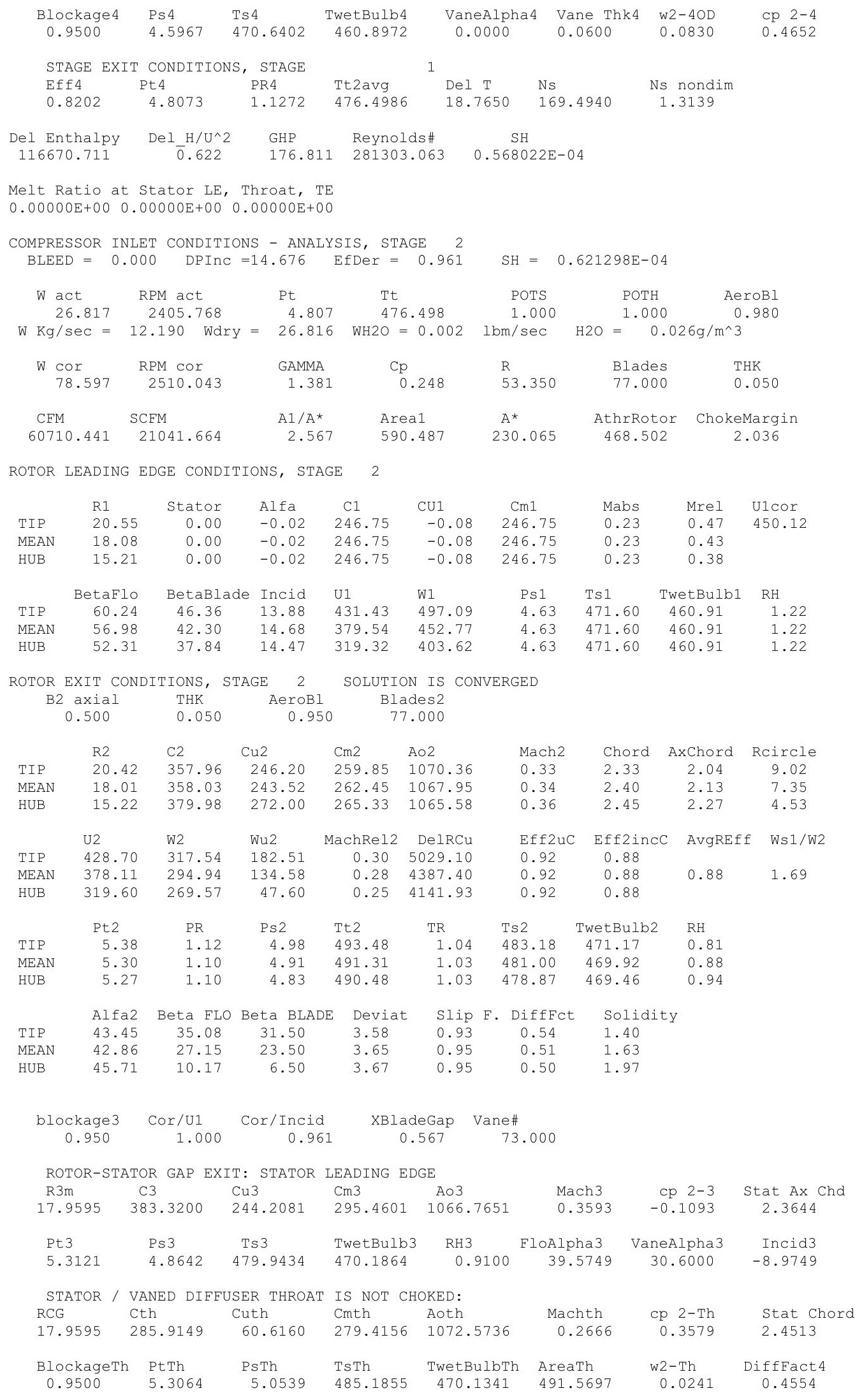




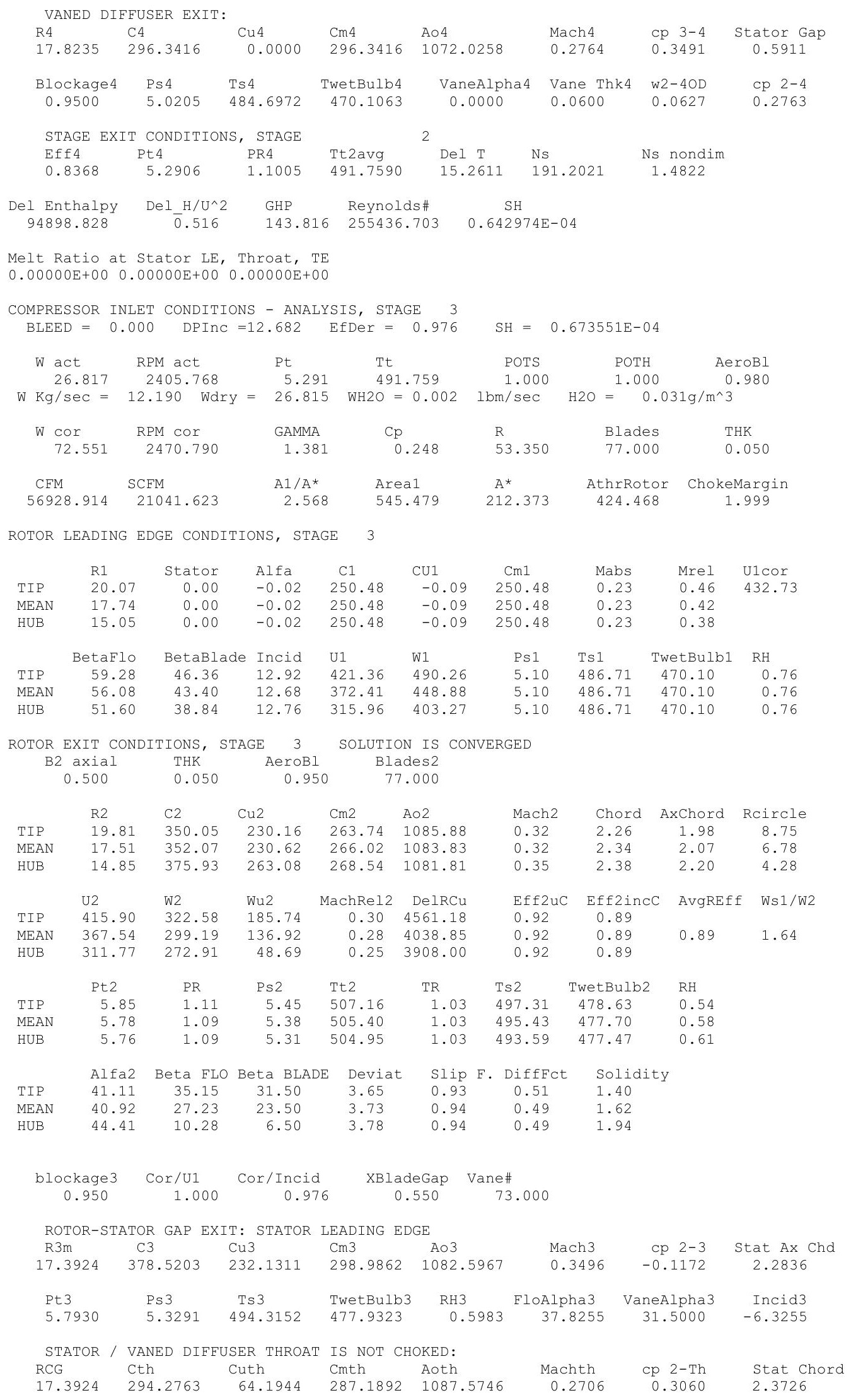




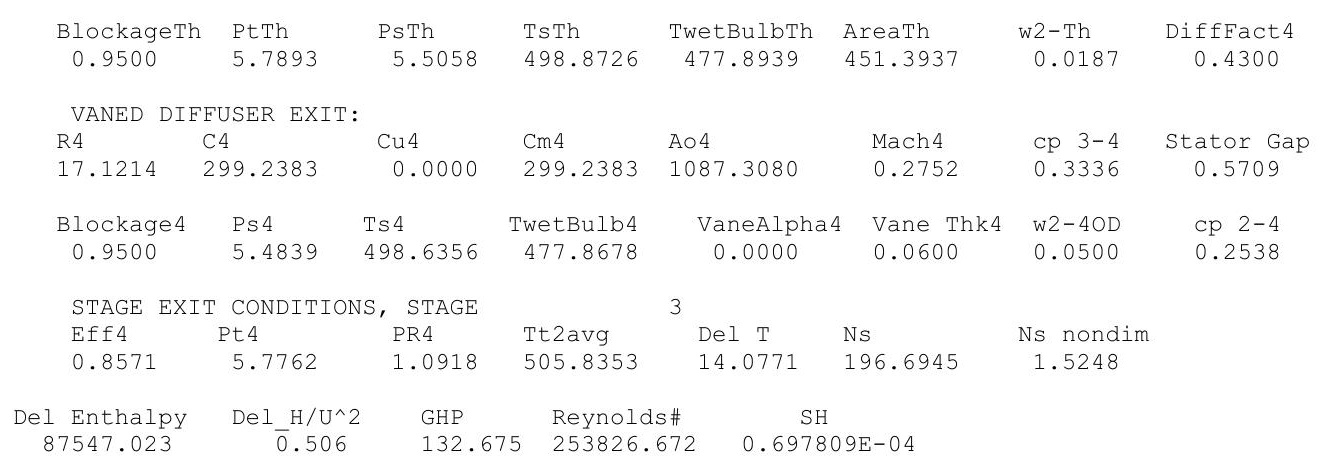

Melt Ratio at Stator LE, Throat, TE

$0.00000 \mathrm{E}+000.00000 \mathrm{E}+000.00000 \mathrm{E}+00$

COMPRESSOR INLET CONDITIONS - ANALYSIS, STAGE 4 BLEED $=0.000 \quad$ DPInC $=11.087 \quad$ EfDer $=0.986$

$\begin{array}{llllll}W & \text { Ret } & \text { Pt } & \text { POTS } & \text { POTH } & \text { AeroBI }\end{array}$

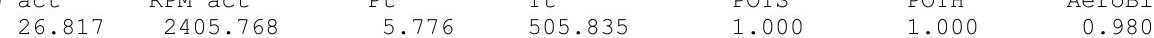

$\mathrm{W} \mathrm{Kg} / \mathrm{sec}=12.190$ WdrY $=26.815$ WH2O $=0.002 \quad \mathrm{lbm} / \mathrm{sec} \quad$ H2O $=0.035 \mathrm{~g} / \mathrm{m}^{\wedge} 3$

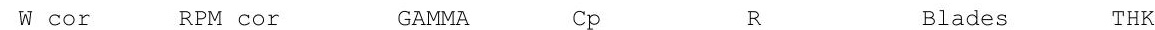

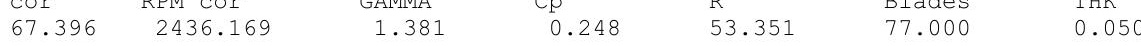

$\begin{array}{ccccc}\text { CFM } & \text { SCFM } & \text { A1/A* } & \text { Area1 } & \text { A* AthrRotor ChokeMargin }\end{array}$

$\begin{array}{lllllll}53596.461 & 21041.572 & 2.602 & 513.330 & 197.287 & 395.845 & 2.006\end{array}$

ROTOR LEADING EDGE CONDITIONS, STAGE 4

$\begin{array}{lcclcccccc} & \text { R1 } & \text { Stator } & \text { Alfa } & \text { C1 } & \text { CU1 } & \text { Cm1 } & \text { Mabs } & \text { Mrel } & \text { U1cor } \\ \text { TIP } & 19.26 & 0.00 & -0.02 & 250.58 & -0.09 & 250.58 & 0.23 & 0.44 & 409.45 \\ \text { MEAN } & 16.97 & 0.00 & -0.02 & 250.58 & -0.09 & 250.58 & 0.23 & 0.40 & \\ \text { HUB } & 14.32 & 0.00 & -0.02 & 250.58 & -0.09 & 250.58 & 0.23 & 0.36 & \\ & & & & & & & & & \\ & & & & & & & & & \\ \text { TIP } & 58.22 & 46.36 & 11.86 & 404.35 & 475.77 & 5.57 & 500.79 & 477.86 & 0.52 \\ \text { MEAN } & 54.89 & 43.80 & 11.09 & 356.29 & 435.65 & 5.57 & 500.79 & 477.86 & 0.52 \\ \text { HUB } & 50.20 & 37.84 & 12.36 & 300.64 & 391.44 & 5.57 & 500.79 & 477.86 & 0.52\end{array}$

ROTOR EXIT CONDITIONS, STAGE 4 SOLUTION IS CONVERGED

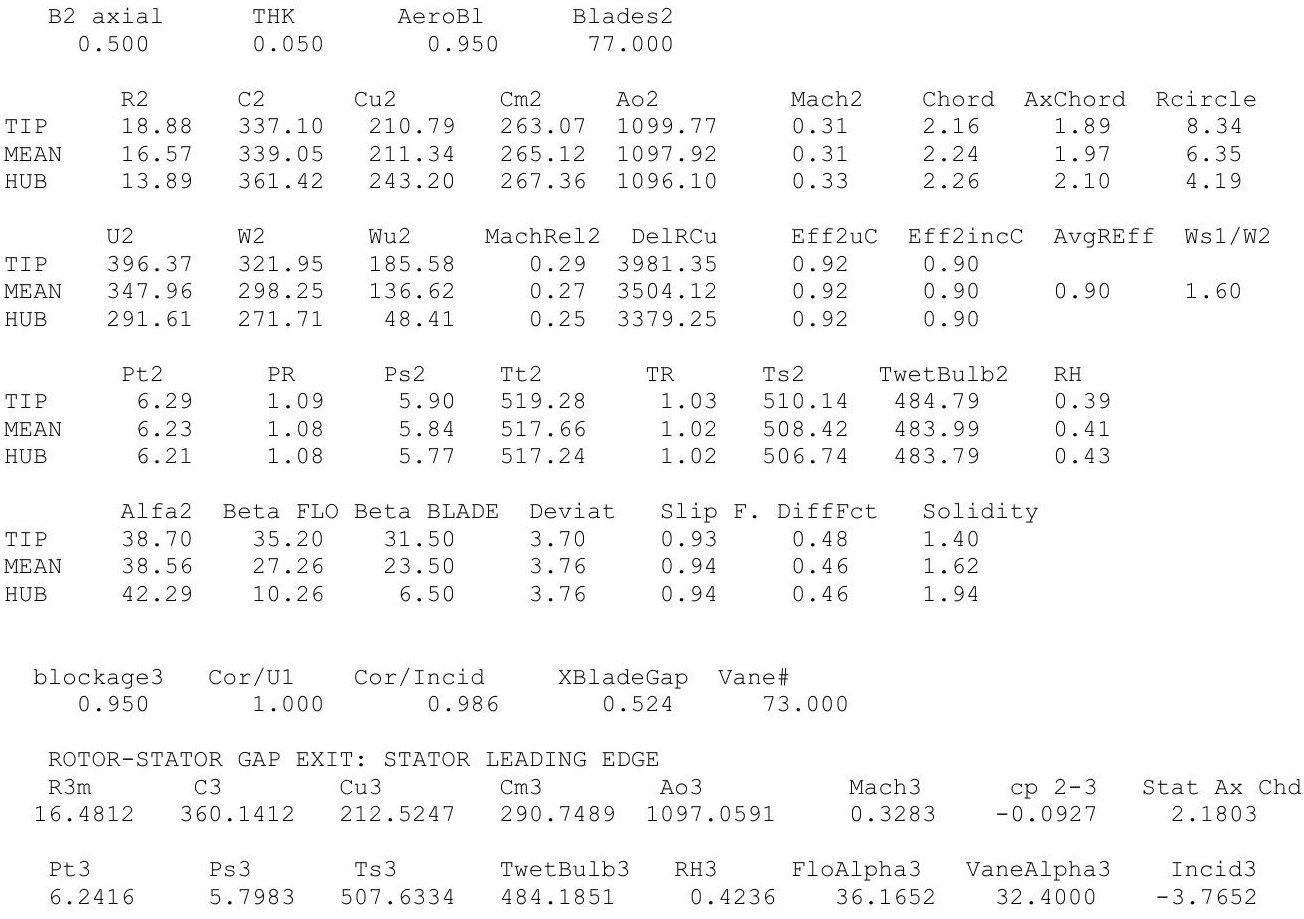




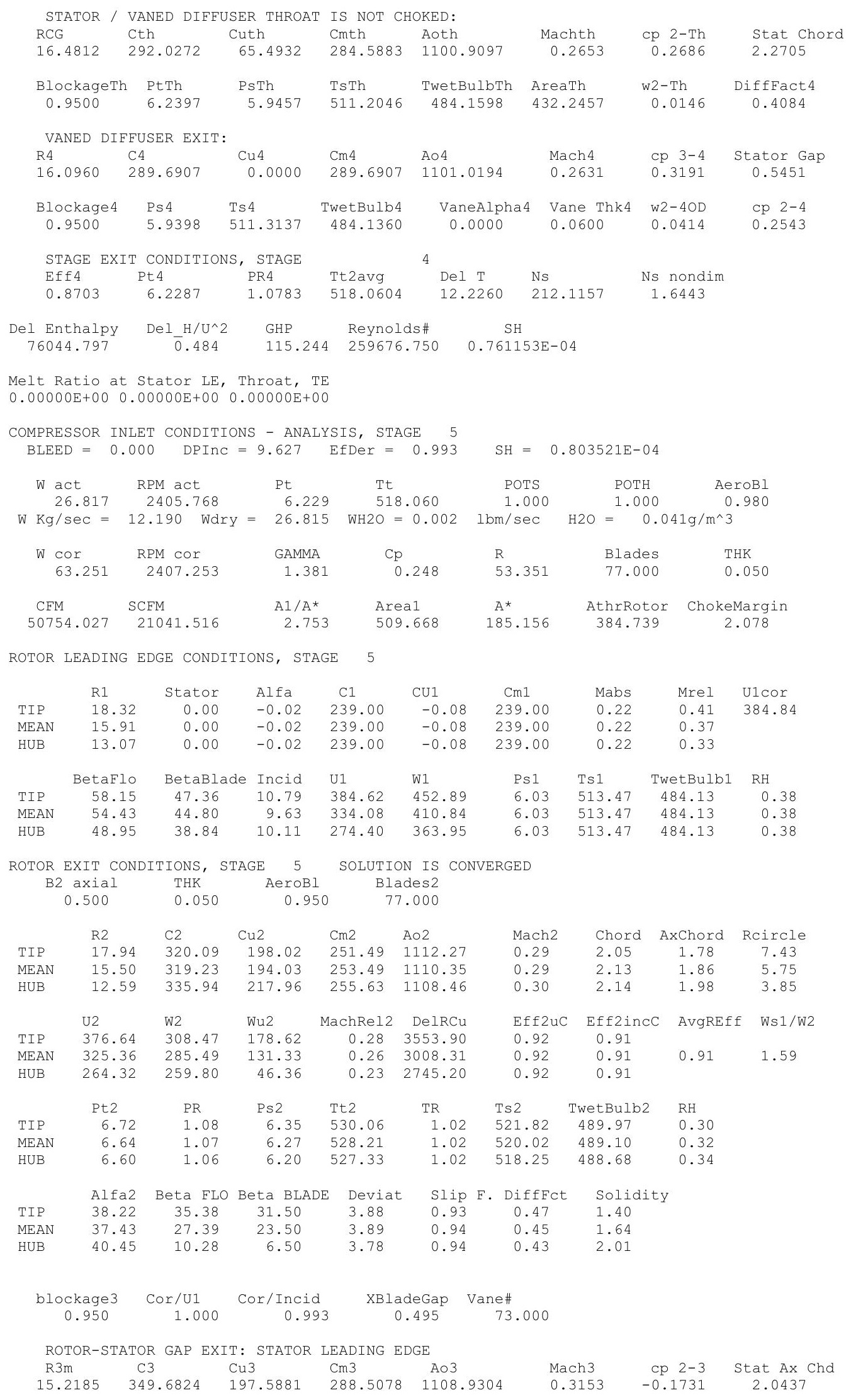




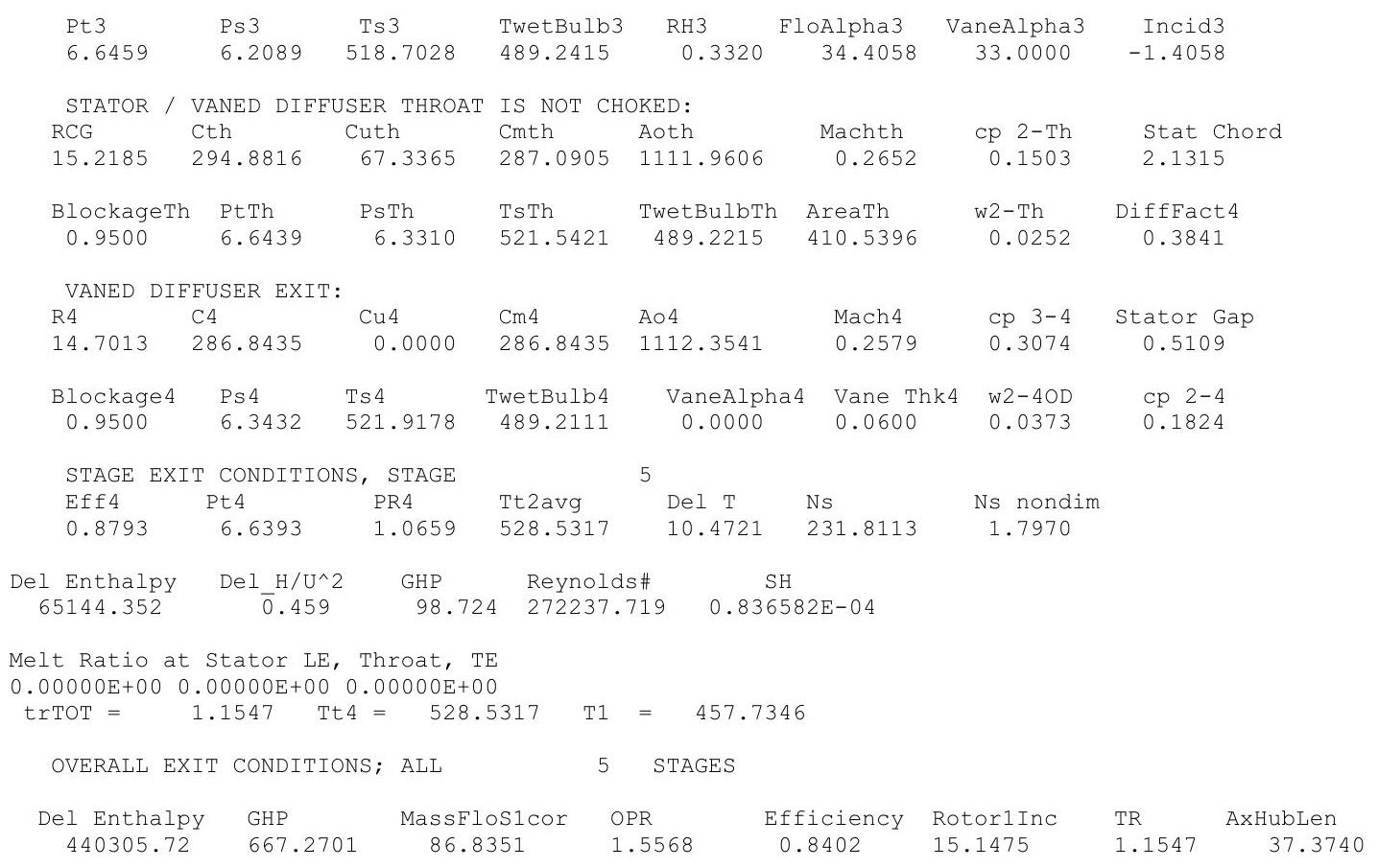




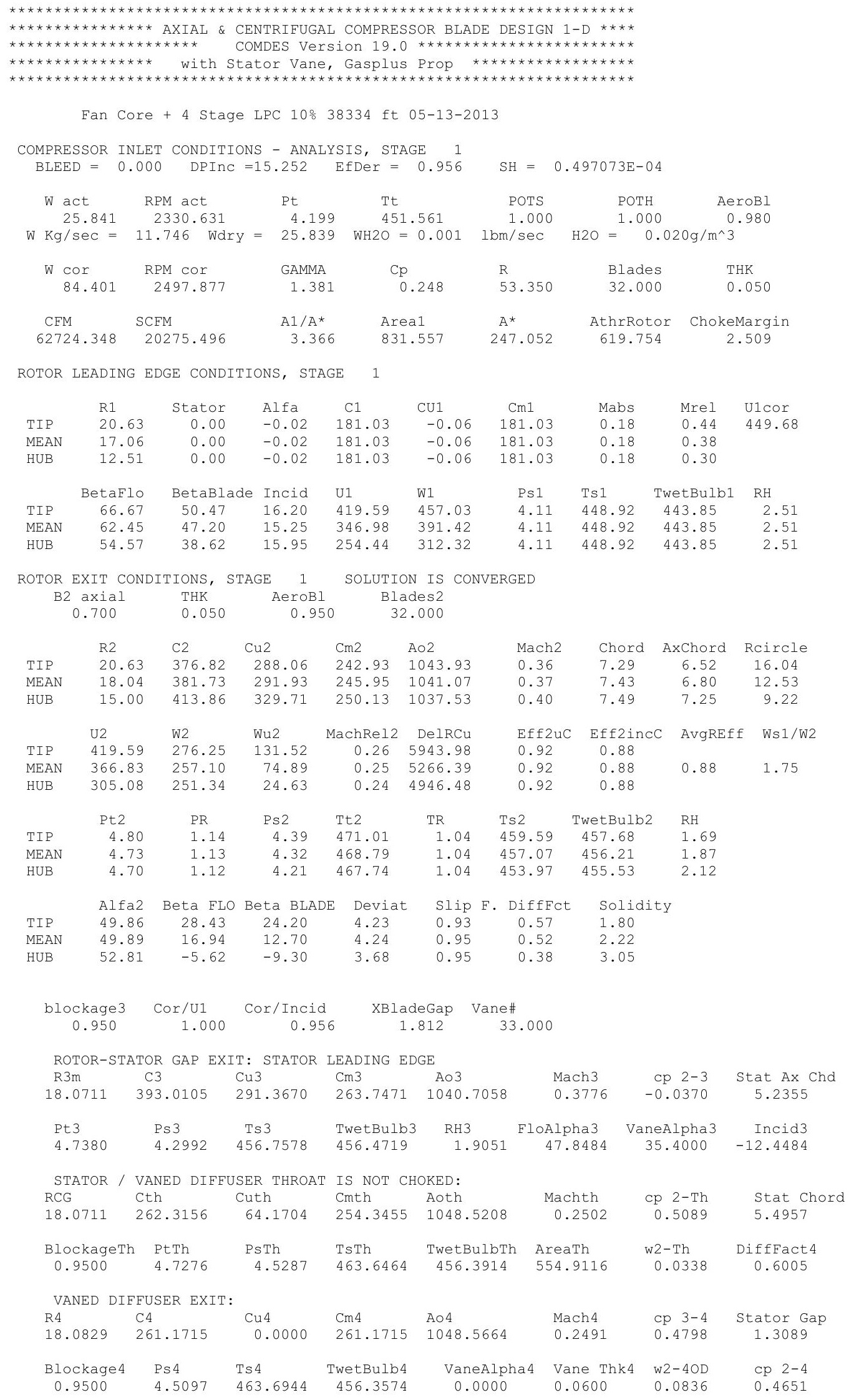




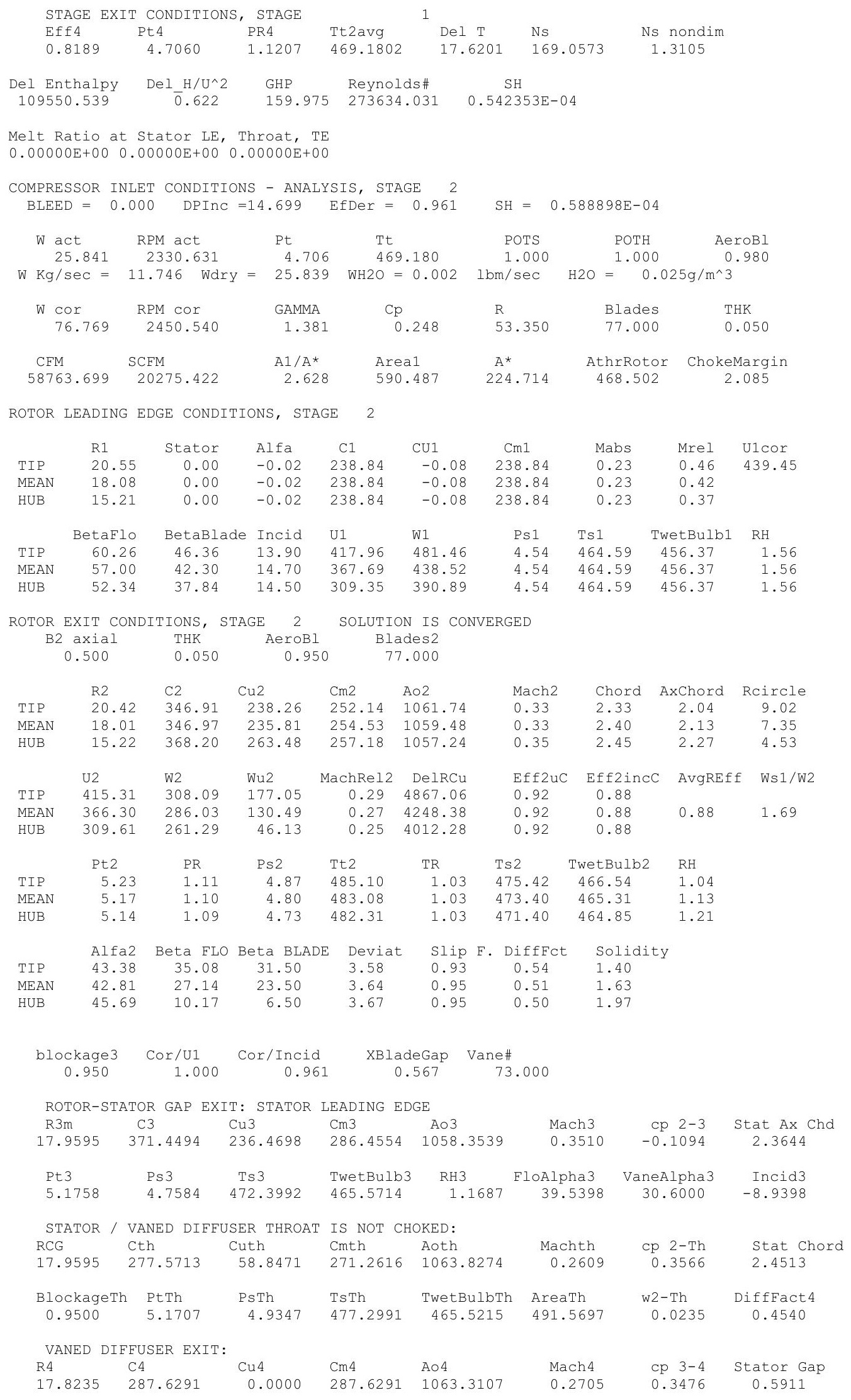




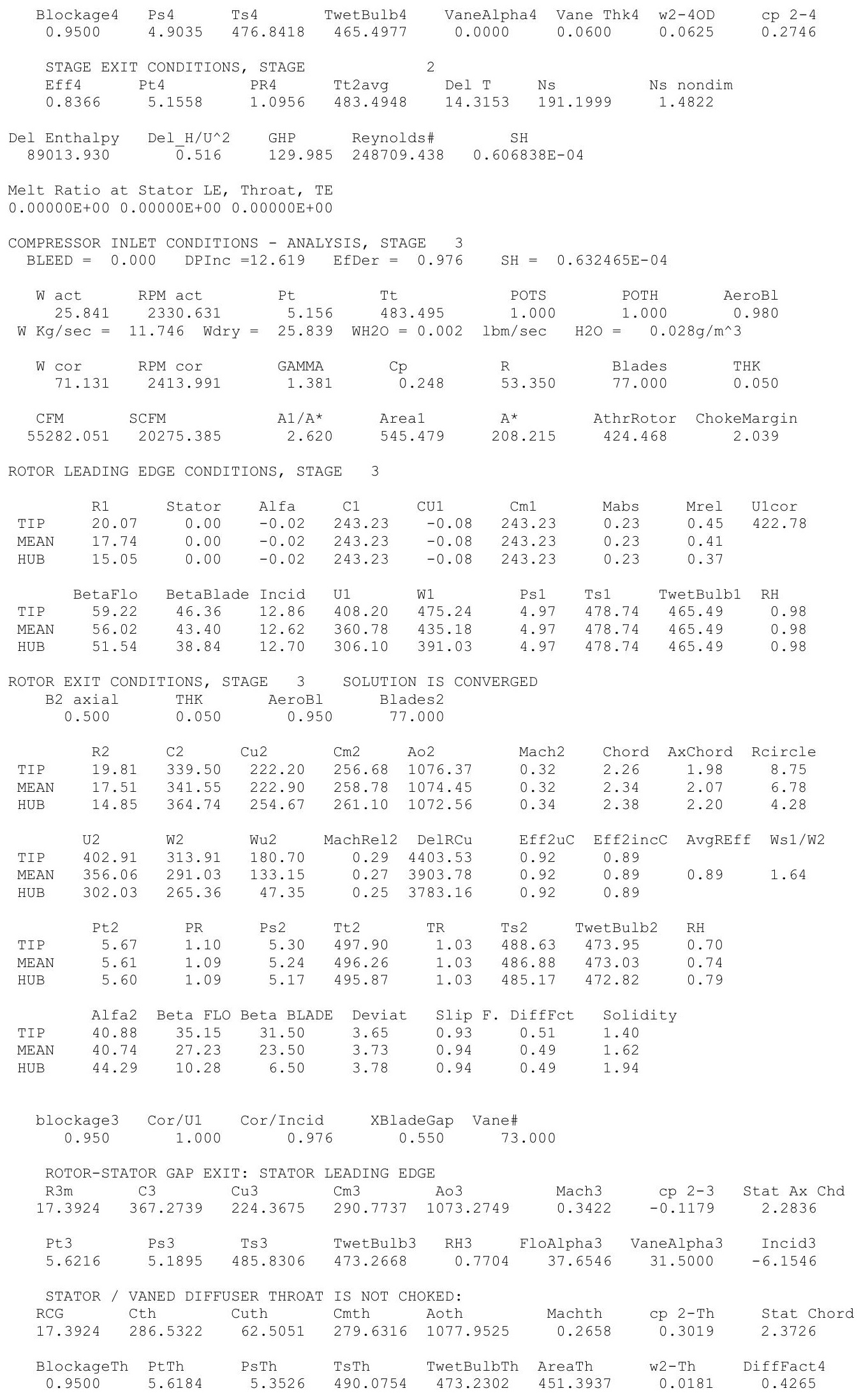




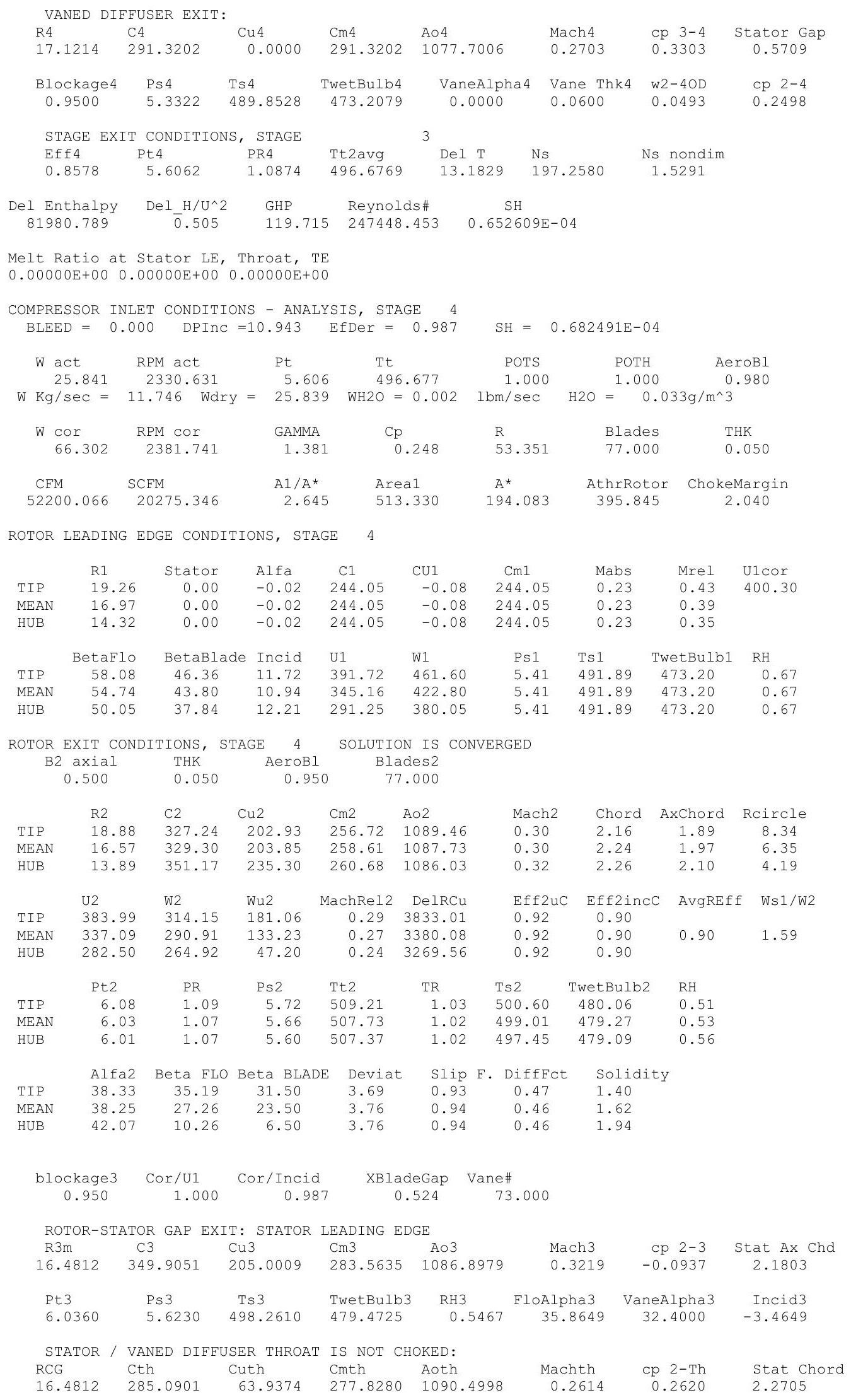




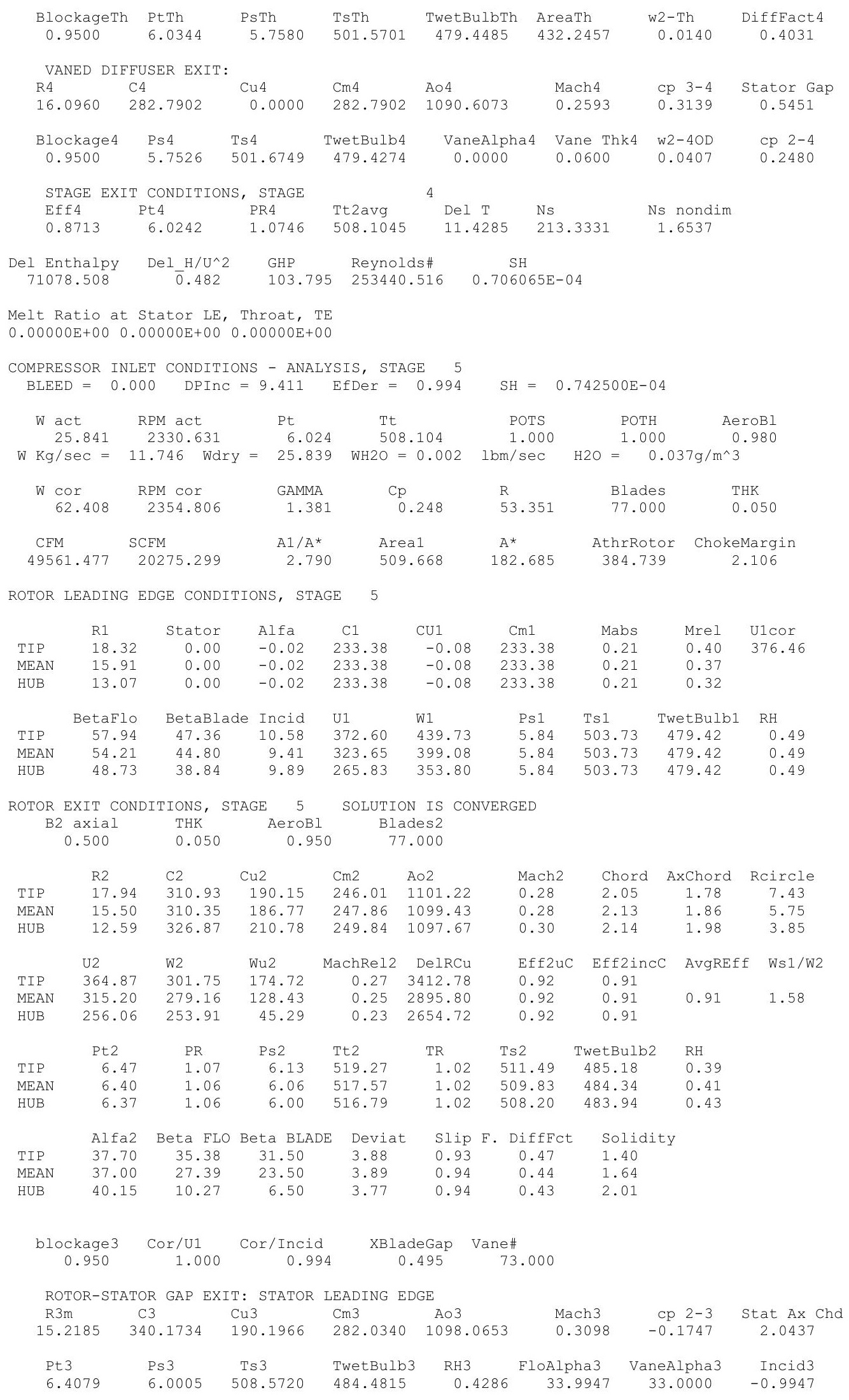




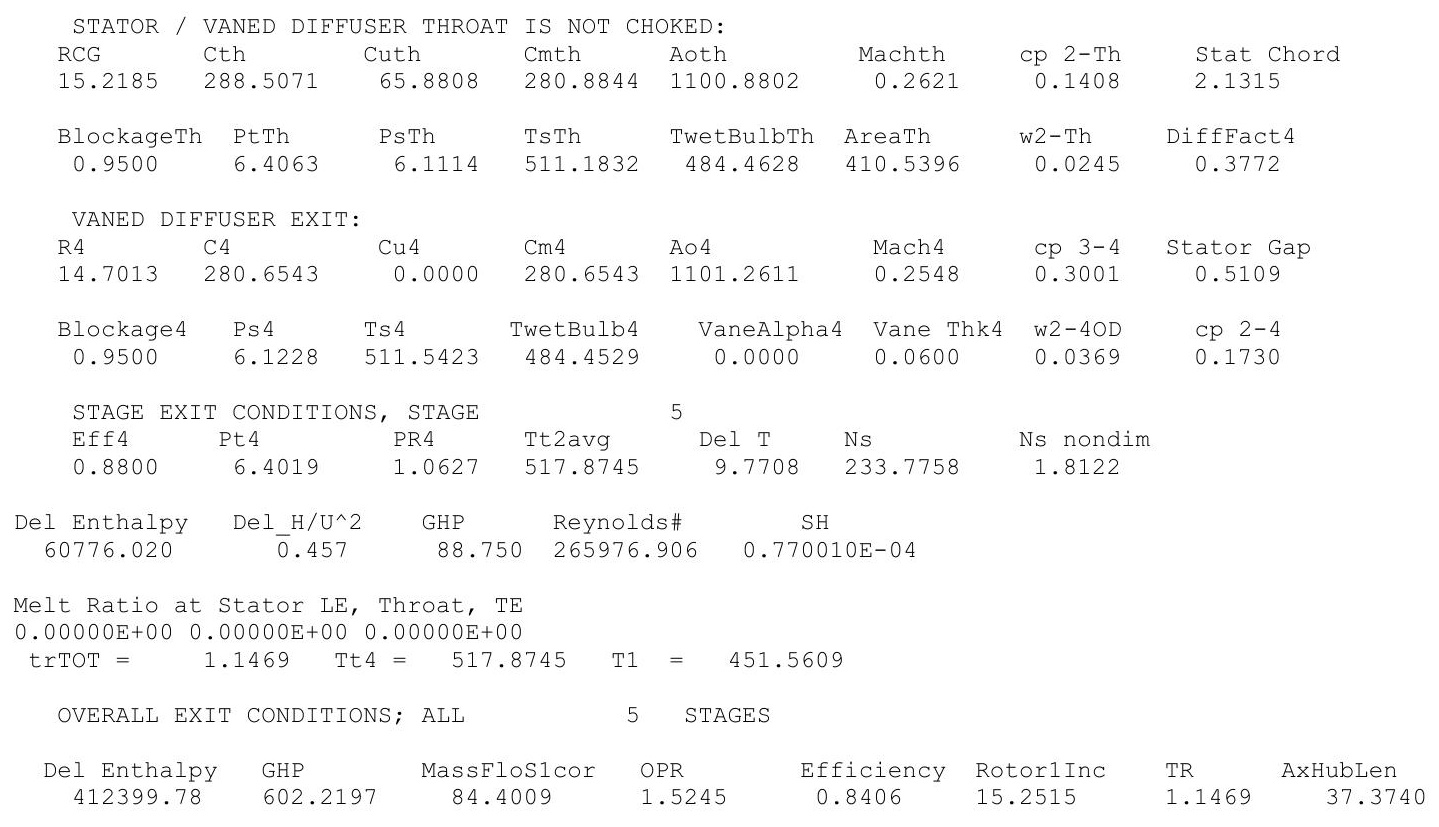




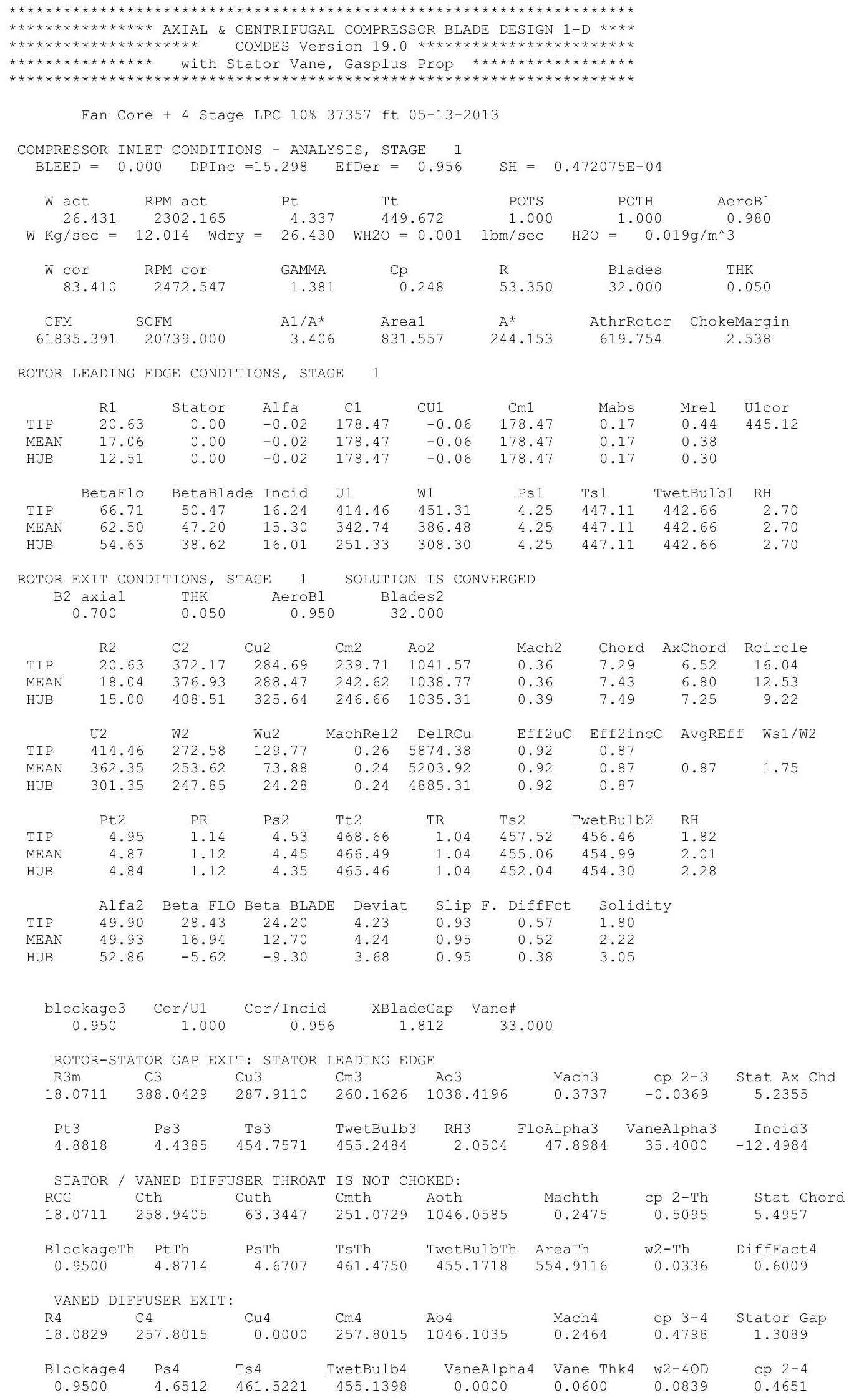




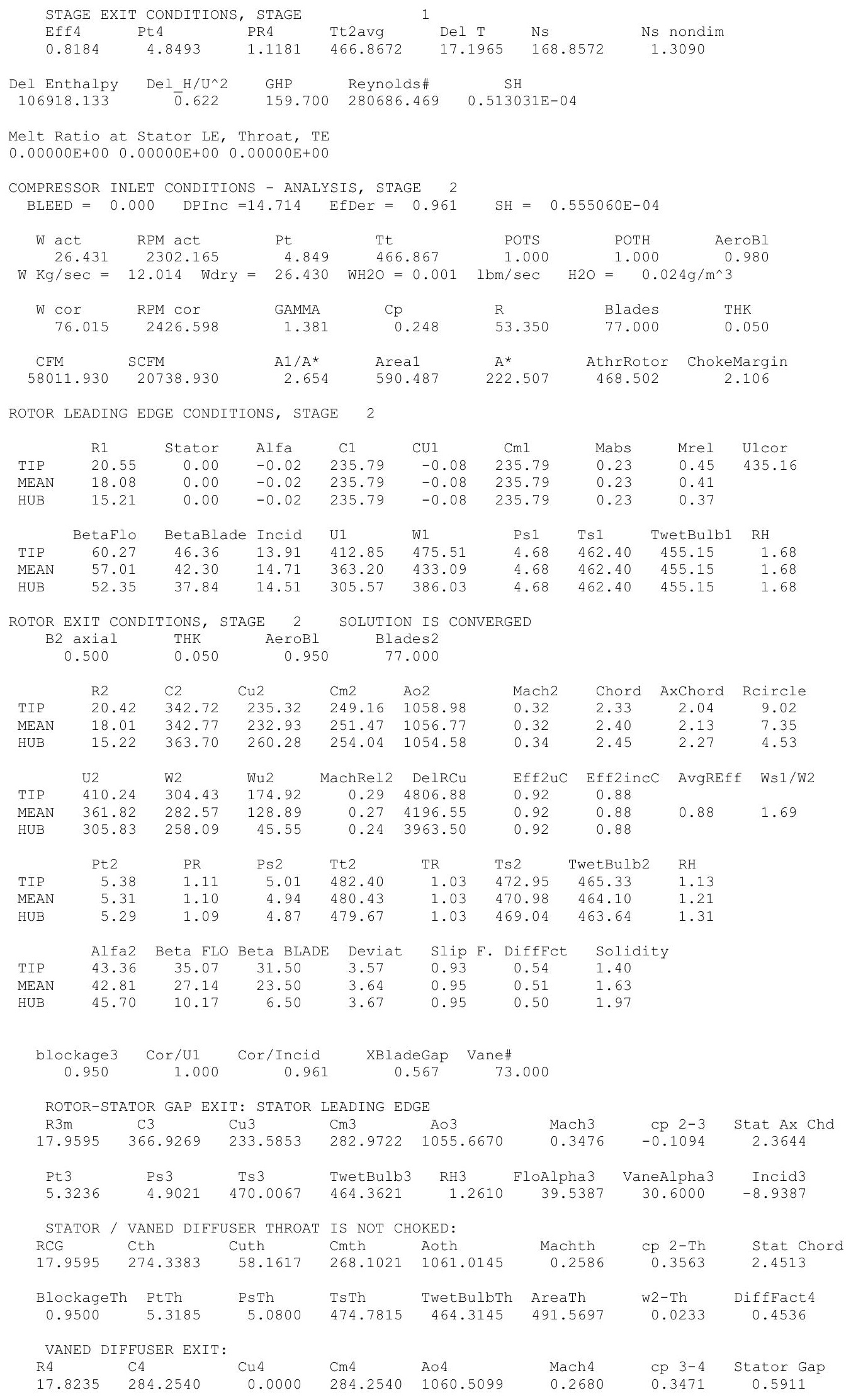




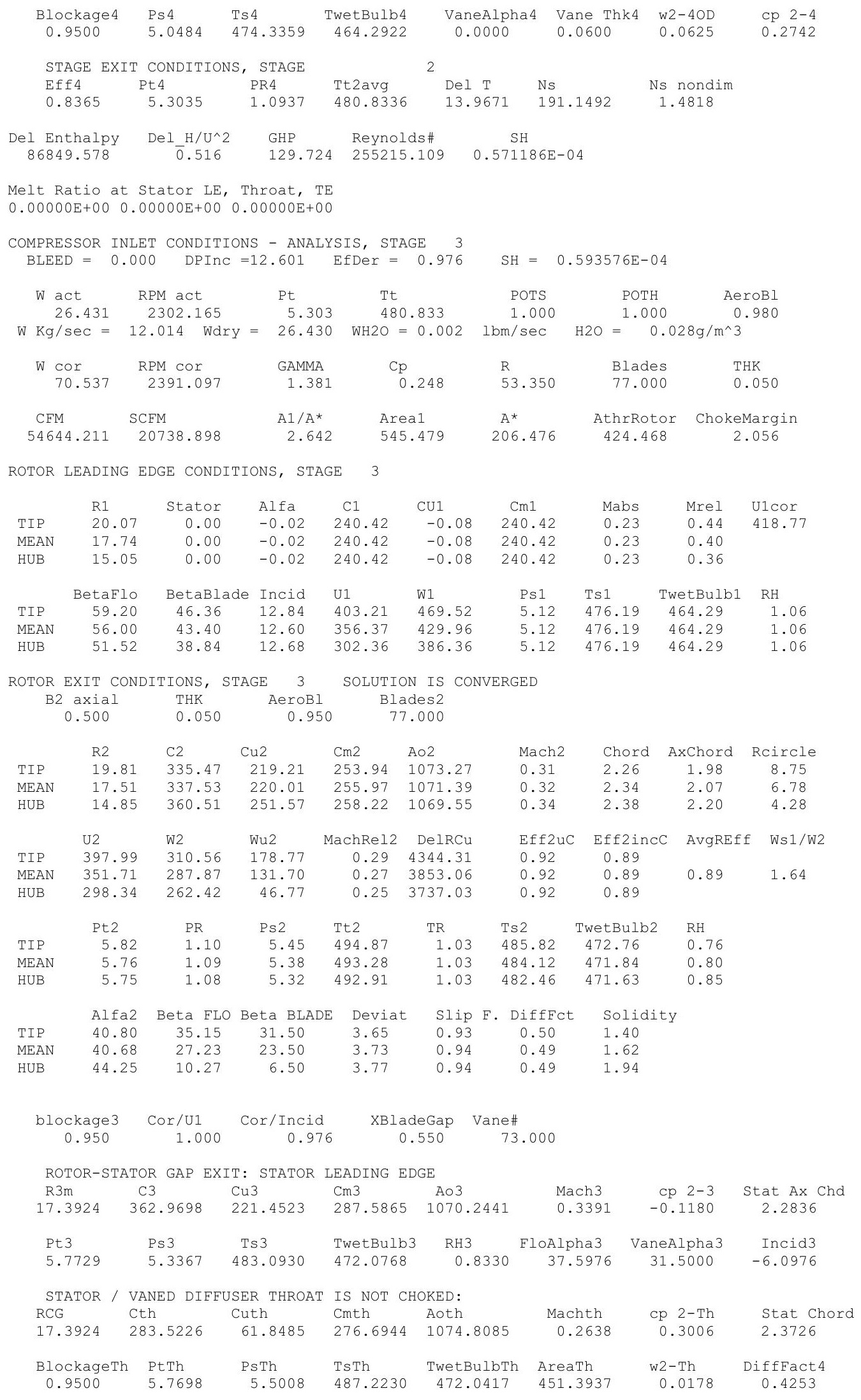




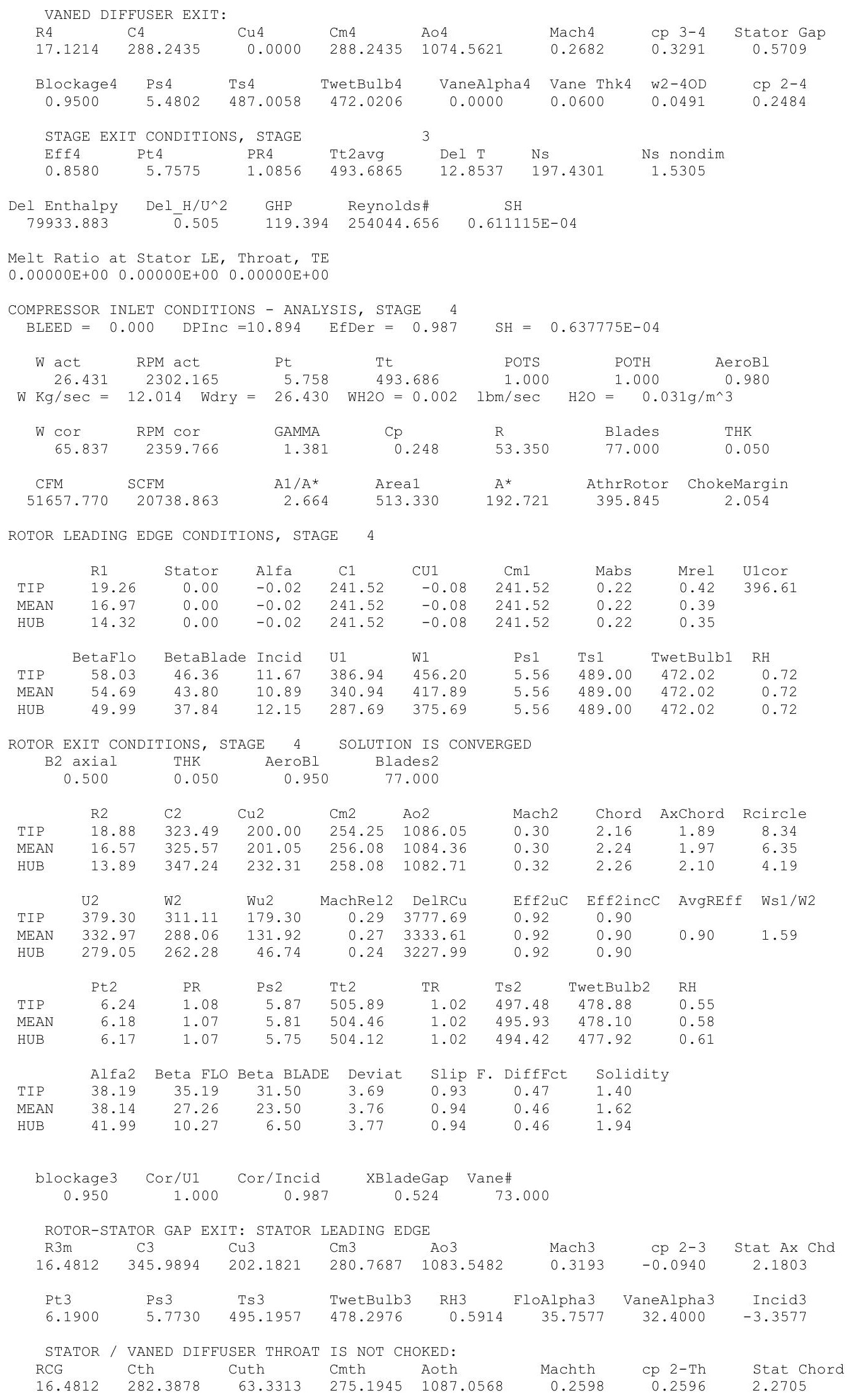




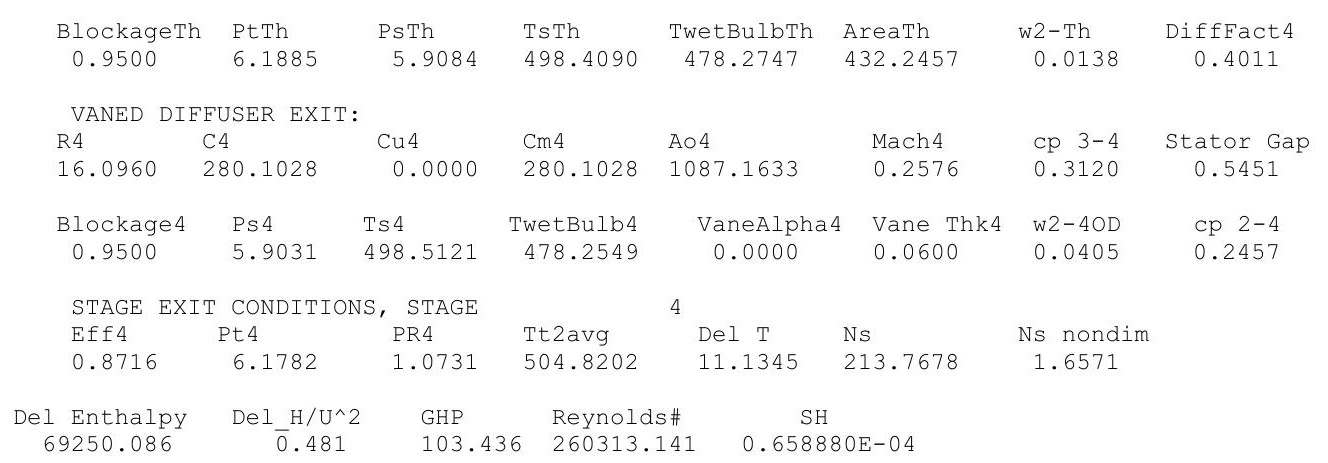

Melt Ratio at Stator LE, Throat, TE

$0.00000 \mathrm{E}+00 \quad 0.00000 \mathrm{E}+00 \quad 0.00000 \mathrm{E}+00$

COMPRESSOR INLET CONDITIONS - ANALYSIS, STAGE 5 BLEED $=0.000 \quad$ DPInC $=9.333 \quad$ EfDer $=0.994$

$\begin{array}{llllll}W \text { act } & \text { RPM act } & \text { Pt } & \text { POTS } & \text { POTH } & \text { AeroBl }\end{array}$ $\mathrm{W} \mathrm{Kg} / \mathrm{sec}=12.014$ Wdry $=26.430 \quad \mathrm{WH} 2 \mathrm{O}=0.002 \quad \mathrm{lbm} / \mathrm{sec} \quad \mathrm{H} 2 \mathrm{O}=0.036 \mathrm{~g} / \mathrm{m}^{\wedge} 3$

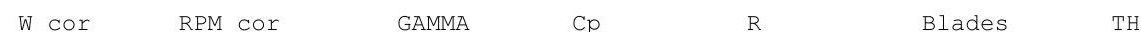

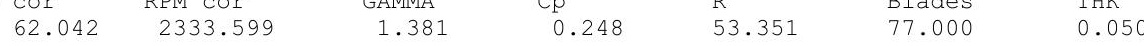

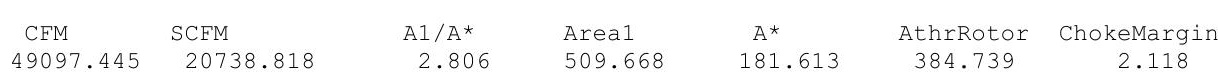

ROTOR LEADING EDGE CONDITIONS, STAGE 5

\begin{tabular}{|c|c|c|c|c|c|c|c|c|c|}
\hline & R1 & Stator & Alfa & $\mathrm{C} 1$ & CU1 & $\mathrm{Cm} 1$ & Mabs & Mrel & U1cor \\
\hline TIP & 18.32 & 0.00 & -0.02 & 231.20 & -0.08 & 231.20 & 0.21 & 0.40 & 373.07 \\
\hline MEAN & 15.91 & 0.00 & -0.02 & 231.20 & -0.08 & 231.20 & 0.21 & 0.36 & \\
\hline HUB & 13.07 & 0.00 & -0.02 & 231.20 & -0.08 & 231.20 & 0.21 & 0.32 & \\
\hline & BetaFlo & BetaBlade & Incid & U1 & W1 & Ps 1 & Ts 1 & TwetBulb1 & $\mathrm{RH}$ \\
\hline TIP & 57.87 & 47.36 & 10.51 & 368.05 & 434.71 & 5.99 & 500.52 & 478.25 & 0.53 \\
\hline MEAN & 54.13 & 44.80 & 9.33 & 319.70 & 394.60 & 5.99 & 500.52 & 478.25 & 0.53 \\
\hline HUB & 48.65 & 38.84 & 9.81 & 262.58 & 349.92 & 5.99 & 500.52 & 478.25 & 0.53 \\
\hline
\end{tabular}

ROTOR EXIT CONDITIONS, STAGE 5 SOLUTION IS CONVERGED

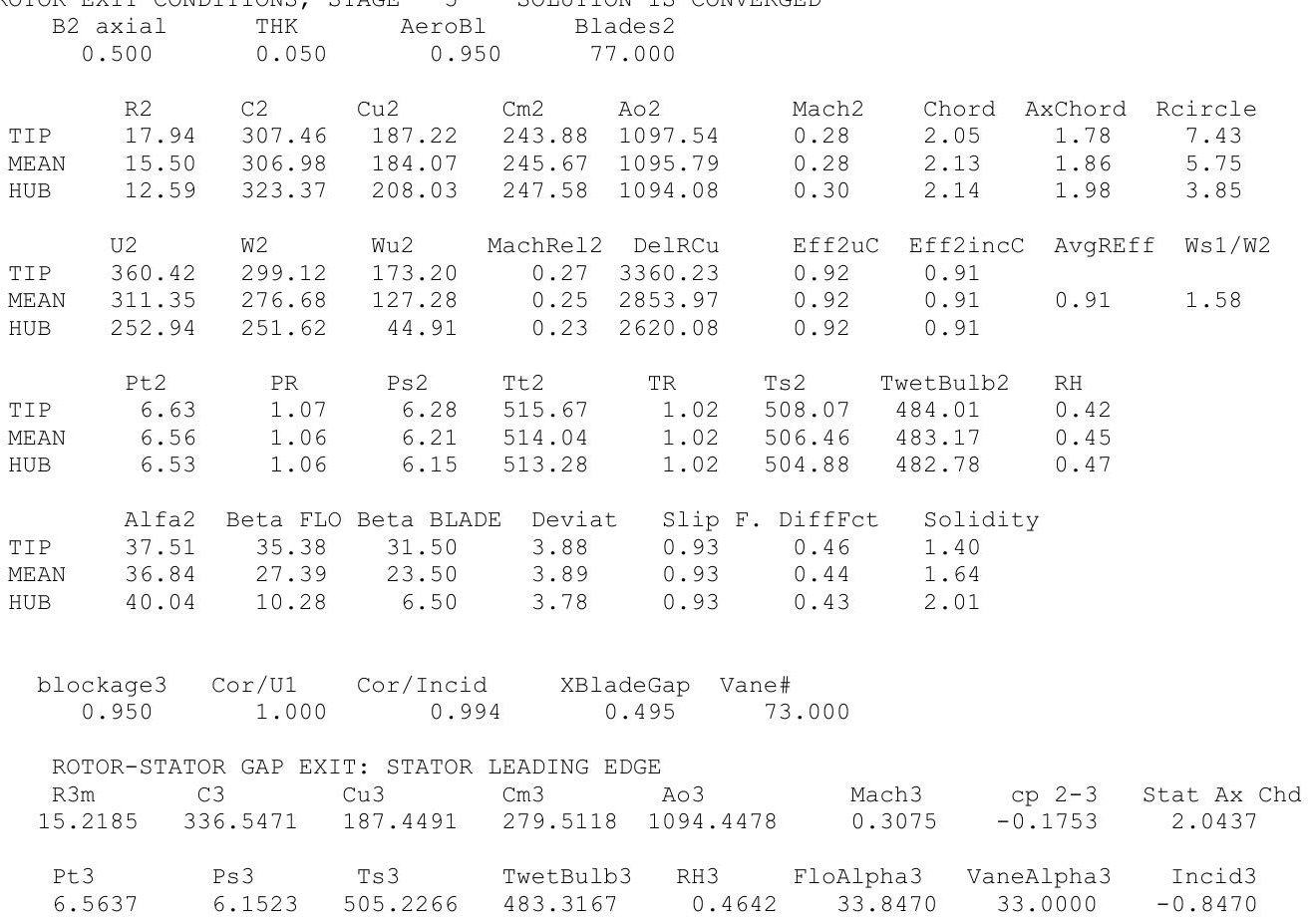




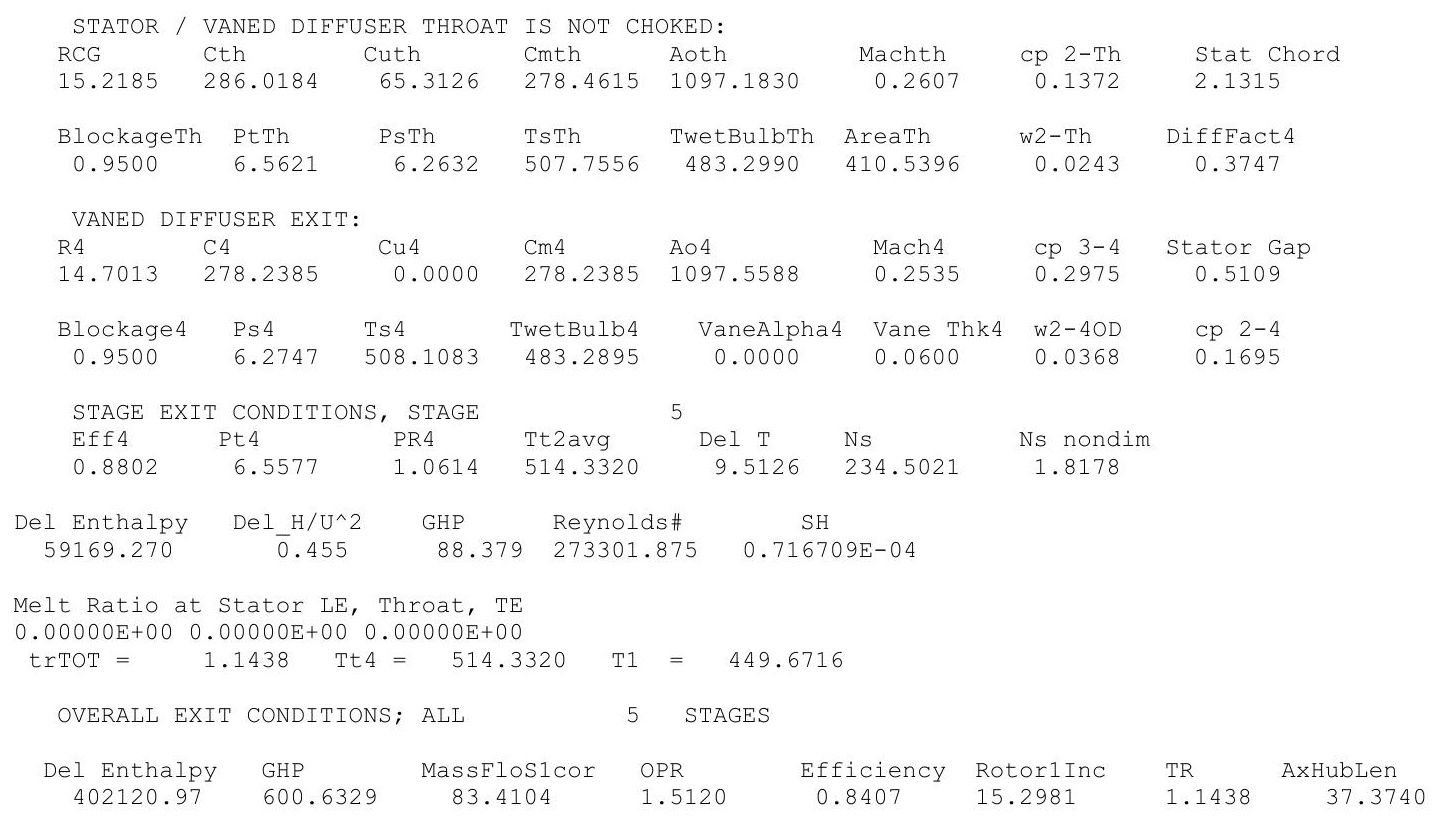




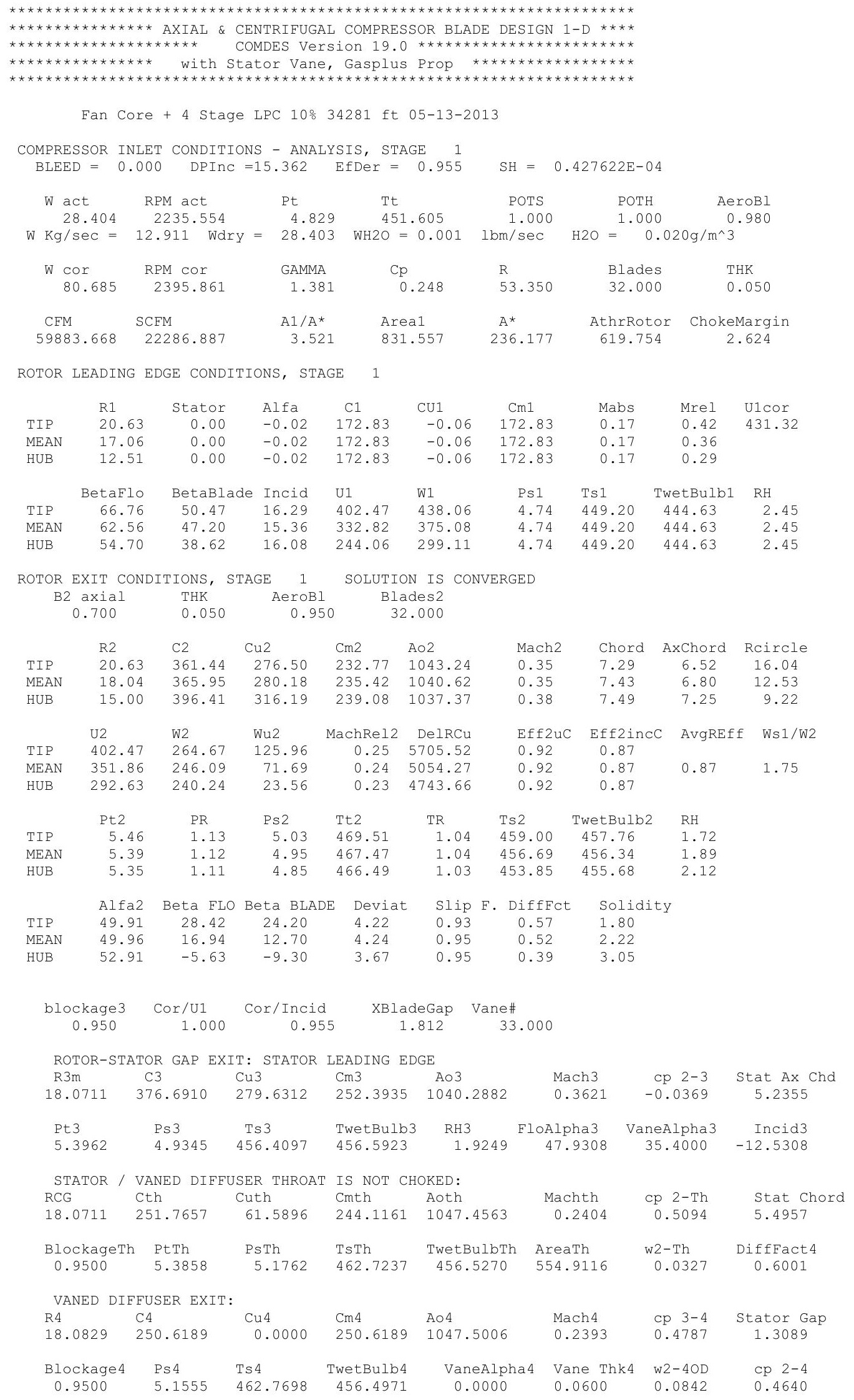




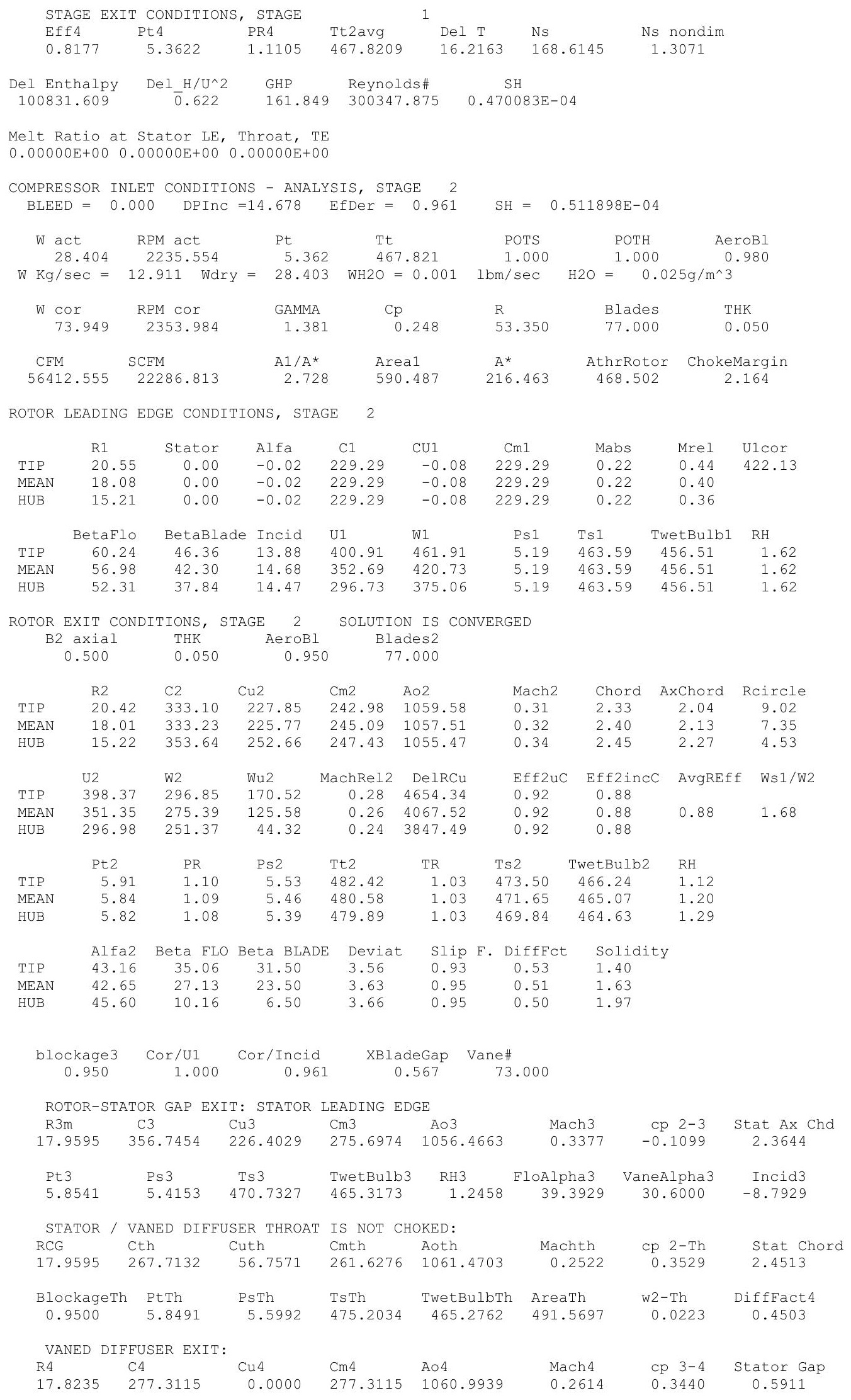




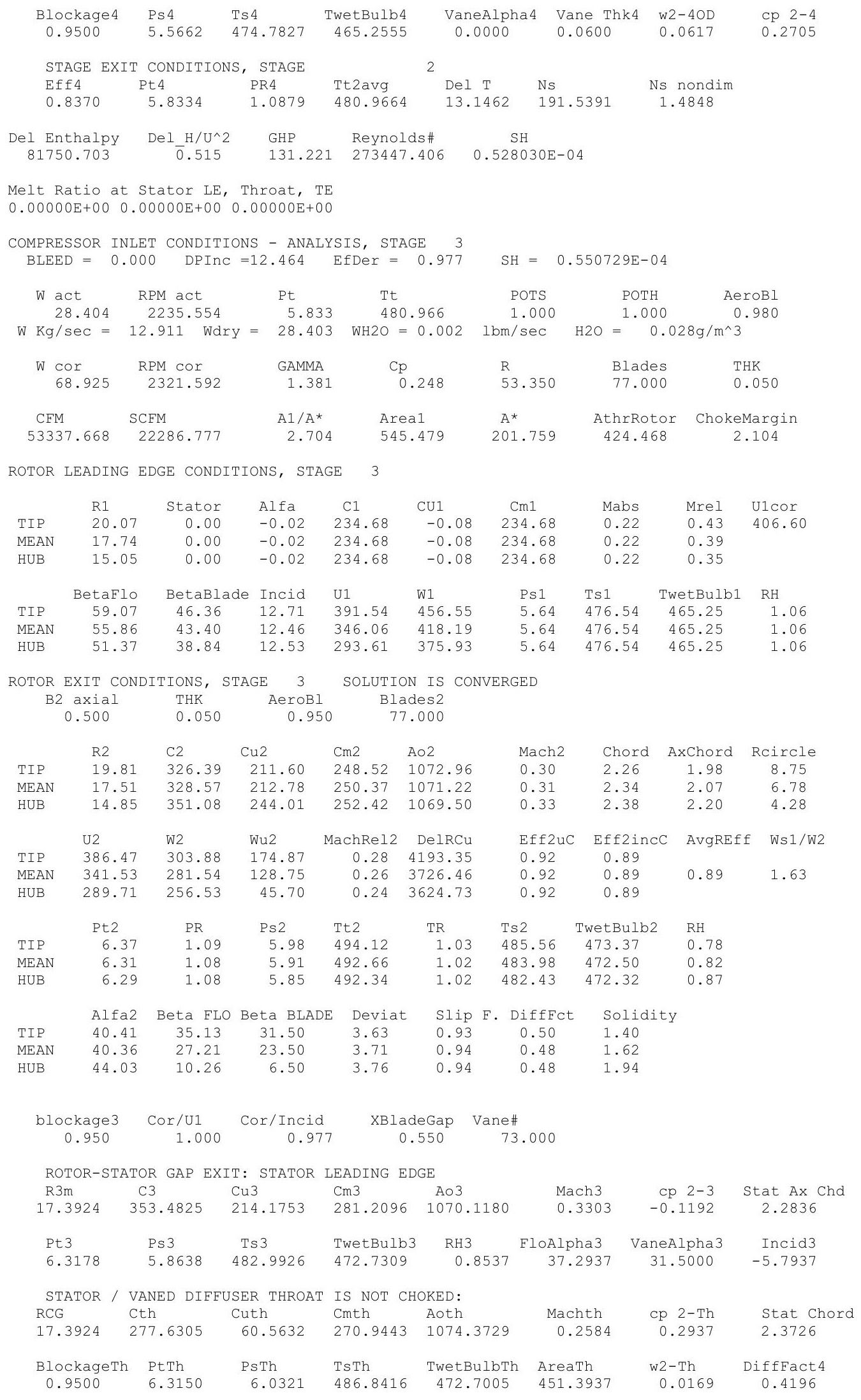




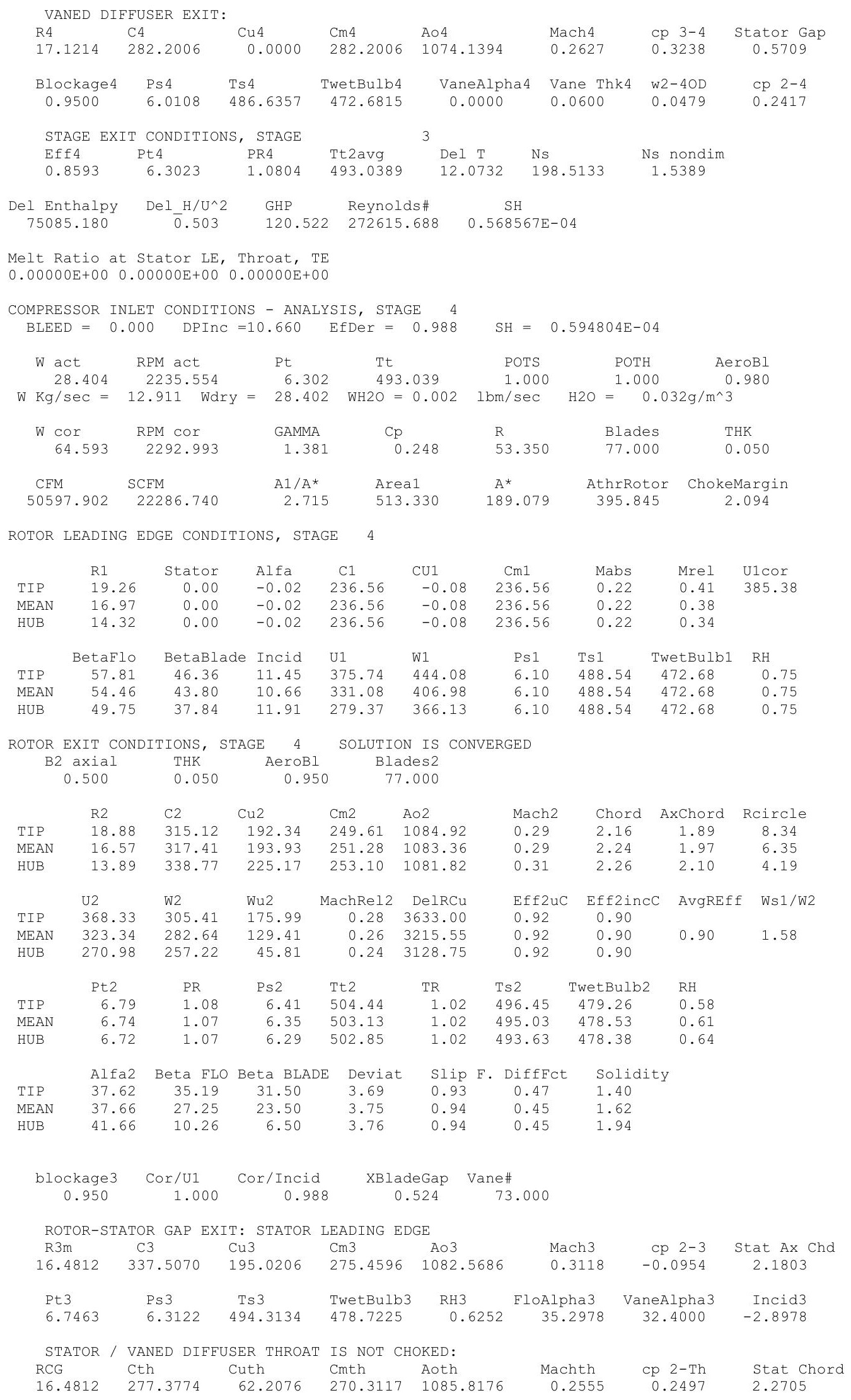




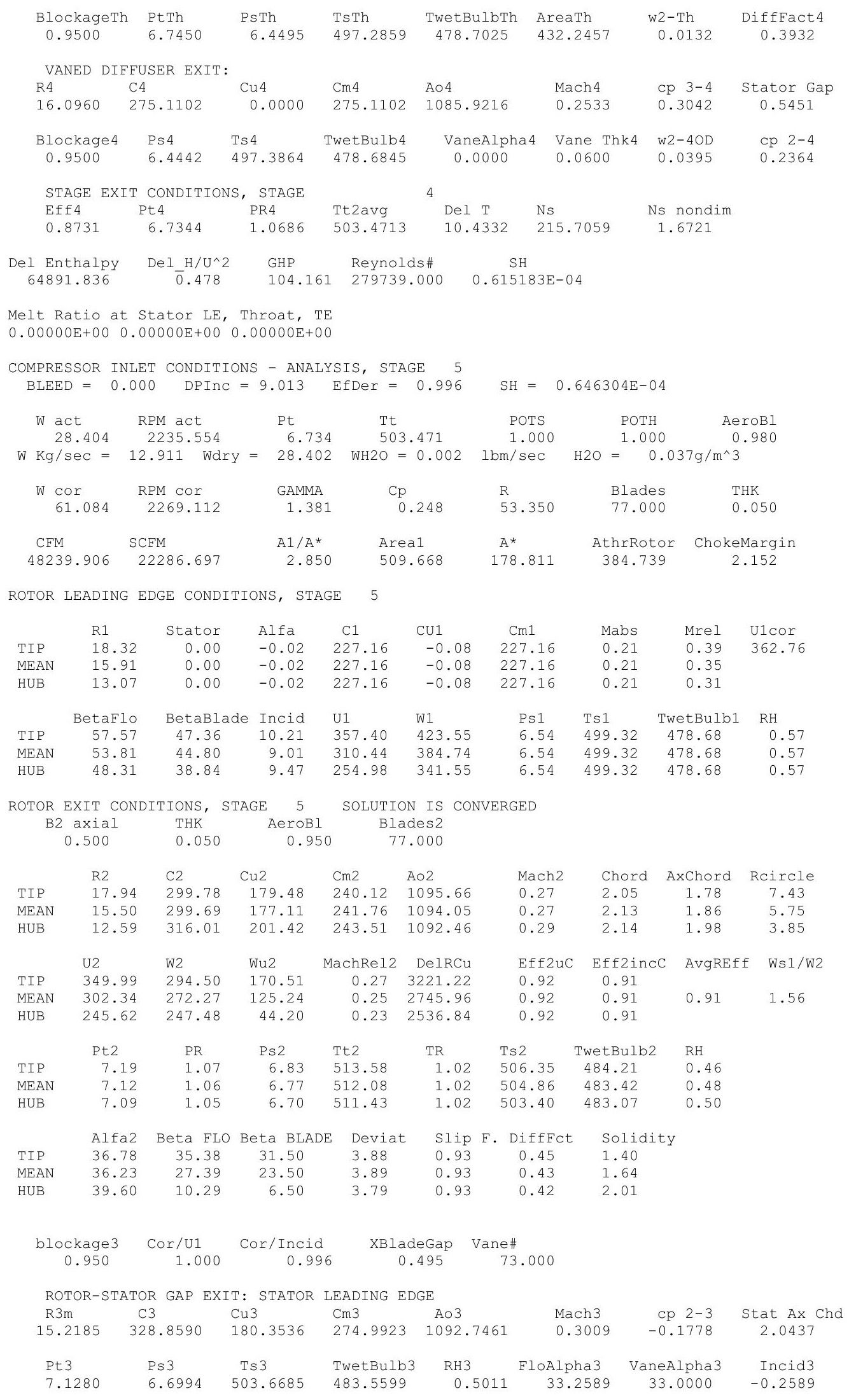




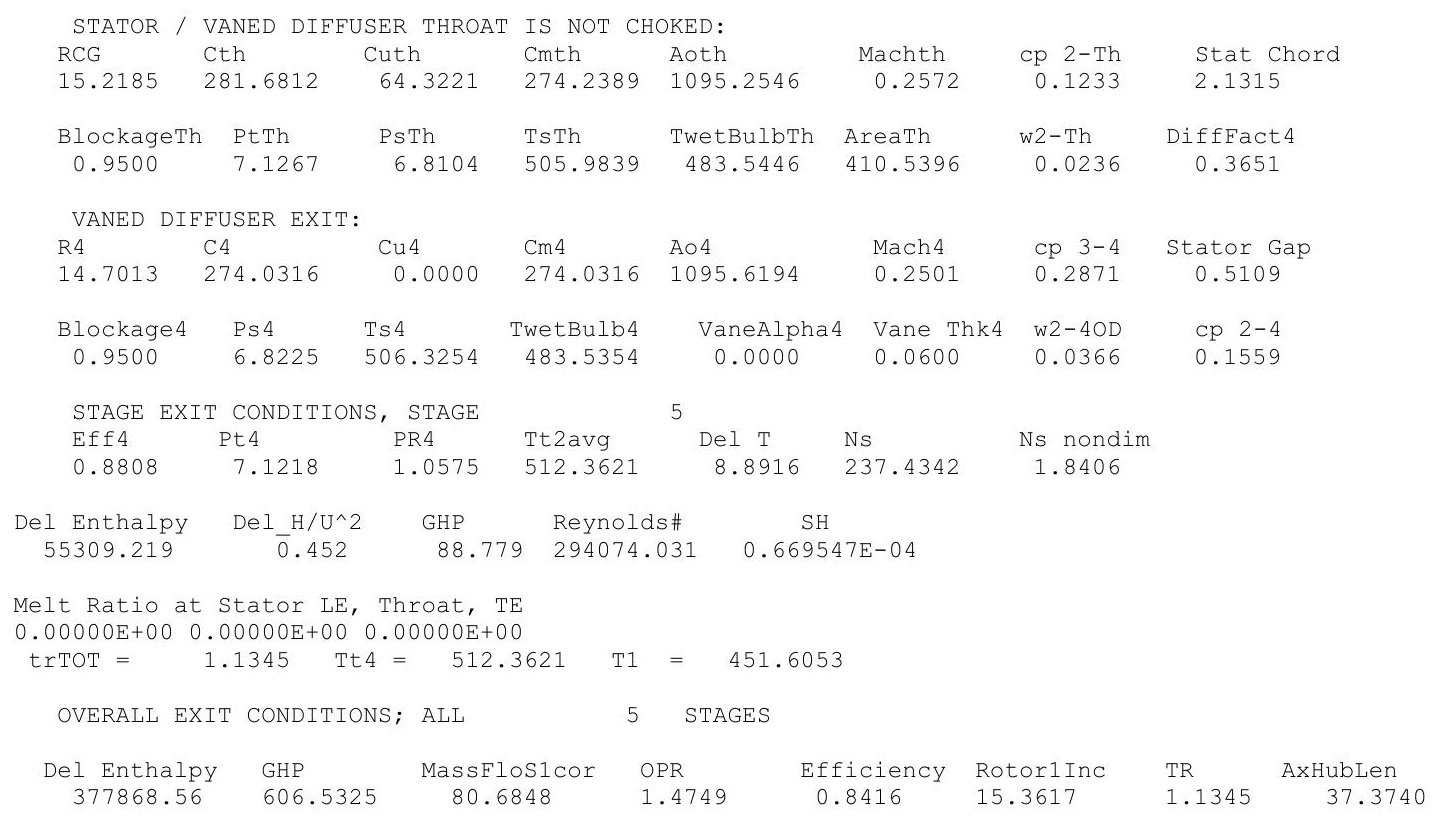




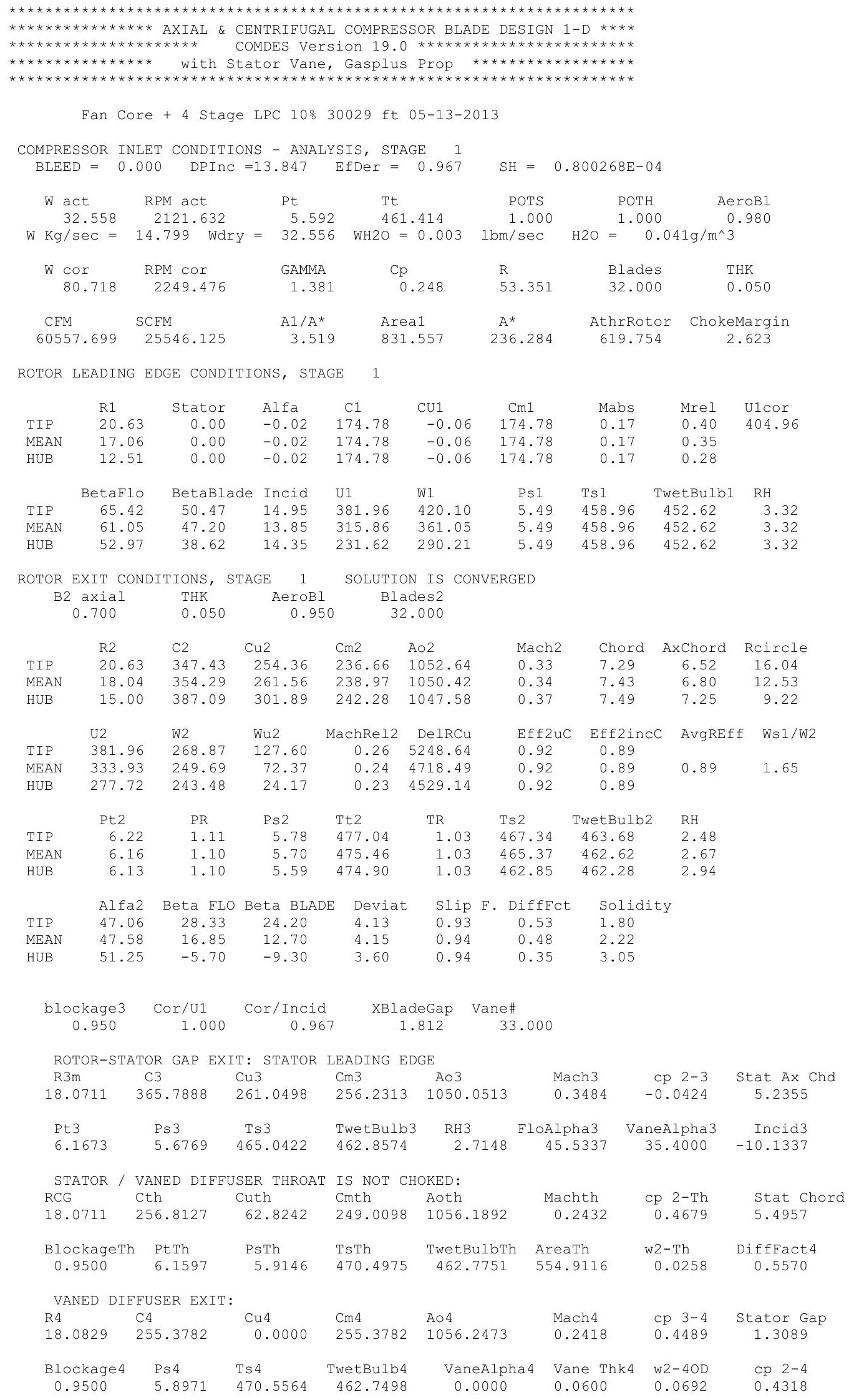




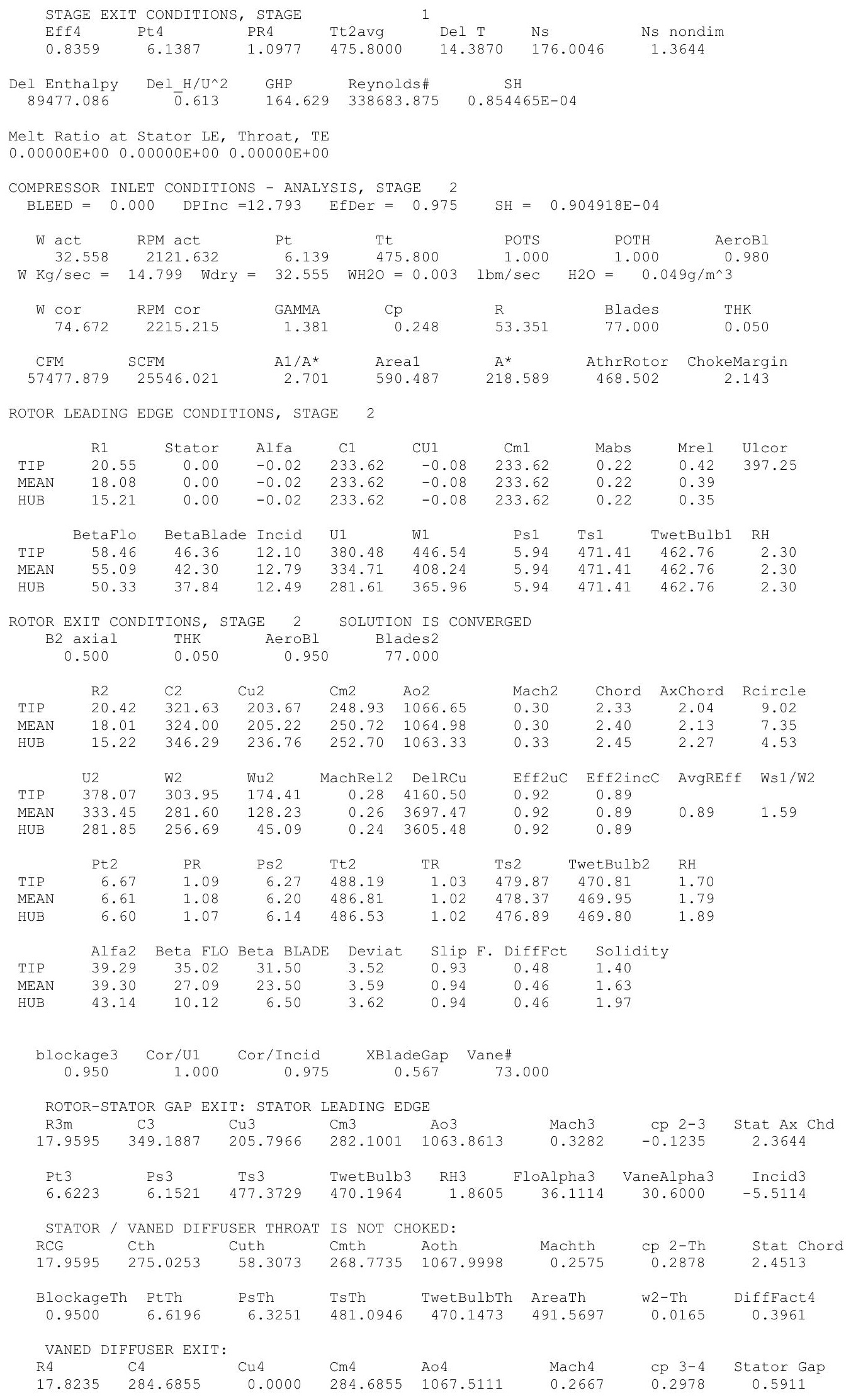




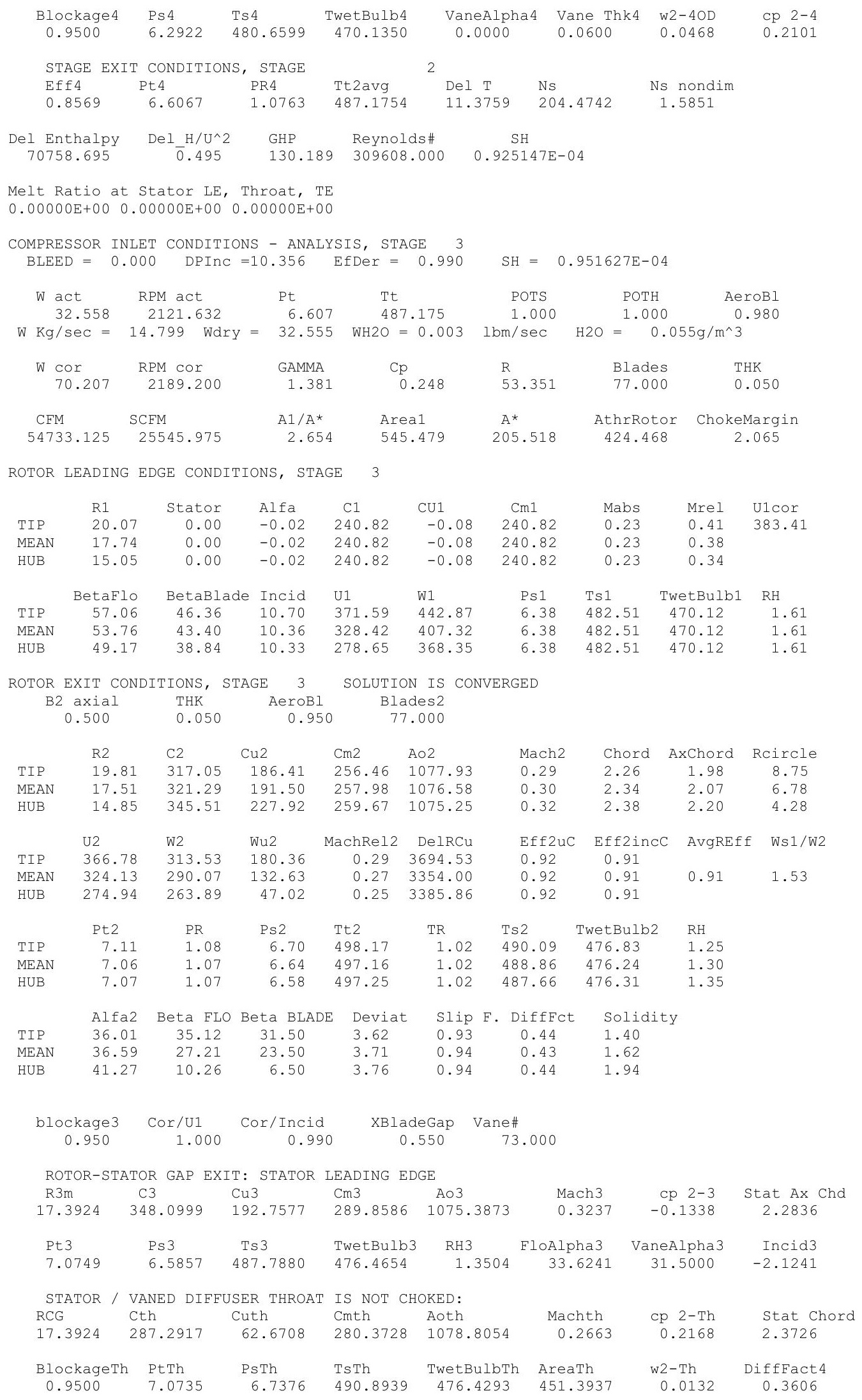




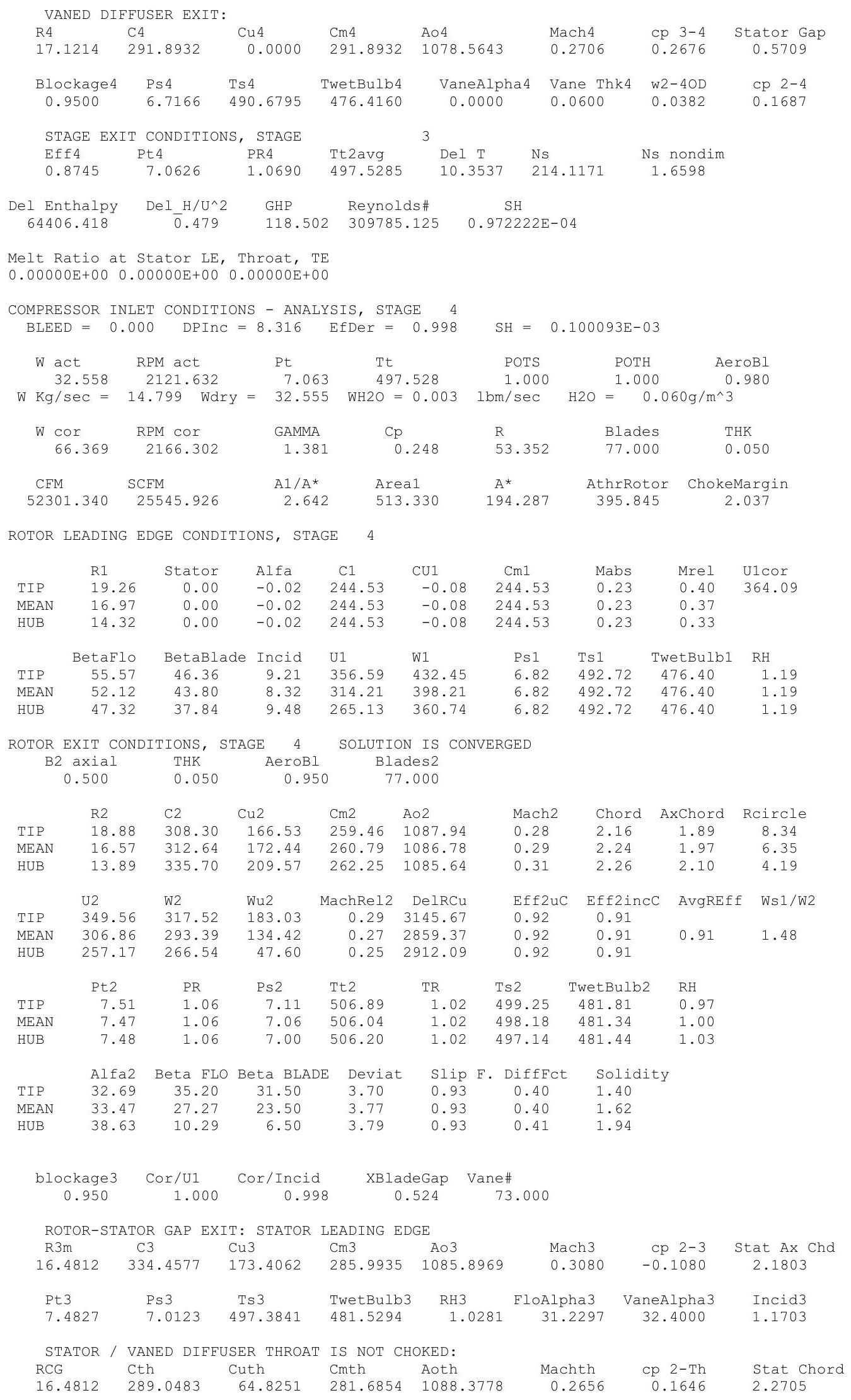




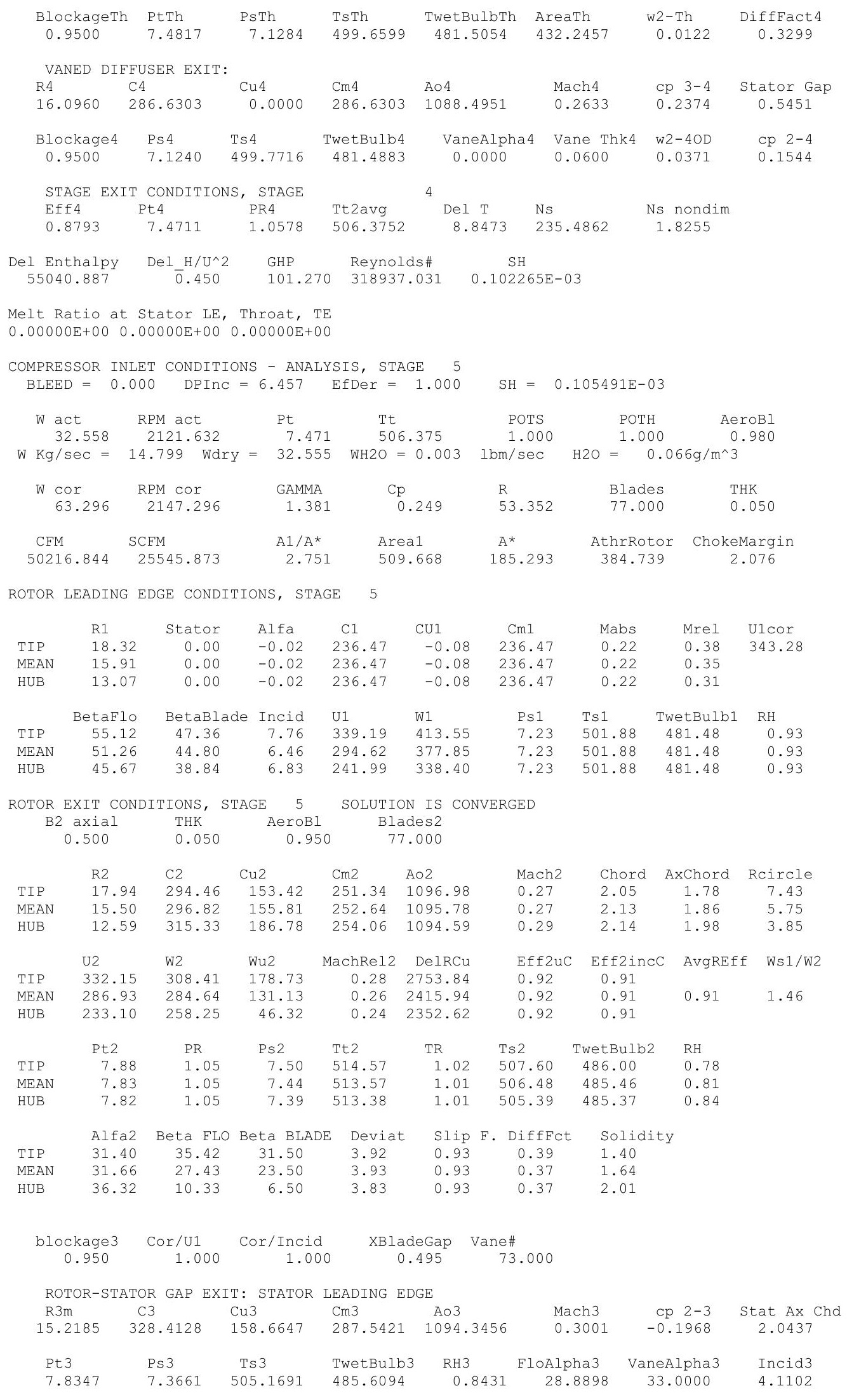




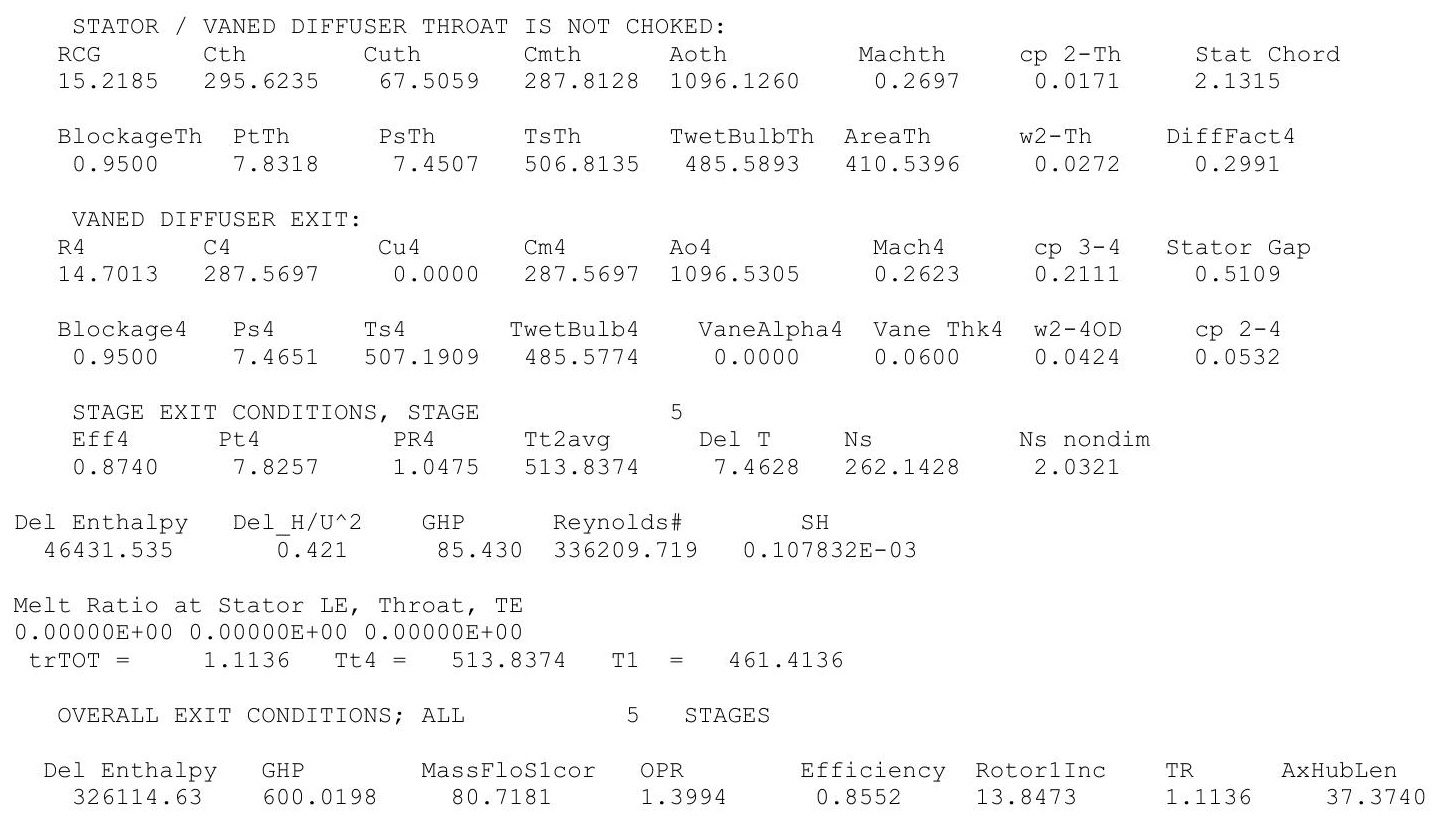




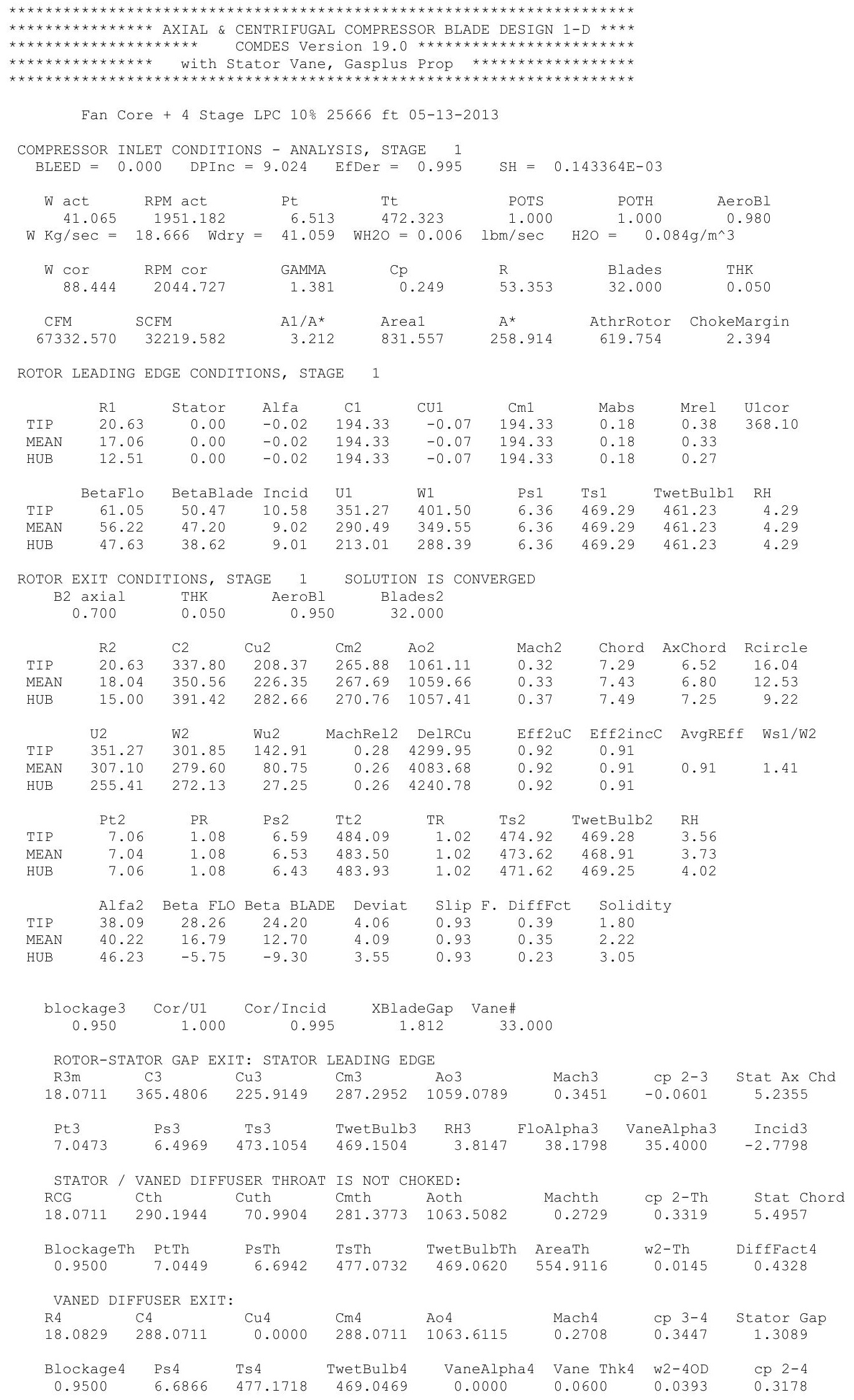




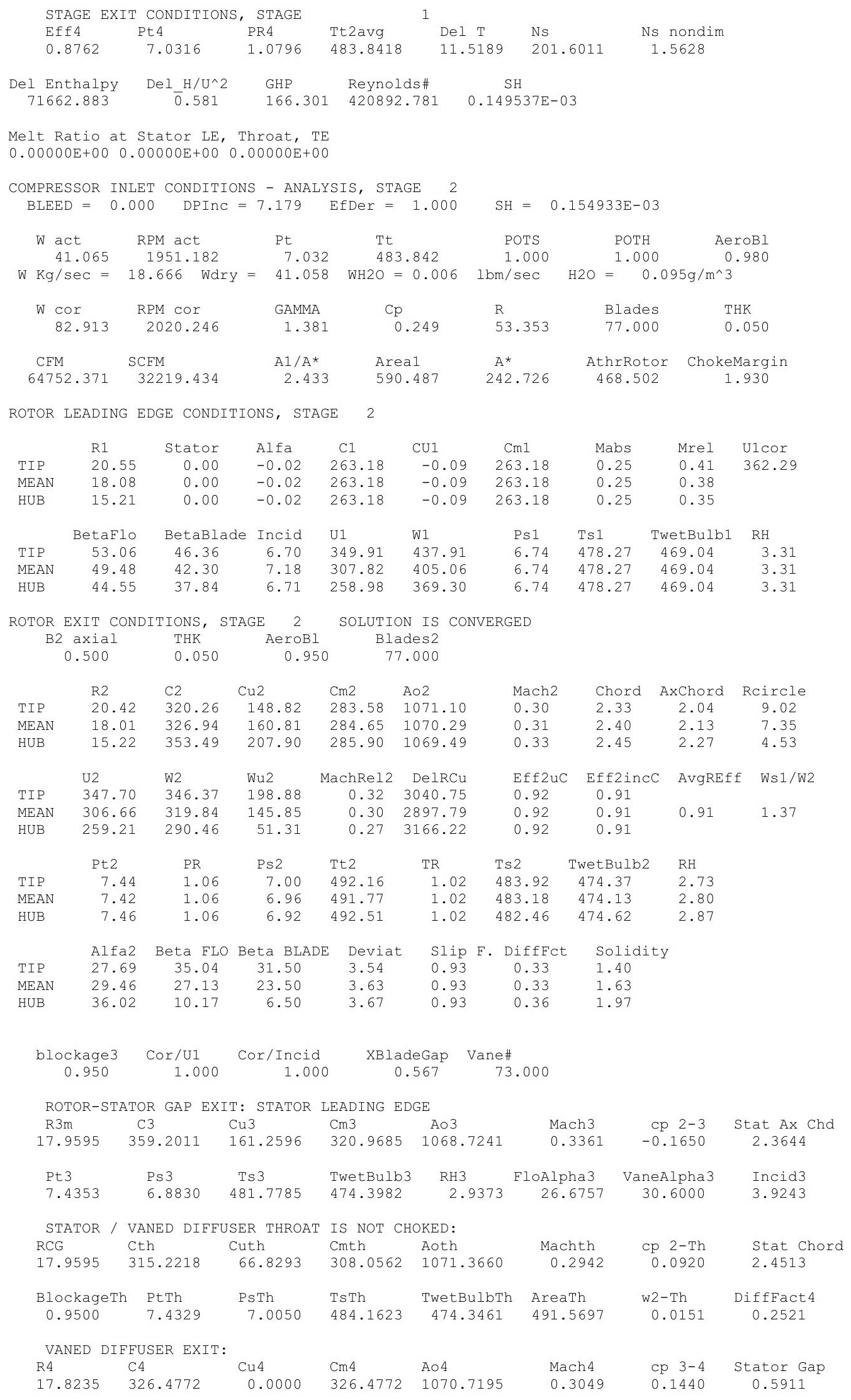




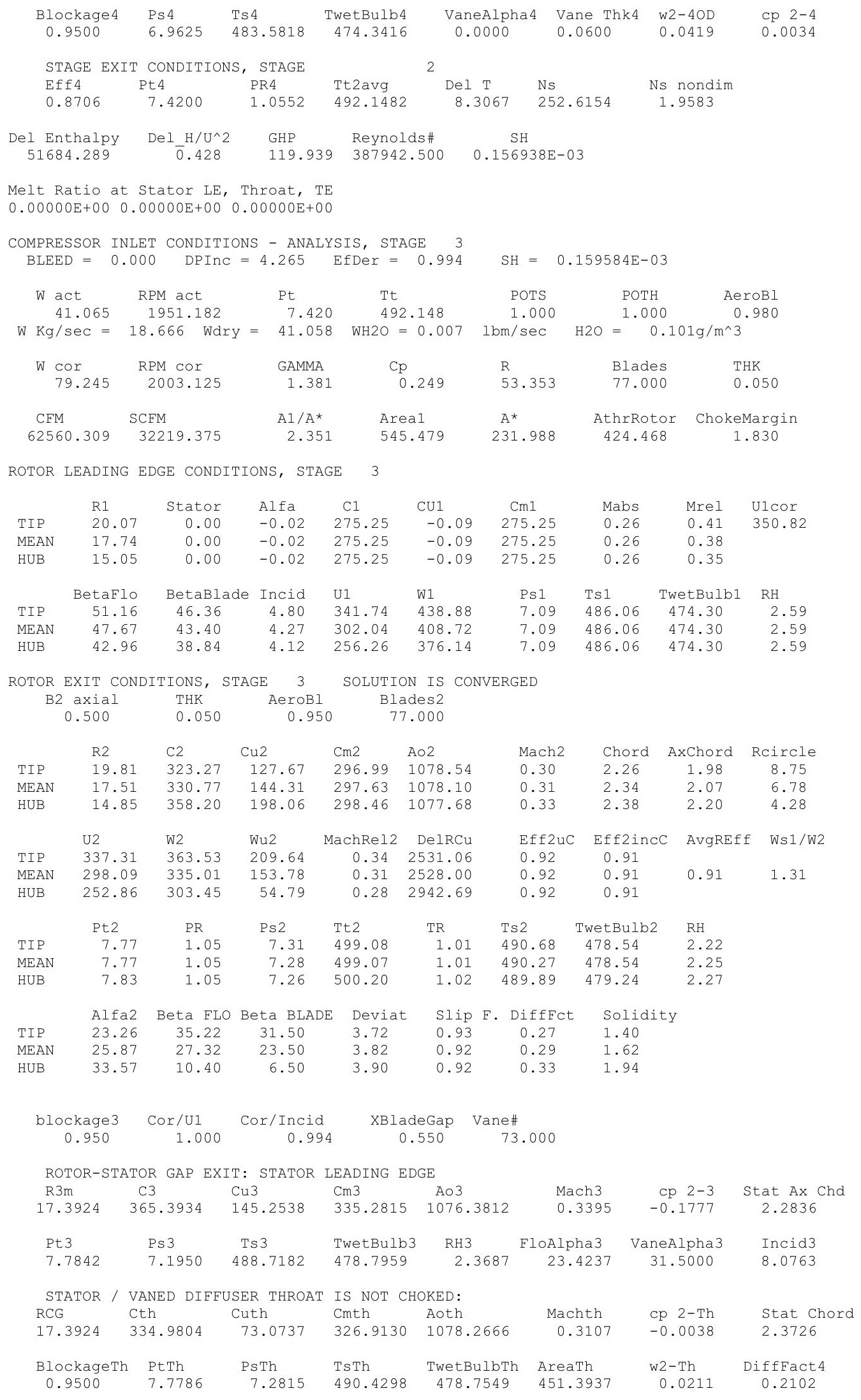




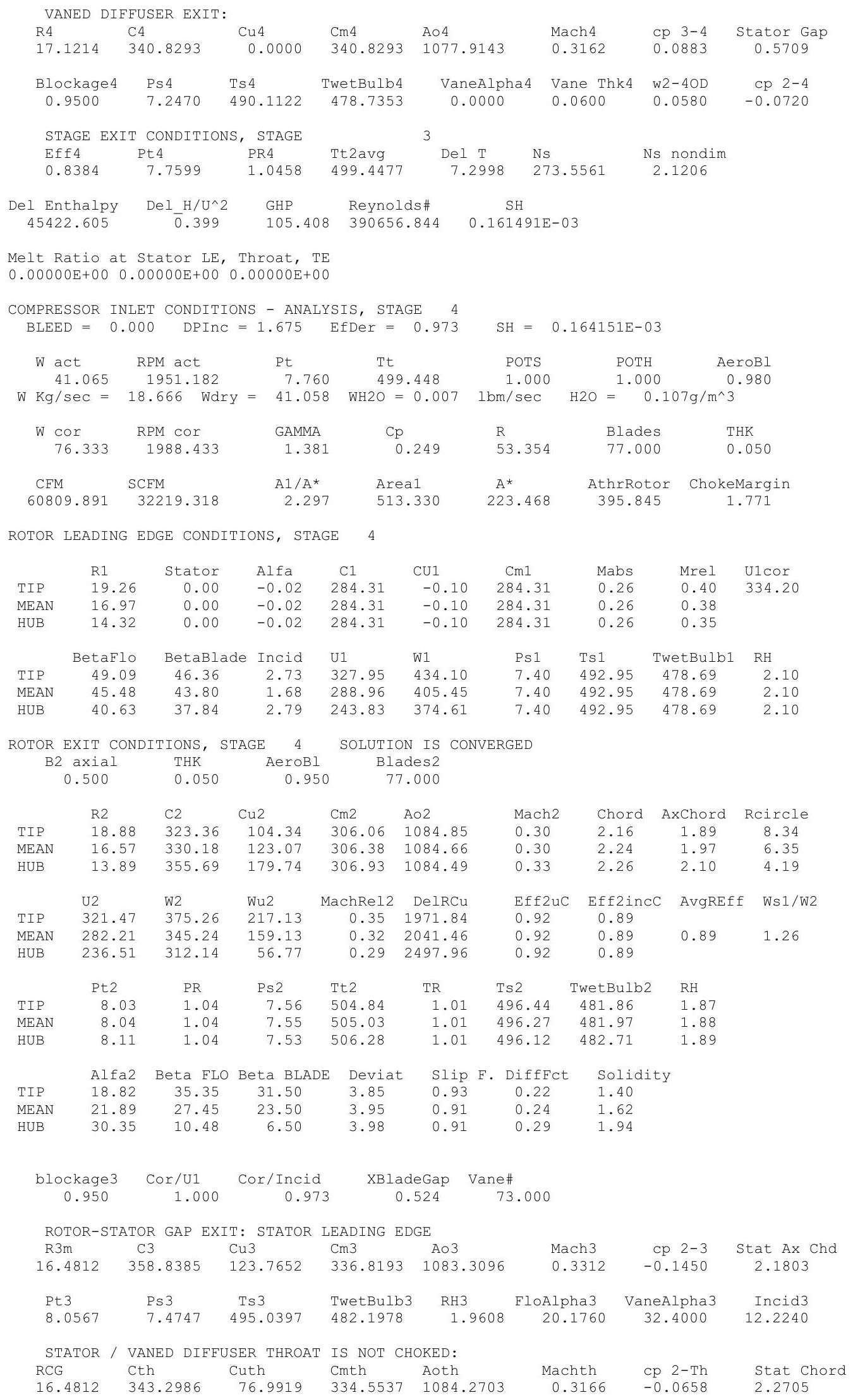




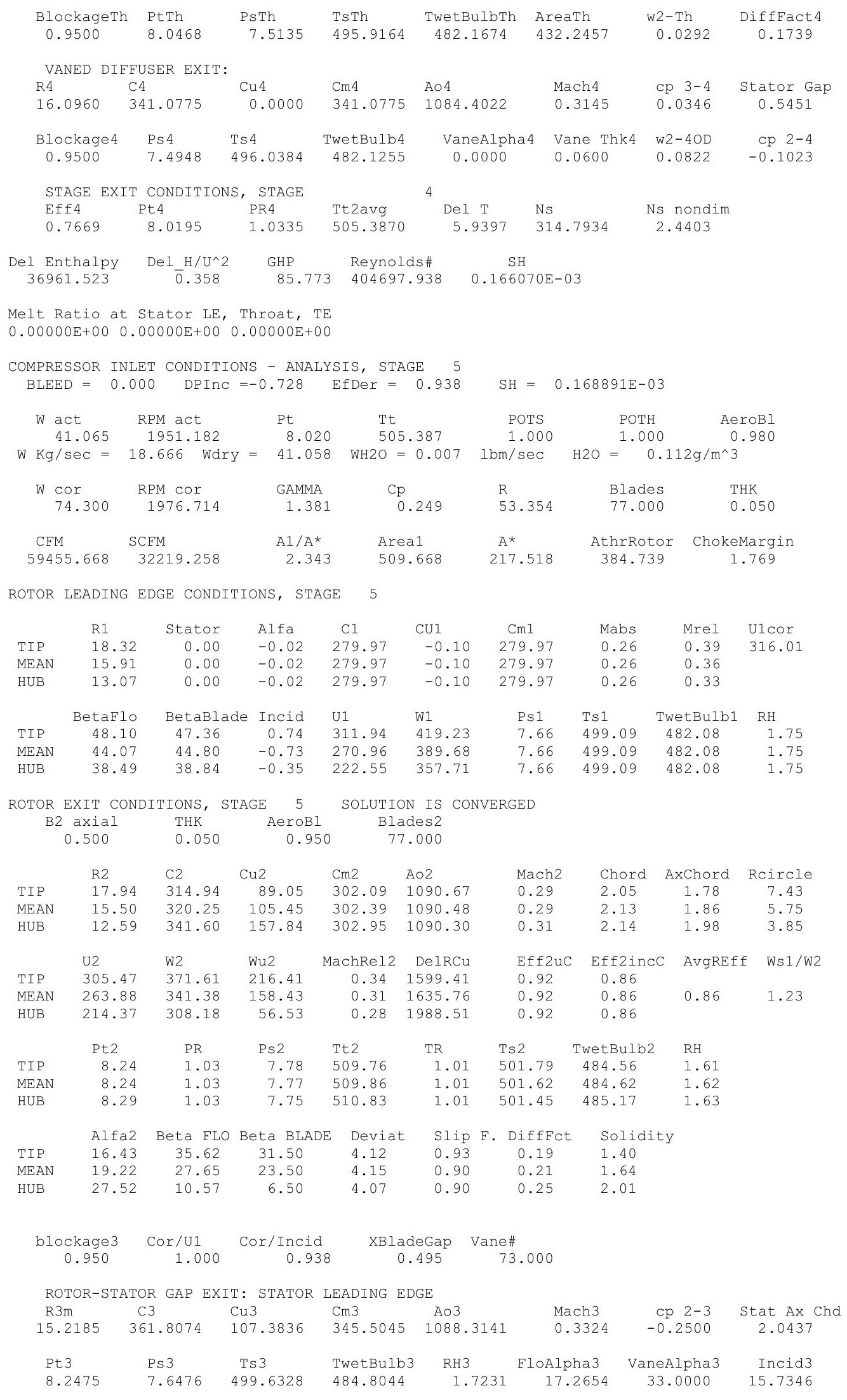




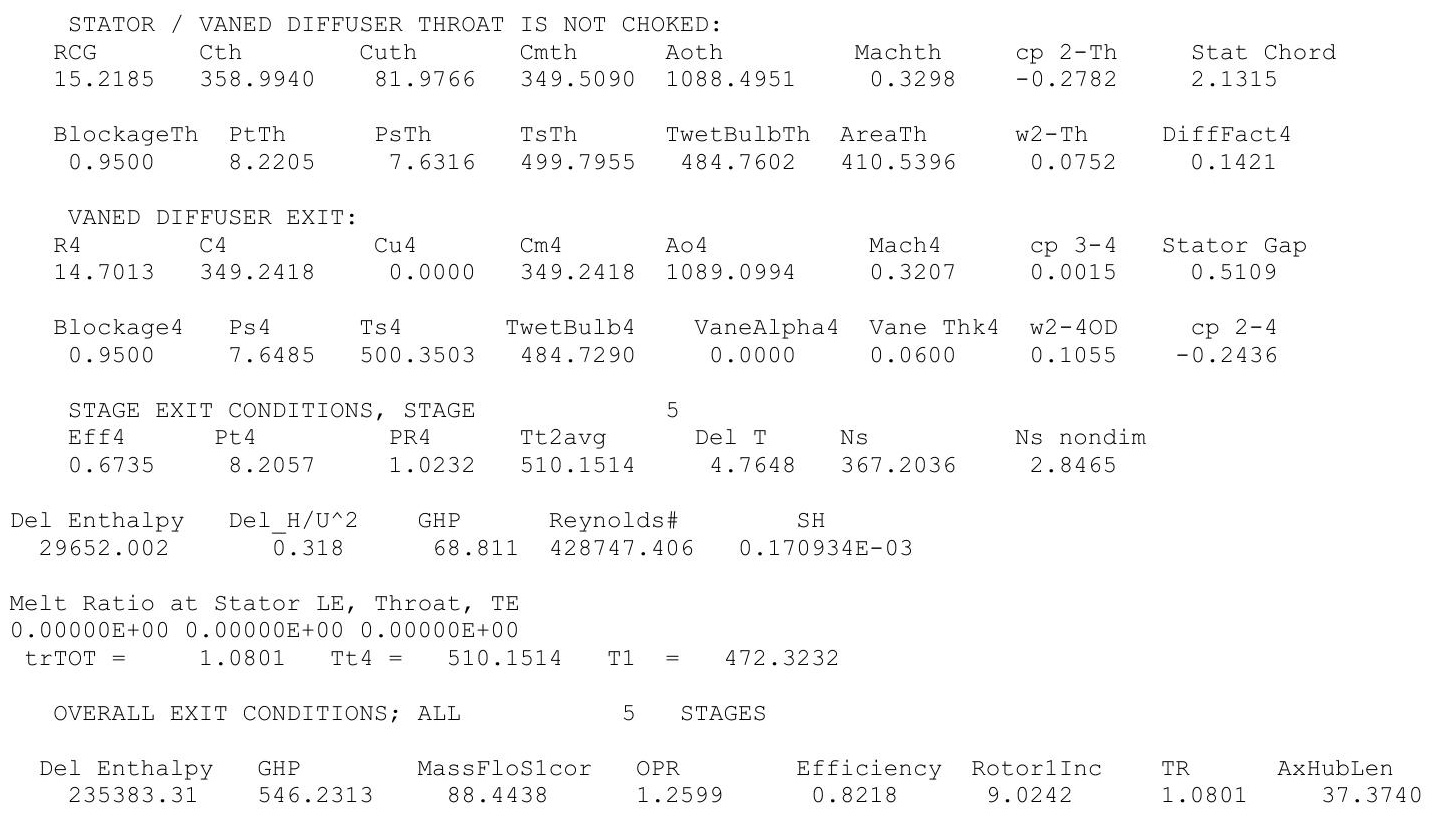




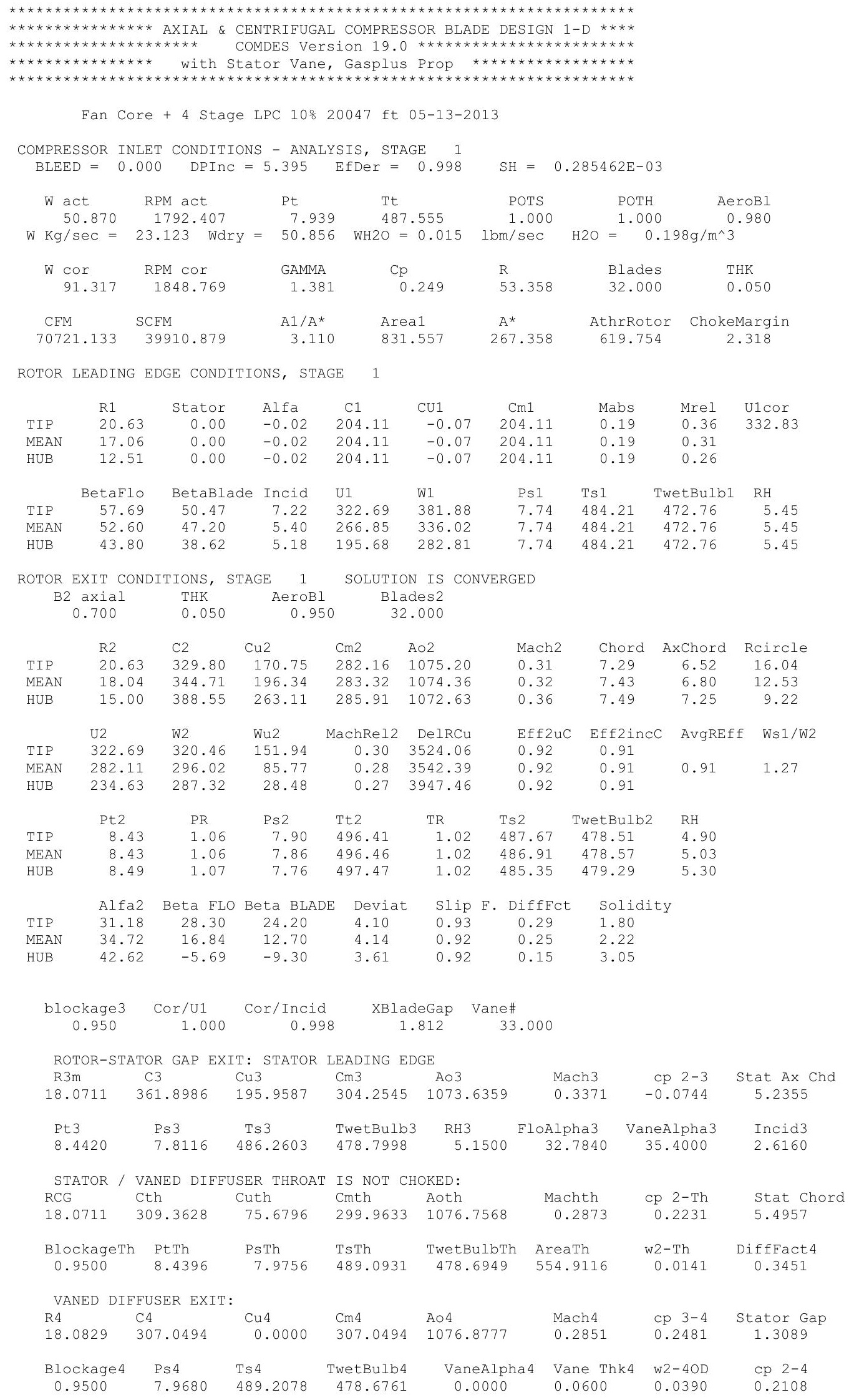




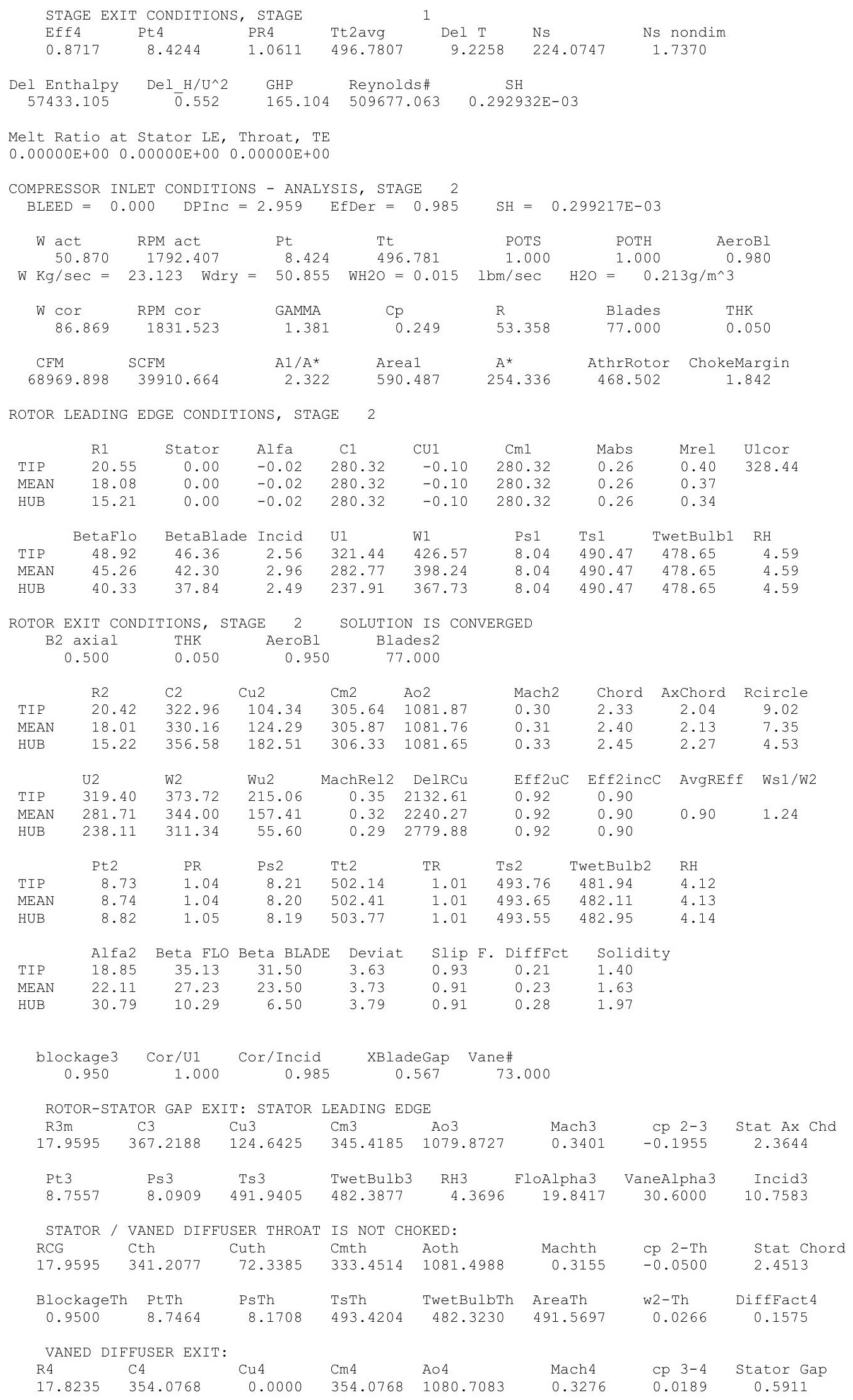




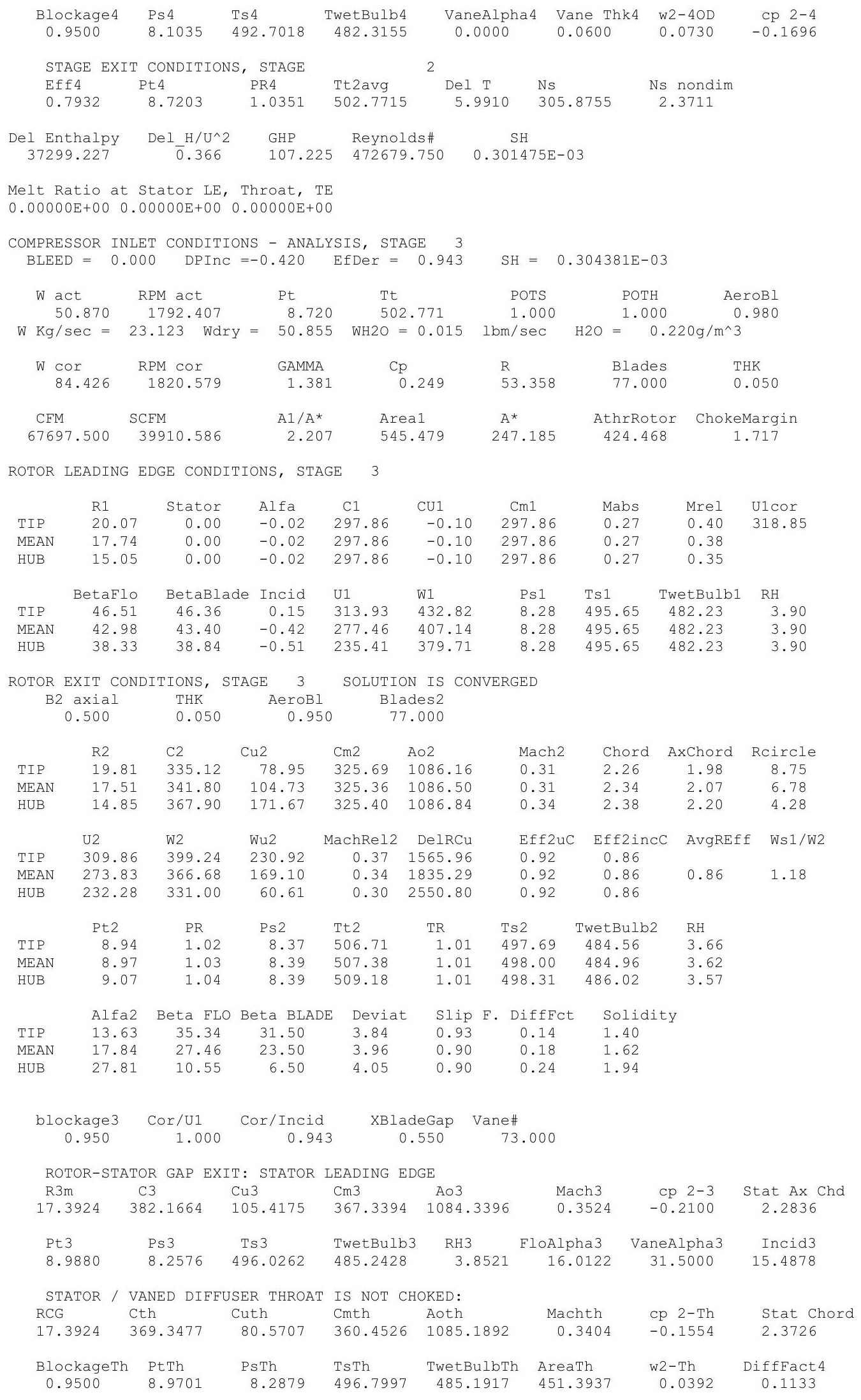




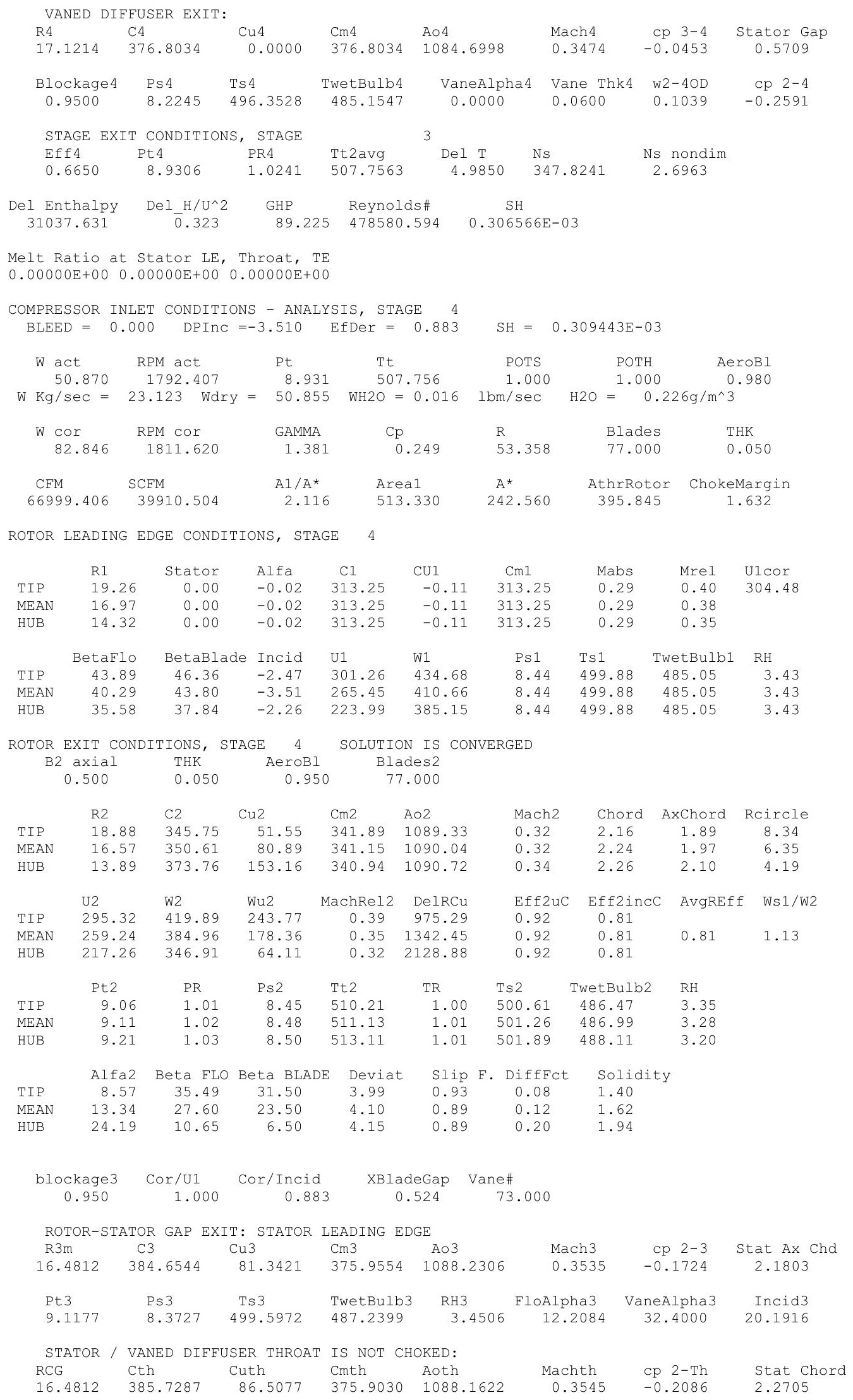




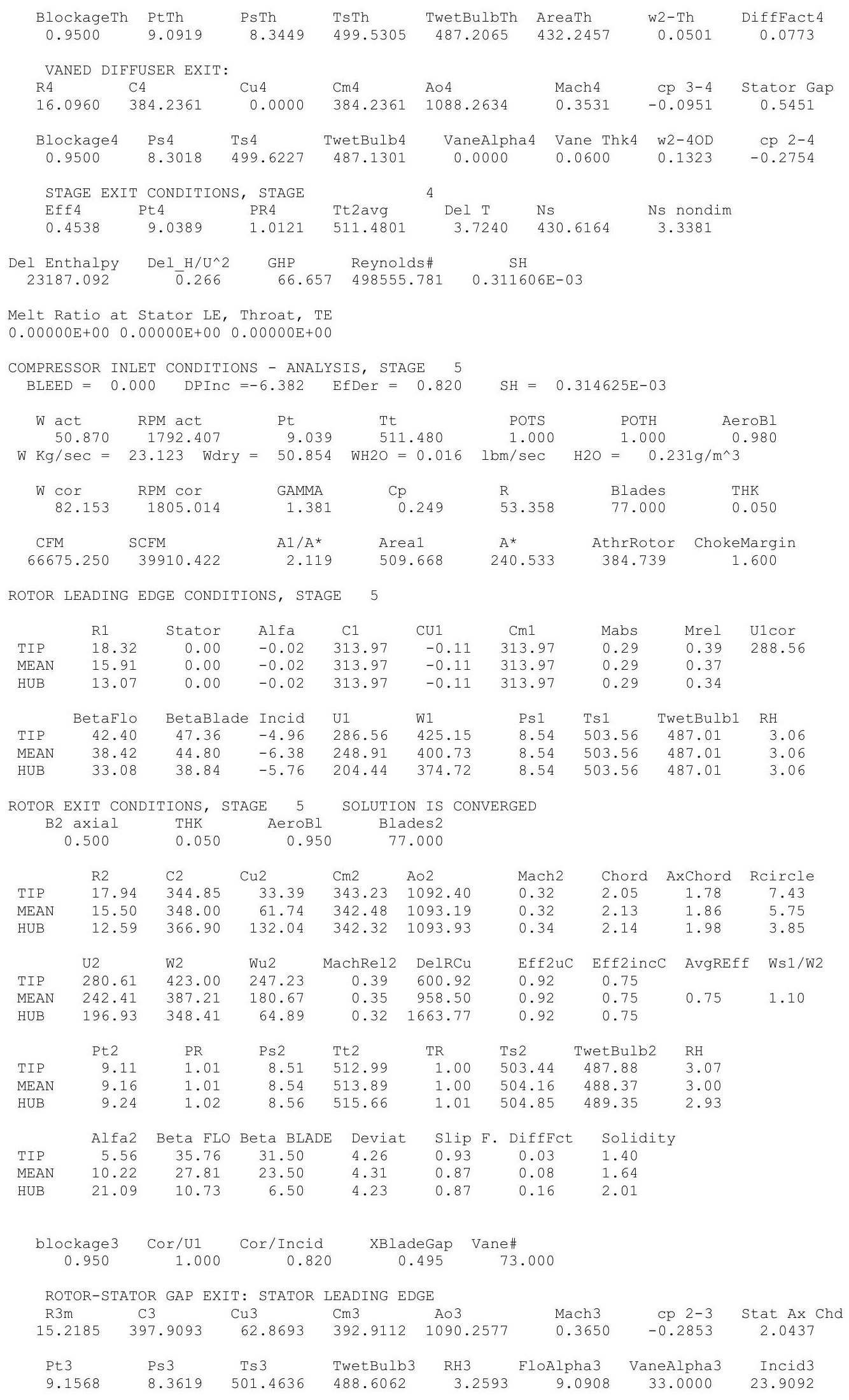




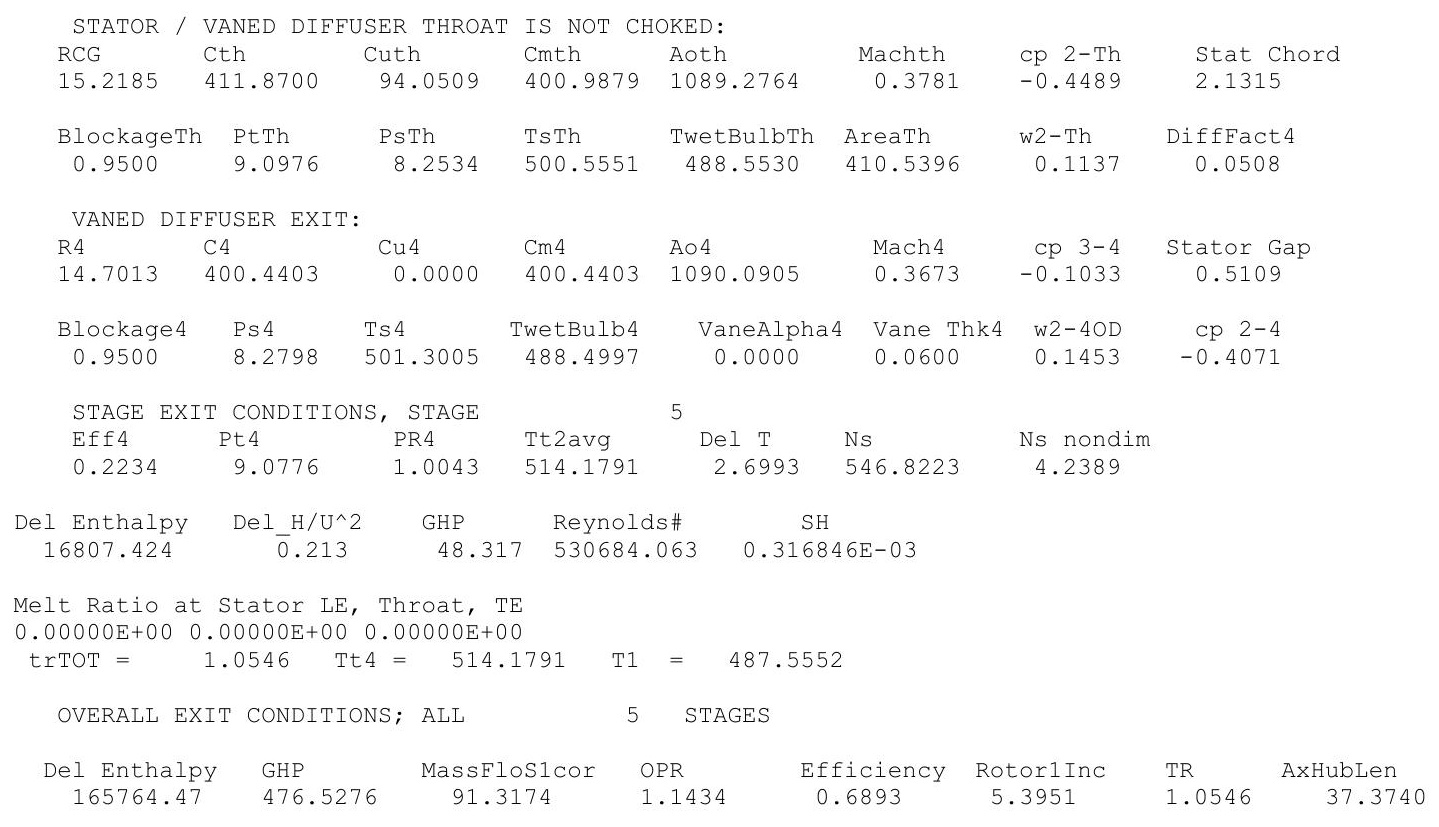




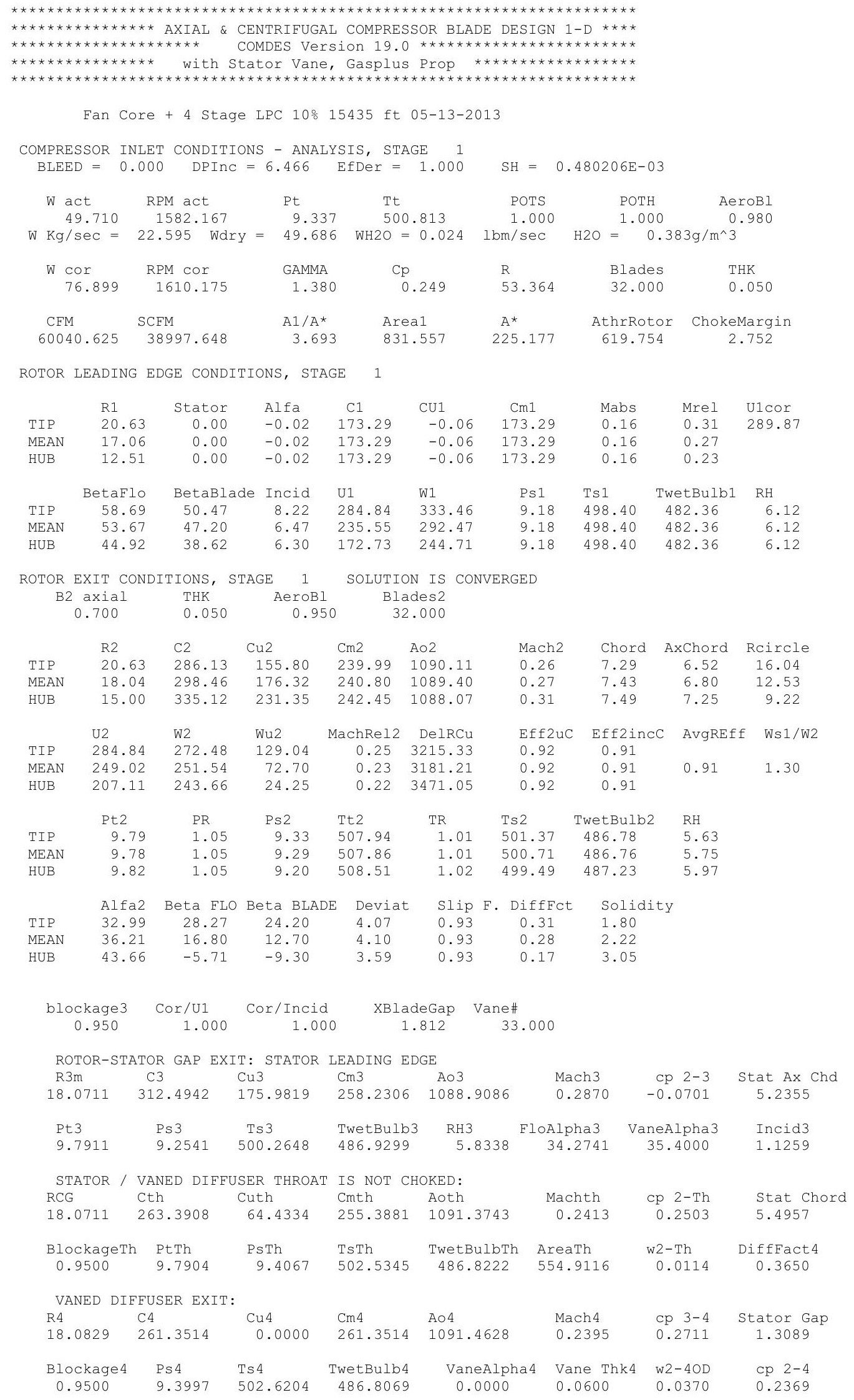




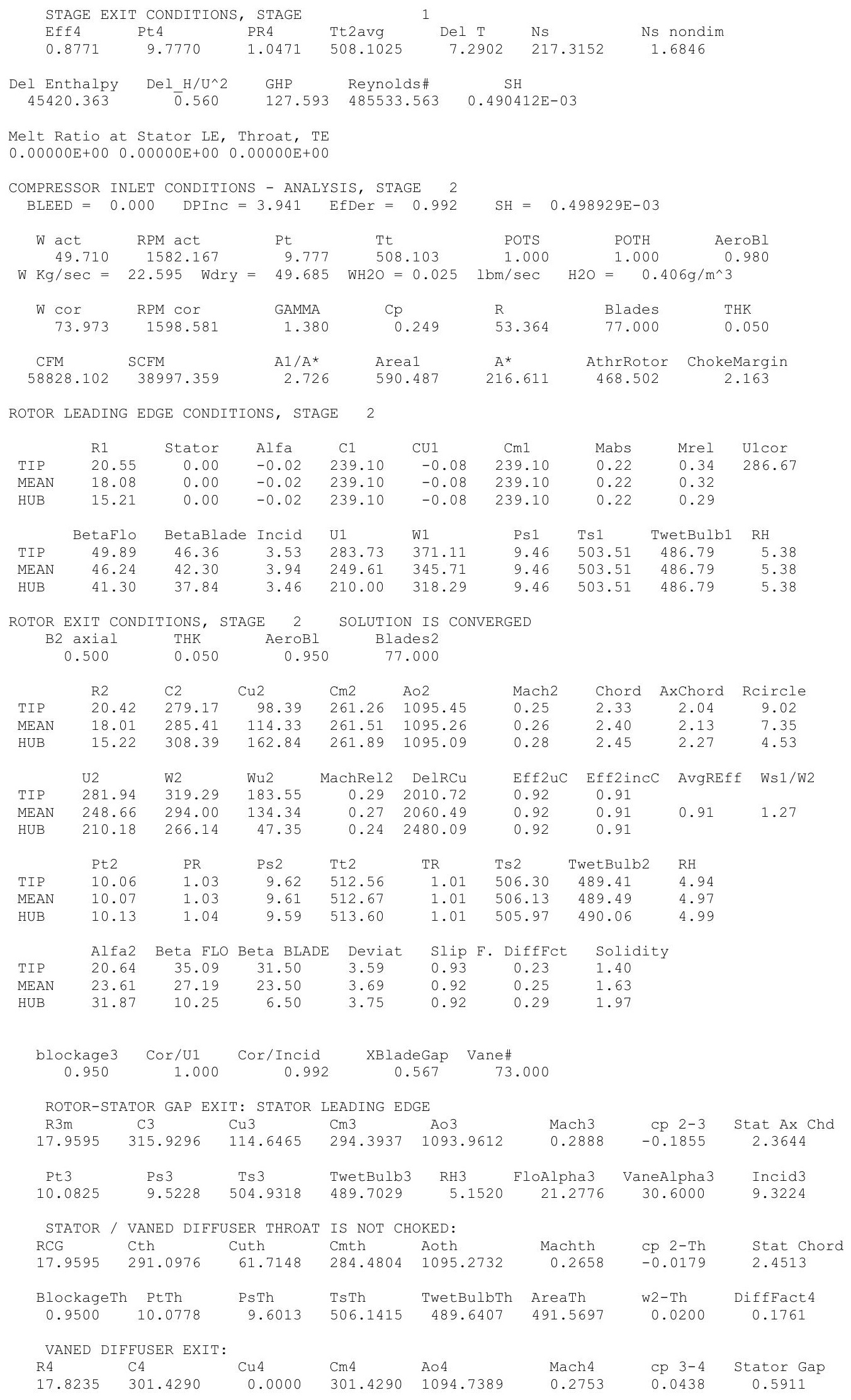




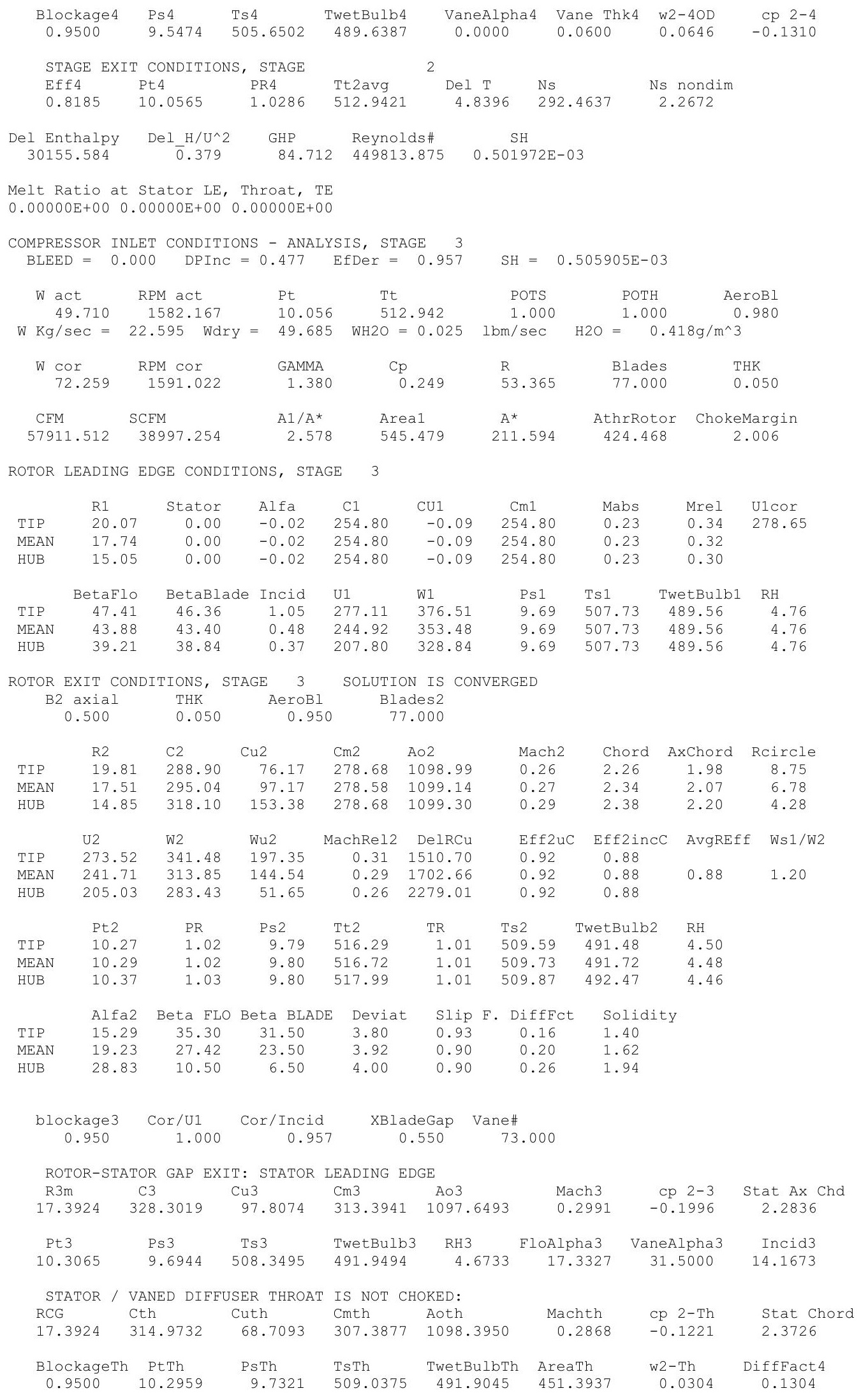




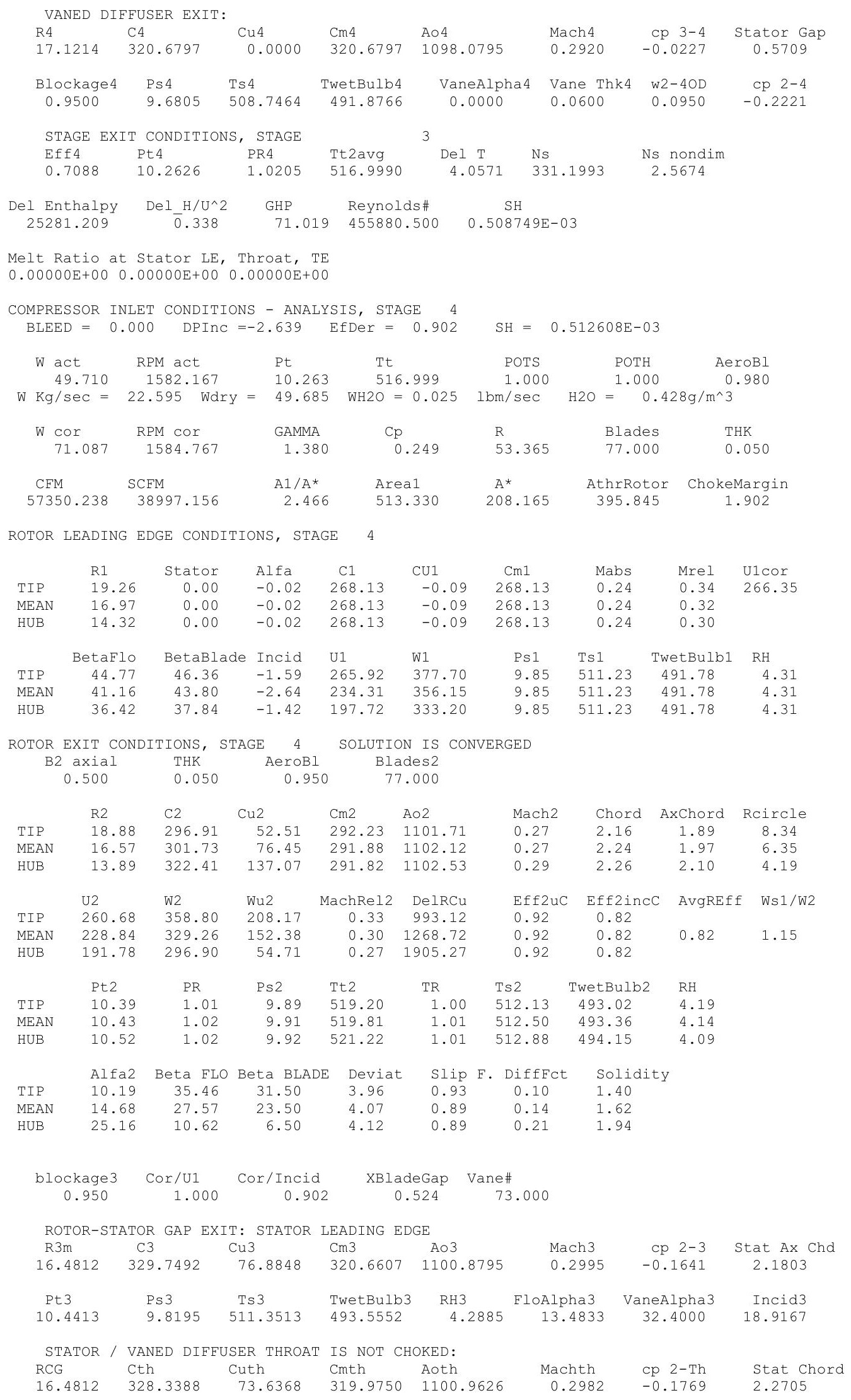




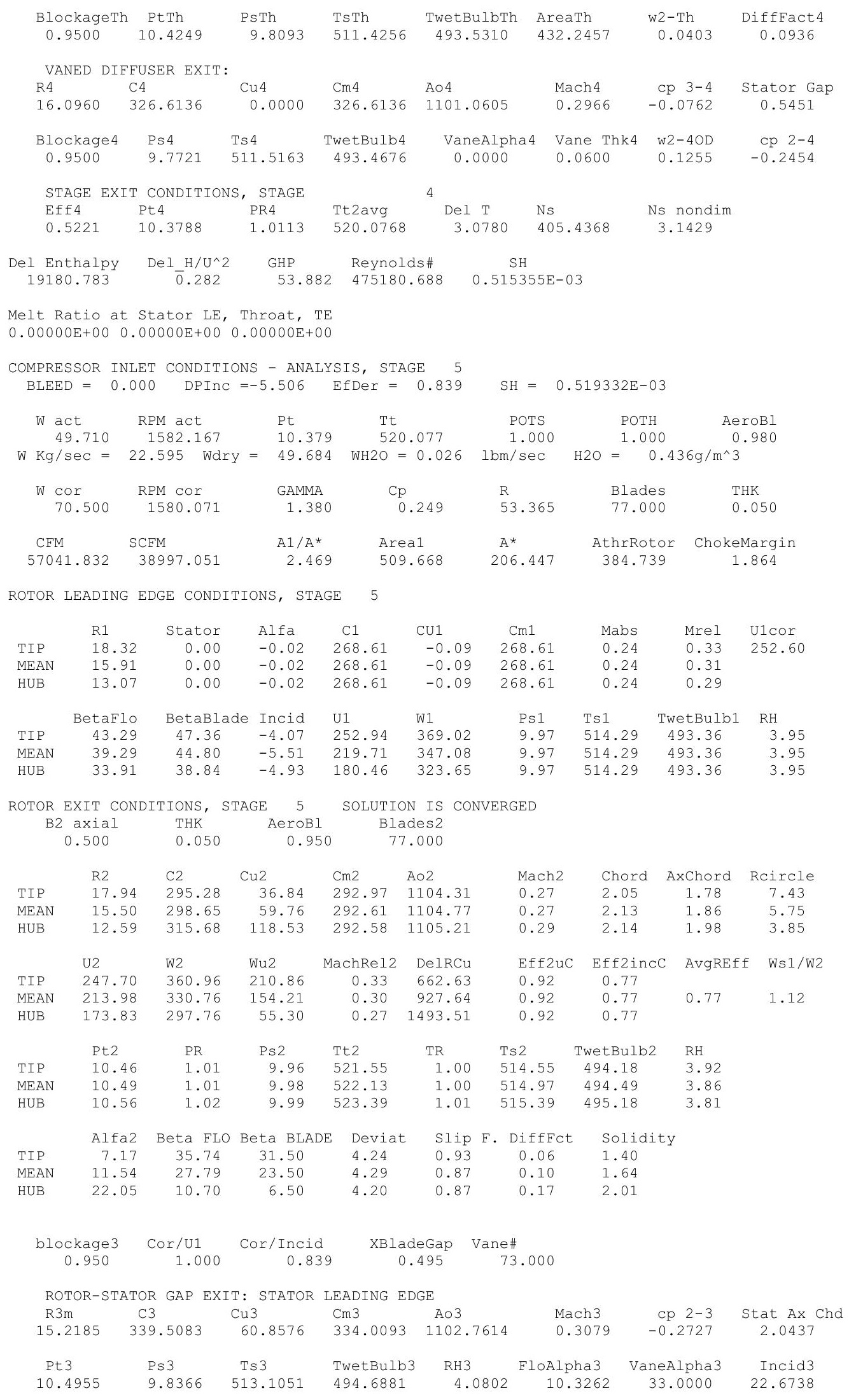




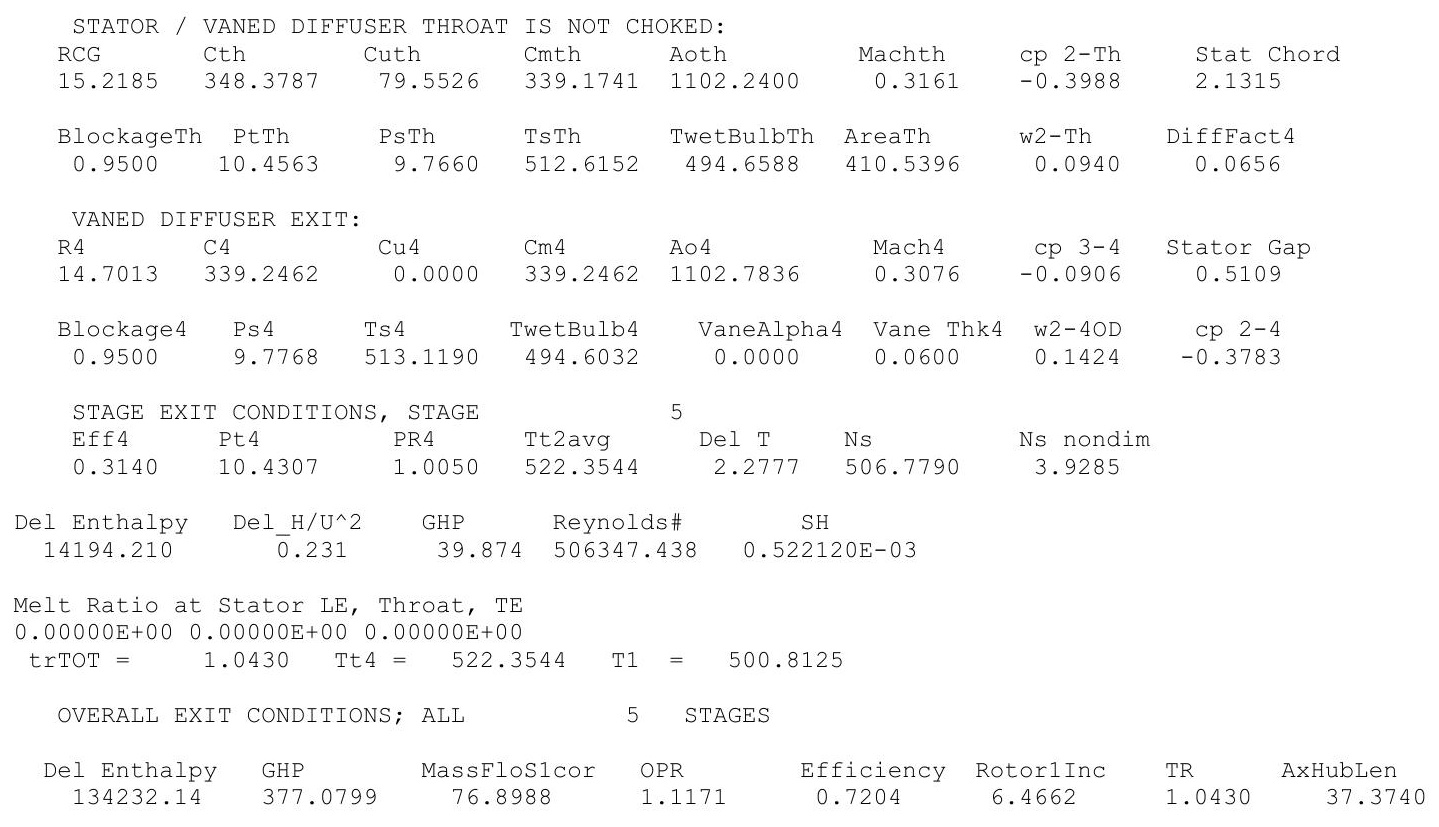


$50 \mu \mathrm{m}$, ISA $+36 \mathrm{R}$

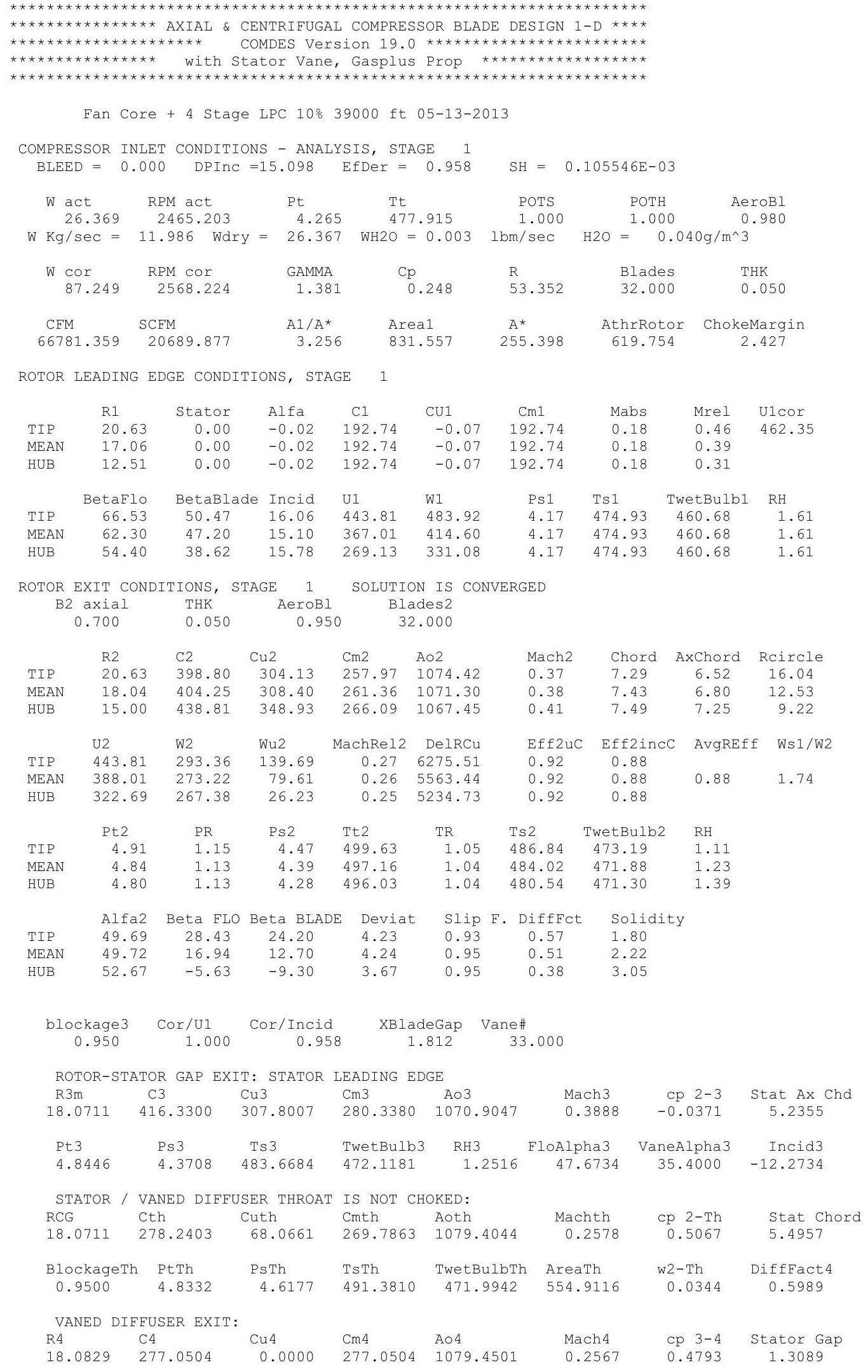




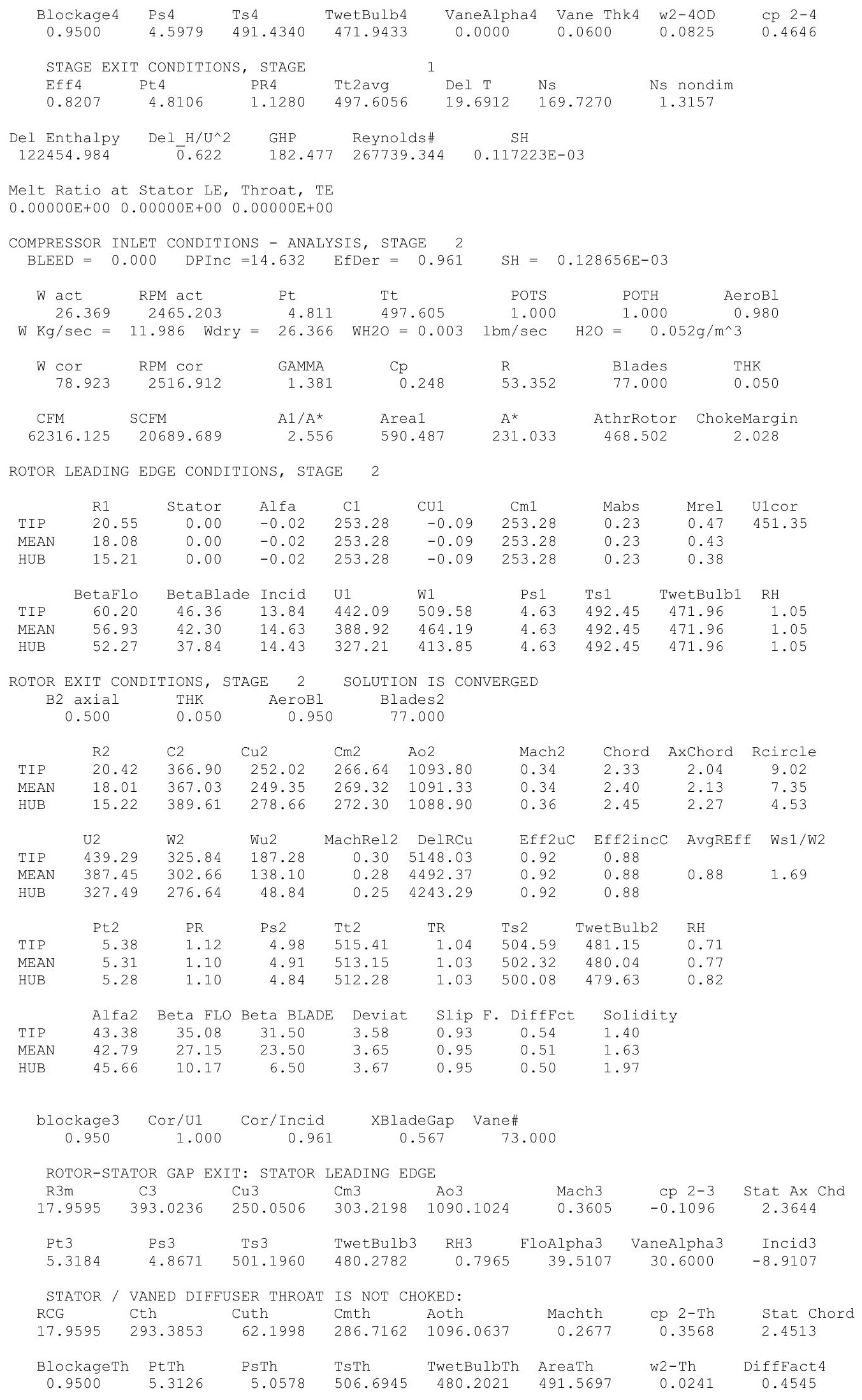




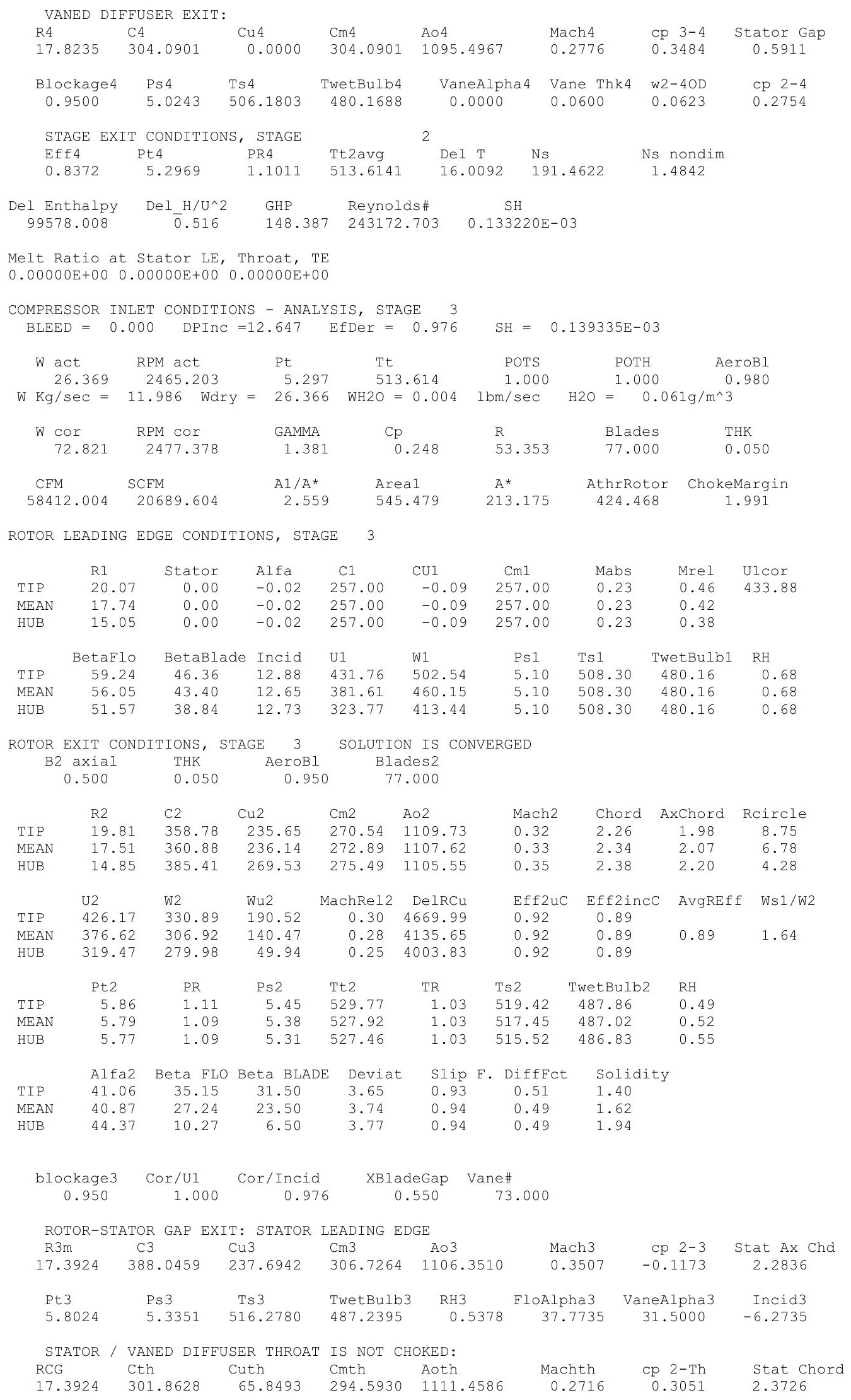




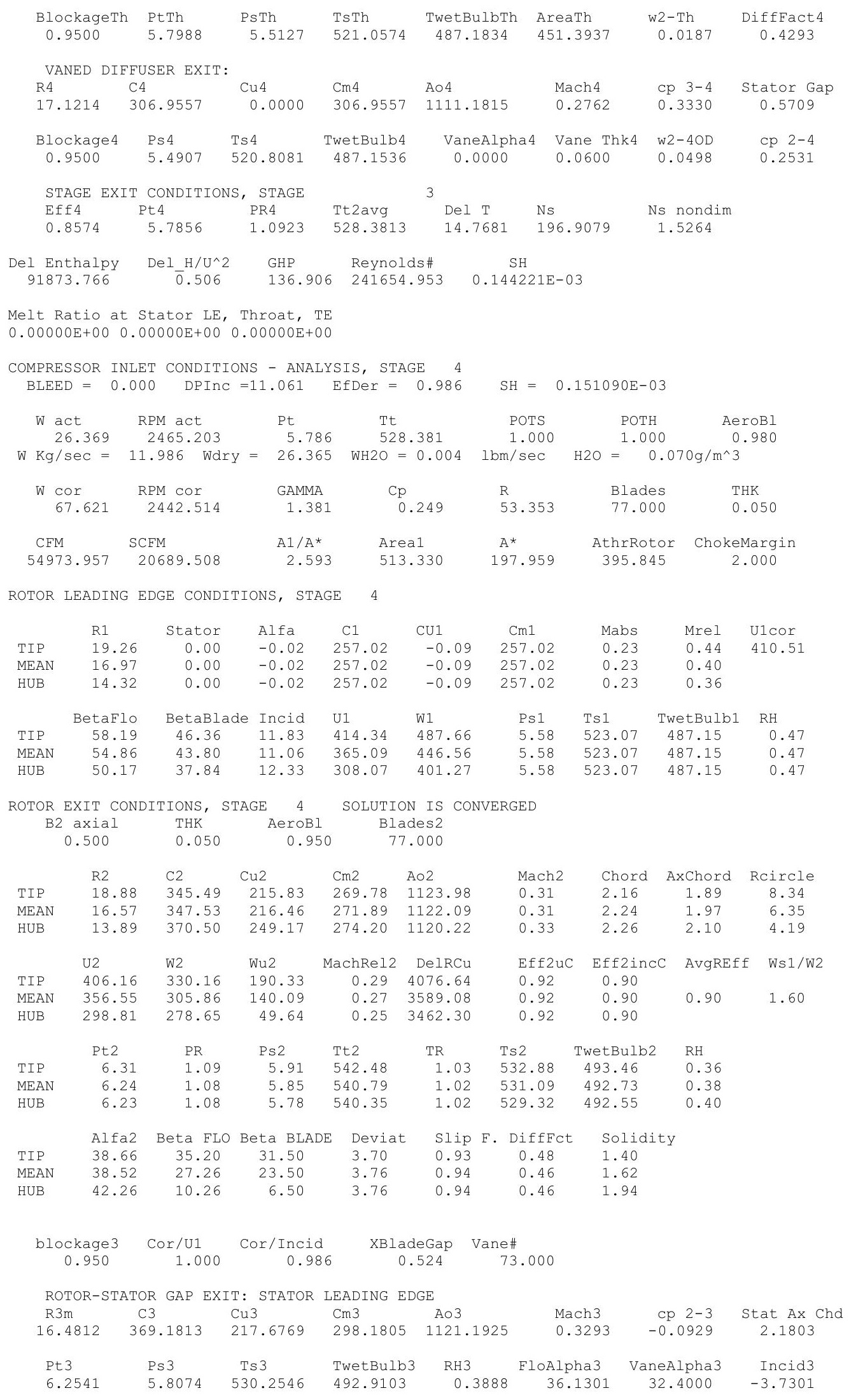




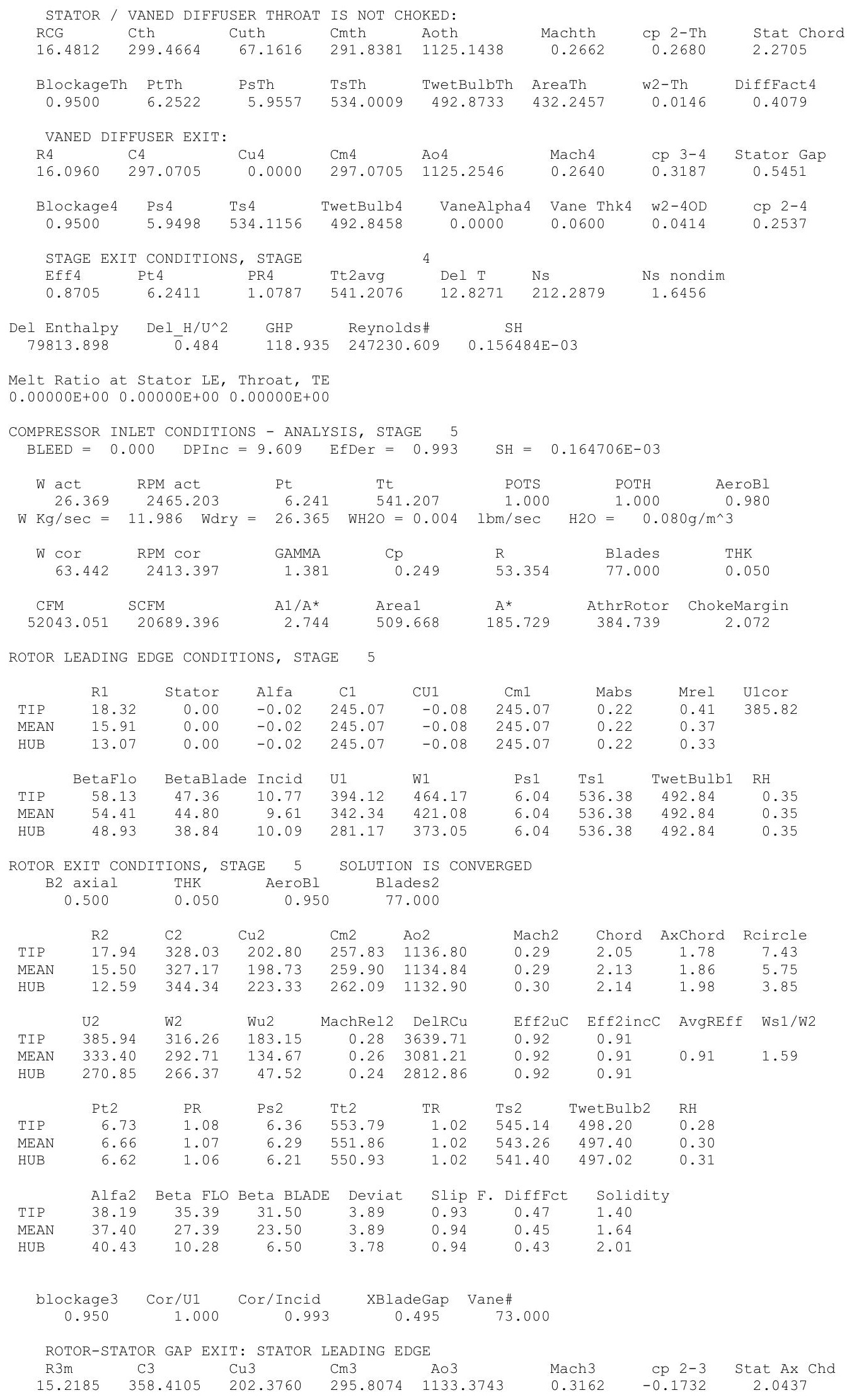




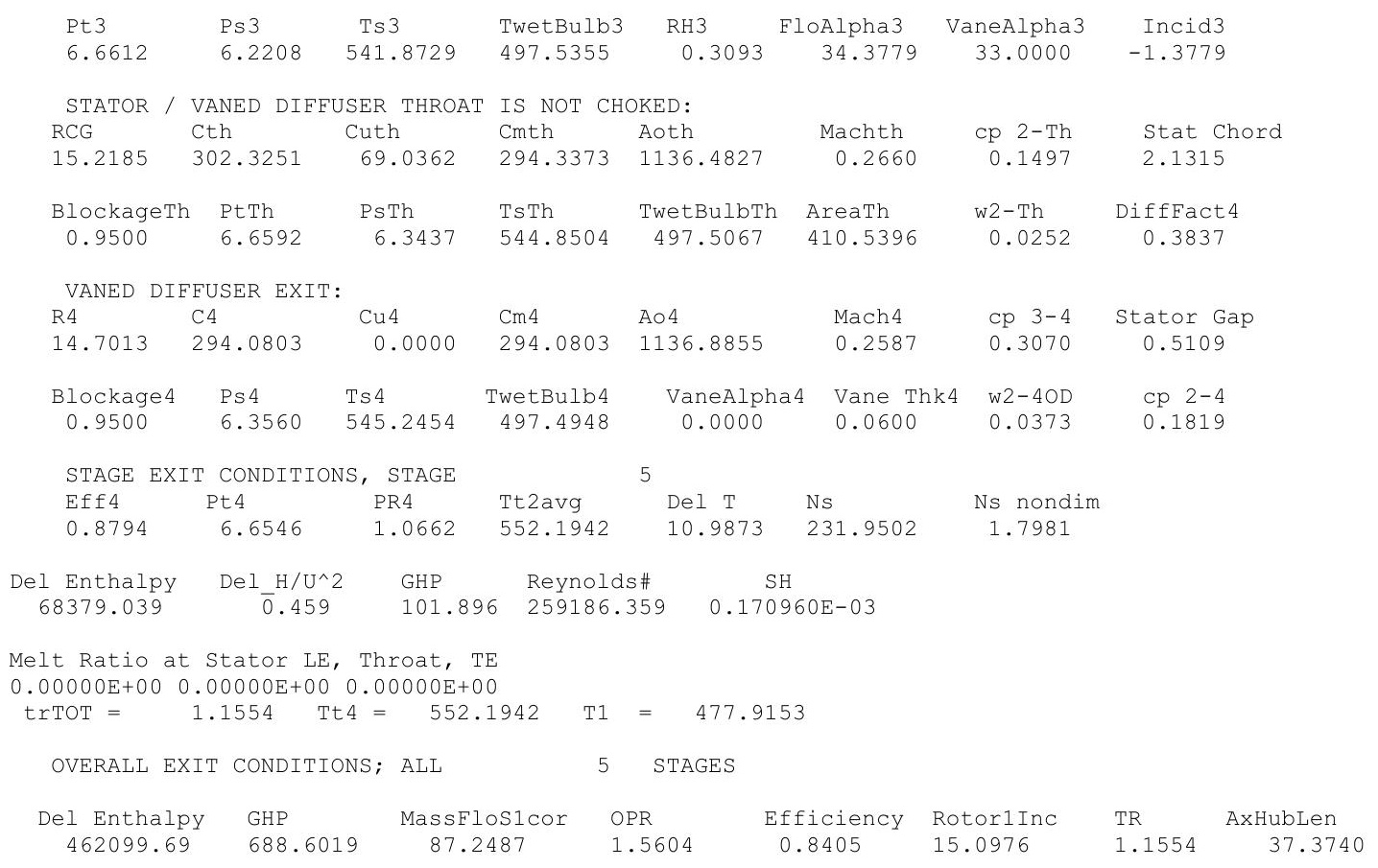




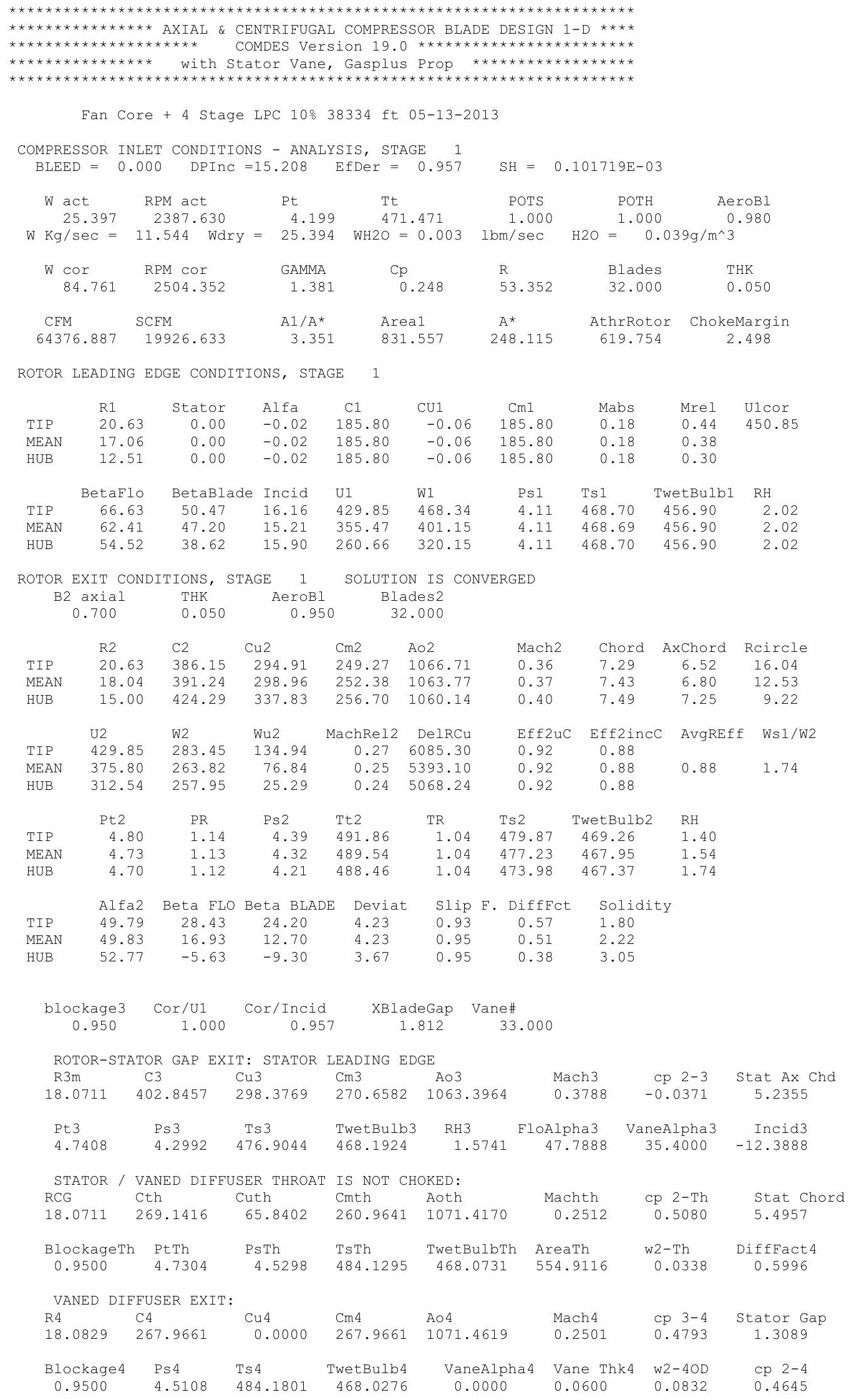




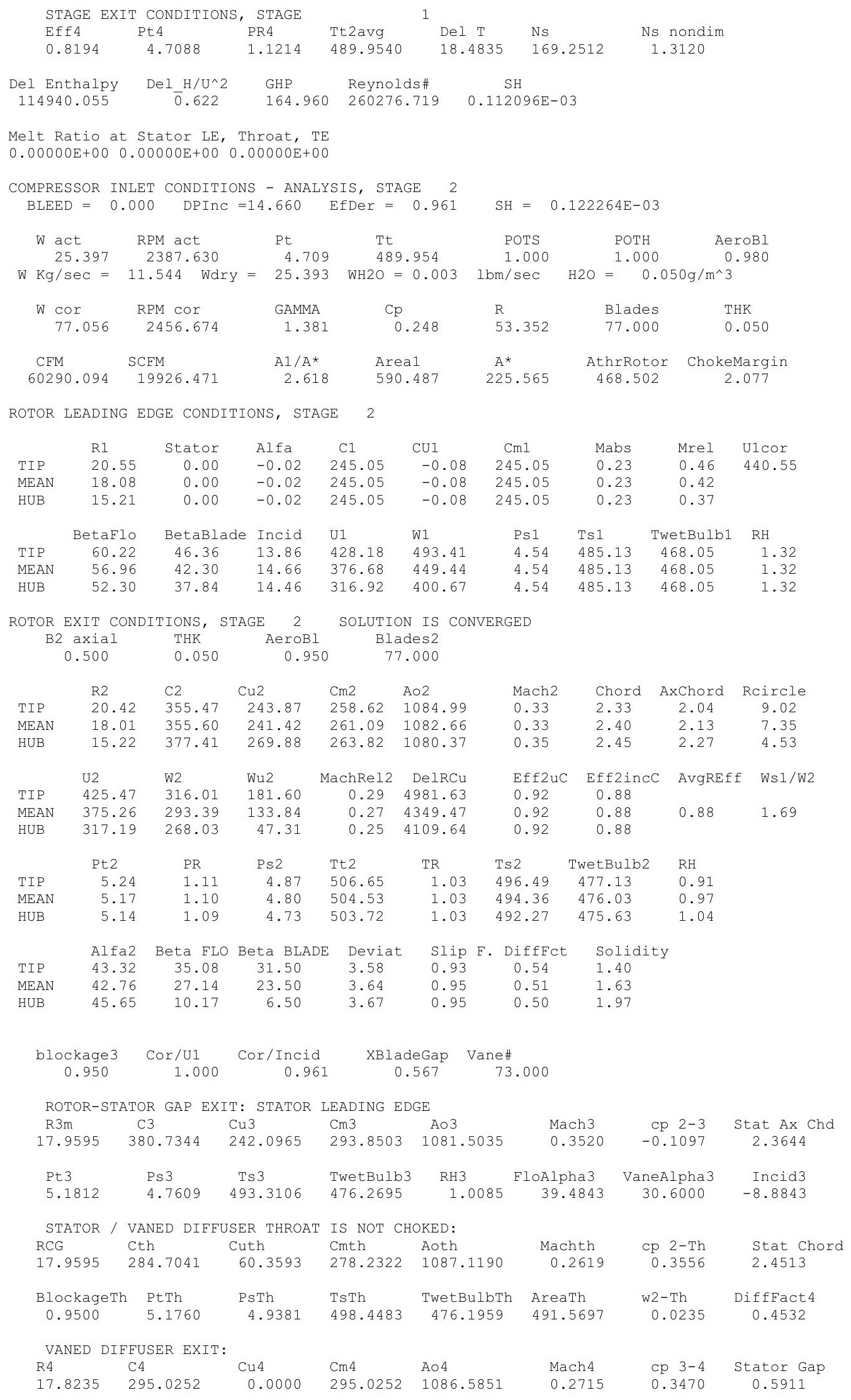




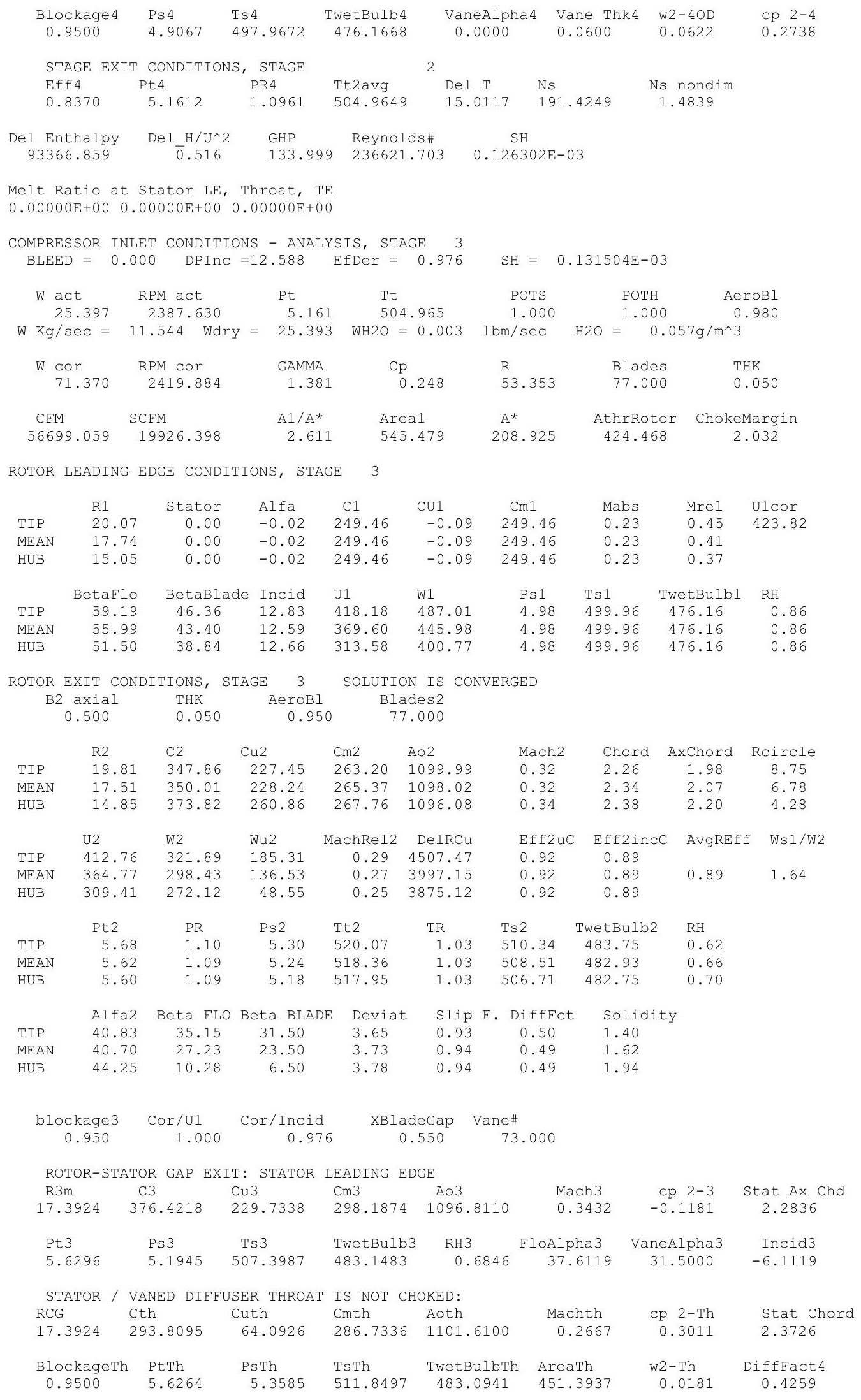




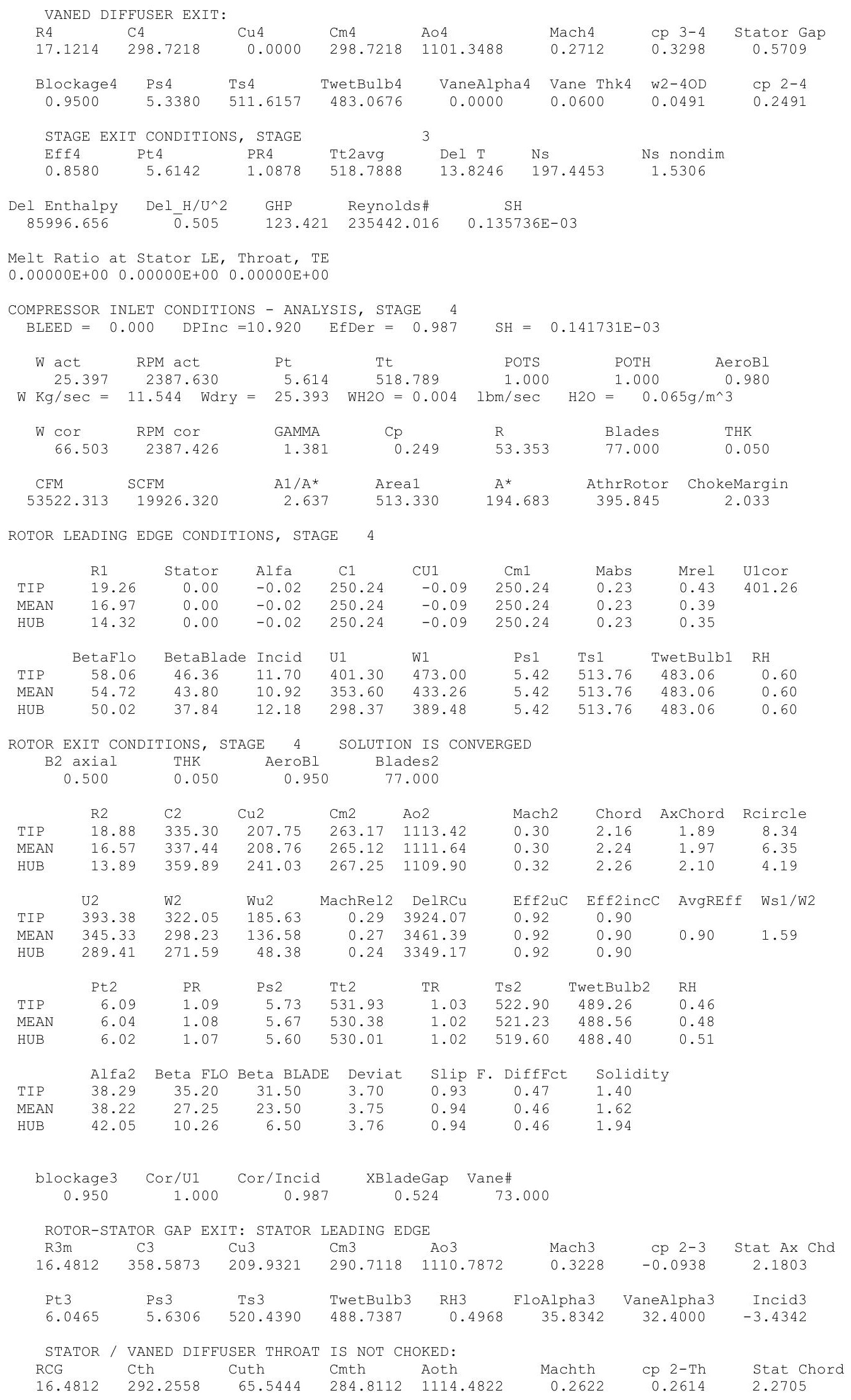




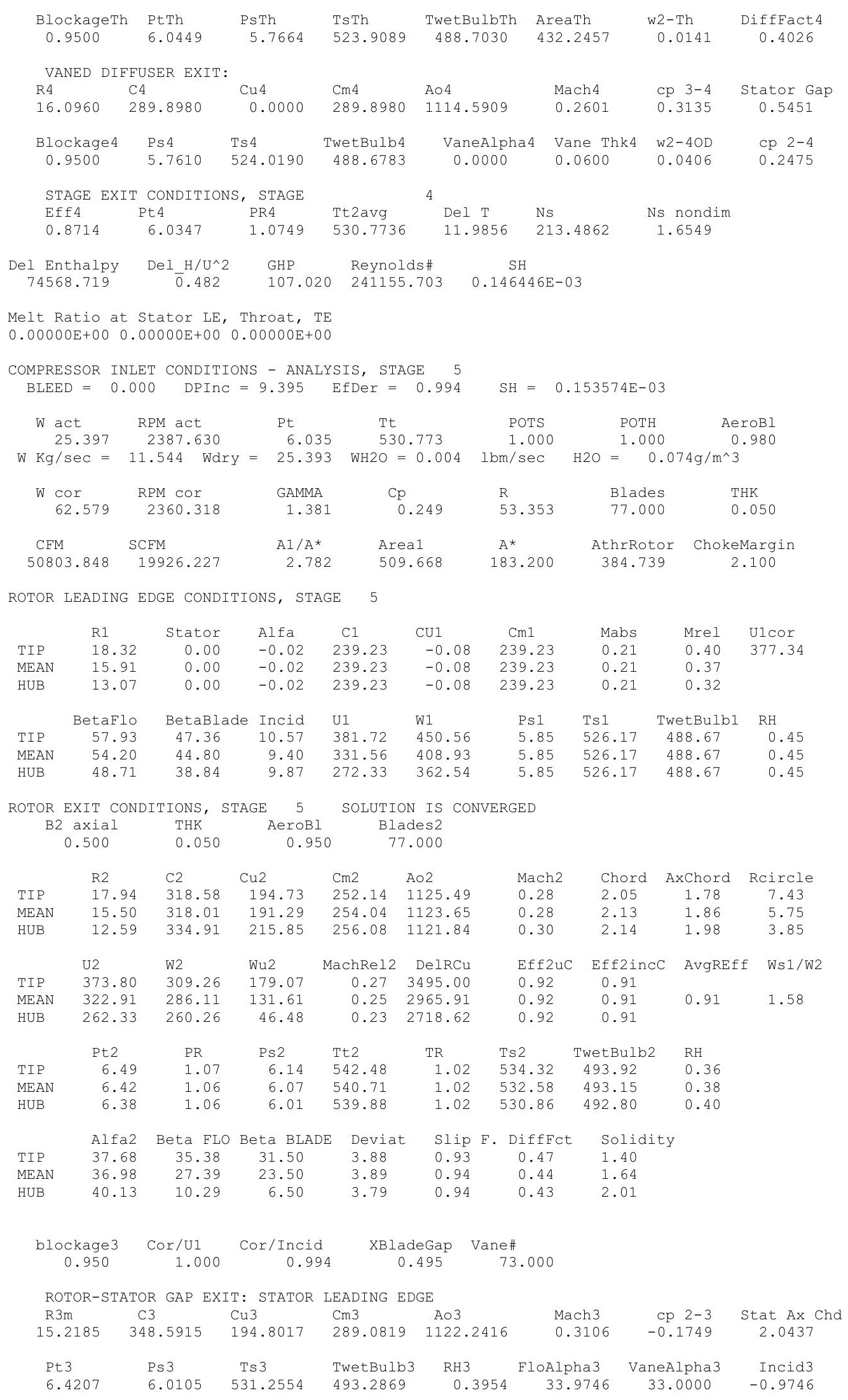




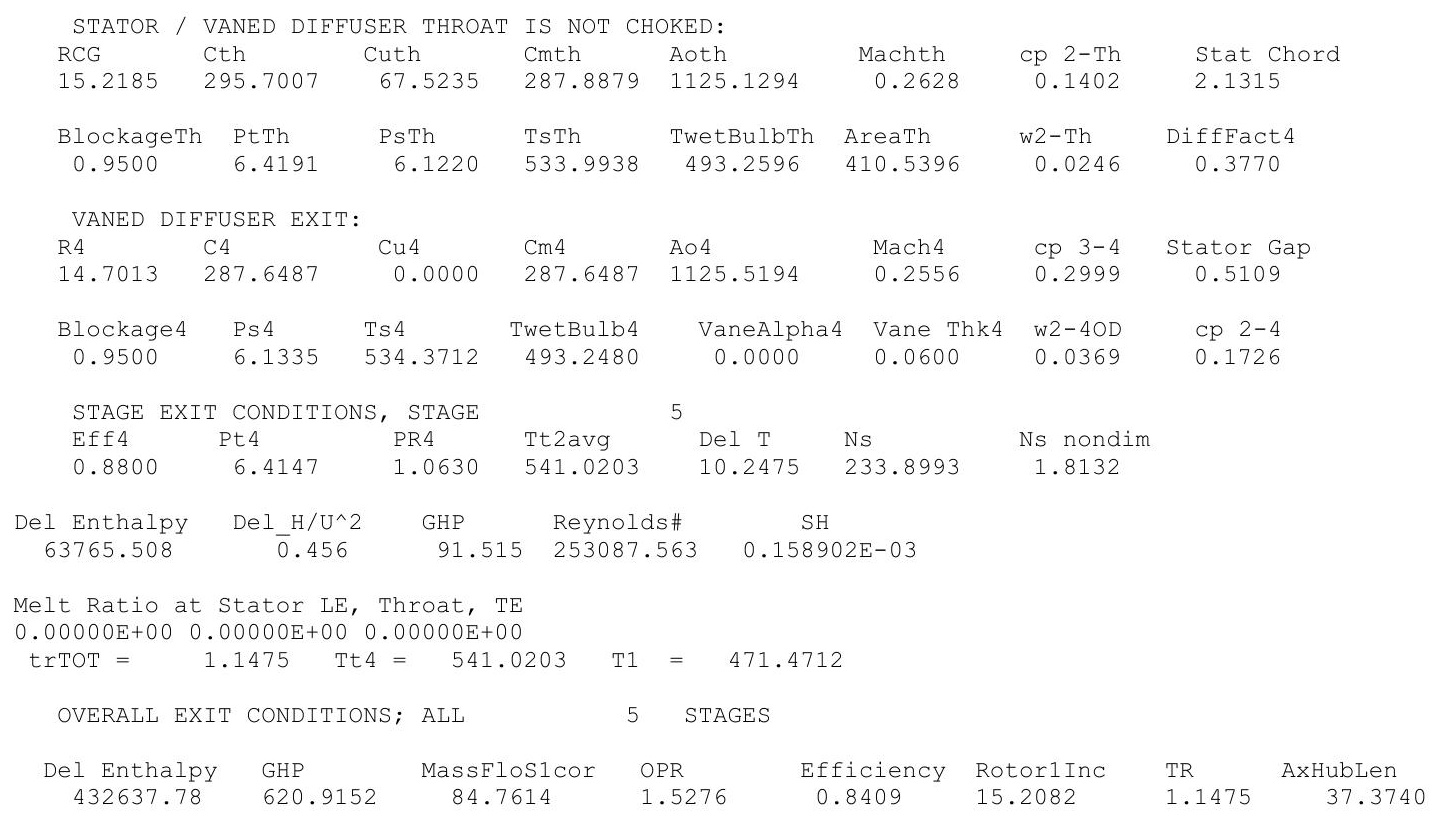




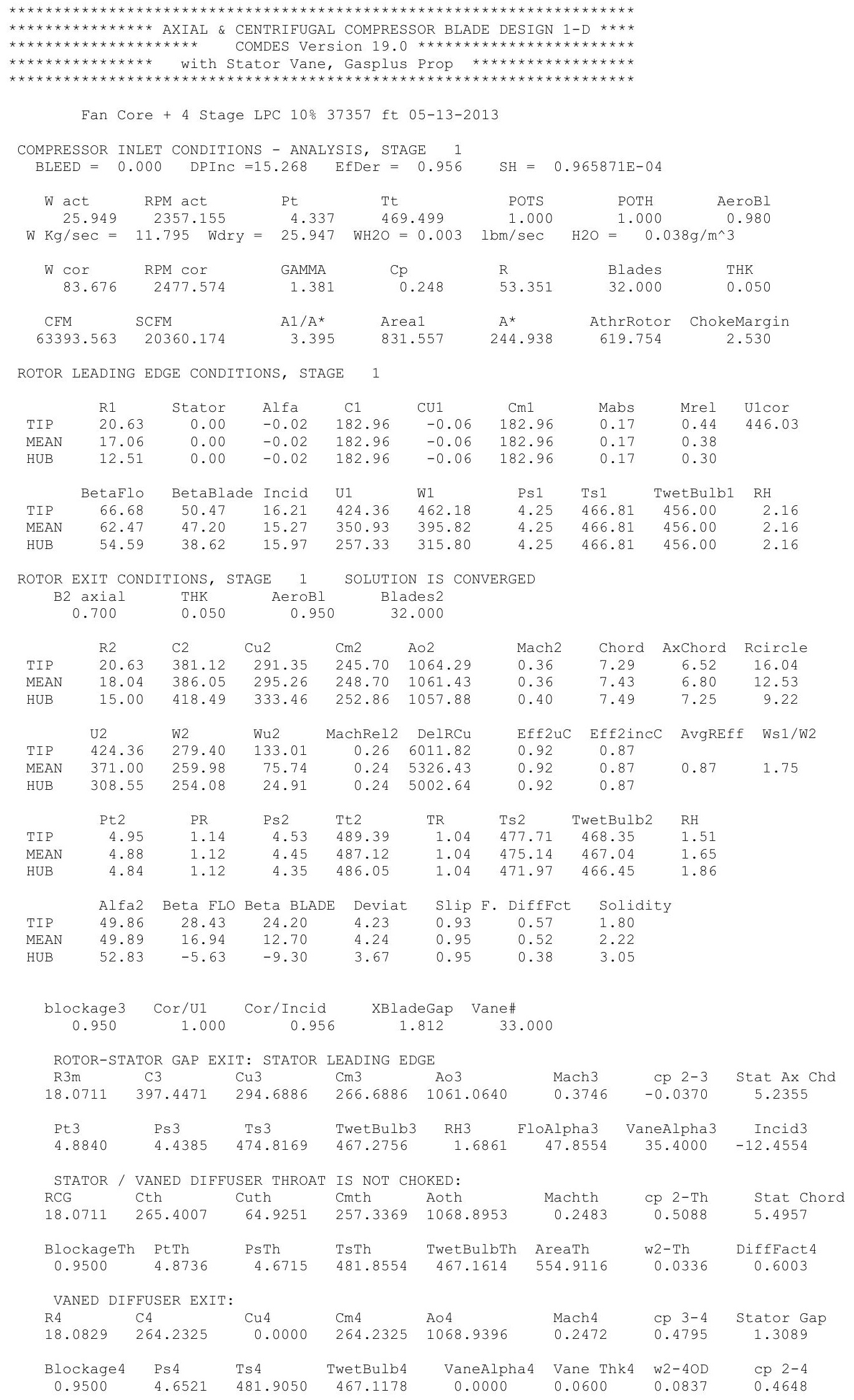




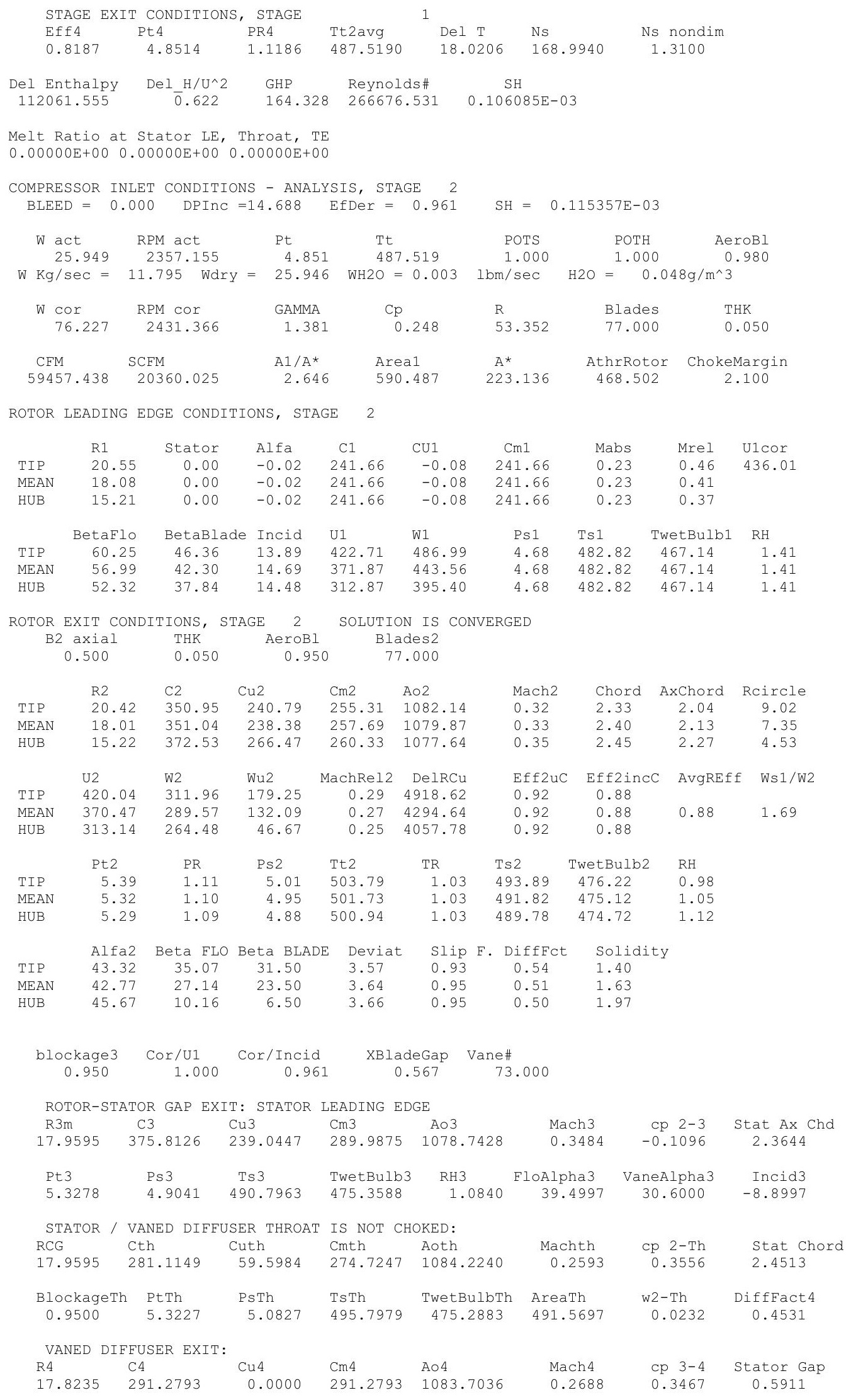




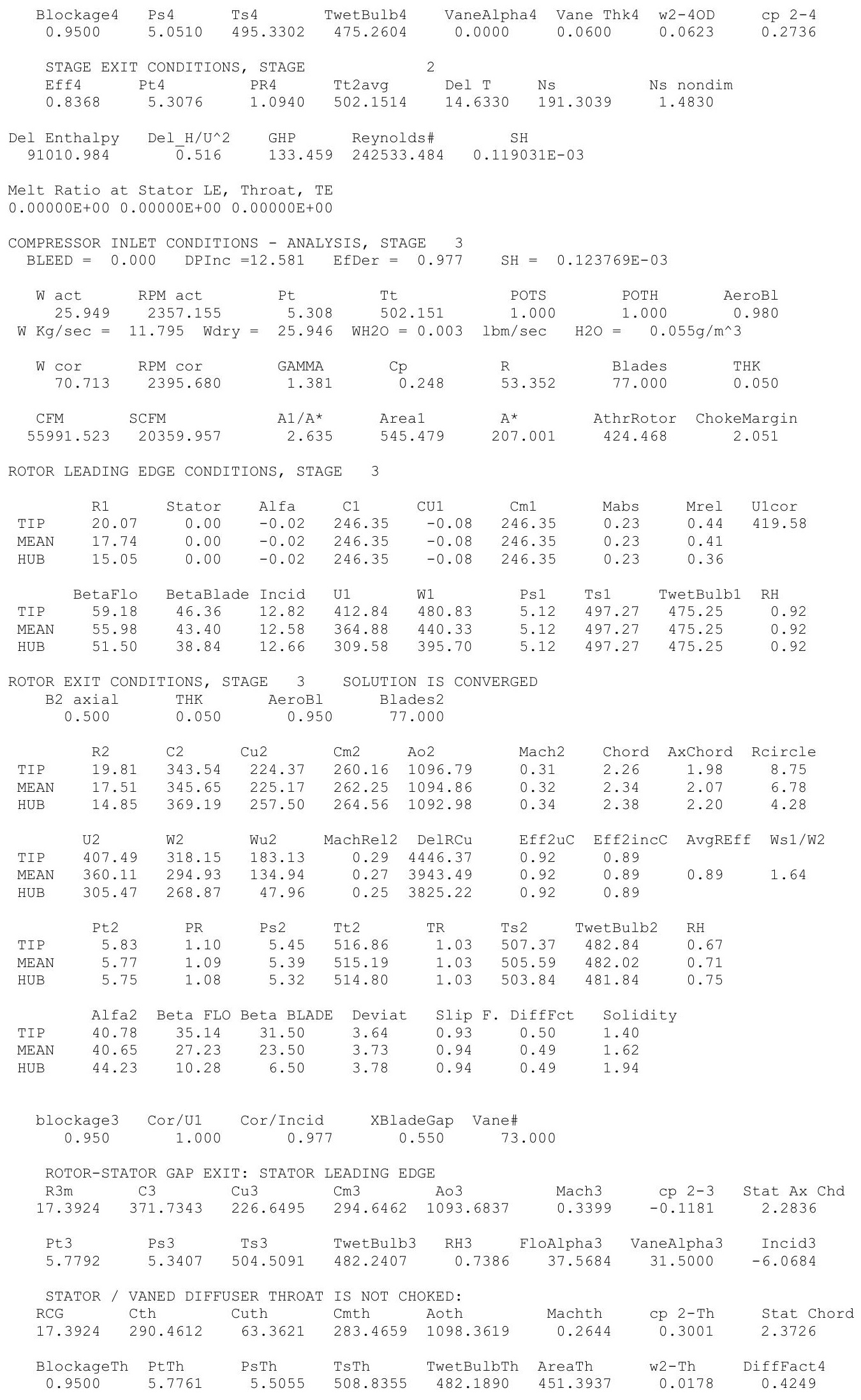




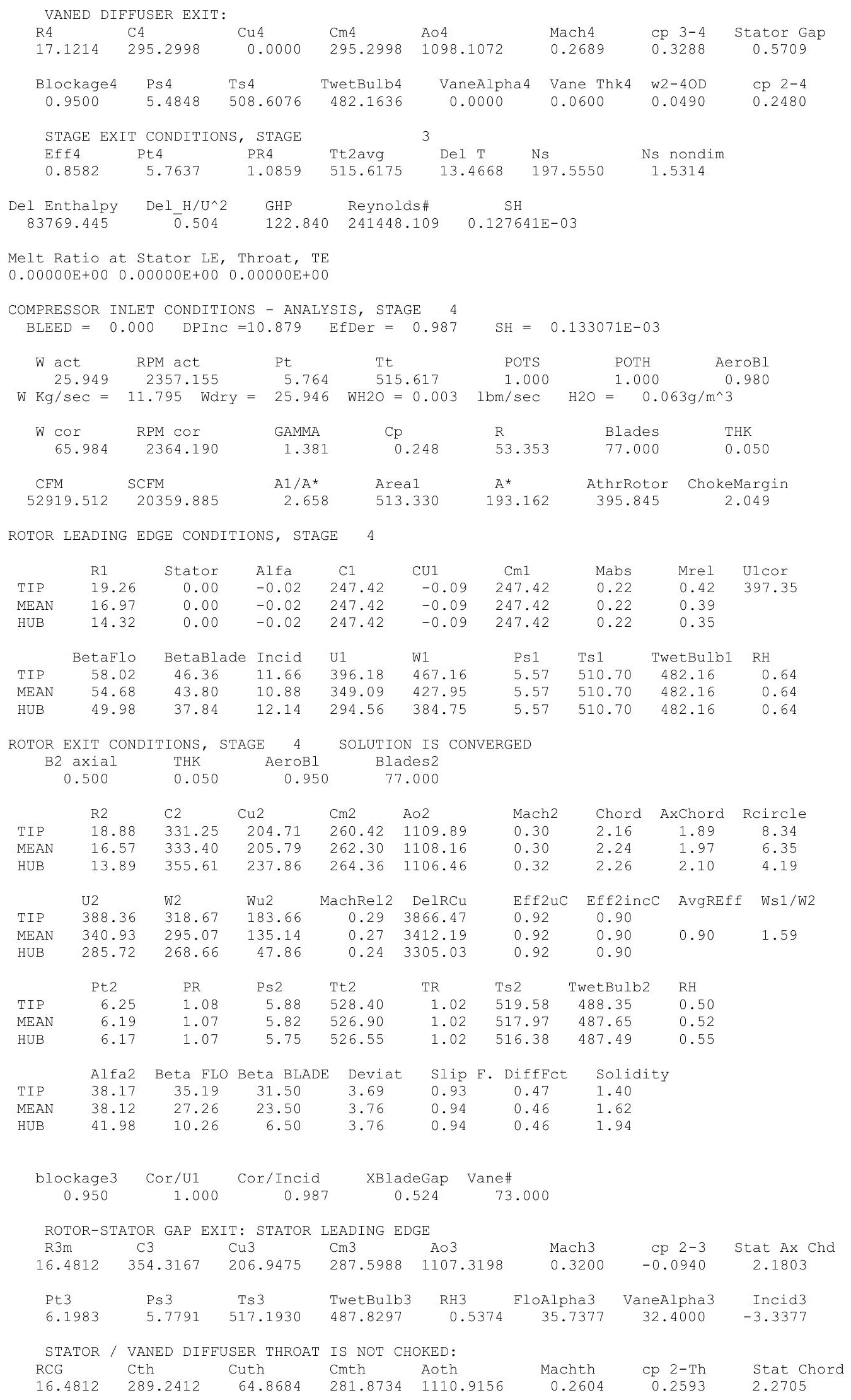




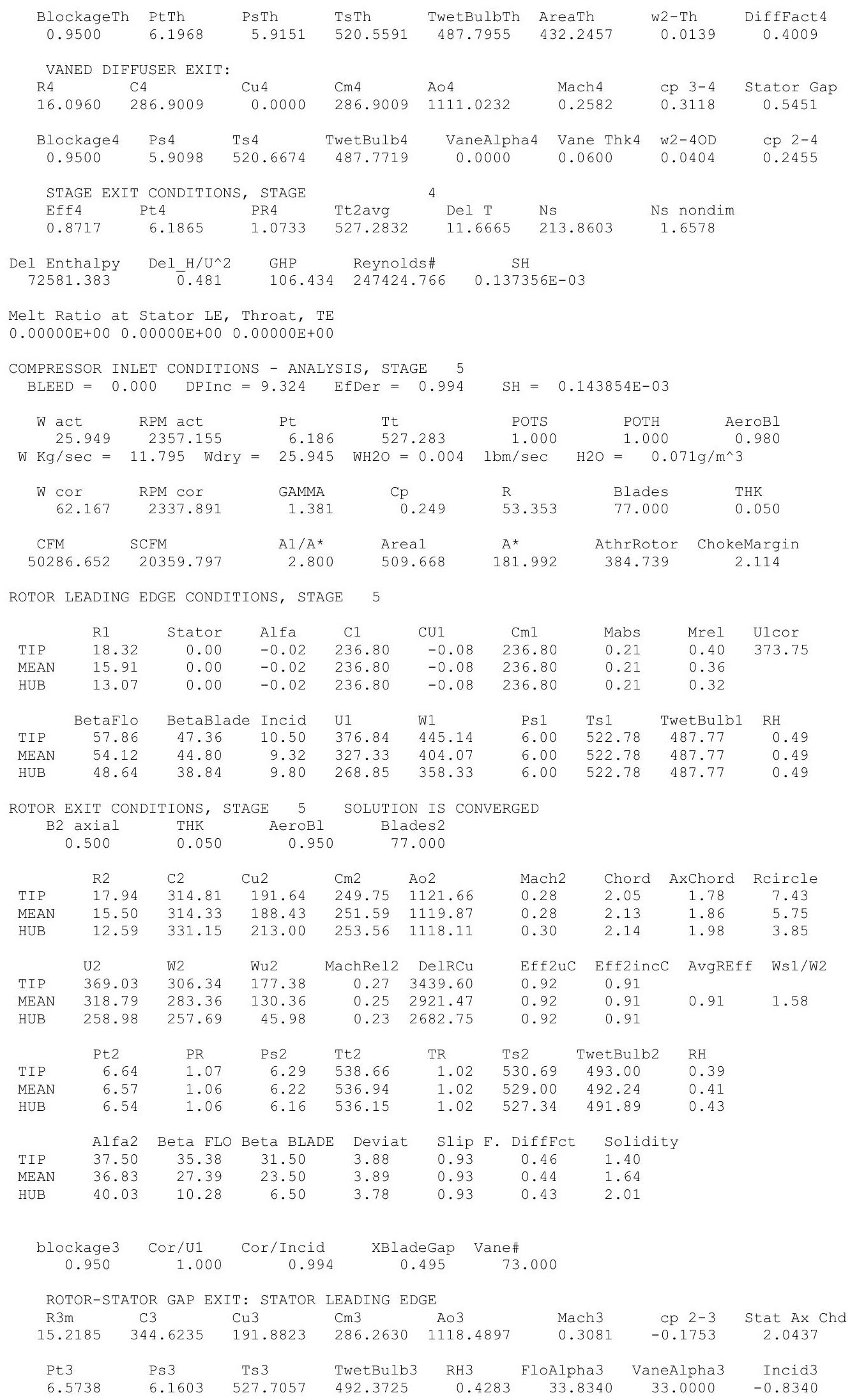




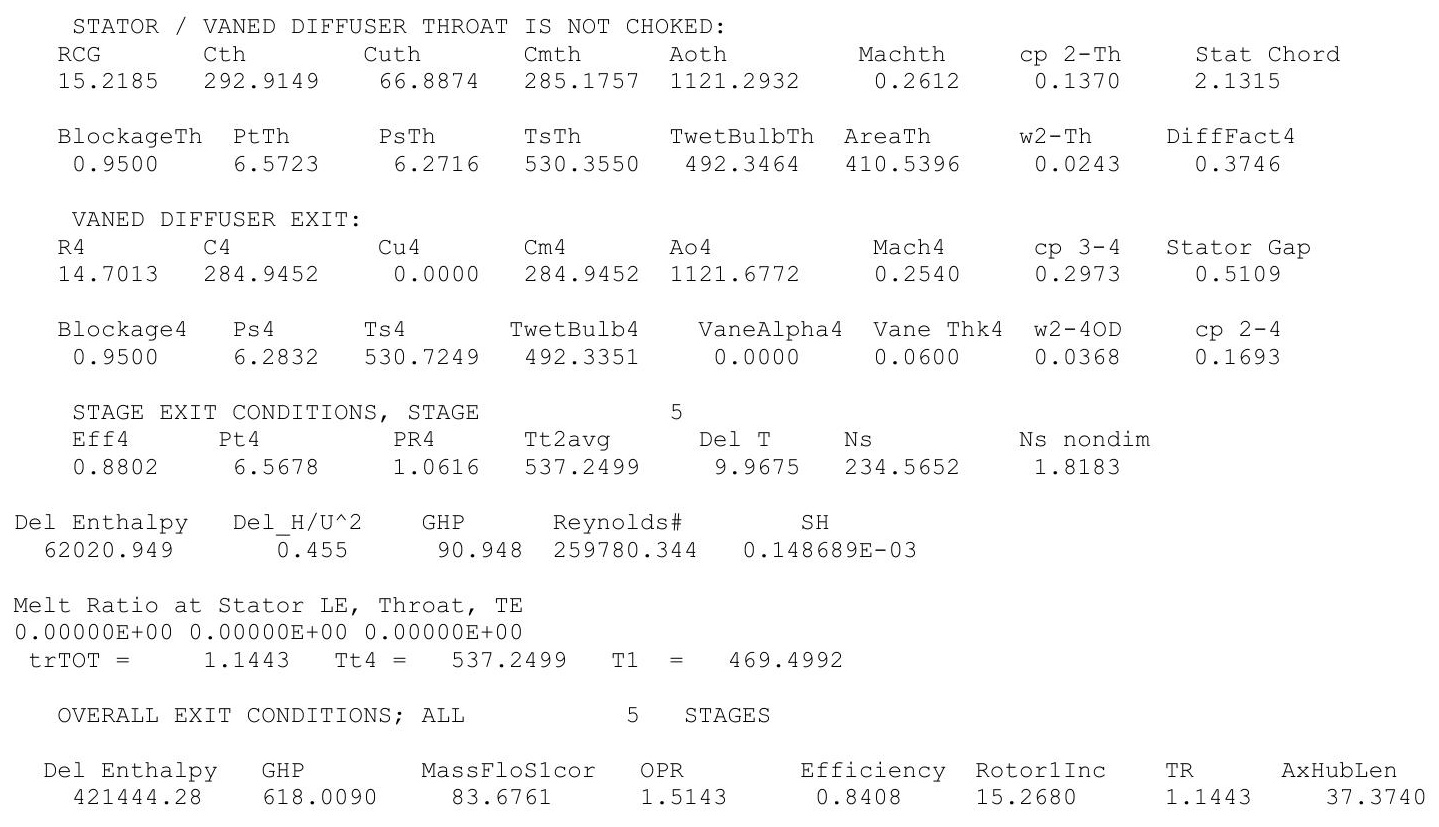




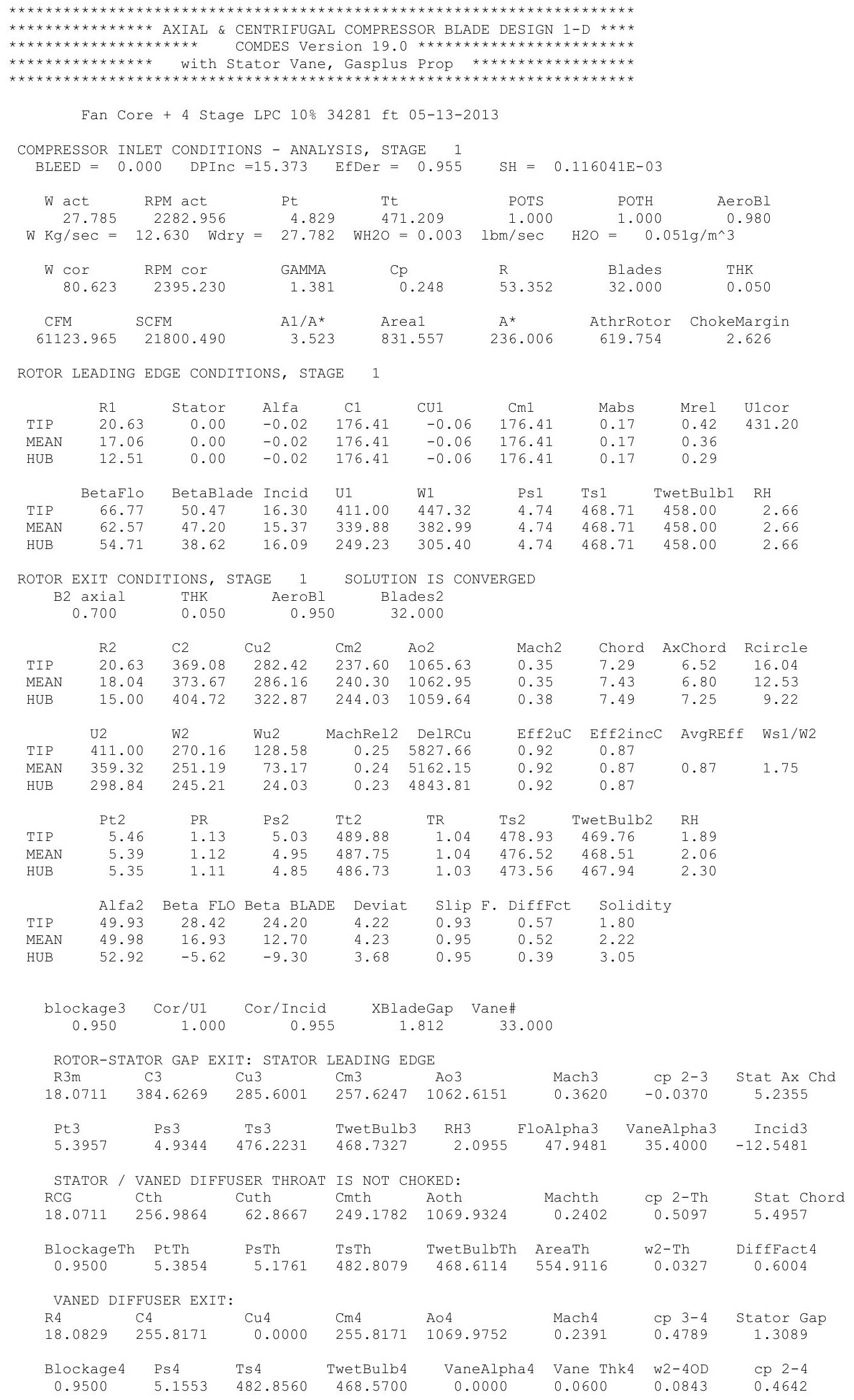




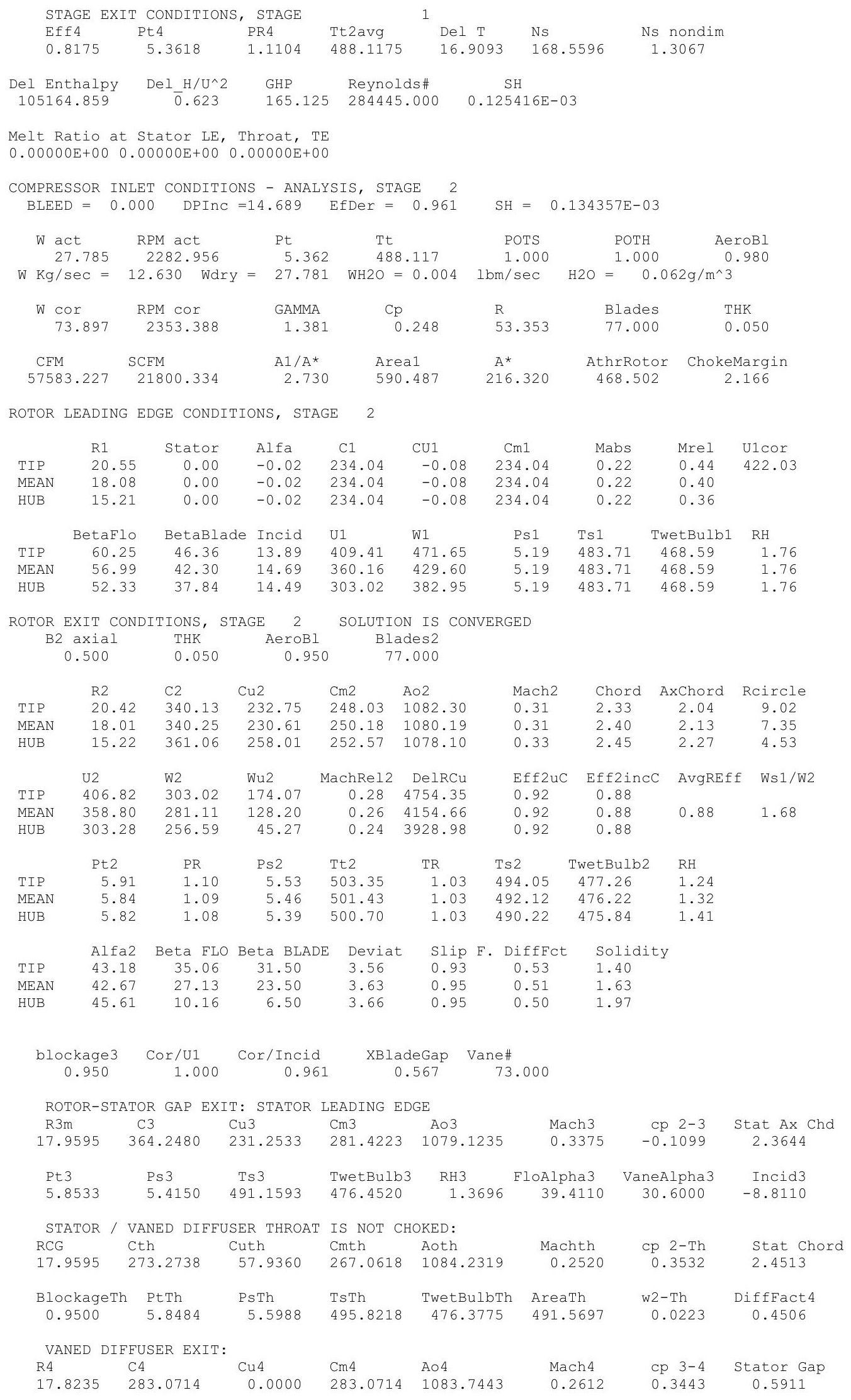




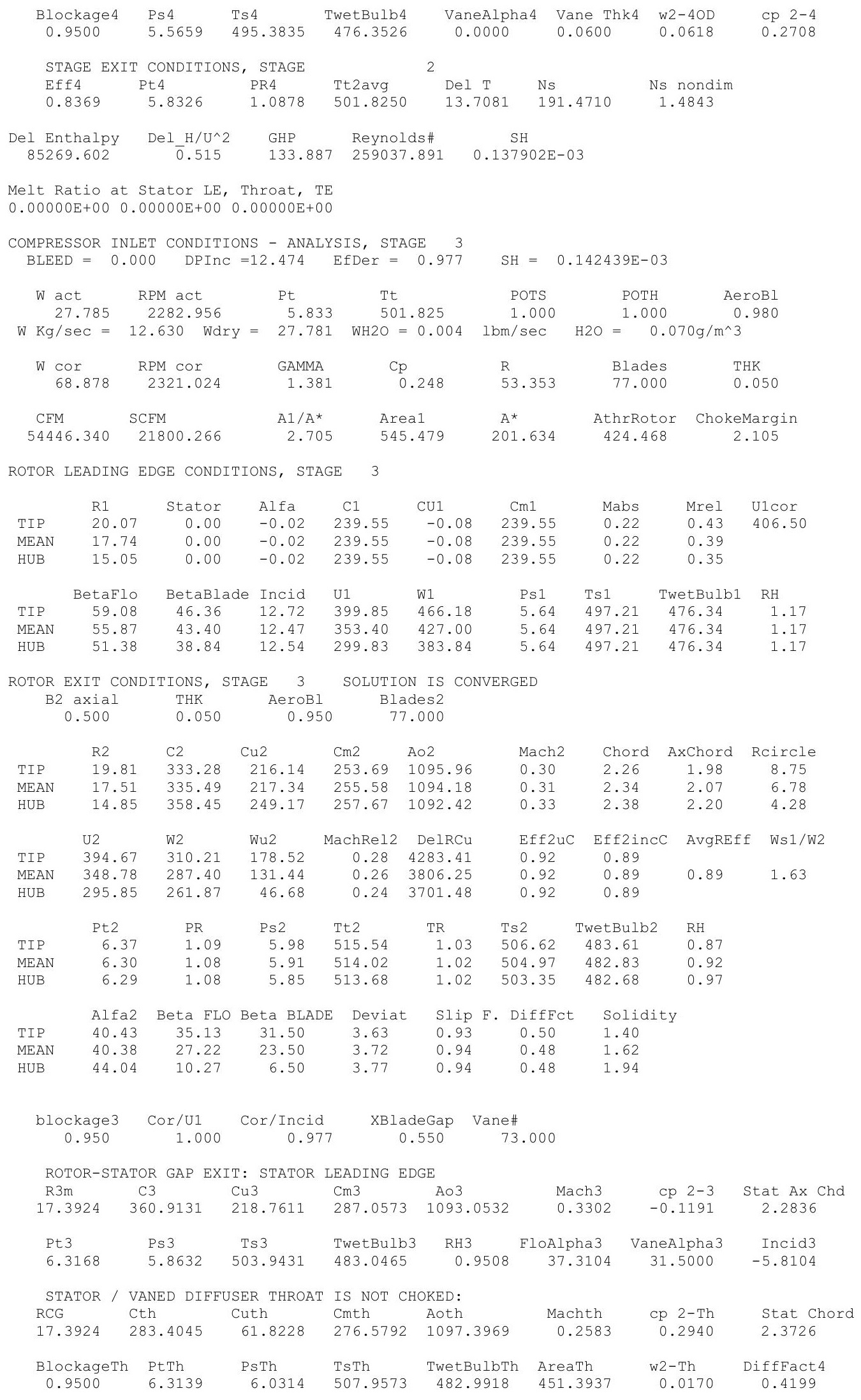




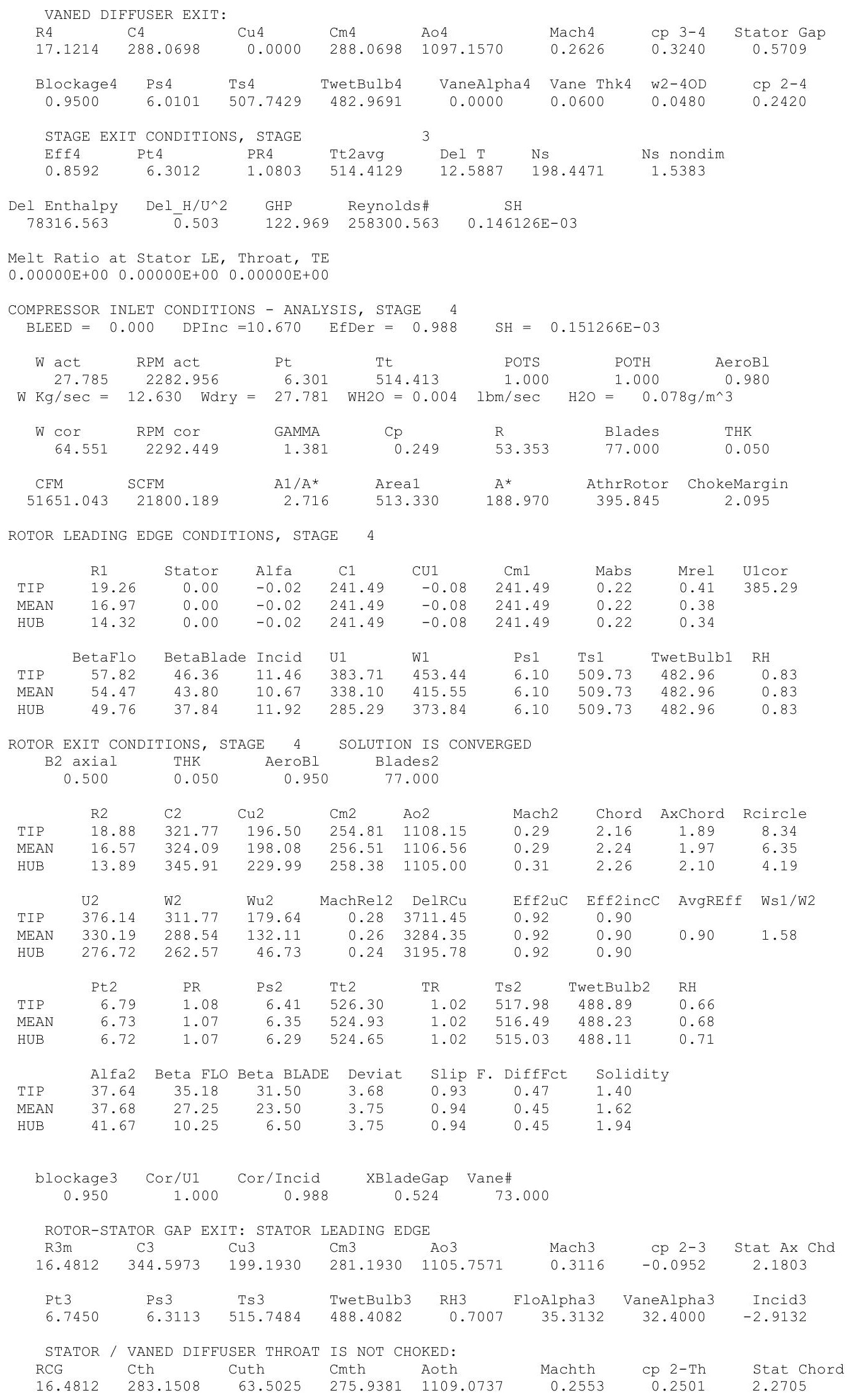




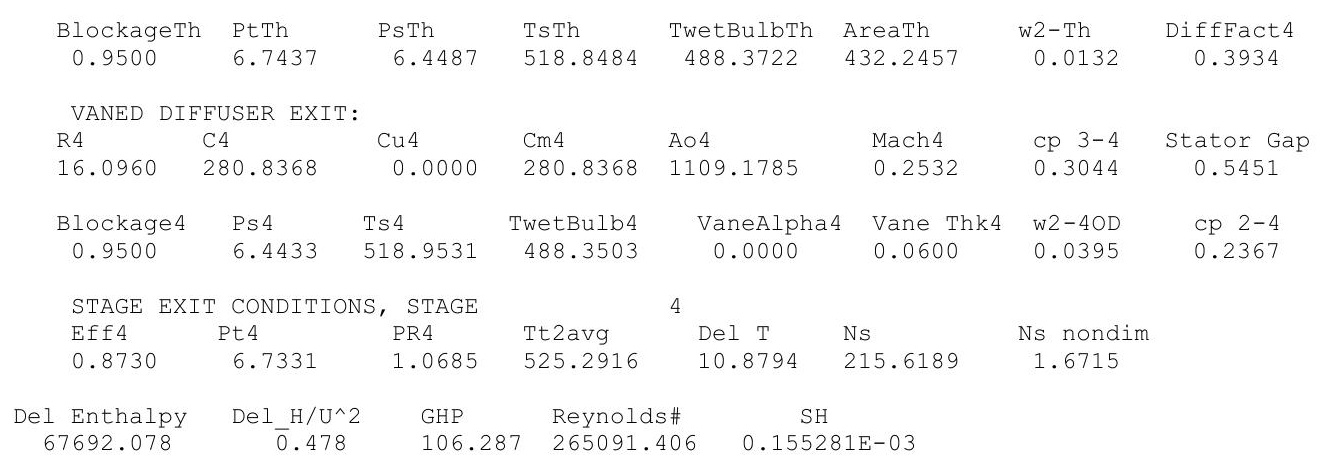

Melt Ratio at Stator LE, Throat, TE

$0.00000 \mathrm{E}+00 \quad 0.00000 \mathrm{E}+00 \quad 0.00000 \mathrm{E}+00$

COMPRESSOR INLET CONDITIONS - ANALYSIS, STAGE 5 BLEED $=0.000 \quad$ DPInC $=9.023 \quad$ EfDer $=0.995$

$\mathrm{SH}=0.161333 \mathrm{E}-03$

$\begin{array}{llllll}\text { W act } & \text { RPM act } & \text { Pt } & \text { POTS } & \text { POTH } & \text { AeroBl }\end{array}$ $\mathrm{W} \mathrm{Kg} / \mathrm{sec}=12.630$ Wdry $=27.781 \quad$ WH2O $=0.004 \quad 1 \mathrm{bm} / \mathrm{sec} \quad \mathrm{H} 20=\begin{gathered}1.000 \\ 0.088 \mathrm{~g} / \mathrm{m}^{\wedge} 3\end{gathered}$

$\begin{array}{ccccccc}\text { W Cor } & \text { RPM cor } & \text { GAMMA } & \text { CP } & \text { R } & \text { Blades } & \text { THK } \\ 61.045 & 2268.587 & 1.381 & 0.249 & 53.354 & 77.000 & 0.050 \\ & & & & & & \\ \text { CFM } & \text { SCFM } & \text { A1/A* } & \text { Areal } & A^{*} & \text { AthrRotor } & \text { ChokeMargin } \\ 49244.773 & 21800.104 & 2.852 & 509.668 & 178.711 & 384.739 & 2.153\end{array}$

ROTOR LEADING EDGE CONDITIONS, STAGE 5

$\begin{array}{lccccccccc} & \text { R1 } & \text { Stator } & \text { Alfa } & \text { C1 } & \text { CU1 } & \text { Cm1 } & \text { Mabs } & \text { Mrel } & \text { U1cor } \\ \text { TIP } & 18.32 & 0.00 & -0.02 & 231.89 & -0.08 & 231.89 & 0.21 & 0.39 & 362.67 \\ \text { MEAN } & 15.91 & 0.00 & -0.02 & 231.89 & -0.08 & 231.89 & 0.21 & 0.35 & \\ \text { HUB } & 13.07 & 0.00 & -0.02 & 231.89 & -0.08 & 231.89 & 0.21 & 0.31 & \\ & & & & & & & & & \\ \text { BIP } & \text { BetaFlo } & \text { BetaBlade } & \text { Incid } & \text { U1 } & \text { W1 } & \text { Ps } 1 & \text { Ts1 } & \text { TwetBulb1 } & \text { RH } \\ \text { MEAN } & 57.58 & 47.36 & 10.22 & 364.98 & 432.48 & 6.53 & 520.97 & 488.34 & 0.63 \\ \text { HUB } & 48.32 & 44.80 & 9.02 & 317.03 & 392.85 & 6.53 & 520.97 & 488.34 & 0.63 \\ & & 38.84 & 9.48 & 260.39 & 348.74 & 6.53 & 520.97 & 488.34 & 0.63\end{array}$

ROTOR EXIT CONDITIONS, STAGE 5 SOLUTION IS CONVERGED

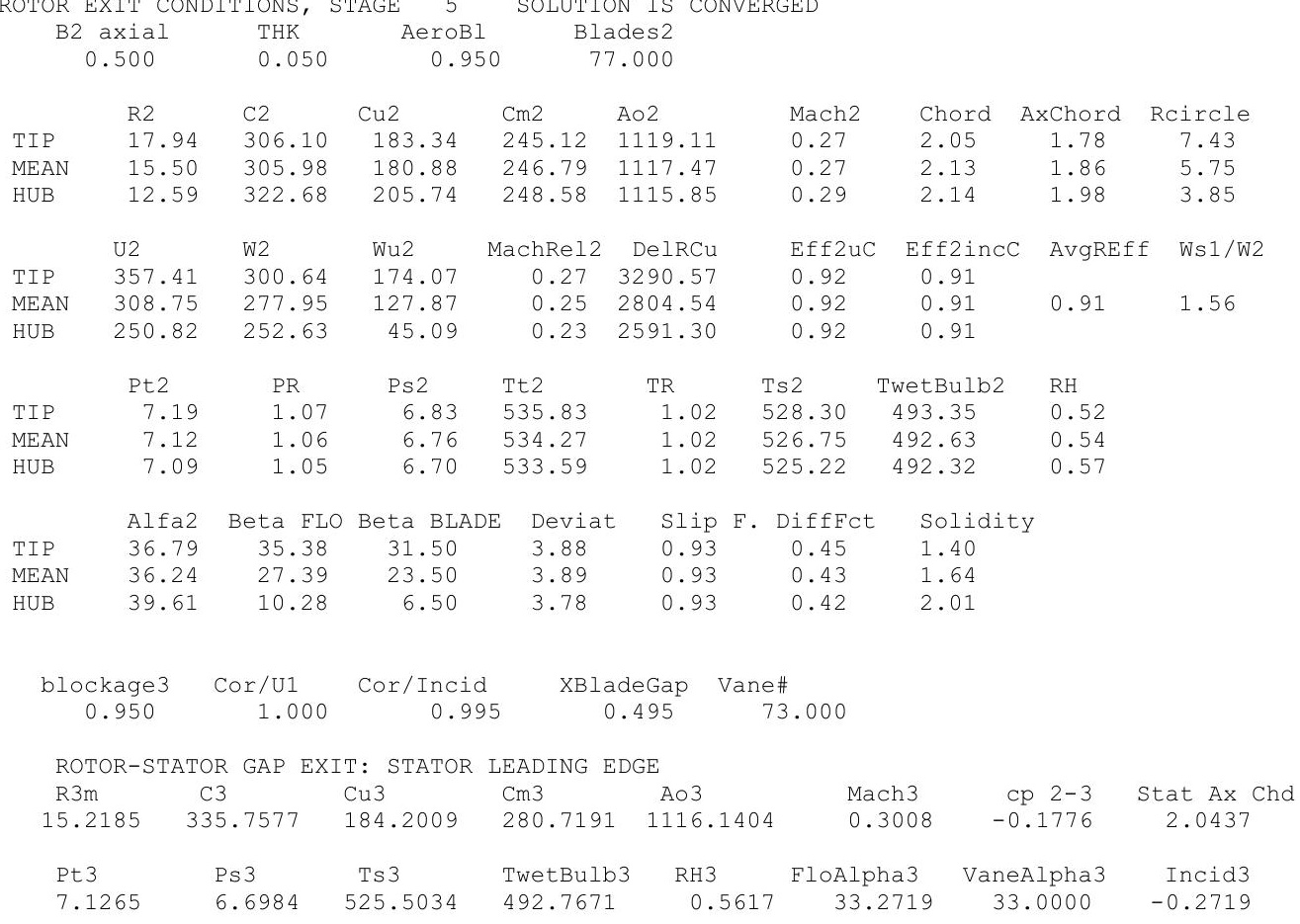




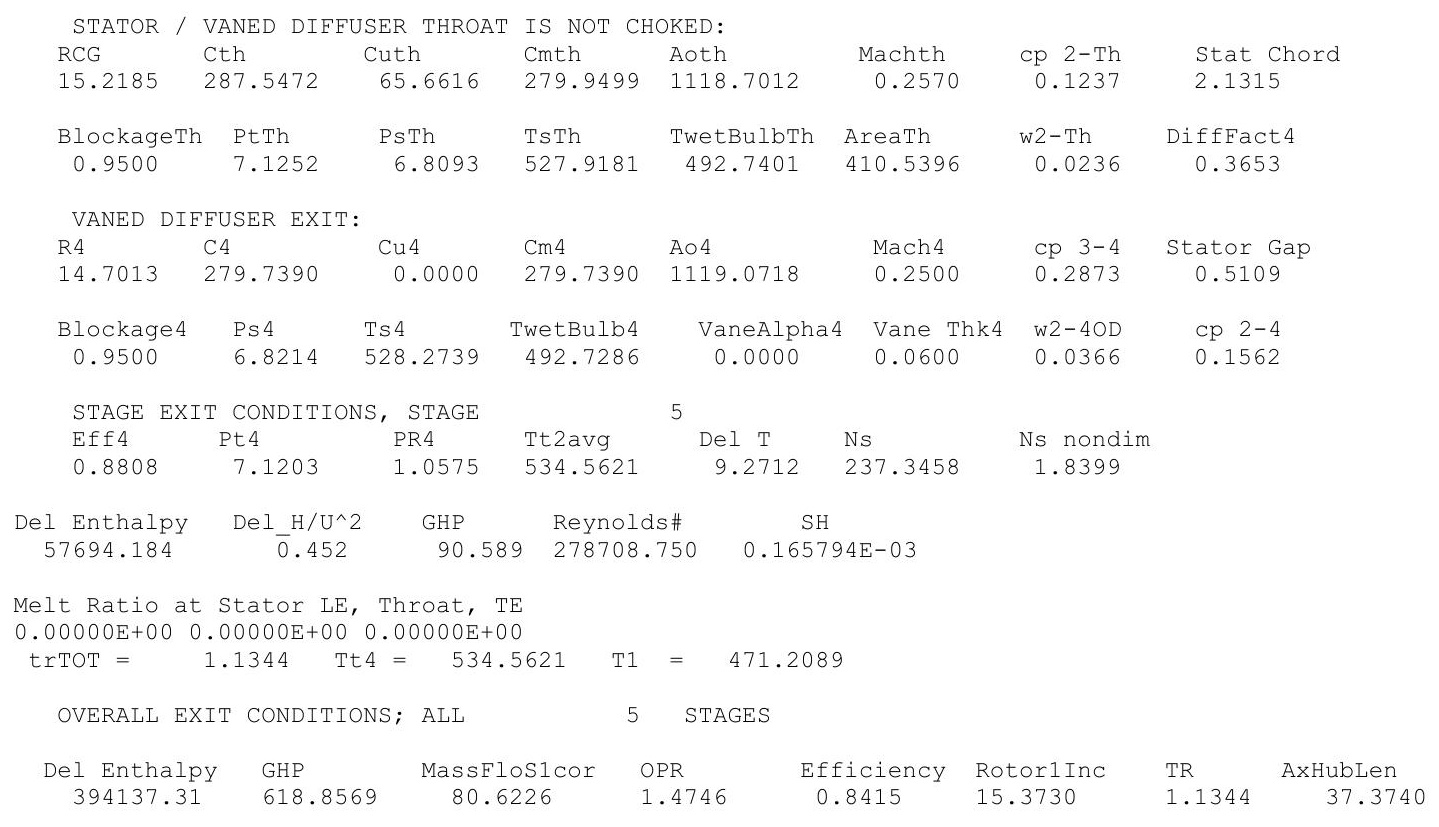




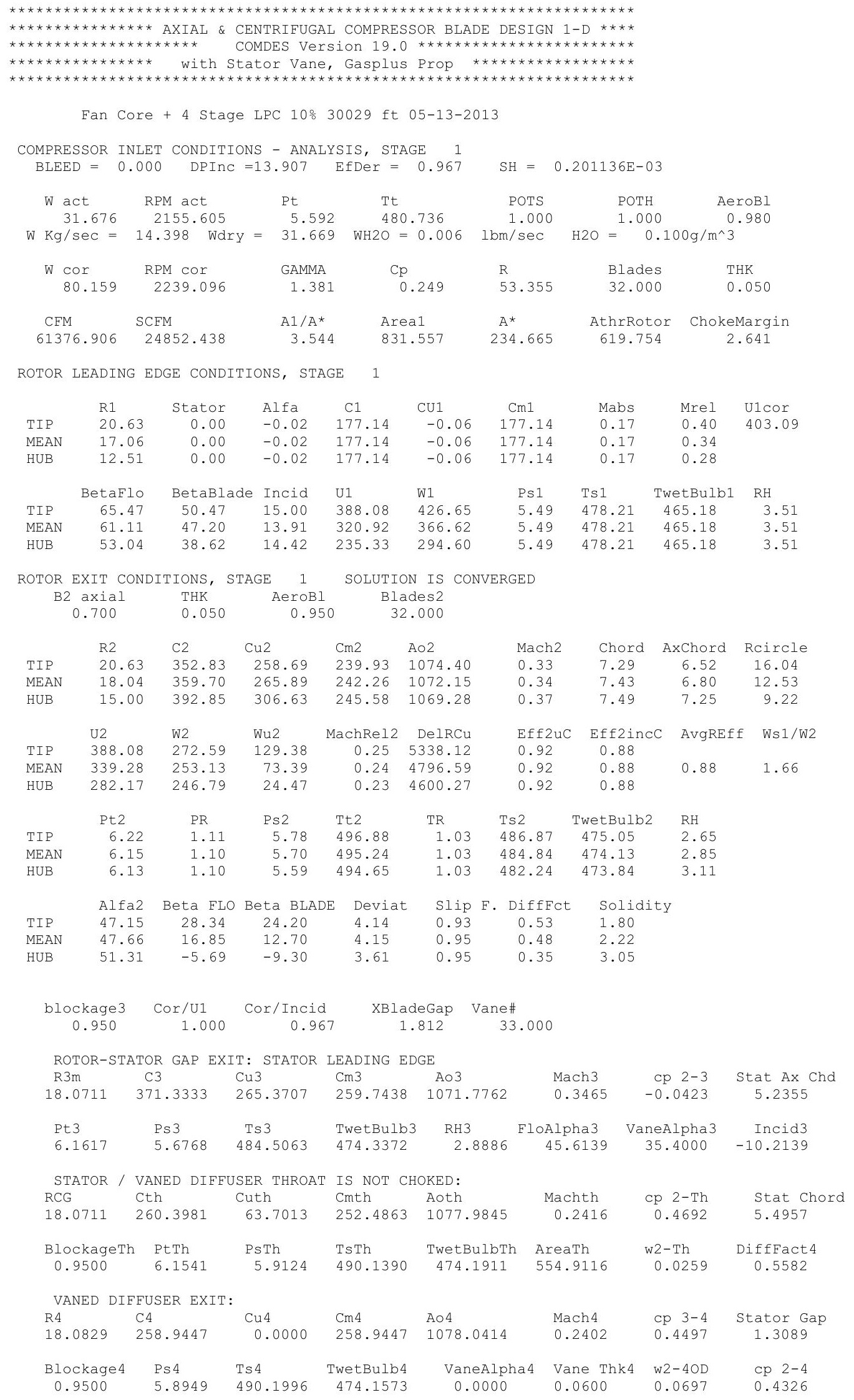




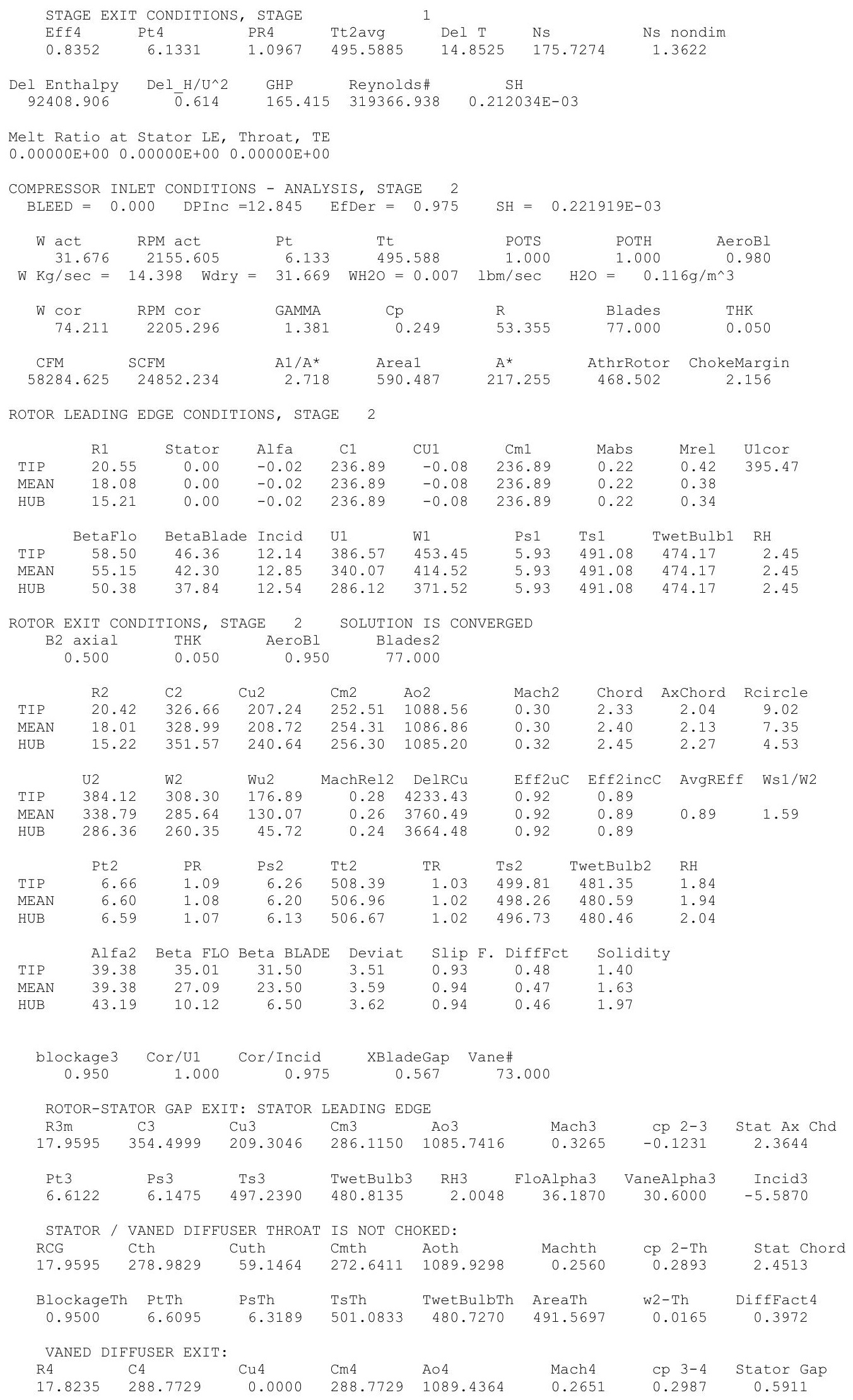




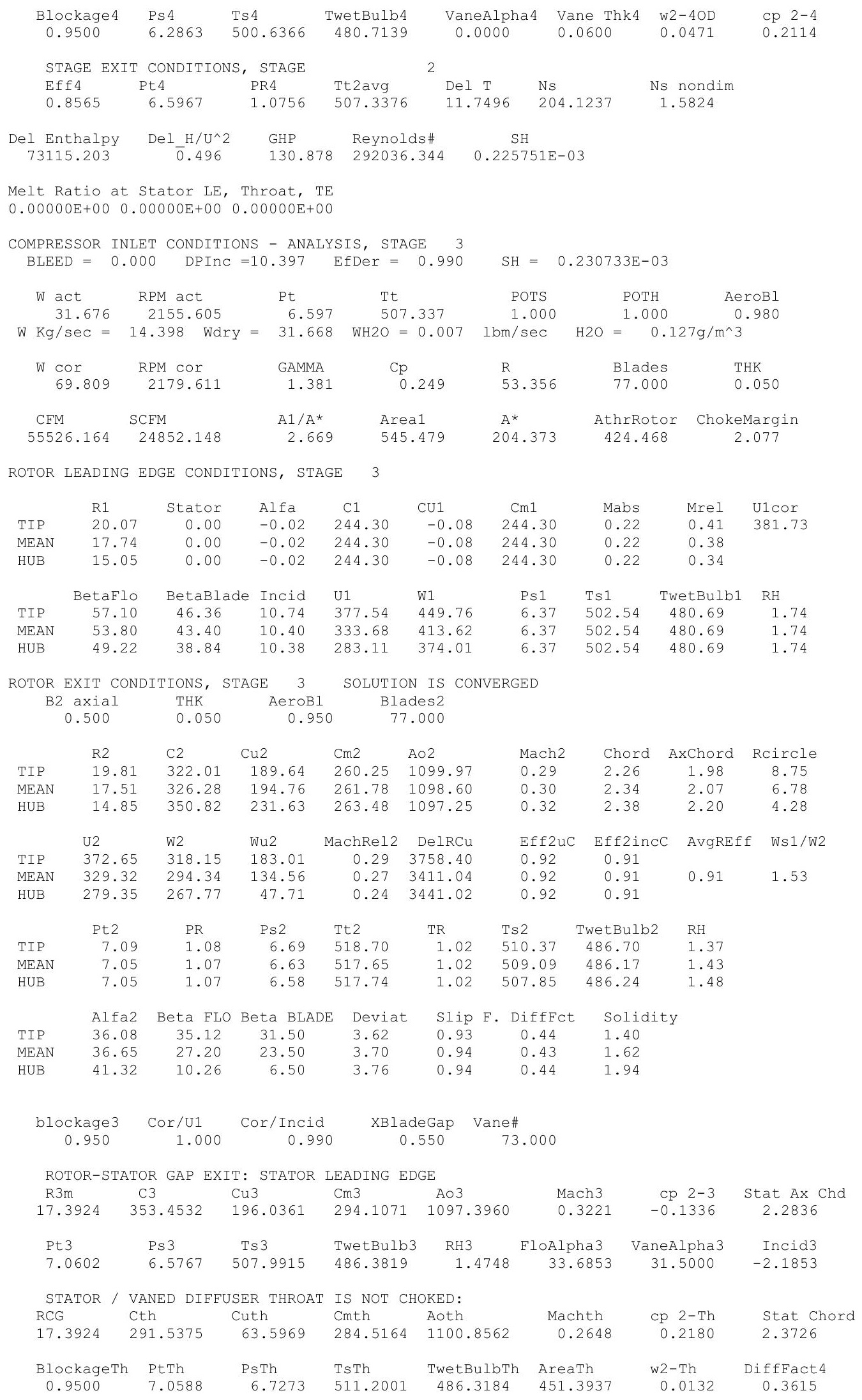




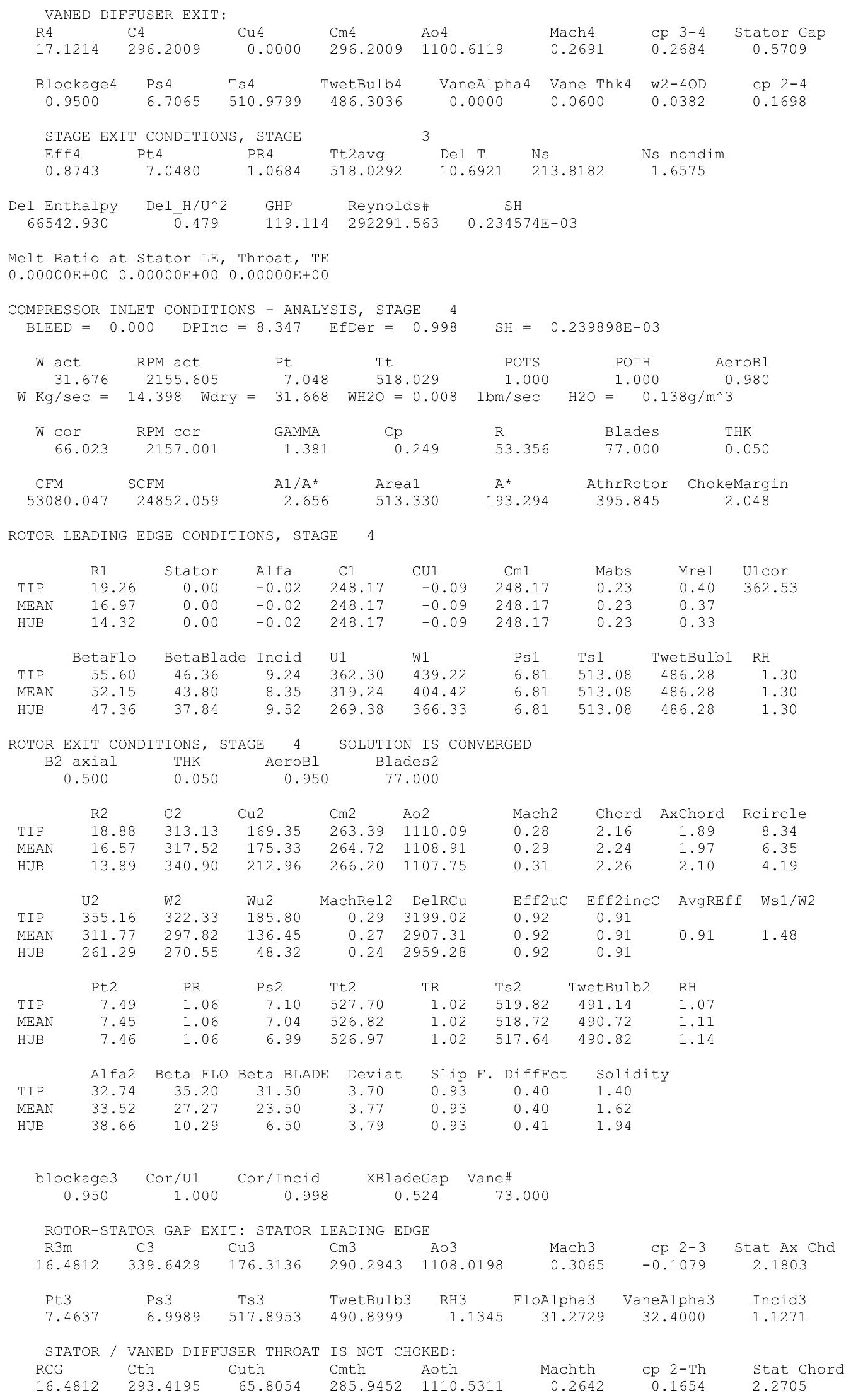




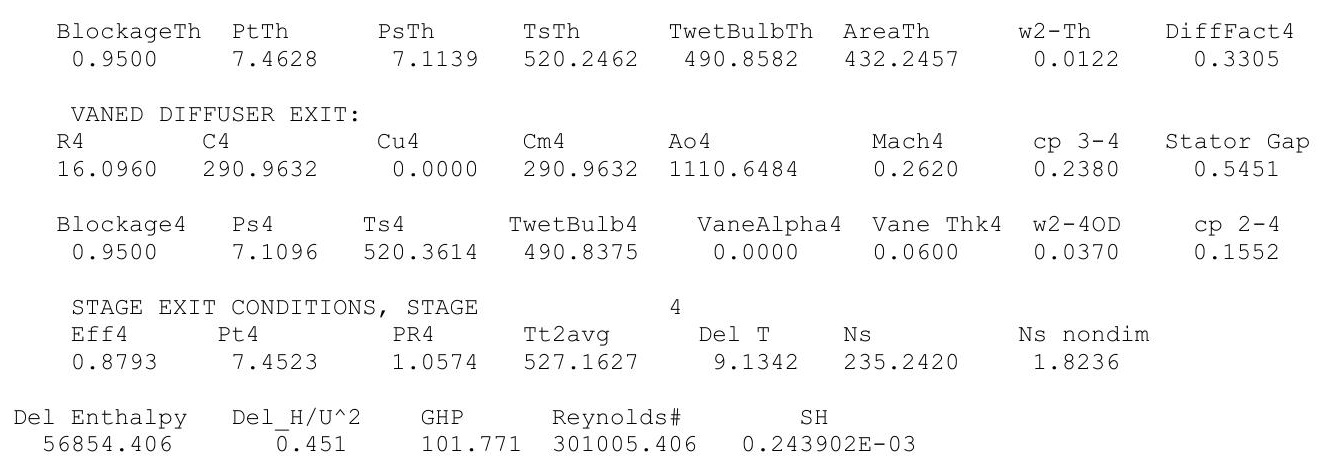

Melt Ratio at Stator LE, Throat, TE

$0.00000 \mathrm{E}+000.00000 \mathrm{E}+000.00000 \mathrm{E}+00$

COMPRESSOR INLET CONDITIONS - ANALYSIS, STAGE 5 BLEED $=0.000 \quad$ DPInC $=6.478 \quad$ EfDer $=1.000$

$\begin{array}{llllll}W & \text { Ret } & \text { Pt } & \text { POTS } & \text { POTH } & \text { AeroBI }\end{array}$

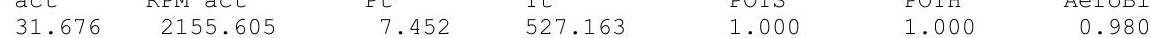

$\mathrm{W} \mathrm{Kg} / \mathrm{sec}=14.398 \mathrm{WdrY}=31.668 \mathrm{WH} 2 \mathrm{O}=0.008 \mathrm{lbm} / \mathrm{sec} \quad \mathrm{H} 2 \mathrm{O}=0.150 \mathrm{~g} / \mathrm{m}^{\wedge} 3$

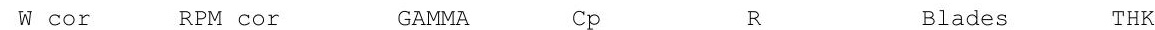

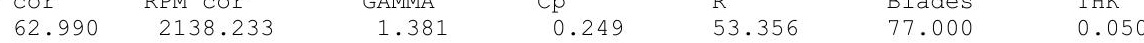

$\begin{array}{clccc}\text { CFM } & \text { SCFM } & \text { A1/A* A } & \text { Area1 } & \text { A* AthrRotor ChokeMargin }\end{array}$

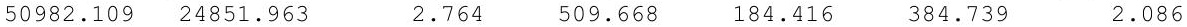

ROTOR LEADING EDGE CONDITIONS, STAGE 5

$\begin{array}{lccccccccc} & \text { R1 } & \text { Stator } & \text { Alfa } & \text { C1 } & \text { CU1 } & \text { Cm1 } & \text { Mabs } & \text { Mrel } & \text { U1cor } \\ \text { TIP } & 18.32 & 0.00 & -0.02 & 240.07 & -0.08 & 240.07 & 0.22 & 0.38 & 341.83 \\ \text { MEAN } & 15.91 & 0.00 & -0.02 & 240.07 & -0.08 & 240.07 & 0.22 & 0.34 & \\ \text { HUB } & 13.07 & 0.00 & -0.02 & 240.07 & -0.08 & 240.07 & 0.22 & 0.31 & \\ & & & & & & & & & \\ & & & & & & & & & \\ \text { TIP } & 55.14 & 47.36 & 7.78 & 344.62 & 420.07 & 7.22 & 522.53 & 490.82 & 1.02 \\ \text { MEAN } & 51.28 & 44.80 & 6.48 & 299.34 & 383.78 & 7.22 & 522.53 & 490.82 & 1.02 \\ \text { HUB } & 45.69 & 38.84 & 6.85 & 245.86 & 343.69 & 7.22 & 522.53 & 490.82 & 1.02\end{array}$

ROTOR EXIT CONDITIONS, STAGE 5 SOLUTION IS CONVERGED

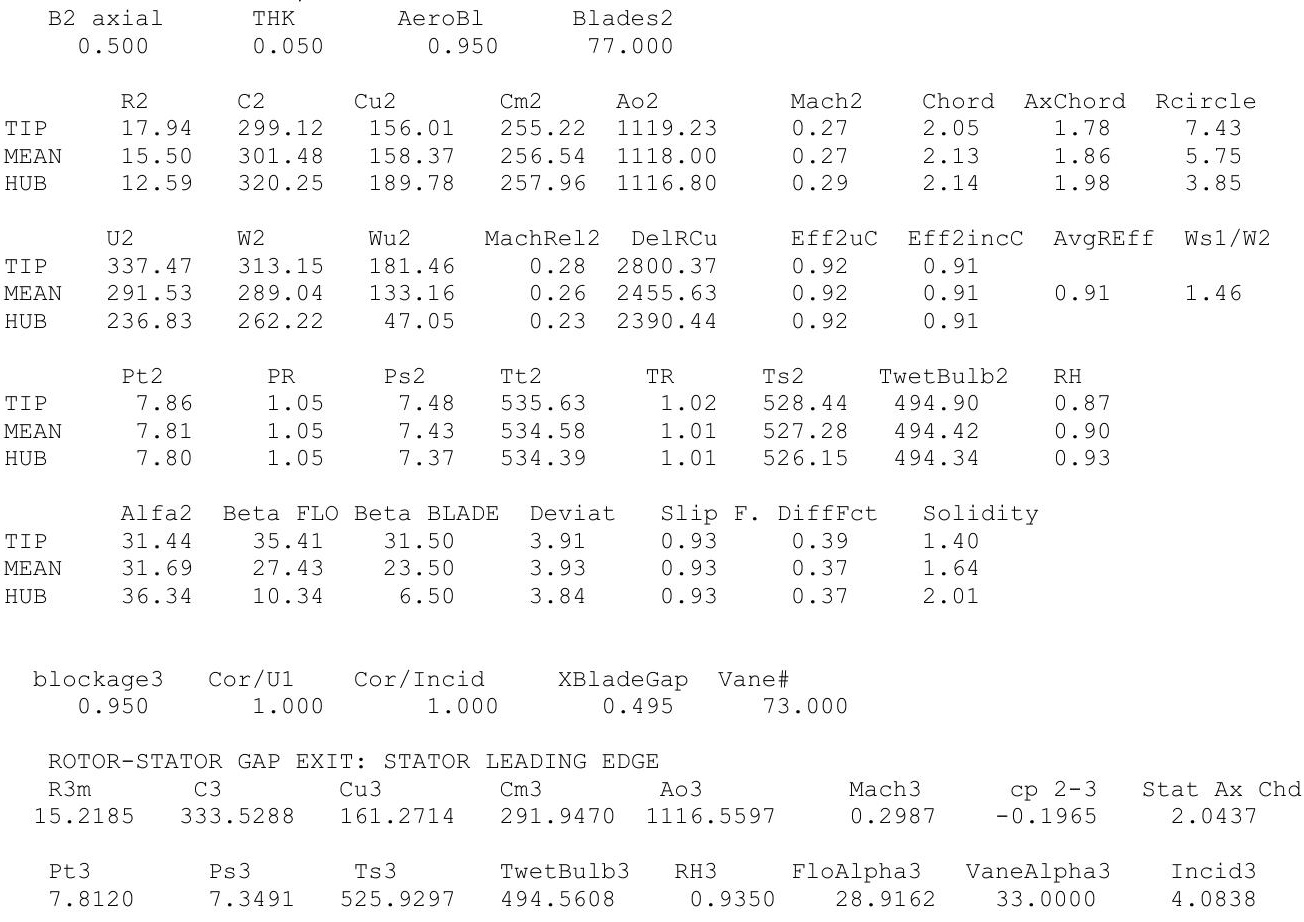




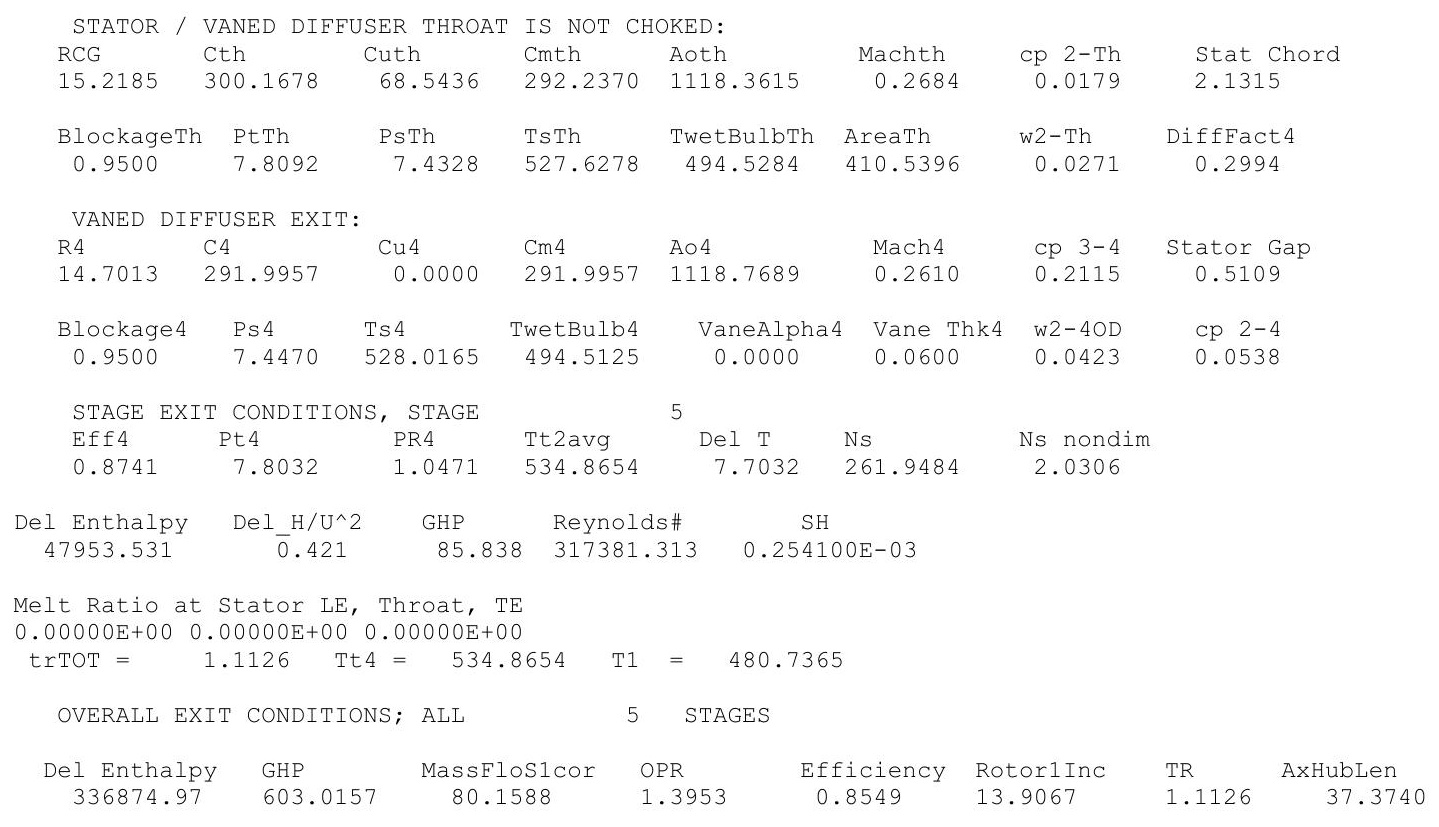




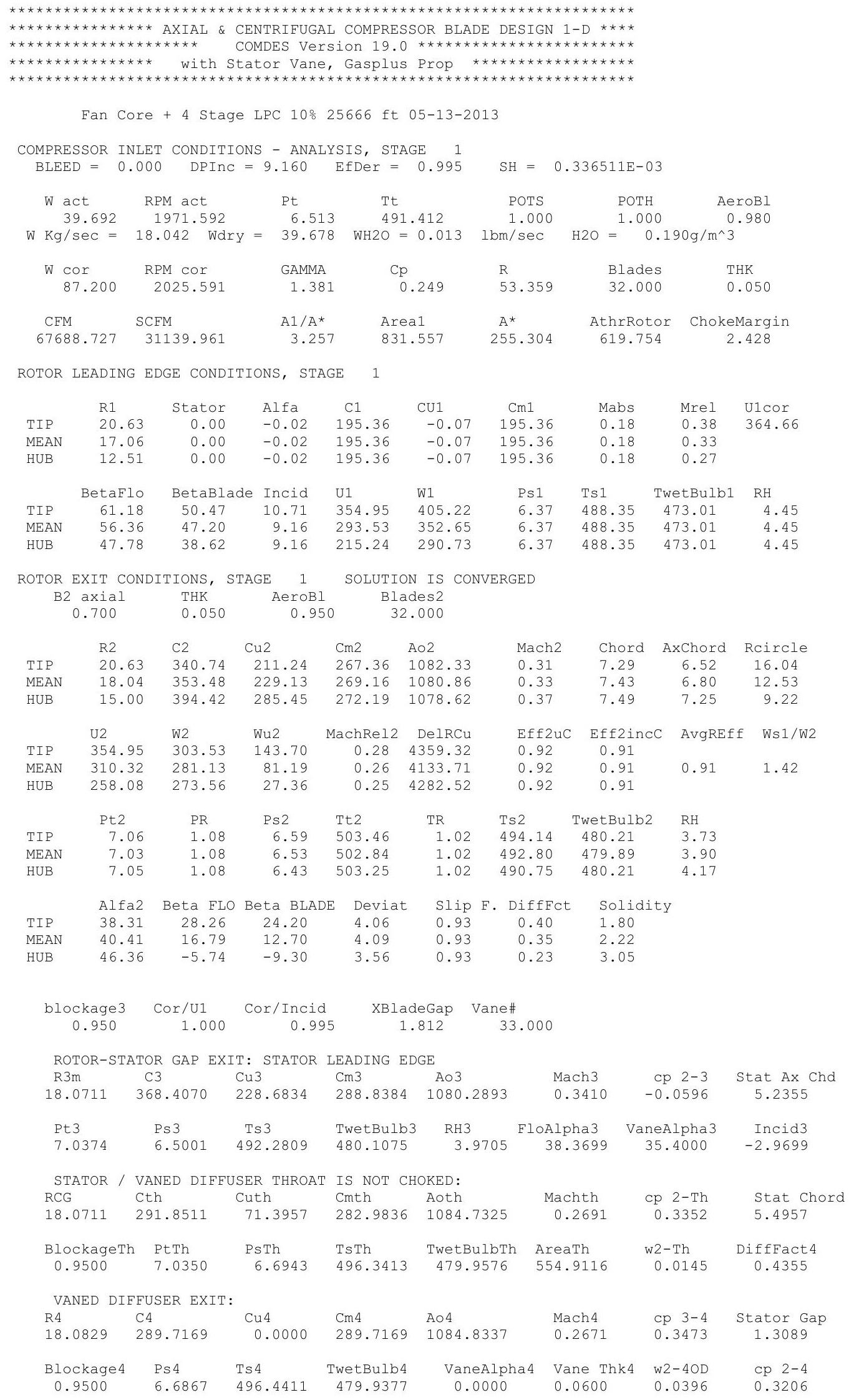




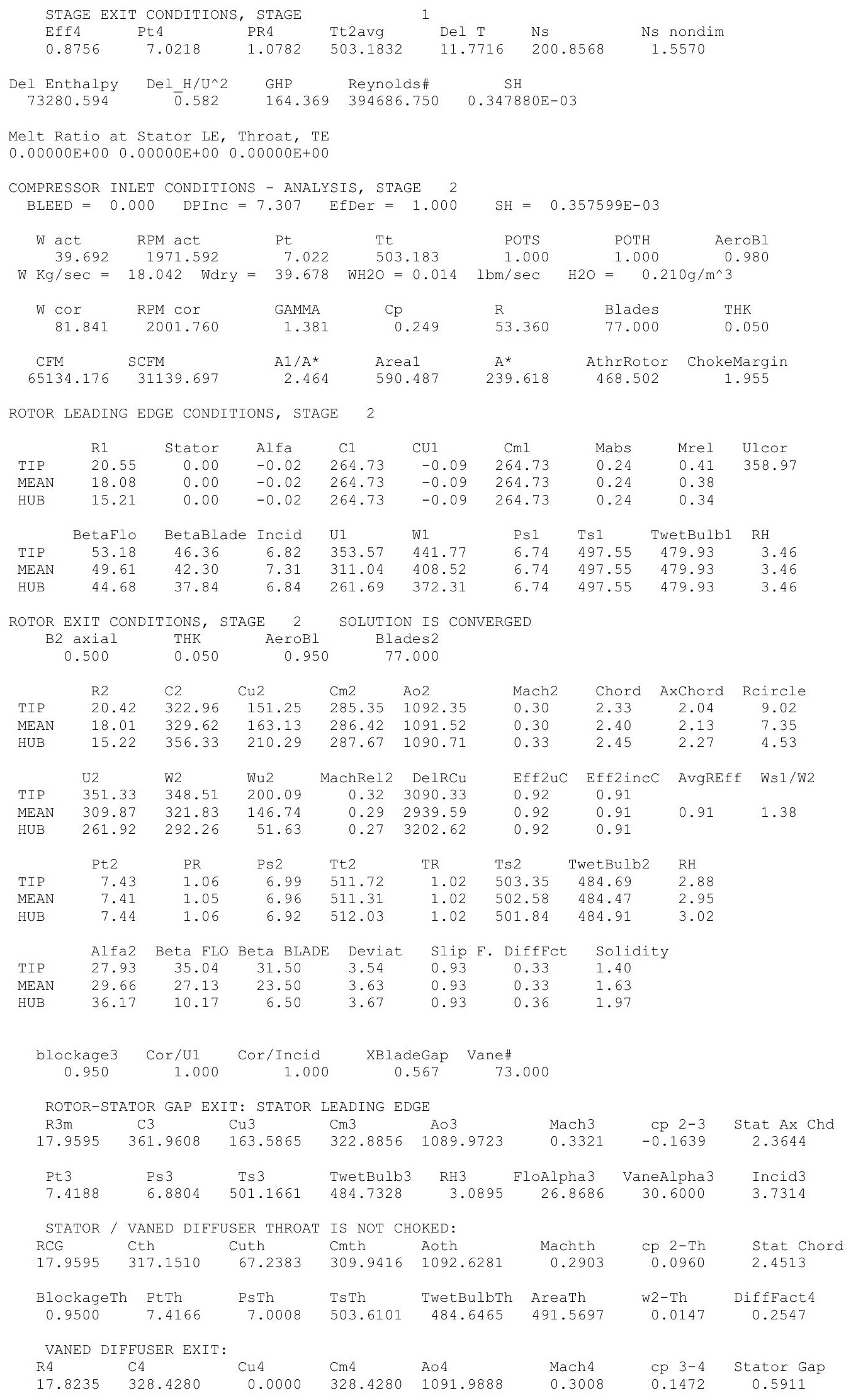




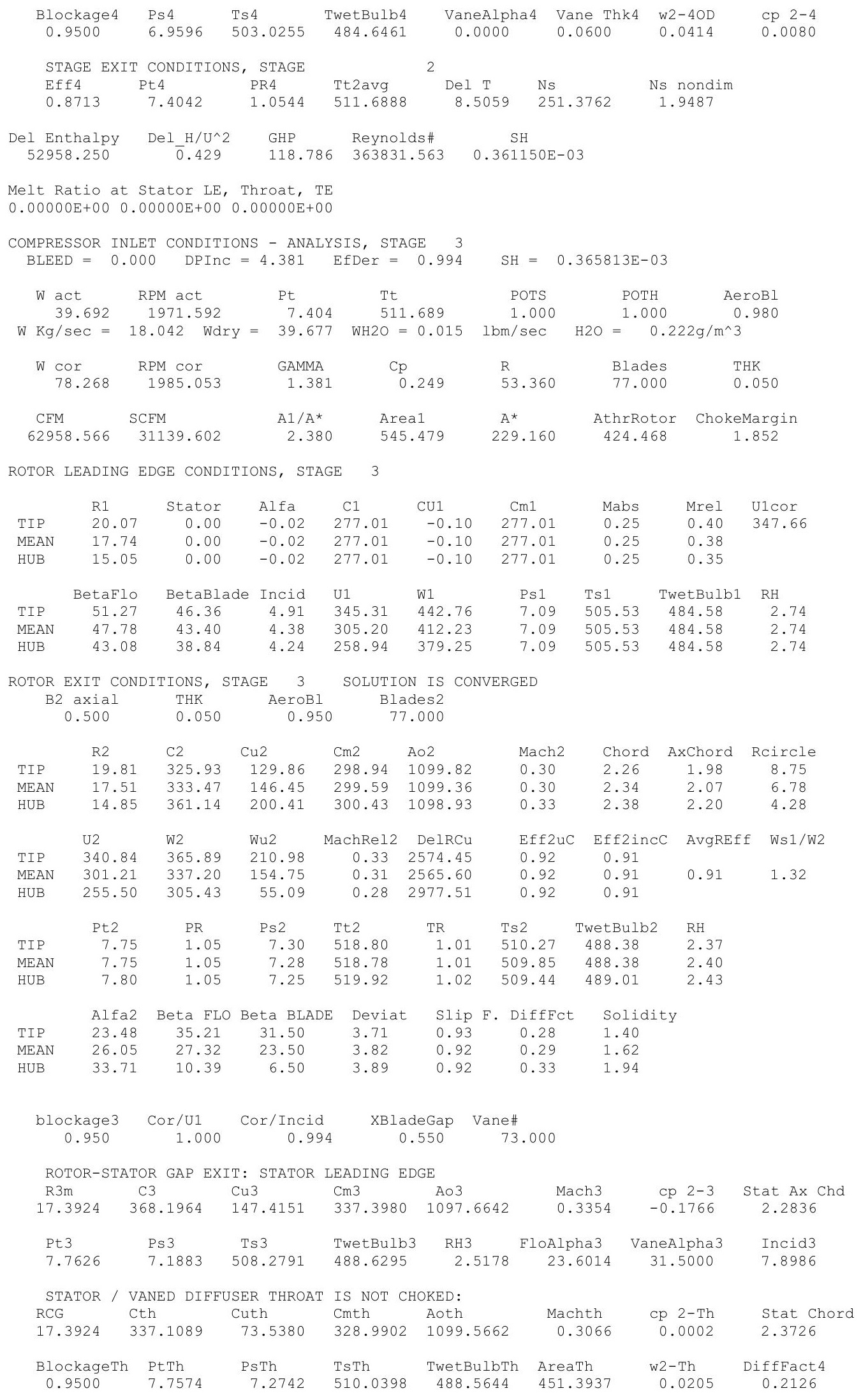




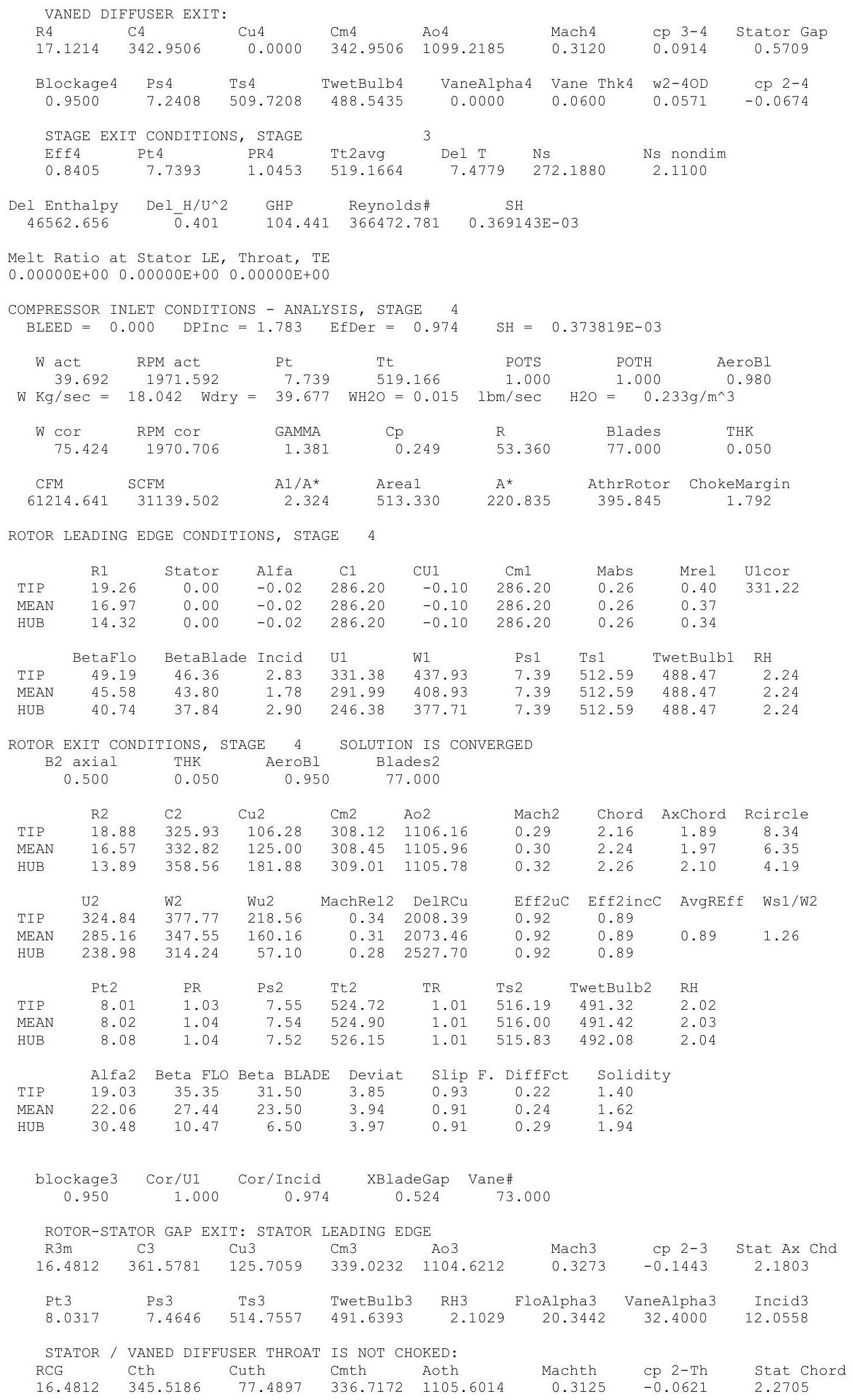




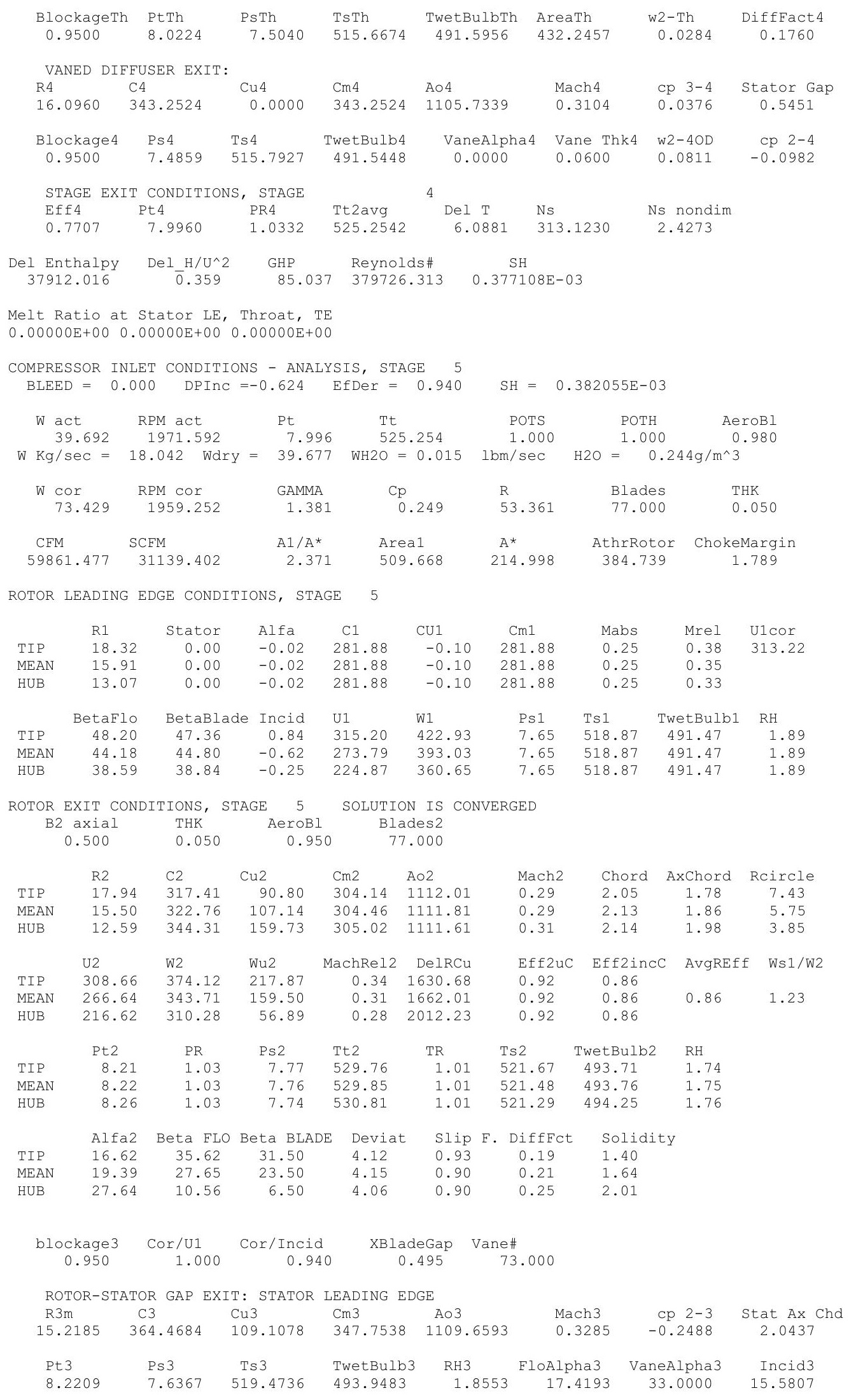




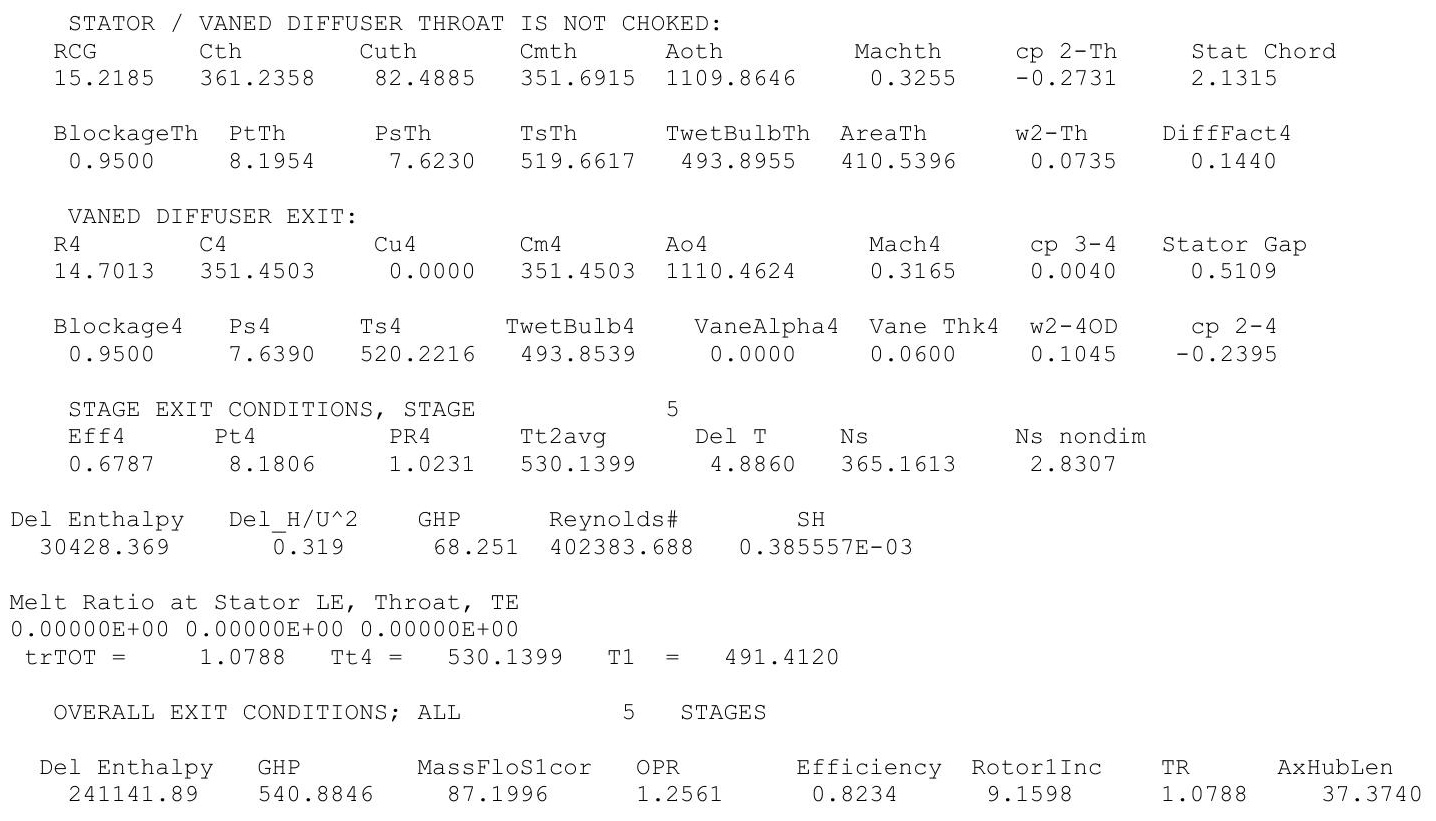




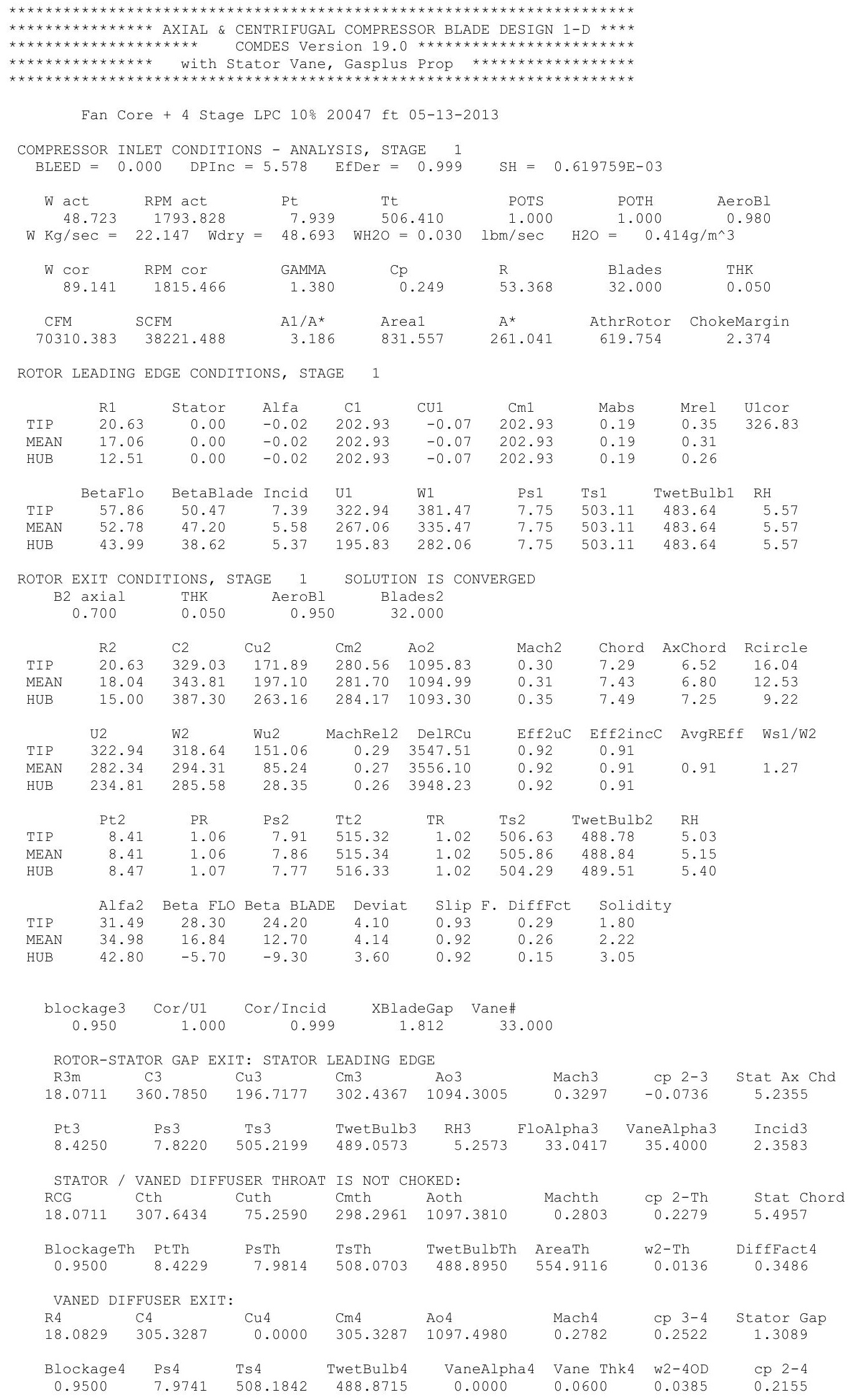




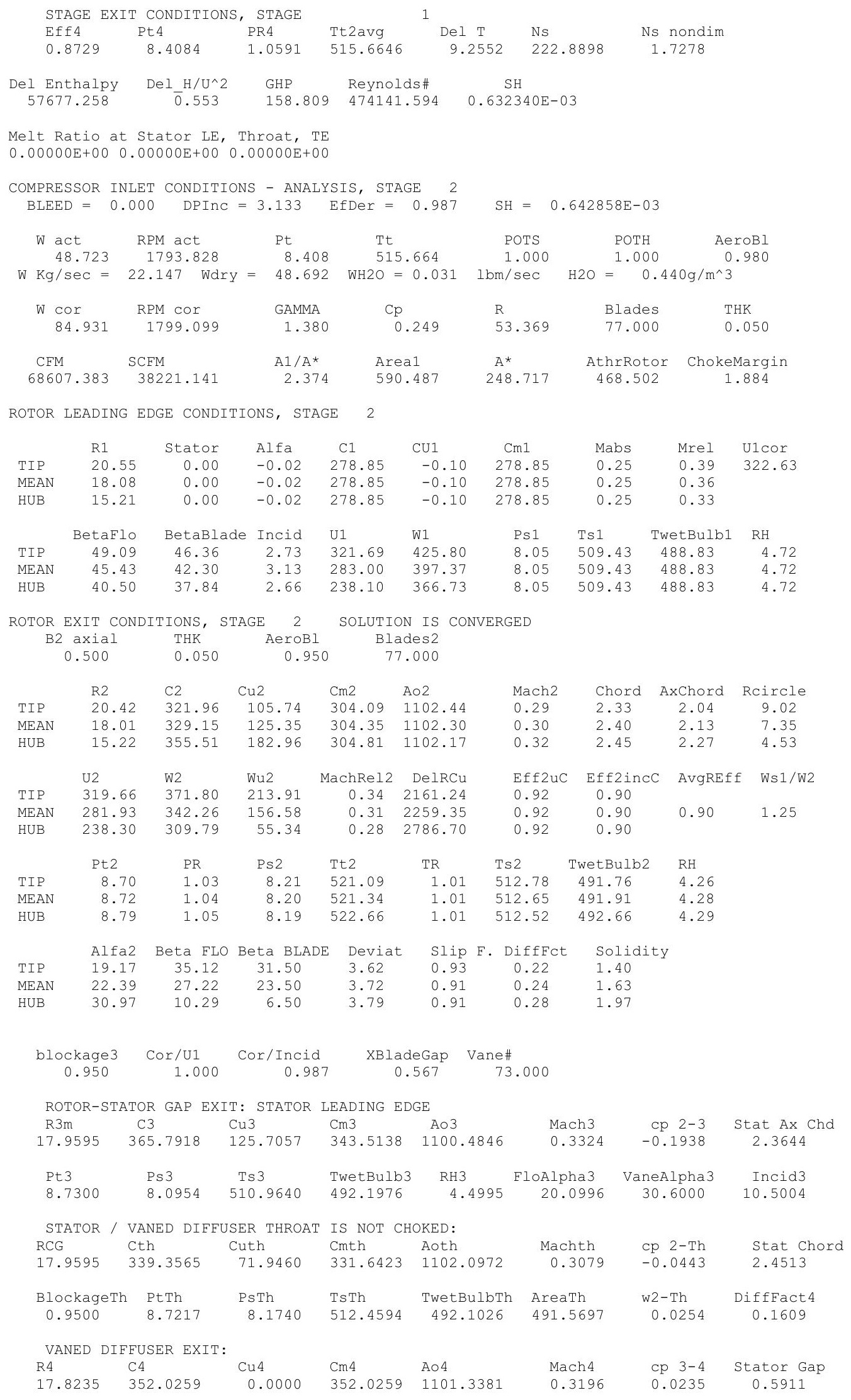




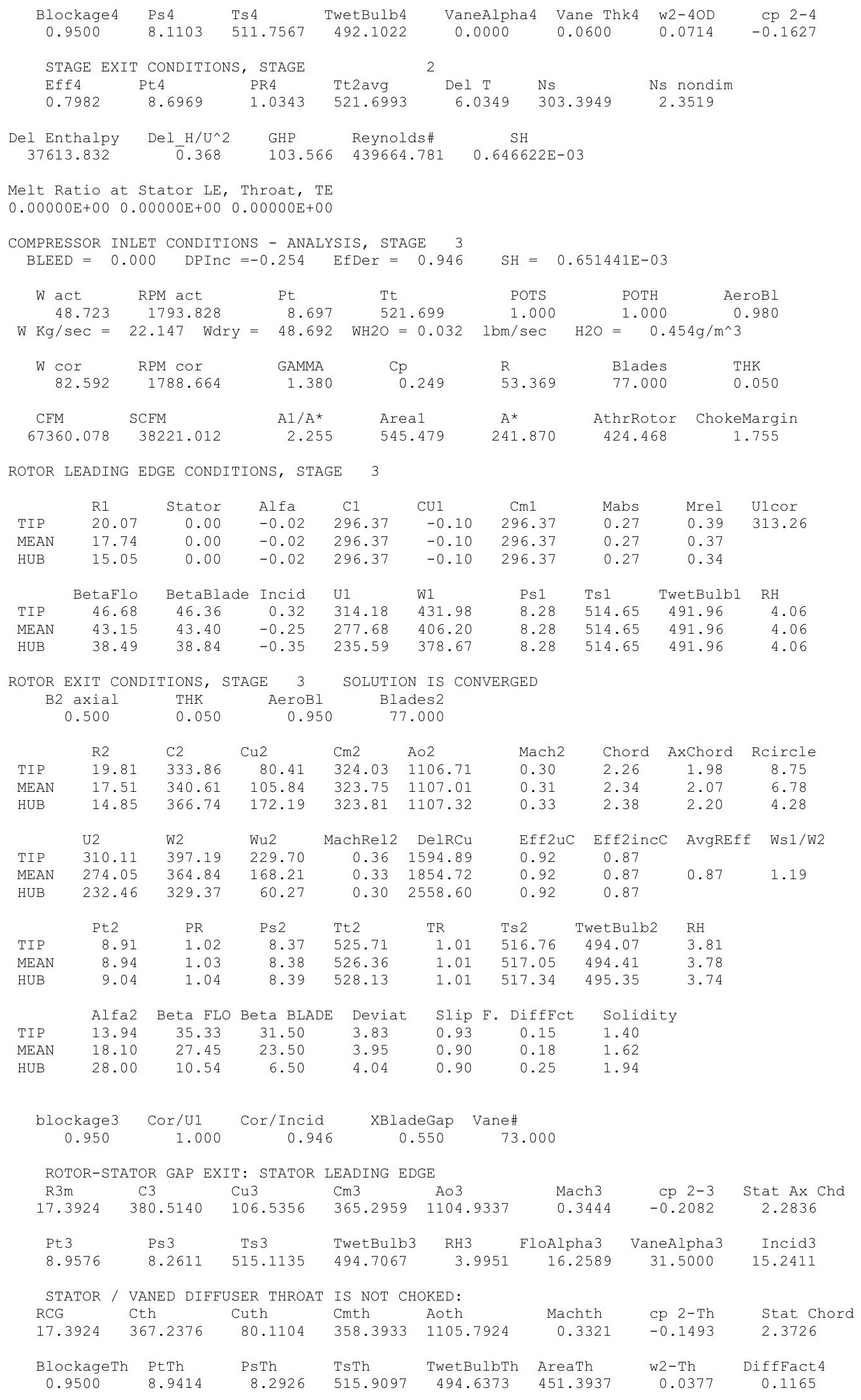




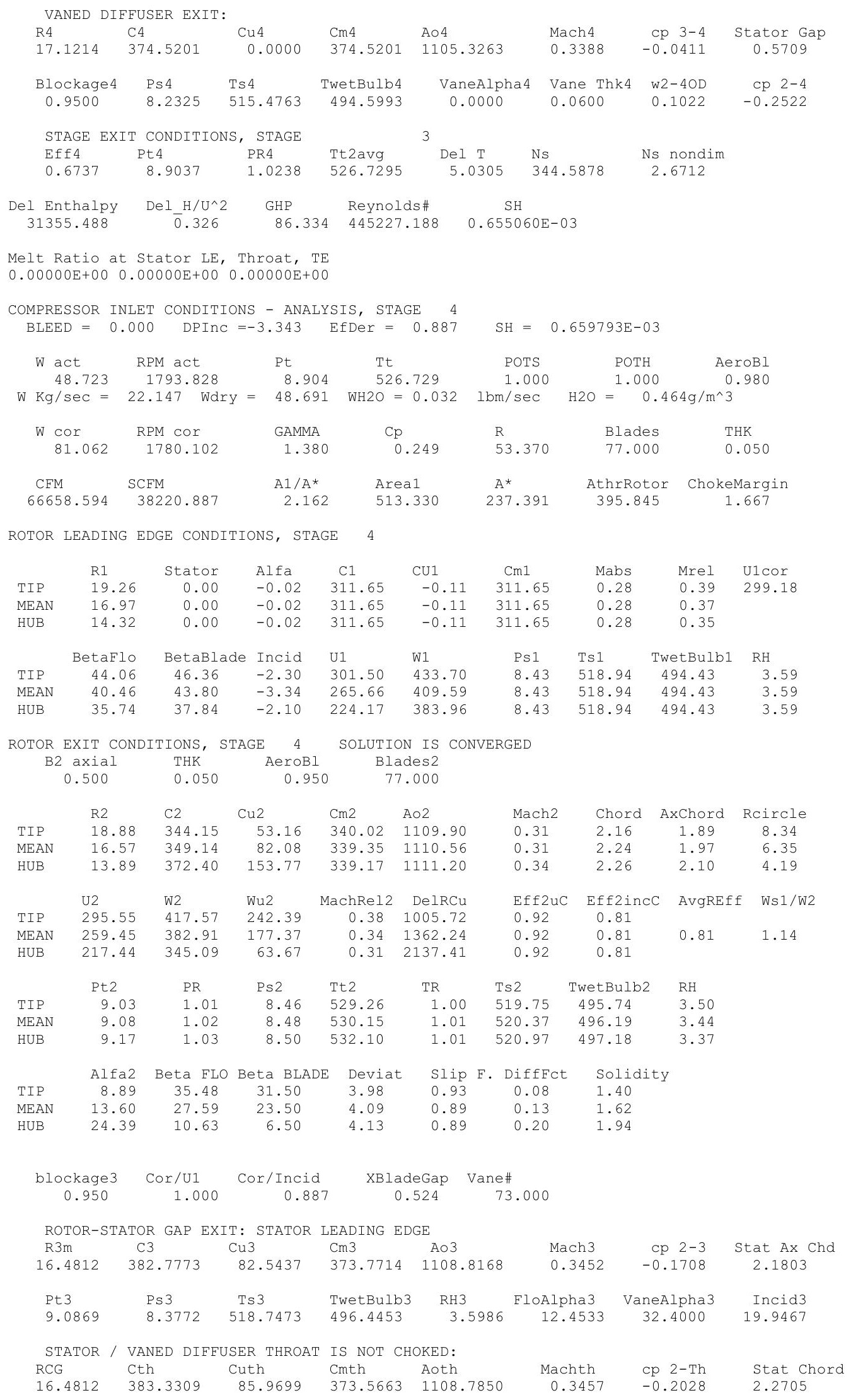




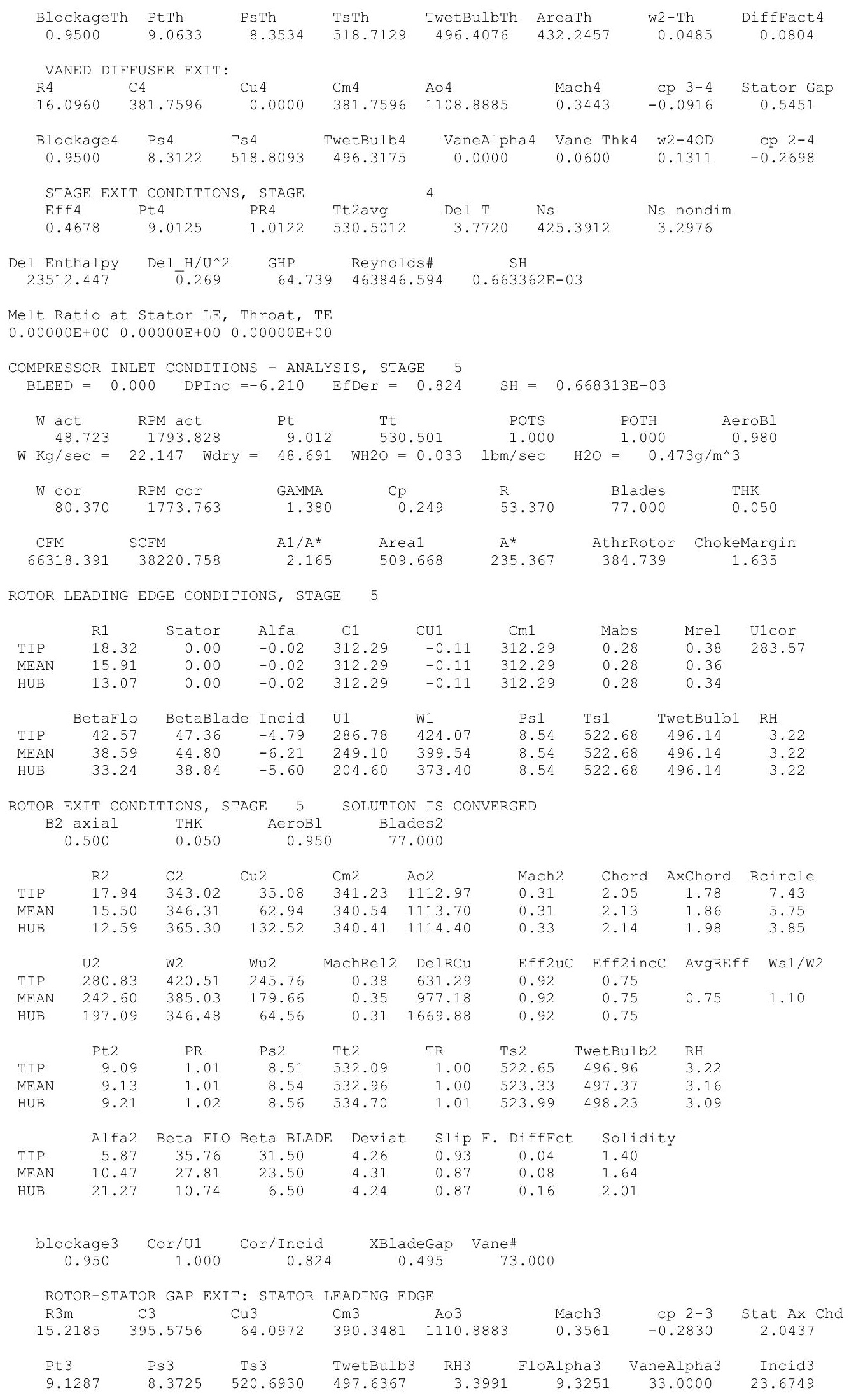




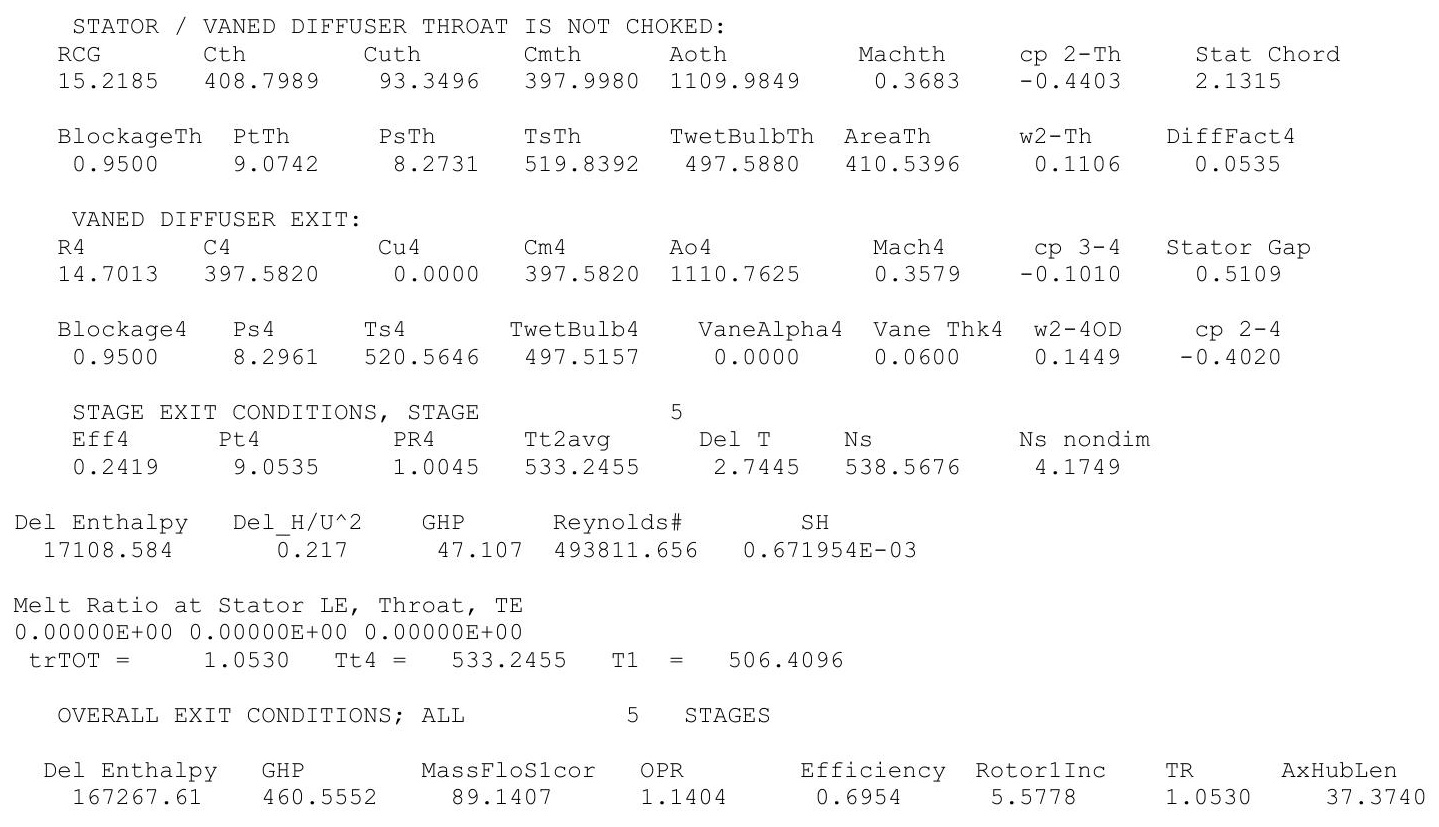




\title{
$100 \mu \mathrm{m}$, ISA $+18 \mathrm{R}$
}

\begin{abstract}
A

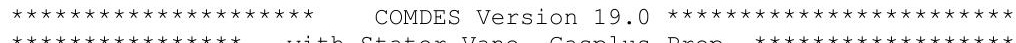

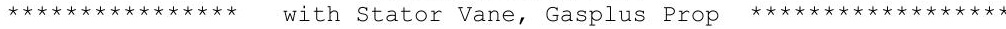

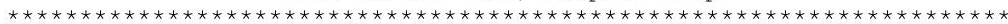

Fan Core + 4 Stage LPC 10\%39000 ft 05-13-2013

COMPRESSOR INLET CONDITIONS - ANALYSIS, STAGE 1

$\mathrm{BLEED}=0.000 \quad$ DPInC $=15.145 \quad$ EfDer $=0.957$

$\mathrm{SH}=0.454452 \mathrm{E}-04$

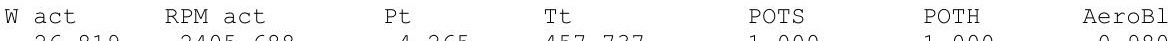

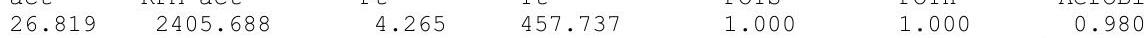

$\mathrm{W} \mathrm{Kg} / \mathrm{sec}=12.191$ Wdry $=26.818 \mathrm{WH} 2 \mathrm{O}=0.001 \mathrm{lbm} / \mathrm{sec} \quad \mathrm{H} 2 \mathrm{O}=0.018 \mathrm{~g} / \mathrm{m}^{\wedge} 3$

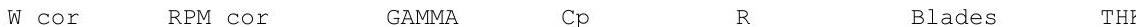

$\begin{array}{llll}1.381 & \mathrm{CP} & \mathrm{R} & \mathrm{B} \\ & 0.248 & 53.350 & 32.000\end{array}$

CFM SCFM A1/A* Area1 A* AthrRotor ChokeMargin

$\begin{array}{lrrrrrr}65038.973 & 21043.451 & 3.271 & 831.557 & 254.197 & 619.754 & 2.438\end{array}$

ROTOR LEADING EDGE CONDITIONS, STAGE 1

\begin{tabular}{|c|c|c|c|c|c|c|c|c|c|}
\hline & R1 & Stator & Alfa & $\mathrm{C} 1$ & CU1 & $\mathrm{Cm} 1$ & Mabs & Mrel & U1cor \\
\hline TIP & 20.63 & 0.00 & -0.02 & 187.71 & -0.06 & 187.71 & 0.18 & 0.45 & 461.02 \\
\hline MEAN & 17.06 & 0.00 & -0.02 & 187.71 & -0.06 & 187.71 & 0.18 & 0.39 & \\
\hline HUB & 12.51 & 0.00 & -0.02 & 187.71 & -0.06 & 187.71 & 0.18 & 0.31 & \\
\hline & BetaFlo & BetaBlade & Incid & U1 & W1 & Ps 1 & Ts 1 & TwetBulb1 & $\mathrm{RH}$ \\
\hline TIP & 66.57 & 50.47 & 16.10 & 433.10 & 472.09 & 4.17 & 454.90 & 448.21 & 1.7 \\
\hline MEAN & 62.34 & 47.20 & 15.14 & 358.15 & 404.42 & 4.17 & 454.90 & 448.21 & 1.7 \\
\hline HUB & 54.45 & 38.62 & 15.83 & 262.63 & 322.87 & 4.17 & 454.90 & 448.21 & 1.7 \\
\hline
\end{tabular}

ROTOR EXIT CONDITIONS, STAGE 1 SOLUTION IS CONVERGED

\begin{tabular}{|c|c|c|c|c|c|c|c|c|c|}
\hline & $\begin{array}{l}\text { axial } \\
700\end{array}$ & $\begin{array}{l}\text { THK } \\
0.050\end{array}$ & $\begin{array}{r}\text { AeroBl } \\
0.950\end{array}$ & $\begin{array}{r}\text { Bla } \\
32\end{array}$ & $\begin{array}{l}\text { ades } 2 \\
2.000\end{array}$ & & & & \\
\hline & R2 & $\mathrm{C} 2$ & $\mathrm{Cu} 2$ & $\mathrm{Cm} 2$ & $\mathrm{~A} \circ 2$ & Mach2 & Chord & AxChord & Rcircle \\
\hline TIP & 20.63 & 389.08 & 297.03 & 251.31 & 1051.48 & 0.37 & 7.29 & 6.52 & 16.04 \\
\hline MEAN & 18.04 & 394.32 & 301.11 & 254.60 & 1048.45 & 0.38 & 7.43 & 6.80 & 12.53 \\
\hline HUB & 15.00 & 427.87 & 340.45 & 259.17 & 1044.69 & 0.41 & 7.49 & 7.25 & 9.22 \\
\hline & U2 & W2 & Wu2 & MachRel2 & DelRCu & Eff $2 u C$ & Eff 2 incC & AvgREff & Ws $1 /$ W2 \\
\hline TIP & 433.10 & 285.78 & 136.07 & 0.27 & 6128.98 & 0.92 & 0.88 & & \\
\hline MEAN & 378.64 & 266.14 & 77.53 & 0.25 & 5431.98 & 0.92 & 0.88 & 0.88 & 1.74 \\
\hline HUB & 314.90 & 260.43 & 25.54 & 0.25 & 5107.52 & 0.92 & 0.88 & & \\
\hline & Pt2 & PR & Ps2 & Tt2 & TR & Ts2 & TwetBulb2 & $\mathrm{RH}$ & \\
\hline TIP & 4.91 & 1.15 & 4.47 & 478.44 & 1.05 & 466.26 & 462.20 & 1.11 & \\
\hline EAN & 4.83 & 1.13 & 4.39 & 476.08 & 1.04 & 463.58 & 460.71 & 1.23 & \\
\hline JB & 4.80 & 1.12 & 4.28 & 474.99 & 1.04 & 460.26 & 460.03 & 1.40 & \\
\hline & Alfa2 & Beta FLO & Beta BLADE & Deviat & Slip & F. DiffFct & Solidity & & \\
\hline P & 49.77 & 28.43 & 24.20 & 4.23 & 0.93 & 0.57 & 1.80 & & \\
\hline AN & 49.78 & 16.94 & 12.70 & 4.24 & 0.95 & 0.51 & 2.22 & & \\
\hline $\mathrm{B}$ & 52.72 & -5.63 & -9.30 & 3.67 & 0.95 & 0.38 & 3.05 & & \\
\hline
\end{tabular}

$\begin{array}{ccccc}\text { blockage3 } & \text { Cor/U1 } & \text { Cor/Incid } & \text { XBladeGap } & \text { Vane\# } \\ 0.950 & 1.000 & 0.957 & 1.812 & 33.000\end{array}$

ROTOR-STATOR GAP EXIT: STATOR LEADING EDGE

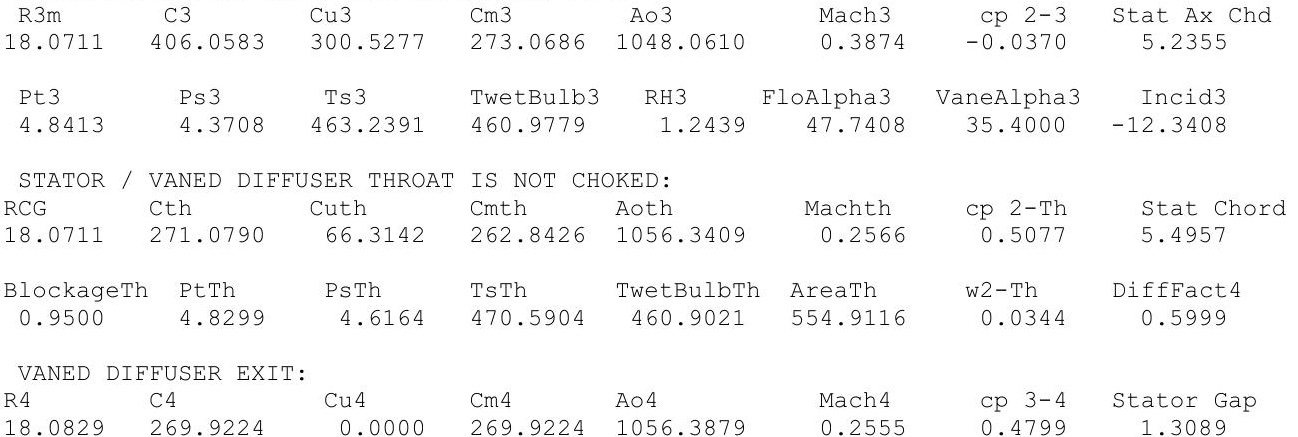

$\begin{array}{llllllll}18.0829 & 269.9224 & 0.0000 & 269.9224 & 1056.3879 & 0.2555 & 0.4799 & 1.3089\end{array}$ 


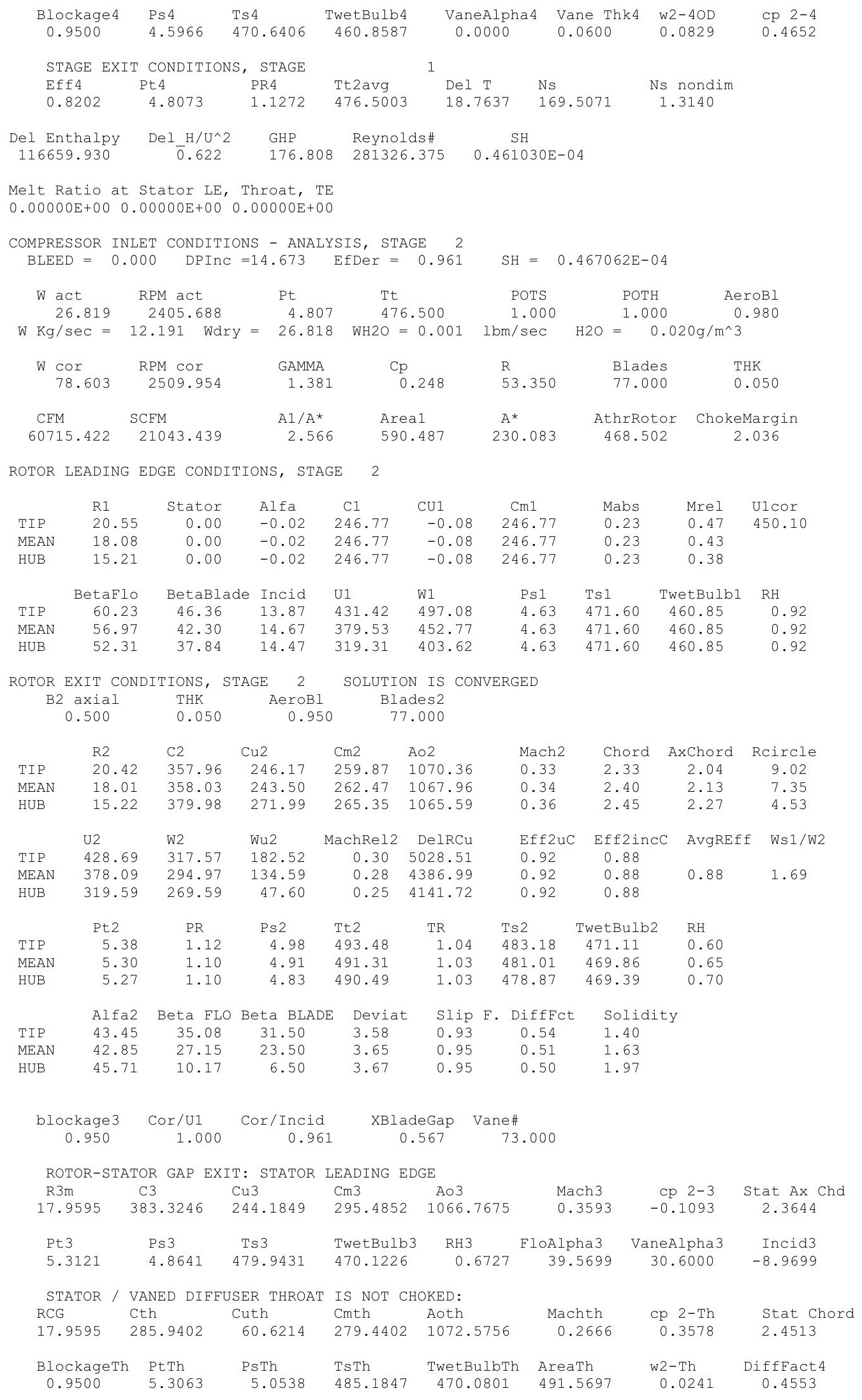




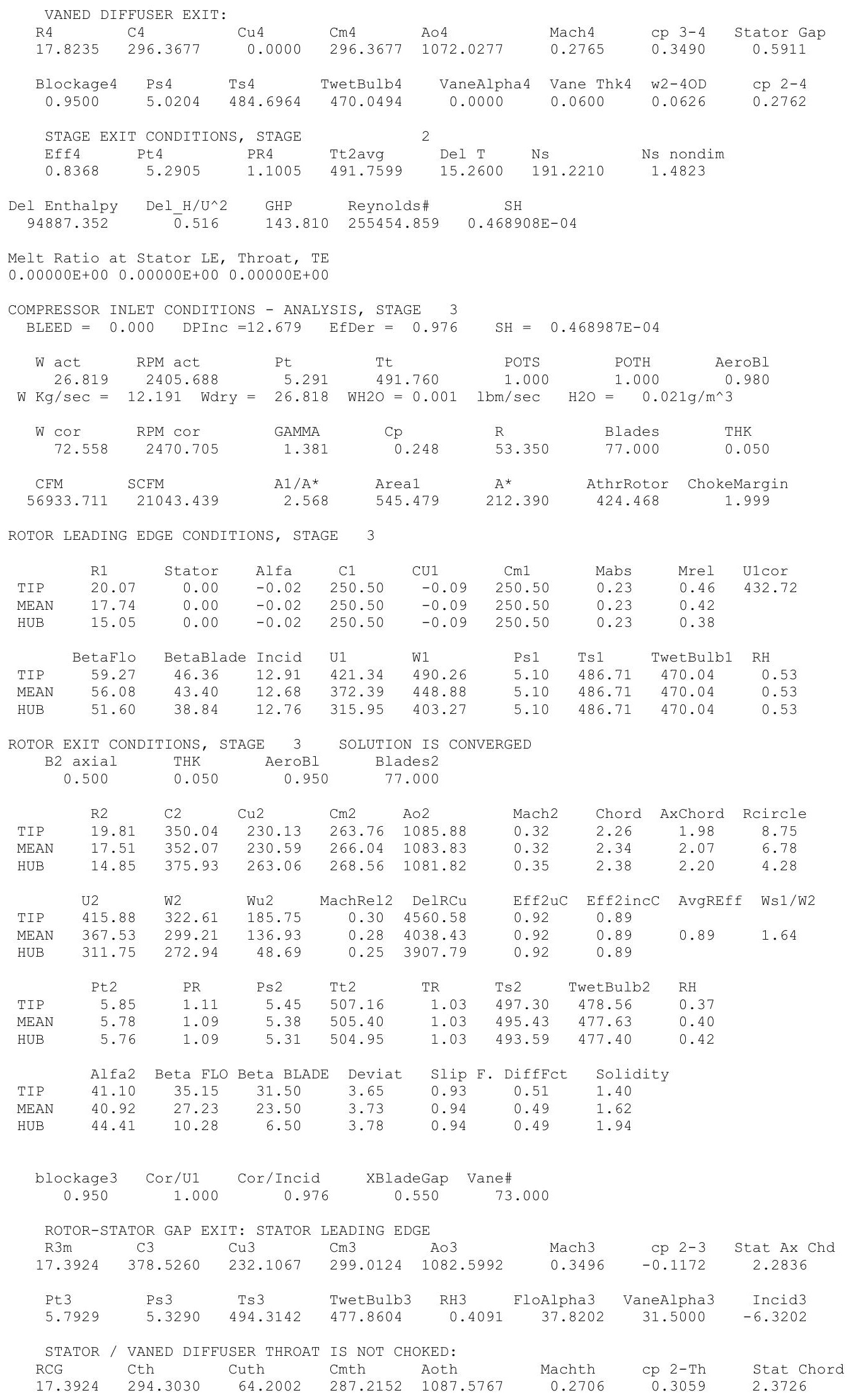




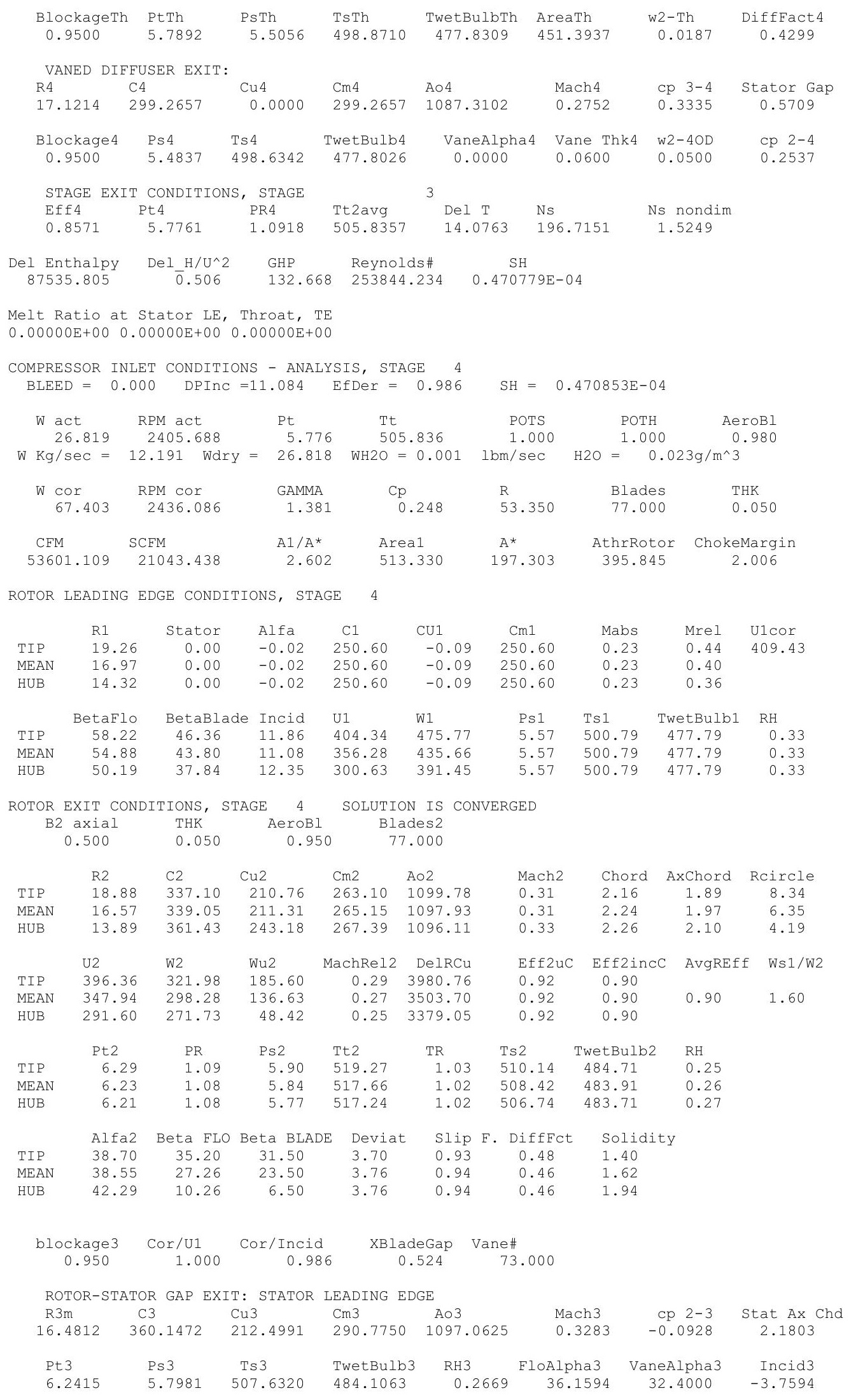




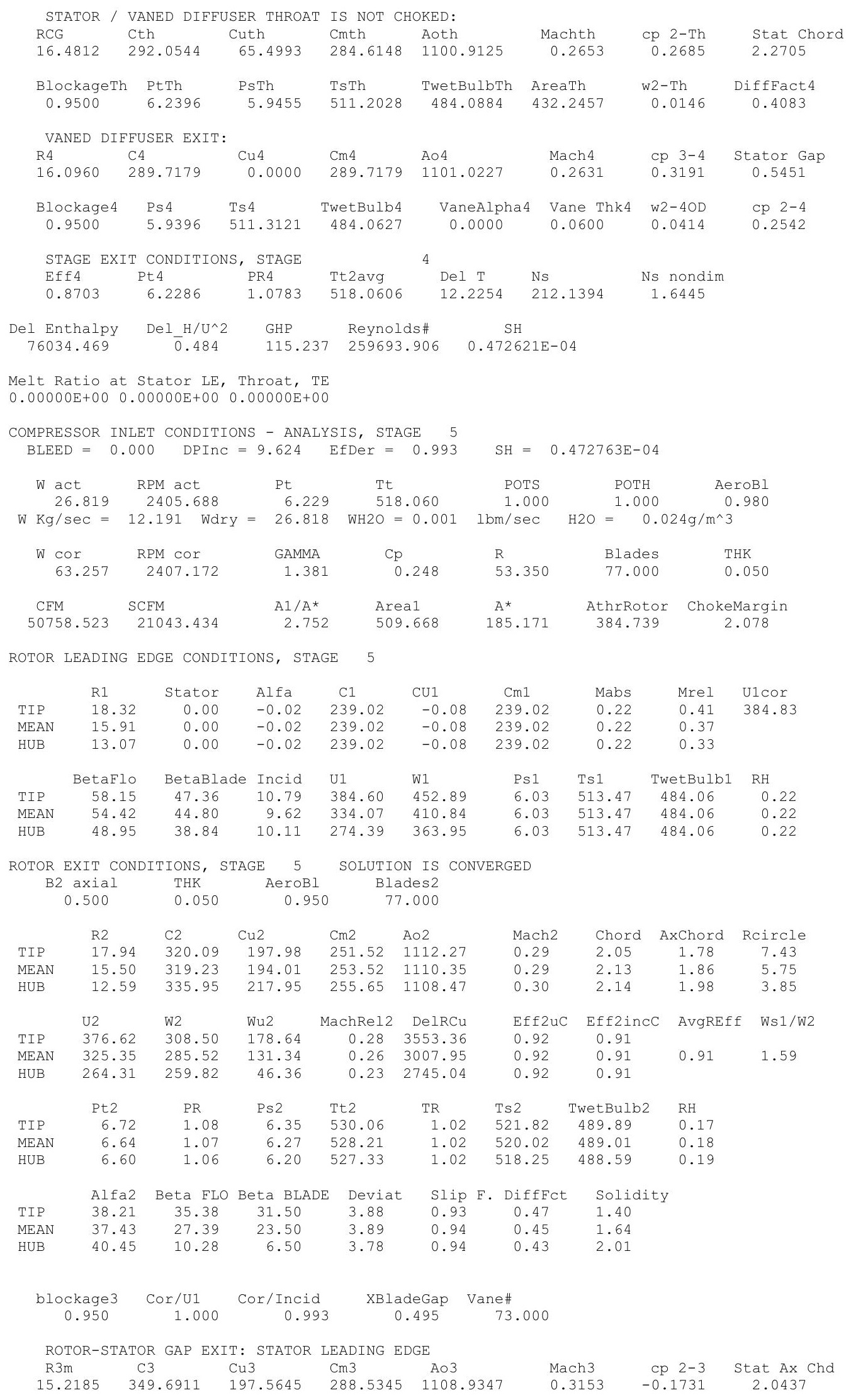




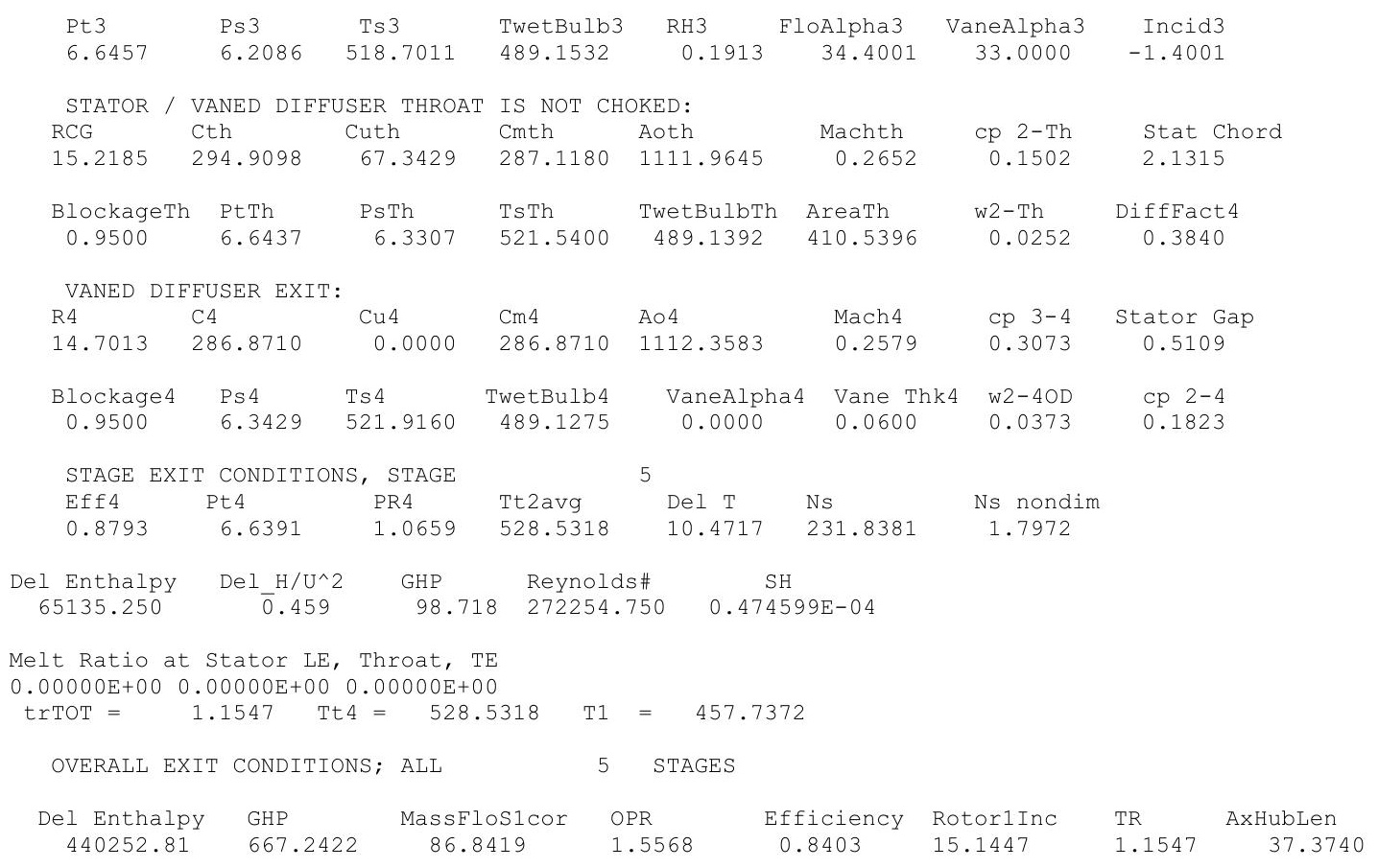




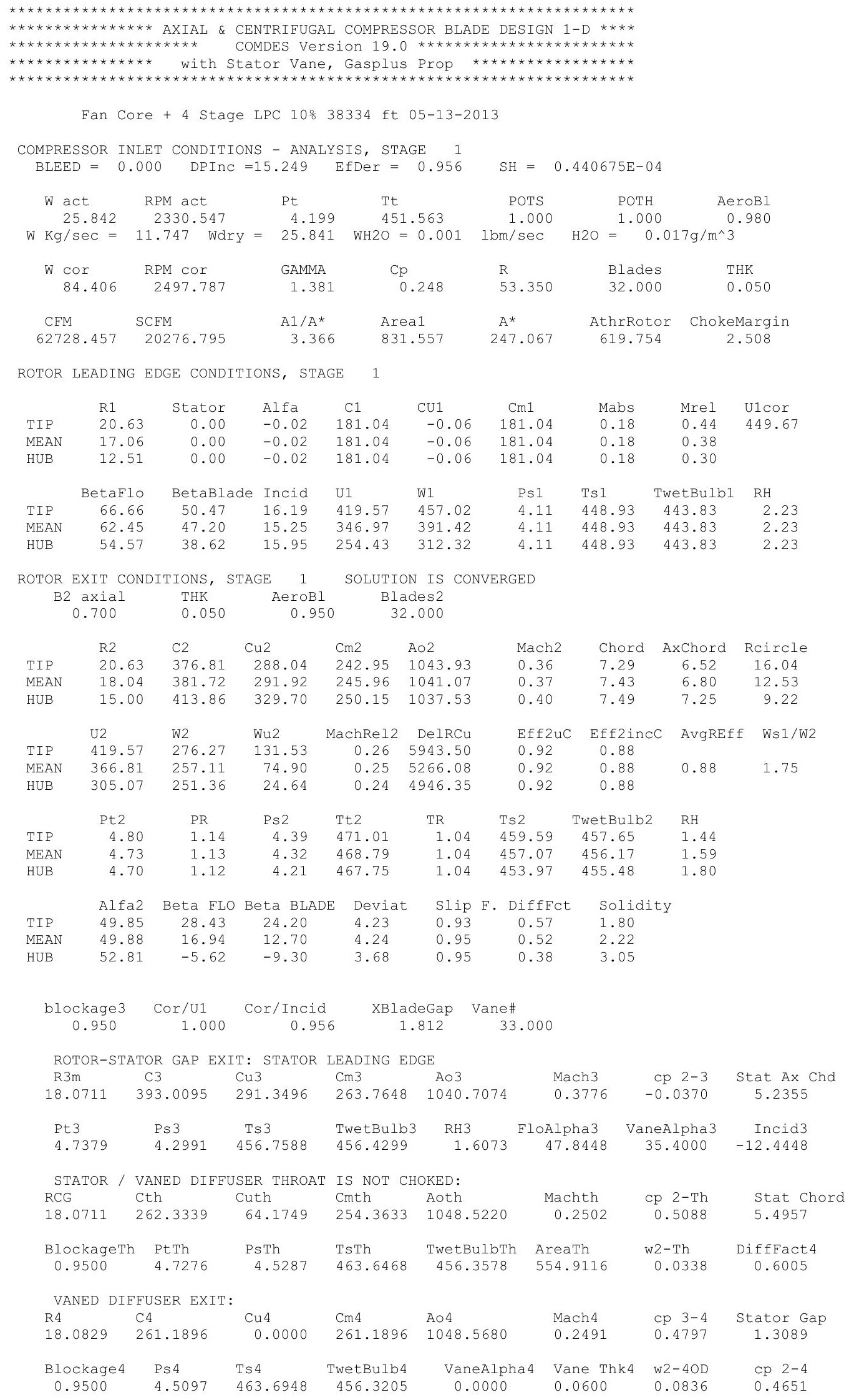




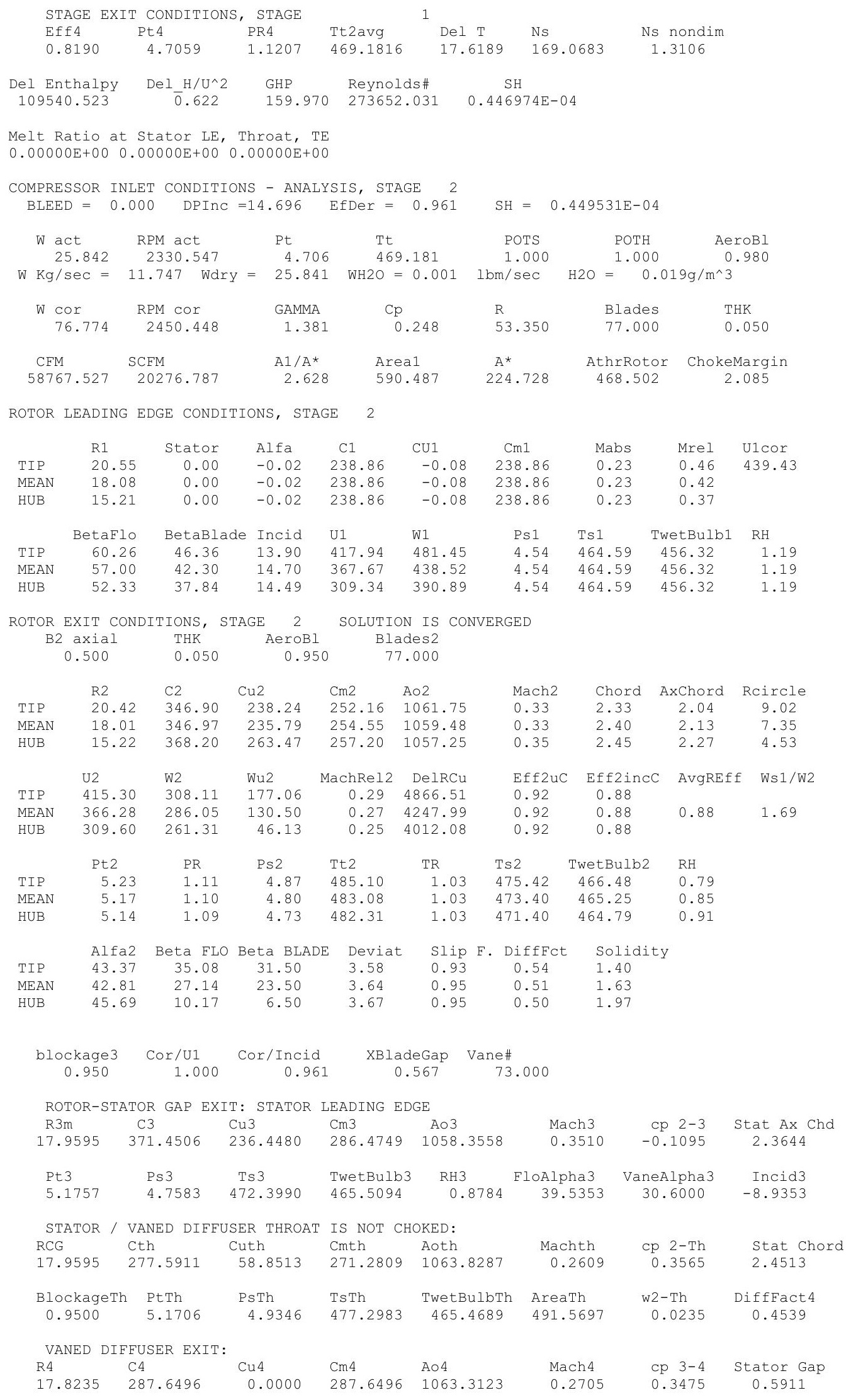




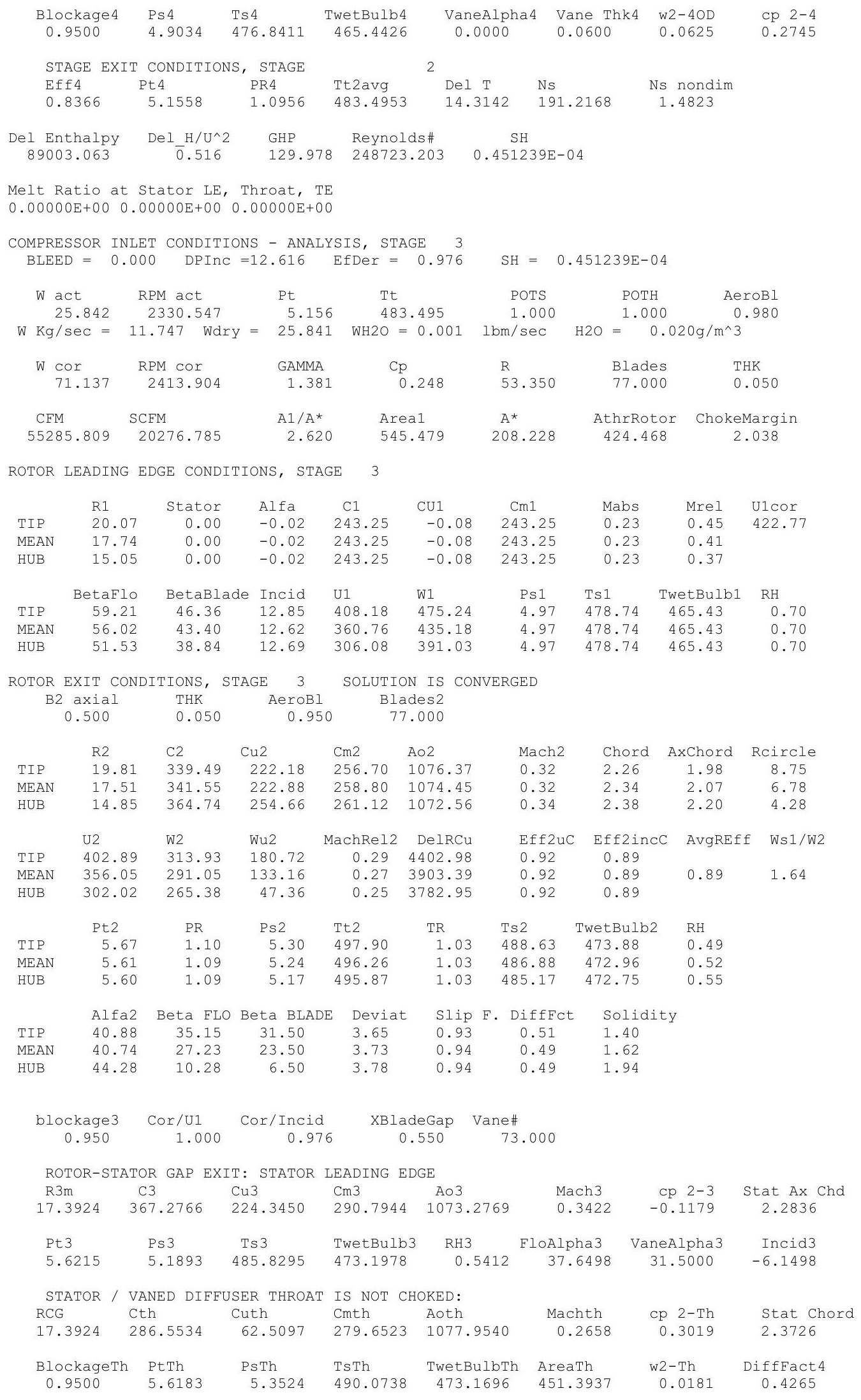




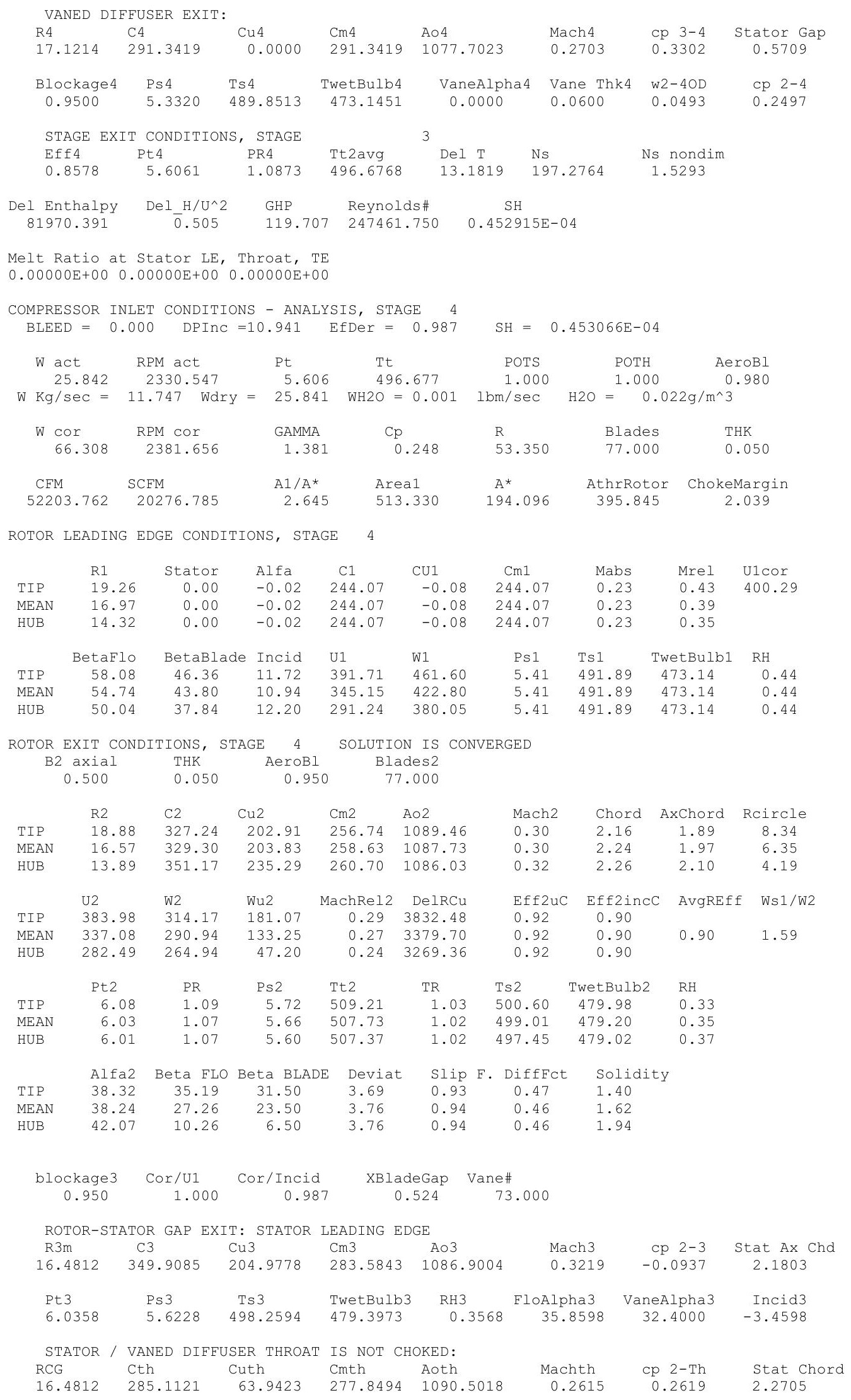




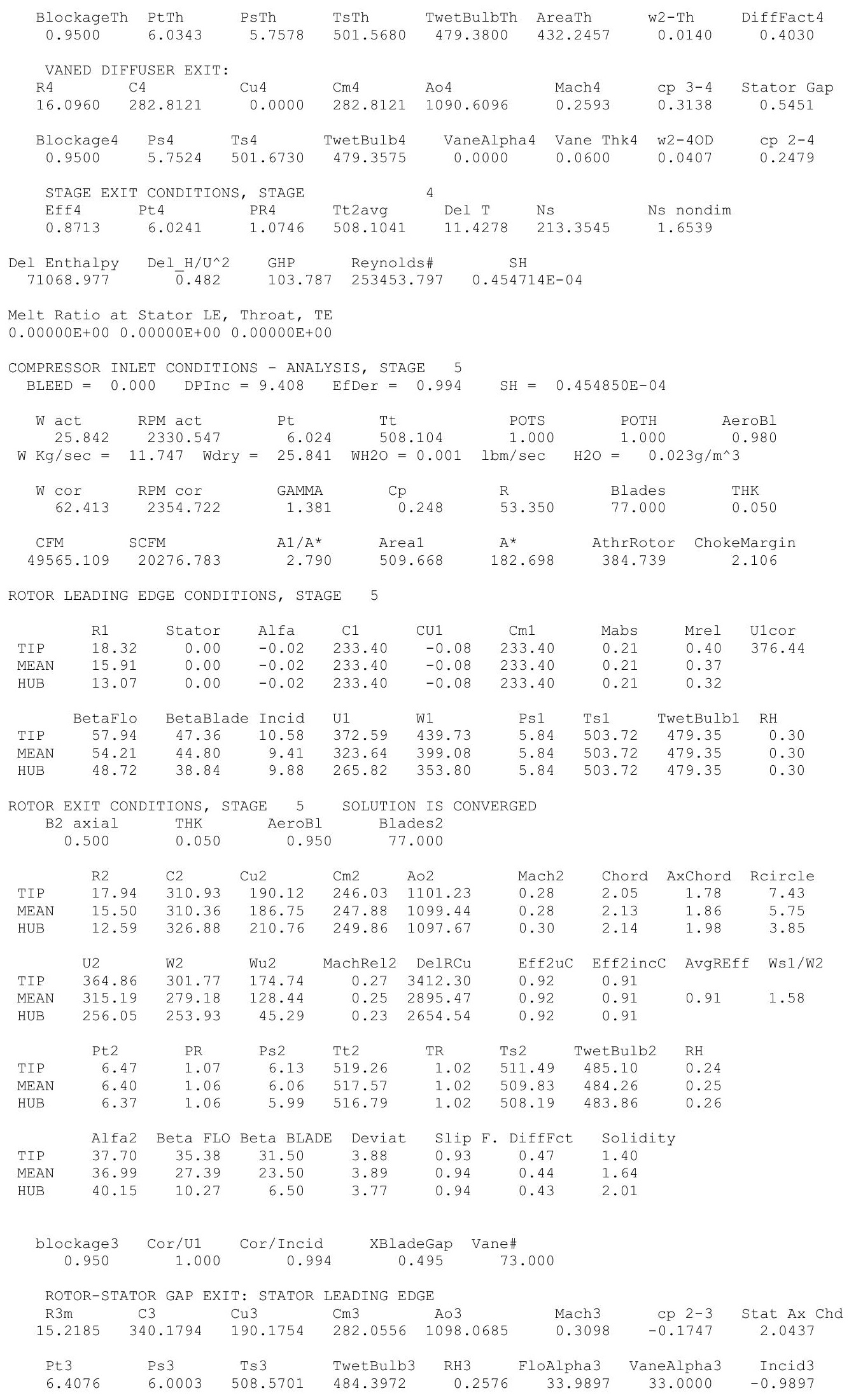




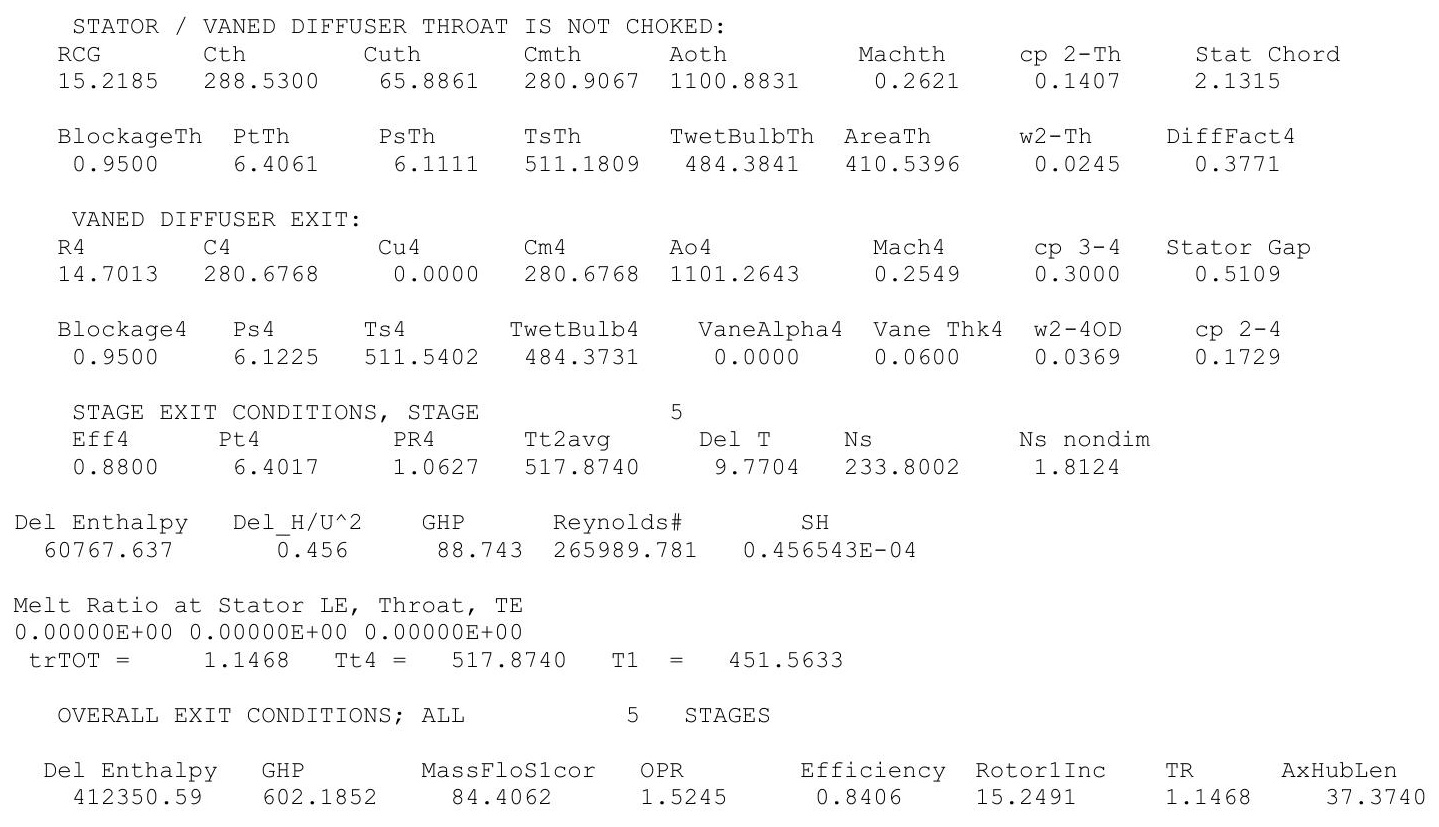




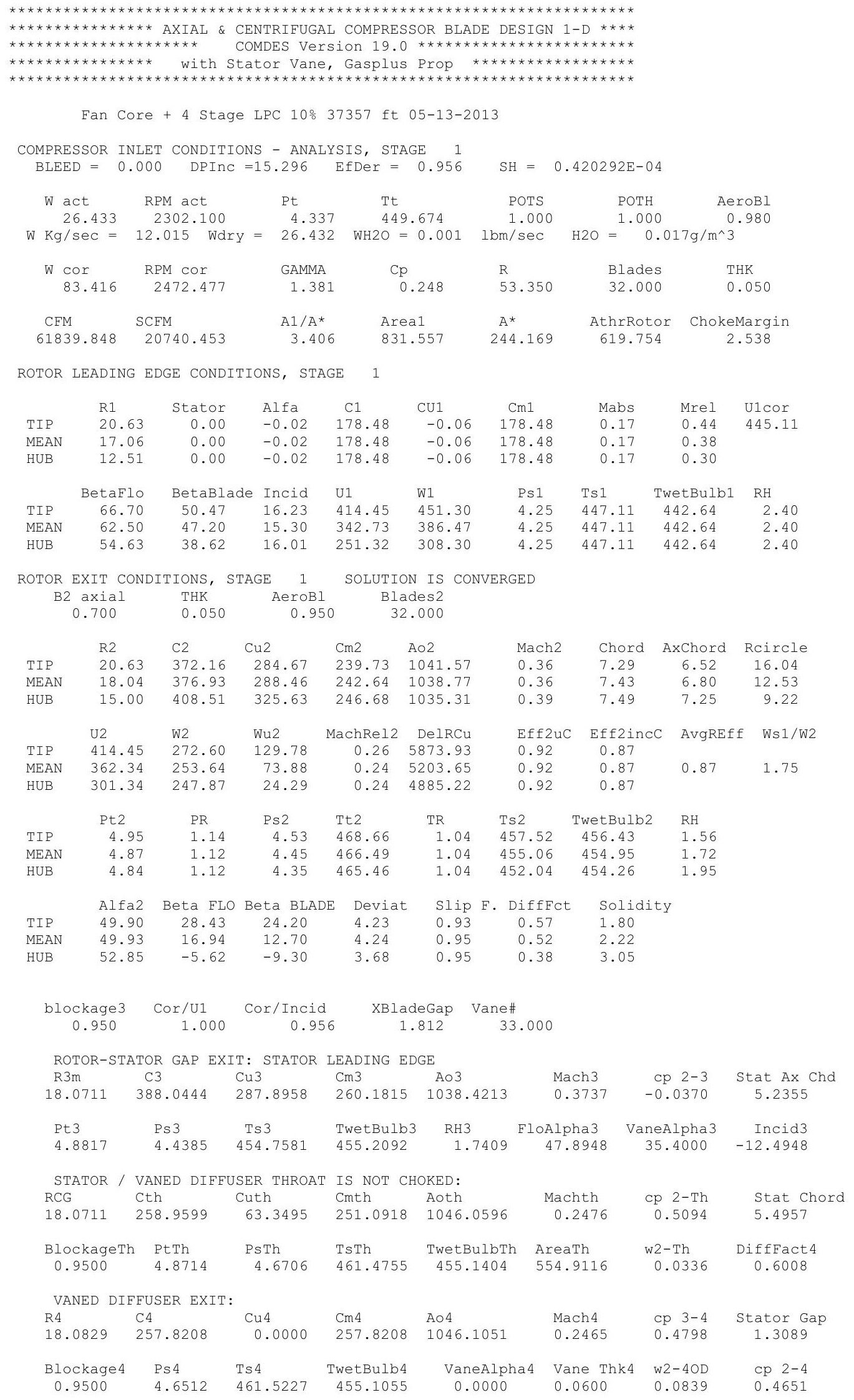




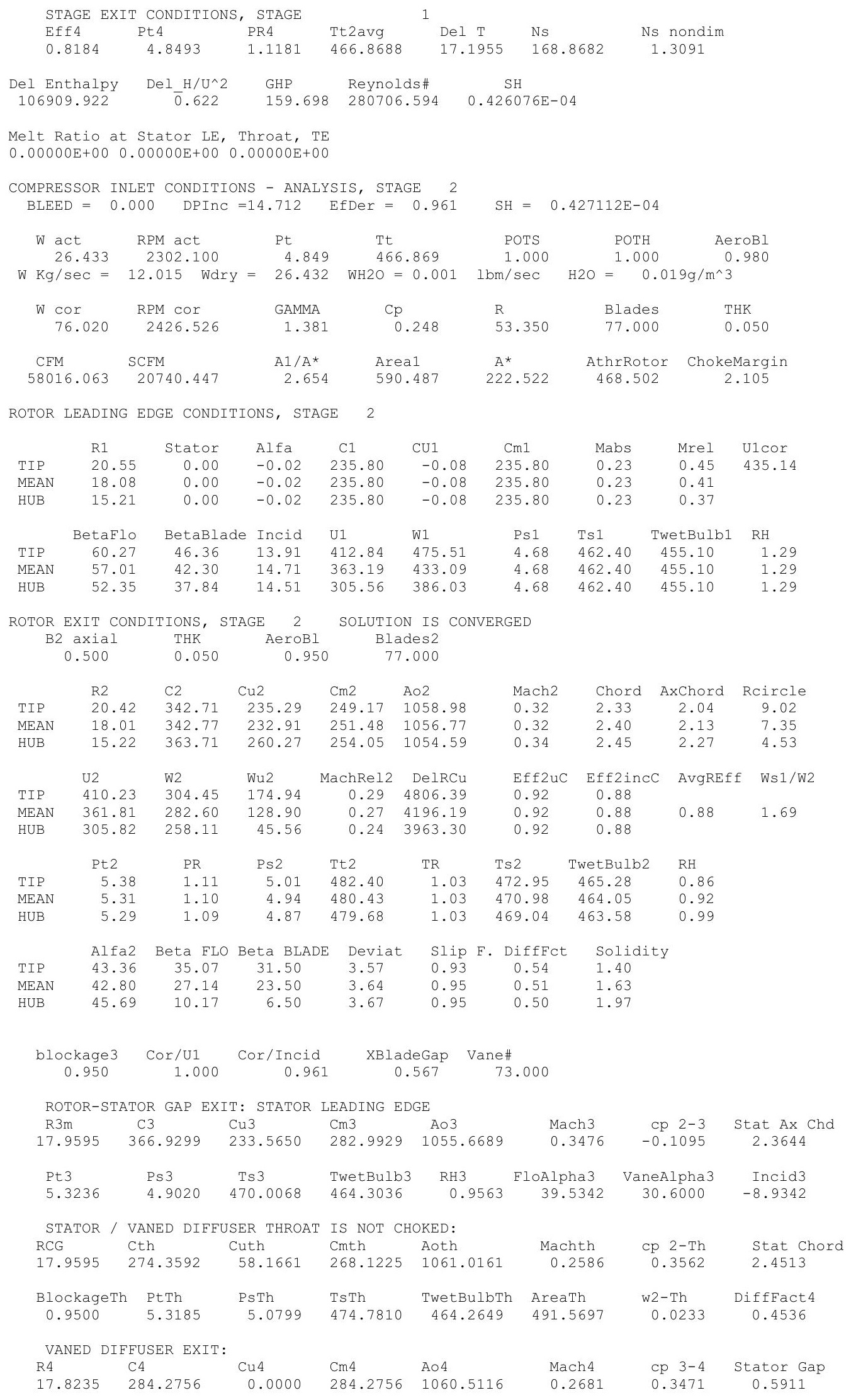




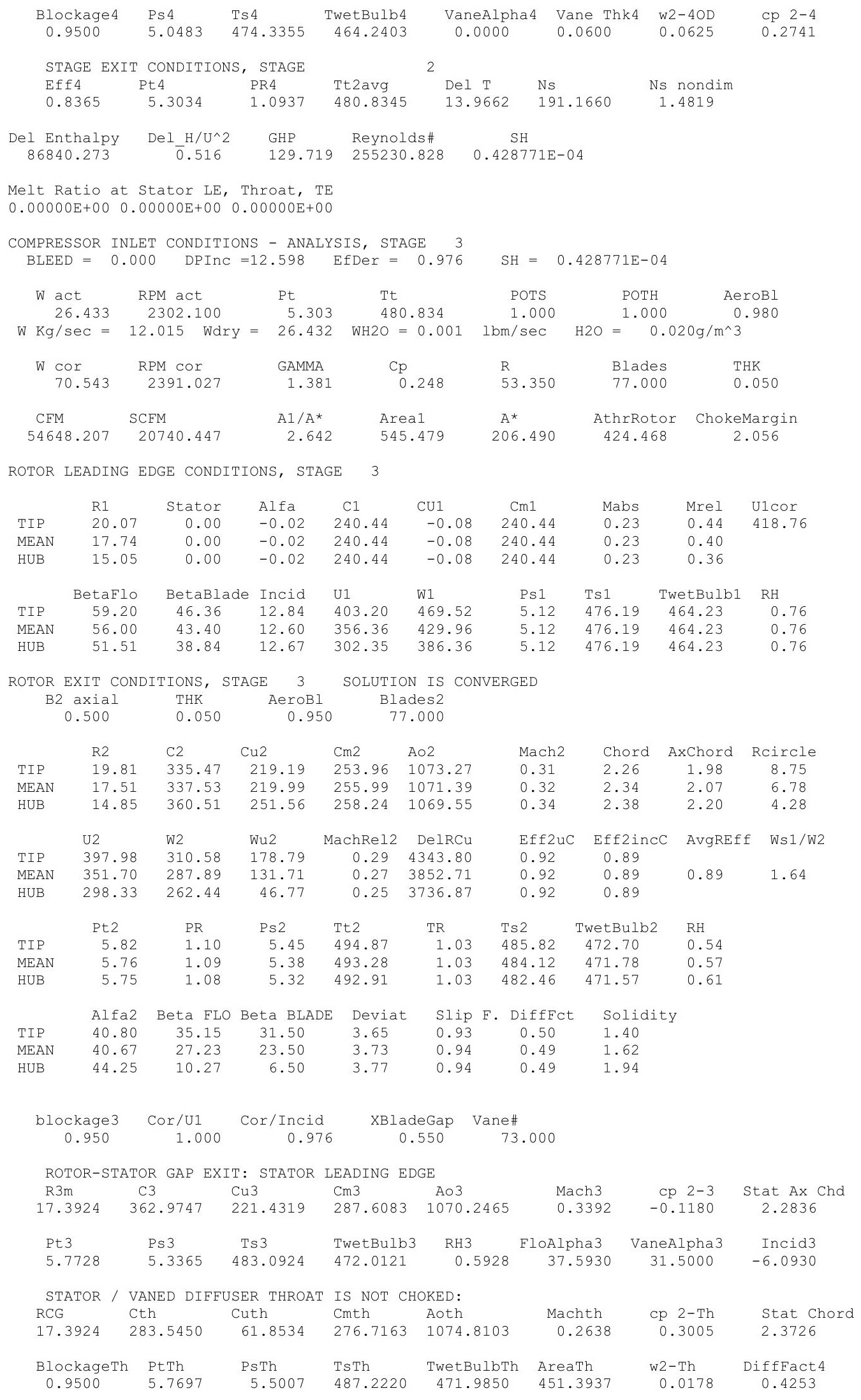




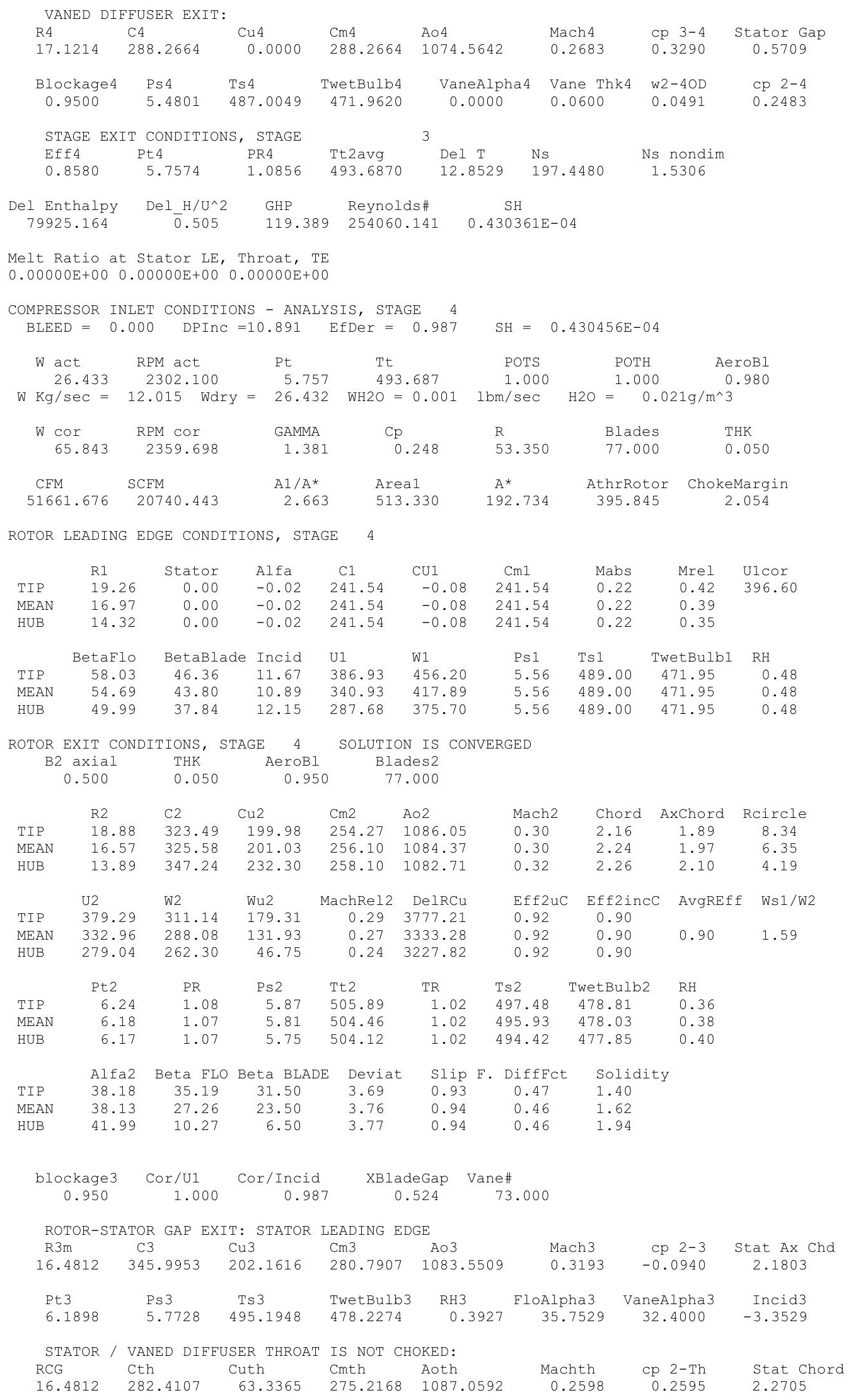




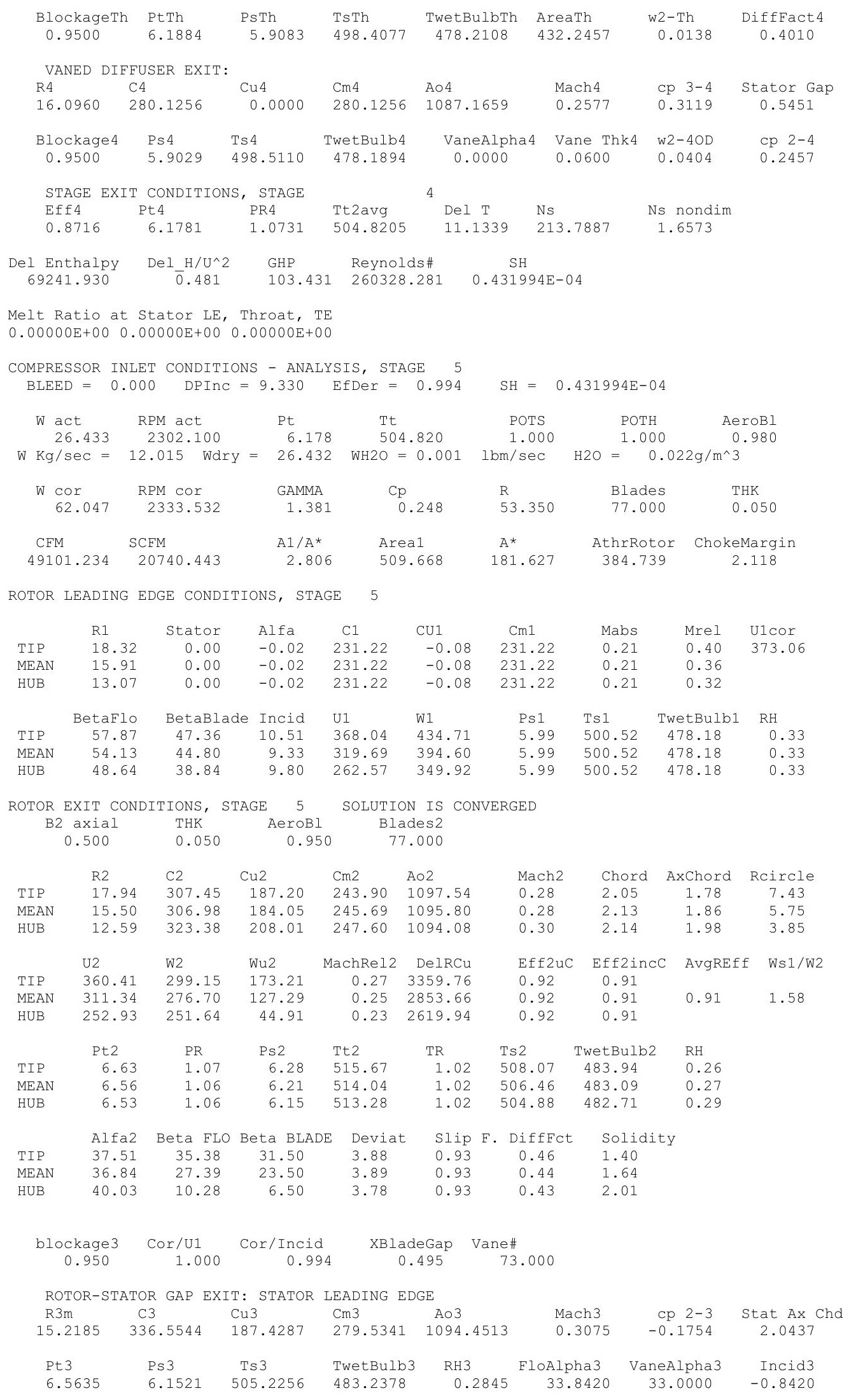




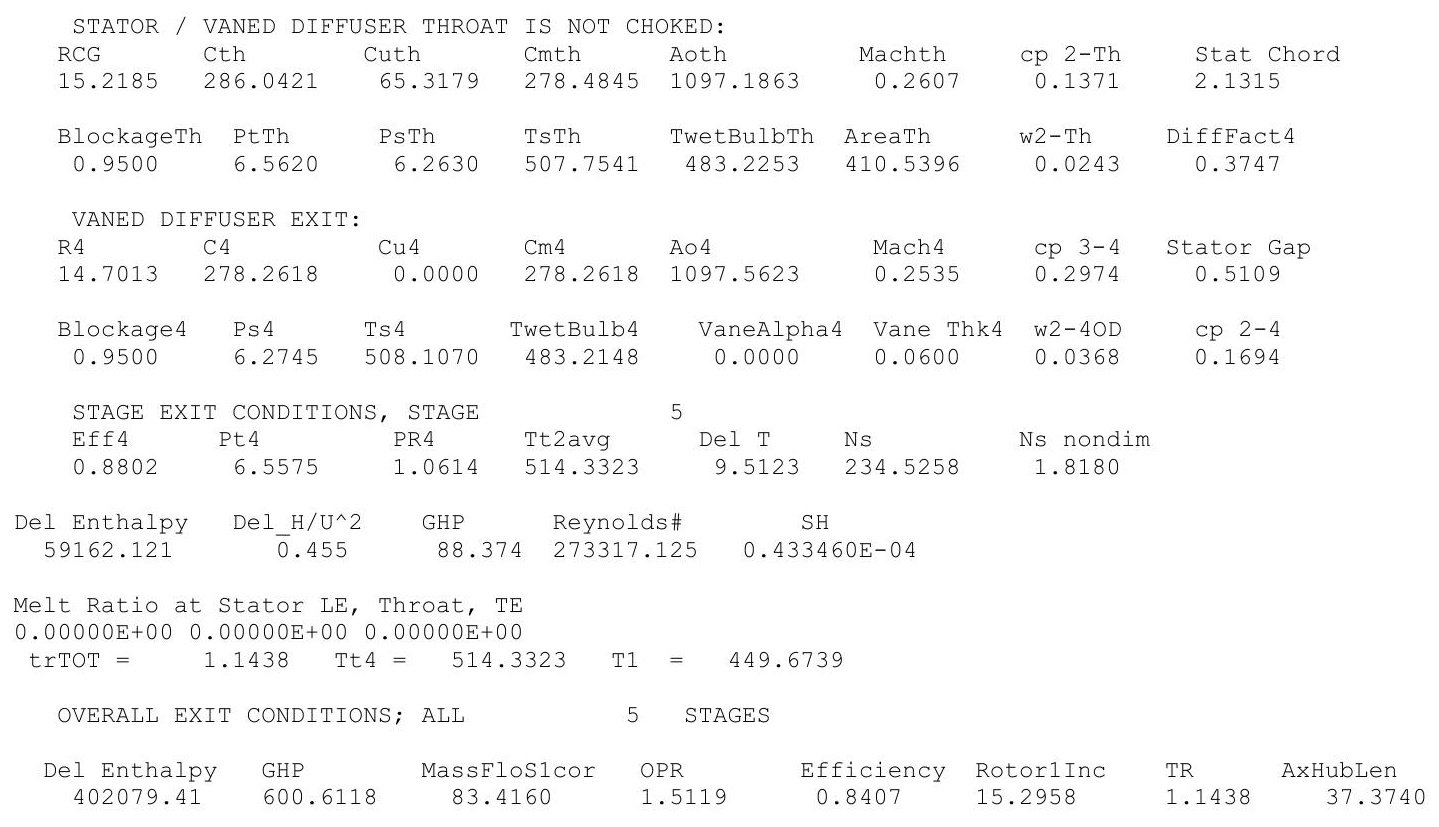




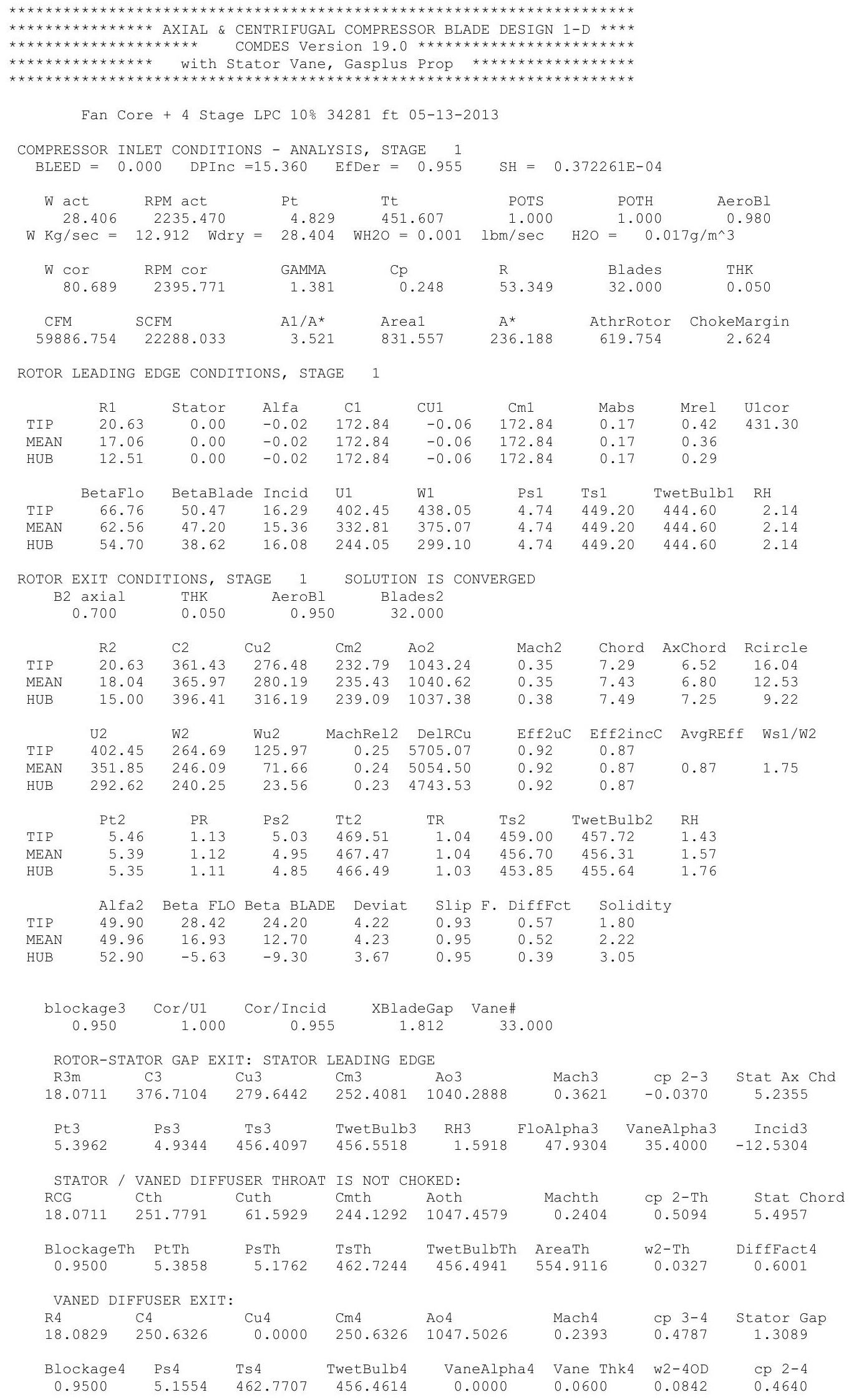




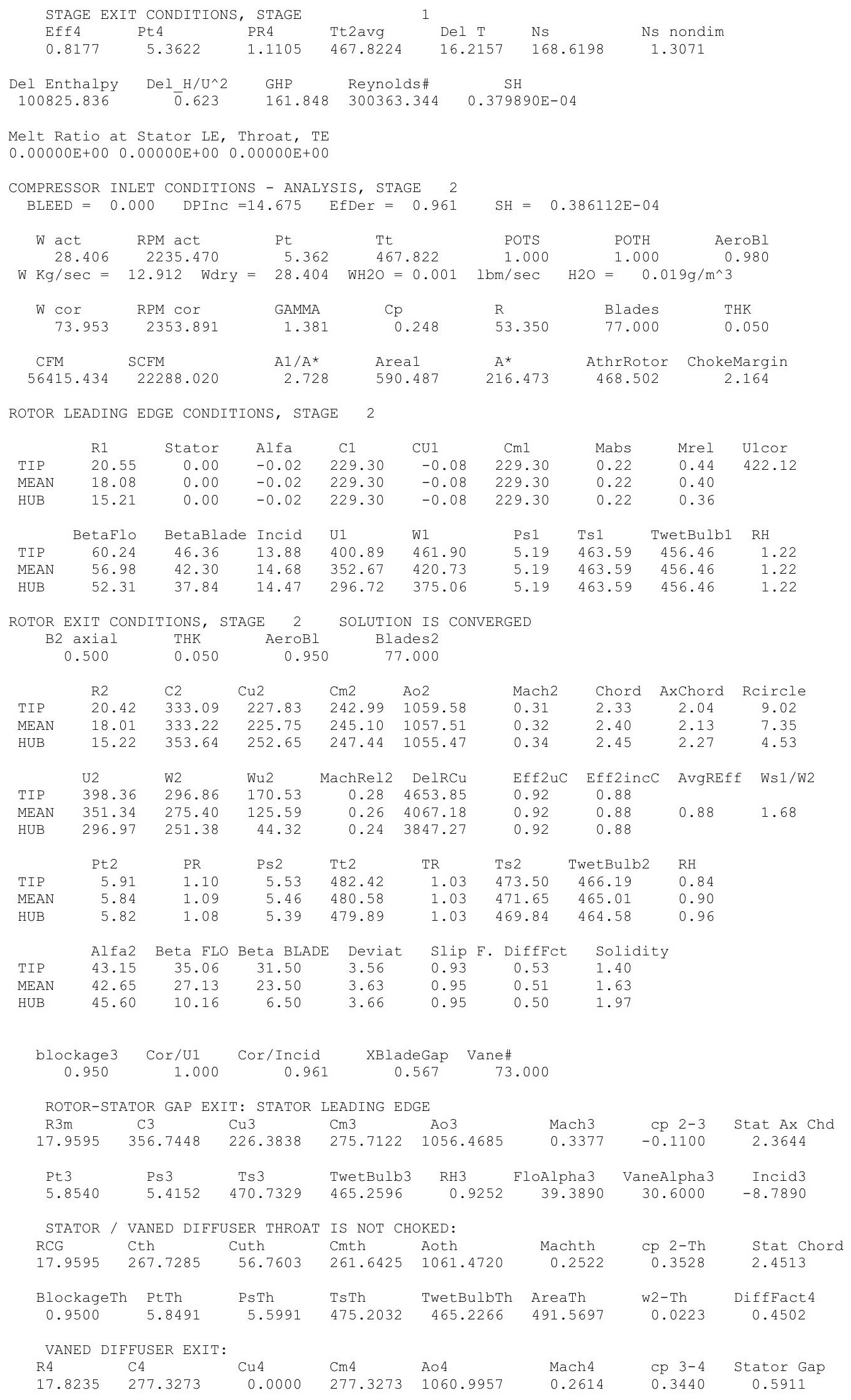




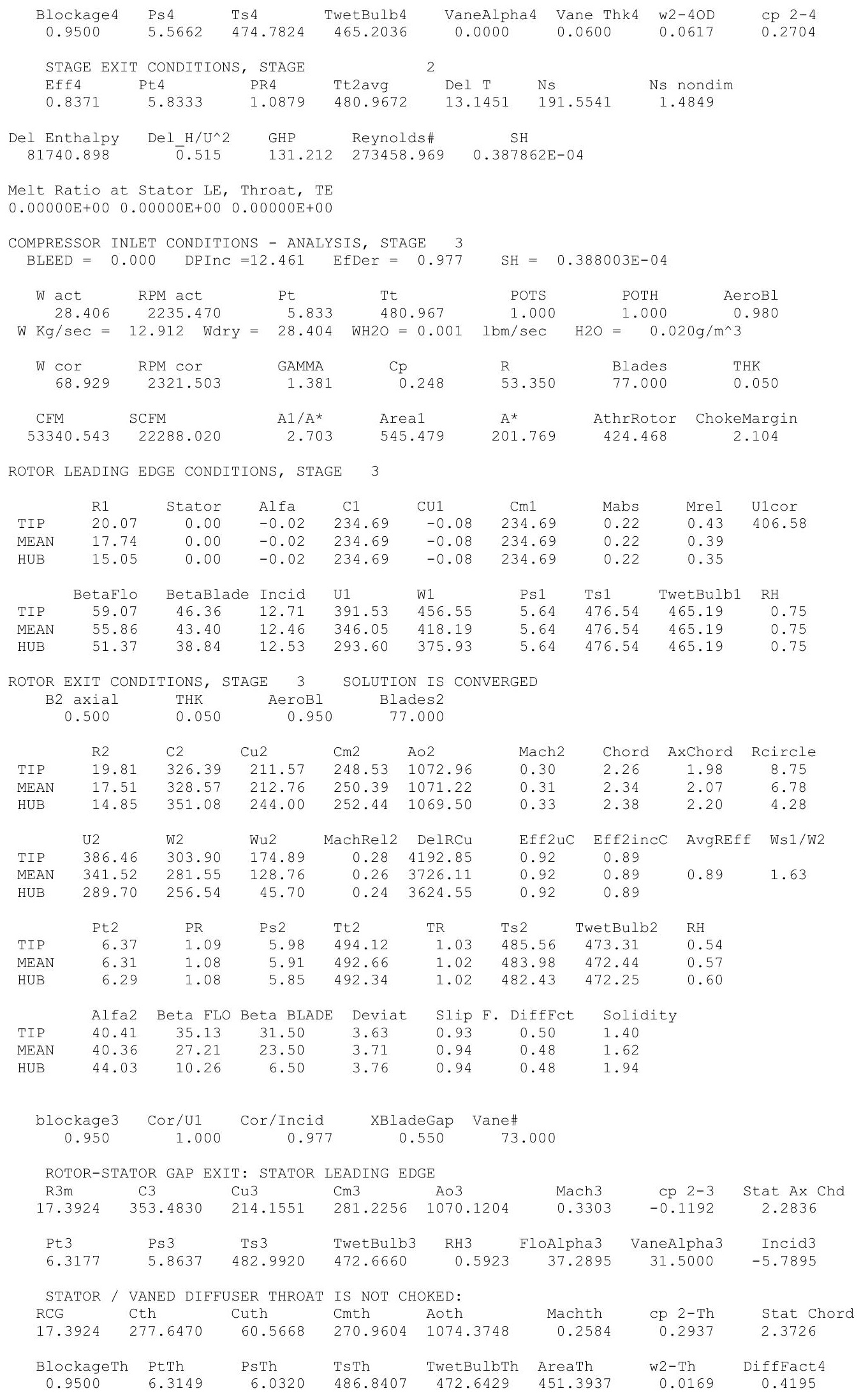




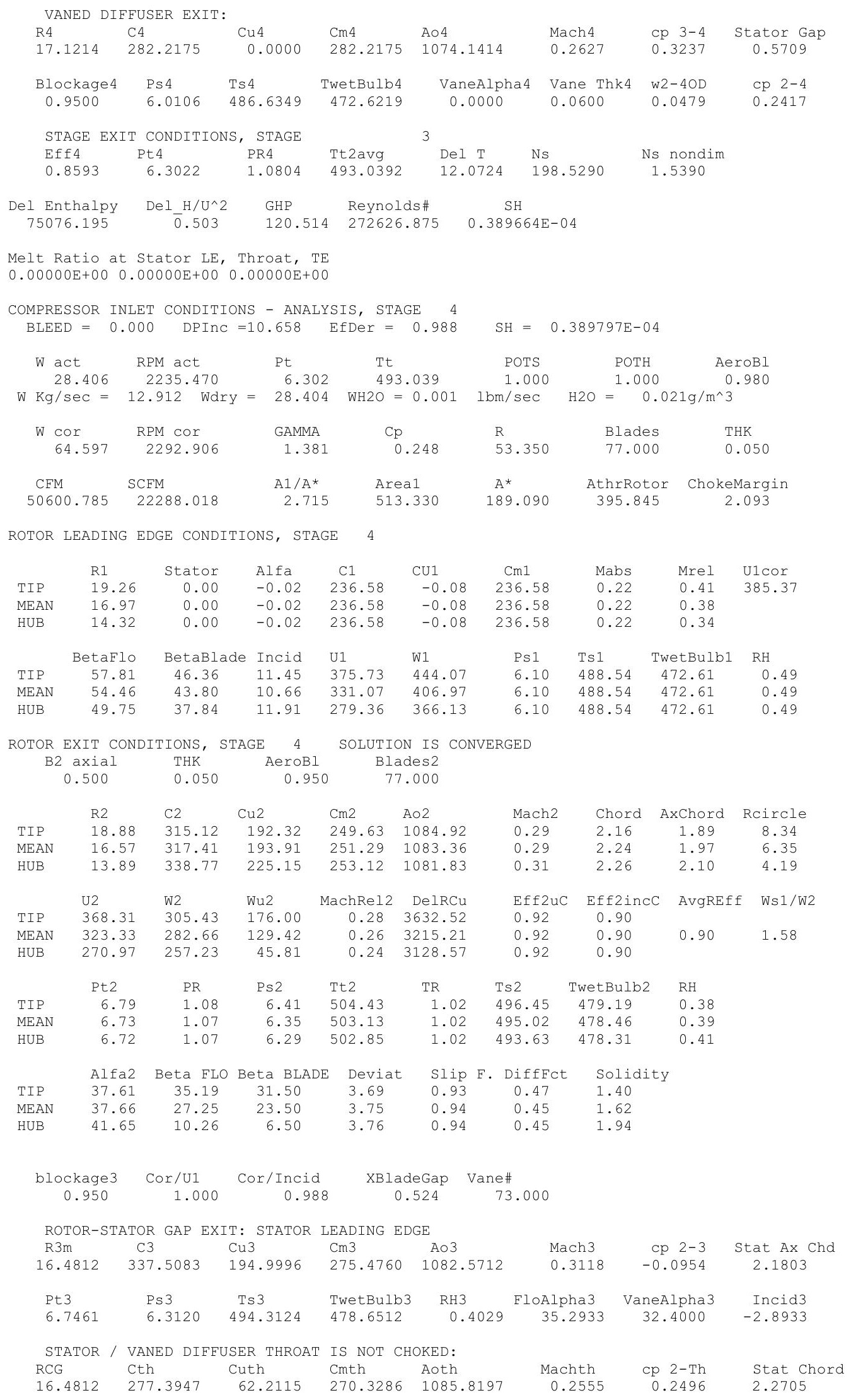




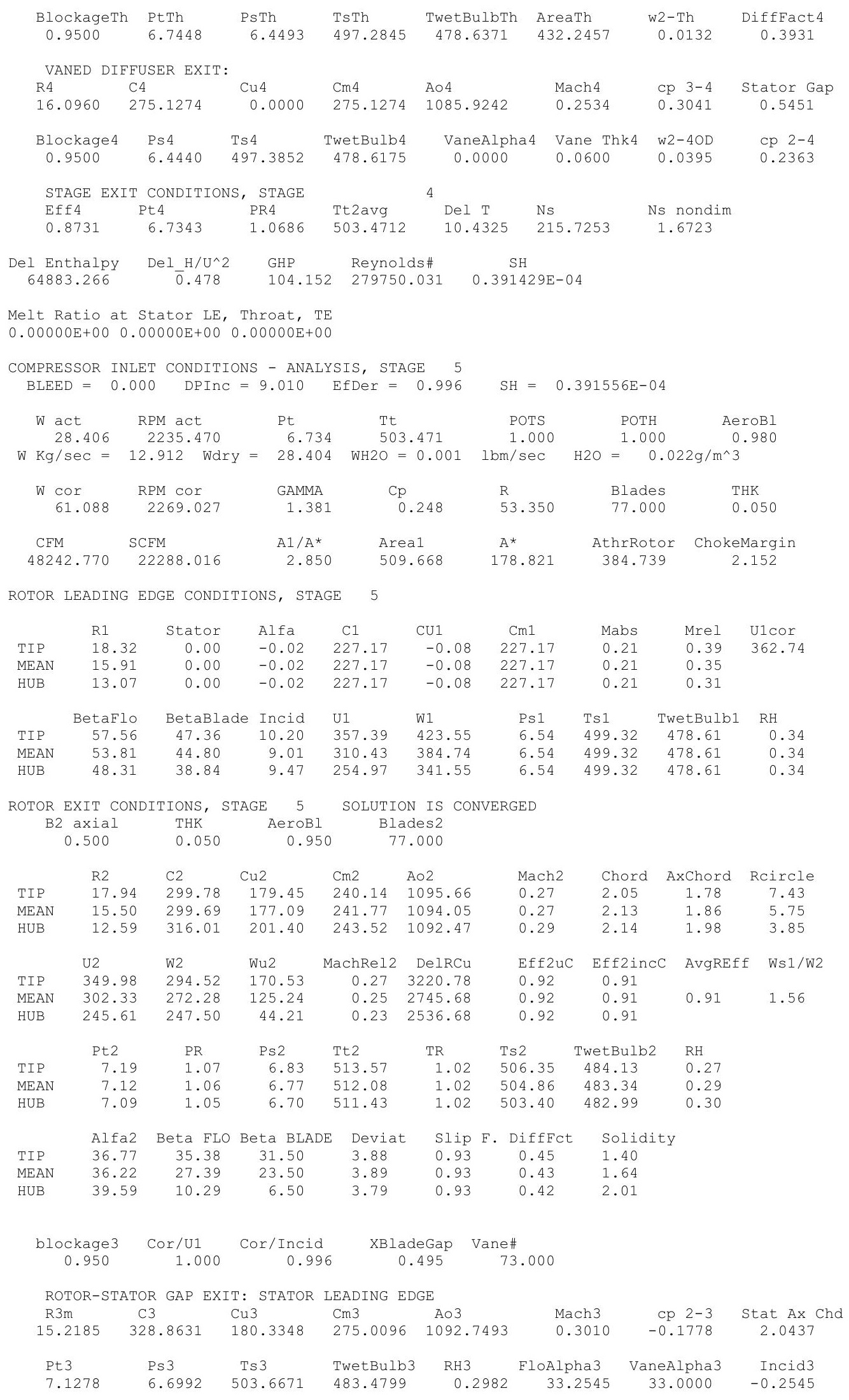




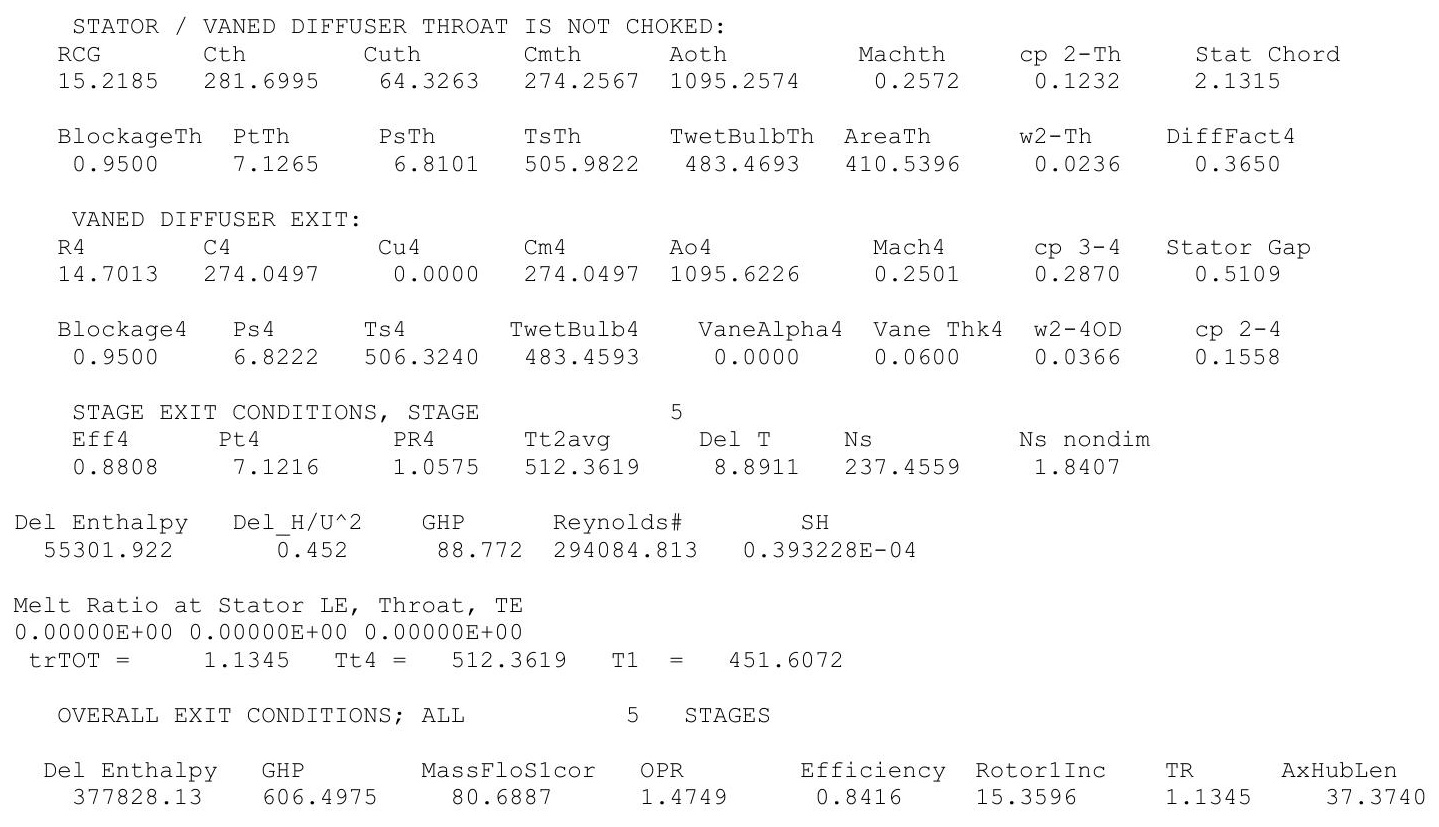




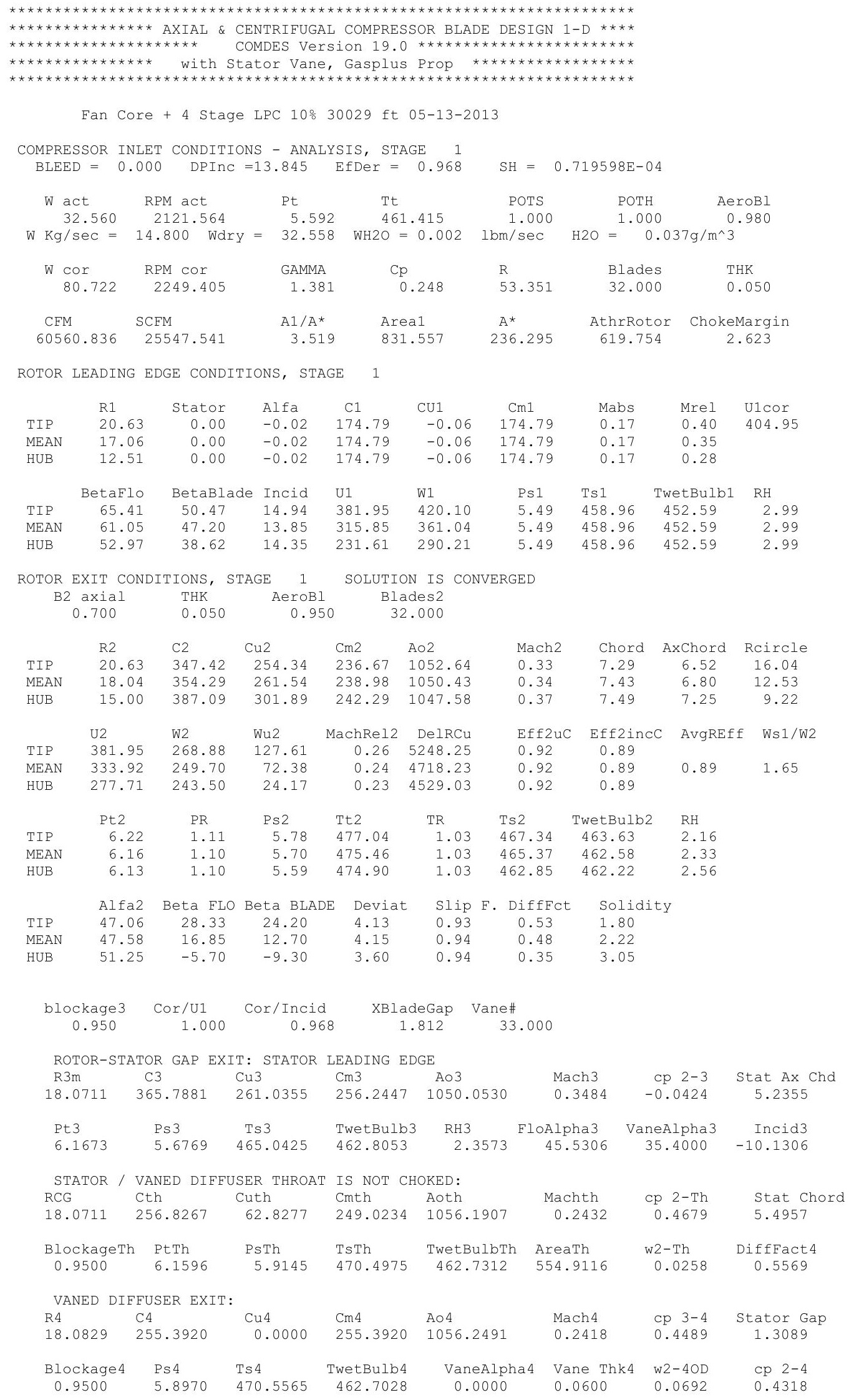




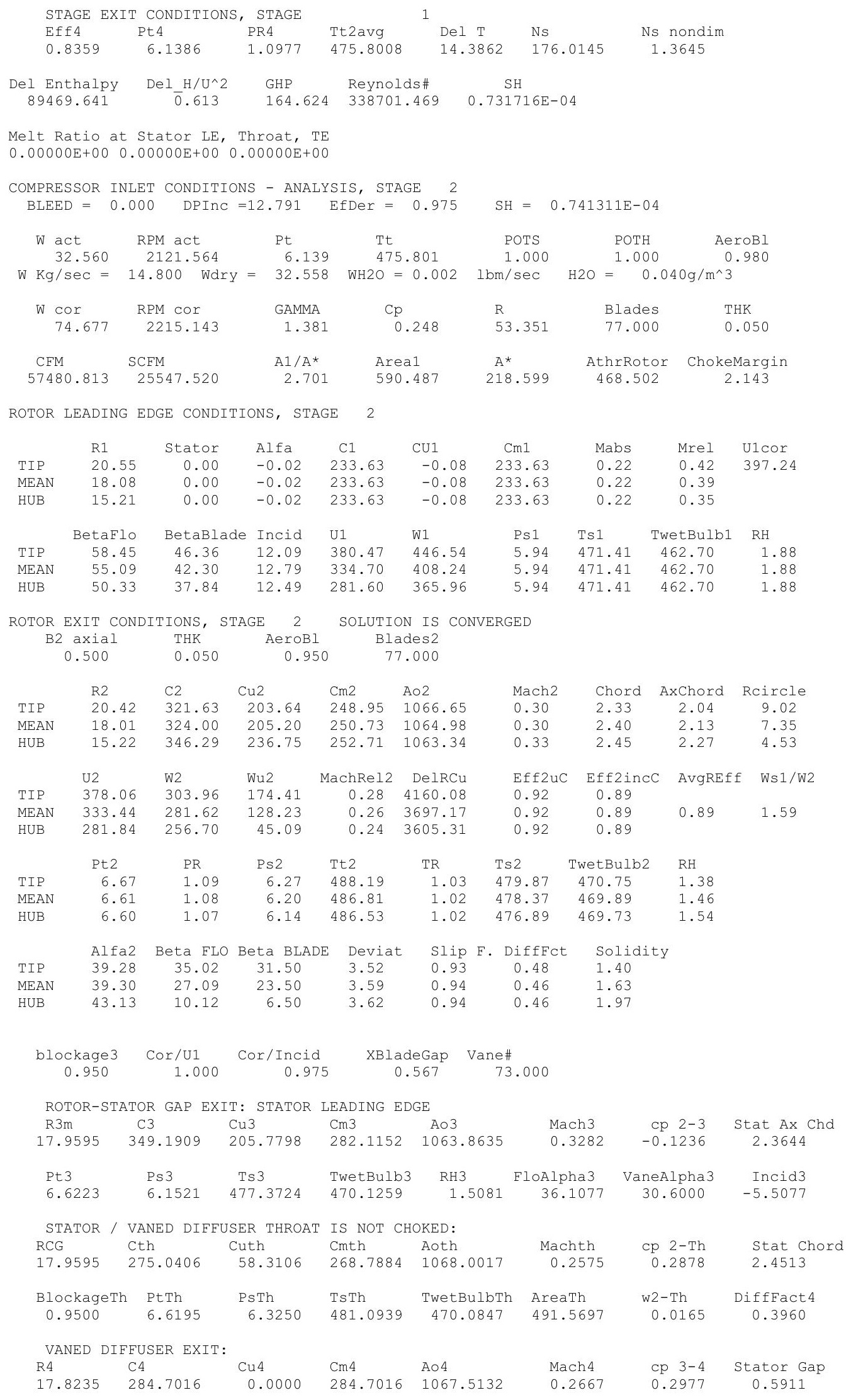




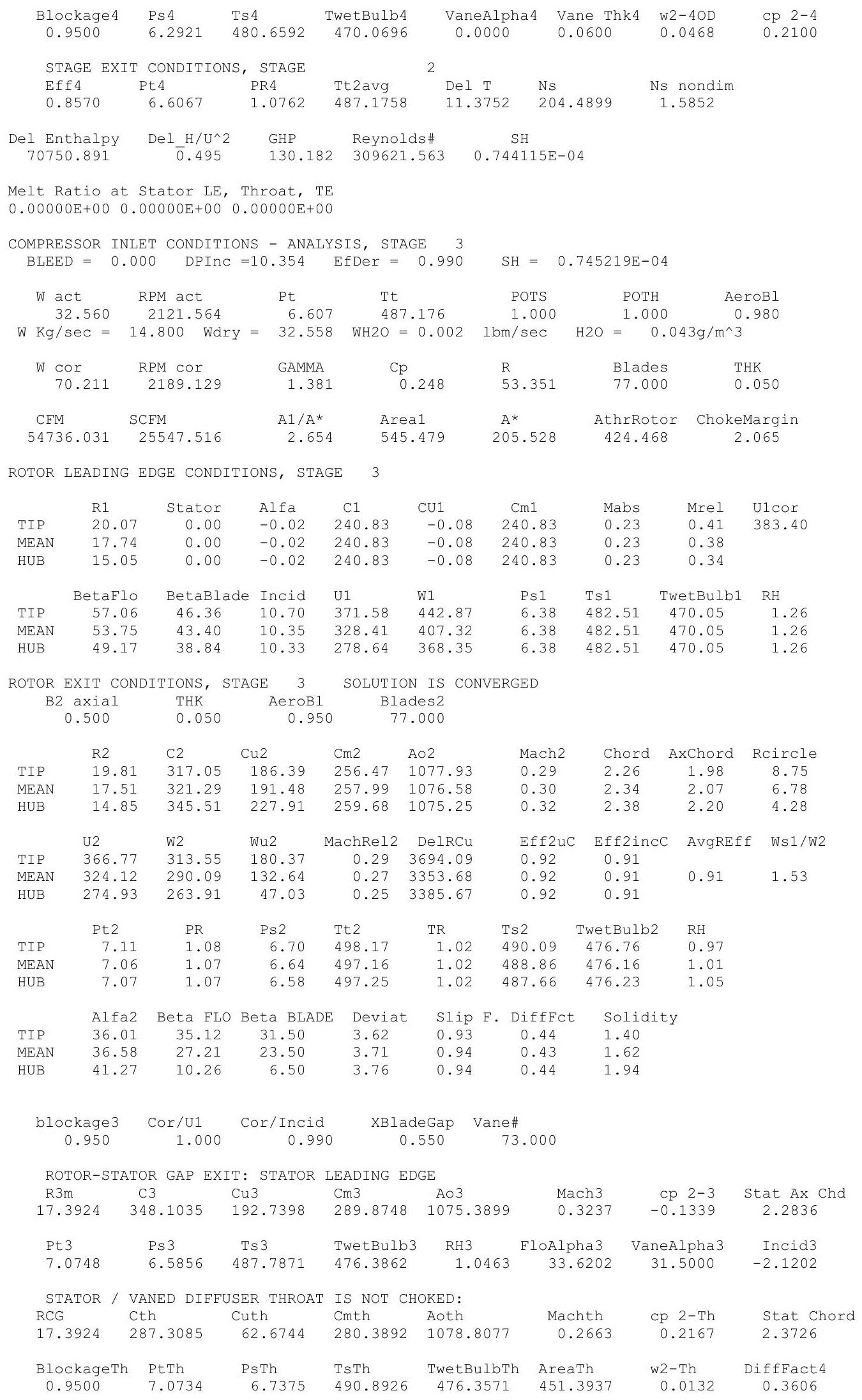




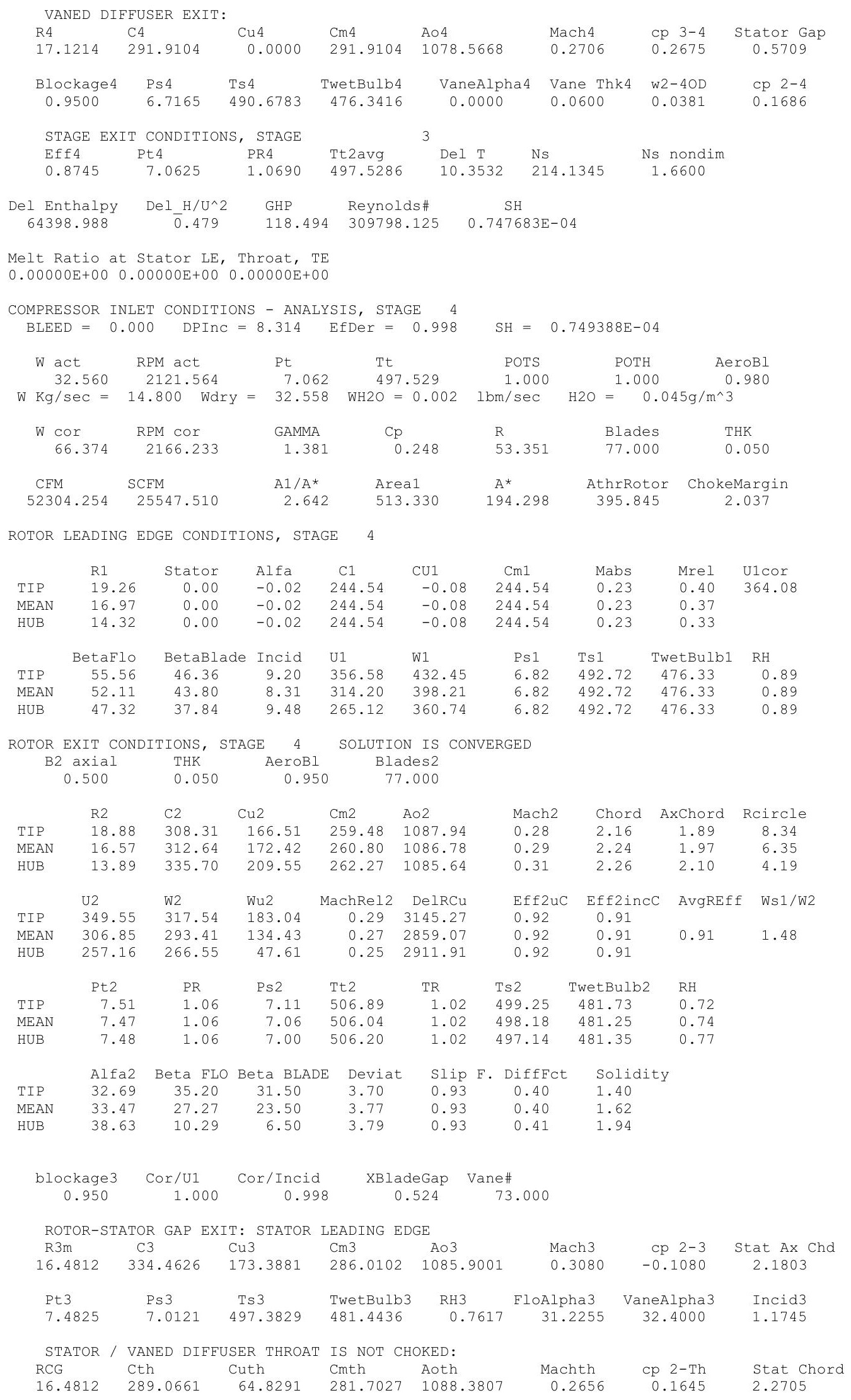




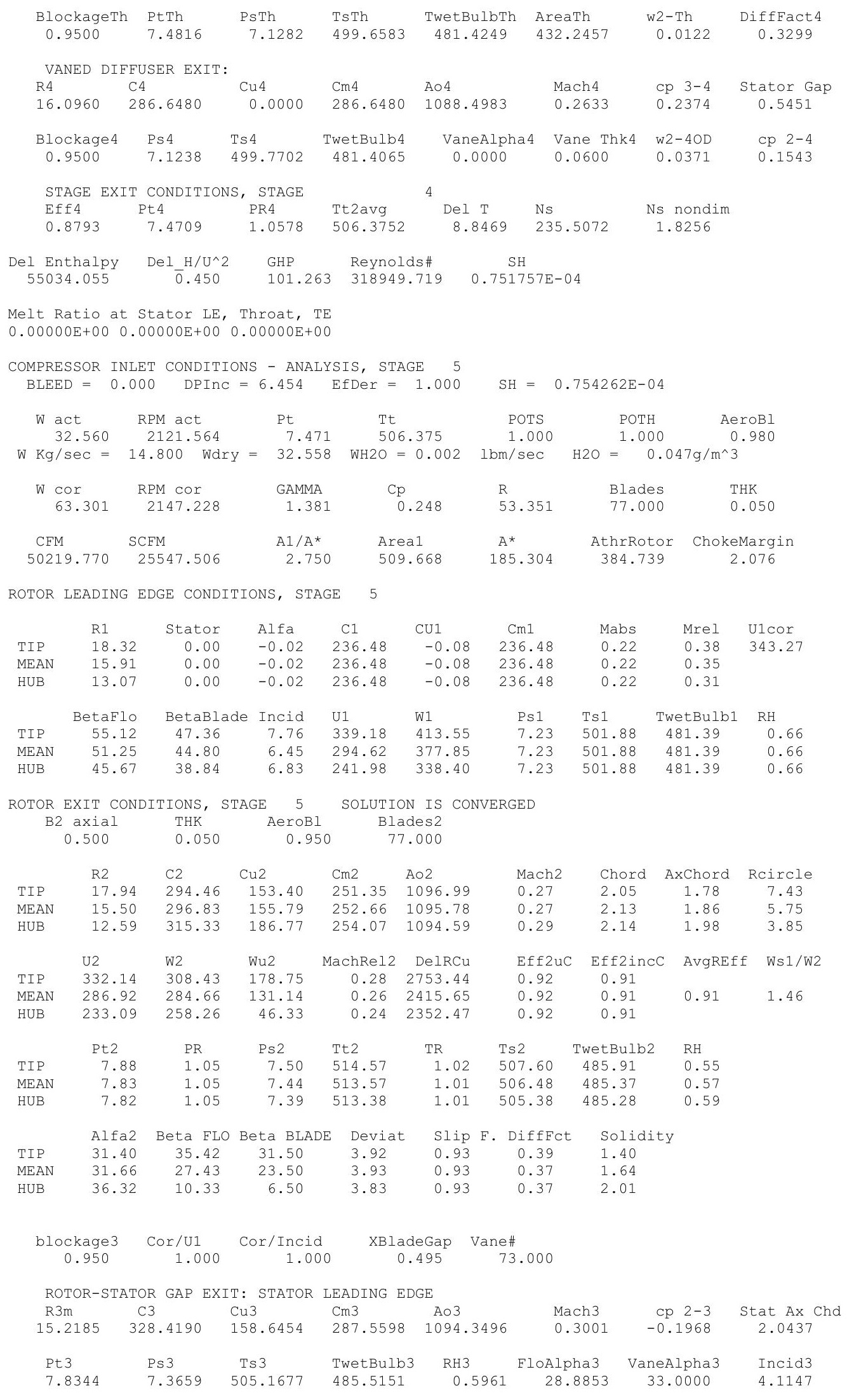




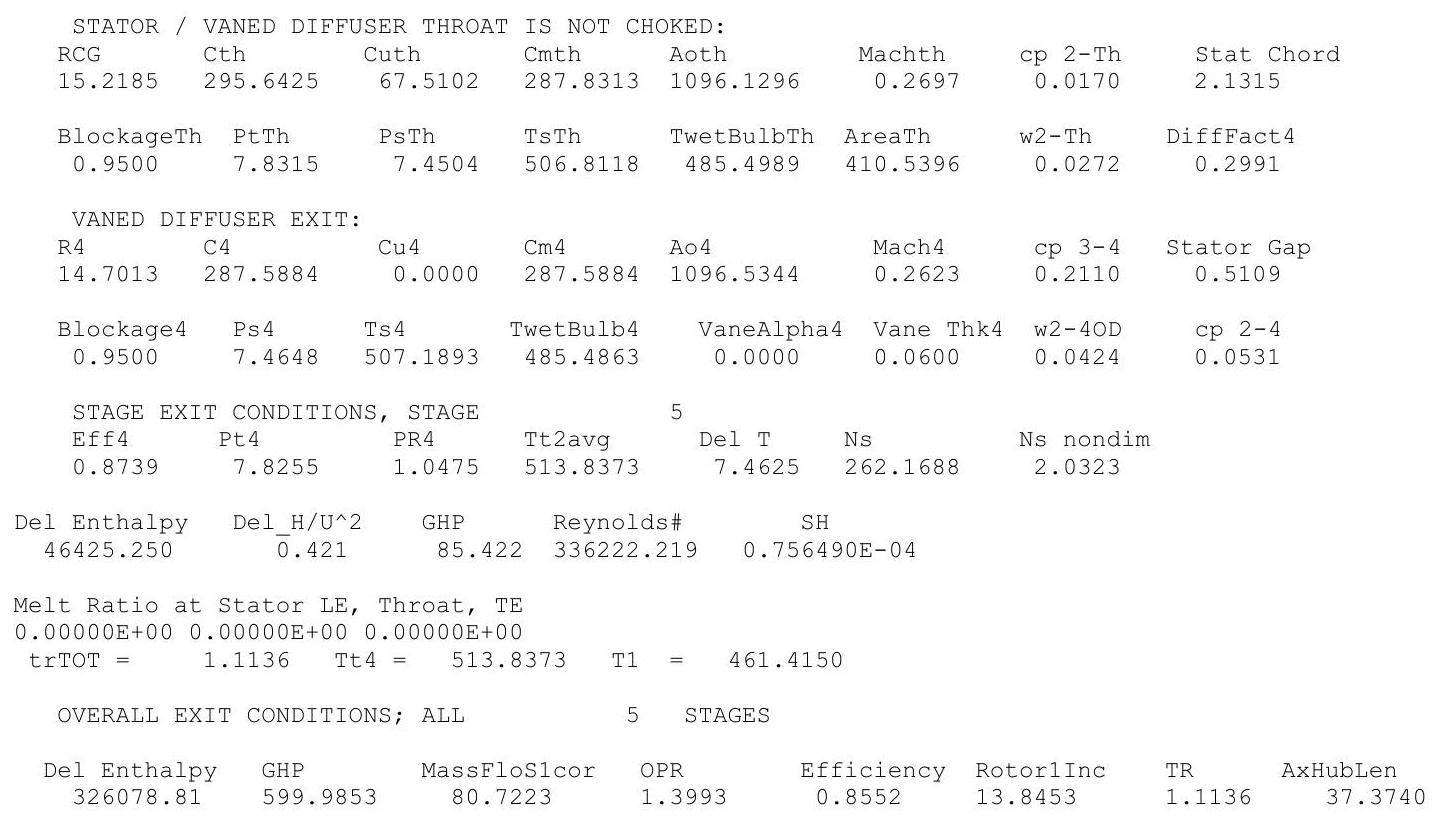




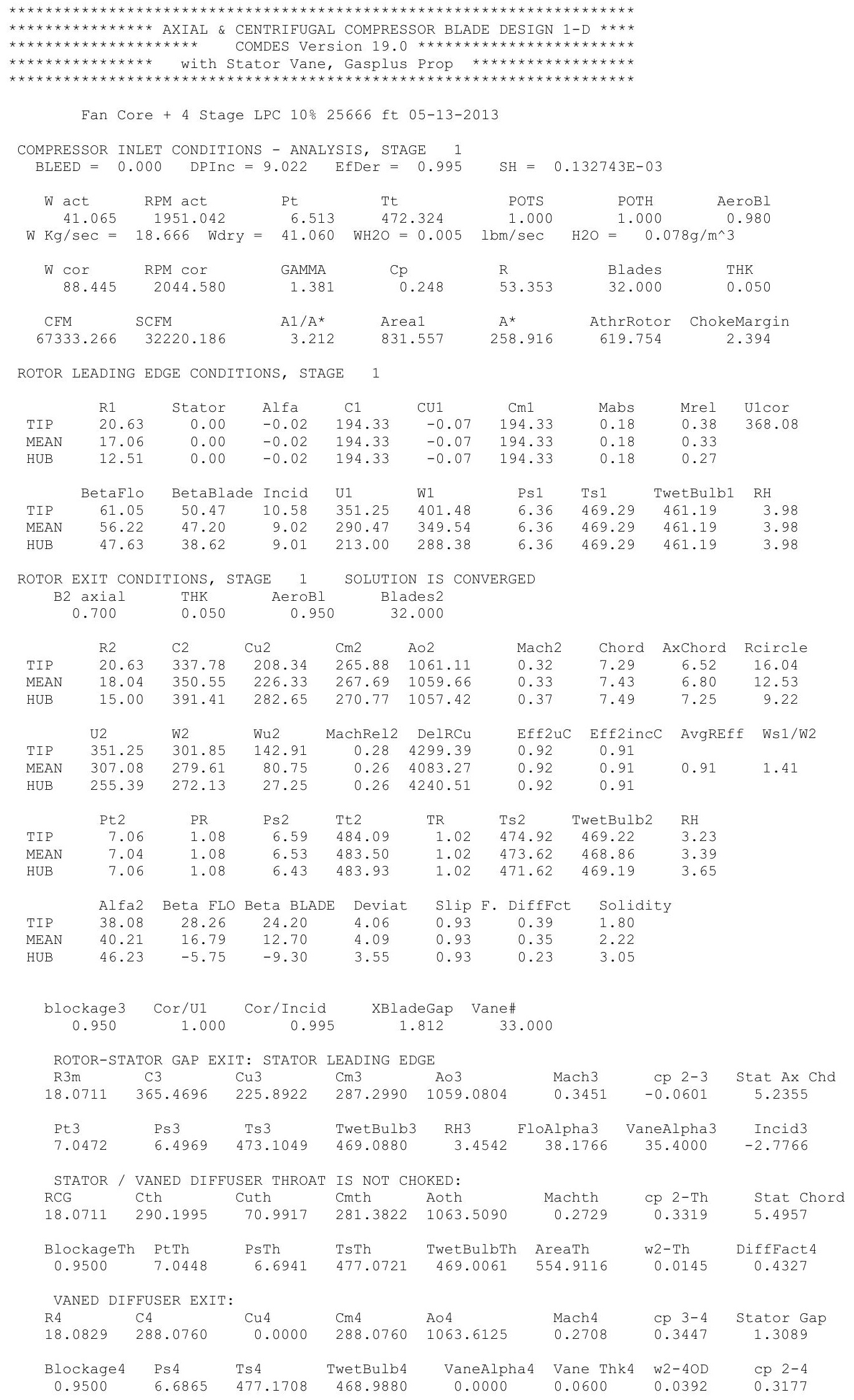




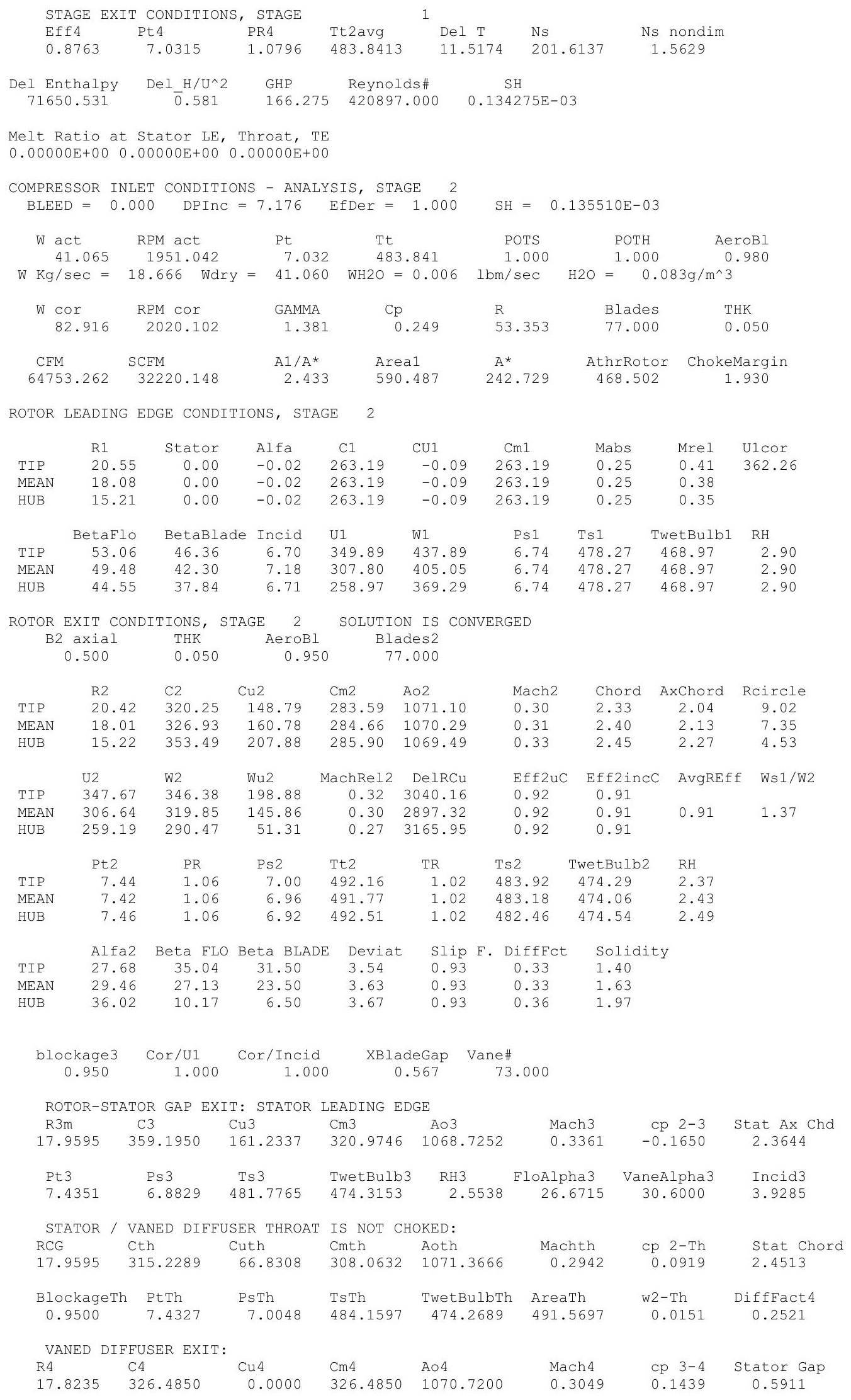




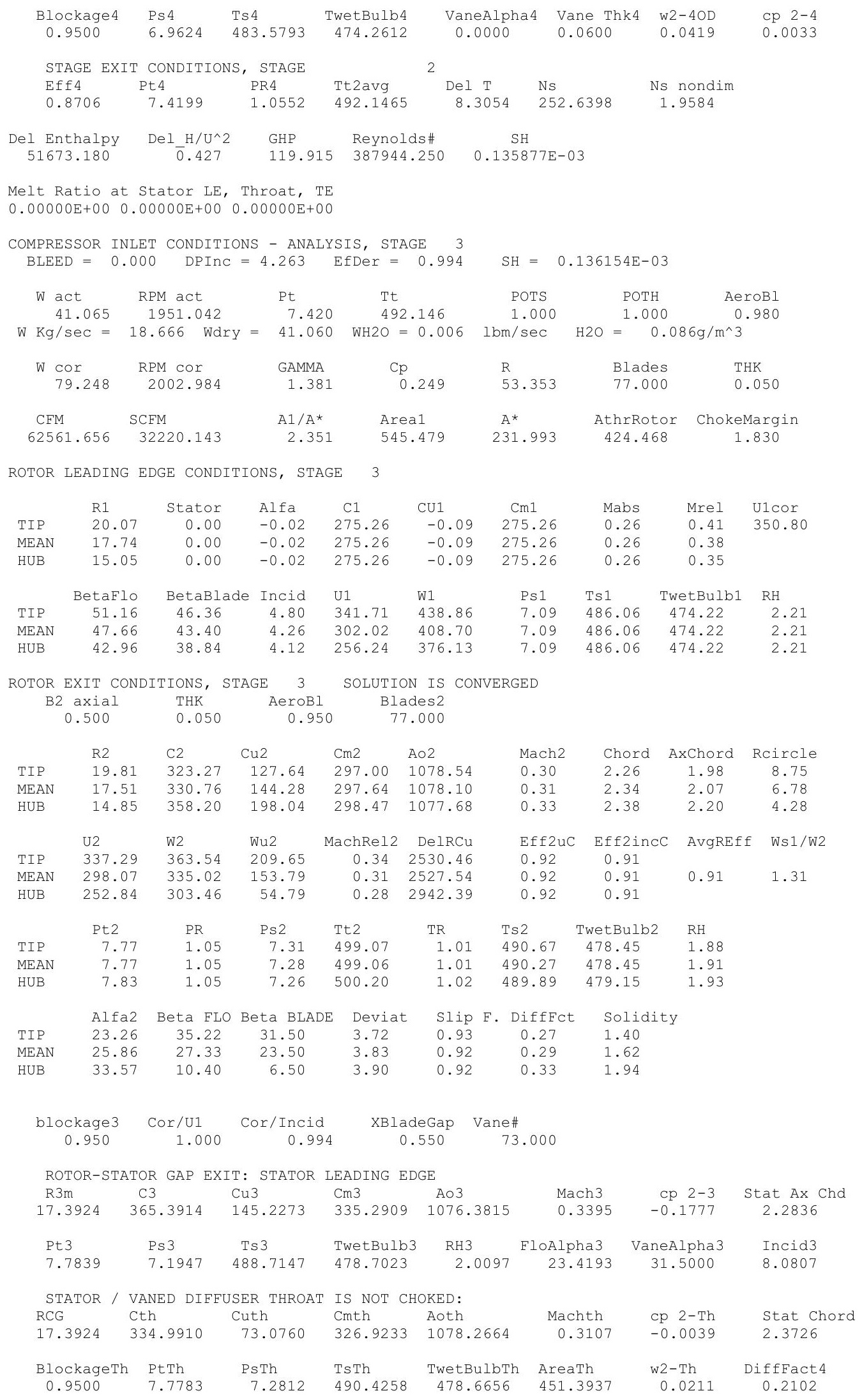




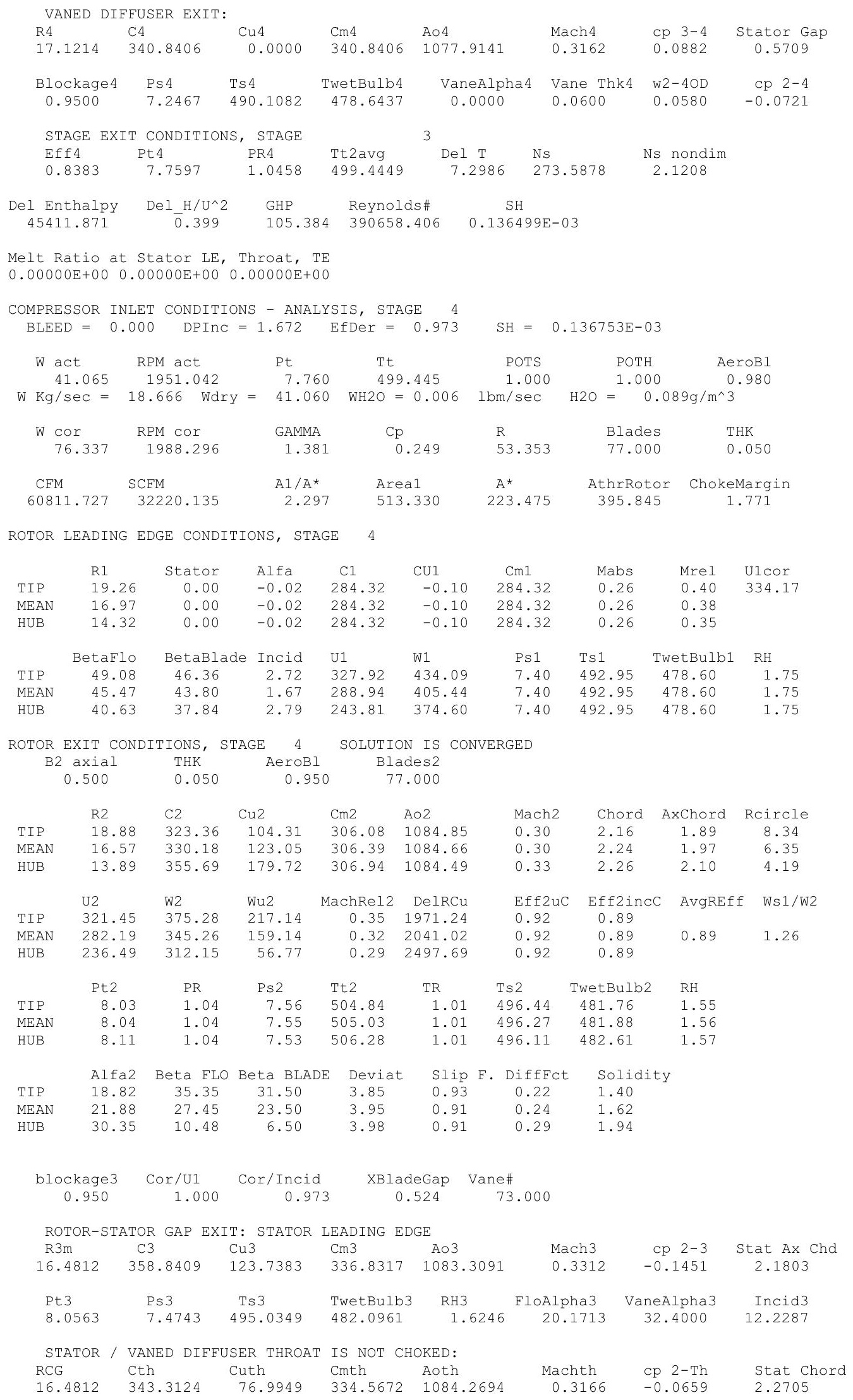




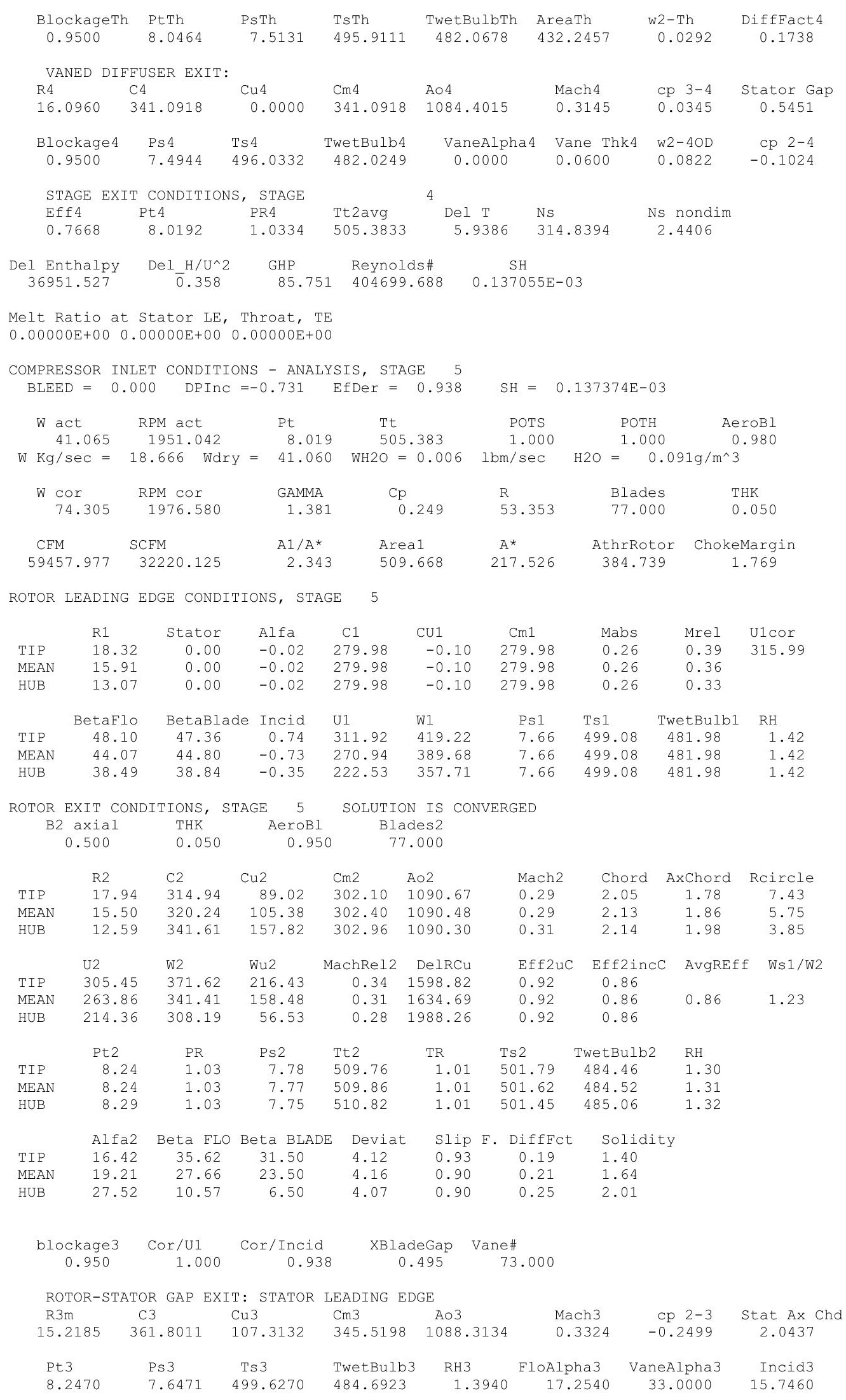




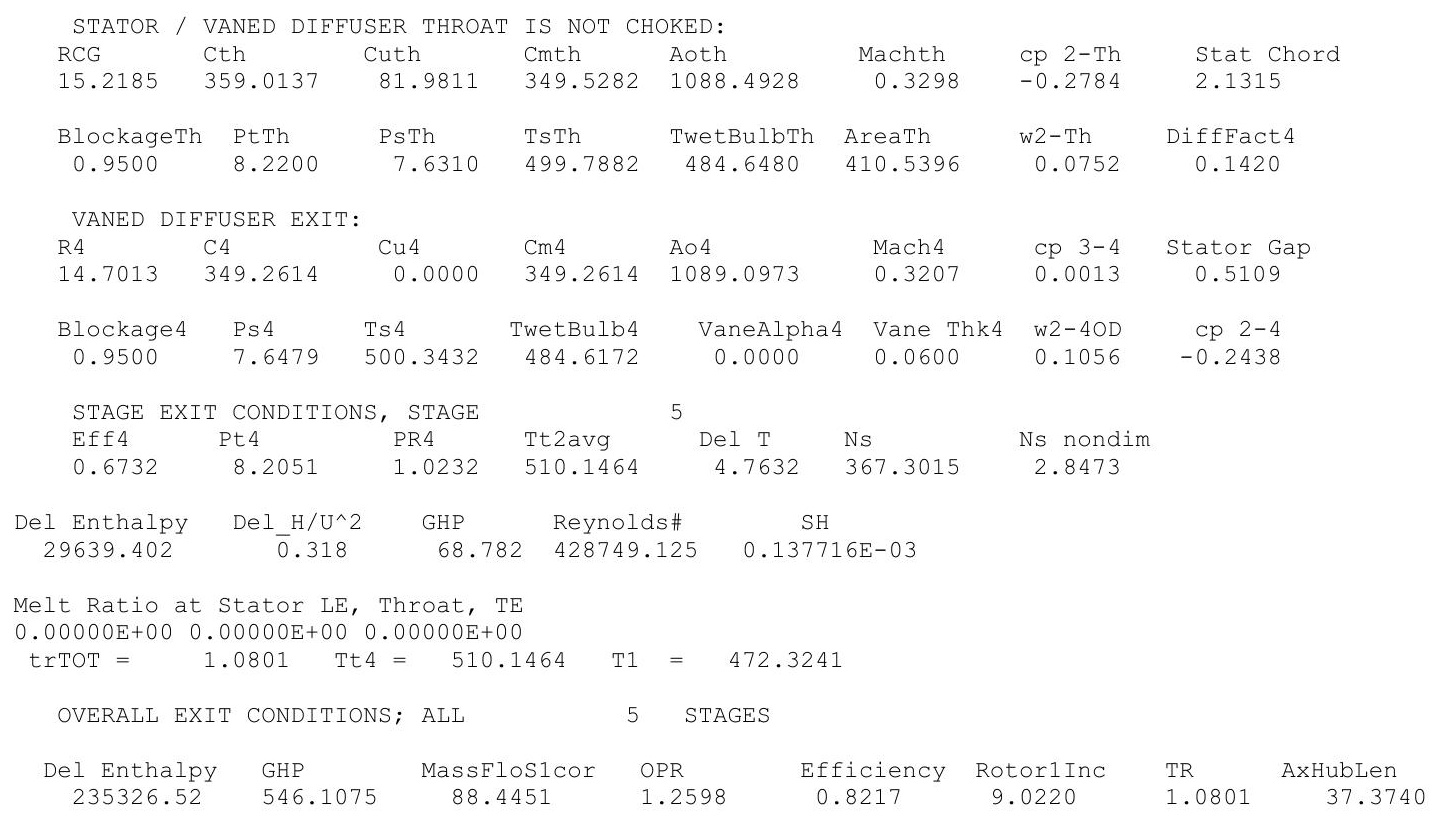




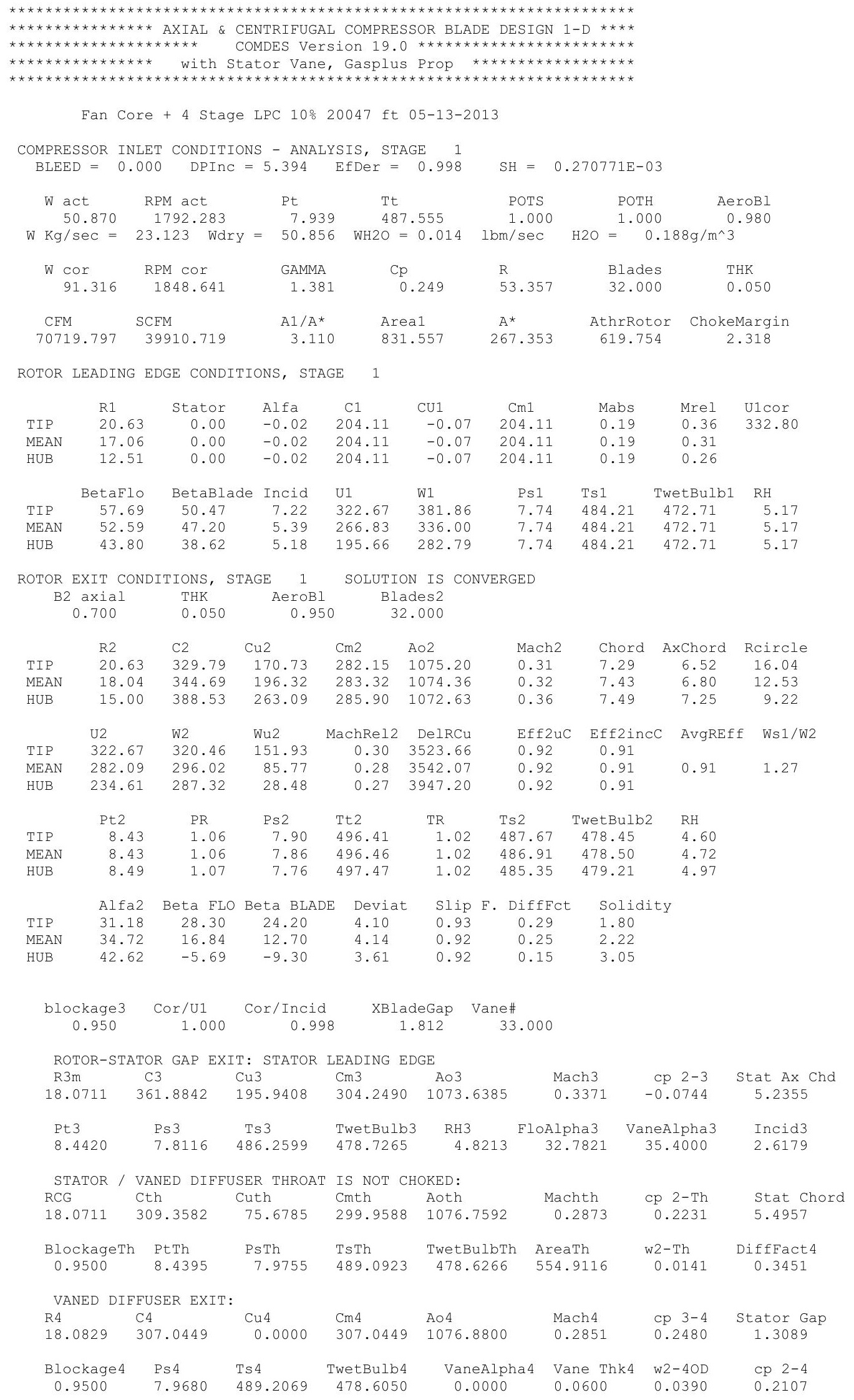




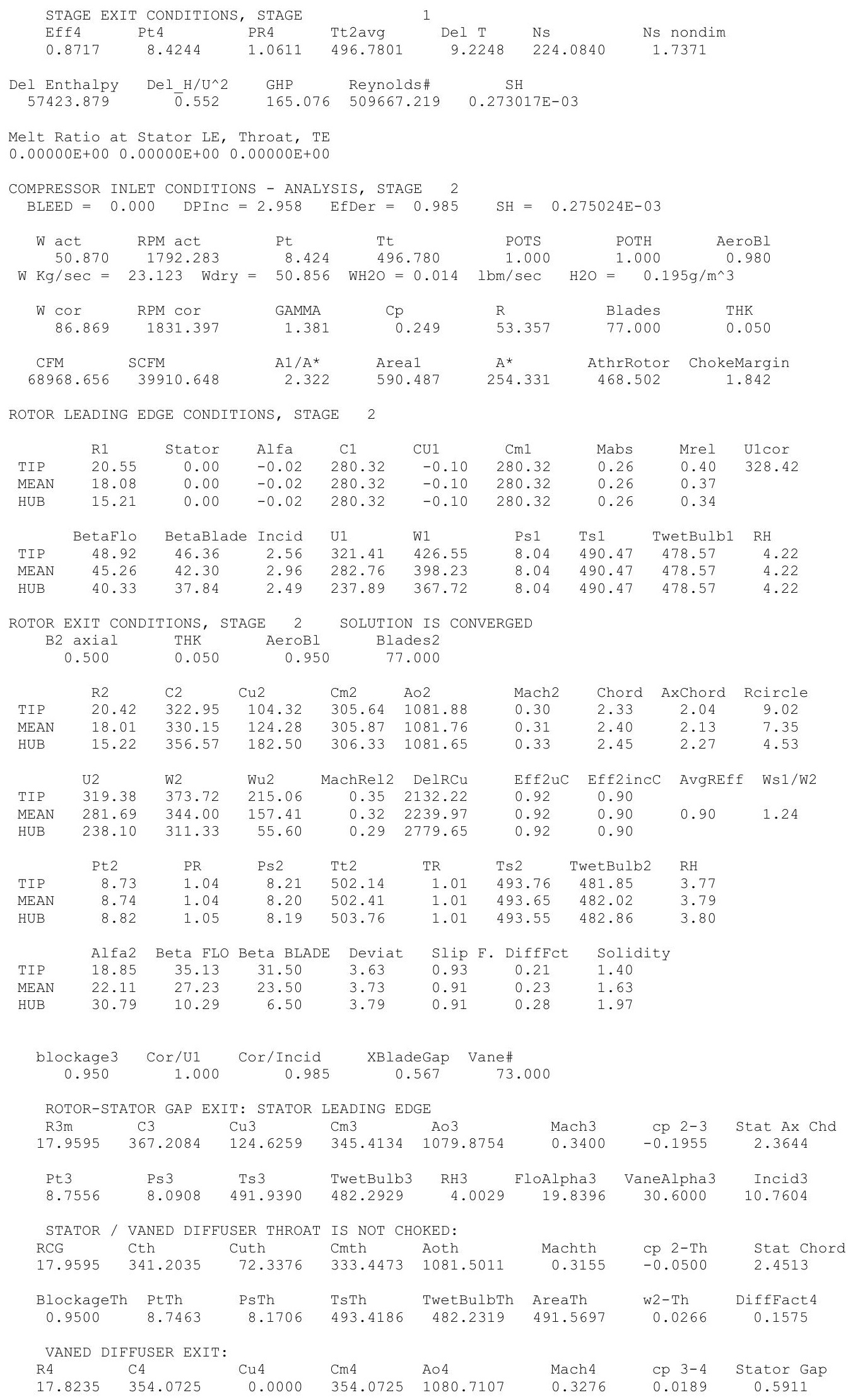




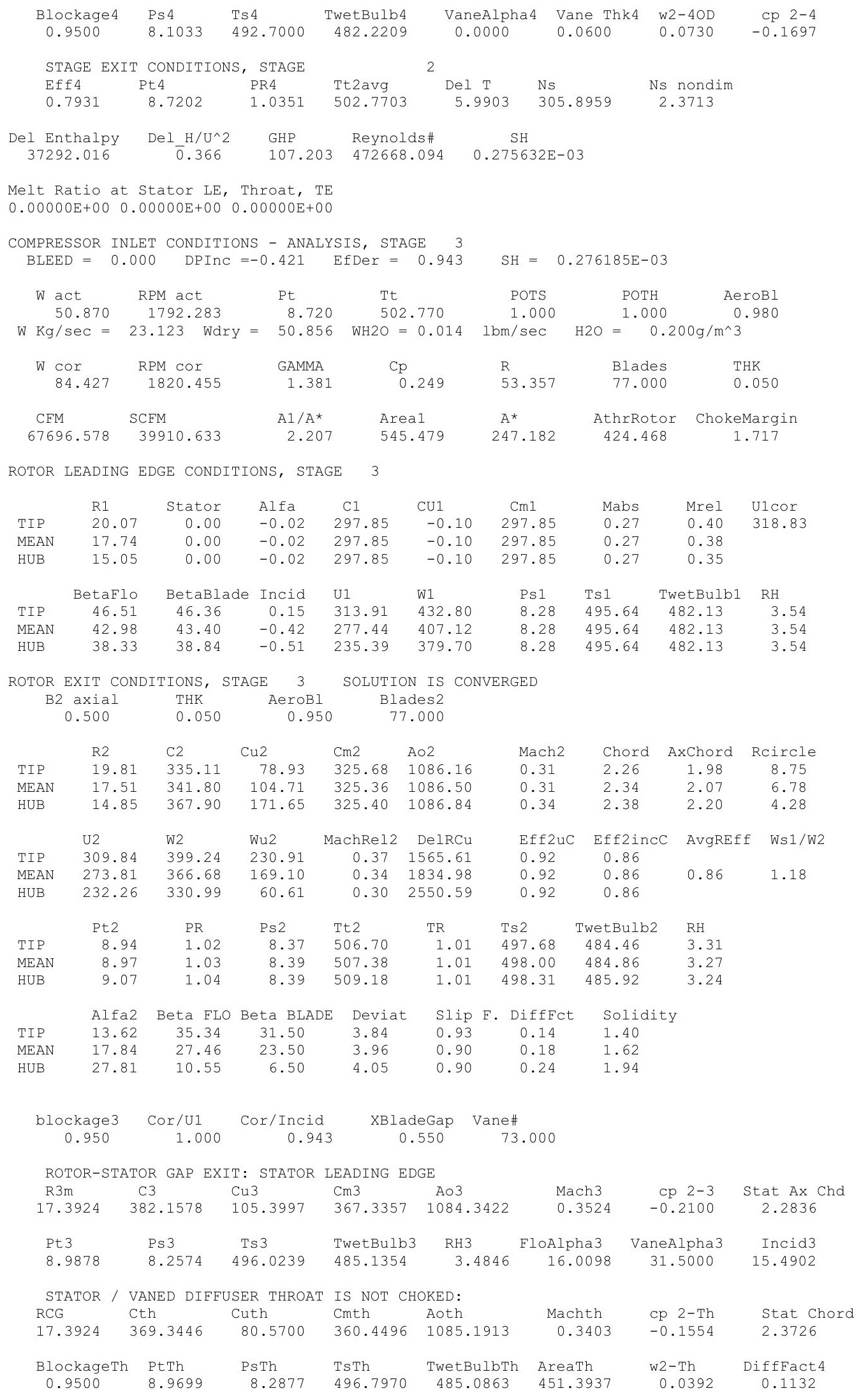




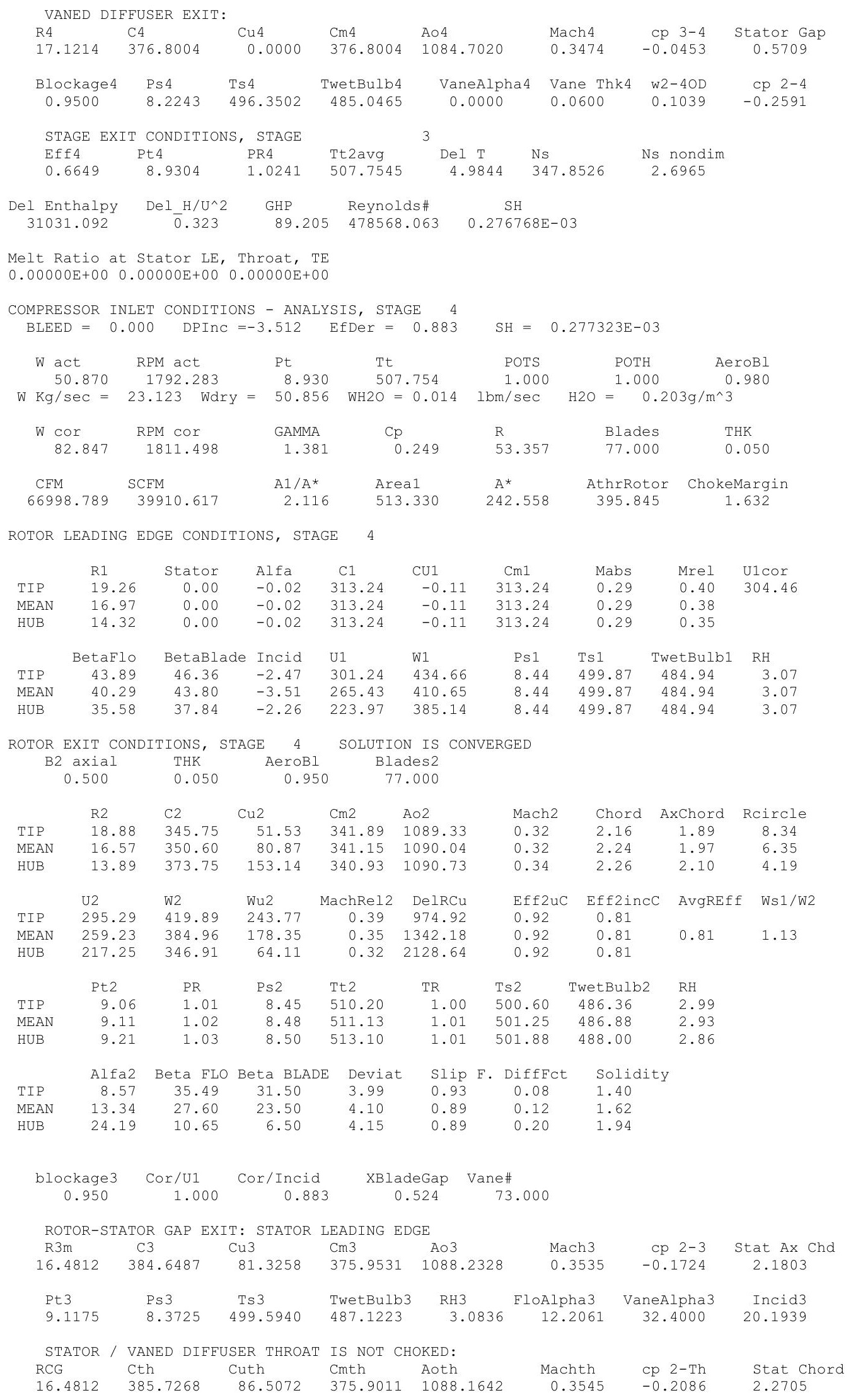




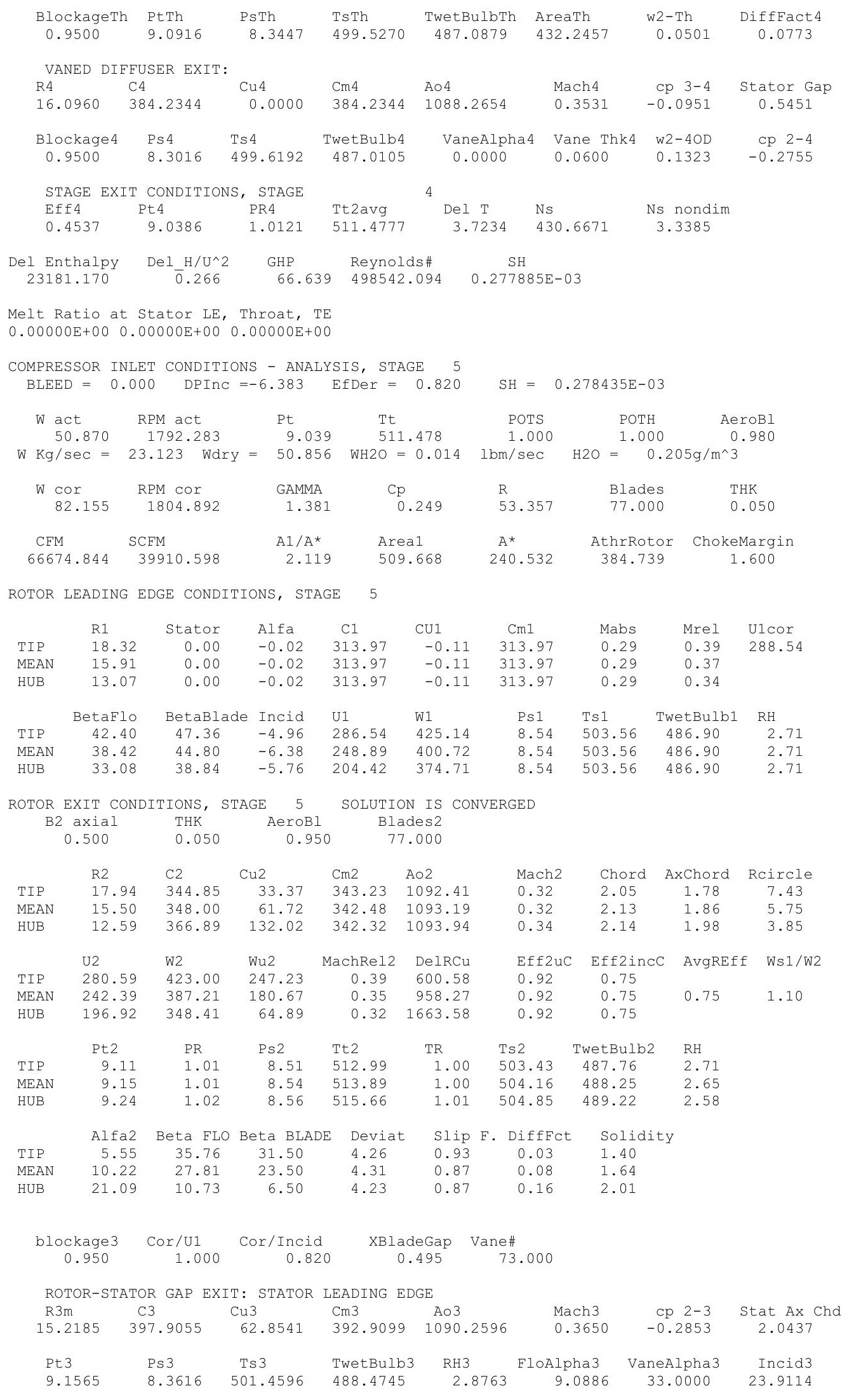




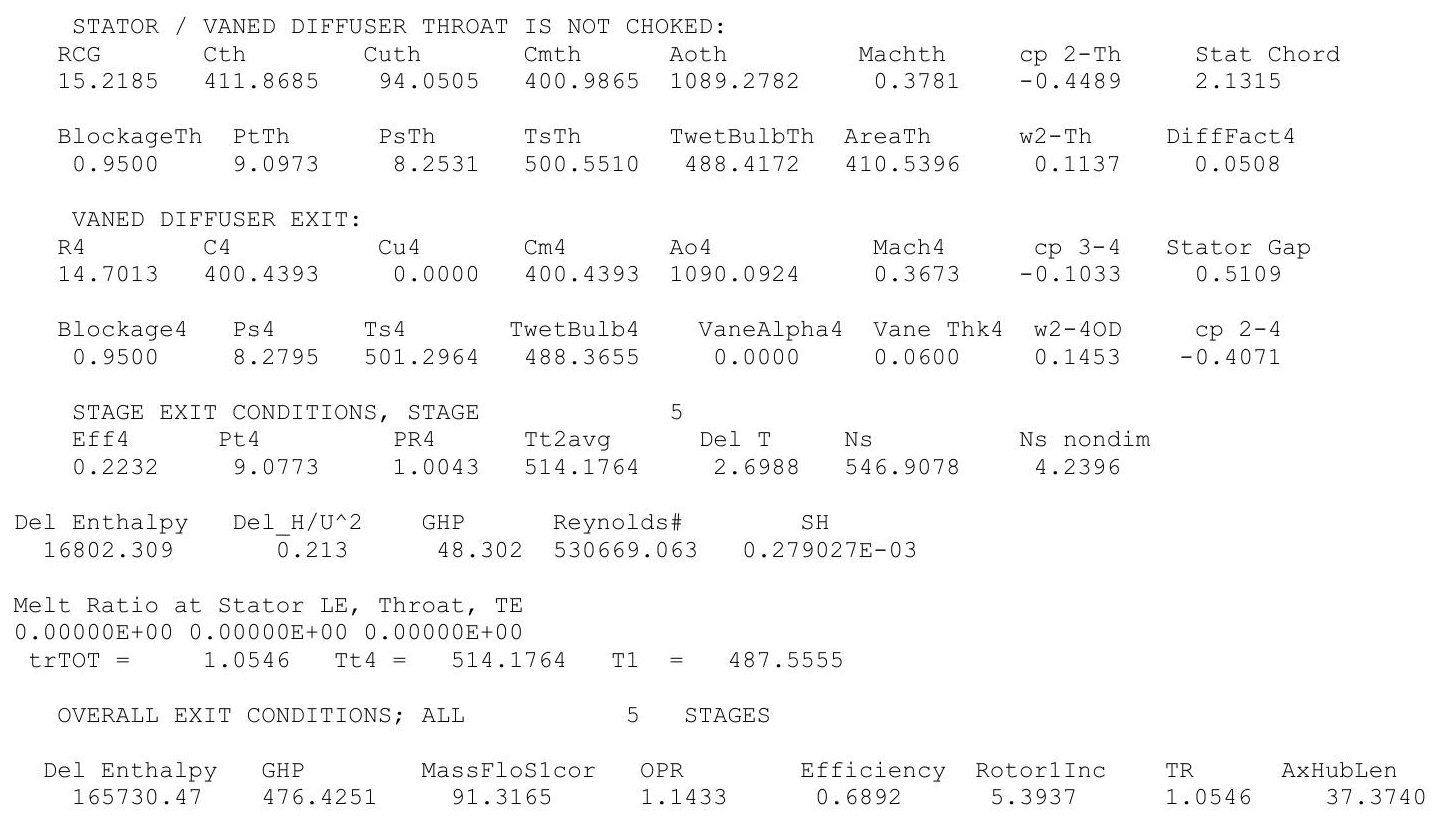




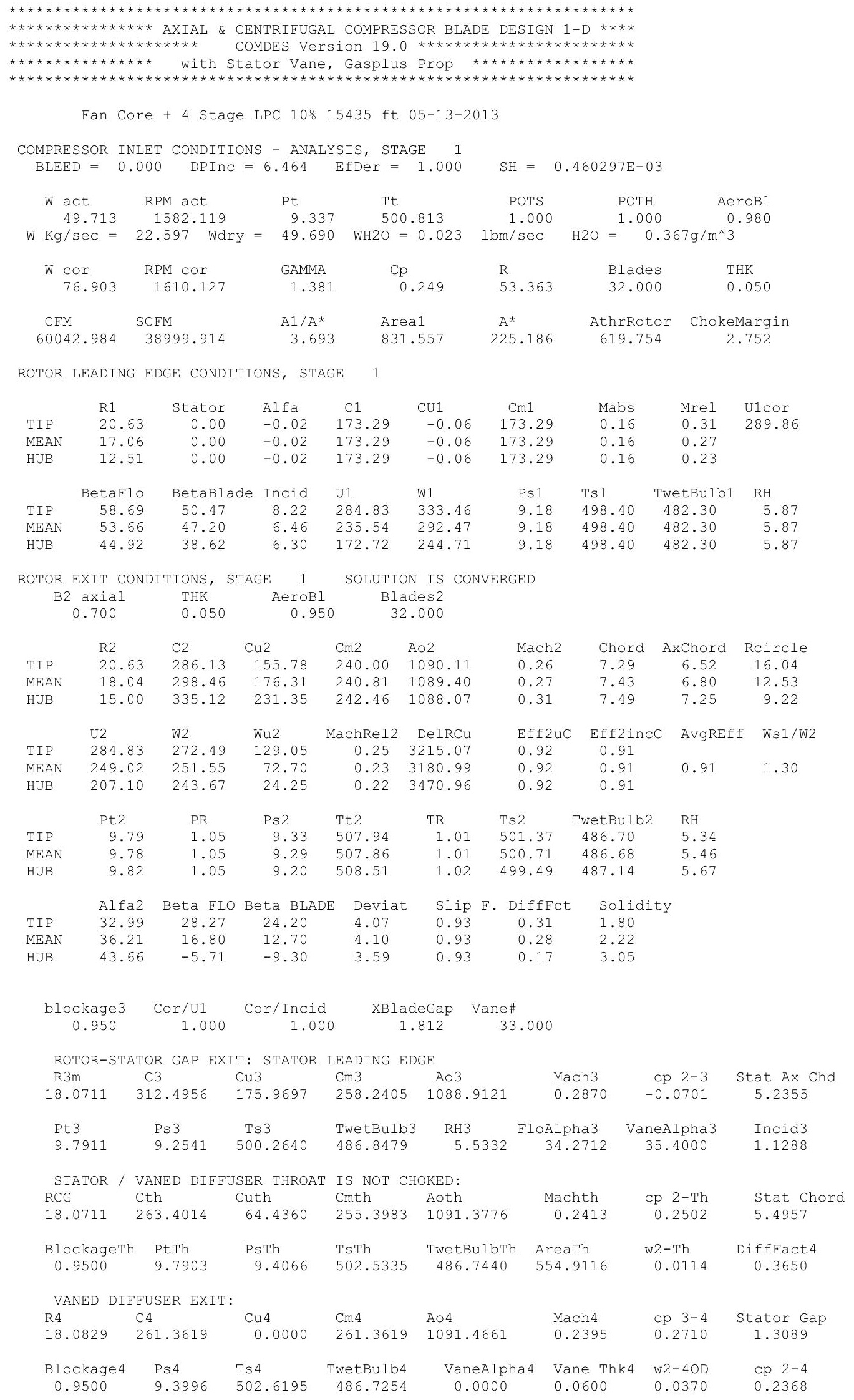




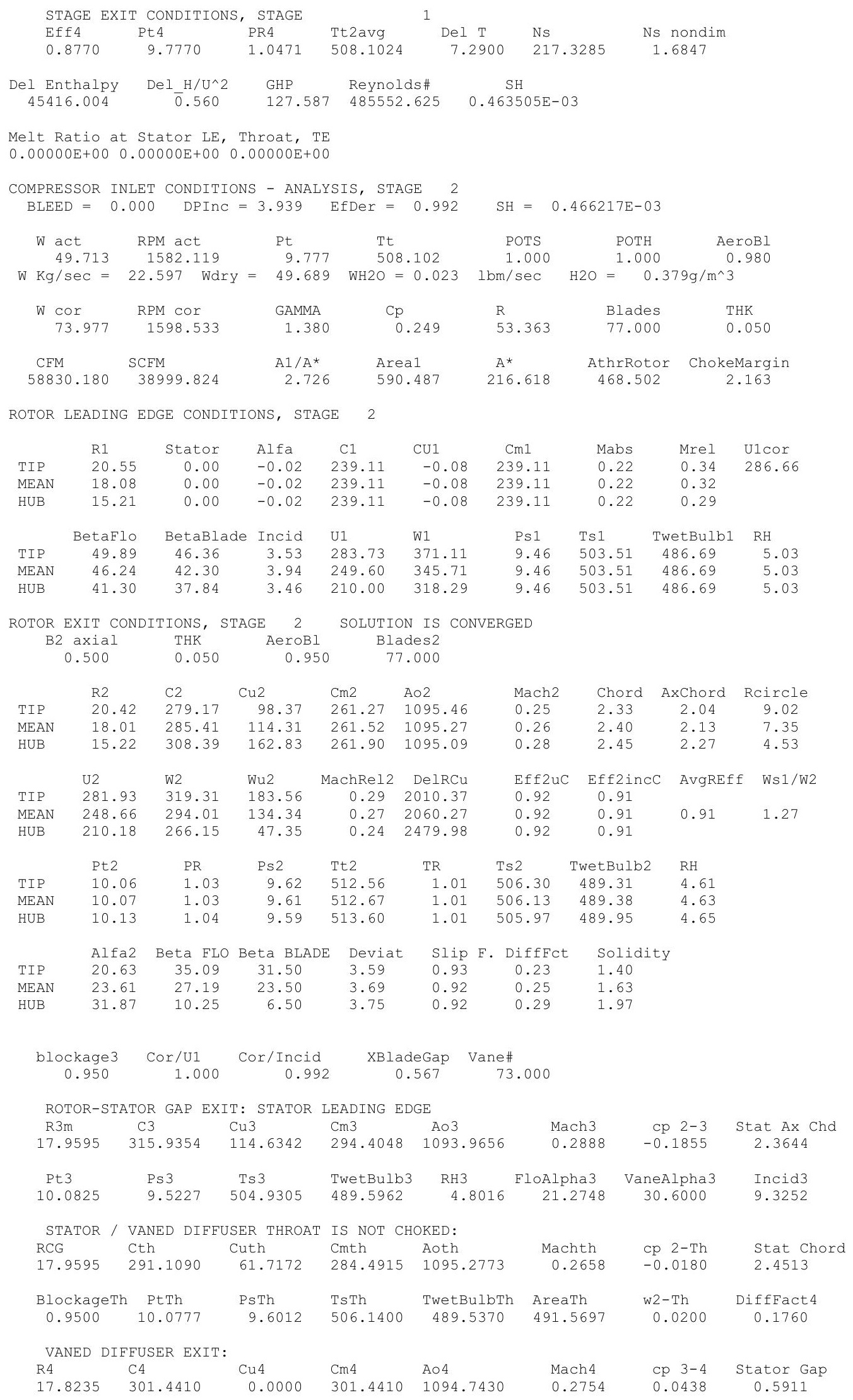




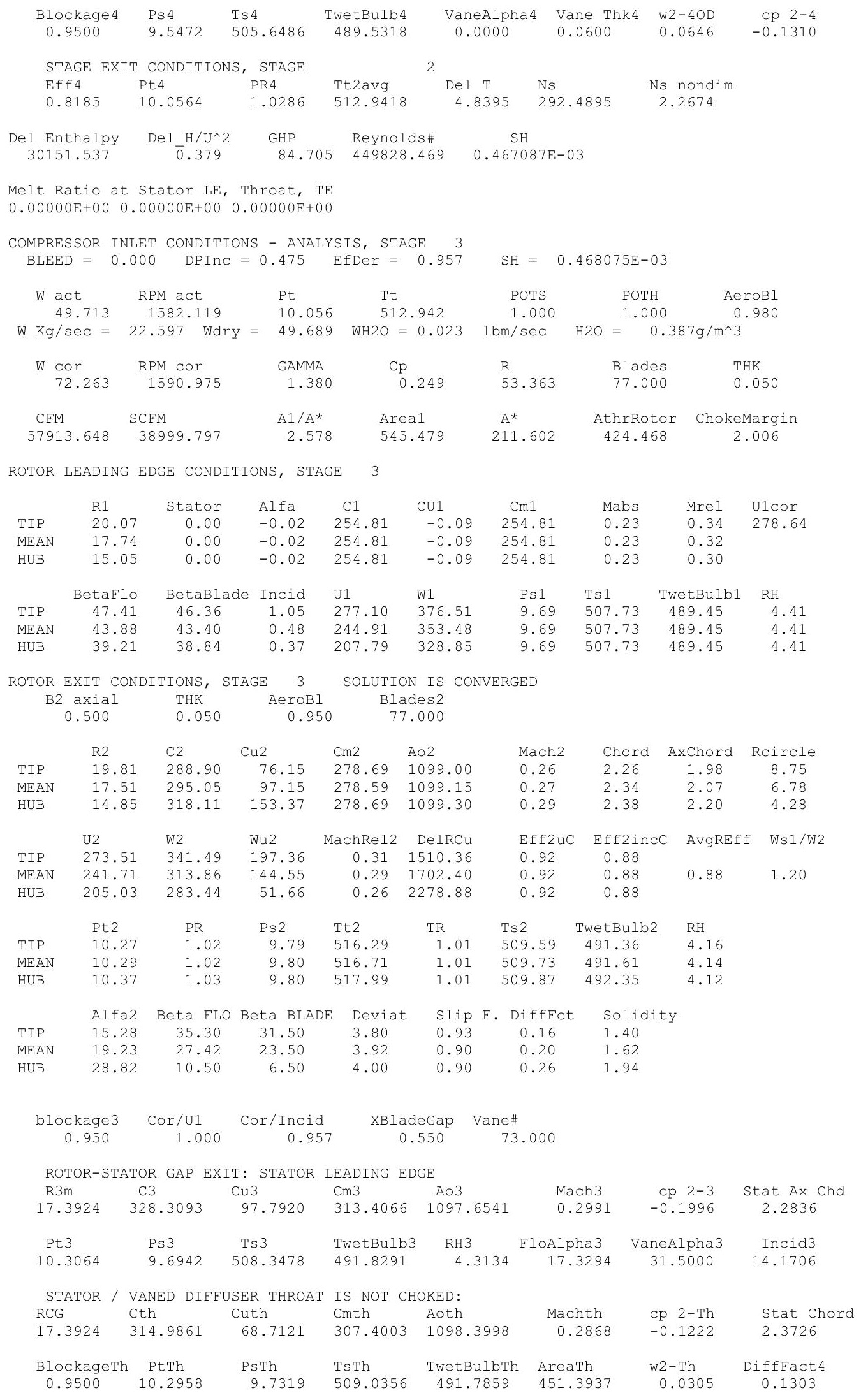




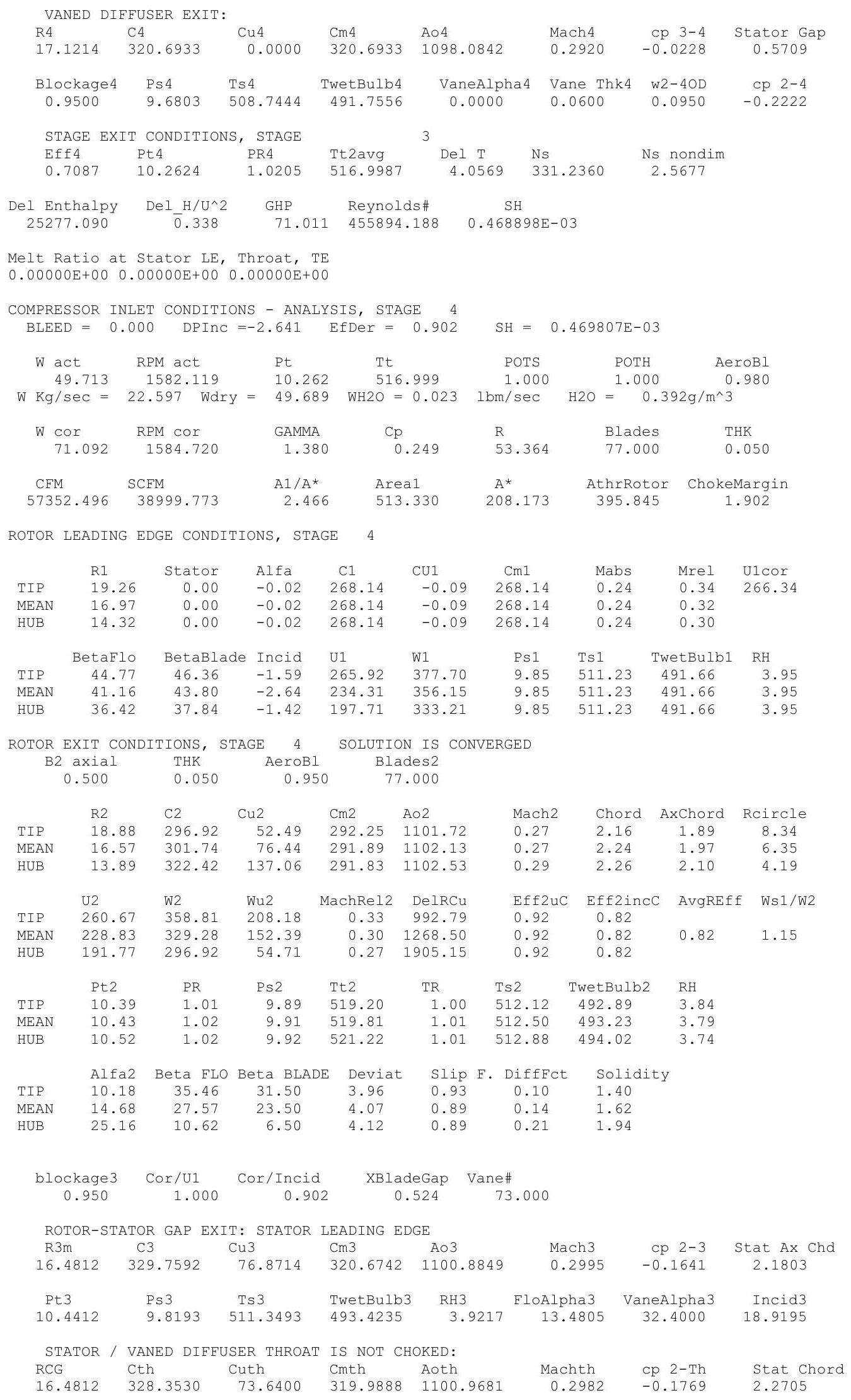




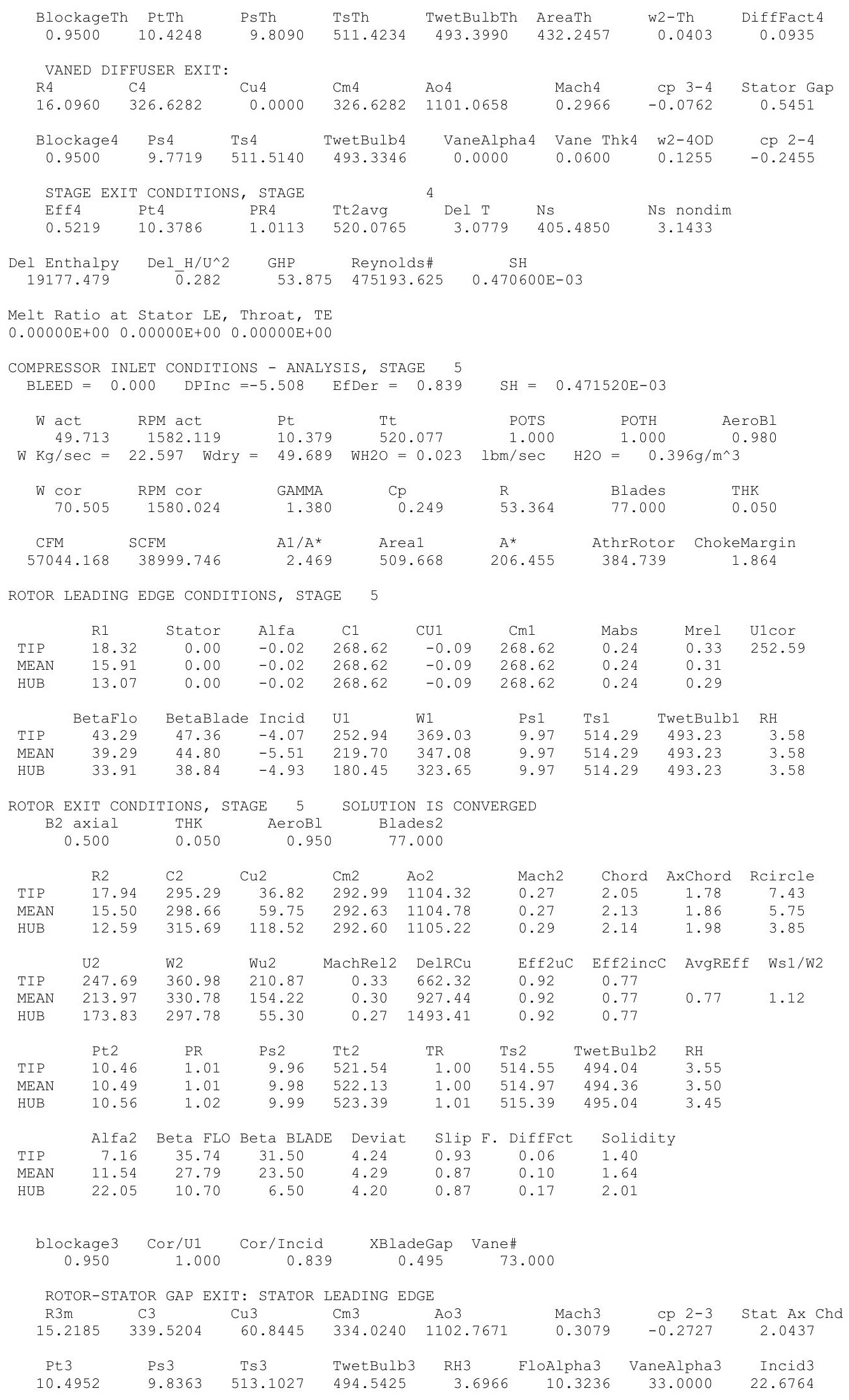




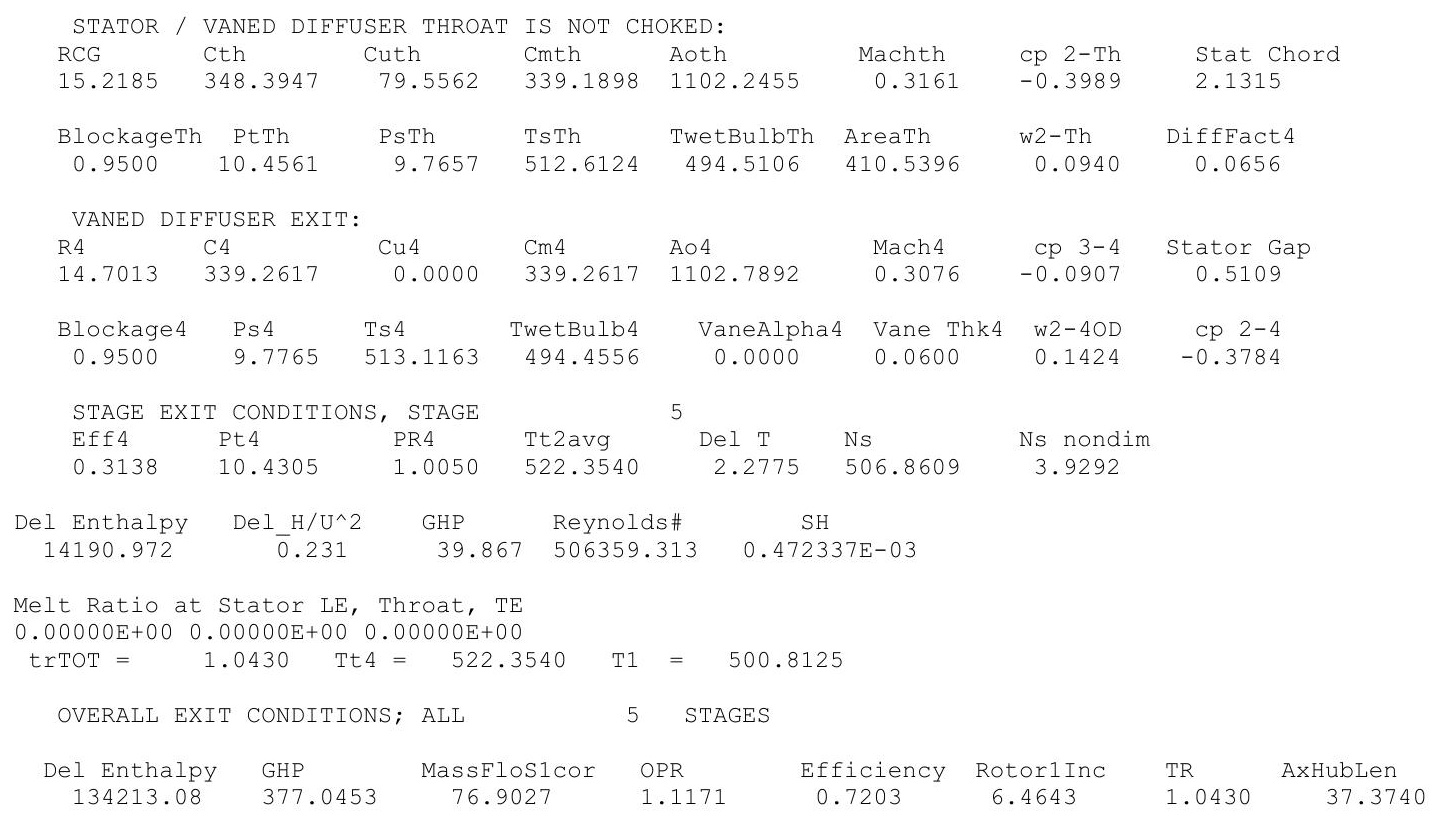




\title{
$100 \mu \mathrm{m}, \mathrm{ISA}+36 \mathrm{R}$
}

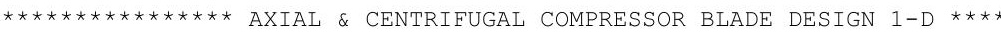

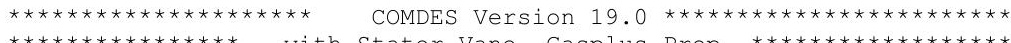

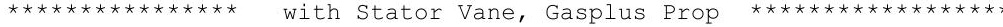

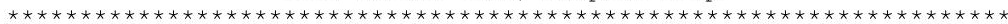

Fan Core + 4 Stage LPC 10\%39000 ft 05-13-2013

COMPRESSOR INLET CONDITIONS - ANALYSIS, STAGE 1

$\mathrm{BLEED}=0.000 \quad$ DPInC $=15.093 \quad$ EfDer $=0.958$

$\mathrm{SH}=0.908425 \mathrm{E}-04$

$\begin{array}{lrrlllr}W \text { act } & \text { RPM act } & \text { Pt } & \text { Tt } & \text { POTS } & \text { POTH } & \text { AeroBl } \\ 26.373 & 2465.077 & 4.265 & 477.918 & 1.000 & 1.000 & 0.980\end{array}$

$\mathrm{W} \mathrm{Kg} / \mathrm{sec}=11.988$ Wdry $=26.371 \mathrm{WH} 2 \mathrm{O}=0.002 \mathrm{lbm} / \mathrm{sec} \quad \mathrm{H} 2 \mathrm{O}=0.035 \mathrm{~g} / \mathrm{m}^{\wedge} 3$

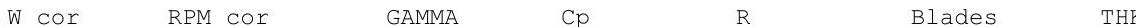

$\begin{array}{lll}1.381 & \mathrm{CP} & \mathrm{R} \\ & 0.248 & 53.351\end{array}$

CFM SCFM A1/A* Area1 A* AthrRotor ChokeMargin

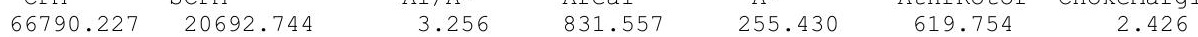

ROTOR LEADING EDGE CONDITIONS, STAGE 1

$\begin{array}{lccccccccc} & \text { R1 } & \text { Stator } & \text { Alfa } & \text { C1 } & \text { CU1 } & \text { Cm1 } & \text { Mabs } & \text { Mrel } & \text { U1cor } \\ \text { TIP } & 20.63 & 0.00 & -0.02 & 192.77 & -0.07 & 192.77 & 0.18 & 0.46 & 462.32 \\ \text { MEAN } & 17.06 & 0.00 & -0.02 & 192.77 & -0.07 & 192.77 & 0.18 & 0.39 & \\ \text { HUB } & 12.51 & 0.00 & -0.02 & 192.77 & -0.07 & 192.77 & 0.18 & 0.31 & \\ & & & & & & & & & \\ & \text { BetaFlo } & \text { BetaBlade } & \text { Incid } & \text { U1 } & \text { W1 } & \text { Ps1 } & \text { Ts1 } & \text { TwetBulb1 } & \text { RH } \\ \text { TIP } & 66.52 & 50.47 & 16.05 & 443.79 & 483.91 & 4.17 & 474.93 & 460.63 & 1.39 \\ \text { MEAN } & 62.29 & 47.20 & 15.09 & 367.00 & 414.60 & 4.17 & 474.93 & 460.63 & 1.39 \\ \text { HUB } & 54.39 & 38.62 & 15.77 & 269.11 & 331.08 & 4.17 & 474.93 & 460.63 & 1.39\end{array}$

ROTOR EXIT CONDITIONS, STAGE 1 SOLUTION IS CONVERGED

\begin{tabular}{|c|c|c|c|c|c|c|c|c|c|}
\hline & $\begin{array}{l}\text { axial } \\
700\end{array}$ & $\begin{array}{l}\text { THK } \\
0.050\end{array}$ & $\begin{array}{l}\text { AeroBl } \\
0.950\end{array}$ & $\begin{array}{r}\mathrm{Bla} \\
32\end{array}$ & $\begin{array}{l}\text { des } 2 \\
2.000\end{array}$ & & & & \\
\hline & $\mathrm{R} 2$ & $\mathrm{C} 2$ & $\mathrm{Cu} 2$ & $\mathrm{Cm} 2$ & $\mathrm{~A} \circ 2$ & Mach2 & Chord & AxChord & Rcircle \\
\hline TIP & 20.63 & 398.81 & 304.11 & 258.01 & 1074.42 & 0.37 & 7.29 & 6.52 & 16.04 \\
\hline MEAN & 18.04 & 404.26 & 308.37 & 261.40 & 1071.31 & 0.38 & 7.43 & 6.80 & 12.53 \\
\hline HUB & 15.00 & 438.82 & 348.91 & 266.13 & 1067.45 & 0.41 & 7.49 & 7.25 & 9.22 \\
\hline & $\mathrm{U} 2$ & W2 & Wu2 & MachRel2 & DelRCu & Eff $2 u C$ & Eff2incC & AvgREff & Ws $1 /$ W2 \\
\hline TIP & 443.79 & 293.39 & 139.68 & 0.27 & 6275.13 & 0.92 & 0.88 & & \\
\hline MEAN & 387.99 & 273.26 & 79.62 & 0.26 & 5562.90 & 0.92 & 0.88 & 0.88 & 1.74 \\
\hline HUB & 322.68 & 267.42 & 26.24 & 0.25 & 5234.54 & 0.92 & 0.88 & & \\
\hline & Pt2 & PR & Ps2 & Tt2 & TR & Ts2 & TwetBulb2 & $\mathrm{RH}$ & \\
\hline TIP & 4.91 & 1.15 & 4.47 & 499.63 & 1.05 & 486.84 & 473.12 & 0.91 & \\
\hline SAN & 4.84 & 1.13 & 4.39 & 497.16 & 1.04 & 484.02 & 471.80 & 1.00 & \\
\hline JB & 4.80 & 1.13 & 4.28 & 496.03 & 1.04 & 480.54 & 471.21 & 1.13 & \\
\hline & Alfa2 & Beta FLO & Beta BLADE & Deviat & Slip & F. DiffFct & Solidity & & \\
\hline & 49.69 & 28.43 & 24.20 & 4.23 & 0.93 & 0.57 & 1.80 & & \\
\hline & 49.71 & 16.94 & 12.70 & 4.24 & 0.95 & 0.51 & 2.22 & & \\
\hline & 52.67 & -5.63 & -9.30 & 3.67 & 0.95 & 0.38 & 3.05 & & \\
\hline
\end{tabular}

$\begin{array}{ccccc}\text { blockage3 } & \text { Cor/U1 } & \text { Cor/Incid } & \text { XBladeGap } & \text { Vane\# } \\ 0.950 & 1.000 & 0.958 & 1.812 & 33.000\end{array}$

ROTOR-STATOR GAP EXIT: STATOR LEADING EDGE

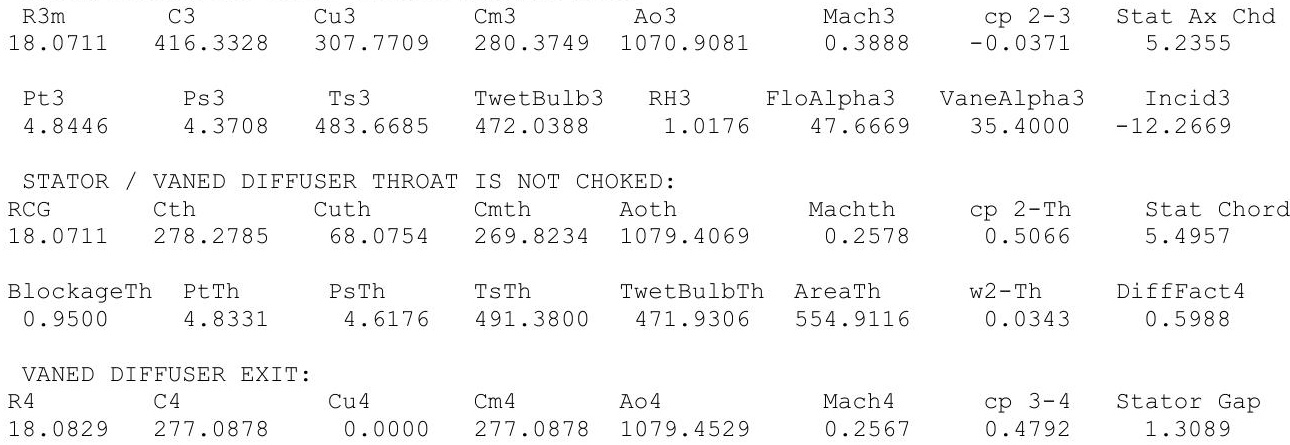




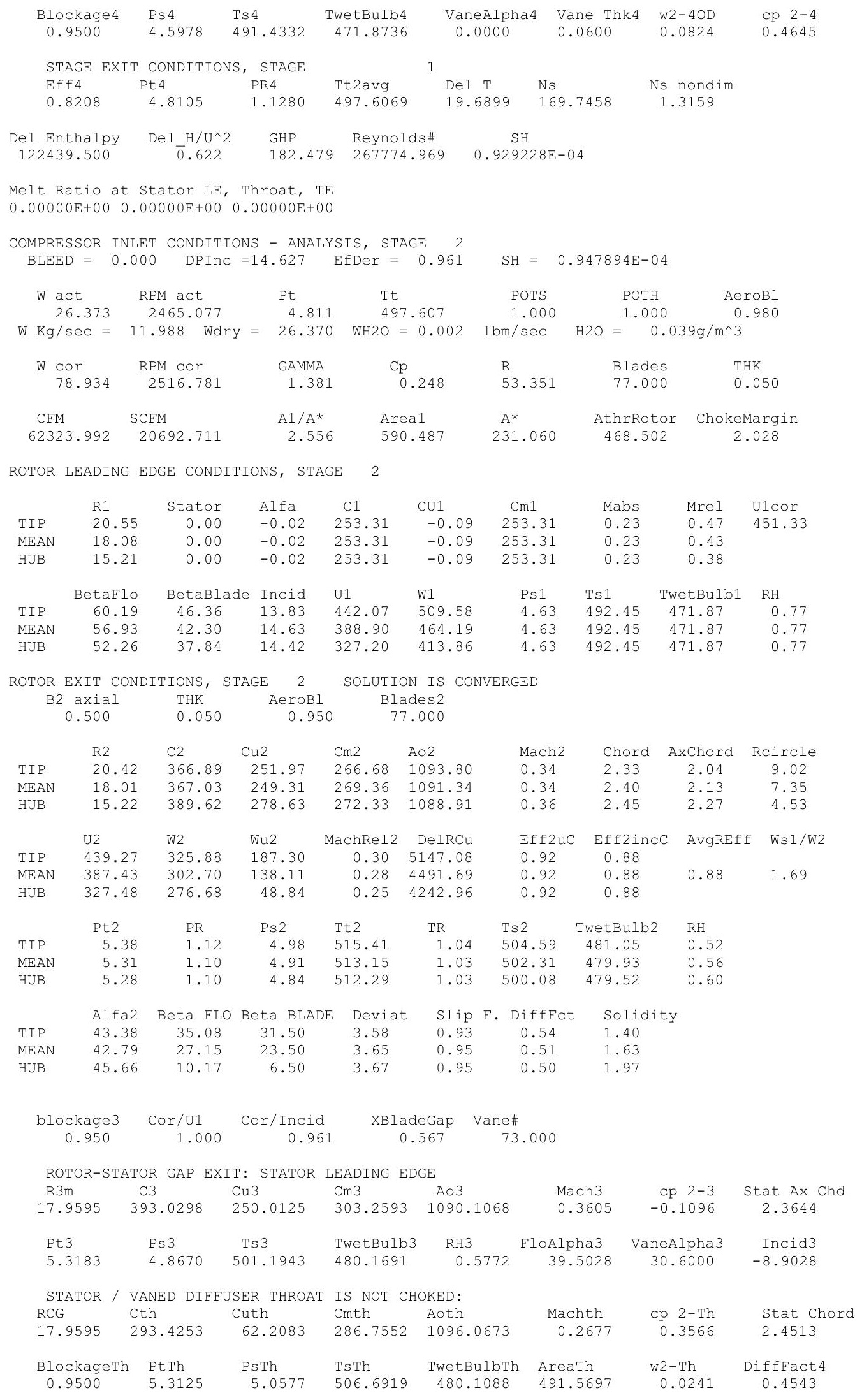




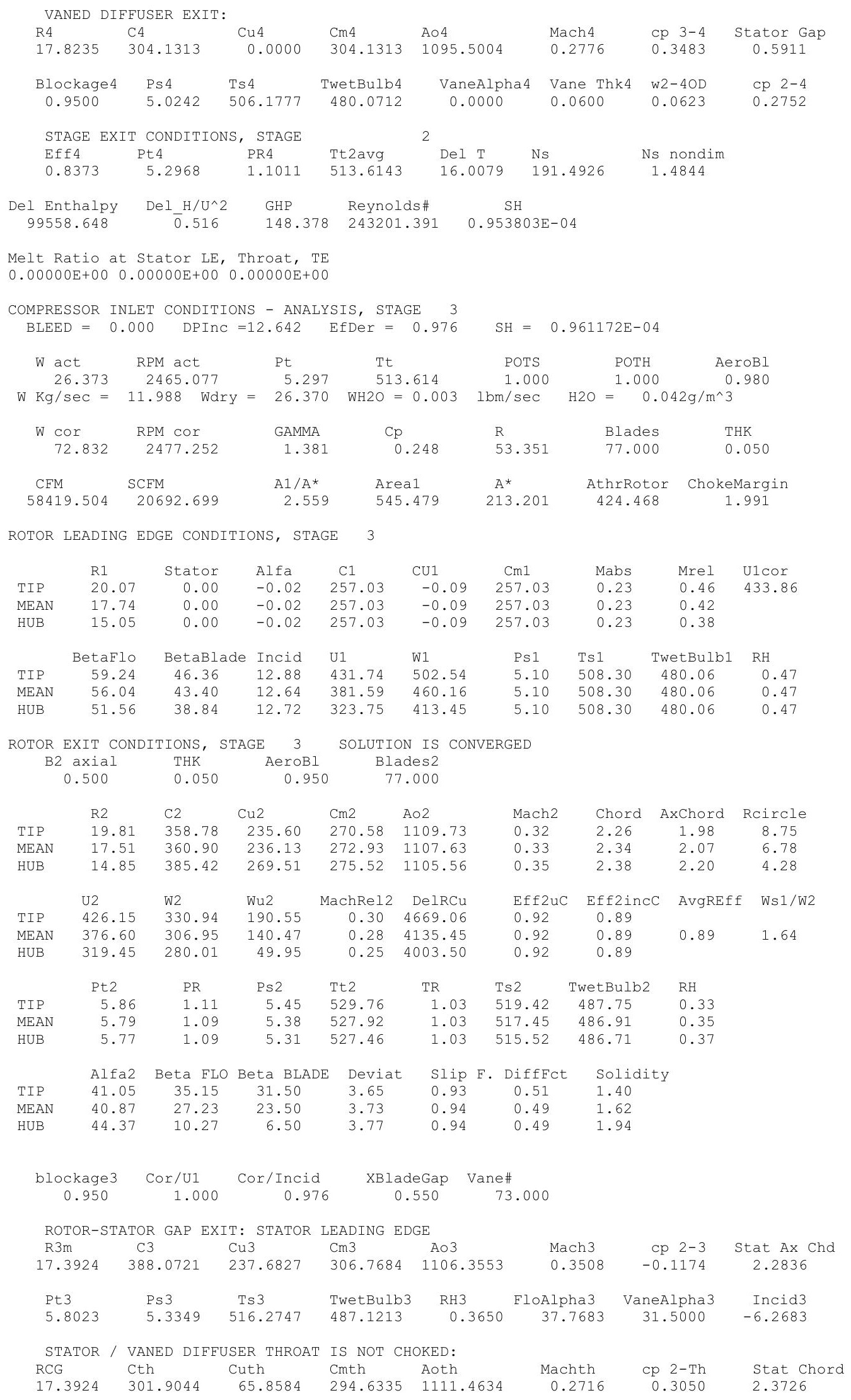




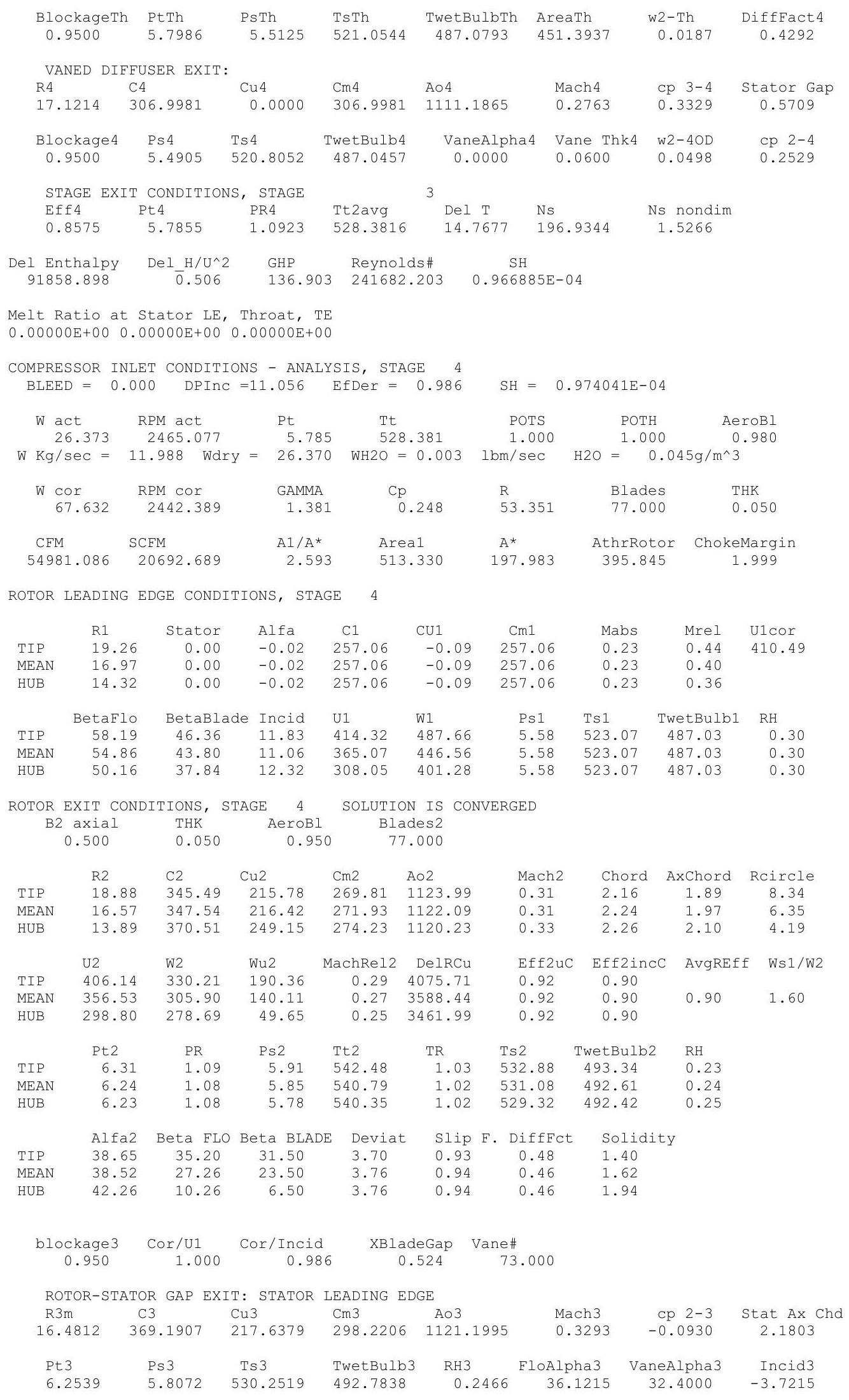




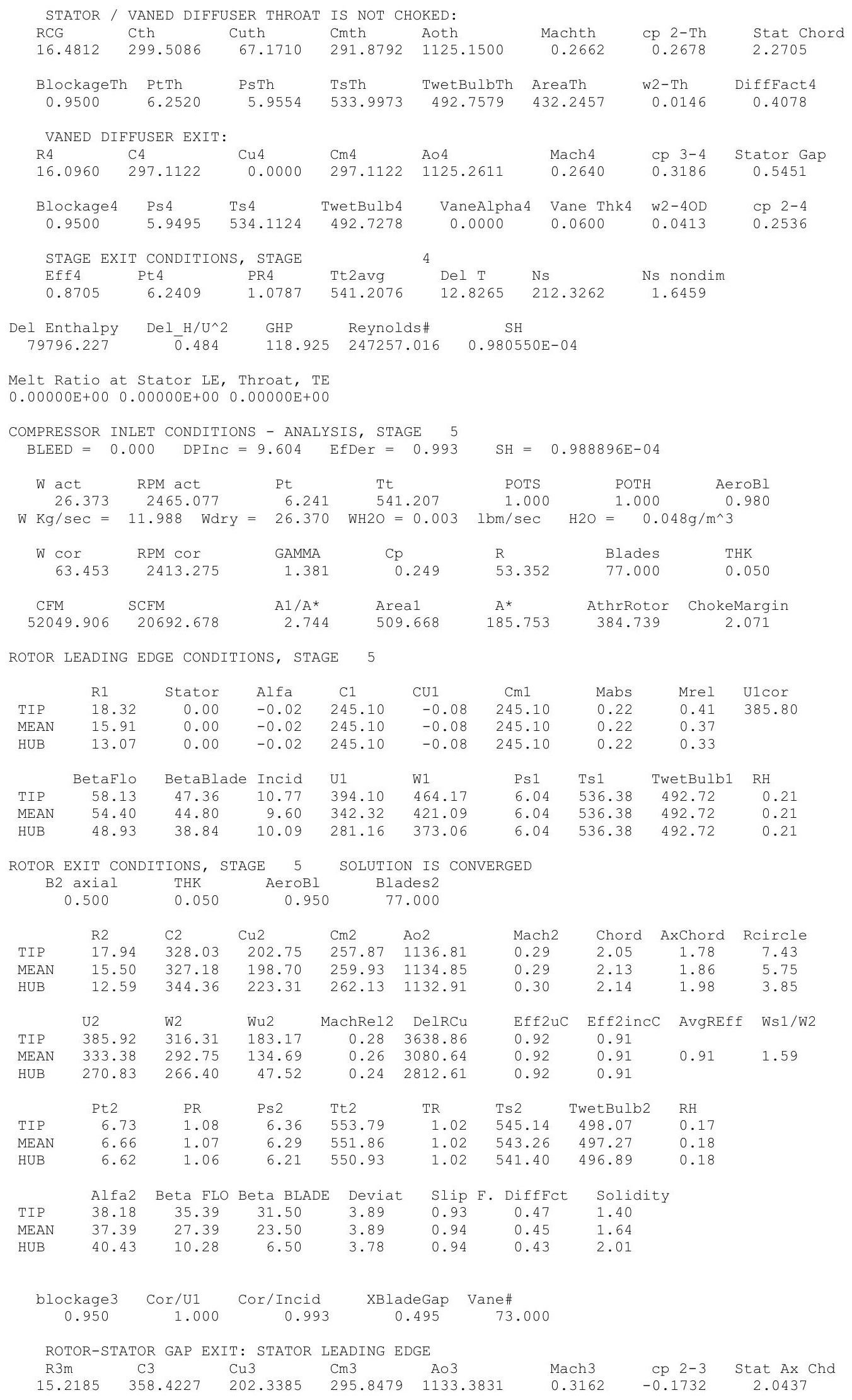




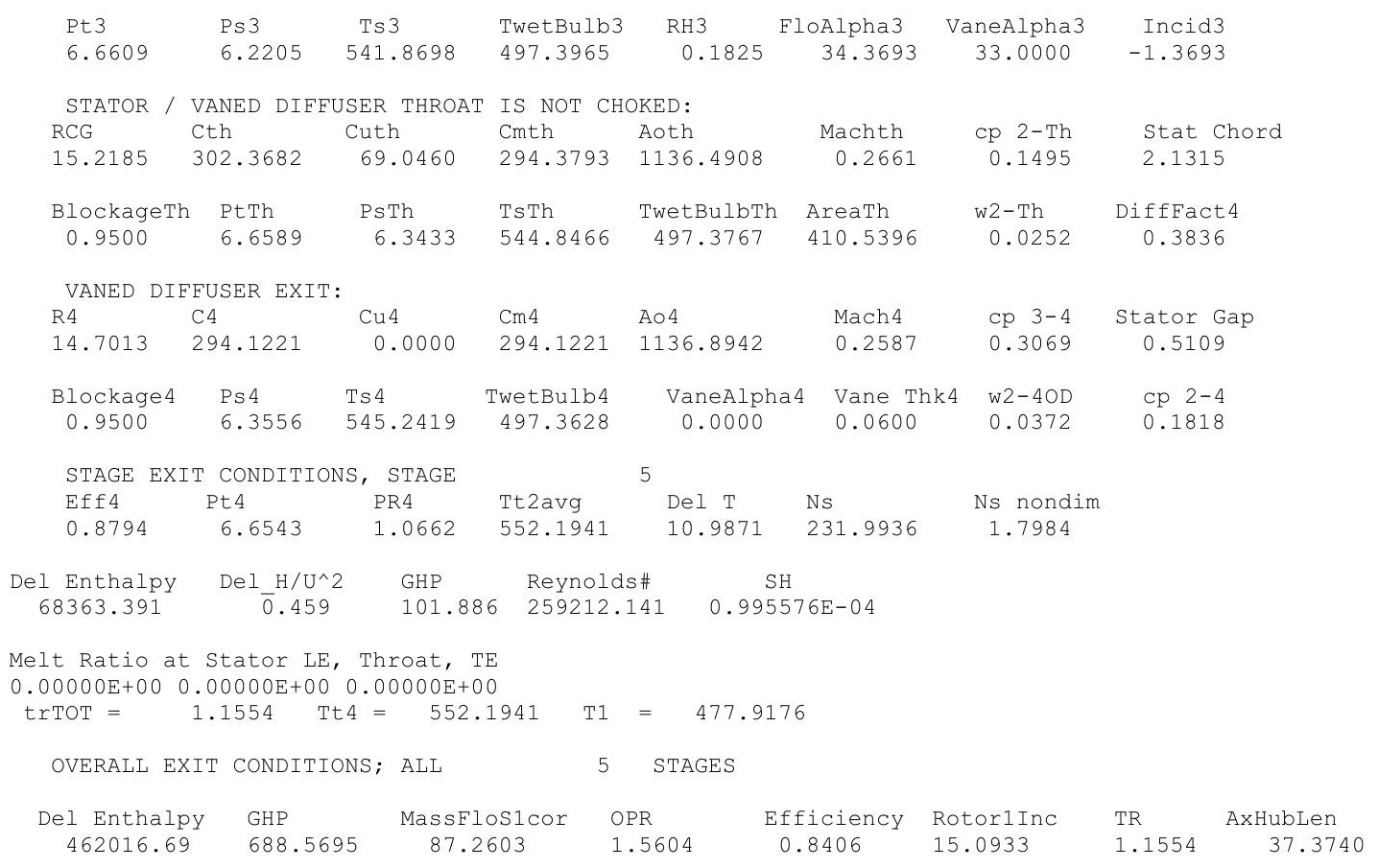




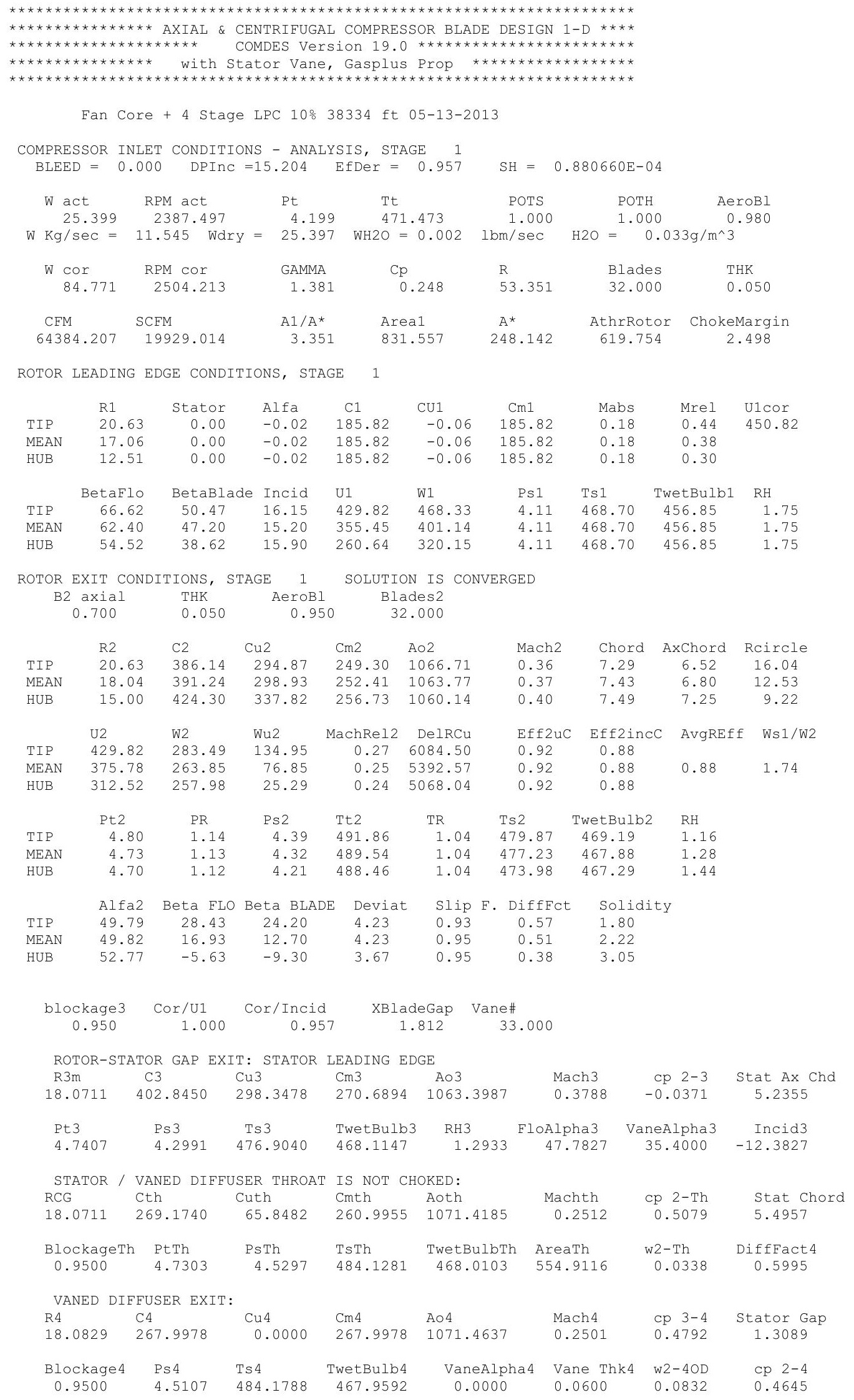




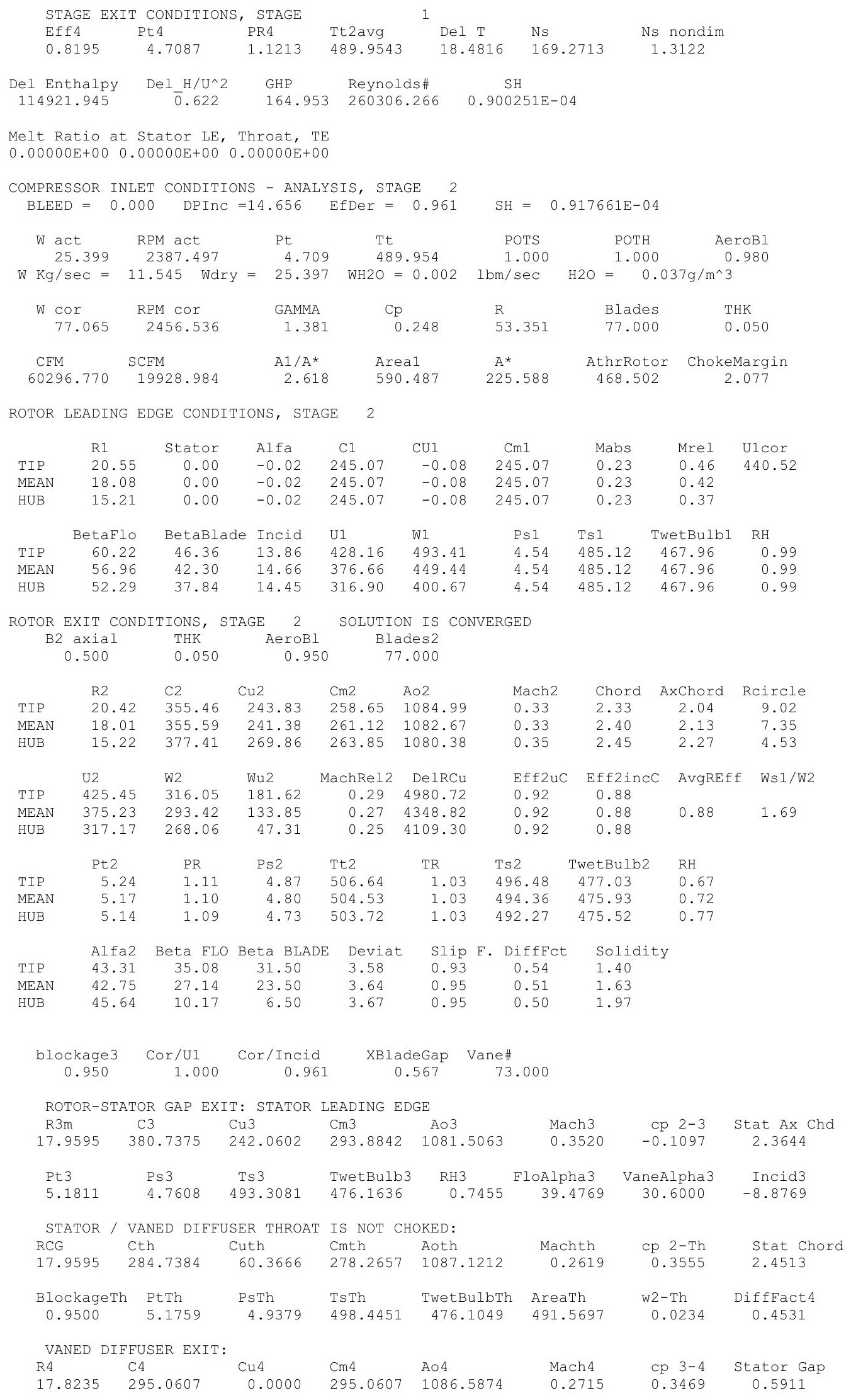




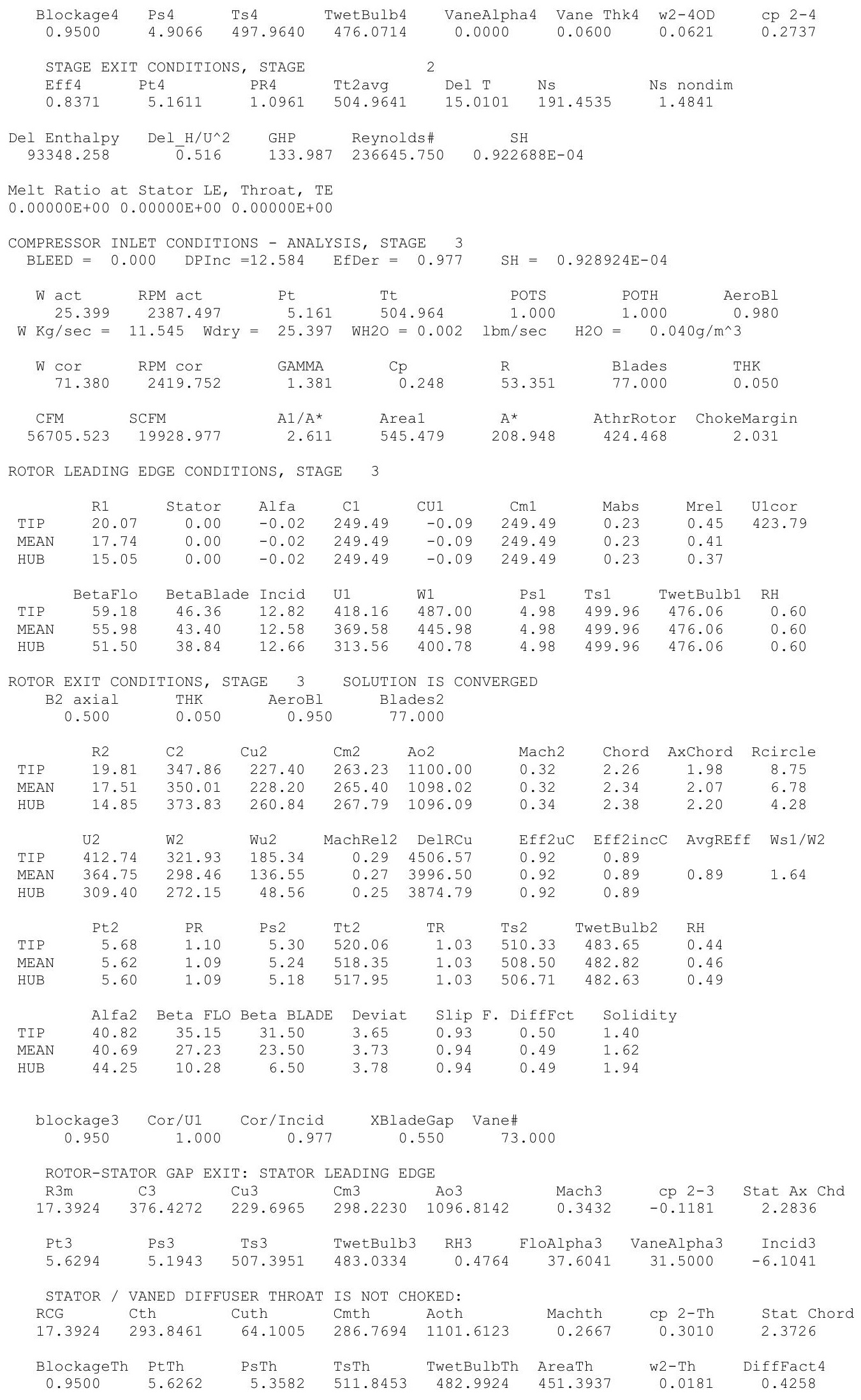




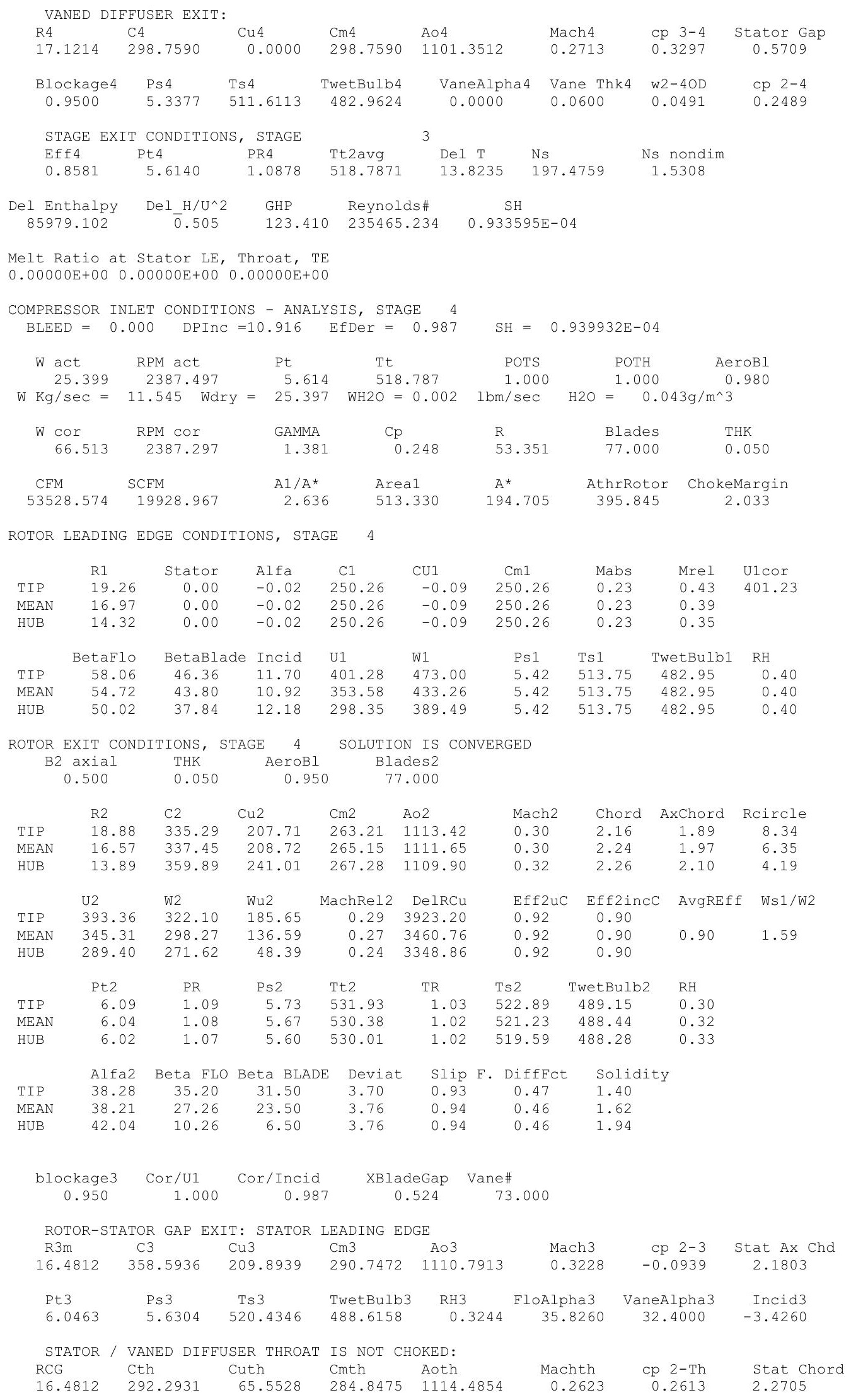




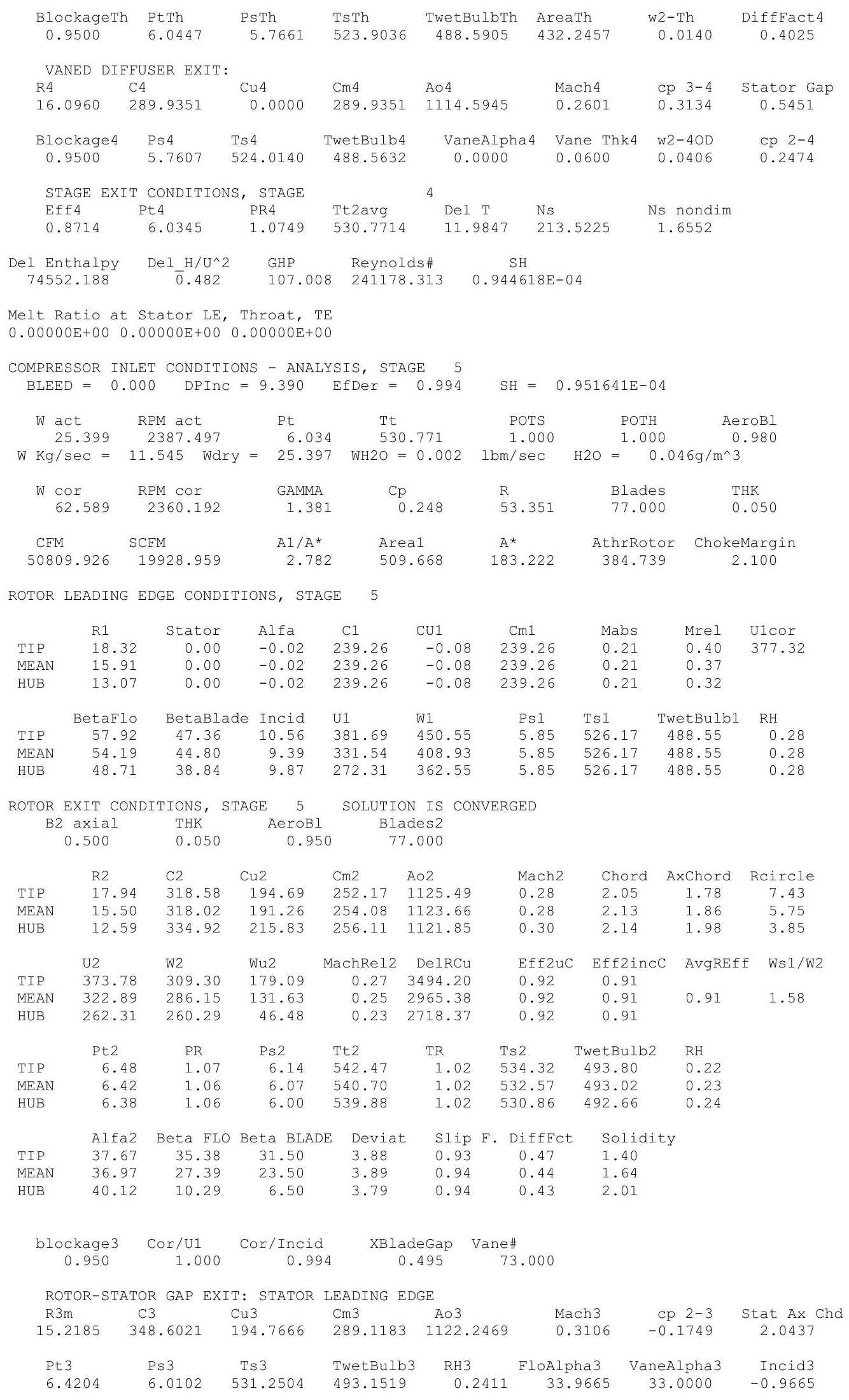




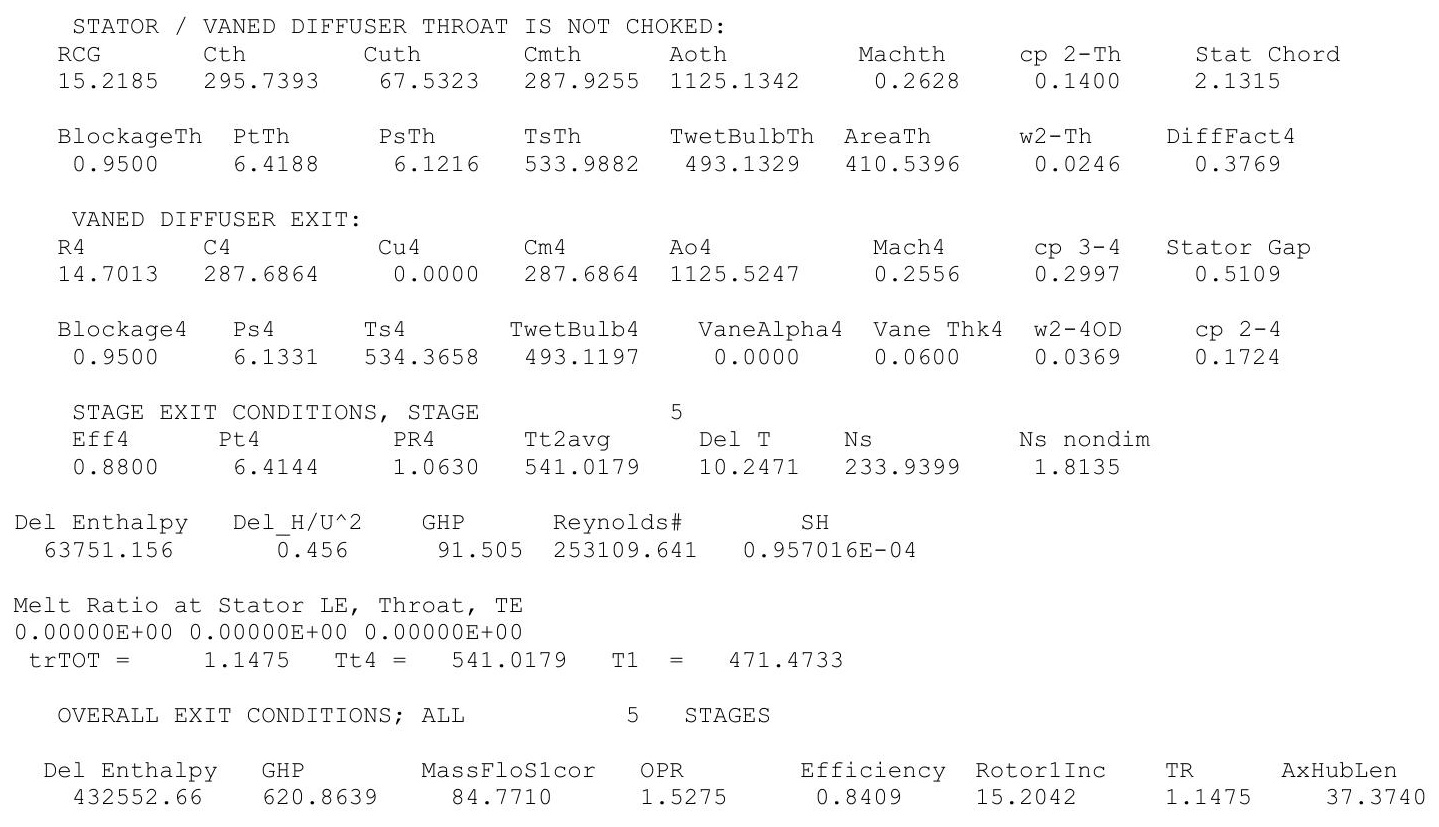




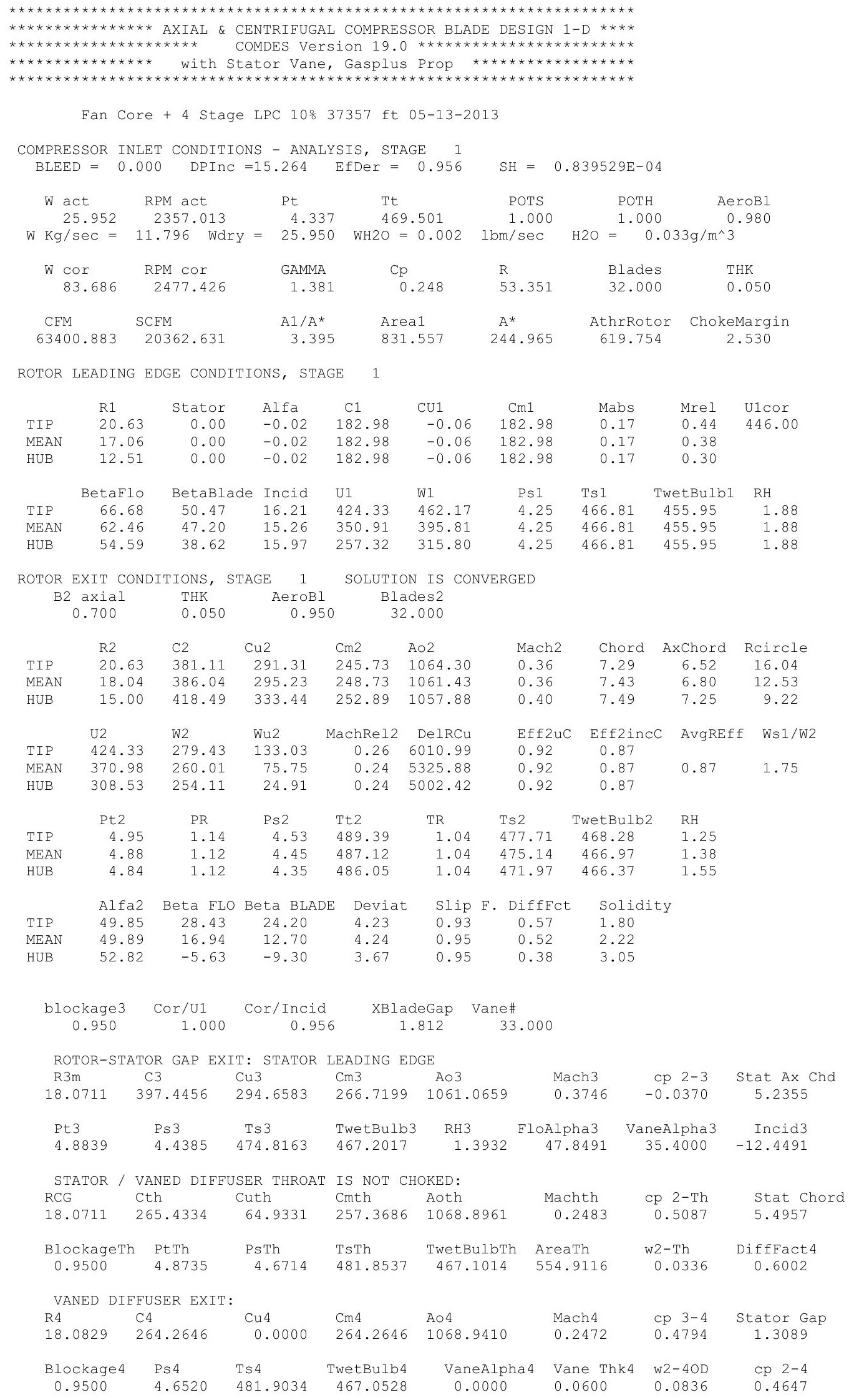




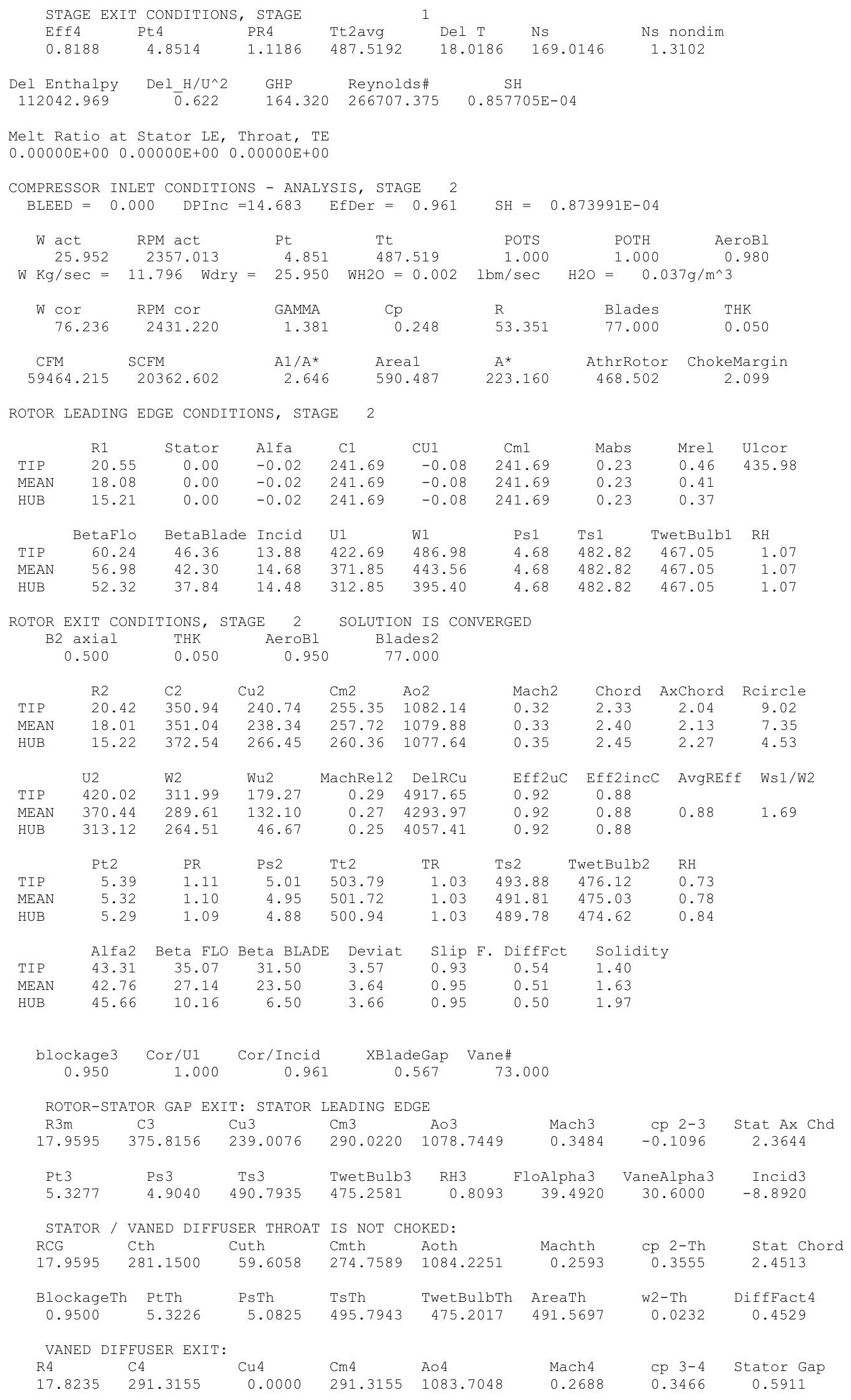




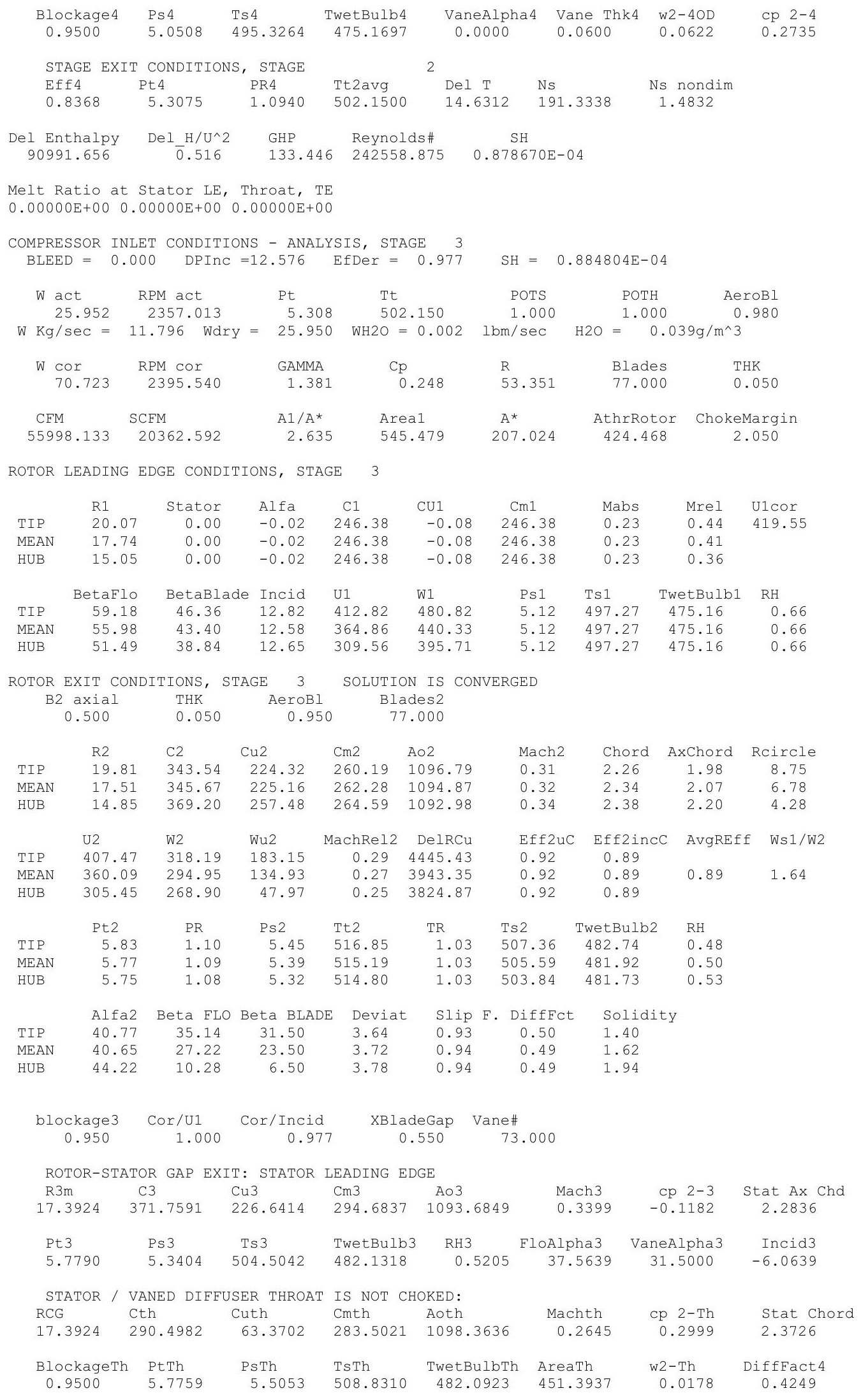




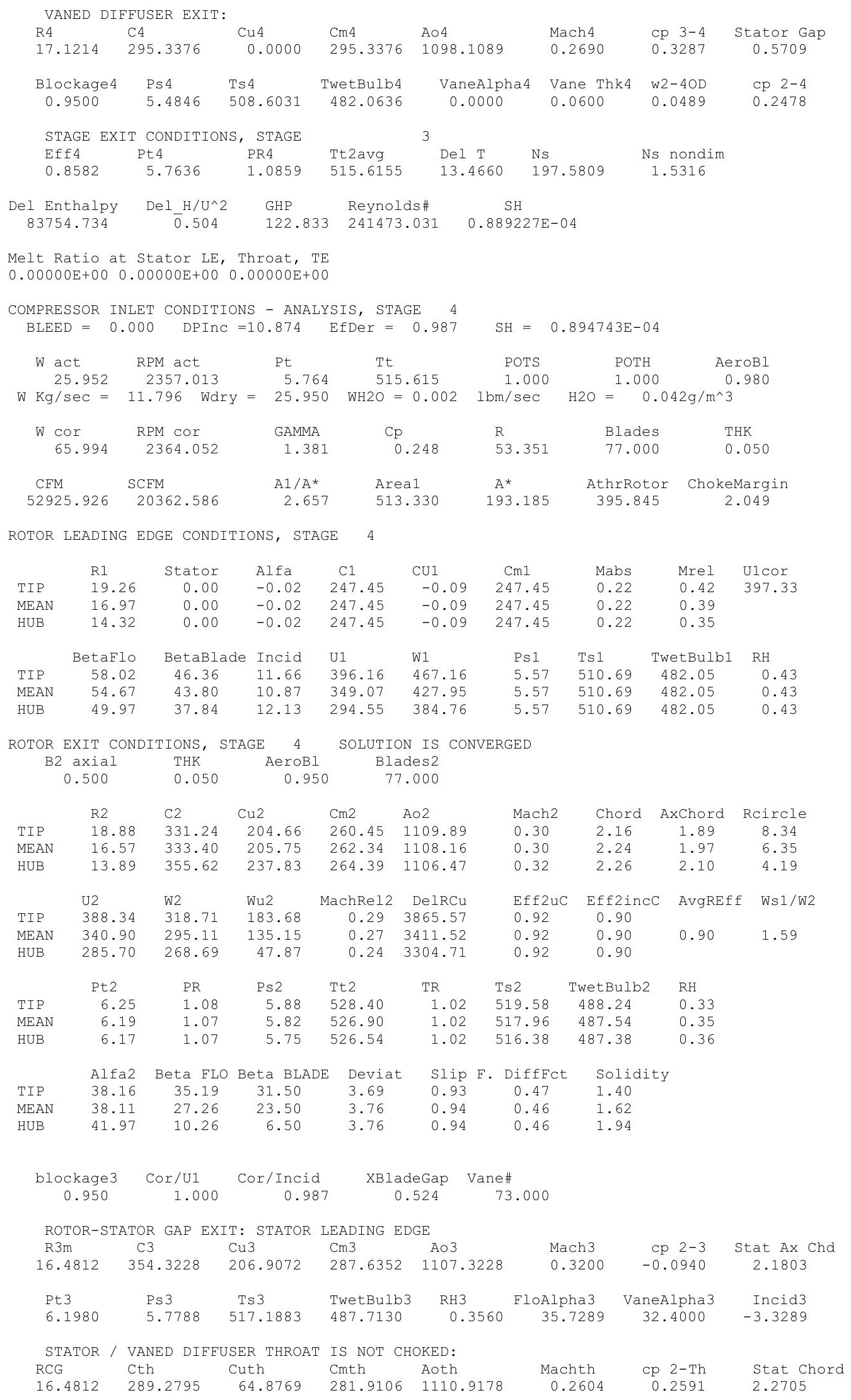




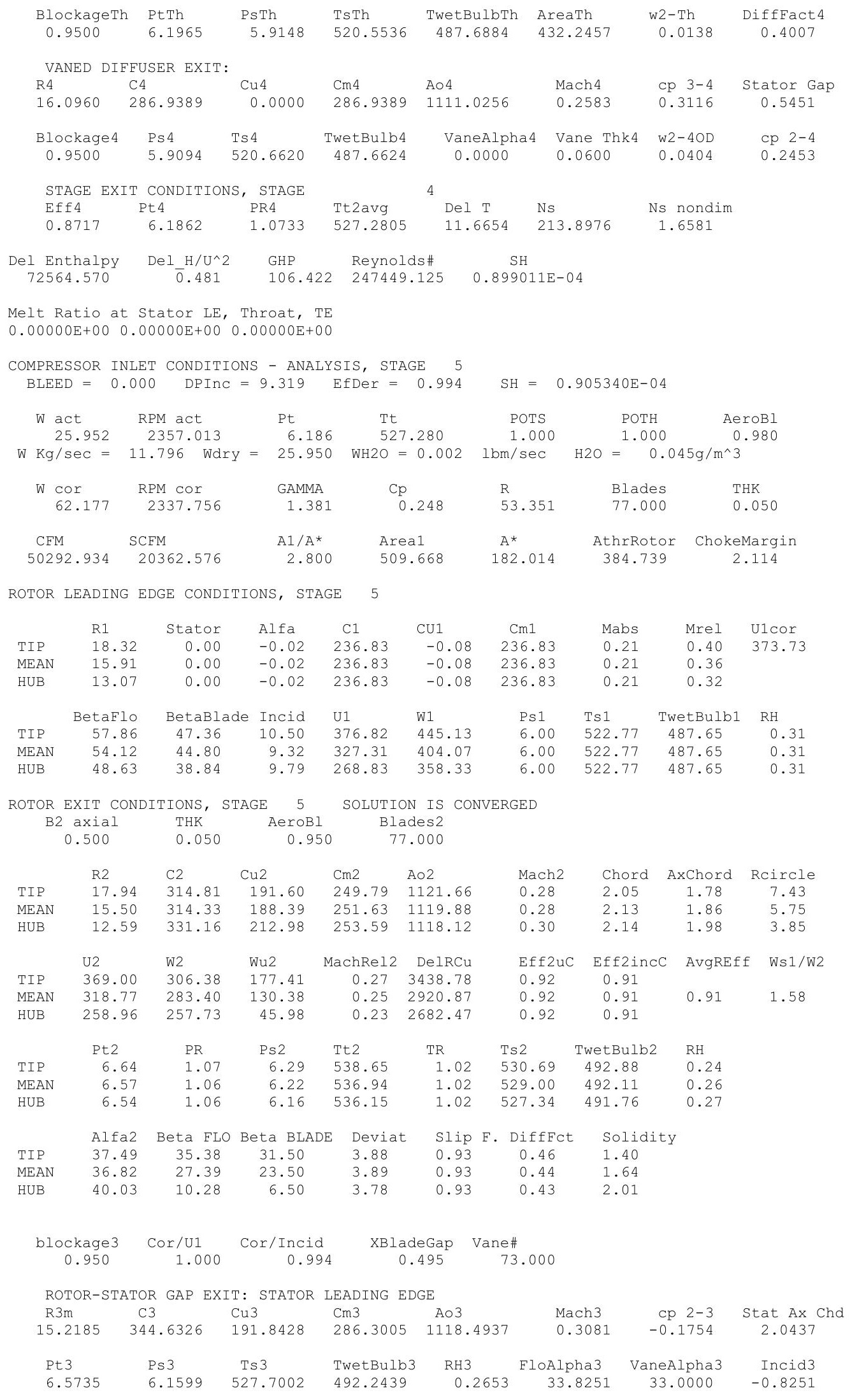




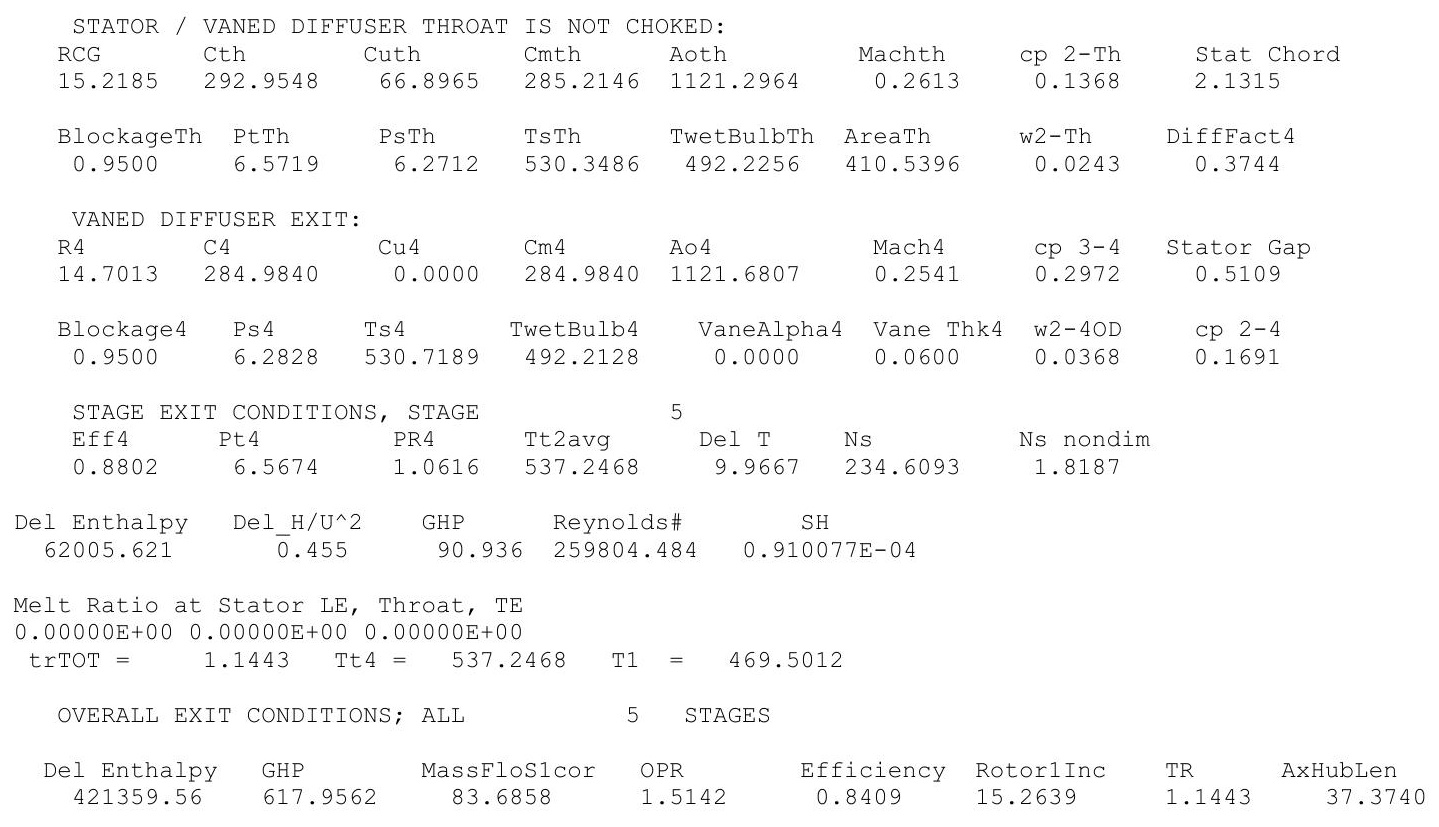




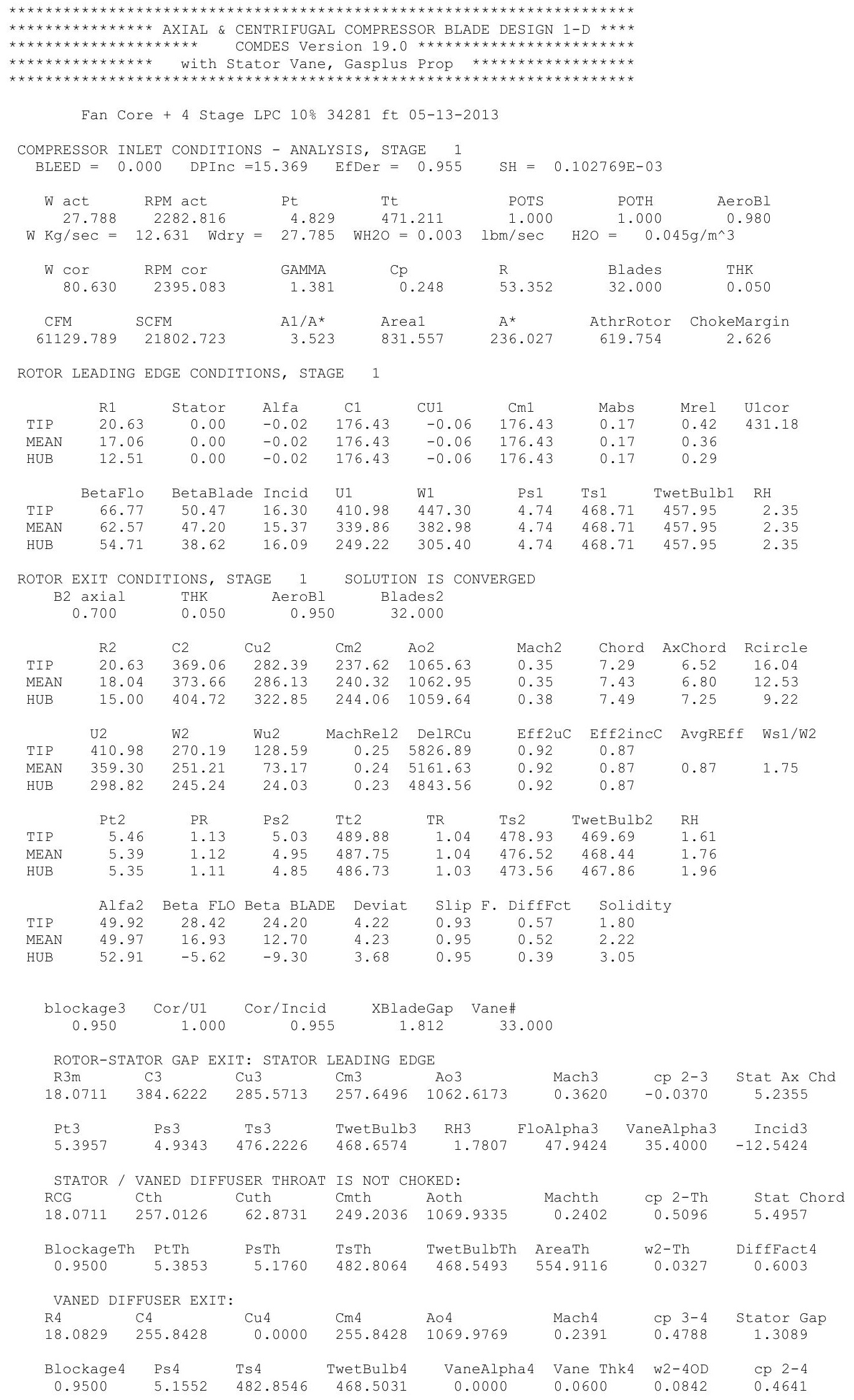




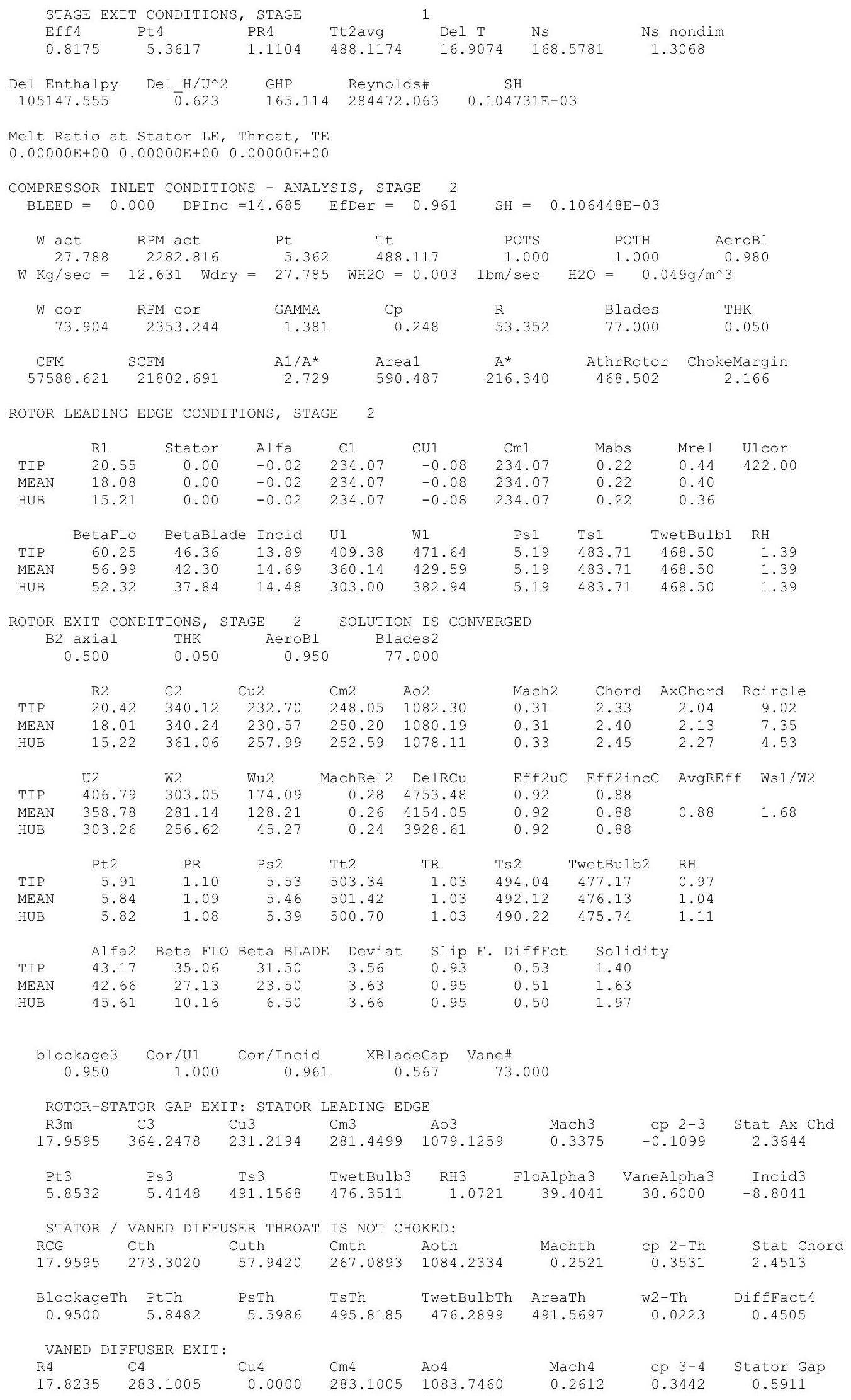




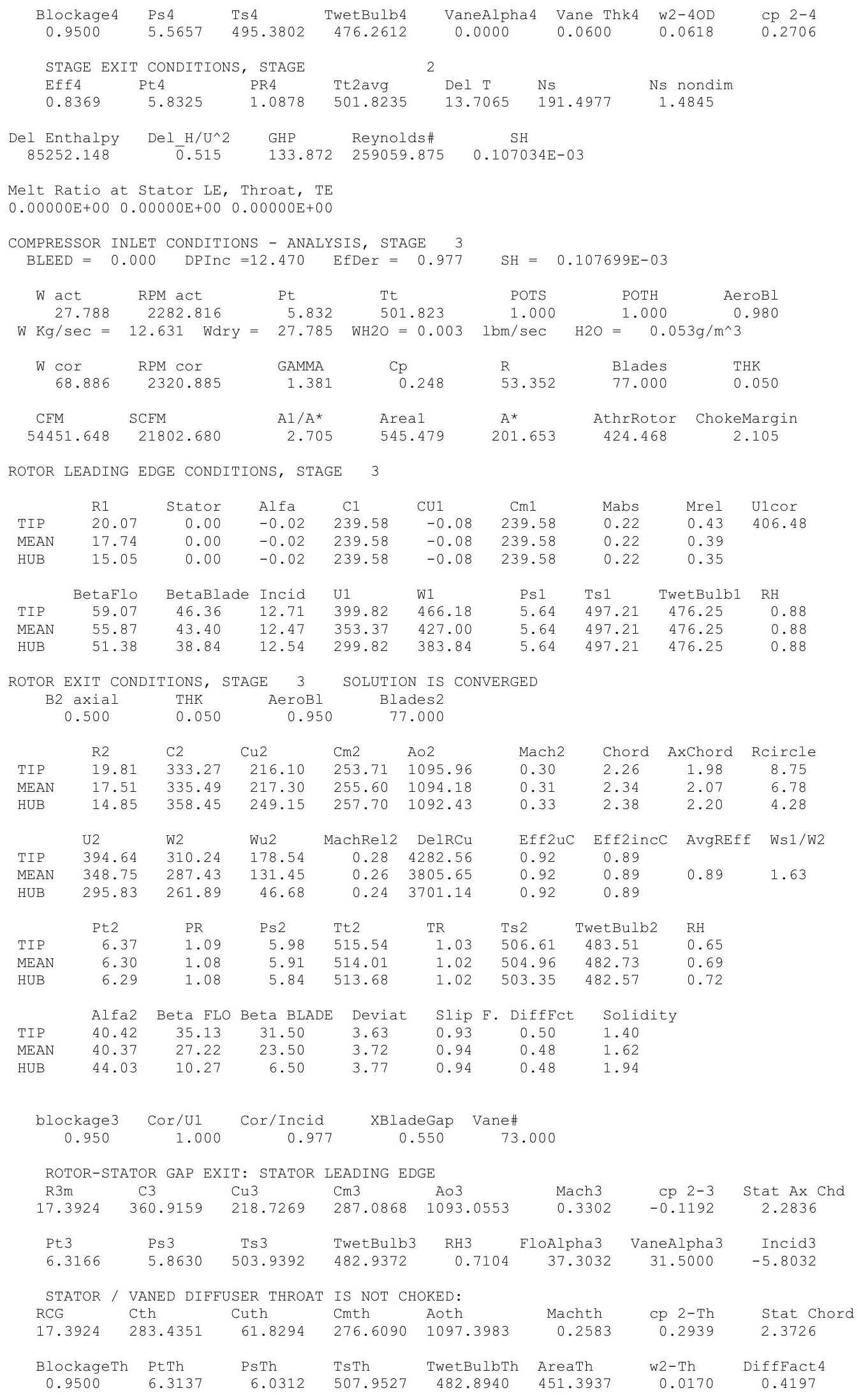




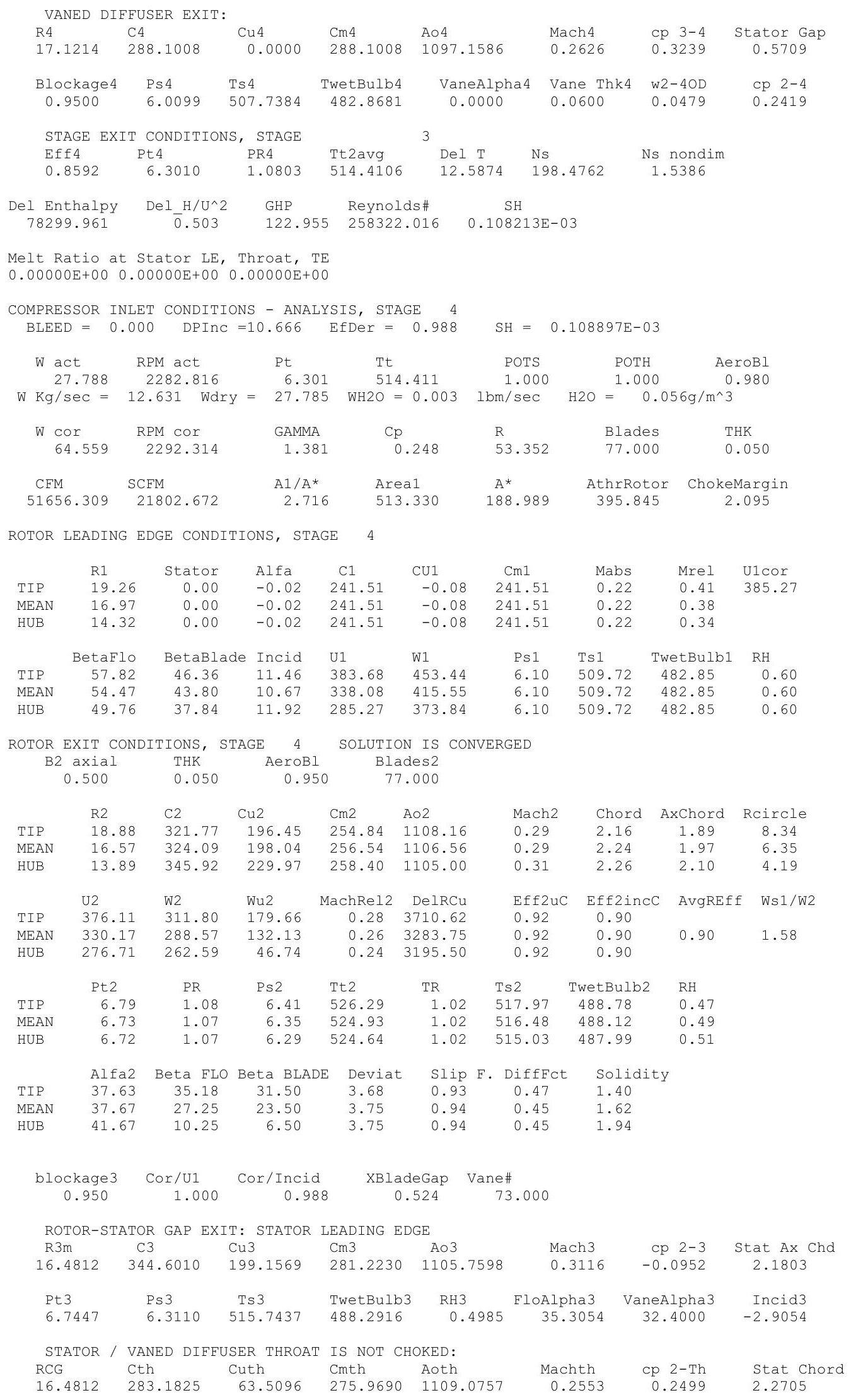




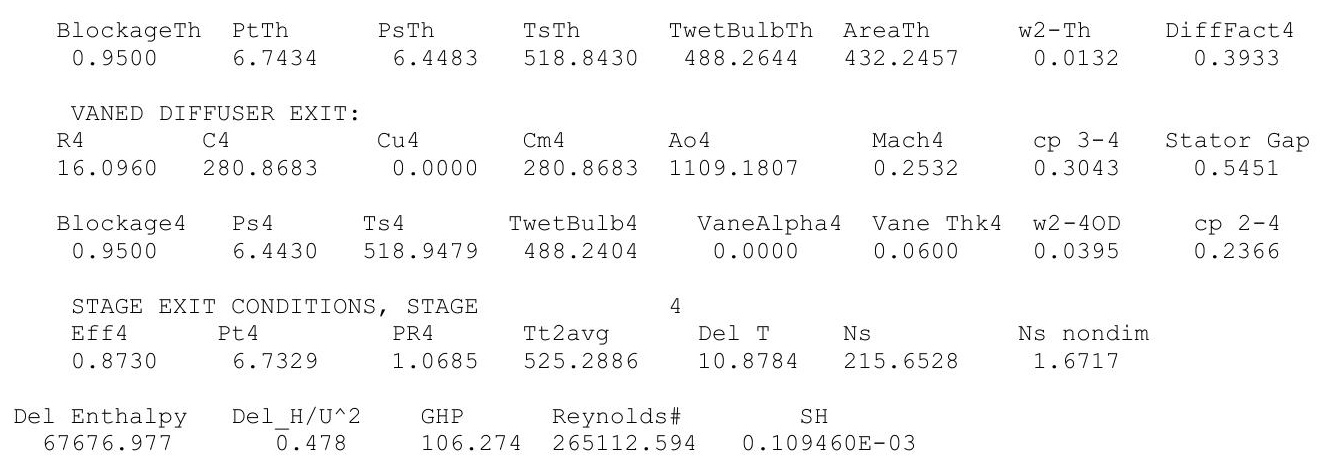

Melt Ratio at Stator LE, Throat, TE

$0.00000 \mathrm{E}+00 \quad 0.00000 \mathrm{E}+00 \quad 0.00000 \mathrm{E}+00$

COMPRESSOR INLET CONDITIONS - ANALYSIS, STAGE 5 BLEED $=0.000 \quad$ DPInC $=9.019 \quad$ EfDer $=0.995$

$\begin{array}{lccllrr}\text { W act } & \text { RPM act } & \text { Pt } & \text { Tt } & \text { POTS } & \text { POTH } & \text { AeroBI } \\ 27.788 & 2282.816 & 6.733 & 525.288 & 1.000 & 1.000 & 0.980\end{array}$

$\mathrm{W} \mathrm{Kg} / \mathrm{sec}=12.631$ Wdry $=27.785 \mathrm{WH} 2 \mathrm{O}=0.003 \mathrm{lbm} / \mathrm{sec} \quad \mathrm{H} 2 \mathrm{O}=0.060 \mathrm{~g} / \mathrm{m}^{\wedge} 3$

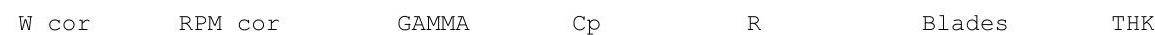

$\begin{array}{ccccccc}61.054 & 2268.455 & 1.381 & 0.249 & 53.352 & 77.000 & 0.050\end{array}$

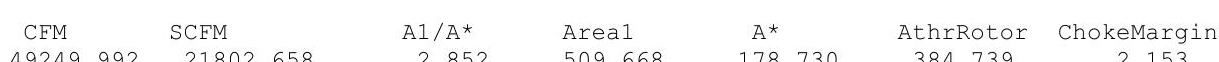

$\begin{array}{lllllll}49249.992 & 21802.658 & 2.852 & 509.668 & 178.730 & 384.739 & 2.153\end{array}$

ROTOR LEADING EDGE CONDITIONS, STAGE 5

\begin{tabular}{|c|c|c|c|c|c|c|c|c|c|}
\hline & R1 & Stator & Alfa & $\mathrm{C} 1$ & CU1 & $\mathrm{Cm} 1$ & Mabs & Mrel & U1cor \\
\hline TIP & 18.32 & 0.00 & -0.02 & 231.92 & -0.08 & 231.92 & 0.21 & 0.39 & 362.65 \\
\hline MEAN & 15.91 & 0.00 & -0.02 & 231.92 & -0.08 & 231.92 & 0.21 & 0.35 & \\
\hline HUB & 13.07 & 0.00 & -0.02 & 231.92 & -0.08 & 231.92 & 0.21 & 0.31 & \\
\hline & BetaFlo & BetaBlade & Incid & U1 & W1 & Ps1 & Ts1 & TwetBulb1 & RH \\
\hline TIP & 57.57 & 47.36 & 10.21 & 364.96 & 432.48 & 6.53 & 520.97 & 488.23 & 0.43 \\
\hline MEAN & 53.82 & 44.80 & 9.02 & 317.01 & 392.85 & 6.53 & 520.97 & 488.23 & 0.43 \\
\hline HUB & 48.32 & 38.84 & 9.48 & 260.37 & 348.74 & 6.53 & 520.97 & 488.23 & 0.43 \\
\hline
\end{tabular}

ROTOR EXIT CONDITIONS, STAGE 5 SOLUTION IS CONVERGED

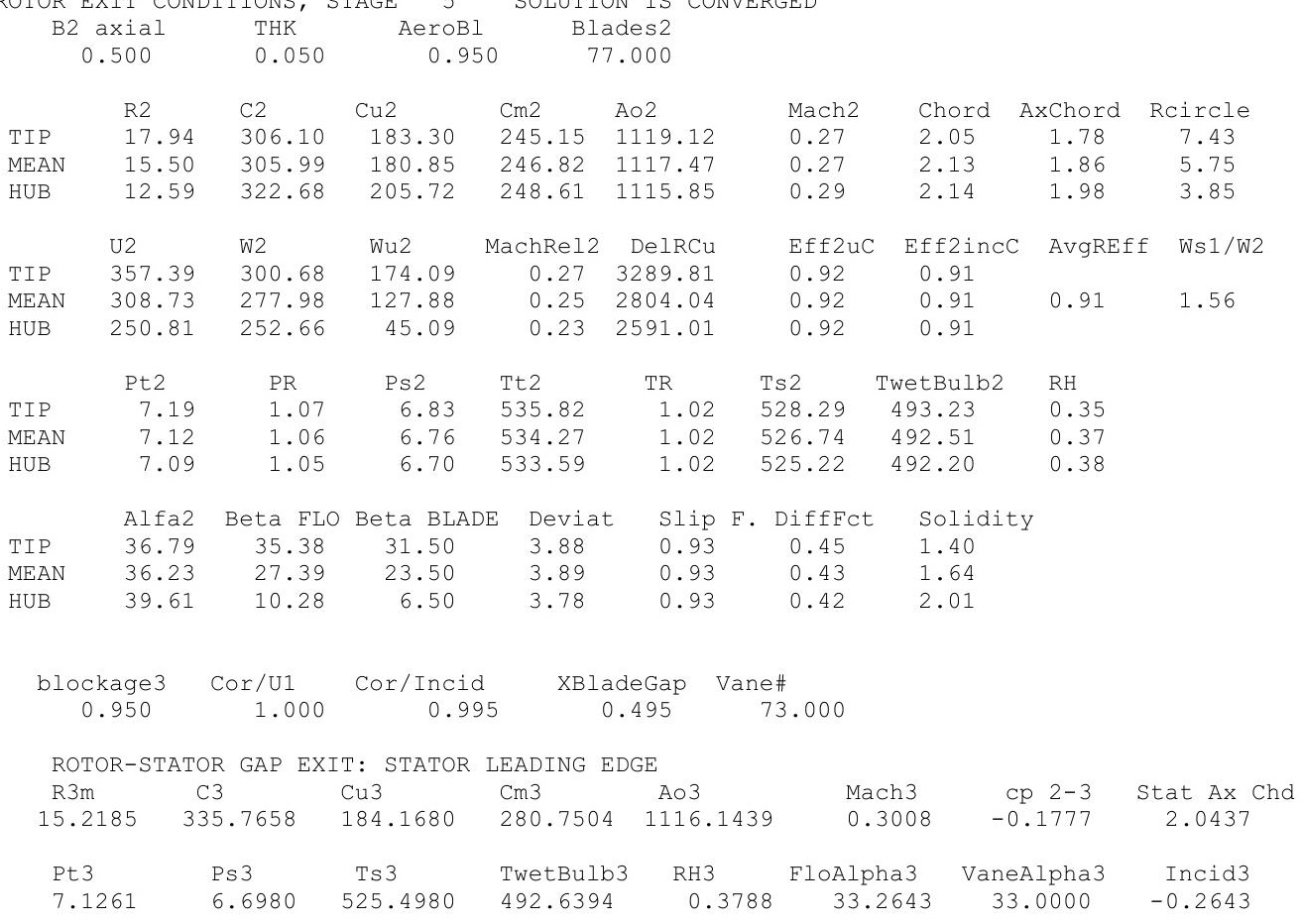




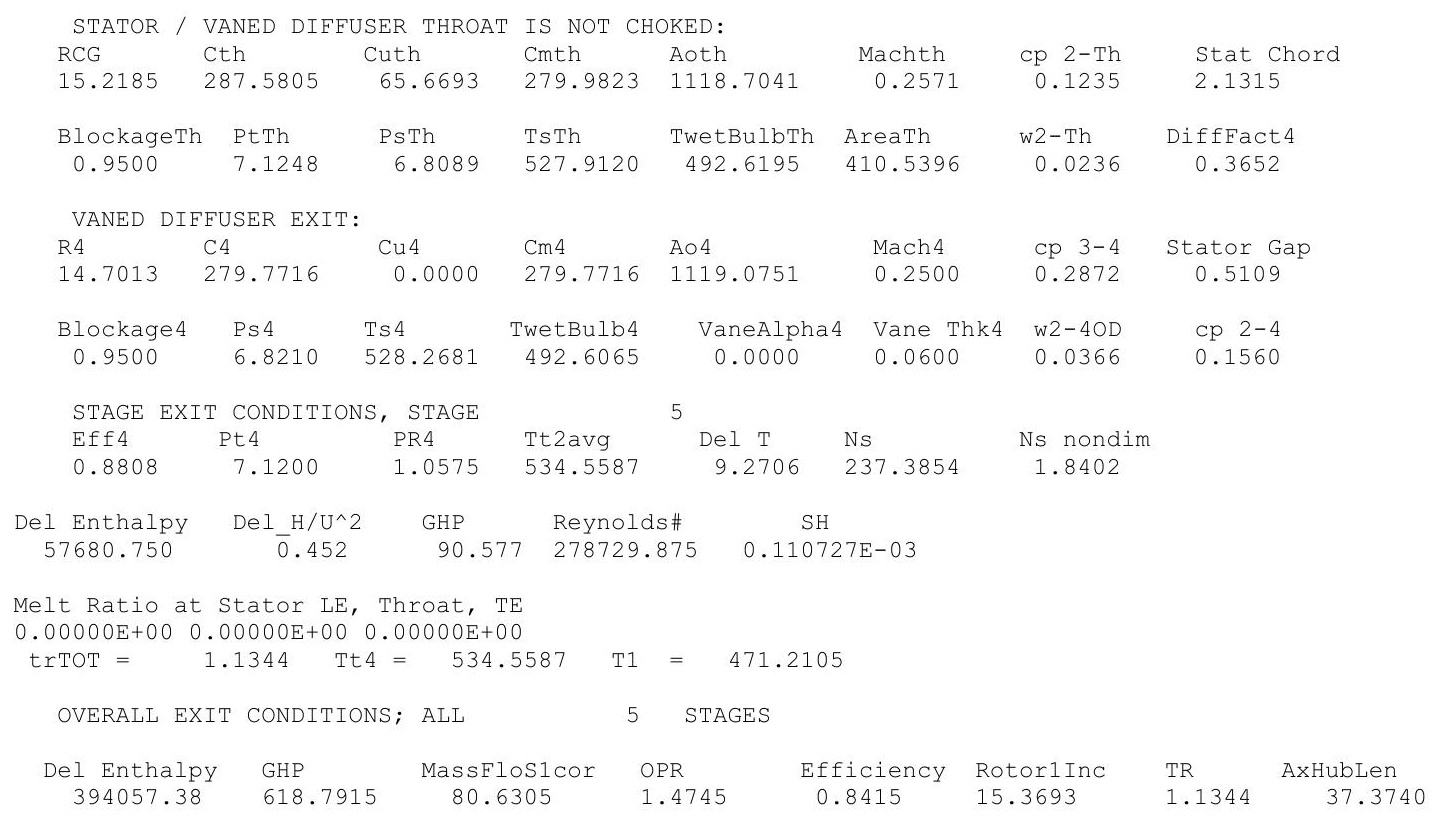




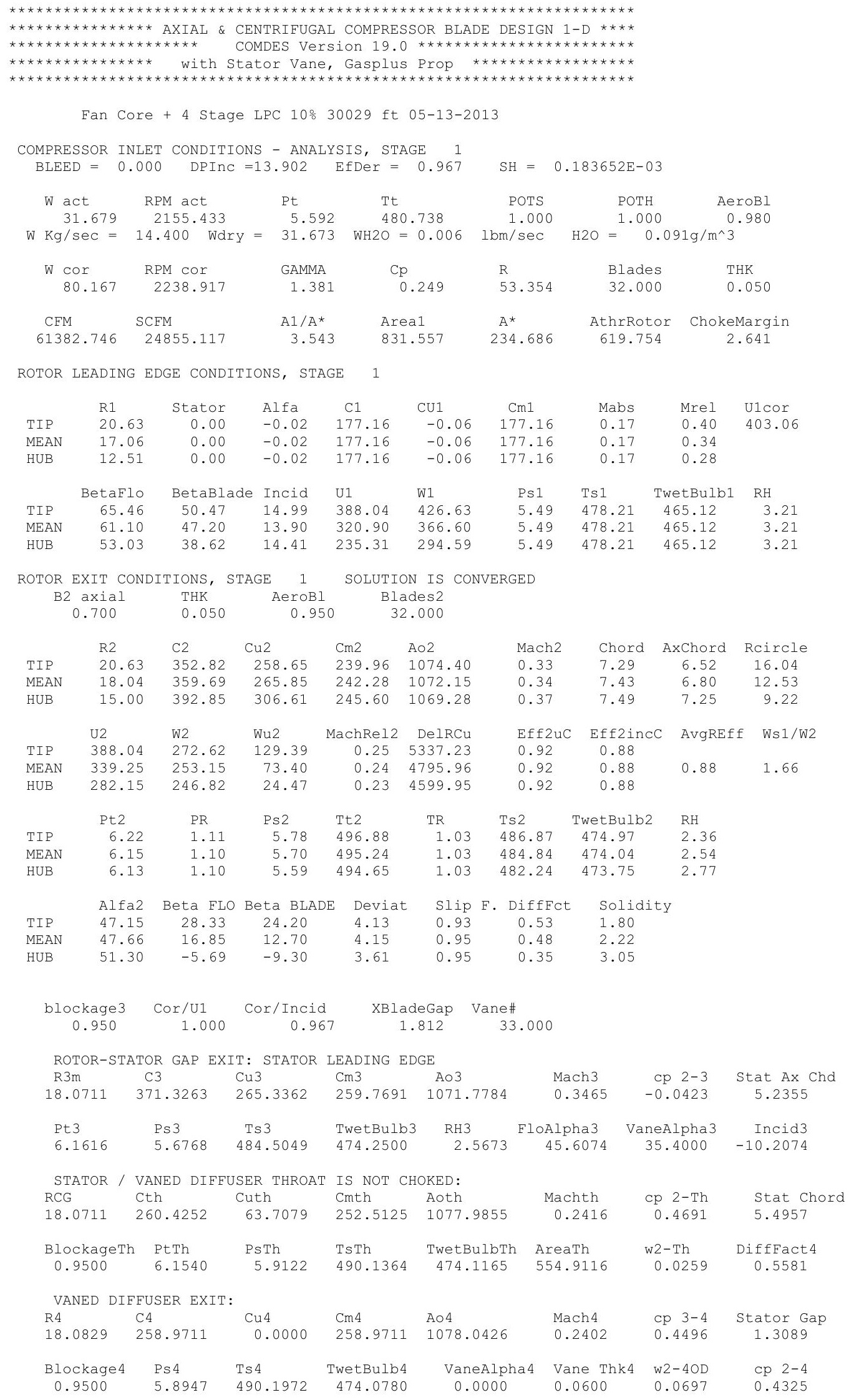




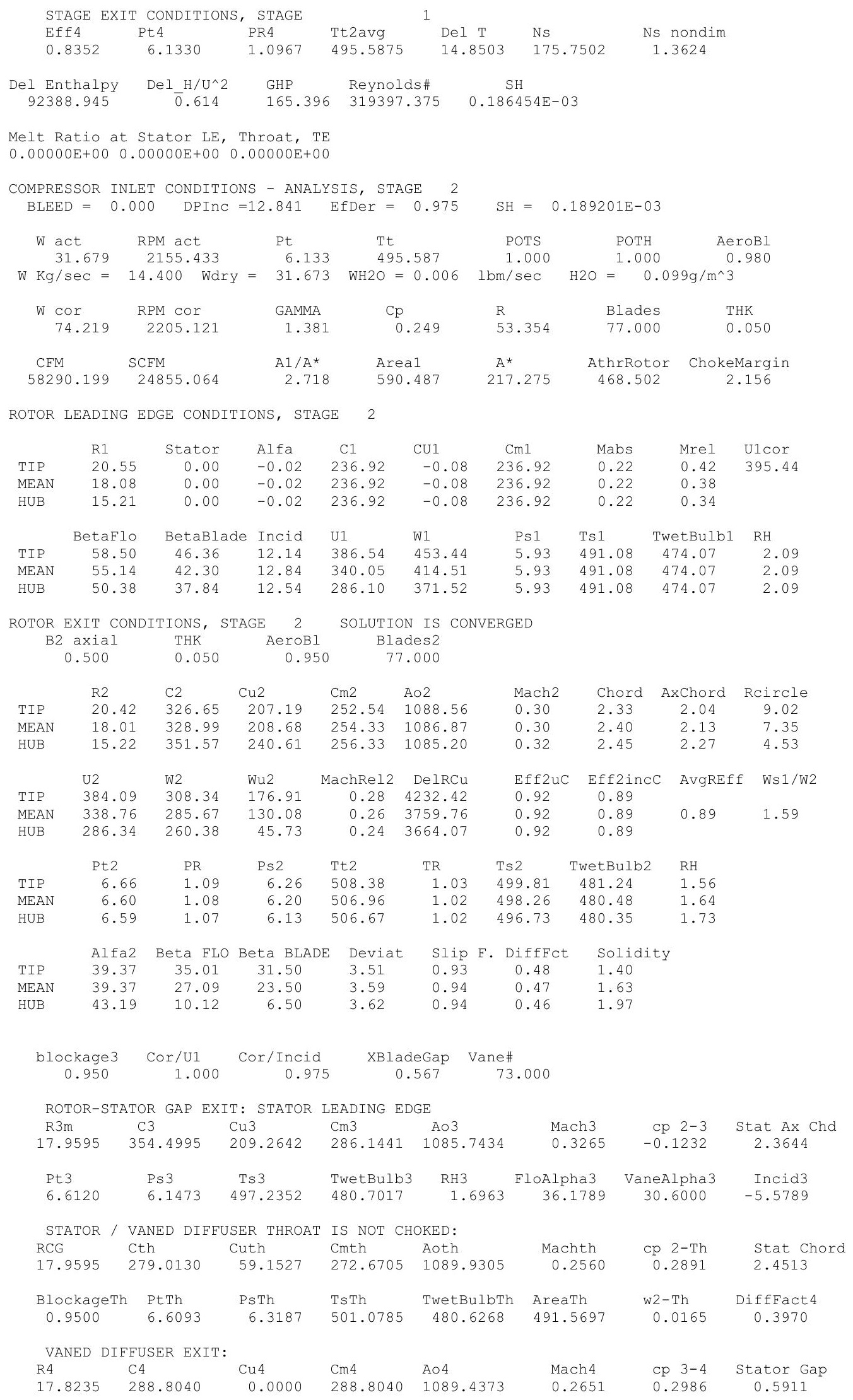




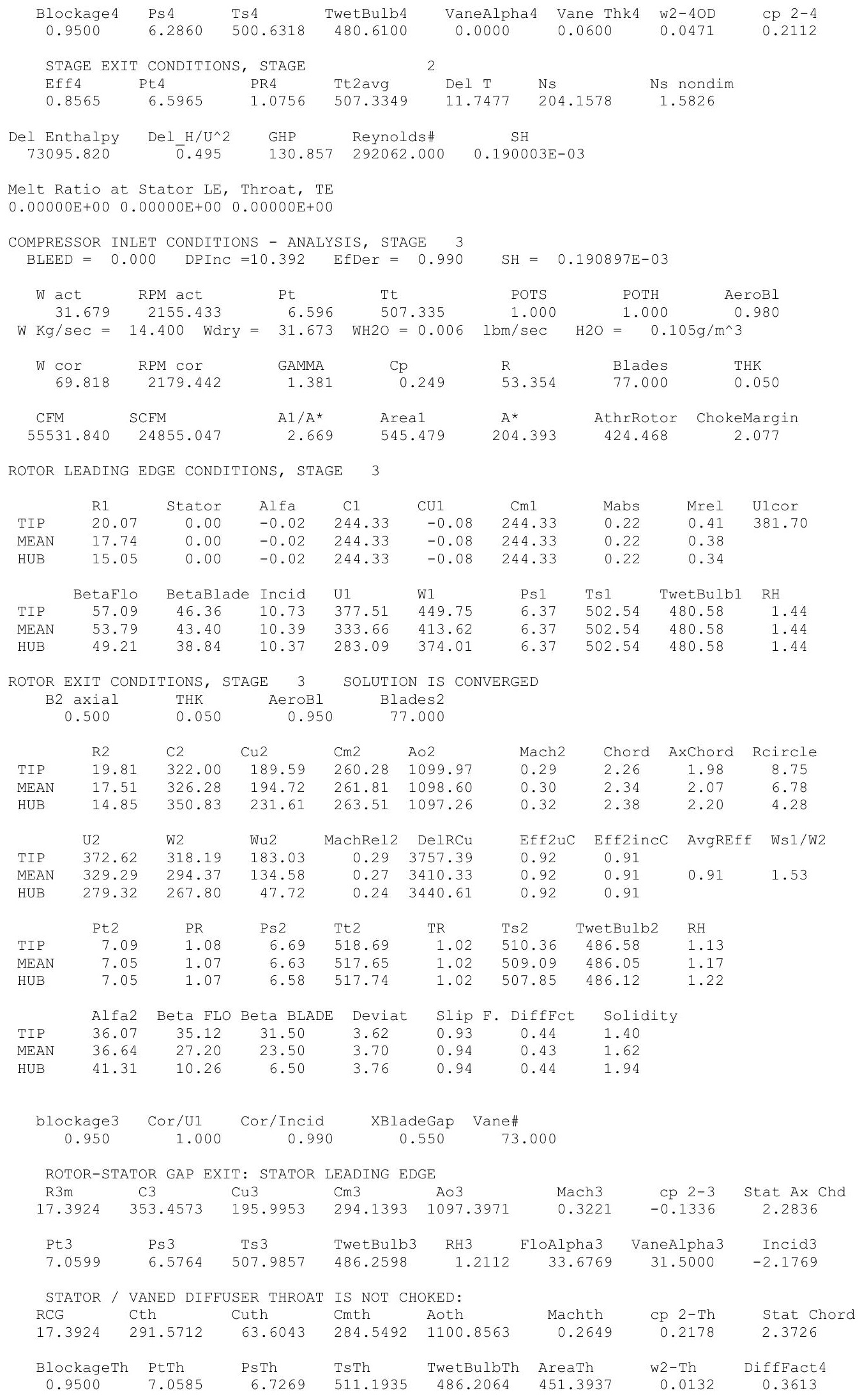




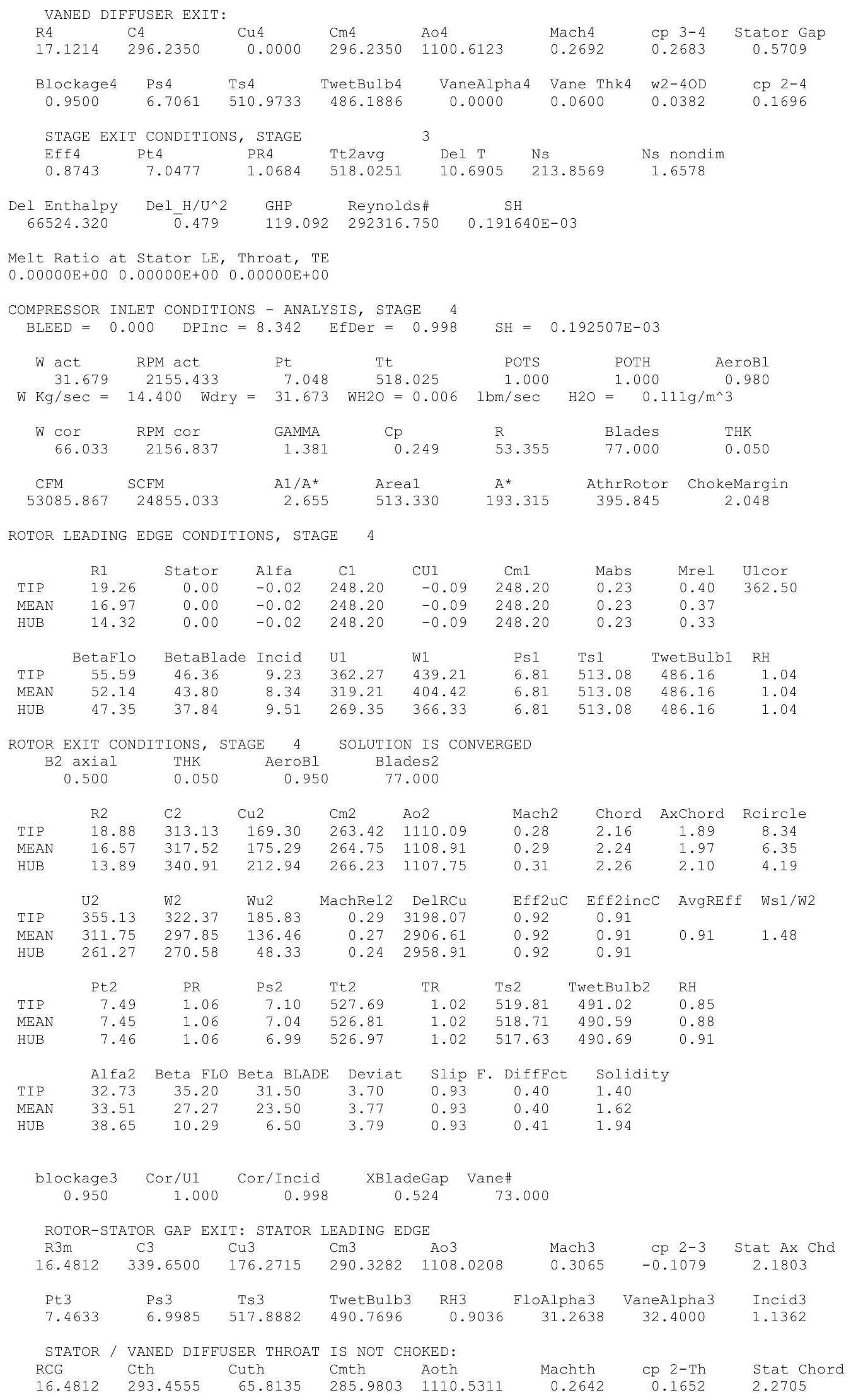




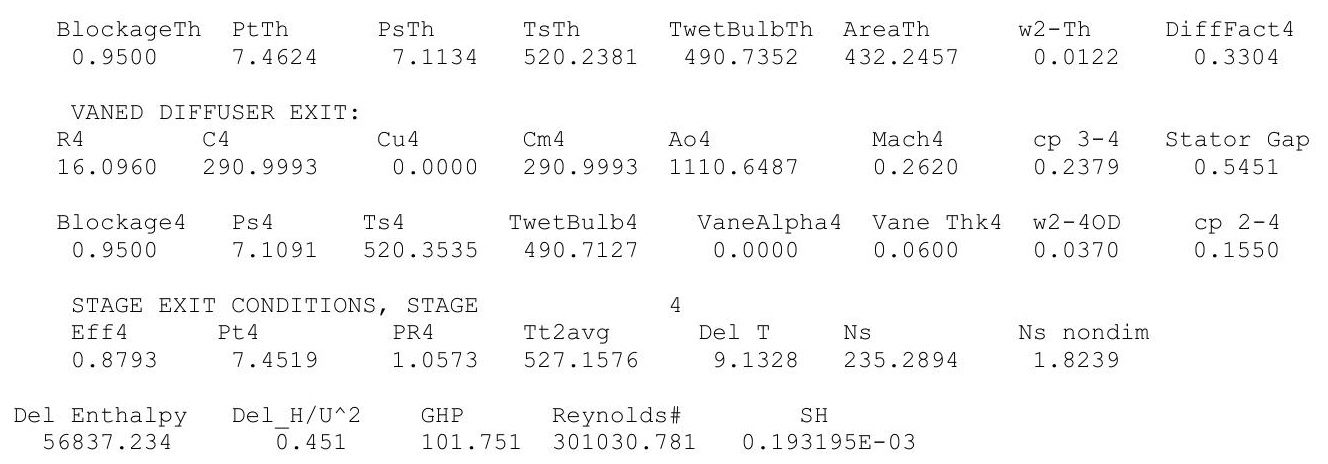

Melt Ratio at Stator LE, Throat, TE

$0.00000 \mathrm{E}+000.00000 \mathrm{E}+000.00000 \mathrm{E}+00$

COMPRESSOR INLET CONDITIONS - ANALYSIS, STAGE 5 $\mathrm{BLEED}=0.000 \quad$ DPInC $=6.473 \quad$ EfDer $=1.000$

$\mathrm{SH}=0.194071 \mathrm{E}-03$

$\begin{array}{llllll}\text { W act } & \text { RPM act } & \text { Pt } & \text { POTS } & \text { POTH } & \text { AeroBl }\end{array}$ $\begin{array}{lll}1.000 & 1.000 & 0.980\end{array}$ $\mathrm{W} \mathrm{Kg} / \mathrm{sec}=14.400$ Wdry $=31.673 \mathrm{WH} 2 \mathrm{O}=0.006 \mathrm{lbm} / \mathrm{sec} \quad \mathrm{H} 2 \mathrm{O}=0.116 \mathrm{~g} / \mathrm{m}^{\wedge} 3$

$\begin{array}{ccccccc}\text { W Cor } & \text { RPM cor } & \text { GAMMA } & \text { Cp } & \text { R } & \text { Blades } & \text { THK } \\ 62.999 & 2138.072 & 1.381 & 0.249 & 53.355 & 77.000 & 0.050 \\ & & & & & & \\ \text { CFM } & \text { SCFM } & \text { A1/A* } & \text { Areal } & A^{*} & \text { AthrRotor } & \text { ChokeMargin } \\ 50988.102 & 24855.018 & 2.763 & 509.668 & 184.438 & 384.739 & 2.086\end{array}$

ROTOR LEADING EDGE CONDITIONS, STAGE 5

$\begin{array}{lccccccccc} & \text { R1 } & \text { Stator } & \text { Alfa } & \text { C1 } & \text { CU1 } & \text { Cm1 } & \text { Mabs } & \text { Mrel } & \text { U1cor } \\ \text { TIP } & 18.32 & 0.00 & -0.02 & 240.10 & -0.08 & 240.10 & 0.22 & 0.38 & 341.81 \\ \text { MEAN } & 15.91 & 0.00 & -0.02 & 240.10 & -0.08 & 240.10 & 0.22 & 0.34 & \\ \text { HUB } & 13.07 & 0.00 & -0.02 & 240.10 & -0.08 & 240.10 & 0.22 & 0.31 & \\ & & & & & & & & & \\ & \text { BetaFlo } & \text { BetaBlade } & \text { Incid } & \text { U1 } & \text { W1 } & \text { Ts1 } & \text { TwetBulb1 } & \text { RH } \\ \text { TIP } & 55.14 & 47.36 & 7.78 & 344.59 & 420.06 & 7.22 & 522.53 & 490.69 & 0.80 \\ \text { MEAN } & 51.27 & 44.80 & 6.47 & 299.32 & 383.78 & 7.22 & 522.53 & 490.69 & 0.80 \\ \text { HUB } & 45.69 & 38.84 & 6.85 & 245.84 & 343.70 & 7.22 & 522.53 & 490.69 & 0.80\end{array}$

ROTOR EXIT CONDITIONS, STAGE 5 SOLUTION IS CONVERGED

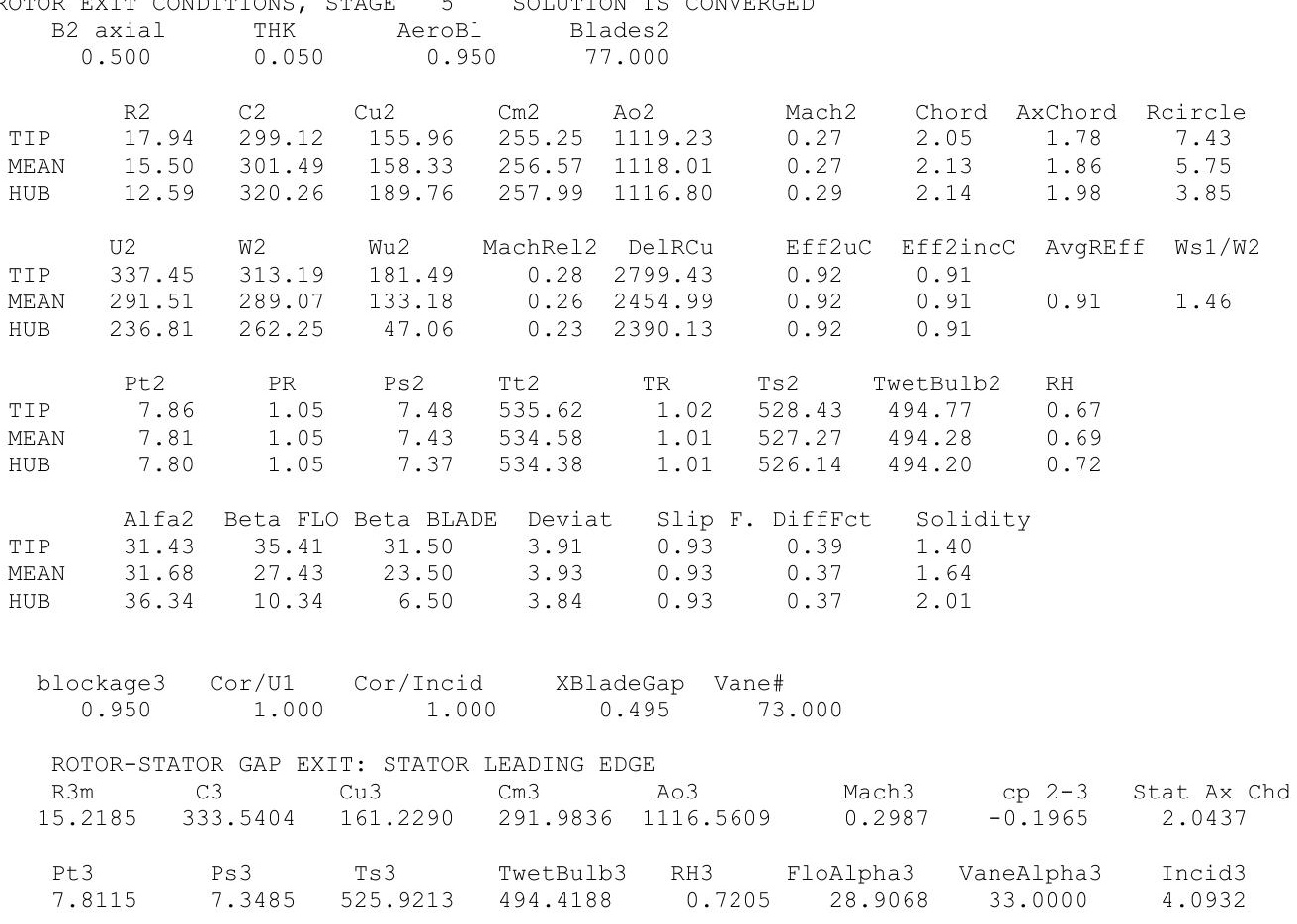




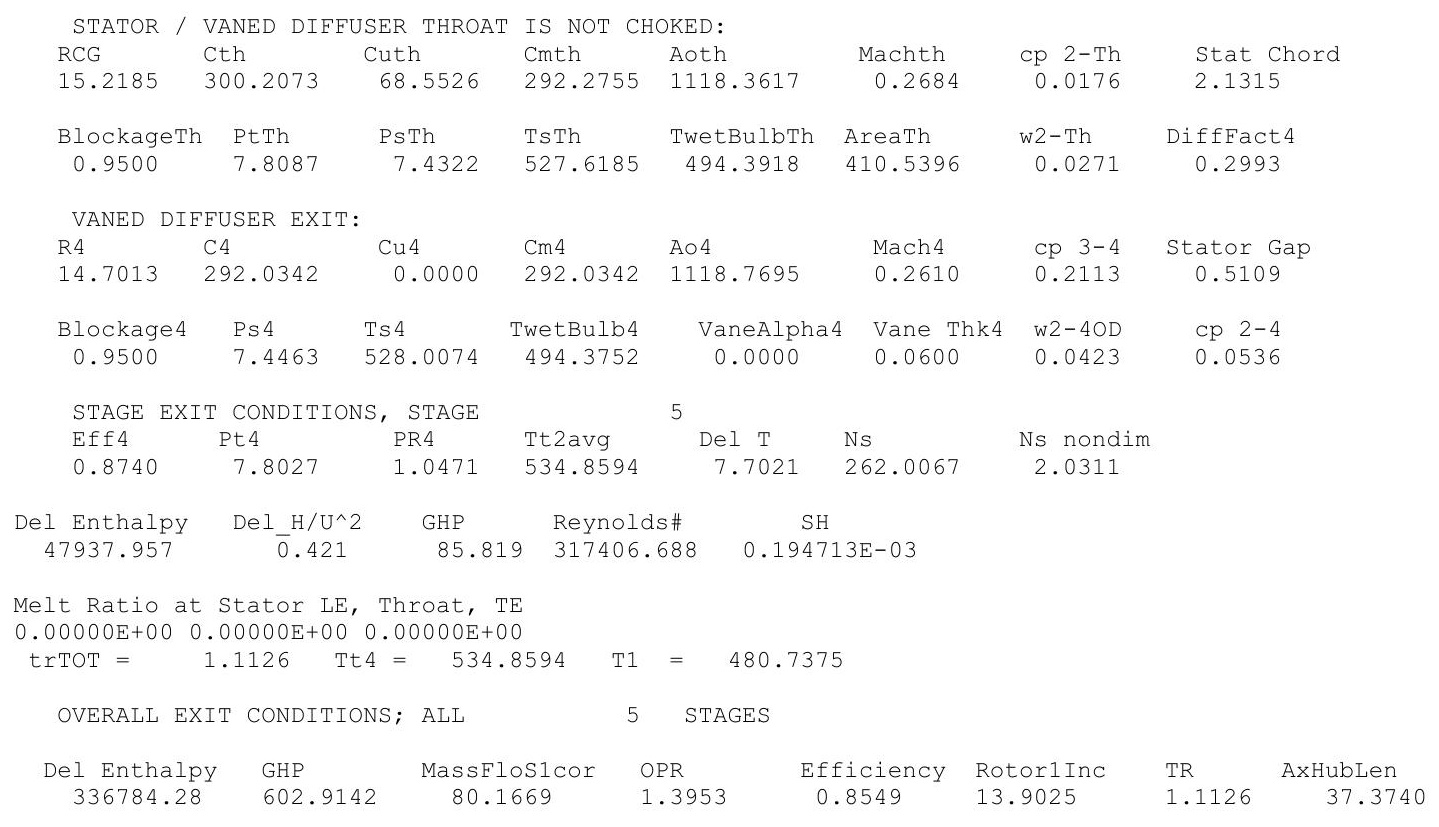




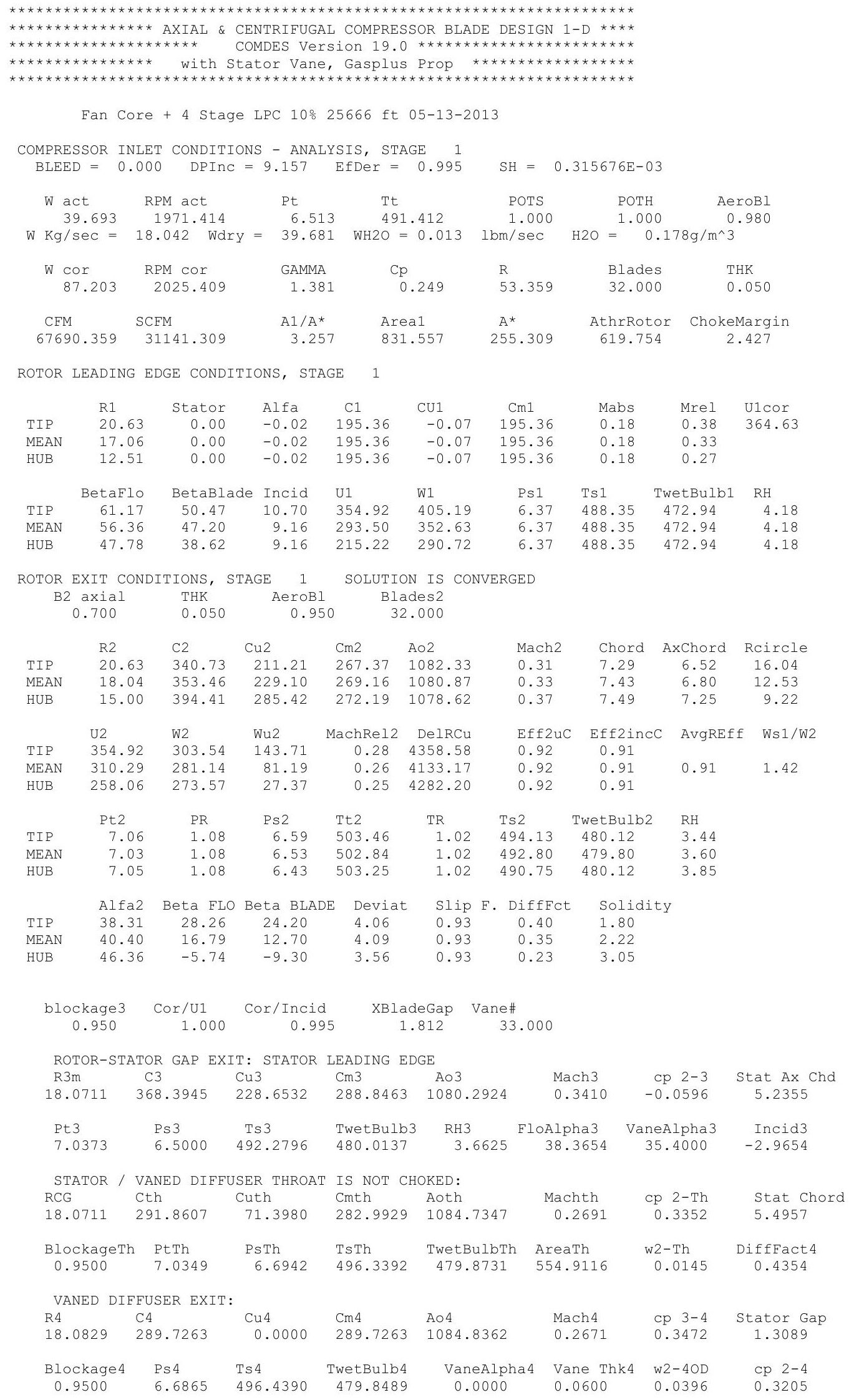




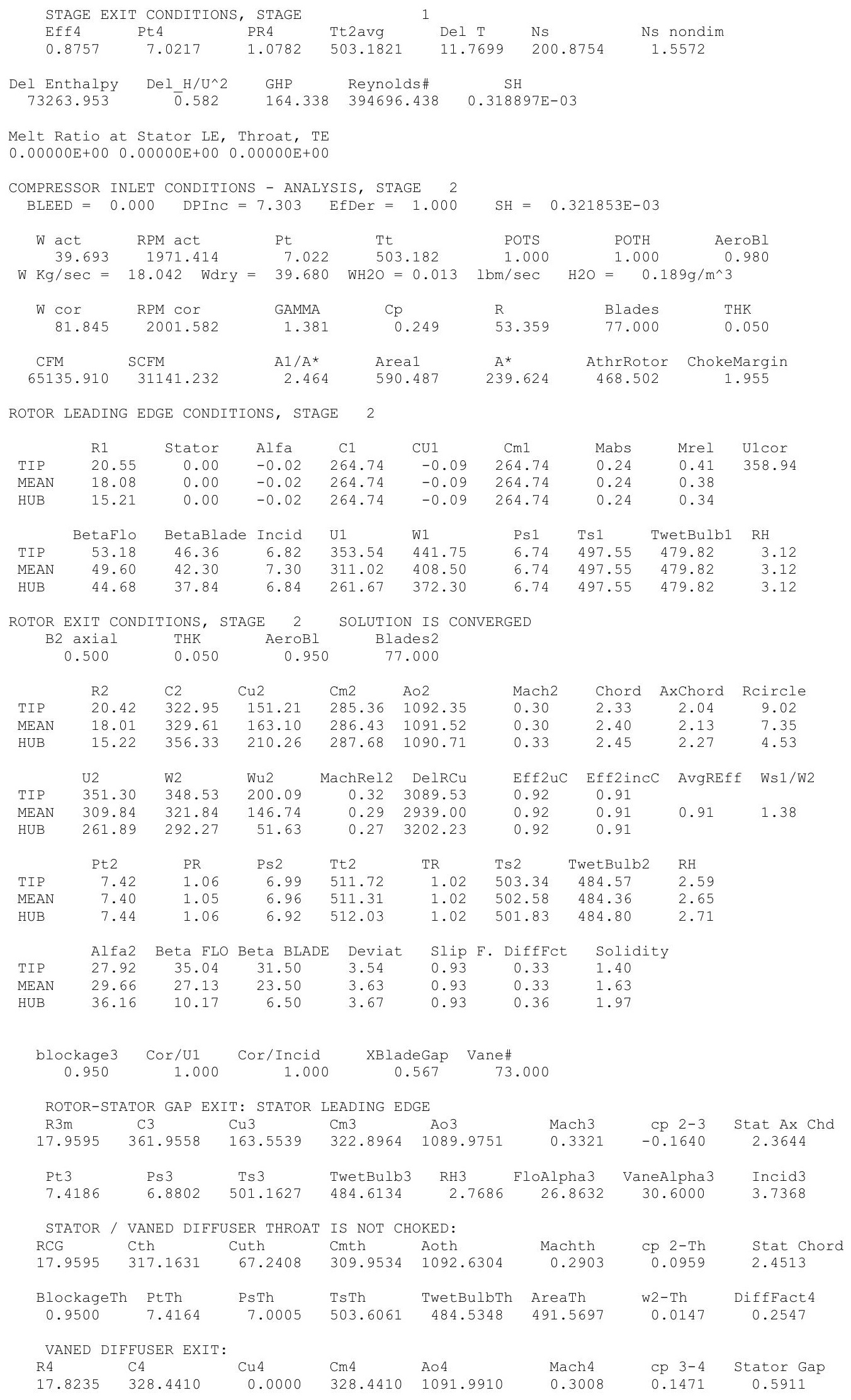




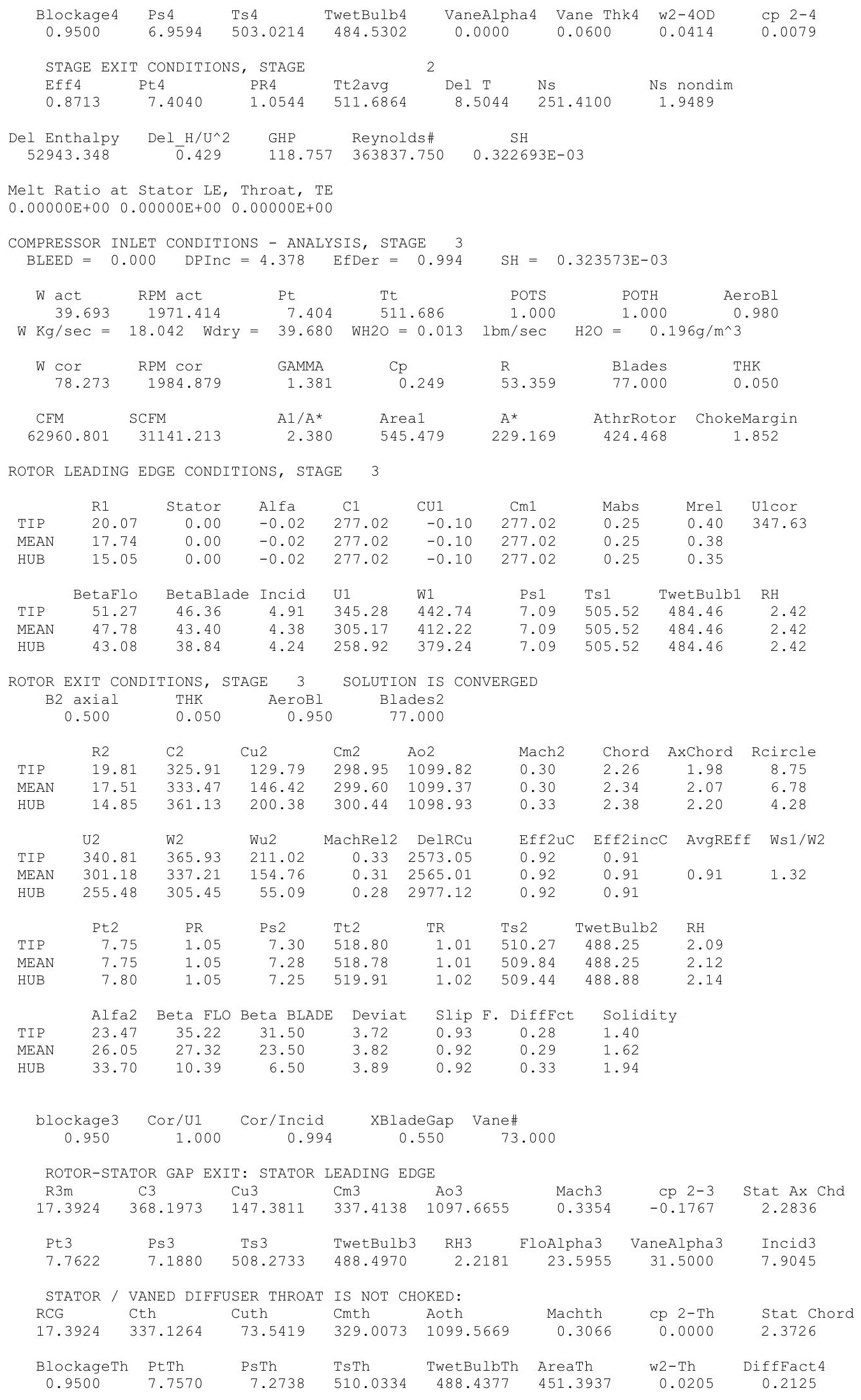




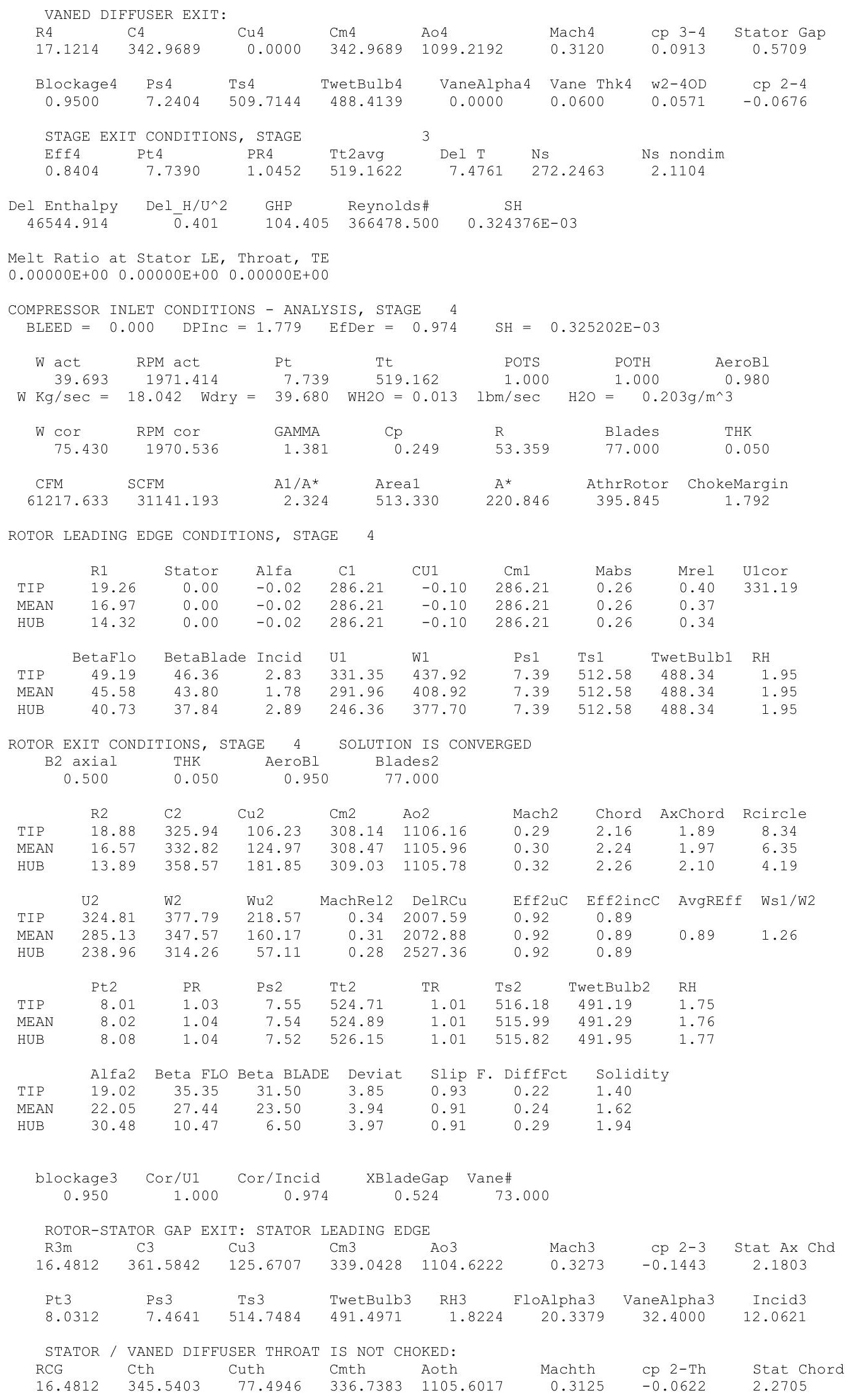




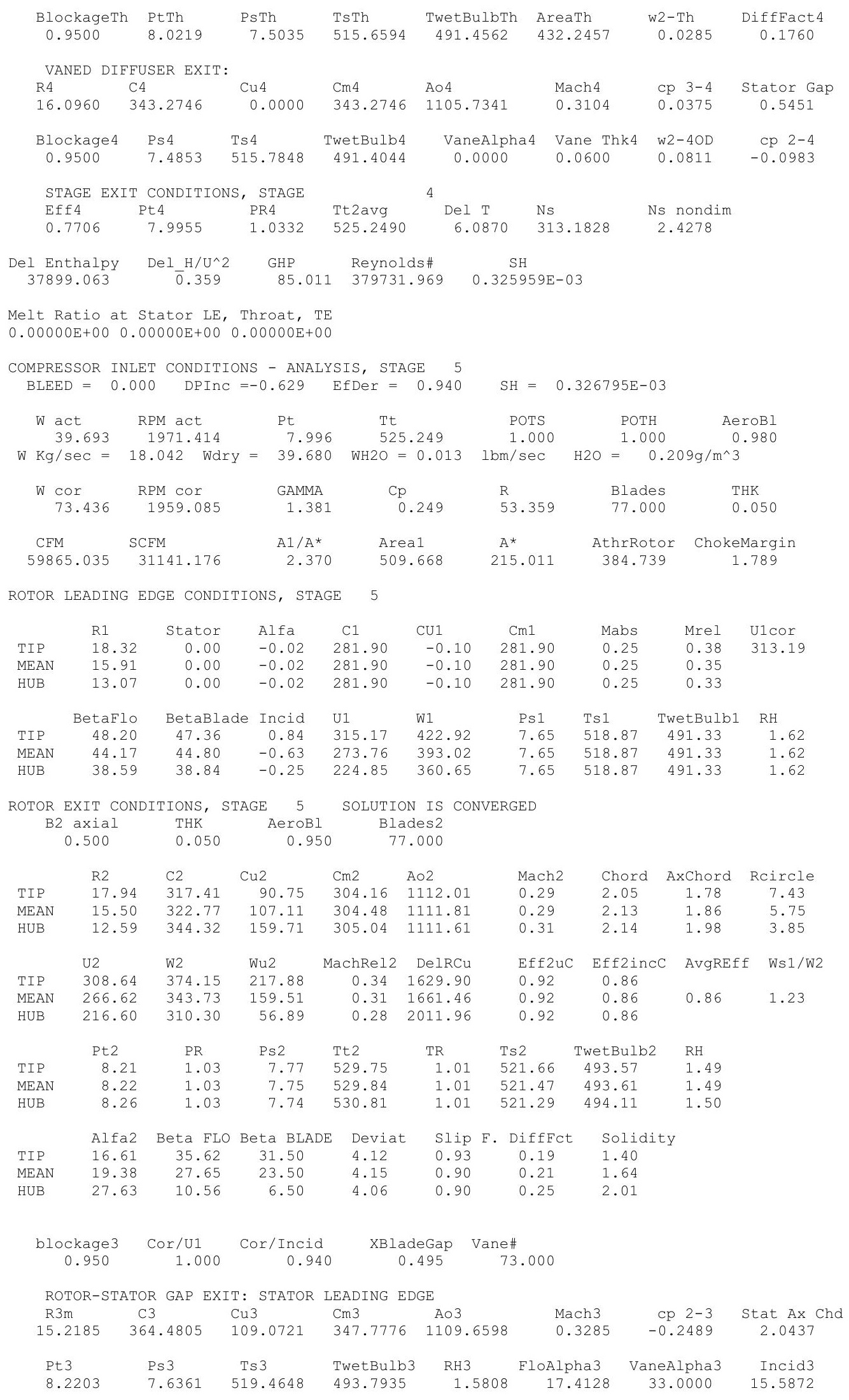




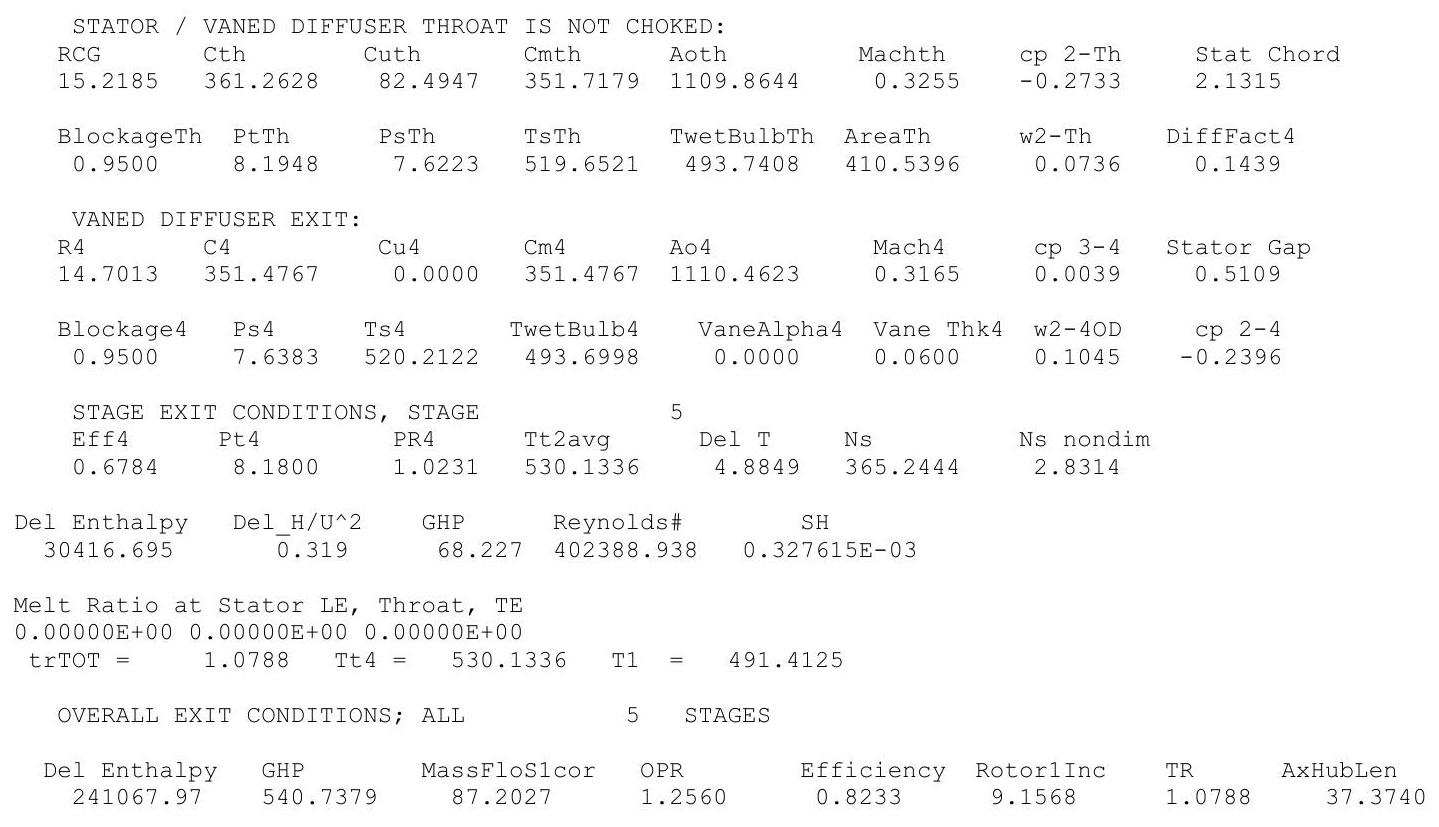




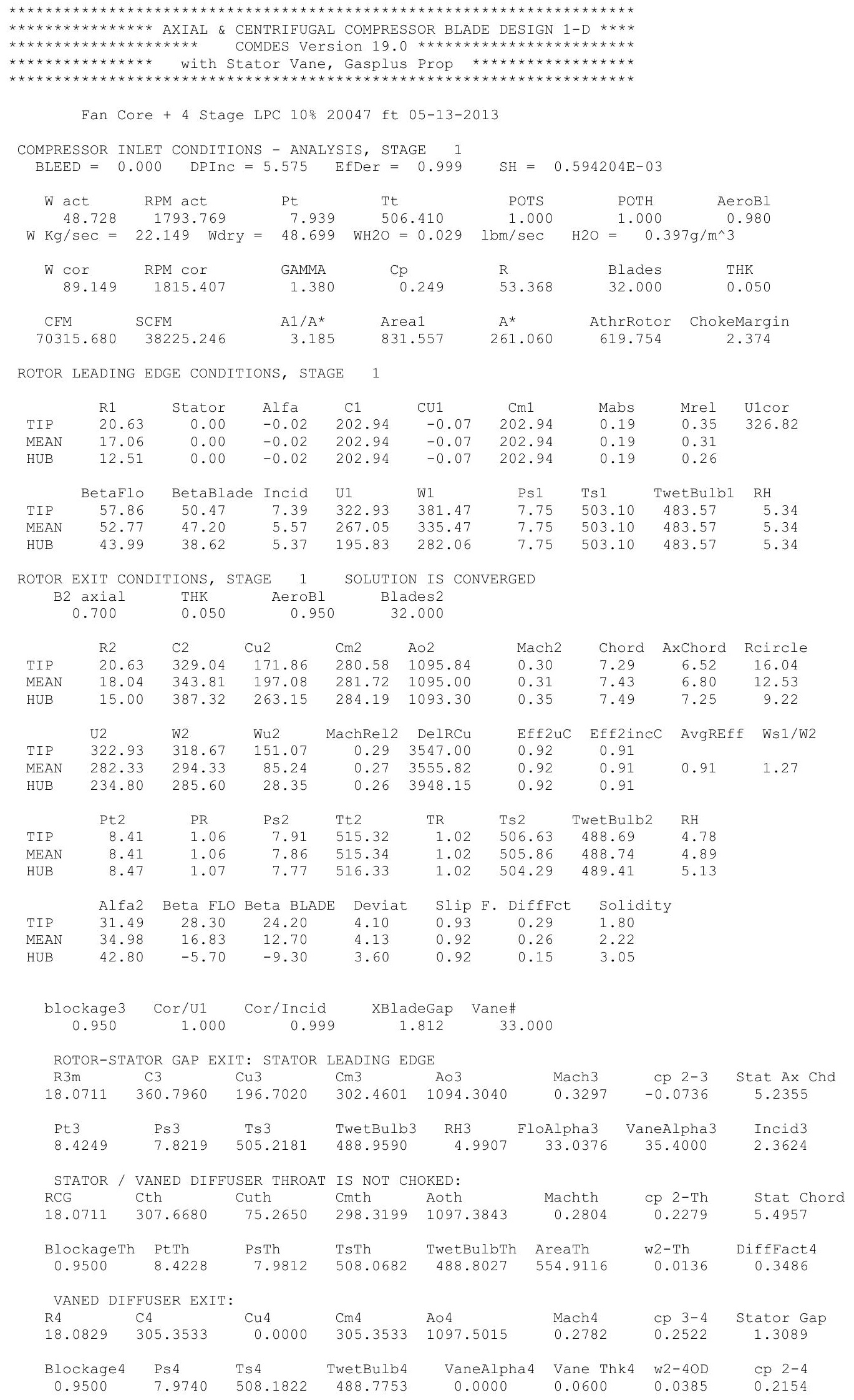




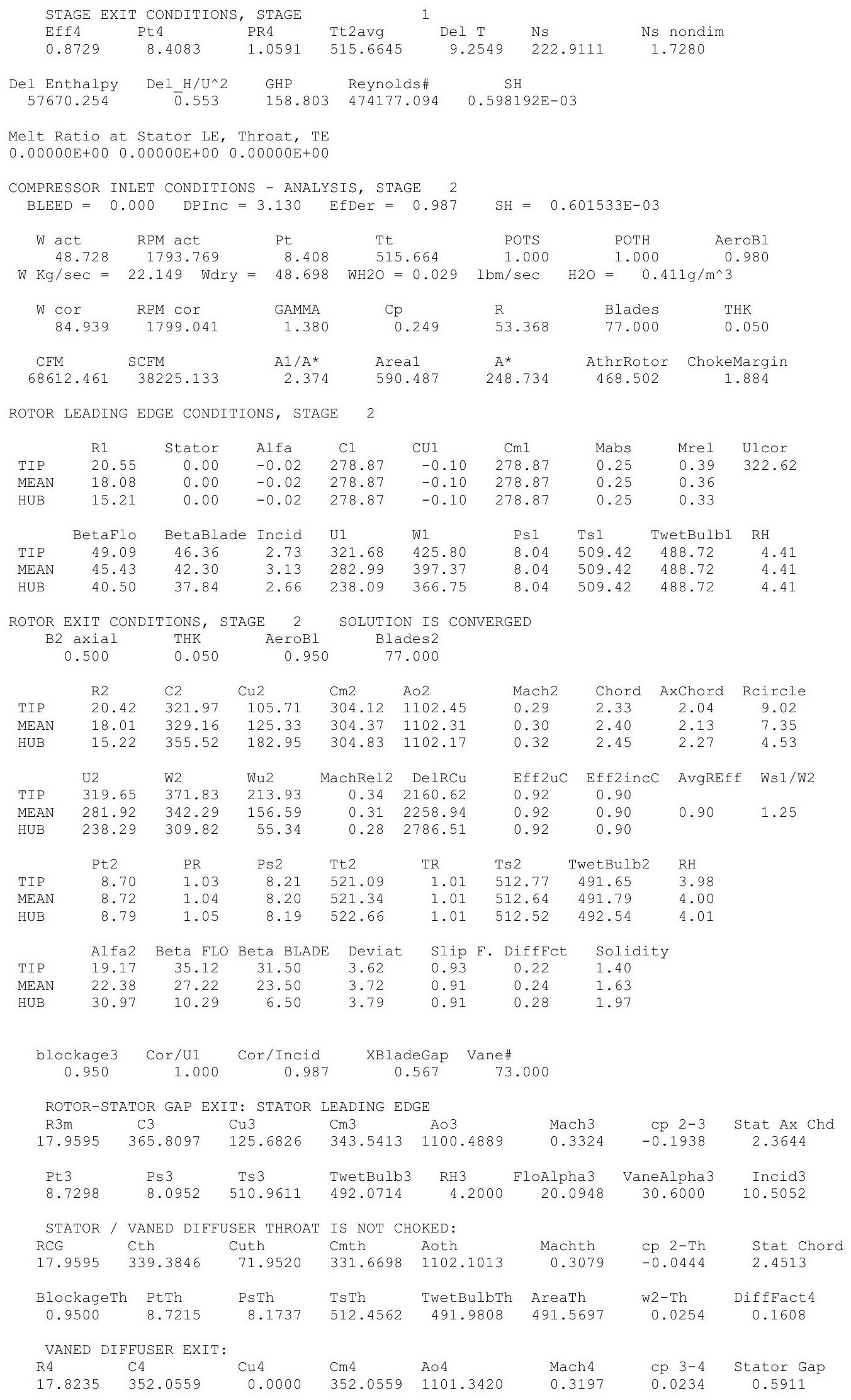




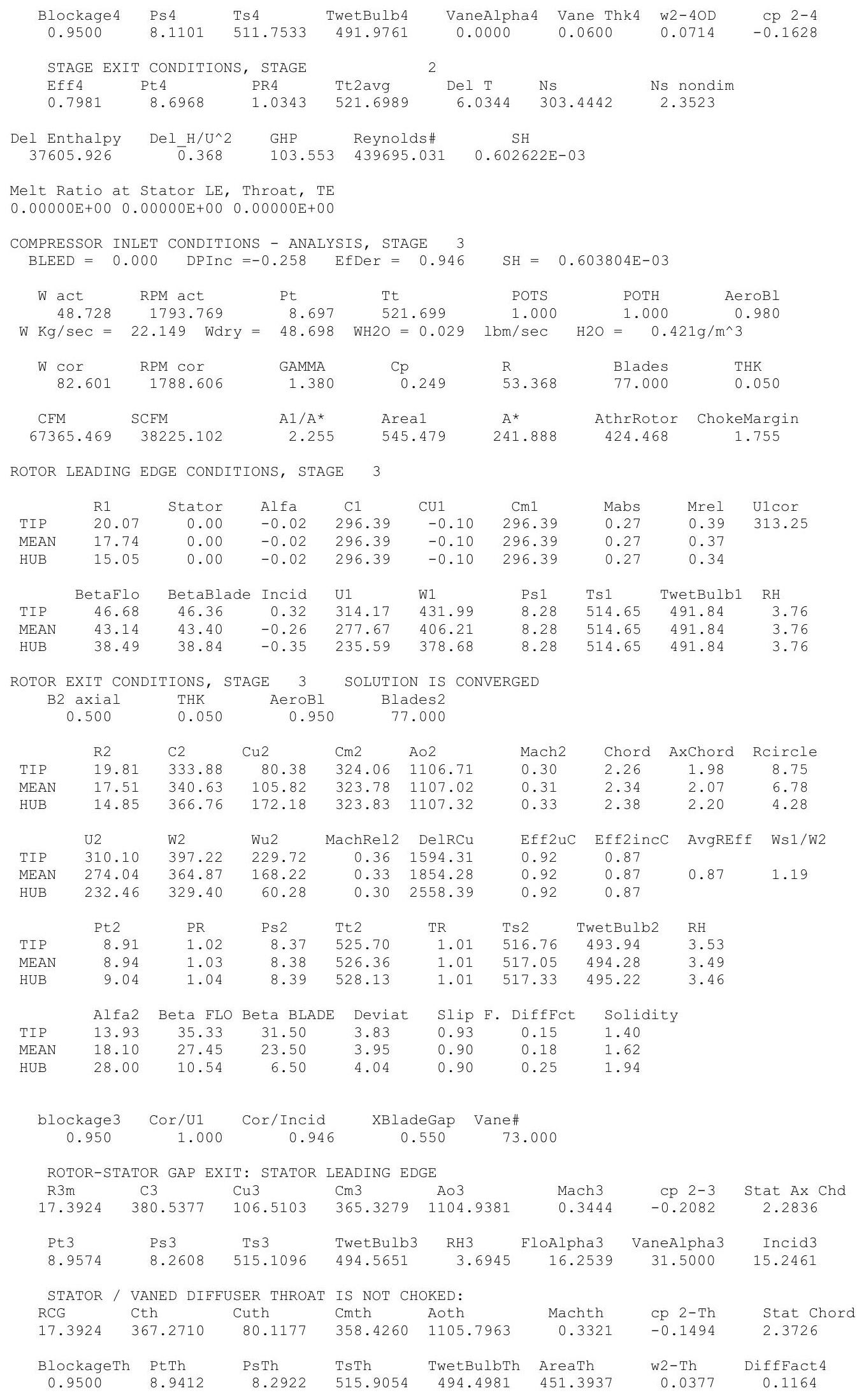




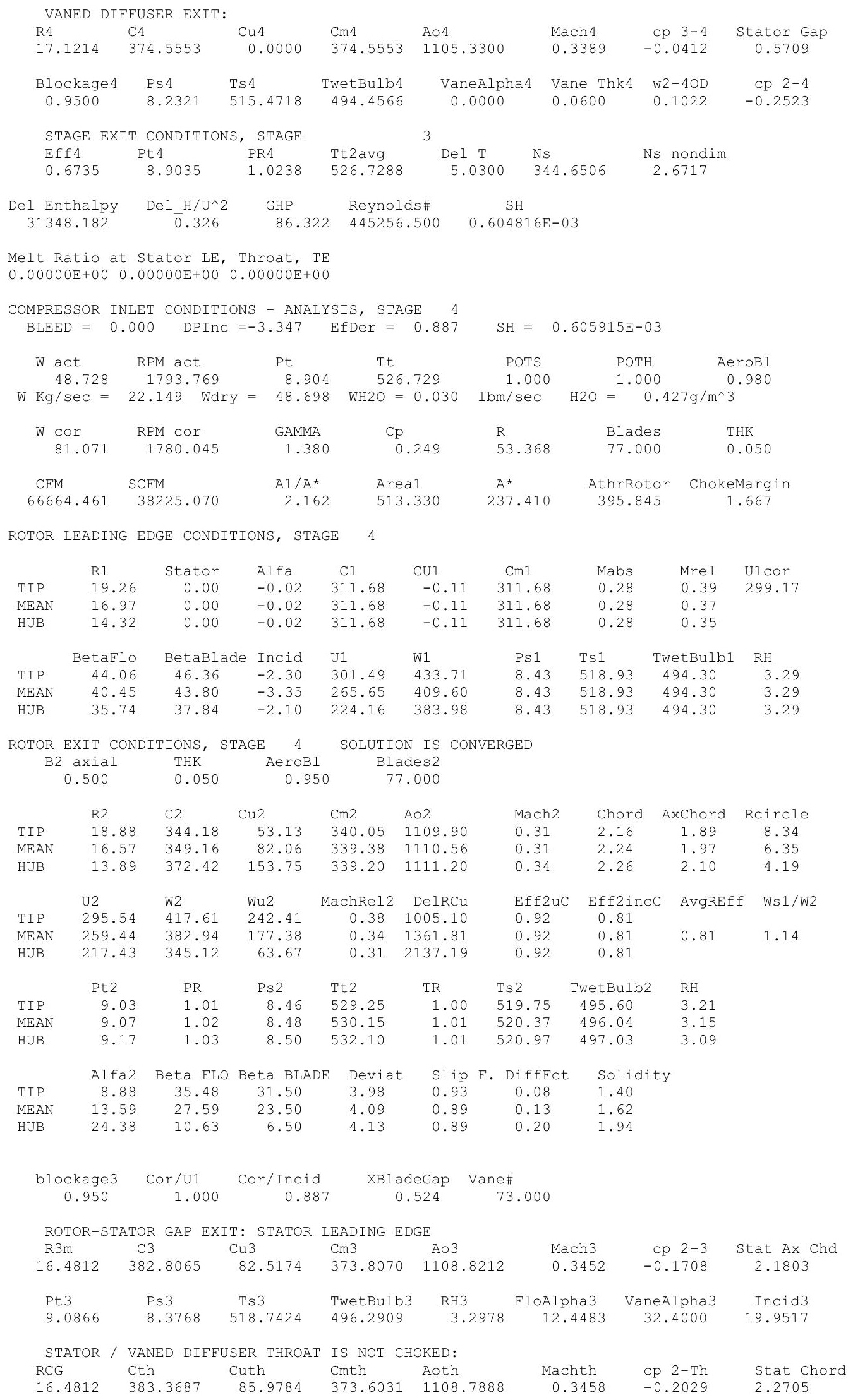




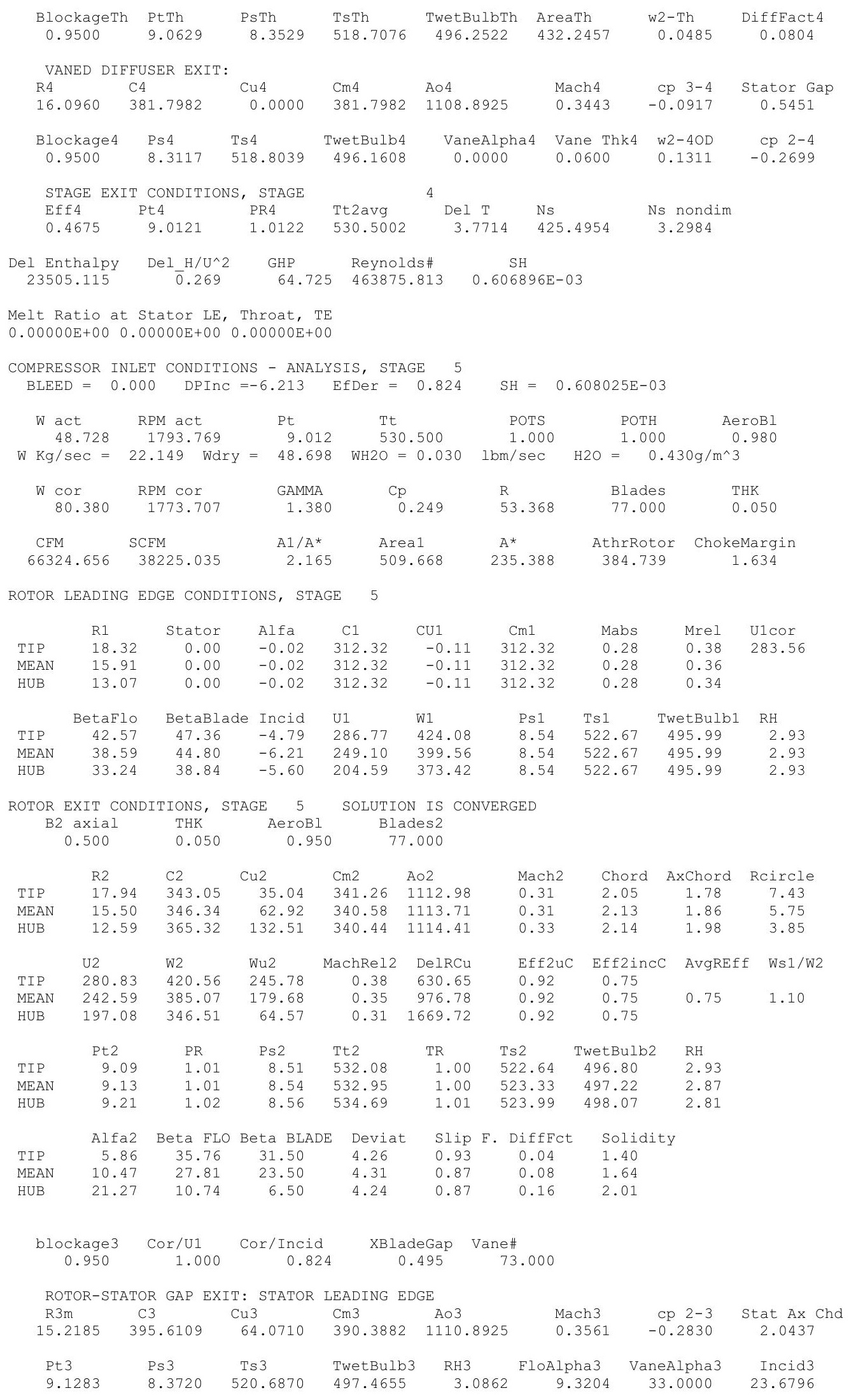




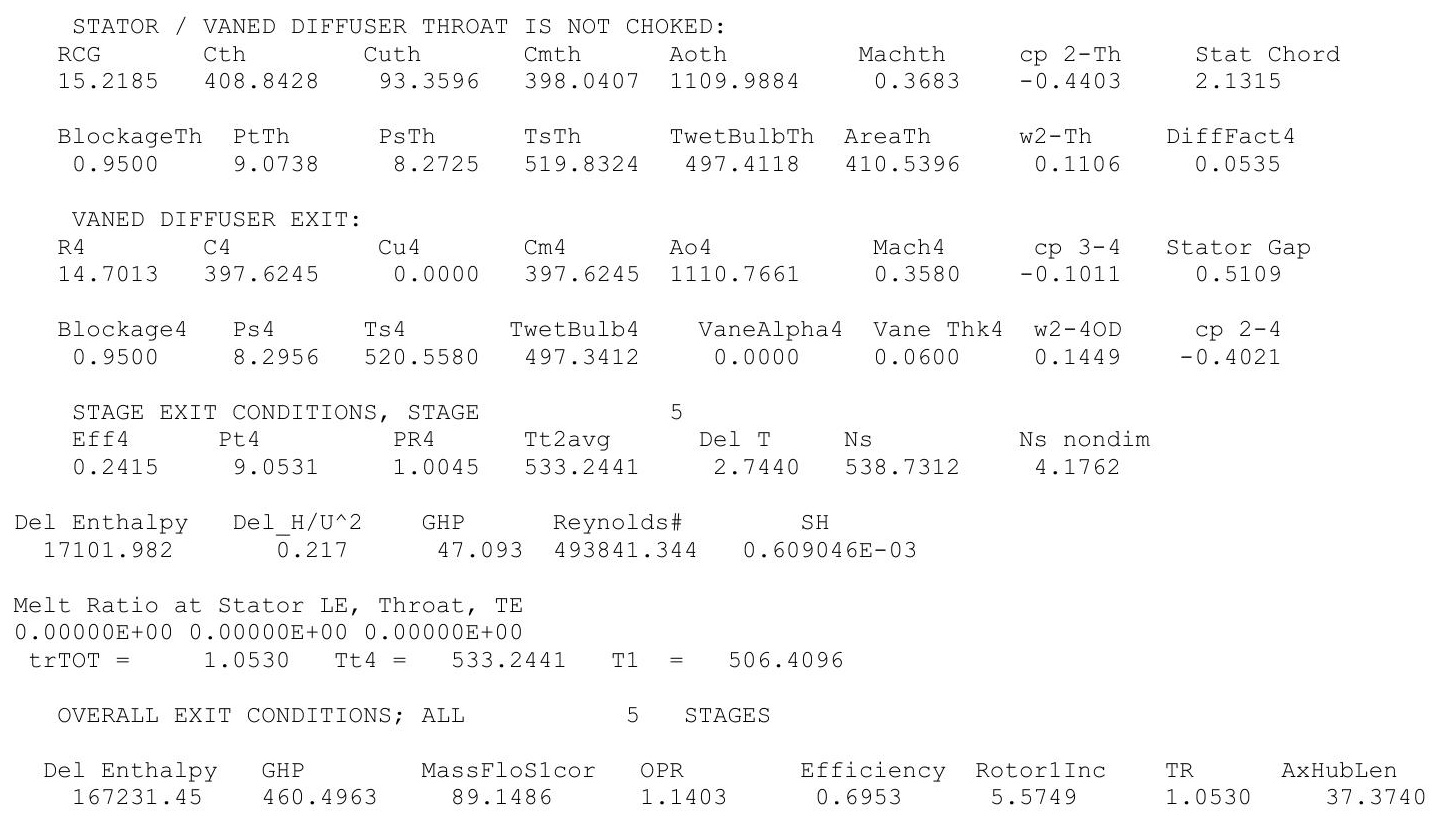




\section{References}

${ }^{1}$ Mason, J. G., Strapp, J. W., Chow, P., “The Ice Particle Threat to Engines in Flight,” AIAA-2006-206-739.

${ }^{2}$ Mason, J. G., Chow, P., Fuleki, D. M., "Understanding Ice Crystal Accretion and Shedding Phenomenon in Jet Engines Using a Rig Test," GT2010-22550.

${ }^{3}$ Mason, J. G., Grzych, M., "The Challenges Identifying Weather Associated With Jet Engine Ice Crystal Icing," SAE 2011-380094.

${ }^{4}$ Jorgenson, P. C. E., Veres, J. P., Wright, W. B., May, R. D., “Engine Icing Modeling and Simulation (Part I): Ice Crystal Accretion on Compression System Components and Modeling its Effects on Engine Performance," SAE International, 2011-380025 .

${ }^{5}$ Veres, J. P., Jorgenson, P. C. E., Wright, W. B., "Modeling the Effects of Ice Accretion on the Low Pressure Compressor and the Overall Turbofan Engine System Performance,” NASA/TM-2013-217034.

${ }^{6}$ Jones, S. M., "An Introduction to Thermodynamic Performance Analysis of Aircraft Gas Turbine Engine Cycles Using the Numerical Propulsion System Simulation Code,” NASA/TM-2007-214690.

${ }^{7}$ Veres, J. P., “Axial and Centrifugal Compressor Mean Line Flow Analysis Method,” AIAA-2009-1641, NASA/TM-2009215585.

${ }^{8}$ Wright, W. B., Potapczuk, M. G., and Levinson, L. H., "Comparison of LEWICE and GlennICE in the SLD Regime," AIAA-2008-0439.

${ }^{9}$ Wright, W. B., Jorgenson, P. C. E., Veres, J. P., "Mixed Phase Modeling in GlennICE with Application to Engine Icing," AIAA-2010-81093.

${ }^{10}$ Struk, P., Currie, T., Wright, W. B., Tsao, J.-C., Broeren, A., Vargas, M., Knezevici, D., and Fuleki, D., "Fundamental Ice Crystal Accretion Physics Studies," SAE-2011-38-0018 or NASA/TM-2012-217429, 2011.

${ }^{11}$ Fowler, J. R., “GASPLUS User's Manual,” NASA LEW-15091, 1994.

${ }^{12}$ Incropera, F. P., and DeWitt, D. P., Fundamentals of Heat and Mass Transfer, John Wiley \& Sons, New York, 1990.

${ }^{13}$ Çengel, Y. A., and Boles, M. A., Thermodynamics: An Engineering Approach, McGraw-Hill, New York, Inc., 1989.

${ }^{14}$ Currie, T. C., Struk, P. M., Tsao, J.-C., Fuleki, D., Knezevici, D., "Fundamental Study of Mixed-Phase Icing with Application to Ice Crystal Accretion in Aircraft Jet Engines," AIAA 2012-3035.

${ }^{15}$ McCullers, L. A., "Aircraft Configuration Optimization Including Optimized Flight Profiles," Proceedings of the Symposium on Recent Experiences in Multidisciplinary Analysis and Optimization, NASA CP 2327, April 1984.

${ }^{16}$ Veres, J. P., Jorgenson, P. C. E., Wright, W. B., Struk, P., “A Model to Assess the Risk of Ice Accretion due to Ice Crystal Ingestion in a Turbofan Engine and its Effects on Performance", AIAA 2012-3038. 


\begin{tabular}{|c|c|c|}
\hline \multicolumn{2}{|c|}{ REPORT DOCUMENTATION PAGE } & $\begin{array}{l}\text { Form Approved } \\
\text { OMB No. 0704-0188 }\end{array}$ \\
\hline \multicolumn{3}{|c|}{ 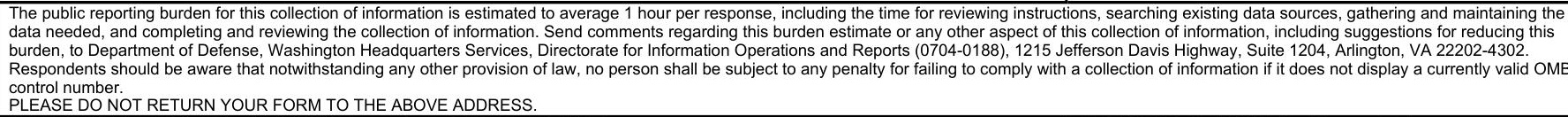 } \\
\hline $\begin{array}{l}\text { 1. REPORT DATE (DD-MM-YYYY) } \\
01-11-2013\end{array}$ & $\begin{array}{l}\text { 2. REPORT TYPE } \\
\text { Technical Memorandum }\end{array}$ & 3. DATES COVERED (From - To) \\
\hline \multirow{3}{*}{\multicolumn{2}{|c|}{$\begin{array}{l}\text { 4. TITLE AND SUBTITLE } \\
\text { A Model to Assess the Risk of Ice Accretion Due to Ice Crystal Ingestion in a Turbofan } \\
\text { Engine and its Effects on Performance }\end{array}$}} & 5a. CONTRACT NUMBER \\
\hline & & 5b. GRANT NUMBER \\
\hline & & 5c. PROGRAM ELEMENT NUMBER \\
\hline \multicolumn{2}{|c|}{$\begin{array}{l}\text { 6. AUTHOR(S) } \\
\text { Jorgenson, Philip, C. E.; Veres, Joseph, P.; Wright, William, B.; Struk, Peter, M. }\end{array}$} & 5d. PROJECT NUMBER \\
\hline \multicolumn{2}{|c|}{$\begin{array}{l}\text { 7. PERFORMING ORGANIZATION NAME(S) AND ADDRESS(ES) } \\
\text { National Aeronautics and Space Administration } \\
\text { John H. Glenn Research Center at Lewis Field } \\
\text { Cleveland, Ohio 44135-3191 }\end{array}$} & $\begin{array}{l}\text { 8. PERFORMING ORGANIZATION } \\
\text { REPORT NUMBER } \\
\text { E-18790 }\end{array}$ \\
\hline \multirow{2}{*}{\multicolumn{2}{|c|}{$\begin{array}{l}\text { 9. SPONSORING/MONITORING AGENCY NAME(S) AND ADDRESS(ES) } \\
\text { National Aeronautics and Space Administration } \\
\text { Washington, DC 20546-0001 }\end{array}$}} & $\begin{array}{l}\text { 10. SPONSORING/MONITOR'S } \\
\text { ACRONYM(S) } \\
\text { NASA }\end{array}$ \\
\hline & & $\begin{array}{l}\text { 11. SPONSORING/MONITORING } \\
\text { REPORT NUMBER } \\
\text { NASA/TM-2013-218094 }\end{array}$ \\
\hline
\end{tabular}

\section{DISTRIBUTION/AVAILABILITY STATEMENT}

Unclassified-Unlimited

Subject Categories: 01, 07, 03, and 02

Available electronically at http://www.sti.nasa.gov

This publication is available from the NASA Center for AeroSpace Information, 443-757-5802

\section{SUPPLEMENTARY NOTES}

This report is a revised and expanded version of AIAA-2012-3038 presented at the Atmospheric Space Environments Conference sponsored by the American Institute of Aeronautics and Astronautics, New Orleans, Louisiana, June 25-28, 2012.

\section{ABSTRACT}

The occurrence of ice accretion within commercial high bypass aircraft turbine engines has been reported under certain atmospheric conditions. Engine anomalies have taken place at high altitudes that were attributed to ice crystal ingestion, partially melting, and ice accretion on the compression system components. The result was one or more of the following anomalies: degraded engine performance, engine roll back, compressor surge and stall, and flameout of the combustor. The main focus of this research is the development of a computational tool that can estimate whether there is a risk of ice accretion by tracking key parameters through the compression system blade rows at all engine operating points within the flight trajectory. The tool has an engine system thermodynamic cycle code, coupled with a compressor flow analysis code, and an ice particle melt code that has the capability of determining the rate of sublimation, melting, and evaporation through the compressor blade rows. Assumptions are made to predict the complex physics involved in engine icing. Specifically, the code does not directly estimate ice accretion and does not have models for particle breakup or erosion. Two key parameters have been suggested as conditions that must be met at the same location for ice accretion to occur: the local wet-bulb temperature to be near freezing or below and the local melt ratio must be above 10 percent. These parameters were deduced from analyzing laboratory icing test data and are the criteria used to predict the possibility of ice accretion within an engine including the specific blade row where it could occur. Once the possibility of accretion is determined from these parameters, the degree of blockage due to ice accretion on the local stator vane can be estimated from an empirical model of ice growth rate and time spent at that operating point in the flight trajectory. The computational tool can be used to assess specific turbine engines to their susceptibility to ice accretion in an ice crystal environment. 15. SUBJECT TERMS

Flow simulation; Aeropropulsion; Engine icing; Aircraft turbine engine system simulation; Turbine engine; Compressor

\begin{tabular}{|c|c|c|c|c|c|}
\hline \multicolumn{3}{|c|}{ 16. SECURITY CLASSIFICATION OF: } & $\begin{array}{l}\text { 17. LIMITATION OF } \\
\text { ABSTRACT } \\
\text { UU }\end{array}$ & $\begin{array}{l}\text { 18. NUMBER } \\
\text { OF } \\
\text { PAGES } \\
166\end{array}$ & $\begin{array}{l}\text { 19a. NAME OF RESPONSIBLE PERSON } \\
\text { STI Help Desk (email:help@ } \text { sti.nasa.gov) } \\
\text { 19b. TELEPHONE NUMBER (include area code) } \\
\text { 443-757-5802 }\end{array}$ \\
\hline
\end{tabular}



